\title{
Abstracts from the 2016 Society of General Internal Medicine Annual Meeting
}

\section{SCIENTIFIC ABSTRACTS}

'IT'S BEEN AN EXPERIENCE, A LIFE LEARNING EXPERIENCE': HOSPITALIZATION AS A REACHABLE MOMENT FOR ADULTS WITH SUBSTANCE USE DISORDERS Honora Englander ${ }^{1,}{ }^{4}$; Christine Velez Klug ${ }^{3}$; Philip T. Korthuis $^{2}$; Sarann Bielavitz ${ }^{2}$; Christina Nicolaidis ${ }^{3}, 2$. ${ }^{1}$ Oregon Health \& Science University, Portand, OR; ${ }^{2}$ Oregon Health \& Science University, Portland, OR; ${ }^{3}$ Portland State University, Portland, OR; ${ }^{4}$ Central City Concern, Portland, OR. (Control ID \#2469883)

BACKGROUND: People with substance use disorders (SUD) have high rates of chronic illness, hospitalization and readmission. Frequently, people are admitted with direct medical complications of their SUD. Despite this, many are not engaged in addiction treatment. And though hospitalization addresses acute medical illness, it often fails to address the underlying cause: the SUD. Hospitalization may be a reachable moment to initiate and coordinate addiction care. However, little is known about hospitalized patients' perceptions of their SUD and the mechanisms that may support or impede engagement and success in recovery. Our study explores the experiences of hospitalized adults with SUD to inform future health system improvements.

METHODS: We conducted in-person interviews of hospitalized adults reporting highrisk alcohol or drug use on the AUDIT-C and/or single item screener between September 2014 and April 2015. This study was nested in a larger needs assessment at a large academic medical center. We excluded non-English speakers, incarcerated individuals, those who were too sick or confused to participate, and those admitted to an ICU or psychiatric ward. We interviewed all participants in their hospital room using a semi structured interview guide. Audio recordings were transcribed, de-identified and transferred to Atlas.ti (Version 7.5.9) for data analysis and retrieval. The coding team - comprised of two general internists and one social worker — conducted a thematic analysis using an inductive approach at the semantic level. Using an iterative process, we selected a preliminary coding schema, independently coded transcripts, and then met as a group or in dyads to discuss and reconcile codes, identify emergent themes, and to resolve discrepancies through consensus. All three coders coded the initial 7 transcripts and established a coding schema. One coder coded all transcripts; the other two coders divided the remaining transcripts. We employed a constructivist approach to data analysis in that we acknowledged no one reality; people are experts on their own lives.

RESULTS: Thirty-two hospitalized adults participated in our study. Mean age was 43 years. Seventy-five percent were male and $68 \%$ identified as white. Participants reported moderate to high-risk alcohol (56\%), amphetamine (56\%), and opioid (56 \%) use, indicating some polysubstance use across the sample. All participants had health insurance. Participants described hospitalization as a wake up call. One participant said, "I wish I didn't put myself in the predicament of coming to the hospital so much. It's been an experience, a life-learning experience. But then again, coming here so much has helped me notice and realize - to wake up. It's time to stop doing' what I'm doing, to get to the sober and clean path." Many described near death experiences, and that mortality was motivating for change. As one woman stated, "I knew it was to that point where I almost died the other day and I have a 3 year old little boy and it's not where I want to be." Participants also described that hospitalization interrupted drug use and allowed for fresh insight. Despite hospitalization being a reachable moment and highly motivating for change, participants described important barriers that impeded the 'opportunity' of the moment. Many described an overwhelming compulsion to use. "My whole addiction to heroin, it's going to end up killing me, and I still know that I'm going to go back out there and do it, and that's just so $f^{* *} d$ up..." Participants described physical pain, trauma, life stressors, and poverty as significant barriers to recovery that are unchanged by acute hospitalization. One participant who underwent medical detoxification in the hospital and expressed strong desire to quit reflected, "I almost see it like being futile... I have nowhere to go and nothing." Participants provided insight as to how healthcare providers and health systems can best leverage a reachable moment. Many emphasized the importance of choice: "when you tell me what to do, I'm a mule. I dig my hooves in and I'm like uh-uh [shaking head], I make my own decisions. But if I have somebody to talk to that could understand where I'm coming from, yeah, I could see that helping people. "Some felt that being given a list of resources (often with long wait times and limited access) without support felt dismissive. She felt providers think: "give her the list of resources, if she wants help she'll figure it out'...they feel it's useless to waste their time when all I want is help." Participants wanted providers that understand SUD - in particular to treat withdrawal to prevent people from leaving the hospital against medical advice and to provide access to medication-assisted treatment. And many noted the importance caring, non-judgmental staff.

CONCLUSIONS: Our findings suggest that hospitalization may be a reachable moment to initiate and coordinate addiction care. Hospitalization is often a wakeup call that is highly motivating. While some barriers such as poverty and trauma may be difficult to address through acute hospitalization, providers and systems can better align to engage patients. Systems can support a culture of non-judgment, train staff about SUD, and provide patients with choice, including access to medication-assisted treatment.

"I'M NOT A TEXTBOOK PATIENT": HOW BEING LISTENED TO CAN AFFECT HEALTH CARE TRAJECTORIES FOR OLDER ADULTS WITH MULTIPLE CHRONIC CONDITIONS AND FREQUENT HOSPITALIZATIONS Melissa Dattalo $^{1,2}{ }^{2}$; Korey Kennelty ${ }^{1,2}$; Elizabeth Chapman ${ }^{2}$; Andrea Gilmore- 
Bykovskyi $^{1,}$; Julia Loosen $^{2}$; Emily Schmitz ${ }^{2,}$; Mary F. Wyman $^{2}$; Nicole Rogus-Pulia ${ }^{1,}$ ${ }^{2}$; Nicole Werner ${ }^{2}$; Amy J. Kind ${ }^{2,}{ }^{1}$; Barbara Bowers ${ }^{2} .{ }^{1}$ William S. Middleton VA Hospital, Madison, WI; ${ }^{2}$ University of Wisconsin-Madison, Madison, WI. (Control ID \#2456862)

BACKGROUND: High-cost, high-need patients account for disproportionate health care spending, with most costs accrued through hospital admissions. A threshold of 3 or more hospital admissions in a year can identify those in the top $5 \%$ of health care spending. Within the Medicare population, $5 \%$ of fee-for-service beneficiaries account for $39 \%$ of total spending. The complex medical and social needs of these individuals are often mismatched with traditional health services. Person-centered care has been associated with greater trust and improved medication adherence, but high-cost, high-need patients are underrepresented in research studies. Understanding the goals, values, and perceptions of this population is critical to designing cost-effective interventions. The purpose of this grounded theory study was to identify the complex needs of high-cost, high-need patients by developing a theoretical model of factors contributing to their hospital utilization patterns.

METHODS: Grounded theory is a qualitative research method that can both describe the complex interactions contributing to a process and generate theories that are grounded in the lived experiences of participants. This qualitative method is designed to analyze indepth information from specific individuals, using small sample sizes. We conducted indepth interviews with high-cost, high-need patients and any caregivers these patients identified as important to their health care. Participants were recruited from four sites: a complex case management organization, a home health agency, an academic hospital, and a managed care organization for dual-eligible patients. Patient eligibility criteria included: age $\geq 55, \geq 2$ chronic health conditions, and $\geq 3$ hospitalizations in a 12 month period. Consistent with grounded theory, data is being collected through theoretical sampling and analysis will continue until theoretical saturation is reached. An interdisciplinary team conducted dimensional analysis and identified "being listened to" as a core category. We then conducted comparative analysis of times when participants perceived themselves as "being listened to" and times when they perceived themselves as "not being listened to." We used memoing, member checking, and peer debriefing to enhance rigor.

RESULTS: We completed interviews with 11 patients and 3 caregivers $(n=14)$. Patients had an average age of 72.4 years, 7 self-reported chronic conditions, and 6.1 hospitalizations in their highest-utilizing year. Demographic questionnaires showed that $54.5 \%(n=$ 6) of patients were female, $45.5 \%(n=5)$ lived alone, $54.5 \%(n=6)$ reported financial strain, and $81.8 \%(n=9)$ had at least a high school reading level. All participants reported managing serious illnesses that they perceived to be intermittently life-threating. Participants who experienced reductions in hospitalization frequency attributed "being listened to" by health care providers as a critical factor contributing to longer periods of stable outpatient management. Patients and caregivers described unique information that did not exist in the medical record, and perceived this information as "being listened to" if it influenced their diagnoses or treatment plans. Categories of unique information included: goals and preferences, early warning signs, positive or negative effects of treatment, and anticipated feasibility of treatment plans. Participants who perceived that this information was "not listened to" either engaged in self-advocacy strategies or tried to address the unique issues on their own. Conditions facilitating "being listened to" included higher severity of symptoms (versus mild to moderate "warning signs"), longer duration of the patient-provider relationship, and use of self-advocacy strategies by patients or caregivers. Effective self-advocacy strategies included preparing for clinic appointments by writing questions and concerns, validating verbally-expressed concerns with written information in the medical record, and engaging other health care providers in advocating on the patient's behalf. Conditions inhibiting a perception of "being listened to" were having symptoms perceived as atypical, having concerns that conflicted with established treatment protocols, and having a deferential approach to interactions with health care providers.
CONCLUSIONS: High-cost, high-need patients in this study reported having unique information that led to longer periods of stable outpatient management if that information was used to individualize their treatment plans. Person-centered care interventions that facilitate this information exchange have the potential to improve quality and reduce hospital utilization for high-cost, high-need patients.

"IT'S LIKE TEXTING AT THE DINNER TABLE": A QUALITATIVE ANALYSIS OF ELECTRONIC HEALTH RECORDS' IMPACT ON PATIENTPHYSICIAN INTERACTIONS Rebekah Gardner ${ }^{1}$; Kimberly Pelland ${ }^{3}$; Rosa Baier $^{2}$. ${ }^{1}$ Alpert Medical School of Brown University, Providence, RI; ${ }^{2}$ Brown University School of Public Health, Providence, RI; ${ }^{3}$ Healthcentric Advisors, Providence, RI. (Control ID \#2465397)

BACKGROUND: Electronic health records (EHRs) have the potential to reduce medical errors and improve care, but their use may complicate the clinical encounter. Understanding how EHRs impact patient-physician interactions can inform the development of interventions to improve how physicians incorporate EHRs when caring for patients. The objective of this study is to describe office- and hospital-based physicians' perceptions of the effect of EHRs on patient-physician interactions using a large statewide sample.

METHODS: We performed a qualitative analysis of free-text comments submitted in response to the 2014 Rhode Island Health Information Technology (HIT) Survey, using a combination of deductive and inductive coding. The survey was administered via mail and email (if addresses available) to all physicians licensed in Rhode Island, in active practice, and located in Rhode Island and adjacent states. Themes and subthemes among both office- and hospital-based physician were derived from responses to the free-text survey question, "How does using an EHR affect your interaction with patients?"

RESULTS: The Rhode Island HIT Survey's response rate was $68.3 \%$. Among the respondents, 2236 (87.1 \%) had EHRs; and among those, 744 (33.3\%) responded to question asking how EHRs affected their interaction with patients. Five main themes emerged in our qualitative analysis. Overall, physicians generally perceived EHRs as negatively altering patient interactions, although the rank-order of the top two (most common) themes was reversed for office- and hospital-based physicians: office-based physicians commented most frequently on EHRs worsening the quality of their interactions and relationships with patients, while hospital-based physicians commented most frequently that they spend less time with patients because they have to spend more time on computers. A smaller proportion of physicians commented that using an EHR has no effect on their interaction with patients. One sub-theme, more common among hospital-based physicians, was that EHRs truly did not affect their interaction. A second sub-theme, more common among office-based physicians, was that EHRs do not affect patient interactions because the physicians have altered their workflow to prevent the EHR from having a negative impact. For example, physicians noted that they document in their EHR before and after an encounter to avoid using a computer during their time with the patient. Positive responses focused on information access and use in patient care. Comments that were generally positive and comments that detailed the patient's perspective comprised the least commonly observed themes for both settings.

CONCLUSIONS: Our analysis of the impact of EHRs on patient interactions, from a large sample of physicians across most physician specialties, demonstrates that physicians in office- and hospital-based settings generally perceive EHRs as negatively altering patient interactions, although they emphasize different reasons. Although our analysis also describes benefits ranging from better information access to improved patient education and communication, the unintended consequences are more frequent themes. These findings can shape interventions to improve how EHRs are used in specific clinical settings and to tailor those interventions to specific specialties. Ultimately, by changing the ways physicians use EHRs with patients, we hope to affect both physician satisfaction and patient experience. 
“SWEATIN' TO THE OLDIES!" ATTITUDES OF OLDER HEMODIALYSIS PATIENTS REGARDING PHYSICAL ACTIVITY AUTHORS: DEBORA AFEZOLLI, MD JANET SEO, BA, ASHISH UPADHYAY MD, JASVINDER BHATIA, MD, CHRISTINE LIU, MD BOSTON UNIVERSITY SCHOOL OF MEDICINE, DIVISION OF NEPHROLOGY BOSTON UNIVERSITY SCHOOL OF MEDICINE, DIVISION OF GERIATRICS Debora Afezolli; Janet Seo; Ashish Upadhyay; Jasvinder Bhatia; Christine Liu. Boston Medical Center, Boston, MA. (Control ID \#2467447)

BACKGROUND: Muscle wasting is common in older hemodialysis patients due to the metabolic acidosis associated with kidney failure. Older hemodialysis patients are weak and have very limited endurance, which predisposes them to falls. Increasing physical activity is one intervention proven to prevent falls. To assess whether a program of increased physical activity for falls prevention is acceptable to older hemodialysis patients, our goal was to elucidate older hemodialysis patient attitudes regarding their physical function, falls, and physical activity.

METHODS: A convenience sample of hemodialysis patients aged 65 or older followed at an urban academic medical center underwent $30-40 \mathrm{~min}$ semi-structured interviews in their homes. Questions included open-ended and Likert style questions about general physical function, falls, and physical activity. The Falls Efficacy Scale-International (FESI) was administered. Interviews were audiotaped and transcribed. Three research team members simultaneously reviewed interview data and determined dominant themes by consensus discussion.

RESULTS: A total of 5 older hemodialysis patients (mean age $75 \pm 6.7$ years, $60 \%$ female, $100 \%$ African-American, mean years on hemodialysis treatments $4.3 \pm$ 3.5 years) were interviewed between November 2015 and January 2016. Mean FES-I score was $22.3 \pm 9.3$ points, representing a moderate level of concern for falling. Major themes that emerged were a near universal desire to increase physical activity, with knowledge that it can improve well-being. Most did not think hemodialysis was a contraindication to physical activity. Sixty percent expressed willingness to undertake physical activity three times a week. The most common barrier to undertaking physical activity was a lack of motivation; other limitations included time constraints due to hemodialysis treatments, fatigue, and the impact of co-morbidities such as arthritis (Table).

CONCLUSIONS: In this predominantly African-American sample, older hemodialysis patients view themselves as capable of undertaking physical activity. Older hemodialysis patients understand physical activity has health and psychological benefits. Although hemodialysis treatments are time intensive and physically draining, the majority is still willing to undertake physical activity multiple times a week. The primary barrier to undertaking physical activity is a lack of motivation. Strategies for patient motivation, such as incentives, will need to be included if physical activity will be utilized as a potential intervention for fall prevention in this patient population. Future studies will focus on exploring the efficacy of various approaches to bolster motivation for physical activity in older hemodialysis patients.

Major barriers to physical activity in older hemodialysis patients

\begin{tabular}{|c|c|}
\hline Theme & Examples \\
\hline \multirow{3}{*}{$\begin{array}{l}\text { Lack of } \\
\text { motivation }\end{array}$} & "I would move more if I had a grandchild to pick up and get ready" \\
\hline & "Having somewhere to be motivates me to move" \\
\hline & $\begin{array}{l}\text { "It would be good if there was something to do after hemodialysis, especially in } \\
\text { the summer" }\end{array}$ \\
\hline \multirow[t]{2}{*}{ Time limitation } & "If I was not on hemodialysis I would do more" \\
\hline & "I do not walk on dialysis days" \\
\hline \multirow[t]{2}{*}{ Fatigue } & "Hemodialysis just drains me out" \\
\hline & "Fatigue prevents me from moving" \\
\hline Co-morbidities & $\begin{array}{l}\text { "My back pain and knee pain prevent me from moving, regardless of whether I } \\
\text { have hemodialysis" }\end{array}$ \\
\hline
\end{tabular}

"WHY I WENT INTO MEDICINE": USING TRANSFORMATIVE LEARNING THEORY TO UNDERSTAND THE IMPACT OF INTERNATIONAL HEALTH ELECTIVES ON RESIDENTS' PROFESSIONAL IDENTITY FORMATION Adam P. Sawatsky; Hannah C. Nordhues; Stephen P. Merry; M. U. Bashir; Frederic Hafferty. Mayo Clinic, Rochester, MN. (Control ID \#2465354)

BACKGROUND: Medical trainees are increasingly interested in global health, and a majority of residency programs offer international health elective (IHE) experiences for their residents. There are numerous benefits to the residents involved in these experiences, including gaining experience with a wide variety of pathology, learning to work with limited resources, developing clinical and surgical skills, and participating in resident education. Residents who participate in IHEs seem more likely to eventually practice in underserved and international settings during their career, suggesting these can be transformative experiences. While much has been studied about the benefits to medical knowledge and patient care, less is understood about the transformative effects of these experiences on professional identity formation. We used the lens of transformative learning theory to analyze resident reflections on the challenges to professional identity posed by the "disorienting dilemma" of IHEs. METHODS: We conducted a qualitative analysis, using constructivist grounded theory with sensitizing concepts of professional identity formation and transformative learning theory to guide analysis. Each resident at the Mayo Clinic who participates in an IHE is required to complete a reflective essay on their experience, and one paragraph about systems improvements based on their experience. Residents from all specialties and across all three Mayo Clinic training sites are eligible to participate, and applications are reviewed by a selection committee. Residents may go to one of the 14 Mayo International Health Program designated partnership sites, set up a rotation through an MIHP contact elsewhere, or choose an independent site with appropriate onsite mentoring. For this study, we analyzed all reflective reports for all residents who participated in an IHE between 2001 and 2014. We created a codebook a priori based on the six ACGME core competencies and three coders independently coded the first 3 years of reflective reports in duplicate and reconciled differences through consensus. Once we reached substantial agreement, we individually coded the remaining reflective reports. Within the code of professionalism, we identified a major theme of reflection around "why I went into medicine." Using the lenses of professional identity formation and transformative learning theory, we identified themes identified by residents around this theme, and then expanded our analysis across all 377 reports to expand our understanding of the themes. We used the constant comparative method to refine our understanding of how IHE experiences influenced professional identity formation. This study was approved by the Mayo Clinic Institutional Review Board.

RESULTS: We analyzed 377 reflective reports by residents and fellows from 40 medical and surgical specialties across three Mayo Clinic training sites who traveled to 56 countries. We identified three main domains of professional identity: making a difference; experiencing a meaningful patient-doctor relationship; and discovering the purest form of medical practice; Within the domain of "making a difference," residents discussed the clearly apparent need of their patients, the sense that their patients have little or no other options, and the experience of altruism. Within the domain of the patient-doctor relationship, residents discussed their experience of gratitude and trust from their patients, and the resilience of their patients in the face of need. This was contrasted to their experiences with patients in the US training environment, and called into question the "true needs of patients." Within the domain of "purest form" of medicine, residents discussed the limitations of medicine and technology and identified the key role of clinical skills in the practice of medicine. They discussed freedom from documentation, billing, and other "distractions" from patient care. They reflected on what was necessary for practice, and what was "just extra." These reflections were facilitated by the stark contrast of their experiences between their IHE and normal practice and the role modeling of physicians they worked with on their IHE. These reflections brought humility, frustration and transformation for their future careers. 
CONCLUSIONS: Through IHEs, residents found meaning in making a difference, experiencing a meaningful patient-doctor relationship, and recognizing "medicine in its purest form." These findings help to understand the transformative power of IHEs on residents' professional identity, and why these experiences remain so popular among trainees. It also prompts discussion about the current resident training environment that causes them to forget "why [they] went into medicine." Faculty mentors can use these findings to help guide resident reflections on their IHE experiences as they deal with the disorientation of these experiences, and facilitate true transformation.

"YOU ARE PULLED IN TWO DIFFERENT DIRECTIONS": THE HOSPITALIST PERSPECTIVE ON OPIOID PRESCRIBING Susan L. Calcaterra ${ }^{2}$, ${ }^{\text {; }}$ Anne Drabkin $^{2,}{ }^{1}$; Sarah E. Leslie ${ }^{6}$; Reina Doyle ${ }^{6}$; Steve Koester ${ }^{4}$; Joseph W. Frank ${ }^{1,5}$; Ingrid A. Binswanger ${ }^{3} .{ }^{1}$ University of Colorado Department of Medicine, Aurora, CO; ${ }^{2}$ Denver Health Medical Center, Denver, CO; ${ }^{3}$ Kaiser Permanente Colorado, Dever, CO; ${ }^{4}$ University of Colorado Denver, Denver, CO; ${ }^{5}$ VA Eastern Colorado Health Care System, Denver, CO; ${ }^{6}$ Center for Health Systems Research, Denver Health Medical Center, Dever, CO. (Control ID \#2448012)

BACKGROUND: Under the Patient Protection and Affordable Care Act, value-based incentive payments to hospitals are calculated based on Hospital Consumer Assessment of Healthcare Providers and System (HCAHPS) survey scores. In the survey, patients are asked to rate their experiences with pain control during their hospitalization. Hospitalists, dedicated inpatient physicians, are encouraged to achieve high patient satisfaction scores for pain to maximize federal incentive payments. Opioid stewardship, the practice of managing opioid prescribing in high-risk patients, may be at odds with current hospital care, which focuses on in hospital pain control rather than long term consequences of opioid use. We aimed to understand physicians' attitudes and practices towards opioid prescribing during hospitalization and discharge.

METHODS: We conducted semi-structured interviews from January 2015 to August 2015 with hospitalists who worked in one of two university hospitals, a safety-net hospital, a Veterans Affairs hospital, or a private hospital located in Denver, Colorado or Charleston, South Carolina. Hospitalists were recruited via email solicitation. We used purposive sampling to achieve an even distribution with respect to gender and years in practice. We developed an interview guide informed by the Theory of Planned Behavior. Interviews were recorded, transcribed, and entered into qualitative software (ATLAS.ti). During regular group meetings, we systematically analyzed interview transcripts and identified emerging themes using a team-based mixed inductive and deductive approach.

RESULTS: Of the 25 participants, 16 (64\%) were women and the majority were nonHispanic White, 21 (84\%). The largest proportion, twelve physicians (48 \%), completed Internal Medicine residency within the past 5 to 10 years. Emergent themes which most impacted physicians' attitudes and practices towards opioid prescribing included 1) unrewarding experiences with patient-perceived pain control, 2) institutional and systemic pressure between opioid prescribing and meeting patient satisfaction metrics and 3) competing demands between opioid prescribing and maintaining efficiency (Table 1).

CONCLUSIONS: We identified important experiences and competing demands related to opioid prescribing which may have unintended consequences on opioid prescribing practices. Future work will focus on understanding how hospitalists overcome and respond to these challenges. Strategies to provide pain relief to patients which allow hospitalists to practice opioid stewardship while maintaining efficiency are urgently needed.
Table 1. Emergent Themes

\begin{tabular}{|c|c|c|}
\hline $\begin{array}{l}\text { Experiences with } \\
\text { Pain Control }\end{array}$ & $\begin{array}{l}\text { Treating chronic, noncancer pain } \\
\text { left hospitalists feeling frustrated } \\
\text { and unsatisfied. }\end{array}$ & $\begin{array}{l}\text { "I have a hard time feeling like I'm } \\
\text { successful with people who have } \\
\text { chronic noncancer pain who come in } \\
\text { for an exacerbation. Unless I can } \\
\text { figure out clear reasons for that } \\
\text { exacerbation, I rarely succeed in } \\
\text { having the patient, nurses and } \\
\text { providers be happy. It is an } \\
\text { unrewarding situation." }\end{array}$ \\
\hline $\begin{array}{l}\text { Opioid Prescribing } \\
\text { and Patient } \\
\text { Satisfaction }\end{array}$ & $\begin{array}{l}\text { Hospitalists were concerned that } \\
\text { institutional expectations to achieve } \\
\text { high satisfaction scores altered } \\
\text { opioid prescribing. }\end{array}$ & $\begin{array}{l}\text { "I'm well aware I'm being watched } \\
\text { for my patient satisfaction scores. I } \\
\text { don't doubt in the least that patient } \\
\text { satisfaction scores are a driver of } \\
\text { opioid prescribing." }\end{array}$ \\
\hline $\begin{array}{l}\text { Opioid Prescribing } \\
\text { and Efficiency }\end{array}$ & $\begin{array}{l}\text { Some hospitalists prescribed } \\
\text { opioids to prevent readmissions and } \\
\text { reduce costs. }\end{array}$ & $\begin{array}{l}\text { "Sometimes it feels utterly } \\
\text { pragmatic [opioid prescribing at } \\
\text { discharge]. If the patient comes back } \\
\text { and gets readmitted because they } \\
\text { don't have pain medicine, it's a } \\
\$ 3,000.00 \text { two-day stay in the hos- } \\
\text { pital that was unnecessary." }\end{array}$ \\
\hline
\end{tabular}

"EVALUATING STUDENT PERCEPTIONS OF PRIMARY CARE AFTER PARTICIPATION IN A STUDENT-FACULTY COLLABORATIVE CLINIC" Meissa M. Jones ${ }^{2}$; Marya J. Cohen ${ }^{1} .{ }^{1}$ MGH, Boston, MA; ${ }^{2}$ Massachusetts General Hospital, Boston, MA. (Control ID \#2468621)

BACKGROUND: Due to an aging population and over 3 million people being insured under the Affordable Care Act, the need for primary care services has drastically increased. However, studies have shown that the number of medical students choosing primary care residencies will not satisfy this high demand. The Crimson Care Collaborative (CCC), a student-faculty collaborative clinic, was created in the hopes of establishing a pipeline to primary care at Harvard Medical School. To determine the effectiveness of $\mathrm{CCC}$, we have evaluated student perceptions of primary care before and after participation in CCC.

METHODS: We compared data from surveys aimed at assessing attitudes toward and knowledge about primary care that were administered to students before and after participation in CCC. CCC students were asked to rate the frequency of positive and negative comments about primary care heard from faculty/administration, residents, and peers during medical school. They also rated the extent to which they agreed or disagreed with the comments. In addition, we also asked students to share the comments that they have heard regarding primary care.

RESULTS: A total of 47 matching pre/post surveys were collected. After participation in CCC, students heard more positive comments about primary care from residents $(t=-2.470, p=0.018)$. In addition, students significantly agreed more with the positive comments from faculty/administration, residents, and peers after participation in CCC $(t=-2.150, p=0.038)$. There were no significant differences in the frequency of negative comments heard from faculty/administration, residents, and peers.

CONCLUSIONS: After participation in a primary care based student-faculty collaborative clinic, positive perceptions towards primary care increased. Programs designed to offer students early exposure to primary care, such as CCC, are needed to meet the high demand for primary care services. 
Themes about Primary Care

\begin{tabular}{ll}
\hline \hline Positive Themes about Primary Care & Negative Themes about Primary Care \\
Long-term Relationships with Patients & High Burnout \\
Meaningful and Innovative Work & Boring \\
Holistic Care & Low Salary \\
\hline
\end{tabular}

List of positive and negative themes about primary care gathered from comments by CCC medical students.

\section{ASSOCIATION OF TEAMWORK WITH PATIENT SATISFACTION AND PHY-} SICIAN WORKFLOW DURING PRIMARY CARE REDESIGN Paul Di Capua ${ }^{1,4}$; Robin Clarke 3, 2; Renee Sednew ${ }^{5}$; Chi-Hong Tseng ${ }^{4}$; Holly Wilhalme ${ }^{4}$; Samuel A. Skootsky ${ }^{2,3}$; Neil Wenger ${ }^{4}$. ${ }^{1}$ Baptist Health Medical Group, Coral Gables, FL; ${ }^{2}$ David Geffen School of Medicine at UCLA, Los Angeles, CA; ${ }^{3}$ UCLA, Los Angeles, CA; ${ }^{4}$ University of California, Los Angeles, Los Angeles, CA; ${ }^{5}$ ucla, Los Angeles, CA. (Control ID \#2469827)

BACKGROUND: Despite the enthusiasm and focus on team-based care in the national conversation on the transformation of primary care, relatively few studies directly measure and report measures of teamwork as a metric of primary care redesign. Relational coordination has emerged as a dominant model for measuring team-based care. At the same time, health systems recognize the importance of optimizing the primary care patient experience as they adopt population health models of care delivery. Relational coordination has been associated with higher levels of patient satisfaction in multiple settings. The health system for the University of California, Los Angeles (UCLA Health) recently implemented a redesign of its primary care system, and sought to measure team-based care to better understand the potential value of focusing on this metric in its redesign. In this study, we hypothesized that teams with higher relational coordination would have lower emergency room visits. We hypothesized that patients in practices with higher relational coordination would report better experiences, and that doctors in those practices would report lower perceived burden of tasks that do not require their medical expertise.

METHODS: UCLA Health is an urban academic system with 26 primary care practices caring for adult patients (internal medicine, family medicine, and geriatrics). Primary care practices within UCLA Health are primarily traditional community-based practices with full time physicians; four practices also have trainees. The health system uses the Clinician and Group Survey by Consumer Assessment of Healthcare Providers and Systems (CGCAHPS $^{\circledR}$ ) 12-Month Survey to measure and track patient experience. The pre/post differences in the six patient experience outcomes between the CC practices and non$\mathrm{CC}$ practices were compared using an adjusted linear mixed effects model with fixed effects for the presence of a care coordinator, time period (pre/post), and care coordinator by time interaction, and a random clinic effect. Relational coordination is defined as a mutually reinforcing web of communication and relationships carried out for the purpose of task integration. Composite relational coordination scores were compared between CC practices and non-CC practices using an adjusted linear mixed effects model with a practice-level random effect and a fixed effect for the presence of a care coordinator. Five questions assessed physicians' perceived burden of non-medical care coordination tasks, each was scored on a 5-point scale where 5 was lowest perceived burden. Differences in scores of the barriers to coordinated care between the CC practices and non-CC practices were compared using an adjusted linear mixed effects model.

RESULTS: A total of 7279 patients completed the patient experience survey of which $65 \%$ were female, $65 \%$ were white, $87 \%$ were not Hispanic, and $65 \%$ had at least a
4 year college degree. The survey for physicians and health professionals had a total of 458 respondents, of which 131 were physicians (response rate $75 \%$ and $64 \%$, respectively). Four of the 26 practices were training sites, median staff size was 13 people, median number of physicians was 4 .

CONCLUSIONS: This study reports an assessment of baseline relational coordination scores across one health care system, and the association of these scores with measure of patient satisfaction and with physician-perceived barriers to efficient workflow. Relational coordination has been shown to be associated with patient satisfaction in multiple settings. However, the one other study examining this relationship in primary care also did not find an association between patient experience and relational coordination. This may be because primary care is somehow different than other settings previously examined, differences in statistical modeling, or publication bias. The lack of association of relational coordination with physician-perceived burden of care coordination may suggest that relational coordination is not associated with physicians practicing at the top of their license. Future research can focus on how to quantitatively evaluate how well systems are designed to facilitate physician workflow and the best use of their time. In order to develop and strengthen a "team-based" approach to care, many focus on workflow processes, team structure, or adding new members to teams. However, most transformation efforts do not directly measure or track the working relationships which underlie team-based care. In order to achieve the envisioned synergies of team-based care in primary care redesign, there must be a purposeful effort to measure and monitor team dynamics.

Association of Relational Coordination with Patient Experience

\begin{tabular}{|c|c|c|c|c|c|c|}
\hline & Access & $\begin{array}{l}\text { Coordinated } \\
\text { care }\end{array}$ & Doctor & Staff & $\begin{array}{l}\text { Overall } \\
\text { doctor } \\
\text { rating }\end{array}$ & $\begin{array}{l}\text { Recommend } \\
\text { doctor }\end{array}$ \\
\hline $\begin{array}{l}\text { Relational } \\
\text { coordination } \\
\text { (unadjusted } \\
\text { model) }\end{array}$ & $\begin{array}{l}6.6 \\
(4.5)\end{array}$ & $3.9(5.7)$ & $\begin{array}{l}1.9 \\
(2.4)\end{array}$ & $\begin{array}{l}5.4 \\
(3.8)\end{array}$ & $\begin{array}{l}-0.9 \\
(2.3)\end{array}$ & $3.2(2.8)$ \\
\hline $\begin{array}{l}\text { Relational } \\
\text { coordination } \\
\text { (adjusted model) }\end{array}$ & $\begin{array}{l}4.2 \\
(6.6)\end{array}$ & $-2.8(7.2)$ & $\begin{array}{l}1.2 \\
(3.1)\end{array}$ & $\begin{array}{l}3.0 \\
(5.7)\end{array}$ & $\begin{array}{l}-2.4 \\
(3.0)\end{array}$ & $-0.2(4.0)$ \\
\hline
\end{tabular}

Scores reported as estimated (std error). Model adjusted for age, race/ethnicity, gender, overall health rating, education, total staff and percent $\mathrm{md} \mathrm{fte}$

Association of relational coordination and physician-perceived barriers to care coordination

\begin{tabular}{llllll}
\hline \hline & $\begin{array}{l}\text { Difficulty } \\
\text { scheduling } \\
\text { specialists }\end{array}$ & $\begin{array}{l}\text { Familiarity } \\
\text { with } \\
\text { outstanding } \\
\text { gaps in quality }\end{array}$ & $\begin{array}{l}\text { Phone calls } \\
\text { requiring } \\
\text { medical } \\
\text { expertise } \\
-0.06\end{array}$ & $\begin{array}{l}\text { Filling } \\
\text { out } \\
\text { forms }\end{array}$ & $\begin{array}{l}\text { Orders } \\
\text { requiring prior } \\
\text { authorization }\end{array}$ \\
$\begin{array}{lllll}\text { Relational } \\
\text { coordination } \\
\text { (unadjusted }\end{array}$ & $0.75(0.47)$ & $0.97(0.39)^{*}$ & -0.63 & $1.55(0.53)^{*}$ \\
$\begin{array}{l}\text { model) } \\
\begin{array}{l}\text { Relational } \\
\text { coordination } \\
\text { (adjusted }\end{array}\end{array}$ & & & $(0.40)$ & $(0.45)$ & \\
model) & $1.0(0.80)$ & $-0.02(0.76)$ & -0.74 & 0.36 & $0.32(0.94)$ \\
\hline
\end{tabular}

Association reported as estimates (std error)

$* p$-value $<0.05$

Model adjusted for age, gender, years working at clinical site, years practicing as an MD, $\%$ MD FTE, and total clinic staff. 


\section{EFFECT OF AVASCULAR NECROSIS ON HOSPITALIZATION IN PATIENTS} WITH SICKLE CELL CRISIS Keri Holmes-Maybank; William P. Moran; Kit N. Simpson. Medical University of South Carolina, Charleston, SC. (Control ID \#2467614)

BACKGROUND: Sickle cell disease is the most common inherited blood disease worldwide. It affects 1 in 375 African Americans in the United States, approximately 70,000-100,000 individuals. Patients with sickle cell disease suffer from chronic anemia, chronic pain, and acute pain known as vaso-occlusive crises or pain crises. The survival from childhood into adulthood has increased from 79 to $89 \%$ with an increased projected life expectancy of 53.5 years. As patients with sickle cell disease are living longer, they are experiencing more complications including end organ damage, some of which manifest as progressive bone disease and daily chronic pain. Bones are the second most common organ affected. Avascular necrosis (also known as osteonecrosis, aseptic necrosis, and bone infarct) is bone death, the end result of severe and prolonged ischemia. The prevalence of avascular necrosis in adults with sickle cell disease is estimated up to $50 \%$ by 35 years of age. A study identifying risk factors for avascular necrosis found a significantly higher rate of painful crises and hospitalizations per year in sickle cell disease patients with avascular necrosis. We studied the effect of avascular necrosis in patients hospitalized with sickle cell crisis on number of hospitalizations, hospital days, and cost. METHODS: We examined the 2011 Agency for Healthcare Research and Quality's Healthcare Cost and Utilization Project (HCUP) State-specific Inpatient Database in four states representing four geographic regions of the United States: New York, California, Mississippi, and Iowa. The HCUP database is derived from administrative data and contains encounter-level, clinical and nonclinical information including all listed diagnoses and procedures, discharge status, patient demographics, and charges for all patients, regardless of payer (e.g. Medicare, Medicaid, private insurance, uninsured) beginning in 1988. We described the number of hospitalizations, number of hospital days, and cost in patients admitted with sickle cell crisis with and without avascular necrosis as well as joint replacement in those with avascular necrosis 18 years of age and older. Cost outcomes utilized hospital-level charge data and publically available hospital-specific cost-to-charge ratios to estimate cumulative costs for each admission.

RESULTS: We found 11,879 hospital admissions for 4357 patients with sickle cell crisis in 2011 in Iowa, California, New York, and Mississippi. The average age was 33 years and $55.37 \%$ were female. $14.8 \%$ of patients (644) had a diagnosis code indicating AVN. Patients with AVN had more admissions per year, 6.2 compared to 3.3 for patients without AVN $(p<.0001)$. Similar patterns were observed for total number of hospital days per year $(43.8$ vs. $18.7, p<.0001)$, and mean cost per admission $(\$ 50,648$ vs. $\$ 33,783$, $p<.0001$ ). Total annual hospital charges were $\$ 259,442$ for AVN patient and $\$ 113,231$ for sickle cell patients without an AVN diagnosis $(p<.0001)$. However, only $4.0 \%(28)$ of patients with $\mathrm{AVN}$ received a hip or shoulder replacement done during the year. The mean annual charge for the AVN subgroup who received a joint replacement was $\$ 257,763$, similar to the mean charges of $\$ 259,512(p=.6379)$ for patients with AVN who did not have a joint replacement. The mean number of hospitalizations in individuals with AVN and joint replacement was 6 compared to 6.2 for those with AVN and no replacement $(p=.7074)$. There was a slight but insignificant difference in the mean charge per admission $(\$ 42,553)$ in individuals with AVN with joint replacement compared to those without replacement $(\$ 50,998, p=.2886)$. Lastly, the mean number of hospital days for patients with AVN and joint replacement was marginally less than those with joint replacement (38.5 vs. $44, p=.3339$ ) respectively.

CONCLUSIONS: Avascular necrosis was present in approximately $15 \%$ of the sample population of patients admitted for sickle cell crisis in Iowa, California, New York, and Mississippi in 2011. Avascular necrosis in sickle cell crisis patients has a major impact on the patients and the health care system. Sickle cell patients with avascular necrosis experienced twice as many hospitalizations, over twice as many hospital days, and over twice as much cost per year. Previous research has determined joint replacement is a successful, definitive treatment for all cause avascular necrosis. However, in our study we found no difference in cost, hospitalizations, and hospital days for sickle cell patients with avascular necrosis who had a joint replacement compared to those without a joint replacement. Identifying avascular necrosis as significant predictor of longer hospital stay in individuals experiencing sickle cell crisis may be helpful in anticipating length of stay/treatment and discharge planning. It may also be helpful in counseling patient expectations about length of hospitalization and treatment. Due to the significant impact of avascular necrosis on sickle cell crisis it should be further investigated as a marker of disease severity and to determine the best modality of treatment.

\section{HOME BASED PRIMARY CARE INTEGRATED WITH SUPPORTIVE SERVICES} AVOIDS LONG TERM INSTITUTIONALIZATION IN FRAIL, MEDICALLY COMPLEX ELDERS Christine Patterson ${ }^{2}$; Peter Boling ${ }^{2}$; George Taler ${ }^{3}$; Jean Yudin ${ }^{1}$; Karl Eric Dejonge $^{3}$; Bruce Kinosian ${ }^{1} .{ }^{1}$ University of Pennsylvania, Philadelphia, PA; ${ }^{2}$ Virginia commonwealth University, Richmond, VA; ${ }^{3}$ Washington Hospital Center, Washington, DC. (Control ID \#2469551)

BACKGROUND: To determine if home-based primary care (HBPC) when integrated with supportive services can lower long term institutionalization (LTI) in frail, medically complex elders. Efforts to integrate medical and supportives services for frail elders have frequently focused on payers, such as managed care organizations (MCOs), or providerpayers such as PACE. The Mid-Atlantic Consortium, comprised of long operating home based primary care programs in Washington DC (WHC-20 years), Richmond (VCU30 years) and Philadelphia (UPENN-20 years), deploy mobile, interdisicplinary teams to care for frail, homelimited elders. Medically complex, high cost HBPC cases $(N=721)$, managed under a FFS/shared savings arrangement, represent $35 \%$ of the total patients served by these hbpc programs. Each program uses a different form of integration with their Area Agency on Agency (AAA), which provide community long term services and supports (LTSS). WHC has its social workers employed as AAA agents; U Penn has formed a dedicated interagency interdisciplinary team with their AAA, and VCU uses a collaborative model with dedicated payer support.

METHODS: Cohort study comparing death and LTI among frail elders receiving HBPC in Philadelphia, Richmond and DC (2012-2015) with matched controls from the 2012 to 13 Medicare $5 \%$ Beneficiary, NPI-linked files. Selection criteria included fee-for-service (FFS) beneficiaries with a hospitalization in the prior 12 months, post-acute care episode in prior 12 months, 2 or more chronic conditions, and JEN frailty index [JFI] $>6$, (equivalent to 2+ impairments in Activities of Daily Living). ADL data were summarized into expected LTSS expenditures using the Minnesota Case Mix Classification (MN) and VA locally adjusted class-specific payments. Observed LTSS expenditures were available for the Philadelphia site. Rates were adjusted for time observed; LTI and death were modeled as competing risks. Primary comparison used matched controls who had not received housecalls $(\mathrm{HC})(n=600)$, with a secondary comparison using controls who had received HC ( $n=103)$, as the Richmond and DC programs enrolled 30-50\% of 5\% sample patients, respectively.

RESULTS: Average monthly census for this frail subgroup rose from 247 in Y1 to 356 in Y3, with mortality averaging $25.8 \%$ (range $22.6-29 \%$ ). The LTI rate declined from $8.9 \%$ (Y1) to $3.2 \%$ (Y3), with a 3-year average of $6.4 \%$ (95 \% CI 4.63-8.17). Mean MN class was "D" (4-6 ADL impairments), with expected LTSS expenditures of \$2382 pbpm, and observed expenditures of \$2151 (CI \$1891-\$2408) for the $64 \%$ of patients receiving LTSS services. Controls who did not receive HCs had an annual mortality of $24.9 \%$, with an LTI rate of $17.1 \%$, while among controls who received HCs the mortality was $26.9 \%$ and LTI was $17.7 \%$, demonstrating modest adverse selection into housecall practices.

CONCLUSIONS: Integrated HBPC with social supports was associated with a $63 \%$ reduction in LTI compared to matched local controls. Expected costs of such supports are 
$\$ 2300$ pbpm, with observed expenditures for successful nursing home diversion consistent with expected costs. Integration of social supports with home based primary care in a FFS, shared savings environment can reduce long term institutionalization among frail Medicare Beneficiaries, providing a provider managed FFS alternative to MCO-operated LTSS

SPECIFIC CUT-OFF POINTS FOR WAIST CIRCUMFERENCE AND BODY MASS INDEX AS PREDICTORS OF METABOLIC SYNDROME COMPONENTS Taiju Miyagami. Juntendo University of school, Bunkyo-ku, Japan. (Control ID \#2467781)

BACKGROUND: The aim of this study was to determine statistical cut-off points for waist circumference (WC) and body mass index (BMI) as predictors of metabolic syndrome (Mets) components.

METHODS: Participants were individuals who received medical health checkups at NTT-Higashi Nihon Kanto Hospital from May 2006 to February 2011. Patients who were treated for hypertension, dyslipidemia, diabetes mellitus, or hyperuricemia were excluded from the study. Metabolic disorders were defined in accordance with the Mets diagnosis criteria of Japan as follows: three classic components of Mets (elevated blood pressure, dyslipidemia, and elevated blood glucose concentration) and elevated uric acid ( $>7.0 \mathrm{mg} /$ dl). A receiver operating characteristics curve was constructed to determine optimal cutoff points of WC/BMI for two or more, three or more, and four components of Mets by $\operatorname{sex}$

RESULTS: A total of 23,945 male (mean age, 47 years) and 9552 female participants (48 years) were considered eligible. Optimal WC/BMI cut-off points for two or more, three or more, and four Mets components were $85 \mathrm{~cm} / 23.7 \mathrm{~kg} / \mathrm{m}^{2}, 85.8 \mathrm{~cm} / 24.6 \mathrm{~kg} / \mathrm{m}^{2}$, and $87.2 \mathrm{~cm} / 24.5 \mathrm{~kg} / \mathrm{m}^{2}$, respectively, among men, and $81 \mathrm{~cm} / 22.7 \mathrm{~kg} / \mathrm{m}^{2}, 82.1 \mathrm{~cm}$ $21.8 \mathrm{~kg} / \mathrm{m}^{2}$, and $90.5 \mathrm{~cm} / 25.3 \mathrm{~kg} / \mathrm{m}^{2}$, respectively, among women.

CONCLUSIONS: Optimal WC/BMI cut-off points for two or more Mets components were $85 \mathrm{~cm} / 23.7 \mathrm{~kg} / \mathrm{m}^{2}$ for men and $81 \mathrm{~cm} / 22.7 \mathrm{~kg} / \mathrm{m}^{2}$ for women. Our results underscore the importance of maintaining ideal body weight for the prevention of lifestyle-related risks.

THROUGH THE PATIENT'S EYES: IDENTIFYING RISK FACTORS FOR HOSPITAL READMISSIONS Prakrati Acharya ${ }^{1}$; Aliya Laeeq ${ }^{1}$; Matthew Carmody ${ }^{1}$; Beth A. Lown ${ }^{2} .{ }^{1}$ Mount Auburn Hospital, Cambridge, MA; ${ }^{2}$ Schwartz Center for Compassionate Healthcare, Boston, MA. (Control ID \#2469195)

BACKGROUND: One in 5 Medicare patients return to hospital within 30 days costing the country $\$ 12$ billion annually. We conducted a study to analyze the patient's perspective regarding factors that caused readmission and to identify methods to prevent them. METHODS: We recruited a convenience sample of English speaking patients $>18$ year old who were readmitted from home within 30 days of discharge for this mixed method study. We compared the questionnaire responses of 22 non-readmitted patients to those of 30 patients readmitted from home over a 5 month period. The 30 -question survey queried patient satisfaction, readiness at time of discharge, understanding of diagnosis, treatment and medication changes, and knowledge about seeking medical attention after discharge. Two of the authors conducted semi-structures interviews of 30 of the readmitted patients from this sample. We excluded 3 patients with cognitive impairment or insufficient responses to analyze. Interview questions queried events between the previous and present admission, experiences in hospital, concerns at time of discharge or at home, and help at home. Interviews were audio-recorded, transcribed, analyzed and coded by 4 physicians using the constant comparison approach
RESULTS: Semi-structured interview results Twelve themes emerged from patient's conversation about readmission. They were: understanding [or not] their problem; describing their physical state; problems with medication or equipment; ability to recall; relationship with inpatient care team members; their hopes and wishes; family involvement [or not], assigning fault to themselves, providers or no one; their emotional reactions; their sense of agency [or not] in their own care; post-hospital care; and systems issues. The most frequently coded themes were statements about physical and emotional states. Patients' descriptions of physical states ranged from symptoms alone and to detailed discussion of diagnosis and treatment interventions. Most patients stated they felt physically well on discharge and many felt confident about their ability to manage themselves with a strong sense of personal agency. Many described family and/or home care services. When these resources failed to provide support matching their needs, patients felt fearful and frustrated. Patients' emotional reactions included frustration at not understanding their diagnosis or having a solution to their problem at discharge [Example: “(I am)...discouraged because I do want a diagnosis so I can live like I was before.”]. Unstable symptoms were associated with fearful anticipation [(“I'm) afraid it's going to happen again."] Some patients described confidence in inpatient providers ["I have a good team because they all work together.”]; others expressed dissatisfaction [“...they were adding too many medications and weren't explaining anything."'] Few patients mentioned their primary care physician's (PCP) role during hospitalization or post-discharge. As patients experienced deterioration in their medical condition, they rarely connected with their PCP or home care services; rather they more frequently called 911. \#Quantitaive results in table CONCLUSIONS: Readmitted patients were significantly more likely to be elderly, felt less ready to go home at the time of their first discharge, hadn't discussed their symptoms with their PCP, and had significantly less understanding of their medications. Interviewed patients expressed frustration at not having a clear idea of their diagnosis, were fearful of their condition deteriorating, and tended to call emergency services rather than their PCPs. These findings suggest that patients, particularly in this age group, require a clear understanding of the diagnosis as well as tactics to execute and people to call if they deteriorate. Patients had fears and feelings of uncertainty about their diagnoses. Both during hospitalization and at discharge, providers should assess patients' emotions and concerns in a systematic fashion. As suggested in extant literature, a call from patients' PCP practice within $48 \mathrm{~h}$ of discharge, and a visit within 7 days may optimize care and reduce readmissions.

Quantitative results

\begin{tabular}{llll}
\hline \hline & NON & READMITTED & $P$ \\
& $\begin{array}{l}\text { READMITTED } \\
(N=22)\end{array}$ & VALUE \\
& $\begin{array}{l}\text { (100\% } \\
\text { Felt ready to go home on discharge }\end{array}$ & $79.3 \%$ & 0.03 \\
$\begin{array}{l}\text { Discussed symptoms to call PCP } \\
\text { Understood medication changes on }\end{array}$ & $96.4 \%$ & $59.3 \%$ & 0.03 \\
discharge & $90.9 \%$ & $69 \%$ & 0.048 \\
PCP office went over discharge plan on & $85.7 \%$ & $54.5 \%$ & 0.024 \\
phone & & & \\
80+ age group & $25 \%$ & $41.7 \%$ & 0.038 \\
Needed more information at discharge & $19 \%$ & $34.5 \%$ & 0.13 \\
Social support & $95.5 \%$ & $85.2 \%$ & 0.36 \\
\hline
\end{tabular}

TRAINEES' KNOWLEDGE AND APPLICATION OF GUIDELINE RECOMMENDATIONS FOR COLORECTAL CANCER SCREENING AND SURVEILLANCE; A GAP THAT NEEDS TO BE BRIDGED. Abhishek Karwa; Rushad Patell; Rocio Lopez; Carol A. Burke. Cleveland Clinic, Cleveland, OH. (Control ID \#2466359)

BACKGROUND: Screening and surveillance have proven to decrease the incidence and mortality of colorectal cancer (CRC). Unfortunately, data demonstrates clinicians in practice do not follow the published guidelines for CRC screening and surveillance. Many factors contribute to this hiatus including knowledge, complexity and acceptance of 
guidelines by clinicians. To date, no assessment of the knowledge of CRC screening or surveillance guidelines nor the correct application of the guidelines has been performed in medical trainees. Our objective was to assess and compare the knowledge gap in CRC screening and surveillance guidelines in medical trainees.

METHODS: An IRB approved 16 question anonymous web based survey was created requesting information on medical specialty, level of training, knowledge of and perceived confidence in applying CRC screening and surveillance guidelines (US Multi-Society Task Force [2012], American College of Gastroenterology [2009], US Preventive Services Task Force [2008]). The 4 clinical vignettes presented included 2 screening scenarios (a 40 year/old Caucasian whose mother had CRC at age 65; and a 45 year/old African American with no additional risk factors) and 2 surveillance scenarios (a 63 year/old with a $5 \mathrm{~mm}$ tubular adenoma and an $8 \mathrm{~mm}$ tubulovillous adenoma with high grade dysplasia; and a 58 year/old with a $7 \mathrm{~mm}$ sessile serrated polyp without dysplasia). Respondents were also asked to pick the specific factors utilized by guidelines in CRC screening (5 of 15-age, family history of CRC, personal history of CRC, IBD or polyps) and surveillance (4 of 9-polyp number, size, pathology and piecemeal resection). Program directors of all ACGME approved internal medicine; family medicine, surgery, ob/gyn, urology, gastroenterology and colorectal surgery training programs were sent an email request to forward the survey hyperlink to faculty, fellows and residents in their departments. Univariable analysis assessed whether respondents' attitude and/or knowledge of CRC screening and surveillance varied by gender, specialty or experience. Pearson's chi-square tests were used to assess all associations. A comparison was made between primary care (family medicine, internal medicine and geriatrics), gastroenterology fellows, surgery (general and colorectal) and others (gynecology and urology). Respondents were also compared based on stage of training. A $p<0.05$ was considered statistically significant.
RESULTS: Six hundred ninety-eight responses were received, which consisted of 575 trainees and 119 practicing physicians among 9 specialties: internal medicine (178), family medicine (179), primary care (24), gastroenterology (119), general surgery (57), ob/gyn (90), urology (17) and colorectal surgery (19). Eighty-five percent reported they ordered and $26 \%$ performed colonoscopies. While $95 \%$ reported they followed guidelines when making recommendations only 69 and $51 \%$ were confident in their ability to recall screening and surveillance guidelines respectively. SCREENING: $27 \%$ identified all 5 factors used to determine onset of CRC screening, and $56 \%$ got both vignettes correct. Combined, the accuracy decreased to $17 \%$. SURVEILLANCE: $17 \%$ identified all 4 factors and $31 \%$ got both surveillance vignettes correct. Combined, the accuracy decreased to $8 \%$. There is a striking difference between specialties in their reported confidence recalling screening (44-88\%) and surveillance (22-91\%) guidelines. Correct responses to clinical vignettes was poor and varied by specialty (34-64\% for screening and $26-51 \%$ for surveillance). The respondents with the highest accuracy to identify all factors used in guidelines to determine age to initiate CRC screening was the PCP group at $32 \%$ and the lowest was ob-gyn/urology at $18 \%$. Similarly, the lowest group to identify factors used for surveillance intervals was ob-gyn/urology at $3 \%$ and the highest was GI at $65 \%$ (Table 1 ). The proportion that correctly answered all vignettes and identified all factors used in the guidelines varied from 3 to $22 \%$ and 0 to $39 \%$ for screening and surveillance respectively. Trainees had lower confidence in recalling screening guidelines vs. practicing clinicians (68 vs. $77 \%$ ). However, they fared better in accurately answering the screening vignettes and identifying factors used in the guidelines (18 vs. $10 \%$ ).

CONCLUSIONS: Primary care and subspecialty trainees exhibit a substantial gap between their reported confidence in recalling $\mathrm{CRC}$ screening and surveillance guidelines and the application of them on a survey. This discordance crosses specialties and stage of practice. Interventions need to be designed to assist care providers in making guidelineconcordant $\mathrm{CRC}$ screening and surveillance recommendations.

Comparison of knowledge and attitudes of CRC screening and surveillance guidelines by specialty and experience.

\begin{tabular}{|c|c|c|c|c|c|c|c|c|c|}
\hline & & $\begin{array}{l}\text { PCP } \\
N=383 \text { (\%) }\end{array}$ & $\begin{array}{l}\text { GI } N= \\
119(\%)\end{array}$ & $\begin{array}{l}\text { Surgery } \\
N=76(\%)\end{array}$ & $\begin{array}{l}\text { OBGYN/GU } \\
N=119 \text { (\%) }\end{array}$ & $P$ value & $\begin{array}{l}\text { Trainees } \\
N=575 \text { (\%) }\end{array}$ & $\begin{array}{l}\text { Staff } \\
N=119 \text { (\%) }\end{array}$ & $P$ value \\
\hline Felt confident recalling & Screening & $71^{[4]}$ & $88^{[1,3,4]}$ & $68^{[2,4]}$ & $44^{[1,2,3]}$ & $<0.001$ & 68 & 77 & 0.04 \\
\hline guidelines & Surveillance & $44^{[2,3,4]}$ & $91^{[1,3,4]}$ & $72^{[1,2,4]}$ & $22^{[1,2,3]}$ & $<0.001$ & 50 & 58 & 0.11 \\
\hline Answered both vignettes & Screening & $64^{[2]}$ & $33^{[1,3,4]}$ & $57^{[2]}$ & $53^{[2]}$ & $<0.001$ & 59 & 44 & 0.03 \\
\hline correctly & Surveillance & $27^{[2]}$ & $51^{[1,3,4]}$ & $29^{[2]}$ & $26^{[2]}$ & $<0.001$ & 31 & 31 & 0.99 \\
\hline Accurately identified factors & Screening & $32^{[4]}$ & 20 & 29 & $18^{[1]}$ & 0.03 & 28 & 24 & 0.30 \\
\hline to determine start/interval & Surveillance & $5^{[2,3]}$ & $65^{[1,3,4]}$ & $20^{[1,2,4]}$ & $3^{[2,3]}$ & $<0.001$ & 18 & 11 & 0.07 \\
\hline Answered both vignettes & Screening & $22^{[2]}$ & $3^{[1,3]}$ & $17^{[2]}$ & 13 & $<0.001$ & 18 & 10 & 0.03 \\
\hline $\begin{array}{l}\text { identified all factors } \\
\text { correctly }\end{array}$ & Surveillance & $1^{[2,3]}$ & $39^{[1,3,4]}$ & $5^{[1,2]}$ & $0^{[2]}$ & $<0.001$ & 8 & 3 & 0.06 \\
\hline
\end{tabular}

[1]: Significantly different from PCP;

[2]: Significantly different from GI;

[3]: Significantly different from Surgery;

[4]: Significantly different from OBGYN/GU;

A significance level of 0.008 was used for pairwise ad-hoc comparisons.

\section{HYPERCALCEMIA IN IN-HOSPITAL PATIENTS; IS IT REALLY UNDER DIAGNOSED? A RETROSPECTIVE CHART REVIEW Maliha Naseer ${ }^{1}$; Samina Qamar ${ }^{2}$. ${ }^{1}$ Wayne State University, Rochester, MI; ${ }^{2}$ Hospital Community Regional Med- ical Center, Fresno, CA. (Control ID \#2469582)}

BACKGROUND: Hypercalcemia is a common metabolic abnormality that is often under-diagnosed. Mild hypercalcemia often asymptomatic, but can have long term complications such as renal calculi, depression, hypertension and osteoporosis. The variability in prevalence reported in the literature as well as the lag time in establishing the diagnosis in many patients suggests that the true prevalence of hypercalcemia is unclear and likely underestimated. Therefore, the objective of this study is to find out the prevalence of undiagnosed and unrecognized hypercalcemia in hospitalized patients.

METHODS: Retrospective chart review was done of patients $>21$ years admitted during April- May 2013 in a community hospital. Out of 7684 patients screened 51 patient (1.94 \%) were found to have Calcium $>10.3 \mathrm{mg} / \mathrm{dL}$ on different occasions. All patients were reviewed for underlying cause of hypercalcemia and associated risk factors. Data entry and analysis was done using Statistical Package for Social Sciences (SPSS) version 21. Descriptive analysis was performed to categorize calcium and corrected calcium 
according to laboratory values obtained, associated diseases and percent of patients having parathyroid hormone (PTH) in each calcium group $(\mathrm{mg} / \mathrm{dL})$.

RESULTS: Prevalence of hypercalcemia in hospitalized population is found to be $1.96 \%$ (153/7684). However only $28 \%$ patient (43 out of 153 ) were documented and further evaluated for the potential management. Demographic and clinical characteristics of the patients with documented and undocumented hypercalcemia were compared. Significant differences were observed in terms of age, length of stay and co-morbid conditions. Age $>50$ years, corrected calcium level $>11 \mathrm{mg} / \mathrm{dL}$, length of stay $>3$ days and having at least 1 co-morbid condition were found to be significantly associated with documentation of hypercalcemia and further evaluation in our patient population. Most common cause of hypercalcemia in patient who had diagnostic work up done was parathyroid disorders (44.18\%), followed by malignancy, chronic kidney disease and sarcoidosis. Conditions that were commonly found in patients with corrected calcium level $>10.3 \mathrm{mg} / \mathrm{dL}$. Out of 153 patients who were found to be hypercalcemic, approximately $25 \%$ were asymptomatic (calcium level in the range of 10.3-11 mg/dl). Thirty-two percent patient had constipation, $21.56 \%$ had diagnosis of depression and around $12 \%$ patients have history of osteoporosis. CONCLUSIONS: Prevalence of hypercalcemia in hospitalized population is greater than published literature and majority of patients with mild hypercalcemia go unrecognized and undocumented. Therefore, they are not evaluated further for potential contributing etiologies. To prevent long term complications of hypercalcemia steps should be taken at institutional and primary care community level which includes, but not limited to continuous medical educational programs for physicians to improve their knowledge base and up gradation of existing EMR system that can alert physicians whenever there is high level of serum calcium on laboratory data

30-DAY READMISSIONS: THE INFLUENCE OF PHYSICIAN ATTENDING TYPE AND SOCIAL CONNECTEDNESS Tafadzwa Muguwe ${ }^{3}$; Nathalie Bloch ${ }^{3}$; Shani Legore ${ }^{5}$; Orissa Viza ${ }^{1}$; Carey C. Thomson ${ }^{4}$; Joanne P. Kerwin ${ }^{2}$; Valerie E. Stone ${ }^{1}$ ${ }^{1}$ Mount Auburn Hosp,Harvard Medical School, Cambridge, MA; ${ }^{2}$ Mount Auburn Hospital, Watertown, MA; ${ }^{3}$ Mount Auburn Hospital, Cambridge, MA; ${ }^{4}$ Mt Auburn Hospital, Cambridge, MA; ${ }^{5}$ University of Connecticut, Hartford, CT. (Control ID \#2469586)

BACKGROUND: In this era of healthcare reform, there is great incentive to understand drivers of readmission for patients with chronic illness. In this 2014 retrospective study of patient-level data, we sought to contextualize and characterize the 30-day readmission experience of an academic community hospital in Massachusetts.

METHODS: A mixed methods approach compared readmitted and non-readmitted medical patients $(N=8,054)$. The first level of quantitative analysis examined demographic and delivery system factors including type of attending physician, primary diagnosis, length of stay (LOS), marital status and discharge disposition. For the second level of qualitative analysis, a telephone interview $(N=56)$ of a matched sample of readmitted and non-readmitted patients with diagnoses of CHF, COPD, and Pneumonia characterized and contextualized patient differences in social connectedness, functional status, and transition of care experiences that may be associated with 30 day readmission.

RESULTS: We found significant variation in readmission rates by attending of record category. The readmission rate for those managed by geriatricians was lower than for the overall medical service readmission rate (10 vs $13.6 \%$, $p$-value $<0.05)$ whereas the readmission rate for primary care physicians (PCP) attendings was significantly higher compared with the overall readmission rate (18 vs $13.6 \%, p$-value 0.003$)$. Hospitalists' readmission rates were not significantly different from the overall medical service. Notably, however, $17.6 \%$ of hospitalists' readmissions went to other hospitals versus $10.6 \%$ for PCP attendings and $6.5 \%$ for geriatricians. Accounting for this outside hospital data effectively erased the difference in readmission rates between hospitalists (15.1\%) and PCPs (14.9\%). Readmitted patients had a higher median LOS (4 days) on initial admission when compared to the overall population (3 days). Patients discharged to home with services were as likely to be readmitted as those sent to another facility, and significantly more likely to be readmitted than those sent home without services ( $p$-value $<0.0001$ ). We did not find an effect of race/ethnicity on readmissions. Being divorced (17\%; $p<0.0065)$, legally separated $(31 \%, p<0.002)$, or widowed $(15 \% ; p<0.038)$ were associated with significantly higher readmission rates. Our telephone interview of patients with primary diagnoses of CHF, COPD, and Pneumonia revealed several measures that distinguished readmitted from non-readmitted patients. Readmitted patients were more than twice as likely to have a first language other than English (17.9 versus $7.1 \%$ ). They were more likely to lack an outpatient connection to a PCP (10.7 versus $0 \%$ ). They were 1.6 times more likely to lack the support of family members, a significant other or close friends during their inpatient stay (17.9 versus $10.7 \%$ ). They were more likely to say they did not recall reviewing their discharge instructions prior to discharge (14.3 versus $0 \%$ ), and they did not know whom to call with questions or concerns about their health following discharge ( 10.7 versus $0 \%$ ). They were more than 5 times likely to say they did not agree with changes made to their medications during hospitalization (60 versus $11.1 \%$ ). They were 3 times more likely to say they did not manage their own medications at home ( $35.7 \mathrm{vs} 10.7 \%$ ). They were almost 3 times more likely to indicate not being able to complete one or several activities of daily living without assistance (bathing, toileting, feeding, dressing) (32.1 vs $11.1 \%$ ). They were 3 times more likely to be discharged to a facility ( $21.4 \mathrm{vs} 7.1 \%$ ), and when discharged to home they were more than twice as likely to report lacking the support of family and/or friends (9.5 vs $4 \%$ ).

CONCLUSIONS: This quantitative and qualitative analysis highlights individual and healthcare system drivers of 30 day readmissions. On the individual level, language barriers and inadequate discharge information were risk factors for readmission. Lack of adequate social support both during and after the inpatient stay was significantly more common among the readmitted. Patients with needs around activities of daily living were at increased risk of readmission. These data suggest the need for rigorous identification of individual risk factors for readmission and tailoring of discharge planning. At the system level, we noted significantly lower readmissions for patients admitted to the geriatric service which supports the notion that continuity of care from outpatient to inpatient settings helps to lower readmissions, but may also reflect the importance of non-medical aspects of geriatric care. We are motivated to further study geriatricians to define their effective strategies that result in lower 30 day readmissions. While it was surprising to find that PCPs had a higher readmission rate than hospitalists, inclusion of outside hospital readmissions erased this difference. We know from statewide data that up to $18 \%$ of readmissions are absorbed by facilities other than the index facility, and we have shown here that this readmission "leakage" varies by attending category. Our data suggest that PCPs and geriatric attendings who likely have long-term relationships with their patients have less readmission "leakage", resulting in greater continuity of care for their patients.

SIX-MONTH OUTCOMES AMONG OLDER ADULTS OF COMMUNITY ENGAGEMENT VERSUS TECHNICAL ASSISTANCE TO IMPLEMENT DEPRESSION COLLABORATIVE CARE Adriana Izquierdo $^{2,3}$; Esmeralda Pulido ${ }^{3,4}$; Kenneth B. Wells ${ }^{1}$; Marina Berkman ${ }^{5}$; Barbara Linski ${ }^{6}$; Vivian Sauer ${ }^{5}$; Jeanne Miranda ${ }^{1}$. ${ }^{1}$ UCLA, Los Angeles, CA; ${ }^{2}$ University of California Los Angeles, Los Angeles, CA; ${ }^{3}$ UCLA Center for Health Services and Society, Semel Institute for Neuroscience and Human Behavior, Los Angeles, CA; ${ }^{4}$ LA Care, Los Angeles, CA; ${ }^{5}$ Jewish Family Service, Los Angeles, CA; ${ }^{6}$ St. Barnabas Hollywood Senior Multipurpose Senior Center, Los Angeles, CA. (Control ID \#2467438)

BACKGROUND: Among older adults, depression is a common and disabling condition associated with increased morbidity and mortality. Although effective treatments and collaborative primary care models for late-life depression exist, older depressed adults, particularly minority and/or under-resourced older adults, remain undertreated in primary 
care settings. A more comprehensive strategy may be needed to improve mental health outcomes for this population. Recent work shows that depression collaborative care implementation using community engagement and planning (CEP) across diverse social and community programs improves depression outcomes in safety-net communities, compared with technical assistance to individual programs (resources for services [RS]). However, outcomes among older adults have not yet been described. In this study, we compare the effects of CEP and RS to implement depression collaborative care on mental health-related outcomes at 6 months among depressed older adult clients.

METHODS: Matched health and community programs $(n=93)$ in Los Angeles, California were randomized to receive one of two interventions to implement depression collaborative care: CEP or RS (ClinicalTrials.gov: NCT01699789). The study and community engagement intervention were implemented using community-partnered participatory research (CPPR). Three hundred ninety-four clients aged 50 and older with depressive symptoms (PHQ- 8 score $\geq 10$ ), $87 \%$ of whom were ethnic minorities, completed baseline or 6-month follow-up and comprise the analytic sample for this study. Measures include self-reported mental health-related quality of life (MHRQL) and mental wellness. We conducted intention-to-treat, comparative-effectiveness analyses with intervention status as the independent variable, using logistic regression for dichotomous measures and log-linear models for counts. We adjusted for baseline status of dependent variables and covariates (e.g., education, race/ethnicity and community). We weighted data for 394 clients to characteristics of the eligible sample, with item-level imputation for missing data and wave-level imputation for missing surveys. Weights account for nonenrollment among eligible clients and attrition. All analyses were conducted using SUDAAN Version 10.0 (http://www.rti.org/sudaan/) and accounted for clustering (clients within programs), weighting and multiple imputations. Significance of comparisons by intervention status was based on regression coefficients. Results of regression models are presented as between-group difference for linear odds ratios (OR) for logistic and incidence-rate ratios (IRR) for Poisson with $95 \%$ confidence intervals (CI)

RESULTS: CEP was more effective than RS at reducing poor MHRQL and increasing mental wellness (each $p<0.05$ )

CONCLUSIONS: CEP to implement collaborative depression care across diverse programs was more effective than RS for individual programs in improving mental healthrelated outcomes, and may offer an expanded community health-home model to address mental health disparities among older depressed safety-net clients.

\section{A COMPARISON OF PATIENT HEALTH LITERACY LEVELS AND GRADE LEVEL READABILITY OF ELECTRONIC HEALTH RECORD PATIENT ED- UCATION MATERIALS IN A RESIDENT PRIMARY CARE CLINIC Omoye E. Imoisili $^{1}$; Benjamin A. Howell ${ }^{1}$; Shoshana Streiter ${ }^{1}$; Erik Levinsohn ${ }^{2}$; Cassie $\overline{\text { Pan }^{2} \text {; Julie }}$ R. Rosenbaum ${ }^{2} .{ }^{1}$ Yale New Haven Hospital, New Haven, CT; ${ }^{2}$ Yale School of Medicine, New Haven, CT. (Control ID \#2469224)}

BACKGROUND: Low health literacy is associated with worse health outcomes and correlated with less effective engagement with the health care system. Limited health literacy accounts for approximately $\$ 29$ billion in excess annual healthcare expenditures. Many health institutions may not provide patient education materials (PEM) that reflect the reading grade level of their patient population. This disparity in literacy and PEM readability renders patients vulnerable to poorer understanding of their health conditions and treatment plans, and may lead to worse health outcomes. This study aimed to assess the health literacy of the patient population in an urban resident primary care clinic and to compare it to the readability of two categories of PEM provided within the electronic health record ("standard" and "easy-to-read").

METHODS: Rapid Estimate of Adult Literacy in Medicine Short Form (REALM-SF) is one of the most commonly used and well validated tools to assess health literacy. The
REALM-SF categorizes health literacy as low ( $\leq 6$ th grade), marginal (7th-8th grade), or adequate ( $\geq 9$ th grade) based on word recognition. While no consensus exists for how best to evaluate readability, the Center for Medicare and Medicaid Services recommends using the Simple Measure of Gobbledygook (SMOG), which applies a pre-set formula to a text based on polysyllabic words for evaluation of readability by grade level. The FleschKincaid tool also uses a formula based on syllables, words, and sentences to determine readability by grade level. Generally, more than one readability tool is used to improve validity. All eligible English-speaking continuity care clinic patients during a 2 week period were asked to participate in the survey. Patients were deemed ineligible if they did not read or speak English due to significant language barrier, or had physical or cognitive disability which prevented them from reading or speaking (ex. blindness or profound intellectual development disorder). Consenting subjects completed a basic demographics questionnaire; subsequently health literacy was evaluated by REALM-SF. The independent association on literacy of demographic categories, including age, educational attainment, English proficiency, race, income, and sex, was assessed using a logistic regression model in STATA. Readability assessments using SMOG and Flesch-Kincaid were performed on PEM (provided by Elsevier's ExitCare, which is integrated into the Epic electonic health record) for the five most common health conditions in the clinic; these were determined based on the most frequently billed clinician visits over a 12 month period.

RESULTS: Of 213 patients who had clinic appointments during the study period, 186 patients were eligible for participation, and of these 175 patients consented to study participation. There were 66 males (37.7\%) and 108 females (61.7\%). Racial composition was 43 white (24.6\%), 96 black (54.9\%), 27 Latino (15.4\%), and 9 other (5.2\%). For education, 54 (30.9\%) did not finish high school, 70 (40\%) completed high school, $25(14.3 \%)$ attended some college, and 25 (14.3\%) graduated college. According to REALM-SF assessments, health literacy levels were $\geq 9$ th grade for 76 patients $(43.4 \%$ ), 7 th-8th grade level for 66 patients $(37.7 \%)$, and $\leq 6$ th grade for 30 patients $(17.1 \%)$. There was a significant correlation for higher REALM-SF score with educational attainment $(p<0.005)$, being a native English speaker $(p=0.02)$, and female sex $(p<0.005)$. These associations remained independently associated in the logistic regression model. The top five most frequent clinic diagnoses, in order, were hypertension, diabetes mellitus, hyperlipidemia, back pain, and depression. Readability assessments of their respective PEM by SMOG showed that for standard PEM, average readability grade level was 9.2 (range 7-11); for easy-to-read PEM readability average grade level was 6.8 (range 6-7). By Flesch-Kincaid, standard PEM average readability grade level was 6.9 (range 6.3-9); easy-to-read PEM average readability grade level was 4.2 (range 2.8 to 5.1).

CONCLUSIONS: The majority of subjects in this study (54.8\%) had low or marginal health literacy, reading at or below an 8 th grade level, as determined by the REALM-SF. For the five most common clinic diagnoses, the average readability grade level by SMOG of standard and easy-to-read PEM were 9.2 and 6.8, respectively. Based on this assessment, standard PEM are written at a level that is inappropriately high for more than half of the clinic population. While easy-to-read PEM are written at a middle school level, this is still too high for almost $20 \%$ of the clinic population. If the concept of universal precautions is applied to health literacy, selecting the "easy-to-read" option, when available, may help maximize comprehension of health information material across the clinic population. Notably, the Flesch-Kincaid assessment graded readability for standard and easy-to-read PEM at lower grade levels. The discrepancy between the results of two different readability tools highlights the difficulty of standardizing readability assessments. Healthcare providers should prioritize distributing PEM at a reading level that is appropriate for the patients they are serving. Targeted interventions that account for the health literacy level in a patient population may have a positive effect on doctor-patient communication, patient satisfaction, and health outcomes. 
A CROSS-SECTIONAL ASSESSMENT OF THE QUALITY OF PHYSICIAN QUALITY REPORTING SYSTEM MEASURES Brittney Frankel; Tara F. Bishop. Weill Cornell Medical College, New York, NY. (Control ID \#2435771)

BACKGROUND: In 2015, Center for Medicare and Medicaid Services (CMS) began requiring all providers treating Medicare patients to report quality measures through Physician Quality Reporting System (PQRS) or incur $1.5 \%$ financial penalty. Recent research indicates that physicians believe quality reporting is expensive to participate in, does not lead to high quality care and does not apply to specialty care. However, little research has examined what PQRS actually measures or how this affects patients and physicians. For this reason, we analyzed the 2011 and 2015 measures to see what kinds of measures are used, what medical specialties they are assessing, and how they changed over time.

METHODS: We conducted an analysis of the publicly available 2011 and 2015 PQRS measures. Two reviewers categorized measured along three domains: physician specialty being measured, type of measure, and National Quality Strategy (NQS) priority category. All analyses were performed using Stata software.

RESULTS: Between 2011 and 2015, the number of measures increased from 198 to 254 (Table). The type of measures changed significantly, with fewer processes ( 85.4 vs. $66.5 \%, p<0.001)$ and more outcomes $(12.6$ vs. $29.1 \%, p<0.001)$. The measures showed no significant specialty or NQS category differences. In 2015, internal medicine accounted for the highest percentage of measures by specialty $(57.5 \%)$ and effective clinical care accounted for the highest percentage of measures by NQS category $(54.3 \%)$. For subcategories within each specialty in 2015 , differences in measure type were statistically significant: surgery had the highest percentage of outcomes (61.1\%) compared to $21.7 \%$ of internal medicine and $5.9 \%$ of obstetrics gynecology. For NQS categories, internal medicine had the highest percentage of effective clinical care measures $(68.5 \%$ ), compared to $22.2 \%$ in surgery. Surgery had the highest percentage of patient safety $(31.9 \%)$ and communication and care coordination measures $(27.8 \%$ ) compared with internal medicine (5.4 and $6.5 \%$ ).

CONCLUSIONS: We found that PQRS measures assess quality for many specialties and assess more outcomes in 2015 than in 2011, particularly for surgery. PQRS has fewer outcome measures for internal medicine specialties and obstetrics/gynecology and did not equally assess all six NQS domains.
A CROSS-SECTIONAL STUDY OF LEVELS OF BURNOUT AND RESILIENCE IN EACH YEAR OF MEDICAL SCHOOL: IMPLICATIONS FOR TIMING A MEDICAL STUDENT WELLNESS INTERVENTION Rachel H. Kon; Justine Owens; Tabor E. Flickinger; Walker Redd; Danielle Oliver; Margaret Plews-ogan; John Schorling. University of Virginia, Crozet, VA. (Control ID \#2468466)

BACKGROUND: Physician burnout contributes to poor physician health, loss of empathy, poor job performance and ultimately medical errors and reduced patient satisfaction. Burnout starts in medical school and may be present in the pre-clinical years. Resilience is a dynamic trait that is correlated with protection against mental health problems, interpersonal conflicts, and poor academic performance in medical students. Defined as the ability to remain positive despite adversity, resilience can be nurtured into a stronger attribute and thus is a potential target for wellness curricula. To reduce burnout among medical students, wellness curricula are being developed to improve resilience but the optimal timing for this intervention is unknown. We conducted a cross-sectional study of medical students at different stages of their 4 year degree using validated survey tools to assess levels of burnout and resilience.

METHODS: Medical students at the University Of Virginia School Of Medicine were sent an online anonymous survey that contained 128 items including demographic information, quality of life measures, and validated questionnaires for anxiety and depression screening, burnout, resilience, well-being, and wisdom. This report will focus on the burnout and resilience measures. Three domains of burnout (emotional exhaustion, depersonalization, and personal accomplishment) were measured using the 22-item Maslach Burnout Inventory (MBI). Using cut offs for high burnout consistent with other studies of medical students, we considered high burnout to be a score of 27 or greater for emotional exhaustion (EE) or a score of 10 or greater for depersonalization (DP). We considered combined burnout to be a high score on either the EE or DP scales. We used the 25-item Connor-Davidson Resilience Scale (CD-RISC) to measure resilience. Statistical analysis was conducted using chi-square testing to compare MBI scores between cohorts as discrete variables with score cutoffs as above. ANOVA testing was used to compare CD-RISC scores between cohorts as continuous variables. Pearson correlation was used to measure the relationship between $\mathrm{MBI}$ and CD-RISC scores.

Table. PQRS measure characteristics, 2011 and 2015

\begin{tabular}{|c|c|c|c|}
\hline \multirow[t]{2}{*}{ Measure characteristics } & \multicolumn{2}{|c|}{ No. (\%) } & \multirow[t]{2}{*}{ p-value } \\
\hline & $\begin{array}{c}2011 \\
(n=198)\end{array}$ & $\begin{array}{c}2015 \\
(n=254)\end{array}$ & \\
\hline Specialty $^{*}$ & & & 0.48 \\
\hline Internal Medical & $126(63.6)$ & $146(57.5)$ & \\
\hline Surgery & $36(18.2)$ & $60(23.6)$ & \\
\hline Obstetrics/Gynecology & $10(5.1)$ & $14(5.5)$ & \\
\hline Pediatrics & $23(11.6)$ & $30(11.8)$ & \\
\hline Other & $55(27.8)$ & $75(29.5)$ & \\
\hline Type & & & $<0.001$ \\
\hline Structure & $4(2.0)$ & $10(3.9)$ & \\
\hline Process & $169(85.4)$ & $169(66.5)$ & \\
\hline Outcome & $25(12.6)$ & $74(29.1)$ & \\
\hline Other & $0(0.0)$ & $1(0.4)$ & \\
\hline NQS priorities & & & 0.20 \\
\hline Effective clinical care & $129(65.2)$ & $138(54.3)$ & \\
\hline Patient safety & $18(9.1)$ & $34(13.4)$ & \\
\hline $\begin{array}{l}\text { Communication and care } \\
\text { coordination }\end{array}$ & $28(14.1)$ & $37(14.6)$ & \\
\hline $\begin{array}{l}\text { Person and caregiver centered } \\
\text { experience }\end{array}$ & $6(3.0)$ & $14(5.5)$ & \\
\hline Efficiency/cost reduction & $7(3.5)$ & $16(6.3)$ & \\
\hline $\begin{array}{l}\text { Community and population } \\
\text { health }\end{array}$ & $10(5.1)$ & $15(5.9)$ & \\
\hline
\end{tabular}

- Sum of the number and percentage of measures is greater because some measures are classified under multiple specialties. 
RESULTS: Six hundred thirty students were eligible to participate, and 378 responded to at least part of the questionnaire ( $60 \%$ response rate). Participants had a mean age of 25 years, with $51.3 \%$ being female and $48.7 \%$ male. Burnout: 307 medical students completed the MBI (49\% response rate). Mean scores for EE were 21.49 (SD 10.91, $95 \%$ CI 20.2-22.7). Mean scores for DP were 7.52 (SD $6.27,95 \%$ CI 6.8-8.2). Mean scores for personal accomplishment were 36.61 (SD $6.87,95 \%$ CI 35.8-37.4). The prevalence of high combined burnout for first, second, third, and fourth year medical students was $28.7,39.7,65.9$, and $42.2 \%$ respectively $\left(\chi^{2}=24.8, p<.000\right)$. Emotional exhaustion domain: prevalence of high EE was 20.3 and $34.6 \%$ in 1 st and 2nd year students respectively, and then was $50.0 \%$ in the third year. $9.1 \%$ of 4 th year students had high EE scores $\left(\chi^{2}=\right.$ 34.3, $p<.000)$. Depersonalization domain: prevalence of high DP was 15.6 and $14.1 \%$ in 1 st and 2 nd year students respectively, and then was $56.5 \%$ in the third year. $40.3 \%$ of 4 th year students had high DP scores $\left(\chi^{2}=46.6, p<.000\right)$. Resilience: 284 medical students completed the CD-RISC (45\% response rate). Mean resilience score was 66.15 (SD 9.46, 95 \% CI 65.0-67.3). Resilience scores varied by year in medical school $(F=5.066, p=.002)$ with scores highest in the fourth year students with a mean score of 69.23 (SD 8.17, $95 \%$ CI 67.1-71.3) and lowest in third year medical students with a mean score of 63.99 (SD 9.43, $95 \%$ CI 60.8-65.2). First and second year medical students had mean scores of 66.51 and 66.34 respectively. Resilience and personal accomplishment were highly positively correlated $(r=.539, p=.000)$. Resilience and emotional exhaustion were moderately negatively correlated $(r=-.371, p=.000)$. Resilience and depersonalization were more weakly negatively correlated $(r=-.198, p=.001)$.

CONCLUSIONS: Prevalence of burnout in these cohorts is highest and resilience scores are lowest in the third year students. All three burnout subscales have lower scores in the first and second year classes compared with burnout scores that are significantly higher in the third year students. This may be expected with the rigor of third year clerkships and initial exposure to severely ill and dying patients. Personal accomplishment and emotional exhaustion subscales revealed lower burnout in the fourth year class close to or lower than in the first and second year classes, but depersonalization in the fourth year students showed high scores similar to the third year scores. Resilience was moderately to highly correlated with emotional exhaustion and personal accomplishment as expected, but more weakly correlated with depersonalization. It is unclear why depersonalization remained prevalent in fourth year students while other domains of burnout decreased when the rigor of the academic schedule decreased. The relationship between resilience and depersonalization was not strongly correlated and may be more complex than the other domains of burnout. These results point to the third year of medical school as a critical time when depersonalization increases. The beginning of the third year appears to be a good target to implement curricula to limit depersonalization and improve wellness in medical students.

\section{A HEPATITIS C SCREENING PROGRAM FOR THE HOMELESS IN NEW} ORLEANS Stephanie Preston ${ }^{2}$; Stephanie Heaney ${ }^{1}$; Jane Andrews ${ }^{1} \cdot{ }^{1}$ Tulane School of Medicine, New Orleans, LA; ${ }^{2}$ Tulane University School of Medicine, New Orleans, LA. (Control ID \#2458235)

BACKGROUND: Chronic hepatitis $\mathrm{C}$ virus $(\mathrm{HCV})$ infection affects over 3.2 million people in the U.S. This figure underestimates the true prevalence of 4-5 million because most studies exclude populations with a high HCV burden including intravenous drug users (IVDU), the homeless, and incarcerated persons. Given the advent and increased availability of curative $\mathrm{HCV}$ treatments, it is crucial for us to treat all infected individuals so the disease can be eradicated.
METHODS: We implemented a voluntary point-of-care HCV screening program at the Ozanam Inn (Oz) and New Orleans Mission (NOM), two homeless shelters in New Orleans. We then followed patients' retention through the treatment cascade for $\mathrm{HCV}$ in this setting. Patients who test positive at $\mathrm{Oz} / \mathrm{NOM}$ are seen at Healthcare for the Homeless (HCH) for an initial visit and to have labs and imaging ordered. On completion of these studies, patients are seen at the University Medical Center (UMC) ambulatory hepatitis clinic. Here we describe patient demographics, self-reported risk factors, and retention in care, using survey data collected during the initial screening session and review of electronic medical records at our referral sites.

RESULTS: Demographics and risk factors - In 9 months, 186 individuals opted into $\mathrm{HCV}$ screening, with a positive HCV antibody rate of $22 \%(n=41)$. Prevalence was highest among baby boomers (37\%), followed by generation $\mathrm{X}$ and millennials (15\%). Regarding risk factors, $22 \%$ of those tested self-reported IVDU, $81 \%$ time spent in jail or prison, $15 \%$ incidental blood exposure, $9 \%$ receiving a blood transfusion, $36 \%$ a serious psychiatric condition, $72 \%$ having a tattoo, $45 \%$ substance abuse, $35 \%$ alcohol abuse. Of those who self-reported IVDU, $49 \%$ were HCV antibody positive. Retention in care-Of the 41 preliminary positive patients, one was co-infected with $\mathrm{HIV} / \mathrm{HCV}$ and referred to the HIV outpatient clinic, and three declined follow-up. Of the 37 patients who entered the treatment cascade, $10(27 \%)$ attended their appointment at $\mathrm{HCH}, 4(11 \%)$ were seen at UMC for labs and studies, and one was ultimately referred to the UMC HCV clinic. During this study, two patients were screened in the ED and obtained expedited referral to the UMC HCV clinic independent of our screening and treatment cascade.

CONCLUSIONS: In this study, we describe an HCV screening program at a homeless shelter in New Orleans in which approximately $25 \%$ of shelter residents opted-in for testing. With an HCV antibody prevalence of $22 \%$, this study further demonstrates the high disease burden of $\mathrm{HCV}$ in the homeless population. The reason for our observed low retention rates is likely multifactorial. These patients face numerous barriers to care. Furthermore, many of our patients are asymptomatic and may understandably prioritize activities of daily living. To improve our patients' retention in care, we are investigating a number of follow-up strategies, including phone calls for appointment reminders, electronic medical record modification to notify other physicians of patients' need for $\mathrm{HCV}$ lab work and referral, and client reminders and transportation assistance by the Homeless Assistance Unit of the New Orleans Police Department

\section{A LONGITUDINAL EVALUATION OF MEDICAL STUDENT SPECIALTY} CHOICE AT JOHNS HOPKINS UNIVERSITY Paul O'Rourke²; Eva Tseng ${ }^{2}$; Sean Tackett $^{1}$; Robert Shochet ${ }^{3}$; Scott Wright ${ }^{3}$. ${ }^{1}$ Johns Hopkins Bayview Medical Center, Baltimore, MD; ${ }^{2}$ Johns Hopkins University, Baltimore, MD; ${ }^{3}$ Johns Hopkins University School of Medicine, Baltimore, MD. (Control ID \#2469872)

BACKGROUND: Medical students' initial specialty preference at matriculation and whether this changes by the time they graduate has not been extensively studied. The choices that students make determine the distributions of physicians within the medical profession and influence workforce excess or shortages. Understanding the factors that may be associated with changing or maintaining a specified interest within medicine may be invaluable, particularly among schools that are trying to accept students with specific interests, such as those keen on primary care. This study set out to: (1) describe and evaluate trends in the evolution of student specialty preferences from the start to the end of medical school, and (2) explore the factors that may be associated with students who maintain versus change their preferred area within medicine across their time in medical school

METHODS: Graduating Johns Hopkins medical students filled out crosssectional surveys from 2007 to 2014. Embedded within an instrument that focused primarily on the medical school learning environment, students were 
asked about their preferred career specialty choice at the start and end of medical school. Additional variables collected included demographics and the presence of medical school debt. We performed descriptive analyses to evaluate trends in maintaining and changing career preferences in medicine. We also conducted logistic regression to determine which student variables are associated with maintaining versus changing career interest from the start to the end of medical school.

RESULTS: Of 923 eligible students, 684 (74\%) medical students completed the survey between 2007 and 2014. After excluding students with missing data on specialty choice and the covariates of interest, we included 606 students in our analyses. Of these, 402 (66\%) students changed their initial specialty interest by the end of medical school while 204 (34\%) students maintained their preference. Specialties demonstrating the greatest increase in medical student interest from the start to the end of medical school were anesthesiology (+6 \%), ophthalmology $(+4 \%)$, emergency medicine $(+3 \%)$, and radiology $(+3 \%)$. In contrast, specialties associated with the greatest decrease in student interest from the start to the end of medical school were general surgery $(-6 \%)$, pediatrics $(-3 \%)$, and obstetrics/ gynecology $(-1 \%)$. Students with children were significantly less likely to maintain their initial specialty interest compared to those who were not parents (OR 0.42, $95 \%$ CI 0.20 to 0.88 ). Other covariates (age, gender, race, marital status, presence of medical school loan debt) were not significantly associated with maintenance of initial specialty interest. Of the 252 students who expressed initial interest in a field that could result in a primary care career (i.e. family medicine, internal medicine, pediatrics, or internal medicine/pediatrics) at the beginning of medical school, $56 \%$ ( $n=140)$ maintained this interest and the remainder chose other fields that they preferred. Similar to above, we found that students with children were less likely to maintain interest in fields that might lead to primary care careers (OR 0.37, $95 \% \mathrm{Cl}$ 0.14 to 0.95$)$.

CONCLUSIONS: The experiential education that occurs in medical school affects students' preconceived ideas about how they might want to spend their career in the medical profession. Certain specialties attract medical students whereas others are unable to maintain the appeal of those initially interested. Ongoing analysis of this rich database may provide insight into student career preferences. These findings have relevant implications for medical school administrators, policymakers, and researchers interested in physician workforce issues.

A LONGITUdINAL PSYCHOSOCIAL CURRICULUM TO ENHANCE RESIDENTS' SELF-EFFICACY IN DELIVERING CULTURALLY COMPETENT CARE Johanna Martinez ${ }^{1}$; Angela Ghesquiere ${ }^{3}$; Anthony T. Pho ${ }^{2}$; Cathy Jalali ${ }^{2}$; Susana Morales $^{2}$; Carla Boutin-Foster ${ }^{2}$; Joseph Conigliaro ${ }^{1} .{ }^{1}$ North Shore LIJ Health System, New Hyde Park, NY; ${ }^{2}$ Weill Cornell Medical College, New York, NY; ${ }^{3}$ Hunter College, New York, NY. (Control ID \#2468323)

BACKGROUND: By 2050 it has been estimated that more than $50 \%$ of the US population will consist of racial and ethnic minorities. Yet even as minority populations grow, health inequities for these patients' persist. There are a plethora of documented health disparities for racial and ethnic minority populations when compared to Whites. These disparities affect care from prevention to treatment, and occur among many disease states. Factors leading to these disparities are often grouped as related to provider, patient, and/or system-based, and often are multifactorial. The Institute of Medicine and the Accreditation Council for Graduate Medical Education have proposed training providers to deliver culturally competent care as a way to address these disparities. The impact of a cultural competency resident curriculum is examined here.
METHODS: Participants were first year Internal Medicine residents at a large New York urban medical center. The study was conducted during the intern resident's ambulatory care mandatory rotation, in their year-long psychosocial course. Several sessions were dedicated to delivering culturally competent care. The curriculum included topics addressing social determinants of health, taking an advanced social history, use of an interpreter, health literacy, health disparities, mistrust and implicit bias. In order to enforce the content each week, the interns were asked to practice the skills learned in the small group sessions during their clinical practice, and to report back at upcoming sessions on those experiential learning opportunities. A time-series study was conducted to compare changes in interns' selfefficacy (preparedness and skillfulness) regarding their ability to deliver culturally competent care. The Cross-Cultural Care Survey was utilized to survey the residents. The pre-survey was administered before any of the psychosocial content was taught. The post-survey was administered 12 months later, at the end of their internship year. The survey has been previously validated and contains questions regarding resident's attitudes towards cross-cultural care and a self-assessment of preparedness and skillfulness to deliver care for diverse patient populations. Survey responses were measured on a 5 point Likert scale, with higher scores indicating higher preparedness and skillfulness. There were 8 preparedness and 10 skillfulness items. The survey responses were de-identified. Statistical analysis included Chi-squares and t-tests, for categorical and continuous variables respectively. IBM SPSS version 22 was used to carry out analyses, with a $p$-value $\leq .05$ as a level of significance.

RESULTS: The pre and post survey response rates were 90.0 and $75.0 \%$ respectively. The participants were $53 \%$ female, $53 \%$ White and predominantly $(72 \%)$ born in the United States. Fifty-six percent of the participants reported speaking a language in addition to English, with Spanish being the most common and Chinese the second most common. At baseline, over $90 \%$ of the participants reported receiving prior instruction on 8 of the 9 cultural competency instructional items surveyed. Over $95 \%$ of the residents reported receiving formal evaluation regarding their ability to handle cross-cultural issues during doctor-patient communications. At baseline only 3 of the 8 preparedness items had more than $80 \%$ of residents reporting being prepared for that item. None of the 10 skillfulness items had more than $60 \%$ of residents report as being very skillful at that item. After course completion, post-survey responses increased for total preparedness, from 24 to 28 points, with possible scores ranging from 8 to 40 points ( $p$-value 0.003 ). Post-survey responses increased from pre-survey responses for the individual skill of being able to identify whether a patient is mistrustful of the health care system or the physician. There were no gender differences for total level of preparedness and skillfulness in delivering cross-cultural care pre or post course. Time was reported by $94 \%$ of residents as being a moderate to large problem/barrier for delivering cross-cultural care.

CONCLUSIONS: Just as our patients come from a diverse background, our residents enter clinical practice with a diverse set of prior experiences and priorities regarding crosscultural care. Despite efforts to prioritize health equity through educational initiatives focused on health disparities and cross-cultural care, this study suggests that more is still needed to achieve enhanced skills in delivering high quality cross-cultural care. The results suggest that not all domains of cross-cultural care are being taught. Despite residents' report of receiving high amounts of instruction and evaluation regarding delivering cultural competent care, few residents report feeling as prepared or skillful when delivering cross-cultural care. This course enhanced residents' self-efficacy, measured by preparedness and skillfulness, around delivering cross-cultural or cultural competent care, although the effect was small. As the medical education community continues to invest in decreasing health disparities through provider education research, identifying which methods are most effective for providers, and more importantly patients is needed. 


\section{A MIXED METHODS STUDY OF INITIAL ENGAGEMENT IN BEHAVIORS} TO PREVENT DIABETES AMONG EMPLOYEES WITH PREDIABETES Jeff Kullgren $^{1}$; Megan Knaus ${ }^{3}$; Kristi Jenkins ${ }^{3}$; Michele Heisler ${ }^{2} .{ }^{1}$ Ann Arbor VA Healthcare System and University of Michigan, Ann Arbor, MI; ${ }^{2}$ Ann Arbor VA/University of Michigan, Ann Arbor, MI; ${ }^{3}$ University of Michigan, Ann Arbor, MI. (Control ID \#2469547)

BACKGROUND: Many employers use workplace screenings to identify and recommend modifications of employees' health risk factors. Such screenings represent an opportunity to help individuals at risk for chronic conditions like type 2 diabetes mellitus (T2DM) better understand and reduce their risks. However, little is known about how often at-risk individuals subsequently engage in behaviors recommended during such screenings and what factors influence engagement. The objective of this study was to describe the frequency of, facilitators of, and barriers to initial engagement in recommended behaviors to prevent T2DM among employees found to have prediabetes during a workplace screening.

METHODS: We conducted a mixed methods study of University of Michigan (U-M) employees found to have prediabetes during workplace screenings conducted in early 2014. In the screenings, employees whose fasting blood glucose was in the prediabetes range were counseled by a health coach to reduce their risk for T2DM by losing weight and getting at least $150 \mathrm{~min}$ of moderate physical activity per week, discussing metformin for T2DM prevention with their primary care provider, or participating in a Diabetes Prevention Program (DPP). We then invited these employees to be surveyed about their demographic characteristics, engagement at 3 months in behaviors to prevent T2DM, and potential behavioral mediators of this engagement. After we collected all survey data, we conducted semi-structured telephone interviews with purposive samples of employees who were and were not initially engaged in a recommended behavior to identify facilitators of and barriers to engagement. For our quantitative analyses, we used Wilcoxon ranksum tests for continuous variables and Chi-square or Fisher's exact tests for categorical variables to compare demographic characteristics and potential behavioral mediators between participants who had or had not engaged in a recommended behavior. For our qualitative analyses, we transcribed interviews and used modified grounded theory to identify salient themes.

RESULTS: Three months after screening, $63 \%$ of the 82 U-M employees with prediabetes who we surveyed were attempting to lose weight and getting recommended levels of physical activity, had asked their primary care provider about metformin for prevention of T2DM, or had attended a DPP. These employees had higher median levels of motivation to prevent T2DM ( $9 / 10$ vs. $7 / 10, P=0.01)$ and lower median estimations of their risk for T2DM ( 40 vs. $60 \%, P=0.04$ ). Among 22 employees who had initially engaged in a recommended preventive behavior, key facilitators of engagement were high motivation and social and external supports. Among 18 employees who had not initially engaged in a recommended behavior, key barriers to engagement were lack of motivation, lack of resources, and competing demands.

CONCLUSIONS: Most employees found to have prediabetes through a workplace screening initially engaged in a recommended behavior to reduce their risk for T2DM. This engagement could perhaps be enhanced by approaches to optimize motivation and risk perception as well as leverage social networks and external supports. More research is needed to measure longer-term engagement in behaviors to prevent $\mathrm{T} 2 \mathrm{DM}$ among employees with prediabetes and test approaches to sustain their ongoing engagement.

\section{A MODEL FOR A SUCCESSFUL WEIGHT LOSS PROGRAM IN A PRIMARY \\ CARE CLINIC Raveen Chawla; Susan Wolver; Puneet Puri; Terence Darcy; Miao-Shan} Yen; Karen Stewart. VCUHS, Richmond, VA. (Control ID \#2466695)
BACKGROUND: Obesity is a major health crisis in the industrialized world. Nearly 80 million adult Americans are obese and have significant associated comorbidities resulting in a substantial health and economic burden. Non-pharmacological interventions (NPI) i.e., diet and exercise remain the cornerstone of medical management of obesity. Primary care physicians (PCP) are at the forefront of delivering and coordinating NPIs. Success rates for sustained weight loss in clinical practice are generally dismal and often associated with high recidivism. Thus there is a large unmet need for efficacious weight loss approaches. The main objectives of the present study were to evaluate the feasibility and success of a pragmatic diet based weight loss program in a primary care setting. METHODS: Adult obese patients, with body mass index (BMI) of $\geq 30 \mathrm{~kg} / \mathrm{m}^{2}$, who elected to participate in a pragmatic weight loss program centered around a low carbohydrate ketogenic diet (LCKD) from May 2013 to July 2015 were included for analyses. This unique weight loss program was developed under the supervision of a single PCP at Virginia Commonwealth University Medical Center. The LCKD used was adapted from the diet used by the Duke Lifestyle Medicine Clinic. The program structure included an initial group class taught by the PCP, followed by group visits every $2-4$ weeks. The group visits were facilitated by a post-doctoral psychology fellow during which each patient was also seen individually by the PCP. There was a private Facebook ${ }^{\circledR}$ page used for recipes and support. The patients were categorized according to the duration of active participation in the program and were followed up longitudinally for 18 months. The main outcome was loss of body weight (in kilograms and percent change) at 18 months. The data were collected retrospectively and statistical analyses were performed. A linear mixed-effect repeated measures ANOVA model with active program length and month at follow up as fixed effects was used to assess the participants' weight change. $P$-values for multiple comparison were adjusted using Bonferroni correction. The study was approved by the institutional review board of VCU Medical Center

RESULTS: A total 173 patients participated in the weight loss program with 142 females ( $82 \%$ ), a mean age of $53.6 \pm 11$ (years) and mixed ethnic backgrounds. The mean baseline weight was $108.1 \pm 23.6 \mathrm{~kg}$ and mean BMI was $39.4 \pm 8.5 \mathrm{~kg} /$ $\mathrm{m}^{2}$. The patients were categorized based on their duration of active participation in the program (Fig. 1). Over $60 \%$ patients (105/173) were in the program for up to 3 months while about $40 \%$ (68/173) were active in the program for $>3$ months. More pronounced weight loss was observed in patients ( $>3$ months vs. $\leq 3$ months) at 6-month $(-10.2 \mathrm{~kg}$ vs. $-4.9 \mathrm{~kg}, p<0.0001)$ and 12 -month $(-13.0 \mathrm{~kg}$ vs. $-2.7 \mathrm{~kg}, p<0.0001)$ follow up. Given the retrospective nature of analysis, the weight measurements at predefined 6- and 12-months were available in 22/105 subjects that participated for $<3$ months. In contrast, among $>3$ month participants, 45/68 patients at 6 -months and 30/68 patients at 12 -months had weight measured. Overall 65 patients ( $38 \%$ ) had 6 month weights available. Among these, $67 \%$ $(n=45)$ achieved $>5 \%$ weight loss. Weight at 12 months was available in 45 patients, of these $34 \%(n=18)$ achieved $>10 \%$ weight loss. Sustained weight loss at 18 months was only noticeable in groups where patients actively participated for $>3$ months. At 18 months weight loss was significantly higher in patients that actively participated in the program for 4-6 months, 6-9 months, 9-12 months and longer than 1 year (all $p$-values of multiple comparison $<0.001$ vs. $\leq 3$ months). CONCLUSIONS: Our pragmatic multidisciplinary low carbohydrate ketogenic diet weight loss program is feasible and sustained weight loss $>10 \%$ is achievable in a primary care clinic even with limited resources. Active participation in the program for $>3$ months is crucial for successful sustained weight loss. Overall compliance with weight loss programs is generally poor; however, in our pilot study the $40 \%$ adherence rate for more than 3 months in the program is very encouraging. Our study is very promising and can serve as a model to incorporate into primary care practice. The predictors of success and challenges with improving adherence to diet based weight loss programs merit further investigation.

Figure 1 


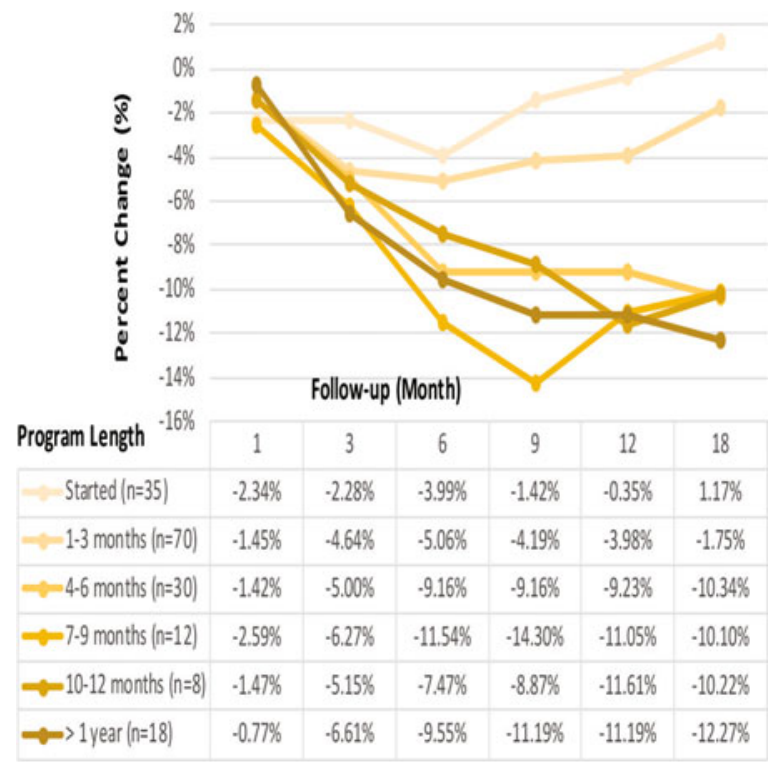

A MULTI-CENTER STUDY OF PRE-OPERATIVE NON-INVASIVE CARDIAC TESTING IN OLDER PATIENTS WITH HIP FRACTURE Ankita Sharma ${ }^{1}$; Liron Sinvani ${ }^{1}$; Joanna Fishbein ${ }^{2}$; Guang Qui ${ }^{1}$; Roman Zeltser ${ }^{3}$; Amgad Makaryus ${ }^{3}$; Christian Nouryan ${ }^{1}$; Gisele Wolf-Klein ${ }^{1} .{ }^{1}$ Northwell Health System, Manhasset, NY; ${ }^{2}$ Feinstein Institute of Medical Research, Manhasset, NY; ${ }^{3}$ Nassau University Medical Center, East Meadow, NY. (Control ID \#2469414)

BACKGROUND: Current guidelines for use of pharmacologic stress tests in preoperative non-invasive cardiac testing need further clarification, especially in older patients with elevated risk and poor functional capacity. This study evaluated the use of these tests in patients admitted for hip fracture for time to surgery (TTS), and time to discharge (TTD), measured in days.

METHODS: A 2 year retrospective chart review of patients over 65, admitted after hip fracture, was conducted across 15 hospitals from 1/01/13 to 6/30/15. Fishers exact test was used to assess associations between having a nuclear stress test (NST), an adverse event, 30 day readmission and other categorical factors. Wilcoxon rank sum or Kruskal-Wallis for factors over 2 levels, was employed as appropriate to assess association between each categorical predictor of interest and TTS or TTD.

RESULTS: Of the 2335 patients admitted, the first 88 who underwent hip repair were analyzed. Median age was 81.5 years (IQR: 76-89), median LOS was 6.6 days (IQR: 4.79.8). No demographic data were significantly associated with having NST. Only $15 \%$ had a NST while $50 \%$ had a transthoracic echocardiogram (TTE). NST and TTE did not significantly change TTS and TTD. None of the 13 NST subjects underwent cardiac catheterization (CCT), compared with 14 of 75 subjects without NST. While $7 \%$ with a TTE had a CCT, $25 \%$ of those without TTE had a CCT $(p=0.0385)$. Patients on the surgical or orthopedic services had lower median TTS and TTD than those admitted to medicine $(1.0,1.5,2.0 ; p=0.0479 ; 4.2,6.7,8.0 ; p=0.0106$, respectively). Males had a longer TTS when compared with females ( 3 vs $1, p=0.0061$ ).

CONCLUSIONS: The study highlights a striking inconsistency in pre-operative noninvasive cardiac testing in older patients with hip fractures. While the rate of NST was low, a surprising and unsupported finding was the $50 \%$ use of preoperative TTE. Yet, neither NST or TTE changed hip fracture outcomes with regard to TTS and TTD. Of interest, patients on the surgical services had lower TTS and TTD, compared to patients admitted to medicine. These data support the urgent need for standardization in the care of older adults with hip fracture.
A MULTICOMPONENT TRANSITIONAL CARE INTERVENTION TO IMPROVE CLINICAL OUTCOMES AMONG PATIENTS BEING DISCHARGED AFTER A HEART FAILURE ADMISSION: A PILOT RANDOMIZED CLINICAL TRIAL Kristen Reilly ${ }^{2}$, 3; Erin West'; Sangmin Jung ${ }^{2}$; Jennifer P. Friedberg ${ }^{2}$; Sundar Natarajan ${ }^{1} .{ }^{1}$ VA New York Harbor Healthcare System, New York City, NY; ${ }^{2}$ VA New York Harbor Healthcare System, New York, NY; ${ }^{3}$ SUNY Downstate College of Medicine, Brooklyn, NY. (Control ID \#2470316)

BACKGROUND: Patients hospitalized with congestive heart failure (CHF) are required to adhere to many health behaviors after discharge. Unstructured education during the inpatient stay is insufficient to ensure that patients are adequately prepared to properly care for themselves following discharge. Augmenting structured inpatient CHF-specific patient education with weekly counseling calls to improve self-care may increase CHF knowledge and adherence to treatment. We conducted a pilot randomized clinical trial (RCT) to evaluate if a multicomponent health education intervention (HEI) that combines a) an interactive computer-based $\mathrm{CHF}$ education video (Kognito Inc, New York) geared toward helping patients understand CHF and its management, b) Educational mailings to reinforce the Kognito video, and c) phone counseling using motivational interviewing (MI) techniques improves knowledge, enhances quality of life (QOL), increases adherence to diet and medication regimens and lowers CHF recurrence.

METHODS: This pilot RCT evaluated the impact of the multicomponent HEI compared to current best practice (CBP) on post-discharge outcomes in Veterans hospitalized with CHF. We enrolled 60 in patients with CHF and randomized them 1:1 to either a) the HEI, which included viewing the HF education video while hospitalized, weekly mailings and 4 weekly post-discharge counseling sessions, or b) current best practice (CBP), which consisted of $\mathrm{CHF}$ treatment as usual. The outcomes assessed 1 month post-discharge were knowledge using the Atlanta HF Knowledge Score, HF- specific quality of life using the Minnesota Living with Heart Failure questionnaire (HF-specific), general quality of life using the SF-36 physical component score, diet adherence using sodium intake estimated from 24-h dietary recall, and medication adherence using the Morisky Medication Adherence Scale. Days to readmission was from data censored at 6 months. Except for HF-specific quality of life, for all other outcomes, higher values indicate better outcomes. Since this was a small pilot study, results are primarily reported as point estimates (medians with interquartile range) or change in score (mean with standard deviation [SD]) without formal hypothesis testing.

RESULTS: There were no differences between groups at baseline. Change in knowledge scores in the HEI group improved slightly after the 1 month program (0.95 [2.38] in HEI vs. 0.88 [2.67] in CBP) as did change in general quality of life (3.91 [9.15] vs. 2.50 [8.92]), change in HF-specific quality of life $(-25.33$ [18.72] vs -21.24 [24.36]) and median sodium intake $(1701[1300,2071] \mathrm{mg}$ vs. $2207[1367,3868] \mathrm{mg}$,). The median days to readmission in HEI was 35 [20, 49] while it was 26.5 [10, 99.5] in CBP. Medication adherence improved more in the control arm after 1 month (0.05 [1.13] vs. 1.13 [2.32]). However, except for sodium intake, which was of borderline significance $(p=.088)$, none of the other comparisons reached statistical significance.

CONCLUSIONS: Comprehensive web-based CHF education during the inpatient stay coupled with mailings and tailored counseling telephone calls to improve patient knowledge of HF and its management as well as self-care shows promise and resulted in improvements in the right direction. However, in this small pilot study ( $n=30$ in each group), no outcome reached statistical significance. Future research with a larger sample size needs to be conducted.

\footnotetext{
A MULTIDISCIPLINARY APPROACH TO IMPACT ACUTE CARE UTILIZATION AMONG INDIVIDUALS WITH SICKLE CELL DISEASE Rhea E. Powell ${ }^{1}$; David Axelrod ${ }^{1}$; Elizabeth D. Pulte ${ }^{2}$; Paris Lovett ${ }^{3}$; Albert G. Crawford ${ }^{1}$; John McAna ${ }^{1}$; Lawrence Ward ${ }^{1} .{ }^{1}$ Thomas Jefferson University, Philadelphia, PA; ${ }^{2}$ German Cancer Research Center, Heidelberg, Germany; ${ }^{3}$ Jackson Memorial Hospital, Miami, FL. (Control ID \#2469875)
} 
BACKGROUND: Sickle cell disease (SCD) is an inherited red blood cell disorder marked by anemia, unpredictable episodes of pain, end organ damage, and early mortality. While many acute pain episodes can be managed at home and in the outpatient setting, acute uncomplicated pain episodes often result in unplanned care use including emergency department use and inpatient admissions, associated with high cost and burden to patients. Evidence-based health system approaches for prevention and management of acute uncomplicated pain episodes are needed.

METHODS: We performed a retrospective study of unplanned care use (ED visits and inpatient admissions) pre- and post- initiation of a multidisciplinary care team intervention at a single urban academic health center. The intervention comprised a monthly multidisciplinary team conference with providers from primary care, hospitalist medicine, hematology, the ED, and social work. During the meeting, every ED visit and admission for uncomplicated sickle cell pain episode in the month prior is reviewed, with attention to presenting symptoms, timeliness and appropriateness of pain management, and a comparison to trends in prior 6 months. Potentially precipitating factors are discussed, such as insurance-lapses or changes, medication adherence, housing stability, mental health issues, or new medical conditions; an individualized care plan is created and updated and made available to providers across the health system. We hypothesized that multidisciplinary care team intervention would lead to decreased ED utilization among patients with a history of high utilization patterns. We used a quasi-experimental design and performed a retrospective review of ED electronic medical record data. The study population included individuals with a SCD-related ED visit from 11/1/2012-6/30/ 2014. We calculated intra-individual change in ED visit rate and inpatient admission and pre- and post- intervention. A paired $t$-test and analysis of variance were used to evaluate differences across strata of age, gender, insurance status, and high-utilization history in change of ED use and inpatient admission pre-post intervention. Change in utilization pre-post intervention was evaluated using the Wilcoxon rank-sum test.

RESULTS: A total of 242 individuals had any SCD-related visit with a total 1188 ED visits across all patients with SCD over the 20-month study period, including 833 ED visits in the 12 months pre-intervention and $355 \mathrm{ED}$ visits in the 8 months postintervention period. The study population was $57 \%$ female, $100 \%$ African-American, and ranged from age 18-70. Among the group, $33 \%$ had three or more ED visits in the year prior to intervention, and thus were considered to have a history of high utilization. Pre-intervention, participants had mean 3.7 ED visits per year, while post intervention the mean was 2.1 ED visits per year. The mean number of inpatient admissions was smaller, with a mean 1.2 admissions for the population pre-intervention, and 0.6 admissions per year post-intervention. There was a significant difference pre-post in utilization among the total population. The mean intra-individual decrease in ED visits was -1.2 visits/year, while the mean intra-individual decrease in inpatient admissions was -0.7 admissions/ year. When stratified by utilization, this statistically significant difference was only noted among those patients with a history of high ED utilization. Stratified analysis of changes in utilization by other demographic characteristics showed no difference across strata of age group, gender, and insurance type.

CONCLUSIONS: Following implementation of a multidisciplinary care team intervention for management of uncomplicated SCD pain crises associated, we identified a significant decrease in ED utilization among those individuals with a history of high utilization. These findings highlight the potential strength of leveraging shared resources for multidisciplinary interventions, and suggest that the most value can be found from targeting high utilizing subpopulations. A limitation of note is that the analysis included only utilization at one health system, as such it is unknown if individuals included in analysis accessed other health systems for acute care services in the study period. This study suggests that multidisciplinary care teams targeting management of uncomplicated SCD pain episodes have potential to reduce the burden of unplanned acute care utilization; further studies could use claims data to assess the impact on utilization across all health centers, and explore patient experiences of such interventions.
A MULTIFACETED PRIMARY CARE PRACTICE-BASED INTERVENTION TO REDUCE ED VISITS AND HOSPITALIZATION FOR COMPLEX PATIENTS Tara O'Brien ${ }^{2,}{ }^{3}$; Ian Stanaitis ${ }^{3}$; Noah Ivers ${ }^{2,}{ }^{3}$; Pauline Pariser ${ }^{2},{ }^{1}$; Steven Friedman ${ }^{2}{ }^{4}$; Howard Abrams ${ }^{1}$; Geetha Mukerji², ${ }^{2}$; Laura Pus ${ }^{3}$; Gillian Hawker ${ }^{2}$. ${ }^{1}$ University Health Network, Toronto, ON, Canada; ${ }^{2}$ University of Toronto, Toronto, ON, Canada; ${ }^{3}$ Women's College Hospital, Toronto, ON, Canada; ${ }^{4}$ University Health Network, Toronto, Canada. (Control ID \#2462125)

BACKGROUND: Poor access to timely consultative services, difficulty in navigation of the healthcare system and fragmented care may contribute to avoidable Emergency Department (ED) visits and hospital admissions for complex patients. SCOPE (Seamless Care Optimizing the Patient Experience) is a quality improvement collaboration between acute and community providers located in downtown Toronto, Canada (University Health Network, Women's College Hospital, Toronto Central Community Care Access Centre and solo community primary care physicians (PCPs)). The SCOPE objective is to strengthen relationships between hospitals, primary, and community care to improve care delivery for complex patients who are frequent users of the ED.

METHODS: A multifaceted innovative model of care was designed whereby participating solo PCPs were provided a single access point via phone or email to a range of resources and providers. The SCOPE intervention included a general internist (GIM) oncall for telephone advice or access to a GIM-led short stay medical unit that provided urgent assessment, investigation, and management for patients with acute or chronic medical conditions. SCOPE also offered a nurse navigator and homecare coordinator to link PCPs with hospital and community resources, and online access to hospital-based patient records. Process measures including frequency of utilization, reason, and outcome of PCP contacts were tracked. The primary outcome, rate of ED visits for patients in participating practices, was assessed using an interrupted time series analysis. This was compared to a propensity matched control cohort. Semi-structured one-on-one interviews of participating PCPs with qualitative analysis were also performed.

RESULTS: Twenty-nine PCPs agreed to participate and were able to access the SCOPE intervention over an 18-month time period. In both the SCOPE PCP group and control group the rate of ED visits significantly decreased post intervention, but the pre-post change in per practice rate of ED visits was not significantly different in the SCOPE PCPs compared to controls. However, qualitative analysis demonstrated that the intervention had beneficial effects. PCPs reported that the intervention had improved the quality and coordination of care provided to their patients; heightened awareness, enabled better provision of available services and demonstrated an enhanced ability to practice shared care for their complex patients within an interdisciplinary environment.

CONCLUSIONS: The SCOPE intervention increased understanding of the challenges solo PCPs face in caring for their complex patients. While we did not observe a decline in ED visits related to the SCOPE intervention, qualitative findings indicate the intervention had important effects at the PCP practice level, which may ultimately translate to reduced ED use with longer observation.

\section{A MULTIMODAL INTERVENTION TO REDUCE TREATMENT DISPAR- ITIES FOR EARLY STAGE BREAST AND LUNG CANCER: BARRIERS TO SYSTEMS IMPROVEMENT IN RACE-SPECIFIC CARE Samuel Cykert ${ }^{1}$; Eugenia Eng $^{1}$; Matthew Manning ${ }^{2}$; Linda Robertson ${ }^{3}$; Christina Hardy ${ }^{1}$; Jennifer Schaal ${ }^{4}$; Dwight Heron ${ }^{3} .{ }^{1}$ University of North Carolina, Chapel Hill, NC; ${ }^{2}$ Cone Health, Greensboro, NC; ${ }^{3}$ University of Pittsburgh Medical Center, Pittsburgh, PA; ${ }^{4}$ The Partnership Project, Greensboro, NC. (Control ID \#2467673)}

BACKGROUND: Lung cancer and breast cancer are the leading causes of cancer death in the U.S. Despite the frequency of fatal outcomes, Black patients with potentially curable 
disease consistently either don't receive or don't complete standard treatments more frequently than White patients contributing to higher mortality rates. The Accountability for Cancer Care through Undoing Racism and Equity (ACCURE) Study is an NCI sponsored intervention trial designed to implement system changes to reduce these treatment disparities. In this report, we describe the intervention in 2 cancer centers focusing on early results and factors that might have influenced differences between centers.

METHODS: The intervention was derived using a community based participatory research approach in partnership with a health disparities collaborative formed in 2003 in response to the IOM's Unequal Treatment Report. The ACCURE intervention consists of 4 components: (1) a real time warning system built with uploads from electronic health record data that automatically signals missed patient appointments or unmet milestones in expected care, (2) race-specific data feedback to each cancer center team regarding adherence to standard treatments, (3) a nurse navigator specially trained regarding health equity issues and race-specific barriers to care (randomized to $1 / 2$ the patients), and (4) quarterly health equity trainings for cancer center staff based on concepts of an established undoing racism curriculum and locally acquired data. All data were collected using uploads from electronic sources. Baseline data were obtained from cancer registry and billing records at each cancer center for all patients with early stage breast and lung cancer diagnosed from Jan. 1, 2007 to Dec. 31, 2011. The intervention cohort was enrolled at each cancer center starting in 2013. The primary outcome, "Treatment Complete", is a combined variable consisting of receipt of lung cancer surgery for early stage lung cancer patients, breast cancer surgery for early stage breast cancer patients, and completion of at least $80 \%$ of prescribed adjuvant radiation for lumpectomy patients and $80 \%$ of prescribed adjuvant chemotherapy for breast cancer patients for whom chemotherapy had been started.

RESULTS: At Center \#1, 2532 patients were in the early stage cancer population identified in the retrospective review. Seven hundred twenty-four with lung cancer and 1808 with breas cancer. The mean age was 63 years (+/-12), $21 \%$ Black, $85 \%$ female, $66 \%$ stage I. For the 147 patients in the prospective study, $75 \%$ had breast cancer. The mean age was 62 years, $37 \%$ were Black, $8 \%$ male, and $69 \%$ had stage I disease. At Center $\# 2,5265$ patients were in the early stage cancer population identified in the retrospective review, 1125 with lung cancer and 4140 with breast cancer. The mean age was 61 years (+/-12), $7.5 \%$ Black, $89 \%$ female, $64 \%$ stage I. For the 126 patients in the prospective study, $49 \%$ had breast cancer The mean age was 64 years, $40 \%$ were Black, $21 \%$ male, and $80 \%$ had stage I disease. During the 2 years of the prospective trial, Center 1 experienced an $80 \%$ turnover of the breast and lung oncology group while Center 2 had no turnover. For health equity sessions, all cancer center physicians and staff were invited but attendance was voluntary. At Center 1 , 20 to $30 \%$ of staff attended to date with minimal oncologist involvement while at Center 2, most physicians attended with similar staff involvement. At both Centers, missing data from the baseline data collection prevented calculation of the Treatment Complete variable for $25 \%$ of patients. For Center 1, unadjusted analysis reveals $80 \%$ of White patients completed therapy at baseline compared to $67 \%$ in the intervention period $(p=.002)$. For Black patients, baseline completion was $80 \%$ compared to $81 \%$ in the intervention $(p=.86)$. Logistic regression controlling for age, Charlson Score, clinical stage, race, and cohort (retrospective vs. prospective) showed no improvement attributable to the intervention. For Center 2, the unadjusted analysis reveals Treatment Complete for $85 \%$ of White patients at baseline compared to $89 \%$ with the intervention ( $p=.33$ ). For Black patients baseline completion was $75 \%$ compared to $86 \%$ in the intervention $(p=.11)$. Logistic regression demonstrated a Black-White disparity pre-intervention ( 75 vs. $85 \%, p=.01$ ) that resolved with intervention ( 86 vs. $89 \%$ ).

CONCLUSIONS: A multimodal intervention to optimize treatment completion and reduce racial disparities seemed effective in one cancer center and ineffective in another. The effective center had stable staffing and high physician engagement at Health Equity sessions whereas the other center did not. These results show the current limitations of fully automated data and the negative effect of low physician engagement and high staff turnover on implementation of system change. These issues need to be considered in approaching real world dissemination and implementation projects.

A MYSTERY SHOPPER STUDY OF PRIMARY CARE ACCESS FOLLOWING THE MEDICAID EXPANSION IN A LARGE URBAN AREA David Grande ${ }^{1,1}$; Karin V. Rhodes ${ }^{2}$; Jessica X. Zuo ${ }^{1}$; Rathnam Venkat ${ }^{1}$; Daniel Polsky ${ }^{1} .{ }^{1}$ University of Pennsylvania, Philadelphia, PA; ${ }^{2}$ Northwell Health, Great Neck, NY. (Control ID \#2469605)

BACKGROUND: With implementation of the Affordable Care Act, there is concern that newly insured individuals will be unable to access primary care. In a multi-state mystery shopper study prior to ACA implementation, individuals with Medicaid were much less likely to be offered a new patient appointment than those with private coverage. With Medicaid expansion occurring in many states, it is important to measure primary care access in ways that are similar to the experience of consumers. We conducted a mystery shopper study in a large urban area with the following objectives: 1) determine primary care availability and wait times for newly insured adults and assess differences by insurance type (Medicaid vs. private coverage), and 2) determine whether small area variations in primary care supply are associated with differences in appointment availability and wait times.

METHODS: We conducted a census of all primary care practices in and near Philadelphia using data from SK\&A, the largest commercial and Medicaid insurers in the region, the local department of public health, and organizations representing CHCs. We conducted an initial telephone survey of each practice to quantify the number of primary care providers in order to calculate population to provider ratios for each census tract based on the adult population and number of providers within a 5-min drive time from the centroid of each census tract. We identified six clusters of census tracts with the lowest supply. We then conducted a mystery shopper study by having two simulated patients call practices - one with private insurance (all practices) and one with Medicaid coverage (only for practices participating in a Medicaid plan). In each case, the simulated patien requested the next available new patient appointment. Multiple and logistic regression were used to assess relationships between local primary care supply and experienced access.

RESULTS: Of the 414 practices determined to be eligible for a private insurance call, 362 $(87.4 \%)$ offered the simulated patient a new patient appointment. Of the 317 practices eligible for a Medicaid patient call, $216(68.1 \%)$ offered the simulated patient a new patient appointment. Among practices that offered an appointment, the median wait time for a private insurance appointment was 9 days versus 12 days for a Medicaid appointment. Median wait times were similar for private and Medicaid calls when restricting the analysis to practices that accept Medicaid. Median wait times for private calls were the same for practices that participate in Medicaid compared with those that do not ( 9 days). Appointment availability was similar for practices located in lower primary care supply areas compared to other areas (private: $\mathrm{OR}=0.93, p=0.891$; Medicaid: $\mathrm{OR}=1.63, p=$ 0.279). Similarly, wait times until the first available new patient appointment were similar for practices in lower primary care supply areas compared to other areas (private: -5.4 days, $p=0.135$; Medicaid: -9.6 days, $p=0.063$ )

CONCLUSIONS: We found that new appointment availability was substantially lower for individuals with Medicaid seeking a new patient appointment compared to those with private coverage. However, once offered an appointment, wait times were similar for callers with both insurance types. Surprisingly, appointment availability and wait times did not vary based on the primary care supply in the immediate area. Future studies should explore other factors that contribute to variation in primary care appointment availability and wait times. 
A NATIONAL ASSESSMENT OF HANDOFF CHARACTERISTICS AND THEIR ASSOCIATION WITH PROGRAM DIRECTOR AND HOSPITALIZED PATIENT SATISFACTION Charlie M. Wray ${ }^{4}$; Stephanie Mueller ${ }^{1}$; Amber Pincavage ${ }^{4}$; Reshma Gupta ${ }^{3}$; Saima Chaudhry ${ }^{8}$; Rachel K. Miller ${ }^{6}$; Linda DeCherrie²; Karin Ouchida $^{7}$; Vineet M. Arora ${ }^{5}$. ${ }^{1}$ Brigham and Women, Boston, MA; ${ }^{2}$ Icahn School of Medicine at Mount Sinai, New York, NY; ${ }^{3}$ University of California, Los Angeles, Los Angeles, CA; ${ }^{4}$ University of Chicago, Chicago, IL; ${ }^{5}$ University of Chicago Medical Center, Chicago, IL; ${ }^{6}$ University of Pennsylvania, Philadelphia, PA; ${ }^{7}$ Weill Cornell Medical College, New York, NY; ${ }^{8}$ nslij, Manhasset, NY. (Control ID \#2457425)

BACKGROUND: Inpatient handoffs have been recognized as a vulnerable time during a patient's hospitalization and are widely associated with adverse events and near misses. A variety of strategies have been implemented in order to improve shift handoffs. To date, no study has described how residency program leadership views these strategies, or how the implementation of these strategies would affect the hospitalized patient's experience. Our objective was to characterize the use of best practices in resident handoffs and evaluate the association with internal medicine program director (PD) satisfaction and patient experience scores.

METHODS: We combined and analyzed the 2014 Association of Program Directors in Internal Medicine (APDIM) survey results with the Center for Medicare and Medicaid Service's Hospital Consumer Assessment of Healthcare Provider and Systems (HCAHPS) survey results. APDIM handoff survey items surveyed properties of written and verbal handoffs and educational interventions. Primary outcomes included PD satisfaction with the handoff process, and HCAHPS survey items that assessed the 5-star composite scores regarding doctor communication, pain management, hospital rating, hospital recommendation, and overall summary rating for the primary affiliated hospital for each corresponding residency program. Each outcome variable was measured on a 5-point scale and dichotomized for analysis. Multivariable logistic regression models controlling for institutionspecific characteristics tested associations between PD satisfaction and both handoff properties and HCAHPS patient experience outcomes.

RESULTS: In total, 234/361 (65\%) of all APDIM member programs responded, with 208/361 (58 \%) responding to all of the handoff questions. Most program directors (60\%) were satisfied with the handoff processes that were used during shift changes. Employing a dedicated room (OR 3.18; 1.34-7.58), supervision by a senior resident (OR 2.44; 1.26 4.74), paper copies of sign outs for receivers (OR 2.32; 1.11-4.88), and interactive workshops (OR 2.17; 0.95-4.96) were positively associated with PD satisfaction, but were not associated with any patient experience outcomes. Use of Electronic Health Records (EHR) for the creation of a written handoff was associated with PD satisfaction (OR 4.15; 1.51-11.37) and patient's overall rating of their hospital care (OR 3.07; 1.43 $6.58)$ and Summary Star rating $(2.24 ; 1.00-5.02)$. PD reported application of these measures ranged from $26 \%$ for interactive workshops to $59 \%$ for receiver obtaining a written copy of sign-out.

CONCLUSIONS: While several handoff strategies are related to PD satisfaction, only the use of an EHR-based written handoff was shown to be associated with higher HCAHPS scores. With less than half of all respondent programs utilizing an EHRbased handoff, this may represent an underutilized resource in patient handoffs, and may represent a future target for meaningful use criteria.

A NATIONAL STUDY OF SMOKING CESSATION COUNSELING IN BUPRENORPHINE VISITS Zoe M. Weinstein ${ }^{2,}{ }^{1}$; Phoebe A. Cushman ${ }^{2,}{ }^{1}$; Lewis $\mathrm{Kazis}^{3}$; Howard Cabral ${ }^{3}$. ${ }^{1}$ Boston Medical Center, Boston, MA; ${ }^{2}$ Boston University, Boston, MA; ${ }^{3}$ Boston University School of Public Health, Boston, MA. (Control ID \#2459750)
BACKGROUND: National guidelines recommend that all smokers receive smoking cessation counseling at every medical visit. Patients with opioid use disorder (OUD) have especially high rates of smoking-related morbidity and mortality. Additionally, achieving smoking cessation improves their addiction treatment outcomes. Buprenorphine is an outpatient medication for OUD that is prescribed in both primary care and specialty settings. Buprenorphine visits offer a unique opportunity to assess the provision of smoking cessation counseling for patients with OUD. The aim of our study is to evaluate the frequency of smoking cessation counseling for patients prescribed buprenorphine by analyzing a nationally representative sample of outpatient visits for patients who smoke. Specifically, we examine the frequency of smoking cessation counseling that occurred in visits in which buprenorphine was prescribed compared to visits in which buprenorphine was not prescribed.

METHODS: We performed a cross-sectional analysis of a nationally representative sample of adult smokers. Publicly available data from the National Ambulatory Care Survey (NAMCS) for years 2003-2010 and 2012, combined with data from the National Hospital Ambulatory Care Survey (NHAMCS) for years 2003-2010 were used to generate the sample. Complex survey analysis methods were used to account for weighting and design effects. Descriptive statistics were utilized to characterize the sample of adult smokers as a whole, and then stratified by whether or not the visit was associated with a buprenorphine prescription. Chi square testing was used to compute bivariate analyses of our main predictor (buprenorphine vs. non-buprenorphine visits) and outcome (smoking cessation counseling) variables, first on the sample as a whole and then stratified by physician specialty. Finally, we constructed a multivariable logistic regression model with our main predictor and outcome variables, adjusting for year of visit.

RESULTS: The sample of adult smokers included 47,146 individual patient visits; buprenorphine was prescribed in 242 of these visits. Overall, the patients seen in buprenorphine visits were significantly younger (mean age 35.0 years vs. mean age 48.2 years; $p<0.001)$ and more likely to be insured by Medicaid (39.6 vs. $16 \%$; $p<0.001$ ) than those for whom buprenorphine was not prescribed. Initial bivariate analysis of our study sample as a whole demonstrated that smoking cessation counseling was significantly more likely to occur in visits in which buprenorphine was prescribed $(41.0 \%)$ than in visits in which buprenorphine was not prescribed $(19.5 \% ; p=0.0051)$. However, when results were stratified by physician specialty, we found that visits with psychiatrists and substance abuse specialists were driving the overall trend. Specifically, for primary care physicians (PCPs), there was no difference in the rate of smoking cessation counseling between the buprenorphine (23.9\%) and non-buprenorphine visits $(24.2 \% ; p=0.97)$, whereas for psychiatrists/substance abuse specialists, the rate of smoking cessation counseling was significantly higher in the buprenorphine $(59.2 \%)$ compared to the non-buprenorphine visits $(17.7 \% ; p=0.0004)$. In adjusted analysis, the PCPs' odds of smoking cessation counseling in buprenorphine compared to nonbuprenorphine visits was $0.99(p=0.98)$ and the psychiatrists'/substance abuse specialists' odds of smoking cessation counseling in buprenorphine vs. non-buprenorphine visits was $5.50(p=0.003)$.

CONCLUSIONS: This study demonstrates a low level of smoking cessation counseling overall, which unfortunately, is consistent with current literature of smoking cessation counseling in outpatient settings. PCPs did not demonstrate differential counseling for buprenorphine vs. non-buprenorphine visits. Psychiatrists performed much better than PCPs in offering smoking cessation counseling during buprenorphine visits, but their overall level of counseling was low. It is possible that psychiatrists who prescribe buprenorphine are more attuned to the relationship between opioid use disorder and tobacco use disorder than are PCPs who prescribe buprenorphine. Our study highlights a missed opportunity for all providers to engage in smoking cessation counseling. Given the particular risks of smoking for patients with OUD, further work should address barriers to and facilitators of the performance of smoking cessation counseling by PCPs who prescribe buprenorphine. 
A NEEDS-BASED RESIDENT CLINIC REDESIGN RESULTS IN IMPROVED PATIENT UTILIZATION OF CARE AND INCREASED RESIDENT SATISFACTION Lynn Bowlby ${ }^{1}$; Alex H. Cho ${ }^{1}$; Natasha T. Cunningham ${ }^{1,1}$; Lawrence Greenblatt ${ }^{1}$; Adia K. Ross ${ }^{1,}{ }^{3}$; Mark Sendak ${ }^{2}$; Daniella A. Zipkin ${ }^{1} .{ }^{1}$ Duke University School of Medicine, Durham, NC; ${ }^{2}$ Duke University, Durham, NC; ${ }^{3}$ Duke University Hospital, Durham, NC. (Control ID \#2469741)

BACKGROUND: Ambulatory care has become an increasing priority for medicine residency programs, the cornerstone of which is the continuity clinic, where residents function as primary care doctors. These resident clinics often serve medically complex patients suffering from co-morbid mental health and substance abuse disorders, with adverse home and community environments, and who are often uninsured or underinsured. Continuity is difficult to achieve given the competing clinical demands on trainees and relative infrequency of clinical sessions. In our clinic, these issues were associated with low resident and staff satisfaction and high rates of emergency department and hospital utilization by patients.

METHODS: Using an adapted DMAIC framework (define; measure; analyze; implement; control) in a process that engaged multiple institutional stakeholders, our interdisciplinary clinic leadership team over the course of 2 years identified some of the major unmet needs of our population and trainees, and developed a multifaceted clinic redesign plan supported by our hospital administration, which could improve outcomes and reduce the cost of care, while also improving the trainee experience. In many cases, these efforts built on or leveraged prior work done in the clinic on some of the same issues. Three main interventions were proposed and implemented: 1) creation of a clinic-based behaviora health-primary care coordinated care model (HomeBASE) for psychosocially complex high-utilizing patients; 2) dedication of a team within the clinic and workflow to manage communications with and review of patients being discharged from the hospital; and 3 ) establishment of "firm"-like resident groups within the clinic. Financial modeling was done of decreased inappropriate ED utilization and reduction in hospital days to justify the initial investments required by the hospital and other partners. Importantly, the clinic itself would not need to see an increase in revenue. A dashboard was created a priori of metrics for measuring impact, and the means and process for obtaining and reporting them established.

RESULTS: Over the past 2 years, substantial reductions were achieved in ED visits $(-14.7 \%)$ and hospitalizations $(-16.8 \%)$ for patients receiving primary care at the DOC, translating into direct cost savings of $\$ 767,878$ in $2013-14$ and $\$ 1,704,953$ in $2014-15$. Overall return on investment was estimated at $2.0 \mathrm{X}$ in the first year and $6.3 \mathrm{X}$ in the second. The impact on residents has also been powerful. Previously, residents often expressed feelings of helplessness when working with clinic patients. Now, residents make frequent use of many of the multidisciplinary options available to address social determinants of health, leading to more anecdotally observed willingness to assess barriers to care that complex patients face. Reflecting this improved confidence, resident assessments of the value of the clinic experience increased from $36 \%$ favorable ('Excellent' or 'Very Good') to $83 \%$ favorable over the same 2-year span.

CONCLUSIONS: When medicine residency teaching clinics are supported by their health systems to improve services for their vulnerable populations, both care and resident experience improves - a clear "win-win." General internal medicine educators need to serve as advocates and leaders in shaping the care delivery models of resident continuity clinics.

\section{A PATIENT EMPOWERMENT PROGRAM (PEP) CAN CHANGE PATIENT EXPECTATIONS OF PATIENT-PROVIDER ROLES IN COMMUNICATION} Joseph Plaksin ${ }^{1}$; Adina Kalet ${ }^{1}$; Sondra Zabar ${ }^{1}$; Sarita Kundrod ${ }^{2}$; Kim Nielsen ${ }^{1}$; Lisa Altshuler ${ }^{1} .{ }^{1}$ NYU School of Medicine, New York, NY; ${ }^{2}$ University of Minnesota, Minneapolis, MN. (Control ID \#2467141)
BACKGROUND: Patients who take an active role in their healthcare and participate in shared decision-making (SDM) with their healthcare providers (HCPs) are better able to accomplish their management goals and maintain their health. However, due to patients' perceptions of their role, desires to be a "good patient," and the difference in knowledge and power between patients and HCPs, many patients do not feel empowered to take an active role in their medical care. We developed a Patient Empowerment Program (PEP) with the goal of reframing the roles of the patient and HCP and democratizing the patient-HCP relationship so patients can fully participate in SDM. As part of the training, participants viewed examples of patient-HCP communication and practiced communication skills with Standardized Healthcare Providers (SHPs). We report on how participants' expectations for communication with HCPs changed over the course of the program.

METHODS: Patients with type 2 diabetes (DM) were recruited from Primary Care clinics at two public hospitals in NYC to participate in PEP, a 4-h, two-session, performance-based workshop run by a Health Psychologist (L.A.) with extensive experience in health education. In Session 1, participants discussed shared decision-making (SDM) in the medical encounter and viewed three sets of trigger videos depicting patient-HCP interactions: 1) providing information about a difficulty, 2) asking for more information, and 3) negotiating an agreeable treatment plan. Each set of videos consisted of a less effective interaction followed by a more effective interaction. After each video participants rated both parties using 4point behaviorally-anchored SDM checklists. In Session 2, participants role-played two scenarios with SHPs, the second of which was tailored specifically for each participant based on an aspect of DM that they struggled with and that was difficult to discuss with their HCPs. After the final scenario, participants rated their own performance while SHPs rated the patient on the same 4-point behaviorally-anchored SDM checklist. At the end of each session, participants also rated the session in terms of how much they learned. All SDM checklists and session ratings were visual Likert scales with a range of $0-3$, where anchor term for 0 was "not at all" and the anchor term for 3 was "very well."

RESULTS: We recruited 71 patients to participate in PEP, 45 of which attended Session 1 and 36 of which attended both sessions. Participants were predominately male $(53 \%)$ with a mean age of 59.0 years $(\mathrm{SD}=10.1)$, racial and ethnic minorities (29\% African-American, $40 \%$ Hispanic), and had a low education level (40\% did not complete high school), low socioeconomic status (62\% income under $\$ 10,000 /$ year), and limited health literacy (76\%, as measured by the Newest Vital Sign). In Session 1, participants rated the patient in each video $(\mathrm{M}=0.98-2.40, \mathrm{SD}=0.84-1.13)$ significantly lower than the HCP $(\mathrm{M}=1.86$ $2.74, \mathrm{SD}=0.49-1.07), \mathrm{t}(43)=2.46-6.78, p=<0.001-0.018$, regardless of whether it was the less effective or more effective example of SDM. However, participants rated both parties in the more effective videos $(\mathrm{M}=1.95-2.74, \mathrm{SD}=0.74-1.01)$ significantly higher than the less effective videos $(\mathrm{M}=0.95-2.10, \mathrm{SD}=0.86$ $1.08), \mathrm{t}(43)=2.05-7.78, p=<0.001-0.046$, across all three sets. In Session 2, topics of individualized role-play scenarios included diet (55\%), blood glucose monitoring (17\%), general stress of living with DM (14\%), medications (8\%), and anxiety about complications from DM (6\%). Participants were rated highly by both themselves $(\mathrm{M}=2.51, \mathrm{SD}=0.66)$ as well as SHPs $(\mathrm{M}=2.71, \mathrm{SD}=0.52)$ and there was a significant relationship between participant self-ratings and SHP ratings, $\left(X^{2}=36.16, p<0.001\right)$. Finally, participants rated both Session $1(M=$ $2.77, \mathrm{SD}=0.42)$ and Session $2(\mathrm{M}=2.71, \mathrm{SD}=0.52)$ very highly and there was no difference in rating between sessions, $\mathrm{t}(35)=0.53, p=0.60$

CONCLUSIONS: Participants from our low-income, low education, and low health literacy population were able to recognize differences in both patient and HCP communication skills as they pertain to SDM. However, it appears that participants are more critical of patients' communication skills, perhaps because they can more easily relate, 
than HCPs. This represents an area that can be targeted by behavioral interventions, such as PEP, in order to decrease the perceived power differential between patients and HCPs. Participants also are able to demonstrate the more effective communication strategies discussed in PEP and seen in the videos when put in an individualized role-play scenario. Their performance suggests that by observing the videos and then practicing these skills, patients can be taught how to be more active in their medical encounters, even when discussing issues they find difficult or challenging. Finally, based on participant ratings, both the observational component and performance-based component of PEP are equally important for participant learning.

A PRELIMINARY ASSESSMENT OF KNOWLEDGE, ATTITUDES, AND PRACTICE TOWARD LONG ACTING REVERSIBLE CONTRACEPTION AMONG WOMEN EXPERIENCING HOMELESSNESS Karyn M. Meshbane; Amelia Poquette; Marie Douglass. University of Miami Miller Schools of Medicine, Delray Beach, FL. (Control ID \#2463616)

BACKGROUND: Homeless women face unique barriers to obtaining contraception, including inadequate knowledge, access, storage, lack of control, and victimization in sexual behavior (Gelberg et. al. 2008). Furthermore, it is estimated at any given time that approximately $10 \%$ of homeless women are pregnant, twice the national average of women of reproductive age (Herndon et al. 2003; Bassuk et al. 1996). Nearly all the research on women experiencing homelessness in relation to contraception access has done been in Los Angeles, a city with different demographics, policies, and resources than Miami. (i.e., Gelberg et al. 2001, 2002, 2004, 2008) The inadequate distribution of the most effective methods of contraception has also been shown to be a problem in this population. When women experiencing homelessness are able to access contraceptive services, they usually are provided methods with high user-based failure rate (ACOG, 2010). In contrast, long-acting reversible contraceptives (LARC) are the most effective methods of reversible contraception on the market today, with a less than $1 \%$ failure rate. To this end, we are interesting in exploring knowledge, attitudes, and practice of a variety of birth control methods, including methods such as LARC, which are less widely used in the United States, despite their effectiveness (Hathaway et al., 2014).

METHODS: Eligible residents of Lotus House were offered the opportunity to complete a survey about contraception knowledge, attitudes, and practice. The survey was selfadministered. Participants who self-identify as unable to read had the option of having the survey administered by a study staff member. In our initial assessment, 20 women residing at the Lotus House shelter in Miami were surveyed using an adapted version of the validated Guttmacher Institute's "National Campaign to Prevent Teen and Unplanned Pregnancy Survey" to assess homelessness experiences, prior pregnancies and outcomes, history of contraception use, and knowledge of different types of contraception.

RESULTS: Seventy-eight percent of respondents had previously been pregnant, with $80 \%$ reporting unintended pregnancy in their lifetime. Eighty-five percent of women felt that it was currently important to avoid getting pregnant, and the majority ( $80 \%)$ stated they would want to delay pregnancy for at least 3 years or avoid future pregnancy entirely. A majority of women (55-75\%) indicated that they would be interested in accessing or learning more about LARC, yet up to $85 \%$ stated they did not know different aspects about LARC (i.e., cost, how it works, who can use it).

CONCLUSIONS: Preliminary data suggests that women experiencing homelessness may be excellent candidates for LARC, with a desire to avoid pregnancy for an extended period of time. However, responses also reveal a deficit in LARC knowledge, accompanied by an interest in education about and access to these forms of contraception. Future assessment will be focused on gathering additional participant responses, qualitative assessment using focus groups, and specialized interventions aimed at specifically addressing the unique health care needs and concerns of this population.
A QUALITATIVE ASSESSMENT OF ANTI-ABORTION ATTITUDES AMONG LOW-INCOME WOMEN UNDERGOING ABORTION Amanda Gelman ${ }^{1}$; Elian A. Rosenfeld $^{3}$; Sonya Borrero ${ }^{2} .{ }^{1}$ University of Colorado School of Medicine, Denver, CO; ${ }^{2}$ University of Pittsburgh and VA Pittsburgh, Pittsburgh, PA; ${ }^{3}$ VA Pittsburgh Healthcare System, Pittsburgh, PA. (Control ID \#2466814)

BACKGROUND: Negative attitudes toward elective abortion, including abortion stigma, are common and may be detrimental to women undergoing abortions by contributing to psychological distress and delays from the time of pregnancy confirmation to termination. Anti-abortion attitudes have not been thoroughly explored in low-income women who disproportionately undergo abortion and who may be at an increased risk for poor abortion-related outcomes due to barriers in obtaining timely and safe abortions. Thus, we sought to identify the range and sources of anti-abortion attitudes among low-income women, as well as women's responses to them.

METHODS: Data were drawn from a larger qualitative study that used in-depth interviews to explore socio-cultural and structural factors that shape pregnancy intentions and contraceptive behavior among low-income women aged 18 to 45 . For this analysis, we restricted our study sample to only those women who had either undergone abortion in the 2 weeks prior to interview or who were planning to have an abortion. Women were asked extensively about the circumstances surrounding their current or recent pregnancy as well as the factors that shaped their decision-making about having an abortion. Additionally, participants were asked a series of questions to assess social norms around abortion and family formation. Narratives were analyzed using content analysis.

RESULTS: The sample consisted of 18 women: 8 had already undergone an abortion in the preceding 2 weeks and 10 were planning to have an abortion. All participants had a yearly income of less than $\$ 50,000$ per year; the majority $(n=15)$ had an income of less than $\$ 20,000$ per year. Two-thirds of the sample self-identified as Black or AfricanAmerican $(n=11)$ or biracial $(n=1)$ and the remaining $(n=6)$ were White. Most women $(n=11)$ had other children at the time of their abortion. Three major themes emerged in our sample. The first was that women commonly experienced negative messages about abortion in their social networks, which included partners, family, friends, and their larger social sphere. These negative messages primarily centered on the idea that abortion is morally reprehensible (abortion was depicted as "killing" or "murder") and that abortion represents a rejection of motherhood, which is the expected and natural response to pregnancy. The second theme was that women themselves held anti-abortion attitudes. These attitudes also revolved around the immorality of abortion. Women also voiced that positive attitudes toward motherhood caused internal conflict in decision-making and led to concerns about whether having an abortion would interfere with their ability to have children in the future. Many women also reported that they believed that abortion was uncommon, thereby contributing to their impression that it is deviant behavior. Lastly, women reacted to these external and internal negative attitudes in ways that could perpetuate abortion stigma. In grappling with their own inner conflict, women often rationalized their abortions by citing their specific circumstances, which they perceived would make abortion less unacceptable (e.g. "as long as you're not 5 or 6 months pregnant, you should be able to get one" and "one is okay, God will forgive you"), as opposed to situations in which women used "abortion as contraception." Participants described these other women as "careless," "cruel," and "disgusting." In addition, many women concealed their abortion from others to avoid conflict and/or to preserve their reputation. Several women also reported that they delayed their abortion while trying to reconcile the discrepancy between their abortion-related attitudes and their behavior, while others described resigning themselves to living with "regret" and "guilt."

CONCLUSIONS: We found that low-income women undergoing abortion face substantial negative attitudes towards abortion in their larger social networks as well as internally, suggesting that many women do not have abortions in the context of supportive (or even neutral) attitudes towards abortion, but rather despite anti-abortion sentiments. 
Furthermore, women react to these external and internal negative attitudes in ways that can perpetuate stigma, including by vilifying other women who undergo abortion and maintaining secrecy about their own abortions. Targeted efforts to address abortion stigma and negative attitudes in low-income populations and normalize abortion are needed to attempt to improve women's abortion experiences and outcomes.

\section{A RANDOMIZED CONTROLLED TRIAL OF A RISK BASED MAMMOGRA-} PHY SCREENING DECISION AID FOR WOMEN 39-48 YEARS OF AGE Marilyn M. Schapira ${ }^{1}$; Rebecca Hubbard ${ }^{1}$; Holli Seitz ${ }^{2}$; Emily Conant $^{2}$; MItchell Schnall $^{1}$; Joseph Capella ${ }^{2}$; Tory Harrington ${ }^{1}$; Carrie A. Inge ${ }^{1}$; Katrina Armstrong ${ }^{3}{ }^{4}$. ${ }^{1}$ University of Pennsylvania, Philadelphia, PA; ${ }^{2}$ Annenberg School of Communication, University of Pennsylvania, Philadelphia, PA; ${ }^{3}$ Harvard University, Boston, MA; ${ }^{4}$ Masssachusettes General Hospital, Boston, MA. (Control ID \#2468373)

BACKGROUND: Guidelines recommend that women in their 40's consider individual risk and personal values in deciding the age at which to begin breast cancer screening However, it is unknown whether a decision aid (DA) that includes tailored risk information for younger women will increase the quality of decision making and impact the age at which women choose to start mammography screening

METHODS: We conducted a RCT in 2014-2015 to evaluate a web based decision aid (DA) delivered within a clinical practice network compared to usual care on knowledge, decisional conflict, and mammography intentions. Women were eligible if aged 39 to 48 , enrolled in a participating primary care clinics (family practice, obstetrics and gynecology, and internal medicine), and had not had a previous mammogram. Recruitment took place in-clinic directly prior to the visit with randomization using concealed assignments occurring after obtainment of informed consent. A follow-up survey was conducted at 6 weeks. The DA tool was self-navigated by the patient, included risk estimates based on the NCI Breast Cancer Risk Assessment Tool, comparative risk information, value elicitation, and coaching to encourage shared decision making. Bivariate analysis was conducted to compare 3 pre-specified primary outcomes between groups; knowledge as measured on a 5 -item scale ( 0 -low to 5-high), decisional conflict as measured by the 16 item Decision Conflict Scale (DCS), and intended number of years before having a first mammogram. A $p$-value of 0.017 was considered significant. Exploratory analyses were conducted on outcomes of DC subdomains (Uncertainty, Informed, Values, Support, Effective Decision Making), anticipated regret, and breast cancer worry.

RESULTS: There were 204 participants enrolled in the study; DA group (102) and control group (102) with $54.9 \%(n=112)$ completing the follow-up survey. The median age at the time of the follow-up survey was 40.1 years (range 39-49), $48 \%$ were white, and $41 \%$ were black. Ninety-one percent $(91 \%, n=49)$ of women in the intervention group who completed the follow-up survey also completed the DA. At follow-up, knowledge (range 0-5) was greater in the DA vs. Control group (mean; difference, $95 \%$ CI). (3.07 vs. 2.52 , Diff: $0.57,95 \%$ CI: 0.14 to 0.98 ). A trend towards lower DCS scores was found in the DA vs. Control group (1.99 vs. 2.29 , Diff: $-0.30,95 \%$ CI: -0.63 to 0.38 ). There was no difference between groups in the intended number of years before having a first mammogram (2.6 vs. 1.6 , Diff: $1.0,95 \% \mathrm{CI}:-0.29$ to 2.31 ). In exploratory analyses, women in the DA group trended towards decreased DCS scores in the Values domain ( 2.08 vs. 2.43 , Diff: $-0.36,95 \% \mathrm{CI}$ : -0.73 to 0.01$)$ and Support domain (1.83 vs. 2.13, Diff:- $0.30,95 \% \mathrm{CI}:-0.62$ to 0.03 ). There was no difference in the other DCS subdomains. There was no difference between groups in anticipated regret ( 7 point scale from 1-low to 7-high) of not having a mammogram in your 40's and having cancer detected at a later date $(5.45$ vs. 5.70 , Diff: $-0.25,95 \% \mathrm{CI}$ : -0.97 to 0.46$)$ or having a mammogram in your 40's and facing unnecessary follow up tests or procedures: (3.47 vs. 3.28, Diff: $0.19,95 \% \mathrm{CI}:-0.55$ to 0.93 ). Breast cancer worry (1-low to 12 -high) did not differ between groups (5.42 vs. 5.07 , Diff: $0.36,95 \% \mathrm{CI}:-0.36$ to 1.07 ).
CONCLUSIONS: A web based DA tailored to individualized risk and delivered in a practice setting increased knowledge and trended towards a decrease in decisional conflict when compared to a control of usual care. This study supports the use of a risk based DA to improve the quality of decision making regarding mammography initiation among younger women.

\section{A RANDOMIZED TRIAL OF A BEHAVIORAL ECONOMIC INTERVENTION} TO DECREASE OVERUSE OF LOW-VALUE HEALTH SERVICES Jeff Kullgren $^{1}$; Erin Krupka ${ }^{2}$; Abigail A. Schachter ${ }^{2}$; Ariel Linden ${ }^{3}$; Jacquelyn Miller ${ }^{2}$; James Alford ${ }^{4}$; Julia Alder-Milstein ${ }^{2} .{ }^{1}$ Ann Arbor VA Healthcare System and University of Michigan, Ann Arbor, MI; ${ }^{2}$ University of Michigan, Ann Arbor, MI; ${ }^{3}$ Linden Consulting, Ann Arbor, MI; ${ }^{4}$ IHA, Ann Arbor, MI. (Control ID \#2469685)

BACKGROUND: Clinicians' decisions to order low-value health care services are typically made during clinical encounters, when their thinking can be rushed and susceptible to factors like patient demands. Inviting clinicians to commit to avoid ordering lowvalue services ahead of encounters when their thinking is often slower, and then providing point-of-care (POC) supports to promote adherence to this commitment, could potentially decrease such orders. The objective of this study was to test whether this behavioral economic strategy could decrease orders for low-value services for 3 common conditions. METHODS: We conducted a mixed-methods, stepped wedge cluster randomized trial in 3 internal medicine and 3 family medicine clinics of a private, multispecialty group practice. At the start of the control period, clinicians were shown Choosing Wisely ${ }^{\circledR}$ recommendations to avoid imaging for uncomplicated low back pain, imaging for uncomplicated headaches, and unnecessary antibiotics for acute sinusitis. At the start of the intervention period, clinicians who consented to study participation were invited to commit to following these recommendations by signing a document. Clinicians who made this commitment received POC reminders of their commitment, POC patient education handouts, and weekly emails with resources to improve communications with patients about low-value care. After the 6-month intervention period there was a 3-month follow-up period. The primary outcome was the difference between the intervention and control periods in the proportion of visits with orders for the targeted low-value services. Secondary outcomes were the difference between the intervention and control periods in the proportion of visits with potential substitute orders for each condition, and the difference between the follow-up and control periods in the proportion of visits with orders for the targeted low-value services. We estimated differences in proportions using linear mixed models with random effects for providers nested in practices, adjusted for patient characteristics, time, and diagnosis. During the follow-up period, we interviewed study clinicians about their experiences with the intervention.

RESULTS: Forty-five clinicians ( $85 \%$ of eligible clinicians) participated. All committed to following the 3 Choosing Wisely recommendations. The intervention was associated with a $1.4 \%$ decrease in the proportion of visits with orders for the targeted services $(95 \%$ $\mathrm{CI},-2.7$ to $-0.2 \% ; P=0.02$ ), but also a $1.7 \%$ increase in the proportion of visits with potential substitute orders ( $95 \% \mathrm{CI}, 0.2$ to $3.2 \% ; P=0.02)$. There was no significant difference in the proportion of visits with orders for the targeted services in the follow-up period compared to the control period. In interviews with 24 study clinicians, 14 felt the intervention changed their conversations with patients about low-value care and 10 felt the intervention changed their practice styles.

CONCLUSIONS: We found that a behavioral economic intervention that paired an opportunity to commit to following Choosing Wisely recommendations with supports to promote adherence to this commitment was highly acceptable to primary care clinicians in a private practice environment, and associated with fewer orders for targeted low-value services. However, to promote sustained improvements in value this strategy may need to be fully integrated into routine care as well as account for potential substitute services. 
A RANDOMIZED TRIAL OF WORK CONDITION INTERVENTIONS IN PRIMARY CARE: EFFECTS ON CLINICAL OUTCOMES Mark Linzer ${ }^{3}$; Sara Poplau $^{3}$; Roger Brown ${ }^{9}$; Ellie Grossman ${ }^{1}$; Anita B. Varkey ${ }^{4}$; Steven H. Yale ${ }^{6}$; Eric Williams $^{7}$; Lanis Hicks ${ }^{8}$; Jill Wallock ${ }^{4}$; Diane Kohnhorst ${ }^{5}$; Michael Barbouche ${ }^{2}$. ${ }^{1}$ Cambridge Health Alliance, Somerville, MA; ${ }^{2}$ Forward Health Group, Inc., Madison, WI; ${ }^{3}$ Hennepin County Medical Center, Minneapolis, MN; ${ }^{4}$ Loyola University Medical Center, Maywood, IL; ${ }^{5}$ Marshfield Clinic Research Foundation, Marshfield, WI; ${ }^{6}$ North Florida Regional Medical Center, Gainesville, FL; ${ }^{7}$ University of Alabama, Tuscaloosa, $\mathrm{AL} ;{ }^{8}$ University of Missouri, Columbia, MO; ${ }^{9}$ University of Wisconsin, Madison, WI. (Control ID \#2467873)

BACKGROUND: Primary care work conditions are associated with adverse clinician outcomes, including stress, burnout, and turnover. In previously published work (the Healthy Workplace Study), we showed that clinics that perform specific types of quality-improvement interventions can reduce provider burnout and improve clinician professional satisfaction. However, the relationship between work conditions and patient clinical outcomes remains elusive. In this analysis, we explored whether work condition interventions that lead to better clinician outcomes result in improved patient care.

METHODS: We performed a cluster randomized controlled trial of a work-life intervention at 34 clinics in the upper Midwest and New York City. At baseline, we collected information about work conditions, clinician and patient attitudes and satisfaction, and quality of care for select chronic diseases (diabetes and hypertension) and health-care maintenance. 'Intervention' clinics received the results of their baseline data, and were encouraged to take on quality-improvement projects related to these topics. We measured outcomes (similar to the data collected at baseline) at approximately 12 months after the intervention period began. Clinical quality outcomes of interest were: achieving blood pressure $<140 / 90$ (or $<135 / 80$ for patients with diabetes) or achieving HbAlc $\leq 7.5 \%$. Errors were defined as a lack of clinical action (as documented in the medical record) for standard quality indicators for patients with hypertension or diabetes, or a lack of attention to breast, cervical, or colorectal cancer screening. Multilevel regression analyses assessed the impact of work condition changes on clinician professional attitudes and satisfaction and patient clinical outcomes. Subgroup analyses assessed impact by intervention type Structural equation modeling (SEM) confirmed relationships between work conditions, clinician outcomes, and patient outcomes.

RESULTS: There were 165 clinicians (143 physicians and 22 nurse practitioners or physician assistants) and 1131 patients enrolled in the study. Of 888 patients who underwent chart audits for quality and errors at the start of the trial, 735 were available for auditing at the end of the trial (17.4\% attrition). Patient age and gender were similar across both arms, with mean age 65 years $(\mathrm{SD}=12.9)$ and $40 \%$ males for intervention clinics, and 64 years $(\mathrm{SD}=11.8)$ and $38 \%$ males for control. Clinicians were also similar in age and gender across both arms, with mean age 48.8 years (SD $=$ 9.09 ) with $47 \%$ males for intervention clinics, and 46.6 years $(\mathrm{SD}=8.53)$ with $52 \%$ males for control. At baseline, $59 \%$ of the patients in the intervention arm and $44 \%$ of those in the control arm had chart-review evidence of meeting at least one diabetes or hypertension quality measure. At follow-up, $86 \%$ in intervention arm and $85 \%$ in control arm had chart-review evidence of meeting at least one of these quality measures. At 1-year-follow-up, there were no differences in error reduction (19 vs $11 \%$; Odds Ratio [OR] of improvement 1.84, $95 \%$ Confidence Interval (CI) 0.70 $4.82, p=0.21)$ or clinical quality improvement ( $19 \%$ improved vs. $44 \%$; OR 0.62 , $95 \%$ CI $0.58-1.21, p=0.42$ ) between intervention and control clinics. The conceptual model linking work conditions, clinician outcomes, and error reduction showed significant relationships between work conditions and clinician outcomes $(p<0.05)$, and a trend toward a reduced rate of errors in providers with lower burnout (OR 1.39, $p=0.08)$.
CONCLUSIONS: Work-life interventions that improve clinician satisfaction and wellbeing do not necessarily reduce errors or improve quality. Longer and more focused interventions may be one way to produce meaningful improvements in patient care.

\section{A RANDOMIZED, CONTROLLED TRIAL OF ALTERNATIVE MESSAGES TO} INCREASE ENROLLMENT IN A HEALTHY FOOD PROGRAM AMONG INDIVIDUALS WITH DIABETES Anjali Gopalan ${ }^{1,2}$; Jithen Paramanund ${ }^{6}$; Pamela Shaw $^{4}$; Deepak Patel ${ }^{6}$; Joelle Friedman ${ }^{5}$; Christine Brophy ${ }^{3}$; Kevin G. Volpp ${ }^{2,5}$. ${ }^{1}$ Kaiser Permanente Northern California, Oakland, CA; ${ }^{2}$ Corporal Michael J. Crescenz VA Medical Center, Philadelphia, PA; ${ }^{3}$ Vitality USA, New York, NY; ${ }^{4}$ Perelman School of Medicine at the University of Pennsylvania, Philadelphia, PA; ${ }^{5}$ University of Pennsylvania, Philadelphia, PA; ${ }^{6}$ Discovery Vitality, Johannesburg, South Africa. (Control ID \#2464610)

BACKGROUND: Increasing healthy food consumption among people with diabetes is important for improving disease management and preventing adverse outcomes. However, enrollment in programs designed to promote healthy eating is often lower than desired. Vitality is a South Africa-based health promotion program with over 1 million members. One of the largest Vitality initiatives is the HealthyFood (HF) benefit, a tiered incentive program designed to encourage healthier eating by offering members monthly cash back payments for healthy food purchases. In this study, we compared the effectiveness of several types of diabetes-focused messaging at increasing HF enrollment amongst Vitality members with diabetes. We hypothesized that messages that were more tailored and relatable, as well as those that prompted immediate action, would result in increased enrollment.

METHODS: For this RCT, we identified adult members of Vitality with a 1) history of diabetes, 2) who had not signed up for $\mathrm{HF}$, and 3) who had previously registered on the Vitality website (a marker of Internet and email access). We then randomized these eligible members to one of five study arms: 1) non-active control arm (received no intervention), 2) a diabetes-specific message 3) a message with a recommendation of HF written from the perspective of another Vitality member with diabetes, 4) a message with a physician's recommendation for the program, or 5) the diabetes-specific message used in Arm 2 paired with an "enhanced active choice" to enroll or not enroll. In an "enhanced active choice", not only are readers asked to make an immediate choice (in this case, to enroll or not enroll), but the pros and cons associated with the preferred (in our case, enrollment) and non-preferred options are highlighted. Enrollment in the program was assessed 1 month following the first emailed message.

RESULTS: Three thousand three hundred ninety-six members were randomized to one of the five arms. Due to non-working email addresses and departures from the Vitality program, 3654 members were included in the analysis. All four intervention arms had higher rates of HF enrollment compared to the non-active control ( $p<0.0001$ for all comparisons) (Figure 1). Compared to those who received the physician's recommendation of HF (6.9 \%), those who received the recommendation of HF written from the perspective of another member with diabetes had significantly higher HF enrollment rates $(10 \%, p=0.04)$. The diabetes-specific message with the embedded "enhanced active choice" (12.5\%) had significantly higher enrollment rates than the diabetes-specific message alone $(7.6 \%, p=$ $0.002)$.

CONCLUSIONS: Messages focused on diabetes were effective at increasing enrollment in a healthy food program. The addition of an "enhanced active choice" embedded within a message significantly raised enrollment rates in this population of adults with diabetes. Targeted messaging, coupled with "enhanced active choice" opportunities, represents a low cost, but effective approach to nudging people with diabetes in the direction of better health. 


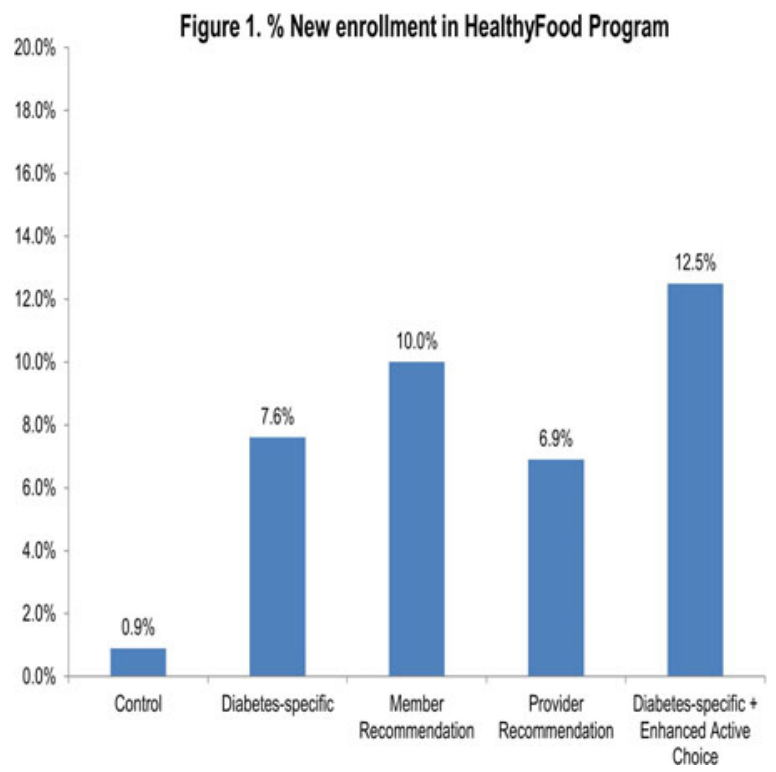

A RETROSPECTIVE ANALYSIS OF THE IMPACT OF WEIGHT LOSS ON RENAL FUNCTION Tiffany E. Schwasinger-Schmidt ${ }^{1}$; Georges Elhomsy ${ }^{3}$; Bobbie G. Paull-Forney ${ }^{2}$; Fanglong Dong ${ }^{4} .{ }^{1}$ University of Kansas School of Medicine, Andover, KS; ${ }^{2}$ Via Christi Weight Management Clinic, Wichita, KS; ${ }^{3}$ University of Kansas School of Medicine, Wichita, KS; ${ }^{4}$ Western University of Health Sciences, Pamona, KS. (Control ID \#2434693)

BACKGROUND: Approximately $66 \%$ of Americans are overweight with approximately half classified as obese. Obesity is associated with increased morbidity and mortality and is an independent risk factor for the progression of chronic kidney disease. Weight loss is correlated with improved renal function and reductions in obesity related glomerulonephropathy. This study investigated the effect of a medically supervised weight loss program on renal function among program participants at baseline and following 12 weeks of therapy.

METHODS: This study was a retrospective analysis of adult patients voluntarily enrolled in a physician-directed community-based weight management program from 2009 to 2014 Patients consumed at least $800 \mathrm{kcal}$ per day, attended weekly behavioral education classes, and expended approximately $300 \mathrm{kcal}$ per day in physical activity. The primary outcome of improved renal function was assessed by using statistical analysis to compare weight loss and GFR. Secondary outcomes included changes in fasting blood sugar, HbAlc, total cholesterol, LDL, HDL, triglycerides, systolic blood pressure, and the number of diabetic and anti-hypertensive medications.

RESULTS: A total of 71 patients with an average weight of $286 \mathrm{lb}$, BMI of 53, and baseline GFR of 29 (stage 4 chronic kidney disease) were included. Following 12 weeks of therapy, $80 \%$ of participants improved in stage, $19 \%$ remained within the same stage, and $1 \%$ progressed to a higher stage $(p=0.017$ ). Analysis revealed a positive correlation of 0.29 between weight loss and increased GFR $(p=0.029)$. Approximately $64 \%$ of patients required fewer antihypertensive medications and $83 \%$ of patients required fewer diabetic medications.

CONCLUSIONS: Organized weight loss programs are a viable treatment modality for the prevention of co-morbid disease progression. This study indicated a positive correlation between weight loss and improved renal function as evidenced by increased GFR, with the majority of participants exhibiting an improvement in chronic kidney disease stage. Analysis of secondary outcomes resulted in improvements in $\mathrm{HbAlc}$, blood pressure, LDL, total cholesterol, and triglycerides. The majority of patients within the study required fewer diabetic and antihypertensive medications following weight loss. When controlling for both diabetes and hypertension, the effect of improved renal function with weight loss persisted
A STAR BY ANY OTHER NAME: COMPARING RATINGS ACROSS FIVE POPULAR PHYSICIAN REVIEW SITES Joy L. Lee ${ }^{1}$; James Colbert ${ }^{4}$; Zackary Berger $^{2}$; Albert W. Wu ${ }^{3} .{ }^{1}$ Johns Hopkins Bloomberg School of Public Health, Baltimore, MD; ${ }^{2}$ Johns Hopkins School of Medicine, Baltimore, MD; ${ }^{3}$ Johns Hopkins University, Baltimore, MD; ${ }^{4}$ Newton-Wellesley Hospital, Newton, MA. (Control ID \#2470287)

BACKGROUND: A number of consumer-directed physician rating websites have proliferated in recent years in order to provider consumers with relevant information on physician quality. Yet there is little guidance for patients and physicians on how to interpret these rating; little is known about how these sites compare with one another. This study sought to compare ratings for a sample of physicians across 5 popular rating websites to assess consistency in ratings and users across sites.

METHODS: A cross sectional content analysis of physician rating sites. A sample of the 20 most-reviewed physicians on Yelp in three specialties (internal medicine, gastroenterology, OB-GYN) and three large metropolitan areas (Los Angeles, New York, Chicago) was compiled in January, 2016. Ratings for the 177-physician sample was abstracted from Yelp, Healthgrades, Vitals, ZocDoc, and DrScore. Key site features were also noted. Descriptive statistics and hypothesis testing were performed to compare ratings across sites.

RESULTS: Table 1 describes a few distinguishing features of the 5 sites. The visibility of the ratings differed across sites. When a specific physician is searched online (i.e. Googled), Yelp, Healthgrades, and ZocDoc all display ratings in the search results while Vitals and DrScore require consumers to click on the particular physician's page for the specific information. The analysis also revealed several inconsistencies across sites. The percentage of Yelp's most reviewed physicians not listed on other sites ranged from 1 to $12 \%$. For those listed, nearly half of Yelp's most reviewed physicians had no ratings on ZocDoc and DrScore (48 and $47 \%$, respectively). The physicians in the sample tended to have high ratings but there was variability in the ratings and number of raters across sites and geographic regions (Table 2). In two out of three regions (New York and Chicago), there were statistically significant differences in the average ratings of the same physicians across the 5 sites. Physician ratings on ZocDoc, for example, were significantly higher than those of the other sites in each region. Significant differences were also noted in the number of reviews physicians received across sites-DrScore was the least used, with an average of 1 rating for each physician in our sample, in all three regions. In contrast, both Vitals and ZocDoc had higher averages - and both sites included physicians who had thousands of reviews.

CONCLUSIONS: Conclusions: This analysis revealed inconsistencies across 5 popular consumer-directed physician rating sites and the need for more patient guidance on physician rating systems. These inconsistencies may be driven by the distinguishing characteristics of each site.

Review Site Characteristics

\begin{tabular}{|c|c|c|c|c|}
\hline $\begin{array}{l}\text { Yelp } \\
\text { - Popular } \\
\text { consumer site } \\
\text { with reviews } \\
\text { for many } \\
\text { businesses, not } \\
\text { just healthcare } \\
\text { - Both } \\
\text { consumers and } \\
\text { businesses can } \\
\text { add a listing to } \\
\text { Yelp } \\
\text { - Each review } \\
\text { includes rating } \\
\text { and comments }\end{array}$ & $\begin{array}{l}\text { Healthgrades } \\
\text { - Healthcare- } \\
\text { specific site with } \\
\text { comprehensive list } \\
\text { of physicians } \\
\text { - Overall rating } \\
\text { derived from } \\
\text { ratings on } 8 \\
\text { domains } \\
\text { - Majority of } \\
\text { reviews only } \\
\text { include } \\
\text { rating - only a } \\
\text { few has comments }\end{array}$ & $\begin{array}{l}\text { Vitals } \\
\text { - Healthcare- } \\
\text { specific site with } \\
\text { comprehensive list } \\
\text { of physicians } \\
\text { - Overall rating } \\
\text { driven by ratings } \\
\text { on } 8 \text { domains } \\
\text { - Some ratings } \\
\text { come from 3rd } \\
\text { party sites such as } \\
\text { DrScore and } \\
\text { PatientFusion }\end{array}$ & $\begin{array}{l}\text { ZocDoc } \\
\text { • Online } \\
\text { medical } \\
\text { scheduling } \\
\text { service } \\
\text { • Many } \\
\text { physicians are } \\
\text { listed, but only } \\
\text { those who use } \\
\text { ZocDoc have } \\
\text { patient reviews } \\
\text { • Patients are } \\
\text { invited to } \\
\text { review via } \\
\text { email after each } \\
\text { visit- so each } \\
\text { patient may } \\
\text { leave multiple } \\
\text { reviews }\end{array}$ & $\begin{array}{l}\text { DrScore } \\
\text { • Healthcare- } \\
\text { specific site } \\
\text { - Overall } \\
\text { rating } \\
\text { derived from } \\
\text { ratings on } 4 \\
\text { domains } \\
\text { - Uses a } \\
\text { 10-point } \\
\text { scale } \\
\text { - No } \\
\text { comment } \\
\text { function in } \\
\text { the reviews }\end{array}$ \\
\hline
\end{tabular}


Average Ratings and Average Number of Raters

\begin{tabular}{lllll}
\hline \hline \multirow{4}{*}{ Ratings } & Los Angeles $(n=60)$ & New York $(n=60)$ & Chicago $(n=57)$ \\
& Yelp & $4.40(0.54)$ & $3.84(0.72)$ & $4.00(0.68)$ \\
& Healthgrades & $4.22(0.55)$ & $4.55(3.82)$ & $4.23(0.59)$ \\
& Vitals & $4.35(0.70)$ & $4.16(0.61)$ & $4.26(0.65)$ \\
& ZocDoc & $4.83(0.19)$ & $4.72(0.24)$ & $4.73(0.33)$ \\
& DrScore & $3.88(1.22)$ & $4.00(1.47)$ & $4.06(1.29)$ \\
Raters & & $<0.001$ & 0.01 \\
& Yelp & $47(23)$ & $22(15)$ & $19(16)$ \\
& Healthgrades & $21(17)$ & $24(15)$ & $16(11)$ \\
& Vitals & $81(236)$ & $40(129)$ & $20(29)$ \\
& ZocDoc & $28(76)$ & $40(129)$ & $20(29)$ \\
& DrScore & $1(2)$ & $1(2)$ & $1(2)$ \\
& $<0.001$ & $<0.001$ & $<0.001$ \\
\hline
\end{tabular}

A SUSTAINABLE LONGITUDINAL STANDARDIZED PATIENT PROGRAM: A QUALITATIVE STUDY OF MEDICAL STUDENTS, FACULTY, AND STANDARDIZED PATIENTS Lauren Block ${ }^{1}$; Rivkah Darabaner ${ }^{1}$; Andrzej Kozikowski ${ }^{2}$. ${ }^{1}$ Johns Hopkins University School of Medicine, Baltimore, MD; ${ }^{2}$ Northwell Health, Manhasset, NY. (Control ID \#2469616)

BACKGROUND: Longitudinal standardized patient (SP) experiences have been described as a means to provide structured opportunities for practice, assessment, and feedback in clinical skills while cultivating an appreciation for continuity of care. The role of LSP experiences in building self-efficacy among students has not been explored. We sought to characterize student, faculty, and SP perceptions of a LSP program in terms of continuity, relationship-building, and feedback.

METHODS: A longitudinal standardized patient (LSP) model was adapted use with first and second year medical students. Students encountered two standardized patient cases portrayed by different actors six times each over 2 years. Student-SP and student-faculty pairings varied by encounter. During that time, the two LSP cases each had a consistent character with an evolving medical and social history. Faculty and SPs provided assessment and feedback with the medical students in real time. We conducted focus groups with 16 students, 10 SPs, and 8 faculty who participated in this model and analyzed results qualitatively using a program evaluation approach

RESULTS: Nine to ten themes emerged from focus groups with each stakeholder group, with considerable overlap between groups. Common themes included the perception that the LSP program facilitated a focus on interval history taking and development of key communication skills such as breaking bad news. Faculty, SPs, and students noted that changing student-SP pairings at each visit was an obstacle to relationship-building and decreased comprehensiveness of feedback. Students expressed that the ability to practice skills with SPs was extremely important for their competency development, and they acknowledged the challenge of carrying information forward across visits. Students requested framing by faculty in how to approach these cases, and felt their own involvement in encouraging health behavior change in the characters, for example recovery from alcohol abuse, might strengthen the experience. Students recommended a more comprehensive door chart to facilitate recall of patient history. SPs felt wedded to their characters and empowered to advocate for changes to make the story line more realistic. SP suggested improving fidelity by pairing students with demographically similar actors (e.g. age) for each longitudinal SP visit. Faculty noted summative assessment sometimes precluded the opportunity to mentor students. Focusing faculty effort in these encounters on coaching rather than summative assessment was recommended as a way to strengthen the mentoring potential of the experience.

CONCLUSIONS: The LSP experience facilitated mastery experiences, role-modeling, and feedback, suggesting a role for self-efficacy in development of medical student competency in continuity of care. Framing goals and learning objectives of a LSP experience may help prepare students for such an experience. Continuity pairings of students, SPs, and faculty in LSP experiences are preferable to build relationships where feasible. Faculty and SPs have complimentary roles in feedback and assessment in a clinical skills arena. Interventions to facilitate familiarity by allowing students to review the longitudinal patient's "chart" and to encourage students to coach patients through behavior change might improve the authenticity of the experience.

A SYSTEMATIC REVIEW OF THE TYPES AND CAUSES OF PRESCRIBING ERRORS GENERATED FROM USING COMPUTERIZED PROVIDER ORDER ENTRY SYSTEMS IN BOTH PRIMARY AND SECONDARY CARE Clare L. Brown $^{3}$; Helen Mulcaster ${ }^{1}$; Katherine Triffitt ${ }^{1}$; Andrew K. Husband ${ }^{1}$; Sarah P. Slight ${ }^{2}$. ${ }^{1}$ Durham University, Teesside, United Kingdom; ${ }^{2}$ Durham University, Stockton on Tees, United Kingdom; ${ }^{3}$ University of Durham, Sunderland, United Kingdom. (Control ID \#2457364)

BACKGROUND: Computerized Provider Order Entry (CPOE) systems are used to order medicines electronically for patients. A range of potential benefits support the use of CPOE systems with clinical decision support, such as improved patient safety and potential cost savings. However, there have also been reports that CPOE systems contributed to new types of medication errors. This review aims to identify the types and causes of prescribing errors associated with the use of CPOE systems.

METHODS: We performed a systematic review of the literature. Eligibility criteria included studies that discussed the types of prescribing errors that were generated from using a CPOE system and their underlying causes. The search included English language articles that were published since 2004. All clinical settings and types of CPOE systems were included. Non peer-reviewed publications, editorials and commentaries were excluded. Three large databases, the Cumulative Index Nursing and Allied Health Literature (CINAHL), Embase and Medline were searched. Broad search terms related to ' $\mathrm{CPOE}$ ', 'Electronic Prescribing' and 'Errors' were used. Duplicate articles were removed. Three authors (CLB, HLT, and KLM) independently reviewed the titles; abstracts and full texts were then reviewed independently by two authors (HLT and KLM) with another author (CLB) reviewing all abstracts and full texts, and thus acting as a constant across all publications. Disagreements were resolved by discussion with a fourth author (SPS). Data was extracted onto a customised data extraction sheet and a narrative synthesis of all eligible studies was undertaken.

RESULTS: The search returned 1185 publications; after reviewing titles, abstracts and full texts, 1128 were excluded. A total of 55 publications were included comprising of 49 full texts and 5 abstracts. Errors were found to occur throughout the prescribing process including: wrong patient, drug, dose, strength, formulation, route, frequency and unintentional treatment omission. Design features of the CPOE system contributed to different types of prescribing errors: (1) miss-selection from drop-down menus, particularly from alphabetical lists; (2) screen layout, such as fragmented order screens where clinicians used multiple screens to prescribe or where the patient's full medication list was not displayed; and (3) default settings, such as in-built order sentences, which led to unintentional doses or drugs being prescribed. Causes of prescribing errors included a reduced scrutiny of electronic orders, the generation of inappropriate clinical decision support alerts, and an incorrect assumption that refill prescriptions were correct. Technical issues, such as a slowness in system speed, also resulted in excessive clicking and an increased risk of miss-selection. Inconvenient login processes discouraged some prescribers from working under their own account and resulted in some clinicians unintentionally prescribing for the wrong patient. Clinicians also ran the risk of becoming more reliant on $\mathrm{CPOE}$ systems over time.

CONCLUSIONS: This review outlines the main prescribing errors that occurred with the use of CPOE systems. Although many errors, for example wrong dosage and prescribing 
for the wrong patient, may occur with handwritten prescriptions, the mechanisms by which these occur in electronic systems differ. The design of the system should be carefully considered and prescriber training surrounding proper use of these systems conducted to safeguard against future prescribing errors.

A TEAM-BASED APPROACH TO POPULATION MANAGEMENT OF DIABETIC PATIENTS IN AN URBAN RESIDENCY CLINIC Swathi Sangli²; Joseph M. Wiener ${ }^{1}$; Rupa Iyengar ${ }^{2}$; Iazsmin Ventura ${ }^{1}$; Shaun Noronha ${ }^{2}$; Adedapo Iluyomade ${ }^{2}$; Suma Chandrasekaran ${ }^{2} .{ }^{1}$ Ryan Chelsea Clinic, New york city, NY; ${ }^{2}$ St. Luke's-Roosevelt Hospital Center, New York, NY. (Control ID \#2469603)

BACKGROUND: In New York State, an estimated 1.6 million people live with Diabetes Mellitus. This epidemic is concentrated in vulnerable populations, including low-income urban minority groups where the diabetes prevalence is $16-18 \%$. The Department of Health has launched a major initiative to improve glycemic control in this vulnerable population, including an explicit goal to reduce the portion of patients with $\mathrm{HbA} 1 \mathrm{C}$ levels over $9 \%$. However, the best approach to achieving this goal remains to be determined. As part of a longitudinal quality improvement curriculum, residents of our training program studied the diabetic population they serve in their primary care clinic and designed a teambased intervention to improve glycemic control.

METHODS: Our prospective study took place in a community-based health center in New York City. Starting in June 2014, a disease registry was created, consisting of patients with at least one $\mathrm{HbA} 1 \mathrm{C}$ level greater than $9.0 \%$ during the preceding 12 -month period. A multi-pronged, team-based intervention was designed to improve patient engagement in diabetes care. Key elements included: (a) Medical Assistants called patients to prompt them to keep appointments and obtain missing labs; (b) a Nurse Care Manager called patients in between visits to reinforce a diabetes care plan; and (c) Resident Physicians led daily huddles in which they coordinated care for diabetic patients in the registry. After 18 months of follow-up, patient charts were again reviewed in December 2015. The primary outcome measured was a decrease in $\mathrm{HbAl}$ c level below $9.0 \%$. A paired $t$-test was used to assess for an association between the combined intervention and primary outcome. A one-sample t-test was used to assess the association between the combined intervention and secondary outcomes.

RESULTS: Fifty-four patients were identified with HbA1C levels above $9 \%$. Of the 54 patients enrolled, 39 (72.2\%) remained engaged in care at the end of the study period and completed all bloodwork. Baseline HbA1C levels ranged from 9.1 to $16.6 \%$, with a mean $\mathrm{HbA1C}=10.6 \%$ (SD 1.6). With respect to the primary outcome, 24 patients (62\%) achieved an HbA1C level below $9.0 \%$ within 18 months $(p<0.001)$. Average HbA1C for the total population improved by $1.55 \%$ (SD 2.31) $(p<0.001)$. A subset of 19 patients $(49 \%)$ showed HbA1C improvements of $2 \%$ or more $(p<0.001)$.

CONCLUSIONS: Our team-based approach to population management achieved significant improvement in glycemic control for a group of high-risk patients with diabetes. At baseline, the patients enrolled in this study were characterized by long-standing hyperglycemia, poor medical follow-up and limited responses to traditional methods of care. After a year and a half, these patients were more engaged in care and exhibited a clinically profound improvement in glycemic control. Key elements of the intervention included the use of a disease registry to monitor the progress of the study population, appropriate outreach to reinforce patient follow-up, team-based efforts to preserve continuous engagement in care, and regular updates to the medical team. These methods were feasible and effective in an urban residency training clinic and likely reproducible to similar settings. It remains to be determined how best to help those patients who did not respond to the team-based methods introduced in this study. Future work is warranted to understand how to serve this subset of patients better

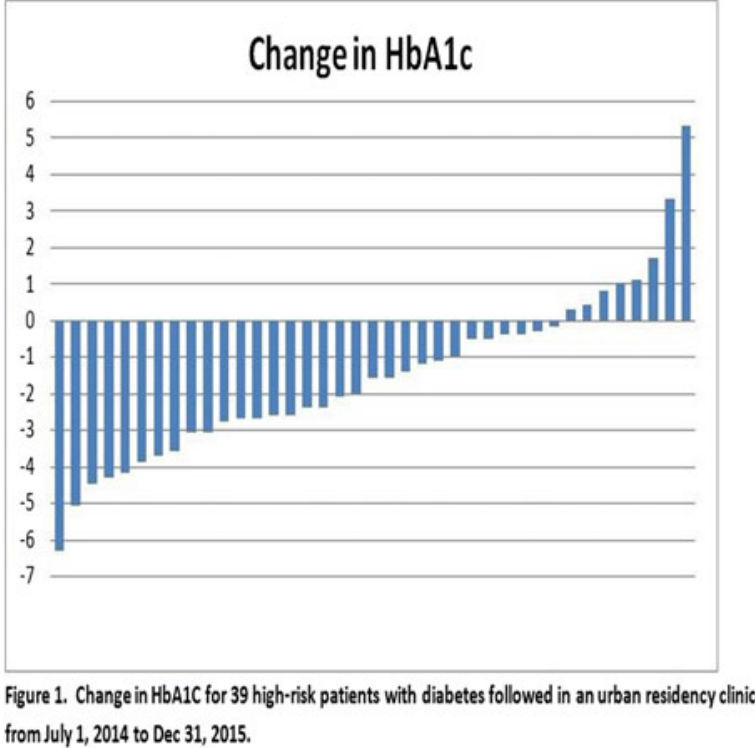

A WATSON GENERATED PROBLEM LIST: RESTORING ORDER TO THE EMR ENTROPY? Neil Mehta ${ }^{3}$; John E. Jelovsek ${ }^{2}$; Julie Tebo ${ }^{2}$; Murthy Devarakonda ${ }^{3}$; Jennifer Liang ${ }^{3}$; Ching-huei Tsou ${ }^{3}$; Charles Steiner ${ }^{1}$; Amy S. Nowacki ${ }^{1}{ }^{1}$ Cleveland Clinic, Cleveland, $\mathrm{OH} ;{ }^{2}$ Cleveland Clinic Lerner College of Medicine, Cleveland, OH; ${ }^{3}$ IBM, New York, NY. (Control ID \#2459677)

BACKGROUND: Despite the potential to improve our healthcare system, Electronic Medical Records (EMRs) have failed to significantly improve patient outcomes Physicians struggle to assimilate vast amounts of data, and continue to report workflow disruptions and decreased productivity using EMR systems. A simple but key function of any medical record is to present a comprehensive problem list that summarizes a patient's medical conditions. Existing EMR systems allow for manua creation and maintenance of such problem lists but often these lists are inaccurate or incomplete particularly when managed in a multi-provider health system. Cognitive computing systems based on natural language processing, information retrieval, knowledge representation, and machine learning have the potential to improve the use of EMRs by automatically generating such lists for clinician review. We hypothesized that clinicians would perceive the such automatically generated problem lists as more useful than the manually-entered problem lists. To study this, we compared 1) manually-entered problem lists from an integrated health care EMR system to 2) lists automatically generated by the IBM Watson cognitive computing system and to 3) lists generated by individual physicians participating in this study.

METHODS: We selected EMRs from 17 randomly selected, de-identified patients from a multi-provider large integrated healthcare system and analyzed them using IBM Watson. Each selected EMR was required to have a minimum of 3 encounters and 200 clinical notes to ensure sufficient data for analysis. We invited 10 internal medicine attending physicians and senior residents to each review 5 randomly selected EMRs out of these 17 records. For each patient record, physicians were asked to first, review the record in the healthcare system's current EMR system and create a problem list (P) as they would for a comprehensive health assessment. Participants then sequentially reviewed the existing manually entered problem list in the EMR (E) and then the IBM Watson generated problem list (W). Each participant was then asked to evaluate the accuracy and clinical significance of each Watson generated problem and to rate the overall usefulness of each of three problem lists $(\mathrm{P}$, $\mathrm{W}$, and $\mathrm{E}$ ) on a 10 point Likert scale. Finally, participants identified whether Watson 
generated any problems that physician missed but were deemed clinically important by them, as rated on a 4-point scale. The full patient record in the institution's current EMR system was available to the participating physicians as a reference source during all parts of this study. Pairwise comparisons among the ratings of the three list types were performed using Wilcoxon signed-rank test (Figure).

RESULTS: Ten physicians were approached for the study, 5 attending physicians completed review of all 5 of their assigned EMRs, 1 senior resident physician completed 2 of the 5 assigned EMRs, and the remaining 4 senior residents did not complete any review. As expected, physicians rated their own lists (P) higher than $\mathrm{W}$ and E. However, participants rated W higher than E (mean (standard deviation) Likert scale scores of 8.4 (1.2), 7.4 (1.6) and 5.8 (2.5), respectively). All comparisons between the three groups (P-W: $p=0.005$; P-E: $p<0.0001$ and W-E: $p=$ 0.02 ) were significant. Out of the 15 records, when compared to $\mathrm{E}, \mathrm{W}$ was rated higher in 10 cases, the same in 2 cases, and lower in 3 cases. In $89 \%(24 / 27)$ assessments, Watson identified at least one problem that the physicians missed and was rated by them as either 'very important' or 'important.'

CONCLUSIONS: Physicians rated the IBM Watson problem list more useful than an existing manually entered problem list in an institution's EMR. This pilot study demonstrates that cognitive computing systems may complement physicians' efforts by assimilating important problems efficiently and identify existing problems that may be missed. Of note, IBM Watson is able to create this summary by analyzing both the unstructured data in the clinical notes and the discrete data like medication lists and laboratory values. These factors highlight the potential of cognitive computing systems to improve the quality of patient care by providing efficient and accurate summarization of patient records, creating a foundation for effective clinical decision support and supporting efforts in quality improvement and research.

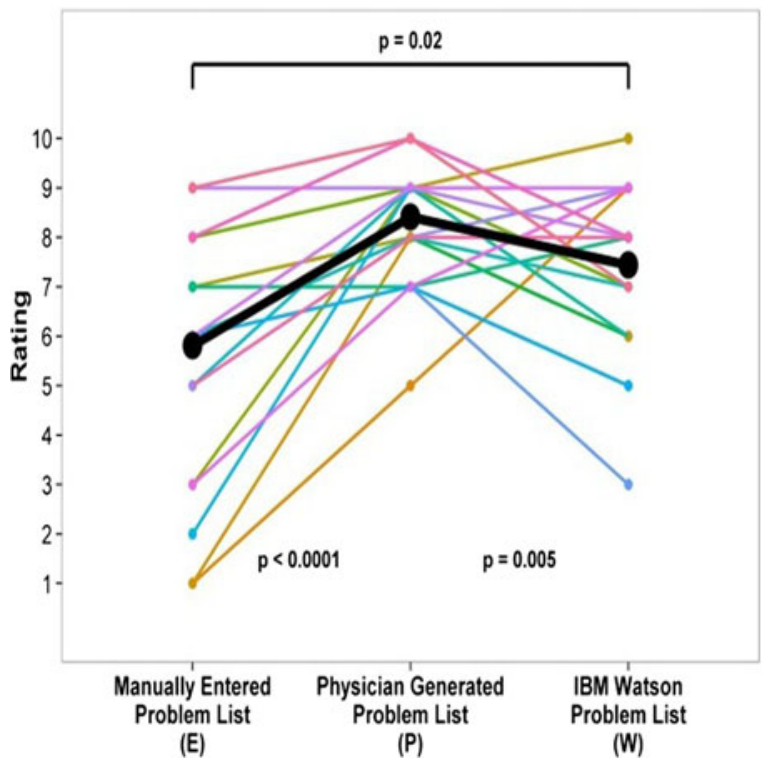

Each colored line represents a single patient record. Each point on the line represents the rating of that particular problem list for that record. The large black points represent the average rating of all patient records for each of the three problem lists. Higher ratings represent lists that are more useful.

ACCESS TO AND SUCCESS WITH NEW HEPATITIS C THERAPY IN AN URBAN PRIMARY CARE CENTER Lesley Miller ${ }^{1}$; Kristi Quairoli ${ }^{2}$; Jennifer Lom ${ }^{1}$; Shelly-Ann Fluker ${ }^{1} .{ }^{1}$ Emory University School of Medicine, Atlanta, GA; ${ }^{2}$ Grady Health System, Atlanta, GA. (Control ID \#2469578)
BACKGROUND: Chronic hepatitis $\mathrm{C}$ is prevalent in primary care practices. While curable, it remains a leading cause of liver cancer, liver transplant and death from liver disease in the United States. Earlier generations of antiviral therapy had suboptimal cure rates, were difficult to tolerate and difficult to access, especially for underserved patients. The introduction of new second generation direct-acting antivirals (DAAs) for hepatitis C has substantially improved both tolerability and cure rates. Access to care remains an issue, but is achievable with new DAAs combined with innovative care models involving generalists. Grady Health System (Atlanta, GA, USA) houses a unique hepatitis $\mathrm{C}$ treatment venue, the primary care-based Grady Liver Clinic (GLC). The GLC provides needed access to curative therapy for a largely African American (AA), uninsured population. We present data from a successful generalist-run program aimed at introducing new DAAs to the GLC population.

METHODS: We conducted a retrospective observational study of all GLC patients who started a sofosbuvir-containing DAA regimen between February 1, 2014 and February 28, 2015. Patients' charts were abstracted for demographics, liver-related characteristics, DAA regimen, and response rates to treatment. Patients who achieved an SVR12 (sustained virologic response 12 weeks after treatment completion) were considered cured. An intention-to-treat analysis was performed.

RESULTS: Ninety-five patients initiated treatment during the 13-month study period, or 7.3 patients/month. By comparison, only 76 patients initiated treatment with first generation DAAs in the 30-month period prior, or 2.5 per month. Eighty-three percent were AA, $84 \%$ were genotype 1, $24 \%$ were treatment experienced, and $74 \%$ (of 72 patients with data available) had advanced fibrosis or cirrhosis. Eighty-three percent of the patients received an all-oral regimen. All 95 patients reached an SVR12 time point and 79 patients (83\%) were cured. Of the remaining 16 patients, 12 failed treatment and 4 did not complete testing to determine SVR12. Of the treatment failures, most patients relapsed (75\%), one patient was non-compliant and one patient stopped therapy due to adverse effects.

CONCLUSIONS: In this underserved, primarily AA population with hepatitis $\mathrm{C}$, many patients accessed, initiated and completed new DAA therapy. Despite a real-world setting with high rates of advanced fibrosis and prior treatment failure, cure rates were close to those reported in clinical trials. Hepatitis $\mathrm{C}$ treatment with new DAAs was feasible and successful in this primary care-based hepatitis C clinic. The GLC can serve as a model for other sites interested in improving access to hepatitis $\mathrm{C}$ care and treatment, especially in resource-limited settings.

ACCESS TO CARE FOR THE LESS ADVANTAGED: DIFFERENTIAL EXPERIENCES IN EUROPE AND THE UNITED STATES Jamie Ryan; Pamela Riley. The Commonwealth Fund, New York, NY. (Control ID \#2470351)

BACKGROUND: As the United States continues implementation of the sweeping health reforms inherent in the Affordable Care Act, it is important to continue to investigate the ability of all Americans to access quality care, not just those with the loudest voices. In the U.S., low-income adults experience substantial health and health care inequities when compared with adults with above-average income. In this study, we explore limitations to accessing quality care among the low-income in the U.S. and compare this with the experiences of low-income adults in France, Germany, and the U.K. to see whether different country policies may have an effect.

METHODS: Data come from the 2013 Commonwealth Fund International Health Policy Survey, which was conducted among nationally representative samples in 11 countries using a common questionnaire that was translated and adjusted for country-specific wording as needed. The 2013 survey was conducted by phone, February-June 2013. This analysis was restricted to the data from France, Germany, the United Kingdom, and the United States. This analysis used as its base the weighted samples from France $(n=1,406)$, Germany $(n=1,125)$, the U.K. $(n=1,000)$, and the U.S. $(n=2,002)$. (response rate of YY percent). We restricted comparisons to those reporting below-average income and those reporting above-average income. 
RESULTS: Adults age 19 and older with below average income are generally more likely than those with above average income to experience cost-related access problems, including having a medical problem but not going to a doctor; skipping a recommended test, treatment, or follow-up; or not filling a prescription or skipping a medication dose because of cost. This difference is most pronounced in the U.S., where half (49 \%) of lowincome adults reported such problems, compared with one-quarter (24\%) of high-income adults. There was no difference in the U.K. Low-income adults also face challenges physically accessing care. In the U.S., $29 \%$ of low-income adults reported ease in accessing care after hours, compared to $45 \%$ of high-income adults. There was no difference in after-hours access by income in France or Germany. Low-income adults were also generally less likely to report being able to get an appointment the same or next day when sick, ( 36 vs. $55 \%$ in the U.S.), though there was no difference by income in the U.K. When low-income adults are able to access the care they need, they are less likely to rate its quality as "excellent" or "very good" in France (59 vs. 66 \%), Germany (55 vs. $65 \%$ ), the U.K. (67 vs. $73 \%$ ), and the U.S. (62 vs. $81 \%$ ).

CONCLUSIONS: Low-income adults are generally more likely than higher-income adults to encounter cost-related access problems and difficulty getting appointments when they need them, and less likely to rate the care they do receive as "excellent" or "very good." France provides for either low or no cost sharing for treatments that fall within care plans for chronically ill patients. Germany limits out-of-pocket spending relative to income, with lower thresholds for sicker patients. Countries whose health systems operate on a "National Health Service" modelsuch as the United Kingdom, allow for lower administrative costs than other countries. The U.S. could learn a lot from these countries

ACCESS TO THE VA: WHICH VETERANS ARE SEEKING PRIMARY CARE SERVICES? Matthew R. Augustine ${ }^{1}$; Karin M. Nelson ${ }^{1}$; Stephan D. Fihn ${ }^{1,2}$; Edwin Wong ${ }^{1} .{ }^{1}$ Department of Veterans Affairs, Seattle, WA; ${ }^{2}$ University of Washington, VA Puget Sound, Seattle, WA. (Control ID \#2468914)

BACKGROUND: Improving timely access to primary care services is a priority within the Veterans Health Administration (VHA) and is a key objective of the patient centered medical home (PCMH) model, the Patient Aligned Care Team (PACT) initiative. Although resources are being directed to increase availability of primary care services for all veterans; models of health behavior suggest that patients seek and access health services differently based upon demographic, health, and socioeconomic factors as well as previous healthcare use. Identifying characteristics of veterans who seek more timely care may help direct resources and improve quality and outcomes. This study aims to identify patient and health system characteristics of veterans who report seeking 5 different types of VHA health care services.

METHODS: In this observational study, we utilized data from the previously validated Consumer Assessment of Healthcare Providers and Systems Clinician \& Group - Patient Centered Medical Home (CAHPS-PCMH) survey that was administered to a nationally weighted, random sample of veterans who received outpatient care in fiscal year (FY) 2012. Five questions assessed whether veterans seek 5 different health services-general checkups, immediate care, and after hours care in addition to medical questions during or after office hours - in the last 12 months. For each access question, we used bivariate analyses to identify differences among patients who report seeking and not seeking these health care services. Using survey responses and VHA administrative data, we determined individual respondent characteristics as suggested by previous models of access - including demographics, health status, mental health diagnoses, barriers to access (distance to the nearest VA facility and copayment status), economic characteristics (county level poverty and unemployment rates), and previous healthcare use in FY2011 (primary care visits, phone visits, nurse visits, emergency department visits, and hospitalizations). Distance to clinic was categorized to less than 10 miles, 10 to less than 40 miles, and greater than 40 miles - the current definition for VA Choice qualification. We used multivariable logistic regression to identify characteristics associated with seeking health services, while accounting for clustering of observations by clinic. In all analyses, responses to questions were adjusted for survey response rates by clinic, gender, and age to reflect the national VHA population.

RESULTS: Veterans who completed one of the five CAPHS-PCMH questions were representative of the national VA population: mean age 62.4 years male $(92.7 \%)$, predominantly White $(70.4 \%)$, and majority with less than college degree $(80 \%)$. In the final multivariable analyses, veterans who were younger, diagnosed with PTSD, and used more primary care services in past year are more likely to seek all services. Veterans who are exempt from copayment, with higher co-morbidity, and visited in the ED in the last year were also more likely to report seeking immediate care, care after hours, and answers to questions during and after hours, yet less likely to report seeking a general checkup. Further, seeking a general checkup in last year was less likely among veterans who are male (OR 0.73, CI 0.63-0.86) with lower education (OR 0.64, CI 0.57-0.72) and who reside in a poverty area (OR 0.89 , CI $0.81-0.99)$. Veterans who are male (OR $0.79, \mathrm{CI}$ 0.69-0.90), have lower education (OR 0.76, CI 0.69-0.84) and reside in areas of higher unemployment (OR 0.98 per $1 \%$ increase, CI $0.97-1.00$ ) were less likely to call with questions during office hours. Although veterans who are less educated (OR 0.69, CI $0.58-0.83$ ), married (OR 0.85, CI 0.77-0.93), and live more than 40 miles away (OR 0.65, CI $0.51-0.84)$ were less likely to call with questions after hours, veterans who are male (OR 1.26, CI 1.03-1.53), of non-white race/ethnicity (Black/AA OR 1.59, CI 1.35-1.86; Hispanic/Latino OR 1.45, CI 1.16-1.80), and reside in areas of higher unemployment (OR 1.03 , CI $1.00-1.05)$ were more likely. Non-white veterans are more likely to report seeking immediate care (Black/AA OR 1.50, CI 1.37-1.64; Hispanic/Latino OR 1.33, CI 1.15-1.54) and care after hours (Black/AA OR 1.42, CI 1.28-1.57; Hispanic/Latino OR 1.31, CI 1.13-1.52). Seeking care after hours was less likely among veterans who live more than 10 miles and less than 40 miles from VA facility (OR 0.91, CI 0.84-0.98) and more likely among veterans living in poverty areas (OR 1.14, CI 1.05-1.24).

CONCLUSIONS: Veterans with different characteristics are seeking care differently. For example, not only are veterans with more co-morbidity and higher clinic use more likely to report seeking healthcare services, but age, race/ethnicity, socioeconomic factors, and fewer barriers to access, particularly co-pay exemption, show a preference for more immediate and after hours care. Directing delivery of these healthcare services within the context of the PACT initiative to these populations of veterans may improve timeliness and quality of care. Future studies are needed to evaluate how the VHA and PACT initiative are meeting the needs of these healthcare services and the impact upon healthcare outcomes.

\section{ACCOUNTING FOR CARE: PRIMARY CARE PROVIDER SATISFACTION WITH DELIVERY SYSTEM REFORM UNDER THE AFFORDABLE CARE ACT Rose Kleiman; Jamie Ryan. The Commonwealth Fund, New York, NY. (Control ID \#2469218)}

BACKGROUND: As the Affordable Care Act (ACA) seeks to promote new payment and delivery models that increase the quality and lower the costs of care, the accountable care organization (ACO) has become a leading model for the delivery of integrated care. This analysis examines the views and experiences of providers working in ACOs with recent changes in health care delivery, compared with providers who do not participate in an ACO model.

METHODS: Data come from the nationally representative Commonwealth Fund/Kaiser Family Foundation 2015 National Survey of Primary Care Providers. Survey responses were collected by mail and online between January 5 and March 30, 2015. The full sample 
consisted of 1624 primary care physicians (34\% response rate), 439 of whom reported that they are a part of an ACO. This analysis assesses opinions and experiences with the ACA and new delivery system reforms, changes in practice characteristics, and job satisfaction among primary care physicians participating with an ACO and those who do not work in an ACO

RESULTS: On several measures of practice capacity and patient interaction, providers working in ACOs were more likely to report improvement since 2014 than non-ACO providers. Twenty-five percent of ACO providers reported improvement in their ability to provide high quality care, compared with $18 \%$ of those not in an ACO. Twenty-three percent of ACO providers reported that their patients' satisfaction with care improved, compared with only $16 \%$ of non-ACO providers. Though few providers reported improvement in the amount of time they have to spend with each patient (12\% of those in ACOs, $8 \%$ of those not in ACOs), ACO providers were more likely than others to report sometimes or frequently discussing end-of-life issues (79 vs. $61 \%$ ), out-of-pocket costs ( 85 vs. $77 \%$ ), and how to obtain health insurance ( 52 vs. $39 \%$ ) with their patients. ACO providers were also more likely to report a favorable opinion of the ACA overall (55\%) compared with non-ACO providers (44\%). Nearly one-third (31\%) of ACO providers reported that the ACA has had a positive impact on their medical practice overall, compared with one in five (20\%) non-ACO providers. More than one-quarter of providers working in ACOs reported that the ACA has had a positive impact on the cost ( $27 \%$ ) and quality ( $27 \%$ ) of health care their patients receive, compared with non-ACO providers ( 18 and $14 \%$, respectively). Additionally, ACO providers were more likely than others to report a positive impact of individual components of the ACA, such as the increased use of medical homes ( 40 vs. $30 \%$ ), the increased use of health information technology (56 vs. $48 \%$ ), and Medicaid expansion (44 vs. $33 \%$ ). As far as provider satisfaction and burnout, ACO providers were no more likely to report dissatisfaction with their medical practice overall $(17 \%)$ or their income from their medical practice $(36 \%)$ than non-ACO providers ( 17 and $35 \%$, respectively). ACO providers were also somewhat less likely than others to report that recent trends in health care are causing them to consider retiring early ( 44 vs. $48 \%$ )

CONCLUSIONS: Primary care providers who reported working in an ACO were more likely to say that their medical practice improved since 2014 and that the ACA had a positive impact on their practices. These providers perform better on several measures of doctorpatient relationship, which is especially important as more ACO providers $(46 \%)$ reported an increase in the number of chronically ill patients they treat since 2014 than did non-ACO providers (33\%). Further analyses also indicate that working in an ACO is correlated with increasing numbers of medical assistants, case managers, and social workers, who may help lighten the load. ACOs enroll nearly 25 million patients. As physician organizations and health systems decide whether or not to take part in ACO arrangements, it is important to consider providers' views of changes in their practice quality and job satisfaction. The widespread transition to the ACO model is ongoing; even among physicians who participate in ACOs, one of four are still unsure of their impact on providers' ability to provide quality care to their patients. Many hope that ACOs will promote better, more integrated care while controlling costs, but physician buy-in is critical to successful implementation.

ACUTE CARE UTILIZATION IN PATIENTS WITH CONCURRENT BEHAVIORAL HEALTH AND COMPLEX CHRONIC MEDICAL CONDITIONS Karen Abernathy; Jingwen Zhang; Elisha L. Brownfield; Patrick D. Mauldin; William P. Moran; Kimberly S. Davis. Medical University of South Carolina, Charleston, SC. (Control ID \#2464056)

BACKGROUND: Patient-Centered Medical Homes (PCMH) are responsible for accessible care and coordination to reduce preventable ED visits, hospitalizations and rehospitalizations. At the population level, patients with both complex medical illness and behavioral health conditions (BHCs), including depression, have higher rates of acute care services utilization compared to patients with complex medical illness alone. We sought to assess the incremental association of BHCs on acute care utilization (emergency department use, hospitalization and re-hospitalization within 30 days) at the practice level for our level 3 certified PCMH population of approximately 12,000 patients.

METHODS: Using previously published risk stratification and clustering methodologies, we compared our PCMH patients by disease cluster with and without BHCs, and used multivariate regression with first order interactions to examine the association between complex medical illness and BHCs on acute care utilization at a University Hospital. Adult patients $\geq 18$ years of age were included for the study if they were seen at least twice in University Internal Medicine (UIM) primary care clinic from 10/1/2010 through 9/30/ 2013. Patients who died before $9 / 30 / 2013$ were excluded. Ten thousand four hundred eight patients met inclusion criteria. Acute care utilization was a cumulative count of ED visits, hospitalizations and re-hospitalizations.

RESULTS: Controlling for demographic and other significant covariates, there was a significant and positive association between aggregated acute care utilization and BHCs $(p<0.0001)$. Significant positive associations were also demonstrated for 3 independent disease clusters centering on COPD, renal disease, and the complex/CVD cluster of patients (all $p<0.0001$ ). First order interactions were then assessed between BHCs and disease clusters, and significantly positive interactions were revealed for BHCs and 2 independent clusters: COPD $(p<0.0001)$ and renal disease $(p<0.0001)$. Similar associations were seen for ED and re-hospitalization $(p<0.0001)$, but with no interaction. Other variables significantly associated with increased utilization included lower outpatient visit compliance, male gender, higher age, non-white race, and Medicare/Medicaid insurance. CONCLUSIONS: Although we cannot demonstrate causation, the significant association between acute care utilization and BHCs supports the need for co-located behavioral health care as a component of PCMH. Recognizing that many patients will only present to their primary care physician for behavioral health care, PCMH provides an opportunity for improving patient access to behavioral health care services in the primary care clinic, which could have a positive impact on utilization. A collaborative care approach utilizing integration of behavioral health care into the primary care clinic has been shown to be effective in improving outcomes, patient satisfaction, and quality of life and may lead to implementation of more effective ways to manage BHCs in complex patients. This study was limited to a single Academic clinical site, and measures of acute care were from a single hospital. We likely underestimate BHC prevalence (especially for depression) and acute care utilization, both of which are subject to ascertainment bias. Because there are no temporal relationships in the data, the study demonstrates association not causation.

\section{ADAPTING A POST-DISCHARGE INTERVENTION FOR HOSPITALIZED SMOKERS TO ENHANCE SCALABILITY: THE HELPING HAND 2 RANDOM-} IZED CONTROLLED TRIAL Nancy A. Rigotti ${ }^{2}$, 1; Hilary Tindle ${ }^{3,}$; ; Susan Regan ${ }^{2,1}$; Douglas Levy ${ }^{2,}$; Y Yuchiao Chang ${ }^{2,1}$; Kelly M. Carpenter ${ }^{4}$; Elyse R. Park ${ }^{2}$, ; Jennifer H. Kelley $^{2}$; Joanna Streck ${ }^{6}$; Zachary Reid ${ }^{2}$; Thomas Ylioja ${ }^{3}$; Michele Reyen ${ }^{2}$; Daniel E. Singer ${ }^{2}{ }^{1}$. ${ }^{1}$ Harvard Medical School, Boston, MA; ${ }^{2}$ Massachusetts General Hospital, Boston, MA; ${ }^{3}$ University of Pittsburgh, Pittsburgh, PA; ${ }^{4}$ Alere Wellbeing, Seattle, WA; ${ }^{5}$ Vanderbilt University, Nashville, TN; ${ }^{6}$ University of Vermont, Burlington, VT. (Control ID \#2456074)

BACKGROUND: Hospitalization provides a good opportunity for smokers to quit. To be effective, tobacco cessation interventions started in the hospital must continue after discharge, yet sustaining tobacco treatment in the transition from hospital to home is a challenge for health care systems. We previously demonstrated the effectiveness of a postdischarge tobacco treatment intervention in a single-site randomized controlled trial (RCT) (JAMA 2014;312:719). It used interactive voice response (IVR) telephone calls to remind smokers to quit and triaged smokers seeking counseling or medication support to hospital- 
based research staff. To improve scalability for future dissemination, we adapted this intervention to triage smokers directly from IVR calls to a telephone quitline, a nationallyavailable cessation resource, rather than to hospital staff.

METHODS: A 3-site RCT compared Sustained Care, a 3-month post-discharge tobacco treatment intervention, to Standard Care among hospitalized adult smokers who wanted to quit smoking and had received a tobacco treatment consultation in the hospital. Sustained Care participants received 5 automated telephone calls and their choice of free FDAapproved cessation medication for 90 days. Each automated call advised cessation, supported medication adherence, and triaged smokers seeking additional support, medication management or refills to a telephone quitline. Standard Care patients received only recommendations for post-discharge cessation medication and counseling from the inpatient smoking counselor. We assessed self-reported tobacco abstinence and tobacco cessation treatment use at 1,3 , and 6 months (mo) after discharge. The primary outcome was biochemically-confirmed past 7-day tobacco abstinence at 6 mo.

RESULTS: One thousand three hundred fifty-seven adult daily smokers admitted to 3 hospitals in MA and PA from December 2012-July 2014 were randomly assigned to Sustained Care $(n=680)$ or Standard Care $(n=677)$. Groups were comparable at baseline in demographic factors (mean age 50 years, $51 \%$ male, $72 \%$ nonhispanic white), medical and smoking history, primary discharge diagnosis, and length of stay. Survey completion was $81 \%$ (1 month), $77 \%$ (3 months), and $75 \%$ (6 months) and did not differ by group. Smokers who were offered Sustained Care, compared to those offered Standard Care, reported more use of pharmacotherapy (79 vs. $59 \%$ [1 month], 86 vs. $66 \%$ [3 months], both $p<.001$ ) and any counseling (18 vs. $12 \%$ [ 1 month], $p=.002 ; 30$ vs. $21 \%$ [3 months], $p<.001$ ) after discharge and had higher rates of self-reported past 7-day tobacco abstinence at 1 month (43 vs. $32 \%, p<.0001)$ and 3 months (37 vs. $30 \%, p=.008)$. At 6 months the difference between groups for self-reported past 7-day tobacco abstinence narrowed (31 vs. $27 \%$, $p=.09$ ) and biochemically-confirmed 7-day abstinence, the primary outcome, did not differ by group ( 17 vs. $16 \%, p=.58$ ). Counseling use post-discharge in the Sustained Care group was lower than in our previous trial that used a dedicated in-house counselor instead of referral to a quitline ( 18 vs. $37 \%$ [ 1 month], 30 vs. $58 \%$ [ 3 months])

CONCLUSIONS: An adapted version of a post-discharge tobacco treatment intervention for hospitalized adult smokers who planned to quit smoking increased self-reported tobacco abstinence rates for 3 months after hospital discharge compared to standard care, but the effect was not sustained at 6 months as it had been in our previous trial. The durability of the intervention effect may have been weakened by the modification made to increase its scalability. Future work should extend the intervention beyond 3 months and explore ways to improve patient engagement with state quitlines or other communitybased cessation resources that offer counseling support.

\section{ADDRESSING THE CHALLENGES OF PALLIATIVE CARE FOR HOMELESS} VETERANS Evelyn Hutt ${ }^{1,}{ }^{5}$; Jacqueline Jones ${ }^{2}$; Mary Weber $^{2}$; Karen Albright ${ }^{4,}$; Hannah R. Dischinger ${ }^{3}$; Thomas O'Toole ${ }^{7,8}$; Daniel Doan ${ }^{9}$; David Gruenewald ${ }^{9} .{ }^{1}$ Denver VAMC, Denver, CO; ${ }^{2}$ University of Colorado AMC, Aurora, CO; ${ }^{3}$ University of Colorado School of Medicine, Aurora, CO; ${ }^{4}$ University of Colorado, Anschutz Medical Campus, Aurora, CO; ${ }^{5}$ University of Colorado, Aurora, CO; ${ }^{6}$ University of Denver Denver, CO; ${ }^{7}$ Providence VAMC, Providence, RI; ${ }^{8}$ Brown University, Providence, RI; ${ }^{9}$ Seattle VAMC, Seattle, WA. (Control ID \#2470574)

BACKGROUND: Homeless adults in the United States are aging; those born between 1954 and 1965 face an elevated risk of homelessness, and in that cohort those who are homeless have health problems similar to the general population in their 70s and 80s: high rates of cardio-metabolic diseases and substance use complicated by cognitive deficits, visual and hearing impairments, urinary incontinence, mobility challenges, and the need for assistance with activities of daily living. The purpose of this study was to develop a deep and broad understanding of the issues, barriers and facilitators to excellent end of life (EOL) care for homeless veterans (HV) and define the key structural, clinical and policy elements required to improve that care. Approximately 50,000 Veterans are estimated to be homeless on any night. Veterans who are both homeless and at EOL do not fit readily into programs for homeless Veterans (HV) or those at end of life (EOL).

METHODS: Using a mixed methods sequential design, we surveyed Department of Veterans Affairs (VA) homeless and EOL programs. We then conducted key informant interviews and focus groups with chronically ill homeless veterans, multidisciplinary providers, and community and VA leadership at four geographically dispersed VA facilities. An iterative, inductive and deductive approach to qualitative analysis drew on field notes, memoing, qualitative content analysis, consultative and reflexive team analysis, and audit and member checking. Finally, a national Program and Policy Development Forum with representation from each of the focus groups and national leadership in homeless care and palliative care was convened to address the challenges uncovered, using Nominal Group Technique and a modified Delphi process

RESULTS: The email survey indicated that VAMCs treated an average of 9.4 homeless veterans at EOL annually. Key informant interviews with homeless Veterans with chronic illness $(n=33)$, decision makers in palliative care and homelessness inside and outside the VA $(n=21)$, and 10 focus groups with front line providers and middle managers emphasized: (1) Declining health militates against independent living or realistic plans to abstain, but housing options are too often limited to places that insist on functional independence and sobriety. (2) Pain management within the context of addiction, unstable housing and behavioral health problems is challenging. (3) Discontinuities within and between care systems restrict care delivery. (4) VA regulations pose significant challenges to collaboration with community providers. (5) Care providers for HV at EOL collaborate informally, but must compete for attention and resources with other pressing challenges. A National Policy and Program Development Forum brought together select focus group participants from each site with national VA palliative and homelessness care leadership to develop policies, collaborations, and programs to facilitate high quality EOL care for HV The Forum developed a series of educational priorities, policy and policy dissemination recommendations that are currently being finalized. Recommendations are likely to include (1) enhanced education of palliative care providers regarding homelessness, harm reduction, pain and symptom management in the setting of substance use disorders; (2) enhanced education of homeless care providers on the basics of primary palliative care; (3) priority for and greater flexibility of housing eligibility for HV at EOL; (4) collaborative development of best practices in palliative care for people with unstable housing.

CONCLUSIONS: Lack of housing suited to HV with rapidly declining health, and isolation of homeless and EOL providers from each other are among the most pressing challenges to care. A multifaceted approach to improving care will require more flexible housing criteria for homeless or formerly homeless Veterans who need palliative care; facilitation of ongoing informal communication and collaboration among VA and non-VA homeless and palliative care providers; and enhanced education of providers across systems and spectra of care.

\section{ADHERENCE TO AGE-/GENDER-SPECIFIC PREVENTIVE CARE PAT- TERNS IN ADOLESCENTS AND ADULTS WITH DOWN SYNDROME Kristin M. Jensen $^{3,}{ }^{2}$; Elizabeth J. Campagna ${ }^{2}$; Elizabeth Juarez-Colunga ${ }^{2}$ 2 ; Edward R. McCabe ${ }^{4}$; Allan V. Prochazka ${ }^{3,}$; Desmond K. Runyan ${ }^{3}$. ${ }^{1}$ Denver VAMC, Denver, CO; ${ }^{2}$ University of Colorado, Aurora, CO; ${ }^{3}$ University of Colorado School of Medicine, Aurora, CO; ${ }^{4}$ March of Dimes Foundation, White Plains, NY. (Control ID \#2468686)}

BACKGROUND: The life-expectancy for persons with Down syndrome (DS) has dramatically improved, such that many DS patients now have risks of typical age- and gender-specific health conditions in addition to their DS-specific comorbidities. 
Increasingly, their medical care is being provided by adult-focused providers who may be less familiar with the special health risks of DS. In this retrospective cohort study, we tested whether adolescents and adults with DS received similar patterns of age- and gender-specific preventive care compared to Medicaid controls without intellectual, physical, or psychological disabilities.

METHODS: Using longitudinal Medicaid billing data (2006-2010) in CA, CO, MI, and PA, we defined our cohorts from patients $\geq 12$ yo with $\geq 45 / 60$ months of enrollment in Medicaid who were not also enrolled in Medicare. DS cases were identified by ICD- $9=$ 758.0 for non-obstetric claims. We matched each DS case $(n=3,951)$ with 2 non-disabled controls $(n=7902)$ by duration of enrollment in Medicaid, urban/rural residence, race/ethnicity, year of birth, gender, and state of residence using propensity-score matching. We identified non-disabled controls among Medicaid beneficiaries without visits coded for intellectual, physical, or psychological disabilities as defined by Chien et al. (Pediatrics 2015) and Lin et al. (JIDR 2013). Adherence to age-/gender-specific preventive screening activities were compared between these cohorts over our 5 year study period with stratified conditional logistic models. Absolute change is defined as the difference in adherence rates between the DS cohort and the control cohort.

RESULTS: In this retrospective cohort study with age-matched cohorts, the median age was 23 years with $15 \%$ over 40 years old. Overall, the DS cohort had better vaccination patterns than controls. Adherence to recommended vaccinations was $<25 \%$ for both cohorts for all vaccinations except for $35 \%$ of the DS cohort receiving at least 1 influenza vaccination during the study period (Table). Adherence to preventive screening activities was remarkably low for both cohorts, exceeding $40 \%$ in only cholesterol (both), breast cancer (both), prostate cancer (DS), and cervical cancer (controls). We observed clinically similar screening patterns between cohorts for diabetes, breast cancer, and colorectal cancer. Lab-related preventive screenings for cholesterol and prostate cancer were markedly higher in the DS cohort, while cervical cancer screening was markedly lower in the DS cohort despite a small, but real, presence of sexually-transmitted infections in both populations (DS $1.3 \%$, Controls $6.4 \%, p<0.001)$.

CONCLUSIONS: We observed poor adherence to preventive care in both the DS and non-disabled cohorts over this 5 year study. Adherence was slightly better in the DS cohort than non-disabled controls in most domains with the notable exception of cervical cancer screening, suggesting a lack of gynecologic care in the DS population. This is concerning given the increased life-expectancy and increased engagement in all aspects of life among persons with DS. Further studies must evaluate ways to integrate age-/gender-specific preventive care with DS-specific care.

\begin{tabular}{|c|c|c|c|}
\hline Preventive Care Activity & $\begin{array}{c}\text { DS } \\
n=3,951\end{array}$ & $\begin{array}{l}\text { Controls } \\
n=7,902\end{array}$ & $\begin{array}{c}\text { Absolute } \\
\text { Change }\end{array}$ \\
\hline \multicolumn{4}{|l|}{ Vaccinations } \\
\hline Tdap, 12-18yo* & $21 \%$ & $17 \%$ & $4 \%$ \\
\hline HPV \#1, Females 12-18yo & $21 \%$ & $21 \%$ & $0 \%$ \\
\hline Meningococcal, 12-18yo* & $24 \%$ & $18 \%$ & $6 \%$ \\
\hline$\geq 1$ Influenza, all ages* & $35 \%$ & $14 \%$ & $20 \%$ \\
\hline \multicolumn{4}{|l|}{ Screening } \\
\hline Diabetes, all ages* & $36 \%$ & $32 \%$ & $3 \%$ \\
\hline Cholesterol, $\geq 18 \mathrm{yo}^{*}$ & $64 \%$ & $43 \%$ & $21 \%$ \\
\hline Prostate Cancer, $\geq 40 \mathrm{yo}^{*}$ & $52 \%$ & $30 \%$ & $22 \%$ \\
\hline Cervical Cancer, $\geq 21 y 0^{*}$ & $29 \%$ & $64 \%$ & $-35 \%$ \\
\hline Breast Cancer, $\geq 40$ yo & $43 \%$ & $47 \%$ & $-4 \%$ \\
\hline Colon Cancer, $\geq 50$ yo & $25 \%$ & $20 \%$ & $4 \%$ \\
\hline
\end{tabular}

* p-value $<0.05$

\section{ADHERENCE TO GUIDELINES IN PATIENTS WITH ACUTE PANCREATI-} TIS ! Hussam Sabbagh ${ }^{2}$; Sarwan Kumar ${ }^{1}$; MHD Hussam Al-Jandali ${ }^{1} .{ }^{1}$ Crittenton Hospital/Wayne state university, Rochester, MI; ${ }^{2}$ Wayne State University, Rochester Hills, MI. (Control ID \#2470304)

BACKGROUND: Acute pancreatitis is a frequent inpatient diagnosis that internists, gastroenterologists, and surgeons all confront. The most common causes are gallstones and heavy alcohol intake. Its management is typically straightforward: intravenous fluids, analgesia, and nothing by mouth. Several major gastroenterological and surgical societies have issued guidelines on how to manage acute pancreatitis, based on evidence from high-quality randomized trials and nonrandomized studies. Information is limited on how well physicians in the United States comply with these guidelines. A suspicion is raised that many US physicians are not following the guidelines either.

METHODS: The Plan Do Study Act (PDSA) cycle was the theoretical framework used in this project. Pre-intervention data was collected from patient charts through EMR. An 80 patients who were admitted to the hospital with a diagnosis of acute pancreatitis were analyzed. We looked into the percentage of patients who were assessed for the severity of acute pancreatitis, the percentage of patients who received adequate fluid resuscitation which is according to ACG guidelines is ranging between $(250-350 \mathrm{cc} / \mathrm{h})$ and the percentage of patients who had amylase and lipase levels ordered daily.

RESULTS: The data collected showed that $100 \%$ of the patients were not assessed initially or daily for the severity of their symptoms, almost $93 \%$ of the patients had amylase and lipase levels ordered daily and $96 \%$ of the patients did not receive adequate fluid resuscitation. This is a significant problem for the number of patients in this study and may mark a sizeable amount of financial loss to the admitting hospital in addition to the effect on the quality of care in patients admitted to the hospital with a diagnosis of acute pancreatitis. CONCLUSIONS: Understanding the benefits of adherence to guidelines in the management of acute pancreatitis is crucial not only due to the financial aspect of it on the hospital but also due to the significant effect on the quality of patient care and safety. Efforts should be made to increase the adherence to these guidelines in order to prevent adverse patient outcomes.

\section{ADHERENCE TO JNC 8 GUIDELINES IN PATIENTS WITH HYPERTENSION AND CHRONIC KIDNEY DISEASE AT AN URBAN AMBULATORY CARE} CLINIC Waleed Al-Darzi; Joyce Philip; Sanah Rana; Elsheikh Abdelrahim; Jeffrey P. Yanez. Henry Ford Health Systems, Detroit, MI. (Control ID \#2466812)

BACKGROUND: In December 2013, the Joint National Committee published new guidelines (JNC 8) regarding blood pressure goals and treatment. In patients with hypertension and chronic kidney disease (CKD), JNC 8 recommended a blood pressure goal of less than 140/90, which was relaxed from a previous blood pressure goal of less than 130/ 80 in JNC 7 (2003). JNC 8 also recommended the use of an ACEI or ARB in patients with $\mathrm{CKD}$ as primary therapy. Our aim is to evaluate adherence of patient treatment to national guidelines at an urban Internal Medicine residency continuity clinic.

METHODS: The Henry Ford Health System electronic database was used to create a retrospective cohort of patients seen between September 2014 and September 2015 in the ambulatory internal medicine residency continuity clinic. Patients with an allergy to ACEI/ARB, acute kidney injury, hyperkalemia or pregnant patients were excluded. Patients having a blood pressure of less than 140/90 and on ACEI/ARB were considered adherent to JNC 8 guidelines. We studied patient socio-demographic and medical conditions associated with likelihood of adherence to guidelines.

RESULTS: Five hundred ten patients were included in our study. Of these, $36.5 \%$ met the criteria for following the guidelines of having controlled blood pressure and being on ACEI/ARB, while $63.5 \%$ did not. Patients who have diabetes mellitus $(p<0.001)$ or congestive heart failure $(p=0.02)$ were more likely to be treated according to the 
guidelines. When compared to other races, treatment of African American patients was less likely to be adherent to JNC 8 guidelines $(p=0.002)$. Age, gender, number of visits to the ambulatory clinic, having coronary artery disease, or having end stage renal disease did not have a statistically significant influence on the likelihood of following JNC 8 guidelines. $33.5 \%(n=141)$ of African American patients were treated according to guidelines, while $50 \%(n=45)$ of other races combined were treated according to guidelines. $43.7 \%(n=115)$ of diabetic patients and $45.4 \%(n=54)$ of CHF patients who have hypertension and CKD were treated in adherence to the JNC8 recommendations. Average patients' age and number of clinic visits were about the same in both groups without a statistical significance.

CONCLUSIONS: In our resident based urban medical center, adherence to the JNC 8 guidelines was found to be suboptimal and there is room for improvement. Patients with diabetes and congestive heart failure were more likely to be treated according to the guidelines while African Americans were less likely to be treated according to the guidelines. Future studies can be done investigating why the majority of our patients were not treated according JNC 8 and how we can improve compliance to the JNC 8 guidelines.

ADHERENCE TO REMOTE PATIENT MONITORING AFTER DISCHARGE OF HOSPITALIZED HEART FAILURE PATIENTS: THE BETTER EFFECTIVENESS AFTER TRANSITION - HEART FAILURE (BEAT-HF) RANDOMIZED CONTROLLED TRIAL Michael Ong ${ }^{4}, 6$; Patrick S. Romano ${ }^{2}$; Sarah Edgington ${ }^{4}$; Harriet U. Aronow $^{7}$; Andrew D. Auerbach ${ }^{5}$; Jeanne T. Black ${ }^{7}$; Teresa De Marco ${ }^{8}$; Jose J. Escarce ${ }^{4}$, 12 ; Lorraine S. Evangelista ${ }^{9}$; Barbara H. Hanna ${ }^{2}$; Theodore G. Ganiats ${ }^{10}{ }^{11}$; Barry H. Greenberg $^{10}$; Sheldon Greenfield ${ }^{9}$; Sherrie H. Kaplan ${ }^{9}$; Asher Kimchi ${ }^{7}$; Honghu Liu $^{4}$; Dawn Lombardo $^{9}$; Carol Mangione ${ }^{1}$; Bahman Sadeghi ${ }^{4}$; Banafsheh Sadeghi ${ }^{2}$; Majid Sarrafzadeh ${ }^{4}$ Kathleen Tong ${ }^{2}$; Gregg Fonarow ${ }^{3}$. ${ }^{1}$ David Geffen School of Medicine at UCLA, Los Angeles, CA; ${ }^{2} \mathrm{UC}$ Davis, Sacramento, CA; ${ }^{3} \mathrm{UCLA}$ David Geffen School of Medicine, Los Angeles, CA; ${ }^{4}$ UCLA, Los Angeles, CA; ${ }^{5}$ UCSF Division of Hospital Medicine, San Francisco, CA; ${ }^{6}$ VA Greater Los Angeles Healthcare System, Los Angeles, CA; ${ }^{7}$ Cedars Sinai Medical Center, Los Angeles, CA; ${ }^{8} \mathrm{UC}$ San Francisco, San Francisco, CA; ${ }^{9} \mathrm{UC}$ Irvine, Irvine, CA; ${ }^{10} \mathrm{UC}$ San Diego, La Jolla, CA; ${ }^{11}$ University of Miami, Miami, FL; ${ }^{12}$ RAND, Santa Monica, CA. (Control ID \#2465761)

BACKGROUND: It remains unclear if telemonitoring approaches provide benefits for heart failure (HF) patients following hospitalization. The Better Effectiveness After Transition - Heart Failure (BEAT-HF) study aimed to evaluate the effectiveness of a care transition intervention using remote patient monitoring at reducing 180-day all-cause readmissions among a broad population of older adults hospitalized with HF.

METHODS: This analysis focused only on the intervention arm of the BEAT-HF randomized control trial. Between October 2011 and September 2013715 of 1437 individuals hospitalized at 6 academic medical centers in California age 50 or older who received active treatment for decompensated HF were randomized to intervention instead of usual care and followed for 180 days. The intervention combined telemonitoring and health coaching telephone calls. Telemonitoring used Bluetooth-enabled equipment that collected daily information about blood pressure, heart rate, symptoms, and weight. Telephone coaching calls occurred on a weekly basis during the first month postdischarge. After the first month, telephone coaching calls were made monthly until the end of the 6-month study period. Centralized registered nurses conducted telemonitoring reviews, protocolized actions, and calls. The primary outcome was readmission for any cause within 180 days after discharge. Secondary outcomes included all cause readmission within 30 days, all cause mortality at 30 and 180 days. Adherence was measured separately for telemonitoring and telephone coaching calls, based on the total days alive during each period. Multivariable logistic regressions controlled for age, gender, race/ethnicity, insurance, comorbidities, year and quarter of enrollment, social isolation, income level, education, self-care behaviors, and enrollment site.
RESULTS: Median age of participants was 73 years; $45.4 \%$ were female, and $22.1 \%$ were African American. Telemonitoring adherence was $51.7 \%$ and telephone coaching adherence was $68.0 \%$ at 180 -days. The intervention and usual care groups did not differ significantly on the readmission or mortality endpoints, except on 30 day mortality which was deemed not related to the intervention. Intervention participants with greater than either $50 \%$ total day adherence at 30 days to telemonitoring or telephone coaching had significantly $(p<0.001)$ fewer 30 -day readmissions ( 13.0 vs. $34.6 \%$ and 14.9 vs. $34.7 \%$ ) and lower 30 -day mortality ( 0.7 vs. $6.6 \%$ and 0.6 vs. $8.7 \%$ ), and those with greater than $50 \%$ total day adherence at 180 days to telemonitoring or telephone coaching had significantly $(p<0.001)$ lower 180 -day mortality ( 6.6 vs. $21.4 \%$ and 8.3 vs. $26.0 \%$ ). However, only intervention participants with greater than $50 \%$ total day adherence at 180 days to telemonitoring had significantly $(p<0.001)$ fewer 180 -day readmissions $(41.3$ vs. $61.1 \%$, telephone coaching: 49.6 vs. $54.0 \%$ ).

CONCLUSIONS: BEAT-HF is one of the largest randomized controlled trials of remote patient telemonitoring in a $\mathrm{HF}$ patient population, and was designed to determine the effectiveness of the intervention using a broad population of patients hospitalized with $\mathrm{HF}$ that would be consistent with actual practice. Similar to other large randomized controlled trials of telemonitoring, we did not find significant effects of the BEAT-HF intervention on all cause readmission. Adherence to the BEAT-HF intervention appears to be a critical factor. Newer approaches could increase adherence or provide better information to identify problems following discharge.

\section{ADOPTION OF END OF LIFE CARE PLANNING PROCESSES IN ACCOUNT- ABLE CARE ORGANIZATIONS: EARLY RESULTS FROM THE NATIONAL SURVEY OF ACCOUNTABLE CARE ORGANIZATIONS Sangeeta Ahluwalia ${ }^{1}$ Benjamin Harris ${ }^{2}$; Carrie H. Colla ${ }^{2} .{ }^{1}$ RAND Corporation, Encino, CA; ${ }^{2}$ The Dartmouth Institute for Health Policy \& Clinical Practice, Geisel Medical School, Hanover, NH. (Control ID \#2469922)}

BACKGROUND: End of life (EOL) care planning has been shown to increase patient family satisfaction with care and decrease healthcare costs, yet organizational adoption of processes and protocols for EOL care planning continues to be stymied by a fragmented delivery system, workforce shortages, and insufficient provider education. Accountable care organizations (ACOs), where provider groups work collaboratively to improve quality and lower spending growth, are well-positioned to support adoption of EOL care planning processes. We sought to understand the extent of EOL care planning processes in place in ACOs, and the types of organizations that have such processes.

METHODS: Cross-sectional analysis using data from 3 waves ( $n=399$ respondents) of the National Survey of Accountable Care Organizations, a nationally representative survey fielded annually between October 2012 and June 2015. Target respondents were ACOs who were participants in 1) the Medicare Sharing Savings and Pioneer ACO programs, 2) state Medicaid ACO programs, or 3) commercial ACO contracts. Our primary outcome variable evaluated the extent to which the ACO had established processes and protocols in place for identifying, counseling and planning for EOL care (few or no processes, some processes, advanced processes). Logistic regression analyses were used to estimate the odds of having established processes for EOL care planning according to organization size, location, composition, leadership, risk-bearing structure, shared savings distribution, palliative care experience, medical home experience, emphasis on patient involvement in decision making, and care management, utilization management, and information technology capabilities.

RESULTS: A fifth of ACOs ( $21 \%$ ) had few or no processes in place for EOL care planning, $60 \%$ had some processes and $19.6 \%$ had advanced processes in place. ACOs with a hospital in their system were significantly more likely to have EOL care planning processes in place than those that did not (OR: 2.87; $p=0.02$ ). ACOs whose shared savings were contingent on performance on quality metrics were more likely to have EOL care planning processes than 
ACOs whose savings were not contingent on quality (OR: $8.89 ; p=0.00$ ). ACOs with comprehensive programs to encourage patient involvement in decision making were more likely to have processes in place for EOL care planning compared to ACOs with few or no processes for encouraging patient involvement in decision making (OR: 16.2, $p=0.000$ ). Finally, ACOs who had advanced care management (OR: $1.43 ; p-0.02)$ and utilization management (OR: $1.58, p=0.001)$ capabilities were significantly more likely to have EOL care planning processes in place than those who had few capabilities.

CONCLUSIONS: ACOs may rely on hospital-based palliative care clinicians to implement EOL care planning process. ACOs with advanced care and utilization management processes may be better equipped to establish EOL care planning processes, which often requires communication and transfer of patient information across settings and providers. Understanding the characteristics of ACOs that have adopted EOL care planning processes can provide guidance for policymakers and ACO leadership as they continue to refine their structures and policies, and can inform future efforts to integrate EOL care planning into emerging healthcare models.

ADVANCE CARE PREFERENCES IN THE PHYSICIAN ORDERS FOR LIFE SUSTAINING TREATMENT AMONG CALIFORNIA NURSING HOME RESIDENTS Lee A. Jennings; David Zingmond; Rachel Louie; Chi-Hong Tseng; Neil Wenger. UCLA, Los Angeles, CA. (Control ID \#2468056)

BACKGROUND: As part of the effort to implement Physician Orders for Life Sustaining Treatment (POLST) in California, the state requires nursing homes to document in Section S of the Minimum Data Set (MDS) whether residents complete a POLST and the cardiopulmonary resuscitation (CPR) and other orders contained in the POLST. This affords the opportunity to examine resuscitation orders among a large, racially diverse population of nursing home residents.

METHODS: We described the physician orders concerning resuscitation contained in the California MDS for all 289,753 California nursing home residents for whom Section S was completed in 2011. We used multinomial models to predict whether a resident had a Do Not Attempt Resuscitation (DNAR) order, CPR order, or no POLST completed using resident age, gender, racial or ethnic group, functional status, cognition, and length of nursing home stay. Models were stratified by quartile of POLST completion at the nursing home (proportion of residents with a completed POLST) and were adjusted for clustering within facility. We then used the models to predict the probability of not having a POLST or having a CRP or DNAR order using different combinations of resident characteristics and high and low levels of POLST completion at the nursing facility.

RESULTS: Of the 142,677 California nursing home residents (49 \%) who completed a POLST in 2011, $50.4 \%$ had an order for DNAR order and $49.6 \%$ had an order for CPR. Resuscitation order differed by length of stay with $60 \%$ of long stay residents $(>100$ days in the nursing home) having a DNAR order compared with $45 \%$ of short-stay residents. Ninety-six percent of residents with a DNAR order also had orders for limited medical interventions or comfort measures. Few residents (4.6\%) had a POLST with conflicting orders (CPR and limited medical interventions or comfort measures). In adjusted multivariate models, older age, long length of stay, and greater functional and cognitive impairment predicted POLST DNAR orders, while being a racial or ethnic minority predicted CPR orders. (Table 1) Predicted probabilities of a DNAR order, CPR order, or no completed POLST varied widely by the level of POLST completion at a nursing facility. (Table 2)

CONCLUSIONS: Nursing home residents with greater care needs were more likely to have DNAR orders. However, racial and ethnic differences in POLST orders and variability in POLST completion across nursing home facilities may represent areas for improvement.

Table 1: Relative Risk Ratios (RRR) Associated with Having a DNAR Order in a POLST

\begin{tabular}{|c|c|c|c|}
\hline \multirow[t]{3}{*}{ Resident Characteristics } & \multicolumn{3}{|c|}{ Quartile of POLST Completion at Nursing Home } \\
\hline & $\begin{array}{l}<25 \text { th, } N= \\
66,622 \\
\text { (306 facilities) }\end{array}$ & $\begin{array}{l}25-75 \text { th }, N= \\
148,785 \\
\text { (607 facilities) }\end{array}$ & $\begin{array}{l}>75 \text { th }, N= \\
60,198 \\
\text { (306 facilities) }\end{array}$ \\
\hline & \multicolumn{3}{|c|}{ RRR $(95 \% \mathrm{CI})$ for DNAR } \\
\hline Long-stay resident & $1.09(0.83,1.42)$ & $1.38(1.30,1.47)$ & $1.78(1.64,1.94)$ \\
\hline Severe Cognitive Impairment* & $1.80(1.36,2.39)$ & $1.89(1.80,1.99)$ & $2.02(1.90,2.15)$ \\
\hline $\begin{array}{l}\text { ADL Score 21-28 (most } \\
\text { dependent)* }\end{array}$ & $2.25(1.35,3.74)$ & $1.66(1.46,1.89)$ & $1.93(1.72,2.16)$ \\
\hline \multicolumn{4}{|l|}{ Race/ethnicity } \\
\hline White, non-Hispanic & REF & REF & REF \\
\hline Hispanic & $0.49(0.38,0.62)$ & $0.45(0.42,0.48)$ & $0.43(0.38,0.48)$ \\
\hline Black & $0.37(0.27,0.51)$ & $0.31(0.28,0.34)$ & $0.28(0.25,0.32)$ \\
\hline Asian & $0.49(0.35,0.69)$ & $0.49(0.46,0.56)$ & $0.49(0.40,0.61)$ \\
\hline
\end{tabular}

Reference outcome used for comparison in the multinomial model is having an order for CPR. *Reference group is no impairment. RRR are adjusted for all variables listed, age, gender, and clustering within facility. RRR in bold are significantly different from reference at $p<0.05$. Model excludes $4.9 \%$ of residents with at least one missing covariate.

Table 2: Predicted Probabilities of DNAR Order, CPR Order, or No Completed POLST for a 78 Year-Old Female Long-Stay Nursing Home Resident

\begin{tabular}{|c|c|c|c|c|c|c|}
\hline \multicolumn{2}{|c|}{ Resident Characteristics } & \multirow{2}{*}{$\begin{array}{l}\text { Facility POLST } \\
\text { Completion }\end{array}$} & \multirow{2}{*}{$\begin{array}{l}\text { White, non- } \\
\text { Hispanic }\end{array}$} & \multirow{2}{*}{$\begin{array}{l}\text { African } \\
\text { American }\end{array}$} & \multirow[t]{2}{*}{ Hispanic } & \multirow[t]{2}{*}{ Asian } \\
\hline \begin{tabular}{|l|} 
ADL \\
Dependence \\
\end{tabular} & \begin{tabular}{|l|} 
Cognitive \\
Impairment
\end{tabular} & & & & & \\
\hline & & & \multicolumn{4}{|c|}{ Predicted Probability of DNAR Order } \\
\hline Minimal & None & \multirow[t]{2}{*}{ Lowest Quartile } & $2.3 \%$ & $1.6 \%$ & $1.7 \%$ & $1.2 \%$ \\
\hline Total & Severe & & $9.4 \%$ & $6.5 \%$ & $7.2 \%$ & $5.3 \%$ \\
\hline Minimal & None & \multirow[t]{2}{*}{ Highest Quartile } & $51.1 \%$ & $23.4 \%$ & $31.3 \%$ & $34.8 \%$ \\
\hline \begin{tabular}{|l|} 
Total \\
\end{tabular} & Severe & & $78.4 \%$ & $52.8 \%$ & $62.1 \%$ & $66.3 \%$ \\
\hline & & & \multicolumn{4}{|c|}{ Predicted Probability of CPR Order } \\
\hline Minimal & None & \multirow[t]{2}{*}{ Lowest Quartile } & $2.5 \%$ & $4.5 \%$ & $3.8 \%$ & $2.7 \%$ \\
\hline Total & Severe & & $2.5 \%$ & $4.7 \%$ & $3.9 \%$ & $2.8 \%$ \\
\hline Minimal & None & \multirow[t]{2}{*}{ Highest Quartile } & $46.0 \%$ & $74.2 \%$ & $65.8 \%$ & $63.3 \%$ \\
\hline Total & Severe & & $18.1 \%$ & $42.9 \%$ & $33.5 \%$ & $30.9 \%$ \\
\hline & & & \multicolumn{4}{|c|}{ Predicted Probability of No Completed POLST } \\
\hline Minimal & None & \multirow[t]{2}{*}{ Lowest Quartile } & $95.2 \%$ & $93.9 \%$ & $94.5 \%$ & $96.1 \%$ \\
\hline \begin{tabular}{|l|l} 
Total \\
\end{tabular} & Severe & & $88.1 \%$ & $88.8 \%$ & $88.9 \%$ & $91.9 \%$ \\
\hline Minimal & None & \multirow[t]{2}{*}{ Highest Quartile } & $2.9 \%$ & $2.4 \%$ & $2.9 \%$ & $1.9 \%$ \\
\hline Total & Severe & & $3.5 \%$ & $4.3 \%$ & $4.5 \%$ & $2.9 \%$ \\
\hline
\end{tabular}


ADVERSE OUTCOMES DURING THE TRANSITION TO A NEW ELEC TRONIC HEALTH RECORD Michael L. Barnett ${ }^{2,}{ }^{1}$; Ateev Mehrotra ${ }^{1}$; Anupam B. Jena ${ }^{1} .{ }^{1}$ Harvard Medical School, Boston, MA; ${ }^{2}$ Brigham and Women's Hospital, Boston, MA. (Control ID \#2468702)

BACKGROUND: In the last decade, most US hospitals have adopted an electronic health record (EHR) or switched from one EHR to another. Evidence on the longterm impact of inpatient EHRs on quality and patient safety is largely positive. However, there have been concerns that EHR implementation, arguably one of the most disruptive planned events a hospital can experience, may increase mortality in the short term. These concerns for patient safety are plausible given that potentially less disruptive changes such as off-hours admissions or the "July effect" of new residents have been associated with increased mortality. To examine the hypothesis that EHR implementations are associated with a short-term decline in patient safety, we exploited the natural experiment of "go live" date transitions to new EHRs and assessed the short-term impact of inpatient EHR implementation on inpatient mortality, readmissions and adverse safety events.

METHODS: Using American Hospital Association survey data and internet searches of publicly available documents, we identified all hospitals implementing a new inpatient EHR or switching EHR vendors during 2011-2012 with a verifiable "go live" date. After identifying these hospitals, we constructed a control group of all other hospitals in the same hospital referral region as the EHR implementing hospitals. We identified admissions and measured rates of outcomes of 30-day mortality, 30-day all-cause readmission and events used in the Patient Safety for Selected Indicators (PSI)-90 composite measure with a $100 \%$ sample of Medicare discharge claims from 2011 to 2012 . We measured these outcomes for the 90-day period before and after the date of EHR implementation for each implementing hospital as well as its regional control hospitals. To assess the impact of EHR implementation, we used difference-in-differences analysis comparing each outcome for the implementation group versus control hospitals before and after the go-live date. All analyses were adjusted for beneficiaries' sociodemographic and clinical characteristics

RESULTS: We identified 17 hospitals with a "go live" date from 2011 to 2012 ( $n=$ 24,071 and 22,790 admissions 90 days before and after go-live) and 399 regional control hospitals ( $n=237,305$ and 231,534 admissions before and after). Before and after implementation, characteristics of admissions were similar in both the study hospitals and control group. Among study hospitals, unadjusted 30-day mortality $(6.74 \%$ [95 \% CI $6.44,7.03]$ to $7.15 \%$ [95 \% CI 6.84, 7.46]) and adverse safety event rates per 1000 admissions (10.5 [95 \% CI 9.3, 11.7] to 11.4 [95\% CI 10.1, 12.7]) did not significantly change after EHR implementation. In difference-in-differences analysis, this trend persisted and there was no significant change in any outcome between pre-and postimplementation periods (all $p \geq 0.10$ ). In pre-specified subgroup analyses, there was no differential effect of EHR implementation across patients with differing mortality risk or among hospitals implementing a new EHR versus switching EHR vendors.

CONCLUSIONS: Despite concerns that EHR implementation may adversely impact patient care during the acute transition period, we found no overall negative impact of EHR implementation across 17 hospitals on short-term mortality, adverse safety events, or readmissions.

AGREE TO DISAGREE-EXAMINING RISK ASSESSMENT CONVERGENCE IN THE UNDERSTANDING PHYSICIAN SIGNOUT RISK PERCEPTIONS (UPS) STUDY Jon R. Grace ${ }^{1}$; Alexander Davis ${ }^{2}$; Baruch Fischhoff ${ }^{2}$; Jonathan Arnold $^{1}$; Andrew Klobuka ${ }^{3}$; Emmanuelle B. Yecies ${ }^{1}$; Deepika Mohan ${ }^{1}$; Janel Hanmer ${ }^{1}$. ${ }^{1}$ University of Pittsburgh, Pittsburgh, PA; ${ }^{2}$ Carnegie Mellon University, Pittsburgh, PA; ${ }^{3}$ University of Pittsburgh Medical Center, Pittsburgh, PA. (Control ID \#2463554)
BACKGROUND: Rapid response teams (RRTs) are a widely adopted approach to expanding critical care services outside of the intensive care unit. These teams have been shown to reduce in-hospital mortality and cardiopulmonary arrests. There is also evidence that delayed activation of RRTs results in increased mortality and morbidity, which makes recognizing patients that are likely to decompensate an important and poorly studied task. It is generally assumed that more experienced individuals (from final year residents to attendings with decades of practice) are more able to predict which patients are at risk for decompensation than more junior trainees, representing a natural progression of medical knowledge and previous experiences. To date no studies have examined how individual physicians or physician teams assess decompensation risk and how those assessments change over time within teams. We report a longitudinal prospective observational study of physician ability to predict RRT activation in an academic general internal medicine setting. We hypothesized that physicians within teams will converge on their perceptions of decompensation risk as they become more experienced and spend more time with the same team members.

METHODS: The project was approved by the local IRB and all physicians gave verbal consent to participate in the study. Data was collected by clinical researchers in a non-blinded fashion 2-3 days per week (separated by at least 2 days) over 5 months at a single tertiary care academic medical center. Study participants included interns, residents, and attendings that were rotating on an inpatient medicine teaching service at the time of collection. Physician team structure consisted of two interns, one senior (second or third year) resident, and one attending. Interns and residents remained on a single team over a 4 week period while, generally, attendings rotated in 2 week cycles. Respondents were privately asked a standardized question $1-2 \mathrm{~h}$ prior to sign-out to assess each patient's percent chance of RRT activation or ICU transfer over the next $24 \mathrm{~h}$. Raw percentages were converted to $\mathrm{Z}$ scores to standardize risk perception across individuals then grouped by level of experience (intern, resident attending). Correlations were assessed by week of the rotation.

RESULTS: Risk judgments were made by 60 interns, 36 residents, and 40 attending physicians, resulting in 1242 intern-resident judgment pairs, 1192 intern-resident judgment pairs, and 1447 resident-attending judgment pairs. Results showed that although physicians generally agreed with each other on which patients were at greatest risk (interns-residents: $r=$ 0.57 ; interns-attendings: $r=0.57$; residents-attendings: $r=0.51$ ), that agreement did not change over time. There was no change in the simple correlation between interns, residents, and attendings from the first week on a team to the second, third, or fourth week of their rotation block, nor was there greater agreement between more experienced physicians (residents and attendings). The results were the same in sensitivity analyses using raw risk scores. However, physicians did adjust their use of the probability scale with more experience with the task-the average judged probability of decompensation of $15 \%$ (among all physicians) in the first week decreased to $10 \%$ for interns and attendings and $8 \%$ for residents in the fourth week. The actual rate for decompensation at our institution is about $2 \%$. CONCLUSIONS: Our data do not support the hypothesis that physician risk perception converges, either with years of experience or time spent within a group. All experience groups had moderate agreement on patient deterioration risk. This finding reinforces the importance of adequate supervision of physician trainees since they are not reliably mirroring the same risk judgments of their attendings. A limitation of this study is not stratifying attending physicians by number of years of practice since those with less experience may have been more likely to converge with senior residents. The finding that physician teams did not converge on risk assessments over time conflicts with conventional group psychology tenets that individuals within groups generally strive to achieve harmony and similar beliefs. The collection of data in a private setting away from other group members likely enabled this unusual finding and suggests that perception of risk is highly individualized and resistant to influence by other physicians. This could also be a reflection of ascertainment bias propagated by more senior physicians that results in less discussion or concern about risk of decompensation in certain patients. Furthermore the substantial discrepancy between predicted and actual base rate (despite its improvement over time) is an example of anchoring bias in clinical practice that could possibly influence 
clinical decision-making. Overall, these findings add to the growing body of evidence of the prevalence of cognitive biases in clinical reasoning and also provides objective evidence to support the traditional supervisory model of internal medicine training.

\section{ALTERNATIVE TOBACCO PRODUCT AND ELECTRONIC CIGARETTE USE} IN A SAMPLE OF HOMELESS SMOKERS IN BOSTON Travis P. Baggett ${ }^{1,2}$; Eric Campbell ${ }^{1}$; Yuchiao Chang ${ }^{1}$; Nancy A. Rigotti ${ }^{1} .{ }^{1}$ Massachusetts General Hospital, Boston, MA; ${ }^{2}$ Boston Health Care for the Homeless Program, Boston, MA. (Control ID \#2469526)

BACKGROUND: Three-quarters of homeless adults smoke cigarettes, but less is known about the extent to which homeless smokers use other tobacco products or electronic cigarettes (e-cigarettes). The concurrent use of cigarettes and alternative tobacco products such as cigars and smokeless tobacco could compound the health risks of smoking and complicate efforts at quitting. We determined the prevalence and correlates of alternative tobacco product and ecigarette use in a clinic-based sample of homeless cigarette smokers in Boston.

METHODS: In April-July 2014, we used stratified time-location sampling to conduct a cross-sectional, in-person survey of 306 currently homeless adult cigarette smokers recruited from 5 high-volume clinics operated by Boston Health Care for the Homeless Program (BHCHP) in an emergency shelter, a daytime drop-in center, and an academic medical center. Participants were sampled in proportion to the estimated number of eligible patients seen at each site in the prior year according to BHCHP administrative and clinical data. After obtaining verbal informed consent, trained interviewers verbally administered the 159-item questionnaire using an electronic tablet. We assessed past-month use of large cigars, little cigars, smokeless tobacco, and e-cigarettes. Among participants who had used e-cigarettes, we asked them to endorse any of 8 reasons for doing so. We used design-adjusted logistic regression analysis to identify the sociodemographic, behavioral health, and cigarette smoking characteristics associated with the use of each product.

RESULTS: Eighty-six percent of eligible individuals participated in the survey. Threequarters of participants were male. The mean age was 48 years. Thirty-six percent were white, $41 \%$ were black, and $18 \%$ were Hispanic. In the past month, $37 \%$ of respondents used large cigars, $44 \%$ used little cigars, $8 \%$ used smokeless tobacco, $24 \%$ used an ecigarette, and $68 \%$ used any of these products. Frequently-endorsed reasons for e-cigarette use included curiosity ( $85 \%$ ) and to help quit conventional cigarettes $(69 \%)$. In multivariable regression analyses, homeless smokers with greater subsistence difficulties were more likely to use little cigars (AOR 1.39, $95 \%$ CI 1.09-1.79, per standard deviation [SD]) and less likely to use e-cigarettes (AOR 0.60, $95 \%$ CI $0.45-0.81$, per SD). Non-Hispanic black (AOR 2.23, 95 \% CI 1.21-4.13), Hispanic (AOR 4.12, 95 \% CI 1.91-8.90), and non-shelter-using (AOR 2.35, $95 \%$ CI 1.05-5.23) participants were more likely to use large cigars. Readiness to quit was not associated with alternative tobacco product use but was significantly associated with e-cigarette use to help quit smoking (AOR 1.28, $95 \%$ CI 1.04-1.57, per SD). CONCLUSIONS: Alternative tobacco product and e-cigarette use was very common in this sample of homeless smokers. Relative to cigarette smokers in the general population, homeless cigarette smokers in this study had a 10-fold higher prevalence of past-month cigar use and a 4-fold higher prevalence of past-month smokeless tobacco use. Health care providers serving homeless populations should consider routine screening for the use of these products to guide cessation discussions and treatment planning.

\section{AN ANALYSIS OF MESSAGES SENT BETWEEN NURSES AND PHYSICIANS ABOUT DETERIORATING INTERNAL MEDICINE PATIENTS TO HELP IDENTIFY ISSUES IN FAILURES TO RESCUE Robert $\mathrm{Wu}^{1,2}{ }^{2}$; Rachel Bierbrier ${ }^{2}$; Philip $\mathrm{Ma}^{2}$; Hannah Wong ${ }^{3}$; Sherman Quan ${ }^{4}$; Sannie Lai ${ }^{2} .{ }^{1}$ University of Toronto, Toronto, ON, Canada; ${ }^{2}$ University Health Network, Toronto, ON, Canada; ${ }^{3}$ York}

University, Toronto, ON, Canada; ${ }^{4}$ Trillium Health Partners, Mississauga, ON, Canada. (Control ID \#2455579)

BACKGROUND: To avoid failing to rescue deteriorating patients in hospital, there has been increased recognition of the importance of escalating their care using rapid response teams. While there are established criteria for activation of rapid response teams, little is known about the actual communication when nurses notify physicians about deteriorating patients. Analysis is difficult as communication still often occurs through paging and telephone calls. The recent move in some hospitals from paging to text messaging allows for analysis of these critical messages which may help identify issues with communication of deteriorating patients.

METHODS: Objectives: In patients who deteriorate on the General Medicine Wards and require a transfer to the intensive care unit, how many have critical messages prior and what is the quality of these messages? Is the quality of the message, the response or the timeliness of rapid response team activation related to death? We conducted a retrospective chart review and analysis of patients on General Internal Medicine (GIM) wards from January 2012 until August 2014 who were transferred to the Intensive Care Unit (ICU) for clinical deterioration. All messages sent from nurses to physicians in the $48 \mathrm{~h}$ prior to ICU transfer were analyzed for rapid response team calling criteria. For those messages with calling criteria, the following messaging characteristics were obtained: the timing of the message to rapid response team activation, the message quality as defined by the presence of Situation, Background, Assessment, and Recommendations (SBAR) and the presence of vitals signs, and the quality and timeliness of physician response. Analysis was performed to look for correlations between message characteristics and in hospital death. RESULTS: Of the 236 patients in the study, and $93 \mathrm{had}$ a critical message in the $48 \mathrm{~h}$ prior to deterioration. Of these 93 patients, 17 already had rapid response team involved, $63 \mathrm{had}$ them involved after the critical message, and 13 did not have any involvement prior to ICU transfer. For the subset of 76 patients who did not have rapid response involved prior to the critical message, the mean time from critical message to rapid response team activation and ICU transfer was $13.6 \mathrm{hs}$ (SD 1.7) and $18.4 \mathrm{~h}$ (SD 1.5 ) respectively. Only $45 \%$ of critical messages contained 2 or more vitals and only $3 \%$ of messages contained all four SBAR components. Responses from physicians were usually timely (median $3 \mathrm{~min}$, IQR 2-17 $\mathrm{min}$ ). In the responses to critical messages, physicians indicated they would call back or see the patient urgently in only $49 \%$ of the cases. Of all message characteristics, only the number of SBAR elements was inversely correlated with in-hospital death $(P=0.047)$. CONCLUSIONS: The communication about critically ill patients between nurses and physicians could be improved. There appear to be significant gaps in the quality of the sent messages and responses as well as a delay in rapid response team activation. Educational efforts and improved communication systems could improve the activation of rapid response teams as well as improving the quality of communication both from nurses and physicians.

Critical messages and responses for patients with delayed rapid response team activation

\begin{tabular}{|c|c|c|}
\hline & & Total $n=76$ \\
\hline \multirow{6}{*}{$\begin{array}{l}\text { Calling criteria from } \\
\text { critical message: }\end{array}$} & Desaturation & $24(32 \%)$ \\
\hline & Worried about patient & $17(22 \%)$ \\
\hline & $\begin{array}{l}\text { Systolic blood pressure } \\
<90\end{array}$ & $17(22 \%)$ \\
\hline & Altered mental status & $8(11 \%)$ \\
\hline & Heart rate $>130$ & $6(8 \%)$ \\
\hline & Respiratory Distress & $4(5 \%)$ \\
\hline \multirow{4}{*}{$\begin{array}{l}\text { Requested response } \\
\text { by nurse: }\end{array}$} & Call back now & $13(17 \%)$ \\
\hline & Text message now & $23(30 \%)$ \\
\hline & Text message within specified period & $4(5 \%)$ \\
\hline & No response requested & $36(47 \%)$ \\
\hline Vital signs: & $\begin{array}{l}\text { Message contains at least } \\
2 \text { vitals signs }\end{array}$ & $34(45 \%)$ \\
\hline
\end{tabular}




\section{(continued)}

\begin{tabular}{lll}
\hline $\begin{array}{l}\text { SBAR components } \\
\text { in message: }\end{array}$ & 1 & $36(47 \%)$ \\
& 2 & $28(37 \%)$ \\
& 3 & $10(13 \%)$ \\
Response content: & 4 & $2(3 \%)$ \\
& $\begin{array}{l}\text { Indicated they will call } \\
\text { back now }\end{array}$ & $3(4 \%)$ \\
& $\begin{array}{l}\text { Indicated they will come } \\
\text { to assess shortly }\end{array}$ & $34(45 \%)$ \\
& $\begin{array}{l}\text { Ordered a treatment } \\
\text { Acknowledged message }\end{array}$ & $7(9 \%)$ \\
& No response & $18(24 \%)$ \\
& $14(18 \%)$ \\
\hline
\end{tabular}

AN EVIDENCE-BASED PATIENT HANDOUT FOR BREAST CANCER SCREENING SHARED DECISION MAKING IN PRIMARY CARE Eloho Ufomata $^{1}$; Yan Huang ${ }^{2}$; Jennifer Corbelli ${ }^{1} .{ }^{1}$ University of Pittsburgh Medical Center, Pittsburgh, PA; ${ }^{2}$ University of Pittsburgh, Pittsburgh, PA. (Control ID \#2460777)

BACKGROUND: Breast cancer screening guidelines directly conflict regarding the recommended screening age of initiation and frequency of mammography. Specifically the United States Preventive Services Task Force recommends biennial screening starting at age 50, the American Cancer Society recommends yearly screening from age 45-50, and then biennially from age 50-74, and the American Congress of Obstetricians and Gynecologists recommend yearly screening at age 40 . This controversy has created ongoing confusion among physicians and patients as to the optimal screening schedule for average-risk patients. Furthermore, many patients do not understand the potentia harms associated with mammography, and research has shown that physicians are five times more likely to discuss potential screening benefits in comparison to harms with patients. Patient preferences, values and risk also vary widely, which further complicates patient counseling as to the optimal screening regimen on an individual level. Patients and physicians, therefore, are both poised to benefit from tools and processes to facilitate evidenced-based shared decision-making regarding breast cancer screening. Objectives Of Intervention: Increase patient knowledge of the risks and benefits of screening mammography Improve the processes of breast cancer screening shared decision making between patients and physicians

METHODS: We created an evidence based patient handout for our General Internal Medicine Clinic at the university hospital, which consists of residents and attending physicians, and serves a large diverse patient population with respect to education, ethnicity, age and socioeconomic status. The handout details the benefits and harms of screening mammography in patient friendly language, which corresponds to an 8th grade Flesch-Kincaid grade level. It discusses key issues for a patient to consider when deciding to be screened between ages $40-50$, including absolute risks of false positives leading to breast biopsy and further testing, over-diagnosis, and mortality benefit from screening mammography. It also discusses whether patients ages $50-74$ should be screened yearly or biennially, but emphasizes that available guidelines consistently recommend that women in this age group should be screened. The handout was explicitly designed with the intention not to endorse one set of guidelines. Medical assistants distributed the handout to women ages 40 to 74 , prior to the physician encounter, who were noted in the healthcare maintenance section of the medical record to be due for a mammogram discussion or who were scheduled for their annual exam. Patients were also given a survey to complete following their visit with the physician. The checkout staff collected the surveys which were independently completed by patients after the visit. If the patient decided to get a mammogram after leaving the clinic,, they had the option to call clinic to have the mammogram order placed. Physicians were surveyed online after completion of the study.
RESULTS: Patient Outcome Results 124 women completed the survey: 64 \% Caucasian, $29 \%$ Black, $3.2 \%$ Asian, $0 \%$ Hispanic, and the remainder identified as other. Seventytwo percent of the respondents were 50-74 years, $20 \% 40-50$ years; $8 \%$ were either older than 75 or did not identify their age. The population was more highly educated than the general population: $73 \%$ had at least some college education, with $32 \%$ having postgraduate degrees. For women aged 50-74, $38 \%$ who planned to get annual mammograms, decided to screen biennially after using the handout in discussion with their physician, while $14 \%$ of women who planned to get biennial mammograms, changed their mind to opt for annual screen Women who changed their minds were significantly more likely to opt for biennial screening vs. annual screening $(p=0.04)$. $64.2 \%$ reported that they learned new information from the handout, and $74.8 \%$ reported that the handout increased their comfort with the discussion of screening mammography. Physician Outcome Results 20 physicians completed the survey: $65 \%$ were attendings, and $70 \%$ were women. Eighty percent of respondents agreed that they would use this handout in the future, $80 \%$ of respondents reported that the handout was helpful when counseling patients aged $40-49$, compared to $100 \%$ of respondents who reported that the handout was helpful when counseling patients aged 50-74. Eighty-five percent of respondents agreed handout was informative for them. One hundred percent of physicians reported that breast cancer screening required shared decision-making.

CONCLUSIONS: This intervention was well received by both patient and physicians, who found it helpful in facilitating mammography shared decision-making, which was the primary aim of the study. The purpose of the intervention was explicitly not to endorse one set of guidelines; given this, it is particularly notable that over half of women ages 50-74 changed their minds about their desired screening interval after reading the handout. Most participants who changed their minds opted for decreased screening. These results are consistent with prior literature, which shows that patients are not adequately informed about mammography options and potential harms of screening. Overall, this intervention was very low cost and can be implemented at practices of varying size and type to facilitate processes of shared decision-making regarding breast cancer screening. Our results indicate that both patients and physicians benefit from decision aids when discussing breast cancer screening.

\section{ANTIBIOTIC RESISTANCE IN OLDER ADULTS WITH URINARY TRACT} INFECTIONS Theresa A. Rowe ${ }^{1}$; Lee Lindquist ${ }^{1}$; Xiaoling Xiang ${ }^{2} .{ }^{1}$ Northwestern University, Chicago, IL; ${ }^{2}$ Northwestern University Feinberg School of Medicine, Chicago, IL. (Control ID \#2455031)

BACKGROUND: Clinicians empirically treat urinary tract infection (UTI) in older adults using guidelines intended for younger patients. This is problematic because older adults may be at higher risk for having an infection from a resistant organism. Additionally, uropathogens responsible for UTI in older adults may be different than in younger patients. The aim of this study was to describe the distribution of uropathogens in adults and to compare resistance patterns across age groups.

METHODS: Retrospective analysis of a cohort of adults $\geq 18$ years from 2010 to 2014 who had $\geq 1$ positive urine culture defined as $\geq 10^{5}$ colony forming units $/ \mathrm{ml}(\mathrm{CFU} / \mathrm{mL})$ of $\leq 2$ uropathogens. Urinary isolates were tested for resistance to commonly prescribed antibiotics. Descriptive analysis including frequency, counts and percentages were used to summarize the sample across different age groups $(\geq 18-40,>40-54, \geq 55$ years). The percentage of resistant uropathogens was calculated by dividing the number of isolates that were resistant to each antibiotic by the total number of organisms that were tested against the agent in each age group. Logistic regression with clustering was used to detect differences in patterns of uropathogens and antibiotic resistance across age groups. RESULTS: A total of 4362 urine cultures were obtained of which 884 (20\%) were positive. An additional 906 (21\%) were identified as contaminates ( $>2$ uropathogens). 
Escherichia coli (E.coli) was the most common organism identified in all age groups 457 $(52 \%)$. However, adults aged $\geq 55$ years were significantly more likely to have a positive urine culture from non-E.coli gram negative $\operatorname{rod}(\mathrm{OR}=1.81$, CI 1.14-2.8, $p=.01)$ compared to those aged $<55$ years after adjustment for sex, cognitive impairment, and diabetes. Resistance rates of E.coli isolates across all age groups were highest to: 1) Ampicillin/Sulbactam (40 \%), 2) Trimethoprim/Sulfamethoxazole (26\%) and 3) Ciprofloxacin $(18 \%)$. Urinary isolates from adults aged $\geq 55$ years were more than twice as likely to have resistance to Ciprofloxacin compared to adults $<55(\mathrm{OR}=2.6$, CI 1.25-5.8, $p=.01)$. Resistance rates for non-E.coli gram negative rods were highest to: 1) Nitrofurantoin (55\%) 2) Ampicillin/Sulbactam (36\%), and 3) Trimethoprim/ Sulfamethoxazole (13\%). There were no significant differences across age groups. Of the 906 patients who had a urine specimen contaminate, at least $22 \%$ still received an antibiotic.

CONCLUSIONS: There are differences in the types of organisms causative of UTI and their antibiotic resistance patterns across age groups. Clinicians should be aware of these differences when empirically prescribing antibiotics for suspected UTI. A significant proportion of adults had contaminated urine specimens however, almost a quarter still received antibiotic therapy. Antibiotics in this setting may potentially be unnecessary.

\section{APPLYING LEAN PRINCIPLES TO REDUCE WAIT TIMES IN THE EMER-} GENCY DEPARTMENT Anita A. Vashi ${ }^{1}$; Farnoosh Haji-Sheikhi ${ }^{3}$; Lisa A. Nashton ${ }^{4}$; Jennifer Ellman ${ }^{5}$; Priya Rajagopal ${ }^{5}$; Steven Asch $^{2} .{ }^{1}$ VA PALO ALTO, San Francisco, CA; ${ }^{2}$ VA/Stanford, Menlo Park, CA; ${ }^{3}$ Stanford University, Palo Alto, CA; ${ }^{4}$ William Jennings Bryan Dorn VA Medical Center, Columbia, SC; ${ }^{5}$ VA Palo Alto Health Care System, Palo Alto, CA. (Control ID \#2467769)

BACKGROUND: Waiting time to see an emergency department (ED) provider has long been recognized as a potential quality problem, however few interventions have successfully addressed this. The objectives of this is study are to (1) describe the extent and depth of the use of Lean quality improvement methodologies to rapidly redesign ED front-end operations that aimed at reducing waiting times to be seen by a provider and (2) evaluate if the intervention achieved its stated aim using quasiexperimental controls and a differencein-differences approach.

METHODS: ED staff at a VA tertiary care referral facility participated in a Rapid Process Improvement Workshop (RPIW) based on Lean system redesign methods in January 2014. Key changes made as a result of the RPIW included standardizing and streamlining evaluation and hand-off processes, better delineating roles for nurses and physicians, and more efficiently utilizing beds and improving team communication. We collected 13 months of pre-intervention and 13 months of post-intervention data. The primary outcome was the change in "Door to Doc" time between the pre-intervention and postintervention periods at the intervention facility compared to contemporaneous national control facility sites $(n=11)$. Secondary outcomes included the change in "Door to Triage" time and the rate at which patients left without being seen (LWBS). We extracted ED workload and performance flow data retrospectively from the VHA Support Service Center (VSSC). We used a regression adjusted difference-in-difference approach to analyze the study outcomes. A linear mixed effect model was utilized to examine this difference in "Door to Doc" time, "Door to Triage" time, and LWBS rate between the preintervention and post-intervention periods at the intervention site versus control sites. This model allowed us to account for within class correlation between sites and across time.

RESULTS: Overall, "Door to Doc" time decreased $12.6 \mathrm{~min}$ after the intervention, compared to $3.7 \mathrm{~min}$ in the control sites. Regression-adjusted difference-in-differences estimates for "Door to Doc" time showed a statistically significant reduction at the intervention site compared to control sites ( $8.9 \mathrm{~min}$ ), during the same time period [standard error $(\mathrm{SE})=3.5 \mathrm{~min} ; p=0.01]$. Regression-adjusted difference-in-differences estimates for "Door to Triage" time showed a statistically significant reduction at the intervention site compared to control sites $(5.0 \mathrm{~min})$, during the same time period $[\mathrm{SE}=1.7 \mathrm{~min} ; p=$ 0.004]. Regression-adjusted difference-in-differences estimates for LWBS rates showed the intervention site's LWBS did not significantly change compared to control sites $(0.1$ vs. $0.3 \%$ ), during the same time period ( $p=0.8$ )

CONCLUSIONS: Using Lean principles, the facility improved patient flow in the ED. Our findings suggest that the ED staff was successful in reducing "Door to Doc" time by standardizing and streamlining work processes, clearly delineating roles for nurses and physicians, efficiently utilizing beds and improving team communication. Lean methods encourage interdisciplinary teams and problem solving across departments and are one approach to addressing systemic factors and contributors to ED crowding.

APPLYING SYSTEMS ENGINEERING TO ADDRESS COMPLEX HEALTH CARE DELIVERY CHALLENGES Vimal Mishra ${ }^{1}$; Shin-Ping Tu ${ }^{1}$; Joseph A. Heim²; Allison E. Phillips ${ }^{1}$; Richard Storch ${ }^{2}$; Heather Masters ${ }^{1}{ }^{1}$ Virginia Commonwealth University, Richmond, VA; ${ }^{2}$ University of Washington, Seattle, WA. (Control ID \#2469419)

BACKGROUND: Hospital health care delivery depends on coordinating a complex, dynamic, interdependent and distributed system of providers, information, technology, as well as physical and financial resources. In academic hospitals with high occupancy rates, an unintended consequence is that general medicine patients are admitted to nursing units scattered throughout the hospital. Effective and timely communication between physicians and nurses who are at the front lines of patient care are critical to high value, safe, and proficient delivery of medical care. Yet an increasingly recognized barrier to efficient, patient-centered care is the geographic dispersion of these primary medicine team members. The purpose of this study was to apply discrete event simulation (DES) to evaluate alternative scenarios of hospitalized patients on the General Internal Medicine (GIM) teams at an academic medical center with an occupancy rate of over $92 \%$ for the past 3 years.

METHODS: We applied Kelton and Law's framework first extracting admission and discharge data for GIM patients admitted from the Emergency Department (ED) during 2013. These records were used to estimate hourly admission rate, discharge rate, nursing unit distribution and length of stay for model data input. We also conducted time and motion studies to capture the current state of the GIM teams' rounding times (including travel between nursing units). We built DES models using SIMIO ${ }^{\circledR}$ Simulation Software and performed verification and validation modeling of the present state. Building on the present state model, we constructed these what-if scenarios: Future State 1-an alternative geographical patient assignment; Future State 2-increased GIM bed capacity; Future State 3-an additional team of physicians; and Future State 4-a combination of the two latter scenarios with an increased admission rate. Our analysis examined the impact of these alternatives on 11 healthcare delivery metrics listed on Table 1.

RESULTS: In 2013, 7902 patients were admitted to the GIM teams with 7222 patients remaining in the sample when the model reached steady state at $800 \mathrm{~h}$. As noted on Table 1, reassigning patients to be more geographically matched with the house staff teams on one nursing unit decreased patient dispersion and rounding travel time; however, this resulted with an increase in the number of patients waiting in the ED and the wait time (Future State 1). Increasing the number of beds available to GIM teams significantly improved the ED metrics; however, patient dispersion did not change. Increasing a GIM team neither improved ED metrics nor patient dispersion. An additional team decreased team utilization (i.e. percent of the maximum number of patients on a team). Future State 4 resulted in increased capacity of GIM patients, improved ED wait time, and numbers of patients discharged. 
CONCLUSIONS: In the age of big data, simulation modeling provides a powerful technique to examine existing data of the complex, dynamic, interdependent and distributed system of providers and physical resources in large hospitals with high occupancy rates. Examination of potential future states may provide valuable information to guide data driven decisions.

Table 1: Discrete Event Simulation Models of Present and Future States

\begin{tabular}{|c|c|c|c|c|c|}
\hline & $\begin{array}{l}\text { Present State } \\
(95 \% \mathrm{CI})\end{array}$ & $\begin{array}{l}\text { Future State l } \\
\text { Geography* } \\
\text { (95\% CI) }\end{array}$ & $\begin{array}{l}\text { Future State } 2 \\
+26 \text { beds } \\
(95 \% \mathrm{CI})\end{array}$ & $\begin{array}{l}\text { Future State } 3 \\
+ \text { Team } 6 \\
(95 \% \text { CI })\end{array}$ & $\begin{array}{l}\text { Future State } 4 \\
+26 \text { beds + Team } 6 \\
+\eta \text { Admit Rate** } \\
(95 \% \text { CI })\end{array}$ \\
\hline Total Pt Admissions/Year & 7222 & 7222 & 7222 & 7222 & 8301 \\
\hline Total Pt Discharges/Year & $7215(2713,7217)$ & $7209(7204,7212)$ & $7222(7221,7224)$ & $7214(7212,7216)$ & $8296(8283,8309)$ \\
\hline General Medicine Pts in System***/Day & 133 & 136 & 125 & 134 & 151 \\
\hline General Medicine Pts ${ }^{* * * *} /$ Day & 119 & 119 & 120 & 119 & 137 \\
\hline Pts Waiting in Emergency Dept & $11(10,12)$ & $13(12,14)$ & $2(2,2)$ & $11(10,12)$ & $10(9,11)$ \\
\hline Wait Time in Emergency Dept (Hrs) & $12(11,13)$ & $14(13,16)$ & $2(2,2)$ & $12(11,13)$ & $9(9,10)$ \\
\hline Change in \# Pts Discharged/Year & Not Applicable & -6 & 0 & -1 & 1081 \\
\hline $\begin{array}{l}\text { Housestaff Team Utilization } \\
\% \text { of max \# Pts/Team }\end{array}$ & $75-77 \%$ & $60-64 \%$ & $75-77 \%$ & $68-70 \%$ & $78-80 \%$ \\
\hline $\begin{array}{l}\text { Hospitalist Team Utilization } \\
\% \text { of max \# Pts/Team }\end{array}$ & $71-74 \%$ & $84-88 \%$ & $72-74 \%$ & $65-67 \%$ & $75-78 \%$ \\
\hline $\begin{array}{l}\text { Nursing Unit Utilization } \\
\% \text { of max \# Pts/NU }\end{array}$ & $42-88 \%$ & $42-88 \%$ & $35-73 \%$ & $42-88 \%$ & $40-84 \%$ \\
\hline Patient Dispersion (NU) & 6.8 & 3.9 & 6.9 & 7.0 & 7.5 \\
\hline Rounding Travel Time (min) & 34.0 & 20.9 & 34.4 & 34.8 & 37.5 \\
\hline \multicolumn{6}{|l|}{ Pts $=$ patients } \\
\hline \multicolumn{6}{|l|}{$\mathrm{NU}=$ Nursing Unit } \\
\hline \multicolumn{6}{|l|}{ Dept $=$ Department } \\
\hline \multicolumn{6}{|l|}{$\#=$ Number } \\
\hline \multicolumn{6}{|c|}{${ }^{*}$ Geography $=$ Pts selectively assigned to 3 NUs for House-staff Teams } \\
\hline \multicolumn{6}{|c|}{$* *$ Increased admission rate $=$ from $21 \mathrm{Pts} /$ day to $25 \mathrm{Pts} /$ day } \\
\hline \multicolumn{6}{|l|}{ ***All NUs and Emergency Department } \\
\hline$* * * *$ All NUs & & & & & \\
\hline
\end{tabular}

\section{APPROPRIATENESS OF PRIMARY CARE PROVIDERS' MANAGEMENT OF GENOME SEQUENCING RESULTS: RESULTS FROM AN EXPERT PANEL} $\underline{\text { Jason L. Vassy }}^{2,}{ }^{1}$; Erica F. Schonman ${ }^{1}$; Michael F. Murray ${ }^{3}$; Joel B. Krier ${ }^{1}$; Carrie L. Blout ${ }^{1}$; David W. Bates ${ }^{1}$; Robert C. Green ${ }^{1}$. 'Brigham and Women's Hospital, Boston, MA; ${ }^{2}$ VA Boston Healthcare System, Boston, MA; ${ }^{3}$ Geisinger Health System, Danville, PA. (Control ID \#2458887)

BACKGROUND: Clinical and research programs like the Precision Medicine Initiative will increasingly generate information about patients' genetic make-up, some of which may be actionable. There are concerns that primary care providers' (PCPs) limited experience with rapid developments in genomics may result in medical mismanagement, particularly since insufficient empiric evidence exists to develop practice guidelines for most variants identified by genome sequencing. We used quantitative expert review to determine the appropriateness of PCPs' management of real clinical cases of genome sequencing and to begin to inform guideline development for genomic medicine contexts with limited evidence.

METHODS: The MedSeq Project is a trial of genome sequencing among generally healthy adults in a network of primary care practices at one urban academic medical center. Patients were enrolled into the study along with their PCPs. Before patient enrollment, the 9 PCP participants underwent a 6-h genetics education curriculum consisting of case-based online modules and 2 1-h group classes. For each sequenced patient, the PCP received a genome report, which described variants identified in genes associated with monogenic disease, along with evidence for their pathogenicity. The PCPs used their clinical judgment to manage the sequence results, and management was documented through audio-recorded PCP-patient discussions, PCP checklists, and review of the medical record. To assess the appropriateness of PCP management, we created a clinical vignette for each patient with a potentially pathogenic variant in a monogenic disease gene. Each vignette summarized the patient's personal and family history, variant information from the genome report, and the PCP's clinical management. We recruited a national sample of 11 expert geneticists with internal or family medicine training. In an online survey, the experts individually rated the PCP's management in each vignette, using the validated 9-point RAND/UCLA Appropriateness Scale (RAS), and provided free-text reasons for their ratings. After completing all the vignettes, the experts were asked to list what they considered to be core components of guidelines for PCPs managing genetic variants in ostensibly healthy patients. In analysis, the management of each vignette was categorized according to the median RAS among the 11 experts: inappropriate (1-3), neither appropriate nor inappropriate (4-6), or appropriate (7-9). Agreement among the experts was defined quantitatively with disagreement indices, calculated using interpercentile ranges adjusted for symmetry.

RESULTS: Of 50 sequenced patients, 11 received a potentially pathogenic variant associated with monogenic disease (Table 1). Experts agreed on the overall management ratings of all 11 cases. Eight cases (73\%) were judged as managed appropriately. Two cases were judged as managed inappropriately: one where the PCP under-evaluated a pathogenic $\mathrm{LXH} 4$ variant associated with combined pituitary hormone deficiency and one where the PCP miscommunicated the inheritance pattern of a factor V Leiden variant. One case was judged to be managed neither appropriately nor inappropriately: experts thought the PCP could have done more to evaluate subclinical manifestations of a variant of uncertain significance in the ARSE gene, potentially associated with chondrodysplasia punctata. Six major recommendations for clinical guidelines emerged from the summary of expert responses (Table 2). 
CONCLUSIONS: Non-geneticist PCPs at one academic medical center generally managed the genome sequencing results of their patients appropriately, according to expert review. Guidelines for the primary care management of sequencing results can be created to target misinformation about genetics concepts and errors of omission in evaluating a variant's clinical significance for a patient.

\begin{tabular}{|c|c|c|c|c|c|c|c|c|}
\hline \multirow[b]{2}{*}{ Patient ID } & \multirow[b]{2}{*}{ Gene } & \multirow[b]{2}{*}{ Disease } & \multirow[b]{2}{*}{ Classification } & \multicolumn{5}{|c|}{ Appropriateness of Clinical Management } \\
\hline & & & & $\begin{array}{r}\text { Inappropriate } \\
\mathrm{N}(\%)\end{array}$ & $\begin{array}{r}\text { Uncertain } \\
\text { Appropriateness } \\
\mathrm{N}(\%)\end{array}$ & $\begin{array}{r}\text { Appropriate } \\
\text { N (\%) }\end{array}$ & $\begin{array}{r}\text { Median } \\
\text { Score }\end{array}$ & Agreement \\
\hline 1 & ARSE & Chondrodysplasia punctata & VUS: FP & $3(27.3)$ & $5(45.5)$ & $3(27.3)$ & 4 & Yes \\
\hline 2 & KCNQ1 & Romano-Ward syndrome & LP & $0(0)$ & $2(18.2)$ & $9(81.8)$ & 7 & Yes \\
\hline 3 & PPOX & Variegate porphyria & $P$ & $1(9.1)$ & $2(18.2)$ & $8(72.7)$ & 8 & Yes \\
\hline 4 & LHX4 & $\begin{array}{l}\text { Combined pituitary hormone } \\
\text { deficiency }\end{array}$ & $P$ & $9(81.8)$ & $2(18.2)$ & $0(0)$ & 3 & Yes \\
\hline 5 & TNNT2 & Hypertrophic cardiomyopathy & VUS: FP & $1(9.1)$ & $1(9.1)$ & $9(81.8)$ & 7 & Yes \\
\hline 6 & F5 & $\begin{array}{l}\text { Factor V Leiden } \\
\text { Thrombophilia, Multifactorial }\end{array}$ & Risk allele & $6(54.5)$ & $5(45.5)$ & $0(0)$ & 3 & Yes \\
\hline 7 & PDE11A & $\begin{array}{l}\text { Primary pigmented } \\
\text { micronodular adrenocortical } \\
\text { disease }\end{array}$ & VUS: FP & $2(18.2)$ & $3(27.2)$ & $6(54.5)$ & 7 & Yes \\
\hline 8 & COL2A1 & $\begin{array}{l}\text { Spondyloepiphyseal dysplasia } \\
\text { congenita }\end{array}$ & LP & $2(18.2)$ & $2(18.2)$ & $7(63.6)$ & 7 & Yes \\
\hline 9 & ANK2 & $\begin{array}{l}\text { Ankyrin-B related cardiac } \\
\text { arrhythmia }\end{array}$ & LP & $0(0)$ & $5(45.5)$ & $6(54.5)$ & 7 & Yes \\
\hline 10 & HFE & Hereditary Hemochromatosis & $P$ & $2(18.2)$ & $3(27.3)$ & $6(54.5)$ & 7 & Yes \\
\hline 11 & RDH5 & Fundus albipunctatus & $\mathrm{P}$ & $1(9.1)$ & $0(0)$ & $10(90.9)$ & 9 & Yes \\
\hline
\end{tabular}

Expert recommendations for guidelines for the primary care management of a genetic variant in an ostensibly healthy patient

1. Obtain additional personal and family history information, targeting subtle phenotypic associations with the identified variant and keeping in mind the possibility of variable expressivity.

2. Evaluate pathogenic variants through relevant physical examinations, laboratory testing, and imaging if not previously performed, even if the variant seems unrelated to patient's phenotype. Consider referring the patient to a specialist in the associated condition.

3. Seek genetics consultation for pathogenic variants, including genetic counseling for implications for family members.

4. Do not make medical decisions about a variant of uncertain significance, but counsel the patient that its classification should be followed over time.

5. Consult resources such as GeneReviews, OMIM, and the medical literature for more information about conditions of concern.

6. Understand key concepts: inheritance patterns, penetrance, variable expressivity, and the classification of variant pathogenicity.

Expert appropriateness ratings (range 1-9) of primary care provider management of genetic variants identified among patients in the MedSeq Project. LP, likely pathogenic; $\mathrm{P}$, pathogenic; VUS:FP, variant of uncertain significance, favor pathogenic.

APPROPRIATENESS OF STRESS ULCER PROPHYLAXIS IN HOSPITALIZED PATIENTS Patrick Chen ${ }^{1}$; Nikhil Reddy ${ }^{4}$; Erin Loesch ${ }^{2}$; Sangeeta Agrawal ${ }^{3}$. ${ }^{1}$ Wright State University, Dayton, $\mathrm{OH} ;{ }^{2}$ dayton VA, Dayton, OH; ${ }^{3}$ Dayton VA, Dayton, $\mathrm{OH} ;{ }^{4}$ Vanderbilt, Nashville, TN. (Control ID \#2433265)

BACKGROUND: The rise in proton pump inhibitor (PPI) prescribing over the past years has raised concerns pertaining to overutilization of this class of medications and an associated increase in costs. As healthcare providers become more conscious of erroneous expenditures, there are concerns with the increasing numbers of hospitalized patients being started on PPIs, incorrect dosages, excessive lengths of therapy, and inappropriate continuation of PPIs upon discharge. Additionally, newly published literature reviewing adverse drug events of PPIs include the risk for infection, such as $C$. difficile and pneumonia, increased bone fractures, potential reduction of clopidogrel efficacy via cytochrome P450 (CYP) $2 \mathrm{C} 19$ inhibition, and b12 deficiency which questions the safety of this previously assumed benign medication class. A medication use evaluation was performed to determine the appropriateness of stress ulcer prophylaxis for the Veterans Affairs (VA) hospital in Dayton, Ohio. 
METHODS: A retrospective chart review was performed on 963 patients admitted to the Dayton Veteran Affairs Medical Center (DVAMC), during a 4 month period, who were prescribed PPIs during hospital admission. The data recorded included patient demographics, PPI type, dose, route, frequency of medication administration, admitting diagnosis, PPI started prior to admission, PPI upon discharge, documented reason for PPI, and if patient met criteria for stress ulcer prophylaxis (SUP). The American Society of Health-System Pharmacists (ASHP) guidelines published in 1999 were used to determine if patients met SUP criteria.

RESULTS: The most frequently prescribed PPI was omeprazole $20 \mathrm{mg}$ once daily. Only $6 \%$ of patients were prescribed a PPI via intravenous route, with pantoprazole $40 \mathrm{mg}$ twice daily being the most common. The vast majority of the patients were admitted to the medical ward, while only $3 \%$ were admitted to the ICU. Sixty-seven percent of the patients were on a PPI prior to admission and $75 \%$ of patients were continued on a PPI at the time of discharge. For $65 \%$ of the patients, the documented reason for PPI use was continuation of an outpatient medication upon admission. SUP was documented in $32 \%$ of patients with only $10 \%$ meeting SUP criteria; $22 \%$ did not meet SUP criteria. The remaining $67 \%$ of patients were excluded because they were on prescription PPIs as an outpatient, prior to admission.

CONCLUSIONS: Inappropriate PPI administration was found in this retrospective review. There were approximately twice as many patients who did not meet SUP criteria compared to patients with appropriate SUP criteria. This retrospective review highlights the inappropriate overuse of SUP at the DVAMC and the need for future cost-saving initiatives and collaborative team methods to increase patient safety by curbing inappropriate use of the PPI medication class

\section{ARE CROWD-SOURCED POPULATIONS SIMILAR TO UNITED STATES ADULTS?: A COMPARISON OF DEMOGRAPHIC AND HEALTH DATA COLLECT- ED FROM AMAZON MECHANICAL TURK AND NATIONAL SURVEYS Veronic Yank $^{1}$; Sanjhavi Agarwal ${ }^{3}$; Pooja Loftus ${ }^{3}$; Steven Asch ${ }^{2}$; David Rehkopf ${ }^{3} .{ }^{1}$ UCSF, Menlo Park, CA; ${ }^{2}$ VA/Stanford, Menlo Park, CA; ${ }^{3}$ Stanford, Stanford, CA. (Control ID \#2462687)}

BACKGROUND: Use of computer-based crowd-sourcing for data collection is an attractive alternative to traditional national health surveys because it is cheaper, faster, and less resource-intensive. The use for health research has skyrocketed but claims of generalizability to the United States (US) population have not been well validated. If not generalizable, crowd-sourced data may mislead researchers, policy makers, and patients. We sought to identify areas of similarity or difference between crowd-sourced respondents and the US adult population on characteristics pertinent to long-term health.

METHODS: We used weighted data from two US nationally-representative, phone-based health surveys, the Behavioral Risk Factor Surveillance System survey (BRFSS, 2014) (of adults age 18 years or greater) and the Health and Retirement Study (HRS, 2012) (of adults age 50 years or greater) to identify characteristics of the US population. From these studies we adapted questions on individual characteristics known to influence cardiovascular morbidity and mortality - specifically, socio-demographical characteristics (age, gender, race/ethnicity, educational attainment, income) and lifestyle- and disease-related (smoking, physical activity, obesity, diabetes mellitus, hypertension, hyperlipidemia) characteristics. We used the world's largest crowd-sourcing platform, Amazon Mechanical Turk (MTurk), to collect crowd-sourced respondent data on these characteristics from a convenience sample of US-based adults registered with MTurk. We stratified data from crowd-sourced and national surveys by age and used logistic regression models to identify characteristics that differed significantly between groups. We applied geocoding software to identify the nearest zip code location of de-identified crowd-sourced respondents and compared these by visual inspection to census-determined population density maps.

RESULTS: We collected and compared data from 2015 crowd-sourced respondents to data from 428,211 BRFSS and 14,343 HRS respondents. Locations of crowd-sourced respondent mapped to 49 US states and generally corresponded with US population densities. [Figure] Crowd-sourced respondents were younger (mean age 33 years (range 18-75; SD 11)) than
US adults (mean 44 (SD 16)) and more likely to be non-Hispanic White and collegeeducated. [Table] With respect to lifestyle-related characteristics, they were less likely to be physically active and more likely to be former smokers. Among 40-59 year olds, crowdsourced respondents were similar to US adults in disease-related characteristics of obesity, diabetes, hypertension, and hyperlipidemia, whereas among 18-39 year olds crowd-sourced respondents were more likely to have diabetes. Those 60-75 years old comprised a small proportion of crowd-sourced respondents ( $3 \%$ ) and were more likely than the age-matched population to be current smokers but were similar in chronic disease characteristics.

CONCLUSIONS: Crowd-sourced health-related data may be collected from and generalizable to some subpopulations of US adults, particularly 40-59 year olds who are White, college-educated, and sedentary. However, studies focused on younger or older adults, racial and ethnic minorities, or populations with lower educational attainment should be cautious about relying on or generalizing from crowd-sourced data until determining that their target populations can be reached by this method. Future studies should investigate whether data collected from other digital platforms such as smartphones, social networking sites, and online search engines should have similar caveats for their use in health-related research.

Odds Ratios of Crowd-sourced Respondents vs. US Population for Demographic and Health-related Characteristics

\begin{tabular}{llllll}
\hline \hline Age range, years & $18-29$ & $30-39$ & $40-49$ & $50-59$ & $60-75$ \\
Total N & 51,088 & 56,072 & 68,716 & 101,130 & 146,087 \\
(Crowd-sourced N) & $(992)$ & $(587)$ & $(223)$ & $(151)$ & $(62)$ \\
Male & $1.22^{*}$ & $1.43^{*}$ & 0.90 & 0.79 & $0.58^{*}$ \\
White & $1.41^{*}$ & 1.25 & 1.94 & $4.97^{*}$ & 0.72 \\
Black & $0.55^{*}$ & $0.31^{*}$ & 0.86 & 0.84 & 0.39 \\
Asian & $1.95^{*}$ & 0.85 & 1.06 & $\wedge$ & 0.31 \\
Hispanic & $0.44^{*}$ & $0.34^{*}$ & $0.27^{*}$ & $0.26^{*}$ & 0.38 \\
Attended college & $5.69^{*}$ & $5.16^{*}$ & $9.67^{*}$ & $6.03^{*}$ & $10.41^{*}$ \\
Income & $1.00^{*}$ & $1.00^{*}$ & $1.00^{*}$ & $0.99^{*}$ & $0.99^{*}$ \\
Former smoker & 1.02 & $1.40^{*}$ & $1.78^{*}$ & 1.12 & $2.28^{*}$ \\
Current smoker & $0.48^{*}$ & 0.82 & 1.13 & 0.96 & $2.71^{*}$ \\
Physically active & 0.90 & $0.62^{*}$ & $0.64^{*}$ & $0.42^{*}$ & $0.36^{*}$ \\
Obesity & 0.99 & $0.98^{*}$ & 0.97 & 1.01 & 1.02 \\
Diabetes & $2.19^{*}$ & $2.22^{*}$ & 1.40 & 1.44 & 0.70 \\
Hypertension & 0.82 & 0.88 & 0.77 & 0.93 & 0.99 \\
Hyperlipidemia & $1.34^{*}$ & 0.86 & 0.88 & 0.77 & 0.71 \\
\hline
\end{tabular}

Logistic regression models used BRFSS data and included covariates shown in the table as well as age and age2 (not shown). Models using HRS data (age ranges 50-59 \& 60-75) were similar but omitted in the interest of space. ${ }^{*} p<0.05$. ^ There were too few crowdsourced Asian respondents to include in model.

Location of Crowd-sourced Respondents on US Population Density Map Location of Crowd-sourced Respondents on US Population Density Map

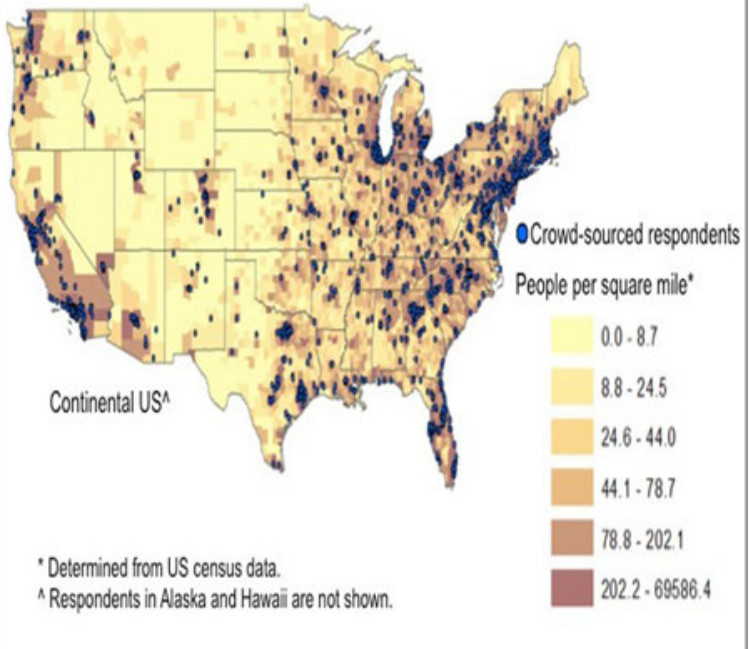


ARE PROVIDERS PREPARED TO ENGAGE YOUNGER WOMEN IN SHARED DECISION MAKING FOR MAMMOGRAPHY? Kathryn A. Martinez ${ }^{1}$ ${ }^{1}$; Abhishek Deshpande ${ }^{1,}{ }^{1}$; Allison L. Ruff ${ }^{4}$; Shari Bolen ${ }^{3}$; Kathryn Teng ${ }^{2}$; Michael B. Rothberg ${ }^{1,1}$. ${ }^{1}$ Cleveland Clinic, Cleveland, OH; ${ }^{2}$ MetroHealth Medical Center, Cleveland, $\mathrm{OH} ;{ }^{3}$ MetroHealth/Case Western Reserve University, Cleveland, $\mathrm{OH} ;{ }^{4} \mathrm{University}$ of Michigan, Cleveland, OH. (Control ID \#2468546)

BACKGROUND: For mammography, the U.S. Preventive Services Task Force recommends providers engage average risk women aged 40-49 years in shared decision making (SDM), which requires provider knowledge, time, and competence in discussing screening. However, it is unknown whether providers possess these prerequisites. It is also not known how providers' estimates of harms and benefits of screening affect whether they believe the decision to screen should be patient-centered.

METHODS: An online survey of primary care providers (MDs, NPs, and PAs) was conducted at four major health centers in greater Cleveland in 2015. The survey inquired about provider knowledge of harms and benefits of screening in younger women, including: the impact of screening on breast cancer-specific mortality, risk of additional imaging, biopsy, or overtreatment following a mammogram, and likelihood of a mammogram detecting a truepositive cancer. Key knowledge was defined as correct estimation of both the mortality benefit of screening and the risk of overtreatment. The survey also assessed provider-reported time typically spent with patients discussing mammography (dichotomized to $<5$ min versus $\geq 5 \mathrm{~min}$ ). Perceived competence (low, somewhat, high) at discussing mammography with patients was assessed via a composite measure comprised of 1) whether the provider felt they had sufficient knowledge to counsel patients (yes or no) and 2) confidence in discussing screening with patients (none, moderate, a lot). Among providers possessing key knowledge, the percentage who also reported feeling at least somewhat competent discussing screening and typically spending $5 \mathrm{~min}$ or more with patients was assessed. Multivariable logistic regression was then used to examine the odds of reporting the decision to screen should be patient-centered (versus not) by provider knowledge of harms and benefits of mammography, as well as provider sex, years of post-training experience, and practice setting (academic versus community-based)

RESULTS: Of 612 providers invited, 220 completed the survey (response rate: $36 \%$ ); $38 \%$ practiced in a community-based setting. Knowledge of common harms of screening was low: 90 and $82 \%$ of providers underestimated the risk of a patient being called back for additional imaging or a breast biopsy, respectively. Sixty-two percent correctly estimated the impact of screening on breast cancer-specific mortality; $16 \%$ underestimated and $21 \%$ overestimated the risk. Most (77\%) reported typically spending less than 5 min with patients discussing mammography. Only $48 \%$ said that they were at least somewhat competent at discussing screening. Of the $51 \%$ of providers who demonstrated key mammography knowledge, only $20 \%$ both felt at least somewhat competent at discussing mammography with patients and also typically spent five or more minutes doing so. In the multivariable logistic regression model, overestimating the impact of screening on mortality was associated with lower odds of reporting that the decision to screen should be patient-centered (OR:0.31, $p=0.003$ ), as was practicing in a community-based setting (OR:0.42, $p=0.021)$.

CONCLUSIONS: The majority of providers in our sample appeared to be inadequately equipped to engage in SDM with younger patients regarding mammography decisions. Providers who overestimate the mortality benefit of screening may be less inclined to engage patients in SDM

ARE RACIAL DIFFERENCES IN OBESITY AND INSULIN RESISTANCE RELATED TO AGGRESSIVE BREAST CANCER? Emily Gallagher ${ }^{1}$; Derek LeRoith ${ }^{1}$; Sheldon Feldman ${ }^{4}$; Elisa Port ${ }^{1}$; Neil Friedman ${ }^{5}$; Melissa Pilewskie ${ }^{6}$; Alison Estabrook ${ }^{7}$; Susan Boolbol ${ }^{8}$; Rebeca Franco ${ }^{3}$; Kezhen $\mathrm{Fei}^{2}$; Nina A. Bickell ${ }^{1}{ }^{1}$ Icahn School of
Medicine at Mount Sinai, New York, NY; ${ }^{2}$ Ichan School of Medicine at Mount Sinai, New York, NY; ${ }^{3}$ Mount Sinai School of Medicine, New York, NY; ${ }^{4}$ Columbia University Medical Center, New York, NY; ${ }^{5}$ Mercy Medical Center, Baltimore, MD; ${ }^{6}$ Memorial Sloan Kettering Cancer Center, New York, NY; ${ }^{7}$ Mount Sinai West, New York, NY; ${ }^{8}$ Mount Sinai Beth Israel, New York, NY. (Control ID \#2470305)

BACKGROUND: Black women are more likely to die of breast cancer and have more aggressive type cancers than white women. Black women are also more likely to have obesity and insulin resistance than white women. Insulin resistance has been associated with faster tumor growth in mouse models of breast cancer, but has not been studied as a potential mediator of racial disparities in women with breast cancer. We hypothesized that black women would have more aggressive breast cancer at presentation and this would be associated with obesity and insulin resistance.

METHODS: We recruited 281 (80\% white, $20 \%$ black) women with a new primary breast cancer and measured pre-operative fasting blood glucose and insulin, BMI, waist circumference (WC), Nottingham prognostic index (NPI). We classified aggressive breast cancer as a NPI $>4.4$. We calculated HOMA-IR (HOMA) scores and classified insulin resistance a HOMA $>2.8$, the upper quartile of insulin resistance in the US population. Patients self-identified race.

RESULTS: Of 281 women with new breast cancer, average age was 58 years ( $\mathrm{SD}=$ 12.5). One hundred eighty-one (64 \%) were stage I; 89 (32\%) stage II; 11 (4\%) stage III at time of diagnosis. Seventeen percent of cancers had an NPI $>4.4$. There was no racial difference in stage or aggressive breast cancer: $22 \%$ of blacks vs. $16 \%$ of whites ( $p=0.3$ ) had cancers with NPI $>4.4$. Eight percent of women were insulin resistant: $11 \%$ blacks vs. $7 \%$ whites $(p=0.4)$. There were also racial differences in BMI ( 32.3 in B vs. 26.0 in $\mathrm{W} ; p<.0001)$, WC (110 cm in B vs $94 \mathrm{~cm}$ in $\mathrm{W}, p<0.001)$, HOMA score (1.7 in B vs. 1.2 vs $\mathrm{W} ; p=0.002)$, NPI score ( 4.0 in B vs. 3.6 in $\mathrm{W}, p=0.01)$ and triple negative breast cancer ( $15 \%$ in B vs $6 \%$ in W, $p=0.04)$. HOMA score was positively associated with NPI score $(r=0.18 ; p=0.02)$. HOMA-IR partially mediates the effect of black race with more aggressive cancer $(\beta=0.07 ; 95 \% \mathrm{CI}$ : 0.02-0.15).

CONCLUSIONS: Among women with newly diagnosed breast cancer, insulin resistance partially mediates the relationship between black race with more aggressive cancer suggesting that hyperinsulinemia may promote breast cancer progression and contribute to racial disparities in breast cancer survival.

\section{ARE THERE GENDER-BASED DIFFERENCES IN PRESCRIBING STATINS IN FEDERALLY QUALIFIED HEALTH CARE CENTERS? EVIDENCE FROM} OFFICE-GUIDELINES APPLIED TO PRACTICE (OFFICE-GAP) Ade B. Olomu $^{2}{ }^{2}$; NAZIA NAZ S. KHAN ${ }^{1}$; Karen Kelly-Blake ${ }^{2}$; Shilpa Kavuturu ${ }^{3}$, ; Zhehui Luo $^{2}$. ${ }^{1}$ MICHIGAN STATE UNIVERSITY, East Lansing, MI; ${ }^{2}$ Michigan State University, East Lansing, MI; ${ }^{3}$ Michigan state university, East Lansing, MI. (Control ID \#2469514)

BACKGROUND: Statin therapy is equally efficacious for the prevention of major vascular events in men and women at equivalent risk for cardiovascular disease (CVD). Studies evaluating the sex disparity among the acute myocardial infarction survivors demonstrated that women are significantly less likely to be prescribed statin; and have disproportionately poor prognosis. The objective of this study is to explore statin's eligibility and the rate of statin's prescription based on new and old Cholesterol guidelines by gender in Federally Qualified Health Centers (FQHCs).

METHODS: The Office Guidelines Applied to Practice (Office-GAP) study is a quasiexperimental, two-center study designed to improve collaboration between patients and providers and to improve outcomes for low-income populations in outpatient clinical settings. The Office-GAP Program included 1) Physician training for patient activation/ 
engagement 2) Patient activation /engagement intervention during a group visit and 3) Use of the Office-GAP checklist tool during follow-up provider visits (3 and 6 month). We enrolled patients with coronary heart disease (CHD) and Diabetes Mellitus (DM) from October 2010 to March 2014. We computed 10 year- atherosclerotic cardiovascular disease (ASCVD) risks scores for men and women based on American College of Cardiology guidelines. We measured the rate of prescription of Statin to patients with DM based on new and old Cholesterol guidelines by gender in FQHCs.

RESULTS: Of the 206 patients ( 85 men and 121 women), 68 had ASCVD, 192 had DM, and 54 had both ASCVD and DM. The mean age for men was 58.4+11.0 and for women 56.0+11.6. Among men, 31 (36.5\%) were white, 36 (42.5\%) were black, and 18 $(21.2 \%)$ were other races. Among women, 55 (45.5\%) were white, 41 (33.9 \%) were black and 25 (20.7\%) were other races. Based on old guidelines, $36(42.4 \%)$ men and 32 (26.4 \%) women with ASCVD and 49 (57.6 \%) men and 89 (73.6 \%) women with DM and no ASCVD were eligible for Statin, whereas based on new guidelines, 36 (42.4\%) men and 32 (26.4\%) women with ASCVD and $46(54.1 \%)$ men and 77 (63.6\%) women with DM and high risk scores without ASCVD were eligible for Statin. For rate of statin's prescription based on old and new guidelines, out of 68 patients with ASCVD eligible for statin, 24/36 (66.7 \%) men vs. 20/32 (62.5\%) women were on statin. Based on old guidelines out of 138 patients with DM eligible for statin, 33/49 (67.4 \%) men vs. 49/89 $(55.1 \%)$ women were prescribed statin. Based on new guidelines, 31/46 (67.4 \%) men and 45/77 (58.4\%) women with DM and high risk scores without ASCVD were prescribed Statin. We found the 10-year ASCVD risk score was significantly higher in black men (0.29) than black women (0.18) ( $p=0.004$ based on the Wilcoxon rank sum test). Black males had higher ASCVD risk scores compared to their white counterpart ( $p$ value $=0.043)$. However, there was no difference in the risk score between men $(0.21)$ and women $(0.16)$ of white or other races $(P=0.100)$

CONCLUSIONS: Our study revealed under prescription of Statin to both men and women with ASCVD and DM in FQHCs. Under both the old and new guidelines, men with ASCVD and DM were prescribed Statin more than women. There was a significant difference in the distribution of ASCVD risk score between black diabetic men and women whereas there was no difference between men and women in white and other races

ASSESSING PROBLEMATIC SUBSTANCE USE IN HIV CARE: WHICH QUESTIONS ELICIT ACCURATE PATIENT DISCLOSURES? Wynne Callon ${ }^{3}$; Mary Catherine Beach ${ }^{4}$; Somnath Saha ${ }^{6}$; Geetanjali Chander ${ }^{4}$; Ira Wilson ${ }^{1}$; Michael B. Laws $^{1}$; Victoria L. Sharp ${ }^{7}$; Jonathan A. Cohn ${ }^{8}$; Richard D. Moore ${ }^{2}$; Philip T. Korthuis ${ }^{5}$. ${ }^{1}$ Brown University, Providence, RI; ${ }^{2}$ Johns Hopkins, Baltimore, MD; ${ }^{3}$ Johns Hopkins School of Medicine, Baltimore, MD; ${ }^{4}$ Johns Hopkins University, Baltimore, MD; ${ }^{5}$ Oregon Health \& Science University, Portland, OR; ${ }^{6}$ Portland VA Medical Center, Portland, OR; ${ }^{7}$ St. Luke-s Roosevelt Hospital, New York, NY; ${ }^{8}$ Wayne State University, Detroit, MI. (Control ID \#2437684)

BACKGROUND: Substance use is associated with higher rates of antiretroviral nonadherence and poor HIV outcomes. This study examined how HIV care providers assess substance use, and which questions elicit accurate patient disclosures.

METHODS: We conducted a conversation analysis of audio-recorded encounters between 56 providers and $162 \mathrm{HIV}$ patients reporting active substance use in post-encounter interviews (cocaine or heroin use in the past 30 days, $>4$ days intoxicated in past 30 days, or AUDIT score $\geq 8$ ). We characterized the types of questions used to assess substance use, and evaluated the frequency with which each question type elicited accurate disclosure.

RESULTS: In 55 reports of active substance use, providers already knew about the use $(n=16)$ or patients disclosed unprompted $(n=39)$. Among the remaining 155 instances of substance use in which providers had the opportunity to elicit disclosure, 78 reports (50\%) of substance use were not discussed. Of the remaining 77 reports in which the provider asked about substance use, 55 (71 \%) patients disclosed and 22 (29\%) did not disclose. Questions were classified as: open-ended ( $n=18$, “How's the drinking going?"); normalizing ( $n=14$, “When was the last time you used?"); closed-ended ( $n=36$, "Have you used any cocaine? "); leading towards non-use ( $n=9$, "Have you been clean? "). Accurate disclosure followed $100 \%$ of open-ended and normalizing questions, $58 \%$ of closedended questions, and $22 \%$ of leading questions. After adjusting for drug type, closedended questions were $41 \%$ less likely ( $p<0.001$ ), and 'leading' questions $78 \%$ less likely ( $p=0.016)$ than broad and normalizing questions to elicit disclosures.

CONCLUSIONS: Providers in this sample missed almost half of the opportunities to identify and discuss substance use with HIV patients. Providers can increase the probability of patient disclosure by using open-ended or normalizing questions that ask about the "last time" that the patient used drugs or alcohol.

ASSESSING THE ABILITY OF DAY INTERNS TO COMMUNICATE THEIR CONCERN OF PATIENT DETERIORATION RISK TO NIGHT INTERNS IN THE UNDERSTANDING PHYSICIAN SIGNOUT RISK PERCEPTIONS STUDY Emmanuelle B. Yecies ${ }^{2}$; Alexander Davis ${ }^{3}$; Baruch Fischhoff ${ }^{3}$; Jonathan Arnold $^{2}$; Jon R. Grace ${ }^{2}$; Andrew Klobuka ${ }^{2}$; Deepika Mohan ${ }^{1}$; Janel Hanmer ${ }^{1} .{ }^{1}$ University of Pittsburgh, Pittsburgh, PA; ${ }^{2}$ University of Pittsburgh Medical Center, Pittsburgh, PA; ${ }^{3}$ Carnegie Mellon University, Pittsburgh, PA. (Control ID \#2469151)

BACKGROUND: To ensure high quality patient care in an inpatient setting, providers must be able to communicate their concerns about patient risk of deterioration to other providers. With new duty hour restrictions, there has been an increase in handoffs between day and night physicians, and many studies have been performed to identify how to best teach resident physicians to communicate relevant information. However, few studies have been conducted to identify whether inherent aspects of training improve handoff quality. Our hypothesis is that certain intern experiences, such as previous night coverage experience, previous ICU experience, and time spent on general medicine wards, would be associated with greater agreement between day and night team assessments of patient decompensation risk.

METHODS: The project was approved by the local IRB and all physicians gave verbal consent to participate in the study. Data was collected by clinical researchers in a nonblinded fashion 2-3 days per week (separated by at least 2 days) over 5 months at a single tertiary care academic medical center. Study participants included day and night interns that were rotating on an inpatient medicine teaching service at the time of collection. Day physician team structure consisted of two interns, one senior (second or third year) resident, and one attending. Day interns remained on a single team over a 4 week period while night interns rotated in 2 week cycles. Day interns were privately asked a standardized question 1-2 h prior to sign-out to assess each patient's percent chance of rapid response team activation or ICU transfer over the next $24 \mathrm{~h}$. Night interns were privately asked the same question 1-2 $\mathrm{h}$ after sign-out. Raw percentages were converted on an individual basis to $\mathrm{Z}$ scores to standardize risk perception then correlations were compared for each week of the rotation, previous night coverage experience, and ICU experience. RESULTS: Risk judgments were made by 60 interns on the day team and 52 interns on the night team, resulting in 977 day-night judgment pairs. Results showed that although day and night team interns generally agreed with each other on which patients were at greatest risk ( $r=0.43$ overall), that agreement did not increase over the intern's monthlong rotation (First week $r=0.48$; Second week $r=0.47$; Third week $r=0.35$; Fourth week $r=0.35$ ). The results were the same in sensitivity analyses using raw risk scores. Neither previous night coverage experience nor previous ICU experience was associated with increased agreement between the day and night team. Interns did adjust their use of the probability scale with more experience, going from an average judged probability of 
decompensation of $15 \%$ among day interns and $20 \%$ among night interns in the first week to $10 \%$ for day interns and $5 \%$ for night interns in the fourth week. This was still well above the base rate of about $2 \%$ of patients deteriorating.

CONCLUSIONS: Our data do not support the hypothesis that interns' ability to convey risk concern would improve with night coverage experience, ICU experience, or handoff practice. This finding reinforces the importance of active refinement of handoff procedures in the inpatient setting as there is no evidence for passive learning with experience. Effective communication of clinical concern across team handoffs is a prerequisite to ensure consistent care for those patients at greatest risk of deterioration.

\section{ASSESSING THE IMPACT OF COLONOSCOPY COMPLICATIONS ON USE} OF COLONOSCOPY AMONG PRIMARY CARE PHYSICIANS AND OTHER CONNECTED PHYSICIANS Nancy L. Keating ${ }^{1,4}$; A. James O'Malley ${ }^{2}$; Jukka-Pekka Onnela ${ }^{3}$; Bruce E. Landon ${ }^{1,5}$. ${ }^{1}$ Harvard Medical School, Boston, MA; ${ }^{2}$ Dartmouth, Hanover, NH; ${ }^{3}$ Harvard School of Public Health, Boston, MA; ${ }^{4}$ Brigham and Women's Hospital, Boston, MA; ${ }^{5}$ Beth Israel Deaconess Medical Center, Boston, MA. (Control ID \#2466038)

BACKGROUND: Psychological biases can distort treatment decision making. The availability heuristic is one such bias, wherein events that are recent, vivid, or easy to imagine are readily "available" to memory and are therefore judged to be more likely to occur than would be expected based on epidemiologic data. Using Medicare administrative data, we assessed if the occurrence of a serious complication related to screening colonoscopy for a primary care physician's patient influenced the likelihood that the physician's other patients underwent colonoscopy screening in subsequent months. We also assessed if the event changed screening behaviors for other primary care physicians within that primary care physician's practice.

METHODS: Using $100 \%$ Medicare administrative data for patients living in 50 representative hospital referral regions across the U.S. during 2005-2010, we identified all patients aged $\geq 65$ who were continuously enrolled in parts $\mathrm{A}$ and $\mathrm{B}$ of fee-for-service Medicare. We assigned all patients to a primary care physician based on office visits during the current and prior year. We identified all patients who underwent screening colonoscopy and also identified 2 serious complications of which the primary care provider would very likely be aware: gastrointestinal bleed or perforation leading to hospitalization or death within 14 days of the colonoscopy. For each physician in each month, we calculated the proportion of patients assigned to them who had a colonoscopy in that month. We then used a difference-in-difference design with fixed effect Poisson regression models to assess the impact of the colonoscopy adverse event on quarterly rates of screening for patients in future months.

RESULTS: We identified 3,201,604 patients assigned to 31,329 physicians. The mean age of physicians was $50.6(\mathrm{SD}=10.7) ; 74.4 \%$ were male, and they had an average of 133 Medicare patients assigned $(\mathrm{SD}=122)$. Four thousand eight hundred seventy physicians had at least 1 patient with an adverse event between December 2006 and December 2010. Using fixed effects models, the estimated change in the quarterly number of colonoscopies among physicians' patients following an adverse colonoscopy event was significantly lower in quarter 2 following the adverse event (Table), before returning to the rate that would be expected in the absence of an adverse event. There was no detectable decrease in the colonoscopy rates among other primary care physicians in the physicians' practice (all $P>.15$ ).

CONCLUSIONS: Having a patient experience an adverse event from colonoscopy was associated with a small and temporary decline in rates of colonoscopy among a physician's other patients. These findings provide empirical evidence for the influence of notable adverse events, possibly due to the availability heuristic, on care, although the negative impact on care is relatively modest for this clinical condition, wherein screening generally is supported by strong evidence.
Change in quarterly number of colonoscopies among physicians' patients following an adverse colonoscopy event among a physician's patient

\begin{tabular}{lll}
\hline \hline Time after adverse event & Change from baseline (SD) & $P$ value \\
Quarter 1 & $-.007(.007)$ & .34 \\
Quarter 2 & $-.020(.007)$ & .002 \\
Quarter 3 & $-.009(.007)$ & .18 \\
Quarter 4 & $.000(.007)$ & 1.00 \\
\hline
\end{tabular}

ASSESSING THE IMPORTANCE OF DEVELOPING CLINICALLY RELEVANT AMBULATORY QUALITY MEASURES FOR USE IN A PRIMARY CARE POPULATION HEALTH MANAGEMENT PROGRAM Steven J. Atlas ${ }^{2}$; Jeffrey M. Ashburner ${ }^{2}$; Daniel M. Horn ${ }^{2}$; Adrian $\mathrm{Zai}^{2}$; Neil W. Wagle ${ }^{3}$; Sreekanth K. Chaguturu ${ }^{2}$; Timothy G. Ferris ${ }^{1} .{ }^{1} \mathrm{MGH}$, Boston, MA; ${ }^{2}$ Massachusetts General Hospital, Boston, MA; ${ }^{3}$ Brigham and Women's Hospital, Boston, MA. (Control ID \#2465292)

BACKGROUND: Many provider-insurer contracts include withholds for achieving ambulatory quality measures that use insurer claims data and patient attribution to providers. Problems with using insurer data from the provider perspective include inaccurate patient-provider attribution, excluding patients seen who are not covered by a contract, varying definitions across insurers, outcomes that lack clinical relevance, and delayed feedback for monitoring progress and taking timely action. As a result, providers may focus more on measurement deficiencies instead of how they performed. As part of an effort to implement an information technology (IT) tool for population health management (PHM) within an accountable care organization (ACO), we developed and implemented clinically relevant registries for chronic disease management meant to address these shortcomings. We hypothesized that more clinically relevant measures would increase provider buy-in, support investment in IT tools and infrastructure, improve internally defined measures and result in better outcomes of care. We compared overall outcomes and change over time using clinically relevant registries and outcomes with those based upon Healthcare Effectiveness Data and Information Set (HEDIS) definitions within a primary care practice network

METHODS: ACO administrators proposed approaching quality metrics from an allpayer, all-patient perspective using a single set of metric definitions that relied on clinical data derived from electronic health records. To enable this approach, the ACO implemented an IT tool with registries for diabetes mellitus (DM) and cardiovascular disease (CVD). The IT tool identified all network patients and attributed them to providers and/or practices, assigned patients meeting criteria to chronic disease registries, and tracked goal attainment in near-real time. We assessed process and outcomes 6 months after implementing the program. Measures included low density level (LDL) cholesterol in the current calendar year (for patients with DM or CVD), and hemoglobin A1c (A1c) for patients with diabetes between 7/1/14 and 12/31/14. Patients with DM or CVD were considered at goal for LDL cholesterol if it was $<100 \mathrm{mg} / \mathrm{dL}$ or were prescribed a statin (high dose for CVD, moderate or high dose for DM). Patients with DM were considered at goal if Alc $<9 \%$. For each measure, the number of eligible patients and the number at goal were obtained from the IT tool at the start (July 1, 2014) and end (December 31, 2014) of the study period. We adapted HEDIS criteria definitions for numerators and denominators used in the registry tool. The number of eligible patients included those listed in the registry and any recorded denominator exclusions (based upon registry specific exclusions such as misdiagnosis, or patient attribution such as patients entering or leaving the network). The number of patients at goal included: meeting HEDIS criteria, on maximal medical therapy for LDL measures, or a numerator exception (such as competing comorbidity or terminally ill). Overall outcomes and changes in quality measures over a 6 month period were assessed along with numerator and denominator factors that accounted for these outcomes. 
RESULTS: Between the start and end of the follow-up period, the number of patients with DM decreased from 13,850 to 13,190, a difference of 660 fewer patients due to 913 exclusions and 253 new diabetics. The number of patients with CVD decreased from 14,725 to 13,701 , a difference of 1024 fewer patients due to 846 exclusions and 178 leaving the network. Table 1 presents the percent of patients at goal for each measure at the end of the study period and categorizes whether the goal was obtained based upon modified HEDIS criteria, receiving maximal therapy or having a recorded exception. HEDIS criteria underestimated overall goal attainment for DM and CVD LDL measures, but was similar for the DM A1c measure. Table 2 presents the change in the percent at goal over time for each measure and attributes the percent change to numerator or denominator factors. Change in HEDIS criteria accounted for less than $60 \%$ of the overall change in $\%$ at goal for each measure over time.

CONCLUSIONS: We developed and implemented an all-payer, all-patient performance improvement program for primary care chronic disease management within an ACO using clinically relevant patient selection and process/outcome criteria. These results indicate that adding clinical criteria such as the patient receiving maximal medical therapy and numerator exceptions contribute to overall ambulatory quality performance beyond standard HEDIS measures. The impact of such clinically relevant criteria is even more important when assessing change in goal attainment over time.

Table 1: \% at Goal at 6 Months

\begin{tabular}{lllll}
\hline \hline Measure & Overall & $\begin{array}{l}\text { Numerator } \\
\text { HEDIS } \\
\text { criteria }\end{array}$ & $\begin{array}{l}\text { Maximal } \\
\text { Rx }\end{array}$ & Exceptions \\
Diabetes: & $9384 / 13,190$ & 7866 & 1067 & 451 \\
LDL & $(71.1 \%)$ & $(59.6 \%)$ & $(8.1 \%)$ & $(3.4 \%)$ \\
Diabetes: & $9482 / 13,190$ & 9207 & & 275 \\
Alc & $(71.9 \%)$ & $(69.8 \%)$ & & $(2.1 \%)$ \\
CVD: & $10,403 / 13,701$ & 8391 & 1428 & 584 \\
LDL & $(75.9 \%)$ & $(61.2 \%)$ & $(10.4 \%)$ & $(4.3 \%)$ \\
\hline
\end{tabular}

Table 2: Change in $\%$ at Goal Over Time

\begin{tabular}{lcllll}
\hline \hline Measure & Overall & $\begin{array}{l}\text { Numerator } \\
\text { HEDIS } \\
\text { Criteria }\end{array}$ & $\begin{array}{l}\text { Maximal } \\
\mathrm{Rx}\end{array}$ & Exception & $\begin{array}{l}\text { Denominator } \\
\text { Total }\end{array}$ \\
Diabetes: & $7.8 \%$ & $4.4 \%$ & $1.1 \%$ & $3.05 \%$ & $-0.8 \%$ \\
LDL & & & & $1.8 \%$ & $1.1 \%$ \\
Diabetes: Alc & $6.8 \%$ & $3.9 \%$ & $1.0 \%$ & $4.05 \%$ & $0.04 \%$ \\
CVD: LDL & $10.8 \%$ & $5.7 \%$ & & &
\end{tabular}

ASSOCIATION BETWEEN DIRECT ALCOHOL BIOMARKERS AND BENZODIAZEPINES DURING ALCOHOL-RELATED HOSPITALIZATION jasmim

bir $^{2}$; Regina Makdissi ${ }^{1}$; Scott H. Stewart ${ }^{2}$. ${ }^{1}$ State University of New York at Buffalo, Buffalo, NY; ${ }^{2}$ University at Buffalo, Buffalo, NY. (Control ID \#2470314)

BACKGROUND: Nationwide, approximately $17 \%$ of medical and surgical hospitalizations are alcohol-related. The risk for acute alcohol withdrawal is elevated during hospitalization, but unnecessary prophylaxis or mistaken diagnosis can result in adverse events. Newer alcohol consumption biomarkers are predictive of the magnitude and recency of alcohol use, and have the potential to risk stratify for severe withdrawal. We are conducting this study to evaluate the associations between benzodiazepine use, a brief patient-report instrument, and the alcohol biomarkers phosphatidylethanol in blood and ethyl glucuronide in urine. Results will help determine if these tests have the potential to supplement or serve in lieu of self-reported drinking and clinical suspicion in assessing withdrawal risk and determining the need for prophylaxis. We report preliminary findings for this ongoing study.
METHODS: Medically-hospitalized patients with alcohol-related conditions were approached for research participation during their first hospital day. The Alcohol Use Disorders Identification Test (AUDIT) was completed, and blood and urine samples were collected for measurement of phosphatidylethanol (PEth) and ethyl glucuronide (EtG) respectively. Following discharge, the total dose of lorazepam equivalents received during hospitalization was determined from pharmacy records. The distribution of each biomarker and the AUDIT and AUDIT-C was compared between high and low benzodiazepine groups. RESULTS: The first 30 subjects had a median age of 48, were largely male (79\%), nonHispanic white $(79 \%)$ and were admitted for alcohol withdrawal or alcohol detoxification (70 \%). Half abused multiple substances. The median dose of lorazepam equivalents was $20 \mathrm{mg}$ (interquartile range 13-44). Urine EtG was marginally higher in patients receiving higher benzodiazepine dosing (Wilcoxon $p=0.08$ ). PEth was also higher in this group but was not significant or marginally significant $(p=0.31)$. The AUDIT and AUDIT-C results were essentially identical in the high and low benzodiazepine groups.

CONCLUSIONS: The direct alcohol biomarker urine EtG, which imperfectly reflects alcohol use in the prior days, may predict the need for higher benzodiazepine dosing during hospitalization for alcohol-related conditions, but statistical significance was marginal. PEth, the AUDIT, and AUDIT-C were not significantly associated with benzodiazepine dosing, although observed differences in PEth are intriguing. These are preliminary conclusions, and we are continuing to accrue patients to more precisely characterize the predictive capacity of blood PEth and urine EtG at the time of hospital admission.

\section{ASSOCIATION BETWEEN POSTERIOR REVERSIBLE ENCEPHALOPATHY SYNDROME AND RENAL ARTERY STENOSIS: A LARGE RETROSPECTIVE} ANALYSIS Hardik Chhatrala ${ }^{1}$; Monoj K. Konda ${ }^{3}$; Shreya Ghetiya ${ }^{5}$; Christopher Di Felice $^{2}$; Sourabh Aggarwal ${ }^{4}$. ${ }^{1}$ Homer Stryker MD WMU School of Medicine, Kalamazoo, MI; ${ }^{2}$ Western Michigan Universiry Homer Stryker M.D. School of Medicine, Portage, MI; ${ }^{3}$ Western Michigan University Homer Stryker M.D. School of Medicine, Kalamazoo, MI; ${ }^{4}$ Western Michigan University School of Medicine, Kalamazoo, MI; ${ }^{5}$ Jersey Shore Medical Center, Neptune, NJ. (Control ID \#2468077)

BACKGROUND: Posterior Reversible Encephalopathy Syndrome (PRES) which manifests as headaches, visual disturbance, altered sensorium, and seizures is characterized by posterior cerebral white matter edema on MRI. It is commonly associated with hypertensive encephalopathy. Bilateral renal artery stenosis (RAS) as an underlying etiology is underrecognzied. The association between PRES and RAS has not been studied.

METHODS: We queried Nationwide Inpatient Sample (NIS) for all patients discharged from US hospitals between 2008 and 2012 with discharge diagnosis of PRES and RAS using International Classification Code 9 codes 348.39 and 440.1. A total of 100,823 cases of PRES and 74,169 cases of RAS were identified. Proportions were compared using chisquared testing. Findings were also stratified by age, gender, hypertension, diabetes mellitus. RESULTS: There was a significant $(p<0.0001)$ association between PRES and RAS, with an odds ratio of 1.37 (95\% confidence interval 1.16-1.48). Patients with PRES having RAS were more likely to be females ( $64.6 \%$ vs $51.5 \%, p<0.001)$. Diabetes did not affect the association between PRES and RAS. The crude prevalence of RAS in patients with PRES was $0.26 \%$, and the crude prevalence of PRES in patients with RAS was $0.35 \%$.

CONCLUSIONS: The results of this large retrospective analysis support the existence of an association between PRES and RAS. However, the percentage of patients with both conditions was low, and this may explain why small studies may fail to demonstrate such an association. It could also represent a vast number of undiagnosed cases of RAS in patients who have PRES. These findings underline the importance of screening for RAS with renal duplex ultrasound in patients with PRES who presented with hypertensive emergency. These results suggest the need for prospective studies to further explore the association between PRES and RAS. 
ASSOCIATION BETWEEN PSYCHIATRIC DISORDERS AND THE PHYSICIAN'S PRACTICE PATTERNS OF THE NEW OUT-PATIENTS WHO VISITED AN UNIVERSITY HOSPITAL OFF-HOUR IN JAPAN. Norio Fukumori ${ }^{1}$; Masaki Hyakutake ${ }^{2}$; Yuta Sakanishi ${ }^{1}$; Tsuyoshi Kurata ${ }^{1}$; Itaru Kyoraku ${ }^{2}$; Motosuke Tomonaga $^{2}$; Akihiko Ogushi ${ }^{2}$; Shu-ichi Yamashita ${ }^{2}$; Takashi Sugioka ${ }^{1} .{ }^{1}$ Faculty of Medicine, Saga University, Saga-shi, Japan; ${ }^{2}$ Saga University Hospital, Saga, Japan. (Control ID \#2470226)

BACKGROUND: Since patients with psychiatric disorders (PPD) visit hospitals with various chief complaints, the clinical decision making for PPD is often difficult. Thus, the medical cares patients received may be different by the presence or absence of psychiatric disorders. We examined the association between presence of psychiatric disorders and the contents of medical care of patients visiting an university hospital off-hour.

METHODS: We reviewed the medical charts of patients visiting the emergency department of Saga University Hospital off-hour from April 2013 to March 2015. Their chief complaints and final diagnoses were encoded by the international classification of primary care, second edition (ICPC-2). PPD was defined by their already-known diagnoses from the medial chart and the physician's interview. Patient's sex, age, whether performed blood tests, imaging studies such as X-ray or CT or MRI, consultations to specialists and admissions were obtained from the medical charts. Multivariate logistic regression analysis was performed to calculate the odds ratio of returning home without any examinations and consultations to specialists.

RESULTS: Two thousad thirty-three patients were reviewed. The mean age was $46.1 \pm$ 20.3, 962 patients ( $45.8 \%$ ) were male. Physicians less likely performed consultations to specialists or hospitalized on PPD (odds ratio $0.53,95 \%$ CI. 0.39-0.73, $p<0.001$; odds ratio $0.5595 \%$ CI. $0.34-0.90, p=0.017$; respectively). Further, PPD more often returned home without any examinations and consultations to specialists (odds ratio $1.73,95 \% \mathrm{CI}$. $1.35-2.22, p<0.001)$. After adjusted sex, age, final diagnoses, post graduated year of physicians, statistical significance remained (adjusted odds ratio $1.42,95 \%$ CI. 1.05-1.93, $p=0.025$ ). (Table)

CONCLUSIONS: Among patients who visited an university hospitals off-hour, PPD may more likely returned home without any examinations and consultations to specialists than patients without psychiatric disorders. These results raise the possibility that PPD received not enough care at off-hour hospital.

The odds ratio of physician's practice pattern for PPD by logistic regression analysis

\begin{tabular}{lccllll}
\hline \hline & OR & $95 \% \mathrm{CI}$ & $P$ value & AOR $\dagger$ & $95 \%$ CI & $P$ value \\
& 1.73 & $1.35-2.22$ & $<0.001$ & 1.42 & $1.05-1.93$ & 0.025 \\
$\begin{array}{l}\text { Return home without } \\
\text { any examination }\end{array}$ & & & & & & \\
$\begin{array}{l}\text { Examinations } \\
\quad \text { Blood test }\end{array}$ & 1.10 & $0.88-1.38$ & 0.383 & 0.90 & $0.67-1.21$ & 0.482 \\
$\quad$ X-ray & 0.87 & $0.68-1.10$ & 0.237 & 1.07 & $0.80-1.45$ & 0.643 \\
CT & 0.74 & $0.54-1.01$ & 0.058 & 0.88 & $0.60-1.28$ & 0.508 \\
MRI & 0.75 & $0.25-2.21$ & 0.599 & 0.57 & $0.10-3.29$ & 0.531 \\
$\quad$ Endoscopy & 1.04 & $0.34-3.19$ & 0.941 & NA & NA & NA \\
$\quad$ Consultations to & 0.53 & $0.39-0.73$ & $<0.001$ & 0.65 & $0.45-0.93$ & 0.020 \\
specialists & & & & & & \\
$\quad$ Hospitalization & 0.55 & $0.34-0.90$ & 0.017 & 0.58 & $0.34-1.00$ & 0.050 \\
\hline
\end{tabular}

PPD, patients with psychiatric disorders; OR, odds ratio; CI, confidence interval; AOR, adjusted odds ratio; $\mathrm{CT}$, computed tomography; $\mathrm{MRI}$, magnetic resonance imaging $\dagger$, adjusted by sex age final diagnoses postgraduated year of physicians

ASSOCIATION BETWEEN RECEIPT OF CANCER SCREENINGS AND PRIOR EXPERIENCE WITH CANCER SCREENINGS Glen B. Taksler; Michael B. Rothberg. Cleveland Clinic, Cleveland, OH. (Control ID \#2468633)
BACKGROUND: An individual's decision to screen for various cancers may be influenced by his/her prior experience with cancer screenings. Bad experiences, such as a false positive test result, might increase or decrease an individual's willingness to continue screening for cancer in the future.

METHODS: Using 8 years of electronic medical records data, we identified individuals aged 50-75 years who had received regular primary care (visits in $\geq 3$ different calendar years across $\geq 5$ years) from 45 locations of a single healthcare system between 2006 and 2013. In each year, we defined a patient's experience with cancer screening as his/her prior receipt of colorectal, breast, cervical and prostate cancer screenings, as well as previous false positive test results and cancer diagnoses. Using logistic regression models, we examined the association between patients' prior experience with cancer screening for each type of cancer, and future receipt of colorectal, breast, cervical and prostate cancer screenings (4 separate outcomes - one per cancer type). We adjusted statistical significance for multiple comparisons using a Bonferroni correction; $5 \%$ significance corresponded to $P=0.0125$. Results were stratified by gender and adjusted for demographics (age, race, ethnicity, marital status, zip code median income); cardiovascular risk factors (tobacco, body mass index, blood pressure, lipids, HbA1c); comorbidity (Charlson score $=0, \geq 1$ ); anticoagulants (for colorectal cancer screening only); type of health insurance; primary care physician specialty (internal medicine, family medicine); number of primary care visits in the previous 365 days; and calendar year.

RESULTS: We identified 78,188 individuals ( $58 \%$ female, $42 \%$ male) aged $50-75$ years; 66,89 , and $86 \%$ of at-risk individuals were up-to-date with colorectal, breast, and cervical cancer screenings, respectively, and $66 \%$ of males had received prostate cancer screening in the prior 2 years. Individuals who received one cancer screening were more likely to obtain other cancer screenings. For example, women who had received breast cancer screening in the prior 2 years were more likely to be up-to-date with colorectal cancer screening $(\mathrm{AOR}=1.86,95 \% \mathrm{CI}=1.75-1.97)$ and cervical cancer screening $(\mathrm{AOR}=$ $2.96,95 \% \mathrm{CI}=2.76-3.17)$, and men who had received prostate cancer screening in the prior 2 years were more likely to be up-to-date with colorectal cancer screening $(\mathrm{AOR}=$ $1.62,95 \% \mathrm{CI}=1.54-1.70)$ (all $P<0.001)$. Associations were stronger for individuals who previously had a false positive test. Women were more likely to be up-to-date with breast cancer screening if they previously had a false positive mammogram $(\mathrm{AOR}=2.47$, $95 \% \mathrm{CI}=2.32-2.64, P<0.001)$. The same women were also more likely to be up-to-date with colorectal cancer screening $(\mathrm{AOR}=1.37,95 \% \mathrm{CI}=1.31-1.43)$ and cervical cancer screening $(\mathrm{AOR}=1.38,95 \% \mathrm{CI}=1.32-1.44)$ (both $P<0.001)$. Coefficients were similar for women who previously underwent a breast biopsy. Additionally, women were more likely to be up-to-date with cervical cancer screening if they previously had a false positive FOBT test $(\mathrm{AOR}=1.14,95 \% \mathrm{CI}=1.08-1.20, P<0.001)$. Similarly, men who previously had a false positive PSA test were more likely to be up-to-date with colorectal cancer screening $(\mathrm{AOR}=1.32,95 \% \mathrm{CI}=1.23-1.41, P<0.001)$. Women who were previously diagnosed with breast cancer but did not undergo mastectomy were also more likely to be up-to-date with colorectal cancer screening $(\mathrm{AOR}=1.35,95 \% \mathrm{CI}=1.23-1.49)$ and cervical cancer screening ( $\mathrm{AOR}=1.45,95 \% \mathrm{CI}=1.31-1.59$ ). However, women who underwent mastectomy were no more likely to undergo these screenings than women who had not been previously diagnosed with breast cancer $(P=0.15-0.68)$. Men who were previously diagnosed with prostate cancer were more likely to be up-to-date with colorectal cancer screening $(\mathrm{AOR}=1.43$ absent radical prostatectomy and $\mathrm{AOR}=1.80$ with radical prostatectomy, both $P \leq 0.009$ ). However, prior diagnosis with colorectal or cervical cancer screening was not associated with increased receipt of screening at other cancer sites $(P=0.26-0.93)$.

CONCLUSIONS: A patient's prior experience with cancer screenings, particularly for breast and prostate cancer, may influence his/her future willingness to obtain cancer screenings. Patients who previously had received a false positive test result or cancer diagnosis were more likely to engage in future screening for all cancers. 


\section{ASSOCIATION BETWEEN SOCIOECONOMIC STATUS AND BREAST CAN-} CER TREATMENT Marie Dreyer; Liliana E. Pezzin; Emily L. McGinley; Ann Nattinger. Medical College of Wisconsin, Milwaukee, WI. (Control ID \#2466129)

BACKGROUND: Traditionally, population incidence and mortality rates were both lower among women of lower socioeconomic status (SES). During the past three decades this situation has changed: while the incidence rate remained lower among women of lower SES, mortality among women in this group is now significantly higher than that of their high SES counterparts. We sought to understand mechanisms that might contribute to differences across the groups. We hypothesized that poor and near-poor women with breast cancer are less likely to receive recommended initial treatment (e.g. axillary surgery, breast surgery) and adjuvant therapy (e.g. chemotherapy, radiation, hormonal therapy) than women of higher socioeconomic status.

METHODS: Multivariate regression analyses were applied to 11,368 women (aged 6690 years old) identified from SEER-Medicare database as having invasive breast cancer diagnosed in 2006-2009. Socioeconomic status was defined as poor (Medicaid or State-BuyIn beneficiaries), near poor (non-poor women living in neighborhoods ranking in the lowest quartile of per capita income in each state), and wealthy (non-poor women living in neighborhoods ranking in the highest quartile of per capita income in each state). All analyses controlled for demographic (e.g. age, race/ethnicity), clinical (e.g. extent of disease), health status (e.g. comorbidities), as well as market, spatial and health care system characteristics. RESULTS: Significant differences were observed in receipt of recommended initial and adjuvant breast cancer treatment between poor, near-poor and wealthy women with breast cancer. Poor and near-poor women were less likely than wealthy women to receive state-ofthe-art sentinel lymph node biopsy (SLNB) $(p<0.0000 ; p<0.0000)$ and post-breast conserving surgery radiation (OR $0.56,95 \%$ CI $0.46-0.69$; OR $0.7995 \%$ CI $0.62-0.99$ ) and were more likely to undergo a mastectomy instead of recommended breast-conserving surgery (BCS) (OR 1.69, 95 \% CI 1.50-1.90; 1.38, $95 \%$ CI 1.21-1.58). Poor women were less likely than near-poor or wealthy women to receive axillary surgery (OR $0.687,95 \% \mathrm{Cl}$ $0.59-0.80$ ) and guideline-compliant adjuvant chemotherapy (OR 0.80, $95 \%$ CI 0.68-0.93) Unexpectedly, no significant differences were observed in use of aromatase inhibitors (AI) between poor and wealthy women. However, near-poor women who initiated hormonal therapy were less likely than poor or wealthy women to use an $\mathrm{AI}$ and were more likely to use the less costly and less effective tamoxifen (OR 1.72, $95 \%$ CI 1.18-2.50).

CONCLUSIONS: This study confirms that women of lower SES are indeed not as likely to receive recommended breast cancer treatments, as compared to women of higher SES, even if covered by Medicare. The focus on socioeconomic disparities, as opposed to racial, is important as it captures the effect on a broader population of women and may be more amenable to policy changes. In addition, because this study included consistency in insurance status, we are able to disregard coverage effects, a common source for treatment and outcome disparities. To determine the contribution of treatment disparities to overall outcome disparities, future studies will focus on the relationship of these processes of care to mortality while controlling for socioeconomic status.

Odds of receiving treatment, relative to wealthy breast cancer patients

\begin{tabular}{lll}
\hline \hline Process of care & Poor & Near-Poor \\
Axillary surgery & $0.69[0.59-0.80]^{* *}$ & $0.99[0.83-1.20]$ \\
$\begin{array}{l}\text { Sentinel lymph node } \\
\text { biopsy (SLNB) }\end{array}$ & $-1.03^{* *}$ & $-0.85^{* *}$ \\
$\begin{array}{l}\text { Mastectomy } \\
\begin{array}{l}\text { Neoadjuvant } \\
\text { chemotherapy }\end{array}\end{array}$ & $1.69[1.50-1.90]^{* *}$ & $1.38[1.21-1.58]^{* *}$ \\
$\begin{array}{l}\text { Adjuvant } \\
\text { chemotherapy }\end{array}$ & $0.83[0.61-1.10]$ & $1.09[0.78-1.50]$ \\
$\begin{array}{l}\text { Post-BCS radiation } \\
\text { Tamoxifen }\end{array}$ & $0.80[0.68-0.93]^{* *}$ & $1.03[0.87-1.20]$ \\
\hline
\end{tabular}

${ }^{*} p p<0.010 ; * p<0.050$
ASSOCIATION BETWEEN SUBCLINICAL THYROID DYSFUNCTION AND CHANGE IN BONE MINERAL DENSITY - AN INDIVIDUAL PARTICIPANT DATA ANALYSIS OF PROSPECTIVE COHORTS Carole E. Aubert ${ }^{1}$; Drahomir Aujesky $^{1}$; Douglas Bauer ${ }^{2}$; Heike Bischoff-Ferrari ${ }^{3}, 4$; Manuel R. Blum ${ }^{1}$; Anne R.Cappola ${ }^{5}$; Jane A. Cauley ${ }^{6}$; Tinh-Hai Collet ${ }^{7,8}$; Bruno Da Costa ${ }^{9}$; Richard Eastell ${ }^{10}$; Howard A. Fink ${ }^{11}$, ${ }^{12}$; Karina Fischer ${ }^{4,3}$; Apostolos Gogakos ${ }^{13}$; Jacobijn Gussekloo ${ }^{14}$; Kim Naylor ${ }^{10}$; Robin P. Peeters $^{15}$; Fernando Rivadeneira ${ }^{15}$; John Robbins ${ }^{16}$; Nicolas Rodondi ${ }^{1}$; Ann Schwartz ${ }^{2}$; Daniel Segna ${ }^{1}$; André Uitterlinden ${ }^{15}$; Anette van Dorland ${ }^{1}$; Graham R. Williams ${ }^{13}$. ${ }^{1}$ Inselspital, Bern University Hospital, Bern, Switzerland; ${ }^{2}$ University of California, San Francisco, CA; ${ }^{3}$ University Hospital Zurich, Zurich, Switzerland; ${ }^{4}$ University of Zurich, Zurich, Switzerland; ${ }^{5}$ University of Pennsylvania, Philadelphia, PA; ${ }^{6}$ University of Pittsburgh, Pittsburgh, PA; ${ }^{7}$ Lausanne University Hospital (CHUV), Lausanne, Switzerland; ${ }^{8}$ Wellcome Trust-Medical Research Council Institute of Metabolic Science, Addenbrooke's Hospital, Cambridge, United Kingdom; ${ }^{9}$ University of Bern, Bern, Switzerland; ${ }^{10}$ University of Sheffield, Sheffield, United Kingdom; ${ }^{11}$ Veterans Health Care Center, Minneapolis, MN; ${ }^{12}$ University of Minnesota, Minneapolis, MN; ${ }^{13}$ Imperial College London, London, United Kingdom; ${ }^{14}$ Leiden University Medical Center, Leiden, Netherlands; ${ }^{15}$ Erasmus University Rotterdam, Rotterdam, Netherlands; ${ }^{16} \mathrm{UC}$ Davis Comprehensive Cancer Center, Women's Center for Health, Sacramento, CA. (Control ID \#2462671)

BACKGROUND: Subclinical hyperthyroidism (SHyper) is associated with an increased risk for hip and other fractures, but the mechanism is not established. Previous populationbased cohort studies found an average annualized bone loss of $0.2-0.6 \%$ at the femoral neck or total hip in the general population with even higher rates in the elderly. Overt hyperthyroidism decreases bone mineral density (BMD), but data on the association between subclinical thyroid dysfunction (SCTD) and bone loss are controversial. Considering the heterogeneity of current literature, we aimed to clarify whether SCTD was associated with bone loss by pooling individual participant data from population-based prospective cohort studies.

METHODS: Two independent reviewers conducted a systematic literature search in MEDLINE/ EMBASE (from inception to May 2015) without language restrictions identifying prospective cohorts with baseline thyroid status and serial BMD measurements. We collected individual participant data by contacting the authors of each cohort. We classified thyroid status into euthyroidism (Thyroid Stimulating Hormone [TSH] $0.45-4.49 \mathrm{mIU} / \mathrm{L}$ ), SHyper (TSH $<0.45 \mathrm{mIU} / \mathrm{L}$ ) and subclinical hypothyroidism (SHypo, $\mathrm{TSH} \geq 4.50-19.99 \mathrm{mIU} / \mathrm{L}$ ), both with normal free thyroxine concentrations. For our primary outcome of bone loss, defined as annualized percentage change in BMD (\% $\%$ BMD), we pooled location-specific data on serial dual x-ray absorptiometry scans of the femoral neck, total hip and lumbar spine. For the main analysis, we excluded individuals taking bone-active medications at baseline, and conducted two sensitivity analyses: 1) additional exclusion of patients on bone-influencing drugs during follow-up; and 2) excluding patients on bone- or thyroid-influencing medications at any time point. We used established methods with a 2-step approach, first analyzing the association in each cohort of SCTD with bone loss using multivariable linear regression models controlling for age, gender, body mass index, smoking, diabetes mellitus and menopausal status, and in a second step calculating pooled estimates using random-effects models. We used $\mathrm{I}^{2}$ indices for assessing heterogeneity across cohorts.

RESULTS: In our individual data analysis of 6 prospective cohorts (from the United States, United Kingdom, Germany, France and the Netherlands) including 7636 participants (median age: 72 years, $56.9 \%$ women), the prevalence was $5.9 \%$ for SHyper and $7.8 \%$ for SHypo at baseline. During follow-up, pooled mean \% $\%$ BMD was -0.59 (95\% confidence interval $[\mathrm{CI}]:-0.63,-0.54)$ at the femoral neck, -0.55 (CI: $-0.61,-0.49)$ at the total hip and 0.32 (CI: $-0.21,0.84$ ) at the lumbar spine. Relative to euthyroidism, SHyper was associated with greater annualized bone loss at the femoral neck (pooled multivariable $\% \Delta \mathrm{BMD}=-0.18$; CI: -0.34 , $-0.02 ; \mathrm{I}^{2}=0.0 \%$ ), with a similar pattern at the total hip ( $\% \Delta \mathrm{BMD}=-0.14$; CI:-0.38,0.10; 
$\left.\mathrm{I}^{2}=52.7 \%\right)$, but not at the lumbar spine ( $\% \Delta \mathrm{BMD}=0.03$; CI: $\left.-0.30,0.36 ; \mathrm{I}^{2}=24.8 \%\right)$. In contrast, SHypo was not associated with changes in $\% \triangle \mathrm{BMD}$ at the femoral neck $(0.00 ; \mathrm{CI}$ : $\left.-0.12,0.13 ; \mathrm{I}^{2}=0.0 \%\right)$, total hip $\left(\% \Delta \mathrm{BMD}=0.02\right.$; CI: $\left.-0.08,0.12 ; \mathrm{I}^{2}=0.0 \%\right)$, or lumbar spine ( $\left.\% \Delta \mathrm{BMD}=-0.01 ; \mathrm{CI}:-0.34,0.32, \mathrm{I}^{2}=37.7 \%\right)$. In stratified analyses (Table), there was a significant trend for increased bone loss at the femoral neck associated with lower TSH subgroups ( $\mathrm{p}$ for trend $=0.03$ ), especially in the subgroup with $\mathrm{TSH}<0.1 \mathrm{mIU} / 1(\% \Delta \mathrm{BMD}=$ -0.58 ; CI: $-0.96,-0.20$ ), and a comparable, but non-significant trend at the total hip (p for trend $=0.11$ ). Bone loss in SHyper relative to euthyroidism was greater among patients not on any thyroid- and bone-influencing medication at any time during follow-up, bone loss at the femoral neck $\left(\% \Delta \mathrm{BMD}=-0.36\right.$; $\left.\mathrm{CI}:-0.71,-0.00 ; \mathrm{I}^{2}=45.9 \%\right)$ and total hip $(\% \Delta \mathrm{BMD}=$ -0.40 ; CI:-0.96,0.16; $\mathrm{I}^{2}=81.9 \%$ ) was increased, but SHypo remained not associated with bone loss at any BMD site.

CONCLUSIONS: Compared to euthyroid individuals, hip bone loss was increased in those with SHyper, which may account for the observed increase in fracture risk, whereas SHypo was not associated with bone loss at any measured body site. These results reinforce the importance of fracture risk assessment and initiation of preventive strategies among older adults with SHyper. Randomized trials are needed to confirm that treatment of SHyper prevents bone loss and reduces fractures.

Stratified Analyses by TSH Subgroups for the Association between Subclinical Thyroid Dysfunction and Annualized Percentage Change in Bone Mineral Density-Femoral Neck

\begin{tabular}{lllll}
\hline \hline TSH subgroups & $\mathrm{N}$ & $\% \Delta \mathrm{BMD}$ & $95 \% \mathrm{CI}$ & $p$ for trend \\
$<0.10 \mathrm{mIU} / \mathrm{l}$ & 53 & -0.58 & $-0.96,-0.20$ & 0.03 \\
$0.10-0.44 \mathrm{mIU} / 1$ & 225 & -0.11 & $-0.19,-0.04$ & \\
$0.45-4.49 \mathrm{mIU} / 1$ & 4661 & reference & reference & \\
$4.50-6.99 \mathrm{mIU} / 1$ & 298 & -0.01 & $-0.10,0.09$ & \\
$7.00-19.99 \mathrm{mIU} / 1$ & 150 & 0.04 & $-0.29,0.36$ & \\
\hline
\end{tabular}

$\% \triangle B M D$ : annualized percentage change in bone mineral density; $95 \% \mathrm{CI}: 95 \%$ confidence intervals; N: number of participants; TSH: thyroid-stimulating hormone

\section{ASSOCIATION OF HEALTH LITERACY AND SOCIAL SUPPORT WITH RE- ADMISSION RISK Amanda S. Mixon ${ }^{1}$; Kathryn Goggins ${ }^{3}$; Samuel K. Nwosu' ${ }^{3}$ Jon- athan S. Schildcrout ${ }^{3}$; Katharine Donato ${ }^{5}$; Kathleene Wooldridge ${ }^{4}$; Frank Harrell ${ }^{3}$; Ken Wallston ${ }^{2}$; Sunil Kripalani ${ }^{3}$. ${ }^{1}$ VA Tennessee Valley Healthcare System and Vanderbilt University, Nashville, TN; ${ }^{2}$ Vanderbilt University Medical Center, Pisgah Forest, NC; ${ }^{3}$ Vanderbilt University Medical Center, Nashville, TN; ${ }^{4}$ Vanderbilt University Medical Center and the VA Tennessee Valley Healthcare System, Nashville, TN; ${ }^{5}$ Vanderbilt University, Nashville, TN. (Control ID \#2469230)}

BACKGROUND: The period following hospital discharge is a vulnerable time for patients when increased self-care requirements are common. Low levels of health literacy and social support are thought to contribute to poor post-discharge outcomes. However, little research has specifically examined the role of health literacy alone or in combination with social support in predicting readmission rates.

METHODS: The Vanderbilt Inpatient Cohort Study (VICS) is a prospective, longitudinal study of adults hospitalized with acute coronary syndromes (ACS) and/or acute decompensated heart failure (ADHF). During hospitalization we assessed demographic characteristics (age, gender, race, education, income), health literacy/numeracy (Brief Health Literacy Screen, short Test of Functional Health Literacy in Adults (sTOFHLA), Subjective Numeracy Scale), and social support (ENRICHD Social Support Inventory, living alone, contact with family, friends, and neighbors). The combined outcome of interest was all-cause readmission to any acute care hospital or emergency department (ED) visit, which was determined at 30 and 90 days after discharge. We constructed logistic regression models predicting readmission or ED visits using health literacy/numeracy and social support as predictors, adjusted for demographics and diagnosis.

RESULTS: The sample of 2603 patients included 1628 (62.5\%) with ACS, 804 (30.9\%) with ADHF, and 171 (6.6\%) with both conditions. The 30-day incidence of readmissions or ED visits was $23.7 \%(N=616)$, and the 90 -day incidence was $39.5 \%(N=1027)$. In unadjusted analyses, lower levels of health literacy/numeracy and social support were each associated with unplanned health care utilization. In multivariable models, neither health literacy nor social support remained significantly associated with 30-day readmission or ED visits. Lower health literacy measured by the sTOFHLA was significantly associated with 90-day readmissions and ED visits $(p=0.033)$ and explained about $2.6 \%$ of the variation in readmissions/ED visits at 90 days. Fewer years of education and lower household income were also independently associated with health care utilization.

CONCLUSIONS: Health literacy, as measured by the sTOFHLA, was independently associated with 90 -day readmissions/ED visits, but the effect was relatively small when taking other characteristics into account. Social support was not independently associated with readmissions at 30 or 90 days in this sample.

\section{ASSOCIATION OF MULTIMORBIDITY AND SPECIFIC COMORBIDITIES} WITH POLYPHARMACY IN UNIVERSITY PRIMARY CARE SETTINGS. Carole E. Aubert ${ }^{1}$; Sven Streit ${ }^{2}$; Bruno Da Costa ${ }^{1}$; Tinh-Hai Collet ${ }^{3}$, ${ }^{\text {; Jacques Cornuz }}{ }^{5}$; Jean-Michel T. Gaspoz ${ }^{6}$; Douglas Bauer ${ }^{7}$; Drahomir Aujesky ${ }^{1}$; Nicolas Rodondi ${ }^{1}$. ${ }^{1}$ Inselspital, Bern University Hospital, Bern, Switzerland; ${ }^{2}$ University of Bern, Bern, Switzerland; ${ }^{3}$ University Hospital of Lausanne, Lausanne, Switzerland; ${ }^{4}$ Addenbrooke's Hospital, Cambridge, United Kingdom; ${ }^{5}$ University of Lausanne, Lausanne, Switzerland; ${ }^{6}$ Geneva University Hospital, Geneva, Switzerland; ${ }^{7}$ University of California, San Francisco, CA. (Control ID \#2432108)

BACKGROUND: Among the ageing population, a high proportion of individuals develop multiple chronic conditions, leading to polypharmacy to treat these diseases or prevent complications based on disease-specific guidelines. Polypharmacy is increasing in prevalence and is associated with adverse drug events, drug-drug interactions, unplanned hospitalizations and sometimes fatal outcomes. In this study, we aimed to assess the extent of polypharmacy in ambulatory care in Switzerland, its risk factors and association with multimorbidity, as well rates of potentially inappropriate prescribing (PIP) and potentially prescribing omission (PPO).

METHODS: We analyzed a retrospective cohort of 1002 randomly selected patients aged 5080 years and followed in Swiss university primary care settings. We defined polypharmacy as $\geq 5$ chronically prescribed drugs and multimorbidity as $\geq 2$ comorbidities based on a large study by Higashi et al. (NEJM 2007) and the Charlson index. We used a logistic mixed-effects regression to assess the association of polypharmacy with the presence of multimorbidity, the number of comorbidities and specific sets of comorbidities. We used a multilevel mixed-effects Poisson regression to assess the association of the number of drugs with the same parameters. PIP and PPO were assessed using a subset of the screening tool of older people's prescriptions (STOPP) and screening tool to alert to right treatment (START) criteria Version 2.

RESULTS: Mean age was 63.5 (SD 8.3) years, with $44 \%$ women. Participants had a mean of 3.9 (range $0-17$ ) drugs and 2.6 (range $0-10$ ) comorbidities. Two thirds received $\geq 5$ drugs and $67.5 \%$ had multimorbidity. Age, number of outpatients visits, BMI, number of comorbidities, multimorbidity, presence of cardiovascular diseases, diabetes mellitus, hypertension and chronic kidney disease were significantly and independently associated with polypharmacy. The association was particularly strong for hypertension (OR 7.96, $95 \% \mathrm{CI} 4.91,12.90$ ), multimorbidity (OR 6.13, $95 \% \mathrm{CI} 4.10,9.15)$ and oldest age category $(75-80$ years: OR $5.19,95 \%$ CI $2.63,10.22$ vs. lowest age category of $50-$ 54 years). The number of chronic drugs increased with the number of comorbidities (Figure). For example, $84.9 \%$ of patients with 7 or more comorbidities had polypharmacy 
(Figure). PIP and PPO were present among 6.7 and $27.5 \%$ of the participants respectively. The prevalence of PIP, but not of POP, was significantly higher among participants with polypharmacy $(p<0.0001)$.

CONCLUSIONS: Polypharmacy is common in Swiss university primary care settings and strongly associated with multimorbidity, the number of comorbidities, PIP, cardiovascular risk factors and cardiovascular diseases. Given the association of polypharmacy with adverse consequences, indications for each drug should be carefully reviewed, particularly in patients with multimorbidity. Criteria developed to avoid PIP and PPO (e.g. STOPP/START criteria) may help improving pharmacological management of these patients. As the prevalence of polypharmacy and multimorbidity is increasing, future randomized trials should include multimorbid patients to be able to develop guidelines adapted to this particular population to help avoiding PIP and adverse drug events.

Percentage of patients in ambulatory medicine receiving a particular number of drugs 10 to $\geq 10$ ), stratified by number of comorbidities ( 0 to $\geq 7)$, out of a list of 17 selected comorbidities, based on a large study by Higashi et al (NEJM 2007) and the Charlson index, as previously defined (Streit et al, PLoS One 2014).

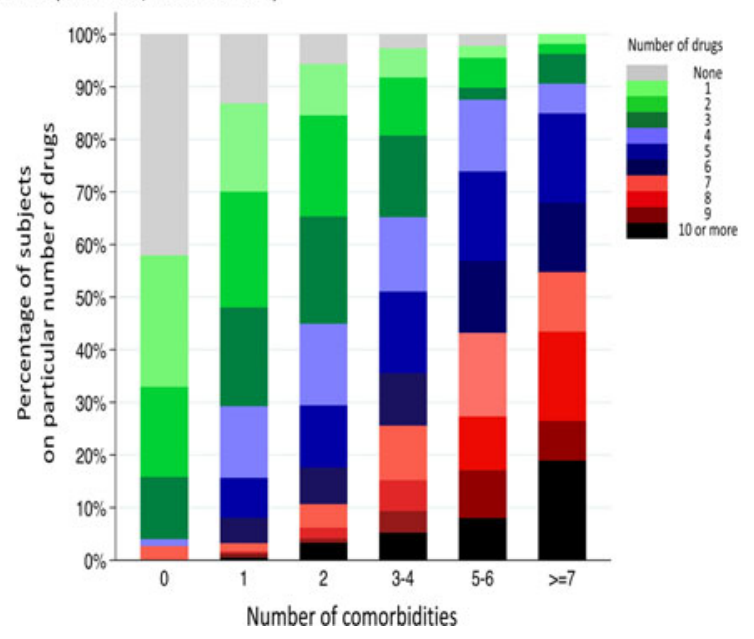

ASSOCIATION OF URINE DRUG TEST SCREENING DURING INITIATION OF CHRONIC OPIOID THERAPY WITH RISK OF OPIOID OVERDOSE. Marc Larochelle $^{2}$; Jane M. Liebschutz ${ }^{1}$; James F. Wharam ${ }^{3}$; Fang Zhang ${ }^{5}$; Dennis RossDegnan ${ }^{4}$. ${ }^{1}$ Boston University School of Medicine, Boston, MA; ${ }^{2}$ Boston University School of Medicine and Boston Medical Center, Boston, MA; ${ }^{3}$ Harvard Medical School, Boston, MA; ${ }^{4}$ Harvard Medical School and Harvard Pilgrim Health Care Institute, Boston, MA; ${ }^{5}$ Harvard Pilgrim Health care Institute, Boston, MA. (Control ID \#2468557)

BACKGROUND: Overdose deaths due to prescription opioids more than quadrupled between 1999 and 2010, mirroring increases in prescription opioid sales. Observational data suggest that urine drug tests (UDT) produce actionable information that could prevent inappropriate opioid prescribing; between one-third and one-half of UDTs identify potential misuse or diversion. However, UDTs are challenging to interpret and surveys suggest providers often err in UDT interpretation. Despite these obstacles, guidelines commonly recommend UDT as a risk monitoring tool for patients prescribed opioids for chronic noncancer pain. Trends in adoption of UDT and the association between UDT and opioid overdose are unknown. We hypothesized that by screening out patients with active drug misuse, receipt of UDT during initiation of chronic opioid therapy is associated with lower risk for opioid overdose compared with patients not screened with UDT.

METHODS: We conducted a retrospective cohort study of commercially insured members aged 18-64 years from a large national United States health insurer. We included members with new chronic opioid use defined as $\geq 90$ days without an opioid prescription followed by three or more opioid prescriptions $\geq 21$ days apart, lasting $\geq 84$ days with $\geq 84$ days supplied. We excluded members with cancer except non-melanoma skin cancer. The exposure of interest was a medical claim for UDT in the period from 30 days before to 90 days after the start of new chronic opioid use. The primary outcome was time to opioid overdose beginning 90 days after the start of chronic opioid use. Patients were followed for up to 2 years and censored for opioid discontinuation, health plan disenrollment, reaching age 65 , or end of 2012. Opioid overdose was identified using ICD-9 codes from emergency department or inpatient claims. We developed a multivariable Cox regression model of time to overdose including UDT exposure as the predictor of interest. We adjusted for opioid dosage and active benzodiazepine prescription as daily time-varying covariates, age, sex, and diagnosis of mental health or substance use disorder.

RESULTS: We identified 179,385 members with new chronic opioid use for non-cancer pain between 2002 and 2011. The rate of UDT during initiation of chronic opioids was $1.3 \%$ in 2002 , increased slightly to $1.8 \%$ in 2006 , then increased more rapidly to $9.5 \%$ by 2011. Over a median follow-up of 159 days, members experienced 165 opioid overdose events. The adjusted hazard ratio of time to overdose for members screened with a UDT was 1.36 (95\% CI $0.79-2.34)$. Younger age, higher daily opioid dosage, active daily benzodiazepine prescription, and presence of mental health and substance abuse diagnoses were all associated with increased risk of overdose (Table)

CONCLUSIONS: In a large cohort of commercially insured members with new chronic opioid use, UDT prior to or early in treatment episodes was not associated with reduced risk of opioid overdose. Although we controlled for several known individual risk factors for opioid overdose, unmeasured confounding is likely substantial. Future studies are needed to understand how providers respond to concerning UDT results and determine the causal relationship between UDT and overdose. Such research could inform interventions that may improve the effectiveness of UDT as a risk mitigation strategy. Multivariable Cox regression model results for time to opioid overdose

\begin{tabular}{ll}
\hline \hline Variable & Adjusted Hazard Ratio $(95 \% \mathrm{CI})$ \\
Urine drug test (UDT) & $1.36(0.79-2.34)$ \\
Male & $1.06(0.78-1.45)$ \\
Age & \\
$-18-24$ years & $6.04(3.05-11.97)$ \\
$-25-34$ years & $2.71(1.61-4.55)$ \\
$-35-44$ years & $1.69(1.05-2.73)$ \\
$-45-54$ years & $1.20(0.75-1.92)$ \\
$-55-64$ years & 1.00 (Referent) \\
Daily dosage in morphine equivalents & \\
- Large $(\geq 100 \mathrm{mg})$ & $3.00(1.92-4.68)$ \\
- Moderate $(\geq 50$ mg to $<100 \mathrm{mg})$ & $2.12(1.27-3.55)$ \\
- Low $(<50 \mathrm{mg})$ & $1.00($ Referent $)$ \\
Daily benzodiazepine prescription & $1.89(1.28-2.79)$ \\
Mental health diagnosis & $2.75(1.99-3.79)$ \\
Substance use disorder diagnosis & $2.17(1.50-3.15)$ \\
\hline
\end{tabular}

ATTITUDES ABOUT ADULT VACCINES AND REMINDER/RECALL IN A LOW-INCOME POPULATION Karen Albright ${ }^{1,3}$; Laura P. Hurley ${ }^{1,2}$; Simran K. Sabherwal $^{1}$; Steven Lockhart ${ }^{1}$; Dennis Gurfinkel ${ }^{1}$; Alison Saville ${ }^{1}$; Brenda Beaty ${ }^{1}$; L Miriam Dickinson ${ }^{1}$; Anne Libby ${ }^{1}$; Allison Kempe ${ }^{1} .{ }^{1}$ University of Colorado and Children's Hospital Colorado, Aurora, CO; ${ }^{2}$ Denver Health, Denver, CO; ${ }^{3}$ University of Denver, Denver, CO. (Control ID \#2469374)

BACKGROUND: In an average year, $95 \%$ of the 20,000 to 50,000 Americans who die of a vaccine preventable disease are adults. In the U.S. the direct health care burden of adult vaccine-preventable disease is approximately $\$ 10$ billion annually. Healthy People 2020 goals for adult vaccines include increasing the proportion of non-institutionalized adults 18-64 and seniors $(\geq 65)$ who are vaccinated against seasonal influenza, and the proportion of high-risk adults $18-64$ and seniors who are vaccinated against 
pneumococcal disease. In order to achieve better immunization rates, the Community Preventive Services Task Force recommends interventions that involve patient reminder/ recall $(\mathrm{R} / \mathrm{R})$, whereby patients receive a reminder for upcoming immunizations or a recall notice for overdue immunizations by letter, postcard, auto-dialer or telephone call. However, relative to pediatric vaccination, little is known about patient attitudes toward adult vaccination, barriers and facilitators to adult vaccination, and adult preferences for $R / R$. The objective of this study was to determine patient perspectives on adult vaccines and identify preferences for adult $\mathrm{R} / \mathrm{R}$.

METHODS: As part of a larger study, 12 focus groups (FGs) were conducted with lowincome patients served by the Denver Health and Hospital Authority, a safety net system that includes 8 community health centers. The sample was stratified by participants' primary language; with half the groups conducted in English and half conducted in Spanish. The English- and Spanish-speaking samples were each further stratified by age and health status, according to the following categories: patients aged 18-64 years with chronic conditions, patients aged 18-64 years without chronic conditions, and patients $65+$ years of any health status. FG discussions included general attitudes about vaccinations; attitudes about and experiences with Tdap, pneumococcal polysaccharide (PPSV23), and seasonal influenza vaccines specifically; attitudes about and experiences with adult R/R; and preferences for adult R/R delivery. All FGs were digitally recorded, transcribed, translated from Spanish to English (where applicable), and analyzed for thematic content by experienced analysts using established qualitative content analysis techniques.

RESULTS: Thirty eight English-speaking patients and 30 Spanish-speaking patients each participated in one FG. The overwhelming majority of patients expressed positive feelings about vaccines generally, reporting that they considered them to be beneficial and, especially among participants with chronic conditions, a top priority in their health care. However, when asked about specific adult vaccines, many expressed confusion or opposition. Most participants were not familiar with the Tdap vaccine, although almost all had heard of tetanus. Similarly, most participants $65+$ and/or with chronic health conditions were not familiar with the pneumococcal vaccine. Most participants reported that they had not received information about these vaccines from their providers and, while they were open to the possibility of receiving them, they wanted to first learn more information. This was particularly true for the English-speaking participants across categories. In contrast, all participants reported familiarity with the seasonal influenza vaccine. However, opinions were divided about the benefits of this vaccine. Many participants, particularly those from English-speaking groups, reported negative experiences with the seasonal influenza vaccine and/or disbelief that it was necessary for adult health. Patients with chronic health conditions were much more likely to describe the seasonal influenza vaccine as beneficial. All patients expressed a strong preference for more information about vaccines from providers, as well as better communication with and improved access to their primary care providers generally. Critical issues in deciding whether to receive adult vaccinations included cost, convenience of location, trust in vaccine deliverer, and specific information about the vaccine, including its relevance to their particular health status. While no participant had previously received a reminder about vaccinations for themselves, all were strongly in favor of the idea, and most reported that a combination of delivery methods (e.g., mailed letters plus text messages) would be most effective for their lifestyles. Most participants preferred straightforward, positive reminders that contained specific information about which vaccines were needed and were clear about the next steps required of the patient. Most disliked reminders that required patients to respond.

CONCLUSIONS: Low-income adults in this study generally expressed positive feelings about vaccines. While none reported previous experiences with receiving $\mathrm{R} / \mathrm{R}$ notices for vaccines, all were in favor of receiving $R / R$ notices for needed vaccines. However, participants had little knowledge of vaccines other than influenza, about which they expressed concerns. These results suggest potential patient interest in greater adoption of evidence-based methods to improve immunization rates, and highlight the need for improved communication between primary care providers and patients about adult vaccines. Data from this study informed an ongoing study evaluating centralized R/R from a state immunization information system compared to usual adult vaccine delivery in this safety net system.

BARRIERS TO EFFECTIVE TEAMING FOR PAIN: TASK REDUNDANCY AND CHALLENGES WITH SPECIALTY COORDINATION Karleen Giannitrapani $^{4}$; Sangeeta Ahluwalia ${ }^{3}$; Roger T. Day ${ }^{4}$; Maura Pisciotta ${ }^{2}$; Steven Dobscha ${ }^{2}$; Erin E. Krebs ${ }^{1}$; Karl Lorenz ${ }^{4,5}$. ${ }^{1}$ Minneapolis VA Health Care System, Minneapolis, MN; ${ }^{2}$ Portland VA Health Care System, Portland, OR; ${ }^{3}$ RAND Corporation, Encino, CA; ${ }^{4}$ Veterans Health Administration, Menlo Park, CA; ${ }^{5}$ Stanford University, Stanford, CA. (Control ID \#2469911)

BACKGROUND: Interdisciplinary team based approaches can improve outcomes for patients with chronic pain. Integrating providers across disciplines, however, is a complex undertaking with varied success. We qualitatively assess primary care team roles and processes around pain screening and management to understand responsibility distribution in a team based approach as well as identify where "teaming for pain" fails.

METHODS: Setting: The Veterans Health Administration (VA) has implemented a team based model for primary care in which a PCP is supported by a core interprofessional team of nurses and clerks as well as an ancillary group of pharmacists, mental health providers, and other specialists. Design: 60 members of VA primary care teams at 6 sites in two states participated in 9 interdisciplinary focus groups. A semi-structured interview guide elucidated provider experiences with screening for and managing chronic pain. Qualitative analysts evaluated transcribed interviews using the method of constant comparison and produced mutually agreed upon themes.

RESULTS: PCPs described themselves as holding principal responsibility for pain assessment and management, but they saw potential for redistributing some tasks across a team: "if we develop processes to better coordinate services [including pain assessment, patient education, and specialty coordination]... a lot of this stuff doesn't need me, necessarily, to be front and center." Redundancy: Some PCPs viewed pain screening as "a team effort" and relied on nurse assessments to save provider time. Others felt, "it doesn't really matter what the nurse charts, you're going to get your own history, it's just a repetitive operation. " Lack of clarity around task division can lead to provider-nurse task redundancy, which beyond being inefficient has consequences including: patients not taking initial nurse assessments seriously, patients having the opportunity to provide inconsistent information "they'll tell the RN something different and by the time they get to the doctor... It's a different story," and patient frustration "Well, I just told your colleague...Why are you asking me again?" Challenges with specialty coordination: Specific challenges include insufficient notification by external providers about specialty appointments ( "those are all encapsulated visits. They're not alerted to their PCP"), poor specialty follow up ("they send them back to us... no follow up"), poor communication ("It's a pain calling the pain clinic"), and referral delays ("we need help to get in touch with the pain clinic... it takes days sometimes"). Case management rounds ("a team... talking about the patient's limitations and different services"), joint visits ("maybe you can, as a team, come up with a new approach"), and nurses following up with e-consults were strategies proposed to mitigate specialty coordination challenges.

CONCLUSIONS: Understanding task distribution related to chronic pain assessment and management can inform recommendations to improve team based care. Our findings stress the importance of clearly defined roles and team processes for pain care as well as highlight specific scenarios, such as role redundancy, that lead to inefficiencies, poor team functioning, and suboptimal care. Establishing relationships with clear and shared role expectations between PCPs and ancillary specialists may facilitate better team processes for coordinated pain care. 
BARRIERS TO HEPATITIS B SCREENING AND VACCINATION WITHIN THE VIETNAMESE COMMUNITY OF NEW ORLEANS EAST Michelle Fleshner $^{1,}{ }^{1}$; Anna Le ${ }^{1}$; Hieu Nguyen ${ }^{1} ;$ Anh Le ${ }^{1}$; Cecile Dinh ${ }^{1}$; Catherine Jones ${ }^{2} .{ }^{1}$ Tulane University, New Orleans, LA; ${ }^{2}$ Tulane University SOM, New Orleans, LA. (Control ID \#2470439)

BACKGROUND: The Hepatitis B Virus (HBV) is a growing problem among Vietnamese Immigrants in the United States, particularly within Louisiana, with an estimated prevalence of $12.5 \%$. HBV screening and vaccination rates within this community remain low, and further research is needed to identify barriers to screening and vaccination within the community.

METHODS: Cross-sectional, convenience-sample survey study among adult Vietnamese individuals attending health fairs within the community in New Orleans, Louisiana between 2012 and 2013.

RESULTS: Eighty-eight subjects completed surveys at three separate health fairs within the Vietnamese community in New Orleans. Knowledge that HBV is preventable and knowledge that there is treatment for HBV were significant predictors of being screened and vaccinated for HBV. Insurance status and having a primary care physician were not associated with whether or not a subject was screened

CONCLUSIONS: The strongest barriers to HBV screening and vaccination within the Vietnamese community in New Orleans were knowledge about HBV as opposed to demographic and financial characteristics. The findings will help direct communitybased public health initiatives towards implementing more cost-effective public information campaigns, rather than resource-intensive work, to combat insurance and financial barriers. More robust and culturally appropriate HBV education is needed within this Vietnamese population.

BARRIERS TO HIGH QUALITY CODING OF HOSPITAL CHART INFORMATION TO ADMINISTRATIVE DATA: A QUALITATIVE STUDY Karen Tang; Kelsey Lucyk; Hude Quan. University of Calgary, Calgary, AB, Canada. (Control ID \#2468537)

BACKGROUND: Administrative data are widely used in research, evaluation of health care delivery, and health policy decision-making processes. Given the downstream consequences of low quality data, there has been much work to assess the validity of administrative data. Though coding of the major or most responsible diagnosis is generally accurate, there are issues with under-reporting of secondary or minor diagnoses, lack of specificity and precision in coding, and errors in the coding of diagnostic types. These issues arise despite standard international disease classification systems and national coding guidelines. We undertook a qualitative study to explore the barriers to high quality coding at the level of coders in Canada. METHODS: Our study design is qualitative. We recruited professional coders and data users working across the province of Alberta, Canada, using a multimodal recruitment strategy. We conducted an in-depth, semi-structured interview with each participant. All interviews were audio-recorded and transcribed. We conducted thematic analysis (e.g., line-by-line open coding) of interview transcripts. Codes were then collated into themes and compared across our dataset to ensure accurate interpretations of the data. The study team met to discuss these themes, modify them, and determine how they might be interpreted regarding the barriers faced in coding administrative data, from the perspective of coders and data users.

RESULTS: Of the 30 participants, 27 were coding specialists and 3 were data users. Though data users emphasized the importance of valid and specific data for use in research, surveillance, and decision-making, they described a lack of understanding of the actual coding process. Three themes emerged regarding their perspectives on barriers to high quality coding: 1) Coders are responsible for the accuracy of coded data, 2) Coders do not understand the importance of downstream uses of administrative data and therefore do not place sufficient importance on accurate coding, and 3) Coders are not sufficiently valued and therefore have low motivation to perform well. In contrast, our interviews with coders revealed that they had high job satisfaction, believed themselves to be adequately trained, and consistently made extra effort in searching charts for additional information to accurately reflect the patient journey. Barriers to high quality coding from the coder perspective included: 1) Incomplete and inaccurate information in physician progress notes and discharge summaries; 2) Difficulty navigating a complex hybrid of paper and electronic medical records; 3) Focus on productivity rather than quality by the employer, resulting in inconsistent instructions for coding secondary diagnoses and discordant expectations between the employer and the coders' professional standards.

CONCLUSIONS: There are misconceptions that the quality of coded administrative data is the sole responsibility of coding specialists. We have found that coders are highly motivated to accurately code chart data, but are primarily limited by their dependence on physician documentation, the unintended consequences of transitioning to an electronic medical record, and productivity expectations. Future interventions to improve the quality of administrative data should focus on physician education of necessary components in charting, evaluation of electronic medical records not just for health care professionals but also for those attempting to abstract data from the electronic medical record, and evaluation of the implications on data validity when creating productivity guidelines and benchmarks for coders.

\section{BARRIERS TO UPTAKE OF NON-PHARMACOLOGIC PAIN MANAGEMENT} STRATEGIES Karleen Giannitrapani ${ }^{4}$; Sangeeta Ahluwalia ${ }^{3}$; Matthew McCaa ${ }^{4}$; Steven Dobscha $^{2}$; Erin E. Krebs ${ }^{1}$; Karl Lorenz ${ }^{4}, 5$. ${ }^{1}$ Minneapolis VA Health Care System, Minneapolis, MN; ${ }^{2}$ Portland VAMC, Portland, OR; ${ }^{3}$ RAND Corporation, Encino, CA; ${ }^{4}$ Veterans Health Administration, Menlo Park, CA; ${ }^{5}$ Stanford University, Stanford, CA. (Control ID \#2469976)

BACKGROUND: In light of the current epidemic of opioid use in the United States, there is increasing interest in reducing reliance on opioid therapy and increasing uptake of non-pharmacologic strategies for the management of chronic pain. Our objective is to understand providers' perceptions of barriers to non-pharmacologic pain management strategies for patients with chronic pain.

METHODS: Sixtykey informants including primary care providers, registered nurses, licensed practical nurses, psychologists, and social workers at two large academicallyaffiliated VA Medical Centers participated in 9 interdisciplinary focus groups. A semistructured interview guide elucidated provider experiences with assessing and managing pain. Qualitative analysts evaluated transcribed interviews using the method of constant comparison and produced mutually agreed upon themes.

RESULTS: The most frequently mentioned barrier to non-pharmacologic pain management strategies was failure to align with patient preferences. Additional barriers to nonpharmacologic pain management strategies clustered around insufficient 1) availability, 2) accessibility, and 3) provider buy-in. Providers highlighted insufficient availability to multiple types of services including: surgical services ("I have no good resources for neurosurgery. I might as well forget about it. You know, it takes too long"), complementary and integrative medicine modalities such as acupuncture, massage, chiropractic, or biofeedback ("I've just found such an incredible lack of integrated medicine here. I think that it's... really key for chronic pain... getting massage therapy, chiropractic... it's really frustrating"), physical and occupational therapy ("if a patient is not a specific service connected amount, then we cannot get them physical therapy in the community no matter what"), as well as behavioral and mental health services ("the VA has been a little slower to catch up... in the participation of mental health in pain management"). Barriers to access included the following sub-themes: patient distance from service ("Oftentimes our 
patients travel long distances. I have patients who come 130 miles to see me... it would be nice if they could get physical therapy at home"), direct cost to Veterans ("if they could get massage therapy at home... that the VA would give them, even if it's just an allotted amount of money every year to spend on these alternative programs, then they at least had the option"), and treatment time delays ("I will offer to get them into pool therapy, though that's a one-time thing. And they can wait 6 months to get into pool therapy"). Barriers to provider buy-in included: insufficient resources ('Here we... don't have resources, all we have to give them as providers are pain medications"), brief appointments that limit time to explore a range of pain management options ("I have a lot of things to do on this short visit.. and then there is this pain.. oh gosh I didn't address it"), confrontation avoidance ("there are times we're pinned down where the choice is, I give this guy 30 days' worth of medicine so I can get him out of the office without having to fight him... Because otherwise it's a confrontation that's involved - a major confrontation"), and increased provider workload associated with non-pharmacologic options ("we need more resources and education... it takes a huge psychological toll on providers and teams to deal with a lot of these challenging cases... And I think that's part of the reason that we've seen opioids mis-prescribed and continue to be prescribed in ways that aren't safe or beneficial")

CONCLUSIONS: Reducing opioid therapy use for chronic pain requires viable alternatives. In order for non-pharmacologic pain management strategies to truly be viable options they need to first be available, accessible, and offered to patients by their providers. Our findings provide actionable information for reducing the barriers to multiple non-pharmacologic pain management strategies.

BASELINE MEDICATION RECONCILIATION RESULTS AMONG FEDERALLY QUALIFIED HEALTH CENTER (FQHC) PATIENTS WITH UNCONTROLLED HYPERTENSION PARTICIPATING IN THE NORTHWESTERN AND ACCESS COMMUNITY HEALTH NETWORK MEDICATION EDUCATION STUDY (NAMES) Elisha M. Friesema ${ }^{1}$; John M. Wagner ${ }^{1}$; Ji Young Lee ${ }^{1}$; Danielle Lazar ${ }^{2}$; Michael S. Wolf ${ }^{1}$; Stephen D. Persell ${ }^{1}{ }^{1}$ Northwestern University, Chicago, IL; ${ }^{2}$ ACCESS Community Health Network, Chicago, IL. (Control ID \#2468773)

BACKGROUND: Patients with chronic conditions frequently use complicated medication regimens which may result in medication errors and adverse drug events. Clinicians may not know how patients are using their medications, and patients may erroneously believe their clinicians have a complete understanding of which medications patients are using when they do not. Medication reconciliation provides clinicians with an accurate medication list and an opportunity to review adherence. It facilitates care coordination, enables medication safety software to be used, and ultimately may decrease adverse drug events and improve disease control.

METHODS: We performed a comparison of patient-reported medication use with the EHR-recorded medication list among patients at baseline enrolled in a randomized clinical trial in a network of FQHCs in Chicago, IL. Eligible patients were $\geq 18$ years of age with $\geq 3$ prescribed medications and not well-controlled hypertension $(\geq 130 / 80 \mathrm{mmHg}$ if they are diabetic or $\geq 135 \mathrm{mmHg}$ systolic or $\geq 85 \mathrm{mmHg}$ diastolic if they are not). We used blinded physicians review to compare baseline medication lists in the electronic health record (EHR) with corresponding patient self-reported medications on the same date. Three levels of reconciliation (all medications reconciled, one or more medications reconciled with discrepancies, and no medications reconciled) were assessed within the following medication subgroups: all medications, chronic disease medications, antihypertensive medications, diabetes medications, and lipid-lowering medications. Medications were designated as reconciled if the drug names, descriptions, and dosage instructions provided by patients in self-reported interviews were recognizable to the physician reviewer as the same medications on the EHR medication list and the patient reported information for dose or frequency (if it was provided) did not directly contradict the data recorded in the EHR. Primary outcomes for this analysis include medication reconciliation rates for chronic disease medications and anti-hypertensive medications while secondary outcomes consisted of reconciliation rates for all medications, diabetes medications, and lipid-lowering medications.

RESULTS: A total of 920 participants enrolled in NAMES with a mean age of 52.3 years, $67.9 \%$ were female, $87.1 \%$ were self-identified as African American, $96.9 \%$ were predominantly English speakers, and $67.4 \%$ had a high school education or less. Newest Vitals Sign scores indicated that most participants were unlikely to have adequate health literacy $(80.6 \%)$ and the mean self-reported adherence score (1.4) on the Morisky Medication Adherence Scale (MMAS-4) showed that many participants were not likely adherent to their current medications. Patients who did not have any medications recorded in the EHR were excluded ( $n=41 ; 4.5 \%)$. Analysis of patients with one or more chronic disease medications recorded in the EHR medication list $(n=879)$ revealed $22.8 \%$ were completely reconciled for chronic disease medications, $66.1 \%$ were reconciled with discrepancies, and $12.1 \%$ had no recognizable match between those listed in the EHR and those reported by the patient. Among patients prescribed anti-hypertensive medications $(n=846), 43.5 \%$ of patients were completely reconciled, $36.2 \%$ partially reconciled, and $20.3 \%$ had no medications reconciled. Secondary outcome medication reconciliation rates at the patient level showed that $55.4 \%$ of diabetes medications were completely reconciled (185/334), 51.6\% of lipid-lowering medications completely reconciled among patients prescribed lipid-lowering medications (200/388), and $8.5 \%$ of patient had their entire medication lists reconciled among all eligible patients $(75 / 879)$. Of patients with inadequate health literacy, only $36.59 \%$ were able to fully reconcile their hypertension medications $(146 / 399, p=0.0004)$ and $18.62 \%$ were able to fully reconcile their chronic disease medications $(78 / 419, p=0.0061)$. Common reasons for discrepancies were: medications included on one list but not another, patients not being able to identify the names of medications they take, and patients using medications at a dose or frequency that differed from what was recorded in the EHR (e.g. taking a multiple-timesa-day medication only once daily). Particularly problematic discrepancies included patients taking duplicate medications (e.g. taking both amlodipine and Norvasc), two drugs in the same drug class (e.g. two ACE inhibitors), and NSAIDs at a higher dose or frequency than what was prescribed.

CONCLUSIONS: Preliminary analysis of medication reconciliation rates among this population showed that large proportions of FQHC patients with hypertension had discrepancies between their self-reported medication lists and the lists in the EHR on the same date. Those with limited health literacy were significantly less likely to fully reconcile their chronic disease and hypertension medications. These discrepancies are likely to have negative influences on chronic disease outcomes and may put patients at risk for adverse drug events. This trial will determine whether nurse-led and EHR-led interventions lead to better medication management and hypertension control.

BASELINE PATIENT CHARACTERISTICS AND EARLY IMPACT OF COLLABORATIVE DEPRESSION CARE MANAGEMENT ON CHRONIC DISEASE OUTCOMES IN AN URBAN ACADEMIC PRIMARY CARE CLINIC Iris Huang; Glennis M. Rodriguez; Katherine Small; Lauren Peccoralo. Mount Sinai, New York, NY. (Control ID \#2469500)

BACKGROUND: Depression increases the risks and adversely affects the course and outcomes of other chronic diseases. Depression is an independent risk and prognostic factor for cardiovascular disease and is associated with decreased glycemic control and increased diabetic vascular complications. Several studies have evaluated the effects of collaborative depression care management (DCM) on diabetes and cardiovascular outcomes and found mixed results. DCM uses non-physician care managers who work closely with primary care providers (PCPs) and psychiatrists. Interventions vary from 
development of care management plans that improve medication adherence to multifaceted models involving intensive management in psychotherapy programs. DCM has been shown to improve depression outcomes in numerous clinical settings. Prior analyses of the DCM program at Mount Sinai found that DCM patients improved in their depression symptoms as demonstrated by significant decreases in Patient Health Questionnaire 9 (PHQ9) scores. However, little is known about the impact of DCM on chronic disease outcomes in academic training clinics. The purpose of this study is to compare baseline characteristics and chronic disease outcomes between patients enrolled in DCM and patients who completed a PHQ9 but not enrolled in DCM.

METHODS: Patients at the Internal Medicine Associates (IMA) practice at the Mount Sinai Medical Center meeting the following criteria can be referred by their PCPs to enroll in DCM: age over 18, clinically significant depression as measured by PHQ9 score greater than 9 and meeting DSM IV criteria for major depressive disorder. DCM consists of universal depression screening, short-term bi-weekly to monthly sessions with a behavioral health social worker and weekly case reviews with a psychiatrist consultant. In a retrospective review, all IMA patients who completed a PHQ9 questionnaire from February 23 to September 30, 2015 and with at least one of the following diagnoses were included in the study: diabetes, hypertension, cardiovascular disease and chronic kidney disease. PHQ9s were completed by a PCP either because of a positive screening PHQ2 or as follow-up to a depression diagnosis. The following baseline variables were extracted from the electronic health record: age, gender, race, ethnicity, primary language, number of ED visits and psychiatry consults prior to initial PHQ9, presence of cardiovascular (CV) (includes cardiovascular disease and hypertension), metabolic (MET) (includes diabetes and chronic kidney disease), substance use (SU) and mental health (MH) conditions in the problem list. A baseline value was defined as the first value after February 23. A follow-up value was defined as the last value before September 30. Baseline and follow-up values were collected for body mass index (BMI), hemoglobin A1C (HbAlc), blood pressure (BP), total cholesterol and low density lipoprotein (LDL). Characteristics of DCM and non-DCM patients were compared using $t$-tests and chi-square tests. Baseline and followup values within groups were compared using paired t-tests.

RESULTS: The DCM group included 52 patients and non-DCM group included 449 patients. There were no differences between groups in the following characteristics: age, race, ethnicity, primary language, number of ED visits prior to initial PHQ9 and presence of $\mathrm{CV}$, MET or SU conditions. The groups differed significantly in four ways: DCM patients had a higher percentage of females ( $92 \mathrm{vs.} 73 \%, p=0.002$ ), higher percentage of MH diagnosis ( 87 vs. $54 \%, p<0.001$ ), higher mean baseline BMI ( 34 vs. $31, p=0.004$ ) and were more likely to have prior visits with mental health providers ( 7.5 vs. 0.35 , $p<0.001)$. Mean baseline PHQ9 scores were similar across groups (12.1 vs. 11.2, $p=$ 0.33 ) but significantly more DCM patients had scores above 9 ( 71 vs. $57 \%, p=0.049$ ). Within the DCM group, there were no significant changes in BMI, HbA1C, BP, total cholesterol or LDL from baseline to follow-up. Within the non-DCM group, there were significant decreases in mean systolic and diastolic blood pressures (138 to 132, $p<0.001$ and 74 to $71, p<0.001$, respectively) from baseline to follow-up. However, changes in BMI, HbA1C, BP, total cholesterol and LDL from baseline to follow-up were not significantly different between groups.

CONCLUSIONS: Patients enrolled in DCM and patients who completed a PHQ9 questionnaire but not enrolled in DCM were significantly different across gender, BMI, percent with PHQ9 score above 9, current MH diagnosis and history of mental health visits. The two groups were not significantly different from each other in terms of changes in BMI, HbA1C, BP, total cholesterol and LDL from baseline to follow-up. Differences in baseline characteristics of the two groups may limit meaningful comparisons between them. Additional limitations of the study include small sample sizes particularly in the DCM group and short follow-up period. Additionally, many patients enrolled in DCM towards the middle or end of the study period and thus may not have been in the program long enough to experience significant changes in chronic disease outcomes. Future study will include a more comprehensive chart review and analysis of depression, utilization and chronic disease outcomes for all patients enrolled in the DCM program over 2 years with comparison to a matched control group.

BEERS POTENTIALLY INAPPROPRIATE MEDICATIONS IN THE ELDERLY CONTRIBUTING TO HOSPITAL ADMISSIONS: LACK OF PREVENTION WITH COMPUTERIZED CLINICAL DECISION SUPPORT ALERTS Mary Amato $^{1,2}{ }^{\text {; Olivia Dalleur }}{ }^{1,3}$; Adrian Wong ${ }^{1,2}$; Julie Fiskio ${ }^{1,4}$; Diane L. Seger ${ }^{1,4}$; Patrick E. Beeler ${ }^{1,5}$; Tewodros Eguale ${ }^{1,2}$; patricia dykes ${ }^{1,6}$; Elizabeth R. Silvers ${ }^{1,4}$; Sarah P. Slight ${ }^{1,7}$; David W. Bates ${ }^{1,6}$. ${ }^{1}$ Brigham and Women's Hospital, Boston, MA; ${ }^{2}$ MCPHS University, Boston, MA; ${ }^{3}$ Université catholique de Louvain, Brussels, Belgium; ${ }^{4}$ Partners Healthcare System, Wellesley, MA; ${ }^{5}$ University Hospital, Zurich, Switzerland; ${ }^{6}$ Harvard Medical School, Boston, MA; ${ }^{7}$ Durham University, Stockton on Tees, United Kingdom. (Control ID \#2469214)

BACKGROUND: Medications listed in the Beers Criteria for Potentially Inappropriate Medication (PIM) Use in Older Adults should generally be avoided in the elderly as they have been associated with adverse events including falls and altered mental status.(1,2) Computerized clinical decision support (CDS) alerts can be used to prompt prescribers to substitute these medications with safer alternatives. The objectives of this study were to determine the frequency of use of PIMs in patients aged 75 years and older admitted to an academic medical center, assess how frequently these medications contributed to the admission, and whether a CDS alert existed for the medications.

METHODS: A retrospective chart review was conducted for 301 consecutive patients aged 75 or over admitted to any service at Brigham and Women's Hospital during the period October 27-November 9, 2014. Scheduled admissions were excluded. Reason for admission, home medications, co-existing conditions, and demographic data were recorded, and patients' admission notes were reviewed by two clinical pharmacists to determine if the patient was prescribed medications on the Beers 2012 and 2015 PIM lists and whether these medications had potentially contributed to the admission.

RESULTS: Two hundred twenty-six (75\%) of 301 consecutive patient admissions were unplanned. The patients' median age was 82 years and $54 \%$ were female. Overall, $42 \%$ (96/226) of patients were prescribed 132 medications from the Beers 2012 PIM list. The five most commonly prescribed medications from this list were benzodiazepines $(n=35)$, NSAIDS $(n=15)$, amiodarone $(n=11)$, zolpidem $(n=11)$, and antipsychotics $(n=8)$. Beers 2012 PIMs were thought to contribute to admission in 16 patients (7\%). Reasons for admission and implicated medications for these admissions included: falls ( $n=7$, including two fractures and one subdural hematoma resulting from fall) resulting from alprazolam $(n=2)$, clonazepam, doxazosin, lorazepam + haloperidol, phenobarbital, and quetiapine; altered mental status $(n=4)$ from belladonna, clonazepam, diazepam + clonidine, and sliding scale insulin; dizziness $(n=2)$ from alprazolam and clonazepam; GI bleed/anemia from diclofenac; near syncope from propafenone; and functional bowel syndrome from hyoscyamine. Inpatient and outpatient CDS alerts were provided for all of the medications implicated in admissions except for lorazepam, propafenone, and sliding scale insulin, and for $76 \%$ of all of the PIMs. Utilizing the recently published Beers 2015 list of PIMs, 94 patients were admitted taking 101 medications on the 2015 list that were not included in the 2012 list. Of these, 92 were proton pump inhibitors, 6 meclizine, 2 paroxetine, and 1 nortriptyline. Chart review revealed that these 2015 additional medications potentially contributed to four admissions (fall with fracture with omeprazole, omeprazole + nortriptyline, and omeprazole + paroxetine; and altered mental status with meclizine). The only CDS warning for these medications was for nortriptyline.

CONCLUSIONS: Nearly half of older patients with unplanned admissions in late 2014 were taking Beers 2012 PIMs, and in $7 \%$ of cases PIMS were reported to have contributed to or caused the admission. Alerts were present for most of the PIMs that were prescribed, 
indicating that alerts for these medications were often overridden. Methods to improve effectiveness of CDS to assist in promoting appropriate prescribing in the elderly should be studied to identify interventions to reduce preventable hospital admissions in this population. References 1. The American Geriatrics Society 2012 Beers Criteria Update Expert Panel. American Geriatrics Society Updated Beers Criteria for Potentially Inappropriate Medication Use in Older Adults. JAGS 2012;60:616-621. 2. The American Geriatrics Society 2015 Beers Criteria Update Expert Panel. American Geriatrics Society 2015 Updated Beers Criteria for Potentially Inappropriate Medication Use in Older Adults. JAGS 2015;63:2227-2246. This study was funded by grant \#U19HS021094 from the Agency for Healthcare Research and Quality (AHRQ)

BEHAVIORAL HEALTH INTEGRATION AT YOUR FINGERTIPS: A DESCRIPTIVE ANALYSIS OF ELECTRONIC CONSULTATION FROM PRIMARY CARE TO PSYCHIATRY Margaret Lowenstein ${ }^{3}$; Olusinmi Bamgbose ${ }^{4}$; Nathaniel Gleason ${ }^{1}$; Mitchell D. Feldman $2 .{ }^{1} \mathrm{UC}$ San Francisco, Nathaniel Gleason, CA; ${ }^{2} \mathrm{UCSF}$, San Francisco, CA; ${ }^{3}$ University of California San Francisco, San Francisco, CA; ${ }^{4}$ University of California, San Francisco, San Francisco, CA. (Control ID \#2467435)

BACKGROUND: In the course of managing behavioral health issues, primary care providers (PCPs) frequently face challenges accessing specialty mental health care. Electronic consultation (eConsult) permits asynchronous communication between the generalist and subspecialist, conducted securely as part of the electronic medical record. eConsults have been implemented in a number of settings and have been found to improve timely access to specialty care and reduce the need for in-person specialist evaluation, allowing for management of clinical issues to remain within primary care. To improve collaborative care and access to psychiatric consultation, the University of California, San Francisco (UCSF) developed a program for eConsults to psychiatry. To our knowledge, this is the first program using eConsults for behavioral health. Our aim was to assess the types of consult questions, efficiency of responses, and the need for in-person consultation.

METHODS: We abstracted data elements from the first 50 eConsults to psychiatry from PCPs in UCSF primary care clinics, including clinical category, specialist response time, recommendation for in-person consultation, and implementation of consultant recommendations. We categorized consults using a previously developed instrument for eConsult classification, modified with Ely's taxonomy of clinical questions. We classified questions and responses into one or more of two major domains: diagnosis and management. We also identified several key subcategories within management questions. One reviewer analyzed all the consult text, and a psychiatrist acted as a second independent reviewer for a subset of the data to ensure accuracy.

RESULTS: The first 50 psychiatry eConsults were completed between October 2014 and August 2015. The most common diagnoses were depression (40\%), anxiety disorders (16\%), psychotic disorders (14\%), and bipolar disorder (6\%). Fifty-eight percent of consults were initiated because patients had failed one or more therapies. The majority (98\%) of questions pertained to management; $6 \%$ asked about diagnosis. Seventy-five percent of management questions involved medication choice, with $32 \%$ inquiring about drug side effects or interactions. The remaining management questions included queries about navigating the mental healthcare system, psychotherapy, and whether the patient required in-person consultation. In their responses, psychiatrists recommended in-person consultation for $25 \%$ of patients - primarily for patients with bipolar disorder and psychotic disorders. All responses included management recommendations, with $75 \%$ focusing on medications. Psychiatrists commented on diagnosis for $56 \%$ of consults and provided multiple therapeutic options for the PCPs in $50 \%$. Specific triggers for starting/ adjusting treatment (32\%), medication titration instructions (34\%), and information about side effects $(42 \%)$ were other major themes in the responses. The average consult response time was 1.4 business days, and PCPs implemented psychiatrist recommendations in $74 \%$ of cases.

CONCLUSIONS: PCPs utilized psychiatry eConsults for assistance with a wide variety of psychiatric issues, particularly in complex cases in which patients had failed initial therapy in primary care. PCPs generally felt comfortable with psychiatric diagnosis, primarily initiating eConsults for medication management. For the majority of patients, psychiatrists supported ongoing management within primary care and provided a range of strategies that facilitated treatment without an in-person referral. eConsults hold promise as one aspect of closer integration of behavioral health in primary care, enhancing and extending traditional psychiatric consultation and allowing for efficient management of complex psychiatric issues in a primary care setting.

BEHAVIORAL HEALTH INTEGRATION IN RESIDENT PRIMARY CARE CONTINUITY CLINICS: A MULTI-INSTITUTIONAL SURVEY OF INTERNAL MEDICINE AND FAMILY MEDICINE RESIDENTS Patrick Hemming²; Amber Hewitt ${ }^{4}$; Joseph J. Gallo ${ }^{5}$; Rodger Kessler ${ }^{3}$; Rachel Levine ${ }^{1} .{ }^{1}$ Johns Hopkins University School of Medicine, Baltimore, MD; ${ }^{2}$ Duke University, Durham, NC; ${ }^{3}$ American Academy of Family Physicians, Leawood, KS; ${ }^{4}$ University of Massachussetts, Worchester, MA; ${ }^{5}$ Johns Hopkins Bloomberg School of Public Health, Baltimore, MD. (Control ID \#2456635)

BACKGROUND: Behavioral Health Integration (BHI) is a practice arrangement where behavioral health $(\mathrm{BH})$ clinicians provide counseling within a primary care practice for conditions including mental health and substance abuse disorders. These BH clinicians include: clinical social workers, psychologists, psychiatrists, and marriage and family therapists. BHI in residency clinics can improve mental health outcomes for patients, and may also improve primary care residents' training in BH. The present study examined whether increased co-management with $\mathrm{BH}$ providers in practices with $\mathrm{BH}$ integration is associated with medical residents having greater perceived ability to successfully treat their patients' BH conditions.

METHODS: For this cross-sectional study, we surveyed residents from 2 Internal Medicine (IM) and 3 Family Medicine (FM) residency training programs. Each practice surveyed had BHI in the residents' continuity clinic. The 47-item survey assessed the following domains: (1) level of co-management with BHI providers; (2) confidence in managing behavioral health conditions; (3) barriers to $\mathrm{BH}$ provision; (4) perception of autonomy when working with integrated $\mathrm{BH}$ providers; (5) satisfaction with clinic; and (6) perceived educational value of different modes of learning behavioral health. To determine whether co-management was associated with the other domains, we used mixed model linear regression analysis.

RESULTS: One hundred seventeen residents responded (73.1\% response rate). Residents who had co-managed more patients with integrated $\mathrm{BH}$ providers reported significantly higher confidence (on a 0 to 10 numeric scale) than those who had co-managed managed fewer than 5 total patients for the following conditions: major depression, suicidal ideation, anxiety, substance abuse and chronic pain. The association remained significant after adjustment for multiple co-variates, including year of training and specialty. Co-management was not significantly associated with greater confidence in managing tobacco dependence. The reported frequency of encountering three common barriers - lack of time, lack of knowledge, lack of access to a BH provider — was not independently associated with residents' level of co-management with $\mathrm{BH}$ providers. Perceptions of decreased autonomy when co-managing patients were significantly higher among IM residents than among FM residents ( 40.0 vs. $14.8 \%, p<.001$ ); however, there was no association between decreased autonomy and numbers of co-managed patients. Residents' overall satisfaction with their continuity clinic was also not significantly associated with co-management, but FM residents were more likely than IM residents to 
rate satisfaction as 8 out of 10 or higher ( 41.6 vs. $19.2 \%, p=.01$ ). Residents rated two educational modes for teaching behavioral health most highly: collaboration with $\mathrm{BH}$ providers ( $68.2 \%$ of residents rating value 8 out of 10 or higher) and observation with feedback by preceptors ( $55.4 \%$ of residents)

CONCLUSIONS: Our study suggests that behavioral health integration within residency practices enhances residents' learning and confidence in providing behavioral health care to their primary care patients.

Multiple linear regression of residents' confidence (10-point numeric scale) with increase in number of patients co-managed $(0-5,6-10,>10)$

\begin{tabular}{lllll}
\hline \hline & $\begin{array}{l}\text { Unadjusted linear } \\
\text { regression }\end{array}$ & $\begin{array}{l}95 \% \\
\text { Confidence } \\
\text { Interval }\end{array}$ & $\begin{array}{l}\text { Adjusted linear } \\
\text { regression }\end{array}$ & $\begin{array}{l}95 \% \\
\text { Confidence } \\
\text { interval } \\
.41-1.08\end{array}$ \\
$\begin{array}{l}\text { Major- } \\
\text { depressive } \\
\text { disorder }\end{array}$ & $0.75 * *$ & $0.51^{*}$ & .92 \\
$\begin{array}{l}\text { Anxiety- } \\
\text { disorders }\end{array}$ & $0.85^{* *}$ & $.53-1.17$ & $0.61 * *$ & $.23-.99$ \\
$\begin{array}{l}\text { Suicidal } \\
\text { Ideation } \\
\text { Substance }\end{array}$ & $0.67 * *$ & $.26-1.08$ & $0.61 *$ & $.09-1.13$ \\
$\begin{array}{l}\text { Abuse } \\
\text { Tobacco }\end{array}$ & $1.05 * *$ & $.67-1.43$ & $1.08 * *$ & $.63-1.54$ \\
Addiction & $0.60 * *$ & $.17-1.03$ & 0.43 & $-.09-.94$ \\
Chronic pain & $1.14 * *$ & $.74-1.54$ & $0.86 * *$ & $.39-1.32$ \\
\hline
\end{tabular}

** $p<.01 * p<.05$ Adjusted linear regression for gender, age, race (white/non-white), specialty, year of training, international medical graduate status, plan to practice primary care (yes or no), and influence of integrated behavioral health on residency choice (yes or no)

Scatter plot of residents' conf'ts

Figure 1: Residents' confidence by level of co-management

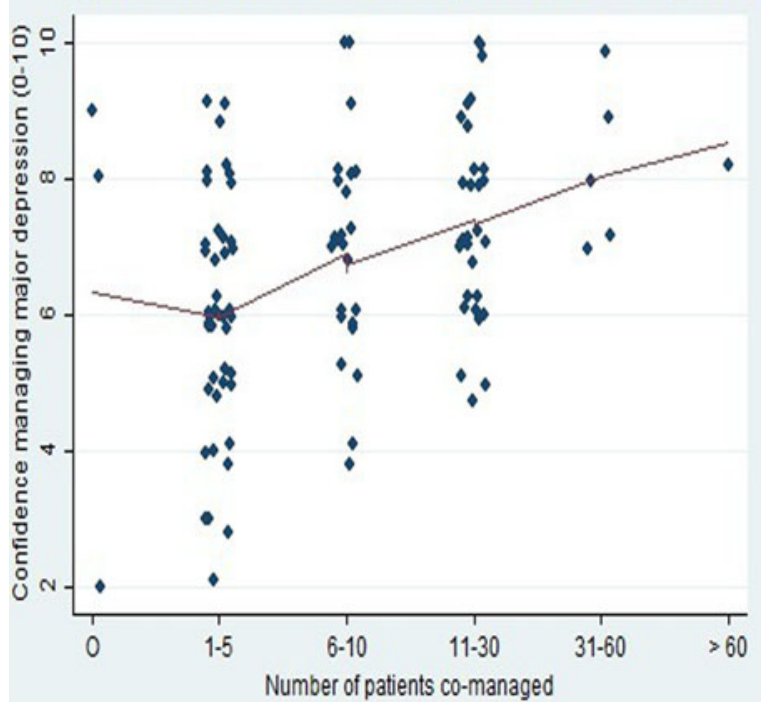

BEHAVIORAL WEIGHT MANAGEMENT INTERVENTION IN UNDERSERVED OVERWEIGHT POSTPARTUM WOMEN, A RANDOMIZED CONTROLLED FEASIBILITY TRIAL: THE RENEW STUDY Priya Joshi ${ }^{1}$; Lisa Quintiliani $^{1}$; Ashley C. Bourland ${ }^{1}$; Ashley Cuellar ${ }^{2}$; Mufaddal Mahesri ${ }^{1}$; Lisa Sullivan ${ }^{3}$; Caroline Apovian ${ }^{1} .{ }^{1}$ Boston Medical Center, Boston, MA; ${ }^{2}$ Indiana University, Indianapolis, IN; ${ }^{3}$ Boston University School of Public Health, Boston, MA. (Control ID \#2463548)
BACKGROUND: The rate of overweight and obesity among women of childbearing age in the United States is increasing at an alarming rate. This age group is most vulnerable to gain the greatest amount of weight in life, mostly due to postpartum weight retention (PWR). African American (AA) women of low socioeconomic status are at higher risk for postpartum obesity and long-term weight retention, and this health disparity has yet to be adequately addressed with scientific research. Previous research has shown the role of poor diet and inadequate exercise in supporting PWR in this population. Such findings document the need for a clinic-based, behavioral intervention to promote healthy diet and exercise among postpartum AA women with overweight or obesity. The postpartum period may offer an ideal opportunity to reach AA women who are overweight or obese for behavioral intervention, as women are connected to clinical care and motivated to lose weight during this time. We developed a pilot behavioral intervention, adapted from the Diabetes Prevention Program (DPP), to address a major racial/ethnic disparity for postpartum AA women with overweight or obesity and improve health outcomes for mothers with obesity and their children. We aimed to assess the feasibility, receptivity, and utility of this pilot intervention for postpartum AA women with overweight or obesity, in an underserved community. Secondary outcomes measured were participant attitudinal changes in eating behaviors, body image and physical activity, to further tailor behavioral intervention programs for this population.

METHODS: The RENEW study was a randomized, controlled trial conducted at Boston Medical Center (BMC) in which postpartum African American women with overweight or obesity were randomly assigned to either a culturally-tailored, in-person, group-based behavioral weight loss program or the control group. There were 3 study visits in addition to the 8-lesson curriculum, where screening, pre- and post-study assessments were conducted. Prenatal inclusion criteria included: pre-pregnancy BMI $\geq 25 \mathrm{~kg} / \mathrm{m}^{2}$, readiness to change and English-speaking. Prenatal exclusion criteria were signs of moderate to severe depression based on the PHQ-9 questionnaire and enrollment in the hospital-based peer health worker program (called Birth Sisters) prior to the study. All subjects provided written, informed consent prior to participation (Visit 1). Approximately 6 weeks postpartum (Visit 2), anthropometrics were objectively assessed and participants completed questionnaires assessing diet, physical activity, attitudes about eating, and body image. Randomization into the intervention or control group was performed via a 1:1 allocation based on permuted blocks of size 4 . The intervention was comprised of 8 weekly culturally relevant lessons on diet modification and supervised exercise sessions. Postintervention measurements were repeated 8 weeks later (Visit 3). Continuous outcome variables were analyzed as the change from baseline to follow-up, and the effect of the intervention was assessed by a student's t-test comparing changes from baseline between treatment groups. Analyses on change from baseline to follow-up include data from participants who completed both baseline and follow-up assessments.

RESULTS: Sixty-two women were randomized (32 intervention/30 control). The mean age of study participants was 30 years. The majority of subjects had a BMI $\geq 30$, reported low annual incomes, and all were African American. The groups were balanced at baseline (Mean BMI 35.4 (SD 7.6) intervention vs. 34.6 (SD 5.3) control). Thirty-five women (16 intervention/19 control) completed the study. Compared to control group participants, intervention completers reported enjoying participating in the weight loss program (58 vs. $100 \%$ 'very much' respectively), thought the program met their diet and exercise needs and weight loss goals ( 26 vs. $60 \%$ 'very much' respectively) and thought the program should be continued at the hospital ( 58 vs. $93 \%$ 'definitely yes' respectively) $(P<0.05$ for all). The final assessment revealed more low-fat eating in the intervention group vs. control, though this was not statistically significant. Both groups gained weight (intervention $2.4 \mathrm{~kg}$, control $2.2 \mathrm{~kg}$ ) from Visit 2 to Visit 3. The difference in weight change between the intervention and control groups was not significant $(P=0.89)$.

CONCLUSIONS: There is currently a paucity of effective and affordable non-surgical weight loss interventions for low-income postpartum AA women living in underserved, urban communities. We evaluated the feasibility of an adaptation of the intensive lifestyle 
behavioral program used in the DPP by instituting a culturally-tailored curriculum of weight loss strategies specific to improving nutrition, physical activity attitudes and behaviors in postpartum AA women diagnosed with overweight or obesity in an underserved urban community. While RENEW intervention study participants reported the program as highly favorable, women anecdotally reported challenges to attending the program, and ultimately gained weight postpartum. Interventions for postpartum weight reduction that overcome barriers to attendance need to be further examined for this population of women. Furthermore, initiating behavioral interventions earlier, such as prenatally, or during pregnancy, may help reduce postpartum weight retention and ultimately reduce associated adverse health outcomes.

BEHIND THE TIMES: INTERNISTS' USE OF IMMUNIZATION INFORMATION SYSTEMS Laura P. Hurley ${ }^{3,2}{ }^{\text {; }}$ Mandy Allison ${ }^{2}$; Cristina Cardemil ${ }^{1}$; Megan C. Lindley $^{1}$; Michaela Brtnikova ${ }^{2}$; Brenda Beaty ${ }^{2}$; Allison Kempe ${ }^{2} .{ }^{1}$ Centers for Disease Control and Prevention, Atlanta, GA; ${ }^{2}$ University of Colorado and Children's Hospital Colorado, Aurora, CO; ${ }^{3}$ Denver Health, Denver, CO. (Control ID \#2467476)

BACKGROUND: Forty-nine U.S. states and several cities have immunization information systems (IISs) for consolidating information from vaccinating providers. The 2014 National Vaccine Advisory Committee Standards for Adult Immunization call for vaccinating providers to understand how to access and to document receipt of vaccines in IISs. Our objectives were to assess among general internists 1) awareness and use of IISs; 2) barriers to using IISs; 3) demographic and practice characteristics and physician beliefs associated with submitting immunization data to IISs; and 4) functionalities that would increase interest in using IISs among those not currently using them.

METHODS: We administered an Internet and mail survey from February to April of 2015 to a national network of 433 GIM physicians representative of the American College of Physicians membership. In addition to descriptive analyses, we performed multivariable logistic modeling to examine associations between submitting adult immunization data to IISs (dependent variable) and demographic and practice characteristics and physician beliefs about barriers to IIS use. Two GIM physicians from New Hampshire were excluded from the analysis because New Hampshire does not have an IIS

RESULTS: The response rate was $63 \%(272 / 431)$. Forty-three percent reported their state/region had an IIS, $10 \%$ reported no IIS, and $48 \%$ reported they did not know. Twenty-one percent reported submitting adult immunization data and $16 \%$ reported looking up individual patients' vaccine needs using an IIS. The top reported 'major' barriers to using IISs were: the IIS not updating immunization information in practices' electronic health records (EHR) (35\%), having to upload immunization data manually (30\%), and the IIS not capturing immunizations given outside the region or state (19\%). Internists were more likely to report submitting immunization data to the IIS if they were younger (OR-1.3, $95 \%$, CI 1.1-1.6 per 5 years), believed the IIS not capturing out-of-state immunizations was a 'major' barrier (OR-3.6, 95 \% CI 1.6-8.0), or believed the IIS not updating the immunization information in their EHR was not a 'major' barrier (OR-3.7, $95 \%$ CI 1.7-8.3). Of internists not using an IIS, the majority reported their interest in using an IIS would increase 'moderately' or 'a lot' if they knew it could generate lists of patients needing immunizations for reminder recall (59\%), generate overall vaccine coverage reports $(56 \%)$, identify patients' immunization needs during clinic visits $(58 \%)$, and forecast vaccine due dates $(51 \%)$.

CONCLUSIONS: Despite their widespread existence, most internists are not aware of IISs or their functionalities and only a minority is submitting adult immunization data to IISs. Informing internists of existing IIS capabilities, encouraging interoperability between IISs and EHRs, and promoting or adapting existing IIS functionalities for use in adult populations could increase internists' interest in IISs.
BEING IN A RELATIONSHIP IS ASSOCIATED WITH WORSENED WELLBEING AMONG FEMALE MEDICAL STUDENTS COMPARED TO THEIR MALE COUNTERPARTS John Schorling ${ }^{2}$; Danielle Oliver ${ }^{1}$; Margaret L. Plews$\mathrm{Ogan}^{2}$; Justine Owens ${ }^{2} .{ }^{1}$ University of Virginia, Charlottesville, VA; ${ }^{2}$ University of Virginia School of Medicine, Charlottesville, VA. (Control ID \#2466554)

BACKGROUND: The well-being of medical students, residents and practicing physicians is a topic of great interest, especially as the rate of burnout among practicing physicians has increased significantly recently. Some measures of stress and well-being differ among male and female physicians. For example, a recent study found women to be at higher risk of self-reported alcohol abuse and dependence than men (Oreskovich, et al. Am J Addictions 2015;24:30). The reasons for these differences are not well defined, nor is how early they begin. One possible contributing factor is the impact that other roles may have on well-being. In this same study, substance misuse disorders were found to be more common among those who reported being married or partnered. Women in particular may feel additional stresses due to their roles as prinicipal care providers for others outside of their professional or educational responsibilities. The purpose of this study was to further investigate the impact of relationship status on well-being among medical students at a single institution.

METHODS: All medical students at the University of Virginia School of Medicine were sent an online anonymous survey that contained 128 items including demographic information, well-being measures, and validated questionnaires for anxiety and depression screening, burnout, resilience, and wisdom. This report will focus on the quality of life and anxiety and depression domains. Quality of life and fatigue were measured using a 10point scale from 0 (as bad as it could be) to 10 (as good as it could be) across several domains, including overall, emotional, mental and physical quality of life. Anxiety and depression were measured using the four question PHQ-4. Statistical analyses were conducted using multivariate ANOVA testing to compare the mean scores for each variable by gender, relationship status (single versus married or partnered), year of medical school, and an interaction term for genderXrelationship status.

RESULTS: Six hundred thirty students were eligible to participate, and 365 responded to at least part of the questionnaire (58\% response rate). Participants had a mean age of 25 years, with $51.3 \%$ being female and $48.7 \%$ male. A total of 305 completed all the well-being measures (48\%). Overall, female students had significantly lower levels of well-being compared to their male counterparts on several measures, including mental quality of life ( $6.7 \mathrm{vs} 7.1, p=.047)$ fatigue ( $5.6 \mathrm{vs} 6.0, p=.023)$ and the total score on the PHQ-4 (3.0 vs 2.1, $p=.004)$. There was also a significant interaction between gender and being married or partnered for a number of variables, including overall, mental and emotional quality of life, and the PHQ-4, with women having worse scores if they were married or partnered, and men having better scores if they were married or partnered ( $p<.01$ for all variables), as well as for fatigue $(p=.049)$. Overall, most measures of wellbeing varied by year of medical school with the lowest levels occurring in the third year and the highest in the fourth year. The gender differences associated with relationship status persisted after controlling for year of medical school.

CONCLUSIONS: Overall, female medical students had more fatigue, anxiety and depression than male students. In addition, women students who were married or partnered had even worse scores in these domains as well as worse overall, mental and emotional quality of life. For all these variables, being in a relationship was associated with improved scores for men and worsened scores for women. These results demonstrated significant gender differences in quality of life, anxiety and depression among medical students that are worsened for women who are in relationships. The reasons for these differences are unclear, although it is possible that being in a relationship results in increased role responsibilities outside of school for women and decreased responsibilities for men. These findings are particularly concerning given the increasing rates of stress and burnout in medicine which may ultimately have a disproportionate effect on women in 
relationships. These results also have important implications for helping to provide support for women medical students who might find that their personal relationships increase rather than decrease their stress.

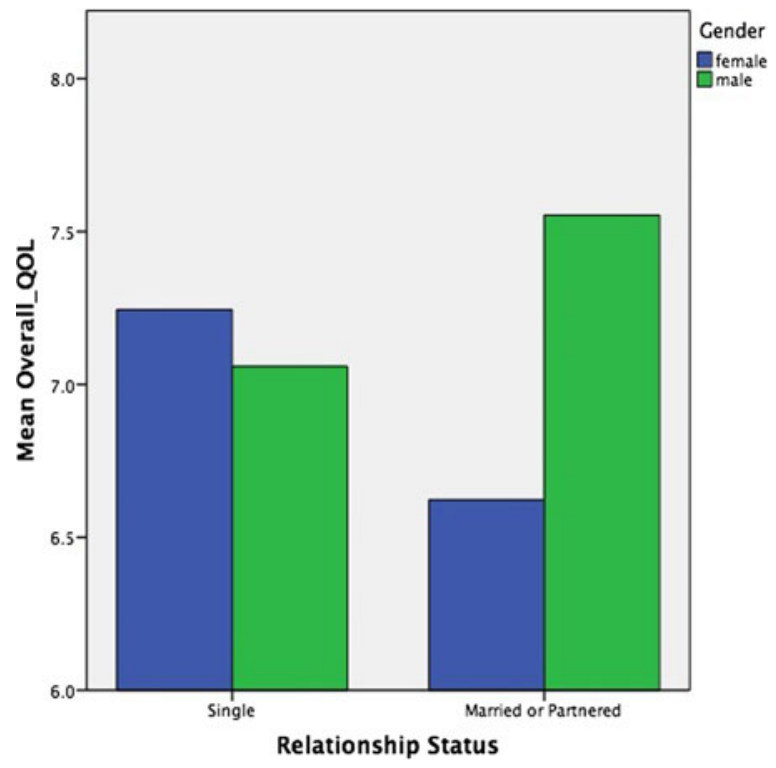

Association between overall well-being and relationship status for female and male medical students $(p=.004)$

BENEFITS AND HARMS OF TREATING BLOOD PRESSURE IN OLDER ADULTS: A SYSTEMATIC REVIEW AND META-ANALYSIS Devan Kansagara ${ }^{2}$, ${ }^{1}$; Michele Freeman ${ }^{2}$; Allison Low ${ }^{2}$; Makalapua Motu'apuaka ${ }^{2}$; Jessica W. Weiss ${ }^{1}$; Robin Paynter $^{2}$; Karli Kondo ${ }^{2}$; Rochelle Fu ${ }^{1}$; Amy L. Kerfoot ${ }^{1}$. ${ }^{1}$ Oregon Health and Science University, Portland, OR; ${ }^{2}$ VA Portland Healthcare System, Portland, OR. (Control ID \#2469360)

BACKGROUND: The optimal blood pressure treatment target in older individuals is controversial. We conducted a systematic review and meta-analysis to clarify the balance of benefits and harms of more intensive blood pressure treatment in adults over age 60

METHODS: We searched Medline, EMBASE, and Cochrane databases through January 2015, and updated our search for large blood pressure treatment trials through November 2015. To assess health outcomes, we included trials of hypertensive adults with mean age over 60 comparing different blood pressure treatment targets, or more versus less intensive treatment strategies. We included trials or observational studies examining potential longterm harms such as cognitive function, falls, and fractures. Two investigators used established criteria to independently assess the risk of bias of included studies, and the strength of evidence (SOE) supporting each finding. We conducted meta-analyses using the profile-likelihood random-effects model to assess all-cause mortality, fatal/non-fatal myocardial infarction (MI), and fatal/non-fatal stroke. We used Cochran's chi-square test and the $\mathrm{I}^{2}$ statistic to assess statistical heterogeneity. We qualitatively synthesized results for all other outcomes

RESULTS: We reviewed 10,627 titles and abstracts, selected 319 for full-text review, and included 21 trials and 3 observational studies that provided relevant primary data. Across 20 trials, more intensive treatment reduced all-cause mortality (RR 0.91, $95 \%$ CI 0.85-0.96; I I $=29 \%$ ), fatal/nonfatal MI (RR 0.80, $95 \%$ CI $0.74-0.86 ; \mathrm{I}^{2}=14 \%$ ), and fatal/non-fatal stroke (RR $0.77,95 \%$ CI $0.71-0.83$; $\mathrm{I}^{2}=14 \%$ ) compared to less intensive treatment (moderate SOE). Most of the benefit was contributed by studies in which patients achieved systolic blood pressures above $140 \mathrm{mmHg}$ and had baseline systolic blood pressure (SBP) above $160 \mathrm{mmHg}$. Low SOE from five treat-to-target trials comparing SBP targets $<140 \mathrm{mmHg}$ or lower to higher targets, showed no consistent impact on mortality reduction (RR $0.87,95 \%$ CI $0.68-1.13 ; \mathrm{I}^{2}=28 \%$ ), but a stronger-albeit nonsignificant - trend toward fatal/nonfatal MI reduction (RR $0.87,95 \%$ CI 0.68 $1.08, \mathrm{I}^{2}=0 \%$ ), and fatal/non-fatal stroke reduction (RR $0.79,95 \%$ CI $0.56-1.03$, $\mathrm{I}^{2}=32 \%$ (low SOE). In patients with prior stroke, moderate SOE showed that targeting SBP as low as $130 \mathrm{mmHg}$ reduced stroke (RR 0.76, $95 \%$ CI $0.67-0.85$, $\mathrm{I}^{2}=0 \%$ ) and fatal/non-fatal MI (RR $0.78,95 \%$ CI $\left.0.63-0.96, \mathrm{I}^{2}=0 \%\right)$, but not all-cause mortality (RR $0.96,95 \%$ CI $0.86-1.12, \mathrm{I}^{2}=0 \%$ ). More patients (RR ranged 1.44-2.00) assigned intensive blood pressure treatment withdrew due to adverse events, most frequently cough and hypotension (low SOE). More intensive treatment did not increase the risk of cognitive impairment ( 7 trials, moderate SOE), falls or fractures ( 3 trials, moderate SOE), advanced kidney disease (12 trials, low SOE), or worsen quality of life (4 trials, moderate SOE). We found insufficient evidence to determine how age and comorbidity burden affected treatment outcomes. Patients with frailty, dementia, advanced chronic kidney disease, and other life-limiting comorbidities such as cancer and liver disease were excluded from most trials.

CONCLUSIONS: In this systematic review, the first to our knowledge to incorporate the recently published SPRINT trial, we found overall that lowering blood pressure over 2 5 years in older patients reduces all-cause mortality (NNT of about 80-90, across different sensitivity analyses), fatal/non-fatal MI (NNT 80-110), and fatal/non-fatal stroke (NNT $80-90)$. We found most of this benefit in trials of patients with higher baseline SBP $(>160 \mathrm{mmHg}$ ) achieving moderate levels of blood pressure control $(>140 \mathrm{mmHg})$. We found that more aggressive treatment (targets below SBP $140 \mathrm{mmHg}$ ) had the potential to reduce MI and stroke occurrence in patients with mild hypertension, but the absolute effect was smaller (NNT of about 200). Moreover, the effect estimates were less precise and did not reach statistical significance, likely because there were fewer trials, relatively low event rates in several of the trials, and inconsistencies in findings among these trials. The SPRINT trial provided the most substantial evidence of benefit among the treat-to-target trials. Whether this was related to the higher cardiovascular risk profile of the patient population is unclear, as we found inconsistent results among three trials conducting subgroup analyses according to cardiovascular risk profile. While more aggressive blood pressure treatment was associated with higher medication burden and increased rates of adverse events such as hypotension, it did not increase the risk of longer term harms such as cognitive impairment or falls. The lack of evidence that blood pressure lowering among older adults causes long-term harms may provide some comfort and flexibility for providers to work with patients in crafting individualized treatment plans. There is little data to assess the balance of risks and benefits of antihypertensive treatment among the frail elderly.

\section{BEST PRACTICES FOR COMMUNICATING PROPORTION DATA TO PA-} TIENTS Claire F. Snyder ${ }^{1,}{ }^{4}$; Elliott E. Tolbert ${ }^{3}$; Katherine Smith ${ }^{3}$, ${ }^{4}$; Elissa Bantug ${ }^{4}$; Amanda L. Blackford ${ }^{4}$; Michael Brundage ${ }^{2}$; PRO Data Presentation Stakeholder Advisory Board ${ }^{5} .{ }^{1} \mathrm{~J}$ ohns Hopkins School of Medicine, Baltimore, MD; ${ }^{2}$ Queen's University, Kingston, ON, Canada; ${ }^{3}$ Johns Hopkins Bloomberg School of Public Health, Baltimore, MD; ${ }^{4}$ Sidney Kimmel Cancer Center at Johns Hopkins, Baltimore, MD; ${ }^{5}$ Group Author, Other States and, MD. (Control ID \#2462834)

BACKGROUND: Patient-reported outcomes (PRO) (e.g., symptoms, quality-oflife) are frequently measured in clinical trials and research studies comparing treatment options. Endpoints are commonly communicated as proportions (e.g., 
$\%$ improved, stable, worsened), but little is known regarding how to graphically present these data to patients most effectively. We tested the accuracy of interpretation and clarity ratings of three types of graphic displays for communicating PRO data expressed as proportions in patient educational materials and decision aids.

METHODS: This mixed-methods study included an internet survey circulated to distribution lists of cancer patients/survivors (age 21+), cancer providers, and PRO researchers using snowball techniques, supplemented with one-on-one in-person interviews with cancer patients/survivors and providers. The one-on-one interviewees were recruited from the Johns Hopkins Clinical Research Network (a consortium of academic and community-based health systems in the mid-Atlantic) and were purposively sampled based on education and cancer type (patients) and specialty (providers). For both the internet survey and one-on-one interviews, respondents were shown three approaches for presenting proportions: pie charts, bar graphs, icon arrays. Format presentation order was randomized to control for order effects. For each format, respondents were asked questions evaluating the accuracy of data interpretation ( 2 questions on the first format seen and 1 question on each of the following two formats), and also rated the format's clarity (very clear, somewhat clear, somewhat confusing, very confusing). At the end, respondents were asked to pick the proportion format "most useful for showing...patient's results." The one-onone interviewees were asked to "think aloud" as they responded to the online survey. The quantitative internet data were summarized descriptively. Multivariable logistic regression models estimated using generalized estimating equations (GEE) were used to analyze differences in accuracy of interpretation and clarity ratings by format. Chisquare tests evaluated differences in whether proportions were selected as "most useful." The one-on-one interview qualitative data informed our interpretation of the quantitative internet survey results.

RESULTS: Internet survey data from 649 respondents included 362 patients, 104 providers, and 183 researchers. Patients were 57 years old, on average, $95 \%$ white, $85 \%$ female, $26 \%<$ college graduate, and $48 \%$ breast cancer. Providers had a mean age of 43 with a mean 15 years in practice. Researchers were an average age of 44 with $49 \%$ having $>10$ years experience. One-on-one interviews were conducted with 10 patients $(30 \%<$ college graduate) and 5 providers representing various cancer specialties. The table shows the descriptive results of the percentage of patients, providers, and researchers who got both questions correct for the first format seen, the percentage rating each format "somewhat" or "very" clear, and the percentage selecting each format as "most useful". In the multivariable logistic regression GEE models, across the 4 accuracy questions for each format, bar graphs were less accurately interpreted than pie charts (odds ratio $[\mathrm{OR}]=0.49 ; 95 \%$ confidence interval $[95 \% \mathrm{CI}]=0.35-0.67 ; p<.0001)$ and icon arrays $(\mathrm{OR}=0.43 ; 95 \% \mathrm{CI}=0.31-$ $0.60 ; p<.0001)$. There were no statistically significant differences between icon arrays and pie charts in accuracy of interpretation. Bar graphs and icon arrays were less likely to be rated clear than pie charts $(\mathrm{OR}=0.35 ; 95 \% \mathrm{CI}=0.25$ 0.49 and $\mathrm{OR}=0.19 ; 95 \% \mathrm{CI}=0.13-0.25$, respectively; both $p<.0001)$, and bar graphs were more likely to be rated clear than icon arrays $(\mathrm{OR}=1.90 ; 95 \%$ $\mathrm{CI}=1.47-2.45 ; p<.0001)$. Pie charts were most often selected as "most useful" by patients $(67 \%$; chi-square $p<.001)$ and providers $(43 \%$; chi-square $p=.02)$, with no significant preference found for researchers.

CONCLUSIONS: Pie charts were more accurately interpreted than bar graphs, and were more likely to be rated clear and to be selected as "most useful" than either bar graphs or icon arrays. These data suggest that pie charts are the most effective approach for conveying proportion data accurately and clearly. Further research should investigate the generalizability of these findings beyond patient-reported outcomes data and in noncancer populations.
Accuracy of Interpretation (1st Format Seen), Clarity Ratings, and Percentage Selecting as Favorite

\begin{tabular}{llll}
\hline \hline N (\%) & $\begin{array}{l}\text { PIE } \\
\text { CHART }\end{array}$ & $\begin{array}{l}\text { BAR } \\
\text { GRAPHS }\end{array}$ & $\begin{array}{l}\text { ICON } \\
\text { ARRAYS }\end{array}$ \\
$\begin{array}{l}\text { PATIENTS }(n=362) \\
\begin{array}{l}\text { Both Accuracy Questions } \\
\text { Correct-1st Format Seen* }\end{array}\end{array}$ & $88(73 \%)$ & $68(56 \%)$ & $96(79 \%)$ \\
$\begin{array}{l}\text { Rating "Very Clear" or } \\
\text { "Somewhat Clear" }\end{array}$ & $278(91 \%)$ & $218(71 \%)$ & $176(58 \%)$ \\
$\begin{array}{l}\text { Selected "Most Useful" } \\
\text { PROVIDERS ( }=104)\end{array}$ & $196(67 \%)$ & $65(22 \%)$ & $32(11 \%)$ \\
$\begin{array}{l}\text { Both Accuracy Questions } \\
\text { Correct-1st Format Seen* }\end{array}$ & $32(91 \%)$ & $24(69 \%)$ & $26(77 \%)$ \\
$\begin{array}{l}\text { Rating "Very Clear" or } \\
\text { "Somewhat Clear" }\end{array}$ & $86(91 \%)$ & $71(74 \%)$ & $63(66 \%)$ \\
$\begin{array}{l}\text { Selected "Most Useful" } \\
\text { RESEARCHERS }(n=183)\end{array}$ & $40(43 \%)$ & $34(37 \%)$ & $19(20 \%)$ \\
$\begin{array}{l}\text { Both Accuracy Questions } \\
\text { Correct-1st Format Seen* }\end{array}$ & $57(93 \%)$ & $41(67 \%)$ & $110(66 \%)$ \\
$\begin{array}{l}\text { Rating "Very Clear" or } \\
\text { "Somewhat Clear" }\end{array}$ & $145(87 \%)$ & $138(83 \%)$ & $44(27 \%)$ \\
\hline \begin{tabular}{l} 
Selected "Most Useful" \\
\hline
\end{tabular} & $60(37 \%)$ & $57(35 \%)$ &
\end{tabular}

* $\mathrm{N}$ is approximately $1 / 3$ of total sample, as respondents were randomized to which format they saw first

BLOOD TRANSFUSION PATTERN IN ACUTE GASTROINTESTINAL BLEEDING: A US POPULATION BASED STUDY Raju Khanal ${ }^{1}$; DILLI R. POUDEL $^{1}$; Ranjan Pathak ${ }^{1}$; Sushil Ghimire ${ }^{1}$; Anthony A. Donato ${ }^{2}$. ${ }^{1}$ Reading Health System, Wyomissing, PA; ${ }^{2}$ The Reading Hospital and Medical Center, W. Reading, PA. (Control ID \#2454808)

BACKGROUND: Over the last two decades concerns have been raised about the potential risk of blood transfusion over the benefits. General restrictive red blood cell (RBC) transfusion approach for anemic hospitalized patients (thresh hold of $7 \mathrm{~g} \%$ ) has been adopted by American hematology society (AHS) in 2012. It is important to recognize that guidelines should be individualized with the patients, and its strict implementation may harm if the physician fails to review the patient comprehensively. Our study aims to look at the trend of blood transfusion in acute gastrointestinal bleeding (GIB) using a large US inpatient database.

METHODS: We used the Nationwide Inpatient Sample (NIS) database for years 20062012 to identify adult patients $>=18$ years of age with acute GIB (combination of International Classification of Diseases, 9th Revision, Clinical Modification (ICD-9-CM) codes 578.0, 578.1, 578.9 for GIB and previously validated procedure clinical classification software (CCS code 68 for endoscopy as a surrogate for acuity)). Recipient of blood transfusion were defined based on procedure CCS code 222 . NIS is the largest publicly available all-payer inpatient care database in the United States and is sponsored by the Agency for Healthcare Research and Quality as a part of Healthcare Cost and Utilization Project. Data analysis was done using STATA version 13.0 (College Station, TX).

RESULTS: We found 351,489 (weighted $N=1,735,813$ ) adults with acute GI bleed across all years. The proportions of patients with acute GIB who received transfusion showed an increasing trend from $41.06 \%$ in 2006 to $48.61 \%$ in 2012 . Mean age of adults with acute GIB was consistently higher among transfused patients in comparison with those not $(p<0.0001)$ transfused throughout all years. Similarly mortality was consistently higher among adults with acute GIB who received blood transfusion. (Table 1)

CONCLUSIONS: Our study clearly shows increasing trend of transfusion among adult patients with GIB even after excluding patients with myocardial infarction and Coronary artery disease although the latter group had $2 \%$ lower rate of transfusion across all year. This is in contrast to the expected decrease in trend 
with the adoption of restrictive transfusion pattern. This may be due to presentation being more severe secondary to rising use of anticoagulants leading to increase in candidates for restrictive transfusion or persistent GI bleeding or recurrent GI bleeding or overall increasing complex comorbidities requiring higher threshold of transfusion. Analysis including post adoption period data (after 2012) might give more insight regarding the topic

\begin{tabular}{|c|c|c|r|r|r|r|}
\hline Year & $\begin{array}{c}\text { Aate } \\
\text { GIB } \\
\text { (n) }\end{array}$ & $\begin{array}{c}\text { Inadence of } \\
\text { acute GIB } \\
\text { (per 10000) }\end{array}$ & $\begin{array}{c}\text { Acute GIB } \\
\text { (we ginted, } \\
\text { N) }\end{array}$ & $\begin{array}{c}\text { No. of } \\
\text { trar fusion } \\
\text { (weighted) }\end{array}$ & $\begin{array}{c}\text { \% of aatte } \\
\text { GIB } \\
\text { transfused }\end{array}$ & $\begin{array}{c}\text { \% transfued } \\
\text { (After excluding } \\
\text { A.MI and CA.D) }\end{array}$ \\
\hline 2006 & 47671 & 59 & 233083 & 94615 & 41.06 & 39.59 \\
\hline 2007 & 48745 & 61 & 240885 & 103564 & 43.43 & 41.75 \\
\hline 2008 & 54060 & 67 & 265083 & 112379 & 42.86 & 40.88 \\
\hline 2009 & 50297 & 65 & 254409 & 116317 & 46.12 & 44.05 \\
\hline 2010 & 49429 & 64 & 246628 & 113033 & 46.39 & 44.58 \\
\hline 2011 & 53427 & 67 & 256425 & 123199 & 48.42 & 46.50 \\
\hline 2012 & 47860 & 66 & 239300 & 115230 & 48.61 & 46.25 \\
\hline
\end{tabular}

Table 1: Trend of blood transfus ion among patients hos pit alized for adt e gas trointes tinal bleedirg (2006 - 2012)

BREAST CANCER RISK PREDICTION USING A COMBINED CLINICAL RISK CALCULATOR AND POLYGENIC RISK SCORE Yiwey Shieh ${ }^{1}$; Donglei $\mathrm{Hu}^{1}$; Lin Ma ${ }^{1}$; Charlotte C. Gard ${ }^{2}$; Jessica Leung ${ }^{3}$; Jeffrey A. Tice ${ }^{1}$; Celine M. Vachon ${ }^{4}$; Steven R. Cummings ${ }^{5}$; Karla Kerlikowske ${ }^{1}$; Elad Ziv ${ }^{1} .{ }^{1}$ University of California, San Francisco, San Francisco, CA; ${ }^{2}$ New Mexico State University, Las Cruces, NM; ${ }^{3}$ Department of Diagnostic Radiology, MD Anderson Cancer Center, Houston, TX; ${ }^{4}$ Department of Health Sciences Research, Division of Epidemiology, Mayo Clinic, Rochester, MN; ${ }^{5}$ San Francisco Coordinating Center, California Pacific Medical Center Research Institute, San Francisco, CA. (Control ID \#2464504)

BACKGROUND: Personalized assessment of breast cancer risk can potentially inform the use of screening and prevention modalities. We previously developed and validated the Breast Cancer Surveillance Consortium (BCSC) risk calculator, which uses age, race/ethnicity, family history of breast cancer, history of breast biopsy, and mammographic breast density to provide individualized estimates of 5- and 10-year risk. Genetics may further refine estimates of risk. A polygenic risk score represents the cumulative effect on the risk of invasive breast cancer conferred by multiple single nucleotide polymorphisms (SNPs). Adding a polygenic risk score to the BCSC risk calculator was recently shown to improve the discrimination between breast cancer cases and controls in a primarily Caucasian population. We sought to replicate these findings in an independent population including approximately $20 \%$ non-Caucasian women.

METHODS: This was a nested case-control study within a cohort of women undergoing screening mammography at a single imaging facility in the San Francisco Mammography Registry. Participants provided passive permission for inclusion in the registry and signed consent to provide blood samples for genotyping. Cases were randomly selected from women with breast cancer, and controls were matched by age and race/ethnicity. Ninetyone SNPs with genome-wide significant $\left(p<5 \times 10^{-8}\right)$ associations with breast cancer from previous studies were genotyped. The polygenic risk score for each woman was calculated as the product of the likelihood ratios for breast cancer associated with each genotype, with the assumption that the SNPs are independent of one other. Multivariate logistic regression was used to explore the contributions of polygenic risk, family history, breast density, and other variables in the BCSC model to breast cancer risk. The area under the receiver operating characteristic curve (AUROC) was used to compare discrimination between models

RESULTS: Cases $(n=486)$ and controls $(n=495)$ were evenly matched by age and race/ethnicity, with the most prevalent being Caucasian (80 \%) and Asian (11\%). The mean unadjusted polygenic risk score was higher in cases $(1.65,95 \% \mathrm{CI} 1.48$ to 1.81$)$ than controls (1.11, $95 \%$ CI 1.01 to 1.65 ). Each increasing quartile of PRS was positively associated with breast cancer risk compared to the lowest quartile (Table). The strength of association between polygenic risk score quartile and breast cancer did not differ between univariate and multivariate analyses adjusted for risk factors in the BCSC model. Likewise, family history and breast density remained strong risk factors with the inclusion of the polygenic risk score in the model. The combined model had better discriminatory ability compared with the BCSC model alone (AUROC 0.65 vs $0.62, p<0.001$ ). The polygenic risk score alone had an AUROC of 0.61 ( $95 \%$ CI 0.56 to 0.64 ) across all subjects, and its performance was preserved in the subset of Asian participants with an AUROC of 0.64 (95\% CI 0.53 to 0.75 ).

CONCLUSIONS: The polygenic risk score was an independent risk factor for breast cancer whose effect was not substantially attenuated by family history or mammographic breast density. Adding the polygenic risk score to the BCSC improved discrimination, suggesting the score can be an important part of risk stratification and likely exerts an effect that is distinct from traditional risk factors. The performance of the polygenic risk score was preserved in Asians, the largest non-Caucasian group in our study, but larger studies in this and other racial/ethnic groups are warranted. The declining cost and increasing accessibility of genetic-based assays may make it possible to use the polygenic risk score in conjunction with other models to risk-stratify women, and to adapt the age of initiation, frequency, and modality of screening based on this estimate. Studies of this approach to screening are underway in the U.S. and in Europe.

Association between polygenic risk score and other risk factors on development of breast cancer

\begin{tabular}{|c|c|c|c|c|}
\hline & Unadjusted* & & Adjusted $^{\dagger}$ & \\
\hline Characteristic & $\begin{array}{l}\text { Odds Ratio ( } 95 \\
\% \text { CI) }\end{array}$ & $\begin{array}{l}P \text { - } \\
\text { value }\end{array}$ & $\begin{array}{l}\text { Odds Ratio (95 } \\
\% \text { CI) }\end{array}$ & $\begin{array}{l}P \text { - } \\
\text { value }\end{array}$ \\
\hline \multicolumn{5}{|c|}{ Polygenic risk score quartiles } \\
\hline$<0.45$ & referent & & referent & \\
\hline $0.45-0.84$ & $1.63(1.10-2.40)$ & 0.014 & $1.78(1.19-2.68)$ & 0.005 \\
\hline $0.84-1.41$ & $1.80(1.23-2.64)$ & 0.003 & $1.86(1.24-2.77)$ & 0.002 \\
\hline$>1.41$ & $2.71(1.87-3.93)$ & $<0.001$ & $2.69(1.82-3.98)$ & $<0.001$ \\
\hline $\begin{array}{l}\text { First degree relative with } \\
\text { breast cancer }\end{array}$ & $1.86(1.38-2.52)$ & $<0.001$ & $1.68(1.23-2.30)$ & 0.001 \\
\hline History of breast biopsy & $1.54(1.14-2.08)$ & 0.005 & $1.52(1.11-2.09)$ & 0.009 \\
\hline Breast density, BIRADS & & & & \\
\hline $\begin{array}{l}\text { a, almost entirely fatty } \\
\text { b, scattered areas of } \\
\text { fibroglandular density }\end{array}$ & $\begin{array}{l}0.90(0.53-1.54) \\
\text { referent }\end{array}$ & 0.70 & $\begin{array}{l}0.80(0.45-1.42) \\
\text { referent }\end{array}$ & 0.49 \\
\hline $\mathrm{c}$, heterogeneously dense & $1.43(1.08-1.90)$ & 0.013 & $1.66(1.21-2.26)$ & 0.001 \\
\hline d, extremely dense & $1.67(1.11-2.49)$ & 0.013 & $2.01(1.28-3.15)$ & 0.002 \\
\hline
\end{tabular}

*Unadjusted measures of association from univariate regression of breast cancer on each individual risk factor. †Adjusted measures of association from a multivariate model including polygenic risk score quartile, first degree relative with breast cancer, history of breast biopsy, breast density, age and body mass index 
BUILDING AWARENESS OF HEALTHCARE TEAMS AMONG MEDICAL STUDENTS AND NURSE. Nicholas R. Mercado ${ }^{1}$; Lauren Tesoriero ${ }^{1}$; Mary A. McNamee ${ }^{3}$; Launette Woolforde ${ }^{3,}$; Lauren Block ${ }^{2} .{ }^{1}$ Hofstra Northwell School of Medicine, Hempstead, NY; ${ }^{2}$ North Shore-LIJ Health System, Lake Success, NY; ${ }^{3}$ North Shore University Hospital, Manhasset, NY. (Control ID \#2468395)

BACKGROUND: Inter-professional education in the health professions is recommended to facilitate acquisition of knowledge and skills collaboration. Collaboration between nurses and physicians has been associated with higher job satisfaction and with improved patient outcomes. Studies have shown that medical students have positive attitudes towards learning from other healthcare professionals, and value teamwork and communication as essential to patient care. However, professional cultures may limit the effectiveness of inter-professional teamwork. To foster interprofessional collaboration we implemented a three-part educational experience for medical students about to begin their third year rotations. We sought to understand the impact of this experience on attitudes and knowledge toward nurse-physician collaboration among medical students and nurses.

METHODS: Rising third year medical students at Hofstra North Shore-LIJ School of Medicine took part in three educational activities to promote interprofessional understanding and collaboration: 1 . Intensive shadowing experience, paired one-on-one with nurse preceptors in medicine and surgery units 2 . Transitions in care workshop highlighting discharge skills and role of interprofessional team in safe discharge from the hospital 3. IV/ phlebotomy training in the Emergency Department, led by triage nurses and nurse educators A pre-post survey design was used to measure attitudes and knowledge toward interprofessional collaboration among medical students and nurses participating in the experience. Outcome measures included the previously validated Jefferson scale of nursephysician collaboration and attitudes toward healthcare teams scale as well as a novel scale of knowledge of nurse roles and responsibilities. A reflection question was used to generate qualitative data on the experience. Medical students and nurse preceptors completed the surveys at the beginning and end of their 3-week transitions course. Preand post-surveys were paired by participant and analyzed using paired t-tests.

RESULTS: A total of 56 of $73(77 \%)$ students who participated in the course and 38 of 46 nurses $(83 \%$ ) contributed pre- and post-survey data and were included in the analysis. Student scores on attitudes toward healthcare teams improved subsequent to participation (mean 4.15 on $1-5$ scale pre-test vs. 4.49 post-test, $p<0.01$ ) as did scores on the Jefferson scale of nurse-physician collaboration ( 4.28 vs. $4.47, p<0.01)$. Among nurse preceptors, scores on attitudes toward healthcare teams improved subsequent to participation (mean 4.41 vs. $4.54, p=0.02$ ) but scores on the Jefferson scale did not change. Medical student knowledge of roles of nurses did not change following participation. Qualitative reflections by medical students spoke to the variety of responsibilities held by nurses, role in protecting patient safety, collaboration with other professionals, and amount of time spent in direct patient care.

CONCLUSIONS: A three-part experience designed to introduce students to the role of nurses in inpatient care was successful in improving attitudes towards healthcare teams and interprofessional collaboration among medical students and improved attitudes toward healthcare teams among nurses. The experience did not improve knowledge of the variety of nurse roles and responsibilities, perhaps since students were not able to witness all nurse responsibilities during the experience. Notably, medical students reported gaining appreciation for the role of nurses in protecting patient safety

BUILDING RESILIENT TEAMS: SENIOR RESIDENT EXPERIENCE WITH DIFFICULT CLINICAL EVENTS Michelle Martinchek ${ }^{1}$; Amber Bird ${ }^{2}$; Amber Pincavage ${ }^{1}$. ${ }^{1}$ University of Chicago, Chicago, IL; ${ }^{2}$ University of Pennsylvania, Philadelphia, PA. (Control ID \#2467684)
BACKGROUND: Building resilience is one promising method to help mitigate burnout and help physicians overcome career challenges, such as difficult clinical events and medical errors. This study assesses residents' baseline resilience and experiences with difficult clinical events.

METHODS: Cross-sectional surveys were given to IM residents. Baseline resilience was assessed with the Connor-Davidson Resilience scale and categorized as low $(<70)$, intermediate (70-79), and high (80-100). Residents were surveyed on burnout and reflection after difficult clinical events.

RESULTS: Forty-one of 62 (66.1\%) IM residents completed the survey. $14.6 \%$ had high resilience, $46.3 \%$ intermediate, and $39.0 \%$ low. $26.8 \%$ reported at least one burnout symptom. There was no association between high or low resilience and burnout ( $p=0.27$ ). $51.2 \%$ of residents experienced difficult clinical events several times a month, $24.4 \%$ several times a week, and $9.8 \%$ daily. One hundred percent of residents reported stress after these events. $22.0 \%$ prefer to discuss events with their team immediately, $56.0 \%$ later that day, $19.6 \%$ in the following days to weeks, and only $2.4 \%$ prefer not to discuss them. Residents individually reflect on these events very often (41.5\%), often (29.2\%), or sometimes $(19.5 \%)$, whereas only $9.8 \%$ rarely reflect. After events, residents talk with interns and students very often (17.1\%), often (39.0\%), or sometimes (39.0\%), but with attendings only rarely ( $41.5 \%$ ) or sometimes $(41.5 \%)$. Only $14.6 \%$ talk with attendings often. $68.3 \%$ of residents think difficult events affect their well-being at work and $58.6 \%$ want more training to help their teams cope.

CONCLUSIONS: IM residents experience difficult clinical events regularly. Most find these events stressful, prefer to discuss them with their teams, and would like more training to help their teams cope. Residents discuss these events with interns and students frequently, but not with attendings. Curricula on leadership skills and promoting team resilience after difficult clinical events are needed, as is faculty development.

CAN DISCRIMINATION EXPLAIN VULNERABLE SMOKERS' MISTRUST OF NICOTINE REPLACEMENT THERAPY? Elisheva Danan ${ }^{1,}{ }^{4}$; Steven Fu ${ }^{1,4}$; Barbara Clothier ${ }^{2}$; Siamak Noorbaloochi ${ }^{1}$; Diana Burgess ${ }^{3} .{ }^{1}$ Minneapolis VA Health Care System, Minneapolis, MN; ${ }^{2}$ Mpls VAHCS, Minneapolis, MN; ${ }^{3}$ University of Minnesota and Minneapolis Veterans Affairs Health Care System, Minneapolis, MN; ${ }^{4}$ University of Minnesota, Minneapolis, MN. (Control ID \#2469126)

BACKGROUND: African American smokers are less likely than whites to quit successfully despite higher motivation and readiness to quit levels. Racial and ethnic minorities are less likely to use evidence-based quit support such as nicotine replacement therapy (NRT) even though research has demonstrated that it is equally effective in minority populations as in whites. It is unclear if this is due to access, cost of treatment, mistrust of NRT, or higher self-reliance/self-efficacy. Minorities are also more likely to experience discrimination than whites, which has been associated with increased negative health behaviors, including smoking, and decreased positive health behaviors and use of preventive services. We hypothesized that the experience of discrimination among smokers would be associated with more negative attitudes toward NRT.

METHODS: We conducted a secondary analysis of the Veterans Victory Over Tobacco Study (VICTORY), a multicenter pragmatic randomized controlled trial of a proactive telephone-based smoking cessation intervention. After initial randomization of 5123 smokers to either usual care or proactive outreach (treatment group), participants were surveyed at 1-year follow-up. We measured experiences of day-to-day discrimination as a continuous variable with a 9-question scale (Kessler 1999), and used the advantages and drawbacks of NRT subscales (ANRT-12) to measure attitudes toward NRT. We performed a complete case analysis and used multiple linear regression to evaluate the relationship between perceived discrimination and attitudes toward NRT, all measured at follow-up after controlling for baseline attitudes toward NRT, demographic characteristics including 
age, race, gender, income, education, marital status, and employment status, and study design variables including treatment group and facility.

RESULTS: Follow-up survey results were available for 3382 study participants (66 \% response rate). There was no difference in experiences of discrimination between treatment groups, but more vulnerable demographic groups (including African Americans, women, those with lower incomes and those unemployed or unable to work) reported more experiences of discrimination than their counterparts. After controlling for these demographic characteristics and other variables, there was a non-significant association between increased perceived discrimination and more negative attitudes toward the advantages of NRT $(\beta=-0.03 ; p=0.12)$ and a statistically significant association between increased perceived discrimination and more concern for the drawbacks of NRT ( $\beta=-0.07 ; p<0.001)$. Regression models explained a significant proportion of variance of these attitude measures $\left(\mathrm{R}^{2}=0.18, \mathrm{~F}(21,1965)=20.7, p<0.0001 ; \mathrm{R}^{2}=0.23\right.$, $\mathrm{F}(21,1989)=28.5, p<0.0001$; respectively). There was no statistically significant interaction between treatment group and the relationship between discrimination and attitudes toward NRT

CONCLUSIONS: In a large, multicenter smoking cessation trial, perceived discrimination was significantly associated with increased concern for the potential drawbacks of NRT, including concern for the side-effects and risk for dependence on these products. This finding suggests that the experience of discrimination may explain the known mistrust of NRT among vulnerable smokers. A telephone-based intervention using trained counselors did not affect the relationship between discrimination and attitudes toward NRT. Interventions to offer NRT to minority smokers should utilize trusted sources, such as personal physicians or community leaders, to overcome the negative effects of discrimination in these populations

CAN PRIMARY CARE PHYSICIANS ACCURATELY PREDICT THE LIKELIHOOD OF HOSPITALIZATION IN THEIR PATIENTS? Andrew S. Hwang ${ }^{1}$; Jeffrey M. Ashburner ${ }^{1}$; Clemens S. Hong ${ }^{2}$; Wei $\mathrm{He}^{1}$; Steven J. Atlas ${ }^{1} .{ }^{1}$ Massachusetts General Hospital, Boston, MA; ${ }^{2}$ Los Angeles County Department of Health Services, Los Angele, CA. (Control ID \#2432254)

BACKGROUND: Prospectively identifying community dwelling patients at increased risk for hospital admissions is a critical step in reducing unnecessary acute care utilization and improving value in healthcare. Primary care physicians (PCPs), with their comprehensive understanding of the clinical and psychosocial needs of their patients, may be uniquely qualified to identify risk factors often missed by existing predictive algorithms. We hypothesized that PCPs could accurately identify patients in their panels at high risk for hospitalization in the subsequent year.

METHODS: We recruited PCPs from the Massachusetts General Hospital (MGH) Primary Care Practice-Based Research Network (PBRN). To identify PCPs' patients, we linked all patients seen in our PBRN in the previous 3 years to specific PCPs using a previously validated attribution algorithm. PCPs managing a panel of at least 100 primary care patients in 2013 were invited to review a list of 100 randomly selected patients. After verifying that the patient was theirs, PCPs were asked, "Would you be surprised if this patient was admitted to the hospital in the next year?" Patients were categorized as high or low admission risk based on whether PCPs answered "No" or "Yes," respectively, to the above question. We compared baseline demographics and Charlson comorbidity scores between patients in the PCP-designated high vs. low admission risk groups using chisquare or $\mathrm{t}$ tests, as appropriate. Unadjusted rates of MGH hospitalization in the prior and subsequent 1-year were compared using zero-inflated Poisson (ZIP) regression while accounting for clustering by PCP. To determine if PCP assessment of hospitalization risk remained an independent predictor of future hospitalization, we used ZIP regression to adjust for patient demographics (age, gender, race, insurance, income), PCP characteristics (age, gender, years since graduation from medical school, years working at MGH PBRN), past acute care utilization, and Charlson comorbidity score while accounting for clustering by PCP. We also calculated the c-statistic for PCPs' prediction of subsequent hospitalization.

RESULTS: Among eligible PCPs, 54 \% (99/182), representing $90 \%$ (17/19) of network practices, reviewed their patient list. PCPs who participated were on average 48.8 years old, $59 \%$ female and worked an average of 14.4 years in the network. These characteristics did not differ from non-participating PCPs. Of the 9900 patients provided to participating PCPs, 9832 patients were reviewed and 238 (2.4\%) were designated as not their patient. Among the remaining 9594 patients, PCPs designated $2037(21.2 \%)$ as high admission risk and $7538(78.6 \%)$ as low admission risk. Nineteen (0.2\%) patients were unassigned and excluded from analyses. Compared to patients in the low admission risk group, patients in the high admission risk group were significantly $(P<0.001$ for all $)$ older ( 66 vs. 50 years), more likely to be white ( 80 vs. $77 \%$ ), insured by Medicare ( 52 vs. $13 \%$ ), live in neighborhoods with low median household income ( $\$ 68,930$ vs. $\$ 77,207)$, have a higher Charlson comorbidity score (2.43 vs. 0.79 ), and have increased unadjusted rates of acute care utilization in the past year (286 vs. 22 hospitalizations/1000 patients). Over the following year, patients in the high admission risk group had higher unadjusted rates of acute care utilizationthan patients in the low admission risk group ( 286 vs. 38 hospitalizations/1000 patients, $P<0.001)$. In addition, adjusted analyses demonstrated that compared to patients in the low admission risk group, patients in the high admission risk group had an adjusted relative rate of (2.72 [95\% confidence interval (CI) 1.95-3.78]) for hospitalization in the subsequent 1-year. The c-statistic for PCP assessment of admission risk was 0.77 [95\% CI 0.75-0.79]. There was no major difference in c-statistics for subsequent hospitalization when patients were stratified by the presence vs. absence of past hospitalization $(0.77$ [95 \% CI $0.72-0.81$ ] for those with hospitalization and 0.75 [95\% CI 0.73-0.77] for those without hospitalization in the past year).

CONCLUSIONS: PCPs' qualitative assessment of future hospitalization risk among patients in their panels was an independent predictor of subsequent hospitalization, identifying a group of patients with a $172 \%$ increased rate of hospitalization. Predictive accuracy of PCP assessment in our study was comparable to the reported c-statistic of other commonly used risk stratification instruments, such as Adjusted Clinical Groups and Hierarchical Condition Categories (Am J Manag Care. 2013;19(9):725-732). There was no major difference in c-statistic among those with or without hospitalization in the prior year, highlighting the fact that PCPs were not simply identifying those with past hospital admissions. Given the predictive accuracy of PCPs' clinical assessment, efforts to identify patients at high risk for future hospitalization should aim to incorporate the unique insight that PCPs have about predisposing biopsychosocial factors. Tailoring interventions to prospectively identified high-risk patients may help improve the quality of care and reduce costly yet preventable hospitalizations.

\section{CAN SECURE ELECTRONIC MESSAGING SERVE AS AN EFFICIENT PAR-} TICIPANT RECRUITMENT TOOL? THE PATH EXPERIENCE. Anuradha Paranjape $^{8}$; Kathleen M. McTigue ${ }^{10}$; Michael Becich ${ }^{10}$; Wendy L. Bennett ${ }^{2}$; Cynthia H. Chuang $^{4}$; Jeanne M. Clark ${ }^{3}$; Daniel E. Ford ${ }^{1}$; Sharon J. Herring ${ }^{7}$; Jennifer Kraschnewski ${ }^{6}$; Christopher Sciamanna ${ }^{5}$; Rachel Hess ${ }^{9} .{ }^{1}$ Johna Hopkins University, Baltimore, MD; ${ }^{2}$ Johns Hopkins School of Medicine, Baltimore, MD; ${ }^{3}$ Johns Hopkins University, Baltimore, MD; ${ }^{4}$ Penn State College of Medicine, Hershey, PA; ${ }^{5}$ Penn State Hershey, Hershey, PA; ${ }^{6}$ Penn State Hershey Medical Center, Hershey, PA; ${ }^{7}$ Temple University, Philadelphia, PA; ${ }^{8}$ Temple University School of Medicine, Philadelphia, PA; ${ }^{9}$ University of Utah, Salt Lake City, UT; ${ }^{10}$ University of Pittsburgh, Pittsburgh, PA. (Control ID \#2469083)

BACKGROUND: Established in 2014 through funding from PCORI, the PaTH Clinical Data Research Network (CDRN) initially brought together 4 academic medical centers (University of Pittsburgh (Pitt), Penn State Hershey (PSU), Temple Health/LKSOM (TU) 
and Johns Hopkins Medicine (JHU) and 2 health care plans covering patients in 7 states and the District of Columbia). Together, PaTH sites are developing an outstanding network through which to explore clinical outcomes and improve methods for sharing and analyzing data. The overarching goal of this project is to build the infrastructure to share patient outcomes data across health systems so that clinical questions can be answered in a real world setting. One of the mandates of the first 18 months of funding was to test novel and efficient recruitment techniques to enroll observational cohorts of patients for one common condition (Atrial Fibrillation), one rare condition (Idiopathic Pulmonary Fibrosis) and one PCORI specified condition (the weight cohort). No incentive was offered to patients for participating in the study. Our a priori expectation was that inperson recruitment would result in the highest participation rate, but that electronic methods (secure email and electronic health record messaging would be an efficient approach for recruiting large numbers of patients with minimal staff effort). We report on the recruitment efforts of the weight cohort who were, on average, expected to be a generally healthy cohort with little engagement with the health condition being studied. METHODS: Patients of participating practices who met inclusion and exclusion criteria for the weight cohort (two weights and a height recorded in the electronic health record (EHR)) were invited to participate over a 2 month period in 2015. Each site used a range of recruitment methods including electronic (email or patient portal), paper mailing as well as very limited in person recruitment at the time of a routine office visit. Each participant contacted electronically or by mail recieved at least two follow up messages. Informed consent was obtained from all participants. For participants invited using electronic means, a short consent quiz was used to ensure participant understanding of recruitment materials. Two of the four health systems were able to complete recruitment and consent through their EHR.

RESULTS: We contacted 33,475 primary care patients recruited from General Medicine practices at the four sites. Of these 22,410 were contacted using electronic means for initial contact, 10,837 received a traditional paper mailing and 228 were recruited at the visit. As noted in the table below, while the rates of consent were the highest among participant recruited during a visit, the electronic approach yielded the largest total number of participants recruited. Of the 835 participants recruited to this cohort, 524 (62.7\%) were enrolled through initial electronic messaging.

CONCLUSIONS: Recruiting patients to join a simple observational protocol through electronic channels is an efficient means of reaching large numbers of participants, however recruitment yield is modest. Even though the four academic health centers are located in very different geographic areas and serve a diverse population, they yielded similar rates of consent by electronic means. We will explore if sociodemographic factors might influence rates of consent and reasons for refusal to participate.

Recruitment rates by site and strategy.

\begin{tabular}{|c|c|c|c|c|c|c|c|c|}
\hline \multirow[t]{2}{*}{ Initial contact method } & Pitt & PSU & $\mathrm{TU}$ & JHU & & & & \\
\hline & Contacted & Consent (\%) & Contacted & Consent (\%) & Contacted & Consent (\%) & Contacted & Consent $(\%)$ \\
\hline Electronic means & 15,121 & $243(1.6 \%)$ & - & - & 3,434 & $115(3.3 \%)$ & 3,855 & $166(4.3 \%)$ \\
\hline Mail & 1,636 & $6(0.4 \%)$ & 8,187 & $177(2.2 \%)$ & 352 & 12 (3.4\%) & 662 & $0(0 \%)$ \\
\hline In person & 119 & 63 (52.9 \%) & - & - & 7 & $3(42.9 \%)$ & 102 & $50(49.2 \%)$ \\
\hline Totals & 16,876 & 312 & 8187 & 177 & 3,793 & 130 & 4,619 & 216 \\
\hline
\end{tabular}

\section{CANADIAN DATA SOURCES ON ETHNIC CLASSIFICATIONS Karen Tang,} Kelsey Lucyk; Hude Quan. University of Calgary, Calgary, AB, Canada. (Control ID \#2469454)

BACKGROUND: In 1971, Canada adopted a policy of multiculturalism to solidify value and respect to all Canadian citizens regardless of ethnic origin. As of 2011 , nearly $21 \%$, or 6.8 million people, were foreign-born. A thorough understanding of the health status of Canadians must therefore take into account their ethnicity, given the genetic, social, and cultural effects of race and ethnicity on health. Our objective was to describe Canadian data sources that collect ethnicity data and the granularity of the ethnic classifications within these sources.

METHODS: Our methods were informed by those of an environmental scan. We searched publicly available government documents, articles in the peer-reviewed literature, and also contacted key informants, to gain a comprehensive understanding of the sources collecting ethnicity data in a nationally representative Canadian sample. Two investigators, using qualitative content analysis, analyzed these sources independently. The study team met to discuss findings, interpretations, and themes gathered from these sources.

RESULTS: There are four main sources of ethnicity data in Canada used in health research: 1) Provincial health insurance registries, 2) Canadian Health Measures
Survey, 3) Canadian Community Health Survey, and 4) Census. Of these, ethnicity data are most limited in the provincial health insurance registries, flagging only Aboriginal status. These registries also ask that new or returning residents specify the country from which they are moving, used as a proxy for ethnicity. Those immigrating to Canada from other countries also have a Permanent Resident Landing File stating their country of origin and country of citizenship, which can be linked to the provincial health insurance registries. The other three data sources are nationally administered, with all three asking individuals to select, out of 11 categories, self-identified racial or ethnic groups. The latter two data sources also have a separate question about ethnic ancestry. The categories for selfidentified racial and ethnic groups are broad and lacking in granularity, and the categories presented are inconsistent across data sources and years. There is much greater granularity collected for ethnic ancestry, with the ability to use free-text to describe ethnic ancestry in the Census. An important lesson to be learned from other non-health related sources of ethnicity data in Canada is that ethnicity is a social and cultural concept; a minority of Canadians feel a sense of belonging with their reported ethnic group and ancestry. How this impacts the validity of current ethnicity data remains unclear.

CONCLUSIONS: Despite the multiculturalism of the Canadian population, current data sources in health care collect only broad information about ethnicities. There 
is a need for greater granularity in ethnic classifications to reflect the diversity of the Canadian population, and to accurately represent individuals of mixed races and those who are non-first generation Canadians. To reflect ethnicity as a social and cultural concept, consideration should also be made to incorporate questions about sense of belonging with the identified ethnic ancestry, rather than relying solely on reported ethnic origin and race.

\section{CANCER SYMPTOM RECOGNITION AND ANTICIPATED DELAY IN HELP-} SEEKING IN A POPULATION SAMPLE OF U.S. ADULTS. Katharine Rendle ${ }^{1}$; Samantha Quaife ${ }^{2}$; Jerry Suls ${ }^{1}$; Heather Edwards ${ }^{1}$; Richard P. Moser ${ }^{1}$; Kelly D. Blake ${ }^{1}$; Sarah Kobrin ${ }^{1} .{ }^{1}$ National Cancer Institute, Bethesda, MD; ${ }^{2}$ University College London, London, United Kingdom. (Control ID \#2469113)

BACKGROUND: Across many organ sites, early cancer diagnosis continues to be one of the strongest predictors of survival. Delays in cancer diagnosis have been linked to individual, social, and structural factors, including failure to recognize early cancer symptoms. As such, there is growing international interest in understanding how individual recognition of potential cancer symptoms is linked to anticipated time to seeking physician-based care. Using data from a novel population-based survey, we examine associations between cancer symptom recognition and anticipated delays in helpseeking in U.S. adults.

METHODS: We conducted computer-assisted telephone interviews with a populationrepresentative sample of English-speaking adults (aged 50 or older) in the United States $(N=1,425)$, using an instrument adapted from the international Awareness and Beliefs about Cancer $(\mathrm{ABC})$ survey. We assessed hypothetical time to seeking physician-based care for persistent cough, rectal bleeding, changes in mole appearance, and breast changes (females only), and categorized "delay" as waiting 2 weeks or longer. Recognition of associated symptoms as potential cancer symptoms was assessed dichotomously. We used multivariate logistic regression models to examine the association (odds ratio $=$ OR; $95 \%$ $\mathrm{CI}=95 \%$ confidence interval) between anticipated time to seeking care and recognition of related cancer symptom, adjusting for age, gender, partner status, race, education, cancer experience (self, friend, or family member), and healthcare access.

RESULTS: For two symptoms, persistent cough and rectal bleeding, the odds of seeking help from a physician within 2 weeks was significantly less among those individuals who did not recognize the symptom as a potential sign of cancer, after adjusting for potential confounders (persistent cough: $\mathrm{OR}=0.59,95 \%$ CI 0.44 , 0.79 ; rectal bleeding: $\mathrm{OR}=0.38,95 \% \mathrm{CI} 0.22,0.65$ ). For changes in mole appearance and breast changes, however, there was no significant relationship between symptom recognition and help-seeking. Across all four symptoms, African Americans were significantly less likely to seek physician-based care within 2 weeks than other races (persistent cough: $\mathrm{OR}=0.27,95 \% \mathrm{CI} 0.16,0.43$; rectal bleeding: $\mathrm{OR}=0.35,95 \% \mathrm{CI} 0.13,0.99$; changes in mole appearance: $\mathrm{OR}=0.42$, $95 \%$ CI $0.26,0.68$; breast changes: $\mathrm{OR}=0.30,95 \%$ CI $0.12,0.76$ ).

CONCLUSIONS: Our results indicate that for some, but not all, cancers, lack of symptom recognition is associated with anticipated delay in seeking physician-based care. Differences between cancers may be driven in part by screening practices or level of interference a specific symptom may cause. For example, mole changes are likely to have less impact on daily functioning than rectal bleeding or persistent cough, and are commonly discussed in routine care as a potential warning sign. Further research is needed to understand the specific role of cancer symptom recognition in help-seeking behaviors and particular focus should be placed on understanding additional factors contributing to helpseeking delay in African Americans.

CARDIAC RISK, SURGICAL RISK, AND OUTPATIENT PREOPERATIVE MEDICAL EVALUATION PRIOR TO ELECTIVE SURGERIES Kevin R. Riggs; Eric B. Bass; Jodi B. Segal. Johns Hopkins University School of Medicine, Baltimore, MD. (Control ID \#2467543)

BACKGROUND: The benefit of preoperative medical evaluations-including preoperative testing and medical office visits - is uncertain. Recent guidelines discourage routine preoperative testing, instead recommending that assessment of individual patients should guide subsequent evaluation. However, little is known about the current practice of preoperative medical evaluations and what factors are associated with receipt of particular services. The aims of this study were to determine the incidence of preoperative medical evaluations and their associated costs, and to measure the association of patient cardiac risk and surgical risk with the receipt of preoperative medical evaluations.

METHODS: We constructed a retrospective cohort from a large administrative claims database consisting of US patients with employer-sponsored private insurance (MarketScan Commercial Claims and Encounters) from 2010-2013. We included those aged 18-64 years who were continuously enrolled for 1 year prior to receiving one of ten common operations. We chose one lower-risk operation (e.g., transurethral resection of the prostate) and one higher-risk operation (e.g., radical prostatectomy) from five surgical specialties. Outcomes were receipt of medical office visits (office visits with internal medicine, family medicine, geriatrics, cardiology and pulmonology) and outpatient tests, and the total costs for these services in the 30 days prior to operation. Costs included the total amoun reimbursed by insurance and the patient out-of-pocket costs. Patient cardiac risk was measured as the Revised Cardiac Risk Index (RCRI). We constructed multivariable logistic regression models to determine whether cardiac risk and surgical risk were independently associated with receipt of each service.

RESULTS: Of 654,788 patients undergoing elective surgery, $79.1 \%$ of patients had an RCRI score of zero. The percentage of patients receiving testing and the cost of those tests are shown in Table 1. $43.4 \%$ of patients had a medical office visit and $65.0 \%$ had at least one test in the 30 days prior to surgery. Among all patients, the mean cost for all of the preoperative tests was $\$ 111.15$. Results of the logistic regression models are shown in Table 2. Higher RCRI score was weakly associated with receipt of preoperative medical office visits and most tests. Higher RCRI score was strongly associated with receipt of stress testing. Undergoing the higher-risk operation was strongly associated with preoperative medical office visits and each test.

CONCLUSIONS: Higher cardiac risk was weakly associated with use of most preoperative medical services, but having the higher-risk operation was strongly associated with use of these services. This pattern is most consistent with certain tests being primarily obtained based on the surgical procedure, rather than selectively based on patient risk. More evidence is needed about which preoperative medical services improve patient outcomes. Better standardization of preoperative medical evaluations has the potential for significant resource savings. 
Table 1. Receipt of Preoperative Services and Their Costs

\begin{tabular}{|l|c|c|c|}
\hline & $\begin{array}{c}\text { Patients } \\
\text { receiving } \\
\text { service, \% }\end{array}$ & $\begin{array}{c}\text { Cost if service } \\
\text { received, } \\
\text { mean \$ }\end{array}$ & $\begin{array}{c}\text { Cost per } \\
\text { patient, } \\
\text { mean \$ }\end{array}$ \\
\hline Office Visit & 43.4 & 143.16 & 62.11 \\
\hline Blood Count & 46.7 & 28.77 & 13.44 \\
\hline Metabolic Panel & 40.5 & 50.47 & 20.43 \\
\hline Coagulation Test & 14.6 & 31.66 & 4.62 \\
\hline Urinalysis & 22.8 & 13.83 & 3.15 \\
\hline Electrocardiogram & 38.9 & 66.59 & 25.93 \\
\hline Stress Test & 2.4 & 931.82 & 22.31 \\
\hline Chest Radiograph & 18.7 & 85.82 & 16.01 \\
\hline $\begin{array}{l}\text { Pulmonary } \\
\text { Function Test }\end{array}$ & 0.9 & 72.67 & 0.63 \\
\hline
\end{tabular}

Table 2. Association of Surgical and Cardiac Risk with Preoperative Medical Evaluations ${ }^{\text {a }}$

\begin{tabular}{|c|c|c|c|c|c|c|}
\hline & Surg & Risk & & Cardi & Risk & \\
\hline & $\begin{array}{l}\mathrm{Hig} \\
\mathrm{OR} \text { ( }\end{array}$ & $\begin{array}{l}\mathrm{sk}^{\mathrm{b}} \\
6 \mathrm{Cl})\end{array}$ & $\begin{array}{l}\mathrm{RC} \\
\mathrm{OR} \\
\end{array}$ & $\begin{array}{l}=1^{\mathrm{c}} \\
\% \mathrm{Cl})\end{array}$ & $\begin{array}{l}\mathrm{RCF} \\
\mathrm{OR}\end{array}$ & $\begin{array}{l}2+c \\
\% \mathrm{Cl})\end{array}$ \\
\hline Office Visit & 1.96 & $\begin{array}{l}(1.93- \\
1.99)\end{array}$ & 1.35 & $\begin{array}{l}(1.33- \\
1.37)\end{array}$ & 1.71 & $\begin{array}{l}(1.67- \\
1.76)\end{array}$ \\
\hline Blood Count & 2.27 & $\begin{array}{l}(2.24- \\
2.30) \\
\end{array}$ & 1.15 & $\begin{array}{c}(1.13- \\
1.17) \\
\end{array}$ & 1.18 & $\begin{array}{c}(1.14- \\
1.21) \\
\end{array}$ \\
\hline Metabolic Panel & 2.31 & $\begin{array}{l}(2.28- \\
2.34)\end{array}$ & 1.30 & $\begin{array}{l}(1.28- \\
1.32) \\
\end{array}$ & 1.29 & $\begin{array}{l}(1.25- \\
1.33) \\
\end{array}$ \\
\hline Coagulation Test & 4.90 & $\begin{array}{c}(4.80- \\
5.00)\end{array}$ & 1.14 & $\begin{array}{l}(1.12- \\
1.16)\end{array}$ & 1.19 & $\begin{array}{l}(1.15- \\
1.23)\end{array}$ \\
\hline Urinalysis & 3.82 & $\begin{array}{l}(3.76- \\
3.88)\end{array}$ & 1.13 & $\begin{array}{l}(1.10- \\
1.15)\end{array}$ & 1.16 & $\begin{array}{l}(1.13- \\
1.20)\end{array}$ \\
\hline Electrocardiogram & 2.47 & $\begin{array}{l}(2.43- \\
2.50)\end{array}$ & 1.17 & $\begin{array}{l}(1.16- \\
1.19)\end{array}$ & 1.00 & $\begin{array}{l}(0.97- \\
1.02)\end{array}$ \\
\hline Stress Test & 2.50 & $\begin{array}{l}(2.41- \\
2.60)\end{array}$ & 2.29 & $\begin{array}{l}(2.20- \\
2.38)\end{array}$ & 3.78 & $\begin{array}{l}(3.59- \\
3.99)\end{array}$ \\
\hline Chest Radiograph & 4.00 & $\begin{array}{l}(3.93- \\
4.07)\end{array}$ & 1.14 & $\begin{array}{c}(1.12- \\
1.16) \\
\end{array}$ & 1.15 & $\begin{array}{l}(1.11- \\
1.18) \\
\end{array}$ \\
\hline $\begin{array}{l}\text { Pulmonary } \\
\text { Function Test }\end{array}$ & 1.55 & $\begin{array}{l}(1.45- \\
1.65)\end{array}$ & 1.18 & $\begin{array}{l}(1.11- \\
1.27)\end{array}$ & 1.25 & $\begin{array}{l}(1.13- \\
1.39)\end{array}$ \\
\hline
\end{tabular}

${ }^{a}$ models include patient demographics, RCRI, comorbidities, procedure type, hospitalization in prior 30 days

${ }^{b}$ compared to reference group low risk surgery

${ }^{c}$ compared to reference group RCRI $=0$

CASE SERIES: AN INVESTIGATION OF 30-DAY PNEUMONIA READMISSIONS AT AN ACADEMIC MEDICAL CENTER. Asad Ali ${ }^{1,2}$; Aparna Kamath ${ }^{2}$ Sameet Sangha ${ }^{2}$. ${ }^{1}$ University of Iowa Hospitals and Clinics, Iowa City, IA; ${ }^{3}$ Veterans Affairs Medical Center, Iowa City, IA. (Control ID \#2469564)

BACKGROUND: Thirty day pneumonia readmission is one of the 5 core measures monitored and compared nationwide by the Center for Medicare \& Medicaid Services (CMS). Pneumonia has a high mortality rate amongst inpatients and is a significant health care burden, thus a target for quality improvement efforts. With the advent of the CMS Hospital Readmission Reduction Program, there has been an increased focus on reducing the 30 day risk-standardized readmission rates (RSRRs) for the core measures of pneumonia, chronic obstructive airway disease, acute myocardial infarction, congestive heart failure and stroke. A 30-day RSRRs for pneumonia admissions have been greater than expected $(18.7 \%$ ) vs predicted $(17.4 \%$ ) at our institution. In an effort to better understand the factors influencing pneumonia readmissions, we conducted a chart review of adult patients readmitted within 30-days of a diagnosis of pneumonia. The goal of this study was to identify potentially modifiable risk-factors which may be a target for future quality improvement measures.

METHODS: We conducted a retrospective chart review to identify and describe the prevalence of potentially modifiable risk factors associated with 30-day pneumonia readmissions at a 711-bed academic medical center. We reviewed 67 cases randomly chosen from a total of 120 charts from 2012 to 2014 for all adult patients readmitted for any reason, within 30 days of an index admission for pneumonia. Patients aged less than 18 years, those with sickle cell disease, patients with planned admissions or re-admissions per CMS 
guidelines, those who signed out against medical advice during the index hospitalization, or patients who were transferred to other acute care facilities were excluded from our study. Three physicians divided the 67 charts for review. An inter-observer reliability was calculated to be $92 \%$ upon review of five similar charts. We evaluated demographics, treatment-specific factors, and patient-specific co-morbidities as potential risk factors for readmission.

RESULTS: According to CMS data all-cause 30-day observed risk standardized pneumonia readmission rate for our hospital from 2012 to 2014 was $18.7 \%$, greater than the predicted $17.4 \%$ readmission rate. Overall, majority of these patients were Caucasian males $(55.7 \%)$ over 40 years of age ( $89 \%$ ) and were readmitted within 14 days of discharge from the hospital (74\%). Risk factors for readmission include current/former smokers (70 \%), non-immunization status to both Flu and Pneumovax (36\%), drugs notably proton pump inhibitors (45\%), opioids (32.7\%), steroids ( $24 \%$ ), immunosuppressant $(30 \%)$. Healthcare associated pneumonia/hospital acquired pneumonia was the most common admitting diagnosis for these patients $(62.3 \%)$, although the majority of these patients were admitted from their home. About $20 \%$ of these patients did not have a radiological confirmation of the pneumonia. There was a high prevalence of anemia (50\%), malignancy (36 \%), CHF (27\%), and an elevated Pneumonia Severity Index (PSI) $>3(84 \%)$. Many of the patients had a prolonged index hospital stay ( $>5$ days) Sixty-five percent of antibiotics prescribed met the IDSA guidelines. About half of the patients had unresolved medical issues at the time of discharge. More than half of these patients were discharged home with no follow up phone call and did not follow in the clinic prior to readmission. Readmission data revealed $42 \%$ of the readmissions were related to index admission and $27.9 \%$ were admitted to intensive care unit. Nearly $74 \%$ of readmissions were discharged within 7 days.

CONCLUSIONS: Our case series suggests patients who were readmitted within 30 days were sick with high PSI on index admission and had a prolonged length of stay on index hospitalization. Majority of these patients were discharged home, had lack of follow up after discharge, and did not receive appropriate antibiotics. Future quality improvement initiatives could target early identification of patients who are at a potential risk for readmission, such as those with multiple co-morbidities especially cancer, $\mathrm{CHF}$, and anemia, and elevated PSI during their index admission. Further interventions could include improving accuracy of pneumonia diagnosis, assessing readiness for discharge with close follow up, and appropriate antibiotic prescription. These common interventions have been target for improvement in many studies; however, the tools to improve these metrics have yielded variable results with its own set of challenges for implementation (e.g. Lace model) and focus is shifting towards predicting readmissions with institution specific models. Thus our findings warrant further validation by performing case-control studies to better elucidate the risk factors and propose interventions that are practical and effective for our institution.

CERVICAL CANCER SCREENING GUIDELINE ADHERENCE IN PENNSYLVANIA MEDICAID Natasha Parekh${ }^{1}{ }^{3}$; Julie M. Donohue ${ }^{2}$; Aiju Men ${ }^{2}$; Jennifer Corbelli ${ }^{1}$; Marian Jarlenski ${ }^{2} .{ }^{1}$ University of Pittsburgh Medical Center, PIttsburgh, PA; ${ }^{2}$ University of Pittsburgh Graduate School of Public Health, Pittsburgh, PA; ${ }^{3}$ University of Pittsburgh School of Medicine, Pittsburgh, PA. (Control ID \#2465168)

BACKGROUND: Over the last decade, each update to the American College of Obstetricians and Gynecologists' evidence-based screening guidelines has recommended less frequent cervical cancer screening in average-risk women. In 2009, the recommended cervical cancer screening interval increased from every 1 year to every 2 years for $<30$-year-olds, and from every $2-3$ years to every 3 years in $\geq 30$ year-olds, while the age for initiation changed from 3 years after the first sexual intercourse to 21 years old. While under-screened women are more likely to develop cervical cancer, over-screened women undergo unnecessary, painful, costly, and potentially harmful procedures. Additionally, guideline-concordant screening would result in an estimated $\$ 490$ million annual reduction in screening and treatment costs nationally. Literature on adherence to cervical cancer screening guidelines is scant and contradictory. Self-report data suggest that inadequate screening is more prevalent, while clinic-based studies suggest a higher prevalence of excessive screening. Among females enrolled in Medicaid, $40 \%$ are in the cervical cancer screening age range, but little is known about the implementation of cervical cancer screening guidelines in this population. The purpose of this study was to assess adherence to cervical cancer screening guidelines before and after the November 2009 guideline change among women enrolled in Pennsylvania (PA) Medicaid.

METHODS: We performed an observational study of medical claims data for all continuously enrolled women 18 to 65 years of age with at least one office visit in PA Medicaid between 2007 and 2013. Women were excluded if they were dually enrolled in Medicare and/or had pre-existing conditions requiring more frequent screening (abnormal pap smear history, history of HIV/AIDS, receipt of immunosuppression). To accurately assess adherence to different guideline-based time intervals, we evaluated only women who had index Papanicolou exams (paps) in pre-specified time periods (pre-guideline period was 1/1/07-6/30/07 and post-guideline period was 11/1/09-4/30/10). We categorized adherence to the 2009 guideline change using a comparison of the time interval between paps with a guideline-based interval between paps (with a window of + /3 months). We considered screening inadequate, appropriate, or excessive if the interval between paps was longer, within, or shorter than the guideline-based window period, respectively. Women who had only 1 pap in the period were considered inadequately screened, and women $<21$ years old who had a pap after 2009 were considered excessively screened. We calculated the mean and standard deviation of time intervals between paps and used regression models to assess differences in screening categories before and after the guideline change.

RESULTS: Our sample included 29,650 women (52\% White, $35 \%$ Black, $11 \%$ Hispanic, and $91 \%$ urban). Mean age was 23 years old among $<30$-year-olds and 39 years old among $\geq 30$-year-olds. When comparing adherence pre- and postguideline changes, inadequate screening decreased in $<30$-year-olds (60 to $26 \%$ ) but slightly increased in $\geq 30$-year-olds ( 28 to $34 \%$, see Table). Among women who were inadequately screened, $44 \%$ had only one pap in their respective enrollment periods. Appropriate screening decreased in both $<30$-year-olds ( 26 to $6 \%$ ) and $\geq 30$ year-olds (18 to $3 \%$ ). Excessive screening increased in both age groups pre- and postguidelines (from 15 to $68 \%$ in $<30$-year-olds and from 54 to $63 \%$ in $\geq 30$-year-olds). Among the $<30$-year-olds who were excessively screened after the guideline change, $46 \%$ were $<21$ years old. All differences were statistically significant $(p<0.001)$. Of women who had two paps in their respective enrollment periods, the mean number $(+/-$ standard deviation) of days between paps were: $355(+/-143)$ for the $<30$-yearold pre-guideline group (guideline 365 days); $458(+/-209)$ for the $<30$-year-old post-guideline group (guideline 760 days); 519 (+/-260) for the $\geq 30$-year-old preguideline group (guideline 760-1095 days); and 533 (+/-270) for the $\geq 30$-year-old post-guideline group (guideline 1095 days).

CONCLUSIONS: Our findings demonstrate that among women enrolled in PA Medicaid, the frequency of appropriate cervical cancer screening significantly decreased after the 2009 guideline change, using a window period to define "appropriate" screening. While inadequate screening remains an issue, especially among $\geq 30$-year-olds, over-screening increased and was more prevalent after the guideline change. The significant increase in excessive screening among women of all ages carries concerning implications including costs of screening and treatment and harms of unnecessary testing/procedures. The dichotomy in our results suggest that different interventions will be necessary to address the separate and prevalent issues of both inadequate and excessive cervical cancer screening in PA Medicaid. 
Cervical Cancer Screening Guideline Adherence, Pre- and Post-Guideline Change*

\begin{tabular}{|c|c|c|c|c|c|c|}
\hline & \% Inadequate Pre & \% Inadequate Post & \% Appropriate Pre & \% Appropriate Post & \% Excessive Pre & \% Excessive Post \\
\hline$<30$-year-olds & 60 & 26 & 26 & 6 & 15 & 68 \\
\hline$\geq 30$-year-olds & 28 & 34 & 18 & 3 & 54 & 63 \\
\hline
\end{tabular}

*All differences are statistically significant; Pre $=$ Pre-guideline, Post $=$ Post-guideline

CHANGE IN GENERIC MEDICATION PRESCRIBING RATES AFTER HEALTH SYSTEM-WIDE REDESIGN OF DEFAULT OPTIONS WITHIN THE ELECTRONIC HEALTH RECORD Mitesh Patel ${ }^{2}, 3$; Susan C. Day ${ }^{1}$; Scott Halpern ${ }^{1}$; Bill Hanson ${ }^{1}$; Joseph Martinez ${ }^{1}$; Steven Honeywell' ${ }^{1}$; Kevin G. Volpp ${ }^{2,3}$. ${ }^{1}$ University of Pennsylvania, Philadelphia, PA; ${ }^{2}$ University of Pennsylvania, New York, NY; ${ }^{3}$ Philadelphia VA Medical Center, Philadelphia, PA. (Control ID \#2467527)

BACKGROUND: Default options have a significant influence on behavior and exist throughout the electronic health record (EHR). However, the optimal design of default options to improve the delivery of higher value care has not been well examined. In 2014, the University of Pennsylvania Health System (UPHS) changed EHR prescription defaults among all specialties across the entire health system by adding an opt out checkbox that was required to choose the brand name medication, otherwise the generic was ordered even when the brand name was initially selected. The objective of this study was to evaluate the impact of this intervention on physician prescribing behaviors.

METHODS: Monthly prescription data from all UPHS outpatient clinics between January 2014 and June 2015 were obtained using Clarity, an EPIC reporting database. New prescriptions for oral medications often prescribed for 10 common medical conditions were included in the sample. Medications without an available generic-equivalent and combination pills were excluded. Generic prescribing rates were compared between the pre-intervention period (January to October 2014) and the post-intervention period (December 2014 to June 2015) using chi square tests. A difference-in-differences approach was used to test whether levothyroxine, a medication known to often have differing levels of thyroid hormone in its generic and brand name formulations, had a different change in trend than other medications with similar baseline generic prescribing rates. If a different trend existed for levothyroxine than it may indicate that physician's preference for the brand name was preserved when warranted.

RESULTS: The overall generic prescribing rate increased significantly from $75.3 \%$ (611,068/811,561 prescriptions) during the 10-month pre-intervention period to $98.4 \%$ $(644,587 / 655,011)$ during the 7 -month post-intervention period $(P<0.001)$ (Figure). Compared to six other medications with similar baseline generic prescribing rates, there was less of an increase for levothyroxine representing a greater proportion of opt-outs (Adjusted Difference-in-Difference: -15.6 percentage points, $95 \%$ Confidence Interval: $-16.4,-14.8, P<0.001)$.

CONCLUSIONS: The change in default options lead to a significant and immediate change in physician prescribing behavior and resulted in an increase in generic prescribing throughout the health system. The difference in trend for levothyroxine indicates that the effectiveness of defaults is appropriately mitigated in the setting of strongly held preferences for the brand name medication.

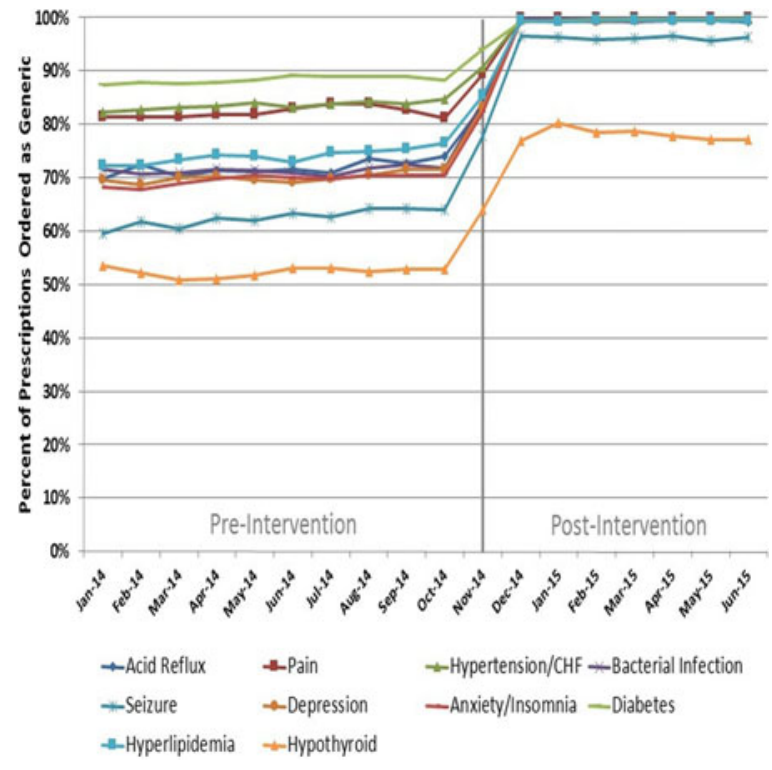

Percentage of Medications Prescribed as Generic by Drug Class and Month.

CHANGES IN CLINICIAN-PATIENT COMMUNICATION WITH SAFETY NET EHR IMPLEMENTATION Neda Ratanawongsa ${ }^{1}$; Jennifer Barton ${ }^{2}$; Courtney Lyles $^{1}$; Ed Yelin ${ }^{1}$; Nathan B. Sackett ${ }^{1}$; Marie Sears ${ }^{1}$; Diana Martinez ${ }^{1}$; Dean Schillinger ${ }^{1}$. ${ }^{1}$ UCSF, San Francisco, CA; ${ }^{2}$ VA Portland Health Care System, Portland, OR. (Control ID \#2466669)

BACKGROUND: EHR implementation may affect patient-provider communication, particularly for complex safety net patients. Different clinicians may adapt differently to the same EHR. Some may multi-task by typing and talking with patients during the visit, some may alternate conversation with periods of silent computer use, and others may chart after their encounters. In addition, changes in the amounts or patterns of computer use triggered by EHR implementation may affect clinician communication behaviors. We investigated the associations between changes in computer use before and after EHR implementation and changes in communication behaviors during safety net primary and specialty care visits.

METHODS: We conducted an observational study before and after the implementation of a certified electronic health record system in 5 academically-affiliated 
U.S. public hospital clinics. At baseline, study clinics used a basic electronic health record system for reviewing test results, tracking health care maintenance, prescribing medications, referring patients, and optional visit note documentation. We video-recorded encounters between English- or Spanish-speaking patients with diabetes, rheumatoid arthritis, and/or congestive heart failure and their primary and specialty care clinicians. We assessed the amount of computer use by summing observer ratings (Cronbach $\alpha=0.67$, score range $0-12$ ) for amount of computer data review, typing or mouse-clicking, lack of eye contact with patients, and noninteractive pauses. We assessed communication behaviors using the Roter Interaction Analysis System, coding each clinician or patient statement and summing into categories: rapport-building, biomedical talk (illness and therapy), or psychosocial/lifestyle talk (patient experience and life situation). Rapport building included positive (e.g., laughter or agreement), negative (e.g., criticism or disagreement), emotional (e.g., empathy or partnership), and social (“chit-chat") talk. Positive affect was a summation of observer ratings of emotional tone. We restricted this analysis to dyads with visits both before and after EHR implementation. We first describe the amount of clinician computer use before and after EHR implementation: low (0-4), moderate (5-7), and high (8-12). We then categorize change in computer use as increased (dyads with $\geq 3$ point increase in computer use score), decreased (dyads with $\geq 3$ point decrease in computer use score), or unchanged (dyads with -2 to +2 point change in computer use score) We calculated change in communication behaviors by subtracting the before-EHR communication counts from the after-EHR communication counts. Finally, we investigated the association between change in computer use and change in communication behaviors, accounting for clustering by clinician and controlling for clinician years in practice, clinic, and change in visit length.

RESULTS: The sample included 28 visits before and 28 visits after EHR implementation involving 21 patients and 21 clinicians. Patients averaged 57 years in age, and $9(43 \%)$ were women. Ten (48 \%) were Hispanic/Latino, 7 (33\%) reported education below high school graduation, 4 (19\%) had limited English proficiency, and $5(24 \%)$ had limited health literacy. Among clinicians, $14(67 \%)$ were women, $12(57 \%)$ were primary care providers, $9(43 \%)$ were specialists, and $18(85 \%)$ were physicians. They averaged 16 years since earning degrees. The median visit length was 26 min before and 29 min after implementation, and $25 \%$ of encounters were conducted in Spanish in both periods. Before implementation, $6(21 \%)$ of visits had low computer use, 10 (36\%) had moderate computer use, and 12 (43\% had high computer use). After implementation, 4 (14\%) had low computer use, 5 (18\%) had moderate computer use, and 19 (68\%) had high computer use. Computer use remained the same in 12 dyads (43\%), decreased in 4 dyads $(14 \%)$, and increased in 12 dyads (43\%). Compared with no change in computer use, an increase in clinician computer use was associated with less positive clinician affective tone $(-6.4, p<0.01)$. Compared with no change in computer use, a decrease in clinician computer use was associated with less chit-chat $(-19.6, p=0.03)$ and more lifestyle/psychosocial questions $(+4.4, p=0.01)$ by patients and fewer biomedical questions $(-49.2, p=0.02)$ from clinicians.

CONCLUSIONS: In the transition from a basic to a fully functional EHR, most safety net clinicians maintained or increased their amount of computer use during visits; an increase in computer use was associated with less positive affective tone by clinicians. Some clinicians adapted to the new EHR by decreasing their use of computers, which was associated with more patientcentered visit content. However, by reducing electronic charting during visits, clinicians likely shifted this work after or between visits, potentially affecting provider satisfaction and burnout. Future research should explore how to design EHR interfaces, trainings, and implementations to foster relationship-centered communication and support sustainability for safety net clinicians.

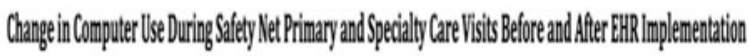
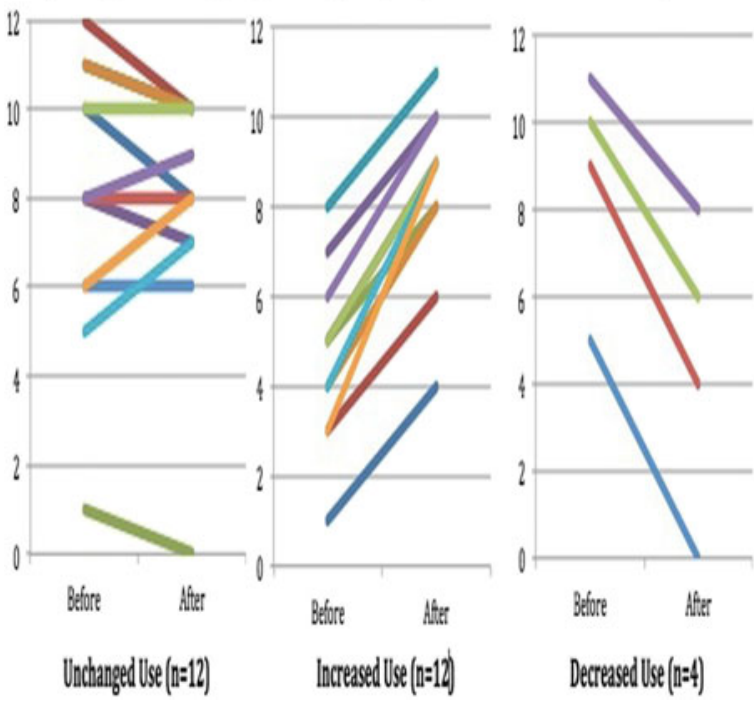

CHANGES IN QUALITY OF CARE AND MEDICAL SPENDING FOR HIGH VERSUS LOW SOCIOECONOMIC STATUS POPULATIONS UNDER GLOBAL PAYMENT Zirui Song ${ }^{1,2}$; Sherri Rose ${ }^{2}$; Michael E. Chernew ${ }^{2}$; Dana G. Safran ${ }^{3,4}$. ${ }^{1}$ Massachusetts General Hospital, Boston, MA; ${ }^{2}$ Harvard Medical School, Boston, MA; ${ }^{3}$ Blue Cross Blue Shield of MA, Boston, MA; ${ }^{4}$ Tufts University School of Medicine, Boston, MA. (Control ID \#2467997)

BACKGROUND: Across the country, payers are increasingly entering population-based payment arrangements with physicians and hospitals contracting as accountable care organizations (ACOs). Meanwhile, disparities in quality of care along socioeconomic and demographic lines persist. Global payment contracts may influence such disparities, particularly when they reward providers for improving quality of care for all patients in addition to establishing accountability for spending. Understanding the impact of global payment on disparities is crucial. On the one hand, payment models that reward quality as part of a population-based budget may motivate ACOs to focus more efforts on improving quality for disadvantaged populations, who tend to have lower quality scores at baseline, thereby helping to reduce disparities. On the other hand, such payment models could fail to address or even exacerbate disparities, as providers who serve disadvantaged populations face greater social or economic challenges in achieving higher quality scores for their patients. To date, evidence on changes in disparities under global payment is lacking. We studied changes in quality and spending among lower- and higher-socioeconomic status (SES) populations associated with the Blue Cross Blue Shield of Massachusetts Alternative Quality Contract (AQC), a large-scale two-sided global budget contract with substantial quality incentives.

METHODS: We analyzed 2006-2012 data on quality of care and medical claims for enrollees whose primary care physicians (PCPs) belonged to organizations in the AQC (intervention) and enrollees whose PCPs belonged to organizations not in the AQC (control). We assigned each enrollee to a lower-SES or higher-SES subgroup based on characteristics of the location of residence by census block group using a principal component analysis of data from the U.S. Census Bureau. The intervention cohort comprised 299,285 individuals in the lower-SES subgroup and 244,415 in the higherSES subgroup. Our primary comparison was between these two subgroups. In secondary analyses, we compared intervention subgroups to control, which were 1,053,089 and 650,041 non-AQC enrollees in lower-SES and higher-SES subgroups, respectively, as well as national and New England averages in Healthcare Effectiveness Data and 
Information Set (HEDIS) performance. For spending analyses, controls comprised 966,813 similar commercially-insured individuals from Truven Health Analytics Commercial Claims and Encounters data

RESULTS: Compared to AQC enrollees in higher-SES areas, those in lower-SES areas were more likely to be nonwhite ( 29.3 vs. $8.6 \%$ ), less likely to complete college ( 33.7 vs. $50.4 \%$ ), and more likely to have lower median annual household incomes ( $\$ 62,050$ vs. $\$ 100,263)$, higher unemployment rates (9.0 vs. $6.3 \%$ ), and higher expected spending as reflected by their DxCG risk scores (1.16 vs. 1.01), all $p<0.001$. The magnitudes of these differences among control enrollees were similar. Improvement in aggregate process quality was on average 1.2 percentage points larger among lower-SES AQC enrollees as compared with higher-SES AQC enrollees over the first 4 years of the contract $(p<0.001)$. Improvement in aggregate outcome quality was no different between lower-SES and higher-SES AQC enrollees (0.11 percentage point average difference, $p=0.82)$, nor were their relative changes in aggregate medical spending $(-\$ 5.74$ per enrollee per quarter average difference, $p=0.43$ ). Sensitivity analyses were consistent with our main results. Compared to their respective control groups, improvement in process quality measures was equal or larger among lower- than higher-SES AQC enrollees: $4.0(p<0.001)$ vs. $3.4(p<0.001)$ percentage points in chronic disease management; 0.7 ( $p=0.047)$ vs. $0.3(p=0.36)$ percentage points in adult preventive care; $1.6(p<0.001)$ vs. $1.6(p<0.001)$ in pediatric care. Both lower-SES and higher-SES AQC enrollees showed sustained improvements relative to HEDIS national and New England averages. Spending grew $\$ 70.53$ per enrollee per quarter less $(7.6 \%$ savings on claims, $p<0.001)$ among lower-SES AQC enrollees relative to control, compared to $\$ 55.73$ less $(6.2 \%$ savings, $p<0.001)$ for higherSES AQC enrollees relative to control.

CONCLUSIONS: Improvements in quality were comparable or greater among lower-SES AQC enrollees than among higher-SES AQC enrollees over the first 4 years of the contract, suggesting that the AQC was likely associated with a narrowing of disparities in quality. This likely reflects the sizeable quality incentives inherent in the AQC. Meanwhile, changes in spending were not significantly different between lower- and higher-SES AQC enrollees. In the broader debate concerning adjustment of quality measures for SES, proponents argue that SES adjustment recognizes greater resources necessary to provide high quality care for disadvantaged populations and protects safety net providers from being unfairly penalized for poorer outcomes, while opponents contend that SES adjustment establishes different standards of care for different populations and may undercut improvement by masking disparities in quality. Our findings suggests that, in a population-based global budget model, combining sufficiently large quality incentives with a budget that gives providers a realistic opportunity to reap shared savings may provide them the resources necessary to intensify efforts to improve quality of care for disadvantaged populations without explicit adjustment of performance for SES

CHANGES IN SELF-REPORTED HEALTH, HEALTHCARE ACCESS AND SMOKING CESSATION AMONG LOW-INCOME MICHIGAN ADULTS AFTER MEDICAID EXPANSION UNDER THE AFFORDABLE CARE ACT Emily

Arntson; John Z. Ayanian; HwaJung Choi; Renuka Tipirneni. University of Michigan, Ann Arbor, MI. (Control ID \#2468930)

BACKGROUND: In April 2014, Michigan implemented the Healthy Michigan Plan (HMP), an expansion of the state's adult Medicaid program under the Affordable Care Act. Since then, 600,000 low-income adults have enrolled. In this study, we aimed to examine changes in self-reported health, access, preventive services utilization and healthy behaviors after Medicaid expansion.
METHODS: We used 2011-14 Michigan data from the Behavioral Risk Factor Surveillance System (BRFSS), a national CDC survey that is administered at the state level with a response rate that ranged from 35 to $50 \%$. Our sample included 5711 BRFSS participants, ages 18-64 and under $250 \%$ of the Federal Poverty Level (FPL), excluding those who were pregnant or on Medicare. The group newly eligible for Medicaid with expansion included those with incomes between 0 and $138 \%$ FPL. The comparison group included adults with incomes between 139 and $250 \%$ FPL who were newly eligible to buy a subsidized plan on the health insurance marketplace, but not eligible to enroll in Medicaid. With these two groups, we analyzed changes in BRFSS measures of self-reported health, healthcare access, preventive services utilization and healthy behaviors. We also evaluated longitudinal trends using a difference-in-difference analysis to compare adults above and below the income threshold for Medicaid eligibility.

RESULTS: After Medicaid expansion, the uninsured rate decreased for both the Medicaid-eligible (32 vs. $38 \%$ ) and comparison (15 vs. $21 \%$ ) groups, but these reductions did not differ significantly. After expansion, Medicaid-eligible participants also had increased rates of reporting less than a week of poor physical health in the last month (73 vs. $66 \%$ with "good physical health") compared with no appreciable change in the Marketplace-eligible group ( 81 vs. $82 \%$ ). Rates of check-ups also increased among Medicaid-eligible participants after expansion ( 65 vs. $57 \%$ ) with less significant changes in the comparison group ( 65 vs. $62 \%$ ). Smoking rates in the Medicaid-eligible group also decreased after expansion (58 vs. $64 \%$ ) compared to no significant change in the Marketplace-eligible group (51 vs. $52 \%$ ). Overall in the year after expansion, Medicaid-eligible participants had a greater decrease in the odds of having more than a week of poor physical health in the last month (adjusted OR 0.67, $p=0.05$ ), a greater increase in the odds of having a checkup in the last year (adjusted OR 1.46, $p=0.04$ ), and a greater decrease in the odds of smoking (adjusted OR 0.78, $p=0.04$ ), compared to no significant changes in the Marketplace-eligible group.

CONCLUSIONS: Medicaid expansion in Michigan was associated with improved healthcare access, smoking cessation and health among Michigan's low-income adults. Further evaluation beyond the initial year of Medicaid expansion will be necessary to evaluate the longer term success of the program.

CHANGES IN THE QUALITY OF CARE DURING PROGRESS FROM STAGE 1 TO STAGE 2 OF THE ELECTRONIC HEALTH RECORD MEANINGFUL USE INCENTIVE PROGRAM David M. Levine; Michael Healey; Adam Wright; David W. Bates; Jeffrey A. Linder; Lipika Samal. Brigham and Women's Hospital, Boston, MA. (Control ID \#2467884)

BACKGROUND: The US health care system often delivers low-quality care. The American Recovery and Reinvestment Act of 2009 included \$30 billion for implementation of the Electronic Health Record (EHR) Meaningful Use (MU) Incentive Program, a three-stage program with the ultimate goal of improving the quality of care for patients through EHR use. MU stage 1 (MU1) began in 2011 and focused on data capture and data sharing. MU stage 2 (MU2) followed in 2014 with an emphasis on clinical processes. MU stage 3 (MU3) begins in 2017 with a concentration on improved outcomes. Critics argue MU has been complicated, expensive, and may distract from care quality. Proponents note that MU should ultimately improve the quality of care for patients, perhaps most significantly during MU3 and when aligned with payment incentives. We examined whether quality changed as physicians progressed from MU1 to MU2.

METHODS: We performed a retrospective cross-sectional evaluation of outpatient physicians at Brigham and Women's Hospital (BWH)-affiliated ambulatory practices from September to November 2012 (MU1) and October to December 2014 (MU2). Physicians used an advanced, homegrown EHR certified for MU1 and MU2 during 2012 and 2014, respectively. Per the MU program, our Physicians Organization chose 
from a list of federal "core" and "menu" objectives to establish an eligible physician as a "meaningful user" and chose from a list of federal clinical quality measures to monitor physician quality. The Physicians Organization used federal definitions to include or exclude physicians for each quality measure. Each quality measure was calculated as the frequency with which an eligible physician delivered high quality care to her/his eligible patients. For example, if a MU-eligible physician saw 100 influenza vaccineeligible patients between October and December 2014 and vaccinated 80, her influenza quality was $80 \%$. We report each measure as the mean quality of physicians. We examined the 7 quality measures that remained consistent in both MU1 and MU2: hypertension control (blood pressure $<140 / 90 \mathrm{mmHg}$, age 18-85); diabetes control (hemoglobin A1c $<9 \%$ ); influenza immunization; assessment and counseling of tobacco use; Chlamydia screening (age 16-24); weight screening and follow-up (age 18-64); and weight screening and follow-up (age $>=65$ ). Of all eligible physicians, $75 \%$ reached MU1 in 2012 and $99 \%$ reached MU2 in 2014. We included only physicians who were meaningful users during MU1 and MU2 (76 \% of all eligible physicians). As a sensitivity analysis, we repeated our analysis including those who did not reach MU1 in 2012 but did reach MU2 in 2014; this did not affect our findings (results not shown). We analyzed whether the same physician's quality of care varied between MU1 in 2012 and MU2 in 2014 with a paired t-test. We did not adjust for physician or patient characteristics because quality measures are not risk-adjusted in the MU program and the goal for most MU2 measures is $100 \%$ adherence. We also stratified our analysis to examine quality among primary care physicians (PCPs) and non-PCPs.

RESULTS: In 2014, 678 physicians were meaningful users during MU1 and MU2; 40 \% were female with mean age of 52 years (standard deviation [SD], 10 years). Physicians had worked at BWH for a mean of 16 years (SD, 10 years) and practiced for a mean of 25 years (SD, 11 years). In 2014, $16 \%$ were PCPs, $16 \%$ were surgeons, $36 \%$ were medical specialists, and $33 \%$ were other specialists. PCPs and non-PCPs had similar demographic characteristics, except $50 \%$ of PCPs were female, whereas $39 \%$ of nonPCPs were female. Between MU1 and MU2, 4 of 7 quality measures improved: hypertension control (35\% during MU1 versus $41 \%$ during MU2, $n=667 ; p<0.01$ ), diabetes control (92\% during MU1 versus $95 \%$ during MU2, $n=503 ; p<0.01$ ), influenza immunization (63\% during MU1 versus $68 \%$ during MU2, $n=89 ; p<0.01$ ), and assessment and counseling of tobacco use ( $86 \%$ for MU1 versus $96 \%$ during MU2, $n=637 ; p<0.01)$. One of 7 quality measures worsened: weight screening and follow-up among those 65 and older (54\% during MU1 versus $49 \%$ during MU2, $n=635$; $p<0.01$ ). Two of 7 quality measures were unchanged: Chlamydia screening ( $10 \%$ during MU1 versus $9 \%$ during MU2, $n=604 ; p=0.1$ ) and weight screening and follow-up among those $18-64$ years old ( $32.0 \%$ during MU1 versus $32.4 \%$ during MU2, $n=672$; $p=0.3$ ). In stratified analysis considering only PCPs, findings were the same as for all physicians, except hypertension quality was unchanged (67.4 \% during MU1 versus $66.7 \%$ during MU2, $n=117 ; p=0.5$ ). In stratified analysis considering only non-PCPs, two metrics improved: hypertension (29\% during MU1 versus $35 \%$ during MU2, $n=$ $550 ; p<0.01)$ and tobacco assessment and counseling (87\% during MU1 versus $95 \%$ during MU2, $n=520 ; p<0.01)$. Other quality metrics were unchanged for non-PCPs. CONCLUSIONS: As physicians progressed from MU1 in 2012 to MU2 in 2014, the quality of care improved for most measures. PCPs improved on more measures than nonPCPs. Although further quality monitoring is needed as the EHR incentive program progresses to MU3 in 2017, these data suggest that continuing MU-engagement may yield further quality improvements.

CHANGING PATIENTS' PERSPECTIVES ON SHARED DECISION-MAKING (SDM): A QUALITATIVE APPROACH Kendi Tang ${ }^{2}$; Joseph Plaksin ${ }^{1}$; Nadiya Pavlishyn ${ }^{1}$; Adina Kalet ${ }^{1}$; Sondra Zabar ${ }^{1}$; Lisa Altshuler ${ }^{1}$. ${ }^{1}$ NYU School of Medicine, New York, NY; ${ }^{2}$ New York University, New York, NY. (Control ID \#2467578)
BACKGROUND: Patients with type 2 diabetes mellitus (DM) who take an active role in their healthcare and participate in shared decision-making (SDM) with their healthcare providers (HCPs) are better able to accomplish disease management goals and maintain their health. However, due to patients' perceptions of their role, desires to be a "good patient," and the perceived power difference between patients and HCPs, many patients do not feel empowered to take an active role in their medical care. Our Patient Empowerment Program (PEP) aims to address this mindset and develop patients' skills needed to better communicate with their HCPs and enhance engagement in their own healthcare.

METHODS: We recruited patients with DM from Primary Care clinics at two public hospitals in NYC that primarily serve a low-income, low health literacy population. Eligibility criteria included English-speaking adults with the diagnosis of DM for at least 1 year and a current hemoglobin A1c between 6.5 and $11 \%$. Participants were then invited to attend a two-session PEP. In session one, participants discussed patient/HCP roles in medical encounters and rated the quality of communication in videos of patient-HCP interactions. In session two, participants first role-played a standardized scenario with Standardized Healthcare Providers (SHPs) and then "played themselves" in a second scenario, designed based on self-reported difficulties with DM. After completion of PEP, participants were invited to focus groups in order to 1) obtain feedback about each component of PEP, 2) determine what participants learned from the program, and 3) discuss how participants plan to apply what they learned to future interactions with HCPs. All focus groups were audio-recorded and professionally transcribed. Using a directed-content analysis, three independent readers developed a coding scheme that was applied to all transcripts using Dedoose software to assist with coding and summary of the analysis into themes.

RESULTS: Of a total of 71 PEP participants, 36 attended both PEP sessions and 33 returned to participate in one of 12 focus groups. Focus group participants were $58 \%$ female with a mean age of 57.5 years $(S D=9.6)$, a majority where racial or ethnic minorities ( $30 \%$ AfricanAmerican, $42 \%$ Hispanic), $37 \%$ did not finish high school, $66 \%$ had an income less than $\$ 10,000 /$ year, and $73 \%$ had limited health literacy. After completing PEP, participants reported changes in attitude and behaviors that empowered them to be more involved in their healthcare. A major theme was a greater sense of responsibility for shaping healthcare visits, with some expressing a newfound confidence to ask questions or express concerns. Others realized that it was proper to stop the doctor when they did not understand or to engage in SDM when the options the doctor provided were not optimal for them. Bringing out these underlying issues allowed participants to see that they could voice their concerns to their HCPs and could use their communication skills to actively take control of their healthcare. A related theme was the value of behavioral rehearsal. Patients felt that the experience of practicing communication skills in role-plays with SHPs gave them a standard for how HCP-patient interactions should go and the sense that they could influence those interactions. One participant, who had a visit with his HCP shortly after completing PEP, reported that "I wanted to see if she would be like [the SHP] in the role-play. And we had a very stimulating conversation about my health and everything where she really shut the door and was like 'Okay. I'm not gonna rush you, let's talk about everything.' And I was like wow, maybe I opened up a little more from doing [the PEP classes] and realized that that's what I needed, to be more assertive. I felt like [PEP] helped me a lot." Finally, many participants mentioned a social benefit to attending PEP, where they realized that all patients with DM share similar struggles. Being able to talk about these issues, share stories, and listen to others inspired participants to improve their health.

CONCLUSIONS: PEP provided participants with the skills needed to improve their interactions with their doctors, enhanced their reported DM self-management skills, and may in turn enhance their diabetes care process and outcomes. Patients with significant medical, economic, and literacy challenges became more active in their healthcare and saw the value in doing so. While this analysis has limited generalizability, it informs refinement of PEP, including creation of an interactive web application to enable reinforcement of learning, ongoing behavioral rehearsal, and scaling this intervention to a larger population of DM patients and other chronic diseases where self-management is critical. 
CHARACTERISTICS AND HEALTH CARE PREFERENCES OF WOMEN VETERANS AT RISK FOR CARDIOVASCULAR DISEASE Karen M. Goldstein ${ }^{2,1}$; Eugene Oddone ${ }^{2,1}$; Lori A. Bastian ${ }^{3,4}$; Su S. Mor ${ }^{6}$; Donna L. Washington ${ }^{6,5}$. ${ }^{1}$ Duke University School of Medicine, Durham, NC; ${ }^{2}$ Center for Health Services Research in Primary Care, Durham, NC; ${ }^{3}$ VA Connecticut Healthcare System, West Haven, CT; ${ }^{4}$ Yale University, West Haven, CT; ${ }^{5} \mathrm{UCLA}$ Geffen School of Medicine, Los Angeles, CA; ${ }^{6} \mathrm{VA}$ Greater Los Angeles Health Services Research and Development Center for the Study of Healthcare Innovation, Implementation \& Policy, Los Angeles, CA. (Control ID \#2468692)

BACKGROUND: For women in the United States, cardiovascular disease (CVD) is the leading cause of death. Compared with civilian women, veteran women may be at increased risk for CVD due to the elevated prevalence of certain risk factors, both traditional (e.g. smoking and obesity) and non-traditional (e.g. depression and low social support). Among women Veterans at risk for CVD, characteristics and healthcare preferences are unknown, but, if clarified, could inform prevention efforts. Our objectives were to describe characteristics and healthcare preferences of female veterans not at-risk for CVD, those at risk for CVD (without a history of CVD), and those with a history of CVD. METHODS: We examined survey responses from participants in the population-based National Survey of Women Veterans, which included 3611 women aged 18 years and older. Participants were both users and non-users of the Veterans Health Administration (VA). Three mutually exclusive populations were defined: 1) At risk for CVD: women without a CVD history, but with hypertension, and/or diabetes, and/or current smoking, and/or with a calculated BMI $\geq 30$ (one of four criteria needed); 2) History of CVD: congestive heart failure, myocardial infarction, or cerebrovascular accident; and, 3) Not at risk for CVD: did not meet criteria for groups 1 or 2. Descriptive analysis was conducted to estimate the prevalence of relevant health behaviors, mental health conditions, health care preferences, and barriers to care.

RESULTS: $36.4 \%$ of women veterans were not at risk for CVD, $53.8 \%$ at risk for CVD, and $9.8 \%$ had a history of CVD. VA users were more likely to be at risk for CVD than those who do not use the VA ( 60.9 vs. $52.6 \%, p<0.05$ ), though similar proportions of VA users and VA nonusers had a history of CVD. Age, marital status, education level, employment, income, insurance status, and military service era differed significantly across groups (all at least $p<0.01$ ). Those with a history of CVD were more likely than the other groups to be age 65 or older, widowed or never married, service-connected for a military disability, and have low income. Those at risk for CVD were less likely than those who were not at risk to be a college graduate or employed. $34.6 \%$ of women at risk reported a diagnosis of depression compared with $21.8 \%$ for those with a history of CVD for CVD and $23.6 \%$ not at risk for CVD $(p<0.05)$. Low physical activity was reported by $16.6 \%$ of those not at risk for CVD compared with $27.1 \%$ for those at risk for CVD, and $54.5 \%$ with a history of CVD $(p<0.001)$. Very good health was reported by $70.3 \%$ of those not at risk, $41.5 \%$ of those at risk and $25.8 \%$ of those with CVD $(p<0.001)$. Those at risk for CVD were more likely to consider availability of a gender-specific clinic very important (26.9\% compared with $15.3-15.5 \%$ for each of the other two groups, $p<0.01)$.

CONCLUSIONS: Over half of women veterans are at risk for developing CVD, and those who receive care within the VA are more likely to be at risk than those who do not. As the majority of women veterans receive care outside the VA, these findings are relevant to both VA and non-VA providers. We identified differences in mental health diagnoses, health behaviors, and healthcare preferences that could inform the design and delivery of CVD prevention and care for women Veterans. The greater preference for gender specific clinics among those at risk for CVD, compared to those not at risk, suggests that women's health clinics may be good starting points for CVD prevention interventions for women veterans. We recommend further exploration of the differences we have identified in order to better inform the development of CVD prevention services.
CHARACTERISTICS AND OUTCOMES OF HOSPITALIZED COMMUNITY DWELLING GERIATRIC PATIENTS DISCHARGED TO A SKILLED NURSING FACILITY Jennifer L. Carnahan ${ }^{1,2}{ }^{2}$; Christopher M. Callahan ${ }^{1,}{ }^{2}$; Roberta Ambuehl$^{3}$; Alexia M. Torke ${ }^{1,2}$. ${ }^{1}$ Indiana University, Indianapolis, IN; ${ }^{2}$ Regenstrief Institute, Inc, Indianapolis, IN; ${ }^{3}$ Regenstrief Institute, Inc., Indianapolis, IN. (Control ID \#2469086)

BACKGROUND: In recent years, older hospitalized patients have faced shorter hospital stays and an increasing likelihood of discharge to a skilled nursing facility (SNF) prior to returning home. Little is known about this population including the quality and outcomes of their care. There are limited guidelines regarding care after the SNF stay, especially regarding transitional care or follow up services after returning home. Post-SNF care by primary care practitioners is hampered by limited information about the patient's medical needs in the SNF and limited knowledge of what distinguishes the post-SNF care as opposed to care for older adults discharged directly home from a hospitalization.

METHODS: We constructed a cohort of older adult patients who received care in the safety net health system of a single metropolitan area between 2007 and 2010. Data from Medicare and Medicaid billing, Minimum Dataset (MDS), and the local electronic medical record were used to identify community dwelling patients who transitioned to a SNF and back to home after a hospitalization. The MDS is a mandated tool that nursing homes and SNFs use to collect data about the facility's patients. Characteristics of this patient population were identified and outcomes after discharge from the SNF to home were examined. We also examined differences between patients discharged to home with home healthcare, home without services, and assisted living using chi-square, student ttest, and ANOVA.

RESULTS: We identified 1543 patients who transitioned from hospital to a SNF to home. The mean age was $78.0,69.3 \%$ were women, $46.3 \%$ were dual eligible and $31.5 \%$ were African American. The mean length of stay (LOS) in the hospital was 8.9 days. There were $31.8 \%$ of patients from this cohort who stayed in the ICU. The mean LOS in the ICU was 5.7 days. The mean LOS in the SNF was 43.7 days. Characteristics of the cohort collected by SNFs in the MDS include multiple frailty and geriatric syndrome indicators such as BMI (mean $=28.1)$, polypharmacy (mean number of meds $=14)$, falls $(43.8 \%$ in 6 months prior to admission), hearing impairment (16.3\% of cohort) and urinary incontinence (33.2\%). Approximately $90 \%$ of the patients reported at least one new medication initiated in the 3 months prior to admission to the SNF. Over a quarter of patients $(27.6 \%)$ were on risky medications for seniors (antipsychotic, antianxiety, and hypnotic medications). Just over a quarter of the patients (26.3\%) scored cognitively impaired on the Cognitive Performance Scale. This group had high health care utilization before and after SNF admission and their mean hospital LACE score was 12.0, indicating a high likelihood of readmission to the hospital. There were 849 patients $(55 \%$ of the cohort) readmitted to the hospital in the year following their index SNF stay. Only $18 \%$ of the patients visited the ED during their SNF stay versus $90 \%$ prior to admission and $66 \%$ visiting the ED at least once in the year after their discharge from the SNF. The mean number of Emergency Department (ED) visits both before and after a SNF stay was greater than the number during their stay in the SNF ( 2.89 and 3.24 versus 1.41 visits during the SNF stay). Overall acute healthcare utilization increased after the SNF stay including mean number of ER visits and hospitalizations. Most (71 \%) were discharged home with home care, $7 \%$ went to assisted living and $22 \%$ went home without services. Those discharged to assisted living were significantly older with a mean age of 82.5 . Those discharged home with home care were significantly more likely to be dual eligible. There was no significant difference in the hospital LOS for the three groups. In the SNF, however, there was a significant difference in LOS between those discharged without home care (34.4 days) versus those discharged with home care or to assisted living (44.8 and 48.9 days). 
CONCLUSIONS: This study describes the characteristics of a racially and economically diverse group of community- dwelling patients discharged to a SNF following a hospitalization. These patients are characterized by a history of severe illness as indicated by a high proportion of ICU stays during hospitalization, as well as older age, longer LOS in the hospital than the national average, and a high incidence of geriatric syndromes. Two thirds had an ED visit and over half had a hospitalization in the 12 months after SNF discharge, suggesting a possible opportunity to reduce unnecessary acute care utilization. There were some significant differences between the patients who were discharged home with and without home care and the patients discharged to an assisted living facility. Many important pieces of information regarding patients' health and functionality that are not typically identified in other health care contexts such as inpatient or in clinic are collected reported in the MDS by the SNF. These key pieces of information would be useful to primary care clinicians caring for patients post-SNF discharge home. Knowledge of the characteristics of these patients can help guide care by the primary care practitioner. Future interventions can utilize this data to better address post-SNF health care needs and risks.

\section{CHARACTERISTICS AND OUTCOMES OF PATIENTS DISCHARGED FROM} THE EMERGENCY DEPARTMENT AFTER REFERRAL FOR HOSPITALIST ADMISSION Christopher Caulfield ${ }^{1}$; John Stephens ${ }^{1}$; Zarina Sharalaya ${ }^{2}$; Jeffrey P. Laux $^{3}$; Daniel Jonas ${ }^{2}$; Edmund A. Liles ${ }^{1} .{ }^{1}$ UNC Hospitals, Chapel Hill, NC; ${ }^{2}$ University of North Carolina Chapel Hill, Chapel Hill, NC; ${ }^{3}$ University of North Carolina School of Medicine, Chapel Hill, NC. (Control ID \#2465350)

BACKGROUND: Hospitalization is costly, associated with the potential for adverse medical events and may have deleterious health effects. Hospitalist physicians are uniquely positioned to help patients avoid unnecessary hospitalizations Our attending-only hospitalist practice in a tertiary academic center admits approximately 350 patients monthly, the majority of which are referred through the emergency department (ED). Our group has the option of providing consultation and discharging patients from the ED if appropriate. Characteristics and outcomes for patients discharged from EDs by hospitalist physicians have not yet been described.

METHODS: We performed an uncontrolled retrospective observational study of patients discharged from the ED after referral for hospitalist admission. Patients were identified via Current Procedural Terminology (CPT) billing codes for consultation performed in the ED linked to physicians in our hospitalist group. The study period was April 2011 to April 2014. We recorded basic demographics and International Classification of Disease, version 9 (ICD9) primary diagnosis codes. Similar codes (i.e., chest pain NOS and musculoskeletal chest pain) were grouped together. Primary outcomes included return to the ED within 30 days and hospitalization within 30 days. Rates were calculated using a generalized linear mixed-effects model (GLMM) to account for the nonindependence of the data, along with Wald confidence intervals.

RESULTS: We identified 1074 patients. Mean age was 55.2 years and $54.7 \%$ were female. Chest pain was the most common diagnosis $(29.7 \%$, Table 1). For the entire cohort, the rate of returning to the ED within 30 days was $17.5 \%$ (CI 15.2-20.1, Table 2). The rate of hospitalization within 30 days was $6.0 \%$ (CI 4.7-7.6). The three diagnosis groups with the highest rates of return to the ED were sickle cell disease with crisis (74.6\%, CI 41.9-92.3), alcohol related diagnoses (41.2\%, CI 29.6-53.8), and abdominal pain (34.3\%, CI 24.7-45.4). Patients with chest pain had a rate of return to the ED similar to that of the entire cohort (17.2\%, CI 13.1-22.2)

CONCLUSIONS: The large majority of patients discharged from the ED after referral for hospitalist admission did not return to the ED within 30 days and the risk of hospitalization within 30 days was low. Almost one-third of the discharged patients were evaluated for chest pain. Patients with sickle cell disease, alcohol related diagnoses and abdominal pain had higher rates of ED revisits when compared with the entire cohort, though relatively few patients in our study had these diagnoses. These results have significant implications for hospitals and physicians seeking to improve patient care by avoiding unnecessary hospitalizations.

Table 1: Primary diagnosis groupings for patients discharged from the emergency department after referral for hospitalist admission

\begin{tabular}{|l|c|}
\hline Diagnosis (or chief complaint) & Number (\%) \\
\hline Chest Pain & $319(29.7 \%)$ \\
\hline Abdominal Pain & $84(7.82 \%)$ \\
\hline Alcohol Related Diagnoses & $63(5.87 \%)$ \\
\hline Syncope and Collapse (and/or Dizziness/Giddiness) & $60(5.59 \%)$ \\
\hline Pneumonia & $51(4.76 \%)$ \\
\hline Nausea (and/or Vomiting) & $42(3.92 \%)$ \\
\hline Sickle Cell Disease with Crisis & $18(1.68 \%)$ \\
\hline
\end{tabular}

Table 2: Estimated proportion of the sampled population expected to return to the ER within 30 days based upon diagnosis (or chief complaint)

\begin{tabular}{|l|l|}
\hline & Mean Proportion (95\% CI) \\
\hline All Diagnoses & $17.5 \% \quad(15.2-20.1)$ \\
\hline Specific Diagnosis (or chief complaint) & \multicolumn{2}{|l|}{} \\
\hline Sickle Cell Disease with Crisis & $74.6 \% \quad(41.9-92.3)$ \\
\hline Alcohol Related Diagnoses & $41.2 \%(29.6-53.8)$ \\
\hline Abdominal Pain & $34.3 \%(24.7-45.4)$ \\
\hline Nausea (and/or Vomiting) & $22.8 \%(12.0-39.2)$ \\
\hline Chest Pain & $17.2 \%(13.1-22.2)$ \\
\hline Pneumonia & $7.8 \%(3.0-19.1)$ \\
\hline Syncope and Collapse (and/or Dizziness) & $5.0 \%$ \\
\hline
\end{tabular}


CHARACTERISTICS AND PREDICTORS OF MORTALITY AMONG FREQUENT USERS OF THE EMERGENCY DEPARTMENT Judith L. Griffin ${ }^{1}$; Marie Yersin Engelberger ${ }^{1}$; Venetia - Sofia Velonaki ${ }^{1,2}$; Stéphanie Baggio ${ }^{3}$; Katia Iglesias ${ }^{4}$; Karine Moschetti ${ }^{5}{ }^{1}$; Ornella Ruggeri ${ }^{6}$; Bernard Burnand ${ }^{1}$; Jean-Blaise Wasserfallen ${ }^{7}$; Francis $\mathrm{Vu}^{1}$; Corine Ansermet ${ }^{8}$; Olivier Hugli ${ }^{9}$; Jean-Bernard Daeppen ${ }^{1}{ }^{10}$; Patrick Bodenmann ${ }^{1} .{ }^{1}$ Lausanne University Hospital, Lausanne, Switzerland; ${ }^{2}$ Institue of Higher Education and Research in Healthcare, Lausanne, Switzerland; ${ }^{3}$ Lausanne University, Lausanne, Switzerland; ${ }^{4}$ University of Neuchâtel, Neuchâtel, Switzerland; ${ }^{5}$ Health Assessment Unit, Lausanne, Switzerland; ${ }^{6}$ University Hospital of Lausanne (CHUV), Lausanne, Switzerland; ${ }^{7} \mathrm{CHUV}$, Lausanne, Switzerland; ${ }^{8}$ Policlinique Médicale Universitaire, Lausanne, Switzerland; ${ }^{9}$ Lausanne university hospital, Lausanne, Switzerland; ${ }^{10}$ Alcohol Treatment Center, Lausanne, Switzerland. (Control ID \#2457786)

BACKGROUND: Emergency department (ED) frequent users account for a disproportionately high number of ED visits. Frequent users are a highly vulnerable subset of ED users, with a significant burden of chronic medical diseases, psychiatric conditions and substance use disorders. Frequent users have an elevated mortality risk compared to the general population and non-frequent users of the ED. However, little is known about risk factors for mortality among frequent users. Our aim was thus to characterize frequent users who died during a randomized trial and to determine predictors of mortality among frequent ED users.

METHODS: This is a post-hoc analysis of a previously conducted randomized clinical trial on case management for frequent ED users (5 or more ED visits during the previous 12 months) conducted from May 2012 to July 2014 at the Lausanne University Hospital (Switzerland). Frequent users with an estimated survival of fewer than 18 months were excluded from the study. At baseline, we collected information on participant socio-demographics, social determinants of health (e.g. financial hardship, unemployment), somatic (e.g. medical comorbidity, severe chronic disease) and mental health (e.g. depression), at-risk behaviors (e.g. interpersonal violence) and health care utilization (e.g. having a primary care physician) using validated scales. We monitored participants over a 12-month period, and all-cause mortality was collected for those who died during this time. Cause of death information was obtained from the electronic medical record or by report from the participants' medical provider. We performed descriptive statistics to compare participants who died during the study period with those who survived using median and interquartile range for continuous variables and frequency and percentages for categorical variables. We examined predictors of all-cause mortality using univariate logistic regressions with death as the dependent variable and age, sex, nationality, country of origin, education, study group (intervention or control), number of visits at enrollment (including index visit), social determinants, somatic determinants, mental determinants, and at-risk behaviors as the independent variables. We repeated all regressions adjusting for age (i.e., multivariate regressions with two regressors - age and all other variables individually). RESULTS: Of the 250 enrolled frequent users, 20 participants ( $8 \%$ ) died during the 12 month follow-up period (10 in the intervention and control groups, respectively). Seven deaths (35\%) were due to cardiac causes, $6(30 \%)$ due to cancer, $2(10 \%)$ due to sepsis (in the setting of urinary tract infection and pneumonia), and 1 death (5\%) each from stroke, hepatic failure, and an unspecified terminal illness (while in palliative care). Compared with surviving participants, deceased participants were older, with a median age of 71.5 years (vs. 43.9 years), were more likely to report any somatic determinant (90 vs. $67 \%$ ), to suffer from a severe chronic illness ( 80 vs. $57 \%$ ), and to report medical comorbidity (55 vs. $20 \%$ ). There were no differences between living and deceased frequent users in terms of sex, education, nationality, number of ED visits, social determinants, mental health conditions or substance use. In the univariate analysis, age $(\mathrm{OR}=$ 1.07 one-year increase, $95 \%$ confidence interval (CI) 1.04-1.11) and medical comorbidity
(OR 4.76, $95 \%$ CI 1.86-12.15) were identified as significant predictors of mortality. Reporting any somatic factor (OR 4.35, $95 \%$ CI 0.98-19.26) or severe illness $(2.97,95 \%$ CI 0.96-9.16) both approached significance as predictive factors. Reporting any risk behavior predicted a lower risk of death (OR 0.22, $95 \%$ CI 0.05-0.96). After adjustment, no factors remained statistically significant predictors except age.

CONCLUSIONS: Although frequent users with an estimated survival of fewer than 18 months were excluded from enrollment, $8 \%$ of participants died during the 12 month study. Two thirds of the deaths were due to heart disease or cancer. Deceased frequent users were older and reported more somatic health problems, including medical comorbidity and severe illness. Likewise, we found age and medical comorbidity to be significant predictors of 12 -month all-cause mortality. The risk of death increased by $7 \%$ with each 1 -year increase in age, with the median age of deceased participants being 71-years-old. Conversely, reporting any at-risk behavior predicted a lower mortality risk. Only age remained a significant mortality risk factor in the multivariate analysis. Our small sample size (20 of 250) may have limited our ability to identify other risk factors. Although these findings are intuitive and consistent with clinical experience, these factors may be used to risk stratify the diverse group of patients that comprise ED frequent users. Case management interventions for frequent ED users should target older patients with multiple medical conditions, particularly cardiovascular disease or oncologic conditions, as they are at increased risk of death and warrant individualized support.

CHARACTERISTICS OF ELDERLY MALE VETERANS ASSOCIATED WITH PHYSICAL ACTIVITY Mackenzie Erdmann ${ }^{3,1}$; Melanie Mercer ${ }^{3,1}$; Tarin Dhaliwal ${ }^{3,1}$; Kia Semons-Booker ${ }^{1}$; Daniel Lans ${ }^{1,2}$; Jeff Whittle ${ }^{1,2}$. ${ }^{1}$ Zablocki VA Medical Center, Milwaukee, WI; ${ }^{2}$ Center for Patient Care Outcomes Research, Milwaukee, WI; ${ }^{3}$ Division of General Internal Medicine, Medical College of Wisconsin, Wauwatosa, WI. (Control ID \#2466231)

BACKGROUND: Despite evidence that physical activity (PA) protects against cognitive and functional decline, few Americans, and especially few older Americans, exercise at recommended levels. Efforts to increase PA have focused on increasing convenience or developing more attractive activities, but prior studies of factors associated with PA suggest intrinsic motivation, is important, along with self-efficacy and having a workout partner. But these studies include few older men, who may differ in their motivation. We analyzed baseline data from a study of exercise and cognition to identify characteristics associated with PA among older male veterans.

METHODS: Male veterans aged 65-89 years old were eligible if they received primary care at the Milwaukee VA Medical Center and scored $>15$ on the Montreal Cognitive Assessment (MoCA). We measured PA in Kcal/week using the International Physical Activity Questionnaire (IPAQ) and Community Health Activities Model Program for Seniors (CHAMPS), and in minutes of aerobic activity/week using the Stanford Brief Activity Survey (SBAS). We measured self-efficacy, social support, social activity, and depression using validated instruments, which we scored using standard method. The Motives for Physical Activity Measure-Revised (MPAM-R) identifies five different forms of motivation for PA: appearance, social, challenge/competence, enjoyment, and fitness. We further categorized these motivational factors into intrinsic (fitness, challenge/ competence, enjoyment) and extrinsic (appearance, social) factors. We also gathered age, race, annual income and years of education. We used linear regression or ANOVA to identify univariate associations between each measure of PA and each potential predictor. We then built three parallel linear regression models to show the impact of motivation on each measure of PA, adjusted for demographics and fixed patient characteristics (self-efficacy, depression, and social support). We first included demographic and fixed characteristics in the model, regardless of significance, then used backwards selection to select the MPAM-R scales that were significant predictors of PA. 
RESULTS: Of 118 enrollees, 115 provided complete data for the present analysis. Their mean age was $75.21(7.6) ; 14 \%$ were black, $83 \%$ were white, and $3 \%$ were identified with another race. Our measures of weekly PA were only weakly correlated - IPAQ and SBAS were not $(r=0.445,0.05$, and 0.346 , respectively for IPAQ:CHAMP, IPAQ:SBAS, and CHAMP:SBAS). Mean PA was $5663.35 \mathrm{kcal} /$ week by IPAQ, which would correspond to approximately $18 \mathrm{~h}$ of walking, if walking were the only exercise. Participants with fewer depressive symptoms, more social support, increased cognitive ability, and higher income tended to report more PA. Neither age nor BMI predicted any measure of PA. Specific motivations for exercise were associated with each of the PA measures, but the specific category varied somewhat by measure. Those who were motivated to exercise based on intrinsic factors reported more PA on all measures, while those motivated by extrinsic factors reported more PA on some. Social support also appeared to positively contribute to CHAMPS and IPAQ measures. Higher income levels were shown to have more impact on IPAQ scores, but were not associated with the other PA measures.

CONCLUSIONS: The results agree with previous literature identifying social support and intrinsic motivation as predictors of PA. Moreover, even in this volunteer population, depressive symptoms, cognitive function, and social support associated with PA, suggesting these characteristics may also be important influencers on PA levels. Since depression and cognitive functioning are relatively unmodifiable features, educational interventions regarding the health benefits of PA at older ages. Our observation that different measures of PA can lead to different conclusions provides a cautionary note for studies relying on single self-report measures.

Best Fit Models for PA measures

\begin{tabular}{llllll}
\hline \hline $\begin{array}{l}\text { Measure of } \\
\text { PA }\end{array}$ & Predictors of PA & SD & $\begin{array}{l}\text { Change in PA* } \\
(\%)\end{array}$ & $\begin{array}{l}P \text { - } \\
\text { value }\end{array}$ & $\begin{array}{l}\text { Adjusted R- } \\
\text { square }\end{array}$ \\
CHAMPS & MOCA & 2.76 & $\begin{array}{l}28.98 \\
0.0134\end{array}$ & 0.237 \\
& SOCIAL SUPPORT & 6.12 & 32.36 & 0.0058 & \\
& MPAM: & 1.32 & 57.42 & $<.0001$ & \\
IPAQ & INTERNAL & & & & \multirow{2}{*}{0.2628} \\
& INCOME & & & & \\
& 50,000+ & - & 119.17 & 0.0087 & \\
& 30,000-49,000 & - & 69.20 & 0.0757 & \\
& Less than 30,000 & - & - & - & \\
& SOCIAL SUPPORT & 6.12 & 37.29 & 0.0058 & \\
& MPAM: & 1.32 & 33.24 & 0.0143 & \\
& INTERNAL & & & & \\
Stanford & DEPRESSION & 5.47 & -28.20 & 0.0234 & \\
& SCORE & & & & \\
& MPAM: & 1.32 & 44.84 & 0.0397 & 0.0284 \\
& INTERNAL & & & & \\
\hline
\end{tabular}

*Percent increase of PA measure for every increase in unit (SD for continuous variables) of predictor variable. Remaining predictors were selected using a backwards selection process with a criterion of $p<.05$. Estimates are adjusted for other variables in the model.

\section{CHARACTERISTICS OF ESOPHAGEAL CARCINOMA IN A VETERAN POP-}

ULATION. Teresa Ratajczak ${ }^{2,}$ 1; Heidar Albandar ${ }^{1,}$ 2; Parker Redlien ${ }^{1,2}$; Sara E. Yacyshyn $^{3}$; Ronald J. Markert ${ }^{3}$; Geetika Kumar ${ }^{1}{ }^{2}$. ${ }^{1}$ Dayton Veteran Affairs Medical Center, Dayton, OH; ${ }^{2}$ Wright State University, Dayton, OH; ${ }^{3}$ Wright State University Boonshoft School of Medicine, Dayton, OH. (Control ID \#2438170)

BACKGROUND: Esophageal cancer is projected to increase by approximately $35 \%$ through 2025 in the U.S. We investigated the characteristics of esophageal carcinoma in a veteran population.

METHODS: Records of patients diagnosed with esophageal carcinoma between 1996 and 2014 were reviewed. Information on demographics, social and environmental factors, hereditary associations, location, length, grade, stage of tumor, treatment, and survival was collected. IBM SPSS Statistics 22.0 and proper data-dependent statistical testing was used.
RESULTS: Eighty-one patients were classified as having adenocarcinoma (EAC) and 37 as having squamous cell carcinoma (SCC). Age at diagnosis for EAC and SCC was 67.1 \pm 9.7 vs. $67.0 \pm 10.8$ years, $(p=0.83$ ). Compared to SCC, patients with EAC were more likely to be white than African American ( 98.8 vs. $59.5 \%, p<0.001$ ). Veterans with EAC were more likely to have $\mathrm{a} \geq 60$-pack year history of smoking than with SCC ( 46 vs. $24 \%$ ( $p=0.027$ ). They did not differ in regards to heavy alcohol user ( 69 vs. $56 \%, p=0.23$ ). The two groups did not differ regarding family history of malignancy of any kind $(62.2 \%$ SCC vs. $65.4 \% \mathrm{EAC}, p=0.73)$ or of solid tumors ( $35.1 \% \mathrm{SCC}$ vs. $35.8 \% \mathrm{EAC}, p=$ $0.94)$, GI cancer ( $13.5 \%$ SCC vs. $18.5 \%$ EAC, $p=0.50)$ or head and neck cancer $(24.3 \%$ SCC vs. $22.2 \% \mathrm{EAC}, p=0.80)$. SCC was distributed nearly equally in the upper (33.3\%), middle $(36.1 \%)$ and lower third (30.6 \%) of the esophagus while EAC almost always occurred in the lower third $(93.7 \%)[p<0.001]$. Approximately three-fourths of tumor grades were moderately or poorly differentiated $(79.5 \% \mathrm{SCC}$ vs. $71.6 \%)$. The mean length of tumors was $62.9 \pm 37.0 \mathrm{~mm}$. Stage II was the most frequent classification ( $42.4 \%$ for SCC vs. $48.1 \%$ for EAC). Only $6.1 \%$ of SCC and $2.5 \%$ of EAC were stage 0 or I. Combination of chemotherapy and radiation was the most common therapy (46.1\%). SCC and EAC patients did not differ in months of survival (SCC median $=7.0$, interquartile range $[\mathrm{IQR}]=13.5 \mathrm{vs}$. $\mathrm{EAC}$ median $=8.6, \mathrm{IQR}=11.9, p=0.72$ ).

CONCLUSIONS: EAC patients were more likely to be white, have a longer smoking history, and have their tumor located in the lower third of the esophagus. Patients with the two cancers did not differ on age, alcohol use, and family history of malignancy and were similar in regard to grade, length, and stage of tumor, treatment, and survival. While EAC and SCC did not differ in diagnostic findings in our study, the changing epidemiology of esophageal carcinoma (EAC increasing and SCC decreasing), suggests that the relationships between the two types of cancer should be reassessed periodically to improve prognosis among veterans.

Patient characteristics

\begin{tabular}{llll}
\hline \hline Cancer type & adenocarcinoma (EAC) & squamous cell (SCC) & $P$ value \\
$\begin{array}{l}\text { Number of patients } \\
\text { Age at diagnosis in years }\end{array}$ & 81 & 37 & \\
White & $97.1 \pm 9.7$ & $67.0 \pm 10.8$ & $p=0.83$ \\
$\begin{array}{l}\text { African American } \\
\text { Z60-pack year }\end{array}$ & $1.2 \%$ & $59.5 \%$ & $p<0.001$ \\
$\begin{array}{l}\text { history of smoking } \\
\text { heavy alcohol user }\end{array}$ & $46 \%$ & $40.5 \%$ & $p=0.027$ \\
$\begin{array}{l}\text { family history of: } \\
\text { any malignancy } \\
\text { family history of: }\end{array}$ & $69 \%$ & $56 \%$ & $p=0.23$ \\
$\begin{array}{l}\text { solid tumors } \\
\text { family history of: }\end{array}$ & $35.8 \%$ & $62.2 \%$ & $p=0.73$ \\
$\begin{array}{l}\text { GI cancer } \\
\text { family history of: }\end{array}$ & $18.5 \%$ & $35.1 \%$ & $p=0.94$ \\
$\begin{array}{l}\text { head and neck cancer } \\
\text { location: }\end{array}$ & $22.2 \%$ & $13.5 \%$ & $p=0.50$ \\
$\begin{array}{l}\text { upper esophagus } \\
\text { location: } \\
\text { middle esophagus }\end{array}$ & $2.5 \%$ & $24.3 \%$ & $p=0.80$. \\
$\begin{array}{l}\text { location: } \\
\text { lower esophagus }\end{array}$ & $3.8 \%$ & $33.3 \%$ & \\
\hline
\end{tabular}

Comparison of grade, stage, treatment and survival for the two types of cancers.

\begin{tabular}{lll}
\hline \hline & $\begin{array}{l}\text { adenocarcinoma } \\
\text { (EAC) } \\
10.8 \% n=8\end{array}$ & $\begin{array}{l}\text { squamous cell } \\
\text { cancer (SCC) } \\
5.9 \% n=2\end{array}$ \\
$\begin{array}{l}\text { Tumor Grade: } \\
\text { not assessed }\end{array}$ & $17.6 \% n=13$ & $14.7 \% n=5$ \\
$\begin{array}{l}\text { Tumor Grade: } \\
\text { well differentiated }\end{array}$ & $37.8 \% n=28$ & $47.1 \% n=16$ \\
$\begin{array}{l}\text { Tumor Grade: } \\
\text { moderately differentiated } \\
\begin{array}{l}\text { Tumor Grade: } \\
\text { poorly differentiated }\end{array}\end{array}$ & $33.8 \% n=25$ & $32.4 \% n=11$ \\
& & \\
\hline
\end{tabular}


(continued)

\begin{tabular}{lll}
\hline \hline $\begin{array}{l}\text { Tumor Stage: } \\
\text { Stage 0 or 1 }\end{array}$ & $2.5 \% n=2$ & $6.1 \% n=2$ \\
Stage II & $48.1 \% n=38$ & $42.4 \% n=14$ \\
Stage III & $24.1 \% n=19$ & $24.2 \% n=8$ \\
Stage IV & $25.3 \% n=20$ & $27.3 \% n=9$ \\
Treatment: & $1.3 \% n=1$ & $11.4 \% n=4$ \\
Surgery & & \\
Chemotherapy & $9.0 \% n=7$ & $8.6 \% n=3$ \\
Radiation & $11.5 \% n=9$ & $20.0 \% n=7$ \\
Surgery and radiation & $1.3 \% n=1$ & $0 \% n=0$ \\
Chemotherapy and radiation & $44.9 \% n=35$ & $48.6 \% n=17$ \\
Surgery, chemotherapy & $19.2 \% n=15$ & $5.7 \% n=2$ \\
radiation & & \\
Comfort care, declined treatment & $12.8 \% n=10$ & $5.7 \% n=2$ \\
Survival: & $33.3 \% n=27$ & $37.8 \% n=14$ \\
6 months or less & & \\
7-12 months & $24.7 \% n=20$ & $21.6 \% n=8$ \\
13-24 months & $21.0 \% n=17$ & $21.6 \% n=8$ \\
More than 24 months & $21.0 \% n=17$ & $18.9 \% n=7$ \\
\hline
\end{tabular}

CHARACTERISTICS OF PATIENTS WHO ENGAGE THE DISCHARGE APPEALS PROCESS Ernie L. Esquivel; Stacy Chu; Ashley Giambrone; Hsiang-Wei Ma; Jesse Bastiaens; Alexandra M. Satty; Linda M. Gerber. Weill Cornell Medical College, New York, NY. (Control ID \#2470362)

BACKGROUND: Since July 2007, hospitals are required to notify hospitalized Medicare and Medicaid beneficiaries of their rights to appeal their discharge. Should a patient decide to appeal the care team's discharge decision, federally appointed Quality Improvement Organizations review the medical record and decide either to uphold or overturn the discharge. During this time, the patient is protected from liability for hospital charges until the day after a decision is reached. The discharge appeals process is a time-consuming process, frequently perceived as a breach of the trust inherent to the physician-patient relationship and its effect on length of hospitalization and quality of care measures have not been reported. Similarly, the characteristics of patients that may predict engagement in the discharge appeals process are unknown.

METHODS: We conducted a retrospective case-control analysis of Medicare/Medicaid beneficiaries admitted to the Medicine Service at New York-Presbyterian Hospital/Weill Cornell who appealed their discharge over a 30 -month period. Data regarding patient demographics, such as gender, ethnicity, income status and language were gathered, along with potential psychosocial factors, such as place of residence, social support network, home care, alcohol and drug use and psychiatric history. The reasons behind the appeals were noted and the proposed disposition at the time of discharge were analyzed. Additionally, we hypothesized that certain medical factors, including comorbid conditions, procedural interventions, inpatient narcotic use, isolation and ICU stay, prior hospitalization and ER visits, may be important determinants. Further, we analyzed the impact of discharge appeals on added length of stay, 30-day readmissions and follow-up at discharge. Age- and discharge date-matched controls were identified and similarly analyzed. Multivariable regression analysis was performed to identify factors that independently predict engagement in the discharge appeals process.

RESULTS: During a 30-month period, 159 patients appealed their discharge and 15 considered, but canceled, the appeal. Of these, review by the QIO upheld the hospital's decision to discharge in $94.3 \%$ of cases. The reasons behind the discharge appeal could be categorized into disagreements with the care plan (48.3\%) and displeasure with the disposition at discharge, e.g. skilled nursing facility (51.7\%). Analysis showed that those who appealed due to the latter were older, had been in the hospital longer, had a higher Charlson Comorbidity Index, more likely to be isolated for infectious reasons and to have undergone a change in their living situation at the time of discharge. Multivariate regression analysis was performed comparing discharge appeals cases with controls and demonstrate important psychosocial and medical factors that predict engagement in the appeals process. Finally, among patients who appealed their discharge, length of hospitalization was increased by a median of 4 days; however, there was no increase in 30-day readmissions

CONCLUSIONS: The decision to appeal the discharge bears significantly on length of hospitalization, although it invariably leads to decisions in favor of the hospital. Psychosocial factors, particularly support systems, and a change in a patient's living situation are important predictors of engagement in the discharge appeals process.

CHARACTERIZING HEART FAILURE PATIENTS WITH 30-DAY READMISSIONS AT AN URBAN SAFETY-NET HOSPITAL Jamie Carter; Amanda K. Johnson; Katie Raffel; Christine Soran; Michelle Schneidermann. University of California, San Francisco \& San Francisco General Hospital, San Francisco, CA. (Control ID \#2468147)

BACKGROUND: In 2012, Medicare launched the Hospital Readmissions Reduction Program, listing heart failure as one of its inaugural penalty diagnoses. In addition to being costly to the health care system, readmissions are also hazardous and disruptive for patients. In response, San Francisco General Hospital (SFGH), a safety-net hospital serving a diverse urban population, launched initiatives to facilitate the transition from the hospital back into the community and primary care home including an interdisciplinary care transitions program. While this program did lead to reduction in heart failure readmission, in 2014, heart failure exacerbation 30-day readmissions remained above the national and local average at $33.7 \%$ of patients.

METHODS: To inform future quality improvement initiatives targeting heart failure readmissions, a group of residents sought to characterize demographic and health systemrelated factors of patients who had a 30-day readmission for heart failure to SFGH compared with patients admitted over the same time period for heart failure but without readmission. We used data obtained from the hospital discharge database from June 2014 to June 2015 and performed descriptive statistical analyses and logistic regression. We evaluated race, language, gender, age, homelessness, provision of home-health services on discharge, length of stay of index admission, having an established PCP, and type of insurance.

RESULTS: Among 288 patient admissions for heart failure, 84 were associated with at least one 30-day readmission. The typical patient with a 30-day readmission for heart failure was male (74.2\%), African-American (50.5\%), English-speaking (87.6 \%), and established in care with a PCP $(91.8 \%)$. Almost a quarter $(22.7 \%)$ of readmitted patients were homeless. After adjustment for the above variables, English-speaking patients had 2.95 times the odds of being readmitted compared to non-English speakers (OR 2.95, $95 \%$ CI 1.29 to 6.78 ). Homeless patients had 2.36 times the odds of being readmitted compared to housed patients (OR 2.36, $95 \%$ CI 1.12 to 4.95). Patients with a PCP had 3.49 times the odds of being readmitted compared to patients without a PCP (OR 3.49, $95 \%$ CI 1.24 to 9.84 ). Finally, patients with Medicare had 0.45 times the odds of being readmitted compared to patients with MediCal (OR 0.45, $95 \%$ CI 0.22 to 0.92). The average age (60) and index admission length of stay (4.6 days) were similar among readmitted and non-readmitted patients. Provision of home health services upon discharge was low in both groups ( $7.2 \%$ of readmitted patients versus $3.1 \%$ of non-readmitted patients), but the difference was not statistically significant.

CONCLUSIONS: Our next iteration of heart failure readmission interventions will be designed with an eye toward the unique needs of our homeless population, but the limited claims and utilization information available in the discharge database does not fully explain the vulnerabilities that contribute to our heart failure readmission rate. The second phase of our resident-led readmission analysis, currently underway, is a representative 
chart review that further characterizes the readmission population-including mental health and treatment, identified substance use disorders, heart failure severity and the nature PCP and cardiology follow-up - and qualitatively describes the events, both preventable and unpreventable, that preceded the readmission.

CHECKING IN ON CHECK-OUT: PERCEPTIONS AND EXPECTATIONS OF RESIDENTS DURING THE CONTINUITY CLINIC CHECK-OUT PROCESS Yvonne N. Covin ${ }^{3}$; Shannon Scielzo ${ }^{2}$; Lynne Kirk ${ }^{1}$; Blake R. Barker ${ }^{2} .{ }^{1}$ UT Southwestern Medical Center, Dallas, TX; ${ }^{2}$ UT Southwestern Medical Center at Dallas, Dallas, TX; ${ }^{3}$ University of Texas Southwestern Medical Center, Arlington, TX. (Control ID \#2465053)

BACKGROUND: Despite focus on increasing the quality of ambulatory education training, few studies have examined residents' perceptions of learning during case discussions with their preceptors (i.e., the "check-out" process). Our objective was to assess the difference between residents' and preceptors' perceptions of behaviors that should occur during check-out discussions. Specifically, we wanted a) to identify areas of deficiency in training from the residents' perspective, b) to examine the correspondence of resident and preceptor perceptions, and finally c) to examine the impact of clinical experience of the correspondence of scores.

METHODS: We conducted a cross-sectional anonymous survey of 81 categorical Internal Medicine and Family Medicine residents and 22 primary-care preceptors utilizing a 5-point Likert questionnaire (1-5, larger numbers $=$ more important or more prevalent) The survey was distributed electronically and assessed 22 components of the check-out process. Respondents from three continuity clinics completed the survey within 2 weeks Completed survey response rates were $61 \%$ of preceptors and $48 \%$ of residents. Resident non-responders were more likely to be post-graduate year 3 .

RESULTS: For residents, we identified discrepancies in desired and perceived check-out behaviors. Specifically, utilizing a dependent samples $t$-test, residents felt that all 22 areas needed additional teaching during check-out $(p<0.05)$. Billing discussions received the highest disparity of delivery (Mdiff $=-2, \mathrm{SD}=1.378)$. Most $(82.9 \%)$ residents believed billing discussions were either important or very important whereas $44.2 \%$ suggested it was never discussed during check-out. Comparing preceptors to residents, several discrepancies were observed. For example, preceptors believed that demonstrating physical exam skills in the patient room during check-out was significantly more important than did residents $(p=0.01)$. Furthermore, we examined the moderating role of preceptor experience on the consistency of beliefs of importance between residents and preceptors Increasing years of clinical experience did not statistically relate to their valuation of components important to residents: management of chronic pain, diagnosis and management of mental illness, discuss age-appropriate vaccinations, discuss age-appropriate cancer screening, and encourage resident to propose plan before offering input.

CONCLUSIONS: Our research highlighted a major deficiency in training in the checkout process - with residents desiring more patient management education in all components. Moreover, faculty and residents do not necessarily agree with what is an important focus in the "teachable moment", and preceptor clinical experience may have a small (yet not statistically significant) impact on this agreement. Our results serve as a training needs assessment for future faculty development seminars and highlight the need to consider resident learning needs in general.

CHOICE OF RISK EQUATION INFLUENCES PREDICTED SOCIOECONOMIC AND RACIAL INEQUITIES IN CARDIOVASCULAR RISK AND THE DEMOGRAPHIC COMPOSITION OF WHO IS ELIGIBLE FOR STATIN THERA-

PY IN THE U.S. Adam K. Richards; Nicholas Jackson; Arleen F. Brown; Jose J. Escarce. UCLA, Los Angeles, CA. (Control ID \#2468864)
BACKGROUND: Cardiovascular disease (CVD) risk equations play an increasingly prominent role in clinical decision-making and provide the primary outcomes for studies used to inform health policy and planning. This study explores whether and how choice of CVD risk equation would impact estimates of socioeconomic (SES) and racial inequities in CVD and alter the racial and SES composition of who is and is not recommended for statin therapy under current guidelines.

METHODS: Data from the National Health and Nutrition Examination Survey (NHANES 1999-2010) were used to define a nationally representative sample between the ages of 45 and 75 years free of cardiovascular disease and diabetes. Predicted 10-year CVD risk for each participant was calculated using four different risk equations: the Pooled Cohort Equation developed for the 2013 lipid guidelines (PCE); the Framingham risk score for CVD $\left(\mathrm{FRS}_{\mathrm{CVD}}\right)$, the FRS for coronary heart disease $\left(\mathrm{FRS}_{\mathrm{CHD}}\right)$ and the Reynolds Risk Score (RRS). Eligibility for statin therapy was defined according to 2013 lipid guidelines. Relative and absolute racial inequities between black and non-black participants were calculated as the ratio and difference, respectively, in, first, the agestandardized 10-year CVD risk predicted by each published equation and, second, the proportion of the population eligible for statin therapy. SES inequities in predicted risk and statin eligibility according to poverty-income-ratio and educational attainment were quantified using relative and absolute concentration indices. The concentration index is a summary measure of health inequity that increases in magnitude as adverse health outcomes become more concentrated among the less well off. Demographic characteristics of individuals eligible for statin therapy, according to alternative risk equations, were compared. Parametric and non-parametric models and bootstrapping methods were used to account for the complex survey design.

RESULTS: Alternative risk equations predicted 10-year CVD event rates that varied overal and by race and SES; and these patterns of risk translated into widely divergent populations eligible for statin treatment in the US. Among 8676 NHANES participants representative of approximately 64 million adults between the ages 45 to 75 years old, absolute CVD risk predicted by studied equations ranged from a low of $5.8 \%$ to a high of $13.5 \%$; and the number of individuals eligible for statin therapy ranged from 21 million ( $33 \%$, for the RRS) to 42 million ( $65 \%$, for the FRS). The PCE predicted the largest age-standardized racia inequities in 10-year CVD risk, on both relative (Black/non-Black ratio 1.63) and absolute scales ( 4 percentage point difference); and this translated into the largest racial disparities in statin eligibility (Black/non-black ratio 1.44; 17.7 percentage point difference; $p<0.05$ for each parameter). In contrast, the Framingham CHD equation predicted that Blacks and nonBlacks would experience similar rates of CVD in the next 10 years (Black/non-Black ratio $1.04, p>0.05$ ), and that a similar proportion would be eligible for statin eligibility (Black/nonBlack ratio 0.97, $p>0.05$ ). Due to clustering of CVD risk factors in low SES groups all equations predicted the presence of SES inequities in 10-year risk and would preferentially allocate statins to lower SES individuals ( $p<0.05$ for all equations). However, patterns of SES inequity varied by equation, and equations that predicted a larger SES gradient in CVD risk compared to other CVD risk equations did not necessarily predict a larger SES gradient in treatment eligibility. For example, the income-related relative concentration indices for CVD risk was largest for the RRS and smallest for the $\mathrm{FRS}_{\mathrm{CHD}}$; whereas the relative concentration index for statin eligibility was largest for the $\mathrm{FRS}_{\mathrm{CVD}}$ and smallest for the RRS ( $p<0.05$ for 4 out of 6 comparisons with SES inequity estimated by the PCE). Compared to the population eligible for treatment using PCE-defined CVD risk, participants newly eligible for treatment according to the Framingham risk scores for CVD (FRS $\mathrm{CVD}$ ) and $\mathrm{CHD}\left(\mathrm{FRS}_{\mathrm{CHD}}\right)$ were younger and more likely to be male and non-Black, and to report higher incomes and educational attainment ( $p<0.05$ for each comparison)

CONCLUSIONS: CVD risk equations predicted qualitatively and quantitatively divergent estimates of racial and socioeconomic inequities in CVD. Clinical application of alternative risk equations would influence whether or not millions of US residents are eligible for statin treatment, as well the demographic composition of who is eligible according to age, gender, race, income and educational attainment. Researchers, 
policymakers, clinicians and patients may choose to take into account the racial and SES dimensions of CVD risk or treatment eligibility when interpreting CVD risk estimated by a particular equation. Until evidence demonstrates the superior accuracy of a new or existing risk equation across a range of racially and socioeconomically diverse US populations it may be reasonable to calculate more than one estimate of CVD risk to establish that risk equation choice does not materially influence modeling study conclusions, or affect clinical decisions to initiate or withhold statin therapy, among disadvantaged populations

CHRONIC BETA BLOCKERS USE AT HOME IS INDEPENDENTLY ASSOCIATED WITH INCREASED IN-PATIENT MORTALITY IN PATIENTS PRESENTING WITH ACUTE CORONARY SYNDROME Yan Zhou; Junhong Gui; Khalid Abusaada. Florida Hospital Orlando, Orlando, FL. (Control ID \#2466299)

BACKGROUND: Beta blockers (BB) are recommended for patients with coronary artery disease and patients presenting with acute coronary syndrome (ACS). However, the effects of chronic BB use at home in patients presenting with ACS are unknown.

METHODS: This is a retrospective cohort study of patients presenting with ACS in Florida Hospital Orlando from January 2013 to December 2014. Data were collected prospectively, as part of the Acute Coronary Treatment and Intervention Outcomes Network (ACTION) Registry.

RESULTS: One thousand one hundred twenty-eight patients were included in the analysis, with 354 patients on BB at home and 774 patients not. Patients on chronic BB were older (67.95 Versus 61.98 years, $P<0.001)$ and more patients had history of hypertension $(94.35$ VS $63.05 \%, P<0.001$ ), diabetes mellitus (51.69 VS $29.84 \%, P<0.001$ ), prior myocardial infarction (MI) (42.66 VS $14.21 \%, P<0.001$ ), stroke (19.77 VS $8.66 \%, P<0.001$ ), lower glomerular filtration rate (75.93 VS 93.23, $P<0.001$ ), and more on aspirin at home (67.23 VS $25.32 \%, P<0.001)$. Patients on chronic BB had higher incidence of Non-ST elevation MI than ST elevation MI (66.1 VS 45.6\%, $P<0.001)$, decompensated heart failure (9.89 VS $3.62 \%, P<0.001)$, and less percutaneous coronary intervention $(\mathrm{PCI})$ use $(73.16 \mathrm{VS}$ $81.27 \%, P<0.05)$ during hospitalization. One thousand eighty-two patients were included for multivariable logistic regression analysis for mortality, $\mathrm{BB}$ use at home was independently associated with increased in-patient mortality (OR 2.83; $95 \%$ CI. 1.28 to $6.25, P=0.01$ ) Other factors, including cardiac arrest, shock on admission etc. were also independently associated with increased in-patient mortality. (See Table 1)

CONCLUSIONS: In patients presenting with ACS, chronic BB use at home was independently associated with increased in-patient mortality. The underlying reason for this association is not clear and more studies are needed to investigate it.

Table 1. Multivariable Logistic Regression Analysis for In-patient Mortality

\begin{tabular}{llll}
\hline \hline Variable & Odds ratio & $P$ value & $95 \% \mathrm{CI}$ \\
Beta blockers use at home & 2.83 & 0.01 & $1.28-6.25$ \\
Peak troponin value & 1.03 & 0.001 & $1.01-1.06$ \\
Creatinine clearance & 0.99 & 0.017 & $0.97-0.10$ \\
Systolic blood pressure & 0.98 & 0.000 & $0.97-0.99$ \\
Left ventricle ejection fraction & 0.96 & 0.001 & $0.94-0.98$ \\
Shock on admission & 3.32 & 0.031 & $1.11-9.91$ \\
Congestive heart failure on admission & 3.65 & 0.006 & $1.46-9.14$ \\
Cardiac arrest & 10.9 & 0.000 & $4.20-28.29$ \\
PCI & 0.42 & 0.026 & $0.20-0.90$ \\
\hline
\end{tabular}

CHRONIC CONDITIONS AND PHYSICAL HEALTH-RELATED QUALITY OF LIFE: EXTERNAL VALIDATION OF THE MULTIMORBIDITY WEIGHTED INDEX Melissa Y. Wei ${ }^{2}$; Mohammed U. Kabeto ${ }^{2}$; Kenneth Mukamal ${ }^{1}$; Kenneth Langa ${ }^{2}$. ${ }^{1}$ BIDMC, Brookline, MA; ${ }^{2}$ University of Michigan, Ann Arbor, MI. (Control ID \#2469773)
BACKGROUND: We developed and internally validated a multimorbidity index that weights 81 diseases and conditions by their impact on physical healthrelated quality of life (HRQOL) in the Nurses' Health Study (NHS), NHS II, and Health Professionals Follow-up Study (HPFS) cohorts. Diseases varied several-fold in their impact on HRQOL. This study assesses the external validity of the multimorbidity weighted index (MWI) in an independent, nationally-representative cohort.

METHODS: The Health and Retirement Study (HRS) is a prospective cohort of more than 38,000 adults aged 51 years and older followed from 1992 to 2012 . We included participants who completed an interview in 2010 on physician-diagnosed diseases and conditions and physical functioning. Nine physical functioning items resembling the 10-item Short Form-36 physical functioning scale were assessed in HRS and rescaled $0-100$ (where the maximum score of 100 corresponds to no physical limitations). Multivariable regression was used to obtain coefficients for the relationship of 16 morbidities on physical functioning. The MWI was formed by weighting morbidities by these coefficients. The inverse weightings were summed to form a MWI for each participant and compared with rankings based upon the original derivation cohorts. Spearman's rank correlation coefficient was used to compare the MWIs from the development and validation cohorts.

RESULTS: The 2010 HRS interview was completed by 22,032 participants, of whom 20,805 (94\%) were eligible for analysis. We excluded participants born after 1959 or not cohort-eligible $(N=1,227)$ and those missing one or more health condition variables $(N=473)$ for a final sample of 20,332 participants. The mean HRS physical functioning scale score was 65 (SD 31). The associations between health conditions and the HRS physical functioning scale varied several-fold (median coefficient 7.8, range 3.3-21.9). Similar to the development cohorts, end-stage organ diseases had the most negative association with physical functioning. These included dementia, congestive heart failure, lung disease, stroke, and hip and knee replacement. The mean disease count was 0.75 (median 1 , range 0-5). Values for the MWI based upon weightings observed in the derivation and validation cohorts were highly correlated (Spearman's $r=0.81$, $p=0.0002)$

CONCLUSIONS: The MWI performed nearly identically in a nationally representative independent cohort of adults as it did in the original larger but less representative cohorts and thus appears to be a valid measure of multimorbidity in community-dwelling adults. In both the development and validation cohorts, the MWI captured a greater range of morbidity than a simple disease count.

CIGARETTE SMOKING STATUS AND RECEIPT OF AN OPIOID PRESCRIPTION AMONG OEF/OIF/OND VETERANS Lori A. Bastian ${ }^{6}$; Mary Driscoll ${ }^{4}$; William Becker ${ }^{5}$; Joseph L. Goulet ${ }^{3}$; Robert D. Kerns ${ }^{4}$; Eric C. DeRycke ${ }^{4}$; Shaina Lynch ${ }^{1}$; Kristin Mattocks ${ }^{7}$; Aimee Kroll-Desrosiers ${ }^{7}$; Cynthia Brandt ${ }^{4}$; Melissa Skanderson ${ }^{4}$; Harini Bathulapalli ${ }^{4}$; Sally G. Haskell ${ }^{2}$. ${ }^{1}$ University of Connecticut, Farmington, CT; ${ }^{2}$ Yale University, VA CT, and VA Central Office, Madison, CT; ${ }^{3} \mathrm{VA}$, West Haven, CT; ${ }^{4}$ VA Connecticut, West Haven, CT; ${ }^{5}$ VA Connecticut Healthcare System, West Haven, $\mathrm{CT} ;{ }^{6}$ Yale University/VA Connecticut, West Haven, CT; ${ }^{7}$ UMass, Worcester, MA. (Control ID \#2468674)

BACKGROUND: Cigarette smoking is highly prevalent among Veterans recently separated from active duty as well as a major cause of excess morbidity, mortality, and loss of productivity worldwide. The highest rates of smoking are observed among the youngest Veterans, born between 1985 and 1989 (40\% men; $44 \%$ women), who served in support of Operations Enduring Freedom, Iraqi Freedom, and New Dawn (OEF/OIF/OND). Smoking rates are higher among individuals with mental health diagnoses, such as 
anxiety, depression, post-traumatic stress disorder, and substance use disorders which are also highly prevalent among Veterans in VA care. In addition, pain is reported by more than one third of OEF/OIF/OND Veterans and opioids are commonly prescribed for noncancer chronic pain in VA settings. There is growing concern about long-term deleterious effects of opioids, many of which are more common among individuals who smoke, including osteoporosis, hypogonadism, and development of an opioid use disorder. This study aims to examine whether Veterans of OEF/OIF/OND who smoke cigarettes are more likely to receive an opioid prescription than non-smokers.

METHODS: A cross-sectional analysis of a national cohort study that included OEF/OIF/OND Veterans who had at least one visit to a VA primary care clinic between 2001 and 2012 and information in their electronic health record for smoking status, pain intensity, and opioid use. Smoking status was defined as current, former, and never. Pain intensity, based on the $0-10$ numeric rating scale, was categorized as no pain/mild (0-3) and moderate/severe (4-10). The dependent variable, opioid receipt, was defined as at least one prescription fill $+/-6$ months of smoking status assessment. Multivariable logistic regression was used to assess the association of smoking status with receipt of an opioid prescription, controlling for potential confounding factors such as age, gender, mental health diagnoses, and pain intensity.

RESULTS: We identified 406,954 OEF/OIF/OND Veterans in the database: the mean age was 30 years; $12.5 \%$ were women $(n=50,988) ; 66.3 \%$ reported no pain or mild pain intensity and $33.7 \%$ moderate or severe pain intensity; $37.2 \%$ were current smokers $16 \%$ were former smokers, and $46.8 \%$ were never smokers. Overall, 33,960 (8.3\%) Veterans received one or more opioid prescription. Results of adjusted models revealed that current smoking [Odds Ratio $(\mathrm{OR})=1.56(95 \%$ Confidence Interval $(\mathrm{CI})=1.52$ 1.61)] and former smoking $[\mathrm{OR}=1.27(95 \% \mathrm{CI}=1.22-1.32)]$ were associated with a higher odds of opioid receipt after controlling for age $[\mathrm{OR}=0.99(95 \% \mathrm{CI}=0.99-0.99)]$ male gender $[\mathrm{OR}=1.16(95 \% \mathrm{CI}=1.11-1.20)]$, service-connection $[\mathrm{OR}=1.96(95 \%$ $\mathrm{CI}=1.92-2.03)$, substance use disorders $[\mathrm{OR}=1.00(95 \% \mathrm{CI}=0.96-1.04)], \operatorname{mood}$ disorders $[\mathrm{OR}=1.22(95 \% \mathrm{CI}=1.17-1.25)]$, anxiety disorders $[\mathrm{OR}=1.33(95 \% \mathrm{CI}=$ $1.28-1.38)], \mathrm{PTSD}[\mathrm{OR}=1.44(95 \% \mathrm{CI}=1.39-1.48)], \mathrm{BMI}>30[\mathrm{OR}=1.04(95 \% \mathrm{CI}=$ $1.01-1.07)]$, and moderate/severe pain intensity $[\mathrm{OR}=6.55(95 \% \mathrm{CI}=6.38-6.73)]$ compared to never smokers. We found a statistically significant interaction between smoking status and gender. Women (both former and never smokers) were less likely than men to have received an opioid prescription; however, among current smokers, no gender differences were observed.

CONCLUSIONS: To our knowledge, this analysis is one of the first studies to examine the association of cigarette smoking and opioid receipt among OEF/OIF/OND Veterans. As hypothesized, and consistent with studies in non-Veteran samples, we that found current smokers were more likely to receive an opioid prescription compared to nonsmokers even after controlling for salient covariates. In our study, this effect was attenuated, but nonetheless present, among former smokers. Due to the cross-sectional design of this investigation, the exact correlation remains unclear and the question remains; Does smoking lead to an increase in opioid use or does the use of opioids result in continued smoking among Veterans with pain? Our study highlights the importance of understanding reported pain intensity and receipt of an opioid prescription in OEF/OIF/OND Veterans who continue to smoke. Our results help identify patients at risk for opioid use. The next step is to determine whether smoking cessation is associated with a reduction in opioid use among Veterans and to develop smoking cessation interventions for this population.

CLINIC UTILIZATION IN YOUTH AND YOUNG ADULTS WITH CHRONIC ILLNESS BEFORE AND AFTER TRANSFER TO ADULT-ORIENTED CARE Laura Hart ${ }^{1}$; Gary R. Maslow ${ }^{2}$; Nirmish Shah ${ }^{2}$; Richard J. Chung ${ }^{2}$; Audrey Brown ${ }^{2}$;
Richard Sloane $2 .{ }^{1}$ University of North Carolina at Chapel Hill, Chapel Hill, NC; ${ }^{2}$ Duke University, Durham, NC. (Control ID \#2468668)

BACKGROUND: The care of young adults with childhood-onset chronic conditions (COCC) is often fragmented. As young adults age out of pediatric care they are either smoothly transferred to an adult provider, drop out of care, or remain in pediatric care well beyond the age of 18 . Little is known about the patterns of clinic utilization by young adults with COCC as they move from pediatric to adult care and how to keep these patients engaged with care during this critical time period.

METHODS: This study utilized medical records data from a large southeastern health center to examine the patterns of care for patients with one of 6 childhood-onset chronic conditions (cystic fibrosis (CF), diabetes, HIV, inflammatory bowel disease (IBD), lupus, sickle cell disease (SCD)) aged 15-27 years over a 13-year period (January 2002 to December 2015). Descriptive statistics were used to describe the different patterns of care for this population of patients including patients active in pediatric care, patients transferring from pediatric to adult care, patients dropping out of pediatric care, and patients dropping out of adult care after transfer. Being lost to follow-up was defined as not having a visit in the clinic for greater than 1 year.

RESULTS: Overall there were 2470 patients in the sample, $5 \% \mathrm{CF}, 54 \%$ diabetes, $5 \% \mathrm{HIV}, 18 \%$ IBD, $8 \%$ lupus, and $10 \% \mathrm{SCD}$. Participants ranged in age from 15 to 27 . Forty-five percent were male. With regard to race, $44 \%$ were black, and $49 \%$ were white. As shown in figure, of the 2470 patients seen for at least 2 visits in a pediatric subspecialty clinic after the age of $15,34 \%$ were lost to follow-up prior to transfer to adult care. Twenty-eight percent of the group was over the age of 18 and active in pediatric care, and $15 \%$ of the group was less than 18 and active in pediatric care. A total of $22 \%$ had transferred to adult care over the time period of the study. Among those who had transferred, $36 \%$ (8\% of total) were lost to follow-up, and $64 \%$ (14\% of the total) were active in adult care. For those who transferred from pediatric to adult care the mean gap between the last pediatric encounter and first adult encounter was 207 days, ranging from 135 days for cystic fibrosis clinic transfers to 411 days for diabetes transfers. The CF, HIV, Lupus, and SCD clinics have formal transition programs, while the diabetes and IBD clinics do not. The two clinics with the longest gap in care were the two without transition programs (IBD-216 days and diabetes-411 days). The number of patients lost to follow-up ranged from $36 \%$ for $\mathrm{CF}$ and Lupus to $58 \%$ for diabetes. The lupus clinic had the highest percentage of patients over the age of 18 still seen in pediatrics ( $21 \%$ ). Sixty-three percent of sickle cell disease patients transferred to adult care, which was the only clinic to have a majority of patients transfer to adult care. Cystic fibrosis had $44 \%$.

CONCLUSIONS: This study examined administrative data over a 13-year period to examine the clinic utilization of patients with one of six childhood-onset conditions. The findings that more young adults over 18 are still seen in pediatrics than transfer to adult care is consistent with other findings in the literature. The large percentage of youth lost to follow-up is of concern; however this data does not allow us to determine if they are lost to care entirely or have transferred their care to another institution or clinic. Clinics with a formal transition program had shorter intervals between last pediatric and first adult clinic visit and had higher rates of transfer to adult care, suggesting that these programs may have a role in successful transfer of youth to adult-oriented care and could have utility if applied in the primary care setting. Guidelines from the National Center for Healthcare Transition recommend the use of registries for tracking patients through the process of transition from identification, to preparation through successful transfer. This study demonstrates the importance of examining all groups of patients, not just those who remain in pediatrics or successful transfer, but also those who drop out of care. 


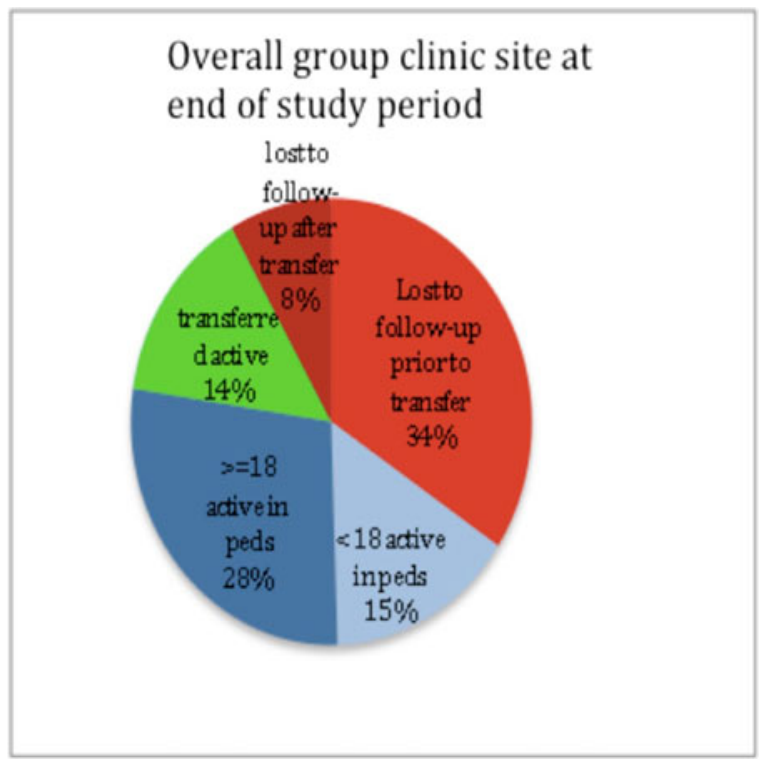

CLINICIAN PERSPECTIVES ON THE MANAGEMENT OF ABNORMAL SUBCRITICAL TESTS IN AN URBAN ACADEMIC SAFETY-NET HEALTHCARE SYSTEM Cassidy Clarity; Urmimala Sarkar; Jonathan S. Lee; Margaret Handley; Lauren Goldman. University of California San Francisco, San Francisco, CA. (Control ID \#2469173)

BACKGROUND: The lack of standardization in the management of abnormal subcritical tests which do not require immediate medical attention poses significant patient safety risks, with concomitant poor patient outcomes. Safety-net systems with limited resources and socially complex patients may be particularly vulnerable to safety gaps resulting from uncertainty in management of abnormal subcritical tests. We sought clinician perspectives to identify and understand system challenges, vulnerable situations, and solution characteristics to inform an intervention to improve abnormal subcritical test management.

METHODS: We conducted a qualitative analysis of 5 semi-structured focus groups with purposefully sampled multidisciplinary clinicians from radiology, hospital medicine, emergency medicine, risk management, and ambulatory care leadership $(n=34)$ from an urban, academic, integrated, safety-net health system in San Francisco. The goals of the focus groups were to characterize and understand challenges of current management of abnormal subcritical tests by different stakeholders and to identify solution characteristics. The transcripts were analyzed using an inductive framework to identify emergent themes specific to vulnerabilities of current abnormal subcritical test management processes and solution attributes.

RESULTS: We identified 3 themes: First, clinicians cited challenges in assigning responsibility for follow-up on abnormal subcritical tests in the context of rotating providers, differing beliefs about who should take responsibility, and acuity of results. Second, clinicians described significant concern about closing the loop in the context of pending tests, patients without primary care providers, inaccurate patient and/or provider contact information, incidental findings, and transitions of care, which they perceived as highly vulnerable situations. Third, clinicians desired solutions that would not increase current workloads, would engender trust in the system through redundant processes that support test management, and would involve integrated technology systems for accurate tracking. CONCLUSIONS: Safety-net clinicians in an urban, academic health setting described challenges in assigning responsibility for the management of abnormal subcritical tests, vulnerable situations that make closing the loop on a test difficult, and a need for system solutions that would fit within existing workflows and technological systems.
Interventions to address the subcritical test result management process should outline clear chains of responsibility and communication, provide tools for ameliorating vulnerable situations, and employ simple, integrated technological solutions that allow for tracking and management of tests.

Illustrative quotes by theme

\begin{tabular}{ll}
\hline \hline Theme & Quote \\
Assigning & "We have no idea who to call. When we call we finally get through the phone \\
responsibility & $\begin{array}{l}\text { tree, they don't know who to contact, no one takes responsibility for the } \\
\text { patient." }\end{array}$ \\
Closing the loop & $\begin{array}{l}\text { "I mean we put it in their discharge sheet but we don't always have the } \\
\text { opportunity to easily close the loop." } \\
\text { Integrated solutions }\end{array}$ \\
$\begin{array}{l}\text { "I would argue that if you create a new system that utilizes the database to } \\
\text { determine-captures certain number of abnormals and you say these need to } \\
\text { be tracked and followed and it's somehow a database that is inherently part of } \\
\text { ECW, it has to integrate with ECW. It has to be a seamless click over." }\end{array}$ \\
\hline
\end{tabular}

CLINICIANS' CONCEPTIONS ABOUT PROGNOSTICATION FOR THE ELDERLY: QUALITATIVE PROJECTIONS ARE MORE RELEVANT THAN QUANTITATIVE ONES John M. Thomas ${ }^{2,}{ }^{1}$; Terri Fried ${ }^{2,}{ }^{1}$. ${ }^{1}$ Veterans Affairs Connecticut Healthcare System, West Haven, CT; ${ }^{2}$ Yale School of Medicine, New Haven, CT. (Control ID \#2466339)

BACKGROUND: A growing literature recognizes the importance of prognostication for older adults, for the purposes of both providing patients with as much information as possible and guiding clinicians' approach in the care of patients. Recent efforts to advance prognostication through research have focused on the development of mortality risk indices. However, little is known about the ways clinicians prognosticate in practice, and the types of guidance they prefer in terms of prognostic tools and research data.

METHODS: Five focus groups comprising 30 primary care clinicians (21 physicians, six nurse practitioners, two physician assistants, one health psychologist) were conducted in two community-based primary care practices, one geriatrics practice, and two Veterans Affairs primary care practices. Participants were asked open-ended questions about the way they make projections about the future health of their patients, and how this informs their approach to caring for patients. Participants were also asked what they believe patients most value about their future health. Then clinicians were asked whether and how they use research data to assist in clinical prognostication. They were also shown two existing indices designed to predict 4-year and 9-year mortality risk, and were asked whether they use these tools, or would use these tools, in their clinical practice. Audio recordings from the focus groups were professionally transcribed. Using a content analysis approach, two analysts independently coded two transcripts and inductively developed a coding structure. They then met to discuss differences in the identification of codes, organization of the coding structure, and the application of codes to text until agreement was achieved. A single analyst coded the remaining transcripts, then two analysts met to discuss the relationships of the codes within and across transcripts to identify emerging themes.

RESULTS: Clinicians had difficulty discussing the question of how they make projections about the future health of their patients until they were asked what they believe patients value most about their future health. They cited quality of life, symptom burden, maintaining independence, being present for family members, avoiding hospitalization, and life expectancy in cases of terminal illness, and then described thinking about their patients' future health in these terms. They characterized successful prognostic discussions as resulting in a clarification of goals and redefining the care plan to achieve those goals. All groups cited the inherent uncertainty in anticipating future health as a major barrier to 
prognostic discussions with patients, except for the group of geriatricians, who were comfortable discussing prognosis but instead focused on the challenges of implementing an alternative plan of care. While clinicians were open to the concept of applying prognostic tools in clinical practice, they expressed many reservations about using risk indices that generate mortality risk estimates, including limited time, limited applicability of cohort-level data to individual patients, concerns that quantitative information would not be well-received by patients, and lack of clear clinical applications. They expressed interest in research aimed at identifying modifiable risk factors they could target in interventions to improve outcomes valued most by patients, and developing tools designed to guide specific clinical questions, like the CHADS score or CURB-65 score.

CONCLUSIONS: In contrast to prevailing attempts in research to advance prognostication through the generation of mortality risk estimates, practicing clinicians conceptualize prognostication for their patients more in qualitative terms rather than in terms of life expectancy, believing that this is aligned with patients' values and expectations. They differed in terms of how they felt about the inherent uncertainty in anticipating future health. While clinicians cited many limitations to applying available prognostic tools in clinical practice, they were optimistic about research that would guide them in applying prognostic projections to care decisions

\section{COGNITIVE DECLINE AND ITS ASSOCIATIONS WITH HEALTH LITERA-} CY AND SELF-MANAGEMENT SKILLS AMONG OLDER ADULTS Laura M

Curtis; Rachel O'Conor; Rebecca Mullen; Derin J. Cobia; William Revelle; Michael S. Wolf. Northwestern University, Chicago, IL. (Control ID \#2470323)

BACKGROUND: The number of adults over 65 is expected to double over the next few decades, and health systems are now seeking ways to adapt to this trend. A relevant and formidable concern is the confluence of two well-known, age-related problems: increased medical morbidity that results in greater and more complex patient self-management demands, and cognitive decline, which might adversely affect an older patient's ability to perform these changing self-management tasks. We sought to determine the prevalence of various forms of cognitive decline over a 3-year period, and to examine associations with requisite health literacy and self-management skills at follow-up among a cohort of older adults. We hypothesized that 'fluid' cognitive abilities that support active learning and problem-solving would decline, but not 'crystallized' abilities reflective of one's accumulated experience and knowledge. Decline in fluid abilities, in turn, would be associated with poorer health literacy and self-management skills.

METHODS: Nine hundred adults between the ages of 55 and 74 receiving care at either an academic general internal medicine ambulatory care clinic or 1 of 5 federally qualified health centers in Chicago, Illinois were recruited for a National Institute of Aging study, referred to as 'LitCog' (R01AG03611). In-depth, structured interviews were conducted at baseline and on average $3.2(\mathrm{SD}=0.4)$ years later among at present, 545 participants. Interviews included a detailed neuropsychological battery consisting of 3 tests in each of 5 cognitive domains: processing speed, working memory, inductive reasoning, long-term memory, and verbal ability. In addition, 3 measures of health literacy were administered to patients (Test of Functional Health Literacy (TOFHLA), Rapid Estimate of Adult Literacy in Medicine (REALM), and the Newest Vital Sign (NVS)) along with the Comprehensive Health Activities Scale (CHAS) - a validated measure of self-management skills wherein patients demonstrate competencies to perform a range of written, spoken, medicationrelated, and multimedia tasks. Cognitive decline was defined for each domain if any test within that domain decreased more than $1 \mathrm{SD}$ from the baseline mean to the second interview. Health literacy and self-management skills as measured at follow-up were then modeled using linear regression with cognitive decline as the main independent variable of interest, controlling for baseline scores, age, and the amount of time between interviews.
RESULTS: LitCog participants were $69 \%$ female, $37 \%$ African American, $57 \%$ White, and had a mean (SD) age of 66 (5.3), ranging 58-79 years of age at follow-up. Fluid cognitive abilities - processing speed, working memory, inductive reasoning, and longterm memory - declined among $16,20,22$, and $25 \%$ of participants, respectively. Only $3 \%$ of patients were found to demonstrate meaningful decline in crystalized verbal abilities. In multivariable models, those who declined in processing speed $(\beta-2.66$, $95 \%$ Confidence Interval $(\mathrm{CI})-4.44,-0.88 ; p=0.003$ ) or in inductive reasoning ( $\beta$ $-2.04,95 \% \mathrm{CI}-3.63,-0.44 ; p=0.01$ ) performed more poorly on the TOFHLA health literacy measure at follow-up. Decline in long term memory was associated with poorer self-management skills ( $\beta-3.26,95 \% \mathrm{CI}-4.96,-1.55 ; p<0.001)$. Cognitive decline was not associated with performance on the REALM or the NVS assessments.

CONCLUSIONS: As many as 1 in 4 older primary care patients exhibited cognitive decline within a 3 year period in our study, and degeneration specific to speed of processing and reasoning skills were most closely linked to health literacy skills necessary to process, understand, and make informed health decisions. Decline in long-term memory, however, was the strongest indicator of skills necessary for routine engagement in self-managing personal health. Future studies should examine whether health literacy and self-management skills reside in the causal pathway between cognitive decline and poorer health. Furthermore, our findings suggest that chronic disease self-management interventions, in particular that are aimed at supporting older adults might see ways to reduce the cognitive demands placed on patients when performing routine personal health tasks in order to increase patients' ability to achieve optimal health.

COMBATTING COGNITIVE BIAS: A CLINICAL REASONING CURRICULUM FOR INTERNAL MEDICINE RESIDENCY EDUCATION Deborah DiNardo $^{3}$; Sarah A. Tilstra ${ }^{4}$; William Follansbee ${ }^{2}$; Amber E. Barnato ${ }^{1}$; Shanta M. Zimmer ${ }^{1}$; Coreen Farris ${ }^{5}$; Melissa McNeil ${ }^{2}$. ${ }^{1}$ University of Pittsburgh, Pittsburgh, PA; ${ }^{2}$ University of Pittsburgh Medical Center, Pittsburgh, PA; ${ }^{3}$ University of Pittsburgh School of Medicine, Pittsburgh, PA; ${ }^{4}$ University of Pittsburgh School of Medicine/ Medical Center, Pittsburgh, PA; ${ }^{5}$ RAND, Pittsburgh, PA. (Control ID \#2469435)

BACKGROUND: To date, the roles that flawed clinical reasoning and cognitive bias play in medical errors have been under-emphasized. Recently, the importance of combatting diagnostic error has been increasingly recognized; in a fall 2015 report entitled "Improving Diagnosis in Healthcare," the IOM highlighted the importance of targeting the diagnostic process during quality improvement efforts, and called for development and deployment of approaches to reduce diagnostic error. While some experimental data suggest that cognitive forcing strategies can be used to reduce diagnostic error, whether such strategies can be successfully applied the clinical setting for decision-making and teaching is unknown. Furthermore, while components of clinical reasoning are infused into standard residency education, formal instruction regarding these principles is often lacking. We therefore developed a curriculum to teach residents about the principles of clinical reasoning and cognitive bias, and to introduce a novel diagnostic checklist for use in the clinical setting in order to combat cognitive bias and diagnostic error.

METHODS: We delivered our curriculum during a workshop immediately prior to the start of the inpatient Internal Medicine rotation during 2 consecutive months at one of our training sites in 2014. The workshop included an introduction to the principles of clinical reasoning, case-based interactive discussions regarding cognitive bias and diagnostic error, and instruction regarding use of a diagnostic checklist in the real clinical setting as a cognitive forcing strategy. Inpatient Medicine residents at 2 other training sites during the same time period served as controls. We evaluated acceptability and usability of the curriculum and the checklist via survey of participating faculty and residents. In addition, residents in both groups were asked to diagnose written cases both before and after the intervention period, and to rate their confidence in their final diagnosis for each case. We 
scored diagnostic accuracy and calculated calibration (relationship between confidence and accuracy) on the cases, and compared change in performance between intervention and control groups. We used general linear models to predict diagnostic accuracy or calibration while taking into account learner level and case complexity. Finally, we performed direct observation of randomly selected teaching teams at the intervention site both before and after curriculum implementation in order to assess the fidelity of our intervention.

RESULTS: A total of 91 residents participated in the study (41 intervention, 50 control). In a general linear model, participation in the curriculum was not associated with change in resident diagnostic accuracy or in calibration on written cases. In direct observation of rounding sessions, we found that use of the diagnostic checklist even after curriculum implementation was relatively infrequent, highly variable between individual teaching teams, and was noted in most cases to be used by attendings rather than residents. In addition, we observed more frequent and varied use of clinical reasoning vocabulary by both attendings and residents after curriculum implementation. In post-curriculum surveys, attending feedback regarding the utility of the checklist/curriculum content was more favorable than resident feedback. Attendings also reported using the checklist and curriculum content more frequently than residents.

CONCLUSIONS: In this single-center study of internal medicine residents in the US, exposure to a clinical reasoning curriculum with training in the use of a diagnostic checklist did not lead to significant improvements in diagnostic accuracy or in diagnostic calibration on written clinical cases. Use of the checklist and curriculum content was highly variable; it was used more frequently and received more favorably by faculty than residents, suggesting that faculty development may be the best way to target resident learners. Faculty and residents provided valuable insights regarding barriers to the use of cognitive forcing strategies in the real clinical setting for patient care and teaching.

COMMUNICATION QUALITY PREDICTS PSYCHOLOGICAL WELLBEING AND SATISFACTION IN FAMILY SURROGATES OF HOSPITALIZED OLDER ADULTS Alexia M. Torke ${ }^{1,3}$; Christopher M. Callahan ${ }^{1,3}$; Greg A. Sachs ${ }^{1,}$ 3; Lucia D. Wocial ${ }^{1,}{ }^{4}$; Paul R. Helft ${ }^{1}$, ; Patrick Monahan ${ }^{2}$; James E. Slaven ${ }^{2}$; Kianna Montz $^{3}$; Emily Burke ${ }^{3}$; Lev Inger ${ }^{3}$. ${ }^{1}$ Indiana University, Indianapolis, IN; ${ }^{2}$ Indiana University School of Medicine, Indianapolis, IN; ${ }^{3}$ Regenstrief Institute, Indianapolis, IN; ${ }^{4}$ Fairbanks Center for Medical Ethics, Indianapolis, IN. (Control ID \#2468667)

BACKGROUND: Nearly half of hospitalized older adults require family surrogates to make decisions due to conditions such as delirium or dementia. Although there is preliminary evidence from the ICU that high quality surrogate/clinician communication can reduce surrogates' psychological distress, little is known about the aspects of communication that matter most or whether this relationship holds outside of the ICU. We measured communication quality and examined associations with important outcomes for surrogates in three diverse hospitals.

METHODS: We conducted a longitudinal cohort study in three hospitals within one metropolitan area (tertiary referral hospital, urban safety net hospital, and university affiliated suburban hospital) between April 2012 and June 2015. Participants were hospitalized older adults (age $65+$ ) who were unable to make their own medical decisions and the family surrogate for each enrolled patient (patient/surrogate dyads). Patients who lacked decision making capacity were identified using the EMR and a brief survey with the patient's inpatient physician. At baseline (between days 3-10 of hospitalization), we administered the 30-item Family Inpatient Communication Survey (FICS) (available at (http://medicine.iupui.edu/IUCAR/research/tools/FICS). The survey includes two subscales, Information (18 items, Cronbach's alpha 0.94) and Emotional Support (12 items; 0.90). At 6-8 week follow-up, we administered measures of posttraumatic stress (Impact of Events Scale-R), anxiety (Generalized Anxiety Disorder-7), depression (Patient Health Questionnaire-9) and satisfaction (single $0-10$ overall rating of the hospital stay from the
HCAHPS). We conducted separate regression analyses for each outcome. We used logistic regressions for Anxiety and Depression outcomes using widely used, published cutoffs (moderate to severe anxiety, GAD-7 scores of 10+; depression, PHQ-9 scores of 10+). We used multivariable regression using a Maximum Likelihood Robust to skewness (MLR) estimator for posttraumatic stress, satisfaction and decisional conflict and report standardized slope parameters (B). For each adjusted analyses, we controlled for demographic variables (patient and surrogate age, education, race and SES). We also controlled for baseline GAD-7 scores, baseline PHQ-9 score, and Kessler-6 scores (a general measure of psychological distress), in respectively, the regression models of anxiety, depression, and posttraumatic stress. We also controlled for patient death between admission and 6-8 week follow-up due to added stress of a death.

RESULTS: The 360 patients had a mean age of 81.89 (SD 8.34), were $62 \%$ female, $69 \%$ white and $28 \%$ African American. The 6-8 week follow-up rate was $91 \%$ (326 surrogates). Surrogates' mean age was 58.25 (SD 11.23). They were $71 \%$ female, $68 \%$ white and $29 \%$ African American. The majority (67\%) were the patient's adult children; $17 \%$ were spouses. Baseline FICS scores ranged from 38 to 150 with a median of 124 . At follow-up, $11.5 \%$ percent of surrogates had mod-high (10+) anxiety and $13.9 \%$ had mod-high depression. Posttraumatic stress scores ranged from 0 to 80 with a median of 4 . Satisfaction scores ranged from 0 to 10 with a median of 9 . In unadjusted analysis, Emotional Support was associated with lower odds of Anxiety at follow-up ( $\mathrm{OR}=0.92$ (95\% CI; 0.87, 0.97), $p=0.0017)$. These results persisted when controlling for Information, demographics, baseline Anxiety and patient death $(\mathrm{OR}=0.81$ $(0.71,0.92), p=0.0016)$. Information was not significantly associated with Anxiety (adjusted model OR $=1.06(0.99,1.12), p=0.0772)$. Similarly, the odds of Depression was lower with higher Emotional Support in unadjusted $(\mathrm{OR}=0.94(0.89,0.98), p=0.0092)$ and adjusted models $(\mathrm{OR}=0.90,(0.81,0.99), p=0.0393)$. Information was not associated with depression. In regression analyses, Emotional Support was associated with lower Posttraumatic Stress scores $(\mathrm{B}=-0.13, p=0.038)$ while Information was not $(B=-0.01, p=0.873)$. When both dimensions were entered into the model, higher Emotional Support predicted lower stress $(B=-0.32$, $p=0.003)$ while higher Information was associated with higher stress $(B=0.23, p=0.014)$. This remained significant when adjusted for covariates (Emotional Support $B=-0.23, p=0.026$; Information $B=0.21, p=0.022$ ). In unadjusted analysis, Satisfaction was associated with both Emotional Support $(B=0.41 p<0.000)$ and Information $(B=0.49, p<0.001)$; in adjusted models, only Information remained significant $(B=0.52 p<0.001)$.

CONCLUSIONS: Emotional support of hospital surrogates is consistently associated with reduced anxiety, depression and posttraumatic stress symptoms. For every 1 point decline in the Emotional Support subscale of the communication survey, the odds of clinically significant anxiety increased by $23 \%(1 / 0.81=1.23)$ Although an observational study cannot prove causation, our results show that emotional support is associated with better psychological outcomes. Good information may not be enough; emotional support may be a key feature of the family member's wellbeing. Educational materials and interventions that emphasize expressions of empathy and relationship building may be especially important. In contrast, high quality information is associated with overall satisfaction with the hospital stay, which may be of particular interest to hospital administrators. Interventions that improve the communication experiences of surrogates are badly needed.

COMMUNICATION SCORES IN 3RD YEAR OSCE PREDICT CHOICE OF DIRECT VS. INDIRECT PATIENT CARE SPECIALTY Felise Milan ${ }^{1,2}$; Joseph Grochowalski ${ }^{3}$; Robert Ostfeld ${ }^{2}$; Rosemarie Conigliaro ${ }^{1,2}$. ${ }^{1}$ Albert Einstein College of Medicine, Bronx, NY; ${ }^{2}$ Montefiore Medical Center, Bronx, NY; ${ }^{3}$ Fordham University, Bronx, NY. (Control ID \#2467361)

BACKGROUND: While there are reports that relate personality to medical career choice $^{1}$, little is known about students' specialty choice and observed communication skills. Harsh ${ }^{2}$ found that students with lower empathy scores on the Hogan ${ }^{3}$ empathy scale 
tended to choose specialties without direct patient contact. Tsao ${ }^{4}$ found no significant difference in mean communication ratings by clerkship directors for 3rd year students entering person versus technique-oriented specialties. Purpose: To compare students' communication skills during their 3rd year OSCE, as rated by standardized patients (SPs), with their subsequent specialty choice.

METHODS: For medical students graduating 2005-2014 $(N=1412)$ we compared NRMP match specialty with their communication score generated using our 12-item, behaviorallyanchored communication skills checklist scored by SPs during their 3rd year 8-station OSCE. SPs undergo a 1-h session with the checklist using frame of reference rater training. The checklist produces highly reliable communication scores. We divided specialty choices into 4 domains: primary care (PC) (IM, pediatrics, FM), direct patient care/non-primary care (NPC) (psychiatry, ER, dermatology, neurology, PM\&R), indirect patient care (IPC) (anesthesia, radiology, pathology), and surgery. We compared differences in mean peformance of the 4 specialty choice groups using ANOVA and post-hoc pairwise comparisons.

RESULTS: Both direct patient care groups (PC and NPC) had significantly higher communication skills scores as compared to the IPC group ( $p=.004, p=.01$ respectively) (Table 1) Thirty-four percent of the IPC students had communication scores in the bottom quartile. Students choosing surgery or surgical subspecialties were not different from the other groups whether analyzed as a single group or separated into general and specialty surgery.

CONCLUSIONS: In a large cohort spanning 10 years, students choosing indirect patient care specialties demonstrate poor communication skills as compared to their peers choosing direct patient care specialties. . There are many variables that influence specialty choice, most of which we were unable to control for. In addition, more competitive specialties may match students who are strong in all areas. Students may choose specialties where the physicians are most similar to themselves regarding communication skills and/or styles. While our data set is large, it is limited to one institution. However, communication skills may be another variable that helps us understand more about how students make their specialty choice.. With regard to milestone tracking and competency assessment, knowledge of students', baseline communication skills may serve as a starting point to enable residency program directors to target skills assessment and focus training for their incoming trainees. 1.Borges NJ, Stratton T, Wagner PJ, Elam CL. Emotional Intelligence and medical specialty choice: findings form three empirical studies. Medical Education 2009; 43:565-572. 2. Harsch HH, The role of Empathy in Medical Students' choice of specialty. Academic Psychiatry 1989; 13 (2): 96-98. 3. Hogan R. Development of an empathy scale. Journal of Consulting and Clinical Psychology, Vol 33(3), Jun 1969, 307-316. 4. Tsao CIP, Simpson D, Treat R. Medical Student Communication Skills and Specialty Choice. Academic Psychiatry July 19, 2014 (online)

\begin{tabular}{llll}
$\begin{array}{l}\text { Specialty } \\
\text { groups }\end{array}$ & $\begin{array}{l}\text { Score } \\
\text { difference } \\
(\% \text { points })\end{array}$ & $\begin{array}{l}95 \% \\
\text { Confidence } \\
\text { Intervals }\end{array}$ & P values \\
\hline DPC-IPC & 3.4 & $(0.8,6.0)$ & .004 \\
\hline NPC-IPC & 3.4 & $(0.6,6.2)$ & .010 \\
\hline SUR-IPC & 1.5 & $(-1.8,4.9)$ & .648 \\
\hline DPC-SUR & 1.9 & $(-0.9,4.7)$ & .301 \\
\hline NPC-SUR & 1.9 & $(-1.1,4.9)$ & .377 \\
\hline DPC-NPC & 0.3 & $(-2.0,2.1)$ & 1.00
\end{tabular}

COMMUNITY HEALTH WORKER SUPPORT FOR SELF-MEASURED BLOOD PRESSURE MONITORING AMONG URBAN AFRICAN AMERICANS WITH UNCONTROLLED HYPERTENSION $\underline{\mathrm{S} \text {. Alison } \mathrm{Kraemer}^{5} \text {; Patti Ephraim }}{ }^{3,6}$; Jane Pendergast $^{1}$; Hui Jie Lee ${ }^{1}$; Jessica Ameling ${ }^{5,}$; LaPricia Lewis-Boyer ${ }^{5}{ }^{5}$; Debra J. Gayles ${ }^{5}$, ${ }^{6}$; Valerie Lee $^{3}$; Hema Ramamurthi ${ }^{3}$; Dwyan Y. Monroe ${ }^{4}$; James Valderrama ${ }^{5,}{ }^{6}$; Nuha Muktar $^{5,6}$; Kathryn A. Carson ${ }^{3,6}$; Lee R. Bone ${ }^{2}$; David M. Levine ${ }^{5}$; Lisa A. Cooper ${ }^{5,6}$; L. Ebony Boulware ${ }^{1} .{ }^{1}$ Duke University, Durham, NC; ${ }^{2}$ Johns Hopkins Bloomberg School of Public health, Baltimore, MD; ${ }^{3}$ Johns Hopkins Bloomberg School of Public Health, Baltimore, MD; ${ }^{4}$ Institute for Public Health Innovation, Columbia, MD; ${ }^{5}$ Johns Hopkins University School of Medicine, Baltimore, MD; ${ }^{6}$ Welch Center for Prevention, Epidemiology, and Clinical Research, Baltimore, MD. (Control ID \#2467866)

BACKGROUND: African Americans are disparately affected by uncontrolled hypertension, resulting in poor clinical outcomes. Community health workers (CHWs) have been employed to improve patients' hypertension self-management, but their effectiveness in encouraging self-measured blood pressure (SMBP) monitoring among African Americans has not been well-studied. The objective of this study is to evaluate the factors associated with adherence to SMBP monitoring among urban African American patients receiving CHW support.

METHODS: We examined frequency of and barriers to SMBP monitoring among 159 African Americans with uncontrolled hypertension (systolic BP $\geq 140 \mathrm{mmHg}$ or diastolic BP $\geq 90 \mathrm{mmHg}$ ) who received care from a CHW as part of the Achieving Blood Pressure Control Together (ACT) study, a randomized controlled trial. At study enrollment (October 2013 to June 2014), participants, who were all receiving primary care in East Baltimore, Maryland, received a digital home BP monitor and training on BP monitor use from $\mathrm{CHWs}$ in their homes. Enrolled participants received ongoing CHW support throughout the study. This included scheduled follow-ups to assess use of and problem-solve difficulties with the home $\mathrm{BP}$ monitor and reminder telephone calls to bring their BP monitor to routine visits with their primary care provider (PCP) for download and review of their BP measurements. We assessed participants' adherence to SMBP monitoring behaviors during the first 120 days after receiving the BP monitor and CHW training on its use. Specifically, we evaluated selfreported SMBP monitoring with participants reporting $\mathrm{BP}$ measurements from their monitors and participants bringing their $\mathrm{BP}$ monitors to routine $\mathrm{PCP}$ visits. We also investigated selfreported barriers to SMBP monitoring. Participant characteristics (age, gender, poverty status, co-morbidities, literacy, employment, medication adherence, hypertension knowledge, tobacco use, alcohol use, depression, self-efficacy, social isolation, instrumental support, and family functioning) were included in multivariable logistic regression analysis to model predictors of SMBP monitoring behaviors.

RESULTS: Prior to receiving the home BP monitor from CHWs, $64 \%$ of the 159 enrolled participants reported they rarely or never checked their BP at home. A total of 114 (94\%) of the 121 participants completing a follow-up survey with the CHWs reported SMBP monitoring at home and 91 (78\%) of the 117 participants seen for routine PCP visits brought their BP monitor to their visit. Among those participants measuring BP at home $(N=114)$, a majority $(N=106$, $93 \%$ reported daily or every other day SMBP monitoring. Self-reported barriers to SMBP monitoring included difficulty with the monitor (11\%), forgetfulness (6\%), and being too busy (5\%). Participants who reported greater (versus less) social isolation (adjusted OR 1.16, $95 \% \mathrm{CI}$ 1.05-1.31) were more likely to measure BP at home. Among those reporting at least some SMBP monitoring, those with high (versus low) medication adherence were 3.6 times more likely to measure BP at home with greater frequency (adjusted OR 3.60, 95 \% CI 1.29-11.11). Each additional year of age was associated with a $17 \%$ increase in the odds of bringing the BP monitor to the PCP visit (OR 1.17, $95 \%$ CI 1.05-1.35).

CONCLUSIONS: After receiving home visits from a CHW, a majority of participants with uncontrolled hypertension performed SMBP monitoring behaviors during a 4-month period. Participants reporting greater social isolation, with greater medication adherence, and who were older were more likely to perform SMBP monitoring behaviors. Studies on sustained SMBP monitoring behaviors in response to $\mathrm{CHW}$ interventions are needed. 


\section{COMPARATIVE INVESTIGATION OF DIFFERENCE IN HEALTHCARE AND} POST-GRADUATE EDUCATION BETWEEN THE UNITED STATES AND JAPAN: A QUALITATIVE STUDY Hirotaka Kato ${ }^{1}$; Alfred Burger ${ }^{1}$; Robert Yanagisawa ${ }^{2}$; Jenny J. Lin ${ }^{2} .{ }^{1}$ Mount Sinai Beth Israel, New York, NY; ${ }^{2}$ Icahn School of Medicine at Mount Sinai, New York, NY. (Control ID \#2468220)

BACKGROUND: Whereas Japan cerebrated 50 years of universal health coverage with an improving equity in 2011, healthcare in Japan faces a challenge of sustainability with a rapidly aging society. The United States spends highest healthcare expenditure in GDP, however, care quality and cost effectiveness have been incorporated into fee schedule, education, and advocated by professional societies. Even though two countries take different approaches to issues in their healthcare systems, Japan has much to learn from the United States. In an effort to elucidate the problems in need of improvement, we sought to identify the differences in healthcare and post-graduate training from the perspective of Japanese international medical graduates (IMGs), who train or have trained in both Japan and selected residency programs in New York City, known as 'N program scholars'.

METHODS: A cross-sectional study was conducted from July 2015 to September 2015. One hundred fifty-two eligible Japanese IMGs were identified and invited to participate in an online survey which consisted of both open- and closed- ended questions related to differences between the US and Japan regarding post-graduate training, health insurance, approach to preventive care, and primary care clinic system. Grounded theory was used to analyze narrative scripts and identify themes.

RESULTS: 118/152 (77.6 \%) completed the survey. Respondents consisted of 20 current residents (17\%), 95 graduates ( $81 \%$ ) and three no answers ( $2 \%$ ). Of the graduates, $26 \%$ graduated within 5 years, $30 \%$ between 6 and 10 years ago, $24 \%>10$ years ago. The most common specialties included internal medicine (67\%) and pediatrics (18\%). The themes regarding post-graduate training revealed that US training was more standardized, evidence based with better evaluation, protected learning/teaching, scheduling, diverse experience and career options, whereas Japan's training had more procedural exposures. Regarding health insurance, the respondents feel that the US insurance causes limited access and inequity due to medical decisions dictated by insurance, complexity of the system and unequal coverage. In contrast, the Japan's universal insurance enables open access and affordable healthcare, but there is a concern about sustainability due to an aging society and abuse of the system. Prohibition of mixed billing was considered as a drawback, delaying the access to state-of-the-art treatments. A different approach to preventive medicine was found between two systems. The US is primary care- based, which is more tailored, evidence based/standardized with continuity but may miss more people without PCP, whereas Japan employs mass screening, which efficiently screens more people but is not tailored. Some respondents felt that immunizations and health maintenance were widely accepted in the US, as compared to Japan, in which efforts towards preventive care were limited. As for primary care clinic system, the US is more physician-friendly with manageable patient flow and longer time for each encounter, but the access is limited, whereas Japan is more patient-friendly because of open access creating disorganized patient flow, short time for visits, and long waiting time. Some respondents mention that the difference comes from expectation and function of the clinics because the clinic in Japan is incentivized to see more patients due to payment cut and it functions in part as urgent care.

CONCLUSIONS: Medical education is often overlooked but an important factor to train future physicians with capabilities to meet increasing social needs. The study findings suggest several implications for medical education in Japan. Firstly, Japan can learn standardization of post-graduate training from the United States. Secondly, preventive medicine including immunization should be weighed and taught early in medical education. At the same time, outcome evaluation of preventive care practice is necessary to optimize utilization of healthcare resources. Lastly, it appears important to prepare trainees for effective provision of preventive/primary care in a limited time for each encounter.
COMPARING CLINICAL EXPERIENCES IN VALUE-BASED CARE AMONG INTERNAL MEDICINE RESIDENCY PROGRAMS Reshma Gupta ${ }^{3,}$; Adam Schickedanz ${ }^{3}$; ; Vineet M. Arora ${ }^{2}$; Clarence H. Braddock ${ }^{1} .{ }^{1}$ Department of Medicine, David Geffen School of Medicine, University of California Los Angeles, Los Angeles, CA; ${ }^{2}$ University of Chicago Medical Center, Chicago, IL; ${ }^{3}$ VA/ UCLA Robert Wood Johnson Clinical Scholars Program, VA Greater Los Angeles Health System, Los Angeles, CA; ${ }^{4}$ Division of Pediatrics, David Geffen School of Medicine, University of California Los Angeles, Los Angeles, CA. (Control ID \#2467797)

BACKGROUND: While graduate medical education (GME) faces increasing pressure to meet society's need for physicians trained to deliver high value care, there is no standard metric to compare residency training program's performance based on how well they train residents to practice high quality care at lower cost. Such a metric could help policymakers guide decision on resource allocation, employers choosing applicants, students deciding where to train, and GME programs identifying areas for improvement. In this study, we compare and rank internal medicine (IM) GME programs using a composite measure of value-based care performance among their affiliated teaching hospitals to better understand the training environment.

METHODS: We conduct a secondary data analysis using hospital-level clinical quality and cost data from 2012 to 2014 for Medicare patients from the Centers for Medicare and Medicaid Services (CMS) Hospital Value-based Purchasing (VBP) Program and Hospital Compare, Medicare Impact Files, and program data on formal high value care curricula from the Association of Program Directors of Internal Medicine (APDIM) 2012 annual survey. We assess the top 100 IM residency programs defined by U.S. News and World Report in 2014 based on the CMS VBP score and its component measures for patient satisfaction, clinical process of care, patient outcomes, and efficiency.

RESULTS: IM program rankings by U.S. News and World Report and CMS VBP scores showed a weakly positive association with each other (Spearman rank correlation 0.268 , $p$-value $=0.008$ ). However, rank lists based upon these two methods showed little resemblance to each other. Compared to national hospital averages, teaching hospitals affiliated with the top 100 IM residency programs had lower risk-adjusted scores for VBP total performance $(p=0.002)$, cost effectiveness $(p<0.001)$, and patient satisfaction $(p<0.001)$. The teaching hospitals were on par with average national process of care scores (11.0, SD 3.1, vs national average of 11.7, SD 4.9, $p$-value NS) and patient outcome scores (14.1, SD 5.4, vs national average of 13.7, 5.8, $p$-value NS). The top 20 IM GME programs in VBP total performance score showed a trend toward being more likely to have a high value care curriculum in place or in development compared to the bottom 20 programs ranked based on VBP score $(88$ vs. $65 \%, p$-value $=0.10)$. However, program VBP score did not correlate with the number of teaching modalities used in formal curricula to train residents in high value care or program directors' perceptions of their residents' readiness to incorporate value and costs into their medical decision making. Lower VBP scores were associated with public safety net hospital affiliation.

CONCLUSIONS: Program rankings based on CMS' VBP total performance score, a widely accepted value measure, weakly correlated with IM residency program rankings by U.S. News and World Report. Overall GME programs fared slightly worse in delivering high value care based on the aggregate scores of their affiliated teaching hospitals compared to the nationwide average hospital performance on the VBP total performance score and a multiple sub-domains. Within individual programs, training sites varied widely in their performance measures of cost and quality, suggesting that internal medicine residents' exposure to high value care training environments is highly variable both across programs and within programs. Programs affiliated with hospitals that have higher DSH payment index scores predicted poorer program rankings, which is explained by well-documented lower patient satisfaction scores at safety net hospitals and lack of CMS adjusting VBP scores for patient socioeconomic status or race/ethnicity. High value care training within safety net hospitals can be assessed separately since these hospitals may 
provide unique teaching opportunities for trainees. Our findings highlight opportunities for GME programs to adapt and lead improvements in measurement and performance to ensure that residents are prepared to practice high value care

COMPARING HIV QUALITY MEASURES BY HIV CARE MODEL Corinne $\underline{\text { Rhodes }^{1} \text {; Yuchiao Chang }}{ }^{1}$; Susan Regan ${ }^{1}$; Daniel E. Singer ${ }^{1}$; Virginia Triant ${ }^{1,2}$. ${ }^{1}$ Massachusetts General Hospital, Harvard Medical School, Boston, MA; ${ }^{2}$ Massachusetts General Hospital, Boston, MA. (Control ID \#2463309)

BACKGROUND: Persons living with HIV/AIDS (PLWHA) engage in several types of HIV primary care models. These include Infectious Disease provider only models (ID), generalist provider only models (generalist), and both ID and generalist provider models (ID\&generalist). Despite HIV Medicine Association longitudinal care provider standards for PLWHA, there is little US-based literature comparing HIV quality measures by different HIV Care Models. Our aim was to compare quality of care in several HIV domains by HIV care models using these measures.

METHODS: We developed a cohort of adult PLWHA who received all HIV and primary care within the Partners Healthcare System (PHS) in 2012. We categorized providers as ID or generalists by electronic medical record labeling and online biography verification. Patient HIV care model exposure was categorized as ID, generalists, or ID\&generalists based on 2-year clinic visit pattern and targeted chart review, with a random sample $(N=100)$ chart validation showing $>94 \%$ correct categorization. Outcomes were 14 quality of care measures endorsed by 5 major medical and quality associations. These are presented in 4 composite domain scores based on processes of care ( 2 measures: retention and CD4 laboratory draw frequency), screening (6 infectious diseases), immunization (3 communicable disease vaccinations), and HIV management (3 measures: antiretroviral prescription, viral load suppression, and pneumocystis jiroveci pneumonia prophylaxis if CD4 count $<200$ ). Composite scores were assessed for each patient as a percentage of completed/eligible measures as of 12/31/2012. Our adjusted analysis used generalized estimating equations to account for clustering by the most frequent provider in 2012. The multivariable models for each composite outcome included pre-determined predictors (age, sex, race, primary language [English vs other], median estimated household income, and Charlson score). Additionally, a sensitivity analysis included number of 2012 provider visits in the model.

RESULTS: In a cohort of 1565 patients, the breakdown by HIV care models was: 875 ID (56 \%), 90 generalists (6\%), and 600 ID\&generalists (38\%). Figure 1 shows the adjusted mean percentage of met quality measures for each domain in each HIV care model. In adjusted analysis processes of care and screening domain outcomes were similar among groups, however, patients receiving care from ID\&generalist models had higher mean percentage composite scores for immunization (+6.1 \% $95 \%$ Confidence Interval (CI) 1.0-11.1\%) and HIV management $(+8.0 \% 95 \%$ CI $4.1-12.0 \%)$ than ID providers. There were no significant differences comparing generalists to either ID or ID\&generalist models. Results adjusting for number of 2012 visits were similar.

CONCLUSIONS: In a system-wide cohort, PLWHA engaged in HIV care models with ID\&generalist providers achieved significantly higher mean quality measure scores in immunization and HIV management domains than PLWHA cared for by ID providers. There were no significant differences comparing generalists to either ID or ID\&generalists, although the study could be underpowered to detect these differences. These significant differences in domain quality measures suggests that HIV care model may affect the quality of HIV care patients receive in immunization and HIV management domains. These results should prompt additional study among HIV Care Models in other system and national contexts.

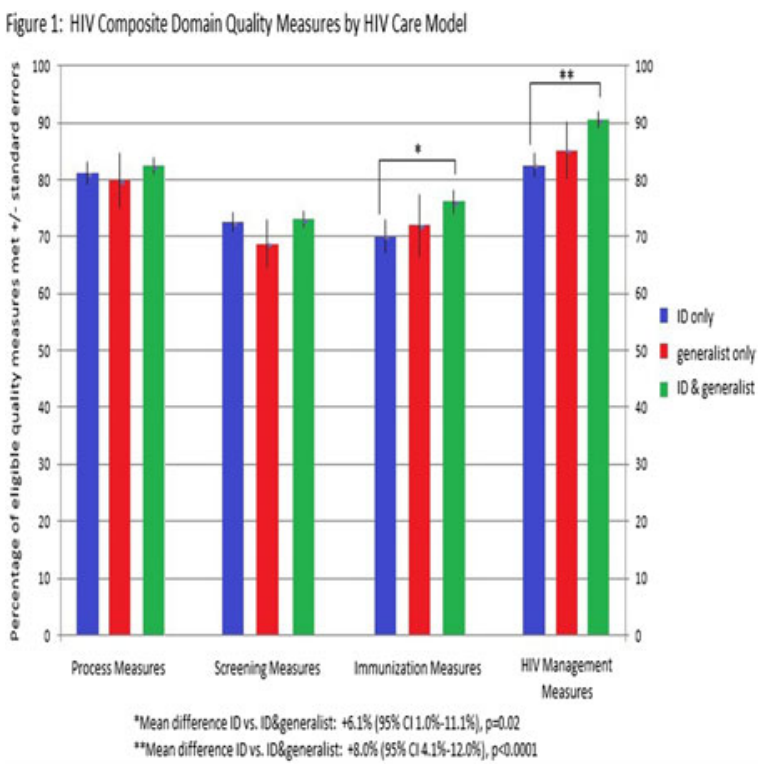

COMPARING KNOWLEDGE AND INTEREST IN LUNG CANCER SCREENING AMONG LATINO AND NON-LATINO CURRENT AND FORMER SMOKERS Sanja Percac-Lima ${ }^{1,2}$; Jeffrey M. Ashburner ${ }^{1}$; Elyse R. Park ${ }^{1}$; Nancy A. Rigotti $^{1,2}$; Lydiesther Martinez ${ }^{3}$; Steven J. Atlas ${ }^{1,2}$. ${ }^{1}$ Massachusetts General Hospital, Boston, MA; ${ }^{2}$ Harvard Medical School, Boston, MA; ${ }^{3}$ University of Illinois College of Medicine at Chicago, Chicago, IL. (Control ID \#2469618)

BACKGROUND: Annual computed tomography lung screening can decrease lung cancer mortality in high-risk smokers. Low socioeconomic status and difficulty accessing care are major obstacles to achieve equity in lung cancer care. These, along with underlying beliefs and knowledge, are also likely to apply to receipt of screening for lung cancer. As part of a study to develop a lung cancer screening program for community health centers $(\mathrm{CHC})$ within a primary care practice network, we surveyed patients who might be eligible for screening to assess beliefs about, knowledge of and interest in lung cancer screening. We compared the responses of Latino and non-Latino former and current smokers who might be eligible for screening. We hypothesized that Latino patients would have less knowledge and interest in lung screening than non-Latinos.

METHODS: From July to September 2015, we conducted a telephone survey of randomly selected current and former smokers 50-80 years old receiving care at one of five CHCs affiliated with an academic primary care network. We oversampled Latinos and excluded patients who did not speak English/Spanish or had a history of lung cancer. Demographic data was obtained from the electronic medical record. Individual survey items asked patients to selfreport their risk of developing lung cancer, their worry about lung cancer, knowledge about screening and patients' willingness to have lung cancer screening. We compared beliefs, knowledge and interest in screening among Latino and non-Latino CHC patients using logistic regression and adjusted for age, sex, education and smoking status (current or former).

RESULTS: Overall, 254 patients completed the survey ( $68 \%$ response rate). The average age was 62.1 years (SD 7.6), $57 \%$ of responders were women, $52 \%$ were current smokers and $46.5 \%$ were Latino. Ninety-two percent of non-Latino patients were white. Table 1 compares patient characteristics among Latino and non-Latino patients. Latino patients were more likely to be younger, male, less educated, and have poorer self-reported health. Table 2 presents patient responses to survey items among Latino and non-Latino patients. Latino patients were more likely to believe that smokers were at higher risk for developing lung cancer and thought that they could do something to prevent lung cancer. Although they were less worried about developing lung cancer, Latino patients were more likely to say they would get screening if offered. 
CONCLUSIONS: Latino high-risk current and former smokers in our CHCs are more likely than non-Latinos to believe that lung cancer can be prevented and that smokers are at high risk of getting the disease. Adjusting for smoking status, Latinos worry less about getting the lung cancer and their perceived risk of getting the disease is lower than in nonLatinos. Overall, less than half of surveyed patients were aware about the ability to screen for lung cancer. If informed and offered lung cancer screening, Latinos may be more likely to get screened. For a lung cancer screening programs to be successful all patients need to be educated about lung cancer and screening options. Our results suggest that non-Latino high-risk former and current smokers receiving care in community health centers might require more outreach to get screened for lung cancer.

Table 1. Demographic characteristics and smoking behavior among Latino and non-Latinos

\begin{tabular}{|c|c|c|c|}
\hline $\begin{array}{l}\text { Characteristic, } \\
N(\%)\end{array}$ & $\begin{array}{l}\text { Latino } \\
(n=118)\end{array}$ & $\begin{array}{l}\text { Non-Latino } \\
(n=135)\end{array}$ & $\begin{array}{l}\text { Odds Ratio } \\
(95 \% \mathrm{CI})^{*}\end{array}$ \\
\hline Age $\geq 65$ & $\begin{array}{l}39 \\
(33.1 \%)\end{array}$ & $\begin{array}{l}58 \\
(42.7 \%)\end{array}$ & $0.58(0.34-0.99)$ \\
\hline Female & $\begin{array}{l}56 \\
(47.5 \%)\end{array}$ & $\begin{array}{l}89 \\
(65.4 \%)\end{array}$ & $0.47(0.28-0.78)$ \\
\hline $\begin{array}{l}\text { Education, } \\
\geq \text { high school } \\
\text { graduate }\end{array}$ & $\begin{array}{l}50 \\
(42.4 \%)\end{array}$ & $\begin{array}{l}93 \\
(68.4 \%)\end{array}$ & $0.33(0.20-0.56)$ \\
\hline $\begin{array}{l}\text { Clinic visits, } \\
>10 \text { over } 3 \text {-years }\end{array}$ & $\begin{array}{l}63 \\
(53.4 \%)\end{array}$ & $\begin{array}{l}71 \\
(52.2 \%)\end{array}$ & $0.96(0.58-1.59)$ \\
\hline Charlson Score $\geq 2$ & $\begin{array}{l}84 \\
(71.2 \%)\end{array}$ & $\begin{array}{l}102 \\
(75.0 \%)\end{array}$ & $0.74(0.42-1.31)$ \\
\hline $\begin{array}{l}\text { Self-Rated Health, } \\
\text { Excellent/very good/ } \\
\text { good }\end{array}$ & $\begin{array}{l}46 \\
(39.3 \%)\end{array}$ & $\begin{array}{l}93 \\
(69.4 \%)\end{array}$ & $0.26(0.15-0.44)$ \\
\hline E-Cigarette Smoker & $\begin{array}{l}6 \\
(5.1 \%)\end{array}$ & $\begin{array}{l}15 \\
(11.0 \%)\end{array}$ & $0.51(0.19-1.40)$ \\
\hline Smoking in household & $\begin{array}{l}26 \\
(22.0 \%)\end{array}$ & $\begin{array}{l}48 \\
(35.3 \%)\end{array}$ & $0.56(0.32-1.00)$ \\
\hline
\end{tabular}

* Adjusted for smoking status (current or former, 1 missing data)

Table 2. Beliefs about lung cancer, knowledge and interest in lung cancer screening among Latino and non-Latinos

\begin{tabular}{|c|c|c|c|}
\hline $\begin{array}{l}\text { Beliefs, knowledge, } \\
\text { interest } \mathrm{N}(\%)\end{array}$ & $\begin{array}{l}\text { Latino } \\
(n=118)\end{array}$ & $\begin{array}{l}\text { Non-Latino } \\
(n=135)\end{array}$ & $\begin{array}{l}\text { Odds Ratio } \\
(95 \% \mathrm{CI})^{*}\end{array}$ \\
\hline $\begin{array}{l}\text { Likely develop } \\
\text { lung cancer }\end{array}$ & $56(47.5 \%)$ & $77(58.3 \%)$ & $\begin{array}{l}0.83 \\
(0.46-1.49)\end{array}$ \\
\hline $\begin{array}{l}\text { Worry about getting } \\
\text { lung cancer }\end{array}$ & $44(37.3 \%)$ & $78(58.7 \%)$ & $\begin{array}{l}0.43 \\
(0.24-0.77)\end{array}$ \\
\hline $\begin{array}{l}\text { Can do something } \\
\text { to prevent lung } \\
\text { cancer }\end{array}$ & $88(74.6 \%)$ & $75(56.0 \%)$ & $\begin{array}{l}2.08 \\
(1.17-3.72)\end{array}$ \\
\hline $\begin{array}{l}\text { Smokers have } \\
\text { high risk } \\
\text { of getting lung } \\
\text { cancer }\end{array}$ & $85(72.7 \%)$ & $78(58.7 \%)$ & $\begin{array}{l}1.83 \\
(1.03-3.27)\end{array}$ \\
\hline $\begin{array}{l}\text { Aware of lung } \\
\text { cancer } \\
\text { screening test }\end{array}$ & $52(44.1 \%)$ & $52(38.2 \%)$ & $\begin{array}{l}1.02 \\
(0.59-1.76)\end{array}$ \\
\hline $\begin{array}{l}\text { Would get } \\
\text { screened } \\
\text { if offered }\end{array}$ & $96(81.4 \%)$ & $60(44.1 \%)$ & $\begin{array}{l}6.23 \\
(3.28-11.80)\end{array}$ \\
\hline
\end{tabular}

* Adjusted for age, sex, education and smoking status (current or former, 1 missing data)

COMPARING PATIENT-CENTERED MEDICAL HOME IMPLEMENTATION IN URBAN AND RURAL VHA CLINICS: RESULTS FROM THE PATIENT ALIGNED CARE TEAM (PACT) INITIATIVE. Victoria Johnson ${ }^{2,}{ }^{3}$; Edwin Wong ${ }^{2}$; Gregory J. Schwartz ${ }^{2}$; Idamay Curtis ${ }^{2}$; John C. Fortney ${ }^{2}$; Peter Kaboli ${ }^{1}$; Stephan D. Fihn ${ }^{2,3}$; Karin M. Nelson ${ }^{2,3}$. ${ }^{1}$ Department of Veterans Affairs, Iowa City, IA; ${ }^{2}$ Department of Veterans Affairs, Seattle, WA; ${ }^{3}$ University of Washington, Seattle, WA. (Control ID \#2467948)
BACKGROUND: Rural primary care clinics in the Veterans Health Administration (VHA) are smaller, have lower levels of staffing and serve more rural patients compared to urban VHA primary care clinics. This may lead to different challenges to implementation of the VHA's Patient-Centered Medical Home (PCMH) model, the Patient Aligned Care Team (PACT). The present study sought to assess whether PACT implementation varied by rural or urban clinic location.

METHODS: This was an observational study of 905 VHA primary care clinics. VHA primary care clinics in the U.S. and Puerto Rico were categorized as rural or urban based on Rural-urban Commuting Area (RUCA) codes corresponding to clinic address or latitude and longitude $(\operatorname{Urban}=1.0,1.1$; Rural $=$ all others $)$. We used a previously developed measure, the PACT Implementation Progress Index $\left(\mathrm{PI}^{2}\right)$, to measure $\mathrm{PCMH}$ implementation in fiscal year 2012. $\mathrm{PI}^{2}$ is a composite score reflecting eight core PCMH domains: access, continuity, coordination, comprehensiveness, self-management support, patient-centered care and communication, shared decision-making and team-based care. The $\mathrm{PI}^{2}$ score is the number of domains in which a clinic is in the top quartile minus the number of domains in which a clinic is in the bottom quartile (range -8 to 8 ). We compared overall $\mathrm{PI}^{2}$ scores and domain scores for urban and rural clinics. We categorized clinics by degree of PACT implementation based on the following PI ${ }^{2}$ scores: 5 to 8 (highest performing clinics), 2 to $4,-1$ to $1,-4$ to -2 and -8 to 5 (lowest performing clinics). We compared trends across $\mathrm{PI}^{2}$ categories for rural and urban clinics using a non-parametric test of trend for ranks across ordered groups.

RESULTS: We analyzed data for 905 clinics, including 350 rural and 555 urban clinics. Rural clinics had significantly higher mean overall $\mathrm{PI}^{2}$ scores compared to urban clinics $(0.39$ vs. $-0.24, p<0.005)$. Trends across $\mathrm{PI}^{2}$ implementation categories were significantly different $(p<0.003)$, with higher proportions of rural clinics in the highest implementation categories compared to urban clinics. Rural clinics had higher scores on measures of team-based care ( 0.16 vs. $-0.08, p<0.001)$, care coordination $(0.07$ vs. $-0.05, p<0.019)$, shared decision-making $(0.06$ vs. $-0.04, p<0.044)$ and patient-centered care $(0.10$ vs. $-0.06, p<0.001)$. Urban clinics had a higher score for care continuity $(0.05$ vs. -0.06 , $p<0.036)$.

CONCLUSIONS: Overall, PCMH implementation in a large, integrated health care system was significantly greater in rural compared to urban primary care clinics. Rural primary care clinics generally provide care to fewer patients and have fewer staff providers compared to urban clinics, and smaller clinics may have fewer challenges to PCMH implementation. Rural/urban differences in PCMH implementation have implications for continued PACT integration and may contribute to variation in PACT-related outcomes across VHA clinics nationwide.

\section{COMPARISON OF THE EFFICACY OF GOREISAN AND PROBIOTICS AMONG JAPANESE ADULTS WITH ACUTE INFECTIOUS GASTROENTER- ITIS: A RANDOMIZED CONTROLLED TRIAL Fujiko Morita ${ }^{3}$; Naoto Matsuda ${ }^{2}$; Akihiro Inui ${ }^{2}$; Yuki Uehara ${ }^{2}$; Kazutoshi Fujibayashi ${ }^{4}$; Hirohide Yokokawa ${ }^{2}$; Hiroyuki

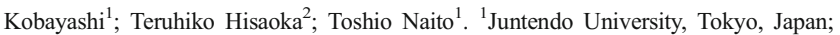 ${ }^{2}$ Juntendo University School of Medicine, Bunkyoku, Japan; ${ }^{3}$ Juntendo University School of Medicine, Bunkyo-ku, Japan; ${ }^{4}$ School of Medicine,Juntendo University, Tokyo, Japan. (Control ID \#2465575)}

BACKGROUND: While acute infectious gastroenteritis is usually self-limiting, dehydration and aspiration pneumonia may lead to severe outcomes, especially among immunocompromised and elderly patients. Outbreaks due to contiguous infection underscore the importance of early stage intervention and infection prevention. Goreisan is a traditional Chinese medicine (Kampo) commonly used in Japan to improve diarrhea and 
vomiting. This study compared the efficacy of Goreisan with that of probiotics in Japanese adults with acute infectious gastroenteritis.

METHODS: Outpatients aged 20 years or older with acute infectious gastroenteritis were enrolled in this randomized controlled trial from December 2014 to December 2015. Seventy-five patients were randomly assigned to receive Goreisan or probiotics (Bifidobacterium). The primary outcomes were duration (hours) and frequency of diarrhea as well as vomiting. The secondary outcomes were duration (days) of concomitant symptoms such as nausea, abdominal pain, fever, fatigue, and anorexia. Patients were instructed to record their symptoms on evaluation postcards for 5 days and to send the postcards back to us.

RESULTS: A total of 53 patients returned their evaluation postcards (follow up rate: $65.3 \%$ ). Four were excluded for diagnoses of bacterial gastroenteritis. Thus, 49 patients were included in the analysis (24 and 25 in the Goreisan and probiotics groups, respectively). Baseline characteristics, including age, sex, duration of diarrhea and/or vomiting before treatment, history of overseas travel, and recent antibiotics use, were similar between the two groups. The duration of anorexia $(2.0 \pm 1.1$ vs. $2.9 \pm 1.3, P=0.03)$ and abdominal pain $(1.9 \pm 1.2$ vs. $2.8 \pm 1.2, P=$ 0.01 ) were significantly shorter in the Goreisan group. The duration of diarrhea and vomiting was not significantly different between the Goreisan group and the probiotics group (diarrhea: $44.2 \pm 30$ vs. $44.2 \pm 40 \mathrm{~h}$; vomiting: $15.0 \pm 9$ vs. $12.2 \pm$ $12 \mathrm{~h}$ ). The duration of other concomitant symptoms was also not significantly different between the two groups (nausea: $1.7 \pm 0.9$ days vs. $2.1 \pm 1.5$ days; fever: $1.4 \pm 0.5$ vs. $1.7 \pm 0.6$; fatigue: $2.4 \pm 0.9$ vs. $3.0 \pm 1.2$ )

CONCLUSIONS: Among Japanese adult patients with acute gastroenteritis, the recovery times from anorexia and abdominal pain were significantly shorter in the Goreisan group than in the probiotics group, although there were no significant differences in the durations of diarrhea and vomiting between groups. The effects of calming gastric and intestinal disorders and pain relief in the Goreisan group may be associated with early improvement of abdominal pain and anorexia. Our data indicate that Goreisan may have the potential to improve general symptoms and prevent progression of serious conditions.

CONTENT AND OUTCOMES OF SOCIAL WORK CONSULTATION FOR PATIENTS WITH DIABETES IN OUTPATIENT PRIMARY CARE PRACTICE Andrew J. Rabovsky ${ }^{3}$; Michael B. Rothberg ${ }^{2}$; Andrei Brateanu ${ }^{1}$; Susannah L. Rose ${ }^{2}$; Lei $\mathrm{Kou}^{2}$; Anita D. Misra-Hebert ${ }^{2} .{ }^{1} \mathrm{CCF}$, Cleveland, $\mathrm{OH} ;{ }^{2} \mathrm{Cleveland}$ Clinic, Cleveland, $\mathrm{OH} ;{ }^{3}$ Case Western Reserve University School of Medicine, Cleveland, OH. (Control ID \#2468468)

BACKGROUND: Social determinants of health may significantly influence chronic disease management in patients with diabetes. While the social worker may be the key member of the clinical team poised to identify and address social determinants of health, the role of the social worker in outpatient primary care practice has not been well described. The purpose of our study was 1) to describe the specific patient needs addressed in social work consultations, categorized using an established framework of understanding the social determinants of health, and 2) to assess the impact of social work consultations on diabetes and cardiovascular risk factor control.

METHODS: A social worker is available on-site to provide outpatient services at three primary care sites in the Cleveland Clinic Health System. Using our electronic medical record, we identified 977 patients with diabetes who completed a social work consultation in 2014 at these sites. Three hundred of the 977 records were randomly selected for chart review of social work progress notes. For each note, we identified patient insurance status and needs addressed in the social work encounter using a standardized data abstraction sheet modeled after the social determinants of health categories established by Wilkinson and Marmot. For all 977 patients, measures of diabetes and cardiovascular risk factor control [glycosylated hemoglobin (HbA1c), low density lipoprotein (LDL), systolic blood pressure (SBP), and Body Mass Index (BMI)] were abstracted at two time points. The baseline (Time 1) was the patient's last biometric value or test result prior to their first social work encounter after January 1, 2013. The second time point (Time 2) was the first clinical or biometric value obtained at least 3 months following the patient's first social work encounter in 2014. We examined 4 subsets of patients: those with HbA1c $\geq 9 \%$, those with $\mathrm{SBP} \geq 160$, those with $\mathrm{BMI} \geq 30$, and those with $\mathrm{LDL} \geq 130$. Each group was matched to a comparison group of patients with diabetes who had not had a social work encounter in 2014. Matching was based on propensity scores, generated using age, gender, race, insurance status (Medicare, Medicaid, Commercial, Self-Pay), and estimated income by zip code as covariates. After matching, comparison patients were assigned a dummy visit date to correspond with the first social work visit in the matched pair, and baseline and follow-up values were extracted from the same calendar time points as Time 1 and Time 2 defined above. A paired t-test was used to compare the Time 1 and Time 2 values for the intervention and comparison groups.

RESULTS: For the 300 charts reviewed, the mean age of patients was $61,36 \%$ were White, $58 \%$ African American, and $6 \%$ Other/Refused. Patient insurance status included $59 \%$ Medicare, $34 \%$ Medicaid, $8 \%$ commercial insurance and $0 \%$ SelfPay. The social determinants of health addressed during the social work encounter are summarized in Table 1. The most common social determinants addressed included issues related to the Social Gradient or Social Support. Regarding the Social Gradient, the social worker assisted the patient in obtaining medications, particularly insulin, or addressed insurance issues in $93 \%$ of these encounters. Regarding Social Support, the social worker most often assessed Activities of Daily Living and assisted patients in acquiring different levels of home care assistance. For the subgroups of patients with poorly controlled diabetes and cardiovascular risk factors prior to socia worker consultation, significant clinical improvement was observed in diabetes control at least 3 months after the social work consult as compared to similar patients with diabetes who did not have a social work consultation. Significant improvements were not noted in LDL, SBP, or BMI (Table 2).

CONCLUSIONS: In our study, for patients with poor diabetes control, we found a temporal association of improved disease control related to consultation with a socia worker embedded in outpatient primary care settings in our health system. We also found that social work visits in the outpatient setting often focus on issues related to obtaining medications, particularly insulin, or health insurance, which may affect diabetes control. Our results suggest the need for further understanding of the role of the social worker in primary care practice and the effect of social work consultation on long-term clinical outcomes in patients with diabetes.

Table 1: Social Determinants of Health Addressed by Social Worker

\begin{tabular}{ll}
\hline \hline Social Determinants of Health & Frequency \% (N) (Total $N=300$ ) \\
The Social Gradient (Concerns about poverty, place in society) & $67 \%(200)$ \\
Social Support & $25 \%(75)$ \\
Transport (for appointments, ability to walk in safe environments) & $14 \%(43)$ \\
Psychiatric issues/need for referral to mental health provider & $12 \%(37)$ \\
Unemployment & $5 \%(14)$ \\
Food (access to healthy foods) & $4 \%(12)$ \\
Stress & $4 \%(11)$ \\
Social exclusion (related to financial issues, other) & $3 \%(8)$ \\
Addiction & $2 \%(6)$ \\
Work (work-related stressors) & $1 \%(3)$ \\
Early life (previous experiences) & $1 \%(2)$ \\
\hline
\end{tabular}


Table 2: Measures of Diabetes and Cardiovascular Risk Factor Control at Time 1 and Time 2

\begin{tabular}{|c|c|c|c|c|c|c|}
\hline Measures of Diabetes and Cardiovascular Risk Factor Control & $\begin{array}{l}\text { Time } 1 \\
\text { Intervention }\end{array}$ & $\begin{array}{l}\text { Time } 2 \\
\text { Comparison }\end{array}$ & $p$ & Intervention & Comparison & $p$ \\
\hline Mean glycosylated hemoglobin \% $(N=188)$ & 11.2 & 11.1 & 0.49 & 9.5 & 10.0 & 0.03 \\
\hline Mean low density lipoprotein mg/dL $(N=125)$ & 155.9 & 155.5 & 0.71 & 121.9 & 134.2 & 0.05 \\
\hline Mean systolic blood pressure mmHg $(N=89)$ & 175.1 & 173.5 & 0.28 & 149.8 & 149.0 & 0.58 \\
\hline Mean Body Mass Index $(N=523)$ & 39.2 & 38.2 & 0.14 & 39.0 & 37.9 & 0.98 \\
\hline
\end{tabular}

$N=$ number of matched pairs

COORDINATING VA-BASED CARE AND VA-PURCHASED CARE FOR GYNECOLOGIC MALIGNANCIES: CHALLENGES AND BEST PRACTICES Jessica L. Zuchowski ${ }^{2}$; Joya G. Chrystal ${ }^{3}$; Alison B. Hamilton ${ }^{3}$; Laurie C. Zephyrin ${ }^{1}$; Kristina M. Cordasco ${ }^{4}$. ${ }^{1}$ Department of Veterans Affairs, Washington, DC; ${ }^{2}$ US Dept of Veterans Affairs, North Hills, CA; ${ }^{3}$ VA Greater Los Angeles Healthcare System, Los Angeles, CA; ${ }^{4}$ VA Greater Los Angles Healthcare System/ UCLA, Los Angeles, CA. (Control ID \#2465554)

BACKGROUND: Last year, widely publicized delays in Veterans' access to VA care led to Congress authorizing multi-billion dollar legislation to increase the amount of care VA purchases for Veterans to use in their communities. Such VA-purchased care is designed to supplement VA-based care and reduce access delays. As a result of the legislation, more VA patients are receiving care both in VA and in community-based settings. Little attention has been paid to the care coordination challenges associated with bridging VA-based care and VA-purchased care. We assessed care coordination practices used in the care of women Veterans with gynecologic malignancies receiving both VA-based and VA-purchased care. METHODS: We conducted 21 semi-structured interviews in 15 VA locations nationally with VA gynecologists, women's health medical directors, and others directly involved in the coordination of care for women with gynecologic malignancies. We asked interviewees to describe resources and processes used at their facilities for the coordination of gynecologic cancer care (ovarian, uterine, endometrial, cervical, vaginal, vulvar cancers) in VA and community-based settings. We also asked about care coordination challenges and best practices. Interviews were recorded, transcribed and summarized in a template of key domains. We generated results using a team-based hybrid deductive/ inductive thematic analytic process in which initial categories were generated a priori from the summaries, and refined with a constant comparative approach.

RESULTS: Interviewees reported patients concurrently receiving a complex patchwork of cancer care across VA-based and VA-purchased care settings. Care coordination challenges included threats to accountability (e.g., unclear who is responsible for tracking patient progress), administrative barriers (e.g., delays associated with medical record transfer and VA-purchased care authorization), and communication barriers (e.g., VA and non-VA provider communication, and patient and provider communication). Care coordination best practices included having one or more people fulfill four key care coordination roles: (1) a care tracker, who brings together information from both VAbased and VA-purchased care, and provides accountability for the patient's overall progress through all stages of gynecologic oncology care; (2) a provider point-ofcontact, who is a direct connection to VA for non-VA gynecology oncology providers to facilitate provider-to-provider communication; (3) a patient liaison, who provides gynecology oncology patients with a VA-based source of information and answers questions about authorizations and appointments; (4) and an administrator, who ensures that authorizations for VA-purchased gynecology oncology care are completed and patient medical records move between VA and community-based systems in a timely fashion.

CONCLUSIONS: Although recent legislation made strides in ensuring that Veterans receive timely, convenient care, the infrastructure to support care coordination between VA and non-VA healthcare systems still needs development. Care for women with gynecologic malignancies is fragmented across VA-based and VA-purchased community care, with resulting challenges of accountability, administrative delays, and communication barriers. Gynecologic oncology care is one example of a situation in which Veterans' care is commonly provided simultaneously across VA and non-VA settings; our findings may be applicable to other conditions in which care is similarly organized. Fragmentation of care is a threat to effective care coordination, and therefore potentially diminishing Veterans' receipt of care that is high quality, efficient, comprehensive, and patientcentered. Delineating key care coordination roles, and strengthening resources and processes to support them, may begin to address these challenges.

CORTICOSTEROID INJECTION USE FOR TREATING ACUTE RESPIRATORY TRACT ILLNESSES IN ADULT PRIMARY CARE OUTPATIENT ENCOUNTERS. Evan L. Dvorin ${ }^{2}$; Marie Claire Lamb ${ }^{2}$; Qingyang Luo ${ }^{1}$; Eboni PriceHaywood ${ }^{1} .{ }^{1}$ Ochsner Clinic Foundation, Harvey, LA; ${ }^{2}$ Ochsner Medical Center, Jefferson, LA. (Control ID \#2464982)

BACKGROUND: Most acute respiratory tract illnesses (ARTIs), including upper respiratory tract infections and bronchitis, are self-limiting illnesses, for which antibiotics and systemic steroids have not been shown to provide clear benefit. Systemic steroids use is associated with some acute adverse effects and a multitude of long-term adverse effects when used chronically or repeatedly over time. Given the potential acute and long-term adverse effects of systemic corticosteroids, the lack of proven efficacy for treating acute respiratory tract illness (ARTIs) and the cost, their use should be rare. On the contrary, many providers in Louisiana use systemic corticosteroid injections for treating ARTIs and many patients have come to expect that such injections will be part of their care. In an unpublished communication with the largest non-Medicare commercial insurer in Louisiana, Blue Cross/Blue Shield of Louisiana, $29.9 \%$ of 252,076 unique encounters for acute respiratory tract illnesses (ARTI) in 2014 included steroid injections. In this study we examine factors associated with systemic corticosteroid injection use for ARTI in adult primary care.

METHODS: This is a retrospective observational study of adult primary care clinics in the Ochsner Health System - Louisiana's largest, nonprofit, academic, integrated delivery system. We examined the rate of corticosteroid injection administration for patients age $>=18$ with acute respiratory tract illnesses (ARTI) diagnosed during clinic encounters occurring between January and December 2014. Using multivariate logistic regression, we examined associations between receipt of corticosteroid injection and patient (number ARTI encounters, race, age, past medical history diagnoses, insurance), provider (degree, mostly urgent care provider, foreign medical graduate, Louisiana medical school, provider age), and encounter characteristics (day of week, urgent care visit, visit diagnoses). This retrospective observational study was approved by the Ochsner institutional review board.

RESULTS: There were 40,510 unique patient encounters for ARTI in 2014 in adult primary care. Steroid injections were used during $20.7 \%$ of these encounters. Provider 
specific injection rate for ARTI encounters varied widely from 0 to $83 \%$. After univariate analysis, all patient, provider, and encounter characteristics were statistically associated with injection use except patient characteristic gender. After multivariate analysis, characteristics associated with increased odds of steroid injection were patient race (white versus black, O.R. [95\%CI]: OR 1.13 [1.07-1.20]), middle age groups 30-49 and 50-69 versus under 30 years old (OR 1.18 [1.09-1.28] and 1.15 [1.06-1.25] respectively), past medical history of chronic obstructive pulmonary disease (OR 1.44 [1.30-1.61]); provider NP degree versus MD/DO (OR 1.96 [1.82-2.11]); and encounter factors of visit diagnoses sinusitis (OR 2.16 [2.02-2.32]), bronchitis (OR 1.62 [1.51-1.74]), and allergic rhinitis (OR 1.34 [1.23-1.46]), all $p<0.001$ ). After multivariate analysis, factors associated with decreased odds of steroid injection were patient past medical history of diabetes (OR 0.69 [0.64-0.75]) and Medicare insurance compared to commercial insurance (OR 0.82 [0.75$0.88]$ ); non-urgent care visit type (OR 0.34 [0.31-0.37]); and urgent care provider type (OR $0.83[0.78-0.90]$ ), all $p<0.0000$ ).

CONCLUSIONS: To our knowledge this is the first known study documenting steroid injection use for ARTIs in adult primary care. Despite unproven effectiveness of steroid injections for ARTI, during $20 \%$ of such visit encounters in our health system steroid injections are used. We elucidate patient, provider and encounter level characteristics associated with steroid use. Our findings provide guidance on potential areas to target in quality improvement initiatives to improve value-based care for ARTI. Our ultimate goal is to create a "Choosing Wisely"-like campaign, similar to such campaigns on appropriate antibiotic use, to educate providers about the lack of proven effectiveness and known short-term and long-term risks with using corticosteroid injections for ARTI

COST-EFFECTIVENESS OF BRIDGING ANTICOAGULATION AMONG PATIENTS WITH NONVALVULAR ATRIAL FIBRILLATION Matt Pappas ${ }^{3,2}$; Geoffrey Barnes ${ }^{2}$; Sandeep Vijan ${ }^{1,2}$. ${ }^{1}$ Ann Arbor VA HSR\&D, Ann Arbor, MI; ${ }^{2}$ University of Michigan Health System, Ann Arbor, MI; ${ }^{3}$ Ann Arbor VA, Ann Arbor, MI (Control ID \#2469856)

BACKGROUND: Bridging anticoagulation is commonly prescribed to patients with atrial fibrillation who are initiating therapy or require interruption of anticoagulation for procedures. Current guidelines recommend bridging for patients at high risk of stroke, but no data guide this recommendation. Meanwhile, although the BRIDGE trial suggested forgoing bridging to be noninferior to bridging, theory and earlier data suggests potential benefit to carefully selected patients. We examined the risks and benefits of bridging anticoagulation across patients at varying risks of thromboembolic and hemorrhagic stroke.

METHODS: We performed a Monte Carlo simulation to predict the cost-effectiveness of bridging anticoagulation among patients with non-valvular atrial fibrillation. We used a combination of primary data and published medical literature to simulate the impact of bridging on death and disability, as mediated by ischemic stroke, intracranial hemorrhage, and extracranial major hemorrhage. Simulations were performed from a third-party payer perspective, with multi-way sensitivity analyses.

RESULTS: When stratified by thromboembolic and hemorrhagic risk scores (such as CHADS2 and HAS-BLED), outpatient bridging anticoagulation reached commonly used cost-effectiveness thresholds in small proportions of simulated patients at high risk of stroke and low risk of hemorrhage. Hospital admission for bridging rarely meets commonly accepted cost-effectiveness thresholds. Results are sensitive to the costs of longterm care and utility weights.

CONCLUSIONS: Bridging that requires hospitalization is unlikely to be cost-effective from the perspective of a third-party payer. Outpatient bridging may be reasonable, if patients are carefully selected by thromboembolic risk, hemorrhagic risk, and patient preference, but the likely benefit is modest. Incorporating small treatment-related disutilities leads to predicted net harm in most simulations. From a third-party payer perspective, hospitalization specifically for bridging is inefficient, as is outpatient bridging in most patients.

COST-EFFECTIVENESS OF INDIVIDUALIZING GLYCEMIC GOALS FOR U.S. ADULTS WITH TYPE 2 DIABETES Neda Laiteerapong ${ }^{2}$; Jennifer Cooper'; Rochelle N. Naylor ${ }^{2}$; Elbert S. Huang ${ }^{2} .{ }^{1}$ The University of Chicago, Chicago, IL; ${ }^{2}$ University of Chicago, Chicago, IL. (Control ID \#2470280)

BACKGROUND: Current guidelines for Type 2 diabetes recommend that providers choose glycemic goals ( $\mathrm{A} 1 \mathrm{C}<6.5 \%,<7 \%$ or $<8 \%$ ) depending on patient age, life expectancy, comorbidity, duration of diabetes, and history of diabetic complications. These guidelines are based on inconsistent evidence on the benefits of intensive control for older patients with established diabetes. Based on guidelines, glycemic goals for patients with Type 2 diabetes should increase (e.g., from an $\mathrm{A} 1 \mathrm{C}<6.5$ to $7 \%$, and then to $8 \%$ ) as they age and develop complications. This strategy of glycemic control has important tradeoffs; higher $\mathrm{A} 1 \mathrm{C}$ values increase the risk of diabetic complications but decrease the risk of hypoglycemia and polypharmacy. To date, the clinical and economic implications of a national strategy of individualizing glycemic control have not been examined.

METHODS: We developed the U.S. Type 2 Diabetes Policy Model based on modifications to the United Kingdom Prospective Diabetes Study (UKPDS) Outcomes Model v2 (OM2), using a societal perspective. The UKPDS-OM2 is a Markov-Monte Carlo simulation model of lifetime complications of patients with type 2 diabetes based on 25 years of UKPDS data. We modified the UKPDS-OM2 by adding a hypoglycemic event model, an American Diabetes Association (ADA)-based algorithm for medications, U.S. 2015 estimated costs for diabetes care (medications, self-monitoring, complications, hypoglycemic events, and health care utilization), and a disutility model for complications, hypoglycemic events, and medications. We modeled 10,000 hypothetical patients for each of 569 adults with self-reported Type 2 diabetes in the National Health and Nutrition Examination Survey (NHANES) 2011. We compared three strategies: 1) goal A1C $<7 \%$ (base-case), 2) goal $\mathrm{A} 1 \mathrm{C}<8 \%, 3$ ) individualized goal (A1C $<6.5,<7$, or $<8 \%$ based on ADA guidelines). For all strategies, medications were added or discontinued in order to maintain patients within $1 \%$ less than their A1C goal. For the individualized goal strategy, A1C goals increased as patients aged and developed complications. We report U.S.weighted projected lifetime average costs, remaining life-years and quality-adjusted life years (QALY) and calculated incremental cost-effectiveness ratios (ICER). Costs and QALYs were discounted at $3 \%$. In sensitivity analyses, we assumed no disutility from medications and a discount rate of $1 \%$.

RESULTS: A U.S. strategy of individualized goal setting dominated the goal $\mathrm{A} 1 \mathrm{C}<7$ and $<8 \%$ strategies. A U.S. strategy of individualized goal setting would be a cost-saving strategy compared to strategies of pursuing universal A1C goal of $<7$ or $<8 \%$ (total costs: $\$ 99,715$ vs. $\$ 113,362$ or $\$ 111,060$; difference $=-\$ 13,647$ or $\$ 11,345$, respectively), due to lower medication costs $(\$ 34,492$ vs. $\$ 48,767$ or $\$ 45,324$; difference $=-\$ 14,275$ or $-\$ 10,832$, respectively). The individualized strategy would also produce more QALYs than a goal $\mathrm{A} 1 \mathrm{C}<7 \%$ or $<8 \%$ (16.6920 vs. 16.6171 or 16.5429 ; difference $=+27.4$ or +54.5 days, respectively). However, individualized goals would be associated with fewer remaining life-years than goal $\mathrm{A} 1 \mathrm{C}<7 \%$ (20.6107 vs. 20.7365 years, difference $=$ -45.9 days) but more life-years than goal $\mathrm{A} 1 \mathrm{C}<8 \%$ (20.6062, difference $=+1.6$ days). In sensitivity analyses, assuming no disutility from medications, the $\mathrm{A} 1 \mathrm{C}<7 \%$ and $\mathrm{A} 1 \mathrm{C}$ $<8 \%$ goal strategies had more QALYs than the individualized strategy (17.0851 and 17.0838 vs. 17.0639 ; difference $=-7.7$ and -7.3 days, respectively). With a discount rate of $1 \%$, the individualized strategy dominated the goal A1C $<7 \%$ and $<8 \%$ strategies by costing less with more QALYs. 
CONCLUSIONS: Individualizing glycemic goals for U.S. adults with Type 2 diabetes is cost-saving and increases QALYs when compared to A1C $<7 \%$ goals; however, this strategy is associated with shorter life expectancy and is sensitive to patient preferences for medications. Decisions to endorse individualized glycemic goals should be tailored to individual patient preferences regarding the tradeoffs between taking medications, quality of life, and length of life.

\section{COST-EFFECTIVENESS OF INTENSIVE BLOOD PRESSURE CONTROL Ilana} B. Richman $^{2,1}$; Michael Fairley ${ }^{3}$; Mads Joergensen ${ }^{3}$; Alejandro Schuler ${ }^{1}$; Emma Smith Rastatter $^{3}$; Douglas K. Owens ${ }^{2,}{ }^{1}$; Jeremy Goldhaber-Fiebert ${ }^{1} .{ }^{1}$ Stanford University School of Medicine, Stanford, CA; ${ }^{2}$ Palo Alto Veterans Affairs Health Care System, Palo Alto, CA; ${ }^{3}$ Stanford University, Stanford, CA. (Control ID \#2468047)

BACKGROUND: Hypertension is a modifiable risk factor for cardiovascular disease that affects nearly 1 in 3 US adults. In the Systolic Blood Pressure Intervention Trial (SPRINT), intensive blood pressure control with a target systolic blood pressure of $<120 \mathrm{mmHg}$ reduced cardiovascular morbidity and mortality compared to a higher target. Achieving this lower target, though, incurs additional expenditures from medications, physician management, and side effects. We sought to evaluate the incremental costeffectiveness of intensive blood pressure treatment compared to standard management among hypertensive adults.

METHODS: We developed a Markov cohort model to estimate costs and benefits of intensive and standard blood pressure control. Our model followed a hypothetical cohort of 68 year-olds over 30 years and tracked the development of heart failure, myocardial infarction (MI), stroke, and death. The model also accounted for both serious and minor adverse events from both treatment strategies, using data from the SPRINT trial to estimate treatment effects and adverse event rates. We applied the cardiovascular benefits of intensive control reported in the trial to our modeled cohort only for the first 3.27 years, the duration of the trial. We used CDC life tables to project age- and cause-specific mortality in our cohort. We used population-based published incidence rates to model development of heart failure, MI, and stroke as well as all-cause mortality associated with those conditions. Costs of treatment of hypertension, cardiovascular complications, and adverse events were derived published sources, from Medicare data, and from the Health Care Cost and Utilization Project. We performed a series of deterministic sensitivity analyses varying the duration of benefit and the magnitude and frequency of adverse events.

RESULTS: The quality adjusted life expectancy for the standard treatment group was 11.58 and 11.78 years in the intensive treatment group. Total costs were $\$ 170,257$ in the standard treatment group and $\$ 172,865$ in the intensive treatment group. Intensive treatment cost $\$ 14,153$ per QALY gained compared to standard treatment. In sensitivity analyses, varying the duration of benefit from minimal (duration of the trial) to maximal (lifetime) did not substantively alter our results - intensive treatment cost \$14,912 per QALY gained. Altering the burden associated with minor adverse events did affect our conclusions - intensive blood pressure control would no longer be the preferred strategy if common adverse events were roughly 10 -fold more severe than estimated for the base-case.

CONCLUSIONS: Intensive blood pressure control, as described in SPRINT, is cost-effective at typical value thresholds in healthcare. Since more severe patient experiences of minor adverse events can make intensive control substantially less cost-effective, it will be important monitor adverse events as intensive treatment becomes more widely used and to continue to identify patients for whom minor adverse events are less tolerable as early as possible.

COST-EFFECTIVENESS THRESHOLD ANALYSIS OF RECENT HYPERTENSION GUIDELINES: THE CVD POLICY MODEL Nathalie Moise ${ }^{4}$; Chen Huang ${ }^{4}$; Anthony Rodgers ${ }^{5}$; Keane Zhong ${ }^{4}$; Pamela G. Coxson ${ }^{3}$; Kirsten Bibbins-Domingo ${ }^{2}$; Lee
Goldman ${ }^{1}$; Andrew Moran ${ }^{4} .{ }^{1}$ Columbia University, New York, NY; ${ }^{2}$ UCSF, San Francisco, CA; ${ }^{3}$ University of California at San Francisco, San Francisco, CA; ${ }^{4}$ Columbia University Medical Center, New York, NY; ${ }^{5}$ The George Institute for Global Health, Sydney, QLD, Australia. (Control ID \#2468584)

BACKGROUND: Results from the Systolic Blood Pressure Intervention Trial (SPRINT) suggest that systolic blood pressure reduction below 2014 hypertension guideline ('JNC8') recommended goals prevents more deaths in high cardiovascular disease risk patients. It remains unclear if net benefits of treating to $\mathrm{SBP}<140 \mathrm{mmHg}$ extends to primary prevention in the general population, however. We previously showed that implementing the intensive treatment goals of the 2003 "JNC7" hypertension guidelines for primary prevention in the general population would be cost-saving compared to 2014 "JNC8" guidelines. Here, we seek to assess the cost-effectiveness threshold at which $\mathrm{JNC7}$ is no longer considered cost-effective compared to JNC8, which remains largely unknown.

METHODS: The Cardiovascular Disease Policy Model was calibrated and validated using randomized controlled trial data. Cardiovascular disease events and treatment costs averted, quality adjusted life years (QALY) gained, and drug and monitoring costs incurred were simulated over 2014-2024 for hypertensive patients aged 35-74 years. In order to explore the impact of diminished BP lowering benefits below JNC8 treatment goals might have on previous cost-effectiveness of JNC7 over JNC8, we progressively attenuated the effectiveness of blood pressure lowering in the systolic/diastolic range 130$139 / 80-89 \mathrm{mmHg}$ in all patients with diabetes or chronic kidney disease, and in the systolic range 140-149 $\mathrm{mmHg}$ in patients aged 60-74 years without diabetes or chronic kidney disease until the JNC7 to JNC8 incremental cost-effectiveness ratio reached $\geq$ $\$ 50,000$ per QALY gained.

RESULTS: JNC7 would no longer be cost-effective compared to JNC8 if lowering BP in the interval systolic $140-149 \mathrm{mmHg}$ yielded one-third the expected effectiveness in adults aged 60-74 years old without diabetes or chronic kidney disease (the equivalent of attenuating coronary heart disease relative risk from 0.77 to 0.83 and stroke relative risk from 0.69 to 0.77 per $10 \mathrm{mmHg}$ lower systolic blood pressure). In patients with diabetes or chronic kidney disease, JNC7 was no longer more cost-effective if treatment in the intervals $130-139 \mathrm{mmHg}$ systolic or $80-89 \mathrm{mmHg}$ diastolic was half as effective in individuals aged 35-59 years (attenuating coronary heart disease relative risk from 0.73 to 0.85 and stroke relative risk from 0.64 to 0.79 per $10 \mathrm{mmHg}$ lower systolic blood pressure or $5 \mathrm{mmHg}$ diastolic blood pressure or one-fourth as effective in those 60-74 years (attenuating the relative risk of coronary heart disease from 0.77 to 0.81 and the relative risk of stroke from 0.69 to 0.75 ).

CONCLUSIONS: The effect of blood pressure control on CVD outcomes would have to be substantially diminished in the general population for more intensive JNC7 blood pressure goals to be considered less cost-effective than conservative blood pressure goals of JNC8. Our study provides a framework through which the cost-effectiveness of future hypertension trials can be assessed and lends support to the potential impact of lower blood pressure goals for primary prevention. Our future analyses will assess the cost-effectiveness of SPRINT.

CREATION AND VALIDATION OF AN INTERPROFESSIONAL 3600 CLINIC EVALUATION C. Scott Smith $^{3}$; Rick Tivis ${ }^{1}$; Donna M. Lowther ${ }^{2}$; Donovan Victorine ${ }^{1}$; Jill Hedt $^{1}$; Elena Speroff ${ }^{1}$; Deborah Smith ${ }^{1}$; Amber Fisher ${ }^{1}$; Adam Brotman ${ }^{1}$; Henry S. Elzinga ${ }^{1} .{ }^{1}$ Boise VA Medical Center, Boise, ID; ${ }^{2}$ Boise VA Medical Center, Meridian, ID; ${ }^{3}$ University of Washington, Boise, ID. (Control ID \#2467791)

BACKGROUND: All clinical health professions have identified discipline-specific core competencies. In addition, the interprofessional educational collaborative has identified desirable interprofessional competencies. However, with the development of team based 
care and training, there is an emerging need for interprofessional competency-based assessment instruments.

METHODS: Development: A group of nurses, physicians, pharmacists, psychologists, and nurse practitioners with an interest and background in education were selected to review competencies and create a universal ambulatory assessment instrument. For phase one, the group devised two questions each for faculty, peers, and staff and anchored them to each professions' core competencies using a developmental framework. These groups also determined Performance Dimension/Frame of Reference expected score ranges for their trainees. For phase two, the assessment instrument was iteratively modified based on testing with uninitiated faculty, staff, and trainees to improve understandability and ease of use. Validation: We performed construct validation of the developmental framework by comparing the scores for each academic level (such as 'student', 'resident') by means of a linear regression with least squares using the PROC REG function in SAS, where the response levels were the average scores and the regressor variable was academic level. Criterion-based validation was accomplished by dividing all primary care providers (physicians, NPs) into "High performer" and "Low performer" overall scores. These rankings were compared to the number of patient complaints over the first 6 months of the academic year by Chi square.

RESULTS: All instruments have five developmental columns consisting of "Critical Deficiency", "Direct Supervision”, "Indirect Supervision”, "Ready for Independent Practice", and "Aspirational" with behavioral descriptors. The clinic staff evaluates "Team Communication" and "Responsible Teamwork". Peers evaluate "Trust in Communication" and "Quality Improvement Participation". Faculty evaluates "Interprofessional Collaboration" and "Self-Improvement". The developmental construct was confirmed. There was a clear trend toward decreased supervisory needs (higher scores) with increasing academic level $\left(F(1,464)=119.7, p<0.0001, \mathrm{r}^{2}=.21\right)$. Criterion-based validation was also confirmed, with statistically fewer complaints and requests for change of provider in higher scoring practitioners $(p=0.02)$

CONCLUSIONS: We have created a universal $360^{\circ}$ evaluation instrument to assess interprofessional ambulatory trainees and teams. This instrument discriminates between academic levels and inversely correlates with provider complaints as expected. Ou preliminary data demonstrates great promise in evaluating interprofessional competencies and facilitating improved interprofessional communication/ discussion.

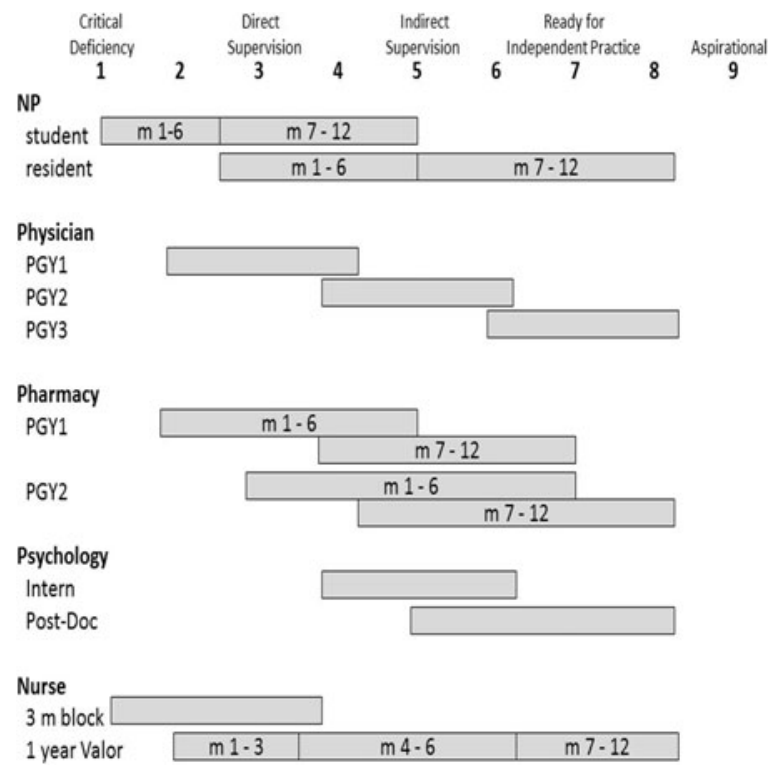

Expected score ranges by academic level for each profession $(\mathrm{m}=$ training month)
CULTURALLY TARGETED STRATEGIES FOR DIABETES PREVENTION IN MINORITY POPULATIONS: A SYSTEMATIC REVIEW AND FRAMEWORK Pooja A. Lagisetty $^{2}$; Shubadra Priyadarshini ${ }^{2}$; Stephanie Terrell ${ }^{2}$; Jessica Landgraf ${ }^{2}$; Vineet Chopra ${ }^{2}$; Michele Heisler ${ }^{1} .{ }^{1}$ Ann Arbor VA/University of Michigan, Ann Arbor, MI; ${ }^{2}$ University of Michigan, Ann Arbor, MI. (Control ID \#2467098)

BACKGROUND: Ethnic minority groups worldwide have high rates of morbidity and mortality from diabetes. The US Diabetes Prevention Program (DPP) along with similar interventions in Finland, India, and China used modest lifestyle changes including diet modification and physical activity to reduce the risk of progression of pre-diabetes to diabetes. While promising, none of these interventions explicitly used cultural tailoring to target ethnic minority groups. Prevention strategies specifically tailored to the culture of such patients may help reduce the incidence of diabetes. We aimed to (a) assess the effectiveness of culturally tailored diabetes prevention interventions in minority populations and (b) introduce a novel framework (FiLLM) to characterize four key domains of culturally tailored interventions.

METHODS: We searched PubMed, EMBASE, and CINAHL for English-language, randomized controlled trials (RCTs) or quasi-experimental (QE) trials testing culturally tailored interventions to prevent diabetes in minority populations. Two reviewers independently extracted data and assessed risk of bias. Inductive thematic analysis was used to develop a framework with four domains (FiLLM: Facilitating [i.e., delivering] Interventions through Language, Location and Message). The framework was used to assess the overall effectiveness of culturally tailored interventions.

RESULTS: Twenty-three trials met eligibility criteria. Five studies were randomized controlled trials, and 18 were quasi-experimental trials. Twenty out of 23 studies significantly improved HemoglobinA1c, fasting glucose, and/or weight loss. Of the 20 successful interventions, 15 (75\%) incorporated at least three culturally targeted domains The seven studies that used all four domains were all successful. In contrast, two of the three unsuccessful studies, only tailored on two domains. The least utilized domain was targeted content (14/23) and delivery (3/23) of the intervention's key educational message. CONCLUSIONS: Culturally tailored interventions improve risk factors for progression to diabetes among ethnic minority adults. Our study identifies and develops a framework (FiLLM) that can easily be adopted and utilized when designing and evaluating culturally targeted interventions. By targeting interventions across all four of these domains, future interventions can more effectively help ethnic minority populations adopt healthier behaviors to reduce their risk of diabetes and other preventable conditions. As diabetes prevalence continues to increase worldwide, sound research design and evaluation of culturally targeted diabetes prevention needs to be a key priority amongst researchers and policy makers.

\section{CURRICULUM DEVELOPMENT FOR A FORMAL PERSONAL AND PRO- FESSIONAL DEVELOPMENT PROGRAM FOR UNDERGRADUATE MEDI- CAL STUDENTS Christopher Mattson; Wei Wei Lee; James N. Woodruff. University of Chicago, Chicago, IL. (Control ID \#2466331)}

BACKGROUND: In 2013, the Association of American Medical Colleges released the Physician Competency Reference Set (PCRS), which included "Personal and Professional Development" (PPD) as a competency domain to be taught and assessed in medical school. Instruction in this domain has traditionally relied primarily on the hidden curriculum. However, there has been increasing interest in formally teaching and assessing these skills. While most medical schools have PPD programming in place, few have comprehensive programs, curricula or assessment methods. We aim to 1) conduct a PPD needs assessment by surveying first year medical students 2) Catalogue the existing PPD programming at the Pritzker School of medicine to identify gaps 3) Use this data to develop a comprehensive PPD program and provide students and their advisors with the tools to improve PPD competencies. 
METHODS: A 46-item PPD Skills Assessment was developed based on a literature review. The survey consisted of 39 skill-based items that asked students to use a five point Likert scale to rate their proficiency on 15 personal skills, 15 professional skills and 9 academic skills. Examples of questions include "Incorporating exercise into my weekly routine", "Seeking advice from advisors and mentors" and "Learning from lecture based oral presentations" respectively. The remainder of the Skills Assessment included 5 questions that used a five point Likert scale to assess student attitudes on reflection and personal and professional development as well as 2 free response questions that asked students to list three goals for personal development and three goals for professional development. The survey was administered to first year medical students (MS1s) at the Pritzker School of Medicine in October 2014 and again in February 2015. Students that completed both surveys were included in our analysis and descriptive statistics were summarized and compared. Next, we mapped the PPD-related events in the 2014-15 academic year to the PPD skills assessed in the survey. Lastly, we mapped the students' self-reported personal and professional goals to the PPD skills.

RESULTS: Seventy-five out of 90 MS1s ( $83 \%$ ) completed both surveys. The 3 lowest rated skills on the baseline survey were "Incorporating exercise into my weekly routine" (mean = 3.39), "Seeking advice from advisors and mentors" (mean = 3.44), and "Balancing demands of studying with other commitments" (mean=3.49); the 3 lowest rated skills on the follow-up survey were "Seeking advice from advisors and mentors" (mean=3.39), "Negotiating difficult conversations" (mean = 3.45), and "Learning from lecture based oral presentations" (mean $=3.48$ ). A significant increase between baseline and follow-up was found for "Balancing demands of studying with other commitments", "Learning from reading materials" and "Learning from one on one preceptorships". No statistically significant decrease was seen on any of the skills assessed between baseline and follow-up. Overall, 65 events from the 2014-15 academic year were mapped to the PPD Skills Assessment skills. Examples of these events as well as the skills to which they mapped include the Career Advising at Pritzker series, which addressed "Seeking advice from advisors and mentors" and Yoga... Movement, Breathwork and Meditation for Stress Relief, which addressed "Incorporating exercise into my weekly routine". Free response goals were analyzed for all students who completed both surveys as well as students who just completed the initial or the follow-up. The 2 personal skills that were amongst the top 3 most commonly listed free response personal goals on both the initial and follow-up surveys were "Incorporating exercise into my weekly routine" ( $n=25$ (18.0\%) on initial; $n=30$ (15.3\%) on follow-up) and "Maintaining relationships with loved ones outside of medical school" ( $n=21$ (15.1\%) on initial; $n=27$ (13.8 \%) on follow-up). The 1 professional skill that was amongst the top 3 most commonly listed free response professional goals on both the initial and follow-up surveys was "Seeking advice from advisors and mentors" ( $n=16$ (11.4\%) on initial; $n=29$ (15.8 \%) on follow-up).

CONCLUSIONS: The high survey response rate indicates that surveying medical students twice per academic year may be a feasible way to track evolving class specific PPD needs while also allowing students the opportunity to reflect on their development and set goals. Additionally, the presence of statistically significant positive changes over the course of a few months was encouraging. By identifying students' self-reported weaknesses and goals as well as gaps in existing PPD programming, medical schools can develop programming to specifically target these needs. PPD programming can be effective in helping medical students acquire the skills necessary to handle the personal and professional demands of careers in medicine.

DAY OF THE WEEK AND PHYSICIAN DECISION FATIGUE Michael L. Barnett $^{1,}{ }^{2}$; Jeffrey A. Linder ${ }^{1}$; Anupam B. Jena ${ }^{2} .{ }^{1}$ Brigham and Women's Hospital, Boston, MA; ${ }^{2}$ Harvard Medical School, Boston, MA. (Control ID \#2468815)

BACKGROUND: Physicians make dozens of clinical decisions daily. In some situations, this may create the potential for "decision fatigue," whereby a physician's decisionmaking effort decreases over the course of a work week. One example is the prescription of antibiotics for upper respiratory infections (URIs), which can be an "easy" way to resolve a visit quickly, despite usually being inappropriate. Recent evidence shows that antibiotic prescribing in this setting increases during the course of a workday, supporting the existence of clinically meaningful decision fatigue among physicians. However, there is little evidence exploring this phenomenon across the week and in a broader set of clinical scenarios. In this study, we examine the hypothesis that physicians display increasing decision fatigue during a typical week, with higher rates of inappropriate prescribing and lower rates of time-intensive patient counseling at the end of a week versus the beginning.

METHODS: We performed a cross-sectional analysis of the nationally representative National Ambulatory Medical Care Survey (NAMCS) of office visits by adults from 2005 to 2012. The main analysis was restricted to "full-time" physicians who saw patients for $\geq 4$ weekdays in the surveyed week. The key exposure was a visit occurring the beginning of the week (Monday/Tuesday) versus the end of the week (Thursday/Friday). For outcomes, we measured the rates of 5 clinical measures with the potential for decision fatigue: 3 inappropriate prescribing measures (antibiotics for URIs; narcotics for acute low back pain (LBP); and sedatives [benzodiazepines or barbiturates] for any cause in older patients $\geq 65$ years old) and 2 time-intensive counseling measures (smoking cessation for active smokers and weight reduction counseling for obese patients). We hypothesized increases in inappropriate prescribing as well as decreases in time-intensive counseling over the work week. We estimated the rates of each outcome in the beginning vs. the end of the week using visit-level logistic regression adjusting for patient characteristics including age, sex, race, number of chronic conditions, and visit urgency. As a sensitivity analysis, we replicated the analysis above with "part-time" physicians seeing patients for $\leq 3$ weekdays in the week who should be less vulnerable to decision fatigue. Because of their shorter work week, we hypothesized that this group of physicians would be less vulnerable to decision fatigue. All rates, regression estimates and standard errors were calculated taking account of the multistage probability design of the surveys.

RESULTS: From 2005 to 2012, the study sample contained 82,725 office visits to 5738 physicians by adults eligible for at least one of the outcomes examined, representing over 2.4 billion visits. Office visits in the beginning versus end of the week did not exhibit clinically meaningful differences, though some differences were statistically significant. For example, patients with visits at the end of the week were slightly younger (53.7 vs. $54.1, p<0.001)$, slightly more likely to be black ( 13.8 vs. $12.7 \% p=0.01)$, and slightly more likely to have private insurance ( 51.0 vs. $50.2 \%, p=0.002)$. Comparing the end of the week to the beginning, after adjusting for patient characteristics, physicians were significantly more likely to prescribe narcotics for LBP (OR 1.16, $95 \%$ CI 1.01-1.33, $p=$ 0.04 ) and sedatives to older patients (OR 1.17, $95 \%$ CI 1.05-1.32, $p=0.01$ ). In contrast, physicians were less likely to perform time-intensive patient counseling, such as smoking cessation counseling (OR $0.82,95 \%$ CI $0.66-1.02, p=0.08$ ) or weight reduction counseling (OR 0.92, $95 \%$ CI 0.84-1.01, $p=0.09$ ), though both of these results were only marginally significant. There was no significant difference in antibiotic prescribing for URIs by day of the week $(p=0.74)$. In a sensitivity analysis examining physicians working $\leq 3$ days in the week, we observed no statistically significant differences in any of the outcomes above between the beginning and end of the week.

CONCLUSIONS: We observe evidence consistent with "decision fatigue" among physicians over the work week. This is supported by statistically significant increases in potentially inappropriate prescribing of narcotics and sedatives across the week for "fulltime", but not "part-time" physicians. We also observe weaker, though directionally appropriate, evidence for decreasing rates of time-intensive counseling for smoking cessation or weight loss. Contrary to prior work, we do not observe strong evidence for increasing antibiotic prescribing across the week, which may be more influenced by time of day than day of the week. These results suggest that strategies to address decision fatigue, such as mandatory breaks, may be effective methods to improve the quality of care delivery in appropriate clinical scenarios. 
DECISION SUPPORT, ALLOPURINOL USE, AND URIC ACID TESTING IN PATIENTS WITH GOUT-RELATED VISITS AT ONE VA MEDICAL CENTER $\underline{\text { Joel C. Boggan }}{ }^{3,}{ }^{4}$; Ryan D. Schulteis ${ }^{1}$; David L. Simel ${ }^{2}$. ${ }^{1}$ Duke University Medical Center, Durham, NC; ${ }^{2}$ Durham VAMC and Duke University, Durham, NC; ${ }^{3}$ Durham Veterans Affairs Medical Center, Durham, NC; ${ }^{4}$ Duke University Health System, Durham, NC. (Control ID \#2466578)

BACKGROUND: Current American College of Rheumatology (ACR) guidelines recommend lowering of serum uric acid to at least $<6 \mathrm{mg} / \mathrm{dL}$. The Department of Veterans Affairs (VA) health system is well-positioned for analysis of the quality of care of gout management given its disproportionately male and elderly patient population. In this study, we investigated gout management within Durham VA Medical Center before and after institution of decision support guidance about allopurinol use and uric acid monitoring.

METHODS: We incorporated data on allopurinol dosing and serum uric acid goals from the 2012 ACR Guidelines for the Management of Gout into our electronic ordering system for allopurinol orders at our facility in consultation with primary care and rheumatology experts and as a quality improvement intervention in January 2014. We measured patient visits for gout-related diagnoses (ICD-9 codes 274.xx), allopurinol prescriptions, and uric acid levels in fiscal year 2013 (Oct. 2012-Sep. 2013) and again in fiscal year 2015 (Oct. 2014-Sep. 2015) using the corporate data warehouse. Our primary outcome was the fraction of patients with a gout-related visit with uric acid levels at goal. Secondary outcomes included number of patients with gout-related visit receiving allopurinol from our facility and patients receiving allopurinol at doses $<=300 \mathrm{mg} /$ day. Differences in frequencies in the pre-intervention and post-intervention periods were investigated using chi-square statistics

RESULTS: The number of patients with gout-related visits increased by $22 \%$ across the study period, from 2150 to 2616 . The frequency of patients with gout-related visits receiving allopurinol prescriptions from DVAMC did not change $(57.3 \%$ of patients pre-intervention vs. $57.1 \%$ post-intervention, $p=0.87$ ). Of these patients receiving allopurinol, the majority received prescriptions with intended daily doses of $<=300 \mathrm{mg}$ daily ( 1166 of 1233 receiving prescriptions in the pre-intervention phase vs. 1408 of 1494 in the post-intervention phase, $p=0.72$ ). Uric acid testing frequency did not increase in the patient population across the study period ( $54.5 \%$ of patients pre-intervention vs. $54.8 \%$ post-intervention, $p=0.83$ ), nor did the proportion of patients with at least one uric acid level at goal during the course of each fiscal year (27.8 vs. $28.2 \%, p=0.78$ ).

CONCLUSIONS: Incorporation of guideline information regarding allopurinol dosing and serum uric acid goals into ordering prompts for allopurinol throughout a VA hospital system did not affect the frequency with which allopurinol was prescribed, the proportion of patients undergoing serum uric acid testing, nor the proportion of patients with a serum uric acid at goal. Overall, significant opportunities exist for improvement in gout management in this population with high rates of gout.

DECISION-MAKING AND GOAL-SETTING IN A RANDOMIZED CONTROLLED TRIAL OF PATIENT-CENTERED SUPPORT FOR CHRONIC DISEASE MANAGEMENT Shreya Kangovi ${ }^{4}$; Nandita Mitra ${ }^{3}$; Robyn A. Smith ${ }^{1}$; Raina Kulkarni ${ }^{3}$; Lindsey N. Turr ${ }^{3}$; Hairong $\mathrm{Huo}^{3}$; Karen Glanz ${ }^{3}$; David Grande ${ }^{3}$; Judith Long ${ }^{2,3}$. ${ }^{1}$ Perelman School of Medicine at the University of Pennsylvania, Philadelphia, PA; ${ }^{2}$ Philadelphia VA Medical Center, Philadelphia, PA; ${ }^{3}$ University of Pennsylvania, Philadelphia, PA; ${ }^{4}$ University of Pennsylvania, Phialdelphia, PA. (Control ID \#2453599)

BACKGROUND: Collaborative goal-setting is a promising strategy for engaging low socioeconomic status (SES) patients in chronic disease management, yet is hampered by knowledge gaps. First, providers may feel reluctant to cede any decision-making to patients for fear that patients' choices may deviate from what providers would advise. Few prior studies have explored whether these concerns are justified. Second, a large body of goal-setting literature demonstrates the importance of setting goals that are challenging, yet attainable. Again, few studies have examined the difficulty of chronic disease management goals. Finally, goal-setting theory suggests that chronic disease management goals need to be deconstructed into short-term goals and concrete action plans. In most prior studies, action planning has been facilitated by clinicians or computerized programs focusing on narrow domains and not on what patients think will help them reach chronic disease goals. As a result, current approaches to collaborative goal-setting may not be as patient-centered as intended. Our objectives were to describe decisions, goals and strategies for chronic disease management among low-SES patients with multiple chronic conditions.

METHODS: We analyzed baseline data from a two-armed, single blinded RCT $(n=302)$ of collaborative goal-setting versus collaborative goal-setting plus 6 months of support from an IMPaCT community health worker (CHW). Participants were recruited from two urban, academically affiliated internal medicine clinics. Eligible patients were: 1) established patients of study clinics; 2) residents of a high-poverty region; 3) diagnosed with $\geq 2$ of: hypertension (HTN), diabetes, obesity and tobacco dependence; 4) uninsured or publicly insured. At enrollment, patients used a low-literacy aid to choose one of their chronic conditions to focus on during the study. Then they collaboratively set a chronic disease management goal for that condition with their primary care provider. Patients randomized to a CHW deconstructed this goal into patient-driven action plans.

RESULTS: The mean age of the cohort was 56 (SD 13.1), $76 \%$ were female, $95 \%$ were black, $56 \%$ had an annual household income $<\$ 15,000$ and $96 \%$ had a history of a traumatic event on the Trauma History Questionnaire. Participants had an average of 3 (SD 4.4) emergency room visits and 1 (SD 2.6) hospital admissions in the prior 12 months Participants had an average 2.5 of the eligibility chronic conditions: 279 (92\%) had a diagnosis of HTN; 235 (78 \%) were obese; 175 (58 \%) had a diagnosis of diabetes and 55 (18\%) smoked (Figure 1). The most popular choice of condition to focus on during the study was obesity (chosen by $62 \%$ of obese patients), followed by tobacco (chosen by $56 \%$ of smokers), diabetes (chosen by $42 \%$ of patients with diabetes) and HTN (chosen by $18 \%$ of patients with hypertension). In multivariate analysis, patient's choice of HTN or diabetes was influenced by having a higher systolic blood pressure (SBP) or HbA1C (respectively). For every $1 \mathrm{mmHg}$ increase in SBP the odds of selecting HTN was 1.05 (95\% CI 1.03-1.07, $p<0.0001)$. For every increase in HbA1c of $1 \%$ the odds of selecting diabetes was 1.71 (95 \% CI 1.50-2.17, $p<0.0001)$. Additionally, patients who chose to work on weight loss had a marginally higher BMI than those who did not $(p=0.17)$. Chronic disease management goals were ambitious: the mean weight loss goal was $-16.8 \mathrm{lb}$ (SD 19.5), goal HbA1C reduction was $-1.3 \%$ (SD $1.7 \%$ ) and goal SBP reduction was $-9.8 \mathrm{mmHg}$ (SD $19.2 \mathrm{mmHg}$ ). Patients' self-rated commitment to achieving these goals was high: 4.9 (SD 3.0) on a scale ranging from 3 to 24 , where 3 is highly committed. Patients' self-efficacy for goal achievement was also high: 3.8 (SD 2.4) on a scale ranging from 3 to 16 , where 3 is the highest. Patient-driven action plans focused on non-clinical issues including behavior change (e.g. "start a weight-loss contest with my daughters") (59\%) and psychosocial issues (e.g. "join a rec center to help me cope with my brother's death") (24\%). Fewer action plans related to health system navigation (e.g. "meet with pharmacist for medication teaching") (9\%) or medical issues (e.g. "talk to doctor about foot pain that limits my exercise.") $(1 \%)$.

CONCLUSIONS: Patients with poorly controlled blood pressure or poorly controlled diabetes were most likely to choose working on conditions in poor control. Collaboratively set chronic disease management goals may have been unrealistic, suggesting that providers require additional training in order to help patients to better gauge goal difficulty. Patients and CHWs created tailored action plans to address the behavioral and psychosocial determinants of patients' chronic disease management. 


\section{Prevalence, choice and baseline control}

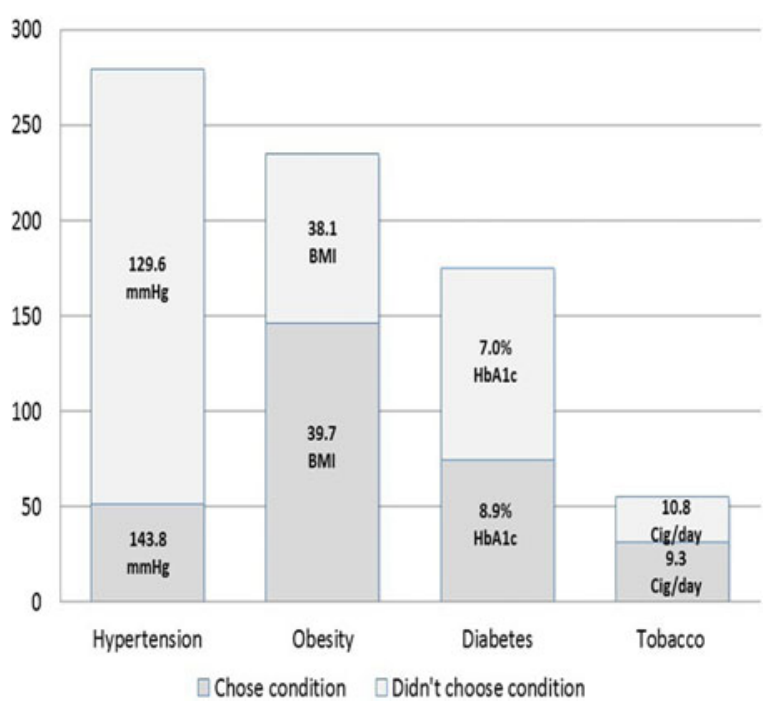
PORTAL AHEAD OF A SCHEDULED CLINIC VISIT Kenzie A. Cameron ${ }^{1}$; Tiffany Brown $^{1}$; Ji Young Lee'; Mita S. Goel ${ }^{1}$; Alpa Patel $^{2}$; Ryan $\mathrm{Chmiel}^{2}$; Paul Botsford ${ }^{2}$; Sameem Samad ${ }^{2}$; Darren Kaiser ${ }^{2} .{ }^{1}$ Northwestern University, Chicago, IL; ${ }^{2}$ Northwestern Medicine, Chicago, IL. (Control ID \#2458869)

BACKGROUND: We previously reported on a pneumococcal vaccination $(\mathrm{PnVx})$ education video, which was well received by patients who viewed the video during a clinic visit. However, this delivery channel required staff involvement to play the video. Patient portals, a feature of many electronic health records (EHRs), present an alternate channel through which to deliver timely, relevant patient education without burdening clinic staff. We implemented an EHR alert to send a patient portal message with a link to a video to patients newly eligible for $\mathrm{PnVx} 1$ week prior to a scheduled clinic visit.

METHODS: The study occurred between April-November 2015 at an academic, internal medicine clinic that uses an EHR for all clinical encounters (Epic) and has approximately $61.7 \%$ of patients active on its patient portal (MyChart). Programmers created and verified accuracy of EHR code that ran nightly to query eligible patients and trigger automatic message delivery. Clinicians consented for their patient panels to be included; the Northwestern Institutional Review Board approved the study with a waiver of patient informed consent. Eligible patients were age 65 or 66 , had an upcoming clinic visit scheduled at least a week in advance, were a registered patient portal user, and had no record of receipt or refusal of PnVx in the EHR. Patients with a preferred language other than English, visual or hearing impairments, or cognitive disorders were excluded. The patient portal message, sent 7 days prior to a scheduled visit, appeared as a message from their primary care provider, highlighting that the patient was now eligible for $\mathrm{PnVx}$ and requesting that the patient click a link to view a 2-min video prior to their visit. Patients who had not clicked the video hyperlink within 4 days were sent a reminder message 3 days prior to their scheduled visit. Patients who replied to the message or canceled their visit were not sent the reminder. Primary outcomes were message status (opened or unread) and video status (viewed, unviewed). A secondary outcome was PnVx status (yes, no, patient or medical exception) following their scheduled visit. All outcomes were assessed via EHR query. Descriptive statistics were used to describe the study population and primary outcomes, logistic regression explored the relationship between watching the video (yes/no) and vaccination status (yes/no).
RESULTS: Mean age of patients receiving the initial message $(n=116)$ was $65.0(\mathrm{SD}=$ 2.8), $29.3 \%$ were male, and $57.8 \%$ were white. The majority of patients ( $n=86,74.1 \%$ ) opened the MyChart message; only 22 (19.0 \%) clicked the hyperlink within 4 days. Among 77 patients ( 3 canceled their visit, 14 directly replied to their provider and were ineligible for reminder) who received a reminder message, $45(58.4 \%)$ opened the message and over a quarter ( $n=23,29.9 \%$ ) clicked the hyperlink. Among those who clicked the hyperlink (either message), $11.1 \%$ did not start the video, $24.4 \%$ watched a portion of the video, and $64.4 \%$ watched the entire video. Of the 40 who watched at least part of the video, 32 (80\%) received a PnVx at their subsequent clinic visit. Patients who watched the video were more likely to receive $\mathrm{PnVx}(\mathrm{OR}=2.97, p=0.03)$ than those who did not view the video.

CONCLUSIONS: Most patients who received a patient portal message opened it prior to their appointment; over a third watched the video. Those viewing the video were significantly more likely than those who did not to receive PnVx. Using the patient portal to deliver patient education about a preventive service to eligible patients ahead of a scheduled clinic visit appears to be both a viable and effective channel. Patient portal delivery requires no on-site staff time to deliver the message (e.g. start the video), and clear documentation of the delivery attempt is available. A disadvantage to patient portal delivery is that unregistered patients or those who are unable to navigate electronic messages are excluded from message exposure. Future research should continue to explore varying delivery channels for health education messages to assess feasibility and effectiveness

DELIVERY OF A DIETARY INTERVENTION USING TEXT MESSAGING AMONG ENGLISH OR SPANISH-SPEAKING ADULTS Tracie C. Collins ${ }^{1}$; Maria Gabriela Valverde ${ }^{1}$; Kelsey Lu ${ }^{1}$; Mugur Geana ${ }^{2} .{ }^{1} \mathrm{KU}$ School of Medicine - Wichita, Wichita, MN; ${ }^{2} \mathrm{KU}$, Lawrence, KS. (Control ID \#2469756)

BACKGROUND: We sought to determine the feasibility and potential efficacy of increasing patient engagement with the dietary component of the NIH-funded Look AHEAD (Action for Health in Diabetes) by using text messaging with overweight/ obese adults, English or Spanish-speaking.

METHODS: We conducted a 6-week pilot study using a pre/post design, We adapted content from Look AHEAD, both individual and group sessions, and created a dietary guide which included a logbook for participants to record meals and daily caloric intake. Thirty adapted text messages, based on the content included within the Look AHEAD dietary intervention, were used for the study. Through a fully automated system specifically developed for this study, we delivered one text message per day for 5 days per week. During the baseline visit, participants completed informed consent and a validated medical history survey, an assessment of weight and height, and validated surveys to assess self-efficacy for eating fruits and vegetables (F\&V); fiber; fat; and self-efficacy for weight loss. Responses to the surveys were based on the Likert scale with maximal self-efficacy scores of 30; 40;25; and 99, respectively. The participant guide, surveys, and all text messages were translated from English to Spanish by a team of community members with expertise in translation. The protocol also included a 1-week supply of meal replacement bars from HMR, Inc. ( 2 per day for 5 days)

RESULTS: We enrolled 25 overweight or obese adults with one or more additional risk factors for atherosclerosis (i.e., age 70 years or older; or age 50 years or older with a current or past history of smoking; hypertension; diabetes mellitus; and/or hyperlipidemia). Among the 25 participants, the mean age was 60.4 years (SD 4.33 ); $76 \%$ were female. Racial/ethnic distribution was $40 \%$ non-Hispanic white, $56 \%$ Hispanic, and $4 \%$ African American; $48 \%$ were Spanish speaking. Among the 25 participants, $36 \%$ were overweight and $64 \%$ were obese. At baseline, mean self-efficacy scores for dietary habits and weight loss were as follows: F\&V 15.88 (SD 3.75); fiber 21.88 (SD 4.24); fat 13.48 (SD 3.74; and weight loss 55.12 (SD 9.68). Self-efficacy scores at 6 weeks were as follows: F\&V 25.2 (SD 2.92); fiber 34.28 (SD 3.25); fat 20.36 (SD 2.64); and weight loss 82.76 (SD 10.39) $P$-value $<0.0001$ for each change. 
CONCLUSIONS: This study demonstrates that using text messages as a component to increase participant engagement with weight loss protocols are a viable course of action. It also validated our ability to successfully translate the dietary component of Look AHEAD into text messages in both English and Spanish and successfully conduct this intervention. Our protocol will be implemented as part of a larger trial which, if efficacious, can be used to improve population health.

DEMYSTIFYING THE ASSOCIATION BETWEEN DEPRESSIVE SYMPTOMS AND CARDIOVASCULAR DISEASE IN BLACK AND WHITE ADULTS: THE REGARDS STUDY Nathalie Moise ${ }^{1}$; Yulia Khodneva ${ }^{2}$; Joshua S. Richman ${ }^{2}$; Daichi Shimbo ${ }^{1}$; Ian M. Kronish ${ }^{1}$; Monika Safford ${ }^{2} .{ }^{1}$ Columbia University Medical Center, New York, NY; ${ }^{2}$ University of Alabama at Birmingham, Birmingham, AL. (Control ID \#2457040)

BACKGROUND: Prior studies on the association between depression, which is a relapsing and remitting disease, and incident cardiovascular disease (CVD) have been limited by single measurements of depression at baseline, and few if any have assessed the effect of depression on incident coronary heart disease (CHD) compared to stroke, particularly in a large, biracial national cohort. In addition, prior studies suggest that racial and ethnic minorities face unique barriers to depression treatment in the United State, but research remains inconsistent as to whether race truly moderates the relationship between depression and CVD. We aimed to assess 1) whether time-varying depressive symptoms similarly confer increased risk of stroke and CHD while adjusting for multiple confounders in a large national cohort and 2) whether this effect is more pronounced in blacks compared to whites.

METHODS: Between 2003 and 2007, black and white participants ( $\geq 45$ years) of the REasons for Geographic and Racial Differences in Stroke (REGARDS) study without a history of CVD at baseline were recruited. Cox proportional hazard regression analyses were used to assess the association between time-varying (up to 3 measurements of) elevated depressive symptoms (4-item Center for Epidemiologic Studies Depression scale $\geq 4$ ) and incident CHD, stroke, and CVD death adjusting for demographic (age, gender, region, income, health insurance, education), physiological (blood pressure, cholesterol, medication, obesity, diabetes, kidney disease, C-reactive protein, QTc interval, atrial fibrillation and left ventricular hypertrophy), behavioral (smoking, alcohol, physical inactivity, medication adherence) and treatment risk factors (antidepressant use). The proportionality assumption was tested by assessing depressive symptoms*log of followup time interactions and was satisfied for all endpoints. Missing data in covariates were imputed using chained equation final estimates were derived by bootstrapping across the 5 imputed datasets. Analyses were conducted using SAS software version 9.4 (SAS Institute, Cary, NC) and STATA version 12 (STATA incorporated, College Station, TX)

RESULTS: Of the 22,663 eligible participants, 2267 (10.0\%) had elevated depressive symptoms at baseline (CES-D $\geq 4)$. The average age was $63.4 ; 58.8 \%$ were female, $41.7 \%$ black, $18.6 \%$ had diabetes, $32.7 \%$ were physically inactive, and $29.2 \%$ were nonadherent to their medication regimen. Depressed patients were more likely to be younger, female, black, have less than a high school education, have an income below $\$ 20,000$, be uninsured, have higher systolic blood pressure, higher total cholesterol, have high-density lipoprotein, be obese, have diabetes, have higher self-reported pack years, be physically inactive, report medication non-adherence, have higher C-reactive protein levels, use antihypertensive and finally have a prolonged QTc interval at baseline. Time-varying depressive symptoms were associated with fatal and nonfatal stroke $(\mathrm{HR}=1.26 ; 95 \% \mathrm{CI}$ 0.99-1.60) and CVD death (HR = 1.30; $95 \%$ CI 1.04-1.63), but were not associated with fatal and nonfatal CHD (HR $=1.11 ; 95 \%$ CI $0.89-1.38)$. Race did not moderate the association between depression and CVD.
CONCLUSIONS: Proximal depressive symptoms were associated with incident fatal and nonfatal stroke and CVD death even after controlling for multiple risk factors. This is one of few large cohort studies to use multiple measurements of depression to examine the timing of the effect of depressive symptoms on CVD and to more definitively clarify that race does not appear to moderate this relationship, which had heretofore been inconsistently shown in the literature. This study further supports the urgent need to treat depression in a timely matter, even in patients without a history of CVD. Understanding these population level associations is key to guiding treatment in the primary care setting, where depression is not often treated as effectively as other independent CVD risk factors.

DENSE BREAST NOTIFICATIONS: VARYING CONTENT, READABILITY, AND UNDERSTANDABILITY BY STATE Nancy R. Kressin ${ }^{1,2}$; Christine M. Gunn ${ }^{2}$; Tracy A. Battaglia ${ }^{2} .{ }^{1}$ VA Boston Healthcare System, Boston, MA; ${ }^{2}$ Boston University School of Medicine, Boston, MA. (Control ID \#2468647)

BACKGROUND: Along with their screening mammogram results, women in 23 states now also receive notifications of breast density. Dense breasts can mask cancer on mammography (masking bias), and are an independent cancer risk factor, but evidence does not yet indicate whether or what supplemental screening is appropriate. Rather, risk stratification is proposed to determine who may benefit from supplemental screening. Dense breast notification (DBN) text may affect women's ability to understand their message. Thus, we examined DBN characteristics across states, to inform future policy. METHODS: We compared the content, readability, and understandability of DBNs across states, noting the mention of supplemental screening, masking bias, density as a cancer risk factor, and required recipients. We measured readability using the FleschKincaid reading level in MS Word and the Dale-Chall readability score, and assessed understandability using the Patient Education Materials Assessment Tool. We obtained the proportion of adults in each state lacking basic prose literacy skills from available statistics, comparing DBN readability with state population literacy level.

RESULTS: Most states $(n=19,83 \%)$ mandate specific language; while 4 (17\%) only mandate required components. Seven states $(30 \%)$ require a generic DBN for every woman receiving a screening mammogram (others only require notification to those with dense findings). All DBNs mention masking bias, 17 (74 \%) mention the association with increased cancer risk, 21 (91\%) mention 'supplemental screening' as an option (using at least that term), advising women to consult their physician. Of 17 states requiring specific language regarding supplemental screening, 6 (35\%) inform women that they might benefit from such screening; 4 mention specific modalities. Flesch-Kincaid readability levels ranged from grades 7-19.4 (mean: 11.1), most exceeding the recommended literacy level (grades 7-8); about $20 \%$ of the population reads below a grade 5 level. Dale-Chall readability scoring produced slightly higher scores overall (range: grades 9-10-13-15). All DBNs scored poorly on understandability (PEMAT; range: 11-33\%). There was widespread discordance between states' DBN readability and corresponding basic literacy levels. Only 3 states' DBN literacy level fell <8th grade; some of the highest DBN readability levels occurred where state literacy levels are lowest.

CONCLUSIONS: We found wide variation in 23 states' DBN content, with generally poor readability and understandability, and discordance with states' average literacy. Such incomprehensible patient notifications may create uncertainty for women attempting to make personalized decisions about supplemental screening, leading to increased supplemental screening utilization for higher literacy populations, which has the potential to further exacerbate existing disparities in breast cancer diagnosis and outcomes. These findings add to other expressed concerns regarding DBN reporting laws. Inconsistencies in DBN content may reflect the lack of evidence regarding supplemental screening, and may confuse women comparing health information across state lines (e.g., sisters with similar anatomy receiving different information). Many DBNs appropriately encourage 
discussions and shared decision making between patients and doctors. However, this alone is insufficient to produce shared decision making, enable time-pressured physicians to explain results and conduct personalized risk assessments, or provide clinical decision support to do so. Given the generally poor readability of the current DBNs, and incompatibility with documented literacy of the general population, efforts should focus on enhancing the understandability of such messages, so that all women are clearly and accurately informed about their density status, its impact on their breast cancer risk, and the harms and benefits of supplemental screening.

\section{DEPRESSION CARE MANAGEMENT: RESULTS FROM THE SECOND YEAR} OF A COLLABORATIVE CARE MODEL IN AN URBAN ACADEMIC PRIMARY CARE CLINIC Glennis M. Rodriguez ${ }^{1}$; Iris Huang ${ }^{1}$; Susan Truong ${ }^{2}$; Lauren Peccoralo ${ }^{1}$. ${ }^{1}$ Mount Sinai, New York, NY; ${ }^{2}$ Jefferson, Philadelphia, PA. (Control ID \#2469170)

BACKGROUND: Despite the high prevalence of major depressive disorders in primary care patients, primary care providers cite limited access to mental health services and providers as barriers to providing optimal depression care. Collaborative care models were designed to address this growing need. The model consists of interventions that utilize non-physician care managers working closely with primary care physicians and psychiatrists. Interventions vary from development of care management plans that improve medication adherence to multifaceted models involving intensive management in psychotherapy programs. While the success of these models has been shown in numerous clinical settings, little is known about their impact in academic training clinics where primary care providers range in experience from trainees to attendings. The purpose of this study is to determine whether the implementation of a collaborative care program improves depression outcomes for patients in an urban academic primary care clinic and whether program outcomes are sustainable over time.

METHODS: Patients at the Internal Medicine Associates (IMA) practice at the Mount Sinai Medical Center meeting the following criteria are invited to participate in the Depression Care Management Program: age over 18, clinically significant depression as measured by a Patient Health Questionnaire 9 (PHQ9) score greater than 10 and meeting DSM IV criteria for major depressive disorder. The program consists of universal depression screening, bi-weekly to monthly sessions with a behavioral health social worker trained in Problem Solving Therapy, Interpersonal Therapy, Motivational Interviewing and Behavioral Activation Therapy. Symptoms are measured by PHQ9 questionnaires at each follow up visit and the end of treatment. New and complex cases are reviewed weekly with two primary care physicians and a psychiatrist consultant. Recommendations and updates regarding patients' statuses are relayed back to their primary care providers. Success of the program was assessed with the following outcomes: depression screening rates, positive screens, depression diagnoses, program referrals, program enrollments and PHQ9 improvement rates. Descriptive data from years 1 and 2 of the program were compared. Because of a transition of care managers in the program, year 1 was analyzed as July 2013 to June 2014 and year 2 as January 2015 to September 2015. Initial and follow up PHQ9 scores were compared using a paired T-test. RESULTS: During the first year of the program, screening rates improved from $22 \%$ in July 2013 to $72 \%$ in June 2014. From January to June 2014, 7076 patients were screened for depression (72\% screening rate), with 1802 screening positive by PHQ2 (25\%) and 648 diagnosed with depression ( $9 \%$ ). Five hundred twenty-seven patients were referred to the program and 124 patients enrolled. Data was available for 115 patients by the end of the study period. Seventy-five patients $(60 \%)$ were in active treatment and $49(40 \%)$ completed treatment and were in relapse prevention. The mean initial PHQ9 score was 13.5 (SD 3.7) and the mean follow up PHQ9 score was 7.8 (SD 4.1) for a significant decrease in PHQ9 score of $5.7(p<0.001)$. Eighty-two percent of patients had an improvement in PHQ9 score. Forty percent had $>50 \%$ reduction in PHQ9 score and $60 \%$ had PHQ9 score $<10$. During the second year of the program, from January to September 2015, 9723 patients were screened for depression ( $84 \%$ screening rate), with 2009 screening positive by PHQ2 (21 \%) and 562 diagnosed with depression (6\%). From January to November 2015, 233 patients were referred to the program and 147 patients enrolled. By September 2015, 66 patients (45\%) were in active treatment, $11(1 \%)$ were in relapse prevention and $70(48 \%)$ were discharged. Of the discharged patients, 49 (70\%) were discharged due to multiple no shows to appointments. The mean initial PHQ9 score was 16 and the mean follow up PHQ9 score was 12.4 for a significant decrease in PHQ9 score of 3.6 ( $p<0.001$, CI 2.49-4.46). Sixty-seven percent of patients had an improvement in PHQ9 score. Twenty-eight percent had $>50 \%$ reduction in PHQ9 score and $27 \%$ had PHQ9 score $<10$. Thirty-one percent of patients either had $>50 \%$ reduction in PHQ9 score or PHQ9 score $<10$.

CONCLUSIONS: Patients in the Depression Care Management Program improved in their depression symptoms as demonstrated by decreases in PHQ9 scores. In year 2, fewer patients had clinically significant improvements in PHQ9 scores, which may be due to the large number of patients discharged because of no shows. Also, some patients may not have been in the program long enough to experience symptom improvement. Nonetheless, the improvements in PHQ9 scores in year 2 demonstrate sustainability of the program. These results demonstrate that collaborative care models for depression treatment can be successfully applied and sustained in urban academic primary care settings. Further research is needed to determine the program's long term effects on patients' mental health outcomes as well as the impact on patients' other medical comorbidities.

DERIVATION AND VALIDATION OF A BLEEDING RISK SCORE FOR ELDERLY PATIENTS WITH VENOUS THROMBOEMBOLISM Eva Seiler ${ }^{8}$; Andreas Limacher ${ }^{7}$; Marie Méan ${ }^{8,}{ }^{9}$; Juerg-Hans Beer ${ }^{10}$; Joseph J. Osterwalder ${ }^{6}$; Beat Frauchiger $^{4}$; Marc Righini ${ }^{5}$; Markus Aschwanden ${ }^{3}$; Christian M. Matter ${ }^{2}$ 11; Marc Husmann $^{2}$; Martin Banyai ${ }^{1}$; Nils Kucher ${ }^{8}$; Daniel Staub ${ }^{3}$; Bernhard Lämmle ${ }^{8}$ 12; Anne Angelillo-Scherrer ${ }^{8}$; Nicolas Rodondi ${ }^{8}$; Drahomir Aujesky ${ }^{8} .{ }^{1}$ Cantonal Hospital of Lucerne, Lucerne, Switzerland; ${ }^{2}$ University Hospital Zurich, Zurich, Switzerland; ${ }^{3}$ Basel University Hospital, Basel, Switzerland; ${ }^{4}$ Cantonal Hospital of Frauenfeld, Frauenfeld, Switzerland; ${ }^{5}$ Geneva University Hospital, Geneva, Switzerland; ${ }^{6}$ Cantonal Hospital of St. Gallen, St. Gallen, Switzerland; ${ }^{7}$ Institute of Social and Preventive Medicine, University of Bern, Bern, Switzerland; ${ }^{8}$ Bern University Hospital, Bern, Switzerland; ${ }^{9}$ Lausanne University Hospital, Lausanne, Switzerland; ${ }^{10}$ Cantonal Hospital of Baden, Baden, Switzerland; ${ }^{11}$ University of Zurich, Zurich, Switzerland; ${ }^{12}$ University Medical Center, Mainz, Germany. (Control ID \#2463577)

BACKGROUND: Current guidelines recommend extended anticoagulation ( $>3$ months) in patients with acute venous thromboembolism (VTE) who have a high risk of recurrence. However, the benefits must be weighted against the risk of prolonged anticoagulant treatment, especially in elderly patients who have an increased bleeding risk and for whom no accurate tools exist to quantify the risk of bleeding. The goal of our work was to develop a clinical prognostic score that accurately identifies elderly patients with VTE who are at high risk of bleeding when receiving extended anticoagulation.

METHODS: This prospective cohort study included 743 patients aged $\geq 65$ years from 9 Swiss hospitals who received extended anticoagulation ( $>3$ months) with vitamin $\mathrm{K}$ antagonists for acute VTE between September 2009 and December 2013. Potential predictor variables comprised 19 previously described clinical predictors of bleeding. The outcome was the time to a first major bleeding up to 36 months of follow-up. We derived our score using competing risk regression, accounting for non-bleeding related deaths as a competing event. We used backward selection to identify bleeding risk variables, retaining variables with a $P$-value $<0.2$. We converted beta-coefficients of the variables retained into integer point 
scores and classified patients into 3 categories of increasing risk of major bleeding (low, moderate, high). We assessed discrimination and calibration of the score using the C-statistic and the Gronnesby and Borgan goodness-of-fit test, respectively. We internally validated the score using bootstrapping.

RESULTS: Our final score consisted of 7 routinely available variables: active cancer, low physical activity level (mostly sitting or lying, or avoid climbing stairs or carrying weight $>5 \mathrm{~kg}$ ), history of major bleeding within the first 3 months of anticoagulation, anemia (hemoglobin $<13 \mathrm{~g} / \mathrm{dl}$ in men and $<12 \mathrm{~g} / \mathrm{dl}$ in women), low platelets $(<150,000 / \mathrm{mm} 3)$, use of platelet inhibitors/NSAIDs, and $<30 \%$ of INR values in the therapeutic range (2.0-3.0) after the initial 3 months of anticoagulation. Overall, $48 \%$ of patients were classified as lowrisk ( $0-1$ points), $37 \%$ as moderate-risk ( $2-3$ points), and $15 \%$ as high-risk of major bleeding ( $\geq 4$ points). The incidence rate of major bleeding was 1.4 events/100 patient-years for low-risk patients, 5.0 events/100 patient-years for moderate-risk patients, and 12.2 events/100 patientyears for high-risk patients. The C-statistic was 0.72 (95\% CI 0.65-0.78) in the derivation sample and did not markedly change in the internal validation sample $(0.68,95 \% \mathrm{CI} 0.61$ $0.74)$. The calibration of the score was good $(P=0.93)$.

CONCLUSIONS: This simple clinical score identified $15 \%$ of patients with VTE who have a high risk of major bleeding and who potentially may not benefit from extended anticoagulation. Independent external validation is important prior to the clinical application of this score.

DERIVING A CLAIMS-BASED FRAILTY INDICATOR WITH THE GOLD STANDARD MEASURE Jodi B. Segal ${ }^{1,2}{ }^{1}$ Hsien-Yen Chang ${ }^{1}$; Yu Du ${ }^{1}$; Ravi Varadhan ${ }^{1}$ 2 . 'Johns Hopkins University Bloomberg School of Public Health, Baltitmore, MD;

${ }^{2}$ Johns Hopkins University School of Medicine, Baltimore, MD. (Control ID \#2466781)

BACKGROUND: Fried et al., in 2001, described a frailty phenotype measured in older adults enrolled in the Cardiovascular Health Study (CHS). This phenotype is manifest when 3 of the following 5 conditions exist: low grip strength, low energy, slowed waking speed, low physical activity, and unintentional weight loss. The CHS frailty phenotype is the most widely accepted definition of frailty. These measures, however, are often unavailable in many types of existing data. We developed a claims-based frailty indictor that is based on readily available administrative information, and that is strongly predictive of the measured frailty phenotype.

METHODS: This was a retrospective cohort study. We used the CHS cohort data linked to Medicare claims. We reviewed published literature that identified frail individuals with a mixture of clinical, administrative, and measured data. We prioritized a candidate set of 40 diagnoses that could be operationalized with claims data alone, and which we expected to correlate with measured frailty. The reference standard was the frailty phenotype as measured in the CHS study, at visits 5 and 9. Using the inpatient and outpatient billing data from the 6 months preceding the study visit, we identified claims for these 40 diagnoses. With alternative methods, including penalized logistic regression, logic regression, and random forest; we identified the optimal set of codes for predicting frailty which provides a good trade-off of positive (PPV) and negative predictive values (NPV). The modeling included internal validation.

RESULTS: There were 5888 CHS cohort participants from 4 clinical sites. Eightyfour percent were white, $58 \%$ were women and their mean age was 72 years at enrollment. Approximately $10 \%$ of the cohort was frail at visits 5 and 9 . Of the 40 diagnoses, those most strongly associated with measured frailty in bivariate analyses were claims in the past 6 months for congestive heart failure, cognitive impairment, delirium, paranoia, impaired mobility, nutritional deficiency, stroke, depression, and falls; each with unadjusted odds ratio exceeding 2.5 (with $p<0.0001$ ). The Deyomodified Charlson comorbidity index was also strongly associated with of frailty $(p<.00001)$. The penalized logistic model had an area under the AUC curve of 0.74; with a cutoff of 0.5 , it had a LR + of 2.2 and a LR - of 0.5 and classified $6 \%$ of the sample as frail.

CONCLUSIONS: Against a validated reference standard, we demonstrated that claims data alone can be used to classify individuals as frail and non-frail. Immediate next steps include validity testing to assure that the claims-based frailty indicator predicts outcomes in this dataset. We have planned external validation using the National Health and Aging Study linked to Medicare claims. The claims-based frailty indicator can be used in research with large datasets for confounding adjustment or for exploration of heterogeneity of treatment effect. The indictor may be useful as well for emergency preparedness planning as it will allow identification, with Medicare data alone, of regions enriched with frail individuals.

DESCRIBING THE LEMIERRE SYNDROME - A PATIENT SURVEY Robert M. Centor; Alexus B. Perry. UAB, Huntsville, AL. (Control ID \#2465792)

BACKGROUND: The Lemierre Syndrome (as Dr. Lemierre described in 1936) presents as a sore throat sequel primarily in adolescents and young adults. The syndrome follows the sore throat onset after approximately 5 days. Patients develop suppurative internal jugular thrombophlebitis, which leads to septic emboli (most commonly septic pulmonary emboli). Septicemia and shock often accompany this syndrome. Previous studies have estimated $5 \%$ mortality in the antibiotic era. This relatively rare complication of a common episodic care presentation could influence whether we should consider empiric antibiotics for some sore throat patients in the commonly affected age group. Because of the syndrome's rarity, we have no data describing the patient's experience with this syndrome.

METHODS: Anonymous volunteers responded to a survey request placed on the Facebook group -"Lemierre's Syndrome Fighters and Survivors". Responders answered questions using the survey program REDCap. The survey included questions concerning demographics, presentation of illness, hospital course, management, and complications. The results include all completed surveys done during a 2-month period.

RESULTS: Eighty-eight volunteers completed the survey. Demographically, the respondents included $77 \%$ women and $90 \%$ Caucasian. Their average age was 24 (range 15-59) with a median age of 20 . They come from 8 countries, with 60 from the USA, 13 from the UK, 6 Canada, 3 Netherlands and 1 each from Norway, Germany, Sweden and Australia Prior to their hospital admission, $60 \%$ had had a rapid antigen strep test-all were negative. $36(41 \%)$ had received a variety of empiric antibiotics (1 cephalosporin, 6 penicillins, 2 clindamycin, 1 tetracycline, 17 macrolides, 7 unknown). Only $21 \%$ noted any improvement after their initial visit (which were predominantly with primary care physicians or family physicians). Their admission presentation included worsening sore throats and $90 \%$ reported rigors. Sixty-eight percent had unilateral and $19 \%$ had bilateral neck swelling. Seventeen percent percent reported that they also had infectious mononucleosis. These patients had long hospital stays - mean 25 days and median 16.5 days. Seventy-four percent required intensive care, with an average of 7.4 days in the ICU overall and 10.9 days on average for those who required intensive care. Their reported complications included, $82 \%$ with lung involvement, $9 \%$ had brain involvement and many reported lasting psychological burdens. Sixty percent reported having septic shock. Seventy-eight percent of the patients reported a positive blood culture with $90 \%$ growing Fusobacterium necrophorum. Ninety percent required either a PICC line or a central line. Ninety percent received antibiotics after discharge for an average of 40 days (median 30 days). Sixty percent of those receiving antibiotics received them intravenously. Seventy-one percent received prolonged anticoagulation.

CONCLUSIONS: While rare, the Lemierre Syndrome has a profound patient impact - financial, physical, and emotional burden. This survey gives some insights on how patients perceive this syndrome. The survey also defines several management unknowns including choice of appropriate antibiotics, duration of antibiotic therapy, and whether to 
anticoagulate these patients. Given the complexity and severity of this syndrome, we should strive to develop prevention strategies for this rare complication of a common symptom, sore throat.

\section{DETECTION OF PREDIABETES AND ITS PROGRESSION TO DIABETES: A} POPULATION BASED STUDY Ramona S. DeJesus; Jennifer St. Sauver; Debra Jacobson; Patrick Wilson. Mayo Clinic, Rochester, MN. (Control ID \#2465546)

BACKGROUND: Thirty seven percent of US adults 20 years and older has pre-diabetes. Our primary care clinics serve over 6000 diabetic patients but the prevalence of prediabetes is unknown and time of progression to diabetes is unclear. It is imperative to identify both so interventions can be proactively initiated to prevent diabetes and optimize risk reduction. In this retrospective cohort study, we determined the prevalence of prediabetes among primary care empanelled patients and estimated rate of progression to diabetes at 1,5 and 10 years.

METHODS: Records of adult community dwelling patients 20 years or older empanelled in a primary care practice in Jan.2005, who met criteria for pre-diabetes based on either hemoglobin A1c or fasting blood sugar, were reviewed to identify prevalence. Incidence rates at 1,5 and 10 years were determined. Modified population attributable risk (PAR) statistics to estimate the impact of reducing fasting glucose on incidence (progression) of diabetes was applied.

RESULTS: Of 66,510 paneled patients who had at least one appropriate lab test (FBS or A1C), $16.2 \%(n=10,786)$ had pre-diabetes. Forty percent were 65 years and older; $80 \%$ were either overweight or obese. At 1 year, number of new diabetics was 404 (3.7\%); at 5 years, it jumped to $17 \%$ and at 10 years, $25.5 \%$ of the cohort had diabetes. The prevalence of patients with baseline glucose of 110-119 is $2743(25.8 \%)$ vs. 633 (6.0\%) of patients with baseline glucose $>=120$ : $633(6.0 \%)$. PAR for a 10 unit reduction in the 110-119 group with $50 \%$ treatment adherence was $\sim 15 \%$; if $50 \%$ of this group is able to lower FBS by 10 units, it is estimated that diabetes incidence would be reduced by $15 \%$. In contrast, PAR for a 10 unit reduction in the $\Rightarrow>120$ group with $50 \%$ adherence was $\sim 6 \%$; if $50 \%$ of this group is able to lower FBS by 10 units, it is estimated that diabetes incidence would be reduced by only $6 \%$. [Figure 1]

CONCLUSIONS: In this cohort, the likelihood of progression from prediabetes to diabetes appears to increase over time; at 5 years, $17.1 \%$ had diabetes. PAR statistics suggest that the greatest reduction in new cases of diabetes may be achieved by targeting interventions among those with fasting glucose between 100 and 119 .
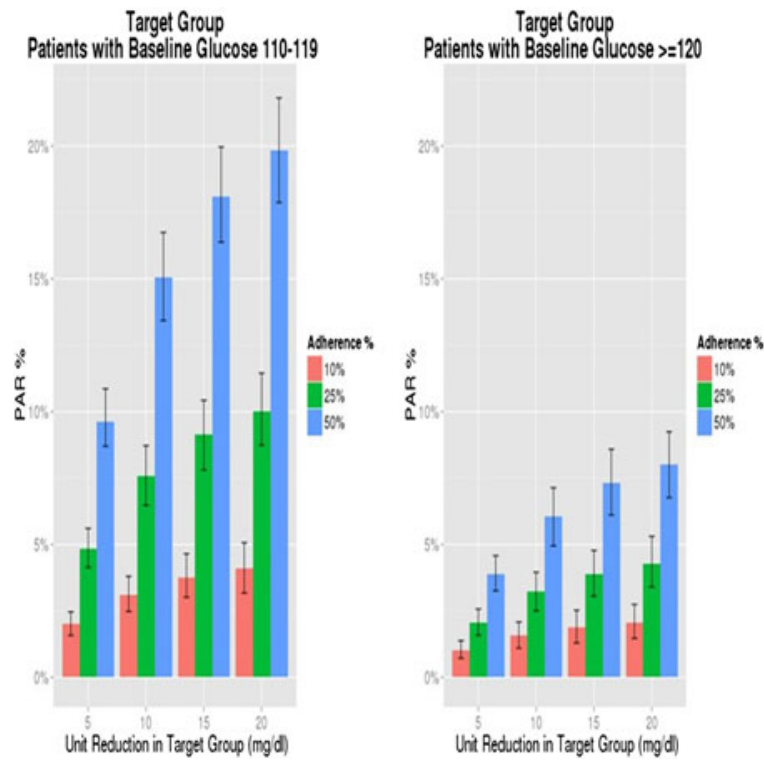

DETERMINING MAXIMUM ACHIEVABLE LIFE EXPECTANCY: A MATHEMATICAL MODELLING APPROACH Elizabeth R. Stevens ${ }^{2,}{ }^{3}$; Glen B. Taksler ${ }^{1}$; Qinlian Zhou ${ }^{2}$; Kimberly Nucifora ${ }^{2}$; R. Scott Braithwaite ${ }^{2} .{ }^{1}$ Cleveland Clinic, Cleveland, $\mathrm{OH} ;{ }^{2} \mathrm{New}$ York University School of Medicine, New York, NY; ${ }^{3}$ New York University College of Global Public Health, New york, NY. (Control ID \#2465510)

BACKGROUND: Little is known about the maximum achievable life expectancy for a population given its unique characteristics, which is important for estimating actionable population health metrics such as potential life-years lost. Our objective is to address the question: Given the current state of health science and technology, what could be the average life expectancy in the United States in the idealized scenario in which modifiable risk factors were eliminated and adherence to evidence-based therapies was perfect.

METHODS: We developed a Monte Carlo microsimulation model that explicitly represented 19 conditions representing the top causes of mortality in each age decile and the 28 risk factors associated with their onset that had consistent directions of effect as well as clinical and statistical significance. Risk factors were grouped by their likelihood of causality using the Grading of Recommendations Assessment, Development and Evaluation (GRADE). Each month individuals can randomly develop new risk factors and/or new conditions, have existing risk factors or conditions resolve (e.g. through treatment), or die. We simulated a birth cohort of one million patients with characteristics resembling the population of the United States. We then compared current health with an idealized scenario in which all modifiable risk factors were eliminated and adherence to evidence-based therapies was perfect.

RESULTS: We estimated that the maximum life expectancy in the United States would be 84.3 years (an increase of 5.5 years) in the idealized scenario in which modifiable risk factors were eliminated and adherence to evidence-based therapies was perfect. Life expectancy for men increased from 76.4 to 82.1 years, and life expectancy for women increased from 81.3 to 86.5 years. The proportion of men surviving past ages 65,75 , and 85 increased from 84.3 to $86.7 \%, 69.3$ to $75.3 \%$, and 41.5 to $51.6 \%$, respectively. The proportion of women surviving past ages 65,75 , and 85 increased from 84.3 to $91.6 \%, 69.3$, to $83.4 \%$ and 41.5 to $64.1 \%$, respectively. Considering only those modifiable risk factors with greater evidence of causality lessened the life expectancy gain to 81.5 years (an increase of 2.7 years) and 80.1 years (an increase of 1.3 years) for level of evidence (GRADE scores) $\geq \mathrm{B}$ and A, respectively. Limitations include only considering the top causes of mortality.

CONCLUSIONS: The highest achievable life expectancy in the United States given current state of health science and technology is 84.3 years, an increase of 5.5 years above current life expectancy. This estimate can inform efforts to improve population health.

\section{Maximum Achievable life-Expectancy by Evidence level}

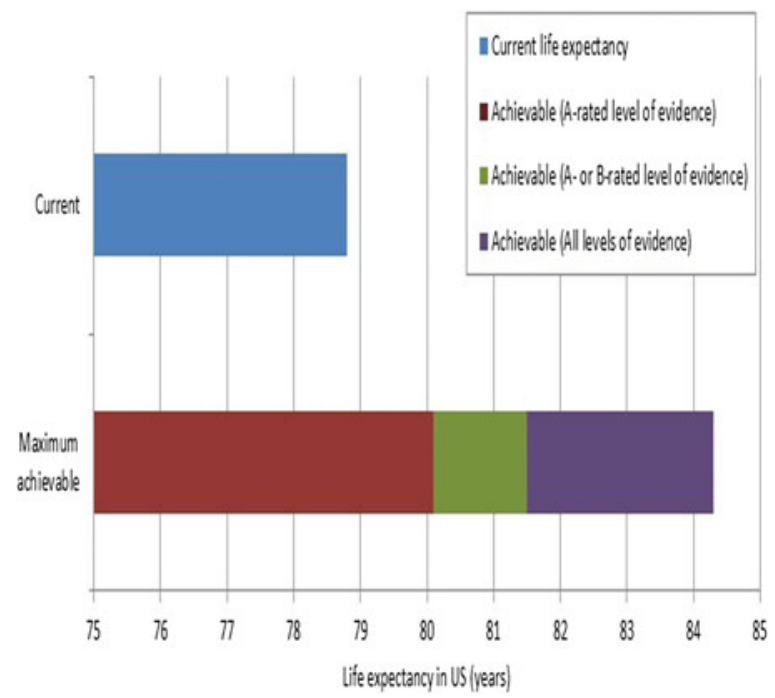

Maximum achievable life expectancy given the use of level of evidence filters 


\section{DETERMINING PREVENTABLE READMISSIONS IN AN URBAN ACADEM-}

IC MEDICAL CENTER Kayley M. Ancy ${ }^{1}$; Zaid I. Almarzooq ${ }^{1}$; Parimal A. Patel ${ }^{1}$; Michelle E. Unterbrink ${ }^{2}$; Jim Brian I. Estil ${ }^{3}$; Jennifer I. Lee ${ }^{1} .{ }^{1}$ NewYork-Presbyterian Hospital/Weill Cornell Medical Center, New York, NY; ${ }^{2}$ Weill Cornell Medical College, Brooklyn, NY; ${ }^{3}$ Weill Cornell Medical College, New York, NY. (Control ID \#2466498)

BACKGROUND: Causes for readmissions are multifactorial and poorly understood, making preventability difficult to define. In a previously completed study, health care providers and caregivers (collectively defined as the patient care circle) identified several issues (minor themes) contributing to readmissions on the general medicine service that we classified into five major themes: healthcare teamwork (T), illness severity and health needs (I), medication-related issues (M), psychosocial stability $(\mathrm{P})$ and health systems management support (S). In this phase of our study, our objectives were to measure (1) agreement within the patient care circle (PCC) on the themes contributing to readmissions of general medicine patients and (2) perception on whether contributing themes were preventable.

METHODS: General medicine patients with same-site 30-day readmissions were randomly identified through a centralized database programmed to flag patients at the time of their readmission. Each patient was used to define one PCC and we identified all relevant members (family member or other primary care giver, floor nurses, emergency department physicians, inpatient attendings, primary care physicians and social workers) to complete the PCC for each patient included in the study. The survey was designed to assess each respondent's agreement about themes contributing to the readmission of a specific patient and contained 27 statements. Each survey statement represented a minor theme identified in our earlier study and was coded to correspond to one of the five major themes. Patients and family member/care givers were asked if the issues (1) affected the patient's prior hospitalization and (2) how strongly they perceived it contributed to the readmission. Providers were asked if the issues (1) contributed to the patient's readmission and (2) were preventable. During the pilot (June-December 2015) we interviewed 12 patients as well as members of their respective PCCs. All providers were combined for provider data analysis. Data were included for only those care circles that included at least one provider interview resulting in ten PCCs included in the final analysis (10 patient surveys and 29 provider surveys). Patient data were analyzed by ranking frequency of agreement for each survey item. The percentage of those reporting they felt an issue contributed to the patient's readmission was determined for each item. Provider responses were clustered by PCC. If at least one provider identified an issue as either (1) a problem or (2) preventable, the responses were reported as identified by the PCC.

RESULTS: The highest reported issue associated with readmission by both patients and care circles was within the theme of illness severity — patients felt unable to manage their medical problems at home and were more comfortable returning to the hospital. Seven patients (70 \%, CI 42-98 \%) agreed with this statement and six of these seven patients ( $86 \%$, CI $60-100 \%)$ felt it contributed to their readmission. In addition, eight PCCs ( $80 \%$, CI $55-100 \%$ ) had at least one provider identify this as a problem but only two of these eight believed it was preventable (25\%, CI $15-55 \%)$.

CONCLUSIONS: Our study was designed to help identify the estimated importance and preventability of previously identified major and minor themes contributing to readmissions. Agreement was noted on only one of the top five issues identified by both patients and providers. Issues relevant to patients focused on the transition period and lack of resources to navigate the healthcare system, supporting the need for further understanding of barriers faced after hospital discharge.
Top 5 Patient Responses $(n=10)$

\begin{tabular}{|c|c|c|}
\hline Survey Item & $\begin{array}{l}\text { \# Reported as a } \\
\text { Problem }\end{array}$ & $\begin{array}{l}\text { \# Reported as } \\
\text { Contributed }\end{array}$ \\
\hline $\begin{array}{l}\text { Unable to manage medical problems at home } \\
\text { (I) }\end{array}$ & 7 & 6 \\
\hline $\begin{array}{l}\text { Unable to go to appointments after discharge } \\
\text { (S) }\end{array}$ & 5 & 3 \\
\hline $\begin{array}{l}\text { Hospital care team not coordinated with care } \\
\text { plan (T) }\end{array}$ & 3 & 3 \\
\hline Family feels safer with me in the hospital (I) & 3 & 2 \\
\hline Follow-up appointments not arranged (S) & 3 & 2 \\
\hline
\end{tabular}

Top 5 Care Circle Responses $(n=10)$

\begin{tabular}{lll}
\hline \hline Survey Item & \# Reported as a & \# Reported as \\
& Problem & Preventable \\
Unable to manage medical problems at home & 8 & 2 \\
(I) & & \\
Feels safer in hospital (S) & 6 & 3 \\
Medical team did not answer all questions (T) & 5 & 5 \\
$\begin{array}{l}\text { Did not know who to contact after discharge (I) } \\
\text { Home medications not continued during }\end{array}$ & 5 & 4 \\
admission (S) & 5 & 4 \\
\hline
\end{tabular}

DETERMINING THE OPTIMAL VACCINATION SCHEDULE FOR HERPES ZOSTER: A COST-EFFECTIVENESS ANALYSIS Phuc H. Le; Michael B. Rothberg. Cleveland Clinic, Cleveland, OH. (Control ID \#2468995)

BACKGROUND: The Advisory Committee on Immunization Practices (ACIP) recommends that a single dose of the live attenuated $\mathrm{HZ}$ vaccine be administered for all adults aged $\geq 60$ years. However, a recent study suggests that efficacy of the vaccine declines to zero over 10 years which would result in a majority of adults lacking protection after age 70 when PHN is most prevalent. A booster dose administered after 10 years might extend protection, but the cost-effectiveness of a booster strategy has not been examined. We conducted a cost-effectiveness analysis to determine the optimal schedule to administer herpes zoster (HZ) vaccine among immunocompetent adults aged 60 years.

METHODS: We built a Markov model to follow patients over their life-time. From the societal perspective, we compared costs and quality-adjusted life years (QALY) saved for 11 strategies: no vaccination, one-time vaccination at 5 -year intervals from 60 to 80 years, vaccination at 5-year intervals from 60 to 75 years with a booster after 10 years, and vaccination at 60 years with 2 booster doses 10 years apart. The entire cohort entered the model in the 'Healthy' state at age 60 and moved between health states with transition probabilities based on published literature. The model considered cases of HZ, PHN, and other complications of HZ (ophthalmic and otic complications, hospitalization and death). Compared to the unvaccinated group, vaccinated patients had a reduction in disease incidence and complications proportional to vaccine efficacy, which waned over time. Following a booster, vaccine efficacy increased and then waned at the same rate as following initial vaccination. Model inputs were derived primarily from US-based studies to reflect the epidemiology, utilities, and quality-adjusted life years (QALYs) of a general US population. Vaccine efficacy was based on the Shingles Prevention Study and its longterm follow up data. Costs, which were drawn from the medical literature, included both direct medical costs and indirect costs due to lost productivity. All costs were expressed in 2014 US dollars.. Outcomes included costs and effectiveness (the number of HZ cases, PHN cases, and QALYs) for each strategy. Results were presented as incremental costeffectiveness ratio (ICER) per QALY saved. Both costs and QALYs were discounted at $3 \%$ per year. Probabilistic sensitivity analysis with 10,000 Monte Carlo iterations was performed. 
RESULTS: At a \$100,000/QALY threshold, "vaccination at 70 plus one booster" was the most cost-effective strategy, with an incremental cost-effectiveness ratio (ICER) of \$41,774/QALY. All single dose and other one-booster strategies were dominated. "Vaccination at 60 plus 2 boosters" was the most effective strategy, but had an ICER of $\$ 160,785 /$ QALY. In deterministic sensitivity analysis, "vaccination at 60 plus 2 boosters" cost $<\$ 100,000 / \mathrm{QALY}$ if compliance rate was $>68 \%$, vaccine cost was $<\$ 152$ per dose, or the booster was more effective than the initial dose. In probabilistic sensitivity analysis, "vaccination at 70 plus one booster" was most likely to be preferred at a willingness-topay of up to $\$ 140,000 /$ QALY.

CONCLUSIONS: Based on currently available information, initiating $\mathrm{HZ}$ vaccine at age 70 years with one booster dose 10 years later appears optimal.

\section{DETERMINING THE THRESHOLD FOR VENOUS THROMBOEMBOLISM} PROPHYLAXIS FOR HOSPITALIZED MEDICAL PATIENTS: A COSTEFFECTIVENESS ANALYSIS Phuc H. Le; Michael B. Rothberg. Cleveland Clinic, Cleveland, OH. (Control ID \#2468997)

BACKGROUND: Venous thromboembolism (VTE) is a common preventable condition in hospitalized medical patients. Chemoprophylaxis with heparin or fondaparinux has been recommended for all hospitalized patients who are not at low risk, and appropriate thromboprophylaxis is a hospital quality measure. However, the specific probability of VTE that requires prophylaxis has not been defined. In addition, the threshold may be different for patients at high risk of bleeding or with short life expectancies. To determine a threshold for prophylaxis, we conducted a cost-effectiveness analysis comparing prophylaxis with low-molecular-weight heparins (LMWHs) versus no prophylaxis in hospitalized adult medical patients. We also considered the impact of bleeding risk and life expectancy on the threshold.

METHODS: We constructed a decision model consisting of two consecutive modules: a simple decision-tree that followed patients up to 3 months after hospitalization, and a lifetime Markov model with 3-month cycles. The model tracked symptomatic deep vein thromboses and pulmonary emboli, bleeding events and heparin-induced thrombocytopenia. Long-term complications included recurrent VTE, post-thrombotic syndrome, and pulmonary hypertension. Compared to the no-prophylaxis group, patients in the prophylaxis group experienced fewer VTEs and more bleeding events proportional to the efficacy of LMWHs. For the base-case analysis, we considered a patient similar to those in clinical trials of VTE prophylaxis: age 72.5 years, life expectancy of 9 years, and a $2.2 \%$ risk of VTE during hospitalization. Model inputs were derived primarily from US-based studies to estimate transition probabilities, utilities, and quality-adjusted life years (QALYs). The efficacy and bleeding complications of LMWH were based on a meta-analysis of randomized trials. Costs drawn from medical literature included both direct medical costs and indirect costs due to lost productivity and were expressed in 2015 US dollars. The study was conducted from the societal perspective. Costs and QALYs were discounted at $3 \%$ /year. After calculating the incremental cost-effectiveness ratio (ICER) per QALY saved for the base case, we conducted a series of sensitivity analyses to determine the threshold for VTE prophylaxis assuming a willingness-to-pay of $\$ 100,000 / \mathrm{QALY}$.

RESULTS: In the base-case, prophylaxis with LMWHs had an ICER of $\$ 35,974$ / QALY saved compared to no prophylaxis. In sensitivity analysis, prophylaxis would cost $>\$ 100,000 /$ QALY if VTE risk was $<1.22 \%$, bleeding risk was $>13.4 \%$, life expectancy was $<3.75$ years or the cost of LWMH exceeded $\$ 98$ / dose. If VTE risk was $<0.31 \%$ or bleeding risk was $>42.8 \%$, the harms of prophylaxis outweighed the benefits. The prophylaxis threshold was relatively insensitive to the cost of LMWH and bleeding risk, but very sensitive to life expectancy (figure). For example, the prophylaxis threshold was $2.5 \%$ for patients with a life expectancy of 3 years.

CONCLUSIONS: Prophylaxis seems to be cost-effective for patients represented in clinical trials. However, its cost-effectiveness depends primarily on the risk of VTE. For most hospitalized patients, offering prophylaxis at a threshold of $1 \%$ appears to represent reasonable value. Patients at very low risk $(<0.31 \%)$ may experience net harm from prophylaxis.

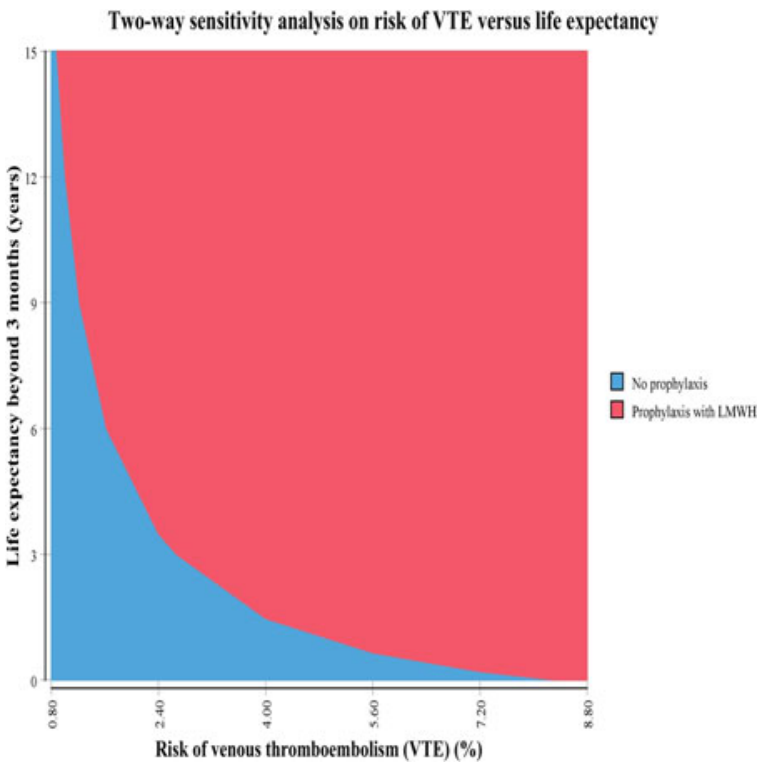

DEVELOPING A CLINICAL PREDICATION RULE FOR FIRST ONSET HOSPITAL-ACQUIRED C. DIFFICILE INFECTIONS Anne Press ${ }^{2}$; Benson $\mathrm{Ku}^{2}$; Lauren McCullagh ${ }^{2}$; Lisa Rosen ${ }^{3}$; Safiya Richardson ${ }^{2}$; Thomas McGinn ${ }^{1} .{ }^{1}$ Hofstra North SHore-LIJ, Manhasset, NY; ${ }^{2}$ Hofstra North Shore LIJ School of Medicine, Manhasset, NY; ${ }^{3}$ Feinstein Institute for Medical Research, Manhasset, NY. (Control ID \#2464165)

BACKGROUND: The healthcare burden of hospital-acquired Clostridium difficile infection (CDI) demands attention and calls for a solution. However, it is often unclear which patients are at risk for primary nosocomial CDI. Identifying patients' risk of developing a primary CDI, as they are admitted to the hospital, is a critical first step in reducing the development of new cases of CDI and the overall incidence and spread of the disease. Clinical prediction rules (CPRs) are tools that quantify the effect an individual patient's characteristics have toward their diagnosis, prognosis, or likely response to treatment. We derived a CPR which can predict a patient's risk of acquiring a primary CDI based on a limited amount of clinical risk factors, which are easily accessible upon admission. Thus, allowing for early interventional strategies in these patients, and providing a novel method of curbing infection.

METHODS: A retrospective chart review was conducted, utilizing data from our electronic health records (EHR), between 2013 and 2014. All patients over the age of 18 were included in the study. Patients with a past medical history of CDI, or community acquired CDI, were excluded. After applying the inclusion and exclusion criteria, we identified 61,482 subjects, which were divided into a training and validation set. The risk factors were identified utilizing administrative and EHR data. The primary outcome was a CDI diagnosed after 3 days of hospitalization defined as a positive PCR result. The CDI CPR was derived using univariate and multivariate analysis to identify those risk factors associated with hospitalacquired CDI. Best subset analysis was then performed to reduce the number of 
variables to 6 . The $\beta$-coefficients for the variables significantly associated with inhospital CDI were used to assign a numerical value to each risk factor and summed to create a total risk score for each patient in the derivation and validation sample. Positive likelihood ratios and predicted probabilities at each score level were calculated. Receiver operating characteristic (ROC) curves were created to compare the overall diagnostic accuracy of the tool. The optimal risk score cutoff for predicting patients at risk for hospital-acquired CDI was calculated. Lastly, we dichotomized patients into a high risk and low risk category and compared the frequencies of bleeding in the derivation and validation samples at these two different levels.

RESULTS: Among 61,482 subjects, CDI occurred in $0.6 \%$. CDI outcome was significantly associated the following: admission in past 60 days, a past or current diagnosis of congestive heart failure (CHF) or diverticulitis, age, sex, race, current dialysis or mechanical ventilation, and antibiotic or protein pump inhibitor (PPI) usage. After best subset analysis was applied 6 factors remained, including: age, admission in the past 60 days, mechanical ventilation, dialysis, history of CHF and use of antibiotic medications (Table 1). As a patient's score increased their risk of acquiring a primary CDI increased along a continuum (Table 2). For example, a patient's risk of CDI was $0.06 \%$ with a risk score of $0,10 \%$ with a score of 7 and $32 \%$ with a score of 9 . Similarly, the positive likelihood ratios for patients with a score of 0 was 1 and increased to 144 with a score of 9 . The optimal cut-off point was determined to be a score of $>=3$. The sensitivity and specificity of the tool using this cut-off, were 82 and $75 \%$, respectively. The AUC was 0.85 , for both the training and validation samples.

CONCLUSIONS: This study successfully derived and validated a CPR that wil help identify patients at high risk for primary CDI infection. The tool showed good discriminatory characteristics in both the validation and derivation sample and risk of CDI increased across a continuum at each score level. This tool will allow physicians to systematically recognize those at risk for CDI and will allow physicians to intervene to prevent the onset of infection. Possible interventions include modification to either the patients' individual risk factors or their environment; such as decreasing the usages of proton pump inhibitors and high-risk antibiotic treatment in these patients. Furthermore, the high NPV of this tool $(99.9 \%)$ will allow physicians to identify low risk patients, who may not need further CDI testing. Further work needs to be done to validate this rule in a prospective environment.

Table 1: Risk Factors Associated with the Clinical Prediction Rule

\begin{tabular}{lc}
\hline \hline Risk Factor & Score \\
Age $(>=65)$ & 1 \\
Admission in the past 60 days & 1 \\
CHF & 1 \\
Dialysis & 2 \\
Mechanical Ventilation & 2 \\
Antibiotics Usage & 2 \\
\hline
\end{tabular}

Table 2: Positive Likelihood ratios and Predicted Probabilities at each score level

\begin{tabular}{ll}
\hline \hline Score & Positive Likelihood Ratio \\
0 & Predicted Probability \\
1 & $0.06 \%$ \\
2 & $0.13 \%$ \\
3 & $0.27 \%$ \\
4 & $0.55 \%$ \\
5 & $1.2 \%$ \\
6 & $2.4 \%$ \\
7 & $5 \%$ \\
8 & $10 \%$ \\
9 & $18 \%$ \\
& $32 \%$ \\
\hline
\end{tabular}

DEVELOPING A PATIENT EMPOWERMENT PROGRAM (PEP) TO ACHIEVE BETTER PATIENT OUTCOMES BY PREPARING PATIENTS TO PARTICIPATE IN MEDICAL ENCOUNTERS Joseph Plaksin ${ }^{2}$; Nadiya Pavlishyn ${ }^{2}$; Adina Kalet ${ }^{2}$; Sondra Zabar ${ }^{2}$; Andrew B. Wallach ${ }^{1,2}$; Sarita Kundrod ${ }^{3}$; Lisa Altshuler ${ }^{2}$. ${ }^{1}$ Bellevue Hospital, New York, NY; ${ }^{2}$ NYU School of Medicine, New York, NY; ${ }^{3}$ University of Minnesota, Minneapolis, MN. (Control ID \#2467329)

BACKGROUND: Ideally, patients with type 2 diabetes mellitus (DM) would receive patient-centered care characterized by their participation as full partners with their healthcare providers (HCPs) in medical decision-making. However, even when providers are skillful in facilitating shared decision-making (SDM) it can be difficult for patients to engage. Patients who are more active in the medical encounter are better able to participate in SDM and more likely to perform DM self-management behaviors. We developed a Patient Empowerment Program (PEP) with the goal of increasing patient activation, preferences for control in medical decision-making, and DM self-management.

METHODS: English-speaking, adult patients with DM whose most recent hemoglobin A1c was between 6.5 and $11 \%$ were recruited from the Primary Care clinics at two public hospitals in NYC to participate in PEP, a two-session, performance-based communication skills workshop. In Session 1 of PEP, participants discussed SDM in the medical encounter, viewed trigger videos depicting patient-HCP interactions, and rated the patient and HCP in each video using a behaviorally-anchored checklist. In PEP Session 2, participants role-played two scenarios with Standardized Healthcare Providers (SHPs), the second of which was individually tailored to each participant based on self-reported difficulties with $\mathrm{DM}$, and then debriefed as a group with research personnel to create a SMART goal-based action plan targeted to improve SDM and DM self-care. At the time of enrollment, each participant completed a set of questionnaires comprised of demographic and clinical information, health literacy as measured by the Newest Vital Sign (NVS), and a series of outcome measures including the Patient Activation Measure (PAM), the Krantz Health Opinion Survey (Krantz), a Preference Control Scale (PCS), and the Diabetes Self-Management Questionnaire (DSMQ). Due to the low literacy of our patient population, Research Assistants (RAs) read each participant all of the questions and recorded their responses. Prior to participant enrollment, RAs developed a standardized method of asking and clarifying all questions. Participants were then called on the phone by RAs 1 week and 3 months after completing PEP to obtain follow-up data. At 1 week post-PEP, participants completed the PAM, Krantz, and PCS. At 3 months post-PEP, they completed all outcome measures assessed at the time of enrollment.

RESULTS: We enrolled a total of 80 participants between both sites, 45 of which attended PEP Session 1 and 36 of which attended the complete PEP Intervention. Participants who attended at least PEP Session 1 were $53 \%$ male with a mean age of 59.0 years ( $\mathrm{SD}=10.1$ ) and most were racial or ethnic minorities (29\% African-American, $40 \%$ Hispanic). They predominately had a low educational status ( $40 \%$ did not complete high school), low socioeconomic status (65\% had an annual income less than $\$ 10,000 /$ year), and limited health literacy (76\%). Multiple attempts were made to collect follow-up data from all participants who attended at least one PEP session. The 1 week post-PEP follow-up was completed an average of 9.5 days after the last PEP Session $(\mathrm{SD}=5.8)$ and 44 out of 45 participants (98\%) completed this assessment. Results are shown in Table 1 and the only significant difference was a higher score on the Information subscale of the Krantz. The 3 month post-PEP follow-up was completed an average of 94.9 days after the last PEP Session ( $\mathrm{SD}=7.0)$ and 33 out of 45 participants ( $73 \%$ ) completed this assessment. Results are shown in Table 2 and at this time there were significantly higher scores on the Glucose Management and Healthcare Utilization subscales of the DSMQ, which were large enough to create a significant difference on the DSMQ Sum Scale. 
CONCLUSIONS: Participants who completed PEP had transient increases in their preference for receiving information from HCPs and longer term increases in self-reported DM self-management behaviors such as blood glucose monitoring, adhering to medications, and attendance at HCP visits. However, there were no differences in patient activation and no effect of these behavior changes on hemoglobin A1c. These results are limited by several factors, including the potential effect of our population's low literacy on questionnaire validity, the limited follow-up interval, and the small sample size. An important next step would be to assess the actual changes in patient engagement during patient-HCP visits by audiotaping those encounters.

Table 1. One Week Follow-Up

\begin{tabular}{llll}
\hline \hline Outcome Measure & $\begin{array}{l}\text { Enrollment Mean } \\
\text { (SD) }\end{array}$ & $\begin{array}{l}\text { 1-week Post-PEP Mean } \\
(\mathrm{SD})\end{array}$ & $\begin{array}{l}\text { Paired Sample t- } \\
\text { test } \\
\mathrm{t}(43)=4.00^{*}\end{array}$ \\
$\begin{array}{l}\text { Krantz: } \\
\text { Information }\end{array}$ & $3.70(1.89)$ & $4.61(1.67)$ & $\mathrm{t}(43)=0.79$ \\
Krantz: Behavior & $2.09(1.87)$ & $1.82(1.81)$ & $\mathrm{t}(43)=1.41$ \\
Krantz Total Score & $5.82(2.84)$ & $6.43(2.55)$ & $\mathrm{t}(40)=0.50$ \\
PCS & $2.78(1.04)$ & $2.88(0.98)$ & $\mathrm{t}(42)=0.36$ \\
PAM & $65.57(15.75)$ & $66.41(12.93)$ & \\
\hline
\end{tabular}

$* p<0.001$

Table 2. Three Month Follow-Up

\begin{tabular}{llll}
\hline \hline Outcome Measure & $\begin{array}{l}\text { Enrollment Mean } \\
\text { (SD) }\end{array}$ & $\begin{array}{l}\text { 3-month Post-PEP } \\
\text { Mean (SD) }\end{array}$ & $\begin{array}{l}\text { Paired Sample t- } \\
\text { test }\end{array}$ \\
Krantz: Information & $3.85(1.84)$ & $4.42(1.75)$ & $\mathrm{t}(32)=1.74$ \\
Krantz: Behavior & $2.21(1.99)$ & $1.76(1.74)$ & $\mathrm{t}(32)=-1.04$ \\
Krantz Total Score & $6.09(2.82)$ & $6.18(2.47)$ & $\mathrm{t}(32)=0.16$ \\
PCS & $2.90(1.01)$ & $2.84(1.00)$ & $\mathrm{t}(32)=-0.25$ \\
PAM & $64.82(15.86)$ & $63.02(12.44)$ & $\mathrm{t}(32)=-0.73$ \\
DSMQ: Glucose & $6.74(2.97)$ & $8.14(2.26)$ & $\mathrm{t}(31)=2.32^{*}$ \\
Management & & & $\mathrm{t}(31)=0.76$ \\
DSMQ: Dietary Control & $6.48(2.06)$ & $6.80(1.81)$ & $\mathrm{t}(31)=1.44$ \\
DSMQ: Physical Activity & $7.24(2.35)$ & $7.82(2.23)$ & $\mathrm{t}(31)=2.41^{*}$ \\
DSMQ: Healthcare & $7.72(2.09)$ & $8.73(1.34)$ & $\mathrm{t}(31)=4.28^{*}$ \\
Utilization & & & $\mathrm{t}(29)=0.79$ \\
DSMQ Sum Scale & $7.66(2.01)$ & $9.24(1.23)$ & \\
Hemoglobin Alc & $8.31(1.31)$ & $8.11(1.31)$ &
\end{tabular}

$* p<0.05$

DEVELOPING ELECTRONIC HEALTH RECORD ALGORITHMS TO IDENTIFY PATIENTS WITH PROBLEM LIST GAPS AND IMPROVE QUALITY OF

CARE Angela $\mathrm{Ai}^{1}{ }$; Skye Aaron ${ }^{1}$; Dustin S. McEvoy ${ }^{2}$; Salman T. Hussain ${ }^{1}$; Thu-Trang T. Hickman ${ }^{1}$; Adam Wright ${ }^{1,3}$. ${ }^{1}$ Brigham and Women's Hospital, Boston, MA; ${ }^{2}$ Partners Healthcare, Boston, MA; ${ }^{3}$ Harvard Medical School, Boston, MA. (Control ID \#2465982)

BACKGROUND: The clinical problem list within an electronic health record (EHR) plays an important role in patient care. A complete and accurate problem list allows for rapid assessment of past medical history, facilitates communication between providers, and enables clinical decision support (CDS) tools such as drug/condition alerts. Despite its potential benefits, the problem list is often incomplete and inaccurate, threatening quality of care and patient safety. Our previous research has demonstrated that problem inference alerts can improve problem list completeness within the EHR by finding problems a patient actually has that are missing from the problem list (gaps). These EHR alerts use medication lists, laboratory results, and billing diagnosis codes from a patient's chart to infer the existence of problems and then prompt physicians to add these problems to the patient's problem list if they are missing (Figure 1). For example, an algorithm for congestive heart failure (CHF) might suggest adding the problem if the patient is taking a loop diuretic and has been billed at least once with a heart failure diagnosis code, but does not have CHF on their problem list. In this study, we sought to refine algorithms for seven conditions which we had previously developed, and to develop new algorithms for five conditions which we had not previously researched. These algorithms have the potential to improve problem list completeness and, ultimately, quality of care and patient safety by prompting providers to add problems when a patient meets certain criteria.

METHODS: To expand on our prior work, we selected twelve chronic conditions based on their prevalence and their association with CDS, with input from a team of clinical and content experts. We worked to refine our previously developed algorithms for asthma, coronary artery disease, congestive heart failure, chronic obstructive pulmonary disease, hypertension, sickle cell disease, and stroke. In addition, we sought to develop and refine novel algorithms for atrial fibrillation, hyperlipidemia, myocardial infarction, sleep apnea, and tuberculosis. To do so, we identified associated clinical data elements, including medications, lab results, billing diagnosis codes, and problem list codes for each of the twelve aforementioned conditions. We collected structured patient data from a random set of 100,000 patient charts and assigned data elements to specific conditions. Clinical content experts from Brigham and Women's Hospital $(\mathrm{BWH})$ used this information to formulate initial problem inference rules for each of the conditions. Using the initial rules, we randomly selected up to 100 rule positive and 100 rule negative charts per condition for manual review (2182 unique charts). These charts were then used as a training set to adjust the initial rules to optimize positive predictive value (PPV) and sensitivity. Final estimates of algorithm performance were obtained by conducting additional rounds of manual chart review (3000 unique charts) and iteratively adjusting the rules.

RESULTS: Results from the final chart review for each condition showed high estimated PPVs across the twelve conditions (average: $92.3 \%$, range: 81.4-99.1\%). Average sensitivity, specificity, and negative predictive value were estimated at 72.5, 99.3, and $96.7 \%$ respectively.

CONCLUSIONS: We developed and validated clinical algorithms which can accurately identify patients who are likely to have particular heart, blood, and lung related problems using structured data elements embedded within an EHR, even when they do not have the documented problem on the problem list. These rules have the potential to improve problem list completeness, which has a variety of important implications, including improved CDS and better identification of patients for research purposes. Future steps include enhancing the performance of our algorithms through the use of natural language processing to analyze unstructured data, such as progress notes and discharge summaries. Additionally, we are working to implement these algorithms in different EHR systems across the United States to determine their impact on problem list completeness and quality of care 


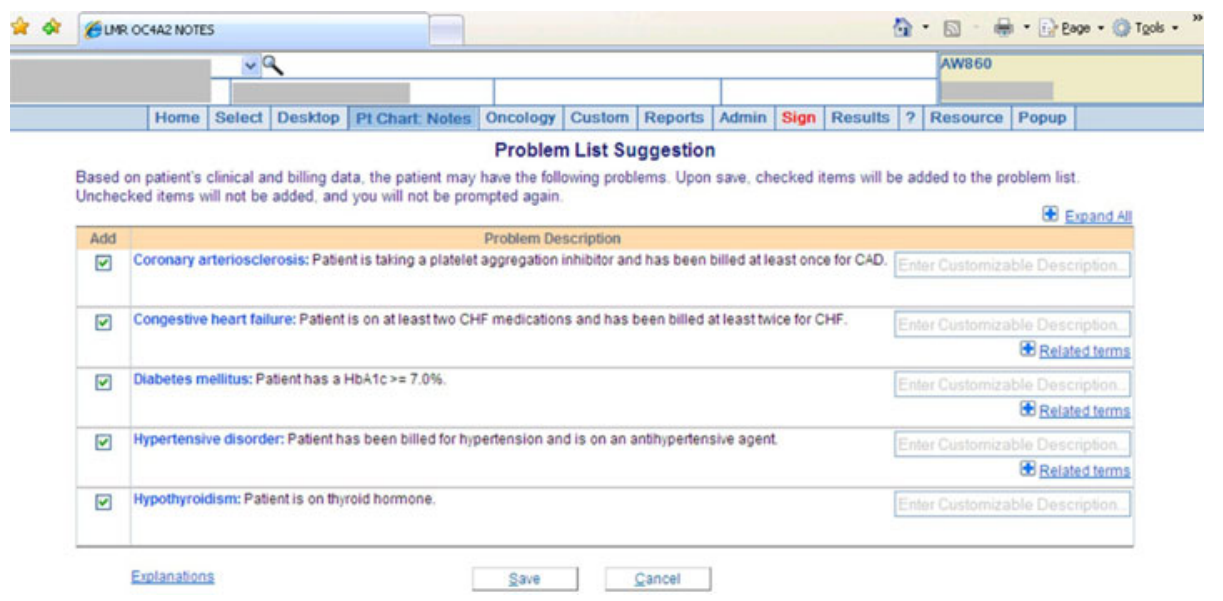

Figure 1. Screenshot of problem inference alerts in the outpatient Longitudinal Medical

Record (LMR) at Brigham and Women's Hospital (BWH).

DEVELOPMENT AND IMPLEMENTATION OF A DATA COLLECTION INSTRUMENT FOR A NATIONAL INFLUENZA EMERGENCY Elizabeth White'; Sarah Collins ${ }^{1,2}$; Beatriz Rocha ${ }^{1,2}$; Satish Bhagwanjee ${ }^{3}$; Raquel Bartz ${ }^{4}$;. Perren Cobb ${ }^{5}$; Roberto A. Rocha ${ }^{1,2}$. ${ }^{1}$ Brigham and Women's Hospital, Norfolk, MA; ${ }^{2}$ Harvard Medical School, Boston, MA; ${ }^{3}$ University of Washington, Seattle, WA; ${ }^{4}$ Duke University School of Medicine, Durham, NC; ${ }^{5}$ Keck Hospital of the University of Southern California, Los Angeles, CA. (Control ID \#2468729)

BACKGROUND: National preparedness efforts for public health emergencies, such as seasonal and pandemic influenza, are important to address challenges to healthcare and communications systems ${ }^{1}$. The United States Critical Injury and Illness Program for Emergency Preparedness (USCIIT-PREP) has been developing systems to facilitate the prospective collection of clinical data during emergent events in order to define the burden of the outbreak, leading to more effective treatment for patients ${ }^{2}$. The objective of this project was to create and validate a consensus-driven electronic data collection instrument in preparation for real-time data collection during a national influenza emergency.

METHODS: A paper case report form (CRF) for collecting data on critically ill influenza patients was developed by clinical and informatics experts on the USCIITPREP CRF Committee. The CRF was implemented using research electronic data capture (REDCap) ${ }^{3}$. REDCap was identified as the software solution for creating the electronic case report forms (eCRFs) because of its wide availability, security, and intuitive design features. To ensure data consistency, data fields from the paper CRF were configured in REDCap with attention to field type, range validation, and logic. We used REDCap's longitudinal project model to define time points for data collection. Some forms required use of REDCap's advanced logic and calculation capabilities, such as the Acute Physiology and Chronic Health Evaluation (APACHE) II form $^{4}$. Once the eCRFs were implemented, they were tested by informatics experts on our team for usability and data consistency. We then conducted a review with USCIITPREP clinical subject matter experts (SMEs) via teleconference. SME feedback on content, workflow, and clarity was incorporated into the final version of the instrument.
RESULTS: The data collection instrument we developed in REDCap consisted of 13 eCRFs with 236 total data elements collected over 17 events (see Table 1). The 236 data elements included 126 (53.4\%) single-select, 7 (3.0\%) multiselect, 77 (32.6\%) free text, 18 (7.6\%) calculated, and 8 (3.4\%) descriptive fields. All fields were marked as required. Conditional logic was applied to 137 (58.1\%) fields, causing them to be hidden or displayed based on previous responses. Value set size for single-select fields ranged from 1 to 12 values (median=2). For multi-select fields, value sets ranged from 3 to 32 values (median $=7)$. Of 77 free text fields, $48(62.3 \%)$ contained at least one validation rule to promote data consistency, such as specific formats (i.e. date, YYYY-MM-DD), minimum and maximum allowed values, or designated units. The complexity of calculated fields varied, with a range of 1 to 17 (median $=2)$ variables per calculation. The most complex calculation was the APACHE II Acute Physiology Subscore, which used 17 variables.

CONCLUSIONS: This project defined a feasible process for achieving consensus and validation for a data collection instrument to be used prospectively during a public health emergency. Clinical data defined by the committee was able to be configured in REDCap in a consistent manner due to the program's functionality allowing for the specification of data type and other validation rules. Further, the wide availability and familiarity of REDCap facilitated collaboration and testing across multiple institutions. This process was instrumental in prospectively defining the data and data capture formats that will be important to understand a future emergent event. While REDCap demonstrated many effective solutions, limitations to its calculation and logic capabilities prevented more dynamic functionality from being implemented in a few instances. Suitability of data points identified for an influenza outbreak was not established in this process. Future efforts include testing this instrument during an influenza emergency to refine the forms and data collection process. References 1. Lurie, N, Manolio, T, Patterson, AP, Collins, F, Frieden, T. (2013) Research as a Part of Public Health Emergency Response. The New England Journal of Medicine, 368(13), 1251-1255. 2. (2016) USCIITG-PREP Public Emergency Medical Countermeasures. Retrieved from https://mcm.usciitg-prep. 
org 3. Harris, PA, Taylor, R, Thielke, R, Payne, J, Gonzalez, N, Conde, J. (2009) Research electronic data capture (REDCap) - A metadata-driven methodology and workflow process for providing translational research informatics support. Journal of Biomedical Informatics, 42(2), 377-381. 4. Knaus, WA, Draper, EA, Wagner, DP, Zimmerman, JE. (1985) APACHE II: A severity of disease classification system. Critical Care Medicine, 13(10), 818-829. This project was funded by the Food and Drug Administration (FDA) and the Biomedical Advanced Research and Development Authority (BARDA).

Table 1

\begin{tabular}{|c|c|c|}
\hline eCRF Section Name & $\begin{array}{l}\text { Number of Data } \\
\text { Elements }\end{array}$ & $\begin{array}{l}\text { Number of eCRF } \\
\text { Recurrences } \\
\text { (out of } 17 \text { events) }\end{array}$ \\
\hline $\begin{array}{l}\text { Demographics and Admission } \\
\text { Information }\end{array}$ & 11 & 1 \\
\hline Initial Assessment & 36 & 1 \\
\hline Influenza Initial Assessment & 30 & 1 \\
\hline Comorbidities & 7 & 1 \\
\hline ICU Admission & 7 & 1 \\
\hline APACHE II & 34 & 1 \\
\hline SOFA Score & 19 & 14 \\
\hline Berlin Criteria Day 1 & 7 & 1 \\
\hline Berlin Criteria Daily & 8 & 13 \\
\hline ARDS Details & 44 & 1 \\
\hline Other Conditions & 2 & 1 \\
\hline ICU Discharge & 25 & 1 \\
\hline Outcomes & 6 & 1 \\
\hline
\end{tabular}

DEVELOPMENT AND INITIAL VALIDATION OF SCALES TO MEASURE PROVIDER SELF-EFFICACY WITH MANAGEMENT OF CHRONIC ILLNESS, MENTAL ILLNESS, AND TEAM-BASED CARE Danielle F. Loeb ${ }^{2}$; Erin Leister $^{7}$; L Miriam Dickinson ${ }^{4}$; Evette Ludman ${ }^{8}$; Ingrid A. Binswanger ${ }^{3,2}$; Danielle M. Kline $^{2}$; Elizabeth Juarez-Colunga ${ }^{4}$; Frank V. deGruy ${ }^{6}$; Donald E. Nease ${ }^{5}$; Elizabeth A. Bayliss ${ }^{3}$; Lori A. Crane ${ }^{1} .{ }^{1}$ University of Colorado Anschutz Medical Campus, Aurora, $\mathrm{CO} ;{ }^{2}$ University of Colorado School of Medicine, Aurora, CO; ${ }^{3}$ Kaiser Permanente and University of Colorado, Denver, $\mathrm{CO} ;{ }^{4}$ University of Colorado, Aurora, $\mathrm{CO} ;{ }^{5} \mathrm{University}$ of Colorado - Denver, Aurora, CO; ${ }^{6}$ University of Colorado SOM, Aurora, CO $;{ }^{7}$ University of Colorado School of Public Health, Aurora, $\mathrm{CO} ;{ }^{8}$ Group Health Research Institute, Seattle, WA. (Control ID \#2468772)
BACKGROUND: Team based care improves outcomes for patients with comorbid mental and physical conditions. Implementing effective team-based care requires Primary Care Providers (PCPs) with the skills and confidence to manage this population. We developed and initially validated a measure to assess PCP self-efficacy for these tasks.

METHODS: Based on Bandura, we developed three scales for the instrument: teambased care (Team), mental illness management (MI), and medical illness (Medical) management. After cognitive and pilot testing, we administered the instrument via mail survey to 900 randomly selected family medicine and internal medicine PCPs from Colorado primary care practices. Response rate was calculated excluding surveys returned for incorrect addresses. We conducted an exploratory factor analysis on the survey items, constructed self-efficacy scales based on the resulting factors, and calculated standardized Cronbach's alpha coefficients of reliability for each of the scales. After the factor analysis, we constructed outcome factor scores by calculating means of the items in each factor. Lastly, we calculated correlation coefficients (CC) between the MI and Team scales and the following validated measures to evaluate construct validity: the Mental Health Knowledge and Management Instrument, the Communication Skills Self Assessment, the Attitudes Toward Health Teams Scale, the Team Climate Inventory, and the Team approach subscale of the Practice Monitor.

RESULTS: Four hundred two (response rate $=49 \%$ ) PCPs from diverse practice settings completed surveys. On an 11-point Likert scale, mean scores (standard deviation) were 7.7 (1.7), 7.1 (1.4), and 9.1 (0.8) for the Team, MI, and Medical scales respectively. Figures 1 and 2 show the distributions for the Team and MI scales. In factor analysis of the survey questions, items grouped into factors as expected. Eigenvalues were 6.915, 3.212 and 2.411 for the Team ( 7 items), MI (10 items), and Medical (6 items) scales. Variance explained was 44,20 , and $15 \%$ respectively. The three scales had high reliability with standardized Cronbach's alpha coefficients of $0.94,0.88$; and 0.83 . The Team scale correlated with communication skills $(\mathrm{CC}=0.30)$, Attitudes Toward Health Care Teams $(0.40)$, Practice Monitor (0.28), and Team Climate Inventory (0.25). The MI scale correlated with communication skills (0.40), experience managing mental illness (0.48), commonness of mental illness $(0.35)$, and preparedness to manage mental illness after residency $(0.40)$.

CONCLUSIONS: This early validation study of an instrument to measure provider selfefficacy with management of chronic illness, mental illness, and team-based care showed high internal validity. Although further testing of the correlation of the instrument with actual practice patterns, it represents a promising tool in the assessment of provider selfefficacy prior to implementation of team-based care interventions for chronic medical and mental illness in the primary care settings.

Figure 1. Distribution of team-based care self-efficacy items

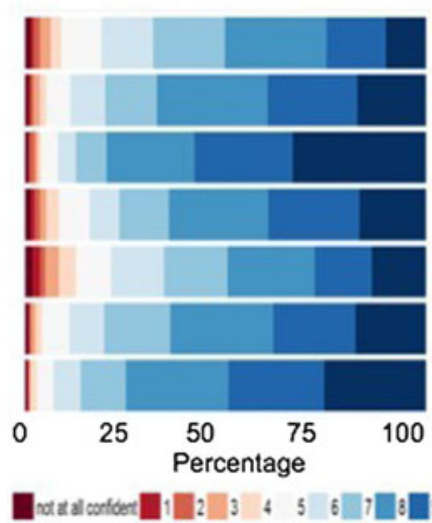

How Confident are you that you can...

1) rely on the care manager to work with your patients to develop specific plans to meet their goals for their chronic medical or mental illness(es)?

2) rely on the care manager to contact your patients to monitor their progress toward meeting their goals for their chronic medical or mental illness(es)?

3) rely on the care manager to contact your patients in your panel with diabetes who are over due for their $\mathrm{HbA1c}$ to schedule them to come in to clinic for follow-up testing?

4) collaborate with the care manager who uses treatment algorithms to make medication adjustments to improve blood pressure control in your patients with uncontrolled hypertension?

5) collaborate with the care manager who uses treatment algorithms to make medication adjustments to improve symptom control in your patients with uncontrolled major depressive disorders?

6) encourage team members to contribute to creative solutions to improve patient care within your practice?

7) establish a positive atmosphere among the members of the clinical team?

hot tal oxidert 


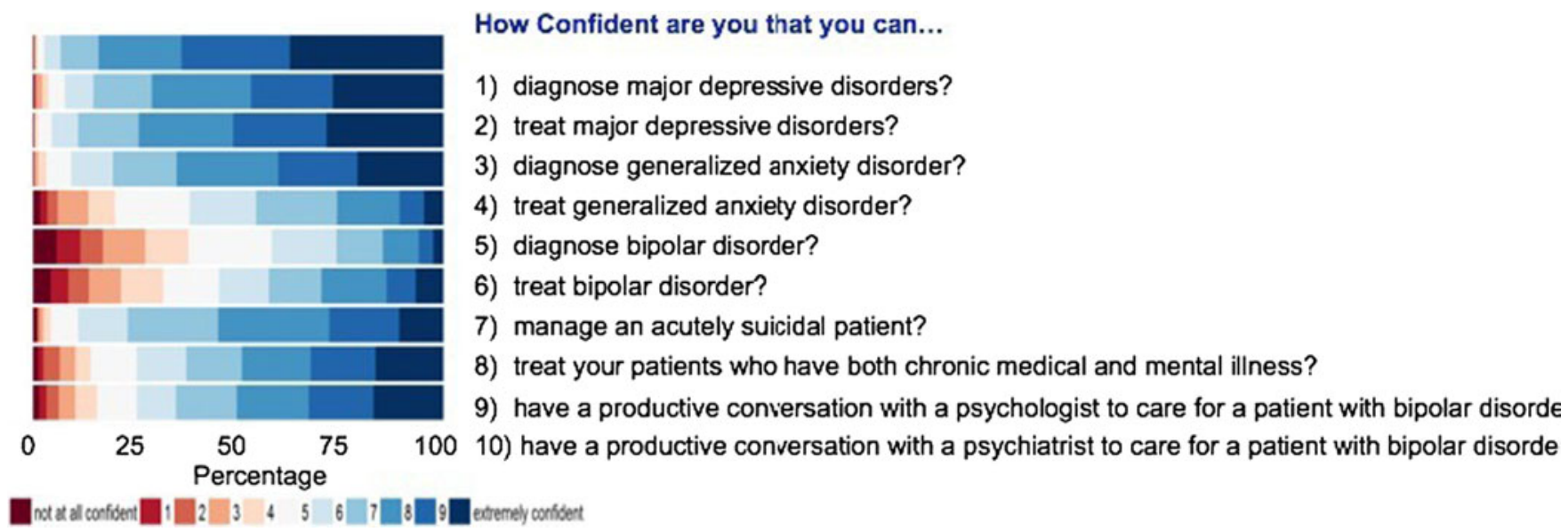

Figure 2. Distribution of mental illness management self-efficacy items

DEVELOPMENT AND VALIDATION OF E-CLINICAL EVALUATION EXERCISE (E-CEX) TOOL TO ASSESS PATIENT-CENTERED ELECTRONIC MEDICAL RECORD USE Maureen D. Lyons; Wei Wei Lee; Lollita Alkureishi; Kristen Wroblewski; Jeanne M. Farnan; Vineet M. Arora. University of Chicago, Chicago, IL. (Control ID \#2456685)

BACKGROUND: Despite widespread adoption of the Electronic Medical Record (EMR) and identification of specific EMR-related behaviors and skills that can enhance patient-physician interactions, providers receive minimal formalized education and feedback on these skills. The American Medical Association and the Alliance for Clinical Education have both recently highlighted the importance of addressing this issue in undergraduate and graduate medical education; however, methods by which to evaluate medical student proficiency in patient-centered EMR use are both essential and lacking. Using a previously developed patient-centered EMR use curriculum, the investigators aimed to develop and validate the electronic-Clinical Evaluation Exercise (e-CEX) tool as a method by which to assess and reassess medical student EMR specific communication skills over time.

METHODS: The investigators developed a patient-centered EMR use curriculum provided to second year pre-clinical medical students (MS2) during their Clinical Skills course at the University of Chicago. The curriculum included a lecture on EMR use barriers and best practices and videotaped Group Objective Structured Clinical Examinations (GOSCEs) with a Standardized Patient (SP) immediately after the lecture and 1 year later. Third year medical students (MS3) who had not received the curriculum also performed in the same patient-centered EMR use OSCE, however as individual participants and not in group format. The investigators created the e-CEX, a 10-item 90-point tool, for use in direct observation assessment by trained observers as well as an SP evaluation tool (16 items, 80 points total) based on best communication practices identified in the literature. Inter-rater reliability of the e-CEX was established using intraclass correlation coefficient and trained observers evaluated the videotaped encounters using the e-CEX. The investigators evaluated internal consistency using Cronbach's alpha and concurrent validity between the e-CEX tool and our SP evaluation tool, a surrogate for patient experience, using Pearson correlation coefficient.

RESULTS: A total of 70 students and 85 encounters were rated using the e-CEX: 20 trained MS2s, 50 untrained MS3s, and 15 MS3s who were trained 1 year prior as MS2s. Cronbach's alpha for the e-CEX was 0.89 , indicating high reliability. Trained MS2 students $(n=20)$ scored significantly higher on the e-CEX than untrained MS3 students $(n=50)[55(\mathrm{SD}=10.7)$ vs. $44.9(\mathrm{SD}=12.7), p=0.003]$. Trained MS2 $(n=20)$ average scores using the SP evaluation tool were significantly higher than untrained MS3 ( $n=88)$ students $[70.8(\mathrm{SD}=4.3)$ vs $58.1(\mathrm{SD}=13.1), p<0.001]$. The e-CEX tool rating correlated with the SP evaluation tool (Pearson correlation 0.74 ). As third year students ( 1 year after receiving the curriculum as MS2s), e-CEX scores were not significantly changed (average change $=-0.9, \mathrm{SD}=15.4 ; p=0.83$ ) although their $\mathrm{SP}$ evaluation scores deteriorated [71.6 $(\mathrm{SD}=3.4)$ vs $62.9(\mathrm{SD}=14.5), p=0.027]$.

CONCLUSIONS: The e-CEX tool was a reliable and valid method of evaluating medical student skills and behaviors surrounding patient-centered EMR use. The curricular intervention was effective in improving students' patient-centered skills while using the EMR and this effect largely remained 1 year after the intervention. Future work would include evaluating the tool with residents and patients in real clinical scenarios and as a faculty development tool.

DEVELOPMENT OF A HIGH-VALUE CARE CULTURE SURVEY Reshma Gupta $^{1,2}{ }^{\text {; Christopher Moriates }}{ }^{3,4}$; James Harrison ${ }^{3}$; Victoria Valencia ${ }^{3}$; Michael Ong ${ }^{1}$, ${ }^{2}$; Robin Clarke ${ }^{1}$; Neil Steers ${ }^{1,2}$; Ron D. Hays ${ }^{1}$; Clarence H. Braddock ${ }^{1}$; Robert M. Wachter ${ }^{3}$. ${ }^{1}$ Department of Medicine, David Geffen School of Medicine, University of California Los Angeles, Los Angeles, CA; ${ }^{2}$ VA/ Robert Wood Johnson Clinical Scholars Program, VA Greater Los Angeles Health System, University of California Los Angeles, Los Angeles, CA; ${ }^{3}$ Division of Hospital Medicine, University of California San Francisco, San Francisco, CA; ${ }^{4}$ University of California San Francisco Center for Healthcare Value, San Francisco, CA. (Control ID \#2467733)

BACKGROUND: Efforts to improve value within hospitals must engage frontline clinicians for organizational culture change. Culture surveys have been used to drive improvements in care, but a survey assessing high-value care culture is needed. We developed a High-Value Care Culture Survey (HVCCS) for use by healthcare leaders and training programs to target future improvements in delivering value-based care.

METHODS: We conducted a modified Delphi process to identify items for inclusion in the survey, and administered the survey to internal medicine residents and hospitalists at two large academic medical centers (AMCs) with different institutional VBP scores. The two-phase modified Delphi process included frontline clinicians and nurses who generated survey questions and evaluated content validity. This comprehensive list was then sent to a national panel of 28 high-value care experts who rated the importance of each item for survey inclusion. Experts represented 
different regions of the country, types of medical centers (academic, county, community, VA), training levels, key stakeholders, and 11 subspecialties. We evaluated survey data collected from 100 internal medicine hospitalists and 170 residents. We conducted exploratory and confirmatory factor analyses. We also estimated productmoment correlations of HVCCS scores with the Center for Medicare and Medicaid Services institutional Value-based Purchasing (VBP) scores.

RESULTS: In the modified Delphi process, 26 (93\%) of experts responded to the first phase survey and $22(85 \%)$ to the second phase survey. Of 79 potential survey items, 37 items were included in the final survey because at least $70 \%$ of the experts were in favor of retaining them. Seventy-nine (98\%) UCLA residents, 62 (77\%) UCSF residents, 39 ( $83 \%$ ) UCLA hospitalists, and 34 (77\%) UCSF hospitalists completed the survey. The exploratory factor analyses suggested 6 underlying domains: 1) Leadership and Health System Messaging ( $\alpha=0.9)$; 2) Data Transparency and Access ( $\alpha=0.8)$; 3) Comfort with Cost Conversations $(\alpha=0.7)$; 4) Blame-free Environment $(\alpha=0.7)$; 5) Beliefs about Healthcare Value $(\alpha=0.6)$; and 6) Patient-centered Decision Making $(\alpha=0.5)$. [Refer to Table 1 for example survey items.] Since the reliability of the latter two scales was suboptimal (i.e., <0.70), we estimated a four-factor confirmatory factor analysis representing the first four domains. This model fit the data well according to the adjusted goodness-of-fit index (0.97). Three of the four institutional HVCCS domain scores differed significantly between the two AMCs. The High-value Care Culture Survey was significantly positively correlated with VBP scores including Leadership and Health System Messaging $(r=0.56, p=0.00)$, Data Transparency and Access $(r=0.27, p=$ $0.00)$, and Blame-free Environment $(r=0.37, p=0.00)$.

CONCLUSIONS: This is the first survey developed to assess high-value care culture among frontline healthcare providers. It was developed from input of frontline experts in value, training, practice, and policy, and tested on internal medicine hospitalists and residents. Our results provide initial support for the reliability and validity of the HVCCS, which can be used by provider groups who aim to improve their high-value care culture.

DEVELOPMENT OF A PREDICTION MODEL FOR 2-YEAR RISK OF OPIOID OVERDOSE AMONG PATIENTS PRESCRIBED CHRONIC OPIOID THERAPY Komal Narwaney ${ }^{1}$; Jason M. Glanz ${ }^{1,2}$; Shane Mueller ${ }^{1}$; Edward M. Gardner ${ }^{3}$; Joseph W. Frank ${ }^{4,}$; Kristin Breslin ${ }^{3}$ Ingrid A. Binswanger ${ }^{1,4}$. ${ }^{1}$ Kaiser Permanente Colorado, Denver, CO ${ }^{2}$ Colorado School of Public Health, Aurora, CO; ${ }^{3}$ Denver Health and Hospital Authority, Denver, CO; ${ }^{4}$ University of Colorado Denver, Denver, CO; ${ }^{5} \mathrm{VA}$ Eastern Colorado Health Care System, Denver, CO. (Control ID \#2467414)

BACKGROUND: Pharmaceutical opioid overdose is a significant problem in the United States. Myriad policies and interventions are being developed and tested to reduce the risk of opioid overdose among patients prescribed chronic opioid therapy for pain. Many of these interventions will target patients at highest risk for overdose, given the large numbers of people on chronic opioid therapy. For instance, one promising intervention is overdose education and naloxone prescribing, which may both enhance risk perception and prevent death from opioid-induced respiratory depression. While individuals at risk can be reached through general medical settings, our prior work suggests that health care providers are uncertain about who to target for naloxone. Thus, the aim of this study was to develop a model to predict fatal and nonfatal opioid overdose among people prescribed chronic opioid therapy in a large health care system.

METHODS: We conducted a retrospective cohort study to identify baseline characteristics that predict opioid overdose in patients on chronic opioid therapy at Kaiser Permanente Colorado. Chronic opioid therapy was defined as prescription of at least 3 opioid medications within 90 days between Jan 1, 2006 and December 31, 2013. Patients were followed to the day of the event (fatal or non-fatal overdose), death, disenrollment or the end of follow-up period ( 2 years). Nonfatal opioid overdoses were identified from electronic health records using ICD-9 codes in emergency department and inpatient settings; fatal overdoses were identified using ICD-10 codes from death records. We considered predictors previously found to be associated with opioid overdose, including demographics, patient medication features, and patient diagnoses. Multivariable Cox regression was performed, using opioid overdose as the outcome of interest. We used a backward elimination method with a $p$-value $>0.05$ for removal of variables. We evaluated model discrimination using concordance index (c-statistic) and calibration using the Greenwood-D'Agostino-Nam test of calibration. The model was validated internally with a bootstrapping resampling technique.

RESULTS: Among 39,311 patients on chronic opioid therapy, we identified 111 patients with fatal or nonfatal opioid overdose during 65,509 person-years of follow-up (169.4 per 100,000 person-years). The mean age of patients was 54.9 years and more than half were women $(57.9 \%)$. Several factors were associated with opioid overdose including age, tobacco use, mental health diagnosis, substance use disorder, antidepressant prescriptions, and high-dose opioid prescriptions. The interaction between substance use disorder diagnosis and tobacco use was statistically significant (Table 1). The model c-statistic was 0.76 and the predicted 2 -year risk deciles were similar to the observed risks $\left(\chi^{2}\right.$ test 3.53 , $\mathrm{df}=8, p=0.9$ ). On bootstrap resampling for model validation, the optimismcorrected calibration slope was 0.9076 , indicating little evidence of overfitting the data. The bootstrap-corrected c-statistic of 0.74 derived from the Cox model shows effective discrimination between those at high and low risk of opioid overdose.

CONCLUSIONS: We developed a prognostic model that could be used to obtain valid predictions of opioid overdose in patients on chronic opioid therapy. Applying this model in clinical practice may help providers target high risk patients for naloxone prescription and other preventive interventions.

Table 1. Multivariable-adjusted Cox proportional hazards regression coefficients for 2 year risk of opioid overdose

\begin{tabular}{llll}
\hline \hline & $\beta$ coefficient & SE & $P$ Value \\
Age & -0.08559 & 0.02548 & 0.0008 \\
Age $^{2}$ & 0.00072 & 0.00024 & 0.003 \\
Mental health diagnosis & 0.74489 & 0.23369 & 0.001 \\
Substance use disorder & 1.58326 & 0.30760 & $<0.0001$ \\
Antidepressant & 0.68893 & 0.23612 & 0.004 \\
Tobacco use & 0.73144 & 0.23486 & 0.002 \\
High dose opioids & 1.03603 & 0.35003 & 0.003 \\
Substance use disorder x tobacco use & -0.96923 & 0.44826 & 0.03 \\
\hline
\end{tabular}

2 year baseline survival S0 (2) $=0.9989$

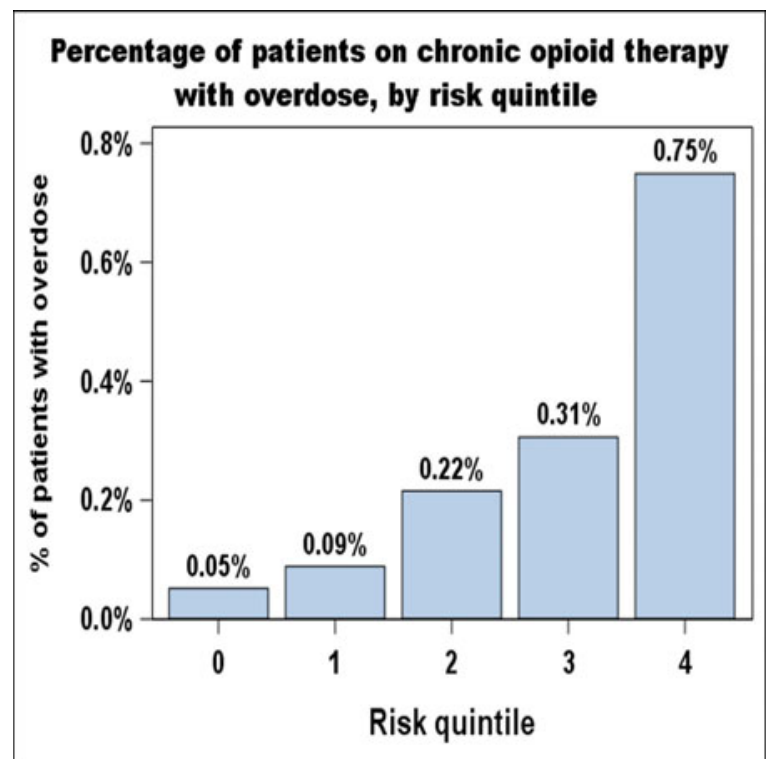


DEVELOPMENT OF A WOMEN'S HEALTH CURRICULUM FOR INTERNAL MEDICINE RESIDENTS: A MIXED-METHODS APPROACH Laura D. Hallett²; Rachel S. Casas ${ }^{1}$; Megan R. Gerber ${ }^{3}$; Catherine A. Rich ${ }^{5}$; Tracy A. Battaglia ${ }^{4}{ }^{1}$ Boston Medical Center, Boston, MA; ${ }^{2}$ Boston University, Needham, MA; ${ }^{3}$ Boston University, Jamaica Plain, MA; ${ }^{4}$ Boston University Medical Center, Boston, MA; ${ }^{5}$ Boston University School of Medicine, Boston, MA. (Control ID \#2468978)

BACKGROUND: Nationally, no standard approach exists to deliver women's health education to internal medicine residents, and survey data suggests trainee deficits in comfort and knowledge of women's health topics. At Boston Medical Center (BMC), there is no coordinated curriculum to train internal medicine residents to provide comprehensive, ambulatory care to female patients. To address this deficit and inform development of a women's health curriculum, we assessed faculty and resident perceptions of resident competency, topic priorities, and optimal teaching methods in women's health, using a mixed-methods approach.

METHODS: We conducted semi-structured interviews of primary care residents and core faculty clinicians in the BMC Internal Medicine residency program. These in-person interviews were transcribed and qualitative analyses used grounded theory. We developed an anonymous, cross-sectional 45 item survey from themes that emerged from these interviews. All internal medicine residents (primary care and categorical) and faculty preceptors at BMC were invited to participate in an electronic survey via email. The survey assessed perceptions of resident competency to manage women's health conditions. Descriptive statistics were used to compare resident and faculty responses.

RESULTS: Qualitative interviews of 7 (of 15) primary care residents and 6 (of 13) core faculty clinicians revealed that residents and faculty are supportive of an increase in women's health education and endorse achievement of a minimum level of proficiency in women's health topics for all residents, regardless of career plans. Interviews identified inconsistency in resident opportunity to participate in women's health training as a major barrier to knowledge acquisition in women's health. Heterogeneity in exposure to women's health education varied with residents' gender, personal interest, and exposure to individual preceptors. Survey data from 46 of 146 residents (32\%) and 23 of 48 (48 \%) faculty revealed areas of discrepancy in resident and faculty perceptions. While $36 \%$ of residents reported they were comfortable managing women's health conditions, only $6 \%$ of faculty perceived that the residents they worked with were comfortable with women's health topics. Similarly, $20 \%$ of residents reported their knowledge of women's health topics was good or very good, while only $12 \%$ of faculty agreed. There was no statistically significant difference between male and female residents' perceived knowledge (chi-squared analysis, $p=0.07)$ or comfort $(p=0.36)$.

CONCLUSIONS: Overall, internal medicine residents' knowledge and comfort in managing women's health conditions are low at BMC. Our mixed methods study suggests that successful development of a women's health curriculum must address the inconsistencies in resident exposure and opportunity, as well as the discrepancy between faculty and resident perceptions of resident skills and comfort. These findings have informed the development and implementation of a new curriculum at BMC covering high-priority women's health topics, including workshops, pre-clinic conferences, and a mini-elective.

DIABETES OUTCOME AND PROCESS MEASURES AMONG PATIENTS WITH LIMITED ENGLISH PROFICIENCY IN MINNESOTA PRIMARY CARE PRACTICES Jane Njeru² ${ }^{2}$ Deborah Boehm ${ }^{1}$; Debra Jacobson²; Laura M. GuzmanCorrales $^{1}$; Chun Fan ${ }^{2}$; Scott Shimotsu ${ }^{1}$; Mark L. Wieland ${ }^{2}$. ${ }^{1}$ Hennepin County Medical Center, Minneapolis, MN; ${ }^{2}$ Mayo Clinic, Rochester, MN. (Control ID \#2466596)

BACKGROUND: Immigrants and refugees with diabetes are less likely to meet recommended disease management goals than the general US population. Those with limited
English proficiency (LEP) may be at an even higher risk for encountering barriers to optimal diabetes management. While most patients with LEP receive their diabetes care in primary care clinics, little is known about the association between LEP and diabetes outcomes in these settings. The objective of this study was to determine adherence with diabetes process and outcomes measures among patients with LEP compared to Englishproficient (EP) patients in primary care settings.

METHODS: Study Design: This was a retrospective cohort study of patients with type II diabetes at two large primary care networks in Minnesota from January 1, 2012 through December 31, 2013. Patients: Patients with a provider-generated diagnosis of type II diabetes in electronic medical records. Patient-identified need for interpreter services was used as a proxy for LEP. Measures: Measures of guideline adherence were based on Minnesota Community Measures for Diabetes Management at the time of the study. Diabetes outcome measure goals were defined as hemoglobin $\mathrm{A} 1 \mathrm{C}<8 \%$, low density lipoprotein cholesterol (LDL-C) $<100 \mathrm{mg} / \mathrm{dL}$, and blood pressure $<140 / 90 \mathrm{mmHg}$. Diabetes process measure goals were defined as hemoglobin A1C measured within the previous 6 months and LDL-C measured within the previous 12 months.

RESULTS: Compared to the EP patients, patients with LEP were less likely to meet guideline outcome recommendations for hemoglobin A1C (66.9 versus $73.9 \%$; $p<0.0001)$ and LDL-C (59.3 versus $71.4 \% ; p<0.0001)$, but more likely to meet guideline outcome recommendations for blood pressure (83.3 versus $75.9 \%$; $p<0.0001)$. In contrast, the LEP patients were more likely than the EP patients to meet guideline recommendations for the two diabetes process measures assessed: hemoglobin A1C measurement within 6 months $(68.4 \%$ versus $60.0 \% ; p<0.0001)$, and LDL-C measurement within 12 months ( $78.5 \%$ versus $72.6 \% ; p<0.0001)$ In logistic regression analyses, adjusted for age, sex, site and number of outpatient visits, LEP patients were more likely to meet guideline outcome recommendations for blood pressure $<140 / 90$ (OR $[95 \% \mathrm{CI}]=2.02[1.7,2.4])$, and more likely meet guidelines for both diabetes process measures examined; completion of A1C testing within 6 months $(\mathrm{OR} ;[\mathrm{CI}]=1.23 ;[1.08$ 1.4]), and completion of LDL testing within 12 months (OR; $[\mathrm{CI}]=1.4 ;[1.2,1.62])$ compared to EP patients.

CONCLUSIONS: Healthcare system solutions are needed to bridge the gap from diabetes process to outcomes among patients with LEP in primary care settings.

DIABETES SCREENING IN A LARGE, SAFETY-NET HEALTHCARE SYSTEM: FREQUENCY AND OUTCOMES OF GLYCEMIC TESTING IN REALWORLD CLINICAL PRACTICE Michael E. Bowen ${ }^{1}$; Lei Xuan ${ }^{1}$; Joanne Sanders ${ }^{1}$; Noel O. Santini ${ }^{2}$; Ildiko Lingvay ${ }^{1}$; Ethan Halm ${ }^{1} .{ }^{1}$ University of Texas Southwestern Medical Center at Dallas, Dallas, TX; ${ }^{2}$ Parkland Health and Hospital System, Dallas, TX. (Control ID \#2469860)

BACKGROUND: Population-based surveys indicate that only $50 \%$ of US adults report being screened for diabetes, and screening is less frequent in ethnic minorities and those with lower socioeconomic status. However, screening patterns and screening rates in clinical practice may differ from those in the general population. Additionally, screening rates in vulnerable populations and ethnic minorities who receive care in healthcare systems designed to care for the underserved may differ from those in the general population. In this study, we sought to characterize glycemic testing patterns in routine clinical practice and determine the prevalence and outcomes of gold-standard diabetes screening in a high-risk, underserved population.

METHODS: We conducted a retrospective cohort study using comprehensive electronic medical record (EMR) data from a large, integrated, indigent healthcare system. Eligible patients were non-pregnant, age 18-65 and had body mass index (BMI) available. All participants had an index visit between January 2012 and June 2013, with the index visit defined as the first visit occurring during this period. All patients were established in 
primary care with 2 or more primary care visits in the 2 years following their index visit. Using a look-back period of 18 months, we excluded patients coded for diabetes on or before their index visit. We further excluded those having a normal gold standard diabetes test result in the 18 months prior to the index visit. By doing so, we created a cohort of patients eligible for screening between the index visit and the end of 2014. Gold standard diabetes testing was defined as a resulted $\mathrm{A} 1 \mathrm{C}$, fasting glucose, or oral glucose tolerance test (OGTT). We describe the frequency of glycemic testing with random glucose, A1C, fasting glucose, and OGTT in clinical practice. We describe the outcomes of gold standard diabetes testing and examine characteristics associated with the completion of gold standard screening tests. We also describe the frequency of guideline-indicated screening according to American Diabetes Association (ADA) screening criteria.

RESULTS: In the total cohort $(N=28,488)$, the mean (SD) age was 45.1 (11.6) years. The mean BMI was 30.9 (7.4) with $80 \%$ having a BMI $\geq 25 \mathrm{~kg} / \mathrm{m}^{2}$. Over $85 \%$ of patients were non-white, with the majority of patients being Hispanic (44\%) or Black (35\%). Patients were $64 \%$ female and $79 \%$ were uninsured. The prevalence of hypertension, hyperlipidemia, and cardiovascular disease was 33,26 , and $3.8 \%$ respectively. In the total cohort of 28,488 patients, $86 \%$ completed one or more glycemic tests during the followup period. Among those with one or more resulted glycemic tests $(N=24,573), 83 \%$ had a random glucose, $59 \%$ had an A1C, $28 \%$ had a fasting glucose, and $0.7 \%$ had an OGTT. Sixty-two percent $(N=17,657)$ of the total cohort completed gold standard diabetes testing during the follow-up period. When glycemic status was classified by goldstandard test results, $51 \%$ were normal, $37 \%$ had prediabetes identified during the follow-up period, and $12 \%$ had diabetes identified during the follow-up period. A total of $49 \%$ of those completing gold-standard tests had dysglycemia, defined as having either prediabetes or diabetes. Those completing gold standard diabetes screening tests were older, had higher BMIs and were more likely to have commercial insurance or Medicaid/ Medicare ( $p<0.001$ for all). However, no difference in screening was observed among the uninsured $(p=0.16)$. Hispanics and Asians were more likely to receive gold standard screening $(p<0.001)$; however no difference was observed among blacks $(p=0.46)$. In the total cohort of 28,488 patients, $89 \%$ of met ADA screening criteria. However, only $64 \%$ of those meeting ADA screening criteria completed gold standard diabetes screening tests during the follow-up period.

CONCLUSIONS: Glycemic testing is common in real-world clinical practice within a large, safety net healthcare system, and the percent of the population completing gold standard diabetes screening tests is higher than that reported on population based surveys. Healthcare systems designed to care for underserved patients may improve diabetes screening rates in high-risk populations. However, in this high-risk population, only two-thirds of those meeting ADA screening criteria completed gold standard glycemic testing. System-level interventions are needed to improve diabetes screening rates.

DIAGNOSTIC DISCORDANCE AND INTER-HOSPITAL TRANSFER OUTCOMES Michael G. Usher ${ }^{2}$; Nishant Sahni ${ }^{2}$; Dana Herrigel ${ }^{1}$; Andrew Olson ${ }^{2}$. ${ }^{1}$ Rutgers-Robert Wood Johnson Medical School, New Brunswick, NJ; ${ }^{2}$ University of Minnesota, Minneapolis, MN. (Control ID \#2468100)

BACKGROUND: Transitions of care, whether between or within institutions, are an important source of medical errors and waste. Inter-hospital transfers, which account for approximately 1.6 million admissions yearly, are complicated by incongruent information systems, indirect and asynchronous communication, and geographical distance, all occurring in settings of high patient complexity and acuity. Risk stratification of this population is challenging, particularly given the potential impact of miscommunication, disease progression, and diagnostic error. We developed a database of patients transferred between hospitals to identify predictors of risk, and to test the hypothesis that information transfer between hospitals during this process is an important determinant of clinical outcomes.
METHODS: A large dataset of transferred patients was generated using Health Care Utilization Project's State Inpatient and Emergency Department databases from 5 states (FL, IA, NY, UT, VT) between 2011 and 2013. Transfers were included only when both referring and receiving hospitalizations could be identified. The primary independent measure was the overall agreement of 29 chronic conditions based on Elixhauser's comorbidities that were considered unlikely to change over the course of a transfer and known to impact inpatient outcomes. The agreement, referred to as the Diagnostic Concordance Index (DCI), is a percentage of total number of comorbidities where each diagnoses was either present or absent at both referring and receiving hospital stays. To capture miscommunication, we looked at the loss of a subset of four specific diagnoses based on ICD9 coding: chronic systolic failure, chronic atrial fibrillation, DVT/PE, and seizure disorder: defined as being present on admission at the referring hospital and absent on admission at the receiving hospital. Inpatient mortality was the primary outcome with length of stay and total cost as secondary outcomes. Each measure was adjusted for age, comorbidities, demographics, and aspects of the prior admission by multivariate logistic regression linear regression when indicated.

RESULTS: We identified 180,337 inter-hospital transfers in the dataset for which both the referring and receiving hospital stay could be identified. Transferred patients had higher adjusted inpatient mortality compared to non-transferred patients (OR 1.39, 95 \% CI 1.36$1.42 p<0.001)$, longer length of stay at the receiving facility $(p<0.001)$, and higher total $\operatorname{cost}(p<0.001)$. The mean DCI of transfer patients was $78 \%$. Higher DCI was associated with reduced adjusted inpatient mortality (OR $0.57,95 \%$ CI $0.39-0.83, p=0.003$ ), reduced LOS $(p<0.001)$, and reduced total cost $(p<0.001)$. Loss of information regarding specific diagnoses was commonly observed. For example, 7373 patients had a prior diagnosis of deep venous thrombosis at the referring hospital. Of these patients, only 3587 (49\%) had a diagnosis of DVT on admission at the receiving hospital. Loss of diagnosis of DVT was associated with an increase in adjusted inpatient mortality (OR $1.2995 \%$ CI $1.17-1.43, P<0.001)$, prolonged LOS $(p<0.001)$, and higher total cost $(p<0.001)$. Similarly, loss of other chronic conditions was associated with increased mortality including seizure disorder (OR $2.195 \%$ CI 1.69-2.42, $p<0.001$ ), chronic atrial fibrillation (OR 1.40, $95 \%$ CI 1.29-1.50, $p<0.001$ ), and chronic systolic heart failure $(1.68$ $95 \%$ CI $1.56-1.86, p<0.001)$.

CONCLUSIONS: Patients transferred between hospitals have high cost and inpatient mortality. We used concordance rates of chronic conditions to illustrate how frequently information is lost during the transfer process. This loss is associated with higher rates of adjusted mortality, higher cost, and greater length of stay. These data suggest augmenting communication by improving infrastructure and standardizing the hand-off process has the potential to mitigate this information loss, improve patient outcomes, and provide higher value care for transferred patients.

DIAGNOSTIC PITFALLS: A NEW PARADIGM TO UNDERSTAND AND PREVENT DIAGNOSTIC ERROR Gordon D. Schiff ${ }^{3}$, ${ }^{2}$; Mayya Volodarskaya ${ }^{3}$; Harry Reyes Nieva ${ }^{3,2}$; Hardeep Singh ${ }^{1,} \overline{4}$; Adam Wright ${ }^{3,}{ }^{2}$. ${ }^{1}$ Michael E. DeBakey Veterans Affairs Medical Center, Houston, TX; ${ }^{2}$ Harvard Medical School, Boston, MA; ${ }^{3}$ Brigham and Women's Hospital, Boston, MA; ${ }^{4}$ Baylor College of Medicine, Houston, TX. (Control ID \#2469104)

BACKGROUND: The Institute of Medicine's Committee on Diagnostic Error in Health Care has recently released a report highlighting the importance of diagnostic errors in medicine. Although studies have shown that diagnostic errors are a leading patient safety problem, particularly in primary care settings, progress in understanding and preventing them has been modest. To increase understanding of common diagnostic challenges, we further refined a paradigm previously developed by our study team - "diagnostic pitfalls" and systematically reviewed multiple sources to identify high-risk clinical situations. 
METHODS: We used a mixed-methods approach to develop the construct of "diagnostic pitfalls," which we define as clinical situations with patterns of, or vulnerabilities to, errors that lead to missed, delayed, or wrong diagnosis. We utilized the following data sources: a) Closed diagnosis-related malpractice claims from 2010 to 2014 , from the primary care and family medicine practices of a major malpractice insurer; b) Institutional risk management patient safety event reports from 2010 to 2014; c) Institutional ambulatory Morbidity and Mortality (M\&M) Rounds of a large integrated academic network from 2006 to 2014; d) AHRQ web-based M\&M presentations from 2003 to 2014; e) A systematic literature review with a pre-defined snowball strategy to identify additional relevant literature. From our data sources, we identified all disease-specific, diagnosisrelated cases and extracted key variables from each including diagnoses (correct and erroneous) and presenting signs and symptoms. We also classified breakdown(s) in the diagnostic process using two previously published taxonomies, Diagnosis Error Evaluation and Research (DEER) and Reliable Diagnosis Challenges (RDC), assigning up to 3 DEER and RDC codes to each case. From this compilation, we identified disease-specific clinical challenges and used the emerging themes to derive general diagnostic pitfalls.

RESULTS: We identified 572 relevant cases consisting of 288 closed ambulatory malpractice claims, 75 diagnosis/treatment risk management reports, 24 instructional network ambulatory M\&Ms, 40 AHRQ web M\&Ms, and 145 scientific articles. Diagnoses were missed in 108 cases and delayed in 242 cases (mean delay, 1.6 years). Misdiagnosis occurred in 258 cases. The most common initial or erroneous diagnoses were in the fields of neurology, infectious disease and pulmonology, while the final or correct diagnoses were related to oncology, neurology, and cardiology. From all of these cases, we identified 325 disease-specific pitfalls across 400 unique diseases/diagnoses. Commonly missed/delayed diagnoses included lung cancer, colorectal cancer, and myocardial infarction, while the most frequent presenting symptoms were pain (abdominal, general, and chest), emesis, fever, headache, and altered mental status. Using the DEER taxonomy we identified failure/delay in ordering needed tests (154/850, $18 \%$ ), failure/delay in considering the diagnosis $(99 / 850,12 \%)$, and failure/delay in follow-up of abnormal test results $(73 / 850,9 \%)$ as the most frequently problematic steps in the diagnostic process. The RDC classification system highlighted issues with test follow-up (87/738, $12 \%)$, recognition of acuity/severity of illness $(58 / 738,8 \%)$, and the performance/interpretation of laboratory tests $(44 / 738,6 \%)$. A number of overarching pitfall paradigms emerged from our analysis including: a) one disease repeatedly misdiagnosed as another (e.g. colon cancer mistaken for celiac disease); b) failure to appreciate the limitations of a test or exam; c) failure to follow-up on incidental findings; d) attributing new symptom to chronic disease rather than suspecting new diagnosis; e) failure to monitor evolving symptom. Illustrative disease-specific pitfalls included: a) misreading lung mass as pneumonia on chest $\mathrm{x}$-ray; b) ordering screening, instead of diagnostic, mammogram in evaluation of breast lump; c) attributing intermittent hematuria to urinary tract infections despite negative urine cultures, missing bladder cancer; d) misinterpreting facial flushing as rosacea, delaying diagnosis of carcinoid syndrome; e) under-weighing possibility of transient ischemic attack in patient with bilateral neurological symptoms.

CONCLUSIONS: "Diagnostic pitfalls" represent a new and useful construct to engage the interface between the disease, systems, and cognitive factors which contribute to misdiagnosis. We were able to identify and classify such pitfalls using the different cross-sectional lenses of locally reported cases, regional malpractice claims, and published reports. The two tools deployed (DEER and RDC) were useful for classifying pitfall features. Recurring patterns of diagnostic pitfalls were seen that illustrate both generic and disease-specific types of errors. Identifying such pitfalls offers potential advantages for increasing awareness and preventing diagnostic errors in medicine.
DIASTOLIC BUT NOT SYSTOLIC HEART FAILURE IS ASSOCIATED WITH MULTIPLE ABNORMALITIES ON PLATELET AGGREGATION TESTING Dharmesh Gopalakrishnan; Heesun J. Rogers; Paul Elson; Keith McCrae. Cleveland Clinic, Cleveland, OH. (Control ID \#2469562)

BACKGROUND: The effect(s) of co-morbid medical conditions on platelet function is poorly understood. In this retrospective EMR-based study, we analyzed the influence of various diseases on in vitro measures of platelet function-platelet function analyzer-100 (PFA-100) closure times, platelet aggregation (using light transmission aggregometry (LTA)), platelet dense granule release (using lumiaggregometry), and platelet flow-cytometry for surface glycoproteins. We also examined their influence on VWF testing.

METHODS: Four hundred ninety seven patients who had platelet aggregation testing performed using LTA between August 2008 and August 2013 were included in our study. Co-morbidities at the time of testing were recorded. Propensity score matching for each individual disease was used to adjust for relevant covariates. We used a 1:1 nearest neighbor match without replacement, with caliper width set to 0.2 times the standard deviation of the logit of the propensity score. Following matching, Fisher's exact test or Chi square test was used as appropriate to assess the association between categorical variables, while the Mann-Whitney test was used to test the association between categorical and continuous measures. Pearson co-efficient was used to assess the correlation between continuous variables. $P<0.05$ was considered significant.

RESULTS: 1$)$ Congestive heart failure $(n=44)$ was associated with impaired platelet aggregation in the presence of arachidonic acid $(p=0.001)$ and collagen $(p=0.009)$, as well as impaired dense granule release in the presence of collagen $(p=0.002)$ and epinephrine $(p=0.012)$. It was also associated with abnormal aggregation $(p=0.024)$ and release $(p=0.028)$ in the presence of $\geq 2$ agonists in the respective panels. Diastolic heart failure $(n=25)$ was found to be associated with impaired aggregation in the presence of $\operatorname{ADP}(p=0.007)$, collagen $(p=0.001)$, or arachidonic acid $(p=0.007)$, and to $\geq 2$ agonists in the aggregation panel $(p=0.008)$. Systolic heart failure $(n=26)$ was not associated with abnormalities in aggregation or release. 2) Severe aortic stenosis ( $n=$ 17) was associated with prolonged collagen/ADP $(p=0.003)$ and collagen/epinephrine $(p<0.001)$ closure times with PFA-100, but not with any abnormalities in the platelet aggregation/release panels. Severe aortic stenosis was associated with a decreased ristocetin cofactor / VWF antigen ratio $(0.66 \pm 0.17$ vs. $0.90 \pm 0.37 ; p=0.030)$, but not with any other abnormalities in VWF testing. 3) Diabetes mellitus $(n=65)$ was associated with impaired platelet aggregation in the presence of collagen $(p=0.034)$ and impaired platelet release in the presence of epinephrine $(p=0.027)$. However, glycated hemoglobin level $(\mathrm{HbA} 1 \mathrm{C})$ was not found to correlate with impairments in either aggregation or release in the presence of any agonist. Hypothyroidism $(n=71)$ or vitamin D deficiency $(n=39)$ were not found to be associated with abnormalities in any of the platelet function assays. Finally, biochemical parameters reflecting hepatic or renal function did not correlate with any abnormalities in platelet function assays. However, the total number of co-morbidities in any patient correlated with the number of abnormalities in the platelet aggregation as well as release panels.

CONCLUSIONS: Diastolic heart failure was associated with impaired platelet aggregation in the presence of multiple agonists. Though the mechanism remains unclear, we postulate that this could be related to shear stress to which the platelets are subjected in the non-compliant ventricles. Severe aortic stenosis was associated with prolonged collagen/ ADP as well as collagen/epinephrine PFA-100 closure times and with lower ristocetin cofactor/VW antigen ratio suggesting functional impairment of VWF. Though diabetes mellitus was associated with impaired platelet aggregation in the presence of collagen and impaired dense granule release in the presence of epinephrine, no correlation was found between these abnormalities and $\mathrm{HbA1C}$ levels, making the significance of the association unclear. 
DID THE CHANGE IN THE US PREVENTATIVE SERVICES TASK FORCE BREAST CANCER SCREENING GUIDELINES CHANGE PHYSICIAN BEHAVIOR? Johanna Martinez ${ }^{1}$; Erica Phillips ${ }^{2}$; Ginger J. Winston ${ }^{3}$; Carla BoutinFoster $^{2}$; Joseph Conigliaro ${ }^{1}{ }^{1}$ North Shore LIJ Health System, New Hyde Park, NY; ${ }^{2}$ Weill Cornell Medical College, New York, NY; ${ }^{3}$ George Washington University, Washington, DC. (Control ID \#2469208)

BACKGROUND: In 2009 the United States Preventative Services Task Force (USPSTF) updated its guidelines regarding screening mammography, and in April 2015 a draft recommendation was released, which is still in the process of finalization. These updated guidelines differ from other major national organizations recommendations. The updated guidelines have been the topic of much controversy, given mammograms have been shown to decrease the mortality related to breast cancer. It is also known that amongst the risk factors for breast cancer age is the strongest predictor of risk. Since these updates little has been published on the actual impact of these guidelines.

METHODS: We conducted a cross sectional analysis of 6640 women ages 39-49. These women were patients who received their primary care at a large urban New York academic medical center. Both Internal Medicine and Gynecology primary care practices were included in the analysis. In order to isolate physician behavior around adherence to the updated USPSTF guidelines, mammograms ordered, whether completed or not, was considered the primary outcome. To determine if physicians had changed their behavior regarding mammography screening for women 40-49 years of age, we compared the proportion of mammograms ordered for women ages 39-49 in 2007 to those ordered in 2011. Several patient socio-demographic data and number of appointments in that respective year were collected, as well as provider specialty. We used paired t-tests to compare continuous variables and a chi-square test to compare categorical variables. For the nonparametric variables of age, and number of appointments in that respective year, we used Wilcoxon rank-sum test and negative binomial regression. All variables in the bivariate analyses with a $p$-value of $\leq 0.05$ were included in the multivariate analyses. Logistic regression was used for the multivariate model to account for directionality. All data was analyzed using STATA 12.1 .

RESULTS: Among the cohort of women, the average age was 43.8. The majority was single $(52 \%)$, had private health insurance (56\%), spoke English $(70 \%)$ and when race/ethnicity was identified the majority was White $(25 \%)$. The patients seen within the two specialty groups, Internal Medicine and Gynecology, were socio-demographically different. When separated, Internal Medicine patients were predominantly a privately insured group ( $62 \%$ ), opposed to the Gynecology group being predominantly a Medicaid group (71\%). This difference is attributed to the Gynecology group being a resident-only practice. In the bivariate analysis, older age, being married, identifying as White, having insurance and having English as the preferred language were significant ( $p$-value $<0.05$ ) for having a mammogram ordered both before and after the updated guidelines. A logistic regression analysis of all women, those seen both in the Internal Medicine and/or Gynecology practices revealed that women had a 1.8 times more likelihood of getting a mammogram ordered in 2011 after the updated guidelines were released, as compared to 2007 (CI 1.6-1.9, $p<.000$ ). The Gynecology (65 \%) group had a higher proportion of mammograms ordered when compared to the Internal Medicine group (55\%). Underinsured women had a 2.3 (CI 1.8-3.1, $p<.000)$ times more likelihood of not having a mammogram ordered.

CONCLUSIONS: Our study shows that the updated 2009 US Preventative Services Task Force guidelines to not routinely screen women between the ages of 40-49 did not decrease the rates of these women getting mammograms. It also continues to support that lack of health insurance continues to be associated with lack of screening. The study demonstrates that Internists and Gynecologists have different rates of agreement with the updated USPSTF guidelines, as evidenced by different rates of ordering mammograms within this age group. Given that lack of insurance continues to predict mammography screening, what impact the USPSTF guidelines may have on insurer's decisions to cover screening mammograms and that decision's impact subsequently on patients must be assessed in future research

DIFFERENCES IN EXPECTED AND REPORTED SYSTOLIC BLOOD PRESSURE MEASUREMENTS AND THE IMPACT ON USER EXPERIENCE FROM A POPULAR, INACCURATE SMARTPHONE BLOOD PRESSURE APPLICATION Timothy B. Plante ${ }^{1}$; Anna C. O'Kelly ${ }^{2}$; Satish Misra ${ }^{3}$; Bruno Urrea ${ }^{3}$; Seth S. Martin ${ }^{3}$. ${ }^{1}$ Johns Hopkins University, Baltimore, MD; ${ }^{2}$ Johns Hopkins University School of Medicine, Baltimore, MD; ${ }^{3}$ Division of Cardiology, Johns Hopkins University, Baltimore, MD. (Control ID \#2469650)

BACKGROUND: Instant Blood Pressure (IBP) is a popular mobile health application (mHealth; app) that measures blood pressure using a smartphone's integrated sensors and no external devices (e.g., cuff). Between its release on June 5, 2014 and removal on July 30, 2015 , it spent 156 days as a top 50 best-selling app on iTunes, with $\geq 950$ copies of this $\$ 4.99$ app sold on each of those days. We previously described a clinical trial in which IBP attained the lowest achievable accuracy grade and misclassified $78 \%$ of hypertensive blood pressure measurements as non-hypertensive. The reason for IBP's commercial success is unclear. We hypothesize that lower-than-expected IBP systolic blood pressure readings may improve user experience by falsely reassuring patients, which may drive app uptake.

METHODS: Adult outpatients were recruited for an IBP validation study from 5 ambulatory clinics. Participants reported their expected BP then had measurements by IBP (unblinded) and an automated sphygmomanometer (blinded). Participants completed a survey addressing 6 domains: IBP user enjoyment, likelihood for peer recommendation, perceived accuracy, perceived appropriateness of IBP in medical decision making, motivation for future mHealth app use, and motivation for future BP self-monitoring. Participants were divided into 3 groups defined by the difference between expected and IBPreported systolic BP measurements: IBP lower (IBP $>10 \mathrm{mmHg}$ below expected), IBP similar (IBP within $10 \mathrm{mmHg}$ of expected), or IBP higher (IBP $>10 \mathrm{mmHg}$ above expected). The outcomes were the proportion scoring $\geq 4$ (agree or strongly agree) in each of the 6 domains. Analysis used unadjusted and adjusted logistic regression models on Stata 13.1. Odds were converted to proportions.

RESULTS: Full sets of blood pressure measurements were collected in 81 individuals (IBP lower $n=25$, similar $n=33$, higher $n=23$ ). There was a younger mean [SD] age in IBP lower (53.3 [16.6] years) than IBP higher (62.7 [13.5] years; $P=0.04)$ but no difference from IBP similar (56.3 [17.6] years; $P=0.51)$. There were fewer men in IBP lower ( $44 \%)$ than IBP similar ( $61 \% ; P=0.02)$ but not IBP higher $(57 \% ; P=0.07)$. More patients owned a smartphone in IBP similar (91\%) than IBP lower and higher $(80 \%$ and $78 \% ; P<0.05$ for both). Groups had a similar prevalence of hypertension (63\%) and mean BMI $(27.5 \mathrm{~kg} / \mathrm{m} 2)$. In the unadjusted model, there were no significant differences between the groups in the proportion rating $\geq 4$ in any of the domains. After adjusting for age, sex, race, and history of hypertension, there were larger proportions for IBP user enjoyment for IBP lower $(0.44 ; 95 \%$ CI $0.38-0.49)$ and IBP similar $(0.46 ; 95 \%$ CI $0.43-0.49)$ than IBP higher $(0.35 ; 95 \%$ CI $\left.0.25-0.43 ; \mathrm{Chi}^{2} \mathrm{P}=0.03\right)$. There was a non-significant trend towards larger proportion motivated for future blood pressure self-monitoring in IBP lower $(0.43$; $95 \%$ CI $0.36-$ $0.48)$ and IBP similar $(0.42 ; 95 \%$ CI $0.35-0.46)$ than IBP higher $(0.31 ; 95 \%$ CI $0.20-0.40$; $\mathrm{Chi}^{2} \mathrm{P}=0.09$ ). This reached statistical significance upon comparing a combined IBP lower and similar group to IBP higher $(P=0.02)$.

CONCLUSIONS: Similar to expected or lower than expected blood pressure measurements are associated with a positive user experience and a possible increase in motivation to self-measure blood pressure. These relationships may preferentially drive uptake of inaccurate mHealth apps that provide reassuring measurements. 
DIFFUSION OF DIGITAL BREAST TOMOSYNTHESIS AMONG WOMEN IN PRIMARY CARE PRACTICE Cheryl R. Clark ${ }^{1}$; Tor Tosteson ${ }^{2}$; Anna N. Tosteson ${ }^{2}$; Tracy Onega ${ }^{2}$; Julie Weiss ${ }^{2}$; Kimberly A. Harris ${ }^{1}$; Jennifer Haas ${ }^{1} .{ }^{1}$ Brigham and Women's Hospital, Boston, MA; ${ }^{2}$ Geisel School of Medicine at Dartmouth, Lebanon, NH. (Control ID \#2468661)

BACKGROUND: Digital breast tomosynthesis (DBT) is an emerging technology that has shown early potential to improve breast cancer screening and diagnostic testing compared to digital mammography (DM), including a reduction in false-positive results. The FDA approved DBT in 2011, with coverage approved by the Centers for Medicare and Medicaid Services in 2015. The transition from DM to DBT is costly for radiology practices, but many radiology practices adopted DBT before coverage approval. Given this complex backdrop, it is important to examine the diffusion of DBT use for screening or diagnostic testing by women seen in primary care, and to examine whether use differed by insurance status over time.

METHODS: We performed a longitudinal analysis of DBT use in 20 primary care practices that participate in the Dartmouth-Brigham and Women's Hospital Populationbased Research Optimizing Screening through Personalized Regimens (PROSPR) breast cancer research center. Data sources include clinical and demographic data reported from electronic medical records (EMR), administrative claims data, and census data based on patient residential addresses. Mammogram type (DBT vs. DM) and indication for testing (screening vs. diagnostic) were defined using EMR and claims data. Insurance type was assigned to each mammogram based on patient information in the EMR. Covariates thought to influence DBT utilization included: age, breast density, race or ethnicity, and median household income for zip code of residence. We analyzed data for all mammograms performed between June 2011 and September 2014, among women aged 40 to 89 years. We examined use at early (June 2011-June 2013), middle (July 2013-June 2014) and late (July 2014-September 2014) phases of DBT uptake. Logistic regression was used to estimate the probability of having DBT as a proportion of mammograms performed, analyzed separately for screening and diagnostic exams. Because women may have had multiple mammograms during the study period, a repeated measures analysis was conducted using generalized estimating equations.

RESULTS: During this study period, 93,182 mammograms were performed on 48,234 women. Of these exams, 16,506 DBT tests were performed for screening $(17.7 \%$ of all screens) and 2537 were performed for diagnosis ( $15.7 \%$ of all diagnostic exams). Over the entire study period, DBT was used most frequently for women with private insurance coverage (19.5\%), compared to women with Medicaid coverage (17.7\%), or Medicare coverage (13.3\%), and was less frequently performed for uninsured women (7.8 \%). DBT was also used more frequently for women with extremely dense breasts $(19.2 \%)$ compared to almost entirely fatty breasts $(9.5 \%)$, where women lived in lower income zip codes (< $\$ 61,000$ median, $19.1 \%$ DBT use) compared to higher income areas ( $>\$ 100,000,16.0 \%$ DBT use), and where women were of non-Hispanic white (19.6\%) or non-Hispanic Asian/ Pacific Islander race (20.6\%), compared to non-Hispanic black (11.4\%) and Hispanic women (12.7\%). Table 1 shows early, middle, and late longitudinal trends of DBT utilization associated with insurance status for screening and diagnostic use. DBT use increased substantially for all groups across the study period. During the early period, a higher proportion of screening mammograms were performed as DBT among Medicaid (5.4\%) and Medicare beneficiaries (3.5\%) compared to privately insured $(2.8 \%)$ or uninsured $(1.1 \%)$ women [test for insurance trend $p<0.001$.] However, by the latest period, DBT was used more frequently under private insurance (43.4\%) than Medicaid (36.2 \%) or Medicare (38.3\%) [insurance trend $P<0.001$.] No differences in use of DBT for diagnostic testing were seen by insurance status in later phases of DBT uptake.

CONCLUSIONS: DBT is increasingly used for breast cancer screening and diagnosis. Use of DBT for screening may relate to insurance status. Surveillance is required to ensure that disparities in breast cancer screening are minimized as DBT becomes more widely available.
Proportion of DBT $(95 \% \mathrm{CI})$ performed by insurance type and exam indication

\begin{tabular}{|c|c|c|}
\hline & $\begin{array}{l}\text { Screening Indication } \\
\text { Proportion DBT }(95 \% \mathrm{CI})\end{array}$ & $\begin{array}{l}\text { Diagnostic Indication } \\
\text { Proportion DBT }(95 \% \mathrm{CI})\end{array}$ \\
\hline \multicolumn{3}{|l|}{ Insurance Type } \\
\hline Early: June 2011-June 2013 & $P<0.0001$ & $P<0.0001$ \\
\hline Private & $2.8(2.6-3.0)$ & $3.8(3.4-4.4)$ \\
\hline Medicaid & $5.4(2.6-3.0)$ & $5.1(3.5-7.3)$ \\
\hline Medicare & $3.5(3.0-4.2)$ & $5.4(4.2-6.9)$ \\
\hline Uninsured & $1.1(0.7-1.6)$ & $1.5(0.9-2.4)$ \\
\hline Mid: July 2013-June 2014 & $P<0.0001$ & $p=0.57$ \\
\hline Private & $16.8(16.2-17.4)$ & $21.9(20.5-23.3)$ \\
\hline Medicaid & $14.0(12.4-15.8)$ & $25.1(21.3-29.4)$ \\
\hline Medicare & $13.9(12.9-14.9)$ & $21.7(19.2-24.5)$ \\
\hline Uninsured & $12.5(9.8-15.8)$ & $25.6(13.9-42.2)$ \\
\hline Late: July 2014-September 2014 & $P=0.001$ & $P=0.95$ \\
\hline Private & $43.4(42.0-44.9)$ & $36.1(33.0-39.4)$ \\
\hline Medicaid & $36.2(31.0-41.7)$ & $35.6(27.4-44.9)$ \\
\hline Medicare & $38.3(35.6-41.1)$ & $37.7(32.3-43.3)$ \\
\hline Uninsured & $34.7(23.3-48.2)$ & $30.9(14.8-53.5)$ \\
\hline
\end{tabular}

Adjusted for age at mammogram, race/ethnicity, region of care (Brigham and Women's Hospital, Dartmouth-Hitchcock), breast density, and neighborhood zip code median household income

DIRECT ORAL ANTICOAGULANT USAGE IN THE TREATMENT OF VENOUS THROMBOEMBOLISM ACROSS RACIAL GROUPS IN DURHAM COUNTY, NC Bhavana P. Singh ${ }^{1}$; Andrea Sitlinger ${ }^{1}$; Ibrahim Saber ${ }^{1}$; Elizabeth Thames $^{1}$; michele beckman ${ }^{2}$; Nimia Reyes ${ }^{2}$; Ryan D. Schulteis ${ }^{3}$; Thomas Ortel ${ }^{1}{ }^{1}$ Duke University, Durham, NC; ${ }^{2}$ Centers for Disease Control and Prevention, Atlanta, GA; ${ }^{3}$ Veteran's Affairs Medical Center, Durham, NC. (Control ID \#2459847)

BACKGROUND: Thromboembolic diseases are of major clinical concern due to their high prevalence and significant comorbidities. The standard of care in the past for venous thromboembolism (VTE) involved the use of unfractionated heparin, low-molecular weight heparin, fondaparinux, and oral vitamin $\mathrm{K}$ antagonists. This landscape has changed with the development of direct oral anticoagulants (DOACs). Following Food and Drug Administration approval for rivaroxaban in 2012, DOACs are becoming more common due to their convenience, absence of significant drug and food interactions, and lack of necessity for lab monitoring. Despite increasing availability, there is little information on the usage of DOACs in different racial groups. It is our hypothesis that significant barriers exist in the usage of DOACs in African American (AA) populations. Furthermore, we also suspect that increased prevalence of certain comorbid conditions in AAs may contribute to differences in DOAC usage.

METHODS: All patients with VTE from April 2012 through March 2014 in Durham County, North Carolina (NC) were identified as part of a VTE Surveillance Project supported by the Centers for Disease Control and Prevention. Patients from Duke University Hospital and Duke Regional Hospital were identified by searching the electronic medical record. Data was abstracted from patient charts into the REDCap database, including information on patient demographics, VTE risk factors, and treatment methods. A similar strategy was applied at the third site, the Durham Veterans Affairs Medical Center. Durham County census data was obtained to determine incidence. Odds ratios and $95 \%$ confidence limits were obtained using logistic regression for both univariate and multivariate models. The data was analyzed using R v3.2.3.

RESULTS: Over the 2-year period we identified 402 of cases of incident VTE among AAs and 313 in non-African-Americans (non-AAs). The incidence of VTE, over the study period was approximately double among patients identified as AA ( $\sim 3.5$ per 1000) versus those identified as non-AA (1.8 per 1000). We found that $9.3 \%$ (29 of 313) of non-AAs received a DOAC following diagnosis of VTE, compared to only $4.5 \%$ (18 of 402) of AAs (OR 0.46, $95 \%$ CI $\left.0.25-0.84, \chi^{2}=6.6, p=0.01\right)$. Using univariate analyses, we measured the 
association of a. priori identified demographic and medical covariates with DOAC usage. Using a cutoff of $p<0.10$, we identified 4 covariates associated with DOAC usage: renal failure (OR 0.16, $\chi^{2}=4.4, p=0.04$ ), hypertension (OR 0.58, $\chi^{2}=3.2, p=0.07$ ), active cancer (OR 0.08, $\chi^{2}=9.9, p=0.002$ ) and insurance status (OR 4.87, $\chi^{2}=3.0, p=0.09$ ). As each covariate had the potential to confound the relationship between race and DOAC usage, we adjusted the estimated association between race and DOAC usage in a stepwise approach, testing each covariate alongside AA status using bivariate logistic regression (Table 1). In each case, AA status was found to be associated with reduced DOAC usage even after adjustment. Of these four variables, only "active cancer" after adjusting for AA status remained significantly associated with reduced DOAC use (OR 0.19, $p=0.017$ )

CONCLUSIONS: We saw less treatment for VTE with DOAC among AA patients with VTE compared to non-AA patients using a population of incident VTE events across a single county in NC. Certain potential confounders, such as renal failure, may be both 1) more common in AA patients; and, 2) inversely associated with DOAC use. To see if the reduction in DOAC use amongst AA patients was due to an independent effect of race versus a consequence of confounding, we adjusted for the effects of renal failure, hypertension, active cancer and insurance status. In each case, we found that the effect of race was independent, even after adjustment for these potential confounders. Future studies with a larger sample size might examine the independent effect of race after adjusting, simultaneously, for more than 1 confounder. These data suggest that AA patients may have less access to DOAC agents than their non-AA counterparts even after adjusting for other potential confounding factors. Future larger studies are needed to confirm these findings and determine strategies that are are effective in lowering barriers to access to safe, affordable, and effective treatments for minority populations. DOACs provide physicians with new therapeutic options in the management of VTE. By understanding the importance of different factors in the usage of DOACs, physicians may be able to modify their prescribing practices in different racial groups.

Table 1: A bivariate analysis of DOAC usage among AA controlling for select covariates

\begin{tabular}{|c|c|c|c|c|}
\hline Variable & $\begin{array}{l}\text { OR AA } \\
(95 \% \mathrm{CI})^{\text {a }}\end{array}$ & $\begin{array}{l}P \text {-Value } \\
\text { AA }^{\text {b }}\end{array}$ & $\begin{array}{l}\text { OR VAR } \\
(95 \% \mathrm{CI})^{c}\end{array}$ & $\begin{array}{l}P \text {-Value } \\
\text { VAR }^{\mathrm{d}}\end{array}$ \\
\hline Renal Failure & $\begin{array}{l}0.50 \\
(0.27-0.92)\end{array}$ & 0.027 & $\begin{array}{l}0.18 \\
(0.02-1.36)\end{array}$ & 0.097 \\
\hline Hypertension & $\begin{array}{l}0.48 \\
(0.26-0.89)\end{array}$ & 0.020 & $\begin{array}{l}0.64 \\
(0.35-1.16)\end{array}$ & 0.138 \\
\hline Active cancer & $\begin{array}{l}0.51 \\
(0.27-0.93)\end{array}$ & 0.030 & $\begin{array}{l}0.09 \\
(0.01-0.65)\end{array}$ & 0.017 \\
\hline Insurance status & $\begin{array}{l}0.47 \\
(0.25-0.86)\end{array}$ & 0.014 & $\begin{array}{l}4.69 \\
(0.63-34.7)\end{array}$ & 0.130 \\
\hline
\end{tabular}

a - OR AA - the effect of AA vs. nonAA after adjustment with covariate,

$\mathrm{b}-P$-Value $\mathrm{AA}-p$ value for the effect of AA vs. nonAA after adjustment with covariate,

c - OR VAR - OR for covariate,

$\mathrm{d}-P$-Value VAR $-P$ value for covariate

\section{DIRECT-OBSERVATION STUDY OF SHARED DECISION MAKING IN A PRI-} MARY CARE INTERNAL MEDICINE CLINIC Jeffrey L. Jackson ${ }^{4}$, 5; Derek Storch $^{4}$; Patrick G. O'Malley ${ }^{1}$; Dorothy Becher ${ }^{2}$; Wilkins Jackson ${ }^{3}$; sheila Scott ${ }^{4}$. ${ }^{1}$ Uniformed Services University, Washington, DC; ${ }^{2}$ Uniformed Services University of the Health Sciences, Bethesda, MD; ${ }^{3}$ University of Wisconsin Milwaukee, Milwaukee, WI; ${ }^{4}$ Zablocki VAMC, Milwaukee, WI; ${ }^{5}$ medical college of wisconsin, Milwaukee, WI. (Control ID \#2468928)

BACKGROUND: Shared decision making is considered a preferred mode of reaching patient decisions, yet little research has directly observed the nature of primary care decision making. We sought to describe decision making in an adult primary care clinic.
METHODS: Adult patients with an established primary care relationship (defined as at least 2 visits in the previous year with the same provider) and at least 3 co-morbid medical conditions were eligible to participate. Prior to a routine visit with their primary care provider, patients completed surveys that assessed their expectations for care, functional status (Medical Outcomes Study SF6), somatization (PHQ-15), mood and anxiety disorders (PRIME-MD) pain severity (0-10 scale), adherence (using both a pill count and the Morisky scale), literacy (REALM), health locus of control and serious illness worry. Prior to their visit, patients were asked to provide their preferred style of shared decision making style, post-visit, patient surveys assessed the degree of shared decision making they experienced during the encounter (using the same scale as before the visit), patient satisfaction (Rand-9 item survey), and trust (Trust in Clinician 11 item instrument). Providers also ated the amount of shared decision making they experienced during the encounter. Visits were audiotaped and coded using the Roter Interactive Assessment System. In addition, three coders coded the transcripts for the type of decision-making that occurred, assessed the complexity of decision making, and rated each decision for the degree of shared decision-making observed for each decision.

RESULTS: One hundred five patients, averaging 65.8 years (52\% female, $57 \%$ black) participated. The encounters (mean, $29 \mathrm{~min}, 7.4$ decisions per visit) involved mostly basic decisions surrounding diagnostic tests $(27 \%)$, medications (26\%), follow-up (14\%), referrals (14\%), treatments $(8 \%)$ and lifestyle changes $(8 \%)$. The interactions were largely balanced between patients and providers (average encounter comprised of $57 \%$ provider and $43 \%$ patient statements). Before the visit, $54 \%$ of patients expressed a desire for equally shared medical decision making, $24 \%$ physician dominated decision making and $18 \%$ preferred that they make all the decisions. After the visit, they reported actually experiencing shared decision making in $57 \%$ of encounters. Objective measures of decision making indicated $52 \%$ of encounters having shared decision-making which varied significantly by complexity of decision making, where more complex decisions had higher rates of both paternalistic and consumeristic styles. Post-visit ratings of satisfaction with care or trust in one's physician did not vary by any preferences or behaviors of decision making, or patient and physician characteristics.

CONCLUSIONS: Decisions are common during adult internal medicine primary care visits; and most tend to be basic. Most patients preferred shared decision making, and their perceptions of the visit decision making style were concordant with their preferences. Actual decision-making varied significantly by complexity of the decision, indicating a need for flexibility in decision making styles. Preferences and behaviors surrounding decision-making did not correlate with patient satisfaction, trust or medication adherence.

DISCORDANCE IN PERCEIVED TASK RESPONSIBILITY BETWEEN NURSES AND PRIMARY CARE PROVIDERS AND BURNOUT IN VETERANS AFFAIRS PATIENT ALIGNED CARE TEAMS Samuel T. Edwards ${ }^{3,4}$; Christian D. Helfrich $^{2}$; Elizabeth Hulen ${ }^{3}$; Jeffery L. Williams ${ }^{3}$; Walter L. Clinton ${ }^{1}$; Gordon B. Wood ${ }^{2}$; Greg Stewart ${ }^{5}$. ${ }^{1}$ US Department of Veterans Affairs, Seattle, WA; ${ }^{2}$ VA Puget Sound Healthcare System, Seattle, WA; ${ }^{3}$ VA Portland Health Care System, Portland, OR; ${ }^{4}$ Oregon Health and Science University, Portland, OR; ${ }^{5}$ VA Iowa Coty Health Care System, Iowa City, IA. (Control ID \#2469830)

BACKGROUND: The Veterans Affairs (VA) patient-centered medical home model Patient Aligned Care Teams (PACT) was designed to promote team-based patient care, with discrete roles for each primary care team member. However, overlap in perceived 
responsibilities among PACT members is common and may lead to inefficiency and overwork. Discordance between primary care providers' (PCPs) and primary care nurse care managers' (NCMs) perceptions of responsibility for clinical tasks is particularly common. We investigated the importance of discordance in reported task responsibility between PCPs and NCMs, examining factors that might contribute to PCP-NCM task responsibility discordance and whether such discordance was associated with PCP and NCM burnout.

METHODS: We performed a cross sectional analysis of a national survey of VA PCPs and primary care staff fielded in November 2014. Questions asked respondents how much PCPs relied on staff for 15 commonly performed primary care tasks on a four point Likert scale (not at all, a little, somewhat, a great deal). Tasks included gathering preventive service history, disease screening, evaluating patients and making treatment decisions, assessing and intervening on lifestyle factors, educating patients about self-care activities and medications, refilling prescriptions, receiving and resolving patient messages, completing forms, tracking diagnostic data, referral tracking, and arranging home health care. We developed a measure of task responsibility discordance for PCP-NCM dyads by calculating the difference between the PCP reported reliance and the $\mathrm{NCM}$ reported reliance for each task. We took the absolute value of this difference, and calculated the mean across all 15 tasks. Survey questions also included a single item measure of burnout (5 point scale, dichotomized at $\geq 3$ burnout, $<3$ no burnout). We performed multivariate logistic regression to test the association between PCP-NCM task responsibility discordance and burnout, controlling for perceived appropriate clinic staffing, recent staff turnover, presence of a PACT coach, minutes/week spent in PACT huddles, and length of VA employment.

RESULTS: In 2809/8114 PACT teamlets surveyed, at least one team member responded, a $34.6 \%$ teamlet level response rate. We restricted our analysis to 777 teamlets in which both a PCP and NCM responded. In this sample $48 \%$ of PCPs and $35 \%$ of NCMs reported burnout. Mean PCP-NCM task responsibility discordance was 0.92 (SD 0.43, range $0-3)$. Appropriate staffing ( $\beta-0.155$, $p<0.01$ for PCPs, $\beta-0.08, \mathrm{p} 0.02$ for $\mathrm{NCMs}$ ) and minutes spent in huddles ( $\beta$ $-0.007, p<0.01$ for PCPs, $-0.004, p<0.01$ for NCMs) were negatively associated with PCP-NCM task responsibility discordance. PCP-NCM task responsibility discordance was significantly associated with burnout for PCPs, but not for NCMs (Table). In multivariate analysis, perceived appropriate staffing was negatively associated with burnout for PCPs and NCMs, while staff turnover, and length of VA employment were positively associated with PCP and NCM burnout (Table). CONCLUSIONS: Nearly half of VA PCPs and over a third of NCMs report burnout. Discordance in reported task responsibility between PCPs and nurses is associated with burnout for PCPs, but not for nurse care managers. Appropriate staffing levels and time spent in daily huddles were associated with less task responsibility discordance, and may represent ways to improve task allocation within teamlets, and consequently reduce PCP burnout.

Association between PCP-NCM task responsibility discordance, staffing and clinic factors and PCP and NCM burnout

\begin{tabular}{lll}
\hline \hline & PCP Burnout (Adjusted OR, & NCM Burnout (Adjusted OR, \\
& $95 \% \mathrm{CI})$ & $95 \% \mathrm{CI})$ \\
Discordance (1 unit increase) & $1.53(1.07-2.19)$ & $0.96(0.68-1.37)$ \\
Appropriate Staffing & $0.56(0.40-0.78)$ & $0.70(0.50-0.97)$ \\
Staff Turnover & $1.74(1.29-2.35)$ & $1.42(1.04-1.94)$ \\
Minutes of Huddle Time & $0.99(0.98-1.01)$ & $0.85(0.69-1.02)$ \\
Presence of PACT Coach & $0.67(0.47-0.94)$ & $1.02(1.00-1.04)$ \\
Length of VA employment & $1.05(1.02-1.07)$ & \\
(per year) & &
\end{tabular}

DISPARATE PROCESSES OF CARE AMONG MULTI-ETHNIC PATIENTS WITH OBESITY IN A GROUP PRIMARY CARE SETTING Shamol R. Williams'; Jose Melendez-Rosado ${ }^{2}$; Elizabeth Pantoja ${ }^{1}$; Michael Heckman ${ }^{1}$; Emily Vargas ${ }^{1}$; Loren Murray ${ }^{1}$; Richard O. White ${ }^{1} .{ }^{1}$ Mayo Clinic, Jacksonville, FL; ${ }^{2}$ Mayo Clinic Florida, Jacksonville, FL. (Control ID \#2466857)

BACKGROUND: This study examined the presence of racial/ethnic disparities in care processes and the quality of dietary and physical activity (PA) counseling for patients with obesity during general medical encounters in a large multigroup primary care practice.

METHODS: Patients seeking primary care at the Mayo Clinic Florida between January 2013 and April 2015 were randomly selected for inclusion in this retrospective study. Patients were oversampled by race/ethnicity to balance the distribution across the sample and allow for adequate comparisons. Patients were included for $\mathrm{BMI} \geq 30,>1$ general medical encounter within the study window, and if their self-reported race/ethnicity data was available in the electronic health record (EHR). Trained abstractors recorded encounter data and applied a set of a priori defined quality metrics informed by national guidelines to assess the quality of dietary and PA counseling. Quality of Dietary and PA counseling was categorized as "no counseling", "inadequate", "fair", "thorough", or "ideal" based upon the degree to which providers assessed behavioral targets, set goals, and arranged for specific follow-up or referral to nutrition/bariatric services when appropriate. Encounter information abstracted included patient demographics, general health information (e.g. obesity-related conditions, comorbidity index (CI)), medications, relevant vitals/lab data (SBP, DBP, LDL, A1C), and provider characteristics. Bivariate comparisons between groups were made using Kruskal-Wallis rank sum test or Fisher's exact test. Proportional odds logistic regression was used to assess the association between patient and provider factors and the quality of dietary and PA counseling with Bonferroni correction to account for multiple testing. Statistical inquiries were performed using SAS (version 9.2; SAS Institute, Inc., Cary, North Carolina). RESULTS: Seven hundred thirty-one unique encounters were reviewed (341 White patients, 214 Black patients, 112 Asian patients, and 64 patients of other races). Two hundred forty-seven patients (34\%) self-identified as Hispanic/ Latino. Asian patients were younger $(p<0.001)$ and had higher rates of diabetes ( $p=0.013)$ despite statistically significant lower BMI $(p<0.001)$ compared to White patients. Asian patients also had impairments of glucose (A1C 6.4 vs 6.1 ), and diabetes rates ( 32.1 vs $34.1 \%$ ) comparable to Black patients, but were much less likely to have had an A1C measured in the last 12 months $(p=0.03)$. Nominally significant differences $(p=0.046)$ were noted for documentation of obesity in the patient's problem list with rates being highest for Blacks $(52.8 \%)$ and lowest for Asians (37.5\%), but this did not withstand Bonferroni correction. Overall, we observed low rates (16.5-24.3\%) of high quality dietary and PA counseling (i.e. Fair-Ideal) across the sample with no significant differences noted by race; however, Hispanic/Latino patients were more likely to receive better PA counseling $(p=0.039)$, referral to nutrition services $(p=0.016)$, and attendance at the referral appointment $(p=0.033)$ compared to non-Hispanic/ Latino patients, but again this did not withstand Bonferroni correction.

CONCLUSIONS: Our study highlights the need for improved patient-provider interactions regarding weight management at the point of care, and confirms the importance of addressing diabetes and cardiovascular risk among Asian patients at lower BMI levels as reflected in the recent ADA position statement. Greater efforts are warranted to ensure high quality lifestyle counseling and equity of process measures for all patients at risk of obesity-related adverse health outcomes. 


\begin{tabular}{|c|c|c|c|c|c|c|}
\hline \multirow[b]{2}{*}{ Variable } & \multirow[b]{2}{*}{ All patients $(N=731)$} & \multirow{2}{*}{$\begin{array}{l}\text { White patients } \\
\qquad(\mathrm{N}=341)\end{array}$} & \multirow{2}{*}{$\begin{array}{c}\text { Black patients } \\
\qquad(N=214)\end{array}$} & \multirow{2}{*}{$\begin{array}{c}\text { Asian patients } \\
(N=112)\end{array}$} & \multirow{2}{*}{$\begin{array}{l}\text { Patients of other } \\
\text { races }(N=64)\end{array}$} & \multirow[b]{2}{*}{ P-value } \\
\hline & & & & & & \\
\hline Age & $56(19.87)$ & $61(20,85)$ & $56(19,87)$ & $44(21,80)$ & $51(20,83)$ & $<0.001$ \\
\hline BMII & $33.8(30.0,70.8)$ & $33.3(30.0 .57 .6)$ & $35.1(30.0,70.8)$ & $32.6(30.1,65.3)$ & $35.0(30.0,54.5)$ & $<0.001$ \\
\hline Charlson comorbidity index & $1(0,16)$ & $1(0,16)$ & $1(0,13)$ & $0(0,10)$ & $0(0,5)$ & $<0.001$ \\
\hline$\leq 1$ & $496(67.9 \%)$ & $208(61.0 \%)$ & $139(65.0 \%)$ & $98(87.5 \%)$ & $51(79.7 \%)$ & \\
\hline$>1$ & $235(32.2 \%)$ & $133(39.0 \%)$ & $75(35.0 \%)$ & $14(12.5 \%)$ & $13(20.3 \%)$ & \\
\hline Systobic BP & $129(86,199)$ & $127(86,197)$ & $132(94,199)$ & $129(99,175)$ & $121(94,157)$ & $<0.001$ \\
\hline Diastolic BP & $79(44,123)$ & $78(44,123)$ & $80(52,120)$ & $80(57,107)$ & $78(55,98)$ & 0.003 \\
\hline Diabetes & $201(27.5 \%)$ & $76(22.3 \%)$ & $73(34.1 \%)$ & $36(32.1 \%)$ & $16(25.0 \%)$ & 0.013 \\
\hline A1C measured within the last 12 months & $424(58.0 \%)$ & $200(58.7 \%)$ & $137(64.0 \%)$ & $54(48.2 \%)$ & $33(51.6 \%)$ & 0.033 \\
\hline Most Recent A1C & $6(4.1,15.0)$ & $5.8(4.1,11.8)$ & $6.1(4.6,15.0)$ & $6.4(5.0,12.1)$ & $5.7(4.9 .8 .8)$ & 0.003 \\
\hline Triglycerides & $115(25,1377)$ & $137(44,1377)$ & $83(25,575)$ & $130(41,565)$ & $111(49.290)$ & $<0.001$ \\
\hline HDL cholester of & $52.0(4.1,164.4)$ & $51.0(22.0,144.0)$ & $58.0(28.0,164.4)$ & $49.0(4.1,91.0)$ & $52.0(5.5,141.0)$ & $<0.001$ \\
\hline Patient taking medication for high blood pressure & $430(58.8 \%)$ & $208(61.0 \%)$ & $140(65.4 \%)$ & $\$ 3(47.3 \%)$ & $29(45.3 \%)$ & 0.002 \\
\hline Patient taking medication for high cholesterol & $311(42.5 \%)$ & $177(51.9 \%)$ & $86(40.2 \%)$ & $34(30.4 \%)$ & $14(21.9 \%)$ & $<0.001$ \\
\hline Overweight/obesity listed in problem list & $332(45.4 \%)$ & $149(43.7 \%)$ & $113(52.8 \%)$ & $42(37.5 \%)$ & $28(43.8 \%)$ & $0.046^{*}$ \\
\hline
\end{tabular}

The sample median (minimum, maximum) is given for continuous variables. Information was unavailable regarding systolic $B P(N=4)$, diastolic $B P(N=4)$, most recent $A \mid C$ $(\mathrm{N}=307)$, total cholesterol $(\mathrm{N}=50)$, triglycerides $(\mathrm{N}=50), \mathrm{LDL}(\mathrm{N}=57), \mathrm{HDL}(\mathrm{N}=50)$, AST $(\mathrm{N}=97)$, ALT $(\mathrm{N}=329)$, fasting blood glucose within 2 years $(\mathrm{N}=36)$, A1C within 2 years $(\mathrm{N}=285)$, and pre-diabetes documented in record $(\mathrm{N}=1)$. P-values result from a Kruskal-Wallis rank sum test or Fisher's exact test. *P-values $\leq 0.0025$ were considered as statistically significant after applying a Bonferroni adjustment for multiple comparisons.

\begin{tabular}{|l|c|c|}
\hline Variable & $\begin{array}{c}\text { Hispanic/Latino patients } \\
(\mathrm{N}=247)\end{array}$ & $\begin{array}{c}\text { Non-Hispanic/ Latino patients } \\
(\mathrm{N}=484)\end{array}$ \\
\hline $\begin{array}{l}\text { Quality of documentation/education provided to patient regarding physical } \\
\text { activity (i.e. quality of physical activity counseling) }\end{array}$ & & \\
\hline No counseling made & $104(42.1 \%)$ & $237(49.0 \%)$ \\
\hline Incomplete & $94(38.1 \%)$ & $175(36.2 \%)$ \\
\hline Fair & $27(10.9 \%)$ & $47(9.7 \%)$ \\
\hline Thorough & $16(6.5 \%)$ & $14(2.9 \%)$ \\
\hline Ideal & $6(2.4 \%)$ & $11(2.3 \%)$ \\
\hline Patient referred to nutrition counseling or bariatric assessment & & $449(92.8 \%)$ \\
\hline Neither & $225(91.1 \%)$ & $11(2.3 \%)$ \\
\hline Nutrition & $16(6.5 \%)$ & $19(3.9 \%)$ \\
\hline Bariatric & $5(2.0 \%)$ & $5(1.0 \%)$ \\
\hline Nutrition \& Bariatric & $1(0.4 \%)$ & $15(42.9 \%)$ \\
\hline If referred to nutrition or bariatric assessment patient showed up & $16(72.7 \%)$ & 0.016 \\
\hline
\end{tabular}

P-values result from a Kruskal-Wallis rank sum test of Fisher's exact test. P-values $\leq 0.0025$ were considered as statistically significant after applying a Bonferroni adjustment for multiple comparisons.

DISPARITIES IN PATIENTS' ELECTRONIC HEALTH ENGAGMENT AT AN URBAN HIV CLINIC. Mohammed F. Dabbagh; Kassem Bourgi. Henry Ford Hospital / Wayne State University, Detroit, MI. (Control ID \#2453824)

BACKGROUND: Patient-accessible electronic medical records (PEMR) enable patients to access and manage their clinical information. This tool provides patients with a better understanding of their health status and increases their engagement in medical decisions. Several studies have highlighted the positive impact of PEMR in health maintenance and chronic disease management. We aimed to evaluate the current rates and trends in electronic health engagement within an HIV clinic.

METHODS: We constructed an electronic cohort of patients seen at our comprehensive HIV clinic between January 2013 and June 2015. We evaluated the association between socio-demographic characteristics, medical conditions and provider-specific factors with the patient's electronic health engagement.

RESULTS: A total of 1450 patients were seen during the study period of which 490 $(33.7 \%)$ were enrolled in the electronic portal. Among enrolled patients the median age 
was 44.9 years, $90(18.3 \%)$ were female, 278 (56.7 \%) were African American, and 344 (70 \%) had AIDS. Enrollment in the PEMR was less common among African Americans (Odds Ratio (OR), 0.53; $95 \%$ confidence interval (CI), 0.42-0.66; $P<0.001$ ), Medicaid beneficiaries (OR, $0.51 ; 95 \% \mathrm{CI}, 0.40-0.64 ; P<0.001$ ), patients who required hospitalization during the study period (OR, $0.77 ; 95 \% \mathrm{CI}, 0.60-0.99 ; P<0.05)$, and those with history of drug or alcohol abuse (OR, 0.60; $95 \% \mathrm{CI}, 0.48-0.76 ; P<0.001)$. On the contrary, patients were more likely to engage in the PEMR if they had an established primary care physician (OR, 2.94; $95 \%$ CI, 2.17-4; $P<0.001)$ and if they were seen more than once (OR, 1.78; $95 \%$ CI, 1.37-2.30; $P<0.001)$. Age and gender had no significant influence on electronic health engagement.

CONCLUSIONS: While engagement in the PEMRs is increasing, vulnerable populations are left behind with significantly lower access. Disparities in the use of the PEMR may potentially reinforce and amplify existing health disparities. Further studies are needed to evaluate potential barriers to electronic engagement in these underserved patients

Table 1. Association of Sociodemographic and Clinical Variables with Electronic Health Engagement

\begin{tabular}{llll}
\hline \hline Variable & Odds Ratio & $95 \%$ Confidence Interval & $P$-value \\
Female Gender & 0.86 & $0.65-1.13$ & 0.29 \\
African American Race & 0.53 & $0.42-0.66$ & $<0.001$ \\
AIDS & 1.26 & $0.99-1.59$ & 0.056 \\
History of Drug or Alcohol Abuse & 0.60 & $0.48-0.76$ & $<0.001$ \\
Hospitalization during study period & 0.77 & $0.60-0.99$ & 0.04 \\
More than 2 office visits & 1.78 & $1.37-2.30$ & $<0.001$ \\
Established Primary Care Physician & 2.94 & $2.17-4.00$ & $<0.001$ \\
Patients with Insurance Coverage & 1.30 & $0.99-1.70$ & 0.62 \\
Medicaid Beneficiaries & 0.51 & $0.40-0.64$ & $<0.001$ \\
\hline
\end{tabular}

DISPARITIES IN PRENATAL AND POSTPARTUM CARE IN PENNSYLVANIA MEDICAID Natasha Parekh ${ }^{1,2}$; Marian Jarlenski ${ }^{3}$; David Kelley ${ }^{4} .{ }^{1}$ University of Pittsburgh Medical Center, PIttsburgh, PA; ${ }^{2}$ University of Pittsburgh School of Medicine, Pittsburgh, PA; ${ }^{3}$ University of Pittsburgh Graduate School of Public Health, Pittsburgh, PA; ${ }^{4}$ Pennsylvania Department of Human Services, Harrisburg, PA. (Control ID \#2467827)

BACKGROUND: State Medicaid programs comprise an important source of pregnancyrelated health care for low-income women. Nearly 2 of every 3 women enrolled in Medicaid are of childbearing age, and Medicaid finances about $48 \%$ of all births in the United States. Half of Medicaid beneficiaries are racial and ethnic minorities. In Pennsylvania (PA), there is a need to improve adverse pregnancy outcomes. Maternal mortality, infant mortality, and preterm birth rates rank 24th, 35th, and 40th in the country, respectively, and racial and ethnic disparities have been noted. Provision of high-quality prenatal and postpartum (perinatal) care represents one way to improve maternal and child health outcomes through earlier assessment and treatment of disease. Disparities in access to perinatal care have not been assessed in PA's Medicaid population. The objective of our study was to assess the extent of disparities by race, ethnicity, and region in the provision and timeliness of perinatal care for women enrolled in PA Medicaid.

METHODS: We performed a cross-sectional evaluation of a representative sample of women of all ages who delivered live births between November 2013-November 2014 and were continuously enrolled in PA Medicaid managed care plans. Independent variables of interest included race, ethnicity, and region. The outcome measures were 3 Healthcare Effectiveness Data and Information Set (HEDIS $\left.{ }^{\circledR}\right)$ binary effectiveness-ofperinatal care measures: 1 ) attendance of $\geq 81 \%$ of expected prenatal visits (i.e. frequency of prenatal care (FPC), defined by American College of Obstetricians and Gynecologists' recommendations for frequency of visits by gestational age and stage of pregnancy at time of enrollment); 2) timeliness of prenatal care (PNC) in the 1st trimester or within 42 days of enrollment; and 3) timeliness of postpartum care (PPC) within 21-56 days after delivery. Sampling was performed and validated per National Committee for Quality Assurance HEDIS ${ }^{\circledR}$ standards, and both administrative data and medical records were reviewed to assess compliance with measures. We analyzed disparities by race, ethnicity, and region within each measure using univariate and multivariable logistic regression. We also assessed interactions between covariates.

RESULTS: Complete data were available for 2991 women, who were $47 \%$ white, $31 \%$ Black, $4 \%$ Asian, and $18 \%$ Other/Not Volunteered/No Race Indicator. With regard to ethnicity, $81 \%$ were non-Hispanic, $17 \%$ were Hispanic, and $2 \%$ had no ethnicity indicator. Race was a significant predictor of compliance across all 3 HEDIS ${ }^{\circledR}$ measures $(p<0.001)$, while region was a significant predictor for both FPC and PNC $(p<0.05)$. Ethnicity was not a significant predictor for the 3 measures. There was no significant interaction between covariates in all models. Comparisons between individual groups demonstrated significant racial and regional disparities. In univariate and adjusted models, Whites were significantly more likely to have FPC (AOR 1.9, $p<0.001$ ) and PPC (AOR $1.3, p=0.004)$ compared to Blacks, and Asians were significantly more likely to have FPC (AOR 2.0, $p=0.003$ ), PNC (AOR 1.9, $p=0.049$ ), and PPC (AOR 1.6, $p=0.025$ ) compared to Blacks (see Table 1). A disparity between Whites and Blacks in PNC became non-significant when adjusting for region. In both univariate and adjusted models, the Southeast performed significantly worse than the Southwest in FPC (AOR 1.5, $p=0.001$ ) and PNC (AOR 1.4, $p=0.025$ ), and significantly worse than Lehigh/Capital in FPC (AOR $1.6, p<0.001)$. Disparities between the Southeast and Lehigh/Capital in PNC and PPC became non-significant after adjusting for race (see Table 2).

CONCLUSIONS: This study identified racial and regional disparities in provision and timeliness of perinatal care, measured by compliance with 3 HEDIS ${ }^{\circledR}$ measures. Our data demonstrate that the magnitude of disparities varies across quality measure, and that both racial and regional disparities (such as frequency of prenatal visits and timeliness of postpartum care in Blacks, and frequency of prenatal visits and timeliness of prenatal care in Southeastern PA) must be addressed in equity-promoting measures.

Table 1: Racial Disparities: Adjusted Analyses^

\begin{tabular}{llll}
\hline \hline & $\begin{array}{l}\geq 81 \% \text { of prenatal } \\
\text { visits (FPC) } \\
\text { Adjusted OR }(95 \%\end{array}$ & $\begin{array}{l}\text { Timeliness of Prenatal } \\
\text { Care (PNC) } \\
\text { Adjusted OR }(95 \% \text { CI) }\end{array}$ & $\begin{array}{l}\text { Timeliness of Postpartum } \\
\text { Care (PPC) } \\
\text { Adjusted OR }(95 \% \text { CI) }\end{array}$ \\
$\begin{array}{l}\text { Comparison } \\
\text { Blacks }\end{array}$ & $1.9(1.5-2.3)^{* *}$ & $1.3(0.9-1.6)$ & $1.3(1.1-1.6)^{* *}$ \\
$\begin{array}{l}\text { Asians vs. } \\
\text { Whites } \\
\text { Asians vs. }\end{array}$ & $1.1(0.7-1.7)$ & $1.5(0.8-3.0)$ & $1.2(0.8-1.9)$ \\
Blacks & $2.0(1.3-3.1)^{* *}$ & $1.9(1.0-3.7)^{*}$ & $1.6(1.1-2.5)^{*}$ \\
\hline
\end{tabular}

${ }^{\wedge}$ Race adjusted for region, ${ }^{* *} p<0.005,{ }^{*} p<0.05$

Table 2: Regional Disparities: Adjusted ${ }^{\wedge}$ Analyses

\begin{tabular}{|c|c|c|c|}
\hline & $\geq 81 \%$ of Prenatal & Timeliness of Prenatal & Timeliness of \\
\hline & Visits (FPC) & Care (PNC) & Postpartum Care (PPC) \\
\hline \multirow[t]{2}{*}{ Comparison } & Adjusted OR (95 & Adjusted OR (95 \% & Adjusted OR (95\% \\
\hline & $\% \mathrm{CI})$ & $\mathrm{CI})$ & $\mathrm{CI})$ \\
\hline Southwest (SW) vs. & $1.5(1.2-1.9)^{* *}$ & $1.4(1.1-1.9)^{*}$ & $1.1(0.9-1.4)$ \\
\hline \multicolumn{4}{|l|}{ Southeast (SE) } \\
\hline Lehigh/Capital vs. SE & $1.6(1.3-2.0)^{* *}$ & $1.3(0.9-1.6)$ & $1.1(0.9-1.3)$ \\
\hline Lehigh/Capital vs. SW & $1.1(0.8-1.4)$ & $0.9(0.6-1.2)$ & $1.0(0.8-1.3)$ \\
\hline
\end{tabular}

${ }^{\wedge}$ Region adjusted for race, ${ }^{* *} p<0.01,{ }^{*} p<0.05$

DIVERSE PATIENT PERSPECTIVES ON RESPECT IN HEALTHCARE: A QUALITATIVE STUDY Somnath Saha ${ }^{2,3}$; Emily Branyon ${ }^{1}$; Mary Catherine Beach ${ }^{1}$. ${ }^{1}$ Johns Hopkins University, Baltimore, MD; ${ }^{2}$ VA Portland Health Care System, Portland, OR; ${ }^{3}$ Oregon Health \& Science University, Portland, OR. (Control ID \#2470589) 
BACKGROUND: Respect is fundamental to all human interactions, and especially important in health care, where some people (patients) are dependent upon others (physicians). Respect allows for dependency without fear of mistreatment or abuse. In Western bioethics, the dominant view of respect focuses almost exclusively on respect for patient autonomy. Other dimensions of the broad concept of respect are either vague or sparsely discussed in bioethics. They are also conceptualized primarily from the perspective of academic theorists, who draw their observations about morality from different experiences than those of patients. Finally, common conceptualizations of respect often do not explicitly include the perspectives of minority groups whose cultural construction of respect may differ from that of the majority. We conducted this study to understand, from the perspective of patients from different racial/ethnic groups, what it means for patients to be treated with respect in healthcare settings.

METHODS: We conducted focus groups with African American, Latino, and white patients with diabetes and/or hypertension in urban and rural communities in the Northwestern U.S. Focus groups were community-based, stratified by race and gender, and facilitated by race/gender concordant moderators from the communities of the focus group participants. The focus groups were designed to elicit patients' perspectives on their relationships and interactions with physicians. The focus group guide included questions asking participants to describe physician behaviors that made them feel respected or disrespected. Responses to these questions, as well as spontaneous references to respect in other parts of the focus group transcripts, were reviewed and coded for: 1) definitions of respect and 2) specific behaviors that convey respect or disrespect. Codes were applied by one author and then reviewed and discussed by all 3 authors. Themes representing definitions and specific behaviors were generated through discussion and consensus.

RESULTS: We conducted 26 focus groups, 5 each with African American men and women, 4 each with Latino men and women, and 4 each with white men and women. We identified two primary definitions of respect described by all three racial/ethnic groups. These were: 1 ) being treated like a person ("like you're a person not just a statistic, or another patient"), and 2) being treated as an equal ("treat me as an equal, like I matter"). When exploring specific behaviors that convey respect or disrespect, there were similarities in themes identified by all racial/ethnic groups. These were: 1) being known as a particular individual ("they know who I am"); 2) stereotyping ("some doctors feel like because you're black, you're on drugs" and "maybe I haven't been able to shave, maybe my jeans are dirty...I've got a bachelor's degree, don't treat me like I'm stupid"); 3) being treated politely vs. rudely (“just receiving you in a welcoming manner" vs. "basically it's the tone they use when they talk to you...you can tell when somebody's talking down to you"); 4) eye contact ("one doctor never looked at me"); 5) honest explanations of medical issues ("help us understand our problems"); and 6) how lateness is handled ("when you guys put me in a room, just stick your head in to tell me it's gonna be a minute"). There were also some differences across racial/ethnic groups. The most prominent physician demonstration of respect mentioned among black participants were: 1) for physicians to hear vs. dismiss what patients say ("you're listening but you're not hearing"); and 2) trusting the patient's knowledge of him/herself ("you know what's wrong with you"). Trusting patient's self-knowledge did not come up in any of the focus groups with Latino or white patients, and hearing/not dismissing what patients say was mentioned by others but far less frequently than by African Americans. The most prominent demonstration of respect discussed in the Latino focus groups, which did not arise in any of the white or African American focus groups, was having the provider show concern by asking questions about the patient's clinical condition ("the questions that they ask—if they ask in a serious manner").

CONCLUSIONS: Our study found that patients have insights that are not specifically included in popular definitions of respect in medical ethics. We also found that deliberate inclusion of participants from three racial/ethnic groups increased the number of themes that emerged. Whether these differences in how respect is described and experienced represent true cultural differences in constructions of respect, or are merely differences in priorities within a universal construction, is unclear from our study but is a possible hypothesis to explore further. Understanding what makes patients from different backgrounds feel respected and disrespected, from the perspectives of patients themselves, is vital to delivering care that is truly patient-centered.

DO BEDSIDE INTERPRETER PHONES IMPROVE HOSPITAL DISCHARGE PREPAREDNESS FOR LIMITED ENGLISH PROFICIENT PATIENTS? A NATURAL EXPERIMENT Jonathan S. Lee ${ }^{2}$; Steven Gregorich ${ }^{2}$; Sunita Mutha ${ }^{2}$; Anna M. Napoles $^{2}$; Eliseo J. Perez-Stable ${ }^{1}$; Jennifer Livaudais-Toman ${ }^{2}$; Leah S. Karliner ${ }^{2}$. ${ }^{1} \mathrm{Na}-$ tional Institute on Minority Health and Health Disparities, Bethesda, MD; ${ }^{2}$ University of California San Francisco, San Francisco, CA. (Control ID \#2467976)

BACKGROUND: Effective communication regarding new diagnoses, medication changes, and follow-up plans is a critical component of the hospital discharge transition of care that may reduce post-discharge adverse events, emergency department visits and hospital readmissions. The rapidly rising population of limited English proficiency (LEP) patients in the United States is at particularly high-risk of sub-optimal discharge communication and post-discharge adverse events because of language barriers and inaccessible and underutilized interpreter services. We investigated the impact of a systems intervention to increase access to professional interpreters on hospital discharge preparedness for LEP patients.

METHODS: In December 2012, a large academic medical center placed dual handset phones at every adult bedside, enabling $24-\mathrm{h}$ access to professional interpreter services. We prospectively recruited Chinese and Spanish speaking LEP patients age $\geq 50$ admitted to the cardiology, general surgery and orthopedic surgery floors during the 6 months before (June-November 2012) and after (March-August 2013) implementation. Patients were interviewed in person while hospitalized (baseline) and by telephone 3 weeks after discharge. Primary outcomes were: 1) patient-reported knowledge of discharge medications; 2) patient awareness of new medications prescribed at discharge; and 3) overall discharge preparedness as assessed by the Care Transitions Measure (CTM), a validated 15 -item questionnaire scored from 0 to 100 . We compared outcomes for the pre- and postimplementation groups in a bivariate analysis and with a propensity analysis adjusting for propensity to be in the pre- or post-implementation group. To contextualize the quantitative results, we conducted two post-implementation qualitative focus groups with nurses to assess utilization patterns and barriers to use of the phones.

RESULTS: Of 214 patients enrolled, 189 completed follow-up post-discharge interviews (94 pre- and 95 post-implementation). Overall, patients had a mean age of $69,56 \%$ were women and $64 \%$ were Chinese speaking. Patients in the two groups did not differ significantly in demographic characteristics, education level or discharge diagnoses. Patient-reported knowledge of discharge medications was high overall for medication purpose and dosing, lower for side effects, and not significantly different between groups (Table). Among the 148 patients prescribed a new medication at discharge, 112 (76\%) were aware of this new prescription at follow-up, and the proportion did not differ significantly between groups $(p=1.0)$. Mean CTM scores before and after phone implementation did not differ significantly ( 72.3 pre vs 75.8 post, $p=0.26$ ). Similar results were obtained for all outcomes in propensity-adjusted analyses. In the focus group analysis, nurses reported positively on ease of use and accessibility of the interpreter phones and their utility for pain and symptom assessment. However, nurses reported using phones infrequently for discharge communication, instead preferring in-person interpreters for complex discharges, and direct communication with family members for routine discharges.

CONCLUSIONS: In this natural experiment study, implementation of a bedside interpreter phone did not improve patient-reported measures of discharge preparedness or knowledge of new discharge medications for LEP patients. This lack of improvement may be due to lack of professional acceptance of interpretation by phone for discharge communication and highlights the importance of healthcare provider education for future interpreter interventions. 
Medication Knowledge Pre and Post Bedside Interpreter Phone Implementation

\begin{tabular}{|c|c|c|c|}
\hline \multirow[b]{2}{*}{$\begin{array}{l}\text { Medication Knowledge at } \\
\text { Discharge }<\mathbf{b} /\end{array}$} & \multicolumn{3}{|c|}{ Strongly Agree/Agree with Knowledge Statement $<$ b/ } \\
\hline & $\begin{array}{l}\text { Pre- } \\
\text { implementation } \\
(N=94) \\
N(\%)<\text { b/ }\end{array}$ & $\begin{array}{l}\text { Post- } \\
\text { implementation } \\
(N=95) \\
N(\%)<\text { b/ }\end{array}$ & $\begin{array}{l}p \text {-value }< \\
\text { b/ }\end{array}$ \\
\hline $\begin{array}{l}\text { I clearly understood the } \\
\text { purpose for taking each of my } \\
\text { medicines }\end{array}$ & $75(85.2)$ & $88(93.6)$ & 0.06 \\
\hline $\begin{array}{l}\text { I clearly understood how to take } \\
\text { each of my medicines }\end{array}$ & $77(87.5)$ & $87(92.5)$ & 0.25 \\
\hline $\begin{array}{l}\text { I clearly understood the possible } \\
\text { side effects of each of my } \\
\text { medicines }\end{array}$ & $58(65.2)$ & $57(62.0)$ & 0.65 \\
\hline
\end{tabular}

DO COMMUNITY-LEVEL MEASURES OF PATIENT FUNCTIONAL STATUS, SOCIAL SUPPORT AND SOCIOECONOMIC STATUS AFFECT PATIENT RISK OF READMISSION FOR PNEUMONIA AND HEART FAILURE? Jennifer Meddings $^{1,2}$; Heidi Reichert ${ }^{1}$; Shawna N. Smith ${ }^{1}$; Jack Iwashyna ${ }^{1,}{ }^{1}$; Mary A. Rogers ${ }^{1}$; Kenneth Langa ${ }^{1}$; TImothy Hofer ${ }^{1,2}$; Laurence F. McMahon ${ }^{1,1}$. ${ }^{1}$ University of Michigan, Ann Arbor, MI; ${ }^{2}$ Ann Arbor VA Medical Center, Ann Arbor, MI. (Control ID \#2470610)

BACKGROUND: Hospital rates of unplanned Medicare readmissions within 30 days of discharge for pneumonia (PNA) and heart failure (HF) patients determine hospital penalties. Centers for Medicare and Medicaid Services (CMS) risk-adjust readmission rates by age, gender and medical comorbidities using claims data. We have shown that patient functional status, social support and socioeconomic status add additional explanatory power to the existing CMS risk-adjustment, but their effect differs between PNA and HF. Because patient-level measures of these characteristics are rarely available in existing datasets, we examined the effect of more readily available community-level measures of functional status, social support and wealth.

METHODS: In this retrospective cohort study, we linked patient-level hospital discharge data with ZIP Code Tabulation Area data on the patient's residence from the American Community Survey. We studied adults age $65+$ with index admissions for pneumonia $(n=$ $27,297)$ or heart failure $(n=37,612)$ at acute care hospitals in Florida and Washington in 2012. The impact of these community-level variables upon an individual's readmission for pneumonia or heart failure was assessed using multilevel logistic regression models with offsets for current CMS risk-adjustments. Community-level functional status was defined as the percentage of those aged $65+$ reporting difficulty dressing or bathing. Communitylevel social support was defined as the percentage of those $65+$ respondents that were married. Socioeconomic status was measured as percentage of those $65+$ in the highest nation-wide income quartile and, separately, the percentage 65+ with Medicaid. Other controls included hospital-level percentage Black/African-American patients and individual race (white, African-American and other non-whites). Likelihood ratio tests were used to compare nested models. $P<0.05$ was considered significant.

RESULTS: Among pneumonia admissions, there were 3640 (13.3\%) unplanned readmissions within 30 days of discharge. Community-level prevalence of $65+$ with ADL difficulties was significantly associated with readmission $(\mathrm{OR}=1.2$ for each $10 \%$ increase, $95 \% \mathrm{CI}: 1.06$ 1.35). Self-identified race was also positively associated with readmission for other non-whites $(\mathrm{OR}=1.14, \mathrm{CI}: 1.001,1.30)$. A likelihood ratio test comparing this model to a model with only clinical comorbidity adjustment showed significant improvement $(p<0.001)$. Among heart failure admissions, there were 6697 (17.8\%) unplanned readmissions within 30 days of discharge. Community-level measures of functional status, social support, wealth and the hospital's racial mix were not associated with readmission, however self-identified race was associated (OR $=1.17$ [CI:1.06, 1.29] for African-Americans and 1.14 [CI: 1.04, 1.26) for other non-whites, respectively). A likelihood ratio test showed significant improvement compared to only clinical comorbidity adjustment $(p<0.001)$.
CONCLUSIONS: Community-level functional status was positively associated with the odds of readmission in the PNA cohort, but not the HF cohort. Patient-level race was associated with higher odds of readmission for the PNA and HF cohorts. Community-level proxies for patient-specific functional status may add value for predicting a patient's risk of readmission and evaluating hospital performance. The differential impact of communitylevel functional status on readmission for pneumonia and heart failure suggest that CMS riskadjustment models should include publicly-available functional status measures.

DO PRIMARY CARE CLINICS WITH HIGHER ADOPTION LEVELS OF PATIENT CENTERED MEDICAL HOME FEATURES HAVE LOWER ED RETURN VISITS? Zayd A. Razouki ${ }^{2}$; Chris Beadles ${ }^{3}$; Sandra Woolson ${ }^{2}$; Valerie Smith ${ }^{1}$; George L. Jackson ${ }^{1}$; Susan N. Hastings ${ }^{1}$. ${ }^{1}$ Durham VA Medical Center, Durham, NC; ${ }^{2}$ Durham VA Medical Center, Chapel Hill, NC; ${ }^{3}$ Research Triangle Institute, Durham, NC. (Control ID \#2467729)

BACKGROUND: The Patient Centered Medical Home (PCMH) is a transformational care delivery model emphasizing care continuity, a whole person orientation, care coordination, and enhanced access. Recent evidence suggests that implementation of PCMH is associated with decreased total ED use. However, it is unknown if these results are transferable to ED return visits for those patients who were discharged after an earlier ED index visit. The objective of this study is to examine if primary care clinics with greater adoption of PCMH features are associated with lower ED return visits at 7 and 30 days in patients who were discharged from an earlier ED visit.

METHODS: We linked two datasets: 1) a $20 \%$ national, random sample of established Veteran Health Administration (VHA) users who were "discharged" after an ED index visit between October 2007 and June 2008; and 2) data from the American College of Physician (ACP) Home builder biopsy survey that assessed the level of PCMH adoption (127 items) for 850 VHA primary care clinics in 2009. Our independent variables included the total PCMH adoption score and, separately, two sub-scores Care Coordination and Access scores. Using a multivariable generalized linear mixed model with a random effect for primary care clinics, we examined the associations between our independent variables and our study outcomes including ED return visits within 7 and 30 days from an earlier ED index visit. The final model was adjusted for various demographic variables, variables that reflect prior patterns of health use. Including previous ED visits, hospitalizations and Diagnostic Cost Group, in addition to other variables

RESULTS: Our sample $(N=98,777)$ was predominately male $(92 \%)$. The mean age and the standard deviation (SD) of our cohort was 59.05 (14.94), .19\% were African-American, and $(3 \%)$ were Hispanic. Among 770 primary care clinics where data was available, the overall mean PCMH score was $88.65(\mathrm{SD}=12.96)$ with a range of $(37-127)$; the mean score for Care Coordination was 12.01 (3.60), with a range of (2-18); and the mean for the Access score was 6.15 (1.28) (SD) with a range of (2-9).ED return visits occurred in $(8.26 \%)$ within 7 days and $(17.74 \%)$ within 30 days in our total sample. Clinics with higher overall PCMH scores were not associated with lower ED return rates within 7-days, $\mathrm{OR}=1.00(95 \% \mathrm{CI}=$ $0.97-1.04)$ and 30-days, $\mathrm{OR}=1.00(95 \% \mathrm{CI}=0.98-1.03)$. Similarly, clinics with higher scores on Care Coordination were not associated with lower ED return visits within 7 days $\mathrm{OR}=1.01(95 \% \mathrm{CI}=0.97-1.04)$ or within 30 days $\mathrm{OR}=1.00(95 \% \mathrm{CI}=0.97-1.02)$ Unexpectedly, clinics with higher Access scores were associated with marginally statistically significantly higher ED return visits on 7-days, $\mathrm{OR}=1.04(95 \% \mathrm{CI}=1.00-1.75)$ and 30 days, $\mathrm{OR}=1.03(95 \% \mathrm{CI}=1.00-1.06)$.

CONCLUSIONS: Greater adoption of the PCMH model alone may be insufficient to reduce ED return visits for patients discharged from an earlier ED visit. These results should re-focus the attention on incorporating new strategies in the current PCMH model that targets the unique social and economic challenges for this population. 
DO RESIDENT ATTITUDES TOWARDS CONTINUITY AFFECT THEIR ACTUAL CONTINUITY? Michael Aylward ${ }^{1}$; Sophia Gladding ${ }^{2} .{ }^{1}$ University of Minnesota, Minnespolis, MN; ${ }^{2}$ University of Minnesota, MInneapolis, MN. (Control ID \#2469266)

BACKGROUND: Physician-patient continuity is associated with important outcomes including physician and patient satisfaction, resident interest in primary care, and improved health outcomes. The Accreditation Council on Graduate Medical Education (ACGME) stresses the importance of resident patient continuity and requires continuity clinics for residents in primary care residencies (internal medicine, family medicine, and pediatrics). Creating continuity in resident clinic can be a challenge because of competing resident scheduling priorities, patient preferences, and health system priorities. Previous studies have investigated a range factors that may affect patient continuity in a residency clinic setting. Several studies examined how structural factors, such as time in clinic and size of patient panel, may affect continuity. These studies found that continuity increases with increased time spent in clinic, increasing clinic frequency, and with decreasing patient panel size. A study of the scheduling system in a resident clinic showed that implementation of an open access system led to decreased continuity, suggesting that system factors play a role in continuity. The provider a resident works with has also been shown to significantly influence residents' continuity of care. A recent qualitative study of internal medicine residents' perceptions of discontinuity identified many of these same factors, as well as factors related to patients such as level of health care literacy and patient preferences. Many of these studies emphasized barriers to continuity focusing on structural and system factors beyond residents' control. We hypothesized that residents' attitudes towards continuity were related to the degree of continuity that they experienced. METHODS: We conducted a written survey of 42 medicine-pediatric residents asking their perceptions of multiple factors related in creating continuity with their patients. . The survey asked residents to rate how important specific factors were in creating continuity on a 4-point Likert-type scale ranging from $1=$ not important to $4=$ essential. These factors included: "resident" factors, which we defined as factors which are predominately enacted by the resident; "relationship" factors, defined as factors that are collaborative between the resident and the patient; clinic factors; and patient factors. We conducted a retrospective data analysis of resident self reported continuity clinic logs to determine residents' rate of continuity. We defined a patient visit as a "continuity visit" if the resident indicated they had seen the patient previously. The relationship between residents' perceptions and actual rates of continuity was determined using Spearman correlations.

RESULTS: Thirty-six residents (86\%) completed the survey. Residents indicated that three relationship factors: "following up with patients between visits" (3.4/4), "patient rapport" (3.4), "getting to know the patient as a person" (3.3) and one clinic factor: "the scheduling system" (3.3) were the most important factors in creating continuity. Spearman correlation coefficients demonstrated significant moderate relationships between the perceived importance of three factors and the residents' actual patient continuity, namely, patient rapport (.42), therapeutic alliance with the patient (.36), and getting to know the patient as a person (.36)

CONCLUSIONS: Residents felt that several relationship factors were most important in creating continuity with their patients. Additionally, higher rates of continuity were associated with a subset of these factors. This suggests that resident attitudes towards the patient relationship are associated with continuity. Curriculum that addresses the importance of the patient relationship may contribute to improved continuity.

DOES CERTIFICATION IN VASCULAR ACCESS MATTER? INSIGHTS FROM THE NATIONAL PICC 1 SURVEY Valerie M. Vaughn ${ }^{1,2}$; Latoya Kuhn ${ }^{3,1}$; David Ratz ${ }^{3,1}$; Scott Flanders ${ }^{2}$; Sarah Krein ${ }^{3,}$; Vineet Chopra ${ }^{3,1}$. ${ }^{1}$ University of Michigan Health System and VA Ann Arbor Health System, Ann Arbor, MI; ${ }^{2}$ University of Michigan, Ann Arbor, MI; ${ }^{3} \mathrm{VA}$ Ann Arbor Health System, Ann Arbor, MI. (Control ID \#2457888)
BACKGROUND: Growing use of peripherally inserted central catheters (PICCs) has led to the development of certification programs for PICC operators. Much like the debate regarding certification in medicine, whether such certification actually influences clinical care is unknown. This knowledge gap is relevant for health systems that hire operators based on certification status and for internists who work closely with these providers in securing venous access. Therefore, we use data collected through a national survey to compare practices between certified and uncertified vascular access providers.

METHODS: A web-based survey was distributed through the listservs of two vascular access societies, with a combined estimated membership of 8385 (of which an estimated 5662 are PICC operators). Questions regarding experience, practice, and knowledge related to PICC insertion and care were posed. Bivariate comparisons between certified and non-certified practitioners were conducted using chi-square, Fisher's exact, or t-tests, as appropriate.

RESULTS: Of the 2762 potential respondents who accessed the survey, 1698 $(61.5 \%)$ were deemed eligible and completed the survey. Those who did not place PICCS or had taken the survey previously were excluded. Of the 1450 respondents who provided certification status, 1020 (70.3\%) stated they held a vascular-access certification. Roles and settings in which certified and noncertified providers practiced varied considerably (Table 1). Compared to noncertified operators, certified operators had more years of experience placing PICCs $(p<0.001)$, had placed more PICCs $(p<0.001)$, and were more likely to be the vascular access lead for their organization $(p<0.001)$. Self-reported adherence to evidence based practices was variable (Table 1). Certified operators were more likely to report they re-advanced dislodged devices $(p<0.001)$ and did not follow certain sterile insertion practices. They were also less likely to recommend removing a PICC due to signs of phlebitis $(p=0.02)$. However, compared to noncertified operators, they were more likely to recommend the team continue to use the PICC while obtaining an ultrasound if they suspected a PICC-related deep venous thrombosis $(p<0.001)$. Notably, certified operators were also more likely to view themselves as consultants whose views were not valued by members of the team $(p=0.007)$.

CONCLUSIONS: Important differences between certified and non-certified vascular access operators exist. Moreover, reported variability and potentially even less compliance with certain evidence-based practices among those certified suggests that further evaluation to better understand the sources of such variation is necessary. Given ongoing debate regarding the role of certification in other fields, understanding the impact of vascular access certification on PICC use and management is important for ensuring safe and effective care for many patients.

Difference Between Certified and Non-Certified PICC Operators

\begin{tabular}{llll}
\hline \hline Question & $\begin{array}{l}\text { Non- } \\
\text { certified }\end{array}$ & Certified & $\begin{array}{l}p \text { - } \\
\text { value }\end{array}$ \\
Hospital Beds (mean) & 287 & 319 & 0.01 \\
Work at Hospital Associated with Medical School (\%) & 68.1 & 73.5 & 0.03 \\
Work in Facilities with >100 PICCs Inserted per Month & 22.1 & 29.5 & 0.004 \\
Have Placed >3000 PICCs in Career & 13.3 & 26.4 & $<0.001$ \\
Average Years Placing PICCs & 7.9 & 11.5 & $<0.001$ \\
Are the Vascular Access Lead (\%) & 43.6 & 55.8 & $<0.001$ \\
Place PICCS in Pediatric Patients (\%) & 22.3 & 38.5 & $<0.001$ \\
Place PICCs in Neonatal Patients (\%) & 5.6 & 10.2 & 0.004 \\
Work at VA Hospital (\%) & 3.7 & 8.0 & 0.003 \\
Review PICCs for necessity on daily basis (\%) & 12.1 & 17.5 & 0.01 \\
Responsible for Daily Dressing Changes (\%) & 35.1 & 43.6 & 0.003 \\
Evidence Based Practices & & & \\
Would Advance a Dislodged PICC (\%) & 1.2 & 6.5 & $<0.001$ \\
Wear Sterile Gloves During Insertion (\%) & 94.0 & 87.6 & $<0.001$ \\
Wear Gown During Insertion (\%) & 95.6 & 91.5 & 0.006 \\
& & & \\
\hline
\end{tabular}




\section{(continued)}

\begin{tabular}{llll}
\hline \hline Wear Cap During Insertion (\%) & 90.7 & 90.3 & 0.846 \\
Wear Mask During Insertion (\%) & 91.4 & 85.7 & 0.002 \\
Use Patient Drape During Insertion (\%) & 91.2 & 82.2 & $<0.001$ \\
Barriers Used During Insertion, 0-5 count (mean) & 4.6 & 4.4 & $<0.001$ \\
Remove PICC for Suspected Phlebitis (\%) & 15.8 & 11.4 & 0.02 \\
Continued PICC use while Assessing For DVT (\%) & 33.8 & 53.6 & $<0.001$ \\
Ensure Approval Prior to Placing PICC in a Patient on & 88.0 & 92.4 & 0.05 \\
Dialysis (\%) & & & \\
Feel Like a Consultant, but Views Not Valued (\%) & 16.7 & 23.0 & 0.007 \\
\hline
\end{tabular}

Abbreviations: PICC, peripherally inserted central catheter; VA, Department of Veteran Affairs; DVT, deep venous thrombosis

DOES EARLY FEEDING REDUCE LENGTH OF HOSPITAL STAY IN ACUTE PANCREATITIS? A SYSTEMATIC REVIEW AND META-ANALYSIS Valerie M Vaughn $^{1,2}$; Dmitry Shuster ${ }^{1}$; Mary Rogers ${ }^{1,2}$; Jason Mann ${ }^{1,2}$; Marisa Conte ${ }^{4}$; Sanjay Saint ${ }^{2}$, 3; Vineet Chopra ${ }^{2,3}$. ${ }^{1}$ University of Michigan Health System, Ann Arbor, MI; ${ }^{2}$ University of Michigan and VA Ann Arbor Health System, Ann Arbor, MI; ${ }^{3}$ VA Ann Arbor Health System, Ann Arbor, MI; ${ }^{4}$ University of Michigan, Ann Arbor, MI. (Control ID \#2457403)

BACKGROUND: Acute pancreatitis is among the most common and costly reasons for hospitalization in the United States. While bowel rest, pain control, and intravenous fluids are the cornerstone of treatment, recent evidence suggests that early rather than delayed feeding may be beneficial. Guidelines, however, continue to recommend waiting for clinical improvement before feeding. We thus performed a systematic review and meta-analysis to evaluate the effect of early versus late enteral feeding on duration of hospitalization and complications in patients with acute pancreatitis.

METHODS: We searched for randomized controlled trials in the following databases Medline via Ovid (1950-present), Embase (1946-present), Cochrane CENTRAL (1948 present), the CINAHL (1960-present), and Web of Science via Scopus. Two author independently reviewed and abstracted studies if they: (a) included adults hospitalized with acute pancreatitis and (b) compared early (within $48 \mathrm{~h}$ ) to delayed enteral (oral or nasoenteric) feeding. Our primary outcome of interest was length of hospital stay. Secondary outcomes included mortality, readmissions, feeding intolerance, complications of pancreatitis and gastrointestinal symptoms (e.g., nausea, vomiting). Risk of bias was assessed using the Cochrane Collaboration tool. Mean differences in hospital length of stay and cumulative risk differences for secondary outcomes were pooled using the Hartung-Knapp-Sidik-Jonkman random effects method. Subgroup analysis was performed to determine whether severity of illness, geographic region, study quality, or route of nutrition affected the association with length of stay. Sensitivity analyses included fitting fixed effects models.

RESULTS: Of 991 citations identified, 7 studies with 598 patients met eligibility criteria. Early feeding was associated with a 1.71 day reduction in length of hospital stay $(95 \% \mathrm{CI}$ : -2.34 days, -1.08 days; $p<0.001, \tau^{2}=0.0 ; \mathrm{I}^{2}=0 \%$ ). Length of stay was less in early (vs delayed) feeding in both nasoenteric and oral feeding subgroups ( $p=0.04, p=0.03$ respectively). Hospital readmission occurred in $6.4 \%$ of patients with early and $10.3 \%$ of patients with late feeding $(p=0.16)$. Feeding intolerance developed in $6.3 \%$ of the early compared to $23.6 \%$ in the late group $(p=0.15)$. Rates of death, nausea, vomiting, necrotizing pancreatitis, and recurrent abdominal pain were similar across both groups.

CONCLUSIONS: Early initiation of feeding in acute pancreatitis is associated with reduced hospital stay and does not result in increased rates of readmission, complications or death. Greater adoption of early feeding in acute pancreatitis may produce substantial cost savings for hospitals in the United States.

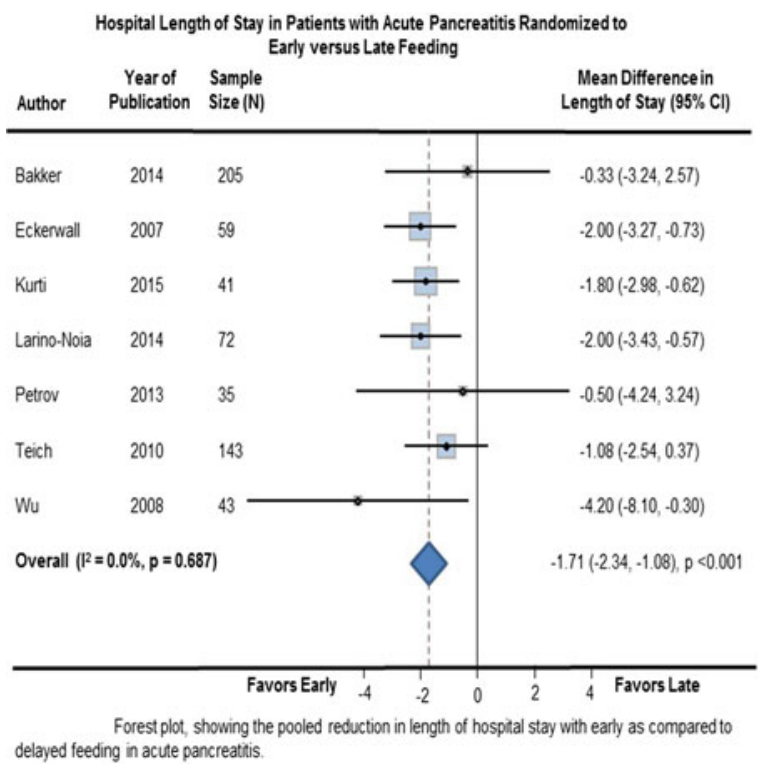

DOES INTENSIVE AMBULATORY MANAGEMENT OF HIGH RISK PATIENTS REDUCE ACUTE CARE UTILIZATION? A PILOT STUDY Brook Watts $^{1,}{ }^{2}$; Renee H. Lawrence ${ }^{1}$; Cameron Carter ${ }^{1}$; Kimberley Schaub ${ }^{1}$; Melissa Klein ${ }^{1}$, ${ }^{2}$; Brigid Wilson ${ }^{1}$; Susan Kirsh ${ }^{1,2}$. ${ }^{1}$ Louis Stokes Cleveland VAMC, Cleveland, OH; ${ }^{2}$ Case Western Reserve School of Medicine, Cleveland, OH. (Control ID \#2468711)

BACKGROUND: The health care burden associated with medically complex, high-risk patients has been well documented. To identify and address the unmet needs of these patients, the Veterans Health Administration (VHA) funded the Patient Intensive Management (PIM) demonstration projects to pilot improvements in the interface between patients and the health system in order to reduce acute care utilization, defined as both emergency department visits and hospitalizations. METHODS: As part of the PIM program, a population of 144 patients at our urban VHA teaching hospital was randomly selected from the veteran population with a Care Assessment Needs (CAN) score (a risk model which has been shown to be an accurate predictor of morbidity and mortality in veteran populations) in the top $10 \%$ of overall 3-month risk for morbidity or mortality and at least one acute care visit in the last 6 months. The intervention, PIM care, is designed to support and extend the VHA primary care structure, which relies on a patient-centered medical home model. Our local PIM program is a team-based, shared-practice model that focuses on care coordination, interdisciplinary treatment planning, home visits, and health coaching. We emphasize the development of a personalized relationship with veteran patients which is supported via frequent (sometimes daily) contacts with team providers. Based on similar efforts from the private sector that leverage unlicensed personnel as key members of outreach teams for high-cost patients with complex health issues, we sought to integrate former military medics into the team as health coaches and patient navigators. Here we present results of VHA acute care utilization 6 months after randomization to the PIM intervention.

RESULTS: Of the 144 patients with CAN scores in the top $10 \%$ randomized to the PIM intervention, 5 patients were found to be deceased, enrolled in hospice, or had moved out of the program catchment area prior to enrollment. The 139 intervention patients had $233 \mathrm{ED}$ visits in the 6 months pre-intervention (mean $+/-\mathrm{SD}=1.68$ visits $+/-1.54$, range 0 to 9 ) and 68 hospital admissions $(0.49+/-0.83$, range 0 to 4$)$. In the 6 months post intervention, there were $140 \mathrm{ED}$ visits $(1.0+/-1.55$, range 0 to 9$)$ and 46 hospital admissions $(0.33+/-0.77$, range 0 to 4$)$ respectively. CONCLUSIONS: In the 6 months after enrollment in the PIM intervention, ED and hospital admissions in a high risk patient group were lower than in the 6 months prior to enrollment, though overall acute care usage remained relatively high. Though this early data is encouraging, further investigation is needed to determine which patients benefit most from the PIM intervention. Better understanding of the needs which prompt acute care utilization (i.e. appropriate versus inappropriate) is also needed. 


\section{DOES OPIOID USE IMPEDE SHARED DECISION MAKING FOR LOW BACK}

PAIN? Emily Wendell; Mahima Mangla; Thomas Cha; Steven J. Atlas; Leigh H. Simmons; Karen R. Sepucha. Massachusetts General Hospital, Boston, MA. (Control ID \#2468407)

BACKGROUND: Low back pain is among the most common non-cancerous conditions for which patients are prescribed opioid therapies. While increasing use of opioids has been well documented, the evidence on the impact opioid use has on patient engagement in their care is not clear. Existing literature suggests that opioid users have a strong desire to be listened to and have their level of pain understood. However, studies suggest that many opioid users have negative views of their provider-patient interaction and feel they are not heard by their providers. The purpose of this study is to examine whether patients who are using opioids for their low back pain treatment report less shared decision making (SDM) and lower ratings of their specialists' communication efforts compared to those who do not use opioid therapy.

METHODS: This study is a secondary data analysis from a prospective shared decision making study of patients with low back pain due to herniated disc or spinal stenosis. Patients aged 21 and older, who were English-speaking, and had not previously undergone surgery for these conditions were surveyed 1 week following an initial visit with an orthopedic specialist. Some patients received decision aids from their specialist shortly after the consult. Respondents completed the 7-item Decision making Process Scale (DPS) that assesses specific SDM behaviors in the visit such as discussion of the treatmen options, the pros and cons of options, and patient's preferences. A total DPS score ( 0 $100 \%$ ) was calculated, with higher scores indicating more SDM. Participants also completed the 3-item CollaboRATE scale, which measures patients' perception of the amount of effort put forth by providers in engaging in SDM (total scores range from 0 to 10). Finally, patients completed demographics and a disease-specific (Oswestry Disability Index) quality of life survey. Treatments used for back pain were abstracted from the medical record. Respondents who had one or more opioid prescriptions in the 6 months prior to the visit were considered opioid users and those with no documented prescriptions were considered non-users. Our hypotheses were that opioid users would have lower DPS and CollaboRATE scores than non-users. We compared scores of the groups using t-tests, and used linear regression models to control for other factors as needed.

RESULTS: About one third of the participants, 63/199 (32\%), were using opioids. Those who used opioids did not differ from non-users on age (58 vs. 60 years old), education ( 52 vs. $57 \%$ with college degree or more), or race (92 vs. $87.5 \%$ White). A similar percentage of patients with herniated disc and spinal stenosis came into the visit using opioids ( 36 vs. $28 \%, p=0.3$ ). Patients who used opioids were more likely to be female ( 56 vs. $36 \%, p=0.01$ ) and reported more disability (ODI scores 44.6 vs. 33.7, $p<0.001)$. Patients who used opioids were less likely to receive a patient decision aid ( 22 vs. $38 \%, p=0.03$ ). The DPS scores were similar for users and non-users ( 59 vs. $64 \%, p=0.3$ ) with similar percentages reporting that the provider discussed surgery as an option (73 vs. $74 \%$ ), discussed non-surgical options (84 and $80 \%$ ) and asked for their preferred treatment (52 and $54 \%$ ). The CollaboRATE scores were similar for users and non-users ( 7.8 vs. $8.0, p=0.4)$. Linear regression models controlling for gender, decision aid use and ODI scores confirmed no significant relationship between DPS or CollaboRATE scores and opioid use. In the regression models, female gender ( $p=$ 0.007) was the only factor significantly associated with lower DPS scores, and the ODI score $(p=0.04)$ was the only factor significantly associated lower CollaboRATE scores. Although the treatment preferences were similar (43\% of opioid users and $32 \%$ of non-users stated a preference for surgery, $p=0.09$ ), opioid users were more than twice as likely to have surgery in the 6 months following the visit compared to non-users (41 vs. $19 \%, p=0.002$ )
CONCLUSIONS: Opioid use was prevalent among female patients and patients with higher self reported disability who came to a specialist for treatment for herniated disc or spinal stenosis. Patients using opioids reported similar frequency of discussion of treatment options and associated risks and benefits. Contrary to published literature, we did not find that opioid users reported more negative ratings of their provider's communication efforts compared to non-users.

DOSE REDUCTION AND DISCONTINUATION OF LONG-TERM OPIOID THERAPY: A SYSTEMATIC REVIEW Joseph W. Frank ${ }^{4,}{ }^{6}$; Travis Lovejoy ${ }^{7}$; William Becker ${ }^{5}$; Benjamin J. Morasco ${ }^{7}$; Christopher J. Koenig ${ }^{3}$; Hannah R. Dischinger ${ }^{6}$; Steven Dobscha ${ }^{2}$; Erin E. Krebs ${ }^{1} .{ }^{1}$ Minneapolis VA Health Care System, Minneapolis, MN; ${ }^{2}$ Portland VAMC, Portland, OR; ${ }^{3}$ University of California, San Francisco \& San Francisco Veterans Affairs, San Francisco, CA; ${ }^{4}$ University of Colorado School of Medicine, Aurora, $\mathrm{CO} ;{ }^{5} \mathrm{VA}$ Connecticut Healthcare System, West Haven, CT; ${ }^{6} \mathrm{VA}$ Eastern Colorado Health Care System, Denver, CO; ${ }^{7}$ Portland VA Medical Center, Portland, OR. (Control ID \#2468888)

BACKGROUND: Approximately 9 million U.S. adults are prescribed long-term opioid therapy (LTOT) for chronic pain in spite of inadequate evidence of long-term benefit and growing evidence of risk. Potential harms, including overdose death, increase with escalating opioid dose. Expert guidelines recommend dose reduction or discontinuation (“opioid tapering") when risks exceed benefits, but do not identify evidence-based opioid tapering strategies. To address this gap, we systematically reviewed studies of strategies to reduce or discontinue LTOT.

METHODS: We developed three key study questions, which assessed (1) the effectiveness of strategies to reduce or discontinue LTOT; (2) the comparative effectiveness of strategies to reduce or discontinue LTOT (i.e., controlled or comparative studies); (3) the effect of LTOT dose reduction or discontinuation on patient outcomes of pain severity, function, quality of life, withdrawal, substance use or adverse events. We conducted searches in MEDLINE, EMBASE, PsycINFO, CINAHL and the Cochrane Database of Systematic Reviews from inception through July 1, 2015. We also examined reference lists from included studies, published expert guidelines, and relevant review articles. Two investigators independently reviewed all abstracts and, when necessary, full manuscripts to determine inclusion. We included studies of adults (age $\geq 18$ years) prescribed LTOT (defined as opioid use for $>3$ months) for chronic pain that addressed one or more key questions. Two investigators independently extracted data using a standardized instrument and assessed study quality using criteria developed by the United States Preventive Services Task Force (USPSTF). Discrepancies were resolved by consensus.

RESULTS: Database searches identified 2993 abstracts and 6 additional articles were identified from reference lists. Of these 2999 studies, 46 met inclusion criteria. These studies included 8 randomized trials, 37 cohort studies and 1 pre-post study. Excluding a single pre-post study that examined all patients in a single healthcare system, studies presented data on 8191 patients (range, 6-1323 patients). Study duration ranged from 5 days- 85 months. Interventions included interdisciplinary pain programs (18 studies), buprenorphine-assisted tapering ( 9 studies), detoxification with or without symptomatic therapies (8 studies), other non-pharmacologic interventions (3 studies), acupuncture (3 studies) and detoxification under anesthesia (2 studies). Among 36 studies involving outpatient care, 2 studies involved primary care settings. To address KQ1, we identified 45 studies that assessed the effectiveness of strategies to reduce or discontinue LTOT. Across studies, opioid discontinuation rates ranged from 14 to $100 \%$.When reported, among participants who discontinued LTOT, rates of opioid re-initiation ranged from 0 to $66 \%$. To address KQ2, we identified a subset of 10 studies that directly compared 
strategies to reduce or discontinue LTOT, 7 of which were randomized trials. Six studies tested opioid or other medication dosing strategies, 3 studies compared nonpharmacologic interventions, and one study compared inpatient and outpatient pain programs. Two of ten studies reported statistically significant effects on LTOT dose reduction or discontinuation. For example, in one study, $21 \%$ of participants in a 4 month interactive voice response program discontinued LTOT at 8 month follow-up compared to a $20 \%$ increase in opioid medication use in the usual care control group. To address KQ3, we identified 33 of 46 studies that assessed one or more patient outcomes of LTOT dose reduction or discontinuation. Outcomes examined included pain severity (30 studies), function (14 studies), quality of life (10 studies), opioid withdrawal (17 studies), substance use (4 studies), and adverse events ( 9 studies). Among 30 studies examining pain severity, 25 studies reported decreased pain following dose reduction, 2 studies reported no change, and 3 studies reported increased pain. Among 14 studies examining pain-related function, 9 studies reported increased function following dose reduction, 4 studies reported no change, and 1 study reported decreased function. Among 9 studies measuring adverse events, mortality was assessed in 5 studies with a single opioid overdose-related death in 1 study. Using USPSTF criteria, one study was rated as 'good' quality, 11 studies were rated 'fair' and 34 were rated 'poor'. Excluding the prepost study, all studies were limited by sampling bias due to the voluntary nature of intervention participation. Nine studies excluded patients based on substance use or aberrant medication-taking behavior criteria.

CONCLUSIONS: Based on our systematic review of 46 studies examining LTOT dose reduction or discontinuation in more than 8000 patients, generalizable evidence-based guidance in each of 3 key questions is currently lacking. Important limitations include the poor study quality of a majority of studies, sampling bias due to the voluntary nature of study participation in 45 of 46 studies, and poor generalizability to primary care settings, where the majority of opioid medications are prescribed. Future work should utilize pragmatic study designs to examine opioid tapering in real-world primary care settings and leverage existing data to assess important patient outcomes such as overdose.

EARLY DISENGAGEMENT AND SUBSEQUENT RE-ENGAGEMENT IN OFFICE BASED OPIOID TREATMENT (OBOT) WITH BUPRENORPHINE IN A LARGE URBAN HOSPITAL David Hui ${ }^{2}$; Zoe M. Weinstein ${ }^{2,1}$; Debbie M. Cheng ${ }^{3}$; Emily Quinn $^{3}$; Colleen T. LaBelle ${ }^{1,2}$; Jeffrey H. Samet ${ }^{2,1}$. ${ }^{1}$ Boston Medical Center, Boston, MA; ${ }^{2}$ Boston University School of Medicine, Boston, MA; ${ }^{3}$ Boston University School of Public Health, Boston, MA. (Control ID \#2465086)

BACKGROUND: Opioid use disorder can be a chronic relapsing disease with individuals requiring multiple treatment attempts before achieving stable recovery. In Office Based Opioid Treatment (OBOT) programs, up to one-third of patients do not remain in treatment even short term, for $>1$ month (early disengagement). Much of the literature focuses on describing factors that predict treatment retention, but less attention is given to what happens to those patients with early disengagement. The primary goal of this analysis of the Disenrollment and Re-engagement in an OBOT Program (DROP) study, was to estimate: 1) the proportion of patients in an urban safety net hospital OBOT program who leave care in less than 1 month (early disengagement); and 2) the proportion of those with early disengagement who re-engage in care with the same clinic within 2 years. The secondary goal is to explore patient characteristics associated with disengagement from care and reengagement within 2 years.

METHODS: We performed a retrospective cohort study of adult patients treated with buprenorphine at the OBOT Program at Boston Medical Center from January 1, 2002 to February 28, 2014. Electronic medical records review included demographics, clinical characteristics and substance use history. We calculated proportions and $95 \%$ confidence intervals for disengagement (lack of clinic contact or an active prescription for 60 days or more) and reengagement (new buprenorphine prescription after disengagement). Characteristics including basic demographics, psychiatric comorbidities, substance use history and results of urine drug tests were compared by disengagement status (yes vs. no) using Chi-square tests for dichotomous variables and T-test or Wilcoxon test for continuous variables. Among the subgroup of early dis-engagers, we compared characteristics by re-engagement status within 2 years (yes vs. no).

RESULTS: This DROP cohort analysis included the first presentation to this clinic for 1234 patients. The mean age on entering the OBOT program was 38 years (range 18-76), and the majority were male (62\%), white race (67\%), and had at least completed high school (64\%). Of the 1234 patients, 104 ( $8 \%, 95 \%$ CI: 7-10\%) had early disengagement. Those with early disengagement appeared less likely to be white (51 vs $69 \%$, $p=.006$ ) and less likely to be transferring from another buprenorphine provider at the time of enrollment ( 2 vs $21 \% p<.0001$ ). Patients with early disengagement appeared more likely to have a diagnosis of psychotic disorder ( 9 vs $3 \%, p=0.002$ ), and be transferring from a methadone provider at the time of enrollment (14 vs $7 \%, p=0.004$ ). In the first month of initiating treatment, patients with early disengagement were more likely to have one or more urine drug tests positive for opiates ( 74 vs $47 \%, p<0.0001$ ), cocaine ( 33 vs $21 \%, p=0.008$ ), amphetamine ( 30 vs $11 \%, p<0.0001$ ), or oxycodone ( 28 vs $12 \%, p=0.0004$ ). Self-reported opioid use history differed between patients with early disengagement versus patients who were retained in treatment (heroin only: 54 vs $39 \%$, opioid pills only: 9 vs $13 \%$, heroin and opioid pills: 37 vs $47 \% ; p=0.02$ ). We did a subgroup analysis of patients with early disengagement who had at least 2 years of follow-up time available to assess for re-engagement. In this subgroup of 84 patients, 10 patients $(12,95 \%$ CI: $6-21 \%)$ re-engaged in the same OBOT program within 2 years. We did not identify any statistically significant different characteristics between those who re-engaged and those who remained out of care

CONCLUSIONS: Patient early disengagement from an OBOT program (i.e., leaving care within 1 month of initiating treatment) was an uncommon event at this established clinic $(8 \%)$, but few $(12 \%)$ re-engaged in the OBOT program within the subsequent 2 years. Non-white race, transferring from methadone maintenance treatment and a diagnosis of psychotic disorders were characteristics associated with early disengagement suggesting these patients may benefit from additional support within the first month of care to enhance retention.

EARLY EXPERIENCES OF MICHIGAN'S MEDICAID EXPANSION BENEFICIARIES WITH COST-SHARING AND FINANCIAL INCENTIVES FOR HEALTHY BEHAVIORS Edith C. Kieffer ${ }^{3}$; Aaron M. Scherer ${ }^{4}$ J Jeffrey $\underline{\text { Kullgren }}^{1,}{ }^{2}$; Sarah J. Clark ${ }^{1,}{ }^{6}$; Zachary Rowe ${ }^{5}$; Erin Beathard ${ }^{1}$; Tolulope Olorode ${ }^{4}$; Lisa Szymecko ${ }^{1,}{ }^{4}$; Mirella Villalpando ${ }^{3}$; Erica Solway ${ }^{1}$; John Z. Ayanian ${ }^{1}$; Susan D. Goold ${ }^{1}$. ${ }^{1}$ University of Michigan, Ann Arbor, MI; ${ }^{2}$ VA Center for Clinical Management Research, Ann Arbor, MI; ${ }^{3}$ Univerity of Michigan School of Social Work, Ann Arbor, MI; ${ }^{4}$ Center for Bioethics and Social Sciences in Medicine, Ann Arbor, MI; ${ }^{5}$ Friends of Parkside, Detroit, MI; ${ }^{6}$ Child Health Evaluation and Research Unit, Ann Arbor, MI. (Control ID \#2468800)

BACKGROUND: Michigan is among the earliest of seven states to have received a CMS-approved waiver allowing demonstrations of alternative approaches to Medicaid expansion. Michigan's Healthy Michigan Plan (HMP) features cost-sharing tied to income level, with opportunities to reduce cost-sharing by completing a Health Risk Assessment (HRA) and documenting intention to engage in healthy behaviors. There is little research regarding Medicaid beneficiaries' experiences with these novel benefit designs, and their impact on beneficiaries' behaviors. This study presents insight into beneficiaries' perceptions of, and reaction and responses to, cost sharing and financial incentives for healthy behaviors. 
METHODS: We conducted in-depth cognitive interviews with 32 HMP beneficiaries reviewing the statements they received from HMP describing their costs and health services in the previous quarter. Interviewees resided in rural, suburban and urban areas in five regions of the state. Thirteen had incomes $\geq 100 \%$ FPL requiring both copays for services and monthly contributions (like premiums); 9 reported incomes at 35-99 \% FPL requiring only copays for services; and 10 reported incomes $<35 \%$ FPL with no required cost-sharing. Interviews were recorded, transcribed verbatim and coded using Dedoose qualitative analysis software. Major themes and illustrative quotes were retrieved for analysis.

RESULTS: Many beneficiaries perceived their required cost-sharing to be fair and affordable. Some commented on their sense of responsibility for paying a share of the cost of health care. Required payments seemed reasonable, even to those with higher costsharing requirements, particularly in relation to the benefits received. Most were relieved that required payments were relatively small. Although most reported they had already paid or intended to pay, a few beneficiaries found payments unaffordable or difficult due to competing financial demands. Most beneficiaries were unfamiliar or unsure about the financial incentives for healthy behaviors and did not make the connection between completing the HRA and earning a financial incentive. Rather, the desire to be healthy, or to get needed health care was the primary reasons beneficiaries gave for engaging in health behaviors. Many beneficiaries reported participating in screening and assessment with a primary care provider; many had received immunizations, new diagnoses and related treatment

CONCLUSIONS: HMP beneficiaries with cost-sharing requirements generally found payments fair and affordable but rarely understood the connection between adopting a behavior change goal and financial incentives. Diverse methods to increase beneficiary awareness and understanding of financial incentives for healthy behaviors may increase their effectiveness. Use of videos, social media and in-person review with community health workers or navigators may increase understanding of written materials, especially with beneficiaries with low educational levels or low health literacy. Many states have implemented or are considering Medicaid expansions that feature higher cost-sharing and incentives for healthy behaviors. This study provides one of the first glimpses into beneficiaries' initial reactions and responses to these novel benefit designs. Follow up and larger-scale evaluation of this and other recent Medicaid expansions is needed to ascertain whether, with longer-term experience, beneficiaries continue to perceive cost-sharing as fair and affordable, and whether they better understand and are motivated by financial incentives to engage in healthy behaviors

\section{EARLY IMPACTS OF THE AFFORDABLE CARE ACT ON ACCESS TO CARE} FOR PATIENTS WITH CHRONIC DISEASES: A NATIONAL PERSPECTIVE Elisabeth Poorman ${ }^{2}$; Hugo A. Torres ${ }^{2}$; Lenore Azaroff ${ }^{2}$; Lauren Campbell ${ }^{2}$; Chin Ho Fung $^{2}$; nicole Mushero ${ }^{2}$; Cynthia Schoettler ${ }^{2}$; Uma S. Tadepalli ${ }^{2}$; Gaurab Basu ${ }^{1}$; Danny McCormick ${ }^{3}$. ${ }^{1} \mathrm{CHA}$, Cambridge, MA; ${ }^{2}$ Cambridge Health Alliance, Cambridge, MA; ${ }^{3}$ Harvard Medical School / Cambridge Health Alliance, Cambridge, MA. (Control ID \#2469506)

BACKGROUND: For patients with chronic diseases, access to medical care improves disease control and outcomes. The Affordable Care Act (ACA), the main provisions of which were implemented in January 2014, was intended to increase insurance coverage and thereby improve access to care. It also was intended to reduce racial and ethnic disparities in access. We therefore examined the early impact of the ACA on people with chronic diseases. Specifically, we assessed: 1) whether the ACA was associated with improvements in insurance coverage; 2) whether access to care improved; and 3) whether racial and ethnic disparities in these outcomes improved.
METHODS: We analyzed nationally representative data from the Behavioral Risk Factor Surveillance Survey (BRFSS) in the 3 years prior to ACA implementation (2011-2013) and the first year post-implementation (2014). The BRFSS collects data through telephone interviews of adults age 18 and older using a population-based probability sample that is representative of the US non-institutionalized population. The study population for our analysis includes adults who reported having at least one of eight chronic medical conditions (heart disease, cancer stroke, diabetes, asthma, chronic obstructive lung disease, kidney disease, arthritis or depression). We compared pre- and post-ACA levels of and changes in health insurance coverage as well as access to care using three measures: 1) having a personal doctor; 2) not foregoing a doctor visit due to cost in the last year; and 3) having a check-up in the past year. We employed a naturally occurring quasi-experimental difference-in-differences design, treating adults 18-64 (the target age group of the reform) as the exposed group and adults 65 years of age or older as a control group. We estimated pre- to post-ACA changes using adjusted linear probability models controlling for demographic and economic characteristics. We also conducted subgroup analyses to estimate changes in pre-existing white-black and white-Hispanic disparities for each outcome RESULTS: The sample included 604,634 adults with chronic diseases aged 18 to 64 (463,163 in the pre-reform period and 141,471 in post-reform period) and 482,050 aged 65 and older. We found pre- to post-ACA increases in the proportion of individuals having insurance coverage (80.1 to $85.0 \%$; adjusted percentage point difference (AD), $4.9 \%$ $[4.5,5.4])$. We also found small but statistically significant changes in measures of health access: 1) not foregoing a doctor visit due to cost (74.4 to $77.1 \%$; AD, $2.6 \%[2.1,3.1])$; and 2) having a check-up in the past year (69.6 to $72.4 \%$; AD, $1.8 \%$ [1.1-2.6]). There was not a significant difference in having a personal doctor $(81.7$ to $81.8 \%$; $\mathrm{AD}, 0.3 \%[-0.2$ $0.8]$ ). Insurance and each measure of access improved for white, black and Hispanic people after reform, and we found small improvements in black-white and Hispanic-white disparities in insurance coverage (adjusted percentage point change in disparity, $-1.7 \%$ $[-0.1,-3.2]$ and $-2.5 \%[-1.9,-3.2]$, respectively). For not having to forgo a physician visit the decrease in black-white disparity was $-1.5 \%(-0.2,-3.3)$ and in Hispanic-white disparity was $-2.4 \%[-1.7,-3.1]$. For having a personal doctor, we noted declines in the white-Hispanic disparity $(-1.4[-0.7,-2.1])$ and in the white-black disparity $(-3.4[-1.8$, $-4.9])$. There was no change in black-white disparitiy for having a physician visit in the past year $(-0.5 \%,[-2.2,1.1])$, but there was a decrease in the Hispanic-white disparity $(-2.1 \%,[-1.2,-2.8])$. Finally, we found that post-ACA implementation, the proportions of white, black and Hispanic people that remained uninsured were 11.7, 17.6 and 29.9\%, while the number who reported forgoing a physician visit due to cost were 20.0, 27.1 and $32.9 \%$, respectively.

CONCLUSIONS: Among patients with chronic diseases nationally, the implementation of the ACA was associated with a moderate improvement in insurance coverage and small improvements in access to care in the first year. Some pre-existing racial and ethnic disparities in coverage and access improved minimally, while others did not change. High proportions of Americans with chronic diseases, particularly racial minorities, continued to face access barriers and a lack of insurance coverage.

\section{EASY ACCESS TO PROFESSIONAL INTERPRETERS IN THE HOSPITAL DECREASES READMISSION RATES AND HEALTHCARE EXPENDITURES FOR LIMITED ENGLISH PROFICIENT PATIENTS Leah S. Karliner'; Eliseo J. Perez- Stable ${ }^{1,2}$; Steven Gregorich ${ }^{1} .{ }^{1} \mathrm{UCSF}$, San Francisco, CA; ${ }^{2} \mathrm{NIH}$, Washington, DC. (Control ID \#2468050)}

BACKGROUND: Twenty-five million people in the U.S. have limited English proficiency (LEP); when this population is hospitalized they have trouble communicating with clinicians and experience worse outcomes. Federal requirements that hospitals provide language access services are very challenging to implement in the fast-paced, 24/7 hospital 
environment. We set out to determine if increasing access to professional interpreters improves hospital outcomes for older LEP patients.

METHODS: This was a natural experiment with varying intervention intensity on the medicine floor of an academic medical center. Participants were patients age $\geq 50$ discharged between Jan 15, 2007-Jan 15, 2010. The medical center placed a dualhandset telephone at every bedside starting July 15, 2008, providing easy access to a telephonic professional interpreter for any healthcare team member entering the room of any LEP patient. The main outcome measures were: 30-day readmission, length of stay (LOS), healthcare expenditures. The main predictor variable was intervention time period as moderated by patient language (primary non-English language-LEP/English-EP). Because there was no expectation that the bedside interpreter intervention would have an effect on the hospital outcomes of the EP group, this group was included as a nonequivalent control group to assess for secular trends in the outcomes. Additionally, we included the following patient-level characteristics as covariates: age ( $\geq 50$ years continuous), sex (M/F), and insurance (Medicare/Medicaid/county/private/none); and the following discharge-level characteristics as covariates: severity of illness index (SOI) (minor/ moderate/major/extreme), intensive care unit (ICU) stay (yes/no), principal diagnosis (categories according to Healthcare Cost and Utilization Project), and calendar month.

RESULTS: Of 8077 discharges, 1963 were for LEP, and 6114 for English-proficient (EP) patients. We defined three intervention periods: pre-intervention ( $0-5$ phones; mean 1.7 calls/LEP discharge), high-intensity (45-66 phones; mean 7.9 calls/LEP discharge), moderate-intensity ( $32-44$ phones; mean 5.9 calls/LEP discharge). There was a significant decrease in observed 30-day readmission rates for the LEP group during the high-intensity period compared to pre-intervention (17.8 vs. $13.4 \%$ ); at the same time EP readmission rates increased (16.7 vs. $19.7 \%$ ). In adjusted analyses, the effect of intervention intensity on readmission rates was significantly modified by patient language $(p=.04)$. This improved readmission outcome for LEP patients was not maintained during the moderate-intensity period. There was no significant impact on LOS in either unadjusted or adjusted analyses. After accounting for interpreter services costs, the estimated 119 readmissions prevented during the high-intensity period were associated with projected monthly healthcare expenditure savings of $\$ 161,404$.

CONCLUSIONS: A systems intervention to provide easy access to professional interpreters was associated with lower 30-day readmission rates for patients with a language barrier. While there are modest costs associated with professional interpretation, this is offset by healthcare expenditure savings which will be increasingly meaningful in the era of population-based health and accountable care organizations.

EFFECT OF A HANDOVER TOOL ON EFFICIENCY OF CARE AND MORTALITY FOR INTERHOSPITAL TRANSFERS Cecelia N. Theobald ${ }^{2}$; Neesha N. Choma ${ }^{1}{ }^{1}$; Jesse M. Ehrenfeld ${ }^{1}$; Stephan Russ ${ }^{1}$; Sunil Kripalani ${ }^{1}$. ${ }^{1}$ Vanderbilt University Medical Center, Nashville, TN; ${ }^{2}$ Vanderbilt University, Nashville, TN. (Control ID \#2465096)

BACKGROUND: Interhospital transfers constitute a significant proportion of hospital admissions, yet transferred patients commonly experience delays in the provision of care and higher mortality rates when compared to patients admitted directly. The interhospital handover is often prolonged, discontinuous, and unstructured, representing a key opportunity to improve patient safety that has not been evaluated to date. Our objective was to determine the effect of a universal handover tool on timeliness of initial care, length of stay, and mortality among interhospital transfer patients.

METHODS: After obtaining IRB approval, we conducted a retrospective cohort study of patients transferred into a large academic medical center over an 18-month period using an interrupted time series design. Patients were excluded if they were initially routed through the Emergency Department or admitted to the cardiology, obstetrics, burn, or trauma services. The intervention was a one-page handover tool containing only information critical for immediate patient care. The handover tool was completed by the transferring physician and available for review before patient arrival. Outcome measures included intervention adherence, timeliness of initial inpatient care (e.g., time to admission order entry and antibiotic order entry), length of stay, and in-hospital mortality. Patients routed through the cardiac transfer center who did not adopt use of the transfer sheet were used as a concurrent control group. Analyses adjusted for patient characteristics, co-morbidities, ICU care, and transferring hospital volume

RESULTS: The handover tool was completed for $85 \%$ of patients in the postintervention period. There was no significant change in either the time to admission order entry after implementation ( 47 vs. $45 \mathrm{~min}$, adjusted $P=0.48$ ) or time to antibiotic order entry (199 vs. $202 \mathrm{~min}$, adjusted $P=0.62$ ). There was a nonstatistically significant reduction in length of stay after implementation (6.5 vs. 5.8 days, adjusted $P=0.18$ ). In-hospital mortality for transfer patients declined significantly in the post-intervention period from 12.0 to $8.9 \%$ (adjusted odds ratio $0.68,95 \%$ confidence intervals $0.47-0.99, P=0.04)$. There was no significant change in mortality for the concurrent control group in the post-intervention period. CONCLUSIONS: Implementation of a standardized handover tool for interhospital transfer patients was feasible and well accepted by transferring physicians. Although implementation did not improve the timeliness of initial care, we observed a trend toward reduced length of stay post-intervention. Furthermore, we noted a significant reduction in mortality among transfer patients after implementation that persisted even after adjustment for comorbid illness and several other hospital and patient-level confounders. Standardizing handover communication for transfer patients may result in improved patient safety in this high-risk population

Effect of handover tool implementation on timeliness of care, length of stay, and mortality

\begin{tabular}{lllll}
\hline \hline Outcome & $\begin{array}{l}\text { Pre- } \\
\text { intervention } \\
(n=1105)\end{array}$ & $\begin{array}{l}\text { Post- } \\
\text { intervention } \\
(n=610)\end{array}$ & $\begin{array}{l}\text { Unadjusted } \\
P \text { value }\end{array}$ & $\begin{array}{l}\text { Adjusted } \\
P \text { value }\end{array}$ \\
$\begin{array}{l}47(20,92) \\
\begin{array}{l}\text { Time to admission } \\
\text { order entry (min) }\end{array}\end{array}$ & $199(18,87)$ & 0.36 & 0.48 \\
$\begin{array}{l}\text { Time to antibiotic } \\
\text { order (min) }(n=1117)\end{array}$ & $6.47(3.4,11.7)$ & $5.81(3.1,10.9)$ & 0.18 & 0.18 \\
$\begin{array}{l}\text { Hospital LOS in days } \\
\text { ICU LOS in days }(n=\end{array}$ & $4.34(2.2,9.0)$ & $4.55(2.7,8.8)$ & 0.38 & 0.34 \\
$793)$ & $12.0 \%$ & $8.9 \%$ & 0.04 & 0.04 \\
\hline In-hospital mortality & & & & \\
\hline
\end{tabular}

Values for LOS and time are presented as median (IQR). Multivariate model includes adjustment for age, gender, payer, admitting team, ICU status, time of admission, modified Elixhauser index, duration of transfer, and volume of transferring hospital; timeliness outcomes were further adjusted for calendar month. Time to antibiotic order was limited to only those patients who received antibiotic order within first $36 \mathrm{~h}$ of admission.

EFFECT OF A WEB-BASED HANDOFF TOOL AND PROVIDER TRAINING ON PREVENTABLE ADVERSE EVENTS Stephanie Mueller ${ }^{1,2}$; Jeffrey L. Schnipper ${ }^{1,2}$. ${ }^{1}$ Brigham and Women, Boston, MA; ${ }^{2}$ Harvard Medical School, Boston, MA. (Control ID \#2465064)

BACKGROUND: Failures in communication among healthcare personnel are known threats to patient safety. Communication is particularly vulnerable to error when patient care responsibility is transferred from one provider to another (i.e., handoff). In this study we implemented a web-based handoff tool and provider training, and evaluated the impact on preventable adverse events (AEs).

METHODS: We performed a prospective cohort analysis of patients on any of 3 general medicine or 2 general surgery teams before and after implementation of a web-based 
handoff tool and provider training. Patients were eligible for inclusion if they were cared for by any of the participating teams between November 1, 2012-February 1, 2013 (Time period 1, pre-implementation), April 1-June 18, 2013 (Time period 2, postimplementation), or November 1, 2013-February 1, 2014 (Time period 3, postimplementation). Between Time periods 2 and 3, Medicine services additionally underwent restructuring to regionalized care teams, daily admitting, and scheduled handoffs between day and evening teams. Surveys to screen for potential AEs were administered during the 3 time periods to nightfloat interns following their shift (to evaluate end-of-shift handoffs), and residents and attendings 2 days after starting on service (to evaluate end-of-service handoffs). Positive survey responses triggered chart review to determine presence and preventability of AE, harm level and attribution of AE to failure in communication, using previously validated scales. Ratings were adjudicated by trained physicians. We performed Poisson regression to obtain incident rate ratio (IRR) of preventable AEs per 100 patient-days in time periods 1 vs time periods $2+3$, accounting for clustering by provider and controlling for service and patients' demographics, length of stay, and comorbidities. Secondary outcomes included comparison of preventable AE rates in each time period, by service, and attributable to communication issues.

RESULTS: Of the 5407 included patients, adjusted preventable AE rates per 100 patientdays significantly decreased from 3.56 in Time period 1 to 1.76 in Time periods $2+3$ combined $(\mathrm{IRR}=0.49,95 \% \mathrm{CI} 0.44,0.55)$, and AEs due to communication failures decreased by $60 \%$, while no difference was seen in non-preventable AEs (Table). Subgroup analysis showed additional reduction in AE rates on medical services between Time periods 2 and 3 from 1.84 to 0.74 ( $p=0.04)$

CONCLUSIONS: We found that implementation of a web-based handoff tool and provider training significantly decreased preventable $\mathrm{AE}$ rates, including those due to communication failures. The additional benefit seen on medicine services between Time periods 2 and 3 may have been due to an additive or synergistic effect of restructuring of medical teams.

\section{Table: Impact of Intervention on Adjusted Adverse Event Rates}

\begin{tabular}{|l|c|c|c|c|}
\hline \multirow{2}{*}{ Outcomes } & \multicolumn{2}{|c|}{${ }^{\dagger}$ Adjusted AE rates per 100 patient days (95\% CI) } & \multirow{2}{*}{ IRR (95\% CI) } \\
\cline { 2 - 5 } & $\begin{array}{c}\text { Time Period 1 } \\
\mathbf{n = 2 4 0 6} \\
\text { (Pre-Intervention) }\end{array}$ & $\begin{array}{c}\text { Time Periods 2 and 3 } \\
\mathbf{n = 3 0 0 1} \\
\text { (Post-Intervention) }\end{array}$ & $1.76(0.93,3.31)$ & $0.49(0.44,0.55)$ \\
\hline Total Preventable AEs & $3.56(1.70,7.44)$ & $1.15(0.76,1.74)$ & $0.40(0.26,0.62)$ \\
\hline $\begin{array}{l}\text { Preventable AEs due to } \\
\text { Errors in Communication }\end{array}$ & $2.88(1.22,6.82)$ & $0.74(0.44,1.15)$ & $<0.001$ \\
\hline${ }^{\text {tNon-Preventable AEs }}$ & $0.39(0.18,0.73)$ & $<0.001$ \\
\hline
\end{tabular}

'Clustered by provider and controlled for service and patients' age, sex, race, length of stay, and diagnosis related group (DRG) weight.

tUnadjusted results shown, as low number of events precluded adjusted analyses.

EFFECT OF AN INPATIENT ADDICTION CONSULTATION ON THE ADDICTION SEVERITY INDEX OF HOSPITALIZED SUBSTANCE USERS AFTER DISCHARGE Sarah E. Wakeman ${ }^{3},{ }^{4}$; Nancy A. Rigotti ${ }^{2}$; Yuchiao Chang ${ }^{1}$; Grace E. Herman ${ }^{5}$; Joshua Metlay ${ }^{3} .{ }^{1}$ Harvard Medical School, Boston, MA; ${ }^{2}$ Massachusetts General Hospital, Boston, MA; ${ }^{3}$ Massachusetts General Hospital, Charlestown, MA; ${ }^{4}$ Harvard medical School, BOSTON, MA; ${ }^{5}$ Massachusetts General Hospital, BOSTON, MA. (Control ID \#2457372)

BACKGROUND: Twenty-two million Americans and $22 \%$ of hospitalized patients have a substance use disorder (SUD). Several interventions have demonstrated efficacy among hospitalized patients with SUD, the challenge is implementing these interventions into real-world hospital practice. To address this, we implemented an addiction consult team to provide consultation for hospitalized general medical patients. The team consisted of a Certified Addictions Registered Nurse - Advanced Practice, a physician (the majority of whom are board-certified in Addiction Medicine), social workers, and recovery coaches. The team offered assessment, initiation of treatment including pharmacotherapy, and linkage to longitudinal treatment following discharge. This abstract examines the effects of receiving consultation on addiction severity and self-reported abstinence 30 days post-discharge.

METHODS: This prospective cohort study assessed the 30-day post-discharge out comes of patients with SUD admitted to 10 general medical floors of one hospital, comparing those who were or were not seen by an addiction consult team during hospitalization. The study enrolled adult patients with an AUDIT-C of 8 or higher, a positive NIDA single question drug screen, or identified to have SUD by routine nursing assessment at the time of admission. The addiction consult team was available on 8 of 10 floors. The decision to consult the addiction team was made by the primary clinicians independent of this study. Intervention patients were those who were evaluated by the consult team and control patients were those who were not, either because they did not receive a referral or were admitted to a floor without the service. Participants were assessed in-person at baseline and 30 and 90 days later by telephone or mail survey. The characteristics assessed included the composite addiction severity index, days abstinent, self-reported utilization of acute care services, overdose, selfefficacy, quality of life, motivation for abstinence, mutual help attendance, medication adherence, and treatment engagement. Primary outcomes were the addiction severity index composite scores for alcohol and drugs and self-reported days abstinent 30 days post-discharge. Secondary outcomes included the number of self-reported emergency room visits, hospitalizations, and overdoses in the past 30 days. Two-sample t-tests were used to compare mean change over time between the two groups. Due to concern for selection bias between those who did and did not receive an addiction consult, we also conducted a matched analysis with a subgroup of patients from interventions and controls matching on major type of substance used and corresponding baseline ASI score within 0.05 . Paired t-tests were used to compare mean change over time between the two groups within the matched analysis.

RESULTS: Enrollment began April, 2015. Among 288 subjects enrolled to date, we report preliminary analysis for the 176 subjects (61\%) who completed 30-day follow-up. The rate of completion of 30 -day follow-up was $60 \%$ for the intervention group and $63 \%$ for controls. Among these 176 subjects, 107 were in the intervention group and 69 were in the control group. Baseline ASI composite scores were higher in the intervention group (ASI-alcohol 0.45, ASI-drug 0.13) than in the control group (ASI-alcohol 0.26, ASI-drug 0.09). At baseline, subjects in the intervention group reported fewer days abstinent in the 30 days prior to admission (12.1 vs. 19.2 days) and greater utilization of the ER (1.55 vs. 0.65 visits) and inpatient hospitalization ( 1.32 vs. 0.48 admissions). Baseline mean 
frequency of overdose was identical at 0.2 events in both groups. Primary and secondary outcomes improved in both groups however the magnitude of improvement was greater in the intervention group for nearly all outcomes (see table): mean ASI-alcohol decreased by 0.22 vs. 0.07 ( $p=0.0002)$; mean ASI-drug decreased by 0.04 vs. $0(p=0.002)$; days abstinent increased 12.6 vs. $5.1(p<0.0001)$; self-reported inpatient admissions decreased 64 vs. $46 \%(p=0.003)$, and ED visits decreased 61 vs. $46 \%(p=0.01)$. Self-reported overdose decreased by 80 vs. $65 \%(p=0.82)$. To control for baseline differences, a subgroup analysis was limited to 44 pairs of patients matched on major substance and baseline ASI. The magnitude of improvement remained greater in the intervention group (though only significant for ASI-drug score, see table): mean ASI-alcohol decreased 0.17 vs. $0.13(p=0.25)$, ASI-drug 0.05 vs. 0.01 ( $p=0.015)$, days abstinent 10.1 vs. $7.4(P=$ $0.24)$, inpatient admission 75 vs. $45 \%(P=0.06)$, ER visit 73 vs. $48 \%(P=0.33)$, and overdose by 86 vs. $36 \%(p=0.41)$.

CONCLUSIONS: In a nonrandomized controlled longitudinal study of hospitalized general medical patients with substance use disorder, inpatient addiction consultation appears to significantly decrease severity of drug use at 30 days post-discharge.

\begin{tabular}{|c|c|c|c|c|c|c|}
\hline & Cohort & & & $\begin{array}{l}\text { Matched } \\
\text { samples }\end{array}$ & & \\
\hline Outcome & No consult & Consult & $p$-value & No consult & Consult & $p$-value \\
\hline $\begin{array}{l}\text { Change in } \\
\text { ASI-major } \\
\text { substance }\end{array}$ & -.09 & -.24 & $<0.001$ & -.15 & -.19 & 0.25 \\
\hline $\begin{array}{l}\text { Change in } \\
\text { ASI-alcohol }\end{array}$ & -.07 & -.22 & $<0.001$ & -.13 & -.17 & 0.33 \\
\hline $\begin{array}{l}\text { Change in } \\
\text { ASI-drug }\end{array}$ & 0 & -.04 & 0.002 & -.01 & -.05 & 0.015 \\
\hline $\begin{array}{l}\text { Change } \\
\text { in days } \\
\text { abstinent }\end{array}$ & 5.1 & 12.6 & $<0.001$ & 7.39 & 10.14 & 0.24 \\
\hline $\begin{array}{l}\text { Change in } \\
\text { inpatient } \\
\text { admissions }\end{array}$ & $-46 \%$ & $-64 \%$ & 0.003 & $-45 \%$ & $-75 \%$ & 0.06 \\
\hline $\begin{array}{l}\text { Change in ER } \\
\text { visits }\end{array}$ & $-46 \%$ & $-61 \%$ & 0.011 & $-48 \%$ & $-73 \%$ & 0.33 \\
\hline $\begin{array}{l}\text { Change in } \\
\text { overdose }\end{array}$ & $-65 \%$ & $-61 \%$ & 0.82 & $-36 \%$ & $-86 \%$ & 0.41 \\
\hline
\end{tabular}

EFFECT OF DIDACTIC LECTURES ON OBESITY DOCUMENTATION AND COUNSELLING AMONG INTERNAL MEDICINE RESIDENTS Vicky Ren ${ }^{2}$; Kathleen E. Ellison ${ }^{2}$; Kiran Busireddy ${ }^{2}$; Miller Jonathan ${ }^{1}$; Mukta Panda ${ }^{2}$; Rehan Qayyum ${ }^{2} .{ }^{1}$ Univ of Tenn College of Medicine, Ooltewah, TN; ${ }^{2}$ University of Tennessee, Chattanooga, TN. (Control ID \#2466691)

BACKGROUND: With obesity in the United States continuing to be a major public health concern, primary care physicians have become key players in addressing this nationwide epidemic. Currently, the United States Preventive Services Task Force (USPSTF) recommends screening all adult patients for obesity and treating them appropriately for weight loss. However, while healthcare providers may be able to accurately identify obesity, many feel ill-equipped to address this topic.

METHODS: Obesity documentation and management were examined in December 2014 through retrospective chart review of patients seen at internal medicine (IM) and family medicine (FM) resident clinics at the University of Tennessee College of MedicineChattanooga. Results indicated that approximately half of the patients presenting to primary care clinics were obese. Of these patients, only $50 \%$ had obesity documented in their chart, and less than $20 \%$ received weight loss counseling. To increase the documentation and counselling of obesity, we devised an intervention that included sharing these findings and the importance for addressing obesity with the IM and FM residents at two didactic presentations in May 2015. To examine the effect of this intervention, we conducted a retrospective medical record review of patients seen by IM residents at outpatient continuity clinic 1 month prior to and 1 month after the intervention. We abstracted data on patient's
BMI, gender, race, comorbid conditions, documentation of and counselling for obesity if $\mathrm{BMI} \geq 30 \mathrm{~m} 2 / \mathrm{Kg}$. We used multivariable logistic regression models to examine the relationship of obesity documentation and counseling with intervention, adjusting for patient age and race, number of diagnoses, and residents' gender and training level.

RESULTS: Of the 513 patients seen in IM resident clinic, 262 were seen prior to and 251 following the intervention. We did not find any effect of intervention on either documentation of obesity or counseling for obesity with or without adjustment for confounding variables ( $P>0.05$ for both). In post-hoc analyses, we found significant differences in rates of obesity counseling by resident training year independent of study intervention and other variables. Post-graduate year (PGY) 2 and 3 residents were $60 \%(P=0.02)$ and $68 \%(P=0.005)$ less likely to perform obesity counseling for obese patients than PGY-1 residents, respectively. In addition, each additional ICD-9 code listed for obese patients increased the odds of obesity documentation by $8 \%(P<0.001)$.

CONCLUSIONS: Obesity management is critically important but inadequately addressed in primary care settings. A brief presentation of data illustrating the lack of appropriate obesity documentation andcounseling by residents in continuity clinic did not cause a significant change in residents' behaviors. Likelihood of addressing obesity during patient visits varied inversely with residents' level of training both pre- and postintervention. Differences among resident levels may be due to variations in patient load as well as burnout among more senior residents. The results suggest that short presentations may be too passive and/or superficial to effect change in resident behavior and improved documentation. Future research should employ more interactive and lengthy interventions as well as potential changes to the electronic medical record (EMR) and office forms.

EFFECT OF ELECTRONIC HEALTH RECORD IMPLEMENTATION ON RESIDENT TIME IN HOSPITAL PATIENT ROOMS Marianne Zachariah ${ }^{1,2}$; Erin L. Duffy ${ }^{1,2}$; Michael A. Pfeffer ${ }^{1}$; Douglas S. Bell ${ }^{1,2} \cdot{ }^{1}$ UCLA, Los Angeles, CA; ${ }^{2}$ UCLA Clinical \& Translational Science Institute, Los Angeles, CA. (Control ID \#2467545)

BACKGROUND: Electronic health records (EHRs) are rapidly gaining adoption in the U.S. If EHRs increase the time that providers need to spend on documentation and ordering, this might reduce the time available for patient interactions and learning activities. We sought to determine how the implementation of a commercial EHR affected the amount of time residents on inpatient rotations spent: 1) with patients and 2) on didactic activities.

METHODS: At Ronald Reagan UCLA Medical Center, 21 first-year residents were recruited to wear AwarePoint real-time location system devices from October 2012 through June 2013. These devices recorded movement throughout the hospital. Using AwarePoint data, time spent in patient rooms and daily noon conference attendance was determined for each resident. To validate AwarePoint data, nine interns were shadowed over the course of 10 days, before and after UCLA's implementation of the Epic EHR system on March 1, 2013. Shadowing was performed by trained undergraduate students who recorded their assigned resident's location every minute between the hours of 7 am and $5 \mathrm{pm}$. To assess AwarePoint's accuracy for each patient room, all spaces on floors 5-8 of Ronald Reagan UCLA Medical Center were categorized as patient rooms or non-patient rooms, and shadowing and AwarePoint data were compared. Patient rooms were considered valid if they had a positive predictive value (PPV) $\geq 80 \%$ and capture (sensitivity) $\geq 35 \%$. Sixty-five out of 279 patient rooms ( $23 \%$ ) on floors 58 showed AwarePoint activity. Including valid rooms from all AwarePoint data, we used Ttests to compare the mean visits per day to a patient room, the mean time per visit, and the mean total time per room per day for each resident before versus after Epic implementation. Analysis of variance (ANOVA) was used to determine if total time spent with patients per day differed by intern. A mixed effects linear regression model was used to test whether time spent in patient rooms differed: 1) pre- versus post-Epic implementation, 2) based on a proxy for patient load, and 3) based on the presence of a shadower, accounting for clustering by resident. 
Finally, the proportion of observed days in which noon conference was attended was compared between the pre- and post- Epic period using Chi-square test for residents on the general medicine wards rotation.

RESULTS: AwarePoint data was available from all 21 subjects on wards rotation, and 26 ( $40 \%$ ) of these patient rooms met the validity criteria. The final data set included 559 distinct resident-patient room-days pre-Epic implementation and 564 post-Epic implementation. On average, residents made fewer visits to a given patient room per day ( $1.9 \mathrm{vs}$. 2.4 visits, $p<0.001$ ), but they spent more time in each visit to a patient room post-Epic implementation ( 4.2 vs. $2.4 \mathrm{~min}, p<0.001$ ), and with greater net patient room time per day post-Epic implementation ( 8.1 vs. $5.8 \mathrm{~min}, p<0.001)$. By resident, the mean minutes per patient room per day ranged from 4.0 to $10.3 \mathrm{~min}$. ANOVA indicated that the means between residents were significantly different from each other. Mixed effects linear regression model showed that the minutes per patient room per day post-Epic implementation increased by $1.43 \mathrm{~min}(p<0.0001)$. There was no association between the minutes per patient room per day and a resident's daily patient load. Overall, proportion of days of noon conference attendance was significantly higher post- Epic implementation for residents on wards $(74 \%)$ versus pre- Epic implementation $(62 \%)(p<0.01)$. However, time trend by month in noon conference attendance showed seasonal variation, with a nadir in December and a peak in February (just prior to Epic go-live), and a modest downtrend in May and June. By contrast, patient room time was at its lowest from November to February, increased to a peak in April, and then downtrended in May and June.

CONCLUSIONS: After EHR implementation, time spent in patient rooms per day increased while the number of visits to patient rooms per day decreased, suggesting that residents became more efficient post-Epic implementation. Although, there seems to be seasonal variation in the amount of time residents spent, both in patient rooms and in noon conference attendance, with a tapering effect towards the end of the year, the magnitude and timing of the increase in patient time suggests that Epic implementation may have had a beneficial effect, or it at least had no discernable adverse effect.

EFFECT OF GLOBAL HEALTH AND UNDERSERVED POPULATIONS TRACK ON GRADUATES' CAREER CHOICES FOLLOWING INTERNAL MEDICINE RESIDENCY Zachary G. Jacobs ${ }^{3}$; Amy J. Kennedy ${ }^{3}$; Timothy Anderson'; Thuy Bui ${ }^{2}$ ${ }^{1}$ UPMC, Pittsburgh, PA; ${ }^{2}$ University of Pittsburgh, Pittsburgh, PA; ${ }^{3}$ University of Pittsburgh Medical Center, Pittsburgh, PA. (Control ID \#2463087)

BACKGROUND: Although an increasing number of residency programs have instituted tracks or pathways dedicated to training physicians in global health and international service, the impact of these changes on career choice and care of underserved populations remains unknown. The University of Pittsburgh Medical Center (UPMC) Internal Medicine Residency Program developed a separate track for residents interested in global health and underserved populations in 2007. This track exposes residents to 6 months of global and underserved healthcare -including up to 4 months of clinical work overseas, 1 month with the Indian Health Service, and a 1 month preparatory seminar -all over the course of a standard 3-year residency. The aim of this study is to assess how participation in this track has affected graduates' career choices as compared to categorical residents in the same program.

METHODS: Occupational data were obtained from the UPMC Office of Graduate Medical Education on all categorical and global health track residents graduating between 2010 and 2015. These data included fellowship specialty, as well as whether the graduate pursued a career in hospitalist, primary care, and/or underserved settings. Graduates of the global health track were also questioned via follow up survey regarding their perceptions of the track, as well as their practice patterns since graduation, including demographics of patient populations, community service, volunteerism and advocacy activity.
RESULTS: A total of 188 graduates were included, 165 from the categorical program and 23 from the global health track. During the first 1-2 years after finishing residency, as compared to categorical residents, graduates of the global health track were more likely to pursue careers in primary care (44 vs $20 \%$ ), and to work with underserved populations (52 vs $4 \%$ ); they were less likely to specialize (44 vs $55 \%$ ), become hospitalists ( 26 vs $34 \%$ ), or work for the Veterans Affairs health system (4.3 vs $9.7 \%$ ). Data from the survey of global health graduates have yet to be analyzed.

CONCLUSIONS: Immediately following graduation, internal medicine residents who completed 6 months of global health and underserved training were more likely than their peers to pursue careers in primary care and to work with underserved populations. As career paths often change in the initial years following residency, going forward, long-term outcomes of global health track trainees will be evaluated via additional survey data

EFFECT OF LACTATION ON MATERNAL HYPERTENSION: A SYSTEMATIC REVIEW Eliana Bonifacino ${ }^{1}$; E. Bimla Schwarz ${ }^{2}$; Hyejo Jun ${ }^{4}$; Charles B. Wessel ${ }^{3}$; Jennifer Corbelli ${ }^{4}{ }^{1}$ UPMC, Pittsburgh, PA; ${ }^{2}$ University of California, Davis, Sacramento, CA; ${ }^{3}$ University of Pittsburgh, Pittbsurgh, PA; ${ }^{4}$ University of Pittsburgh Medical Center, Pittsburgh, PA. (Control ID \#2469134)

BACKGROUND: Though the health benefits of breastfeeding on infants and children has extensively been investigated, less is known about the benefits to maternal health, including the amount of breastfeeding required to incur potential maternal benefits. There has been a growing body of literature that suggests that lactation during a woman's lifetime could be protective against several cardiovascular risk factors including diabetes, hyperlipidemia, and the metabolic syndrome. Hypertension and hypertension-associated conditions are a leading preventative cause of morbidity and mortality in women. Though there are individual studies indicating that there may be an effect of lactation on blood pressure, studies are of varying type and quality, and there has not been a systematic review of the literature addressing this issue. We systematically reviewed the literature on lactation and effects on blood pressure and future development of hypertension in parous women. We aimed to identify and summarize the literature regarding the effect of breast feeding and development of hypertension.

METHODS: We included studies that reported on the association between breastfeeding of any duration and maternal risk of hypertension at any future time point outside of subsequent pregnancies, including cross-sectional, cohort, case-control or randomized controlled trials that were published in a peer-reviewed source. All included studies had a comparison group of either women who had not breastfed, or shorter durations of breastfeeding compared to the lactation group. We identified studies by searching PubMed and EMBASE in August 2015. A health sciences librarian developed and translated the systematic literature searches. Keywords included the concepts of hypertension, metabolic risk and/or blood pressure, and lactation or breastfeeding

RESULTS: Our search located 5342 references. After duplicate citations were identified and removed, 4817 citations were screened, and 4771 of these were eliminated by title or abstract. The remaining 46 full-text citations were reviewed, 19 of which met our inclusion criteria. Overall, though the follow up length of each trial varied from 4 days to 35 years, fourteen of nineteen studies showed a protective effect of breastfeeding on future blood pressure. The duration of lactation assessed in each study varied from 2 days post-partum to greater than 24 months of lactation. Ten were cohort studies, seven were case control, one was a randomized control trial and one was a cross-sectional study. The quality of studies was addressed using New Castle- Ottawa Quality Assessment Scale for casecontrol and cohort studies. The studies were of variable quality, with lower quality observed more commonly among older studies.

CONCLUSIONS: The majority of studies which assessed the association between lactation and maternal hypertension found a protective effect. The wide variability in 
breastfeeding duration and participant follow up for hypertension assessment makes it difficult to quantify a minimum duration of lactation to achieve a protective effect. It is notable that even short durations of breastfeeding, including 1-6 months, were found to significantly decrease a woman's future risk of hypertension. Limitations of studies include the lack of ascertainment of exclusivity of breast feeding, and the lack of a clinically relevant period of time in which to address blood pressure effects. Overall, this systematic review finds that breastfeeding is likely to significantly decrease the lifetime incidence of maternal hypertension, and supports the growing body of literature which shows a protective effect of breastfeeding on maternal cardiovascular risk factors.

\section{EFFECT OF THE CONTRACEPTIVE PROVISION OF THE AFFORDABLE} CARE ACT ON INSERTION RATES AND OUT-OF-POCKET COST FOR THE INTRAUTERINE DEVICE Cynthia H. Chuang ${ }^{2}$; Douglas L. Leslie ${ }^{1}$; Guodong Liu ${ }^{1}$; Ashley H. Snyder ${ }^{2}$; Carol S. Weisman ${ }^{1} .{ }^{1}$ Penn State College of Medicine, Hershey, PA; ${ }^{2}$ Penn State Milton S. Hershey Medical Center, Hershey, PA. (Control ID \#2469604)

BACKGROUND: The intrauterine device (IUD) is a highly effective yet expensive form of long-acting reversible contraceptive. The Affordable Care Act's (ACA) contraceptive provision became effective August 1,2012 and requires private health insurance plans to cover all Food and Drug Administration-approved contraceptive methods, including IUDs, with no out-of-pocket costs. Prior to the implementation of this provision, the high upfront cost of IUDs discouraged women from choosing IUDs in favor of less effective birth control methods with lower out-of-pocket cost. We aim to evaluate the impact of this policy on trends in IUD insertion rates and out-of-pocket costs for IUDs among privately insured women.

METHODS: Data from Truven Health MarketScan ${ }^{\circledR}$, a claims database from private insurance companies nationally, was used to examine rates of claims for new IUD insertions and IUD-related out-of-pocket costs before and after implementation of the contraceptive provision of the ACA in August 2012. Study cohorts were created for each calendar year between 2005 and 2013 that included women 13-45 years of age who had prescription coverage during that year with no requirement for continuous enrollment. The study cohorts included 5.58 million women in 2005, 5.91 million women in 2006, 6.79 million women in 2007, 8.53 million women in 2008, 8.43 million women in 2009, 9.09 million women in 2010, 10.32 million women in 2011, 10.6 million women in 2012, and 9.03 million women in 2013. We report the rate of IUD insertion pre- and post- implementation of the contraceptive provision of the ACA. Out-of-pocket cost for device and IUD insertion was estimated by combining cost of copayment, coinsurance and deductible payments. All costs were adjusted for inflation to the year 2013 dollars using the Consumer Price Index. A $t$-test was used to compare the average difference in out-ofpocket cost for IUD between 2011 (the year before the ACA's contraceptive provision became effective) and 2013 (the year after the ACA's contraceptive provision became effective). A Wilcoxon rank sum test was used to compare the median difference in out-ofpocket cost for IUD between 2011 and 2013.

RESULTS: The rate of claims for IUD insertions increased over time from $0.36 \%$ in 2005 to $1.17 \%$ in 2011 (the year before the ACA's contraceptive provision became effective) to $1.54 \%$ in 2013 (the year after the ACA's contraceptive provision became effective). We also observed that the average out-of-pocket cost for the IUD nearly doubled from $\$ 49.7$ (standard deviation \$106.9) in 2005 to $\$ 107.0$ (standard deviation $\$ 216.3$ ) in 2012. The median cost throughout this same time remained stable at around \$20 (range \$17.9 to \$23.1). In 2013, the year following the implementation of the contraceptive provision, the average out-of-pocket cost for IUD decreased to $\$ 21.5$ $(p<.0001)$ while the median decreased to $\$ 0(p<.0001)$.

CONCLUSIONS: We observed a dramatic reduction in the average out-of-pocket cost for the IUD following implementation of the contraceptive provision of the ACA. While most women had no out-of-pocket cost post-ACA implementation of the contraceptive provision, the mean cost was still $\$ 21.50$ (standard deviation $\$ 107$ ). These costs may be attributable to grandfathered plans, employers with religious exemptions, or noncompliance with the ACA provision. The rate of claims for IUD insertions increased over each year in the study, including the year following the implementation of the contraceptive provision of the ACA. Since our sample only includes data for 1 year post-ACA contraceptive provision, it is unclear if the small increase in the rate of claims for IUD insertion in 2013 is consistent with the secular trend for increasing IUD insertions or is a direct effect of the provision. Time trend analysis will be possible when more data are available. To our knowledge, ours is the first study to look at trends in both IUD insertion rates and out-of-pocket costs following the implementation of the contraceptive provision of the ACA using a large national database of privately insured women. Because cost is often a leading indicator, we may see an increase in the use of IUDs in coming years following implementation of the ACA contraceptive provision.

\section{EFFECTS OF A MULTI-FACETED INTERVENTION TO IMPROVE CARE} TRANSITIONS WITHIN A PIONEER ACCOUNTABLE CARE ORGANIZATION Jeffrey L. Schnipper $^{1,2}$; Nyryan V. Nolido ${ }^{1}$; Anuj K. Dalal ${ }^{1,2}$; Cherlie Magny-Normilus ${ }^{1}$; Lipika Samal $^{1,2}$; Asaf Bitton ${ }^{1,2}$; Ryan Thompson ${ }^{3,2}$; Stephanie Labonville ${ }^{1}$; Gwen Crevensten ${ }^{3,2}$. ${ }^{1}$ Brigham and Women's Hospital, Boston, MA; ${ }^{2}$ Harvard Medical School, Boston, MA; ${ }^{3}$ Massachusetts General Hospital, Boston, MA. (Control ID \#2469108)

BACKGROUND: Transitions from hospitals to the ambulatory setting are high risk periods for patients. The advent of the Patient-Centered Medical Home (PCMH) and Accountable Care Organizations (ACOs) provide an opportunity for true collaboration in which both inpatient and outpatient providers contribute to improving transitions in care. The goal of this study was to develop, implement, refine, and evaluate a multi-faceted, multi-disciplinary transitions intervention across two hospitals and 18 PCMHs within a Pioneer ACO

METHODS: We developed an intervention with the following components: inpatient pharmacist-led medication reconciliation and patient counseling; coordination of care and patient education from an inpatient Discharge Advocate and PCMH Responsible Outpatient Clinician; a structured visiting nurse intervention; structured post-discharge phone calls; timely follow-up visits; tools to improve communication among care team members; and home pharmacist visits for selected patients. The study used a "stepped wedge" design in which each PCMH practice started in the usual care arm and then at a randomly selected point in time changed to the intervention. Outcomes included 30-day hospital readmissions using medical records and telephone follow-up; and new or worsening symptoms in the 30 days after discharge based on telephone follow-up. The two outcomes were analyzed by multivariable logistic and Poisson regression, respectively, adjusted for study month, patient age and sex, and clustered by primary care practice.

RESULTS: One thousand six hundred seventy-eight patients were enrolled, including 758 assigned to usual care and 920 to the intervention. Receipt of different components of the intervention varied by component (Table 1) and in some cases by hospital, unit, and practice. Thirty-day readmission rates were $14.9 \%$ in the intervention arm and $14.4 \%$ in usual care ( $p=0.70$; Table 2). The number of new or worsening symptoms was 0.67 per patient in the intervention arm and 0.76 per patient in usual care $(p=0.16)$.

CONCLUSIONS: Results showed no difference in 30-day readmission rates among patients in the two study arms, likely due to lower than expected intervention fidelity and the low proportion of readmissions that are truly preventable in this patient population. The intervention was associated with a non-significant trend towards reduced new or 
worsening symptoms in the post-discharge period, an outcome more sensitive to change than readmissions. As with readmissions, efficacy was likely limited by intervention fidelity. Further study is needed to further explore the causes and effects of low intervention fidelity, determine the most important components of the intervention, and determine patient subgroups most likely to benefit from these types of interventions.

Table 1. Intervention Fidelity Among Patients in the Intervention Arm

\begin{tabular}{|c|c|c|}
\hline Intervention Component & $\begin{array}{l}\text { Hospital } 1 \\
(n=360)\end{array}$ & $\begin{array}{l}\text { Hospital } 2 \\
(n=\mathbf{2 2 2})\end{array}$ \\
\hline Inpatient pharmacist, n (\%) & $131 / 292(44.9 \%)$ & $34 / 222(15.0 \%)$ \\
\hline $\begin{array}{l}\text { Discharge Advocate note in } \\
\text { EMR (Site 1) or Email } \\
\text { facilitating Nurse-Nurse } \\
\text { Communication (Site 2), } \\
\text { n (\%) }\end{array}$ & $118 / 285(41.4 \%)$ & 76/98 (77.6 \%) \\
\hline $\begin{array}{l}\text { Post-discharge phone call by } \\
\text { Responsible Outpatient } \\
\text { Clinician within } 2 \text { business } \\
\text { days }\end{array}$ & 49/285 (17.2\%) & $30 / 98(30.6 \%)$ \\
\hline Visiting Nurse & $23 / 112(20.5 \%)$ & 16/98 (16.3\%) \\
\hline Network VNA & $21 / 112(18.8 \%)$ & 27/98 (27.6 \%) \\
\hline Non-network VNA & $68 / 112(60.7 \%)$ & $55 / 98(56.1 \%)$ \\
\hline Not discharged with VNA & & \\
\hline Outpatient pharmacist visit & $70 / 140(50.0 \%)$ & 19/41 (46.0\%) \\
\hline Patient Accepted Service & $70 / 140(50.0 \%)$ & $22 / 41(54.0 \%)$ \\
\hline
\end{tabular}

Table 2. 30-Day Post-Discharge Outcomes

\begin{tabular}{|c|c|c|c|c|}
\hline Outcome & Intervention & $\begin{array}{l}\text { Usual } \\
\text { Care }\end{array}$ & $\begin{array}{l}\text { Adjusted and } \\
\text { Clustered } \\
\text { Relative Risk } \\
(95 \% \mathrm{CI})^{*}\end{array}$ & $P$ value \\
\hline $\begin{array}{l}\text { Readmitted to a Partners } \\
\text { hospital within } 30 \text { days } \\
\text { post discharge, n (\%) }\end{array}$ & $\begin{array}{l}122 / 920 \\
(13.3 \%)\end{array}$ & $92 / 758(12.1 \%)$ & $\begin{array}{l}\text { AOR } 1.11 \\
(0.89,1.37)\end{array}$ & 0.35 \\
\hline $\begin{array}{l}\text { Readmitted to a non- } \\
\text { Partners hospital within } \\
30 \text { days post discharge, } \\
\text { n (\%) }\end{array}$ & $\begin{array}{l}21 / 920 \\
(2.3 \%)\end{array}$ & $\begin{array}{l}20 / 758 \\
(2.6 \%)\end{array}$ & $\begin{array}{l}\text { AOR 0.84 } \\
(0.48,1.50)\end{array}$ & 0.55 \\
\hline $\begin{array}{l}\text { Total 30-day Readmissions, } \\
\text { n }(\%) \dagger\end{array}$ & $\begin{array}{l}137 / 920 \\
(14.9 \%)\end{array}$ & $\begin{array}{l}109 / 758 \\
(14.4 \%)\end{array}$ & $\begin{array}{l}\text { AOR } 1.04 \\
(0.85,1.27)\end{array}$ & 0.70 \\
\hline $\begin{array}{l}\text { New or Worsening } \\
\text { symptoms within }\end{array}$ & $\begin{array}{l}308 / 449 \\
\text { patients }\end{array}$ & $\begin{array}{l}370 / 472 \\
\text { patients }\end{array}$ & $\begin{array}{l}\text { IRR } 0.88 \\
(0.73,1.06)\end{array}$ & 0.16 \\
\hline $\begin{array}{l}30 \text { days post discharge, } \mathrm{N} \\
\text { per patient }\end{array}$ & $\begin{array}{l}0.67 \text { per } \\
\text { patient }\end{array}$ & $\begin{array}{l}0.76 \text { per } \\
\text { patient }\end{array}$ & & \\
\hline
\end{tabular}

AOR: adjusted odds ratio; IRR: (adjusted) incidence rate ratio

*Adjusted for study month, patient age and sex, and clustered by primary care practice.

$\dagger$ Patients may have had readmissions to both Partners and non-Partners hospitals

EFFECTS OF A PHYSICIAN-SPECIFIC HOSPITAL BOUNCEBACK POLICY TO REDUCE READMISSIONS Nathan Moore ${ }^{2}$; Emily D. Fondahn ${ }^{1}$; Melvin Blanchard ${ }^{3}$. ${ }^{1}$ Washington University, St Louis, MO; ${ }^{2}$ Washington University, Saint Louis, MO; ${ }^{3}$ Washington University in St. Louis, St. Louis, MO. (Control ID \#2438622)

BACKGROUND: Unplanned hospital readmissions are a major quality issue for the US healthcare system. Resident and hospitalist medical services across the country commonly use a bounceback policy to determine the admitting physician for readmitted patients in an attempt to improve quality of care and reduce readmissions. Bounceback policies vary widely by institution in duration (e.g., the policy only applies for 7 days after discharge) and application (e.g., the policy only applies if the senior resident is still on service). Despite their widespread use, to date there have been no studies of bounceback policies or their effect on patient outcomes. In July 2011, the Internal Medicine residency program at Washington University in St. Louis changed the bounceback policy for the general medicine teaching service. Prior to 2011, only patients who were readmitted within 2 days of discharge were placed back on the discharging team's service. The new rule extended the duration of the policy to 28 days post-discharge. Our hypothesis, based on direct observation of clinical decision-making by residents, was that the extension of the bounceback policy would strengthen residents' incentive to avoid readmissions, leading to a reduction in readmissions. We also hypothesized that residents may keep patients in the hospital longer as one strategy to avoid readmissions, leading to an increased length of stay.

METHODS: The primary objective of this retrospective cohort study was to determine the effect of a stronger bounceback policy for the general medicine teaching service on 30day readmission rates, length of stay (LOS), and adjusted LOS index. The secondary objective was to assess the effect of time within clinical rotations on the same outcomes. Participants included patients admitted to the teaching and nonteaching general medicine services at Barnes Jewish Hospital, a large, tertiary-care, academic medical center, from $6 / 15 / 09$ to $6 / 16 / 13$; total number of hospitalizations analyzed was 61,149 . We utilized a difference-in-differences design to compare outcomes on the teaching and nonteaching medical services with adjustment for age, severity of illness, age, gender, and race. We used the nonteaching service as a control group because the two services care for similar patient populations, share facilities and non-physician staff, and the bounceback policy on the nonteaching service did not change during the study period. The strength of this quasiexperimental difference-in-differences design is that it controls for secular trends that are correlated with patient outcomes, impacting both residents and hospitalists similarly. The analyses were repeated for patients with a diagnosis of heart failure, acute MI, pneumonia, or COPD only.

RESULTS: When comparing patients discharged after the bounceback policy was implemented to those discharged before, the teaching service had a $7.92 \%$ lower relative risk ratio of 30-day readmission compared to the nonteaching service $(p=.007)$, a change in mean LOS of -0.28 days ( $p=0.008)$, and no significant difference in LOS index. For patients with a diagnosis of heart failure, acute MI, pneumonia, or COPD, there was no significant difference in LOS or readmission rates, but LOS index decreased by 0.09 on the nonteaching service when compared to the teaching service $(p=0.03)$. LOS and readmission rates were not significantly correlated to the number of days from the start of a rotation to the patient's discharge before or after the bounceback rule was implemented, but LOS did exhibit a weekly pattern with lowest LOS on Mondays.

CONCLUSIONS: A stronger bounceback policy for the teaching service resulted in a small but significant decrease in 30-day readmission rates and LOS, without a significant difference in LOS index. Physician-specific hospital bounceback policies may be an effective tool to reduce readmissions but further study is needed to validate our findings in different clinical settings.

EFFECTS OF NEW FUNDING MODELS FOR PATIENT-CENTERED MEDICAL HOMES ON PRIMARY CARE PRACTICE FINANCES AND SERVICES: RESULTS OF A MICRO-SIMULATION MODEL Sanjay Basu ${ }^{1,2}$; Russell Phillips²; Zirui Song ${ }^{2}$; Bruce Landon ${ }^{2}$; Asaf Bitton ${ }^{2} .{ }^{1}$ Stanford University, Stanford, CA; ${ }^{2}$ Harvard Medical School, Boston, MA. (Control ID \#2459435)

BACKGROUND: Payer-sponsored patient-centered medical home (PCMH) initiatives offer new opportunities to achieve financial stability among primary care clinics that provide medical home services. We sought to assess financial and service delivery implications for practices of participating in new PCMH payment initiatives.

METHODS: We created and validated a microsimulation model, tabulating practice revenues and costs from a practice manager perspective. Revenue and cost data were obtained from national practice surveys. Simulated clinics reflecting the national range of practice size, location, and patient population were subjected to each of three PCMH funding approaches: increased fee-for-service (FFS) payments, standard FFS with 
additional per member per month (PMPM) payments, or standard FFS with additional PMPM and pay-for-performance payments. Changes in clinic utilization and net annual revenue were estimated after clinics expanded services to include: investing in patient tracking, communications, and quality improvement systems; increasing support staff; altering visit templates to accommodate longer visits, telephone visits, and/or electronic visits; and extending service delivery hours. The primary outcome measure was practicelevel change in net annual revenue per full-time physician following new PCMH funding and associated service changes.

RESULTS: Under the 'status quo' of traditional FFS payments, clinics would need to minimally change their service delivery strategies to maximum net annual revenue, suggesting they respond strongly to existing financial forces. Practices gained substantial additional net annual revenue per full-time physician under PMPM or PMPM with payfor-performance bonuses (\$113,300 per year, $95 \%$ CI: \$28,500 to \$198,200) while losing net revenue under increased FFS $(-\$ 53,500,95 \% \mathrm{CI}:-\$ 69,700$ to $-\$ 37,200)$, after accounting for the costs of meeting minimum PCMH funding requirements. Expansion of services beyond minimum required levels resulted in net revenue losses among clinics. Even at high PMPM payment rates, expanding services such as telephone or electronic visits reduced net annual revenue by between $\$ 18,000$ and $\$ 23,700$ per full-time physician from the level meeting minimum PCMH funding requirements, due to opportunity costs of lost traditional FFS revenue.

CONCLUSIONS: In a model of primary care practices, PCMH funding through PMPM with or without pay-for-performance bonuses improved practice finances. Yet no PCMH funding initiatives offered sufficient incentives to expand services beyond minimum requirements, or change the service delivery approach that they had developed under usual fee-forservice payments. Achieving goals of the medical home model may require more radical payment reforms to specifically target funding towards the delivery of desired services.

EFFICACY OF INTERVENTIONS TO REDUCE RESIDENT PHYSICIAN BURNOUT: A SYSTEMATIC REVIEW Kiran Busireddy; Miller Jonathan; Kathleen E. Ellison; Vicky Ren; Mukta Panda; Rehan Qayyum. University of Tennessee, Chattanooga, TN. (Control ID \#2466677)

BACKGROUND: Burnout, characterized by emotional exhaustion, depersonalization, and reduced personal accomplishment, poses significant challenges to the optimal delivery of healthcare and is associated with a variety of negative consequences including depression, stress related health problems, decreased empathy, suicidal ideation, low career satisfaction, risk of medical errors, and negative effects on patient safety. Studies have examined the incidence and prevalence of burnout as well as common factors associated with burnout (e.g., work hours, sleep deprivation, staff conflicts, etc.); however, less is known about how to reduce burnout. Since resident physicians have the highest prevalence of burnout, and there is little consensus about how to address it, a systematic review of the literature was used to investigate the presence of previously conducted interventions, and analyze their efficacy at reducing burnout

METHODS: PubMed, EMBASE, and Web of Science databases were searched, without time or geographical limitations, using the following keywords: burnout and (resident or intern or residency). Two reviewers independently evaluated and screened for relevant articles. Peer-reviewed studies that were published in medical journals and reported an identifiable intervention and outcome were included for review. We excluded review articles, editorials, letters to the editors, and articles published in non-English language. Data on study characteristics, and population, interventions, and outcomes were abstracted. Study quality was assessed using Newcastle-Ottawa scale for cohort studies and Jadad scoring scale for randomized controlled trials (RCT). The data was pooled from these studies using random-effects meta-analysis that accounted for between-study heterogeneity. These studies reported data in different forms; when studies reported data on the number (or percentage) of residents with high levels of burnout we pooled their odds ratio and when studies reported mean burnout scores we pooled mean differences. Betweenstudy heterogeneity was assessed by standard tests.

RESULTS: Of the 1294 retrieved articles, 19 articles describing (6 RCTs and 13 cohort studies) 2030 number of residents in 12 different types of interventions fulfilled our inclusion and exclusion criteria. Maslach Burnout Inventory (18) Tedium Index were used to measure burnout in 19 articles. Work hour restrictions, particularly related to the Accreditation Council for Graduate Medical Education (ACGME) 2003 and 2011 duty hour rules (DHR), was the most common type of intervention ( 8 of the 19 studies). Of the 13 studies that were assessed using New Castle-Ottawa Scale, 2 were rated high quality and 6 moderate quality. Of the 6 RCTs that were assessed using Jadad scale, 4 were highquality studies (score $\geq 3$ ). DHRs showed a reduction in overall burnout in only one study; however, three additional studies found a significant improvement in emotional exhaustion domain and one study in depersonalization domain. In addition to DHRs, Respiratory One method of relaxation, self-care workshops and self-development groups showed an improvement in resident burnout. DHR were associated with a $42 \%$ lower odds of the percentage of residents reporting high levels of emotional exhaustion component (odds ratio $=0.58 ; 95 \%$ confidence interval $=0.43$ to $0.77 ; P<0.001)$ and with a decrease in mean score $($ mean difference $=-2.73 ; 95$ confidence interval $=-4.12$ to -1.34 ; $P<0.001)$. DHR had no effect on the number of residents with high level of depersonalization (odds ratio $=0.83 ; 95 \%$ confidence interval $=0.62$ to $1.11 ; P=0.20$ ) although there was a small but statistically significant decrease in mean depersonalization score (mean difference $=-1.73 ; 95 \%$ confidence interval $=-3.00$ to $-0.46 ; P=0.008$ ). Similarly, there was no effect of DHR on number of residents with high levels of personal accomplishment (odds ratio $=1.01 ; 95 \%$ confidence interval $=0.67$ to $1.54 ; P=0.95$ ) or with mean personal achievement score (mean difference $=0.93 ; 95 \%$ confidence inter$\mathrm{val}=-0.19$ to $2.06 ; P=0.10$ ). The I2-statistic for heterogeneity between the studies varied from $0 \%$ for some outcomes to up to $63 \%$.

CONCLUSIONS: ACGME duty-hour limitations, the Respiratory One Method of relaxation, self-care workshops and self-development groups demonstrated decrease in resident burnout although the results of studies were not consistent. Further research is needed in exploring interventions that can consistently decrease resident burnout.

ELEMENTS OF THE PATIENT CENTERED MEDICAL HOME ASSOCIATED WITH CLINICAL QUALITY: EVIDENCE FOR ACCESS, CONTINUITY AND CARE COORDINATION Karin M. Nelson ${ }^{2}$; Philip W. Sylling ${ }^{5}$; Leslie Taylor ${ }^{6}$; Idamay Curtis $^{5}$; Danielle Rose ${ }^{3}$; Gordon Schectman ${ }^{4}$; Richard Stark ${ }^{1}$; Stephan D. Fihn ${ }^{5,} 2$. ${ }^{1}$ Department of Veterans Affairs, Washington, DC; ${ }^{2}$ University of Washington, VA Puget Sound, Seattle, WA; ${ }^{3}$ VA GLA Healthcare System, Sepulveda, CA; ${ }^{4}$ Veterans Affairs Central Office, Milwaukee, WI; ${ }^{5}$ Veterans Health Administration, Seattle, WA; ${ }^{6}$ VA Puget Sound, Seattle, WA. (Control ID \#2469727)

BACKGROUND: A cornerstone of the patient centered medical home (PCMH) model is the provision of accessible, continuous, and team-based primary care. Our previous work has shown that more effective implementation of the PCMH model (the Patient Aligned Care Team (PACT) initiative) in over 900 Veterans Health Affairs (VHA) primary care clinics was associated with lower health care use, decreased staff burnout and higher clinical quality and patient satisfaction. By incorporating features of the chronic care model, PCMH models may be more effective in managing patients with chronic conditions. The goal of the current study is to determine which domains of PACT implementation are most associated with improved clinical quality indicators for preventive care and among cohorts of veterans with chronic medical conditions (e.g., diabetes, hypertension, $\mathrm{CHF}$ ).

METHODS: We conducted an observational study using FY2012-2014 data from the VHA national performance evaluation program, the External Peer Review Program (EPRP), to 
evaluate the quality of preventive care and chronic disease management among 672,177 Veterans who received primary care at VHA. Clinic-level outcomes were percentage of patients meeting 48 clinical quality indicators based on chart abstraction. To measure PCMH implementation, we utilized a previously validated instrument, the PACT implementation progress index $\left(\mathrm{PI}^{2}\right)$ comprised of 53 items from 8 core PCMH domains: access, continuity, care coordination, comprehensiveness, self-management support, patient-centered care and communication, shared decision-making and team-based care. Each clinic received a standardized domain score (mean of 0 ) for each of the 8 domains and were also categorized into quartiles. Domain scores for care coordination, comprehensiveness, self-management support and patient centered care and communication are derived from the Consumer Assessment of Health Plans (CAPHS)-PCMH survey ( $n=75,101$ responses administered to a weighted, random sample of Veterans who received outpatient care in 2012). Access, continuity and care coordination included items from the CAPHS-PCMH survey in addition to administrative data for 5,632,188 unique Veterans who received primary care at 640 VHA hospital- and community-based outpatient clinics (CBOCs). We used binomial regression to assess the odds of patients at higher implementation clinics obtaining better clinical quality, adjusting for a clinic- level random effects and testing for interactions between each EPRP measure and the $\mathrm{PI}^{2}$ domain, enabling comparisons of the mean EPRP success rate for the clinics in the lowest 25th percentile compared to clinics the highest 25 th percentile for each domain. Within each domain, we also calculated the difference in percentage of patients of meeting each clinic quality indicator from clinics in the top quartile compared to clinics in the lowest quartile RESULTS: We found that top-quartile clinics had significantly higher odds of meeting clinic quality indicators for the access (OR 1.11,95\% CI 1.07-1.16, $p<0.001$ ), continuity (OR 1.10, $95 \%$ CI 1.05-1.14, $p<0.001$ ), care coordination (OR 1.10, $95 \%$ CI 1.06-1.15, $p<0.001$ ), and team-based care (OR 1.04, $95 \%$ CI 1.002-1.08, $p=0.04$ ) domains. Sites with high access scores performed significantly better on 32 out of 48 quality measures compared to sites with low access scores. Similar results were noted for sites with higher continuity scores (37/48 measures), care coordination (34/48 measures), and team-based care (16/48). Overall clinical quality was high and differences between high and low domain scores were modest. For example, compared to sites with lower access scores, sites with higher access scores had the largest difference in rates of osteoporosis screening for women 65 years and older (65\% in high scoring clinics compared to $55 \%$ in low scoring clinics, difference $10.4 \%$ ), patients with diabetes prescribed aspirin $(5.8 \%$ difference), and patient counseling for alcohol misuse (5.0\% difference). Compared to sites with lower continuity scores, clinics with greater continuity had $9.5 \%$ higher rates of appropriate disposition for veterans who screened positive for PTSD.

CONCLUSIONS: Overall, we found that access, continuity and care coordination were associated with better clinical quality. Initiatives that support these elements of the medical home model may improve clinical care.

ENABLING SENIORS TO PLAN FOR THEIR POST-HOSPITAL DISCHARGE NEEDS BEFORE A HOSPITALIZATION OCCURS: PLANYOURLIFESPAN.ORG Lee Lindquist $^{2}$; Vanessa Ramirez-Zohfeld ${ }^{2}$; Priya Sunkara ${ }^{2}$; Chris Forcucci ${ }^{1}$; Kenzie A. Cameron ${ }^{2} .{ }^{1}$ Aging \& In-Home Services of NE IN, Fort Wayne, IN; ${ }^{2}$ Northwestern University, Chicago, IL. (Control ID \#2467475)

BACKGROUND: Most hospital discharge/transitional care planning occurs during the final days of a hospitalization. When seniors are hospitalized, they are frequently too sick to make informed decisions about their post-discharge care. Subsequently, family members and friends must scramble to make support choices (e.g. skilled nursing facility placement, home-based caregiver hiring). We sought to advance the timeline for posthospital care decisions to before a hospitalization ever occurs. To help seniors plan ahead for their post-discharge care, we developed and tested PlanYourLifespan.org (PYL) - an online tool that provides education on support options following health crises (e.g. hospitalizations, falls). Within PlanYourLifespan.org, videos of seniors present the reallife decisions that they had to make and advice they wish they had following a hospitalization. PYL facilitates choosing a skilled nursing facility and connects seniors to local community-based resources. Users can make personalized choices and email their choices to loved ones in case a health crisis occurs.

METHODS: We conducted a two-arm randomized controlled trial of the PYL tool as compared to an attention control arm (Go4Life website). Participants in Chicago, IL; Fort Wayne, IN; and Houston, TX were randomly assigned to one of the two interventions using equal (1:1) allocation and random permuted block design. Participants were English-speaking, 65 years and older who currently live independently in the community. Eligible participants completed an in-person survey, followed by exposure to the intervention or attention control websites (minimum 15 min., maximum 45 min.). Participants then completed both a 1- and 3month telephone survey. Knowledge was measured at all three time points with six items pertaining to areas such as understanding home services and rehabilitation options. We employed linear mixed modeling with random participant effects to explore differences in knowledge score over time (between baseline and 1 month) among arms, while controlling for significant baseline covariates.

RESULTS: Among 385 participants randomized, mean age was $71.9(\mathrm{SD}=5.6)$, with $79.5 \%$ female; and $64.4 \%$ identified as White and $24.2 \%$ as African-American. At 1 month, participants in the intervention group had a 0.6 point change in knowledge score $(\mathrm{SD}=1.6)$ and the control group had $\mathrm{a}-0.1$ point change in knowledge score $(\mathrm{SD}=1.4)$. Linear mixed modeling results suggested that level of education and income were significant predictors of knowledge score over time, with higher income and education resulting in higher knowledge scores. Controlling for these variables, treatment effect remained significant $(p=0.0005)$, with participants in the treatment group having a change in knowledge score that was, on average, 0.28 points higher than those in the control group.

CONCLUSIONS: Seniors who used PlanYourLifespan.org demonstrated an increased understanding of post-hospitalization and home services compared to the control group. The implications of seniors realizing their post-hospitalization needs prior to a hospitalization are substantial: Loved ones may experience less burden, Hospital length of stays may be reduced, Referrals to rehabilitation facilities may be expedited, and ultimately, seniors can have a voice in their future care. PlanYourLifespan.org is nationally available, free-to-use, and being widely disseminated to health systems nationwide.

END-OF-LIFE EXPERIENCE OF OLDER ADULTS DYING OF END-STAGE RENAL DISEASE VERSUS CANCER Melissa W. Wachterman ${ }^{3,}{ }^{4}$; Zhonghe $\mathrm{Li}^{6}$; Stuart R. Lipsitz ${ }^{4}$; Karl Lorenz ${ }^{5}$; Edward R. Marcantonio ${ }^{1}$; Nancy L. Keating ${ }^{2,}{ }^{4}$. ${ }^{1}$ Beth Israel Deaconess Medical Center, Boston, MA; ${ }^{2}$ Harvard Medical School, Boston, MA; ${ }^{3}$ VA Boston Healthcare System, Jamaica Plain, MA; ${ }^{4}$ Brigham and Women's Hospital, Boston, MA; ${ }^{5}$ Stanford University School of Medicine, Palo Alto, CA; ${ }^{6}$ Harvard School of Public Health, Boston, MA. (Control ID \#2455868)

BACKGROUND: Older adults with end-stage renal disease (ESRD) are a rapidly growing group of seriously-ill patients. Yet, despite higher mortality rates for older adults with ESRD than for many advanced cancers, little is known about their end-of-life experience. Understanding this experience is critical for primary care physicians since primary care providers' role in the care of seriously-ill patients becomes even more important near the end of life. The objective of this study is to compare advanced care planning, treatment intensity, and symptoms, including pain, for older adults dying of ESRD with those dying of cancer. We chose cancer as a comparator both because it is the condition that has been most extensively studied in end-of-life care research and because it is appreciated to cause severe symptoms at the end of life; thus it provides important benchmarks for key outcomes. 
METHODS: We used data from the 2002-2010 Health and Retirement Study (HRS), a nationally representative longitudinal survey of community-dwelling adults $\geq 51$ years old. Our data source was the "Exit Interview", conducted with the proxy (usually a family member) after a participant's death. Our analyses compared the following proxy-reported outcomes between ESRD and cancer decedents: 1) documentation of advanced care planning; 2) ability to participate in end-of-life care decisions; 3) death in the hospital; 4) intensive care unit use within 2 years of death; and 5) symptoms including moderatesevere pain, dyspnea, depression, and confusion. Cause of death was determined by proxy report. We used propensity score weighting to balance covariates (age, gender, race, marital status, education, net worth, and comorbidity) among ESRD and cancer decedents and minimize confounding. We used chi-square statistics to compare outcomes by cause of death after propensity weighting.

RESULTS: Our sample included 358 patients who died of end-stage renal disease (ESRD), representing 1.2 million ESRD decedents nationally, and 1525 patients who died of cancer, representing 5.4 million cancer decedents nationally. Before propensity weighting, ESRD decedents were older than cancer decedents (mean age 77 vs. 73, $P<.001)$ and less often married ( 39 vs. $48 \%, P=.01$ ) or White ( 82 vs. $86 \%, P=.05$ ). After propensity weighting, the groups were well-matched on all covariates. As shown in the Table, in propensity-weighted analyses, significantly less, about a third, of patients dying of ESRD had written end-of-life instructions compared to half of those dying of cancer. Similarly, there was a trend towards less, only about a third, of ESRD decedents being able to participate in end-of-life care decisions, compared to closer to half of cancer decedents. In terms of treatment intensity, significantly more, over half, of patients dying of ESRD, died in the hospital, compared to less than a third of cancer decedents. Furthermore, significantly more, over half, of patients dying of ESRD used the intensive care unit in the last 2 years of life, compared to just over a third of cancer decedents. In the last year of life, over half of patients dying of either ESRD or cancer had moderate/severe pain. Both groups suffered from not only similarly high rates of pain, but also other symptoms including dyspnea, depression, and confusion.

CONCLUSIONS: Older adults dying of ESRD had significantly lower rates of advanced care planning than those dying of cancer, which is particularly concerning since two-thirds of ESRD patients were not able to participate in medical decision-making near the end of life. Those dying of ESRD also had higher treatment intensity near the end of life than those dying of cancer and had similarly high rates of pain and other symptoms. Our findings suggest the need for improvements in both symptom management and communication about goals of care for the rapidly increasing number of older adults dying of ESRD, a patient population whose end-of-life care needs have been underappreciated.

Adjusted* Comparisons of Advanced Care Planning, Treatment Intensity, and Symptoms Near the End-of-Life for Those Dying of ESRD and Cancer

\begin{tabular}{llll}
\hline \hline OUTCOME & $\begin{array}{l}\text { Adjusted* } \\
\text { Proportion of } \\
\text { Patients with } \\
\text { ESRD }(n=358)\end{array}$ & $\begin{array}{l}\text { Adjusted* Proportion } \\
\text { of Patients } \\
\text { with Cancer } \\
(n=1525)\end{array}$ & $P$ Value \\
$\begin{array}{l}\text { ADVANCED CARE PLANNING } \\
\text { Had written end-of- }\end{array}$ & 38.6 & 49.8 & .006 \\
life care instructions & 33.2 & 42.5 & .10 \\
$\begin{array}{l}\text { Able to participate in } \\
\text { end-of-life care } \\
\text { decisions }\end{array}$ & & & $<.001$ \\
$\begin{array}{l}\text { TREATMENT } \\
\text { INTENSITY }\end{array}$ & & & $<.001$ \\
$\begin{array}{l}\text { Died in the hospital } \\
\text { Used the intensive } \\
\text { care unit in the last }\end{array}$ & 52.6 & 29.0 & \\
$\begin{array}{l}2 \text { years of life } \\
\text { SYMPTOMS DURING } \\
\text { THE LAST YEAR OF }\end{array}$ & 56.9 & 37.2 & .28 \\
$\begin{array}{l}\text { LIFE } \\
\text { Often troubled } \\
\text { by moderate/ } \\
\text { severe pain }\end{array}$ & & & \\
\hline
\end{tabular}

\section{(continued)}

\begin{tabular}{lccc}
\hline \hline $\begin{array}{l}\text { Dyspnea for at } \\
\text { least } 1 \text { month }\end{array}$ & 61.7 & 53.5 & .14 \\
$\begin{array}{l}\text { Depression for at } \\
\text { least } 1 \text { month }\end{array}$ & 52.8 & 54.5 & .79 \\
$\begin{array}{l}\text { Periodic confusion } \\
\text { for at least } 1 \text { month }\end{array}$ & 40.8 & 44.2 & .45 \\
\hline
\end{tabular}

*Adjusted for age, gender, race, marital status, education, net worth, and comorbidity using propensity weighting

ENHANCING PATIENT ENGAGEMENT IN INTENSIVE OUTPATIENT MANAGEMENT: ADDRESSING NEIGHBORHOOD BARRIERS AND CHALLENGES Colin W. O’Brien ${ }^{1}$; Jessica Y. Breland ${ }^{2,3}$; Cindie Slightam²; Andrea Nevedal ${ }^{2}$; Donna M. Zulman ${ }^{2,4}$. ${ }^{1}$ Stanford University, San Francisco, CA; ${ }^{2}$ VA Palo Alto, Menlo Park, CA; ${ }^{3}$ Stanford University, Stanford, CA; ${ }^{4}$ Stanford Unversity, Stanford, CA. (Control ID \#2464636)

BACKGROUND: Intensive Outpatient Care Programs (IOCPs) are designed to improve care and reduce costs for patients with complex medical and social needs, but these programs' effects are often diminished due to patient engagement challenges. We sought to 1) understand how stressors related to patients' neighborhoods (e.g., housing, transportation, safety) influence their participation and engagement in IOCPs; and 2) identify IOCP strategies to address these challenges.

METHODS: We conducted a qualitative study with 20 IOCP leaders and clinicians (e.g., physicians, nurses, psychologists, case workers) to investigate patient engagement challenges and strategies. Participants represented 12 diverse Northern California programs operated by county healthcare systems $(n=4)$, Veterans Affairs facilities $(n=2)$ community health centers $(n=3)$, private healthcare systems $(n=2)$, and a public payer $(n=1)$. In a pre-interview survey, we assessed the proportion of patients facing neighborhood-level barriers to engagement. Then, in semi-structured interviews we asked participants to describe specific barriers and the strategies that their programs use to overcome them. Using standard content analysis methods, two investigators coded each transcript to examine how neighborhood barriers influence patient engagement, and to identify strategies that IOCPs employ to address neighborhood challenges.

RESULTS: Over half of participants reported that the following neighborhood-related barriers to patient engagement affected most or all of their complex patient panel: 1) marginalized neighborhood or poor neighborhood conditions; 2) lack of access to positive neighborhood destinations; and 3) poor social environment or lack of social connections. Specific challenges included housing instability, safety concerns, inadequate transportation, social stressors, poor living conditions, food insecurity and the presence of drugs. Strategies to address these barriers included connecting patients with social services ("We can call adult protective services to say that this person is at risk of losing their housing and they will help in many situations"), and helping patients establish housing more suitable to their needs and goals ("Financially, it was really hard for her, so we started helping her look for other places ... so it was easier for her to mobilize out of the apartment."). A key facilitator of the success of these strategies was staff familiarity with local neighborhoods ("People who are not familiar with neighborhoods or don't actually come from those neighborhoods is a little bit of a barrier so we are recruiting (for staff that live) within those neighborhoods").

CONCLUSIONS: Programs designed to meet the needs of patients with complex medical and social needs should be attuned to challenges arising from patients' neighborhoods, including the location and quality of their housing, safety and transportation obstacles, and social stressors. While these issues are rarely addressed in traditional 
healthcare settings, many IOCPs have identified creative solutions, primarily by providing adequate staff time and harnessing knowledge about local communities and resources.

ENVISIONING OPPORTUNITIES: IDENTIFYING VISION CARE NEEDS AMONG INPATIENTS WITH DIABETES Janaki Patel ${ }^{2}$; Vineet M. Arora ${ }^{1}$; Allison J. Louis ${ }^{1}$; Victoria I. Moreira ${ }^{3}$; Seenu M. Hariprasad ${ }^{1}$; David Meltzer ${ }^{1}$; Valerie G. Press ${ }^{1}$. ${ }^{1}$ University of Chicago, Chicago, IL; ${ }^{2}$ Pritzker School of Medicine, Chicago, IL; ${ }^{3}$ Medical College of Georgia, Augusta, GA. (Control ID \#2466087)

BACKGROUND: In 2012, over 22 million Americans were diagnosed with diabetes; patients with diabetes accounted for over 40 million days of inpatient hospitalization. Diabetes carries a known increased risk for visual impairment, prompting recommendations for regular outpatient vision care and appropriate self-management. However, studies show less than ideal attainment of outpatient vision assessments, suggesting the need for novel ways to increase vision care engagement. Over one-third of hospitalized general medicine patients fail a vision screening. Therefore, the hospital setting may be an opportunity to identify patients with diabetes who may need further vision care after discharge, but few studies have evaluated vision status among inpatients with diabetes Beyond the post-discharge vision care needs that could be identified and intervened on for inpatients with diabetes, inadequate vision during hospitalization may put patients at increased risk for falls or delirium. Our study aimed to identify the prevalence of insufficient vision among inpatients with diabetes and evaluate their level of empowerment and knowledge in managing their disease, specifically in relation to vision care. METHODS: The Vision in Senior Inpatients: Outcomes and Needs (VISION) study is an ongoing, prospective, observational study of adult ( $\geq 18$ years), English-speaking, cognitively intact general inpatients (IRB \#9967). Trained research assistants measured visual acuity using a pocket Snellen chart (insufficient vision defined as worse than 20/40 in both eyes). Participants completed a survey about access to, and need for, vision care Participants with diabetes were identified by medical record and self-report; a subset completed the Diabetes Empowerment Scale-Short Form (DES-SF), the Diabetes and Vision Knowledge Test (DVKT), and a brief history of their disease management Wilcoxon Mann-Whitney $U$ tests and $\chi^{2}$ tests were used to analyze the data.

RESULTS: From June 2012- November 2015, 2660 participants were enrolled with a mean age of 52 years. The majority were female (59 \%) and African American (78\%) Over one-third $(36 \%, 959 / 2660)$ had diabetes, with a mean HbAlc of $8.9 \%$ and average blood glucose level of 167 . Those with (versus without) diabetes were more likely to be older (56 vs. 49 years, $p<0.001$ ) and African American (46 vs $76 \%, p<0.001$ ). Insufficient vision was found in almost half of participants $(43 \%, 1139 / 2660)$, and was significantly more prevalent in those with (vs without) diabetes (56 \%, 537/959 vs 35\%, $602 / 1701 ; p<0.001)$. Excluding those with self-reported eye disease, participants with diabetes were still more likely to have insufficient vision (53\%, 368/693 vs $31 \%$, 438/ 1433; $p<0.001$ ). Of note, nearly $1 / 4$ of those with diabetes were wearing their corrective lenses, but still had insufficient vision ( $24 \%, 226 / 949 ; p=0.02)$. Regarding diabetesrelated access, control, empowerment, and knowledge, there were significant differences based on vision status among inpatients with diabetes. Participants with insufficient vision (vs. sufficient) were more likely to believe they needed to see an eye doctor now (59\%, $266 / 454$ vs $50 \%, 159 / 317 ; p=0.001$ ), and, after excluding those with known eye disease, were more likely to have had an eye exam within the past year (74 \%, 204/276 vs $55 \%$, $114 / 209 ; p, 0.001)$. Those with insufficient vision were also more likely to be older (60 vs 51 years, $p<0.001$ ). Younger participants with diabetes were more likely to have a higher HbAlc $(b=-0.04 ; p<0.001)$; controlling for vision status, there was no significant difference in $\operatorname{HbA} 1 \mathrm{c}(p=0.2)$. Among participants completing the diabetes empowerment and knowledge tools $(n=494)$, the mean DES score was 4.14/5 (IQR 3.75-4.75) and $49 \%$ (466/959) correctly answered all the questions of the DVKT. Those with insufficient vision (vs sufficient) scored lower on the DES (3.96/5, IQR 3.37-4.63 vs 4.5/5, IQR 4.39$4.75 ; p<0.001)$, but were more likely to correctly answer all DVKT questions ( $55 \%, 293$ / 537 vs $41 \% 173 / 422 ; p<0.001)$. There was no significant difference in mean A1c based on DES scores $(p=0.2)$ or DVKT results $(p=0.1)$.

CONCLUSIONS: sion screening. Despite the high prevalence of insufficient vision, most participants with diabetes self-reported regular eye exams. These findings suggest that self-reported attainment of vision care and wearing of corrective lenses may be inadequate markers of sufficient visual acuity during hospitalization or adherence to recommended guidelines of care. Interestingly, although participants with poor vision reported lower empowerment scores, they were actually more knowledgeable about vision-related complications and more often reported seeking regular vision care. Vision loss may be related to feelings of lower self-efficacy, affecting attitude toward diabetes care, but not necessarily tangible measures of self-care. Further work should evaluate interventions to improve inpatient screening and referrals for improved diabetes-related vision care.

\section{ESTABLISHING CONSENSUS ON RESIDENCY EDUCATION IN WOMEN'S} HEALTH Amy H. Farkas ${ }^{1}$; Sarah A. Tilstra ${ }^{3}$; Sonya Borrero ${ }^{4}$; Melissa McNeil ${ }^{2} .{ }^{1} \mathrm{UPMC}$, Pittsburgh, PA; ${ }^{2}$ University of Pittsburgh Medical Center, Pittsburgh, PA; ${ }^{3}$ University of Pittsburgh School of Medicine/Medical Center, Pittsburgh, PA; ${ }^{4}$ University of Pittsburgh and VA Pittsburgh, Pittsburgh, PA. (Control ID \#2468568)

BACKGROUND: The American College of Graduate Medical Education (ACGME) expects internal medicine residents to be able to provide "gender-specific" care upon graduation, yet literature suggests that residents often fall short of this goal. This may be in part to a lack of clear standards for women's health residency education. The objective of this project is to develop a consensus list of core knowledge domains and procedural skills in women's health that every categorical internal medicine resident should possess upon graduation.

METHODS: We conducted a two-round Delphi with a panel of women's health experts identified using the 2015 Directory of Residency and Fellowship Programs in Women's Health and the Society of General Internal Medicine's Women's Health Education Interest Group membership list. Nationally recognized women's health leaders were also recruited to participate. In the first round, participants were presented with a list of 54 women's health topics and asked to: 1) assess the importance of each topic for internal medicine residents to be proficient in upon graduation in order to provide high-quality care to women using a 1-5 Likert scale, 2) to prioritize the topics for a women's health curriculum by identifying them as "critical" topics. Additionally, participants could list any topics they felt were critical but not included in the initial list in a free-response section. Those who completed the first round of the Delphi were asked to participate in the second round. Participants were presented with the list of 54 topics plus two additional topics identified in the free response section of the first round. Mean rating and standard deviation from the first round were shown. Again, participants were asked to rate the importance of each topic with the same 1-5 Likert scale. Participants were also presented with the list of topics identified as "critical" by at least $50 \%$ of the participants in the first round and asked to indicate whether or not they agreed with the designation using a 1-5 Likert scale. Mean importance rating for all topics and mean agreement rating for the critical topics were calculated. Topics with a mean importance rating of $\geq 4$ or a mean agreement rating of $\geq 4$ in the second round were included in the consensus list of core women's health topics and skills. Stata SE was used for all calculations. The University of Pittsburgh IRB approved our study.

RESULTS: Of the 41 individuals we invited to participate in our Delphi, 19 (46\% response rate) agreed to participate and completed both rounds. Our experts represented 17 different programs from across the country, including both VA and non-VA programs. The majority $(62.5 \%, n=35)$ of topics received an importance rating of $\geq 4$ indicating that they were "very 
important" or "essential." The highest-ranking topics included cervical cancer screening, screening for osteoporosis, and diagnosis of sexually transmitted infections, (mean rating of 4.95/each). Other highly rated topics included those related to contraception/reproductive planning, benign and malignant breast disease, menopause, and performing breast and pelvic exams. Our expert panel identified $60.7 \%$ of the topics $(n=34)$ as "critical" for a women's health curriculum. The only topic that received an importance rating of $>4$ but was not identified as a critical topic was the initiation of hormone replacement therapy for menopause, indicating very little discrepancy between the "critical" topics and those deemed "very important" or "essential" on the Likert scale. Many of the topics that were deemed less important or non-critical were related to management (as opposed to diagnosis) of various conditions including: chronic pelvic pain, endometriosis, sexual dysfunction, eating disorders, and polycystic ovarian syndrome. Additionally, pregnancy related topics, such as comanagement of pregnant patients and management of post-partum complications, were deemed less important and non-critical.

CONCLUSIONS: Our study generated a consensus list of 35 core women's heath knowledge domains. As residency programs have struggled to equip trainees with basic women's health education, our consensus list provides guidance on which topics programs should focus their resources to train residents in "gender-specific" care.

EVALUATING INDIVIDUAL FEEDBACK AND PARTNER FEEDBACK TO IMPROVE STATIN MEDICATION ADHERENCE Ashok Reddy ${ }^{4}$; Tiffany Huseman $^{1}$; Anne Canamucio ${ }^{1}$; Steven C. Marcus ${ }^{3}$; David A. Asch ${ }^{2}$; Kevin G. Volpp ${ }^{1}$; Judith Long ${ }^{1}$. ${ }^{1}$ Philadelphia VA Medical Center, Philadelphia, PA; ${ }^{2}$ University of Pennsylvania, Philadelphia, PA; ${ }^{3}$ university of pennsylvania, Philadelphia, PA; ${ }^{4}$ University of Washington, Seattle, WA. (Control ID \#2467338)

BACKGROUND: Among patients who have had a heart attack, nearly half stop taking their statin medications within a year. Simple nudges such as individual adherence feedback or social incentives (peer pressure) might facilitate adherence.

METHODS: We conducted a 6-month randomized controlled trial testing two different feedback approaches to improve medication adherence among veterans with CAD who had been prescribed a statin but were poorly adherent (defined as a medication possession ratio of $<80 \%$ ). Participants were randomly assigned to one of three groups: 1. a control group that received an electronic pill monitoring device with no alarms or feedback; 2. an individual feedback group that received a daily alarm and a weekly medication adherence feedback report; and 3. a partner feedback group that received an alarm and a weekly feedback report that was shared with the participant and a selected partner (a friend, family member, or a similar peer struggling with statin adherence). The intervention continued for 3 months; participants were followed for an additional 3 months post-intervention. The primary outcome was adherence during the 3 months of the intervention. Secondary outcomes included change in LDL $\mathrm{mg} / \mathrm{dl}$, patient activation, and social support.

RESULTS: We enrolled 126 patients. We used a stratified 2:2:3 block assignment to assign extra participants into the partner feedback arm since participants were being offered a choice of a family member or a peer (Control: 36, Individual Feedback: 36, Partner Feedback: 54). Demographic characteristics were similar across control and treatment groups. During the 3 month intervention period, medication adherence in both feedback arms was higher than in the control arm (89\% in the individual feedback; $86 \%$ in the partner feedback; $67 \%$ in the control group - $p$-value for comparison with control $=$ 0.0011 and 0.0004 , respectively). There was no significant difference in adherence between the 2 feedback groups. In the post-intervention period, when all feedback mechanisms were stopped, adherence rates dropped in both feedback groups. At 6 months there was no differences in medication adherence between the feedback groups and control $(60 \%$ in individual feedback group; $52 \%$ in partner feedback group; $54 \%$ in the control group $-p$-value for comparison with control $=0.75$ and 0.97 , respectively). There were no changes relative to control in any of the other secondary outcomes. CONCLUSIONS: Daily alarms combined with individual feedback reports or individual and partner feedback improved statin medication adherence in veterans with CAD and poor adherence. While neither individual feedback nor a partner feedback strategy created a sustainable medication adherence habit, the intervention itself is relatively easy to implement and low cost. If improved adherence continues if the intervention remains active, remote monitoring with adherence feedback could be a relatively simple way to improve long-term medication adherence.

GloCaps Weekly Adherence by Arm

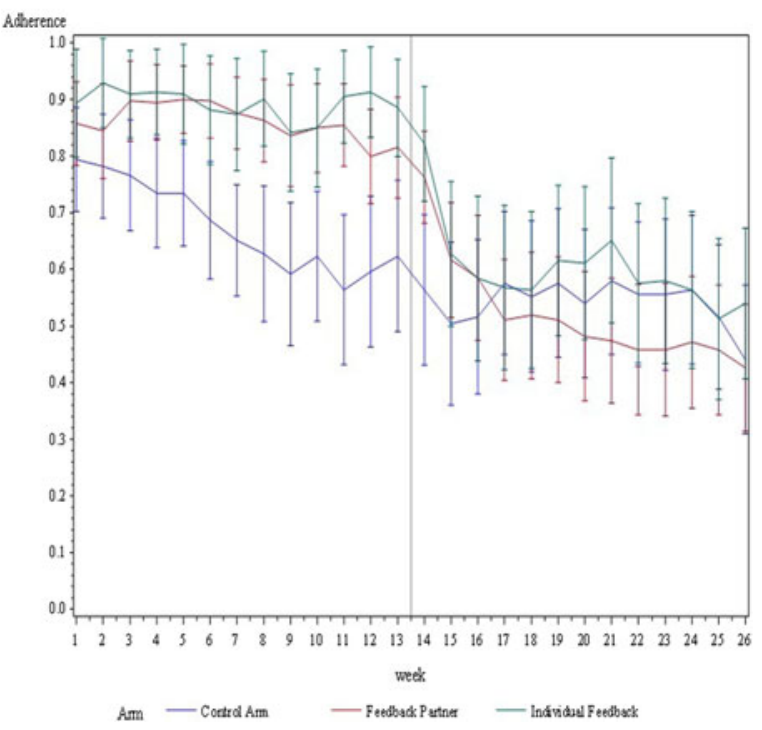

EVALUATING SHARED DECISION-MAKING DURING PRIMARY CARE VISITS IN A COMMUNITY HEALTH CENTER USING COLLABORATE Sanja Percac-Lima $^{1,2}{ }^{1}$; Rachel C. Forcino ${ }^{3}$; Rachel Thompson ${ }^{3}$; Elissa Ozanne ${ }^{3}$; Paul Barr ${ }^{3}$; Glyn Elwyn $^{3} .{ }^{1}$ Massachusetts General Hospital, Boston, MA; ${ }^{2}$ Harvard Medical School, Boston, MA; ${ }^{3}$ Geisel School of Medicine at Dartmouth, Lebanon, NH. (Control ID \#2469401)

BACKGROUND: Patient engagement and shared decision-making have become health care policy priorities in the US. Our health care systems are designed to maximize profit and efficiency sometimes neglecting the concerns and preferences of patients. Efforts to implement and evaluate health system changes designed to increase patient engagement and shared decision-making require valid, reliable and scalable measurement tools that adequately reflect patients' views and priorities. In 2013, CollaboRATE - a three-item, multi-platform, patient-reported measure - was developed to evaluate patient engagement, and specifically, shared decision-making, during clinical encounters. The objective of this study was to assess the feasibility of routinely administering CollaboRATE to measure patient engagement during primary care visits in a community health center. METHODS: The study was conducted at Massachusetts General Hospital Chelsea HealthCare Center Adult Medicine practice (MGH Chelsea) serving predominantly low-income, ethnically diverse populations. We asked all adult patients who had a visit at MGH Chelsea during May 2015 to complete either an English or Spanish version of CollaboRATE. Data were also collected on age, gender, whether patients had assistance completing the survey, and whether an interpreter was present during the visit. Surveys were returned in a secure box. The primary outcome was the proportions of respondents who experienced shared decision-making, defined as those who gave the highest possible 
score (9) on all three CollaboRATE questions. Chi square tests were used to compare this proportion across patient demographics, provider and interpreter use.

RESULTS: Of 1687 patients that were seen by 18 providers at the practice during the study period, 1230 completed the survey ( $73 \%$ response rate). Of respondents, 848 (69 \%) were female and $375(30 \%)$ were male. Six hundred twenty-four patients (51\%) used English and 606 (49\%) used Spanish versions of the survey. An interpreter was used in 131 (7.8\%) encounters (62 in Spanish, 69 in other languages). One thousand fifty-nine patients ( $86 \%$ ) experienced shared decision-making. The proportion of responders who experienced shared decision-making ranged from 76 to $99 \%$ across providers. Similar rates of shared decision-making were observed among men and women ( 85 and $87 \%$, respectively, $p=0.319$ ), and by those who used the English and Spanish versions ( $86 \%$ for both groups). However, different rates of shared decision-making were observed among different age groups (e.g., $78 \%$ among 18 24-year-olds vs. $95 \%$ among 65-74-year-olds, $p=0.003$ ). No significant difference was observed based on interpreter presence ( $92 \%$ with an interpreter vs. $86 \%$ without an interpreter, $p=0.173$ ).

CONCLUSIONS: CollaboRATE was found to be a feasible measure for administration during primary care visits in a diverse population. The patients' response rate was high. While patients' perceptions of levels of engagement and shared decision-making were generally very high, considerable variation was observed by provider and among some patient subgroups, at least partially addressing questions about 'ceiling effects' in measurement. These questions will continue to be addressed in related work assessing the validity and reliability of the measure in real-world clinical practice.

EVALUATING THE EFFECT OF BREAST DENSITY NOTIFICATION LEGISLATION ON BREAST CANCER STAGE AT DIAGNOSIS Ilana B $\underline{\text { Richman }}^{2,}{ }^{1}$; Steven Asch ${ }^{2,1}$; Eran Bendavid ${ }^{1}$; Jay Bhattacharya ${ }^{1}$; Douglas K. Owens ${ }^{2}$, ${ }^{1}$. ${ }^{1}$ Stanford University School of Medicine, Palo Alto, CA; ${ }^{2}$ Palo Alto Veterans Affairs Health Care System, Palo Alto, CA. (Control ID \#2468033)

BACKGROUND: Breast density is an independent risk factor for breast cancer and also reduces the sensitivity and specificity of mammography. Nearly half of states now require physicians to inform patients when they are found to have dense breasts on screening mammography. In some states, physicians must also tell women with dense breasts that they may benefit from supplemental screening with MRI or ultrasound Advocates have suggested that this kind of legislation will facilitate earlier breast cancer diagnosis among women with dense breasts, while critics worry that the optimal screening strategy for such women is unknown. We sought to evaluate the effect of this legislation on stage at diagnosis.

METHODS: We used data on stage-specific breast cancer incidence from the Surveillance, Epidemiology, and End Results (SEER) program from 2005 to 2012. Among the states included in SEER, one (Connecticut), had enacted breast density notification legislation during the period for which data were available. Using a difference-in-differences framework, we compared changes in stage-specific breast cancer incidence in counties in Connecticut to counties in states that ho such legislation. We used a mixed effects negative binomial model with county-level random effects to estimate changes in stage-specific breast cancer incidence and to evaluate the legislative effect. We also evaluated the legislative effect specifically among women ages $40-49$, who have the highest prevalence of dense breasts. Models were adjusted for state fixed effects and selected time-varying county characteristics including median household income, physician density, county racial composition, and uninsured rate. RESULTS: Our study included data from 613 counties in 18 SEER geographies over an 8 year period. The eight counties in Connecticut were subject to breast density notification legislation beginning in late 2009. In adjusted models, counties subject to breast density notification legislation did not see a decline in the incidence of metastatic disease post legislation, compared to control counties (incidence rate ratio 1.03, $95 \%$ CI 0.90-1.19, $p=0.67)$. Breast density notification legislation was not associated with a decrease in the incidence of lymph node positive disease at presentation (IRR 1.00, $95 \%$ CI 0.94-1.07, $p=0.94$ ). We also did not observe an increase in early stage diagnosis with passage of breast density notification legislation. Among counties in Connecticut, there was no change in the incidence of localized disease (IRR $1.010 .96-1.06,95 \% \mathrm{CI}$ ) or ductal carcinoma in situ (IRR $0.94,95 \%$ CI $0.88-1.01, p=0.09$ ) compared to control counties. Findings were similar when we limited our analysis to women $40-49$, who are most likely to have dense breasts.

CONCLUSIONS: We found that passage of breast density notification legislation was not associated with a reduction in the incidence of late-stage diagnosis in counties in Connecticut compared to control counties. Our study is limited by a relatively short follow up period and small number of intervention counties in a single state. Further work may examine a broader group of intervention counties over a longer period as additional data become available.

EVALUATING THE EFFECTIVENESS OF A PILOT COMMUNITYTAILORED CARDIOVASCULAR HEALTH PROGRAM AMONG IMMIGRANT CHINESE AND VIETNAMESE COMMUNITIES IN UPTOWN, CHICAGO Kevin Zheng ${ }^{1}$; Katherine Mueller ${ }^{2}$; Edwin Chandresekar ${ }^{2}$; Namratha R. Kandula ${ }^{1} .{ }^{1}$ Northwestern University, Feinberg School of Medicine, Chicago, IL; ${ }^{2}$ Asian Health Coalition, Chicago, IL. (Control ID \#2464225)

BACKGROUND: Traditional approaches to health education and disease managemen have not been effective for Asian immigrants, a population in Chicago that has both cardiovascular and diabetes in its top five causes of death. Barriers to health care faced by Asian immigrants include financial and linguistic barriers to the implementation of public health interventions, limited access to and poor usage of preventive medical care, and cultural and religious attitudes that are poorly understood by Western health professionals The Asian Health Coalition has adapted an evidence-based cardiovascular health selfmanagement program for the underserved and limited English-proficient Chinese and Vietnamese communities in Uptown, Chicago. The program targets at-risk individuals and uses culturally and linguistically appropriate methods for health education. The objective of this study is to evaluate the overall effectiveness of the program by looking at changes in knowledge and patient-centered outcomes, which focus around the intention and selfconfidence to change at-risk behavior.

METHODS: Lower-income Chinese and Vietnamese immigrants $(n=136)$ living in the Uptown neighborhood of Chicago were recruited through word-of-mouth, notices in ethnic media, and flyers posted in community locations such as grocery stores and hair/nail salons. Participants underwent a 6-week long series of workshops from the National Heart, Lung and Blood Institute's Your Health is Golden module, an evidencebased health promotion program which has been tested and tailored for the Asian community. Sessions were taught by bilingual community health workers well-known in the neighborhood. Participants were asked to complete surveys before and after the program that assessed attitudes, knowledge, readiness to change cardiovascular health behaviors, and participants' locus of control. Clinical measurements (e.g., body mass index, $\mathrm{HbA} 1 \mathrm{c}$, cholesterol) were taken at the start of the program and at 16 weeks after baseline assessment. Simple descriptive statistics were performed on demographic and clinical data. Chi-squared tests of independence were performed on responses to survey questions.

RESULTS: Overall, participants were 61.6 (SD 13.1) years of age and $100 \%$ were limited English-proficient. At baseline, $78.7 \%$ had at least one cardiovascular disease risk factor; $46.6 \%$ were overweight/obese, $44.3 \%$ had high cholesterol, $65.6 \%$ had pre- 
diabetes or diabetes, and $41.2 \%$ had high blood pressure. Pre- and post-intervention survey results demonstrated that participants' heart health knowledge increased significantly $(p<0.001)$. Participants' readiness to adopt heart healthy behaviors increased significantly as well $(p<0.01)$, with progression towards determination and action stages. There was no significant change in participants' locus of control or clinical outcomes. CONCLUSIONS: A trusted community organization was able to successfully deliver a culturally tailored cardiovascular disease prevention program. Information learned from this study will help us gain a greater understanding of how to implement effective health outreach programs in limited English-proficient immigrant populations and for wider dissemination into other Asian immigrant communities in Chicago.

EVALUATING THE EFFECTIVENESS OF A POLICY THAT ENCOURAGES CLINICAL REASONING BASED ON CLINICAL GUIDELINES IN THE DIAGNOSIS AND MANAGEMENT OF ANDROGEN DEFICIENCY IN THE VA PITTSBURGH HEALTHCARE SYSTEM (VAPHS). Stephanie Karmo ${ }^{1}$; Alexandra Clark $^{1}$; Laura Potoski ${ }^{3}$; Ronald Codario ${ }^{3}$; Erika L. Hoffman ${ }^{2}$; Harsha Rao ${ }^{3}$. ${ }^{1}$ UPMC, Pittsburgh, PA; ${ }^{2}$ VAPHS/Univeristy of Pittsburgh, Pittsburgh, PA; ${ }^{3}$ VA Pittsburgh Healthcare System, Pittsburgh, PA. (Control ID \#2464217)

BACKGROUND: Testosterone (T) prescriptions, which increased 3-fold in the U.S from 2000 to 2011, are most commonly prescribed by Primary Care Physicians (PCPs). However, assessments of prescribing practices show poor adherence to guidelines, compared to Endocrinologists (ENDOs). The Endocrine Society's Clinical Practice Guidelines recommend that diagnosis be established with $\geq 2$ morning $T$ values, using a reliable assay (e.g. LC/MS/MS), and with bioavailable T (BAT) if diagnosis is uncertain or an alteration in SHBG is suspected (e.g. obesity, nephrotic syndrome, DM, older age, liver disease, HIV). The guidelines also recommend monitoring Hct, PSA and T levels within 6 months, and performing a rectal exam for age $>40$ and PSA $>0.6$. They identify prostate cancer, PSA $>4$, and Hct $>50 \%$ as relative contraindications to T therapy, and advise caution in patients with untreated obstructive sleep apnea (OSA). A history of stroke and acute coronary syndromes has been recently added to the list of precautionary comorbidities, and the FDA now requires product labels to inform of possible increased risk of heart attack and stroke in patients taking $\mathrm{T}$, although the relationship of $\mathrm{T}$ therapy to cardiovascular risk is not clearly established.

METHODS: Since 2008, the Endocrine Division at VAPHS has followed a policy that encourages clinical reasoning for diagnosing androgen deficiency and managing $\mathrm{T}$ replacement therapy. It adheres to all Endocrine Society guidelines, but also recommends active restraint in men aged $>65$ years, or with a history of thromboembolic events (TEE), recent ischemic heart disease (IHD) and stroke (CVA). By contrast, Primary Care at VAPHS does not have a specific policy governing T therapy, so that every PCP is required to individually determine diagnostic thresholds and prescribing constraints for $\mathrm{T}$ therapy. The objective of this study was to determine whether a policy encouraging clinical reasoning to diagnose and manage androgen deficiency was associated with adherence to recommended clinical guidelines. To that end, we conducted chart reviews of 425 male veterans prescribed T between 2008 and 2014 at VAPHS by either ENDOs $(n=125)$ or PCPs $(n=300)$ to assess adherence to guidelines for initiating and managing $\mathrm{T}$ therapy in a number of areas. These included clinical justification for checking $\mathrm{T}$ (documented reason for suspecting hypogonadism), how the diagnosis was made (time of blood draw, confirmation with two $\mathrm{T}$ levels, assay method, and diagnostic threshold), how $\mathrm{T}$ therapy was monitored (T level, Hct, PSA), and whether age $>65$, contraindications (prostate cancer, BPH) and precautionary comorbidities (TEE, recent IHD or CVA) were considered before starting $\mathrm{T}$ therapy.

RESULTS: Prior to starting T therapy, T levels were drawn without valid justification (i.e. as part of "routine" labs, by patient request, or without a documented reason) in 65/300
( $22 \%)$ of PCP patients, whereas justification was documented in all ENDO patients (125/ $125[100 \%])$. PCPs were also significantly less likely $(p<0.0001)$ to check T levels twice (115/229 [50.2\%] vs ENDO 88/101 [87.1\%]), use an LC/MS/MS assay (99/226 [44 \%] vs ENDO 80/101 [79\%]), or check it between 7 and 9 am (53/226 [23\%]) vs ENDO 53/ $101[52 \%]$. They were also less likely to use strict diagnostic criteria for androgen deficiency $(\geq 1$ total $\mathrm{T} \leq 200$ or BAT $<100$ : PCP 144/226 [64 \%] vs ENDO 79/101 [78\%] $(p=0.009)$. After starting T therapy, significantly fewer PCP patients $(p<0.0001)$ had a follow-up T level drawn (PCP 199/300 [70 \%] vs ENDO 115/125 [92 \%]), and even fewer had it within 6 months (PCP 127/300 [40 \%] vs ENDO 101/125 [81 \%]). PCP patients were also significantly less likely $(p<0.0001)$ to meet the 6-month recommendation for monitoring either Hct (PCP 139/300 [46 \%] vs ENDO 92/125 [74 \%],) or PSA (PCP 151/300 [50 \%] vs ENDO 80/125 [64 \%]). Finally, PCPs were far more likely to initiate $T$ therapy in patients age $\geq 65$ years (PCP 106/300 [35\%] vs ENDO (20/125 [16\%], $p=0.0004$ ), without querying for OSA (PCP 224/300 [75\%] vs ENDO 64/125, [51\%], $p<0.0001$ ), in the presence of relative contraindications like Hct $>50 \%$, PSA $>4, \mathrm{BPH}$, and prostate cancer (PCP 83/300 [28 \%] vs ENDO 15/125 $[14 \%], p=0.0004)$, or when precautionary comorbidities, like TEE, or IHD/CVA in prior year, were present (PCP 100/300 [33 \%] vs ENDO 20/125 [16\%], $p=0.0003$ ).

CONCLUSIONS: Our results show that PCPs were less likely to check T levels with valid clinical justification, at the correct time, using a reliable assay and a second $\mathrm{T}$ level for confirmation. They were also more likely to initiate $\mathrm{T}$ therapy in men over 65 or with contraindications or precautionary comorbidities, but less likely to obtain follow-up labs in the recommended time frame after initiating $\mathrm{T}$ therapy. A policy governing the diagnosis and management of androgen deficiency according to recommended guidelines may support PCPs in diagnosing androgen deficiency appropriately and initiating $\mathrm{T}$ therapy with appropriate monitoring and in fewer patients with contraindications or precautionary comorbidities. It remains unclear, however, whether such policy-driven practices alter outcomes but, until the relationship of $\mathrm{T}$ therapy to cardiovascular outcomes is clarified, it appears wise to exercise restraint in offering $\mathrm{T}$ replacement without clear justification, appropriate diagnostic testing, or careful monitoring.

EVALUATION OF CHRONIC HEPATITIS B (CHB) TREATMENT AND ASSESSMENT OF CHB CARE AT AN ASIAN COMMUNITY HEALTH CENTER IN NYC, 2012 Christopher Su ${ }^{1}$; Vivian Huang ${ }^{2} .{ }^{1}$ Montefiore Medical Center, New York, NY; ${ }^{2}$ Charles B. Wang Community Health Center, New York, NY. (Control ID \#2469823)

BACKGROUND: Chronic hepatitis B (CHB) is a significant chronic disease in Asia and among patients of Asian descent in the United States. In recent years, there has been an increased focus on the screening, identification, and treatment of patients with CHB. Various treatment guidelines have been established by different expert panels, and a novel treatment guideline proposed exclusively for Asian Americans ("Tong guidelines") lowers the laboratory treatment threshold for CHB compared to widely adapted guidelines such as those proposed by the Association for the Study of Liver Diseases (AASLD). The Tong guidelines (which also mirror recent European recommendations) have the potential to significantly impact the number of patients eligible for treatment, particularly at health centers serving large Asian communities. We propose to examine treatment rates using the new Tong guidelines compared with the AASLD guidelines at a major Asian community health center in New York City. In addition, patients with newly-diagnosed CHB require a comprehensive work-up at the time of diagnosis. Therefore, we also wish to review longitudinal care for patients with $\mathrm{CHB}$ at this health center during our study period by employing a comprehensive review of provider notes, clinical visits, follow-ups, laboratory tests, imaging, referrals, and demographic information. The aims of this study are 
fourfold: 1) to assess treatment eligibility for patients with $\mathrm{CHB}$ at a large Asian community health center using the novel treatment guidelines for Asian Americans proposed by Tong et al.; 2) to assess the treatment initiation rate for patients with $\mathrm{CHB}$ at a large Asian community health center using the Tong guidelines compared to the standard AASLD guidelines; 3 ) to determine demographic and laboratory factors affecting treatment initiation using the Tong guidelines; 4) to assess the quality of outpatient CHB care provided to patients at a large Asian community health center in New York City.

METHODS: A retrospective cohort study was conducted on new patients presenting with chronic hepatitis B to a major Asian community health center in New York City in $2012(n=677)$. Chronic hepatitis B treatment eligibility was determined via AASLD guidelines and the Tong guidelines proposed for Asian Americans. The final patient group of interest for this study included all patients falling under the treatment and "gray zone" categories under the Tong guidelines $(n=385)$. Treatment initiation under both Tong guidelines and AASLD guidelines were assessed. Logistic analysis was used to determine predictors of treatment initiation. Extensive chart review was performed for our final patient group of interest. Internal medicine provider notes were fully reviewed for at least 1 year following initial visit to $\mathrm{CBWCHC}$, with careful attention to $\mathrm{CHB}$ follow-up care performed at the health center

RESULTS: Patients in our study group of interest following application of the Tong guidelines $(n=385)$ were $53 \%$ male, $94 \%$ Chinese, and average 37.3 years old. Ninetythree percent of patients were either uninsured or on public insurance (Medicaid or Medicare), and most patients received hepatitis B testing in the context of an initial physical exam Treatment initiation rates following AASLD and Tong guidelines were $75 \%$ (39 out of 52 patients eligible) and $34 \%(63 / 183)$, respectively. Following logistic analysis, insurance status ( $p=0.01)$, positive HBeAg serology $(p<0.001)$, ALT level $(p<0.001)$ and HBV DNA vira load $(p<0.001)$, and platelet count $(p=0.01)$ were significant predictors of treatment initiation Heath center performance indicators of CHB care are presented in Table 1.

CONCLUSIONS: We report a comparable, if slightly higher, AASLD treatment initiation rate for eligible patients compared to other studies conducted at the community leve in the United States. Adopting Tong guidelines significantly increased treatment eligibility given the lower treatment thresholds; however, patients should be observed for persistent elevation of laboratory values prior to treatment initiation. We report high rates of CHB coinfection screening and HCC surveillance care at the study center compared to other published community studies. Significantly, primary care providers directly manage CHB care for the vast majority of patients and liver biopsy rates remain very low in this population.

Health Center Performance Indicators of CHB Care, 2012

\begin{tabular}{ll}
\hline \hline Criteria & Performance \\
Treatment rate under Tong guidelines & $63 / 183,34 \%$ \\
Treatment rate under AASLD guidelines & $39 / 52,75 \%$ \\
At least one CHB follow-up visit after & $358 / 385,93 \%$ \\
initial visit (avg: 70 days) & \\
At least two CHB follow-up visit after & $304 / 385,79 \%$ \\
initial visit (avg: 155 days) & \\
At least three CHB follow-up visit after & $246 / 385,64 \%$ \\
initial visit (avg: 143 days) & \\
Average visits to internal medicine clinic & $2.7 \pm 2.0$ \\
in the first 12 months & \\
Still active at the end of clinic \\
last visit<12 months) by 2015
\end{tabular}

EVALUATION OF INTERNET-BASED PATIENT EDUCATION MATERIALS FROM INTERNAL MEDICINE SUBSPECIALTY ORGANIZATIONS - WILL PATIENTS UNDERSTAND THEM? Elizabeth S. John ${ }^{1}$; David R. Hansberry ${ }^{4}$; Ann $\mathrm{John}^{3}$; Nitin Agarwal ${ }^{5}$; Ranita Sharma ${ }^{2} .{ }^{1}$ Rutgers Robert Wood Johnson, Montville, NJ; ${ }^{2}$ RWJ, New Brunswick, NJ; ${ }^{3}$ NJMS, Newark, NJ; ${ }^{4}$ Jefferson, Philadelphia, PA; ${ }^{5}$ University of Pittsburgh Medical Center, Pittsburgh, PA. (Control ID \#2469541)

BACKGROUND: The prevalence of the Internet is becoming more prominent in our society, and is used on a daily basis by the majority of Americans for a variety of reasons, but most notably, for health information. Of the $78 \%$ of Americans who use the Internet, approximately $80 \%$ rely on it for health information. This is, in part, propagated by the ease of accessing the Internet from numerous portable interfaces such as mobile phones, tablets, and personal laptops. While many patients will use the information to measure their own personal health or the health of a family member, others will use it to evaluate a newly prescribed medication or treatment suggestions offered by physicians. In fact, $69 \%$ of surveyed patients used online health information as a second opinion and $11 \%$ relied on Internet sources instead of visiting a physician. While this online information can be a powerful tool to supplement physician's visits and strengthen the patient to physician relationship, it can also be a source of anxiety and confusion if the information is not conveyed properly. The American Medical Association (AMA) and National Institutes of Health (NIH) have recommended that health information should be written between a 3 rd and 7 th grade level. The purpose of this study was to evaluate the level of readability of online patient education materials written specifically for patients from professional organization websites of 14 major internal medicine subspecialties to determine if they were effectively meeting the guidelines.

METHODS: Ten well established quantitative readability scales were used to assess written text from 540 patient education articles from the major professional organizations representing internal medicine subspecialty groups: Allergy and Immunology, Cardiology, Endocrinology, Gastroenterology, Geriatrics, Hematology, Hospice and Palliative Care, Infectious Disease, Nephrology, Oncology, Pulmonology and Critical Care, Rheumatology, Sleep Medicine, and Sports Medicine. The level of readability of each website was compared to each other using a one-way ANOVA and Tukey's Honestly Significant Difference (HSD) post hoc analysis with a $p<0.05$. Statistical analysis was performed with OriginPro (Northamptom, MA)

RESULTS: The 540 articles from the 14 different websites were collectively written at an 11.0 grade level (standard deviation of 1.4). The most readable webpages were from Sleep Medicine and Nephrology, which were written at an academic grade level of $8.5 \pm 1.5$ and $9.0 \pm 0.2$, respectively. The material from the Infectious Disease webpage was written at the most difficult level with an average level of readability corresponding to a grade level of $13.9 \pm 0.3$.

CONCLUSIONS: With the widespread accessibility of the Internet, it is becoming ever more crucial to ensure that health information is indeed written at levels that will maximize patient understanding. No society in this study provided patient education materials that were consistently written at the AMA and the NIH guidelines that patient education articles be written between a 3rd and 7 th grade levels. The AMA, NIH, and Centers for Disease Control and Prevention (CDC) offer guidelines to ensure articles are written at levels patients can understand, but making these changes is often tedious and ignored by most. If these online resources were rewritten, it is likely that a greater population would derive benefit from reading them. 
EVALUATION OF MEDICATION-RELATED CLINICAL DECISION SUPPORT OVERRIDES IN THE INTENSIVE CARE UNIT Adrian Wong ${ }^{3,2}$; Mary Amato ${ }^{3,2}$; Diane L. Seger ${ }^{1,3}$; Patrick E. Beeler ${ }^{5,3}$; Olivia Dalleur ${ }^{6,6}$; patricia dykes $^{3,7}$; Tewodros Eguale ${ }^{2,3}$; Julie Fiskio ${ }^{4,3}$; Elizabeth R. Silvers ${ }^{1,3}$; Sarah P. Slight ${ }^{8,3}$; David W. Bates ${ }^{3,7}$. ${ }^{1}$ Partners HealthCare, Brighton, MA; ${ }^{2}$ MCPHS University, Boston, MA; ${ }^{3}$ Brigham and Women's Hospital, Boston, MA; ${ }^{4}$ Partners Healthcare, Wellesley, MA; ${ }^{5}$ University Hospital, Zurich, Switzerland; ${ }^{6}$ Université catholique de Louvain, Brussels, Belgium; ${ }^{7}$ Harvard Medical School, Boston, MA; ${ }^{8}$ The University of Durham, Stockton on Tees, Durham, United Kingdom. (Control ID \#2467355)

BACKGROUND: Clinical decision support (CDS) has been shown to reduce hospital length of stay, costs and the incidence of medication errors. $(1,2)$ However, it is also clear that medication-related CDS alert overrides are very frequent in many systems. $(3,4)$ This high proportion is due to poor alert design and the underlying alert databases which include too many clinically insignificant warnings. Data also suggest that many overrides are inappropriate which represents a safety concern. Critically ill patients are particularly susceptible to adverse drug events (ADEs) for multiple reasons, including the high number of medications administered, including high-risk medications, altered pharmacokinetics secondary to altered organ systems, and relatively long length of stays. Given the lack of data regarding medication-related alerts in the intensive care unit (ICU) and potential for increased patient harm it is important to evaluate alert overrides in this setting.

METHODS: We performed a retrospective evaluation of CDS alert overrides at an academic medical center and included alerts from 1/2009 to 12/2011. The CDS alert overrides occurred while the patient was being treated in an adult ICU and were focused on specific alert types (allergy, drug-drug interaction [DDI], geriatric and renal). Geriatric alerts were for patients who were 65 years of age or older. The primary outcome was the appropriateness of the override, assessed by two independent reviewers with a set of predetermined criteria specific for each type of alert. A multidisciplinary group, including pharmacists, physicians and nurses reviewed and agreed on this set of criteria. Orders for home medications at the same or a reduced dose, with no evidence of intolerance, were determined to be appropriate for override. A random sample of 400 overrides (100 from each type of alert) was then selected for evaluation of appropriateness. Secondary outcomes included the documented override reason for each alert, and the incidence of ADEs that resulted from the overridden alert, based on documentation in the patient's medical record.

RESULTS: A total of 40,267 overridden alerts were identified as relevant to the ICU setting Alerts that were excluded from the evaluation at this point were triggered by orders based on institutional policies, e.g. those for short-term laxatives in geriatrics. A total of 28,978 alerts were considered relevant for evaluation $(72.0 \%)$. The majority of the overrides took place in the cardiac and medical ICUs. Regarding allergy alerts, the most common allergens were $\beta$-lactams and opiates. The majority of the documented reasons for drug allergy overrides were due to "patient has taken previously" ( $47.5 \%)$. The medications that accounted for the most DDIs were tacrolimus and amiodarone. "Will monitor" was the most common reason given for overriding DDI alerts (68.6\%). The median age for the geriatric alerts was 72 (IQR 68-79), with long acting benzodiazepines being the most common alerting medication (38.9\%). Nonsteroidal antiinflammatory drugs were the most commonly implicated medication for renal alerts $(26.6 \%)$. Within the randomized sample, 261 overrides were determined to be appropriate $(65.3 \%)$, with the proportion varying by type of alert (allergy: $94.0 \%$, DDI: $82.0 \%$, geriatric: $59.0 \%$, renal: $26.0 \%$ ). The kappa coefficient between reviewers was $87.5 \%$. Inappropriate overrides of allergy alerts were largely due to $\beta$-lactams with a documented unspecified reaction ("unknown") $(n=3)$. Simvastatin was the most common medication inappropriately overridden in DDI alerts and was administered at doses greater than recommended with CYP3A4 inhibitors ( $n=7,36.8 \%$ ). Identification of ADEs potentially associated with CDS overrides were rare $(n=$

7) and limited due to lack of documentation and their rare occurrence.

CONCLUSIONS: The appropriateness of medication-related CDS overrides varied substantially by the type of alert, with allergy alerts found to be the most appropriately overridden, while renal alert overrides were rarely appropriate. The risk for alert fatigue may be minimized by increasing the specificity and clinical significance of displayed alerts, while decreasing the number of insignificant alerts. Specific recommendations which could help to improve the accuracy of these alerts include the differentiation of medication allergy vs. intolerance, and the consideration of patient-specific factors such as comorbidities, ordered renal replacement therapy, and ordered monitoring parameters. References: 1. Bates DW, Leape LL, Cullen DJ, et al. Effect of computerized physician order entry and a team intervention on prevention of serious medication errors. JAMA. 1998;280:1311-6. 2. Bates DW, Teich JM, Lee J, et al. The impact of computerized physician order entry on medication error prevention. J Am Med Inform Assoc. 1999;31321. 3. Cho I, Slight SP, Nanji KC, et al. The effect of provider characteristics on the responses to medication-related decision support alerts. Int J Med Inform. 2015;84:630-9. 4. Nanji KC, Slight SP, Seger DL, et al. Overrides of medication-related clinical decision support alerts in outpatients. $J$ Am Med Inform Assoc. 2014;21:487-91. This study was funded by grant \#U19HS021094 from the Agency for Healthcare Research and Quality (AHRQ)

EVALUATION OF THE QUALITY OF CONFERENCE ABSTRACTS, PUBLISHED ABSTRACTS, AND MANUSCRIPTS IN MEDICAL EDUCATION USING THE MERSQI SCORE Christopher Stephenson; Brianna E. Vaa; Darrell Schroeder; Thomas J. Beckman; Darcy Reed; Adam P. Sawatsky. Mayo Clinic, Rochester, MN. (Control ID \#2469463)

BACKGROUND: Assessing the quality of an abstract is an important step in deciding whether the abstract is accepted for conference presentation or reviewed for potential publication. However, abstracts may omit information included in the manuscript, diminishing their quality. Additionally, there is little evidence in medical education scholarship to demonstrate whether abstract quality compares to manuscript quality. The Medical Education Research Study Quality Instrument (MERSQI) has been developed to evaluate the quality of medical education literature. In order to support the use of MERSQI to evaluate abstract quality in medical education research, we sought to investigate the relationships among the quality of conference abstracts, published abstracts, and manuscripts.

METHODS: We compared the quality of 46 medical education conference abstracts submitted to the 2009 Society of General Internal Medicine (SGIM) Annual Meeting to the quality of their published abstracts and manuscripts. Quality was assessed using the MERSQI, a 10-item tool that evaluates quality with 6 domains: study design, sampling, type of data, data analysis, validity of evaluation instrument, and outcome measures. The MERSQI score ranges from 5 to 18 with higher scores signifying higher quality. The MERSQI has been evaluated and showed validity to content, relationship to other variables, and internal structure. We included medical education studies involving education interventions, curriculum development, assessment tools, and education surveys at all levels of medical training. We excluded qualitative research, meta-analysis, and systematic reviews, since these were excluded in the MERSQI validation study. The quality of conference abstracts was reviewed and scored in a preceding study. The quality of the corresponding 46 published abstracts and manuscripts was scored using the MERSQI. Prior to scoring, two authors evaluated medical education literature outside of this study and demonstrated good inter-rater reliability using the MERSQI. We compared the overall MERSQI score and individual MERSQI item score among the conference abstracts, published abstracts, and manuscripts. Comparisons were performed using the signed rank test.

RESULTS: The mean total MERSQI score did not significantly differ between the conference abstracts or published abstracts $(9.67 \mathrm{vs} 9.96, p=0.299)$. However, manuscripts showed a significant increase in MERSQI scores compared to conference abstracts (9.67vs 11.33, $p<0.001)$ and published abstracts (9.96vs 11.33, $p<0.001)$. Published 
abstracts had higher MERSQI scores compared to conference abstracts in the domains of type of data ( 2.39 vs $2.31, p=0.031)$ and data analysis $(0.89$ vs $0.54, p<0.001)$. Manuscripts had higher MERSQI scores compared to conference abstracts in the domains of data analysis ( $1.00 \mathrm{vs} 0.54, p<0.001)$ and validity, specifically content validity $(0.59 \mathrm{vs}$ $0.13, p<0.001)$ and relationships to other variables validity $(0.20$ vs $0.04, p=0.039)$. Manuscripts also had higher MERSQI scores compared to published abstracts in the domains of data analysis (2.00 vs $1.80, p=0.004)$ and validity, specifically internal structure validity $(0.26$ vs $0.07, p=0.012)$, content validity ( 0.59 vs $0.20, p<0.001)$, and relationships to other variables validity $(0.20$ vs $0.00, p<0.004)$. The response rate, included in the sampling domain, was significantly increased for manuscripts compared to conference abstracts $(0.83$ vs $0.60, p=0.008)$ and published abstracts $(0.83$ vs 0.54 , $p<0.001$ ). Only $4 / 46$ manuscripts either had response rates less than $50 \%$ or did not report a response rate compared to $21 / 46$ for published abstracts.

CONCLUSIONS: We report the first study to compare the quality of medical education abstracts to manuscripts using the MERSQI. Overall, quality increased from abstracts to manuscripts. The differences between the conference abstracts and published abstracts were minimal and their mean total MERSQI scores did not significantly differ. However, compared to conference abstracts, published abstracts were more likely to report higher quality data, such as objective measurements and more complex data analysis. This may be because data collection or analysis was either not complete or not as thoroughly discussed in a conference abstract compared to the published abstracts More differences were seen comparing abstracts to manuscripts. Manuscripts were more likely to include a validity evaluation of assessment tools, possibly related to abstract brevity. However, the mean scores for validity evaluation were low for manuscripts, suggesting a limited discussion of validity in this sample of medical education literature Additionally, manuscripts were more likely to report a response rate compared to abstracts, again possibly related to abstract brevity or incomplete data collection. Overall, we demonstrated similar quality between medical education conference abstracts and published abstracts while showing a significant increase in quality of the published manuscript. As suspected, many of these differences were due to missing information in the abstracts such as discussions of validity evidence, and reports of response rate and data analysis. Future research to develop a modified MERSQI for abstracts that omit these items could be considered.

EVIDENCE BASED MEDICINE AND CLINICAL REASONING ON THE FLY: PARADOXICAL OPINIONS FROM RESIDENTS AND FELLOWS Deepa R. Nandiwada $^{2}$; Kenneth J. Smith ${ }^{2}$; Carla Spagnoletti ${ }^{2}$; Megan McNamara ${ }^{1}$. ${ }^{1}$ Louis Stokes Cleveland VAMC, Cleveland, $\mathrm{OH} ;{ }^{2}$ University of Pittsburgh, Pittsburgh, PA. (Control ID \#2469533)

BACKGROUND: Evidence Based Medicine (EBM) is comprised of critical appraisal skills and the application of evidence to clinical decision making. Consult services could provide a key interface to communicate and teach EBM. We conducted a needs assessment to evaluate behaviors and attitudes of residents, fellows, and attendings about their EBM practices while on consult services.

METHODS: Surveys were designed and sent to first year subspecialty fellows, all internal medicine residents, and subspecialty attendings who spent at least 2 weeks on the consult services. The surveys assessed four domains: teaching practices, communication of evidence based practices, communication of clinical reasoning, and ideas to promote daily practice of evidence based medicine. Surveys for fellows contained questions that overlapped with both attendings' and residents' surveys. Residents answered questions from two perspectives: as the primary team placing the consult and as residents on a consult service rotation. Survey items were assessed on a 5 point agreement Likert scale (strongly disagree to strongly agree), a frequency scale (almost never, seldom, half the time, usually, almost always), and a free text option for ideas on how to promote EBM on a daily basis. ANOVA was used for comparison of means and paired t-tests for within group comparisons. Free text comments divided into categories based on content. RESULTS: Response rates for attendings, residents, and fellows were $45 \%$ (64 of 141 attendings), $40 \%$ (63 of 159 residents), and $84 \%$ (41 of 49 fellows) respectively. Overall, attendings agreed it was more important to communicate clinical reasoning (4.56) than evidence $(4.05, p=.0001)$, although both were rated highly. Fellows reported communicating the evidence behind their clinical decisions about half the time (mean score 3.0), while residents did so seldomly (mean 2.3). From the perspective of the primary team placing the consult, residents agreed that they value evidence in the note (mean 4.56) and that they are more likely to follow a consult recommendation if evidence is provided (mean 4.06). Attendings and fellows felt that residents poorly communicated evidence in notes (mean 2.0 and 2.2 respectively, correlating with seldom). Conversely, residents felt they communicated evidence more frequently (mean 2.6), significantly higher than attendings' perceptions (mean 2.0; $p=0.0028$ ). Providing evidence on rounds without prompting was also rated at 2.3 and 2.43 by attendings and fellows respectively. Attendings and fellows perceived residents communicated clinical reasoning in their notes with means of 3.1 and 3.4 (about half the time) compared to residents who rated themselves at 4.7 (usually to almost always; $p=0.001$,). Five themes facilitating the daily practice of EBM on consult services were generated: brevity, accountability, expectations on rounds, transparency in notes, and online shared resources

CONCLUSIONS: This needs assessment shows that although EBM and clinical reasoning are deemed important by all groups, reported communication behaviors vary drastically. Residents report communicating more clinical reasoning than evidence. Residents felt that providing evidence is educationally valuable and impacts clinical management when placing a consult, although they do not practice this behavior when responding to consults when on their consult electives. Residents also perceive that they communicate clinical reasoning and evidence more often than their supervisors observe them to be doing. Brief curricular interventions to address these issues that promote accountability and transparency of both of components should be developed.

\section{EXAMINING HEALTH SOCIAL NETWORKS IN AN EMERGING CARIBBEAN COHORT Terri Ann Thompson; Carol Oladele; Marcella Nunez- Smith. Yale University, New Haven, CT. (Control ID \#2468757)}

BACKGROUND: Studies have shown social networks influence health behaviors, including those related to noncommunicable diseases, and play an important role in disease management. This study analyzed health social networks to determine the role healthcare providers play in health decisions in an emerging cohort in the eastern Caribbean

METHODS: We surveyed 378 residents aged 40 and older who lived in Barbados and the U.S. Virgin Islands for at least 10 years. Participants were asked to name individuals in their network who they talk to about health, whose opinions on healthcare matter to them, and who they would trust to make a healthcare decision if they were unable. Analyses were conducted to describe features of the health social network in this setting

RESULTS: A total of 2154 health social contacts were provided by participants. Of this number, only $7 \%$ were healthcare providers. Approximately $54 \%$ of the providers were named as an important source of health information versus $38 \%$ who were named as a source whose opinion on healthcare mattered. Eight percent of providers were named for all three questions. In contrast, $47 \%$ of the persons named were family members, of which $22 \%$ were named for all three questions

CONCLUSIONS: Findings from our study suggest that healthcare providers play a smaller role than family members in the healthcare decisions of this emerging Caribbean cohort. Next steps will involve examining this phenomenon in the larger cohort 
EXAMINING THE EFFECT OF CANCER RISK AND PATIENT PREFERENCES ON THE NET BENEFIT OF LUNG CANCER SCREENING: A PERSONALIZED LUNG CANCER SCREENING MODEL Tanner Caverly ${ }^{2}$; Pianpian $\mathrm{Cao}^{4}$; Rodney A. Hayward ${ }^{1,3}$; Rafael Meza ${ }^{4} .{ }^{1}$ U. Michigan, Ann Arbor, MI; ${ }^{2}$ University of Michigan Medical School, Ann Arbor, MI; ${ }^{3}$ Ann Arbor VA Center for Clinical Management Research, Ann Arbor, MI; ${ }^{4}$ University of Michigan School of Public Health, Ann Arbor, MI. (Control ID \#2468696)

BACKGROUND: Most low-dose computed tomography (LDCT) lung cancer screening guidelines recommend shared-decision making (SDM) before initiating screening. Indeed, Medicare even requires documentation of SDM as a condition of payment. The clinical benefit of screening, however, varies dramatically across eligible patients. Also, the harms of LDCT can be quite substantive, such as fear, biopsies resulting from false positive results, and even unnecessary treatment. Thus, clinicians and health systems need guidance on how to communicate about screening to patients with different levels of estimated benefit. To examine how screening recommendations can be personalized to facilitate the delivery of a patient-centered screening program, we developed the Personalized Lung Cancer Screening Model, a microsimulation model that generates individual-specific health gain estimates with LDCT and can evaluate the potential effects of patient preferences on health gains across low- and high-benefit groups.

METHODS: We estimated the effects of LDCT screening on lung cancer outcomes and quality-adjusted life years (QALYs). The natural history model probabilities were based on previously validated lung cancer incidence, other-cause mortality, and lung cancer detectability models, as well as individual-level data from 2 large randomized trials on lung cancer screening (NLST and PLCO) and data from the Surveillance, Epidemiology and End-Results (SEER) cancer registry. We generated a nationally representative sample of 1 million patients eligible for LDCT screening for analyses. Simulated patients and their risk profiles were derived from adult smokers within the 2010 National Health Interview Study (NHIS). We quantified patient preferences using literature-derived utilities. Because there is uncertainty regarding some utility estimates and also because our primary aim in this study was to understand the effect of varying patient preferences on the net benefit of screening, we varied utilities across a plausible range in sensitivity analysis.

RESULTS: Using individual-level data from the NLST to provide validity evidence for our model, we compared model predictions with observed outcomes. Model predicted lung cancer incidence and mortality outcomes were consistent with those observed in the NLST. Similarly, average incremental QALY gains were consistent with that found in an NLST-based costeffectiveness analysis. We then used the simulated population of 1 million eligible smokers from the National Health Interview Study (NHIS) for our main analyses. First, we examined the effect of baseline lung cancer risk. As expected, incremental QALY gains varied significantly across eligible smokers due to differing baseline risks of developing lung cancer (range in base-case analysis: 2 QALYs lost per 100 people screened to 5 QALYs gained per 100 screened). Next, to clarify the importance of patient preferences in determining the net benefit of LDCT screening, we varied key model utilities over a plausible range (ie, utilities that quantify patient preferences about the burden of testing, false-positive diagnoses, treatment, and complications that result from the screening and treatment process). We found that the magnitude of net benefit is highly sensitive to patient's views of the burdens and harms of testing and treatment: most patients experienced net benefit when assuming favorable views (lower screening-related disutilities and higher benefit-related utilities) of the pros and cons of LDCT and most patients experienced net harm when assuming views unfavorable to LDCT (higher screening-related disutilities and lower benefit-related utilities). For instance, across all ages in the 3rd quintile of risk, QALYs ranged from net benefit with favorable preference assumptions (eg, 4 QALYs gained per 100 screened) to net harm with unfavorable preference assumptions (eg, 2 QALYs lost per 100 screened).

CONCLUSIONS: Results from our Personalized Lung Cancer Screening Model provide further support for the importance of an individual's estimated baseline lung cancer risk in determining net benefit from LDCT screening. In addition, we found that patient preferences are important for determining whether screening is indicated. Thus, our results support a policy of shared decision making, and the use of a decision-support tool, for most patients eligible for LDCT screening, rather than strong recommendations to screen or not screen.

EXPLORING FACULTY PERSPECTIVES ON FACILITATING INSTRUCTION IN THE RECOGNITION AND MANAGEMENT OF RACIAL AND ETHNIC IMPLICIT BIAS FOR MEDICAL STUDENTS Cristina M. Gonzalez $z^{2}$; Ramya J. Garba $^{1}$; Alyssa R. Liguori ${ }^{1}$; Paul R. Marantz ${ }^{3}$; M. Diane McKee ${ }^{1} .{ }^{1}$ Albert Einstein College of Medicine, Bronx, NY; ${ }^{2}$ Albert Einstein College of Medicine/Montefiore Medical Center, Bronx, NY; ${ }^{3}$ Albert Eisntein College of Medicine, Bronx, NY. (Control ID \#2466701)

BACKGROUND: Evidence suggests that individual physicians' actions contribute to health disparities. Some disparities in clinical practice may be due to implicit (subconscious) bias rather than explicit (conscious) bias. Implicit bias can impact clinical decision-making and could therefore contribute to health disparities. A curriculum on recognition and management of implicit bias may mitigate provider contributions to health disparities. There are currently no comprehensive, developmentally appropriate curricula published for medical students. The development of such curricula will require dialogue that is potentially emotionally charged. To optimize faculty involvement in such a course, our study explored faculty perceptions of instruction in implicit bias recognition and management.

METHODS: Using a semi-structured interview guide, the PI conducted individual interviews with faculty members at Albert Einstein College of Medicine, in Bronx NY. We used typical case sampling to identify participants. Interview questions explored experiences and suggestions about facilitating discussions on race, prejudice, or other related topics. Interviews were digitally recorded and professionally transcribed. Qualitative analysis was performed using grounded theory. Two investigators independently analyzed two transcripts and created a list of codes, which were applied to two other transcripts to refine and finalize the codebook. The final codebook was applied to the remaining transcripts by two investigators each. Any disputes in coding were resolved through consensus, with a third party available when needed. Interviews were conducted until analysis revealed saturation of themes.

RESULTS: Seventeen interviews were conducted with participants representing both basic and clinical sciences. Four themes emerged through the data analysis. These themes included components of faculty's personal identity, institutional factors, obstacles to instruction and perceived opportunities to enhance instruction. Faculty's personal identity, including previous lived experience and past teaching experiences could influence their skill and comfort in facilitating instruction. "As a junior faculty member, I've had particular challenges co-facilitating with much more clinically experienced faculty when they use certain offensive terms i.e.: oriental. This is where it gets really uncomfortable, because it's often in the presence of students, and I don't want to have that conversation in front of them." Institutional factors (i.e.: competing priorities and the potential negative effects of the hidden curriculum) could influence the development of successful curricula: "Where I struggle with this teaching point is when students go out to clinical settings, they see the exact opposite." Participants identified a myriad of obstacles to instruction, including varied levels of buy-in to the concept of implicit bias, limited opportunities for faculty development, and potentially strong emotions that might limit open and honest faculty and student engagement with the material: "People are afraid to appear as racist; it is very scary to be in a room where there is racism coming up. It feels extremely uncomfortable, either because of our own backgrounds or sensitivities, or because of our experience with conflict- it is all fear." Finally, our participants elucidated several perceived opportunities to enhance instruction such as the importance of ongoing faculty development and role modeling: "part of being a role model is saying I don't know, or I 
made a mistake and this is what I learned. It's not always easy, but I think it's part of our job and I think you gain credibility and respect by doing so." The themes that emerged led to the development of a conceptual model (Figure). This model links the four themes and their influence on each other and on the faculty's instructional experience, which in turn would affect and be affected by the students' experience, all of which eventually would influence student engagement in future instruction.

CONCLUSIONS: Medical school faculty identified obstacles to and opportunities for the successful implementation of a comprehensive, developmentally appropriate curriculum in implicit bias recognition and management. Faculty at other medical schools may have similar concerns. Their insights led to our conceptual model that will inform future curricula, faculty development, and facilitator guides.

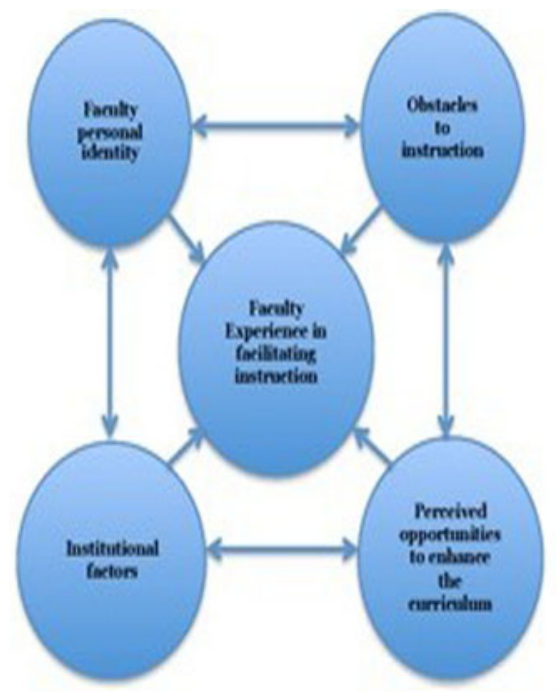

Figure: Conceptual Model Linking Faculty Experience in Facilitating Instruction with Identified Themes.

EXTENDED WEIGHT LOSS MAINTENANCE FOR INDIVIDUALS IN A NATIONAL NONPROFIT WEIGHT LOSS PROGRAM Nia S. Mitchell ${ }^{1,}$; Emmanuel A. Seyoum ${ }^{3}$; Anna L. Furniss ${ }^{2}$; Victoria Catenacci ${ }^{1,1}$. ${ }^{1}$ University of Colorado Anschutz Medical Campus, Aurora, CO; ${ }^{2}$ University of Mississippi Medical Center, Jackson, MS; ${ }^{3}$ University of Colorado, Denver, CO. (Control ID \#2467498)

BACKGROUND: The Institute of Medicine (IOM) defines significant weight loss as weight loss of $\geq 5 \%$ of baseline weight, because it is associated with improvements in weight-related comorbidities, including diabetes, hypertension, and obstructive sleep apnea. The IOM defines long-term weight loss as weight loss that is maintained for at least 1 year, because extended weight loss maintenance is uncommon in lifestyle modification programs. For example, while almost $66 \%$ of weight loss in lifestyle modification programs is maintained at 1 year, only $20 \%$ of weight loss is maintained at 5 years. Unfortunately, the health benefits of weight loss disappear once weight is regained. Take Off Pounds Sensibly (TOPS) is a national, nonprofit, lifestyle modification weight loss program. Observational data demonstrate that the average weight loss for TOPS participants who consecutively renew their annual memberships is $6 \%$ at 1 year and $8 \%$ at 7 years. The purpose of the current study is to examine the annual follow up weight change categories of individuals who achieved significant weight loss in TOPS and to determine what percentage remain in the significant weight loss category in subsequent years.

METHODS: Participants were those individuals who joined TOPS from 2005 to 2010 and who had at least two consecutive annual membership renewals. Based on the year they joined the program, individuals could be followed for 2 to 7 years. For the first year, weight change was calculated as percentage change from baseline weight at initial TOPS enrollment and placed into one of three categories: 1) significant weight loss (SWL, weight loss $\geq 5 \%$ of baseline weight); 2 ) weight stable (WS, weight change of 0 to $<5 \%$ of baseline weight); and 3) weight gain (WG, weight gain from baseline weight). Individuals were eligible for follow up in subsequent years if they met the following criteria: 1) they were in the in the SWL category; 2) they renewed their annual membership; and 3) they were eligible for follow up based on the year they joined the program. Weight change category for those with SWL was then calculated for subsequent years of enrollment to determine if they continued to maintain significant weight loss. For each year of follow up, cumulative percentage weight change was calculated based on the participants' follow up weight relative to their baseline weight (not to the previous year's weight).

RESULTS: The results are presented in Figure 1. At the time of the first annual renewal (Year 1), the weight change categories for the 65,559 participants were as follows: $50 \%$ had SWL, $32 \%$ were WS, and $18 \%$ were in the WG category. Of the 22,292 individuals with SWL at year one, who renewed their membership at year two and who were also eligible for follow up based on the year they joined, $80 \%$ were still in the SWL category; $16 \%$ moved to the WS category (i.e., they gained enough weight from their previous renewal that their cumulative weight change was from 0 to $<5 \%$ of their baseline weight); and $4 \%$ moved into the WG category (i.e., they gained enough weight from their previous renewal that their cumulative weight change was above their baseline weight). Approximately $80-95 \%$ of the individuals with SWL at year one, who renewed their membership subsequent years, and who were eligible for follow up based on the year they joined, continued to maintain SWL for up to 7 years. Between 4 and $16 \%$ of the individuals with SWL at year one moved into the WS category during subsequent years. From $<1$ to $4 \%$ of individuals moved into the WG category during subsequent years of follow up after achieving or maintaining significant weight loss

CONCLUSIONS: Our study demonstrates that significant weight loss can be maintained for up to 7 years in a subset of individuals participating in the TOPS program. Given the difficulty in maintaining weight loss in lifestyle modification programs for extended periods of time and that health benefits of weight loss disappear when weight is regained, maintaining long-term weight loss is a critical challenge in treating obesity and weightrelated comorbidities. Therefore, further studies of the TOPS program should examine which populations will succeed at weight loss and weight loss maintenance and determine the factors that can improve these outcomes.

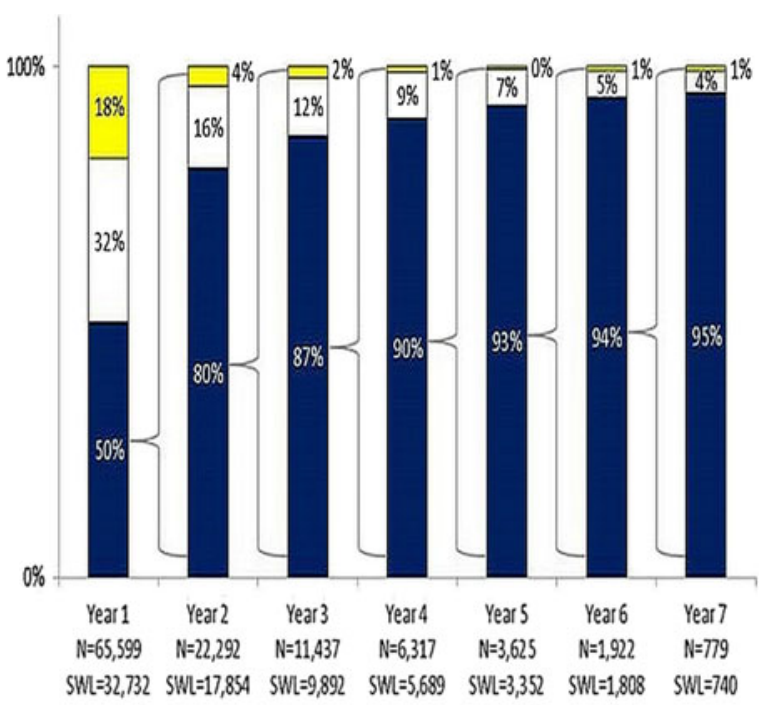

ESignificant Weight Loss DWeight Stable aWeight Gain 
Annual follow up weight change categories for individuals with significant weight loss. SWL, significant weight loss. WS, weight stable. WG, weight gain.

\section{EXTRACURRICULAR CLASSES OF ENGLISH FOR MEDICAL PURPOSES} PROMOTE CONFIDENCE IN UNDERGRADUATE MEDICAL STUDENTS IN JAPAN Yuka Naito ${ }^{2}$; Naoko E. Furukawa ${ }^{2}$; Masaki Tago ${ }^{2}$; Norio Fukumori ${ }^{1}$; Shu-ichi Yamashita ${ }^{2}$. Faculty of Medicine, Saga University, Saga-shi, Japan; ${ }^{2}$ Saga University Hospital, Saga, Japan. (Control ID \#2455430)

BACKGROUND: English ability is an essential skill for physicians of all countries in studying global-level medical science and in sharing information in medical meetings and journals. Over half of all physicians in Japan use English daily to read and write articles regardless the type of hospital to which they belong. Teaching staff at Japan's undergraduate medical institutions require that students improve their skills in English reading and listening. However, some medical students wish to improve their English communication ability further, such as in developing conversation skills. In particular, students who have undergone training in English-speaking countries are often keen to have more opportunities to speak English. The Japan Society for Medical Education and Japan Society for Medical English Education have been developing programs and guidance related to the teaching of English for medical purposes (EMP). Furthermore, many universities and colleges in Japan are reportedly making attempts to teach EMP. Few studies have examined the current situation regarding Japanese undergraduate medical students' interest and confidence in using EMP and the effects of EMP education. This study evaluated the effectiveness of our extracurricular EMP classes on medical students' interest and confidence in using medical English.

METHODS: The Department of General Medicine of Saga University Hospital, Japan, introduced the EMP class in October 2011. The class takes place after school once a week as an extracurricular activity, and the class tutor is the professor of the department faculty and a general practitioner with sufficient ability in EMP. Participants discuss a clinical case of general medicine taken from an English-language textbook, and only English is used in the class. The class is open to all students, residents, and young doctors at Saga University Hospital. The decision to attend is entirely left to the individual. The majority of participants are fifth- and sixth-year students of Saga University, School of Medicine. Usually, 10-20 people physically attend each session. We conducted a self-completed questionnaire survey in December 2014 among 168 fifth- and sixth-year medical students who had completed their clinical training as student doctors in our department and were eligible to attend the EMP class. The questionnaire included items related to interest and confidence on a scale of 1 (not at all interested or confident) to 5 (very interested or confident): studying in English-speaking countries; attending international conferences; gathering information from English-language articles; writing articles in English; acquiring an international medical license; communicating with English-speaking patients, physicians, or students; and making presentations in English. We divided the subjects into three groups according to the number of times they had taken part in the EMP classes $(0-1,2-5$, and over 6 times). We analyzed the relationship between the number of times they had attended and degree of interest and confidence using the Jonckheere-Terpstra trend test.

RESULTS: Students with greater class attendance were more interested in communicating with English-speaking patients $(p=0.048)$ and students $(p=0.049)$. Students with greater class attendance were more confident about acquiring an international medical license $(p=0.017)$, communicating with English-speaking patients $(p=0.007)$, physicians $(p=0.015)$, and students $(p=0.001)$, and making presentations in English $(p=$ $0.018)$

CONCLUSIONS: This study found that students who had attended our EMP classes more often were more confident in acquiring an international medical license as well as communicating and making presentations in English. The EMP class did not augment confidence in attending international conferences; however, we hope that it may exert a positive influence on the students' confidence once they become medical practitioners and gain more experience.

FACILITATING LIFESTYLE CHANGES AMONG VETERANS IN PRIMARY CARE: RESULTS OF USABILITY TESTING OF A TECHNOLOGY-ASSISTED WEIGHT MANAGEMENT AND GOAL SETTING TOOL Natalie L. Ricci ${ }^{2,}{ }^{1}$; Pich Seekaew $^{2,}{ }^{1}$; Katrina F. Mateo ${ }^{2,}{ }^{1}$; Natalie B. Berner ${ }^{2,}{ }^{1}$; Raymond Manalo ${ }^{1}$; Scott Sherman $^{2,}{ }^{1}$; Melanie Jay ${ }^{2,}{ }^{1}$. ${ }^{1} \mathrm{NYU}$ School of Medicine, New York, NY; ${ }^{2}$ VA NY Harbor Healthcare System, New York, NY. (Control ID \#2462862)

BACKGROUND: Veterans are disproportionately affected by obesity and its associated diseases. Veterans Affairs (VA) Medical Centers offer MOVE!, an intensive weight management program to treat patients with obesity. Some VA sites use an expert system software program called the MOVE!11, an intake tool for MOVE! delivered on a desktop computer that evaluates current behaviors and stage of change, and also provides individualized advice and handouts. Our prior research found that the current MOVE!11 software does not facilitate goal setting for lifestyle behavior change and is not currently used in primary care (PC). Based on formative research, we enhanced the MOVE!11 program to promote goal setting and facilitate weight management counseling. This online tool is one of the components of a PC-based weight management intervention called MOVE! Towards Your Goals (MTG). We conducted usability testing of the new MTG tool in order to evaluate its utility and inform iterative improvements.

METHODS: We recruited overweight/obese Veterans in 2 ways: In Phase 1, we invited Veterans who had previously participated in a focus group study. In Phase 2, we recruited Veterans with upcoming PC appointments through mailings and follow up phone calls. In each phase, a research assistant observed participants individually as they used the MTG tool. Veterans were asked to verbalize their thoughts and reactions as part of a "ThinkAloud" protocol, and a second research assistant took field notes. Each session was digitally recorded and professionally transcribed. A coding guide was derived from the International Standards Organization (ISO) definition of usability and focused on positive and negative interactions between four domains (Tool, Task, User, Context). Two researchers reviewed and coded the transcripts independently, meeting frequently to resolve differences with the help of a third coder when needed.

RESULTS: A total of 21 Veterans participated in the study. In Phase 1, 10 Veterans participated, of which $60 \%$ were male, 1 identified as Hispanic, 6 identified as Black or African American, 3 identified White, and 1 identified as Other (multiracial). In Phase 2, 11 Veterans were seen, of which $90.9 \%$ were male. Five identified as Black, 3 White, 2 Asian, 1 American Indian or Alaskan Native, and 1 Other. Veterans had a mean age of 62.2 years $(\mathrm{SD}=10.50)$ and a mean $\mathrm{BMI}$ of $30.58(\mathrm{SD}=3.5)$. Veteran comments regarding the tool generally focused on tool functionality, personal experiences related to weight management, and tailored advice from the tool. Veterans identified areas of poor functionality (e.g. sensitivity of the touch screen and navigation of the tool) that impeded independent use, and many required a stylus to navigate the tool. Veterans discussed their past experiences while using the tool to help them answer questions about barriers and facilitators to lifestyle behavior change and current behaviors. They appreciated having adequate answer choices to these questions. Participants were most satisfied with the individual tailoring of the advice and personalized binders generated by the tool. Participants with diabetes (4/21) felt that the tool should include additional questions and advice about diabetes to make the tool more relevant to them. All identified at least one nutrition goal and a physical activity goal that they wished to pursue.

CONCLUSIONS: The usability data obtained from this study were used to make iterative changes to the MTG tool for a pilot randomized controlled study of the MTG 
intervention. Future studies will also test usability of the MTG tool in non-Veteran populations to inform tailoring for specific populations.

FACTORS ASSOCIATED WITH A CAREER IN PRIMARY CARE MEDICINE: RELATIONSHIPS MATTER Irina Kryzhanovskaya ${ }^{2}$; Beth Cohen ${ }^{1}$; Jeff Kohlwes ${ }^{3}$. ${ }^{1}$ San Francisco VA/University of California, San Francisco/ San Francisco VA Medical Center, San Francisco, CA; ${ }^{2}$ UCSF, San Francisco, CA; ${ }^{3}$ VAMC/UCSF, San Francisco, CA. (Control ID \#2469954)

BACKGROUND: A recent survey of University of California, San Francisco (UCSF) internal medicine alumni reported that $35 \%$ of graduates from primary care programs lost their initially stated interest in primary care during residency. The majority of those alumni who lost interest noted that their continuity clinic experience negatively influenced their final career choice. Another study demonstrated that $43 \%$ of internal medicine graduates were less likely to pursue a career in primary care because of their continuity clinic experience. Neither study examined what factors within the residency clinic experience were associated with the atrophy of resident interest in a primary care career. The purpose of this study was to identify the specific aspects of the residency clinic experience that positively or negatively affected the residents' ultimate career choice.

METHODS: We conducted a cross-sectional electronic survey of a cohort of UCSF primary care internal medicine residency alumni who graduated between 2000 and 2015 Survey questions included demographics, current clinical duties, debt level, and how aspects of the ambulatory and inpatient experience during residency influenced their career choice. Items were rated on a 5-point Likert scale from strong negative influence away from primary care to strong positive influence towards primary care. We performed Chi-Square analyses comparing responses of those who pursued primary care careers at the conclusion of residency and those who did not.

RESULTS: Of the 305 alumni contacted, 188 responded (62\%) with 103 reporting current careers in primary care and 85 in other fields. While $174(93 \%)$ of primary care alumni were interested in a primary care career prior to residency, 128 (68\%) remained interested at the conclusion of residency, and 103 (55\%) currently practice primary care Among those who went into primary care, 61 out of 103 (59\%) said their continuity clinic experience positively influenced them toward their final career path. Personal factors, including patient-provider relationship, patient population, and access to role models were the most commonly endorsed positive influences toward a career in primary care in both groups, though these were reported more often by those in primary care jobs than by those in other fields ( $p<0.01$ for each factor). The patient-provider relationship was the most commonly reported positive influence, endorsed by $95 \%$ of those in primary care and $76 \%$ of those in other fields. Alumni noted that experiences with support staff (46\%), clerical duties (60\%), and documentation (46\%) negatively influenced them away from primary care careers. The proportion endorsing these factors as negative influences was similar for those in primary care and in other fields ( $p>0.05$ for each factor). Pressure to see patients in a short time was the most commonly reported negative factor, reported by $75 \%$ of alumni not in primary care. Level and importance of resident debt did not differ in those pursuing primary care or non-primary care careers.

CONCLUSIONS: Alumni who responded to our survey confirmed previous studies reporting a high rate of attrition away from primary care jobs amongst residents initially interested in pursuing a career in primary care. Interpersonal factors such as patientprovider relationships, patient population, and interaction with mentors were the strongest positive influences toward a primary care career. These factors compensated for the reported frustrations of clinic such as time pressure, clerical, or documentation demands, which negatively impacted all alumni similarly. These factors seem to indicate that alumni who stay in primary care are less influenced by the burdens of clinic and place more importance on their relationships with patients and role models. Enhancing patient and mentor relationships and improving support for administrative tasks may increase the retention of primary care residents.

\section{FACTORS ASSOCIATED WITH MAINTENANCE OF BODY MASS INDEX IN} THE JACKSON HEART STUDY Brandon Auerbach ${ }^{2}$; Ronit Katz'; Edward J. Boyko $^{2}$; Adam Drewnowski ${ }^{2}$; Katherine L. Tucker ${ }^{3}$; Alain Bertoni ${ }^{4}$; Patricia M. Dubbert $^{5}$; DeMarc Hickson ${ }^{6}$; Adolfo Correa ${ }^{7},{ }^{8}$; Bessie Young ${ }^{1} .{ }^{1} \mathrm{UW}$, Seattle, WA; ${ }^{2}$ University of Washington, Seattle, WA; ${ }^{3}$ University of Massachusetts Lowell, Lowell, MA; ${ }^{4}$ Wake Forest University, Winston Salem, NC; ${ }^{5}$ University of Arkansas for Medical Sciences, Little Rock, AR; ${ }^{6}$ Jackson State University, Jackson, MS; ${ }^{7}$ University of Mississippi Medical Center, Jackson, MS; ${ }^{8}$ Jackson Heart Study, Jackson, MS. (Control ID \#2465392)

BACKGROUND: The United States has the highest prevalence of obesity of any industrialized nation, and our obesity epidemic disproportionately affects African Americans and Southern states. In Mississippi, where the Jackson Heart Study (JHS) is based, $36 \%$ of adults and $43 \%$ of African Americans are obese. In order to determine the highest value interventions to prevent obesity and other chronic diseases, we need to know who maintains their body mass index (BMI) and who does not. Our objective was to measure factors associated with BMI maintenance in adults free of obesity at baseline in the JHS. The JHS is a prospective cohort study of 5301 African Americans living in the Jackson, Mississippi area. We evaluated participants with a BMI $<30.0$ at enrollment. The primary outcome was maintenance of a non-obese BMI over 8 years of follow-up. We hypothesized that maintaining a non-obese BMI would be most strongly associated with diet quality and physical activity (PA) level.

METHODS: The JHS enrolled adult subjects between 2000 and 2004, and conducted two follow-up visits between 2005-2008 and 2009-2013. Of the 5301 enrollees, 1932 met eligibility criteria and were included in this analysis. Participants were excluded who had a BMI of $>30.0$ at enrollment $(n=2,251)$, had missing weight data at both follow-up visits $(n=612)$, or died prior to the first follow-up visit $(n=497)$. Baseline information was collected on subjects' height, weight, socio-demographic factors, health and lifestyle habits, and prevalent comorbidities. Independent variables were PA level, diet quality, built environment measures, neighborhood safety, and food access. Socioeconomic position, cigarette smoking, age, sex, problem alcohol drinking, and prevalent comorbidities were covariates of interest. A food frequency questionnaire assessed diet. Diet quality was measured at baseline on a 5 point scale, with 1 point given for meeting each of 5 dietary goals of the American Heart Association's (AHA) Life's Simple Seven (LSS) recommendations: (1)>4.5 cups/day of whole fruit, vegetables, or $100 \%$ fruit juice; (2) > two $3.5 \mathrm{oz} \mathrm{servings/week} \mathrm{of} \mathrm{fish;} \mathrm{(3)>}$ three $1 \mathrm{oz}$ servings/day of whole grains (4) $<36$ fluid oz/week of sugar-sweetened beverages; and (5) $<1500 \mathrm{mg}$ of sodium/day. Participants completed a baseline PA survey and were grouped into three categories per AHA guidelines: (1) Recommended: $\geq 150 \mathrm{~min} /$ week of moderate PA or $\geq 75 \mathrm{~min} /$ week of vigorous PA; (2) Intermediate: 1-149 min/week of moderate PA or 1-74 min/week of vigorous PA; and (3) Poor: $0 \mathrm{~min} /$ week of PA. Baseline differences in demographics and health status between participants who maintained their BMI $<30$ versus had a BMI increase to $>30.0$ were evaluated using Student's t-test, chisquare test, or ANOVA. Poisson regression was used to examine the association of each independent variable and the outcome of maintenance of a non-obese BMI. Regression models were adjusted sequentially for the following known obesity risk factors and potential mediators: age, sex, income status, education level, prevalent depression, prevalent diabetes, prevalent cardiovascular disease, cigarette smoking, and problem alcohol drinking. We calculated incidence rate ratios (IRR) of factors associated with BMI maintenance. IRRs of $>1.0$ are positively associated with BMI maintenance.

RESULTS: Of the 1932 subjects included in analyses, $55 \%$ were women, the mean age was 55 years, and the mean BMI was $26.2 \mathrm{~kg} / \mathrm{m}^{2}$. More than $70 \%$ of subjects were overweight at 
baseline, $14 \%$ were current smokers, and $5 \%$ met criteria for problem alcohol drinking. At baseline $8 \%$ of subjects met criteria for major depression, $12 \%$ had diabetes, and $8 \%$ had cardiovascular disease. Participants who maintained a non-obese BMI were older (mean age 56 v. 52 years, $P<0.001$ ), more likely to be male ( 46 v. $39 \%$ male, $P=0.014$ ), and had lower mean baseline BMI (25.6 v. 28.4, $P<0.001)$. In unadjusted analyses, having poor access to a grocery store was associated with a $9 \%$ increase in BMI maintenance $<30.0$, which lost significance after adjustment. Male sex (IRR $=1.05,95 \%$ CI 1.01, 1.11), age per 10 year increase (IRR 1.06, $95 \%$ CI 1.04, 1.08), and baseline BMI per SD increase of 2.71 (IRR = $0.83,95 \%$ CI $0.82,0.85$ ) were associated with BMI maintenance. All other factors were not significant in both unadjusted and adjusted analyses. No assessed independent variables were associated with the primary outcome of maintenance of a BMI $<30.0$.

CONCLUSIONS: In this prospective cohort study of African American adults, no lifestyle factor or measure of the built environment, food environment, or neighborhood safety was significantly associated with maintenance of a non-obese BMI in adjusted analyses. Participants who maintained a non-obese BMI were older, more likely to be male, and had a lower mean baseline BMI. There are multiple explanations for these null results. The JHS is an older cohort with an average baseline age of 55 years. Restricting our analyses to non-obese subjects at baseline could have led to differential selection bias of persons likely to maintain their weight. Dietary exposure misclassification may be present, and limitations in power to determine an association may exist. We are cautious to draw conclusions from this data. While it is possible that AHA's LSS physical activity and diet guidelines are not associated with weight maintenance in this population, it likely that selection bias or differential exposure misclassification affected our results.

FACTORS ASSOCIATED WITH RECOMMENDATION OF ROUTINE MAMMOGRAPHY FOR WOMEN AGED 40-49: PROVIDER CHARACTERISTICS AND SCREENING INFLUENCES Abhishek Deshpande ${ }^{1,1}$; Kathryn A. Martinez ${ }^{1,1}$; Allison L. Ruff ${ }^{1,}$; Shari Bolen ${ }^{3}$; Kathryn Teng ${ }^{2}$; Michael B. Rothberg ${ }^{1,1}$. ${ }^{1}$ Cleveland Clinic, Cleveland, OH; ${ }^{2}$ MetroHealth Medical Center, Cleveland, OH; ${ }^{3}$ MetroHealth/Case Western Reserve University, Cleveland, OH; ${ }^{4}$ University of Michigan, Cleveland, OH. (Control ID \#2468536)

BACKGROUND: Despite the revised U.S. Preventive Services Task Force (USPSTF) recommendation against routine screening mammography in average risk women younger than 50 years, rates remain high, suggesting provider screening recommendations for younger women may be motivated by factors other than USPSTF guidelines. The objective of this study was to understand provider-reported influences on mammography screening recommendations for average-risk women aged $40-49$

METHODS: An online survey of primary care providers (MDs, NPs, and PAs) was conducted at 4 major health centers in greater Cleveland in 2015. Provider-reported routine recommendation of mammography screening for average risk women aged 40-49 was the primary outcome. Independent measures included provider perceptions of the importance of the following influences on their screening recommendations: electronic health records, national guidelines, institutional policy, patient preferences, concerns about overtreatment, concerns about false positive findings, and interest in early detection of cancer. Each was scored on a 4-point scale and dichotomized to "heavily influencing screening recommendations" versus "not heavily influencing screening recommendations." The survey also assessed providers' beliefs about the balance of benefits versus harms of mammography in average risk younger women, categorized as "benefits exceed harms," "harms exceed benefits," or "benefits and harms are equivalent." Multivariable logistic regression was used to estimate the odds of routinely recommending screening by provider-reported screening influences, controlling for provider characteristics, including sex, years of post-training experience (resident, $<5$ years, $\geq 5$ years), and practice setting (academic versus community-based), as well as provider belief regarding the balance of benefits vs. harms of screening in this age group.
RESULTS: Of 612 providers invited, 220 completed the survey (response rate: $36 \%$ ); $62 \%$ practiced in academic settings and $56 \%$ reported having $\geq 5$ years of post-training experience. The majority $(69 \%)$ reported routinely recommending screening for average risk women aged $40-49$. While $43 \%$ felt that the benefits of screening outweighed the harms in this age group, $24 \%$ felt that harms outweighed the benefits. Reporting being heavily influenced by institutional policy was associated with higher odds of routinely recommending screening (OR: $4.19, p=0.013)$, as was interest in early detection of cancer (OR: $5.09, p=0.004$ ). Conversely, reporting being heavily influenced by national guidelines was associated with lower odds of recommending screening (OR: $0.25, p=0.010$ ). Providers in community-based settings were significantly more likely to routinely recommend screening for younger average-risk women than those in academic settings (OR: $4.26, p=0.001$ ), as were female providers (OR:3.89, $p=0.007$ ). Compared to providers with five or more years of post-training experience, residents were significantly less likely to routinely recommend screening (OR: $0.14, p=0.004$ ). While $59 \%$ of providers reported being heavily influenced by patient preferences, the influence of patient preferences was not associated with screening recommendation in the adjusted model.

CONCLUSIONS: Providers face competing influences on screening recommendations for mammography for women aged 40-49. Some influences may be at odds with providers' professional beliefs. Focusing on USPSTF guidelines and changing institutional policy to allow for individually-tailored screening discussions may improve patientcentered care.

FACTORS ASSOCIATED WITH SUSTAINED UNCONTROLLED HYPERTENSION IN A LOW-INCOME, LARGELY HISPANIC PRIMARY CARE COHORT WITH DIABETES Leah L. Zullig ${ }^{1,}{ }^{4}$; Yuanyuan Liang ${ }^{2}$; Shruthi Vale $^{3}$; Hayden Bosworth ${ }^{1,}{ }^{4}$; Barbara J. Turner ${ }^{3}$. ${ }^{1}$ Durham Veterans Affairs Medical Center, Durham, NC; ${ }^{2}$ UT Health Science Center at San Antonio, San Antonio, TX; ${ }^{3}$ University of Texas Health Science Center at San Antonio, San Antonio, TX; ${ }^{4}$ Duke, Durham, NC. (Control ID \#2467056)

BACKGROUND: In the U.S., hypertension (HTN) is less likely to be controlled in Hispanics than non-Hispanic whites. Given Hispanics' high prevalence of diabetes mellitus (DM), it is critical to identify factors associated with gaps in blood pressure (BP) control for this population. In a longitudinal cohort of low income, majority Hispanic primary care patients with DM and uncontrolled HTN at baseline, we examined effects of sociodemographic, clinical, and healthcare adherence factors on temporal changes in BP over a 2-year period. Our goal was to identify specific groups in greater need of case management to improve HTN control and reduce cardiovascular morbidity and mortality.

METHODS: For a Medicaid waiver project, we developed a longitudinal database of patients aged 18+ with DM in two academic-affiliated primary care Central Texas practices from clinical, laboratory, and demographic data in the electronic medical record (EMR) and scheduling data. Patients were seen in two consecutive years (1/2012-12/ $2013)$ and had mean uncontrolled BP (>140/90) in 2012. Linear mixed-effects models were used to examine the following predictors of the trajectory of systolic BP (SBP) over a 2 year timeframe: age $(<40,40-64.9, \geq 65)$, race-ethnicity/gender (White men, Hispanic men, Black men, White women, Hispanic women, Black women), insurance status, baseline SBP category $(<140,140-149.9,150-159.9,160-169.9, \geq 170 \mathrm{mmHg})$, mean A1c in 2012 (missing, $<7,7-7.9,8-8.9, \geq 9 \%$ ), number of scheduled visits, adherence ( $\%$ attending scheduled visits $<60 \%$ [Low], 60-74.5\% [Moderate], $\geq 75 \%$ [High]), and clinic type (internal medicine or family medicine). Statistical analyses were performed using Stata 14 (College Station, TX).

RESULTS: Our analysis included 8014 SBP measurements from 870 unique patients with baseline mean SPB $>140 \mathrm{mmHg}$. The study cohort was primarily Hispanic $(76.5 \%$, $n=684)$, women $(61.4 \%, n=549)$, uninsured $(41.0 \%, n=357)$ and with a mean age 
59.8 years $(\mathrm{SD}=11.2)$. On average, $\mathrm{SBP}$ decreased by 0.5 per month $(p<0.001)$. After adjustment, SBP trajectory was significantly $(p<0.05)$ affected by mean A1c in 2012, baseline SBP, and race-ethnicity/gender. Compared to patients with controlled DM (A1c $<7$ at baseline), patients with uncontrolled DM (A1c $\geq 9$ at baseline) had a slower rate of decline in SBP $(p=0.01)$. Hispanic women had less improvement than White women $(p=0.02)$. While SBP declined for all women, Hispanic women with controlled DM had less improvement than White women with uncontrolled DM (Figure). Change in BP was not associated with: insurance status, adherence to scheduled visits, number of visits, age, and clinic type. While SBP control improved over time, the majority (58\%) of patients did not reach SBP control $(<140)$ at 24 months.

CONCLUSIONS: In a predominantly Hispanic cohort, more complex patients who had poor control of both DM and HTN at baseline showed less improvement in SBP control over time, but this effect was most apparent for Hispanic women. Adjustment for patient adherence to scheduled visits did not modify this effect. In subsequent years, we will target patients with case management to improve outcomes, focusing first on Hispanic women and clinically complex patients with uncontrolled DM and HTN.

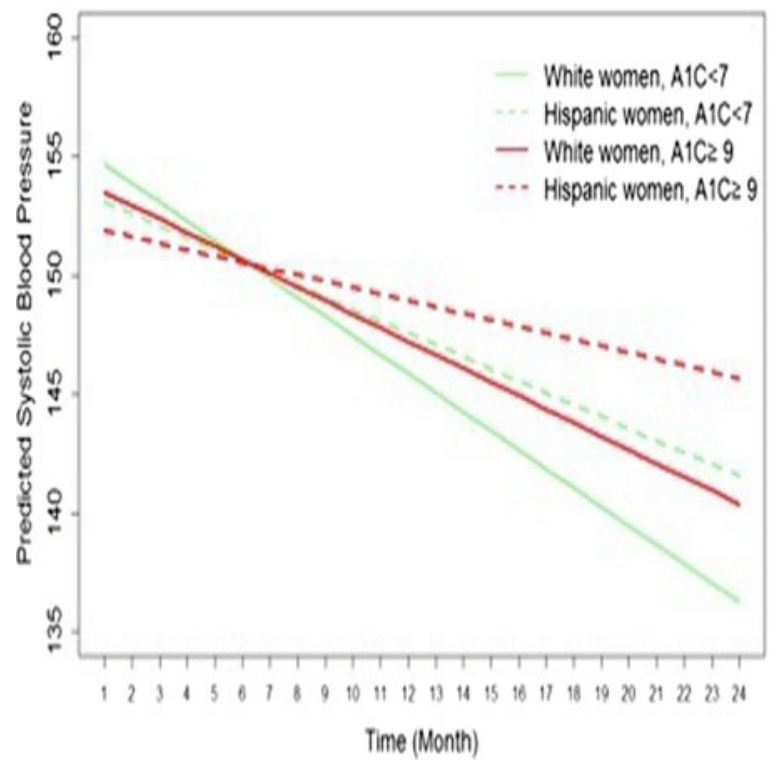

Change in SBP over time by race/ethnicity and gender

FACTORS PREDICTING IMPROVED GLYCEMIC CONTROL IN RESPONSE TO BEHAVIORAL SUPPORT INTERVENTIONS FOR POORLY CONTROLLED TYPE 2 DIABETES William S. Fuller ${ }^{1}$; Judith Long ${ }^{2} .{ }^{1}$ Hospital of the University of Pennsylvania, Philadelphia, PA; ${ }^{2}$ Philadelphia VA Medical Center, Philadelphia, PA. (Control ID \#2470705)

BACKGROUND: Management of type 2 diabetes is complicated by socioeconomic status, duration and severity of diabetes, and medical comorbidities including mental health issues. Prior studies have associated many of these factors with poor glycemic control. However, these factors have not previously been analyzed with regard to how they may influence the efficacy of behavioral support interventions.

METHODS: Two hundred eighty diabetic patients with their last two glycosylated hemoglobin (HbAlc) values $>8 \%$ were recruited from outpatient medical practices. Participants were randomized to four arms: usual care, peer mentoring, financial incentives, or a combination of mentoring and incentives. A baseline questionnaire asked about demographics, comorbidities, depression (via PHQ-2), medication adherence and diabetes-related attitudes.
$\mathrm{HbAlc}$ was measured at baseline, at the conclusion of the 6 month intervention period, and at 12 month follow up. We performed multiple logistic regression to determine which baseline characteristics were predictive of a reduction in $\mathrm{HbAlc}$ of at least $1 \%$.

RESULTS: Participants were $70 \%$ female and $83 \%$ African American. The mean age was 53.1 years (SD 8.6). The mean baseline HbAlc was 9.6 (SD 1.8). At 6 months, odds of having a decrease in $\mathrm{HbAlc}$ of at least $1 \%$ were 1.6 (95\% CI 1.3-2.0) for each $1 \%$ increase in baseline $\mathrm{HbAlc}$ and $4.0(95 \% \mathrm{CI} 1.1-15.1)$ for those with health literacy of 7 th- 8 th grade level as compared to $\geq 9$ th grade. These associations persisted at 12 month follow up. At 12 months, a baseline PHQ-2 score $<4$, as compared to $\geq 4$, was predictive of lower odds of HbAlc reduction $\geq 1 \%(0.3: 95 \% \mathrm{CI} 0.1-0.8)$. Also predictive of $\mathrm{a} \geq 1 \%$ drop in $\mathrm{HbAl} \mathrm{c}$ at 12 months were reporting less diabetes-related distress (OR 2.9, $95 \%$ CI 1.2-6.8) and self-identifying more barriers to medication adherence (OR 1.12, $95 \%$ CI 1.0-1.2).

CONCLUSIONS: Higher baseline score on the PHQ-2 depression screening questionnaire, lower health literacy, lower diabetes-related distress, and perceiving more barriers to medication adherence were predictive of improved responses to behavioral support intervention even after adjusting for baseline $\mathrm{HbAlc}$. These groups represent vulnerable populations in which diabetes control has historically been poor. Behavioral support interventions may have increased efficacy in these patients.

FACULTY EXPERIENCE WITH A LONGITUDINAL CLINIC MODEL FOR TEACHING 1ST AND 2ND YEAR MEDICAL STUDENTS Mark Mayer ${ }^{2}$; J. H. Isaacson ${ }^{1}$; Samina Yunus ${ }^{1} .{ }^{1}$ Cleveland Clinic, Cleveland, $\mathrm{OH} ;{ }^{2}$ Cleveland Clinic Lerner College of Medicine, Cleveland, OH. (Control ID \#2463713)

BACKGROUND: Longitudinal learning experiences are increasingly common for 3rd year medical students. Studies suggest that when compared to traditional block models, students gain equivalent content knowledge, but that empathy may be better preserved in a longitudinal setting. There is less known about longitudinal teaching of year 1 and 2 medical students, and about faculty satisfaction in this setting. Since the inception of our medical school in 2004, each medical student has had a longitudinal clinic (LC) during their first 2 years, with 43 sessions over 20 months with a single primary care physician. The focus of the experience is development of interviewing and physical exam skills, professional identity formation, and gradual learning of clinical reasoning. Continuity of teaching and care are emphasized. Course evaluations and student promotions committee reviews support development of solid communication skills, clinical skills, and professionalism. We have had a high retention rate of faculty teachers in $\mathrm{LC}(>80 \%)$, and a high attendance at yearly faculty development sessions $(>85 \%)$. We wondered what led to such high rates of retention and engagement, in an era of increasing difficulty recruiting primary care teachers. Our objectives were to understand how intrinsic and extrinsic motivators influence satisfaction with teaching; challenges to teaching in this model; and how satisfaction/preference compare for longitudinal vs. block model teaching. METHODS: We developed a 19-item survey for faculty teaching years 1 \& 2 students in our longitudinal clinics during 2014-2015 $(n=61)$. The survey included questions about intrinsic and extrinsic motivators of participation in this teaching model, challenges to this role, and comparison of satisfaction between teaching in a longitudinal vs. a block setting. The survey instrument was vetted with our Medical Education Research Committee and our IRB, which granted exempt status. We solicited participation by email; data were collected in the RedCAP system. Bivariate analysis with Chi Square was used to compare demographics and experience teaching in this system with measures of satisfaction.

RESULTS: Forty-two of 61 faculty members (69 \%) participating in LC teaching responded to our survey. Twenty-seven respondents were under 50 years old, 15 were over 50; 22 were male, 20 female; 15 were Family Physicians, 27 Internists. Faculty responding had taught medical students 12 years on average. Twenty-one had taught in our years $1 \& 2$ longitudinal clinic for 6 years or less, and 21 for over 6 years. Thirty-nine of 42 preceptors were satisfied or very satisfied with their teaching role. Twenty-five taught in blocks as well as in the LC model. Thirty-seven responded that they would prefer 
longitudinal teaching to block teaching, 2 preferred a block model, and 3 were neutral. Among professional roles, LC teaching was a major satisfier in 38. For the following extrinsic satisfiers, the following preferences were noted (neutral responses omitted): for salary support/clinical RVU target reduction, 24 felt well supported, 4 did not; for support from institutional leaders, 26 felt well supported, 3 did not; for faculty development, 33 felt well supported, 3 did not; and for other resources, such as books and reminders, 28 felt well supported, 2 did not. Thirty-five felt that being an LC preceptor helped improve their own clinical skills, 6 felt there was no effect. Twenty-six faculty members felt their involvement in this program fostered clinical scholarship, 15 did not. Forty-one of 42 felt they functioned as a mentor to the student they taught in longitudinal clinic. There were no significant differences between groups stratified by age, gender, specialty, or years having taught, in relation to any of the satisfaction ratings. Open-ended questions included queries about faculty development sessions. Aspects most valued by faculty were debriefing with colleagues about best practices, role playing feedback, practice teaching clinical skills and clinical reasoning to early learners, and expectation setting. Suggestions about topics to further explore in faculty development included giving tools to teach the approach to a difficult patient; how to teach best use of technology in clinical encounters; and teaching reflection. Common themes that emerged from responses about satisfaction teaching in the LC model included being able to foster skills improvement over time, and teaching the value of patient follow up. Faculty preceptors valued being able to help in the professional growth and development of students. Common themes about challenges with this model included time management, and completion of assessments.

CONCLUSIONS: The level of professional satisfaction was high among faculty precepting 1st and 2nd year medical students in longitudinal clinics. Extrinsic satisfiers were important, including support of institutional leaders and faculty development. Intrinsic satisfiers appeared to be even more important in motivating faculty to teach in our system: these included fostering student clinical skills development over time, promoting professionalism, and mentoring students. It may be that support of the institution and faculty development are necessary, though not sufficient for retention of dedicated clinical teachers; intrinsic motivators appear to be even more highly valued. In this study, the majority of faculty preferred longitudinal teaching over block teaching - important to note as faculty willingness to teach competes with other demands.

FAVORABLE CLINICAL OUTCOMES DESPITE INSUFFICIENT ANTIMICROBIAL THERAPY IN PATIENTS WITH CAMPYLOBACTER FETUS BACTEREMIA Hiromizu Takahashi ${ }^{5}$; Yuji Hirai ${ }^{2}$; Kiyozumi Suzuki ${ }^{2}$; Fujiko Morita ${ }^{4}$; Yuki Uehara ${ }^{3}$; Teruhiko Hisaoka ${ }^{3}$; Toshio Naito ${ }^{1} .{ }^{1}$ Juntendo University, Tokyo, Japan; ${ }^{2}$ Juntendo University Faculty of Medicine, Bunkyo, Japan; ${ }^{3}$ Juntendo University School of Medicine, Tokyo, Japan; ${ }^{4}$ Juntendo University School of Medicine, Bunkyo-ku, Japan; ${ }^{5}$ Juntendo university, Tokyo, Japan. (Control ID \#2462405)

BACKGROUND: Campylobacter fetus (CF) belongs to the Enterobacteriaceae family, and causes bacteremia more frequently than Campylobacter jejuni. CF can cause severe disseminated infections, including meningitis and mycotic aneurysm, in immunosuppressed patients. However, the clinical features of Campylobacter fetus bacteremia (CFB) remain unclear.

METHODS: Herein, the medical records of patients with CFB between April 1997 and March 2014 admitted to the Juntendo University Hospital (a 1020-bed university hospital) were retrospectively reviewed. For the purpose of the study, CFB was defined as CF detected on a blood culture, identified using an automated analyzer (API Campy, bioMérieux, France). Adult patients ( $>18$ years old) with CFB were included in the study. The data included age, sex, underlying diseases, initial presentation, systemic antimicrobial treatment, period between blood cultures and detecting CF and between the blood culture and initial intravenous antimicrobial treatment, antimicrobial susceptibility, month of disease onset, complications of CFB, and 90-day survival rate.

RESULTS: We enrolled 15 patients with CFB on the basis of positive blood cultures during the study period. The median age of patients was 43 years old (range, 26-86 years old), and $53.3 \%$ were women. $73.3 \%$ of the cases occurred in summer and winter. Underlying diseases included systemic lupus erythematosus (20\%) and malignancy (26.6 \%), and 3 patients $(20 \%)$ were immunocompetent. The initial presentations were fever $(100 \%)$, lumbago (26.7 \%), headache (13.3\%), and diarrhea (13.3\%). The complications of CFB observed were soft tissue infection (33.3\%), such as cellulitis and skin abscess, and meningitis $(6.6 \%)$. No mycotic aneurysms were found among 15 patients. The median time from performing the blood culture to detecting CF was 8.5 days (range, 3-13 days). No carbapenem-resistant CF strains were identified. However cefotaxime-resistant (20\%) and fluoroquinolone-resistant (levofloxacin $26.7 \%$, nalidixic acid $100 \%$ ) strains were detected. The average period from performing the blood culture to administering the initial appropriate intravenous antimicrobial therapy was 4.43 days (range, $0-13$ days). Furthermore, $26.6 \%$ of patients received outpatient antimicrobial therapy (OPAT), and patients received an inappropriate selection of definitive antimicrobial agents (30.7\%) for an insufficient duration ( $<7$ days; $30.7 \%$ ). The 90 -day survival rate was $100 \%$.

CONCLUSIONS: Our results suggest that $\mathrm{CFB}$ has a seasonal distribution, and favorable clinical outcomes can be achieved despite delayed initial intravenous antimicrobial therapy. However, we did observe a few cases of severe disseminated infections, such as meningitis and abscess, which required intensive treatment. Although the underlying disease was associated with developing complications related to CFB and the prognosis, the virulence of $\mathrm{CF}$ appeared to vary. Further investigation, including an epidemiological analysis and virulence determination, is therefore necessary.

Summary of the clinical data and outcome of patients with Campylobacter fetus bloodstream infections

\begin{tabular}{|c|c|c|c|c|c|c|}
\hline No. & Age (year) & Sex. & Underlying disease & Duration of IV ABx & Treatment & 90 days Outcome \\
\hline 1 & 41 & M & None & ND & ND & Cured \\
\hline 2 & 35 & $\mathrm{~F}$ & $\begin{array}{l}\text { SLE, Sjogren } \\
\text { Synd, Corticosteroid therapy, } \\
\text { Hypogammaglobulinemia }\end{array}$ & 5 & inappropriate & Cured \\
\hline 3 & 25 & $\mathrm{~F}$ & None & 14 & appropriate & Cured \\
\hline 4 & 27 & M & None & 19 & appropriate & Cured \\
\hline 5 & 26 & $\mathrm{~F}$ & Pregnancy & 3 & inappropriate & Cured \\
\hline 6 & 32 & $\mathrm{~F}$ & Anorexia nervosa & 11 & inappropriate & Cured \\
\hline 7 & 36 & $\mathrm{~F}$ & Gastric cancer, Chemo therapy & 7 & inappropriate & Cured \\
\hline 8 & 86 & M & Lymphoma (NHL) in CR & 14 & appropriate & Cured \\
\hline 9 & 35 & $\mathrm{~F}$ & SLE (Lupus nephritis), Corticosteroid therapy & 18 & inappropriate & Cured \\
\hline 10 & 34 & $\mathrm{~F}$ & SLE (Lupus nephritis), Corticosteroid therapy & 18 & inappropriate & Cured \\
\hline 11 & 65 & M & Pancreatic cancer (OEP + Chemo therapy) & 6 & inappropriate & Cured \\
\hline 12 & 60 & M & None & 15 & appropriate & Cured \\
\hline 13 & 31 & $\mathrm{~F}$ & MCTD & $\mathrm{ND}$ & ND & Unknown \\
\hline 14 & 57 & M & Diabetes & 27 & inappropriate & Cured \\
\hline 15 & 60 & M & Pancreatic cancer (Chemo therapy) & 6 & inappropriate & Cured \\
\hline
\end{tabular}


FELLOWSHIP APPLICANTS COMPLETE MORE AND BETTER SCHOLARLY ACTIVITIES Ana M. Palacio; Leonardo Tamariz; Deidre T. Campbell. University of Miami, Miami, FL. (Control ID \#2470087)

BACKGROUND: Scholarly work in internal medicine is a residency accreditation requirement. A motivation to complete scholarly activities may include fellowship application. Our aim was to evaluate if residents pursuing fellowships publish more than residents pursuing primary care or hospitalist career paths.

METHODS: We conducted a retrospective cohort study at the internal medicine residency program at the University of Miami among all internal medicine residents who trained between 2007 and 2013. At the end of residency, we asked all residents if they applied for fellowship. We collected data on all manuscripts published by each resident up to a year post graduation. We defined publication as a manuscript published in a peer reviewed journal listed in pubmed. The quality of the publications was defined as the mean impact factor of the publications. We calculated the odds ratio (OR) of publication by fellowship status adjusted for age, gender and biostatistical knowledge.

RESULTS: We included 221 internal medicine residents. Twenty six percent of the residents did not do fellowships. Those who applied for fellowship were less likely to be women $(34 \%)$. Fifty three percent of those who applied for fellowship published at least one manuscript compared to $15 \%$ of those who did not apply for fellowship $(p<0.01)$. The mean number of publications for those who applied for fellowship was $1.48+/-2.7$ compared to $0.13+/-0.38(p<0.01)$ among those who did not. The a mean impact factor for those who publish and did not apply for fellowship was $1.3+/-1.7$ compared to $2.8+/-1.3(p<0.01)$ for those who applied for fellowship. The OR for publication for those who applied for fellowship was 5.42;95 \% CI 2.3-12.6.

CONCLUSIONS: Internal Medicine residents applying for fellowships are more likely to complete better scholarly activities. Strategies to incentivize the production of knowledge among physicians wishing to deliver primary or hospital care will be key to promote the culture of quality improvement required by the Affordable Care Act, to impact outcomes and to reduce disparities in care.

\section{FLIPPING THE QUALITY IMPROVEMENT CLASSROOM IN RESIDENCY} EDUCATION Thomas J. Beckman ${ }^{1}$; Sara Bonnes $^{1}$; Kimberly Carter ${ }^{2}$; Luke T. Hafdahl ${ }^{1}$; Andrew J. Halvorsen ${ }^{1}$; Jayawant N. Mandrekar ${ }^{1}$; Amy Oxentenko ${ }^{1}$; John T. Ratelle ${ }^{1}$; Amy T. Wang ${ }^{3}$; Christopher M. Wittich ${ }^{1}$. ${ }^{1}$ Mayo Clinic, Rochester, MN; ${ }^{2}$ University of Kansas Medical Center, Kansas City, KS; ${ }^{3}$ Harbor-UCLA Medical Center, Torrance, CA (Control ID \#2463259)

BACKGROUND: Experts in medical education have observed that in-depth learning and retention requires teaching approaches that are electronically accessible, learner-focused, and interactive. Graduate medical training traditionally entails countless hours of attending lectures and completing assignments, yet research suggests that learners' engagement and knowledge retention are limited by this pedagogical approach. The flipped classroom (FC) has the potential to engage resident physicians on core topics, yet there are limited studies of the FC model in medical education. The FC involves delivering curricular content independently prior to class and devoting class-time to discussing and applying the content. Our goals were to 1) develop and validate an instrument to measure residents' perceptions of a FC during a quality improvement (QI) curriculum, 2) determine residents' perceptions of the FC curriculum, and 3) determine associations between resident characteristics, FC perception scores, and QI knowledge.

METHODS: This was a prospective validation study of all internal medicine residents $(N=143)$ at Mayo Clinic in 2014-2015. A FC perception instrument (FCPI), an 8 item instrument, was developed based on existing literature and iterative revision. Postgraduate year (PGY) 1 and 3 residents participated in the newly developed FC QI curriculum. PGY-2 residents participated in a non-flipped QI patient safety curriculum and served as controls. Pre-class worked involved Institute for Healthcare Improvement (IHI) online modules completed independently by the residents. In-class time was devoted to discussing the IHI content and applying it to a group QI project. QI knowledge was assessed by the previously validated Quality Improvement Knowledge Assessment Tool (QIKAT). The FCPI and QIKAT were administered before and after the FC QI curriculum. RESULTS: Factor analysis revealed a two-factor structure (Cronbach alpha): "pre-class activity" (0.81) and "in-class application" (0.88). Post-course FCPI scores increased for 3 of 8 items including 1) Online modules enhanced my learning, 2) I participate and engage in projects in-class, and 3) Working on a team enhances my learning (all $p<0.05$ ). Additionally, when asked whether they preferred a traditional or flipped classroom environment, more of the residents preferred a flipped classroom experience after completing the flipped QI curriculum (traditional classroom 22 [35\%] versus flipped classroom 41 [65\%]) compared to before completing the curriculum (traditional classroom 31 [49 \%] versus flipped classroom 32 [51\%], $P<0.0001$ ). QI knowledge scores (maximum score $=27)$ increased by $5.1(3.2)$ points $(P<0.0001)$ among residents who experienced the FC. The percentage of modules completed was associated with a significant change in FCPI score (mean; SD): 0-74 \% completed $(-0.06 ; 0.36) ; 75-100 \%$ completed $(0.19$, $0.47), P=0.04$. Previous FC exposure was associated with a significant difference in in post FC QI knowledge score: prior exposure $(3.1,2.7)$; no prior exposure $(6.1,3.0), P=$ 0.002. Post-course surveys of the PGY-1 and PGY-3 residents indicated that $7 \%$ of residents felt that their QI knowledge was most enhanced by online modules, $36 \%$ felt it was most enhanced by in-class sessions, and $56 \%$ felt it was from online modules and inclass sessions equally.

CONCLUSIONS: To our knowledge, this is the first study of flipped classrooms in residency QI education. Validity evidence for the FCPI includes content, internal consistency reliability, and an intuitive, bidimensional structure. The FC curriculum was associated with improved resident attitudes toward FC and increased QI knowledge. Residents valued the in-class application sessions more than the online component. These findings highlight the benefit of combining both online and in-class learning as a way to promote active learning. The findings of the current study have important implications for QI curricula and graduate medical education in general, as residency training programs increasingly utilize flipped classroom models. We are hopeful that the validated flipped classroom perception instrument that was developed in this study will be useful when applied to future flipped classroom curricula and research in graduate medical education.

FLUID RESUSCITATION FOR GENERAL WARD INPATIENTS: A PRELIMINARY AUDIT OF THE NON-INVASIVE FLUID ASSESSMENT AND CHALLENGE TOOL (NI-FACET) Amos Lal ${ }^{2}$; Kay C. See ${ }^{1} .{ }^{1}$ National University Hospital, Singapore, Singapore, Singapore; ${ }^{2} \mathrm{Ng}$ Teng Fong General Hospital, Singapore, Singapore, Singapore. (Control ID \#2457137)

BACKGROUND: Patients admitted to the general ward may develop hypo tension/ shock (defined as systolic blood pressure $<90 \mathrm{mmHg}$ or lactate $>4 \mathrm{mmol} / \mathrm{L}$ or presence of symptoms/signs of hypo perfusion). However, few protocols exist to guide appropriate therapy for administering fluids in the general ward patients with hypo tension/shock. We thus created the Non-Invasive Fluid Assessment and ChallengE Tool (NI-FACET) and investigated its clinical safety and efficacy.

METHODS: From May 2014 to September 2015, two groups of general ward inpatients with hypo tension/shock were compared. For Group 1, we used the NIFACET for fluid resuscitation of patients. Group 2 had usual care (non-protocolised fluid resuscitation). Multiple linear regression was used for hospital length of stay (LOS) (log-transformed for normality) as the outcome. Logistic regression was used 
for hospital mortality as the outcome. Multivariate analyses adjusted for age, sepsis diagnosis, initial lactate level and co morbidity.

RESULTS: One hundred seventy-nine patients were studied (mean age $71.0 \pm$ 16.4 years, 70/39.1 \% female, median hospital LOS 13 days [interquartile range, IQR, 6-25 days], hospital mortality $18.4 \%$ ). One hundred thirty-three patients $(74.3 \%)$ had sepsis and $43(24 \%)$ were in Group 1. Unadjusted analysis yielded no statistical difference in median hospital LOS for Group 1 vs. Group 2 (11.5 days, IQR $4-25$ vs. 13 days, IQR $7-25, P=0.265)$ or in hospital mortality (19.1 vs. $18.3 \%, P=0.543)$. Using multiple linear regression, the adjusted coefficient for NI-FACET use was 0.881 (95\% confidence interval [CI] $0.598-1.30, P=0.522$ ) for $\log$ (hospital LOS). Using logistic regression, the adjusted odds ratio for NI-FACET use was 0.544 (95 \% CI $0.138-2.141$, $P=0.384$ ) for hospital mortality.

CONCLUSIONS: In this preliminary audit, there was a trend towards shorter hospital LOS (average decrease of $12 \%$ ) and decreased hospital mortality (average decrease of $46 \%$ ) when the NI-FACET was used for patients with hypo tension/shock. Greater use and continued audit will be needed to more firmly establish the clinical superiority of NI-FACET.
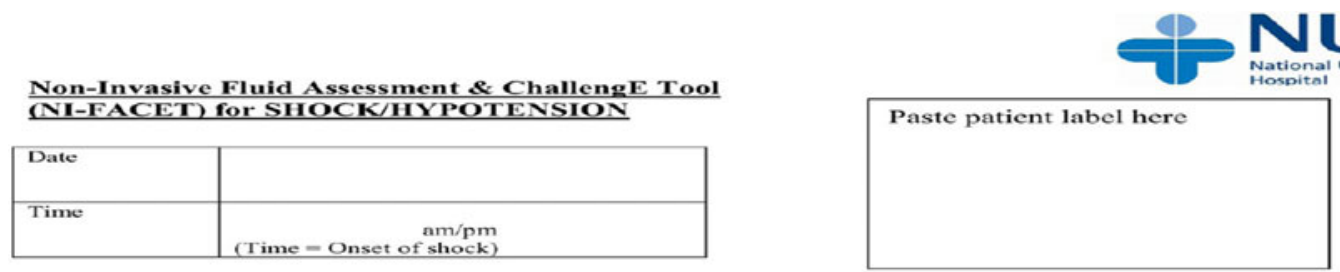

SHOCKSHYPOTENSION IS M MEDICAT, EMERGENCY
IF THIS PATIENT IS FOR ACTIVE MANAGMENT, PROCEED WITHOUT DELAY

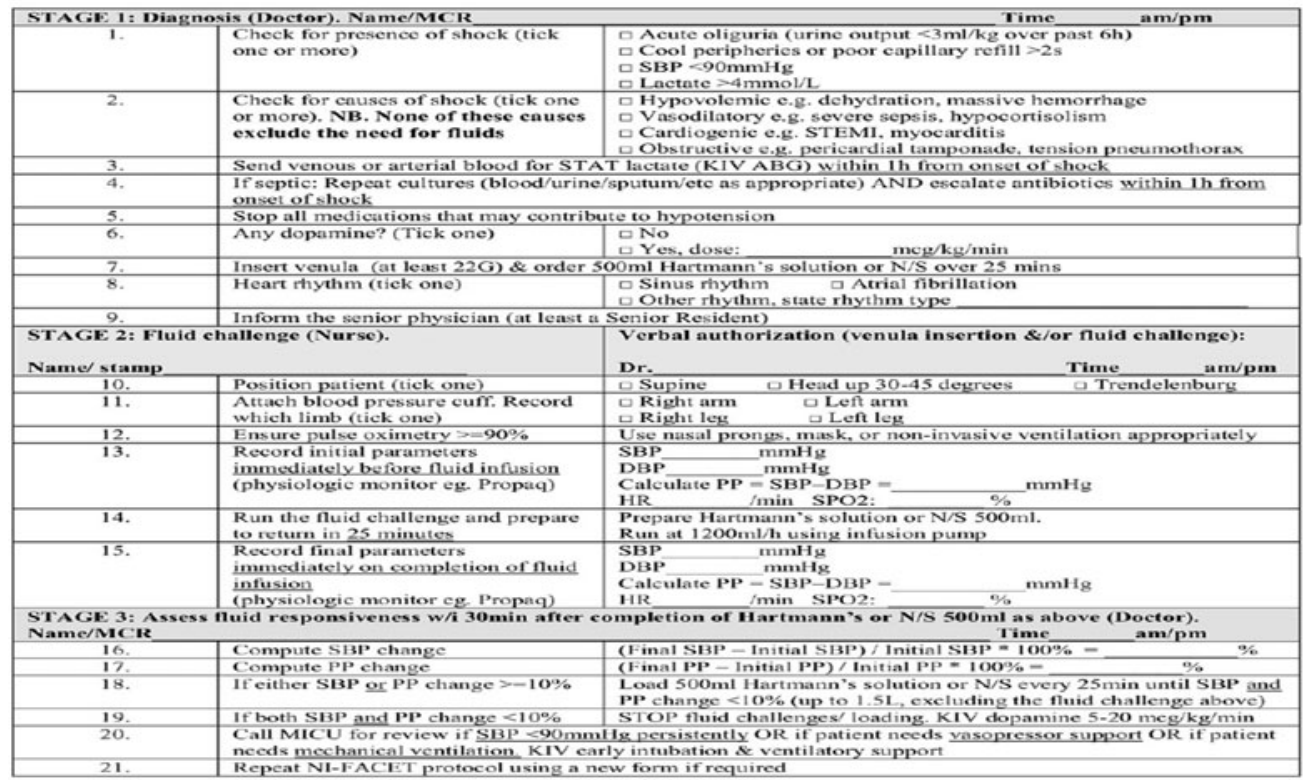

FAX COMPLETED FORM TO MA: Attn Louis Widjaja (6775-6757) A member of the NUHS

Non-Invasive Fluid Assessment and ChallengE Tool (NI-FACET) Protocol

FOLLOW-UP OF ABNORMAL COLON CANCER SCREENINGS Linda J. Canty $^{1,}{ }^{1}$; David J. Desilets ${ }^{1,4}$; Rony Ghaoui ${ }^{1,}{ }^{4}$; Paul Visintainer ${ }^{1,}$, Joseph Rozell ${ }^{1,4}$; Donielle F. Sliwa ${ }^{1,4}$; Elizabeth Tarbox ${ }^{3}$; Michael B. Rothberg ${ }^{2} .{ }^{1}$ Baystate Medical Center, Springfield, MA; ${ }^{2}$ Cleveland Clinic, Cleveland, $\mathrm{OH} ;{ }^{3}$ University of New England College of Osteopathic Medicine, Biddeford, ME; ${ }^{4}$ Tufts University School of Medicine, Boston, MA. (Control ID \#2467934)
BACKGROUND: Colon cancer is a leading cause of cancer death in the United States. Colorectal cancer screening can identify and remove precancerous polyps such as tubular adenomas years before they develop into malignancy. Despite the effectiveness of colon cancer screening, follow-up of abnormal tests is often inadequate. In this study, we aimed to determine the proportion of patients who undergo timely follow-up and evaluate factors associated with follow-up. 
METHODS: We performed a retrospective cohort study at Baystate Medical Center, an academic hospital in western Massachusetts. We included all subjects ages 40-75 years who had an endoscopy performed by a Baystate gastroenterologist with removal of at least one tubular adenoma between June 20, 2005 and November 20, 2007 or at least one tubulovillous adenoma between June 20, 2005 and November 20, 2009. Subjects with colon cancer at time of the index colonoscopy and those who died before the follow-up colonoscopy was due were excluded. This earliest qualifying procedure within the timeframe was considered the index procedure. The primary outcome was timely follow-up, defined as a follow-up study within 6 months of the recommended due date. Follow-up was ascertained using the electronic medical record, a comprehensive system that includes outpatient records of primary care notes, gastroenterology notes, endoscopy notes and histology reports. For each patient we also recorded age, gender, race, language, and histopathology of polyp.

RESULTS: During the study period, 556 unique patients underwent an endoscopy with removal of at least one adenomatous polyp. Median age was 57 years, $43 \%$ were female, and $65 \%$ were Caucasian. The majority of the patients spoke English (68\%) followed by Spanish (23\%.) Polyps were primarily tubular adenomas $(97 \%$.) Of all procedures, $170(31 \%)$ had follow-up colonoscopies within 6 months of the recommended time in the medical record system. Another 89 (16\%) had their follow-up done more than 6 months after the recommended due date. The remaining 297 (53\%) had no documented evidence of a repeat colonoscopy. Of these, $56 \%$ appeared to have no primary care visits in at least 2 years Factors such as patient race and language were not correlated with timely followup. More females $(37 \%)$ had timely follow-up compared with males (26\% $p<0.01$.)

CONCLUSIONS: Over two-thirds of patients with abnormal colonoscopies did not receive follow-up colonoscopies within 6 months of the recommended due date. Quality improvement efforts aimed at increasing colon cancer screening should also focus on repeat screening for patients at high risk due to previous polyps.

\section{FOLLOW-UP RATES AND CARE COORDINATION FOR HOMELESS PERSONS DISCHARGED FROM THE HOSPITAL Andrew Coyle. Icahn School of Medicine at Mount Sinai, New York, NY. (Control ID \#2466795)}

BACKGROUND: Homelessness is associated with poor access to care, high ED and hospital utilization, and increased mortality. Numerous studies note a high burden of chronic conditions amongst the homeless, yet homeless persons are far less likely to report having a PCP than housed peers. Without adequate primary care, homeless persons are more likely to have frequent ED visits or hospitalizations. Those who are hospitalized have more costly hospitalizations, longer stays, and more frequent readmissions. Several studies have shown improvements in outcomes and reductions in utilization through programs for homeless persons that employ longitudinal social work involvement, care coordination, and intensive primary care. However, as many institutions do not have homeless-specific interventions, there is no data about how often these patients are enrolled in more general care coordination programs. Additionally, no studies have examined post-discharge follow-up, nor have they looked at show-rates for follow-up appointments made in traditional clinics. This study explores post-discharge follow-up in homeless patients as compared to domiciled patients and how often patients actually show up to these appointments. It also examines recruitment rates of homeless patients into existing care coordination programs.

METHODS: A retrospective review was performed of all adult discharges from medicine services (general medicine, liver medicine, oncology, cardiology) from 1/1/
15-6/30/15 at an academic urban tertiary care hospital, excluding elective and direct admissions. An EMR keyword search for homeless-related terms was performed to identify homeless patents, followed by manual chart review of social work notes and discharge summaries to confirm homeless status (defined by discharge location and/ or self-report). A random sample of domiciled patients was created from the list of all discharges using a random number generator. All selected charts were evaluated for an identified PCP, clear follow-up plans, and participation in care coordination programs (examples include social work-run care coordination programs and a highrisk patient clinic). Follow-up plans were identified in discharge orders and summaries, with credit given for a follow-up plan if it included a physician's name, location, or phone number or appointment dates/times. Categorical associations were tested by comparing homeless and domiciled individuals using the Fisher exact test, and continuous associations were tested using independent-samples t-tests. All statistical analysis was done using SPSS 21.0 for Windows.

RESULTS: We identified 173 unique homeless patients discharged from the hospital during the 6-month time period and 346 domiciled patients as controls. Table 1 reports descriptive characteristics of homeless and domiciled patients. Homeless patients were more likely to be male and younger than domiciled patients. Homeless persons had higher readmission rates, with a 30-day readmission rate of $28.3 \%$. During the 6-month period from January to June 2015, homeless patients had more ED visits (3.0 vs. 2.1) and hospital admissions (1.7 vs. 1.4) on average. Despite this, homeless persons were less likely to be enrolled in a care coordination program (16.8 vs. $24.2 \%$ ). Table 2 reports characteristics of patients' identified hospital discharge and transition planning. Homeless patients were far more likely to leave the hospital against medical advice ( 18.5 vs. $1.4 \%$ of domiciled patients). On discharge, only $47.4 \%$ of homeless persons had a PCP identified by name (vs. $82.9 \%$ ). Homeless persons were also much less likely to have clear follow-up arranged on discharge (64.7 vs. $92.8 \%$ ). Homeless persons had a low show-rates of $39.7 \%$ for follow-up appointments made within the hospital system.

CONCLUSIONS: As compared with domiciled patients discharged from medicine services, homeless patients had higher rates of utilization and lower rates of outpatient follow-up. Poor follow-up may be driven by increased rates of AMA discharges and unclear transition plans. Even for those who do have clear follow-up plans on discharge, very high no-show rates in clinic limit the ability to engage patients in care. Despite this, homeless patients were less likely to be enrolled in care coordination programs. This data suggests several possible interventions. Transition planning for homeless patients should begin on admission, starting with clear PCP identification. At discharge, communication with outpatient providers (and homeless shelters) should be prioritized. Care coordination programs should specifically target homeless persons given higher rates of utilization and difficulties with follow-up. Clearly a multi-pronged approach targeting homeless persons is needed to better engage these patients in primary care after hospital discharge.

Patient Characteristics

\begin{tabular}{llll}
\hline \hline & $\begin{array}{l}\text { Homeless Patients } \\
(n=173)\end{array}$ & $\begin{array}{l}\text { Domiciled Patients } \\
(n=346)\end{array}$ & $P$-Value \\
Age (Years) & 51.7 & 63.7 & $<0.001$ \\
Male Gender & $74.5 \%$ & $48.1 \%$ & $<0.001$ \\
$\begin{array}{l}\text { 30-Day Readmission } \\
\text { Rate }\end{array}$ & $28.3 \%$ & $16.8 \%$ & 0.002 \\
$\begin{array}{l}\text { Mean \# of Admissions } \\
\text { During 6-Month }\end{array}$ & 1.7 & 1.4 & 0.035 \\
$\begin{array}{l}\text { Period } \\
\text { Mean \# of ED Visits }\end{array}$ & 3.0 & & $<0.001$ \\
$\begin{array}{l}\text { During 6-Month } \\
\text { Period }\end{array}$ & & 2.1 & \\
Care Coordination & $16.8 \%$ & & 0.02 \\
$\begin{array}{l}\text { Enrollment During } \\
\text { 6-Month Period }\end{array}$ & & $24.2 \%$ & \\
\hline
\end{tabular}


Discharge Characteristics and Transition Planning

\begin{tabular}{llll}
\hline \hline & $\begin{array}{l}\text { Homeless Patients } \\
(n=173)\end{array}$ & Domiciled Patients $(n=346)$ & $P$-Value \\
Discharge Against Medical Advice & $32(18.5 \%)$ & $5(1.4 \%)$ & $<0.001$ \\
PCP Identification on Discharge & $82(47.4 \%)$ & $287(82.9 \%$ & $<0.001$ \\
Follow-Up Arranged & $112(64.7 \%)$ & $321(92.8 \%)$ & $<0.001$ \\
Show Rate for Scheduled Follow- & $34 / 88$ & $209 / 279$ & $<0.001$ \\
Up Appointments & Appointments & Appointments & \\
& $(39.7 \%)$ & $(74.9 \%)$ & \\
\hline
\end{tabular}

FROM SEEING TO DOING: TRANSLATING A PROBLEM INTO PRACTICENURSES' INPUT INTO IMPLEMENTING INPATIENT VISION SCREENING Victoria I. Moreira ${ }^{2}$; Allison J. Louis ${ }^{1}$; Ashley Hull ${ }^{1}$; Seenu M. Hariprasad ${ }^{1}$; David Meltzer ${ }^{1}$; Vineet M. Arora ${ }^{1}$; Valerie G. Press ${ }^{1} .{ }^{1}$ University of Chicago, Chicago, IL; ${ }^{2}$ Medical College of Georgia, Augusta, GA. (Control ID \#2466076)

BACKGROUND: Insufficient vision is an under-recognized risk factor in inpatient care that affects patient safety and self-care. In fact, the Agency for Healthcare Research and Quality (AHRQ) recommends that all patients have their glasses within reach for the duration of their hospital stay. However, research shows that nearly one-third of general medicine inpatients fail a basic bedside vision screening due to a myriad of factors including inadequate prescriptions, unavailable lenses, and limited access to vision care providers. To fill identified gaps in preventive care, the hospital setting is increasingly being utilized to incorporate preventive screening for patients. Nurses have been among the key providers in implementing preventive hospital-based care. For example, hospital nurses screened inpatients to identify second-hand tobacco smoke exposure, in one study. Therefore, nurses may be the ideal provider to address the need for inpatient vision screening. The present study aimed to assess nurses' perceptions of vision problems among general medicine inpatients, nurses' attitudes toward vision screening, and the feasibility of making vision screening part of standard inpatient care.

METHODS: Research Assistants (RAs) at the University of Chicago Medicine (UCM) verbally consented and conducted single in-person semi-structured interviews with voluntarily recruited registered nurses (RNs) from both day and night shifts from two different general medicine hospital units (IRB \#15-0593). Interviews were audio recorded, transcribed, and then immediately destroyed after statistical analyses. Participants were given a $\$ 5$ gift certificate to a hospital eatery in compensation for their time. Descriptive statistics, including means and proportions, were employed using Stata v.14.

RESULTS: While only two-thirds $(68 \%, 17 / 25)$ of RNs believed that vision problems are "moderately common" among their patients, almost half $(44 \%, 11 / 25)$ of RNs agree that poor vision interferes with risk of adverse events in the hospital. When asked about making vision screening part of standard inpatient care, the majority $(80 \%, 20 / 25)$ of RNs believed that this testing would be moderately $(48 \%, 12 / 25)$ or very $(32 \%, 8 / 25)$ important. Moreover, RNs believe that patient quality of life $(100 \%, 25 / 25)$ and quality of care $(88 \%, 22 / 25)$ would improve if vision were tested. Additionally, most $(80 \%, 20$ / 25 ) of the RNs felt that it would be very or extremely useful for a nurse or doctor to know their patients' vision results. More than half of RNs also felt that it would be very (36\%, $9 / 25)$ or extremely $(32 \%, 8 / 25)$ useful to help correct patient vision, when possible, using non-prescription readers. Regarding the feasibility of implementing vision screening in the inpatient setting, almost half $(48 \%, 12 / 25)$ of RNs believed that it would be "very feasible" and the majority $(88 \%, 22 / 25)$ of RNs felt that there was a reasonable amount to time during a normal day to test patients' vision. They believed that this testing would be most appropriate either within the first $24 \mathrm{~h}$ of admission $(28 \%, 7 / 25)$ or upon admission vital sign completion $(28 \%, 7 / 25)$. Interestingly, despite support for RN-led feasibility of vision test, more than half $(56 \%, 14 / 25)$ of RNs believed an MD would be the ideal staff member to test patients' vision, as half $(52 \%, 13 / 25)$ of RNs reported that they do not feel like they know how to administer a vision screening test using a basic eye chart. Surprisingly, despite this discomfort with vision screening, theee-quarters $(76 \%, 19 / 25)$ of RNs have actually administered a vision test using a basic eye chart before.

CONCLUSIONS: Vision problems are an under-identified risk in the inpatient setting as even nurses, who have primary patient interaction, are largely unaware of its prevalence in this setting. As nurses acknowledge that vision problems in the inpatient setting may increase the risk of adverse events and vision screening would be important in standard inpatient care, it is important to identify strategies to use the inpatient setting to test vision. Nurses may be a critical component of this inpatient screening effort, and especially with training, this effort may be even more feasible and acceptable for nurses. Regardless, an inter-professional approach should be explored for best practice implementation of inpatient vision screening.

FROM SOCIAL SERVICE AGENCIES TO ACADEMIC RESEARCH INSTITUTIONS: SUCCESSFULLY AND MEANINGFULLY ENGAGING STAKEHOLDERS FROM ALL SECTORS IN HEALTHCARE RESEARCH Rebecca J. Schwei ${ }^{1}$; Laura Pinsoneault ${ }^{3}$; Jerica Broeckling ${ }^{3}$; Katy Allen ${ }^{4}$; Jennifer Frumer $^{5}$; Vivian Sauer ${ }^{6}$; Elba A. Simpson ${ }^{4}$; Erika Zambrano Morales ${ }^{6}$; Elizabeth Jacobs ${ }^{2} .{ }^{1}$ School of Medicine and Public Health, University of Wisconsin, Madison, WI; ${ }^{2}$ University of Wisconsin School of Medicine and Public Health, Madison, WI; ${ }^{3}$ Alliance for Strong Families and Communities, Milwaukee, WI; ${ }^{4}$ Community Place of Greater Rochester, Rochester, NY; ${ }^{5}$ Alpert Jewish Family and Children's Service, West Palm Beach, FL; ${ }^{6}$ Jewish Family Service of Los Angeles, Los Angeles, CA. (Control ID \#2464486)

BACKGROUND: In 2010 the United States Congress authorized the creation of the Patient Centered Outcomes Research Institute (PCORI), a large funding agency, whose mission is, in part, to produce and promote high-integrity, evidence-based information that comes from research guided by patients, caregivers and the broader healthcare community. A core component of this agency's mission is to meaningfully engage stakeholders in healthcare research, and thus the questions of who are appropriate stakeholders and what does meaningful engagement look like have become topics of great importance to many physician-researchers. Our objective was to qualitatively assess what meaningful engagement is and what strategies supported this engagement in our PCORI funded research study of peer-to-peer support of older adults and its impact on promoting aging in place in 3 communities across the US

METHODS: The University of Wisconsin-Madison, the Alliance for Strong Families and Communities, Alpert Jewish Family and Children's Service of Palm Beach, the Community Place of Greater Rochester, and Jewish Family Services of Los Angeles received funding from PCORI in 2014. The research team includes twenty four people who are academic researchers, leaders of social services organizations, older adults who receive the services we are studying, caregivers and friends of older adults that receive the services we are studying, and community members who have a passion for promoting health in their community. From the beginning stages of this partnership understanding what meaningful engagement means to all the members of our research team has been a priority. We conducted one on one interviews and group disscusions with members of the team. Our semi-structured questions asked them to reflect on our engagement on several different occasions to explore what meaningful engagement looks like and what concrete activities have facilitated personal engagement in this project. We conducted a thematic analysis of concepts and ideas that were frequently cited and identified key strategies that were repeated and we shared them with the research team to validate our conclusions.

RESULTS: We identified 4 key themes that provide the foundation for meaningful engagement and 6 strategies that facilitated this engagement. Meaningful engagement themes included (1) Mutual respect, (2) Equal partnership, (3) Willingness to push boundaries (stakeholders and investigators allow themselves to work and think outside their comfort 
zones to accomplish rigorous and patient centered research) and (4) Shared goals. Strategies include (1) Collaborating and agreeing on roles and responsibilities of everyone on the team, (2) Frequent, transparent communication, (3) Collaboratively developed and agreed upon plan for how to communicate when there is a problem with the engagement, (4) Including cultural brokers as part of the team to help both investigators and stakeholders listen to and understand one another, (5) Financially compensating team members in all roles and (6) Embedding research staff in community organizations participating in research.

CONCLUSIONS: Respect, equal partnership, willingness to push boundaries and shared goals have provided the foundation for meaningful engagement for all team members for this project. Furthermore, we identified key strategies for achieving meaningful engagement. Other research teams can use some of the strategies we have identified to meaningfully engage members of their own research team who may work across many different domains

GASTROINTESTINAL CARRIAGE OF POTENTIAL BACTERIAL AND VIRAL PATHOGENS IN HOSPITALIZED PATIENTS ADMITTED WITH NON-GASTROINTESTINAL SYMPTOMS, DETECTED BY TARGET ENRICHED MULTIPLEX POLYMERASE CHAIN REACTION (TEM-PCR) Khushdeep S. Chahal ${ }^{2}$; Esmeralda B. Gutierrez Asis ${ }^{1}$; Ali Hassoun ${ }^{3} .{ }^{1}$ UAB Huntsville, Huntsville, AL; ${ }^{2}$ University of Alabama Birmingham, Huntsville Regional Campus, Huntsville, AL; ${ }^{3}$ Alabama Infectious disease center, Huntsville, AL. (Control ID \#2469832)

BACKGROUND: To determine the frequency of potentially pathogenic bacterial and viral organisms in stool specimens of hospitalized patients with non-gastrointestinal symptoms and signs.

METHODS: Retrospective chart review was performed for 199 patients admitted to a tertiary medical center in north Alabama who had stool testing done from January 2015 to April 2015. One hundred fifty-four patients who had gastrointestinal symptoms were excluded. Stool samples were obtained and screened for 3 viral, 10 bacterial and 2 parasitic targets using TEM-PCR

RESULTS: Study population included 55 patients of which $49 \%$ were females, mean age was 67 years, $25 \%$ aged $61-70$ years followed by $22 \%$ aged $71-80$ years. Pertinent positive history included immunosuppression in $20 \%$, recent antibiotic use in $24 \%$, recent hospitalization in $45 \%$, Proton pump Inhibitor (PPI) use in $44 \%$, history of GI disorders in $29 \%$. Most stool samples were collected for occult blood testing (82\%) in anemic patients and $51 \%$ were positive for occult blood. Positive isolates were observed in $9 \%(n=5$ of 55$)$ of patients of which $60 \%(n=3$ of 5$)$ were Norovirus and $20 \%(n=1$ of 5) each of Enterpathogenic Escherichia Coli and Clostridium Difficile. Eighty percent of the carriers ( $n=4$ of 5 ) were more than 65 years and $80 \%(n=4$ of 5$)$ were males. There was no co detection amongst isolates. There was no detection of giardia or cryptosporidium targets. In addition we screened for bacterial/toxins targets including Enterohemorrhagic E. Coli, Shiga toxin 1, Shiga toxin 2, Shigella, Enteroinvasive E. Coli, Salmonella, Campylobacter Jejuni, Vibrio parahaemolyticus and viral targets including Adenovirus and Rotavirus which were negative. In-hospital testing of stool for Culture and Ova and Parasites was done in $9 \%(n=5$ of 55$)$ and was all negative.

CONCLUSIONS: Hospitalized patients admitted for non-gastrointestinal conditions were rarely a carrier for pathogenic bacterial organisms which cause gastroenteritis. Nine percent patients had Norovirus detected, which needs further studies to assess clinical implications of carriage of this virus which is one of the most common causes of gastroenteritis.

GENDER AND RACE DIFFERENCES IN PHYSICIAN-PATIENT INTERACTIONS Harry B. Burke ${ }^{2}$; Patrick G. O'Malley ${ }^{1}$; Jeffrey L. Jackson ${ }^{3}$. ${ }^{1}$ Uniformed Services University, Washington, DC; ${ }^{2}$ Uniformed Services University of the Health Sciences, Bethesda, MD; ${ }^{3}$ Zablocki VAMC, Milwaukee, WI. (Control ID \#2468758)
BACKGROUND: Although there is a significant literature regarding the total time physicians and patients spend interacting, little is known about how patient gender and race are related in terms of the functional units "interactional time" and "topic." We hypothesize that there are gender-specific and race-specific patient differences in the length of time spent interacting, the number of topics in the interaction, and the length of time spent within a topic.

METHODS: Sixty-six audio-recorded and transcribed encounters of established primary care outpatient physician-patient interactions were coded in terms of the duration of the physician-patient interaction and the occurrence of topics in each interaction. Topic types were: discussing a new medical problem; discussing an existing medical problem; discussing wellness and screening; reviewing what has happened to the patient since the last visit; administrative tasks; summarizing the interaction; obtaining participant agreement; and discussing social topics. We examined differences in self-identified gender and race $(\mathrm{CA}=$ Caucasian-American, $\mathrm{AA}=$ African-American $)$. The Student's t-test was used to determine significant differences $(<0.05)$.

RESULTS: Table 1. Male patients interacted with their physicians longer than female patients, both used the same number of topics, and the males stayed on a topic significantly longer than the females. Table 2. Caucasian-American patients interacted with their physicians significantly longer than African-American patients, Caucasian-American patients used slightly more topics than African-American patients, and CaucasianAmerican patients stayed on a topic significantly longer than African-American patients. CONCLUSIONS: We found that male patients and female patients used the same number of topics during their interaction but that male patients stayed on a topic longer than female patients. We found that Caucasian-American patients spent more time with their physicians than African-American patients and that Caucasian-American patients stayed on a topic longer than African-American patients. In summary, there were significant gender-specific and race-specific patient differences in terms of time spent in the physician-patient interaction and in the duration of topics

Table 1. Gender (mean, confidence interval)

\begin{tabular}{llll}
\hline \hline Patients & Minutes* & Topics & Mins/Topic** \\
Male $(n=32)$ & $32.42(28.57-36.27)$ & $22(19-25)$ & $1.57(1.37-1.78)$ \\
Female $(n=34)$ & $27.92(24.70-31.14)$ & $22(19-24)$ & $1.31(1.19-1.42)$ \\
\hline
\end{tabular}

*Male vs. female, $p=0.071 ; * *$ male vs. female, $p=0.026$

Table 2. Race (mean, confidence interval)

\begin{tabular}{llll}
\hline \hline Patients* & Minutes** & Topics & Mins/Topic*** \\
CA $(n=26)$ & $33.97(29.91-38.03)$ & $23(19-26)$ & $1.63(1.40-1.86)$ \\
AA $(n=35)$ & $28.01(24.58-31.44)$ & $21(19-23)$ & $1.32(1.20-1.44)$ \\
\hline
\end{tabular}

* Five "other" patients were not included. ${ }^{* *} \mathrm{CA}$ vs. AA, $p=0.025 ;{ }^{* * *} \mathrm{CA}$ vs $\mathrm{AA}, p=$ 0.010

GENDER DIFFERENCES IN FIREARM STORAGE MOTIVATIONS AND LEVEL OF COMFORT WITH FIREARM SAFETY INTERVENTION COUNSELORS Joseph A. Simonetti ${ }^{1,}{ }^{1}$; Ali Rowhani-Rahbar ${ }^{1}$; Cassie King ${ }^{2}$; Elizabeth Bennett ${ }^{2}$; Frederick Rivara ${ }^{1} .{ }^{1}$ University of Washington, Seattle, WA; ${ }^{2}$ Seattle Children's Hospital, Seattle, WA. (Control ID \#2469359)

BACKGROUND: On average, more than 100,000 individuals sustain firearm-related injuries each year in the United States. Safe firearm storage is associated with a lower risk of such injuries and effective interventions to promote safe storage exist. To increase the effectiveness of those interventions, it may be necessary to improve our understanding of motivations for firearm storage practices and design safety 
interventions to be participant-centered. The aims of this study were to assess levels of comfort with potential firearm safety counselors, motivations for firearm storage practices, and gender differences in such characteristics among participants in a communitybased firearm safety event.

METHODS: We studied event participants who completed onsite and follow-up phone surveys 4-8 weeks afterwards that asked respondents who they "would feel comfortable discussing gun safety with at future events" and listed several potential options for safety counselors (e.g., physicians). The survey also assessed risk of unintentional or intentionally self-inflicted firearm injury among household members as a motivator for firearm storage practices. Response options were based on five-point Likert scales. We summarized responses and compared differences by gender using chi-squared tests.

RESULTS: Of 398 event participants, 195 completed both surveys and were included. The mean age of the study sample was 39 years (SD 13.6 years). About one-half were female, $73.3 \%$ were married or in a significant relationship, $78.0 \%$ had children less than 19 years of age living in their household, and $81.4 \%$ reported keeping a firearm in the home. Among those who reported keeping a firearm in the home, male respondents were more likely than females to report that they were primarily responsible for the manner in which the firearm was stored (76.6 vs. $14.8 \%, p<0.001)$. A majority of men and women, respectively, agreed they would be "comfortable" or "very comfortable" discussing firearm safety with their child's pediatrician ( 70.8 vs. $68.4 \%, p=0.75)$, their doctor (70.1 vs. $72.4 \%, p=0.73$ ), a law enforcement officer (91.9 vs. $94.4 \%, p=0.49$ ), a firearm retailer ( 88.5 vs. $72.9 \%, p<0.01)$, and members of firearm clubs or firearm advocacy groups $(87.2$ vs. $71.4 \%, p<0.01)$. A small proportion of both men and women, respectively, agreed that concerns of family members accidentally (51.4 vs. $42.3 \%, p=0.26$ ) or intentionally ( 31.2 vs. $25.6 \%, p=0.57$ ) injuring themselves with a firearm were "very" or "extremely" important in their decisions on how to store firearms. CONCLUSIONS: Though most respondents reported being comfortable with several potential firearm safety counselors, women were less likely to do so with members of firearm advocacy groups or retailers. A small proportion of all respondents reported firearm injury risk among household members as reasoning for their firearm storage practices suggesting that raising awareness about injury risk may be an important element of future firearm safety interventions.

GENDER DIFFERENCES IN PATIENT REPORTED HOSPITAL EXPERIENCES AND INFORMATION SHARING Rosana Gonzalez-Colaso; Tara Rizzo; Carol Oladele; Baylah Tessier-Sherman; Marcella Nunez-Smith. Yale University, New Haven, CT. (Control ID \#2469718)

BACKGROUND: Prior studies have documented that patient reported hospital care experiences differ by gender. Women tend to report more negative and less positive experiences than men overall. These differences are worse in hospitals serving large populations of women. Most recently, two large national studies found gender differences among several processes of care. When compared to men, women had less positive experiences with regards to communication about medicines, discharge information, cleanliness, pain management, and behavior of nurses. To further explore specific process of care that consider individual patient preferences, needs, and values possibly sensitive to gender differences, our study objective is to compare the hospital care experiences between women and men for specific information sharing processes that promote patient centered care.

METHODS: Data from the Patient-Reported Experiences of Discrimination in Care Tool (PreDict) study was used for analyses. The PreDict study includes a self- administered survey of recently discharged adult patients from a national sample of hospitals. This cross-sectional study describes patients' most recent hospital experiences using the novo items to evaluate the patient experiences with information sharing. Patients were invited to participate via mailed postcard if they were discharged within the past 30 days from a medical service to home, 21 years and older, and English- or Spanish-speaking. We examined associations between inpatient experiences, doctorpatient communication, experiences of discrimination, and the outcomes primary care appointment within 30-days of discharge and emergency department visit within 30 days of discharge. Data for 1503 participants who completed the survey as of December 31, 2015 were included in analyses. We used bivariate to compare experiences by gender and multiple logistic regression to test whether gender differences were associated to specific process of care under for information sharing.

RESULTS: Multivariable model results showed significantly associated with gender differences in patient reported experiences of care for one specific item. Women were less likely to answer positively to the question: 'The doctors asked me what name I wanted to be called' (adjusted $\mathrm{OR}=0.58 ; 95 \%$ C.I. 0.47-0.72), after adjusting for marital status and general health status.

CONCLUSIONS: Our findings stress the importance of analyzing different process contributing to the patient reported hospital experience as an opportunity to improve quality of care and provide equitable care for all. Our results are consistent with existing literature that shows that women tend to report worse care experiences at hospital than men. Given that women represent the majority of hospital populations, improving the specific experiences they are sensitive to can also improve the overall.

GENERAL INTERNISTS' ATTITUDES, PRACTICES AND PREPAREDNESS RELATED TO SUBSTANCE USE DISORDER Sarah E. Wakeman ${ }^{2,3}$; Genevieve Pham-Kanter ${ }^{4}$; Karen Donelan ${ }^{1} .{ }^{1}$ Massachusetts General Hospital, Boston, MA; ${ }^{2}$ Massachusetts General Hospital, Charlestown, MA; ${ }^{3}$ Harvard medical School, BOSTON, MA; ${ }^{4}$ Drexel University Dornsife School of Public Health, Philadelphia, PA. (Control ID \#2457492)

BACKGROUND: Despite the growing incidence, morbidity, and mortality of substance use disorder (SUD), previous research has shown that most primary care physicians do not regularly screen for SUD, feel unprepared to treat SUD, and find caring for patients with SUD challenging and unsatisfying. Healthcare providers have been shown to have negative attitudes towards patients with SUD, which negatively impacts patients and treatment outcomes. Confidence in SUD management has been associated with improved clinical practice and interaction with a person with a history of SUD has been shown to reduce stigma. The goal of this study was to evaluate inpatient and outpatient general internists' attitudes, preparedness, and clinical practices related to SUD.

METHODS: This study consisted of a cross-sectional email survey with leadership endorsement and \$5 postpaid incentive. The sampling frame included 290 general internists practicing in a major academic medical center in 2014. Physicians were asked to rate their agreement with eight attitude statements; their preparedness to deliver each component of screening, brief intervention and referral to treatment (SBIRT), to make a diagnosis, and to discuss treatment options, overdose prevention, and harm reduction with patients; the frequency with which they currently provide SUD care; and their exposure to SUD in clinical practice.

RESULTS: One hundred forty-nine general internists responded, a response rate of $51 \%$. Although $46 \%$ frequently cared for patients with SUD, only $16 \%$ frequently 
referred patients to treatment, $6 \%$ frequently prescribed a medication to treat SUD, and $5 \%$ frequently prescribed naloxone to a patient at risk of overdose. Negative attitudes towards SUD were endorsed by some respondents. Thirty-one percent felt that SUD is different from other chronic diseases because they believe using substances is a choice. Fourteen percent felt treatment with opioid agonists was replacing one addiction with another. Twelve percent of hospitalists and $6 \%$ of PCPs felt that someone who uses drugs is committing a crime and deserves punishment. A minority of respondents felt very prepared to deliver SUD care. Twenty percent felt very prepared to screen for SUD, $9 \%$ to provide a brief intervention, $7 \%$ to discuss behavioral treatments, and $9 \%$ to discuss medication treatments. Preparedness was significantly associated with evidence-based clinical practice and favorable attitudes. Frequently caring for patients with SUD was significantly associated with preparedness, clinical practice, and favorable attitudes.

CONCLUSIONS: A substance use disorder is a treatable and prevalent disease, yet a majority of general internists do not feel very prepared to screen, diagnose, provide a brief intervention, refer to treatment, or discuss treatment options with patients. Very few frequently prescribe medications to treat SUD. Some physicians view substance use as a crime and a choice. Physician preparedness and exposure to SUD is associated with improved clinical practice and favorable attitudes towards SUD. Physicians may need education and support to provide better care for patients with SUD

GERIATRICS IN JAIL: EDUCATING PROFESSIONALS TO IMPROVE THE CARE OF OLDER INMATES Meera Sheffrin; Cyrus Ahalt; Irena Stijacic Cenzer; Brie Williams. University of California, San Francisco, San Francisco, CA. (Control ID \#2468020)

BACKGROUND: While the number of older prisoners is rising precipitously, few professionals in jails and prisons have geriatrics expertise. Following a needs assessment, we developed a geriatrics training for correctional clinicians and officers (guards) in the San Francisco Jail and evaluated its acceptability, relavence, and potential effect on practice change at Rikers Island Jail (NY) and Oahu Correctional Center (HI)

METHODS: Both correctional clinicians and officers received an "aging in correctional settings" lecture, followed by hands-on trainings on dexterity, mobility, and medication management. The 2-h officer training focused on identifying and reporting geriatric emergencies, such as falls and confusion. The 2day clinician training included additional sessions on geriatrics tailored to jail, such as balance exercises in small cells and assessing the need for assistive devices in high security settings. Participants rated the overall quality of the training and their geriatrics knowledge using a retrospective pre-post survey (5point scale; 1-poor, 5-excellent). In open-ended responses, participants described the training's anticipated effect on future practice.

RESULTS: Overall, 30 clinicians and 68 officers received the training. All participants rated the training highly (mean $4.8 / 5$ clinicians, 4.4/5 officers). Knowledge scores increased significantly for both groups in all domains assessed. Clinicians reported improved knowledge of geriatrics principles (2.6 to 4.3), evaluating unique functional impairments in jail (2.8 to 4.4), and eliciting goals of care (2.6 to 4.4; all $P<0.01)$. Officers reported greater knowledge recognizing falls as a critical medical event (2.7 to 4.3), recognizing the effect of dementia on behavior and judgment (3.0 to 4.3 ), and detecting signs of elder abuse $(2.6$ to 4.2 , all $P<0.01)$. All participants reported a high likelihood of applying new knowledge to their work (clinicians
$90 \%$, officers $84 \%$ ). In open-ended responses after the training, correctional officers reported they would slow down and be more patient with elderly inmates. Openended responses suggested geriatric mental health, pain management, and palliative care are important for areas for additional clinician training.

CONCLUSIONS: We developed geriatrics trainings for correctional officers and clinicians to "geriatricize" jail. Both correctional officers and correctional clinicians rated the training highly and indicated it would affect their interactions with older adults. Educating professionally diverse groups of learners in prison and jail is an opportunity to improve the care of medically vulnerable older adults in the criminal justice system.

GETTING HIV PRE-EXPOSURE PROPHYLAXIS (PREP) INTO PRIMARY CARE PRACTICE: TRAIN ALL PROVIDERS, DESIGNATE AN ON-SITE PREP SPECIALIST, OR REFER OUT? E. J. Edelman ${ }^{1}$; Brent A. Moore ${ }^{1}$; Gail Berkenblit $^{2}$; Sarah K. Calabrese ${ }^{3}$; Chinazo Cunningham ${ }^{4}$; David A. Fiellin ${ }^{1}$; Theresa $\mathrm{Katz}^{1}$; Viraj V. Patel ${ }^{4}$; Karran A. Phillips ${ }^{5}$; Jeanette M. Tetrault ${ }^{1}$; Minesh Shah ${ }^{6}$; Oni J. Blackstock ${ }^{4}$. ${ }^{1}$ Yale University School of Medicine, New Haven, CT; ${ }^{2}$ Johns Hopkins, Baltimore, MD; ${ }^{3}$ Yale University, New Haven, CT; ${ }^{4}$ Montefiore Medical Center/ AECOM, New York, NY; ${ }^{5}$ National Institute on Drug Abuse, National Institutes of Health, Baltimore, MD; ${ }^{6}$ University of Illinois-Chicago, Chicago, IL. (Control ID \#2469553)

BACKGROUND: Once daily oral use of tenofovir/emtricitabine (Truvada ${ }^{\circledR}$ ) for HIV pre-exposure prophylaxis (PrEP) represents a novel, yet underutilized HIV prevention intervention. While all primary care practices should be able to provide PrEP, the preferred PrEP implementation model among primary care physicians (PCPs) remains unknown. Among a sample of PCPs, we sought to determine preferred PrEP implementation models as well as differences in model preference based on provider and practice characteristics.

METHODS: From April to May 2015, we conducted an online survey among a convenience sample of Society of General Internal Medicine members. In addition to provider and practice characteristics, we asked participants: "In your opinion, which approach do you think would be most feasible to implement PrEP into your clinical practice setting?" Response options were: 1) all providers in the practice receive training to provide PrEP and prescribe to eligible patients; 2) one provider in the practice is appointed as a PrEP specialist; or 3) no providers in the practice receive training or provide PrEP; rather, patients are referred outside the practice. We used chi-square and t-tests to determine the association between provider and practice characteristics and preferred model for PrEP implementation.

RESULTS: Among our sample $(N=245)$, participants reported a mean (standard deviation) age of 41 (9) years and were mostly White (71\%), female (62\%), and heterosexual (93\%). Seventy-eight percent were attending physicians, with $51 \%$ reporting $<10$ years in practice. On average, participants spent $43 \%$ of their time providing direct patient care. Most practices were located in the Northeast (49\%) and in urban settings $(85 \%)$. Most reported their practices were primary care-based (94\%) and located at academic medical centers (69\%). Participants most commonly reported caring for 1-10 HIV-infected patients (53\%). Equal proportions of participants favored having all providers PrEP-trained (42\%) or an on-site PrEP specialist (42\%); $16 \%$ indicated that patients should be referred out for PrEP $(p<0.0001$ for comparison to onsite options). Participants who preferred that all providers prescribe 
PrEP were more likely to spend a greater proportion of their time delivering direct patient care $(48 \%$ of time) than providers who preferred having an on-site PrEP specialist (41\% of time) or referring patients out (34\% of time) $(p=0.02)$. Also, compared to providers who preferred having an on-site PrEP specialist (45\%) or referring patients out $(33 \%)$, participants who preferred that all providers prescribe PrEP were more likely to practice in the Northeast $(58 \%)$ compared to other regions $(p=0.04)$. There were no differences in preferred implementation model based on other characteristics.

CONCLUSIONS: The majority of PCPs thought that PrEP integration into primary care was favorable, with equal levels of support expressed for all providers prescribing PrEP vs. having an onsite PrEP specialist. Models for PrEP implementation that focus on training all providers may be most feasible for PCPs who spend most of their time delivering direct patient care. Geographic differences in primary care organizational culture may affect the feasibility of various PrEP implementation models. Future studies should evaluate the feasibility, acceptability, and effectiveness of implementing these models to optimize PrEP access and uptake.

\section{GOAL SETTING PROCESSES IN A TECHNOLOGY-ASSISTED HEALTH} COACHING INTERVENTION AMONG VETERANS IN PRIMARY CARE: A QUALITATIVE ANALYSIS Natalie B. Berner ${ }^{1,2}$; Katrina F. Mateo ${ }^{1,2}$; Natalie L. Ricci $^{1,}{ }^{2}$; Gail Schechter ${ }^{1}$; Adina Kalet ${ }^{2}$; Scott Sherman ${ }^{1,}$ 2; Melanie Jay ${ }^{1,2}{ }^{2}{ }^{1}$ VA NY Harbor Healthcare System, New York, NY; ${ }^{2}$ NYU School of Medicine, New York, NY. (Control ID \#2462888)

BACKGROUND: Obesity is a growing concern in the Veteran population. Veteran Affairs (VA) Medical Centers provide obesity screening and lifestyle counseling delivered by Patient Aligned Care Teams (PACT) in the primary care (PC) setting, and our formative work with Veterans and PACT staff have demonstrated the acceptability of goal setting for weight management. Goal setting is a common technique that is recommended as part of the $5 \mathrm{As}$ framework for obesity management and is part of several behavior change theories, including the Theory of Planned Behavior. Yet, there has been little research regarding optimal goal setting processes or assessment of whether/how these goals impact weight management outcomes. As part of our developed PC-based weight management intervention called MOVE! Toward Your Goals (MTG), Veterans make initial weight loss and behavior change goals with the help of an online tool (MTG tool) and then work with a health coach to guide them in making them "SMART" (Specific, Measurable, Attainable, Relevant, Timely). The aim of this study was to qualitatively characterize goals made by Veterans participating in a pilot study of the MTG intervention, as well as evaluate goal setting processes.

METHODS: Overweight/obese Veteran PC patients from an urban VA were recruited to pilot test the MTG intervention. The first round of testing was lab-based (Round 1) and the second was clinic-based (Round 2) where Veterans met with their PCP immediately after the intervention. At baseline, each Veteran completed pre-surveys, used the online MTG tool to create initial goals around weight loss, nutrition, and physical activity. This was followed by a health coaching session delivered by a member of the research team trained in motivational interviewing and health coaching protocols, and post-surveys. All health coaching sessions were audio-recorded. Review of pre-/post-surveys, audio-recordings, and health coaching notes were used to categorize goals and compare them to data from MTG tool output.
RESULTS: Twenty-one Veterans that participated in Rounds $1(n=10,60 \%$ female) and 2 ( $n=11,9 \%$ female) of testing had a mean age of 58.57 years $(\mathrm{SD}=10.50$ years $)$, and mean $\mathrm{BMI}$ of $30.58(\mathrm{SD}=3.5)$. Prior to completing the MTG tool and receiving health coaching, $33 \%$ of Veterans (7/21) indicated "not at all true of me" or "only a little true of me" that that they had a specific plan to lose weight, whereas only $19 \%$ of Veterans indicated "very true of me". In both Round 1 and 2, the MTG tool facilitated $100 \%$ of patients to choose a weight loss, 1-2 nutrition, and a physical activity goal. During health coaching, $85.71 \%$ set a weight loss SMART goal, $85.71 \%$ set at least 1 nutrition SMART goal, and $71.43 \%$ set a physical activity SMART goal. Regardless of goal choice on the MTG tool, the most commonly occurring SMART goal was derived from "I will eat more servings of vegetables" (53\% of all SMART goals created in Round 1, $44 \%$ in Round 2). Barriers to making health coach-facilitated SMART goals included running out time before PCP visit and Veteran resistance to discussing goals. Facilitators included tailored patient education materials generated by the MTG tool, SMART goal worksheets, and feeling supported by the health coach. CONCLUSIONS: Preliminary results from this study suggest that the MTG intervention facilitates goal setting for weight loss and lifestyle change to manage weight. Findings were used to develop best practices, health coaching guides, and trainings for future studies.

GOT SDM?: A MULTIMODAL INTERVENTION TO IMPROVE SHARED DECISION-MAKING DURING INPATIENT ROUNDS ON MEDICINE AND PEDIATRIC SERVICES. Rebecca Blankenburg ${ }^{3}$; Stephanie Rennke $^{4}$; Debbie S. Sakai ${ }^{1}$; Stephanie M. Harman ${ }^{1}$; Patrick Yuan ${ }^{4}$; Poonam Hosamani $^{2}$; lijia xie ${ }^{1}$; Lisa Shieh ${ }^{1}$; ian chua ${ }^{1}$; Adeena Khan ${ }^{4}$; Eric Huynh ${ }^{1}$; Jason Satterfield ${ }^{4}$; Bradley Monash ${ }^{4}$. ${ }^{1}$ Stanford University, Stanford, CA; ${ }^{2}$ Stanford University School of Medicine, Stanford, CA; ${ }^{3}$ Stanford University School of Medicine, Palo Alto, CA; ${ }^{4}$ University of California, San Francisco, San Francisco, CA. (Control ID \#2468215)

BACKGROUND: Shared decision-making (SDM) has been shown to be an important tool for improving patient engagement and health care outcomes. Despite the demonstrated value of SDM across disciplines, little is known about how to increase SDM in general inpatient settings. We sought to evaluate the effectiveness of an educational "bundle" on inpatient resident teams' abilities to demonstrate shared decision-making during morning rounds.

METHODS: We completed an IRB-approved pre/post intervention study of SDM on inpatient ward rounds on pediatrics and internal medicine services at two large university-based hospitals. Twelve observers were trained to use a validated, 9-item behavior observation tool, the Rochester Participatory Decision-Making Scale (RPAD, Figure 1) and observed the occurrence of SDM behaviors on inpatient rounds. Interrater reliability was achieved through a series of blinded video calibrations until average $\mathrm{SD}<1$ on a 9-point scale, and $\mathrm{SD}<0.5$ per item on a 1 -point scale. Data was collected for 12 weeks prior to and 12 weeks following a multimodal educational intervention. This education "bundle" included three parts: 1) a series of train-the-trainer workshops for residents, chief residents and hospitalist faculty, with interactive role-play exercises and video scenarios to evaluate SDM using the RPAD 2) an educational "campaign" including pocket cards, screensavers on hospital computers, posters, and email reminders; and 3) an audit and feedback program during morning rounds with observations followed by real-time assessment and feedback. Teams received follow-up 
electronic communications to reinforce SDM behaviors. Mean scores across all nine RPAD items were calculated, and performance across pediatrics and internal medicine services was compared using ANOVA.

RESULTS: In total, 32 teams, 95 ward rounds, and 268 unique patient encounters were observed pre-intervention and 34 teams, 100 ward rounds, and 391 unique patient encounters were observed post-intervention. Both pre- and postgroups were split approximately evenly between pediatrics and internal medicine services. A statistically significant improvement was seen in the four services, with 1.8 mean improvement in RPAD total score on a 9-point scale (range: 0.5 to $2.8, p=<0.05$ ) (Figure 2).

CONCLUSIONS: A SDM educational bundled intervention launched over an 8-week period was effective in improving shared decision-making skills in inpatient resident teams across all 4 observed services.

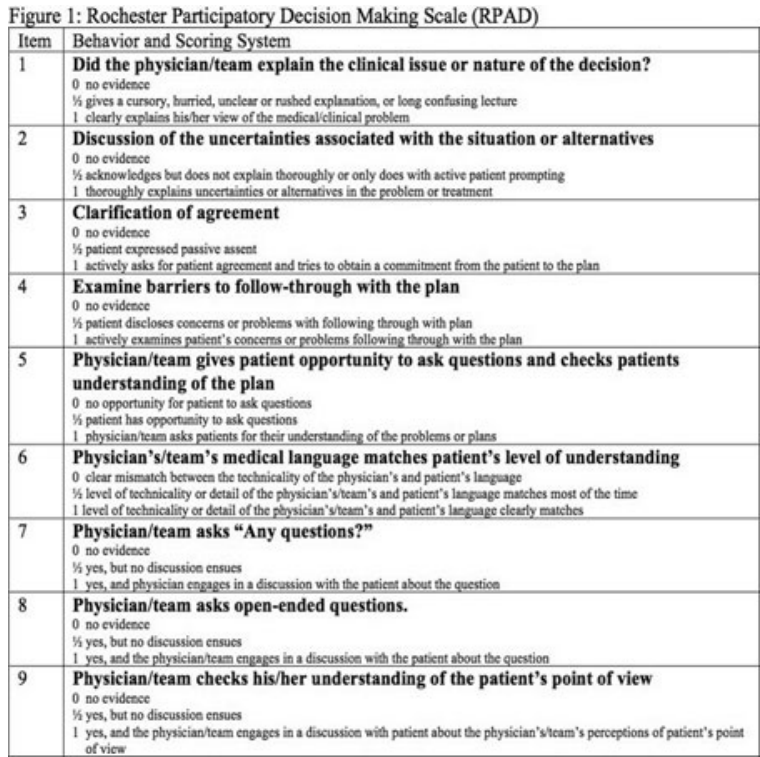

Figure 2: RPAD Composite Scores Pre- and Post-Intervention (by site and total)

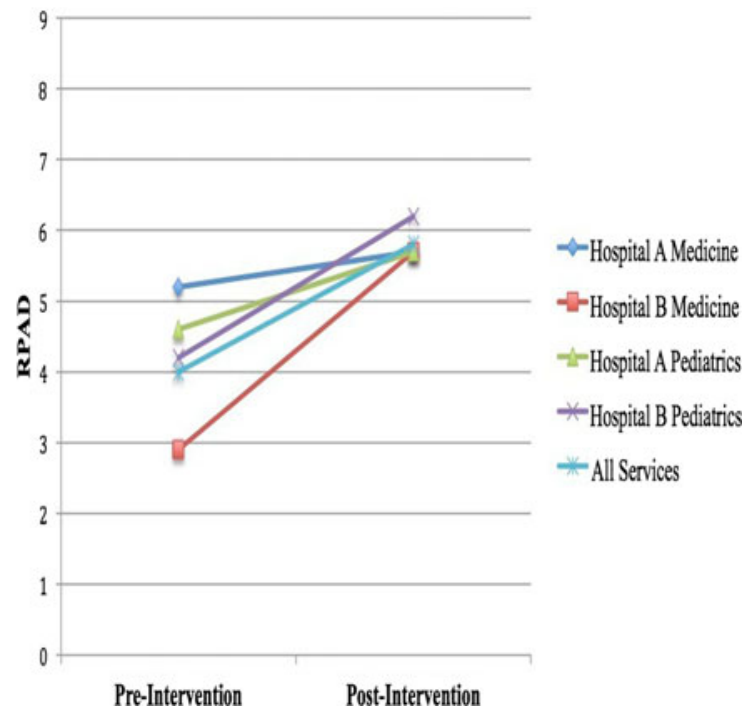

GRADUATE MEDICAL TRAINEE INTEREST IN PROGRAMS TO ADDRESS WELL-BEING AND BURNOUT Susan Maya ${ }^{2}$; Robert Fallar ${ }^{1}$; Michael Leitman ${ }^{1}$; Jonathan Ripp ${ }^{3}$. ${ }^{1}$ Icahn School of Medicine at Mount Sinai, New York, NY; ${ }^{2}$ Mount Sinai Hospital, New York, NY; ${ }^{3}$ Mount Sinai School of Medicine, New York, NY. (Control ID \#2467848)

BACKGROUND: Job burnout is common among graduate medical trainees and may lead to depression, poor academic performance and possibly medical errors. A number of interventions, such as mindfulness training and facilitated discussion, have been found to reduce job burnout in self-selected practicing physicians, but few have been studied in trainees. Little is known about the feasibility of implementing well-being programs in trainee populations and whether such programs would be of interest to trainees if made available. We conducted an assessment to determine trainee acceptance of well-being programs and whether there might be system-level work intensity factors associated with job burnout in graduate medical trainees.

METHODS: We administered an electronic survey to all resident physicians and physician fellows across a large multi-site academic health system over a 4-week period in December 2015 and January 2016. Our instrument measured job burnout using 2 validated single-item measures of emotional exhaustion and depersonalization from the Maslach Burnout Inventory. We also measured self-reported work hours, patient service size and interest in several programs aimed at reducing job burnout, including mindfulness training, resiliency training, peer- or expert-facilitated discussion, and narrative writing. Based on convention, we considered responses of weekly or more frequent to be "high" on the emotional exhaustion or depersonalization survey items. Data analysis included basic cross tabulations and chi-square tests after variables were dichotomized.

RESULTS: Of 2505 resident and fellow trainees eligible to participate in our study, 527 (21\%) completed the survey. Among the five interventions offered, trainees were most interested in mindfulness and resiliency training and least interested in narrative writing (somewhat or very likely to participate, 43 vs 46 vs $26 \%$, respectively, $p<0.05$ ). Thirtyfour percent of respondents reported working more than $70 \mathrm{~h}$ per week and $12 \%$ reported caring for more than 20 patients per day. Burnout was significantly related to working more than $70 \mathrm{~h}$ per week compared to fewer ( 64 vs $46 \%, p=0.0002$ ) and to caring for more than 20 patients per day compared to fewer ( 74 vs $50 \%, p=0.0006$ ). Respondents also indicated that lack of time was a barrier to participation in any of the proposed programs to decrease burnout.

CONCLUSIONS: Our assessment of graduate medical trainees found that a near majority of respondents indicated considerable interest in programs aimed at promoting wellbeing and decreasing job burnout. Given that these interventions were previously found to be effective in a self-selected volunteer population of practicing physicians, our findings suggest that trainees might choose to participate in such programs if offered. Novel strategies need to be explored for implementing these programs given the time barriers placed on graduate medical trainees. Furthermore, the correlation we identified between work hours, patient service size and burnout suggests that programs to improve well-being should be included alongside measures aimed at decreasing resident work intensity.

H(IGH)-B-P NOT A-B-CS: HYPERTENSION, BUT NOT HEALTH LITERACY, IS ASSOCIATED WITH PATIENT AWARENESS OF THEIR CKD AMONG OLDER ADULTS Milda R. Saunders ${ }^{2}$; Ashley M. Snyder ${ }^{1}$; Marshall Chin ${ }^{1}$; David Meltzer ${ }^{1}$; Vineet M. Arora ${ }^{2}$; Valerie G. Press ${ }^{1} .{ }^{1}$ University of Chicago, Chicago, IL; ${ }^{2}$ University of Chicago Medical Center, Chicago, IL. (Control ID \#2469248)

BACKGROUND: The aging population with chronic kidney disease (CKD) is large and increasing. In the US, approximately $33 \%$ of individuals over 60 meet criteria for CKD. Patient awareness of CKD is important to for patient self-care (e.g., avoiding nephrotoxic 
medication) and patient planning (e.g. selecting dialysis modality, transplant, or conservative management) if CKD progresses. Patients over the age of 65 have been shown to have low awareness of their CKD diagnosis and low health literacy (HL). We sought to determine if $\mathrm{HL}$ and other demographic, clinical, and healthcare factors were associated with patient awareness of CKD in older adults.

METHODS: We conducted a retrospective study using survey and medical record data from an urban academic medical center. Our study population included general medicine inpatients over age 65 discharged between June 2011 and July 2013 with CKD, defined as having at least one CKD ICD-9 code (585.0-585.9) among their first 20 admission diagnoses. Our primary outcome was patient awareness of their $\mathrm{CKD}$, defined as correct patient self-report of "kidney problems." We used the Brief Health Literacy Screen (BHLS), a three-item verbal questionnaire to assess HL. Descriptive statistics, chisquared tests, and t-tests were used to determine and compare means and proportions. Logistic regression was used to analyze the influence of demographic, HL, clinical, and healthcare utilization covariates on the likelihood of a patient reporting CKD awareness. Stata 14.0 was used for all analyses.

RESULTS: In this sample of older adult inpatients with CKD $(n=668)$, patient awareness of CKD was only $29 \%$ overall (196/668). Of the 100 patients with advanced CKD, patient awareness of CKD was $56 \%$ in patients with Stage 4 CKD (eGFR 15-29) and $69 \%$ in patients with Stage $5 \mathrm{CKD}(\mathrm{eGFR}<15)$. In addition, more than half of patients $(58 \%$, $319 / 551$ ) had inadequate HL. However, patient awareness of CKD was not associated with HL. Married patients, those with diabetes, hypertension, or increasing CKD stage, patients who had been hospitalized in the past 12 months, and patients who had visited a provider 2-3 times per year had greater awareness of their CKD, all $p$-values $<0.05$. In multivariable analysis, factors that remained significantly associated with CKD awareness were age $>80$ years old (OR 0.48 , compared to 65-69 year olds), having hypertension (OR 2.7) and having advanced CKD (Stage 4, OR 5.6, or Stage 5, OR 13.1), all $p<0.05$. In the stratified analysis shown in Table 1, patients with hypertension had greater awareness of their CKD, regardless of HL status. In Table 2, patient awareness of CKD was highest in patients with both diabetes and hypertension (reference group), followed by hypertension only. In addition, patient awareness of CKD was significantly lower in patients without diabetes, independent of diabetes status, all $p<0.05$.

CONCLUSIONS: Conclusion: Among hospitalized older adults with CKD, both CKD awareness and HL are low and inadequate. Regardless of patient health literacy status, patients with pre-existing diagnoses of hypertension were more likely to be aware of their CKD diagnosis. Patients with hypertension may be receiving (or retaining) more education related to $\mathrm{CKD}$ than those without hypertension.

Table 1. Association of Health Literacy $(\mathrm{HL})^{\mathrm{a}}$ and Hypertension on Patient CKD Self-Report

\begin{tabular}{|c|c|c|c|}
\hline \multirow{7}{*}{ 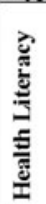 } & \multirow[t]{2}{*}{$\mathrm{N}=668$} & \multicolumn{2}{|c|}{ Hypertension } \\
\hline & & Yes & No \\
\hline & \multirow[t]{2}{*}{ Low } & REF & $0.31 * *(0.16,0.63)$ \\
\hline & & $\mathrm{N}=254,46 \%$ & $\mathrm{~N}=65,12 \%$ \\
\hline & \multirow[t]{3}{*}{ High } & $0.86(0.58$ & $0.26 * *(0.12,0.57)$ \\
\hline & & 1.28) & $\mathrm{N}=56,10 \%$ \\
\hline & & $\mathrm{N}=176,32 \%$ & \\
\hline
\end{tabular}

Table 2. Association of Diabetes and Hypertension on Patient CKD Self-Report

\begin{tabular}{|c|c|c|c|}
\hline & $\mathrm{N}=668$ & & ertension \\
\hline & & Yes & No \\
\hline & Yes & $\begin{array}{l}\text { REF } \\
\mathrm{N}=244 \quad 37 \%\end{array}$ & $\begin{array}{l}0.17 * * *(0.09,0.33) \\
\mathrm{N}=100\end{array}$ \\
\hline مَّ & No & $\begin{array}{l}0.61 *(0.42, \\
0.90) \\
\mathrm{N}=208,31 \%\end{array}$ & $\begin{array}{l}0.09 * * *(0.04,0.20) \\
N=116,17 \%\end{array}$ \\
\hline$* \mathrm{p}$ & .05 & $0<0.01 \quad * * *$ & 0.001 \\
\hline
\end{tabular}

HANDOFF PRACTICES IN A RESIDENT CONTINUITY CLINIC Stephanie Nothelle; Laura Hanyok; Scott Wright; Colleen Christmas. Johns Hopkins University, Baltimore, MD. (Control ID \#2469409)

BACKGROUND: The Accreditation Council for Graduate Medical Education has called for internal medicine programs to minimize conflicts between inpatient and outpatient rotation requirements. Subsequently, when beginning inpatient rotations, residents commonly transition the care of their continuity practice to a covering resident who is on an outpatient rotation. Transitions of care can result in medical errors and thus a formal "handoff" of information about the patient has been recommended for use during these periods. Most prior literature on handoffs in graduate medical education has focused on the inpatient setting, but there is growing concern and literature about transitioning outpatient panels at the end of a resident's training. The temporary transition of responsibility for patients when a resident transitions from the outpatient to the inpatient settings is common, yet unstudied. We conducted a study to explore this transition of care, to describe the current ambulatory handoff practices at a single institution, and to examine if there are transition factors that may correlate with use of health care resources.

METHODS: Internal Medicine residents at Johns Hopkins Bayview Medical Center in Baltimore, MD who were seeing patients in the residency continuity practice during the 3month study period were approached to participate in the study $(N=40)$. Participating residents were given a list of all patients for whom they were designated as primary care physician and a second list naming all patients they saw during the last outpatient rotation prior to switching to an inpatient rotation. Residents were asked to identify on both lists patients who met of one of more of the following three criteria: patients for whom they completed a handoff, patients who they were concerned would have an issue requiring the covering provider's attention, and patients who had an issue arise addressed by a covering provider. Charts of all patients identified were systematically abstracted during the time the resident was on the outpatient and inpatient rotation for the following: (1) demographic data (2) medication changes and tests ordered prior to the transition (3) follow up on tests that resulted while resident was unavailable (4) telephone calls to the office by the patient (5) specialty clinic office visits and visits to our general internal medicine (GIM) office practice and (6) visits to the emergency department (ED) and hospital admissions. The Elixhauser index, a validated measure of comorbidity, was calculated for each patient based on his or her problem list. Descriptive statistics of the data abstracted was performed.

RESULTS: Twenty-six (65\%) of the eligible Internal Medicine residents participated. Most participants were female $(n=16)$ and in the categorical residency track. There was a statistically significant increase in continuity panel size with post-graduate year (PGY) $(p<0.01)$. Of the three criteria used to identify patients, patients were most commonly identified because the resident was concerned about them $(n=216,90 \%)$. Less commonly, residents identified patients because a handoff was completed $(n=37,15 \%)$. There was no difference in residents' report of concern about patients they handed off and those they did not hand off. Patients for whom a handoff was completed were significantly more likely than those not handed off to have been seen in the office prior to the trainee's time away from the practice ( $70 \% \mathrm{v} 48 \%, p<0.02)$. Signed out patients were significantly more likely to interact with the health care system as measured by telephone calls ( $p<0.03)$, specialty office visits $(p<0.02)$, and GIM office visits $(p<0.001)$ during the time their outpatient physician was away. Residents did not behave differently with respect to hand off completion based on post-graduate year or residency track. Similarly, having recently adjusted patients' medicines or ordered tests did not influence likelihood of handing off a patient. There were no differences in patients' age, gender, mean Elixhauser index, ED visits, or hospital admissions between the two groups.

CONCLUSIONS: Although residents suspect that many of their continuity panel patients will need the help of a colleague while they are away and less available, few actually hand off or inform their covering resident about these matters. While a pervasive part of the 
culture in the inpatient setting, handing over responsibility for ambulatory care may need to catch up through formalized expectations in resident ambulatory practices.

\section{HARNESSING THE POTENTIAL OF INTERPROFESSIONAL HANDOFF} COMMUNICATION David Serksnys ${ }^{2}$; Rahul Nanchal ${ }^{1}$; Kathlyn Fletcher ${ }^{1} .{ }^{1}$ Medical College of Wisconsin/Milwaukee VAMC, Tbd, AL; ${ }^{2}$ Medical College of Wisconsin, Milwaukee, WI. (Control ID \#2465542)

BACKGROUND: Many hospitalized patients experience adverse events, and handoffs are a particularly vulnerable period for such incidents. Both physicians and nurses routinely engage in handoffs, and there is significant overlap in their content. However, the data typically remain in their respective silos. Merging these silos would likely result in both safer and more efficient care, but there is little data to suggest how that might be accomplished. Consequently, the objective of our study was to better understand current practice and future opportunities for incorporating inter-professional input into handoffs. Towards that end, we pursued 2 aims. 1) To identify key areas of interest for physicians and nurses related to inter-professional input into handoffs. 2) To identify facilitators and barriers for gaining inter-professional input into handoffs.

METHODS: We performed our prospective observational study in the medical intensive care unit (ICU) at a tertiary care teaching hospital. We chose to start in the ICU because the nurses and physicians are geographically co-located. We conducted field observations of both physician and RN handoffs as well as afternoon rounds. We also surveyed and conducted semi-structured interviews with stakeholders We audio recorded and transcribed the interviews. We used grounded theory to open code the field notes and transcripts to identify themes of importance. Before completing data analysis, we took our preliminary findings back to the participants in the ICU for feedback. We then synthesized the identified themes into theoretical constructs to explain opportunities, faciitators and barriers to inter-professional handoff communication.

RESULTS: We conducted 9 observations and interviews with 4 residents, 1 fellow, 3 faculty, 2 advanced practice providers, and 6 nurses. The surveys from interview participants indicated a high degree of interest in incorporating inter-professional input into handoff communication. One hundred percent of the physicians and advanced practice providers were at least somewhat interested in having RN input into their hand-offs. Eightthree percent of nurses were interested in having MD input into their hand-offs. From the qualitative analysis, we describe theoretical constructs explaining the information physicians and nurses desired from each other. We also identified the facilitators and barriers to exchanging that information. The primary point of interest to physicians was the nurses' ability to put patient data (vital signs, degree of pain, mental status) into context. This was primarily related to the fact that nurses spent significantly more time with patients and were therefore able to identify patterns and contributing factors that were not observable in the short physician visits. Nurses were generally interested in gaining a better understanding of the physicians plan of care and also gaining access to the physicians' knowledge and thought processes about the patients' conditions and hospital courses. Additionally, both physicians and nurses expressed an interest in nurses being provided with anticipatory guidance from physicians, similar to that which is already routinely included in handoffs between physicians. We also identified a number of facilitators to obtaining interprofessional input into handoffs. Participants were near unanimous in stating that close proximity and face to face communication were key to facilitating communication. Use of the EHR was identified by a number of participants as another vehicle, but some also expressed concerns about the accuracy of its content and noise in the system. The primary barrier to communication, identified by nearly all participants, was limited time and busy schedules. Finally, a few participants indicated that negative attitudes, specifically nurses' views not being valued by physicians, as a barrier.
CONCLUSIONS: Both physicians and nurses were able to identify key areas where input from the other profession would improve their handoff communication. Additionally, both professions indicated that it would be beneficial for physicians to give anticipatory guidance to nursing staff. Future quality improvement projects to improve handoff communication should capitalize on these areas of interest and on providing anticipatory guidance to nursing staff. They should also seek to utilize existing work flow structures for facilitating such communication. Possibilities include structuring rounds to frontload inter-professional content and making handoff instruments accessible from within the EHR. Finally, this study was conducted in an ICU, but the principles identified are likely to be applicable to general medical wards as well. Conducting a similar study in that setting is an important next step.

HAVE YOU MET YOUR PATIENT? PERFORMANCE OF A BRIEF BEDSIDE ASSESSMENT OF PHYSICAL FUNCTION Bonnie J. Konkowski ${ }^{2}$; Hafizur Rahman²; Hilary Mosher ${ }^{1,2}$. ${ }^{1}$ University of Iowa Hospitals and Clinics, Iowa City, IA; ${ }^{2}$ Iowa City VA Health Care, Iowa City, IA. (Control ID \#2470227)

BACKGROUND: Functional decline during hospitalization of older adults is wellrecognized and potentially preventable. Recent studies have suggested that clinically significant functional decline also affects hospitalized adults under 65 years of age, and that physical function is an important predictor of hospital readmissions. Despite the importance of function, we lack simple, efficient, and standardized measures to assess physical function in the hospital. Therefore, our objective was to apply a brief screening maneuver to assess ambulation (Dionne's Egress Test) in a general adult inpatient population. We developed a schema for scoring performance on the maneuver, where scores ranged from 0 (bedbound) to 11 (independently ambulating), allowing us to measure day-to-day changes in patient performance. The purposes of this modified Egress Test (mET) were to determine 1) if the test was safe and acceptable to medical inpatients and 2) if the test was sensitive to changes in physical function occurring during the hospital course.

METHODS: All patients admitted to a single medical team on an adult medicine inpatient service during the 10 -week study period were eligible. The mET was offered to patients in their hospital rooms on a daily basis (Monday-Friday) during daytime hours ( $8 \mathrm{am}-4 \mathrm{pm})$. If patients were not available, were occupied, or declined the test, the test was not performed on that day. The assessment was performed as part of a Quality Improvement initiative, and was determined to be Human Subjects Research Exempt by our Institutional Review Board. Performance on the mET and changes in function as measured by the novel scoring system were analyzed using simple descriptive statistics.

RESULTS: Of the 194 patients eligible for the mET, 188 (96.9\%) completed the assessment on at least one hospital day, and 118 (60.8 \%) completed the mET on multiple days. There were no falls or injuries during the assessments. The mean mET score was 9.4 (standard deviation 2.22; median 11, range 8). Among the 118 patients with more than one measured $\mathrm{mET}$, the mean change in $\mathrm{mET}$ score between first day measured and last day measured was 0.67 (standard deviation, 2.8). Fourteen patients (11.9\%) had declines between first and last measured mET, 66 (56.0 \%) had no change, and 34 (28.8 \%) had increases. When only highest and lowest mET scores were considered, 50 patients ( $42.4 \%$ of patients with $>1 \mathrm{mET}$ measured, or $26.6 \%$ of all patients assessed at least once) had changes in their physical function as measured by the mET during their hospitalization. CONCLUSIONS: The mET, a brief beside assessment of physical function, is sensitive to change during medical hospitalization. Approximately 1 in 4 hospitalized adults experienced changes in function that were measured by the mET. Further study is needed to investigate the external validity (i.e. self-reported ADLs, physical therapy assessment) and predictive ability (i.e. of hospital readmission, falls) of this brief bedside assessment. 
The mET, which could be considered an extension of the daily physical exam of adult inpatients, is a potentially valuable bedside assessment of changes in physical function.

HAVING MULTIPLE SOURCES OF PAYMENT IS ASSOCIATED WITH RISKY OPIOID THERAPY AMONG MILITARY/VA William Becker ${ }^{2}$; Brenda Fenton $^{2}$; Erin Doyle ${ }^{4}$; Brent A. Moore ${ }^{3}$; Cynthia Brandt $t^{2}$ Joe Francis ${ }^{5}$; Robert D. Kerns ${ }^{1}$; Peter Kreiner ${ }^{4} .{ }^{1}$ VA Connecticut, West Haven, $\mathrm{CT} ;{ }^{2} \mathrm{VA}$ Connecticut Healthcare System, West Haven, CT; ${ }^{3}$ Yale University School of Medicine, New Haven, CT; ${ }^{4}$ Brandeis University, Waltham, MA; ${ }^{5}$ VA Central Office, Washington, DC. (Control ID \#2469664)

BACKGROUND: Opioid overdose and other related harms are a major source of morbidity and mortality among US military and veterans (VA), in part due to high-risk opioid prescribing. In response, military/VA health systems have implemented myriad approaches attempting to limit access to risky opioid therapy; however, out-of-system access may be more difficult to control. We sought to determine whether having multiple sources of payment for opioids - as a marker for out-of-system access - is associated with risky opioid therapy among military/VA.

METHODS: This study was part of a quality improvement project and determined not to be human subjects research by the VA Connecticut Investigational Review Board. Using data supplied by the Kentucky All Schedule Prescription Electronic Reporting (KASPER) system, we identified all individuals with military/VA source of payment for controlled substance prescriptions in Kentucky during fiscal year 2014-15. We performed descriptive statistics and then divided the sample into two categories: those for whom the only source of payment was military/VA ("sole source") and those for whom sources of payment were military/VA plus at least one other source: Medicare, Medicaid, private insurance, and/or cash ("multiple source"). We used t-tests to compare differences between groups on proportion of two independent metrics of risky opioid therapy: combination opioid/benzodiazepine therapy and high-dose opioid therapy, defined as morphine equivalent daily dose (MEDD) $\geq 100 \mathrm{mg}$. We then performed two multivariable models, both controlling for gender and age: (1) regression analysis to examine the association between multiple sources of payment and percentage of opioid prescription days with overlapping benzodiazepine prescriptions; and (2) logistic regression to examine the association between multiple sources of payment and high-dose opioid therapy.

RESULTS: Of the 16,497 individuals included in the analyses, there were 10,393 sole source participants and 6104 multiple source. Sole source participants' prevalence of combination opioid/benzodiazepine therapy was $10.6 \%$ compared to $17.6 \%$ prevalence for multiple source participants $(p<0.0001)$. In terms of highdose opioid therapy, the prevalence among sole source participants was $3.0 \%$ compared to $5.6 \%$ among multiple source participants $(p<0.0001)$. We also performed Satterthwaite and Cochran tests, which assume unequal variances, with no material difference in significance. On multivariable analyses controlling for age and gender, having multiple sources of payment was independently associated with percentage of opioid prescription days with overlapping benzodiazepine prescriptions ( $\mathrm{t}$ value $14.9 ; p<.0001$ ) and odds of high-dose opioid therapy (OR 1.8, $95 \% \mathrm{CI}$ $1.5-2.1)$

CONCLUSIONS: In this analysis of individuals with military/VA source of payment for controlled substances, having additional sources of payment was associated with risky opioid therapy, even after controlling for gender and age. Providers should consider the safety of opioid therapy from within-system as well as out-of-system sources. Two recent policy changes-VA pharmacies reporting to state prescription drug monitoring programs and mandatory provider checks of these databases - may help improve monitoring capability.
HEALTH CONDITIONS OF A 1990-1991 GULF WAR ERA VETERAN COHORT - THE GULF WAR ERA COHORT AND BIOREPOSITORY Karen $\underline{\text { M. Goldstein }}^{1,2}{ }^{2}$; Rebecca B. McNeil ${ }^{3}$, ${ }^{4}$ Lara Khalil ${ }^{3}$; Kristina Felder ${ }^{3}$; Catherine Thomas $^{3}$; Richard L. Whitley ${ }^{3}$; Mary E. Grewe ${ }^{3}$; Elizabeth Hauser ${ }^{3,2}$; Kendrick Gray ${ }^{3}$; Kellie J. Sims ${ }^{3}$; Grant Huang ${ }^{5}$; Dawn Provenzale ${ }^{3,2} .{ }^{1}$ Center for Health Services Research in Primary Care, Durham, NC; ${ }^{2}$ Duke University School of Medicine, Durham, NC; ${ }^{3} \mathrm{VA}$ Cooperative Studies Program Epidemiology Center, Durham, NC; ${ }^{4}$ Medical University of South Carolina, Charleston, SC; ${ }^{5}$ VA Cooperative Studies Program, Office of Research and Development, Washington, DC. (Control ID \#2469466)

BACKGROUND: During the 1990-1991 Gulf War (Operation Desert Shield/Operation Desert Storm), the U.S. Armed Forces deployed just under 700,000 service members to Southwest Asia and other locations. Veterans of this era have reported increased fatigue as well as musculoskeletal, gastrointestinal and neurologic symptoms when compared to the general population, but equivalent or lower disease-related mortality rates. As this veteran population ages, questions remain about the impact of possible exposures incurred in the Gulf on chronic disease development. Previously, studying the health conditions of this veteran cohort has been challenging due to the small sample sizes of past studies. To facilitate ongoing research and enable clinicians to better address the health concerns of Gulf War veterans, the VA's Cooperative Studies Program (CSP) is creating the longitudinal research cohort: Gulf War Era Cohort and Biorepository (GWECB, CSP 585). Data and specimens will be available for research on the health and health conditions of veterans of the 1990-1991 Gulf War. We describe the demographic characteristics and health concerns of the first 1180 participants in the GWECB.

METHODS: Eligible veterans are those who were in the Uniformed Services during August 1990-July 1991, regardless of deployment status or current use of VA health care. Recruitment is based on a random sample of 90,000 veterans of the 1990-1991 Gulf War era, stratified by deployment in theater (50\%), active duty (50\%), Army (50\%), officer grade $(10 \%)$, race (20\% non-white), and sex (20\% non-male). The recruitment process includes a mailed invitation packet with project information, consenting documents, and a survey of health conditions and potential exposures. After returning all required documents, a blood sample is collected and stored in a central VA laboratory; DNA is extracted. RESULTS: There are currently 1180 participants. Of these, $533(45.2 \%)$ have used VA for health care in the previous 12 months, $892(75.6 \%)$ are male, and $857(72.6 \%)$ are white. The median age is 52.9 years, and 594 (50.3\%) are ever-smokers. Service branches are primarily Army (617, $52.3 \%$ ), Navy (215, 18.2 \%), and Air Force (190, $16.1 \%) ; 827$ (70.1\%) were deployed in support of the Gulf War. A total of 528 (44.7\%) participants hold at least a Bachelor's degree, $836(70.8 \%)$ are married/partnered, and $682(57.8 \%)$ are currently employed full-time. Participants generally report overall health as "good", with $395(33.5 \%)$ reporting fair or poor overall health. The most commonly reported everdiagnoses pertain to the circulatory $(829,70.3 \%)$, musculoskeletal $(652,55.3 \%)$, digestive $(612,51.9 \%)$, and neurologic $(550,46.6 \%)$ systems. In addition, $574(48.6 \%)$ reported vision/hearing problems and $488(41.4 \%)$ reported mental health disorders. Those who report using VA health care had higher prevalence of conditions in all systems: circulatory 78.4 vs $63.0 \%$; musculoskeletal 69.8 vs $42.8 \%$; digestive 59.3 vs $45.7 \%$; neurologic 61.4 vs $33.6 \%$; vision/hearing 58.3 vs $40.3 \%$; mental health 55.5 vs $29.4 \%$. CONCLUSIONS: In this diverse cohort of Gulf War era veterans, participants report a variety of conditions, with a higher prevalence of all conditions among those who currently use the VA healthcare system. Through collection of extensive individual-level clinical information, symptoms, deployment-related exposure history, and blood specimens, we are creating a new resource for research on the health issues of this veteran population. The inclusion of approximately $55 \%$ non-users of VA care is a particular strength of this cohort. This work is positioned to support the discovery of new treatments and improved healthcare approaches for the prevalent and incident health issues found among veterans who served during the 1990-1991 Gulf War era. 
HEALTH DISPARITIES IN HEPATITIS C SCREENING IN INTERNAL MEDICINE CLINICS Gina Maki; Kassem Bourgi; Amal Hejab; Mikhail Shteynvil; Zorawar Singh; Jay Yarlagadda; Kimberly Baker-Genaw. Henry Ford Hospital / Wayne State University, Detroit, MI. (Control ID \#2452757)

BACKGROUND: The Centers for Disease Control and Prevention along with the United States Preventive Task Force recommend screening for Hepatitis C (HCV) in patients born between 1945 and 1965. We aimed to evaluate the HCV screening rates and trends in internal medicine clinics at our health system.

METHODS: We used Henry Ford Health System's electronic medical records (EMR) to create a cohort of all patients born between 1945 and 1965 seen at 21 internal medicine clinics between July 2014 and June 2015. Patients previously screened for $\mathrm{HCV}$ and those with established $\mathrm{HCV}$ disease were excluded. We examined the association between patients' socio-demographic factors, clinical comorbidities, engagement in electronic heath along with provider specific factors and the likelihood of screening. Univariate and multivariate logistic regression was performed.

RESULTS: Forty-seven thousand three hundred four patients were included in our study cohort and 40,561 patients met inclusion criteria. A total of 8657 (21.3\%) were screened. On univariate and multivariate analyses, screening rates were higher among men $(p<0.001)$, African Americans $(p<0.001)$ and patients seen more than once during the study period $(p<0.0001)$. However, patients with multiple comorbidities $(p<0.001)$ were less likely to be screened $(p<0.001)$. Practice setting influenced screening rates as patients seen in residency teaching clinics were more likely to be screened $(p<0.001)$. Patient electronic health engagement, through subscribing to the patient's portal of the EMR, was associated with higher screening rates $(p<0.001)$ on both univariate and multivariate analyses.

CONCLUSIONS: HCV screening rates continue to be suboptimal with significant impact of multiple socio-demographic and provider specific factors. This reiterates the necessity of more robust interventions to improve screening rates. Increasing patients' electronic health engagement emerges as a promising tool in achieving that.

Univariate Analysis Comparing Patients Screened for HCV to those who were Not Screened

\begin{tabular}{|c|c|c|c|}
\hline Variable & $\begin{array}{l}\text { Non-Screened } \\
(N=31904)\end{array}$ & $\begin{array}{l}\text { Screened } \\
(N=8657)\end{array}$ & $\begin{array}{l}\text { Comparison } \\
P \text {-value }\end{array}$ \\
\hline Age in Years & $59.3 \pm 5.9$ & $59.5 \pm 5.7$ & $0.007 *$ \\
\hline African Americans & $\begin{array}{l}11616 \\
(42.0 \%)\end{array}$ & $\begin{array}{l}3538 \\
(46.8 \%)\end{array}$ & $<0.001 *$ \\
\hline Male Gender & $\begin{array}{l}12901 \\
(40.4 \%)\end{array}$ & $\begin{array}{l}3684 \\
(42.6 \%)\end{array}$ & $<0.001 *$ \\
\hline $\begin{array}{l}\text { Patients Engaged } \\
\text { with Electronic }\end{array}$ & $\begin{array}{l}16769 \\
(52.6 \%)\end{array}$ & $\begin{array}{l}4929 \\
(56.9 \%)\end{array}$ & $<0.001 *$ \\
\hline Health (MyChart) & & & \\
\hline HIV Positive & $144(0.5 \%)$ & $20(0.2 \%)$ & $0.004 *$ \\
\hline Drug Use & $1104(3.5 \%)$ & $311(3.6 \%)$ & 0.552 \\
\hline $\begin{array}{l}\geq 1 \text { visit in Residency } \\
\text { Teaching Clinic (RTC) }\end{array}$ & $5471(20.7 \%)$ & $1677(23.2 \%)$ & $<0.001 *$ \\
\hline Total Number of Visits & $2.14 \pm 1.55$ & $2.36 \pm 1.66$ & $<0.001 *$ \\
\hline $\begin{array}{l}\text { Charlson Comorbidity } \\
\text { Index }\end{array}$ & $0.91 \pm 1.51$ & $0.86 \pm 1.38$ & 0.878 \\
\hline $\begin{array}{l}\text { Median Household } \\
\text { Income (USD) }\end{array}$ & $\begin{array}{l}52008 \pm \\
20256\end{array}$ & $\begin{array}{l}52146 \pm \\
20766\end{array}$ & 0.894 \\
\hline
\end{tabular}

Multivariate Analysis Comparing Patients Screened for HCV to those who were Not Screened

\begin{tabular}{lll}
\hline \hline Variable & Odds Ratio & $P$-value \\
African American Race & 1.28 & $<0.001^{*}$ \\
Male Gender & 1.177 & $<0.001^{*}$ \\
Electronic Health Engagement & 1.244 & $<0.001^{*}$ \\
(My Chart) & & \\
Residency Clinic Visit & 1.202 & $<0.001 *$ \\
More than 1 Office Visit & 1.421 & $<0.001^{*}$ \\
Charlson Comorbidity Index & 0.868 & $<0.001^{*}$ \\
\hline
\end{tabular}

HEALTH INSURANCE DISPARITIES OF PATIENTS TRANSFERRED

BETWEEN HOSPITALS Dana Herrigel ${ }^{1}$; Madeline E. Carroll ${ }^{2}$; Christine Fanning ${ }^{1}$; Michael G. Usher ${ }^{2}, 3$. ${ }^{1}$ Rutgers Robert Wood Johnson Medical School, Whitehouse station, NJ; ${ }^{2}$ Rutgers-Robert Wood Johnson Medical School, New Brunswick, NJ; ${ }^{3}$ University of Minnesota, Minneapolis, MN. (Control ID \#2469761)

BACKGROUND: Healthcare disparity research indicates that uninsured patients receive less aggressive and less costly care, particularly in the setting of critical illness, and have higher adjusted mortality rates. The Emergency Medical Treatment and Labor Act (EMTALA) requires patient stabilization and treatment regardless of insurance coverage, but this mandate is inconsistently applied outside of the emergency department (ED). Interhospital transfer is known to improve mortality rates for indications such as trauma and myocardial infarction, but may be associated with increased mortality in other groups of patients. Prior data suggests that uninsured patients are less likely to undergo this care transition for unclear reasons. The impact of patient demographics, timing of transfer, and insurance coverage of patients undergoing inter-hospital transfer remain poorly understood. We sought to illustrate the characteristics of inter-hospital transfer patients, comparing the impact of insurance coverage on transfer patterns and outcomes, to test the hypothesis that interhospital transfers may exacerbate economic disparities in health care delivery.

METHODS: A large dataset was generated using Health Care Utilization Project's State Inpatient (SID) and Emergency Department (SEDD) databases from 5 states (FL, IA, NY, UT, VT) between 2011 and 2013. Patients were included only when both referring and receiving acute care hospitalizations could be identified. Patients who underwent interhospital transfer were compared with inpatients who did not undergo transfer from the same statewide database. Patients were identified by insurer and separated into four categories: Private, Medicare, Medicaid, and Uninsured. Comorbidities were assessed using the Charleson comorbidity index and by weighted Elixhauser comorbidities. The primary outcome was inpatient mortality, with secondary outcomes of referring and receiving facility length of stay (LOS), total cost, $24 \mathrm{~h}$ mortality, and discharge home within $48 \mathrm{~h}$ of transfer. Outcomes were adjusted for patient age, race, geography, and comorbidities.

RESULTS: We identified 173,654 inter-hospital transfers where the referring and receiving hospital stay could be identified and insurance payor information was available and known. Overall, uninsured patients composed $6.3 \%$ of all patients transferred between hospitals, which was slightly higher than the general population of non-transferred patients (4.8\%), but similar when adjusting for age, demographics, and comorbidities. The majority of uninsured patients $(55 \%)$ were transferred directly from referring EDs, contrasting with patients who had private insurance (39.6\%), Medicare (28.5\%), and Medicaid (37.2\%), who were more likely to be admitted prior to transfer. If admitted, uninsured patients were transferred earlier in the hospitalization $(p<0.001)$. Uninsured patients had a higher adjusted inpatient mortality (OR $1.3195 \%$ CI 1.16-1.49, $p<0.001$ ), and 24 h mortality (OR $2.895 \%$ CI 1.91-3.97, $p<0.001$ ). Conversely, this group exhibited a reduced LOS $(p<0.001)$, lower cost $(p<0.001)$, and were more likely discharged home within $48 \mathrm{~h}$ (OR 1.3, $95 \%$ CI 1.32 95\%CI 1.24-1.41, $p<0.001$ ).

CONCLUSIONS: Uninsured patients transferred between hospitals receive less costly care and have higher adjusted mortality similar to other populations. Despite EMTALA, uninsured patients are more likely to be transferred without admission, and are more likely die within $24 \mathrm{~h}$ of arrival. These data suggest a continued impetus to transfer uninsured patients in order to defray cost, which has the potential to exacerbate health disparities of the uninsured.

Results

\begin{tabular}{|c|c|c|c|c|}
\hline & Private & Medicare & Medicaid & Uninsured \\
\hline $\begin{array}{l}\text { Number of transferred } \\
\text { patients }\end{array}$ & 41,401 & 98,169 & 23,060 & 11,024 \\
\hline $\begin{array}{l}\text { Charleson Comorbidity } \\
\text { Index (SD) }\end{array}$ & $3.35(2.7)$ & $3.53(2.98)$ & $2.38(2.77)$ & $1.61(2.13)$ \\
\hline $\begin{array}{l}\text { LOS (days) at referring } \\
\text { hospital (SD) }\end{array}$ & $2.28(5.6)$ & $3.1(6.1)$ & $3.2(8.2)$ & $1.27(5.15)$ \\
\hline
\end{tabular}




\section{(continued)}

\begin{tabular}{|c|c|c|c|c|}
\hline $\begin{array}{l}\text { Number of patients } \\
\text { transferred from ED } \\
\text { (\% of total transfers) }\end{array}$ & $\begin{array}{l}16,399 \\
(39.6 \%)\end{array}$ & $\begin{array}{l}28,954 \\
(28.5 \%)\end{array}$ & $\begin{array}{l}8,578 \\
(37.2 \%)\end{array}$ & $\begin{array}{l}6016 \\
(54.6 \%)\end{array}$ \\
\hline $\begin{array}{l}\text { Adjusted Inpatient } \\
\text { Mortality of } \\
\text { transferred } \\
\text { patients }(95 \% \mathrm{CI})\end{array}$ & 1.00 (ref) & $\begin{array}{l}0.70 \\
(0.66-0.74)\end{array}$ & $\begin{array}{l}1.03 \\
(0.94-1.12)\end{array}$ & $\begin{array}{l}1.31 \\
(1.16-1.49)\end{array}$ \\
\hline
\end{tabular}

HEALTH LITERACY AS A MEDIATOR OF RACE AND ETHNICITYASSOCIATED CHILDHOOD ASTHMA HEALTH DISPARITIES: A LONGITUDINAL ANALYSIS David M. Washington ${ }^{1}$; Laura M. Curtis ${ }^{2}$; Katie Waite ${ }^{1}$; Michael S. Wolf ${ }^{2}$; Michael Paasche-Orlow ${ }^{1} .{ }^{1}$ Boston Medical Center, Boston, MA; ${ }^{2}$ Northwestern University, Chicago, IL. (Control ID \#2469369)

BACKGROUND: Race and ethnicity-related disparities in childhood asthma are well established. While socioeconomic status and associated measures explain a portion of ethnic disparities in asthma quality of life, asthma severity, and asthma control, they do not explain these disparities in totality. Health literacy has been proposed as a one such mediator. The objective of this study is to characterize the longitudinal effect of race/ethnicity on a range of asthma outcomes, and to what extent health literacy mediates these associations.

METHODS: Children ages 8-14 and their caregivers were recruited from public and archdiocese schools in Chicago, Illinois for the Chicago Initiative to Raise Asthma Health Equity (CHIRAH) cohort study. Caregivers $(n=544)$ completed a face-to-face interview and were followed by phone every 3 months for 1.5 years. Health literacy was measured at baseline with the Rapid Estimate of Adult Literacy in Medicine (REALM). Other covariates include sex, age, education level, income, cigarette smoke exposure, asthma duration, parental employment status, and insurance status. We conducted a series of regression models to evaluate health literacy as a mediator of the relationship between race/ethnicity and the following longitudinal asthma-related outcomes across 7 time points: 1) asthma knowledge (mean(sd): 8.05(1.43), range 2-11 over the course of the study); 2) asthma-related quality of life, as measured by the modified Children's Health Status for Asthma (CHSA), (median: 4.4, IQR: 3.6-4.9, range 1-5 over the course of the study). 3) moderate/severe vs. intermittent/mild asthma severity; and 4) poor vs. not well/well asthma control based on NAEPP 2007 guidelines. Briefly, models for each outcome were run using general estimating equation methodology, first including race and the covariates mentioned above, as appropriate. Health literacy (limited vs adequate) was then added and changes in estimates were examined.

RESULTS: African-American race and Hispanic ethnicity were significantly associated with all four outcomes when compared to Whites in initial, unadjusted models: asthma knowledge ( $\beta=0.58$, CI: -0.82 to -0.34 , and $\beta=-0.64$, CI: -0.94 to $-0.34 ; p<0.001$ ); asthma-related quality of life ( $\beta=-0.38, \mathrm{CI}:-0.49$ to -0.28 , and $\beta=-0.30, \mathrm{CI}:-0.42$ to $-0.17 ; p<0.001$ ); asthma severity (RR: $1.82, \mathrm{CI}: 1.49$ to 2.22 and RR: $1.57, \mathrm{CI}: 1.25$ to $1.98 ; p<0.001$ ), and asthma control (RR: $1.87, \mathrm{CI}: 1.45$ to 2.40 and 1.42 , CI: 1.22 to 2.16 ; $p<0.001)$. All outcomes' associations with African-American race and Hispanic ethnicity were attenuated with the addition of health literacy to the models. The association of asthma knowledge with African-American race and Hispanic ethnicity remained significant with the incorporation of health literacy: asthma knowledge ( $\beta=0.28, \mathrm{CI}:-0.54$ to -0.02 , and $\beta=-0.37$, CI: -0.67 to $-0.06 ; p<0.01$ ). African-American race alone remained statistically significant for the outcomes of asthma-related quality of life ( $\beta=$ -0.12 , CI: -0.23 to $-0.01 ; p<0.01$ ), asthma severity (RR: 1.30 , CI: 1.06 to 1.60 , $p<0.01$ ), and asthma control (RR: 1.31 ; CI: 1.01 to $1.69 ; p<0.05$ ).

CONCLUSIONS: African-American race and Hispanic ethnicity are significantly associated with worse asthma outcomes within child-caregiver dyads compared to Whites. Limited health literacy was identified as a partial mediator of these disparities. This study is one of the first investigations to our knowledge to demonstrate how health literacy may in part explain racial and ethnic disparities in prospective health outcomes over time. as a contributor to such disparities in longitudinal childhood asthma outcomes; this strengthens observations made in prior cross-sectional studies. Efforts to improve health literacy, through evidence based patient education and counseling interventions, may be important to reducing racial and ethnic asthma disparities.

HEALTH REFORM AND MEDICARE: A GROWING MARKET FOR CHERRIES OR LEMONS? Sachin J. Shah ${ }^{1,}{ }^{2}$; Mary Price ${ }^{1}$; John Hsu ${ }^{1,}$. ${ }^{1}$ Massachusetts General Hospital, Boston, MA; ${ }^{2}$ Harvard Medical School, Boston, MA. (Control ID \#2469202)

BACKGROUND: In recent years, many beneficiaries have switched from traditional feefor-service Medicare (TM) to Medicare Advantage (MA), generally accepting narrower physician choice for enhanced benefits. The Medicare Improvements for Patients and Providers Act (MIPPA) and Affordable Care Act (ACA) added regulatory requirement and mandated sizable MA reimbursement cuts starting in 2011, that should decrease the generosity of MA benefits. Despite this, in 2015, MA enrollment increased to $31 \%$ of all beneficiaries - a historic high. Rising enrollment in the face of these changes is seemingly a paradox. We examined the switching patterns of beneficiaries between TM and MA, to assess whether MA has cherry picked healthier beneficiaries.

METHODS: We examined Medicare enrollment data from 1999 to 2012 in a $20 \%$ random TM sample of all beneficiaries. Beneficiaries also enrolled in Medicaid were excluded. We divided the time into three categories: T1 (2000-05), during which payments were adjusted mostly for age and gender, and beneficiaries could switch between TM and MA monthly; T2 (2006-10), during which payments were adjusted for beneficiary comorbidities (i.e., risk-adjusted based on diagnoses), and the phasedin restrictions on switching; and T3 (2011-12), during which there were no further additional to risk adjustment or enrollment processes, but MA reimbursement decreased. We examined switches to MA and from MA separately. Medicare projects expected cost of caring for beneficiaries using the hierarchical condition category (HCC) model where a score of 1.0 represents average cost. Among beneficiaries switching from TM to MA, we report HCC scores from the population with 1 year of TM enrollment in the prior year. Among beneficiaries switching from MA to TM, we report HCC scores from the population with 1 year of TM enrollment in the year after the switch. We performed pairwise comparisons for each group in each time period using chi-squared tests.

RESULTS: On average, 85,596 beneficiaries per year switched from TM to MA in T1, 266,409 beneficiaries per year switched in T2, and 232,387 beneficiaries per year switched in T3. In contrast, 96,793 beneficiaries per year switched from MA to TM in $\mathrm{T} 1,63,114$ beneficiaries per year switched in $\mathrm{T} 2$, and 78,280 beneficiaries per year switched in T3. Among those switching from TM to MA, the mean HCC scores was higher in $\mathrm{T} 2$ than in $\mathrm{T} 1(0.974$ vs $0.873, p<0.001)$, but the mean scores in T3 and T2 were not significantly different $(0.977$ vs $0.974, p>0.05)$. Among those switching from MA to $\mathrm{TM}$, the mean HCC scores was higher in T2 than in T1 (1.078 vs 0.960, $p<0.001)$, and higher in T3 than in T2 $(1.143$ vs $1.078, p<0.001)$.

CONCLUSIONS: There was substantial net movement of beneficiaries from TM to MA over time; there was no major reductions in this movement in the years after the MA reimbursement cuts. Importantly, despite cuts in reimbursement and increased program costs, beneficiaries switching in recent years from TM to MA were not substantially healthier compared to those switching the early, pre-risk adjustment period. Said differently, recent years saw less cherry picking by the MA program. Nevertheless, beneficiaries moving from MA to TM continue to be sicker than average, i.e., lemon dropping, particularly in recent years. This suggests the possibility that some of the sickest beneficiaries or those experiencing recent changes in their health might be experiencing 
discontinuities in their care (changing physicians and formularies) as they move from MA to TM.

\begin{tabular}{|c|c|c|c|c|c|c|c|c|}
\hline & \multicolumn{3}{|c|}{ From MA to TM } & \multirow[b]{2}{*}{$\begin{array}{c}P \\
\text { value }\end{array}$} & \multicolumn{3}{|c|}{ From TM to MA } & \multirow[b]{2}{*}{$\begin{array}{c}P \\
\text { value }\end{array}$} \\
\hline & $\begin{array}{c}2000-05 \\
\text { (T1) }\end{array}$ & $\begin{array}{c}2006 \cdot 10 \\
(T 2)\end{array}$ & $\begin{array}{c}2011 \cdot 12 \\
(T 3)\end{array}$ & & $\begin{array}{c}2000-05 \\
\text { (T1) }\end{array}$ & $\begin{array}{c}2006 \cdot 10 \\
(\mathrm{~T} 2)\end{array}$ & $\begin{array}{c}2011 \cdot 12 \\
(T 3)\end{array}$ & \\
\hline Beneficiaries per year & 96,793 & 0,114 & 78,280 & & 85,596 & 266,409 & 232,387 & \\
\hline Age & 72.7 & 72.2 & 71.6 & $<0001$ & 68.8 & 69.7 & 68.4 & $<0001$ \\
\hline Female & $55.0 \%$ & $55.1 \%$ & $53.3 \%$ & $<0001$ & $53.5 \%$ & $53.4 \%$ & $51.4 \%$ & $<0001$ \\
\hline \multicolumn{9}{|l|}{ Race } \\
\hline White & $86.05 \%$ & $81.77 \%$ & $83.52 \%$ & $<0001$ & $79.85 \%$ & $83.78 \%$ & $82.1 \%$ & $<0001$ \\
\hline Blad & $9.58 \%$ & $13.17 \%$ & $10.85 \%$ & & $12.02 \%$ & $10.68 \%$ & $10.76 \%$ & \\
\hline Other & $4.37 \%$ & $505 \%$ & $5.63 \%$ & & $8.13 \%$ & $5.54 \%$ & $7.14 \%$ & \\
\hline \multicolumn{9}{|c|}{ Original Reason for Enrollment } \\
\hline Aged & $85.61 \%$ & $79.03 \%$ & $76.91 \%$ & $<0001$ & $79.49 \%$ & $78.55 \%$ & $77.00 \%$ & $<0001$ \\
\hline Dsabled & $14.26 \%$ & $20.7 \%$ & $22.85 \%$ & & $20.26 \%$ & $20.83 \%$ & $22.67 \%$ & \\
\hline ESRD & $0.04 \%$ & $0.08 \%$ & $0.06 \%$ & & $0.15 \%$ & $0.1 \%$ & $0.11 \%$ & \\
\hline Boh dsabled and ESRD & $0.07 \%$ & $0.19 \%$ & $0.18 \%$ & & $0.1 \%$ & $0.12 \%$ & $0.15 \%$ & \\
\hline Mean HCC score & $0.960^{\circ}$ & 1.0785 & 1.1433 & $<0001$ & $0.873^{\circ}$ & $0.974^{\circ}$ & 0.977 & $<0001$ \\
\hline
\end{tabular}

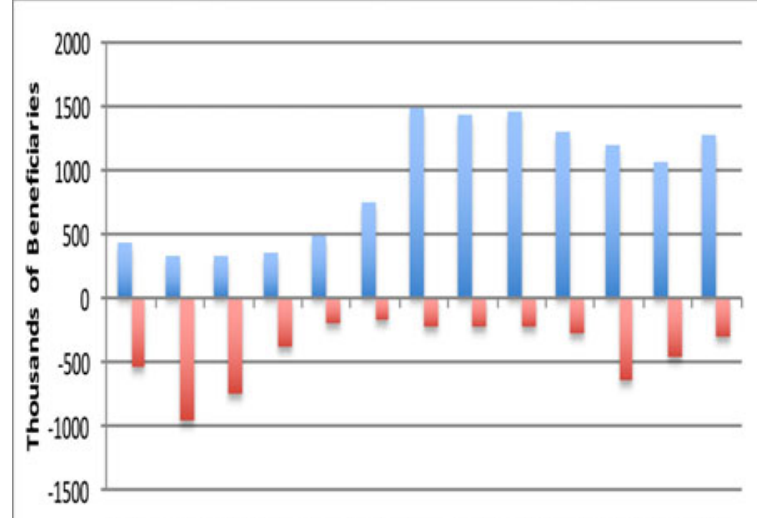

2000200120022003200420052006200720082009201020112012

Year

«Move from TM to MA "Movefrom MA to TM

HEALTH REFORM, COVERAGE CHANGES, AND INDIAN HEALTH SERVICE USE AMONG NATIVE AMERICANS Molly Frean ${ }^{1}$; Shelbie Shelder'; Meredith Rosenthal ${ }^{1}$; Thomas D. Sequist ${ }^{2}$; Benjamin D. Sommers ${ }^{1,3}$. ${ }^{1}$ Harvard School of Public Health, Brookline, MA; ${ }^{2}$ Partners Healthcare System, Boston, MA; ${ }^{3}$ Brigham \& Women's Hospital, Boston, MA. (Control ID \#2467181)

BACKGROUND: Native Americans experience substantial health-care related disparities. The Affordable Care Act (ACA) sought to increase health coverage for Native Americans through the Medicaid expansion, creation of health insurance Marketplaces with tax credits for coverage, and permanent reauthorization of the Indian Health Care Improvement Act, which funds the Indian Health Service (IHS). We evaluated changes in insurance and IHS use among Native Americans following the ACA's first year of coverage expansion.

METHODS: Using 2012-2014 data from the U.S. Census Bureau's American Community Survey, we compared pre-ACA (2012-2013) and post-ACA (2014) changes in outcomes among Native Americans in all states. Then we compared changes between states that expanded Medicaid and those not expanding Medicaid, using a differences-indifferences approach. The sample consisted of non-elderly (aged 0-64) individuals who reported "American Indian or Alaska Native" race. We specified three different levels of geography for our analysis, from broadest to narrowest: 1) national (all 50 states + Washington DC; $n=168,654) ; 2$ ) states with IHS facilities and Native American prevalence of at least the national average (referred to hereafter as "IHS states"; $n=108,163$ ); and 3) Native American reservations containing populated land in both a Medicaid expansion and non-expansion state $(n=24,575)$. Outcomes included four mutuallyexclusive categories of primary health insurance coverage-Medicaid, Private, Other, and uninsured. Each insurance outcome was evaluated with and without concurrent use of IHS (which the federal government does not consider health insurance), as well as overall rates of IHS use. Models adjusted for socio-demographic characteristics and used Census survey weights and robust standard errors clustered by state.

RESULTS: Native Americans living on or near reservations were poorer, younger, and less likely to be employed than the national population. Nationally, the Native American uninsured rate dropped from $24.8 \%$ in $2012-2013$ to $20.6 \%$ in $2014(p<0.001)$. The differences-in-differences estimates for the Medicaid expansion were declines in the uninsured rate of -2.9 percentage-points nationally $(p<0.001),-3.8$ in IHS states $(p=$ $0.002)$ and -8.6 for Native Americans living on or near reservations $(p=0.02)$. Medicaid coverage increased at the national, IHS state, and reservation levels by $4.2,5.4$, and 10.4 percentage points, respectively (all $p<0.001$ ). Nationally, Medicaid gains were approximately equally divided between IHS users and non-IHS users, while on reservations, most coverage gains occurred among IHS users. We did not observe significant changes in private coverage or in overall rates of IHS use.

CONCLUSIONS: The ACA was associated with significant increases in coverage for Native Americans in 2014, primarily in Medicaid expansion states, and gains were largest among those living on or near reservations. There was no net change in IHS use, indicating that the ACA's coverage expansion is supplementing rather than replacing IHS's role in this population. This finding suggests that the coverage expansion among IHS users will bring much-needed additional revenue into IHS and tribal health services, which have traditionally suffered from budgetary shortfalls. Meanwhile, states still considering the Medicaid expansion should consider the potential implications for access to care and health disparities among Native Americans, both within and outside of the Indian Health Service.

HEALTHCARE FRAGMENTATION AND THE FREQUENCY OF RADIOLOGY AND OTHER DIAGNOSTIC TESTING Lisa M. Kern; Joanna K. Seirup; Lawrence P. Casalino; Monika M. Safford. Weill Cornell Medicine, New York, NY. (Control ID \#2467433)

BACKGROUND: Healthcare fragmentation is an emerging concept that can refer to a pattern of ambulatory care that is spread across multiple providers and that lacks a dominant provider. Having fragmented ambulatory care has been associated with high rates of emergency department visits and hospitalizations, but effects on other types of utilization are not clear. We sought to determine whether more fragmented care is associated with more radiology and other diagnostic testing, compared to less fragmented care.

METHODS: We conducted a cross-sectional study in a 7-county region of New York State, using administrative claims for 2010 that had been aggregated across 5 commercial payers. We included 126,801 adult patients who were insured through one of the participating payers, had been attributed to a primary care physician in the region, and had $\geq 4$ ambulatory visits that year. For each patient, we calculated fragmentation of care using the Bice-Boxerman Index, which captures both "dispersion" (the spread of a patient's care across multiple providers) and "density" (the relative share of a visits by each provider). We then transformed this Index by reversing its scale (so that higher scores reflected more 
fragmentation) and by dividing the scores into 7 categories (to address the non-normal distribution). We used negative binomial regression to determine the association between fragmentation category and rates of testing, stratified by number of chronic conditions and adjusting for patient age, patient gender, and number of visits

RESULTS: Patients with the most fragmented care had approximately twice the number of radiology and other diagnostic tests than patients with the least fragmented care, across all 4 subgroups of patients stratified by number of chronic conditions. Because patients with more chronic conditions had more tests than those with fewer chronic conditions, a doubling of the number of tests had the largest absolute effect for those with the most chronic conditions. For example, among patients with no chronic conditions, those with the least fragmented care had 71 tests per 100 patients, while those with the most fragmented care had 139 tests per 100 patients ( +68 tests per 100 patients, $+95 \%$, adjusted $p<0.0001$ ). Among patients with 5 or more chronic conditions, those with the least fragmented care had 258 tests per 100 patients, while those with the most fragmented care had 542 tests per 100 patients ( +284 tests per 100 patients, or $+110 \%$, adjusted $p<0.0001$ ). Similar results were observed among those with 1 or 2 chronic conditions and among those with 3 or 4 chronic conditions.

CONCLUSIONS: More fragmented care was independently associated with higher rates of radiology and other diagnostic testing compared with less fragmented care. This has important implications for population management, as providers become increasingly responsible for all of their patients' care, not just the care that they themselves provide.

\section{HEALTHCARE UTILIZATION AND CLINICAL WORKLOAD ASSOCIATED} WITH CHRONIC NONCANCER PAIN Joanne Bernstein ${ }^{2}$; Erica M. Wozniak ${ }^{1}$; Sara Koller $^{4}$; Audrey Ye ${ }^{4}$; Cynthia Kay ${ }^{3,}{ }^{4} .{ }^{1}$ Medical College of Wisconsin, Milwaukee, WI; ${ }^{2}$ Medical College of Wisconsin, Whitefish Bay, WI; ${ }^{3}$ Zablocki VA Medical Center/ Medical College of Wisconsin, Milwaukee, WI; ${ }^{4} \mathrm{MCW}$, Milwaukee, WI. (Control ID \#2456941)

BACKGROUND: Past studies have shown that chronic pain is associated with increased health care utilization. It is unknown whether chronic pain is also associated with increased workload, such as answering service calls, patient calls, and secure messages. While health care utilization often is a reimbursable cost, workload is not usually compensated. Our study's objectives are to examine healthcare utilization and clinic workload associated with chronic noncancer pain, pain contracts, and narcotic classification.

METHODS: This was a retrospective chart review. Subjects were internal medicine clinic patients at an academic medical center who were either on a pain contract or were prescribed at least 3 months of narcotics between April 1, 2014 and April 1, 2015. Patients within 6 months of surgery, with active cancer, and those not seen at least once in the established time frame were excluded. The study's main subjects were matched to patients not on a pain contract or chronic narcotics by age and gender and served as controls. Data collected included patient demographics, medical diagnoses, pain contract status; healthcare utilization and workload (ER visits, hospitalizations, clinic visits, answering service calls, telephone/secure messages); and current narcotic regimen for pain patients. Descriptive statistics and univariate analyses (chi-square or Fisher's exact test, where appropriate) were used to compare instances of healthcare utilization as counts. Comparisons were made between chronic pain patients and non-pain controls, as well as contract and non-contract pain patients. Logistic regression models with ER visits and hospitalizations (any/none) as binary outcomes were used to examine the effect of chronic pain status on utilization, controlling for other relevant covariates, such as age, gender, and the presence of psychiatric disorders.

RESULTS: A total of 836 patients were on chronic narcotics for noncancer pain, with 342 (41\%) on a pain contract with the clinic. Patients with chronic noncancer pain were likely to have more ER and clinic visits, hospitalizations, answering service calls, and telephone and secure messages compared to patients without chronic pain $(p<0.001)$. When examined by pain contract status, there were no differences in the number of ER and clinic visits, hospitalizations, and answering service calls between patients with and without a contract. The pain contract group, however, did have more telephone calls and secure messages than the no contract group $(p=0.007)$. Regardless of pain contract status, patients on schedule II narcotics were more likely to have 6 or more clinic visits in a year $(p=0.003), 1$ or more answering service calls $(0.017)$, and 4 or more telephone or secure messages $(p=0.001)$ compared to patients on schedule III or IV narcotics. Model-based results show that, controlling for relevant demographic and clinical characteristics, the odds of a chronic pain patient having one or more ER visits are 2.7 times the odds of a non-pain control patient (95\% CI 2.13.5). Similarly, the odds of a chronic pain patient being hospitalized one or more times are 5.4 times (95\% CI 4.0-7.5) the odds of a non-pain patient.

CONCLUSIONS: Patients with chronic pain utilized significantly more health care resources and produced more workload compared to patients without chronic pain. While one might expect a pain contract to lessen provider or clinic workload, our findings actually suggest the opposite. The explanation for this remains unclear. The increased phone calls and secure messages may reflect increased patient complexity or greater pain severity of those on pain contracts. Alternatively, it may indicate close follow up or open physician-patient communication. Schedule II narcotics are considered to have higher abuse potential than schedule III-IV, which may be one reason the pain contract group had more clinic visits and telephone or secure message contact. After controlling for demographics and comorbidities, chronic pain patients were significantly more likely to be hospitalized or have an emergency room visit as compared to non-chronic pain controls. This finding may imply that chronic pain has important effects on other comorbidities, the impact of which we may not fully realize. Further research is needed to examine whether more effective pain management can reduce healthcare utilization and workload associated with chronic noncancer pain.

\section{HEPATITIS B PREVALENCE IN THE MILWAUKEE HMONG COMMUNITY}

Timothy Wang; Heather Chou; Kia Saeian. Medical College of Wisconsin, Milwaukee, WI. (Control ID \#2466765)

BACKGROUND: Although the United States is considered a low endemicity region for hepatitis B and has had low hepatitis B prevalence rates $(0.3 \%)$ since the implementation of routine vaccination for newborns in 1991, the Hmong population in the U.S. has rates of up to $20 \%$ according to prior studies in California, making hepatitis B a prominent ethnic health disparity affecting the Hmong-American community. Based on the 2010 Census, the Hmong population in the Milwaukee-Waukesha-West Allis area numbers 11,904, making it the fourthlargest Hmong population of all major metropolitan areas in the nation. However, prior to our efforts, there had never been an attempt to determine the prevalence of hepatitis B in the Milwaukee Hmong community. Our aims were two-fold: to conduct Milwaukee's first-ever series of free hepatitis B screening and education events in the Hmong community, and to determine the prevalence of hepatitis B in this high-risk population.

METHODS: From 2013 to 2015, we organized a total of five hepatitis B screenings in the Milwaukee Hmong community. We utilized various locations such as local Hmong markets, churches, and festivals in order to maximize our number of screening participants. To advertise the screenings, we posted fliers at local restaurants/markets and aired hepatitis B segments on the local Hmong radio station. At the screenings, participants filled out a personal information and demographic form, answered a survey about basic hepatitis B knowledge, received a 10-15 min educational session from a health care provider or trained coordinator, and had their blood drawn. All blood samples were centrifuged on-site and sent to LabCorp in Chicago to be tested for hepatitis B surface antigen (HBsAg) and hepatitis B surface antibody (HBsAb).

RESULTS: In total, 176 participants were screened over five events. Eighteen participants (10.2\%) tested positive for hepatitis B infection (HBsAg +, HBsAb -). 51 (29\%) 
were non-immune and designated "susceptible" to infection (HBsAg -, HBsAb -). 102 $(58 \%)$ were immune (HBsAg -, HBsAb + ) and $5(2.8 \%)$ were designated as "gray zone" (HBsAg -, low HBsAb titers).

CONCLUSIONS: Based on our screening results, the prevalence of hepatitis B in our screening population was $10.2 \%$. Although lower than the $20 \%$ prevalence rate documented in prior studies, there is still a disproportionately high prevalence of hepatitis B in the Milwaukee Hmong community compared to the general U.S. population. Equally important, $29 \%$ of our screening population was still susceptible to being infected with hepatitis B. Given these data, it is clear that hepatitis B is an important health disparity worth addressing in the Milwaukee Hmong community, and efforts to promote hepatitis B awareness, education, and when indicated immunization are warranted.

\section{HEPATITIS C LINKAGE TO CARE AT AN URBAN INTERNAL MEDICINE}

CLINIC Jay Yarlagadda; Kassem Bourgi; Zorawar Singh; Amal Hejab; Mikhail Shteynvil; Gina Maki; Kimberly Baker-Genaw. Henry Ford Hospital / Wayne State University, Detroit, MI. (Control ID \#2457407)

BACKGROUND: Despite the recent advances in Hepatitis C (HCV) treatment and the availability of direct acting antiviral, challenges remain in linking HCV patients to care. We assessed the access to care and treatment rates for patients who screened positive for HCV. METHODS: We identified patients who were screened positive for HCV screening in 21 Internal Medicine clinics at Henry Ford Health System between July 2014 and June 2015. We assessed the $\mathrm{HCV}$ care cascade within this cohort. We further evaluated the association of patient's socio-demographic factors and HCV care access.

RESULTS: Of 40,561 patients eligible for HCV screening, 8657 (21.3\%) patients were screened during the study period and $117(1.4 \%)$ patients tested positive. Eleven patients had no evidence of active viremia. 83 (78\%) patients were referred to a Hepatitis C specialist and 53 (50\%) were successfully evaluated. On follow-up, $30(27 \%)$ of $\mathrm{HCV}$ positive patients received treatment with Direct Acting Antivirals. After excluding 6 patients with active drug use, currently ineligible for therapy as per insurance constraints, we compared the characteristics of the patients who received treatment (30) to those who were not treated (70). In adjusted modules, we identified that female patients were more likely to be treated $(p<0.05)$. However Medicaid patients were less likely to receive treatment when compared to patients with Medicare or private insurance $(p<0.05)$. There was a trend towards a decline in treatment rates among patients with lower income. Electronic health engagement, through subscribing to the patient's portal of the electronic medical records, was a significant factor that improved the odds of treatment $(p<0.05)$.

CONCLUSIONS: Patients who were screened positive for HCV had suboptimal linkage to care. Socioeconomic factors were significant determinants of care access with a major disadvantage for Medicaid patients. These findings highlights the need for a more equitable care delivery system with a particular focus on vulnerable underserved patient groups Univariate Analysis Comparing HCV Positive Patients who Received Treatment Compared to Untreated Patients

\begin{tabular}{llll}
\hline \hline Variable & Treated Patients & Untreated Patients & $p$-Value \\
Number of Subjects & 30 & 70 & \\
Age & $62.2 \pm 4.7$ & $61.1 \pm 4.7$ & 0.28 \\
Female Gender & $16(53.3 \%)$ & $22(31.4 \%)$ & $0.04^{*}$ \\
African American Race & $21(70 \%)$ & $54(78.3 \%)$ & 0.38 \\
Electronic Health Engagement & $14(46.7 \%)$ & $17(24.3 \%)$ & $0.03^{*}$ \\
Residency Clinic Visit & $16(53.3 \%)$ & $38(54.3 \%)$ & 0.93 \\
Income $\leq \$ 50,000$ & $24(80 \%)$ & $65(92.9 \%)$ & 0.06 \\
Medicaid Insurance & $2(6.7 \%)$ & $18(25.7 \%)$ & $0.03^{*}$ \\
Total Number of Visits & $2.90 \pm 1.77$ & $2.84 \pm 2.51$ & 0.41 \\
Charlson Comorbidity Index & $1.77 \pm 1.01$ & $1.46 \pm 1.71$ & $0.04^{*}$ \\
Fibrosis (FIB-4) Score & $2.48 \pm 2.15$ & $2.37 \pm 1.78$ & 0.94 \\
\hline
\end{tabular}

HEPATITIS C SCREENING: THE DOWNSTREAM DISSEMINATION OF EVOLVING GUIDELINES IN A RESIDENT CONTINUITY CLINIC Kamraan Madhani; Ali Aamar; David Chia. Waterbury Hospital, New Haven, CT. (Control ID \#2432406)

BACKGROUND: In 2012 the CDC published guidelines supporting one-time screening for hepatitis C (HCV) in all persons born between 1945 and 1965. It is estimated that $75 \%$ of adults infected with $\mathrm{HCV}$ fall within this cohort. Furthermore, it is projected that this preventative health intervention would lead to the diagnosis of 800,000 unknown cases and the prevention of 120,000 deaths. The primary objective is to measure adherence to $\mathrm{HCV}$ screening in a continuity practice staffed by internal medicine residents and attending physicians. The secondary objectives include: [1] whether insurance or provider status effects adherence to HCV screening and [2] adherence to HIV screening.

METHODS: We performed a retrospective chart review of a random sample of patients born between 1945 and 1965. In order to meet inclusion criteria, the patients must have had an HCV status that was unknown and must have been seen by a primary care provider $\geq 2$ times between January 1 and December 31, 2013. Data extracted included whether HCV and HIV testing was ordered, whether the testing was performed primarily for screening purposes, demographic information, insurance status, number of clinic visits, and whether the primary provider was a resident or attending physician. Data was analyzed using chi-squared testing.

RESULTS: Out of 294 patients reviewed, 200 met inclusion criteria. Forty-four percent of whom were insured by Medicaid, $38.5 \%$ by Medicare, $16 \%$ by private insurance and $1.5 \%$ were self-pay. One hundred twenty-one $(60.5 \%)$ patients had resident physicians and 79 (39.5\%) patients had attending physicians as their primary providers. Only $17(8.5 \%)$ patients were offered screening for HCV of which $13(76.5 \%)$ patients completed the test and none tested positive. In comparison, the rate of HIV screening rates was $15 \%$. There was no statistically significant difference in HCV screening rates when comparing insurance status $(p=0.96)$, provider status $(p=0.72)$ or number of clinic visits $(p=0.15)$.

CONCLUSIONS: Adherence to HCV screening guidelines in a resident continuity practice is low. Dissemination of evolving guidelines requires practice-based improvement and vigilance of the ever-changing landscape of evidence-based medicine for both resident and attending physicians alike.

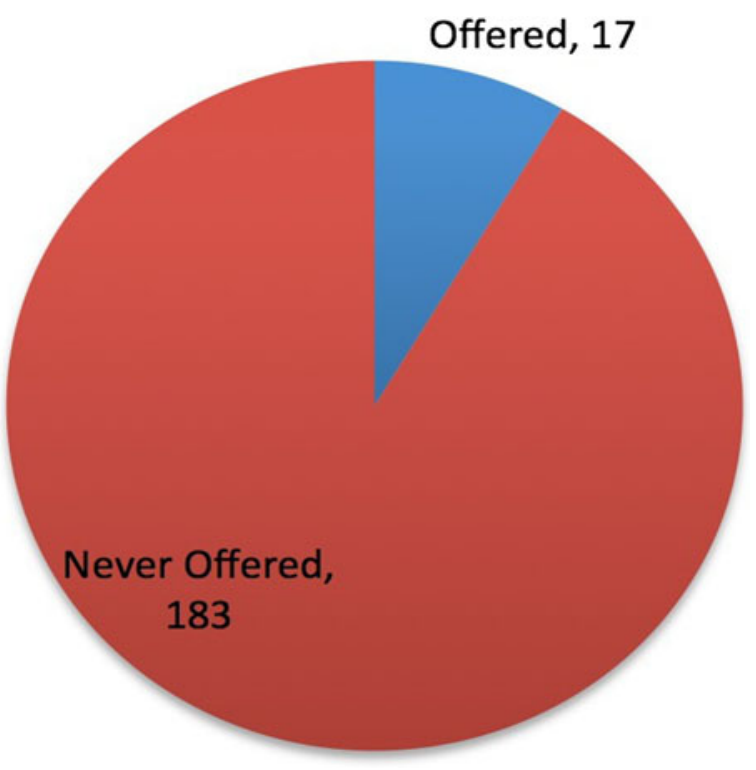


HIGH HCV CURE RATES FOR DRUG USERS TREATED AT AN URBAN PRIMARY CARE CLINIC Julia Fleming ${ }^{1}$; Meredith Steinman ${ }^{1}$; Chinazo Cunningham $^{3}$; Alain H. Litwin ${ }^{2}$; Brianna L. Norton ${ }^{3} .{ }^{1}$ Montefiore Medical Center, Bronx, NY; ${ }^{2}$ Albert Einstein College of Medicine, Bronx, NY; ${ }^{3}$ Albert Einstein College of Medicine \& Montefiore Medical Center, Bronx, NY. (Control ID \#2469633)

BACKGROUND: In the United States, approximately $60 \%$ of all people infected with $\mathrm{HCV}$ are people who inject drugs. $\mathrm{HCV}$ is the leading cause of end stage liver disease and hepatocellular carcinoma, and accounts for over 19,000 deaths annually. This disease burden is predicted to increase 3 -fold over the next decade without imminent action. In clinical trials, new direct-acting antivirals have shown high cure rates for the majority of HCV-positive patients; however, it is unknown whether high cure rates will be obtained in clinical practice, particularly among persons who use drugs (PWUDs). In addition, HCV treatment is now being offered more widely in primary care settings so as to remove barriers to care for marginalized populations. We investigated the effectiveness of onsite treatment with care coordination for patients who access primary care at a federally qualified health center $(\mathrm{FQHC})$. We hypothesized that $\mathrm{HCV}$ cure rates would be similar to registration trials and that there would be no difference in cure rates between PWUD versus non-PWUD.

METHODS: Onsite HCV treatment occurred once weekly by an HCV specialist at an FQHC in the Bronx, NY. An HCV care coordinator was responsible for patient scheduling, reminder calls, health education, and obtaining prior authorizations. We identified 114 patients with an HCV evaluation from January 2014-February 2015, and reviewed medical records for patients who initiated HCV treatment. Patients were categorized as PWUD if they were receiving opioid agonist therapy (OAT) or noted to have active drug use in the medical chart or urine toxicology. Chi-square testing was performed to determine differences in HCV cure between PWUD and non-PWUD.

RESULTS: One hundred fourteen patients were evaluated for $\mathrm{HCV}$, and 67 (59\%) initiated HCV treatment during the study time frame. Treatment patients were mostly male (64 \%), Latino or African-American ( $82 \%$ ), with a median age of 60 . Twenty-one percent were HCV treatment experienced, $22 \%$ were HIV/HCV co-infected, and $24 \%$ had cirrhosis. Over half of the patients were PWUD (52 \%). Twenty-eight patients were on OAT (15 on methadone, 13 on buprenorphine), and 24 patients were actively using drugs during $\mathrm{HCV}$ care. The majority of the patients were genotype $1(93 \%)$ and all were treated with sofosbuvir-based regimens. The overall HCV cure rate was $96 \%(64 / 67)$, and there were no differences in cure rates for PWUD ( $97 \%$, 34/35) versus non-PWUD ( $94 \%, 30 / 32, p=0.5)$.

CONCLUSIONS: Primary care-based HCV treatment at an urban FQHC resulted in high cure rates, and cure rates for PWUD were indistinguishable from non-PWUD patients. Suboptimal HCV treatment of PWUD contributes to growing HCV-related morbidity and mortality, and maintains a continued reservoir for HCV infection. Many insurers, including Medicaid, restrict access to the new direct-acting antivirals for PWUD by requiring periods of drug abstinence. These data do not support these restrictions. On-site treatment with care coordination may help to mitigate barriers to specialty care and improve $\mathrm{HCV}$ cure rates for PWUD. Similar treatment models should be replicated and tested throughout the $1200 \mathrm{FQHCs}$ in the United States, settings that are known to serve high numbers of PWUD.

HIGH RATES AND LOW DOCUMENTATION OF HOSPITAL ACQUIRED ACUTE KIDNEY INJURY IN AN URBAN ACADEMIC MEDICAL CENTER Tanya Rudakevych; David Meltzer; Marshall Chin; Milda R. Saunders. University of Chicago, Chicago, IL. (Control ID \#2469931)

BACKGROUND: Acute kidney injury is a common condition that affects between 5 and $20 \%$ of hospitalized patients. Prior work has demonstrated that hospital-acquired acute kidney injury (HA-AKI) is associated with increased morbidity, mortality and health care costs. In addition, HA-AKI is often under-recognized and under-documented by treating clinicians which may lead to poor continuity of care. We sought to determine the incidence of HA-AKI as well as AKI documentation rates among general medicine inpatients in a single, urban academic medical center.

METHODS: Our initial cohort included 1345 general medical admissions between January and June 2012. We excluded all patients with a history of transplant (996.81, V42.0, $n=62)$ or ESRD $(585.6, n=128)$. Our final sample included 1164 individual patients. From medical records, we obtained demographic information, discharge ICD-9 codes, serum creatinine (SCr) and eGFR values. Estimated glomerular filtration rate (eGFR) was calculated by the modified MDRD equation estimated (GFR $(\mathrm{mL} / \mathrm{min} /$ $\left.1.73 \mathrm{~m}^{2}\right)=175 \times\left(\mathrm{S}_{\text {creatinine }}\right)^{-1.154} \times(\text { Age })^{-0.203} \times(0.742$ if female $) \times(1.212$ if African American) recommended by the National Kidney Disease Program (NKDEP). First, we examined the prevalence of kidney dysfunction, eGFR $<60$ on at least one occasion during hospitalization. In addition, we calculated at the incidence and severity of acute kidney injury based on the Kidney Disease Improving Global Outcomes (KDIGO) and creatinine kinetics-based definition of AKI (CK-AKI) definitions. AKI by KDIGO criteria was defined as an increase in SCr by $0.3 \mathrm{mg} / \mathrm{dl}$ within $48 \mathrm{~h}$ or a $50 \%$ increase in $\mathrm{SCr}$ from the baseline within 7 days. The CK-AKI is defined by an increase in $\mathrm{SCr} \geq 0.3 \mathrm{mg} / \mathrm{dl}$ within $24 \mathrm{~h}$ or $\geq 0.5 \mathrm{mg} / \mathrm{dl}$ within $48 \mathrm{~h}$. The baseline was defined dynamically as the average of the SCr values from the previous 7 days. We used all available $\mathrm{SCr}$ values with corresponding dates to adhere specifically to the two AKI definitions and staging systems. Finally, we investigated the AKI documentation rates using ICD-9 codes. We used Fisher's Exact Test to test the bivariate associations between characteristic variables and AKI by either CK-AKI or KDIGO. Logistic regression was used to analyze the influence of demographic and clinical covariates on the likelihood of a patient developing HA-AKI. Stata 14.0 was used for all analyses.

RESULTS: In our sample of urban adult hospitalized general medicine patients, the average age was 56.2 and consisted of $67 \%$ African-Americans and $56 \%$ females. The baseline prevalence of kidney dysfunction was $47 \%$. The incidence of hospital-acquired AKI by KDIGO criteria was (243/1164) $21 \%$ with $83.5 \%$ Stage I AKI, $6.6 \%$ Stage II AKI, and $9.9 \%$ Stage III AKI. The incidence of AKI using the CA-AKI definition was $17 \%$ with $54 \%$ with Stage I AKI, $25.5 \%$ Stage II AKI, and $20.5 \%$ Stage III AKI. All individuals with AKI by CA-AKI definitions also met KDIGO criteria. In multivariable analysis, factors associated with developing HA-AKI include prior CKD (OR 3.8), length of stay 4 days or longer (OR 4.8), diabetes (OR 1.6), hypertension (OR 1.6), age greater 64 years (OR 1.9 compared to age under 40 years), hospitalization within the last 12 months (OR 1.5), all $p<0.05$. AKI was coded in discharge ICD-9 codes in only $38 \%$ of cases, the rates increased to $71 \%$ for those with Stage III AKI.

CONCLUSIONS: Among general medicine inpatients in an urban academic medical center, the incidence of hospital-acquired AKI is high $(>20 \%)$. Rates of discharge documentation of HA-AKI are low, even among the highest stage AKI. Recognition and documentation of HAAKI is important for appropriate referral and monitoring in the outpatient setting.

HIGH SERUM LEVELS OF STRESS BIOMARKERS ARE ASSOCIATED WITH 30-DAY UNPLANNED READMISSION AND DEATH AFTER ACUTE CARE HOSPITALIZATION Carole E. Aubert ${ }^{1}$; Antoine Folly ${ }^{2}$; Marco Mancinetti ${ }^{2}$; Daniel Hayoz ${ }^{2}$; Jacques Donze ${ }^{1}$. Inselspital, Bern University Hospital, Bern, Switzerland; ${ }^{2}$ Fribourg Cantonal Hospital, Fribourg, Switzerland. (Control ID \#2456882)

BACKGROUND: The theory of a physiological stress that patients experience in the hospital has been advocated to explain the transient period of vulnerability following hospital discharge, and often named "post-hospital syndrome". After discharge, physiological systems are still impaired, and reserves depleted from the acute illness and the global stressful situation, making patients more susceptible to new health threats. Stress 
biomarkers, such as copeptin and cortisol, may help to quantify this stress. Some studies showed an association between stress biomarkers and adverse outcome in a wide range of situations, such as stroke, myocardial infarction, heart failure, pneumonia, chronic obstructive pulmonary disease exacerbation, pneumonia or septic shock. Some biomarkers have also been associated with length of hospitalization, in-hospital death and all-cause mortality. We hypothesized that high level of stress biomarkers at discharge of an acute care hospitalization may be associated with readmission and death, and therefore help identifying those high-risk patients.

METHODS: In a prospective cohort study, we included all consecutive patients aged $\geq 50$ years and admitted to the division of general internal medicine at a large community hospital in Switzerland (Fribourg Hospital), between April and September 2013. Exclusion criteria were death before discharge, refusal to give informed consent, and transfer to another acute care hospital, to a rehabilitation center, or to a palliative care center. The primary outcome was the first unplanned readmission or death within 30 days after discharge of index admission. The secondary outcome was the same as the primary outcome but within 90 days after discharge. We measured the area under the receiver operating characteristic (ROC) curves to assess the discriminatory power of 2 important stress biomarkers, copeptin and cortisol levels at discharge, to predict unplanned readmission and death. We selected the cutoff levels by taking the best compromise between sensitivity and specificity according to the ROC curves. To assess the association of these biomarkers with the primary and secondary outcomes, we used multivariate logistic regression analyses adjusted for age, Charlson comorbidity index, number of hospitalizations in the previous 6 months and diagnosis of infection during hospitalization. Patients taking systemic corticosteroids were excluded from the cortisol analysis.

RESULTS: Among the 346 included patients, 40 (11.6\%) had an unplanned readmission or died within 30 days after discharge. Mean age was 73.4 years (SD 11.5), and $49.7 \%$ were men. Median length of hospitalization was 7 days (IQR 4-12). Areas under the curve for predicting the primary outcome were 0.60 for copeptin and 0.59 for cortisol. Cutoff levels with the best compromise between sensitivity and specificity were $9 \mathrm{pmol} / \mathrm{l}$ for copeptin (66.7 and $59.7 \%$, respectively) and $590 \mathrm{nmol} / 1$ for cortisol (54.6 and $76.4 \%$, respectively). Copeptin (odds ratio [OR] 2.23, $95 \%$ confidence interval [CI] $1.04-4.90$ ) and cortisol (OR 2.76, 95\% CI 1.07-7.10) were significantly associated with 30-day unplanned readmission or death in multivariate analysis. At 90 days after discharge, copeptin only was still significantly associated with unplanned readmission or death (OR 1.96, 95 \% CI 1.09-3.55).

CONCLUSIONS: High levels of copeptin or cortisol at discharge were associated with a 2 to 3 times increased risk of unplanned readmission or death within 30 days after discharge in a general medical population. These findings support the hypothesis that a physiological stress seen in an acute care hospitalization may be associated with a higher risk of early readmission or death. The use of these biomarkers may provide an interesting measure to help identifying patients at high risk for readmission.

Univariate and multivariate logistic regression for unplanned readmission or death within 30 days after discharge of index admission.

\begin{tabular}{|c|c|c|c|}
\hline Biomarker & $\begin{array}{l}\text { Univariate } \\
\text { analysis, } \\
\text { OR }(95 \% \mathrm{CI})\end{array}$ & $\begin{array}{l}\text { Model A, } \\
\text { OR }(95 \% \mathrm{CI})^{\circ}\end{array}$ & $\begin{array}{l}\text { Model B, } \\
\text { OR }(95 \% \mathrm{CI}) *\end{array}$ \\
\hline $\begin{array}{l}\text { Copeptin }>9 \\
\text { pmol/l } \\
\text { at discharge }\end{array}$ & $\begin{array}{l}2.62 \\
(1.28-5.39)\end{array}$ & $\begin{array}{l}2.20 \\
(1.02-4.73)\end{array}$ & $\begin{array}{l}2.23 \\
(1.044 .90)\end{array}$ \\
\hline $\begin{array}{l}\text { Cortisol }>590 \\
\mathrm{nmol} / 1 \text { at discharge }\end{array}$ & $\begin{array}{l}3.35 \\
(1.35-8.31)\end{array}$ & $\begin{array}{l}3.01 \\
(1.20-7.61)\end{array}$ & $\begin{array}{l}2.76 \\
(1.07-7.10)\end{array}$ \\
\hline
\end{tabular}

${ }^{\circ}$ Model A: adjusted for age and Charlson comorbidity index.

* Model B: adjusted for age, Charlson comorbidity index, number of hospitalizations in the 6 months before index admission, diagnosis of infection.

Abbreviations: OR, odds ratio; CI, confidence interval.
HOMEBOUND PATIENTS' PERSPECTIVES ON TECHNOLOGY AND TELEMEDICINE: A QUALITATIVE ANALYSIS Kristin Huang ${ }^{1}$; Tracy $\mathrm{Lu}^{2}$; Arash Mostaghimi ${ }^{3} .{ }^{1}$ Tufts Medical Center, Boston, MA; ${ }^{2}$ Harvard Medical School, Boston, MA; ${ }^{3}$ Brigham and Women's Hospital, Boston, MA. (Control ID \#2465159)

BACKGROUND: Homebound patients make up $20 \%$ of the community-dwelling Medicare population, and many face burdensome medical conditions, limitations in access to health care, and financial strain in the context of isolation. Telemedicine holds promise in bridging the gap between homebound patients and high quality health care, but uptake of telehealth technologies remains limited. The goal of this study was to characterize the attitudes of homebound patients towards technology and telemedicine and to identify potential barriers to adoption.

METHODS: Qualitative interviews were conducted from May to July 2015, with 17 participants recruited from a home care agency and a chronic wound clinic in the Boston area. Participants were English-speaking, $\geq 18$ years old, met the Medicare definition of homebound, and had received home care services for a minimum of 1 month. Interviews were conducted by two trained interviewers using a semi-structured questionnaire. Interviews were videotaped, transcribed, and analyzed inductively using immersion/ crystallization methods for central themes.

RESULTS: Six key takeaways organized around two major themes emerged regarding the main barriers to the adoption of telemedicine by homebound patients. First, participants who lack familiarity with technology are hesitant about telemedicine: 1) Baseline use of technology at home was limited; 2) Participants did not feel capable of learning how to use telehealth technology; 3) The advantages of telemedicine were unclear. Second, homebound patients place a high value on in-person office visits: 4) Participants felt inperson medical evaluation was more thorough and could provide more therapeutic benefit than virtual visits; 5) They preferred face-to-face communication; 6) Participants enjoyed the social aspect of medical appointments and getting out of the house.

CONCLUSIONS: In our study, homebound participants had low familiarity with technology, which contributed to a lack of confidence and desire to adopt telemedicine approaches. Similarly, participants value in-person office visits, despite the effort it takes to get to clinic. Addressing these two main concerns will be critical to pave the road for broad adoption of telemedicine as a routine part of the care of homebound patients.

HOSPICE CARE AND LENGTH OF STAY IN HOSPICE FOR PATIENTS DYING WITH MELANOMA. Rebecca N. Hutchinson; F L. Lucas; Mary Becker; Heidi R. Wierman; Kathleen Fairfield. Maine Medical Center, Portland, ME. (Control ID \#2465825)

BACKGROUND: Timely hospice referral is an indicator of high quality end-of-life care for cancer patients. While the ideal duration of hospice care is not known, there is some evidence from patient reported outcomes that 3 months of hospice improves the patient and caregiver experience when compared with shorter stays. Socioeconomic and racial disparities in hospice utilization and length of stay have been demonstrated in prior studies of other malignancies. There is limited knowledge about referral patterns in the melanoma population. METHODS: We used the Surveillance, Epidemiology, and End Results (SEER)-Medicare database to identify 7556 melanoma patients who were 66 and older at the time of diagnosis and enrolled in part A and B fee for service Medicare for the year prior to diagnosis, and who died by the end of 2010. These patients were diagnosed between 2000 and 2009. The primary outcome was enrollment in hospice prior to death. The secondary outcome was hospice length of stay. Given that ideal length of stay has not been defined, we categorized duration of hospice care using three definitions. We first analyzed patients who enrolled in hospice in the last 3 days of life, as this is an accepted marker of poor quality end of life care. We then looked at patients who started hospice care within the last week of life as this is a 
common duration of hospice care, yet it is likely too short to achieve optimal end of life care. Next, we examined characteristics of patients achieving a more ideal length of stay of at least 3 months. We also examined possible disparities in hospice enrollment, including age, race, marital status, and rural residence. Socioeconomic disparities were investigated by using census-tract median income and education. Finally, we examined how hospice utilization and length of stay changed over time.

RESULTS: Among 7556 patients who died with melanoma, 4386 (58 \%) received any hospice care. Late hospice referral, within the last 3 days of death, was observed in 733/ $4386(17 \%)$. Less than a third of patients $(1374 / 4386,31 \%)$ who received hospice care were referred within 7 days of death, while 554/4386 (13\%) patients had at least 90 days of hospice care. Patients who were older at the time of diagnosis were more likely to receive hospice services than were younger patients ( $64 \%$ of those 85 and older vs $45 \%$ of 66-69 year olds; $p<0.0001)$. Although not statistically significant, there was a modestly lower rate of hospice services received by black patients compared to white patients (48 vs $58 \%, p=0.25$ ). Patients with a higher Charlson comorbidity index were more likely to have hospice than those with a lower index ( $70 \%$ for patients with scores of 1 and 2 or more vs $52 \%$ for a score of $0 ; p<0.0001)$. We did not observe differences in hospice enrollment by gender, marital status, rurality or census tract median income or education levels. Late hospice referral, within 3 days of death, was observed at higher frequencies among younger, male patients. Late hospice referral was also more common in patients residing in census tracts with higher percentage of college graduates ( $18 \%$ of those in census tracts with $>20 \%$ completing at least 4 years of college vs $15 \%$ in census tracts with $\leq 20 \%$ with at least 4 years of college; p 0.01). Higher median income levels were also associated with higher rates of late hospice referral ( $19 \%$ for the highest median income tertile vs $13 \%$ for the lowest tertile; p 0.0001). Black patients had a lower, though not statistically significant, rate of late hospice referral (7 vs $17 \%$; p 0.29). Finally, urban patients were more likely to be referred within 3 days of death compared with those in rural areas (17 vs $8 \%$; $p$-value 0.01$)$. Similar associations were noted when we analyzed patients referred to hospice within the last week of life. Older, female, unmarried patients and patients coming from census tracts with lower average income levels were more likely to have at least 90 days of hospice care. Finally, we noted that the number of patients receiving hospice care increased significantly over time from no patients who died in 2000 (though the total number of deaths in 2000 was small), to $70 \%$ of patients who died in $2010(p<0.0001, p$ trend $<0.0001)$. We did not observe a decline in the percent of patients referred to hospice within 3 days of death (range 14-19\%) or 7 days (range 29-35\%). There was a modest increase in the number of patients who achieved a more ideal length of stay of at least 90 days ( $3 \%$ in 2001 vs $16 \%$ in 2010; $p<0.0001, p$ trend $<0.0001$ ).

CONCLUSIONS: Rates of hospice enrollment have increased over time among melanoma patients. However, late hospice enrollment has not improved and many patients are being referred in the last 3 days or last week of life. Few patients achieve a more ideal length of stay of at least 3 months, though this improved over time. Disparities remain evident in overall hospice enrollment amongst melanoma patients. However, the differences in late enrollment show surprising trends, where patients from census tracts with the lowest median income levels were more likely to receive at least 90 days of hospice care. Ongoing efforts to decrease disparities in hospice care and increase hospice length of stay for patients dying with melanoma is essential.

HOSPITAL IN HOME PATIENTS SUFFER FEWER PATIENT SAFETY EVENTS THAN COMPARABLE HOSPITAL INPATIENTS Jonathan Liu ${ }^{1,}{ }^{4}$; Ming Jang ${ }^{2}$, ${ }^{\text {; }}$ Haggerty Mary-Ann ${ }^{4}$; Laurence Buxbaum ${ }^{4}$; Rajesh Makineni ${ }^{5}$; Edna Schwab $^{4}$; Bruce Kinosian ${ }^{2,3}$. ${ }^{1}$ Hospital of the University of Pennsylvania, Philadelphia, PA; ${ }^{2}$ University of Pennsylvania, Philadelphia, PA; ${ }^{3} \mathrm{Cpl}$. Michael J Crescenz VAMC, Philadelphia, PA; ${ }^{4} \mathrm{Cpl}$ Michael J Crescenz VAMC, Philadelphia, PA; ${ }^{5}$ Providence VAMC, Providence, RI. (Control ID \#2469112)
BACKGROUND: Frail geriatric patients are at high risk to suffer adverse outcomes during hospitalization. The Hospital in Home $(\mathrm{HiH})$ care model was created to mitigate the occurrence of these iatrogenic, costly adverse outcomes by providing hospital level care in the home. The $\mathrm{HiH}$ program at the Corp. Michael J. Crescenz VA Medical Center (CMC VAMC) in Philadelphia, operational since 2012, consists of 3 MDs, a NP director, a contracted homehealth agency (providing nursing, therapies, social work, and aides) and infusion pharmacy. HiH provides services that include daily physician and nursing care, phlebotomy, radiology, medication administration, parenteral therapy and DME/home oxygen coordination. The most common diagnoses include CHF, COPD, pneumonia and cellulitis, with patients admitted from the emergency department and outpatient settings (Substitutive) and as transfers from inpatient services (Complementary). Recent $\mathrm{HiH}$ evaluations have shown benefits in patient satisfaction and cost, but not risk of patient safety events (PSEs).

METHODS: Matched case-control study, using all HiH admissions (March, 2012June 2015), and inpatient controls directly matched for admission month, DRG, age, gender, JEN frailty index (JFI), and CAN score. JFI is a claims-based grouping of the number of geriatric syndromes, with an ROC area of .81 to identify $3+$ ADL impairments. CAN is a VA proprietary algorithm that predicts 90 day and 1 year risks of death and hospitalization (ROC areas .8-.85). We used the 90d CAN hospitalization score, computed post discharge, to control for readmission risk. Rates of adverse events, including central line associated blood stream infections, falls, delirium, catheter-associated urinary tract infections, venous thromboembolism, pressure ulcers (PU) and 30-day readmissions were documented via review of progress notes, medication lists and other documentation in the VA electronic medical record (CPRS), for all $\mathrm{HiH}$ admissions, and for matched controls (where the $\mathrm{HiH}$ admission had an assigned DRG, restricted to Complimentary patients). A composite Patient Safety Event (PSE) rate was constructed using events/days at risk. Comparison of PSE rates between HiH patients, matched controls and facility benchmarks were by Poisson regression.

RESULTS: The $115 \mathrm{HiH}$ patients had 732 risk days, with 56 patients (48 \%) being Complementary, thus with a coded DRG. Of these, 50 patients with 328 risk days could be directly matched on JFI/CAN as controls. The HiH composite PSE rate was 1.3/1000d (1/732), with a control rate of 9/1000d (3/328), and a facility benchmark of $12.6 / 1000 \mathrm{~d}$. ( $\mathrm{HiH}<\operatorname{control} p=.0026)$ Readmissions were $8.7 \%$ for $\mathrm{HiH}, 8 \%$ for controls, and $11.3 \%$ for the facility benchmark. There were $3 \mathrm{HiH}$ transfers to a higher level of care ( $2 \mathrm{MICU}, 1$ inpatient), or $2.6 \%$, compared to 5 floor-to-unit transfers among controls (10\%).

CONCLUSIONS: Patients in $\mathrm{HiH}$ experience fewer adverse safety events compared to matched controls, making $\mathrm{HiH}$ a preferred setting for appropriate patients with both higher quality and lower cost (previously shown). Lower event rates for matched controls compared to hospital benchmarks suggest that $\mathrm{HiH}$ patients are a lower-risk subset of all hospital inpatients. Hospital coding of $\mathrm{HiH}$ admissions, as with usual inpatients, would facilitate comparative effectiveness studies involving $\mathrm{HiH}$

HOSPITAL MORTALITY IN PATIENTS WITH UPPER GASTROINTESTINAL BLEEDING DUE TO VARICES David Mossad ${ }^{1}$; Ronald J. Markert ${ }^{1}$; Sangeeta Agrawal ${ }^{1,2}$. ${ }^{1}$ Wright State University, Dayton, OH; ${ }^{2}$ Dayton VA Medical Center, Dayton, OH. (Control ID \#2456007)

BACKGROUND: Patients presenting with upper gastrointestinal bleeding (UGIB) due to varices areacutely at high risk for morbidity and mortality. Urgent therapy is paramount as these patients can reach hemodynamic instability with brisk bleeding. Multiple scoring systems stratify risk in patients with UGIB to assess the need for endoscopic intervention, chances of re-bleeding, and mortality risk. The purpose of this study was to assess the effects of patient demographics, comorbidity burden, and hospital characteristics on inhospital mortality in patients with UGIB due to varices.

METHODS: We used ICD-9 diagnosis codes from the 2001-2010 National Hospital Discharge Survey to identify cases of UGIB due to varices. Comparisons on 
demographics, number of comorbidities, hospital length of stay (LOS), and selected health system characteristics were made between those who died in-hospital and those discharged. SPSS was used for chi-square and Mann-Whitney analysis.

RESULTS: Among 2484 cases of UGIB due to varices, 252 died in-hospital and 2232 were discharged. The mortality group was older (mean age of 56.8 vs 54.4 years, $p=0.01$ ) but did not have a significantly longer LOS ( 9.1 vs 6.1 days, $p=0.77$ ). There was no difference in mortality between genders ( $10.7 \%$ of males vs $8.9 \%$ of females, $p=0.17)$. Race had a significant impact on mortality, with African Americans (15.3\%) having a higher rate compared to Caucasians $(9.9 \%)$ and others $(9.2 \%)(p=0.022)$. Patients with seven or more comorbidities had a higher mortality rate (11.5\%) compared to those with six or fewer comorbidities $(5.0 \%)[p<0.001]$. Neither coronary artery disease (CAD) $(p=0.51)$ nor chronic kidney disease (CKD) $(p=0.82)$ impacted mortality. There was no difference in mortality between the Midwest (9.7\%), Northeast (13.3\%), South (9.1\%), and West $(9.9 \%)$. Neither hospital size $(p=0.45)$ nor ownership $(p=0.60)$ was associated with mortality. Principal source of payment affected mortality rate, with Medicare (16.4\%), Medicaid (14.5\%), and other government programs (16.3\%) having the highest mortality rates and self pay $(5.5 \%)$ having the lowest $(p=0.002)$. Type of admission did not affect mortality $(p=0.81)$.

CONCLUSIONS: Prompt and accurate risk stratification in UGIB due to varices is important due to the clinical urgency and potential need for endoscopy, as these patients are most at risk for mortality in the first two days after variceal hemorrhage. This study found that age, race, comorbidity burden, and payment method impacted in-hospital mortality. While comorbidity burden was related to mortality, neither CAD nor CKD specifically led to increased mortality.

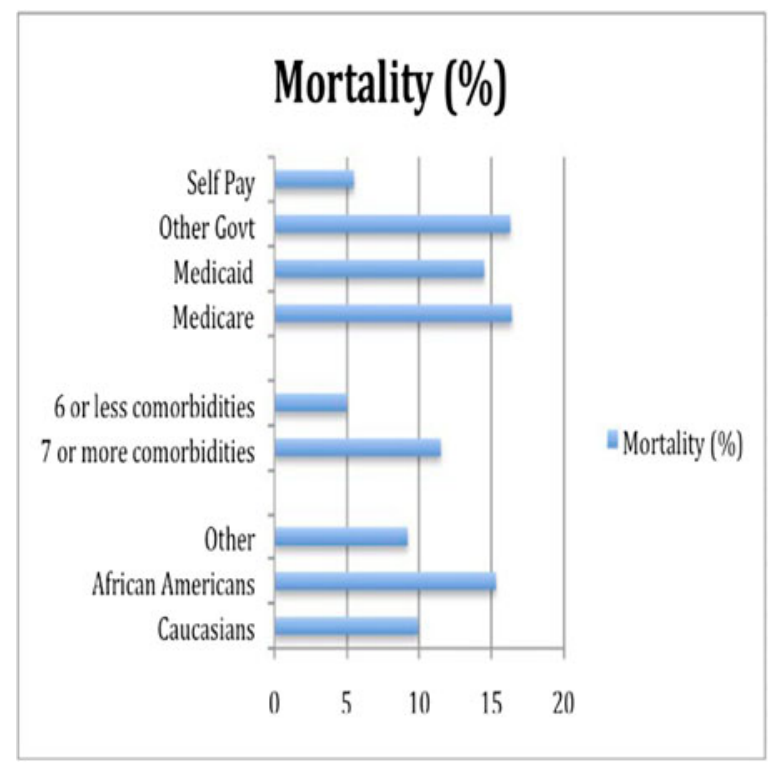

HOSPITAL PAYER AND RACIAL/ETHNIC MIX AT PRIVATE ACADEMIC MEDICAL CENTERS IN BOSTON AND NEW YORK CITY - A TALE OF TWO CITIES Roosa S. Tikkanen ${ }^{7}$; Steffie Woolhandler ${ }^{7}$; David Himmelstein ${ }^{7}$; Nancy R. Kressin ${ }^{1,2}$; Amresh D. Hanchate 2, 3; Meng-Yun Lin ${ }^{1,4}$; Chieh Chu ${ }^{1,2}$; Danny McCormick $^{6,5}$; Karen E. Lasser ${ }^{1,2}$. ${ }^{1}$ Boston Medical Center, Boston, MA; ${ }^{2}$ Boston University School of Medicine, Boston, MA; ${ }^{3}$ VA Boston Healthcare System, Boston, MA; ${ }^{4}$ Boston University School of Public Health, Boston, MA; ${ }^{5}$ Harvard Medical School, Boston, MA; ${ }^{6}$ Cambridge Health Alliance, Cambridge, MA; ${ }^{7}$ City University New York School of Public Health at Hunter College, New York City, NY. (Control ID \#2469777)
BACKGROUND: Racial and ethnic minorities' access to health services lags behind that of other Americans, in part because minorities are more often uninsured or covered by Medicaid. Academic Medical Centers (AMCs) play a unique role in the health care system, contributing to medical training and research. They are also often viewed as providing the highest quality care. We investigated differences in racial and payer mix at private AMCs in the Boston metropolitan area and in New York City (NYC), to explore the extent to which those facilities serve minority and lowincome patients, the degree of racial segregation, and recent changes in patterns of care in NYC.

METHODS: We analyzed discharge data on adults ( $\geq 18$ years old) at acute care hospitals in Boston and NYC in 2009, and the most recent (2013), publicly available data for NYC (2013 data was not publicly available for Boston). Our focus was on the behaviour of private AMCs, (referred to as just "AMCs" in results), which we defined as privately-owned hospitals listed as "integrated" members of the Council of Teaching Hospitals, and their affiliated hospitals. We categorized patient race/ethnicity as "white", "black" or "other minority." The inconsistent classification of Hispanic patients across hospitals precluded reliable analyses of this group. Hence, we classified patients identified as "white Hispanics" as "other minority" and black Hispanic patients as "black". We assessed differences in racial and payer mix between private AMCs and all other hospitals, including public AMCs (1 in NYC, 0 in Boston), using Pearson's chi-square tests. We performed multivariable logistic regression analyses on patient-level data to explore the association of race with the likelihood of being hospitalized in a private $\mathrm{AMC}$, after controlling for insurance, sex and age. We also calculated the Index of Dissimilarity (ID) for each city, which compares the evenness of distribution of majority (white) and minority (black or other minority) patients across hospitals. The ID ranges between 0 (races represented at all hospitals in the same proportions as in the city overall), and 1 (complete segregation).

RESULTS: The Boston sample consisted of 170,640 discharges at 6 AMCs and 64,272 discharges at 7 non-AMCs. The NYC sample included 288,084 discharges at 10 AMCs, and 750,841 discharges at 48 non-AMCs in 2009; and 274,997 discharges at 10 AMCs and 663,037 discharges at 54 non-AMCs in 2013. In NYC, uninsured patients accounted for a far smaller proportion of discharges at AMCs than at other hospitals $(1.5$ vs. $5 \%, p<0.001)$; Medicaid patients were also underrepresented at AMCs ( 9 vs. $22 \% p<0.001$ ). Conversely, privately insured patients (54 vs. $42 \%, p<0.001)$ and Medicare patients (35 vs. $30 \%, p<0.001)$ were overrepresented at AMCs. In Boston there were no significant differences in average payer mix between AMCs and non-AMCs for Medicaid (15 and $14 \%, p=0.243$ ) or uninsured ( $1 \%$ for both, $p=0.053)$ patients. However, AMCs in Boston cared for significantly more privately insured (42 and $30 \%, p<0.001$ ) but fewer Medicare patients $(38$ vs. $51 \%, p<0.001)$ than other hospitals. In Boston, AMCs had more black ( 15 vs. $9 \%, p<0.001)$ and other minority $(10$ vs. $9 \%, p<0.001)$ patients, but fewer white patients (75 vs. $83 \%, p<0.001$ ) than non-AMCs. In NYC, AMCs cared for more white ( 52 vs. $32 \%, p<001)$ but half as many black patients (18 vs. $36 \%, p<0.001)$ as other hospitals. In multivariable analyses, race was a significant predictor of AMC hospitalization in both cities, but with opposite effects. In Boston, black patients were much more likely (OR 2.05, $95 \%$ confidence interval [CI] 1.99 2.12), and other minorities somewhat more likely (OR 1.23, $95 \%$ CI 1.19-1.28) to be discharged from an $\mathrm{AMC}$ than white patients. In contrast, in NYC, black patients were much less likely (OR $0.35,95 \% \mathrm{CI} 0.35-0.36$ ), and other minorities somewhat less likely (OR $0.65,95 \% \mathrm{CI} 0.64-0.66$ ) to be hospitalized at an $\mathrm{AMC}$ than whites. Hospital racial segregation, as measured by the ID, was relatively low in Boston (blacks $=0.33$; other minorities $=0.20)$ but moderately high in NYC $(0.55$ and 0.46 respectively). By 2013, hospital racial segregation in NYC improved modestly (blacks $=0.52$; other minorities $=0.42$ ). However, payer segregation worsened: compared to other hospitals, AMCs in NYC had about one-quarter as many Medicaid 
(5.5 vs. $20 \%, p<0.001)$ and one-thirteenth as many uninsured $(0.3$ vs. $3.9 \%$, $p<0.001)$ discharges.

CONCLUSIONS: In 2009, minority, uninsured and Medicaid patients were underrepresented among adult inpatients at private AMCs in NYC, a pattern not present in Boston. Differences in insurance did not account for NYC's racial disparities. Massachusetts' 2006 health reform is unlikely to fully explain lower levels of segregation at Boston's private AMCs. We did not control for the distance between patients' residence and hospitals, although some private AMCs in both cities are located in minority neighborhoods. Boston lacks NYCs' extensive network of public hospitals, which may relieve pressure on NYC's private AMCs to care for disadvantaged patients. Overall, our findings suggest that low-income and minority New Yorkers face substantial barriers to care at private AMCs, resulting in de facto racial segregation in hospital care.

\section{HOSPITALIZATION RATES FOR DIABETIC KETOACIDOSIS INCREASE} DURING THE TRANSITION TO ADULTHOOD Andrea S. Christopher ${ }^{1,3}$; David Bor $^{1,3}$; Steffie Woolhandler ${ }^{2,1}$; David Himmelstein ${ }^{2,1}$; Danny McCormick ${ }^{1,3}$. ${ }^{1}$ Cambridge Health Alliance, Boston, MA; ${ }^{2}$ City University of New York School of Public Health, New York, NY; ${ }^{3}$ Harvard Medical School, Boston, MA. (Control ID \#2469928)

BACKGROUND: Access to outpatient care for patients with chronic medical conditions improves outcomes. The transition from adolescence to young adulthood is commonly accompanied by changes in circumstances that could affect access to care such as the loss of eligibility for Medicaid (at ages 18-19 in most states), the transition from living with parents to independent living (often with the loss of parental health insurance) and a change in primary care physician (from pediatrician to an adult medicine physician). However, little is known about whether the transition to adulthood is associated with changes in access to outpatient care. Ambulatory care sensitive conditions, such as diabetic ketoacidosis (DKA), are conditions that can often be managed with timely and effective treatment in an outpatient setting, thereby preventing hospitalization. Hospitalization rates for these conditions, therefore, provide an indirect measure of access to outpatient care. We examined age trends in hospitalization rates for DKA as an indicator of outpatient access to care for patients with diabetes at the transition from adolescence to young adulthood.

METHODS: We analyzed hospitalization rates for DKA for a nationally representative sample of adolescents and young adults. We first estimated DKA hospitalization counts nationally from the Agency for Healthcare Research and Quality's Nationwide Inpatient Sample, a $20 \%$ sample of discharges from community hospitals in the United States (1998-2012). To calculate hospitalization rates, we divided these hospitalization counts by population counts from the US Census Bureau's Current Population Survey Annual Social and Economic Supplement (1999-2013). We estimated and plotted the mean hospitalization rates at each age from 12 to $30(n=439,339)$. We also compared the mean hospitalization rates immediately prior to age 18 (ages $15-17 ; n=57,457$ ) and after age 18 (ages 19 $21 ; n=93,631)$ using a z-test. We also obtained US uninsurance rates at each age from 12 to 30

RESULTS: We found that uninsurance rates rise markedly from $13.9 \%$ among US adolescents age $15-17$ to $28.9 \%$ among young adults age $19-21$ ( $p$-value $<0.0001$ ). US hospitalization rates for DKA rose from 5.8 (per 10,000 people) for ages 15-17 to 10.6 for ages 19-21 ( $p$-value $<0.0001)$, an $83 \%$ rise (Figure 1$)$.

CONCLUSIONS: Co-incident with rising uninsurance rates, DKA hospitalization rates in the US rise sharply at the transition to adulthood, suggesting that many adolescents suffer a loss of access to outpatient care. Efforts to minimize losses in access to care at the transition to adulthood would likely improve outcomes of DKA, such as preventable hospitalizations

\section{Figure 1. Uninsurance Rate}

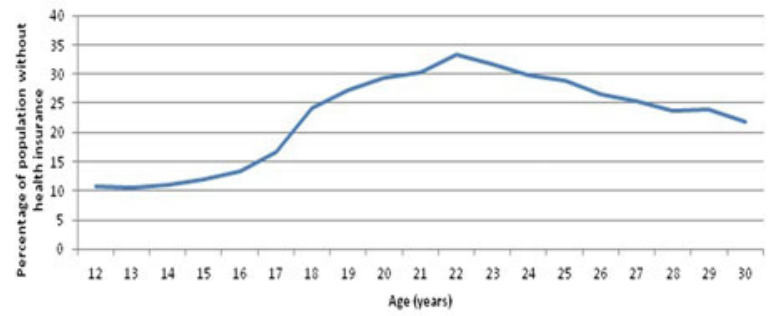

Figure 2. DKA Hospitalization Rate

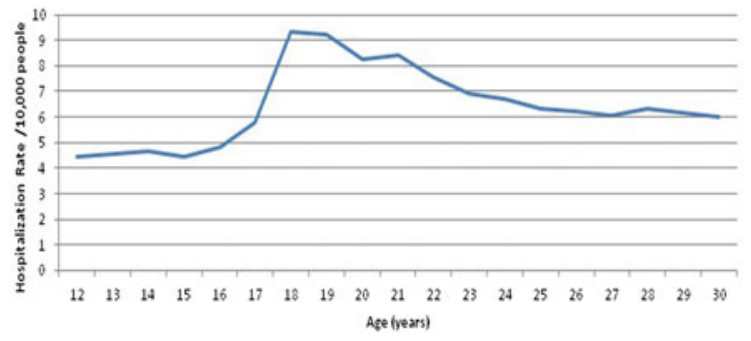

HOW ARE MASSACHUSETTS' SAFETY-NET HOSPITALS FARING IN THE POST-HEALTH CARE REFORM ERA?: VIEWS OF SAFETY NET CEOS Maria Nardell; Galina Tan; Andrew Moore; Susan Inonog; David Scales; Jyothi Ravindra; Nihan K. Cannon; Anna Goldman; Gaurab Basu; Danny McCormick. Cambridge Health Alliance, Cambridge, MA. (Control ID \#2469701)

BACKGROUND: Safety net hospitals (SNH) play a vital role in the care of the poorest and most vulnerable patients in the United States. The Affordable Care Act (ACA) mandates substantial cuts in Medicaid Disproportionate Share (DSH) Hospital payments to SNHs that were historically used to offset financial losses associated with the provision of uncompensated and undercompensated care. Partly as a way improve the financial status of their safety net hospitals, several states have recently participated in Medicaid incentive programs to transform delivery systems, referred to as the "Delivery System Transformation Initiative" (DSTI) and the similar "Delivery System Reform Incentive Payment" (DSRIP) programs. In these programs, SNHs receive financial incentive payments in return for implementing intensive hospital system delivery reforms and reform evaluations that meet specific criteria. It is anticipated that many other states will turn to these programs as ACA mandated funding cuts grow in future years. Because the main provisions of the ACA were only implemented in 2014, little is known about the impacts of health reform and DSTI/DSRIP programs on SNHs. Massachusetts' (MA) 2006 health reform served as a template for the ACA, and seven SNHs in MA have been involved in DSTI/DSRIP programs since 2010. Therefore, MA provides an opportunity to examine a similar but more mature reform in order to understand challenges and opportunities that may emerge for other SNHs under national health reform. We explored these issues by conducting qualitative interviews with Chief Executive Officers (CEOs) of MA SNHs.

METHODS: Six of the seven CEOs of the DSTI/DSRIP-participating SNHs in MA agreed to participate through email invitations. We developed an interview guide based on our a priori knowledge of and literature review on MA health reform and SNHs. From February to April 2015, we conducted confidential semi-structured 30-60 min interviews. Interviews were transcribed verbatim, and two trained researchers used the qualitative data analysis software program Dedoose for coding and analysis. We used a modified grounded theory approach, developing a coding structure that focused on the impact of the MA reform on SNH finances and organizational structure.

RESULTS: Based on our analysis of the CEOs' perspectives on how their hospitals were impacted by MA reform and the DSTI/DSRIP programs, we identified the following themes: Financial implications: (a) Medicaid reimbursement rates have historically been substantially below actual cost of care, therefore SNHs continue to struggle financially given the even higher 
proportion of Medicaid patients in SNHs as a result of health reform; (b) DSTI/DSRIP programs have enabled SNHs to refocus their care delivery efforts to better support their patients and promote long-term financial sustainability; (c) while DSTI/DSRIP programs gave SNHs a necessary infusion of funding, much of this is consumed by costly administrative burdens of implementing delivery system improvement programs and reporting outcomes. Organizational impacts: (a) SNHs have been able to preserve their mission of providing care to vulnerable populations; (b) DSTI/DSRIP programs have spurred innovative efforts to increase primary care access and decrease emergency room utilization; (c) transformations in mental health delivery have not been feasible through DSTI/DSRIP given its short timeline requirement for SNHs to demonstrate returns on investments in waiver projects. Health reform lessons for the nation: (a) SNHs continue to be important through their provision of comprehensive care to socially complex patient communities; (b) DSTI/DSRIP's patchwork payment scheme is irrational and unsustainable for SNHs; (c) policy change around insurance reimbursement rates is vital for the future financial stability of SNHs.

CONCLUSIONS: MA health reform and DSTI/DSRIP programs have had both positive and negative impacts on SNHs. The DSTI/DSRIP incentive payments have enabled SNHs to maintain short-term financial solvency in the era of health reform mandated cuts in DSH payments while promoting innovative delivery system transformations within these institutions. However, DSTI/DSRIP programs also add substantial financial and administrative burdens on these hospitals that are already strained due to inadequate reimbursement rates and a system that does not adequately support mental health services. As SNHs will continue to be important to the health of the nation's most vulnerable patient communities, ongoing efforts are needed to ensure the financial wellbeing of these institutions.

HOW CAN CLINICAL PRACTICES PRAGMATICALLY INCREASE PHYSICAL ACTIVITY FOR PATIENTS WITH TYPE 2 DIABETES? A SYSTEMATIC REVIEW Kelsey Luoma $^{1}$; Ian M. Leavitt ${ }^{1}$; Joel C. Marrs ${ }^{2}$; Andrea Nederveld ${ }^{1}$; Judith G. Regensteiner ${ }^{1}$ ${ }^{3}$; Andrea L. Dunn ${ }^{4}$; Russell E. Glasgow'; Amy G. Huebschmann ${ }^{1,3}$. ${ }^{1}$ University of Colorado School of Medicine, Aurora, CO; ${ }^{2}$ University of Colorado Skaggs School of Pharmacy and Pharmaceutical Sciences, Aurora, CO; ${ }^{3}$ University of Colorado Center for Women's Health Research, Aurora, CO; ${ }^{4} \mathrm{AL}$ Dunn Health Consulting, LLC, Loveland, CO; ${ }^{5}$ University of Colorado, Aurora, CO. (Control ID \#2465075)

BACKGROUND: The prevalence of type 2 diabetes (T2D) has increased rapidly. Although physical activity (PA) is a cornerstone of treatment for T2D that improves glycemic control, reduces cardiovascular risk, and enhances physical function, most people with T2D are sedentary. Several randomized controlled trials (RCTs) have demonstrated the effectiveness of behavioral interventions to increase PA for people with T2D, but few such RCTs were in clinical practice settings. To address this gap, we conducted a systematic review to identify effective and pragmatic PA interventions for patients with T2D in clinical practice settings. Our specific aims were to: 1) Assess the potential of PA interventions for implementation in clinical practice settings; and 2) Assess the prevalence of intervention characteristics related to implementation and effectiveness in the highest-ranking interventions from Aim 1.

METHODS: An initial PubMed search yielded 4813 articles. Two independent raters identified studies meeting our inclusion criteria: behavioral PA interventions coordinated by healthcare clinics for patients with T2D. For Aim 1, we used an existing tool to assess the 9 domains of the Pragmatic-Explanatory Continuum Indicator Summary 2 (PRECIS-2) scale and to assess 5 external validity issues from the Reach, Effectiveness, Adoption, Implementation, Maintenance model (RE-AIM). The PRECIS-2 domains were: participant eligibility criteria, participant recruitment efforts, trial setting, organizational resources of the intervention, flexibility of delivery, flexibility of adherence, participant follow-up intensity, relevance of primary outcome, and use of intention-to-treat analysis. The RE-AIM external validity issues were: participant engagement with the intervention, extent of adaptation of intervention during study, program sustainability, unintended intervention effects, and monetary costs of intervention. Independent raters scored each domain on a scale of 1 to 5 , with 5 representing the best potential for future implementation. We defined effectiveness of interventions based on a statistically significant increase in a valid PA behavior outcome as compared to the control group $(P<0.05)$

RESULTS: We ultimately identified 36 RCTs ( $n=13,358$ participants) that met inclusion criteria. The RCTs included had a mean PRECIS-2 score $=3.90$ (SD: 0.40 ). To compare the pragmatism and effectiveness of the RCTs, we plotted the PRECIS- 2 scores of the RCTs stratified by the presence/absence of effectiveness to increase physical activity behavior (Figure 1). In the most pragmatic quartile of interventions (mean PRECIS-2 score $=4.38$, SD: 0.19), $70 \%$ used tailored behavioral counseling; $20 \%$ tracked PA levels; $20 \%$ used technology and personal counseling; and $20 \%$ significantly increased PA behavior. Only 36 and $8 \%$ of RCTs, respectively, reported on RE-AIM issues of participant engagement and intervention cost. CONCLUSIONS: A minority of PA interventions for adults with T2D were both effective and highly pragmatic for future implementation in clinical practice settings. Interventions that are both effective and highly pragmatic should be tested for widespread use with implementation trial designs. Such future testing should assess outcomes relevant to key stakeholders of clinicians, patients, and health care systems - clinical effectiveness, patient-reported outcomes such as quality of life, and intervention costs.

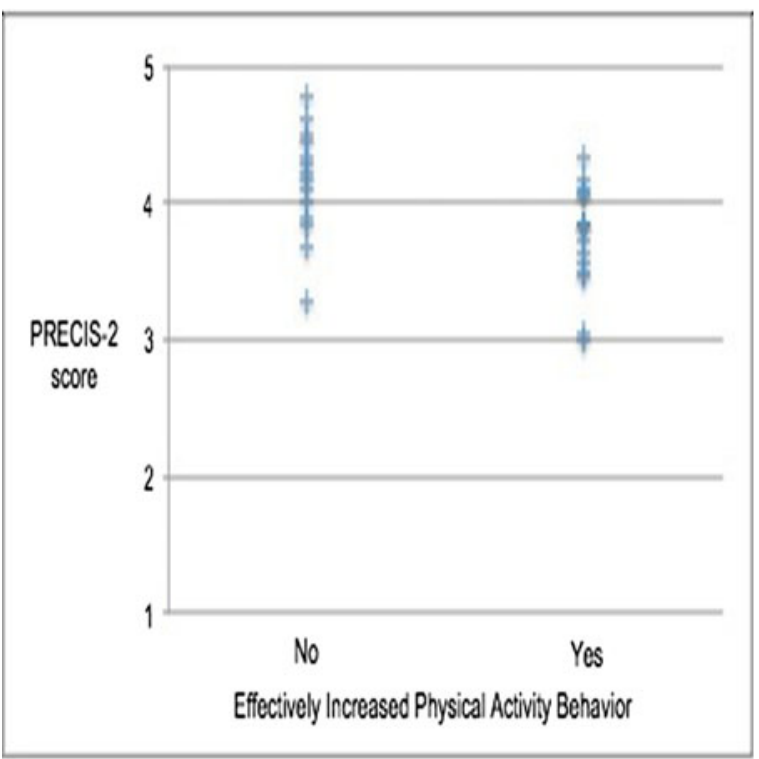

Figure 1. Display of Effectiveness and PRECIS-2 scores

HOW DO BURNOUT, EMOTIONAL STYLE, AND EMPATHY RELATE? A PRELIMINARY CORRELATION STUDY. Mariah A. Quinn ${ }^{1}$; Amy Zelenski ${ }^{2}$. ${ }^{1}$ University of Wisconsin, Middleton, WI; ${ }^{2}$ University of Wisconsin School of Medicine and Public Health, Madison, WI. (Control ID \#2470211)

BACKGROUND: Empathy is a critical skill in the practice of medicine and growing evidence demonstrates that it can be taught. Empathy comprises internal domains including the ability to modulate personal distress in the face of another's suffering and fantasy, the imaginative projection of oneself into the shoes of another. Externally or other-focused domains of empathy include empathic concern and perspective-taking. Personality, coping styles and affective states likely interact highly with the domains that comprise empathy. We designed a pilot curriculum for medical interns to increase empathy and reduce distress, evaluation of which is ongoing. At baseline, we assessed empathy using the Interpersonal Reactivity Index (IRI), burnout using the Maslach Burnout Inventory (MBI), and an inventory designed to assess multiple components of emotional style Emotional Styles Inventory (ESI)1,2. The domains of the three instruments are 
shown in Table 1. Here we present the correlations between the domains of these instruments. This protocol was Exempted by the UW IRB.

METHODS: We assessed pairwise correlations between all subscales of these instruments. Data analysis was performed using SPSS (version 22.0)

RESULTS: Twenty-seven subjects completed baseline data collection, 26 of them completed all instruments. $(\mathrm{RR}=93 \%)$. There were moderately strong correlations between resilience and self-awareness, attention, emotional exhaustion, and personal distress. Fantasy was correlated moderately with social intuition and personal distress. Selfawareness was notle only correlated with resilience but also perspective-taking. Table 2 shows correlations between domains.

CONCLUSIONS: These preliminary results support empathy and well-being as connected, and suggest specific domains that are moderately strongly correlated. This provides evidence that interventions that impact well-being may impact empathy and vice versa, as is starting to be seen in both the medical education and neurobiology literature3-5. These relationships should be considered when designing interventions. References 1. Davidson RJ, Begley S. The Emotional Life of Your Brain: How Its Unique Patterns Affect the Way You Think, Feel, and Live-and How You Can Change Them. New York: Plume; 2013. 2. Davis MH. Measuring individual differences in empathy: Evidence for a multidimensional approach. J Pers Soc Psychol. 1983;44(1):113-126. doi:10.1037/0022-3514.44.1.113. 3. Weng HY, Fox AS, Shackman AJ, et al. Compassion Training Alters Altruism and Neural Responses to Suffering. Psychol Sci. May 2013. doi:10.1177/0956797612469537. 4. Krasner MS, Epstein RM, Beckman H, et al. Association of an Educational Program in Mindful Communication With Burnout, Empathy, and Attitudes Among Primary Care Physicians. Jama-J Am Med Assoc. 2009;302(12):1284-1293. 5. Beckman HB, Wendland M, Mooney C, et al. The Impact of a Program in Mindful Communication on Primary Care Physicians. Acad Med. 2012;87(6):815-819. doi:10.1097/ACM.0b013e318253d3b2.

Table 1. Domains of instruments assessming empathy, burnout, and emotional style.

\begin{tabular}{|c|c|c|}
\hline IRI & MBI & ESI \\
\hline Personal Distress (PD) & $\begin{array}{l}\text { Emotional Exhaustion } \\
\text { (EE) }\end{array}$ & $\begin{array}{l}\text { Resilience (Res) - Speed of recovery from } \\
\text { adversity }\end{array}$ \\
\hline Fantasy (FS) & Depersonalization (DP) & Outlook - Ability to sustain positive emotion \\
\hline Perspective Taking (PT) & $\begin{array}{l}\text { Personal Accomplishment } \\
\text { (PA) }\end{array}$ & $\begin{array}{l}\text { Social Intuition - Adeptness at picking up } \\
\text { social signals }\end{array}$ \\
\hline Empathic Concern (EC) & & $\begin{array}{l}\text { Self-Awareness - How well one perceives } \\
\text { bodily feelings reflecting emotion } \\
\text { Sensitivity to Context - Self-regulation in } \\
\text { light of social context } \\
\text { Focused Attention - How and clear focus is }\end{array}$ \\
\hline
\end{tabular}

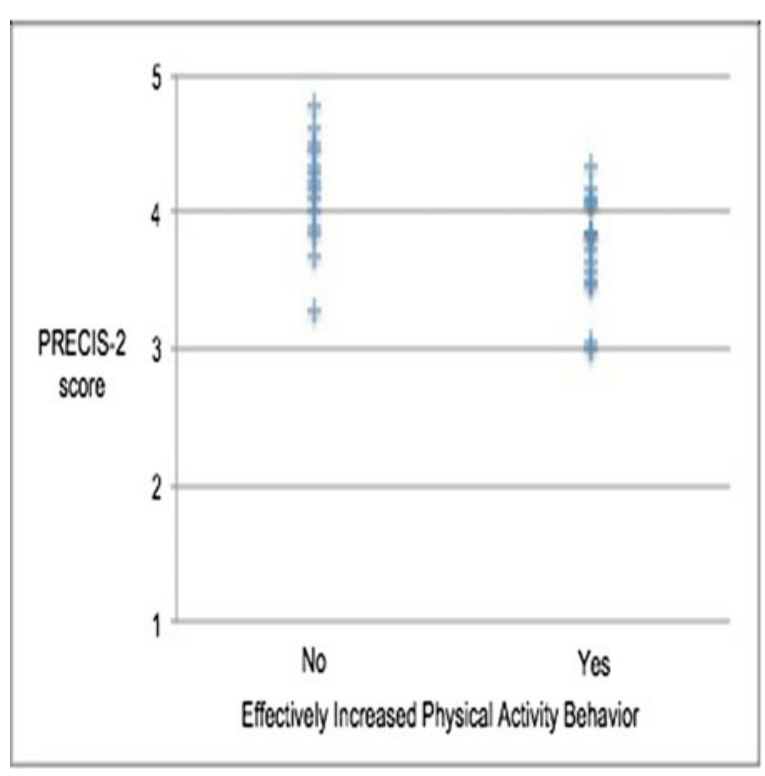

Table 2. Two-Tailed Pearson Correlations
HOW DO WE MEASURE CVD RISK? A CROSS-SECTIONAL ANALYSIS OF CVD RISK FACTORS AND CVD RISK PREDICTIONS IN HIV-POSITIVE AND HIV-NEGATIVE PERSONS IN RURAL UGANDA Mara E. Murray Horwitz ${ }^{1}$; Gene F. Kwan ${ }^{1}$; Yukari Manabe ${ }^{2} .{ }^{1}$ Boston Medical Center, Cambridge, MA; ${ }^{2}$ Johns Hopkins University, Baltimore, MD. (Control ID \#2470333)

BACKGROUND: Both low socioeconomic status and HIV infection have been strongly associated with increased cardiovascular disease (CVD) mortality. Many risk models have been created to identify persons at increased risk of CVD. However, data and tools to accurately predict CVD risk in developing regions of the world - such as sub-Saharan Africa, where the vast majority live in poverty, and where three-quarters of the world's HIV-infected persons reside-are lacking. This study aims to (1) describe CVD risk factor prevalence in HIV-positive and HIV-negative individuals in rural Uganda, and (2) estimate CVD risk in this population using various prediction models.

METHODS: We enrolled 540 HIV-positive ART-naïve persons with CD4 $=<250$ cells $/ \mu \mathrm{L}$ in an HIV treatment study in Kiboga District, Uganda. Each subject underwent vital sign measurement, anthropometrics, and fasting blood sugar and lipid panel testing prior to ART initiation. Using a stratified random sample of 165 of the 540 HIV-positive participants, we recruited 165 age- and sex-matched HIV-negative individuals from the same district for a one-time CVD risk factor screening. Five- and ten-year CVD risk was estimated for all participants, using the following CVD risk models: Framingham risk score, ASCVD, SCORE, WHO risk charts, and Globorisk. Analysis was restricted to the age range for which the above risk models have been validated (40-74 years old).

RESULTS: After age restriction, 193 HIV-positive and 96 HIV-negative participants were included. Women composed half of each group. Age distributions were similar, with $75 \%$ of subjects aged 43 to 55 years old. HIV-positive participants reported significantly lower socioeconomic status and significantly more tobacco and alcohol use. HIV-negative participants had significantly higher rates of overweight and obesity, elevated waist circumference, hypertension, diabetes, and higher total and LDL cholesterol. HIV-positive participants had significantly lower HDL cholesterol. Based on the study results, $29 \%$ of the HIV-negative group met criteria for metabolic syndrome compared to $11 \%$ of the HIV-positive cohort. In general, CVD risk predictions in this population were low. All risk models predicted more HIV-negative persons to be at "high risk" than HIV-positive persons. Correlation between risk scores varied; Framingham and ASCVD scores correlated well, while WHO risk scores correlated poorly with all other scores. In general, correlations were stronger for the HIV-negative group. According to the 2013 ACC/AHA guidelines, at least $23 \%$ of the HIV-positive and $29 \%$ of HIV-negative participants met criteria to consider statin therapy to prevent CVD.

CONCLUSIONS: In this rural Ugandan community, individuals without HIV had worse traditional CVD risk factors and seemingly higher CVD risk than their age- and sex-matched HIV-positive counterparts. Clearly the relationship between HIV infection and CVD mortality is not explained by traditional risk factors, nor is it reflected in the most commonly used CVD risk prediction models. Some potential "emerging" CVD risk factors that we identified in this HIV-positive cohort include low socioeconomic status, increased tobacco and alcohol use, underweight, anemia, chronic kidney disease, low HDL cholesterol, and high triglycerides. Further research should be conducted to explore associations between these potential risk factors and CVD mortality. In this population, there was marked variation between CVD risk prediction models in the identification of "high risk" individuals. A few of the risk models were consistent about which HIV-negative persons were high risk, but was very poor agreement about which 
HIV-positive persons were high risk. This weak agreement may have been due to small numbers in this study. A larger cohort study involving HIV-positive and HIV-negative individuals may help validate existing CVD risk prediction models, or develop new ones, for such underserved populations.

HOW DOES DEPRESSION AFFECT THE UTILIZATION OF HIGH- AND LOW-VALUE CARE? Brian Halbert; Roger B. Davis; Christina C. Wee. Beth Israel Deaconess Medical Center, Brookline, MA. (Control ID \#2465839)

BACKGROUND: Persons with depression under-utilize high-value cardiovascular services. However, they over-utilize low-value opioids for chronic pain. It is unclear if patients with depression over- or under-utilize other low-value ambulatory services. METHODS: We conducted a secondary analysis of the National Ambulatory Medical Care Surveys from 2006 to 2012 to compare high- and low-value care between US adults with and without depression and antidepressant use during primary care visits. After each visit, providers indicated if the patient had depression and submitted lists of medications and diagnoses. We derived quality measures from consensus guidelines and available data in the survey. High-value measures included: aspirin, statin, and beta-blocker use for coronary disease; ace-inhibitor and betablocker use for heart failure; pharmacotherapy for osteoporosis; and tobacco cessation counseling for smokers. Low-value measures included prostate cancer screening for men $\geq 75$ years old; electrocardiogram, complete blood count, X-ray, and urinalysis during routine general medical exams; opioid prescription for back pain; CT/MRI for acute back pain; and antibiotics for uncomplicated upper respiratory tract infections. We used logistic regression to compare visits by depressed patients with or without antidepressant use to a reference group of visits by patients without depression or antidepressant use. We controlled for age, sex, race, year, region, number of prior visits, and the presence of arthritis, asthma, cancer, stroke, heart failure, chronic kidney disease, COPD, diabetes, hyperlipidemia, hypertension, coronary disease, and obesity. Analyses accounted for the complex survey design. RESULTS: We identified 64,698 visits by adult patients to their primary care physicians from 2006 to 2012 . Overall, $80.6 \%$ of visits were by patients without depression not on antidepressants (reference), $5.9 \%$ of visits were by patients with depression not on antidepressants, and $6.8 \%$ of visits were by patients with depression on antidepressants. An additional $6.6 \%$ of visits were by patients without depression on antidepressants (data not shown). Baseline characteristics varied significantly across groups, including more female patients, more white patients, and more prior visits among the depression groups, regardless of antidepressant use. Compared to reference, visits by patients with depression not on antidepressants had lower rates of many high-value measures, although only statin use for coronary disease, beta-blocker use for coronary disease, and pharmacotherapy for osteoporosis were significantly lower after adjustment. Of the low valuemeasures, only opioid use for back pain was significantly higher (Figure 1). In contrast, visits by patients with depression on antidepressants did not differ significantly in any high-value measures compared to the reference group. Of the lowvalue measures, opioid use for back pain and CBC during GME were signficantly higher (Figure 2).

CONCLUSIONS: Visits by patients with depression not on antidepressants were associated with lower utilization of several high-value ambulatory services. This finding was not demonstrated among visits by patients with depression on antidepressants. Visits by patients with depression were associated with opioids for back pain regardless of antidepressant use; however, there was no associations for most other low-value services. Depressed patients not on antidepressants may be an important target population to improve ambulatory care.
Adjusted odds ratios: Depression without antidepressant use

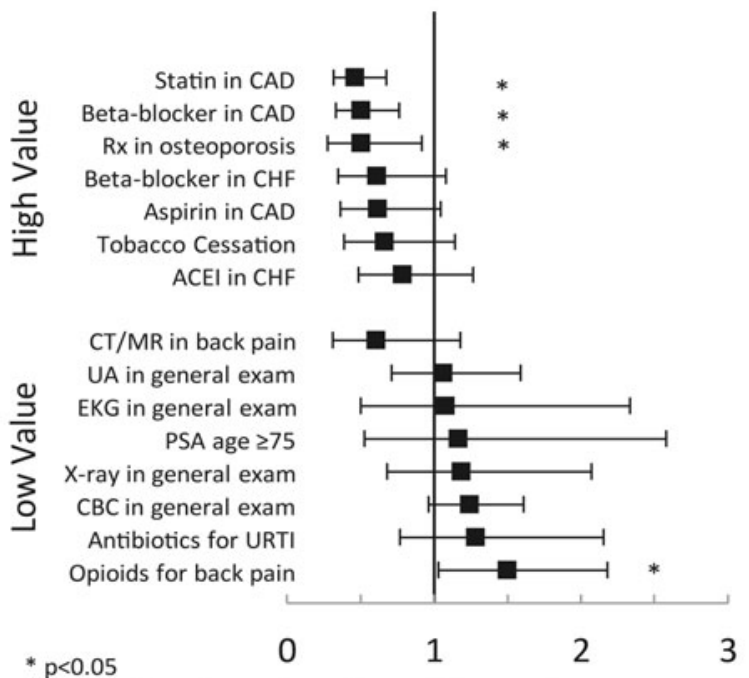

Reference is no depression and no antidepressant use Figure 1

Adjusted odds ratios: Depression with antidepressant use

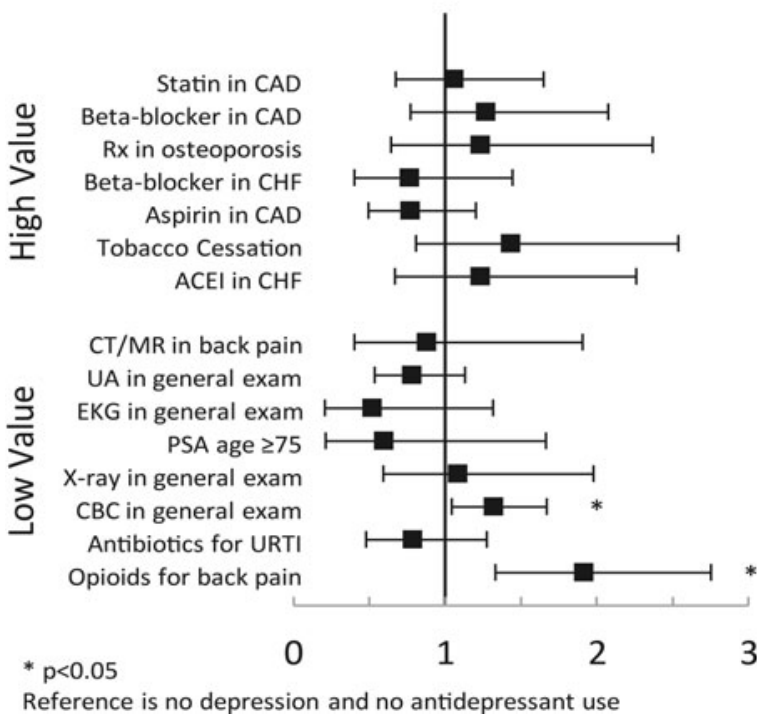

Figure 2

HOW DOES HOSPITAL PERFORMANCE FOR READMISSION RATES PUBLICLY DISPLAYED ON MEDICARE'S 'HOSPITAL COMPARE' WEBSITE COMPARE TO WHETHER A HOSPITAL WAS PENALIZED FOR READMISSION RATES BY THE HOSPITAL READMISSION REDUCTION PROGRAM? Jennifer Meddings; Heidi Reichert; Shawna N. Smith; Laurence F. McMahon. University of Michigan, Ann Arbor, MI. (Control ID \#2470616)

BACKGROUND: Hospital rates for unplanned readmissions within 30 days for 5 conditions (heart failure, pneumonia, heart attack, chronic obstructive pulmonary disease, total hip or knee arthroplasty) are publicly reported on Medicare's 'Hospital Compare' website for consumers using 3 potential grades: "No different than the National Rate," "Worse than the National Rate," or "Better than the National Rate;" the grade assigned accounts for the margin of uncertainty for this measurement. By the recently implemented Hospital Readmissions Reduction Program, the Centers for Medicare and Medicaid 
Services (CMS) financially penalize hospitals by a percentage reduction called the "Readmissions Adjustment Factor" in all Medicare hospitalization payments based on a calculation of "excess readmission ratio" (ERR) and magnitude of readmission payments for all 5 conditions; this calculation does not account for measurement uncertainty. Our objective was to assess how often hospitals that were financially penalized for readmission rates had readmission grades of "No different" or "Better than the National Rate" displayed on Medicare's 'Hospital Compare' website for consumers.

METHODS: Retrospective secondary data analysis of publicly reported hospital data from the 'Hospital Compare' website from July 1 2010-June 30 2013. We compared each hospital's readmission grade for each of the 5 conditions as displayed on the website for consumers to whether the hospital received a financial penalty overall for readmissions from this time period by the downloadable data file for Readmissions Adjustment Factor. RESULTS: Overall, 2591 (77\%) of the 3375 hospitals with data reported on 'Hospital Compare' received a financial readmission penalty by the Readmissions Adjustment Factor; 1507 (58 \%) of 2591 hospitals were graded as "No different" or "Better than the National Rate" on the 'Hospital Compare' display for all 5 conditions; 209 (8\%) were graded as "Worse than the National Rate" for only 1 of the 5 conditions; only 69 (3\%) were graded as "Worse than the National Rate" on more than 1 condition. Of the 3375 hospitals with data reported, half $(1802,53 \%)$ were graded as "No different" or "Better than the National Rate" for readmission rates for all 5 conditions; 1507 (84\%) of these 1802 hospitals received a financial penalty by the Readmission Reduction Program.

CONCLUSIONS: Many hospitals received financial penalties by the Readmissions Reduction Program despite being reported and displayed for consumers as having readmission rates for all 5 conditions as being "No different" or "Better than the National Rate" on Medicare's 'Hospital Compare' website. Two of the most heralded CMS programs for publicly reporting value and linking value to payment yield vastly different results about readmission performance for individual hospitals. The underlying uncertainty in the measurement of readmission rates is reflected in the consumer-targeted "Hospital Compare" website grades, but not in the assigned CMS financial penalties for readmission. Uncertainty in measurements used to assess quality must be reflected in any credible payment system that purports to embrace the value-based purchasing paradigm.

HOW INTERNATIONAL HEALTH ELECTIVES FULFILL ACCREDITATION COUNCIL FOR GRADUATE MEDICAL EDUCATION CORE COMPETENCIES: A QUALITATIVE STUDY. Hannah C. Nordhues; M. U. Bashir; Stephen P. Merry; Adam P. Sawatsky. Mayo Clinic, Rochester, MN. (Control ID \#2467510)

BACKGROUND: Residents have shown an increased desire to participate in international health electives (IHE). Studies have concluded that IHEs offer a unique, identifiable set of educational opportunities. Within the field of medical education, there has been focus on competency-based education (CBE). Multiple experts have called for development of competencies for the field of global health and for individual IHEs in order to establish consistency across these experiences and to develop assessment tools. We aimed to explore the perspectives of a large sample of residents across multiple specialties of how IHEs fulfill the six ACGME core competencies.

METHODS: We conducted a thematic analysis of resident perspectives to assess how IHEs fulfill the six ACGME core competencies. Data was collected from residents from all specialties across three Mayo Clinic training sites who participated in an IHE through the Mayo International Health Program. Upon return from the IHE, each resident was required to submit a reflective essay outlining details of the trip and how it impacted him or her personally and professionally. We analyzed all reflective reports for participants between 2001 and 2014. The authors created a codebook a priori from the six ACGME core competencies: Medical Knowledge (MK), Patient Care and
Procedural Skills (PC), Interpersonal and Communication Skills (ICS), Practice-Based Learning and Improvement (PBLI), and Professionalism (PROF). Using a deductive strategy, three coders independently coded the first 3 years of reflective reports in duplicate and reconciled differences through consensus. Once the coders reached substantial agreement (intercoder reliability, $k>0.60$ ), the remaining reports were individually coded. The authors inductively coded data corresponding to each of the six deductive codes in duplicate using a constant comparative method to identify significant themes within each code. The authors used meetings, discussion, and memoing to organize the identified themes into categories that explain larger domains of the six ACGME core competencies. This study was approved by the Mayo Clinic Institutional Review Board.

RESULTS: We analyzed 377 resident reports, including participants from 40 medical and surgical specialties who visited 56 countries. We identified multiple themes within each of the six ACGME core competencies. Within the domain of MK, we identified 5 themes, including knowledge of: tropical diseases; non-tropical diseases rarely seen in developed countries; advanced and atypical presentations of common diseases; diseases of poverty; and socioeconomic and epidemiologic determinants of health. Within PC we identified 7 themes: time management and efficiency in patient care; provision of care in a limited resource setting; flexibility and resourcefulness; unique procedural approaches; differential diagnosis building skills; physical examination skills and clinical acumen; and understanding and respecting patients' cultural beliefs and values. Within PBLI we identified 3 themes: identifying limitations and employing self-directed efforts to lessen gaps in knowledge; participating in education of patients, families, and health-care providers; and participating in research, quality improvement, and protocol development. Within SBP we identified 2 themes: identifying systems errors and implementing systems solutions; and incorporating considerations of cost awareness and risk-benefit analysis into care. Within ICS we identified 5 themes; working with translators, or as a translator; working as an effective part of multidisciplinary teams; communicating with patients from different cultures, socioeconomic status and backgrounds; appreciation for nonverbal communication skills; and the opportunity and ability to act in a consultative role. Within PROF we identified 5 themes; responding to patient needs above self-interest; displaying sensitivity and responsiveness to a diverse patient population; exhibiting compassion and empathy; balancing ethical principles with practical care; and rejuvenating their decision to pursue medicine. These experiences provided a contrast between the host country and the home institution, presenting a stimulus for reflection. Residents experienced a new set of common diagnoses, an increased level of autonomy, a high volume of patient care, a unique set of limited resources, experience with varied cultural and ethnic groups, exposure to different models of healthcare delivery, and a model within host-site mentors.

CONCLUSIONS: Through IHEs residents advanced their knowledge, skills, and attitudes in each of the six ACGME core competencies. To our knowledge, this is the first multi-specialty study to investigate how IHEs fulfill the ACGME core competencies. These data can be used in conjunction with input from host sites for development of global health competencies and milestones for resident assessment. It may help mentors and residents develop goals and objectives prior to an IHE and guide post-rotation debriefing. Our findings emphasize how IHEs enhance resident training within prespecified ACGME educational competencies.

\section{HOW INTERNATIONAL HEALTH ELECTIVES IMPACT RESIDENTS'} PERCEPTIONS ON DEALING WITH DEATH AND DYING M. U. Bashir; Hannah C. Nordhues; Stephen P. Merry; Adam P. Sawatsky. Mayo Clinic, Rochester, MN. (Control ID \#2469632)

BACKGROUND: There has been a progressive increase in residents' desire to participate in international health electives (IHE). Studies demonstrate that they provide a number of 
opportunities and experiences which are valuable to a trainee. Some of these benefits include varying manifestations of common diseases, increased exposure to uncommon diseases, and an increased reliance on clinical skills in managing and treating various conditions. These electives can serve as transformative experiences which have the potential to dramatically alter an individual's future practice. Given the potential to influence education and careers, more information is needed to determine how these experiences can affect trainees, so that educators can better prepare them for and support them after these experiences. We aimed to analyze resident's personal reflections regarding perceptions on death and dying, to form a better understanding of the range of residents' responses and attitudes.

METHODS: The Mayo International Health Program (MIHP) allows residents from all specialties at all three Mayo training sites the opportunity to participate in IHEs. Upon return from an IHE, each resident is required to submit a reflective essay outlining details of the trip and how it impacted him or her personally and professionally. For this study the authors analyzed all reflective reports for participants between 2001 and 2014. We conducted a thematic analysis of resident perspectives to assess how IHEs impacted residents perceptions on death and dying. We created a codebook a priori from the six ACGME core competencies. All three coders independently coded the first 3 years of reflective reports in duplicate and reconciled differences through consensus. Once a predetermined level of agreement was reached, the remaining reports were coded individually. Within the code of professionalism, we looked specifically at the residents experiences with death and dying and attempted to identify major emerging themes that recurred throughout the narratives. We met as a team to discuss coding and categorize the major themes. This study was approved by the Mayo Clinic Institutional Review Board. RESULTS: We analyzed 377 resident reports, including participants from 40 medical and surgical specialties who visited 56 countries. We identified 5 major themes regarding residents' perceptions on dealing with death and dying: increased frequency of patient death; needless or preventable deaths; lack of closure regarding cause of death; difficulty with triaging limited healthcare resources; and differing cultural expectations and beliefs about death. Many of the reflections discussed an increased exposure to death, but this was often accompanied by a sense of helplessness, especially when deaths were considered preventable either by earlier access to healthcare or access to more resources such as could be found at the residents' home institutions. This feeling of helplessness was often compounded by lack of closure as the circumstances surrounding patients' deaths often remained a mystery. Residents discussed distress when being faced with the need to reserve vital healthcare resources for those who would benefit most often at the expense of those who were deemed "futile." Not only did the residents feel ill-prepared to make such decisions, they struggled with the emotional impact of making value judgments about who should live and who should die. Another theme was that of differing cultural views toward death. Notably, there was often a more accepting view of death, with patients and practitioners in the host countries accepting it as an inevitability, and patients were grateful to providers whether or not there was anything that could be offered medically. "Death was seen as an accepted outcome, not a failure of medical management." Moreover, many residents struggled with cultural differences regarding end-of-life care. Specifically, the paternalistic view toward medical decision-making at the end of life was particularly difficult as it seemed at odds with the way medicine is practiced in the United States. This was exemplified by a case in which a resident and his host mentor fundamentally disagreed on whether it was in the best interest of the patient to disclose or withhold his terminal diagnosis.

CONCLUSIONS: We have identified multiple themes regarding the impact of IHEs on residents' perspectives of dealing with death and dying. Understanding these perspectives will be beneficial in ensuring that residents get the most out of IHEs while minimizing potential harms. IHEs can offer a variety of benefits to residents and can have a transformative impact, but can also evoke feelings of fear and helplessness, particularly when dealing with death and dying. We must ensure that residents have the appropriate support to process the more difficult aspects of these IHEs. Further investigation is needed to determine the optimal method for how best to address these concerns. However, our data may serve as a useful platform to help directors develop IHE curricula and guide discussion about death and dying before and after IHEs.

HOW MANY PRIMARY CARE DOCTORS DO WE REALLY HAVE? ESTABLISHING A PRECISE STATEWIDE COUNT OF PRIMARY CARE PHYSICIAN FULL TIME EQUIVALENTS Theodore Long ${ }^{2,}{ }^{1}$; Sandra Powell²; Margaret I. Gradie ${ }^{2}$; Tracy L. Jackson ${ }^{2}$; Emily Silvia ${ }^{2}$; Russell Phillips ${ }^{3}$; Nicole Alexander-Scott ${ }^{2} .{ }^{1}$ Yale University School of Medicine, Providence, RI; ${ }^{2}$ Rhode Island Department of Health, Providence, RI; ${ }^{3}$ Harvard Medical School Center for Primary Care, Boston, MA. (Control ID \#2469502)

BACKGROUND: National workforce projections predict a shortage of 31,000 adult primary care providers by the year 2025, but with considerable uncertainty. Making an accurate assessment of the existing primary care workforce is essential. The Centers for Disease Control and Prevention estimates Rhode Island to have approximately 701 physicians in the state. In 2010, the Graham Center estimated RI to have 830 physicians The highest estimate is from the federal Health Resources and Services Administration (HRSA), which approximates 1002 primary care physicians in the state. In the context of estimates that varied by as much as $40 \%$, we collected primary data to determine the primary care workforce capacity in order to base state healthcare planning on accurate assessments.

METHODS: The Rhode Island Department of Health (RIDOH) Primary Care Practice survey was designed to capture comprehensive information about primary care practices including providers and their full time equivalents (FTE), Medicaid access to care, and FTEs of nurse practitioners and physician assistants. A definition of primary care was used that is consistent with the primary care workforce literature and federal definition. This included general internal medicine, geriatrics, family medicine, or pediatrics. Using the RIDOH licensure database, a list of potential primary care physicians was created and sorted by location to establish a list of practices. Telephone communication confirmed that they were primary care practices The survey was sent to practice administrators. For the primary care physicians for whom data was unable to be collected, 1.0 FTE was assumed.

RESULTS: The total FTEs of primary care physicians in Rhode Island was found to be 602.7. A total of 341 primary care practice locations were identified in Rhode Island. Data were collected for 719 out of 803 identified primary care physicians, yielding a response rate of $90 \%$ of physicians. On average, there was one primary care physician FTE for every 1718.1 Rhode Islanders. The median age among physicians was 52 with $11 \%$ being age 65 or above. In calendar year 2014, the mean number of physician clinical visits for family medicine, internal medicine, and pediatric physicians was 6118,6076 , and 7665 , respectively. Sixty-six percent of practices accepted new adult patients, and $45 \%$ of practices accepted new pediatric patients. In terms of patients covered by Medicaid, $45 \%$ of practices accepted new adult Medicaid patients, and $38 \%$ of practices accepted new pediatric Medicaid patients. Forty percent of practices reported having nurse practitioners, with a mean FTE of 1.78 and a mean number of clinical visits in 2014 of 4063 per practice. Eighteen percent of practices reported having physician assistants, with a mean FTE of 1.33 and a mean number of clinical visits in 2014 of 2798 per practice CONCLUSIONS: The precise counting of 602.7 full time equivalents of primary care physicians in Rhode Island is substantially lower than other previous estimates by up to $40 \%$. Based on the Henry J. Kaiser Family Foundation specific recommendation for Rhode Island and the Health Resources and Services Administration evaluation methodology using 1500:1 population to physician ratio, the data indicate an approximate $10 \%$ shortage of full time equivalents of primary care physicians. 
While there may have been an improvement over recent years, focused efforts should be made to continue educating, recruiting, and retaining primary care physicians and providers to address primary care needs in Rhode Island.

\section{HOW MEN CHOOSE SPECIALISTS WHEN DIAGNOSED WITH CANCER: A} QUALITATIVE STUDY OF MEN WITH PROSTATE CANCER Tammy Jiang ${ }^{3}$; Christian H. Stillson $^{2}$; Craig E. Pollack ${ }^{1}$; Linda B. Crossette ${ }^{2}$; Michelle E. Ross ${ }^{2}$; Archana Radhakrishnan ${ }^{1}$; David Grande ${ }^{2,}{ }^{2}$. ${ }^{1}$ Johns Hopkins University School of Medicine, Baltimore, MD; ${ }^{2}$ University of Pennsylvania, Philadelphia, PA; ${ }^{3}$ Brown University School of Public Health, Providence, RI. (Control ID \#2468806)

BACKGROUND: Prostate cancer is one of the most common cancers among men in the United States. Previous studies suggest that the specific physician patients see at the time of their prostate cancer diagnosis influences the type of treatment that they ultimately receive. However, little is known about how men with prostate cancer actually choose specialists for treatment. To address this gap, we conducted a qualitative study of men diagnosed with prostate cancer to understand how they choose specialists.

METHODS: We conducted semi-structured, qualitative interviews with 47 patients who were diagnosed with prostate adenocarcinoma between 2013 and 2015 in the Greater Philadelphia region. We recruited participants from a larger survey study. Men were purposefully sampled based on whether they lived in areas with high or low potential access to urologists and radiation oncologists, as defined by the number of clinics within a 30 min drive. Telephone interviews were recorded, professionally transcribed, and imported to NVivo software for analysis. After coding all transcripts, content was discussed among the full study team and grouped into common themes.

RESULTS: Forty-seven men participated in qualitative interviews between June and August 2015. The mean age of participants was 67 years (range 59-86). Most participants were white $(81 \%), 19 \%$ were Black, and $47 \%$ of participants had at least a college degree. Fifty-three percent had private insurance, $43 \%$ had Medicare, and $4 \%$ had Medicaid. Three main patient profiles emerged for choosing specialists: activated $(21 \%)$, passive $(26 \%)$, and partially activated $(53 \%)$. Activated patients conducted substantial research when choosing a specialist: they searched online, consulted other men with prostate cancer, and/or visited multiple specialists for opinions. "I looked things up on the internet and chose my own specific urologist... I also researched on the internet with [the hospital] and got all the documentation from them... [data] that I actually paid for from [the hospital].” When describing how they evaluated specialists, activated patients often used physician's case volume, job title, involvement in research, and years of experience as proxies for quality. In contrast, passive patients relied exclusively on referrals from their primary care provider (PCP). "I just went with what my family doctor said...I just went with what was suggested, and I was satisfied." They often emphasized trust in their referring PCP and their specialist as well as a sense of urgency to begin treatment which limited their time to even consider seeking a second opinion. "But at the time, I was so devastated that I had [cancer], and more upset, and wanted to hurry up and just get it out of me, so I chose to just have it taken out." The third patient profile, partially activated patients, took an additional step to find a specialist on their own after receiving a referral from their $\mathrm{PCP}$, but similar to passive patients, relied heavily on their PCP. They frequently described high levels of trust in their referring PCPs and emphasized trustworthiness and bedside manner when describing their specialist.

CONCLUSIONS: Although there were highly varied patterns of specialist-seeking behaviors, most patients relied almost exclusively on their PCPs for referrals to specialists after being diagnosed with prostate cancer. The results underscore the central role PCPs play in the process of choosing a specialist, which in many cases has a large and unanticipated impact on treatment decisions.
HOW RISKY IS WATCHING AND WAITING? A PILOT STUDY COMBINING CLINICAL TRIAGE AND AUTOMATED RISK MODELING TO ENHANCE ALLOCATION OF AMBULATORY INTENSIVE CASE MANAGEMENT RESOURCES FOR HIGH-RISK PATIENTS Brook Watts ${ }^{1,2}$; Renee H. Lawrence ${ }^{1}$; Cameron Carter ${ }^{1}$; Melissa Klein ${ }^{1,2}$; Kimberley Schaub ${ }^{1}$; Brigid Wilson ${ }^{1}$; Susan Kirsh ${ }^{1,2}$. ${ }^{1}$ Louis Stokes Cleveland VAMC, Cleveland, OH; ${ }^{2}$ Case Western Reserve School of Medicine, Cleveland, OH. (Control ID \#2469120)

BACKGROUND: Automated risk models that incorporate clinical and socio-demographic parameters are potentially useful tools to identify high-risk, high-utilizer populations. However, there are still substantial knowledge gaps on how best to use risk modeling to identify patients who will benefit from intensive interventions to augment usual care. In addition, though targeting additional clinical resources to high-risk patients may reduce long term costs, up-front expenses and capacity constraints necessitate efficient interventions. As part of the Veterans Health Administration (VHA) Patient Intensive Management (PIM) pilot program to improve outcomes for patients at high risk for hospitalization or death, we developed and evaluated a clinical team triage method for use in conjunction with the Care Assessment Needs (CAN) score, a validated risk model for veteran populations. Our goal was to distinguish high-risk patients for whom continuing usual care might be sufficient in order to efficiently target those patients for whom the PIM intervention might be of greatest benefit

METHODS: One hundred thirty-nine patients at our urban VHA teaching hospital were randomly identified for our local PIM program based on CAN scores in the top $10 \%$ of overall 3-month risk for morbidity or mortality and at least one acute care visit in the last 6 months. To assess appropriateness for PIM (a team-based model that focuses on care coordination, home visits, and health coaching), each patient's chart was reviewed by an interdisciplinary health care team using a standard protocol to evaluate for factors which might benefit from PIM team intervention (e.g., medication adherence, impaired access to health services). For unanswered questions after chart review, primary care providers were contacted. Based on this process, patients were triaged to either "Continue with Usual Care, PIM Watch and Wait" or "PIM Team Intervention". Charts of patients in the Continue Usual Care group were re-reviewed every 1-2 months for factors indicating "PIM Team Intervention" was now appropriate.

RESULTS: Of the 139 patients with high CAN scores, the team felt 93 patients $(66.9 \%)$ were appropriate to Continue with Usual Care. Through monitoring in the 6 months following triage, we identified four of the 93 patients $(4.3 \%)$ as having a change in circumstance that indicated PIM Team Intervention was appropriate (e.g., 2 patients with hospitalizations for potentially modifiable factors). Of the 93 patients triaged to Continue Usual Care, 43 patients (46.2 \%) had at least one ED visit (mean visits $+/-\mathrm{SD}=0.90+/-$ 1.43, range 0 to 9). Analysis of ED chart documentation suggested that frequently these visits were for basic primary care needs.

CONCLUSIONS: Using our team triage method to review patients identified by a riskbased model, in 6 months of follow-up, few patients required re-classification suggesting that a watch and wait strategy safely identified patients for whom Usual Care was an acceptable approach. Although almost half of Usual Care patients used the ED during this time period, the usage reflected issues appropriate for basic ambulatory care services - i.e. would not be efficient to engage an entire PIM team to address. Further investigations are needed to determine the optimal process and metrics to target and monitor high risk patients for team-based care interventions.

HUMAN VS. AUTOMATED PREDICTION OF CLINICAL DETERIORATION: RESULTS FROM THE UNDERSTANDING PHYSICIAN SIGNOUT RISK PERCEPTIONS (UPS) STUDY Jonathan Arnold ${ }^{2}$; Alexander Davis ${ }^{3}$; Baruch Fischhoff $^{3}$; Jon R. Grace ${ }^{2}$; Andrew Klobuka ${ }^{2}$ Emmanuelle B. Yecies²; Deepika Mohan ${ }^{1}$; Janel Hanmer ${ }^{1} .{ }^{1}$ University of Pittsburgh, Pittsburgh, PA; ${ }^{2}$ University of Pittsburgh Medical Center, Pittsburgh, PA; ${ }^{3}$ Carnegie Mellon University, Pittsburgh, PA. (Control ID \#2467959) 
BACKGROUND: Prediction of clinical deterioration is an essential aspect of hospitalized patient management and an area of interest for automation and expert system development. If clinicians can identify patients at high risk for clinical deterioration they can develop contingency plans and attempt preventative measures. If they can effectively communicate this concern across team handoffs they can ensure continuity of care for those at greatest risk. There have been previous attempts to develop simple algorithms for predicting clinical deterioration and, with the widespread implementation of electronic health records (EHR), there has been the development of more complex proprietary systems for automated prediction. Prior studies have evaluated the accuracy of clinician predictions and algorithms independently, however these have not been directly compared. The UPS study is a prospective observation study comparing physician judgment and a proprietary automated system in predicting clinical deterioration.

METHODS: The UPS study was conducted on the general internal medicine teaching floors of a tertiary care academic medical center. Twice a week for 5 months the daytime medical teams were queried regarding the likelihood of clinical deterioration for each patient under their care. Each physician member of the team was asked to give a rating from 0 (definitely will not deteriorate) to 100 (definitely will deteriorate) for each patient. Data were collected just prior to signout to the overnight team. This medical center had recently installed an automated system for predicting clinical deterioration, the Rothman Index. This system received real-time updates from the electronic medical record and classified each patient as high, medium or low risk of clinical deterioration and assigned a color value of red, yellow or blue. It also assigned a variability flag as none (not flagged), medium, high, or very high separate from the color assignment. The automated system's color and flag assignment were collected for each patient on the medical teams jus following data collection from the physicians. Predictions from the physicians and the automated system were collapsed into three categories of high, medium, and low risk. For the automated system we considered a patient high risk if their color was yellow or red and they had any variability flag set; medium risk if they their assigned color was yellow or red and they had no flag set; and low risk if their color was blue regardless of the flag. For the physicians we ranked their clinical predictions for the day and assigned patient risk category by matching the marginal distribution of the automated system. At a later time we collected the time of any rapid response team call or ICU transfer, our outcome events, in the 7 days following data collection of the predictions. We calculated the odds ratio for each category of team member (intern, resident, and attending) as well as for the automated system, comparing the odds of an outcome event in high risk versus low risk patients. We also performed a logistic regression with physician prediction and automated system prediction when both were available.

RESULTS: We collected data for 2231 patient observation times in 1252 unique patients. We collected 1517 risk assessments from interns, 1836 from residents, 1748 from attending physicians, and 2120 from the automated system. There were a total of 34 outcome events at 1 day $(24 \mathrm{~h})$, for a rate of $1.5 \% ; 70$ at 3 days $(72 \mathrm{~h})$ for a rate of $3.1 \%$; and 105 at 7 days $(168 \mathrm{~h})$ for a rate of $4.7 \%$. A high risk prediction from the automated system predicted clinical deterioration vs. a low risk prediction with an odds ratio of 34 [95\% CI: 8, 155] at 1 day, 13 [95\% CI: 6, 28] at 3 days and 10 [95 \% CI: 6, 17] at 7 days. Interns performed best among the physicians, where a high risk prediction from an intern predicted clinical deterioration vs. a low risk prediction with an odds ratio of $6[95 \% \mathrm{CI}: 2,16]$ at 1 day, 7 [95\% CI: 3, 15] at 3 days, and 8 [95 \% CI: 4, 15] at 7 days. When the automated system and interns were both included in a logistic regression model, a high risk prediction from the automated system was significantly better (OR: $18[95 \% 4,88])$ than the interns (OR: 3 [95 \% CI: 1,9]) at 1 day, but they were similar at 3 and 7 days.
CONCLUSIONS: Both physicians and the automated system were able to predict clinical deterioration at $24 \mathrm{~h}$ and up to 1 week. At $24 \mathrm{~h}$ the automated system significantly outperformed physician judgment, at longer time periods this advantage decreased and there was no significant difference at 3 or 7 days. In combined models the automated system and physician judgments had statistically significant and independent predictive power for clinical deterioration, suggesting they used different cues to predict deterioration.

HYPERTENSION AS A MAJOR BURDEN OF HEALTH CARE SYSTEM - A NATIONAL INPATIENT SAMPLE STUDY OF EMERGENCY DEPARTMENT VISITS OVER A PERIOD OF 7 YEARS Monoj K. Konda ${ }^{2}$; Hardik Chhatrala ${ }^{1}$; Yashwant Agrawal ${ }^{2}$; Sandeep Patri ${ }^{3} .{ }^{1}$ Homer Stryker MD WMU School of Medicine, Kalamazoo, MI; ${ }^{2}$ Western Michigan University Homer Stryker M.D. School of Medicine, Kalamazoo, MI; ${ }^{3}$ Western Michigan University school of medicine, Kalamazoo, MI. (Control ID \#2467963)

BACKGROUND: Almost 29 to $31 \%$ of adults in the US have hypertension and a significant portion of population has undiagnosed hypertension. Poor access to health care and medications as well as lack of adherence to treatment are main reasons for inadequate control of blood pressure which results in elevation of BP severe enough to visit emergency department (ED). The aim of this abstract is to assess trends in ER visit over a 7-year period using the data from National Inpatient Sample (NIS)

METHODS: Using the data on NIS database, we were able to assess the trends in total number of visits, percent admitted to the same hospital, percent discharged from ED, percent died during ED visit, percent died during hospital stay and also the trends in population demographics, location, teaching status of hospitals and insurance payers.

RESULTS: The total number of ED visits gradually increased from 559,934 to 759,320 over 7 year period. There is a gradual decrease in percent of patients admitted to the same hospital (10.55 to $7.96 \%$ ) and a corresponding increase in percent discharged from ED. Deaths during ED visit remained constant at $0.02 \%$ while the deaths during hospital stay decreased from $0.18 \%$ in 2006 to $0.08 \%$ 2012. The male and female percentages remained constant through this period at around 40 and $59 \%$ respectively. The percent of patients $85+$ years old increased from 5.83 to $6.75 \%$ steadily and a decrease was observed in age group 18-44 years. The percent costs paid by Medicare and Medicaid increased from $45.72 \%$ in 2006 to $50.49 \%$ in 2012. A shift of patients to teaching hospitals was noted over the period and almost half of the hypertension visits are noted in the south region of United States.

CONCLUSIONS: From the above statistics, it is known that overall burden of hypertension has been increasing over health care and effective outpatient control is very essential to cut the expenses. For unknown reasons the southern region of United States has almost half of the country's total ED visits for hypertension. There has been a shift in the age towards elderly reflecting better health care resulting in increased life expectancy and survivals. It also reflects more compliance among the younger population. There has been no change in the sex ratio over the 7-year period and women continue to visit ED with hypertension in higher numbers than men. An overall increase in burden over Medicare and Medicaid can also be noted from above observations. Hence, it is important to focus on aggressive control of hypertension and also patient education regarding compliance to address this major and one of the most common medical illnesses. It has to be borne in mind that NIS database includes data from only hospitals participating in the NIS (around $20 \%$ of all hospitals in US) 
National Statistics of All ED Visits for Hypertension (ICD 9 code 401.9)

\begin{tabular}{|c|c|c|c|c|c|c|c|}
\hline & 2006 & 2007 & 2008 & 2009 & 2010 & 2011 & 2012 \\
\hline Total number of visits & 559,934 & 587,588 & 617,969 & 669,950 & 708,435 & 735,792 & 759,320 \\
\hline $\begin{array}{l}\text { Admissions to } \\
\text { same hospital }\end{array}$ & 59,057 (10.55 \%) & $63,458(10.80 \%)$ & 60,254 (9.75 \%) & 66,956 (9.99 \%) & 66,592 (9.40 \%) & 66,203 (9.00 \%) & 60,434 (7.96 \%) \\
\hline Died during hospital stay & $106(0.18 \%)$ & $98(0.15 \%)$ & $102(0.17 \%)$ & * & $65(0.10 \%)$ & * & $49(0.08 \%)$ \\
\hline Died during ED visit & $88(0.02 \%)$ & $81(0.02 \%)$ & $69(0.01 \%)$ & $140(0.02 \%)$ & $132(0.02 \%)$ & $136(0.02 \%)$ & $151(0.02 \%)$ \\
\hline Sex M F & $39.88 \% 60.12 \%$ & $40.00 \% 59.98 \%$ & $40.23 \% 59.75 \%$ & $40.52 \% 59.35 \%$ & $40.69 \% 59.30 \%$ & $40.21 \% 59.78 \%$ & $40.86 \% 59.13 \%$ \\
\hline $\begin{array}{l}\text { Age 5+ } \\
8-44\end{array}$ & $5.83 \% 24.52 \%$ & $6.44 \% 23.74 \%$ & $6.34 \% 23.45 \%$ & $6.38 \% 23.28 \%$ & $6.34 \% 23.57 \%$ & $6.74 \% 22.47 \%$ & $6.75 \% 21.93 \%$ \\
\hline Payer Medicare Medicaid & $35.94 \% 9.78 \%$ & $35.02 \% 9.96 \%$ & $35.10 \% 10.16 \%$ & $34.73 \% 10.98 \%$ & $35.04 \% 11.92 \%$ & $37.11 \% 13.07 \%$ & $37.59 \% 12.90 \%$ \\
\hline
\end{tabular}

Statistics based on estimates with a relative standard error (standard error / weighted estimate) greater than 0.30 or with standard error $=0$ in the nationwide statistics (NIS, NEDS, and KID) are not reliable. These statistics are suppressed and are designated with an asterisk $\left({ }^{*}\right)$

\section{IDENTIFICATION AND REFERRAL OF EMERGENCY DEPARTMENT PATIENTS WITH A PRESENTATION SUSPICIOUS FOR UNDERLYING MALIGNANCY Sara Chen ${ }^{1}$; Onita Bhattasali ${ }^{2}$; Renzhong Ran ${ }^{1}$; Margaret Crawford ${ }^{1}$; Martha Mims ${ }^{1}$; Cedric Dark ${ }^{1} .{ }^{1}$ Baylor College of Medicine, Houston, TX; ${ }^{2}$ Kaiser Permanente, Los Angeles, CA. (Control ID \#2468037)}

BACKGROUND: In 2010 more than 1.5 million new cases of cancer were diagnosed in the United States. The same year the Centers for Disease Control and Prevention reported that neoplasms accounted for 255,000 or $0.2 \%$ of all United States emergency department primary diagnoses. A large percentage of cancer diagnoses occur in the emergency setting, but limited literature is published describing this phenomenon in detail. One Michigan study reported that among elderly patients, $23 \%$ of colorectal cancers and $19 \%$ of lung cancers were diagnosed during an emergency department visit. Patients diagnosed with cancer in the emergency setting frequently present with late stage disease and perform poorly compared to patients diagnosed earlier in the disease process. First-time cancer diagnoses in the emergency department are likely a result of a multitude of factors. The most common malignancies in the United States include prostate, breast, lung, and colon cancer. With the exception of lung cancer, there are routine screening algorithms for each of these malignancies that have enabled physicians to diagnose cancer at earlier stages in the absence of symptoms and when chance for remission or cure is high. Unfortunately, recommended screening practices are not consistently employed. White patients and patients insured privately or through Medicare are significantly more likely to receive screening examinations compared to non-white patients and patients uninsured or insured through Medicaid, respectively. In addition, patients without primary care doctors are less likely to undergo routin screening for cancers. These inequalities often fail to provide crucial screening services to the highest risk groups and allow disease to progress without detection. A study in England identified age, social deprivation status, and male gender to be significantly associated with first-time diagnosis in the emergency department. The study also noted geographic differences in rates of diagnosis by emergency admission, though these results were not statistically significant. Geographic differences may be secondary to regional average socioeconomic status, inadequate primary care physician coverage, or other factors. Identification of regional differences in our patient population would allow us to create geographically tailored interventions to decrease emergency department cancer diagnoses.

METHODS: A retrospective chart review at a county hospital with 182,099 annual visits in 2014 was conducted between August 18th, 2014 and May 23rd, 2015. Patients who presented to the ED and were suspected of having an underlying malignancy with subsequent referral to the IRC were included in our analysis. Descriptive statistics were computed for patient demographic characteristics and two-tailed Fisher's exact test was used to calculate patient outcomes.

RESULTS: Overall, 122 patients were referred from the ED to the IRC for a suspected underlying malignancy and were included in our final analysis. The mean age at presentation was 51 (range 18-81) and $52.5 \%$ were women. Of all patients referred to the IRC,
$103(84.4 \%)$ presented for further work-up of their suspected malignancy and 87 (71.3\%) were successfully referred to further specialists. Mean time between ED presentation and IRC visit was 11.4 days (range 1-48). Sixty-nine (56.6\%) patients were ultimately diagnosed with cancer; of these diagnoses, $33(47.8 \%)$ were metastatic at time of presentation. The most common malignancies diagnosed were colorectal (14), breast (12), and pancreatic cancer (10). The most common malignancy diagnosed in patients with and without a PCP was pancreatic cancer and colorectal cancer, respectively. Patients without a PCP were more likely to be diagnosed with a malignancy than those with a PCP (63.8 vs. $42.9 \%$; OR 2.34 [CI 1.09,5.03]; $p=0.035$ ). However, the proportion of patients diagnosed with metastatic cancer on presentation did not significantly vary with PCP status (21.4 \% with PCP vs. $30.0 \%$ without PCP; OR 1.57 [CI 0.65,3.78]; $p=0.393$ ).

CONCLUSIONS: This study found that patients without a PCP were twice as likely to be diagnosed with a malignancy versus those with a PCP. In this sample, there was no significant difference in percentage of cancers that were metastatic on presentation. The natural course of cancer varies depending on primary site, and several of the malignancies observed in this study often have metastases on presentation. The most common malignancies diagnosed among patients without a PCP were ones for which there is a national screening protocol (i.e. colorectal or breast cancer). This aligns with our hypothesis that patients without a PCP are more likely to present to the ED with a malignancy that would otherwise have been detected in a primary care setting. Savage et. al found colorectal, lung, and CNS malignancies to be the most common cancers presenting in a London-based ED. Our study population excludes patients who were direct hospital admissions. As a result, CNS and hematologic malignancies may not be appropriately represented in our study. Interestingly, over half of patients referred to the IRC were ultimately found to have a malignancy, suggesting that the IRC is an important diagnostic resource.

IDENTIFICATION OF HIGH UTILIZATION INPATIENTS ON INTERNAL MEDICINE SERVICES Marc Heincelman; Samuel O. Schumann; Jenny Riley; Patrick D. Mauldin; Jingwen Zhang; Justin Marsden; Don Rockey; William P. Moran. Medical University of South Carolina, Charleston, SC. (Control ID \#2452720)

BACKGROUND: As health care reform moves toward value based care, hospitals must continue to focus on high quality care for their patients, yet be considerate of costs whenever appropriate. Available data suggest that a relatively small number of patients consume a disproportionately large proportion of medical resources. In order to intervene on these select patients and provide optimal value care, identification early in the hospital stay is needed. The objective of this study is to identify patient features associated with high utilization in inpatients and to develop a predictive model to identify these potentially high cost patients on admission. 
METHODS: An administrative, hospital-based, retrospective observational study was performed on all adult patients $>18$ years old admitted to internal medicine services from July 1st, 2013-June 30th, 2014. We identified the top $10 \%$ highest cost patients admitted to internal medicine services over this 1 -year period. We examined 45 admission variables, including demographic, clinical, and laboratory features to determine variables associated with high inpatient costs that are available at the time of admission. Using multivariate logistic regression analyses, we developed a predictive model that could be used on admission to identify potential high utilization patients. The primary study outcome was total hospital costs. Secondary outcomes included length of stay and patient discharge status.

RESULTS: The top $10 \%$ highest cost patients represented our high utilizer patients $(n=757)$. The median total hospital cost for high utilization patients was $\$ 53,430 \pm$ 60,679 compared to $\$ 8,431 \pm 7,245$ in the control group $(p<0.0001)$. The median length of stay for high utilization patients was $19.5 \pm 32.5$ days compared to $3.8 \pm$ 3.9 days in the control group $(p<0.001)$. Variables associated with high utilization included transfer from an outside hospital (OR 1.6), admission to pulmonary/medical intensive care unit (OR 2.4), admission to cardiology (OR 1.8), chronic or active coagulopathy (OR 2.6), and fluid and electrolyte disorders (OR 2.1). The receiveroperating characteristics (ROC) curve of this prediction model yielded an area under the curve (AUROC) of 0.80

CONCLUSIONS: High resource utilization patients appear to have a specific phenotype. Specific admission variables can be used to develop a predictive model that identifies high cost patients in real-time.

IDENTIFICATION OF HIGH UTILIZATION INPATIENTS ON INTERNAL MEDICINE SERVICES USING TRAJECTORY VARIABLES Samuel O $\underline{\text { Schumann }^{2}}$; Marc Heincelman ${ }^{2}$; Jingwen Zhang ${ }^{1}$; Justin Marsden ${ }^{2}$; Patrick D. Mauldin ${ }^{2}$; Don Rockey ${ }^{2}$; William P. Moran ${ }^{2} .{ }^{1}$ MUSC, Charleston, SC; ${ }^{2}$ Medical University of South Carolina, Charleston, SC. (Control ID \#2470079)

BACKGROUND: The value based care movement places considerable pressure on healthcare systems to deliver high quality medical care while decreasing overall costs. Numerous studies have demonstrated that a small number of patients disproportionately consume an exceedingly large amount of medical resources. In order to intervene on these patient's excessive resource use and provide high value care, identification of these patients early in their hospitalization is crucial. However the ability to identify these patients in real time has remained challenging. The objective of this study is to identify patient features associated with high utilization in inpatients and to develop a predictive model, using both static and trajectory variables, to identify these potentially high cost patients on admission.

METHODS: An administrative, hospital-based, retrospective observational study was performed on all adult patients $>18$ years old admitted to internal medicine services from July 1st, 2013-June 30th, 2014. We identified the top $10 \%$ highest cost patients admitted to internal medicine services over this 1-year period. We examined 45 admission variables, including demographic, clinical, and laboratory features to determine variables associated with high inpatient costs that are available at the time of admission. Using multivariate logistic regression analyses, we developed a predictive model using both static and trajectory variables that could be used on admission to identify potential high utilization patients. The primary outcome for the multivariate analysis was binary and represented whether or not a patient was in the top $10 \%$ of total hospital costs

RESULTS: The top $10 \%$ highest cost patients represented our high utilizer patients $(n=757)$. The median total hospital cost for high utilization patients was $\$ 53,430 \pm$ 60,679 compared to $\$ 8,431 \pm 7,245$ in the control group $(p<0.0001)$. The median length of stay for high utilization patients was $19.5 \pm 32.5$ days compared to $3.8 \pm$ 3.9 days in the control group $(p<0.001)$. Variables significantly associated with high utilization included transfer from an outside hospital (1.70 OR: 1.35-2.14 95\% CI), admission to pulmonary/medical intensive care unit (2.50 OR: $1.96-3.2095 \% \mathrm{CI})$, admission to cardiology (1.71 OR: 1.27-2.31 $95 \% \mathrm{CI}$ ), chronic or active coagulopathy (2.37 OR: 1.95-2.89 $95 \% \mathrm{CI}$ ), fluid and electrolyte disorders (1.99 OR: 1.62-2.45 $95 \% \mathrm{CI}$ ) and a rising BUN slope from days 0 to 3 (1.57 OR: 1.14-2.18 $95 \%$ CI). The Receiver-Operating Characteristics Curve of this prediction model yielded an Area Under the Curve of 0.81, suggesting good model accuracy.

CONCLUSIONS: High resource utilization patients appear to have a specific phenotype. Both static and trajectory variables obtained early in a patients hospital course can be used to develop a predictive model that identifies high cost patients in real-time.

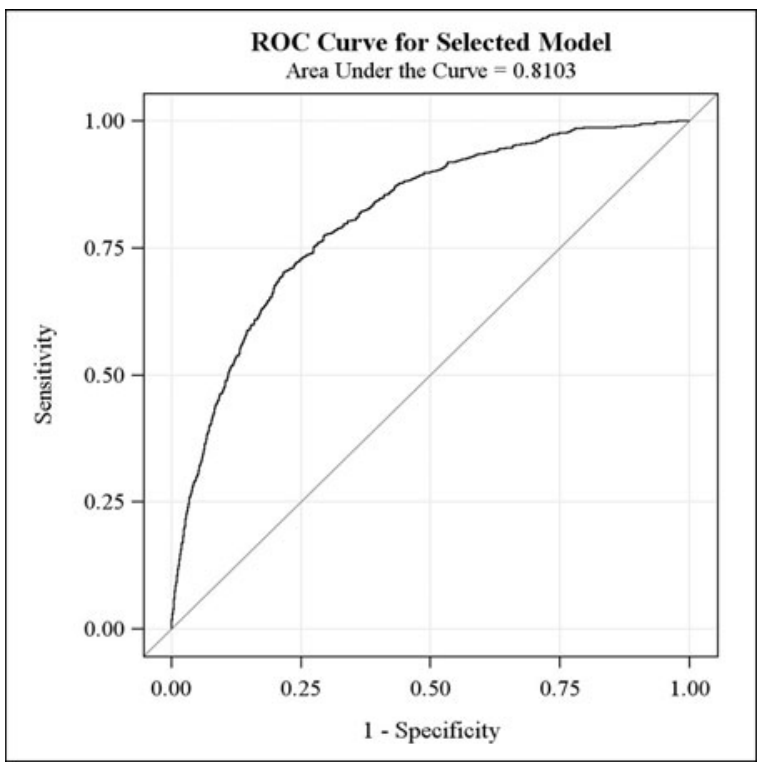

ROC Curve

IDENTIFY OPPORTUNITY TO IMPROVE CLINIC PERFORMANCE AND REDUCE PATIENT SERVICE TIME IN A COUNTY PRIMARY CARE CLINIC: A QUALITY IMPROVEMENT PROJECT Kin Wai Hung ${ }^{1}$; Anna Shvartsur ${ }^{3}$; Steven Hadawar ${ }^{3}$; John $\mathrm{Sy}^{2}$; Richard Tennant ${ }^{2} .{ }^{1}$ Olive View - UCLA Medical Center, Sherman Oaks, CA; ${ }^{2}$ Olive View-UCLA Medical Center, Sylmar, CA; ${ }^{3}$ University of California Los Angeles, Los Angeles, CA. (Control ID \#2463418)

BACKGROUND: Objective: Identify opportunity to improve the clinic performance and reduce patient service time in a Los Angeles county hospital primary care clinic Study Design: Operational process analysis

METHODS: Methods: 1. Map out the critical processes for a clinic visit 2.Identify the key sources of arrival and service variability at these processes 3 . Examine the patient flows through these processes to determine the process times 4.Calculate the capacity and utilization and identify potential bottlenecks 5 . Identify how best to improve the clinic performance and reduce patient service time by reducing variability, improving capacity, and reducing utilization at each process

RESULTS: Results: A total of 64 patient visits were compiled for analysis. Four critical clinic processes were identified in the sequence: 1. Check-in 2. Vitals 3. Physician 4. Dispo. Total service time for each clinic visit averaged $88.73 \mathrm{~min}$, of which $58.64 \mathrm{~min}$ 
contributed to wait time. An estimated 24.50 min of wait time were behind-the-scenes (unmeasured) processes, resulting in a total clinic process time of $54.59 \mathrm{~min}$. Process time of Physician averaged $34.15 \mathrm{~min}$, followed by Dispo (12.89 $\mathrm{min}$ ), Vitals (5.33 $\mathrm{min}$ ), and Check-in (2.22 min). Wait time was highest between Vitals and Physician (24.59 min), followed by wait time between Check-in and Vitals (17.61 min), and least between Physician and Dispo (16.44 min). Physician process was identified as the bottleneck, with the lowest capacity (10.54 patient - served per hour) and the highest utilization (75.88\%). Significant wait time totaling $35 \mathrm{~min}$ was due to variability occurred prior to the Physician process. Variability included arrival of patient time, interruptions of LVNs for translation, complexity of patients, room assignment and availability, printer malfunctioning, and multiple order forms. Wait time after Physician process was primarily due to behind-thescenes processes (13.43 min). Behind-the-scenes processes included writing orders, printing prescriptions, processing orders, scheduling and documentation.

CONCLUSIONS: Conclusions: Opportunities to reduce variability, improve capacity, and reduce utilization are identified. System-wide proposal to target the reduction of variability include enforcing on- time appointment policy, establishing volunteer translators program, improving allocation of room within the clinic, scheduling regular check-up for printers and simplifying ordering forms. Establishing a dedicated health care coordination program is proposed to offload the bottleneck to target behind-the-scenes processes by assisting with writing orders, printing prescriptions, processing orders and reviewing treatment plan. Potential of a total 48.43 min of service time can be reduced to improve performance in the clinic.

IDENTIFYING DISCREPANCIES BETWEEN RECOMMENDATIONS AND REALITY: FIRST TIME SCREENING COLONOSCOPY AGES FOR AN AVERAGE RISK POPULATION IN AN INNER CITY TERTIARY CARE HOSPITAL: AN OBSERVATIONAL STUDY Joshua Jackson ${ }^{2}$; Jared Okun ${ }^{1}$; Snow Trinh T. Nguyen ${ }^{2}$; Hassan Shazhad ${ }^{2} .{ }^{1}$ New York Medical College, Flushing, NY; ${ }^{2}$ Brookdale Medical Center, Brooklyn, NY. (Control ID \#2470282)

BACKGROUND: Despite goals for colorectal cancer screening, it remains the second leading cause of death related to cancer in the United States, and the third most common type of cancer overall. The burden of these statistics are carried disproportionately, as minority populations carry a greater degree of incidence and mortality secondary to colorectal cancer. This health disparity gap persists, despite an overall decline in incidence over the last 20 years in the United States.

METHODS: As part of an initiative to identify persistent health disparity gaps, a retrospective review of patient data at a major inner city teaching hospital in New York City was performed. Data was collected from average risk first time screening colonoscopies over a 2-month period. Ninety-five charts were identified. The analysis compared ages of males to females and African Americans (AA's) to Non-AA's. In addition, incidence of screening was divided into age ranges of 50-54, 55-59, 60-64, 65-69 and 70-75, in accordance with the United States Preventive Services Task Force screening recommendations of age 50-75 for individuals of average risk.

RESULTS: A total of 95 patients in the hospital service were analyzed. The data showed an average first time screening age for average risk AA's $(n=70)$ of 59.34. Non-AA's $(n=25)$ had an average age of 59.76. The average male $(n=42)$ was 59.86 while the average female $(n=53)$ was aged 59.38. By age range, $10 \%$ of all AA's received their colonoscopy at an age $70-75(n=7), 19 \%(n=13)$ were aged $65-69,16 \%(n=11)$ were aged $60-64,21 \%(n=$ $15)$ were aged $55-59$, and $34 \%(n=24)$ were aged 50-54. Of non-AA's, $4 \%(n=1)$ received their colonoscopy at an age 70-75, $20 \%(n=5)$ were aged $65-69,20 \%(n=5)$ were aged $60-64,40 \%$ (10) were aged $55-59$, and $16 \%$ (4) were aged $50-54$.

CONCLUSIONS: This study highlights a disparity between the recommended age for a first time screening colonoscopy in average risk patients and the observed age among patients in an inner city hospital. The incongruity could be a manifestation of an even broader health disparity gap. The significantly higher incidence of age 50-54 screenings in AA's could be explained by the American College of Gastroenterology's recommendation to begin screening in African Americans at age 45 years. There were no major differences in average age found between gender or race, implicating geography and geo-socio-economics as a formidable risk factor and greater predictor of screening patterns than race or gender. The advent of this data emphasizes the need for stronger investments in preventive medicine in inner city populations, and its implications are the current focus of an initiative to fortify preventive services in a lower income community of minority predominance.

IDENTIFYING PATIENT-CENTERED CARE TEAMS USING ELECTRONIC HEALTH RECORDS ACCESS DATA AND SOCIAL NETWORK ANALYSIS Xi $\underline{Z h u}^{1}$; Nengliang $\mathrm{Yao}^{2}$; Vimal Mishra ${ }^{3}$; Allison E. Phillipss; Alan Dow ${ }^{3}$; Shin-Ping $\mathrm{Tu}^{3}$. ${ }^{1}$ University of Iowa, Iowa City, IA; ${ }^{2}$ University of Virginia, Charlottesville, VA; ${ }^{3}$ Virginia Commonwealth University, Richmond, VA. (Control ID \#2469388)

BACKGROUND: Electronic Health Records (EHR) systems are broadly used to support the delivery of patient care and to facilitate communication and coordination among providers. The use of EHR has been demonstrated to improve health care quality and efficiency. However, the impact of how providers utilize EHRs and communicate information in care delivery remains unclear. Specifically, how do EHR systems shape the patterns of communication between providers? Does information exchange in EHR indicate emergence of care teams? What types of outcomes are likely to be affected by communications taking place in EHR systems? The purpose of this study was to 1) test the feasibility of using EHR access data to identify care teams and 2) characterize communication patterns in care teams using social network analysis.

METHODS: This study was conducted at a large academic medical center, and was approved by its Institutional Review Board. A random sample of 100 surgical colorectal cancer patients receiving treatment between 2013 and 2014 were selected. We extracted detailed access logs information from the EHR system to create a chronological dataset of provider access to the sample patients' EHR records. The data include encrypted patient identity, encrypted user (i.e., provider) identity, clinical role of the user, date and time of access, and action performed on the record. For each patient, we created a communication tie between two providers who accessed the patient's EHR records consecutively, and used such communication ties to construct a patient-centered communication network. We used Gephi, a social network visualization tool, to visualize the communication networks. We calculated betweenness centrality, which is a structural indicator of how central a provider was in a network based on his/her position on communication pathways between other providers, for selected patients' communication networks; and identified care teams that consisted of providers with the highest centrality.

RESULTS: For the 100 patients, there were 5075 unique providers (physicians, nurses, technicians etc.) involved in their care, and 267,826 communication ties. Figure 1 shows the communication network of all providers who accessed patient A's EHR records. This network consists of 433 providers and 1826 communications ties. The average path length (i.e., the average minimum number of ties needed to connect any two providers) is 4.2. Figure 2 shows communication ties between 12 distinguishable providers who have the highest centrality in the communication network, which we identify as the care team for patient A. Examining the network structure in Figure 2, we find that: 1) Attending MD 3 is the most central provider in this network; 2) most frequent communications (indicated by the width of the tie) does not involve the most central provider; and 3) the team can be structurally divided into two components - the outpatient component (lower half of the network) and the inpatient component (upper half of the network) that are primarily connected through Attending MD 3. 
CONCLUSIONS: The EHR access data provide rich information on patterns of communication between providers. Using social network analysis, it is feasible to identify and examine structural characteristics of care teams that emerge from the large communication networks. Future study is needed to examine the implications of team characteristics on care coordination and patient outcomes.
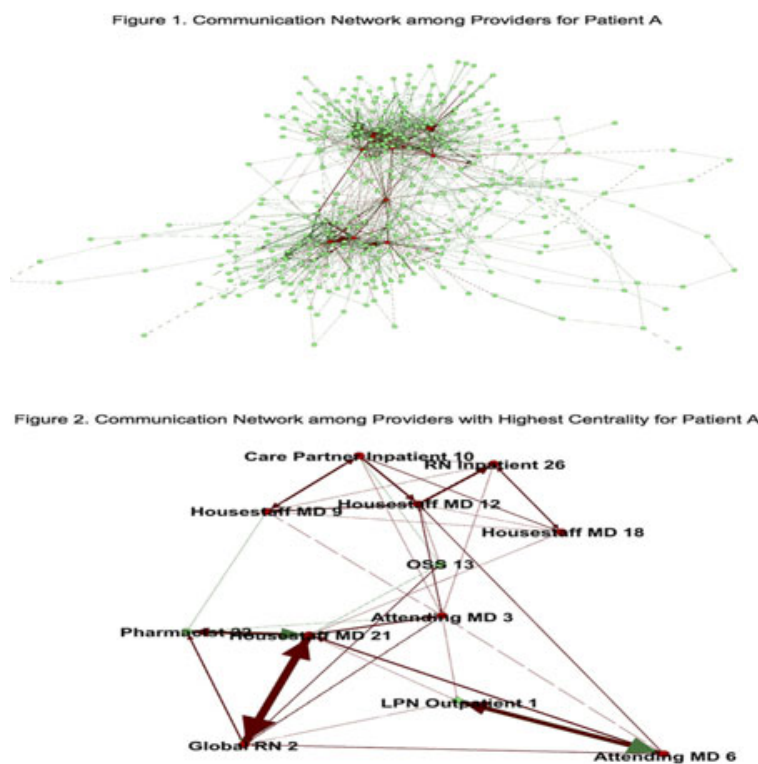

IMPACT OF A \$15 ci INSURANCE ELIGIBILITY Lea J. Selitsky ${ }^{1}$; Rajiv Bhatia $^{2,3}$ ${ }^{1}$ Wayne State University School of Medicine, Detroit, MI; ${ }^{2}$ The Civic Engine, Berkeley, CA; ${ }^{3}$ UCSF, San Francisco, CA. (Control ID \#2463800)

BACKGROUND: The Affordable Care Act of 2010 expanded insurance opportunities for low-income childless adults both by broadening eligibility for Federal Medicaid subsidies and by providing tax credits for individuals purchasing private insurance in State and Federal health insurance exchanges. However, 20 states have not expanded Medicaid insurance eligibility for poor working-aged adults, leaving at least 3 million adults in households earning less than the federal poverty line without affordable health insurance options. Paradoxically, these 3 million adults are not eligible to receive subsidies for insurance purchased on the exchanges. Our objective was to predict at a state-by-state level how a $\$ 15$ Federal minimum wage would change eligibility for Medicaid or subsidized health plans available through the health insurance exchanges.

METHODS: Using an existing quantitative model that predicted the elasticity of family income to minimum wage levels as a function of the position of an individual in the U.S. family income distribution, we estimated how a change in the minimum wage would change family income for individuals categorized by both family income and household poverty status. In states that had chosen to expand Medicaid, we then estimated the number of individuals moving from below to above $138 \%$ of the federal poverty line (FPL), the threshold at which an individual would lose Medicaid eligibility and gain exchange eligibility. In states that did not expand Medicaid, we estimated of the number of individuals moving from below to above $100 \%$ FPL.

RESULTS: Increasing the federal minimum wage to $\$ 15$ would make 2.7 million adults residing in the 20 states that did not expand Medicaid newly eligible to purchase subsidized health insurance. Raising the minimum wage to $\$ 15$ will also impact people in the states that expanded Medicaid. We estimate that 2.9 million adults in these states would lose eligibility for Medicaid and gain eligibility for subsidies for insurance purchased through the health exchange. CONCLUSIONS: Increasing the federal minimum wage would increase health insurance access for low-income working aged adults in states that did not expand Medicaid.
IMPACT OF A COMMUNITY BASED SERVICE LEARNING EXPERIENCE IN GERIATRICS FOR INTERNAL MEDICINE RESIDENTS Rachel K. Miller'; Jennifer Michener ${ }^{2}$; Phyllis Yang $^{2}$; Karen M. Goldstein ${ }^{1}$; Jennine GroceMartin $^{2}$; Gala True ; Jerry Johnson ${ }^{2} .{ }^{1}$ Durham VA; Duke University, Durham, NC; ${ }^{2}$ University of Pennsylvania, Philadelphia, PA; ${ }^{3}$ Southeast Louisiana Veterans Health Care System, New Orleans, LA. (Control ID \#2469675)

BACKGROUND: Internal Medicine (IM) residency programs face challenges to establish effective experiences in geriatrics for their trainees. Community based service learning (CBSL) programs are a potential way to address this gap in training. Our primary objective was to provide IM residents with a CBSL experience in geriatrics that increases understanding of the home, community environment, and resources of seniors and the skills required to care for the geriatric population. Secondary objectives were to build capacity for future CBSL experiences and to provide community seniors with health information. METHODS: From 2011 to 2014, IM residents at the University of Pennsylvania $(n=80)$ participated in a CBSL experience one half day per week during their ambulatory blocks. Residents attended senior housing units or multi-purpose senior centers and gave an interactive talk about a geriatric health related topic. The residents toured the facility, interacted with the seniors, and received information about local resources for the elderly from a geriatrics education coordinator. Residents and seniors evaluated the program via IRB approved surveys dispersed to them after the site visit.

RESULTS: Eighty University of Pennsylvania PGY3 IM residents participated in the program and delivered 64 sessions to the community. To evaluate the learning experience, residents completed an online survey which contained 5-point Likert scale questions ranging from Strongly Disagree (1) to Strongly Agree (5) and open-ended questions The survey $(71 / 80$, response rate $=88.8 \%)$ showed that the residents found the program useful for learning about the geriatric population outside of the hospital setting and felt this experience would change their practice in the future. Multiple comments said they would benefit from having earlier access to this knowledge. Most participating medicine residents felt the experience increased their ability to communicative effectively with older adults (mean 3.91, SD 0.73), increased their knowledge of senior resources and community living (mean 4.09, SD 1.01), and expanded their knowledge of health topics as it pertains to aging (mean 3.48, SD 1.09). From 2012 to 2014, 36 of 47 sessions were evaluated by survey with $461 / 815$ seniors responding, (57\% response rate). Seniors answered two questions with a 5 point Likert Scale with Strongly Disagree (1) to Strongly Agree (5) and five open-ended questions about the general format and content delivered. Participating seniors offered a favorable view of the program. Most surveyed seniors felt that IM residents delivered health information clearly (mean 4.55, SD 0.88) and that the health topics were relevant to them (mean 4.26, SD 0.92). These results led to expansion of the topics and sites.

CONCLUSIONS: This CBSL program was an effective educational tool for teaching IM residents to appreciate the community context in which seniors reside and to understand the resource needs of community living seniors. IM residents and seniors valued the program. Next directions include expansion of sites and topics and continued evaluation of impact for both residents and seniors.

IMPACT OF AN ATRIAL FIBRILLATION DECISION SUPPORT TOOL (AFDST) ON THROMBOPROPHYLAXIS FOR ATRIAL FIBRILLATION Mark $\underline{\text { H. Eckman }^{1}}{ }^{\text {; Gregory Y. Lip }}{ }^{2}$; Ruth E. Wise ${ }^{1}$; Barbara Speer ${ }^{1}$; Megan D. Sullivan ${ }^{3}$; Nita Walker $^{1}$; Brett M. Kissela ${ }^{1}$; Matthew L. Flaherty ${ }^{1}$; Dawn Kleindorfer ${ }^{1}$; Peter Baker ${ }^{1}$; Robert Ireton ${ }^{1}$; David Hoskins ${ }^{1}$; Brett M. Harnett ${ }^{1}$; Carlos Aguilar ${ }^{1}$; Anthony C. Leonard $^{1}$; Lora Arduser ${ }^{1}$; Dylan Steen ${ }^{1}$; Alexandru Costea ${ }^{1}$; John Kues ${ }^{1} .{ }^{1}$ University of Cincinnati, Cincinnati, $\mathrm{OH} ;{ }^{2}$ University of Birmingham, Birmingham, United Kingdom; ${ }^{3}$ UC Health, Cincinnati, OH. (Control ID \#2455527) 
BACKGROUND: Appropriate thromboprophylaxis for patients with atrial fibrillation (AF) remains a national challenge.

METHODS: We hypothesized that provision of decision support in the form of an Atrial Fibrillation Decision Support Tool (AFDST) would improve thromboprophylaxis for AF patients. We conducted a cluster randomized trial involving 15 primary care practices and 1493 adults with non-valvular AF in an integrated healthcare system between April 2014 and March 2015. Physicians in the intervention group received patient-level treatment recommendations made by the AFDST. Our primary outcome was the proportion of patients with antithrombotic therapy that was discordant from AFDST recommendation. RESULTS: Treatment was discordant in $42 \%$ of 801 patients in the intervention group Physicians reviewed reports for 240 patients. Among these patients thromboprophylaxis was discordant in $63 \%$, decreasing to $59 \% 1$ year later $(p=0.02)$. In non-stratified analyses changes in discordant care were not significantly different between the intervention group and control groups. In multivariate regression models assignment to the intervention group resulted in a non-significant trend towards decreased discordance

\begin{tabular}{l|} 
Name: \\
Gender: Female \\
Age: 67 \\
Medical record: \\
Physician name: \\
Current Tx plan: Rivaroxaban
\end{tabular}

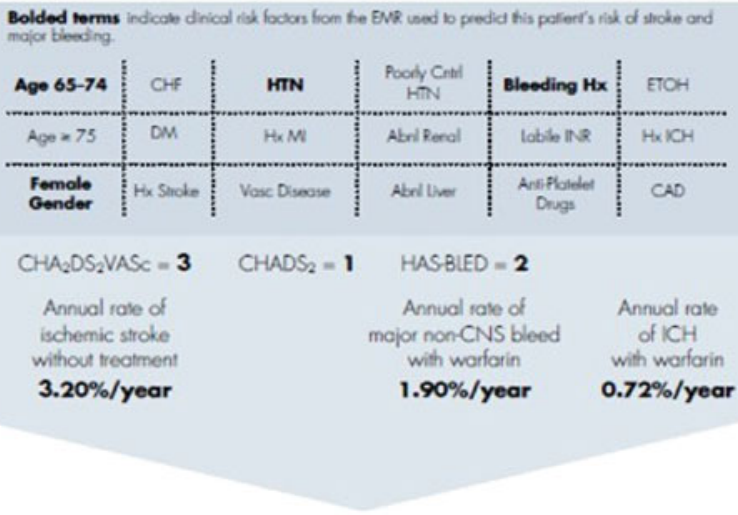

Difference -0.14 GAIY,

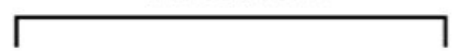

No Tx Oral Anticoagulant * ASA 13.45 QALYS 13.92 QALYs 13.59 QALYs Current Tx Plan Recommended

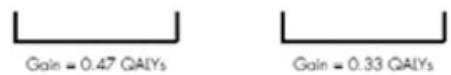

$(p=0.29)$, being a patient of a resident physician $(p=0.02)$, and a higher HASBLED score predicted decreased discordance $(p=0.03)$, while female gender $(p=0.01)$ and a higher CHADSVASc score $(p=0.10)$ predicted increased discordance.

CONCLUSIONS: Among patients whose physicians reviewed recommendations of the decision support tool discordant therapy decreased significantly over 1 year. However, in nonstratified analyses the intervention did not result in significant improvements in discordant antithrombotic therapy. These findings suggest next steps we must take to decrease barriers to the convenient and more effective use of the AFDST, perhaps by improving its integration into the EHR as a fully embedded application; by better targeting high yield clinical situations (i.e., generating best practice alerts within the EHR only when evolution in clinical risk factors results in a recommendation change by the decision support tool) instead of asking physicians to review all $\mathrm{AF}$ patients with discordant therapy; and finally to consider targeting additional clinician groups as decision makers, such as cardiologists and clinical pharmacists in addition to primary care physicians; and focusing on decision-making for incident as well as prevalent $\mathrm{AF}$, when initial therapeutic decisions are first being made.

\begin{tabular}{|c|c|}
\hline \multicolumn{2}{|c|}{$\begin{array}{l}\text { Recommendations for patients with AF considered for } \\
\text { long-term oral anticoagulation therapy (AHA/ACC/HRS 2014) }\end{array}$} \\
\hline Stroke risk & Recommended ansithrombotic therapy \\
\hline $\mathrm{CHA}_{2} \mathrm{DS}_{2} \mathrm{VASC}=0$ & $\begin{array}{l}\text { It is reasonable to omit ontithrombotic theropy } \\
\text { ICloss llo, level of Evidence: BI. }\end{array}$ \\
\hline $\mathrm{CHA}_{2} \mathrm{DS}_{2} \mathrm{VASC}=1$ & $\begin{array}{l}\text { No ontihnombotic therapy or reatment with on orol anticoogulant } \\
\text { or ospirin moy be considered (Closs llb, level of Evidence: Cl. }\end{array}$ \\
\hline$C H A_{2} D S_{2} V A S C=2$ & $\begin{array}{l}\text { Oral anticoogulants are recommended ICloss I1. Options include: } \\
\text { - wartorin (INR } 2.0 \text { b } 3.0 \text { ) (level of Evidence: A) } \\
\text { - dabigation (level of Evidence: B) } \\
\text { - ivoroxaban (level of Evidence: B) } \\
\text { - opviaban (level of Evidence: B) }\end{array}$ \\
\hline
\end{tabular}

\section{Classification and Level of Evidence}

\begin{tabular}{|c|c|}
\hline $\begin{array}{l}\text { Closs I - Benefit } \gg>\text { Risk } \\
\text { Treotment Should Be Pertormed. }\end{array}$ & $\begin{array}{l}\text { Level A - Dato derived from mult ple RCTs } \\
\text { or Metoonolyis. }\end{array}$ \\
\hline $\begin{array}{l}\text { Class lla - Beneitit >> Risk } \\
\text { it is Reosonable to Perform Treatment. }\end{array}$ & $\begin{array}{l}\text { Level B - Dato derived from a single RCT } \\
\text { or multple nonrondomized studies. }\end{array}$ \\
\hline $\begin{array}{l}\text { Class Ilb - Beneft > Risk } \\
\text { Treatment Moy Be Considered. }\end{array}$ & $\begin{array}{l}\text { Level C - Only consensus opinion of ex } \\
\text { pents, case sudies, or sandond of core. }\end{array}$ \\
\hline
\end{tabular}

In patients with AF, antithrombotic therapy should be individualized based on shared decisionmaking afer discussion of the absolute and relative risks of stroke and bleeding and the patients values and preferences [Closs I, level of Evidence: C)

Notes:

Patient report containing review of clinical data and risk factors, $\mathrm{CHA}^{2} \mathrm{DS}^{2} \mathrm{VASc}, \mathrm{CHADS}{ }^{2}$, and HAS-BLED scores, AFDST treatment recommendation, and AHA/ACC/HRS guideline.

IMPACT OF AN OBESITY EDUCATION INTERVENTION ON OBESITY DIAGNOSIS AND WEIGHT MANAGEMENT PLAN DOCUMENTATION IN AN INTERNAL MEDICINE RESIDENT OUTPATIENT CLINIC Kevin Brough ${ }^{2}$; John Bundrick ${ }^{2}$; Jill Huber ${ }^{2}$; Karen F. Mauck ${ }^{1}$; Jason Post ${ }^{1}$; Darrell Schroeder ${ }^{1}$; Kris G. Thomas $^{1}$; Matthew Thomas ${ }^{2}$; Mark L. Wieland ${ }^{1}$; Majken T. Wingo ${ }^{1}$. ${ }^{1}$ Mayo Clinic, Rochester, MN; ${ }^{2}$ Mayo Clinic Rochester, Rochester, MN. (Control ID \#2469019)
BACKGROUND: Medical societies recommend routine monitoring and counseling for obesity. Physician documentation of obesity as a medical diagnosis is associated with improved documentation of a weight management plan. It is important for resident physicians to develop skills to recognize obesity and to develop a management plan; however, obesity is under-recorded in internal medicine (IM) resident continuity clinics. Prior educational interventions to improve resident knowledge on obesity have corresponded to more effective 
counseling, but rates of documentation remain low. The objective of this study was to assess the impact of an obesity education intervention in an outpatient IM resident clinic on rates of documented obesity diagnosis and weight management counseling.

METHODS: Education Intervention: An obesity education intervention was delivered in the Mayo Clinic IM resident outpatient clinic as half-day, small group sessions facilitated by a clinic preceptor to groups of 6-10 IM residents across all training levels from October through November 2013. Each available resident attended a single session $(N=145)$. The curriculum included an interactive presentation on obesity epidemiology, health consequences, screening \& prevention, diagnosis \& treatment. Residents then self-audited clinical documentation on their most recent 10 general medical exams (GMEs) to estimate their frequency of obesity documentation for diagnosis and treatment. The self-audit exercise was followed by a $30 \mathrm{~min}$ facilitated reflection on barriers to diagnosis at the individual (patient and provider), care team, and system levels with corresponding discussion on solutions. Data Collection: A chart review was performed of all GMEs in the IM resident outpatient clinics from July 2013 through September 2013 for the pre-intervention period and December 2013 through May 2014 for the post-intervention period. The frequency of documentation of overweight (body mass index (BMI) $\geq 25-<30 \mathrm{~kg} / \mathrm{m}^{2}$ ) or obesity (BMI $\geq 30 \mathrm{~kg} / \mathrm{m}^{2}$ ) and documentation of a weight management plan in the clinical note of eligible patients were assessed. Data Analysis: Data are summarized using mean \pm SD for continuous variables and frequency percentages for categorical variables. The post-intervention study period was divided into 2 time intervals as the outcomes were highest in the first month following the intervention: early (12/1/2013-2/28/ 2014) and late (3/1/2014-5/31/2014). Outcomes were analyzed using generalized estimating equations (GEE) to take into account the correlation of outcomes for patients seen by the same provider. For these models, the outcome of interest was the dependent variable, intervention period was the independent variable, and additional covariates included patient age, sex and BMI category (overweight vs obese), and resident sex and year of training. Results are summarized by presenting the odds ratio (OR) and $95 \%$ confidence interval (CI) for each of the post-intervention time periods compared to the pre-intervention time period. In order to identify patient and provider characteristics associated with the outcomes of interest an additional GEE analysis was performed that included only data from the post-intervention time periods. In all cases, two-tailed $p$-values $\leq 0.05$ were considered statistically significant. RESULTS: Data were analyzed from 2208 GMEs identified as overweight or obesity (708, 693 and 807 GMEs during the pre, early-post and late-post intervention periods, respectively). During the pre-intervention phase, overweight/obesity was designated on the problem list for $32.6 \%$ of eligible patients. This rate increased to $44.2 \%$ during the early post-intervention phase $(\mathrm{OR}=1.94,95 \%$ C.I. $1.49-2.53, p<0.001)$ and $37.7 \%$ during the late post-intervention phase $(\mathrm{OR}=1.34,95 \%$ C.I. $1.10-1.76, p=0.042)$. The percentage of patient encounters with a weight management plan documented was $57.8 \%$ during the pre-intervention phase, $77.5 \%$ during the early post-intervention phase $(\mathrm{OR}=$ $2.58,95 \%$ C.I. $2.04-3.27, p<0.001$ ), and $69.1 \%$ during the late post-intervention phase $(\mathrm{OR}=1.65,95 \%$ C.I. $1.31-2.09, p<0.001)$. Similar results were obtained when the analyses were repeated separately for overweight and obese patients. From a multivariate analysis restricted to data from the post-intervention time period, the likelihood of having overweight/obesity designated on the problem list was significantly increased if the patient was obese compared to overweight $(\mathrm{OR}=5.64,95 \%$ C.I. $4.28-7.42, p<0.001)$ and significantly decreased for older patients $(\mathrm{OR}=0.44,95 \%$ C.I. $0.32-0.59, p<0.001$ for those 70 years or older compared to 50-59 years). These characteristics were also found to be associated with having a plan documented $(\mathrm{OR}=1.26,95 \%$ C.I. $1.84-2.85, p<0.001$ for obese compared to overweight; $\mathrm{OR}=0.38,95 \%$ C.I. $0.27-0.53, p<0.001$ for those 70 years or older compared to 50-59 years). There were no significant differences in documentation based on resident training level or gender.

CONCLUSIONS: This intervention significantly improved both the frequency of overweight/obesity diagnosis and weight management plan documentation by IM residents of all training levels. The effect of the intervention was most robust in the early postintervention period but improvement from baseline was maintained even in the late intervention period. Future research is needed to determine impact of the intervention on weight management counseling quality and corresponding patient weight loss.

IMPACT OF CIRRHOSIS AND HCV COINFECTION ON MORTALITY OF HIV POSITIVE PATIENTS IN THE ICU Maria A. Corcorran ${ }^{1}$; Jason Goldman ${ }^{1,2}$; Shireesha Dhanireddy ${ }^{1}$; Kristina Crothers ${ }^{1} .{ }^{1}$ University of Washington, Seattle, WA; ${ }^{2}$ Fred Hutchinson Cancer Research Center, Seattle, WA. (Control ID \#2444468)

BACKGROUND: HIV/HCV coinfection is known to accelerate progression towards cirrhosis and death due to liver disease. While limited studies on HIV and HCV suggest ICU mortality is higher in the coinfected population, none control for cirrhosis. We aimed to describe ICU and 30-day mortality among critically ill HIV monoinfected vs. HIV/HCV coinfected populations, and to determine the association between cirrhosis and outcomes. METHODS: We identified $446 \mathrm{HIV}+$ patients admitted to the medical and surgical ICUs at two academic hospitals between 10/2007 and 12/2013. HCV coinfection was identified by antibody and RNA testing and ICD-9 codes. On chart review, cirrhosis was identified by abdominal ultrasound, liver biopsy, FibroSure and/or clinical history. Primary outcomes were ICU and 30-day mortality. Secondary outcomes included acute kidney injury (AKI), hemodialysis and 30-day readmission. AKI was defined as an increase in serum creatinine by $>0.3 \mathrm{mg} / \mathrm{dL}$ and/or $50 \%$ from baseline within $48 \mathrm{~h}$ of admit. Results were compared using Chi-square, Fisher Exact and Mann-Whitney U tests.

RESULTS: Of the $446 \mathrm{HIV}+$ patients, 149 (33\%) were coinfected with HCV. Most recent CD4 count, HIV viral load, use of HAART and APACHE scores were not statistically different between monoinfected and coinfected groups. $18 \%$ (27) of coinfected patients had cirrhosis. There was no difference in mortality among monoinfected and coinfected groups in the ICU ( 7 vs. $8 \%, p=0.81$ ) or at 30 days ( 11 vs. $12 \%, p=0.76$ ). ICU and 30 -day mortality was highest among those coinfected with cirrhosis (15 and $22 \%$ ), but not statistically different when compared to those coinfected without cirrhosis ( 7 and $10 \%, p=0.23, p=0.10$ ). Among coinfected patients, death or readmission at 30 days was $44 \%$ in the cirrhosis group vs. $28 \%$ in those without cirrhosis $(p=0.09)$. Death or 30 day readmission between all coinfected patients and monoinfected controls was statistically insignificant ( 31 vs. $28 \%, p=0.52$ ). $29 \%$ of coinfected patients vs. $20 \%$ of monoinfected patients developed AKI $(p=0.038)$, with no difference in the proportion that underwent hemodialysis. There was no difference in AKI among those with and without cirrhosis.

CONCLUSIONS: Our study suggests that HIV/HCV coinfection alone does not confer a higher likelihood of ICU or 30-day mortality among critically ill HIV+ persons, but may be associated with increased risk of AKI. There was a trend towards increased risk of mortality in $\mathrm{HIV} / \mathrm{HCV}$ coinfected patients with cirrhosis that warrants further study.

\section{IMPACT OF CONSULTATION ON HOSPITAL OUTCOMES AND RESOURCE} UTILIZATION FOR PATIENTS WITH ACUTE CHF Syed Rafay Ali Sabzwari; Khalid Abusaada; Zoltan Varga. Florida Hospital Orlando, Winter Park, FL. (Control ID \#2457625)

BACKGROUND: Consultation in hospital is an important tool for acquiring subspecialty support when managing patients with acute congestive heart failure (CHF). The effect of consult utilization on hospital outcomes and resource utilization of acute CHF is unknown.

METHODS: Discharge data was obtained for patients with a principal diagnosis of acute CHF over 3 year period from a tertiary care center. Data on demographics, number of consultations, length of stay (LOS), readmissions within 30 days of discharge, cost of care, and mortality were compared according to number of subspecialty consultations. Premier's Care Science methodology was used for risk adjustment. 
RESULTS: One thousand five hundred fifty-four patients were included in the analysis. $103(6.6 \%)$ patients had no consultation, 482 (31\%) patients had 1 consult, $365(23.5 \%)$ patients had 2 consults, and 229 (14\%) patients had 3 consults, and 375 (24\%) had 4 or more consults. On multivariate linear regression analysis teaching service, age and black race were independently associated with decreased consultation ( $p<0.001$ for all of them) while case mix index (CMI) was associated with increased consultation $(p<0.001)$. Risk adjusted LOS and cost significantly increased with increased number of consults $(p<0.001$ for both). This remained significant after adjusting for age, gender, CMI, practice type, and insurance type $(P<0.001$ for both). There was no difference in risk adjusted mortality or 30 day readmission rate based on number of consults ( $p=0.35$ and 0.98 respectively).

CONCLUSIONS: Consultation occurs frequently in patients admitted with acute CHF. Increased consultation is associated with increased cost and LOS that is not explained by increased complexity of patients. Decreased utilization of consultation by teaching service in our institution suggests that there is an opportunity to decrease utilization of health care resources by streamlining the utilization of consultations in the inpatient setting.

Table 1. Variation of characteristics with number of consults and their statistical significance

\begin{tabular}{|c|c|c|c|c|c|c|}
\hline Baseline characteristics & $\begin{array}{l}\text { No consult } \\
(n=103)\end{array}$ & $\begin{array}{l}1 \text { consult } \\
(n=482)\end{array}$ & $\begin{array}{l}2 \text { consults } \\
(n=365)\end{array}$ & $\begin{array}{l}3 \text { consults } \\
(n=229)\end{array}$ & $\begin{array}{l}4 \text { or more } \\
\text { consults }(n=375)\end{array}$ & Significance \\
\hline Age (Years) & 68.6 & 70.2 & 70.3 & 68.3 & 68.7 & $P=0.21$ \\
\hline Gender (\% Male) & 46.6 & 56.8 & 56.7 & 57.6 & 61.8 & $P=0.08$ \\
\hline Case mix index & 1.10 & 1.23 & 1.50 & 1.77 & 2.82 & $P<0.001$ \\
\hline LOS (Days) & 3.40 & 4.58 & 6.07 & 7.58 & 14.17 & $P<0.001$ \\
\hline Cost (Dollars) & 3551 & 4753 & 6923 & 10356 & 22385 & $P<0.001$ \\
\hline Readmission in 30 days (\%) & 0.17 & 0.19 & 0.19 & 0.21 & 0.20 & $P=0.87$ \\
\hline In-hospital mortality (\%) & 0.01 & 0.01 & 0.01 & 0.03 & 0.08 & $P=0.89$ \\
\hline LOS risk adjusted $(\mathrm{O} / \mathrm{E}$ ratio) & 0.99 & 1.25 & 1.49 & 1.73 & 2.55 & $P<0.001$ \\
\hline Cost risk adjusted (O/E ratio) & 0.54 & 0.65 & 0.88 & 1.14 & 1.78 & $P<0.001$ \\
\hline Readmission risk adjusted $(\mathrm{O} / \mathrm{E}$ ratio) & 1.04 & 1.11 & 1.02 & 1.13 & 1.08 & $P=0.98$ \\
\hline Mortality risk adjusted $(\mathrm{O} / \mathrm{E}$ ratio) & 1.66 & 1.46 & 0.22 & 7.24 & 3.29 & $P=0.36$ \\
\hline
\end{tabular}

$\mathrm{O} / \mathrm{E}$ : Observed/Expected value based on care science risk adjustment methodology

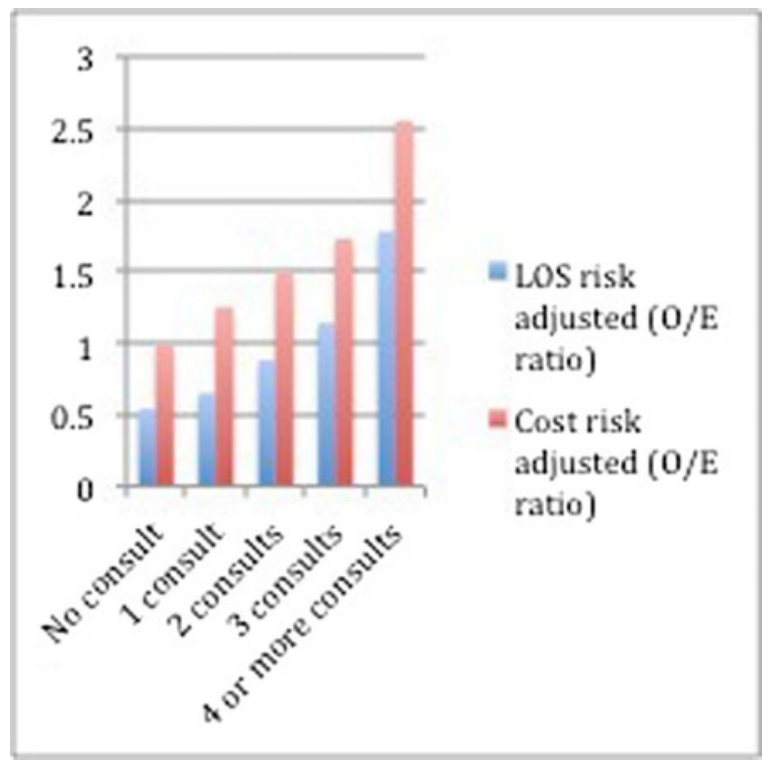

Figure 1. Effect of number of consults on risk adjusted LOS and cost

\section{IMPACT OF ELECTRONIC HEALTH RECORDS INTEROPERABILITY ON} INTER-HOSPITAL TRANSFER OUTCOMES. Michael G. Usher'; Nishant Sahni ${ }^{2}$; Dana Herrigel ${ }^{1}$; Andrew Olson ${ }^{2}$. ${ }^{1}$ Rutgers-Robert Wood Johnson Medical School, New Brunswick, NJ; ${ }^{2}$ University of Minnesota, Minneapolis, MN. (Control ID \#2468112)

BACKGROUND: Electronic health records (EHR) are nearly ubiquitous, yet their impact on patient outcomes remains unknown. One barrier to realizing the potential of improved information systems is their fragmented and variable adoption and lack of interoperability between health systems. Regional health information exchanges (HIE) are being developed to address this problem, but the impact of HIEs on health care delivery is also unclear. Patients transferred between hospitals, accounting for 1.6 million admissions yearly, have a disproportionately high mortality. Communication is vital for high quality transition of care between hospitals and barriers, such as the lack of real-time comprehensive information to augment hand-offs, likely impede optimal care. Thus, this population is uniquely suited to study EHR interoperability and outcomes. We tested the hypothesis that improved integration through adoption of health information exchanges improves clinical outcomes.

METHODS: We used two parallel approaches to assess the impact of health information exchanges on the outcomes of patients transferred between hospitals. First, we performed a single center observational study of EHR data from all patients transferred to an academic tertiary care facility from August 2011 through March 2015. The degree of EHR interoperability between the transferring and accepting hospitals was dichotomized into two categories. Complete integration was defined as fully accessible electronic records at any point during the transfer. Incomplete integration was defined as patient level data accessible only after arrival at the accepting institution (i.e. Epic CareEverywhere functionality or paper charts). The primary outcome was adjusted 90day all-cause mortality; length of stay (LOS) and inpatient mortality were secondary outcomes. Data were adjusted for age, demographics, transfer timing, and severity of illness employing MPM0-III or Apache-II using multivariate regression. Next, to confirm these results were not limited to a single tertiary referral center, we developed a database of patients transferred between acute care facilities based on Health Care Utilization Project's State Inpatient and ED database from 5 states (NY, FL, VT, IA, UT) between 2011 and 2013. Hospital level data including EHR adoption and HIE participation was collected from the American Hospital Association IT survey. Inpatient mortality was the primary outcome in addition to LOS and total hospital cost while adjusting for age, comorbidities, distance transferred, payer, and were assessed by multivariate regression.

RESULTS: From our initial observational study we identified 4689 patients whom arrived from hospitals with complete EHR integration. An additional 5184 patients arrived from facilities with partial or no integration. Patients transferred between hospitals with complete EHR integration were more stable on admission with lower MPM0-III and Apache-II scores $(p<0.001)$, and were less likely to be in shock $(p<0.001)$. Complete EHR integration was associated with a significant reduction in adjusted 90 day all-cause mortality (HR 0.78, $95 \%$ CI $0.57-0.86, p<0.001$ ), inpatient mortality (OR 0.68, $95 \%$ CI $0.49-0.78, p<0.001$ ), and reduced length of stay $(5.5 \mathrm{vs} 8.5, p<0.001)$. Results were validated against 130,539 patient transfers from 439 hospitals identified in the state inpatient databases. $50.4 \%$ of referring and $63.7 \%$ of receiving hospitals reported participation in a HIE. Single hospital HIE 
involvement did not alter patient outcomes, but patient transfer between hospitals where both reported HIE participation (39\% of all transfers) was associated with reduced adjusted inpatient mortality (OR .87, $95 \%$ CI $0.83-0.91, p<0.001)$, reduced total cost $(p<0.001)$, but no change in length of stay $(p=0.334)$

CONCLUSIONS: Seamless communication and information sharing between regional hospitals through information exchanges remains incomplete. Data from this single center observational study, further validated in a large administrative database, suggest that more complete information system interoperability is associated with improved mortality and more cost effective care for transferred patients.

IMPACT OF HOSPITAL OCCUPANCY ON IN- AND OUT-OF-HOSPITAL MORTALITY FOR OLDER MEDICARE PATIENTS Mahshid Abir ${ }^{2,3}$; Rosalie J. Malsberger ${ }^{3}$; Jason goldstick ${ }^{2}$; Claude Setodji ${ }^{4}$; Neil Wenger ${ }^{1}{ }^{1}$ University of California, Los Angeles, Los Angeles, CA; ${ }^{2}$ University of Michigan, Ann Arbor, MI; ${ }^{3}$ RAND Corporation, Santa Monica, CA; ${ }^{4}$ RAND Corporation, Pittsburgh, PA. (Control ID \#2469090)

BACKGROUND: Hospital crowding is a challenge confronting many U.S. healthcare systems. Crowding in the emergency department has been shown to be associated with adverse patient outcomes and many assume that this is true in the hospital as well, but there are scant data on the impact of high occupancy in the inpatient setting on patient outcomes.

METHODS: We performed a retrospective analysis of administrative discharge data from the California (CA) Office of Statewide Planning and Development (OSHPD) for the years 1998-2012. Using admission date and hospital length-of-stay for each observation in the dataset we calculated a daily census for each hospital. For each hospital day, we reviewed the hospital census for the 60 days prior and 60 days following and used rankings within that timeframe, represented as percentiles, to quantify occupancy. We chose this relative measure of hospital occupancy because what constitutes a high occupancy day likely depends on the hospital considers "normal" and absolute number of staffed beds is not reliably available. Only acute medical and surgical beds were included in the measure of occupancy (excluding obstetric, psychiatric and long-term beds). We constructed patient-level variables representing average occupancy during hospitalization and occupancy on discharge day. We then evaluated inpatient mortality and 30-day out-of-hospital mortality among Medicare recipients over the age of 64 admitted to an acute medical/surgical bed from the emergency department with hospital length of stay $<50$ days and a primary discharge diagnosis of myocardial infarction, congestive heart failure or pneumonia. Using logistic regression clustered by hospital, we predicted the impact of hospital occupancy on mortality, adjusting for patient characteristics (age, race, gender, socioeconomic status, and severity of illness), hospital characteristics (size, geography, ownership, urbanicity, and teaching status) and day of the week, month and year. For ease of interpretation, odd ratios were transformed into adjusted proportions, also known as recycled predictions. Predicted probabilities for every value of the predictor were calculated from the model output.

RESULTS: Among 1,741,145 hospitalizations in 382 CA acute care facilities, increasing average occupancy during hospitalization was associated with significantly lower in-hospital mortality (OR 0.82 , CI $0.79-0.85$ ), but did not significantly impact 30-day out-of-hospital mortality (OR 1.04, CI 0.99-1.08). Increasing hospital occupancy on discharge day was associated with significantly decreased in-hospital mortality (OR 0.61 , CI $0.59-0.63$ ) and significantly increased 30-day out-of-hospital mortality (OR 1.26, CI 1.23-1.31). Predicted probabilities for in-hospital and out-of-hospital mortality as a function of average occupancy and occupancy on discharge are depicted in Figures 1 and 2, respectively.

CONCLUSIONS: In a large sample of discharges from CA hospitals, when adjusted for key covariates, increased occupancy is associated with significantly lower in-hospital mortality, and increased occupancy on discharge day is associated with significantly higher 30-day out-of-hospital mortality. These findings suggest that patients expected to die may be discharged from crowded hospitals. The complex relationship between inpatient occupancy and patient outcomes requires much greater study.

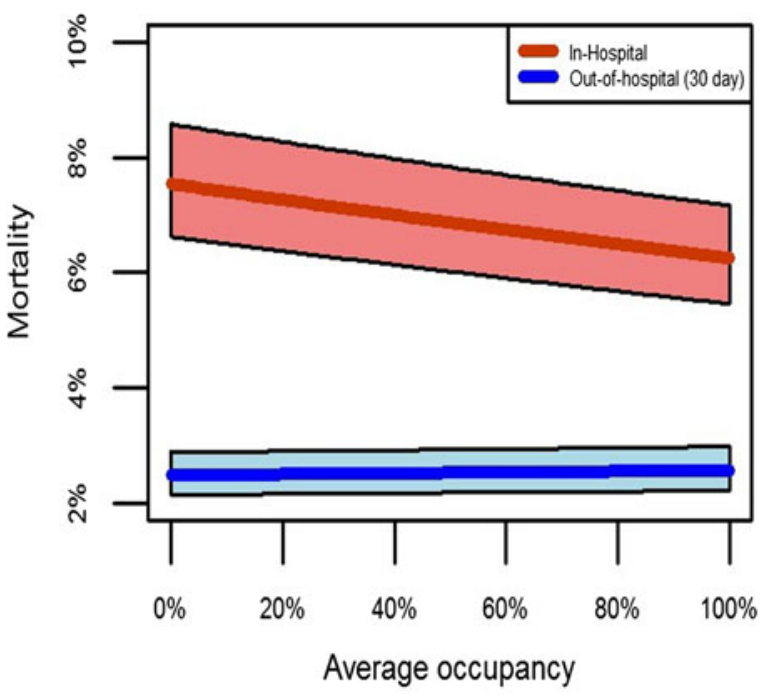

Figure 1: Predicted probabilities of in-hospital and 30-day out-of-hospital mortality as a function of average occupancy

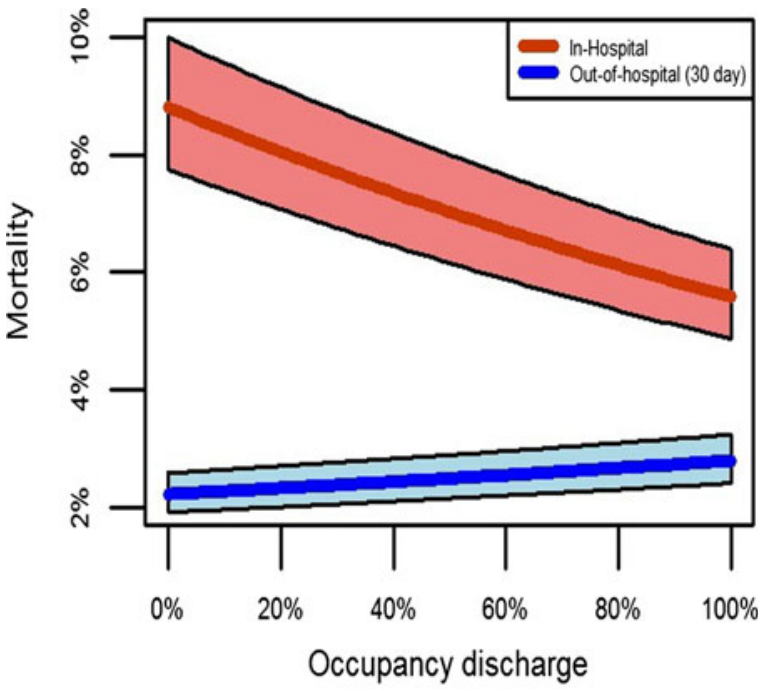

Figure 2: Predicted probabilities of in-hospital and 30-day out-of-hospital mortality as a function of discharge day occupancy

IMPACT OF NUMERACY ON UNDERSTANDING OF PROSTATE CANCER RISK REDUCTION IN PSA SCREENING Kevin Koo; Charles D. Brackett; Ellen Eisenberg; Kelly A. Kieffer, Elias S. Hyams. Dartmouth-Hitchcock Medical Center, Lebanon, NH. (Control ID \#2469131)

BACKGROUND: PSA screening in men of average risk remains controversial. Results from randomized trials are widely cited in patient education materials, but patients' ability to incorporate probability and risk data in decision-making may depend on their numeracy, or facility with quantitative concepts. This study aimed to assess patients' numeracy and its impact on their understanding of risk reduction benefits in PSA screening. 
METHODS: We used a randomized, cross-sectional survey design. All men 40-75 years old attending a general internal medicine clinic were invited to complete 1 of 4 surveys. All surveys included demographics, personal PSA and prostate cancer (PCa) history, and a validated 3-item numeracy test. Surveys differed in their presentation of PSA testing risk reduction data derived from the European Randomized Study of Screening for PCa (absolute (ARR) vs relative risk reduction (RRR), with or without baseline risk (BR)). Respondents were asked to adjust their perceived risk of PCa mortality using the risk reduction data presented. Numeracy was scored as the number of items correctly answered (score range $0-3$ ). Accuracy of risk reduction was evaluated based on how data was presented.

RESULTS: One hundred thirty-nine men completed the survey (61\% response rate). Mean age was 60 years, and $91 \%$ were high-school graduates. Fifty-one percent had received a PSA test, and $4 \%$ reported a PCa diagnosis. Demographic characteristics were not significantly different among the 4 groups. The majority of respondents incorrectly answered 1 or more of the 3 numeracy items; half could not convert " 1 in 1000 " to a percentage, and one-third were unable to calculate $1 \%$ of 1000 . Overall accuracy of adjustment in perceived risk was $22 \%$ among all groups. Accuracy varied with data presentation format: when presented with RRR, respondents were $12 \%$ accurate without $\mathrm{BR}$ and $39 \%$ accurate with $\mathrm{BR}$; when presented with ARR, they were $0 \%$ accurate without BR and $36 \%$ accurate with BR. Including BR data significantly improved accuracy for both RRR and ARR groups $(p<0.05)$. Accuracy was significantly related to numeracy: respondents were $0 \%$ accurate with a numeracy score of $0,6 \%$ accurate with a score of $1,9 \%$ accurate with a score of 2 , and $57 \%$ accurate with a score of 3 $(p<0.05)$. Neither PSA testing history nor PCa history was associated with accuracy.

CONCLUSIONS: Numeracy was associated with the accuracy of interpreting quantitative benefits of PSA screening. Although accuracy improved when the presentation of risk reduction data was framed by baseline risk, numeracy in this screening population of men was poor overall, suggesting that alternative methods of communicating these data are needed to facilitate shared decision-making.

\section{IMPACT OF PATIENT-ENTERED WELLNESS DATA AND TAILORED EMR RECOMMENDATIONS ON DELIVERY OF PREVENTIVE CARE Julie Foucher;} Michael B. Rothberg. Cleveland Clinic, Cleveland, OH. (Control ID \#2470251)

BACKGROUND: Modifiable lifestyle behaviors account for the leading causes of morbidity and mortality in the US, yet counseling and education are delivered only $18 \%$ of the time in the primary care setting. We investigated whether a novel tool using patient-entered wellness data to generate tailored EMR recommendations improved preventive care delivery.

METHODS: We conducted a mixed-methods retrospective cohort study of primary care encounters from a single community internal medicine clinic that utilized our patiententered wellness data tool from 6/25/2013-9/4/2013. Encounters were matched 1:1 using propensity scores to control encounters from the same site from 2/11/2013-4/24/2013. Changes were compared to matched encounters at two similar clinics within the same health system during the same two time periods. The tool synthesizes patient-entered data with that of the EMR (EPIC Systems) to generate a risk score for the patient in each of the following areas: nutrition, stress, exercise, depression, insomnia, sleep apnea, and breathing. Accompanying each risk score are prompts for the provider to order associated screening tests, consults, or treatments, and provide relevant counseling information. The primary outcome was the number of preventive consults, screening tests, and treatments ordered. Secondary outcomes included perceptions of the tool obtained through patient and provider surveys and qualitative individual semi-structured interviews conducted with each provider. We also conducted an electronic chart review to determine whether patients followed through on selected orders.
RESULTS: Five providers conducted 863 patient encounters using the patient-entered wellness tool. Patients were mostly older $(60.7 \pm 14.9$ years), female $(67.6 \%)$, white

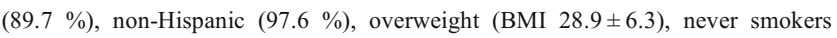
(56.4\%), and with a variety of comorbidities. Patients found the tool easy to use (99\%), did not require help (96\%), and would use it again (94\%), even those greater than 80 years of age. In most wellness categories, patients scored "low risk," while a majority of patients scored at least "mild risk" in both the nutrition (89\%) and stress $(53 \%)$ categories. Following encounters that used the tool, providers placed a greater proportion of orders for consults to smoking cessation ( 2.43 vs. $0.46 ; p<0.001)$, online nutrition $(2.43$ vs. $1.39 ; p=0.039)$ and stress $(5.45$ vs. $0.93 ; p<0.001)$ programs, lifestyle medicine ( 2.43 vs. $0 ; p<0.001)$, and psychology $(2.32$ vs. $1.04 ; p=0.037)$. They also ordered more spirometry tests ( 5.91 vs. $1.74 ; p<0.001)$, polysomnograms ( 6.26 vs. $1.28 ; p<0.001)$, and antidepressant ( 7.18 vs. $3.94 ; p=0.001)$ and hypnotic ( 2.20 vs. $0.70 ; p=0.011)$ medications There were no significant changes in any of these orders at the control clinics. With the exceptions of spirometry $(N=31,58 \%$ completed), nutrition therapy $(N=6,27 \%$ completed), and polysomnogram $(N=13,31 \%$ completed) orders, patient follow-through remained low $(<12 \%$ completed). Providers found the tool easy to use, but it increased visit time and they perceived little overall impact on patient behaviors.

CONCLUSIONS: Patients are willing to enter wellness data prior to their primary care visit, and this data influences the placement of preventive care orders by providers. Patient-entered data may be useful for improving large-scale delivery of preventive care, particularly screening tests such as spirometry and polysomnograms. Further work is required to decrease the time requirement during the visit as well as patient follow-through.

IMPACT OF PHARMACISTS ON THE PRIMARY CARE TEAM ON EMERGENCY ROOM VISITS AND HOSPITALIZATIONS FOR POORLY CONTROLLED PATIENTS WITH DIABETES Gerardo Moreno ${ }^{2}$; Jeffery Y. Fu ${ }^{1}$; Janet Chon $^{1}$; Natalie Whitmire ${ }^{1}$; Chi-Hong Tseng ${ }^{4}$; Jonathan Grotts ${ }^{1}$; Shawn Lee ${ }^{1}$; Douglas S. Bell ${ }^{3}$; Robin Clarke ${ }^{2}$; Samuel A. Skootsky ${ }^{1}$; Carol Mangione ${ }^{1}$. ${ }^{1}$ David Geffen School of Medicine at UCLA, Los Angeles, CA; ${ }^{2}$ UCLA, Los Angeles, CA; ${ }^{3}$ UCLA David Geffen School of Medicine, Los Angeles, CA; ${ }^{4}$ ucla, Los Angeles, CA. (Control ID \#2469237)

BACKGROUND: Patients with diabetes account for $12 \%$ of emergency room (ER) visits and $26 \%$ of hospitalizations. Pharmacists are effective at improving control of cardiovascular (CVD) risk factors, but it is not known whether these improvements translate into less ER use and fewer hospitalizations. In 2012, UCLA Health implemented "Managing Your Medications for Education and Daily Support" (MyMeds) program that embeds clinical pharmacists in 14 of 28 geographically dispersed primary care offices in the Los Angeles basin. The MyMeds program provided an opportunity to assess whether clinical pharmacists who are integrated into primary care teams can decrease use of the ER and hospital for patients with diabetes who have poor control of one or more CVD risk factors.

METHODS: The analytic sample included patients with diabetes with one or more of the following: hemoglobin A1c >9 \%, blood pressure $>140 / 90 \mathrm{mmHg}$, LDL-cholesterol $>130 \mathrm{mg} / \mathrm{dL}$ or five or more prescribed medications who had one or more visits in either a MyMeds ( $N=630$ patients from 14 practices $)$ or control practice $(N=2520$ patients from 14 practices) between April 2013 and April 2014. We used a patient-level propensity model to identify comparable control patients (1:4 match) based on the Nearest Neighbor Matching . "Treatment" was defined as receipt of 1 or more visits with a MyMeds pharmacist. We compared the monthly ER and hospitalization rates before and after the MyMeds consultation between MyMeds and control patients. A Poisson Generalized Estimating Equations (GEE) model with robust variance estimator was used to estimate the adjusted predicted change in hospitalization and ED monthly use rates before and after 
the pharmacist consultation. The Average Treatment Effect on the Treated (ATET) were estimated, specifically the predicted rates of each outcome with exposure to MyMeds compared to the predicted rates had those same patients not had a MyMeds consultation. RESULTS: We found that patients who received one or more visits with a clinical pharmacist had a mean age of $69(+/-13)$ years, $54 \%$ were females, and $87 \%$ had hypertension. The demographic characteristics of the matched controls were not significantly different than patents in MyMeds. In models adjusted for the clinical and demographic differences between MyMeds patients and controls, the adjusted mean (SD) predicted number of ED visits/month during the year prior to consultation was 0.09 among MyMeds patients. During the year after initiating the care with the pharmacists, this rate decreased to an adjusted mean monthly rate of 0.06 , with an ATET $=.034(p=0.003)$, a predicted reduction of approximately $34 \%$ in ED visits associated with the clinical pharmacist consults. There was a non-significant predicted reduction in hospitalizations over time for patients in the MyMeds program ATET $=0.014(p=0.077)$, a predicted reduction of $14 \%$.

CONCLUSIONS: In this study, we found that clinical pharmacists are an important addition to clinical care teams in primary care practices and significantly decrease utilization of the emergency room among patients with poorly controlled diabetes.

\section{IMPACT OF PRIMARY CARE PROVIDERS ON TREATMENT DECISIONS} FOR MEN WITH LOCALIZED PROSTATE CANCER Archana Radhakrishnan ${ }^{1}$; David Grande ${ }^{2}$; Linda Crossette ${ }^{2}$; Justin Bekelman ${ }^{2}$; Craig E. Pollack ${ }^{1}{ }^{1}$ Johns Hopkins University, Baltimore, MD; ${ }^{2}$ University of Pennsylvania, Philadelphia, PA. (Control ID \#2465973)

BACKGROUND: Despite increasing calls for greater integration of primary and cancer care, the role of primary care providers (PCPs) across the cancer care continuum remains poorly defined. Often having longitudinal relationships with their patients, PCPs may ideally help patients make cancer treatment decisions that are better in line with patients' goals and beliefs. We sought to evaluate how frequently men with localized prostate cancer discuss treatment with their PCP and whether those men who do, are more likely to forgo definitive treatment. Localized prostate cancer is an important clinical scenario to examine the PCP role: it is the leading cancer diagnosed among men, has multiple treatment options that are available, and many men receive definitive treatment who are unlikely to experience clinical benefit.

METHODS: We performed a mailed survey of men in the greater Philadelphia area diagnosed with localized prostate cancer. Men diagnosed between 2012 and 2013 were identified from the Pennsylvania Cancer Registry. We excluded patients with advanced disease and those who did not report having a PCP at the time of their cancer diagnosis. Patients were asked whether their PCP helped decide how to treat their cancer. Definitive treatment was defined as having radical prostatectomy or radiation therapy. Unadjusted and multivariable logistic regression analyses were used to compare sociodemographic and clinical characteristics of patients who did and did not discuss treatment with their PCP. We then performed multivariable logistic regression analysis to determine whether PCP involvement was associated with the receipt of definitive treatment, adjusting for patient characteristics. We then performed sub-group analyses on men who may be least likely to benefit from definitive treatment including those $>70$ years, with limited life expectancy ( $>50 \% 10$-year mortality risk), and with low-risk prostate cancer.

RESULTS: Our response rate was $50.6 \%$; 1735 men were eligible for analyses. Overall, $675(38.9 \%)$ men reported discussing treatment with their PCP and a large majority $(79.9 \%$ ), received definitive treatment. In adjusted analyses, non-Hispanic black men (Odds Ratio [OR] 1.74; 95 \% Confidence Interval [CI] 1.24-2.45) and Hispanic men (OR 2.74; $95 \%$ CI 1.13-6.66) were more likely than non-Hispanic white men to discuss treatment with their PCP. However, men who had treatment discussions with their PCP were not more likely to forgo definitive treatment ( $p=$ 0.96 ) overall or among the subgroups of men who may be least likely to benefit from definitive treatment.

CONCLUSIONS: Though a substantial proportion of men reported engaging in treatment discussions with their PCP, these discussions were not associated with differences in the receipt of definitive treatment among men with localized prostate cancer. Further effort is needed to determine how to optimize primary care physicians' role in supporting preference-sensitive decisions in cancer care.

IMPACT OF RACE/ETHNICITY AND SOCIOECONOMIC STATUS ON RISKADJUSTED READMISSION RATES: IMPLICATIONS FOR THE CMS HOSPITAL READMISSIONS REDUCTION PROGRAM Claudia Steiner ${ }^{1,}{ }^{1}$; Grant Martsolf ${ }^{2}$; Marguerite L. Barrett ${ }^{3}$; Audrey Weiss ${ }^{4}$; Raynard Washington ${ }^{5}$; Ateev Mehrotra $^{2}$; Rosanna M. Coffey ${ }^{4} .{ }^{1}$ Agency for Healthcare Research and Quality, Rockville, MD; ${ }^{2}$ RAND, Pitssburgh, PA; ${ }^{3}$ M.L. Barrett, Inc,, Del Mar, CA; ${ }^{4}$ Truven Health Analytics, Santa Barbara, CA; ${ }^{5}$ Council for Affordable Quality Healthcare, Washington DC, DC. (Control ID \#2470308)

BACKGROUND: The Centers for Medicare \& Medicaid Services (CMS) Hospital Readmissions Reduction Program (HRRP) penalizes hospitals with excess readmissions for select conditions and procedures. The HRRP methodology does not currently risk adjust readmission rates for race/ethnicity or socioeconomic status (SES), factors that are significantly related to readmissions. We therefore examined the extent to which the addition of race/ethnicity and SES in the Centers for Medicare \& Medicaid Services (CMS) Hospital Readmissions Reduction Program (HRRP) risk-adjustment algorithm impacts hospitals' excess readmissions ratios and potential penalties under the HRRP.

METHODS: We calculated the excess readmission ratio, with and without race/ethnicity and SES included in the model, for the five HRRP measures: acute myocardial infarction, heart failure, pneumonia, chronic obstructive pulmonary disease, and total hip or knee arthroplasty. We then estimated the overall impact on penalty payments of including race/ethnicity and SES in the model for select hospital characteristics. We included hospitals from 15 geographically dispersed states with a minimum of 25 Medicare discharges for at least one of the five HRRP measures using hospital discharge data from the 2011-2012 Healthcare Cost and Utilization Project (HCUP) State Inpatient Databases (SID).

RESULTS: For each measure, Medicare payments would be affected for a majority of hospitals by including race/ethnicity and SES in the risk-adjustment approach used to calculate readmission rates in the HRRP. Adding race/ethnicity and SES to the risk adjustment had a moderate effect on the size of penalties (-14.4 to $25.6 \%$ change in the penalty amount) but an extremely small effect on overall Medicare base payments (because the penalty amount is currently a very small percentage of base payments). Safety net and rural hospitals were disproportionately favorably affected when race/ethnicity and SES were included in the calculation, being much more likely to experience a decrease in penalties.

CONCLUSIONS: Adding race/ethnicity and SES to the HRRP risk-adjustment calculation would affect many hospitals, some favorably and others unfavorably. The financial impact, however, would likely be small for hospitals and the Medicare program, given the current size of the HRRP penalties. Safety net and rural hospitals would fare better although again the penalty impact itself would be small. While adding race/ethnicity and SES to the HRRP impacts a large number of hospitals, the overall financial impact is small. However, hospitals may perceive the addition of race and SES into the risk adjustment and the impact as important. Therefore, policy makers should continue to consider the effect of race/ethnicity and SES on HRRP payments as the program evolves over time. 
IMPACT OF RESIDENT WORKLOAD REDUCTION ON A MEDICAL CONSULTATION ROTATION Ethan Kuperman ${ }^{1}$; Eric Linson ${ }^{1}$; Manish Suneja ${ }^{1}$; Michele Fang ${ }^{1,2}$. ${ }^{1}$ University of Iowa Carver College of Medicine, Iowa City, IA; ${ }^{2}$ University of Pennsylvania, Penn valley, PA. (Control ID \#2469103)

BACKGROUND: Duty hour regulations have led to decreased resident patient contact hours. Evidence for the efficacy of these changes on educational value, resident satisfaction, and workload has been mixed. We developed an alternative method of decreasing work load without altering resident patient contact hours by adding an additional staff member to offload the resident service. The objective was to determine whether this intervention would improve the educational value and resident satisfaction on an internal medical consultation/surgical comanagement (SCM) rotation.

METHODS: In Fiscal Year (FY) 2014, an additional staff member was hired to work on the SCM rotation at a single US teaching hospital. Resident workload was assessed using billing data and compared to the baseline year of FY2013. Residents' perception of their workload and education were measured using an anonymous electronic survey tool (Medhub, Dexter, MI) administered at the end of each ward rotation. FY2014 and FY2015 resident survey responses to adequacy of patient volume, appropriate balance between responsibility and supervision, and resident perception of educational value of the rotation were compared to the baseline year of FY2013.

RESULTS: The average total number of patients seen per resident per day decreased from 8.0 (SD 3.3) in FY2013 to 5.0 (SD 1.9) in FY2014 $(p<0.001)$ and 5.7 (SD 2.0) in FY2015 $(p<0.001)$. In FY2013 $64.4 \%$ of residents felt that volume was just right versus $91.7 \%$ in FY $2014(p<0.001)$ and $96.7 \%$ in FY2015 ( $p<0.001)$. In FY2013, $79.9 \%$ of residents felt that curricular goals were met (percentage agree or strongly agree); however, after the intervention, this increased to $95.0 \%$ in FY2014 $(p<0.001)$ and $97.2 \%$ in FY2015 $(p<0.001)$. The overall educational value of the rotation was improved after the intervention ( $40.0 \%$ in FY2013 felt that the rotation was very good or excellent) versus $72.2 \%$ in FY2014 $(p<0.001)$ and $72.6 \%$ in FY2015 ( $p<0.001) .75 .5 \%$ felt that there was always or usually an appropriate balance between responsibility and supervision in FY 2013 and after the intervention, this improved to $81.6 \%$ in FY2014 $(p<0.001)$ and $93.3 \%$ in FY2015 $(p<0.001)$

CONCLUSIONS: Adding an extra staff member helped offload resident workload and was associated with increased resident satisfaction with the educational value, perception of appropriate workload, and supervision on a medical consultation rotation.

IMPACT OF TEACHING SERVICE ON COPD EXACERBATION MANAGEMENT AND OUTCOMES IN A COMMUNITY HOSPITAL Cai Yuan; Khalid Abusaada; Leen Alsaleh; Hassan Baig; Idljona Gllava; Sunil Adwani; Julie W. Pepe. Florida Hospital, Orlando, FL. (Control ID \#2465586)

BACKGROUND: Identifying factors that affect the length of stay for patients with acute exacerbation of COPD (AECOPD) may aid in better utilization of hospital resources. Multiple studies looked at patient-related factors affecting length of stay (LOS) for patients with AECOPD. However, there is wide variation in AECOPD outcomes among hospitals that is not explained by patient characteristics. No studies addressed the impact of physician practice pattern on outcomes of AECOPD and the role of teaching versus non-teaching practices on LOS remains unknown. This study aims to investigate the association between teaching vs non-teaching practice and LOS for AECOPD patients in the same hospital as well as difference in practice patterns between teaching and non-teaching service.

METHODS: Retrospective cohort study of patients admitted to Florida Hospital with principle diagnosis of COPD between 2007 and 2012. Patients with acute respiratory failure on presentation or a principle diagnosis other than AECOPD were excluded. Bivariate analysis was conducted for baseline characteristics and outcomes using student $t$ test and chi squared test as appropriate. The following variables were included in the multivariable linear regression analysis: BAP65 score, number of comorbidities, homelessness, need for social work consult, insurance status, associated pneumonia or acute decompensated CHF, need for Home Oxygen at baseline, low eosinophils

RESULTS: A total of 283 patients were included in the analysis. One hundred twenty-nine in the teaching service and 154 in the non-teaching service. LOS was significantly shorter for the teaching service compared to non-teaching service ( 2.80 vs 5.04 days, $p<0.001$ ). There was significantly less utilization of consultations, intravenous steroids and antibiotics in the teaching service compared to non-teaching service. On a multivariate linear analysis that adjusted for the variables mentioned in the methods section, being on the non-teaching service was independently associated with decreased LOS (B coefficient - 1.75). When number of consultations, use of IV steroids and IV antibiotics as intervening variables were added to the multivariate model, number of consults (B coefficient 1.04, $p<0.001$ ) and use of intravenous antibiotics (B coefficient $0.93, p=0.024$ ) were independently associated with increased LOS and being on the teaching service was no longer a significant factor. Use of oral steroids was not independently associated with prolonged LOS.

CONCLUSIONS: Teaching service in our institution was independently associated with significant decrease in AECOPD LOS. Decreased utilization of consultation and intravenous antibiotics explained most of the decrease in LOS in the teaching service. Type of inpatient physician practice is a significant factor in AECOPD LOS within the same institution and is associated with different patterns of utilization of resources. Streamlining consultation process and the use of intravenous antibiotics and steroids for AECOPD patients could result in improvement in utilization of hospital resources.

Patient characteristics and outcomes

\begin{tabular}{|c|c|c|c|}
\hline Characteristics & $\begin{array}{l}\text { Teaching service } \\
\text { (n-129) }\end{array}$ & $\begin{array}{l}\text { Non-teaching } \\
\text { service } \\
(n=154)\end{array}$ & $p$ value \\
\hline Age (mean/SD) & $58.3 / 0.98$ & $69.6 / 1$ & $<0.001$ \\
\hline $\begin{array}{l}\text { Number of comorbidities } \\
\text { (mean/SD) }\end{array}$ & $1.7 / 0.12$ & $2 / 0.13$ & 0.01 \\
\hline Hemoglobin (mean/SD) & $13.5 / 0.18$ & $12.9 / 0.16$ & 0.007 \\
\hline Creatinine & $0.94 / 0.05$ & $0.94 / 0.04$ & 0.99 \\
\hline Systolic BP on presentation & $134 / 7$ & $128 / 2$ & 0.32 \\
\hline $\begin{array}{l}\text { Number of consults per } \\
\text { patient }\end{array}$ & $0.3 / 0.06$ & $1.4 / 0.09$ & $<0.001$ \\
\hline BAP65 score & $1.6 / 0.07$ & $1.9 / 0.06$ & $<0.001$ \\
\hline Uninsured (\%) & 30.32 & 5.84 & $<0.001$ \\
\hline Homeless patients (\%) & 17.83 & 4.55 & $<0.001$ \\
\hline Use of oxygen at home (\%) & 17 & 35 & $<0.001$ \\
\hline Eosinopenia (\%) & 22.3 & 24.8 & 0.58 \\
\hline $\begin{array}{l}\text { Patients received oral steroids } \\
\text { since admission (\%) }\end{array}$ & 85 & 8.9 & $<0.001$ \\
\hline $\begin{array}{l}\text { Need for social work } \\
\text { intervention (\%) }\end{array}$ & 36 & 41 & 0.39 \\
\hline $\begin{array}{l}\text { Associated decompensated } \\
\mathrm{CHF}\end{array}$ & 4.65 & 4.55 & 0.96 \\
\hline Associated Pneumonia & 13.48 & 13.64 & 0.91 \\
\hline $\begin{array}{l}\text { Need for mechanical } \\
\text { ventilation }(\%)\end{array}$ & 0.78 & 0.65 & 0.9 \\
\hline Mortality & 0 & 1.95 & 0.110 \\
\hline
\end{tabular}

Factors associated with length of stay in patients with AECOPD

\begin{tabular}{llll}
\hline \hline Factor & B coefficient & Significance & $95 \%$ CI \\
Number of comorbidities & 0.38 & 0.039 & $0.017-0.64$ \\
Teaching service & -1.74 & $<0.001$ & $(-2.5)-(-0.92)$ \\
Need for social work consult & 1.3 & 0.001 & $0.54-2.13$ \\
Eosinopenia & 1.33 & 0.004 & $0.41-2.25$ \\
\hline
\end{tabular}

IMPACT OF THE PATIENT-CENTERED MEDICAL HOME ON PATIENTS WITH DEPRESSION Amy W. Baughman ${ }^{4}$, ${ }^{\text {; }}$ Zhonghe $\mathrm{Li}^{6}$; Mark W. Friedberg ${ }^{3}$; Sara Singer ${ }^{2}$; Eric Schneider ${ }^{5}$; David W. Bates ${ }^{1}$; Meredith Rosenthal ${ }^{6}$. ${ }^{1}$ Brigham and Women, Boston, MA; ${ }^{2}$ Harvard School of Public Health, Boston, MA; ${ }^{3}$ RAND, Boston, MA; ${ }^{4}$ VA Boston Healthcare System, Cambridge, MA; ${ }^{5} \mathrm{BWH}$, Boston, MA; ${ }^{6}$ Harvard T. H. Chan School of Public Health, Boston, MA. (Control ID \#2469822) 
BACKGROUND: The patient-centered medical home (PCMH) is a team-based primary care model that focuses on enhanced access, care coordination, and quality improvement. Evidence to date on the impact of the PCMH model regarding quality, utilization and cost outcomes has been mixed, but recent studies suggest that patients with more complex health needs may benefit the most from participation in a PCMH. One important example is in patients with depression, a common comorbid illness that is strongly associated with worse health outcomes and increased costs of care. Collaborative care, a variation of the medical home that includes mental health services, has showed improved outcomes for patients with depression. Whether a PCMH without extensive mental health resources could also improve outcomes for patients with depression has not been established. Given this, we evaluated whether receiving care in primary care practices participating in a large, statewide medical home implementation pilot affected overall healthcare utilization and quality of care among adult patients with depression.

METHODS: We performed a retrospective cohort analysis of a large PCMH pilot among small and medium sized primary care sites across Colorado from 2007 to 2009 . The study cohort included $\sim 98,000$ patients who could be attributed to one of the 15 PCMH pilot or 66 control sites. We identified patients with depression based on prior documented depression or antidepressant use. We measured outcomes including healthcare utilization (emergency visits, admissions, and office visits) and HEDIS-derived quality measures of diabetes care and cancer screening. To estimate the impact of the PCMH pilot, we used a difference-indifferences approach to estimate the change in each outcome attributable to PCMH pilot participation. Given the non-random selection of $\mathrm{PCMH}$ pilot practices, we used propensity score methods to match PMCH pilot practices to non-pilot practices using patient characteristics from the 2-year baseline period prior to the implementation. We used encounterlevel negative binomial regression models to estimate the differences in the rates of each outcome between the PCMH pilot practices and matched controls in the post-intervention period, accounting for clustering on the patient level. All estimates were adjusted for patient characteristics, including age, sex, and comorbidities using the Elixhauser index.

RESULTS: At baseline, for patients with depression there were no statistically significan differences between patient characteristics, baseline utilization rates or quality measures between patients attributed to pilot PCMH sites vs. propensity matched control sites (al $p>0.06$ ). Patients in the pilot practices had a mean age of 42 , were $70 \%$ female and had an average of 3 comorbidities. Patients with depression in the pilot practices had significant reductions in inpatient admissions than comparison practices (incidence rate ratio (IRR)= 0.59 [ $95 \% \mathrm{CI}, 0.37$ to 0.96 ]. There were no other significant differences in utilization: ED visits $\mathrm{IRR}=1.02$ [CI, 0.86-1.22], ambulatory care sensitive (ACS) ED visits IRR $=1.11$ [CI, $0.72-1.73]$, ACS inpatient admissions $\mathrm{IRR}=1.28[0.45,3.64], \mathrm{PCP}$ visits $\mathrm{IRR}=1.02[\mathrm{Cl}$ $0.93,1.02]$, specialist visits IRR $=1.07$ [CI $0.97,1.17]$. We found no significant differences in 6 HEDIS quality measures for rates of cancer screening (colon, breast and cervical) and diabetes care (hemoglobin $\mathrm{A} 1 \mathrm{C}$, lipid testing, and dilated eye exams).

CONCLUSIONS: Identifying target populations that benefit from the resource intensive PCMH model is crucial for the success of medical homes. We found that patients with depression in the PCMH pilots experienced reduced in-patient admissions than comparison practices, suggesting that patients with depression may disproportionately benefit from care in the PCMH model. We did not see impact on other utilization measures or HEDIS quality measures of care.

IMPACTS OF THE 2014 MEDICAID EXPANSION ON COVERAGE, ACCESS TO CARE, AND HEALTH Benjamin D. Sommers ${ }^{1,2}$; Brandy Lipton ${ }^{3}$; Sandra Decker ${ }^{3}$. ${ }^{1}$ Harvard School of Public Health, Brookline, MA; ${ }^{2}$ Brigham \& Women's Hospital, Boston, MA; ${ }^{3}$ Agency for Healthcare Research \& Quality, Rockville, MD. (Control ID \#2467161)

BACKGROUND: Under the Affordable Care Act (ACA), 26 states and Washington D.C. expanded Medicaid to cover adults with family income up to $138 \%$ of the federal poverty level (FPL) during 2014. In addition, the establishment of premium tax credits to purchase Marketplace coverage likely affected individuals in both Medicaid expansion and non-expansion states. Previous research on the Medicaid expansion using nongovernmental surveys has shown significant gains in coverage and some access measures, though these data sources have low response rates and other limitations. Our objective was to assess the initial impacts of the ACA's Medicaid expansion on coverage, access to care, and self-reported health using a richer set of outcome measures and validated federal interview survey data.

METHODS: We used National Health Interview Survey (NHIS) data from 2008 to 2014 to analyze U.S. adults aged 19-64 with family income below $138 \%$ of FPL $(n=92,500)$. We assessed the changes in outcomes within expansion and non-expansion states, and then used a difference-in-differences design to compare outcomes for expansion compared to non-expansion states. Models adjusted for demographic and economic characteristics, linear time trends, and state fixed effects. Outcomes included insurance coverage, primary care and specialist visits, Emergency Department (ED) visits, cost-related delays in care, self-reported health, and psychological distress.

RESULTS: The uninsured rate for low-income adults dropped in both expansion $(-11.5$ percentage points, $p<0.01)$ and non-expansion states ( -5.8 percentage points, $p<0.01$ ) Coverage gains in expansion states were primarily from Medicaid ( +9.8 percentage points, $p<0.01)$, while gains in non-expansion states were primarily from private insurance $(+4.8$ percentage points, $p<0.01)$. Overall, the decline in the uninsured rate was significantly greater in expansion states (difference-in-differences estimate -5.3 percentage points, $p<0.01)$. Low-income adults in expansion states experienced larger increases in the likelihood of having seen a primary care doctor in the past year $(+3.9$ percentage points, $p<0.05)$ and having seen a specialist in the past year $(+3.2$ percentage points, $p<0.05)$ compared to those in non-expansion states. Adults in both expansion and non-expansion states experienced significant increases in having a usual source of care and reductions in cost-related delays in care, but the difference-in-differences estimates for these outcomes were not significant. We did not detect significant effects on ED visits, self-reported health, or psychological distress in expansion or non-expansion states.

CONCLUSIONS: The first year of the ACA's Medicaid expansion was associated with significant gains in insurance coverage, primary care visits, and specialist care visits among low-income adults in expansion compared to non-expansion states, but with no significant changes in self-reported physical or mental health. As 20 states still consider whether or not to expand Medicaid under the ACA, our study provides further evidence on the positive impacts of the expansion on access to care, though longer-term follow-up may be needed before changes in health become apparent.

\section{IMPLEMENTATION OF AN INTERPROFESSIONAL, TEAM-BASED PROTOCOL TO ENHANCE MAMMOGRAPHY SCREENING IN PRIMARY CARE, JOHNS HOPKINS GREEN SPRING GENERAL INTERNAL MEDICINE, 2015 Samantha I. Pitts ${ }^{1}$; Melinda Sawyer ${ }^{2}$; Nisa Maruthur ${ }^{1}$; Candace Nigrin $^{1}$; Renee Grimes ${ }^{1}$; Heather Sateia ${ }^{1}$; Kimberly Curreri ${ }^{1}$; Nicklaine Paul ${ }^{1}$; Xiao Wang ${ }^{1}$; Jill A. Marsteller ${ }^{3}$; Jeanne M. Clark ${ }^{1}$; Kimberly S. Peairs ${ }^{1}{ }^{1}$ Johns Hopkins University School of Medicine, Baltimore, MD; ${ }^{2}$ Johns Hopkins University, Baltimore, MD; ${ }^{3}$ Johns Hopkins University, School of Public Health, Baltimore, MD. (Control ID \#2469612)}

BACKGROUND: Despite the availability of preventive care services for nine of the top ten causes of mortality in the United States, many adults do not receive these evidencebased interventions. Although the United States Preventive Services Task Force recommends screening mammography every 2 years for women $50-74$ years old, approximately $30 \%$ of women nationally and within our Accountable Care Organization have not had documented screening, potentially leading to missed opportunities to detect and treat breast cancer. Objectives: 1 . To redesign the process of care delivery to improve 
documentation and delivery of evidence-based preventive care services, using screening mammography as the initial prototype; 2 . To increase the proportion of women aged 50 74 years old seen at Green Spring Station General Internal Medicine (GIM) who have had documented screening mammography within the prior 24 months.

METHODS: During 2014-2015, an interprofessional team from Green Spring GIM developed and implemented a protocol to standardize documentation and referral for mammography. The protocol increased participation by non-physician staff, defined clear roles for each participant in the process of care (including medical office staff, certified medical assistants, and physicians), established the expectation that mammography would be reviewed at every visit, initiated a workflow to obtain data on outside mammograms through the Maryland health information exchange, created standardized messaging about mammography for patients, and instituted a review of protocol failures. Women aged 50 74 years old with a visit to Green Spring GIM from March 9 to September 4, 2015 were eligible for the protocol. Women who were not candidates for mammography (e.g., history of bilateral mastectomy) were excluded. We defined women as up to date on mammography if they had documentation of a screening or diagnostic mammogram within the previous 24 months. We abstracted data by week from the electronic medical record of eligible women during December 14, 2014-September 4, 2015, including 1) the \% of women up to date on mammography at the time of their visit, 2) the $\%$ of women not up to date who had mammography addressed at their visit, and 3 ) the $\%$ of women who were up to date on mammography at 8 weeks following their visit. Data were evaluated by statistical process control and by pre- and post-implementation comparison, using a chi-squared test.

RESULTS: Following implementation, the proportion of eligible women who were up to date on mammography at the time of the clinical visit increased significantly ( 84.5 versus $76.9 \%, p<0.001$ ), as did the proportion who had mammography addressed during their visit ( 73.5 versus $46.2 \%, p<0.001$ ), and the proportion who were up to date at 8 weeks following the visit ( 88.6 versus $82.5 \%, p=0.002$ ). Beginning in week 13 of protocol implementation, the proportion of women who were not up to date at 8 weeks post-visit fell consistently below the pre-intervention mean

CONCLUSIONS: Greater involvement of non-physician staff, clearly defined roles for each participant in the process of care, and the acquisition of health information exchange data improved our documented completion of mammography both at the time of a clinical visit and at 8 weeks post-visit. This highlights the importance of interprofessional collaboration and inclusion of preventive care services within a health information exchange. These data will now allow us to focus on targeted interventions to reduce barriers to completion of mammography, such as same day mammograms.

IMPLEMENTING A CENTRAL POPULATION HEALTH CHRONIC DISEASE MANAGEMENT PROGRAM IN A PRIMARY CARE NETWORK: 6-MONTH OUTCOMES Jeffrey M. Ashburner ${ }^{1}$; Daniel M. Horn ${ }^{1}$; Sandra OKeefe ${ }^{1}$; Adrian Zai ${ }^{1}$; Neil W. Wagle ${ }^{2}$; Steven J. Atlas ${ }^{1} .{ }^{1}$ Massachusetts General Hospital, Boston, MA; ${ }^{2}$ Northwestern University, Brookline, MA. (Control ID \#2465899)

BACKGROUND: Provisions of the Affordable Care Act support population health management (PHM) activities as part of fostering accountable care organizations. Few studies have evaluated the impact of implementing a PHM program as part of routine care within primary care networks. We developed a program for chronic disease management using central population health coordinators (PHCs) assigned to specific practices within a large, diverse, academic primary care network. We conducted a quasi-experimental evaluation of the program and compared quality of care process and outcome measures over the first 6 months of the PHM program for patients with diabetes mellitus (DM), cardiovascular disease (CVD), and hypertension (HTN) in practices assigned a central PHC or not.

METHODS: An information technology (IT) tool was previously implemented for PHM of preventive cancer screening in the primary care network in 2011. In 2014, this PHM tool was expanded to include patients with DM, CVD, and HTN. The tool identified network patients, assigned them to chronic disease registries, and tracked goal attainment in near-real time. A central PHM program was developed, and PHCs were hired and trained in the first half of 2014. PHCs were embedded into their practice's workflow and regularly "huddled" with physicians. The PHCs managed lists of patients not at goal and performed administrative tasks including appointment scheduling, ordering overdue laboratory testing, and obtaining home blood pressure values. Due to funding constraints, the program was implemented in 8 of 19 network practices. Practices were invited to participate and PHCs were non-randomly assigned to achieve inclusion of community health center, and community-based private and on-campus practice sites within the network. In practices not assigned a central PHM coordinator, training and support was provided for practice staff already using the PHM tool for preventive cancer screening to permit its use for chronic disease management. Staff in these practices remained responsible for managing administrative tasks. The evaluation period for the PHM program was between July 1 and December 31, 2014. Table 1 describes criteria selected for process and outcome measures. Blood pressure (BP) outcomes utilized the most recent or the average of the most recent 3 BPs over 18 months. We compared the difference in differences over the 6-month follow-up period for each outcome measure between PHC and non-PHC practices. Because PHCs were not randomly assigned to practices, we controlled for patient characteristics (age, gender, language, race, insurance, practice type [community health center or not], and patient-physician continuity) and practice site among PHC and non-PHC practices using a logistic regression model with a time-byPHC-practice interaction term and accounting for clustering within patients using the general estimating equations approach. Since all practices used the PHM IT tool for preventive cancer screening without central PHC input, we examined these outcomes as a way to control for the non-random assignment of the central PHC.

RESULTS: At the start of the program, there were 13,850 patients with DM (4770 and 9080 in PHC and non-PHC practices), 14,725 patients with CVD (4705 and 10,020 in PHC and non-PHC practices) and 44,852 patients with HTN (15,883 and 28,969 in PHC and non-PHC practices). Outcomes at baseline and follow-up are shown in Table 2. Patients in PHC practices had greater increases for all outcome measures. They started with lower scores on all measures and ended with higher scores on 3 of 5 measures. All differences between PHC and non-PHC practices persisted after adjustment for baseline characteristics $(p<0.001)$. Differences among patients in PHC and non-PHC practices were also observed for measures of process as well as for outcomes (data not shown). In contrast to outcomes on chronic disease measures, differences in cancer screening (breast, cervical, colorectal) measures were similar in $\mathrm{PHC}$ and non-PHC practices (range of differences: 0.11-1.04 favoring non-PHC practices).

CONCLUSIONS: The implementation of a PHM program for chronic disease management led to large improvements in process and outcome measures in a diverse primary care practice network. Though not assigned randomly, practices assigned central personnel to support administrative tasks achieved larger increases in quality than practices that did not receive this support.

Table 1: At Goal Criteria for Process and Outcome Measures

\begin{tabular}{|c|c|c|c|}
\hline Measure & Time Period & $\begin{array}{l}\text { Based on Lab } \\
\text { or BP Values }\end{array}$ & $\begin{array}{l}\text { Based Upon Maximal } \\
\text { Med Rx }\end{array}$ \\
\hline $\begin{array}{l}\text { Diabetes: LDL } \\
\text { cholesterol }\end{array}$ & $\begin{array}{l}1 / 1 / 14-12 / \\
31 / 14\end{array}$ & $<100 \mathrm{mg} / \mathrm{dL}$ & $\begin{array}{l}\text { Moderate or high } \\
\text { dose statin }\end{array}$ \\
\hline Diabetes: Alc & $\begin{array}{l}7 / 1 / 14-12 / \\
31 / 14\end{array}$ & $<9 \%$ & \\
\hline Diabetes: BP & $7 / 1 / 14-12 / 31 / 14$ & $\begin{array}{l}\mathrm{SBP}<140, \\
\mathrm{DBP}<90 \text { or } \\
\mathrm{Age} \geq 60: \mathrm{DBP}<70\end{array}$ & $\begin{array}{l}\geq 3 \text { meds from } \\
\text { different anti- } \\
\text { hypertensive classes }\end{array}$ \\
\hline $\begin{array}{l}\text { CVD: LDL } \\
\text { cholesterol }\end{array}$ & $\begin{array}{l}1 / 1 / 14-12 / \\
31 / 14\end{array}$ & $<100 \mathrm{mg} / \mathrm{dL}$ & High dose statin \\
\hline HTN: BP & $7 / 1 / 14-12 / 31 / 14$ & $\begin{array}{l}\mathrm{SBP}<140, \mathrm{DBP}<90 \text { or } \\
\text { Age } \geq 60: \mathrm{DBP}<70 \text { or } \\
\text { Age } \geq 60: \mathrm{SBP}<150, \\
\mathrm{DBP}<90\end{array}$ & $\begin{array}{l}\geq 3 \text { meds from } \\
\text { different anti- } \\
\text { hypertensive classes }\end{array}$ \\
\hline
\end{tabular}


Table 2: \% Achieving Process and Outcome Measures Among Practices with or without Central PHCs*

\begin{tabular}{|c|c|c|c|c|c|c|}
\hline \multirow[t]{5}{*}{ Measure } & \multirow{4}{*}{\multicolumn{3}{|c|}{$\begin{array}{l}\text { Patients } \\
\text { in Practices } \\
\text { with Central } \\
\text { PHC }\end{array}$}} & \multirow{4}{*}{\multicolumn{3}{|c|}{$\begin{array}{l}\text { Patients } \\
\text { in Other } \\
\text { Practices }\end{array}$}} \\
\hline & & & & & & \\
\hline & & & & & & \\
\hline & & & & & & \\
\hline & Baseline & $\begin{array}{l}\text { Follow- } \\
\text { Up }\end{array}$ & Difference & Baseline & $\begin{array}{l}\text { Follow- } \\
\text { Up }\end{array}$ & Difference \\
\hline Diabetes: LDL & 59.5 & 70.6 & 11.1 & 65.3 & 71.4 & 6.1 \\
\hline Diabetes: BP & 74.5 & 81.2 & 6.7 & 78.2 & 80.5 & 2.3 \\
\hline Diabetes: A1c & 63.2 & 73.6 & 10.3 & 66.0 & 71.0 & 5.0 \\
\hline CVE: LDL & 64.6 & 77.5 & 12.9 & 65.4 & 75.2 & 9.8 \\
\hline HTN: BP & 69.0 & 75.1 & 6.2 & 72.1 & 76.3 & 4.2 \\
\hline
\end{tabular}

IMPLEMENTING AN ELECTRONIC CLINICAL DECISION SUPPORT TOOL TO REDUCE LOW VALUE IMAGING FOR BACK PAIN IN A LARGE SAFETY NET HEALTH SYSTEM John N. Mafi ${ }^{1,2}{ }^{2}$; Jennifer N. Sayles ${ }^{3}$; Maitraya K. Patel ${ }^{4}$; Deborah Kahaku ${ }^{3}$; Carmen A. Carillo ${ }^{1}$; John F. Brunner ${ }^{5}$; Anish Mahajan ${ }^{3}$; Laura Sarff ${ }^{3}$; Susan Ettner ${ }^{1}$; Katherine L. Kahn ${ }^{1}$; Catherine Sarkisian ${ }^{1}$. ${ }^{1}$ David Geffen School of Medicine at UCLA, Los Angeles, CA; ${ }^{2}$ RAND Corporation, Santa Monica, CA; ${ }^{3}$ LAC+USC Medical Center, Los Angeles, CA; ${ }^{4}$ UCLA Olive View Medical Center, Los Angeles, CA; ${ }^{5}$ USC Norris Comprehensive Cancer Center and Hospital, Los Angeles, CA. (Control ID \#2466141)

BACKGROUND: Back pain costs the U.S. over $\$ 100$ billion annually, and much of this spending is wasteful due to the overuse of advanced diagnostic imaging. Despite widespread dissemination of clinical guidelines and the Choosing Wisely campaign discouraging use of costly and low value imaging, magnetic resonance imaging (MRI) and computed tomography $(\mathrm{CT})$ studies remain frequently overused. Real-time electronic clinical decision support (CDS) at the point of care has been increasingly emphasized as an important strategy to improve the value of back pain management; however, studies suggest that CDS at best only modestly influences practice patterns. Few studies have rigorously investigated the causes of CDS' limited influence on care or examined interventions to improve CDS' impact on reducing low value imaging.

METHODS: We evaluated the first year of the implementation of an electronic decision support tool to reduce low value MRI and CT studies for back and neck pain at a large safety net health system. The CDS tool provides real-time clinician education and feedback at the point of care by using American College of Radiology (ACR) appropriateness scores with links to clinical guidelines and studies. Clinicians answer clinical questions and select the indication for imaging and the CDS tool provides an ACR Appropriateness Criteria score, with high (appropriate) scores ranging from 7 to 9 , medium (borderline) scores ranging from 4 to 6 , and low (inappropriate) scores ranging from 1 to 3 . We obtained electronic health record order entry data and our primary outcomes were high scoring exams, canceled/changed exams, as well as unscored exams (CDS' biggest pitfall). Early observation of CDS implementation and continuous refinement revealed that one department at LAC+USC (the largest practice site among the 16 sites studied) had a large percentage of unscored exams. In response, for that site (the intervention site), we provided a "nudge" incentive grounded in behavioral economic theory ("nudging" preserves freedom of choice and gently encourages clinicians to order more appropriate tests without hard restrictions) where clinicians ordering high scoring exams could bypass the usual protocol of a mandatory phone call to radiology. To evaluate this natural experiment, we constructed logistic regression models to run a pre-post quasi-experimental difference-in-differences (DinD) analysis to measure whether appropriate imaging orders increased and unscored studies decreased in the intervention site compared to 15 control sites. RESULTS: From February 2nd, 2015-January 7th, 2016, 979 clinicians ordered 4866 spinal MRIs and 2540 spinal CTs (out of 7613 initiated attempts) for patients presenting with back pain (mean patient age 48.3 years; $41.4 \%$ female). Among all order attempts, 1642 (21.6\%) orders were high scores, $582(7.6 \%)$ orders were medium scores, $552(7.3 \%)$ were low scores, and 4837 (63.5\%) were unscored. Unscored studies occurred more frequently among specialists than among generalists (e.g., 74.0\% unscored studies for orthopedic and neurosurgeons vs. $60.2 \%$ for internal/family medicine, $p<0.001$ ). The major causes of unscored exams included clinicians overriding CDS by typing a custom free-text indication ( $65.9 \%$ of the time), CDS recommending a different study that the clinician ignored (19.9\%), clinicians not answering a question (7.8 \%), or a software error $(6.4 \%)$. Among all order attempts initiated that were not completed $(n=603), 173$ ( $2.3 \%$ of all attempted orders) were cancelled by the clinician after a low score, and 430 (5.7 \% of all attempted orders) were changed to different tests, resulting in significant improvement in scores, increasing from a mean score of 2.4 to 7.2 after changing the order per the ACR recommendation, $p<0.001$. After implementing the natural experiment at the intervention site, we found reductions in unscored exams (DinD $-2.2 \%, p<0.001)$ and large increases in high scoring exams $(\operatorname{DinD}+20.5 \%$, $p<0.001)$ compared to controls over time (see Figure)

CONCLUSIONS: The implementation of real-time CDS using ACR appropriateness scores to reduce low value imaging for back pain resulted in mostly unscored studies mainly because most orders were bypassed by clinicians, undermining CDS' effectiveness. These findings provide insight into CDS's modest impact on improving care in the U.S. to date and are particularly salient for other safety net systems struggling to improve quality while lowering costs. Our results also encouragingly suggest that implementing a "nudge" to clinicians (such as eliminating a phone call so that CDS streamlines rather than interrupts workflow) can substantially enhance CDS' impact in improving the value of care.

\section{Figure. Proportion of High Scoring and Unscored Imaging Orders Over Time at the Intervention Site vs. Control Sites}

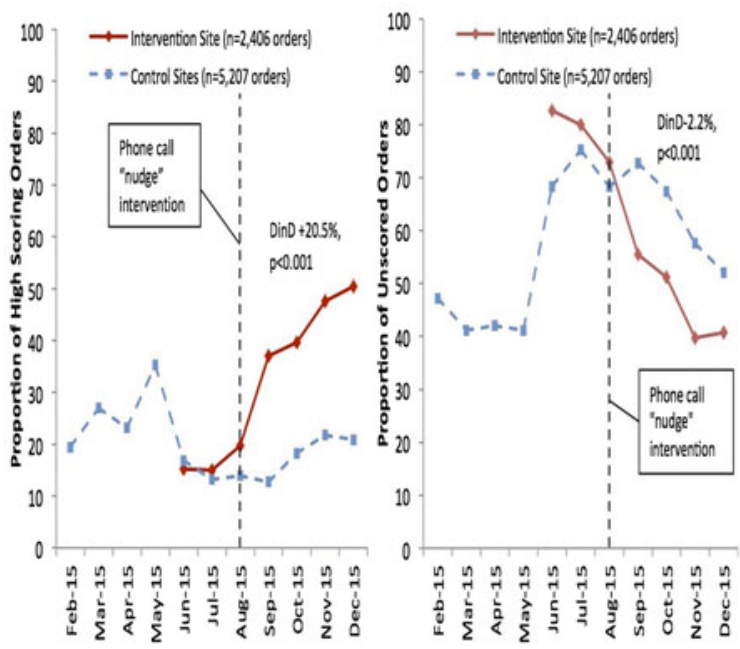

DinD $=$ Difference in Difference

IMPLICIT BIAS IN UNDERGRADUATE MEDICAL EDUCATION: DEVELOPMENT AND VALIDATION OF THE IMPLICIT BIAS ATTITUDE SCALE Cristina M. Gonzalez ${ }^{1}$; Ramya J. Garba ${ }^{3}$; Joseph Grochowalski ${ }^{4}$; Paul R. Marantz ${ }^{2} .{ }^{1}$ Albert Einstein College of Medicine/Montefiore Medical Center, Bronx, NY; ${ }^{2}$ Albert Eisntein College of Medicine, Bronx, NY; ${ }^{3}$ Albert Einstein College of Medicine, Bronx, NY; ${ }^{4}$ Fordham University, Bronx, NY. (Control ID \#2466733)

BACKGROUND: Implicit bias (the unintentional, unconscious assumptions we make about each other) has been demonstrated to influence physician's clinical decisionmaking and may contribute to racial and ethnic health disparities. In an effort to diminish health disparities, medical schools are developing curricula to help mitigate the influence of medical student's implicit bias on their clinical decision-making. To achieve this, a comprehensive, developmentally appropriate curriculum would need to 
be designed that enhances students' knowledge, attitudes, and skills regarding implicit bias recognition and management in clinical encounters. To our knowledge, there exists no validated instrument to assess medical students' attitudes on the topic. The purpose of this study was to develop and validate a novel scale to assess learners' attitudes towards racial and ethnic implicit bias in oneself and in others, its influence in clinicaldecision making, and participating in instruction during their medical education. METHODS: We first developed a list of survey items among our team. We then identified and invited experts in the field of implicit bias to participate in the refinement of the survey items using a modified Delphi Technique, where we corresponded via web-conference and email to design the survey items instead of in-person. We convened the experts for two rounds in order to design the wording and order of survey items. The preliminary scale was administered to medical student volunteers, along with four other validated scales measuring convergent and discriminant constructs identified from the literature. The two convergent scales were chosen after review of the survey items demonstrated measurement of similar constructs. We chose the Health Beliefs Attitudes Survey scale (related to cultural competency training) and the Seeking Professional Help Attitude Scale (related to stigma tolerance and interpersonal openness). The two discriminant scales chosen were the Integrative Medicine Attitude Questionnaire and the Groningen Reflective Ability Scale because those survey items measured constructs unrelated to implicit bias. Upon completion of the scales, mini-cognitive interviews were held discussing each item on our scale, leading to the final scale. We then estimate score reliability (internal consistency, $n=156$ ); and assessed validity $(n=20)$. The participants' scores on the different scales were examined for validity via a multitraitmultimethod correlation matrix.

RESULTS: The final scale had 27 items. Constructs assessed included attitudes toward: 1 . implicit bias as a valid concept, 2. implicit bias existing within oneself, 3. the potential for implicit bias to influence clinical care, 4. the value of implicit bias in medical student education, and 5. their self-perceived confidence level in recognizing and managing one's own implicit biases. Our scale had very high internal consistency $(r=.90)$. The scores were moderately convergent with measures of health beliefs and attitudes and seeking professional help $(r=.28$ and .37 , respectively) suggesting that scores on these scales and the implicit bias scale generally increased and decreased together, signifying some similarity in the constructs they measure. The scores were discriminant with measures of reflective ability and integrative medicine attitudes ( $r=.03, .05$, respectively) suggesting that there is no relationship between scores from the implicit bias scale and these scales, and that they measure different constructs.

CONCLUSIONS: Our novel scale is internally reliable, and with our current sample, is valid. Implicit bias education requires the recognition of bias in oneself, and the humility to be open to learning to mitigate its effect on clinical decision-making. The required components of a successful curriculum are still in development. Program evaluation of curricula depends, in part, on the ability to aggregate student assessments (e.g. regarding attitudes). While further research is needed, our scale may contribute to the assessment of students' attitudes as they progress through a curriculum on implicit bias recognition and management, potentially enhancing future curricular interventions.

\section{IMPROVED TEAM FUNCTION ASSOCIATED WITH LOWER HEALTH} CARE USE Karin M. Nelson ${ }^{2}$; Philip W. Sylling ${ }^{6}$; Leslie Taylor ${ }^{7}$; Christian D. Helfrich ${ }^{4}$; Danielle Rose ${ }^{3}$; Idamay Curtis ${ }^{6}$; Richard Stark ${ }^{1}$; Gordon Schectman ${ }^{5}$; Stephan D. Fihn ${ }^{6,2}$. ${ }^{1}$ Department of Veterans Affairs, Washington, DC; ${ }^{2}$ University of Washington, VA Puget Sound, Seattle, WA; ${ }^{3}$ VA GLA Healthcare System, Sepulveda, CA; ${ }^{4}$ VA Puget Sound Healthcare System, Seattle, WA; ${ }^{5}$ Veterans Affairs Central Office, Milwaukee, WI; ${ }^{6}$ Veterans Health Administration, Seattle, WA; ${ }^{7}$ VA Puget Sound, Seattle, WA. (Control ID \#2469705)

BACKGROUND: The foundation of the patient centered medical home (PCMH) model is the provision of accessible, continuous, and team-based primary care. Our previous work has shown that more effective implementation of the PCMH model (the Patient Aligned Care
Team (PACT) initiative) in over 900 Veterans Health Affairs (VHA) primary care clinics was associated with lower health care use, decreased staff burnout and higher clinical quality and patient satisfaction. The previous findings assessed implementation effectiveness using an overall index comprising 8 PCMH domains: access, continuity, care coordination, comprehensiveness, self-management support, patient-centered care and communication, shared decision-making and team-based care. The goal of the current analysis is to determine which of these domains have the strongest associations with lower health care use.

METHODS: We conducted an observational study to assess the association between PACT implementation in 2012 with patient-level health care use (ED/urgent care visits or hospitalization) during 2012-2014, aggregated to the clinic level. We used VHA clinical and administrative data from 6,379,418 million Veterans who received primary care at 640 VHA hospital- and community-based outpatient clinics (CBOCs). PACT implementation scores for 8 domains were derived from a previously validated instrument, the PACT implementation progress index $\left(\mathrm{PI}^{2}\right)$, which includes patient-ratings of care $(n=75,101$ responses to the Consumer Assessment of Health Plans PCMH module administered to a weighted, random sample of Veterans who received outpatient care in 2012) and primary care provider and staff PACT experiences (5404 responses to a survey distributed to all VHA primary care providers and staff). Each clinic received a domain score for each of the 8 domains for $\mathrm{PI}^{2}$. The domain scores were standardized to have mean zero and also categorized into quartiles. We modeled clinic-level annual counts of all-cause admissions and emergency and urgent care visits from FY2012-2014 using negative binomial regression model, adjusting for clustering by clinic, average clinic-level co-morbidity, patient age and patient gender, and if the clinic was a community based outpatient clinic (CBOC) or VA medical center. Clinic level predictors included the 8 domains of the $\mathrm{PI}^{2}$ score from 2012 (the highest $25 \%$, middle $50 \%$, lowest $25 \%$ ) due to a hypothesized lag between PACT implementation and potential effects. We performed a separate model for each domain of $\mathrm{PI}^{2}(N=1,744$ observations over 3 years for clinics where data was available: 492 clinics have 3 observations, 122 clinics have 2 observations, and 27 clinics have one observation). For these analyses, we report the percent difference in the either all-cause hospitalization or emergency room/ urgent care visits associated with a clinic being in the highest $25 \%$ compared to the lowest $25 \%$ for the domain as well as the percent change in the outcome associated with a one standard deviation increase in the continuous domain score. Limitations to our study include low response rate (33\%) on the provider survey, which is used to derive the team-based care variables.

RESULTS: Comparing clinics in the highest 25th percentile to those in the lowest, higher scores on the team-based care domain were positively associated with lower all-cause admission $(-8.41 \%, p=0.067)$ and $-16.18 \%$ lower emergency room and urgent care use $(p=0.017)$. An increase in one-standard deviation on the team-based care measure was associated with lower all-cause admission $(-3.4 \%, p=0.035)$ and emergency room use $(-7.0 \%, p=0.002)$. The single items included in the team-based domain with the largest associations with lower ED use included increased delegation of primary care providers (PCPs) to other team members in following up on referral $(-22.39 \%, p=0.004)$, responding to prescription refill requests $(-21.91 \%, 0.007)$ and in tracking patient diagnostic data $(-19.49 \%, p=0.031)$. Higher scores on the access domain were significantly associated with increased hospitalization. This association was driven by the increased association with the two patient reported questions about improved after hours care.

CONCLUSIONS: Improved team-based care, with increased delegation of tasks by PCPs to staff, was associated with lower health care use.

IMPROVEMENT IN PATIENT CENTERED MEDICAL HOME ELECTRONIC HEALTH RECORD DOCUMENTATION BY PRIMARY CARE PRACTICES SEEKING NATIONAL COMMITTEE FOR QUALITY ASSURANCE RECOGNITION Sara Myers; Lynn A. Volk; Elizabeth R. Silvers; Catherine Liang; Colleen Blanchette; Amy Feeney; Eric Weil. Partners HealthCare, Boston, MA. (Control ID \#2465360) 
BACKGROUND: The Patient Centered Medical Home (PCMH) model, originating from a pediatric model of care, seeks to improve care coordination and selfmanagement, particularly for patients with complex, high-risk health conditions. The Population Health Management team at Partners Healthcare supports both pediatric and adult primary care practices seeking National Committee for Quality Assurance (NCQA) $\mathrm{PCMH}$ recognition, and the process is rigorous. The $\mathrm{PCMH}$ chart documentation requirements were some of the most challenging areas for our 2014 cohort of practices. Based on feedback from these practices, we implemented Electronic Health Record (EHR) interventions and developed new educational materials to support documentation efforts for our 2015 cohort. This study aims to determine whether chart review scores changed from our 2014 cohort to our 2015 cohort

METHODS: We conducted a comparative cohort study to assess changes in chart review scores for primary care practices undergoing PCMH transformation in 2014 and 2015. Because the applicable NCQA standards changed between these 2 years, we evaluated two factors for which the documentation standards did not change and for which less than $90 \%$ of practices passed: documentation of treatment goals and providing information about new medications to patients. In order for a practice to meet these two requirements, at least $75 \%$ of charts must have clearly documented treatment goals and at least $80 \%$ of charts must have included documentation indicating that information about new medications was given to the patient. We also compared scores of pediatric and adult practices within and between the 2014 and 2015 cohorts RESULTS: As shown in Table 1, the proportion of total practices passing these two documentation requirements improved between the 2014 and 2015 cohorts. The adult and pediatric practices both scored higher in 2015 , but pediatric practices showed greater improvement than adult practices on both of the documentation factors evaluated.

CONCLUSIONS: Supporting the 2014 cohort through their PCMH transformation granted us the opportunity to evaluate our PCMH practice support program. Many practices found chart documentation requirements burdensome on workflow because providers were asked to document conversations that they were already having with their patients. Additional challenges that practices noted include time demands, a lack of understanding of PCMH required factors, difficulty with obtaining provider buy-in, and limitations with EHR functionality. We used this opportunity to refine and develop educational materials including step-by-step manuals, examples of best practices, and informational webinars. We also implemented new EHR functionality to address documentation challenges. We developed EHR templates for free-text notes that satisfied many $\mathrm{PCMH}$ chart documentation requirements, including heading for "Treatment Goals" to remind providers to include this documentation in their charting. Additionally, we added a "Patient Educated" checkbox to the medications tab in the EHR, which clinicians may check off to indicate they provided information about a new medication to a patient. Practices that used these EHR interventions and our educational materials scored highest and found the process less taxing. While EHR interventions coupled with enhanced educational materials improved chart review scores, pediatric practices improved more than adult practices did. This may be due to an emphasis in pediatrics on harnessing support from family and school resources to develop self-management plans with goals that focus on continuity of care. We found that practices seeking PCMH NCQA recognition could improve their scores and chances of recognition by implementing EHR interventions that satisfy PCMH documentation requirements and are easily incorporated into existing workflow.

Table 1. Proportion of adult and pediatric practices meeting PCMH documentation requirements in the 2014 and 2015 cohorts ( $n=$ number of practices).

\begin{tabular}{lllllll}
\hline \hline $\begin{array}{l}\text { PCMH Documentation } \\
\text { Requirement }\end{array}$ & Adult & \multicolumn{5}{c}{ Pediatric } \\
& & & & & Total & \\
& 2014 & 2015 & 2014 & 2015 & 2014 & 2015 \\
& $n=28$ & $n=14$ & $n=5$ & $n=10$ & $n=33$ & $n=24$ \\
$\begin{array}{l}\text { Treatment goals } \\
\text { Information about }\end{array}$ & $82 \%$ & $86 \%$ & $60 \%$ & $100 \%$ & $79 \%$ & $92 \%$ \\
$\begin{array}{l}\text { new medications } \\
\text { given to patient }\end{array}$ & $46 \%$ & $64 \%$ & $40 \%$ & $80 \%$ & $45 \%$ & $71 \%$ \\
\hline
\end{tabular}

IMPROVING ADHERENCE TO NEW LIPID MANAGEMENT GUIDELINES IN RESIDENT CONTINUITY CLINICS: AN INTERNAL MEDICINE RESIDENCY QUALITY IMPROVEMENT INITIATIVE Vignesh Shettar ${ }^{1}$; Jennifer M. Schmidt ${ }^{1,2}$. ${ }^{1}$ Medical College of Wisconsin, Milwaukee, WI; ${ }^{2}$ Clement J Zablocki VA Medical Center, Milwaukee, WI. (Control ID \#2467708)

BACKGROUND: In 2013, the ACC/AHA implemented changes to lipid management for the primary prevention of atherosclerotic cardiovascular disease. Previous guidelines focused on achieving target values while the new guidelines focused on primary prevention, determining risk using a cohort calculator. As the new guidelines presented an opportunity for marked practice change, our Internal Medicine residency program formulated a year-long quality improvement project which aimed to increase the use of these new guidelines in resident continuity clinics.

METHODS: A QI steering team led this Project. The team surveyed categorical Internal Medicine residents regarding their continuity clinic practice (including academic and community clinics). Each resident reported data from the last 10 patients they saw in clinic via an Excel spreadsheet created by the study team. Data included use of the atherosclerotic cardiovascular disease (ASCVD) cohort calculator, review of each patient's current statin regimen, and an explanation of why patients meeting new guideline criteria were not on statin therapy. The intervention was an educational presentation during a weekly didactic session and biweekly emails focused on the importance of lipid management. Residents also received the results of the initial data collection regarding the group's statin usage. Analysis included descriptive and univariate analyses using 2tailed tests with significance at $p<0.05$.

RESULTS: Pre-intervention data was gathered on 765 patients; 1077 patients were included in post-intervention analysis. One hundred percent of residents submitted data (fewer patients were included in the pre-analysis as some first year residents had not yet seen 10 clinic patients). A statistically significant decrease in patients with an ASCVD risk of $>7.5 \%$ who were not on a statin was seen $(p=0.038)$. There was also a statistically significant decrease in "resident didn't know" as a reason for appropriate patients not being on statin therapy ( $p=$ 0.046). No significant difference was seen in "preceptor preference," "patient refused" or "contraindicated" as reasons why statin therapy was not initiated.

CONCLUSIONS: This project focused on increasing resident adherence to the 2013 cholesterol management guidelines. Education was the primary intervention, however, the act of residents' collecting data on their own clinic patients also served as an intervention by focusing residents' attention on their personal lipid management. The QI team set out to use interventions other than education, however, because of the diversity of residen clinics and time restraints, general resident education was the most appropriate and feasible intervention. Despite its known weaknesses, our intervention yielded statistically significant results. Efforts to increase our interventions' reach included using multiple forms of communication as well as including literature on the impact of appropriate statin management. An important outcome of this project was the development of an Epic dot phrase that it is now being used by the staff community physician group in their clinics.

IMPROVING IDENTIFICATION OF PATIENTS AT HIGH-RISK FOR READMISSION USING SOCIO-BEHAVIORAL PATIENT CHARACTERISTICS Amol S. Navathe ${ }^{1,3}$; Feiran Zhong ${ }^{2}$; Victor Lei ${ }^{2}$; Frank Y. $\mathrm{Chang}^{2}$; Roberto A. Rocha ${ }^{2}$; Li Zhou ${ }^{2} .{ }^{1}$ University of Pennsylvania, Philadelphia, PA; ${ }^{2}$ Brigham and Women's Hospital, Boston, MA; ${ }^{3} \mathrm{CMC}$ VA Medical Center, Philadelphia, PA. (Control ID \#2467524)

BACKGROUND: Identifying patients at high risk for readmission is a health system priority because Medicare penalizes up to $3 \%$ of annual inpatient payments for excess readmissions and includes readmissions as an accountable care organization (ACO) 
quality measure. Patients with socio-behavioral issues are known to be at high risk for readmission but detecting them through billing codes may be incomplete.

METHODS: Our objective was to assess whether using information from physician notes would increase the detected prevalence of social-behavioral issues in a hospital patient population and improve identification of patients at high risk for readmission. Using 3 years of administrative and electronic health record (EHR) data from a large multihospital academic health system, we compared the frequency of eight socio-behavioral factors (poor social support, housing instability, tobacco use, alcohol abuse, drug use, depression, and fall risk) among 93,606 patients with cardiovascular disease based on ICD-9 codes, structured EHR data such as problem lists and heath maintenance registries, and physician documentation in narrative notes. Physician narrative notes were analyzed using MTERMS, a validated natural language processing (NLP) tool, to extract sociobehavioral characteristics of each patient. We then used multivariate regression techniques to assess the association between 30-day all cause readmissions and each socio-behavioral factor based on the three data types. Finally, we constructed a multivariate risk adjusted model using detailed clinical data to evaluate whether the enhanced social-behavioral factors using information in physician notes were associated with higher readmission risk. RESULTS: The frequency of identifying socio-behavioral factors among hospitalized patients was increased in 7 of 8 socio-behavioral factors using clinical text analysis versus either ICD-9 codes, structured EHR data, or combining those sources. We identified an additional 33,674 patients ( $36 \%$ of the entire sample) with chronic wounds, 27,912 patients $(29.7 \%$ ) with alcohol abuse, 22,030 patients (23.4 \%) with fall risk, 17,449 (18.5\%) with tobacco use, 14,675 patients $(15.6 \%)$ with poor social support, 6458 patients $(6.9 \%)$ with depression, 5236 ( $5.6 \%$ ) with drug abuse, and 242 patients $(0.3 \%)$ with housing instability. Patients with each of the eight socio-behavioral factors detected in physician documentation were more likely to be readmitted than those without (Figure 1), with a range in significant Odds Ratios of 1.05 1.50. Four factors were associated with readmission after detailed risk-adjustment: housing instability (OR 1.42; $95 \%$ CI 1.20-1.67; P<0.001), depression (OR 1.13; 95 \% CI 1.06-1.21; $P<0.001$ ), poor social support (OR 1.09; $95 \%$ CI 1.03-1.16; $P=0.02$ ), and presence of chronic wound (OR 1.08, $95 \%$ CI 1.03-1.13; $P<0.001)$.

CONCLUSIONS: Patients with socio-behavioral factors are substantially more prevalent than represented in health system administrative data. Automated methods for analyzing physician notes may enable better identification of patients with high socio-behavioral need who are at increased risk for readmission and improved targeting of care management resources.

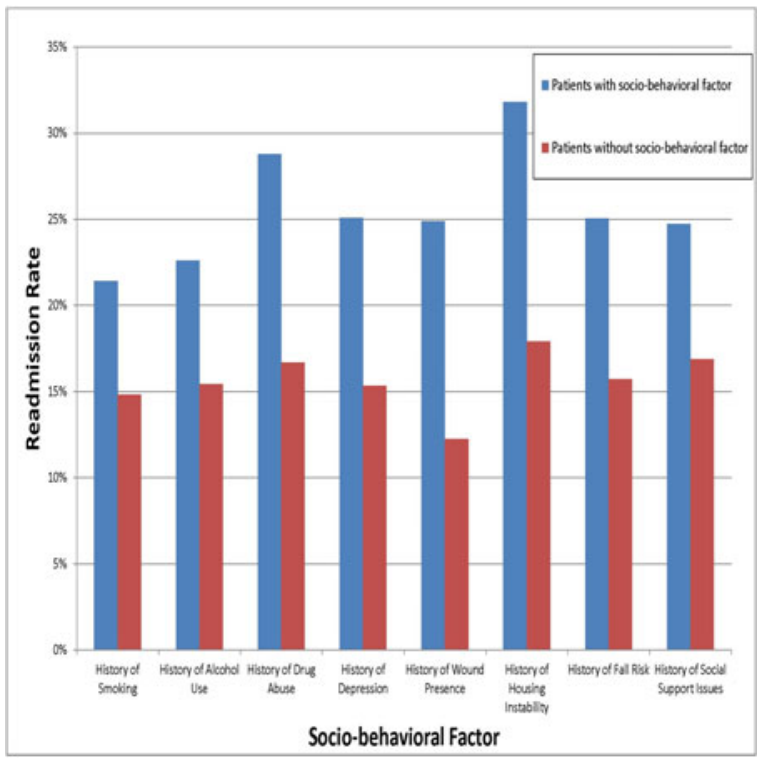

Figure 1: Readmission Rate by Presence of Socio-behavioral Factor in Physician Note
IMPROVING INCLUSION OF RACIAL AND ETHNIC MINORITIES IN A RANDOMIZED CANCER CLINICAL TRIAL USING A DIVERSE AND MULTI-LINGUAL RESEARCH TEAM Sharon $\mathrm{Bak}^{4}$; Tracy A. Battaglia ${ }^{4,}{ }^{1}$; Maria Castano $^{1}$; Kate Festa ${ }^{1}$; JoHanna Flacks ${ }^{5}$; Christine M. Gunn ${ }^{1,3}$; Naomi Y. Ko ${ }^{2,}{ }^{1}$; Samantha Morton ${ }^{5}$; jennifer Pamphile ${ }^{1}$. ${ }^{1}$ Boston Medical Center, Boston, MA; ${ }^{2}$ Durham VA Medical Center, Boston, MA; ${ }^{3}$ Boston University, Boston, MA; ${ }^{4}$ Boston University Medical Center, Boston, MA; ${ }^{5}$ Medical-Legal Partnership| Boston (MLP), Boston, MA. (Control ID \#2468697)

BACKGROUND: Randomized clinical trials provide the evidence base for new advances in treating and preventing cancer. The representation of racial and ethnic minorities in clinical trials remains persistently low, ranging from 1.5 to $5 \%$. This lack of ethnic and racial diversity among study participants hinders the ability to generalize findings and deliver the best care possible. Studies without adequate minority representation may diminish opportunities for identifying the health needs of minority groups, and may contribute to the disproportional burden of cancer in these populations. It is essential to include a diverse sample of participants in clinical trials. This study describes best practices for engaging diverse populations in clinical trials

METHODS: Project SUPPORT is a randomized controlled clinical trial that seeks to recruit 374 low-income and racially and ethnically diverse breast cancer patients at Boston Medical Center (BMC), an urban academic safety net hospital. The Project SUPPORT study objective is to determine whether patient navigation supported by medical-legal partnership improves timely cancer treatment. Participants randomized to the control arm receive a patient navigator, who coordinates patient care and addresses barriers to care. Participants randomized to the intervention arm receive a patient navigator who systematically consults with lawyers from Medical-Legal Partnership| Boston (MLP) to address socio-legal barriers to care such as housing insecurity. This descriptive study aims to explore how enrollment of a diverse patient population into a randomized controlled trial is achieved with bilingual research assistants who recruit patients in their primary language: English, Spanish or Haitian Creole. Recruitment protocols required that primary spoken language be determined at the time of eligibility, so that all eligible participants were matched with a research assistant who spoke their primary language at the time of recruitment. We report here retention and enrollment rates by both race and primary spoken language.

RESULTS: Of the 186 eligible women approached for enrollment, 39 declined participation and 147 (79\%) enrolled. The median age of those enrolled was 56 years, 77 (52\%) were Black, 32 (22\%) Hispanic, 30 (20\%) White, and 8 (6 \%) other. Primary spoken language among participants: 31 (21\%) Spanish, 14 (10\%) Haitian Creole and 102 (69\%) English. The majority ( $73 \%$ ) of subjects had public health insurance. Enrollment rates by race/ethnicity were: 77 out of 91 (84\%) Black, 32 out of 38 (84\%) Hispanic, and 30 out of $44(70 \%)$ White patients. Enrollment rates by primary spoken language include: 31 out of 37 (84 \%) Spanish, 14 out of 20 (70\%) Haitian Creole and 102 out of $128(80 \%)$ English speakers. Research assistants survey patients at enrollment, 3, 6 and 12 months post-enrollment. Retention rates are high with $94 \%$ at 3 months, $91 \%$ at 6 months and $82 \%$ at 12 months.

CONCLUSIONS: These preliminary data suggest that having culturally diverse research staff who can communicate with study participants in their primary language aids in the successful recruitment and retention of historically underrepresented populations and should be a topic of further investigation.

IMPROVING INFLUENZA VACCINATION RATE WITH HEALTH MAINTENANCE TABLES Sue-Wei Luu ${ }^{1}$; Maryam Sattari ${ }^{2} .{ }^{1}$ University of Florida, Gainesville, FL; ${ }^{2}$ University of Florida College of Medicine, Gainesville, FL. (Control ID \#2467662) 
BACKGROUND: More than 200,000 people in the United States are hospitalized each year for illnesses associated with seasonal influenza virus infections. Influenza vaccination is associated with decreased mortality, hospitalization rates, and medical costs, yet only $43.6 \%$ of adults older than 18 reported flu vaccination during the 2014-2015 influenza season. Effective interventions are needed to increase flu vaccination coverage. To improve flu vaccination rates, we created a tool referred to as the health maintenance table (HMT) for use in our electronic medical record (EMR) at the University of Florida (UF). The goal of this study was to evaluate the impact of this tool on rate of influenza vaccination.

METHODS: We performed a retrospective review of medical charts for patients who visited one of UF Internal Medicine clinics during the month of August 2014 and extracted the following information for 238 consecutive patients: (a) whether HMT was included in the clinical encounter documentation, (b) patient's age at the time of clinic visit, (c) patient's gender, (d) whether the patient was eligible for flu vaccination, and (e) whether flu vaccination was administered. We considered a patient eligible for flu vaccination if he/ she was 18 years of age or older and there was not any documentation of anaphylactic reaction after previous flu vaccine or to a vaccine component, moderate or severe acute illness, fever, or history of Guillain-Barré syndrome within 6 weeks of previous influenza vaccination. We used SPSS software version 16 (SPSS, Chicago, IL) to determine and compare the rate of influenza vaccination in HMT and non-HMT users.

RESULTS: Of the 238 patients included in this study, 162 were female and 76 were male The average age of patients was 54.73 (range 20-90). All the patients were eligible for influenza vaccination and $112(47.1 \%)$ received the vaccination. Vaccination rate was $41.1 \%(67 / 163)$ for HMT non-users and $60.0 \%$ (45/75) for HMT users. The difference in vaccination rates between the two groups was statistically significant $(p=0.007)$. We did not find statistically significant associations between flu vaccination and patients' gender or the provider level (faculty versus housestaff). However, there was a statistically significant difference in vaccination rates among different patient age groups, with older patients more likely to be vaccinated.

CONCLUSIONS: Association of increased influenza vaccination rates with HMT use suggests that routine implementation of HMT might be a cost-effective method for increasing vaccination rates and therefore, decreasing hospitalizations, morbidity and mortality, and medical costs due to influenza virus infections. While we developed and implemented the HMT in an outpatient clinic in an academic center, this tool can be easily adapted and incorporated in private/community settings as well. The other advantages of the HMT are that it is simple, intuitive, and medical provider-friendly and can be incorporated into paper-based medical charts as well as EMR. Future research is warranted to elucidate the effectiveness of HMT implementation in different healthcare settings as well as its downstream effects on patient outcomes.

IMPROVING NURSE-PHYSICIAN COMMUNICATION THROUGH A RESIDENT-LED COMMUNICATION DEVICE Alexis Wickersham $^{3,2}$; Krista M. Johnson ${ }^{1}$; Aparna Kamath ${ }^{4}$; Peter Kaboli ${ }^{2}$. ${ }^{1}$ University of Iowa Hospitals and Clinics, Iowa City, IA; ${ }^{2}$ Iowa City VAMC, Iowa City, IA; ${ }^{3}$ University of Iowa Hospitals and Clinics, Iowa City, IA, IA; ${ }^{4}$ Duke University Medical Center, Durham, NC. (Control ID \#2467154)

BACKGROUND: Communication deficits are a leading cause of medical errors and many simple errors can cause considerable, potentially life-threatening, harm. Two drivers for inconsistent communication amongst providers at our institution are resident duty hour regulations and lack of geographical bed placement, both of which create unpredictable rounding time and location of the inpatient resident teams. Our aim was to improve communication by implementing a quality improvement (QI) project to increase nursing participation in bedside rounds.
METHODS: An online survey was developed and delivered to all nursing staff and attending and resident physicians who worked on the general medicine inpatient units in the prior 2 months. The pre-implementation survey included questions in the domains of communication, teamwork, and care coordination using a 5-point Likert scale. Survey responses were dichotomized for reporting and assessed using the chi-square statistic. Qualitative survey data were grouped into themes. For the first Plan-Do-Study-Act (PDSA) cycle, senior residents on 3 inpatient teams were equipped with a Vocera badge to broadcast a message when the physician team was rounding on a unit. All nursing staff logged into a unit via their personal Vocera badge received the broadcast and the patient's bedside nurse was expected to join rounds. We used direct observation to asses nursing presence at bedside rounds

RESULTS: The overall survey response rate was $52 \%: 53 \%$ of nurses $(n=38)$ and $52 \%$ of physicians $(n=22)$. In the domain of communication, responses to 8 of 11 survey questions were discordant. For example, physicians were more likely to agree that communication with nursing staff prior to $(p=0.01)$, during $(p=0.02)$ and after rounds $(p=0.006)$ was occurring, compared with nurses' perceptions. Both agree that communication between nurses and physicians was not efficient $(p=0.35)$, though $84 \%$ of nurses believed that communication between team members was adequate, compared to only $47 \%$ of physicians $(p<0.001)$. Half of physicians believed they alert a nurse when rounds are occurring, compared to only $3 \%$ of nurses $(p<0.001)$. In the teamwork domain, both physicians $(100 \%)$ and nurses $(92 \%)$ agreed that bedside nurse-physician rounds are not a part of hospital's culture $(p=0.18)$. Nurses felt their input was not encouraged $(p=0.02)$ nor well received $(p<0.001)$ on rounds and patients' daily care plans lack clarity $(p=0.02)$. Physicians were more likely to agree $(68 \%)$ than nurses $(26 \%)$ that it is difficult to locate a nurse on the unit for rounds $(p=0.001)$. Qualitative data themes focused on improving communication, rounding, physician paging, teamwork and work-environment. Rounding observation in the pre-intervention phase demonstrated that physician teams were including nurses during bedside rounds only $21 \%$ of the time.

CONCLUSIONS: Effective communication is essential in improving patient safety and reducing medical errors. Prior to implementing our QI project aimed at increasing nursing contribution during patient bedside rounds, nursing and physician perceptions about communication, teamwork and care coordination differed largely. Data suggest that a culture change amongst physicians and nurses must occur in order to promote nursing contribution during bedside rounding and improve efficient communication. It is our goal to alert nurses with the Vocera device prior to rounding on their patients, allowing opportunity for face-to-face communication amongst providers. Post-intervention surveys and round observations will be used to assess the effectiveness of the intervention and design future PDSA cycles.

\section{IMPROVING RESIDENT SKILL IN PROVIDING CONTRACEPTION} COUNSELING USING A MULTI-RESOURCE MODEL Shannon K. Boerner; Kara Markin. University of Nebraska Medical Center, Omaha, NE. (Control ID \#2469834)

BACKGROUND: Contraception knowledge and patient access to contraceptive counseling is an important aspect of primary care. Misconceptions exist among patients and providers about contraceptive options and their risks, benefits and effectiveness. The CHOICE Project, a study published by researchers at Washington University, has shown that appropriate counseling techniques and subsequent provision of chosen contraception free of charge can result in higher rates of patient adherence to contraceptive methods and is associated with lower rates of unintended pregnancy and abortion within the studied community. However, there is little information on best practices for teaching residents effective contraceptive counseling techniques. We aimed to meet a perceived need for additional education and clinic resources for residents by providing targeted education and 
introducing a contraceptive counseling toolkit at the clinics. Instruction on patient counseling is a required part of an institution's educational program and a competency that must be integrated into its curriculum. We describe our effort to build a new educational curriculum that fills a knowledge gap and meets an ACGME requirement. We delivered a targeted curriculum regarding contraceptive counseling to all Internal Medicine and Medicine/Pediatrics residents. The curriculum included didactic teaching (1 h noon conference) and a hands-on resource (contraceptive counseling toolkit). The contraceptive counseling toolkit contains physical models of various contraceptive devices and an illustrated quick reference for providers. The study authors created a two-sided, spiralbound book, professionally designed and printed on heavy-weight paper. One half of the book is intended for patients to use to learn about various contraceptive options and the other half is intended for providers to use as a reference for prescribing. The specific objective of this study was to implement and assess a new contraceptive counseling curriculum for residents. Our study sought to assess attitudes surrounding contraception management, as well as perceptions of the role of residents in provision of contraceptive care. Lastly, we sought to improve knowledge needed to safely and competently provide contraception to patients.

METHODS: This study utilized a pre/post design to measure the impact of a new curriculum on residents by using a survey to assess attitudes, perceptions and knowledge The study was conducted among 83 residents enrolled in 2 residency programs at a large tertiary care center. The survey was made available to all residents via email 1 month prior to and 5 months following the introduction of the curriculum. All residents were recruited to the didactic teaching conference via typical methods for our residency program. Toolkits were provided in resident clinics for use in patient care and education. Pre- and post-intervention surveys were analyzed using Wilcoxon rank sum test and Fisher's exact test. We also conducted focus groups evaluating residents' perceptions regarding their comfort levels with contraceptive counseling.

RESULTS: A total of 83 residents were included in the study; 60 responses to the surveys were recorded. Analysis of pre/post responses did not identify a statistically significant improvement in residents' perceived confidence in prescribing various methods of contraception nor knowledge-based questions regarding most of the methods of contraception studied. Improvement in knowledge regarding one specific method (emergency contraception) did approach significance $(P=0.051)$ following the intervention. Of note, residents described a high level of confidence in their ability to provide contraceptive counseling in general; across all methods, $58.4 \%$ described themselves as "comfortable" or "very comfortable". However, knowledge regarding specific methods was suboptimal; residents' ability to correctly identify the most efficacious vs least efficacious method was only $60 \%(33 / 55)$. A minority of residents $(15 / 54,27.7 \%)$ were able to consistently identify an important side effect of one method (delay in return of fertility due to injectable contraceptive). Qualitative data obtained from involved residents indicated a range of perceptions regarding their comfort levels in providing contraception; some residents endorsed lack of familiarity with contraceptive options as well as a perceived division of responsbilities amongst primary care providers and OBGYN providers. However, some residents (those in a dedicated primary care track) indicated a higher comfort level with provision of contraceptive care in particular and women's health concerns in general.

CONCLUSIONS: We found no significant improvement in resident knowledge of several contraception methods before and after our curriculum was delivered. Of concern is the suboptimal knowledge base in light of the residents' high perceived level of confidence in counseling regarding contraception. Our use of a hands on toolkit coupled with didactic teaching may not have adequately met residents' needs for use in the ambulatory setting, but we anticipate improvement with next steps. Qualitative data regarding resident experiences with our curriculum have indicated varying degrees of comfort with contraceptive care; a dedicated primary care track appears to impact residents' reported capacity for prescribing contraception. Translating our printed materials into an app-based format is another potential outgrowth of this project.
IN BOSTON AND NYC, SAFETY-NET HOSPITALS ARE MINORITYSERVING HOSPITALS: IMPLICATIONS FOR DELIVERY SYSTEM REFORM Roosa S. Tikkanen ${ }^{7}$; Steffie Woolhandler ${ }^{7}$; David Himmelstein ${ }^{7}$; Nancy R. Kressin ${ }^{1,}$; Amresh D. Hanchate ${ }^{2,} 3$; Meng-Yun $\operatorname{Lin}^{1,}{ }^{4}$; Chieh $\mathrm{Chu}^{1,}$ 2; Danny McCormick ${ }^{5,}{ }^{6}$; Karen E. Lasser ${ }^{1,}{ }^{2}$. ${ }^{1}$ Boston Medical Center, Boston, MA; ${ }^{2}$ Boston University School of Medicine, Boston, MA; ${ }^{3}$ VA Boston Healthcare System, Boston, MA; ${ }^{4}$ Boston University School of Public Health, Boston, MA; ${ }^{5}$ Cambridge Health Alliance, Cambridge, MA; ${ }^{6}$ Harvard Medical School, Boston, MA; ${ }^{7}$ City University New York School of Public Health at Hunter College, New York City, NY. (Control ID \#2469843)

BACKGROUND: Medicaid, uninsured and minority patients often rely on safety-net hospitals (SNHs), but no recent studies have quantified the extent of that reliance in major urban areas. These hospitals often lack adequate resources, which may contribute to racial disparities in care. Since Medicaid reimbursement rates remain below the costs of care, many SNHs rely on public subsidies, including Disproportionate Share Hospital (DSH) funds. The Affordable Care Act (ACA) will reduce DSH payments to SNHs, potentially undermining the quality of care at SNHs. We examined the role of SNHs in the inpatient care of minorities in the Boston metropolitan area and New York City (NYC) and assessed whether the patterns of care had changed in NYC in recent years.

METHODS: We analyzed discharge data on adults ( $\geq 18$ years old) hospitalized at acute care hospitals in Boston and NYC in 2009, and the most recent (2013), publicly available data for NYC (2013 data was not publicly available for Boston). SNH hospitals were defined as those in the top quartile by the proportion of Medicaid plus uninsured hospitalizations. We categorized race/ethnicity as "white", "black" or "other minority". The inconsistent classification of Hispanic patients across hospitals precluded reliable analyses of this group. We classified patients identified as "white Hispanics" as "other minority" and black Hispanic patients as "black". In addition to descriptive analyses, we conducted multivariable logistic regression analyses to explore the association of race with the likelihood of being hospitalized in a SNH (vs. non-SNH), after controlling for sex and age. We also calculated an Index of Dissimilarity (ID) for each city, which compares the evenness of distribution of majority (white) and minority (black or other minority) patients across hospitals. The ID ranges between 0 (races represented at all hospitals in the same proportion as in the city overall), and 1 (complete segregation).

RESULTS: The Boston sample consisted of 43,252 discharges at three SNHs and 191,660 discharges at ten non-SNHs. The NYC sample consisted of 210,383 discharges at 15 SNHs, and 828,542 discharges at 43 non-SNHs in 2009; and 193,321 discharges at 14 SNHs and 744,713 discharges at 40 non-SNHs in 2013. In 2009, Medicaid insurance was three times more common among black inpatients compared with whites (Boston: 30 vs. $9 \%$; NYC: 25 vs. $8 \%$ ). Uninsured hospitalizations were uncommon in Boston for all races $(0.9-1.4 \%)$. In NYC, there were nearly twice as many uninsured discharges among blacks (4.6\%) and other minorities (4.7\%) compared to whites $(2.4 \%)$. Nearly half ( $45 \%$ ) of all black patient discharges in Boston were at SNHs, compared to $12 \%$ of white patient discharges. In NYC, nearly one-third (30\%) of all black discharges were at SNHs compared to $7 \%$ of white discharges. In multivariable analyses race was a significant predictor of SNH hospitalization: black patients were 5-6 times more likely (Boston OR $5.87,95 \%$ confidence interval [CI] 5.71-6.04; NYC OR 5.09, $95 \%$ CI 5.02-5.17), and other minorities 2-3 times more likely (Boston OR 2.03, $95 \%$ CI 1.97-2.11; NYC OR $3.36,95 \% \mathrm{CI} 3.30-3.41)$ to be hospitalized at a $\mathrm{SNH}$, relative to white patients after adjusting for sex and age. Segregation by race across all hospitals was moderate-to-high in NYC (ID 0.55 for blacks, 0.46 for other minorities), and lower in Boston (ID 0.33 and 0.20 , respectively). By 2013, racial segregation at NYC hospitals decreased minimally; $24 \%$ of black discharges were at SNHs vs. $11 \%$ of white discharges, and the IDs were 0.52 for blacks and 0.42 for other minorities. 
CONCLUSIONS: In both Boston and NYC, SNHs care for a large share of minority inpatients. Hence, the ACA's reductions in DSH that will negatively impact the financial position of many SNHs, disproportionately threatens minority patients. Our finding of high levels of segregation, particularly in NYC, is especially worrisome since a body of evidence has suggested that patients at racially segregated hospitals may have worse outcomes. In the short term, improving SNH funding by raising Medicaid payment rates and increasing supplemental funding may help protect the care of minority patients. In the longer term, fundamental policy changes are needed to address the de-facto segregation of hospital care.

INCARCERATION HISTORY AS AN INDEPENDENT PREDICTOR OF UNINSURANCE AND POOR HEALTH Tyler N. Winkelman ${ }^{1,2}$; HwaJung Choi ${ }^{1}$; Matthew M. Davis ${ }^{1,}{ }^{1}$. ${ }^{1}$ University of Michigan, Ann Arbor, MI; ${ }^{2}$ VA Ann Arbor Healthcare System, Ann Arbor, MI. (Control ID \#2465412)

BACKGROUND: Previously incarcerated individuals have high rates of uninsurance in the year following release from prison. Nationally representative data indicate that uninsured rates are between 40 and $60 \%$ in the year after release from prison or jail. Uninsurance after release is a critical issue for justice-involved individuals because they have mortality rates 12 times higher than the general population in the 2 weeks following release. Justice-involved individuals also have higher rates of mental illness, substance use disorders, and chronic disease, and they are hospitalized more frequently than then general population. Whether high rates of uninsurance persist beyond the first year post-release is unknown. It is also unclear whether socio-demographic factors alone (ie. employment and income) account for high uninsurance rates or whether a history of incarceration is an independent predictor for uninsurance. We hypothesized that incarceration would be an independent risk factor for uninsurance due to state policies that terminate Medicaid upon incarceration and other life disruptions associated with incarceration. We also evaluated whether self-reported health status and substance use were independently associated with incarceration after accounting for socio-demographic factors and health insurance status. METHODS: We used the National Survey of Family Growth (NSFG) to investigate the associations between incarceration and health insurance, self-reported health, and substance use. NSFG is a nationally representative survey of men and women ages $15-44$. We limited our study to men between the ages of 19-44 because incarceration data were not collected for female respondents, and because men account for $85 \%$ of people in jail and over $90 \%$ of people in prison. Individuals between 19 and 45 years account for approximately $70-80 \%$ of those in jail or prison. We pooled two waves of survey data, 2006-2010 and 2011-2013, for our analyses. Incarceration history was determined by responses to the questions "In the last 12 months, have you spent any time in a jail, prison, or a juvenile detention facility?" and "Have you ever spent time in a jail, prison, or juvenile detention center?". Health insurance variables were coded using standard national survey categories. We tabulated results with weighted percentages and obtained unadjusted odds ratios using logistic regression. We used multivariable logistic regression to obtain adjusted odds ratios of uninsurance, controlling for age, race/ethnicity, income, education, employment status, marital status, biological children, health status, drug and alcohol use, and survey wave. We also used multivariable logistic regression to obtain adjusted odds ratios of fair/poor health and drug or alcohol use in the last 12 months, controlling for age, race/ethnicity, income, education, employment status, marital status, and survey wave.

RESULTS: Our sample consisted of 12,368 men between the ages of 19 and 45 at the time of interview. Over one quarter of the sample had a history of incarceration; $19.8 \%$ had a distant history of incarceration (incarcerated more than 12 months ago) and $6.2 \%$ had a recent history of incarceration (incarcerated within the last 12 months). Compared to men who have never been incarcerated, men with a history of distant or recent incarceration were more likely to be black $(9.9 \%$ (never incarcerated) vs $14.2 \%$ (distant incarceration) vs $19.6 \%$ (recent incarceration)), below $138 \%$ of the federal poverty level ( 23.0 vs 28.7 vs $39.5 \%$ ), without a high school diploma (12.7 vs 22.3 vs $34.9 \%$ ), unmarried (55.0 vs 60.7 vs $82.5 \%$ ), unemployed ( 8.7 vs 15.8 vs $22.9 \%$ ), uninsured (23.6 vs 34.5 vs $47.7 \%$ ), and have had recent drug or alcohol use. In both unadjusted and adjusted analyses we found that distant and recent incarceration were both independent predictors of uninsurance ( $p \leq 0.005$ for all models) (Table 1). In adjusted analyses, distant and recent incarceration were both associated with fair/poor self-reported health (OR 1.43 (1.09-1.88), OR 1.46 (1.04-2.03), respectively), drug use in past 12 months (OR 2.75 (2.15-3.53), OR 4.10 (3.03-5.56), respectively), and binge drinking in past 12 months (OR 1.78 (1.50-2.12), OR 2.25 (1.78-2.85), respectively).

CONCLUSIONS: A history of incarceration is an independent risk factor for uninsurance and persists for more than 1 year after release from prison or jail. While those with a distant history of incarceration have lower uninsured rates than their recently incarcerated peers, their uninsured rates remain significantly higher than men without a history of incarceration. Justice-involved men are in poorer health and have more health risk factors than men without a history of incarceration after controlling for sociodemographic factors and would benefit from improved access to care. Additional outreach to justice-involved individuals is needed to assist with insurance enrollment prior to or following release, particularly in states that terminate Medicaid upon incarceration.

Table 1. Odds of Uninsurance by Incarceration History for Young Men Ages 19-44

\begin{tabular}{lll}
\hline \hline & Uninsured Rates & \\
Outcome measure & Unadjusted OR & Adjusted OR \\
& $(95 \% C I)$ & $(95 \% C I)^{\mathrm{a}}$ \\
Never incarcerated & 1.0 & 1.0 \\
Distant incarceration & $1.71(1.45-2.01)^{* *}$ & $1.29(1.08-1.53)^{* *}$ \\
Recent incarceration & $2.95(2.39-3.63)^{* *}$ & $1.45(1.14-1.85)^{* *}$ \\
\hline
\end{tabular}

a Adjusted for age, race, income, education, employment status, marital status, biological children in household, self-reported health, drug use, binge drinking, and survey wave; * $p<0.05 ; * * p \leq 0.005$

INCORPORATING MEDICATION INDICATION INTO CPOE ORDERING: VIEWS OF PHYSICIANS AND PHARMACISTS Kevin W. Kron ${ }^{5}$; Aaron Nathan ${ }^{2}$; Sam Karmiy ${ }^{5}$; Lynn A. Volk ${ }^{4}$; Mary Amato ${ }^{3}$; Pamela M. Neri ${ }^{6}$; Alejandra Salazar ${ }^{2}$; Enrique Seoane-Vazquez ${ }^{3}$; Adam Wright ${ }^{1}$; Tewodros Eguale ${ }^{2}$; Sarah K. McCord ${ }^{7}$; Rosa RodriguezMonguio $^{8}$; Gordon D. Schiff ${ }^{2} .{ }^{1}$ Brigham and Women, Boston, MA; ${ }^{2}$ Brigham and Women's Hospital, Boston, MA; ${ }^{3}$ Brigham and Womens Hosptial/MCPHS, Boston, MA; ${ }^{4}$ Partners HealthCare, Wellesley, MA; ${ }^{5}$ Partners Healthcare, Boston, MA; ${ }^{6}$ Partners Healthcare Systems, Inc., Wellesley, MA; ${ }^{7}$ Massachusetts College of Pharmacy and Health Science University, Boston, MA; ${ }^{8}$ University of Massachusetts—Amherst, Amherst, MA. (Control ID \#2467203)

BACKGROUND: Currently medication orders lack information about the drug indication, which many have argued could help with patient education and preventing medication errors. As part of a larger national project to advance the incorporation of indications in the electronic prescribing process, we surveyed key stakeholders about their views of the benefits and barriers. Physicians and pharmacists may have differing opinions as to the importance or difficulty of these factors that need to be considered when exploring the possibility of incorporating indications into the prescribing process.

METHODS: An online survey was administered via email to 575 experts and stakeholders involved in various aspects of the prescribing process including physicians, pharmacists, and others (e.g. nurse practitioners, vendors, patients, educators). Participants were asked to rate the importance of the benefits of incorporating indications into the prescribing process, and how difficult they perceived various barriers to be, each on a scale of 1 (least important/difficult)-5 (most important/difficult). T-tests were used to 
measure the significance of the difference between the answers provided for each question by physicians and pharmacists compared to the rest of the participant sample.

RESULTS: One hundred sixteen of the 575 (20.2\%) invitees responded to the survey including 23 physicians, and 46 pharmacists. The data indicates strong overall agreement across all respondents with the importance of incorporating indications into prescriptions (4.49) with statistically significant differences $(p<.05)$ between physicians and pharmacists (3.96 vs. 4.74 , respectively). There was also strong support across participants for incorporating indications for a number of reasons including decreasing medication errors (4.58), facilitating patient education/counseling/instructions (4.43), and enhancing team communication (4.26). There were differences between pharmacists and physicians regarding the importance of various benefits (Table 1). However, there was more agreement between these two groups with respect to the level of difficulty of overcoming barriers (Table 2). Results that were statistically significantly different for physicians were all lower than the rest of the participant sample, and those that were statistically significantly different for pharmacists were all higher than the rest of the participant sample.

CONCLUSIONS: A select group of experts and stakeholders involved in this study perceive significant benefits to incorporating drug indication into prescriptions and the prescribing process. While potentially biased by the limited sample size and the fact that participants had an interest in improving prescribing using drug indications, it included representatives from key stakeholders and organizations related to the prescribing process. Physicians expressed less support than pharmacists regarding a number of potential benefits. Both groups agreed that a number of barriers were important to overcome including interoperability and technical issues, policy issues in overcoming market fragmentation, and concerns about physicians' time and billing issues.

Table 1

\begin{tabular}{lll}
\hline \hline Potential Benefits & $\begin{array}{l}\text { Physician } \\
(n=23)\end{array}$ & $\begin{array}{l}\text { Pharmacist } \\
(n=46)\end{array}$ \\
$\begin{array}{l}\text { Decreasing medication errors } \\
\text { Facilitating patient education/ }\end{array}$ & $4.23^{*}$ & 4.72 \\
counseling/instructions & 4.35 & 4.54 \\
Enhancing team communication & $3.68^{*}$ & $4.44^{*}$ \\
$\begin{array}{l}\text { Integrating problem list with } \\
\text { medications Rx }\end{array}$ & 3.91 & 4.26 \\
$\begin{array}{l}\text { Identifying duplicate medications } \\
\text { Empowering patients to ask } \\
\text { questions about their medications }\end{array}$ & $3.61^{*}$ & $4.24^{*}$ \\
$\begin{array}{l}\text { Increasing prescribing transparency } \\
\text { Aiding medication reconciliation }\end{array}$ & 3.73 & 4.09 \\
$\begin{array}{l}\text { Decreasing wrong-patient errors } \\
\text { Reduce inappropriate prescribing }\end{array}$ & 3.57 & 4.07 \\
$\begin{array}{l}\text { Aiding care transitions } \\
\text { Improving medication adherence }\end{array}$ & 3.83 & $4.24^{*}$ \\
$\begin{array}{l}\text { Supporting quality and outcomes/ } \\
\text { effectiveness research }\end{array}$ & $3.52^{*}$ & 4.24 \\
$\begin{array}{l}\text { Reorganizing medication list for } \\
\text { more logical grouping }\end{array}$ & $3.24^{*}$ & $4.2^{*}$ \\
$\begin{array}{l}\text { Reducing calls to and from } \\
\text { pharmacy }\end{array}$ & 3.57 & 4.15 \\
$\begin{array}{l}\text { Facilitating reimbursement coding } \\
\text { and prior authorization }\end{array}$ & 3.48 & 3.91 \\
Prescribing speed/efficiency & 3.61 & 3.89 \\
\hline & 3.70 & 3.67 \\
\hline & $2.96^{*}$ & $3.98^{*}$ \\
& $2.91^{*}$ & $3.72^{*}$ \\
& 2.57 & $3.26^{*}$ \\
\hline
\end{tabular}

Evaluation of the importance of the benefits of indications-based prescribing on a scale of $1-5$, Unimportant - Very important. (* significantly different $(P<.05)$ than rest of the respondents) Table 2

\begin{tabular}{lll}
\hline \hline Perceived Barriers & Physician $(n=23)$ & Pharmacist $(n=46)$ \\
$\begin{array}{l}\text { Interoperability issues } \\
\text { Overcoming policy \& market } \\
\text { fragmentation (EHR/PBM/payer) }\end{array}$ & 3.65 & 3.72 \\
$\begin{array}{l}\text { Technical problems in } \\
\text { implementing }\end{array}$ & 3.39 & 3.40 \\
$\begin{array}{l}\text { Legal and billing issues (e.g., } \\
\text { off FDA label use) }\end{array}$ & 3.26 & 3.62 \\
$\begin{array}{l}\text { Third parties second-guessing } \\
\text { clinical decisions }\end{array}$ & 3.00 & 3.49 \\
\hline
\end{tabular}

(continued)

\begin{tabular}{lcc}
\hline \hline $\begin{array}{l}\text { Concerns about extra prescriber } \\
\text { time/effort }\end{array}$ & 3.65 & 3.20 \\
$\begin{array}{l}\text { Complexities in differentiating } \\
\text { billing diagnoses vs. indications }\end{array}$ & 3.55 & 3.28 \\
\hline
\end{tabular}

Evaluation of the difficulty to overcome various barriers to implementing the addition of indications to the prescription process on a scale of 1 (very easy) - 5 (very difficult). (* significantly different $(P<.05)$ than rest of the respondents)

INCORPORATING MORE SYSTEMS-BASED PRACTICE: A HEALTH POLICY AND PUBLIC HEALTH COURSE FOR 4TH YEAR MEDICAL STUDENTS AND HOUSESTAFF IMPROVES KNOWLEDGE, BEHAVIORS AND ATTITUDES Manik N. Aggarwal ${ }^{1}$; Jack Penner ${ }^{2}$; Rachel H. Kon ${ }^{3} .{ }^{1}$ Medstar Georgetown University Hospital, Washington, DC; ${ }^{2}$ Georgetown University School of Medicine, Washington, DC; ${ }^{3}$ University of Virginia Health System, Charlottesville, VA. (Control ID \#2438112)

BACKGROUND: Healthcare is rapidly changing and it takes more than clinical acumen and medical decision-making to make an effective physician. Clinicians must have knowledge of health policy (HP) and public health (PH) principles to help their patients navigate the health system, and there has been much emphasis on adding this material to the medical education curriculum as a means of better preparing future physicians for medical practice. To improve physician training, our institution put together an elective course using a combination of didactics, small group projects, and site visits that exposes 4th year medical students, residents and fellows to the fields of PH and HP, with the goal of increasing their knowledge base and sparking an interest in advocacy and PH. The aim of our study is to assess the change in knowledge, behaviors and attitudes of medical trainees regarding systems based practice (SBP) after completing such a course.

METHODS: Annually, the Department of Medicine at Medstar Georgetown University Hospital, a single, large academic center, offers a 2 to 4 week elective course in SBP, particularly covering topics in PH and HP. In 2015, there were a total of 25 participants in the course, which included 4th-year medical students $(N=11)$ and housestaff (residents and fellows, $N=14$ ). The trainees were selected based on self-enrollment in the course. A pre-test/post-test survey to assess acquisition of knowledge and changes in behaviors and attitudes regarding PH and HP topics was developed based on course objectives. The questionnaire consisted of 20 objective and 20 subjective multiple choice questions. In addition to on-site didactics, there were guest lecturers and site visits. The data was anonymous and confidential and did not impact trainees' performance or evaluation. Statistical analyses were performed using Numbers for Mac OS X (Apple Inc., Cupertino, CA). Significance was determined at the $P<0.05$ level using unpaired, two-tailed T-tests for comparisons across participant groups, or paired, twotailed T-tests for comparisons between each participant's pre and post-survey results. RESULTS: -For all participants, there was a significant score improvement of $7 \%$ between objective pre- and post-test scores $(P<0.05$, for this and all further mentions of significance). -Those with an objective pre-test score below $75 \%$ showed a significant improvement between pre-and post-surveys with an average increase of $10.1 \%$. -The entire cohort showed significant improvement across all measures, which included comprehension of the Affordable Care Act, implementation of health policy and strategies for health promotion and disease prevention. -Frequency of reading HP literature significantly increased for 4th- year medical students. -Both medical students and housestaff showed significant improvements across all categories measuring subjective knowledge, including an individual's comprehension of financial trends in healthcare, billing and insurance policies and how to handle medical issues from a population health and public health perspective. 
CONCLUSIONS: There has been a recent emphasis by the LCME and ACGME to incorporate SBP into the undergraduate and graduate medical education curriculum. Prior studies did not evaluate knowledge acquisition after a particular SBP intervention was implemented but this research provides evidence that we can increase HP and PH knowledge amongst trainees with an elective course. The course implemented for trainees was efficacious in three key areas. First, there is a significant increase in knowledge based on objective outcomes, particularly among those with lower baseline knowledge. Second, there is an increase in perceived knowledge and confidence in discussing or implementing topics related to PH and HP. Finally, trainees demonstrated increased engagement in the SBP literature. These findings validate the efficacy of a didactic, experiential course to positively impact the knowledge, behaviors, and attitudes of trainees in the fields of $\mathrm{PH}$ and HP, with particular efficacy for 4 th year medical students. Future trainees can benefit from a similar course that introduces health policy, public health and other system-based practice.

\section{INCREASING ACCESS: PATIENTS' PERSPECTIVES ON VOTER} REGISTRATION IN URBAN HEALTH CENTERS Marce E. Abare; Matthew Anderson; Asiya Tschannerl. Montefiore Medical Center, Bronx, NY. (Control ID \#2467837)

BACKGROUND: Voting is a right of U.S. citizenship and offers the potential to influence societal conditions that affect health. Voter turnout rates are at historically low levels, particularly in working class neighborhoods. Among the first barriers to voting is registration itself. In communities consistently underrepresented in the electoral process, some physician groups have suggested that opportunity for nonpartisan voter registration be made available in the healthcare setting. Federally qualified health centers are legally able to offer voter registration. Yet little research has been done regarding how patients feel about voter registration in clinics. This study examines perspectives on voting and voter registration among patients in primary care settings in the Bronx, NY

METHODS: Patients at three community health centers were asked six question regarding their current voter registration status, recent voting behavior, beliefs about the importance of voting and the acceptability of voter registration services in the healthcare center. These questions were quantitative with the option to add open-ended comments The three clinics are FQHCs operated by Montefiore Medical Center in the Bronx, NY and serve as teaching sites for residency programs in Family Practice, Internal Medicine and Pediatrics. All patients presenting for care at any of the three clinics were eligible for participation in the study. Patients were excluded if they were presenting for an emergency visit or unable to provide oral informed consent. Surveys were conducted in either English or Spanish depending on patient preference. Data were analyzed using independent sample t-test in SPSS 15. Qualitative analysis of the comments involved development of themes by joint review and iterative processing using consensus among authors.

RESULTS: Two hundred eighty-three patients participated. Most (91.3\%) patients reported that they believe voting is important to their community, and 206 (72.8\%) patients were registered to vote. Regardless of registration status, most patients, 246 $(89.45 \%)$ said that it would be appropriate to make voter registration available in the healthcare center. Among the 75 patients not already registered to vote, $42(70 \%)$ reported that they would be more likely to register if they could do so at the healthcare center People who believe that voting is important for their community were more likely, than those who do not, to declare that it would be appropriate for the healthcare center to help people register to vote $(61(93.1 \%)$ vs $4(57.1 \%)$ and were more likely to indicate interest in registering to vote at the healthcare center if available (83 (83\%) vs $1(25 \%)$. Of those registered to vote, only $55(26.1 \%)$ reported having voted in the most recent local elections. Responses were similar across clinical sites, types of practice and gender with the exception of perceived difference in difficulty of voter registration, which varied by clinic site and gender. Optional comments (offered by $22 \%$ of respondents) expressed skepticism of the utility of voting and mentioned practical barriers to both registering to vote and voting. Roughly $16 \%$ of comments express concern that other members of their community do not understand the registration process.

CONCLUSIONS: A large majority of patients believe is it appropriate and useful to register to vote at their health center. These results should be interpreted cautiously in the context of potential response, reporting and researcher biases typical of survey research; however, the unambiguous nature of the responses suggests reliability of these data. Given its broad favorability among patients, voter registration in healthcare centers could address barriers to voting in communities such as the Bronx, where voter disparity and low voter turnout undermine the electoral process by leaving society's most vulnerable citizens unrepresented. Voting has implications for health policy and health resource distribution; thus, facilitating civic participation by enabling registration in the healthcare center could be understood as an intervention derived from social determinants analysis, with broad implications for health equity. This study indicates that the feasibility of implementing onsite registration should be explored further. Importantly, voter turnout was low despite the percentage of respondents who were already registered, implying that factors aside from registration status contributed to voter turnout in this most recent election. An important next step is to identify other influences on voter behavior and the potential for local heath care centers to address barriers beyond registration. A larger sample size, focused inquiry, and pilot programs might elucidate further avenues for health systems to promote civic engagement related to health

INCREASING ALCOHOL USE IN WOMEN ASSOCIATED WITH WORSE ANTIRETROVIRAL ADHERENCE OVER TIME Nikita Barai ${ }^{2}$; Anne Monroe ${ }^{2}$; Catherine R. Lesko ${ }^{1}$; Bryan Lau ${ }^{1}$; Heidi Hutton ${ }^{3}$; Cui Yang ${ }^{4}$; Mary E. McCaul ${ }^{3}$; Anika A. Alvanzo $^{2}$; Geetanjali Chander ${ }^{2} .{ }^{1}$ Johns Hopkins University, Baltimore, MD; ${ }^{2}$ Johns Hopkins University School of Medicine, Baltimore, MD; ${ }^{3}$ The Johns Hopkins Hospital, Baltimore, MD; ${ }^{4}$ Johns Hopkins Bloomberg School of Public Health, Baltimore, MD. (Control ID \#2468443)

BACKGROUND: Heavy alcohol use is common among women with HIV infection and may adversely affect clinical outcomes, including antiretroviral therapy (ART) adherence and viral load suppression. Our aim was to determine the association between changes in alcohol use, measured as the average number of drinks per week and number of binge drinking days, and both ART adherence and HIV RNA.

METHODS: This was a secondary analysis of data from a randomized control tria designed to test the effectiveness of a brief alcohol intervention for reducing hazardous drinking among women with HIV infection. Additional data were collected from a group of women concurrently recruited from the same HIV clinic who did not initially report at-risk drinking. Alcohol use data, measured using the Timeline Follow Back (TLFB) method, was collected during the initial assessment (visit 0), at 6 months (visit 1), and at 12 months (visit 2) and collapsed into average number of standard drinks per week and number of binge drinking days ( $\geq 4$ drinks/day) in the 90 days preceding each study visit. A clinically significant change in alcohol use was defined as an increase in alcohol consumption by an average of $\geq 1$ drink per week or $\geq 1$ additional binge drinking day in the 90 days preceding each study visit. The outcomes of interest were ART adherence, defined as medication possession ratio $(\mathrm{MPR}) \geq 0.8$, and undetectable viral load, defined as $<400$ copies $/ \mathrm{mL}$. Data from 234 women with 420 assessments of change in adherence and viral load were included. Analysis was stratified based on initial medication adherence (adherent or non-adherent), frequency of binge drinking (frequent binge drinking $\geq 3$ episodes/90 days), and average number of drinks per week (heavy drinking $>7$ drinks/week). We used logistic regression (with generalized estimating equations to account for repeated measures) to estimate the odds of a change in medication adherence and viral load suppression associated with an increase in alcohol consumption between assessments. 
RESULTS: Medication Adherence (Table 1): Increased number of binge drinking days was associated with increased odds of worsening medication adherence in women who were initially adherent and frequent binge drinkers (OR 1.02, $95 \% \mathrm{CI}: 1.00,1.05)$ and decreased odds of improved medication adherence in women who were initially nonadherent and non-frequent binge drinkers ( $\mathrm{OR}=0.90,95 \% \mathrm{CI}: 0.82,0.99)$. An increase in the average number of drinks per week was associated with increased odds of worsening medication adherence in women who were initially adherent and non-heavy drinkers $(\mathrm{OR}=1.05,95 \%$ CI: 1.01, 1.08). Viral load suppression (Table 2): Increased number of binge drinking days was associated with decreased odds of achieving viral load suppression in women who had a detectable viral load and non-frequent binge drinking initially $(\mathrm{OR}=0.81,95 \% \mathrm{CI}: 0.67,0.98)$. An increase in the average number of drinks per week Association Between Increased Alcohol Consumption and Change in ART Adherence was associated with increased odds of developing a detectable viral load in women who were initially undetectable and non-heavy drinkers $(\mathrm{OR}=1.03,95 \% \mathrm{CI}: 1.00,1.07)$ and decreased odds of achieving an undetectable viral load in women with an initially detectable viral load and non-heavy drinking $(\mathrm{OR}=0.75,95 \% \mathrm{CI}: 0.59,0.95)$.

CONCLUSIONS: We found increases in alcohol use are associated with reduced medication adherence and viral load suppression in several subgroups of HIV-infected women. Women without a recent history of frequent binge drinking or heavy alcohol use may be particularly vulnerable to the negative impact of increasing alcohol use on medication adherence and viral load suppression. These results highlight the importance of integrating proactive alcohol counseling strategies into routine HIV-care, inclusive of women who do not have a prior history of heavy or binge drinking behavior.

\begin{tabular}{|c|c|c|c|c|c|}
\hline Baseline adherence status & Baseline alcohol status & Exposure of interest & Outcome & N observations & $\begin{array}{l}\text { Odds ratio (MV } \\
\text { analysis) }\end{array}$ \\
\hline Adherent & Frequent binge* & $\begin{array}{l}\text { Effect of increasing binge } \\
\text { drinking by } \geq 1 \text { binge day }\end{array}$ & Adherent to nonadherent & 54 & $1.02(1.00,1.05)$ \\
\hline Adherent & Non-frequent binge & $\begin{array}{l}\text { Effect of increasing binge } \\
\text { drinking by } \geq 1 \text { binge day }\end{array}$ & Adherent to nonadherent & 183 & $0.65(0.35,1.23)$ \\
\hline Nonadherent & Frequent binge & $\begin{array}{l}\text { Effect of increasing binge } \\
\text { drinking by } \geq 1 \text { binge day }\end{array}$ & Nonadherent to adherent & 56 & $1.02(0.99,1.06)$ \\
\hline Nonadherent & Non-frequent binge & $\begin{array}{l}\text { Effect of increasing binge } \\
\text { drinking by } \geq 1 \text { binge day }\end{array}$ & Nonadherent to adherent & 122 & $0.90(0.82,0.99)$ \\
\hline Adherent & Heavy drinker** & $\begin{array}{l}\text { Effect of increasing drinking } \\
\text { by average of } \geq 1 \text { drink per } \\
\text { week }\end{array}$ & Adherent to nonadherent & 37 & $1.01(0.99,1.02)$ \\
\hline Adherent & Non-heavy drinker & $\begin{array}{l}\text { Effect of increasing drinking } \\
\text { by average of } \geq 1 \text { drink per week }\end{array}$ & Adherent to nonadherent & 200 & $1.05(1.01,1.08)$ \\
\hline Nonadherent & Heavy drinker & $\begin{array}{l}\text { Effect of increasing drinking by } \\
\text { average of } \geq 1 \text { drink per week }\end{array}$ & Nonadherent to adherent & 41 & $0.99(0.98,1.01)$ \\
\hline Nonadherent & Non-heavy drinker & $\begin{array}{l}\text { Effect of increasing drinking } \\
\text { by average of } \geq 1 \text { drink per week }\end{array}$ & Nonadherent to adherent & 137 & $0.94(0.87,1.01)$ \\
\hline
\end{tabular}

This table describes the relationship between change in drinking and ART adherence status. * $\geq 3$ binge days over 90 day period ** > average of 7 drinks/week calculated over 90 day period.

Association Between Increased Alcohol Consumption and Change in HIV RNA

\begin{tabular}{|c|c|c|c|c|c|}
\hline Baseline virologic status & Baseline alcohol status & Exposure of interest & Outcome & N observations & $\begin{array}{l}\text { Odds ratio (MV } \\
\text { analysis) }\end{array}$ \\
\hline Undetectable & Frequent binge* & $\begin{array}{l}\text { Effect of increasing binge } \\
\text { drinking by } \geq 1 \text { binge day }\end{array}$ & Undetectable to detectable & 81 & $1.01(0.98,1.05)$ \\
\hline Undetectable & Non-frequent binge & $\begin{array}{l}\text { Effect of increasing binge } \\
\text { drinking by } \geq 1 \text { binge day }\end{array}$ & Undetectable to detectable & 250 & $0.94(0.75,1.18)$ \\
\hline Detectable & Frequent binge & $\begin{array}{l}\text { Effect of increasing binge } \\
\text { drinking by } \geq 1 \text { binge day }\end{array}$ & Detectable to undetectable & 29 & $1.00(0.96,1.04)$ \\
\hline Detectable & Non-frequent binge & $\begin{array}{l}\text { Effect of increasing binge } \\
\text { drinking by } \geq 1 \text { binge day }\end{array}$ & Detectable to undetectable & $\mathbf{5 5}$ & $0.81(0.67,0.98)$ \\
\hline Undetectable & Heavy drinker** & $\begin{array}{l}\text { Effect of increasing drinking } \\
\text { by average of } \geq 1 \text { drink per week }\end{array}$ & Undetectable to detectable & 58 & $0.99(0.97,1.01)$ \\
\hline Undetectable & Non-heavy drinker & $\begin{array}{l}\text { Effect of increasing drinking } \\
\text { by average of } \geq 1 \text { drink per week }\end{array}$ & Undetectable to detectable & 273 & $1.03(1.00,1.07)$ \\
\hline Detectable & Heavy drinker & $\begin{array}{l}\text { Effect of increasing drinking by } \\
\text { average of } \geq 1 \text { drink per week }\end{array}$ & Detectable to undetectable & 20 & $1.00(0.98,1.02)$ \\
\hline Detectable & Non-heavy drinker & $\begin{array}{l}\text { Effect of increasing drinking } \\
\text { by average of } \geq 1 \text { drink per week }\end{array}$ & Detectable to undetectable & 64 & $0.75(0.59,0.95)$ \\
\hline
\end{tabular}

This table describes the relationship between change in drinking and change viral load status. * $\geq 3$ binge days over 90 day period $* *>$ average of 7 drinks/week calculated over 90 day period.

INCREASING USE OF HOME HEALTH CARE SERVICES AMONG PATIENTS ADMITTED WITH HEART FAILURE IN THE UNITED STATES $\underline{\text { Ranjan Pathak }}^{2}$; Anene Ukaigwe ${ }^{4}$; Smith Giri ${ }^{5}$; Paras Karmacharya ${ }^{2}$; Madan R. Aryal ${ }^{3}$; Anthony Donato ${ }^{1} .{ }^{1}$ Reading Health System, W. Reading, PA; ${ }^{2}$ Reading Health System, West Reading, PA; ${ }^{3}$ The Reading Hospital and Medical Center, Wyomissing, PA; ${ }^{4}$ Hershey Medical Center, Hershey, PA; ${ }^{5}$ University of Tennessee Health Science Center, Memphis, PA. (Control ID \#2469753)
BACKGROUND: Despite recent advancements in the medical management of acute and chronic Heart Failure (HF), smooth transitions of care for patients hospitalized with HF remain elusive for most health care systems. Use of home health services and other ancillary services have been promoted in recent years as a means of reducing readmission rates among HF patients, which still remain as high as $25 \%$ in the first 30 days of discharge. Using a large national database, we aimed to study the recent trends in discharge outcomes/disposition among patients discharged with HF. 
METHODS: Using National Inpatient Sample, we studied all hospitalizations among patients $\geq 18$ years with a primary diagnosis of HF from 2005 to 2011 . We studied the trends in discharge disposition during this time period. Annual rates were calculated for each year (2006-2011), fitted into a log-linear model and compared using Monte Carlo permutation test to study the changes in trend. Analysis of trends in the rates of various discharge outcomes/disposition and calculation of average annual percent change were done using the Joinpoint Regression Program (v 4.0.4, National Cancer Institute, Bethesda, Maryland) in conjunction with STATA version 13.0 (College Station, TX).

RESULTS: An estimated 7,572,400 HF hospitalizations were identified during the study period. The mean age was $72 \pm 14$ years. Of these, $51 \%$ were females and $68 \%$ were Caucasians. From 2005 to 2011, the inpatient mortality rate dropped from 3.78 to $3.09 \%$, at an average annual percentage change of $-3.04 \%(95 \%$ CI $1.5-4.6)$ per year $(P<0.05)$. During the same period, the proportion of patients discharged with home health services increased at an average annual percentage change of $+4.83 \%(95 \%$ CI 3.7-6.0) per year $(P<0.05)$ while transfers to skilled nursing facility or intermediate care steadily increased by $+1.82 \%(1.1-2.6)$ per year $(P<0.05)$. CONCLUSIONS: From 2005 to 2011, there was a nationwide increase in inpatient discharge dispositions that included home health services or discharge to skilled nursing facility/intermediate care facilities. These changes reflect increasing emphasis on use of home health services as means of reducing readmissions and improving patient outcomes. Cost-benefit analyses of these services and their effects on readmissions are areas for future study

Trends in Discharge Disposition among patients admitted with Heart Failure in the US, 20052011

\begin{tabular}{llll}
\hline \hline Year & $\begin{array}{l}\text { Mortality } \\
\text { (\% hospitalizations) }\end{array}$ & $\begin{array}{l}\text { Home health } \\
(\% \text { hospitalizations })\end{array}$ & $\begin{array}{l}\text { SNF or } \\
\text { intermediate } \\
\text { care transfers } \\
(\% \text { hospitalizations) }\end{array}$ \\
2005 & $3.78 \%$ & $16.31 \%$ & $18.57 \%$ \\
2006 & $3.48 \%$ & $17.12 \%$ & $18.35 \%$ \\
2007 & $3.22 \%$ & $17.33 \%$ & $19.19 \%$ \\
2008 & $3.29 \%$ & $18.83 \%$ & $19.24 \%$ \\
2009 & $3.21 \%$ & $18.94 \%$ & $19.29 \%$ \\
2010 & $3.09 \%$ & $20.77 \%$ & $20.02 \%$ \\
2011 & $3.09 \%$ & $21.82 \%$ & $20.67 \%$ \\
Average & $3.04 *$ & $4.83 *$ & $1.82 *$ \\
annual & & & \\
percent & & & \\
change & & & \\
(2005 & & & \\
to 2011) & & & \\
\hline
\end{tabular}

$\mathrm{SNF}=$ skilled nursing facility; *statistically significant at $P<0.05$.

\section{INDIVIDUAL CHARACTERISTICS OF AND BARRIERS EXPERIENCED BY FEMALE JUNIOR FACULTY APPLYING FOR K-AWARDS AND R01 GRANTS Lyndonna M. Marrast ${ }^{1}$; Rene Carapinha ${ }^{2}$; Emorcia Hill ${ }^{2}$; Joan Reede ${ }^{2} .{ }^{1}$ Hofstra North Shore LIJ School of Medicine, Kew Gardens, NY; ${ }^{2}$ Harvard Medical School, Boston, MA. (Control ID \#2470160)}

BACKGROUND: Applying for and obtaining grants are measures of academic success. The K-award and R01 grant are 2 competitive awards that can positively impact career advancement. Studies describing factors related to grant application and receipt may inform faculty development programs. The objective of this study is to assess the individual characteristics and barriers experienced by female instructors and assistant professors in academic medical centers with regards to applying for and receiving K-awards and R01 grants.

METHODS: We conducted a secondary analysis of survey data from the Women and Inclusion in Academic Medicine (WIAM) study, a multi-site study designed to assess the professional and personal experiences of women in academia. The outcomes of interest were ever having (1) applied for and (2) received a K-award or R01 award. Individual factors included average time at the institution and number of publications, having completed a fellowship or an additional degree, having served as a PI or co-investigator and having spent $>=20 \%$ time in research. Respondents were asked to rate the extent to which a series of barriers were typical in the pursuit of their academic interests (i.e. teaching, research and/or service) in the past 12 months (based on a five-point agreement Likert scale). In addition to calculating descriptive statistics, chi-square and t-tests were performed to assess mean differences among groups. We calculated mean scores across each barrier and performed a Mann-Whitney $U$ test to assess differences across the outcomes. Lastly, we examined the odds of having the outcomes in multivariable models that included individual characteristics, race/ethnicity, degree to which mentoring needs are met and significant barriers experienced

RESULTS: In a sample of 3127 women, 1943 were at the instructor or assistant professor rank. Among these faculty, $160(8 \%)$ applied and 74 (3.8\%) were funded for a K or R01. The majority $[n=1285(66 \%)]$ have undergone fellowship training and one-third have an additional degree $[n=646(33 \%)]$. The majority $1683(86.6 \%)$ were in a clinical department. In the bivariate analyses, the significant individual factors that differentiated those who applied for a grant were: 1) completion of a fellowship, 2) lack of an additional degree, 3) role as a coinvestigator, 4) having a primary appointment in a clinical department and 5) length of time at one's institution. The significant factors that differentiated those who received funding from those who did not included: 1) completion of fellowship training, 2) having served as a coinvestigator, 3) primary appointment in a clinical department and 4) length of time at one's institution. Number of publications, role as a principal investigator, percent time spent in research and race-ethnicity were not related to application for or receipt of an award. The following career barriers were significantly related to applying for an award: inadequate funding, time committed to clinical activities and family, and limited access to mentors and students. Time committed to patient care and family and access to students remained significant among those who received funding. In the fully adjusted multivariate logistic regression model, those who served as co-investigator had greater odds of having applied for $(\mathrm{OR}=5.44$; $\mathrm{CI}=2.15-13.78)$ and received $(\mathrm{OR}=8.87 ; \mathrm{CI}=2.27-34.71)$ grant funding.

CONCLUSIONS: K-award and R01 grants are increasingly competitive and understanding the factors that are related to successful grant application could focus the program offerings of faculty development offices. This study highlights the importance of fellowship training and role as a co-investigator in grant application and receipt. Future studies can outline the key elements within these characteristics that facilitate female junior faculty applying for and receiving these government grants.

INFORMING RCT PROTOCOL DEVELOPMENT BY PILOTING A TECHNOLOGY-ASSISTED WEIGHT MANAGEMENT INTERVENTION AMONG VETERANS WITHIN PRIMARY CARE Katrina F. Mateo ${ }^{2,1}$; Natalie L. Ricci $^{2,1}$; Natalie B. Berner ${ }^{2,1}$; Pich Seekaew ${ }^{2,1}$; Adina Kalet ${ }^{1}$; Scott Sherman ${ }^{2,1}$; Melanie Jay ${ }^{2,1}$. ${ }^{1}$ NYU School of Medicine, New York, NY; ${ }^{2}$ VA NY Harbor Healthcare System, New York, NY. (Control ID \#2462896)

BACKGROUND: Obesity affects over one-third of Veterans receiving care at Veterans Affairs (VA) Medical Centers. Despite offering all Veterans screening and referral to an intensive weight management program called MOVE!, the majority of eligible Veterans choose not to attend and often do not receive further counseling. The United States Preventive Services Task Force endorses the use of the 5As framework (Assess, Advise, Agree, Assist, Arrange) to deliver obesity counseling within primary care (PC). We recently developed a technology-assisted, weight management intervention called MOVE! Toward Your Goals (MTG) to deliver 5As weight management counseling within the VA's patient centered medical home model of care (referred to as Patient Aligned Care Teams (PACT)). To participate in the MTG intervention, Veterans arrive early to their PC appointment to use an online goal-setting tool (MTG tool) delivered on an iPad that generates a personalized binder of tailored materials. The Veteran then meets with a health coach to further refine 
weight loss and lifestyle goals, address barriers, and suggest other weight management resources. PACT teams then endorse goals and provide brief motivational interviewing as needed. Veterans receive follow-up phone coaching calls from their health coach to document progress and adjust goals. This study aimed to assess the feasibility and acceptability of this intervention among Veterans and PACT staff in order to inform protocol development for a pilot randomized controlled trial (RCT).

METHODS: Three PACT teams - including 3 primary care providers (PCPs), 3 registered nurses (RNs), and 3 licensed practical nurses (LPNs) - at one VA were recruited to participate in the MTG intervention and received a training on study protocols and brief 5As weight management counseling. We then recruited overweight/obese Veterans with upcoming PC appointments to participate. During the baseline visit, Veterans completed pre-surveys, received the MTG intervention, attended their PCP visit, and completed a post-survey. PACT teams completed a survey to evaluate intervention feasibility and acceptability within their PC practice.

RESULTS: We successfully recruited a representative sample of 11 Veterans from an urban VA (91\% male, $46 \%$ Black, $27 \%$ White, $27 \%$ Hispanic, mean age $=55.36$ years $(\mathrm{SD}=$ $15.10)$, mean $\mathrm{BMI}=30.1 \mathrm{~kg} / \mathrm{m} 2(\mathrm{SD}=4.47))$. On average, Veterans completed the MTG tool in less than $20 \mathrm{~min}$ and health coaching sessions lasted less than $30 \mathrm{~min}$. All 11 Veterans agreed that the length of time to complete the MTG tool was "just right" as opposed to "too much" or "too little." Feedback about the MTG intervention from Veterans was very positive, and they appreciated receiving tailored counseling from the health coach. After completing the baseline visit, confidence and motivation to successfully achieve goals related to weight loss, nutrition, and physical activity increased among all Veterans. Several Veterans commented on how the MTG tool needed some improvements to fix the lag time and iPad sensitivity. Almost all PACT members (8/9) indicated that the intervention fit well with current workflow, and all (9/9) indicated that it did not prevent them from seeing their patients on time. All (9/9) endorsed that the intervention filled a needed gap in obesity care in the VA and improved the quality and efficiency of weight management counseling. Most (8/9) felt that the intervention is feasible for patients. Suggestions by PACT members for improvement included better communication/ coordination between research staff and PACT with regards to the study visit time and better integration of VA MOVE! weight management program staff/dieticians into the intervention. CONCLUSIONS: This study supports the feasibility of our recruitment and baseline visit intervention protocols and demonstrates acceptability of the MTG intervention for both Veterans and PACT staff. Recruitment for the pilot RCT of the intervention began in December 2015 to further refine study protocols in preparation for a larger, cluster-RCT of the MTG intervention at two VA sites.

\section{INITIATION OF NALTREXONE FOR MAINTENANCE OF SOBRIETY IN PATIENTS HOSPITALIZED FOR ALCOHOL DETOXIFICATION John Stephens $^{1}$; Kelly Stepanek ${ }^{1}$; Edmund A. Liles ${ }^{1}$; Carlton R. Moore ${ }^{2}$; Daniel Jonas ${ }^{2}$; Lisa M. Walston ${ }^{3}$; Dianna Trudeau ${ }^{3}$; James C. Garbutt ${ }^{3}$. ${ }^{1} \mathrm{UNC}$ Hospitals, Chapel Hill, NC; ${ }^{2}$ University of North Carolina Chapel Hill, Chapel Hill, NC; ${ }^{3}$ University of North Carolina, Chapel Hill, NC. (Control ID \#2465595)}

BACKGROUND: Alcohol use disorders (AUDs) are common among hospitalized patients. Naltrexone has been proved to assist patients with AUDs in maintenance of sobriety after a short period of abstinence. The initiation of naltrexone has been studied primarily in the outpatient setting, with less exerience among hospitalized patients. Our hospitalist program lacked a process for initiation of naltrexone for maintenance of sobriety among inpatients with AUDs.

METHODS: We created an algorithm for evaluating hospitalized patients with AUDs for naltrexone, including medication dosage and contraindications. We targeted patients who were admitted specifically for detoxification or withdrawal from alcohol. The algorithm was disseminated to the group via email, posting of paper versions at computer work stations, presentation at a monthly group meeting and the creation of "smart phrases" in the electronic medical record. Patients hospitalized for alcohol-related indications before intervention were identified using Diagnosis Related Groupings (DRGs) 896 and 897, which are "alcohol/drug abuse or dependence without rehabilitation therapy," with and without major co-morbidity or complication, respectively. Post-intervention patients were identified prospectively as they were admitted for alcohol withdrawal or detoxification. Discharge summaries were reviewed for documentation of counseling for and/or initiation of naltrexone. Process measures included the percentage of patients hospitalized for alcohol withdrawal or detoxification who were counseled on naltrexone by time of discharge, before and after intervention. Consecutive samples of ten patients were plotted sequentially on a control chart. Outcome measures included 30-day emergency department (ED) revisit rate and 30-day re-hospitalization rate. RESULTS: There were 165 patients identified before and 28 after implementation of the new process. After implementation, the percentage of patients hospitalized for alcohol detoxification or withdrawal who were counseled on natrexone before discharge rose from 3.0 to $75 \%$. $(p<.001$, Table 1$)$ The increase also met control chart rules for special cause variation. (Figure 1) The 30-day ED revisit and re-hospitalization rates declined, but did not achieve statistical significance. (Table 1)

CONCLUSIONS: Our hospital medicine group demonstrated the successful creation and implementation of an algorithm for evaluating patients for naltrexone during hospitalization for alcohol detoxification or withdrawal. Implementation of the new process correlated with trends toward decrease in 30-day ED revist and re-hospitalization rates. Further data are needed to ensure sustainment of process measures and to determine statistical significance of outcome measures.

Table 1

\begin{tabular}{|c|c|c|c|}
\hline Outcome & $\begin{array}{c}\text { Pre-Intervention } \\
(\mathbf{n = 1 6 5})\end{array}$ & Post-Intervention & $\begin{array}{c}\text { p- } \\
\text { value }\end{array}$ \\
\hline $\begin{array}{c}\text { \% Patients Counseled } \\
\text { on Naltrexone }\end{array}$ & $3.0 \%$ & $75.0 \%(\mathrm{n}=28)$ & $<.001$ \\
\hline $\begin{array}{c}\text { \% Patients Started on } \\
\text { Naltrexone }\end{array}$ & $3.0 \%$ & $39.3 \%(\mathrm{n}=28)$ & $<.001$ \\
\hline 30-Day ED Revisit Rate & $27.9 \%$ & $11.1 \%(\mathrm{n}=18)$ & .125 \\
\hline $\begin{array}{c}\text { 30-Day } \\
\text { Re-hospitalization Rate }\end{array}$ & $13.9 \%$ & $5.6 \%(\mathrm{n}=18)$ & .317 \\
\hline
\end{tabular}

Figure 1

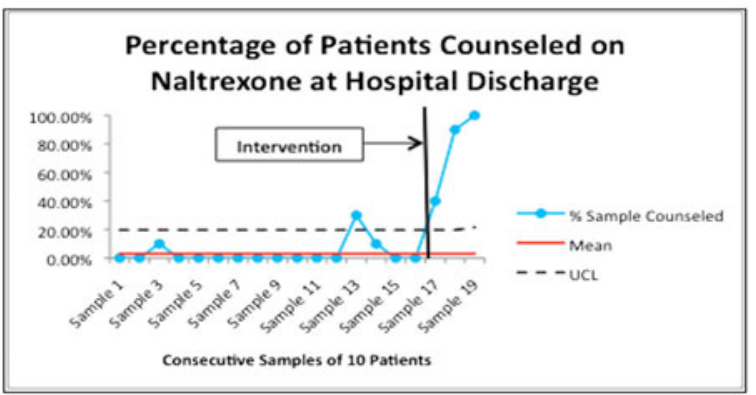

INSIGHTS FROM PREGNANT WOMEN AND PROVIDERS ABOUT HEALTHY LIFESTYLE GOALS IN PREGNANCY: A QUALITATIVE INDEPTH INTERVIEW STUDY Manasa Ayyala ${ }^{2}$; Haley Kreft ${ }^{2}$; Janice L. Henderson ${ }^{1}$; Janelle W. Coughlin ${ }^{2}$; Lindsay Martin² ${ }^{2}$ Jeanne M. Clark ${ }^{2}$; Lawrence J. Appel ${ }^{2}$; Wendy L. Bennett ${ }^{2} .{ }^{1} \mathrm{JHU}$, Baltimore, MD; ${ }^{2} \mathrm{Johns}$ Hopkins University, Baltimore, MD. (Control ID \#2469510)

BACKGROUND: Pregnancy provides a unique opportunity to engage women in improving their health behaviors, as they are motivated to protect the well-being of their babies and have frequent interactions with the healthcare system. Our goal is to explore women's perspectives about setting and reaching health goals in pregnancy to inform the development of a technology-enhanced, remotely delivered pregnancy health coaching program. To complement patient perspectives, we also explored providers' perspectives 
on the interface between health coaching and clinical care to enhance the program's integration into care.

METHODS: We conducted 20 in-depth interviews with patients and 10 in-depth interviews with obstetrics providers from 2 prenatal care clinics at an urban, academic hospital. Interviews were audiorecorded then transcribed verbatim. Two investigators independently coded transcripts for thematic content using editing style analysis. We applied the PRECEDE-PROCEED program development framework to our findings to facilitate interpretation of our results.

RESULTS: Patient perspectives: Using the PRECEDE-PROCEED framework we identified predisposing, enabling and reinforcing factors to improve women's healthy behaviors in pregnancy. These factors are presented below followed by illustrative quotations: Predisposing Factor: Motivation to have a healthy baby “...I just want to make sure that I have a healthy delivery... and the baby will just be health[y] — both of us will be healthy... And, of course take care of him. You know-all throughout his life, and you know.. [I] have to be healthy first so I can take care of him. " Enabling Factor: Use of technology and features of a coaching program "They [my providers] will know where I'm coming from I think, and they can work with me better. I think that would help them on their end. The stuff that I'm talking with my coach about... Bigger picture of what's going on with me." "[Sometimes] I forgot to ask my doctor [questions]. But I can ask my health coach because my health coach is going to call me once a week [versus] seeing my doctor right now every 2 weeks... [because] then I forget. Reinforcing Factor: Social support and sense of community "But the fact that I'm starting to see more and more that people care it makes me want to stop [unhealthy behaviors]." "And it's good to have-not just inside support but outside support -[someone] that is getting to know you, to support you" Provider perspectives: Providers commented on the need for the health coaching program to have broad eligibility criteria and include normal to obese women and to be integrated with the electronic medical record (EMR) to enhance provider referrals, engagement and communication.

CONCLUSIONS: In this qualitative study, pregnant women described factors that would enable them to make significant health behavior changes in pregnancy to manage their weight gain and achieve health goals. Their perspectives will directly inform the content of learning modules, cadence of health coaching contacts and choice of technology features for a remotely-delivered health coaching intervention for pregnant and postpartum women. Integration into routine clinical care using the electronic medical record should improve program uptake, and enhance the potential for future scalability.

\section{INSURANCE ENROLLMENT AT A STUDENT-RUN FREE CLINIC AFTER THE PATIENT PROTECTION AND AFFORDABLE CARE ACT Megan} McGeehan; Rebecca DeMaria; Lusha Liang; Caroline Pinke; Emily Nash; Shobana Ramasamy; Kristy Krsulich; Lisa Noble; Yun-Han Huang; Pamela Charney; Ashita Batavia. Weill Cornell Medical College, New York, NY. (Control ID \#2469421)

BACKGROUND: Open enrollment under the Patient Protection and Affordable Care Act (PPACA) began in October 2013; nationally over 14 million Americans have gained health insurance under this landmark legislation. Our study aims to understand the effects of the PPACA on insurance enrollment at a student run free clinic. The Weill Cornell Community Clinic (WCCC) is a medical student-run free clinic (SRFC) located in New York City that provides primary care services to uninsured adults earning less that $400 \%$ of the federal income poverty level (less than $\$ 47,080$ per year for an individual). Using chart review and IRB-approved scripted phone interviews, we identified persistent barriers to insurance enrollment among WCCC patients.

METHODS: All WCCC new patient encounters include meeting with a social worker to discuss insurance eligibility and provide a referral to a facilitated enroller if indicated. In total 140 patients were seen at the WCCC between October 1st, 2013 and September 30th, 2015. In addition to demographic information, data was collected on insurance status, method of insurance enrollment, and barriers to enrollment. Data analysis was performed using Stata 13 software (StataCorp LP, TX).

RESULTS: Among the 140 patients seen during the study period, the majority $(84 \%, N=$ $118)$ were seen by a social worker during clinic hours. Of these, $58 \%(N=68)$ were uninsured at the end of the study period. As compared to those who gained coverage, those without insurance tended to be younger ( $p=0.058)$, live in a larger household $(p=0.022)$, and to have an undocumented immigration status $(p=0.01)$. Gender and employment status were not statistically significant predictors of enrollment. The most commonly described barriers to coverage were undocumented immigration status $(N=24)$, inability to afford health insurance premiums $(N=18)$ and lack of interest in obtaining coverage $(N=6)$. Among patients that gained coverage during the study period, the majority enrolled in Medicaid $(N=27)$, a marketplace plan $(N=10)$, or employer-based plan $(N=4)$. The WCCC social work referral to a facilitated enroller was integral in $26 \%$ of cases, with the remainder gaining insurance through either another facilitated enroller, their employer or on their own

CONCLUSIONS: Since the implementation of the PPACA, approximately a third of the patients seen at WCCC have gained health insurance coverage. Persistent barriers to gaining coverage are younger age, large household size, and having an undocumented immigration status. At this time the PPACA does not provide undocumented individuals with avenues for gaining coverage. Nationally, it is estimated that $17.8 \%$ of the uninsured are undocumented, and this is similar to our experience at the WCCC. Immigration status aside, younger individuals and those living in a larger household tended to be less successful in gaining coverage and disproportionately described an inability to afford premiums and a lack of interest. Considering that our on-site social worker was integral in acquiring coverage for $26 \%$, this is an underutilized resource. Restructuring to allow our social worker to perform extended interviews, education and more intensive follow up with patients may lead to higher enrollment rates. We are planning a pilot quality improvement study at the WCCC to investigate this hypothesis. We believe that our experience and findings will be informative to other SRFCs adapting to the PPACA.

INTEGRATING MENTAL HEALTH PATIENT DECISION AIDS INTO PRIMARY CARE PRACTICE Felisha A. Marques; Madeleine Matthiesen; Karen J. Carlson; Lisa Brugnoli-Semeta; Karen R. Sepucha; Leigh H. Simmons. Massachusetts General Hospital, Boston, MA. (Control ID \#2464364)

BACKGROUND: Decision aids (DA) have been shown to increase patients' knowledge and involvement in treatment decisions, and reduce decisional conflict in randomized controlled trials. However, less is known about how to incorporate these tools in routine care. The aim of this study was to implement patient decision aids for mental health conditions in routine primary care practice.

METHODS: We worked with three primary care practices affiliated with an academic health center. These practices were undergoing a hospital-wide effort to improve depression screening and behavioral health integration in primary care. We developed an order sheet with a description of three mental health video/booklet DAs addressing depression, anxiety, and insomnia. Practice staff gave the order sheet to all patients scheduled for preventive care visits at the time of arrival. Patients reviewed the sheet, checked off the program(s) of interest, and returned the sheet to the medical assistant. The medical assistant placed an order for the appropriate decision aid in the electronic medical record (EMR). In one of the practices, for the first 6 weeks of the pilot, medical assistants administered the patient health questionnaire 2 (PHQ-2), a validated screening tool, to identify patients at risk for depression. When a patient screened positive on the PHQ-2 (total score of 3 or greater), medical assistants offered that patient an order form. After 
6 weeks, the pilot was modified so that the medical assistants offered the DAs to all patients at their annual visit regardless of their PHQ-2 score. For all three practices, we tracked the number of patient orders for these programs and compared that with orders placed in the 4 months prior to launching the new process. We also elicited feedback from the staff and clinicians at each site about their experience with the new process.

RESULTS: From August-November 2014, the 4 months prior to the study period, 2 depression, 1 anxiety, and 0 insomnia programs were ordered by clinicians at these practices. The new process was launched in December and from December 2014March 2015, 134 depression, 193 anxiety, and 212 insomnia programs were requested by patients across these three practices. Prior to the new process for decision aid delivery, the medical assistants had never initiated any orders for decision aids. Of note, at the one practice that initially used the PHQ-2 as a trigger for prescription of the decision aids, the low \# of positive PHQ-2 scores meant that very few patients were offered decision aids. Thus, this practice began offering the order form to all patients seen for annual visits. The staff who manage the order sheets and place the orders in the EMR reported that it was easy to incorporate into their workflow. Many of the staff members involved also commented on how patients have expressed their enthusiasm on being offered these resources. The clinicians were pleased that their patients were receiving high quality materials and were pleased that the workflow was not dependent on the clinicians remembering to order programs.

CONCLUSIONS: There was a significant interest from patients in these practices for mental health focused decision aids. A clinician-driven approach to disseminating DAs greatly underestimated patients' desire for information and support. While the PHQ-2 is a validated tool for identifying patients who should be further evaluated for depression, we found that restricting the ordering to patients who screen positive severely limited the use of DAs on depression and other mental health conditions. Further study is ongoing to determine the impact of these programs on patient knowledge and decision making about mental health conditions.

INTERDISCIPLINARY ROUNDS: IMPROVING CARE DELIVERED TO PATIENTS Samit Datta ${ }^{1}$; Sherri Sandel ${ }^{2}$; Nazish Ilyas ${ }^{1} .{ }^{1}$ Lenox HIll Hospital, New York, NY; ${ }^{2}$ Lenox Hill Hospital, New York, NY. (Control ID \#2469402)

BACKGROUND: Length of stay (LOS) and excess days are two quality measures that have been linked to patient outcomes. In addition, Catheter Associated Urinary Tract Infections (CAUTI) and Central Line-Associated Blood Stream Infections (CLABSI) are adverse events that worsen patient outcomes and increase LOS. For example, a large multi-center study in Mongolia recently published showed an increase in hospital stay by 15.1 and 8.2 days for patients with CLABSIs and CAUTIs, respectively. Hospitals are trying innovative methods to decrease LOS and excess days, while delivering improved quality of care and decreasing adverse events. One such intervention is interdisciplinary rounds (IDRs), also known as case management and multidisciplinary rounds. Traditional IDRs focus on discharge readiness and disposition planning. A recent study showed a significant decrease in LOS index and case-mix index (showing decreasing patient complexity); however, $95.8 \%(n=92)$ physicians agreed that quality and safety of care had improved. There has been some research on specific diagnoses showing decreased LOS in total hip arthroplasty and cholecystectomy using IDRs. In this paper, we present research showing a definitive impact of IDRs on hospital LOS and excess day reduction with improvements in patient care measures, including CAUTI, CLABIS, mortality, and readmission rates.

METHODS: Two inpatient medical floors (4URIS and 7URIS) at Lenox Hill Hospital in New York City had IDRs implemented as a separate rounding time. An interdisciplinary care team consists of the following components: Medical residents: present each patients describing the acuity and day-to-day changes and approximate discharge date. In addition, discuss barriers to discharge and address quality measures. Supervising attending: assess and discuss patient readiness for discharge. In addition, address quality measures, including VTE Prophylaxis, need for Foley Catheters and Central Lines, Hepatitis C screening, early mobilization with physical therapy, and patients who require specific education. Nurse manager: provides a list of patients with Foley Catheters and Central Lines and in conjunction with nurse, will discuss patients who are high risk for falls or who have increased supervision needs. Nurse: Address patient PRN medication usage, early mobilization, physical therapy, fall prevention, and provide education, such as Coumadin and Stroke education. Social worker/Case Manager: assesses home/disposition needs for patients ready for discharge and gives updates on progress of placement and insurance issues. Hospital administrator: works to expedite discharge and resolve barriers. Our specific IDRS take place between 10:30 and 11:30 AM with a follow-up meeting at 3 PM to discuss progress throughout the day. Data was analyzed from January 2014 to November 2014 and January 2015 to November 2015 for year over year data. The core metrics of LOS and excess days were analyzed before and after implementation of IDRS. In addition, the rates of mortality, readmission, CAUTI, CLABSI, and falls were also analyzed.

RESULTS: The LOS on 4URIS went from 6.88 average days to 4.6, showing a $33.1 \%$ reduction. The LOS on 7URIS went from 7.37 average days to 4.67 , showing a $35.7 \%$ reduction. Excess days on 4URIS went from -1012 to +1701 , showing a +2713 excess day improvement. Excess days on 7URIS went from -1287 to +1482 , showing a +2769 excess day improvement. Total cases from January 2014-November 2014 was 5571 compared to 6112 from January 2015-November 2015. Meanwhile, readmission rates were 1.11 in 2014 and 1.02 in 2015, while hospital mortality was 1.08 in 2014 and 0.75 in 2015. In addition, case-mix index was 1.340 in 2014 and 1.416, showing increased patient complexity. The CAUTI rate on the above mentioned floors went from 57 to 13 from 2014 to 2015 , showing a $77.2 \%$ reduction. The CLABSI rate went from 11 to 8 from 2014 to 2015 , showing a $27.3 \%$ reduction

CONCLUSIONS: There have been many papers documenting how LOS increase the risk of hospital-acquired infections, which in turn, increase LOS even further. The quality of care delivered to patients hinges on early, appropriate discharge to improve patient outcomes. Prior studies have shown the impact of IDRs on LOS with the primary focus being discharge. The study mentioned above had a decreased LOS, but patient complexity went down as well. The goals of our study was to describe the impacts of a comprehensive approach, focusing on not only discharge, but also core patient quality measures. There was a dramatic change in LOS and excess day showing significant improvement. Concurrently, hopsital readmission rates, mortality rates, and CLABSI and CAUTI rates all decreased, showing improvements in patient care. The hospital acquired infection rate showed vast improvements year over year to significantly improve patient outcomes. Finally, it is especially important to note that the improved care occurred in the context of an increased number of patient cases and increased patient complexity. Our comprehensive model of IDRs provides an approach that not only creates an environment for timely discharge with decreased LOS, but also minimizes the occurrence of adverse events and vastly improves the quality of care delivered to our patients.

INTERNAL MEDICINE RESIDENT ASSESSMENT OF OPIOID OVERDOSE RISK AND WILlINGNESS TO PRESCRIBE NALOXONE J. Deanna Wilson ${ }^{1}$; Natalie Spicyn ${ }^{3}$; Pamela Matson ${ }^{1}$; Anika A. Alvanzo ${ }^{2}$; Leonard S. Feldman ${ }^{1} .{ }^{1}$ Johns Hopkins, Baltimore, MD; ${ }^{2}$ Johns Hopkins University School of Medicine, Baltimore, MD; ${ }^{3}$ Chase Braxton Health Center, Baltimore, MD. (Control ID \#2464512)

BACKGROUND: As part of comprehensive strategies to address the United States opioid epidemic, there has been a growing movement to encourage physicians to prescribe 
naloxone to patients at-risk of overdose. Despite evidence of naloxone efficacy as an overdose prevention strategy and the growing recognition of the importance of physicians in both opioid stewardship and overdose prevention, few physicians have routinely prescribed naloxone during their clinical training, which may limit their ability to incorporate it into their future practice. This study aims to: 1) evaluate current naloxone prescribing patterns and barriers to naloxone prescription among physicians-in-training, 2) evaluate how physicians-in-training assess risk of opioid overdose in their patients, 3) examine concordance between risk assessment and naloxone prescribing, and 4) better understand patient and provider factors that influence naloxone prescription in physician trainees.

METHODS: The HOPE: Hospital-based Overdose Prevention and Education Initiative is a quality improvement initiative to teach internal medicine residents to assess overdose (OD) risk, provide risk reduction counseling, and prescribe naloxone. As part of a needs assessment, internal medicine residents at an academic medical center in Baltimore, MD were surveyed in 2015. Residents were asked about their knowledge of OD risk assessment, naloxone prescribing patterns, attitudes related to naloxone, and barriers to naloxone prescription. Residents were asked to evaluate 7 clinical vignettes representing a range of demographics, substances used, and co-morbidities to determine likelihood of OD risk and if they would prescribe naloxone. Residents were asked to classify patients as being "not likely at all" to overdose or at "low", "moderate" or "high" likelihood of OD and were asked if they would prescribe naloxone to the patient. Data were collected anonymously using Qualtrics. Descriptive statistics and simple logistic regression were completed using Stata.

RESULTS: Ninety-seven internal medicine residents at a single, urban residency program completed the survey. Residents were willing to prescribe naloxone to at-risk patients (90\%), however, only a minority $(15 \%)$ had prescribed naloxone in the past. The residents identified several barriers to naloxone prescription, with $52 \%$ of residents reporting low confidence in their ability to identify patients at-risk for OD. Neither postgraduate year of training, race, or gender of resident was a significant predictor of willingness to prescribe naloxone or having prescribed naloxone in the past ( $p$-value $>$ 0.05 ). Analysis of clinical vignettes showed that even when residents perceived patients to be at moderate or high risk of $\mathrm{OD}$, they were unwilling to prescribe naloxone. The discordance between perceived moderate and high risk of OD and prescribing naloxone was greatest for patients prescribed opioids versus those using illicit opioids. For example, $43.8 \%$ of residents who viewed an 80 year old patient prescribed prescription opioids as being at moderate to high risk of OD would not prescribe naloxone to the patient. This discordant prescribing pattern was also seen for a similar clinical vignette with an 88 year old patient patient prescribed opioids: a majority, $59.1 \%$, of residents who viewed her as moderate to high risk of OD would not prescribe naloxone. The rates of discordant naloxone prescribing for patients at moderate to high risk of OD were less for the clinical vignettes involving patients who injected drugs $(7.0 \%)$ or were described as misusing prescription opioids (16.7\%). The odds of residents being willing to prescribe naloxone were 4.5 times greater in patients with a current substance use disorder (SUD) compared to those without it regardless of clinical risk factors increasing a patient's risk of overdose (95\%CI $2.9,7.1 ; p=<0.0001)$

CONCLUSIONS: Despite residents endorsing an overall willingness to prescribe naloxone to patients, the clinical vignettes revealed that willingness to prescribe was modified by patient characteristics. Analysis of case vignettes demonstrates that even when residents identify patients at moderate or high risk of OD, they are often unwilling to prescribe naloxone. Discordance between risk assessment and willingness to prescribe was greatest for elderly patients and for those who lacked certain risk factors associated with overdose, such as a history of injection drug use or other SUDs. Consistently, residents were much more likely to prescribe naloxone to those with a SUD than those without. Future work should explore reasons for the discordance between risk assessment and intervention. In order to improve rates of physician-prescribing, educational initiatives for residents should target interventions helping physicians more accurately assess OD risk and also better link risk assessment to naloxone prescription.

INTERNAL MEDICINE RESIDENTS' COMFORT IN PROVIDING CARE FOR YOUNG ADULTS WITH COMPLEX CONDITIONS OF CHILDHOOD ONSET Cynthia Peacock $^{4}$; Larry Laufman ${ }^{5}$; Christopher Hanks ${ }^{3}$; Eva Waite ${ }^{2}$; David E. DeLaet ${ }^{1}$. ${ }^{1}$ Icahn School of Medicine at Mount Sinai, New Yor, NY; ${ }^{2}$ Mount Sinai, New York, NY; ${ }^{3}$ The Ohio State University Wexner Medical Center, Hilliard, OH; ${ }^{4}$ baylor college of medicine, Houston, TX; ${ }^{5}$ Baylor College of Medicine, Houston, TX. (Control ID \#2468694)

BACKGROUND: Nationally, it is estimated that more than 1 million youth with special health care needs enter adulthood every year. Adult medical generalists will be increasingly charged with delivering comprehensive care for this diverse and medically complex patient population. Little is known about internal medicine (IM) trainees' comfort and willingness to assume care for such patients. To address this gap and inform future curriculum development, we conducted a survey of IM residents at 11 training institutions. METHODS: Members of the Society of General Internal Medicine "Adults with Complex Conditions Originating in Childhood Task Force" developed a 15-item Webbased survey to assess IM and Internal Medicine/Pediatrics (IM/Peds) residents' personal and professional experience with young adult patients with chronic conditions of childhood onset as well as their comfort and willingness to provide care for these patients. Selfreported measures were evaluated using a 9-point Likert scale. The list of disabilities included autism, cerebral palsy, congenital heart disease, cystic fibrosis, Down syndrome, intellectual disability, learning disability (e.g., dyslexia or attention deficit hyperactivity disorder), muscular dystrophy, pediatric genetic syndromes (e.g., Williams syndrome, Angelman syndrome, Fragile X sydnrome), physical disability that confines the individual to a wheelchair, sickle cell disease, and spina bifida. The survey assessed factors that increased comfort in caring for this patient population. It also evaluated comfort in addressing routine social and behavioral health needs of the general young adult population. Task force members emailed the survey invitation to program directors of 17 residency programs ( $9 \mathrm{IM}$ and $8 \mathrm{IM} /$ Peds) at 11 of their respective institutions: 2 North/ Northeast; 6 South/Southeast; 3 Central. The invitation included a link to the Web-based survey with a request that it be forwarded to the programs' residents. Spearman's rho correlation coefficient and the Mann-Whitney test were used to analyze ordinal ratings. The chi-square test was used to compare respondents by year of training.

RESULTS: The total number of survey respondents was 207 from a potential 928 residents in the respective programs, for a response rate of $22.3 \%$. Cronbach alpha reliability was 0.920 . Pre-training exposure to individuals with complex conditions of childhood onset was generally not associated with comfort or willingness, with weak associations found only for congenital heart disease (willingness: rho $0.206, p=0.01$ ), learning disability (comfort: rho $0.208, p=0.01$; willingness: rho $0.177, p=0.03$ ), and muscular dystrophy (comfort: rho $0.195, p=0.01$ ). Conversely, the self-reported number of patients cared for during residency for each of the assessed conditions was positively correlated with comfort and willingness to provide care for the respective condition. This association was strongest for comfort in providing care for CHD (rho $0.512, p<0.01$ ), cystic fibrosis (rho 0.509, $p<0.01$ ), cerebral palsy (rho 0.494, $p<0.01$ ), pediatric genetic syndromes (rho $0.473, p<0.01$ ), sickle cell disease (rho 0.446, $p<0.01$ ), spina bifida (rho $0.427, p<0.01$ ), and Down syndrome (rho $0.426, p<0.01$ ). IM/Peds residents were more likely than IM residents to report comfort in providing care for each of the conditions except cystic fibrosis and muscular dystrophy as well as in addressing the social and behavioral needs of the general young adult population. The two groups of residents were equally likely to report that improved social worker support, easier access to subspecialists to assist with care management, and additional educational opportunities regarding the 
included conditions would increase willingness to treat this patient population in the future. Greater comfort in providing care for each condition was associated with higher level of post-graduate year of training.

CONCLUSIONS: Our findings indicate that resident comfort and willingness to provide care for medically complex young adults is positively correlated with the number of patients with such conditions cared for during training. IM/Peds residents were more likely than IM residents to report comfort and willingness to provide care for this diverse patient population as well as comfort in addressing the social and behavioral needs of young adult patients in general. Limitations of this study include low survey response rate, its employment of convenience sampling, and self report of exposure to patients with the assessed conditions. However, the information gathered from this study will be useful to inform curriculum development for internal medicine residency training programs in an effort to improve care for this vulnerable patient population.

INTERVENTIONS TO PROMOTE RESIDENT WELLNESS: A NATIONAL SURVEY OF RESIDENCY PROGRAM DIRECTORS Rajiv Bhagat; Patrick M. Cocks; Matthew Vorsanger. New York University School of Medicine, New York, NY. (Control ID \#2469690)

BACKGROUND: Several studies have documented the prevalence of resident burnout during internal medicine residency training $[1,2]$ and the detrimental effect of burnout on resident safety [3] and patient care [4]. Quality of life is also rated as an important factor in choosing residency programs among internal medicine residency applicants [5] Therefore, reducing burnout and improving the quality of life of residents should be an important priority for internal medicine residency programs. At this time, there are only a few published studies on interventions to reduce physician burnout and improve quality of life at the residency level [6]. However, prior research has been done to characterize these interventions at the medical school level [7]. Interventions that do exist at the residency level remain institution-specific and are not generally shared across institutions. The objective of this study was to examine the state of resident wellness programming that currently exists within internal medicine residency programs in the United States.

METHODS: In February of 2015, an electronic survey was sent to program directors of internal medicine programs accredited by the Accreditation Council for Graduate Medical Education (ACGME) via a pre-existing listserv maintained by the Association of Program Directors in Internal Medicine (APDIM). Survey items evaluated the presence of wellness programming, internal evaluation of this programming, and perceptions of residency administration's role in promoting resident wellness. A reminder email was sent in October 2015 to increase participant yield. The study was approved by the New York University Langone Medical Center Institutional Review Board (IRB). Participant consent was waived by the IRB, and survey responders were informed that their responses could be used for research purposes. Results were analyzed using descriptive statistics.

RESULTS: Among the 397 programs, 130 (33\%) responded to our survey. Among respondents, 40 (31\%) indicated they had a unified, comprehensive wellness program for residents. The most common interventions cited to promote wellness were free consultation with mental health staff ( $77 \%$ ), followed by free access to gym/discounted gym membership (66\%). Only $15 \%$ of programs internally evaluated the effectiveness of the interventions that were offered. Among all respondents, $93 \%$ agreed that residency programs have a responsibility to encourage residents to practice healthy lifestyles. However, only $29 \%$ agreed that the level of support mandated by the ACGME was sufficient in achieving this goal of wellness promotion among residents.

CONCLUSIONS: Despite studies that indicate a high prevalence of resident burnout and its adverse effect on patient care $[1,2,4]$, our study shows only $31 \%$ of internal medicine residency programs offered a comprehensive set of interventions to promote resident wellness, with minimal internal evaluation of the interventions offered. Many program directors also felt that the level of support mandated by the ACGME was insufficient in achieving the goal of wellness promotion among residents. Leadership in Graduate Medical Education (GME) must continue to research the impact of specific interventions to promote resident wellness. The ACGME, in collaboration with national organizations in GME, can play an important role characterizing the interventions that do exist and ensuring that a basic and unified set of interventions exist at all programs. Programs already having implemented interventions should assess their impact and publish results to address the shortage of evaluative research on this important topic. References: [1] West CP, Shanafelt TD, Kolars JC. Quality of life, burnout, educational debt, and medical knowledge among internal medicine residents. JAMA. 2011:306(9):952-960. [2] Shanafelt TD, Bradley KA, Wipf JE, Back AL. Burnout and self-reported patient care in an internal medicine residency program. Ann Intern Med. 2002;136(5):358-367. [3] West CP, Tan AD, Shanafelt TD. Association of resident fatigue and distress with occupational blood and body fluid exposures and motor vehicle incidents. Mayo Clin Proc. 2012;87(12):1138-1144. [4] Fahrenkopf AM, Sectish TC, Barger LK, et al. Rates of medication errors among depressed and burnt out residents: prospective cohort study. BMJ. 2008;336(7642):488-491. [5] Flynn TC, Gerrity MS, Berkowitz LR. What do applicants look for when selecting internal medicine residency programs? A comparison of rating scale and open-ended responses. J Gen Intern Med. 1993;8(5):249-254. [6] West CP, Dyrbye LN, Rabatin JT, et al. Intervention to promote physician well-being, job satisfaction, and professionalism: a randomized clinical trial. JAMA Intern Med. 2014;174(4):527-533. [7] Wolf TM, Scurria PL. A survey of health promotion programs in U.S. and Canadian medical schools. Am J Health Promot. 1995;10(2):89-91.

INTERVENTIONS TO REDUCE PHYSICIAN BURNOUT: SYSTEMATIC REVIEW AND META-ANALYSIS Colin P. West; Liselotte Dyrbye; Patricia J. Erwin; Tait Shanafelt. Mayo Clinic, Rochester, MN. (Control ID \#2468993)

BACKGROUND: Physician burnout has reached epidemic levels as documented in national studies of both physicians-in-training and practicing physicians. Consequences include negative effects on patient care, professionalism, physicians' own care and safety, and the viability of the health care system. These concerns have prompted recent calls for increased attention to physician well-being, including efforts targeting burnout. However, relatively few studies have tested interventions to prevent or reduce burnout, and many of these studies have had methodologic limitations such as single-arm, non-randomized designs and inclusion of burnout measures with limited validity evidence. Gaining a more complete understanding of the quality and outcomes of the literature on approaches to prevent and reduce burnout is necessary to establish a strong foundation for further research to fill gaps in this literature. Prior reviews on physician distress have been limited by a combination of factors, including lack of focus on physicians, lack of focus on burnout, and inconsistent adherence to modern methodologic systematic review standards. We applied current rigorous systematic review methodology to examine the literature to date on interventions to prevent and reduce physician burnout.

METHODS: We searched multiple databases (inception to July 10,2015) using a strategy developed by an experienced medical librarian. Using independent duplicate assessment, we included randomized trials evaluating the effect of an intervention on physician burnout measured by a validated instrument. Individual study risk of bias was assessed using established validity criteria. Studies were combined in meta-analysis using randomeffects models, and subgroup analyses were performed to evaluate for differences in results according to study quality, physician category (resident or staff physician), and intervention type.

RESULTS: Our search strategy identified 2430 articles, of which 217 met the criteria for full-text review. A total of 15 randomized trials met eligibility criteria. The randomized studies inconsistently reported details of randomization processes and uniformly lacked 
blinding of participants to the interventions, as would be expected. Other potential biases were generally addressed well. For the 11 randomized controlled trials reporting differences in emotional exhaustion as a continuous variable, the pooled mean difference estimate was a statistically nonsignificant 1.87 -point reduction in emotional exhaustion total domain score ( $\left.95 \% \mathrm{CI}-3.89,0.16 ; p=0.07 ; \mathrm{I}^{2}=22 \%\right)$. For the 7 randomized controlled trials reporting differences in rates of high emotional exhaustion, the pooled mean difference was a statistically significant absolute reduction of $14 \%$ in proportion of participants with a high level of emotional exhaustion ( $95 \% \mathrm{CI}-0.22,-0.06 ; p=0.0009$; $\mathrm{I}^{2}=0 \%$ ). For the 10 randomized controlled trials reporting differences in depersonalization as a continuous variable, the pooled mean difference estimate was a statistically significant 1.42-point reduction in depersonalization total domain score (95\% CI -2.41, $-0.44 ; p=0.004 ; \mathrm{I}^{2}=0 \%$ ). For the 5 randomized controlled trials reporting differences in rates of high depersonalization, the pooled mean difference was a statistically nonsignificant absolute reduction of $4 \%$ in proportion of participants with a high level of depersonalization $\left(95 \% \mathrm{CI}-0.21,0.13 ; p=0.63 ; \mathrm{I}^{2}=61 \%\right)$. Subgroup analysis comparing the two trials reporting rates of high depersonalization involving staff physicians with the remaining three trials involving resident physicians yielded distinct subgroups ( $p=$ 0.002 ), with a statistically significant reduction in rates of high depersonalization of $15 \%$ among staff physicians ( $95 \% \mathrm{CI}-0.23,0.07 ; p=0.0002 ; \mathrm{I}^{2}=0 \%$ ) and a statistically significant increase in rates of high depersonalization of $28 \%$ among resident physicians (95\% CI $0.02,0.53 ; p=0.03 ; \mathrm{I}^{2}=0 \%$ ). Otherwise. results did not differ across the prespecified subgroups (structural or organization-level versus individual-level interventions, resident versus staff physicians, and lower versus higher risk of bias).

CONCLUSIONS: Evidence on interventions to reduce or prevent physician burnout generally suggests that both structural and individual approaches can reduce both the emotional exhaustion and depersonalization domains of burnout. Additional welldesigned trials are needed to more clearly determine which interventions are most effective for specific physician groups. Meanwhile, efforts to reduce burnout and promote physician satisfaction should be bolstered by the growing evidence of their beneficial effects.

INVESTIGATION INTO THE “ONE-SIZE-FITS-ALL” DIURETIC STRATEGY TO HEART FAILURE EXACERBATION MANAGEMENT: A RETROSPECTIVE STUDY Hirotaka Kato; Perry Fisher; Dahlia Rizk. Mount Sinai Beth Israel, New York, NY. (Control ID \#2468226)

BACKGROUND: Diuretic therapy is the mainstay of treatment for heart failure exacerbations but its optimal dosing strategy remains unclear. In 2011, the DOSE trial exhibited a trend toward improvement in patients' global symptom assessments when utilizing a highdose diuretic strategy, which used nearly $80 \mathrm{mg}$ intravenous furosemide three times daily in the first $72 \mathrm{~h}$ on average. To improve heart failure outcomes and test the applicability of this trial to our population, we implemented the initial dose of $80 \mathrm{mg}$ intravenous furosemide three times daily for patients admitted through our emergency department. We hypothesized that standardizing high dose furosemide for all patient populations may lead to some negative inpatient outcomes.

METHODS: Consecutive 333 patients admitted for acute systolic or diastolic heart failure were identified in an urban, academic medical center and reviewed from July 2014 to June 2015 . Total diuretic dose was defined as total diuretics administered in the first $72 \mathrm{~h}$ after hospitalization. Multivariable regression models with stepwise selection method was used to assess the statistical association between variables and inpatient outcomes including length of stay, worsening renal function, 30-day readmission rate, and mortality.

RESULTS: Baseline patient characteristics included average age of 70.4 , male of $57 \%$, White of $31.8 \%$, EF of $35.5 \%$, Cr on admission of 1.37 , and BNP of 1113 on average. Higher total furosemide dose in first $72 \mathrm{~h}$ was significantly associated with longer length of stay (Coefficient $0.0032, p=0.000$ ), higher reduction in GFR (Coefficient -0.00085 , $p=0.000$ ), higher grade of worsening renal function (OR 1.0013, CI 1.0006-1.0019, $p=$ 0.000 ). Importantly, there was no association between total furosemide dose and 30-day readmission rate or inpatient mortality. Higher reduction in GFR was significantly associated with longer length of stay (Coefficient $-11.2, p=0.000$ ). History of stroke and history of heart failure admission in past 12 months were significantly associated with higher 30-day readmission rate (OR 2.4, CI 1.15-5.09, $p=0.019$ and OR 2.4, CI 1.32$4.53, p=0.004$, respectively).

CONCLUSIONS: In patients admitted for acute heart failure exacerbation, higher diuretic dose in first $72 \mathrm{~h}$ was associated with longer length of stay and worsening renal function in the setting of increasing initial diuretic dose in our patient population. Even though a high dose diuretic strategy is advantageous for reducing symptoms early per the DOSE trial, our data suggest that physicians should use clinical judgment, and that other variables may play a role in applying a high dose strategy to the appropriate patients

IS A TITLE VII FUNDED INTERNAL MEDICINE RESIDENCY PROGRAM FOCUSED ON URBAN UNDERSERVED PATIENTS ASSOCIATED WITH A CAREER PROVIDING CARE FOR UNDERSERVED POPULATIONS? A CROSS SECTIONAL ANALYSIS OF A 10-YEAR COHORT. Marion Stanley ${ }^{1}$; Bridget O'Brien ${ }^{2}$; Sharad Jain ${ }^{2}$; Jeff Kohlwes ${ }^{3}$. ${ }^{1}$ Northwestern Memorial Hospital, Chicago, IL; ${ }^{2} \mathrm{UCSF}$, San Francisco, CA; ${ }^{3}$ VAMC/UCSF, San Francisco, CA. (Control ID \#2469828)

BACKGROUND: Title VII seeks to improve heath care for underserved populations by enhancing the quality, diversity, and cultural competency of the primary care workforce. The San Francisco General Primary Care (SFPC) program is supported by Title VII funds and provides culturally sensitive training to internal medicine residents who intend to practice in urban underserved settings. Our study examines whether training in a Title VII funded primary care internal medicine program focused on urban underserved patients is associated with a career working with underserved populations.

METHODS: We conducted a cross-sectional survey of a cohort of internal medicine residency alumni from the University of California, San Francisco (UCSF) who graduated between 2001 and 2010. Alumni responded to an electronic survey regarding demographics and whether a majority of their current clinical work is with underserved populations. We performed Chi-Square analysis to compare the focus of current clinical work among graduates from the SFPC internal medicine track to graduates of all other internal medicine residency tracks, including alumni from the categorical track as well as the Primary Care General Internal Medicine track (UCPC) which has a focus on ambulatory primary care training. RESULTS: We contacted 481 alumni and 322 responded (67\% response rate). We excluded 42 graduates who self-identified as current fellows and 5 graduates from the Molecular Medicine residency. For this analysis, we compared 30 responses from SFPC alumni to 245 responses from all other alumni. Twenty-six out of 30 (87 \%) alumni from the SFPC program agreed that the majority of their current clinical work is with underserved populations vs. 31 out of $76(41 \%)$ of UCPC alumni and 45 out of $169(27 \%)$ of categorical alumni $(p<0.0001)$. Notably, 19 out of 30 (63\%) SFPC alumni agreed that the majority of their current work is in primary care, and 18 out of $30(60 \%)$ agreed that the majority of their current clinical work is both in primary care and with underserved populations

CONCLUSIONS: The majority of graduates from the Title VII funded SFPC training program continued working with underserved populations after graduation as compared to other alumni from the same university program. Calls have been made to re-evaluate Graduate Medical Education funding formulas, arguing that outcomes data of residency programs should be used to further fund programs that supply physicians to areas in which there are shortages. Our results suggest that Title VII funds used to promote population specific clinical training are a wise investment that may help to expand the work force focused on underserved patients. 
IS APPROPRIATE RECONCILIATION OF CARDIOVASCULAR MEDICATIONS AFTER ELECTIVE SURGERY ASSOCIATED WITH FEWER POST-DISCHARGE ACUTE HOSPITAL AND AMBULATORY VISITS? Jonathan S. Lee ${ }^{1}$; Ralph Gonzales ${ }^{1}$; Kirsten E. Fleischmann ${ }^{1}$; Eric Vittinghoff $^{1}$; Neil Sehgal ${ }^{1}$; Kitty K. Corbett ${ }^{2}$; Andrew D. Auerbach ${ }^{1}$. ${ }^{1}$ University of California San Francisco, San Francisco, CA; ${ }^{2}$ University of Waterloo, Waterloo, ON, Canada. (Control ID \#2469148)

BACKGROUND: The post-operative period represents a potentially vulnerable time when chronic cardiovascular medications may not be appropriately reconciled. We investigated the association between appropriate discharge continuity of 4 cardiovascular medication classes and subsequent acute hospital and ambulatory visits for patients undergoing elective non-cardiac surgery.

METHODS: We analyzed data for patients admitted from May 2007 to February 2011 for non-cardiac surgery at a large academic medical center. We included patients $>18$ years of age who were taking medications from at least 1 of 4 cardiovascular medication classes in the pre-operative period: antiplatelet agents, beta-blockers, angiotensin-converting-enzyme inhibitors (ACEI) or angiotensin-receptor blockers (ARB), and statins. Data were collected through chart review and in-person patient interviews while hospitalized and by telephone 1 month after discharge. We defined continuity as chart notation why a medication was not continued, documented perioperative complications contraindicating specific medications (bleeding for antiplatelet agents, hypotension for $\mathrm{ACEI} / \mathrm{ARB}$ and beta-blockers and acute kidney injury for $\mathrm{ACEI} / \mathrm{ARB}$ ) or clear indication that the medication was being prescribed in discharge instructions or discharge summaries. We assessed medication continuity between the pre-operative and discharge periods as an all-or-none complete vs incomplete measure (patients were discharged on all medication classes they were taking pre-operatively) and for each medication class individually. Our primary outcomes were acute hospital visits (emergency department visits or hospitalizations) and unplanned ambulatory visits (primary care or surgical) at 1-month after discharge. We used multivariable logistic regression to assess the independent associations between medication continuity and outcomes, taking into account patient clustering by surgical service and adjusting for baseline patient demographics and comorbidities.

RESULTS: Of 752 patients meeting inclusion criteria, 679 (90.3\%) completed 1month follow-up. Overall, the proportion of patients taking each medication class at baseline was $43.5 \%$ for antiplatelet agents, $83.0 \%$ for beta-blockers, $48.0 \%$ for ACEI or ARB, and $54.0 \%$ for statins. Nearly three-quarters of patients $(73 \%)$ were taking at least 2 medication types. Continuity between the pre-operative and discharge periods for each medication class was $47.7 \%(156 / 327)$ for antiplatelet agents, $81.2 \%(506 / 624)$ for beta-blockers, $69.8 \%(252 / 361)$ for ACEI or ARB, and $74.4 \%$ (302/406) for statins. Among patients completing follow-up, $21.7 \%$ had been seen in the emergency department or hospitalized and $16.6 \%$ had been seen for an unplanned ambulatory visit at 1 month after discharge. In multivariable analyses, continuity of all pre-operative medications was not significantly associated with emergency department visits or hospitalizations (odds ratio, $0.93 ; 95 \% \mathrm{CI}$, $0.65-1.32$ ) or unplanned ambulatory visits (odds ratio, 1.51; $95 \% \mathrm{CI}, 0.75-3.02$ ).

There were no statistically significant associations between continuity of individual medication classes and either outcome (Table).

CONCLUSIONS: In this observational study, gaps in cardiovascular medication continuity were not associated with adverse post-discharge acute outcomes in surgical patients. While residual confounding by indication may have occurred, such confounding would tend to bias our results towards an association not suggested by our observed point estimates and confidence intervals. Further study is needed to identify specific aspects of discharge medication reconciliation that may affect patient outcomes.
Adjusted 1-month outcomes by medication continuity

\begin{tabular}{|c|c|c|}
\hline & Outcome $<$ b/ & \\
\hline & Emergency & Unplanned \\
\hline & department & ambulatory \\
\hline & visit or & visit $<$ b/ \\
\hline & hospitalization $<$ b/ & \\
\hline \multirow{2}{*}{ Continuity $<$ b/ } & Adjusted Odds & Adjusted Odds \\
\hline & Ratio $(95 \% \mathrm{CI})^{*}<\mathrm{b} /$ & Ratio $(95 \% \mathrm{CI})^{*}<\mathrm{b} /$ \\
\hline Complete vs & $0.93(0.65-1.32)$ & $1.51(0.75-3.02)$ \\
\hline \multicolumn{3}{|l|}{ incomplete } \\
\hline Antiplatelet agent & $1.08(0.88-1.32)$ & $1.20(0.50-2.91)$ \\
\hline Beta-blocker & $0.95(0.52-1.75)$ & $1.24(0.72-2.14)$ \\
\hline ACEI or ARB & $1.13(0.81-1.58)$ & $1.51(0.67-3.38)$ \\
\hline Statin & $0.48(0.23-1.01)$ & $1.11(0.57-2.16)$ \\
\hline
\end{tabular}

* Odds ratios represent odds of the outcome for medication continuity (vs discontinuity) and are adjusted for age, sex, race/ethnicity, revised cardiac risk index risk factors, the number of baseline cardiovascular medication classes prescribed and clustering by surgical service.

IS DUAL USE OF VA AND MEDICARE DIABETES SERVICES ASSOCIATED WITH ADVERSE HEALTH OUTCOMES? Thomas R. Radomski ${ }^{2,3}$; Xinhua Zhao ${ }^{3}$; Carolyn T. Thorpe ${ }^{1,3}$; Joshua M. Thorpe ${ }^{1,3}$; Chester $\operatorname{Good}^{3,2}$; Maria Mor ${ }^{3}$; Michael J. Fine ${ }^{3,2}$; Walid F. Gellad ${ }^{3,2}$. ${ }^{1}$ University of Pittsburgh, Pittsburgh, PA; ${ }^{2}$ University of Pittsburgh School of Medicine, Pittsburgh, PA; ${ }^{3}$ VA Pittsburgh Healthcare System, Pittsburgh, PA. (Control ID \#2465532)

BACKGROUND: Many Veterans enrolled in the VA Healthcare System (VA) are also enrolled in other health insurance plans and receive care outside VA (i.e. dual use). Although dual use may improve Veterans' access to care, it could result in care fragmentation and worse health outcomes. We previously developed a novel method to characterize dually-enrolled Veterans based on their patterns of care utilization within VA and Medicare. Our objective was to determine how these patterns of dual use are associated with health outcomes in a national cohort of Veterans with diabetes.

METHODS: We linked VA and Medicare fee-for-service claims data from fiscal years (FYs) 2008-2010 to identify a cohort of dually-enrolled Veterans 65 years or older with type 2 diabetes, who had at least one outpatient visit within VA and received a diabetes medication in FY2009. Using latent class analysis, we previously classified this cohort into 4 groups of dual users. Group 1 (VA predominant users) had a high probability of VA use and low probability of Medicare use across all facets of diabetes care; Groups 2 (VA+ Medicare visits \& labs), 3 (VA+ Medicare test strips), and 4 (VA+ Medicare prescriptions) had high probabilities of VA and Medicare dual use, but differed in the specific VA and Medicare services used. For example, Veterans in Group 3 received test strips exclusively through Medicare and not VA, whereas Veterans in Group 4 received their medications predominantly through Medicare. We compared differences in outcomes (death or having a diabetes-related hospitalization or ED visit in FY2010) between the 4 dual use groups using chi-square tests. We used multinomial logistic regression to determine the independent association between dual use groups and health outcomes, controlling for sociodemographic characteristics and medical comorbidities. For our primary analysis, we adjusted for Elixhauser comorbid conditions excluding diabetes using data from both VA and Medicare, as is standard in research on dual use. Due to known discrepancies in comorbidity coding between VA and Medicare, we also performed a model adjusting for comorbidities exclusively documented in VA.

RESULTS: There were 316,775 Veterans in the final cohort. The median age was 74 years (IQR 69-80), $98.7 \%$ were male, $83.0 \%$ were non-Hispanic white; $53.9 \%$ of Veterans were classified as Group 1 (VA predominant) dual users and 17.2, 21.8, and 7.0 \% of Veterans were in Groups 2, 3, and 4, respectively. As compared to Group 1, dual use Groups 2, 3, and 4 had fewer documented comorbidities using only VA claims (mean number of Elixhauser 
comorbidities: 1.6 vs $1.2,0.9,0.8$ ) but more documented comorbidities when adding Medicare claims (1.8 vs $2.5,2.3,2.6)$. There were significant differences between the 4 dual use groups in the frequencies of death $(5.3,6.2,6.4$ and $7.2 \% ; p<.001)$ and diabetesrelated hospitalizations or ED visits $(17.2 \%, 17.8,15.8$ and $17.7 \% ; p<.001)$. When adjusting for sociodemographics and VA comorbidities, dual use Groups 2, 3, and 4 had significantly increased odds of death, with odds ratios ranging from 1.08 to 1.24 , as compared to Group 1 (VA predominant users) (Table). After adding comorbidities documented in Medicare, Groups 2, 3, and 4 now had significantly decreased odds of death, with odds ratios ranging from 0.89 to 0.94 . Similar patterns and differences between adjustment methods were seen for hospitalizations or ED visits, with the exception of dual use Group 3
(VA + Medicare Test strips), which had lower odds of the outcome compared to Group 1 in both models (Table)

CONCLUSIONS: Dual use of VA and Medicare diabetes services was associated with decreased odds of death and having a diabetes-related hospitalization or ED visit, which is in contrast to most prior studies of dual use. However, these results were highly sensitive to adjusting for comorbidities documented in Medicare, suggesting that bias in ascertainment of comorbid conditions in dual users versus non-dual users could be impacting the results. These results illustrate the importance of developing a risk adjustment method, such as a medicationbased adjustment, that is not susceptible to coding discrepancies between VA and Medicare to better understand the association between dual use and health outcomes.

Odds Ratios of Death or Having a Diabetes-related Hospitalization/ED Visit in FY2010 in Dual Users (Groups 2, 3, 4) as Compared to VA Predominant Users (Group 1)

\begin{tabular}{|c|c|c|c|c|c|c|}
\hline & $\begin{array}{l}\text { Groups } 2 \text { vs } \\
\text { Group } 1\end{array}$ & $\begin{array}{l}\text { Groups } 3 \text { vs } \\
\text { Group } 1\end{array}$ & $\begin{array}{l}\text { Groups } 4 \\
\text { vs Group } 1\end{array}$ & & & \\
\hline Death & OR & $95 \%$ CI & OR & $95 \%$ CI & OR & $95 \%$ CI \\
\hline Unadjusted & 1.18 & (1.14-1.23) & 1.23 & (1.18-1.27) & 1.38 & (1.31-1.46) \\
\hline Adjusted for: & & & & & & \\
\hline Sociodemographics* & 1.07 & $(1.02-1.11)$ & 1.03 & (0.99-1.07) & 1.17 & $(1.10-1.24)$ \\
\hline $\begin{array}{l}\text { Sociodemographics* } \\
\text { VA Comorbidities }\end{array}$ & 1.08 & (1.03-1.13) & 1.10 & $(1.06-1.14)$ & 1.24 & $(1.17-1.31)$ \\
\hline $\begin{array}{l}\text { Sociodemographics* } \\
\text { VA + Medicare } \\
\text { Comorbidities }\end{array}$ & 0.90 & $(0.86-0.93)$ & 0.89 & $(0.86-0.93)$ & 0.94 & $(0.89-0.99)$ \\
\hline $\begin{array}{l}\text { Hospitalization/ } \\
\text { ED Visit }\end{array}$ & OR & $95 \%$ CI & OR & $95 \%$ CI & OR & $95 \%$ CI \\
\hline $\begin{array}{l}\text { Unadjusted } \\
\text { Adjusted for: }\end{array}$ & 1.04 & (1.02-1.07) & 0.90 & $(0.88-0.92)$ & 1.04 & $1.00-1.07)$ \\
\hline Sociodemographics* & 1.06 & (1.03-1.09) & 0.92 & (0.90-0.95) & 1.03 & $(0.99-1.07)$ \\
\hline $\begin{array}{l}\text { Sociodemographics* } \\
\text { VA Comorbidities }\end{array}$ & 1.03 & (1.01-1.06) & 0.95 & $(0.93-0.98)$ & 1.06 & $(1.02-1.10)$ \\
\hline $\begin{array}{l}\text { Sociodemographics* } \\
\text { VA + Medicare } \\
\text { Comorbidities }\end{array}$ & 0.88 & $(0.86-0.91)$ & 0.80 & $(0.78-0.82)$ & 0.84 & $(0.81-0.87)$ \\
\hline
\end{tabular}

*Includes age, race, gender, Medicaid eligibility, and VA priority group. Models that adjust for Elixhauser comorbidities also adjust for diabetes complications and the presence of serious mental illness.

\section{IS MORTALITY FOLLOWING AN ACUTE ILLNESS HOSPITALIZATION} REALLY LOWER AMONG RACIAL/ETHNIC MINORITIES? Amresh D Hanchate $^{3,}$; ; Michael Paasche-Orlow ${ }^{2}$; Lenny Lopez ${ }^{1}$; Nancy R. Kressin ${ }^{3,}$; Eun Ji $\mathrm{Kim}^{5,}$; Jennifer E. Rosen ${ }^{4}$; Chen Feng ${ }^{2}$; Meng-Yun $\mathrm{Lin}^{2}{ }^{1}{ }^{1}$ University of California at San Francisco, San Francisco, CA; ${ }^{2}$ Boston University School of Medicine, Boston, MA; ${ }^{3}$ VA Boston Healthcare System, Boston, MA; ${ }^{4}$ MedStar Washington Hospital Center, Washington, DC; ${ }^{5}$ Bedford VA, Bedford, MA. (Control ID \#2466081)

BACKGROUND: Patient mortality within 30 days of hospitalization for acute high-risk illness is a widely used metric to evaluate hospital quality, and reward or penalize hospitals. Applying this metric to different racial/ethnic groups, a number of studies have found lower 30-day mortality among minority patients compared to non-Hispanic whites; this finding is discordant with the predominant view, and a preponderance of evidence, that racial/ethnic minorities are generally at higher risk of receiving inadequate, delayed or poor quality treatment. We posited that this 'reverse disparity' might be a function of confounding not adequately controlled for in prior studies. We performed multiple analyses to reduce confounding and explored related measures of mortality risk to better understand the underlying disparities. We examined if the reverse disparity is robust to the exclusion of do-not-resuscitate (DNR) discharges (less common among minorities) and discharges with same/next day live discharges (as these may not be true cases of acute illness). We also evaluated if the reverse disparity is found in both inpatient and post- hospitalization mortality. We then examined if the risk of hospitalization for acute illness varied by race/ethnicity, as this may indicate unmeasured differences in patient status and severity.

METHODS: Using the Agency for Healthcare Research Quality (AHRQ) Inpatien Quality Indicators (IQI) protocol we identified all inpatient discharge records for patients aged 18 or older for acute myocardial infarction, congestive heart failure, pneumonia, acute stroke, gastrointestinal bleed, and hip fracture from all California hospitals between 1/1/2010 and 6/30/201 1, excluding discharges for non-state residents. The main outcome measures were mortality: inpatient, 30-day (from date of admission), 90-day and 180-day. Using generalized linear regression models with covariate data on patient demographics, comorbidity and (area-level) socioeconomic indicators, we estimated adjusted rates of mortality outcomes and risk ratios by four race/ethnicity groups: Hispanics, blacks (nonHispanic), Asians (non-Hispanic) and whites (non-Hispanic). We compared these estimates (base model) with corresponding estimates from models excluding admissions with DNR patient status and same/next day live discharges. To assess disparities in survival following hospitalization, we examined mortality rates for patients who survived the index hospitalization, and used Cox proportional hazards regressions to estimate the hazard rate of mortality. After merging with census data, we also estimated the population incidence of hospitalization for selected acute illnesses using a Poisson regression model. As a combined measure of the risk of hospitalization and the risk of inpatient death given hospitalization, we estimated the risk of hospitalization with inpatient death. 
RESULTS: We examined 370,993 patients; $9 \%$ were black, $19 \%$ Hispanic, $9 \%$ Asian and $63 \%$ white. Relative to whites, age-sex adjusted inpatient mortality was $18 \%$ lower for blacks, $14 \%$ higher for Asians and similar for Hispanics; but 30-, 90- and 180-day mortality was lower for all three minority groups compared to whites. DNR was more frequent among whites $(15.4 \%)$ than any minority group $(\leq 8.4 \%)$. Live same/next day discharges were most common among blacks (12.1\%). Exclusion of either or both of these discharges did not change the risk ratios in mortality for minority groups. Among those who survived the index hospitalization, the hazard rate of mortality was lower for all three minority groups, and this trend too was robust to the aforementioned exclusions for DNR and live same/next day discharges. Risk of hospitalization for the selected acute illnesses was 132 admissions per 1000 census population among whites; this risk was $73 \%$ higher among blacks (168 admissions/1000 population), and 15 and $36 \%$ lower among Hispanics (58) and Asians (65). Relative to whites, the population risk of hospitalization with inpatient death was similar for blacks, and 22 and $26 \%$ lower for Hispanics and Asians, respectively.

CONCLUSIONS: We found different trends in disparities in the risk of inpatient mortality versus post-hospitalization mortality: relative to whites, risk of inpatient mortality was lower for blacks, higher for Asians and similar for Hispanics, but risk of posthospitalization mortality was lower for all the minority groups. These trends remained unchanged with the exclusion of DNR and same/next day live discharges. However, risk of hospitalization for acute illness varied widely by race/ethnicity, suggesting that there may be additional unobserved dissimilarities that may confound the comparison of mortality risk differences by race/ethnicity. Additional research is needed, preferably using richer clinical data on patient status and acuity, to confirm if reverse disparity is a real phenomenon indicative of quality of care differences.

\section{IT'S AN OKAY PLACE TO GET STUFF WRONG: HOW GREAT INPATIENT ATTENDING PHYSICIANS CREATE SAFE LEARNING ENVIRONMENTS} Molly Harrod $^{2}$; Karen E. Fowler ${ }^{2}$; Sanjay Saint ${ }^{1,2}$. ${ }^{1}$ University of Michigan, Ann Arbor, MI; ${ }^{2}$ Department of Veterans' Affairs Ann Arbor HealthCare System, Ann Arbor, MI. (Control ID \#2464484)

BACKGROUND: Medical education has changed substantially over the last few decades. The reduction to an 80-h resident workweek - and the more recent 16 -h intern shift cap - has meant that medical education on the wards must be high yield. This responsibility falls to attending physicians, few of whom have had formal training on how to teach. Based on previous studies, numerous recommendations and strategies exist on how to be a better attending such as: create a positive environment, be interested in the learner, read facial expressions, and be student-centered. However, few of these studies provide actual examples of how great attendings do this in practice. Therefore, the aim of this study was to better understand how great inpatient attendings create optimal learning environments, from both their own and learners perspectives.

METHODS: This research was designed as an ethnographically based study. We identified participants using a modified snowball sampling approach along with considering organizational and individual characteristics to ensure variation. We conducted 12 site visits in total, one with each of the attendings that agreed to participate. Data collection consisted of team round observations, focus groups or semi-structured interviews with current and former learners and semi-structured interviews with the attendings. Photos of the attendings engaged with their teams were taken along with any teaching artifacts that were used. Data were analyzed using an inductive, thematic approach.

RESULTS: Many of the learners spoke about the attendings' ability to create "safe learning environments". We found several consistent elements that converged to create this type of environment. First, attendings took a non-judgmental approach to the learners' expressed thoughts and questions. Many learners likened this approach to stimulating the learning environment resulting in them asking more questions. The ability to voice their questions without fear of criticism often opened up the discussions to other learning opportunities. Second, the attendings would encourage learners to say what they thought even though it may be incorrect. Learners appreciated this approach, often comparing it to other experiences in which they were criticized or demeaned in front of others for either not knowing or having the wrong answer. As a result, they would often withdraw from the learning environment. Third, all of the attendings expected learners to challenge and question them. The attendings goal was to develop not only independent thinkers, but those who would advocate for their patient in order to provide the best possible care even if their opinion was in the minority. And, finally, the fourth element was that the attendings were willing to admit when they did not know something and would go and seek out information. They used their own mistakes as teaching points. The learners felt that if the attending could admit when s/he did not know something, then that provided the latitude for them to admit uncertainty.

CONCLUSIONS: Given limited previous guidance for inpatient attendings, identifying the elements great attendings use to create safe learning environments is crucial so that junior attendings and others can learn how to create optimal learning environments.

JEEPERS CREEPERS, WHAT ABOUT THOSE EPERS?: CREATING A CULTURE OF PHYSICIAN EVENT REPORTING Jennifer M. Schmidt ${ }^{2}$; Justin Adams ${ }^{1}$; Kathlyn Fletcher ${ }^{2} .{ }^{1}$ Medical College of Wisconsin, Milwaukee, WI; ${ }^{2}$ Medical College of Wisconsin/Milwaukee VAMC, Milwaukee, WI. (Control ID \#2469536)

BACKGROUND: Adverse event (AE) reporting is an essential part of patient safety. However, literature shows physicians are less likely than other healthcare specialties to report such events. Knowledge of how to report as well as the time reporting requires are commonly cited as event reporting barriers. This quality improvement project seeks to increase the reporting rate among Internal Medicine residents.

METHODS: Participants were Internal Medicine residents in an academic program rotating at the VA hospital. Baseline data on knowledge and perception of event reporting was collected via survey. Questions were "Yes/No", free response or utilized a Likert scale (anchors 1 = very unlikely, 5 = very likely). Data specific to VA event reporting: Electronic Patient Event Report (ePER) was collected (at baseline and after each PDSA cycle) from VA rotating residents using a three question yes/no survey. Interventions included educational presentations, posters and emails, increased event reporting discussion at daily resident case conferences, ePER completion demonstration and feedback on actions resulting from ePERs. Each month long PDSA cycle built on the previous months' cycles. By the third month, residents were receiving an email at the start of their rotation detailing how and where to file an ePER, posters were hung in resident team rooms and ePER discussion as well as simulation were included in daily didactics. Analysis was done with Chi-square test $(P<0.05)$ and run charts.

RESULTS: Baseline data showed that 24 and $54 \%$ of residents had witnessed an adverse event or near miss (NM) during their last rotation at the VA. Forty-nine and $22 \%$ of residents would report an AE or NM if witnessed. However, only $3 \%$ had ever filed an event report at the VA. Residents cited "Time" (45\%), "Don't know how" (43\%) and "Don't think change will occur" (30\%) as reasons why they are unlikely to report. Preliminary data analysis of monthly surveys after three PDSA cycles shows a statistically significant increase across all questions (see Table 1).

CONCLUSIONS: Upon completion of three PDSA cycles, we were able to increase resident physician awareness of and participation in event reporting at our institution. While this intervention required consistent effort, it is clearly sustainable because it involved a small amount of educational time. We believe that the main effect from this intervention came through increasing discussions of ePERs in the context of other ongoing 
conferences. The chief residents made a commitment to take every opportunity in informal and formal settings to suggest the filing of an ePER, when appropriate. We see this as the beginning of a culture change in our program that involves the residents having a more active role in patient safety. Plans to sustain this momentum include completing a final PDSA cycle with presentation on a formally conducted Root Cause Analysis (RCA) that resulted from an ePER filed by a resident. This presentation will be incorporated into a regular morbidity and mortality conference. Also part of the final PDSA cycle will be using 5-10 $\mathrm{min}$ at noon reports throughout the month to update the residents on actions spurred from other ePERs. We plan to spread this successful intervention to our affiliates in the coming academic year.

Monthly Resident Survey Results

\begin{tabular}{|c|c|c|c|c|c|c|}
\hline & $\begin{array}{l}\text { Do you know } \\
\text { what an ePER is? }\end{array}$ & $\begin{array}{l}\text { Do you know how } \\
\text { to file an ePER? }\end{array}$ & $\begin{array}{l}\text { Did you file an } \\
\text { ePER this month? }\end{array}$ & & & \\
\hline & No. of Residents & $\%$ of Residents Who & No. of Residents & $\%$ of Residents & No. of Residents & $\%$ of Residents \\
\hline & Who Said Yes & Said Yes & Who Said Yes & Who Said Yes & Who Said Yes & Who Said Yes \\
\hline Sept & 11 & $68.8 \%$ & 8 & $50 \%$ & 0 & $0 \%$ \\
\hline Dec & 24 & $96.0 \%$ & 20 & $80 \%$ & 6 & $24 \%$ \\
\hline$p$-value & 0.016 & & 0.044 & & 0.034 & \\
\hline
\end{tabular}

KICK-STARTING A CULTURE OF SAFETY: HOW TEAMSTEPPS AND SIMULATION TRANSFORMED ATTITUDES ON THE MEDICINE SERVICE Katherine A. Hochman ${ }^{3}$; Nicole Adler ${ }^{4}$; Frank Volpicelli²; Benjamin Wertheimer ${ }^{3}$; Sondra Zabar ${ }^{1}$; Demian Szyld ${ }^{1} .{ }^{1}$ NYU School of Medicine, New York, NY; ${ }^{2}$ New York University, New York, NY; ${ }^{3}$ New York University School of Medicine, New York, NY; ${ }^{4}$ nyu, New York, NY. (Control ID \#2469046)

BACKGROUND: TeamSTEPPS (Strategies and Tools to Enhance Performance and Patient Safety) has been shown to improve patient safety by improving teamwork and communication skills. Our objective was to use TeamSTEPPS training to enhance teamwork across the interdisciplinary team on the Medicine service.

METHODS: We developed a 4-h TeamSTEPPS multi-disciplinary training session, conducted in our simulation center. We invited 90 core members of the medicine service to participate. Participants represented the following 5 disciplines: hospitalists, nurses, care managers, social workers, and trainees/nurse practitioners/physician assistants. We trained 15 participants each session ( 3 participants per discipline). We held 6 identical sessions over a 4 month period. Each session consisted of a short didactic session on patient safety and TeamSTEPPS training delivered by department of medicine leadership. Participants were divided into three groups, each consisting of a hospitalist, a nurse, a care manager, a social worker and a trainee/NP/PA. Each group rotated through three different scenarios that were devised to mimic real-life: interdisciplinary rounds, a team huddle and an end-of-life discussion with a standardized patient at the bedside. Each participant was given some but not all of the relevant information and asked to play his or her own role (so a social worker would play a social worker) in the scenarios. A facilitator gave feedback and taught one of three TeamSTEPPS strategies for improved communication: CUS (I am concerned/uncomfortable/stop the line for safety), SBAR (situation, background, assessment, recommendation) or check back. For the final session, participants were introduced to and practiced IPASS as a method of signout. Change in participant's skill confidence was assessed after each workshop using a retrospective pre post survey using a 4 point scale from "not confident" to "very confident". Participants completed commitment to change (CTC) statements after each workshop. The primary outcome was a comparison of participants' comfort levels with a.) being an effective team member and b.) speaking-up before and after training as determined by a survey (filled out anonymously by participant before leaving the session). The secondary outcome was the percentage of participants who fulfilled their CTC after at least 1 month.

RESULTS: Eighty-seven health-care professionals participated in the TeamSTEPPS training session. Seventy-six percent would "definitely" and $20 \%$ would "probably" recommend the session to a colleague. Participant confidence, as measured by the retrospective pre-post assessments, improved in the ability to be an effective team member from 36 to $72 \%$ "very confident" and in the ability to speak up in the case of a patient safety concern from 46 to $81 \%$ "very confident". Figure 1. Of the 87 participants, 32 reported back on their CTC after at least 1 month. Of these, $88 \%$ fulfilled their commitment.

CONCLUSIONS: TeamSTEPPS training helped participants feel more comfortable in being effective team members and in speaking up.

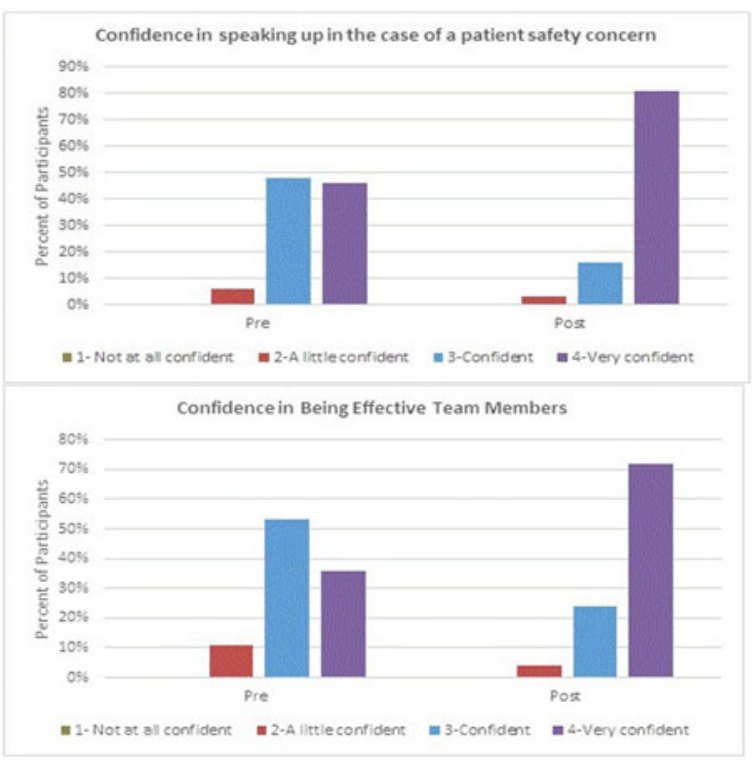

Figure 1

KNOWLEDGE, ATTITUDES, AND BELIEFS ASSOCIATED WITH HIV PREEXPOSURE PROPHYLAXIS (PREP) UPTAKE AMONG PRIMARY CARE PHYSICIANS Oni J. Blackstock ${ }^{1}$; Brent A. Moore ${ }^{5}$; Gail Berkenblit ${ }^{2}$; Sarah K. Calabrese $^{4}$; Chinazo Cunningham ${ }^{1}$; David A. Fiellin ${ }^{5}$; Viraj V. Patel ${ }^{1}$; Karran A. Phillips $^{3}$; Minesh Shah ${ }^{6}$; Jeanette M. Tetrault ${ }^{5}$ E. J. Edelman ${ }^{5} .{ }^{1}$ Albert Einstein College of Medicine \& Montefiore Medical Center, Bronx, NY; ${ }^{2}$ Johns Hopkins, Baltimore, MD; ${ }^{3}$ National Institute on Drug Abuse, National Institutes of Health, Baltimore, MD; ${ }^{4}$ Yale University, New Haven, CT; ${ }^{5}$ Yale University School of Medicine, New Haven, CT; ${ }^{6}$ University of Illinois, Chicago, Chicago, IL. (Control ID \#2469508)

BACKGROUND: Oral HIV pre-exposure prophylaxis (PrEP), the daily use of oral antiviral medications in conjunction with screening for HIV and other sexually transmitted infections and counseling for risk reduction and adherence, is a new, evidence-based HIV prevention strategy. Despite its demonstrated efficacy, uptake of PrEP has been low 
among eligible individuals. Given their central role in disease prevention, primary care physicians (PCPs) are optimally positioned to increase uptake by educating and offering PrEP to their patients. Yet, little is known about how PCP's knowledge, attitudes, and beliefs may be associated with their PrEP uptake.

METHODS: From April to May 2015, we conducted an online anonymous survey among a convenience sample of Society of General Internal Medicine members. The survey collected data about self-rated knowledge, attitudes, and beliefs about PrEP and PrEP uptake (defined as prior prescription or referral for PrEP [yes/no]). We conducted ttests to assess the association between PrEP-related knowledge ( $1=$ poor to $5=$ excellent $)$, attitudes and beliefs ( $1=$ not at all to $4=$ extremely), and PrEP uptake.

RESULTS: Among our sample $(n=266), 33 \%$ reported PrEP uptake with $19 \%$ having ever prescribed PrEP and $18 \%$ having ever referred a patient for PrEP. Mean age (standard deviation) was 41 (10) years. Most participants were White (72\%), female (62\%), heterosexual (92\%) and attending physicians (vs. fellows or residents) (75\%). Fifty percent were in clinical practice less than 10 years, $75 \%$ provided care to at least one HIVpositive patient, and $35 \%$ practiced at clinics with on-site HIV care. Compared with those who did not report PrEP uptake, participants who reported PrEP uptake endorsed greater self-rated knowledge of PrEP ( $3.4 \pm 1.0$ vs. $2.5 \pm 1.0, p<0.001)$ and its side effects $(3.3 \pm$ 1.2 vs. $2.2 \pm 1.2, p<0.001)$, greater perceived safety of PrEP $(3.2 \pm 0.6$ vs. $3.0 \pm 0.5$, $p<0.001)$, greater comfort with prescribing $\operatorname{PrEP}(3.0 \pm 1.0$ vs. $2.1 \pm 1.0, p<0.001)$, and greater perceived importance of PrEP in combination with condoms ( $3.2 \pm 0.7$ vs. $2.9 \pm$ $0.8, p<0.001)$. Compared to those who reported PrEP uptake, those who did not report PrEP uptake endorsed greater belief that PrEP increases risk behaviors ("risk compensation”) ( $2.1 \pm 0.8$ vs. $1.9 \pm 0.7, p=0.007)$. PrEP’s perceived effectiveness was not significantly associated with PrEP uptake $(p=0.97)$.

CONCLUSIONS: Only one-third of PCPs reported PrEP uptake. Compared to PCPs who did not report PrEP uptake, those who reported PrEP uptake endorsed greater selfrated knowledge, comfort prescribing, and perceived benefit of PrEP in combination with condoms. In contrast, PCPs who did not report PrEP uptake more strongly believed that PrEP use led to risk compensation. Efforts to expand PrEP uptake should focus on improving PCP knowledge about PrEP, including its safety, as well as the potential benefits of PrEP in combination with condom use. Importantly, as risk compensation was not observed in any PrEP clinical trials or studies of PrEP in real-world clinical practice, misconceptions among PCPs about risk compensation related to PrEP must be addressed.

LABORATORY TESTING: UNDERSTANDING THE PATIENTS' PERSPECTIVE Takae Brewer; Maryam Sattari. University of Florida, Shawnee, KS. (Control ID \#2431976)

BACKGROUND: Colorectal cancer (CRC) is the third-leading cause of cancer death for adults in the USA. Despite consensus recommendations for screening, only $59 \%$ of eligible adults have undergone screening. Similarly, despite the 2006 Centers for Disease Control and Prevention (CDC) guidelines for standardized, non-targeted opt-out HIV testing for individuals aged 13 to 64 years in all healthcare settings, these guidelines have not been universally adopted. Both CRC and late HIV diagnosis are associated with increased mortality and healthcare costs. We conducted this survey study to determine rates of colonoscopy in our outpatient internal medicine clinic and to assess patients' believes about colonoscopies and routine laboratory testing.

METHODS: The study was approved by the IRB at the University of Florida. As part of a 26-part questionnaire to assess patient's attitudes about colonoscopies, we developed 3 items to assess patient's believes about the nature of routine labs ordered by their health care providers: (1) "I think my doctor checks me for colon cancer every time he/she checks my blood," (2) "I think my doctor checks me for diabetes every time he/she checks my blood," and (3) "I think my doctor checks me for HIV/AIDS every time he/she checks my blood." Participants were asked to choose either "strongly agree," "agree," "neither agree nor disagree," "disagree," or "strongly disagree," in response to these items. All patients visiting our internal medicine clinic were eligible to participate. Participation in the study was anonymous and voluntary. We analyzed the data to determine patients' believes about routine laboratory testing, including testing for HIV, colorectal cancer, and diabetes mellitus. RESULTS: Seventy-eight patients participated in the study. The average age of the patients was 52.8 years (range 19-87). Sixty-one participants (78.2\%) reported a flu vaccination within the past 12 months and $45(84.9 \%)$ of those over 50 reported having had a colonoscopy. Forty-seven participants (50.3\%) either "disagreed" or "strongly disagreed" that routine laboratory results detect colorectal cancer (CRC). Thirty-two $(41.0 \%)$ selected similar options for diabetes (DM) and 39 (50\%) for HIV (Table 1). The selection "neither agree nor disagree" was chosen by 24 (30.8\%) for CRC, 22 (28.2 \%) for DM, and 26 (33.3\%) for HIV. Five participants (6.4\%) agreed or strongly agreed that routine laboratory testing detects CRC, $20(25.6 \%) \mathrm{DM}$, and $10(13.3 \%) \mathrm{HIV}$. CONCLUSIONS: While we found higher than expected rates for health maintenance behavior (colonoscopy and flu vaccination) in our study population, we were surprised by the patients' incomplete understanding of routine laboratory tests. The incorrect assumptions about routine laboratory testing might lead to false reassurance and influence patient's decisions not to undergo appropriate screening procedures (e.g. CRC screening), disclose high-risk behavior to their providers, alter high-risk behavior, or seek appropriate STD testing. These factors can potentially contribute to delayed diagnosis of disease (e.g. CRC or HIV). Our study highlights the need for better communication between healthcare providers and patients about the laboratory tests they order as well as the results of those tests.

LACK OF INDEPENDENT AMBULATION ABILITY OR REQUIREMENT OF INVALID DIET BEFORE ADMISSION CAN PREDICT POOR PROGNOSIS OF INPATIENTS OF 90 YEARS OLD AND OVEr Hidetoshi Aihara ${ }^{2}$; Masaki Tago ${ }^{1}$; Naoko E. Furukawa ${ }^{3}$; Yoshinori Tokushima ${ }^{5}$; Shu-ichi Yamashita ${ }^{4}$; masanori nishiyama ${ }^{6}$. ${ }^{1}$ Saga Medical School Hospital, Saga, Japan; ${ }^{2}$ Saga University Hospital, Saga, Japan; ${ }^{3}$ Saga University Hospital, Saga-shi, Japan; ${ }^{4}$ Saga medical university, Saga, Japan; ${ }^{5}$ saga university, Kashima, Japan; ${ }^{6}$ YuaiKai Foundation \& Oda Hospital, Kashima, Japan. (Control ID \#2454927)

BACKGROUND: In step with progressive growth of the elderly population in Japan, the ratio of highly aged inpatients is increasing in Japan. When hospitalizing markedly aged patients, it is imperative to predict their prognosis, which has a major impact on the option of treatments along with the diagnosis itself, presence of dementia and malignancies, or their activity of daily living.

METHODS: We retrospectively analyzed charts of all hospitalized patients of 90-yearold and over in the department of Internal Medicine of Yuai-kai Foundation and Oda Hospital, Saga, Japan from April 2013 to June 2014, total 15 months. We divided the patients into two groups: Group A; those who died during hospitalization, and Group B; those who were discharged alive. By investigating both groups on age, sex, vital signs and laboratory data on admission, walking ability, requirement of invalid diet before admission, presence of referral letters from other hospitals, use of ambulances, and presence of Do-Not-Resuscitate order on admission, we clarified the relationship between above mentioned factors and patients' death in hospital.

RESULTS: A total of 261 Japanese patients were enrolled (men; $28 \%$, age; $94.0 \pm$ 3.0 years). Forty-three patients (16.5\%) died in our hospital (Group A), and 218 patients $(83.5 \%)$ were discharged alive (Group B). By chi-square test, group A had higher rate of the patients lacking walking ability (odds ratio (OR) 2.19, 95\% CI 1.10-4.37, $p=0.024$ ), requiring invalid diet (OR 2.11,95\% CI 1.06-4.21, $p=0.032$ ), and having altered mental 
status (OR 2.88, 95\%CI 1.45-5.70, $p=0.002$ ) than group B. By independent sample ttest, group A had significantly lower levels of blood pressure (systolic; $120.9 \pm 23.8 \mathrm{vs}$. $133.9 \pm 29.2 \mathrm{mmHg}, p=0.007$, diastolic; $66.3 \pm 14.7$ vs. $71.0 \pm 16.1 \mathrm{mmHg}, p=0.004)$, oxygen saturation $(93.1 \pm 5.6$ vs. $95.1 \pm 3.2 \%, p=0.030)$, hemoglobin $(10.6 \pm 2.0$ vs $11.3 \pm 1.9 \mathrm{mg} / \mathrm{dl}, p=0.038)$, platelets $\left(16.4 \pm 8.0\right.$ vs. $\left.19.3 \pm 6.7 \times 10^{4} / \mathrm{mm}^{3}, p=0.031\right)$, total protein $(6.2 \pm 0.9$ vs. $6.6 \pm 0.7 \mathrm{~g} / \mathrm{dl}, p=0.002)$ and albumin $(3.0 \pm 0.6 \mathrm{vs} .3 .4 \pm 0.5 \mathrm{~g} /$ $\mathrm{dl}, p=0.001)$, and significantly higher levels of pulse rate $(92.9 \pm 22.3$ vs. $83.9 \pm 18.1$ $/ \mathrm{min}, p=0.005)$, C-reactive protein $(8.55 \pm 7.72 \mathrm{vs} .4 .31 \pm 5.89 \mathrm{mg} / \mathrm{dl}, p=0.001)$ and blood urea nitrogen $(33.9 \pm 22.5$ vs. $25.7 \pm 15.9 \mathrm{mg} / \mathrm{dl}, p=0.007)$.

CONCLUSIONS: Lack of independent ambulation ability or requirement of invalid diet before admission can be a predictor of in-hospital death of patients of 90 years old and over in Japan.

\section{LANGUAGE USED IN MEDICAL RECORDS MAY REFLECT} (UNCONSCIOUS) BIAS Mary Catherine Beach ${ }^{1}$; Katie O'Conor ${ }^{1}$; Carlton Haywood $^{3}$; Sophie Lanzkron ${ }^{1}$; Somnath Saha ${ }^{2} .{ }^{1}$ Johns Hopkins University, Baltimore, MD; ${ }^{2}$ Portland VA Medical Center, Portland, OR; ${ }^{3}$ The Johns Hopkins School of Medicine, Baltimore, MD. (Control ID \#2470492)

BACKGROUND: Notes within medical records may reflect healthcare providers' conscious or unconscious biases, misconceptions, and stigmatizing attitudes. Language used by one provider can in turn influence the attitudes of subsequent providers caring for the patient. Although this may occur in any clinical setting, the acute treatment of persons with sickle cell disease (SCD) - who are typically African American and in need of opioids to manage painful crises - is particularly laden with interpersonal tensions, biases, and negative attitudes. We sought to characterize how these tensions and biases might manifest within the language of SCD patient medical records.

METHODS: We conducted a discourse analysis of language contained within the emergency department records of 25 SCD patients who reported experiencing discrimination, and the inpatient medical records of $57 \mathrm{SCD}$ patients who were admitted within the study period. Two authors reviewed language within the progress notes written by nurses and physicians, and abstracted any comments that might reflect potential biases. We then synthesized these comments and organized them into themes agreed upon by consensus among research team members.

RESULTS: We identified four themes where language was used that might convey negative perspectives: 1) casting doubt on the validity of patients' pain, 2) perpetuating negative stereotypes, 3) blaming a patient for their symptoms, and 4) equating a patient with their disease. Doubt on validity of patients' pain was conveyed directly ("patient displays 'narcotic seeking behavior"') or indirectly by juxtaposing patient reports of pain with discrediting comments ("patient chatting on phone, requesting pain medication"). Providers perpetuated negative stereotypes overtly by describing patients in unpleasant terms ("Patient observed to be screaming, cursing, and verbally abuse to staff" or "Patient snoring loudly") or more subtly by using quotation marks to highlight patient comments that reveal low education or health literacy and make the patient appear unsophisticated ("patient not able to keep food down because 'it goes straight through me in the diarrhea'"). Providers can convey blame when they highlight patients' nonadherence or cooperativeness ("patient found not wearing mandatory oxygen" or "patient declared she wanted to leave AMA [against medical advice]"). Finally, patients are depersonalized with terms that conflate the person with the disease identity ("sickler" vs. "woman with sickle cell disease").

CONCLUSIONS: Bias towards patients can be found in the medical record in both overt and subtle ways that capitalize on the illusion of clinician objectivity. Although it is natural for physicians and nurses to have both positive and negative reactions to patients, and legitimate medical reasons to document concerns about drug addiction and nonadherence, the effect that these reactions have on how information is transmitted in the medical record, and how this information influences others caring for these patients, may be unrecognized. We suggest that language use in medical records should be more carefully considered, and potential biases should be acknowledged and addressed.

LEADERSHIP CURRICULA IN HEALTH DISPARITIES: WHAT DO STUDENTS LEARN IN STUDENT- INITIATED VS. ASSIGNED FIELD EXPERIENCES? Joy Williams; Andy Haig; Patricia Mullan; Brent C. Williams. University of Michigan, Ann Arbor, MI. (Control ID \#2466236)

BACKGROUND: Leadership training is now advocated as an essential component of health professions' education. While most leadership curricula are based on 'best concepts' from business or organizational leadership literature $(1,2)$, optimal curricula include self-perceived needs assessment of targeted learners (3). Our leadership curriculum is based on experiential learning with reflection-on-action, consistent with adult learning theory. To inform our leadership curriculum we sought to describe medical students' perceptions of high-value leadership lessons derived from field experiences. We were particularly interested in determining whether lessons derived from experiences designed by the students themselves differed from those in program-assigned projects.

METHODS: Ninety second-year medical students in the University of Michigan Global Health and Disparities Path of Excellence during 3 academic years (2012-15) formed teams to complete field projects. In Years 1 and 3 students pursued student-directed questions of interest through engagement with an organization or person in health disparities selected by the students. In Year 2 we experimented with a different model, in which teams collaborated with pre-selected community-based organizations (CBOs) to deliver a 'product' useful to the CBO. In all years students were introduced to the GRP (Goals, Roles, Processes, and Interpersonal Relations) model of project development (4) Content analysis of team reports was performed by two authors (5). Open-text narratives described "lessons in physician leadership" in Years 1 and 3 and "lessons/reflections" in Year 2, the subject of this report. Year 2 reports also included a structured narrative analysis using the GRPI categories. Open-text responses from all years were abstracted and phrases describing lessons in leadership were extracted. Responses were sorted into categories by consensus among the authors.

RESULTS: In all years students identified 3 categories (with representative concepts and phrases) of leadership themes: 1) Personal Commitment (finding inspiration, continuing through failure; “...work in health disparities requires a lot of passion and commitment”; 2) Personal Leadership Skills (motivating others, team building; "fight the urge to micromanage!"); and 3) Organizational Leadership (strategic planning, coalition building; "identify the problem before creating an unnecessary solution", "importance of involving all stakeholders"). In Years 1 and 3 four additional categories were identified: 4) Career Development (choosing a specialty, scholarship; "it is important to connect with physicians outside your field"); 5) Work-Life Balance (sacrificing or postponing family commitments; "there is no work-life balance (in global health\}"); 6) Interdisciplinary Work (working with non-physicians; "medicine...is a team sport"); and 7) PatientCentered Care (maintaining commitment to individual patients as well as populations; "a commitment to providing quality care to every patient").

CONCLUSIONS: Medical students' high-value learning in health disparities leadership occurs across a broader range of domains than curricula based on business models. Students' leadership learning appears inextricably combined with their exploration personal values and characteristics, and of career paths and options. Student-directed, field-based projects allow students to learn across a wider range of domains than program-directed curricula. These findings, if validated in additional cohorts of students at our and other institutions, suggest that leadership curricula should provide opportunities for students to explore both inner and outer paths, and include exposure to role models, opportunities for testing skills in collaboration and organizational development, 
and reflection. Other areas of leadership learning of high value to medical students may be identified in future research. 1. Webb AMB, Tsipis NE, McClellan TR, McNeil MJ, et al. A first step toward understanding best practices in leadership training in undergraduate medical education: A systematic review. Acad Med 2014; 89:1563-70. 2. Academy of Medical Royal Colleges. Medical Leadership Competency Framework: Enhancing Engagement in Medical Leadership. 3rd ed. Coventry, UK: NHS Institute for Innovation and Improvement; 2010. 3. Thomas PA, Kern, DE, Hughes, MT, Chen BY. Curriculum Development for Medical Education: A Six-Step Approach. 3rd ed. Johns Hopkins University Press; 2015. 4. Raue S, Tang S-H, Weiland C, Wenzlik C. The GRPI Model—An approach for team development. Systemic Excellence Group. Independent Think Tank for Leading Practice. Feb 2, 2013 5. Hsieh H-F, Shannon SF. Three approaches to qualitative content analysis. Qualitative Health Research 2005; 15:1277-88.

\section{LEARNING PATIENT-CENTERED PERSPECTIVES IN FRAGMENTED} HEALTHCARE SYSTEMS: A QUALITATIVE ANALYSIS OF MEDICAL STUDENT PERCEPTIONS OF VALUE-ADDED ROLES WITHIN THE HEALTHCARE SYSTEM Jed Gonzalo; Daniel R. Wolpaw; Deanna Graaf; Britta M. Thompson. Penn State College of Medicine, Hershey, PA. (Control ID \#2470300)

BACKGROUND: Despite recommendations for enhancing skills in Health Systems Science (HSS) and creating value-added roles for students, early experiential roles still primarily focus on the clinical sciences. In contrast to these experiences that are primarily designed to enhance learning in physical examination and communication skills, valueadded roles, defined as “...experiential roles that can positively impact health outcomes while also enhancing student education," have been proposed as a method to develop students' skills and knowledge in HSS. While this is intuitively appealing, there has been little in the way of published reports on these kinds of programs that would be helpful in critically appraising the rationale and outcomes. Following implementation of a largescale program to embed students in value-added roles, we explored the question-What do students perceive as educationally valuable in value-added roles?

METHODS: In the 2014-15 ( $n=85$ students) and 2015-16 ( $n=138$ students) academic years, our 1st-year students have been integrated into interprofessional care teams in health systems to serve as patient navigators. Activities include being linked with patients to identify root causes preventing optimal health, and exploring action plans to improve health, including medication reconciliation, home safety assessments, coordinating communication during transitions, and motivational interviewing to improve adherence. Following each experience, students completed an electronic log about the patient, barriers to care, activities undertaken, and educational benefits. In May 2015, we additionally performed six, one-on-one, audio-recorded student interviews to explore educational benefits. Using a thematic analysis with several grounded theory techniques, investigators analyzed an initial transcript, compared codes for inconsistency/agreement, and jointly updated the codebook. Remaining transcripts were independently coded, with regular adjudication sessions. Disagreements were discussed, additional codes created/collapsed, and final themes identified.

RESULTS: Between September 2014-January 2016, a total of 769 logs (307 in Year 1, 485 in Year 2) were completed about 363 patients, and six interviews were completed (range 35-85 min); three students were male and three were female. Students reported seven different themes related to the educational benefits from value-added roles as patient navigators. These included: (1) enhanced understanding and appreciation for the patients' perspective on healthcare and his/her health, (2) barriers and social determinants of health, (3) healthcare delivery and system, (4) presence and need for interprofessional collaboration and teamwork, (5) health system improvement needs, (6) clinical medicine (e.g. diagnosis and therapeutics), and, (7) approach to communicating with patients.
CONCLUSIONS: These results suggest that embedding students in value-added roles has great potential to enhance learning in Health Systems Science concepts, including population and public health, social determinants of health, health system delivery, valuebased care, and teamwork. With the changes in the expectations of clinical environments, healthcare organizations are increasingly regarding HSS and team skills as essential physician training. Based on these results, students engaging in patient-centered systems roles were provided with first-hand learning opportunities that allowed them to engage in experiential learning of HSS. Value-added roles focused on HSS can potentially begin to shift professional role formation in the direction of patient-centered, collaborative care models aimed at achieving better patient outcomes.

LEDIPASVIR-SOFOSBUVIR BASED THERAPY FOR HEPATITIS C HAS HIGH EFFICACY BUT POTENTIAL FOR RENAL IMPAIRMENT Omar Sadiq $^{1}$; Adrienne Lenhart ${ }^{1}$; William J. Kane ${ }^{2}$; Alexander Weick ${ }^{1}$; Vinay Kathukuri ${ }^{3,1}$; Syed-Mohammed Jafri ${ }^{3,} 1$. ' Henry Ford Hospital, Detroit, MI; ${ }^{2}$ Wayne State University, Detroit, MI; ${ }^{3}$ Henry Ford Health System, Detroit, MI. (Control ID \#2469723)

BACKGROUND: Ledipasvir-sofosbuvir combination pill for the treatment of Hepatitis C was approved for use by the FDA in October, 2014. The use of the drug is not indicated in patients with severe renal impairment due to its renal excretion. There is limited data studying the effects of ledipasvir-sofosbuvir on renal function in patients with normal or mildly impaired renal function.

METHODS: All patients without history of transplant treated with ledipasvir-sofosbuvir therapy between January 1st, 2015 and October 1st, 2015 were evaluated for efficacy of therapy at a single center. Detailed demographic and laboratory evaluation was reviewed for all patients. Patients reaching 12 weeks' post-therapy were included. Sustained viral response (SVR) was evaluated at weeks 4 (SVR4) and 12 (SVR12) after treatment. Glomerulus filtration rate (GFR) was calculated using the Modification of Diet in Renal Disease (MDRD) equation.

RESULTS: Five hundred six patients were started on treatment, 82 completed therapy and were at least 12 weeks out from treatment with appropriate laboratory follow up. Fifty-nine percent were male, mean age was 61 (range 34-86) and mean BMI was 28.19 (range 15.36-37.29). Thirty-nine percent were African American and $46 \%$ white. Fifty-four percent had genotype 1a, $39 \%$ genotype $1 \mathrm{~b}$. Fifteen percent of patients were treated appropriately for 8 weeks, $73 \%$ for 12 weeks and the $12 \%$ for 24 weeks. Eleven percent were treated with 12 weeks of therapy in combination with ribavirin. Twenty-nine patients $(35 \%)$ had cirrhosis. Seventy-seven patients (94\%) achieved SVR12. 40 (98\%) of patients with cirrhosis achieved SVR12. Five patients failed therapy. Four were on 12 weeks of ledipasvir-sofosbuvir alone, one had addition of ribavirin. No patients treated for 8 weeks due to treatment naïve with viral load $<6$ million failed therapy. 33 (40\%) patients were previous treated, of which 7 were previously treated with boceprevir and 3 with telaprevir. 32 (97\%) of previously treated patients achieved SVR12. 14 (100\%) previously treated cirrhotics achieved SVR12. One patient with prior treatment failure on boceprevir failed ledipasvir-sofosbuvir therapy. Average reduction in hemoglobin without ribaviran was $1.1 \mathrm{~g} / \mathrm{dL}$. Average reduction in hemoglobin on ribavirin was $2.0 \mathrm{~g} /$ dL. $27(33 \%)$ patients had initial reduction in GFR by $25-50 \% .5(19 \%)$ patients recovered function by completion of therapy. 3 (4\%) patients, including 1 cirrhotic, had initial reduction in GFR greater than $50 \%$. GFR for all these patients improved but did not completely recover by the end of treatment.

CONCLUSIONS: In our initial experience, combination therapy of ledipasvir-sofosbuvir is well tolerated in the management of HCV. Therapy achieved high rates of SVR with low rates of acute complication with or without ribavirin. This regimen was $94 \%$ effective at achieving SVR12 in our population. Despite over a third having a transient worsening of renal function 
during therapy, none of our patients had over $50 \%$ reduction of GFR by the end of therapy. Longer term follow-up will reveal if renal function recovers after completion of therapy.

LEFT ATRIAL APPENDAGE EXCLUSION IN PATIENTS WITH ATRIAL FIBRILLATION IS ASSOCIATED WITH A DECREASED RISK OF STROKE William T. Love ${ }^{1}$; Victor A. Abrich ${ }^{2}$; Aalap D. Narichania ${ }^{1}$; Dan Sorajja ${ }^{2} .{ }^{1}$ Mayo Clinic Arizona, Phoenix, AZ; ${ }^{2}$ Mayo Clinic Arizona, Scottsdale, AZ. (Control ID \#2465568)

BACKGROUND: Atrial fibrillation is the most common cardiac arrhythmia worldwide and is becoming increasingly prevalent due to improved survival of an aging population. It is associated with up to a five-fold increased risk of ischemic stroke due to thrombus formation in the left atrium, with resultant embolism to cerebral circulation accounting for $15-20 \%$ of all ischemic strokes. Surgical left atrial appendage exclusion (LAAE) is often performed with the intention of decreasing the risk of stroke in patients with atrial fibrillation (AF) who are undergoing cardiac surgery; however its effect on stroke risk remains controversial due to limited data. The objective of this study was to determine if, through retrospective chart review, a benefit could be identified in the reduction of stroke or transient ischemic attack (TIA) following LAA exclusion during cardiac surgery in patients with a known history of atrial fibrillation.

METHODS: This was a retrospective cohort study assessing patients from Mayo Clinic Arizona and Rochester with AF who had undergone coronary artery bypass graft surgery or mitral valve surgery between 2001 and 2014. Patients were screened electronically using ICD-9 codes. The first 500 patients that met the inclusion criteria were selected for analysis. The Chi-Square test and student's t-test were used to calculate $p$-values for differences between the two groups. A Cox proportional hazards regression model was used to derive the hazard ratio (HR) and corresponding $95 \%$ confidence intervals (CI) for the association between LAAE and stroke/TIA via univariate and multivariate analysis. RESULTS: Among patients who underwent LAAE, there were more females and a lower prevalence of hypertension, diabetes mellitus, systolic dysfunction and vascular disease compared to patients who did not undergo LAAE. More patients in the former group were on anticoagulation and their mean CHA2DS2VASc score was lower (3.1 vs 3.6, $p=$ 0.0008). A total of 6 cerebrovascular events occurred in the LAAE group compared to 16 cerebrovascular events in the non-LAAE group $(\mathrm{HR}=0.33[0.12-0.80], p=0.0129)$. This difference persisted in the multivariate analysis, controlling for anticoagulation use and CHA2DS2VASc score $(\mathrm{HR}=0.34$ [0.12-0.84], $p=0.0192)$.

CONCLUSIONS: In this retrospective study of 500 patients with atrial fibrillation, LAAE was associated with a significantly decreased risk of stroke and TIA within the first year. Although there were important baseline differences between each cohort, the hazard ratio remained significant in our multivariate analysis. Our data may support routine $\mathrm{LAAE}$ in patients with $\mathrm{AF}$ undergoing cardiac surgery. Prospective, randomized data are needed to confirm our findings.

LENGTH OF STAY AND COST-CONSCIOUS CARE IN SYNCOPE ADMISSIONS AT A TERTIARY HOSPITAL Elena Gkrouzman ${ }^{1}$; Jeffrey Kluger ${ }^{2}$. ${ }^{1}$ UCONN Health, Hartford, CT; ${ }^{2}$ Hartford Hospital, Hartford, CT. (Control ID \#2437149)

BACKGROUND: Syncope comprises a considerable percentage of emergency department (ED) visits and hospitalizations with significant implications on costs related to hospital stay and number of unnecessary tests or consultations performed. In an effort to administer evidence-based but also cost-conscious care, the effect of the introduction of a syncope order set and of a series of educational activities on syncope were assessed with particular focus on length of hospital stay.

METHODS: This is a retrospective, case-control study. Patients of a large, tertiary, teaching hospital with admission and discharge diagnosis of syncope were identified for two time periods, 1 year before $(n=167)$ and after $(n=210)$ the intervention. Patients could have been admitted under Cardiology, Hospitalist, Housestaff or Other service. The intervention consisted of the introduction of a syncope order set based on the OESIL risk score, in addition to a series of educational lectures on syncope management. The length of stay (LOS) of the identified patients was compared before and after the intervention.

RESULTS: The LOS of patients with admission and discharge diagnosis of syncope was calculated to be significantly lower after the intervention among all services combined [mean LOS before: 3 days (95 \% CI 2.6-3.3); mean LOS after: 2.4 days (95\% CI 2.1-2.5); $p=0.045]$. When broken into different services, Hospitalist's service achieved a significant reduction in $\operatorname{LOS}(p=0.026)$ compared to Cardiology $(p=0.38)$ and Housestaff $(p=0.41)$. The syncope order set in the Hospitalist service was not as heavily used (15\% of admissions) as in Cardiology (47\% of admissions) and Housestaff (38\% of admissions) underlining the importance of educational activities in reducing LOS in syncope admissions.

CONCLUSIONS: Education on syncope management based on current guidelines and use of a syncope order set for admission of patients with syncope as chief complaint may reduce length of stay and associated costs in tertiary hospitals.

LEVERAGING COMMUNITY HEALTH WORKERS TO SCREEN AND MANAGE HYPERTENSION IN A REMOTE TRIBAL REGION OF INDIA Sujatha Sankaran ${ }^{1}$; Prema Ravi ${ }^{2}$; James Harrison ${ }^{1}$; Raman Khanna ${ }^{1} .{ }^{1}$ University of California San Francisco, Mill Valley, CA; ${ }^{2}$ Tribal Health Initiative, Sittilingi, India. (Control ID \#2467359)

BACKGROUND: Adverse outcomes due to hypertension are the leading causes of death and disability in the world, including under-resourced regions Numerous studies have implicated hypertension as causing a tremendous amount of morbidity and mortality in India, where the leading cause of death is cardiovascular disease. Community health workers (CHWs) with little formal education have been identified as a key component of successful public health interventions in India and other developing nations that have a shortage of trained medical personnel. CHW models offer an excellent opportunity for effecting systems-wide change in hypertension-related burden of disease by training CHWs to diagnose and manage chronic hypertension with limited interaction from higher-level clinicians. We investigated whether diagnosis and management of hypertension provided by trained CHWs with limited physician interaction is an effective mechanism for achieving hypertension control in an under-resourced rural region of Southern India.

METHODS: We partnered with a local non-governmental organization in the Sittilingi Valley of Southern India, the Tribal Health Initiative, to train 24 literate and semi-literate CHWs with no previous chronic disease identification and management experience to diagnose, manage, and refer hypertensive patients appropriately. We worked with local nurses to train the CHWs to check blood pressure, document blood pressure values in a log, refer patients with uncontrolled hypertension to the central clinic, and provide patients with well-controlled hypertension with continuing medications and lifestyle advice. After a 7-month training program that included didactics and practical teaching about blood pressure measurement and medical management, the CHWs began the hypertension intervention with patients in their villages and their progress was assessed after 1 year. Every week, visiting nurses provided evaluation and monitoring of CHWs as they delivered hypertension care in the field to ensure blood pressure values were being adequately measured, documented, and managed. The primary outcome measured was blood pressure control at 1 year. 
RESULTS: After a year of this program, CHWs have screened 598 patients over age 60 and 245 patients have been identified as hypertensive, defined as blood pressures greater than 140/90. We found a baseline hypertension prevalence rate of $44 \%$ in the over-60 population. All patients who were initially screened as hypertensive received initial evaluation and medication initiation by a physician and were then followed in the field by CHWs. The CHWs only referred patients with uncontrolled hypertension back for physician management. After 1 year, $51.8 \%$ of hypertensive patients had blood pressures less than 140/90. An average of 82 days were needed to first achieve blood pressure control in hypertensive patients, an average of 2.8 visits were needed to first achieve blood pressure control, and $12 \%$ of patients never achieved blood pressure control up to 11 visits later.

CONCLUSIONS: A teaching program educating community health workers about hypertension followed by screening, diagnosis, and management of hypertension by community health workers in the field is a feasible mechanism for achieving hypertension control in a remote tribal community in India. These promising results show that community health worker training is a potentially good avenue for decreasing hypertension rates in remote global settings with physician shortages.

\section{LOCAL IMPRESSIONS OF THE EXPATRIATE PHYSICIAN AND TRAINEE} WORKFORCE IN MALAWI: A QUALITATIVE STUDY Natasha Parekh ${ }^{3,4}$; Adam P. Sawatsky ${ }^{1}$; Ihunanya Mbata ${ }^{5}$; Adamson Muula ${ }^{6}$; Thuy Bui ${ }^{2} .{ }^{1}$ Mayo Clinic, Rochester, $\mathrm{MN} ;{ }^{2}$ University of Pittsburgh, Pittsburgh, PA; ${ }^{3}$ University of Pittsburgh Medical Center, PIttsburgh, PA; ${ }^{4}$ University of Pittsburgh School of Medicine, Pittsburgh, PA; ${ }^{5}$ Oak Ridge Methodist Medical Center, Oak Ridge, TN; ${ }^{6}$ University of Malawi College of Medicine, Blantyre, Malawi. (Control ID \#2467689)

BACKGROUND: Expatriate physicians and trainees serve diverse roles abroad in clinical care, education, mentorship, and research, and a significant proportion of physicians from high-income countries have global health experiences. Despite published benefits of global health experiences for expatriates, little is known about host physician and trainee impressions of their expatriate counterparts. The goal of this study was to explore University of Malawi College of Medicine (CoM) physicians' and trainees' impressions toward expatriate physicians and trainees.

METHODS: We conducted a cross-sectional qualitative study using semi-structured interviews with CoM medical students, interns, residents, and faculty at the two CoM teaching hospitals in Malawi: the Queen Elizabeth Central Hospital (QECH) in Blantyre, Malawi, and Kamuzu Central Hospital $(\mathrm{KCH})$ in Lilongwe, Malawi. For residents and faculty, we sampled from across several specialties to obtain a broad perspective. Interviews were audio-recorded and transcribed verbatim. Through open coding and shared adjudication of differences, we developed a codebook that we applied to interview transcripts. Once coding was completed, we reviewed codes, performed thematic analysis that determined major domains and themes, and selected representative quotations.

RESULTS: We interviewed 46 participants comprising of $28 \%$ medical students, $26 \%$ interns, $22 \%$ residents, and $24 \%$ faculty physicians. Seventy-eight percent were from $\mathrm{QECH}$ and $22 \%$ were from $\mathrm{KCH}$. Of the faculty and registrars, $33 \%$ were from the Department of Medicine, $19 \%$ were from Pediatrics, $14 \%$ were from Obstetrics and Gynecology, $14 \%$ were from Surgery, $7 \%$ were from Ophthalmology, and $2 \%$ were from Anesthesia and Intensive Care. Sixty-five percent were male. Participants discussed themes within the following domains: perceived benefits of expatriate physicians and trainees in Malawi, perceived challenges, past contributions of expatriate physicians and trainees, and perceived roles that expatriate physicians should play going forward. Host faculty and trainees appreciated the approachability, enthusiasm, perspectives, and contribution to education that expatriates have provided to the CoM. They felt that expatriates offer diverse perspectives and clinical skills expertise, especially in teaching students varied approaches to diagnosis and management. Others commented that their presence has helped them feel connected to a "greater medical community," and have appreciated their roles in helping to relieve a dramatic physician shortage in the country. On the other hand, some expatriate physicians and trainees have been perceived as aggressive, unable to relate to patients and trainees, and deficient at adapting to the low-resource setting. Some have given the impression that they are in Malawi solely for their own academic benefit in terms of research. Potential roles that host physicians and trainees feel expatriates should serve include those in education, mentorship, clinical training, capacity-building, and the provision of exchange opportunities for local physicians and trainees.

CONCLUSIONS: This study highlights perceived benefits and challenges that physicians and trainees at the CoM have experienced with their expatriate counterparts, and suggests roles that expatriates should begin and continue. Our data can be used to help inform existing guidelines for expatriates abroad, assist with the establishment of host institution expectations for global health programs, and guide individual expatriate physicians who hope to optimize their roles abroad

LONGER-TERM SAFETY AND TOLERABILITY OF CANAGLIFLOZIN IN PATIENTS WITH TYPE 2 DIABETES: A POOLED ANALYSIS Dainius Balis ${ }^{1}$; $\underline{\text { Michael J. Davies }}{ }^{2}$; Mehul Desai ${ }^{1}$; Gary Meininger ${ }^{1}$; Rong Qiu ${ }^{1}$; John Xie ${ }^{1} .{ }^{1}$ Janssen Research \& Development, LLC, Raritan, NJ; ${ }^{2}$ Janssen Scientific Affairs, LLC, Raritan, NJ. (Control ID \#2468394)

BACKGROUND: Canagliflozin (CANA), a sodium glucose co-transporter 2 (SGLT2) inhibitor approved for the treatment of adults with type 2 diabetes mellitus (T2DM), lowers the renal threshold for glucose, thereby increasing urinary glucose excretion and resulting in a mild osmotic diuresis and net caloric loss. Across Phase 3 studies of 26 to 104 weeks in duration, CANA improved glycemic control, body weight, and systolic blood pressure, and was generally well tolerated, with an increased incidence of adverse events (AEs) related to the mechanism of SGLT2 inhibition. In this post hoc analysis, the longer-term safety and tolerability of CANA 100 and $300 \mathrm{mg}$ were evaluated in a broad population of patients with T2DM using pooled data from 7 placeboand active-controlled studies.

METHODS: Patients $(N=5598)$ received CANA 100 or $300 \mathrm{mg}$ or non-CANA (placebo, sitagliptin, or glimepiride) as monotherapy or in combination with various background antihyperglycemic agents over 52 or 104 weeks. Mean duration of exposure was $65.1,59.4$, and 56.4 weeks with CANA 100 and $300 \mathrm{mg}$ and non-CANA, respectively; 34,26 , and $22 \%$ of patients had $\geq 102$ weeks of exposure. Safety was assessed based on AE reports, with additional data collection prespecified for selected AEs.

RESULTS: The incidence of overall AEs, AEs leading to discontinuation, and serious AEs was similar across groups (Table). AEs with an incidence $\geq 5 \%$ are reported in the Table. Compared with non-CANA, CANA 100 and $300 \mathrm{mg}$ were associated with a higher incidence of AEs related to the mechanism of SGLT2 inhibition (eg, urinary tract infections, genital mycotic infections, and polyuria/pollakiuria). Volume depletionrelated $\mathrm{AE}$ rates were low across groups $(2.4,2.6$, and $1.8 \%$, respectively).

CONCLUSIONS: CANA 100 and $300 \mathrm{mg}$ were generally well tolerated in a broad population of patients with T2DM in studies up to 104 weeks in duration, with a safety profile consistent with previous studies. 
Table. Summary of Overall Safety and Common AEs

\begin{tabular}{|c|c|c|c|}
\hline Patients, n (\%) & $\begin{array}{l}\text { Non-CANA } \\
(n=2109)\end{array}$ & $\begin{array}{l}\text { CANA } 100 \mathrm{mg} \\
(n=1557)\end{array}$ & $\begin{array}{c}\text { CANA } 300 \mathrm{mg} \\
(\mathrm{n}=1932)\end{array}$ \\
\hline Overall AEs & $1555(73.7)$ & $1148(73.7)$ & $1440(74.5)$ \\
\hline AEs leading to discontinuation & $102(4.8)$ & $79(5.1)$ & $123(6.4)$ \\
\hline Serious AEs & $186(8.8)$ & $128(8.2)$ & $146(7.6)$ \\
\hline \multicolumn{4}{|l|}{ Most common AEs } \\
\hline Nasopharyngitis & $198(9.4)$ & $144(9.2)$ & $192(9.9)$ \\
\hline Urinary tract infection & $146(6.9)$ & $150(9.6)$ & $150(7.8)$ \\
\hline Vulvovaginal candidiasis & $35(1.7)$ & $116(7.5)$ & $138(7.1)$ \\
\hline Back pain & $97(4.6)$ & $90(5.8)$ & $108(5.6)$ \\
\hline Polyuria/pollakiuria & $39(1.8)$ & $88(5.7)$ & $101(5.2)$ \\
\hline Influenza & $84(4.0)$ & $81(5.2)$ & $89(4.6)$ \\
\hline
\end{tabular}

$\geq 5 \%$ in any treatment group

LOS OUTLIERS: A CHALLENGING PROBLEM FOR BOTH THE TEACHING AND PRIVATE NON-TEACHING GENERAL MEDICINE SERVICES AT STANFORD HOSPITAL Jeffrey Ketchersid ${ }^{1}$; Lisa Shieh ${ }^{1}$; Neera K. Ahuja ${ }^{4}$; Jeffrey $\mathrm{Chi}^{3}$; Jason Hom ${ }^{2} .{ }^{1}$ Stanford, Mountain View, CA; ${ }^{2}$ Stanford Health Care, Stanford, CA; ${ }^{3}$ Stanford School of Medicine, Sunnyvale, CA; ${ }^{4}$ Stanford University Medical Center, Palo Alto, CA. (Control ID \#2469558)

BACKGROUND: Given the high correlation between patient length of stay (LOS) and hospital cost, there has been significant interest in studying LOS outliers. Indeed, in a large-scale analysis of Australian public hospitals, LOS outliers were shown to have a dramatic effect on overall LOS statistics [1]. Prior research suggests that large teaching hospitals have a higher number of LOS outliers compared to other hospitals, with a variety of possible explanations including differences in $\mathrm{CMI}$ and socioeconomic factors [2, 3] While these prior studies have compared the proportion of LOS outliers between large teaching hospitals and other hospitals, we make a unique comparison within Stanford Hospital, where inpatients on General Medicine can be admitted to either the teaching service or a separate non-teaching service, with other features (such as nursing staff, therapy staff, accommodations and laboratory and radiology facilities) the same for both services.

METHODS: We obtained a dataset of all patients discharged from Stanford University Hospital between September 1, 2014 and March 31, 2015.We extracted basic hospitalization data for those patients admitted to the teaching and private non-teaching general medicine services. For each Diagnosis-Related Group, geometric means and standard deviations of length of stay were calculated. If an individual patient's length of stay was greater than the geometric mean plus two times the standard deviation for their Diagnosis-Related Group, they were defined as a high LOS outlier, using a previously validated definition [4]. Two-tailed t-tests were used for statistical comparisons.

RESULTS: Case Mix Index between patients on the university service and the private service was not statistically different. There were a total of 1868 discharges from the teaching service and 858 discharges from the private non-teaching service during the study period. Of these discharges, 121 (6.48 \%) of those discharged from the teaching service and $58(6.76 \%)$ of those discharged from the private nonteaching service were high LOS outliers. There was no difference in outlier proportion between the two services $(p=0.80)$. On the teaching service, high LOS outliers accounted for a total of 1660 hospital-days (20.2\%), compared to 748 hospital-days $(19.7 \%)$ on the private non-teaching service. There were a total of 303 patients with the most common DRG, severe sepsis with major complication. Of these, 10/201 (4.97\%) on the teaching service, and 6/102 (5.88\%) on the private non-teaching service were LOS outliers $(p=0.74)$. We also found no significant difference in outlier burden when restricting our focus to extreme outliers, defined as an LOS 4 standard deviations greater than the geometric mean. There were $14(0.75 \%)$ such outliers on the teaching service and $3(0.35 \%)$ on the private non-teaching service $(p=0.20)$. These extreme outliers represented $3.7 \%$ of hospital-days on the university service and $2.7 \%$ of hospital-days on the private service.

CONCLUSIONS: In general, our research confirms the disproportionate impact of LOS outliers, with less than $7 \%$ of the patients accounting for approximately $20 \%$ of total hospital-days. Moreover, there is no difference in the incidence of high LOS outliers between the teaching and private non-teaching general medicine services at Stanford Hospital, though there does seem to be a trend toward an increased number of extreme LOS outliers on the university service. Further research using both automated methods and manual chart review will be used to create multi-variable analyses exploring 1) the risk factors for LOS outliers at Stanford and 2) the effects of new interventions aimed to reduce them.

\section{LOW-INCOME COMMUNITIES WITH HIGH WELL-BEING: IDENTIFYING} POSITIVE DEVIANCE Anita S. Arora ${ }^{3}$; Erica S. Spatz ${ }^{2}$; Brita Roy ${ }^{2}$; Carley Riley ${ }^{4}$; Jeph Herrin ${ }^{1}$; Kenneth Kell ${ }^{5}$; Elizabeth Y. Rula ${ }^{5}$; Carter R. Coberley ${ }^{5}$; Harlan M. Krumholz $z^{2} .{ }^{1}$ Yale University, Charlottesville, VA; ${ }^{2}$ Yale University School of Medicine, New Haven, CT; ${ }^{3}$ Yale University, New Haven, CT; ${ }^{4}$ Cincinnati Children's Hospital, Cincinnati, OH; ${ }^{5}$ Healthways, Franklin, TN. (Control ID \#2467859)

BACKGROUND: Many studies have demonstrated that living in a poor neighborhood is negatively associated with overall well-being and its components of physical, mental and social health. However, poverty is not the only determinant of health and some low-income neighborhoods may report high well-being. Though collective well-being among lowincome communities may vary, this has not been studied. Finding variation and identifying poor communities with high-well-being may present an opportunity to characterize and share successful methods for improving well-being among low-income communities.

METHODS: We defined low-income communities as counties in the top quartile for poverty rates in the U.S. In these counties, the poverty rate was $>20.1 \%$, consistent with the U.S. Census definition of a poverty area. We characterized the well-being of lowincome counties using data from the 2010-2012 Gallup-Healthways Well-Being Index (GHWBI), a national survey of individuals $\geq 18$ years. The survey evaluates 6 domains of well-being: physical health; healthy behaviors; basic access (including perception of safety, access to housing and healthcare); work environment; emotional health; and life evaluation. Domain and composite well-being scores range from 0 to 100 , with 100 representing the highest well-being. County-level scores were derived from the average scores of respondents residing in that county. We divided low-income counties into those with high well-being and those with low well-being, based on whether county well-being was above or below the median well-being score in the U.S. We examined the distribution of well-being and each of the domain scores within the high and low well-being groups. We then used standardized differences to explore which domain scores varied the most between the high and low well-being groups.

RESULTS: Among 770 low-income counties, the well-being score ranged from 46.2 to 81.3 (mean 64.4, SD 4.4). This was compared to all counties in the U.S. for which the well- 
being score ranged from 39.9 to 90.6 (mean 66.5, SD 5.4). The median well-being score among all counties in the U.S. was 66.7. There were 217 low-income counties with wellbeing scores above this median (high well-being group) and 553 low-income counties with well-being scores below this median (low well-being group). All of the 6 domain scores were higher in the high well-being group, but differences were not uniform. The standardized differences between domain scores in the high and low well-being group ranged from 0.58 to 0.92 . These differences were highest when the life evaluation domain $(0.92)$ and emotional health domain (0.84) were compared between the two groups and lowest when the work environment domain (0.58) and healthy behaviors domain ( 0.59$)$ were compared. CONCLUSIONS: In this national study, 217 low-income communities had high wellbeing. Compared to low-income communities with low well-being, the domain scores that varied the most were the life evaluation and emotional health domains and those that varied the least were the work environment and healthy behaviors domains. Future analysis in this study will identify community factors that explain variation in overall well-being and the domain scores among low-income communities. These findings may influence neighborhood-level investments focused on improving well-being of lowincome communities.

LOW-VALUE BACK IMAGING: ARE DOCTORS AND PATIENTS LEARNING TO CHOOSE WISELY? Arthur Hong ${ }^{1}{ }^{2}$; Dennis Ross-Degnan ${ }^{1}$; Fang Zhang ${ }^{1}$; James F. Wharam ${ }^{1} .{ }^{1}$ Harvard Medical School and Harvard Pilgrim Health Care Institute, Boston, MA; ${ }^{2}$ Harvard Medical School Fellowship in General Medicine and Primary Care, Boston, MA. (Control ID \#2468727)

BACKGROUND: In April 2012, the American Board of Internal Medicine released highly publicized guidelines - called "Choosing Wisely" - targeting potentially unnecessary health care. Importantly, Choosing Wisely partnered with consumer groups to disseminate the recommendations not only to doctors, but also to patients. To assess Choosing Wisely's impact, we examined rates of the most widespread form of low-value care, inappropriate low back pain imaging. We then probed for stronger effects of Choosing Wisely amongst high-deductible health plan (HDHP) and low-income members.

METHODS: We started with claims data for 46 million health plan members enrolled in a large national insurer. We applied an interrupted time-series study design, and our primary outcome was the monthly rate of low-value imaging for acute low back pain. Based on previous algorithms, we defined inappropriate imaging as a plain film, CT scan, or MRI, 7 days before or 42 days after an index visit for acute low back pain. We excluded patients with red flag symptoms such as major trauma, cancer, fever, or neurologic deficits. We plotted monthly low-value back imaging rates (standardized for age and gender) from 27 months before, to 7 months after the April 2012 introduction of Choosing Wisely. We then applied aggregate-level segmented regression, adjusting standard errors for autocorrelation. Finally, we stratified by high deductible ( $\geq \$ 1,000$ annual deductible) and low annual household income ( $\leq$ $\$ 49,000)$, to probe for differing trends among members with strong incentives to reduce low-value care.

RESULTS: Our sample included 806,437 patients with 938,019 back pain visits from January 2010 to December 2012. At the beginning of 2010, $27.5 \%$ of visits for low back pain included a low-value imaging exam. Prior to Choosing Wisely, there was a small downward trend in low-value imaging ( -4.5 images/ $10,000$ visits/month [95 \% CI: $-2.9,-6.1]$ ), and this downward trend was unchanged after Choosing Wisely in April 2012 (Figure). Even after we stratified by high deductible membership and household income, there was no significant difference in trend after Choosing Wisely's release.

CONCLUSIONS: We detected no short-term changes in low-back pain imaging after the Choosing Wisely campaign of 2012 , both overall and among patients with strong incentives to reduce low-value care. Future studies should assess the longer-term effects of Choosing Wisely and other targeted low-value services. Policymakers might need to consider alternative approaches to reducing low-value care if our null findings persist over time.

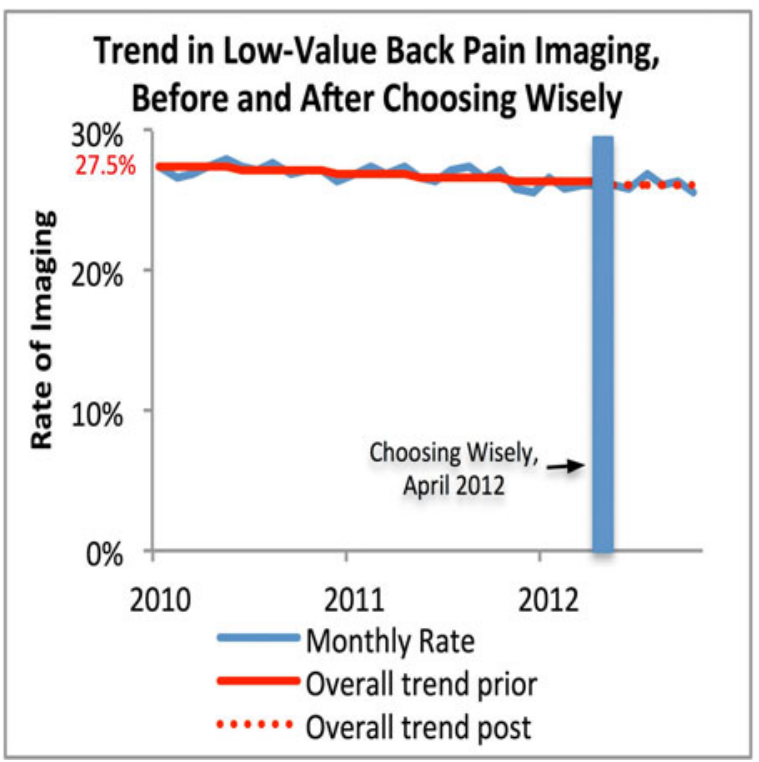

LUNG CANCER AMONG HIV-INFECTED INDIVIDUALS AT SINGLE URBAN INSTITUTION IN RECENT ANTIRETROVIRAL ERA Kristen Hysell' ${ }^{1}$; Lydia A. Barakat ${ }^{2}$; Michael Virata ${ }^{1}$; Brinda Emu ${ }^{1} .{ }^{1}$ Yale New Haven Hospital, New Haven, CT; ${ }^{2}$ Yale University, New Haven, CT. (Control ID \#2466033)

BACKGROUND: Lung cancer is the leading cause of non-AIDS defining cancer deaths among patients with HIV infection and large cohort studies have found that HIV is an independent risk factor for lung cancer incidence. However, studies are needed to better understand specific characteristics of the HIV-infected patients who develop lung cancer, particularly to ensure adequate screening and prevention in the primary care setting. This study sought to characterize demographics, HIV disease status and treatment, substance abuse, and lung cancer outcomes among HIV-infected individuals diagnosed at a single urban institution in the recent antiretroviral era.

METHODS: All patients with HIV who were diagnosed with lung cancer between 2001 and 2015 at Yale New Haven Hospital (New Haven, CT) were analyzed through electronic medical records for demographical information and characteristics of HIV infection, HIV treatment, lung cancer stage and pathology, cancer treatment, and mortality. RESULTS: A total of 28 patients were identified from 2001 to 2015 . Sixteen patients were Black (57\%), 10 were Caucasian (36\%), 2 Hispanic. The majority (71 \%) was male. Median age at time of cancer diagnosis was 52 (range 40 to 65 ). The median interval of time from HIV diagnosis to lung cancer diagnosis was 16 years. Mode of HIV transmission included IVDU (46.5\%), MSM (14\%), heterosexual sex (18\%), or unknown (21.5\%). Only 6 patients $(21 \%)$ had a diagnosis of AIDS by CDC stage at time of lung cancer diagnosis. The median CD4 count at time of lung cancer diagnosis was 498 (range 26-1240). Eighty-two percent of patients were on anti-retroviral treatment at time of cancer diagnosis. All patients had a past or present smoking history with median of 30 pack years (range 7-100). Sixty-four percent of patients had documented past or present alcohol use and $79 \%$ had a documented history of past or present drug use with cocaine and/or heroin. Ninety-three percent of patients had non- 
small cell lung carcinoma (NSCLC) while $7 \%$ had small cell lung cancer. Of those with NSCLC, 13 were identified with adenocarcinoma, 8 with squamous cell, and 2 had unspecified NSCLC. Fifty percent of all patients had stage IV lung cancer at time of diagnosis. The 1 year mortality was $60 \%$ and was associated with male gender, advanced stage of cancer, and the presence of AIDS at the time of cancer diagnosis. There was no statistical significance in HIV viral load, use and choice of ARVs, or histology type.

CONCLUSIONS: This study provides detailed characterization of patients with HIV infection and lung cancer in an urban population. At the time of cancer diagnosis, patients presented with relatively high CD4 count, with the majority on anti-retroviral therapy and virally suppressed. As seen in other cohorts, presentation occurred at a younger age with high rates of smoking, as well as high rates of illicit drug use, suggesting that smoking cessation may be especially important to target in this patient population. This study also suggests that the current lung cancer screening guidelines may not be capturing a significant proportion of HIV patients who may be at increased risk for lung cancer and requires further analysis with larger cohorts.

\section{MANAGEMENT AND CLINICAL OUTCOMES OF CANCER PATIENTS} WITH HUMAN IMMUNODEFICIENCY VIRUS AFTER CANCER THERAPY Christian Donato-Santana ${ }^{3}$; Nora T. Oliver ${ }^{4}$; Andrea Gabriela Barbo ${ }^{2}$; Heather Lin $^{2}$; $\underline{\text { Jessica T. Foreman }}{ }^{3}$; Harrys Torres ${ }^{3}$; Bruno Granwehr ${ }^{2}$; Elizabeth Chiao ${ }^{4}$; Jessica P. Hwang ${ }^{1} .{ }^{1} \mathrm{MD}$ Anderson Cancer Center, Houston, TX; ${ }^{2} \mathrm{UT}$ MD Anderson Cancer Center, Houston, TX; ${ }^{3}$ University of Texas MD Anderson Cancer Center, Houston, TX; ${ }^{4}$ Baylor College of Medicine, Houston, TX. (Control ID \#2465981)

BACKGROUND: Human immunodeficiency virus (HIV) is a significant chronic illness in the US where more than 1.2 million of persons are infected. Persons with HIV infection are at high risk for malignancy due to immunosuppression, co-infection with oncogenic viruses, and environmental factors. However, HIV patients on HAART have better clinical outcomes than patients without HAART. The purpose of this study is to describe the management and clinical outcomes of HIV-infected patients after cancer therapy.

METHODS: This is a retrospective cohort study where we identified HIV-positive adults with any type of cancer who registered at MD Anderson Cancer Center between January of 2004 through April of 2011 and received systemic cancer therapy. We used institutional databases to establish a cohort of HIV-positive patients using HIV 1/2 antibody test and confirmatory Western blot test. If confirmatory Western blot test was not available, then viral load was used as confirmation. We also examined demographics, cancer type, comorbidities, and cancer treatment including chemotherapy, radiation, and surgery. We determined the date of HIV diagnosis, HAART regimen initiation date, modifications to HAART treatment, history of opportunistic infections (OI), as well as baseline viral load and CD4 count 2 months before first chemotherapy until second chemotherapy. Clinical outcomes included frequency and duration of inpatient hospitalizations, occurrence of neutropenic fever, and mortality. Frequencies and percentages as well as means, standard deviations and medians were used to describe the characteristics of HIV-positive patients. Chi-square test and Fisher's exact tests of association were used to assess the relationship between certain clinical attributes and outcomes of the patients. Log-rank test was performed to test the difference in mortality between groups.

RESULTS: We identified 38 patients who were HIV-positive. Mean age was 42 ( \pm 10 years). There were $74 \%(n=28)$ patients with hematological malignancies and $26 \%(n=10)$ with solid tumors. The most common type of cancer was non-Hodgkin lymphoma ( $55 \%, n=21)$. Most patients $(61 \%)$ had advanced cancer. Over $23 \%$ of patients had 1 or more comorbidity. Five (13\%) patients received radiation therapy, and another 5 had surgery within the first year of chemotherapy. Twenty-four (63\%) patients had known HIV at presentation. Only 9 (24\%) patients had a history of OI in the past. Twenty-two patients, $58 \%$, had at least one neutropenic fever episode within the first year of chemotherapy. Patients with past history of OI were more likely to have at least one episode of neutropenic fever within the first year of chemotherapy than patients who did not have history of OI ( 89 vs $48 \%, p=0.05$ ). Mean baseline viral load was 29,805 $( \pm 58,858)$ and mean baseline CD4 count was $241( \pm 238)$. Among the study patients, $84 \%$ $(n=32)$ had at least one hospitalization within the first year of chemotherapy, with a median length of stay of 9 days. Nearly $90 \%$ of all hospitalizations included an infectious disease diagnosis, not including HIV. Twenty patients (53\%) were initiated on HAART prior to chemotherapy, and of these $20 \%(n=4)$ had a change in the HAART therapy during the first year of chemotherapy. Seventeen ( $45 \%$ ) patients died within 2 years after completing chemotherapy.

CONCLUSIONS: Overall, HIV-positive patients with cancer have frequent hospitalizations and episodes of neutropenic fever, and they have high rates of mortality. HIV-positive patients who have past history of OI were associated with having neutropenic fever episodes during the first year after chemotherapy. Future research goals include determining predictors and clinical outcomes compared to cancer patients without HIV infection.

MAPPING THE EVIDENCE: INTERVENTION SEX EFFECTS FOR DEPRESSION, DIABETES, AND CHRONIC PAIN Wei Duan-Porter ${ }^{3,2}$; Karen M. Goldstein $^{3,}$; Jennifer McDuffie ${ }^{2}$; Jaime M. Hughes ${ }^{4}$; Megan Clowse ${ }^{1}$; Ruth Klap ${ }^{5}$; Varsha Masilamani ${ }^{2}$; Nancy Allen LaPointe ${ }^{1}$; Avishek Nagi ${ }^{2}$; Jennifer M. Gierisch ${ }^{3,2}$; John W. Williams ${ }^{3,2}$. ${ }^{1}$ Duke University, Durham, NC; ${ }^{2}$ Durham VA Health Services Research \& Development, Durham, NC; ${ }^{3}$ Duke University School of Medicine, Durham, $\mathrm{NC}$; ${ }^{4}$ University of North Carolina, Chapel Hill, NC; ${ }^{5}$ VA Greater Los Angeles Healthcare System, Los Angeles, CA. (Control ID \#2464493)

BACKGROUND: Treatment effects may differ importantly by sex or gender (termed "sex effects"). By synthesizing current clinical evidence, systematic reviews (SRs) may significantly contribute to the evaluation of sex effects. We used evidence mapping to describe if and how SRs report sex effects for interventions addressing depression, type 2 diabetes mellitus (DM), and 3 chronic pain conditions: chronic low back pain (CLBP), knee osteoarthritis (OA), and fibromyalgia.

METHODS: We searched PubMed and Cochrane Database of Systematic Reviews for SRs published from January 1, 2009 to October 31, 2014, and included relevant SRs evaluating medications, behavioral interventions, supervised exercise, quality improvement (QI), and some condition-specific treatments (e.g., bariatric surgery for DM). Abstracted data included: SR characteristics, analysis methods (e.g., meta-regression or individual patient data [IPD] meta-analysis), characteristics of primary studies, and sex effects. For selected interventions and conditions (e.g., QI and psychotherapy for depression), a sample of primary randomized controlled trials (RCTs) were also examined for reporting of sex effects.

RESULTS: From 2531 unique citations, we identified 313 eligible SRs $(n=86$ for depression, $n=159$ for DM, $n=68$ for chronic pain conditions). SRs addressing medications and psychotherapy for depression, and medications for DM included the greatest number of primary studies (see Figure). SR reporting of sex distribution in primary studies varied from a low of $31 \%(n=8)$ for CLBP to highest of $68 \%(n=23)$ for fibromyalgia. Sex effects were rarely reported by SRs $(16 \%, n=14$ SRs for depression; $8 \%, n=13$ for DM; and $8 \%, n=2$ for CLBP); no SRs on knee OA or fibromyalgia reported sex effects. Overall, sex effects were most commonly reported for medications $(n=17)$, although some SRs also addressed sex effects for psychotherapy and behavioral interventions $(n=6)$, bariatric surgery $(n=2)$, guided self-help $(n=1)$, and QI or organizational interventions ( $n=2$; see Table). The majority of SRs used meta-regression to evaluate sex effects, while 9 SRs used subgroup analysis or IPD metaanalysis. Primary RCTs were examined for the largest, most recent, eligible SRs for the following interventions and conditions: 1) collaborative care and psychotherapy for depression; 2) dietary, mixed behavioral, and culturally tailored psychoeducation interventions for DM; 3) exercise for knee OA; and 4) behavioral interventions for CLBP. All primary RCTs $(n=21)$ 
included in the SR examining collaborative care had at least 75 participants per arm, and thus, may have been adequately powered to evaluate interaction between sex and treatment effects. However, only 2 of these RCTs reported evaluation for sex effects. Few of the remaining primary RCTs for other interventions and conditions met this minimum size criterion (e.g., only $11 \%$ of psychotherapy RCTs for depression and $30 \%$ of dietary RCTs for DM had at least 75 participants per arm). Overall, among primary RCTs meeting the minimum sample size, only $14 \%(n=7)$ reported evaluation for sex effects.

CONCLUSIONS: SRs on depression, DM, and chronic pain conditions rarely examined sex effects; when sex effects were evaluated, SRs often used suboptimal analysis approaches. Primary RCTs often lacked adequate sample size to examine sex effects, but even RCTs of sufficient size reported sex effects infrequently. Larger RCTs and/or IPD meta-analyses are likely needed to establish moderation of treatment effects by sex or gender; prioritization of interventions and conditions for these future efforts could be based on several considerations, including results from basic biomedical, preclinical, and/ or observational studies that indicate potential for important treatment sex effects. In the shorter term, systematic and unbiased reporting by RCTs of subgroup effects by sex could support pooled analyses that accurately establish treatment effects for women separately from men. All SRs should report the proportion of women enrolled in primary studies, and, when primary data permit, evaluate sex effects using appropriate methods. Table: Summary of Intervention Sex Effects Reported by Systematic Reviews

\begin{tabular}{|c|c|c|}
\hline \multirow[t]{2}{*}{ Conditions } & Interventions & \\
\hline & $\begin{array}{l}\text { Possible differences } \\
\text { between men and } \\
\text { women }\end{array}$ & $\begin{array}{l}\text { Possibly no differences } \\
\text { between men and women }\end{array}$ \\
\hline Depression & $\begin{array}{l}\text { - Selective serotonin } \\
\text { reuptake inhibitors } \\
\text { in older adults } \\
\text { \& Duloxetine } \\
\text { \& Cognitive behavioral } \\
\text { therapy } \\
\text { \& Paroxetine* }\end{array}$ & $\begin{array}{l}\text { \& Combined } \\
\text { antidepressants } \\
\& \text { psychotherapy } \\
\text { \& Quality improvement } \\
\text { \& Self-help, guided } \\
\text { \& Antidepressants, overall* }\end{array}$ \\
\hline Diabetes & $\begin{array}{l}\text { - Metformin } \\
\text { \& Sulfonylureas* } \\
\text { \& Pioglitazone* }\end{array}$ & $\begin{array}{l}\text { - Medications, overall } \\
\text { - Incretin mimetics } \\
\text { - Bariatric surgery }\end{array}$ \\
\hline $\begin{array}{l}\text { Chronic low } \\
\text { back pain }\end{array}$ & \&uality improvement & \& Duloxetine \\
\hline
\end{tabular}

* Sex and gender differences for adverse effects of interventions

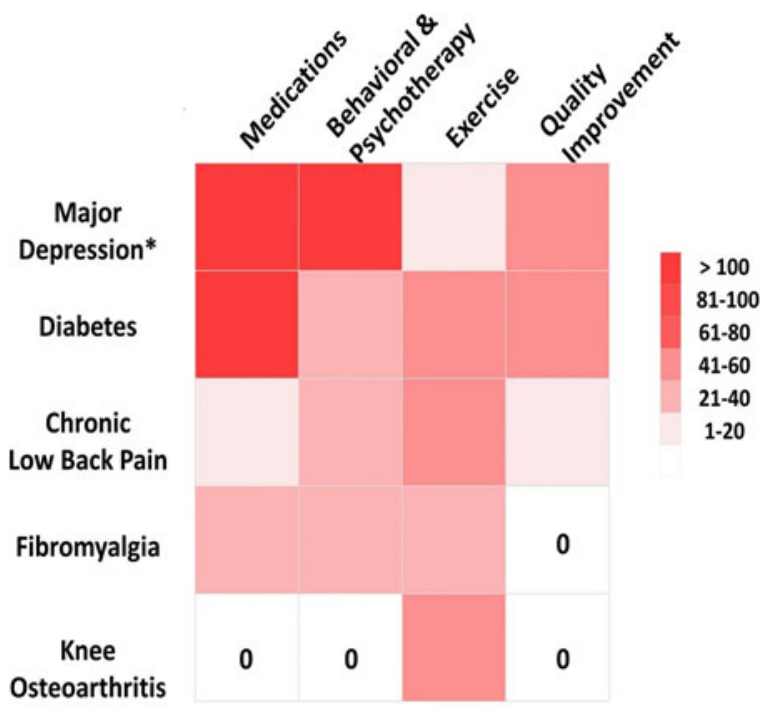

*Some reviews included studies with other depressive disorders.

Figure: Number of Included Primary Studies for Largest Eligible Systematic Reviews.
MARIJUANA USE IS ASSOCIATED WITH LOW PRESCRIPTION OPIOID ANALGESIC (POA) USE AMONG HIV-INFECTED PATIENTS WITH CHRONIC PAIN. Laila Khalid ${ }^{2}$; Joanna L. Starrels ${ }^{1}$; Nancy Sohler ${ }^{3}$; Julia H. Arnsten ${ }^{2,1}$; john jost ${ }^{1}$; Chinazo Cunningham ${ }^{1} .{ }^{1}$ Albert Einstein College of Medicine \& Montefiore Medical Center, Bronx, NY; ${ }^{2}$ Montefiore Medical Center, Bronx, NY; ${ }^{3}$ City College of New York, NYC, NY. (Control ID \#2465311)

BACKGROUND: Approximately $50 \%$ of HIV-infected individuals have chronic pain and of these, $30 \%$ are prescribed opioid analgesics. Because substance use including marijuana, and mental health disorders are prevalent in HIV-infected individuals, chronic pain management with POAs can be complicated. Research indicates that states with medical marijuana laws have lower POA overdose mortality. However, few studies focus specifically on POA and marijuana use among HIV-infected individuals. We describe demographic, clinical and substance use characteristics of HIV-infected patients taking POAs, and explored factors associated with taking POAs.

METHODS: We conducted a cross-sectional secondary data analysis using screening interviews from a parent study, Project FIRST (a randomized trial of abstinence-reinforcing financial incentives to improve HIV outcomes among HIV-infected drug users). From 2012 to 2015, we recruited adults from a variety of sources, including Bronx (NYC borough) drug treatment programs, Bronx HIV clinics, and newspaper advertisements. This analysis included a subset of HIV-infected individuals with chronic pain. Interview data included questions regarding demographic characteristics, presence of chronic pain ( $>3$ months), patterns of POA use, current marijuana and other substance use (within the past 30 days), comorbid illnesses, and current utilization of and adherence to antiretroviral therapy (ART). Our dependent variable was participants' self-report of current POA use during the previous month. To explore factors associated with POA use, we first conducted bivariate analyses using chi-square and t-tests. Variables with $p<0.10$ on bivariate analyses were then entered into a multivariate logistic regression analysis.

RESULTS: Of the 1317 individuals screened, 459 were HIV-infected with chronic pain. The mean age was 52 years, and most were male (57.1\%), and Hispanic (46.4\%) or non-Hispanic black (44.9\%). While $94 \%$ reported utilizing ART, only $20 \%$ reported $100 \%$ ART adherence. Hepatitis C virus co-infection was common (40.8\%), as was cigarette smoking (75.1\%), any alcohol use (57.0\%), methadone (40\%) and illicit drug use with marijuana (39.4\%), cocaine (32.2\%), heroin (16.6\%) or illicit opioid analgesics (19.0\%). Nearly half (47.1\%) reported taking POAs, and of them, $44.9 \%$ reported taking POAs more frequently or in higher doses than prescribed. In bivariate analyses, compared to those not taking POAs, individuals taking POAs were significantly more likely to be older $(51.2$ vs. 52.8 years, $p<0.05)$ and report $100 \%$ ART adherence ( 15.4 vs. $25.1 \%, p<0.05$ ), and significantly less likely to report current use of any alcohol (62.7 vs. $52.7 \%, p<0.05)$ or marijuana (46.5 vs. $31.5 \%$, $p<0.05$ ). In multivariate analysis adjusted for age, ART adherence, alcohol, and heroin use, only marijuana use remained significantly associated with lower odds of taking POAs $(\mathrm{aOR}=0.57,95 \% \mathrm{CI}: 0.38-0.87)$.

CONCLUSIONS: Among a convenience sample of HIV-infected individuals with chronic pain in the Bronx, POA use and misuse was common. The only factor we found independently associated with POA use was marijuana use, with marijuana users nearly half as likely to report POA use as non-marijuana users. It is not clear whether patients with marijuana use were prescribed less POAs or POA use led to decreased marijuana use. As the use of medical marijuana across the US continues to grow, examining how marijuana use is associated with POA use and chronic pain management in larger prospective studies will be important, particularly among HIVinfected individuals. 
MASSACHUSETTS HEALTH REFORM WAS ASSOCIATED WITH A REDUCTION IN HOSPITALIZATIONS RELATED TO DRUG, BUT NOT ALCOHOL USE. Karen E. Lasser ${ }^{1,}{ }^{4}$; Amresh D. Hanchate ${ }^{5,}$; ; Danny McCormick ${ }^{3}$; Alexander Y. Walley ${ }^{1}$; Richard Saitz ${ }^{1,2}$; Meng-Yun Lin ${ }^{1}$; Chieh Chu ${ }^{1}$; Nancy R. Kressin ${ }^{5}$,

${ }^{1}$. ${ }^{1}$ Boston University School of Medicine, Boston, MA; ${ }^{2}$ Boston University and Boston Medical Center, Boston, MA; ${ }^{3}$ Harvard Medical School / Cambridge Health Alliance, Cambridge, MA; ${ }^{4}$ Boston University School of Public Health, Boston, MA; ${ }^{5}$ VA Boston Healthcare System, Boston, MA. (Control ID \#2461507)

BACKGROUND: Substance use (alcohol and drug) disorders are often underdiagnosed and undertreated. Massachusetts (MA) health reform provided insurance to previously uninsured patients, potentially allowing them to obtain access to substance use disorder treatment. Assessment of hospital admission rates for substance use-related conditions (e.g pneumonia with comorbid alcohol abuse) and substance use disorders (e.g. drug dependence) is a method of assessing changes in access to care for treatment of substance use disorders. We hypothesized that there would be a small decline in substance-use related acute hospitalizations in the years following MA health reform. Prior work examining the impact of MA health reform on access to outpatient care has shown slight improvements or no change in a number of other outcomes following reform, including 30-day readmissions, hospitalizations for ambulatory care sensitive conditions, and procedure use

METHODS: We examined whether MA health reform (enacted 2006-07) affected population-based rates of alcohol and other drug-related inpatient hospitalizations (as primary or secondary diagnosis) in general acute care hospitals, or, secondarily, whether such rates differed between areas with high- or low-baseline uninsurance. We used 2004 2010 all-payer inpatient discharge data from MA. These data include all non-Federal shortterm acute care hospitals; inpatient psychiatric and addiction treatment facilities are not included in the dataset. Using difference-in-differences analysis to identify post-reform (2008-2010) changes, we adjusted for secular trends unrelated to reform. Participants included 91,546 medical and surgical hospital discharges where a substance use diagnosis was either a primary or secondary discharge diagnosis. We measured pre- to post-reform changes in age and sex standardized population-based rates of alcohol and other drug-related inpatient medical and surgical hospitalizations (discharges per 100,000 population).

RESULTS: Population-based rates of substance-use-related hospital discharges increased after MA health reform. In analyses comparing adults age 18-64 (target population of the reform) with adults age $>65$ (control population), MA health reform was associated with $14.8 \%$ (95\% CI 11.8 to 17.7$)$ reduction in the rate of drug-related discharges. Similarly, in analyses comparing adults age 18-64 residing in MA counties with the highest uninsurance rates to those residing in counties with lower uninsurance rates, MA health reform was associated with a $5.4 \%(95 \%$ CI 3.5 to 7.3$)$ reduction in the rate of drugrelated discharges. In analyses utilizing the same comparison groups (age 18-64 vs. age $>65$ and MA counties with high vs. low uninsurance), MA health reform resulted in no change in the rate of alcohol-related discharges when making comparisons by age group (1.6 \% [95\% CI -0.7 to 3.8$]$ ), or when making comparisons by county rates of uninsurance $(1.7 \%[95 \% \mathrm{CI}-0.2$ to 3.6$])$.

CONCLUSIONS: MA health reform was associated with a reduction in hospitalizations related to drug, but not alcohol use. In order to reduce alcohol-related hospitalizations, states may need to go beyond simply expanding insurance coverage to ensuring the availability of high quality alcohol treatment.

MEASURING LEVEL OF INTEGRATION OF AN EARLY STAGE BEHAVIORAL HEALTH PROGRAM IN AN ACADEMIC PRIMARY CARE CLINIC Mara Terras ${ }^{1}$; Neda Laiteerapong ${ }^{1}$; Lisa M. Vinci ${ }^{1}$; Daniel Yohanna ${ }^{2}$; Erin M. $\mathrm{Staab}^{1}$; Pooja Dave ${ }^{2}$; Nancy Beckman ${ }^{2}$; Daviel Thomas ${ }^{1} .{ }^{1}$ University of Chicago, Chicago, IL; ${ }^{2}$ University of Chicago Medicine, Chicago, IL. (Control ID \#2465151)
BACKGROUND: The benefits of integrating behavioral health in primary care are wellproven. Although several evidence-based models exist, effective implementation is a complex and challenging process that requires collaboration across multiple disciplines. However, few studies have systematically examined the attitudes, knowledge, and beliefs of primary care providers and staff during the process of behavioral health integration.

METHODS: Early in the launch of a co-located behavioral medicine clinic at the University of Chicago's primary care practice, we administered the validated Level of Integration Measure (LIM) to survey primary care (PCP) and behavioral medicine (BMP) providers and clinical staff on their perceptions, attitudes, and beliefs. The LIM includes six domains of integrated primary care: systems integration, beliefs and commitment, integrated practices, relationships, training, and leadership. Participants were asked their level of agreement (strongly disagree-strongly agree) on a 5-point Likert scale or "Don't Know" for the 32-item LIM. Descriptive analyses were performed; responses were categorized into agree (strongly agree/agree), disagree (strongly disagree/disagree), or don't know (don't know, undecided). Domains of integrated primary care were calibrated to a score of (0 (low)-100 (high)). Based on prior literature, an overall score $\geq 80$ represents an established highly integrated clinic.

RESULTS: Our sample $(n=46)$ had an overall response rate of $79 \%$; response rates were similar between providers and clinical staff $(p>.05)$. All respondents agreed that integrated care was a superior form of patient care (100\%), and the vast majority $(87 \%)$ agreed that integrated care ensured that patients receive appropriate care and was a worthwhile investment for the clinic (96\%). However, half of respondents disagreed that there were sufficient behavioral health specialists on-site $(50 \%)$, that behavioral health was integrated into the workflow (46\%), that the clinic systematically triaged (52\%) or detected and served $(56 \%$ ) behavioral health needs of its patients, or tracked the progress of behavioral health treatment $(50 \%)$. Many respondents did not know or were unsure as to whether the scheduling system allowed for primary care patients to be seen by behavioral medicine on the same day (48\%), and whether PCPs and BMPs were treated as equals (63\%), reviewed each other's notes (61\%), did warm hand-offs (41\%), or regularly consulted each other (56\%). Also, $74 \%$ of respondents disagreed that integrated care was supported by a viable financial system. Our clinic scored lowest on the integrated practice domain (31/100 points) (e.g., "PCPs and BMPs do "warm hand-offs" according to patient needs") and highest on the leadership domain (75/100 points) (e.g., "The clinic administrator(s) value integrated care"). Overall, the clinic scored $41 / 100$ points on the LIM.

CONCLUSIONS: Despite a strong enthusiasm for integrated behavioral health in primary care in general and in our clinic, we identified several areas in which the reality of clinical practice did not meet the basic requirements for a truly integrated system by using a validated tool that measures behavioral health integration. By identifying key systemic, practical, and cultural areas of need, we are developing a more effective strategy for implementing a system that merges physical and behavioral health care in our academic primary care clinic.

MEDICAL EXPENDITURES EXACERBATE INCOME INEQUALITY Andrea S. Christopher $^{1,3}$; David Bor ${ }^{1,3}$; Danny McCormick ${ }^{1,3}$; Steffie Woolhandler ${ }^{2,}, \overline{\text {; David }}$ Himmelstein ${ }^{2,1}$. ${ }^{1}$ Cambridge Health Alliance, Boston, MA; ${ }^{2}$ City University of New York School of Public Health, New York, NY; ${ }^{3}$ Harvard Medical School, Boston, MA. (Control ID \#2469920)

BACKGROUND: Prior research has demonstrated that poverty and income inequality increase mortality. Unlike other developed nations that fund virtually all health care through taxes, the U.S. health system requires substantial household payments in the form of premium contributions, copayments and deductibles. However, little is known about the relationship between families' medical payments and income inequality. We sought to determine the impact of medical expenses on family poverty and population-wide income inequality. 
METHODS: We analyzed data from the March 2014 Current Population Survey (CPS), a nationally-representative survey of the U.S. population. We (and the CPS) defined medical out-of-pocket payments (MOOP) broadly, as the total that each family paid for medical services, drugs and supplies, as well as household contributions to health insurance premiums. We used family income before and after subtracting MOOP to calculate income in relation to the federal poverty line (FPL), income distribution by deciles, and the Gini coefficient, an aggregate measure of income inequality. Finally, we repeated all calculations under the assumption that employer payments for health insurance add to families' pre-MOOP income.

RESULTS: For 7.26 million Americans, MOOP lowered effective income from above to below the FPL. After accounting for MOOP, the median family income was reduced by $52 \%$ in the poorest decile versus a $2 \%$ drop for the richest 99 th percentile. As measured by the Gini coefficient, MOOP caused income inequality to rise by $3 \%$. Taking into account employer-paid insurance premiums, medical payments increased the Gini coefficient by $4 \%$, from 0.351 to 0.365 .

CONCLUSIONS: The health care financing system in the U.S. deepens income inequality and drives many into poverty. Policymakers should consider reducing or eliminating cost-sharing, especially for low and middle-income Americans.

\section{MEDICAL STUDENT LOAN BURDEN AND HEALTH AND WELLNESS} DURING MEDICAL SCHOOL: A SINGLE CENTER LONGITUDINAL STUDY Padmini Ranasinghe ${ }^{1}$; Brandyn D. Lau ${ }^{2} .{ }^{1}$ Johns Hopkins School of Medicine, Baltimore, MD; ${ }^{2}$ The Johns Hopkins University School of Medicine, Baltimore, MD. (Control ID \#2470149)

BACKGROUND: Health and wellness vary over time, and are influenced by a variety of internal and external factors. Earlier studies have found that medical students experience symptoms of stress, depression and burnout at a higher rate compared to the general population. Medical students may also have a higher rates of depression including suicidal ideation. Medical education is also associated with increases in stress and symptoms. A cross sectional study reported that approximately one-third of all students reported experiencing stress during medical school. One study found that $53 \%$ of medical students reported experiencing high emotional exhaustion and/or patient depersonalization. Another study reported that $10 \%$ of medical students experienced emotional exhaustion and $6 \%$ reported patient depersonalization. To our knowledge there are no published studies where students are followed longitudinally to examine these variables. Similarly, there is a lack of evidence about the effect of student loan burden on the mental and physical health outcomes of medical students. The aim of this study is to longitudinally evaluate student loan burden across 4 years of medical education and its relation to selfreported depression, stress, burnout, leisure time activity, and satisfaction.

METHODS: In this longitudinal survey study, we evaluated the health and wellness of medical students across 4 years of medical school using a combination of validated and adapted assessment tools. Standardized surveys were administered annually between 2009 and 2014 at the Johns Hopkins University School of Medicine in Baltimore, Maryland. This study was approved by the Johns Hopkins Medicine Institutional Review Board and consent was obtained at the time each survey was administered. All students continuously enrolled in the undergraduate medical education program at the Johns Hopkins University School of Medicine who matriculated in 2009, 2010, or 2011 were included in this study. Participants were recruited during medical student orientation. Each participant completed 5 surveys: one at the beginning of each year of medical school and an exit survey postgraduation. Students were sent up to four reminders via email that ceased with completion of the survey.

RESULTS: Three hundred sixty (360) students matriculated to the undergraduate medical education program at the Johns Hopkins University School of Medicine between 2009 and
2011. 284 (78.9\%) were continuously enrolled during pre-clinical and clinical years, and were eligible for inclusion in this study. One hundred fifty three medical students (53.9\%) completed the health and wellness survey each year. Upon enrollment, the mean age of the participants was 28.1 (standard deviation $[\mathrm{SD}]=2.4$ ) years. Approximately half of the student body was female $(52.0 \%)$ and white $(50.0 \%)$. The majority of students $(82.3 \%)$ reported some level of loan burden on enrollment with $29.4 \%$ feeling very burdened by loans. The mean perceived stress scores were $17.1(\mathrm{SD}=5.9)$ during first year and 19.3 $(\mathrm{SD}=7.5)$ during second year, $19.5(\mathrm{SD}=6.1)$ in third year, and $20.0(\mathrm{SD}=7.3)$ during fourth year. Perceived stress was associated with self-reported loan burden during the first year. Students with no loan burden reported significantly lower perceived stress than those with loan burden (14.2 vs. 18.9, $p=0.0394)$. During the first year of medical school, $4.7 \%$ of respondents reported symptoms of at least minor depression. Each year, an increasing proportion of respondents reported symptoms of depression. Satisfaction with life was highest during the first year of medical school, dropped during second year and remained unchanged during third and 4 years. The proportion of respondents who self-reported at least one symptom of burnout was low during first year (4.2\%), increased dramatically in second year $(18.8 \%)$, remained high in third year (17.7\%) and then increased dramatically again in fourth year (31.3\%). Depression satisfaction with life and burnout were not associated with loan burden during any of the 4 years.

CONCLUSIONS: In this single institution study the majority of medical students reported some level of loan burden on enrollment with $29.4 \%$ feeling very burdened by loans. Mean perceived scores were gradually increased during medical school and it was associated with self reported loan burden during first year. Each year, an increasing proportion of respondents reported symptoms of depression measured by PHQ-9. Burnout has increased gradually and highest during fourth year. It is important to understand these trends among medical students by academic generalists who are involved in undergraduate medical education to become effective teachers and evaluators

\section{MEDICAL STUDENT PERCEPTION OF FORMATIVE FEEDBACK ON OSCE PERFORMANCE FROM STANDARDIZED PATIENTS Samina Yunus ${ }^{1}$; Mark} Mayer ${ }^{2} .{ }^{1}$ Cleveland Clinic Lerner College of Medicine, Chagrin Falls, OH; ${ }^{2}$ Cleveland Clinic Lerner College of Medicine, Cleveland, OH. (Control ID \#2469641)

BACKGROUND: The Objective Structured Clinical Examination (OSCE) was designed to test clinical skills and competence and has been widely utilized in the training of medical school students. The OSCE is an integral part of the Clinic Lerner College of Medicine (CCLCM) since the inception of the medical school in 2002. Our OSCEs provide formative feedback to first and second year medical students on their communication and physical examination skills; students incorporate feedback from the OSCEs into a plan for self-improvement within a learning portfolio. We have utilized standardized patients (SPs) during our OSCEs, but for several years, utilized feedback only from physician observers. We sought to determine whether feedback on communication skills by SPs would be useful in augmenting that obtained from physician observers.

METHODS: Thirty-three second year medical students at CCLCM were invited to complete a survey to help us identify their perception of the utility of SP feedback on their communication skills from an OSCE station. Participation was voluntary; the study was reviewed by the IRB at the Cleveland Clinic Foundation and granted exempt status. Students were contacted via email and data were collected in the RedCAP system. Bivariate analysis with Chi Square was used to evaluate student perception of the standardized patient feedback. Twenty-six students $(78.87 \%)$ completed the survey and provided the data presented in this analysis. The survey consisted of 7 yes/no questions: Did you find the feedback from the SP useful? Did it provide information distinct from that provided by your preceptors? Did the feedback target a behavior or action? Did that make it easier to formulate a plan for change? Did your preceptor (physician observer) 
identify areas for improvement in communications? Did your preceptor identify the same areas for improvement as the SP? Will you utilize feedback from the SP in your portfolio? RESULTS: The majority of the students answering the survey felt the feedback received from the SP was useful ( $88.5 \%$ ) and that it provided information distinct from that provided by the preceptor $(80.8 \%)$. Most students $(80.8 \%)$ thought that the feedback targeted a behavior or action. $73.1 \%$ of students thought that SP feedback made it easier to formulate a plan for change. Although the preceptors (physician observers) also targeted areas for improvement in communication during the OSCE in $92.3 \%$ of the students answering the survey, concurrence in the areas identified was only $50 \%$ between physician preceptors and the SPs. Only $34.6 \%$ of students indicated that they would utilize feedback from the SP in their portfolio.

CONCLUSIONS: Since the introduction of the OSCE In medical schools in 1975 it has been increasingly adopted as a reliable and valid test of student competence in a clinical setting However, it is a resource intensive endeavor. To offset some of the cost, SPs are being used not only as simulated patients but also as examiners in some settings. While the reception from students has generally been positive, the validity of the feedback related to domains such as medical knowledge has been questioned. Correlation between SP and faculty evaluation of communication skills appears to be stronger. Results from our study, in which there was only a $50 \%$ concordance in feedback on communication skills on the OSCE between physician observer and SP, suggests that there could be an additive effect of formative feedback from the physician observer and the SP. There are some limitations to our observations. The overall sample size is small. While only $35 \%$ of survey participants indicated that they would utilize SP feedback in their learning portfolio, this may be an underestimate, as the SP feedback was delayed till after their second year portfolios were complete during their spring 2015 porfolio submission process. More timely turnaround of SP feedback on OSCEs to students, providing them with this before their final second year portfolio is due, should help clarify this.

MEDICARE CASE MIX TRENDS ACCORDING TO HOSPITAL CHARACTERISTICS: FOR-PROFIT AND NON-TEACHING HOSPITALS APPEAR TO BE WINNING THE CODING RACE Anna Goldman ${ }^{2}$; Samuel L. Dickman $^{4}$; David Bor ${ }^{2}$; Steffie Woolhandler ${ }^{3}$; David Himmelstein ${ }^{1}$; Danny McCormick ${ }^{5}$ 2 . ${ }^{1}$ CUNY School of Public Health, New York, NY; ${ }^{2}$ Cambridge Health Alliance, Cambridge, MA; ${ }^{3}$ City University of New York School of Public Health, New York, NY; ${ }^{4}$ Harvard Medical School, Boston, MA; ${ }^{5}$ Harvard Medical School / Cambridge Health Alliance, Cambridge, MA. (Control ID \#2465169)

BACKGROUND: Medicare payments, which account for more than one-quarter of U.S. hospital revenues, substantially affect hospitals' profitability. Medicare pays for inpatient care using set fees (bundled payments) based on Diagnosis Related Groups (DRGs). DRGs group patients into relatively homogeneous categories based mostly on discharge diagnoses, with higher payments provided for patients with more severe diagnoses. Since its inception, the case mix index (CMI), a measure of a hospital's average per-case DRG billings intended to reflect patients' acuity and the resources required for their care, has risen faster than expected. Substantial evidence suggests that much of this excess growth is caused by hospitals' efforts to code more (and more severe) diagnoses. However, little research has assessed the relationship of hospital characteristics to changes in CMI.

METHODS: We linked data on hospital characteristics reported in annual Medicare Cost Reports to Case Mix Index Files for all U.S. hospitals receiving DRG-based payment from 1998 to 2013. Using random effects multivariate linear regression, we assessed the relationship between hospital characteristics and annual change in CMI. Each .01 increase in CMI represents approximately a $1 \%$ increase in reimbursement.

RESULTS: CMI for all hospitals grew by an average of 0.0103 annually over the 15 -year study period. CMI rose faster at hospitals that were: non-teaching $(0.0128$ vs. 0.0108 per year); urban (0.0187 vs. 0.0128$)$; non-recipients of Disproportionate Share Hospital funds (0.0148 vs. 0.0110); and caring for fewer Medicaid patients
( 0.0153 vs. 0.0097$)(p<0.0001$ for all comparisons). Ownership had a large influence on CMI growth: for-profit hospitals increased their CMI by 0.014 (95\% CI $0.0135-0.0145)$ annually vs. 0.0103 (95\% CI $0.0100-0.0106)$ at non-profit facilities and 0.0078 (95\% CI $0.0073-0.0083)$ at public hospitals. This pattern persisted in models controlling for other hospital characteristics, including two proxy measures of acuity — changes in the share of care provided in intensive care units and in hospital length-of-stay. Differences in CMI growth rate according to hospital ownership widened after the 2008 implementation of the MS-DRG system, a revision of the DRG system designed to better account for severity of illness. In the period after the 2008 introduction of MS-DRGs, each 0.01 increase in CMI was associated with a $0.64 \%$ increase in hospitals' profit margin.

CONCLUSIONS: For-profit hospitals - as well as non-teaching facilities, and those serving a small proportion of low-income patients - increased their DRG billings more rapidly than others. The differences in CMI growth were likely dues to differences in coding, since they were unrelated to other measures of patient severity that are independent of coding. This pattern suggests that DRGs and other bundled payment schemes based on provider-reported diagnoses, may shift funding to more business-savvy institutions, while penalizing equally efficient but more mission-driven ones. Annual Rate of CMI Change by Hospital Characteristic

\begin{tabular}{lll}
\hline \hline & $\begin{array}{l}\text { Annual Rate } \\
\text { of Change }\end{array}$ & $\begin{array}{l}95 \% \text { Confidence } \\
\text { Interval }\end{array}$ \\
All hospitals & 0.0103 & $0.0101-0.0105$ \\
Public & 0.0078 & $0.0073-0.0083$ \\
Nonprofit & 0.0103 & $0.0100-0.0106$ \\
For-Profit & 0.0140 & $0.0135-0.0145$ \\
Teaching & 0.0108 & $0.0104-0.0111$ \\
Non-teaching & 0.0128 & $0.0125-0.0131$ \\
Percent Medicaid & 0.0153 & $0.0147-0.0158$ \\
patients, bottom & & \\
$\begin{array}{l}\text { 10\%* } \\
\text { Percent Medicaid }\end{array}$ & & $0.0095-0.0098$ \\
patients, top 90 \% & 0.0097 & \\
Disproportionate & & $0.0108-0.0112$ \\
Share Hospital & 0.0110 & \\
(DSH) Funding & & $0.0143-0.0152$ \\
Non-DSH & 0.0148 & \\
\hline
\end{tabular}

*Corresponds to $<2.5 \%$ of bed-days

Annual Case Mix Index Growth Before and After Implementation of the MS-DRG Program

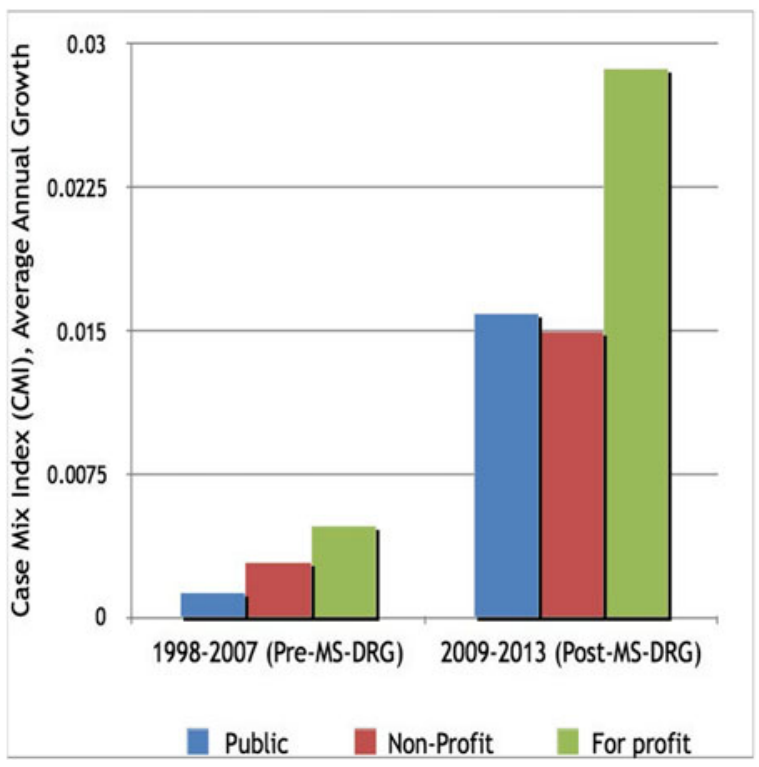




\section{MEDICATION REGIMEN COMPLEXITY AND PREDICTION OF} MEDICATION DISCREPANCIES Chirag Patel ${ }^{2}$; Kristin Zimmerman ${ }^{4}$; Jennifer R. Fonda ${ }^{1}$; Amy Linsky ${ }^{1,3}$. ${ }^{1}$ VA Boston Healthcare System, Boston, MA; ${ }^{2}$ Massachusetts College of Pharmacy \& Heath Sciences, Boston, MA; ${ }^{3}$ Boston University School of Medicine, Boston, MA; ${ }^{4}$ Virginia Commonwealth University School of Pharmacy, Richmond, VA. (Control ID \#2468797)

BACKGROUND: Complex medication regimens are associated with adverse events, non-adherence, and an increased risk of hospitalization and mortality. Nearly $25 \%$ of adverse drug events in ambulatory care settings are associated with medication discrepancies. The Medication Regimen Complexity Index (MRCI) is a validated and accepted tool that integrates information about dosage form, frequency, and additional directions to provide a numerical score for medication complexity. We sought to evaluate the relationship MRCI scores with medication discrepancies in US Veterans seen in ambulatory care, hypothesizing that increased MRCI scores will be associated with increased discrepancies.

METHODS: We conducted a retrospective cohort study of a convenience sample of patients $(N=104)$ seen in ambulatory care clinics at VA Boston, $4 / 2010-7 / 2011$, by fourth-year medical students. Students performed medication reconciliation to a computer-generated medication list ("List") that included all medications and nondurable medical supplies dispensed at VA Boston (i.e., locally-dispensed), from other VA facilities (i.e., remotely-dispensed) and non-VA sources. Our primary outcomes were 1) the presence of any medication discrepancy, and 2) a specific subtype of discrepancies, commissions (where the medication is active on the list but the patient is not taking). We calculated the primary exposure-MRCI scores - utilizing medication lists before medication reconciliation as these are comparable to the prescribed medication list available in the medical record. We describe the distributions of the outcomes, primary exposure, and covariates [patient age ( $>65$ vs. $<65$ years), sex, care provided at other VA facilities (yes vs. no) and presence of non-locally dispensed medications (yes vs. no)]. There are no established thresholds for MRCI to predict discrepancies; thus, we dichotomized MRCI scores at the median for use in analyses. Separate multivariable logistic regression models estimated the associations between dichotomized MRCI scores and any medication discrepancies or commissions. We also examined the sensitivity and specificity of other cutoffs to predict discrepancies using receiver operating characteristic (ROC) curves.

RESULTS: Of the 104 patients analyzed, 99 (95\%) were male, 59 (57\%) were over age $65,16(15 \%)$ had care at a VA system other than Boston, and $50(48 \%)$ had documentation of non-locally dispensed medications. The median total number of medications on the list was 8 (interquartile range [IQR] 5-13), and the median MRCI score was 25 (IQR 14.25-42.50). Of these patients, 62 (60 \%) had any discrepancy and 37 (36 \%) had a commission. In unadjusted analyses, having a high MRCI score was not statistically associated with the presence of any discrepancy (OR 1.73, $95 \%$ CI 0.78-3.81); however, patients with a high MRCI score were more likely to have commissions (OR 2.90, 95\%CI 1.25-6.74). In analyses adjusting for age, sex, multiple sites of care, and multiple dispensing facilities the results were similar, remaining non-significant for any discrepancy (OR 1.63, 95\% CI 0.68-3.88) and significant for commissions (OR 3.64, 95 \% CI 1.419.41). In unadjusted ROC curves, using the median MRCI score of 25 as a cutoff had a sensitivity of $68 \%$ and a specificity of $58 \%$ of detecting a commission.

CONCLUSIONS: We found that patients with MRCI scores greater than 25 were more likely to have commissions. This index may serve as a screening tool to detect patients with potential medication discrepancies who would benefit from a more intensive medication review. As team-based care expands in primary care, this could potentially be performed by other members of the team, such as a clinical pharmacist. Identifying discrepancies and other problematic prescribing in a proactive, systematic manner has the potential to reduce adverse drug events and hospitalizations, leading to improvements in overall health outcomes.
METABOLIC CHARACTERISTICS OF ESOPHAGEAL CARCINOMA IN A VETERAN POPULATION Teresa Ratajczak ${ }^{1,2}$; Heidar Albandar ${ }^{1,2}$; Parker Redlien ${ }^{1,2}$; Sara E. Yacyshyn ${ }^{3}$; Ronald J. Markert'; Geetika Kumar ${ }^{1,2}$. ${ }^{1}$ Wright State University, Beavercreek, OH; ${ }^{2}$ Dayton Veteran Affairs Medical Center, Dayton, OH; ${ }^{3}$ Wright State University Boonshoft School of Medicine, Dayton, OH. (Control ID \#2438202)

BACKGROUND: Esophageal cancer is projected to increase by $35 \%$ through 2025 , representing $1.0 \%$ of all new cancers and becoming the 18 th most common malignancy in the U.S. Studies have shown a relationship between increased body mass index (BMI) and risk of adenocarcinoma (EAC), but decreased risk of squamous cell carcinoma (SCC). SCC was shown to occur more in patients with hypertension. We investigated whether the presence of metabolic syndrome components differed between these two types of esophageal cancer in our veteran population. History of gastroesophageal reflux disease (GERD) was also evaluated. METHODS: Records of patients diagnosed with esophageal carcinoma between 1996 and 2014 were reviewed. Patients without biopsy proven carcinoma and incomplete documentation were excluded. Information on demographics, BMI before diagnosis, hypertension, hyperlipidemia, hyperglycemia, GERD, and diabetes mellitus (DM) type 2 was collected from charts. Statistical analysis was performed using IBM SPSS Statistics 22.0 software and proper data-dependent statistical testing.

RESULTS: Of the 118 eligible patients, 81 were classified as having adenocarcinoma (EAC) and 37 as having squamous cell carcinoma (SCC). Age at diagnosis for EAC and SCC was $67.1 \pm 9.7$ vs. $67.0 \pm 10.8$ years, $(p=0.83)$. Compared to SCC, patients with EAC were more likely to be white than African American ( 98.8 vs. $59.5 \%, p<0.001$ ). Veterans with EAC had a higher mean pre-diagnosis BMI than those with SCC $(29.7 \pm 6.5$ vs. $25.0 \pm 8.0 \mathrm{~kg} / \mathrm{m} 2, p<0.001)$. Patients with EAC were more likely to have hyperlipidemia ( 59 vs. $35 \%, p=0.015$ ), GERD ( 51 vs. $22 \%, p=0.003$ ), hyperglycemia ( 44.4 vs. $27.0 \%, p=0.07$ ) and type $2 \mathrm{DM}(37$ vs. $19 \%, p=0.049)$ than those with SCC. The two groups did not differ on HTN (59.3 vs. $56.8 \%, p=0.80)$. Pre-diagnosis BMI (OR=1.11; $95 \% \mathrm{CI}=1.01$ to 1.21$)$ and $\mathrm{GERD}(\mathrm{OR}=3.60 ; 95 \% \mathrm{CI}=1.27$ to 10.19$)$ were independent predictors of type of cancer-i.e., significant after controlling for the other variables. Hyperlipidemia ( $\mathrm{OR}=1.94$ [0.63 to 5.92]) and Type $2 \mathrm{DM}(\mathrm{OR}=1.08$ [0.32 to 3.67]) were not independent predictors of type of esophageal cancer.

CONCLUSIONS: We found that type of cancer (EAC or SCC) was associated with presence of certain components of metabolic syndrome (i.e., pre-diagnosis BMI, GERD, hyperlipidemia, and diabetes mellitus type 2) with the former two being independent predictors of EAC when compared to SCC. With the increasing incidence of obesity, GERD, and metabolic syndrome in the Western world, it is important to evaluate the relationship of common morbidities to esophageal carcinoma, which for EAC has also risen. Characteristics of the two malignancies

\begin{tabular}{llll}
\hline \hline Cancer type & $\begin{array}{l}\text { adenocarcinoma } \\
\text { (EAC) }\end{array}$ & $\begin{array}{l}\text { squamous cell } \\
\text { carcinoma } \\
(\mathrm{SCC})\end{array}$ & $P$ value \\
Number of patients & 81 & 37 & \\
$\begin{array}{l}\text { Age at diagnosis } \\
\text { in years }\end{array}$ & $67.1 \pm 9.7$ & $67.0 \pm 10.8$ & $p=0.83$ \\
White & $98.8 \%$ & $59.5 \%$ & \\
African American & $1.2 \%$ & $40.5 \%$ & $p<0.001$ \\
pre-diagnosis & $29.7 \pm 6.5$ & $25.0 \pm 8.0$ & $p<0.001$ \\
BMI kg/m2 & $59 \%$ & $35 \%$ & $p=0.015$ \\
Hyperlipidemia & $51 \%$ & $22 \%$ & $p=0.003$ \\
GERD & $44.4 \%$ & $27.0 \%$ & $p=0.07$ \\
Hyperglycemia & $37 \%$ & $19 \%$ & $p=0.80$ \\
type 2 DM & $59.3 \%$ & $56.8 \%$ & \\
HTN & & & \\
\hline
\end{tabular}

MULTIVARIATE ANALYSIS:

\begin{tabular}{ll}
\hline \hline Pre-diagnosis BMI & $\mathrm{OR}=1.11 ; 95 \%(\mathrm{CI}=1.01$ to 1.21$)$ \\
GERD & $\mathrm{OR}=3.60 ;(95 \% \mathrm{CI}=1.27$ to 10.19$)$ \\
Hyperlipidemia & $\mathrm{OR}=1.94[0.63$ to 5.92$])$ \\
Type 2 DM & $1.08[0.32$ to 3.67$])$ \\
\hline
\end{tabular}


METHODS FOR MEASURING PRIMARY CARE PROVIDERS' QUALITY OF CARE FOR URINARY INCONTINENCE Lueng S. Tcheung ${ }^{1,2}$; Kristina M. Cordasco ${ }^{1 \text {, }}$ 2; Marjorie Danz ${ }^{1,3}$; LaShawnta Bell-Lewis ${ }^{1}$; Donna L. Washington ${ }^{1,}{ }^{2}$. ${ }^{1}$ VA Greater Los Angeles Healthcare System, Los Angeles, CA; ${ }^{2}$ University of California Los Angeles Geffen School of Medicine, Los Angeles, CA; ${ }^{3}$ RAND, Santa Monica, CA. (Control ID \#2468648)

BACKGROUND: Efficient methods are needed for adapting clinical guidelines and standards of care for use in program evaluation of the care documented in electronic medical records (EMRs). Despite the high prevalence of urinary incontinence in primary care practice, there are insufficient EMR-based tools to evaluate the quality of care for urinary incontinence in primary care. We formulated methods for identifying, operationalizing, and developing tools for applying quality indicators (QIs) to measure the assessment and management of urinary incontinence in women from VA EMR abstractions.

METHODS: We first performed a literature scan to identify existing evidence-based guidelines and QIs for the primary care management of urinary incontinence in women. Then, to define the clinical encounters, or "episodes of care," to which identified QIs would be applied, we defined: (a) "index events"-points of care that define inclusion in the sample and are an anchoring point for measurement; and (b) appropriate timeframes relative to the index event for applicable clinical actions to occur. In determining these timeframes, we solicited input from two urinary incontinence specialists, accessed web-based resources from professional societies, and scanned the literature for related reviews and guidelines. Third, for each QI, we determined specifications - the numerator, denominator, inclusion and exclusion parameters, and relevant defined terms. We then developed tools for utilizing these QIs by drafting two abstraction forms - one that assesses episodes of care for sample inclusion/exclusion criteria, and a second that collects from these episodes information needed for each QI. Forms consist of a series of questions, with closed-response options, for a medical record abstractor to answer based on reviewing a medical record. We used branching logic so that abstractors will only answer questions relevant to the clinical scenario. For each question on the form, we specified which EMR elements (e.g., progress notes, orders, labs) are to be used in answering the question. We also developed guidelines for the abstractors to use as a reference in answering each question. Finally, the form was programmed into an electronic data entry platform (REDCap-VA Research Electronic Data Capture) and iteratively revised by testing on a sample of EMR extracts.

RESULTS: Our literature yielded 8 sources of guidelines and 1 source of QIs. The QI source (Anger, et al.) included evidence-based QIs that were developed using expert panel methodology supported by a literature review that incorporated the evidence underlying the guidelines; therefore, we selected that source for form development. From the 28 QIs that are in the Anger article, we identified 11 as being measurable and applicable to the primary care setting, detailed enough to adapt for use with EMRs, and spanning the breadth of assessment and management of the various types of urinary incontinence (e.g., stress, urge incontinence). Of the 11 identified QIs, four pertain to obtaining details from the medical history, two pertain to elements of the physical exam, one pertains to laboratory evaluation, and four pertain to treatment of urinary incontinence. To operationalize these QIs, we defined two index events: (1) the day a primary care provider referred the patient to specialty care for management of UI; (2) the day a PCP ordered medication to treat UI. We specified that points of care occurring within the 18 months prior to or on the Day of the Index Event would be assessed for the occurrence of applicable clinical actions. The form developed to determine if the patient record meets inclusion/exclusion criteria has 14 questions and the form to determine adherence with quality indicators has 11 questions; with branching logic, only a subset of questions are applicable to each patient's EMR extract.

CONCLUSIONS: Using published quality indicators, we developed tools to determine the quality of primary care assessment and management of urinary incontinence in VA women patients. These tools may be utilized to measure adherence of primary care practice with evidence-based guidelines, as well as potential variations by providers, practice settings, and patient populations. Such data may guide quality improvement initiatives. Further, our methods for adapting existing measures of quality may be applied to other conditions for use in program evaluation and health services research using electronic medical records.

MINDFULNESS TO IMPROVE MENTAL HEALTH AMONG HOMELESS YOUTH: A PILOT INTERVENTION STUDY Leah Swanson ${ }^{1}$; Scott B. Harpin ${ }^{2}$. ${ }^{1}$ UNIVERSITY OF COLORADO ANSCHUTZ MEDICAL CAMPUS, Louisville, CO; ${ }^{2}$ University of Colorado, Aurora, CO. (Control ID \#2463638)

BACKGROUND: Approximately two million U.S. youth experience homelessness yearly. These youth suffer from high rates of mental illness, substance use, and sleep disorders. This is compounded by reticence to access traditional care and transiency among homeless youth. With few effective interventions available, there is need for new, holistic approaches to address these issues. Mindfulness, a practice of present moment awareness, is effective for mental health and sleep-related disorders in adults, yet little is known about its utility among homeless youth. Given the overlapping symptomatology of mental illness, sleep deficit and substance use, this pilot study was designed to evaluate the feasibility of Mindfulness as an alternative therapy for these problems in homeless youth.

METHODS: This pilot study employed a prospective single group design. Participants were 22 homeless English speaking youth ages 18-24. Study settings were two long-term housing units run by Urban Peak, Denver's non-profit serving homeless youth. The intervention consisted of 4 weekly 1-h Mindfulness sessions designed by a certified Mindfulness instructor and adapted for homeless youth during summer 2015. Pre- and post-surveys measured the following primary outcomes: mental distress (Center for Epidemiologic Studies Depression Scale [CES-D]), overall sleep quality (Pittsburgh Sleep Quality Index [PSQI-Q6]), and dispositional Mindfulness (Mindful Attention Awareness Scale-Adolescent [MAAS-A]). Self-compassion (Self-Compassion ScaleShort Form [SCS-SF]) was evaluated as an additional measure of Mindfulness. Scores were analyzed using paired t-tests.

RESULTS: Twenty-two youth consented at the beginning of the 4-week intervention period. Among those 22, three youth completed one to two sessions and eight completed three to four sessions. Eight youth (36\%) had no post-surveys or session attendance data. Incomplete data resulted from youth leaving the center or participating in sessions without completing surveys. Both pre- and post-surveys were available for 12 participants ( $55 \%$ ). Among these 12, overall sleep quality improved significantly (PSQI-Q6 change $=0.67$; $t=2.11 ; p<.05$ ). Mental distress and self-compassion trended toward improvement but were not statistically significant (CES-D change $=4.16, p=0.036$; SCS-SF change $=0.11$, $p=0.64)$. Mindful state, however, decreased with the intervention (MAAS-A change $=$ $0.08, p=0.86$ )

CONCLUSIONS: This pilot study demonstrated significant improvement in overall sleep quality. Trends toward improvement in mental distress and self-compassion were observed. Although mindful state as measured by MAAS-A did not show improvement, it is possible the youth had a greater understanding of the meaning of Mindfulness following the training sessions, leading to more reflective responses. Completion rates were acceptable, suggesting feasibility of implementation in this population and setting. The intervention was easily integrated into typical programming and well-received by youth. Overall, these results suggest Mindfulness training may be a useful adjunctive treatment in this vulnerable, transient population. Future studies should take additional steps to increase retention and evaluate the number and types of sessions in addition to feasibility in different housing environments, 
such as overnight shelters and drop-in centers. Larger studies are needed to substantiate effectiveness in this population.

\section{MINORITIES ARE MORE LIKELY TO PARTICIPATE IN COMPLEX} COMPARED TO SIMPLE CLINICAL RESEARCH STUDIES Leonardo Tamariz ${ }^{1}$; Ana M. Palacio ${ }^{1}$; Olveen Carrasquillo ${ }^{1}$; Erin N. Marcus ${ }^{2} .{ }^{1}$ University of Miami, Miami, FL; ${ }^{2}$ University of Miami Miller School of Medicine, Sylvester Comprehensive Cancer Center, Miami, FL. (Control ID \#2470118)

BACKGROUND: Minorities are underrepresented in clinical trials. A potential explanation for this underrepresentation could be lack of willingness to participate. The aim of our study was to evaluate willingness to participate in different hypothetical clinical research scenarios and evaluate the role that predictors like health literacy might have on the willingness of minorities to participate in clinical research studies.

METHODS: We conducted a mixed methods study at the Miami VA Healthcare system and included hypertensive primary care patients. We measured willingness to participate as a survey of 4 clinical research scenarios that evaluate common study designs encountered in clinical research and that increase in complexity. Our qualitative portion included comments provided in the scenarios

RESULTS: We included 123 hypertensive patients in our study. Of the entire sample 93 were minorities. Seventy percent of the minorities were more willing to participate compared to $60 \%$ of the non-minorities. The OR of willingness to participate in simple studies was $1.11 ; 95 \% \mathrm{CI} 0.48-2.53) p=0.79$ and the OR of willingness to participate in complex studies was $5.43 ; 95 \%$ CI 1.20-24.45) $p=0.02$. In complex studies minorities with low literacy cited obtaining benefits $(47 \%)$ as the most common reason to be willing to participate. Minorities not willing to participate cited fear of unintended outcomes as the reason as the reason for being willing to participate.

CONCLUSIONS: Contrary to popular belief minorities are more willing to participate in clinical trials. Low literacy is an important mediator in reasons cited to participate and not participate.

MOBILE APPS FOR VULNERABLE POPULATIONS STUDY Urmimala Sarkar ${ }^{3,2}$; Gato I. Gourley $^{3,2}$; Courtney Lyles $^{3,2}$; Lina Tieu ${ }^{3,2}$; Cassidy Clarity ${ }^{3,2}$; Lisa Newmark ${ }^{4}$; Karandeep Singh ${ }^{4}$; David W. Bates ${ }^{1} .{ }^{1}$ Brigham and Women, Boston, MA; ${ }^{2}$ University of California, San Francisco, San Francisco, CA; ${ }^{3}$ University of California San Francisco, San Francisco, CA; ${ }^{4}$ Partners HealthCare System, Wellesley, MA. (Control ID \#2469434)

BACKGROUND: Mobile applications or 'apps' intended to help people manage their health and chronic conditions are widespread and gaining in popularity. However, little is known about their acceptability and usability for low-income, racially/ ethnically diverse populations who experience a disproportionate burden of chronic disease and its complications. The overall objective of this study was to investigate the gaps between existing mobile applications and the needs of vulnerable populations and to provide recommendations to app developers.

METHODS: Participants were recruited using convenience sampling. Flyers were posted in the San Francisco General Hospital (SFGH) General Medicine Clinic (GMC) waiting room for each of the three categories of the study allowing participants to self-select for one or more of each of the three categories of apps: Type 2 Diabetes; Caregiving; and Depression. Participants were also co-recruited with another study based out of the Center for Vulnerable Populations evaluating the usability of a new patient portal for SFGH patients. Patients that qualified for each study were referred to the other study and vice versa. The third method was through presenting the study to diabetes support groups at SFGH and providing participants there with contact information about the three categories. The fourth method was through provider referral at the
SFGH GMC. Participant word of mouth also helped with recruitment, as participants occasionally informed family members or friends about the study. 26 participants were interviewed, with 3 participants interviewing twice as they qualified for two categories of apps for a total of 29 interviews across all three categories. Interviews were approximately $2 \mathrm{~h}$. The participants were diverse: $58 \%$ African American, $27 \%$ White, $8 \%$ Asian, $8 \%$ Latino with either diabetes, depression, or were caregivers. Usability testing was conducted with participants ( $n=9$ caregivers; $n=10$ patients with depression; and $n=10$ patients with diabetes) on a total of 11 of the most popular health apps ( 4 diabetes apps, 4 depression apps, and 3 caregiver apps) on both iPad and Android tablets. Participants were given a specific task to complete, such as entering a blood glucose value into a diabetes app. Participant interviews were video recorded and were coded to evaluate attempts and completions of tasks. The 3 coders first coded the same interview in order to calibrate their coding and come to agreement on definitions of tasks and codes before coding subsequent videos individually.

RESULTS: On average it took participants about $2 \mathrm{~h}$ to complete all the tasks for each of the four apps in a particular category. Participants completed 76/191 (40\%) of tasks across 11 apps without assistance. Three themes emerged from participant comments: lack of confidence with technology, frustration with design features and navigation, and interest in having technology to support their self-management. Participants expressed lack of confidence with technology: ( "I'm not good at this at all"); frustration with design features and navigation ( "it's like saying, 'haha you pressed the wrong button', so why even have it"), but also expressed an interest in having technology to support their self-management ("Absolutely. I wouldn't have to wait for somebody else to tell me about me')

CONCLUSIONS: App developers should employ participatory design strategies with diverse patients in order to truly have an impact on chronic conditions like diabetes and depression which disproportionately affect vulnerable populations. While patients express interest in using technologies for self-management, current tools are not consistently usable for diverse patients receiving care at San Francisco General Hospital.

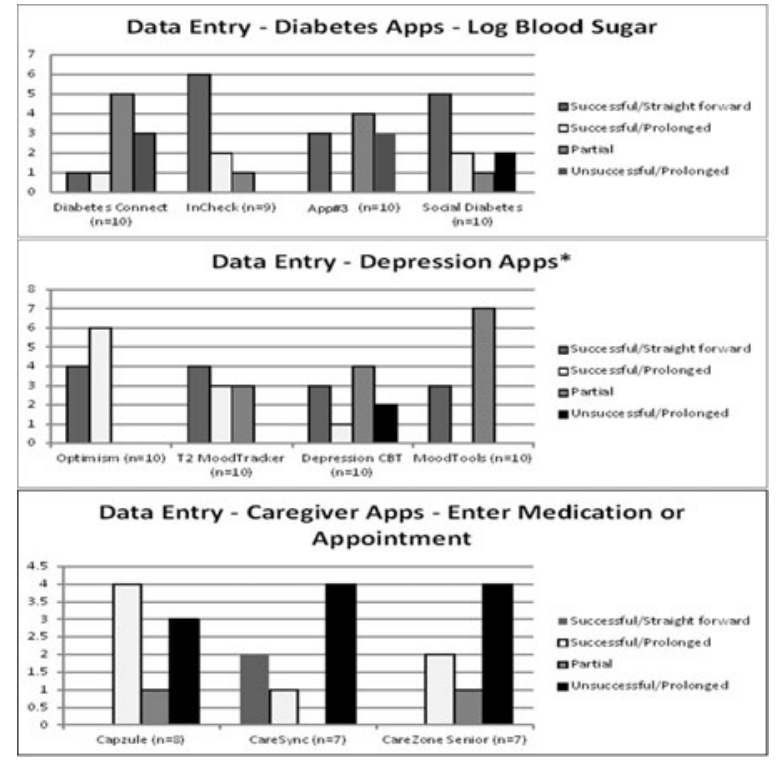

MORBIDITY AND MORTALITY PROJECT: LEARNING FROM UNINTENDED MEDICAL ERRORS AND ADVERSE EVENTS SAAD EMHMED ALI; Adnan Elghezewi. PRESENCE SAINT FRANCIS HOSPITAL, Chicago, IL. (Control ID \#2463733)

BACKGROUND: Morbidity \& Mortality (M\&M) conference provides a safe platform for the residents to discuss unintended medical errors and adverse events, helps 
identifying areas of improvement in patient care and promotes professionalism, ethical integrity and transparency in an academic teaching institution. We conducted a questionnaire-based survey for internal medicine residents every month prior to the $M \& M$ conference. Questions pertaining to the case were asked prior to the discussion, and the answers were reviewed after the didactics at the end of the conference. Resurveys were done a few months later with similar questions to assess the retained knowledge.

METHODS: During the monthly M\&M conference conducted for the past 24 months (2013 to 2015), 4 multiple-choice questions are prepared for each case and answers from internal medicine residents were recorded using an audience response system. This is considered as "Pre-conference data". Then the case was discussed in detail followed by a power point presentation on topics related to the case, highlighting the correct practices and most recent guidelines. The correct answers were revealed at the end of the conference and comprehensive explanations were given for each question. After 3 months, re-surveys were done using the same set of questions and recorded as "post-conference data". Both pre and the post conference internal medicine residents were the same. Collected data was analyzed using the Paired t-test.

RESULTS: Data was collected for 24 M\&M conferences held during the academic year 2013 to 2015 . Pre- conference data was defined as "Population 1 " and Post conference resurvey data was defined as "Population 2". Analysis revealed that the mean for population 1 was $59.06 \pm 14.58(n=35)$, whereas the mean for population 2 was $73.54 \pm 12.56(n=35)$. Paired t-test was applied for population 1 vs. population 2. A significant improvement in correct responses on random resurvey after few months was noted (Mean improvement $-14.48 \pm$ $6.55,95 \%$ CI- $11.84-17.12, p<0.001)$

CONCLUSIONS: There was a significant increase in the percentage of correct responses to the questions after the didactic discussion in the conference even after a few months and the difference was statistically significant. The detailed discussion of the case and the PowerPoint presentation has helped in improving the knowledge among the residents which in turn should help to decrease similar adverse events and improve patient safety. To further improve the house staff awareness and knowledge we uploaded the PowerPoint presentations on the residents portal. We are also planning to start sending the monthly newsletters to involve them more in learning. In conclusion, the monthly M\&M conferences have shown a positive outcome in our hospital by improving upon resident knowledge and retaining important objectives to improve patient safety.

MORNING REPORT FOR MILLENNIALS: RE-EXAMINING A LEARNERDRIVEN EDUCATIONAL CONFERENCE. Timothy Anderson ${ }^{1}$; Eliana Bonifacino $^{1}$; Andrea Carter $^{1}$; Camille M. Webb ${ }^{1}$; Melissa McNeil ${ }^{2}$; Shanta M. Zimmer ${ }^{2} .{ }^{1}$ UPMC, Pittsburgh, PA; ${ }^{2}$ University of Pittsburgh, Pittsburgh, PA. (Control ID \#2465943)

BACKGROUND: Resident report, in which residents present recent clinical cases they have encountered to their peers, faculty and chief residents, has been a cornerstone of internal medicine graduate medical education for many generations. Some training programs have discontinued resident report due to competing curricular demands and a concern that report lacks a formal curriculum to achieve learning objectives. As teaching priorities change, we sought to examine the characteristics of resident report cases and discussion points at a large tertiary academic medical center residency program to better understand the role of resident report in the millennial era.

METHODS: At the University of Pittsburgh Medical Center (UPMC) there are daily resident reports at three residency training sites: UPMC Presbyterian - the primary residency tertiary care hospital, UPMC Shadyside - a community affiliate hospital, and the Pittsburgh VA Medical Center. Conference are attended by internal medicine residents, chief medical residents and one or two invited faculty members. Patients are chosen by residents from those whom they have seen in the hospital in the preceding 6 months, occasionally with the assistance of a chief resident. For this study, the chief medical residents at UPMC compiled data on resident reports for 5 months, from July through November 2015. They collected patient age, gender, chief complaint, final diagnosis, and primary specialty of the case. Additionally, chief residents evaluated each case after the conference to determine which if any of the following topics were discussed: patient safety, high value care, clinical reasoning, identification of bias, social determinants of health, and utility of the physical exam. RESULTS: Two hundred eighty-three morning report cases were recorded across the three educational sites. $43.8 \%$ of patients were female and $40.1 \%$ were over the age of 65 . There were a wide variety of chief complaints with the most common being dyspnea (35), musculoskeletal pain (27), altered mental status (26), abdominal pain (19), fever (19), chest pain (16), skin changes (15), and cough (10). Figure 1 shows the primary specialty of final diagnosis for each case. One third of cases were classified as "Zebras" or very rare diagnoses (101/283), with the remainder being common diagnoses (139) and four cases with no known diagnosis. The majority of case discussions focused on diagnosis of the underlying etiology $(155 / 283$ or $54.8 \%$ ), 51 focused on disease management and 77 on both diagnostics and management. Chest x-rays were reviewed in $32.1 \%$ of cases, electrocardiograms in $14.8 \%$ and arterial blood gases in $8.9 \%$. Figure 2 demonstrates the frequency of topic discussion, with the majority of cases including a discussion of physical exam utility and clinical reasoning.

CONCLUSIONS: In a single-residency, three-site observational study, we find resident report to be a unique learner-directed conference in which peer-to-peer discussion addresses multiple curricular priorities. Report cases included rare and common diseases from all medical specialties. Discussions covered essentia topics such as high value care, patient safety, and clinical reasoning, as well as foundational physical exam and diagnostic test interpretation skills.

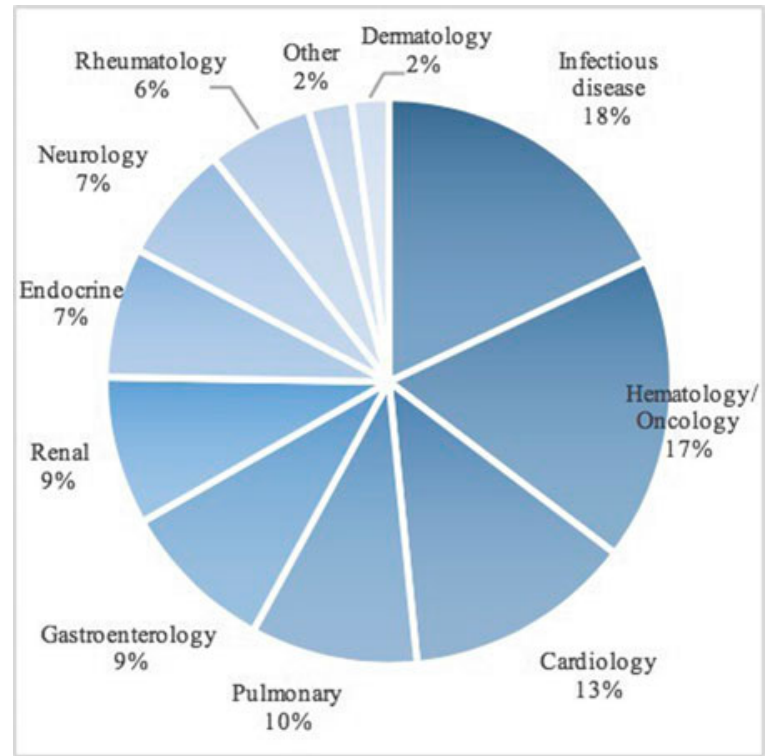

Figure 1: Specialty of final diagnosis of resident report cases. 


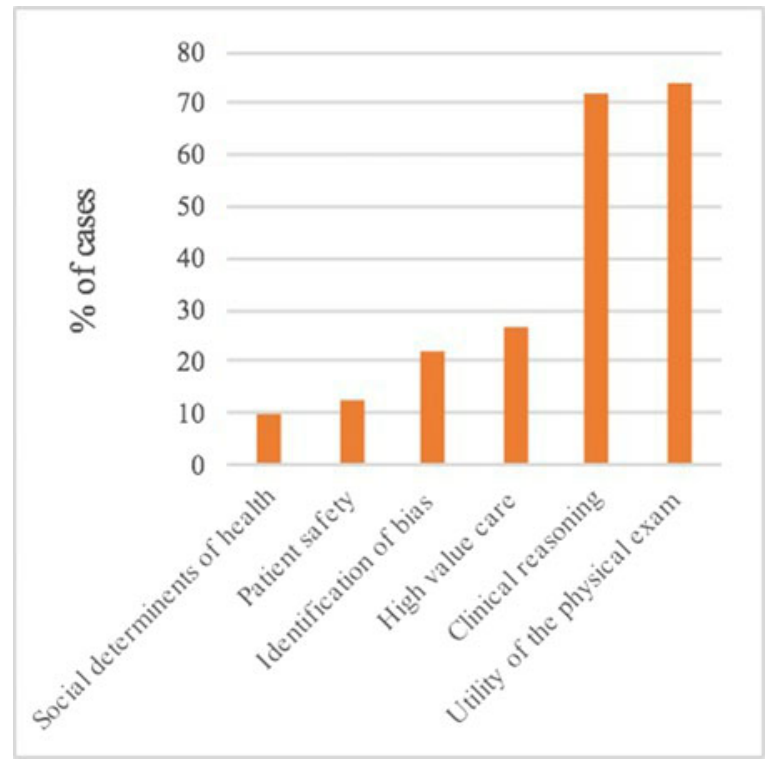

Figure 2: Educational topics discussed in resident report.

\section{MORTALITY AFTER CRITICAL CARE HOSPITALIZATION IN PATIENTS} WITH PSYCHOTIC DISORDERS Daniel Weisberg; Paula Chatterjee; Emily D. Bethea; Maria Yialamas; Kenneth B. Christopher; on behalf of the Brigham and Women's Internal Medicine Housestaff. Brigham and Women's Hospital, Boston, MA. (Control ID \#2468533)

BACKGROUND: Medical inpatients have a high burden of co-morbid psychiatric illness. There is limited understanding of the post-discharge hospital outcomes in patients with serious psychiatric illness. We hypothesized that a diagnosis of a psychotic disorder in patients who survived critical care would be associated with adverse outcomes following hospital discharge. METHODS: We performed a two center observational cohort study of patients treated in medical and surgical intensive care units (ICUs) in Boston, Massachusetts. All data was obtained from the Research Patient Data Registry at Partners HealthCare. We studied 82,583 patients, age $\geq 18$ years, who received critical care between 1997 and 2012 and survived hospitalization. The exposure of interest was a psychotic disorder without depression diagnosed in the 5 years prior to a hospital admission involving critical care. Psychotic disorders were determined by ICD-9 code 259x without ICD-9 $296.20-296.36$ or 300.40 or 311.00 . The primary outcome was mortality in the 90 days following hospital discharge. Secondary outcome was discharge disposition. Adjusted odds ratios were estimated by multivariable logistic regression models with inclusion of covariate terms thought to plausibly interact with both psychotic disorders diagnosis and outcome. Adjustment included age, race (white versus non-white), gender, DeyoCharlson Index, patient type (medical versus surgical), sepsis, and acute organ failure. Changes from the expected hospital length of stay (LOS) were computed as the difference between the actual LOS and the geometric mean LOS for each DRG as determined by the Centers for Medicare \& Medicaid Services.

RESULTS: Two thousand five hundred forty-seven patients had a diagnosis of a psychotic disorder without a concomitant diagnosis of depression. The median [IQR] change from expected LOS was $1.4[-1,6.4]$ and $3.6[-0.1,11.4]$ days in patients with and without psychotic disorders respectively. Fifty-eight percent were male, $78 \%$ were white. The mean age was 61.2 years. the 90 -day post-discharge mortality was $7.2 \% .33 .1 \%$ were discharged to a care facility. The absolute risk of 90-day post-discharge mortality was 7.0 and $13.0 \%$ in patients with and without psychotic disorders respectively. The odds of an increase from the expected length of hospital stay in patients with psychotic disorders fully adjusted were 1.42 (95\%CI $1.29-1.57$; $P<0.001$ ), relative to patients without psychotic disorders. Further, the presence of psychotic disorders is a significant predictor of the adjusted odds of discharge to a care facility rather than to home [OR $1.87(95 \%$ CI $1.72-2.04 ; P<0.001)]$. Psychotic disorders was a robust predictor of post-discharge mortality and remained so following multivariable adjustment. The odds of 90-day post-discharge mortality in patients with psychotic disorders fully adjusted were 1.38 (95\%CI $1.22-1.57 ; P<0.001)$, relative to patients without psychotic disorders.

CONCLUSIONS: Patients with psychotic disorders are among a high-risk group of ICU survivors. In patients treated in a critical care unit who survive hospitalization, a psychotic disorder is a robust predictor of increase in mortality, hospital stay, and discharge to a care facility. These observational findings suggest the need for designing and testing interventions prioritizing safe transitions of care for ICU survivors with psychotic disorders.

MORTALITY ASSOCIATED WITH PEPTIC ULCER BLEEDING- ANALYSIS OF THE NATIONAL HOSPITAL DISCHARGE SURVEY David Mossad ${ }^{1}$; Ronald J. Markert ${ }^{1}$; Sangeeta Agrawal ${ }^{1,2}$. ${ }^{1}$ Wright State University, Dayton, OH; ${ }^{2}$ Dayton VA Medical Center, Dayton, OH. (Control ID \#2456018)

BACKGROUND: Patients presenting with upper gastrointestinal bleeding (UGIB) due to peptic ulcer disease (PUD) are acutely at high risk for morbidity and mortality. Multiple scoring systems stratify risk in these patients to assess the need for endoscopy, chances of re- bleeding, and mortality risk. The purpose of this study was to assess the effects of patient demographics, comorbidity burden, and hospital characteristics on in-hospital mortality in patients with UGIB due to PUD.

METHODS: We used ICD-9 diagnosis codes from the 2001-2010 National Hospital Discharge Survey to identify cases of UGIB due to PUD. Comparisons on demographics, number of comorbidities, hospital length of stay (LOS), and selected health system characteristics were made between those who died in-hospital and those discharged. SPSS was used for chi-square and t test analysis.

RESULTS: Among 12,196 cases of UGIB due to PUD, 499 died in-hospital and 11,697 were discharged. The mortality group was older (mean age of 74.4 vs 67.5 years, $p<0.001)$ and had a longer LOS (13.1 vs 6.2 days, $p<0.001$ ). There was no difference in mortality between genders ( $4.0 \%$ of males vs $4.2 \%$ of females, $p=0.71$ ) or race (Caucasians $4.5 \%$, African Americans $4.0 \%$ and others $3.5 \%, p=0.06$ ). Patients with seven or more comorbidities had a higher mortality rate $(5.1 \%)$ compared to those with six or fewer comorbidities $(1.4 \%)[p<0.001]$. Neither chronic kidney disease (CKD) $(p=0.40)$ nor coronary artery disease (CAD) $(p=0.43)$ impacted mortality. There was no difference in mortality between the Midwest (4.2\%), Northeast (4.9\%), South (3.7\%), and West (3.8\%). While increasing hospital size was associated with higher mortality ( $p=$ $0.001)$, hospital ownership was not $(p=0.89)$. Principal source of payment affected mortality rate, with Medicare having the highest mortality rate $(4.9 \%)$ and self pay $(2.0 \%)$ and Blue Cross/Blue Shield $(2.0 \%)$ having the lowest $(p<0.001)$. Sources of admission showed a significant difference in mortality, with transfers from skilled nursing/ intermediate care facilities $(7.7 \%)$ and other healthcare facilities $(12.3 \%)$ having the highest mortality rates $(p=0.002)$. Type of admission also affected mortality, with emergent admissions $(4.5 \%)$ showing the highest mortality rate $(p=0.038)$.

CONCLUSIONS: Prompt and accurate risk stratification in UGIB due to PUD is important due to the clinical urgency, potential need for endoscopy, and future risk of re-bleeding. This study found that age, LOS, hospital size, payment method, type of admission, and source of admission impacted in-hospital mortality. While comorbidity burden was related to mortality, neither CAD nor CKD specifically led to increased mortality. 
Mortality Rate for Patients Admitted with Upper Gastrointestinal Bleeding due to Peptic Ulcer Disease Based on Hospital Size

\begin{tabular}{ll}
\hline \hline Hospital Size (Number of Beds) & Mortality Rate (\%) \\
$6-99$ & 3.0 \\
$100-199$ & 3.2 \\
$200-299$ & 4.1 \\
$300-499$ & 4.9 \\
$\geq 500$ & 5.1 \\
\hline
\end{tabular}

$\mathrm{p}=0.001$

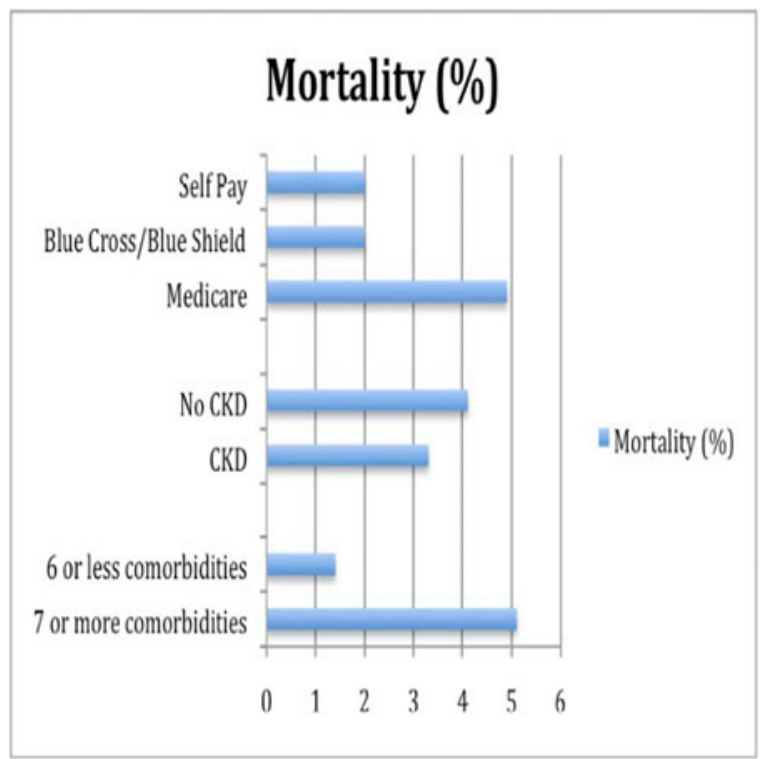

MULTIMORBIDITY AND LONG TERM PHYSICAL AND MENTAL HEALTHRELATED QUALITY OF LIFE IN COMMUNITY-DWELLING ADULTS Melissa Y. Wei ${ }^{2}$; Kenneth Mukamal ${ }^{1} .{ }^{1}$ BIDMC, Brookline, MA; ${ }^{2}$ University of Michigan Medical School, Ann Arbor, MI. (Control ID \#2469805)

Table 1. Change in the Short Form-36 physical and mental health-related quality of life scales and summary measures by multimorbidity weighted index in the Nurses' Health Study, $1992-2000$.

\begin{tabular}{|c|c|c|c|c|c|}
\hline Short Form-36 & Mean (SD) & $\begin{array}{l}\text { Beta coefficient } \\
\text { unadjusted* }\end{array}$ & Standard error & $\begin{array}{l}\text { Beta coefficient } \\
\text { adjusted } * \wedge\end{array}$ & Standard error \\
\hline Vitality & $51.4(9.1)$ & -0.78 & 0.009 & -0.70 & 0.012 \\
\hline Social functioning & $52.8(8.4)$ & -0.67 & 0.009 & -0.61 & 0.011 \\
\hline Role-emotional & $51.0(8.7)$ & -0.46 & 0.009 & -0.36 & 0.012 \\
\hline Mental health & $53.1(7.4)$ & -0.26 & 0.008 & -0.29 & 0.010 \\
\hline Mental Component Summary & $54.4(7.8)$ & -0.11 & 0.009 & -0.16 & 0.011 \\
\hline Physical functioning & 46.6 (10.5) & -1.36 & 0.011 & -1.10 & 0.012 \\
\hline Role-physical & 46.6 (11.5) & -1.21 & 0.012 & -1.02 & 0.015 \\
\hline Bodily pain & $48.0(9.1)$ & -1.01 & 0.009 & -0.91 & 0.011 \\
\hline General health & $52.0(9.5)$ & -1.07 & 0.009 & -0.95 & 0.012 \\
\hline Physical Component Summary & $46.4(10.8)$ & -1.50 & 0.011 & -1.24 & 0.012 \\
\hline
\end{tabular}

^Adjusted for age, race, body mass index, smoking status, geographic region* All $\mathrm{P}<0.001$

MY PATIENT CAN READ MY NOTE? RESIDENT AND ATTENDING PERCEPTIONS OF PATIENT ACCESS TO PHYSICIAN OPEN NOTES Deepa $\underline{\text { R. Nandiwada }}{ }^{1}$; Gary Fischer ${ }^{1}$; Glenn M. Updike ${ }^{2}$; Molly B. Conroy ${ }^{1} .{ }^{1}$ University of Pittsburgh, Pittsburgh, PA; ${ }^{2}$ University of Pittsburgh Medical Center, Pittsburgh, PA. (Control ID \#2467671)
BACKGROUND: Multimorbidity is associated with decreased health-related quality of life (HRQOL) but this has not been quantified using a comprehensive multimorbidity weighted index (MWI) in community-dwelling adults. Prior studies have been limited by the number and scope of chronic conditions assessed, as well as the measure of multimorbidity, often using a simple disease count. The MWI was developed and validated using 81 chronic conditions and weights diseases by their impact on physical HRQOL. This study aims to quantify the impact of the MWI on long term physical and mental HRQOL.

METHODS: The Nurses' Health Study (NHS) is a prospective cohort of 121,700 female nurses aged 30-55 years when data collection began in 1976. In 1992, participants completed a questionnaire on physician-diagnosed chronic diseases and conditions. In 2000, participants completed the Medical Outcomes Study's 36-item Short Form Health Survey (SF)-36 that includes eight scales and two summary measures, the Physical Component Summary (PCS) and Mental Component Summary (MCS). These scales and scores are ranged 0-100 from lowest to highest functioning and standardized with a mean of 50 and standard deviation of 10 . The MWI was assessed as a continuous variable and in quartiles. We used multiple linear regression to measure the association between MWI as a continuous variable and 8-year HRQOL after adjusting for age, race, body mass index, smoking status, and geographic region. RESULTS: Of 103,981 women who returned questionnaires on chronic disease, we included 80,664 women who also completed the SF-36. At baseline, their mean age was 58.4 years (SD 7.2) and mean MWI was 2.7 (SD 3.3). The mean MCS score was 51.8 (SD 8.6) and mean PCS score was 49.6 (SD 9.3), close to general population norms. The mean mental HRQOL scales (social functioning, role-emotional, mental health) were on average higher than the mean physical HRQOL scales (physical functioning, role-physical, bodily pain), with general health and vitality shared between both mental and physical HRQOL dimensions. Each point increase in the MWI was associated with a 1.2 point decrease in long term PCS (coefficient $-1.24,95 \% \mathrm{CI}$ : -1.26 , $-1.21 ; p<0.0001$ ) and 0.16 decrease in long term MCS (coefficient $-0.16,95 \%$ CI: -0.18 , $-0.13 ; p<0.0001)$ in the fully adjusted models

CONCLUSIONS: In this study of over 80,600 women with both a MWI and HRQOL, MWI was associated with over a one-point decline in long-term physical HRQOL scales and PCS score. The association between the MWI and mental HRQOL was of smaller magnitude, with a mild decrease in 8-year predicted MCS. Our results suggest that a MWI may be used to predict future long term HRQOL, where a higher MWI score is associated with decrements in both physical and mental HRQOL, but a greater impact on physical HRQOL. 
Trainees need to be exposed to these EHR features to feel comfortable using them and maximizing their potential. There have been no studies to date assessing internal medicine residents' comfort with open notes as compared to attending physicians. We compared differences between resident and attending physicians' perceptions of open notes to identify barriers in order to facilitate optimal use of this feature.

METHODS: Starting November 2015, clinic notes in our primary academic general internal medicine clinic, in which both residents and attendings see patients, were made available to patients through our secure portal. Physicians had the option to document "hidden" notes as well if they felt there was a compelling reason. Attending physicians had 3 faculty meetings set up in the months prior to roll out of the pilot to provide feedback and address concerns related to implementation of open notes. A tip sheet was provided and reviewed immediately prior to roll out. Residents had a 20 min briefing session immediately before pilot roll out and were also provided a tip sheet. A standardized survey that is publically available on the website www.opennotes.org was given to all physician participants to assess their perceptions of current practices, benefits, patient impact and barriers to use of open notes prior to roll out. Descriptive statistics were generated evaluating the frequency of each response. Fishers Exact and Chi Square tests were used to assess for significant differences between attending and resident perceptions. We collapsed categories of responses for Likert scales on survey items so that "Disagree" included disagree and somewhat disagree and "Agree" included agree and somewhat agree. Concern responses were divided so that "Not Concerned" included "not concerned" and "minimally concerned" while "Concerned" included "moderately", "very", and "so concerned I do not want open notes". Free response comments on additional concerns regarding roll out of open notes were also assessed and categorized.

RESULTS: Survey response rates were $37 \%(n=54$ total) for resident physicians and $72 \%$ ( $n=44$ total) for attending physicians. Eighty percent of the combined group agreed open notes can empower patients and help them better understand their care plan. Sixty-three percent were concerned that access to notes would make their patients worry more. Eighty-two percent were concerned that their patients would contact them with questions about the notes. Residents were more concerned than attending providers about patients being offended by the content of their note ( 50 vs $23 \%, p=.005$ ). Both groups stated they would change their documentation about sensitive topics including cancer, mental health, and substance abuse $(61 \%, 70 \%, 70 \%$ for each topic respectively). Residents felt more likely to change documentation about weight than attendings ( 65 vs $34 \% ; p=0.03$ ). Residents also perceived an increased risk of litigation (50 vs $13 \% ; p=0.01)$. Fifty-three percent of attendings said they communicate almost daily with patients versus $0 \%$ of the residents $(p<.0001)$. Within the survey free response comments, providers expressed concerns about patient confusion regarding medical terminology, how to approach sensitive topics, and for patient requests for corrections of note content. One provider stated that "notes will be longer, less helpful for reference later as may leave out things to make [the patient] happy." In the introduction session with the residents, significant concern about how much additional work this would create for them and how they would have to change large amounts of their documentation was expressed. Many questions came up during the debriefing primarily related to legal concerns and patient misinterpretation of their notes which correlated with survey responses.

CONCLUSIONS: Though resident and attending providers shared some of the same perceptions about open notes, we found some significant differences. Both groups felt open notes have potential to empower patients but were concerned about discussing sensitive topics in notes. This needs assessment identified that residents were more concerned about offending patients and litigation. It is possible some of these differences exist as residents in our practice communicate with their patients significantly less outside of the clinic as compared to our attending physicians. Another contributing factor to resident discomfort may have been the lack of resident input into the roll out process that would allow them a venue to address these concerns. In order to prepare trainees to be comfortable with patient access to provider notes, these perceptions need to be addressed as well as curricula developed highlighting how to use this as a feature to empower patients Reference Article: 1. Delbanco T, Walker J, Bell SK, Darer JD, Elmore JG, Farag N, et al. Inviting Patients to Read Their Doctors' Notes: A Quasi-experimental Study and a Look Ahead. Ann Intern Med. 2012;157:461-470. doi:10.7326/ 0003-4819-157-7-201210020-00002

NARROWING HEALTHCARE DISPARITIES IN A UNIVERSITY OUTPATIENT CLINIC Elizabeth B. Kirkland; Benjamin Bevill; Frank C. Kurzynske; Jingwen Zhang; Patrick D. Mauldin; William P. Moran. Medical University of South Carolina, Charleston, SC. (Control ID \#2467601)

BACKGROUND: While the presence of healthcare disparities in the US is welldocumented in medical literature, there is limited evidence to support specific strategies to narrow the gap in healthcare outcomes as relate to underlying socioeconomic, racial, and other factors. In an attempt to assess the impact of system change on disparities within our clinic population, we utilized hemoglobin A1C (HgbA1C) and systolic blood pressure (SBP) as process measures for markers of population disease control. Baseline values were obtained before designation of our clinic as a patient-centered medical home (PCMH), and annually thereafter. We hypothesize that PCMH-based quality improvement initiatives will help us identify the impact of PCMH status on the magnitude of healthcare disparities between African-Americans (AA) and Caucasians (C) as indicated by improved rates of glycemic control and blood pressure.

METHODS: Data were analyzed from a cohort of patients whose blood pressure was considered "out of control" (criteria $\geq 140 \mathrm{SBP}$ ) and/or patients whose glycemic control was considered "out of control" (criteria HgbA1C $\geq 8$ ) for dates Oct 2011 (PCMH certification) annually to October 2015. Proportions were assessed using Chi-square analysis. Statistical significance was determined at the $5 \%$ level. SAS 9.3 was used for the statistical analysis.

RESULTS: At the time of PCMH certification, the inception cohort had 1531 patients with $\mathrm{SBP} \geq 140(72 \% \mathrm{AA}, 28 \% \mathrm{C})$ and 444 patients with $\mathrm{HgbA1C} \geq 8$ $(82 \%$ AA, $28 \% \mathrm{C})$. These values served as our denominators for subsequent comparisons. We observed significantly decreased rates of "out of control" patients in both races during the first year post-PCMH implementation. We also saw that Caucasians were controlled better at all time points for both measures compared to African-Americans (figure 1). While the longitudinal differences between races fluctuate, there appears to be a trend towards convergence in rates between races over the last 12 month period.

CONCLUSIONS: Through the analysis of HgbA1C and SBP as process markers before and after designation of our University Internal Medicine (UIM) clinic as a level $3 \mathrm{PCMH}$, we are able to identify disparities in rates of chronic disease control. Limitations of our study include that the data was obtained from a single center inception cohort susceptible to selection bias and regression to the mean. Further analysis is in process to assess the effects of team-based outreach to reduce the discrepancy in diabetic and hypertensive control among those of differing racial backgrounds. We propose that establishment of PCMH initiatives such as patient outreach and multidisciplinary care approaches be considered for diverse outpatient clinics in order to reduce well-documented healthcare disparities.

Rates of control of systolic blood pressure and hemoglobin A1c by race from October 2011 (pre-PCMH status) and annually thereafter. 


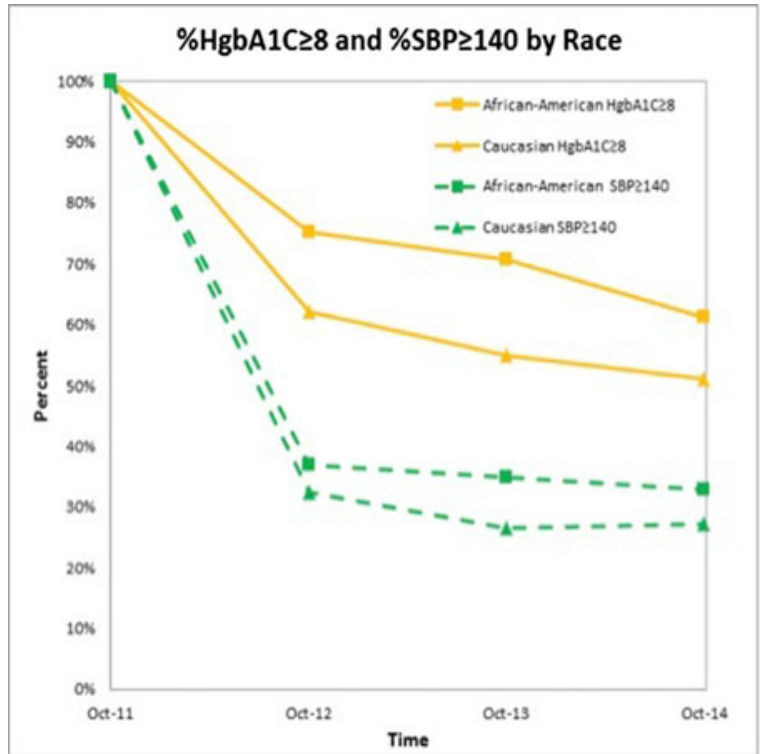

NATIONAL COLORECTAL CANCER SCREENING DISPARITIES AMONG OBESE ADULTS AGED 50-75 Ryan G. Seibert'; Amresh D. Hanchate'2; Paul C. Schroy ${ }^{1}$. ${ }^{1}$ Boston Medical Center, Boston, MA; ${ }^{2}$ Boston University School of Medicine, Boston, MA. (Control ID \#2469694)

BACKGROUND: Colorectal cancer (CRC) is the third leading cause of cancer and cancer mortality in the United States. Obesity is a well-established but under-recognized risk factor for $\mathrm{CRC}$ and $\mathrm{CRC}$-related deaths with stronger associations found in men Despite the mortality benefits of CRC screening, past studies have suggested obese adults receive less CRC screening, though results are mixed. As the prevalence of obesity increases with highest rates among vulnerable populations, addressing screening disparities is essential in minimizing unnecessary CRC morbidity and mortality. Physician screening recommendations are one of the most important factors contributing to screening adherence though little is known about CRC screening advice to the obese. The objective of this study was to elucidate differences in CRC screening and physician recommendations among obese men and women using a nationally representative sample. METHODS: Data from 9782 adults aged 50 to 75 years was obtained from the 2010 National Health Interview Survey (NHIS) and Cancer Control Supplement. NHIS is a self-reported, cross-sectional survey of U.S. citizens collected through stratified, multistage cluster design. Respondents in our study were excluded if they did not complete the Cancer Control Supplement $(n=264)$, had a personal history of CRC $(n=88)$, or were lacking height or weight information $(n=801)$, yielding a final sample size of 8629 . Screening outcomes included 1) overall guideline-adherent CRC screening (colonoscopy within 10 years, sigmoidoscopy within 5 years combined with a home fecal occult blood test (hFOBT) within 3 years, or hFOBT within the past year), 2) guideline-adherent endoscopic-based screening alone, and 3) guideline-adherent stool-based screening alone. Respondents not up-to-date with screening were asked whether they received a physician recommendation to screen within the past year. Analysis was performed using R software accounting for complex survey design. Weighted screening rates and adjusted odds ratios were calculated using multivariate logistic regression to explore the association between body mass index (BMI) category and the above outcomes for men and women. Covariates included sociodemographic factors, insurance status, number of doctor visits in the past year, usual source of care, smoking/alcohol use, number of comorbidities, region, family history of CRC, and history of colorectal polyps.
RESULTS: The overall national CRC screening rate for adults aged 50-75 was 58.8\% and similar between sexes. Among the obese subgroup with $\mathrm{BMI} \geq 30$, this rate was $59.27 \%$, not significantly different compared to the non-obese. When stratified by sex and obesity severity, men with a BMI $\geq 40$ (Obese class III) had significantly lower odds of meeting screening guidelines (OR 0.32, $95 \%$ C.I. 0.14-0.70) and of using endoscopic-based screening (OR 0.33, 95 \% C.I. 0.15-0.72) compared to normal weight men. There was no significant BMI-related difference in the odds of hFOBT use among men and no significant difference in any screening for obese women. Among those not up-to-date, obese women with $\mathrm{BMI} \geq 35$ (Obese class II/III) were more likely to report receiving a physician screening recommendation compared to normal weight women (OR 2.22, 95 \% C.I. 1.12-4.37), while no significant difference was found in obese men.

CONCLUSIONS: Using nationally representative data, this study reveals potential sexspecific CRC screening disparities among the obese. Severely obese men who are at higher risk for CRC and CRC death had significantly lower odds of being adherent to screening guidelines, and this was mostly attributable to decreased endoscopy use. The more equitable screening rates found among obese women may be partially due to the increase in physician screening recommendations. These findings suggest targeting severely obese men with increased counseling on CRC screening might not only increase participation of this high risk group, but also combat healthcare disparities among the vulnerable populations affected by obesity.

NATIONAL EVALUATION OF INTER-HOSPITAL TRANSFERS Stephanie Mueller $^{1,2}{ }^{2}$; Jie Zheng ${ }^{3}$; Endel J. Orav ${ }^{1,}{ }^{3}$; Jeffrey L. Schnipper ${ }^{1,}{ }^{2}$. ${ }^{1}$ Brigham and Women, Boston, MA; ${ }^{2}$ Harvard Medical School, Boston, MA; ${ }^{3}$ Harvard School of Public Health, Boston, MA. (Control ID \#2465399)

BACKGROUND: Care transitions expose hospitalized patients to risks of discontinuity of care. Although various forms of care transitions have been widely studied, little is known about inter-hospital transfers (IHT, the transfer of patients between acute care hospitals). We used national Medicare data to examine the frequency of IHT and compare patient characteristics of transferred versus non-transferred non-critical care medical and surgical patients.

METHODS: We performed a retrospective analysis using $2013100 \%$ Master Beneficiary Summary and Inpatient claims files from CMS. Beneficiaries were eligible for inclusion if age $\geq 65$, continuously enrolled in Medicare A and B, with an acute care hospitalization claim, excluding Medicare managed care and ESRD beneficiaries. Transferred patients were defined as those with a "transfer out" claim in their discharge status, indicating transfer to another acute care hospital. Top diagnoses at time of transfer and most common medical conditions of transferred patients were identified using ICD-9 codes of primary diagnosis and comorbid conditions at time of transfer. Patient and hospital characteristics of transferred versus non-transferred patients were compared using chi-square analyses to calculate relative risk of transfer.

RESULTS: Among 28 million eligible beneficiaries, 7.3 million had an acute care hospitalization. Of these, 153,779 were transferred to another acute care facility $(2.1 \%)$. Among transferred patients, the top 5 primary diagnoses at time of transfer included Acute Myocardial Infarction (AMI) (10.4 \%), Congestive Heart Failure (CHF) (7.0 \%), Sepsis (6.7\%), Arrhythmia (6.2\%), and Pneumonia (4.5\%). Comorbid conditions most commonly present in transferred patients included CHF (50.2\%), renal failure (49.7\%), arrhythmia (47.1\%), and chronic obstructive pulmonary disease $(36.9 \%)$. Younger age white men were more likely to be transferred. Small, non-teaching, rural hospitals in the northeast were more likely to transfer patients. Risk factors associated with transfer are further shown in 
Table 1 . Thirty-day mortality among transferred patients was $12.6 \%$ compared with $9.6 \%$ of non-transferred patients $(p<0.001)$.

CONCLUSIONS: To our knowledge this is the largest cohort examining IHT that has been compiled. Our data demonstrates that IHT occurs at a moderate rate nationwide, with large variation by hospital type. Patients are transferred for high acuity conditions with high frequency of serious comorbid conditions. Characteristics of transferred and non-transferred patients clearly differ. This foundational study is an important initial evaluation of this understudied patient population, and warrants further investigation to examine the benefits, harms, and safety and quality of this care transition.

\begin{tabular}{|c|c|c|c|}
\hline Characteristic & $\begin{array}{l}\text { Frequency of Being } \\
\text { Transferred (\%) }\end{array}$ & $\begin{array}{l}\text { Relative Rikk of Tramser } \\
95 \% \mathrm{CD}\end{array}$ & P value \\
\hline \multicolumn{4}{|l|}{ Patient Characteristics } \\
\hline $\begin{array}{c}65-74 \\
75-84 \\
85+ \\
\end{array}$ & $\begin{array}{l}24 \\
22 \\
15\end{array}$ & $\begin{array}{l}1.55(1.53-1.57) \\
1.46(1.44-1.48) \\
\text { Referent }\end{array}$ & $\infty .001$ \\
\hline Gender: & $\begin{array}{l}24 \\
19\end{array}$ & $\begin{array}{l}1.26(1.25-1.27) \\
\text { Referent }\end{array}$ & $\infty 0001$ \\
\hline $\begin{array}{c}\text { White } \\
\text { Black } \\
\text { Hispanic } \\
\text { Other }\end{array}$ & $\begin{array}{l}21 \\
1.9 \\
1.6 \\
2.3\end{array}$ & $\begin{array}{l}1.29(1.24-1.35) \\
1.15(1.09-1.20) \\
\text { Referent } \\
1.43(1.36-1.51)\end{array}$ & $\infty .001$ \\
\hline $\begin{array}{c}\text { Acute Myocardal Infirirction } \\
\text { Yes } \\
\text { No }\end{array}$ & $\begin{array}{l}5.8 \\
1.8\end{array}$ & $\begin{array}{l}3.27(3.23-3.31) \\
\text { Referent }\end{array}$ & $\infty .001$ \\
\hline $\begin{array}{l}\text { Respirator Dependence/Tracheostomy } \\
\text { Yes } \\
\mathrm{No}\end{array}$ & $\begin{array}{l}53 \\
2.1\end{array}$ & $\begin{array}{l}258(251-260) \\
\text { Referent }\end{array}$ & $\infty .001$ \\
\hline $\begin{array}{ll}\text { Dialysis } & \text { Yes } \\
& \mathrm{No} \\
\end{array}$ & $\begin{array}{l}5.1 \\
21 \\
\end{array}$ & $\begin{array}{c}2.44(2.30-259) \\
\text { Referent }\end{array}$ & $\infty 0001$ \\
\hline \multicolumn{4}{|l|}{ Hospital Characteristics } \\
\hline 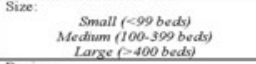 & $\begin{array}{l}5.9 \\
21 \\
0.7 \\
\end{array}$ & $\begin{array}{l}8.13(8.00-8.27) \\
2.87(2.82 \cdot 291) \\
\text { Referent }\end{array}$ & $\infty .001$ \\
\hline $\begin{array}{l}\text { Northeast } \\
\text { Mid West } \\
\text { South } \\
\text { West } \\
\text { Other }\end{array}$ & $\begin{array}{l}2.4 \\
21 \\
20 \\
119 \\
1.8\end{array}$ & $\begin{array}{l}1.29(1.27-1.32) \\
1.144(1.12-1.19 \\
1.09(1.07-1.10) \\
\text { Referent } \\
0.99(0.87-1.12)\end{array}$ & $\infty 0.001$ \\
\hline $\begin{array}{c}\text { Teaching Status: } \\
\text { Non-Teaching } \\
\text { Maimor } \\
\text { Major }\end{array}$ & $\begin{array}{l}3.0 \\
1.5 \\
0.7\end{array}$ & $\begin{array}{l}3.96(3.88-4.05) \\
2.01(1.97-2.20) \\
\text { Referent }\end{array}$ & $<0,001$ \\
\hline 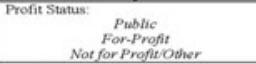 & $\begin{array}{l}29 \\
23 \\
19\end{array}$ & $\begin{array}{c}1.51(1.49-1.53) \\
1.17(1.16-1.19) \\
\text { Referent }\end{array}$ & $\infty .001$ \\
\hline $\begin{array}{l}\text { Rural } \\
\text { Urban }\end{array}$ & $\begin{array}{l}7.1 \\
1.8\end{array}$ & $\begin{array}{c}3.86(3.81-3.91) \\
\text { Referent }\end{array}$ & 20.001 \\
\hline
\end{tabular}

\section{NATIONAL TRENDS IN THE USE OF PERCUTANEOUS ENDOSCOPIC} GASTROSTOMY (PEG) TUBE AMONG ELDERLY PATIENTS WITH DEMENTIA IN THE US FROM 2006-2011 Ranjan Pathak ${ }^{1}$; Smith Giri ${ }^{3}$; Madan R. Aryal $^{2}$; Paras Karmacharya ${ }^{1}$; Vijaya R. Bhatt ${ }^{4}{ }^{1}$ Reading Health System, West Reading, PA; ${ }^{2}$ The Reading Hospital and Medical Center, Wyomissing, PA; ${ }^{3}$ University of Tennessee Health Science Center, Memphis, TN; ${ }^{4}$ University of Nebraska Medical Center, Omaha, NE. (Control ID \#2469734)

BACKGROUND: The use of Percutaneous Endoscopic Gastrostomy (PEG) tubes among patients with dementia is controversial, with multiple studies showing no clear benefit in terms of risks of aspiration, survival or quality of life. Despite this growing evidence, prior studies have shown sharply rising rates in the use of PEG tube among demented patients, however the recent trends remain unknown. We aimed to use a national database to study the nationwide trends in the use of PEG tubes among patients with dementia.

METHODS: We used the Nationwide Inpatient Sample (NIS) database to identify elderly patients ( $\geq 65$ years) with dementia who underwent PEG tube placement from 2006 to 2011 , using appropriate International Classification of Disease version 9-Clinical Modification (ICD-9 CM) codes. NIS is the largest publicly available all-payer inpatient care database in the US containing discharge level data from 5 to 8 million hospital stays per year. Annual rates of PEG tube placement were calculated for each year (2006-2011), fitted into a loglinear model and compared using Monte Carlo permutation test to study the changes in trend. Statistical analysis was done using STATA and Joinpoint Regression Program.
RESULTS: A total of 489,413 elderly patients ( $\geq 65$ years) with a diagnosis of dementia were identified during the study period, out of which 2720 patients underwent placement of PEG tube. The mean age was $81.62( \pm 7,18)$, out of which $58 \%(n=1561)$ were males and $55 \%(n=$ 1266) were whites. The proportion of patients who underwent PEG tube placement decreased from 584 per 100,000 in 2006 to 449 per 100,000 in 2011 with an AAPC of $-4.49 \%$. Join point analysis revealed that from 2009 to 2011, the proportion of elderly individuals with dementia getting PEG tube placement significantly declined from 602 per 100,000 to 449 per 100.000 with an AAPC of $-13 \%$ (95\% Confidence Interval -19.8 to $-5.7 \%$; $p$ value $<0.05$ ).

CONCLUSIONS: Our study shows declining national trends in the placement of PEG tubes among hospitalized elderly individuals with dementia between 2006 and 2011. This trend may have resulted from, change in guidelines from various professional societies (American Geriatrics Society, Alzheimer's association) discouraging the use of PEG tube among elderly patients as well as several high quality studies confirming the lack of benefit of feeding tubes among demented patients.

NATIONWIDE INTERNET SURVEY ON THE SYMPTOM OF COUGH ASSOCIATED WITH COMMON COLD Howard M. Druce ${ }^{1}$; Peter Dicpinigaitis ${ }^{2}$; Ron Eccles ${ }^{3}$; Ronald Turner ${ }^{4}$; Maryann Adeleke ${ }^{5}{ }^{1}$ Rutgers-NJ Medical School, Newark, $\mathrm{NJ} ;{ }^{2}$ Albert Einstein College of Medicine, Bronx, NY; ${ }^{3}$ Cardiff University, Cardiff, United Kingdom; ${ }^{4}$ University of Virginia Health System, Charlottesville, VA; ${ }^{5}$ Pfizer Consumer Healthcare, Madison, NJ. (Control ID \#2455806)

BACKGROUND: Common cold is often accompanied by acute cough, which is poorly characterized. This investigation sought to elucidate individual perspectives and experience of common cold-associated cough.

METHODS: A 15-20 min Internet survey to characterize cough due to common cold was fielded for approximately 20 days in April 2015. Feedback was solicited from US respondents experiencing cough due to cold within the previous 3 months. Respondents with chronic cough or medical illness/treatments predisposing to cough were excluded. Response quotas ensured the respondents were representative of US population demographics. Respondent characteristics, cough attributes and impact, and cough hypersensitivity were explored.

RESULTS: Of 19,530 respondents screened, 2708 met inclusion/exclusion criteria; $58 \%$ were female, $85 \%$ were white, and $45 \%$ worked full-time. Ninety percent rated their health status as good to excellent. Nineteen percent smoked tobacco; $8 \%$ used e-cigarettes. Cough was one of the initial cold symptoms experienced in $23 \%$ of respondents, but most commonly (62\%) cough emerged 12 days after other cold symptoms began. Cough outlasted other cold symptoms in $69 \%$, disappearing most commonly between 1 day and 1 week after other symptoms remitted. Cough was thought to affect other cold symptoms by $45 \%$ of respondents; most commonly this included worsening of sore throat (42\%) and fatigue (41\%). Cough was more frequent in the evening/nighttime (56\%). Spells or bouts involving multiple coughs (50\%) were more common than single-cough spells ( $9 \%)$. Cough was described as irritating, hacking, chesty, tickling, sudden, or painful in $\geq 25 \%$ of respondents each. Changing positions, talking, and dry air were felt to trigger cough in $\geq 30 \%$ each. Overall, 2009/2708 (74 \%) reported attempts to treat the actual cough; most commonly over-the-counter (OTC) drug/syrup (62\%) or lozenges ( $47 \%$ ) were used for this purpose. Mucus was produced during cough in $89 \%$ of respondents and began within the first 2 days of a cold in $66 \%$. Mucus production was consistent over the duration of the cough in $45 \%$, and the quantity was $\leq 1$ teaspoon in $68 \%$. Post-nasal drip was reported in $67 \%$. The urge to cough sensation was endorsed by $2648 / 2708$ ( $98 \%$ ) of our sample. Of those, the inability to control cough (62\%) and throat clearing (40\%) were the most bothersome aspects of urge to cough. In total, 1968/2648 (74 \%) of those with urge to cough attempted some form of treatment; most commonly this involved the use of OTC syrup (59\%) or a throat lozenge (53\%). These specific treatments were reported as somewhat or very effective in 83 and $78 \%$, respectively. Of those who attempted treatment for actual cough $(n=2,009)$ and/or urge to cough $(n=$ 
1,968), healthcare provider recommendations were sought by only $368(18 \%)$ and $324(16 \%)$, respectively. Treatment advice obtained from healthcare providers was similar across the 2 aspects of cough; a prescription drug was recommended in $60 \mathrm{vs} 67 \%$ and an OTC syrup was recommended in 45 vs $46 \%$, respectively, for actual cough vs urge to cough. Despite only a minority of patients seeking healthcare provider advice, over $95 \%$ of those who received a recommendation for either aspect of cough followed that recommendation.

CONCLUSIONS: This survey provides insight into individual experience of cough due to cold and, for the first time to our knowledge, provides detail on the patient experience of the urge to cough sensation. Survey results suggest that the sensation of urge to cough is especially bothersome in cough due to cold, and as a result, it often becomes a focus of treatment. Although medical consultation for cough due to cold was sought in less than one-fifth of survey respondents, the high rate of adherence to healthcare provider recommendations illustrates the importance respondents place on such advice in this clinical setting. ACKNOWLEDGMENTS This study was sponsored by Pfizer Consumer Healthcare. Medical writing support was provided by John H. Simmons, MD, of Peloton Advantage, LLC, and was funded by Pfizer.

\section{NEIGHBORHOOD BROADBAND INTERNET INEQUALITY AND THE} DIGITAL DIVIDE IN PERSONAL HEALTH RECORD USE Adam T. Perzynski ${ }^{2}$; Mary Jo Roach ${ }^{2,3}$; Sarah Shick ${ }^{3}$; Bill Callahan ${ }^{4}$; Douglas Gunzler ${ }^{2,1}$; Randall D. Cebul ${ }^{2,1}$; John D. Thornton ${ }^{5}$; Anne Huml ${ }^{5}$; David Kaelber ${ }^{5,2}$; Douglas Einstadter ${ }^{5,2}$. ${ }^{1}$ Case Western Reserve University, Chagrin Falls, OH; ${ }^{2}$ Case Western Reserve University at MetroHealth, Cleveland, $\mathrm{OH} ;{ }^{3}$ Case Western Reserve University, Cleveland, OH; ${ }^{4}$ Connect Your Community 2.0, Cleveland, OH; ${ }^{5}$ The MetroHealth System, Cleveland, OH. (Control ID \#2469368)

BACKGROUND: Personal health records (PHRs) have shown potential for increasing the quality and efficiency of health care, and federal incentives have led to their increased adoption and use. Neighborhood-based differences in the uptake and use of PHRs could increase or exacerbate health disparities. We sought to examine differences in enrollment and use of PHRs according to sex, race/ethnicity, age, insurance status, and broadband internet coverage at the neighborhood (census tract) level.

METHODS: This observational study combined electronic medical record and national public data (US Census and Federal Communications Commission) for all patients seen in outpatient clinics of an urban safety net system from 2012 to 2015. Uptake of the PHR was defined as a single login occurring during the study period. A total population of 304,142 patients had visits eligible for inclusion (at least one outpatient encounter) and 75,140 (24.7\%) of these patients had logged into the PHR. PHR logs were further queried to describe patients who sent messages to providers. For adults with complete data on all covariates ( $n=204,882$ after listwise deletion) multivariate logistic regression models were used to examine the association between quintiles of fixed neighborhood broadband internet coverage and PHR uptake (yes/no) and having ever sent a message to a provider via the PHR. Covariates included age, sex, insurance type, utilization (total number of visits), and co-morbidity (Charlson Index). Random intercepts were included in the models to adjust for the influence of clustering within census tracts.

RESULTS: Minority patients were less likely to begin using the PHR, with uptake at $30.7 \%$ for whites, $19.0 \%$ for blacks and $18.7 \%$ for Hispanics. A higher portion of females (29.1\%) used the PHR than males (19.1\%), and uptake was lower for Medicaid $(19.9 \%)$ and uninsured patients $(16.4 \%)$ than Medicare $(22.9 \%)$ and commercially insured (35.8\%) patients. The PHR was used by $30.1 \%$ of those $18-64,25 \%$ of those 65 79 and $16.5 \%$ of those age 80 years and over. Figure 1 displays the bivariate relationship between broadband internet and PHR uptake at the zip code (ZCTA) level. Regression results controlling for age, sex, insurance type, healthcare utilization and co-morbidity indicated that neighborhood broadband access was associated with PHR uptake (OR=
1.24 per quintile, $95 \% \mathrm{CI} 1.23-1.24, p<.0001$ ) and sending a message to a provider via the PHR $(\mathrm{OR}=1.15,95 \% \mathrm{CI} 1.09-1.14, p<.0001)$. These associations persist when controlling for income and education $(\mathrm{OR}=1.13,95 \% \mathrm{CI} 1.10-1.16, p<.0001$; $\mathrm{OR}=$ $1.04,95 \%$ CI 1.01-1.07, respectively; $p<.0001$ ). Patients living in neighborhoods where 80 to $100 \%$ of residents have internet were twice as likely to use the PHR as patients living in neighborhoods where $20 \%$ or fewer households had broadband internet.

CONCLUSIONS: According to recent Pew Research data, more than $30 \%$ of U.S. households do not have home broadband internet access. PHR data from a health system serving a large share of patients from disadvantaged backgrounds in Cleveland suggest the presence of a potent digital divide in PHR uptake and use. Uptake is low overall and uptake and use are systematically lower for blacks, Hispanics, older adults, persons of low socioeconomic status, and those living in neighborhoods with low levels of home broadband internet. Policy changes and financial incentives are necessary to combat this emerging inequality.

\section{Figure 1. PHR Use by Broadband Coverage at the Zip Code Level} $(\mathrm{N}=304,142 \mathrm{z}=50)$

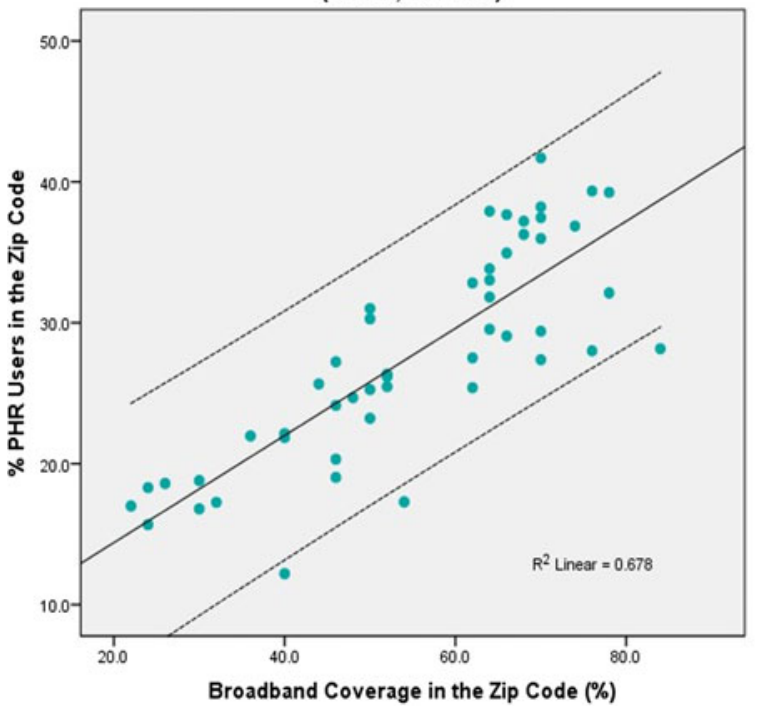

NEPHROLOGY CO-MANAGEMENT VERSUS PRIMARY CARE SOLO MANAGEMENT FOR EARLY CHRONIC KIDNEY DISEASE IN THE ELDERLY Dustin S. McEvoy ${ }^{4}$; Adam Wright ${ }^{2}$; Sushrut S. Waikar ${ }^{1}$; Jeffrey A. Linder ${ }^{3}$; Lipika Samal ${ }^{3} .{ }^{1} \mathrm{BWH}$, Boston, MA; ${ }^{2}$ Brigham and Women, Boston, MA; ${ }^{3}$ Brigham and Women's Hospital, Boston, MA; ${ }^{4}$ Partners Healthcare, Lancaster, MA. (Control ID \#2467133)

BACKGROUND: As we have shown in prior research, elderly chronic kidney disease (CKD) patients are less likely to be referred to a nephrologist than younger CKD patients. In the elderly, it is unknown whether or not patients co-managed by a nephrologist receive different care from PCP solo-managed patients, and whether or not these differences might translate into improved outcomes. We sought to determine whether co-managed CKD patients receive different care than solo-managed patients in the elderly through a retrospective cross-sectional analysis.

METHODS: After screening all patients who had at least one PCP visit at one of 12 primary care clinics at the Brigham and Women's Primary Care Practice Based Research Network during 2009, we included adults aged 75 years and older with laboratory evidence of stage 3 or $4 \mathrm{CKD}$ in the preceding 2 years (defined as two estimated glomerular filtration 
rates (eGFR) between 15 and $59 \mathrm{~mL} / \mathrm{min} / 1.73 \mathrm{~m} 2$ separated by at least 90 days). We assessed two process measures (urine protein/albumin testing and angiotensin converting enzyme inhibitor or angiotensin receptor blocker [ACE/ARB] prescribing) and two intermediate clinical outcomes (mean systolic and diastolic blood pressure). We used multivariable logistic and linear regression to adjust for potential confounders and to produce weighted estimates (weighted to adjust for covariates and to account for clustering by PCP using the LSMEANS feature of the GENMOD procedure).

RESULTS: Of $1558 \mathrm{CKD}$ patients aged 75 years and older, only $7.9 \%$ were co-managed by a nephrologist. Co-management by a nephrologist was associated with younger age, male sex, minority race/ethnicity, diabetes, and more frequent PCP visits (Table 1). After adjustment, elderly patients co-managed by a nephrologist were more likely to receive routine urine protein/albumin testing. However, co-managed patients were not more likely to be prescribed an $\mathrm{ACE} / \mathrm{ARB}$ or have lower blood pressure compared to solo-managed patients (Table 2).

CONCLUSIONS: In the elderly, nephrology co-management was only associated with more urine protein/albumin testing and was not associated with intermediate clinical outcomes compared to solo-management. Though urine protein/albumin is wellsupported by observational studies, those studies generally did not include elderly patients. Therefore, further research is needed to determine whether urine protein/albumin testing translates to better outcomes in the elderly.

\begin{tabular}{|c|c|c|c|c|}
\hline Patient Characteristics & $\begin{array}{l}\text { Total } \\
(N=1,558)\end{array}$ & $\begin{array}{l}\text { Nephrology Co- } \\
\text { Management } \\
(N=124)\end{array}$ & $\begin{array}{l}\text { Solo PCP } \\
\text { Management } \\
(N=1,434)\end{array}$ & $p$ value \\
\hline Patient Age, mean (SD) & $83(6)$ & $81(5)$ & $84(6)$ & $P<0.01$ \\
\hline Male Sex, $N(\%)$ & 537 (34 \%) & $53(43 \%)$ & 484 (34 \%) & $P=0.04$ \\
\hline \multicolumn{5}{|l|}{ Race/Ethnicity, $N$ (\%) } \\
\hline White & $1104(72 \%)$ & 64 (52 \%) & $1040(74 \%)$ & \\
\hline Black & 297 (19\%) & 37 (30 \%) & $260(18 \%)$ & \\
\hline Other & $135(9 \%)$ & $21(17 \%)$ & $114(8 \%)$ & \\
\hline eGFR, mean (SD) & $44(9)$ & $33(9)$ & $46(9)$ & $P<0.01$ \\
\hline Hypertension, $N(\%)$ & $1088(70 \%)$ & $93(75 \%)$ & 995 (69 \%) & $P=0.19$ \\
\hline Diabetes, $N(\%)$ & $392(25 \%)$ & $50(40 \%)$ & $342(24 \%)$ & $P<0.01$ \\
\hline PCP Visits Yearly, mean (SD) & $4.2(3.0)$ & $5.3(3.9)$ & $4.1(2.9)$ & $P<0.01$ \\
\hline
\end{tabular}

Association of Nephrology Co-Management with Quality of Care - Weighted Results

\begin{tabular}{|c|c|c|c|}
\hline \multirow[t]{2}{*}{ Outcomes } & Nephrology & Solo PCP & $p$ value \\
\hline & Co-Management & Management & \\
\hline Urine Protein/Albumin & $75 \%$ & $35 \%$ & $P<0.01$ \\
\hline$A C E / A R B$ prescription & $71 \%$ & $70 \%$ & $P=0.75$ \\
\hline Systolic BP, mmHg & 132.5 & 134.0 & $P=0.43$ \\
\hline Diastolic BP, mmHg & 66.3 & 68.6 & $P=0.10$ \\
\hline
\end{tabular}

Weighted percentage and p-value estimated by multivariate model accounting for clustering by PCP and adjusting for age, gender, race/ethnicity, eGFR, hypertension, diabetes and number of PCP visits.

\author{
NEW MEDICAID ENROLLEES SEE HEALTH AND SOCIAL BENEFITS IN \\ PENNSYLVANIA'S EXPANSION Jeffrey Hom ${ }^{1,3}$; Charlene Wong ${ }^{4,3}$; Christian H. \\ Stillson $^{3}$; Jessica Zha ${ }^{3}$; Carolyn C. Cannuscio ${ }^{2}$; Rachel M. Cahill ${ }^{5}$; David Grande ${ }^{3,3}$. \\ ${ }^{1}$ Crescenz VA Medical Center, Philadelphia, PA; ${ }^{2}$ Perelman School of Medicine, U. of \\ Pennsylvania, Philadelphia, PA; ${ }^{3}$ University of Pennsylvania, Philadelphia, PA; \\ ${ }^{4}$ Children's Hospital of Philadelphia, Philadelphia, PA; ${ }^{5}$ Benefits Data Trust, \\ Philadelphia, PA. (Control ID \#2469669)
}

BACKGROUND: More than 6.8 million low-income Americans have gained health insurance as a result of the Medicaid expansion, a key provision of the Affordable Care Act. Understanding how new Medicaid enrollees are approaching their own health and health care in a shifting health care landscape has implications for future outreach and enrollment efforts, as well as service planning for this population. The objective of this study was to explore the health care experiences, perspectives and expectations of new Medicaid expansion beneficiaries in the immediate post-enrollment period.

METHODS: Semi-structured, qualitative interviews. We interviewed a random sample of 40 adults in Philadelphia, age 19-64, who had completed an application for Medicaid through a comprehensive benefits organization after January 1, 2015, when the Medicaid expansion in Pennsylvania took effect. We conducted an inductive, applied thematic analysis of interview transcripts using the qualitative software NVivo, version 10.0.

RESULTS: Participants were predominantly African-American, unmarried and unemployed, and approximately evenly distributed with regard to age and gender. The new Medicaid beneficiaries described especially high levels of pent-up demand for care. Dental care was a far more pressing and motivating concern than medical care - it was mentioned without prompting by 33 out of 40 participants. Preventive services were also frequently mentioned. Participants anticipated that insurance would reduce both stress and financial strain and improve their experience in the health care system by raising their social standing. Lastly, participants highly valued the support of telephone application counselors in the Medicaid enrollment process to overcome bureaucratic obstacles they had encountered in the past.

CONCLUSIONS: As more states opt-in to the Medicaid expansion, enrolling the uninsured and meeting the needs and expectations of new enrollees will be paramount. Dental care and preventive services appear to be high priorities for new Medicaid enrollees. Telephone outreach and enrollment support services can be an effective way to overcome past experiences with administrative and bureaucratic barriers. Future research should investigate how expanded coverage impacts mental health, financial strain 
and perceived or actual discrimination in health care settings. These factors may contribute to Medicaid beneficiaries' decisions to re-enroll in the program and determine the degree to which Medicaid expansion achieves its goal of greater access and improved health for low-income Americans

Themes, representative quotes, and policy and research implications

\begin{tabular}{|c|c|c|}
\hline Theme & Representative Quotes & $\begin{array}{l}\text { Policy and Research } \\
\text { Implications }\end{array}$ \\
\hline $\begin{array}{l}\text { High demand for } \\
\text { dental and } \\
\text { preventive care }\end{array}$ & $\begin{array}{l}\text { "Once they found out } \\
\text { I didn't have insurance, } \\
\text { I couldn't get the root } \\
\text { canal because I would } \\
\text { have to pay X amount } \\
\text { of dollars to get it, } \\
\text { which I didn't have. } \\
\text { So this bad tooth has } \\
\text { been lingering in my } \\
\text { mouth for X amount } \\
\text { of time." } \\
\text { "The first thing I did } \\
\text { was visit the doctor } \\
\text { just to make sure that } \\
\text { I didn't have high } \\
\text { blood pressure or } \\
\text { diabetes or anything } \\
\text { like that." }\end{array}$ & $\begin{array}{l}\text { Dental coverage was } \\
\text { highlighted by study } \\
\text { participants - people } \\
\text { perceived their dental } \\
\text { health as integral to } \\
\text { their overall health. } \\
\text { Emphasizing and } \\
\text { including dental } \\
\text { benefits may entice } \\
\text { uninsured individuals } \\
\text { to obtain coverage. } \\
\text { Understanding both } \\
\text { demand for dental } \\
\text { services and availability } \\
\text { of providers who serve } \\
\text { Medicaid enrollees is } \\
\text { an important research } \\
\text { and policy priority. }\end{array}$ \\
\hline $\begin{array}{l}\text { Decreasing stress } \\
\text { and financial strain }\end{array}$ & $\begin{array}{l}\text { "Just knowing that } \\
\text { overall I have the } \\
\text { actual insurance } \\
\text { carrier - as simple } \\
\text { as it may seem, is } \\
\text { just a big weight } \\
\text { off my shoulders." } \\
\text { "I have been able } \\
\text { to use [my insurance], } \\
\text { and it's been } \\
\text { wonderful because } \\
\text { now I don't have } \\
\text { to pay cash. I could } \\
\text { just use that cash to } \\
\text { buy the kids some food." }\end{array}$ & $\begin{array}{l}\text { Future research should } \\
\text { focus on the impact of } \\
\text { new insurance on finances, } \\
\text { stress and mental health, } \\
\text { and potential spillover } \\
\text { effects within households. }\end{array}$ \\
\hline $\begin{array}{l}\text { Health insurance as a } \\
\text { social equalizer }\end{array}$ & $\begin{array}{l}\text { "I feel as though I'm } \\
\text { gonna get the proper } \\
\text { care for me and my } \\
\text { child that I'm carrying. } \\
\text { And without any } \\
\text { worries. I also know } \\
\text { that with this health } \\
\text { insurance, there's } \\
\text { more doors that are } \\
\text { gonna open more } \\
\text { quickly." }\end{array}$ & $\begin{array}{l}\text { While stigma in the health } \\
\text { care setting has been } \\
\text { previously described, our } \\
\text { study suggests that new } \\
\text { Medicaid enrollees see } \\
\text { health insurance as a tool } \\
\text { for reducing discrimination } \\
\text { and increasing social } \\
\text { standing, as well as for } \\
\text { improving access to } \\
\text { higher quality health } \\
\text { care. }\end{array}$ \\
\hline $\begin{array}{l}\text { Application assistance } \\
\text { to overcome enrollment } \\
\text { challenges }\end{array}$ & $\begin{array}{l}\text { "Receiving a letter } \\
\text { [about being eligible] } \\
\text { was the first thing that } \\
\text { was helpful...I just } \\
\text { called and she said } \\
\text { she could do } \\
\text { everything over the } \\
\text { phone... She gave } \\
\text { me her name and } \\
\text { number to call back, } \\
\text { if I had any questions, } \\
\text { and she just helped } \\
\text { me with everything." }\end{array}$ & $\begin{array}{l}\text { Application assistance } \\
\text { was valued by new } \\
\text { Medicaid enrollees and } \\
\text { seen as instrumental to } \\
\text { gaining coverage. } \\
\text { Comprehensive benefits } \\
\text { programs could be an } \\
\text { important model for not } \\
\text { only enrollment assistance, } \\
\text { but also outreach to identify } \\
\text { people who may not know } \\
\text { they are eligible. }\end{array}$ \\
\hline
\end{tabular}

NON-MEDICAL USE OF PRESCRIPTION OPIOIDS: CHARACTERISTICS OF USE AND BARRIERS TO CARE David Cheng; Meredith Lynn; Michael Malone; Colleen Vessell. New York University, New York, NY. (Control ID \#2469819)

BACKGROUND: Over the past two decades morbidity and mortality associated with non-medical use of prescription opioids has tripled. Despite this growing epidemic, rates of treatment for prescription opioid abuse remain low. Our study seeks to describe the characteristics of prescription opioid users who want to be in treatment and better understand their barriers to care

METHODS: Self-reported weighted data from the 2013 National Survey on Drug Use and Health was analyzed to compare the demographic information, patterns of opioid use, and medical comorbidities of non-medical prescription opioid users who wanted but did not receive treatment compared to those who received treatment within the past year. Barriers to care were assessed for the group that did not receive treatment. Chi-square testing was used to examine differences between the study groups.

RESULTS: Based on weighted data from the 2013 survey, $4.2 \%$ of respondents ( $n=$ $11,066,219)$ used opioids for non-medical reasons in the past year. Of this population, $3.3 \%$ $(n=364,241)$ reported receiving treatment for non-medical prescription opioid use in the past year, while another $1.2 \%(n=132,668)$ wanted but never received treatment. Compared to those who received treatment, those who never received treatment were more likely to be older than 50 years of age ( 17.7 vs. $15 \%, p=0.0000)$, have a college education $(13.9$ vs. $7.4 \%, p=$ $0.0000)$ and have higher annual family income of $>\$ 75,000(20.8$ vs. $17.1 \%, p=0.0000)$. In addition, those needing treatment were more likely to report fair to poor overall health (30 vs. $12.7 \%, p=0.0000)$ as well as greater unmet mental health needs ( 49.5 vs. $32.7 \%, p=$ 0.0000 ). Of those wanting but not receiving treatment, only $24.1 \%$ reported making an effort to obtain treatment. The most commonly reported barriers to obtaining treatment included being poorly perceived by neighbors ( $45.5 \%$ ), treatment having a negative impact on their job $(40.9 \%)$, and treatment not being covered by insurance ( $28.2 \%)$.

CONCLUSIONS: Our study utilizes one of the few opportunities to characterize prescription opioid users who want but are not receiving treatment among the general population in the United States. Based on this analysis of 2013 data, prescription opioid users who desired but did not receive treatment were more likely to be older, wealthier, college educated and have unmet physical and mental health needs compared to those in treatment. Barriers to treatment included perceived stigma, job disruption and lack of insurance coverage. It is increasing important to better understand this population in the current climate of rising morbidity and mortality associated with non-medical prescription opioid use. Furthermore, by focusing on the population that is seeking but not obtaining treatment more effective interventions could be designed to address this unmet need. Further research on this population is needed to best address this significant public health issue.

NOT A PROBLEM IN MY COMMUNITY: THE ROLE OF FAITH-BASED ORGANIZATIONS IN PROVIDING SUPPORT FOR MUSLIM-AMERICANS WITH SUBSTANCE USE DISORDERS Sarah Mallik ${ }^{2}$; Joanna L. Starrels ${ }^{1}$; Shadi Nahvi ${ }^{1} .{ }^{1}$ Albert Einstein College of Medicine \& Montefiore Medical Center, Bronx, NY; ${ }^{2}$ Montefiore Medical Center, Bronx, NY. (Control ID \#2466676)

BACKGROUND: Despite the mainstream Islamic prohibition of alcohol and other drugs, substance use is prevalent in Muslim-American communities. In separate surveys, almost $50 \%$ of Muslim-American undergraduate students reported drinking alcohol, and $14 \%$ of Muslim-Americans reported binge drinking. Though American faith-based organizations have provided various forms of support to people with substance use disorders, the majority of these groups are rooted in Christian traditions. Muslim faith-based organizations, most commonly mosques, are led by imams. Imams perform many activities including leading prayer, teaching classes and providing psychosocial support to individuals who attend the mosque. Previous studies have not examined how Muslim faith-based organizations perceive their role in addressing substance use. The objectives of this study were to 1) explore imams' perspectives on the extent to which substance use affects their communities and 2) describe their perspectives toward Muslim-Americans dealing with these issues.

METHODS: Imams were recruited by initially contacting a random sample of mosques and later by snowball sampling in two regions of New York City, the Bronx and upper 
Manhattan. Semi-structured interviews were conducted in 2015 with ten imams in their respective mosques. Interview questions addressed imams' perspectives on various substances, their experiences discussing substance use with individuals, and the ways in which they assisted them. Interviews lasted 30 to $60 \mathrm{~min}$ and were audio-recorded, professionally transcribed, and de-identified. After creating an initial coding scheme based on the first three interviews, interview data were imported and coded using the Dedoose web-based application. Data were analyzed iteratively using inductive thematic analysis, applying a modified grounded theory framework to identify prominent themes.

RESULTS: Of ten participants, all ten were male, five were over the age of 50, and nine were born outside the United States (primarily in West Africa and South Asia). Several themes emerged from the interviews. Substance use was not perceived to be a prevalent issue affecting individuals that attend the mosque regularly; rather, it was viewed as primarily affecting nonmosque going Muslims and non-Muslims. However, a less prominent theme reflected an opposing view: substance use is a highly prevalent and stigmatized issue among Muslims, and the Muslim community has not done enough to help those with problems related to substance use. Imams associated substance use with shame, social disruption and withdrawal from the mosque community. Finally, imams emphasized religious concepts such as sin and intoxication when discussing drug use, and they discussed repentance and forgiveness while discussing recovery from addiction.

CONCLUSIONS: In this study, imams held divergent views about the prevalence of substance use among Muslim-Americans. They viewed substance use and addiction primarily in religious terms, and differed in how they perceived their role in addressing it. These findings suggest that there are likely to be opportunities for Muslim faith-based organizations to address substance use within and beyond mosque going communities. Further research is necessary to understand barriers and facilitators and to develop interventions for faith-based organizations to help individuals with substance use disorders.

\section{NOVEL ORAL ANTICOAGULANT AND WARFARIN USE OVER TIME IN A} PRIMARY CARE NETWORK Jeffrey M. Ashburner; Steven J. Atlas; Leila H. Borowsky; Daniel E. Singer. Massachusetts General Hospital, Boston, MA. (Control ID \#2468941)

BACKGROUND: Atrial fibrillation (AF) is the most common significant cardiac arrhythmia, affecting $>3$ million adults in the United States, and is associated with a 5-fold increased risk of stroke. Treatment with warfarin oral anticoagulation therapy (OAC) reduces the risk of stroke by approximately two-thirds. In recent years, novel oral anticoagulants (NOACs) have been introduced as alternatives to warfarin for the prevention of thromboembolic events. In clinical trials, NOACs have shown to be noninferior for prevention of stroke and associated with less intracranial bleeding. There is limited data about the uptake of these new anticoagulants in clinical practice especially in primary care networks, and whether their introduction has led to more patients at high risk of stroke being anticoagulated. We examined the use of OACs among patients with $\mathrm{AF}$ at high risk of stroke in a primary care network following the introduction of NOACs.

METHODS: The study cohort includes adult patients aged 18 and older with AF who were cared for in a 19-practice primary care network between 2010 and 2014. We identified patients by searching billing data for physician-assigned International Classification of Diseases, Ninth Revision, Clinical Modification (ICD-9) diagnostic codes of AF (427.31) and electronic health record problem list entries. We assigned AF status each calendar year and considered patients as having AF if they had at least 1 ICD-9 code plus at least 1 problem list entry in the prior 3 years. Chart review validation of a random sample of 720 records demonstrated a sensitivity of $99.5 \%$ and specificity of $96.8 \%$. In each year, we considered patients to be using a medication if they had at least 1 prescription in that year. These analyses are focused on the years 2010-2014 to show anticoagulation use before and after widespread NOAC introduction (FDA approval for dabigatran: October, 2010). We examined anticoagulation usage over time among all patients with $\mathrm{AF}$, and among patients at high risk of stroke $\left(\mathrm{CHA}_{2} \mathrm{DS}_{2}-\mathrm{VASc} \geq 2\right)$. We assessed trends over time using the Cochran-Armitage test for trend and compared $\mathrm{CHA}_{2} \mathrm{DS}_{2}$-VASc scores among patients prescribed NOACs and warfarin in 2014 using t-tests. RESULTS: In 2014, there were 4959 patients with AF in the primary care network, representing $3.1 \%$ of the total population. The average age of an $\mathrm{AF}$ patient increased from 73.0 years in 2010 to 73.9 years in 2014. Over the study period, NOAC usage increased from $0.31 \%$ of AF patients in 2010 (dabigatran) to $16.4 \%$ (apixaban $2.5 \%$; dabigatran $4.1 \%$; rivaroxaban $9.8 \%$ ) in 2014 ( $p$-value for trend $<0.001$ ). The proportion of AF patients taking warfarin decreased over time from $60.3 \%$ in 2010 to $45.2 \%$ in 2014 $(p<0.001)$. There was little change in the proportion of patients not prescribed any anticoagulation treatment from $2010(39.4 \%)$ to 2014 (38.4\%) ( $p=0.09)$. The mean $\mathrm{CHA}_{2} \mathrm{DS}_{2}$-VASc score among all AF patients increased from 3.38 in 2010 to 3.82 in 2014. Similarly, the mean $\mathrm{CHA}_{2} \mathrm{DS}_{2}$-VASc score increased among anticoagulated (2010: 3.76 and 2014: 4.21) and non-anticoagulated patients (2010: 2.81 and 2014: 3.19). The mean $\mathrm{CHA}_{2} \mathrm{DS}_{2}$-VASc $(3.56$ vs. 4.45 in $2014, p<0.001)$ and age $(72.4$ vs. 76.6 in 2014, $p<0.001$ ) were lower among patients prescribed NOACs compared to patients prescribed warfarin. Among patients with a $\mathrm{CHA}_{2} \mathrm{DS}_{2}-\mathrm{VASc}$ score $\geq 2$, the proportion of patients anticoagulated did not increase following introduction of NOACs (2010: $66.2 \%$ and 2014: $66.4 \%, p=0.99)$.

CONCLUSIONS: The introduction of NOACs in late 2010 provided an alternative to warfarin for stroke prevention in AF. In addition to an increasing adoption of these novel agents among AF patients, o patients using NOACs are younger and have lower stroke risk than patient using warfarin, suggesting that the increased NOAC usage may mainly be among patients with newly diagnosed AF or who are newly anticoagulated. Increased use of NOACs appears to be replacing rather than adding to warfarin use. Anticoagulation rates and the proportion of patients at high risk of stroke that are anticoagulated have not increased over time.

NPH INSULIN FOR THE MANAGEMENT OF GLUCOCORTICOID-INDUCED HYPERGLYCEMIA IN ONCOLOGY PATIENTS Youngjee Choi ${ }^{1}$; Sara Butler ${ }^{4}$; Leigh Boehmer ${ }^{2}$; Michael Elliott ${ }^{3}$; Anna Roshal ${ }^{4}$; Garry Tobin ${ }^{4} .{ }^{1}$ Johns Hopkins School of Medicine, Baltimore, MD; ${ }^{2}$ Mercy Medical Center-North Iowa, Mason City, IA; ${ }^{3}$ Saint Louis University, St. Louis, MO; ${ }^{4}$ Washington University School of Medicine/BarnesJewish Hospital, St. Louis, MO. (Control ID \#2465872)

BACKGROUND: There are no guidelines for the management of glucocorticoid (GC)induced hyperglycemia. Oncology patients frequently receive high dose GCs and would benefit from a protocol for GC-induced hyperglycemia. We evaluated a pharmacistinitiated insulin protocol for oncology patients requiring high dose GCs.

METHODS: We conducted a prospective pilot study at a large tertiary academic center. Patients with a diagnosis of lymphoma, sarcoma, or lung cancer admitted for chemotherapy with a daily minimum of $20 \mathrm{mg}$ prednisone equivalents were eligible if either (1) they were treated with insulin or moderate to high-dose insulin secretagogues prior to admission or (2) they developed a blood glucose $>235 \mathrm{mg} / \mathrm{dL}$ during the hospitalization. The intervention consisted of a weight-based, GC-dose dependent calculation for NPH insulin to be administered with each GC dose. Two oncology pharmacists screened patients for eligibility and calculated and recommended initial insulin orders; an endocrine consult team made subsequent adjustments. All participants received sliding-scale insulin; participants on outpatient insulin or high-dose insulin secretagogues also received basal-bolus insulin during admission. We used historical patients matched for cancer diagnosis, diabetes status, insulin use, GC dose, BMI, and A1C as controls. The primary outcome was the percentage of days that participants experienced hyperglycemia (glucose $>180 \mathrm{mg} / \mathrm{dL}$ ); secondary endpoints included percentage of days with severe hyperglycemia (glucose $>299 \mathrm{mg} / \mathrm{dL}$ ), hypoglycemia (glucose $<70 \mathrm{mg} / \mathrm{dL}$ ), and severe 
hypoglycemia (glucose $<40 \mathrm{mg} / \mathrm{dL}$ ). We used linear regression to analyze the effect of the intervention, controlling for hemoglobin $\mathrm{A} 1 \mathrm{C}$, BMI, and timing of $\mathrm{GC}$ administration

RESULTS: Sixteen patients participated in the study. Five met criteria for enrollment at admission; 11 were enrolled after reaching a blood glucose of $>235 \mathrm{mg} / \mathrm{dL}$ during the hospitalization. Participants did not differ significantly from historical controls in the percentage of days with hyperglycemia or hypoglycemia. Participants had significantly lower percentage of days with severe hyperglycemia compared to historical controls. Neither participants nor historical controls had any severe hypoglycemia.

CONCLUSIONS: We demonstrated that a pharmacist-initiated insulin protocol in conjunction with endocrine consultation could successfully improve rates of severe hyperglycemia in oncology patients receiving high dose GCs. Our prospective study adds to the evidence that a protocol of long-acting insulin can be effective in managing GC-induced hyperglycemia.

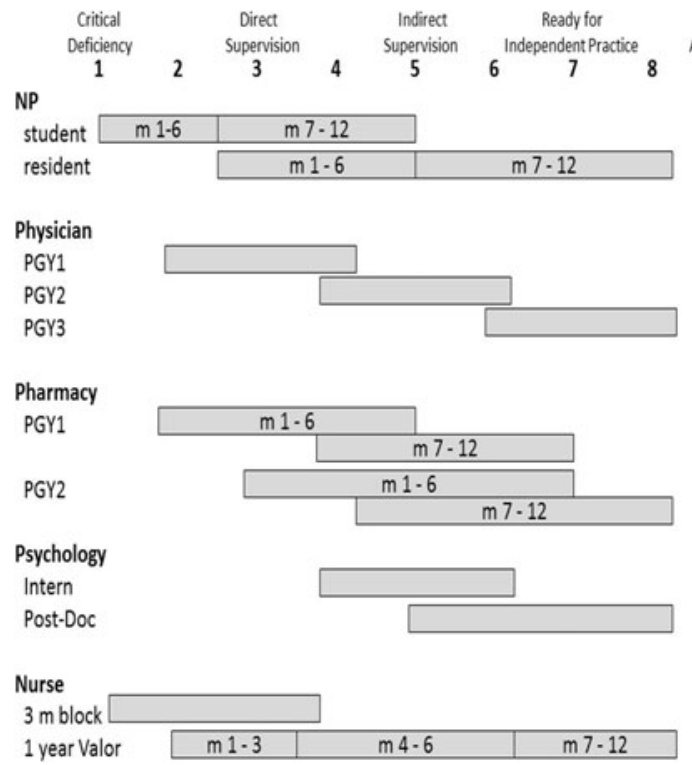

NURSES' PERCEPTIONS IN IMPLEMENTING SMOKING CESSATION GUIDELINES FOR HOSPITALIZED VETERANS David A. Katz ${ }^{5}$ Kenda R. Stewart ${ }^{2}$; Monica Paez ${ }^{4}$; John Holman ${ }^{2}$; Susan Adams²; Mark Vander Weg ${ }^{2}$; Catherine Battaglia $^{3}$; Anne Joseph ${ }^{1}$; Marita Titler ${ }^{7}$; Sarah S. Ono ${ }^{6} .{ }^{1}$ Department of Medicine, University of Minnesota, Minneapolis, MN; ${ }^{2}$ Department of Veterans Affairs, Iowa City, IA; ${ }^{3}$ Eastern Colorado Healthcare System, Denver, CO; ${ }^{4}$ Iowa City VA Health Care System, Iowa City, IA; ${ }^{5}$ University of Iowa, Iowa City, IA; ${ }^{6}$ Center to Improve Veteran Involvement in Care (CIVIC) Portland VA Medical Center, Portland, OR; ${ }^{7}$ School of Nursing, University of Michigan, Ann Arbor, MI. (Control ID \#2470313)

BACKGROUND: Many hospitalized smokers do not receive guideline-recommended tobacco treatment, but little is known about the perceptions of inpatient nurses with regard to tobacco treatment.

METHODS: We used a sequential explanatory mixed methods design to help explain the findings of an academic detailing intervention trial on the inpatient medicine units of four Veterans Affairs (VA) hospitals. We surveyed 164 nurses and conducted semi-structured interviews in a purposeful sample of 33 nurses with different attitudes toward cessation counseling. Content analysis was used to inductively characterize the issues raised by participants. Emerging themes were categorized using the knowledge-attitudes-behavior framework of guideline adherence.

RESULTS: Knowledge-related and attitudinal barriers included perceived lack of skills in cessation counseling and skepticism about the effectiveness of cessation guidelines in hospitalized veterans. Nurses also reported multiple behavioral and organizational barriers to guideline adherence: resistance from patients, insufficient time and resources, the presence of smoking areas on VA premises, and lack of coordination with primary care. CONCLUSIONS: VA hospitals should train inpatient staff how to negotiate behavior change, integrate cessation counseling into nurses' workflow, develop alternative referral mechanisms for post-discharge cessation counseling, and adopt hospital policies to promote inpatient abstinence.

\section{NUTRITION AND EXERCISE EDUCATION FOR HIGH SCHOOL STUDENTS} AS A WAY TO PREVENT ADULTHOOD OBESITY. Kathryn A. Jobbins ${ }^{1}$; Sarah L. Goff ${ }^{2}$; Reva Kleppel ${ }^{1}$; Jane Garb ${ }^{1} .{ }^{1}$ Baystate Medical Center, Springfield, MA; ${ }^{2}$ Baystate Medical Center/Tufts University School of Medicine, Springfield, MA. (Control ID \#2467089)

BACKGROUND: Obesity rates have nearly doubled in the U.S. in the past three decades, with an estimated $35 \%$ of adults now suffering from obesity (1) Rates for adolescents are nearly as high with 1 in 4 high school and middle school students in Massachusetts classified as overweight or obese. (2) According to recent study published in the journal Obesity, over $\$ 3.5$ billion of medical expenses in Massachusetts are due to adult obesity. (8) Health habits attitudes develop during childhood and adolescence and tend to persist into adulthood. Being overweight or obese as a teen can directly affect physical and mental health and increases risk for obesity-associated diseases in adulthood. Once established, obesity is difficult to treat, making prevention critical. (10) School-based programs are one of many approaches to addressing obesity at a population level. Although school-based interventions suggest that teacher-delivered cognitive-behavioral skills-building interventions can positively affect health, psychosocial, and academic outcomes, (11) this research is not commonly translated into practice outside of the research setting. In this study, we sought to test the effectiveness of a pragmatic supplemental nutrition and exercise educational curriculum in a Massachusetts high school.

METHODS: Study design: In this intervention study, we developed a 3 session, $1.5 \mathrm{~h}$ curriculum that focused on nutrition recommendations, dietary habits, drinks high in sugar, exercise recommendations, exercise habits and other health habits including stress, depression and sleep and included at home practice assignments. To evaluate the intervention's effectiveness, we used a pre-/post- questionnaire that included 5 domains related to nutrition and exercise knowledge, behaviors, skills and attitudes. Participants were assigned a unique identifier so that responses were anonymous but pre-post questionnaires could be linked. Measures: Participants were asked to rate their self-perceived nutrition/healthy habits on a 5 -point Likert scale, and to demonstrate knowledge of principles of healthy eating and exercise. The "healthy habits" questions focused on assessing how frequently the students tracked the food they consumed and exercise they performed as well their mindfulness and insight into these behaviors. Identical surveys were completed immediately prior to the first educational session and at the end of the last session. 
Surveys were scored in two parts: 1) a "healthy habit" score was calculated by summing the Likert score for five questions in this domain and 2) changes in selfreported behaviors were assessed. Subjects: High school senior students attending a physical education classes. Data Analysis \& Statistical Considerations: Pre- and post-intervention healthy habits and knowledge scores were reported as means and standard deviation and compared for all students using the paired t-test. We estimated that between 70 and 100 students would be needed to give adequate power $(80 \%$ or greater) to detect a small effect size $(0.3$ or greater $)$ in mean difference in pre-post curriculum scores. A total of 73 students were included. A healthy habits score was generated by summing the ordinal responses to 5 questions on exercise and food habits. Descriptive statistics (mean, standard deviations or percents, as appropriate) were used to describe baseline subject characteristics. The paired t-test was used to compare pre-and post-intervention responses for questions on a continuous scale. McNemar's test of symmetry was used to compare dichotomously categories responses (recommended/not recommended) for habits and knowledge.

RESULTS: Changes in habits and perceived recommendations Fruit and vegetable consumption increased from baseline to follow-up (2.4 servings/day to 3.1 servings/day, $p=0.0004)$ and sweet drink consumption decreased $(12.6 \mathrm{oz} /$ day to $8.1 \mathrm{oz} /$ day, $p=0.0032$ ). The perceived frequency of recommended exercise decreased $(1.6 \mathrm{~h} /$ day to $1.2 \mathrm{~h} /$ day $p=0.01)$ as and healthy habits scores increased (13.9 to $15.6, p=0.0006$ ). There were no significant changes in the knowledge of the recommended \# of daily fruits \& vegetable servings, the recommended ounces of sweet drinks, or in the actual number of hours exercised per day, which was high (almost $2 \mathrm{~h}$ ) at baseline and follow-up

CONCLUSIONS: Interventions that successfully promote healthy eating and exercise habits in youth are critical to reducing obesity rates in adults. Our brief pragmatic schoolbased intervention demonstrated a positive effect on fruit and vegetable consumption and reduction in sugar-sweetened beverages among a group of high school seniors. This brief intervention may be incorporated as a supplement to existing curricula with potential beneficial effect.

\section{OBESITY AND THE RISK OF RECURRENT VENOUS THROMBOEMBOLISM IN} ELDERLY PATIENTS Carolin Mueller ${ }^{1}$; Andreas Limacher ${ }^{2}$; Marie Méan ${ }^{1}$; Marc Righini $^{3}$; Hans-Jürg Beer ${ }^{4}$; Beat Frauchiger ${ }^{5}$; Joseph J. Osterwalder ${ }^{6}$; Christian M. Matter $^{7}{ }^{8}$; Marc Husmann ${ }^{7}$; Martin Banyai ${ }^{9}$; Markus Aschwanden ${ }^{10}$; Nils Kucher ${ }^{1}$; Daniel Staub ${ }^{10}$; Anne Angelillo-Scherrer ${ }^{1}$; Olivier Hugli ${ }^{11}$; Lucia Mazzolai ${ }^{11}$; Nicolas Rodondi ${ }^{1}$; Drahomir Aujesky ${ }^{1} .{ }^{1}$ Bern University Hospital, Bern, Switzerland; ${ }^{2}$ University of Bern, Bern, Switzerland; ${ }^{3}$ Geneva University Hospital, Geneva, Switzerland; ${ }^{4}$ Cantonal Hospital of Baden, Baden, Switzerland; ${ }^{5}$ Cantonal Hospital of Frauenfeld, Frauenfeld, Switzerland; ${ }^{6}$ Cantonal Hospital of St. Gallen, St. Gallen, Switzerland; ${ }^{7}$ Zurich University Hospital, Zurich, Switzerland; ${ }^{8}$ University of Zurich, Zurich, Switzerland; ${ }^{9}$ Cantonal Hospital of Lucerne, Lucerne, Switzerland; ${ }^{10}$ Basel University Hospital, Basel, Switzerland; ${ }^{11}$ Lausanne University Hospital, Lausanne, Switzerland. (Control ID \#2465386)

BACKGROUND: Although obesity is a known risk factor for a first venous thromboembolism (VTE) episode, only sparse data exist on the relationship between obesity and recurrent VTE, particularly in the elderly. We therefore aimed to examine the association between 2 measures of excess body weight, the body mass index (BMI) and the waist circumference (WC), and recurrent VTE in a prospective cohort study of elderly patients.

METHODS: We studied 986 patients aged $\geq 65$ years with an acute VTE from 9 Swiss hospitals between September 2009 and December 2013. At study entry, the BMI was determined and categorized as $<25,25$ to $<30$, or $\geq 30 \mathrm{~kg} / \mathrm{m}^{2}$. The WC was measured at the umbilical line and categorized as $<80 \mathrm{~cm}$ in women (W) / $<94 \mathrm{~cm}$ in men (M), 80 to $<88 \mathrm{~cm}$ (W) / 94 to $<102 \mathrm{~cm} \mathrm{(M),} \mathrm{or} \geq 88 \mathrm{~cm}$ (W) / $\geq 102 \mathrm{~cm}$ (M). The primary outcome was the recurrence of a first symptomatic, objectively confirmed VTE. We examined the association between obesity measures and recurrent VTE using competing risk regression, accounting for non VTE-related death as a competing event. We analyzed BMI and WC both as a categorical and continuous variable. We adjusted the models for other known risk factors of VTE recurrence, including age, gender, unprovoked VTE, active cancer, prior history of VTE, and periods of anticoagulation as a time-varying covariate.

RESULTS: Overall, 122 patients $(12.4 \%)$ had a first recurrent VTE during a median follow-up period of 30 months. Patients with a BMI $<25,25$ to $<30$, and $\geq 30 \mathrm{~kg} / \mathrm{m}^{2}$ had a cumulative incidence of recurrent VTE of 17.6, 11.5, and 17.0\% at 36 months, respectively $(P=0.09)$. Patients with a WC $<80 \mathrm{~cm}$ (W) $/<94 \mathrm{~cm}$ (M), 80 to $<88 \mathrm{~cm}$ (W) $/ 94$ to $<102(\mathrm{M}) \mathrm{cm}$, and $\geq 88 \mathrm{~cm}(\mathrm{~W}) / \geq 102 \mathrm{~cm}(\mathrm{M})$ had a cumulative incidence of recurrent VTE of $21.4,13.8$, and $13.9 \%$, respectively $(P=0.49)$. After adjustment, neither BMI nor WC was associated with recurrent VTE (see Table). Stratified analyses for gender yielded similar results for men and women.

CONCLUSIONS: In our prospective cohort of elderly patients with acute VTE, we did not find an association between obesity measures and recurrent VTE. Excess body weight does not appear to be a risk factor for VTE recurrence in elderly patients with VTE.

Table. Association between obesity measures and recurrent venous thromboembolism

\begin{tabular}{ll}
\hline \hline Measure of obesity & $\begin{array}{l}\text { Adjusted sub-hazard } \\
\text { ratio* } 95 \% \\
\text { confidence interval) }\end{array}$ \\
$\begin{array}{l}\text { Body mass index, } \mathrm{kg} / \mathrm{m}^{2} \\
\text { Categorized }\end{array}$ & \\
$<25$ & Reference \\
25 to $<30$ & $0.78(0.51-1.19)$ \\
$\geq 30$ & $1.11(0.7-1.75)$ \\
Continuous, per unit & $1.02(0.98-1.05)$ \\
Waist circumference, cm & \\
Categorized & \\
$<80(\mathrm{~W}) /<94$ (M) & Reference \\
80 to $<88(\mathrm{~W}) / 94$ to $<102(\mathrm{M})$ & $0.87(0.43-1.74)$ \\
$\geq 88(\mathrm{~W}) / \geq 102(\mathrm{M})$ & $0.99(0.56-1.75)$ \\
Continuous, per unit & $1.01(0.99-1.02)$ \\
\hline
\end{tabular}

*Adjusted for age, gender, unprovoked VTE, active cancer, history of prior VTE, and anticoagulation as a time-varying covariate.

\section{OBJECTIVE NOCTURNAL OXYGEN SATURATION AND HEART RATE INFORMATION IN HEART FAILURE PATIENTS AT HOME Harry B. Burke;}

Cubby L. Gardner; Michael C. Flanagan. Uniformed Services University of the Health Sciences, Bethesda, MD. (Control ID \#2468482)

BACKGROUND: The outpatient management of heart failure involves routine office visits where patients provide retrospective subjective information. But this information may not be highly accurate. Furthermore, a one night or 1 day-and-night study, e.g., 
sleep study or 24-h Holder, many not fully capture patients' nocturnal clinical condition at home because their condition can vary from night to night. We hypothesized that the oxygen saturation and heart rate of heart failure patients could be suboptimal at night and that their oxygen saturations and heart rates could vary both within and between nights.

METHODS: We prospectively recruited 37 patients at their routine heart failure clinic visit. Patients were managed according to the ACCF/AHA guidelines. Exclusion criteria were: NYHA class IV, current participation in a clinical trial, and inability to understand English. Patients were asked to wear a pulse oximeter at night for six nights and they returned their devises on the seventh day.

RESULTS: The average age of the participants was 67.2 (SD, 12.6), 26 (70\%) were male, 24 (65\%) were African-American, NYHA class: I, 11 (30\%); II, 20 (54 \%); and III, $6(16 \%)$. Eight patients were on CPAP at night. The mean oxygen saturation was $92.4 \%$ (SD, 2.4). The mean of the minimum oxygen saturations was $71 \%$. Approximately one fourth of the patients, $24 \%$ ( 9 of 37), experienced minimum oxygen saturations of less than $70 \%$. Furthermore, $44 \%$ ( 4 of the 9 ) of those patients experienced mean oxygen saturations of less than $60 \%$. Many patients exhibited high night-to-night variability in oxygen saturation; the coefficient of variation across nights was high $(>0.03$ to $<0.06)$ for 11 patients, moderate $(>=0.02$ to $<=0.03$ ) for 15 patients, and low $(0.01$ to $<0.02)$ for 11 patients. The mean heart rate was $72.5 \mathrm{bpm}(\mathrm{SD}, 5.9)$. The mean of the minimum heart rates was $36.8 \mathrm{bpm}$ and the mean of the maximum heart rates was $180.4 \mathrm{bpm}$. Almost all of the patients, $86 \%$ (32 of 37), experienced minimum heart rates less than $50 \mathrm{bpm}$ Almost all of the patients, $88 \%$ ( 34 of 37), experienced maximum heart rates greater than $100 \mathrm{bpm}$. Additionally, almost half the patients, $49 \%$ (18 of 37), experienced maximum heart rates greater than $200 \mathrm{bpm}$. Many patients exhibited high night-to-night variability in heart rate; the coefficient of variation across nights was high ( $>=0.10$ to 0.14 ) for 10 patients, moderate $(>=0.05$ to $<0.10)$ for 23 patients, and low $(0.01$ to $<0.05)$ for 4 patients.

CONCLUSIONS: Many heart failure patients exhibited severe and profound hypoxia and bradycardia/tachycardia at night. In addition, some patients exhibited high inter-night variability that suggests that a one-night sleep study or a 24-h Holter may not accurately capture their true clinical condition at home at night. In summary, clinicians need objective physiologic information collected at home over several nights on a regular basis in order to optimally manage their heart failure patients.

\section{OLIGELLA URETHRALIS INFECTION: A CASE SERIES AND REVIEW OF} THE LITERATURE. Sreelakshmi Panginikkod; Dima Dandachi; Venu Pararath Gopalakrishnan; Maaman Bashir. Presence Saint Francis Hospital, Evanston, IL. (Control ID \#2469812)

BACKGROUND: Clinical infection due to Oligella urethralis has been rarely reported in the literature. This may be due to misidentification of this organism or uncertainty of its pathogenicity. Our observation suggested that oligella urethralis is transforming from being a commensal of the genitourinary tract to a drug resistant pathogen and capable of invasive disease. In this case series, the characteristic features, antibiotic susceptibility and clinical outcome were assessed in 16 patients and the literature for previously reported cases of oligella infections were reviewed.

METHODS: The clinical data of patients with Oligella urethralis infection were collected retrospectively from 4 hospitals in the Chicagoland area between January 2010 and December 2015. Totally, there were 16 cases identified during this period. A literature search for case reports of Oligella urethralis published until December 31, 2015, was performed on PubMed, MEDLINE, Scopus Elsevier, Cochrane, and Google Scholar. RESULTS: All 16 patients identified were adults except one patient who is 14 years old, with a mean age of 61 years and ranging from 14 to 93 years of age. Of those 16 patients, 6 were males $(37.5 \%)$ and 10 were females $(62.5 \%)$. Source of infection was identified as urine in 9 patients $(56.25 \%)$ and wound in 4 patients $(25 \%)$. Two patients had abscesses (one axillary and one labial) that grew oligella urethralis. One patient had Oligella urethralis bacteremia with no identified source. He had a chronic Foley catheter, however urine culture did not grow Oligella and renal ultrasound did not show any sign of obstruction or hydronephrosis. Although Oligella urethralis infection is reported to be associated with malignancy and immunosuppression, only two patients $(12.5 \%)$ were found to have active cancer and nine out of 16 patients $(56.25 \%)$ had underlying type 2 diabetes mellitus. There was no evidence of urinary obstruction in any of the patients but urinary incontinence was documented in 2 of them. Antimicrobial susceptibility testing is not routinely performed on Oligella urethralis isolates because of lack of interpretive standardized methods for susceptibility testing by Clinical and Laboratory Standards Institute (CLSI). Out of 7 clinical isolates tested for antibiotic susceptibility, all were found to be resistant to Fluoroquinolones and 2 were found to be resistant to Aztreonam, but all were sensitive to a wide variety of antibiotics including beta-lactams. All patients were treated with at least one drug to which the organism was sensitive and all, except the patient with oligella bacteremia, recovered without any morbidity. That one patient with blood culture positive for Oligella responded initailly but died after 3 months due to sepsis.

CONCLUSIONS: Of the reported cases of Oligella urethralis, most occurred as an opportunistic infection in patients with a source of immunosuppression such as malignancy, HIV or newborns. In the cases we studied, however, there was no major source of immunosuppression, and there was no clinical or laboratory evidence to suggest any abnormality of urinary tract. We also observed that in all seven cases, the organism is sensitive to a wide variety of antibiotics including penicillin, but resistant to fluoroquinolones. According to literature, Oligella urethralis may be an opportunistic pathogen and quinolone resistance may arise in patients for whom quinolones are used extensively. Our review suggest that further studies are necessary to understand this bacterium's clinical significance and antibiotic susceptibility.

\section{ONLINE TREATMENTS FOR MOOD AND ANXIETY DISORDERS IN PRIMARY CARE: A RANDOMIZED CONTROLLED TRIAL Bruce L. Rollman;} Bea Herbeck Belnap; Scott D. Rothenberger; Kaleab Abebe; Armando J. Rotondi; Jordan F. Karp. University of Pittsburgh, Pittsburgh, PA. (Control ID \#2469235)

BACKGROUND: Internet support groups (ISGs) that enable individuals with similar conditions to access and exchange self-help information and emotional support have proliferated in recent years. However, their benefit has not been established in randomized trials. Similarly, several computerized cognitive behavioral therapy (CCBT) programs have been proven as effective as face-to-face CBT at treating mood and anxiety disorders and used by hundreds of thousands of patients outside the U.S., yet CCBT remains largely unknown and little utilized within the U.S. While dozens of trials have proven the effectiveness of various collaborative care strategies at treating mood and anxiety disorders in primary care, we are unaware of any trial that incorporated an online CCBT program or ISGs into its design. Providing depressed and anxious patients with access to these emerging technologies via a collaborative care program linked to their usual source 
of primary care may be an ideal method to deliver effective mental health treatment, especially to those residing in areas with poor access to care resources or have transportation difficulties or work/home-life obligations that make in-person counseling difficult to obtain. We tested the effectiveness of providing CCBT and ISG embedded within a collaborative care intervention in a randomized, controlled, 3-arm, single-blind, effectiveness trial.

METHODS: We enrolled depressed and anxious patients from 26 Pittsburgh-area primary care practices that shared a common electronic medical record (EMR) system (EpicCare). Primary care physicians (PCPs) referred patients to our trial in response to an EMR-generated prompt that automatically launched whenever "depression", "anxiety" or "panic" was entered as a visit diagnosis for patients aged 18-75. We telephone-screened referred patients to confirm the presence of at least moderate levels of mood and/or anxiety symptoms (PHQ-9 or GAD-7 $\geq 10$ ) and reliable Internet access, then randomized protocol-eligible and consenting patients in a 3:3:1 ratio to either: (1) six months of care manager-guided access to the online 8-session U.S. version of the Beating the Blues program (www.beatingthebluesus.com; CCBT-alone); (2) CCBT-alone plus access to a care manager-moderated ISG we created that patients could login to via personal computer or smartphone $(C C B T+I S G)$; or (3) their PCP's "usual care" $(U C)$. We assigned an offsite bachelor's degree-level care manager to each intervention arm who guided patients in use of the CCBT program and assessed their preferences for antidepressant or anxiolytic pharmacotherapy via telephone and secure email, and provided regular feedback and treatment recommendations to their PCP via EMR. In addition, the care manager assigned to the $C C B T+I S G$ arm moderated the discussion boards on our ISG and created weekly group email messages that encouraged patients to log-in to the site. We collected sociodemographic and clinical data at baseline, and blinded telephone assessors administered the SF-12 MCS and PROMIS Depression and Anxiety Short Forms to measure health-related quality of life, mood, and anxiety symptoms, respectively, at baseline, 3 and 6-months follow-up, and at 6-months post-treatment (12-months) to evaluate the durability of our interventions. We used linear mixed models that adjusted for age group and clinic size to estimate between-arm differences at 6- and 12-months in intention-totreat analyses, with exploratory per-protocol analyses to assess outcomes by level of patient engagement with our online treatments.

RESULTS: Between 8/12 and 9/14, we received 2884 electronic referrals and randomized 704 depressed and/or anxious patients (101\% of goal: 301 CCBT-alone, 302 CCBT+ $I S G, 101 U C$ ) whose baseline characteristics were similar by treatment assignment (mean age: 42 years (range: $18-75$ ), $80 \%$ female, $82 \%$ White, $77 \%$ on pharmacotherapy, mean PHQ-9: 13.3 (SD: 5.0), GAD-7: 12.9 (4.4), and SF-12 MCS: 31.4 (9.0)). At 6-months, $86 \%$ of all patients with access to CCBT started the program and $37 \%$ completed all 8 sessions (mean sessions completed: 5.4 ); $75 \%$ of $C C B T+I S G$ patients logged into the ISG at least once, and $46 \%$ provided one or more comments (mean: 9.5 comments/ person). Compared to $U C$ patients at 6-months, those randomized to the CCBT-alone arm reported similar effect size (ES) differences in SF-12 MCS scores, but significant improvements in $\operatorname{mood}(\mathrm{ES}: 0.31 ; P=0.006)$ and anxiety symptoms (ES: $0.26 ; P=0.02)$ that increased with higher levels of program engagement (e.g., completed $\geq 4$ sessions: $0.41 \mathrm{ES}$ mood; completed all 8 sessions: $0.52 \mathrm{ES}$ mood) and persisted 6 months post-treatment. However, $C C B T+I S G$ patients had similar ES improvements as CCBT-alone patients on the SF-12 MCS and PROMIS measures, and exploratory analyses among those who either logged-into or commented on our ISG three or more times within 6 months did not alter these findings.

CONCLUSIONS: Providing online CCBT to depressed and anxious primary care patients embedded within the context of a centralized collaborative care program is an effective, scalable, and durable strategy for delivering mental health care. While offering care manager-moderated access to our ISG did not produce any measurable benefit over CCBT-alone, our study findings have important implications for transforming the way mental health care is delivered in primary care, and may focus further attention on the emerging field of e-mental health by other U.S. investigators

OPIOID ANALGESIA USE AFTER AMBULATORY SURGERY: MISMATCH BETWEEN QUANTITIES PRESCRIBED AND USED Christopher W. Shanahan ${ }^{1}$; Inga Holmdahl' ${ }^{2}$; Olivia Gamble ${ }^{2}$; Julia Keosaian ${ }^{2}$; Ziming Xuan ${ }^{3}$; Jane M. Liebschutz ${ }^{1}$. ${ }^{1}$ Boston University School of Medicine, Boston, MA; ${ }^{2}$ Boston Medical Center, Boston, MA; ${ }^{3}$ Boston University School of Public Health, Boston, MA. (Control ID \#2469334)

BACKGROUND: Prescription opioid misuse is a major public health problem. The single largest source of misused opioids is those that were legitimately prescribed. Anecdotally, opioid prescriptions to treat post-operative pain have been implicated in steering patients toward a path of addiction, or, conversely, are overprescribed and are the source of diversion of opioids. We aimed to characterize post-operative opioid analgesia prescribing practices of surgeons and the patient experience through a prospective study of patients undergoing elective ambulatory surgery.

METHODS: The study design was a longitudinal survey 1 week prior and 1 week after planned ambulatory surgery at an academic safety-net hospital. The surgeries included all specialties (e.g. orthopedic, otolaryngology, general, podiatry, maxillofacial, gynecology, urology), excluding procedures not expected to require post-operative pain management (such as eye procedures or endoscopies). Participants were recruited and interviewed both in-person at pre-operation appointments or via a personalized letter mailed to participants, with an opt-out option, followed by a phone call. Participants were consented and administered baseline questionnaires between 1 and 14 days prior to scheduled surgery. The follow-up assessment was administered 10 14 days post-operatively via telephone. An initial questionnaire collected baseline information on socio-demographics, pain severity and function (the Graded Chronic Pain Scale), and alcohol and drug use disorders (AUDIT and DUDIT). The follow-up questionnaire included assessment of post-operative pain (International Pain Outcomes questionnaire), modified versions of two validated scales to identify opioid medication misuse (the POMI and PMQ), and questions to characterize prescription medication disposal and storage. Lastly, a timeline follow-back tool asked about daily pain levels and medications used each post-operative day for 10 days.

RESULTS: Of the 180 participants recruited from 18 physicians, we obtained follow-up data from 149 participants ( $83 \%$ retention rate); $53 \%$ were female and had a mean age of 49. Participants were $44 \%$ white, $34 \%$ black, and the rest identified as "other" (largely mixed ethnic and race). Sixty-seven percent of participants had some post-high school education. Baseline results of pain severity and disability from the Graded Chronic Pain scale were as follows: $26 \%$ had highly disabling and severely limiting pain, $18 \%$ had highly disabling and moderately limiting pain, $31 \%$ had low-disabling and highly intense pain, $20 \%$ had low-disabling and low intensity pain, and $5 \%$ reported no pain. For alcohol use, $87 \%$ of participants indicated low or no risk and $10 \%$ indicated some level of risk or dependence. At baseline, $19 \%$ of participants reported use of illegal drugs or prescribed drugs for non-medical reasons. Physicians prescribed opioid medications for post-operative pain control to $91 \%$ of participants, $81 \%$ of whom were prescribed either 
oxycodone or oxycodone with acetaminophen. Physicians prescribed an average of 31 pills to participants, while participants took an average of 15 pills over the 10-day period. Physicians prescribed 4655 pills total, and participants reported having only taken $44 \%$ of those (2067 pills total). At follow-up, using a scale of $0-10,32 \%$ of participants rated the effectiveness of their pain relief as complete (10), 36\% as high (7-9), $24 \%$ as moderate (4-6), $5 \%$ as low (1-3), and $3 \%$ as ineffective (0). Twenty-two percent of participants reported that they would have liked more pain treatment than received. In terms of total number of opioid pills taken post-operatively, $14 \%$ reported taking 0 pills, $31 \%$ took $1-7$ pills, $42 \%$ took 8-32 pills, and $13 \%$ took $33-67$ pills over the 10 -day period. Of participants taking opioid pain medication, $14 \%$ reported taking them more often than prescribed and $10 \%$ reported needing an early refill. The majority of participants (71\%) reported that they had pain medication left over, of whom $33 \%$ of participants reported intent to use a safe means of disposal (flushing or giving to police) while $48 \%$ planned to keep (33\%) or continue taking their medication (15\%). The remaining participants (19\%) with leftover medications reported plans to throw them away (6\%), did not know their plans $(5 \%)$, or provided other responses $(8 \%)$.

CONCLUSIONS: For elective ambulatory procedures, most participants reported using significantly less post-operative opioid pain medication than was prescribed Nearly half of participants reported plans to retain their unused medications after the resolution of their pain. To reduce unnecessary opioid prescribing and potential availability for diversion and misuse, improved prescribing and disposal practices are needed.

ORDERING OF LABS AND TESTS: VARIATION AND CORRELATES OF VALUE-BASED CARE IN AN UNANNOUNCED STANDARDIZED PATIENT VISIT Sondra Zabar ${ }^{5}$; Kathleen Hanley ${ }^{2}$; Hillary Lee ${ }^{5}$; Irina Gershgorin ${ }^{3}$; Lisa Altshuler ${ }^{5}$; Barbara Porter ${ }^{4}$; Andrew B. Wallach ${ }^{1}$; Colleen Gillespie ${ }^{6} .{ }^{1}$ Bellevue Hospital, New York, NY; ${ }^{2}$ NYU, New York, NY; ${ }^{3}$ NYU Langone, New York, NY; ${ }^{4}$ NYU SOM, New York, NY; ${ }^{5}$ NYU School of Medicine, New York, NY; ${ }^{6}$ NYU SoM, Brooklyn, NY. (Control ID \#2468828)

BACKGROUND: Value-based care has led to greater scrutiny of test ordering, including processes for ensuring the clinical effectiveness of high cost tests and identifying unneeded or redundant tests, not just to reduce costs but also because inappropriate tests can cause unnecessary patient discomfort, increase the risk of false-positive results, and overload diagnostic services. At the same time, timely access to the right lab results are necessary for patient care and can reduce downstream costs. And yet, little is known about variation in ordering of labs and tests, medical education and training efforts have not focused much on teaching trainees about what to order when, and the costs of tests is rarely discussed in an educational context. Ordering of appropriate labs and tests is not only an essential element of quality care, it is also a clear indicator of physician's clinical reasoning and decision-making skills. Studies have found that resident tend to order more avoidable tests than more senior physicians. However, most studies of test ordering have used chart reviews to identify inappropriate utilization and therefore suffer from the limitation that information not documented in the chart may support the use of tests that otherwise appear unnecessary. We take advantage of the methodological control of such information afforded by Unannounced Standardized Patients (USP) and report on lab and test ordering for a standardized asthma case presented as a real patient to resident's in their clinics.

METHODS: One hundred thirty-eight USPs were delivered to medicine residents in two safety net primary care clinics. Highly trained SPs showed up to the clinic as new patients with a chief complaint of worsening asthma. The SP completed a comprehensive behaviorally anchored assessment of the competence of the resident in the core domains: communication, assessment, counseling, physical exam, treatment plan, and patient activation. A systematic chart review was conducted of the notes written and orders placed for each of these visits. All labs and tests ordered were recorded and compared to a "gold standard" of orders deemed appropriate in this standardized case by a consensus panel of primary care physicians and residency program faculty. Residents' clinical reasoning was assessed based on the overall quality of the note as measured by the chart audit team based on criteria of organization, accuracy, completeness and clarity. The total number of labs/tests ordered was calculated for each visit and then compared to the gold standard of indicated labs/tests to arrive at a value representing the $\%$ labs ordered that were inappropriate (\% inappropriate labs) as well as the total number of unnecessary labs (unnecessary labs). Correlations were calculated to assess the degree of association between residents' ordering of inappropriate labs and their core clinical skills (assessed as \% of items rated as well done within the core domains: communication, assessment, education and counseling, physical exam, treatment plan, and patient centeredness and activation) as well as the overall quality of their patient notes (4-point scale from poor to excellent).

RESULTS: Eight labs or tests were determined to be appropriate for this standardized presentation of asthma symptoms and patient history. Residents ordered a mean of 2.48 tests per visit $(\mathrm{SD}=1.49)$ with a range from 0 to 9 tests. For each visit, on average, $87 \%$ of ordered labs were indicated ( $13 \%$ of labs were inappropriate). This meant that an average of .41 (SD .89) tests/visit were unnecessary with a range of 0 to 6 unnecessary tests ( $n=10$ residents ordered 2 unnecessary tests; $n=4$ residents ordered 3 unnecessary tests; and 1 resident ordered 6 unnecessary tests). The number of unnecessary labs ordered was mildly negatively correlated with communication score skill domains $(-.19, p=.03$, for information gathering; $-.16, p=.08$, for relationship development, and - $.14, p=.10$ for patient education) and with patient centeredness $(-.16, p=.08)$ and patient activating skills (-.16, $p=.07)$. Number of unnecessary labs ordered was not associated with the overall quality of the note $(-.02, p=.89)$. Unnecessary labs ordered were Chest X-Rays $(n=16)$, A1C $(n=4)$, Lipids $(n=14)$, CBC $(n=10)$, Basic Metabolic Panel $(n=8)$, Hcg $(n=3)$, TSH $(n=5)$, Hepatic Function $(n=1)$, Hep B $(n=1))$.

CONCLUSIONS: Residents' orders for this standardized case demonstrated some but not a tremendous amount of variation: while most residents ordered less than 1 unneeded lab, a few residents ordered 3-6). A slight trend for the ordering of unnecessary labs to be negatively associated with core communication and patient engagement (centeredness and activation) suggests that enhancing these skills could reduce the overutilization of labs/tests. The finding that clinical reasoning was not associated with test ordering may illustrate the limited sensitivity of our measure (a simple rating of quality) as well as the somewhat limited overutilization of labs we found; it may also mean that education should focus directly on lab orders and not just on general clinical reasoning. Even given that overutilization doesn't appear to be a large problem in this case, we found that 62 unnecessary labs/tests were ordered - a not insignificant misuse of costs, resources, and time. Future research should explore residents' decision-making in selecting tests and in particular those who ordered many unnecessary tests to help inform educational interventions to support appropriate ordering.

OUTCOMES OF A MULTI-SPECIALTY, SCREENING, BRIEF INTERVENTION, AND REFERRAL TO TREATMENT (SBIRT) RESIDENCY CURRICULUM FOR UNHEALTHY ALCOHOL AND OTHER SUBSTANCE USE Jeanette M. Tetrault ${ }^{1}$; Michael Green ${ }^{1}$; Steve Martino ${ }^{2}$; Sheryl Ryan ${ }^{3}$; Steven L. Bernstein ${ }^{3}$; Jessica Illuzzi ${ }^{3}$; Shara Martel ${ }^{3}$; Michael Pantalon ${ }^{3}$; Patrick O'Connor'; David A. Fiellin ${ }^{1}$; Gail D’Onofrio ${ }^{3}$. ${ }^{1}$ Yale School of Medicine, New Haven, CT; ${ }^{2}$ Yale University School of Medicine, New Haven, CT; ${ }^{3}$ Yale University, New Haven, CT. (Control ID \#2469070) 
BACKGROUND: Alcohol and other substance use cause significant morbidity and mortality; yet, many physicians fail to recognize or address this use. In response, the federal Substance Abuse and Mental Health Services Administration (SAMHSA) funded a Screening, Brief Intervention, and Referral to Treatment (SBIRT) initiative to disseminate a targeted approach for identifying patients with unhealthy alcohol and substance use, providing brief counseling (including motivational techniques), and referring to specialty treatment. In 2008, Yale University was funded by SAMHSA to train residents in Internal Medicine [(IM), Primary Care Internal Medicine (PC), Medicine/Pediatrics (MP)], Emergency Medicine (EM), Pediatrics (Peds), Psychiatry (Psych), and Obstetrics and Gynecology (OB) in SBIRT techniques.

METHODS: Developed through a rigorous curriculum development process, the SBIRT training session included didactic teaching, video examples, and role plays using specialty-specific cases. After the training session, residents were observed performing SBIRT with a standardized patient and skills were assessed using a standardized checklist. Faculty development was offered to encourage faculty observation of the residents performing and documenting SBIRT in their clinical encounters. We present data on resident characteristics, number of brief interventions (BI) performed during residency training, knowledge, and subsequent use of SBIRT skills in clinical practice. Performance of BI was tracked via resident documentation using an online procedure log. Residents completed a knowledge survey pre-training, 30 days post-training and 3 years post-training. At 30 days, and 3 years post-training, we surveyed residents regarding the use of SBIRT in their clinical practices.

RESULTS: Between 2008 and 2013, we trained 554 residents (264 IM, 89 EM, 106 Peds, 54 Psych, 41 OB). Forty-six percent were PGY-1, $41 \%$ PGY-2, $11 \%$ PGY-3, and $2 \%$ PGY-4. The sample was $45 \%$ male and $55 \%$ female. Ninetythree percent of residents completed 30 day and $77 \%$ of residents available for follow up (348/451) completed the 3 year follow-up surveys. Residents documented performance of 1249 BNIs in clinical practice: 561 by IM, 371 by EM, 101 by Peds, 174 by Psych and 42 by OB. Scores on the knowledge survey increased by $14 \%$ from baseline (20/30 questions correct) to 30-days post training (23/30 questions correct) and by $8 \% 3$-years post training (22/30 questions correct). At 3 year follow up, $66 \%(231 / 348)$ of residents reported using SBIRT skills in clinical practice

CONCLUSIONS: Implementation of a residency SBIRT curriculum is feasible in a multi-specialty format. Integrating SBIRT curriculum into graduate medical education has a lasting impact on resident knowledge. Additionally, residents incorporate and document BIs in patient care encounters and continue to use these skills subsequently in clinical practice.

OUTPATIENT MANAGEMENT OF CHRONIC NONCANCER PAIN AND PREDICTORS OF A PAIN CONTRACT Audrey $\mathrm{Ye}^{2}$; Erica M. Wozniak ${ }^{1}$; Sara Koller ${ }^{4}$; Joanne Bernstein ${ }^{2}$; Cynthia Kay ${ }^{3},{ }^{4}$. ${ }^{1}$ Medical College of Wisconsin, Milwaukee, WI; ${ }^{2}$ Medical College of Wisconsin, Whitefish Bay, WI; ${ }^{3}$ Zablocki VA Medical Center/Medical College of Wisconsin, Milwaukee, WI; ${ }^{4} \mathrm{MCW}$, Milwaukee, WI. (Control ID \#2456934)

BACKGROUND: Despite a lack of evidence supporting its use beyond the short term, chronic noncancer pain is routinely treated with narcotics. In fact, pain contracts have become the norm. It is unclear if providers follow guidelinerecommended opioid management practices when prescribing narcotics. Our study's objectives are to characterize the population of internal medicine patients on long-term narcotics for chronic noncancer pain, examine whether providers follow guideline-recommended opioid practices, and explore whether patient or medication factors predict implementation of a pain contract.

METHODS: This was a retrospective chart review of internal medicine clinic patients at an academic medical center who were either on a pain contract or were prescribed at least 3 months of narcotics between April 1, 2014 and April 1, 2015. Patients within 6 months of surgery, with active cancer, and not seen in the established time frame were excluded. The study's main subjects were matched to patients not on a pain contract or chronic narcotics by age and gender and served as controls. Data collected included patient demographics (age, gender, diagnoses); pain contract status; guideline-recommended opioid management practices (documented pain history, physical exam, pain screen, risk stratification, baseline urine drug screen and follow up); and current narcotic regimen. Descriptive statistics and univariate analyses (chi-square/Fisher's exact or t-tests) were used to compare characteristics of the chronic pain and control groups, as well as to compare pain patients by contract status. Predictors of pain contract status were assessed jointly using logistic regression with pain contract (yes/no) as a binary outcome

RESULTS: Approximately $9 \%$ of the clinic population, or 836 patients, were identified as being on chronic narcotics. Of these, 342 patients $(41 \%)$ were on a pain contract. The average age of patients with chronic pain was 61 years (SD $12.8)$ and $552(66 \%)$ were women. Compared to the control group, patients with chronic noncancer pain were more likely to be African American, have depression, diabetes, hypertension, arthritis, be an active tobacco user, have a history of substance abuse, and be obese (all $p<0.001$ ). Fewer demographic and clinical differences were observed between patients on chronic narcotics based on pain contract status. Race and depression were significantly different between contract and no contract groups, with African American patients and those with depression more likely to be on a pain contract ( $p=0.03$ and $p=0.009$, respectively). Guideline-recommended opioid management practices also differed between pain contract and no contract groups. Documentation of a pain history, use of pain screen and risk assessment tools, completion of baseline urine drug screen (UDS), and follow up were all more likely in the pain contract group $(p<0.001)$. Practices were also examined before and after opioid guidelines were published in 2009 , finding that patients who signed a contract after guidelines were published were more likely to have a baseline UDS $(p<0.001)$. No other practices were statistically different. Patients on a pain contract tend to be on higher morphine equivalent doses (average $98 \mathrm{mg}$ vs $48 \mathrm{mg}$ ), on a class II narcotic, and be prescribed more than one narcotic (all $p<0.001$ ). Controlling for demographic and clinical characteristics, logistic regression indicated that the strongest predictors of having a pain contract included being prescribed narcotics for more than 6 years, on class II narcotics, use of risk assessment tools, and presence of baseline UDS. Specifically, patients on class II narcotics were 23 times more likely to be on a contract compared to patients on class III or IV narcotics (95\% CI 10.9-54.6). Those who had their risk assessed were 5 times more likely to be on a contract (95\% CI 2.0-15.0) and having a baseline urine drug screen was associated with a 17 times increase in the odds of being on a contract (95\% CI 4.8-87.1).

CONCLUSIONS: Patients with chronic noncancer pain differ from patients without chronic pain. Fewer differences were observed between patients with chronic pain based on pain contract status, but race and depression remained significant. While no patient characteristic predicted having a pain contract, being on a stronger narcotic class and having had a risk assessment done were significant predictors. Providers do not consistently follow guideline-recommended opioid management practices. However, further research is needed to determine whether guideline-recommended practices improve patient outcomes, and if so, what can assist providers in following them. 
OUTPATIENT PRIMARY CARE UTILIZATION AMONG SERIOUSLY MENTALLY ILL ADULTS IN CALIFORNIA Maria E. Garcia ${ }^{2}$; Dean Schillinger ${ }^{2}$; Eric Vittinghoff ${ }^{2}$; Jennifer Creasman ${ }^{1}$; Christina Mangurian ${ }^{2} .{ }^{1}$ UCSF, San Francisco, CA; ${ }^{2}$ University of California, San Francisco, San Francisco, CA. (Control ID \#2469878)

BACKGROUND: Individuals with serious mental illness (SMI) have premature morbidity and mortality, largely due to cardiovascular disease, diabetes, and respiratory illnesses. Despite high rates of modifiable cardiovascular risk factors, and high use of antipsychotic medications with severe metabolic side effects, many individuals with SMI are not engaged in primary care. This study examined individual-level predictors of non-psychiatric outpatient care utilization among a large cohort of individuals with SMI in California.

METHODS: We used administrative, pharmacy, and billing data from Medi-Cal and the Client and Service Information (CSI) system for a cohort of 56,895 adults with SMI (schizophrenia, bipolar disorder, schizoaffective disorder, and major depressive disorders with psychosis) seen in community mental health clinics from October 1, 2010 to September 20, 2011. Poisson regression was used to estimate the independent association of each predictor of interest (including age, race/ethnicity, gender, Axis I psychiatric diagnosis by mental health providers, use of antipsychotic medication, urban vs. rural-dwelling, and substance or alcohol use disorder) with any non-psychiatric outpatient visit ("outpatient visit") during this 1 year time period (defined using CPT codes for new patient, returning patient and consult visits).

RESULTS: Two thirds of participants ( $66 \%$ ) had at least one outpatient visit during the study period. Half of the individuals had schizophrenia (52\%). Fifty-five percent of the sample was female and aged 38-57 years old. Ninety-seven percent of participants were on second-generation antipsychotics. The largest proportion of participants were white (38\%), followed by Hispanic (20\%) and Black (19\%) individuals. In multivariate analyses, after adjusting for potential confounders for each individual predictor, older individuals were more likely to have at least one outpatient visit (aRR 1.07 and 1.19 respectively for ages $28-47$ and 48-67) when compared to younger adults less than 27 years old. Women were more likely to have at least one outpatient visit than men (aRR 1.29, $95 \%$ CI 1.25, 1.34). Compared to whites, Asian and Pacific Islanders were $9 \%$ more likely to have at least one outpatient visit (aRR $1.09,95 \%$ CI 1.03, 1.16), while Blacks were less likely to have an outpatient visit (aRR $0.93,95 \%$ CI $0.89,0.96$ ). Hispanic ethnicity was neither more nor less likely to be associated with outpatient visits, compared to whites. Rural dwellers were $36 \%$ less likely to have an outpatient visit compared to urban dwellers (aRR 0.64, $95 \%$ CI 0.53, 0.77). Compared to individuals with schizophrenia, individuals with all other SMI diagnoses had greater likelihood of at least one outpatient visit in the 1 year follow up period. Individuals with a diagnosis of substance or alcohol use disorder by a psychiatrist were less likely to have an outpatient visit (aRR 0.95, $95 \%$ CI 0.92, 0.98).

CONCLUSIONS: Despite high morbidity and mortality among individuals with SMI, particularly individuals with schizophrenia, there was relatively low utilization of medical outpatient services among this large cohort. This study identified multiple groups who were less likely to access medical services, including younger patients, men, blacks, individuals with schizophrenia, and rural dwellers, which highlights important disparities that exist in medical care among this vulnerable population. Future research and interventions are needed to understand how best to engage these subgroups at higher risk of premature morbidity and mortality, as well as the impact of external, systems-level factors (such as case management, emergency room visits and hospitalizations) on primary care access and utilization.

OVERUSE OF LOW-VALUE MEDICAL SERVICES IN THE SAFETY-NET POPULATION Michael L. Barnett ${ }^{1}$; Jeffrey A. Linder ${ }^{1}$; Cheryl R. Clark ${ }^{1}$; Benjamin D. Sommers ${ }^{2,}{ }^{1}$. ${ }^{1}$ Brigham and Women's Hospital, Boston, MA; ${ }^{2}$ Harvard School of Public Health, Brookline, MA. (Control ID \#2468846)
BACKGROUND: The overuse of low-value medical services is an important and preventable source of unnecessary medical costs. However, little is known about patterns of overuse for patients with "safety-net" insurance coverage, such as those without health insurance or those covered by Medicaid. Safety-net populations tend to have lower access to high-value services compared to those who are privately insured, but there is little research on patterns of overuse of low-value care in these groups. The extent to which medical overuse in safetynet populations varies by patient or clinician characteristics could have important policy implications for how state Medicaid programs try to reduce lowvalue practices. To understand patterns of overuse in this population, we used nationally representative data to examine patient and clinician level predictors of overuse of low-value care. Additionally, we examined underuse of guideline recommended medical services to determine the extent of access to high-value care in safety-net populations.

METHODS: We performed a cross-sectional analysis of the nationally representative National Ambulatory Medical Care Survey and National Hospital Ambulatory Medical Care Surveys of office visits by adults aged 18 and over from 2005 to 2012 . We categorized patients according to insurance type (those without health insurance or Medicaid coverage ["safety-net patients"] vs. private insurance), and clinicians according to the proportion of safety-net patients seen $(0 \%$ safety-net patients ["non-safety-net physicians" vs. $\geq 20 \%$ safety-net patients ["safety net physicians"]). Using previously published guidelines, we measured the visit-level rates of 9 services likely to represent low-value care (e.g. narcotics for acute low back pain) and 10 services representing highvalue, guideline recommended care (e.g. tobacco cessation counseling), as well as composite measures for any delivery of low or high-value care. After adjusting for all available patient characteristics, including age, sex, race, number of chronic conditions, and urban/ rural setting we used weighted, visit-level logistic regression to examine the extent to which having safety-net insurance coverage or being treated by a safety-net physician was associated with high rates of overuse. All rates, regression estimates and standard errors were calculated taking account of the multistage probability design of the surveys.

RESULTS: From 2005 to 2012, the study sample contained 266,934 office visits to physicians by adults eligible for at least one measure examined, representing over 4.4 billion visits, $16.4 \%$ of which were from safety-net patients. Physicians serving a high proportion of safety-net patients ( $\geq 20 \%$ of all visits) represented only $25.3 \%$ of all clinicians, but provided care for $74.5 \%$ of all safety-net patient visits in the study period. Compared to non-safety-net physicians serving no safety-net patients, safety-net physicians treated individuals who were significantly younger (49.0 vs. 55.9 years old, $p<0.001)$ and more likely to be black ( 17.5 vs. $8.1 \%, p<0.001)$. Overall, the likelihood of receiving low-value and high-value care was similar between safety-net and privately insured patients after adjusting for patient characteristics (37.9 vs. $35.9 \%$ for low-value, $p=0.19 ; 28.4$ vs. $26.5 \%$ for high-value, $p=0.20$ ). In aggregate, after adjusting for patient characteristics, low-value care was not more likely among visits to safety-net physicians vs. non-safety-net physicians ( 36.8 vs. $34.2 \%, p=0.55$ ). However, two specific low-value services were more likely among patients served by safety-net physicians after adjustment: narcotic prescription for acute low back pain (35.9 vs. $25.5 \%, p<0.001)$, and antibiotics for viral upper respiratory infections with borderline significance $(53.2$ vs. $43.4 \%, p=$ 0.06 ). In contrast, safety net physicians were significantly less likely to provide recommended high-value care than non-safety-net physicians in the aggregate ( $27.5 \mathrm{vs.} 30.5 \%$, $p<0.001$ ) with significantly lower rates of high-value care for 6 of 10 measures, including aspirin use in CAD (36.8 vs. $43.4 \%, p=0.03$ ), statin use in CAD (38.1 vs. $47.6 \%, p=$ $0.003)$ and statin use in diabetes ( 26.8 vs. $34.8 \%, p=0.01)$.

CONCLUSIONS: Overall, overuse of low-value care and underuse of high-value care occurred frequently among all patients and physicians in our study. There was little 
difference in the receipt of low or high-value care by patients' insurance status. In contrast, patients seen by safety-net physicians had lower rates of providing high-value care than non-safety-net clinicians but similar or higher rates of overuse. This suggests that, as in the general population, both underuse and overuse are challenges for physicians serving lowincome populations. In addition to improving underuse of high-value care, overuse of lowvalue care is a potentially important focus for state Medicaid programs and safety net institutions to pursue cost-savings and improved quality.

\section{PALLIATIVE CARE AND RESOURCE UTILIZATION AT THE END OF LIFE} IN ADVANCED LIVER DISEASE Arpan A. Patel; Anne M. Walling; Sammy Saab; Neil Wenger. University of California, Los Angeles, Los Angeles, CA. (Control ID \#2468857)

BACKGROUND: There is increasing attention paid to the experiences of patients at the end of life dying of chronic conditions. Patients with end stage liver disease usually are treated for this condition for an extended period before death, yet little is known about how these patients die in the hospital. Furthermore, while use of palliative care services at the end of life is associated with better patient outcomes, palliative care use among patients dying with advanced liver disease remains largely unexplored. We examined palliative care and resource utilization during the terminal hospitalization of patients with advanced cirrhosis using a national database.

METHODS: Patients with advanced liver disease were identified in the 20092013 National Inpatient Sample (NIS) using validated International Classification of Disease, 9th Revision, (ICD-9) codes for cirrhosis and decompensated disease. Only deceased patients and those with lengths of stay greater than 3 days were included. Utilization including procedures, length of stay and total charges was evaluated. We then compared utilization, demographics, hospital characteristics, liver transplant status and comorbidities between patients who received and did not receive palliative care during the hospitalization. Logistic and multiple linear regression analyses were performed to determine independent predictors of palliative care consultation and resource utilization, respectively.

RESULTS: Of the 128,971 decedent patients with advanced liver disease, 12,277 (9.5\%) decedents had advanced liver disease with decompensation during the hospitalization. Among this group, $7.2 \%$ carried a diagnosis of hepatocellular carcinoma, $1.4 \%$ were listed for liver transplant, and $0.76 \%$ had received a liver transplant during the hospitalization. Patients dying in the hospital with decompensated liver disease died after a mean length of stay of 14.2 days (standard deviation (SD) 15.5 days) and mean hospital charges of $\$ 183,558$ (SD \$253,897). Fifty-four percent of these patients received mechanical ventilation, $16 \%$ dialysis, $8 \%$ total parenteral nutrition (TPN), $52 \%$ blood transfusion, and $7 \%$ cardiopulmonary resuscitation (CPR) during the terminal hospitalization. Twenty-nine percent of decedents (3571 patients) with decompensated liver disease received palliative care during the terminal hospitalization. Palliative care consultation increased from $18 \%$ in 2009 to $36 \%$ in $2013(p<0.001)$. Mean total charges increased from $\$ 163,540.50$ in 2009 to $\$ 202,255.10$ in $2013(p<0.001)$, although length of stay did not significantly change (14.6 to 13.9 days, $p=0.2$ ). Patients receiving palliative care had lower rates of mechanical ventilation (48 vs. $57 \%$, $p<0.001$ ), TPN (7.5 vs. $8.3 \%, p<0.001$ ), blood transfusion (49 vs. $53 \%$, $p<0.001$ ), dialysis (13 v $17 \%, p<0.001$ ), and CPR (3 vs. $9 \%, p<0.001$ ), as compared to those who did not receive palliative care. The logistic regression model revealed that patient and hospital characteristics were independently related to receipt of palliative care. Compared to Whites, African-American (odds ratio [OR] 0.73, $95 \%$ confidence interval [CI] 0.64-0.85), Hispanic (OR 0.72, $95 \%$ CI 0.64-0.82) and Asian (OR $0.69,95 \%$ CI $0.52-0.91)$ patients were less likely to receive palliative care, as were patients who had received a liver transplant (OR 0.25, $95 \%$ CI $0.13-$ 0.47). Patients were more likely to receive palliative care if they had more comorbidities (OR 1.03 per Charlson index point, $95 \%$ CI 1.01-1.05) or hepatocellular carcinoma (OR 1.44, 95 \% CI 1.23-1.67). Hospitals in the Northeast, non-teaching status and smaller hospitals were all associated with a lower likelihood of receiving palliative care. In multivariable analyses of the utilization variables, palliative care was consistently independently associated with less utilization, adjusting for patient and hospital characteristics. Receipt of palliative care, compared to not receiving it, was associated with shorter length of stay $(p<0.001)$ lower total charges $(p<0.001)$, and a lower likelihood of mechanical ventilation ( $p<0.001)$, TPN $(p<0.001)$, blood transfusion $(p<0.001)$, dialysis $(p<0.001)$, and CPR $(p<0.001)$.

CONCLUSIONS: Palliative care consultation has increased over time for patients with decompensated liver disease dying in the hospital, although most patients still receive mechanical ventilation and blood transfusions during their terminal hospitalization. While this analysis is limited by not accounting for deaths outside the hospital and the analysis identifies associations rather than causality, the findings suggest substantial unmet need for palliative care among patients dying with liver disease.

PARTICIPANT FEEDBACK AND ITERATIVE IMPROVEMENTS TO A MOBILE HEALTH PROGRAM FOR POSTPARTUM WOMEN AT ELEVATED CARDIOMETABOLIC RISK Jacinda M. Nicklas ${ }^{2}$; Jennifer A. Leiferman ${ }^{1}$; Sheana S. Bull ${ }^{1}$; Cynthia N. Hazel ${ }^{1}$; Susan J. Gilbert ${ }^{3}$; Dean A. Hovey ${ }^{3}$; Linda A. Barbour ${ }^{2}$. ${ }^{1}$ Colorado School of Public Health, Aurora, CO; ${ }^{2}$ University of Colorado School of Medicine, Aurora, CO; ${ }^{3}$ Digifit, Santa Barbara, CA. (Control ID \#2466070)

BACKGROUND: Certain pregnancy complications, including gestational diabetes and preeclampsia, sound an early warning for future cardiometabolic disease, often before traditional risk factors manifest. Nearly one-third of postpartum women have a risk factor demonstrating elevated risk for cardiovascular disease or diabetes. Since women in the postpartum period are traditionally difficult to reach for face-to-face interventions, and smartphone use is highly prevalent among diverse socioeconomic groups of women of reproductive age, the use of mobile health (mHealth) is a novel, scalable approach to reach this population. Our objective was to develop and iteratively test an mHealth program designed to help postpartum women decrease their risk for cardiometabolic disease by enhancing weight loss via healthy eating and physical activity.

METHODS: We developed the theory-based Fit After Baby program for overweight and obese postpartum women with recent gestational diabetes (GDM), hypertensive disorders of pregnancy, pre-term birth, and/or a small-forgestational age (SGA) delivery. We adapted the Diabetes Prevention Program to develop an mHealth program designed to increase postpartum weight loss, improve diet, and increase physical activity. The Fit After Baby program delivers daily evidence-based daily content, and facilitates tracking of weight, diet, and steps. The program has a behavioral science and health communication foundation and relies on common strategies for apps such as points, tracking, and rewards. Participants are encouraged to check in weekly with a lifestyle coach. We initially conducted one round of alpha-testing among women with young children, focused primarily on generating reaction to the concept of using an app and features planned to facilitate postpartum weight loss. We then conducted two rounds of beta-testing in postpartum women with the target pregnancy conditions, focused primarily on usability and navigability of the app. We analyzed feedback and implemented changes to the mobile app and program after each round before conducting subsequent rounds of testing. 
RESULTS: Five women completed alpha testing, and changes were made in response to feedback, including improving the process of downloading and getting started, improving navigation, and adding daily reminders to use the app. Four women completed round one and 13 women completed round two of beta-testing, with mean age 33 (SD 4.5) years; BMI 34 (SD 6.4) kg/m², $94 \%$ White and $6 \%$ Asian. Participants had a recent pregnancy complicated by preeclampsia (41\%), pre-term delivery (35\%), gestational hypertension
(29\%), gestational diabetes (29\%), and/or SGA delivery (12\%). We demonstrated an increase in participant satisfaction and in the number of interactions with the coach from round 1 to round 2. Data from these rounds and the main themes from participant feedback are presented in table 1 .

CONCLUSIONS: Through a process of iterative development and testing we were able to improve the mHealth Fit After Baby program in preparation for a larger randomized trial.

Table 1: Participant Use and Feedback from Beta-testing of the Fit After Baby program

\begin{tabular}{|c|c|c|c|c|c|}
\hline Round & $\mathrm{N}$ & Themes for Development & Mean \# Days Used (of 28) & Mean \# Coach Check- Ins & Overall Satisfaction \\
\hline Beta 1 & 4 & $\begin{array}{l}\text { 1) Increase content specific for postpartum moms } \\
\text { 2) Increase structure for interactions with coach } \\
\text { 3) Make exercise tracker more customizable } \\
\text { 4) More instruction on how to earn points and badges } \\
\text { 5) Explain how to save content }\end{array}$ & 23 & 2 & $50 \%$ \\
\hline Beta 2 & 13 & $\begin{array}{l}\text { 1) Improve navigation through more straightforward homepage } \\
\text { 2) Add a display to show when tasks are completed } \\
\text { 3) Add nutrition content including recipes } \\
\text { 4) Add exercise content including workouts } \\
\text { 5) Simplify exercise tracking }\end{array}$ & 19 & 4.3 & $80 \%$ \\
\hline
\end{tabular}

PATIENT ACtivation: IS IT ALL ABOUt THE PHYSiCIAN? James Rotenberg $^{2}$; Amy S. Tang ${ }^{2}$; Nicole Van Groningen ${ }^{2}$; Natasha Rastogi ${ }^{2}$; Irina Gershgorin ${ }^{1}$; Colleen Gillespie ${ }^{4}$; Sondra Zabar ${ }^{3} .{ }^{1}$ NYU Langone, New York, NY; ${ }^{2}$ NYU Primary Care Internal Medicine Residency, New York, NY; ${ }^{3}$ NYU School of Medicine, New York, NY; ${ }^{4}$ NYU SoM, Brooklyn, NY. (Control ID \#2466164)

BACKGROUND: Primary care delivery is increasingly multidisciplinary with incentives for provider teams made up of physicians and non-physicians. Increased patient initiative and self-management, activation, is an intended outcome of coordinated patient-focused care because patient activation can be validly measured and correlates with positive health outcomes and reduced costs. While physician providers can increase activation, it remains unproven whether activation results from physician behavior or physician-independent aspects of the clinical environment. This exploratory secondary analysis uses data from visits of unannounced standardized patients (USP) to resident physicians in clinic to study the relationship of elements of activation to both physician and non-physician behavior. METHODS: Data are from 492 USP encounters at two primary care clinics from 2009 to 2015. USPs portrayed 6 standardized clinical scenarios and completed a comprehensive, post-visit, behaviorally-anchored assessment of each encounter. Six questions from the Patient Activation Measure assessed the USPs' degree of activation after the visit. Physician-independent variables included the performance of 2 non-physician clinic staff members, visit length and wait times, and global assessments of the clinic environment. Pearson correlation coefficients related individual variables with activation scores. Hierarchical regression modeling examined whether physicians and non-physician factors affected patient activation.

RESULTS: Physician behaviors which positively correlated with patient activation included medical competence in forming a management and treatment plan (Pearson correlation coefficient $r=0.441$ ), performing a thorough patient assessment $(r=0.298)$, using effective communication skills $(r=0.427)$, providing education and counseling $(r=0.504)$, and relationship building $(r=$ 0.540). Physician independent features of the USP visit also positively related to patient activation. Provider team functioning $(r=0.120)$, non-physician professionalism $(r=0.157)$, satisfaction with staff $(r=0.201)$, clinic navigability $(r=0.120)$, visit length $(r=0.154)$, and recommending the clinic to a friend $(r=0.420)$ all positively and significantly $(p<0.05)$ correlated with patient activation. At one site, clinic chaos $(r=0.209)$ also correlated with activation. A multivariate linear regression model with physician variables and non-physician variables did not demonstrate significant association with patient activation, with physician items alone accounting for $93 \%$ of the variance in activation. This study's relatively large sample size and finding of strong positive correlation between activation and physician behavior strengthen the validity of the negative findings for physician-independent items. Limiting the findings are the use of standardized, instead of realworld, patients and the subsequently reduced generalizability. USPs were also likely cued to focus on the physician provider when considering activation. CONCLUSIONS: When controlled for physician factors, physician-independent components of the clinic environment did not directly influence standardized patients' sense of activation. Activation was instead a function of physicianspecific behaviors and skills.

\section{PATIENT ALIGNED CARE TEAM IMPLEMENTATION ASSOCIATION WITH} EMPLOYEE SATISFACTION AND WORKPLACE PERCEPTIONS BEFORE AND AFTER IMPLEMENTATION. Ryan Derickson ${ }^{2}$; Christian D. Helfrich $^{3,5}$; Stephan D. Fihn ${ }^{4,}$; David Mohr ${ }^{1} .{ }^{1}$ VA Boston Healthcare System, Boston, MA; ${ }^{2}$ Department of Veterans Affairs, Cincinnati, OH; ${ }^{3}$ VA Puget Sound Healthcare System, Seattle, WA; ${ }^{4}$ Department of Veterans Affairs, Seattle, WA; ${ }^{5}$ University of Washington School of Public Health, Seattle, WA; ${ }^{6}$ University of Washington School of Medicine, Seattle, WA. (Control ID \#2467893)

BACKGROUND: Efforts to implement patient-centered medical homes require a high level of buy-in and commitment from primary care clinicians and staff. How much is successful implementation of a medical home predicted by a clinic's employee morale and workplace climate? And how stable are these associations over time?

METHODS: We analyzed data from primary care clinics at the Veterans Health Administration. The outcome was a previously validated patient aligned care team implementation index ( $\mathrm{Pi} 2)$. The $\mathrm{Pi} 2$ is an index score based on eight principles of a medical home model, such as comprehensiveness of care and team-based care. Predictor measures were obtained from an annual employee survey and aggregated to a clinic-level. Job satisfaction 
was assessed with 13 items, such as how satisfied employees are overall and satisfaction with workload. Workplace climate was assessed with 31 items, such as whether employees are given an opportunity to develop their skills or how services are designed to meet customer needs. Respondent demographics and clinic characteristics were included as covariates in multivariate linear regression models where Pi2 was regressed on each of the survey domains (job satisfaction and workplace climate) in separate models. Final models were selected for parsimony. Two different study periods were included to examine stability of the associations over time.

RESULTS: We had a total of 17,543 respondents from 306 clinics in 2009 (the year prior to launch of the medical home) and 29,318 respondents from 418 clinics in 2012 (the year the Pi2 was measured). In 2009 (pre-implementation), higher levels of satisfaction with the type of work and perceived customer satisfaction were associated with higher Pi2 scores while higher overall job satisfaction was associated with lower Pi2 scores. Higher ratings on climate measures relating to customer service and coworker relations were both associated with higher Pi2 scores. In 2012 (post-implementation), higher perceived customer satisfaction, greater manager focus on performance planning, and lower ratings of satisfaction compared to 2 years ago were associated with higher Pi2 scores. Adjusted R-squared values for job satisfaction models were .10 and .11, for 2009 and 2012 data, respectively, and for workplace climate were .11 and .06 for 2009 and 2012 data, respectively.

CONCLUSIONS: Job satisfaction and workplace climate were significantly associated with clinic-level medical home implementation a full 3 years later, and showed a relatively large effect. This suggests implementation of a medical home may be more likely to succeed when primary care employees feel there is an existing patient focus, have managers who set and monitor goals, and work in a civil environment. It also suggests that it may be more effective in the long term to attend to workplace climate and morale before engaging in medical home implementation, rather than to expect climate and morale to improve as a result of medical home implementation. We did not set out to test a specific model of job satisfaction and climate, and therefore these findings should be viewed as exploratory

\section{PATIENT AND PHYSICIAN VIEWS OF CULTURE AND RELIGION IN} GOALS OF CARE CONVERSATIONS Jenny J. Lin ${ }^{1}$; Shelli L. Feder ${ }^{3}$; Cardinale B. Smith $^{1}$; Nina A. Bickell ${ }^{1,2}$; Dena Schulman-Green ${ }^{3} .{ }^{1}$ Icahn School of Medicine at Mount Sinai, New York, NY; ${ }^{2}$ Mount Sinai School of Medicine, New York, NY; ${ }^{3}$ Yale University, West Haven, CT. (Control ID \#2467092)

BACKGROUND: Religious and cultural beliefs can shape patient and physician treatment decisions particularly for serious life-threatening conditions and pose challenges to conduct effective goals of care (GoC) conversations. Physicians' views on how patients' culture or religion affects $\mathrm{GoC}$ conversations remain largely unexplored, and it is unknown how patient and physician perspectives may differ.

METHODS: We conducted semi-structured interviews at academic, community, municipal and rural hospitals $(n=4)$ in New York and Connecticut with patients with advanced cancer $(n=19)$ and oncologists $(n=13)$. Interviews were audiotaped and transcribed. Three coders developed a code key and independently coded transcripts. Codes were compared and discrepancies resolved. We analyzed data using interpretive description.

RESULTS: Of the physicians, $85 \%$ were male and the mean age was 47 years. Sixty-nine percent were White, $23 \%$ Asian, and $8 \%$ Hispanic. The mean number of years in practice was 20 and $54 \%$ had received training in GoC. Of the patients, $63 \%$ were female and the mean age was 58 years. A third (32\%) were White, $42 \%$ Black, $16 \%$ Hispanic, $5 \%$ Asian and $5 \%$ Other. Shared cultural issues affecting GoC conversations identified by patients and physicians were family dynamics, gender (male-dominated or men reacting differently than women), religion, complementary medicine remedies, medical mistrust, and patient-physician cultural congruency. For example, a physician stated, "There are some cultures where the males are very dominant... so if you have an older female patient they often don't seem to have a voice in their treatment decisions and that makes it really hard to have a goals of care discussion because I am not sure who I am really having the conversation with." A female patient stated, "There are some cultures where women are not open...if the husband is there they won't talk." Physicians also identified language barriers and treatment-decision making differences and reported that $\mathrm{GoC}$ conversations can be more difficult with patients from cultures that dissuade diagnosis disclosure or that value quantity over quality of life. One stated, "I guess in some, whether it's religions or cultures...the belief that you should continue treatment and prolong life at the expense of quality of life, even if it's by a day or a week-I think those beliefs can sometimes make it difficult to have a goals of care discussion." Patients stated that discussion of culture or religion was an individual issue and reported that some physicians are not interested in discussing culture or religion. While physicians distinguished culture from religion, most patients equated them. Although all physicians believed that culture and religion played a role in GoC conversations, some patients did not feel these topics should be included. One patient stated, "I would say to the extent possible if it is not directly related to the procedures and what is happening on that level, I think...the doctor should refer them to somebody who deals with culture, like social workers, like get it out of your hands, because it's, like how do you answer those? What do you do? Somebody asks you about God healing, what do you say? I can't answer that question."

CONCLUSIONS: We identified several similarities between physicians' and patients' views about the role of culture and religion in GoC conversations. To improve outcomes in patients with advanced cancer, GoC conversations should be individualized and broader in scope than advanced directives. Physicians should be aware of differences in cultural and religious beliefs in order to tailor $\mathrm{GoC}$ conversations and should directly ask patients about their belief systems, assess their desire for information and discuss whether addressing culture or religion in the medical context would be helpful for the patient

Patient and Physician Views on Culture and Religion in GoC Conversations

\begin{tabular}{lll}
\hline \hline Physician and Patient & Physician Only & Patient Only \\
Religion & Language & Culture/religion is \\
Family Dynamics & BarriersFamily doesn't & individual issue \\
Gender Roles & want patient to know & Not physician's role \\
Complementary/Alternative & diagnosis & to discuss \\
Medicine & Treatment & culture/religion \\
Medical Mistrust & decision-making & Physician not \\
Patient-Physician & Valuing quantity over & interested in \\
Cultural/Religious & quality of life & culture/religion \\
Congruency & &
\end{tabular}

PATIENT AND PROVIDER PERSPECTIVES ON THE MANAGEMENT OF OBESITY: BIASES, BLAME, AND THE NEED FOR COMMON GROUND $\underline{\text { Shamol R. Williams }}{ }^{2}$; Jose Melendez-Rosado ${ }^{1}$; Elizabeth Pantoja ${ }^{1}$; Michael Heckman ${ }^{1}$; Emily Vargas ${ }^{1}$; Loren Murray ${ }^{1}$; Richard O. White ${ }^{1}{ }^{1}$ Mayo Clinic, Jacksonville, FL; ${ }^{2}$ Mayo Clinic Florida, Jacksonville, FL. (Control ID \#2469707)

BACKGROUND: Outpatient management of obesity requires a strong interpersonal relationship between the primary care provider and patient. The purpose of this qualitative study was to understand better the specific patient and provider factors that serve as barriers to the management of obesity in the primary care setting. 
METHODS: Within the context of a retrospective study examining the quality of dietary and physical activity counseling across primary care at the Mayo Clinic Florida, we collected real time qualitative data from providers and patients to gain insight into potential drivers and barriers to high quality obesity management. Eligible providers and patients were identified via a Research IT-supported query of the electronic health record (EHR). Two secure online surveys were sent to $N=93$ and $N=746$ providers and patients respectively to assess their experiences including knowledge, barriers, and facilitators of weight management in primary care. All patients were obese $(\mathrm{BMI}>30)$ and each provider practiced in a primary care location within a group practice. Providers were asked to rate their personal knowledge of effective obesity management strategies, how often they provided dietary and/or exercise counseling, and how often they made referrals to nutrition/bariatrics. Providers were further asked to identify significant barriers to counseling obese patients in their practice and to self-report their perceptions of their own weight and level of physical activity. Patients were asked to rate their quality of life, health status, and how often they engaged in moderate to vigorous physical activity. Patients were also asked to determine if their provider ever discussed their weight with them and to what extent, and to select primary barriers and facilitators to adopting a healthier lifestyle. Finally, patients were asked to disclose whether they felt that weight issues impacted upon the quality of care they received or if they were treated differently by providers or staff.

RESULTS: A total of 59 providers (63\%) and 324 patients (43\%) completed the survey. Providers indicated that their personal knowledge of effective obesity management strategies were "fair" $(42.4 \%)$ or "good" $(45.8 \%)$. Providers responded that they provided evidence-based dietary and/or exercise counseling to their patients with obesity "sometimes" (35.6\%) or "often" (49.2\%). Significant barriers to counseling obese patients included lack of time $(72.9 \%)$, patient not motivated to change $(59.3 \%)$, and patient not interested $(35.6 \%)$ or previous failed attempts $(32.2 \%)$. From the patient's perspective, $23.3 \%$ responded that their primary care provider never discussed their weight with them. The major barriers for patients to adopt a healthier lifestyle or achieve a healthier weight included unclear recommendations from their provider $(95.7 \%)$, portion control $(40.4 \%)$, stress $(38.3 \%)$, lack of self-control (37.0\%), lack of motivation (36.7\%), lack of time $(29.6 \%)$, or lack of energy $(26.5 \%)$. Some patients $(20 \%)$ indicated that they often felt marginalized, negatively judged, and made to feel as though they do not care about their own health because of their weight. CONCLUSIONS: Our study demonstrated that there are significant discrepancies between perceptions of primary care providers and patients with obesity regarding weight management in the primary care setting. Despite providers perceiving adequate knowledge and application of weight management strategies, there is a sense of inertia hindering action that is predominated by patient factors. Conversely, patients overwhelmingly reported lack of clear guidance from providers and they echoed many of the provider-reported barriers through tones of self-blame. These results highlight the need for a more collaborative, empowering, and patient-centered approach to weight management and underscore the importance of effective health communication between patients and providers to curb the risk of obesity-related adverse health outcomes.

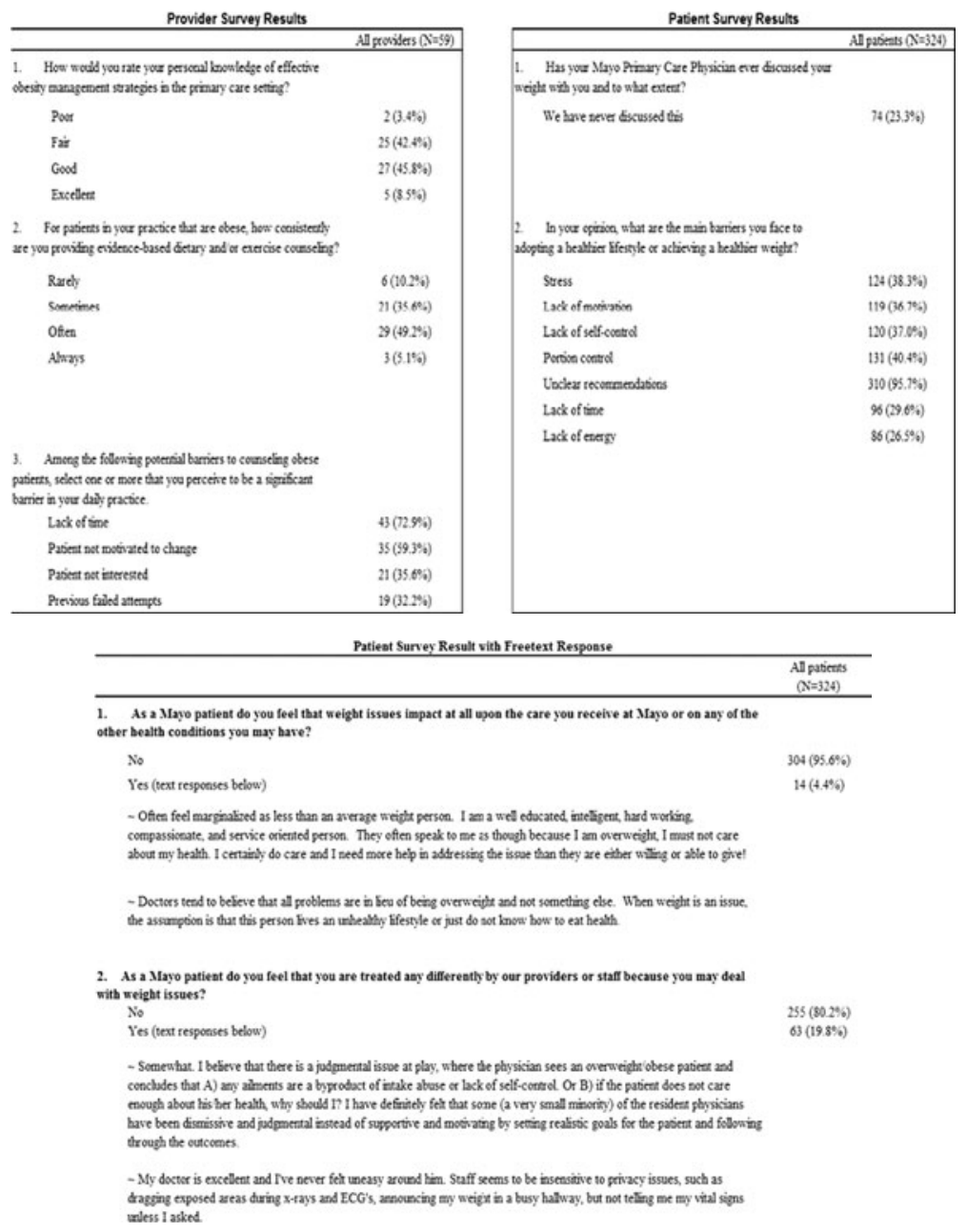


PATIENT ENGAGEMENT PROGRAM LED TO IMPROVED EVIDENCEBASED MEDICATION USE IN DIABETIC AND HEART DISEASE PATIENTS IN FEDERALLY QUALIFIED HEALTH CENTERS: THE OFFICE GUIDELINES APPLIED TO PRACTICE PROGRAM Ade B. Olomu'; NAZIA NAZ S. KHAN $^{1}$; David Todem ${ }^{2}$; Shireesha Bottu ${ }^{1}$; Syeda Quadri ${ }^{1}$; Margaret Holmes-Rovner ${ }^{2}$. ${ }^{1}$ MICHIGAN STATE UNIVERSITY, East Lansing, MI; ${ }^{2}$ Michigan State University, East Lansing, MI. (Control ID \#2469351)

BACKGROUND: Many studies have documented suboptimal care for diabetic/cardiac outpatients, especially for minority and low-income populations. Patient activation/ engagement is an increasingly important component of strategies to improve outcomes for patients and reform health care. The Office-Guidelines Applied to Practice (OfficeGAP) Program is an application of a previous model revised to address a low-income population with diabetes and coronary heart disease (CHD). The program employs decision aids (DAs) and trains health care providers and patients in shared decision making (SDM). Objectives: 1) Determine the feasibility of implementing the OfficeGAP Program in Federally Qualified Health centers (FQHCs). 2) Determine the impact of a patient activation/engagement intervention on guidelines based medication use for diabetic and CHD patients in FQHCs.

METHODS: The Office-GAP Program is a two-center study designed to improve cardiovascular care for minority and low-income populations in outpatient clinical settings. Clinics were assigned to intervention or control arm by tossing the coin. Office-GAP intervention included: 1) Patient activation /engagement intervention during a group visit 2) Physician training for patient activation/engagement and 3) Decision support/Checklist intervention (DSI) used in real time in the office. After a group visit, patients followed up with 2 physician visits using GAP tools. We performed chart abstraction of all enrolled patients with CHD and Diabetes Mellitus (DM) from October 2010 to March 2014 in 2 FQHCs. Logistic Regression analysis was used to examine change over time in the proportion of patients eligible for using Aspirin/Plavix, Angiotensin Converting Enzyme Inhibitors /Angiotensin Receptor Blockers (ACEIs/ARBs), beta-blockers and statins. Propensity score was used to control for cofounders.

RESULTS: Feasibility of the Office-GAP program in the FQHC setting was established. Program elements were consistently used ( $>98 \%$ clinic attendance at training, tool in patient chart; $>80 \%$ of patients attended group visit). Of 243 patients studied, 120 patients were in the intervention (Office-GAP) arm and 123 in the control arm. Using a propensity score method to control for potential confounders, the logistic regression model for correlated data revealed that for all eligible patients, Office-GAP program significantly improved the use of evidence-based medications from baseline: ACEI at 3 months (OR $1.77,95 \% \mathrm{CI}=1.04 ; 2.98), 6$ months ( $\mathrm{OR} 2.04,95 \% \mathrm{CI}=1.30 ; 3.22)$; Statin at 3 months (OR 1.71, 95\% CI $=1.19 ; 2.44), 6$ months $(2.21,95 \% \mathrm{CI}=1.47 ; 3.31), 12$ months (OR $2.00,95 \% \mathrm{CI}=1.24 ; 3.21)$; Aspirin/Plavix at 6 months $(\mathrm{OR}=2.03,95 \% \mathrm{CI}=1.20 ; 3.41)$, 12 months $(\mathrm{OR}=1.73,95 \% \mathrm{CI}=1.11 ; 2.70)$. There was no significant increase in any of the medication use in the control arm.

CONCLUSIONS: The use of Office-GAP program to teach SDM and use of DAs in real time was demonstrated to be feasible in $\mathrm{FQHCs}$. This patient engagement program led to increase use of guidelines based medications for secondary CHD prevention among underserved populations with CHD and diabetes. The Office-GAP program is a brief, efficient platform for delivering patient and provider education in SDM and could serve as a model for implementing guidelines based care for all chronic diseases in outpatient settings. Further study is needed to establish reach, effectiveness, and cost-effectiveness.

PATIENT EXPERIENCES OF DISCRIMINATION AND RISK FACTORS FOR 30-DAY READMISSION Carol Oladele; Tara Rizzo; Marcella Nunez-Smith. Yale School of Medicine, New Haven, CT. (Control ID \#2467492)
BACKGROUND: A growing body of evidence demonstrates the importance of patient experiences in predicting healthcare outcomes and improving quality of care. National priorities are focused on understanding how patient perspectives/experiences influence outcomes that contribute to healthcare disparities. Findings show patient-reported experiences of discrimination as an important determinant of healthcare satisfaction and adverse health outcomes such as leaving against medical advice and increased emergency visits. With the implementation of quality measures such as the hospital-wide all-cause readmission measure, aimed to improve quality of care and reduce costs, hospitals have implemented policies and practices to support these efforts. This study examined the association between patient experiences, including discrimination, communication, and adverse outcomes associated with 30-day readmission to determine quality of care improvements amid policy and practice changes at hospitals.

METHODS: Data from the Patient-Reported Experiences of Discrimination in Care Tool (PreDict) study was used for analyses. The PreDict study includes a self-administered survey of recently discharged patients across the US. This cross-sectional study describes patients' most recent hospital experiences using de novo items and questions from previously validated scales including the Communication Assessment Tool and Everyday Discrimination Scale. Patients were invited to participate via mailed postcard if they were discharged within the past 30 days, 21 years and older, discharged from a medical service, discharged to home, and English- or Spanish-speaking. We examined associations between inpatient experiences, doctor-patient communication, experiences of discrimination, and the outcomes primary care appointment within 30-days of discharge and emergency department visit within 30 days of discharge. Doctor-patient communication was measured using the Communication Assessment Tool and the Everyday Discrimination Scale was used to measure experiences of discrimination. Patient experience questions assessed comfort with providers, discharge processes, and attending physician visits. Data for 1503 participants who completed the survey as of December 31, 2015 were included in analyses. We used multiple logistic regression to determine the association between independent variables and outcomes.

RESULTS: Multivariable model results showed that doctor-patient communication and experiences of discrimination are significantly associated with having a primary care follow up appointment post-discharge from an inpatient admission. Patients with higher doctor-patient communication were $30 \%$ more likely ( $\mathrm{OR}=1.3 \mathrm{CI}$ : $1.04-1.56$ ) to have a follow up appointment post-discharge while patients who reported more everyday discrimination were $23 \%$ less likely (OR $=.77$ CI: $0.63-0.96$ ) to have a follow up appointment. Demographic variables, including sex and socioeconomic status, and variables that assessed other patient experiences were not statistically significant. Model findings for emergency department visits within 30 days of discharge were not statistically significant. CONCLUSIONS: These findings highlight the importance of patient experience in better understanding ways to improve quality and continuity of care. The current findings are consistent with existing literature that shows improved communication and positive healthcare experiences are associated with increased patient satisfaction and better health outcomes. To improve quality of care efforts and reduce adverse outcomes like readmission, it is imperative that we consider patient experiences in developing strategies to address communication and experiences of discrimination in healthcare.

PATIENT LEVEL FACTORS ASSOCIATED WITH POST-EMERGENCY DEPARTMENT OBSERVATION STAYS IN A MEDICARE POPULATION Zayd A. $\underline{\text { Razouki }^{1}}{ }^{\text {; }}$ Timothy Platts-Mills ${ }^{3}$; Richard Sloane ${ }^{4}$; David Edelman ${ }^{2}$, ; Susan N. Hastings ${ }^{2,4}$. ${ }^{1}$ Durham VA Medical Center, Chapel Hill, NC; ${ }^{2}$ Durham VA Medical Center, Durham, NC; ${ }^{3}$ University of North Carolina, Chapel Hill, NC; ${ }^{4}$ Duke University Medical Center, Durham, NC. (Control ID \#2469902)

BACKGROUND: Observation stays have been criticized for their ill-defined role and described as a billing mechanism rather than an effective clinical care pathway. 
Understanding the utility of observation stays is limited in part because of a lack of understanding of how current uses of observation stays differ from other post-Emergency Department (ED) dispositions. Our objective is to examine patient level factors associated with post-ED observation stays, compared with post-ED discharges and post-ED hospitalizations.

METHODS: Data were obtained from the Medicare Current Beneficiary Survey Cost and Use and Access to Care files. We constructed a cohort of all ED visits among Medicare fee-for-service enrollees aged 65 or older from 1999 to 2011. Our independent variables were patient level factors that were selected according to the Behavioral Health Care Use Model including demographics, education and income status, satisfaction with care, access measures, insurance status, comorbid diseases and functional status, as well as ED geographical location and living in a metropolitan area. Two logistic regression models examined the association of these factors with the three possible post-ED dispositions. First, we compared post-ED observation care with post-ED discharge. Second we compared post-ED observation care with post-ED hospitalization.

RESULTS: The full sample included 22,646 ED visits. Overall $55.3 \%$ of ED visits resulted in discharge, $39 \%$ led to hospital admission, and $5.7 \%$ were assigned to an observation stay. Compared to ED visits resulting in hospitalization, specific patient level factors including white race $(\mathrm{OR}=1.26, p<0.001)$ and at least a 1 year established relationship with a provider $(\mathrm{OR}=1.26, p=0.024)$ were more likely associated with post-ED observation stays. In contrast, other factors including younger age (1 year increment $\mathrm{OR}=0.99, p=0.001)$, male gender $(\mathrm{OR}=0.75, p<0.001)$, not having supplemental insurance $(\mathrm{OR}=0.75, p=0.01)$, difficulties in any activities of daily living $(\mathrm{OR}=$ $0.84, p=0.012)$, and living within a metropolitan area $(\mathrm{OR}=0.65, p<0.0010)$ were less likely associated with post ED observation stays. Compared to post ED discharge visits, white race $(\mathrm{OR}=1.23, p=0.04)$ and at least a year established relationship with a provider $(\mathrm{OR}=1.37, p=0.004)$ were more likely associated with observation stays. In contrast, only low annual income was less likely to be associated with observation stays. ED visits in the Northeast were less likely to be associated with post ED observation stays compared to other geographical locations.

CONCLUSIONS: In a large nationally representative sample of Medicare patients, white race and an established relationship with a provider were associated with higher use of observation stays. Whereas financial factors including low household income and the lack of supplemental insurance were associated with lower use of observation care. Additional work is needed to understand how out-of-pocket reimbursements and policies related to observation stays influence the use of this care pathway.

PATIENT PERCEPTION OF THE PRIMARY CARE PROVIDER CONTINUITY VISIT IN AN INPATIENT SETTING Barbara Ogur ${ }^{2},{ }^{1}$; Gabriel Fregoso ${ }^{2}$; Adetaye Adeseye $^{2}$; Dana D. $\mathrm{Im}^{2}$; Richard Pels ${ }^{1}$; David Bor ${ }^{1}{ }^{1}$ Cambridge Health Alliance, Cambridge, MA; ${ }^{2}$ Center for Primary Care, Harvard Medical School, Boston, MA. (Control ID \#2463992)

BACKGROUND: Continuity of care over time from a primary care physician (PCP) in the ambulatory setting results in better outcomes, lower cost of care, and higher patient satisfaction.[i] These improved outcomes appear to be mediated through increased adherence to prescribed medication, higher rates of preventive interventions, and improved coordination of care. The literature suggests that, in the ambulatory setting, patients from certain demographics may be more likely to prefer receiving care from their own PCP, particularly older patients, patients from the non-majority culture, and patients on public insurance, suggesting that trust and concordance of view of the medical issues may be important factors.[ii] Less is known about continuity of care between outpatient and inpatient venues. Inpatient management has increasingly been delegated to hospitalists as physicians of record to decrease length of stay and ensure expertise in the management of acute problems. [iii] But, while some have raised concerns about the fragmentation of care and possible patient dissatisfaction, and have called for new types of interactions to ensure ongoing involvement of PCPs with their patients who are hospitalized[iv], there has been little assessment of patients' attitudes about maintaining PCP involvement during inpatient stays.

METHODS: One hundred ninety-nine in-person and telephone interviews were conducted with patients with a PCP in the Cambridge Health Alliance (CHA) network who were discharged from the inpatient medical service. Patients were asked questions about their demographics, their relationship with their PCP, any PCP continuity visits or telephone encounters that may have occurred during their hospitalization, and their perception of how continuity visits impacted their care or might have impacted their care.

RESULTS: The mean age of patients interviewed was 62 years, with an average length of stay of 3.61 days. Sixty-eight percent of patients self-identified as White, $18 \%$ as Black. The mean length of patient-PCP relationships was 7.1 years. Patients' mean self-reported health was 6.30 out of 10.57 (29\%) of patients reported involvement of their PCP in their inpatient care. $150(77 \%)$ of patients reported that PCP involvement was or would be helpful, including $89 \%$ of those whose PCPs were involved and $73 \%$ of those whose PCPs were not involved. The patients who were more likely to prefer PCP involvement were older and gave higher ratings to their PCP. Race, gender and educational level were not predictive of preference for PCP involvement. Thematic assessment of patients' responses demonstrated that the main reasons patients preferred PCP involvement included that their PCP would provide comfort based upon their personal knowledge of the patient and would have background knowledge relevant for their inpatient care. One patient commented: "I would have received more personal care. Here, they treat disease... I asked one doctor about my care after I leave the hospital. He said .....we took care of the problems in our department so you need to see a psychiatrist for your other problems." CONCLUSIONS: Patients express a preference for PCP involvement in their inpatient care, likely due to the personal nature of their relationship with their provider and to their desire for informational continuity. Further research should explore whether other demographic variables or particular diagnoses, such as psychiatric or end of life issues may identify patients who most prefer inpatient contact with their PCP. This information should inform policy decisions on how to best structure PCP involvement in their patients' hospital care.

\section{PATIENT PERCEPTIONS ABOUT PREDIABETES AND PREFERENCES FOR} DIABETES PREVENTION Matthew J. O'Brien ${ }^{2}$; Margaret R. Moran² ${ }^{2}$ Joyce W. Tang $^{2}$; Maria Vargas ${ }^{2}$; Mary Talen ${ }^{1}$; Laura J. Zimmermann ${ }^{1}$; Ronald T. Ackermann ${ }^{2}$; Namratha R. Kandula ${ }^{2}$. ${ }^{1}$ Erie Family Health Center, Chicago, IL; ${ }^{2}$ Northwestern University, Chicago, IL. (Control ID \#2457552)

BACKGROUND: Adherence to new diabetes screening recommendations will identify millions of patients with prediabetes. While large clinical trials have established that intensive lifestyle interventions (ILI) and metformin can substantially reduce the risk of developing diabetes, neither treatment is being offered to the vast majority of high-risk Americans. Investigating how patients with prediabetes perceive their need for treatment and weigh treatment options represents an important first step to inform prediabetes management. However, little patient-centered research exists to guide clinical practice in this area. The objectives of the current interview study of adult primary care patients with prediabetes were: 1) to examine their knowledge and perceptions about the risk of developing diabetes; and 2) to explore their preferences for treatment to prevent or delay diabetes.

METHODS: We enrolled 35 adults with prediabetes from 2 large Midwest primary care practices. Participants were identified by querying diagnosis codes and laboratory results in clinics' electronic health record systems for impaired fasting glucose, impaired glucose tolerance, or elevated hemoglobin A1C. We excluded patients with diagnosed diabetes, antidiabetic medication use, and those who had a myocardial infarction, stroke, or cancer 
treatment in the previous year. We conducted in-depth interviews using a semi-structured interview guide, which was designed to elicit the following patient perspectives: knowledge about the risk of developing diabetes; reactions to information about future diabetes risk with and without treatment; and treatment preferences as shaped by underlying values, goals, and concerns. The study team developed a codebook that reflected topics from the interview guide, in addition to previously unexpected topics identified inductively. Digital audio files of the interviews were transcribed verbatim. Transcripts were coded and analyzed by at least 2 members of the study team (MO, MM, MK). NVivo ${ }^{\circledR}$ software (v. 10; Victoria, Australia) was used to assist the investigative team with organizing and analyzing the qualitative data.

RESULTS: We identified 3 major themes reflecting participants' perceptions about their diabetes risk and treatment preferences to prevent or delay diabetes. Theme 1: Knowledge gaps about prediabetes and its treatment are pervasive. Most participants overestimated the risk of developing diabetes. ("As soon as I hear prediabetes, I automatically think you are going to get diabetes. I think people usually go that way.") Knowledge about treatment options was also limited. ("The doctor suggested to me to reduce the meals and to exercise. But just that;" and "I've heard that people who have diabetes can control it with medication. But I never heard that if you take a medication, it can prevent diabetes.") Theme 2: Evidence about diabetes risk and treatment options for prediabetes is motivating to patients. For some participants, learning this information during the interview gave them hope that diabetes could be prevented. ("There's still some time and the time is now... I feel a little better seeing the numbers and seeing that there is a possibility to really prevent diabetes.") Learning the diabetes risk reduction associated with ILI and metformin seemed to motivate patients to take action. ("This information is letting me know that I can beat this...that I can try to develop an exercise and eating plan that will get me back to being almost normal.”) Theme 3: Both ILI and metformin are considered acceptable treatments. Most participants preferred ILI as their initial choice to prevent or delay diabetes. They cited some of the following perceptions about ILI to support this preference: it has additional benefits beyond lowering diabetes risk; it constitutes "personal responsibility" and a "natural way" for managing prediabetes; and it is associated with a greater risk reduction than metformin. While many patients preferred to begin treatment with ILI, most believed that they would take metformin if their lifestyle efforts failed and their primary care physician recommended it. ("You have a better chance with working out and losing weight than you would with the medication... But if my doctor said, 'I think you need to,' then I would take it.”) Some participants noted that they would use metformin as an adjunct to ILI, because they presumed the combination of treatments may reduce diabetes risk more than either treatment alone. Only 3 participants reported an unwillingness to take metformin for diabetes prevention under any circumstances.

CONCLUSIONS: This study presents some of the first evidence describing patients" perspectives about prediabetes and its treatment. Our findings suggest that these individuals have inaccurate perceptions of diabetes risk and incomplete knowledge of treatment options. We found that patients with prediabetes want specific information on these topics, which may encourage them to take action to prevent or delay diabetes. This study is the first to report that both ILI and metformin are considered acceptable options for lowering diabetes risk, which should encourage shared decision making approaches by patients with prediabetes and their primary care providers. Further research with patients and providers is needed to promote the widespread adoption of prediabetes treatment.

PATIENT PERCEPTIONS OF DEPRESCRIBING (PPOD): SURVEY DEVELOPMENT AND VALIDATION Amy Linsky ${ }^{1,2}{ }^{1}$; Steven R. Simon ${ }^{1}$; Kelly Stolzmann ${ }^{1}$; Rachel A. Lippin-Foster ${ }^{1}$; Mark Meterko ${ }^{1}$. ${ }^{1}$ VA Boston Healthcare System, Boston, MA; ${ }^{2}$ Boston University School of Medicine, Boston, MA. (Control ID \#2468859)

BACKGROUND: While many patients would prefer to take fewer medications, there is little guidance for providers and patients regarding effective communication and decision- making about medication discontinuation ("deprescribing”). Although deprescribing is often thought of as a provider decision, it is also critical to understand the patient perspective. To address this gap in knowledge, we sought to develop a survey instrument that assesses patients' experience with and attitudes toward medication discontinuation. METHODS: Based on a conceptual framework of patient factors hypothesized to be predictive of willingness to discontinue a medication, we developed a questionnaire that incorporated scales from established instruments, including the Beliefs about Medications Questionnaire, Trust index, CollaboRATE, Patient Attitudes Towards Deprescribing, and Autonomy Preference Index. We composed additional items to address dimensions of the conceptual model not adequately covered by existing measures. The complete instrument included items focused on attitudes toward medication, patients' relationships with their clinical providers, and interest in and experiences with stopping medicines. We surveyed a national sample of 1548 Veterans Affairs patients who at the time of the survey were prescribed $\geqq 5$ medications for 365 days (3/17/14-3/16/15). We used exploratory factor analysis (EFA) and confirmatory factor analysis (CFA) to assess the reliability and validity of the proposed new scales. In the EFA, percent variance accounted for, a scree plot, and conceptual coherence were used to determine the number of factors. In the CFA, proposed factor structures were evaluated using indices of absolute fit (standardized root mean square residual, SRMR), parsimony-corrected fit (root mean square error of approximation, RMSEA), and comparative fit (comparative fit index, CFI).

RESULTS: Respondents ( $n=790 ; 51 \%$ response rate) were randomly assigned to derivation and validation samples of 395 each; there were no significant differences in demographics or number of medications between these groups. The EFA yielded credible fourand five-factor models. In the former, the factors were "Medication Concerns," "Perceived Provider Knowledge," "Interest in Deprescribing," and "Unimportance of Medications." The five-factor model added a "Shared Decision Making" scale, and we elected to test this model in the CFA phase because of its potential to differentiate additional relevant dimensions of patient attitudes. We tested two variations of the five-factor model, moving two items with marginal loadings on the basis of conceptual fit. The baseline model and the modified model had SRMR values of 0.07 and 0.06 , RMSEA values of 0.07 and 0.07 , and CFI values of 0.90 and 0.91 , respectively. Given the virtually identical fit indices observed for both models, we elected to retain the modified 5-factor model based on overall content coherence and consistency. The five new scales demonstrated internal consistency reliability, with Cronbach alphas based on all respondents of: Medication Concerns, 0.82; Perceived Provider Knowledge, 0.86; Interest in Deprescribing, 0.77; Shared Decision Making, 0.61; and Unimportance of Medications, 0.70 .

CONCLUSIONS: The Patient Perceptions of Deprescribing (PPoD) questionnaire is a novel, multidimensional instrument to measure patients' attitudes, knowledge and experiences related to medication discontinuation. Psychometric analyses demonstrated strong empirical support for a 5-factor structure evaluating patients' concerns about medications, perceptions of their providers' knowledge, interest in deprescribing, affinity for shared-decision making and views of the importance of medications. The PPoD questionnaire can be used in future research to determine how to best involve patients in decisions about deprescribing. These data may then facilitate the development of targeted interventions that lead to safe and highquality medication use by discontinuing medications that are no longer appropriate.

PATIENT PERCEPTIONS OF TELEHEALTH VIDEO PRIMARY-CARE

VISITS Rhea E. Powell; Kristin L. Rising; Jeffrey Henstenberg; Grace Cooper. Thomas Jefferson University, Philadelphia, PA. (Control ID \#2469744)

BACKGROUND: Virtual telehealth is emerging as a model of care delivery with potential to increase flexibility and reach of health services. Telehealth is being rapidly adopted in many healthcare settings, including primary care, yet little is known about patient perceptions of this novel delivery model. This study seeks to describe patient opinions about and experiences with telehealth video calls for urgent and routine primary care visits. 
METHODS: This is a prospective qualitative study using individual semistructured interviews with patients who have completed a scheduled syncronous telehealth video visit. Patients from primary care practices at a single academic medical center were recruited and interviewed. Interviews with patients sought to elicit common themes across telehealth experiences. Interviews were coded by two independent reviewers. Themes were identified from the interviews using a modified grounded theory approach.

RESULTS: A total of 16 interviews were conducted with patients. Among these patients, 7 (44\%) had prior experience with video calls for non-health-related purposes, and 13 (81\%) expressed desire to use telehealth as replacement for office visits in the future. Many patients cited convenience as a primary reason for wanting to participate in a virtual visit, and some patients felt that virtual visits are more personal than traditional in-office visits, as video visits require the physician's complete attention and eye contact. A few patients expressed a preference for receiving serious news via virtual visits. Patients also expressed interest in use of telehealth in other health care settings, including for urgent or acute care.

CONCLUSIONS: Among a set of early adopting patients, telehealth primary care visits are acceptable and preferable for many situations as compared to in-person visits. Limitations of this study include that it was performed among a set of patients who completed a telehealth visit, and does not represent opinions of those who opted against use of telehealth virtual visit. Future studies are needed to assess long-term impact of telehealth on patient outcomes, utilization of in-person routine and acute care services, and provider satisfaction.

PATIENT-CENTERED SUBSTANCE USE DISORDER TREATMENT FOR WOMEN VETERANS Karleen Giannitrapani ${ }^{3}$; Alexis K. Huynh ${ }^{2}$; Catherine A. Schweizer $^{4}$; Alison B. Hamilton ${ }^{1,5}$; Katherine J. Hoggatt ${ }^{6}{ }^{1}$ VA Greater Los Angeles Healthcare System, Los Angeles, CA $;{ }^{2}$ Veterans Affairs, Sepulveda, CA; ${ }^{3}$ Veterans Health Administration, Menlo Park, CA; ${ }^{4}$ Veterans Health Administration, Los Angeles, CA; ${ }^{5}$ David Geffen School of Medicine at UCLA, Los Angeles, CA; ${ }^{6}$ Fielding School of Public Health at UCLA, Los Angeles, CA. (Control ID \#2469932)

BACKGROUND: Substance use disorders (SUDs) are damaging to women's health and quality of life. Timely and appropriate treatment, however, can mitigate some of the SUDs' detrimental effects. Women are more likely than men to encounter barriers to seeking and following through with SUD treatments. Previous studies have called for health services research to develop methods for reducing barriers for women seeking SUD treatment in out-patient settings. In this study, we interview interdisciplinary providers to identify the key components of a non-residential patient-centered SUD treatment program for Women Veterans.

METHODS: Design: We conducted semi-structured in person interviews with interdisciplinary providers involved in the care of Women Veterans with substance use disorders. A purposive snowball sampling approach was used to identify key informants. A semistructured interview guide prompted providers to describe the SUD treatment options currently available to Women Veterans as well as ideas for the components of an ideal SUD treatment program. Transcripts were analyzed using a method of constant comparison. We also interviewed Women Veterans and compared provider and Veteran perspectives.

RESULTS: A total of 17 providers (including psychiatrists, psychologists, social workers, women's health primary care providers, and nurses) and 6 Veterans participated. Analyses reveal a range of options pertaining to an ideal outpatient SUD treatment program for Women Veterans: 1) care should be available in a space that feels physically and psychologically safe (often, a women's only environment), "the easy reason for not getting the treatment is," 'Oh, I don't want to be in a group with all of those men...' There's a lot of sexual harassment... if there are a lot of men who do not have good impulse control and you're the only woman, I can see why that doesn't feel like a safe environment." 2) Scheduling and location of services have to be flexible enough to accommodate work and family responsibilities: "some of them, they're the main breadwinner... They know that they have a problem, but they can't take time away from work. That's one of the barriers I see." 3) A program should be sufficiently resourced to prevent restrictions on numbers or types of visits: "They'd like to have therapy, but not the brief interventions that are offered now." 4) The treatment options should be comprehensive, integrative of mental health, and allow for choice (e.g., "women only groups" and "individual therapy"). 5) The range of available treatment options should be understood by providers: "Let's pull together a list of the existing resources, be sure that all the providers have those literally at their fingertips." Providers described existing SUD treatment options in the VA as inconsistent in meeting the care needs of Women Veterans in all 5 categories. Women Veterans concurred with the program components identified by providers.

CONCLUSIONS: Patient-centered gender tailored SUD treatment for Women Veterans should be safe, flexible, sufficiently resourced, comprehensive, and understood by providers. Lessons learned in this Veteran sub-population lend insight into potential strategies that can reduce barriers to SUD treatment for a broader population of women.

\section{PATIENT-CENTERED USE OF EHRS: CAPTURING RESIDENTS' PERCEP-} TIONS Onur Asan ${ }^{3}$; Jeanne T. Tyszka ${ }^{1}$; Kathlyn Fletcher ${ }^{2} .{ }^{1}$ Medical College of Wisconsin, Milwaukee, WI; ${ }^{2}$ Medical College of Wisconsin/Milwaukee VAMC, Tbd, AL; ${ }^{3}$ Medical College of wisconsin, Milwaukee, WI. (Control ID \#2462188)

BACKGROUND: The presence of Electronic Health Records (EHR) in outpatient clinic examination rooms has increased since the adoption of the American Recovery and Reinvestment Act of 2009 (HITECH); this proliferation has given rise to studies on the impact of the use of EHR. Advantages of exam room EHR have been reported as improvements in quality, safety, decision making, information exchange, and efficiency. An obstacle not yet overcome is the adverse effect on physician-patient communication that can occur with the presence of this technology. These studies inform attending physicians of the benefits and downsides of EHR, but the perspective of residents has received little attention. Previous work showed that the effectiveness of residents' communication skills may be impacted when computers are present in exam rooms. In this qualitative study, we wished to gain an understanding of the experiences of internal medicine residents when EHR are used in primary care clinic visits. Specifically, we sought to identify situations in which the residents' verbal and nonverbal communication skills work in tandem with data provided on the EHR; additionally, we looked for residentperceived facilitators and barriers that influence their use of EHR-based communication in clinical encounters.

METHODS: We conducted qualitative, semi-structured interviews of internal medicine housestaff during a 4-month period from September to December 2014. We announced details about the study and sent follow-up email invitations to 125 residents at an urban academic medical college. The criteria for participation: continuity clinic assignment using electronic health records for at least 4 months. Twenty-four responded to the invitation, with 21 completing the interview. All subjects were consented before being interviewed in a private room. Interviews were arranged to be convenient for the resident's schedule. Subjects were offered a $\$ 30$ gift card. We prepared an interview guide, which had 8 questions with probes. Questions centered on the resident's communication style, training, multi-tasking, screen sharing behavior, and experiences with EHR. We held two pilot interviews as we developed the guide to ensure the questions and probes were understandable and suitable. We collected data through interviews and demographic surveys. Interviews were audio-recorded, transcribed, and anonymized. We used NVivo 10 , qualitative analysis software, to analyze the data in the interview transcripts. The study 
team reviewed four transcripts and devised a preliminary coding scheme; each member of the study team then coded one transcript using this scheme. The study team met to revise the scheme, and developed a codebook with defined codes.

RESULTS: Four themes emerged from data analysis; these themes and subthemes were: 1. Resident training a. Medical school provided training in communication with patients $b$. Institutional training occurs for general use of EHR c. Mentors may provide informal training in meaningful use of EHR while communicating with patients 2. Impact of EHR on resident's communication skills a. Nonverbal interaction i. The importance of eye contact is apparent to these providers ii. Other nonverbals may be limited to nodding and gesturing iii. When discussing sensitive subjects, providers give full attention to patient $b$. Verbal interaction i. Residents may use EHR to guide the interview ii. Residents may show screen while explaining content 3. Using EHR as tool for communication/teaching a. Screen sharing i. Increases patient's health literacy ii. Facilitates shared decision making iii. Increases trust $b$. Resident perception of patient's learning style 4. General perceptions of EHR use a. Positive/negative/challenges b. Resident's perceptions of patient's perceptions of EHR

CONCLUSIONS: We found that, while mentors influenced their practice, residents had not received formal training for using electronic health records to educate or communicate with patients. Informal advice, personal experience, observation, and practice helped them develop their approaches to meaningful use of EHR with patients. In spite of the positive aspects that EHR can bring to health care, residents were concerned that its use could diminish their interactions, and make it more difficult to keep the patient at the center of health care.

\section{PATIENT-REPORTED EXPERIENCE WITH THE AFTER VISIT SUMMARY} IN A PRIMARY CARE INTERNAL MEDICINE PRACTICE Gregory B. Summerville ${ }^{3}$; Celia P. Kaplan ${ }^{2}$; Leah S. Karliner ${ }^{1} .{ }^{1} \mathrm{UCSF}$, San Francisco, CA; ${ }^{2}$ University of California San Francisco, San Francisco, CA; ${ }^{3}$ University of California, San Francisco, San Francisco, CA. (Control ID \#2468069)

BACKGROUND: The after visit summary (AVS) is a document provided to patients after a medical encounter which contains actionable and relevant instructions, as well as a variety of other personalized health information to help patients understand the treatment received and physician recommended plan. There is little research documenting patient experience with the AVS. We set out to assess patient recall and use of the AVS after a primary care visit.

METHODS: Patients were recruited as part of a larger study assessing tobacco use in an academic primary care internal medicine practice; those patients who did not endorse tobacco use were automatically directed to the AVS survey as the control survey for the tobacco study. The survey was administered on a computer tablet in the waiting room just prior to a follow-up primary care visit. The survey assessed patient recall about whether they received an AVS after their prior primary care visit, manner of receipt (in-person, via electronic patient portal, or both), whether and how many times they reviewed it postencounter, and whether they shared it with anyone. A subset of patients were additionally asked about the free-text patient instructions section of the AVS, including ease of understanding, usefulness, and which topics were addressed in the instructions.

RESULTS: Of the 393 patients who completed the survey, $50.1 \%$ were women. Of those surveyed, 325 (82.7\%) remembered receiving an AVS after their last appointment, Most $(59.3 \%)$ reported that they received the AVS in-person, $19.4 \%$ received it via the electronic patient portal, and $21.3 \%$ reported receiving it via both modalities. Nearly two-thirds $(64.9 \%)$ reported reviewing the AVS since their last appointment, and almost half $(46.9 \%)$ stated they reviewed the AVS more than two times since their last visit; close to one-third (30.5\%) indicated that they shared the AVS with family members. Among the subset of 141 patients asked about free text instructions, $94.2 \%$ found them very easy or easy to understand, and $92.8 \%$ found them very or somewhat useful. The three most common patient-reported topics addressed in the instructions were: medication information, what to do before their next visit, and lifestyle advice. (Figure)

CONCLUSIONS: A majority of patients in our primary care practice reported reviewing the AVS after their visit, and finding tailored free-text instructions to be both useful and easily understandable. The AVS presents an opportunity to educate patients about their disease and medications, summarize the discussion that took place during the encounter and highlight the most important next steps in a patient's treatment plan. Future investigations should identify which subgroup of patients might benefit most from receiving personalized instructions and whether the AVS enhances adherence to treatment plans and engagement in self-management.

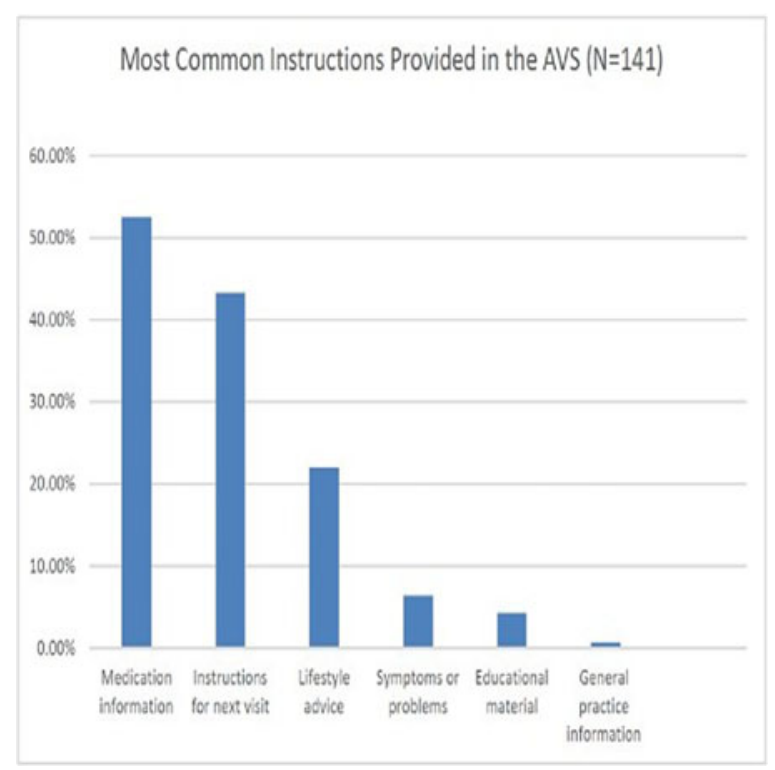

Most Common Instructions Provided in AVS

PATIENT-REPORTED SIDE EFFECTS USING ELECTRONIC PHARMACOVIGILANCE IN PATIENTS NEWLY STARTED ON MEDICATIONS FOR HYPERTENSION, DIABETES, INSOMNIA OR DEPRESSION $\underline{\text { Elissa V. Klinger }}{ }^{3}$; Alejandra Salazar ${ }^{3}$; Jeffrey Medoff ${ }^{3}$; Mary Amato ${ }^{4}$; Lake Walsh ${ }^{3}$; patricia dykes ${ }^{3}$; Jennifer Haas ${ }^{1}$; David W. Bates ${ }^{2}$; Gordon D. Schiff ${ }^{3} .{ }^{1}$ BWH, Boston, MA; ${ }^{2}$ Brigham and Women, Boston, MA; ${ }^{3}$ Brigham and Women's Hospital, Boston, MA; ${ }^{4}$ Brigham and Womens Hosptial/MCPHS, Boston, MA. (Control ID \#2470278)

BACKGROUND: Safe and effective use of prescription drugs in the outpatient setting requires patient monitoring to track outcomes and ensure potential adverse effects are identified and addressed in a timely fashion. Proactive and automated approaches that are acceptable and user-friendly promise that such monitoring could be performed reliably and efficiently. We developed a patient-centered interactive voice response system (IVRS) to actively survey patients taking FDA-approved medications for one of four common chronic conditions (diabetes, hypertension, insomnia, depression), with integrated management support by a clinical pharmacist. We report on patient-reported side effects entered by patients into automated phone calls.

METHODS: We developed an algorithm to identify any adult primary care patient at any of 9 primary care clinics who had been newly started on a medication for one of the conditions. Eligible patients were sent a letter with study information and an opportunity to decline participation. Using automated IVRS phone calls, patients were called 46 weeks following their medication start date and prompted to confirm that they were 
taking the drug as prescribed for the condition of interest. The IVRS then asked a series of questions querying both adherence and whether the patient was experiencing any of 8 common symptoms associated with the medication: (1) stomach/intestinal problems; (2) problems with memory/confusion; (3) muscle aches; (4) skin rash; (5) dizziness/balance; (6) headaches; (7) problems with sexual function; and, (8) weight changes. All patients who inputted information for each of the 8 side effect questions was scored from 0 to 8 , with a score of 0 corresponding to no positive symptoms reported and a score of 8 indicating that the patient had a positive screen for each of the symptoms queried. RESULTS: Over a 16-month period, 4847 eligible intervention patients received automated calls and $640(13.2 \%)$ consented to participate in IVRS surveys. Of the 640 participants, 499 (78.0 \% of participants) answered all side effect questions and an additional 33 (5.2\%) answered at least 1 side effect question without skipping/hanging up the call. A total of $48.1 \%$ of all patients who answered all 8 questions reported at least 1 side effect; $21.6 \%$ reported only 1 side effect; $12.4 \%$ reported 2 side effects; $7.8 \%$ reported 3 side effects; $3.0 \%$ reported 4 side effects; $2.6 \%$ reported 5 side effects; $0.6 \%$ reported 6 side effects; no patients reported 7 or 8 side effects. For patients taking medications for depression, $63.2 \%$ reported experiencing at least 1 side effect, followed by patients treated for diabetes (58.6\%), insomnia ( $41.7 \%$ ), and hypertension ( $40.5 \%$ ). Survey completion by therapeutic indication ranged from $70.6 \%$ for patients on medications to treat insomnia to $81.2 \%$ for patients treated for depression. Patients treated for depression were more likely to "skip" questions in the automated survey. The question most commonly skipped by any treatment group asked about problems with sexual function.

CONCLUSIONS: We demonstrate that an automated, proactive IVRS to capture patientreported side effects is viable, in that more than $70 \%$ of patients who agree to participate in such a system complete a series of questions related to symptoms and side effects which may be related to newly prescribed medications. Consistent with prior studies screening for adverse drug events, nearly $50 \%$ of patients reported at least one symptom on the automated call. Our specially designed system allowed for real time transfer of IVRS calls to a pharmacist who could simultaneously access patient-reported data and the patient's chart, and thereby troubleshoot symptoms that were consistent with adverse drug events Patients prescribed medications to treat depression may require additional assessment to determine whether reported symptoms are drug-related.

PATIENTS AND CLINICIANS COLLABORATING ON THE MEDICAL RECORD: A CONCEPT PAPER ON OURNOTES John N. Mafi ${ }^{1,2}{ }^{2}$ Macda Gerard ${ }^{3}$; Hannah Chimowitz ${ }^{3}$; Melissa Anselmo ${ }^{3}$; Jan Walker ${ }^{3}$. ${ }^{1}$ David Geffen School of Medicine at UCLA, Los Angeles, CA; ${ }^{2}$ RAND Corporation, Boston, MA; ${ }^{3}$ Beth Israel Deaconess Medical Center, Brookline, MA. (Control ID \#2467270)

BACKGROUND: While patient engagement is central to improving our healthcare system, few interventions have successfully empowered patients to participate in their own care. In the OpenNotes trial, clinicians invited their patients to read their visit notes, which resulted in substantial patient enthusiasm, self-reported clinical benefits, and little impact on clinician workflow. But reading notes is passive. In this study, we conduct interviews with prominent thought leaders in healthcare from across the United States to solicit ideas on the concept of patients, families, and clinicians collaborating on the medical record.

METHODS: We conducted a series of in-depth qualitative interviews with expert patient advocates, nurse practitioners, physician assistants, primary care physicians, health services researchers, informaticists, government officials, and health innovators from 3/20/ 2015-12/31/2015. We identified potential interviewees by a research committee discussion and voting process and recruited participants using a form letter via email, with up to two reminder emails as needed. We used standardized and semi-structured interview guides to conduct a $30 \mathrm{~min}$ telephone interview with each participant. Guides were divided into five subject areas: (1) initial reaction to the idea of patients/families contributing to the record and how that might work, followed by questions about specific ways they might generate data (2) before the visit, (3) during the visit, (4) after the visit, and (5) between visits. Interviews were audio-recorded, professionally transcribed, and inductively analyzed using immersion/crystallization methodologies for major themes.

RESULTS: We conducted 28 interviews. Overall, interviewees expressed enthusiasm about the concept of patients, families, and clinicians collaborating on the medical record. Most participants endorsed the concept of patients/families contributing to their records prior to the visit, both in a structured and free-text format. Specifically, most anticipated overall benefit from patients who update their past history or list their agenda items prior to their upcoming visit. Many expressed concern that patient participation during the office visit would be unfeasible as it could increase the length of time of the encounter. The majority of participants agreed with the idea that patients could collaborate on the impression and plan part of the note, with many suggesting that clinicians invite patients to addend the note (as opposed to editing or altering the note). Many could see advantages to patients generating data about chronic conditions between visits as a way to track progress, but few could imagine clinicians monitoring it without additional incentives or staff resources. Overall, participants worried that OurNotes could add to time pressures, though most thought pre-visit data entry and agenda setting hold some promise for saving time.

CONCLUSIONS: As movement towards fully transparent care spreads rapidly across the United States, we have garnered several potential strategies to engage patients, families, and clinicians to collaborate on the medical record. Concepts such as patients and families updating their past history, entering their visit agenda, and addending the plan section of the note hold promise as important strategies to increasingly engage patients in their own care.

PATIENTS' VIEWS OF WORKING WITH MEDICAL STUDENTS: LONGITUDINAL VERSUS SINGLE CONTACT Barbara Ogur ${ }^{3}{ }^{3}$; Amy R. Weinstein ${ }^{1}$; Barbara Gottlieb $^{2,3}$; Kristen Goodell ${ }^{3}$; Steven R. Simon ${ }^{4,3}$; Gabriel Fregoso ${ }^{3}$; Adetaye Adeseye ${ }^{3}$; Penny Wang ${ }^{3}$; Sara Fazio ${ }^{1,3}$. ${ }^{1}$ Beth Israel Deaconess Medical Center, Boston, MA; ${ }^{2}$ Brigham and Women's Hospital, Jamaica Plain, MA; ${ }^{3}$ Harvard Medical School Center for Primary Care, Boston, MA; ${ }^{4}$ VA Boston Healthcare System, Boston, MA; ${ }^{5}$ Cambridge Health Alliance, Cambridge, MA. (Control ID \#2466139)

BACKGROUND: Learning medical science without learning its application to people in their real-life contexts often results in ineffective care[i]. Engaging patients in teaching interactions when they come for healthcare sometimes seems awkward and even slightly coercive, but the literature on patients' perceptions of students' involvement in their care has found almost entirely positive responses.[ii] Longitudinal integrated clerkships (LICs) provide students with opportunities to develop relationships with patients over time, relationships that provide insight into important contextual aspects of care such as patients' values, barriers to accessing services, complexities of the relationship between doctor and patient, and the lived experiences of patients with serious illness.[iii] Despite the growing body of literature on students' perceptions of longitudinal relationships with patients, there has been little written about how patients feel about working with students over time. [iv] We know that patients with continuity relationships with their primary care provider (PCP) have better health outcomes and report both higher satisfaction with care from their "own" doctor and more likelihood of following recommendations,[v] but little is known about whether these same phenomena apply to longitudinal relationships with medical students.

METHODS: We adapted questions from the CAHPS (Consumer Assessment of Healthcare Providers and Systems) to assess patient satisfaction with their visit with their PCP and with the student working with their PCP, and supplemented these questions with 
2 open-ended questions about the medical student's role in their care. Trained interviewers interviewed 35 patients about their experiences working with third-year medical students completing their internal medicine experience in the Cambridge Integrated Clerkship (CIC), a longitudinal clerkship and 10 patients in an Internal Medicine block ambulatory experience at the Beth Israel Deaconess Medical Center (BIDMC). To ensure that there were adequate numbers of patients who had had longitudinal contacts with students, 9 additional patients were interviewed who were identified by CIC students as patients with whom they had formed a strong connection. All interviews were audio or video-taped, and transcribed and independently read by multiple researchers. An initial code-book was developed by consensus. All interviews were then coded, using NVIVO software, and analyzed for differences in themes.

RESULTS: Patients in all groups expressed high levels of satisfaction with working with medical students. The qualities they appreciated in their medical student were: thoroughness, respect, attentiveness, and supportiveness. Patients viewed the student as an extension of their doctor, even in situations where students performed special functions such as coordinating referrals or health counseling. Many patients in both groups positively affirmed the students' warmth and supportiveness, but this was more commonly noted in patients who had seen their students longitudinally. The nature of the emotional support was described differently, as several patients with longitudinal student contact noted that the student had "been there" during a difficult period. No patients from any group specifically endorsed concerns about the extra time involved in working with students; in fact one patient in the single-contact group felt that he received more attention because of the involvement of the student. There were several areas in which patients noted that their students had put in extra effort in their care. While the patients in the single-contact group noted more detailed histories and physicals, patients in the longitudinal-contact group noted that their students went to outside specialty appointments, researched their problems and "thought outside the box". Patients in the longitudinal-contact group frequently mentioned that their students called them between visits to follow up on issues and to coordinate care. This inherent structural distinction between both groups was clearly a source of surprise and enthusiasm for patients. Several patients noted that having a longitudinal student gave them better access to their physician between visits, by calling the student as a bridge to their primary care provider. While none of the patients articulated negative feelings about working with a medical student, more of the single-contact patients expressed reservations about working with a student in the future or recommending that friends or family do so.

CONCLUSIONS: All patients, whether having a single experience with a student or a longitudinal experience, found the students prepared, thorough, knowledgeable, and supportive. They did not feel that the visit took too much time. They felt gratified in being able to provide the service of training future physicians and felt their contribution was important. Patients who experienced longitudinal contacts with students felt more personally supported and found that the students played a more substantial role in their care. They were more likely to want to work with students in the future. As medical schools increase ambulatory educational experiences, involving students in longitudinal relationships with patients can provide experiences highly valued by both students and patients.

PATIENTS' HOPES FOR TREATMENT OF ADVANCED CANCER: A MIXED METHODS APPROACH Jeremy DeMartini ${ }^{1,}{ }^{1}$; Camille Cipri ${ }^{1}$; Joshua Fenton ${ }^{1,}{ }^{1}$;
Ronald Epstein ${ }^{2}$ 2 ; Paul Duberstein ${ }^{2}$ 2 ; Daniel Tancredi ${ }^{1}$; Guibo Xing ${ }^{1}$; Richard L. Kravitz ${ }^{1,1}$. ${ }^{1}$ University of California, Davis, Sacramento, CA; ${ }^{2}$ University of Rochester, Rochester, NY. (Control ID \#2463567)

BACKGROUND: Patients with advanced cancer tend to overestimate their prognosis. This can lead to aggressive treatments without better rates of survival. In their role as patient advocates, primary care physicians (PCPs) frequently help patients with cancer define goals of care. Understanding patients' hopes for treatment is a critical pre-requisite for advance care planning, yet little is known about the nature, prevalence, or antecedents of these hopes.

METHODS: In baseline interviews conducted as part of a randomized trial of cancer communication coaching, 281 adult patients with a poor prognosis from stage III/IV cancer (mean age $64 \mathrm{y} ; 55 \%$ female) recruited from the offices of 38 oncologists in California and New York were asked, "What are you hoping for at this time from your cancer treatment?" Responses were transcribed verbatim and used to develop an inductively-derived taxonomy of hopes consisting of 8 major categories (Table). Two raters independently classified patients' hopes with good reliability (mean $(95 \% \mathrm{CI})$ of 8 category-specific Cohen kappas $0.76(0.71,0.81)$; min-max: $0.38-0.90)$; disagreements were adjudicated by a third investigator. We performed logistic regression analysis to explore whether patient demographic variables (age, gender, race, religious affiliation, committed relationship status, and education), prognostic expectations (likelihood of cure or of living two or more years), and prior discussion of hopes with their PCP or oncologist were related to two particularly salient (and starkly contrasting) hopes for treatment: hopes for improved health related quality of life and hopes for an unqualified cure. RESULTS: Ninety-five percent of the patients shared hopes for treatment. Health related quality of life (HRQOL) was the most prevalent hope category (40 \%) followed by life extension (31 \%) and tumor stabilization (26\%) (Table). Thirteen percent of patients had hopes for an unqualified cure while an additional $5 \%$ hoped for cure but recognized this was likely impossible (cure tempered by realism). Patients represented hopes in different styles. For example, hope for an unqualified cure could be expressed directly as, "[I want to] be cured. So I can enjoy the rest of my life with no worries", or indirectly as, "I want to be fully healed and [have] no recurrence of cancer". Almost 2/3 (64\%) stated they had little to no prior discussion of their hopes with their PCP, while $35 \%$ had little to no prior discussion with their oncologist. HRQOL was not significantly associated with patient demographic variables, prognostic expectations, or prior discussion of hopes with medical providers. Hopes for an unqualified cure were more likely among younger patients (OR 2.68 comparing those in lowest age quartile $[<57$ years] to those $>=57$ years, $95 \%$ CI $1.22-$ 5.89 ), if they believed their likelihood of cure was greater than $50 \%$ (OR 2.63, $95 \%$ CI 1.05-6.57), or if they had little to no prior discussion with their PCP about their hopes (OR 2.77, $95 \%$ CI 1.09-7.06).

CONCLUSIONS: In this study of patients with advanced cancer, the vast majority of patients could articulate hopes for treatment. Desire for an improved HRQOL was the most commonly expressed hope. Hopes for an unqualified cure were less common, but were more prevalent in those patients who were younger, had higher expectations for cure, or had not explored their hopes with their PCP or oncologist. Most patients in this study reported having little to no prior discussion with their PCP about these hopes. A better understanding of patients' hopes for treatment may assist PCPs in counseling patients with advanced cancer regarding goals of care and advance care planning. 


\begin{tabular}{|c|c|c|}
\hline $\begin{array}{l}\text { Hopes for } \\
\text { Treatment } \\
\text { Category }\end{array}$ & $\begin{array}{l}\text { Prevalence } n \\
(\%)\end{array}$ & Examples \\
\hline $\begin{array}{l}\text { Unqualified } \\
\text { cure }\end{array}$ & 37 (13\%) & $\begin{array}{l}\text { "To be cured. So I can enjoy the rest of my } \\
\text { life with no worries", "I will never have a } \\
\text { [recurrence] of cancer. After losing the } \\
\text { last } 13 \text { years of my life, I am ready to have } \\
\text { it back again and I just refuse to have } \\
\text { cancer" }\end{array}$ \\
\hline $\begin{array}{l}\text { Cure tempered } \\
\text { by } \\
\text { realism }\end{array}$ & 14 (5\%) & $\begin{array}{l}\text { "A cure is the main thing I would love, but I } \\
\text { know that is not possible", "Hoping to be } \\
\text { cured. [It's] probably not going to happen, but } \\
\text { that is what hope is" }\end{array}$ \\
\hline HRQOL & $113(40 \%)$ & $\begin{array}{l}\text { "Hoping for comfort", "To be able to walk } \\
\text { strongly and feel normal when I get up in the } \\
\text { morning" }\end{array}$ \\
\hline \multicolumn{3}{|l|}{$\begin{array}{l}\text { Control of } \\
\text { cancer* }\end{array}$} \\
\hline $\begin{array}{l}\text { Life } \\
\text { extension }\end{array}$ & 88 (31\%) & $\begin{array}{l}\text { "To extend my life as long as possible", "Love } \\
\text { life and want to live longer", "Goal is to stay } \\
\text { alive" }\end{array}$ \\
\hline Remission & 59 (21\%) & $\begin{array}{l}\text { "Hoping tumor will go away", "Knocking the } \\
\text { hell out of cancer...put it in remission", "It } \\
\text { will leave me with no evidence of cancer" }\end{array}$ \\
\hline $\begin{array}{l}\text { Tumor } \\
\text { stabilization }\end{array}$ & $72(26 \%)$ & $\begin{array}{l}\text { "Slow down the growth of my metastatic } \\
\text { prostate cancer", "Hope to stabilize the } \\
\text { spread of cancer. Keep it in check", "Hoping } \\
\text { cancer will shrink" }\end{array}$ \\
\hline $\begin{array}{l}\text { Control not } \\
\text { otherwise } \\
\text { specified }\end{array}$ & 26 (9 \%) & $\begin{array}{l}\text { "To get better", “...show improvement from } \\
\text { the treatment", "Hoping for healing” }\end{array}$ \\
\hline Milestone & $40(14 \%)$ & $\begin{array}{l}\text { "I just want to make another two years till my } \\
\text { daughter graduates", "Would like to see [my] } \\
\text { grandchildren, daughter was just married", } \\
\text { "Nephew's wedding is in September and [I] } \\
\text { would like to attend" }\end{array}$ \\
\hline
\end{tabular}

*The prevalence of one or more of the four control of cancer categories was 201 (72\%)

PATIENTS' PERCEPTIONS OF AND PREFERENCES FOR THE OUTPATIENT AFTER-VISIT SUMMARY: A QUALITATIVE STUDY Alex Federman ${ }^{1}$; Angela M. Sanchez Munoz ${ }^{1}$; Lina Jandorf ${ }^{1}$; Nathaniel Cohen ${ }^{1}$; Michael S. Wolf ${ }^{2}$; Joseph Kannry ${ }^{1}$. ${ }^{1}$ Icahn School of Medicine at Mount Sinai, New York, NY; ${ }^{2}$ Northwestern University, Chicago, IL. (Control ID \#2467929)

BACKGROUND: The outpatient after visit summary (AVS) has been widely promulgated through electronic health records (EHR). Although hailed as a tool for promoting patient centered care and enhancing clinician-patient communication, little is known about the patients' experiences with the AVS and their preferences for its content.

METHODS: We conducted a qualitative assessment of the AVS by conducting focus groups and individual semi-structured interviews with patients in ambulatory care practices in New York City, Long Island, NY, and Chicago, IL. Focus groups and interviews were audiotaped. Two members of the research team independently coded the audiotape transcripts using NVIVO and revised the coding by consensus.

RESULTS: We conducted 5 focus groups involving 25 patients and 14 individual interviews from sites that used the Epic (75\%) or Allscripts (25\%) EHRs. The median age of participants was 61 (range 30-90) and $71.8 \%$ were female. Seven themes emerged from the transcript analyses: use and purpose of the AVS, its content, formatting, problems, where it is kept, need for AVS customization, and medication formatting and details. Two distinct uses of the AVS emerged; most saw its value as a summary of what occurred during the visit, but several others found greater value in the document as a general summary of their health and healthcare. The latter group tended to be individuals who used a web portal to access their medical records and yet wanted a paper document which they could carry at all times in the event they needed to share their health information using the AVS to clarify their medication regimen and health problems with their physician. Patients' preferences for content was generally consistent with what is typically found on AVS like follow up and contact information, though there was disagreement about including goals of care, vital signs, test results, and other elements. Several patients said their AVS would be improved by including reasons for referrals, diagnostic tests, and medications. Regarding formatting, patients preferred an AVS with simplified language and appearance, larger font, and limited to 4 pages or less.

CONCLUSIONS: Patients have many consistent but also diverging preferences for the content and formatting of the AVS and its use, with some preferring a visit summary and others a summary of their general medical care. Quantitative research may help refine understanding of patients' AVS preferences and future development of EHR functionality should consider features that enable its customization to meet patient needs.

\section{PCP WEIGHT LOSS ASSISTANCE IN A PRIMARY CARE POPULATION: LESSONS LEARNED FROM PARTICIPANTS WITH RECENT INTENTIONAL WEIGHT LOSS IN THE MAINTAIN-PC STUDY Michael Eng; Bethany Barone Gibbs; Dana L. Tudorascu; Kathleen M. McTigue; Diane Comer; Kimberly A. Huber; Laurey R. Simkin-Silverman; Cindy L. Bryce; Gary Fischer; Molly B. Conroy. University of Pittsburgh, Pittsburgh, PA. (Control ID \#2469040)}

BACKGROUND: Supporting weight loss remains a challenge for primary care providers (PCPs). Patients who desire weight loss are interested in receiving PCP counseling, which can be associated with positive behavior changes. Given time and other constraints, PCPs may target specific patient populations for lifestyle counseling. We investigated characteristics and behaviors associated with report of PCP weight loss assistance among adults with recent, intentional, clinically significant weight loss. We hypothesized that participants who report receiving PCP assistance with their recent weight loss will have a distinct set of weight loss strategies as well as a greater magnitude of weight loss compared to those who report no PCP assistance with recent weight loss.

METHODS: The current study is a secondary analysis of baseline data from Maintaining Activity and Nutrition through Technology-Assisted Innovation in Primary Care (MAINTAIN-pc), a randomized, controlled trial of the use of electronic health record (EHR)-delivered tools and health professional support to help primary care patients successful weight loss maintenance. To be eligible, participants had to have $\geq 5 \%$ weight loss in the 2 years prior to enrollment (i.e., qualifying weight loss), verified by EHR. At screening, participants were asked "Did your PCP assist you with your weight loss efforts?" Participants responding yes were categorized as having reported PCP assistance with recent weight loss. We assessed demographics and body measurements during a baseline study visit and used standardized questionnaires for medical co-morbidities, use of weight loss strategies, and prior PCP weight-related counseling. Weight loss strategies included: self-monitoring, group/commercial support (including commercial weight loss programs), other behavioral skills, and non-PCP professional support (e.g., nutritionists). Prior PCP weight-related counseling included topics of healthy eating, physical activity, and body weight and the time dedicated to each topic. We used Chi-square tests and T-tests 
(or Wilcoxon tests) to determine differences in participant characteristics and responses between those with and without report of PCP assistance.

RESULTS: We included all 194 MAINTAIN-PC participants, who were $73.7 \%$ female, $88.1 \%$ White, $76.3 \%$ college educated, and had a mean age of 53.4 years (SD: 12.1). Participants had an average qualifying \% weight loss of $11.3 \%$ (SD: $6.5 \%$ ). Forty-four participants $(22.7 \%)$ reported receiving PCP assistance with their recent weight loss. Participant report of PCP assistance with recent weight loss did not differ with respect to age, gender, race, education, or marital status. Baseline weight, waist circumference, BMI, or qualifying \% weight loss also did not differ between those with and without PCP assistance with recent weight loss. A higher proportion of participants with diabetes reported PCP assistance with recent weight loss compared to those without diabetes ( 22.7 vs. $10.7 \%, p=.039$ ). Report of PCP assistance with recent weight loss was marginally more frequent in participants having cardiovascular disease (18.2 vs $8.7 \%$, $p=.074$ ). Report of PCP assistance with recent weight loss did not differ comparing those with and without other medical comorbidities including pre-diabetes, high blood pressure, high cholesterol, asthma, or depression/anxiety. Most reported prior weight-related counseling from their PCP with respect to healthy eating ( $83.5 \%$ ), physical activity $(87.1 \%$ ), and body weight $(87.6 \%)$. A higher proportion of those who reported PCP assistance with recent weight loss also reported prior PCP counseling about healthy eating compared to those without report of PCP assistance ( 97.7 vs $79.3 \%, p=.002$ ). However, report of prior PCP counseling on physical activity or body weight was not different in those with and without PCP assistance with recent weight loss. Those that reporting received PCP assistance with recent weight loss also noted greater number of minutes spent receiving prior PCP counseling about body weight ( $7.0 \mathrm{vs} 5.8$, $p=.027)$. Use of weight loss strategies was prevalent among this sample across all categories: self-monitoring (68.6\%), group/commercial programs (72.7\%), other behavioral skills (99.0\%), and non-PCP professional support (70.6 \%). Among weight loss strategies employed, the use of group/commercial support was less frequent among those who reported PCP assistance with recent weight loss to those who did not (61.4 vs $76.5 \%, p=.047)$. PCP assistance with recent weight loss was not related to other weight loss strategies.

CONCLUSIONS: A minority of primary care patients with recent intentional weight loss reported PCP assistance with their recent weight loss effort, despite most having received prior weight-related counseling. This could suggest that either the time spent or information transmitted in the past is not perceived by patients as integral to their most recent weight loss. The increased report of PCP assistance with recent weight loss among participants with diabetes suggests that counseling is either more effectively targeted or received among patients whose disease process is more clearly weight- and diet-related. The negative association between report of PCP assistance with recent weight loss and use of group/commercial strategies may indicate certain patients' desire for increased structure or support with their weight loss efforts, a challenge in routine clinical care

PEER HEALTH COACHING TO IMPROVE MEDICAL STUDENT WELLBEING Jennifer Packard ${ }^{1}$; Neena Natt ${ }^{1}$; Jill Huber $^{2}$; Liselotte Dyrbye ${ }^{1} .{ }^{1}$ Mayo Clinic, Rochester, MN; ${ }^{2}$ Mayo Clinic Rochester, Rochester, MN. (Control ID \#2469226)

BACKGROUND: The value of health coaching to promote positive behavioral change is increasingly recognized. These skills have not routinely been incorporated into traditional medical school curriculum. The purpose of the this study is to describe an innovative blended educational approach to teaching medical students health coaching skills and practicing those techniques to encourage positive health behavior change.

METHODS: All first-year medical students at Mayo Medical School $(n=54)$ completed a required $10.5 \mathrm{~h}$ longitudinal health coaching curriculum plus four peer health coaching sessions in 2015. After receiving classroom-based instruction on peer coaching, including motivational interviewing techniques, students participated in peer coaching sessions. Students worked in triads, taking turns being the health coach, receiving health coaching, and observing the health coach. All students completed post experience surveys assessing attitudes and behavior change.

RESULTS: A majority of students identified exercise (35\%) or sleep (26\%) as the targeted behavior to improve through peer coaching. Eighty-percent thought it very or somewhat important to work on personal improvements in their health behavior. Nearly all students ( $89 \%$ ) thought peer coaching was very or somewhat helpful, $86 \%$ became very or somewhat confident in their ability to make health behavior change, and $89 \%$ reported making a positive change in their behavior as a result of the peer coaching. Seventy-two percent indicated they achieved at least half of their health behavior goal. Two-thirds considered themselves healthier after the activity. Nearly all (95\%) thought the health behavior curriculum was a very or somewhat important part of the medical school curriculum. Qualitative comments provided insights into barriers and facilitating factors for health behavior change, how the experience impacted their well-being and promoted peer support and relationship building, as well as thoughts around how health behavior coaching skills would translate to patient care.

CONCLUSIONS: A health coaching curriculum is a valuable addition as it enables medical students to practice health coaching skills and experience health coaching aimed at changing health behavior valuable for personal wellness as well as in the clinic.

PEER SUPPORT FOR ACHIEVING INDEPENDENCE IN DIABETES (PEERAID): RESULTS OF A RANDOMIZED CONTROLLED TRIAL OF COMMUNITY HEALTH WORKER ASSISTED SELF-MANAGEMENT SUPPORT AMONG LOW-INCOME ADULTS WITH DIABETES Karin M. Nelson ${ }^{1}$; Leslie Taylor $^{3}$; Julie B. Silverman ${ }^{2}$; Meghan M. Kiefer ${ }^{4}$; Paul Hebert ${ }^{3}$; Daniel S. Lessler ${ }^{4}$; Jim Krieger ${ }^{5} .{ }^{1}$ University of Washington, VA Puget Sound, Seattle, WA; ${ }^{2}$ VA Puget Sound, Seattle, WA; ${ }^{3}$ VA HSR\&D Seattle-Denver Center of Innovation for Veteran-Centered and Value-Driven Care, Seattle, WA; ${ }^{4}$ University of Washington, School of Medicine, Department of Medicine, Seattle, WA; ${ }^{5}$ Action for Healthy Food, Seattle, WA. (Control ID \#2458302)

BACKGROUND: The prevalence of diabetes in the United States remains high, and low income and minority populations have an increased burden of disease. Diabetes selfmanagement is an integral component of effective care, yet many people do not have the opportunity to learn these important skills. The use of community health workers (CHWs) may be an effective approach for providing diabetes self-management to disadvantaged populations. A recent meta-analysis of randomized controlled trials of CHWs for patients with diabetes reported modest intervention effects. The majority of previous studies targeted specific racial or ethnic minority groups. Translating effective interventions into diverse community settings remains a public health challenge. Although CHWs interventions improve patient knowledge and self-care behaviors, there is limited data on the association of CHW interventions and patient reported outcomes such as health related quality of life (HRQOL). The objective of this study was to test the effectiveness of a home-based community health worker (CHW) intervention for improving glycemic control among a diverse group of low-income adults with diabetes.

METHODS: Participants in King County, WA were randomized to a 12-month homebased CHW-delivered diabetes self-management intervention $(n=145)$ versus usual care $(n=142)$. Participants were recruited from September 2010 to May 2013 from 3 health systems: Harborview Medical Center (HMC), the VA Puget Sound Health Care System and Sea Mar Community Health Centers. The electronic health records of each of the three clinic sites were queried approximately every 3 to 6 months to identify potentially eligible participants. Eligible participants had a diagnosis of type 2 diabetes, an $\mathrm{HbA} 1 \mathrm{c}$ of $8.0 \%$ or greater at their clinic site during the 3 to 6 month period prior to enrollment, a household income of less than $250 \%$ of the federal poverty level (which in 2011 was an annual income of $\$ 27,225$ or less for a single individual), were between 30 and 70 years old, and 
spoke English or Spanish. Although we utilized clinic laboratory values from the three participating health systems to screen for eligibility, we obtained a baseline enrollment blood sample at the first home visit to collect pre-intervention measures. The intervention took place in participants' homes and was comprised of 4 required home visits by the CHW and one optional home visit. CHWs worked with clients to set behavioral goals, and identify actions that were feasible and likely to help them achieve these goals, then assessed the impact of actions on progress toward goals, and again worked with clients to revise action plans to make them more effective. The primary outcome was Hemoglobin $\mathrm{A} 1 \mathrm{c}(\mathrm{HbA1})$ at 12 months; secondary outcomes included blood pressure and lipid levels, health care use, and HRQOL (as measured by the SF-12 physical component summary (PCS) and mental component summary (MCS) and a previously validated diabetes quality of life measure, the Diabetes-39). Health care used was documented by self-reported outpatient clinic visits, hospitalization and emergency room encounters. RESULTS: All participants were low-income and were from diverse racial and ethnic backgrounds. Intervention and control groups were comparable at baseline on sociodemographic and most clinical characteristics. There was no difference in the pre/post change in $\mathrm{HbAlc}$ in the intervention group (from unadjusted mean of $9.09 \%$ to $8.58 \%$, change of $-0.51 \%$ ), as compared to the control group (from 9.04 to $8.71 \%$, change of $-0.33 \%$ ) ( $p=0.54)$; however there was a significant interaction between baseline $\mathrm{HbA} 1 \mathrm{c}$ and intervention group $(p=0.037)$. In the pre-specified subgroup analysis of individuals with baseline $\mathrm{HbA} 1 \mathrm{c}>10 \%$, the intervention group had a $1.18 \%$ greater decrease in $\operatorname{HbA} 1 \mathrm{c}(p=0.046)$. There was a decrease in self-reported physician visits $(15 \%$ lower in the intervention group, $p<0.001)$ and a trend toward improvement in health related quality of life in the intervention group (increase in PCS score of 0.25 in controls vs 2.4 in intervention group, $p=0.07$, and a decrease in the Social Burden of Disease subscale of the Diabetes-39 in the intervention group $(p=0.051)$ ). There were no differences between the intervention and control groups in blood pressure or lipid levels, MCS scores, or in hospitalizations and emergency department visits.

CONCLUSIONS: A home-based CHW intervention to support diabetes self-managemen was effective for low-income participants with poorly controlled diabetes at baseline, but not for others included in the study. Intervention participants reported significantly less outpatient clinic visits and showed a trend toward improvements in health related quality of life. Further studies are needed to determine if CHWs can improve HRQOL. Our results suggest targeting CHW programs for low-income individuals with diabetes with severe hyperglycemia, despite access to care, may be effective in improving glycemic control.

PERCEIVED EFFECTS OF SOCIAL SUPPORT ON DIABETES MANAGEMENT IN AFRICAN AMERICAN WOMEN WITH ADEQUATELY VERSUS INADEQUATELY CONTROLLED TYPE 2 DIABETES Catherine Gliwa ${ }^{2}$; Keyonna M. King ${ }^{1}$; Juan M. Barron ${ }^{1}$; Arleen F. Brown ${ }^{1}{ }^{1}$ UCLA, Los Angeles, CA; ${ }^{2}$ Geffen School of Medicine at UCLA, Los Angeles, CA. (Control ID \#2469959)

BACKGROUND: African American women have higher rates of type 2 diabetes, poorer glycemic control, and more associated morbidity and mortality than white women. Social support may play an important role in diabetes management for African American women; however, the features of support networks that confer benefit, and those that may contribute to poorer control, are not well characterized. This focus group study compared perspectives on the relationship between diabetes management and social support in African American women with controlled, versus uncontrolled, type 2 diabetes. METHODS: Focus group participants were recruited from community clinics, faithbased organizations, and local businesses in South Los Angeles. Eligible participants were African American woman ages 25-65 years with a diagnosis of type 2 diabetes and hemoglobin A1c level either below 7.5 ("controlled") or 8 or higher ("uncontrolled"). We conducted two focus groups with women who had controlled diabetes $(N=8)$ and two groups with uncontrolled diabetes $(N=13)$. Focus groups were recorded and transcribed, and data were imported into Atlas.ti. The data were analyzed for emergent themes and motifs using grounded theory methodology.

RESULTS: Six key themes emerged in the discussions: actions intended to be supportive, alignment/misalignment with family and friends, supportive vs. scolding interactions, role as caregivers, isolation, and motivation. Food was a prominent sub-theme in all focus groups. The women described many actions taken by family and friends that were meant to be supportive, from calling to check in, to preparing or bringing over food, to encouraging healthier lifestyle choices. Both groups described friends and family who were positive role models and others who were viewed as undesirable role models. Many of the women described stress that came from their role as caregiver to children, grandchildren, parents, siblings, and others and linked this stress to diabetes onset or worsening. Importantly, whether an action was viewed as supportive was based on a woman's understanding of her disease. The actions of friends and family members viewed as being aligned (concordant) with her understanding of her disease were interpreted as supportive, while those actions that were misaligned (discordant) were interpreted as negative or scolding. Many of the women whose diabetes was inadequately controlled perceived a lack of support and a feeling that diabetes put strain on existing relationships. Several of these women described resenting some of the "supportive" actions they received from friends and family. Women with uncontrolled diabetes were more likely to describe misalignment between themselves and their support systems.

CONCLUSIONS: African American women with uncontrolled diabetes endorsed feelings of isolation and misalignment between themselves and their support systems. Alignment was important for supportive actions from family and friends to be perceived as positive and helpful. Education and training for support networks on providing effective assistance with diabetes management may improve alignment between these women and their support systems and contribute to better control of blood glucose levels.

PERCEIVED RISK OF RECURRENCE AND CANCER WORRY AMONG BREAST CANCER SURVIVORS Marilyn M. Schapira; Linda A. Jacobs; Angela DeMichele; Karen Glanz; Abigail Blauch; Donna A. Pucci; Steven Palmer. University of Pennsylvania, Philadelphia, PA. (Control ID \#2469758)

BACKGROUND: Breast cancer (BC) survivors face uncertainty regarding risk of recurrence over time and face both short and long terms risks of recurrence. However, little is known regarding the accuracy of quantitative measures of perceived risk of recurrence across 1,5 , and 10 year time horizon following diagnosis and the predictors of perceived risk. Overestimation of risk of recurrence may increase worry and negatively impact quality of life (QoL).

METHODS: A cohort of breast cancer survivors was identified and enrolled in a prospective study. Participants were eligible if they had a diagnosis of nonmetastatic breast cancer and had completed initial treatment within 1 year. Following enrollment and informed consent, participants completed a baseline survey administered through the patient portal. Factors assessed at baseline included age, education, race, marital status, income, insurance status, stage of disease at diagnosis, hormone receptor status, Oncotype Dx genomic testing and score if tested, and treatment received. Perceived risk of recurrence was assessed on a scale of 0 $100 \%$ over a 1 year, 5 year, and 10 year time horizon. BC worry was assessed using a 5-item Likert scale (1-low to 5-high) with the following items; 1) Because cancer is unpredictable, I feel I cannot plan for the future, 2) I will probably have a relapse (recurrence) within the next 5 years, 3) My fear of having my cancer 
getting worse gets in the way of my enjoying life, 4) I am afraid of my cancer getting worse, 5) I am certain that I have been cured of cancer (reverse scored). Bivariate analyses were done using parametric and non-parametric statistics. A multivariate regression model was used to evaluate predictors of perceived risk of recurrence while controlling for potential socioeconomic status, clinical, treatment, and prognostic factors. A spearman correlation coefficient was used to evaluate the association of perceived risk of recurrence and BC worry.

RESULTS: Ninety-seven (97) participants were in the study; $2 \%$ were stage $0,56 \%$ stage I, $30 \%$ stage II, and $12 \%$ stage 3 at diagnosis. The mean age was 50.6 years, (SD 12.4), $75 \%$ were white, and $19 \%$ black and $77 \%$ had completed college. Seventy-one percent $(71 \%$ ) were ER/PR + and $13 \%$ were triple negative. Of the 32 patients who had Oncotype dx genomic testing, $41 \%(n=13)$ had a score of $<18,56 \%(n=16)$ a score of $18-31$, and $3 \%(n=1)$ a score $>31$. Seventy three percent $(73 \%)$ received chemotherapy, $74 \%$ radiation, $27 \%$ herceptin, $33 \%$ tamoxifen, and $55 \%$ Aromatase Inhibitor; $23 \%$ had a bilateral mastectomy, $26 \%$ a lumpectomy, and $20 \%$ a unilateral mastectomy. The perceived risk of recurrence over a 1,5, and 10 year horizon (median, interquartile range) was $5.0 \%(0-25 \%), 16.5 \%(5-50 \%)$, and $25 \%(5-50 \%)$, respectively. The mean (SD) of BC Worry (1-low to 5-high) was $3.51(0.75)$. In bivariate analysis there was no association between SES factors, clinical, treatment, or prognostic factors with risk perceptions over a 5 or 10 year time horizon. However, perceived risk of recurrence over a 1 year time horizon was higher among women with lower levels of education: high school or some college (27.6\%); completed college (13\%), and graduate studies (10.6\%), $p=0.002$, and among those who had chemotherapy compared to others (18.2 vs. $7.5 \%$, $p=0.019$ ). These finding persisted in multivariate analyses controlling for stage at diagnosis, education, and receipt of chemotherapy. Finally there was a moderate to strong correlation of quantitative estimates of perceived risk of recurrence with affective measures of BC worry over the 1 year $(0.35), 5$ year $(0.56)$ and 10 year $(0.53$, all $p<0.05)$ time horizon.

CONCLUSIONS: BC survivors demonstrate a wide range of quantitative perceived risk of recurrence over a 1,5 , and 10 year horizon with estimates strongly correlated with $\mathrm{BC}$ worry. The absence of an association of biologic and genomic indicators with perceived risk of recurrence may indicate a lack of awareness or understanding of the prognostic value of these measures Further education regarding these prognostic indicators may be warranted especially among those with lower levels of formal education.

PERCEPTION OF BREAST CANCER RISK AND ADHERENCE TO U.S. CANCER PREVENTION GUIDELINES Anees Chagpar; Erin Hofstatter; Natalie H. Wallace. Yale New Haven Hospital, New Haven, CT. (Control ID \#2469563)

BACKGROUND: Among American women, breast cancer is the mostly commonly diagnosed malignancy. It is estimated that there were over 235,000 breast cancer diagnoses and over 40,000 breast cancer deaths in the United States in 2014. Incidence rates of breast cancer in developed countries is approximately 72 per 100,000 , as compared to 12 per 100,000 in developing countries, and these differences are thought to be attributed to lifestyle practices in more affluent populations. Breast cancer is one of a number of malignancies that have been linked to lifestyle factors, such as diet, exercise and alcohol consumption. This divide between the developed and developing world is most clearly seen when immigrant populations are studied. Oropharyngeal, stomach, liver and cervical cancers are far more prevalent in the developing world, while colorectal, prostate and breast cancers are more common in wealthier countries. However, within just one-to-two generations after immigration from lower-income to wealthier countries, immigrant populations exhibit cancer patterns consistent with those observed in their new home countries. It is projected that nearly 200,000 US cancer deaths each year can be attributed to poor diet and physical inactivity. In recognition of the major contribution of lifestyle factors to the development of various malignancies, the American Cancer Society (ACS) releases guidelines regarding physical activity and nutrition every 5 years, most recently in 2012. In our study, we investigated whether women who perceived themselves to be at high risk of breast cancer were more likely to adhere to a number of key guidelines drawn from the ACS report, namely physical activity, alcohol consumption and fruit and vegetable intake.

METHODS: The National Health Interview Survey (NHIS) is a population-based survey conducted each year by the CDC, designed to represent the noninstitutionalized civilian population in the United States. We utilized data from the 2010 NHIS adult and cancer supplements to compare fruit/vegetable intake, alcohol use and exercise habits among women who perceived themselves to be at high risk of developing breast cancer versus those women who perceived themselves to be at average or low risk.

RESULTS: In 2010, 12,055 women without a history of cancer were surveyed, representing 94,990,140 people in the population. Perceived risk of breast cancer was not associated with improved adherence to nutrition and physical activity guidelines (see Table). Less than $5 \%$ of all U.S. women meet the recommended fruit/vegetable intake regardless of perceived risk, and only one-third of all women achieve recommended physical activity. Approximately $95 \%$ of women adhere to guidelines regarding alcohol intake. On multivariate analysis, perceived risk did not affect health habits when controlling for age, ethnicity, education, insurance status, income, region and body mass index (BMI).

CONCLUSIONS: Breast cancer is the most commonly diagnosed cancer in American women, with over 235,000 women being diagnosed in 2014, and the second most common cause of death in women, with 40,000 associated deaths in 2014. As many as $1 / 3$ of the 572,000 annual US cancer deaths are attributed to poor diet, lack of exercise, and associated overweight and obesity. Breast, along with prostate and colorectal, cancers are commonly associated with affluent westernized lifestyles and behaviors, which may be amenable to change. Perceived risk is an important motivating factor in terms of behavior change. In this study, we sought to determine whether adherence to the ACS recommendations around physical activity, alcohol consumption and fruit and vegetable intake were different between women who perceived themselves to be at high as compared to low or average risk of developing breast cancer. We found that there were no statistically significant differences in behaviors regardless of perceived risk, suggesting that women who perceive themselves to be at high risk do not engage in these recommended protective behaviors at a higher rate. The reasons for this could be multifaceted and include lack of knowledge about potential health protective effects of these behaviors, lack of feelings of self-efficacy around implementing these recommendations and various socioeconomic barriers that may affect attitudes, finances or proximity to needed services or safe areas for exercise. There is very strong support for the relationship between increased physical activity and decreased alcohol consumption with respect to decreased breast cancer risk and we feel that these would be important targets of future behavior change interventions. Further research is needed to help women accurately determine their risk of breast cancer as this, along with perceptions of self-efficacy or control over preventing the disease, are important motivators in promoting behavior change. With lifestyle factors contributing to over 80,000 US breast cancer cases annually, behavioral change around such topics as physical activity, alcohol consumption and obesity are essential to decreasing the burden of this very prevalent disease.

Breast cancer risk perception and adherence to American Cancer Society risk reduction guidelines 


\begin{tabular}{|c|c|c|c|c|c|}
\hline & Univariate Analysis & & & Multivariate Analysis & \\
\hline & $\begin{array}{l}\text { Perceived High Risk-\% } \\
\text { meeting guidelines }\end{array}$ & $\begin{array}{l}\text { Perceived Low/Avg Risk-\% } \\
\text { meeting guidelines }\end{array}$ & $p$-value & OR $(95 \% \mathrm{CI})$ & $p$-value \\
\hline Fruit/ Veg Intake ( $>$ /= 5 servings / day) & $4.90 \% a$ & $4.42 \%$ & 0.5679 & $1.14(0.78-1.66)$ & 0.5110 \\
\hline Alcohol Intake $(<=7$ svg/week) & $94.22 \%$ & $95.13 \%$ & 0.3093 & $0.84(0.61-1.17)$ & 0.3076 \\
\hline Physical Activity (75-150 min vig-mod act/week) & $30.11 \%$ & $27.74 \%$ & 0.1465 & $1.02(0.87-1.21)$ & 0.7921 \\
\hline
\end{tabular}

PERCEPTIONS OF FIREARMS AMONG INTIMATE PARTNER VIOLENCE (IPV)-EXPOSED WOMEN Jennifer McCall-Hosenfeld ${ }^{1,}{ }^{2}$; Laura Leuenberger ${ }^{1}$; Erik B. Lehman $2 .{ }^{1}$ Penn State College of Medicine, Hershey, PA; ${ }^{2}$ Pennsylvania State University, Hershey, PA. (Control ID \#2463518)

BACKGROUND: Almost one-half of American women will experience intimate partner violence (IPV) - physical, sexual, or psychological harm by a current or former partner. Intimate partner homicide accounts for $40 \%$ percent of femicide, with firearms as the most commonly used weapon. Not only does the presence of a gun in a woman's home increase her risk of homicide, but the victim's perception of likelihood of lethality also correlates to increased risk. However, there is little evidence describing the factors associated with perception of risk from firearms among IPV-exposed women. We designed a study to better understand the impact of interpersonal trauma exposure and other demographic factors on firearm perceptions among a cohort of IPV-exposed women.

METHODS: Two-hundred sixty-six women with a lifetime exposure to IPV (defined by a modified HARK (humiliation-afraid-rape-kick) screening instrument, a standard 4-item validated tool used to identify IPV exposure), were recruited from ambulatory care clinics and domestic violence shelters. Women were surveyed on demographics, interpersonal trauma exposures, and firearm perceptions: 1) "How easy is it for people who live near you to get a gun?," 2) "Does having a gun around make you feel safer or less safe?," and 3 ) "Are any firearms kept in or around your home?" Four key interpersonal trauma experiences were evaluated: IPV type was categorized as physical ("Rape-Kick”) or nonphysical ("Humiliation-Afraid"). IPV recency was categorized as within the past 12 months or lifetime. Unwanted sexual exposure (defined from a validated tool) was categorized as never, lifetime, or past year. Adverse childhood experiences (ACEs) were taken from the ACE study (an ongoing collaborative research endeavor funded by the CDC and Kaiser Permanente) and stratified by tertile. We also assessed health-related and demographic variables shown in the literature to be associated firearm perceptions (age, race/ethnicity, poverty, rurality, marital status, education, mental health comorbidities, and the presence of a child in the home). Multivariable analyses examined each of the three firearm perception questions controlling for interpersonal trauma exposures and demographics. RESULTS: Demographics: Among the 266 women responding to the survey, the median age was $45,89 \%$ were white, non-Hispanic, $17 \%$ were at $/$ near poverty, $54 \%$ were ruralresiding, $59 \%$ were employed full time, $48 \%$ were married, $77 \%$ had schooling beyond high school, $38 \%$ had a child at home, and $31 \%$ had a mental health diagnosis. Ease of firearm acquisition: Women who reported physical IPV (aOR 2.40, 95\%CI 1.20,4.81), women who reported past year unwanted sexual exposure (aOR 4.01, $95 \% \mathrm{CI}$ $1.00,16.03$ ), and women of ages $47-54$ (aOR $3.5795 \% \mathrm{CI} 1.49,8.55$ ) were more likely to report that guns were easy to acquire, compared to women ages 19-34. Perceived safety in the proximity of a gun: Women with the highest ACE score, indicating a high level of childhood trauma, were less likely to feel safe with a gun nearby (aOR $0.41,95 \% \mathrm{CI}$ $0.21,0.84$ ). Rural women (aOR 4.13, 95\%CI 1.55,11.01), and women ages 35-46 had increased odds (aOR $2.88,95 \% \mathrm{CI} 1.35,6.12$ ) of reporting that the presence of guns made them feel safer. Odds of guns in the home ownership: Women who were divorced or separated (aOR $0.22,95 \% \mathrm{CI} 0.09,0.54)$ and women were widowed or single (aOR 0.22 , $95 \% \mathrm{CI} 0.07,0.68$ ) had lower odds of having a gun in the home, compared to married women. Rural women had higher odds of living in a home with a gun (aOR 3.30, 95\% CI $1.04,10.49)$. We did not find a significant effect of any of the trauma variables on the odds of having a gun at home.
CONCLUSIONS: Trauma exposures, including physical IPV and past-year unwanted sexual exposure, were associated with women's perceptions that it was easier to acquire guns in their community. Additionally, women with more severe childhood trauma were less likely to feel safe around firearms, but trauma exposures did not predict gun ownership. Instead, demographics of being married and rural residence were associated with gun ownership, consistent with findings in the general population. Women with a trauma history are at an elevated risk for mortality from firearms. Healthcare providers should not only be aware of the demographic factors that predict gun ownership (age, marriage), but should also consider that trauma exposure may inform women's perception of their safety around guns. Survivors of interpersonal trauma may benefit from a frank discussion of their own perceptions of firearm-related risk, and providers should attend to their patient's perceptions of this risk during preventive healthcare counseling.

PERCEPTIONS OF PHYSICIANS, RESIDENTS, NURSES, AND MEDICAL OFFICE ASSISTANTS FOLLOWING IMPLEMENTATION OF SCREENING, BRIEF INTERVENTION, AND REFERRAL TO TREATMENT: A MULTI-SITE REVIEW Jeanne Morley ${ }^{1,2}$; Sandeep Kapoor ${ }^{1,2}$; Mark Auerbach ${ }^{3,2}$; Nancy Kwon ${ }^{3,2}$; Jonathan Morgenstern ${ }^{4,}$; Joseph Conigliaro ${ }^{1,2}$. ${ }^{1}$ Northwell Health, Manhasset, NY; ${ }^{2}$ Hofstra Northwell Health School of Medicine, Garden City, NY; ${ }^{3}$ Northwell Health, New Hyde Park, NY; ${ }^{4}$ Northwell Health, Great Neck, NY. (Control ID \#2469167)

BACKGROUND: The U.S. Preventative Services Task Force and Center for Disease Control recommend screening for alcohol and substance misuse in Primary Care (PC) offices and Emergency Departments (ED) yet only $4 \%$ of patients with an alcohol use disorder receive treatment from a health care provider. To address this deficit, a state agency on alcohol and substance abuse services, a large health care delivery system and two academic institutions partnered to implement a Screening, Brief Intervention, and Referral to Treatment (SBIRT) program into four PC offices, including one PCMH, and four ED's in Hurricane Sandy affected areas in New York State(NYS). The focus of this project was to implement SBIRT protocols into healthcare settings, develop effective sustainable models of service delivery and formulate best practices for eventual dissemination throughout NYS. Medical Office Assistants (MOA) or Nurses (RN) conducted pre-screening (AUDIT C and DAST-1) when obtaining vital signs and if positive, a Health Coach completed the AUDIT and/or DAST-10, a brief intervention (BI) and a referral to treatment (RT) if indicated. As of 12/2015, we completed over 131,000 prescreens along with $4651 \mathrm{BI}$ and 1885 RT's among eight sites. Maximizing provider awareness of SBIRT and establishing stakeholder "buy-in" was essential for sustainability. We performed a multi-site evaluation to assess the affect of our implementation on physician and staff beliefs and behaviors, and to understand the effectiveness of our implementation model. METHODS: We used a mixed-methods approach to evaluate the SBIRT program. We surveyed PC and ED Physicians (84[45 IM, 39 ED]), MOAs (24), and RN's (91) using an 18-item, 5-point Likert-scaled survey ( 1 = strongly disagree, $5=$ strongly agree). Surveys were administered via paper/pencil or online between 12/2014 and 6/2015. In addition, patients completed a brief, 8-item Likert-scaled survey, between 12/2014 and 7/2015. We recruited only screen positive patients who received services and were randomly sampled for 6-month follow up. Fifty-eight patients agreed to be surveyed over the phone 1 week after receiving SBIRT services. Qualitative data were obtained from 9 physicians (5 IM, 4 
ED), 7 MOAs, and 12 RN's via individual semi- structured interviews conducted by phone or in-person between 4/2015 and 7/2015.

RESULTS: Attitudes towards SBIRT: Physicians viewed SBIRT positively overall (agree/strongly agree) ( $>90 \%)$, supported universal screening ( $>90 \%)$, believed substance misuse impacts health $(>95 \%)$ and believed substance misuse was a problem among their patients (>85\%). Implementation: Physicians viewed site implementation positively ( $>80 \%$ ) and felt the implementation process was easy $(>70 \%)$. A minority felt implementation increased wait time $(<20 \%)$ or disrupted workflow $(<10 \%)$. Interview data supported survey outcomes in that no physician felt their workflow or patient wait time was negatively affected. Support for SBIRT: Physicians felt worksite staff were supportive $(86 \%)$ and that SBIRT improved patient care $(72 \%)$, however they were uncertain if it improved patient outcomes $(51 \%)$. Interview data were mixed with physicians being split on whether staff supported SBIRT or not due to lack of understanding. Interview data were also mixed regarding patient outcomes with half the physicians believing SBIRT was useful and led to favorable outcomes and half being unsure or negative. For those who were unsure, lack of outcome data was frequently mentioned. RN and MOA results were generally concordant with physicians ( $>80 \%$ believed substance misuse was a problem among patients, $>90 \%$ believed it impacted chronic medical problems and $<90 \%$ believed patients benefited from the discussion). Only $45 \%$ believed SBIRT improved overall patient care. During interviews, it became clear that the pre screen process varied among staff with only half asking the questions exactly as worded. Patients overwhelmingly reported positive experiences in the SBIRT program. They felt staff were respectful (almost $100 \%$ ), were comfortable discussing substance use (90\%), were glad it was discussed $(\sim 80 \%)$, and felt other patients would benefit ( $\sim 80 \%$ ).Patients thought the interaction was helpful $(\sim 80 \%)$, knew more about how substance use affected health ( $70 \%)$, and felt SBIRT made them think differently about substance use $(\sim 68 \%)$. When comparing ED to PC staff, substance misuse was considered more of a problem (mean 4.6 vs 3.3 ) and common enough to screen at every visit ( 4.4 vs 3.4 ), however there was less belief in the value of SBIRT ( 3.4 vs 4.1 ) and treatment programs generally (3.6 vs 4.2 ). All were significant at $P<.05$. ED and PC physicians viewed SBIRT favorably (4.0 vs 4.2), with minimal workflow disruption (2.1 vs 2.2). There was less familiarity with SBIRT among ED compared to PC physicians ( $3.8 \mathrm{vs} 4.3$ ) and a stronger feeling that substance misuse was a problem (4.6 vs $3.8, P<.05)$

CONCLUSIONS: Our current model for delivery of SBIRT service was generally well received and perceived by both $\mathrm{ED}$ and $\mathrm{PC}$ patients and practitioners, although there were differences in how much of a problem substance misuse was and the value of SBIRT on patient outcomes. Our process of implementation was also considered well integrated in both PC and ED sites. More rigorous protocols and surveillance are needed to ensure the fidelity of prescreen techniques by staff. Finally, establishing regular feedback regarding patient outcomes will likely enhance provider commitment and sustainability.

PERCEPTIONS OF SOCIAL SUPPORT IN PERSONS WITH MEDICAL COMORBIDITIES AND ASSOCIATIONS WITH INTENTIONAL WEIGHT LOSS: RESULTS FROM THE MAINTAIN-PC STUDY Sobie Maduka ${ }^{1}$; Dana L. Tudorascu $^{1}$; Kathleen M. McTigue ${ }^{1}$; Cindy L. Bryce ${ }^{1}$; Kimberly A. Huber ${ }^{1}$; Laurey R. Simkin-Silverman ${ }^{1}$; Diane Comer ${ }^{1}$; Rachel Hess ${ }^{2}$; Gary Fischer ${ }^{1}$; Molly B. Conroy ${ }^{1}$. ${ }^{1}$ University of Pittsburgh, Pittsburgh, PA; ${ }^{2}$ University of Utah, Salt Lake City, UT. (Control ID \#2464523)

BACKGROUND: Social support can be a beneficial component of a weight loss program and lead to greater adoption of healthy lifestyle behaviors. Little is known about the association of social support and intentional weight loss in individuals with co-morbid medical conditions. We hypothesized that individuals with medical co-morbidities would report higher perceived social support and greater intentional weight loss compared to those without co-morbidities, perhaps due to greater attention from family and counseling from providers to help them cope with their medical conditions. Secondarily, we hypothesized that the presence of specific comorbidities such as anxiety/depression would affect the association between perceived social support and weight measures.

METHODS: Maintaining Activity and Nutrition through Technology-Assisted Innovation in Primary Care (MAINTAIN-pc) is a randomized, controlled trial to evaluate the use of electronic health record (EHR)- delivered tools and health professional support to help primary care patients successfully maintain intentional weight loss. Patients had to have lost at least $5 \%$ of their body weight in 2 years prior to enrollment (i.e., qualifying weight loss) to be eligible. Perceived social support was measured at baseline using the Interpersonal Support Evaluation List (ISEL), a validated 12-item questionnaire that assesses availability of social support on a four-point scale ( $0=$ "definitely false", 3 = "definitely true"). ISEL scores range from 0 to 36 , with a higher score indicating greater perceived social support. Qualifying weight and \% weight loss were verified in EHR. Medical comorbidities were measured by participant self-report using a standardized questionnaire. For the purpose of this analysis, we focused on conditions shown to be related to weight/obesity: prediabetes/diabetes, hypertension, hyperlipidemia, CVD, sleep apnea, GERD, arthritis, anxiety/depression, asthma, thyroid disease, and migraine. We examined presence vs. absence of co-morbidities, as well as total number of co-morbidities. We used Chi-square (or Fisher's exact) and t-tests to compare the participant characteristics those with and without co-morbidities. We used ANOVA to determine association between perceived social support and total number of co-morbidities, and T-tests for association between specific comorbidities and perceived social support. We used Pearson's correlations to evaluate the association between both qualifying weight and \% weight loss and perceived social support, stratified by co-morbidity subgroup.

RESULTS: All 194 participants in the MAINTAIN-pc study completed the ISEL at baseline. The study population was $74 \%$ female with a mean age of 53.4 (SD: 12.1), mean BMI 30.4 (SD: 5.9), and mean ISEL of 42.4 (SD: 5.5). Of the participants, 171 (88\%) had at least 1 co-morbidity. Participants with co-morbidities were older (54.8 vs. 42.9 years; $p=0.0001$ ), more likely to be white ( 90 vs $74 \% ; p=0.03$ ), and less likely to be employed ( 68 vs. $87 \%$; $p$-value $=0.04)$ than those with no co-morbidities. There was no difference in perceived social support ( 42.3 vs. $43.4 ; p=0.34$ ) when comparing those with and without co-morbidities. Qualifying weight and \% weight loss also did not vary when comparing those with and without co-morbidities ( $190.3 \mathrm{lb}$ vs. $180.4 \mathrm{lb} ; p=0.29$ and 13.1 vs. $11.1 \% ; p=0.17)$. With respect to individual conditions, individuals with knee arthritis $(N=50(26 \%)$ had significantly lower perceived social support than those without arthritis of the knees ( 40.9 ; vs. 43.0; $p=0.02)$, as did individuals with anxiety/depression $(N=53$ $(27 \%) ; 39.5$ vs. $43.6 ; p<0.0001)$. Individuals with higher number of total co-morbidities had significantly lower perceived social support than those with fewer co-morbidities ( $p=$ 0.02). In participants without anxiety/depression, there was a significant correlation between baseline weight and perceived social support ( -0.18 ; $p$-value: 0.03$)$, suggesting those who weighed more had lower perceived social support. No correlation was found between either baseline weight or baseline weight loss and perceived social support in any other individual co-morbidity subgroup.

CONCLUSIONS: Contrary to our hypothesis, presence or absence of co-morbidities was not associated with perceived social support or weight. Individuals with knee arthritis and anxiety/depression had lower perceived social support, but this was not associated with either qualifying weight or $\%$ weight loss. Our results suggest that even individuals with medical co-morbidities can experience intentional weight loss, and factors other than perceived social support may be influential in determining the degree of weight lost. 
PERSONAL ELECTRONIC HEALTH RECORDS AND VULNERABLE PATIENTS: PORTALS TO NOWHERE? Lina Tieu ${ }^{2}$, ; Dean Schillinger ${ }^{2}$, ; Neda Ratanawongsa ${ }^{2}$, 2; Urmimala Sarkar ${ }^{2}$, 2; James Ralston'; Courtney Lyles ${ }^{2}, 2$. ' Group Health Research Institute, Seattle, WA; ${ }^{2}$ University of California, San Francisco, San Francisco, CA. (Control ID \#2467789)

BACKGROUND: Patient portals, websites tethered to electronic health records that provide patients and healthcare proxy agents access to personal health information, are becoming ubiquitous in the US, especially with the support of Meaningful Use financial incentives. Although patient portals will be the first health technology to reach many diverse patient populations across the country, little is known about barriers to accessing these systems among vulnerable patients. The objective of this study was to examine specific usability barriers to patient portal website engagement by vulnerable patients and caregivers in a safety net setting.

METHODS: We conducted 25 observational "think aloud" interviews with patients with 1 or more chronic illnesses receiving primary care at the Richard H. Fine People's Clinic at Zuckerberg San Francisco General Hospital and caregivers of such patients, from February 2015 to July 2015 . We videotaped participants using a test account of the newly launched portal website and asked them to complete 5 tasks independently within 2 attempts: 1) Logging in, 2) Viewing an after-visit summary, 3) Viewing a specifically prescribed health education fact sheet, 4) Viewing a laboratory test result for a fasting glucose test, and 5) Looking up general health information. We offered assistance to the next step of the process if the participant asked a question, became stuck, or gave up. First, we assessed whether participants could complete each task independently without assistance and time to completion. Second, using an iteratively developed coding system, we categorized and recorded the frequency of barriers in completing the tasks. Finally, we assessed the ability of participants to interpret health information on the portal: 1) Within the after-visit summary, participants were asked to find a treatment plan with "next steps after the visit"; 2) After viewing the test result, participants were asked to interpret the result as indicating a "good or bad health outcome"; and 3) Participants were asked if they would ask their provider for further explanation or clarification about the test results. We used a validated one-item question ("How confident are you in filling out medical forms on your own?") to classify respondents as having limited versus adequate health literacy. RESULTS: The mean age of the sample was 58 years, and $60 \%$ had limited health literacy; $76 \%$ were non-white, $68 \%$ were female, and $56 \%$ reported high interest in using the Internet to manage their health. In navigating the portal, participants experienced four types of barriers: (1) novice computer, defined as barriers caused by a lack of basic computer knowledge (e.g., clicking the wrong mouse button); (2) routine computer, defined as barriers commonly experienced by a user with at least basic computer knowledge (e.g., navigational issues, typos); (3) reading and writing; (4) and medical content barriers. The majority of portal navigation barriers were related to routine $(54 \%)$ and novice $(25 \%)$ computer barriers, but reading and writing $(14 \%)$ and medical content (7\%) barriers were also observed. Compared with participants with adequate health literacy, participants with limited health literacy required an average of $2 \mathrm{~min}$ longer to complete each navigation task, were more likely to require assistance to complete navigation tasks (Figure 1), and were more likely to experience each type of barrier (Table 1). Participants with limited health literacy also experienced more inaccuracies in interpreting medical information (Table 1).

CONCLUSIONS: Participants with limited health literacy could complete fewer tasks unassisted, had a higher prevalence of barriers, took longer to complete tasks, and had more problems with accurately interpreting medical information. Our findings suggest a strong need for tailored and accessible training and support to assist all vulnerable patients and/or caregivers with portal registration and use. Measuring the health literacy of a patient population might serve as a strong proxy for identifying patients that need the most support in using health technologies.
Table 1. Barriers to portal navigation and interpretation of health information

\begin{tabular}{|c|c|c|c|}
\hline & Variable & $\begin{array}{l}\text { Adequate } \\
\text { Health Literacy } \\
(n=10)\end{array}$ & $\begin{array}{l}\text { Limited Health } \\
\text { Literacy }(n=15)\end{array}$ \\
\hline \multirow[t]{4}{*}{ Portal Navigation } & $\begin{array}{l}\% \text { With Novice } \\
\text { Computer Barrier }\end{array}$ & $10 \%$ & $69 \%$ \\
\hline & $\begin{array}{l}\text { \% With Routine } \\
\text { Computer Barrier }\end{array}$ & $90 \%$ & $93 \%$ \\
\hline & $\begin{array}{l}\text { \% With Reading/ } \\
\text { Writing Barrier }\end{array}$ & $30 \%$ & $33 \%$ \\
\hline & $\begin{array}{l}\% \text { With Medical } \\
\text { Content Barrier }\end{array}$ & $10 \%$ & $25 \%$ \\
\hline \multirow[t]{3}{*}{$\begin{array}{l}\text { Health Content } \\
\text { Interpretation }\end{array}$} & $\begin{array}{l}\text { \% With Difficulty } \\
\text { Finding Treatment Plan }\end{array}$ & $0 \%$ & $42 \%$ \\
\hline & $\begin{array}{l}\text { \% With Difficulty } \\
\text { Interpreting Lab } \\
\text { Results Correctly }\end{array}$ & $10 \%$ & $40 \%$ \\
\hline & $\begin{array}{l}\text { \% Seeking Explanation } \\
\text { From Provider }\end{array}$ & $90 \%$ & $93 \%$ \\
\hline
\end{tabular}

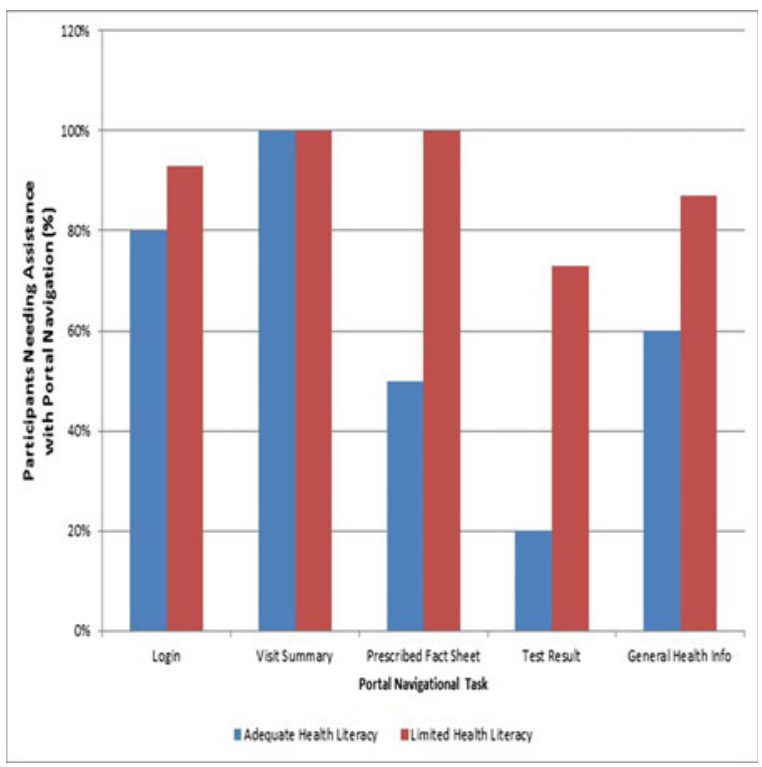

Figure 1. Need for assistance to complete portal navigation tasks

PHYSICAL HEALTH COMORBIDITY ASSOCIATED WITH PRIMARY CARE VISITS FOR SUBSTANCE USE DISORDERS Pooja A. Lagisetty ${ }^{2}$; Amy Bohnert ${ }^{2}$; Donovan Maust ${ }^{2}$; Michele Heisler ${ }^{1} .{ }^{1}$ Ann Arbor VA/University of Michigan, Ann Arbor, MI; ${ }^{2}$ University of Michigan, Ann Arbor, MI. (Control ID \#2467091)

BACKGROUND: Primary care visits provide an opportunity to engage persons with substance use disorders (SUDs) who are not involved in specialty mental health care due to reasons of stigma or limited access. Understanding which health conditions commonly co-occur with SUDs among individuals treated in primary care, and how these differ from other treatment settings, can aid the adaptation and tailoring of treatment for SUDs in primary care. Mental health comorbidity associated with SUD is well-described, but little is known about the physical health comorbidity associated with primary care visits for patients with SUD. We examined the top physical health comorbidities associated with US ambulatory care visits for SUD and compared how these comorbidities differ by provider type (general medicine vs. others). 
METHODS: We analyzed a pooled cohort from the National Ambulatory Medical Care Survey (NAMCS) and the National Hospital Ambulatory Medical Care Survey (NHAMCS) from 2009 to 2011 ( $n=194,884$ visits). We identified visits for patients with an age $\geq 18$ with a diagnoses or complaint of a substance use disorder. Comorbidities were identified either by ICD-9-CM code or if noted as a chronic condition (up to 3 conditions). Among all visits by patients with a SUD, we describe the percentage with common physical health comorbidities present. We evaluated differences in proportions for each comorbidity by provider type.

RESULTS: Across the survey interval, there were 3934 total visits from 2009 to 2011 to ambulatory settings by adult patients with a SUD (1051 visits with general medicine providers and 2883 visits with other providers) representing an estimated 3, 600, 250 million visits per year. The top three physical health comorbidities associated with a diagnosis for SUD seen by general medicine providers were hypertension, hyperlipidemia, and COPD. General medicine providers compared to other providers saw all three of these comorbidities more often: Hypertension (31.68 vs. $15.88 \%, p<001$ ), hyperlipidemia (17.72 vs. $7.49 \%, p<0.001)$, and COPD (10.82 vs. $6.18 \%, p=0.033)$. Hepatitis C and HIV comorbidity together were associated with less than $1.5 \%$ of the SUD related visits for general medicine providers.

CONCLUSIONS: The top comorbidities seen by general medicine providers in ambulatory visits for persons with SUDs are common chronic medical diseases. Infectious disease diagnoses generally associated with drug use and commonly studied in integrated treatment models are very uncommon. Through treatment of these chronic medical conditions, general medicine providers are offered a unique opportunity to screen and manage patients with SUDs who are not engaged in specialty care. While there is robust literature on how to manage and prevent chronic diseases such as hypertension, COPD, and obesity; disease management treatment models that treat both chronic medical conditions and addiction are currently under-developed and may lead to improved health outcomes.

PHYSICIAN ADOPTION OF NEW CARDIOVASCULAR DRUGS: DOES NOVELTY MATTER? Timothy Anderson ${ }^{1}$; Wei-Hsuan Lo-Ciganic ${ }^{4}$; Walid F. Gellad ${ }^{3}$, ${ }^{6}$; Rouxin Zhang ${ }^{5}$; Haiden A. Huskamp ${ }^{2}$; Niteesh K. Choudhry ${ }^{2}$; Joyce Chang ${ }^{6}$; Seth Richards-Shubik ${ }^{7}$; Hasan Guclu ${ }^{5}$; Bobby Jones ${ }^{6}$; Julie M. Donohue ${ }^{5}$. ${ }^{1}$ University of Pittsburgh Medical Center, Pittsburgh, PA; ${ }^{2}$ Harvard Medical School, Boston, MA; ${ }^{3}$ VA Pittsburgh, Pittsburgh, PA; ${ }^{4}$ University of Arizona, Phoenix, AZ; ${ }^{5}$ University of Pittsburgh Graduate School of Public Health, Pittsburgh, PA; ${ }^{6}$ University of Pittsburgh, Pittsburgh, PA; ${ }^{7}$ Carnegie Mellon University, Pittsburgh, PA. (Control ID \#2466506)

BACKGROUND: Whether new drugs lead to changes in patient care and expenditures depends on how quickly physicians adopt and how broadly they prescribe them. Little is known about whether individual physicians bring a consistent approach to adopting new drugs or differentiate based on novelty. In this study we examined patterns and predictors of physician prescribing of three new cardiovascular drugs (from most to least novel, respectively): dabigatran, the first oral anticoagulant since warfarin; aliskiren, a first-inclass antihypertensive; and pitavastatin, the seventh statin.

METHODS: Longitudinal analyses of physician prescribing of anticoagulants, antihypertensives and statins using IMS Health Xponent ${ }^{\mathrm{TM}}$ data capturing retail prescriptions from all payers. We examined the prescribing of primary care physicians (PCPs) and cardiologists practing in Pennsylvania from 2007 to 2011 who prescribed each drug category 12 months pre-introduction and 15 months post-introduction. Group-based trajectory models were used to identify rapid adopters as those with early and sustained monthly prescribing. Physicians were grouped based on rapid adoption of none, some or all three drugs. Predictors of adoption (age, sex, specialty, training, prescribing volume, practice setting, payer mix) were examined using logistic regression models.
RESULTS: Five thousand nine hundred fifty-three primary care physicians (PCPs) and cardiologists met inclusion criteria. The majority of physicians (63.8 \%) adopted none of the new drugs in the first 15 months after introduction, $35.0 \%$ rapidly adopted one or two, and $1.2 \%$ rapidly adopted all three. Physicians were more likely to rapidly adopt the most novel drug dabigatran $(27.3 \%)$, than they were aliskiren $(10.5 \%$ ) or pitavastatin ( $8.0 \%)$. Compared to PCPs, cardiologists were more rapid adopters of dabigatran (Adjusted Odds Ratio (OR) 8.90, $95 \%$ CI [7.42-10.67]; aliskerin (2.05 [1.56-2.69]) and pitavastatin (3.44 [2.60-4.57] $P<0.001$ ). Female physicians were less likely to adopt dabigatran (OR 0.71 [0.59-0.85], $P<0.001$ ) and aliskiren (OR 0.64, [0.49-0.83], $P<0.001$ ). Education at a top-ranked medical school was associated with greater adoption of dabigatran (OR 1.35 [1.08-1.69], $P=0.008$ ) and lower adoption of aliskiren (OR 0.53 [0.36-0.77], $P<0.001)$ and pitavastatin $(\mathrm{OR} 0.65$ [0.430.97], $P=0.03)$.

CONCLUSIONS: Most cardiologists and primary care physicians took a conservative approach to prescribing new cardiovascular medications; although drug novelty and specialty training were associated with greater adoption. This finding may alleviate concerns about high costs owing to rapid, indiscriminate technology adoption but heighten concerns about slow diffusion of more effective new pharmaceuticals.

\section{PHYSICIAN AND PATIENT VIEWS ON PUBLIC PHYSICIAN-RATING} WEBSITES Alison M. Holliday ${ }^{3}$, ${ }^{\text {; }}$ Allen Kachalia ${ }^{3,}{ }^{1}$; Gregg Meyer ${ }^{1,}$; Thomas D. Sequist ${ }^{3},{ }^{1}$. ${ }^{1}$ Harvard Medical School, Boston, MA; ${ }^{2}$ Massachusetts General Hospital, Boston, MA; ${ }^{3}$ Brigham and Women's Hospital, Boston, MA. (Control ID \#2464368)

BACKGROUND: Rating scores and open-ended patient comments about individual physicians are increasingly available on public physician-rating websites (PRWs). This information is collected either by private companies (e.g., Healthgrades) via the internet (“Independent PRWs") or by health care delivery organizations via validated patient experience surveys ("Health System PRWs"). However, data on physician and patient opinions of PRWs remains limited. We surveyed physicians and patients within a large accountable care organization (ACO) to assess their views on PRWs.

METHODS: We conducted two cross-sectional surveys in August 2015 within an ACO that included two academic medical centers and three community hospitals, and their affiliated ambulatory clinics. We emailed invitations to complete a web-based survey to 1916 ACO physicians, along with three follow-up reminders to nonresponders at 1-week intervals, and achieved a response rate of $42 \%(810 / 1916)$. We also mailed a survey to a random sample of 1500 adult patients ( 300 patients per hospital) who had at least one hospitalization or ambulatory clinic visit during May 2015. We achieved a response rate of $33 \%$ (494/1500) after one followup mailing at 4 weeks to nonresponders. For both physicians and patients, the surveys assessed their: 1) use of PRWs, 2) belief in the accuracy of rating scores and comments posted on PRWs, 3) support for making routine physician rating scores and comments publicly available on Health System PRWs, and 4) perceived impact of publishing physician rating scores and comments on Health System PRWs. We fit multivariate logistic regression models to assess the association between views on sharing patient comments online and either physician characteristics (age, sex, specialty, inpatient or ambulatory clinical time) or patient characteristics (age, sex, race, educational level, health status, and internet access).

RESULTS: Among physician respondents, the average age was 49 years, the majority were male (55\%), and $28 \%$ reported having only inpatient clinical time. The most frequent specialties were primary care $(27 \%)$ and medical specialists $(26 \%)$, with the remainder being surgeons $(19 \%), \mathrm{OB} / \mathrm{GYN}(6 \%)$, and other specialties (21\%). Among patient respondents, the average age was 66 years, the majority were female (63\%), white ( $87 \%$ ), attended college $(79 \%)$, and reported access to the internet on most days of the week ( $85 \%)$. Both physicians $(53 \%)$ and patients (39\%) reported having visited a PRW at least once. Physicians were more likely to report that open-ended patient comments on Health System PRWs accurately reflect the quality of care they provide compared to comments on Independent PRWs (62\% versus $36 \%$, 
$p<0.001)$. Patients were more likely to report trust in reviews obtained on Independent PRWs compared to Health System PRWs ( $57 \%$ versus $45 \%, p<0.001)$. Physicians were less likely to support sharing of patient comments obtained from patient experience surveys in increasingly public venues, ranging from support for sharing within their own practice (33\%), within their entire health care organization ( $28 \%$ ), and publicly online for all consumers (21\%). In contrast, $51 \%$ of patients would like patient comments to be shared publicly online. Physician characteristics associated with support for sharing comments publicly included decreasing age (OR $1.01,95 \%$ CI 1.01-1.01), male gender (OR 1.4, 95 \% CI 1.2-1.8), surgical specialty (OR 1.4, $95 \%$ CI 1.3-1.6), and having some ambulatory clinical time (OR 1.6, $95 \%$ CI 1.3-2.6). Among patients, regular internet access was associated with support for sharing comments publicly (OR 3.7, $95 \%$ CI 1.7-7.9). The majority of physicians (78 \%) believed that posting patient comments on Health System PRWs would have a negative effect on physician job stress; while smaller proportions perceived a negative effect on the physician-patient relationship (46\%), health care overuse (34\%), and patient-reported experience of care (33\%). Less than one-third of patients (29\%) believed that publication of their comments on Health System PRWs would cause them to be less honest.

CONCLUSIONS: Physicians and their patients frequently visit physician-rating websites, though their viewpoints on this information are often discordant, including whether Independent or Health System PRWs are the more reliable source of information, and whether such data should be shared on public websites.

PHYSICIAN AND STAFF PERCEPTIONS AFTER IMPLEMENTING A POPULATION HEALTH MANAGEMENT PROGRAM WITHIN A PRIMARY CARE NETWORK Jeffrey M. Ashburner; Daniel M. Horn; Sandra OKeefe; Adrian Zai; Steven J. Atlas. Massachusetts General Hospital, Boston, MA. (Control ID \#2465932)

BACKGROUND: Though many provider-insurer contracts include withholds for achieving ambulatory quality measures, physicians and staff in primary care practices often view such activities as separate from routine clinical care. Factors limiting uptake include contracts that do not include all patients, financial incentives that are small relative to visit-based revenue, measures with definitions that vary and lack clinical relevance, and little ongoing feedback to track progress. As provider groups form accountable care organizations (ACOs) in response to provisions of the Affordable Care Act, population health management (PHM) activities will likely expand the scope of ambulatory quality initiatives. We developed an information technology (IT) tool with clinically relevant registries to support PHM within an ACO and implemented the tool as part of an organized program of chronic disease management and preventive cancer screening. We hypothesized that more clinically relevant measures, an IT tool that provided regular updates on performance, and programmatic support for practice personnel would increase provider buy-in. We surveyed physicians and staff within a primary care network to assess perceptions of the PHM program after the first year.

METHODS: A PHM program was developed and implemented in all network practices in July 2014. As part of a pilot project, 8 of 19 practices received dedicated network support by assigning a central population health coordinator (PHC) who regularly "huddled" with physicians. PHCs managed lists of patients not at goal and performed administrative tasks including appointment scheduling, ordering overdue laboratory testing, and obtaining home blood pressure values. Other PHM practices received training and support for practice staff, but staff in these practices were responsible for managing administrative tasks. In early 2015 , we surveyed all network PCPs who saw patients in 2014 and all non-PCP staff (nurse practitioners, registered nurses, medical assistants and care coordinators) who used the IT system in 2014 and asked them about their experience with the program during the prior year. The survey asked about activities performed, the importance and impact of population health activities, and the amount of work involved with these activities. We compared responses among PCPs and staff in pilot (with central PHCs) and non-pilot PHM practices.

RESULTS: Among PCPs, the survey response rate was $64.8 \%$ (127/196), with a similar response from PCPs in pilot (60.8\%) and non-pilot (62.3\%) practices. The response rate among staff was $59.2 \%(122 / 206)$, with a similar response from staff in pilot $(54.8 \%)$ and nonpilot (57.3\%) practices. Population health activities were performed by most PCPs and staff regardless of whether they were in pilot or non-pilot practices (see Table 1). In pilot practices, $93.3 \%$ of PCPs and $73.9 \%$ of staff reviewed a list of patients appropriate for outreach activities that was prepared and acted on by a central PHC. PCPs in pilot practices with a central PHC were slightly more likely to state that they thought population health activities were "absolutely essential" or "very important" to the care of their panel (62.2 vs. $53.3 \%, p=0.34)$, but were significantly less likely to believe that these population health activities increased the amount of work it takes to care for their panel of patients ( 66.7 vs. $89.3 \%, p=0.002)$. Similar proportions of PCPs in pilot and non-pilot practices believed these activities had a positive impact on the care provided to their patients ( 86.7 vs. $84.0 \%$ ). Most staff, regardless of whether they were in a pilot or non-pilot practice, viewed population health activities as "absolutely essential" or "very important" (Overall: $86.2 \%$ ) and having a positive impact on the care of patients in their practice (Overall: $94.0 \%$ ). Most staff also believed these activities increased the work required to care for patients (Overall: $80.9 \%$ ).

CONCLUSIONS: Following implementation of a PHM program within a primary care network that used an IT tool with clinically relevant registries and programmatic support, we found a high level of engagement in PHM activities among both PCPs and practice staff. PCPs and staff viewed this as important work, but also saw these activities as involving extra work. Among practices assigned central personnel to support administrative tasks, PCPs viewed PHM activities as involving less extra work than PCPs in practices without this extra support.

\begin{tabular}{|c|c|c|c|c|}
\hline & $\begin{array}{l}\text { PCPs } \\
\text { Pilot } \\
(n=45)\end{array}$ & $\begin{array}{l}\text { Staff } \\
\text { Non-Pilot } \\
(n=76)\end{array}$ & Pilot $(n=21)$ & $\begin{array}{l}\text { Non-Pilot } \\
(n=94)\end{array}$ \\
\hline Reviewed a list of patients to exclude those who were not mine/my practice & $39(86.7 \%)$ & $63(82.9 \%)$ & $21(91.3 \%)$ & $53(56.4 \%)$ \\
\hline Reviewed registry of patients with a chronic condition who were "not at goal" or "due for test" & $45(100 \%)$ & $73(96.1 \%)$ & $21(91.3 \%)$ & $75(79.8 \%)$ \\
\hline Reviewed registry of patients overdue for cancer screening & $43(95.6 \%)$ & $70(92.1 \%)$ & $13(56.5 \%)$ & $49(52.1 \%)$ \\
\hline Entered patient data into structured fields in EHR for care done outside network & $37(82.2 \%)$ & $51(67.1 \%)$ & $11(47.8 \%)$ & $52(55.9 \%)$ \\
\hline
\end{tabular}

PHYSICIAN DECISION MAKING FOR CARDIOVASCULAR TREATMENTS IN ADULTS WITH MILD COGNITIVE IMPAIRMENT Deborah A. Levine ${ }^{1,3}$; Kenneth M. Langa ${ }^{1,3}$; Angela Fagerlin ${ }^{4}$; Lewis Morgenstern ${ }^{1}$; Brahmajee K. Nallamothu' ${ }^{1}$ Jane Forman ${ }^{2}$; Andrzej Galecki ${ }^{1}$; Mohammed U. Kabeto ${ }^{1}$; Colleen D. Kollman $^{1,5}$; Tolu Olorode ${ }^{1}$; Bruno Giordani ${ }^{1}$; Lynda Lisabeth ${ }^{1}$; Darin B. Zahuranec ${ }^{1}$. ${ }^{1}$ University of Michigan, Ann Arbor, MI; ${ }^{2}$ VA Center for Clinic'al Management Research,
Ann Arbor, MI; ${ }^{3}$ VA Ann Arbor Healthcare System, Ann Arbor, MI; ${ }^{4}$ University of Utah, Salt Lake City, UT; ${ }^{5}$ Kollman Research Services, Ann Arbor, MI. (Control ID \#2468452)

BACKGROUND: Cardiovascular disease (CVD) is the leading cause of death and serious morbidity in the 5.4 million older adults with mild cognitive impairment (MCI), many of whom enjoys good quality of life and will not progress to dementia. Yet, some 
evidence suggests that older MCI patients less frequently get effective treatments for acute myocardial infarction (AMI) and acute ischemic stroke (AIS) compared with cognitively normal patients. To better understand this disparity, we examined the influence of $\mathrm{MCI}$ on physician recommendations for AMI and AIS treatment in a pilot study.

METHODS: Concurrent mixed methods study at a large academic medical center using surveys and semi-structured interviews of physicians. We conducted a mailed survey among 114 physicians consisting of 1 of 5 clinical vignettes of AMI (cardiologists, $n=53$ ) or AIS (neurologists, $n=61$ ), with the patient in the vignette randomized to 1 of 3 cognitive states: normal cognition, MCI (cognitive difficulties that do not impact daily activities), mild dementia (cognitive difficulties that mildly impact daily activities). The primary outcome was a composite quality measure, calculated by dividing the number of treatments that a physician recommended by the number of effective treatments indicated for each patient. This score ranges between 0 and 1 , with values closer to 1 implying better physician adherence to guidelinerecommended treatments. Secondary outcomes were the individual treatments. We compared physicians' likelihood of recommending treatments (diagnostic testing, revascularization, rehabilitation, secondary prevention medications) for the AMI and AIS cases by patient cognitive status using linear regression before and after adjusting for physician characteristics. In addition, physicians predicted the 5-year risk of dementia in patients with normal cognition or MCI. We also conducted semi-structured interviews with 18 physicians (4 cardiologists, 9 neurologists, and 5 internists) recruited from a convenience sample ( $n=$ 39 ) of faculty in each specialty at a major medical school using email solicitation and a standard guide. We used qualitative content analysis to identify unifying and recurrent themes.

RESULTS: The survey response rate was $72 \%$. Physicians recommended less intensive treatment to patients with cognitive impairment (MCI or mild dementia), but this varied by physician specialty. Neurologists' likelihood of recommending all effective AIS treatments was non-significantly lower in MCI patients (12\% lower; $P=0.15$ ) but significantly lower in patients with mild dementia (36 \% lower; $P<0.001)$ compared to cognitively normal patients. Cardiologists' likelihood of recommending all effective AMI treatments did not differ significantly by patient's cognitive status. Patient's cognitive impairment (MCI or mild dementia) was negatively associated with the likelihood of neurologists to recommend brain MRI, carotid imaging, echocardiogram, carotid revascularization, and inpatient rehabilitation (all $\mathrm{P} \leq 0.01$ ) for AIS. Physicians overestimated dementia risk in the survey patient with MCI. Cardiologists and neurologists both predicted a median absolute 5-year risk of dementia of $50 \%$ (interquartile range, 30-70\%) in the 75 year-old communitydwelling patient with MCI, although actual risk was as low as $10-15 \%$ based on prior studies. Physicians also rated cognitively impaired patients as more likely to miss follow-up appointments $(P=0.01)$ and less likely to participate in treatment $(P=$ 0.03 ) than cognitively normal patients. In the interviews, we found that some physicians were unaware of the definition of MCI and conflated MCI with dementia (e.g., one cardiologist asked, "What's the difference?"). Physicians made assumptions that MCI is associated with older age/shortened life expectancy, frailty/poor functional status, or patient/family preferences for less intensive treatment.

CONCLUSIONS: An MCI diagnosis appeared to influence physician recommendations for CVD treatment, but this varied by physician specialty. Neurologists recommended less intensive treatment for AIS to patients with MCI or mild dementia than to cognitively normal patients, although cardiologists did not for AMI. Our findings further suggest that physicians overestimate dementia risk, conflate MCI with dementia, associate MCI with very poor prognosis and function, and assume patient/family preferences for less intensive treatment. Better understanding of how physician recommendations contribute to underuse of CVD treatments in MCI patients is critical to the design of interventions aimed to improve the quality and outcomes of CVD care.
PHYSICIAN EDUCATION IS LACKING IN INPATIENT MANAGEMENT OF ACUTE AND CHRONIC PAIN Anne Drabkin ${ }^{1}$; Susan Calcaterra ${ }^{1}$; Ingrid A. Binswanger ${ }^{2} .{ }^{1}$ Denver Health, Denver, CO; ${ }^{2}$ University of Colorado Denver, Denver, CO. (Control ID \#2469923)

BACKGROUND: Prescribing opioids for pain control in an inpatient setting is challenging for internal medicine physicians. Multiple factors complicate this process including a limited educational focus on pain management during training. As a result, many physicians rely on their personal medical experience to guide opioid-prescribing decisions, which leaves junior physicians disadvantaged and vulnerable to the potentially negative consequences of overprescribing opioids or prescribing them to the wrong patients. We are in the midst of an opioid epidemic with the highest opioid-related overdoses and deaths in the world (McHugh RK, et al. 2015). Inpatient providers play a crucial role in this epidemic and a recent study has linked opioid prescriptions during hospitalization to chronic narcotic use 1 year after discharge (Calcaterra SL, et al. 2015). In addition to its public health importance, inpatient pain management has significant reimbursement consequences for hospitals and providers through HCAHPS scores. Despite its inarguable importance, many providers feel they have inadequate skills in pain management and never received formal training.

METHODS: In-depth, semi-structured key informant interviews were performed with internal medicine hospitalists and interviewees were questioned about their feelings towards treating acute and chronic pain in an inpatient setting. A broad base of hospital types was targeted. Interviews were conducted either in person or over the telephone then recorded and transcribed. A mixed inductive and deductive team-based approach was used to identify themes to create a central code-book for qualitative analysis using Atlas.ti. RESULTS: Twenty-five internal medicine hospitalists were interviewed who worked at two university hospitals (28\%), a safety net hospital (32\%), a Veterans Affairs hospital (12\%) and community hospital ( $28 \%$ ). $64 \%$ were female, $36 \%$ had less than 5 years of post-graduate training and $16 \%$ had greater than 10 years of training. Many providers (44\%) providers felt they had inadequate training in pain management and felt they had to rely almost exclusively on their personal medical experience in deciding how and when to prescribe narcotics. Providers working with residents in academic facilities felt that $47 \%$ of current residents do a "poor job" of managing inpatient pain and prescribing opioids on discharge. Providers (57\%) feel that a more education and training is needed to provide better care for their patients.

CONCLUSIONS: Designated and focused pain management training during residency and post-graduate education is needed. Specific goals such as exploring more non-opioid pain options with patients as well as basic guidelines on up- and down-titration of opioids is needed. Inpatient physicians should be educated on how to better communicate goals and expectations of pain management with their patients and how to better transition them back to their outpatient providers.

PHYSICIAN PERSPECTIVES ON DISCONTINUING DIABETES MEDICATIONS Natalia Lipin ${ }^{2}$; Robert Sargis ${ }^{2}$; Christopher Masi1 ${ }^{1}$; Aviva G. Nathan ${ }^{3}$; Michael T. Quinn ${ }^{2}$; Elbert S. Huang ${ }^{2}$; Neda Laiteerapong ${ }^{2}$. ${ }^{1}$ NorthShore University HealthSystem, Evanston, IL; ${ }^{2}$ University of Chicago, Chicago, IL; ${ }^{3}$ University of CHicago, Chicago, IL. (Control ID \#2466322)

BACKGROUND: Diabetes care is increasingly complex due to multiple options for medications and uncertainty around the benefits of intensive glycemic control. Multiple care guidelines emphasize best practices for initiation and intensification of therapy and choosing individualized patient-centered hemoglobin $\mathrm{A}_{1 \mathrm{C}}\left(\mathrm{HbA}_{1 \mathrm{C}}\right)$ goals. However, when patients have $\mathrm{HbA}_{1 \mathrm{C}}$ values well below their goals, guidelines do not provide strategies for de-intensifying diabetes medications. As a consequence, some patients may be over- 
treated and risk complications of diabetes treatments, like hypoglycemia. We sought to understand physician perspectives and practices regarding discontinuing glycemic therapy in patients with type 2 diabetes.

METHODS: The "Type 2 Diabetes Care Practices" survey was conducted among primary care physicians and endocrinologists at the University of Chicago Medical Center (UCMC; academic medical center) and at NorthShore University HealthSystem (NUHS; community hospital). Survey content included questions about knowledge of the concept of individualizing glycemic goals, how frequently they individualized goals, and whether they ever initiated conversations about medication discontinuation for patients with low stable $\mathrm{HbA}_{1 \mathrm{C}}$ values, and under what circumstances (e.g., at what $\mathrm{HbA}_{1 \mathrm{C}}$ levels, side effects, costs). Data analyses included descriptive statistics, chi-square analyses, and multiple logistic regression analysis examining the relationships between physician and practice characteristics with physician's 1) knowledge of guidelines for individualizing goals and 2) report of active discontinuation of diabetes treatments.

RESULTS: Response rate was $73 \%$ (155/213); $92 \%$ of respondents were primary care physicians, $8 \%$ were endocrinologists. Forty eight percent of endocrinologists responded compared to $76 \%$ of primary care physicians $(p=0.004)$. Most physicians $(78 \%)$ responded that they were familiar with the concept of individualizing $\mathrm{HbA}_{1 \mathrm{C}}$ goals and did so at least "most of the time" (70\%). The majority of physicians $(80 \%)$ reported that they have initiated a conversation about discontinuing or reducing the dose of diabetes medications for patients with a low stable $\mathrm{HbA}_{1 \mathrm{C}}$. However, only a minority (21\%) of those physicians reported that they initiated this conversation based on individualized $\mathrm{HbA}_{1 \mathrm{C}}$ goals; the remainder used absolute $\mathrm{HbA}_{1 \mathrm{C}}$ goals, including $<6 \%(32 \%),<6.5 \%$ (23\%), and $<5.7 \%$ (15\%). In multivariate analysis, only practicing at the academic medical center was associated with knowledge of individualization guidelines (odds ratio (OR) 12.8; $95 \%$ confidence interval (CI) 1.6-105.2). Also, in multiple logistic regression, female gender (OR 3.4; CI 1.1-8.1), practicing at an academic medical center (OR 4.5; CI 41.1-17.9), and practicing for less than 20 years (OR 2.8; CI 1.0-7.6, $p=.0499$ ) were associated with initiating a conversation about discontinuing medications for patients with low stable HbA1c levels. Physicians also considered discontinuing diabetes medications because of side effects $(93 \%)$, limited life expectancy (75\%), polypharmacy (63\%), medication costs (58\%), and medication non-compliance (53\%).

CONCLUSIONS: Discontinuing diabetes medications based on individualized goals is an important concept for providing patient-centered care. Our study suggests that most physicians individualize $\mathrm{HbA}_{1 \mathrm{C}}$ goals and consider medication discontinuation in patients with low stable $\mathrm{HbA}_{1 \mathrm{C}}$. However, most physicians do not use an individualized $\mathrm{HbA}_{1 \mathrm{C}}$ goal to guide discontinuing diabetes medications. To address this issue, we recommend that treatment guidelines include calculation of each patient's 'delta $\mathrm{HbA}_{1 \mathrm{C}}$ '(i.e., the difference between goal $\mathrm{HbA}_{1 \mathrm{C}}$ and measured $\mathrm{HbA}_{1 \mathrm{C}}$ ). If 'delta $\mathrm{HbA}_{1 \mathrm{C}}$ ' is greater than the $\mathrm{HbA}_{1 \mathrm{C}}$ lowering potential of one of the patient's current diabetes medication (generally $0.8-1.5 \%$ based on prior studies), then discontinuing that medication can be considered, combined with appropriate follow-up

PHYSICIAN SELF-REFLECTION AND BEHAVIOR CHANGE IN HOSPITAL MEDICINE CONTINUING EDUCATION John T. Ratelle ${ }^{2}$; Christopher M. Wittich²; Roger $\mathrm{Yu}^{1}$; James Newman ${ }^{2}$; Sarah Jenkins ${ }^{2}$; Thomas J. Beckman ${ }^{2} .{ }^{1}$ Scripps Health, La Jolla, CA; ${ }^{2}$ Mayo Clinic, Rochester, MN. (Control ID \#2463701)

BACKGROUND: Self-reflection facilitates the identification of performance gaps and is a critical step in the process of behavior change among physicians. However, little is known about reflection and its relation to behavior change in hospital medicine (HM) continuing medical education (CME). Our objectives were to identify associations between validated reflection scores and characteristics of CME presentations, and to measure associations between validated measures of reflection and behavior change among HM CME participants
METHODS: This was a cross-sectional study of 260 participants and 29 presenters delivering 31 presentations at a Mayo Clinic HM CME course in 2015. Participants provided CME reflection ratings, which were obtained from an instrument with known content, internal structure and criterion validity evidence (Wittich et al. Mayo Clinic Proc. 2013). The instrument contains 8 items on 5-point scales (range: $1=$ strongly disagree to $5=$ strongly agree) that address the following 4 levels of reflective thinking described by Kember et al.: habitual action, understanding, reflection and critical reflection. Participants' planned behavior change was assessed with a single item: "As a result of this presentation, I plan to make a change to my clinical practice", which is rated on the same 5-point scale. Associations were measured between CME reflection scores and planned behavior change, and between CME reflection scores and characteristics of presentations and presenters. Scores were compared using linear regression models with generalized estimating equations to adjust standard errors for repeated data within each presentation. The threshold for statistical significance was set at $p<0.05$.

RESULTS: A total of 223 out of 262 participants ( $85.1 \%$ ) returned evaluations, yielding 6083 ratings for statistical analysis. There was a statistically significant correlation between CME reflection scores and behavior change scores across presentations (Pearson coefficient $0.69, p<.0001$ ). Additionally, CME reflection scores (mean [SD]) were significantly associated with the number of audience response opportunities (0:3.62 (0.57) vs. $1-3: 3.70(0.54)$ v. $\geq 4: 3.75(0.55), p=0.02)$ and the use of clinical cases ((3.55 [0.57]) vs. no clinical cases $(3.37$ [3.71]; $p=0.004)$. There were no significant associations between CME reflection scores and presentation length (minutes), number of slides, presenter specialty or academic rank

CONCLUSIONS: To our knowledge, this is the first study to measure the association between validated reflection scores and behavior change in HM CME. Reflection scores were strongly correlated with planned practice change among CME participants. Additionally, our findings indicate that CME presentations which incorporate audience response and clinical cases may lead to greater self-reflection and planned changes in clinical practice among physician learners.

PHYSICIANS AND PHARMACISTS ON OVER-THE-COUNTER CONTRACEPTIVE ACCESS FOR WOMEN Nazanin Kuseh Kalani Yazd ${ }^{2}$; Michelle Gaffaney $^{2}$; Carol A. Stamm ${ }^{3}$; Laura Borgelt ${ }^{2}$ Lilyana Gross ${ }^{2}$; Bryan McNair ${ }^{2}$; Leanne Rupp ${ }^{1}$; Daniel Topp ${ }^{2}$; Christine Gilroy ${ }^{3} .{ }^{1}$ The Colorado Health Foundation, Denver, CO; ${ }^{2}$ University of Colorado Anschutz Medical Campus, Aurora, $\mathrm{CO} ;{ }^{3}$ University of Colorado Anschutz Medical Campus, Denver, CO. (Control ID \#2468999)

BACKGROUND: Recently, legislation has been proposed nationally to provide easier access to oral contraceptive pills. The proposed legislation entitled Allowing Greater Access to Safe and Effective Contraception Act seeks to allow "routine-use contraceptives" to be sold over-thecounter (OTC). This legislation would increase women's access to contraception; however, it could also increase out-of-pocket cost because the Affordable Care Act contraception provision only covers prescription contraception. Alternative legislation entitled Affordability Is Access Act requires health insurance to cover costs of oral contraception when it becomes OTC. California and Oregon have transitioned to allow access to oral contraception through pharmacist provision. Undeniably, political and healthcare leaders are investigating alternative methods of access to oral contraception. The goal of this study was to assess and compare the perceptions of Colorado physicians, pharmacists, and pharmacy technicians on OTC access to progestinonly oral contraception in order to identify specific concerns among health professionals to inform future policy and to guide effective provider education.

METHODS: A physician-specific survey was created for faculty physicians in internal medicine, family medicine, and obstetrics and gynecology at the University of Colorado School of Medicine and emailed to faculty members. A pharmacist and pharmacy technician specific survey was created for pharmacists and pharmacy technicians practicing in Denver 
County outpatient pharmacies. Two student researchers visited 74 randomly selected pharmacies on the list of Denver pharmacies, and they attempted to obtain responses from one pharmacist and one pharmacy technician at each site. Survey responses were compared among respondent groups using chi-square and Fisher's exact tests.

RESULTS: Responses were collected from 56 physicians, 58 pharmacists, and 43 pharmacy technicians. There was a statistically significant relationship between profession (physician, pharmacist, and pharmacy technician) and how confident the survey participant felt about patients correctly using the progestin-only contraception without prior consultation ( $p$-value $=0.0318)$. Surveyed physicians, pharmacists, and pharmacy technicians "somewhat agree" that OTC progestin-only contraception will have a positive impact on women's health $(58.93 \%, 56.14 \%$, and $41.64 \%$ respectively), and there was no statistically significant difference among groups $(p$-value $=0.142$ ). However, there was a significant difference on top concern of OTC access of progestin-only contraception ( $p$-value $<0.001$ ). The biggest concern of physicians was efficacy of progestin-only contraception (35.71\%), while pharmacists and pharmacy technicians both responded that patient self-assessment of contraindications was their top concern, (35.09 and $23.82 \%$ respectively).

CONCLUSIONS: Our study has shown that there are differing views among healthcare professionals on OTC provision of progestin-only contraception. This study builds upon previous literature including the work of Daniel Grossman et. al that indicates that most women are able to self-assess for contraindications using simple checklists. Given recently proposed legislation, it is crucial to seek the perspectives of primary stakeholders, including physicians and pharmacists. Legislators should address the concerns of healthcare professionals before moving forward with OTC provision. While provider/ physician prescribing is a barrier to contraceptive access and adherence, moving oral contraception to OTC status may create unforeseen barriers including removing insurance copays and increasing direct costs to women. Affordable prices, assured insurance reimbursement, and clear packaging may alleviate some concerns.

\section{PHYSICIANS' PERSPECTIVES ON INNOVATIVE PATIENT EDUCATION} CHANNELS Mita S. Goel ${ }^{1}$; Tiffany Brown ${ }^{1}$; Ji Young Lee ${ }^{1}$; Alpa Patel ${ }^{2}$; Ryan Chmiel $^{2}$; Paul Botsford ${ }^{2}$; Sameem Samad ${ }^{2}$; Darren Kaiser ${ }^{2}$; Kenzie A. Cameron ${ }^{1} .{ }^{1}$ Northwestern University Feinberg School of Medicine, Chicago, IL; ${ }^{2}$ Northwestern Medicine, Chicago, IL. (Control ID \#2461608)

BACKGROUND: Physician perceptions of patient education occurring outside of the physician encounter may impact the successful adoption of such quality improvement efforts. As a result, we sought to assess physicians' perceptions of patient education materials regarding pneumococcal vaccination, delivered through innovative channels, on clinic workflow and communication.

METHODS: As part of a larger study, we developed a novel 2 min video for patients newly eligible for the pneumococcal vaccination. We tested the video among 65 and 66year old patients using 2 different delivery channels: (1) point-of-care patient viewing with the video triggered by a rooming nurse or medical assistant during a visit and (2) out-of office viewing delivered to patients via an electronic patient portal message (MyChart). To understand physician perspectives on the perceived effects of each of the delivery channels, all physicians in the general internal medicine practice where the patient education was implemented were asked to participate in a brief on-line survey. Employing Likert scale responses, survey questions solicited perceived effects of the communication channel on patient flow and clinic operations, as well as perceived effects on quality of care. Completion of the survey indicated physician consent to participate and physicians were compensated with a $\$ 25$ gift card for their completion of each survey. The Northwestern University Institutional Review Board approved the study.

RESULTS: For point-of-care communication, 28/32 providers completed the survey for a response rate of $88 \%$. There were few concerns for its impact on workflow: no physicians
$(0 \%)$ reported that it disrupted workflow despite $2 / 28$ (7\%) reporting that they entered the room when the video was playing and 6/28 (21\%) reporting that patients asked more questions about pneumonia and the pneumococcal vaccine than prior to the intervention. Furthermore, 4/28 (14\%) agreed with a statement that the intervention may distract rooming nurses from more important things, but only $1 / 28$ (4\%) agreed that it distracted the physician. Physicians perceived a beneficial effect of the intervention on quality of care: 12/28 (43\%) agreed that the video had a positive impact on the practice, 13/28 (46\%) agreed that the intervention improves patient safety and 9/28 (32\%) agreed that it helped physicians recognize when patients needed a pneumococcal vaccine. For the educational video sent to patients via the patient portal, 23/31 eligible providers completed the survey for a response rate of $74 \%$ (there was a change in the total number of provides at the practice). Overall perceptions of the portal-delivered video indicated minimal concern for adverse effects on workflow or patient outcomes. None of the physicians $(0 \%)$ indicated that the video disrupted workflow and 2/23 (9\%) "slightly agreed" that they perceived an increase in physician workload. When asked about increase in patient anxiety or increase in patient confusion regarding pneumococcal vaccination, only $1 / 23$ physicians (4\%) reported perceiving those effects, and only at the level of "slightly agree" for each of the categories. In contrast, most reported "slightly" or "strongly" agreeing with each of the following effects of the intervention: 16/23 (70\%) perceived an improvement in quality of care and 21/23 (91\%) perceived an increased understanding of the need for pneumococcal vaccination. Most also supported the use of the portal to reach out to patients about pneumococcal vaccination over other modes of outreach by slightly or strongly agreeing with the following statements: 12/23 (52\%) preferred portal outreach over nurse outreach; 13/23 (57\%) preferred the portal over quarterly quality reports; 14/23 (61\%) preferred the portal over mailing letters. The only mode of outreach that was an exception was informing patients at the point-of-care, with 8/23 (35\%) agreeing with this preference. Overall, physicians were comfortable with the use of portals to provide information about pneumococcal vaccination as well as a recommendation to receive a pneumococcal vaccination, 18/23 (78 \%) for both.

CONCLUSIONS: Physicians perceived that novel channels for patient education had a positive impact on quality of care, with minimal concern for their effect on workflow. If effective, these channels would likely garner physician support for broader dissemination.

\section{PLANYOURLIFESPAN.ORG - AN INTERVENTION TO HELP SENIORS MAKE CHOICES FOR THEIR 70'S, 80'S, 90'S: RESULTS FROM A PCORI-FUNDED RANDOMIZED CONTROLLED TRIAL. Lee Lindquist ${ }^{2}$; Vanessa Ramirez-Zohfeld ${ }^{2}$;} Priya Sunkara ${ }^{2}$; Chris Forcucci ${ }^{1}$; Kenzie A. Cameron ${ }^{2} .{ }^{1}$ Aging \& In-Home Services of NE IN, Fort Wayne, IN; ${ }^{2}$ Northwestern University, Chicago, IL. (Control ID \#2467496)

BACKGROUND: Seniors regularly plan for their end of life but very few people consider their support and health needs that they will encounter during their 70's, 80's, and 90's - the "4th Quarter" of life. During this time period, remaining in one's own home is often a high priority for seniors; however, as their functional and cognitive needs increase, seniors are likely to need more support to safely remain in their homes. We developed and tested the www.PlanYourLifespan.org (PYL) tool to assist seniors in making informed choices about issues in their health trajectory that may influence their ability to remain safely in their own homes. PYL is a web-based planning tool that enables seniors to explore issues related to hospitalizations, falls, memory loss, and connect to community-based resources in order to better plan for their future health.

METHODS: We conducted a multi-site, two-arm randomized controlled trial of the PYL tool as compared to an attention control arm, in rural and urban settings in Chicago, IL; Fort Wayne, IN; and Houston, TX. Participants were English-speaking individuals 65 years and older who currently live independently in the community. After consent, participants were randomly assigned to one of the two interventions using equal (1:1) allocation and random permuted block design. Participants completed an in-person survey, followed by exposure to the 
intervention or attention control websites (minimum 15 min, maximum 45 min). Participants then completed a 1- and 3-month telephone survey. Our primary endpoint was a planning behavior score (range 5-25; higher score signifies more planning behavior). We used an Analysis of Covariance (ANCOVA) to assess difference between the two arms on our primary outcome at 1 month, controlling for baseline planning behavior.

RESULTS: Among 385 participants randomized, the mean age was $71.9(\mathrm{SD}=5.6), 79.5 \%$ were female; $64.4 \%$ identified as White and $24.2 \%$ as African American. Mean planning behavior score overall was $16.51(\mathrm{SD}=4.56)$, with comparable scores across arms at baseline. Between baseline and the 1-month follow-up, planning behavior score decreased on average by -0.37 , with the attention control group having an average decrease of 0.93 and the PYL arm having an average increase of 0.22 . After controlling for baseline behavior score, participants in the PYL arm had a 1-month behavior score that was significantly higher than those in the attention control arm (1.25 points, CI 0.37-2.12, $p=0.0054)$. Secondary longitudinal generalized linear mixed modeling suggested four significant baseline covariates: self-reported health rating, self-efficacy score, support score, and power of attorney. Controlling for these variables, the treatment effect remained significant $(p=0.0166)$

CONCLUSIONS: PlanYourLifespan.org demonstrated efficacy in helping seniors plan for their health and support needs that typically follow health crises. This free, nationally available tool may help individuals understand, plan, and communicate their options for their future support needs.

PLEASE OPEN YOUR MOUTH: WHAT DO WE NEED TO TEACH RESIDENTS TO HELP ADDRESS HEALTH DISPARITY IN ORAL HEALTH? Jennifer Adams $^{2}$; Kathleen Hanley ${ }^{1}$; Colleen Gillespie ${ }^{2}$; Matthew R. Augustine ${ }^{3}$; Jasmine A. Ross ${ }^{2}$; Sondra Zabar ${ }^{2} .{ }^{1}$ NYU, New York, NY; ${ }^{2}$ NYU School of Medicine, New York, NY; ${ }^{3}$ New York University Medical Center, New York, NY. (Control ID \#2468858)

BACKGROUND: The burden of oral disease is most significantly borne by vulnerable populations including minorities, the poor, and immigrants. Seventy percent of older adults have no dental insurance, What do we know about percentage of people seen in PC who have not seen dentist? According to a 2000 Surgeon General's Report, Oral Health is integral to overall health and a core primary care competency. Internal medicine residency programs rarely have curriculum on oral health. We created an oral health station as part of our annual primary care comprehensive OSCE to assess our residents competency in oral systemic health and to raise their awareness of this issue.

METHODS: The case involved a 55 year-old former smoker with a oral lesion simulated using moulage. The patient presented with a painless mass in his mouth for the "past few months" that he would like evaluated. He denied any other chronic illness and reported no past medical history and a normal "physical" including labs, 4 months prior. He did have a 60 pack year tobacco history but had quit smoking 5 year prior and used no drugs or alcohol. The clinical challenge for residents was to evaluate a patient with an oral lesion, perform a comprehensive oral-systemic health exam and discuss potential diagnosis and importance of a referral for biopsy. Twenty-four primary care residents completed this case as part of a 11-station annual OSCE. A highly trained standardized patient (SP) rated the core communication, patient history and physical diagnosis skills of the residents as well as specific items tailored to each clinical case which for this case included: asking about oral health problems including any changes in size, color or texture of the oral lesion, dental history, tobacco use, constitutional symptom review and recognizing link between tobacco use and oral cancer and the need for surveillance lung cancer screening. Physical exam skills evaluated included palpation of the neck for adenopathy, inspection of the oral cavity including the tongue, inside of the lips and cheeks, and the floor and roof of the mouth, and palpation of the gums. Assessment items used a behaviorally anchored scale with not done, partly done and well done as response options. Frequencies are reported for specific items assessing oral health competencies and mean communication, physical diagnosis and patient activating scores. SP's comments about the performance of residents are reported, as were resident's comments about the case, collected through a survey administered after the OSCE.

RESULTS: While overall interviewing skills were strong, results suggest both strengths and weaknesses in residents' comfort with and skills in addressing oral systemic health issues. One hundred percent of residents elicited relevant medical information regarding the lesson, $96 \%$ asked about constitutional symptoms and $100 \%$ recognized need for the patient to see the dentist. However, only $61 \%$ directly asked about the patient's dental history, only $65 \%$ examined the neck for lymphadenopathy and only $52 \%$ palpated the gums for additional lessons. Furthermore, $9 \%$ of the residents did not wear gloves and none of the residents ordered a CT for lung cancer screening for the 60 pack year smoking history. The SP reported that $65 \%$ addressed all their questions and concerns, $50 \%$ discussed a clear differential diagnosis of the lesson, $42 \%$ gave information for follow or contacting the them and only $25 \%$ used teach back when educating the patient. The majority (\%) of the residents reported feeling unprepared for this case and wish they knew more about the differential diagnosis. Faculty observer's supervision requirement rating showed 2 residents required direct supervision, 17 residents required indirect supervision, 1 was ready for unsupervised practice and 3 could supervise others.

CONCLUSIONS: Performance in this case and residents' self-reports suggest the need for greater education and training in oral health core competencies. While most residents addressed the core concern faced by this patient and were able to conduct a basic medical interview, fewer residents were able to fully evaluate the patient and complete an appropriate oral health exam and patient education. None thought to address issues beyond the oral concern such as the need for lung cancer screening.

\section{PNEUMOCOCCAL VACCINATION IN SOLID ORGAN TRANSPLANT RECIP-}

IENTS Omar Sadiq ${ }^{1}$; Mansur Pathan ${ }^{1}$; Tommy J. Parraga ${ }^{2}$; Mohammad Arsalan Siddiqui $^{2}$; Zeinab Saleh ${ }^{1}$; Gagandeep Cheema ${ }^{2}$; George Alangaden ${ }^{2} .{ }^{1}$ Henry Ford Health System, Riverview, MI; ${ }^{2}$ Henry Ford Hospital, Detroit, MI. (Control ID \#2468866)

BACKGROUND: As compared to the healthy population the incidence of pneumococcal disease is significantly increased in solid organ transplant (SOT) recipients. Hence pneumococcal vaccination has been recommended in all transplant recipients prior to transplantation. There is a growing body of evidence describing the safety and efficacy of vaccinations among SOT recipients, yet most vaccines are still underutilized.

METHODS: An electronic medical records (EMR) was used to identify adult (age $\geq 18$ years) patients who were SOT recipients from April 1st, 2013 to October 31st, 2014. Using EMR and Michigan Care Improvement Registry (MCIR) the patients' lifetime pneumococcal vaccination data (PCV-13 and/or PPSV-23) was collected. Other information collected included patient demographics, smoking history, number of admissions, history of lung disease, number of flu vaccinations, PCP visits, Charlson comorbidity index, and institutional PCP. Institutional PCP was defined as having 2 or more clinic visits with the internal medicine or family medicine departments within the institution where SOT was performed. Data was analyzed using univariate analysis with Chisquared analysis, t-test, and multivariate analysis with logistic regression.

RESULTS: A total of 348 SOT patients were included, of which $65 \%$ were male, $35 \%$ female. A majority of patients $(81.3 \%)$ were age $<65$. Overall, $51 \%(177 / 348)$ were vaccinated. Pneumococcal vaccination rates among kidney, liver, and lung + heart were 49, 45 and $80 \%$ respectively. There was no significant difference in race, gender, socioeconomic status and Charlston comorbidity index between patients who were and were not vaccinated. Univariate analysis showed three predictors were significantly associated with vaccination rates: number of Flu vaccines OR $=1.54$ (95\% CI 1.133$1.82, p=<.001)$, number of PCP visits $\mathrm{OR}=1.22(95 \% \mathrm{CI} 1.11-1.36, p=<.001)$, and Institutional $\mathrm{PCP}=\mathrm{OR} 3.56(95 \% \mathrm{CI} 2.11-6.18, p=<.001)$. Similar results were obtained when controlling for age group. The multivariate analysis revealed that the number of Flu 
vaccines and Institutional PCP had higher odds of receiving the vaccine [OR 1.43 (95\% CI 1.23-1.71) and OR 1.94 (95\% CI 1.04-3.65). There was no significant association between smoking history $(p=.391)$, hospital admissions $(p=.972)$, or history of lung disease $(p=.091)$ and vaccination status. Finally, there were significant differences between lung and heart transplant compared to other transplant types $(p=0.00005)$.

CONCLUSIONS: Our data demonstrates that the number of influenza vaccines administered is predictive of pneumococcal vaccination status. For every additional one influenza vaccine, the odds of receiving a vaccine increase by $54 \%$, after adjusting for all other covariates. This could be representative of patient attitudes towards vaccination in general, as well as being a marker of primary care physician adherence to vaccination guidelines. A significant association was found between pneumococcal vaccine administration and being seen by a primary care physician (PCP) at the transplant Institution. For every additional one institutional PCP visit, the odds of receiving a vaccine increase by $21 \%$. Current protocols at our institution leave responsibility for vaccination in the hands of the patient as well as the PCP. This approach has significant limitations, including difficult to access guidelines for transplant patients and the overall high complexity of this patient population. This association may therefore be a result of greater ease in information sharing between institutional physicians. In addition, physicians working out of transplant institutions may have greater knowledge about vaccination guidelines as a higher proportion of their patients are transplant recipients. Heart and lung transplant patients were significantly more likely to receive pneumococcal vaccinations. This might indicate that the greater pulmonary disease burden in these patients spurs higher vaccination rates. In addition, we surmise that these patients have more contact with pulmonary and thoracic specialists who would be expected to be more aware of pneumococcal vaccination guidelines than other physicians.

Baseline characteristics of patients with solid organ transplant

\begin{tabular}{llll}
\hline \hline Variable & Response & Vaccine No & Vaccine Yes \\
Transplant & Heart & $6(30 \%)$ & $14(70 \%)$ \\
Type & Kidney & $89(51.45 \%)$ & $84(48.55 \%)$ \\
& Liver & $74(55.22 \%)$ & $60(44.78 \%)$ \\
Sex & Lung & $2(9.52 \%)$ & $19(90.48 \%)$ \\
& Male & $109(48.23 \%)$ & $117(51.77 \%)$ \\
Race & Female & $62(50.82 \%)$ & $60(49.18 \%)$ \\
& African American & $44(47.31 \%)$ & $49(52.69 \%)$ \\
Ethnicity & Other & $105(49.07 \%)$ & $109(50.93 \%)$ \\
& African American & $43(49.43 \%)$ & $44(50.57 \%)$ \\
Age & Other & $85(52.15 \%)$ & $78(47.85 \%)$ \\
Age Group & Mean \pm Std.Dev & $53.6 \pm 13.19$ & $56.73 \pm 9.38$ \\
& $<65$ & $142(50.18 \%)$ & $141(49.82 \%)$ \\
Charlson & $\geq 65$ & $34(52.31 \%)$ & $37(47.69 \%)$ \\
Comorbidity Index & Mean \pm Std.Dev & $5 \pm 3.1$ & $4.88 \pm 3.13$ \\
& & & \\
\hline
\end{tabular}

Multivariable model predicting vaccine at any time, with kidney as the reference group

\begin{tabular}{lll}
\hline \hline Variables & OR (95 \% CI) & $p$-value \\
Transplant Type: Heart & $1.897(0.657,5.98)$ & 0.248 \\
Transplant Type: Liver & $0.766(0.452,1.29)$ & 0.318 \\
Transplant Type: Lung & $8.151(2.133,53.73)$ & 0.007 \\
Age Group: > 65 & $1.507(0.821,2.78)$ & 0.186 \\
Count of FLU Vaccines & $1.434(1.231,1.71)$ & $<0.001$ \\
Charlson Comorbidity Index & $0.997(0.924,1.08)$ & 0.947 \\
Institutional PCP & $1.94(1.04,3.65)$ & 0.038 \\
Admissions & $0.997(0.867,1.15)$ & 0.972 \\
Smoking History: Yes & $0.811(0.5,1.31)$ & 0.391 \\
\end{tabular}

POLYPHARMACY AND RISK OF FALLS AND FRACTURES FOR PATIENTS WITH HIV INFECTION AND SUBSTANCE DEPENDENCE Theresa W. Kim ${ }^{5}$; Alexander Y. Walley ${ }^{5}$; Alicia S. Ventura ${ }^{3}$; Gabriel Lerner $^{2}$; Gregory Patts ${ }^{4}$; Timothy Heeren ${ }^{1}$; Richard Saitz ${ }^{3}{ }^{1}$ Boston University, Boston, MA; ${ }^{2}$ Boston University School of Medicine, Boston, MA; ${ }^{3}$ Boston University and Boston Medical Center, Boston, MA;
${ }^{4}$ Boston University School of Public Health, Boston, MA; ${ }^{5}$ Boston University School of Medicine and Boston Medical Center, Boston, MA. (Control ID \#2467939)

BACKGROUND: Although patients with HIV infection are at higher risk for polypharmacy, there is limited knowledge about potential harms associated with polypharmacy such as falls and fractures. We determined whether the number and type of medication (e.g. sedating and non-sedating) was associated with falls and fractures among patients with HIV infection.

METHODS: We studied a prospective cohort of 250 adults with HIV infection and pastyear substance dependence or ever injection drug use recruited from two HIV primary care settings. The number of medications overall and number of sedating medications were identified by electronic medical record review. Outcomes were past year i) accident or fall requiring medical attention and ii) fracture. We fit separate logistic regression models for the number of medications in each category (overall, sedating, and non-sedating) and each outcome. Receiver operating characteristic curve analyses determined the optimal discriminating number of medications for identifying outcomes (Youden index). We also examined the results of adjusted models with one of the following covariates in each model: age, sex, race/ethnicity, Charlson Comorbidity Index Score, any recent (past 30day) heavy drinking ( $5+$ drinks in a day or $>14$ /week for men, $4+$ or $>7$ for women), recent illicit opioid use, recent illicit sedative use, and recent cocaine use. Number of sedating medications was also included in the model examining non-sedating medications as the main predictor.

RESULTS: Among 250 participants, $37 \%$ were female, median age was 50; median Charlson comorbidity index was two; $72 \%$ had undetectable HIV viral load; and $80 \%$ were prescribed $\geq 1$ sedating medication. Recent substance use included the following: $51 \%$ had heavy drinking, $23 \%$ had illicit opioid use, $9 \%$ had sedative use, and $30 \%$ had cocaine use. Sixteen percent reported an accident/fall and $8 \%$ a fracture in the past year. The median number (IQR) of prescribed medications was: overall $10(7,14)$; sedating 2 $(1,3)$; and non-sedating $8(5,12)$. The odds of an accident/fall requiring medical attention were significantly higher with each additional medication overall (odds ratio [OR], 1.12, $95 \%$ Confidence Interval $[\mathrm{CI}]=1.05,1.18, p<0.001$ ), each additional sedating medication (OR 1.41, 95\% CI $=1.19,1.67, p<0.001)$ and each additional non-sedating medication (OR 1.10, 95\% CI $=1.02,1.18, p=0.01)$. Optimal cutoffs for predicting accident/falls were: $\geq 8$ overall and $\geq 2$ sedating medications. Number of non-sedating medications was not sigificantly associated with accident/falls when adjusted for number of sedating medications (AOR $1.0595 \% \mathrm{CI}=0.98,1.14, p=0.19$ ). Results of adjusted models for overall and sedating medications were not different in magnitude, direction, or statistical significance. Although not statistically significant, the odds of past-year fracture were higher with each medication overall (AOR $1.0595 \% \mathrm{CI}=0.97,1.13)$ and each sedating medication (AOR 1.13, 95\% CI $=0.92,1.39)$.

CONCLUSIONS: Number of medications was associated with accidents and falls. This association appeared to be driven mostly by sedating medications. Polypharmacy in HIV patients with substance dependence warrants further research to determine whether reducing sedating medications will lower risk of accident/falls and whether these associations also hold for fractures.

POPULATION DEMOGRAPHICS AND TRENDS OF PATIENTS DIAGNOSED WITH PULMONARY EMBOLISM: A STUDY INVOLVING 176,320 PATIENTS Rakshita Chandrashekar. Western Michigan school of medicine, Kalamazoo, MI. (Control ID \#2469665)

BACKGROUND: Pulmonary embolism (PE) is the sudden blockage of a major blood vessel (artery) in the lung, usually by a blood clot. In most cases, the clots are small and are not deadly, but they can damage the lung. Large PEs' can cause serious complications like 
shock, pulmonary hypertension, heart failure and/or death. This abstract is to assess the demographics and trends of PE using the latest data released recently in National Inpatient Sample (NIS) database for the year 2013.

METHODS: Using the data on NIS database (contains $20 \%$ of US hospitalizations), we were able to assess the following parameters on PE for 2013-population demographics, number of discharges, insurance payers, location and teaching status of hospitals, hospital regions, and mean length of stays (LOS) in hospitals.

RESULTS: There were a total of 176,320 discharges with overall in-hospital mortality of $3.09 \%$ with $39.22 \%$ of patients being in the $65-84$ year age group. Mortality rate in $85+$ age group was $5.67 \%$. Surprisingly, women constituted $52.39 \%(92,375)$ of cases while men constituted $47.60 \%$ of cases. Medicare paid for $51 \%$ and private insurance paid for $31 \%$ of the discharges. $5.83 \%$ of patients remained uninsured. Thirteen percent cases were treated in rural areas, 40 and $47 \%$ were treated in urban non-teaching and urban teaching hospitals respectively. $37.7 \%$ cases were from the South region and only $18.60 \%$ cases from West. $58.35 \%$ of the cases were treated in hospitals with large bedsize. Mean LOS in hospital was 4.9 days and mean charges were 39,371\$. Upto $40 \%$ of the cases were discharged to nursing homes, rehabilitation facilities or home health care. Maximum number of discharges has been in the year 2012 (179,615 cases). Mean length of stay has been 4.9 days in 2013 but the mean charges have been at an all time high at 39,371\$. In hospital deaths was at $2.95 \%$ in 2012 and $3.09 \%$ in 2013.

CONCLUSIONS: From the above statistics, it is known that close to $35.5 \%$ of patients develop major complications leading to increased mortality. Greater than $46 \%$ of PE cases with major complications are handled by urban teaching hospitals. Greater than $37 \%$ cases are from the South due to unknown factors and LOS in hospitals is greater in complicated cases. It is unknown why there is increased predominance of PE in women when compared to men though the complication rates are similar in both groups. Further research is necessary in this aspect. It has to be borne in mind that NIS database includes data from only $20 \%$ of all hospital admissions in the United States.

POPULATION HEALTH AND ENDING HOMELESSNESS: CAN HOSPITALS RATIONALLY ALLOCATE SOCIAL BENEFITS? Stefan Kertesz ${ }^{1,2}$; Young-il $\mathrm{Kim}^{1,2}{ }^{2}$; Aerin DeRussy ${ }^{3}$; Erika L. Austin ${ }^{2}$; David Pollio ${ }^{2}$; Carol VanDeusen Lukas ${ }^{3}$; Sally Holmes ${ }^{3}$. ${ }^{1}$ Birmingham VA Medical Ctr, Birmingham, AL; ${ }^{2}$ University of Alabama at Birmingham, Birmingham, AL; ${ }^{3}$ Boston VA Medical Center, Boston, MA. (Control ID \#2466097)

BACKGROUND: Medical institutions increasingly are called upon to address social determinants of health, including homelessness. Homelessness, which affects 600,000 Americans, is associated with excess hospital utilization, among other problems. Permanent housing interventions such as Housing First (HF) have been shown to reduce hospital use. However, little research has be done on whether medical institutions can rationally allocate social benefits, such as housing, according to need. In 2009, the Department of Veterans Affairs announced a plan to house $>65,000$ homeless veterans by combining rental subsidies and case management ("HUD-VASH"). In late 2012, VA directed that veterans with greatest vulnerability should be prioritized for this program. Vulnerability criteria included heavy service use and chronic homelessness We examined whether the 2012 requirement led to increased selectivity of HUD-VASH clients based on vulnerabilities cited in the homeless policy literature.

METHODS: We compiled data from veterans seeking homeless assistance at 8 VA Medical Centers from 2011-2014 $(n=15,006)$. We identified 2 markers for excess hospital utilization (top $10 \%$ for emergency department visits and top $10 \%$ for hospital admissions). We also flagged vulnerabilities in these domains: Homelessness (Chronically Homeless, Custody of Children, Literal Homelessness e.g. shelter, outdoors); Clinical status (\# of chronic medical disorders, \# of chronic mental disorders, Alcohol abuse, Drug abuse); Service History (Iraq/Afghan Veteran, Year of Service Entry); and Age, Race, Gender. We compared the percentage of HUD-VASH entrants $(n=5608,37 \%)$ to nonHUD-VASH entrants ( $n=9393,63 \%$ ) for all vulnerabilities. In logistic regressions, HUDVASH Entry (yes/no) was the dependent variable and vulnerability risks were treated as predictors. To assess if selectivity changed after VA's directive we iterated models for the Pre-Housing First (2011-12) and Post-Housing First (2013-14) era.

RESULTS: Markedly increased selectivity was found for Chronic Homelessness (72 vs $40 \%$ of HUD-VASH Entrants vs non-Entrants in 2011-12, 81 vs $37 \%$ in 2013-14) (Table, below). Female gender and custody of children were associated with HUD-VASH entry in both eras. Other vulnerabilities, such as number of mental or medical disorders, had only modest or no association with entry into the HUD-VASH program. Contrary to expectation, frequent hospital admission was inversely associated with HUD-VASH entry (OR 0.6 and 0.7 for Pre-HF and Post-HF, respectively) and ED use was not associated with HUD-VASH entry.

CONCLUSIONS: VA policy changes shifted allocation of housing resources toward chronically homeless persons, especially women and parents. However, although HF reduces excess hospital utilization, a history of frequent hospital admissions or substance abuse was associated with reduced likelihood of access to the program. Heavy healthcare users can present serious challenges for clinical staff. As healthcare delivery systems move toward population health objectives, these data suggest that additional supports are needed to allocate clinical and social resources to especially vulnerable populations.

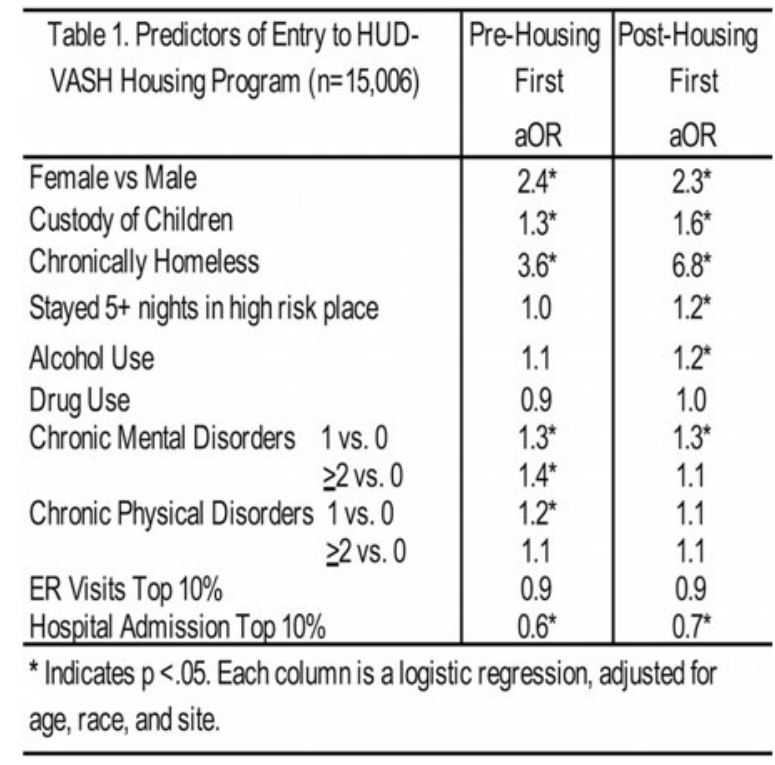

POST-PCI ANTITHROMBOTIC MANAGEMENT AND OUTCOMES IN PATIENTS REQUIRING ORAL ANTICOAGULATION Mohamed Rezik; Michelle Mohyi; Owais Nadeem; Fawad Virk; Akshay Khandelwal; Michael Hudson. Henry Ford Health System, Detroit, MI. (Control ID \#2469691)

BACKGROUND: Dual antiplatelet therapy with aspirin and a $\mathrm{P}_{2} \mathrm{Y}_{12}$ inhibitor is a mainstay following percutaneous coronary intervention (PCI) in the prevention of recurrent disease and other adverse cardiac events. Nearly $20 \%$ of patients who receive dual antiplatelet therapy after PCI also require oral anticoagulation for reasons which include atrial arrhythmia, cardiac thrombus, mechanical valve prosthesis or venous thromboembolism, among others. The combination of the three medications results in an increased bleeding risk to the patient which in turn leads to significant morbidity and mortality. Recent studies have suggested that avoidance of the "triple therapy" regimen and the use of dual therapy with $\mathrm{P}_{2} \mathrm{Y}_{12}$ inhibitor and oral anticoagulation only may improve patient outcomes by significantly reducing bleeding risk while also adequately preventing adverse 
cardiac events. Our study aims to determine prescribing practices of antithrombotic therapy in patients requiring oral anticoagulation after PCI as well as differences in outcomes between the various regimens

METHODS: Retrospective chart review was performed on all patients who received PCI and coronary stenting at a large US tertiary care center and its satellite campuses during the years 2013 and 2014. Patients with an indication for oral anticoagulation at the time of PCI were included in the study and further chart review was performed. Patients with atrial fibrillation with a CHADS ${ }^{2}$-VASc score $<2$ were excluded from the study. Patients were divided into three therapy groups - triple therapy: aspirin $+\mathrm{P}^{2} \mathrm{Y}_{12}$ inhibitor + oral anticoagulant; dual therapy (DT): $\mathrm{P}_{2} \mathrm{Y}_{12}$ inhibitor + oral anticoagulant; and dual antiplatelet therapy (DAPT): aspirin $+\mathrm{P}_{2} \mathrm{Y}_{12}$ inhibitor. Primary outcome was the rate of major adverse cardiovascular events (MACE) and bleeding between the three groups. MACE was defined as death, myocardial infarction (MI), stroke (CVA) and unplanned revascularization after PCI. Bleeding events were classified using the BARC classification system and were included if they met criteria for a score $\geq 2$.

RESULTS: A total of 1891 patient charts were reviewed. Of these patients, 209 (11\%) had an indication for oral anticoagulation at the time of PCI. Atrial fibrillation was the predominant indication $(n=165)$, followed by venous thromboembolism $(n=25)$ and cardiac thrombus $(n=13)$. Other indications included peripheral arterial disease $(n=3)$, mechanical valve prosthesis $(n=1)$, factor $\mathrm{V}$ leiden $(n=1)$ and antiphospholipid syndrome $(n=1)$. Triple therapy, dual therapy and dual antiplatelet therapy was prescribed to 71,9 and $20 \%$ of patients following PCI, respectively. Sixty-four of the 209 (30.1\%) patients included had a major adverse cardiac event with 79 total events. MACE occurred in $28.4,15.8$ and $45.2 \%$ of patients receiving triple therapy, dual therapy, and dual antiplatelet therapy, respectively $\left(\chi^{2}=6.54 ; p=0.038\right)$. The number of total major adverse cardiac events between the three groups was 34.5, 21.1 and $57.1 \%\left(\chi^{2}=9.65, p=0.008\right)$, respectively. Bleeding events occurred in $22.3,21.1$ and $35.7 \%\left(\chi^{2}=3.31, p=0.19\right)$ of the patients, respectively.

CONCLUSIONS: In post-PCI patients requiring oral anticoagulation, there was a statistically significant difference in the rate of major adverse cardiac events between patients receiving triple therapy, dual therapy and dual antiplatelet therapy. The lowest rate of events occurred in patients receiving dual therapy $\left(\mathrm{P}_{2} \mathrm{Y}_{12}\right.$ inhibitor + oral anticoagulation), followed by triple therapy (aspirin $+\mathrm{P}_{2} \mathrm{Y}_{12}$ inhibitor + oral anticoagulation) and then dual antiplatelet therapy (aspirin $+\mathrm{P} 2 \mathrm{Y}_{12}$ inhibitor). While there was no statistically significant difference between bleeding rates between the three treatment groups, dual therapy yielded the lowest bleeding rate between the three groups

Post-PCI Antithrombotic Regimen Outcomes

\begin{tabular}{llll}
\hline \hline & $\begin{array}{l}\text { Triple Therapy } \\
(n=148)\end{array}$ & $\begin{array}{l}\text { Dual Therapy } \\
(n=19)\end{array}$ & $\begin{array}{l}\text { Dual Antiplatelet } \\
\text { Therapy }(n=42)\end{array}$ \\
MI & $8(5.4 \%)$ & $1(5.3 \%)$ & $7(16.7 \%)$ \\
Revascularization & $10(6.8 \%)$ & $1(5.3 \%)$ & $6(14.3 \%)$ \\
CVA & $9(6.1 \%)$ & 0 & $2(4.8 \%)$ \\
Death & $24(16.2 \%)$ & $2(10.5 \%)$ & $9(21.4 \%)$ \\
Total MACE & $51(34.5 \%)$ & $4(21.1 \%)$ & $24(57.1 \%)$ \\
Bleeds & $33(22.3 \%)$ & $4(21.1 \%)$ & $15(35.7 \%)$ \\
\hline
\end{tabular}

MACE - major adverse cardiac events (composite of MI, unplanned revascularization, CVA, death). MI - myocardial infarction. CVA - cerebrovascular accident. Bleeding events - BARC class 2 and above.

POTENTIAL IMPACT OF A BEDSIDE PROCEDURE SERVICE ON TRAINING PROCEDURALLY COMPETENT HOSPITALISTS IN A COMMUNITY-BASED RESIDENCY PROGRAM Anthony S. Montuno ${ }^{1}$; Bijou R. Hunt ${ }^{2}$; May M. Lee ${ }^{1}{ }^{1}$ Mount Sinai Hospital, Chicago, IL; ${ }^{2}$ Sinai Health System, Chicago, IL. (Control ID \#2470245)
BACKGROUND: Hospital medicine has become part of the mainstream delivery of health care in the United States since the mid-1990's, approximately 30,000 hospitalists in 2010. In our residency program, over $60 \%$ of our graduates become hospitalists. In 2006, the Society of Hospital Medicine developed a document of core competencies. These competencies include performing procedures such as lumbar puncture, paracentesis, thoracentesis and vascular access. Despite these core competencies for hospitalists, procedures performed by hospitalists are still in decline. A reason may be traced back to residency training. Research has demonstrated the positive impact of a specialized medical procedure service (MPS) in the training of procedural skills to Internal Medicine residents. Little is known whether or not exposure to an MPS impacts the procedural certification rate in trainees. We sought to determine whether or not exposure to an MPS would impact both the number of procedures performed by residents and the rate of resultant certifications.

METHODS: This retrospective review was performed at Mount Sinai Hospital Medical Center in Chicago (MSH). The medical procedure service (MPS) was established in January of 2014 with 3 pulmonary/critical care faculty along with one procedural hospitalist. The study group included all the internal medicine residents at MSH from July 2010 to August 2015. Procedural data were entered by the residents into myevaluations.com, a medical education training management service provider. Six types of procedures were included: paracentesis, thoracentesis, lumbar puncture, jugular vein, femoral vein, and subclavian vein. Any procedure performed prior to the launch of MPS was labeled "unexposed" and any procedure performed after was labeled "exposed." The procedure data were analyzed comparing months where residents were exposed to the intervention to months where residents were unexposed to the intervention across all 3 post-graduate years. We used the Wilcoxon rank-sum test to test for statistically significant differences between the number of procedures per month in the exposed and unexposed periods. These data were analyzed using SAS 9.3. The certification data were analyzed overall (all six certification types included) and by specific certification type. We compared two proportions: the number of certifications over the number of 6-month unexposed periods and the number of certifications over the number of 6-month exposed periods. We used the two-sample test of proportions to test the hypothesis that there was no statistically significant difference in the proportion of certifications between the exposed and unexposed periods. These data were analyzed using Stata/SE 14.1.

RESULTS: To determine whether exposure to an MPS would increase the number of procedures performed by residents, we calculated the average number of procedures performed per month among exposed versus unexposed groups. We found a statistically significant difference between the groups, with the unexposed group performing 4.3 procedures per month compared to the exposed group performing 6.7 procedures per month $(p=0.0010)$ (Figure 1). In term in the number of certifications obtained by residents, we calculated the 6-month certification rates for the exposed and unexposed groups. We found differences in several categories — overall, paracentesis, femoral central lines, and jugular central lines (Figure 2).

CONCLUSIONS: To our knowledge, this is the first study to demonstrate that exposure to an MPS affects both the number of procedures performed by residents and the rate of resultant certifications. This study demonstrated that resident exposure to an MPS significantly increased the number of procedures performed. These findings suggest that having an accessible attending physician may encourage residents to perform more procedures because help is readily available. This study also showed that overall certification rates were significantly different between the exposed and unexposed groups in both PGY1 and PGY2 classes. We also found significant differences in the certification rates for specific procedures - paracentesis, femoral lines, and jugular central lines. Being certified earlier in training suggests more opportunity to both perform additional procedures and also potentially gain experience in becoming a supervisor for these procedures. Moreover, gaining certification during residency allows for additional clinical privileges when the residents acquire postgraduate employment 


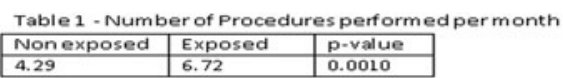

\begin{tabular}{|c|c|c|c|}
\hline \multirow{2}{*}{\multicolumn{4}{|c|}{\begin{tabular}{|l|l|l|} 
Unexnnserd & Fxpnoser & n-value \\
\end{tabular}}} \\
\hline & & & \\
\hline AllPG years & 0.036 & 0.069 & 0.0025 \\
\hline PGY1 & 0 & 0.037 & 0.0000 \\
\hline PGY2 & 0.052 & 0.096 & 0.0396 \\
\hline PGY3 & 0.097 & 0.076 & 0.4576 \\
\hline \multicolumn{4}{|l|}{ Paracentesis } \\
\hline PGY1 & 0 & 0.083 & 0.0056 \\
\hline PGY2 & 0.048 & 0.057 & 0.8373 \\
\hline PGY3 & 0.056 & 0 & 0.1515 \\
\hline \multicolumn{4}{|l|}{ Femoral } \\
\hline PGV1 & 0 & 0.028 & 0.1124 \\
\hline POY2 & 0.045 & 0.188 & 0.0224 \\
\hline PGY3 & 0.175 & 0.143 & 0.7231 \\
\hline \multicolumn{4}{|l|}{ Jugular } \\
\hline PGV1 & 0 & 0.111 & 0.0013 \\
\hline PGV2 & 0.159 & 0.391 & 0.0214 \\
\hline PGY3 & 0.3 & 0.529 & 0.1200 \\
\hline \multicolumn{4}{|c|}{ Thoracentesis } \\
\hline \multirow{2}{*}{\multicolumn{4}{|c|}{$\begin{array}{l}\text { PGY1 } \\
\text { PGY2 }\end{array}$}} \\
\hline & & & \\
\hline \multicolumn{4}{|l|}{ PGY3 } \\
\hline \multicolumn{4}{|l|}{ Subclavian } \\
\hline \multicolumn{4}{|l|}{ PGV1 } \\
\hline PGV2 & 0.015 & 0.029 & 0.6532 \\
\hline PGY3 & 0.049 & 0.029 & 0.6700 \\
\hline \multicolumn{4}{|c|}{ Lumbar puncture } \\
\hline \multicolumn{4}{|l|}{ PGY1 } \\
\hline PGV2 & 0.046 & 0.028 & 0.6502 \\
\hline PGV3 & 0.053 & 0 & 0.1813 \\
\hline
\end{tabular}

PRACTICES AND PERSPECTIVES SURROUNDING PARENTAL/GUARDIAN HEALTH INTERVENTIONS IN PEDIATRIC PRIMARY CARE SETTINGS

Maya S. Venkataramani; Tina :. Cheng; Barry S. Solomon; Craig E. Pollack. Johns Hopkins University School of Medicine, Baltimore, MD. (Control ID \#2465974)

BACKGROUND: Pediatric primary care providers have frequent contact with adult parents and guardians during routine well child visits. The American Academy of Pediatrics (AAP) recommends addressing a number of adult caregiver health issues that affect child health during these visits, including maternal depression, tobacco use and family planning. To date, there has been no comprehensive assessment of pediatric providers' practices and perspectives surrounding adult caregiver health promotion. METHODS: We are conducting a national, mail-based survey of 1000 pediatric primary care providers (500 general pediatric, 250 family medicine and 250 medicine-pediatric (med-peds) physicians). Participants were selected randomly from the American Medical Association database; they were considered eligible if they performed well child visits in the outpatient setting. The 32-item survey includes questions about demographic and practice characteristics, the frequency and type of physician and practice involvement in six areas of adult health promotion (maternal depression, tobacco use, intimate partner violence (IPV), health insurance, family planning and pertussis (Tdap) immunization), billing practices, and barriers and facilitators to adult health promotion. Logistic regression analyses were performed to identify correlates of physician engagement in each area of adult health promotion.

RESULTS: To date, we have received 230 responses (144 pediatricians, 37 family medicine providers, 49 med-peds providers) from the 827 eligible participants (response rate $28 \%$, data collection on-going). Fifty-three percent saw patients in private practice, $21 \%$ in a hospital based clinic and $8 \%$ in a community health center. Forty-eight percent were part of an accountable care organization. A large majority of providers reported addressing tobacco use ( $92 \%$ ), maternal depression ( $82 \%$ ), and Tdap immunization status $(75 \%)$ in at least a quarter of infant or well child visits. Less frequently, providers reported addressing family planning (47\%), IPV (44\%), and parental insurance status (44\%). In adjusted analyses, pediatric providers who were co-located with adult medical services were more likely to address IPV (Odds Ratio [OR] 2.4, $95 \%$ Confidence Interval [CI] 1.2-4.7) and family medicine practitioners were more likely to address family planning compared to pediatricians (OR 5.0, $95 \%$ CI 1.9-13.3). Lack of time was the most frequently reported practice-related barrier to engaging in adult health promotion (endorsed by $85 \%$ of respondents), and approximately half identified lack of referral resources, complex referral mechanisms and inadequate reimbursements as barriers (56, 51 , and $54 \%$, respectively). In contrast, concerns over personal liability and fear that the caregiver would not accept an intervention were endorsed by a relative minority of respondents for each issue. Most commonly endorsed facilitators to engagement in specific issues involved co-location of services including behavioral health specialists for maternal depression, case managers/social workers for health insurance status, and adult health providers for family planning and Tdap immunization.

CONCLUSIONS: In the setting of health care reform and the move towards greater health system integration with a renewed emphasis on population health, this study underscores the potential to leverage pediatric visits to support adult health promotion. Pediatric providers have frequent contact with caregivers and often engage in a variety of adult health promotion activities. Designing interventions that are less time intensive, in addition to taking advantage of co-located services and easing referral pathways, may help increase adult health promotion during pediatric visits.

PRACTICING BETTER POPULATION HEALTH BY USING BETTER CRITERIA TO IDENTIFY PERSISTING, HIGH COST, HIGH NEED MEDICARE BENEFICIARIES Bruce Kinosian ${ }^{1}$; Peter Boling ${ }^{2}$; George Taler ${ }^{3}$; Joanna Kubisiak ${ }^{4}$; Anna Kudryashova ${ }^{4}$; Daniel M. Gilden ${ }^{4}$. ${ }^{1}$ University of Pennsylvania, Philadelphia, PA; ${ }^{2}$ Virginia Commonwealth University, Richmond, VA; ${ }^{3}$ Medstar Washington Hospital Center, Washington, DC; ${ }^{4}$ JEN Associates, Boston, MA. (Control ID \#2469389)

BACKGROUND: To determine the ability of Independence at Home qualifying criteria (IAH-Q) to identify persisting high cost, high need Medicare beneficiaries. Recognition that a large fraction of costly health care episodes occur among a small portion of the Medicare population- "the $5 \%$ of beneficiaries responsible for $50 \%$ of Medicare costs"- has led to programs that focus on such beneficiaries, in an effort to "reduce costs where they are greatest", a core focus of a population health approach. IAH criteria for FFS beneficiaries, which can be determined from claims, are a) hospitalization in prior 12 months, b) post-acute care- $\mathrm{SNF}$, home health, or inpatient rehab-in prior 12 months, c) 2 or more chronic conditions (from the Chronic Condition Warehouse), and 2 or more ADL dependencies (from OASIS or MDS).

METHODS: Population Studied: Fee-for-Service subpopulation of Medicare $5 \%$ beneficiary sample 2011-2013. Cross-sectional adjudication of IAH qualification and prospective classification of costs and outcomes (hospitalization, death, 30-day readmit, and incident Long Term Institutionalization [LTI]). Groups studied were: a) IAH-Q (Medicare beneficiaries similar to those in the IAH demonstration) and b) Dual-Eligible (Medicare and Medicaid qualified). We modified the IAH criteria, using a JEN Frailty Index score of $6+$ as equivalent to $2+\mathrm{ADL}$ dependencies. (JFI ROC area .81 for $2+\mathrm{ADL}$ dependencies). For Dual Status, we used state buy-in, from the enrollment file (includes full and partial duals) on last day of eligibility in 2012. Eligibility criteria (either Medicaid status for Duals, or hospital or PAC use for IAH) were ascertained through 2011 to classify the population at the start of 2012, then incident cases were classified for those who met criteria during 2012. We measured concentration of events or cost as the ratio of the stratum's share of all such FFS events or costs to the stratum's share of all FFS beneficiaries.

RESULTS: The IAH-Q population has a greater concentration of cost (cost : population ratio of 4.4:1) than Duals (1.4:1), representing greater FFS spending (\$93 M vs $\$ 82 \mathrm{M})$ than Dual status, despite a more highly targeted population (6.6 vs. $18 \%$ ). Removing Dual IAH-Q, the non-IAH-Q duals have no increased concentration of cost (1.04:1), hospitalization, death, and readmits, and a modest increased concentration of LTI (1.18:1) (see Table).Among IAH-Q survivors into 
2013 (74 \% of all 2012 IAH-Q), they still represented $15 \%$ of all FFS spending and $22.4 \%$ of all incident LTI, but $4.7 \%$ of the FFS population in 2013 (cost:population ratio of 3.1:1, LTI:population ratio of 4.8:1).

CONCLUSIONS: IAH-Q criteria are superior to Dual Status to identify high concentrations of costs and adverse events among fee for service Medicare Beneficiaries, prospec- tively identifying $6.6 \%$ of the FFS population that account for $30 \%$ of all FFS costs and $39 \%$ of all incident LTI in the subsequent year. IAH-Q criteria can be used to narrowly target a small portion of the FFS Medicare population at substantial risk for experiencing high costs and adverse events over the subsequent year for effective interventions, such as home based primary care.

Event Rates as a \% of the FFS Population

\begin{tabular}{|c|c|c|c|c|c|c|c|c|}
\hline & $\begin{array}{l}\text { Population } \\
\text { Count }\end{array}$ & $\begin{array}{l}\text { \% FFS } \\
\text { Population }\end{array}$ & Mortality & $\begin{array}{l}\text { Acute Care } \\
\text { Hospitalization }\end{array}$ & 30-d Readmits & Incident LTI & AB spending $\mathrm{CY} 2012$ & \% AB spending \\
\hline Total FFS Population & $33,519,620$ & & $1,475,920$ & $6,230,620$ & 929,860 & 681,200 & $\$ 318 B$ & \\
\hline IAH-Q 6+ (all) & $2,220,060$ & $6.6 \%$ & $23.8 \%$ & $25 \%$ & $46 \%$ & $39.4 \%$ & $\$ 93.5 \mathrm{~B}$ & $29.4 \%$ \\
\hline Duals (all) determined at Jan 2012 & $6,228,240$ & $18.6 \%$ & $24.9 \%$ & $24 \%$ & $29 \%$ & $32.6 \%$ & $\$ 82 B$ & $25.8 \%$ \\
\hline IAH-Q Duals & 549,360 & $1.6 \%$ & $5.3 \%$ & $6 \%$ & $13 \%$ & $13 \%$ & $\$ 26 B$ & $8.2 \%$ \\
\hline Non-IAH-Q duals & $5,678,880$ & $16.9 \%$ & $19.7 \%$ & $17 \%$ & $16 \%$ & $20 \%$ & $\$ 56 B$ & $17.6 \%$ \\
\hline
\end{tabular}

PRE-POST RCT OF FOUR TRAINING INTERVENTIONS TO IMPROVE SIGN-OUT QUALITY FOR INTERNS Soo Hoon Lee ${ }^{3,1}$; Christopher P. Terndrup $^{2}$; Phillip Phan ${ }^{1,2}$; Sandra E. Zaeh ${ }^{2}$; Kwame Atsina ${ }^{2}$; Nicole Minkove ${ }^{2}$; Diana Kwong ${ }^{2}$; Idoreyin Montague ${ }^{2}$; Alexander Billioux ${ }^{2}$; Bennett Clark ${ }^{2}$; Andrew Hughes ${ }^{2}$; Souvik Chatterjee ${ }^{2}$; Sanjay Desai ${ }^{1,1}$. ${ }^{1}$ Johns Hopkins University, Baltimore, MD; ${ }^{2}$ Johns Hopkins Medicine, Baltimore, MD; ${ }^{3}$ Old Dominion University, Norfolk, VA. (Control ID \#2435509)

BACKGROUND: Resident duty-hour restrictions have led to more instances of sign-outs, which can increase the potential for preventable harm. Medical schools do not usually teach sign-outs formally, which results in a sign-out process that is largely unstructured and highly variable. This study seeks to evaluate the best intervention that enhances sign-out quality in information exchange, task accountability, and personal responsibility.

METHODS: Four general medical teams at a large Internal Medicine training program were randomly assigned to one of four 1-h interventions. Interns first watched a 3-min video of a standard sign-out. Group 1 was the control group in which interns listened to a 1-h didactic lecture on sign-outs. Group 2 interns listened to a 15 -min lecture with a video showing low and high levels of task specificity, and were given a policy mandate to be accountable for providing specific to-do tasks and rationales during sign-outs. Group 3 interns were taught the IPASS method to exchange information with a video showing low- and high-quality communication practices. They role-played three scenarios using IPASS for a sick, new, and stable patient. Group 4 interns were taught how to conduct a Rapid Improvement Event. Trainees discussed problems encountered in their sign-outs, and identified the provision of contingency plans as an area for improvement. Eight trained observers monitored 10 nights of sign-outs in each group during the pre- and postintervention periods using a standardized sign-out checklist developed from a systematic literature review and JCAHO's National Patient Safety Goal 2E.

RESULTS: One thousand one hundred eighty-three patient sign-outs were observed comprising 563 pre- and 620 post-intervention observations with acceptable kappa scores (coefficient of agreement) of 0.646 and 0.648 respectively. Pre-post intervention differences show that improvement in the number of information elements exchanged during sign-out was most significant with the IPASS group. The policy mandate group reported the highest improvement in task accountability to receivers. The RIE group reported the highest improvement in personal responsibility in which both senders and receivers ensured contingency plans were discussed.

CONCLUSIONS: Each intervention enhanced different dimensions of sign-out quality. There is no single best method. Instead, a combination of interventions will likely yield optimal results. Program directors should incorporate all aspects of information exchange, task accountability, and personal responsibility during sign-out training.

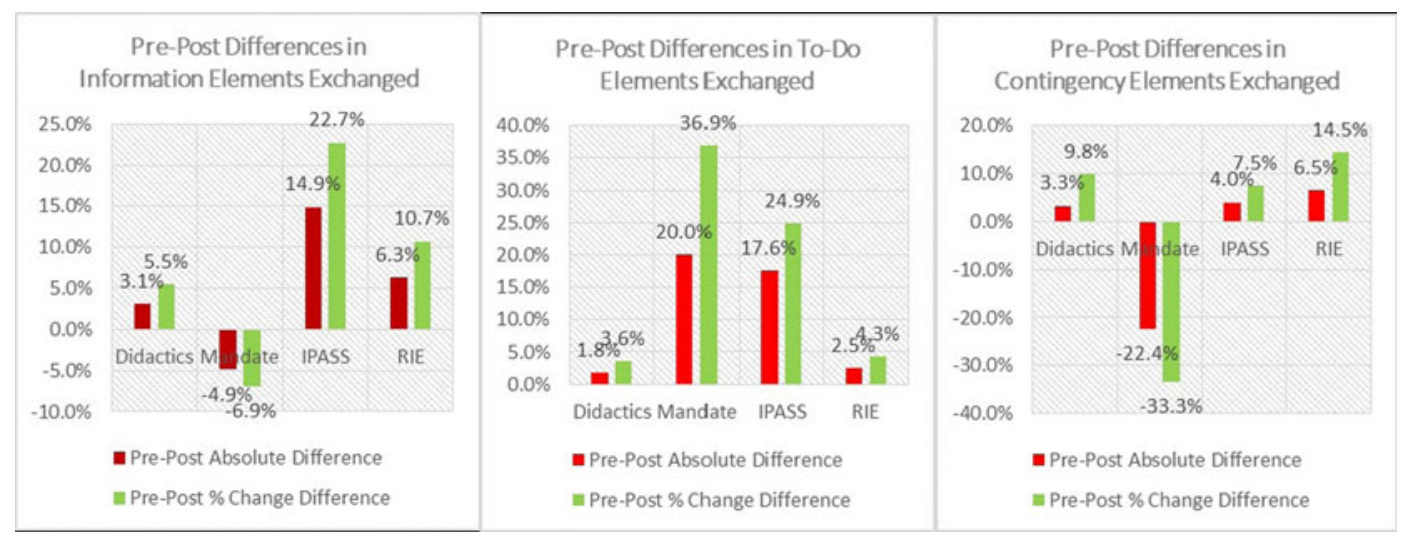

RCT Intervention to Improve Evening Signout 
PRECONCEPTIONAL AWARENESS OF TOBACCO AND ETHANOL USE AMONG DISADVANTAGED WOMEN IN THE DOMINICAN REPUBLIC BASED ON EDUCATIONAL LEVEL AND SOURCE OF MEDICAL INFORMATION Weston J. Bertot ${ }^{1}$; Erin M. Walker ${ }^{1}$; Cristina Rabaza ${ }^{1}$; Erich T. Wyckoff ${ }^{1}$; Luis Esmurdoc $^{2}$; Robert S. Egerman ${ }^{1,1}$. ${ }^{1}$ University of Florida, Gainesville, FL; ${ }^{2}$ Universidad Católica Nordestana (UCNE), San Francisco de Macorís, Dominican Republic. (Control ID \#2464114)

BACKGROUND: Medical schools are increasingly promoting initiatives in global health and firsthand experience through medical mission trips. Medical missions have the potential for benefitting patients if performed responsibly and in a culturally relevant manner. As a means to assess public health needs and to determine appropriate future emphasis, our purpose was to assess baseline knowledge of preconceptional hazards of alcohol and tobacco use among underserved women of child bearing age in the Dominican Republic.

METHODS: We recruited patients seeking care at the Universidad Catolica Nordestena (UCNE) /University of Florida (UF) medical mission in San Francisco de Macoris in the Dominican Republic. Female subjects between the ages of 18 and 50 gave verbal consent to participate in the study. The patients' baseline preconceptional knowledge of smoking and ethanol use was assessed. Women were stratified based on educational level (less than 5th grade education, 5th to 8 th grade education, high school or university education) as well as the primary source of their medical information (family and friends, newspapers and books, TV and radio, or health care professionals). Comparisons between groups were made using Chi square test (with significance defined as $p<0.05)$.

RESULTS: Seventy three women participated with educational levels: $<5$ th grade $(n=13)$, 5 th to 8 th grade $(n=24)$, high school $(n=22)$ and university experience $(n=14)$. There we no significant differences between the educational level groups and the primary resources for obtaining medical information $(p=0.15)$. Most women received medical information through a healthcare professional (53/73 or $72.6 \%$ ). One woman in the lowest educational level did not cite a source and another in the 5 th to 8 th grade educational bracket used the internet as her source. Awareness of the hazards of periconceptional tobacco and ethanol use was present among 97.3 and $98.6 \%$, respectively, of the entire group

CONCLUSIONS: Regardless educational level, underserved Dominican women are knowledgeable regarding the hazards of tobacco and ethanol use during pregnancy. Although not studied, local public health initiatives are seemingly effective in this regard. Alternative aspects of preventative care should be considered for emphasis in this population including periconceptional vitamin supplementation to reduce infant birth defects and awareness of preeclampsia symptoms to reduce maternal mortality.
PREDICTING 30-DAY PNEUMONIA READMISSIONS USING ELECTRONIC HEALTH RECORD DATA FROM THE FIRST DAY OF HOSPITALIZATION: MODEL DEVELOPMENT AND COMPARISON Anil N. Makam ${ }^{1}$; Oanh K. Nguyen ${ }^{1}$; Song Zhang ${ }^{2}$; Bin Xie ${ }^{4}$; Mark A. Weinreich ${ }^{1}$; Ruben Amarasingham ${ }^{4}$; Eric Mortensen ${ }^{3}$; Ethan Halm ${ }^{1} .{ }^{1}$ UT Southwestern Medical Center, Dallas, TX; ${ }^{2}$ University of Texas Southwestern Medical Center, Dallas, TX; ${ }^{3}$ VANTHCS, Dallas, TX; ${ }^{4}$ Parkland Center for Clinical Innovation, Dallas, TX. (Control ID \#2464149)

BACKGROUND: Identifying patients hospitalized for pneumonia at highest risk for readmission can help target resource-intensive interventions to those most likely to benefit. However, current models have only modest predictive ability and have not incorporated detailed information on clinical severity, such as the pneumonia severity index (PSI), which may improve prediction. This study sought to: 1) develop a pneumonia-specific risk prediction model incorporating electronic health record (EHR) data from the first day of hospitalization and 2) compare our 'pneumonia first-day' model performance to other models.

METHODS: This was an observational study using clinically granular EHR data from 6 hospitals in the Dallas-Fort Worth metroplex, including safety net, community, and nonteaching sites. We included hospital discharges from all medicine services among adults ( $\geq 18$ years) between November 1, 2009 and October 30, 2010. We excluded deaths, transfers to other acute care facilities, and those who left against medical advice. For patients who had multiple hospitalizations, we included the first one. The primary outcome was non-elective allcause 30-day readmission to any of 75 acute care hospitals in north Texas ascertained using an all-payer regional hospitalization database. Significant univariate candidate variables were entered into a multivariate logistic regression model using stepwise backward selection with a significance threshold of $\mathrm{p} \leq 0.05$. Internal validation was performed using 5 -fold crossvalidation. For comparison, we used our group's previously developed first-day multicondition model, the CMS pneumonia administrative model, the PSI and the CURB-65 score. RESULTS: Of 1463 pneumonia admissions, $14 \%$ were readmitted. The final model included 7 variables: single (OR 0.61, $95 \%$ CI, 0.40-0.95); Medicare (OR 1.72, $95 \%$ CI, 1.23-2.41); Medicaid (OR 2.16, 95 \% CI, 1.14 4.11); median income per ZIP code $<\$ 30,000$ (OR 1.85, $95 \%$ CI, 1.14-3.02); hospitalizations in the prior year (OR 1.18 per admission, $95 \%$ CI, 1.03 1.36), platelets $>350 \times 10^{3} / \mu \mathrm{L}$ (OR 2.18, $\left.95 \% \mathrm{CI}, 1.46-3.24\right)$, and PSI (OR 1.11 per 10 points, $95 \%$ CI, 1.06-1.17). The pneumonia first-day model had better discrimination (c-statistic of 0.68 vs. $0.58-0.66, p<.05$ for all comparisons), better reclassification (NRI -0.06 to -0.14 ), and stratified individuals across a broader range of readmission risk than other models (Table). CONCLUSIONS: An EHR-based pneumonia-specific readmission risk prediction model outperformed our EHR-based multi-condition model as well as the CMS pneumonia administrative model, PSI, and CURB-65. Future work should examine whether incorporating EHR data from the full hospital stay, including in-hospital clinical severity and complications, further improves model performance.

Comparison of Model Performance of Different Pneumonia-Specific Readmission Models

\begin{tabular}{|c|c|c|c|c|c|}
\hline Model Name & C-statistic & $\begin{array}{l}\text { Comparison of C-statistic } \\
\text { ( } p \text {-value) }\end{array}$ & $\begin{array}{l}\text { IDI, \% } \\
(95 \% \mathrm{CI})\end{array}$ & $\begin{array}{l}\text { Categorical } \\
\text { NRI, } \\
(95 \% \mathrm{CI})\end{array}$ & $\begin{array}{l}\text { Avg predicted risk } \\
\text { from lowest to } \\
\text { highest decile }\end{array}$ \\
\hline First-day pneumonia & $0.68^{\mathrm{b}}$ & [Reference] & [Reference] & [Reference] & $0.04-0.33$ \\
\hline First-day multi-condition & 0.66 & 0.029 & $-0.01(-0.03-0.01)$ & $-0.06(-0.15-0.02)$ & $0.07-0.36$ \\
\hline CMS Pneumonia & 0.64 & 0.014 & $-0.03(-0.04--0.02)$ & $-0.07(-0.15-0.02)$ & $0.08-0.29$ \\
\hline PSI first-day & 0.64 & $<0.001$ & $-0.03(-0.04--0.02)$ & $-0.09(-0.17--0.01)$ & $0.06-0.27$ \\
\hline CURB-65 first-day & 0.58 & $<0.001$ & $-0.05(-0.07--0.04)$ & $-0.14(-0.28--0.05)$ & $0.10-0.18$ \\
\hline
\end{tabular}

Abbreviations: IDI, Integrated

Discrimination Improvement;

NRI, Net Reclassification Index; CMS, Centers for Medicare

Medicaid Services; PSI, pneumonia severity index;

a Categorical NRI is calculated as the highest two risk

quintile versus the bottom 3 risk quintiles

${ }^{\mathrm{b}} \mathrm{C}$-statistic is optimism-corrected using

5-fold cross-validation 


\section{PREDICTING 30-DAY PNEUMONIA READMISSIONS USING ELECTRONIC} HEALTH RECORD DATA FROM THE FULL HOSPITAL STAY: MODEL DEVELOPMENT AND COMPARISON Oanh K. Nguyen ${ }^{1}$; Anil N. Makam ${ }^{1}$; Song Zhang $^{2}$; Bin Xie ${ }^{4}$; Mark A. Weinreich ${ }^{1}$; Ruben Amarasingham ${ }^{4}$; Eric Mortensen ${ }^{3}$; Ethan Halm ${ }^{1} .{ }^{1}$ UT Southwestern Medical Center, Dallas, TX; ${ }^{2}$ University of Texas Southwestern Medical Center, Dallas, TX; ${ }^{3}$ VANTHCS, Dallas, TX; ${ }^{4}$ Parkland Center for Clinical Innovation, Dallas, TX. (Control ID \#2464166)

BACKGROUND: Identifying patients hospitalized for pneumonia at highest risk for readmission can help target resource-intensive interventions to those most likely to benefit. However, current models have only modest predictive ability and have not incorporated clinical severity measures updated at discharge, which may improve prediction. This study sought to: 1) develop a pneumonia-specific risk prediction model incorporating electronic health record (EHR) data from the full hospital stay, and 2) compare our 'full-stay pneumonia' model performance to a first-day pneumonia model, in addition to other models.

METHODS: This was an observational study using EHR data from 6 hospitals in the DallasFort Worth metroplex, including safety net, community, and non-teaching sites. We included hospitalizations from all medicine services among adults ( $\geqq 18$ years) between November 2009 and October 2010. We excluded deaths, transfers to other hospitals, and those who left against medical advice. For patients who had multiple hospitalizations, we included the first one. The primary outcome was non-elective all-cause 30-day readmission to any of 75 hospitals in north
Texas ascertained using an all-payer regional hospitalization database. Significant univariate candidate variables were entered into a multivariate logistic regression model using stepwise backward selection with a significance threshold of $p \leq 0.05$. Validation was performed using 5fold cross-validation. For comparison, we used our group's previously developed 'first-day pneumonia' and 'first-day multi-condition' models, the CMS pneumonia administrative model, and updated PSI and CURB-65 scores using data available within $24 \mathrm{~h}$ of discharge. RESULTS: Of 1463 pneumonia admissions, $14 \%$ were readmitted. Updated PSI score at discharge (OR 1.22 per 10 points, $95 \%$ CI $1.15-1.29$ ); $\geq 1$ vital sign instability at discharge (OR $1.47,95 \%$ CI, 1.05-2.07); and disposition (e.g., hospice, OR $0.23,95 \%$ CI, 0.07-0.83) were significant predictors of readmission in the final model, in addition to predictors available on admission [median income per ZIP code $<\$ 30,000$ (OR 1.92, $95 \%$ CI, 1.18-3.12); platelets $>350 \times 10^{3} / \mu \mathrm{L}$ (OR 2.35, $\left.95 \% \mathrm{CI}, 1.57-3.52\right)$; hospitalizations in prior year (OR 1.26 per admission, $95 \% \mathrm{CI}, 1.10-1.44)$ ). The full-stay pneumonia model had better discrimination (cstatistic of 0.71 vs. $0.60-0.68, p<.05$ for all comparisons), better reclassification (NRI -0.08 to -0.18 ), and stratified individuals across a broader range of readmission risk than other models (Table)

CONCLUSIONS: An EHR-based pneumonia-specific readmission risk prediction model derived using data from the full hospital stay outperformed our group's 'first-day pneumonia' and 'full-stay multi-condition' models, the CMS model, and PSI and CURB-65 scores at discharge. Clinical severity measures at discharge available in the EHR are important prognosticators for readmission.

Comparison of Model Performance of Different Pneumonia-Specific Readmission Models

\begin{tabular}{|c|c|c|c|c|c|}
\hline Model name & C-statistic & $\begin{array}{l}\text { Comparison of C-statistic } \\
\text { ( } p \text {-value) }\end{array}$ & $\begin{array}{l}\text { IDI, \% } \\
(95 \% \mathrm{CI})\end{array}$ & $\begin{array}{l}\text { Categorical NRI, }^{\mathrm{a}} \\
(95 \% \mathrm{CI})\end{array}$ & $\begin{array}{l}\text { Avg predicted risk from } \\
\text { lowest to highest decile }\end{array}$ \\
\hline Full-stay pneumonia & $0.71^{\mathrm{b}}$ & [Reference] & [Reference] & [Reference] & $0.03-0.37$ \\
\hline First-day pneumonia & 0.68 & 0.018 & $-0.02(-0.01--0.03)$ & $-0.08(-0.01--0.15)$ & $0.04-0.33$ \\
\hline Full-stay multi-condition & 0.68 & 0.008 & $-0.01(-0.03-0.01)$ & $-0.09(-0.17--0.01)$ & $0.06-0.39$ \\
\hline CMS Pneumonia & 0.64 & $<0.001$ & $-0.05(-0.04--0.07)$ & $-0.15(-0.23--0.06)$ & $0.08-0.29$ \\
\hline PSI at discharge & 0.67 & $<0.001$ & $-0.04(-0.06--0.03)$ & $-0.09(-0.16--0.01)$ & $0.05-0.30$ \\
\hline CURB-65 at discharge & 0.60 & $<0.001$ & $-0.07(-0.08--0.05)$ & $-0.18(-0.27--0.09)$ & $0.09-0.22$ \\
\hline
\end{tabular}

Abbreviations: IDI, Integrated Discrimination Improvement; NRI, Net Reclassification Index; CMS, Centers for Medicare \& Medicaid Services; PSI, pneumonia severity index; ${ }^{a}$ Categorical NRI is calculated as the highest two risk quintiles versus the bottom 3 risk quintiles; ${ }^{\mathrm{b}} \mathrm{C}$-statistic is optimism-corrected using 5-fold cross-validation

PREDICTING BACTEREMIA IN THE EMERGENCY DEPARTMENT Akane Takamatsu; Haruki Mito. Teine Keijinkai Hospital, Sapporo, Japan. (Control ID \#2463914)

BACKGROUND: Bacteremia is the presence of bacteria in blood. Blood cultures are the gold standard test for proving bacteremia, enabling identification of the causal bacteria and their antibiotic sensitivities. In consideration of the high mortality amongst patients with bacteremia leading to sepsis or septic shock, most physicians have a relatively low threshold for ordering blood cultures in patients with suspected bacteremia. However, the consequence of such obtained blood cultures is the relatively low yield and false positives from bacterial contaminants. These false positive reports, in turn, may lead to unnecessary antibiotic therapy requiring longer hospitalization. Although several previous studies have reported certain clinical prediction rules (CPRs) to predict the risk of bacteremia, these models are frequently comprehensive, including multiple variables with some requiring numerous laboratory data in making the prediction. Due to the nature of blood cultures and blood tests taken concurrently in clinical practice, including laboratory findings in the prediction model is inconvenient and impractical. As such, this study aimed to develop a CPR with high feasibility for predicting bacteremia in the emergency department (ED) combining patient characteristics and vital signs.
METHODS: This single-center retrospective study derived a novel CPR and internal validation was performed. The study used clinical data from adult patients of whom blood cultures were obtained on presentation to the ED. These data were selected from a laboratory database of a 600 bed tertiary teaching hospital in Hokkaido, Japan, from April 2013 to March 2014. Clinical characteristics and vital signs were reviewed from electronic medical records. The main outcome was bacteremia diagnosed by blood culture. We developed a CPR using stepwise logistic regression and $\beta$ coefficients for significant predictor variables. All statistical analyses were performed with EZR (Saitama Medical Center, Jichi Medical University, Saitama, Japan), which is a graphical user interface for R (The R Foundation for Statistical Computing, Vienna, Austria). RESULTS: A total of 170 patients were analyzed; 135 (49 with bacteremia) in the derivation group and 35 (14 with bacteremia) in the validation group. We derived a clinical prediction model with four variables: shaking chills, and medical comorbidities of respiratory, cardiovascular, and endocrine disease. In the derivation group, area under the curve (AUC) was 0.73 (95\% confidence interval [CI]: 0.65-0.82). Sensitivity and specificity were $96.0 \%$ (95\% CI: $86.0-99.5 \%$ ) and $23.0 \%$ (95 \% CI: $14.8-33.6 \%$ ), respectively. Negative predictive value (NPV) was $90.9 \%$ (95 \% CI: 70.8-98.9\%). In the validation group, AUC was 0.63 (95\% CI: $0.44-0.81$ ). Sensitivity and specificity were $92.9 \%$ (95 \% CI: 66.1-99.8 \%) and $19.0 \%$ (95\% CI: $5.441 .9 \%$ ), respectively. NPV was $80.0 \%$ (95 \% CI: $28.4-99.5 \%$ ). 
CONCLUSIONS: In this study, a novel CPR for predicting bacteremia was developed and internally validated. With the high sensitivity and NPV, it could be implemented as a useful screening tool in helping judge clinicians in the ED to rule out those not in need of testing. Further study is needed to externally validate and reveal the impact of this CPR on the anticipated reduction of false positives from bacterial contaminants.

PREDICTORS AND CAUSES OF DEATH IN ELDERLY PATIENTS WITH ACUTE VENOUS THROMBOEMBOLISM Nicolas Faller ${ }^{3}$; Andreas Limacher ${ }^{2}$; Marie Méan ${ }^{1}$; Marc Righini ${ }^{5}$; Daniel Staub ${ }^{10}$; Hans-Jürg Beer ${ }^{7}$; Beat Frauchiger ${ }^{8}$; Joseph J. Osterwalder ${ }^{6}$; Bernhard Lämmle ${ }^{3}$; Jacques Cornuz ${ }^{1}$; Anne Angelillo-Scherrer ${ }^{3}$; Christian M. Matter ${ }^{9}$; Marc Husmann ${ }^{9}$; Martin Banyai ${ }^{4}$; Markus Aschwanden ${ }^{10}$; Lucia Mazzolai $^{1}$; Olivier Hugli ${ }^{1}$; Nicolas Rodondi ${ }^{3}$; Drahomir Aujesky ${ }^{3} .{ }^{1}$ Lausanne University Hospital, Lausanne, Switzerland; ${ }^{2}$ University of Bern, Bern, Switzerland; ${ }^{3}$ Bern University Hospital, Bern, Switzerland; ${ }^{4}$ Cantonal Hospital Lucerne, Lucerne, Switzerland; ${ }^{5}$ Geneva University Hospital, Geneva, Switzerland; ${ }^{6}$ Cantonal Hospital of St. Gallen, St. Gallen, Switzerland; ${ }^{7}$ Cantonal Hospital of Baden, Baden, Switzerland; ${ }^{8}$ Cantonal Hospital of Frauenfeld, Frauenfeld, Switzerland; ${ }^{9}$ Zurich University Hospital, Zurich, Switzerland; ${ }^{10}$ Basel University Hospital, Basel, Switzerland. (Control ID \#2465343)

BACKGROUND: Mortality in patients with venous thromboembolism (VTE), defined as pulmonary embolism and/or deep vein thrombosis, has been extensively studied. However, only a few studies focused specifically on the elderly. We therefore sought to assess the causes of death, and identify predictors of mortality in a prospective cohort study of elderly patients with acute VTE.

METHODS: We studied 991 patients aged $\geq 65$ years with an acute VTE from 9 Swiss hospitals between September 2009 and December 2013. The primary outcome was overal mortality. We examined the association between patient baseline characteristics (demographics, comorbidities, vital signs on admission, and laboratory findings) and mortality using Cox regression analysis, adjusting for baseline variables and periods of anticoagulation as a timevarying co-variate. Causes of death were adjudicated by a blinded, independent committee.

RESULTS: The median age was 75 years and 463 (47\%) patients were women. During a median follow-up period of 30 months, 206 patients ( $21 \%)$ died. Of these, 53 (26\%) died during the first 3 months following the index VTE. Cancer (34\%), pulmonary embolism (17\%), sepsis/infection (16\%) were the most common causes of death, whereas cardiac disease $(8 \%)$ and bleeding-related deaths $(6 \%)$ were less common. Independent predictors of overall death included active cancer (hazard ratio [HR] 5.80, $95 \%$ confidence interval [CI] 4.22-7.97), and systolic blood pressure $<100 \mathrm{mmHg}$ (HR 2.77, $95 \% \mathrm{CI}$ $1.56-4.92$ ), and to a lesser extent, age (HR $1.32,95 \% \mathrm{CI} 1.05-1.65$, per decade), diabetes mellitus (HR 1.50, $95 \%$ CI 1.02-2.22), low physical activity level (HR 1.92, $95 \%$ CI 1.38-2.66), polypharmacy (HR 1.41, $95 \%$ CI 1.01-1.96), anemia (HR 1.48, $95 \%$ CI $1.07-2.05$ ), C-reactive protein $>40 \mathrm{mg} / \mathrm{L}$ (HR $1.88,95 \%$ CI $1.36-2.60)$, troponin $>14 \mathrm{pg}$ ) $\mathrm{mL}$ (HR 1.54, $95 \%$ CI 1.06-2.25), and D-dimer $>3000$ ng/ml (HR 1.45, $95 \%$ CI 1.04 2.01). A body-mass index $\geq 30 \mathrm{~kg} / \mathrm{m}^{2}$ (HR $0.93,95 \%$ CI $0.90-0.96$ ) and recent major surgery (HR 0.34, $95 \%$ CI 0.19-0.59) were inversely associated with mortality.

CONCLUSIONS: In elderly patients with acute VTE, several clinical and laboratory parameters are associated with mortality. Cancer, pulmonary embolism, and sepsis/ infection are the most common causes of death.

PREDICTORS FOR PATIENTS UNDERSTANDING REASON FOR HOSPITALIZATION Himali Weerahandi ${ }^{2}$; Boback Ziaeian ${ }^{1}$; Robert L. Fogerty ${ }^{4}$; Leora I. Horwitz ${ }^{3}$ ${ }^{1}$ David Geffen School of Medicine at UCLA, Los Angeles, CA; ${ }^{2}$ Icahn School of Medicine at Mount Sinai, New York, NY; ${ }^{3}$ NYU School of Medicine, New York, NY; ${ }^{4}$ Yale School of Medicine, New Haven, CT. (Control ID \#2469162)
BACKGROUND: The transition from hospital back to community is a particularly vulnerable time for patients, exacerbated by poor communication. We recently demonstrated that only about $60 \%$ of patients were able to accurately describe their diagnosis in post-discharge interviews. In this study we examine predictors for understanding reason for hospitalization.

METHODS: We analyzed data from a prospective, observational cohort study of patients 65 years or older admitted to Yale-New Haven Hospital for acute coronary syndrome, heart failure, or pneumonia between May 2009 and April 2010 who were subsequently discharged to home. Complete understanding of diagnosis was the primary outcome. We assessed the association of systems-level, modifiable factors with complete understanding of diagnosis: the presence of jargon on discharge instructions, whether outpatient provider knew if the patient was admitted, and whether the patient had more than more than 1 day notice prior to discharge, based on post-discharge patient interviews. Univariate associations between predictors and outcome were assessed using the chi-square or Fisher's exact test. If a significant association was found, a multivariable logistic regression model was performed, controlling for age, race, sex, and diagnosis.

RESULTS: There were 377 patients in this cohort. The mean age was 77.0 years, $45.5 \%$ were women, and $11.4 \%$ were black. Overall, $59.8 \%$ of these patients completely understood their diagnosis. In a univariate analysis, patients with jargon on their discharge instructions were more likely to completely understand their diagnosis compared with patients without jargon on their discharge instructions (70.5 vs $56.3 \%$ respectively, $p=$ $0.01)$. Stratified analysis by diagnosis demonstrated that congestive heart failure (CHF) patients with jargon were more likely to understand their diagnosis ( 66.7 vs $43.5 \%, p=$ $0.05)$. Most of these patients ( $57 \%$ ) had "coronary artery disease/unstable angina" listed on their discharge instructions. In a multivariate model controlling for age, sex, race and diagnosis, jargon was no longer a significant predictor for completely understanding diagnosis (OR 1.51, $95 \%$ CI 0.87-2.64, $p=0.14$ ); however, patients admitted with CHF were less likely to understand their diagnosis (OR 0.37, $95 \%$ CI $0.19-0.69$, $p<0.01)$. Outpatient provider being aware of admission was not associated with completely understanding diagnosis ( 59.0 vs $55.0 \%, p=0.64$ ). In addition, having more than day notice prior to discharge was not associated with completely understanding diagnosis ( 60.3 vs $58.4 \%, p=0.70)$.

CONCLUSIONS: Jargon on discharge instructions, outpatient provider being aware of admission, and having more than 1 day notice prior to discharge were not associated with completely understanding diagnosis. On the other hand, CHF diagnosis was associated with not completely understanding diagnosis. The patients with CHF who did understand their diagnosis largely had concomitant acute coronary syndrome. Our findings highlight that despite outpatient provider being aware of hospitalization and patients having adequate notice for discharge, many patients still do not understand their reason for admission. Given that this is an older cohort, in-hospital delirium may affect patients' ability to understand why they were admitted; however, this study excluded patients who failed a mental status screen. Specific diagnoses such as acute coronary syndrome may initiate a cascade of patient education unique to that diagnosis. Further studies should investigate which educational interventions are effective in the inpatient setting, and if they can be generalized to different diagnoses.

\section{PREDICTORS OF ADMISSION TO AN OBSERVATION VERSUS INPATIENT} SERVICE AFTER THE MEDICARE TWO-MIDNIGHT RULE Shira Yun; Gregory B. Kruse; Jaya Aysola. University of Pennsylvania, Philadelphia, PA. (Control ID \#2467782)

BACKGROUND: Medicare implemented the Two-midnight rule in October 2013, specifying stays less than two midnights should be placed in observation, to ensure consistency in length of stays for observation admissions as a cost savings measure. 
However, implications of this rule are unknown, with CMS projections suggesting that it may lead to reductions in observation admissions. We aim to identify predictors of admission to observation versus inpatient services before and after the Medicare Twomidnight rule.

METHODS: We analyzed data from admissions $(n=285,323)$ to three hospitals in the University of Pennsylvania Health System from October 2010 to December 2014. Our predictors included age, gender, race, socioeconomic status (level of education and median income), insurance type, hospital site, and clinical comorbidities (Elixhauser Index). We fitted multivariate logistic regression models to estimate the relationship between our predictors and primary outcome, admission status to observation versus inpatient. We estimated the difference in Medicare and Managed Medicare, where the rule does not consistently apply, compared to private insurance before and after the Medicare Two-midnight rule.

RESULTS: About $68 \%$ of our patient population was less than 65 years of age, $54 \%$ female, $46 \%$ non-Hispanic white, and $26 \%$ were insured by Medicare. We found that overall patients were significantly more likely to be admitted to an observation service after the Medicare Two-midnight rule, (AOR (Adjusted Odds Ratio): 1.20; $95 \%$ confidence interval (CI): 1.05-1.36). As hypothesized, the differences in admission patterns between private insurance and Managed Medicare before and after the Two-midnight policy change were not significant. In contrast, Medicare patients were $24 \%$ more likely to be admitted to an observation service compared to those with private insurance after the rule (AOR: 1.24 ; $95 \% \mathrm{CI}: 1.17-1.33$ ). Medicaid patients were $34 \%$ less likely to be admitted to an observation service compared to those with private insurance throughout the time period, regardless of rule (AOR: 0.66 ; $95 \%$ CI: $0.62-0.71$ ). Other significant predictors of admission to observation services beyond insurance type included, age, gender, minority status, clinical comorbidity index, and hospital site (physical observation unit versus virtual observation unit). Patients were more likely to be admitted to an observation service where there was a physical observation unit (AOR: 1.19; $95 \% \mathrm{CI}$ : 1.16-1.21) and less likely to be admitted where there was a virtual unit (AOR: 0.53; $95 \%$ CI: $0.52-0.55$ ) compared to a mixed observation service (both virtual and physical units) throughout the time period.

CONCLUSIONS: We found the Medicare Two-midnight rule significantly increased the use of observation services for Medicare patients compared to patients with other insurance types, which is contrary to CMS projections. The implications of increased observation patients on quality of care, reimbursement for care, and cost reductions needs further inquiry, particularly in light of the CMS reduction to inpatient payments in conjunction with the Two-midnight rule given their projected increase in inpatient admissions after the rule.

PREDICTORS OF CULTURAL COMPETENCE EXPERIENCE AMONG HEALTH CARE EMPLOYEES Jaya Aysola; Hairong Huo; Diana Harris; Eve Higginbotham. University of Pennsylvania, Philadelphia, PA. (Control ID \#2469621)

BACKGROUND: A diverse workforce is broadly viewed as essential to delivering culturally effective health care and addressing health care disparities. Inclusiveness and cultural competence within an organization influences both recruitment and retention of diverse employees. Therefore, the objective of our study was to determine key predictors of cultural competence within University of Pennsylvania educational and clinical sites. METHODS: We conducted a cross-sectional sub-analysis of data from a 22 -item validated survey administered to all employees $(n=3500)$ University-wide from February to April 2015. We focused the cultural competence factor (CCF), defined utilizing four survey questions that assess respondents' perceptions on institutional culture and capacity to make creative use of its diverse workforce. Our focus on this measure was that UPENN ranked in the bottom third on this factor score, when benchmarked against other universities administering this survey nationwide. We utilized ANOVA to compare CCF scores by respondent characteristics and chi-square tests to determine significant predictors of being above or below the mean CCF score. Our predictors included, age, gender, race/ethnicity, sexual orientation, belief system, disability status, primary language, position, length of employment, and place of work. Our primary outcome was the CCF score as a binary variable, either above or below the mean score. We fitted multivariate logistic regression models to estimate the associations of our predictors with our primary outcome. RESULTS: The mean (SD) of the CCF score was 15.4 (3.0), with $52.4 \%$ of the respondents $(n=3500)$ characterizing cultural competence of their institution above the mean. Significant predictors of CCF score in adjusted models include gender, sexual orientation, race/ethnicity, site of work. Respondents selfidentifying as non-Hispanic black as compared to non-Hispanic whites were over $60 \%$ less likely to rate CCF score above the mean (Adjusted Odds Ratio (AOR): $0.34 ; 95 \%$ Confidence Interval (CI): $0.28,0.44)$. Females were about $30 \%$ less likely than males to report CCF above the mean (AOR: 0.66 ; $95 \%$ CI: 0.56, 0.78). Respondents identifying as LGBTQ were about $40 \%$ less likely to characterize the CCF score above the mean as compared to heterosexuals (AOR: 0.59; $95 \%$ CI: 0.46, 0.76). Employees of the Children's Hospital of Philadelphia were almost twice as likely to score CCF above the mean as compared to employees from the School of Medicine (AOR: 1.92; $95 \%$ CI: 1.50, 2.47) whereas employees from the Veterinary School were less likely to score CCF above the mean as compared to School of Medicine (AOR: 0.51; $95 \%$ CI: 0.37, 0.69).

CONCLUSIONS: Our findings suggest that there are significant differences in employee perceptions of organizational cultural competency, with minority groups, females, and individuals that identify as LGBTQ significantly less likely to rate workplace cultural competency above the mean. We also find significant variation between workplace sites, which highlights the capacity of organizations to respond to such survey measures and the need for ongoing measurement to improve inclusion. In addition, there remains a need to determine what organizational characteristics distinguish high performing workplace sites in order to inform efforts to improve inclusion and promote recruitment and retention of a diverse workforce.

PREDICTORS OF IN-HOSPITAL MORTALITY IN ACUTE PANCREATITIS David Mossad ${ }^{1}$; Mustafa Musleh ${ }^{2}$; Ronald J. Markert ${ }^{1}$; Sangeeta Agrawal $^{2,}{ }^{3}$. ${ }^{1}$ Wright State University, Dayton, OH; ${ }^{2}$ Wright State University Boonshoft School of Medicine, Dayton, OH; ${ }^{3}$ Dayton VA Medical Center, Dayton, OH. (Control ID \#2456009)

BACKGROUND: Acute pancreatitis presents with significant risk of morbidity and mortality. Multiple scoring systems have looked at several risk factors to predict the severity of acute pancreatitis. In this study, we evaluated the role of demographics, comorbidities, and health system characteristics on in-hospital mortality in acute pancreatitis patients.

METHODS: We used ICD-9 diagnosis codes from the 2001-2010 National Hospital Discharge Survey to identify cases of acute pancreatitis. Comparisons on demographics, number of comorbidities, hospital length of stay (LOS), and selected health system characteristics were made between those who died in-hospital and those discharged. SPSS was used for chi-square and t test analysis at alpha $=0.05$.

RESULTS: Among 27,259 cases of acute pancreatitis, 573 died in-hospital and 26,686 were discharged. The mortality group was older (mean age of 64.5 vs $51.8, p<0.001$ ) and had a longer LOS (12.0 vs 6.4 days, $p<0.001)$. There was no difference in mortality between genders ( $2.2 \%$ of males vs $2.0 \%$ of females, $p=0.10)$, but Asians had the highest mortality ( $4.4 \%$ ). Patients with four or more comorbidities had a higher mortality rate $(2.5 \%)$ compared to those with three or fewer comorbidities $(0.1 \%)(p<0.001)$. Midwest U.S. had a lower death rate (1.6\%) compared to the Northeast (2.5\%), South $(2.2 \%)$, and West $(2.4 \%)(p=0.002)$. Mortality rate was lowest in hospitals with 6-99 beds $(1.3 \%)$, compared to greater than $2 \%$ in all other hospital sizes $(p<0.001)$. Hospital 
ownership, however, did not show any significant differences in mortality ( $2.0 \%$ proprietary, $2.4 \%$ government, $2.1 \%$ nonprofit or church, $p=0.49$ ). Among sources of payment, Medicare (3.6\%) and Medicaid (2.0\%) patients had the highest mortality rates. For source of admission, transfer from ambulatory surgery centers (5.9\%), hospitals (5.3\%), and skilled nursing/intermediate care facilities (4.9\%) had the highest mortality rates. Type of admission (e.g., emergent, elective) did no impact mortality.

CONCLUSIONS: Age, race, LOS, comorbidity burden, region of the U.S., hospital size, source of payment, and source of admission were related to higher in-hospital mortality. The lower mortality rate in small hospitals may be due to patients with a high risk of mortality being transferred to larger hospitals more capable of managing critically ill patients.
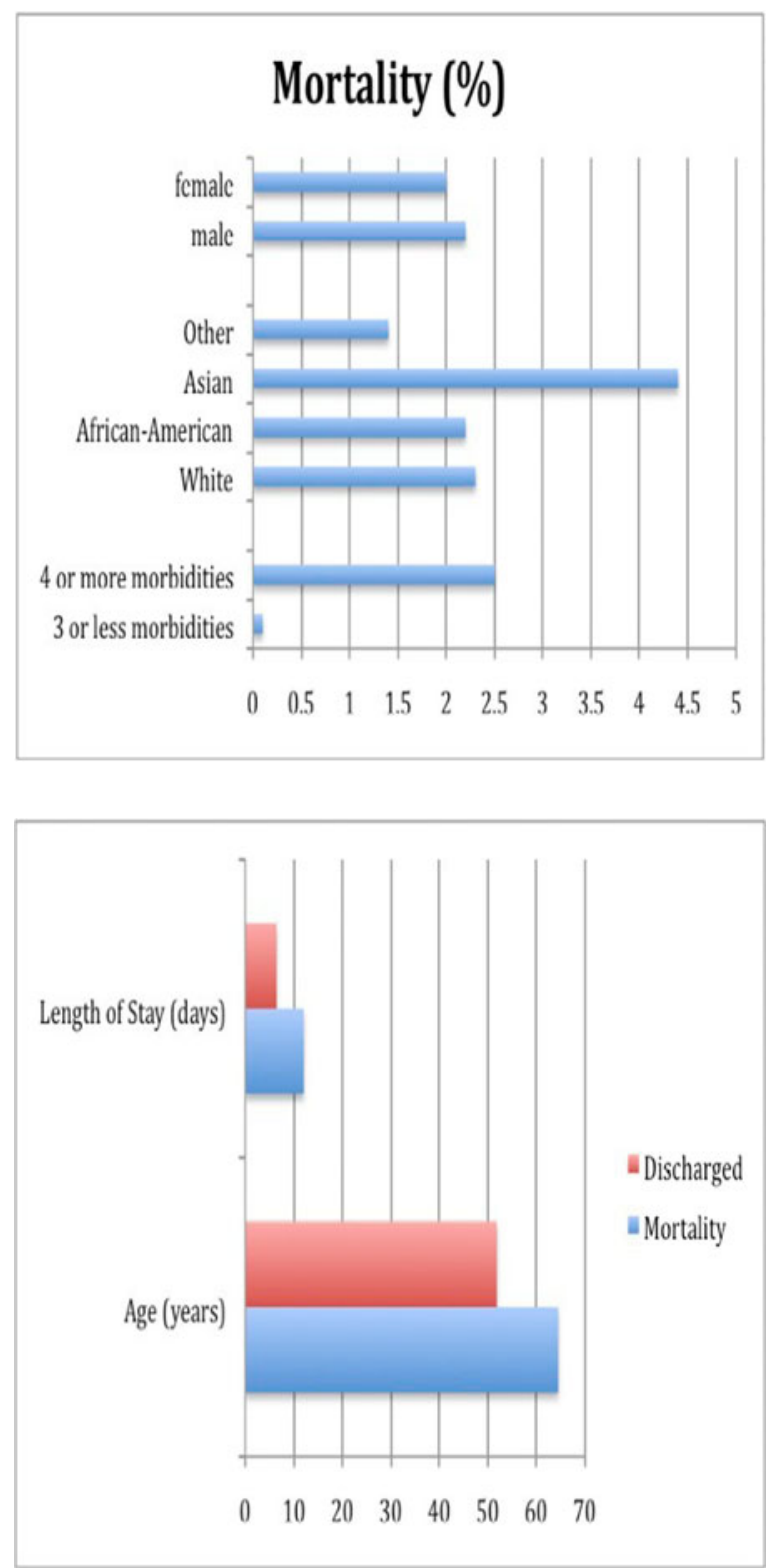

PREDICTORS OF INITIATING OPIOID AGONIST THERAPY IN A LARGE U.S. COHORT OF HIV-INFECTED AND UNINFECTED PATIENTS Jonathan L. Robbins ${ }^{2}$; Kathleen A. McGinnis ${ }^{4}$; E. J. Edelman ${ }^{7}$; Adam Gordon ${ }^{3}$; Ajay Manhapra ${ }^{8}$; David A. Fiellin ${ }^{5}$; Brent A. Moore ${ }^{7}$; Philip T. Korthuis ${ }^{1}$; J. R. Gaither ${ }^{5}$; Kirsha S. Gordon ${ }^{8}$; Declan Barry ${ }^{7}$; Stephen Crystal'; Amy C. Justice ${ }^{6}$; Kevin Kraemer ${ }^{2} .{ }^{1}$ Oregon Health \& Science University, Portland, OR $;{ }^{2}$ University of Pittsburgh Medical Center, Pittsburgh, PA; ${ }^{3}$ University of Pittsburgh and VA
Pittsburgh Healthcare System, Pittsburgh, PA; ${ }^{4}$ VA Pittsburgh Healthcare System, Pittsburgh, PA; ${ }^{5}$ Yale University, New Haven, CT; ${ }^{6}$ Yale University, West Haven, CT; ${ }^{7}$ Yale University School of Medicine, New Haven, CT; ${ }^{8}$ Yale School of Medicine, New Haven, CT; ${ }^{9}$ Rutgers University, New Brunswick, NJ. (Control ID \#2468105)

BACKGROUND: Opioid use disorders (OUD) are prevalent among HIV-infected patients and when untreated, contribute to ongoing HIV transmission and poor health outcomes. Opioid agonist therapy (OAT), in the form of methadone or buprenorphine is the most effective treatment for OUD and is associated with improved health outcomes, including higher CD4 counts, antiretroviral adherence, and viral suppression. To inform clinical practices and policies, we sought to identify patient and facility-level predictors associated with OAT initiation among HIV-infected and uninfected patients with OUD. METHODS: Using the Veterans Aging Cohort Study (VACS), a national prospective, observational cohort of HIV-infected and 1:2 matched uninfected controls, we identified new OUD treatment episodes from 2000 to 2012 among 149,561 individuals (47,172 HIV-infected and 102,389 uninfected) after an inpatient or outpatien encounter with a primary or secondary OUD ICD-9 code diagnosis. To qualify as a new OUD episode, we required 5 months with no OAT or OUD ICD-9 codes, following the method of a RAND capstone report on mental health and addiction services in the VA. After a new OUD episode, initiation of OAT was defined as a methadone clinic visit or a prescription fill for buprenorphine within 14 days, a timeframe used by the National Committee for Quality Assurance as a measure of healthcare quality. Independent variables in the study included patient factors (sociodemographic factors, HIV-status, comorbid alcohol and other substance userelated diagnoses) and rural or urban resident. Bivariate and multivariate models were constructed to identify predictors of OAT initiation. Multiple episodes were accounted for in the multivariate model using mixed effects logistic regression.

RESULTS: In the VACS cohort, $8.7 \%(4,118)$ of HIV-infected and $5.9 \%(6,081)$ of uninfected patients had at least one new OUD episode, while $42.7 \%$ of patients had more than one episode for a total of 19,804 unique OUD episodes. After the first OUD episode, 1349 (13.2\%) of patients initiated OAT within 14 days. From 2000 to 2004 to 2009-2012, methadone initiation decreased from 13.8 to $8.8 \%(p<0.001)$, while rates of buprenorphine initiation tripled from 2.0 to $6.1 \%(p<0.001)$. In bivariate analysis after the first new OUD episode, HIV infection was not associated with methadone initiation (11.6 vs $11.2 \%, p=0.47$ ), but uninfected patients were more likely to receive buprenorphine ( 3.4 vs $2.5 \%, p<0.01$ ). African-Americans were more likely to receive OAT than Caucasians ( 15.3 vs $9.3 \%, p<0.001)$. Urban patients were more likely to receive methadone than rural patients ( 12.7 vs $7.4 \%, p<0.001)$, but equally likely to receive buprenorphine ( 2.8 vs $3.5 \%, p=0.09$ ). In the mixed-effects multivariate logistic regression model allowing for multiple OUD episodes, African-American race (OR 1.7; $95 \%$ CI 1.43-1.92) and comorbid alcohol-related diagnoses (OR 2.1; 95 \% CI 1.88-2.39) were associated with greater odds of initiating OAT, and rural patients were less likely (OR $0.73 ; 95 \%$ CI $0.62-0.86$ ) to initiate OAT. HIV infection was not associated with OAT in the multivariate model (OR $0.91 ; 95 \%$ CI $0.81-1.03$ ).

CONCLUSIONS: Timely initiation of OAT within a cohort of HIV-infected and uninfected patients receiving care in VA facilities remains uncommon. Although buprenorphine therapy increased during the study period, absolute treatment numbers remained low. Urban patients and African-Americans were more likely than their rural, Caucasian counterparts to initiate OAT, particularly methadone. HIV-status was not significantly associated with the initiation of overall OAT. Comorbid alcohol use diagnosis was associated with OAT, which may reflect increased facility utilization and concurrent pharmacotherapy for alcohol use. The results of this study should prompt VA policy makers to increase provision of OAT, particularly buprenorphine, and to increase capacity for OAT in rural areas, which have recently been the source of HIV outbreaks. 


\section{PREDICTORS OF PATIENT INTEREST IN DEPRESCRIBING: RESULTS OF A} NATIONAL SURVEY Rachel A. Lippin-Foster ${ }^{1}$; Amy Linsky ${ }^{1,}{ }^{2}$; Steven R. Simon ${ }^{1}$; Kelly Stolzmann ${ }^{1}$; Mark Meterko ${ }^{1} .{ }^{1}$ VA Boston Healthcare System, Boston, MA; ${ }^{2}$ Boston University School of Medicine, Boston, MA. (Control ID \#2468885)

BACKGROUND: Polypharmacy, often defined as taking 5 or more medications, is associated with adverse clinical outcomes and increased healthcare costs. Many patients who take multiple medications would like to decrease their out-of-pocket medical expenses and the burden of taking multiple pills. Improving clinical processes leading to appropriate and safe medication discontinuation may improve both patient and systemlevel outcomes. Including patient preferences could increase the likelihood of successful medication discontinuation. Therefore, we sought to identify patient characteristics and attitudes associated with a stronger interest in deprescribing.

METHODS: We conducted a national mail survey of 1548 patients who received care from a Veterans Affairs (VA) primary care provider and $\geqq 5$ prescriptions during the year prior to March 2015. The survey explored general attitudes toward medication, patients' relationships with clinical providers, perceptions of provider knowledge, interest in and experiences with stopping medicines, and concerns about medications. In addition, individuals answered general health and demographic questions. The primary outcome, the Interest in Deprescribing Scale, was computed as the mean response to the following three items: "If my doctor said it was possible, I would be willing to stop one or more of my regular medications," "I would like to reduce the number of medications that I am taking;" and "I am taking one or more medications that I would like to stop." Responses were on a 5-point Likert scale, ranging from $1=$ "Strongly Disagree" to $5=$ "Strongly Agree." We began this exploratory analysis by examining the distribution of responses to all variables. To determine the bivariate association of each predictor variable with the primary outcome, we conducted multiple analyses of variance (ANOVAs), comparing scores on the outcome variable across groupings formed by the candidate predictors. We then developed a multivariable regression model by including any predictor from the ANOVA analyses significant at $\mathrm{p} \leq 0.1$ and used backward selection to achieve a more parsimonious model. To create our final model, we added age and sex to the model obtained from the backward regression.

RESULTS: In our sample of 790 Veterans (response rate $51 \%$ ), respondents were predominately white ( $73 \%$; black $18 \%$, other $9 \%$ ), male $(86 \%)$, mean age 67 years $(\mathrm{SD}=10)$, and were prescribed a mean of $10(\mathrm{SD}=3.6)$ medications. Respondents generally expressed moderate Interest in Deprescribing [mean $(\mathrm{m}) 3.4, \mathrm{SD}=0.8$ ]. Responses for the predictor attitudinal scales (5-point Likert scales) were: Beliefs about Medication Overuse $(\mathrm{m}=2.9$, $\mathrm{SD}=0.8)$, Medication Concerns $(\mathrm{m}=3.0, \mathrm{SD}=0.9)$, Perceived Provider Knowledge $(\mathrm{m}=$ $3.8, \mathrm{SD}=0.8)$, and Unimportance of Medications $(\mathrm{m}=2.4, \mathrm{SD}=0.8)$. The model overall was significant $(p<0.0001)$, explaining $42 \%$ of the variance in patient interest in deprescribing. In the final adjusted model, the following variables were significantly associated with Interest in Deprescribing (standardized beta coefficient $[\beta], p$-value): Beliefs about Medication Overuse $(\beta=0.19, p<0.0001)$, Medication Concerns $(\beta=0.41, p<0.0001)$, Perceived Provider Knowledge $(\beta=0.11, p=0.0005)$, and Unimportance of Medications ( $\beta=0.22, p<0.0001)$. Non-White Veterans had a lower interest in deprescribing (black: $\beta=$ $-0.09, p=0.003$; other: $\beta=-0.07, p=0.012$ ).

CONCLUSIONS: Patients who believe that medicines generally are overused, that one or more of their medicines are unimportant, have concerns about their medicines, and are confident in their provider's knowledge are more likely to be interested in deprescribing their own medications. Non-white Veterans were less likely to have interest in deprescribing than white Veterans, a finding that merits further study. Taken together, these findings suggest the use of patient beliefs and attitudes towards medication as a tool to identify individuals who are more amenable to stopping medication. Further research is needed on identifying patients who are motivated toward deprescribing and on approaches to educating those who may not be as predisposed.
PREDICTORS OF PRIMARY CARE PHYSICIAN SELF-EFFICACY IN CARING FOR PATIENTS WITH MENTAL ILLNESS IN A TEAM-BASED MODEL $\underline{\text { Danielle F. Loeb }^{7} \text {; Lori A. Crane }}{ }^{4}$; Erin Leister ${ }^{10}$; Ingrid A. Binswanger ${ }^{8,}{ }^{6}$; Evette Ludman $^{9}$; Frank V. deGruy ${ }^{5}$; Donald E. Nease ${ }^{3}$; L Miriam Dickinson ${ }^{2}$; Danielle M. Kline $^{6}$; Elizabeth Juarez-Colunga ${ }^{10}$; Elizabeth A. Bayliss ${ }^{1,6}$. ${ }^{1}$ Kaiser Permanente and University of Colorado, Denver, CO; ${ }^{2}$ University of Colorado, Aurora, CO; ${ }^{3}$ University of Colorado - Denver, Aurora, CO; ${ }^{4}$ University of Colorado Anschutz Medical Campus, Aurora, $\mathrm{CO} ;{ }^{5}$ University of Colorado SOM, Aurora, CO; ${ }^{6}$ University of Colorado School of Medicine, Aurora, CO; ${ }^{7}$ University of Colorado Denver, Aurora, CO; ${ }^{8}$ Kaiser Permanente Colorado, Aurora, CO; ${ }^{9}$ Group Health Research Institute, Seattle, WA; ${ }^{10}$ University of Colorado School of Public Health, Aurora, CO. (Control ID \#2466024)

BACKGROUND: Team based care improves outcomes for patients with comorbid mental and physical conditions. Implementing effective team-based care requires Primary Care Providers (PCPs) with the skills and confidence to manage this population. We developed and initially validated a measure to assess PCP self-efficacy for these tasks. Our aim was to assess PCP's self-efficacy in a) managing mental illness and b) practicing teambased care for patients with chronic illnesses.

METHODS: We administered a cross-sectional mail survey of 900 randomly selected family medicine (FM) and internal medicine (IM) PCPs in Colorado. We excluded surveys returned for incorrect addresses from response rate. Based on Bandura, we developed two self-efficacy scales for the instrument: team-based care and mental illness management. We performed cognitive testing with health services researchers and pilot tested the survey with 30 PCPs. The dependent variables for the multivariable analysis were scores on each of the two self-efficacy scales. Using separate multivariable linear regression models we assessed performance on each of the two scales as a function of demographic factors, practice characteristics, and multiple adapted validated scales: the Mental Health Knowledge and Management Instrument, the Communication Skills Self-assessment, the Attitudes Toward Health Teams Scale, the Team Climate Inventory, and the Team approach subscale of the Practice Monitor. We, first, performed simple linear regression for demographic, practice characteristics, and the adapted scales. We, then, used a purposeful selection procedure used to select final adjusted linear regression models (inclusion $p=0.25$, retention $p=0.10$ ).

RESULTS: Four hundred two (response rate $=49 \%$ ) PCPs from diverse practice settings completed surveys. On an 11-point Likert scale, mean scores (standard deviation) on the selfefficacy scales were 7.7 (1.7) and 7.1 (1.4) for the team-based care and mental illness management scales respectively. Factors associated with self-efficacy with mental illness in the final multivariable model are shown in Table 1. Notable associations included: positive associations with selfefficacy with communication [estimate $=0.532, p<0.001$ ] and with access to psychology consultation [estimate $=0.394, p=0.002$ ]; and negative associations with feeling "not at all" or "slightly" prepared to manage bipolar disorder at completion of residency [estimate $=-0.534, p=$ 0.001 ] and with "never" or "uncommonly" managing major depressive disorder [estimate= $-0.463, p=0.092]$. Factors associated with self-efficacy with mental illness in the final multivariable model are shown in Table 2. Notable associations included: positive associations with high score on the Attitude Toward Team-Based Care scale [estimate $=1.111, p<0.0001$ ], presence of a health psychologist [estimate $=0.592, p=0.044$ ], access to an Electronic Medical Record [estimate $=0.559, p<0.0001$ ], and self-efficacy with communication [estimate $=0.498$, $p<0.0001$ ]; negative association with practicing in a solo practice [estimate $=-0.629, p=0.028$ ] CONCLUSIONS: This research provides important insights into the factors associated with PCP self-efficacy in managing complex patients with mental illness in a team-based model. In terms of self-efficacy, experience with treating patients with mental illness may be more important than didactic knowledge in treating mental illness. A focus on clinician attitude, communication skills, and practice resources may be crucial to preparing PCPs for a team-based model of care. The negative association between Hispanic ethnicity and self-efficacy in team-based care should be interpreted with caution, as only 14 respondents identified as Hispanic. Further, the generalizability of the results is limited by responses from one state in Rocky Mountain region. 


\begin{tabular}{|c|c|c|c|c|c|}
\hline Variable group & Covariate & Reference & Estimate & SE & P-value \\
\hline \multirow{2}{*}{ Role } & Clinician-Administrator & Clinician & 0.420 & 0.186 & 0.075 \\
\hline & Clinician-Educator/Researcher & Clinician & 0.067 & 0.192 & - \\
\hline Specialty & Family Medicine & Internal & 0.254 & 0.128 & 0.048 \\
\hline Resources & Psychology consult & No access & 0.394 & 0.124 & 0.002 \\
\hline Communication & Factor Score & & 0.532 & 0.063 & $<.001$ \\
\hline MH Knowledge & $10 \%$ increase in score & & 0.140 & 0.046 & 0.002 \\
\hline \multicolumn{6}{|c|}{ Experience - How often managed treatment? } \\
\hline \multirow[t]{2}{*}{ Bipolar Disorder } & Always/Commonly & Somewhat comm & 0.039 & 0.195 & 0.051 \\
\hline & Never/Uncommonly & Somewhat comm & -0.358 & 0.147 & - \\
\hline \multirow[t]{2}{*}{ GAD } & Always/Commonly & Somewhat comm & 0.361 & 0.160 & 0.067 \\
\hline & Never/Uncommonly & Somewhat comm & 0.155 & 0.256 & $\cdots$ \\
\hline \multirow[t]{2}{*}{ MDD } & Always/Commonly & Somewhat comm & 0.214 & 0.188 & 0.092 \\
\hline & Never/Uncommonly & Somewhat comm & -0.463 & 0.247 & $\cdots$ \\
\hline \multicolumn{6}{|c|}{ Preparedness - How prepared to manage at completion of residency? } \\
\hline \multirow[t]{2}{*}{ Bipolar Disorder } & Not at all/Slightly & Somewhat & -0.534 & 0.149 & 0.001 \\
\hline & Very/Completely & Somewhat & 0.262 & 0.264 & - \\
\hline
\end{tabular}

Table 1. Adjusted Association between Clinician Characteristics and Mental Illness Management Self-Efficacy

\begin{tabular}{|c|c|c|c|c|c|}
\hline Variable group & Covariate & Reference & Estimate & SE & P-value \\
\hline Number Providers & 1 (just myself) & 2 and up & -0.629 & 0.285 & 0.028 \\
\hline \multirow[t]{5}{*}{ Clinical Practice Site } & Community Health Center & Private & -0.275 & 0.261 & 0.016 \\
\hline & Non-academic/MCO & Private & -0.128 & 0.208 & $\ldots$ \\
\hline & University & Private & -0.267 & 0.280 & -- \\
\hline & VAMC & Private & -0.272 & 0.284 & $--\cdot$ \\
\hline & Other & Private & -1.573 & 0.478 & $\ldots$ \\
\hline Ethnicity & Hispanic & Non-Hispanic & -0.931 & 0.424 & 0.029 \\
\hline Specialty & Family medicine & Internal & -0.395 & 0.153 & 0.010 \\
\hline \multirow[t]{2}{*}{ Resources } & Health psychologist & No access & 0.592 & 0.175 & $<.001$ \\
\hline & Electronic Medical Record & No access & 0.559 & 0.276 & 0.044 \\
\hline Communication & SE Factor Score & & 0.498 & 0.082 & $<.001$ \\
\hline Attitude Toward Team-based Care & Mean score & & 1.111 & 0.174 & $<.001$ \\
\hline Team Climate Inventory & Mean score & & 0.226 & 0.108 & 0.038 \\
\hline
\end{tabular}

$<$ !-StartFragment- $>$ Table 2. Adjusted Association between Clinician Characteristics and Team Based Care Self-Efficacy $<$ !-EndFragment- $>$

PREOPERATIVE RISK FACTORS PREDICT RISK OF DELIRIUM AND OTHER POSTOPERATIVE COMPLICATIONS AMONG ELDERLY PATIENTS UNDERGOING ELECTIVE SURGERY: SYSTEMATIC REVIEW Jennifer A. Watt Andrea Tricco $^{1,2}$; Catherine Talbot-Hamon ${ }^{3}$; Agnes Grudniewicz ${ }^{4,5}$; Douglas Sinclair ${ }^{3,6}$; Sharon Straus ${ }^{2,3}$. 'Dalla Lana School of Public Health, University of Toronto, Toronto, ON, Canada; ${ }^{2} \mathrm{Li}$ Ka Shing Knowledge Institute, St Michael's Hospital, Toronto, ON, Canada; ${ }^{3}$ Department of Medicine, University of Toronto, Toronto, ON, Canada; ${ }^{4}$ Institute of Health Policy, Management and Evaluation, University of Toronto, Toronto, ON, Canada; ${ }^{5}$ Health System Performance Research Network, Toronto, ON, Canada; ${ }^{6} \mathrm{St}$ Michael's Hospital, Toronto, ON, Canada. (Control ID \#2466735)

BACKGROUND: As elderly patients are increasingly undergoing elective surgery, clinicians have an opportunity to identify patients at higher risk of postoperative complications and to potentially lessen this risk; however, the optimal method for assessing elderly patients' risk of postoperative complications remains unclear. The objective of this study was to identify preoperative risk factors that increase the odds of elderly patients experiencing postoperative complications.

METHODS: A systematic review was conducted to identify preoperative risk factors and assessment tools that predict elderly patients' risk of postoperative complications. Studies were identified by searching electronic databases (MEDLINE, EMBASE, CINHAL, AgeLine, and Cochrane Central Register of Controlled Trials) for articles published between 1948 and June 24, 2014, and by reviewing reference lists of included studies. Authors of conference abstracts were contacted for unpublished work. Prospective studies reporting risk factors for postoperative complications including delirium, functional decline, destination at discharge from hospital, prolonged length of hospitalization, and postoperative mortality among elderly patients ( $\geq 60$ years and mean age $\geq 65$ years) undergoing elective surgery were included. Studies were excluded if they included only risk factors that would not be considered as part of the comprehensive geriatric assessment (i.e. serum interleukin). Two independent reviewers conducted all levels of screening, data abstraction, and quality appraisal; discrepancies were resolved through discussion. The methodological quality of cohort studies was assessed using the Ottawa Newcastle Scale and the risk of bias in the controlled before-and-after study was assessed with the Cochrane Collaboration's Risk of Bias Tool. Data analysis will be completed in February 2016.

RESULTS: Fifty-four cohort studies and 1 controlled before-and-after study (11,596 patients) were included after screening 5165 citations. An additional 14 companion reports were retained for supplementary information. Included studies reported preoperative risk factors for the following outcomes: postoperative changes in cognition (number of included studies $(n)=$ $30)$, prolonged length of hospitalization $(n=14)$, destination at discharge from hospital $(n=5)$, functional decline $(n=5)$, postoperative complications $(n=24)$ and postoperative mortality $(n=$ 10). Several types of surgery were listed among the included studies: gastrointestinal/abdominal $(n=17)$, cardiac $(n=15)$, orthopedic $(n=14)$, urological $(n=8)$, vascular $(n=5)$, thoracic $(n=$ $5)$, gynecological $(n=2)$, and other $(n=7)$. Preoperative assessments among included studies were quite varied; some studies reported complete comprehensive geriatric assessments, but others reported only selected components (i.e. baseline demographics, assessments of physical or cognitive function, investigations). The timing of the preoperative assessment was not reported in 24 studies; however, in studies in which the timing of the preoperative assessment was reported, it ranged from 3 months to the day of admission to hospital for surgery. Twentyeight studies reported varying levels of detail concerning patients' surgical procedures. The duration of patient follow-up in the postoperative period ranged from 2 days to 1 year. Older age, 
functional dependence, cognitive impairment, frailty, history of alcoholism or smoking, presence of severe preoperative pain, lower education level, and a history of depression were significant predictors of changes in cognition (defined as delirium, confusion, postoperative cognitive dysfunction, or cognitive changes) after elective surgery. Poor functional status at baseline, greater comorbidity, smoking status, male sex, and having a diagnosis of cancer were predictive of functional decline. Destination at discharge from hospital was predicted by functional dependence, frailty, history of falls, weight loss $\geq 10 \%$, ASA score $\geq 2$, and cognitive impairment. Older age, frailty, functional dependence, cognitive impairment, smoking status, small arm circumference, slower Timed Up and Go test (TUG), higher ASA score, polypharmacy, and weight loss $\geq 10 \%$ were all predictive of prolonged hospitalization. Older age, cognitive impairment, frailty, falls, greater comorbidity, functional dependence, slower TUG, and low preoperative hematocrit and albumin levels were all predictive of postoperative mortality. CONCLUSIONS: There are a number of identifiable risk factors that predict postoperative complications and these risk factors should be part of a preoperative assessment when considering elective surgery in elderly patients. Further studies are needed to help refine this list of risk factors and to develop interventions that can be implemented to help mitigate this risk.

PREPARING THE NEXT GENERATION: BUILDING AN INTERPROFESSIONAL ORAL HEALTH WORKFORCE Jennifer Adams ${ }^{2}$; Kenneth L. Allen ${ }^{4}$; Judith Haber ${ }^{5}$; Erin Hartnett ${ }^{1}$; Thomas S. Riles ${ }^{5}$; Abigail Bella ${ }^{1}$; Ruth Crowe ${ }^{3} .{ }^{1} \mathrm{NYU}$ College of Nursing, New York, NY; ${ }^{2} \mathrm{NYU}$ School of Medicine, New York, NY; ${ }^{3} \mathrm{NYU}$ School of Mediicne, New York, NY; ${ }^{4}$ New York University College of Dentistry, New York, NY; ${ }^{5}$ NYU, New York, NY. (Control ID \#2469064)

BACKGROUND: The aim of the Teaching Oral-Systemic Health (TOSH) Program Interprofessional Oral-Systemic Health Standardized Patient and Case Study Experience at New York Simulation Center for Health Sciences (NYSIM) is to promote the acquisition of the Core Competencies for Interprofessional Collaborative Practice (IPEC, 2011) and the Interprofessional Oral Health Core Clinical Competencies (HRSA, 2014) among future primary care providers by engaging nurse practitioner (NP), nurse-midwifery (NM), dental (DDS), and medical (MD) students in interprofessional oral-systemic health simulation experiences.

METHODS: During the Fall 2013 and 2014, students and faculty from NYU College of Nursing, College of Dentistry, and School of Medicine participated in an interprofessional simulation experience featuring a standardized patient (SP) and case study teaching-learning strategy to expose students to both an oral-systemic and interprofessional clinical experience in order to advance their competence in both areas. The Interprofessional Collaborative Competencies Attainment Survey (ICCAS) was selected to evaluate the degree to which students, using a pre-test/post-test approach, report a change in perception of their own IP competencies following the interprofessional learning experiences.

RESULTS: For all three student groups in both 2013 and 2014, the changes in ICCAS mean scores from pre to post were significant ( $p<0.001$, two-tailed) (See Figure 1). There was also a statistically significant change in mean scores from pre-test to post-test in each of the six interprofessional competency domains measured by the ICCAS for all participating students $(p<0.001$, two-tailed) (See Figure 2). There were no significant differences in the magnitude of change in ICCAS survey scores among the different student groups.

CONCLUSIONS: These findings suggest that the TOSH Interprofessional Oral-Systemic Health Standardized Patient and Case Study Experience was similarly effective among all three student groups in having a positive impact on students' perceived interprofessional competencies. Topics in which mean post-survey scores were lower are potential opportunities for curriculum refinements to further emphasize those competencies. A clinical simulation approach appears to be an effective strategy for improving students' self-assessed change in level of interprofessional competency.
Figure 2. TOSH Event ICCAS Mean Pre and Post Scores by Topic Area $(2013 \& 2014)(n=618)$

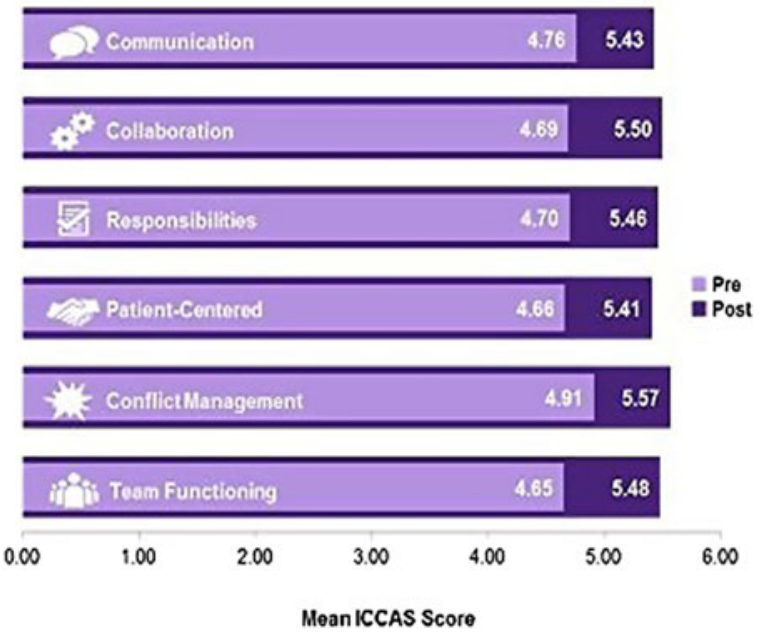

Figure 1. TOSH Event ICCAS Mean Pre and Post Scores by Student Group (2013 \& 2014)

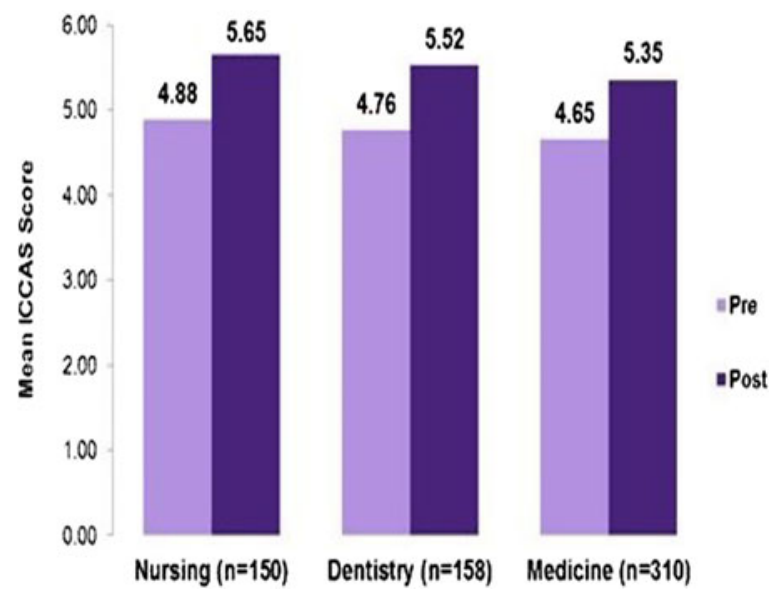

PREVALENCE AND PREDICTORS OF NON-ADHERENCE WITH BREAST CANCER SCREENING: PERSPECTIVE AND PREFERENCES OF HOSPITALIZED WOMEN Waseem Khaliq ${ }^{2}$; Regina Landis ${ }^{1}$; Scott Wright ${ }^{3} .{ }^{1}$ Johns Hopkins, Baltimore, MD; ${ }^{2}$ Johns Hopkins University School of Medicine, Ellicott city, MD; 3 jhusom, Baltimore, MD. (Control ID \#2464235)

BACKGROUND: Disparities in screening mammography persist among low-income women, even those who are insured, despite the proven mortality benefit. We evaluated the prevalence of breast cancer screening non-adherence among hospitalized women and their receptivity to inpatient screening mammography as a novel approach to increase compliance with screening.

METHODS: A cross sectional study was conducted among 250 hospitalized women age 50-75 years, admitted to medicine service at Johns Hopkins Bayview Medical Center in early 2012. Respondent characteristics are presented as proportions and means. Unpaired $t$ test and Chi square tests were used to compare demographic and socioeconomic 
characteristics, barriers to screening mammography, and receptivity to inpatient screening mammography based on adherence to breast cancer screening guidelines. T-tests and chisquare tests determined significance at $p<0.05$. The probit regression model was used for the analysis of contingent valuation data to predict mean willingness-to-pay (WTP). Logistic regression models were used for analyses to predict the odds of non-adherence with breast cancer screening among the hospitalized women. The survey data were analyzed using Stata statistical software (StataCorp LP, Version 12.1).

RESULTS: Approximately one-third of women enrolled were African American, and $61 \%$ of the study population reported an annual household income of less than $\$ 20,000$. Forty-one percent were overdue for screening, $13 \%$ of whom had never had a mammogram. Thirty-two percent of the women were at high risk for breast cancer ( 5 -year risk prediction Gail score $\geq 1.7 \%$ ). The most commonly reported barriers to screening mammograms were failure to remember appointments, and lack of transportation. After adjustment for demographic variables and comorbidities, three variables were found to be independently associated with nonadherence to breast cancer screening: low income ( $\mathrm{OR}=3.81,95 \% \mathrm{CI} ; 1.84-7.89)$, current or ex-smoker $(\mathrm{OR}=2.29,95 \% \mathrm{CI} ; 1.12-4.67)$, and history of stroke $(\mathrm{OR}=2.83,95 \% \mathrm{CI} ; 1.21-6.60)$. Most women (91\%) believed that it is important for healthcare providers to discuss breast cancer screening while patients are in the hospital, and $72 \%$ were willing to pay out of pocket a mean of $\$ 83.41$ (95\% CI; \$71.51-\$95.31) towards inpatient screening mammogram costs.

CONCLUSIONS: A significant number of hospitalized women from lower socioeconomic class are non-adherent and at high risk of developing breast cancer. Because hospitalization creates the scenario wherein patients are in close proximity to healthcare resources, at the same time that they are reflecting upon their health status, strategies could be employed to counsel, educate, and motivate these patients towards health maintenance.

PREVALENCE AND RISK FACTROS OF VITAMIN-D DEFICINECY IN YOUNG HEALTHY NEPALESE POPULATION: A CROSSECTIONAL STUDY AT A UNIVERSITY HOSPITAL IN KATHMANDU, NEPAL. Samiksha Pandey ${ }^{1}$; Louise Spendrup ${ }^{2}$; Lua Rangel ${ }^{2}$; Aseem Bhattarai ${ }^{1} .{ }^{1}$ Institute of Medicine, Kathmandu, Nepal; ${ }^{2}$ University of Gotenborg, Gotenborg, Sweden. (Control ID \#2469535)
BACKGROUND: The serum levels of vitamin D (25 OH-D) are known to be affected by several different factors, including latitude, season, clothing, skin types, duration of exposure to sun, and the intake of food containing vitamin D. Different studies conducted in our region show a prevalence of vitamin D insufficiency in the general population. Very few large scale studies in the context of a developing country like Nepal have been conducted, nevertheless, and the ones that have been finished suggest a general insufficiency of vitamin $\mathrm{D}$, in different cohorts of patients, especially in preschool children. We aimed to determine the association between vitamin D levels in normal healthy Nepalese individuals and the exposure to sunlight, the skin type and dietary intake of vitamin D

METHODS: We performed a cross sectional descriptive study from 24th March to 23rd May, 2015100 apparently healthy adults coming in for general health checkups in the University Hospital at the Institute of Medicine. Serum 25-hydroxyvitamin D (25-OH D) level was measured by standard laboratory technique. The remaining data was collected using a general self-administered questionnaire including dietary habits and sun exposure. Standard Fitz Patrick's scale was used to classify skin types of patients. Subjects were grouped into different categories based on variables and independent sample t-tests and Analysis of Variance (ANOVA) were conducted to compare mean values and to assess the statistical significance.

RESULTS: Among 100 healthy subjects, 53 were female and 47 were male. Mean age was $30.26 \pm 8.0$ years (male $31.23 \pm 8.4$, female $29.40 \pm 7.7$ ). Only $4 \%$ had normal 25 OH D level (Figure 1, panel a-b). The mean 25-OH D level was significantly higher in males $(18.08 \pm 6.0 \mathrm{ng} / \mathrm{ml}$ for males, $15.03 \pm 6.9 \mathrm{ng} / \mathrm{ml}$ for females, $p$-value $=0.029)$. The subjects with vegetarian diet were found to have significantly low 25-OH D level compared to subjects with non-vegetarian diet $(13.0 \pm 4.8 \mathrm{ng} / \mathrm{ml} \mathrm{vs} 16.9 \pm 7.1 \mathrm{ng} / \mathrm{ml})$. Fish intake was found to have similar impact on the level of 25-OH D. However, there was no significant difference in mean 25-OH D levels associated with amount of sun exposure and skin types in the study population.

CONCLUSIONS: Our study shows high prevalence of vitamin D insufficiency in healthy subjects of Nepal. Low dietary intake is likely the cause of the low serum levels of vitamin D in this population as vegetarians have significantly lower level. However, sun exposure and the skin type of Nepalese population did not have any correlation with low vitamin D level contrary to the studies done in developed world.
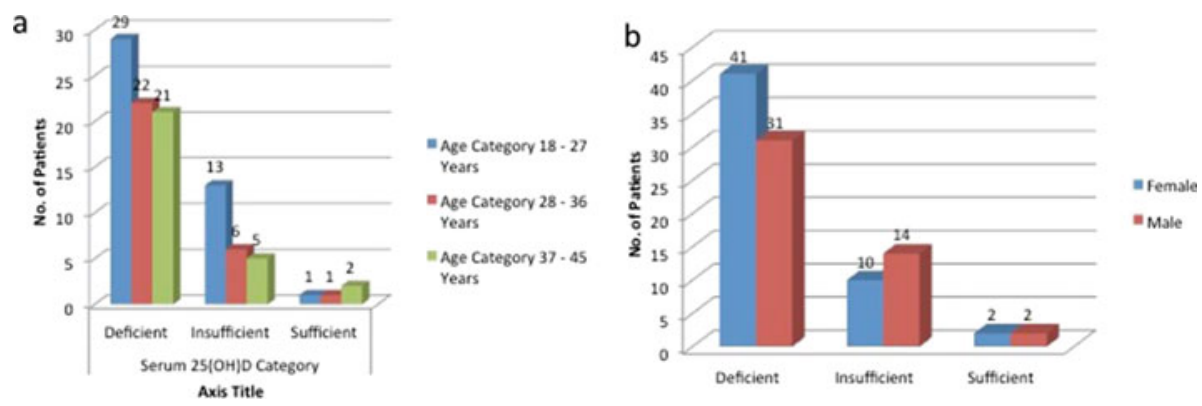

Figure 1: Panel a- age wise distribution, Panel-b sex wise distribution of $25-\mathrm{OH}$ D level (Deficient $<20 \mathrm{ng} / \mathrm{ml}$, sufficient $>30 \mathrm{ng} / \mathrm{ml}$ and insufficient $20-30 \mathrm{ng} / \mathrm{ml}$ ).

PREVALENCE OF PHYSICIAN/PHARMACIST COUNSELING AROUND CONCOMITANT USE OF ACETAMINOPHEN AND RISK OF DOUBLE DIPPING $\underline{\text { Rebecca Mullen }}{ }^{1}$; Laura M. Curtis ${ }^{1}$; Marina Serper ${ }^{2}$; Danielle M. McCarthy ${ }^{1}$; Kara L. Jacobson $^{3}$; Ruth Parker ${ }^{3}$; Michael S. Wolf ${ }^{1}{ }^{1}$ Northwestern University, Chicago, IL; ${ }^{2}$ University of Pennsylvania, Philadelphia, PA; ${ }^{3}$ Emory University, Atlanta, GA. (Control ID \#2469189)
BACKGROUND: Unintentional concomitant use (aka 'double dipping') of prescription and over-the-counter (OTC) acetaminophen-containing products continues to be a significant public health concern. To date, systematic efforts to promote safe use of these products have primarily been limited to labeling changes. The objectives of the present study were 1) to investigate the prevalence of physician and/or pharmacist spoken counseling to patients 
newly prescribed acetaminophen-containing prescription opioids, and 2) to document patients' risk of double dipping of acetaminophen-containing products.

METHODS: Between April and September 2011, 248 adult patients receiving a new prescription for an acetaminophen-containing medication were recruited at discharge from an emergency department in Chicago, IL ( $n=111)$ or from a hospital pharmacy in Atlanta, GA $(n=137)$. Patients were provided a standard, formatted medication diary and for the next 4 days asked to record how they took their new acetaminophen-containing prescription, as well as any chronic prescribed and OTC medications. Patients were asked to return 4-7 days later for a follow-up visit, at which time patients' medication diaries were reviewed to determine whether they simultaneously used their prescribed acetaminophen product with another acetaminophen-containing medication. Patients were then also shown additional, common OTC acetaminophen-containing products and asked to respond if they could take these medications in addition to their actual prescription. Recall of physician and pharmacist counseling specific to concomitant use of acetaminophen was also assessed via patient self-report. A combination of two-sample t-tests, ANOVAs and Fisher's exact tests were used to determine the association of demographic variables with physician or pharmacist counseling and both our double dipping outcomes. Multivariable analyses were performed to examine the independent association of provider counseling with double dipping, controlling for gender, race, education, income, total number of medications and dispensing site.

RESULTS: Participants had a mean (SD) age of 43 (11.8) years; $54 \%$ were female, $67 \%$ African American, $47 \%$ had a high school education or less and $59 \%$ reported earning less than $\$ 20,000$ per year. Fourteen percent of patients reported physician counseling regarding concomitant use for acetaminophen products, while only $3 \%$ reported receipt of such counseling from a pharmacist. White race, college education, self-reported income of more than $\$ 50,000$ per year and the Chicago site were all associated with increased likelihood of either physician or pharmacist counseling. Review of patients' medication diaries revealed that $11 \%$ of patients combined their actual acetaminophen-containing prescription with at least one additional acetaminophen-containing product. Forty-two percent of patients were considered at increased risk for double dipping; this was characterized as having reported they felt it was safe to use their prescription with one or more OTC acetaminophen products presented to them. Gender, education and lack of provider counseling were all associated with demonstrated double-dipping or being at risk for concomitant use. In multivariable models, participants self-reporting either physician or pharmacist counseling related to concomitant use of acetaminophen-products were significantly less likely to demonstrate misuse after controlling for relevant covariates (OR 0.29 , CI $0.12-0.68, p=0.01$ ).

CONCLUSIONS: Overall, counseling for safe use of acetaminophen by either a physician or pharmacist was exceptionally low. A moderate number of participants demonstrated concomitant use with their actual prescribed medications, and nearly half of patients displayed poor understanding and increased risk for double dipping. Those receiving physician or pharmacist counseling explicit to the warning of concomitant acetaminophen use were less likely to be at risk for misuse. Reliance on label information and warnings may not be sufficient to mitigate concomitant use of acetaminophen-containing products. Increased efforts to counsel patients regarding safe use at the time of prescribing may be necessary to appropriately address patient risk.

PREVENTING HOSPITALIZATIONS WITH VETERANS AFFAIRS HOME BASED PRIMARY CARE: WHICH PATIENTS BENEFIT MOST? Samuel T. Edwards ${ }^{1,2}{ }^{2}$; Somnath Saha ${ }^{1,}{ }^{2}$; Julia Prentice ${ }^{3}$; Steven D. Pizer ${ }^{3} .{ }^{1}$ VA Portland Health Care System, Portland, OR; ${ }^{2}$ Oregon Health and Science University, Portland, OR; ${ }^{3}$ VA Boston Healthcare System, Boston, MA. (Control ID \#2464980)
BACKGROUND: Intensive primary care and home-based services have the potential to reduce rates of hospitalization for patients with extensive care needs, but appropriate patient selection is critical to ensure efficacy and cost effectiveness. In this study, we describe how the degree of patient comorbidity modified the relationship between enrollment in Home Based Primary Care (HBPC), a national home care program operated by the US Department of Veterans Affairs (VA), and hospitalizations for ambulatory care sensitive conditions.

METHODS: We performed a national cohort study of Veterans over age 67, who were traditional Medicare beneficiaries and had a diagnosis of diabetes mellitus. Our primary exposure was enrollment in HBPC, defined as a minimum of $3 \mathrm{HBPC}$ encounters. Our outcomes were preventable hospitalizations, i.e., hospitalizations for an ambulatory care sensitive condition (ACSC), as measured by the Agency for Healthcare Research and Quality's Prevention Quality Indicators. Data consisted of VA records supplemented by Medicare claims. Patients were followed from January 1st 2006 through December 31st, 2010. We evaluated the effect of HBPC enrollment on ACSC hospitalizations, using a two-stage discrete time survival model. We used distance from the Veteran's residence to the nearest VA facility that provides HBPC as an instrumental variable, to control for potential unmeasured confounding. We used the results of this regression to calculate risk scores for ACSC hospitalization based on individual Elixhauser comorbidities. We then conducted stratified analyses of the effect of HBPC enrollment on overall ACSC hospitalization rates, by risk score quartile. We subsequently examined the effect of HBPC enrollment on hospitalization rates for specific ACSCs.

RESULTS: We identified 364,941 Veterans with 16,570,421 person-months of followup. Of these, 4542 Veterans enrolled in HBPC and 360,399 did not. HBPC patients were older (mean 76.0 vs. 70.9 years), had more co-existing conditions including congestive heart failure (CHF 39.9 vs. $18.4 \%$ ), and chronic pulmonary disorder (41.0 vs. $26.4 \%$ ), and had a higher prevalence of mental illness (depression 29.5 vs. $15.0 \%$ ). The ACSC hospitalization risk score ranged from -2 to 27 , with a median of 1 (IQR 0-6). A summary of patient characteristics stratified by ACSC risk score quartile is presented in the table. Mean number of comorbidities increased across risk score quartiles. In the first quartile, most patients had hypertension as their only comorbidity, while in the fourth quartile, $64 \%$ of patients had CHF and $71 \%$ had chronic pulmonary disorder. For the entire sample, HBPC enrollment was associated with a significant decrease in the odds of all ACSC hospitalizations (OR 0.35 per person-month, $95 \%$ CI $0.30-0.42$ ). Results of the stratified analysis are presented in the figure. In the first quartile (lowest risk), HBPC enrollment was associated with significantly increased odds of all ACSC hospitalizations (OR 33.18, $95 \%$ CI 4.58-240.12). In the second and third quartiles, no significant association was observed (second quartile HBPC OR 3.56, $95 \%$ CI 0.83-15.31, third quartile HBPC OR $2.11,95 \%$ CI 0.69-6.53). In the highest quartile, HBPC enrollment was associated with a significant decrease in the odds of all ACSC hospitalizations (OR 0.43, 95 \% CI 0.190.93). In analyses of individual ACSC hospitalizations, HBPC enrollment was associated with a significant decrease in the odds of hospitalization for CHF (OR 0.46, $95 \%$ CI 0.35-0.60), bacterial pneumonia (OR 0.21, $95 \%$ CI 0.14-0.31), chronic pulmonary disease (OR $0.64,95 \%$ CI $0.42-0.98$ ), urinary tract infection (OR 0.47, $95 \%$ CI $0.31-0.71$ ) and dehydration (OR $0.28,95 \%$ CI $0.15-$ 0.51). HBPC enrollment was associated with a significant increase in hospitalization for angina (OR 4.46, $95 \%$ CI 1.48-13.43).

CONCLUSIONS: HBPC enrollment is associated with lower odds of hospitalization for ACSC conditions, but this association is limited to patients at highest risk of hospitalization, while for patients at lower risk, HBPC enrollment was associated with increased odds of hospitalization. HBPC was associated with reductions in multiple clinical conditions with varied etiologies, suggesting that HBPC's comprehensive approach is responsible for its impact. These findings have important implications for the design and financing of intensive ambulatory care programs like HBPC. 
Patient Characteristics by ACSC Risk Score Quartiles.

\begin{tabular}{|c|c|c|c|c|}
\hline & $\begin{array}{l}\text { Quartile 1 } \\
(n=83631)\end{array}$ & $\begin{array}{l}\text { Quartile } 2 \\
(n=103695)\end{array}$ & $\begin{array}{l}\text { Quartile } 3 \\
(n=85940)\end{array}$ & $\begin{array}{l}\text { Quartile 4 } \\
(n=92175)\end{array}$ \\
\hline $\begin{array}{l}\text { Risk Score } \\
\text { Range }\end{array}$ & -2 to -1 & $0-2$ & $3-6$ & $7-27$ \\
\hline $\begin{array}{l}\text { Proportion } \\
\text { enrolled in } \\
\text { HBPC }\end{array}$ & $0.3 \%$ & $0.8 \%$ & $1.3 \%$ & $2.5 \%$ \\
\hline $\begin{array}{l}\text { Mean Number } \\
\text { Elixhauser } \\
\text { Comorbidities } \\
\text { Prevalence } \\
\text { of selected } \\
\text { Elixhauser } \\
\text { comorbidites } \\
(\%)\end{array}$ & $\begin{array}{l}1.57 \\
\text { (SD 0.68) }\end{array}$ & $\begin{array}{l}2.30 \\
\text { (SD 1.30) }\end{array}$ & $\begin{array}{l}3.67 \\
\text { (SD 1.52) }\end{array}$ & $\begin{array}{l}6.07 \\
\text { (SD 2.26) }\end{array}$ \\
\hline Hypertension & $98.9 \%$ & $73.39 \%$ & $84.08 \%$ & $88.96 \%$ \\
\hline $\mathrm{CHF}$ & $0 \%$ & $0 \%$ & $11.12 \%$ & $64.00 \%$ \\
\hline $\begin{array}{l}\text { Chronic } \\
\text { Pulmonary } \\
\text { Disorder }\end{array}$ & $0 \%$ & $0 \%$ & $37.19 \%$ & $70.96 \%$ \\
\hline $\begin{array}{l}\text { Cardiac } \\
\text { Arrhythmia }\end{array}$ & $0 \%$ & $12.08 \%$ & $25.59 \%$ & $58.23 \%$ \\
\hline Psychosis & $0.03 \%$ & $15.07 \%$ & $16.37 \%$ & $18.23 \%$ \\
\hline
\end{tabular}

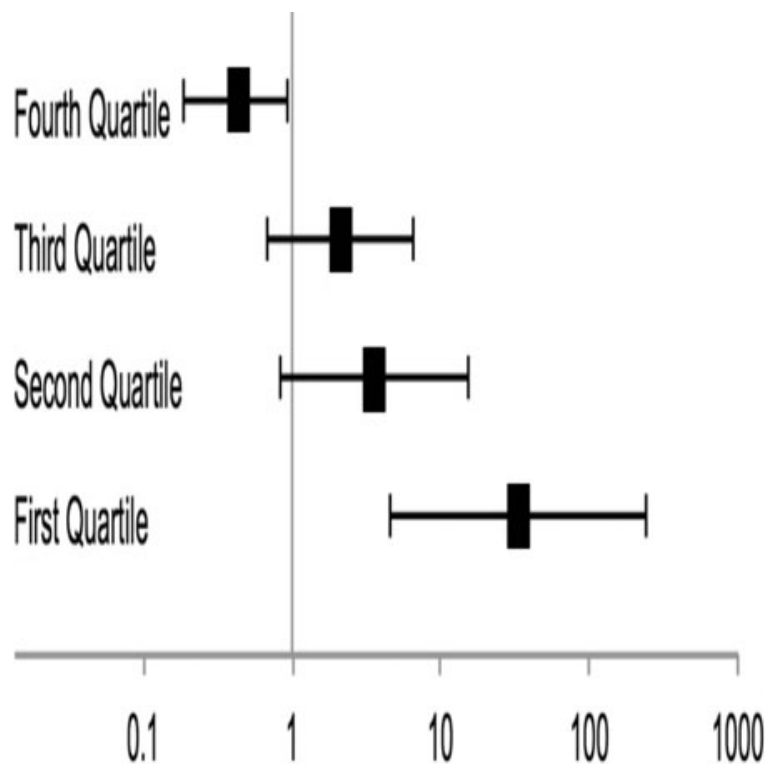

PRIMARY CARE AND OUTCOMES IN ADULT PATIENTS WITH CANCER: A SYSTEMATIC REVIEW Zackary Berger ${ }^{1,3}$; Ya Luan $\mathrm{Hsiao}^{4}$; Victoria G. Riese ${ }^{5}$; Rachel K. Walker ${ }^{6}$; Patricia Davidson ${ }^{7}$; Craig E. Pollack ${ }^{2}$; Claire F. Snyder ${ }^{1} .{ }^{1}$ Johns Hopkins School of Medicine, Baltimore, MD; ${ }^{2}$ Johns Hopkins University School of Medicine, Baltimore, MD; ${ }^{3}$ Johns Hopkins Berman Institute of Bioethics, Baltimore, MD; ${ }^{4}$ Johns Hopkins School of Public Health, Baltimore, MD; ${ }^{5}$ National Institutes of Health Library, Bethesda, MD; ${ }^{6}$ University of Massachusetts-Amherst College of Nursing, Amherst, MA; ${ }^{7}$ Johns Hopkins School of Nursing, Baltimore, MD. (Control ID \#2469702)

BACKGROUND: Previous studies have reported on the importance of primary care provider (PCP) involvement in cancer survivorship. However, little is known about the effect of primary care compared to usual care among adult cancer patients.

METHODS: We evaluated the types of primary care in adult cancer patients and impact on clinical outcomes, patient reported outcomes and health maintenance. Medline and CINAHL were systematically searched for studies published till January 1, 2015. Two independent reviewers assessed the eligibility of each article based on predefined criteria (study design, population, PCP intervention and outcomes). A third reviewer adjudicated disagreements. Data were extracted and outcomes were qualitatively synthesized. PRISMA criteria were used for reporting and GRADE criteria to assess risk of bias. RESULTS: From 4333 titles, 424 abstracts were reviewed and 47 studies met inclusion criteria. A meta-analysis was not performed due to heterogeneity of outcome measures, cancer types, and population, as well as missing data. The studies were randomized control trials $(n=24)$; prospective cohort studies $(n=3)$; retrospective cohort studies $(n=10)$; cross sectional studies $(n=3)$; and case-control studies $(n=3)$; non-controlled trials $(n=2)$ and nonexperimental designs $(n=2)$. The studies included various types of PCPs: 12 studies involved primarily nurses, 25 primarily physicians, and the remainder multidisciplinary arrangements. We categorized endpoints a priori into clinical outcomes, patient reported outcomes and health maintenance. Twenty studies reported clinical outcomes (including physician visits, hospitalizations and costs). Two studies found no difference in cost of care; one study reported a $37 \%$ reduction in cost of care in the outpatient setting. Three studies reported decreased hospitalizations with primary care compared to usual care; three studies reported that there were increased visits to PCPs and fewer visits to specialists with primary care compared to usual care. Twenty studies reported patient reported outcomes. Nine studies reported no improvement for quality of life while four studies with nurse-led primary care showed improvement in quality of life. For health maintenance ( 8 studies), patients who saw PCPs (or both PCPs and oncologists) were more likely to receive mammograms, cancer screening and vaccinations compared to those with only oncologist follow-up. Quality was mixed: 27 studies were associated with a high risk of bias, 11 with a low risk of bias. In 9 studies, the risk of bias was unclear or could not be assessed. Common sources of bias included lack of blinding of personnel, lack of blinding of outcome assessors to intervention status, and lack of blinding of assessors to critical outcomes.

CONCLUSIONS: Cancer care involving PCPs in comparison to non-PCPs was associated with mixed effects. There was no clear conclusion for clinical and patient-reported outcomes. Primary care was associated with greater uptake of health maintenance services compared to no primary care but less uptake compared to follow-up by both PCPs and oncologists. Improvement in quality of life was reported in the studies that involved nurses as PCPs. Evidence for care coordination in cancer survivorship can lead to targeted quality improvement.

PRIMARY CARE ENGAGEMENT IN EMERGENCY DEPARTMENT PATIENTS WITH MULTIPLE CHRONIC CONDITIONS IN A MEDICALLY UNDERSERVED AREA Bianca Jackson ${ }^{1}$; Erik L. Carlton ${ }^{1}$; George Relyea ${ }^{1}$; SangNam $\mathrm{Ahn}^{1}$; James E. Bailey ${ }^{2}$. ${ }^{1}$ University of Memphis, Memphis, TN; ${ }^{2}$ University of Tennessee Health Science Center, Memphis, TN. (Control ID \#2469714)

BACKGROUND: Patients with chronic conditions in medically underserved minority communities are often less engaged in primary care, less likely to get recommended care, often delay or forgo needed care, and experience high emergency department (ED) utilization and adverse clinical outcomes. However, precise estimates of the levels of primary care engagement seen in these vulnerable populations are poorly recorded within hospital registrar records. This study sought to assess patient primary care engagement, assessment of chronic illness care and continuity of care, and interest in self-care interventions to improve primary care engagement among diabetes patients with multiple chronic conditions (MCC), using a major ED in a medically underserved area (MUA).

METHODS: A cross-sectional survey of a convenience sample of $30 \mathrm{ED}$ patients with diabetes, and one or more additional chronic conditions, was conducted in a majority African-American, low-income, and medically underserved community. The survey 
measured patient self-reports of chronic illness care, continuity of care, and primary care engagement. Primary care provider (PCP) status was extracted from hospital registrar data. Demographic and primary care engagement characteristics, and the validity of the local hospital registrar's assessment of PCP status were evaluated using descriptive statistics, chi-square tests of independence, independent sample t-tests, one-way ANOVAs, and bivariate correlations.

RESULTS: The sampled patient population had an average of 3.3 chronic conditions per patient, a mean age of 56 years, $63 \%$ were female, and $90 \%$ non-Hispanic Black. The survey revealed that $83 \%$ of patients reported having a PCP, with nearly half (46.7\%) seeing their provider 3-4 times in the past year. Hospital registrar data assessment showed only moderate agreement with patient reports (Cohen's $\mathrm{K}=.512, p=.004$ ), underestimating PCP status at $72.4 \%$. Many patients reported delaying needed care due to various reasons. Getting a timely appointment (29.6\%), transportation difficulties (26.9\%), and inconvenient office hours ( $22.2 \%$ ) were cited as the most notable barriers to accessing chronic illness care. But delaying needed care was not significantly associated with health literacy level or other patient factors. Most patients reported high levels of continuity with PCPs, however patients also reported many perceived gaps in the quality of their chronic illness care including: never being encouraged to go to health groups or classes $(57.1 \%$ ), never being asked for their ideas on the treatment plan (35.7\%), never being given a written list of things to improve their health (33.3\%), and never being asked about goals $(21.4 \%)$. Furthermore, patients reported high levels of interest in mobile and in-person interventions to improve primary care engagement. Specifically, $76.9 \%$ used a smart phone and $92.9 \%$ used some type of cell phone. Of these users $50 \%$ sent or received text messages one or more times a day, and expressed high levels of interest (73\%) in receiving text messages from their provider's office. Patients $(88 \%)$ also reported a strong interest in health coaching to help them reach specific diabetes self-care goals.

CONCLUSIONS: This study demonstrates that most medically underserved diabetes patients with MCC have PCPs, but difficulty in accessing high quality primary care often contributes to their foregoing of needed care. These patients also report significant gaps in the quality of the chronic illness care they do receive. Patients are highly interested in mobile health and in-person interventions to improve their engagement in primary care. Furthermore, PCP status is underrepresented in hospital registrar documentation. These results suggest that primary care-based text messaging and health coaching have strong potential to improve diabetes self-care and primary care engagement in MUA. In addition, more accurate registrar documentation of PCP status is needed to facilitate patient linkage to essential outpatient care.

PRIMARY CARE ENGAGEMENT IS ASSOCIATED WITH MEDICATIONASSISTED TREATMENT FOR ALCOHOL USE DISORDER. Paul Joudrey ${ }^{2}$; Mathew Kladney ${ }^{2}$; Chinazo Cunningham ${ }^{1} .{ }^{1}$ Albert Einstein College of Medicine \& Montefiore Medical Center, Bronx, NY; ${ }^{2}$ Montefiore Medical Center, New York, NY. (Control ID \#2466611)

BACKGROUND: Alcohol use accounts for up to $12 \%$ of disease and injury burden among American adults and it is one of the leading causes of preventable death in the United States. Primary care providers frequently encounter alcohol use disorders (AUD) and treatment is needed to reduce this disease and injury burden. Evidence based treatment for AUD includes community-based, individual psychological and pharmacologic therapies. Medication-assisted treatment (MAT) for AUD has the potential to reduce harm; naltrexone, acomprasate and topiramate all have strong evidence supporting their effectiveness by reducing high risk drinking and promoting abstinence. The numbers needed to treat for these medications are comparable to medications used to treat tobacco disorders and these medications should be broadly adopted within the primary care setting. Despite the known benefits of MAT for AUD, MAT is underutilized. Only $3.0 \%$ of patients with AUD in the Veteran's Health Administration were prescribed MAT. Young age, female gender and exposure to specialty addiction care were associated with the receipt of MAT. Despite the recognized promise of MAT within primary care settings, it remains unknown if engagement with primary care is associated with receiving MAT for AUD. We therefore aimed to assess the rate of MAT among patients engaged with primary care and the association between primary care engagement and receipt of MAT.

METHODS: We completed a cross-sectional study of patients with AUD seen within a large urban healthcare system in the Bronx, New York from 2010 to 2014. Data were extracted from the health center's clinical information system and included ICD9 codes, problem lists, visits, medications and sociodemographic characteristics. Adults with an ICD9 code or problem list entry indicating a diagnosis of alcohol abuse or dependence during an emergency department (ED), inpatient or outpatient visit were included. Primary care engagement was defined as patients who visited the same primary care facility at least twice more than 90 days apart within a 1 year period. MAT was defined as any outpatient prescription of acamprosate, naltrexone or topiramate during the study duration. To assess the association between primary care engagement and MAT, we conducted a logistic regression analysis. We adjusted for age, gender, socioeconomic status, race, ethnicity, preferred language and presence of substance use disorder or psychiatric comorbidity. The number of ED, inpatient encounters, and whether the patient received outpatient specialty substance use or psychiatric care was also included.

RESULTS: Among the 24,295 adult patients identified with AUD within the study period, the median age was 49 years and the majority were male (71.1\%), non-hispanic $(52.8 \%)$, black or multi-racial (56.2\%) and English speaking (85.8 \%). Among all patients with AUD, $2.6 \%$ were prescribed MAT. In bivariate analysis, patients engaged in primary care were more likely to receive MAT than patients not engaged in primary care (3.5 vs. $2.4 \%, p<0.001)$. Patients receiving specialty substance use care or psychiatric care adopted MAT at a rate of 33.1 and $7.6 \%$ respectively. In multivariate analysis, patients engaged in primary care were more likely to receive MAT $(\mathrm{OR}=1.33,95 \% \mathrm{Cl}[1.09-1.62])$. Patients connected with outpatient substance use specialty care $(\mathrm{OR}=14.2,95 \% \mathrm{Cl}$ [9.83-20.52]) were markedly more likely to receive MAT. Patients with outpatient psychiatric care were also more likely to receive MAT $(\mathrm{OR}=$ $2.32,95 \% \mathrm{Cl}[1.86-2.89])$. Male patients $(\mathrm{OR}=0.80,95 \% \mathrm{Cl}[0.67-0.95])$ and black patients $(\mathrm{OR}=0.55,95 \% \mathrm{CI}[0.43-0.70])$ were less likely to be prescribed MAT. Older patients were less likely to receive MAT $(\mathrm{OR}=0.98,95 \% \mathrm{CI}[0.98-0.99])$.

CONCLUSIONS: Among adult patients with AUD in a large urban healthcare system in the Bronx, New York, MAT was vastly underutilized. Despite its potential to reduce disease and injury burden, adoption of MAT remains poor even among patients engaged in primary care. While engagement with primary care was associated with receiving MAT, the association was modest. Similar to prior studies, we found that receiving MAT was strongly associated with specialty substance use and psychiatric care. These findings suggest moderate adoption of MAT within specialty settings, while MAT is greatly underutilized in primary care. Further research is needed identifying the barriers to adoption and developing implementation strategies specific to primary care.

PRIMARY CARE POPULATION SIZE, DEMOGRAPHICS, \& RISK UNDER VARYING POPULATION INCLUSION CRITERIA Nathaniel Gleason; Priya A. Prasad. UCSF, San Francisco, CA. (Control ID \#2470181)

BACKGROUND: The provision of high-value healthcare to a specific population is part of the core mission for an increasing number of healthcare organizations. As organizations assume accountability for the health of a population, and assume some risk for the costs, the interest and investment in programs to improve Population Health has grown. While an organization may be informed of the individual patients enrolled in certain capitated health plans, the majority of patients in the US are free to seek care in more than one health system. A definition based upon healthcare activity is the only means by which an organization can describe the makeup of its patient population. We lack consensus criteria 
with which organizations may define the patients currently under their care. We anticipated that the size would decrease while median age and HCC risk scores increase for populations with increasingly stringent inclusion criteria. The magnitude of these differences is not known. Further, contacts outside of the office visit, including telephone and secure email via Electronic Health Record "Patient Portals," now serve as an important part of care delivery, and the degree to which inclusion of these out of office contacts in the definition alters the population is not known. We apply different criteria based upon visits, telephone encounters, and secure email at two large academic health systems and describe the resulting populations.

METHODS: We surveyed 9 academic health systems to understand the variability in the population definition. All were either in ongoing discussions or had recently determined their own, activity-based definition. Data gathered from 2 University of California health systems for this analysis included ambulatory office visits (date, provider, practice, and CPT code); telephone encounter dates; email encounter dates; and patient age, sex, and payer between June 2012 and June 2015. Age was calculated using the beginning of the study period (6/1/12). Medicaid status was determined using the active payer during specialty visits that occurred in the final year of the study period. Medicaid was assigned as the payer for a given patient if used for at least one specialty visit during that time period. We applied the following 4 different criteria to this dataset to describe the primary population composed of patients with, over a 3-year period, - At least 1 contact (visit, secure email, or phone) - At least 1 office visit-At least 2 contacts (visit, secure email, or phone)-At least 2 office visits The CMS Hierarchical Condition Category Community summary score (HCC) is used to adjust Medicare payments to capitated Medicare Advantage plans with a higher score indicating greater morbidity and greater likelihood to require high-cost healthcare. The HCC was calculated for all patients using the age, sex, Medicaid status, and ICD-9 codes documented at primary care and specialty care ambulatory visits in the final year of the study period. Neither the reason for Medicaid enrollment nor the number of months on Medicaid was available and therefore the recommended value of 0 was used for these fields in the calculation.

RESULTS: The size of the resulting primary care population decreases with each successive definition while the age, proportion on Medicaid, and HCC risk scores rise with each. The differences between definition 1 (any contact via office visit, secure email, or phone) vs. definition 2 (at least 1 office visit) in age and HCC are relatively small — not statistically significant at one institution and modest at the other. Differences in age, Medicaid status, and HCC risk score are statically significant in nearly all other comparisons between definitions. See table for full results.

CONCLUSIONS: The federally funded Accountable Care Organizations define the population attributable to a given health system in retrospect based upon patient care activity during the prior year. With the growth of such payment mechanisms, healthcare organizations wishing to target efforts to improve population health need to anticipate the size and risk of the population of patients under their care. The size, median age, Medicaid status, and HCC risk scores of a population differ with increasingly stringent inclusion criteria and the magnitude of the differences between definitions are illuminating. At the most sensitive and least specific end of the spectrum, any contact (office visit, secure email, or phone) over 3 years (definition 1) does increase the size of the population when compared with the requirement that a patient have at least one office visit (definition 2) over 3 years ( $6 \%$ at one institution and $13 \%$ at the other), but has a modest effect on the age and HCC risk score. NB: Data from two additional UC Health Systems will be added to the analysis prior to the SGIM National Meeting.

\begin{tabular}{lllll}
\hline UC-Davis & Definition 1 & Definition 2 & Definition 3 & Definition 4 \\
\hline Female (Freq/\%) & $73,265(57 \%)$ & $68,994(57 \%)$ & $67,513(57 \%)$ & $61,080(58 \%)$ \\
Age (median, IQR) & $48.1(34.2,60)$ & $48.2(34.4,60)$ & $48.9(35.3,60.5)$ & $49.5(36.2,61)$ \\
Medicaid (Freq/\%) & $33,891(26 \%)$ & $32,037(26 \%)$ & $32,171(27 \%)$ & $29,877(28 \%)$ \\
HCC (mean (SD)) & $0.396(0.270)$ & $0.393(0.267)$ & $0.399(0.269)$ & $0.401(0.266)$ \\
Total Population & 129,338 & 121,825 & 117,713 & 105,798 \\
& & & & \\
& & & & \\
UC San Francisco & Definition 1 & Definition 2 & Definition 3 & Definition 4 \\
Female (Freq/\%) & $39,877(60 \%)$ & $34,571(59 \%)$ & $34,048(61 \%)$ & $27,002(61 \%)$ \\
Age (median, IQR) & $46.2(31.9,60.4)$ & $46.5(33.1,60.9)$ & $48.2(33.6,61.8)$ & $49.9(35.8,63.4)$ \\
Medicaid (Freq/\%) & $19,038(28 \%)$ & $17,669(30 \%)$ & $17,677(31 \%)$ & $15,980(36 \%)$ \\
HCC (mean (SD)) & $0.404(0.273)$ & $0.404(0.270)$ & $0.414(0.273)$ & $0.426(0.275)$ \\
Total Population & 66,847 & 58,295 & 56,158 & 44,617
\end{tabular}

PRIMARY CARE PROVIDER AND PRACTICE CHARACTERISTICS ASSOCIATED WITH HIV PRE-EXPOSURE PROPHYLAXIS (PREP) UPTAKE Oni J. Blackstock $^{1}$; Brent A. Moore ${ }^{6}$; Gail Berkenblit ${ }^{2}$; Sarah K. Calabrese ${ }^{5}$; Chinazo Cunningham $^{1}$; David A. Fiellin ${ }^{6}$; Viraj V. Patel ${ }^{1}$; Karran A. Phillips ${ }^{3}$; Minesh Shah ${ }^{4}$; Jeanette M Tetrault ${ }^{6}$; E. J. Edelman ${ }^{6} .{ }^{1}$ Albert Einstein College of Medicine \& Montefiore Medical Center, Bronx, NY; ${ }^{2}$ Johns Hopkins, Baltimore, MD; ${ }^{3}$ National Institute on Drug Abuse, National Institutes of Health, Baltimore, MD; ${ }^{4}$ University of Illinois-Chicago, Chicago, IL; ${ }^{5}$ Yale University, New Haven, CT; ${ }^{6}$ Yale University School of Medicine, New Haven, CT. (Control ID \#2469545)

BACKGROUND: HIV pre-exposure prophylaxis (PrEP) — once daily oral tenofovir/ emtricitabine (Truvada ${ }^{\mathbb{R}}$ ) in combination with routine HIV and STI screening and risk reduction and adherence counseling - is a new and effective HIV prevention tool recommended by the Centers for Disease Control and Prevention. Despite its proven efficacy, uptake of PrEP has been low among patients and providers alike. Primary care physicians (PCPs) are uniquely positioned to increase PrEP uptake among their patients at high risk for HIV infection. However, little is known about why PCPs may or may not prescribe or refer a patient for PrEP.

METHODS: From April to May 2015, we conducted an online anonymous survey among a convenience sample of Society of General Internal Medicine members who provide direct outpatient care. Data collected included information about provider characteristics (age, race/ethnicity, gender, sexual orientation, role [attending vs. fellow/ resident], years in practice, percent time allocation [direct care, research, etc.]) and practice characteristics (region of country, rurality/urbanicity, main focus of clinic [e.g., primary care with onsite HIV care], type of clinical setting [e.g., clinic at academic medical center], number of HIV-positive patients) as well as PrEP uptake, defined as ever prescribing or referring a patient for PrEP (yes/no). To assess the association between provider and practice characteristics and PrEP uptake, we conducted multivariate logistic regression. 
RESULTS: Among our sample $(n=266), 33 \%$ reported PrEP uptake with $19 \%$ having ever prescribed PrEP and $18 \%$ having ever referred a patient for PrEP. Mean age (standard deviation) for our sample was 41 (10) years. Most participants were white (72\%), female (62\%), heterosexual ( $92 \%$ ) and attending physicians $(75 \%)$. Fifty percent were in clinical practice less than 10 years. Most clinical practices were in the Northeast (49\%) and the South (20\%); urban settings (85\%); and located at an academic medical center $(68 \%)$. Seventy-five percent of participants provided care to at least one HIV-positive patient and $35 \%$ practiced at primary care clinics with on-site HIV care. In adjusted analyses, PrEP uptake was associated with less years in clinical practice $(<10 \mathrm{vs.}>10-15$ and $>15$ years $)(\mathrm{aOR}=5.55,95 \% \mathrm{CI}: 1.96-16.67 ; \mathrm{aOR}=5.55,95 \% \mathrm{CI}: 2.22-14.29)$, providing care to a higher number of HIV-positive patients ( $>50$ vs. 0 patients) ( $\mathrm{aOR}=5.04,95 \% \mathrm{CI}$ : $1.10-23.2$ ), and practicing at a clinic with onsite HIV care (vs. no onsite HIV care) (aOR=2.66, 95\% CI: 1.10-6.41). CONCLUSIONS: We found that only about one-third of PCPs surveyed reported PrEP uptake. PCPs with less years in clinical practice, with a higher number of HIV-positive patients, and those practicing in clinics with onsite HIV care were more likely to report PrEP uptake. Our results suggest that multiple provider-focused strategies to promote PrEP uptake may be warranted, including: provider-focused PrEP educational initiatives that 1) target PCPs who have been in practice longer as well as those practicing in clinics without comprehensive HIV treatment services, 2) focus on PCPs who are more likely to prescribe (e.g., those in clinics with HIV care) to train "PrEP champions", or 3) target PCPs broadly, regardless of provider and practice characteristics.

PRIMARY CARE PROVIDER APPROACHES AND PERCEPTIONS TO PREVENTIVE HEALTH DELIVERY: A QUALITATIVE STUDY Hemalatha Murugan; Clarence Spigner; Christy McKinney; Christopher Wong. University of Washington, Seattle, WA. (Control ID \#2468042)

BACKGROUND: How best to deliver preventive health in primary care continues to be debated. Office visits may be divided into two categories: separate, stand-alone preventive health appointments or visits for medical conditions such as diabetes or hypertension. There is conflicting evidence regarding the efficacy of preventive health visits, and, yet, the alternative practice of "catching up" on preventive health during other visits has not been shown to be effective. While many studies have addressed perceived barriers to preventive health, less is known regarding how primary care providers have adapted to the current medical environment to address preventive health for their patients. The objective of this qualitative study was to seek decision-making insights at the provider level to gain understanding of the values that shape how providers deliver preventive health in the primary care setting.

METHODS: Subjects were recruited from primary care clinics at an academic medical system. Two interviewers conducted semi-structured, one-on-one interviews. Questions focused on several domains: use of preventive health guidelines, preventive health delivery in preventive health visits versus acute care or chronic disease visits, methods of tracking and documentation, and opinions regarding team-based care. Interviews lasted approximately $20 \mathrm{~min}$, were recorded and then transcribed. A thematic analysis was conducted, and, in keeping with Grounded Theory, no pre-specified hypotheses were established. Reliability and validity was assured by independent coding of transcript content.

RESULTS: Twenty-one subjects from 4 different primary care clinics were interviewed. The following major themes emerged: 1. Longitudinal care is perceived as integral to preventive health: Nearly all providers preferred deferring certain core preventive health services to a patient's own primary care provider, citing the patient-provider relationship as essential in preventive health discussions. 2. Conflict and doubt accompany non-preventive visits: Providers expressed the desire to accomplish some preventive health during these visits, but often felt that they were either not able to accomplish this goal due to time constraints or focused only on brief interventions such as immunizations. 3. Time and patient risk factors provide the framework around which preventive health is delivered, regardless of type of visit: Rather than using a single checklist, providers tailored selection of preventive health services to the patient's medical and social conditions as a whole, as well as other concerns addressed during a clinic visit. Those providers who routinely conducted separate preventive health visits deferred services that require more discussion and shared decision-making to those visits. 4. Adaptation arises: The provider's level of confidence in the electronic medical record (EMR) tended to lead to either adaptation or discomfort. Many providers expressed discomfort with the EMR's adequacy of tracking preventive health and consequently adopted other systems, including having their own separate tracking system in the patient's problem list. Reasons most cited included lack of completeness and inability to express rationale for decision-making in the EMR. 5. Comfort level with teambased preventive health delivery is connected with the degree of shared decision-making required: Nearly all providers expressed that other members of the health care team should increase involvement in preventive health, but there were varying responses based on whether a preventive service was perceived as controversial, or requiring discussion.

CONCLUSIONS: In this qualitative study, the themes that surfaced suggest that primary care providers are resilient in finding ways to deliver preventive health to their patients, despite a system in which they often feel uncomfortable. They use a number of factors in deciding what preventive health to address and when for a given clinic visit, built upon a platform that values time, the patient's medical conditions and expectations, and a strong value that a patient's primary care provider should be involved in complex preventive care decisions. They frequently adapt to an EMR that they lack complete confidence in or find not fully adequate. Together these findings have implications for the primary care practice of the future: flexibility, utilizing multiple methods of delivery, having provider input into EMR design, and supporting the primary care provider's role as leader of a patient's preventive health, even with team-based care, should be critical foundations. Limitations of this study include the inherent nature of its qualitative approach and the academic medical center setting, impacting generalizability. However, it is likely that certain dilemmas are common to the practice of primary care, and the insight into provider behavior found by these qualitative interviews, with answers unable to be gleaned from quantitative data, may provide stimulus for further research.

PRIMARY CARE PROVIDER EXPERIENCE WITH BREAST DENSITY LEGISLATION IN MASSACHUSETTS Christine M. Gunn ${ }^{1,3}$; Nancy R. Kressin' ${ }^{2}$ Kristina Cooper'; Tracy A. Battaglia ${ }^{1} .{ }^{1}$ Boston University Medical Center, Boston, MA; ${ }^{2}$ Dept of Veterans Affairs and Boston University, West Roxbury, MA; ${ }^{3}$ Boston University, Boston, MA. (Control ID \#2465751)

BACKGROUND: Patients and advocacy groups have influenced the adoption of legislation requiring that women be notified of their breast density in 24 states to date. Breast density laws have been advocated on the premise that notification will increase knowledge and empower women to make informed decisions about breast cancer screening, yet there is little evidence on how providers have integrated this into practice, or been affected by it. This study aims to assess primary care providers' awareness, attitudes, knowledge, and impact on clinical practice of breast density legislation enacted in Massachusetts in January 2015.

METHODS: We conducted an anonymous survey at a Boston safety-net medical center. Practicing primary care providers were invited to participate in a brief survey about the implementation of the Massachusetts breast density notification mandate. Data were collected from July through December 2015. Attitudes and clinical impact were assessed on a 5 point Likert scale (Strongly Disagree to Strongly Agree). Three additional questions tested provider knowledge about the mandate provisions and evidence for supplemental screening. Descriptive statistics were performed on the entire sample. For these analyses, Agree/Strongly Agree and Disagree/Strongly Disagree were grouped and these two categories were the basis for comparison. Bivariate associations using chi-square tests tested for differences in attitudes and knowledge by provider gender and years of practice.

RESULTS: Fifty-two providers completed the survey (59\% response rate). Providers were mostly female (58 \%), and had varying levels of experience: $25 \%$ had practiced less than 5 years, $27 \%$ 5-10 years, and $48 \%$ more than 10 years. Attitudes and Awareness: A large majority of providers indicated they were familiar with the dense breast notification law enacted in 
Massachusetts (77\%). Most were not in favor of the legislation (63\%) and few providers perceived the legislation to promote informed decision making (23\%). Impact on Clinical Practice: Forty percent of providers agreed with the statement that the notification mandate has changed how they discuss mammography results with patients. Qualitative descriptions about changes in practice indicated that it takes additional time to explain these results. Less than half felt that counseling women on breast density was the responsibility of primary care providers $(40 \%)$. Yet, more than half ( $52 \%)$ felt unprepared to counsel women about breast density. In line with this, $79 \%$ of those surveyed were interested in additional training in management for dense breasts. Knowledge: Notably, none of the providers correctly identified all components listed in the Massachusetts breast density notification. When prompted to identify supplemental screening tests that evidence suggests may be warranted for women with dense breasts, only $27 \%$ correctly indicated that neither ultrasound, MRI, tomosynthesis, nor genetic testing are evidence-based recommendations. In response to a scenario-based knowledge question, $52 \%$ correctly identified conducting a risk assessment as the appropriate action in response to a finding of dense breasts in a 60 year old woman, which is the currently recommended approach in Massachusetts. In bivariate analyses, male providers were more likely to correctly respond about evidence-based recommendations compared with female providers ( 56 vs. $13 \%, p=0.004$ ). No other associations between knowledge or attitudes and gender or years of practice were statistically significant CONCLUSIONS: Despite legislative efforts to promote informed decision making for women with dense breasts, primary care providers who are tasked with carrying out this mandate are not prepared to engage women in these discussions. This survey indicated that dense breast notification legislation has impacted clinical practice in primary care, but providers require additional guidance to support them in recommending evidence-based care.

PRIMARY CARE PROVIDERS' KNOWLEDGE OF SCREENING AND DIAGNOSIS OF DIABETES AND PREDIABETES Eva Tseng ${ }^{1}$; Hsin-Chieh $\mathrm{Yeh}^{3}$; Maura J. McGuire $^{2}$; Jeanne M. Clark ${ }^{3}$; Nisa Maruthur ${ }^{4} .{ }^{1}$ Johns Hopkins, Baltimore, MD; ${ }^{2}$ Johns Hopkins Community Physicians, Baltimore, MD; ${ }^{3}$ Johns Hopkins University, Baltimore, MD; ${ }^{4}$ Johns Hopkins University School of Medicine, Baltimore, MD. (Control ID \#2466509)

BACKGROUND: One out of three adults (86 million) in the U.S. have prediabetes. Diagnosing prediabetes is an important step in diabetes prevention since 5 to $10 \%$ of individuals with prediabetes will develop diabetes annually. However, evidence suggests that 9 out of 10 people with prediabetes are unaware of their diagnosis. Primary care providers play an essential role in initiating diabetes prevention interventions, but little is known about their knowledge of guidelines for screening for and diagnosing diabetes and prediabetes. METHODS: Primary care providers from a large academically-affiliated multispecialty group (which served 244,000 patients in 2015), completed a written, self-administered survey at their annual retreat in November 2015. Survey items on risk factors and laboratory parameters for the screening and diagnosis of diabetes and prediabetes were based on the American Diabetes Association's guidelines which have been consistent since 2010. We used logistic regression to evaluate the association between clinician characteristics (sex, race, years since training, clinic sessions/week) and knowledge of risk factors and diagnostic criteria for diabetes and prediabetes.

RESULTS: Of 184 adult primary care provider attendees, 141 (77\%) completed the survey. Among the respondents, $99 \%$ were physicians or nurse practitioners. Medicine specialties represented included internal medicine (43\%), family medicine (40\%), and internal medicine-pediatrics $(15 \%)$. The majority of providers were female $(72 \%)$, white (55\%; other races: Asian, $23 \%$, African American, $14 \%$ ) and in practice for at least 10 years (59\%). Number of half-day clinic sessions varied with $16 \%$ reporting $5-6,21 \%$ reporting 7-8, and $40 \%$ reporting $9-10$ per week. Only $6 \%$ of providers correctly identified all the risk factors that should prompt screening for diabetes. The most commonly identified risk factors were family history of diabetes in a first-degree relative (94\%), overweight (89\%), history of gestational diabetes (87\%), dyslipidemia (86 \%), hypertension (77\%), and history of heart disease ( $75 \%)$. The least-commonly identified risk factors were Asian race (38\%) and Hispanic ethnicity (52\%). Roughly two-thirds of providers correctly identified the laboratory parameters for the diagnosis of diabetes using both fasting glucose $(\geq 126 \mathrm{mg} / \mathrm{dL})$ and hemoglobin A1c $(\geq 6.5 \%)$. Only $41 \%$ of providers correctly identified the laboratory parameters for the diagnosis of prediabetes based on fasting glucose (100-125 mg/dL), and even fewer providers (21\%) correctly identified prediabetes based on hemoglobin A1c (HbAlc 5.7-6.4\%). Each additional full day of clinic per week was associated with a 1.4- to 1.5 -fold increased odds (for fasting blood glucose: $\mathrm{OR}=1.35 ; 95 \% \mathrm{CI}: 1.05$ to 1.74 ; for $\mathrm{HbA1c}$ : OR $=1.47$; $95 \% \mathrm{CI}$ : 1.04 to 2.06 ) of correctly choosing the laboratory criteria for the diagnosis of prediabetes. We did not find a significant association between other clinician characteristics and knowledge of diagnostic criteria.

CONCLUSIONS: Primary care providers from a large academically-affiliated multispecialty group demonstrated knowledge of most risk factors for diabetes, but knowledge of Asian race and Hispanic ethnicity as risk factors was low. Fewer than $70 \%$ of providers correctly identified the laboratory parameters for the diagnosis of diabetes. Knowledge of criteria for prediabetes was even lower (41 and $21 \%$ for fasting glucose and $\mathrm{HbAlc}$, respectively). These substantial gaps in knowledge of prediabetes, the high-risk state preceding diabetes, must be addressed if we are to impact the diabetes epidemic meaningfully in primary care.

PRIMARY CARE PROVIDERS' MANAGEMENT AND TREATMENT PRACTICES FOR PREDIABETES Eva Tseng ${ }^{1}$; Hsin-Chieh Yeh $^{3}$; Maura J. McGuire ${ }^{2}$; Jeanne M. Clark ${ }^{3}$; Nisa Maruthur ${ }^{4} .{ }^{1}$ Johns Hopkins, Baltimore, MD; ${ }^{2}$ Johns Hopkins Community Physicians, Baltimore, MD; ${ }^{3}$ Johns Hopkins University, Baltimore, MD; ${ }^{4}$ Johns Hopkins University School of Medicine, Baltimore, MD. (Control ID \#2466511)

BACKGROUND: Prediabetes, the high-risk state preceding diabetes, is a growing public health issue. Strong evidence shows that intervening in patients with prediabetes decreases the incidence of diabetes. Since 2008, the American Diabetes Association (ADA) has recommended an intensive diet and physical activity behavioral counseling program (targeting $7 \%$ weight loss and 150 min of moderate-intensity physical activity per week) for all patients diagnosed with prediabetes; consideration of metformin in patients with additional risk factors; and yearly testing for conversion from prediabetes to diabetes. Limited existing evidence suggests that patients with prediabetes are not receiving this recommended care. Therefore, we sought to understand primary care providers' knowledge and practices regarding the management of prediabetes.

METHODS: Primary care providers from a large academically-affiliated multispecialty group (which served 244,000 patients in 2015), completed a written, self-administered survey at their annual retreat in November 2015. Providers were asked about their knowledge and practices regarding the management of patients with prediabetes, including follow-up visit intervals, repeat laboratory testing, and treatment with a behavioral counseling program and/or metformin. We used logistic regression to evaluate the association between clinician characteristics (sex, race, years since training, clinic sessions/ week) and management of prediabetes.

RESULTS: Of 184 adult primary care provider attendees, 141 (77\%) completed the survey. Among the respondents, $99 \%$ were physicians or nurse practitioners. Medicine specialties represented included internal medicine (43\%), family medicine (40\%), and internal medicine-pediatrics (15\%). The majority of providers were female (72\%), white (55\%; other races: Asian, $23 \%$, African American, $14 \%$ ) and in practice for at least 10 years (59\%). Number of clinic sessions varied with $16 \%$ reporting $5-6,21 \%$ reporting 7-8 and $40 \%$ reporting 9-10 half-day sessions per week. About two-thirds of providers correctly identified 5-10\% as the minimum recommended weight loss for overweight patients with prediabetes. Male (vs. female) providers were less likely (OR 0.39, $95 \% \mathrm{CI}$, 0.17 to 0.86 ), and those with more clinic sessions per week were more likely (OR for each additional session per week $1.31,95 \%$ CI 1.01 to 1.69 ) to know this weight loss recommendation. Less than half of the providers $(45 \%)$ correctly reported the minimum 
amount of physical activity recommended by the ADA. The majority of providers (96\%) reported counseling on diet changes and physical activity as the best recommended initial management for a patient with prediabetes while only $11 \%$ selected referral to a behavioral counseling program as the best initial approach. When asked about their actual treatment practices in their clinical settings, providers reported the following approaches for the management of prediabetes: counseling on diet changes and physical activity (99\%), referral to a nutritionist (32\%), discussing starting metformin (29\%), and referral to a behavioral counseling program (12\%). The majority of providers reported rechecking labs within 6 months ( $87 \%$ ) and arranging for a follow-up visit within 6 months (88 \%) for patients identified as having prediabetes. Metformin use to treat patients with prediabetes was uncommon with $25 \%$ of providers reporting never prescribing metformin; $36 \%$ reporting use in $1-5 \%$ of patients; $26 \%$ reporting use in $5-25 \%$ of patients; $6 \%$ reporting use in $25-50 \%$ of patients; and $8 \%$ reporting use in over $50 \%$ of patients. Knowledge of physical activity recommendations for prediabetes and the use of metformin in the management of patients with prediabetes was not associated with important differences in clinician characteristics.

CONCLUSIONS: Primary care providers from a large academically-affiliated multispecialty group reported following their patients with prediabetes closely with nearly all providing counseling on lifestyle change. However, knowledge of specific lifestyle recommendations for prediabetes was inadequate, particularly for physical activity, and only $11 \%$ of providers selected referral to a behavioral weight loss program as the best recommended initial management of patients with prediabetes. As expected, metformin use for prediabetes appeared low. Primary care providers are in an ideal position to address diabetes prevention using proven interventions, but our results demonstrate that substantial knowledge gaps must be addressed to improve the management of prediabetes in the primary care setting.

PRIMARY CARE PROVIDERS' ROLE IN HIV PREVENTION WITH PREEXPOSURE PROPHYLAXIS (PREP): PERSPECTIVES FROM YOUNG MEN OF COLOR WHO HAVE SEX WITH MEN (YMCSM) Patel V. Viraj ${ }^{1}$; Anthony F. Santoro ${ }^{1 \text {, }}$ 2; Sarit A. Golub ${ }^{3}$; Julia H. Arnsten ${ }^{1} .{ }^{1}$ Montefiore Medical Center/Albert Einstein College of Medicine, New York, NY; ${ }^{2}$ Ferkauf Graduate School of Psychology, Bronx, NY; ${ }^{3}$ Hunter College of the City University of New York, New York City, NY. (Control ID \#2467779)
BACKGROUND: Young men of color who have sex with (YMCSM) have the highest rates of new HIV infections in the United States. Despite the fact that Pre-exposure prophylaxis (PrEP) with tenofovir-emtricitibine is highly effective, is FDA approved, and is recommended by the Centers for Disease Control and Prevention (CDC) as a first-line prevention tool for MSM, uptake has been low in YMCSM. We therefore conducted a qualitative study to explore YMCSM's perspectives on barriers and facilitators to PrEP use and better understand what role primary care providers may have in the PrEP adoption process to inform implementation interventions.

METHODS: We conducted individual semi-structured interviews with young MSM $(N=17)$, recruited through targeted study advertisement on social media (i.e., Facebook). Interviews were conducted in-person between July and November 2015. Inclusion criteria included being 18 29 years of age, HIV negative status (by self-report), living or working in the Bronx, New York, identifying as Black and/or Latino, and fluent in English or Spanish. Interviews were audiorecorded and professionally transcribed and/or translated. We used a modified grounded theory approach to analysis; first a preliminary codebook was developed based on the literature and then iteratively refined by three investigators from an initial analysis of four transcripts. Two investigators then coded the remaining transcripts using Dedoose qualitative software.

RESULTS: Approximately half the participants $(n=9)$ were currently using PrEP, majority had Medicaid $(n=13)$, all reported having a PCP, identified English as their primary language ( $n=15$ ), and all identified as gay. Two overarching themes emerged from our analysis pertaining to PCPs. First, PCPs can be highly influential in facilitating PrEP use by YMCSM. Specifically, participants reported that providers may serve as an important facilitator by a) suggesting $\operatorname{PrEP}$ use, especially if they have a strong rapport with and trust in their PCP, and/or b) if the PCP provides reassurance about PrEP use . Second, PCPs may also serve as a significant barrier to accessing PrEP, due to : a) perceived stigma from providers related to sexual activity or reasons for potential PrEP use, and b) lack of PrEP awareness and knowledge by PCPs. These barriers contributed to some missed opportunities for PrEP education and/or provision to individuals at high risk who had come in for routine and/or sexual health care.

CONCLUSIONS: PCPs appear to be critical in influencing YMCSM's decision making about PrEP and also serve as an important 'gatekeepers' for this prevention tool. Interventions to increase PrEP adoption in YMCSM are urgently needed and should target PCPs with education and support for PrEP and engage YMCSM into culturally-sensitive and competent routine primary care.

Representative Quotes

\begin{tabular}{ll}
\hline \hline Domain & Theme \\
PCP as a facilitator & Having a strong rapport and trust
\end{tabular}

in a primary care provider

Suggestion and reassurance about PrEP use from a PCP

PCP as a barrier
Perceived stigma from providers related to sexual activity or reasons for potential PrEP use Lack of PrEP awareness and knowledge by PCPs

\section{Quotes}

"...I am taking [PrEP] and from what I have heard...I take my doctor's word. I have been here for 25 years, so I must be doing something right if I follow his instruction." "My doctor is very open. He has known me for a billion years. He said, "Well, I am going to tell you about it. It is your preventive care." And I said, "Really, doctor? Seriously?" Now, but he seriously went into it and he explained...you can take this and it will help lower your risk of becoming infected with HIV. I said, "Okay." "I spoke to my doctor about it. This was a time when me and my partner were starting to break up and I heard about. All I knew was that you take a pill a day and it helps lower your risk. I didn't know anything too much about it. So, I went to my primary care and he started explaining things about it..."

"I went to my doctor's office. That was when I got diagnosed with gonorrhea. Then he was like, "Have you thought about taking PrEP? I was like," "Tell me about it, because I keep seeing it. I read a little bit, but I don't know enough." Then, he told me about it. He was like, "Do you have safe sex." I was not going to lie. I was like, "I am a freak. I like [sex]"...I did some more research. I decided to take [PrEP]." “...I didn”

"Then I asked him, "Hey, what do you think about PrEP? I'm interested in getting into it." He didn't even know what it was. What he knew how to treat was diabetes, high blood pressure, etc. He wasn't familiar with HIV drugs as prevention."

"Then I met somebody and my partner was HIV positive, so we were using protection but there were occasions where it would break and then I would be freaking out. I would just try to avoid that, so I went out and looked for doctors that would give me PrEP. Most doctors didn't know what I was talking about or they would tell me that it's not out yet or I can't get it for whatever reason." 
PRIMARY CARE TEAM STAFFING AND TURNOVER HAVE STRONGER ASSOCIATION WITH BURNOUT THAN WORKLOAD AND PATIENT COMPLEXITY. Christian D. Helfrich ${ }^{1}$; Joseph A. Simonetti ${ }^{1,3}$; Walter L. Clinton ${ }^{1}$; Gordon B. Wood ${ }^{1}$; Leslie Taylor ${ }^{1}$; Gordon Schectman ${ }^{5}$; Richard Stark $^{2}$; Stephan D. Fihn ${ }^{1,}{ }^{4}$; Karin M. Nelson ${ }^{1,}{ }^{4}$. ${ }^{1}$ Department of Veterans Affairs, Seattle, WA; ${ }^{2}$ Department of Veterans Affairs, Washington, DC; ${ }^{3}$ University of Washington, Seattle, WA; ${ }^{4}$ University of Washington, VA Puget Sound, Seattle, WA; ${ }^{5}$ Veterans Affairs Central Office, Milwaukee, WI. (Control ID \#2468936)

BACKGROUND: Work-related burnout is common among primary care personnel, and is associated with worse patient safety, patient satisfaction, and employee mental health. Clinical workload and staffing issues (e.g., turnover, inadequate staffing) are risk factors for burnout and have been increasing in primary care. However, the relative importance of team-specific workload and staffing to primary care team-member burnout is unknown. Test the associations of clinician burnout with team-specific staffing and workload characteristics among four team occupations: primary care provider (PCP), nurse care manager, clinical associate, and administrative clerk.

METHODS: We conducted an individual-level cross-sectional analysis of national VA primary-care personnel survey data and administrative data from 2014. The dependent variable was workplace burnout measured with a previously validated single-item measure dichotomized to indicate the presence of burnout. The independent variables were measures of team staffing (having a fully-staffed team, serving on multiple teams, and turnover on the team), and workload (working extended hours during the week and during the weekend, having a panel over-capacity, and average comorbidity of panel). Adjusted analyses were performed using GLIMMIX.

RESULTS: There were 4610 respondents, with an estimated response rate of $22 \%$. Overall reported burnout prevalence was $40.9 \%$, and was highest for PCPs $(49.2 \%)$ and lowest for clinical associates (32.0\%). In adjusted analyses, the strongest associations with burnout were for having a fullystaffed teamlet $(\mathrm{OR}=0.56,95 \%$ CI $0.48-0.65)$, turnover on the teamlet $(\mathrm{OR}=1.62,95 \% \mathrm{CI} 1.40-1.87)$, working extended hours on weekends $(\mathrm{OR}=$ $1.63,95 \%$ CI 1.21-2.19), and having a panel over-capacity (OR $=1.21,95 \%$ CI 1.04-1.41). The estimates of burnout for PCPs working on teamlets that experienced turnover in the previous year were 35 vs. $47 \%$ for PCPs working on a teamlet with no turnover. Predicted burnout for PCPs working on teamlets that were overcapacity was 43 vs. $38 \%$ on PCPs working on teamlets under capacity. Predicted burnout for PCPs who reported working extended weekend hours was 53 vs. $41 \%$ for PCPs who worked extended hours during the week and $30 \%$ for PCPS who reported not working any extended hours. The associations held true across all 4 occupations, but were most significant for PCPs. Associations were additive (table), with predicted prevalence of burnout $27 \%$ lower ( 21 vs. $48 \%$ ) for respondents working on fully-staffed teamlets, with no turnover and serving a panel within capacity, relative to respondents in the inverse condition. Greater length of experience with the VA patient-centered medical home model was also positively associated with burnout.

CONCLUSIONS: Burnout among primary care personnel was strongly associated with team-specific staffing and team-level workload. Future research is needed to determine whether burnout declines as staffing and workload improve.

\begin{tabular}{|c|c|c|c|c|c|}
\hline \multirow[b]{2}{*}{ Adjusted Probability of Burnout: } & \multicolumn{2}{|c|}{ PCPS Nurses } & \multicolumn{2}{|c|}{ CA $A A$} & \multirow{2}{*}{$\begin{array}{c}\text { All } \\
\text { Roles } \\
\text { n33 }\end{array}$} \\
\hline & 0.41 & 0.34 & 0.26 & 0.33 & \\
\hline Fully Staffed + No Team Change & 0.28 & 0.24 & 0.21 & 0.18 & 0.23 \\
\hline Not Fully Staffed + Team Change & 0.52 & 0.48 & 0.43 & 0.39 & 0.46 \\
\hline Not Overcapacity + Fully Staffed & 0.31 & 0.27 & 0.23 & 0.20 & 0.25 \\
\hline Overcapacty + Not Fully Staffed & 0.49 & 0.44 & 0.40 & 0.35 & 0.42 \\
\hline Not Overcapacity + No Team Change & 0.32 & 0.28 & 0.24 & 0.21 & 0.26 \\
\hline Overcapacity + Team Change & 0.48 & 0.43 & 0.38 & 0.34 & 0.41 \\
\hline FST + No Team Change + Not Overcapacity & 0.27 & 0.22 & 0.16 & 0.21 & 0.21 \\
\hline Non-FST + Team Change + Overcapacity & 0.56 & 0.49 & 0.40 & 0.48 & 0.48 \\
\hline 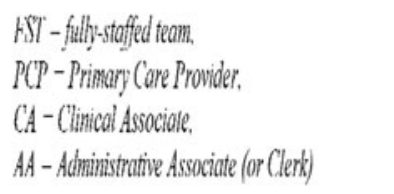 & & & & & \\
\hline
\end{tabular}

PRIMARY PROVIDER INFLUENCE ON PATIENT USE OF AN ELECTRONIC PATIENT PORTAL IN AN ACADEMIC PRIMARY CARE PRACTICE Brian Chan ${ }^{1,}$ 2; Courney R. Lyles ${ }^{3}$; Celia P. Kaplan²; Rosemary N. Lam²; Leah S. Karliner'. ' OHSU, Portland, OR; ${ }^{2}$ UCSF, San Francisco, CA; ${ }^{3}$ University of California San Francisco, San Francisco, CA. (Control ID \#2468871)

BACKGROUND: Electronic patient portals offer the potential for increased patient-physician communication, and improved clinical care beyond the office visit. Patient portals are now integrated into federal "meaningful use" metrics, and adoption is necessary for receipt of financial incentives associated with implementing electronic health records. Adoption of patient portals holds promise for improved patient outcomes, and there is evidence that provider encouragement to use portals is a strong predictor of patient use; however, there have been no investigations of differences in portal use by type of provider. We investigated the role of provider type in use of an electronic patient portal in an academic general internal medicine practice.

METHODS: This is a cross-sectional study using administrative data of all established patients seen at three clinics of an academic internal medicine practice between May 1, 2014 and April 30, 2015. Patients had a primary care provider (PCP) who was either an attending physician, resident physician, or a nurse practitioner (NP). For a small subset of patients who changed PCPs within the practice during the study period, we defined a patient's PCP as the provider listed on the first visit within study period. We defined patient portal 'use' as having accessed any active (secure messaging, medication refill request) or passive (viewing lab reports, after visit summaries, or appointments) patient portal function more than one time over the study period. We defined 'active use' of the patient portal as having accessed any active function more than one time over the study period, and 'passive use' of the patient portal as having accessed any passive function more than one time over the study period. We investigated the association of provider type (attending, resident, NP) with patient portal 'use' (vs. non-enrollment / enrollment but non-use) with bivariate analysis, and examined the odds of patient portal 'use' with multivariate logistic regression adjusted for age, sex, preferred language, race/ethnicity, insurance, and clinic location. Among patient portal users, we further examined the association of provider type with 'active use' of the patient portal using the same method.

RESULTS: The mean age of the study population $(n=19,898)$ was 53.4 (SD 17.4), with $46.9 \%$ White, $23.0 \%$ Asian, $9.6 \%$ Black, $8.5 \%$ Latino, and $12 \%$ other/unknown race/ethnicity. The majority of the population was English speakers $(85.8 \%)$. Over half (61.7 \%) had private insurance, while $23.9 \%$ had Medicare, $10.9 \%$ Medicaid, $2.6 \%$ had 
dual-eligible Medicaid-Medicare, and $0.9 \%$ unknown/other. Over two-thirds of the population had attending PCPs (66.8\%), $22.1 \%$ had resident PCPs, and $7.7 \%$ had NP PCPs. Although $71.7 \%$ of the population enrolled in the patient portal, only $51.7 \%$ met criteria as a patient portal 'user', with $38.3 \%$ 'active users' of patient portal, and $13.4 \%$ 'passive users.' In both unadjusted and adjusted analyses, patients with attending physician and nurse practitioner PCPs had higher odds of both being a 'user' of the patient portal. However, among 'users' of the patient portal, only patients with attending physician PCPs had higher odds of being an 'active user.' (Table 1) CONCLUSIONS: In a cross-sectional sample of an academic general internal medicine practice population, patients with attending physicians and NPs as PCPs were more likely to use the electronic patient portal than those patients with resident physician PCPs; patients with attending PCPs were also more likely to use the electronic patient portal in an active manner. Provider type may be a proxy for clinical experience or a marker for patient-provider relationship that facilitates effective use of patient portals. Even after adjusting for other factors known to be associated with patient portal use, such as preferred language and race/ethnicity, provider type appears to be influential in patient portal use. Future research should explore which elements of the patient-provider interaction affect patient portal use, and how portal use impacts quality of care as measured by patient experience and health outcomes.

Adjusted ORs of being a 'user' of the patient portal, and 'active user' of patient portal by provider type

\begin{tabular}{|c|c|c|}
\hline$\overline{\text { PCP Type }}$ & $\begin{array}{l}\text { Odds of being a 'user' } \\
\text { of the patient portal } \\
\text { compared to non-use } \\
\text { of the patient portal* } \\
N=19,898 \\
\text { OR (95\% CI) }\end{array}$ & $\begin{array}{l}\text { Odds of being an 'active } \\
\text { user' compared to 'passive } \\
\text { user' of the patient portal* } \\
N=10,292 \\
\text { OR }(95 \% \text { CI) }\end{array}$ \\
\hline Attending & $1.88(1.74,2.02)$ & $1.29(1.14,1.46)$ \\
\hline $\begin{array}{l}\text { Nurse } \\
\text { Practitioner }\end{array}$ & $1.32(1.16,1.50)$ & $1.00(0.82,1.23)$ \\
\hline Resident & Reference & Reference \\
\hline
\end{tabular}

*Adjusted for age, sex, preferred language, ethnicity, insurance status, clinic site

PRIOR ANTIBIOTIC USE AND OUTCOMES IN PATIENTS HOSPITALIZED WITH PNEUMONIA Eric Mortensen; David Rotter. VANTHCS, Dallas, TX. (Control ID \#2463118)
BACKGROUND: Despite the growing number of antibiotic-resistant pathogens, the effect of prior use of antibiotics on pneumonia-related outcomes is unclear. Prior studies examining the effect of use of antibiotics prior to hospitalization for pneumonia have had divergent findings. Therefore, the purpose of our study was to examine the association between prespecified groups of prior outpatient antibiotics and clinical outcomes for those subsequently hospitalized with pneumonia.

METHODS: We identified patients $>65$ years of age admitted to any United States Department of Veterans Affairs hospital in fiscal years 2002-2012 with a discharge diagnosis of pneumonia. We examined the impact of prior antibiotic use within 90 days of pneumonia admission on 30-day mortality, intensive care unit (ICU) admission, use of mechanical ventilation, and use of vasopressor therapy. We grouped prior antibiotics as aminoglycosides, antibiotics recommended for the outpatient treatment of communityacquired pneumonia (CAP), other beta-lactams, and all other antibiotics. We performed separate multilevel regression models, adjusting for potential confounders including demographics and comorbidities, to examine the association between the 4 different prior antibiotics groups and the outcomes of interest.

RESULTS: Our cohort was comprised of 94,910 subjects with a mean age was 77.8 (standard deviation 7.4 years). Overall $29.8 \%$ of subjects received at least one antibiotic prior to pneumonia admission. Table 1 demonstrates the results of the multilevel regression models. After adjusting for potential confounders receipt of prior CAP antibiotics was not associated with mortality but was associated with lower risk of ICU admission, mechanical ventilation, and use of vasopressors. Receipt of other beta-lactam antibiotics or other antibiotics were associated with increased morality and other secondary outcomes. Aminoglycoside use was not significantly associated with worse outcomes.

CONCLUSIONS: A large number of patients were found to have antibiotic use prior to hospitalization for pneumonia. While the prior use of outpatient CAP antibiotics was not associated with worse outcomes, the receipt of other betalactams or other antibiotics were associated with worse outcomes. Our study suggests that for patients at risk for pneumonia, or being treated for outpatient pneumonia, should be treated with antibiotics recommend by CAP clinical practice guidelines if possible.

Table 1-Results Of Multilevel Regression Models (odds ratio, $95 \%$ confidence intervals)

\begin{tabular}{lllr}
\hline \hline Prior antibiotic groups & Mortality at 30-days & ICU admission & Mechanical Ventilation \\
Other beta-lactams & $1.14(1.06-1.23)$ & $1.09(1.02-1.16)$ & $1.01(0.90-1.14)$ \\
Aminoglycosides & $1.14(0.70-1.85)$ & $0.93(0.60-1.44)$ & $1.71(0.87-3.38)$ \\
Other antibiotics & $1.14(1.06-1.22)$ & $1.13(1.06-1.21)$ & $0.96(0.86-1.08)$ \\
CAP antibiotics & $1.01(0.96-1.07)$ & $0.85(0.81-0.89)$ & $0.54(0.22-1.31)$ \\
& & & $1.25(1.11-1.42)$ \\
\hline
\end{tabular}

PRIOR HEALTH LITERACY TRAINING, USE OF HEALTH LITERACY TECHNIQUES AND PERCEIVED SKILLS BY RESIDENTS AT AN URBAN ACADEMIC MEDICAL CENTER Nina Song ${ }^{3}$; Lisa Altshuler ${ }^{1}$; Allison Squires ${ }^{2}$; Shonna Yin ${ }^{3}$; Tamasyn Nelson ${ }^{4}$; Sondra Zabar ${ }^{1}$; Adina Kalet ${ }^{3} .{ }^{1}$ NYU School of Medicine, New York, NY; ${ }^{2}$ New York University, New York, NY; ${ }^{3}$ New York University School of Medicine, New York, NY; ${ }^{4}$ Vanderbilt University Medical Center, Nashville, TN. (Control ID \#2469059)

BACKGROUND: Low health literacy (HL) is particularly common in underserved communities. Deficits in $\mathrm{HL}$ are associated with poor health outcomes and reduced patient safety. A "universal precautions" approach is recommended to providers and includes various communication strategies including use of plain language and "teach back" to confirm patient understanding, both associated with better patient health outcomes. Despite this recommendation, a recent study found that only $72 \%$ of the 133 allopathic medical schools in the U.S. included health literacy in their curriculum (Coleman \& Appy, 2012). As part of a curriculum needs assessment, we surveyed 7 Graduate Medical Education programs to determine whether residents received previous training in HL, their current use of HL techniques, perceived skill, and desire for further HL training. METHODS: All residents in the targeted programs were surveyed via an online survey (Qualtrics) during the 2013-2014 academic year. Individuals surveyed included the following programs: Internal Medicine (IM), Primary Care (PC), Obstetrics \& Gynecology (OBGYN), Surgery (SUR), Emergency Medicine (EM), Pediatrics (Peds), and Orthopedics (Ortho). HL questions were adapted from Schwartzberg et al. (2007) and Turner et al. (2009) and assessed the following domains: 1) prior HL training (1Q) assessed 
with a question about whether they had HL training, and if so, where they received it (open text); 2) use of $\mathrm{HL}$ techniques $(8 \mathrm{Q})$ rated on a 5-point Likert scale from 1 (never) to 5 (always); techniques included teach-back, providing easy to read written materials, underlining key points on written materials among others, with an Overall HL Use score calculated by averaging responses (Cronbach's alpha $=.88)$; 3 ) perceived skill in key $\mathrm{HL}$ techniques $(3 \mathrm{Q})$ rated on a 4 point scale from 1 (not at all skilled) to 4 (very skilled); skills assessed included teach back, choosing appropriate written materials, and converting medical terms into plain language with, a HL Skills score calculated by averaging responses (Cronbach's alpha $=.70$ ); 4) desire for HL training (1Q). Independent samples t-test was performed to determine whether there were significant differences in $\mathrm{HL}$ use and HL skills between those that reported having received health literacy training and those who did not.

RESULTS: Completed responses were received from $76 \%$ of residents (253/333 total), $65 \%$ of whom reported prior HL training with the majority of this being in medical school using a variety of educational formats from didactics to OSCEs. Overall HL Use was the same for residents reporting having received HL training compared to those who did not, except that residents who reported having received training were more likely to use "underline, circle, or otherwise highlight key points on written educational materials" $(\mathrm{M}=3.24, \mathrm{SD}=1.049, \mathrm{M}=2.90, \mathrm{SD}=1.074, \mathrm{t}(262)=2.47, p=.014)$. Similarly residents who reported having had prior training rated their HL skills higher than those who did not receiving HL training on each of the skills: using simple jargon (3.47 vs. $3.29, p=.018$ ); using teach-back ( 3.18 vs. $3.00, p=.041$ ); choosing printed handouts that are written at the appropriate literacy level for the patient/family member (3.00 vs. 2.67, $p=.002$ ). There are differences between specialties with respect to self-reported use of and comfort with $\mathrm{HL}$ approaches. In spite of their reported skill level, all groups identified need for further HL training Furthermore, open responses found that many residents stated that they are interested in "finding more resources for patient health education materials in various languages" and having "easy to access databases of printable resources".

CONCLUSIONS: Most residents across multiple specialties report at least some training in HL during medical school and use recommended techniques at least some of the time. Those who reported receiving HL training felt more skilled in HL techniques. Interestingly, although there are significant differences in perceived HL skills, there was no significant difference in actual use of HL techniques. This suggests that even without training residents attempt to use HL techniques and there maybe factors other than training which pose as a barrier to implementing HL techniques with patients. For instance, residents requested more access to culturally and linguistically appropriate health education materials in addition to HL skills training. Resident focused training needs to include skills and raising awareness of available resources to ensure that patients with low health literacy levels are receiving appropriate levels of care

PRIORITIES FOR CHRONIC PAIN CARE: RATINGS FROM CONSUMERSTAKEHOLDERS IN TWO TEXAS RURAL COMMUNITIES VERSUS AN ONLINE SURVEY OF A POPULATION-BASED SAMPLE OF HISPANICS FROM FIVE SOUTHWESTERN STATES Natalia Rodriguez ${ }^{1}$; Melissa Valerio ${ }^{2,1}$; Paula Winkler ${ }^{1,}{ }^{1}$; Jennifer Potter $^{1,}{ }^{1}$; Kay Avant ${ }^{1,}{ }^{1}$; Jason Hill ${ }^{1}$; Barbara J. Turner ${ }^{1,1}$. ${ }^{1}$ University of Texas Health Science Center at San Antonio, San Antonio, TX; ${ }^{2}$ University of Texas School of Public Health, San Antonio, TX. (Control ID \#2470035)

BACKGROUND: Management of chronic pain in the U.S. has serious deficiencies with many sufferers lacking access to evidence-based nonpharmacologic services and treatments. Residents of low resource settings such as rural Texas have significant unmet needs for chronic pain care. To develop research priorities to address these needs, we recruited consumer-stakeholders with non-cancer chronic pain from two majority Hispanic rural counties and used concept mapping to identify, sort, and rate their ideas about pain care. To evaluate the generalizability of their highest priorities, we conducted an online survey of population-based sample of Hispanics from five states.

METHODS: Community members, aged 35-75, with chronic pain lasting $>3$ months and affecting daily activities and/or sleep were recruited using respondent-driven and purposive sampling in 2 rural South Texas counties, respectively. Overall, 60 community members met three times to brainstorm, sort, and rank the importance (5 point Likert Scale) of implementing specific types of services to improve chronic pain outcomes. Highest priority categories of services were identified from mixed methods analysis. Secondary evaluation was conducted via an online survey of Hispanics, aged 35-75, with and without chronic pain from five southwestern states from the nationally representative KnowledgePanel ${ }^{\circledR}$ of GfK Custom Research. Subjects were asked to prioritize the 13 highest ranked community stakeholder-generated categories of services for chronic pain using MaxDiff analysis, which randomizes four services per question and forces the respondent to choose the service most likely and the service least likely to help with pain.

RESULTS: The 60 community stakeholders from two Texas counties and 486 Hispanic survey respondents from 5 states concurred in rating highly the following types of services to improve outcomes of persons with chronic pain: 1) a center with specialized programs for chronic pain, 2) massage therapy, 3) more visits with a physical therapist, 4) professional counseling about managing pain, and 5) water exercises. Community stakeholders rated peer support groups and nutritional programs more highly than the survey respondents while the latter group rated rated chiropractic services more highly.

CONCLUSIONS: Priorities from two rural Texas community stakeholder groups for services to improve chronic pain outcomes were largely endorsed by a population-based sample of Hispanics from 5 southwestern states. Most notably, survey respondents and community stakeholders endorsed the need for multi-professional services focusing on the mind-body effects of chronic pain. In our rural Texas communities, virtually no access to these high priority types of services was available. These data reinforce the need to develop affordable resources within the health care system and/or in the community to provide and support receipt of diverse types of evidence-based interventions to improve outcomes of persons with chronic pain.

PRIORITIES FOR PATIENT CENTERED OUTCOMES RESEARCH: THE VIEWS OF MINORITY AND UNDERSERVED COMMUNITIES Susan D. Goold $^{2}$; c. D. myers ${ }^{3}$; Lisa Szymecko ${ }^{2}$; Carla Cunningham ${ }^{4}$; Sal Martinez ${ }^{5}$; Charo Ledon $^{2}$; Terrance Campbell ${ }^{6}$; Marion Danis ${ }^{1}$; Stephanie Solomon Cargill ${ }^{7}$; Myra Kim² Zachary Rowe ${ }^{8} .{ }^{1}$ National Institutes of Health, Bethesda, MD; ${ }^{2}$ University of Michigan, Ann Arbor, MI; ${ }^{3}$ University of Minnesota, Minneapolis, MN; ${ }^{4}$ Reynolds County Health Department, Centerville, MO; ${ }^{5}$ Community Renewal and Development, Inc., St. Louis, MO; ${ }^{6}$ YOUR center, Flint, MI; ${ }^{7}$ St. Louis University, St. Louis, MO; ${ }^{8}$ Friends of Parkside, Detroit, MI. (Control ID \#2467735)

BACKGROUND: Patient centered outcomes research (PCOR) recognizes that policy makers, scientists and clinicians must incorporate patients' and potential patients' views and values for the priorities of research as well as their views of health outcomes and processes. Engaging patients and the public in priority setting can illuminate and inform decisions and make the PCOR agenda more just, more accountable, and more responsive to patients' needs and values. Yet how to engage communities in priority setting has been a challenge. Academic-community partnerships in Michigan and Missouri used a community-based participatory research (CBPR) approach to develop and test a method for engaging minority and underserved communities in informed deliberations about PCOR priorities. 
METHODS: Academic-community partnerships adapted CHAT for PCOR prioritysetting using existing research agendas and interviews with community leaders, clinicians and key informants. Sixteen groups $(n=183)$ of community members from minority and underserved communities in two states deliberated about PCOR priorities using the simulation exercise CHAT (CHoosing All Together) in English and Spanish. Data collection included pre- and post-deliberation surveys measuring participants' experiences with deliberations, and individual and group priorities. A multilevel logistic regression with groups as random intercepts was used to obtain estimates for independent associations between each priority selection and both various individual-level (e.g., age) and grouplevel characteristics (e.g., urban vs. rural).

RESULTS: Most participants were minority, $1 / 3$ reported income $<\$ 10,000$, and $1 / 4$ reported fair/poor health. Nearly all ( $93 \%$ ) agreed that, during discussion, all positions were considered with equal respect. About 4 of 5 agreed they would "trust a process like this to inform funding decisions." About three-quarters agreed "The way in which the group reached its decision was equally fair to each member of the group," and "I gained understanding of the arguments that opposed my own." About one-quarter said there was too little time to discuss. Asked "how much input do people like you have in setting research priorities?" $20 \%$ said a great deal, $64 \%$ some and $16 \%$ none at all. Asked "How much input should people like you have in setting research priorities?" $44 \%$ said a great deal, $53 \%$ some and $3 \%$ none at all. Individuals and groups prioritized research on quality of life, patient-doctor relations, access, special needs and (by total resources spent) comparing approaches. Those over 70 years old were less likely to prioritize multiple conditions and families and caregivers research than young adults. We found no significant relationship between priorities and gender, rural vs. urban residence, ethnicity, income, or health status. Four categories of research - compare approaches, special needs, access and families \& caregivers - were more often selected after than they were before deliberation.

CONCLUSIONS: Members of underserved communities, in informed deliberations, prioritized research on quality of life, patient-doctor relations, special needs, access and comparative effectiveness.Deliberations about patient centered outcomes research priorities were judged fair and reason-based by participants. Most would support and trust this method for informing funding decisions.

PRIORITIES OF MINORITY AND UNDERSERVED COMMUNITIES FOR HEALTH RESEARCH: THE EFFECT OF DELIBERATION Susan D. Goold ${ }^{2}$; C. D. Myers ${ }^{3}$; Lisa Szymecko ${ }^{2}$; Karen Calhoun ${ }^{4}$; Adnan Hammad ${ }^{5}$; James Bridgeforth ${ }^{6}$; Charo Ledon ${ }^{7}$; Zachary Rowe ${ }^{8}$; Myra Kim ${ }^{2}$; Marion Danis ${ }^{1}$; Julia Abelson ${ }^{9} .{ }^{1}$ National Institutes of Health, Bethesda, MD; ${ }^{2}$ University of Michigan, Ann Arbor, MI; ${ }^{3}$ University of Minnesota, Minneapolis, MN; ${ }^{4}$ Michigan Institute for Clinical and Health Research, Ann Arbor, MI; ${ }^{5}$ Arab-American Medical Association, Dearborn, MI; ${ }^{6}$ Michigan Center for Urban African American Aging Research, Detroit, MI; ${ }^{7}$ Acción Buenos Vecinos, Ann Arbor, MI; ${ }^{8}$ Friends of Parkside, Detroit, MI; ${ }^{9}$ McMaster University, Hamilton, ON, Canada. (Control ID \#2468400)

BACKGROUND: Engaging patients and the public in setting research priorities can illuminate and inform decisions and make the research agenda more just, more accountable, and more responsive to their needs and values. The DECIDERS project (Deliberately Engaging Communities in DEcisions about Research Spending) aimed to develop and evaluate a method to engage communities in deliberations about health research funding priorities, emphasizing the voices of minority and medically underserved communities in the state of Michigan.

METHODS: Academic-community partnerships adapted the simulation exercise CHAT (CHoosing All Together) for research priority-setting using existing funders' research agendas and interviews with community leaders, clinicians and key informants to develop a "menu" of spending options that would be understandable to communities and useful for decision makers. Forty-seven groups $(n=519)$ of community members from minority and underserved communities throughout the state deliberated about health research priorities using CHAT in English and Spanish. Participants received information about health research priorities and then played CHAT by allocated a limited number of markers to 16 different areas of health research spending (Fig 1). Participants made allocations first as individuals, then as part of a deliberating group, and again as individuals after group deliberations. Data collection included pre- and postdeliberation,individual priorities and group priorities selected during deliberations. Within-participant changes in priorities from pre- to post-deliberation are modeled using a mixed-effect logistic regression model accounting for within-participant repeated assessment nested within deliberation groups.

RESULTS: Participants ranged from 18 to 88 years old (Mean 48.3); about 2/3 were women. Half of the participants self-identified as white, 1/3 African-American, $7 \%$ Hispanic, $5 \%$ Native American, and $3 \%$ Arab, Arab-American or Chaldean.. Most had incomes $<\$ 35,000 /$ year, and $17 \%$ reported poor or fair health status. Before deliberation, participants most commonly prioritized research about Mental Health (90.6\%), Child Health $(89.3 \%)$, Causes of Disease (85.6 \%), Promote Health $(82.7 \%)$, Healthcare Quality (82.7\%), Aging (81.1\%), and Environment (81.1\%). Access was the only type of research more likely to be selected after deliberation rather than before ( $77.2 \mathrm{vs.} 84.0 \%$, OR $1.63, p=.005)$. Improving Research (67.5à53.6 \%, OR .51), Policy Research (64.5à51.5\%, OR .52) and Culture and Beliefs (55.6à46.0, OR .58) each became less likely to be selected after group deliberations (all, $p<.001$ ). Groups likewise prioritized Child Health and Mental Health; Not only did nearly all groups (93.6 and $95.7 \%$, respectively) select these types of research at some level of investment, a majority of groups chose the highest level of investment for these two categories. Causes of Disease, Environment, Aging, Access, Promoting Health and were the next most likely to be selected by groups. Culture and Beliefs (selected by $36.2 \%$ ), Policy Research (38.3\%), Improving Research (27.7 \%) and Comparing What Works (38.3\%) were the least likely to be prioritized at any level by deliberating groups.

CONCLUSIONS: Members of underserved communities, in informed deliberations, prioritized research on mental health, child health, access, health promotion, aging and causes of disease. Deliberation had some effect on participant's priorities, in particularly leading to higher prioritization of research on access.

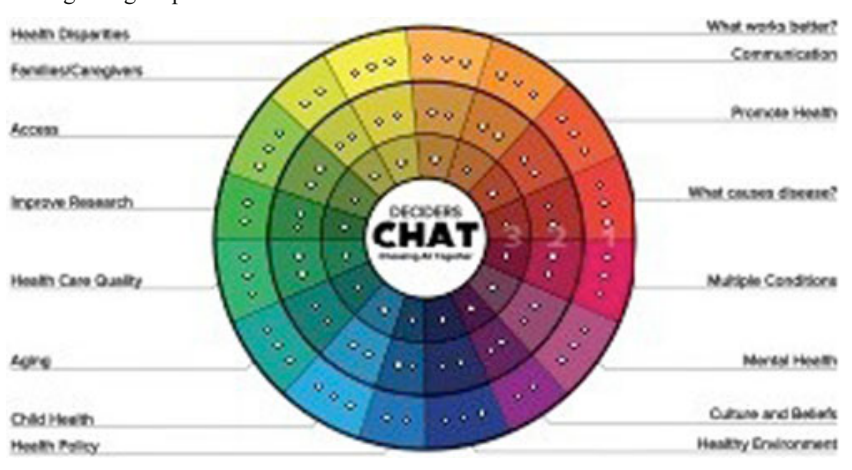

Figure 1. CHAT depiction of research spending options

PRISON OVERCROWDING AND THE RISK OF SELF-HARM IN A PRE-TRIAL DETENTION SETTING IN GENEVA, SWITZERLAND Hans Wolff ${ }^{1}$; Alejandra Casillas $^{1}$; Thomas Perneger ${ }^{3}$; Patrick Heller ${ }^{1}$; Diane Golay ${ }^{1}$; Elisabeth Mouton ${ }^{1}$; Patrick Bodenmann ${ }^{2}$; Laurent Gétaz ${ }^{1} .{ }^{1}$ Geneva University Hospitals, Chêne-Bourg, Switzerland; ${ }^{2}$ Vulnerable Population Center, Lausanne, Switzerland; ${ }^{3}$ Geneva University Hospitals, Geneva, Switzerland. (Control ID \#2457896) 
BACKGROUND: Institutional conditions of detention centers can affect the risk for selfharm among detainees. In particular, prison overcrowding may increase the likelihood of self-harm by creating competition for resources, space, and enhancing a "deprivation state". Prisoner self-harm is a common and dangerous phenomenon within correctional institutions and associated with subsequent suicide. Rates of suicide in male prisoners in high-income countries are estimated to be three to seven times higher than in the general population, and non-lethal acts of self-harm occur even more. The prison of ChampDollon in Geneva, Switzerland has suffered from overcrowding since 1997. Over the last several years, prison health care professionals have expressed concern over the rise of selfharm events, particularly with acts of self-strangulation/hanging. This study examines the association between overcrowding and prisoner acts of self-harm.

METHODS: This cross-sectional study took place at Geneva's pre-trial prison (capacity: 387) between 2006 and 2014. Outcomes were 1) acts of self-harm that required medical attention, and 2) self-strangulation/hanging events (combined into one group, as these are difficult to differentiate). Dichotomous predictors were overcrowding index- annual mean daily population divided by capacity (>200 vs. $<200 \%$ ), and year group (2006-2009 vs. 2011-2014)

RESULTS: Self-harm and self-strangulations/hangings increased in 2011-2014 compared to 2006-2010 (Table 1). Overcrowding in excess of $200 \%$ was associated with self-strangulation/hangings $(p<0.001)$ but not with all selfharm events. Figure 1 shows the occupancy rate fluctuating between $123 \%$ (2011) and $220 \%$ (2014). Self-strangulations/hangings increased even more with more than 2 events per 100 inmate-years as of 2011, and a peak in 2014 when 89 inmates were found strangulated or hanged. In terms of pertinent demographics that would affect self-harm, there was no prison change in gender, area of origin, foreign residency, religion, or psychiatric treatment.

CONCLUSIONS: We observed a significant increase in self-harm and selfstrangulation/hangings over time, and overcrowding was significantly associated with self-strangulation/hangings (but not with all self-harm events). Overcrowding can impose destructive effects on the psychological and behavioral well being of inmates in prison, influencing a myriad of emotional and livelihood factors that predispose to harmful behavior. This report should alert public health authorities to this issue, and garner resources to address such an alarming rise. Next steps should further examine the relation between self-harm, strangulation/hanging and overcrowding, adjusting for pertinent information from individual patient prison records.

Table 2. Association of Surgical and Cardiac Risk with Preoperative Medical Evaluations

\begin{tabular}{|c|c|c|c|c|c|c|}
\hline & \multirow{2}{*}{\multicolumn{2}{|c|}{$\begin{array}{c}\text { Surgical Risk } \\
\text { High Risk }{ }^{\circ} \\
\text { OR }(95 \% \mathrm{Cl})\end{array}$}} & \multicolumn{4}{|c|}{ Cardiac Risk } \\
\hline & & & \multicolumn{2}{|c|}{$\begin{array}{c}\text { RCRI }=1^{c} \\
\text { OR }(95 \% \text { CI) }\end{array}$} & \multicolumn{2}{|c|}{$\begin{array}{l}\text { RCRI }=2+^{c} \\
\text { OR }(95 \% \mathrm{Cl})\end{array}$} \\
\hline Office Visit & 1.96 & $\begin{array}{c}1.93- \\
1.99)\end{array}$ & 1.35 & $\begin{array}{l}(1.33- \\
1.37)\end{array}$ & 1.71 & $\begin{array}{c}(1.67- \\
1.76)\end{array}$ \\
\hline Blood Count & 2.27 & $\begin{array}{l}(2.24- \\
2.30)\end{array}$ & 1.15 & $\begin{array}{c}(1.13- \\
1.17)\end{array}$ & 1.18 & $\begin{array}{c}(1.14- \\
1.21) \\
\end{array}$ \\
\hline Metabolic Panel & 2.31 & $\begin{array}{l}(2.28- \\
2.34)\end{array}$ & 1.30 & $\begin{array}{l}(1.28- \\
1.32)\end{array}$ & 1.29 & $\begin{array}{l}(1.25- \\
1.33)\end{array}$ \\
\hline Coagulation Test & 4.90 & $\begin{array}{l}(4.80- \\
5.00)\end{array}$ & 1.14 & $\begin{array}{l}(1.12- \\
1.16)\end{array}$ & 1.19 & $\begin{array}{l}(1.15- \\
1.23)\end{array}$ \\
\hline Urinalysis & 3.82 & $\begin{array}{l}(3.76- \\
3.88)\end{array}$ & 1.13 & $\begin{array}{l}(1.10- \\
1.15)\end{array}$ & 1.16 & $\begin{array}{l}(1.13- \\
1.20)\end{array}$ \\
\hline Electrocardiogram & 2.47 & $\begin{array}{l}(2.43- \\
2.50)\end{array}$ & 1.17 & $\begin{array}{l}(1.16- \\
1.19)\end{array}$ & 1.00 & $\begin{array}{l}0.97- \\
1.02)\end{array}$ \\
\hline Stress Test & 2.50 & $\begin{array}{l}(2.41- \\
2.60)\end{array}$ & 2.29 & $\begin{array}{l}(2.20- \\
2.38)\end{array}$ & 3.78 & $\begin{array}{l}\text { (3.59- } \\
3.99)\end{array}$ \\
\hline Chest Radiograph & 4.00 & $\begin{array}{l}(3.93- \\
4.07)\end{array}$ & 1.14 & $\begin{array}{l}(1.12- \\
1.16)\end{array}$ & 1.15 & $\begin{array}{l}(1.11- \\
1.18)\end{array}$ \\
\hline $\begin{array}{l}\text { Pulmonary } \\
\text { Function Test }\end{array}$ & 1.55 & $\begin{array}{l}(1.45- \\
1.65)\end{array}$ & 1.18 & $\begin{array}{l}(1.11- \\
1.27)\end{array}$ & 1.25 & $\begin{array}{l}(1.13- \\
1.39)\end{array}$ \\
\hline
\end{tabular}

a models include patient demographics, RCRI, comorbidities, procedure type,

hospitalization in prior 30 days

${ }^{b}$ compared to reference group low risk surgery

${ }^{c}$ compared to reference group RCRI $=0$
Figure 1: Overcrowding and rates of self-harm and self-strangulation/hanging events in Champ-Dollon, 2006-2014

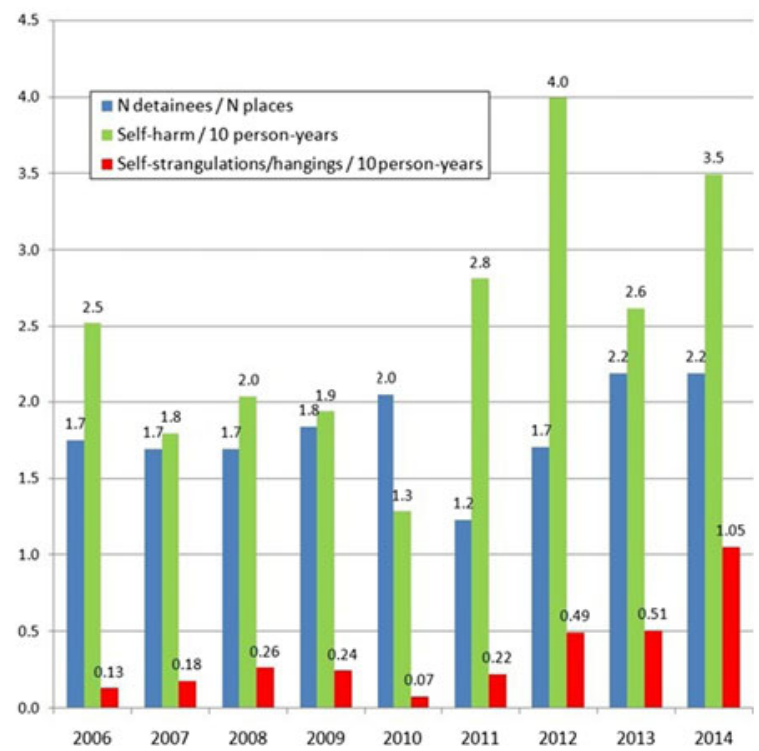

PROCALCITONIN VALUES WITHIN 24 h OF ADMISSION CAN PREDICT SEVERITY OF ACUTE PANCREATITIS: A META ANALYSIS Karthik Ragunathan ${ }^{1}$; Shravana Bheemanathi ${ }^{2} .{ }^{1}$ OSF St.Francis medical center, Peoria, IL; ${ }^{2}$ St. Barnabas Hospital, Bronx, NY. (Control ID \#2469866)

BACKGROUND: Severe acute pancreatitis develops in about $10-15 \%$ patients with acute pancreatitis and is associated with increased morbidity and mortality. Several laboratory markers have been tested to predict severe acute pancreatitis such as Creactive protein, blood urea nitrogen, creatinine but none of these have been shown to be effective in predicting within $24 \mathrm{~h}$. Procalcitonin (PCT) is an acute phase reactant and has been studied as a marker in pancreatitis but mixed results have been reported in the literature. We aimed to do meta analysis to calculate the cumulative sensitivity, specificity, likelihood ratios and diagnostic odds ratio for PCT as a marker.

METHODS: MEDLINE, EMBASE, and SCOPUS were searched to identify potential articles published from 1946 to December 2015. MeSH headings included in the search included severe acute pancreatitis, procalcitonin. All references from the identified articles were further searched to find more studies. Only studies in adult population and studies in English language were included. Studies that reported the predictive value of PCT measured within $24 \mathrm{~h}$ of admission as a marker of severe acute pancreatitis as opposed to mild pancreatitis were only included. We collected sufficient data to construct a $2 \times 2$ contingency table for true- positive, true- negative, false-positive and false negative results. DerSimonian-Laird random-effects model was used to estimate the pooled sensitivity, specificity, and likelihood ratio, and a diagnostic odds ratio. Analyses were performed using Meta-Disc Version 1.4.

RESULTS: A total of 470 articles were identified from the search strategy and from articles identified through references. Of these 9 studies involving 594 patients were included in the final analysis based on our inclusion criteria. Pooled sensitivity and specificity of PCT as a marker of severe acute pancreatitis was $54 \%$ (95\% CI 47 to 60$)$ and $81 \%$ (95\%CI 67-91). The combined positive likelihood ratio was 3.41 (95\% CI $1.57-7.40)$ and negative likelihood ratio was 0.59 (95\% CI $0.41-0.84)$. The pooled odd ratio was 6.51 (95\% CI 2.18-19.47).

CONCLUSIONS: PCT when measured within the first $24 \mathrm{~h}$ can predict severe acute pancreatitis with an odds ratio of 6.51 . With high specificity, positive and negative 
likelihood ratio, high PCT can be used as a reliable marker in acute pancreatitis. PCT can be routinely measured in all patients getting admitted to the hospital for severe acute pancreatitis and proper triaging of these patients and early aggressive management may potentially reduce morbidity and mortality associated with severe acute pancreatitis

PROGNOSTIC UTILITY OF THE HEART SCORE IN PATIENTS EVALUATED FOR A POSSIBLE ACUTE CORONARY SYNDROME IN AN OBSERVATION UNIT Alexander Michaels ${ }^{1}$; Sagger Mawri ${ }^{1}$; Joseph Gibbs ${ }^{1}$; George R. Dirani ${ }^{1}$; Gordon Jacobsen ${ }^{2}$; Richard Nowak ${ }^{1}$; James McCord ${ }^{1} .{ }^{1}$ Henry Ford Hospital, Detroit, MI; ${ }^{2}$ Henry Ford Health System, Detroit, MI. (Control ID \#2469345)

BACKGROUND: The evaluation of patients with possible acute coronary syndrome (ACS) is the most common indication for observation unit (OU) stays in the United States. The HEART score (HS) is a risk stratification tool involving elements of the history, EKG, age, cardiac risk factors, and troponin levels. We applied the HS to patients evaluated for possible ACS in an OU.

METHODS: This was a single-center (Henry Ford Hospital, Detroit, MI), retrospective study in 2013 that evaluated 838 patients placed in an OU who were evaluated for possible ACS and had stress testing. The HEART score was applied, and patients were followed at 30 days for major adverse cardiac events (MACE): death, myocardial infarction (MI), or revascularization procedure.

RESULTS: There were 895 stress tests performed, as some patients had more than one test. At 30 days there were 14 MACEs: 0 deaths, 0 MI, and 14 revascularization procedures ( 3 coronary artery bypass grafting surgeries and 11 percutaneous interventions). Patients with a higher HS had higher MACE rates, and more commonly had positive stress tests (table). All patients with a $\mathrm{HS}<4$ with a positive stress test who underwent coronary angiography demonstrated non-obstructive disease.

CONCLUSIONS: Patients with a HS $<4$ evaluated for possible ACS are at very low risk for MACE at 30 days and may be able to be discharged without stress testing. Patients with an abnormal stress test and HS $<4$ likely have a false positive test. These findings should be validated prospectively in a larger trial.

Association of Intervalized HEART score with Positive Stress Test and MACE at 30 days

\begin{tabular}{llll}
\hline \hline HEART Score & $<4$ & $4-6$ & $>6$ \\
Number of patients & 262 & 557 & 19 \\
Positive stress tests & $8(3 \%)$ & $42(7.5 \%)$ & $4(21 \%)$ \\
MACE & 0 & $12(2.2 \%)$ & $2(10.5 \%)$ \\
\hline
\end{tabular}

PROGRAM DIRECTORS' PERCEPTIONS OF RESIDENT EDUCATION IN WOMEN'S HEALTH: A NATIONAL SURVEY Rachel S. Casas ${ }^{2}$; Laura D. Hallett ${ }^{2}$, 1; Catherine A. Rich ${ }^{2}$; Megan R. Gerber ${ }^{1}$; Tracy A. Battaglia ${ }^{2} .{ }^{1}$ Boston University, Jamaica Plain, MA; ${ }^{2}$ Boston University Medical Center, Boston, MA. (Control ID \#2468966)

BACKGROUND: Despite professional societies' continued emphasis on women's health in internal medicine (IM) resident curricula, national implementation has varied and residents have demonstrated deficiencies in women's health knowledge. This study describes the current state of women's health education in IM residency programs and evaluates program directors' perceptions of educational needs.

METHODS: We recruited 369 categorical and 39 primary care program directors of IM residency programs accredited by the Accreditation Council for Graduate Medical Education (ACGME) to complete an electronic, cross-sectional survey in 2015. Participants were surveyed about desired resident mastery of women's health topics and interest in expansion of women's health education in the context of their program's current characteristics, core curricula, and training opportunities. Twelve priority women's health topics for resident mastery and inclusion in core curricula, such as screening for gender specific cancer and managing menopausal symptoms, were compiled from recommendations from national societies. We compared responses between categorical and primary care program directors and between programs with and without a primary care track.

RESULTS: One hundred and twelve program directors completed the survey; 96 identified as categorical program directors and 16 identified as primary care program directors (response rates 26 and $41 \%$ respectively, $p=0.05$ ). Forty-eight programs $(43 \%)$ were in a university or academic hospital and $39(35 \%)$ had a primary care track. Program directors reported high overall agreement that residents should master topics in women's health (ranging from 48 to $98 \%$ by topic) with higher agreement among topics more likely to be included in current core curricula. A minority of program directors $(N=26,23 \%)$ were very interested in expanding opportunities in women's health. Desired resident mastery and interest in expansion of opportunities did not differ between categorical and primary care program directors. A minority of programs had a women's health concentration or track $(N=8,7 \%)$ or continuity clinic $(N=15,13 \%)$, with more opportunities available in programs with a primary care track ( $p=0.008$ and 0.0002 respectively). While a majority of programs offered women's health electives $(N=76,68 \%), 48$ programs (43\%) reported that $20 \%$ or less of residents participated. The most highly represented women's health topics in current core curricula were gender specific cancer screening $(N=105$, $94 \%)$, osteoporosis $(N=102,91 \%)$, and sexually transmitted infection $(N=99,88 \%)$. The majority of programs $(N=74,66 \%)$ covered eight or more topics. Topics included in the core curriculum did not differ between programs with and without a primary care track. CONCLUSIONS: Surveyed IM program directors agreed that residents should master women's health topics and that their programs should include priority topics in core curricula. However, women's health training opportunities, such as continuity clinics, tracks, and electives, are limited or underutilized and a minority of program directors state a strong interest in expanding these opportunities. Further research is needed to determine barriers to expansion of women's health education in residency programs

PROMOTING PROGRESS OR PERPETUATING PROBLEMS: STRATEGIC PLANNING AND DIVERSITY IN ACADEMIC MEDICINE David M. Washington $^{1,}{ }^{2}$; Michael Paasche-Orlow ${ }^{1,2}$; Jane M. Liebschutz ${ }^{1,2}$. ${ }^{1}$ Boston Medical Center, Boston, MA; ${ }^{2}$ Boston University School of Medicine, Boston, MA. (Control ID \#2469667)

BACKGROUND: Efforts to increase underrepresented minority (URM) representation in medicine have been relatively successful for students but not faculty of medical schools. Strategic planning is a component of Diversity 3.0, the diversity and inclusion improvement framework of the Association of American Medical Colleges (AAMC); however, it is not known whether strategic planning is successful in achieving faculty diversification. We conducted a study to examine whether the existence of a strategic plan for diversity was associated with the proportion of URM faculty in AAMC member institutions.

METHODS: Using their publicly available websites, 141 AAMC-member medical schools were categorized as having a strategic plan with an expressed purpose of increasing institutional diversity and/or inclusion at the faculty level. Using the $A A M C$ Faculty Roster, institutions were stratified by the percent of faculty members who selfreported as Black, Hispanic, Native American, or Pacific Islander in 2014. Statistical outliers ( $N=9, \%$ URMM greater than $16.9 \%)$ were excluded from the final analyses as the URM faculty representation at these institutions was high enough to potentially preclude a full strategic plan for diversity. The remaining 132 institutions (Median $(\mathrm{IQR})=6.3 \%(4.8 \%))$ were divided into those in the first quartile for URM faculty representation $(N=34)$ being defined as low diversity and those that were not $(N=98)$ 
as average-to-high diversity. A Chi-squared test of independence was performed to evaluate whether the proportion of institutions having strategic plans was different for the low diversity and average-to-high diversity groups. The percent of change in URM faculty from 1998 to 2014 was also calculated with for institutions that reported URM numbers in both years excluding aforementioned outliers $(N=119$, Median $(\mathrm{IQR})=1.6 \%(2.08 \%))$. Institutions in the first quartile for \% change in URM faculty were defined as low improvement in diversity institutions $(N=30, \%$ change in URM faculty $<0.35 \%$ ), and institutions that were not in first quartile were defined as average-to-high improvement in diversity institutions. A Chi-squared test of Independence were performed to evaluate if the proportion with strategic plans in the low change in diversity institutions was different to the proportion in the average-to-high change in diversity group. Similar tests were conducted to evaluate: 1) if the proportion of institutions with strategic plans for diversity was different between those with a decrease in \% URM faculty representation from 1998 to 2014 $(N=22)$ and those with an increase $(N=97) ; 2)$ if having a strategic plan in place for a longer period of time $(\leq 3$ years $(N=31)$ or $>5$ years $(N=16))$ was associated with low diversity versus average-to-high diversity institutions; and, 3) if there was a difference between the low improvement in diversity group compared to the averageto-high improvement in diversity group for institutions with a strategic plan in place for a longer period of time.

RESULTS: In 2014, URM faculty representation for the 132 institutions included in the analyses ranged from 1.6 to $16.7 \%$ and 51 (38.6 \%) of these institutions had a faculty diversity plan. Presence of a strategic plan for diversity was not associated with the level of faculty diversity (Chi-squared $=0.067, p=0.79$ ). Presence of a strategic plan for diversity as not associated with the amount of improvement in diversity between 1998 and 2014 (Chi-squared $=1.37, p=0.24$ ). Having a strategic plan was not associated with a decrease or increase in URM faculty representation (Chi-squared $=0.64, p=0.42$ ). Having a strategic plan for a longer period of time was not associated with level of faculty diversity ( $\leq 3$ years: Chi-squared $=0.86, p=0.35 ;>5$ years: Chi-squared $=1.09, p=0.29$ ) or improvement in \% URM faculty from 1998 to 2014 ( $\leq 3$ years: Chi-squared $=0.16, p=0.68$; $>5$ years: Chi-squared $=0.082, p=0.77$ ).

CONCLUSIONS: The existence of a strategic plan for diversity on institutional websites is not associated with the degree of URM faculty representation or the change in URM faculty representation from 1998 to 2014. Future efforts need to determine whether the quality or specific content of strategic plans for diversity influence faculty diversification. The characterization of successful elements of strategic plans for faculty diversity should inform best practices for AAMC member institutions

PROSPECTIVE VALIDATION OF THE HOSPITAL SCORE TO PREDICT PATIENTS AT HIGH RISK OF UNPLANNED READMISSION Carole E. Aubert ${ }^{1}$; Antoine Folly ${ }^{3}$; Marco Mancinetti ${ }^{3}$; Daniel Hayoz ${ }^{3}$ Jacques Donze ${ }^{1,2} .{ }^{1}$ Inselspital, Bern University Hospital, Bern, Switzerland; ${ }^{2}$ Brigham and Women's Hospital, Boston, MA; ${ }^{3}$ Fribourg Cantonal Hospital, Fribourg, Switzerland. (Control ID \#2457208)

BACKGROUND: In order to most efficiently improve transition of care, hospitals need to target intensive discharge interventions at those patients at high risk of unplanned readmission. The "HOSPITAL" score, derived previously in the US, is an easy to use prediction model that accurately identifies medical patients at high risk of readmission. It includes the following predictors: Hemoglobin, discharge from an Oncology service, Sodium level, Procedure during the index admission, Index Type of admission (urgent or elective), number of Admission(s) during the last 12 months and Length of stay. This score showed good performance in an international multicenter retrospective study. We aimed to demonstrate in a prospective study the accuracy of the HOSPITAL score to predict high risk for unplanned 30-day readmission and death.
METHODS: We prospectively screened all consecutive patients aged $\geq 50$ years admitted to the department of general internal medicine of a large community hospital in Switzerland between April and September 2013. Patients who were transferred to another acute care hospital or rehabilitation center, who refused to sign the inform consent, or who died during hospitalization were excluded. The primary outcome was the first unplanned readmission or death that occurred within 30 days from hospital discharge. We calculated the "HOSPITAL" score for all patients. Since the hospital had no specific oncology division, the variable "Oncology" was replaced by a recent diagnosis of cancer ( $<5$ years $)$ on admission. The performance of the prediction model was evaluated according to its overall accuracy (Brier score), its discriminatory power (C-statistic), and its calibration (Pearson goodness of fit test).

RESULTS: Among the 346 included patients, $11.6 \%(n=40)$ had an unplanned readmission or died within 30-days after discharge. Mean age of the patients was 73.4 years (SD 11.5) and median length of stay 7 days (IQR 4-12). The overall accuracy of the "HOSPITAL" score was very good with a low Brier score (0.09). Its discriminatory power to predict unplanned readmission or death was good with a C-statistic of $0.70(95 \% \mathrm{CI}$ 0.61-0.79). Patients were classified into 3 risk categories for the primary outcome: low $(67 \%)$, intermediate $(19 \%)$, and high risk $(14 \%)$. The proportions of unplanned readmission/death for each risk category were 9,12 , and $25 \%$, respectively. The calibration was excellent with a $P$-value of 0.97 . A simplified version of the score without the variable "Procedure during the index admission" showed fair performance, with a Cstatistic of 0.67 (95 \% CI 0.58-0.75)

CONCLUSIONS: The "HOSPITAL" score identified patients at high risk of 30-day unplanned readmission or death with good discrimination when applied prospectively to a cohort of medical patients in Switzerland. The "HOSPITAL" score has the potential to easily and prospectively identify patients in need of more intensive transitional care interventions.

PROSTATE CANCER SCREENING TRENDS IN PRIMARY CARE PRACTICES IN A LARGE INTEGRATED HEALTHCARE SYSTEM: 2007-2015 Anita D. Misra-Hebert; Bo Hu; Eric A. Klein; Glen B. Taksler; Andrew Stephenson; Michael B. Rothberg. Cleveland Clinic, Cleveland, OH. (Control ID \#2468652)

BACKGROUND: Conflicting evidence for the benefit of prostate cancer screening with the prostate specific antigen (PSA) test, concerns for overdiagnosis, and evolving practice guidelines have created confusion for primary care providers. In 2008 The US Preventive Services Task Force (USPSTF) recommended against screening with the PSA for men >75 years and in 2012 recommended against screening in men of any age. In 2013 the American College of Physicians and the American Urologic Association recommended shared decision making for PSA testing for men ages 50's-69 and agreed that men $>70$ should not undergo routine PSA testing. Established risk factors for prostate cancer include age, African American race, and family history of prostate cancer. The purpose of this study is to assess the trends in utilization of PSA testing for prostate cancer screening in the primary care clinics of a large integrated health system as correlated with 1) the release of these evolving guidelines and 2) known risk factors for prostate cancer. METHODS: We conducted a retrospective study using electronic medical record data from January 2007-June 2015. Our study population included all men ages 40 and above with at least 1 visit in an Internal Medicine or Family Medicine clinic in the Cleveland Clinic Health System in any of the study years. As our focus was on PSA screening testing in primary care, we excluded men who had a visit with the urology department prior to the PSA test in any study year and men with a history of prostate cancer. Any "diagnostic PSA" test or PSA tests associated with International Classification of Diseases - Ninth revision (ICD-9) diagnosis codes for urinary symptoms or prostatic hypertrophy with symptoms were also excluded. We calculated PSA screening rates for men in the 
following age groups: $>70,50-69$ and $40-49$. We also calculated rates of screening for men with established risk factors for prostate cancer including African American race and documented family history of prostate cancer.

RESULTS: Prostate cancer screening rates with PSA testing in primary care clinics for men $>70$ years of age decreased steadily from 2007 to 2015 from 10 to $5 \%$, and rates of screening in men ages 40-49 remained low at 3-4\%. For men ages $50-69$, while a small decrease in screening rates was noted in 2012-13, temporally corresponding with the initial release of the USPSTF guidelines, the rates have remained stable at $13 \%$ in both 2007 and 2015 (Figure). Including all men ages 40 and older, the rate of prostate cancer screening in African American men ranged from 6.8 to $8.2 \%$ and remained lower than screening rates in non-African American men from 2007 to 2015 . For all men with a documented family history of prostate cancer, PSA screening rates remained higher than for men without a family history of prostate cancer throughout the study period (Table).

CONCLUSIONS: Physicians in our sample appeared to embrace guideline recommendations against screening older men, but not those recommending against all screening. Men with a family history of prostate cancer were more likely, and African American men less likely, to be screened. Future work should address approaches to risk-based shared decision making for prostate cancer screening, specifically for African American men.

Rates of PSA Screening Testing in Men with High Risk for Prostate Cancer

\begin{tabular}{|c|c|c|c|c|c|c|}
\hline & $\begin{array}{l}\text { African } \\
\text { American } \\
\text { Men } \\
(N=8669)\end{array}$ & $\begin{array}{l}\text { Non- } \\
\text { African } \\
\text { American } \\
\text { Men } \\
(N= \\
81828)\end{array}$ & & $\begin{array}{l}\text { Men with } \\
\text { Family } \\
\text { History } \\
\text { of Prostate } \\
\text { Cancer } \\
(N=1718)\end{array}$ & $\begin{array}{l}\text { Men } \\
\text { without } \\
\text { Family } \\
\text { History of } \\
\text { Prostate } \\
\text { Cancer } \\
(N= \\
88779)\end{array}$ & \\
\hline Year & Rate (\%) & Rate (\%) & $\mathrm{p}$ & Rate (\%) & Rate (\%) & $\mathrm{p}$ \\
\hline 2007 & 6.8 & 7.1 & 0.28 & 10.1 & 7.0 & $<0.001$ \\
\hline 2008 & 7.0 & 7.7 & 0.03 & 11.6 & 7.5 & $<0.001$ \\
\hline 2009 & 7.8 & 8.1 & 0.30 & 12.1 & 8.0 & $<0.001$ \\
\hline 2010 & 8.2 & 9.0 & 0.02 & 12.6 & 8.8 & $<0.001$ \\
\hline 2011 & 8.2 & 9.7 & $<0.001$ & 14.3 & 9.5 & $<0.001$ \\
\hline 2012 & 7.1 & 8.5 & $<0.001$ & 10.3 & 8.4 & $<0.01$ \\
\hline 2013 & 7.4 & 7.8 & 0.23 & 12.1 & 7.7 & $<0.001$ \\
\hline 2014 & 7.9 & 9.5 & $<0.001$ & 13.3 & 9.3 & $<0.001$ \\
\hline 2015 & 8.2 & 9.6 & $<0.01$ & 13.8 & 9.4 & $<0.001$ \\
\hline
\end{tabular}

2015 rates are annualized

\section{PSA Testing Rates 2007-2015 by Age}

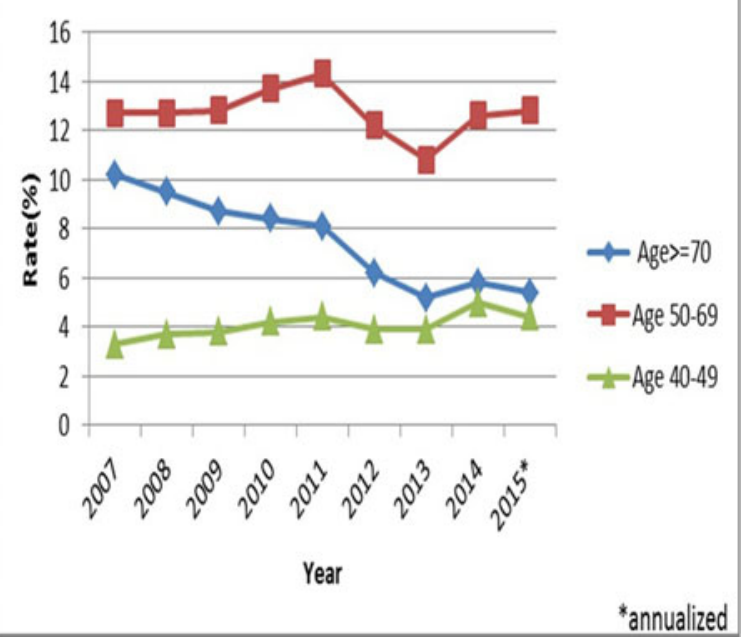

PROVIDER FEEDBACK ON WEIGHT LOSS MAINTENANCE RESOURCES AND A NOVEL INTERVENTION USING THE ELECTRONIC MEDICAL RECORD: RESULTS FROM THE MAINTAIN-PC STUDY Jonathan Arnold ${ }^{2}$; Dana L. Tudorascu $^{1}$; Kathleen M. McTigue ${ }^{1}$; Cindy L. Bryce ${ }^{4}$; Kimberly A. Huber ${ }^{1}$; Laurey R. Simkin-Silverman ${ }^{4}$; Rachel Hess ${ }^{3}$; Gary Fischer ${ }^{1}$; Molly B. Conroy ${ }^{1} .{ }^{1}$ University of Pittsburgh, Pittsburgh, PA; ${ }^{2}$ University of Pittsburgh Medical Center, Pittsburgh, PA; ${ }^{3}$ University of Utah, Salt Lake City, UT; ${ }^{4}$ University of Pittsburgh Graduate School of Public Health, Pittsburgh, PA. (Control ID \#2466630)

BACKGROUND: Interventions targeting weight loss often fail to demonstrate long-term results. Primary care providers (PCPs) can play a critical role in supporting patients' weight loss maintenance efforts, but lack of time and resources are barriers. A novel solution would be to use existing electronic health record (EHR) and linked personal health record (PHR) resources along with non-physician clinical staff to support primary care patients' weight loss maintenance and involve the PCP through timely updates. Understanding the current PCP weight loss maintenance practices and soliciting feedback on integration of EHR tools into existing clinical workflow are key steps in creating weight loss maintenance interventions that will have the greatest potential for dissemination in diverse clinical settings.

METHODS: Maintaining Activity and Nutrition through Technology-Assisted Innovation in Primary Care (MAINTAIN-pc) is a randomized clinical trial of a multidisciplinary weight loss maintenance program for patients who have had intentional weight loss of at least $5 \%$ in the past 2 years. The MAINTAIN-pc intervention uses health coaches and patient-facing PHR tools (including secure messaging, dietary and weight tracking flowsheets, and standardized surveys) to coach patients. MAINTAIN-pc also includes PCP-facing EHR tools including best practice alerts, real-time patient update reports prior to scheduled appointments, and notifications of significant weight changes. We solicited feedback on MAINTAIN-pc via an anonymous email survey from all providers in our ten collaborating practices and any other provider who endorsed participation for a patient referred through other means (e.g., research registry). Surveys were sent in May, 2015, 3 months after completion of patient enrollment, and the initial survey was followed by three reminder messages. Survey respondents were not paid but could voluntarily enter a drawing for a fitness-related prize. We asked all respondents to estimate the proportion of their patient panel that had recent intentional weight loss. We asked them to describe their current practices for management of these patients, identify the availability and use of clinic and community resources, and to indicate their level of comfort with their EHR. For providers who had referred patients to MAINTAIN-pc, we also asked for feedback on the MAINTAIN-pc tools and interventions. We analyzed the results from all providers using descriptive statistics. RESULTS: We received survey responses from 64 of the 126 providers contacted (51\%) and $51(40 \%)$ completed at least $80 \%$ of the survey. Respondents were $63 \%$ female and $84 \%$ white. Most $(67 \%)$ completed training $>=10$ years ago. Respondents were primarily clinician educators (43\%) or full-time clinicians (26\%) working in faculty (42\%) or academically affiliated (25\%) internal medicine practices. Most respondents (61\%) reported that $10-24 \%$ of their patients had experienced intentional weight loss. Over $80 \%$ of respondents reported that their EHR was "easy and intuitive to use". When providers were asked about what they offer to patients with intentional weight loss, $86 \%$ provide "general support and encouragement" and $86 \%$ provide specific advice about diet and exercise. Fifty-one percent used health educators or coaches to communicate with patients online or via email and $23 \%$ used clinical staff (i.e., health coaches, RNs, and MAs) for reminder phone calls. While $51 \%$ referred patients to community or commercial lifestyle programs, only $12 \%$ provided electronic or paper lists of these resources for patients. When asked about resources they did not have but wanted to provide for patients, $95 \%$ reported wanting health educators or coaches for email or online communication and $95 \%$ wanted lists of community resources. Thirty-seven respondents had referred a patient to MAINTAIN-pc and $92 \%$ of them felt that the referral process integrated smoothly into their normal workflow. Thirty referring providers reported receiving a 
patient progress report just prior to an office visit, $93 \%$ felt they integrated smoothly and $97 \%$ found them useful. Twenty-three referring providers reported receiving real-time EHR messages alerting them to patient weight los or gain, $82 \%$ felt they integrated smoothly and $74 \%$ found them useful. Overall, $97 \%$ of referring providers felt that MAINTAIN-pc was a useful addition to the health care of their patients

CONCLUSIONS: The providers who had experience with MAINTAIN-pe reported that the intervention tools integrated smoothly into their workflow and were useful for their patients. The providers we surveyed were largely established in practice, academically affiliated, and had a significant number of patients who had experienced intentional weight loss. They were interested in using clinical staff and electronic resources to support patients' weight management efforts; those who had these resources largely used them while those who did not have them overwhelmingly wanted them. Our survey also identified an opportunity to extend the MAINTAIN-pc tools to provide information about community based programs for lifestyle support.

PROVIDER RESPONSE TO EHR-LINKED PATIENT-REPORTED EFFECTIVENESS OF DIABETIC PERIPHERAL NEUROPATHY TREATMENT Mark Lin; Richard W. Grant; Lin Ma; Alyce Adams. Kaiser Permanente Northern California, San Francisco, CA. (Control ID \#2467423)

BACKGROUND: Recent emphasis on patient-centered care has catalyzed the development and adoption of patient-reported outcomes in clinical research. Integration of patientreported measures into the electronic health record (EHR) may be useful for conditions such as chronic pain that rely on subjective reports of treatment response. However, information about strategies for integrating patient-reported outcomes into clinical practice is lacking. The purpose of this study was to gauge primary care provider (PCP) response to receiving patient-reported information in the EHR from patients newly initiated on diabetic peripheral neuropathy (DPN) treatment as part of an ongoing clinical trial. METHODS: We conducted structured EHR chart-reviews of patients with type-2 diabetes newly started on DPN-related neuropathic pain medications who provided treatment effectiveness and medication adherence information via interactive voice response (IVR) telephone surveys. Surveys were administered between $1 / 1 / 15-7 / 15 / 15$ in a large integrated healthcare delivery system in Northern California as part of the Diabetes Telephone Study, a large-scale clinical trial [NCT02056431]. Patients reporting any of 3 suboptimal treatment outcomes (sideeffects within 7 days, symptom relief dissatisfaction, not taking medication) were included. PCPs were notified via brief secure electronic notifications, along with additional detailed EHR documentation. For our primary outcome, we examined PCP response rates to these notifications, defined as EHR-recorded DPN discussion and/or medication change within 60 days. We compared baseline measures (demographics, utilization, patient-provider communication survey results, health status, medical complexity) between patients with and without evidence of PCP response using Student's t-test or Fisher's exact test.

RESULTS: We included 101 records of patients reporting DPN treatment-related concerns via IVR cared for by 90 PCPs. Nearly half (48/101, $47.5 \%$ ) of EHR notifications had evidence of subsequent PCP response, $70.8 \%$ of which were through EHRdocumented discussions. Comparing patients with and without evidence of $\mathrm{PCP}$ response, we found no statistically-significant differences in baseline patient-reported PCP communication or shared decision-making measures, socioeconomic/demographic characteristics, or baseline annual rates of emergency department $(0.29$ vs $0.42, p=0.57)$ or inpatient visits $(0.06$ vs $0.04, p=0.43)$. There was no difference in self-reported continuous medication use ( 75.0 vs $64.2 \%, p=0.11$ ). Among patients with continuous use, there was no difference in side-effects ( 77.8 vs $82.4 \%, p=0.67$ ) or self-titration (52.8 vs $35.3 \%, p=0.16$ ); however, lack of symptom improvement and/or treatment dissatisfaction were more common in patients with evidence of PCP response (19.4 vs $2.9 \%, p=$ 0.04). Health-related quality-of-life was worse among patients with evidence of PCP response (EQ5D: 0.629 vs 0.668, $p=0.04$ ), but there were no observed differences in number of issues listed in the subjective ( $1.95 \mathrm{vs} 1.72, p=0.39$ ) or assessment/plan (4.34 vs $3.04, p=0.07)$ of post-IVR PCP documentation, diagnosis count (17.2 vs $15.2, p=$ 0.19 ), or total medications ( 13.0 vs $11.8, p=0.17$ )

CONCLUSIONS: Nearly half of PCPs documented DPN-related discussions or medication adjustments within 60 days of receiving patient reports of sub-optimal treatment outcomes. PCPs responded more frequently to reports of inadequate symptom relief and/ or treatment dissatisfaction than presence of side-effects, suggesting that certain types of responses were more likely to elicit PCP reactions. These findings suggest that patientreported responses integrated into the EHR may be useful in helping treat conditions like DPN, especially if actionable items such as treatment efficacy are reported.

PROVIDER VARIATION IN ANTIBIOTIC PRESCRIBING FOR RESPIRATORY TRACT INFECTIONS IN PRIMARY CARE Mahesh Manne ${ }^{3}$; Michael B. Rothberg $^{3}$; Abhishek Deshpande ${ }^{3}$; Bo $\mathrm{Hu}^{3}$; Glen B. Taksler ${ }^{3}$; Anita D. Misra-Hebert ${ }^{3}$; Stacey E. Jolly ${ }^{3}$; Andrei Brateanu ${ }^{1}$; Robert W. Bales ${ }^{2} .{ }^{1} \mathrm{CCF}$, Cleveland, OH; ${ }^{2}$ Cleveland Clinic, Twinsburg, OH; ${ }^{3}$ Cleveland Clinic, Cleveland, OH. (Control ID \#2469497)

BACKGROUND: Inappropriate antibiotic use for respiratory tract infection is an ongoing problem with important consequences. Antibiotic overuse has been linked to the emergence of drug resistance and other adverse effects. Inappropriate prescribing may contribute to higher medical costs and encourage patients to seek unnecessary care in the future. Little is known about the prescribing practices of individual physicians or the impact of physician prescribing habits on patient behavior. We aimed to describe individual health care providers' (HCP) antibiotic (ATB) prescribing pattern for acute respiratory tract infections (RTI) over 2 years.

METHODS: We analyzed adult patients (age $\geq 18$ years) who had a visit for respiratory tract infection (acute upper respiratory infection, otitis media, sinusitis, pharyngitis, bronchitis or bronchiolitis; and pneumonia) to any Internal Medicine or Family Practice providers across the Cleveland Clinic Health System from 1/1/2011 to 12/31/2012. A RTI diagnosis was identified based on the ICD-9 code. We excluded patients with a history of chronic obstructive pulmonary disease, immunocompromised status (history of organ transplant, leukemia, lymphoma, HIV/AIDS), current use of immunosuppressant medications or who had been hospitalized within 14 days prior to initial outpatient RTI visit. Baseline demographics; comorbidities including diabetes, asthma, heart failure, end stage renal disease; primary care provider (PCP), provider for the visit, visit RTI diagnosis, antibiotic prescriptions associated with RTI diagnosis and any subsequent visit to ambulatory provider, emergency room or hospitalization during the study period after index visit were extracted from the electronic medical records. Descriptive statistics were used to summarize continuous variables as means and standard deviations or medians and interquartile ranges; and categorical variables as frequencies and percentages. The frequency of ATB prescribing was analyzed at the provider level. Logistic regression was used to examine predictors for prescribing antibiotics. Models included provider as a random effect. Statistical significance was considered as a two-sided $p$-value $<0.05$.

RESULTS: In our final cohort of 33,174 patients, 18,181 (54.8 \%)) received an ATB for a RTI. The majority of the population was female $(62.9 \%)$ and white $(85.9 \%)$, with a mean age of 45.9 years. Only $40.4 \%$ visits were with patient's listed PCP. Patient factors that were independently associated with ATB prescribing included white race $(\mathrm{OR}=1.55[1.44,1.68])$, age younger than $65(\mathrm{OR}=1.15[1.09,1.22])$, presence of fever $(\mathrm{OR}=2.00[1.66,2.41])$ or hypoxia $(\mathrm{SaO} 2<92 \%, \mathrm{OR}=1.10[1.05,1.15])$, and visit with a provider other than the PCP $(\mathrm{OR}=1.16[1.12,1.22])$. Among the 329 providers with at least 10 RTI visits, ATB prescribing rates ranged from 0 to $95 \%$ (median $48.2 \%$, IQR $27.6 \%, 69.2 \%$ ) (Figure). There were 27 providers $(8.2 \%$ ) with ATB prescribing rates $>80 \%$ and 7 providers $(2.1 \%)$ did not prescribe any ATBs for RTI. When compared to a diagnosis of upper respiratory infection, ATBs were 
more likely to prescribed for acute sinusitis $(\mathrm{OR}=30.2[27.1,33.6])$, bronchitis $(\mathrm{OR}=10.3[9.3$, 11.3]), suppurative otitis media $(\mathrm{OR}=7.3[2.0,26.2])$, pneumonia $(\mathrm{OR}=2.1[1.9,2.4])$ or pharyngitis $(\mathrm{OR}=1.7[1.6,1.8])$. The most commonly prescribed antibiotics were macrolides ( $40.9 \%$ ), penicillins (38\%), and tetracyclines ( $8.1 \%$ ). Patients who were prescribed ATB were more likely to return to office within 30 days for any cause ( 6.4 vs $5.0 \%, p<0.001$ ), as well as after 30 days but within a year for any RTI diagnosis $(21.5 \mathrm{vs} 16.7 \%, p<0.001)$.

CONCLUSIONS: Providers prescribing pattern of ATB for RTI varies widely and appears driven partly by patient factors but predominantly by provider practice style. Patients prescribed antibiotics for RTI were more likely to return for RTI and may have higher health care utilization. Better understanding individual provider factors that influence antibiotic prescribing could inform targeted interventions to reduce this important problem.

Antibiotic prescribing rate by provider

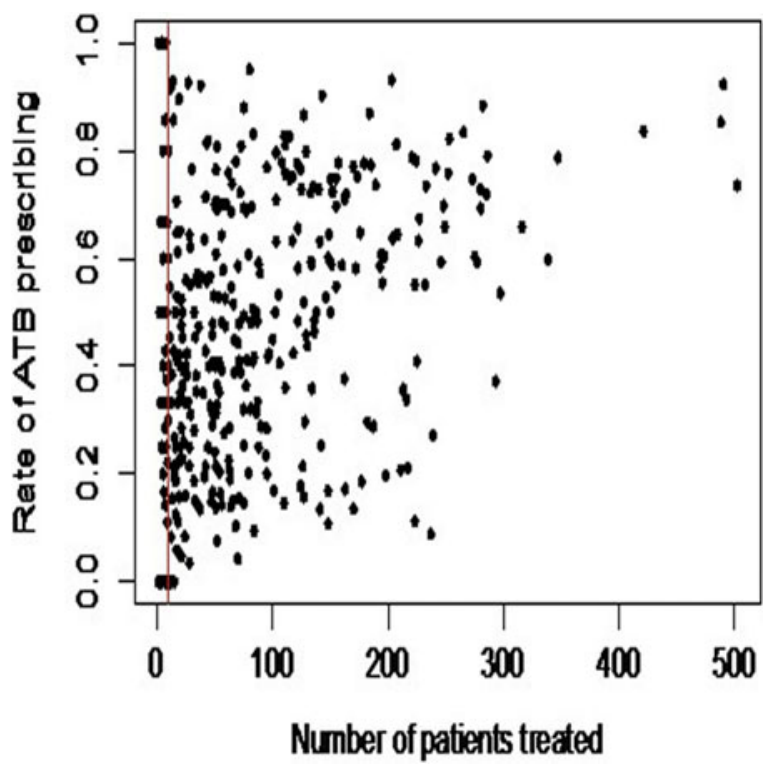

PROVIDERS' COMPARISON BETWEEN CLINICAL DECISION SUPPORT OF A HOMEGROWN ELECTRONIC HEALTH RECORD SYSTEM AND A VENDOR PRODUCT Elizabeth R. Silvers ${ }^{1,2}{ }^{\text {; }}$ Diane L. Seger ${ }^{1,2}$; Adrian Wong ${ }^{1,4}$; Mary Amato ${ }^{1,}{ }^{4}$; Sarah P. Slight ${ }^{1,3}$; Patrick E. Beeler ${ }^{1,}{ }^{6}$; Julie Fiskio ${ }^{1,2}$; Tewodros Eguale $^{1,4}$; Patricia C. Dykes ${ }^{1,5}$; David W. Bates ${ }^{1,5}$. ${ }^{1}$ Brigham and Women's Hospital, Boston, MA; ${ }^{2}$ Partners HealthCare, Wellesley, MA; ${ }^{3}$ Durham University, Stockton on Tees, United Kingdom; ${ }^{4}$ MCPHS University, Boston, MA; ${ }^{5}$ Harvard Medical School, Boston, MA; ${ }^{6}$ University Hospital, Zurich, Switzerland. (Control ID \#2465947)

BACKGROUND: Evidence suggests that clinical decision support (CDS) improves patient care, but the amount of improvement achieved is typically a fraction of what is possible. Computerized provider order entry (CPOE) with advanced CDS has been shown to improve patient safety outcomes ${ }^{1}$, and understanding how providers respond to CDS alerts is a critical factor in improving patient care. Our institution, Brigham and Women's Hospital (BWH), recently adopted a vendor-based system after using a homegrown electronic medical record (EHR) system for over 20 years. While the new system has medication CDS in place, it is not as robust and does not include a tiering of alerts, which existed in our homegrown EHR. This study is an opportunity to learn more about providers' views regarding the design of alerts of the new EHR system and to identify potential areas of improvement

METHODS: We targeted providers that had interacted with both of the mentioned systems to capture their thoughts regarding the changeover, and who frequently overrode medication CDS alerts $(n=1,770)$ in the inpatient setting between January 2009 and
December 2011. Research pharmacists trained in effective counter-detailing techniques conducted academic detailing sessions, which were tailored to the provider's particular overrides. The sessions aimed to identify prevalent concepts related to prescribing behavior and EHR system effects on patient safety and medication errors.

RESULTS: We conducted a total of 34 academic detailing sessions, which included a diverse group of providers of varying professional backgrounds and specialties. Most providers felt the lack of tiering of CDS in the vendor system increased their alert fatigue in comparison with the homegrown EHR. They also found the presentation of all alerts at the time of signing an order, as opposed to at the time of ordering, to be overwhelming. Many providers agreed that drug-drug interactions (DDI) that were high priority alerts in the homegrown system should also be either a hard stop or high priority in the vendor system. The medication search in the vendor EHR presented several lists and formulations, which often led to confusion and an interruption in providers' workflow when compared to the more concise lists in the homegrown EHR. The vendor EHR system recommended default doses for only some medications in comparison to the previous homegrown EHR system, which did so for nearly all medications. The vendor EHR did not offer CDS of renal-based alerts outside of labeling renal insufficiency, whereas the homegrown EHR adjusted the dose or frequency of medications and recommended an alternative medication when appropriate. This lack of CDS in the vendor EHR caused providers to often refer to the pharmacy or literature to make an informed decision. The ability to distinguish between true allergies and intolerances in the new EHR was an improvement when providers were made aware of this functionality. Providers suggested that the new vendor system include the tiering of alerts and draw attention to serious reactions and contraindications.

CONCLUSIONS: A number of insights surrounding CDS in both EHR systems were identified through academic detailing sessions with providers. In the areas of DDIs and renal-based alerts, providers felt the homegrown system provided much more useful information such as a recommended dose adjustment and alternative medications. Drug allergy interaction alerts employed by the new EHR were felt to be more useful than the allergy module in the previous system by allowing providers to distinguish between true allergies and intolerances. However, most were unaware of this functionality, and attention should be drawn to this feature in the training offered to providers so they may more effectively utilize the vendor EHR in the future. These findings will form the basis for several recommendations to senior management on how to improve the vendor system. By incorporating a more robust CDS system, the vendor EHR could be a more useful asset to clinicians in providing more effective and efficient patient care. References: 1. Mishuris RG, Linder JA, Bates DW, Bitton A. Using Electronic Health Record Decision Support is Associated with Improved Quality of Care. Am J Manag Care. 2014 Oct 1;20(10):e445-52. This study was funded by grant \#U19HS021094 from the Agency for Healthcare Research and Quality (AHRQ)

PUBLIC LIBRARIES ADDRESS THE SOCIAL DETERMINANTS OF HEALTH $\underline{\text { Anna U. Morgan }}^{2}{ }^{2}$; Roxanne Dupuis ${ }^{3}$; Kiahana Brooks ${ }^{3}$; Bernadette A. D'Alonzo ${ }^{3}$; Amy Graves ${ }^{3}$; Hillary Bonger ${ }^{1}$; Carolyn C. Cannuscio ${ }^{1,3}$. ${ }^{1}$ Perelman School of Medicine, U. of Pennsylvania, Philadelphia, PA; ${ }^{2}$ University of Pennsylvania, Philadelphia, PA; ${ }^{3}$ Center for Public Health Initiatives, University of Pennsylvania, Philadelphia, PA. (Control ID \#2468934)

BACKGROUND: Over $80 \%$ of adult Americans have visited a public library, with over half of having done so in the past 12 months. Though the public library is a widely used resource among Americans of all socioeconomic backgrounds, low-income and African- 
American individuals are more likely than the general population to ask for help from a librarian in person. The Free Library of Philadelphia is a large public institution, with 61 branches and over 5.5 million in-person and 8 million online visits annually, in a city of 1.5 million residents. In partnership with researchers at the University of Pennsylvania, the Library's strategic plan broadly calls for expansion of health-related programming, with intensive efforts centered on a newly renovated branch in South Philadelphia that is colocated with public and private medical clinics and a recreation center. This project sought to understand both the challenges faced by South Philadelphia residents as well as the current and anticipated health-relevant work of Philadelphia's public library staff. METHODS: Fifty-nine ( $35 \%$ male) interviews were conducted with residents of South Philadelphia by trained staff in collaboration with an experienced urban street photographer. Care was taken to obtain a diverse sample of participants with regard to race, ethnicity, gender, age, and language. Community member participants were asked to discuss the community in which they lived and challenges they faced in their daily lives, as well as their current or anticipated use of the public library system. Over a similar time period, twelve qualitative interviews were conducted with library staff familiar with the South Philadelphia community. Participants were asked to share their daily work practice, with a particular emphasis on health-related topics, including the social determinants of health. They were also asked to describe the demographic profile and perceived medical and social needs of the populations they serve. The research team used an iterative approach to analyze all interviews and collectively identify common themes in each group of interviews. The themes were then compared.

RESULTS: South Philadelphia is a highly diverse neighborhood where over $20 \%$ of the population lives in poverty. In interviews with both residents and library staff, a clear picture emerged of an area with tremendous social needs, as well as a high level of engagement on the part of librarians in addressing the social determinants of health. Community residents stated that their primary concerns included lack of services and treatment for people with mental health disorders, substance use, and homelessness. They also expressed concern about lack of supervision of children and adolescents as well as service gaps for new immigrants. Library staff reported interacting predominantly with vulnerable populations, including people with mental health and substance use disorders, the unemployed, and the homeless - as well as with recent immigrants, children, and the elderly (Figure 1). Notably, residents tended to describe the community in more positive terms than librarians, which may reflect the fact that librarians are often interacting with the most challenging and vulnerable members of the community. In their daily work, library staff provide direct support to these groups, including assisting with job applications, helping patrons navigate the health care system and clarifying housing rights. Many librarians report devising programs specifically tailored to meet the community needs they observed. Library staff noted that the library is often seen as a trusted institution and can be a place of refuge and last resort for vulnerable individuals. Due to the community's profound poverty and social discord, librarians do not always feel wellprepared to deal with the myriad of challenges faced by their patrons. They liken their work to that of social workers and sometimes lament their lack of specialized training to address major social challenges.

CONCLUSIONS: Taken together, findings from this project illuminate many of the challenges facing individuals living in South Philadelphia, as well as the current and potential future work of public librarians to address the social determinants of health and health disparities. Indeed, these results show the library to be a responsive public institution that is highly attuned to the needs of the community at large, with specific emphasis on vulnerable populations. Given their trusted role within the community, location within neighborhoods, deep understanding of local needs and resources, and interest in further healthrelevant training, librarians hold promise as novel contributors to the public health work force.

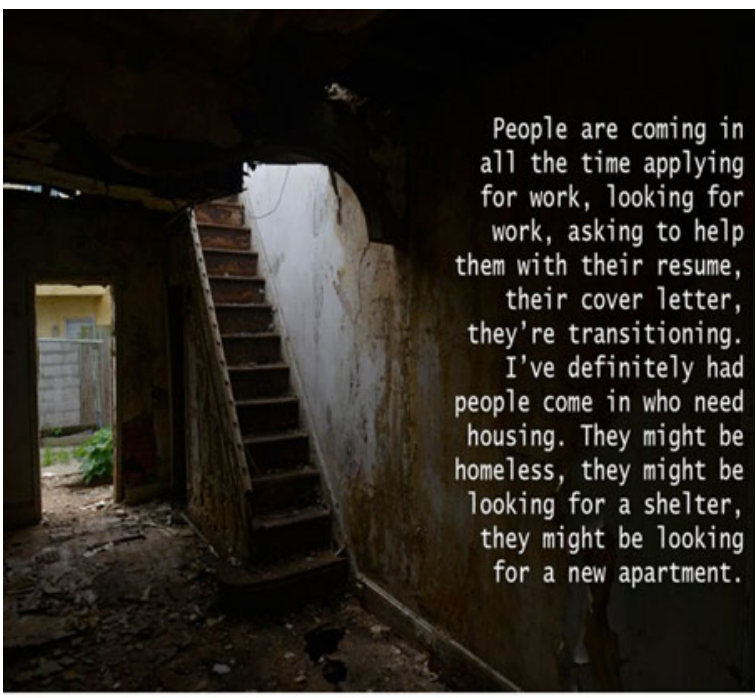

Figure 1. Quote from a South Philadelphia librarian discussing the social needs of patrons. Photograph of abandoned home in South Philadelphia.

PUBLICATION RATES FOLLOWING PRESENTATION AT THE 2009 NATIONAL SGIM CONFERENCE Heidi M. Egloff ${ }^{3}$; Colin P. West ${ }^{2}$; Amy T. Wang ${ }^{1}$; Katie M. Lowe ${ }^{2}$; Jithinraj Edakkanambeth Varayil²; Thomas J. Beckman²; Adam P. Sawatsky ${ }^{2} .{ }^{1}$ Harbor-UCLA Medical Center, Torrance, CA; ${ }^{2}$ Mayo Clinic, Rochester, MN; ${ }^{3}$ Mayo clinic, Rochester, MN. (Control ID \#2464623)

BACKGROUND: Current publication rates of medical literature suggest many abstracts initially accepted at national meetings are never subsequently published. Publication rates have been previously investigated in medical and surgical subspecialties, with reported rates ranging from 32 to $77 \%$. The factors influencing publication, as well as the attributes of studies which are subsequently published, remain unclear. To date, there have only been 2 studies examining publication rates for abstracts from general medicine conferences, both of which were studies outside of the U.S. The primary aim of this study was to determine subsequent publication rates of abstracts accepted at a general internal medicine national conference. The secondary aim was to identify factors associated with publication.

METHODS: We reviewed all abstracts accepted to the 2009 SGIM national conference, excluding clinical vignettes. We determined subsequent full publication by searching title and author in the MEDLINE database. Research was considered published if we identified a full-length manuscript using this search strategy. The title, methods, and results of the published article were compared to the original abstract to confirm corresponding publication. Data were extracted, including: title, authors, institution, research category, number of study sites, sample size, study design, statistical significance (CI and p value) in abstract and final publication, journal of publication, publication date, and journal impact factor. Multiple logistic regression was applied to evaluate multivariable models of factors associated with publication, with a significance threshold set at $p<0.05$.

RESULTS: Five hundred seventy-eight abstracts were analyzed. Overall 274 (47.4\%) of abstracts were subsequently published as a full manuscript in a peer-reviewed journal. In multivariable analyses, publication rates were higher for multicenter studies than single institutions (52.4 vs. $40.4 \%$, OR 1.66, $p=$ 0.04). Randomized controlled trials had higher publication rates than systematic reviews/meta-analyses or observational studies (66.7 vs. 50.0 vs. $45.9 \%, p=$ 
0.03). Abstract topics including Medical Education, Aging/Geriatrics, and Mental Health/Substance Abuse were most likely to be published (OR 5.05, 3.31, 4.16 , respectively, $p=0.01$ ). Systematic reviews/Meta-analyses were associated with a higher impact factor (8.2) compared to randomized controlled trials (3.9). The publication rates did not statistically differ between academic and community-based centers ( 48.4 vs. $25.0 \%, p=0.051$ ). Positive result studies also did not portend higher publication rates than negative studies (OR $0.89, \mathrm{CI}$ $0.6-1.31, p=0.21$ ). Cancer research was associated with the highest median impact factor among topics $(\mathrm{IF}=19)$. The median impact factor of positive result abstracts versus negative results was not statistically different (3.3 vs. $2.8, p=0.05)$.

CONCLUSIONS: This study demonstrated that $47.4 \%$ of abstracts presented at a general internal medicine national conference were subsequently published in a peer-reviewed journal, consistent with the median publication rates cited in a major Cochrane review (44.5\%). Publications across a wide array of topics speak to the diversity of publication among general internists. This study did not demonstrate publication bias in contrast to prior studies in subspecialty literature, which may be a reflection of the diversity of research represented at the conference.

PULSUS ALTERNANS AN ECHO OF THE PAST Judson Alderman ${ }^{1}$; Apostolos Dallas $^{2}$; Katherine Shaver ${ }^{2}$; Marguerite Underwood ${ }^{2} .{ }^{1}$ Virginia Tech Carilion School of Medicine, Roanoke, VA; ${ }^{2}$ Carilion Clinic, Roanoke, VA. (Control ID \#2464564)

BACKGROUND: Since the late 1800s after its discovery by Traube, pulsus alternans (PA) has been characterized as a a phenomenon of alternating strong and weak pulse pressures with constant R-R intervals observed in patients with cardiovascular disease or tachycardia. The magnitude of PA is the difference between the strong and weak systolic pressures. It may be present in patients with a wide variety of cardiovascular complications including: $\mathrm{CHF}$, acute MI, coronary artery disease, catecholamine excess, aortic stenosis, mitral stenosis, cardiomyopathy, pericarditis, electrolyte imbalances, and HTN. The underlying mechanism, prevalence, and clinical significance of PA are undetermined. PA is thought to be a predictor of poor cardiovascular prognosis. Recently, it has been noted that beta-blockers eliminate PA. We hypothesize that a patient's magnitude of PA, as measured by sphygmomanometer, can be used to estimate ejection fraction measured by transthoracic echocardiogram from the bedside.

METHODS: Fifty three patients at an academic tertiary care center and a heart failure outpatient clinic receiving transthoracic echocardiograms with regular rhythm consented to having their heart rate, blood pressure, and the magnitude of PA assessed at bedside using a sphygmomanometer. Bedside measurements were taken the same day as the patient's echocardiogram. Electronic medical records were used to acquire patient data relating to age, gender, medications, and medical history. Ejection fraction was estimated by cardiologist readings of the echocardiogram.

RESULTS: PA was measuresed in 1 out of 53 patients. Average ejection fraction was $52.5 \%$. Fifty-one out of 53 patients had cardiac comorbidities including congestive heart failure (CHF), prior myocardial infarction (MI), valvular disease, or hypertension (HTN). Eighty one percent of patients were taking cardiovascular medications with $64 \%, 47 \%$, and $26 \%$ on beta-blockers, ACE inhibitors/ARBs, and calcium channel blockers respectively. The populations inpatient and outpatient populations in this study were similar in age and vital signs. However, they differed in their medical history and medication use. CONCLUSIONS: The prevalence of PA was lower than expected. With new evidence suggesting beta-blockers eliminate PA, we conclude that PA may not be a relevant physical exam finding in modern clinical practice, but may, instead, have greater applications in low-resource settings where cardiovascular disease is not medically managed.
Further research is needed with a larger sample size to determine if there is a statistically significant link between PA and ejection fraction. However, our study demonstrates the rarity of this finding in patients receiving transthoracic echocardiograms studies within a tertiary care academic hospital and an outpatient heart failure clinic.

Demographics, Vital Signs at Bedside, and Ejection Fraction of Total Study Population $(N=53)$

\begin{tabular}{llll}
\hline \hline Category & Mean & Minimum & Maximum \\
Age (years) & 64 & 21 & 90 \\
Systolic BP (mmHg) & 131 & 80 & 186 \\
Diastolic BP (mmHg) & 71 & 40 & 144 \\
Heart Rate (bpm) & 72 & 43 & 120 \\
Ejection Fraction & $52.5 \%$ & $20 \%$ & $65 \%$ \\
\hline
\end{tabular}

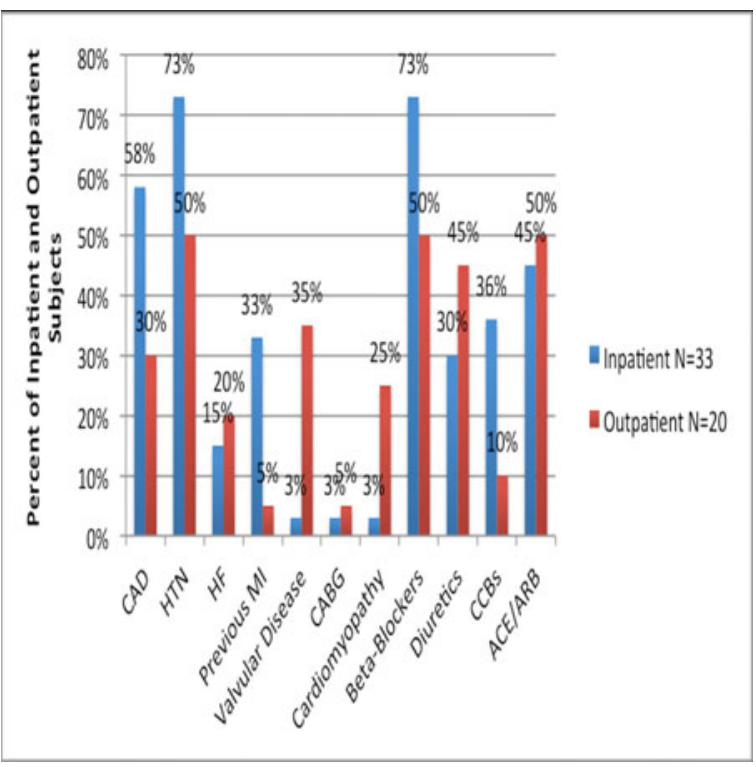

$\mathrm{BP}=$ Blood Pressure; $\mathrm{bpm}=$ beats per minute; lower range of ejection fraction estimate was used for average.

Comparison of Cardiovascular Disease History and Prescription Drug Use of Patients at Inpatient Academic Tertiary Care Facility and Outpatient Heart Failure Clinic

QUALITATIVE ANALYSIS OF OLDER ADULTS' PERSPECTIVES ON INTERPROFESSIONAL HEALTHCARE Andrea Kendall²; Theresa Gattari ${ }^{2}$; Arwa Hasan ${ }^{1}$; Leticia G. Haff ${ }^{1}$; Rania Krayem ${ }^{1}$; Megan Kucemba ${ }^{1}$; Alexandra H. Targan ${ }^{2}$; Jennifer Mendez ${ }^{2}$; Diane L. Levine ${ }^{1} .{ }^{1}$ Wayne State University, Detroit, MI; ${ }^{2}$ Wayne State University, West Bloomfield, MI. (Control ID \#2469492)

BACKGROUND: Communication and interprofessionalism have been outlined by the Interprofessional Education Collaborative (IPEC) Expert Panel which stated the importance of healthcare professionals' ability to "apply relationship-building values and the principles of team dynamics to perform effectively in different team roles to plan and deliver patient-/ population-centered care that is safe, timely, efficient, effective, and equitable."1 Moreover, according to the 1999 Institute of Medicine Report, communication failure was listed as one type of preventable medical error. Furthermore, the report estimated as many as 98,000 annual deaths in hospitals as a result of preventable medical errors.2 One major focus of quality improvement and patient safety is interprofessionalism, which plays an integral role in cost effective and patient-centered care.3 Students from Nursing, Medical, Pharmacy, Social Work, 
Occupational Therapy and Physical Therapy at Wayne State University performed home visits with older adults as part of the existing Interprofessional Team Visit (IPTV) Program. The goal of this activity was to engage patients in conversation about interprofessionalism and quality healthcare. The specific objectives of this research were to gain a general understanding of past experiences and communication issues when working with an interprofessional care team from a geriatric patient perspective, appreciate opinions on positive and negative attributes of healthcare providers from a geriatric patient perspective, and collaborate with healthcare professional students to understand the benefits and challenges of an interprofessional healthcare team.

METHODS: The study design was a qualitative analysis of open-ended responses to the communication survey questions during the IPTV program at Wayne State University. The study was conducted at Wayne State University's Medical School campus. The 12-student interprofessional analysis team consisted of 4 medical students, 2 physician assistant students, 2 nursing students, 2 physical therapy students, 1 occupational therapy student, and 1 pharmacy student. There were three separate coding sessions as follows: first, each student coded individually, then, students met in groups of three to perform focused coding, finally, the entire team met and consolidated codes and found themes that emerged from the data. The communication survey questions are as follows: 1 . What types of healthcare providers have delivered care to you since you were 65 years old? For example, physicians (primary care and/or specialists), physician assistants, nurses, physical therapists, occupational therapists, social workers, pharmacists, etc.? 2a. In your opinion, what qualities does a good provider possess? 2b. A bad provider? 3a. Have you ever had an issue communicating with a healthcare provider? No/Yes If yes, explain. 3b. Have you ever observed miscommunication/lack of communication between providers? No/Yes If yes, explain. 4 . How did this impact your health/healthcare? What recommendations do you have to improve communication.

RESULTS: During the study, 215 older adults were interviewed. For Question 1, the vast majority of older adults received care from an interprofessional team rather than a single provider. Common providers included pharmacist, primary care physician, nurse, physical therapist, and dentist. The codes developed to describe a good provider (Question 2) included: professional, accessible, personable, good listener, competent, empathetic, patient-centered, offers alternatives, good communication, spends enough time [with patient]. Codes developed to describe a bad provider (Question 2) included: unprofessional, inaccessible, impersonable, bad listener, incompetent, not empathetic, and not patient-centered, paternalistic, does not spend enough time, not collaborative. Of the 215 older adults interviewed, 93 (42\%) had experienced communication issues with healthcare providers (Question 3). The impact of communication issues (Question 4) included delayed care, physical manifestations (pain), emotional consequences (fear, anxiety, stress, frustration, confusion), distrust/avoidance of healthcare professionals, switching providers, and wasted resources (duplicate testing, lost time, lost money). CONCLUSIONS: Qualitative analysis of older adults' responses to communication questions helped identify healthcare needs and expectations representative of our aging population sample. In addition, working as an interprofessional team allowed appreciation for various perspectives to patient care. References: 1 . "Core competencies for interprofessional collaborative practice Report of an expert panel." Interprofessional Education Collaborative Expert Panel. Washington, D.C.: Interprofessional Education Collaborative. 2011. 2. Kohn L T, Corrigan J M, Donaldson MS. "To err is human: building a safer health system." Institute of Medicine Washington, DC: National Academy Press, 2000. Pg 1, 36. 3. "Interprofessional Quality and Safety Training Could Transform Healthcare." University of Missouri Health School of Medicine. http://medicine.missouri.edu/news/0176.php April 27, 2013. Accessed January 10, 2016

QUALITY IMPROVEMENT IN THE SAFETY-NET: RESULTS FROM THE BIG AIMS INITIATIVE IN CALIFORNIA Shirin Hemmat ${ }^{2}$; Courtney Lyles ${ }^{2,3}$; Sara Ackerman $^{1,3}$; Gato I. Gourley ${ }^{2,3}$; Margaret Handley ${ }^{2,3}$; Dean Schillinger ${ }^{2,3}$; Urmimala $\underline{\text { Sarkar }^{2}}{ }^{2}{ }^{3}$. ${ }^{1} \mathrm{UCSF}$, San Francisco, CA; ${ }^{2}$ University of California San Francisco, San Francisco, CA; ${ }^{3}$ Center for Vulnerable Populations, San Francisco, CA. (Control ID \#2469323)
BACKGROUND: US health systems are increasingly accountable for measuring and reporting quality metrics. This may be a particular challenge for safety-net health systems that care for low-income populations. In 2011, California's safety-net hospital and health care systems embarked on a state Delivery System Reform Incentive Program (DSRIP), which provided funding to meet the needs of the expanded Medicaid population. Under DSRIP's terms, the California Association of Public Hospital's Safety Net Institute (SNI) collated and reported performance data to the state health department on behalf of the 21 participating health systems (some reported jointly, resulting in 17 reporting units). This was a massive expansion of performance measurement and reporting. While DSRIP did not include specific performance targets, in 2013 the public health care systems participating in DSRIP embarked on a voluntary, statewide quality improvement initiative, called the Big Aims, to improve performance of mammography screening and cholesterol control in diabetes (LDL-C). The goal for the Big Aims was to reach the 90th percentile threshold for commercial Medicare health plans. The objective of our study was to characterize the change in mammography and LDL-C as a result of the Big Aims 1 year after it was initiated.

METHODS: Each health system conducted independent quality improvement projects as part of the Big Aims. SNI supported the initiative by facilitating sharing of best practices and obtaining quarterly performance data. Data may have included duplicate patients across time periods. We conducted a retrospective analysis of aggregated mammography and LDL data. Mammography rates were defined as the percentage of female patients ages 50-74 who had a screening mammography for breast cancer in the past 2 years. LDL-C was defined by health systems as the percentage of diabetes patients ages 18-75 who had their LDL in control $(<100 \mathrm{mg} / \mathrm{dL})$ in the past year. We selected two other DSRIP measures not included in the Big Aims, influenza immunization and blood glucose control for diabetes ( $\mathrm{HbAlc}$ control), as comparison metrics for which there were no performance targets. HbAlc control was defined as the percentage of people with diabetes ages 18-75 who had their $\mathrm{HbAlc}$ in control $(<8 \%)$ in the past year. Influenza immunization rates were measured as the percentage of patients 50 and older who had received an influenza vaccine in the past year. We calculated exploratory statistics for all 4 quality metrics. We first examined each of health system for changes in performance from 6/2011-6/2014 (two tailed paired t-test). We aggregated the data to produce an overall measure of performance for each metric each year. We used a difference-in-differences analysis to compare change between the Big Aims metrics (LDL-C, Mammography) to the DSRIP-only metrics (HbA1C, Immunization) over time, to examine whether the Big Aims metrics changed beyond the overall DSRIP metric changes.

RESULTS: Our analysis showed that overall performance improved over time (Figure 1). Health systems did not reach the performance target of Medicare 90th percentile for any measure. The difference-in-differences analysis suggested that there was no difference in performance between 2013 and 2014 for the Big Aims metrics (mammography and LDL-C) compared to reference DSRIP-only metrics (immunization and $\mathrm{HbA1C}$ control).

CONCLUSIONS: During the DSRIP period, public hospital systems, in aggregate, improved in their performance on all quality metrics examined. This suggests that safety net systems can implement quality measurement and reporting and demonstrate improvement while falling short of an ambitious target. Our study's limitations include its scope, encompassing one large and diverse state, and the examination of aggregated system level rather than individual patient data over time. It is likely that unmeasured patient characteristics influenced each health system's performance on the quality measures. We could not independently verify the accuracy of each hospital's data. These results are consistent with prior large-scale quality improvement initiatives, which also demonstrated variable improvement and data quality across sites. Further work is needed to determine whether more tightly coordinated improvement activities, sustained investment, and/or longer time horizons are needed to yield benefits from quality measurement and improvement efforts. 
Figure 1: California Public Hospitals Performance on Big Aims ${ }^{\mathrm{a}}$ and DSRIP Quality Improvement Measures ${ }^{b}$ : July 2011 to June 2014

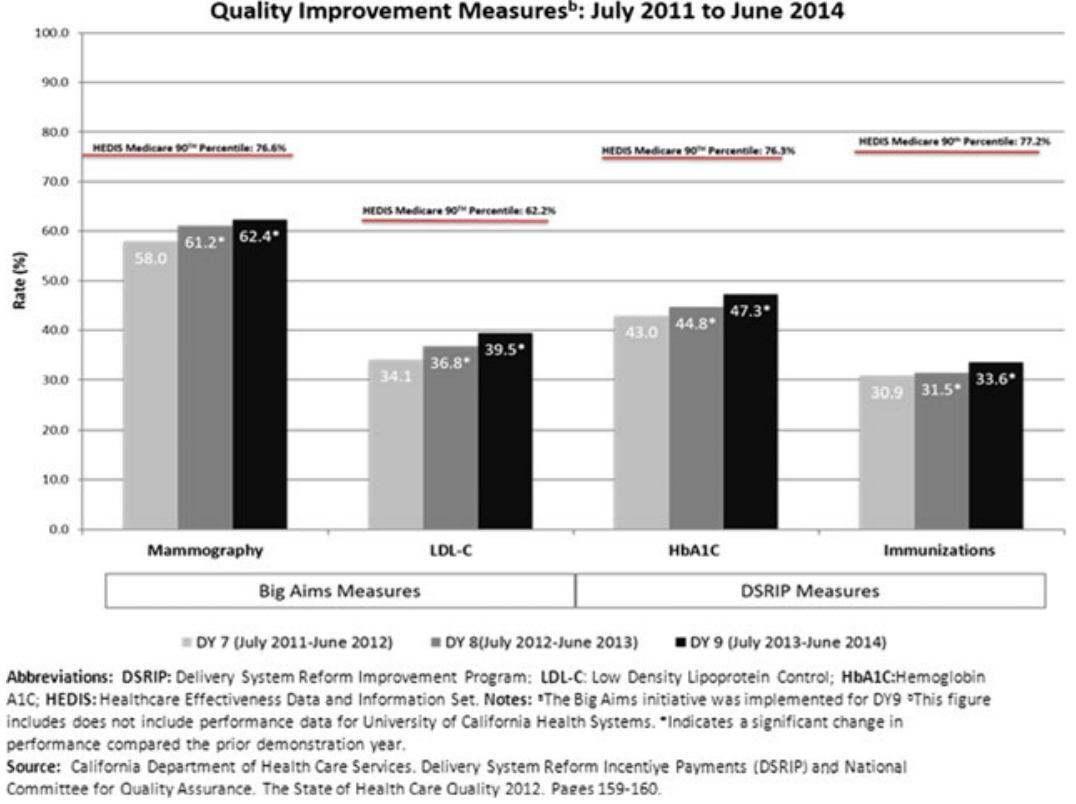

QUALITY MEASURES OF ACCOUNTABLE CARE ORGANIZATIONS: TRANSPARENCY OF THE EVIDENCE BASE Zackary Berger ${ }^{3}$, 13; Stephanie W. $\underline{Z u o}^{3}$; Michael E. Bowen'; James S. Yeh ${ }^{1}$; Harry B. Burke ${ }^{10}$; William Kormos ${ }^{5}$; Deepa R. Nandiwada $^{12}$; Ricardo R. Correa ${ }^{7}$ Jocelyn A. Carter ${ }^{6}$; Daniel J. Elliott'; Heather Sateia ${ }^{4}$; Rebecca Beyth ${ }^{11}$; Beth Smith ${ }^{8} .{ }^{1}$ Brigham and Women, Boston, MA; ${ }^{2}$ Christiana Care Health System, Newark, DE; ${ }^{3}$ Johns Hopkins School of Medicine, Baltimore, MD; ${ }^{4}$ Johns Hopkins University, Lutherville, MD; ${ }^{5}$ Mass General Hospital, Boston, MA; ${ }^{6}$ Massachusetts General Hospital, Boston, MA; ${ }^{7}$ National Institute of Health, Rockville, MD; ${ }^{8} \mathrm{OHSU}$, Portland, OR; ${ }^{9}$ UT Southwestern Medical Center, Dallas, TX; ${ }^{10}$ Uniformed Services University of the Health Sciences, Bethesda, MD; ${ }^{11}$ Univ. of Florida \& Malcom Randall VAMC, Gainesville, FL; ${ }^{12}$ University of Pittsburgh, Pittsburgh, PA; ${ }^{13}$ Johns Hopkins Berman Institute of Bioethics, Baltimore, MD. (Control ID \#2468418)

BACKGROUND: In 2010, the Patient Protection and Affordable Care Act mandated that the Centers for Medicare and Medicaid Services (CMS) create Accountable Care Organizations (ACOs), groups of health care providers that can share in cost savings if they meet specified quality benchmarks and spend wisely. CMS utilizes a core set of 33 measures (ACO-33) to assess ACO performance and determine eligibility for shared savings. Despite a growing participation in ACOs, the end user accessibility of the quality measure evidence base has remained unclear.

METHODS: We designed an Internet search algorithm that imitates the search methodology of a busy, practicing clinician or a well-informed patient. We chose to evaluate 25 of the ACO33 measures in this manner, as they were most pertinent to the daily practice of a primary care provider, and defined by an existing evidence base. First, the "measure steward," or the body charged with defining and calibrating the measure, was identified from the ACO-33 measure summary. Next, a reviewer experienced in evidence-based medicine accessed the steward's website and searched for the measure number using navigation features within the website to find a full description of the measure. If a full description was not identified, the reviewer then performed a Google search for "ACO Measure XX" and/or the given ACO33 measure description and reviewed only the first page of search results. In either case, once a full description of the ACO measure was found, we specifically examined it for citations of systematic reviews or meta-analyses, which we considered the gold-standard for measure evidence. Searches were conducted by 11 evidence-based medicine experts and then doublechecked by a second reviewer for consistency.
RESULTS: Both first and second reviewers agreed on all assessments. The measures reviewed $(n=25)$ fell into three broad categories: care coordination/patient safety (10), preventative health (8), and at risk populations (7). Using our two-step search algorithm, we found a full description for 18 of 25 measures ( $72 \%$ ). However, this description was identified on the steward's website for only $39 \%(n=7)$ of measures. The remaining $61 \%$ of measure descriptions were located using the Google search strategy. We identified links to systematic reviews $(n=5)$ and meta-analyses $(n=3)$ for only $24 \%$ of measure descriptions reviewed. CONCLUSIONS: Using a real-world, pragmatic Internet search strategy, we were unable to identify a rigorous evidence base for a majority of the $33 \mathrm{ACO}$ quality measures. If the ACO-33 measures are to be used widely by clinicians and medical organizations, the evidence base must be of high-quality and readily available without undue inconvenience to the busy clinician or patient.

QUALITY OF DIABETES CARE AND HEALTH INSURANCE COVERAGE: A RETROSPECTIVE STUDY IN A PUBLIC HOSPITAL IN SWITZERLAND Yves Jackson $^{1,}{ }^{2}$; Juan Lozano ${ }^{1}$; Marc Carpentier ${ }^{1} .{ }^{1}$ Geneva University Hospitals, Geneva, Switzerland; ${ }^{2}$ Global Health Institute, University of Geneva, Geneva, Switzerland. (Control ID \#2456536)

BACKGROUND: Socioeconomic disadvantage is associated with increased risk of adverse diabetes outcomes. In Switzerland, people without health insurance face barriers in accessing to care and receiving standard quality of care. The Geneva University Hospitals (HUG) have implemented policies aiming at reducing those gaps. We compared diabetes quality of care and ambulatory healthcare services utilization among insured and uninsured diabetic patients.

METHODS: This retrospective study linked health and administrative data of type 2 diabetic outpatients with at least one HbA1c test performed in 2012-2013 at HUG. Quality of care evaluation relied on processes and outcomes assessment

RESULTS: Of the 198 patients included, 80 (40.4\%) were uninsured. Both groups underwent annual $\mathrm{HbAlc}$, cholesterol and microalbuminuria testing at comparably high rate and had non-significantly different numbers of ambulatory visits. After adjustment for age and sex, there was no significant difference in serum $\mathrm{HbA} 1 \mathrm{c}$ between groups both in those with prevalent or with newly diagnosed diabetes. Initial medical intervention entailed comparable glycaemic improvement after 6 months in incident diabetes. 
CONCLUSIONS: This study highlights the lack of difference in diabetes quality of care between insured and uninsured patients in a public hospital enforcing health-equity policies for universal access to and delivery of standard diabetes care. It confirms the frontline role of public hospitals in contributing to care delivery equity in countries with theoretical universal healthcare coverage.

QUALITY OF LIFE AND WALKING DISTANCE IN AFRICAN AMERICANS WITH PERIPHERAL ARTERY DISEASE Tracie C. Collins ${ }^{1}$; Kelsey Lu ${ }^{1}$; Jianghua $\mathrm{He}^{2}$. ${ }^{1} \mathrm{KU}$ School of Medicine - Wichita, Wichita, MN; ${ }^{2}$ University of Kansas (KU) Medical Center, Kansas City, KS. (Control ID \#2469810)

BACKGROUND: African Americans are two times more likely than non-Hispanic whites to have peripheral artery disease (PAD) - atherosclerosis of the abdominal aorta and arteries of the lower extremities. We sought to determine the correlation between quality of life and walking distance among a cohort of African Americans with PAD. METHODS: We completed recruitment for an ongoing 5-year NIH funded clinical trial in which we seek to determine the efficacy of a behavioral intervention to improve walking distance among African Americans with PAD. We analyzed baseline data from this trial. Trial eligibility includes self-identifying as African American and screening positive for PAD as defined by an ankle-brachial index $(\mathrm{ABI})<0.99$ Variables collected include walking distance (6-min walking test), quality of life (Vascular Quality of Life Questionnaire), and walking impairment (Walking Impairment Questionnaire [WIQ]). The vascular quality of life survey has 4 domains which are symptoms, pain, emotion, activity. There is a maximum possible score of 7 for each domain and the summary score The WIQ is based on a Likert scale with summary scores (0 to 100) for each of the following: walking distance, walking speed, and stair climbing. The range of scores for each of the three components is 0 to $100 \%$. For this analysis, we obtained quality of life scores and mean walking distance, as based on the 6-min walking distance and the WIQ We used Pearson correlation coefficient to assess for correlations of quality of life with each measure of walking distance.

RESULTS: Among the 174 African Americans enrolled, $74 \%$ werre women and the mean age of the cohort was 63.7 years (SD 11.1). Mean ABI was 0.85 (SD 0.15) and mean vascular quality of life scores were as follows: activity 5.04 (SD 1.00), symptoms 5.63 (SD 1.11), pain 4.70 (SD 1.64), emotional 5.89 (SD 1.16), with a mean overall score of 5.39 (SD 1.04). Mean baseline walking distance among the cohort was $1,171 \mathrm{ft}$ (SD 234.2). The Pearson correlation coefficient between each quality of life domain score and walking distance, as measured by the 6-min walk test, were as follows: activity $0.287(P<0.0001)$, symptoms $0.165(P=0.03)$, pain $0.191(P=0.011)$, and emotion $0.195(P=0.01)$. The correlation between the overall vascular quality of life score and walking distance, as measured by the 6-min walk test, was 0.255 $(P<0.0008)$. Mean walking distance score, as measured by the WIQ, was 51.9 (SD 32.5). Correlations between each vascular quality of life score and mean walking distance score, as measured by the WIQ, were as follows: activity $0.552(P<0.0001)$, symptoms 0.461 $(P<0.0001)$, pain $0.505(P<0.0001)$, and emotion $0.408(P<0.0001)$.

CONCLUSIONS: In a cohort of African Americans with PAD, vascular quality of life was highly correlated with walking distance. Our findings support the strength of the association between walking distance and quality of life among African Americans with PAD. In clinical practice, an assessment of quality of life should be considered for use among African Americans following treatment for PAD. NCT01321086

QUALITY OF LIFE, PERCEPTIONS AND HEALTH SATISFACTION IN OLDER ADULTS WITH END STAGE RENAL DISEASE: A SYSTEMATIC REVIEW. Seki

A. Balogun; Rasheed A. Balogun; John T. Philbrick; Emaad M. Abdel-Rahman. University of Virginia Health System, Charlottesville, VA. (Control ID \#2457486)
BACKGROUND: The prevalence of chronic kidney disease (CKD) is increasing as the population ages, with significant proportions of elderly patients with end stage renal disease (ESRD) requiring renal replacement therapies (RRT). Many studies have examined quality of life of ESRD patients on RRT but few have explored this exclusively in older adults. In addition, the perceptions and health satisfaction of these patients are also not fully known. We conducted a systematic review of the literature that address the QOL, perceptions and health satisfaction of older adults with ESRD on renal replacement therapies.

METHODS: A PubMed database search from January 1994 to December 2014 was conducted for articles addressing quality of life, perceptions and health satisfaction of older adults on renal replacement therapies. This was limited to human subjects and older adults age $65+$ years. The authors independently reviewed the identified literature and selected articles based on the following inclusion criteria: articles on ESRD patients on RRT with primary study data addressing perceptions or attitudes, health satisfaction and quality of life, and with separate analysis of data for adults 65 years and older. The methodologic quality of each of the selected articles was assessed using eight standards adapted from well-established research quality review criteria. RESULTS: Of the initial 1401 articles identified, a total of 23 publications met the inclusion criteria. Seventeen studies addressed quality of life (QOL) of older adults on RRT, four addressed perceptions of older adults on RRT and two evaluated health satisfaction of older adults on RRT. No individual study both addressed all of these factors collectively and met the inclusion criteria. The age range of patients in the studies examined was 65 to 90 years. Majority of the publications ( 15 of 23 studies, $78 \%$ ) met six or more of the methodologic standards and only one met less than 4 methodologic standards. Eight of seventeen studies (47\%) found similar or higher overall health- related quality of life (HRQOL) and mental component summary QOL scores in elderly ESRD patients compared to age-matched controls or younger patients. However, the physical component summary QOL scores tended to be lower in elderly patients on RRT. Only six studies addressed heath satisfaction and perceptions of elderly ESRD patients on RRT, with widely variable findings.

CONCLUSIONS: Few studies specifically examine QOL in elderly ESRD patients on RRT and even fewer address issues of perceptions and health satisfaction. However, the limited data from the studies on QOL looks promising with a significant proportion showing similar or higher overall health-related quality of life (HRQOL) and mental component summary scores in elderly ESRD patients compared to age-matched controls or younger patients. This supports the opinion that older adults should not be excluded from renal replacement therapies solely based on age. The very limited data on perceptions and health satisfaction of elderly ESRD patients on RRT makes it difficult to make any generalizable conclusions. Overall, more research is needed to examine these factors in the elderly ESRD population on renal replacement therapies. Knowing the quality of life, perceptions and health satisfaction on RRT would also better inform elderly patients and their physicians about the suitability of these modalities.

R-VA-MARQUIS: IMPLEMENTING BEST PRACTICES IN MEDICATION RECONCILIATION FOR RURAL VETERANS Kathleene Wooldridge ${ }^{5}$; Amy R. Aylor ${ }^{4}$; Susan Byerly ${ }^{5}$; Peter Kaboli ${ }^{1}$; Christianne Roumie ${ }^{3}$; Robert S. Dittus ${ }^{5}$; Amanda S. Mixon ${ }^{2}$. ${ }^{1}$ Iowa City VAMC, Iowa City, IA; ${ }^{2}$ VA Tennessee Valley Healthcare System and Vanderbilt University, Nashville, TN; ${ }^{3}$ Vanderbilt University, Nashville, TN; ${ }^{4}$ VA-Center for Applied Systems Engineering, Indianapolis, IN; ${ }^{5}$ Vanderbilt University Medical Center and the VA Tennessee Valley Healthcare System, Nashville, TN. (Control ID \#2467569)

BACKGROUND: High-quality medication reconciliation is key to reducing medication errors during care transitions. This concept is of paramount importance to Veterans living in rural areas due to less access to clinical pharmacy services. We adapted MARQUIS (Multicenter Medication Reconciliation Quality Improvement Study), in which hospitalists mentored teams in the implementation of best practices for inpatient medication reconciliation, to the VA context. We then implemented and evaluated R-VAMARQUIS in 3 rural VA Medical Centers as a multi-site quality improvement project. 
METHODS: Principal investigators conducted site visits to generate recommendations from the best practices toolkit, then mentored improvement groups through implementation. Trained project pharmacists or nurses completed data collection from January to September 2015. Data were gathered through performance of a best possible medication history and documentation of unintended discrepancies with the primary team's history, admission and discharge orders. Mean discrepancies were compared pre- and postintervention for the sites using Wilcoxon rank sum tests. We compared data pre- and post-intervention from all sites combined and for each site individually using a negative binomial regression model with an outcome of total discrepancies adjusted for age and number of discharge medications.

RESULTS: There were 301 Veterans hospitalized at 3 sites. Veterans were $94 \%$ male and $86 \%$ white with a mean age of 68 (SD 11.7). Median length of stay was 2 days (IQR 2,4), and $58 \%$ of the Veterans had been hospitalized in the prior year. Site populations were similar, including mean number of medications per Veteran. Site A did not implement any toolkit interventions. Site B implemented interventions on admission reconciliation, and Site $\mathrm{C}$ implemented interventions on discharge reconciliation. Mean total discrepancies varied by site (Figure 1). Site A had a baseline mean of 4.9 total discrepancies per Veteran. Site B had an increase in mean total discrepancies from 3 to 3.3 after intervention. Site C reduced mean discrepancies from 4.2 to 2.2 . In adjusted negative binomial regression comparing pre- to post- intervention, mean total discrepancies decreased after the intervention $(\mathrm{IRR}=0.75$, CI $0.58,0.96, p=0.02)$ when all sites were analyzed together. When analyzed separately, results were not statistically significant.

CONCLUSIONS: After the first year, implementation of R-VA-MARQUIS was associated with reduced discrepancies. While sample size limited the ability to draw statistical conclusions about individual intervention effects, analysis will continue in the second year of the project. The early results of R-VA-MARQUIS support that providing smaller, rural VAMCs with tools and subject-matter mentorship may promote high quality care for Veterans.

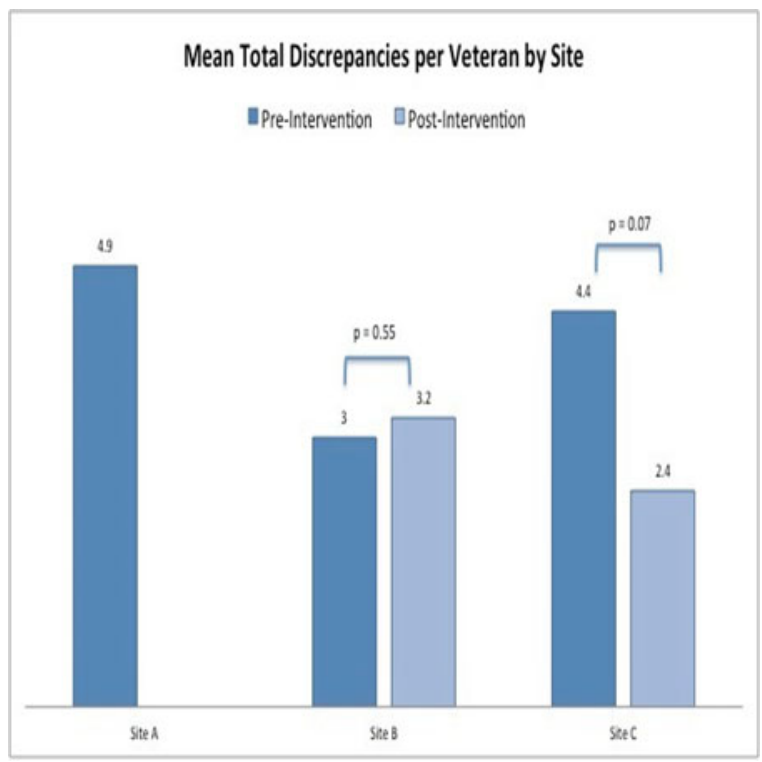

RACIAL DIFFERENCES IN ACCESS TO CANCER CARE: COMBINING A MYSTERY-CALLER SURVEY AND GEOSPATIAL APPROACH TO PROSTATE CANCER CARE David Grande ${ }^{2,}$ 2; Michelle E. Ross ${ }^{2}$; Katrina Armstrong ${ }^{3}$; Charles Branas²; Karin V. Rhodes 2, 2; Justin Bekelman²; Alicia Wentz ${ }^{4}$; Christian H. Stillson $^{2}$; Archana Radhakrishnan ${ }^{1}$; Enny Oyeniran ${ }^{2}$; Craig E. Pollack ${ }^{1} .{ }^{1}$ Johns Hopkins University School of Medicine, Baltimore, MD; ${ }^{2}$ University of Pennsylvania, Philadelphia, PA; ${ }^{3}$ Massachusetts General Hospital, Boston, MA; ${ }^{4}$ Johns Hopkins Bloomberg School of Public Health, Baltimore, MD. (Control ID \#2464879)
BACKGROUND: Prostate cancer is the most commonly diagnosed cancer in men and one of the leading causes of cancer death among men of all races in the United States. However, treatment disparities exist among men of different races and socioeconomic status. Access to healthcare may be an important determinant of men's ability to undergo certain types of treatment and may help explain observed differences in cancer treatment by patient race, socioeconomic status, and type of insurance. We sought to determine whether insurance type influences access to prostate cancer treatment and whether areas with higher proportions of black men have lower potential access to prostate cancer care. METHODS: We performed a 'mystery caller' survey of urology and radiation oncology practices in and around southeastern Pennsylvania. A research assistant, posing as a scheduler from a referring primary care office, attempted to make a new patient appointment. Linear regression models were used to determine the association between time to next available appointment and practice characteristics. Practice locations were mapped and spatial regression was used to examine the association between the number of practices within a 30 -min driving radius and census tract sociodemographic characteristics.

RESULTS: There were 223 practices in the region, of which 151 (67.7\%) were urology and $72(32.3 \%)$ were radiation oncology practices. Radiation oncology practices were more likely to accept Medicaid compared to urology practices (91.3 vs $36.4 \%$ ) and had shorter mean wait times for new patient appointments (9.0 vs 12.8 days). Practices that accepted Medicaid had statistically significantly longer wait times than those that did not (3.9 days, $95 \%$ Confidence Interval 0.0, 7.7). Census tracts with higher proportions of black men had a greater number of urology and radiation oncology practices within a 30min drive compared to census tracts with lower proportions of black men (Figure 1). In adjusted analyses, every $10 \%$ point increase in the proportion of black men was associated with $6 \%$ more urology practices within a 30-min drive (Risk Ratio [RR] 1.06, $95 \%$ Credible Interval $[\mathrm{CI}] 1.04,1.08)$ and $6 \%$ more radiation oncology practices within a 30min drive (RR 1.06, $95 \%$ CI 1.04, 1.08).

CONCLUSIONS: While wait times for prostate cancer specialists were generally short, fewer radiation oncology practices accepted Medicaid and practices that accepted Medicaid tended to have significantly longer wait times. We did not observe racial differences in potential geographic access to care in a large, metropolitan area suggesting the need to examine other dimensions of access beyond location.

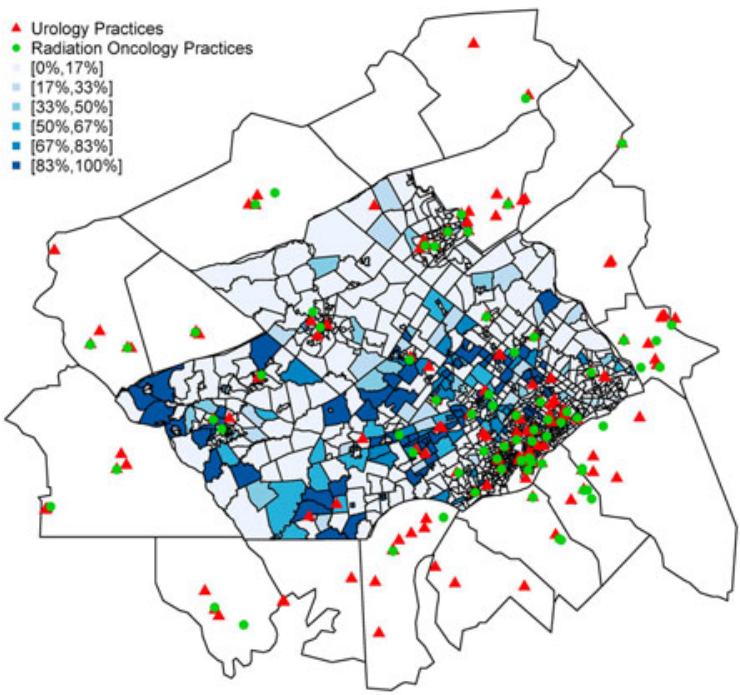

Figure 1. Map of census tracts displaying percent African American over 45 years in 8 counties around Philadelphia and location of urology and radiation oncology practices in surrounding area.

Figure 1. Map of census tracts displaying percent African American over 45 years in 8 counties around Philadelphia and location of urology and radiation oncology practices in surrounding area. 
RACIAL DIFFERENCES IN THE QUALITY OF END-OF-LIFE CARE OVER TIME Rashmi K. Sharma ${ }^{2}$; Vicki Freedman ${ }^{3}$; Judith Kasper ${ }^{4}$; Pedro Gozalo ${ }^{1}$; Vincent Mor ${ }^{1}$; Joan Teno ${ }^{2} .{ }^{1}$ Brown University, Providence, RI; ${ }^{2}$ University of Washington, Seattle, WA; ${ }^{3}$ University of Michigan, Ann Arbor, MI; ${ }^{4}$ Johns Hopkins, Baltimore, MD. (Control ID \#2467993)

BACKGROUND: Previous research has documented striking disparities in bereaved family member perceptions of the quality of end-of-life care. Our goal is to examine whether these differences have persisted more than 10 years later.

METHODS: Using data from the National Health and Aging Trends Study (NHATS), a prospective cohort of Medicare enrollees aged 65 and older, we analyzed responses of bereaved family members and close friends of decedents from rounds 2-4 ( $N=1061$; weighted 4.2 million deaths) on the following topics: unmet needs for symptom management and spiritual support, concerns with communication and decision making, and overall ratings of quality of care. Surveys were conducted between 2012 and 2014 . Analysis with survey weights examined the association between the decedent's race and bereaved family members' perceptions of the quality of care. The multivariate logistic regression model adjusted for age, the family member's relationship to the decedent, the round of data collection $(2,3$, or 4$)$, functional trajectory, and whether the patient had cancer.

RESULTS: Table 1 summarizes the difference between perceptions of bereaved family members of white and African-American decedents.

CONCLUSIONS: Although we no longer see significant differences in the quality of end-of-life care between African-American and white patients, substantial palliative care needs remain for both groups. With less than half of family members rating the overall quality of care received by their loved one as excellent, efforts to improve end-of-life care for all patients are clearly needed.

Table 1. Race and the Quality of End of Life Care

\begin{tabular}{|c|c|c|c|}
\hline$\overline{\text { Bereaved family member report }}$ & White decedents $(n=521)$ & African-American decedents $(n=241)$ & AOR $95 \% \mathrm{CI}^{*}$ \\
\hline Weighted N & $3,342,810$ & 883,245 & \\
\hline Unmet need for pain management & 22.3 & 16.3 & $0.66(0.37-1.2)$ \\
\hline Unmet need for anxiety/depression & 48.2 & 52.5 & $1.2(0.65-2.3)$ \\
\hline Unmet need for dyspnea & 21.8 & 15.6 & $0.70(0.33-1.5)$ \\
\hline Not always treated with respect & 15.9 & 12.8 & $0.66(0.40-1.1)$ \\
\hline Religious/spiritual concerns & 58.4 & 52.8 & $0.84(0.52-1.4)$ \\
\hline Decision made without enough input from the decedent or family & 9.2 & 8.3 & $0.89(0.50-1.6)$ \\
\hline Decision made that the decedent would not have wanted & 13.3 & 10.1 & $0.83(0.50-1.4)$ \\
\hline Family not always kept informed & 22.6 & 21.3 & $0.88(0.58-1.3)$ \\
\hline Overall care was excellent & 49.5 & 46.0 & $0.94(0.65-1.4)$ \\
\hline
\end{tabular}

* AOR: adjusted odds ratio with $95 \%$ confidence intervals. Adjusted for decedent age, respondent relationship to decedent, round of NHATS, pattern of functional decline as indicated by not getting out of bed, and presence of a cancer diagnosis.

RACIAL DIFFERENCES IN WEIGHT PERCEPTION AMONG BREAST CANCER PATIENTS Jenny J. Lin ${ }^{1}$; Kezhen $\mathrm{Fei}^{2}$; Rebeca Franco ${ }^{2}$; Emily J. Gallagher ${ }^{1}$; Derek LeRoith ${ }^{1}$; Nina A. Bickell ${ }^{1,2}$. ${ }^{1}$ Icahn School of Medicine at Mount Sinai, New York, NY; ${ }^{2}$ Mount Sinai School of Medical, New York, NY. (Control ID \#2467311)

BACKGROUND: Black women are more likely than white women to be obese, and obesity is associated with worse breast cancer prognosis. Moreover, compared with white women, black women are more likely to be satisfied with their body size and may be less likely to try to lose weight. Weight perception, however, has not been studied as a potential mediator of racial disparities in women with breast cancer. We describe early findings of the association between weight perception and race among women with newly diagnosed breast cancer.

METHODS: We recruited 351 ( $81 \%$ white, $19 \%$ black) women with a new primary breast cancer and surveyed them about socio-demographics, weight perception, diet and exercise habits. Height and weight were measured at enrollment which occurred at the time of primary surgical treatment. We classified women with a BMI $\geq 25$ who reported that they were "about the right weight" as under-perceivers and women who had BMI $<25$ who reported that they were overweight as over-perceivers. Chi-square (or Fisher's exact) tests were used to assess factors associated with under-perception of weight. Logistic regression models were fit to evaluate the racial difference in under-perception controlling for other covariates. RESULTS: Of 351 women with new breast cancer, the average age was 58 years ( $\mathrm{SD}=$ 12.7). At diagnosis, $63 \%$ had stage I cancer, $33 \%$ had stage II, and $4 \%$ stage III. One hundred eighty (51\%) women had a normal BMI (18.5-24.9 kg/m²), $79(23 \%)$ were overweight (BMI: $25-29.9 \mathrm{~kg} / \mathrm{m}^{2}$ ) and $83(24 \%)$ were obese (BMI $\left.>30 \mathrm{~kg} / \mathrm{m}^{2}\right)$. Overall, 26 (7\%) women under-perceived their weight, 57 (16\%) over-perceived their weight, and $268(76 \%)$ correctly perceived their weight. Black women were more likely to be overweight or obese ( 85 vs. $37 \%, p<.0001)$ and were more likely to be underperceivers $(18$ vs. $5 \%, p<.0001)$ while white women were more likely to overperceive their weight ( 20 vs. $0 \%, p<.0001)$. Black women were less likely than whites to have had a college education ( 56 vs. $72 \% ; p=0.01$ ) and those with less than a college education were more likely to be under-perceivers ( 11 vs. $6 \%, p=0.04)$. Multivariate logistic regression model found that after adjusting for age and education level, black women were still more likely to under-perceive their weight ( $\mathrm{RR}=3.5 ; 95 \% \mathrm{CI}: 1.6-8.0)$. CONCLUSIONS: As black women have higher rates of obesity and since obesity is associated with worse breast cancer outcomes, weight under-perception by black women may contribute to racial disparities in breast cancer. Weight control and increasing the desire to lose weight among overweight and obese women are critical modifiable factors that may help improve breast cancer outcomes. If weight loss can decrease breast cancer incidence, further work should elucidate how to change weight perception among high risk obese and black women.

RATES OF UNINTENDED PREGNANCY, CONTRACEPTIVE USE, AND UNMET CONTRACEPTIVE NEED AMONG WOMEN VETERANS Sonya Borrero ${ }^{4,}{ }^{7}$; Lisa S. Callegari $^{8}$; Florentina Sileanu' ${ }^{7}$ Xinhua Zhao ${ }^{7}$; Maria Mor $^{7}$; Galen E. Switzer ${ }^{3}$; Susan L. Zickmund $^{5}$; Donna L. Washington ${ }^{6}$; Laurie C. Zephyrin ${ }^{1}$; E. Bimla Schwarz $2 .{ }^{1}$ Department of Veterans Affairs, Washington, DC; ${ }^{2}$ University of California, Davis, Sacramento, CA; ${ }^{3}$ University of Pittsburgh, Pittsburgh, PA; ${ }^{4}$ University of Pittsburgh and VA Pittsburgh, Pittsburgh, PA; ${ }^{5}$ University of Pittsburgh/VA Pittsburgh Healthcare System, Pittsburgh, PA; ${ }^{6}$ VA Greater Los Angeles Healthcare, UCLA, Los Angeles, CA; ${ }^{7}$ VA Pittsburgh, Pittsburgh, PA; ${ }^{8}$ VA Puget Sound, Seattle, WA. (Control ID \#2466226) 
BACKGROUND: Unintended pregnancy is a prevalent problem in the US that has been associated with adverse health and social consequences for women and their children. Women veterans who seek health care from the Veterans Administration (VA) are disproportionately from lower income strata, from racial/ethnic minority groups, and have a high prevalence of medical and mental illness - characteristics that have been associated increased risk of unintended pregnancy. This study determined the rates of unintended pregnancy, contraceptive use, and unmet contraceptive need among a national sample of reproductive-aged women veterans.

METHODS: We conducted a cross-sectional, telephone-based survey with a sample of female veterans aged 18-44 years who had received care within VA in the prior 12 months. From April 2014 to December 2015, a random sample of women veterans, representing all regions and Veterans Integrated Service Networks (VISNs) in the US, were invited to participate in the study, "Examining Contraceptive Use and Unmet Need among Women Veterans (ECUUN)" which was designed to collect information on reproductive outcomes and factors affecting risk of unintended pregnancy and contraceptive use. We used descriptive statistics to assess rates of unintended pregnancy and contraceptive use in the entire sample. We also estimated unmet need for contraception, defined as the proportion of women veterans at risk for unintended pregnancy (i.e., sexually active with a man in the prior 3 months, had not had a hysterectomy or other history of infertility, and were not pregnant, postpartum or seeking pregnancy) who were not using prescription contraception. We then examined whether unmet need for contraception varied by race/ethnicity, presence of a chronic medical condition, mental illness, and history of military sexual trauma (MST). For associations significant in bivariate analysis, we then conducted multivariable logistic regression to adjust for potential confounders.

RESULTS: In this national sample of 2165 women veterans, mean age was 34.7 (SD = 6); $52 \%$ had a household annual income of less than $\$ 40,000 ; 52 \%$ were White, $29 \%$ Black, $12 \%$ Hispanic, and $7 \%$ were of other race/ethnicity. In addition, $56 \%$ reported having been diagnosed with $\geq 1$ chronic medical condition, $69 \%$ reported having been diagnosed with $\geq 1$ mental illness, and $55 \%$ reported a history of MST. The annual pregnancy rate in the sample was 65.6 per 1000 women, and the unintended pregnancy rate was 25.4 per 1000 women; $37 \%$ of pregnancies were unintended. Overall, $62 \%$ of women veterans were using contraception at the time of interview. Specifically, $34 \%$ were using a highly effective method [sterilization, intrauterine device (IUD) or implant]; $18 \%$ were using a moderately effective method (contraceptive pill, patch, vaginal ring, or injection); and $10 \%$ were using less effective, non-prescription methods (condoms, withdrawal, and natural family planning). Among the $38 \%$ of contraceptive non-users, $14 \%$ reported not being currently heterosexually active, $7 \%$ reported prior hysterectomy, $4 \%$ reported infertility, and $7 \%$ reported being either pregnant, postpartum, or seeking pregnancy. Therefore, among the 1087 of women at risk for unintended pregnancy, $89 \%$ of women were using some form of contraception. Specifically, $49 \%$ were using a highly effective method (notably, $23 \%$ of women were using an IUD or implant), $23 \%$ were using a moderately effective method, $16 \%$ were using a less effective method, and $11 \%$ were using no method. Thus, $27 \%$ of women at risk for unintended pregnancy were not using prescription contraception, thereby demonstrating a potential unmet contraceptive need. We found that unmet need for contraception did not significantly vary by presence of medical illness $(p=0.24)$, presence of mental illness $(p=0.26)$, or by history of MST ( $p=$ 0.81 ). However, non-Hispanic whites were significantly less likely to have unmet need for contraception than women from other racial/ethnic groups ( 25 vs $31 \%, p=0.03$ ), a difference that persisted in multivariable analysis adjusting for age, income, education, and marital status ( $\mathrm{OR}=0.75$; $95 \% \mathrm{CI}$ : $0.56-0.99)$.

CONCLUSIONS: Over a third of all pregnancies occurring among women VA-users each year are unintended. We also found that over a quarter of women veterans at risk for unintended pregnancy are not using prescription contraception and that significant racial/ ethnic disparities in contraceptive use exist, indicating that further efforts are needed to improve contraceptive service delivery for women veterans.
REASONS AND APPROPRIATENESS OF OVERRIDING ANAPHYLAXIS DRUG ALLERGY ALERTS Diane L. Seger ${ }^{1,2}$; Elizabeth R. Silvers ${ }^{1,}$; ; Sarah P. Slight $^{1,3}$; Mary Amato ${ }^{1,}{ }^{4}$; Julie Fiskio ${ }^{1,2}$; Adrian Wong ${ }^{1,}$; Patrick E. Beeler ${ }^{1,}$; Tewodros Eguale ${ }^{1,4}$; Patricia C. Dykes ${ }^{1,5}$; David W. Bates ${ }^{1,5}$. ${ }^{1}$ Brigham and Women's Hospital, Boston, MA; ${ }^{2}$ Partners Healthcare System, Wellesley, MA; ${ }^{3}$ School of Medicine, Pharmacy and Health, The University of Durham, Stockton on Tees, Durham, United Kingdom; ${ }^{4} \mathrm{MCPHS}$ University, Boston, MA; ${ }^{5}$ Harvard Medical School, Boston, MA; ${ }^{6}$ Research Center for Medical Informatics, University Hospital, Zurich, Switzerland. (Control ID \#2466012)

BACKGROUND: Clinical Decision Support (CDS) systems are designed to provide real-time guidance and support to providers at the point of prescribing. Drug allergy interaction alerts (DAIs) are generated when a known adverse sensitivity inducing substance is prescribed. Anaphylaxis is a serious allergic reaction, and given its lifethreatening nature, this potential allergic reaction is typically a red flag for providers. Surprisingly, a recent study at our institution showed that providers overrode the majority of DAI alerts including those warning against 'anaphylaxis' ${ }^{\text {, }}$ t therefore this study aimed to determine the reasons why providers overrode these alerts, and whether these overrides were appropriate.

METHODS: All DAI alerts where the reaction was anaphylaxis from Jan 2009 to Dec 2011 for inpatients at Brigham and Women's Hospital and 36 primary care practices affiliated with Partners Healthcare were downloaded and analyzed. These interruptive alerts suggested a life threatening interaction and gave the provider the option of either 'cancelling' the order or 'overriding' the alert. A total of 1851 inpatient DAI alerts and 226 outpatient DAI alerts were collected. The downloaded file included the name of the drug and allergen that triggered the DAI alert, and the reasons that the providers gave at the time of overriding the alert. Because the range of cross-sensitization varies greatly among individuals and is often difficult to predict with any certainty according to the World Allergic Organization ${ }^{2}$, we decided to primarily focus on the DAI that were classified as definite matches where the medication being ordered was the same as the allergen listed. A research pharmacist assessed the appropriateness of the DAI overrides by conducting a detailed chart review according to a predetermined set of criteria.

RESULTS: Among the 1851 anaphylaxis DAI alerts in the inpatient setting, 202 of them were classified as 'definite', 1025 were classified as 'probable' where the medication and allergen were in the same drug class (i.e.: ordering ibuprofen in a patient with a NSAID allergy), and 624 were classified as 'possible' where the medication and allergen may have cross reactivity (i.e.: ordering a Cephalosporin for a patient with a Penicillin allergy). In the outpatient setting, we found a total of 226 anaphylaxis DAI where 20 were classified as 'definite', 162 'probable' and 44 'possible'. The rate of definite anaphylaxis DAI overrides was $46 \%(93 / 202)$ in the inpatient setting and $70 \%(14 / 20)$ in the outpatient setting. The appropriateness rate of the definite DAI overrides for inpatients was $83.9 \%$ (78/93) and $100 \%(14 / 14)$ for outpatients. The most common reason for appropriate overrides in the inpatient setting were "administered per desensitization protocol" and for the outpatient setting "patient does not have this allergy -will d/c pre-existing allergy". There were 15 DAI inpatient overrides found to be inappropriate where the provider gave an override reason as "Physician aware" (6), "Low risk cross sensitivity" (3), "No reasonable alternative" (2), "Patient has taken previously and tolerated" (3) and "Pt requested" (1), however these reasons were unable to be verified on chart review. Twelve of these overrides were stopped by pharmacy so the patient never got the medication and the other 3 overrides were all for prochlorperazine suppositories when required, which the patient never needed or received.

CONCLUSIONS: The rate of overrides for definite anaphylaxis DAI alerts were found to be high but appropriate for the majority (83.9\%) or all (100\%) of the overrides in the inpatient and outpatient setting, respectively. Inappropriate drug allergy alerts at best can 
waste clinicians' time, and so it is very important that the information contained in patients' drug allergy lists and the clinical evidence on drug cross-reactivity in the decision support systems are updated. Further research to review appropriateness of overrides of anaphylaxis DAIs for probable and possible matches should be done to ascertain how accurate and relevant these alerts are. References: 1- Topaz M, Seger DL, Slight SP, Goss F, Lai K, Wickner PG, Blumenthal K, Dhopeshwarkar N, Chang F, Bates DW, Zhou L. Rising drug allergy alert overrides in electronic health records: an observational retrospective study of a decade of experience. J Am Med Inform Assoc. 2015 Nov 17. [Epub ahead of print] 2- Drug Allergies. Thong B, Vervloet D (Updated 2014). World Allergy Organization website. Available from: http://www.worldallergy.org/professional/allergic diseases_center/drugallergy This study was funded by grant \#U19HS021094 from the Agency for Healthcare Research and Quality (AHRQ)

REASONS FOR MISSED DOSES OF PHARMACOLOGICAL VENOUS THROMBOEMBOLISM (VTE) PROPHYLAXIS IN MEDICAL INPATIENTS

Jahangir Khan; Suresh K. Subedi; Azza Ahmed; Abdul Mohammed; Binay Kumar; Scott Kaatz. Hurley Medical Center, Flint, MI. (Control ID \#2469494)

BACKGROUND: Guidelines recommend venous thromboembolism (VTE) pharmacological prophylaxis for high risk patients and many patients do not receive prescribed prophylaxis. We aimed to describe reasons for missed doses of unfractionated heparin (UFH) and low molecular weight heparin (LMWH) and compare differences in hospitalized medical patients.

METHODS: Adult patients admitted for a non-surgical condition to the general medical floor who missed at least one dose of VTE prophylaxis on hospital days 3-6 were identified. We interviewed nurses regarding reasons for missed doses and if no reason was obtained or if the reason was patient refusal, we interviewed patients. The study was continued until we found no new information from 10 consecutive cases (saturation). Differences were compared with chi square test.

RESULTS: Eighty-four patients were included and 68 (78.6 \%) were on UFH and 18 $(21.4 \%)$ on LMWH. The top 3 reasons for missed doses were: 1$)$ patient did not want injections, 28/84 (33.3\%); 2) patients were ambulatory, 15/84 (17.9\%) and 3) LMWH/UFH was held for a procedure $8 / 84(9.7 \%)$. Reasons for missed doses were not different between LMWH and heparin except for "ambulatory", which was more common in patients prescribed LMWH [LMWH $44.4 \%$ vs. UFH $10.6 \%(P=0.003)$ ]. CONCLUSIONS: Common reasons for missed doses for VTE prophylaxis included patients not liking injections, being held for procedures and patients being ambulatory. Differences in ambulation between LMWH and heparin prophylaxis are not clear and could represent confounding factors. Nursing and patient education around VTE prophylaxis and reassessment of VTE risk during hospitalization represent improvement opportunities.

\section{REDUCING HEALTH CARE COSTS: A CLOSER LOOK AT ED TRANSFERS} FROM URGENT CARE AT A FEDERALLY QUALIFIED HEALTH CENTER

Sneha Shrivastava. Greater Hudson Valley Family Health Center, White Plains, NY. (Control ID \#2466688)

BACKGROUND: There is a growing need in our country to reduce health care costs by appropriately utilizing the Emergency Department (ED) resources. There are several stateand national reforms to reduce recurrent ED admissions. As a Federally Qualified Health Center (FQHC), our Urgent Care (UC) department plays an important role in the mission to reduce such admissions. Despite having an in-house UC, we still experience high ED transfer rates; this is particularly true for patients who present to our UC with chest pain. While chest pain is an alarming chief complaint, not all patients with chest pain are at risk for medical emergencies. In the absence of established protocols in the US setting for evaluating chest pain, transfer to the ED happens due to clinical caution without clear alarm symptoms adding to the increasing cost of health care. Our objective is to (i) evaluate outcomes of the patients that were transferred to ED from our UC for further evaluation of chest pain that were not at risk for medical emergencies, and (ii) subsequently develop new protocols to reduce these ED transfers.

METHODS: A retrospective chart review was conducted for patients seen in UC with a chief complaint of chest pain between October 1, 2014 and March 31, 2015. The review consisted of evaluating (i) outcomes of patients that were transferred to ED, and (ii) quality of UC documentation including a comprehensive history and physical, an EKG assessment, risk stratification for medical emergencies, and a comprehensive plan.

RESULTS: Patient outcomes: 52 patients were seen in UC in the above-mentioned 6 month period for the chief complaint of chest pain. $37 \%$ (19/52) of these patients were transferred to the ED for further evaluation. Out of these 19 patients, only $15 \%(3 / 19)$ were admitted for possible medical emergencies, and the remaining $85 \%(16 / 19)$ were discharged within a few hours of their ED stay. The diagnosis of these $85 \%$ patients included influenza, anxiety, musculoskeletal pain, gastro-esophageal reflux disease, urinary tract infection, cervical radiculopathy, asthma, and benign essential hypertension, all of which are not medical emergencies. These patients were subsequently followed-up by their primary care providers and remained symptom-free after their initial treatment in the ED. Quality of documentation: Only $48 \%$ of the patient charts that were seen in UC for chest pain had a complete documentation of history, physical exam, assessment and plan. Specifically, we found that $67 \%$ of the patient charts had an EKG, $37 \%$ of the patient charts had a documented cardiac risk stratification, and $10 \%$ of patients received an aspirin $(325 \mathrm{mg})$ in UC.

CONCLUSIONS: The ED transfer rate for patients with chest pain who were not at risk of a medical emergency was high. These patients can be safely evaluated and treated in UC. Based on these observations, we have created a chest pain protocol in our UC that includes (i) early triage and intervention from nurses, (ii) increasing the utilization of Xray, EKG and point of care testing, (iii) improved access to our on-call cardiologist, and (iv) a new electronic chest pain evaluation form. The chest pain evaluation form will be designed to help UC providers improve the quality of their documentation and hence efficiently evaluate patients, risk stratify, and identify medical emergencies. The chest pain protocol will be rigorously analyzed after each stage of its implementation in a Plan-DoStudy-Act cycle format. Efficiently evaluating and managing patients with chest pain who are not at risk for medical emergencies can reduce ED transfers from UC and help significantly reduce overall health care costs.

REDUCING PRENATAL AND CHILDHOOD ENVIRONMENTAL EXPOSURE RISKS AMONG HISPANIC FAMILIES IN SOUTH TEXAS AND NORTH TAMAULIPAS, A BI-NATIONAL APPROACH Kristopher R. Koch ${ }^{1}$; Noe Garza ${ }^{2}$; Beatriz Tapia ${ }^{2}$. University of Texas Health Science Center, San Antonio, TX; ${ }^{2}$ University of Texas - Rio Grande Valley, Harlingen, TX. (Control ID \#2465909)

BACKGROUND: Families in border communities are exposed to various environmental allergens and problems that directly affect the health of their families and put children at risk of developing chronic disease. In this intervention, we sought to decrease the amount of environmental exposure to harmful chemicals and allergens by using a "train the trainer" approach with Promotoras. Promotoras de Salud, also referred to community health workers, have been used to target hard to reach populations, traditionally excluded racial/ethnic groups, and other medically underserved communities while serving as a cultural bridge primary care health centers, community based organizations and their communities. The purpose of this intervention was to educate families in South Texas and North Tamaulipas on reducing hazardous environmental exposures that may affect 
pregnant women and young children and to assess knowledge, attitudes and behavior changes regarding targeted exposures.

METHODS: Promotoras from Nuevo Laredo, Laredo, Brownsville and Reynosa surveyed and educated 392 families (192 in Mexico and 200 in the US) in their respective communities on environmental exposures. Participants were given a 20 item pre-test and behavior assessment to determine preexisting knowledge and recent behaviors towards environmental household factors and allergens. Promotoras collected observations of 17 different household environmental exposures. Thirty-nine families (19 in Mexico and 20 in the US) were then followed up over 9 months at 3-month intervals to provide continual education and monitor changes in reported behaviors and household environmental exposures. Post-tests were given after each education session to measure changes in knowledge gained and changes in attitude as a result of the training. Knowledge, behavior and household environmental exposure indexes were created. Intervention knowledge was assessed using paired t-test to determine educational session. Longitudinal pre-test knowledge, behaviors, and observed environmental exposures were analyzed using Repeated Measures ANOVA to determine baseline differences and changes over time. RESULTS: Environmental exposure knowledge increased from 50.4 to $81.4 \%$ $(p<0.000)$ and increased across all tested categories: asthma \& allergies $(p<0.000)$, pesticides $(p<0.000)$, heavy metal exposure $(p<0.000)$. Mexican households had higher levels of each environmental exposure as compared to households in the United States $(p<0.000)$. Fragrances $(79.9 \%)$, pesticide sprays $(66 \%)$, and combustible products $(60.6 \%)$ were the most common environmental exposures in Mexico as compared to solvents (36.4\%), sprays (33.1\%), and dander (29.1\%) in the United States. Over 9 months, pre-test knowledge in both South Texas and Northern Tamaulipas increased from a mean of $50.3 \%$ to $85.5 \%(p<0.000)$. Knowledge increased across tested all environmental exposures: asthma \& allergies (p, 0.000), pesticides ( $p, 0.000)$, heavy metal exposure (p, 0.000). Environmental health behavior scores increased from 59.6 to $87.8 \%$ $(p<0.000)$. Total observed environmental exposures per household on a 17 -item scale decreased from 4.56 per household (SD 3.65) to 2.60 (SD 2.91) over 9 months.

CONCLUSIONS: This evaluation demonstrates the effectiveness of using promotoras in reducing environmental exposures among a binational community. This intervention was developed with the intention to help reach patient populations who typically have low or minimal contact with the traditional health care system and reduce environmental exposures such as pesticide, lead, and mercury exposure and asthma triggers.

REFLECTIVE WRITING WORKSHOPS LED BY NEAR PEERS DURING THIRD-YEAR CLERKSHIPS: A SAFE SPACE FOR SOLIDARITY, CONVERSATION, AND FINDING MEANING IN MEDICINE Lorenzo R. Sewanan ${ }^{2}$; Daniel Zheng $^{2}$; Priscilla Wang ${ }^{2}$; Xiaoyue M. Guo ${ }^{2}$; Isha M. Di Bartolo ${ }^{1}$; Nareh V. Marukian² Jack L. Turban ${ }^{2}$; Danilo A. Rojas-Velazquez ${ }^{2}$; Anna Reisman ${ }^{1}$. ${ }^{1}$ Yale School of Medicine, New Haven, CT; ${ }^{2}$ Yale University, New Haven, CT. (Control ID \#2467852)

BACKGROUND: Third year medical students (MS3s) often encounter challenging moments that evoke strong emotions, such as death of a patient or questionable behavior of a clinical team member. At the same time, many factors interfere with debriefing these experiences such as time constraints, low level in the clinical hierarchy, and isolation from peers during clerkships. Medical schools are increasingly incorporating reflective writing into their curricula. A growing body of literature demonstrates the potential for positive impact on students, including increased empathy and improved learning. However, reflective sessions are usually conducted by faculty facilitators, whose presence may prevent truly open discussion and limit the effectiveness of sessions. We hypothesized that reflective writing workshops led by upperclassmen ("near peers") built into the third year curriculum would provide an ideally structured outlet for reflection. The objectives of this study were to perform qualitative analyses of: (1) MS3 participant responses to near peer-led reflective writing workshops and their perceptions of helpfulness, surprise, and potential for behavior change. (2) Upperclassmen medical student facilitator responses regarding themes of discussion that emerged, and their perceptions of the strongest benefit of the workshop.

METHODS: Students and near-peer facilitators are medical students at Yale School of Medicine. The study was designed to take place over a 1 year period, as MS3s rotate through four core clerkships. Facilitators are upperclassmen (fourth year or above) volunteers. The study was granted institutional exemption under code 45 CFR 46.101(b) (2). The reflective writing workshops are held at the end of each core clerkship; all students $(\sim 100)$ attend. Facilitators received training from a faculty member and a graduating medical student who designed the session. Each facilitator led a group of 10$15 \mathrm{MS} 3 \mathrm{~s}$, with no faculty present. In each session, rules of engagement are reviewed, including confidentiality, commitment to active participation and listening, and withholding judgment. The workshops follow a structured but flexible series of questions divided into three phases including short and superficial "warm-up" questions and longer questions that challenge students. Each session concludes with an anonymous voluntary paper survey of open ended questions. Students could also opt to submit their written reflections. We used an iterative consensus process with grounded theory to analyze the qualitative data in our open ended questionnaires. Surveys were randomly mixed and a portion of the total was assigned to one of nine reviewers, who read each survey form twice and created a label for each response. The surveys were then reassigned to a different reviewer who relabeled the responses. A central reviewer compiled and identified common labels and categories. An instrument was created to identify the categories from the compiled coding scheme, and each survey was processed to fit within this framework. Major themes and relationships arising from this formal categorization process were used in the final analysis, along with representative quotations from the questionnaires.

RESULTS: Approximately 100 third year medical students participated in the workshop in August 2015 and were facilitated by 8 near peers. Our preliminary results are based on analysis of the questionnaires from students and facilitators. Both students and facilitators found that the near-peer workshop provided a safe space for reflection and sharing, and many students were surprised to discover how willing peers were to share their experiences. Many students valued having the protected time for the workshop and found the format beneficial due to the thoughtful, stimulating focus of the prompts and clear structure of the workshop. Common themes included patient care in difficult situations, medical culture related to hierarchy and interprofessional tension, and personal difficulties with burnout/fatigue. Students found that the workshop format of writing and then sharing provided a sense of solidarity and support within the class. Most students stated that the workshops helped them find closure, vent, and process experiences they had not discussed before, and that the experience encouraged them to focus more attention on empathy and emotions in clinical care. Many students described a desire to write and engage in active reflection as a result of having participated in the workshop.

CONCLUSIONS: A near-peer reflective writing workshop integrated into clerkships provided a protected space to process difficult clerkship experiences, increased solidarity and support within the class, and encouraged MS3s to actively reflect through writing. Increased incorporation of similar activities throughout medical education may have similar benefits and be an appreciated addition to clerkship curricula by medical students.

REGIONAL UTILIZATION PATTERNS OF PERCUTANEOUS VERTEBROPLASTY AND KYPHOPLASTY Claudia Scheuter ${ }^{1}$; Radoslaw Panczak $^{2}$; Alan Haynes ${ }^{2}$; Arnaud Chiolero ${ }^{3}$; Maria Monika Wertli ${ }^{1}$; Eveline Hofmann ${ }^{1}$; Arnaud Perrier ${ }^{4}$; Nicolas Rodondi ${ }^{1}$; Drahomir Aujesky ${ }^{1}$. Division of General Internal Medicine, Bern University Hospital, Bern, Switzerland; ${ }^{2}$ University of Bern, Bern, Switzerland; ${ }^{3}$ Lausanne University Hospital, Lausanne, Switzerland; ${ }^{4}$ Geneva University Hospitals, Geneva, Switzerland. (Control ID \#2462758) 
BACKGROUND: Randomized controlled trials did not show any benefit of percutaneous vertebroplasty in painful osteoporotic vertebral fractures compared to conservative treatment. The American Academy of Orthopedic Surgeons recommends against the routine use of percutaneous vertebroplasty and kyphoplasty in painful osteoporotic vertebral fractures and the Australian government has revoked public funding for these procedures. Nevertheless, these procedures continue to be performed in many countries. We assessed utilization patterns of percutaneous vertebro- and kyphoplasty in Switzerland.

METHODS: We used population-based data from all adult patients who were discharged from a Swiss acute care hospital during calendar year 2012. All Swiss acute care hospitals are mandated by law to provide the Swiss Federal Statistical Office (SFSO) a standard set of data for each patient discharge, including age, gender, place of residence, the principal diagnosis and up to 8 secondary diagnoses based on the International Classification of Diseases-10German Modification, and up to 10 procedural codes. The procedural codes for percutaneous vertebro- and kyphoplasty were compiled from the Swiss procedural codes handbook. We defined Swiss hospital service areas (HSAs) according to Wennberg's 3-step approach. First, all acute care hospitals in Switzerland were identified and assigned to one of the 706 census regions defined by the SFSO. The number of census regions with at least one acute care hospital defined the maximal number of possible HSAs. Second, we determined in which census region the majority of residents were discharged from the hospital. The census region with the highest number of discharges was defined as the corresponding HSA. Lastly, visual examination of the HSA borders was performed to ensure geographical contiguity. We calculated utilization rates per 10,000 persons by HSA using procedure counts and the 2012 Swiss Federal Population Census data, adjusting for age and gender.

RESULTS: Overall, 1.2 million patients were discharged from 155 Swiss acute care hospitals in 2012, of whom 2293 (0.2 \%) underwent vertebro- or kyphoplasty. 1351 (59\%) vertebro- and kyphoplasties were performed as an elective procedure. Overall, 30 patients $(1 \%)$ died during the hospitalization. Our analysis of patient flows yielded 24 HSAs, with a mean population size of 271,000 persons per HSA. The adjusted mean (SD) utilization rate of vertebro- and kyphoplasty was $3.86( \pm 2.61)$ per 10,000 persons and varied between 1.2 and 10.9 per 10,000 persons across HSAs (Figure).

CONCLUSIONS: We found a 8.5 -fold regional variation in the use of percutaneous vertebro- and kyphoplasty in Switzerland, which is unlikely to be explained by regional differences in the prevalence or severity of vertebral fractures or patient preferences. More likely, our findings indicate a widespread overuse of a costly procedure that lacks evidence of benefit. In areas with a high utilization of vertebro- and kyphoplasty, physician and patient education measures and/or policy-level interventions and may be necessary to reduce the use of these interventions.

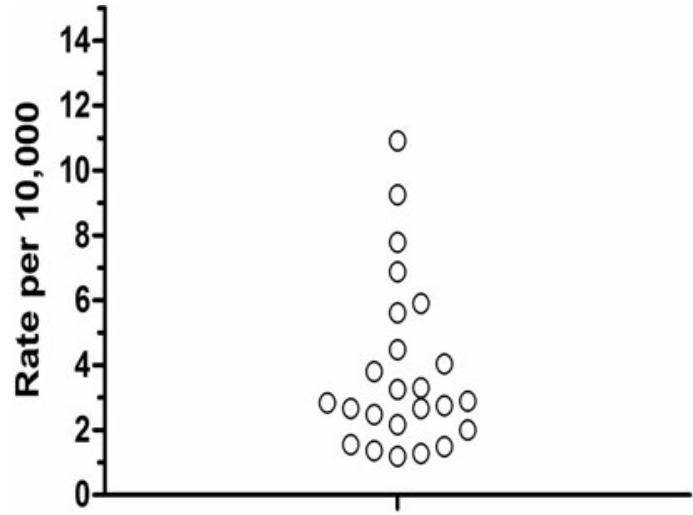

Figure. Utilization rates for vertebroplasty and kyphoplasty per 10,000 persons, adjusted for age and gender. Each dot represents one HSA.
RELATIONSHIP OF ECHOCARDIOGRAPHIC FEATURES WITH DEVELOPMENT OF PULMONARY HYPERTENSION AFTER PULMONARY EMBOLISM. A 10 YEAR RETROSPECTIVE ANALYSIS FROM A TERTIARY CARE FACILITY. Ayush Motwani ${ }^{3}$; Shahzaib Nabi ${ }^{1}$; Fawad Virk ${ }^{1}$; George Samuel ${ }^{1}$; Daryl Sudasena ${ }^{2}$; Adeel Arshad ${ }^{4}$; Philip Kuriakose ${ }^{2} .{ }^{1}$ Henry Ford Health System, Detroit, MI; ${ }^{2}$ Henry Ford Hospital, Detroit, MI; ${ }^{3}$ Henry Ford hospital, Detroit, MI; ${ }^{4}$ Hamad Medical Center, Doha, Qatar. (Control ID \#2466974)

BACKGROUND: Chronic thromboembolic pulmonary hypertension (CTPH) remains rather underdiagnosed. Echocardiography (mainly to assess the right side of the heart) done at the time of pulmonary embolism (PE) has become the standard of care and an important predictor of outcomes in this patient population. We sought to evaluate relationship between different echocardiographic findings with development of CTPH. We also assessed the impact of these findings on mortality of this patient population.

METHODS: A retrospective chart review was carried out to include patients with CTPH in the past 10 years. Only patients with an echocardiogram at the time of PE were included in the study $(n=580)$. For variables with two categories, we used a one-sample t-test. For variables with three or more categories, we used an Analysis of Variance (ANOVA). Finally, for continuous variables, we used a simple linear regression and reported the slope t-test coefficient to see if there is an association.

RESULTS: Out of a total of 580 patients analyzed in this study, 481 patients $(82.9 \%)$ had Pulmonary Hypertension (PH). Presence of right ventricle dysfunction (33.7 vs $21.2 \%$, OR $1.89,95 \% \mathrm{CI} 1.12-3.17, p<0.05)$ and tricuspid regurgitation (63.6 vs $42.4 \%$, OR $2.37,95 \% \mathrm{CI} 1.53-3.68, p<0.05)$ at the time of PE were found to be associated with development of PH later in life. It was also noted that presence of a low ejection fraction $(\mathrm{EF})$ at the time of $\mathrm{PE}$ was related to an earlier development of $\mathrm{PH}$ especially in patients with $\mathrm{EF}$ of less than $20 \%(\mathrm{~F}=3.31, p=0.02)$. Higher pulmonary artery pressures at the time of pulmonary embolism were also related to an earlier development of pulmonary hypertension especially when the PA pressures were above $65 \mathrm{~mm}$ of $\mathrm{Hg}(\mathrm{F}=2.87, p=$ $0.04)$. Time to death after development of pulmonary hypertension was not significantly affected by the echocardiographic features analyzed in this study.

CONCLUSIONS: Echocardiographic evidence of right ventricle dysfunction and tricuspid regurgitation at the time of Pulmonary Embolism are associated with development of Pulmonary Hypertension later in life. In addition, presence of low Ejection Fraction $(<20 \%)$ and higher PA pressures $(>65 \mathrm{mmHg}$ ) were found to be associated with an earlier development of Pulmonary Hypertension.

RELATIONSHIPS BETWEEN PATIENT ACTIVATION, PREFERENCES FOR SELF-DIRECTED CARE, AND DIABETES SELF-MANAGEMENT BEHAVIORS IN A LOW HEALTH LITERACY POPULATION Nadiya Pavlishyn ${ }^{1}$; Joseph Plaksin $^{1}$; Irina Gershgorin ${ }^{1}$; Kendi Tang ${ }^{2}$; Sondra Zabar ${ }^{1}$; Adina Kalet ${ }^{1}$; Lisa Altshuler ${ }^{1}$. ${ }^{1}$ NYU School of Medicine, New York, NY; ${ }^{2}$ New York University, New York, NY. (Control ID \#2467197)

BACKGROUND: Patients with low health literacy face increased challenges in accessing healthcare and often have a higher burden of chronic disease. Low health literacy has also been correlated to lower levels of patient activation, which is independently related to health outcomes. One potential mechanism for these factors to affect health outcomes is through less effective communication with healthcare providers (HCPs) and less involvement in shared decision-making (SDM). We hypothesize that health literacy, patient activation, and patient preferences for communicating with HCPs are inter-related concepts that work together to impact patient behavior during healthcare visits. In turn, we anticipate that these factors will affect self-management behaviors outside of the HCP's office and, ultimately, health outcomes. 
METHODS: English-speaking patients with type 2 diabetes mellitus (DM) were recruited from three NYC public hospitals to participate in a Patient Empowerment Program (PEP), an intervention focused on improving health outcomes by teaching patients how to be more engaged in their care and participate in SDM with their HCPs. At the time of enrollment, participants completed baseline assessment including the Patient Activation Measure (PAM), preference for communication with HCPs (Krantz Health Opinion Survey (Krantz), Preference Control Scale (PCS)), and diabetes self-care (Diabetes SelfManagement Questionnaire (DSMQ)). Their most recent hemoglobin A1c was also obtained from the electronic medical record. Additionally, demographic and clinical information was collected including health literacy, as measured by the Newest Vital Sign (NVS). Due to the low literacy of our patient population, two Research Assistants (RAs) read the entire set of questionnaires to each patient and recorded their responses. Both RAs asked participants the same questions in the same order and a standardized protocol was devised to assure that questions were asked and clarified in the same manner. Data was stored in a secure REDCap database and analyzed using SPSS software.

RESULTS: We enrolled a total of 80 patients across the three sites, 73 of whom completed the full set of questionnaires. Participants were predominately male $(53 \%)$ with a mean age of 59.4 years $(\mathrm{SD}=9.1)$, most were racial or ethnic minorities $(37 \%$ African American, $33 \%$ Hispanic/Latino), $38 \%$ did not finish high school, $63 \%$ had an annual income less than $\$ 10,000$, and $75 \%$ had limited health literacy (NVS score $\mathrm{M}=$ $2.21, \mathrm{SD}=1.67)$. Univariate analyses were conducted using chi-square tests for categorical variables and correlations for continuous variables. Higher health literacy (NVS total score) was correlated with a preference for more active involvement in communication with HCPs as measured by total Krantz total score $(r=0.40, p<0.001)$ as well as both the Information subscale $(\mathrm{r}=0.30, p=0.008)$ and Behavioral Involvement subscale $(r=0.31$, $p=0.007)$. NVS score was not significantly correlated with either PAM score $(\mathrm{r}=0.04$, $p=0.730)$ or the DSMQ sum scale $(r=-0.09, p=0.434)$. Limited health literacy was also not associated with a less cooperative PCS score $\left(X^{2}=3.12, p=0.203\right)$. PAM score was significantly correlated with the DSMQ sum scale $(\mathrm{r}=0.28, p=0.012)$ but both Krantz total score $(r=-0.03, p=0.79)$ and PCS score $(r=0.12, p=0.302)$ were not. Finally, neither NVS score, Krantz score, PAM score, PCS score, or DSMQ sum scale were significantly correlated with hemoglobin A1c $(r=-0.47-0.13, p=0.246-0.809)$. Multivariate analyses were conducted using a series of stepwise linear regression models. With DSMQ sum scale as the dependent variable, we entered participant characteristics (e.g. age, gender, race, education level, socioeconomic status, and health literacy) into the first step of the model, followed by questionnaire scores (e.g. Krantz Information subscale, Krantz Behavior subscale, PAM level, and PCS). The only significant variable in this model was PAM level, $\mathrm{R}^{2}=0.13$, adjusted $\mathrm{R}^{2}=0.12, \mathrm{~F}(1,69)=10.11, p=0.002$. The same stepwise linear regression was repeated with hemoglobin Alc as the dependent variable and DSMQ sum scale added in as a predictor variable with the other questionnaire scores. However, this model was not significant, $\mathrm{R}^{2}=0.02$, adjusted $\mathrm{R}^{2}=-0.06$, $\mathrm{F}(5,69)=0.25, p=0.941$.

CONCLUSIONS: As expected, patient activation was a significant predictor of DM selfmanagement behaviors. Surprisingly, health literacy and patient activation were not significantly correlated, yet higher health literacy was associated with a higher Krantz score, reflecting a preference for more active stance when communicating with HCPs. The concepts of patient activation and patent engagement are sometimes regarded as interchangeable, but our results suggest important differences. The PAM seems to measure the patients' perceived confidence in their capacity to understand and implement their treatment plan while the Krantz measures more concrete preferences toward obtaining information from HCPs and self-directing their care. While patients with lower health literacy in this population are not less activated or engaging less in self-care behaviors, they do prefer to rely on HCPs for more direction and seem less comfortable engaging in SDM. This highlights the importance of exploring patient preference for engagement as we seek to work collaboratively with them in shaping their care.
RELIGION, SPIRITUALITY, AND HIV CLINICAL OUTCOMES: A SYSTEMATIC REVIEW OF THE LITERATURE Benjamin R. Doolittle ${ }^{1}$; Amy C. Justice ${ }^{2}$; David A. Fiellin ${ }^{1} .{ }^{1}$ Yale University, New Haven, $\mathrm{CT} ;{ }^{2}$ Yale University, West Haven, CT. (Control ID \#2469831)

BACKGROUND: Religion and spirituality are important to HIV-infected patients. In a study that evaluated 2266 patients from the HIV Cost and Utilization Study (HCSUS), $85 \%$ responded that spirituality was "somewhat" or "very important" to their lives. Sixtyfive percent of individuals rated the importance of religion similarly. Notably, $72 \%$ responded that they "often" or "sometimes" used religion and spirituality when making decisions; and $65 \%$ responded that they use religion and spirituality when they confront problems. While important to HIV-infected patients, religious and spiritual involvement has also been tightly correlated with improved mood, less depressive symptoms, greater well-being, and less self-blame. While religious and spiritual involvement may correlate with improved emotional wellness, an important question remains: does religious involvement make a difference in HIV clinical outcomes? Several studies have shown that religious involvement is associated with reduced mortality among the general population, those with cardiovascular disease, and potentially those diagnosed with cancer. Among patients with HIV, it is not clear whether religious and spiritual involvement confer the same benefit? More specifically, the association between religion and spirituality with tcell counts, viral load, mortality, and other clinical outcomes is unknown. This systemic review of the literature seeks to address this important question.

METHODS: A systematic literature review was conducted for all English language articles published between 1980 and 2015 in relevant databases, including Medline (medicine), ATLA (religion), psychology (PSYCHinfo), CINAHL (nursing), and Sociological Abstracts (sociology), and the Cochrane Central Register of Controlled Clinical Trials. Search terms included: spirituality, religion, HIV/AIDS, t-cells, CD4 cells, viral load, outcomes, morbidity, and mortality. We included studies that reported on the association a between a measure of religion or spirituality and a measurable clinical or biological outcome.

RESULTS: Five hundred and forty-eight studies were evaluated. Seventeen met our inclusion criteria. The median number of enrolled individuals was 147 with a range of 331138. Twelve (71\%) studies used cohort or convenience samples. 4 (24\%) were longitudinal. One study ( $6 \%$ ) evaluated an intervention. The heterogeneity of the studies precluded aggregate analysis. Thirteen ( $76 \%$ ) studies demonstrated a positive association between religion or spirituality and a clinical HIV outcome, including lower viral loads, greater T cell counts, delayed HIV-disease progression of illness, and better overall physical health. Two (12\%) studies failed to detect such an association and $2(12 \%)$ demonstrated a negative association between religion or spirituality and a clinical HIV outcome.

CONCLUSIONS: Most studies demonstrate a positive association between measures of religion or spirituality and HIV clinical outcomes. Recognizing the religious or spiritual commitments of patients may serve as an important variable in patient care. Large longitudinal studies and interventions are warranted to further clarify the potential impact of religion and spirituality on HIV clinical outcomes.

\section{RESEARCH PARTICIPANTS DO NOT HAVE LONG TERM COMPREHENSION}

OF THE NFORMED CONSENT Leonardo Tamariz; Jimmy S. Rivadeneira; Pamela Vazquez; Ana M. Palacio. University of Miami, Miami, FL. (Control ID \#2470127)

BACKGROUND: Informed consent is key to ethical research. The lack of comprehension right after the informed consent is well documented particularly in subjects with low literacy. The aim of our study was to evaluate the long term comprehension of a clinical trial informed consent and the role of health literacy on long term comprehension.

METHODS: We conducted a prospective cohort study at the Miami VA Healthcare system in a multicenter hypertension clinical trial. We measured informed consent comprehension using 
the brief informed consent intervention protocol (BICEP-II), a higher score indicates lack of comprehension. We measured health litreacy using a validated 3 item questionaire. We calculated the beta coefficient of the BICEP score for each variable.

RESULTS: We included 87 hypertensive Veterans participating in a clinical trial with a mean age of $61.7+/-7.6$ of whom $69 \%$ were minority and $23 \%$ had low literacy. The BICEP score was $12.9+/-1.7$ after $45.7+/-7.0$ months of participating in the clinical trial. Fifty-two percent of research participants did not think the research study voluntary, $98 \%$ thought there was direct or indirect benefit to the research participants and $79 \%$ of participants thought there was no risk involved in participating, Health literacy was associated with lower comprehension (beta coefficient $-0.7 ; 95 \% \mathrm{CI}-1.5$ to -0.5 ) $p=0.03$. Age, gender, minority status, employment and education were not significant $(p>0.05)$

CONCLUSIONS: Lack of comprehension of a clinical trial informed consent is prevalent even after participating in the study for a long time. Therapuetic misconception is a common finding. Health literacy is a predictor of this lack of comprehension. There is a clear need for continued education of research participants

RESIDENT COPING MECHANISMS: STATE OR TRAIT? Brielle Spataro ${ }^{1}$; Sarah A. Tilstra ${ }^{4}$; Doris Rubio ${ }^{2}$; Melissa McNeil ${ }^{3} .{ }^{1}$ UPMC Presbyterian Hospital, Pittsburgh, PA; ${ }^{2}$ University of Pittsburgh, Pittsburgh, PA; ${ }^{3}$ University of Pittsburgh Medical Center, Pittsburgh, PA; ${ }^{4}$ University of Pittsburgh School of Medicine/Medical Center, Pittsburgh, PA. (Control ID \#2463084)

BACKGROUND: Physician burnout is a syndrome characterized by emotional exhaustion, depersonalization, and a low sense of accomplishment. Burnout is a response to chronic emotional and interpersonal stressors. While a significant amount of research has been conducted in the last 10 years regarding physician burnout, little headway has been made in preventing or combating this phenomenon. Coping mechanisms are cognitive and behavioral efforts that people use to deal with stress. They can be loosely grouped into "adaptive" or "maladaptive" based on their usefulness in a given situation. Data show that use of specific coping mechanisms is associated with burnout and that coping can differ by sex. However, little is known regarding the general coping patterns of physicians. We hypothesize that in order to fully understand physician burnout, we must first start by understanding physician coping mechanisms. The objectives of our study were to determine the coping mechanisms used by internal medicine residents during residency. We hypothesized that use of these coping mechanisms would change throughout residency and that there would be differences in coping mechanisms used by residents based on sex, race and international graduate status. METHODS: We performed a retrospective longitudinal analysis of coping mechanisms used by internal medicine residents at a large academic facility. The residents were surveyed using the Brief Cope, a 28-item instrument measuring the frequency of 14 different coping mechanisms on a 4-point Likert-type scale. The Brief COPE is widely cited in the literature and has good reliability in many different populations. The survey is first administered prior to the intern year in June, before any clinical time, and annually thereafter. We analyzed 818 surveys from 377 different residents. The survey items that measured a specific coping mechanism were combined to provide frequency of use data for specific coping mechanisms on a 2-8 point scale. Mean frequency of use was calculated for each coping mechanism based on post-graduate year (PGY). To assess how coping mechanisms changed throughout residency, we compared results from the new intern assessment taken prior to starting residency to those at the end of PGY-1, PGY-2 and PGY-3 year. We calculated significant changes from the new intern assessment using a mixed effects ordered logistic regression model. Models controlled for covariates that were potentially associated with coping mechanisms, including sex, race, and international graduate status. To assess whether the changes were effected by sex, race (white v. nonwhite) and international graduate status, we analyzed interactions between PGY and those variables.

RESULTS: Out of 494 residents from 2010 to 2015, 377 (76\%) completed the survey. One hundred eighty-eight were male (50\%), 267 (70\%) were white, and $346(92 \%)$ were domestic medical school graduates. Of the 818 surveys, 236 new intern assessments, 195 PGY-1, 197 PGY-2 and 190 PGY-3 surveys were analyzed. Using the incoming intern assessment as a baseline, the frequency of use of acceptance $(p<0.001)$, planning ( $p<0.001)$, positive reframing $(p<0.001)$, use of emotional support $(p=0.013)$, use of instrumental support $(p=0.020)$, self blame $(p=0.002)$ and humor $(p=0.002)$ all significantly decreased during residency. The frequency of use of active coping, denial, and religion decreased but returned to baseline at the PGY-3 assessment. After controlling for PGY level, race, and international graduate status, female residents used acceptance ( $p=$ 0.02 ), active coping ( $p=0.043$ ), positive reframing ( $p<0.001)$, use of emotional support $(p<0.001)$, use of instrumental support $(p=0.001)$, self-distraction $(p=0.046)$ and venting $(p<0.001)$ more than their male counterparts. Male residents used humor more frequently $(p=0.032)$. Non-white residents used denial more frequently $(p=0.011)$ and international medical graduates reported less frequent use of emotional support ( $p=$ $0.045)$, humor $(p=0.004)$ and venting $(p=0.048)$. There were no differences in how coping changed over time when results were stratified by sex or international medical graduate status when controlling for all other variables. Race had a significant effect on use of positive reframing $(p=0.0341)$ throughout residency as non white residents had a more significant change from the intern assessment $(-0.70,-1.29$ and -1.41$)$ when compared to white residents ( $-0.83,-0.88$ and -0.47$)$ (PGY-1, PGY-2 and PGY-3 respectively).

CONCLUSIONS: Our data show that the frequency of use of coping mechanisms changes during residency. Use of all but one adaptive coping mechanism significantly decreased throughout residency. The use of maladaptive coping mechanisms were not substantially affected except for self-blame which significantly decreased. These changes do not seem to be affected by sex or international medical graduates but non-white residents did have greater changes in positive reframing. During residency, trainees are using adaptive coping mechanisms less frequently, which should beg the question, how are they dealing with their stress? It is unclear whether a decrease in these coping skills sets the stage for burnout, or if burnout contributes to poor coping once it has set in. More research is needed to further delineate the link between burnout and coping. However, educators should be aware of these changes as they provide guidance to residents or target solutions for wellness.

RESIDENT EDUCATION OF RIGHTCARE RIGHT NOW Jennifer M. Schmidt ${ }^{1,2}$; Arun K. Singavi ${ }^{1}$; Shivtej Kaushal ${ }^{1}$; Rochelle Herre ${ }^{1}$; Ryan Tomlinson ${ }^{1} .{ }^{1}$ Medical College of Wisconsin, West Allis, WI; ${ }^{2}$ Clement J Zablocki VA Medical Center, Milwaukee, WI. (Control ID \#2469673)

BACKGROUND: Medical overuse is a growing problem in the United States, totaling over one trillion dollars in 2014. The RightCare Educator program offers grants to chief residents to develop educational initiatives for residents on mindful care. Using this grant, we created a week-long "RightCare" program for Internal Medicine residents aimed at increasing awareness and knowledge of medical overuse.

METHODS: At an academic Internal Medicine program with two primary training sites, multiple study interventions were utilized. An initial presentation introduced basic concepts and definitions of "RightCare". Further interventions included an overuse count, Do No Harm report, daily emails, clinical vignette contest and reception with gift card incentives for participation. Knowledge and awareness of overuse/costs were assessed with a survey before and after the intervention using multiple choice questions and Likert scale questions with anchors $1=$ never- $6=$ always. Analysis included descriptive and univariate analysis with 2 tailed tests with significance at $p<0.05$. Ward residents were analyzed separately. RESULTS: $8 / 8(100 \%)$ teams at hospital one and 3/9 (33\%) teams at hospital two participated in the overuse count with 24 and 12 counts submitted respectively. Nineteen clinical vignettes were submitted across the two hospitals. For both overall and ward-only analysis, a statistically significant difference in correct answers to $1 / 3$ knowledge questions was seen. No statistically significant difference was seen in the awareness assessment questions. 
CONCLUSIONS: The purpose of our study was to educate residents on proper utilization of care. In both pre and post surveys, the preponderance of answers showed greater than occasional consideration of cost to both patient and healthcare system in resident decisionmaking. This shows that residents already had some awareness of "RightCare" and a 1 week introductory initiative alone is insufficient to influence resident practice significantly. Additionally, residents responded that there was rarely discussion of costs with attending staff, both pre and post-intervention. Taking both of these ideas together, we feel that a cultural shift toward mindful care is necessary. Future plans include continued discussion of RightCare during daily didactics and through residency social media with a follow-up awareness survey.

\section{RESIDENT PHYSICIANS' MANAGEMENT OF BACK PAIN IN AN UNAN-} NOUNCED STANDARDIZED PATIENT VISIT: VICODIN- VS. NON-VICODIN PRESCRIBERS Sondra Zabar; Kathleen Hanley; Hillary Lee; Irina Gershgorin; Colleen C. Gillespie. NYU School of Medicine, New York, NY. (Control ID \#2470180)

BACKGROUND: Use of opioids for management of back pain is a controversial issue in light of mounting evidence of an epidemic of opioid use disorders fueled by over prescribing of prescription opioids. We designed and fielded an Unannounced Standardized Patient case involving vicodin use for a chief complaint of back pain to understand how our primary care and internal medicine residents managed care in such cases. We also wanted to explore how residents' choices in managing pain might be associated with their core clinical skills, focusing especially on communication, assessment, and patient education skills.

METHODS: Forty-eight Unannounced Standardized Patient visits involving a patient with re-emergence of back pain and who was self-treating with Vicodin were delivered to primary care and internal medicine residents in two urban safety net primary care clinics. Highly trained SPs portrayed this case consistently and then completed a comprehensive behaviorally anchored assessment of the competence of the resident physician in the core domains of communication, information gathering, patient education, physical exam, treatment plan, and patient centeredness and activation. Summary scores were calculated for each domain as percent of items rated well done. USPs also recorded the prescriptions and recommended dosages and as part of a systematic chart review, the residents' prescriptions as represented in the chart were also noted to triangulate the accuracy of the USP report. The core question we explored was whether residents who chose to prescribe Vicodin were different from those who did not prescribe Vicodin in terms of their communication skills, specific assessment behaviors, patient education practices, back pain physical exam, and treatment plan and referral patterns.

RESULTS: Nine of the 48 residents prescribed Vicodin. Of the 39 residents who did not prescribe Vicodin, most (63\%) prescribed motrin (400-600 mg or not specified) and the rest prescribed some combination of Tylenol and Aleve. Eleven percent also prescribed a muscle relaxer. One hundred percent of the Vicodin prescribers also ordered physical therapy compared to $74 \%$ of non-vicodin prescribers. Overall, Vicodin prescribers had significantly higher communication scores than non-vicodin prescribers in relationship development ( 80 vs. $57 \%$ well done, $p=.05$ ) and patient education and counseling (59 vs. $31 \%$ well done, $p=.02$ ). Assessment summary scores were also higher ( 51 vs. $38 \%$ ) but not significantly $(p=.07)$ and more Vicodin than non-Vicodin prescribers assessed pain treatment (100 vs. $50 \%$ ), asked about previous Vicodin use (50 vs. $5 \%$ ) and asked about previous imaging (100 vs $42 \%$ ). Vicodin and non-vicodin prescribers were similar in terms of percent providing the patient with a pain management plan (100 vs. $82 \%$ ) and appropriate referrals (44\% for both). Both groups of prescribers also received similar scores in back pain physical exam (Vicodin prescribers $=50 \%$ done compared to $42 \%$ of non-Vicodin prescribers). None of the residents in either group of prescribers fully explained about medication safety (Tylenol/Vicodin interactions).

CONCLUSIONS: Just under $20 \%$ of residents prescribed Vicodin in a case where a new patient reported effective pain relief using Vicodin. These residents demonstrated better communication and assessment skills than the residents who did not prescribe Vicodin, asking more questions and more complete questions about pain treatment and previous medication use and imaging, all while developing a positive relationship with the patient. Residents managed this case similarly regardless of whether or not they prescribed Vicodin. Results suggest that residents may be hesitant to prescribe Vicodin when they either don't feel confident in their assessment and rapport building or lacked the time to effectively assess the situation and build a positive relationship with the patient. Future research should explore physicians' decision-making process in considering opioids for helping manage patients' back pain.

RETENTION IN BUPRENORPHINE MAINTENANCE TREATMENT IS ASSOCIATED WITH LOWER RISK OF INCARCERATION AMONG HIV PATIENTS Aaron D. Fox ${ }^{1,2}$; Daniel P. Riggins ${ }^{2}$; Yuming Ning ${ }^{1}$; Chinazo Cunningham ${ }^{1}$, ${ }^{2}$. ${ }^{1}$ Montefiore Medical Center, Bronx, NY; ${ }^{2}$ Albert Einstein College of Medicine, Bronx, NY. (Control ID \#2464044)

BACKGROUND: Opioid use disorder has become epidemic in the United States and up to one-third of heroin users (200,000 individuals) are incarcerated annually. Because incarceration is destabilizing and increases the risk for opioid overdose upon release, prevention of incarceration is an important treatment goal for opioid use disorder. Both incarceration and opioid use disorder disproportionately affect people living with HIV/AIDS, making this an important population to target with interventions. Buprenorphine maintenance treatment (BMT) is a safe and effective pharmacotherapy for opioid use disorder that may be offered in HIV and primary care settings; however, studies have been conflicting as to whether BMT reduces incarceration risk. Therefore, we investigated whether retention in BMT was associated with incarceration risk in a multi-site longitudinal cohort study.

METHODS: We analyzed data from the Buprenorphine-HIV Evaluation and Support (BHIVES) collaborative, which studied implementation of BMT in HIV primary care settings in 10 different sites across the United States. Between 2004 and 2009, subjects entered the cohort upon initiating BMT and were followed for one year. Study visits were conducted at baseline and then quarterly. Data collection was standardized across sites and included questionnaires and medical record extraction. For this analysis, the main independent variable was retention in BMT. Subjects who received at least one prescription for buprenorphine during a quarter were defined as being retained for that quarter. The dependent variable was self-reported incarceration (i.e., $\geq 1$ day in jail or prison within the 90 days preceding a study visit). We compared incarceration during quarters when subjects were and were not retained in BMT using generalized estimating equations to account for clustering of study visits by individual. Because subjects could have stopped BMT and then restarted in subsequent quarters, we analyzed retention by quarter. We also adjusted for covariates associated with the independent variable $(p<0.10)$ in bivariate testing. Our final model included type of opioid use (heroin vs. opioid analgesics), addiction severity, mental illness, gender, and history of prior incarceration. RESULTS: Of 306 subjects, most were Black (51\%) or Hispanic (23\%), male (67\%), and unemployed (74 \%). Homelessness (25\%), mental illness (55\%), and prior injection drug use (79\%) were common. At baseline, $79 \%$ had ever been incarcerated and $13 \%$ had been incarcerated in the prior 30 days. Following BMT initiation, $74 \%, 66 \%, 58 \%$, and $48 \%$ were retained in BMT through quarters one, two, three, and four respectively. Four percent, $9 \%, 4 \%$, and $7 \%$ of subjects reported incarceration during quarters one, two, three, and four respectively. For those reporting incarceration, the median length of incarceration was 8 days. After adjustments, retention in BMT was associated with a $63 \%$ decreased risk of incarceration $(\mathrm{OR}=0.37,95 \% \mathrm{CI}=0.19-0.70)$. Addiction severity $(\mathrm{OR}=1.03,1.00-1.06)$, male gender $(\mathrm{OR}=2.18,1.01-4.71)$, and prior incarceration $(\mathrm{OR}=2.70,1.06-6.87)$ were also significantly associated with risk of incarceration. CONCLUSIONS: Retention in BMT was strongly associated with lower risk of incarceration in this national cohort of HIV positive individuals. Although our study design cannot determine causality, the direction of the association is likely that retention in BMT reduced incarceration. BMT is known to reduce illicit opioid use and overdose, but this 
study is the first to demonstrate a reduction in incarceration during BMT in a treatmentseeking HIV or primary care population. BMT should be considered for all individuals with opioid use disorder, especially those at risk for incarceration.

RISK FACTORS FOR SINGLE AND RECURRENT URINARY TRACT INFEC TIONS IN KIDNEY TRANSPLANT RECIPIENTS Asaad Nakhle; Kassem Bourgi; Zaid Abdelrahman; George Alangaden. Henry Ford Hospital, Detroit, MI. (Control ID \#2455692)

BACKGROUND: Urinary tract infection (UTI) is the most common infection after kidney transplantation (KT). Previous studies have linked UTI in the first year of transplant to adverse transplant outcomes, however results are incongruent regarding risk factors associated with incidence and recurrence of symptomatic UTIs in this patient population.

METHODS: We constructed a retrospective cohort of patients who underwent KT at Henry Ford Health System between 01/2012 and 12/2013. Patients with symptomatic single and recurrent UTI within the first year of KT were identified. Recurrent infection was defined as having at least 2 UTIs in 6 months or 3 UTIs in 1 year. Data elements including demographic information, medical comorbidities, and transplant variables were assessed for association with single and recurrent UTIs.

RESULTS: One hundred ninety patients underwent KT within the study period. After excluding subjects with asymptomatic bacteriuria, a total of 36 patients (19\%) developed a UTI within the first year, 18 of whom had recurrent UTIs. Factors associated with developing UTI (single or recurrent) were female gender ${ }^{\partial}$ and history of previous $\mathrm{KT}^{*}$. Patients with recurrent UTIs were significantly more likely to be diabetic ${ }^{*}$ and to have higher comorbidity index ${ }^{*}$ when compared to subjects with a single UTI. KT recipients with recurrent UTI had significantly higher incidence of adverse renal outcomes, defined as an increase in serum creatinine by $50 \%$ during the first year of transplant ${ }^{*}$. They were also more likely to have an abnormality on urological ultrasound, though this difference was not statistically significant. Interestingly, there was a significant association" between isolation of Klebsiella pneumoniae in the initial urine culture and the likelihood of recurrent UTI

CONCLUSIONS: UTI is a common problem after KT. It is associated with a high likelihood of recurrence and results in adverse renal outcomes. Diabetes, female gender, and a previous kidney transplant are all predisposing factors for development of single UTI. Recurrece is more likely when the first episode was caused by Klebisella pneumoniae. ${ }^{*} p$-value $<0.05 ;{ }^{\partial} p$-value $<0.001$

Table 1: Comparison of risk factors associated with UTI within first year of transplant

\begin{tabular}{|c|c|c|c|c|c|}
\hline - & Total & Controls & Patients with UTI & $P$-Value & \\
\hline Number of Subjects & 190 & 154 & 36 & . & \\
\hline Recipient Age (mean) & 53.7 & 53.4 & 53.8 & 0.89 & \\
\hline \multirow[t]{2}{*}{ Gender } & Female & 69 & 48 (31\%) & $21(58 \%)$ & $<0.001$ \\
\hline & Male & 121 & $106(69 \%)$ & $15(42 \%)$ & \\
\hline \multirow[t]{3}{*}{ BMI } & $<25$ & 53 & $44(29 \%)$ & $9(25 \%)$ & 0.51 \\
\hline & $25-30$ & 63 & 48 (31\%) & $15(42 \%)$ & \\
\hline & $>30$ & 72 & $60(39 \%)$ & $12(33 \%)$ & \\
\hline Diabetes Mellitus & 92 & $74(48 \%)$ & $18(50 \%)$ & 0.83 & \\
\hline \multirow[t]{5}{*}{ Reason for Transplant } & Diabetic Nephropathy & 60 & 45 (29 \%) & $15(42 \%)$ & 0.07 \\
\hline & Glomerulonephritis & 43 & $31(20 \%)$ & $12(33 \%)$ & \\
\hline & Structural Kidney Disease & 21 & $18(12 \%)$ & $3(8 \%)$ & \\
\hline & Hypertension & 42 & $39(25 \%)$ & $3(8 \%)$ & \\
\hline & Other & 23 & $20(13 \%)$ & $3(8 \%)$ & \\
\hline Concurrent Transplant & 22 & $16(10 \%)$ & $6(17 \%)$ & 0.29 & \\
\hline History of Previous Transplant & 15 & $9(6 \%)$ & $6(17 \%)$ & 0.03 & \\
\hline Donor Age (mean) & 37.4 & 38.6 & 37.2 & 0.61 & \\
\hline \multirow[t]{2}{*}{ Donor Type } & Living & 67 & $58(38 \%)$ & $9(25 \%)$ & 0.15 \\
\hline & Deceased & 123 & $96(62 \%)$ & $27(75 \%)$ & \\
\hline Renal Failure Within First Year & 30 & $21(14 \%)$ & $9(25 \%)$ & 0.18 & \\
\hline CMV Viremia Within First Year & 25 & $20(13 \%)$ & $5(14 \%)$ & 0.9 & \\
\hline BK Viremia Within First Year & 38 & $35(23 \%)$ & $3(8 \%)$ & 0.05 & \\
\hline Double J-Stent & 103 & $81(53 \%)$ & $22(61 \%)$ & 0.35 & \\
\hline
\end{tabular}

Table 2: Comparison of risk factors associated with single vs. recurrent UTI within first year of transplant

\begin{tabular}{lllll}
\hline \hline & Total & Single UTI & Recurrent UTI & $p$-value \\
Recipient Age (mean) & 53.4 & 49.9 & 56.9 & 0.15 \\
Donor Age (mean) & 38.6 & 38.9 & 38.4 & 0.91 \\
Female Gender & 21 & $13(72 \%)$ & $8(44 \%)$ & 0.09 \\
DM & 18 & $5(27 \%)$ & $13(72 \%)$ & 0.007 \\
Renal Failure & 9 & $2(11 \%)$ & $7(39 \%)$ & 0.04 \\
Klebsiella Pneumonia Infection & 6 & $1(6 \%)$ & $5(28 \%)$ & 0.03 \\
Abnormal Ultrasound & 24 & $5(28 \%)$ & $10(56 \%)$ & 0.09 \\
Double J-Stent & 22 & $14(78 \%)$ & $8(44 \%)$ & 0.04 \\
\end{tabular}

RISK OF THROMBOEMBOLISM WITH USE OF HORMONAL CONTRACEPTION IN DIABETES Sarah H. O'Brien ${ }^{1,2}$; Terah $\mathrm{Koch}^{2}$; Sara Vesely ${ }^{3}$; E. Bimla Schwarz ${ }^{4} .{ }^{1}$ The Research Institute at Nationwide Children's
Hospital, Columbus, OH, Columbus, OH; ${ }^{2}$ Nationwide Children's Hospital, Columbus, OH; ${ }^{3}$ University of Oklahoma Health Sciences Center, Oklahoma City, OK; ${ }^{4}$ University of California, Davis, Sacramento, CA. (Control ID \#2468862)

BACKGROUND: A growing number of women of childbearing potential have been diagnosed with diabetes mellitus (DM). Nearly two-thirds of pregnancies conceived by women with DM are unplanned, due at least in part to the fact that women with DM are less likely than women without DM to receive contraceptive counseling or use contraception. One barrier to prescribing hormonal contraception to women with DM is the risk of thromboembolic (TE) complications, which may exacerbate underlying vascular disease resulting from DM. As most prior epidemiologic studies of TE complications of hormonal contraception have excluded women with conditions known to increase risk of TE, such as DM, we investigated the safety of use of hormonal contraceptives by women with DM. 
METHODS: We examined contraceptive claims filed from 2002 to 2011 in the Clinformatics Data Mart, which contains medical claims, outpatient pharmacy claims, lab results, hospitalizations, standard pricing, coverage dates for members, and demographic information on an annual average of 15 million individuals from all 50 states with commercial health plans. Over this 10- year study, 47 million unique individuals contributed information to the Clinformatics database. Inpatient and outpatient services are coded with CTP-4 or HCPCS procedure codes; diagnoses with ICD-9-CM diagnoses codes. All claims include the dates of service. Outpatient pharmacy data include NDC codes, drug brand names, generic classifications, quantity, days supplied, and date dispensed. Postpartum women were removed from analyses for 3 months after delivery. We identified 146,080 unique females aged $14-44$ years who contributed 478,650 women-years of observation who had at least one ICD-9 code for diabetes (250.xx) and a prescription for a diabetes medication or device. We compared time to TE (venous thrombosis, stroke, or myocardial infarction) among women with diabetes who were dispensed progestin-only, combination, or no hormonal contraception using a modification of the Cox proportional hazards which allowed for time-dependent covariates (i.e. changes in contraceptive use). RESULTS: Only $29 \%$ of reproductive-aged women with DM had any claims for hormonal contraception (prescriptions or devices), the majority of whom were prescribed estrogen-containing oral contraceptives. We identified 3012 total thrombotic events in our study population, producing a rate of 6.3 thrombotic events per 1000 woman years of observation. In bivariate analyses, both estrogen and progestin-only products were associated with a modestly increased risk (hazard ratio of 1.87 and 1.54 , respectively) of TE compared to no hormonal contraception, ( $p<0.001$ for both hazard ratios), although the absolute risk of TE among diabetic women using either form of hormonal contraception remained less than 11 per 1000 woman-years of use. The hazard ratio comparing any TE for progestin-only contraception to estrogen-containing products was 0.82 but was not statistically significant $(p=0.08)$. As women age, their overall risk of TE increases, however, the impact of contraception on the risk of TE decreases; risk of TE in women with diabetes $\leq 30$ years of age (with estrogen, $\mathrm{HR}=3.24,95 \% \mathrm{CI}=2.71-3.88$, with progestin-only, $\mathrm{HR}=1.86,95 \% \mathrm{CI}=1.23-2.81$, as compared to similarly-aged women with diabetes who were not using hormonal contraception), decreased to a non-significant relationship among women with diabetes $>40$ years of age (with estrogen, $\mathrm{HR}=1.20$, $95 \% \mathrm{CI}=0.93-1.56$, with progestin-only $\mathrm{HR}=1.11,95 \% \mathrm{CI}=0.64-1.92)$. The lowest rates of TE were seen among women with diabetes who used intrauterine ( $n=2730$ users, 5036 women-years, 17 with TE, rate $=6$ per 1000) and sub-dermal $(n=124$ users, 163 women-years, 0 with TE) contraceptives

CONCLUSIONS: The absolute risk of TE among women with DM using hormonal contraception is low, with fewer than 11 events per 1000 woman years of use. The contraceptives least likely to be associated with TE when used by women with diabetes are the intrauterine and sub-dermal contraceptives. These highly effective reversible contraceptives are excellent options for women with diabetes who hope to avoid the teratogenic effects of hyperglycemia by carefully planning their pregnancies. This work was supported by the American Diabetes Association grant \#1-13-CE-31

\section{RISK STRATIFICATION OF CHEST PAIN PATIENTS USING THE CALCIUM} SCORE Xiaoming Jia ${ }^{1,3}$; Victor Huang ${ }^{3}$; David E. Winchester ${ }^{2} .{ }^{1}$ Baylor College of Medicine, Houston, TX; ${ }^{2}$ University of Florida, Gainesville, FL; ${ }^{3}$ University Florida College of Medicine, Gainesville, FL. (Control ID \#2469504)

BACKGROUND: One of the most important steps in the work-up of chest pain in the emergency room is to distinguish between high-risk patients who warrant inpatient evaluations and those at lower risk who may only need observation in a chest pain evaluation center or who can be discharged from the emergency department outright. Methods to identify this low risk group, however, must be objective and accurate as the consequences of false negatives are significant. One such strategy that is being studied is the calcium score. Here, we examine how calcium score correlates with findings on coronary computer tomography angiogram (CCTA) and on left heart catheterization. We further sought to determine the threshold at which a calcium score can be used as a negative predictor for major adverse cardiac events (MACE).

METHODS: The study design is a retrospective chart review of consecutive patients seen in the chest pain evaluation center of an academic tertiary care center within 1 year, between January 1, 2012 and December 31, 2012. Data from the electronic medical record was gathered by trained study personnel. When examining the relationship between calcium score and CCTA results, a point bi-serial correlation was employed. Linear regression analysis was used to evaluate the relationship between calcium score and coronary artery lesions found by left heart catheterization. Meanwhile, a logistic regression model was used to examine the relationship between calcium score and MACE.

RESULTS: The database contained 1016 patients with complete records, of which 467 had undergone testing for calcium score and CCTA. Of these patients, 172 had lesions found on CCTA, 74 required further evaluation by coronary angiography and 49 had lesions seen on cath. Overall, 18 patients had a MACE within 1 year of admission. The correlation coefficient for calcium score and CCTA was $0.389,95 \%$ confidence interval $[0.309,0.463]$. The correlation coefficient of calcium score and lesions seen on coronary angiography was found to be $0.121,95 \%$ confidence interval $[-0.169,0.392]$. Meanwhile, logistic regression of calcium score and MACE resulted in an odds ratio of 1.002, $95 \%$ confidence interval [1.001, 1.003].

CONCLUSIONS: Based on our study, we found that there is a significant correlation between elevated calcium score and some degree of disease based on CCTA. However, elevated calcium score did not correlate significantly with lesions in the coronary arteries as confirmed by left heart catheterization nor was it a good predictor MACE. Therefore, the results of this study suggest that calcium score cannot be used alone to risk stratify patients for discharge without additional testing. More robust studies encompassing multiple centers as well as prospective studies should be undertaken to further explore this topic.

ROLE OF STATINS IN DECREASING THE RISK OF CONTRAST INDUCED NEPHROPATHY (CIN) IN PATIENTS WITH DIABETES AND/OR CHRONIC KIDNEY DISEASE WHO ARE UNDERGOING CORONARY ANGIOGRAM. $\underline{\text { Sai Prasad Gadapa }}{ }^{1}$; sree V. pilla ${ }^{3}$; Siwadon Pitukweerakul ${ }^{2}$; Daniel Hart ${ }^{4}$; Harvey J. Friedman ${ }^{4}{ }^{1}$ Presence Saint Francis Hospital, Evanston, IL; ${ }^{2}$ Presence St. Francis Hospital, Evanston, IL; ${ }^{3} \mathrm{St}$ Francis Hospital, EVANSTON, IL; ${ }^{4} \mathrm{St}$ Francis Hospital, Evanston, IL. (Control ID \#2468629)

BACKGROUND: Five percent of patients undergoing coronary angiogram develop CIN, risk is even higher in patients with CKD and diabetes. Although, CIN is generally reversible with no permanent sequelae, there is some evidence that its development is associated with adverse outcomes. In patients with chronic kidney disease who develop an episode of AKI, there is an increase in the risk of progression of CKD. The proposed pathophysiology based on animal models is renal vasoconstriction and tubular injury by direct cytotoxic effect and generation of oxygen free radicals. Statins improve endothelial function; reduce arterial stiffness and therefore have anti-inflammatory activity. No definitive treatment is available for contrast nephropathy, prevention is the best strategy. The study objective is to demonstrate the protective effect of statins in contrast induced nephropathy (CIN) in high risk patients.

METHODS: Study design: Retrospective case-control study. Study population: 1216 patients who were admitted at Presence Saint Francis Hospital and underwent coronary angiogram during the time period June 2013 and June 2015. Cases: Patients with CIN. Controls: Patients without CIN. Exposure: Statins. Non-exposure: No statins. CIN inclusion 
criteria: Oliguria/non-oliguria, rise in creatinine $\geq 0.5 \mathrm{mg} / \mathrm{dL}$ or $25 \%$ above baseline within 24-72 h of contrast exposure with concurrent decrease in GFR, FENA $<1 \%$ and urine analysis showing muddy brown granular casts and epithelial cell casts in CKD and/or DM patients. CIN exclusion criteria: Other causes of AKI, UA showing findings suggestive of interstitial nephritis or glomerular disease and patients with insufficient data. Method of data collection: Examine medical records of all the patients who had received normal saline $0.9 \%$ $1 \mathrm{ml} / \mathrm{kg}$ body weight $6-12 \mathrm{~h}$ before the procedure and $6-12 \mathrm{~h}$ after the procedure and identify patients who developed CIN based on above inclusion criteria and exclusion criteria. Medication list of study subjects was then reviewed to identify if they were on statin therapy or not. Out of 1216 patients, 82 patients met the above criteria in which 15 patients had CIN and 67 did not develop CIN. Limitations of study: It is an unmatched case control study. Since it is a retrospective study design, it cannot be determined whether patients were adherant with statin therapy. Further sub group analysis needs to be done if $\mathrm{CIN}$ was more common among older subjects. N-Acetyl cysteine used in some patients could have conferred further renal protection. Strengths of study: Our results are consistent with previous studies. The advantage of this data is that it demonstrates protective effects with statins at any dose. Recollection bias is eleminated by using electronic medical records. Since we examined data of patients who were admitted at our hospital, it ensured that we obtained a random sample from the community. Analysis of the data: Gathered data was analyzed in the database using SPSS software V.23 to calculate the odds ratio and detect the statistical significance.

RESULTS: Out of 63 patients who were on statins 7 (11.1\%) had CIN and $56(88.9 \%)$ had no CIN. Among 19 patients who are not on statins 8 (42.1\%) had CIN and 11 (57.9) had no CIN. Patients who are on statins are less likely to develop contrast induced nephropathy than patients who are not on statins (odds ratio, 0.17; $95 \%$ confidence interval [CI], 0.05 to 0.57 ). $\mathrm{P}$ value is 0.0041 .

CONCLUSIONS: Statins decrease the risk of developing contrast induced nephropathy in diabetic and/or chronic kidney disease patients.

\section{SAD WHITE BLOOD CELLS? THE ASSOCIATION OF MAJOR DEPRESSIVE DISORDER AND MORTALITY IN OLDER VETERANS HOSPITALIZED FOR PNEUMONIA Ami DeWaters ${ }^{1}$; Eric Mortensen ${ }^{2} .{ }^{1}$ University of Texas Southwestern Medical Center, Dallas, TX; ${ }^{2}$ VANTHCS, Dallas, TX. (Control ID \#2461425)}

BACKGROUND: Major depressive disorder has been identified as an independent risk factor for mortality for many comorbid conditions, such as heart failure, cancer and coronary artery disease. In addition, major depressive disorder has been linked to immune dysregulation and suppression by generating a chronic inflammatory state. Despite this, the association between major depression and one of the most common global fatal infections, pneumonia, has not been examined. The aim of this study was to examine the association between major depressive disorder and outcomes, including mortality within 30 and 90 days and ICU admission in patients hospitalized with pneumonia. METHODS: We conducted a retrospective national study using administrative data of patients hospitalized at any Veterans Administration acute care hospital. We included patients greater than 65 years old hospitalized with pneumonia in fiscal years 2002-2012. Major depressive disorder was defined using ICD-9 codes from data that was collected in the 12 months prior to admission. We used generalized linear mixed effect models to examine the association of major depressive disorder with the outcomes of interest after controlling for potential confounders, including sociodemographics (age, race, gender, marital status, and priority status), comorbid conditions based on a Charlson comorbidity score, outpatient health care utilization in the 12 months prior to admission, severity of illness, and the admitting hospital.

RESULTS: Of 103,997 patients who met the inclusion criteria, 9247 carried a diagnosis of major depressive disorder. The general characteristics of the entire cohort was $98 \%$ men, $82.9 \%$ white, with a mean age of 77.8 years (standard deviation of 7.4 ) and a mean
Charlson comorbidity score of 3.2 (standard deviation of 2.6). In the univariate analyses, mortality at 30-days was $14.7 \%$ in those with depression versus $14.3 \%$ in those without ( $p=0.25$ ), and 90 -day mortality was $23.9 \%$ in those with depression vs. $23.1 \%$ in those without $(p=0.07)$. In the multilevel models, after adjusting for potential confounders, patients with depression had a significantly higher 30-day (odds ratio, 1.22; $95 \%$ confidence interval 1.15-1.29) and 90-day (odds ratio, 1.20; $95 \%$ confidence interval 1.12-1.29) mortality. However, patients with major depressive disorder had lower rates of admission to the ICU (odds ratio, 0.72; $95 \%$ confidence interval 0.66-0.78).

CONCLUSIONS: For older veterans hospitalized with pneumonia, a concurrent diagnosis of major depressive disorder was associated with high mortality. It is possible that the decreased probability of admission to the ICU contributed to the increased mortality. This study highlights that major depressive disorder is potentially an independent risk factor for mortality for older adults hospitalized with pneumonia. Clinicians must be aware of the increased risk associated with major depressive disorder so that appropriate care can be ensured for these high-risk patients.

SAFETY NET PATIENT ATTITUDES TOWARDS CLINICIAN EHR USE: IT'S NOT JUST THE AMOUNT THAT MATTERS BUT HOW YOU USE IT Neda Ratanawongsa $^{1}$; Jennifer Barton ${ }^{2}$; Courtney Lyles ${ }^{1}$; Michael Y. Wu ${ }^{3}$; Ed Yelin ${ }^{1}$; Diana Martinez ${ }^{1}$; Dean Schillinger ${ }^{1} .{ }^{1}$ UCSF, San Francisco, CA; ${ }^{2} \mathrm{OHSU}$, Portland, OR; ${ }^{3}$ Univeristy of Hawaii, Honolulu, HI. (Control ID \#2461825)

BACKGROUND: The 2009 HITECH Act catalyzed certified electronic health record (EHR) implementation throughout U.S. safety net clinics. Given the communication challenges experienced by vulnerable patients in safety net settings, it is crucial to understand how EHR implementation affects patient-provider communication behaviors and patient satisfaction with that communication. We investigated the association between communication behaviors and patients' negative attitudes toward computer use after EHR implementation in a safety net health system.

METHODS: We conducted a mixed methods observational study at least 2 months postimplementation of a certified electronic health record system in 5 academically-affiliated U.S. public hospital clinics. We video-recorded encounters between English- or Spanishspeaking patients with diabetes, rheumatoid arthritis, and/or congestive heart failure and their primary and specialty care clinicians. We assessed the degree of computer use by summing observer ratings (Cronbach $\alpha=0.67$, score range $0-12$ ) for amount of computer data review, typing or mouse-clicking, lack of eye contact with patients, and noninteractive pauses. We assessed patient negative attitudes toward clinician computer use in structured post-visit interviews. Negative attitudes comprised responses of "yes definitely" or "yes somewhat" to: "provider's use of a computer make it harder for you to talk with him or her"; "the computer got in the way of me talking with my doctor"; "the doctor looked at the computer more than at me"; "my doctor did not look at me enough because of the computer"; and/or "my doctor did not listen carefully to me because of the computer." We assessed communication behaviors using the Roter Interaction Analysis System, coding each clinician or patient statement and summing into categories: rapportbuilding, biomedical talk (illness and therapy), or psychosocial/lifestyle talk (patient experience and life situation). Rapport building included positive (e.g., laughter or agreement), negative (e.g., criticism or disagreement), emotional (e.g., empathy or partnership), and social ("chit-chat") behaviors. Positive affect was a summation of observer ratings of emotional tone. Verbal dominance was the ratio of clinician to patient talk. For this analysis, we first described the amount of clinician computer use (0-4 low, 5-7 moderate, $8-12$ high) and the proportion of visits with negative patient attitudes in each of these categories. We then investigated the association of negative patient attitudes with specific communication behaviors, controlling for patient education and quality of life; clinician type, years in practice, and clinic; and visit length. 
RESULTS: The sample included 36 visits between 25 patients and 26 clinicians. Patients averaged 57 years in age, and 11(44\%) were women. Twelve (48\%) were Hispanic/Latino, $28 \%$ reported education below high school graduation, 6 (24\%) had limited English proficiency, and 5 (20\%) had limited health literacy. Among clinicians, $73 \%$ were women, $46 \%$ were primary care providers, and $84 \%$ were physicians. They averaged 16 years since earning degrees. The median visit length was $28 \mathrm{~min}$, and clinicians conducted $6(17 \%)$ visits in Spanish without medical interpreters and $1(3 \%)$ with interpretation. Six $(17 \%)$ encounters had low clinician computer use, $6(17 \%)$ had moderate computer use, and 24 had (67\%) high computer use. Twelve patients in 15 encounters had at least one negative attitude toward clinician computer use after the encounter. This rating was offered in exactly half of high clinician use encounters, half of moderate computer use encounters, and no low computer use encounters. Negative patient attitudes were associated with more clinician disagreement with patients $(+1.5, p=0.02)$, less positive clinician affect $(-2.9, p<0.01)$, and greater verbal dominance $(p=0.01)$.

CONCLUSIONS: In a post-EHR implementation sample, most safety net encounters involved high clinician computer use. In half of visits with moderate and high computer users, patients reported negative feelings about that computer use, and these feelings were associated with observable clinician communication behaviors. Since a combination of degree and style of computer use may be important to patient satisfaction, we are currently conducting qualitative analysis to delineate different patterns of computer use during visits, such as multi-tasking EHR use (chatting while typing) vs. compartmentalized EHR use (alternating segments of talking with patients and nonverbal EHR use). Future implementation research should explore how EHR interfaces, infrastructural interventions, and skills-based curricula can foster the communication styles preferred by diverse patients.

\section{SARET: EVALUATION OF EARLY CAREER IMPACT OF INTERPROFES-} SIONAL SUBSTANCE USE RESEARCH TRAINING Sewit Bereket ${ }^{2}$; Marc N. Gourevitch $^{2}$; Kathleen Hanley ${ }^{1}$; Frederick More ${ }^{4}$; Madeline Naegle ${ }^{3}$; Ellen Tuchman ${ }^{5}$. ${ }^{1}$ NYU, New York, NY; ${ }^{2}$ NYU School of Medicine, New York, NY; ${ }^{3}$ NYU College of Nursing, New York, NY; ${ }^{4}$ NYU College of Dentistry, New York, NY; ${ }^{5}$ YU School of Social Work, New York, NY. (Control ID \#2465812)

BACKGROUND: Innovative initiatives are needed to build the ranks of health professionals engaged in conducting research on and treating substance use disorders (SUDs). Though SUD treatment frequently requires an interdisciplinary team approach informed by the recognition that SUDs represent the complex interplay of biological, psychological and social factors, SUD research teams are often grounded in a single discipline. The fundamental goal of the NIDA-funded inter-professional Substance Abuse Research Education and Training (SARET) program is to stimulate dental, medical, nursing and social work students' pursuit of careers in substance use (SU) research. Participants engage in stipend-supported mentored research complemented by weekly didactic seminars, site visits, and an online curriculum previously shown to increase students' interest in SU research. We evaluated early career outcomes and attitudes of 54 students who have participated in these summer $(n=47)$ and year-long $(n=7)$ research fellowships since 2008.

METHODS: In Fall 2015, we conducted a survey of SARET participants from the 20082014 cohorts. The thirteen-question survey employed 4 point Likert scale as well as openresponse questions that were analyzed qualitatively. We assessed participants' current careers and the perceived influence of SARET participation on career choice, attitudes towards other professions, and research and treatment of SUDs. The survey was sent to the 65 (of 68) participants for whom we had valid email addresses. We assessed participants' scholarly work based on self-report as well as through automated monthly Pubmed searches for published journal articles.
RESULTS: The survey response rate was $83 \%(54 / 65 ; 19$ dentistry, 17 medical, 10 nursing, 8 social work). Over half of the past participants were still in training: e.g., completing residency training or various degrees in dentistry, nursing or social work. Nonetheless, $46 \%$ (25/54) report being somewhat or very involved in research, and $64 \%$ of these (30\% overall) were engaged in substance use related research. Past participants had published 40 articles (12 SUDs-related) and 3 book chapters (e.g., "Addressing Unhealthy Alcohol use in Primary Care"), had 6 manuscripts submitted or under review ( 2 tobacco cessation-related), and have completed 50 oral or poster presentations (20 SUDs-related). Six participants have received research funding, including a scholarship for one medical student to investigate naloxone overdose prevention. The overwhelming majority $(96 \% ; 52 / 54)$ of respondents reported that participation in the mentored research program had increased their interest in SU research, their knowledge about SU (100 \% 54/ $54)$ and their appreciation of $(96 \% ; 52 / 54)$ and skill at $(90 \%$; 49/54) communicating with other disciplines. Responses to the open-ended question about the main impact of SARET participation on their career conveyed several themes: increased sensitivity to the issue of SUDs in both clinical and research arenas; increased skills and knowledge about SUDs; acquisition of research skills in general; increased recognition of SUDs as a health issue; and, the need for more research in the field. Respondents also expressed increased appreciation for other health professions and for the value of interdisciplinary collaboration. Regarding impact of SARET participation outside of their career, comments again focused on increase awareness and sensitivity about SUDs in their personal and clinical lives as well as greater confidence about research and inter-professional interactions. All open-ended responses about the impact of SARET participation were positive.

CONCLUSIONS: The SARET program increased interest in SU research and clinical care as well as appreciation for interprofessional connection among dentistry, medicine, nursing and social work students. Participants have demonstrated some early research successes. Longer term follow-up will allow continued assessment for enduring impact of the SARET program on career trajectory.

SCREENING FOR DEPRESSION AT A CO-LOCATED BEHAVIORAL HEALTH STUDENT-FACULTY COLLABORATIVE CLINIC Meissa M. Jones²; Marya J. Cohen ${ }^{1} .{ }^{1} \mathrm{MGH}$, Boston, MA; ${ }^{2}$ Massachusetts General Hospital, Boston, MA. (Control ID \#2468609)

BACKGROUND: According to the Anxiety and Depression Association of America, depression affects 14.8 million American adults in a given year. Many depressed patients seek care from their primary care physicians (PCP), which has increased the need for effective screening tools for primary care settings. Research has shown that co-located behavioral health primary care clinics improve access to care, reduce wait times for referrals, and improve overall health outcomes. The Crimson Care Collaborative (CCC), a network of six student-faculty clinics, was created in the hopes of increasing primary care interest among Harvard Medical School students. Massachusetts General Hospital (MGH) has a community health center that provides multispecialty services to predominantly underserved and refugee/immigrant populations in Chelsea, MA. CCC-Chelsea is the first and only co-located behavioral health student-faculty clinic in the U.S. In accordance with hospital guidelines, CCC-Chelsea uses the Patient Health Questionnaire-2 (PHQ-2) as a screening tool for depression and patients are to be screened annually. To evaluate the incorporation of interdisciplinary services, we designed a project that would examine the behavioral health referral process for patients seen at the clinic. METHODS: A retrospective chart review of all CCC-Chelsea patients, who presented to our clinic between January 2015 and March 2015, was conducted.

RESULTS: Of the 56 patients seen at CCC-Chelsea from January 2015-March 2015, 52 patients $(92.8 \%)$ had received PHQ-2 screenings within the last year. During the time frame, five patients had a positive screening. Four patients received behavior health 
referrals from their PCP and 1 patient declined a referral. Out of the four patients who were referred to behavioral health, two patients met with a mental health provider during their clinical visit and follow-up appointments were scheduled during the consultation. The average wait time for a follow-up appointment was 4.5 weeks. In addition, those who screened positive on the PHQ-2 identified various social service needs such as cash, food, heating, and housing assistance.

CONCLUSIONS: Bridging the gap between mental health and primary care by using shared office space has led to reduced health care costs, increased health care access, and an improvement in health outcomes. Because the clinical process of care at CCC-Chelsea includes a "warm hand-off', mental health appointments are scheduled during the PCP visit allowing for an easier and more effective process. Future efforts will include using CCC-Chelsea's data for internal benchmarking within the MGH system as well as external benchmarking.

SEARCHING FOR MEANING IN MEANINGFUL USE IN THE SAFETY NET: AN IMPLEMENTATION CASE STUDY OF 5 COMMUNITY HEALTH CENTERS IN CALIFORNIA Courtney R. Lyles; Urmimala Sarkar; Lina Tieu; Dean Schillinger; Margaret A. Handley; Gato I. Gourley; Sara Ackerman. University of California, San Francisco, San Francisco, CA. (Control ID \#2468839)

BACKGROUND: US healthcare systems are rapidly implementing electronic health records (EHRs) to meet Meaningful Use certification criteria from the federal government Beginning in 2014 for Stage 2, the Meaningful Use financial incentives were tied to defined patient-level engagement metrics: specifically, $50 \%$ of patients offered online patient portal access and $5 \%$ using portal websites at least once. While safety net healthcare systems previously lagged other healthcare systems in EHR implementation, the majority are now working toward these metrics. This is the first study to our knowledge that has explored how Meaningful Use - particularly the patient engagement metrics - is being implemented in safety net healthcare systems.

METHODS: Between August and October 2015, we conducted 5 site visits with community health centers across California. All 5 sites were engaged in Meaningfu Use Stage 2 reporting to increase patient registration and use of their newly launched online patient portal websites. We selected the health centers to represent Northern and Southern California, rural and suburban sites, and a range in size (single clinic to a $10+$ clinic organization). Sites also represented both high and moderate success in reaching the two Meaningful Use patient-level benchmarks (i.e., 3 had already met the benchmarks and the other 2 were still working on their attestation process). For 4 of the sites, we conducted 1- to 1.5-day site visits that included in-depth interviews with leadership, informal focus groups with frontline staff, observations of clinic practices for patient portal sign-up, and review of marketing materials and clinic workflows. At the 5th clinic, we could not find a full day for a site visit, and therefore we conducted in-depth phone interviews with leadership and frontline staff at that location. Our work utilized a rapid ethnography approach in order to be able to grasp contextual factors, practices, and perceptions of administrators, providers, and staff that were relevant to efforts to meet Meaningful Use metrics.

RESULTS: Overall, all 5 clinics were highly mobilized to add portal registration/sign-up to their existing clinical workflows, which were already utilizing team-based primary care. In most cases, they relied on temporary volunteers during the initial portal "launch" phase to promote the new website and use waiting room time to sign patients up. However, they acknowledged highest success rates when existing staff (almost exclusively front desk or medical assistants rather than physicians) took on the role of offering portal access within the usual workflow. For example, one clinical leader said, "We think it's [portal access] the right thing to do, but...we stopped other work to get our enrollment numbers." Furthermore, after the sign-up phase, ongoing or sustained portal use among patients was not high: "The usage that we're seeing is very low" - with most of the sites averaging around
50 patient messages sent through the portal for every 1000 in-person clinic visits in a given month. Some of the biggest barriers to portal use in these safety net clinics were technology proficiency among patients ("Shifting into the electronic world is very difficult for them"), and the related need to provide extended, time-consuming education to patients about the website and how it might change care.

CONCLUSIONS: Safety net systems were committed to providing patients access to their EHR information through online patient portal websites. Many successful sites created work-around processes to use volunteers or extra front desk staff time to be able to walk patients through the entire registration process on-site, especially since a significant proportion of patients needed technical assistance in setting up accounts. Despite a high number of hours dedicated to the registration process, most of the sites did not have high levels of sustained patient engagement, with very few messages sent through the website. Looking forward to future stages of Meaningful Use, many staff and leaders raised concerns about what meaningful and long-term engagement from their patient population might require.

SECULAR TRENDS IN THYROID CANCER: INDIRECT EVIDENCE OF OVERDIAGNOSIS AND OVERTREATMENT? Sabrina N. Jegerlehner ${ }^{1}$; Drahomir Aujesky $^{1}$; Jean-Luc Bulliard ${ }^{2}$; Arnaud Chiolero ${ }^{2,}{ }^{3}$; Simon Germann ${ }^{2}$, ${ }^{3}$; Isabelle Konzelmann ${ }^{3}$; Nicolas Rodondi ${ }^{1} .{ }^{1}$ Inselspital Bern University Hospital Switzerland, Bern, Switzerland; ${ }^{2}$ IUMSP university instiution for social and preventive medicine, Lausanne, Switzerland; ${ }^{3}$ Observatoire valaisan de la santé, Sion, Switzerland. (Control ID \#2468421)

BACKGROUND: Due notably to increasing detection and screening activities and incidental findings, the incidence of thyroid cancer has increased in several countries during the last decades. However, it is possible that this recent increase reflects the growing detection of indolent forms of thyroid cancers, indicating potential overdiagnosis. We analyzed secular trends in the incidence and mortality of thyroid cancer, as well as of thyroid surgery, in Switzerland to assess for potential overdiagnosis and overtreatment.

METHODS: We used primary invasive thyroid cancer cases (C73, International Classification of Diseases for Oncology [ICD-O-3]) recorded between 1998 and 2012 in all population-based regional (cantonal) cancer registries in Switzerland. Cancer cases were classified by histological subtype (papillary vs. non-papillary) and stage (early vs. advanced) according to recent oncology guidelines using the TNM classification. We assessed thyroid cancer-specific mortality. We also analyzed trends in thyroid surgery between 2001 and 2012 using data from the Swiss Federal Statistical Office's hospital statistics.

RESULTS: We analyzed data of 4907 cases of thyroid cancer (women: 3642; men: 1265). Between 1998-2000 and 2010-2012, the age-standardized incidence of thyroid cancer increased from 6.5 to 11.3 cases per 100,000 women (absolute increase: $+4.8 / 100,000$; relative increase: $+75 \%$ ) and from 2.5 to 3.8 cases per 100,000 men (absolute increase: $+1.2 / 100,000$; relative increase: $+48 \%$ ). The increase was essentially due to an increase in the papillary histologic subtype, the more indolent form of thyroid cancer. No substantial change was observed in other histologic subtypes. The incidence of early stages increased substantially, especially in women, while the incidence of advanced stages increased marginally. During the same period, the thyroid cancer-specific mortality rate slightly decreased, from 0.67 to 0.51 deaths per 100,000 women (absolute decrease: $-0.16 / 100,000$; relative decrease: $-24 \%$ ) and from 0.54 to 0.40 deaths per 100,000 men (absolute decrease: $-0.14 / 100,000$; relative decrease: $-27 \%$ ). Between 2001 and 2012, we observed in Switzerland a 1.5-fold increase in the agestandardized rate of thyroidectomy in both sexes (from 43.6 to 63.3 interventions per 100,000 women and from 11.5 to 18.7 interventions per 100,000 men).

CONCLUSIONS: We observed a substantial increase in the incidence rate of thyroid cancer and thyroid surgery, without a proportional increase in thyroid cancer-specific mortality rate. The increase in thyroid cancer was limited to the more indolent papillary subtype. These findings may represent indirect evidence of overdiagnosis and overtreatment of thyroid cancer 
SEEING IS BELIEVING: THE RELATIONSHIP BETWEEN VISIONRELATED QUALITY OF LIFE AND AGE IN HOSPITALIZED PATIENTS Elizabeth R. Donnelly ${ }^{3}$; Vineet M. Arora ${ }^{1}$; Madeleine Matthiesen ${ }^{2}$; Esther Schoenfeld ${ }^{1}$; Gavin W. Hougham ${ }^{4}$; Seenu M. Hariprasad ${ }^{1}$; David Meltzer ${ }^{1}$; Valerie G. Press ${ }^{1} .{ }^{1}$ University of Chicago, Chicago, IL; ${ }^{2}$ Massachusetts General Hospital, Boston, MA; ${ }^{3}$ Pritzker School of Medicine, Chicago, IL; ${ }^{4}$ Battelle Memorial Institute, Seattle, WA. (Control ID \#2466079)

BACKGROUND: Impaired vision may be a risk factor for poor health outcomes during hospitalization, including falls and delirium. Additionally, hospitalized patients with poor vision may be unable to read critical documents such as consent forms. Vision also has major implications for quality of life (QOL); in a study of older adults, those with visual impairment reported lower satisfaction with their lives and were more likely to experience depression. Our previous work has shown that nearly one-third of hospitalized patients fail an inpatient vision screening. Therefore, our study set out to examine the prevalence and effect of poor vision on vision-related QOL (VRQOL) among hospitalized patients and to determine whether age impacts VRQOL.

METHODS: Hospitalized adult general medicine inpatients at the University of Chicago were enrolled in the Hospitalist Study, an ongoing study of resourceallocation and quality-of-care. Eligible patients ( $\geq 18$ years, English speaking, cognitively intact) were given a vision screening (Snellen Eye Chart) wearing corrective lenses, if applicable; sufficient vision was classified as 20/40 or better visual acuity in the better eye. VRQOL was measured using the validated National Eye Institute 25-item Visual Function Questionnaire (VFQ-25, scale of 0 (lowest) to 100 (highest)) which covers 12 areas of function, including general health, general vision health (near and far sight), social functioning (e.g., visiting people), and mental health (composite of embarrassment, frustration, loss of control, and worry related to vision problems), among others. The Shapiro-Wilk test was used to test for normal distribution of VFQ-25 subscale and composite scores. Because these variables were not normally distributed, median and interquartile range (25th-75th percentile) were used as descriptive statistics. Relationships between VFQ-25 scores and visual acuity (sufficient vs. insufficient vision) and age $(<65$ vs. $\geq 65$ ) were tested using Mann-Whitney $\mathrm{U}$ tests. All tests were two-sided and $p$ values less than 0.05 were considered statistically significant. Statistical computations were performed using Stata 13 (StataCorp, College Station, TX).

RESULTS: Between June 2011 and November 2015, 1172 participants completed both a vision screening and the VFQ-25. The majority of participants were female (55\%) and African American (78 \%) with a mean age of 53 years; $44 \%$ (521/1172) had insufficient vision. Participants with insufficient vision were more likely to be African American (81 vs. $76 \%, p=0.02$ ) and older ( 63 vs. 45 years, $p<0.001$ ). The median VFQ- 25 composite score for all participants was 93 (IQR 84-97). Participants with insufficient vision reported a lower median VFQ composite score compared to those with sufficient vision ( 90 vs 95 , $p<0.001$ ); in addition, participants with insufficient vision reported lower median scores in all subscales except for General Health $(p=0.44)$ and Ocular Pain $(p=0.10)$. The same trend was seen when participants were separated by age $(<65$ and $\geq 65)$; participants $<65$ with insufficient vision scored lower than those with sufficient vision (89 vs. 95 , $p<0.001)$ and participants $\geq 65$ with insufficient vision reported lower scores than those with sufficient vision (91 vs. $94, p<0.001$ ). When all participants were stratified by age $(<65$ and $\geq 65)$ regardless of their visual acuity, median composite scores were not significantly different ( 94 vs. $93, p=0.16$ ). Similarly, among only participants with sufficient vision, there was no significant difference in median composite scores based on age $(<65=95$ vs. $\geq 65=94, p=0.50)$. However, when looking only at those participants with insufficient vision, participants $<65$ years reported a lower median composite score than their older counterparts ( 89 vs. $91, p=0.003$ ) and lower VRQOL related to Near Activities ( 81 vs. $88, p=0.009)$ and Mental Health (88 vs. $94, p<0.001$ ).
CONCLUSIONS: Insufficient vision is prevalent among hospitalized patients and affects both younger and older individuals. Regardless of age, patients with insufficient vision are more likely to have lower VRQOL than their counterparts with sufficient vision. And, while we found that patients with insufficient vision were on average older than those with sufficient vision, the negative impact of insufficient vision on younger patients' VRQOL was more pronounced, with the most notable decline in their mental health. The high number of hospitalized patients with poor vision makes hospitalization a prime opportunity to identify these patients and intervene in order to provide short-term in-hospital as well as longer-term post-discharge improved VRQOL. Future work is needed to test hospital-based interventions that identify patients with impaired vision in order to improve vision and VRQOL. Such interventions should not be targeted only to older individuals because the negative quality of life impacts of poor vision may be even more pronounced in younger patients.

SELF-REPORTED OR SOCIALLY-ASSIGNED SOCIAL CLASS: WHICH MATTERS MORE IN EXPERIENCES OF DISCRIMINATION? Tara Rizzo; Carol Oladele; Baylah Tessier-Sherman; Marcella Nunez-Smith. Yale School of Medicine, New Haven, CT. (Control ID \#2469655)

BACKGROUND: A growing body of research has shown a negative effect of discrimination on health outcomes. Self-reported social class has been associated with discrimination and numerous outcomes, however, a self-reported rating of how others perceive one's social status (self-reported socially-assigned social class) has not been examined. Other models of examining socially-assigned identity (by race and gender) have emerged in recent years and have highlighted the important role that perception of self by others plays in experiences of discrimination and health outcomes. In the present analyses, we compare the association of self-reported social class and self-reported socially-assigned social class with self-reported experiences of discrimination.

METHODS: Data from the field-testing phase of the Patient-Reported Experiences of Discrimination in Care Tool (PreDict) study was used for analyses. The PreDict study includes a self-administered survey of recently discharged patients across the US. Patients were invited to participate if they were over 21 and discharged to home from an inpatient medical service in the past 30 days. The survey is offered in English and Spanish. The survey includes questions about patients' most recent hospital experiences, self-reported outcomes, questions from existing validated communication and discrimination scales, and demographics, including self-reported socioeconomic class ('do you consider yourself low, middle, upper class?') and self-reported socially-assigned class ('do other people usually consider you low, middle, upper class?'). Participants with complete field testing data as of December 31, $2015(n=1503)$ were included in analyses. The Fisher's exact test was used to examine the association between the social class variables and logistic regression was used to determine the association between the social class variables and discrimination

RESULTS: Self-reported social class and socially-assigned social class were independent $(p<.0001)$. Respondents were more likely to report their socially-assigned social class as higher than their self-reported social class. In multivariate analyses self-reported middle and lower class respondents were less likely to report experiencing discrimination in the hospital (middle: $\mathrm{OR}=0.123, p=0.03$; lower: $\mathrm{OR}=0.112, p=0.04$ ). Conversely, participants who reported that other people perceive them as lower class were more likely to report experiencing discrimination in the hospital $(\mathrm{OR}=12.5, p=0.03)$. Gender was not significantly associated with discrimination in any model.

CONCLUSIONS: Self-reported social class and socially-assigned social class were significantly associated with discrimination, but in opposite directions. Our finding that self-reported middle and lower class respondents were less likely to report discrimination is consistent with existing literature showing that higher SES persons are more likely to 
report experiencing discrimination. The converse finding that socially-assigned lower social class is associated with experiencing discrimination may be influenced by other factors. Given that more respondents reported higher socially-assigned class than selfreported class, it is possible that those who do report lower socially-assigned class are particularly marginalized and vulnerable to discrimination, which requires further study. Inclusion of measurement of self-reported socially-assigned social status in health services research should increase. Similar to socially-assigned race and gender conformity, socially-assigned social class is independent of self-reported social status and may play a role, perhaps larger than self-reported social status, in experiences of discrimination and negative health outcomes.

SERIAL CRP TESTING: DOES THIS SELF-FULFILLING PROPHECY BRING HIGH VALUE CARE? Radha Mehta; Patrick L. Gordan. Mount Auburn Hospital, Cambridge, MA. (Control ID \#2467692)

BACKGROUND: The value of medical care relates to benefit relative to cost; higher value care provides higher benefit at a lower cost. Although physician decision making is key to the provision of high value care, many physicians are unaware of the cost of diagnostic testing and therapeutic interventions. In addition to the well documented cost savings of reducing unnecessary tests, patient safety and satisfaction also increases with the reduction of unnecessary tests. For instance, unnecessary blood tests do not just cost more money but they also increase patient discomfort and risk additional unnecessary testing. For these reasons, testing that does not provide value should be avoided. We evaluated the use of serial C-reactive protein (CRP) testing in our intensive care unit (ICU) at Mount Auburn Hospital. Although CRP testing is relatively inexpensive, the added value to patient care is unclear and there is no widely accepted consensus regarding CRP testing best practice. We hypothesized that CRP testing in our ICU is ordered more often than it influences care.

METHODS: The study design included surveys and chart reviews. An online survey was sent to our resident trainees (total of 55) and the ICU attendings (total of 8). The survey included questions addressing the components mentioned in Table 1. The chart review included medical record review of all the patients admitted to the ICU at Mount Auburn Hospital from June 2014 to August 2014 to observe the actual trend of CRP testing. We collected data including admitting service, admitting and discharge diagnosis, presence of other comorbidities such as CHF, malignancies, renal disease with glomerular filtration rate $<60 \%$, liver disease, the site of sepsis (when present). Regarding more specific CRP data, we collected the number of CRPs ordered in the ICU in this time period, the number of CRPs per patient per visit normalized for length of stay, the average frequency of CRP testing per patient, whether the CRP value was mentioned in patient care notes, whether the result of CRP testing was taken into consideration in decision making and whether it was used to change management.

RESULTS: We received a $38 \%$ response rate for the survey, the results of which are summarized in Table 1 . Of the charts reviewed, $81 \%$ of the patients were either primarily intensivist service or transferred to the intensivist service. There was a documented presence of sepsis with a positive microbiology in $24 \%$ of the cases with lung being the most common site. On comparison of the CRP testing accounting for the diagnosis of sepsis and non-sepsis, there was a marginally significant difference with a higher percentage of CRP ordered for sepsis compared to non-sepsis ( $\mathrm{P}$ value of 0.045 ). CRP was ordered much more frequently on intensivist and hospitalist service compared to the surgery and cardiology service (Fisher exact test: P value: 0.0004$)$ Head to head comparison between surgery and intensivist services showed significantly more CRP tests ordered on the intensivist service compared to the surgery service even when accounting for the diagnosis of sepsis (Fisher's test: $p$-value: 0.004 ) There were significantly more CRPs ordered in the ICU compared to the rest of the hospitalization even when normalizing for the length of stay ( $t$-test: $p$-value: 0.000039$)$ Of the total number of times CRP was ordered, its result was mentioned in the resident notes only $45 \%$ of the times and in attending notes only $25 \%$ of the times. The documentation of the CRP in assessment and plan was done in $25 \%$ of the resident notes and $10 \%$ of the attending notes with the most common documentation being trend CRP and follow CRP. CRP value was documented to have changed management in only one out of the 71 charts reviewed.

CONCLUSIONS: The major conclusion of this study was that CRPs were ordered significantly more times than its value influenced patient care. The ICU team ordered significantly more CRPs compared to the surgical team even when comparing for the diagnosis of sepsis. Significantly more CRPs were ordered in the ICU compared to the rest of the hospitalization, even when normalizing it for the length of stay. The CRP test was not charted as often as it was ordered. Most significantly, CRP level did not change management. It is estimated that our hospital spends $\$ 16,000$ per year in the ICU on a test that does not provide high value care.

Table 1: Survey Results

\begin{tabular}{|c|c|c|}
\hline & Attendings & Residents \\
\hline Percentage of respondents & $20 \%$ & $80 \%$ \\
\hline \multicolumn{3}{|l|}{ Perception of interval of CRP testing } \\
\hline Daily & $55 \%$ & $25 \%$ \\
\hline Every alternate day & $15 \%$ & $25 \%$ \\
\hline Every $72 \mathrm{~h}$ & $10 \%$ & $0 \%$ \\
\hline No established interval & $20 \%$ & $50 \%$ \\
\hline \multicolumn{3}{|c|}{ Perception of the Diagnoses prompting serial CRP } \\
\hline First elevated result & $0.00 \%$ & $70 \%$ \\
\hline No improvement in clinical condition & $25 \%$ & $60 \%$ \\
\hline Increasing or steady white count & $25 \%$ & $60 \%$ \\
\hline Fever & $25 \%$ & $45 \%$ \\
\hline Attending request & $25 \%$ & $35 \%$ \\
\hline ID attending recommendations & $25 \%$ & $50 \%$ \\
\hline I don & $50 \%$ & $0.00 \%$ \\
\hline \multicolumn{3}{|c|}{ Perception of change in management due to serial CRP } \\
\hline Rarely & $75 \%$ & $15 \%$ \\
\hline $25 \%$ & $25 \%$ & $55 \%$ \\
\hline $50 \%$ & $0 \%$ & $25 \%$ \\
\hline$>75 \%$ & $0 \%$ & $5 \%$ \\
\hline \multicolumn{3}{|l|}{ Perception of cost } \\
\hline$<37$ dollars (actual cost) & $50 \%$ & $40 \%$ \\
\hline$>100$ dollars & $0 \%$ & $10 \%$ \\
\hline 37-100 Dollars & $25 \%$ & $15 \%$ \\
\hline Don & $25 \%$ & $35 \%$ \\
\hline
\end{tabular}

SERUM PROCALCITONIN AIDS IN THE DIAGNOSIS OF BLOODSTREAM INFECTION REGARDLESS OF WHETHER PATIENTS EXHIBIT THE SYSTEMIC INFLAMMATORY RESPONSE SYNDROME. James P. Campbell ${ }^{2}$; Rashi Arora $^{2}$; Jonathan D. Kirsch ${ }^{2}$; Gyorgy J. Simon ${ }^{1}$; Nishant Sahni ${ }^{2} .{ }^{1}$ Mayo Clinic, Rochester, MN; ${ }^{2}$ University of Minnesota, Saint Paul, MN. (Control ID \#2463833)

BACKGROUND: Physicians frequently rely on the systemic inflammatory response syndrome (SIRS) criteria to detect sepsis and bloodstream infections (BSIs). However, up to 1 in 8 patients with sepsis may be SIRS-negative at the time of presentation. We evaluated the diagnostic performance of procalcitonin (PCT) in detecting BSI in SIRSnegative and SIRS-positive patients.

METHODS: We created a dataset of 7455 PCT measurements along with contemporaneous SIRS scores, serum lactate, and blood cultures. Using this dataset, we tested the association of the SIRS score, PCT, and serum lactate with BSI using logistic regression analysis. Receiver Operating Characteristic (ROC) curves were created to assess the diagnostic performance of these variables.

RESULTS: Seven thousand four hundred fifty-five PCT measurements were reviewed, of which 648 measurements $(8.7 \%)$ were associated with an ongoing BSI. SIRS score, serum lactate, and $\log (\mathrm{PCT})$ were associated with BSIs in univariate regression analysis 
$(p<0.05)$. In multivariate regression analysis, only $\log$ (PCT) was independently associated with BSIs $(p<0.05$ ). The area under the curve (AUC) of PCT in detecting BSI ( $95 \%$ CI: $0.65-0.71$ ) was significantly higher than serum lactate ( $95 \%$ CI: $0.58-0.64)$ and the SIRS score (95\% CI: 0.53-0.58). Elevated PCT levels were associated with BSI irrespective of SIRS status. PCT of less than $0.1 \mathrm{ng} / \mathrm{ml}$ had a negative predictive value of $97.5 \%$ for ruling out BSI in the SIRSnegative sub-group and $96.2 \%$ in the SIRS-positive sub-group.

CONCLUSIONS: PCT is independently associated with BSI and outperforms the SIRS score and serum lactate in detecting BSI in both SIRS-positive and SIRS-negative patients.

\section{SEX DIFFERENCES IN THE POSITIVE PREDICTIVE VALUE OF NON-} INVASIVE STRESS TESTING AT A COMMUNITY HOSPITAL Marianna Sargsyan $^{2}$; N Ranjeeta ${ }^{2}$; QUANG TUNG NGUYEN ${ }^{1}$; Shahriar Dadkhah ${ }^{2} .{ }^{1}$ Presence Health Saint Francis Hospital, Evanston, IL; ${ }^{2}$ Saint Francis Hospital, Evanston, IL. (Control ID \#2466846)

BACKGROUND: Increased attention has been placed on sex differences in diagnosing CAD. The gold standard for diagnosing CAD remains coronary angiography. Noninvasive stress testing (NIST) is often used as the initial assessment of patients with CAD in both males and females. However, the positive predictive value (PPV) of NIST in a community hospital is not well known and shows sex disparities. Objectives: The primary goal of this study was to determine the PPV and correlation between NIST and obstructive CAD in males compared to females. The secondary goal was to identify cardiac risk factors that predict a true positive stress test.

METHODS: This retrospective study included all consecutive patients $(N=352)$ who underwent invasive coronary angiography (ICA) following a positive NIST (myocardial perfusion imaging (MPI) stress test, or stress echocardiogram). Ultimately, 321 patients met inclusion criteria after exclusion of 31 patients who had a history of CABG. The study sample was separated into three groups based on results of the ICA and treatment modality. Group 1 patients had a non-obstructive lesion ( $<50 \%$ luminal stenosis). Group 2 patients had an obstructive lesion or had a percutaneous coronary intervention (PCI). Group 3 patients reported to have an obstructive lesion on ICA but who were not treated with PCI. Angiography images of patients in group 3 were reviewed by 2 senior board certified interventional cardiologist who were blinded to any patient or performer data. Subsequently, this group was reclassified to group 1 or 2 .

RESULTS: Sixty percent of the patients were female with mean age of 62.9 years and for males the mean age was 65.2 years. $96 \%$ had MPI and $4 \%$ had stress echocardiography. The positive predictive value of NIST for males and females were 66 and $37 \%$, respectively. Compared to patients with a false positive NIST, males with a true positive NIST were notably older $(68.8$ vs $60.1, P<0.001)$ and had hypertension $(P<0.029)$. Females with a true positive NIST were also older $(67.8$ vs $60.9, P<0.003)$ and were not obese (BMI of 28.1 vs $32.5, P<0.003$ ). Stress echocardiography had a significantly higher PPV compared to MPI stress test ( 71 vs $35 \%, P<0.005$ ). In-hospital testing vs outpatient testing or specialty of interpreting physician (cardiologist vs radiologist) did not significantly impact PPV. Multi-variable logistic regression analysis revealed that age, sex and cardiac risk factors were found to be significant confounding factors for higher PPV. CONCLUSIONS: Our single center community hospital study revealed that the PPV of NIST is poor, especially in young and obese females (37\%).

\section{SEXUAL BEHAVIORS AND SEXUALLY TRANSMITTED INFECTIONS} AMONG MALE VETERANS AND NON-VETERANS Mark W. Evans ${ }^{1}$; Elian A. Rosenfeld ${ }^{3}$; Sonya Borrero ${ }^{2,3}$. ${ }^{1}$ University of Pittsburgh, Pittsburgh, PA; ${ }^{2}$ University of Pittsburgh and VA Pittsburgh, Pittsburgh, PA; ${ }^{3}$ VA Pittsburgh Healthcare System, Pittsburgh, PA. (Control ID \#2467147)
BACKGROUND: Higher rates of sexually transmitted infections (STIs) and sexual risk behavior have been documented among women veterans compared to the general population of U.S. women. Although males comprise more than $90 \%$ of the U.S. veteran population, less is known about the sexual health of this population than of their female counterparts. This study sought to evaluate sexual behaviors and history of STIs among male veterans compared to male non-veterans.

METHODS: We analyzed male respondent data from the 2011-2013 National Survey of Family Growth (NSFG), a nationally representative sample of men ages 15-44 years old. Our sample included all men 18-44 years old who reported ever having had sex with a woman or man. The key independent variable of interest was veteran status, which was based on the response to the question "Have you ever been on active duty in the Armed Forces for a period of 6 months or more?" Behavioral outcomes included age at first sex (with either a female or a male), $\geq 6$ lifetime female sexual partners, $\geq 10$ lifetime sexual partners (female or male), $\geq 3$ past-year sexual partners (female or male), past-year history of $\geq 1$ female and $\geq 1$ male sexual partner, and condom use at last vaginal sex. STI outcomes included a positive selfreported history of herpes, genital warts, chlamydia, gonorrhea, or syphilis, as well as an aggregate measure capturing any STI history. We first examined whether veteran status was associated with our outcomes using Rao-Scott corrected chi-squared tests. We then used linear or logistic regression analyses to evaluate the associations between veteran status and all of our outcomes without adjustment and after adjusting for age, race, education level, and marital status, which were chosen a priori based on previously observed associations with sexual behaviors and STIs. All analyses were conducted using the sampling weights provided by the NSFG to produce nationally representative data. RESULTS: Our sample consisted of a total of 3860 men: 409 veterans and 3451 non-veterans. Compared to non-veterans, veterans were slightly older, had higher income, and were more likely to be married. There were no significant differences between veterans and non-veterans with regard to race or education level. For behavioral outcomes, there were no significant differences by veteran status for age at first sex, lifetime history of $\geq 10$ sexual partners, and $\geq 3$ past-year partners. However, a greater proportion of veterans had $\geq 6$ lifetime female partners (66.0 vs. $52.1 \%, p<0.01)$ and $\geq 1$ female and $\geq 1$ male partner in the past year (58.3 vs. $23.1 \%, p=0.02)$, and fewer veterans used a condom at last vaginal sex (26.8 vs. $38.4 \%, p=0.01$ ). A greater proportion of veterans reported a history of genital warts compared to non-veterans (5.3 vs. $2.1 \%, p=0.04)$. There were no differences in lifetime prevalence of any other STI diagnoses, nor in the aggregate measure, with $7.6 \%$ of veterans and $4.1 \%$ of non-veterans ( $p=0.07)$ reporting ever having any STI. In adjusted analyses, the associations between veteran status and use of a condom at last vaginal sex and history of genital warts were no longer significant. However, the other associations remained statistically significant including higher odds of having $\geq 6$ lifetime female partners (OR 1.7, $95 \%$ CI: 1.1-2.5) and $\geq 1$ female and $\geq 1$ male partner in the past year (OR 5.0, $95 \%$ CI: 1.1-22.9) among veterans compared to non-veterans. In addition, after adjustment, veterans were found to have higher odds than non-veterans of having $\geq 3$ partners in the past year (OR $1.9,95 \% \mathrm{CI}: 1.2-3.1)$ and of having ever been diagnosed with gonorrhea (OR 2.9, $95 \%$ CI: $1.1-8.1)$.

CONCLUSIONS: Male veterans are more likely than male non-veterans to have risk factors for contracting new STIs, including higher numbers of lifetime female sexual partners and past-year partners of either sex, having partners of both sexes in the past year, and having a history of gonorrhea. These findings indicate that male veterans warrant thorough screening for STIs and sexual behavioral risk factors during routine outpatient visits. 
SHORT-ACTING OPIOIDS PROVIDE EQUIVALENT ANALGESIA TO LONGACTING OPIOIDS IN PATIENTS WITH CHRONIC OSTEOARTHRITIS PAIN WITH A SUBSTANTIALLY REDUCED OPIOID EQUIVALENT REQUIREMENT Paul Chelminski ${ }^{3}$; Ameer Ghodke ${ }^{3}$; Stephanie Barquero ${ }^{1}$; Marc S. Piper ${ }^{2}$; Timothy Ives ${ }^{1} .{ }^{1}$ UNC School of Pharmacy, Chapel Hill, NC; ${ }^{2}$ University of Michigan, Ann Arbor, MI; ${ }^{3} \mathrm{UNC}$ School of Medicine, Chapel Hill, NC. (Control ID \#2456690)

BACKGROUND: With the expansion of opioid treatment for chronic non-cancer pain in the 1990's, the use of long-acting opioids (often combined with short-acting opioids for "breakthrough pain") emerged as a standard of care despite a lack of rigorous clinical trial data to support such an approach. In theory, the provision of potent "basal" opioid analgesia would provide better pain relief than "as-needed" use of equally potent opioids. In response to this standard, a number of short-acting opioids were reformulated to extend their duration of action and the use of one older opioid with a long half-life, methadone, was revived. There have been no long-term trials comparing the effectiveness of shortacting opioids (SAO) vs. long-acting opioids (LAO) in improving outcomes in patients with chronic pain. Adherence to this standard has led to the use of higher doses of opioids this has been linked to the current epidemic of prescription opioid overdoses and death Also, the use of proprietary extended-release preparations is substantially more costly than using generic short-acting opioid preparations. In this study, we examined the relative effectiveness of SAO vs. LAO preparations in a cohort of patients with chronic osteoarthritis pain enrolled in a primary care disease management program at an academic general internal medicine clinic. We also analyzed relative magnitude of opioid therapy (in opioid equivalents) between those receiving SAOs and LAOs.

METHODS: There were 324 active patients in the UNC Internal Medicine Pain Clinic during the fourteen-month study period from April 2014 until June 2015 (Selection of start date was based on implementation of a new EHR at our institution). Of these, 171 met the specified inclusion criteria of chronic pain duration of at least 6 months caused by osteoarthritis of the axial spine or peripheral joints. We excluded 153 patients for the following reasons: 41 with non-osteoarthritis pain; 33 with no opioids prescribed; 26 with pain contract violations; 20 with active malignancy; 21 who did not meet other inclusion criteria; 12 were deceased. Patients were seen every 3 months or more frequently. Pain scores were recorded at each visit using the ten-point Brief Pain Inventory across its four dimensions. Pain scores were averaged across visits. Morphine opioid equivalents were calculated for each patient. We then compared pain scores and opioid equivalents between patients receiving SAOs vs. LAOs. We analyzed the groups for potential confounding factors such as age, gender, race, or adjunctive use of NSAIDs, neuropathic agents, and antidepressants. Bivariate and multivariate analysis was performed. The Institutional Review Board at UNC-Chapel Hill approved this study.

RESULTS: The average age of patients in the study was 60 , and $69 \%$ were female. Seventy-two percent of patients were white; $25 \%$ were African American. Thirty-six percent were smokers. The average number of visits for the 171 patients included in our analysis was 4.5 (range 2 to 8). Sixty-nine percent of patients (118) were taking SAOs only; thirty-one percent (53) were LAOs (or LAOs with SAOs for breakthrough pain). The distribution of osteoarthritis pain generators is show in Table 1. Lumbosacral back pain was the most common pain generator ( $41.5 \%$ ) followed by osteoarthritis of the knees (24.8\%) and hips (16.4\%). The average pain score for patients receiving SAOs was 5.67, and the average score for patients receiving LAOs was $5.35(p=0.201)$. The average low pain score for patients receiving SAOs was 3.83 , and the average low pain score for patients receiving LAOs was $3.52(p=0.296)$. Adjusting for age, gender, race, and the use of other adjunctive pain medications did not affect the results. A trend toward higher pain scores was observed in smokers. The average number of daily morphine opioid equivalents in the SAO group was 52.4; the average in the LAO group was $97.0(p<0.001)$.

CONCLUSIONS: In a cohort of patients with osteoarthritis receiving opioid analgesics for chronic pain, SAOs provided statistically equivalent analgesia to LAOs with a $45 \%$ lower opioid equivalent requirement. The small absolute differences in pain scores between the two groups could not be considered clinically important. Results were unaffected in adjusted analyses. These results do not support the practice standard that "basal" opioid exposure provides superior relief of pain compared to short-acting opioid regimens in patients with chronic non-cancer pain. The use of potent SAOs, rather than LAOs, could decrease the cost of treatment for patients with chronic pain due to osteoarthritis. It may also reduce the risk of overdose that accompanies the use of opioids for chronic pain.

SHOW ME THE DATA: WHERE IS THE NNT? Joel Jorgenson ${ }^{2}$; Allan V. Prochazka ${ }^{1}$; Ravi K. Gopal ${ }^{1} .{ }^{1}$ Denver VAMC, Denver, CO; ${ }^{2}$ University of Colorado, Denver, CO. (Control ID \#2467546)

BACKGROUND: Physicians now commonly focus more on statistical analysis that includes absolute terms. The clinical relevance of an absolute risk reduction (ARR) or number needed to treat (NNT) is easier to interpret than the relative terms that are often reported in trials. Many physicians learn about these differences when studying for their ABIM Board exam. A common study tool is the American College of Physicians Medical Knowledge Self-Assessment Program (MKSAP). In MKSAP 16, under the section on statistical analysis they report "Defining risk in absolute terms and calculating number needed to treat/harm is the best way to understand the magnitude of difference in the sample". Given their recognition of the importance of using absolute terms, we wanted to determine how much the text of MKSAP 16 reflected this approach.

METHODS: The General Internal Medicine (GIM) section of the online version of MKSAP 16 was chosen for analysis since the section contained the previous statement about reporting data in an absolute form. A reviewer examined the entire text of the GIM section and all 168 questions that were associated. Any clinical trial data was noted. After all the data was collected, three physicians examined each statement and determined if the data referenced a therapeutic intervention and if the data provided could be used to calculate an ARR/NNT, whether it included a relative risk (RR) or RRR or if any of these categories were indeterminate.

RESULTS: We identified 189 statements within the GIM section that contained any statistical information. The majority $(81.5 \%, n=150)$ were found in the text of the GIM section and $18.5 \%(n=43)$ came from the GIM questions. These statements contained 271 statistics. We excluded 108 epidemiology statements, 56 diagnostic statements, 4 screening statements, 1 economic statement and one that was not a statistic. Of the included 47 statements deemed to be therapeutic data, six (12.8\%), included a RR, none included an ARR, four ( $8.5 \%)$ included a NNT, seven (14.9\%) provided the raw data, and) $32(67.2 \%)$ were indeterminate. Of those that could not be determined, 17 had data that was not clearly absolute or relative and 15 were continuous variables.

CONCLUSIONS: Using ARR or NNT to estimate effect size is important to gauge the clinical benefit of a study. As physicians study for their certification or recertification, many rely on MKSAP. Currently, the rates of reporting the data required to calculate these numbers or the numbers themselves are low. Given the importance of this topic and widespread use of MKSAP, there is a tremendous missed opportunity to educate physicians. Adding this information in will make the MKSAP an even more valuable tool.

\section{SMOKING CESSATION TREATMENT FOR HOSPITALIZED SMOKERS WITH SERIOUS MENTAL ILLNESS: SECONDARY ANALYSIS OF A RAN- DOMIZED CONTROLLED TRIAL Erin Rogers ${ }^{2}$; Rebecca Friedes ${ }^{2}$; Annika Jakes ${ }^{5}$; Ellie Grossman ${ }^{1}$; Alissa R. Link ${ }^{4}$; Scott Sherman ${ }^{3} .{ }^{1}$ Cambridge Health Alliance, Somer- ville, MA; ${ }^{2}$ VA NY Harbor Healthcare System, New York, NY; ${ }^{3}$ VA New York Harbor HCS, New York, NY; ${ }^{4}$ New York University, New York, NY; ${ }^{5}$ University of Washington, Seattle, WA. (Control ID \#2468360)}


BACKGROUND: Persons with serious mental health illness (SMI) have high rates of tobacco use. Hospitalization provides a unique opportunity to engage smokers with SMI into cessation treatment, but it is unclear how to best help them after discharge.

METHODS: This study is a secondary analysis of a randomized comparative effectiveness trial testing two models of post-discharge counseling for hospitalized patients. Participants included in the secondary analysis were 384 current smokers with a history of schizophrenia, schizoaffective disorder or bipolar disorder. Participants were enrolled during hospitalization and randomized at discharge to receive either: a) seven sessions of telephone counseling from study staff $(n=204)$ or b) referral to the state Quitline for 1-2 counseling sessions $(n=180)$. Logistic regression was used to compare study groups on self-reported 30-day abstinence at 6 months and use of treatment.

RESULTS: There were no significant group differences in abstinence at 6 months ( $28 \%$ Quitline vs. $29 \%$ Intervention; $\mathrm{OR}=1.05,95 \% \mathrm{CI}=0.59,1.85$ ), use of cessation medications ( $42 \%$ Quitline vs. $47 \%$ Intervention; $\mathrm{OR}=1.22,95 \% \mathrm{CI}=0.71-2.08$ ) or receipt of at least one counseling call ( $47 \%$ Quitline vs. $42 \%$ Intervention; $\mathrm{OR}=0.71,95 \% \mathrm{CI}=0.47$ 1.08). Only $6 \%$ of patients in the multi-session counseling arm completed all seven sessions. Patients with hazardous drinking ( $p=.04)$ or good health $(p=.03)$ were less likely to use cessation medications. Homeless patients were less likely to use counseling $(p=.02)$.

CONCLUSIONS: Most patients in both groups did not use cessation treatment after discharge, and very few patients completed multi-session counseling when given the opportunity. The intensive intervention's effectiveness may have been hampered by the very low rate of treatment engagement. Interventions are needed that improve use of tobacco cessation treatment after hospitalization among smokers with SMI, particularly homeless patients and patients with comorbid alcohol abuse who were especially vulnerable to treatment barriers in the current study.

SOCIAL NETWORKS IN ELECTRONIC HEALTH RECORDS Shin-Ping Tu'; Nengliang $\mathrm{Yao}^{2}$; Xi Zhu ${ }^{3}$; Vimal Mishra ${ }^{1}$; Allison E. Phillips ${ }^{1}$; Alan Dow ${ }^{1} .{ }^{1}$ Virginia Commonwealth University, Richmond, VA; ${ }^{2}$ University of Virginia, Charlottesville, VA; ${ }^{3}$ University of Iowa, Iowa City, IA. (Control ID \#2469307)

BACKGROUND: Team-based approaches have been broadly promoted to improve healthcare delivery in the U.S. However, few studies have analyzed the complexities of team-based communication in the current healthcare delivery landscape of increasing electronic health record (EHR) use and the rapidly evolving EHR functionalities. Major hurdles to studying effective communication within and between healthcare teams include: the expansive scope of this research inquiry; detailed communication data between team members; and rigorous methods to analyze communication data. Based upon the large body of research on network analysis, we explored the application of network analysis to visualize healthcare teams at a large, urban academic medical center with EHR data. Based upon the large body of research on network analysis, we explored the application of EHR access data to describe the connectivity and network structure of healthcare teams at a large urban academic medical center.

METHODS: The study was approved by its Institutional Review Board. A total of 100 surgical colorectal cancer patients receiving treatment in 2013 and 2014 were selected at random. We used detailed access logs for the Cerner EHR system to create a table of encrypted patient identity, medical record number, users' identity, clinical position/role of the user, date of access, and several other variables of the access action. We pulled data from 1 month before cancer diagnosis to 9 months post diagnosis. There were approximately 2.45 million records of access logs. More than 6800 unique users, representing over 150 roles or occupations, accessed the health records. Decisions regarding exclusion and categorization were reached through consensus of three physician project team members. After excluding student learners and database analysts, we categorized the remaining users into three groups: core patient care team, support team, and administrative team.
RESULTS: Figure 1 shows the global network of the core patient care team, which depicts regular information transmission ties among core care providers. The network was constructed using 1933 different providers in the medical center who were connected to an average of 5.8 other providers; however, over 20 of them were very highly connected ( $>100$ other providers). There were 13 subgroups based on the closeness of their relationship. The size of subgroups ranged from 3 providers to 320 providers. The largest subgroup was dominated by inpatient nurses and housestaff physicians, and the second largest subgroup was led by outpatient nurses and attending physicians. The longest path length (i.e., the minimum number of information transmission ties needed to connect two providers) between any two nodes in the network was 14 . The average path length was 4.09 .

CONCLUSIONS: This study demonstrates that EHR systems can provide extensive and detailed data to describe the connectivity and network structure of healthcare teams. Future study is needed to validate this method in studying team care.

Figure 1. Global network of core patient care team and clustering

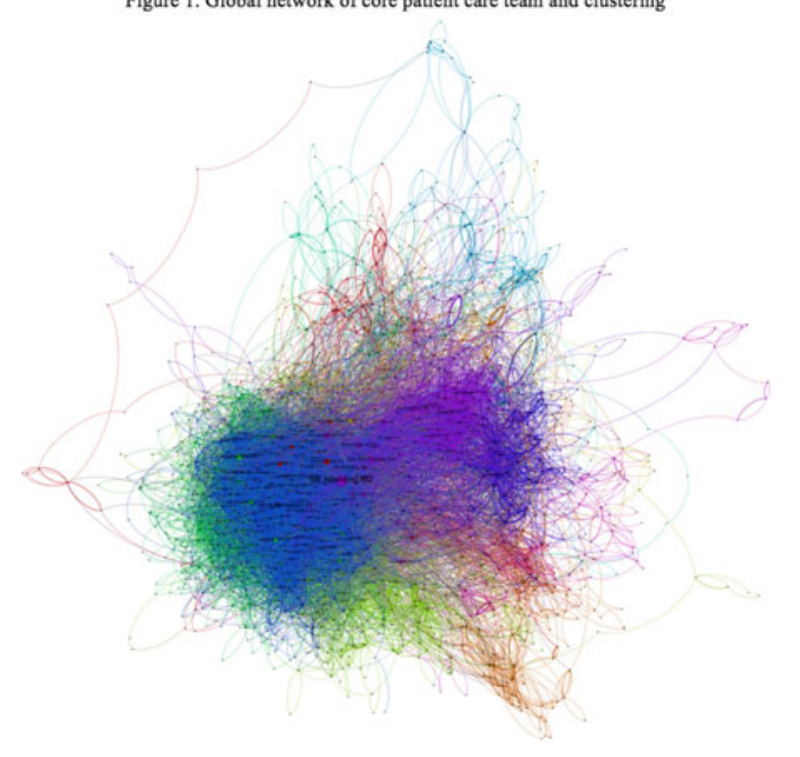

SOCIOECONOMIC DISPARITIES IN COMPLETION OF OPEN ACCESS COLONOSCOPY Jeremy Gentile ${ }^{2}$; Patrick Bradley ${ }^{1,}{ }^{2}$; Kevin S. Fay ${ }^{1}$; Michelle Mohyi ${ }^{1}$; Daniel Hrabec ${ }^{1}$; Kassem Bourgi ${ }^{3}$. ${ }^{1}$ Henry Ford Health System, Livonia, MI; ${ }^{2}$ Henry Ford Hospital, Sterling Heights, MI; ${ }^{3}$ Henry Ford Hospital, Detroit, MI. (Control ID \#2469153)

BACKGROUND: Screening colorectal cancer, a leading cause of death in the US, decreases disease incidence and mortality. While traditionally colonoscopies required specialist consultation, open access colonoscopy allows eligible patients to schedule their colonoscopy with a referral from their primary care physician. We aimed to evaluate current rates and trends in completing colonoscopy screening among patients with open colonoscopy referrals.

METHODS: We constructed an electronic cohort of all patients with an open colonoscopy referral ordered between 6/1/2013 and 6/30/15 and then identified the subjects who successfully completed the colonoscopies. We evaluated the association between multiple patient and provider specific factors and the likelihood of having colonoscopy screening completed. Patients' income was based on the health system's virtual demographic warehouse which uses the 2010 US geocode census. We used descriptive analysis, chisquare and two-sample t-tests to identify the proportion and characteristics of patients who completed screening. We also performed multivariate logistic regression to analyze odds of colonoscopy completion.

RESULTS: A total of 14,399 patients were included in our cohort of which 5062 (35.2\%) had successfully completed their colonoscopy screening. Patients who completed their 
colonoscopy screening had higher income $(58669 \pm 31100$ vs $53711 \pm 28491, p<0.001)$ with an adjusted odds ratio (aOR 1.05, 1.04-1.07, $P<0.001$ ) with each incremental income increase of $\$ 10,000$. Additionally, African Americans (aOR of $1.4,95 \% \mathrm{CI}$ $1.29-1.51)$ and patients who subscribed to the patient portal of the Electronic Medical Records (EMR) (59 vs. $49 \%, p<0.01$, aOR $1.41,95 \%$ CI 1.31-1.52) were more likely to have their colonoscopy order completed. However, female gender (56.9 vs. $59.1 \%$, $p<0.01$, aOR $0.87,95 \%$ CI $0.81-0.94$ ) and being divorced (7 vs. $8 \%, p<0.001$, aOR $0.87,95 \%$ CI $0.76-0.99)$ were associated with lower likelihood of completing colonoscopy screening. Similarly current smokers ( 13 vs. $18 \%, p<0.01$, aOR $0.74,95 \% \mathrm{CI}$ $0.67-0.82$ ) were significantly less likely to complete colonoscopy screening

CONCLUSIONS: Significant gender, racial and economic disparities are associated with the completion of colonoscopy screening among patients with open access colonoscopy referrals. However electronic health engagement, through subscribing to the patient portal of the EMR, is associated with higher likelihood of colonoscopy completion.

SPATIAL ACCESS TO PRIMARY CARE - THE CONTRIBUTION OF COMMUNITY HEALTH CENTERS TO ADDRESSING ACCESS DISPARITIES IN A LARGE URBAN AREA Jane W. Seymour ${ }^{2}$; Daniel Polsky ${ }^{1,}{ }^{1}$; Elizabeth Brown ${ }^{3}$; David Grande ${ }^{1,1}$. ${ }^{1}$ University of Pennsylvania, Philadelphia, PA; ${ }^{2}$ Boston University, Boston, MA; ${ }^{3}$ Christiana Care Health System, Wilmington, DE. (Control ID \#2469529)

BACKGROUND: Several years into implementation of the Affordable Care Act, low income and minority populations are experiencing large gains in coverage. However, they also are at higher risk of living in primary care shortage areas. We previously found that the supply of primary care was lower in and around census tracts with a high fraction of African Americans. Given the growing role of community health centers $(\mathrm{CHCs})$ in the U.S., we examined the geography of primary care in a large urban area with the following objectives: 1) describe CHCs contribution to primary care supply, 2) describe location of CHCs relative to the socio-demographics of the population including racial composition, 3) determine how proximity to a $\mathrm{CHC}$ influences the risk of a census tract being located in a low access area and whether the effects varies based on racial composition. METHODS: We conducted a census of all primary care practices in and near Philadelphia using data from SK\&A, the largest commercial and Medicaid insurers in the region, the local department of public health, and organizations representing CHCs. We conducted a telephone survey of each practice to quantify the number of primary care providers in each practice and to approximate hours worked. We calculated population to provider ratios for each census tract based on the adult population and number of providers within a 5-min drive time from the centroid of each census tract. We identified six clusters of tracts with the lowest supply. Logistic regression was used to assess relationships between sociodemographic factors and proximity to a $\mathrm{CHC}$ and risk of being in a low access cluster.

RESULTS: We identified 472 primary care office sites in 222 census tracts in Philadelphia with a total of 1570 full-time equivalent (FTE) providers. Of these, 35 offices (7.4\%) were CHCs, representing $13.6 \%$ of the primary care provider FTEs. In adjusted analyses, census tracts in close proximity to a $\mathrm{CHC}(<5 \mathrm{~min})$ had higher rates of public insurance (30-39 vs. $<10 \%$ : $\mathrm{OR}=7.84, p=0.001 ; \geq 40$ vs. $<10 \%$ : $\mathrm{OR}=31.06, p<0.001)$ but lower rates of uninsurance $(10-19$ vs. $<10 \%, \mathrm{OR}=0.42, p=0.045)$. Tracts in close proximity to a $\mathrm{CHC}$ had a higher population density (log thousand/square mile; OR 3.49, $p<0.0001$ ) but similar proportions of African Americans and Hispanics. In adjusted analyses, census tracts in close proximity to a $\mathrm{CHC}$ were less likely to be in a low access cluster $(\mathrm{OR}=0.13, p<0.001)$ while those with a high proportion of African Americans $(>80 \%)$ were more likely to be in a low access cluster (OR $=$ $23.16, p<0.001$ ). The effect of proximity to a $\mathrm{CHC}$ on risk of being in a low access cluster did not vary based on the fraction of the population that was African-American.

CONCLUSIONS: We found that the location of CHCs is strongly associated with population density and public insurance rates, negatively associated with uninsurance rates and not associated with racial composition. CHCs have a protective effect - census tracts close to CHCs are less likely to be in a cluster of low primary care supply. However, this protective effect does not vary by a tract's racial composition. In order to address identified racial disparities in access to primary care, areas with higher proportions of racial minorities need to be prioritized.

SPEAKING UP ABOUT TRADITIONAL AND PROFESSIONALISM-RELATED PATIENT SAFETY THREATS: A NATIONAL STUDY OF RESIDENTS William Martinez $^{5}$; Lisa Lehmann ${ }^{7,8}$; Gerald B. Hickson ${ }^{4}$; Donald Brady ${ }^{4}$; Eric J. Thomas ${ }^{2}$; Jason Etchegaray $^{9}$; Julia Shelburne ${ }^{2} ;$ Anneliese Schleyer ${ }^{6} ;$ Jennifer Best $^{6} ;$ Natalie May $^{3}$; Sigall K. Bell ${ }^{1} .{ }^{1}$ BIDMC, Boston, MA; ${ }^{2}$ The University of Texas Medical School at Houston, Houston, TX; ${ }^{3}$ University of Miami Miller School of Medicine, Charlottesville, VA; ${ }^{4}$ Vanderbilt University, Nashville, TN; ${ }^{5}$ Vanderbilt University Medical Center, Nashville, TN; ${ }^{6}$ University of Washington, Seattle, WA; ${ }^{7}$ United States Department of Veterans Affairs, Washington, DC; ${ }^{8}$ Harvard Medical School, Boston, MA; ${ }^{9}$ RAND Corporation, Santa Monica, CA. (Control ID \#2468063)

BACKGROUND: Objectives: 1) To compare residents' attitudes toward and experience with speaking up about traditional patient safety threats (eg, breach of sterile technique) vs. professionalism-related patient safety threats (eg, disruptive behavior). 2) To identify independent predictors of speaking up about traditional and professionalism-related patient safety threats.

METHODS: We anonymously surveyed 1800 medical and surgical residents across six U.S. academic medical centers to assess their attitudes and experiences with speaking up about traditional patient safety threats and professionalism-related patient safety threats. Residents were asked to rate their likelihood of speaking up and the potential for harm to the patient for two vignettes: 1) Vignette A (traditional safety threat): clinician unknowingly contaminates a sterile field while placing a central line; 2) Vignette B (professionalism-related safety threat): clinician is distracted by non-patient related text messaging during ICU rounds. Additional items assessed: a) Respondents' self-reported speaking up about traditional patient safety threats and unprofessional behavior; b) Attitudes toward speaking up about patient safety and unprofessional behavior, c) Patient safety-related climates using validated scales of teamwork, safety, and speaking up for safety and professional climates.

RESULTS: Response rate was $47 \%$ (837/1800). Respondents' gender, specialty, and post-graduate year did not differ significantly from the total population surveyed. The majority of respondents $(82 \%, 683 / 837)$ agreed that speaking up about unprofessional behavior was important for patient safety. During their most recent inpatient month, $75 \%$ (638/837) of respondents reported observing unprofessional behavior, and $49 \%(410 / 837)$ reported observing traditional patient safety threats; $P<0.001$. However, respondents reported speaking up about unprofessional behavior (46\%, 287/638) less commonly than speaking up about traditional patient safety threats (71\%, 291/410); $P<0.001$. When compared to speaking up about traditional patient safety concerns, respondents' had significantly more negative attitudes regarding the possibility of meaningful change, support from colleagues, and norms of behavior regarding speaking up about unprofessional behavior (Table 1). After adjusting for perceived potential for harm to the patient, role of individual being spoken up to (ie, nurse, intern, attending), patient safety-related climates (ie, teamwork, safety, and speaking up for safety and professional climates), respondent characteristics, and study site, residents were significantly less likely to speak up in the professionalism-related safety threat vignette than the traditional patient safety threat vignette (OR 0.05 , $P<0.001$ ). Teamwork climate and speaking up climate for patient safety (SUCSafe) were independently associated with speaking up in the traditional safety 
vignette but not the professionalism-related safety vignette; while only the speaking up climate for professionalism (SUC-Prof) was associated with speaking up in the professionalism vignette (Table 2).

CONCLUSIONS: Residents routinely observe unprofessional behavior, and agree that speaking up about unprofessional behavior is important for patient safety, yet they are less likely to speak up about it compared to traditional patient safety threats. After adjusting for contextual and personal variables, including perceived potential for patient harm, residents were much less likely to speak up in the professionalism-related safety threat vignette than the traditional patient safety threat vignette. Teamwork climate and safety climate alone may not assess important aspects of safety culture that support speaking up, particularly speaking up about unprofessional behavior, and the SUC-Prof may fill an important gap in safety culture assessment. Self-reported patient safety training was not associated with speaking up, suggesting a need to revise existing curricular efforts.

Table 2 - Multivariate Analysis of Predictors of Likelihood of Speaking Up in Two Safety Threat Vignettes

\begin{tabular}{|c|c|c|}
\hline Predictors & $\begin{array}{l}\text { Vignette A } \\
\text { (Traditional): } \\
\text { Breach of Sterile } \\
\text { Field } \\
\text { OR }(99 \% \mathrm{CI})\end{array}$ & $\begin{array}{l}\text { Vignette B } \\
\text { (Professionalism): } \\
\text { I n a t t e n t i v e } \\
\text { Clinician } \\
\text { OR (99\% CI) }\end{array}$ \\
\hline \multicolumn{3}{|l|}{ Vignette Characteristics } \\
\hline \multicolumn{3}{|l|}{ Speaking-up to a: } \\
\hline Nurse & $1.07(0.70-1.65)$ & $0.76(0.56-1.02)$ \\
\hline - Intern & $2.49(1.49-4.16)$ & $2.00(1.48-2.70)$ \\
\hline - Resideint & 1.00 (reference) & 1.00 (reference) \\
\hline Attenidng & $0.18(0.12-0.26)$ & $0.11(0.07-0.16)$ \\
\hline Potential for Harm (high) & $2.80(2.09-3.74)$ & $5.47(4.27-7.01)$ \\
\hline \multicolumn{3}{|l|}{ Patient Safety Climates } \\
\hline $\begin{array}{l}\text { Safety Attitudes Questionnaire - } \\
\text { Teamwork Climate (positive) }\end{array}$ & $1.90(1.36-2.66)$ & $1.31(0.99-1.74)$ \\
\hline $\begin{array}{l}\text { Safety Attitudes Questionnaire - } \\
\text { Safety Climate (positive) }\end{array}$ & $0.97(0.69-1.37)$ & $1.11(0.84-1.47)$ \\
\hline $\begin{array}{l}\text { Speaking Up Climate for Patient } \\
\text { Safety (positive) }\end{array}$ & $1.46(1.02-2.09)$ & $1.01(0.77-1.33)$ \\
\hline $\begin{array}{l}\text { Speaking Up Climate for } \\
\text { Professionalism (positive) }\end{array}$ & $1.84(1.05-3.20)$ & $1.76(1.23-2.50)$ \\
\hline \multicolumn{3}{|l|}{ Respondent Characteristics } \\
\hline Male & $1.20(0.90-1.59)$ & $1.52(1.20-1.93)$ \\
\hline Intern (PGY-1) & $0.37(0.28-0.50)$ & $0.42(0.32-0.55)$ \\
\hline Surgical Specialty & $1.65(1.20-2.28)$ & $3.03(2.34-3.92)$ \\
\hline Moral Courage (high) & $1.72(1.13-2.62)$ & $1.44(1.08-1.92)$ \\
\hline Self-reported Patient Safety Training & $1.02(0.64-1.62)$ & $1.44(0.94-2.20)$ \\
\hline
\end{tabular}

Models included Study Site as a covariate (not shown)

Table 1 - Attitudes Regarding Speaking Up about Patient Safety Concerns vs. Unprofessional Behavior

\begin{tabular}{|c|c|c|c|}
\hline Item & $\begin{array}{l}X= \\
\text { patient } \\
\text { safety } \\
\text { concerns } \\
\% \text { Agree, } \\
N=837\end{array}$ & $\begin{array}{l}\mathrm{X}= \\
\text { unprofessional } \\
\text { behavior } \\
\% \text { Agree, } \\
N=\mathbf{8 3 7}\end{array}$ & $\begin{array}{l}P- \\
\text { value }\end{array}$ \\
\hline $\begin{array}{l}\text { 1. Speaking up about } X \text { results in } \\
\text { meaningful change in my clinical area. }\end{array}$ & $60 \%$ & $40 \%$ & $<0.001$ \\
\hline $\begin{array}{l}\text { 2. In my clinical area, it is difficult to } \\
\text { speak up if I have/observe } X \text {. }\end{array}$ & $16 \%$ & $38 \%$ & $<0.001$ \\
\hline $\begin{array}{l}\text { 3. The culture in my clinical area } \\
\text { makes it easy to speak up about } \mathrm{X} \\
\text { that does not involve me or my } \\
\text { patients. }\end{array}$ & $54 \%$ & $34 \%$ & $<0.001$ \\
\hline $\begin{array}{l}\text { 4. In my clinical area, I observe others } \\
\text { speaking up about } X \text { even if it does not } \\
\text { involve them or their patients. }\end{array}$ & $43 \%$ & $27 \%$ & $<0.001$ \\
\hline $\begin{array}{l}\text { 5. I am encouraged by my colleagues to } \\
\text { speak up about } X \text {. }\end{array}$ & $65 \%$ & $36 \%$ & $<0.001$ \\
\hline
\end{tabular}

SPECIALTY PHYSICIANS AND MEDICAID: PROBLEMS BEYOND LOW REIMBURSEMENT Meredith A. Niess ${ }^{2}$; Irene V. Blair ${ }^{3}$; Anna L. Furniss ${ }^{4}$; Arthur Davidson ${ }^{1} .{ }^{1}$ Denver Health, Denver, CO; ${ }^{2}$ Oregon Health Science University, Portland, OR; ${ }^{3}$ University of Colorado Boulder, Boulder, $\mathrm{CO} ;{ }^{4}$ University of Mississippi Medical Center, Jackson, MS. (Control ID \#2438072)

BACKGROUND: Medicaid insures more than 72 million people, but patients often have difficulty accessing outpatient care because of restrictive acceptance practices. Provider willingness to care for Medicaid patients varies; primary care physicians note particular difficulty finding specialty care for Medicaid patients. Some specialty providers describe discontent with the Medicaid system, its patients, or both. In this study, we measure outpatient specialty physician beliefs about problems with the Medicaid system and its patients, then correlate these beliefs with their willingness to provide care for these patients.

METHODS: In 2014, we surveyed 1600 randomly selected Colorado Medical Society specialist members. Primary care physicians, hospitalists, pediatric specialists, and emergency physicians were excluded, along with retirees and trainees. Using a 4-point scale ranging from no problem (0) to major problem (3), respondents described perceptions of how problematic 8 Medicaid "system factors" and 9 "patient factors" were in caring for Medicaid patients. Using factor analysis, we identified "problem themes" from system and patient factors. To assess potential impact of perceived problems, we used problem theme variables (average score of all problems in each theme) in a logistic regression model with a primary outcome of provider willingness to see Medicaid patients. We derived the willingness outcome from two survey responses: willingness to see Medicaid (1-7) and ideal practice Medicaid percentage (5 levels).

RESULTS: Of 1600 eligible physicians, $810(51 \%)$ returned the completed survey. Respondents were predominantly male (76\%), white non-Hispanic ( $88 \%$ ), practicing for $>10$ years $(80 \%)$, and had ownership interest in their practice $(73 \%)$. Overall, perception of system factors (mean score $=2.06 \mathrm{SD}=0.51$ ) was more problematic than patient factors (mean $=1.72 \mathrm{SD}=0.59)$ with 62 and $35 \%$ of respondents rating these as moderate/major problems, respectively. Among system problems, moderate/major problem rankings were most common for low reimbursement $(97 \%$; mean $=2.77)$ and administrative burden $(82 \%$; mean $=2.28)$. Among patient problems, moderate/major problem rankings were most common for patient non-adherence $(84 \%$; mean $=2.29)$ and late or missed appointments $(82 \%$; mean $=2.32)$. [See Figures] Factor analysis revealed system problems clustered in themes of "opportunity costs," (mean $=2.49 \mathrm{SD}=0.72$ ) "bureaucratic burden," (mean=2.18 SD=0.78) and "patient support needs," (mean=1.89 SD=0.72). Patient problem themes included "irresponsible patient behaviors," (mean $=2.30 \mathrm{SD}=$ 0.72 ) "patient complexity," (mean $=1.91 \mathrm{SD}=0.73$ ) and "undesirable/undeserving patients" (mean $=1.28 \mathrm{SD}=0.79$ ). Highest problem themes included opportunity costs, irresponsible patients, and bureaucratic burden with a moderate/major problem score by $89 \%, 79 \%$, and $73 \%$ of respondents, respectively. Logistic regression yielded association between lower provider willingness to see Medicaid patients and higher problem rating for opportunity costs $(p<0.001)$ and patient irresponsibility $(p=0.014)$.

CONCLUSIONS: Consistent with prior research, reimbursement remains specialists' highest concern regarding Medicaid. However, specialty providers also perceive other major problems in caring for Medicaid patients. In our model, specialty providers' low willingness to accept these patients correlated with concern for high opportunity costs and irresponsible patient behavior. Thus, even with improved reimbursement, Medicaid patients will likely still experience an access gap due to specialty provider concerns. Targeted interventions to address these concerns (e.g., care management to help appointment attendance and non-adherence, thereby attenuating provider assessments of patient irresponsibility) merit further investigation. As we work to improve Medicaid access, lessons from the quality and safety movement suggest addressing issues at the systemlevel holds greater promise than blaming individuals. 


\section{Patient Problem Severity: Moderate/Major}

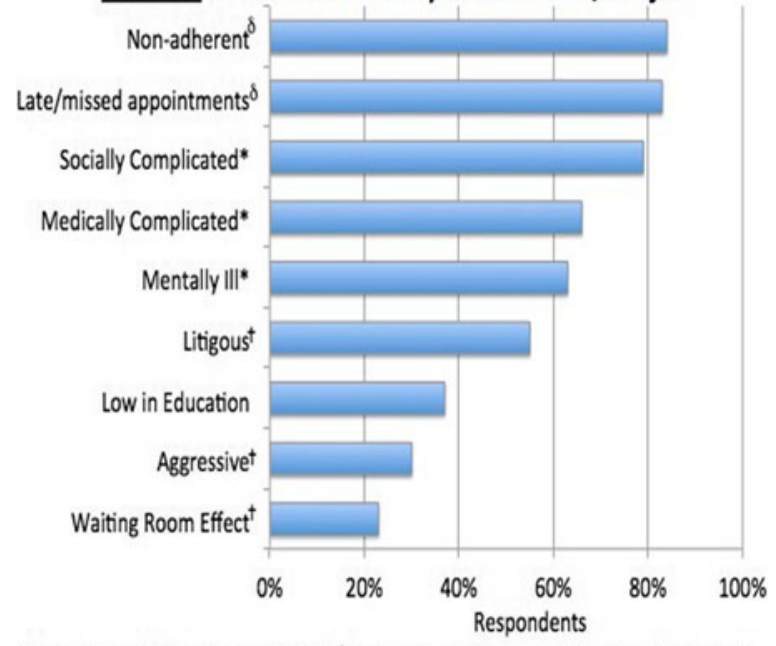

"Factor Group "Patient complexity"; ${ }^{\circ}$ Factor Group "Irresponsible patient behavior"; "Factor Group "Undesirable or undeserving patients"

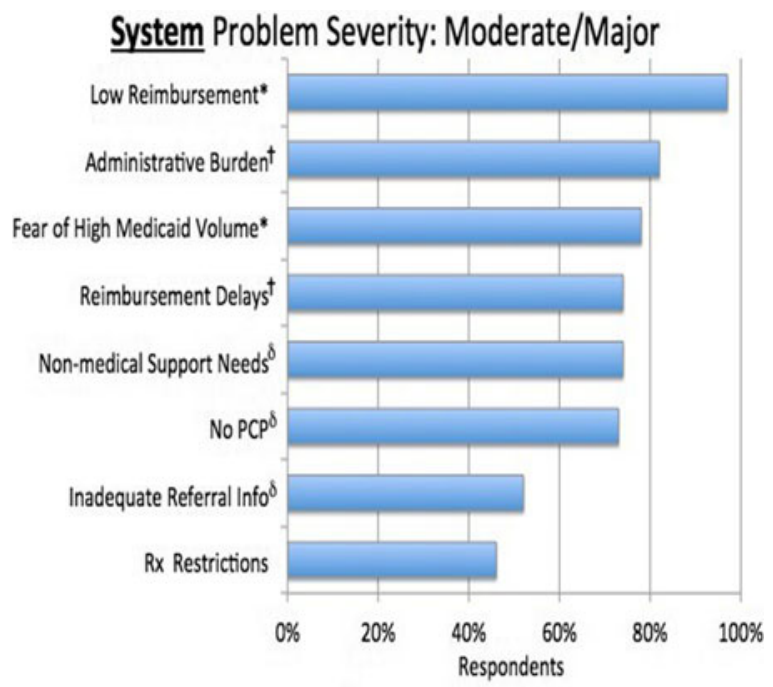

"Factor Group "Opportunity costs"; ${ }^{\dagger}$ Factor Group "Bureaucratic burden"; Factor "Group "Unmet patient support needs"

\section{SPECIALTY REFERRALS FROM THE PRIMARY CARE SETTING AND THE} IMPACT ON DIAGNOSIS Andrew Schreiner ${ }^{2}$; Jingwen Zhang ${ }^{1}$; Patrick D. Mauldin ${ }^{2}$; William P. Moran ${ }^{2} .{ }^{1}$ MUSC, Charleston, SC; ${ }^{2}$ Medical University of South Carolina, Charleston, SC. (Control ID \#2468901)

BACKGROUND: Diagnostic error is "the failure to establish an accurate and timely explanation of the patient's health problem(s) or communicate that explanation to the patient." This dynamic process requires the collaboration and communication of clinicians, patients, and families. Specialty referrals revolve around these same stakeholders, and may play an integral role in reducing diagnostic error. Understanding how specialty referrals contribute to diagnostic accuracy may help in optimizing their value. To help answer this question, we identified referrals placed for the purpose of aiding diagnosis and assessed the concordance of diagnosis at the time of referral and after specialist consultation. Evaluating the frequency of diagnostic change will help to address the value added by the specialty referral process.

METHODS: For this study, we searched the electronic health record of an academic, internal medicine, patient-centered medical home (PCMH) at the Medical University of
South Carolina (MUSC) for ambulatory referral orders to specialty providers during a 6 month period between July and December 2014. These referrals were randomly selected and reviewed with a data abstraction tool. Data abstraction elements included the goal of referral (diagnosis, therapy, procedural need, or other), the attached ICD-9 code at the time of referral, and the ICD-9 code assigned to the issue by the specialist (at initial or subsequent visits). All ICD-9 codes were then characterized as either a symptom or diagnosis. Referrals deemed to have diagnostic influence included those where ICD-9 codes transitioned from symptom to diagnosis or a diagnosis to a new or more specific diagnosis.

RESULTS: PCMH providers placed a total of 2757 referrals for specialty consultation. After random selection, 200 referrals underwent review. Of these, 52 originated for the purpose of diagnostic aid, and $33(63.4 \%)$ received an ICD-9 code that was a diagnosis and differed from that assigned by the primary care provider at the time of referral. Orthopedic surgery and dermatology referrals together made up the majority of these influential diagnostic consultations (24.2 and $21.2 \%$ respectively).

CONCLUSIONS: Specialty physician referrals for the purpose of diagnostic aid do not always achieve this goal. Potential reasons for this include poor communication, misdirection of specialty destination, and diagnostic error. Further analysis is underway, incorporating more patients, to determine the value added benefit attributable to diagnostic referrals. The gravity and frequency of diagnostic error demand investigation into the value of diagnostic specialty referrals. Further steps to enhance the process include better goal clarification by primary providers, identification of frequent symptoms or diagnoses driving referrals for targeted education, and an understanding for the role and value of diagnostic confirmation.

\section{SPECIALTY REFERRALS: A CASE OF WHO YOU KNOW VERSUS WHAT} YOU KNOW? Andrew Schreiner ${ }^{2}$; Jingwen Zhang ${ }^{1}$; Patrick D. Mauldin ${ }^{2}$; William P. Moran ${ }^{2} .{ }^{1}$ MUSC, Charleston, SC; ${ }^{2}$ Medical University of South Carolina, Charleston, SC. (Control ID \#2468906)

BACKGROUND: Specialty referrals serve as the interface between primary care and the specialty physician. Historically, primary care physicians identified the importance of the relationship with particular specialty physicians in making referral decisions. This relationship included the ability to communicate, the medical skill of the specialist, and the overall past experience with previous referrals. As primary care physician practices vertically integrate with hospitals, has this emphasis on individual specialist relationships changed? To help answer this question, we looked at the frequency of specific provider designation in specialty referrals from an academic, hospital-owned, internal medicine, patient-centered medical home.

METHODS: We searched the electronic health record of an academic, internal medicine, patient-centered medical home (PCMH) at the Medical University of South Carolina (MUSC) for ambulatory referral orders to specialty providers during a 6 month period between July and December 2014. These referrals underwent random selection and subsequent chart review. Data abstraction elements included the ordering provider, the specialty designation, and a query for specific provider identification. Univariate analysis (Chi squared test) compared proportions of referrals with a designated provider placed by resident and faculty physicians. SAS 9.3 (SAS Institute Inc., Cary, NC) was used for statistical analysis.

RESULTS: General internists placed 2757 referrals for specialty consultation, and after random selection, 200 charts underwent review. Of these, $25(12.5 \%)$ referrals included a preference for a specific specialty provider. In total, $19 \%$ (18/95) of referrals placed by faculty designated a specific physician versus a non-specific specialty, compared to $6.7 \%$ (7/105) of referrals placed by residents $(p=0.009)$.

CONCLUSIONS: Relationships between primary care and specialty providers used to play an integral role in ambulatory referral decisions. Vertical integration of practices with 
large health systems may diminish the role of these relationships in the referral process. Additional factors to consider in this change include referral education, the physicians' length of tenure with their practice, an increased reliance on electronic communication, and the need for scheduling flexibility. The loss of relationship in the referral process may pose a threat to communication and care coordination, leading to adverse consequences on quality, cost, and patient satisfaction.

SPECIALTY REFERRALS: EXPERIENTIAL LEARNING AND REFERRING Andrew Schreiner $^{2}$; Jingwen Zhang ${ }^{1}$; Patrick D. Mauldin' ${ }^{2}$; William P. Moran ${ }^{2} .{ }^{1}$ MUSC, Charleston, SC; ${ }^{2}$ Medical University of South Carolina, Charleston, SC. (Control ID \#2468947)

BACKGROUND: In 2009, 105 million ambulatory office visits resulted in referral, with the proportion of ambulatory visits resulting in specialty referral nearly doubling (4.8 to $9.3 \%$ ) over the previous decade (1999 to 2009). Estimated annual costs for these specialty visits approach $\$ 98$ billion. Many elements contribute to referral practices, including the goal of referral, patient factors, physician factors, and practice setting. Investigating how these elements interact, and which factors drive referral patterns, may provide a starting point for optimizing value in the referral process. Physician factors include the type of training received, years of experience, comfort with the condition at hand, and diagnostic certainty. This study will look at the impact of years of physician experience on referral patterns. METHODS: We searched the electronic health record of an academic, internal medicine, patient-centered medical home (PCMH) at the Medical University of South Carolina (MUSC) for ambulatory referral orders to specialty providers between July and December of 2014. These referrals were randomly selected and reviewed. Data abstraction elements included the ordering provider and the goal of referral. This required chart review to identify referral patterns of residents to avoid masking with the supervising physician. After chart review, we merged the level of experience of each provider to the referral order placed during the study period. Resident experience ranged from 1 year (PGY-1) to 5 years (PGY-5). Faculty experience groupings include: 0-2 years, 3-5 years, 6-10 years, 1120 years, $21-30$ years, and $>30$ years. Univariate analysis (Chi squared test and Fisher's exact test) was used to compare proportions of referrals placed by the respective levels of experience. SAS 9.3 (SAS Institute Inc., Cary, NC) was used for statistical analysis.

RESULTS: PCMH providers placed 2757 referrals for specialty consultation. After random selection, 200 of these referrals underwent chart review. Resident physicians placed 105 of these referrals and faculty placed 95 . No statistically significant difference arose in the types of referrals (diagnosis, therapy, procedural need, and other) placed by level of experience in residents $(p=0.657)$. Of the 95 specialty referrals placed by faculty physicians during the study period, a statistically significant difference existed in the types of referrals placed $(p=0.0014)$. Further, faculty physicians significantly differed in the number of diagnostic referrals ordered by varying years of experience $(p=0.0024)$.

CONCLUSIONS: Physicians' years of experience appears to play a role in the referral patterns of faculty. Further analyses are ongoing to identify which years of experience drive this difference. Potential reasons for this difference include different training environments (time, location, or population), varying levels of diagnostic and therapeutic comfort, duration of tenure in a single practice, and the patient satisfaction pressures in building or maintaining a practice. Identifying trends in referrals rates related to the primary care physicians' years of experience may provide targets for interventions aiming to optimize quality and lower costs associated with specialty referral.

SPECIALTY REFERRALS: IF YOU BUILD IT, WILL THEY COME? Andrew Schreiner $^{2}$; Jingwen Zhang ${ }^{1}$; Patrick D. Mauldin ${ }^{2}$; William P. Moran ${ }^{2} .{ }^{1}$ MUSC, Charleston, SC; ${ }^{2}$ Medical University of South Carolina, Charleston, SC. (Control ID \#2468883)
BACKGROUND: Referrals from primary care practitioners to specialty providers occur with great frequency in the U.S. and are rapidly accelerating. In 2009, 105 million ambulatory office visits resulted in referral, with the proportion of ambulatory visits resulting in specialty referral nearly doubling (4.8 to $9.3 \%$ ) over the previous decade (1999 to 2009). One in three patients receive specialty referral, and over half of outpatient physician visits are to specialists. Estimated costs for these specialty visits approach $\$ 98$ billion annually. These cost data reflect visits to specialty providers, but the aforementioned frequency of referrals from primary care providers is far greater. Of those referrals placed, the volume of appointments scheduled and visits completed may greatly impact the availability of providers, the efficiency of care delivery, and the health of patients. In this study, we looked at the frequency of specialty referral placement, scheduling, appointment attendance, and the differences in these variables between two clinics of differing socioeconomic status.

METHODS: We searched the electronic health record of an academic, internal medicine, patient-centered medical home (PCMH) at the Medical University of South Carolina (MUSC) for ambulatory referral orders to specialty providers during a 6 month period between July and December 2014. These referrals were then randomly selected and reviewed with a data abstraction tool. Data abstraction elements included the ordering provider, the date of the specialty appointment, and the date of the attended specialty visit. With this data, we compared the rates of scheduling and attendance of specialty visits from the resident and faculty practices in the PCMH. Delineation between practices was based upon the ordering provider. Univariate analysis (Chi squared test) was used for proportions. SAS 9.3 (SAS Institute Inc., Cary, NC) was used for statistical analysis.

RESULTS: Primary care providers ordered 2757 referrals for specialty consultation. After random selection, 200 charts underwent review with the exclusion of 8 podiatry referrals (due to an inability to confirm scheduling or attendance). Of the remaining 192 specialty referrals, $161(83.4 \%)$ were scheduled, and $120(74.5 \%)$ of those scheduled were attended. The referrals placed, scheduled, and attended were compared by the ordering provider. There was no statistically significant difference in the proportion of specialty visits scheduled between faculty $(83.7 \%)$ and resident $(83.2 \%)$ referrals $(p=$ $0.922)$. However, there was a lower proportion of scheduled visits attended for resident referrals $(67.9 \%)$ compared to those placed by faculty $(81.8 \%, p=0.042)$.

CONCLUSIONS: Disparities exist in the attendance of specialty physician consultative visits. This disparity persists despite similar proportions of scheduled referrals, and further analysis is underway to better understand potential causes which may include lack of transportation and poor communication regarding scheduling. This disparity impacts the health of the referral recipients, specialist availability, and health delivery efficiency. Addressing this disparity will potentially help to improve the health of patients, lower cost, and improve access

SSRIS, SNRIS OR TCAS ARE NOT ASSOCIATED WITH ABNORMAL PLATELET AGGREGATION OR RELEASE IN VITRO Dharmesh Gopalakrishnan; Heesun J. Rogers; Paul Elson; Keith McCrae. Cleveland Clinic, Cleveland, OH. (Control ID \#2469518)

BACKGROUND: There is conflicting evidence concerning the effects of various medications on platelet function and their clinical implications. The major groups of medications reported to cause platelet dysfunction are the non-steroidal anti-inflammatory drugs (NSAIDs), selective serotonin re-uptake inhibitors (SSRIs), serotonin and norepinephrine reuptake inhibitors (SNRIs), tricyclic antidepressants (TCAs), vitamin E, vitamin C, antiepileptic drugs, particularly divalproex, and various supplements including fish oil, garlic etc. In this retrospective EMR-based study, we analyzed the effect of some of these and other commonly-used medications on various assays of platelet function - platelet function analyzer-100 (PFA-100) closure times, platelet aggregation assay (using light transmission 
aggregometry (LTA)), platelet dense granule release (using lumi-aggregometry), and platelet flow-cytometry for surface glycoproteins.

METHODS: Four hundred ninety-seven patients who had platelet aggregation testing performed using LTA between August 2008 and August 2013 were included in our study. Medications taken during the time of testing were recorded from electronic medical records. Propensity score matching for each individual medication of interest was used to adjust for relevant covariates. We used a 1:1 nearest neighbor match without replacement, with caliper width set to 0.2 times the standard deviation of the logit of the propensity score. Following matching, Fisher's exact test or Chi square test was used as appropriate to assess the association between categorical measures, while Mann-Whitney test was used to test the association between categorical and continuous variables. $P<0.05$ was considered significant.

RESULTS: 1$)$ NSAIDs $(n=55)$ were associated with impaired aggregation in the presence of $\operatorname{ADP}(p=0.014)$, but not with any other abnormality in PFA-100 analyses, LTA or release assay. NSAIDs were not associated with the total number of abnormalities or with having $\geq 2$ abnormalities in aggregation or release panels. 2) Antidepressant/antianxiety drugs: No association was found between current use of SSRIs $(n=78)$, SNRIs $(n=30)$ or TCAs $(n=11)$ and any abnormality in platelet aggregation/release panels, PFA-100 closure times, or platelet flow-cytometry. 3) Anti-hypertensive drugs: Beta blockers $(n=112)$ were associated with reduced platelet aggregation $(p=0.017)$ and release $(p=0.046)$ in response to arachidonic acid. Calcium channel blockers $(n=54)$ were associated with impaired aggregation in response to arachidonic acid $(p=0.023)$. ACE inhibitors/ARBs $(n=99)$ were not associated with any abnormalities in the panels. None of the anti-hypertensive groups were associated with having $\geq 2$ abnormalities in either the aggregation or release panels, or with any abnormality in ristocetin-induced aggregation, PFA-100, or platelet flow-cytometry analyses. 3) Other medications: Statins $(n=103)$, metformin $(n=23)$, proton pump inhibitors $(n=126)$ or vitamin $\mathrm{E}(n=14)$ use was not associated with any abnormality in PFA-100, LTA, lumi-aggregometry or flow cytometry. Too few patients in our study were on vitamin C, divalproex, or supplements such as fish oil or garlic to analyze their association with the results of any of the platelet function assays.

CONCLUSIONS: Contrary to previous reports, we did not observe a statistically significant increase in the incidence of abnormalities in any of the platelet function assays in patients taking SSRIs, SNRIs or TCAs. The same was true in patients treated with proton pump inhibitors, statins, metformin and vitamin E. Though NSAIDs, betablockers, and calcium channel blockers were associated with impaired aggregation and/ or release in response to individual agonists, none of them showed a consistent association with the number of abnormalities in aggregation or release panels, or with $\geq 2$ abnormalities in any panel. These findings were unexpected, and a potential weakness of the study design was its retrospective nature in which drug ingestion was based on EMR review. However, in some cases, such as SSRI ingestion, these results may also reflect dissociation between in vitro platelet function analyses and the risk of clinical bleeding associated with drug ingestion.

STAFF SUPPORTED DEPRESSION CARE IN PRIMARY CARE PRACTICES: STAFF CAN FILL SOME GAPS, BUT NOT ALL Elizabeth Pfoh ${ }^{1}$; Anita Everett ${ }^{2}$; Sahar Alee Koloukani ${ }^{2}$; Margo Nathan²; Beth McGinty ${ }^{3} .{ }^{1}$ Johns Hopkins School of Medicine, Baltimore, MD; ${ }^{2}$ Johns Hopkins University, Baltimore, MD; ${ }^{3}$ Johns Hopkins Bloomberg School of Public Health, Baltimore, MD. (Control ID \#2469623)

BACKGROUND: Integrating depression care supports into the primary care setting has been shown through randomized control trials to decrease morbidity and mortality. These support systems are so essential that the U.S. Preventive Services Task Force only recommended depression screening when staff-assisted depression care supports are in place. Understanding how to implement staff supported depression care without the infrastructure of a research study is important because depression screening is a required quality metric for Accountable Care Organizations. We interviewed physicians and practice care managers at primary care practices prior to the integration of depression care support staff to to understand 1) current depression care practices; 2) perceived gaps in depression care; 3) how staff support can fill the perceived gaps.

METHODS: We conducted a qualitative study of physicians and practice managers at 4 clinics in the 3 months prior to their having a masters-level worker trained in behavioral health ("HBS") stationed at their practice. The semistructured interviews were conducted by 2 clinicians and a health services researcher. The interview guide was developed by the interviewers, a psychiatrist, and leadership at the health insurer funding the health behavior specialists. The guide included questions about current depression screening, treatment and management practices, barriers to treating patients with depression, and preferences for support structure (e.g. what activities should the HBS undertake?). All interviews were less than $30 \mathrm{~min}$. The transcribed interviews were analyzed using grounded theory analysis. Johns Hopkins Bloomberg School of Public Health IRB approved this study.

RESULTS: We interviewed seven people: 3 physicians, 3 practice managers and 1 social worker. The results were categorized into current practices in delivery of depression care in primary care settings, limitations of current practices, ways the HBS will fill the identified gaps, and remaining gaps. Current practices included screening patients for depression in both systematic and ad-hoc ways, and treating patients using a combination of non- pharmacological and pharmacological (e.g. exercise, talk-therapy) treatments. The two major perceived gaps were lack of time with patients and the dearth of available psychiatrists. HBS were seen as having the ability to address the former gap, and partially address the latter. Specifically, HBS's could assess disease severity, provide short-term therapy, connect patients with psychiatrists and track referrals. Further, there was a desire for the HBS to identify mental health resources in the community for patients. In addition to depression, interviewees thought the HBS could help them meet the needs of people with other behavioral health conditions such as anxiety, substance abuse, cognitive deficits and multimorbidity. While the HBS was seen as a way to connect patients with psychiatrists, the dearth of available psychiatrists is a bigger problem that the HBS would not be able to solve.

CONCLUSIONS: Primary care practices identified a clear need for assistance in treating and linking patients with behavioral health needs to psychiatrists. The depression care staff support could alleviate some but not all gaps in mental health care for primary care patients. Understanding the needs of primary care practices when attempting to meet quality measures associated with ACO status is important, and may be vital to improving the quality of care for patients.

\section{STATUS CHECK: MEDICAL HOMES FOR THOSE WHO NEED THEM MOST} Jamie Ryan; Jordan Kiszla. The Commonwealth Fund, New York, NY. (Control ID \#2469938)

BACKGROUND: U.S. health care costs are disproportionately concentrated among adults with multiple chronic conditions or functional limitations - a population often referred to as "high-need" patients. Additionally, low-income individuals experience substantial disparities in health care and health outcomes when compared with their more well-off counterparts. Among low-income patients in particular, studies have shown that access to primary care is associated with improved quality of care, better management of chronic conditions, and lower health care costs. The medical home model encourages 
better care management in an effort to contain costs. This analysis looks at the interaction of medical homes with the experiences of high-need adults and low-income adults. METHODS: Data come from the 2014 Biennial Health Insurance Survey, which was conducted among a nationally representative sample of 6027 adults age 19 and older. The 2014 survey was conducted by land line (response rate: $16 \%$ ) and cell phone (response rate: $14 \%$ ), July 22-Dec 14, 2014. We created a medical home composite variable, defining survey respondents as having a medical home if they reported the following indicators: they have a regular provider or place of care, they can get clinical advice by phone during regular hours, they believe their provider knows important information about their medical history, and their regular provider helps to coordinate care with other doctors. This study investigates the experiences of high-need adults, defined as those with at least three chronic conditions and limitations in daily activities $(n=467)$. We also examine low-income respondents, defined as those reporting income below $200 \%$ of the federal poverty level $(n=2,603)$.

RESULTS: Overall, $45 \%$ of all adults surveyed reported having all four indicators of a medical home, compared with $53 \%$ of high-need adults and just $40 \%$ of low-income adults. Nine of ten adults reported having a regular doctor or usual source of care, compared with nearly all high-need adults ( $98 \%$ ) but only $85 \%$ of low-income adults. About one-third (32\%) of all adults reported experiencing at least one of four access problems because of cost: did not fill prescription; skipped recommended test, treatment, or follow-up; had a medical problem but did not visit doctor/clinic; did not get needed specialist care. Experiencing any cost-related access problems was more prevalent among both low-income (41 \%) and high-need (48\%) adults. However, only $25 \%$ of adults with a medical home reported such issues. Half ( $49 \%$ ) of all adults rated the quality of their care as excellent or very good; this is consistent with the experience of high-need adults (also $49 \%$ ). Among low-income adults, such satisfaction with care dropped to $39 \%$, while $65 \%$ of adults with a medical home rated their care as excellent or very good.

CONCLUSIONS: While nearly half of all adults have a medical home, this rate is higher among high-need adults and lower among low-income adults. Adults with a medical home seem to fare better than others in terms of access and quality. Further regressions will quantify the protective effect of having a medical home on these two populations. As the implementation of new models of care marches on, it is important to understand the effects of these models on patients who could benefit most, such as the high-need and the lowincome. Examining the experiences of these two populations is of critical importance: Among high-need adults, $69 \%$ reported low income. Further research on this overlap could reveal promising models beyond the medical home for coordinating and improving care for dual Medicaid-Medicare eligible patients.

\section{STRATEGIES TO ENGAGE HIGH-RISK, COMPLEX PATIENTS IN INTEN-} SIVE OUTPATIENT CARE PROGRAMS Donna M. Zulman ${ }^{2,3}$; Colin W. O'Brien ${ }^{1}$; Cindie Slightam ${ }^{2}$; Andrea Nevedal ${ }^{2}$; Jessica Y. Breland ${ }^{2,3}$. ${ }^{1}$ Stanford University, San Francisco, CA; ${ }^{2}$ VA Palo Alto Health Care System, Menlo Park, CA; ${ }^{3}$ Stanford University, Stanford, CA. (Control ID \#2468675)

BACKGROUND: Intensive Outpatient Care Programs (IOCPs) are designed to improve health outcomes and contain costs for high-risk patients with complex medical and social needs. The impact of these programs is often diminished because of challenges related to patient engagement (i.e., patients' active participation and partnership in their care). We sought to understand medical, behavioral, and social factors that influence patient engagement in IOCPs, and to identify strategies that IOCPs use to address these issues. METHODS: We conducted a qualitative study with 20 IOCP leaders and clinicians (e.g., physicians, nurses, psychologists, case workers) from 12 Northern California programs affiliated with diverse settings (academic hospitals, county healthcare systems, Veterans Affairs facilities, community health centers, and private health systems). Participants were asked to identify barriers to patient engagement in their programs. We conducted semistructured interviews to understand the approaches and strategies that programs employ to address these challenges. We used standard content analysis methods to code engagement barriers and program approaches, and to identify IOCP features that facilitate patient engagement

RESULTS: The most common barriers to patient engagement in IOCPs (cited by over half of participants) included lack of social support/feelings of isolation, care fragmentation across providers and/or healthcare systems, mental illness, financial insecurity, physical symptoms and limitations, and poor social and neighborhood conditions. Other challenges that were cited but affected fewer patients included: inadequate transportation, food insecurity, housing instability, health literacy difficulties, and negative feelings about healthcare providers and systems. Patient engagement strategies comprised four categories: 1) Trust and relationship-building efforts ( "We start out by listening, developing that trust, hearing what their identified problems are... and addressing those first"), 2) Concrete resources and services (e.g., transportation vouchers, cell phones, assistance making appointments), 3) Direct counseling to build insight and coping strategies ("We typically start out by doing the problem solving for them and then slowly work our way to them doing it themselves"), and 4) Population-based approaches (e.g., screening for social and environmental stressors and red-flags). Specific program features that were cited as facilitating patient engagement included: 1) Multidisciplinary teams with diverse skills, knowledge, and personalities to facilitate relationship-building, 2) Adequate staffing and resources to handle demands of patient population, and 3) Flexibility to permit creative, time-intensive, patient-centered strategies, as described by one participant: "Recognizing that the patient is the expert in their life and the patient is in charge of their health and healthcare ... ultimately helps engage people because they feel respected."

CONCLUSIONS: IOCPs use a range of clinical, interpersonal, and population-based approaches to engage high-risk patients, and almost universally offer patient-centered, individualized care that may deviate from standard practice. Findings suggest that programs aiming to increase engagement of complex, high-risk patients should seek out creative and proactive staff, and ensure that they have adequate time, resources, and flexibility to address patients' needs on patients' terms.

STRESS ELECTROCARDIOGRAPHY (ECG) VERSUS RADIONUCLIDE MYOCARDIAL PERFUSION IMAGING (RMPI) AMONG PATIENTS ADMITTED FOR CHEST PAIN: HOW OFTEN ARE WE CHOOSING WISELY? Abdulfatah A. Issak ${ }^{2}$; Karishma Samtani ${ }^{2}$; Jared M. Liston ${ }^{1}$; Dean Bricker ${ }^{2}$; Ronald J. Markert ${ }^{1} .{ }^{1}$ Wright State University, Dayton, $\mathrm{OH} ;{ }^{2}$ Wright State University, Kettering, $\mathrm{OH}$. (Control ID \#2469344)

BACKGROUND: Background: Chest pain is one of the most common reasons patients present to the Emergency Department and results in over 600000 hospitalizations annually. The necessity of inpatient stress testing and particularly nuclear medicine perfusion imaging is not thoroughly studied, but may be over-utilized. The American Board of Internal Medicine (ABIM) Foundation's Choosing Wisely initiative has identified the routine use of stress cardiac imaging among low risk patients as an expensive test that should be questioned by both physicians and patients. Objective: To determine how often patients hospitalized for chest pain are studied with stress ECG versus rMPI, and to evaluate to what degree current practice follows the recommended appropriate use of these cardiac tests as described by American Heart Association (AHA) practice guidelines and published recommendations by the ABIM Foundation's Choosing Wisely campaign.

METHODS: Materials and Methods: We examined medical records of selected patients from both the teaching and non-teaching medicine services of a 900 bed university 
affiliated teaching hospital in Dayton, who were admitted between January 1, 2014 and December 31, 2014 with primary diagnosis of chest pain using ICD 9 codes of 786.50 and 786.59. We included patients who met AHA appropriate use criteria for stress ECG and assessed whether they were tested with stress ECG (appropriate) or rMPI (potentially inappropriate).

RESULTS: Results: A total of 130 patients from the teaching service and 103 patients from the non-teaching service were included in the study. Sex, Age, EKG and Troponin changes at admission were similar between the two groups. The frequency of obtaining Stress ECG when appropriate was $34.2 \%$ for teaching service versus $17.9 \%$ for non teaching service $(p=0.015)$. Stress ECG was carried out in only one quarter of the aggregate sample of patients for whom that study would have been appropriate.

CONCLUSIONS: Conclusion: Our study shows substantial inconsistencies with adherence to appropriate use of cardiac tests per AHA guidelines and ABIM Foundation's Choosing Wisely campaign recommendations among low risk patients admitted for chest pain. Many unnecessary rMPI studies were performed when stress ECG would have been more appropriate. Choosing stress ECG when appropriate can translate into substantial future cost savings as the typical cost of a stress ECG is $\$ 200-\$ 300$ compared with rMPI $\$ 500-\$ 2,000$, and can reduce unnecessary exposure of patients to potentially harmful radiation.

SUBOPTIMAL ANTIPLATELET THERAPY SUGGESTED BY PLATELET AGGREGATION STUDIES DOES NOT CORRELATE WITH A CHANGE IN CLINICAL MANAGEMENT Dharmesh Gopalakrishnan; Heesun J. Rogers; Paul Elson; Keith McCrae. Cleveland Clinic, Cleveland, OH. (Control ID \#2469441)

BACKGROUND: With the expanding repertoire of antiplatelet drug targets and therapies, quantifiable parameters to assess their efficacy can prove to be useful in clinical decision-making. In this retrospective analysis we examined patients who had platelet aggregation testing done at our center between August 2008 and August 2013, focusing on those who were on some form of antiplatelet therapy during testing. Our goal was to define the impact of platelet aggregation testing on decision-making regarding continuation or change in antiplatelet therapy.

METHODS: Light transmission aggregometry (LTA) was used to assess efficacy of treatment with antiplatelet agents. Inhibition of platelet aggregation in response to ADP and arachidonic acid are reflective of the therapeutic effect of aspirin, while inhibition of platelet aggregation in response to ADP reflects the effect of $\mathrm{P} 2 \mathrm{Y} 12$ receptor antagonists. As per parameters developed at our center, the combination of arachidonic acid aggregation $<20 \%$ and ADP aggregation (at concentration of $5 \mathrm{uM}$ ) $<70 \%$ is indicative of optimal therapeutic response to aspirin. Though not fully standardized, ADP aggregation $<40 \%$ is considered to be indicative of therapeutic response to clopidogrel. Descriptive statistics for frequency were used. Pearson coefficient was used to assess correlation. RESULTS: We studied results of platelet aggregometry in 117 patients who were on some form of antiplatelet therapy - 81 on a single agent ( 72 on aspirin alone, 9 receiving P2Y12 antagonist alone), 34 on dual therapy (33 on aspirin + P2Y12 antagonist, 1 on aspirin + cilostazol), and 2 patients on triple therapy ( 1 on aspirin + P2Y12 antagonist + cilostazol, 1 on aspirin + dipyridamole + cilostazol). None of our patients were on Gp IIb/ IIIa inhibitors. In total, 108 patients were on aspirin therapy and 43 patients were on P2Y12 inhibitors. In 65 out of these 117 patients, the primary indication for platelet aggregation testing was to monitor the efficacy of antiplatelet therapy, while in the remaining 52, testing was done for other indications. Fifty-nine of these 65 patients were tested in the setting of a recent thrombotic event in the cerebral, coronary, peripheral, or other vascular bed. While 68 (58 \%) patients had optimal therapeutic response, 49 (42\%) patients - 38 of the 108 (35\%) patients on aspirin, and 14 of the 43 (32\%) patients on a P2Y12 inhibitor - had evidence of suboptimal response to the respective agent. However, antiplatelet therapy was changed or adjusted in only 8 of these 49 patients following these sub-optimal test results, and only 3 had repeat testing following the change (all three of whom were shown to have complete response). Among the 108 patients on aspirin therapy, the total daily dose did not correlate either with the PFA-100 closure times (Collagen/ADP or Collagen/epinephrine) or with the degree of platelet aggregation in response to any of the agonists (ADP, arachidonic acid, collagen, epinephrine or ristocetin).

CONCLUSIONS: Most of the patients who underwent platelet aggregation testing to monitor the efficacy of antiplatelet therapy had a recent thrombotic event that prompted the test. Though $42 \%$ of patients on antiplatelet agent(s) had in vitro evidence of suboptimal platelet inhibition, antiplatelet therapy was changed or adjusted in only $16 \%$ of these individuals, and only $6 \%$ had repeat testing following the change. This suggests that, though platelet aggregation testing was potentially useful in monitoring efficacy of platelet inhibition, clinical changes in antiplatelet therapy were guided more by other factors, casting uncertainty upon the cost effectiveness of platelet function testing in this population. No significant increment was found in the in vitro antiplatelet effect of aspirin with increasing daily doses, suggesting lack of a dose-response beyond $81 \mathrm{mg}$ per day.

SUBSISTENCE DIFFICULTIES AND THEIR ASSOCIATION WITH PERCEIVED BARRIERS TO QUITTING SMOKING AMONG HOMELESS SMOKERS IN BOSTON Travis P. Baggett ${ }^{1,}{ }^{2}$; Nancy A. Rigotti ${ }^{1}$; Yuchiao Chang ${ }^{1}$; Eric Campbell ${ }^{1}{ }^{1}$ Massachusetts General Hospital, Boston, MA; ${ }^{2}$ Boston Health Care for the Homeless Program, Boston, MA. (Control ID \#2469576)

BACKGROUND: Three-quarters of homeless adults smoke cigarettes, and this contributes to 3- to 5-fold higher rates of smoking-attributable mortality relative to the general population. Although most homeless smokers want to stop smoking, few are able to do so. Competing priorities for shelter, food, clothing, and other subsistence needs may be one explanation for this. We assessed the association between subsistence difficulties and perceived barriers to quitting smoking in a clinic-based sample of homeless smokers in Boston.

METHODS: In April-July 2014, we used stratified time-location sampling to conduct a cross-sectional, in-person survey of 306 currently homeless adult cigarette smokers recruited from 5 high-volume clinics operated by Boston Health Care for the Homeless Program (BHCHP). Our primary independent variable was a composite measure of past-month subsistence difficulties, assessed using a 5-item scale originally developed for the RAND Course of Homelessness Study. Scale items assessed the frequency ( $0=$ never, $3=$ usually) of past-month difficulty finding shelter, food, clothing, a place to wash, and a place to use the bathroom, with good internal consistency $(\alpha=0.80)$. Covariates included sociodemographic measures, general health status, drug and alcohol use and psychiatric symptom severity assessed with the Addiction Severity Index - 5th Edition, nicotine dependence assessed with the Fagerstrom Test of Nicotine Dependence, and readiness to quit smoking assessed with the Contemplation Ladder. Our dependent variable was a composite measure of perceived barriers to quitting smoking, assessed with 12 items $(\alpha=0.78)$ that we developed and pretested in the target population. The items tapped physiologic (e.g. "cravings to smoke"), psychological (e.g. "loss of a way to cope with stress"), social (e.g. "everyone around me smokes"), and structural (e.g. "don't know where or how to get help with quitting") barriers. For each item, respondents were asked to report whether the issue was a large barrier (2), a small barrier (1), or not a barrier (0) to quitting smoking. Responses were summed across all items to create a composite barrier score. We then used design-adjusted linear regression analysis to assess the association between subsistence difficulties and barriers to quitting smoking, controlling for important confounders.

RESULTS: Eighty-six percent of eligible individuals participated in the survey. Threequarters of participants were male. The mean age was 48 years. Thirty-six percent were 
white, $41 \%$ were black, and $18 \%$ were Hispanic. Considerable proportions of respondents reported any past-month difficulty finding shelter (49\%), food (41\%), clothing (50\%), somewhere to wash (35\%), and somewhere to go to the bathroom (43\%). Participants with greater subsistence difficulties were younger $(p=0.002)$, less likely to have a high school diploma ( $p=0.03)$, more likely to report fair or poor health $(p=0.004)$, and had greater levels of psychiatric symptoms $(p<0.001)$, drug use $(p<0.001)$, alcohol use $(p<0.001)$, and nicotine dependence $(p=0.001)$. Subsistence difficulties were not associated with level of readiness to quit smoking $(p=0.71)$. Overall, the most strongly endorsed barrier to quitting smoking was cravings to smoke (57\% large, $33 \%$ small), while the least strongly endorsed barrier was not knowing where or how to get help with quitting (12\% large, $24 \%$ small). In multivariable analyses adjusted for age, education, general health status, psychiatric symptom severity, drug and alcohol use severity, and nicotine dependence, increasing subsistence difficulties were significantly associated with greater perceived barriers to quitting smoking (effect size $=0.18, p=0.007$ ).

CONCLUSIONS: Subsistence difficulties were common in this sample of homeless smokers. Respondents with greater difficulty meeting subsistence needs were no less ready to quit smoking than those with fewer subsistence difficulties, but they perceived significantly more barriers to doing so. Interventions for homeless smokers should assess competing subsistence needs and assist in identifying resources to help address these needs in order to improve the likelihood of cessation success.

\section{SUCCESSFUL IMPLEMENTATION OF A DIRECT OBSERVATION PROGRAM} IN AN AMBULATORY BLOCK: 2-YEAR FOLLOW-UP Jeremy Smith ${ }^{1}$; David Feldstein $^{2}$; Linda M. Baier ; Bennett Vogelman ${ }^{3} .{ }^{1}$ University of Wisconsin, Madison, WI; ${ }^{2}$ University of Wisconsin School of Medicine and Public Health, Madison, WI; ${ }^{3}$ University of Wisconsin, Madison, Madison, WI; ${ }^{4}$ Univ of Wisconsin, Madison, WI. (Control ID \#2461535)

BACKGROUND: Direct observation of trainees has been shown to increase frequency of feedback, identify clinical deficiencies otherwise unrecognized, increase learner confidence, and improve learners' clinical skills. At our institution, however, direct observation of clinical skills was not being performed regularly. We also recognized that within the ambulatory block rotation, the single end-of-rotation summative evaluation being used by each faculty evaluator was inadequate and potentially lacking in validity, because each resident might interact with multiple different faculty during the rotation. Therefore, we designed a novel evaluation system and implemented it into residents' ambulatory block rotation to address these needs. Our objectives were to: 1 . Increase the amount of direct observation of residents. 2. Improve faculty's attitudes towards direct observation. 3 . Increase residents' feedback and improve the quality of residents' evaluations by moving from a single summative evaluation to multiple formative evaluations throughout the rotation. 4. Begin to develop an institutional culture wherein direct observation is seen by faculty and learners to be an integral part of the training process.

METHODS: Thirty-five residents per year rotate through a university-based ambulatory block rotation. Residents spend 1-3 months seeing outpatients 5 days per week, primarily in a general internal medicine clinic. Fourteen different faculty supervise residents on this rotation. Each resident was required to obtain 9 observations over the course of the ambulatory block, comprised of 3 observations from each of 3 clinical domains: history-taking, physical exam, and counseling/decision-making. We developed single-page, literature-based evaluation tools for each clinical domain, which were given to residents at the start of the rotation. They were instructed to work with their faculty member to select appropriate patients for observation. Residents gave the relevant form to the faculty prior to the encounter, and faculty completed the form while observing the resident. After the encounter, the faculty provided the resident with feedback, and the completed form. The forms were collected at the end of the rotation. These 9 formative evaluations took the place of a single summative end-of-rotation evaluation. End of rotation questionnaires were completed by all participating residents and faculty evaluators.
RESULTS: A total of 507 patient encounters were directly observed over 23 months. Fifty-two of 57 residents (91\%) completed all 9 observations. Residents found the experience valuable, with $89 \%$ reporting they had permanently changed aspects of their history-taking, physical exam, or counseling based on the feedback received. Fifty-six percent of residents reported having previously been directly observed a total of 3 times or less throughout residency; $100 \%$ of residents were directly observed at least 7 times over the course of the rotation. Faculty reported that the program increased their use of direct observation. Fifty-eight percent of the faculty reported conducting direct observation less than 6 times per year prior to the program, and $75 \%$ reported conducting at least 6 observations in the 6 months since the program started $(p=0.01$ for change in number of observations). Faculty attitudes toward direct observation also improved. Sixty-seven percent of faculty felt this new system made it more likely or much more likely that they would conduct direct observation in the future. Faculty rated on a $1-5$ scale how burdensome they thought the observations were going to be, and how burdensome they actually found them to be; there was a significant $(p=0.015)$ improvement in faculty perceptions of the burden of direct observation. When asked to rate how useful they found the direct observations in terms of their ability to evaluate and provide feedback, on a $1-5$ scale $(5=$ extremely useful), $92 \%$ rated 4 or $5.83 \%$ of faculty preferred the new system to the prior evaluation system which used a single summative evaluation.

CONCLUSIONS: The new evaluation system was successful in increasing direct observation of residents, and improving faculty attitudes toward direct observation. Some residents and faculty suggested that the observations should be more learner-centered and flexible, allowing the number of observations in each clinical domain to be modified based on the learner's self-assessed needs. Some residents noted the significant effort required to collect the 9 observations, though we believe putting the onus on the residents to obtain the observations was key to accomplishing them. Future efforts will focus on measuring whether this system leads to increases in direct observation conducted by participating faculty in other venues, and modifying the system so that the observations are conducted based on learner self-identification of areas needing improvement.

Faculty and Residents Differed in Which Domain They Found Most Useful for Direct Observation

\begin{tabular}{lll}
\hline \hline & Residents & Faculty \\
History-Taking & $7 \%$ & $33 \%$ \\
Physical Exam & $26 \%$ & $17 \%$ \\
Counseling & $67 \%$ & $50 \%$ \\
\hline
\end{tabular}

"In which domain did you find the direct observations most useful, in terms of evaluation and feedback: history-taking, physical exam, or counseling/decision-making?"

SUPPORTING MEDICATION DISCONTINUATION: PROVIDER PREFERENCES FOR INTERVENTIONS TO FACILITATE DEPRESCRIBING Amy Linsky $^{1,2}$; Mark Meterko ${ }^{1}$; Kelly Stolzmann ${ }^{1}$; Steven R. Simon ${ }^{1} .{ }^{1}$ VA Boston Healthcare System, Boston, MA; ${ }^{2}$ Boston University School of Medicine, Boston, MA. (Control ID \#2468834)

BACKGROUND: Discontinuing ("deprescribing") medications that are outdated, not indicated, or of limited benefit relative to risk may prevent adverse drug events. Literature supports the clinical feasibility and safety of medication discontinuation but does not provide extensive information for implementation in typical practice. Many factors impede clinicians' ability to deprescribe, including patient complexity, clinical uncertainty, and shared management with other providers. We surveyed prescribers to determine preferences for interventions that would improve their ability to discontinue medications appropriately. 
METHODS: We conducted a national web-based survey of 2475 prescribers [physicians, nurse practitioners (NP), physician assistants (PA), and clinical pharmacy specialists] practicing in Department of Veterans Affairs (VA) primary care clinics. One question presented 15 potential changes to medication-related practices and asked respondents to rank their top three choices for changes that would "most improve [their] ability to discontinue medications." We assigned weights of 3 for first-choice, 2 for second-choice, and 1 for third-choice selections. We then summed the weighted rankings for each of the fifteen response options. Preferences were determined for the whole sample and within subgroups of respondents defined by demographic and background characteristics (e.g., age, gender and provider type), medication-relevant experience (e.g., discontinuing medications initiated by other providers), and beliefs (e.g., about medication overuse) RESULTS: A total of 411 clinical providers (response rate $17 \%$ ) completed the online questionnaire (48\% male; $72 \%$ non-Hispanic white; $74 \%$ physicians, $17 \%$ NP/PAs, $9 \%$ clinical pharmacy specialist; $52 \%$ worked $<8$ clinic sessions per week, and $79 \%$ had prior experience working in a non-VA setting). Among the 326 respondents who provided rankings, the top choice for a change that would help improve their ability to discontinue medications was "Requiring all medication prescriptions to have an associated "indication for use." Next most highly rated was "Assistance with follow-up of patients as they taper or discontinue medications is performed by another member of the Patient Aligned Care Team (PACT)," followed by "Increased patient involvement in prescribing decisions." This combination of options, albeit in varying rank order, was the most commonly selected, with 250 (77\%) respondents who answered the question including at least one of these items in their three highest ranked choices, regardless of prescriber demographics, experience, or beliefs. Other less frequently selected items included "Improved information exchange with all VA and non-VA pharmacies to confirm medication reconciliation," "Use of clinical decision support tools, such as drug-drug alerts, within the electronic health record," and "Improved information exchange with providers located at community facilities."

CONCLUSIONS: Continued efforts to improve clinicians' ability to make medication decisions, especially around deprescribing, have many potential benefits, including decreased pharmaceutical and health care costs, fewer adverse drug events and complications, and improved patient involvement and satisfaction with their care. The consistency of the preferences among the providers in our study, regardless of provider characteristics, experience or beliefs, strongly suggests that these intervention options would be more likely to be accepted by a wider range of providers. Requiring all medication prescriptions have an indication for use has been described elsewhere as an approach to improve medication safety and engage patients more fully in their care. Future work, whether as research or quality improvement, should incorporate clinicians' preferences for interventions, as greater buy-in from front-line staff leads to better adoption of changes.

SUPPORTIVE HOUSING IS ASSOCIATED WITH IMPROVED HIV OUTCOMES IN A CHRONICALLY HOMELESS HIV-POSITIVE POPULATION WITH COMORBID SERIOUS MENTAL ILLNESS AND SUBSTANCE USE DISORDERS Laura Van Metre ${ }^{1,2}$; Nneka Ogbutor ${ }^{3} .{ }^{1}$ Montefiore Medical Center, New York, NY; ${ }^{2}$ Albert Einstein College of Medicine, New York, NY; ${ }^{3}$ City College of New York, New York, NY. (Control ID \#2465600)

BACKGROUND: Homeless HIV-infected people face many obstacles to achieving optimal HIV outcomes, including unstable living environments, substance use disorders, and serious mental illness. Supportive housing has been shown to improve HIV adherence and decrease viral loads, but few studies have specifically examined factors associated with treatment success among chronically homeless HIV-infected individuals with substance use disorders and serious mental illness. To address this, we assess the effectiveness of a housing first model of supportive housing, which includes case management and medication monitoring, for improving HIV outcomes. We hypothesize that housing and case management services, particularly voluntary engagement in medication monitoring, are associated with a greater probability of viral load suppression.
METHODS: We reviewed the medical and psychiatric charts of $26 \mathrm{HIV}$-infected individuals with serious mental illness and/or substance use disorders who reside in supportive housing in the South Bronx. On-site support includes case management, social work and other services, and regular visits from an internist (Dr. Van Metre) and a psychiatrist. To test the hypothesis that supportive housing (with associated case management services) is associated with better HIV outcomes, we fit two multivariate regression models. The first model included the primary outcome of viral load (detectable or undetectable), and the second model included the secondary outcome of CD4 count. The major independent variable of interest was number of years with supportive housing. Other independent variables of interest were whether the patient was enrolled in medication monitoring, Hepatitis C diagnosis, and assignment to an onsite doctor (internist or psychiatrist), and adjustment variables were age, sex, race, and sexual orientation. All charts were reviewed from patient's entry into supportive housing through July 2015. The facility opened in 2010 , and the mean duration in supportive housing was 3.3 years.

RESULTS: On entry to supportive housing, $23 \%(n=6)$ of the cohort had an undetectable viral load. At their most recent available data point, $50 \%$ of the cohort $(n=13)$ had an undetectable viral load, and all those who entered with undetectable status maintained it. Among those who entered supportive housing with a detectable viral load $(n=20), 50 \%(n=10)$ achieved an undetectable viral load at some point, and $35 \%(n=7)$ remained undetectable. The mean change in CD4 count from entry to the most recent data point was 116 . In multivariate analysis, number of years in supportive housing was significantly associated with achieving an undetectable viral load. Specifically, each additional year in supportive housing was associated with a $17 \%$ increased probability of achieving undetectable viral load $(p<0.01)$. In addition, enrollment in medication management was strongly associated with achieving undetectable viral load (coefficient of $0.83, p<0.01$ ). Co-infection with Hepatitis $\mathrm{C}$ was negatively associated with achieving an undetectable viral load (coefficient of $-0.67, p=0.06$ ), though this was of marginal significance.

CONCLUSIONS: We conclude that supportive housing is associated with improved viral load suppression and CD4 count among chronically homeless HIV-positive individuals with cooccurring substance use disorders and serious mental illness. Our data demonstrate larger benefits for patients who engage in medication monitoring. While at present the homeless and triply diagnosed population (HIV, mental illness, substance abuse) experience a heavier burden of disease and worse outcomes, our study suggests that with housing and supportive services, this vulnerable population can achieve outcomes similar to the general HIV-positive population. Results of multivariate linear model: dependent variable is a binary indicator equal to 1 if the patient had an undetectable viral load

\begin{tabular}{llll}
\hline \hline Independent Variable & Coefficient & Std. Err. & $P$-value \\
Number of years in supportive housing & $0.17^{* * *}$ & 0.06 & $<0.01$ \\
Enrolled in medication management & $0.83^{* * *}$ & 0.25 & $<0.01$ \\
Hepatitis C co-infection & $-0.67^{*}$ & 0.33 & 0.06 \\
Assigned to onsite doctor & -0.17 & 0.25 & 0.50 \\
Gender (Female =0) & -0.52 & 0.41 & 0.22 \\
Age & 0.00 & 0.01 & 0.92 \\
Sexual orientation (Heterosexual =0) & 0.23 & 0.26 & 0.39 \\
Constant & 0.39 & 0.61 & 0.54 \\
R-squared & 0.727 & & \\
Number of observations & 20 & & \\
\hline
\end{tabular}

$* * * p<.01, * * p<.05, * p<.10$

SUSTAINING COLLABORATIVE CARE IN PRIMARY CARE AND BEHAVIORAL HEALTH THROUGH AN INNOVATIVE CASE-BASED BUNDLED PAYMENT MODEL Michelle Blackmore ${ }^{1}$; Henry Chung ${ }^{2} .{ }^{1}$ Montefiore Medical Center, Yonkers, NY; ${ }^{2}$ Montefiore Medical Center, New York, NY. (Control ID \#2470367)

BACKGROUND: Substantial empirical research supports the effectiveness of the collaborative care model in integrating behavioral health services in primary care practices (Gilbody, 2006; Thota, 2012). However, evaluation and evidence supporting sustainable 
payment models within collaborative care is limited, particularly within Medicare and Medicaid populations, contributing to a relatively modest uptake of the collaborative care model nationally (Katon, 2012). Innovative case-based bundled payment models may allow support for a range of on and off-site activities not traditionally billable in a FFS environment, helping to accelerate adoption and further clinical impact of the CCM. For instance, utilization of patient centered technology support can help ensure populationbased health care demands are better met and resources maximized to improve care team efficiency. Ensuring sustainability and wider application of CCM also requires demonstrating the model's utility in managing a broad range of behavioral health disorders that commonly present in primary care settings across the lifespan, from pediatrics to geriatrics. To demonstrate the potential sustainability and scalability of CCM, Montefiore Medical Center, serving a population of primarily Medicare and Medicaid recipients with significant racial and ethnic diversity, implemented the Bronx Behavioral Health Integration Program (BHIP). BHIP aims to promote sustainability of CCM through a case-based bundled payment model that allows providers to work collectively and creatively in providing a person-centered approach with enhanced "between visit" care and case supervision, facilitated by the use of technology support.

METHODS: Seven high-volume Montefiore primary care sites are participating in BHIP, beginning enrollment in February 2015. Behavioral health teams consisting of a consulting psychiatrist, licensed social worker, and care manager, were integrated into each site to provide behavioral health services and worked collaboratively with the primary care physicians. Patients were enrolled in BHIP if they met severity criteria for a range of target behavioral health conditions (e.g., depression, panic, generalized anxiety, PTSD, alcohol use), with the goal of each site maintaining an active caseload of 150 patients (total $=1575$ patients enrolled across sites each year). A case-based payment model was financially modeled utilizing data from year 1 outcomes. In the model, each site receives an initial case-based payment to begin providing the behavioral health intervention once a patient is enrolled. The payment covers 3 months of CCM services traditionally billable in a FFS or managed care environment, such as psychotherapy visits with a social worker or medication management by a psychiatrist, as well as non-billable services, including in between visit support and follow up (e.g., psychiatric consultation, coaching, and behavioral activation to support treatment plans). Quarterly maintenance payments are then made contingent on ongoing patient engagement and progress toward primary clinical outcome metrics. Technology support, interactive voice response and smartphone applications, is being implemented in year 2 to maximize behavioral health resources and support between visit care and consultation.

RESULTS: To date, 1770 patients have enrolled in BHIP (20\% male, $80 \%$ female; $3 \%$ pediatric, $79 \%$ adult, and $18 \%$ geriatric). Across sites, a $70 \%$ engagement rate following initial assessment was demonstrated, with $77 \%$ of patients receiving at least monthly contact during a 3-month average time in treatment. Primary clinical outcomes show a $50 \%$ decrease from baseline scores by week 10 in 44 and $49 \%$ of the population on the Patient Health Questionnaire-9 (PHQ-9) and Generalized Anxiety Disorder Screener-7 (GAD-7), respectively. Caseloads across sites exceeded projections in Year 1, providing support for the case-based payment model. Utilizing these engagement and outcome metrics, the virtual case-based payment streams will be implemented in year 2 .

CONCLUSIONS: BHIP demonstrates promising results that $\mathrm{CCM}$ can be utilized to improve population health for a range of behavioral health disorders across the lifespan in a socio-economically challenged high-cost, high-needs population. The model has the needed elements, including patient engagement and clinical outcomes, needed to support an innovated case-based bundled payment system to improve sustainability and scalability of CCM.

SYMPTOM BURDEN IN PATIENTS INITIATING TREATMENT FOR METASTATIC COLORECTAL CANCER Laura B. Cantino; Nicole Thompson; Christine Ritchie; Michael Rabow; Kara Bischoff. University of California San Francisco, San Francisco, CA. (Control ID \#2465193)
BACKGROUND: Despite screening efforts, colorectal cancer remains a prevalent cause of cancer-related morbidity and mortality. Consensus guidelines recommend early, outpatient palliative care (PC) for all patients with metastatic disease, based on studies suggesting that PC can improve quality of life and align care with patients' preferences. However, no study has yet focused on the PC needs of patients with colorectal cancer.

METHODS: We are conducting a single arm trial examining the effect of providing integrated outpatient PC along with usual oncologic care from the time of diagnosis of metastatic colorectal cancer (mCRC). Patients are referred by oncologists at our institution's comprehensive cancer center and are enrolled within 90 days of diagnosis of mCRC or of establishing care for mCRC. The 30 patients enrolled prior to the intervention receive usual care, which includes oncologist referral to outpatient PC as needed. They were surveyed regarding their symptoms, psychosocial needs, functional status and prognostic awareness as a needs assessment. The next 30 patients will be offered the intervention: routine, integrated outpatient PC visits every 1-3 months. Patients from both groups will be followed longitudinally until death or discontinuation of care, with data collected through chart review and surveys. The survey includes selected questions from the Edmonton Symptom Assessment Scale (ESAS), Quality of Life at the End of Life Measure (QUAL-E), Steinhauser Spiritual Assessment, NCCN Distress Thermometer, Paffenbarger Physical Activity Questionnaire, and the Prognosis and Treatment Perceptions Questionnaire. Here, we report on the baseline characteristics and survey results of the control cohort.

RESULTS: To date, 26 patients with $\mathrm{mCRC}$ receiving usual oncology care have been enrolled. Average age at the time of enrollment is 60 (range 42-86), $31 \%$ are women, $65 \%$ are Caucasian, $27 \%$ Asian, and $8 \%$ other races. Eight percent identify as Hispanic or Latino and $15 \%$ have limited English proficiency. Functional status is good in the majority of patients; $62 \%$ of patients reported exercising long enough to work up a sweat at least once per week, and $38 \%$ of patients did so at least 3 times per week. Seventy-three percent of patients had at least 1 moderate or greater symptom ( $\geq 4$ on 10 point scale) and $27 \%$ of patients had at least 1 severe symptom $(\geq 7 / 10)$. Among patients with at least one symptom of moderate or greater severity, the median number of symptoms was three (range 1-7). Rates of specific symptoms are listed in the Table. Seventy-three percent of patients stated that they felt "quite a bit" or "completely" at peace; $77 \%$ of patients stated that their quality of life was good or excellent. Forty-two percent of patients reported problems in at least one psychosocial domain in the past week; $27 \%$ of patients reported problems in at least two domains. The most commonly reported problems were treatment decisions (23\%), insurance or financial (19\%), and transportation (15\%). Thirty-eight percent of patients rated their level of overall distress in the past week as at least moderate $(\geq 4 / 10)$. Most patients $(81 \%)$ believed that they had received "good" or "excellent" quality of information about their prognosis and treatment options. However, $62 \%$ believed it was at least moderately likely ( $>50 \%$ chance) that they would be cured of cancer, and $31 \%$ believed it was extremely likely ( $>90 \%$ chance).

CONCLUSIONS: Symptoms and psychosocial problems are common among our cohort of patients with $\mathrm{mCRC}$, even early in the course of metastatic disease. While management of certain physical symptoms, such as pain, is a commonly recognized aspect of supportive care for cancer patients, we found that other problems, such as anorexia and decreased wellbeing, were actually more common. Despite their overall good functional status, patients also reported significant social needs. These findings highlight the importance of providers screening broadly for symptoms and concerns. Patients indicated that they had received high-quality information about treatment options and prognosis, but over one in five reported difficulty with treatment decisions, and the group had marked optimism about their odds of being cured of cancer. These feelings offer a window into patients' experience during the transitional period in care that our study captures, and present an opportunity for oncologists and primary care providers to provide support and tailored education. 
Frequency and Severity of Symptoms

\begin{tabular}{lll}
\hline \hline Symptom per ESAS & $\begin{array}{l}\text { Percentage with Moderate } \\
\text { Greater Symptom }(\geq 4 / 10)\end{array}$ & $\begin{array}{l}\text { Percentage with Severe } \\
\text { Symptom } \geq 7 / 10) \\
12 \%\end{array}$ \\
Anorexia & $38 \%$ & $4 \%$ \\
Decreased feeling of & $38 \%$ & \\
wellbeing & & $12 \%$ \\
Fatigue & $35 \%$ & $8 \%$ \\
Constipation & $31 \%$ & $0 \%$ \\
Pain & $23 \%$ & $0 \%$ \\
Anxiety & $23 \%$ & $8 \%$ \\
Diarrhea & $15 \%$ & $0 \%$ \\
Shortness of breath & $12 \%$ & $4 \%$ \\
Drowsiness & $12 \%$ & $0 \%$ \\
Nausea & $8 \%$ & $0 \%$ \\
Depression & $8 \%$ & $4 \%$ \\
Other symptom & $12 \%$ & \\
\hline
\end{tabular}

SYSTEM ATTRIBUTES AND USER REQUIREMENTS FOR A CHRONIC KIDNEY DISEASE CLINICAL DECISION SUPPORT TOOL IN PRIMARY CARE Joy Gulla ${ }^{1}$; Pamela M. Neri ${ }^{2}$; David W. Bates ${ }^{1,2}$; Lipika Samal ${ }^{1,3}$. ${ }^{1}$ Brigham and Women's Hospital, Boston, MA; ${ }^{2}$ Partners Healthcare Systems, Inc., Wellesley, MA; ${ }^{3}$ Harvard Medical School, Boston, MA. (Control ID \#2464557)

BACKGROUND: Chronic kidney disease (CKD) represents a prevalent and costly public health issue with high morbidity and mortality. Approaches to improving management of CKD must focus on primary care clinics because $95 \%$ of patients with CKD are in early stages of the disease and are cared for by primary care physicians (PCPs). Many clinical decision support (CDS) interventions have failed in part because they were not incorporated into physician workflow. We sought to gather information about PCP workflow and decision-making for CKD management in order to inform user-centered design of a CKD clinical decision support system.

METHODS: We interviewed PCPs to gather information about CKD management and to identify desirable clinical decision support system attributes. Interviewees were general internists recruited from a network of primary care physicians within 12 clinics affiliated with Brigham and Women's Hospital. We developed a structured interview guide to 1) prompt discussion about evidence-based CKD management, 2) elicit changes in patient status that would prompt referral to a specialist, and 3) identify labs, medications, risk factors and other data the PCP needs to make a decision about referral. Interviews were audio taped and ranged from 30 to $60 \mathrm{~min}$. Interviews were transcribed, de-identified, and coded. Codes were refined into a final coding scheme through iterative review by three investigators (JG, PN, LS). During the user requirements analysis, we first identified system attributes which summarized the needs expressed by the intended end users and then ascribed one or more user requirements to each attribute.

RESULTS: Twelve primary care physicians participated. Participants were $75 \%$ male, mean age 53 (range 37-82), and had a mean of 27 years in practice (range 11-58). Overall, 17 system attributes were identified during the analysis including some specific to CDS: the need for a CDS tool to provide actionable information, links to guidelines and reference materials, and visualization of trends. These attributes resulted in twelve user requirements for a well designed CDS system to address PCP needs. In addition, interviewees expressed a desire for support for PCP management of risk factors, support for interpreting CKD severity, and help in identifying - the cause of CKD. These attributes were translated into four user requirements about medical decision making and PCP management. Other attributes included integration with scheduling and resource systems, integrated documentation tools, efficient ordering, and automated synthesis of information related to disease progression. The attributes were translated into five user requirements concerning PCP support throughout the referral pathway.
CONCLUSIONS: We conducted a user requirements analysis to translate data into user requirements for a CKD clinical decision support system. The results of this user requirements analysis could be used to design an intuitive and usable CDS system that would have the attributes necessary to support PCP management of CKD.

TEN YEARS AFTER THE CENTER FOR DISEASE CONTROL AND PREVENTION'S ROUTINE HIV TESTING RECOMMENDATIONS: HOW MUCH INTERNAL MEDICINE RESIDENTS KNOW AND WHERE DO WE STAND? $\underline{\text { Dima Dandachi }}{ }^{1}$; Navdeep Kaur ${ }^{2}$; Roshanak Habibi ${ }^{1}$; Alvaro Joaquin Altamirano Ufion ${ }^{3}$. ${ }^{1}$ Presence Saint Francis Hospital, Evanston, IL; ${ }^{2}$ Presence Saint Francis hospital, Evanston, Evanston, IL; ${ }^{3}$ Illinois Masonic, Chicago, IL. (Control ID \#2469720)

BACKGROUND: In September 2006, the CDC recommended routine, voluntary HIV screening for all individuals 13 to 64 years of age in all health care settings regardless of HIV-related risk factors. The guidelines also recommend annual HIV testing for persons at high-risk of HIV infection. Separate written consent is no longer required. These recommendations have been made on the basis that when HIV is diagnosed early, appropriately timed interventions can lead to improved health outcomes. However, 10 years since the publication of these recommendations, HIV screening remains very low and many health care providers remain unaware of the recommendation. Limited information is available about the usefulness of specific Interventions aimed at resident physicians to improve universal HIV screening. The aims of this study include- 1) to evaluate HIV- testing related knowledge among Internal Medicine residents at three different residency programs; 2) to collect information about the adherence to the HIV universal testing guidelines at 35 different community health centers across Chicago metropolitan area.

METHODS: We conducted a self-administered anonymous electronic survey of internal medicine residents at three community based residency programs in Chicago metropolitan area. The survey consisted of 17 close-ended questions, including questions about knowledge and barriers to HIV testing. The software "SurveyMonkey" was used to collect and analyze the data. Adherence to HIV screening was measured as a ratio of patient population who received HIV testing to the total number of patients who should have received testing according to $\mathrm{CDC}$ guidelines. This data was collected from 35 community health centers in Chicago metropolitan area as part of quality improvement. Data was collected over a period of 4 months extending from July 2015 to October 2015.

RESULTS: Of the 144 residents, 91 (63.2\%) filled the survey. 60 (65.9\%) were men and $31(34 \%)$ were women. $79(88.8 \%)$ of respondents were between 25 and 34 years of age and $10(11.2 \%)$ are between 35 and 44 years of age. 32 (35.2\%) were in PGY1, 26 $(28.6 \%)$ in PGY2 and 33 (36.3\%) in PGY3 or higher. Only 4 (4.5\%) have graduated from medical school more than 10 years ago. While most of the respondents $73(81.1 \%)$ reported receiving some form of training or education about HIV testing guidelines, only $52(58.4 \%)$ knew that new CDC guidelines recommend universal screening regardless of risk factors. In regards to the frequency of testing, 29 (31.9\%) thought that persons at high risk for HIV acquisition should be tested once in their lifetime. Men having sex with men is the most common mode of HIV transmission in Chicago area. However, 28 (30.8\%) of the internal medicine trainees thought that injection drug use or heterosexual contact are the most common mode of HIV transmission. Despite changes in the law, 44 (50\%) of the residents still believed that a separate written consent for HIV testing is still required by Illinois law. The most common reported barriers for ordering HIV testing were: deferred because of other acute healthcare issues (47.2\%), lack of time (45.3\%) and patients are uncomfortable discussing HIV testing (39.6 \%). HIV testing data collected from 35 federally qualified community health centers spread over Chicago metropolitan area showed a low and steady decline from July to October 2015 in number of HIV testing and did not cross $21 \%$ at any time (Table 1 ) 
CONCLUSIONS: Our study identifies the gap in medical knowledge regarding HIV screening among internal medicine residents and identifies a very low HIV screening rate across Chicago. Lack of awareness regarding the revised CDC recommendation might be a contributing factor to infrequent HIV testing. To realize the goal of universal HIV screening, more efforts are needed to educate internal medicine residents to adopt this approach in their future practice. Further studies are needed to assess if an intervention targeting the residents will help increase the testing. HIV testing data

\begin{tabular}{llll}
\hline \hline Month & Total Encounters & HIV Tests Ordered & $\begin{array}{l}\text { HIV Tests/ } \\
\text { Encounters }\end{array}$ \\
July 2015 & 23,891 & 4,919 & $20.6 \%$ \\
August 2015 & 23,108 & 4,370 & $18.9 \%$ \\
September 2015 & 22,255 & 4,068 & $18.3 \%$ \\
October 2015 & 21,946 & 3,846 & $17.5 \%$ \\
\hline
\end{tabular}

TESTING IS ONLY PART OF THE STORY: DISTINGUISHING BETWEEN C. DIFFICILE INFECTION AND COLONIZATION Michele Yeung; Daniel Luger; Marcelo Mendez; Dana Mazo; Dahlia Rizk. Mount Sinai Beth Israel, New York, NY. (Control ID \#2469581)

BACKGROUND: Clostridium difficile infection (CDI) is the most common cause of health care-associated infection in US hospitals today. In 2011, an estimated 453,000 cases of $C$. difficile occurred in the United States. In 2008, CDI may have resulted in as much as $\$ 4.8$ billion in excess healthcare costs in acute-care facilities alone. According to the Infectious Disease Society of America (IDSA) clinical practice guidelines, the diagnosis of CDI requires both a positive lab test and clinical symptoms of CDI such as diarrhea, abdominal pain, fever, and leukocytosis. A positive laboratory test in an asymptomatic patient is consistent with $C$. difficile colonization, which does not require treatment or contact isolation. Risk factors including recent antibiotic use and recent healthcare facility exposure are similar for CDI and C. difficile colonization. Incorrectly diagnosing colonization as true infection can lead to patient harm by preventing correct diagnosis of cause of diarrhea, inappropriate use of antibiotics and delays in discharge. Since C. difficile colonization rates increase during hospitalization and inpatient laxative use is common, the risk for confusing colonization for infection is especially high for hospital onset C. difficile cases. A campaign to promote appropriate $C$. difficile testing involving education and electronic medical record order support and education was initiated October 2015. The goal of this project was to evaluate whether hospital-onset C. difficile testing was being sent appropriately before this campaign launched.

METHODS: A retrospective chart review of hospital-onset CDI incident cases from 1/1/ 2015 to 9/30/2015 was conducted at an urban academic medical center. Per the Center for Disease Control, the criteria for hospital-onset $C$. difficile is defined as a specimen collected greater than 3 days after admission to a facility. Medical records were reviewed for clinical signs and symptoms consistent with diarrhea and symptoms of infection (fever, leukocytosis, subjective or objective abdominal complaint) within $48 \mathrm{~h}$ prior to submitting stool specimen for testing. CDI testing was deemed appropriate if the patient had documented diarrhea along with at least one other sign or symptom of $C$. difficile infection. All cases that had inappropriately sent samples were evaluated for laxative use within $48 \mathrm{~h}$ prior to ordering $C$. difficile test.

RESULTS: Of the 57 patients with incident HO CDI, $60 \%$ had stool sent for C.difficile testing appropriately. All patients received treatment for CDI. Of the 23 patients with inappropriate testing, 15 (65\%) did not have diarrhea documented in medical or nursing notes and eight ( $35 \%$ ) did not have signs of infection. Of the patients with diarrhea and no clinical symptoms, 4 (50\%) had laxative use within the previous $48 \mathrm{~h}$. There were no significantly differences in prevalence of risk factors among patients whose tests were sent appropriately versus inappropriately. Antibiotic use within 30 days of C. difficile testing was found among $96 \%$ of patients with inappropriate tests and $85 \%$ of those with appropriate tests (Odds ratio 3.79, $95 \%$ Confidence Interval $0.38-187.39, p=0.21$ ). Healthcare facility exposure within 90 days of admission was found among $43 \%$ of those with inappropriate use and $38 \%$ of those with appropriate testing (OR 1.18, $95 \%$ CI $0.35-3.97, p=0.75$ ).

CONCLUSIONS: In our facility, $40 \%$ of HO CDI cases were sent inappropriately according to IDSA guidelines. Therefore, almost half of these cases likely represent C. difficile colonization instead of true infection. Our small sample size did not find differences among risk factors between patients who had tests sent appropriately versus inappropriately. Inappropriately sending $C$. difficile tests leads to over-diagnosis, unnecessary antibiotic use, and increased health care costs. Additionally, in our current model of health care reimbursement, this may have negative consequences for the institution by way of quality reporting and financial penalties. This data highlights the urgent need to for campaigns such as the one started at Mount Sinai Beth Israel for decision support and education on appropriate $C$. difficile testing.

THE 50/50 CONTINUITY CLINIC MODEL: EFFECT ON RESIDENTS' AND PRECEPTORS' PERCEPTIONS, PATIENT OUTCOMES, AND CONTINUITY OF CARE Raquel Buranosky; Michael Elnicki; Rachel Jantea; Deborah M. Simak. University of Pittsburgh Medical Center, Pittsburgh, PA. (Control ID \#2468325)

BACKGROUND: Continuity clinic is a required longitudinal patient experience for medicine residents. Traditionally, residents spend $1 / 2$ day in continuity clinic per week, which creates conflict with inpatient responsibilities on clinic days and may blunt the educational experience. In an effort to minimize this conflict, several innovative clinic models have been developed. We switched to a 50/50 block scheduling model and assessed outcomes for residents, preceptors, and patients.

METHODS: In June 2014, we implemented a 50/50 block scheduling model in our internal medicine residency program, consisting of 3 continuity clinic sites: 2 university hospital-based clinics and 1 Veterans' Affairs hospital-based clinic. Participants were PGY1-3 categorical internal medicine residents and clinic preceptors. Prior to the 50/50 model, residents followed a traditional clinic schedule. Inpatient and elective rotations were divided into thirteen $3-5$ week blocks, with continuity clinic $1 / 2$ day per week, excluding night float and ICU rotations. Consecutive inpatient blocks were often scheduled. Residents attended rotation duties before and after clinic. They were expected to maintain inter-visit care (calls, test results, medication requests) through their physical mailboxes and the ambulatory electronic medical record (EMR). They were to delegate these tasks to fellow residents when unavailable. In our 50/50 model, there were twelve 45 week blocks. Residents alternated between inpatient and elective rotations, with clinic 1 full day per week during elective blocks only. Total inpatient and elective rotations and total clinic sessions were preserved. Residents were expected to maintain inter-visit care as in the traditional model. In addition, they were paired in a cross-coverage "buddy" system to optimize continuity. Residents' patients were managed preferentially by their clinic buddies when they were on inpatient blocks (clinic visits only) or otherwise unavailable (clinic visits and inter-visit care). We distributed electronic surveys to residents and preceptors in June 2014, before the 50/50 model, and June 2015, 1 year postimplementation. Items included 5-point Likert responses to statements about clinic in domains such as time constraints in clinic, work flow, opportunities for ambulatory learning, and competing responsibilities. Pre- and post-implementation responses were compared using t-tests. Visit and inter-visit continuity were measured using a Usual Provider of Care (UPC) index for physician visit encounters and inter-visit encounters, respectively. The UPC was calculated by dividing the number of encounters with the patient's primary care provider by the number of encounters with any physician. Pre- and post-implementation values were analyzed by $X^{2}$ statistics. Residents' ambulatory EMR log-in days were recorded pre- and post-implementation and the monthly means compared 
using t-tests. Blood pressure and hemoglobin Alc values of residents' hypertension and diabetes patients, respectively, were measured pre- and post-implementation and compared using t-tests.

RESULTS: Survey response rates were 52-71\%. Inpatient distractions decreased (residents' Likert responses 3.26 vs. 1.71, $p<0.001$; preceptors 4.27 vs. $1.33, p<0.001$ ) Perceived time for clinic teaching conferences increased (residents 3.33 vs. 4.05, $p<0.001$; preceptors 3.85 vs. $4.52, p<0.01$ ). Residents' felt patient ownership increased ( 3.79 vs. $4.18, p<0.01$ ), they were more functional in clinic ( 3.13 vs. $3.61, p<0.001$ ), and clinic staff worked better together in the new model (3.51 vs. $4.08, p<0.001$ ). Clinic satisfaction scores were unchanged ( 75 vs. $77 \%, p>0.2)$. Visit continuity decreased (UPC 67 vs. $64 \%, p<0.001$ ), but Inter-visit continuity was preserved, as measured by both UPC index ( $73 \%$ in both models, $p>0.2)$ and residents' mean EMR log-in days per month (18 vs. $18.5, p>0.2)$. Systolic blood pressure (135 vs. $136 \mathrm{mmHg}, p>0.2)$ and A1c (7.77 vs. $7.58 \%, p=0.07)$ were unchanged.

CONCLUSIONS: Our 50/50 model reduced inpatient distractions in clinic and increased perceived time for clinic learning. Residents reported improved sense of patient ownership, functionality within the clinic setting, and work relations with clinic staff. Clinic satisfaction was unchanged. Although visit continuity was slightly decreased, inter-visit continuity was preserved, and patient outcomes were not negatively impacted. An unanticipated challenge was scheduling new patients for follow-up with the resident caring for them in the hospital. Although this was a single institution study, it addresses the common issue of competing resident responsibilities and difficulty optimizing the ambulatory learning environment. It is likely generalizable to many residency programs. We support this $50 / 50$ block scheduling model to minimize residents' competing responsibilities and improve clinic learning, without impacting quality of care and minimally impacting continuity. In the future, combined resident pair continuity should be measured to determine the impact of team-based care within this model. Impact on inpatient learning environment and outcomes would also be of interest

THE ACUTE EVALUATION OF SYNCOPE: VARIATION IN HOSPITAL ADMISSION PRACTICES AND IN ORDERING LOW VALUE NEUROLOGICAL DIAGNOSTIC TESTING Zayd A. Razouki ${ }^{2}$; Matthew Maciejewski ${ }^{1}$; Lynn M. Van Scoyoc ${ }^{2}$; George L. Jackson ${ }^{1}$; Susan N. Hastings ${ }^{1}$; David Edelman ${ }^{1}$. ${ }^{1}$ Durham VA Medical Center, Durham, NC; ${ }^{2}$ Durham VA Medical Center, Chapel Hill, NC. (Control ID \#2467688)

BACKGROUND: Syncope (i.e. fainting) is a common presenting symptom to the Emergency Department (ED). A significant proportion of patients with ED visits for syncope receive low value neuro-diagnostic tests (i.e. brain imaging, carotid ultrasound (CUS)) despite evidence-based recommendations (Choosing Wisely) that discourage their routine use. In addition, many patients are admitted to the hospital despite the uncertain value of many syncope hospitalizations. The objective of this study is to describe syncope evaluation practices via neuro-diagnostic tests and hospital admission practices in 20112013 in VA Medical Centers.

METHODS: Using national VA data, we constructed a cohort $(N=42,777)$ of all eligible ED visits with a primary diagnosis of syncope (ICD 9 780.02) in 2011-2013 in 114 VA Medical Centers. We classified ED visits into three groups according to their post ED disposition status: a) discharged from ED b) assigned to observation stay or c) hospitalized. We also calculated the frequency of ordering brain imaging and CUS for each ED visit, and in the 60 days after their final post ED disposition status. Medical sites were marked as outliers in hospital admission or test ordering rates, if the difference between their calculated rates was one standard deviation larger than mean of that particular outcome of all eligible medical sites

RESULTS: A majority (51.8\%) of patients with an ED visit for syncope were discharged from the $\operatorname{ED}(51.77 \%), 37.7 \%$ were hospitalized $(37.70 \%)$ and $10.5 \%$ were admitted for an observation stay. Neuro-diagnostic testing was common, because brain imaging was ordered in $67.4 \%$ of ED visits and CUS was ordered in $17.3 \%$. Brain imaging rates were higher for patients admitted for an observation stay $(77.8 \%)$ or hospitalized $(80.3 \%)$ than for patients discharged from the ED (55.9\%). Further, there was significant variation in brain imaging rates among the $114 \mathrm{VA}$ medical centers, ranging from ( 25 to $100 \%$ per ED visit). We found 15 outlier sites with high ordering rates of brain imaging and 16 outlier sites with low ordering rates of brain imaging. Similarly, we found the use of CUS varied widely across all eligible sites, ranging from ( 0.3 to $50 \%$ per ED visit). We found 19 outlier sites with high rates of ordering CUS and 22 outlier sites with low ordering rates of CUS. Five medical sites were outliers having both, high rates of hospitalization and ordering brain imaging. Three medical sites were outliers having both, high rates of hospitalizations and ordering CUS. Seven medical sites were outliers having both, low rates of hospitalizations and ordering brain imaging. Only one medical site was an outlier, having both low rates of hospitalizations and ordering CUS

CONCLUSIONS: In the acute evaluation of syncope, hospital admission rates and ordering neuro-diagnostic testing are prevalent and vary considerably across VA medical centers. Future studies should identify factors that contribute to variation in syncope evaluation practices in order to streamline its workup and reduce unnecessary hospitalizations and the use of low value diagnostic tests.

THE ALIKI EXPERIENCE WILL GO WITH ME IN ALL WALKS OF MY PROFESSIONAL LIFE: LONG-TERM IMPACTS OF THE ALIKI INITIATIVE ON MEDICAL RESIDENTS Colleen Christmas ${ }^{2}$; Laura Hanyok ${ }^{2}$; Janet Record ${ }^{1}$; kathy dunning ${ }^{4}$; Roy C. Ziegelstein ${ }^{3}$; Cynthia S. Rand ${ }^{3} .{ }^{1}$ Johns Hopkins Bayview Medical Center, Baltimore, MD; ${ }^{2}$ Johns Hopkins University, Baltimore, MD; ${ }^{3}$ Johns Hopkins University School of Medicine, Baltimore, MD; ${ }^{4}$ university of maryland, Baltimore, MD. (Control ID \#2470204)

BACKGROUND: In 2007 our urban internal medicine residency training program started a novel educational intervention known as the Aliki Initiative (AI) with the goals of improving the ability of residents to understand their patients as people and apply this information to tailor treatments and transitions of care. Residents utilized a reordered history and physical admission note that emphasizes individual personal, family, and life circumstances, completed in-depth medication adherence reviews, communicated with caregivers and physicians who cared for the patient in the outpatient settings, completed a patient-centered discharge plan, called patients within a week after discharge, and visited selected patients after discharge in their homes or other care facilities when they rotated onto the AI team, which was one of 4 general ward teams all residents rotated through. Residents identified that the AI provided them with unique experiences that they valued highly. We have previously published the short-term benefits of the AI: reduced readmissions for patients admitted with heart failure, high patient and resident satisfaction, improved self-efficacy in addressing medication adherence and communicating with patients about transitions of care, and improved selfreport of knowing patients as individuals. Medical knowledge outcomes were the same on the AI team compared to other ward teams. In this study, we aimed to understand whether exposure to the AI during residency training had lasting impact on graduates from our residency after they left our program.

METHODS: We sent web-based surveys via e-mail to the doctors who graduated from the Johns Hopkins Bayview Medical Center between the years of 2007 and 2014, inclusively. The survey took asked graduates what, if anything, they felt they utilized in their current practices that is a result of having participated in the AI as a resident, in addition to demographic information about the graduate and their current practice parameters.

RESULTS: One hundred fifty residents completed at least 1 year of training during 2007 2014, for which we had 110 successful e-mail contacts. Ninety-four graduates (85\%) responded to this survey. There was an even distribution across genders and years in 
training of respondents, and 21 (22\%) were still in fellowship training and $71(76 \%)$ were working full time. About half of the graduates reported they participated in the AI both as a resident and as an intern while $16 \%$ participated in one or the other of these times and $15 \%$ either did not participate or were not sure if they had. The majority ( $77 \%$ reported that the AI was valuable to their training as a physician "a great deal" and $18(22 \%)$ "a little" while none responded "not at all" and only 1 reported he or she was "not sure". Similarly, the majority felt that their practice as a physician was influenced by participating in the AI "a great deal" (63\%) or "a little" (31\%) while $3(4 \%)$ says it was "not at all" and $2(2 \%)$ responded "I no longer practice medicine". Themes from open comments about the ways in which the AI influenced their current practice included and enhanced understanding of social determinants of health, impact on communication skills (pausing more, listening more, and explicitly asking about non-medical aspects of the patient), building relationships with patients, and tailoring treatments to individual patients. Representative quotes demonstrating these themes include: "I invest more time with my patients and make it a point to develop a relationship with them. They get better care and I am more fulfilled with my practice." "I think I communicate with patients and their families more. Although it can take more time, I call patients (families) on my way home when I am concerned that something may have not been communicated effectively. I think this really stems from my experiences on Aliki. I will never forget going to a patient's home and realizing that half of the medications we changed were never filled and/or just sitting in a box." "I pause frequently in my busy practice to consider patients as people, and to understand their lives more thoroughly, and how their lives affect their disease and vice versa." "I enjoy getting to know who patients are and find that by getting to know patients' stories, I can better care for them. My listening skills are better because of Aliki." “The Aliki program has not only influenced my career path, it has also had a tremendous impact on the way I interact with every inpatient and outpatient I encounter. Patients are surprised and pleased by how accessible I am to them and primary providers seem to appreciate my eagerness to discuss medically or socially complex cases...I strongly believe that programs like Aliki are key to transforming medical education and healthcare delivery in the U.S."

CONCLUSIONS: This survey of graduates from our residency program demonstrate very high degree of satisfaction with having participated in the AI during training, and identified that this curriculum had a significant and lasting impact even beyond residency training. The "holy grail" of interventions in medical education is to ultimately impact the attitudes and patient care practices in a targeted way for the learners exposed to the intervention; this is one of only a few studies to report such outcomes. Further work is needed to understand whether the AI objectively impacted patient care in the long term.

THE ASAP SCORE: A NOVEL SCORING SYSTEM FOR PROGNOSIS PREDICTION IN TERMINALLY ILL CANCER PATIENTS Naoki Takamatsu. Teine Keijinkai Hospital, Sapporo-shi, Japan. (Control ID \#2469794)

BACKGROUND: Prognosis assessment is critical in management of terminal cancer patients, enabling provision of optimal end-of-life care to patients and their families. Prognostic scoring systems are increasingly used in cancer care, and the Palliative Prognostic Score (PaP score) and Palliative Prognostic Index (PPI) are two most widely used tools. Although extensively validated with high accuracy, these tools include predictors that require clinical experience in evaluating factors such as the clinician's prediction of survival (CPS), and delirium. As such, CPS tends to estimate longer time of remaining life, while PPI is reported to be overpessimistic when predicting survival durations of less than 3 weeks. Consequently, there is the need of a more accurate and feasible tool with high reproducibility, in which evaluation of variables would not differ among clinicians. Accurate prognostication of terminal cancer patients would identify those needing appropriate attention concerning their last period until death. The aim of this study was to develop an easy-to-use support tool providing clinicians with prognosis prediction.
METHODS: This single-center retrospective observational study was performed to develop a validated scoring system for survival prediction with internal validation. The study used clinical data from adult patients hospitalized with terminal cancer and referred to the palliative care team of a 600-bed acute care hospital in Japan, from September 2014 to November 2015. Electronic medical records were reviewed for clinical characteristics, and the main outcome was days to death upon consultation. Stepwise logistic regression analysis was performed, and $\beta$ coefficients were calculated for each variables of prognostic significance. All statistical analyses were performed with EZR (Saitama Medical Center, Jichi Medical University, Saitama, Japan), which is a graphical user interface for R (The R Foundation for Statistical Computing, Vienna, Austria).

RESULTS: We recruited 284 participants, and 115 patients were finally analyzed. The derivation set included 93 patients, and the validation set included 22 patients. The multivariate-adjusted analysis showed that anorexia (odds ratio [OR] 3.52, $95 \%$ confidence interval [CI]: $1.18-10.5, P=0.02$ ), shortness of breath (OR 5.39, $95 \% \mathrm{CI} 1.11-26.1, P=$ 0.03 ), bed-ridden ADL (OR 5.14, $95 \%$ CI 1.45-18.3, $P=0.01$ ), and pulse rate $\geqq 85$ (OR $2.84,95 \%$ CI $1.01-8.00, P=0.04)$ were significantly and independently associated with death within 14 days. $\beta$ coefficients based on logistic regression analysis were used for scoring of variables, allocating each with 1 point. ASAP score was developed using the above four significant predictors: Anorexia, Shortness of breath, ADL, and Pulse rate. Area under the curve (AUC) in the derivation and validation set, was 0.83 (95\% CI 0.75-0.91) and 0.87 (95\% CI 0.71-1.00), respectively. Using ASAP score cutoff points of 2, death within 14 days was predicted with a $73 \%$ sensitivity and $78 \%$ specificity. Stratum-specific likelihood ratio were 0.08 ( 0 point), 0.6 ( 1 point), 1.3 ( 2 points), and 9.0 ( $\geqq 3$ points).

CONCLUSIONS: In this study, a novel scoring system for predicting prognosis of 14 days was successfully developed and internally validated. ASAP score is a feasible tool with 4 predictors, each having equal weight of 1 point. When ASAP score is 0 , it is highly useful for exclusion, and score of 3 points and above would highly suggest death within 14 days. Two weeks would allow adequate time to prepare patients for their impending death, and give family opportunities for last visits, 'as soon as possible'.

THE ASSOCIATION OF PATIENTS' PRIMARY LANGUAGE WITH READMISSION FOR HIGH-VOLUME HOSPITAL CONDITIONS Anika L. Hines ${ }^{1}$; Kevin Heslin ${ }^{3}$; Marguerite L. Barrett ${ }^{2}$; Ernest Moy ${ }^{3}$; Rosanna M. Coffey ${ }^{4} .{ }^{1}$ Johns Hopkins School of Medicine, Baltimore, MD; ${ }^{2}$ ML Barrett, Inc, Del Mar, CA; ${ }^{3}$ Agency for Healthcare Research and Quality, Rockville, MD; ${ }^{4}$ Truven Health Analytics, Inc, Bethesda, MD. (Control ID \#2469924)

BACKGROUND: Hospital readmissions are common and costly-3.3 million readmissions cost $\$ 4.3$ billion in the U.S. in 2011. A potential risk for readmission is the inability of the patient to understand discharge instructions when they do not speak English - a risk that has been studied rarely and with mixed results. Understanding patient and hospital risk factors could help create more tailored interventions designed to reduce readmissions for vulnerable populations. The purpose of this study was to examine the association of patients' primary language with readmission for high-volume hospital conditions while controlling for clinical factors, patient characteristics, and hospital characteristics.

METHODS: We used California discharge data for years 2009-2013 from the Healthcare Cost and Utilization Project (HCUP). We identified 1.2 million patients admitted for any of five high-volume conditions: acute myocardial infarction (AMI), heart failure (HF), gastrointestinal (GI) hemorrhage, pneumonia, and stroke. Readmissions within 30 days of the index admission were identified. Patients' primary language was classified in one of four groups - English, Spanish, one of 26 languages of Asia or the Pacific Islands, or all "other" languages - including 53 languages of Africa and Europe. We calculated riskadjusted rates of readmission across primary language groups. In a phased approach, we used multivariate logistic regression to estimate odds of readmission controlling for 
clinical characteristics, clinical plus patient characteristics, and clinical, patient, and hospital characteristics comparing non-English to English speakers overall as well as within racial/ethnic groups

RESULTS: Across language groups, patients who were readmitted were older, were often insured by Medicare, were less often privately insured, and were more often admitted to private, for-profit hospitals compared to those who were not readmitted. Overall, compared to English speakers and adjusting for patient and hospital factors, non-English speakers had lower or similar odds of readmissions for all conditions, except patients of API language and "other"-language groups with stroke and "other"-language groups with HF who had higher odds of readmission. Patients discharged from minority-serving institutions had higher odds of readmission for all conditions, and patients discharged from non-English-language-serving institutions had higher odds of readmission for heart attack and pneumonia. Patients discharged from hospitals ranked in the lowest quartile for patients' assessments of doctors' communication proficiency had higher odds of readmission for all five conditions; the hospital quartile that was the weakest in terms of patients' assessment of information provided at discharge had higher odds of readmission for four of the five conditions. Similarly, among analyses within race or ethnic group, nonEnglish speakers had similar or lower odds of readmission compared to their English counterparts with the exception of higher odds of readmission for HF and pneumonia among APIlanguage-speaking patients of API ethnicity.

CONCLUSIONS: Patients who spoke non-English languages, other than Spanish, had higher odds of readmission for heart failure and stroke. For some conditions, non-English speakers had lower odds of readmission compared to their English counterparts. This could be due to other patient and hospital factors, such as socioeconomic status, health literacy, access to interpretation services, or lack of other hospital resources. Minorityserving hospitals and non-English-language-serving hospitals, who often suffer from financial strain, experienced a disproportionate number of readmissions. More research is needed to understand the complex interplay of patient and hospital factors and readmissions in the U.S. when a patient's primary language is not English.

Adjusted Odds of Readmission By Language for selected conditions in California, 2009-2013

\begin{tabular}{llllll}
\hline \hline Characteristic & AMI & HF & GI hem & Pneu & Stroke \\
Language (ref: English) & & & & & \\
Spanish & 0.96 & $0.95^{*}$ & 0.95 & $0.93^{*}$ & 1.00 \\
Languages of API & 1.02 & 1.05 & 1.05 & 1.06 & $1.10^{*}$ \\
Other & 1.08 & $1.06^{*}$ & 1.01 & 1.04 & $1.15^{*}$ \\
\hline
\end{tabular}

Note: $\left(^{*}\right)$ are significant at the $p<.05$ level.

Adjusted Odds of Readmission Comparing Non-English-Language Speakers With English-Language Speakers Within Race/Ethnicity Groups for selected conditions in California, 2009-2013

\begin{tabular}{lc}
\hline \hline Race/Ethnicity and Diagnosis & OR \\
Black ("Other" language vs. English) & \\
AMI & 0.48 \\
Heart failure & 1.12 \\
GI hemorrhage & $0.25^{*}$ \\
Pneumonia & $0.48^{*}$ \\
Stroke & 0.97 \\
Hispanic (Spanish vs. English) & \\
AMI & 0.97 \\
Heart failure & $0.96^{*}$ \\
GI hemorrhage & 0.95 \\
Pneumonia & $0.93^{*}$ \\
Stroke & 1.01 \\
Asian/Pacific Islander (API vs. English) & \\
AMI & 1.02 \\
Heart failure & $1.06^{*}$ \\
GI hemorrhage & 1.07 \\
Pneumonia & $1.08^{*}$ \\
Stroke & 1.09 \\
\hline
\end{tabular}

Abbreviation: API, Asian/Pacific Islander

Note: $\left.{ }^{*}\right)$ significant at the $p<.05$ level.
THE BENEFITS OF TRANSITIONAL CARE SERVICES FOLLOWING A DISCHARGE FOR MEDICARE BENEFICIARIES Andrew B. Bindman ${ }^{1}$; Donald $\mathrm{Cox}^{2}$; Jeff Burrell ${ }^{3}$; Jonathan Briskman ${ }^{3}$; Jonathan Daus ${ }^{3}$. ${ }^{1}$ University of California San Francisco, San Francisco, CA; ${ }^{2}$ US Department of Health and Human Services, Washington, DC; ${ }^{3}$ Acumen LLC, Burlingame, CA. (Control ID \#2463141)

BACKGROUND: In 2013, Medicare adopted transitional care management (TCM) payment codes, which pay practitioners an enhanced rate for an office visit if it is provided in combination with transitional care services for 30 days following discharge from a hospital or nursing home. A member of the care team must communicate with the beneficiary or caregiver within 2 business days of the discharge and the practitioner must provide an office visit within 14 days. We determined the frequency of TCM billing over time, the specialty characteristics of practitioners using the TCM code, the health characteristics of the patients it is used for, and whether billing this code is associated with beneficiaries' subsequent health care costs and mortality.

METHODS: We examined claims on all Medicare beneficiaries from January 1, 2013 through June 30, 2015. Based on Medicare payment criteria, we considered live discharges to the community from a hospital, nursing facility or an outpatient facility for an observational stay to be eligible for TCM services provided the individual had not been billed for these services related to a separate eligible discharge in the prior 30 days. From each of these events we extracted information on the beneficiary's age, gender, and diagnosis as well as subsequent billing for TCM or office visits within 14 days of an eligible discharge. We calculated a beneficiary's health risk score using the demographic and diagnostic information. The specialty category of the practitioner billing for TCM services was based on linked information available in Medicare Data on Provider Practice and Specialty (MD-PPAS) We compared differences in total Medicare health care costs (Parts A, B, and D) and mortality rates in the 60 days beyond the potential period in which the beneficiary could receive TCM services (days 31-90 post discharge) based on whether the beneficiary received TCM services, an office visit but no TCM services within 14 days of the discharge, or neither TCM nor an office visit within 14 days of the discharge. RESULTS: Practitioners billed TCM services for only $2.4 \%$ of $47,266,270$ eligible discharges and this billing rate increased slowly over time ( $1.5 \%$ in 2013, $2.6 \%$ in 2014 and $3.3 \%$ in the first 6 months of 2015). Primary care practitioners, who comprised $35 \%$ of Medicare physicians, billed for $88 \%$ of TCM services. As compared to Medicare beneficiaries who did not receive TCM services following an eligible discharge, those who received TCM were older (75.6 years vs 73.9 years; $p<.0001)$ but had lower health risk scores ( 2.09 vs $2.21 ; p<.0001)$. In $30 \%$ of the cases in which an eligible discharge was not associated with the provision of TCM services, the beneficiary had made at least one office visit within 14 days of the discharge. Beneficiaries who received TCM services had significantly lower per capita Medicare costs $(\$ 5,729)$ in the 2 months after receiving these services as compared with those who had just an office visit within 14 days ( $\$ 7,553 ; p<.0001)$ or who had neither TCM services or an office visit within 14 days $(\$ 6,794$; $p<.0001$ ) during the same $31-90$ days following an eligible discharge. Similarly the mortality rate during this same time period was significantly lower among those who received TCM services ( $2.9 \%)$ than those with an office visit within 14 days $(4.5 \% ; p<.0001)$ and those who received neither TCM nor an office visit $(6.2 \% ; p<.0001)$. These differences in health care costs and mortality remained significant after adjusting for the beneficiary's age, sex, and risk score. CONCLUSIONS: More than 2 years after the implementation of a new TCM payment code, its use in Medicare remains very low. Medicare beneficiaries who receive these services following an eligible discharge have lower costs and mortality in the subsequent 2 months as compared to similar beneficiaries who do not receive these services. There are stepwise mortality benefits of transitional care services on top of the benefits of an office visit within 14 days of an eligible discharge. Given the relatively higher rate of billing for routine office visits than TCM services following a discharge, it would be useful to know what the barriers are that have prevented broader uptake of this apparently beneficial service. 


\section{THE CAREER CHOICES OF CATEGORICAL INTERNAL MEDICINE RESI-} DENTS - TIMING AND INFLUENCES Nancy A. LaVine ${ }^{2}$; Johanna Martinez ${ }^{4}$; Lauren Block ${ }^{3}$; Daniel J. Coletti ${ }^{5}$; Nicole Moodhe ${ }^{3}$ Joseph Conigliaro ${ }^{1} .{ }^{1}$ North Shore LIJ Health System, New Hyde Park, NY; ${ }^{2}$ North Shore-LIJ Health System, Lake Success, NY; ${ }^{3}$ North Shore/LIJ, New Hyde Park, NY; ${ }^{4}$ Northwell Health, Lake Success, NY; ${ }^{5}$ The Zucker Hillside Hospital, Glen Oaks, NY. (Control ID \#2468819)

BACKGROUND: The forecasted gap in primary care providers has led to the reexamination of residents' career choices, given the dwindling number of internal medicine residents $(<20 \%)$ who plan to pursue primary care careers. The number of categorical internal medicine PGY1 National Residency Matching Program (NRMP) positions has increased from $21.9 \%$ in 2011 to $24.8 \%$ in 2015 . However, fewer and fewer residents are choosing to enter primary care careers. The increased number of students entering categorical medicine presents a 3 year opportunity to inspire trainees to pursue primary care careers. The purpose of the study was to examine the process by which categorical residents choose their career path. The aims were to identify when career decisions are made, assess the certainty of career decisions, and examine how medical school experiences and satisfaction with residency ambulatory experience may influence career decisions.

METHODS: An electronic survey was administered to all 104 categorical internal medicine residents at the North Shore Long Island Jewish Internal Medicine Residency Program, one of the largest categorical programs in the nation. Survey questions included items on career planning (timing of decision, certainty of decision) and items evaluating interest in three main areas of internal medicine practice (primary care, subspecialty and hospitalist medicine). Items from the Veterans Affairs Learners Perception Survey (2016) were included on ambulatory training satisfaction (satisfaction with preceptors, the learning environment, and personal experience). Residents were asked about the amount of time spent in ambulatory settings during their internal medicine clerkships and the influence these clerkships had on the desirability of different types of internal medicine practice.

RESULTS: Completed surveys were submitted by 65 residents ( $62.5 \%$ response rate). The majority were male $(60 \%)$ and between the ages of 26-30 (78 \%). The racial composition of respondents was $53 \%$ Caucasian, $31 \%$ Asian, $11 \%$ African American, and $4.6 \%$ mixed race. Eight percent of respondents identified as Hispanic/Latino. A majority $(61 \%)$ had debt greater than $\$ 150,000$. Of the 65 respondents, $75 \%$ reported having made a decision about their career. Having made a career choice was associated with PGY level $\left(\mathrm{X}^{2}=10.53, \mathrm{df}=2, p=.005\right)$ and was noted to increase from $43 \%$ of the PGY1 respondents to $88 \%$ of the PGY3 residents. Of those who reported making a decision about their career, $8 \%$ planned to pursue primary care, $14 \%$ hospitalist medicine and $78 \%$ planned to enter a subspecialty. The majority reported making their career choice during residency (60\%) or during the clinical years of medical school (35\%). Just under half $(49 \%)$ reported being very certain about their decision. Overall satisfaction with ambulatory training was high: $89 \%$ were somewhat/very satisfied with ambulatory faculty, $81 \%$ were somewhat/very satisfied with the ambulatory learning environment, and $78 \%$ reported being very/somewhat satisfied with their personal experience in the ambulatory environment. Of all respondents, $34 \%$ reported being extremely, very or moderately interested in primary care. Sixty-five percent were moderately, very, or extremely interested in hospitalist medicine, and $88 \%$ moderately, very, or extremely interested in subspecialty medicine. However, the majority of residents $(64 \%)$ reported spending $25 \%$ or less of their IM clerkship time in the ambulatory setting. They also reported their IM clerkship made a career in primary care seem less attractive $(40 \%)$ or had no influence ( $43 \%$ ). Seventy percent reported that their IM clerkship made a career in subspecialty medicine more attractive.

CONCLUSIONS: This data, drawn from a large categorical internal medicine residency program, indicates that the career choices of residents are largely made during the clinical years of medical school and during residency, and that these choices are weighted towards subspecialty medicine. It was interesting to note that while $75 \%$ of residents reported having made a decision about their career, only $49 \%$ were very certain of that choice. Further, $25 \%$ of respondents had not yet made a career choice. This finding suggests that residency directors and faculty may be able to influence residents' decisions about their career choice, particularly in regard to primary care, as $34 \%$ of residents reported being at least moderately interested in a primary care career. Respondents reported that IM clerkships offered limited exposure to ambulatory medicine, and most felt that a subspecialty career became more attractive during their clerkship time. This data highlights the need for enhanced exposure to positive primary care experiences beginning in medical school and across the training continuum, with particular emphasis on redesigning resident training experiences. Based on these findings, the opportune time for such interventions would appear to be early in residency training, when many residents remain undecided as to their career choice.

THE CONTRIBUTION OF PHYSICIAN CLINICAL SKILLS IN PATIENT ACTIVATION: WHICH SKILLS MATTER? Angela $\mathrm{Chen}^{3}$; Hillary Lee ${ }^{1}$; Irina Gershgorin $^{1}$; Suvam Paul ${ }^{3}$; Sondra Zabar ${ }^{2}$; Colleen Gillespie ${ }^{3} .{ }^{1}$ NYU Langone, New York, NY; ${ }^{2}$ NYU School of Medicine, New York, NY; ${ }^{3}$ NYU SoM, Brooklyn, NY. (Control ID \#2467375)

BACKGROUND: Higher levels of patient activation (PA) have been associated with more appropriate use of the health care system, higher patient satisfaction and an increase in healthy behaviors. Given its association with better health outcomes, PA has been proposed as a patient outcome that is an 'educationally sensitive'. We used unannounced standardized patients (USPs) to evaluate how patient-activating skills relate to other resident competencies.

METHODS: Two USP cases, Fatigue Case (FC) $(N=119$ visits) and Asthma Case (AC) ( $N=144$ visits) were conducted from 2009 to 2015 at 2 urban ambulatory clinics: Trained actors completed a post-evaluation behaviorally anchored checklist. Scores ( $\%$ well done) were calculated for Overall Communication, History Gathering, Management and Treatment Plan, Case-Specific Patient Education, Patient Activation, and Patient Centeredness. Two hundred sixty-three residents participated (58 PC and 5 IM PGY 1, 32 PC and 85 IM PGY 2, 30 PC and 53 IMPGY3). An alpha level of 0.05 was used for all statistical tests. Bivariate analyses were conducted to determine the relationship between scores. For regression analyses and evaluation of different PGY levels, PC and IM residents were analyzed separately due to uneven sample distributions within each training year. Using $\mathrm{R}$ statistical software, Akaike Information Criterion (AIC) provided a guide to finding the model of best fit after performing stepwise multiple linear regressions to evaluate the best predictors of PA. Factors accounted for included the above listed skill domain scores; visit length, and PGY or months in training.

RESULTS: Mean scores for PA in the FC was $27.5 \%$ (SD 40.35) and 45.7 (SD 71.85) well done in the AC. In both cases, PA scores were significantly and positively correlated with all evaluated skill domains $(p<0.001)$, with no significant differences between genders or PGY. For the FC, there was no association between USP performance with visit length or clinical chaos ratings or the resident's PGY level. The AC differed in that visit length had significant positive correlations with History Gathering $(\mathrm{r}(142)=0.21, p=0.01)$, Management and Treatment Plan $(\mathrm{r}(142)=0.17, p=0.01)$, and Case-Specific Patient Education $(\mathrm{r}(142)=0.27, p=$ 0.001). Regression analysis for both cases showed that the following skill domains best predicted PA scores after controlling for resident program: Case-Specific patient education, Patient Centeredness, and Overall Communication. Together for PC, they explained a significant portion of PA scores: $\mathrm{R}^{\wedge} 2=0.54, \mathrm{~F}(3,56)=23.64, p<0.001$ in $\mathrm{FC}$ and $\mathrm{R}^{\wedge} 2=0.66, \mathrm{~F}(3,56)=38.63, p<0.001$ in AC. Similar results were 
obtained for IM. For PC residents, there were no significant differences between PGY groups in scores except for History Gathering in FC $\left(\mathrm{C}^{2}(34, N=60)=49.82, p=\right.$ $0.01)$ and Overall Communication $\left(C^{2}(30, N=60)=49.6, p=0.02\right)$ and Management and Treatment Plan $\left(\mathrm{C}^{2}(8, N=60)=19.11, p=0.01\right)$ in AC. In the fatigue case, PC PGY-3's held the highest scores in Overall Communication, Case-specific Patient Education and Patient Activation, while they held the lowest scores in these areas in addition to Patient Centeredness in AC. For both cases, IM PGY-2 residents outperformed the IM PGY-3 residents. Also in both cases, $\mathrm{PC}$ residents showed a significant difference in visit lengths by PGY. For IM residents, there was no significant difference in scores or visit lengths between PGY-2 and PGY-3 residents in either of the cases as well.

CONCLUSIONS: While PA scores are highly correlated with many of the measured resident competencies, it seems most closely tied to and predicted by the following skills: Overall Communication, Case-specific Patient Education, and Patient Centeredness. These skills alone seem to account for over half of PA scores when evaluating residents. Characterizing physician factors that contribute to patient activation in this way not only provides a potential method of analyzing PA without the influence of patient bias but can also further the understanding of which skills to cultivate from an educational standpoint with the ultimate goal of improving PA

THE CURRENT STATE OF INTERNAL MEDICINE PRIMARY CARE TRAINING IN THE UNITED STATES Paul O'Rourke ${ }^{1}$; Eva Tseng ${ }^{1}$; Rachel Levine ${ }^{3}$; Marc Shalaby ${ }^{2}$; Scott Wright ${ }^{3} .{ }^{1}$ Johns Hopkins University, Baltimore, MD; ${ }^{2}$ Perelman School of Medicine at the University of Pennsylvania, Philadelphia, PA; ${ }^{3}$ Division of General Internal Medicine, Johns Hopkins Bayview Medical Center, Baltimore, MD. (Control ID \#2465868)

BACKGROUND: The United States is projected to face a primary care physician (PCP) shortage with certain geographic areas disproportionately affected. Over one-third of PCPs are internists, and prior research suggests that generalist physicians are more likely to practice close to where they trained. There is currently no comprehensive database of all internal medicine (IM) primary care programs and therefore the geographic distribution of programs is unclear. Our study objectives were: 1) to identify and create a database of all United States IM primary care programs and 2) to compare the locations of these training programs relative to the $\mathrm{PCP}$ to population ratio across the country.

METHODS: Through a systematic and step-wise approach, we identified all U.S. IM primary care programs, which we defined as one that participates in the National Resident Matching Program (NRMP) with a distinct match code, or one that offers an optional primary care track within an IM categorical residency program. We used data from the NRMP 2014 Residency Match to identify IM primary care programs with a distinct NRMP match number, and data from the American Medical Association FREIDA database to identify IM primary care tracks that did not participate in the NRMP match. In addition, we evaluated individual program websites and/or contacted individual programs via email or telephone to verify all IM primary care tracks without a distinct NRMP code. For the second objective, we considered the geographic locations of all identified primary care programs by state and region, and compared these to the PCP to population ratio in these same areas.

RESULTS: We compiled a comprehensive database of 104 IM primary care programs, 64 of which possess a distinct NRMP program code and 40 of which offer an optional primary care track within a categorical program. The majority of programs are located in the Northeast $(51.9 \%)$ where there is also a higher PCP to population ratio (Figure). Only $13.5 \%$ of programs are located in the Southeast and Mountain West collectively, regions with low PCP to population ratios.

CONCLUSIONS: In our comprehensive database, we identified 104 IM primary care programs. These are unevenly distributed by location and by PCP to population ratio across the United States. Because prior research suggests that generalist physicians are more likely to practice close to where they trained, PCP shortages in specific regions may be addressed through augmenting local training opportunities. These data may be useful when considering strategic solutions for the PCP workforce shortage in this country.

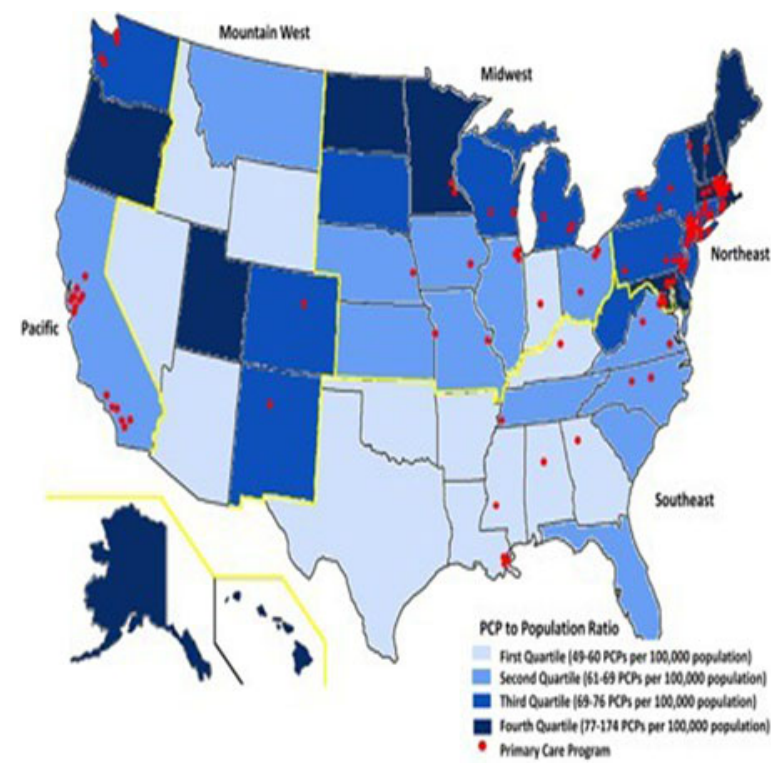

Figure. United States map demonstrating state quartiles of primary care physician to population ratio and the locations of IM primary care programs.

THE EFFECT OF A REGIONAL HEALTH IMPROVEMENT COLLABORATIVE ON THE RATE OF AMBULATORY CARE SENSITIVE HOSPITALIZATIONS FOR PNEUMONIA AND ASSOCIATED COSTS Joseph Tanenbaum ${ }^{2,3}$; Mark E. Votruba ${ }^{3,2}$; Douglas Einstadter ${ }^{1,2}$; Randall D. Cebul ${ }^{1,2}$. ${ }^{1}$ Case Western Reserve University, Chagrin Falls, OH; ${ }^{2}$ MetroHealth Medical Center, Cleveland, OH; ${ }^{3}$ Case Western Reserve University, Cleveland, OH. (Control ID \#2469209)

BACKGROUND: Hospitalization rates for ambulatory case sensitive conditions (ACSC) are a widely accepted and utilized metric of primary care quality. Bacterial pneumonia is an example of an ACSC and one of the most common causes of ACSC hospitalizations $(\mathrm{ACSH})$ in the United States. We have reported a significant increase in utilization of the 23-valent pneumococcal vaccine among residents of Cuyahoga County, Ohio, associated with dissemination of a protocol discovered as part of a county-wide health improvement collaborative in 2008. Although the efficacy of the 23 valent pneumococcal vaccine in preventing bacterial pneumonia has been well established, its effectiveness in reducing population-level ACSH for bacterial pneumonia is unknown. The primary objective of this investigation is to measure the relative change in pneumonia hospitalization rates and associated costs in Cuyahoga County following the introduction of a pneumonia vaccination protocol compared to five other large, urban counties in Ohio.

METHODS: We conducted a difference-in-differences analysis of bacterial pneumonia hospitalization rates in Cuyahoga County, Ohio, before (2003-2008) and after (20092013) dissemination of a region-wide protocol to increase in utilization of the 23 valent pneumococcal vaccine in primary care. Hospitalizations for bacterial pneumonia were defined by the Prevention Quality Indicator (PQI) number 11 of the Agency for Healthcare Research and Quality (AHRQ). We used a population-based, all-payer database comprised of all hospital discharges from all non-federal acute care hospitals in Ohio to measure bacterial pneumonia hospitalization rates. Patients were identifiable according 
to their county of residence, where they were more likely to receive their primary care. Hospital charges, included in the same database, were inflated to 2014 U.S. dollars and converted to direct costs using hospital-level cost-to-charge ratios from the Center for Medicare and Medicaid Services' Provider Specific Files. Year-and county-specific mean costs per pneumonia hospitalization were estimated by dividing the total direct costs for bacterial pneumonia in each county by the total number of hospitalizations for bacterial pneumonia in that county. Yearly averted hospitalizations were estimated for 2009-2013 by multiplying the mean difference in hospitalization rate for Cuyahoga County from 2009 to 2013 by the estimated, year-specific population of Cuyahoga County. Cost savings were estimated by multiplying estimates of year-specific averted pneumonia hospitalizations in Cuyahoga County by year-specific mean cost per pneumonia hospitalization in Cuyahoga County. All models used ordinary least squares linear regression and included county and year specific fixed effects. The estimated population size of each county was used as a county specific analytic weight in all models.

RESULTS: Between 2003-2008 and 2009-2013, the hospitalization rate for pneumonia in Cuyahoga County significantly decreased relative to the next five most populous counties, a reduction of 30.9 pneumonia hospitalizations per 100,000 person years ( $p=$ 0.028). The difference-in-differences estimate implies that an estimated 1964 hospitalizations for bacterial pneumonia were averted in Cuyahoga County over the 2009-2013 period, with implied cost savings from these averted hospitalization of $\$ 7.04$ million. In support of our empirical strategy, we find no evidence for differential trends in the hospitalization rate for pneumonia in Cuyahoga County over the 2003-2008 period ( $p=0.61$ in test for differential linear time trends in Cuyahoga relative to the five comparison counties).

CONCLUSIONS: In the modern era of payment and health care delivery transformation, hospitalization rates for ambulatory case sensitive conditions (ACSC) such as bacterial pneumonia are an increasingly accepted and utilized metric of access to quality primary care. In this investigation, the rate of bacterial pneumonia hospitalization was shown to differentially decrease in Cuyahoga County following the introduction of a primary care-based health improvement collaborative that targetted, in part, an increase utilization of the 23 -valent pneumococcal vaccine in high-risk populations. These findings support the premise that quality improvement initiatives that are primary care focused and that are implemented at the regional level can reduce preventable hospitalizations for ambulatory care sensitive conditions. Our results represent a cause for optimism that more widespread adoption of regional health improvement collaboratives can reduce ACSC hospitalizations and associated costs

\section{THE EFFECT OF LIPOGENESIS ON RESECTED COLORECTAL LIVER} METASTESES Hinda Boutrid ${ }^{1}$; Mary D. Chamberlin ${ }^{2,1}$. ${ }^{1}$ Dartmouth Hitchcock Medical Center, White River Junction, VT; ${ }^{2}$ Dartmouth Hitchcock Medical Center, Lebanon, NH. (Control ID \#2468270)

BACKGROUND: Activation of lipogenesis in cancer cells is known to correlate with a poorer prognosis for many tumor types. Colorectal cancer (CRC) tumors have been shown to express increased levels of molecules involved in the de novo synthesis and hepatic uptake of lipids (S14, LPL, FASN, CD36). However, the expression of these molecules in CRC liver metastases has not been assessed. This study evaluates the expression of lipogenic molecules in CRC liver metastases in comparison with both the primary CRC tumors and the surrounding liver tissue. It further demonstrates the effect of a low fat, low calorie diet on these levels of expression.

METHODS: A retrospective study was performed using available tissue specimens collected from patients with metastatic CRC who were randomized into a group which received a low calorie, low fat Optifast diet for 1 week prior to surgery or to a group with a regular diet. Tissue samples from primary CRC tumors as well as resected CRC liver metastases and liver tissue adjacent to the metastases prior to and following a 1 week diet were evaluated by immunohistochemistry (IHC) for Sp14, LPL, FASN and CD36. All slides were scored by one pathologist. IHC scores for these proteins were compared using Fisher's exact test.

RESULTS: CRC liver metastases in the control group expressed S14 (70 \% of samples), LPL (50\%) and FASN (100\%). There was a statistically significant difference in expression of LPL in CRC liver metastases (50\%) in comparison to adjacent liver tissue samples $(100 \%, p=0.0096)$ and primary tumor samples $(100 \%, p=0.0124)$ in the control group. No statistically significant difference was seen in expression of S14, LPL and FASN between the control and low calorie diet groups.

CONCLUSIONS: CRC liver metastases express molecules involved in lipogenesis. However, these tumors may have a decreased ability to hydrolyze fatty acids from triglycerides present in circulating lipoprotein particles, as indicated by decreased levels of LPL expression. In addition, a short term low fat, low calorie diet, does not appear to have an effect on CRC liver metastases. This information provides a better understanding of the CRC liver metastatic tumor microenvironment and may lead to new therapeutic targets.

THE EFFECT OF RED BLOOD CELL TRANSFUSIONS ON PATIENT FATIGUE LEVELS Micah T. Prochaska; David Meltzer. University of Chicago, Chicago, IL. (Control ID \#2470236)

BACKGROUND: As guidelines increasingly favor limiting red cell transfusion among hospitalized persons to those with lower or "restrictive" hemoglobin (Hb) levels, many also recommend transfusions for hospitalized patients with symptoms of anemia, such as fatigue. These recommendations are based on the premise that the severity of anemia symptoms is influenced by a patient's $\mathrm{Hb}$ concentration, and that raising $\mathrm{Hb}$ levels with transfusion will alleviate symptoms of anemia. However, data on the effects of transfusion is largely limited to post-operative surgical patients and there is little data on how transfusion affects fatigue specifically. Moreover, the association of $\mathrm{Hb}$ levels with fatigue has only been tested in ambulatory patient populations, and not in hospitalized patients who may have fatigue due to their acute illness. Therefore, we sought to measure how anemia-related fatigue changes from hospitalization to 30 days post-discharge, and whether patients' minimum $\mathrm{Hb}$ while hospitalized and receipt of a transfusion are associated with changes in fatigue.

METHODS: From April 2014 through June 2015, hospitalized general medicine patients with a $\mathrm{Hb}<9 \mathrm{~g} / \mathrm{dL}$ at an urban academic medical center were approached for an in-person interview while in the hospital and a 30-day post-discharge phone interview. Consenting patients were administered the Functional Assessment of Chronic Illness Therapy (FACIT) Fatigue Subscale to measure fatigue at both times. FACIT Fatigue scores range from 0 to 52 , with lower scores reflecting greater fatigue. "Change in fatigue" was calculated by subtracting responses on the FACIT while hospitalized from responses on the FACIT at the interview 30-days post-discharge. Positive change in fatigue scores represented decreased (or improved) fatigue from hospital to 30-days postdischarge. Patients' $\mathrm{Hb}$ concentration while hospitalized and whether a transfusion was received was determined using the hospital administrative data. Linear regression was used to test associations between "change in fatigue" score, baseline $\mathrm{Hb}$ concentration, and receipt of a transfusion, controlling for age, sex and length of stay. In these models, an interaction between inpatient fatigue 
level and minimum $\mathrm{Hb}$ while hospitalized was used as a moderator and interacted with whether the patient received a transfusion to predict changes in fatigue 30-days post-discharge.

RESULTS: Five hundred eighteen participants completed the inpatient interview. The mean age of participants was 56 years, $61 \%$ were female, and $64 \%$ were African American. There were no significant differences in these demographics between those who were transfused versus those who were not transfused [(median age 57 vs. $55 ; p=0.07$ ), (percent female 58 vs. $63 \% ; p=0.24$ ), (African American 64 vs. $59 \% ; p=0.54$ ). There were also no significant differences in the mean number of self-reported comorbidities between persons who were and were not transfused ( 4 vs. $4 ; p=0.9$ ). Baseline inpatient FACIT score was 27 in those transfused and was 28 in those not transfused $(p=0.28)$. In linear regression models among patients whose minimum $\mathrm{Hb}$ was less than $9 \mathrm{~g} / \mathrm{dL}$ during their hospitalization, the interaction between inpatient fatigue level, minimum $\mathrm{Hb}$, and receipt of a transfusion while hospitalized was a statistically significant predictor of change in fatigue $(\beta=-0.18, p=0.04)$. This implies that a patient whose fatigue is 3 points greater than average and $\mathrm{Hb} 2-3$ point below average would have their fatigue reduced by $1-2$ points. This interaction remained significant in models that controlled for additional comorbidities including cancer, kidney disease, and a history of anemia

CONCLUSIONS: These findings are consistent with the hypothesis that blood transfusions may improve fatigue in hospitalized anemic patients whose minimum $\mathrm{Hb}$ while hospitalized is $<9 \mathrm{~g} / \mathrm{dL}$, which is within the range of $\mathrm{Hb}$ values in which restrictive approaches to transfusion have been recommended. Our results demonstrate that the greater and more fatigued a patient is (lower FACIT scores) and the lower their Hb while hospitalized, the more likely they are to benefit from a transfusion with improved fatigue levels at 30-days post discharge. This suggests that inpatient providers may be able to combine self-reported fatigue in patients and their $\mathrm{Hb}$ concentration to better target those most likely to benefit symptomatically from a transfusion, while avoiding transfusions and attendant complications in other patients. Prospective studies are warranted to identify the effects of a symptom-guided transfusion strategy on other symptoms of anemia, such as functional capacity, and to evaluate other outcomes of hospitalized patients with anemia.

\section{THE EFFECTS OF MEDICAL STUDENT INTEREST IN HUMANITIES AND} ARTS: A MULTI-INSTITUTIONAL STUDY Salvatore Mangione ${ }^{4}$; Elizabeth Cerceo $^{1}$; Chayan Chakraborti ${ }^{3}$; Megan Voeller ${ }^{5}$; Rebecca A. Harrison ${ }^{2}$; Wendy Bedwell ${ }^{5}$; Keaton Fletcher ${ }^{5}$; Allan Tunkel ${ }^{6}$; Marc J. Kahn ${ }^{3} .{ }^{1}$ Cooper University Hospital, Cherry Hill, NJ; ${ }^{2}$ Orgeon Health and Science University, Portland, OR; ${ }^{3}$ Tulane University School of Medicine, New Orleans, LA; ${ }^{4}$ Sidney Kimmel Medical College of Thomas Jefferson University, Philadelphia, PA; ${ }^{5}$ University of South Florida, Tampa, FL; ${ }^{6}$ The Warren Alpert Medical School of Brown University, Providence, RI. (Control ID \#2466028)

BACKGROUND: Although it has been suggested that exposure to arts and humanities improves desirable characteristics of physicians, there is little evidence to support this hypothesis. Given the current epidemic of physicians' burnout, the medical profession is in crisis. We tested the hypotheses that medical students with greater participation in arts and humanities would score higher on assessments of personal qualities that are conducive to well-being (e.g., wisdom, empathy, self-efficacy, emotional appraisal); but would score lower on qualities that are detrimental to well-being (e.g., intolerance of ambiguity, physical fatigue, emotional exhaustion, and cognitive weariness).

METHODS: To examine the relationship between exposure to humanities and students' psychosocial qualities we developed an online survey administered at five medical schools: 1) The Sidney Kimmel Medical College at Thomas Jefferson University; 2)
Tulane University School of Medicine; 3) Warren Alpert Medical School of Brown University; 4) Oregon Health \& Science University School of Medicine; and 5) Cooper Medical School of Rowan University. A faculty representative emailed the survey to all students enrolled in the 2014-2015 academic year and remained accessible online for 7 months. The survey comprised three parts: 1) Demographic and background information 2) Questions relating to both passive and active exposure to humanities (i.e. listening to music vs playing a musical instrument); and 3) A validated set of assessment tools to measure attributes of physician well being. These included assessments of empathy, tolerance of ambiguity, wisdom, and burnout. We also assessed spatial skills. Demographic and Background Information. demographic data, collegiate major/minor in humanities or non-humanities fields, language proficiency, parental education, and additional work or time off prior to or during medical school. Exposure to Humanities. assessed respondents' active participation or passive exposure to humanities and the arts via a composite score ('Humanities Index' or HI) engaging in visual arts, singing, playing musical instruments, listening to music, dancing, writing for pleasure, reading for pleasure, attending theatre, going to museums/galleries, and attending concerts. Wisdom. measured by the 21-item Brief Wisdom Screening Scale 1 Empathy. measured using the Jefferson Scale of Empathy the 'S' version of the JSE, which was developed for administration to medical students. Tolerance for Ambiguity. measured using the Budner Tolerance for Ambiguity Scale. Emotional Intelligence. measured using two 4-item subscales from Wong and Law's4 Emotional Intelligence Scale: self-emotion appraisals Self-Efficacy. measured using a 10item generalized self-efficacy scale Burnout. measured using the three subscales (physical fatigue, cognitive weariness, emotional exhaustion) of the Shirom-Melamed Burnout Measure. Spatial Skills. measured with the Santa Barbara Solids Test7, comprised of 30 multiple choice questions. The questions consist of 3D geometric shapes that are bisected with a plane.

RESULTS: Of 3107 invited students, 739 (23.8\%) responded and completed $80 \%$ or more of the survey. Regression analyses revealed that humanities exposure positively correlates with wisdom, emotional intelligence, and empathy, tolerance of ambiguity, empathy and self-efficacy and negatively correlates with components of burnout Dependent variables were subject to exploratory factor analysis to determine which variables form coherent subsets that are independent of one another. Using principal componen analysis, with varimax rotation, a four-factor structure emerged. Factor 1 group was wisdom, emotional intelligence subscales, and self-efficacy. Factor 2 group was the burnout subscales. Factor 3 group was tolerance for ambiguity and empathy and factor 4 group was spatial skill abilities. All correlations fell below the .70 range and thus, hypothesis testing was performed based on these four factors. Regression analyses using the general linear model in SPSS (Version 22) were conducted based on the identified factors from the exploratory factor analysis. In support of our hypotheses, exposure to humanities was found to be a significant predictor of all variables of interest. To determine the general effect that humanities exposure had on each of these variables, we calculated Cohen's d using the $\eta p 2$ from the regression analyses. Our results suggest moderate effect sizes for tolerance for ambiguity $(\mathrm{d}=.63)$, wisdom $(\mathrm{d}=.59)$, and empathy $(\mathrm{d}=.46)$. Furthermore, others' emotional appraisals, physical fatigue, and emotional exhaustion were all found to have smaller, but still practically meaningful effect sizes $(d=.29)$. CONCLUSIONS: Greater exposure to humanities is correlated to a significant increases in tolerance for ambiguity, wisdom, empathy, others' emotional appraisals, emotional exhaustion, and physical fatigue suggesting greater exposure to humanities relates to a meaningful increases in these constructs. Additionally, those with an interest in humanities and arts have a higher degree of emotional appraisal, are more tolerant of ambiguity, have lower degrees of cognitive weariness, physical fatigue, and emotional exhaustion and perform better on spatial aptitude tests. These findings support the benefits of the humanities in medical education, and thus have important implications for admission decisions, medical school curricula, and the professional development and well-being of future physicians. 
THE END OF LIFE OPTION ACT PRESENTS NEW OPPORTUNITIES AND CHALLENGES FOR CALIFORNIA Elizabeth Dzeng; Laura A. Petrillo; Barbara Koenig. University of California San Francisco, San Francisco, CA. (Control ID \#2465217)

BACKGROUND: The End of Life Option Act, which gives patients with a 6 month or less prognosis the ability to request medication to end their lives from their doctors, will go into effect in California in 2016. Though similar laws exist in Oregon, Washington, and Vermont, healthcare systems in California face a fresh set of challenges in responding to the law because of California's size and diversity. To anticipate and prepare for these challenges, we convened a 1-day meeting of healthcare, ethics, and policy leaders from across California to guide policy development. We here report major themes that the expert participants identified as areas of necessary policy focus in California.

METHODS: We invited key stakeholders who will be involved in local policy-making regarding California's End of Life Option Act to meet. Participants (111 attendees and 15 volunteers) included clinical and ethics leaders from public and private hospitals, medicine and pharmacy professional society leaders, state officials, researchers, and disability and patient rights advocates. We included participants who had both advocated for and against the law. Six healthcare professionals and researchers from Oregon and Washington also presented their experience with their states' laws. Using interactive panels and smallgroup breakout sessions, we engaged presenters and participants on a range of subjects that healthcare systems will need to consider in crafting policies. Topics included broad subjects such as the interaction of the law with ethics and spirituality, as well as the needs of specific patient populations, such as patients with disabilities or limited social resources. All conversations were recorded with video and/or notes by volunteers, with participants' permission. Two authors conducted independent thematic review of notes and transcripts and inductively determined themes, then met and refined themes to achieve consensus.

RESULTS: An important area of consensus among participants was that every clinic, healthcare dispensary or facility in California that serves adult patients should develop a policy regarding provider activities related to the Act, whether the clinic permits providers to participate or not. Outpatient providers, especially primary care providers, will field the most requests and will need to choose whether or not to prescribe. Inpatient facilities and home-based providers must consider whether and how to provide care when a patient ingests the medication, as well as how to provide bereavement support for families and safe disposal of unused pills. Clinics and facilities must strike a balance between respecting the right of providers to opt out and providing continuity of care to patients. Participants agreed that policies should support and guide providers, embrace interdisciplinary team strengths, and be revised with experience over time. Participants also underscored the value of palliative care for all patients who request a prescription under the End of Life Option Act. This includes thorough evaluation of the meaning behind requests to identify unmet needs, as well as provision of services such as hospice as an alternative or supplement to a prescription. Participants noted that large areas of California have limited access to palliative care and identified the need to increase the palliative care workforce and education for all providers. Participants identified two extremes of harm to patients that could arise; that providers without palliative care training could use the End of Life Option Act indiscriminately in patients whose needs could be met by other means, or not at all in patients who qualify but live in an area without participating providers. Similarly, participants raised concerns that vulnerable patients in California will be at risk of worsened health disparities. This could manifest as either unwelcome pressure to use the law from family, society or healthcare providers or conversely, insufficient access for qualified patients due to high cost of prescriptions. Policies should draw on interdisciplinary team strengths to identify risk and prevent harm to patients. Participants noted tension between the importance of safeguards and the burdensomeness of regulation to patients with limited life expectancy. Research should investigate both the patterns of the law's use, as well as the effect of the law on attitudes about end of life care, including trust in the healthcare system, across diverse populations. Data elements that will be monitored by the state will be insufficient to answer questions about the law's impact on vulnerable populations. Thus, a research network was formed at the meeting to develop future collaborations.

CONCLUSIONS: The End of Life Option Act represents a major shift in the landscape of options available to patients in California. Though the absolute number of patients who will use the law to end their lives in California will likely be small, a much larger number of patients will use the law's existence to initiate conversations with providers about their needs, preferences and fears. In addition to complying with the law, clinics and healthcare facilities must consider many dimensions of the patient and family experience and provide resources to respond to their needs. Our meeting of key stakeholders identified several areas that will require special attention in California when the law takes effect, and began building an infrastructure to support providers in providing patient-centered care.

THE GREAT VANISHING ACT: A LACK OF HIGH QUALITY CLINICAL INSTRUCTION IN PHYSICAL EXAMINATION Allison Taylor ${ }^{1}$; Paul A. Bergl'; Jennifer L. Feagles ${ }^{2}$; Kerrie Quirk ${ }^{2}$; Martin Muntz ${ }^{2}$; Kathlyn Fletcher ${ }^{3} .{ }^{1}$ Medical College of Wisconsin, Wauwatosa, WI; ${ }^{2}$ Medical College of Wisconsin, Milwaukee, WI; ${ }^{3}$ Medical College of Wisconsin/Milwaukee VAMC, Tbd, AL. (Control ID \#2463811)

BACKGROUND: Medical students generally receive formal education in physical examination skills during the preclinical years. By the third and fourth years, schools expect students to refine and apply these skills in clinical contexts. Direct observation and feedback from experienced teachers is one of the most efficient ways for students to improve skills. The AAMC Graduation Questionnaire and other studies suggest that students are observed performing physical exams during their clinical rotations. However these studies rely on self-reporting and do not completely capture how well or how often students are observed. We sought to better characterize the frequency and content of physical exam instruction by directly observing teaching activities on the general medicine ward teams.

METHODS: This prospective observational study used time motion data, checklists on clinical teaching, and reflections written by the observer. Data was collected by a medical student (AT) who was trained by our lead investigator (PB). The team generated a data collection tool based on best practices in clinical teaching described in the literature. Behaviors of inpatient medicine teaching teams were observed during pre-rounds, attending rounds, and admission (i.e. "on call") activities. Our observer recorded bedside patient encounters and dialogues regarding the physical exam.

RESULTS: Sixteen (16) teams were observed. Participants included 21 faculty attendings, 12 residents, 21 interns, 11 fourth year students, and 26 third year students. During attending rounds, the physical exam was presented $44 \%$ of the time $(72 / 163)$ in the conference room, $56 \%$ of the time (24/43) in the hallway, and performed $67 \%$ of the time $(82 / 123)$ at the bedside. The location of teaching activities had no effect on whether teams discussed the clinical significance of exam findings $(p>0.05)$. When the physical exam was discussed away from the patient, the team noted physical findings to verify at the bedside $7 \%(5 / 72)$ of the time when rounding in the conference room and $13 \%(3 / 24)$ of the time in the hallway. Of the 82 patients examined by the attending at the bedside, the teacher noted exam findings for learners $45 \%$ (37/82) of the time, asked students to reexamine the patient before revealing findings $6 \%(5 / 82)$ of the time, and observed the learner's physical exam skills $15 \%$ (12/82) of the time. Bedside experiences significantly increased the probability that the team would highlight a physical finding $(p<0.001)$. During 30 new patient admissions, the student independently examined the patient $53 \%$ $(16 / 30)$ of the time and was observed by a senior team member $20 \%(6 / 30)$ of the time. A senior team member demonstrated exam techniques for the student $7 \%(2 / 30)$ of the time. During 30 patient-student interactions on pre-rounds, the student examined the patient 
$70 \%(21 / 30)$ of the time. When the student performed an exam, it was observed by a senior team member $19 \%(4 / 21)$ of the time.

CONCLUSIONS: Physical exam skills were most likely taught at the patient bedside, although significant discussion about the physical exam occurred away from the bedside. Thus, the presence of the patient appears to influence how the physical exam is taught. As expected, students routinely pesrform physical exams during pre-rounds and new admissions; however, students were rarely observed or guided by senior team members. Our findings suggest that self-reported student-level data do not completely describe the extent to which students are observed performing physical examinations. Our data are particularly relevant as medical schools adopt the Core Entrustable Professional Activities for Entering Residency framework which depends on workplace-based direct observation of skills like physical exam. Based on our findings, teaching of the physical examination could be improved by increasing bedside encounters and encouraging senior team members to directly observe students during times they are likely to examine patients.

THE HEPATITIS C AND HIV CO-INFECTED PATIENT: TIME TO SCALE UP HCV THERAPY AT A LOCAL LEVEL Alireza Meighani ${ }^{1}$; Kassem Bourgi ${ }^{1}$; Rebecca Bajoka ${ }^{1}$; Muhammad Yasser Alsafadi ${ }^{1}$; Indira Brar ${ }^{2} .{ }^{1}$ Henry Ford Hospital / Wayne State University, Detroit, MI; ${ }^{2}$ Henry Ford Hospital, Detroit, MI. (Control ID \#2446216)

BACKGROUND: Since the advent of HAART, HCV-related liver disease has emerged as a significant cause of morbidity and mortality among HIV-infected patients. With traditional therapies patients with co-infection were less likely to be treated and achieve a sustained virologic response (SVR) compared to patients with HCV monoinfection. We reviewed HCV treatment trends from our HIV clinic database to describe the demographics and response to HCV therapy in coinfected patients.

METHODS: In a retrospective study we included patients coinfected with HIV and HCV seen at Henry Ford Hospital (HFH) from 2000 through 2015. Demographic, clinical and laboratory data were collected in addition to the treatment regimens for HIV and HCV. Descriptive analysis was done comparing patients treated with (OT): old therapy (interferon, interferon + ribavirin or Inteferon + ribavirin + telapavir/bocepavir) to those treated with (NT): new therapy of Direct Acting Antivirals.

RESULTS: In the HIV clinic, 1450 HIV infected patients have been followed, of which $11 \%$ are coinfected with HCV. In an analyzed cohort of 67 coinfected patients mean age 45 years, $76 \%$ were men, $34 \%$ were IVDU and $21 \%$ MSM. Two thirds of the patients had AIDS, $82 \%$ had undetectable HIV viral load and median CD4 count was 523 cells/ mm3. Regarding Hepatitis C, Genotype 1a was the most prevalent (52\%) followed by genotype $1 \mathrm{~b}(21 \%)$. Among the 25 coinfected patients who received HCV therapy, 16 were treated with OT of which 9 achieved SVR while 9 of the 9 patients who received NT achieved SVR. Patients who received NT had a higher fibrosis score (FIB-4 score) 2.50 compared to 1.50 .

CONCLUSIONS: With the newer therapies SVR in coinfected patients is comparable to monoinfected patients. Although we demonstrated that the rate of patient treatment has increased since the advent of NT (6 persons/year with NT compared to 1.2 persons/year with OT), HIV-positive patients continue to be undertreated for Hepatitis C. Further prospective appraisal of treatment barriers (host and virus factors as well as drug coverage) is needed.

THE HISTORICAL DEVELOPMENT OF HETEROGENEITY IN MEASURING ETHNICITY AND THEIR IMPLICATIONS FOR HEALTH: FINDINGS FROM THE CANADIAN CENSUS, 1871-PRESENT Kelsey Lucyk; Karen Tang; Hude Quan. University of Calgary, Calgary, AB, Canada. (Control ID \#2469806)
BACKGROUND: Since 1971, when the Trudeau Government formally introduced and adopted a policy of multiculturalism, Canada has affirmed the value and dignity of all Canadians. Specifically, the Multiculturalism Policy of Canada ensured that all citizens could maintain their ethnic and racial identities and take pride in their ancestry. Today, Canada remains ethnically diverse, with over one fifth of its population being foreignborn. Classifying ethnicity is important to understanding the health status of Canadians, for population health outcomes may vary significantly by ethnic, cultural, racial, or ancestral groups. Prior to 1971, Canada maintained a long history of acculturation and intolerance of ethnic diversity, which is reflected in the changes that have been implemented over the last 140 years in the census of the population regarding how data on ethnicity has been collected. The purpose of this project is to review how ethnic classifications in Canada have developed into their present state as heterogeneous indicators that are widely used for population health research and surveillance. We explore how these developments have occurred in their social and political context.

METHODS: Using the unique interdisciplinary lens of population health, health services research, and history, we conducted a review of documents produced by Statistics Canada (i.e., decennial censuses and their question guides) from 1871 to the present day. Informed by history methodology, we used conventional content analysis to extract data relating to ethnicity (e.g., race, ethnic origins, colour, ancestry, place of birth). We then collated key changes relating to ethnic classifications to construct an historical narrative. Using social sciences literature, we mapped how these changes in the census regarding ethnicity related to Canada's changing social and political landscape. Finally, we drew on contemporary literature from the health sciences to consider the implications of these changes for population health. RESULTS: In Canada, the heterogeneity of ethnic classifications has significantly reflected changing social and political circumstances. The changing government policies of this young nation have influenced ethnic classifications to become increasingly more inclusive and granular. Three periods emerge as particularly important in this evolution: (1) early attempts at nation-building during the late nineteenth and early twentieth centuries; (2) social changes in post-World War II Canadian society and the introduction of multiculturalism as a national policy; (3) present-day efforts to preserve representative ethnic classifications for the purposes of research and preserving cultural diversity. In the context of health, ethnic classifications have impacts for population health research and surveillance. There remains the need, for example, to develop consistent approaches to ethnic classifications that can be used by those involved in population health surveillance. Additionally, ethnicity data may be important in identifying health disparities in health status or access to health services. Therefore, data that sufficiently capture ethnic differences would be of great benefit to decision-makers in government and health, as well as those involved in developing interventions to reduce health inequalities.

CONCLUSIONS: Despite the increasingly inclusive approach to heterogeneity that has occurred in Canada over the last 140 years, there remains significant room for improvement regarding the precision of classifying ethnicity. Currently, health data sources only collect broad information about ethnicity. Efforts should be made to capture ethnicity as both a social and cultural concept, especially in large data sources, such as the census of the population, that are used for population health research and surveillance and to influence health decision-making.

THE HOSPITAL SCORE PREDICTS 30-DAY POTENTIALLY AVOIDABLE READMISSIONS IN CONDITIONS TRACKED BY CMS Jacques Donze ${ }^{1,2}$; Mark V. Williams ${ }^{7}$; Edmondo Robinson ${ }^{3}$; Eduard E. Vasilevskis ${ }^{6}$; Sunil Kripalani ${ }^{6}$; Joshua Metlay ${ }^{4}$; Grant S. Fletcher ${ }^{8}$; Andrew D. Auerbach ${ }^{5}$; Jeffrey L. Schnipper ${ }^{2} .{ }^{1}$ Bern University Hospital, Bern, Switzerland; ${ }^{2}$ Brigham and Women, Boston, MA; ${ }^{3}$ Christiana Care Health System, Wilmington, DE; ${ }^{4}$ Massachusetts General Hospital, Boston, MA; ${ }^{5} \mathrm{UCSF}$ Division of Hospital Medicine, San Francisco, CA; ${ }^{6}$ Vanderbilt University, Nashville, TN; ${ }^{7}$ University of Kentucky, Lexington, KY; ${ }^{8}$ Harborview Medical Center, Seattle, WA. (Control ID \#2457220) 
BACKGROUND: The Center for Medicare and Medicaid Services (CMS) instituted the Readmissions Reduction Program to incentivize improvements in care transitions for patients with several common and serious illnesses, including heart failure, acute myocardial infarction, pneumonia, and more recently COPD exacerbations. The HOSPITAL score has been shown to accurately identify medical patients at high-risk for 30-day potentially avoidable readmission, but has never been validated specifically for the medical diagnoses targeted by CMS. We aimed to assess whether the HOSPITAL score would perform well specifically in patients with the primary CMS conditions listed above.

METHODS: We retrospectively included all consecutively discharged patients from 6 US hospitals between January and December, 2011. We excluded patients who died before discharge or who were transferred to another acute care facility. The primary outcome was any 30-day readmission that was classified as potentially avoidable using the previously validated SQLape algorithm. By comparing ICD-9 diagnosis codes from the two hospitalizations, SQLape excludes foreseen readmissions such as chemotherapy and readmissions for new diseases unknown during the preceding hospital stay unless a complication of care. The performance of the HOSPITAL score was evaluated according to its overall accuracy (Brier score), its discrimination (C-statistic, representing the area under the ROC curve) and its calibration (based on the Pearson goodness-of-fit statistic).

RESULTS: During the study period, 9181 patients were discharged with a main diagnosis of pneumonia $(n=3,335,36.3 \%)$, heart failure $(n=3,189,34.7 \%)$, acute myocardial infarction ( $n=767,8.4 \%)$, or COPD ( $n=1,890,20.6 \%)$. Overall, the 30-day potentially avoidable readmission rate was $13.6 \%$, and the HOSPITAL score had a Brier score of 0.11 (indicating very good accuracy) and a C-statistic of $0.68(95 \% \mathrm{CI} 0.66-0.70)$ in the entre cohort, indicating fair to good discrimination. The Pearson goodness-of-fit test showed a $\mathrm{p}$ value of 0.77 , indicating very good calibration. The accuracy and the calibration were good for all 4 diagnoses tested, although calibration seemed to be higher for pneumonia and COPD than for acute myocardial infarction or heart failure. In terms of discriminatory power, the C-statistic ranged from 0.67 to 0.71 for each of the diagnoses (table).

CONCLUSIONS: The HOSPITAL score performed well in identifying 30-day potentially avoidable readmission in patients hospitalized with a main diagnosis of pneumonia, heart failure, acute myocardial infarction, and COPD. This score has the potential to easily identify patients in need of more intensive transitional care interventions when hospitalized for one of these main diagnoses.

Score performance by main diagnosis

\begin{tabular}{|c|c|c|c|c|c|}
\hline & $\begin{array}{l}\text { Number of patients } \\
(\mathrm{N})\end{array}$ & $\begin{array}{l}\text { 30-day potentially avoidable } \\
\text { readmission rate }\end{array}$ & Brier score & C-statistic (95\%CI) & Pearson Goodness-of-fit test \\
\hline Entire cohort & 9,181 & $13.6 \%$ & 0.11 & $0.68(0.66-0.70)$ & 0.77 \\
\hline Pneumonia & 3,335 & $11.3 \%$ & 0.10 & $0.68(0.65-0.71)$ & 0.76 \\
\hline Heart failure & 3,189 & $15.4 \%$ & 0.12 & $0.68(0.65-0.70)$ & 0.17 \\
\hline Acute myocardial infarction & 767 & $12.7 \%$ & 0.11 & $0.67(0.61-0.72)$ & 0.16 \\
\hline COPD & 1,890 & $15.1 \%$ & 0.12 & $0.71(0.67-0.74)$ & 0.81 \\
\hline
\end{tabular}

THE IMPACT OF COMMUNITY ENGAGEMENT ON HEALTH, SOCIAL, AND UTILIZATION OUTCOMES IN DEPRESSED IMPOVERISHED POPULATIONS: SECONDARY FINDINGS FROM A RANDOMIZED TRIAL Christine Lam ${ }^{2,3}$; Cathy Sherbourne ${ }^{1}$; Lingqi Tang ${ }^{2}$; Thomas R. Belin ${ }^{2}$; Pluscedia Williams ${ }^{4}, \overline{\text {; Angela Young-Brinn }}{ }^{6}$; Jeanne Miranda ${ }^{2}$; Kenneth B. Wells ${ }^{2,2}$. ${ }^{1}$ The RAND Corporation, Santa Monica, CA; ${ }^{2}$ UCLA, Los Angeles, CA; ${ }^{3}$ VA Greater Los Angeles Healthcare System, Los Angeles, CA; ${ }^{4}$ Healthy African American Families II, Los Angeles, CA; ${ }^{5}$ Charles R. Drew University of Medicine and Science, Los Angeles, CA; ${ }^{6}$ J.D. Pacada Foundation, Los Angeles, CA. (Control ID \#2467812) BACKGROUND: Disparities in depression care exist among the poor. The Community Partners in Care (CPIC) intervention developed a networked-collaborative intervention to improve depression care in under-resourced communities. We examine whether this intervention impacts health, social, and utilization outcomes in depressed impoverished groups.

METHODS: An analysis of clients sampled above $(N=268)$ and below $(N=750)$ the federal-poverty level, and, among the poor, three non-overlapping subgroups: justiceinvolved $(N=158)$, homeless but not justice-involved $(N=298)$, and other poor $(N=$ 294). Matched programs $(N=93)$ from health and community sectors were randomly assigned to community engagement and planning (CEP) or resources for services (RS). Primary outcome measures included poor mental-health-quality-of-life and PHQ-9 scores. Secondary measures included community-prioritized outcomes, social outcomes and service utilization outcomes. Advantages favoring CEP over RS within subgroups were scrutinized using false-discovery-rate-adjusted $p$-values to account for multiple comparisons.

RESULTS: Consistent with positive results in the entire CPIC sample, CEP showed advantages over RS in the large majority of community-prioritized secondary outcomes.
Primary outcomes did not reveal significant differences. Evidence favoring CEP on secondary outcomes was less convincing after adjustment for multiple comparisons. Among poor clients, the strongest evidence favoring CEP emerged on mental wellness at 6 months $(p=0.004)$. Within subgroups of the poor, the strongest evidence favoring CEP was among justice-involved clients, which suggested the intervention may reduce the percentage of currently homeless or homelessness risk factors $(p=0.036$ ), reduce the total number of life difficulties $(p=0.017)$, reduce the number of mental-health-specialty medication visits $(p=0.038)$, and reduce the number of mental-health counseling visits $(p=0.007)$ at 6 months.

CONCLUSIONS: Although evidence from those below the federal poverty line is not as strong as in the broader CPIC sample, the favorable profile of CEP extends to impoverished depressed clients.

\section{THE IMPACT OF COMORBIDITIES ON DEVELOPMENT OF CHRONIC THROMBOEMBOLIC PULMONARY HYPERTENSION AFTER ACUTE PUL- MONARY EMBOLISM. A 10 YEAR RETROSPECTIVE ANALYSIS FROM A TERTIARY CARE FACILITY Fawad Virk ${ }^{1}$; Shahzaib Nabi ${ }^{1}$; Daryl Sudasena ${ }^{2}$; George Samuel $^{2}$; Ayush Motwani ${ }^{3}$; Adeel Arshad ${ }^{4}$; Tarun Jain ${ }^{2}$; Philip Kuriakose ${ }^{1}$. ${ }^{1}$ Henry Ford Health System, Detroit, MI; ${ }^{2}$ Henry Ford Hospital, Detroit, MI; ${ }^{3}$ Henry Ford hospital, Detroit, MI; ${ }^{4}$ Hamad Medical Center, Weill Cornell University, Doha, Qatar. (Control ID \#2466278)}

BACKGROUND: Pulmonary hypertension (PH), a cause of significant morbidity and mortality, is estimated to develop in $1-5 \%$ of the patients who survive acute pulmonary 
embolism. It carries a poor prognosis with a very high mortality rate which rises proportionally with the severity of $\mathrm{PH}$ and right ventricular dysfunction. We aimed to study various comorbidities and their impact on development of $\mathrm{PH}$ after venous thromboembolism (VTE) and subsequent effect on mortality.

METHODS: We conducted a retrospective chart review of 481 patients with $\mathrm{PH}$ secondary to VTE for analysis of a number of comorbid conditions and their relationship with the time to development of PH after VTE $(n=481)$. We also analyzed the effect of these comorbid conditions on mortality i.e. time to death after PH development $(n=210)$. Data from the past 10 years was collected from the electronic medical records. One-sample ttest was used for variables with two categories. For continuous variables, we used a simple linear regression and reported the slope t-test coefficient to see if there was an association. RESULTS: Patients developed PH after an average of $28.1 \pm 36.9$ months of developing VTE. Patients with VTE were more likely to develop PH earlier if they had congestive heart failure ( $\mathrm{t}=3.68, p=0.0003)$, an active malignancy $(t=3.21, p=0.001)$, diabetes mellitus $(t=3.12, p=0.002)$, peripheral vascular disease $(t=2.94, p=0.004)$, chronic obstructive airway disease $(t=2.91, p=0.004)$, coronary artery disease $(t=2.13, p=0.03)$ or dementia $(t=2.00, p=0.04)$. Out of the 481 patients, 210 died after an average $31.4 \pm$ 25.3 months of developing PH. Patients survived for shorter periods after development of PH if they had chronic obstructive airway disease $(t=3.94, p=0.0001)$, congestive heart failure $(t=2.24, p=0.03)$ or dementia $(t=2.22, p=0.03)$.

CONCLUSIONS: In patients who develop PH after VTE, presence of malignancy, coronary artery disease, diabetes, peripheral vascular disease, congestive heart failure, chronic obstructive airway disease or dementia is related to an earlier development of $\mathrm{PH}$. Presence of COPD, CHF and dementia in these patients is also related to decreased survival as compared to those without these conditions.

THE IMPACT OF GENERIC COMPETITION ON ATORVASTATIN USE AND COSTS TO PATIENTS Jing Luo; John Seeger; Macarius Donneyong; Joshua Gagne; Aaron Kesselheim. Brigham and Women's Hospital and Harvard Medical School, Boston, MA. (Control ID \#2469220)

BACKGROUND: When blockbuster drugs lose their patent protection, competition by generics may be delayed or prevented, leading to unnecessary spending. We examined the transition to generic availability of atorvastatin (Lipitor), the highest selling prescription drug of all time. Atorvastatin's transition from being available as a brand-name-only to a competitive generic market was delayed by several factors, including patent litigation and a period of limited generic competition, during which the first generic competitor challenging a brand-name patent receives an additional 180 days of exclusivity protection. We sought to identify trends in utilization and out-of-pocket spending for atorvastatin around the time of generic entry. We also estimated the financial impact of Pfizer's patent litigation settlement with an Indian generic manufacturer named Ranbaxy.

METHODS: Our cohort study used commercial claims data from Optum (UnitedHealth Group) from December 2010 to May 2013. Participants were adults who had at least 180 days of continuous enrollment and had been dispensed one or more statin medications during the study period. We examined the effect of generic competition on trends in the monthly utilization of different versions of atorvastatin using an interrupted-times series analysis. We defined two interruption points in our analysis: loss of market exclusivity for the brandname version (December 1, 2011) and full generic competition (June 1, 2012). We called the segment between these points the period of limited generic competition, during which Pfizer only competed against a single independent generic. A priori, we divided atorvastatin dispensations into three categories: brand-name, independent generic, or authorized generic (a brand-name product sold as a generic). We calculated the additional costs attributable to Pfizer's patent litigation settlement by comparing actual spending for brand-name atorvastatin against counterfactual spending estimated from our empiric data
RESULTS: Our study included 1,968,709 beneficiaries who filled 13,285,223 statin prescriptions. Limited generic competition reduced monthly brand-name atorvastatin fills by 20,869 (level change, $p=0.03$ ), an $18.1 \%$ change compared to the month preceding loss of exclusivity. During the 6-month period of limited generic competition, brand-name atorvastatin fills decreased by 1346 prescriptions per month, however this trend was not significantly different from the baseline trend $(p=0.33)$. Full generic competition further reduced brandname fills by 54,944 (level change, $p<0.001$ ), a $47.6 \%$ change relative to baseline. The monthly trend of 3006 fewer brand-name fills per month after full generic competition was significantly different from the baseline trend $(p=0.04)$. Generic atorvastatin fills did not surpass brand-name atorvastatin until 7 months after loss of exclusivity. There were no meaningful differences in out-of-pocket spending comparing brand-name and generic atorvastatins during limited generic competition. After full generic competition, monthly out-of-pocket spending for brand-name atorvastatin (median \$30.00, IQR: \$15.00-\$91.38) was substantially higher than spending for either generic atorvastatin (median $\$ 5.10$, IQR: \$3.36-\$19.98) or authorized generic atorvastatin (median \$5.52, IQR: \$3.48-\$19.98). A 20month delay in any generic competition resulted in excess costs of $\$ 5.74$ billion.

CONCLUSIONS: Numerous strategies, including lawsuits, support periods of extended exclusivity protection for pharmaceutical products nearing patent expiration. A delay in the introduction of robust generic competition may lead to billions in unnecessary spending for blockbuster drugs like atorvastatin.

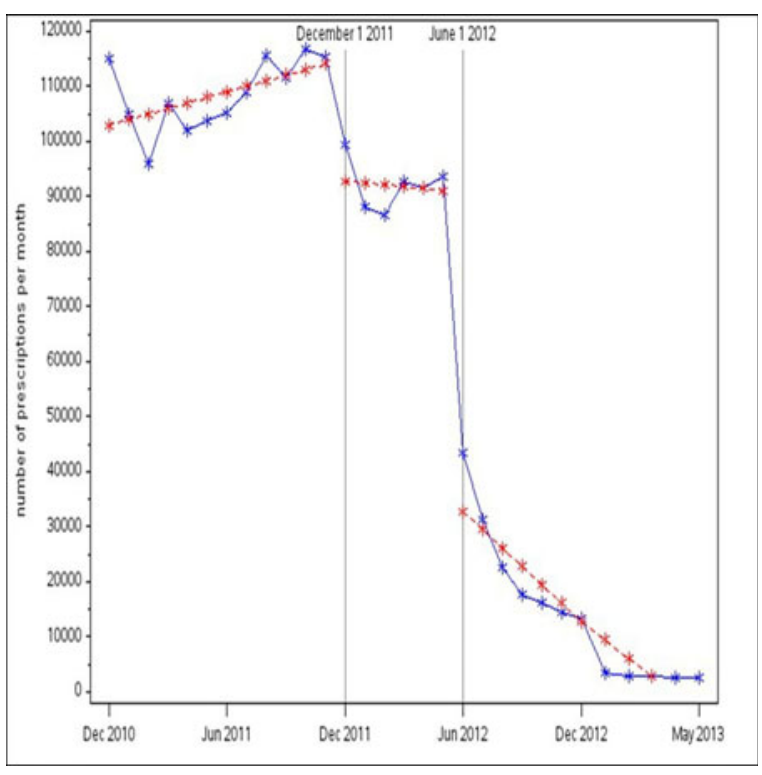

Observed and predicted monthly fills for brand-name atorvastatin between December 2010 and May 2013. Vertical bars indicate pre-specified cut-points (loss of exclusivity and start of full generic competition) for the segmented regression analysis.

\section{THE IMPACT OF LANGUAGE CONCORDANCE ON CANCER SCREENING IN LIMITED ENGLISH PROFICIENT PATIENTS Lisa C. Diamond ${ }^{2}$; Mohammed Imran Mujawar $^{2}$; Margaux C. Genoff ${ }^{1}$; Warren Ferguson ${ }^{3}$; Sukyung Chung ${ }^{4}$; Francesca Gany $y^{2}$. ${ }^{1}$ Memorial Sloan Kettering Cancer Center, New York City, NY; ${ }^{2}$ Memorial Sloan- Kettering Cancer Center, New York, NY; ${ }^{3}$ University of Massachusetts Medical Center, Worcester, MA; ${ }^{4}$ Palo Alto Medical Foundation Research Institute, Palo Alto, CA. (Control ID \#2469672)}

BACKGROUND: Language concordance between limited English proficient (LEP) patients and their clinicians has been shown to be beneficial. Clinicians who are partially fluent in Spanish, e.g. who use their limited Spanish skills to communicate the risks and 
benefits of cancer screening, may be providing worse care to their Spanish-speaking patients than to their Korean-speaking patients with whom they use an interpreter for all interactions. The few studies that have examined the relationship between patient-clinician language concordance and cancer screening have not included a valid measurement of clinician language proficiency. We sought to evaluate if LEP patients with truly bilingual clinicians have higher cancer (colorectal (CRC), breast, and cervical) screening rates compared to LEP patients with partially language concordant clinicians.

METHODS: Primary care providers (PCPs) who reported using non-English language skills to communicate with patients were recruited from the Palo Alto Medical Foundation and from Massachusetts Community Health Centers. PCPs completed the Clinician Cultural and Linguistic Assessment (CCLA), a validated oral-proficiency exam. We calculated rates of breast, cervical, and colon cancer screening completion by physician from the organizations' electronic records for the appropriate patient population based on current guidelines. We included covariates to control for provider-, patient-, and systems-level factors that could influence cancer screening. We used hierarchical/multilevel modeling to account for patient, clinician and system level factors (patients nested within clinicians and clinicians within sites) and performed separate models for each cancer screening type.

RESULTS: Ninety-eight PCPs participated in this study. The majority were women (75.5\%), identified as White/Caucasian (62.2\%), with Spanish (81.6 \%) as their nonEnglish language. The average CCLA score was 78/100 and 69 (70\%) participants passed the CCLA. For colorectal cancer (CRC) screening, English speaking patients were less likely to have completed screening ( $\beta=0.82,95 \%$ CI 0.7-0.97) compared to language discordant patient-physician pairs. Non-English speaking patients with a language concordant clinician were more likely to have CRC screening compared to English speakers ( $\beta=1.5,95 \%$ CI 1.3-1.8). Similar findings were seen with breast and cervical cancer screening. Non-English speaking patients with a language concordant clinician were twice as likely to have completed mammography ( $95 \%$ CI 1.6-2.6) and 1.5 times as likely to have had a Pap smear (95 \% CI 1.3-1.7) compared to English speakers.

CONCLUSIONS: Our findings showed that non-English language speaking patients with language concordant clinicians were more likely to complete CRC, breast, and cervical cancer screening. These findings may be due to unmeasured cultural connections between patients and their bilingual providers, or to differences in the English speaking patients studied. It is essential to understand the relationship of use of limited non-English skills by clinicians and its impact on cancer screening rates. Such knowledge is critical to the development of evidencebased standards for appropriate language services for patients with LEP.

THE IMPACT OF PARTNERSHIP STATUS ON DIABETES CONTROL AND SELF-MANAGEMENT BEHAVIORS Lindsay K. Haines ${ }^{1}$; Natalie Coppa ${ }^{1}$; Janey James $^{1}$; Margaret Yoon ${ }^{1}$; Yael T. Harris ${ }^{2}$; Juan Wisnivesky ${ }^{3}$; Jenny J. Lin ${ }^{1}{ }^{1}$ The Icahn School of Medicine at Mount Sinai, New York, NY; ${ }^{2}$ Hofstra North Shore-LIJ School of Medicine, Great Neck, NY; ${ }^{3}$ Icahn School of Medicine at Mount Sinai, New York, NY. (Control ID \#2467536)

BACKGROUND: Being married or partnered has protective health effects for patients with diabetes, such as improving adherence to recommended diet regimens. However, few studies have evaluated the relationship between diabetes control and partnership status. We aim to assess the effect of partnership status on changes in Hemoglobin A1c (HbAlc) levels, medication adherence, and diabetes self-management behaviors.

METHODS: Patients with type 2 diabetes who were taking at least one oral hypoglycemic agent were invited to participate. To measure medication adherence, participants were given an electronic medication monitoring pill bottle (ECaps $\left.{ }^{\circledR}\right)$ for 3 months. Patients who took their medication as prescribed $>80 \%$ of the time were considered adherent. Diabetes self-management behaviors were evaluated using the Summary of Diabetes Self-Care Activities. Participants were surveyed at baseline and after 3 months, and HbAlc levels were measured at baseline and 3 months. Descriptive and univariate analyses were used to assess the association of partnership status with diabetes control and self-management behaviors at 3 months.

RESULTS: Of the 94 patients who have completed the 3 month interview to date, $45.7 \%$ were married/partnered, $46.8 \%$ were female, and mean age was 62 years. Among married/partnered patients, 24 (56\%) experienced stable or decreased HbAlc values at 3 months while 25 (49\%) of un-partnered patients had a stable or decreased HbAlc value $(p=0.5)$. Partnered patients trended towards being more likely to be adherent to their diabetes medication ( 75 vs. $55 \%, p=0.09$ ) and more likely to participate in a specific exercise session at least 4 times in the past week (34 vs. $18 \%, p=0.08$ ). Partnered and unpartnered patients were equally as likely to be adherent to a healthy eating plan at least 4 times in the past week ( 69 vs. $66.7 \%, p=0.8$ ). Partnered patients were less likely than unpartnered patients to check fingerstick blood glucose monitoring at least four times per week (39.5 vs $60 \%, p=0.06)$.

CONCLUSIONS: There were no significant differences in $\mathrm{HbA} 1 \mathrm{c}$ change, medication adherence, exercise frequency, or diet adherence among patients with type 2 diabetes with or without a partner/spouse. This data suggests that partner status may not impact diabetes management. However, while not statistically significant, partnered patients were more likely to participate in more diabetes self management behaviors such as exercise, healthy diet habits, and medication adherence but were less likely to monitor blood glucose levels. Further investigation with a larger cohort should assess the roles that partners play in helping patients with their diabetes self-management behaviors.

THE IMPACT OF REDUCED ACCESS TO CARE ON CHRONIC DISEASE OUTCOMES: EVIDENCE FROM A NATURAL EXPERIMENT Aaron Baum ${ }^{1,4}$; Ashley E. Jensen ${ }^{3,5}$; Mark D. Schwartz ${ }^{2}$. ${ }^{1}$ Columbia University, New York, NY; ${ }^{2}$ NYU School of Medicine, New York, NY; ${ }^{3}$ VHA and NYU School of Medicine, New York, NY; ${ }^{4}$ University of Pittsburgh School of Medicine, Pittsburgh, PA; ${ }^{5}$ University of Calgary, Calgary, AB, Canada. (Control ID \#2463126)

BACKGROUND: What are the health consequences of reduced access to health care services? The Oregon Health Insurance Experiment, which focuses on access to insurance rather than to care, concludes that increased Medicaid coverage has no effect on most health outcomes, including blood pressure and glycated hemoglobin levels. Observational studies examining this issue face the econometric challenge of recovering unbiased estimates in the absence of experimentally induced random assignment. For example, studies using hospital closure to instrument for reduced access generate biased estimates to the extent closure is correlated with unobserved differences, such as declining economic conditions or decreasing hospital performance, which influence health outcomes and hospital closure simultaneously. This study isolates the health effects of reduced access to care by exploiting an unexpected, large and plausibly exogenous reduction in access caused by a natural disaster. Damages from Superstorm Sandy, the largest North Atlantic hurricane on record, forced the Manhattan campus of the Veterans Affairs (VA) New York Harbor Healthcare System to close for 6 months. To estimate the impacts of reduced access, I compare differences in changes to health outcomes across patients that had been going to VA hospitals differentially impacted by the storm. All VA facilities use an integrated electronic medical records system, allowing me to follow patients as long as they visited any VA facility in the 2 years after the storm.

METHODS: I estimate concurrent and longer-term impacts of a transitory reduction in health access through a generalized difference-in-difference model on a balanced panel of patients. I use data from the electronic medical records of the universe of patients who visited the Manhattan, Bronx, Brooklyn, and New Haven VA Hospitals from 2010 to 2014 (100,000 patients, $6 \mathrm{~m}$ prescriptions, and $10 \mathrm{~m}$ appointments). The specification includes individual, zip code, and month fixed effects, and zip code specific time trends. Estimates 
can be interpreted as the average treatment effect (ATE) under a common pre-trend assumption, which I assess descriptively (Fig. 2, top) and directly test for based on coefficient estimates for time periods preceding the storm (Fig. 2, bottom).

RESULTS: The hospital's unexpected closure caused a complete loss of service at that facility (Fig. 1, top). For the cohort of patients that usually went to the VA Manhattan prior to the storm, the number of visits to any VA medical facility dropped by $50 \%$ during the 6 month closure (Fig. 1, bottom). Figure 2 illustrates trends for and effects on systolic blood pressure over time, displaying raw data (top panel) and difference-in-difference estimates of the ATE (bottom panel) for each month (95\% CIs in dashes, standard errors clustered by zipcode). The ATE during the 6 months when the hospital was closed on blood pressure and glycated hemoglobin levels was $6.55 \mathrm{mmHg}$ and $0.25 \%$, respectively $(p<.001)$, resulting in an absolute increase in the prevalence of hypertension of $10 \%$ and diabetes of $11 \%(p<.001)$. These effects persist after access was restored, with an ATE 18 months after access was restored on blood pressure of $4.09 \mathrm{mmHg}(p<.01)$ and on glycated hemoglobin of $0.21 \%(p<.10)$.

CONCLUSIONS: This study provides evidence that reductions in health access worsen chronic disease outcomes concurrently and that effects persist for 18 months after access is restored.
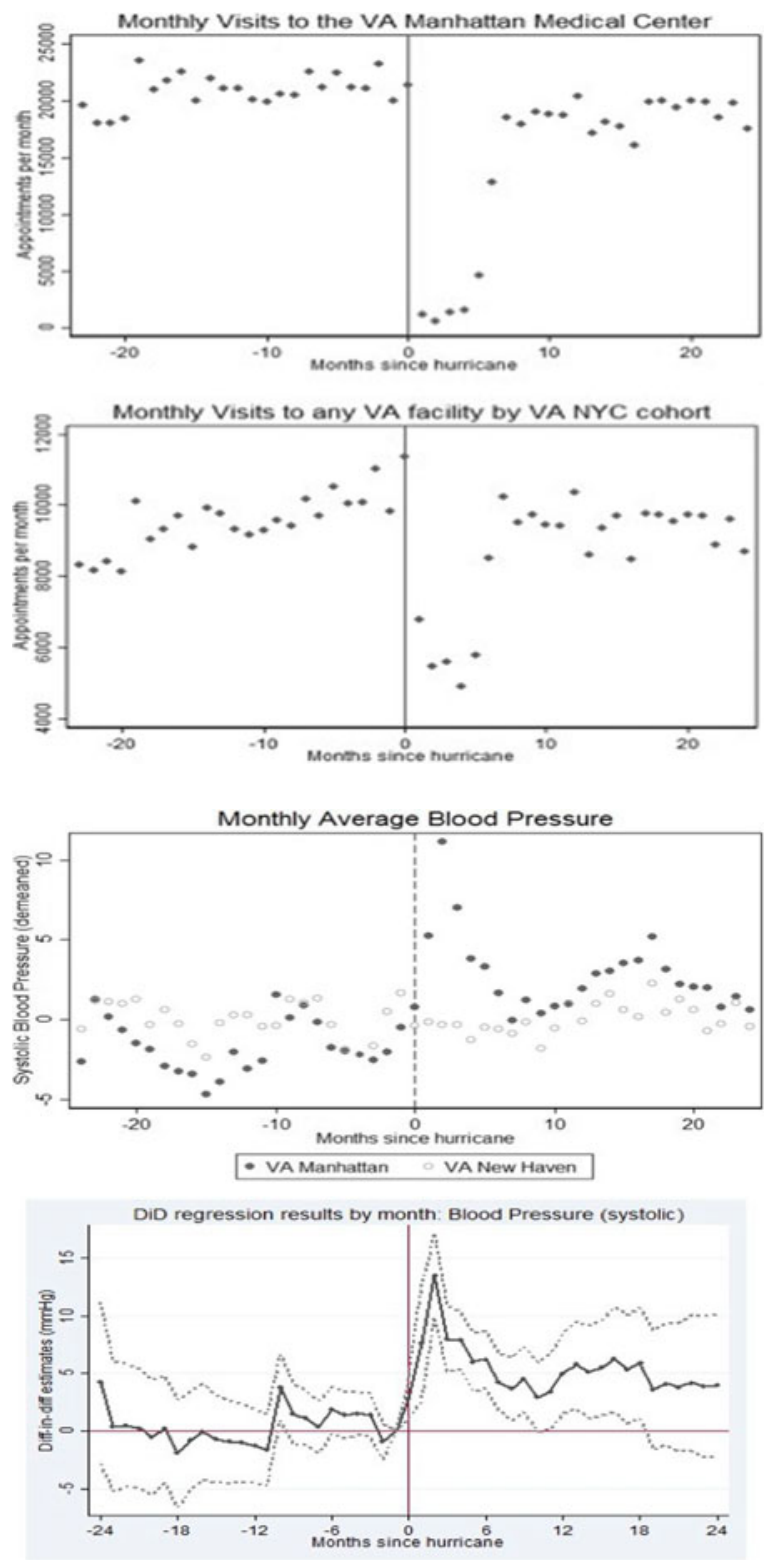

THE IMPACT OF RESIDENT HOLDOVER ADMISSIONS ON INPATIENT LENGTH OF STAY AND RISK OF TRANSFER TO AN INTENSIVE CARE UNIT Deepshikha C. Ashana; Vincent K. Chan; Sitaram Vangala; Douglas S. Bell. UCLA David Geffen School of Medicine, Los Angeles, CA. (Control ID \#2469300)

BACKGROUND: ACGME duty hour standards have led to the creation of novel staffing systems such as the "holdover" system, whereby residents admit patients at night and transfer care to daytime teams who provide longitudinal care. Despite growing literature describing differences between holdover and traditional staffing models, it remains unknown whether patient outcomes are ultimately affected. Thus, we conducted this study to investigate whether patients admitted by holdover teams at a large academic health center experience worse outcomes than those admitted by traditional teams that provide longitudinal care. In particular, we hypothesized that these patients would have a longer length of stay (LOS) and higher rate of transfer to the ICU within $72 \mathrm{~h}$ of admission.

METHODS: We conducted a retrospective cohort study including patients admitted to the general internal medicine wards service at Ronald Reagan Medical Center at the University of California, Los Angeles from July 1, 2013 to June 6, 2015. Primary outcomes included LOS and transfer to an ICU within $72 \mathrm{~h}$ of admission. Secondary outcomes were any transfer to an ICU, inpatient mortality, discharge to home (versus discharge to post-acute care facility), and inpatient readmission within 30 days of discharge.

RESULTS: Five thousand five hundred and eighteen patient encounters met criteria for inclusion. Of these, $64 \%$ were admitted by the holdover team. LOS was significantly longer for holdover encounters, with a geometric mean LOS of $4.95(4.78,5.12)$ days for non-holdover patients and $5.18(5.04,5.31)$ days for holdover patients $(p=0.037)$. Rates of $72 \mathrm{~h} \mathrm{ICU}$ transfer (OR 1.30, CI 0.58-2.93), any ICU transfer (OR 1.29, CI 0.78-2.14), inpatient mortality (OR 1.21, CI 0.27-5.37), home discharge (OR 1.27, CI 0.74-2.18), and 30-day hospital readmission (OR 0.97 , CI $0.83-1.12$ ) were not statistically different between study groups.

CONCLUSIONS: The holdover system at our institution results in longer LOS, perhaps due to inefficiencies and delays in care delivery, but patient safety outcomes are not affected. Modest increases in LOS, when aggregated, can have a substantial impact on bed capacity, patient throughput, and hospital financial performance. Therefore, additional work to understand the drivers of this increase should be undertaken at hospitals that utilize a holdover staffing model.

THE IMPACT OF SELF-ESTEEM ON CAREER ADVANCEMENT AMONG WOMEN AND UNDERREPRESENTED MINORITIES IN ACADEMIC MEDICINE Jennifer M. Manne-Goehler ${ }^{1,7}$; Karen Freund ${ }^{5}$; Anita Raj ${ }^{6}$; Samantha E. Kaplan² Norma Terrin ${ }^{4}$; Janis L. Breeze ${ }^{4}$; Phyllis Carr ${ }^{3}$. ${ }^{1}$ Beth Israel Deaconess Medical Center, Boston, MA; ${ }^{2}$ Boston University School of Medicine, Boston, MA; ${ }^{3}$ Massachusetts General Hospital, Boston, MA; ${ }^{4}$ Tufts Medical Center, Boston, MA; ${ }^{5}$ Tufts University School of Medicine, Boston, MA; ${ }^{6}$ UCSD, San Diego, CA; ${ }^{7}$ Harvard Medical School, Boston, MA. (Control ID \#2468189)

BACKGROUND: Despite efforts to advance gender equality among physicians, differential career outcomes by gender persist in academic medicine. One factor unexplored as a reason for gender differences in career outcomes is the impact of self-esteem on physician outcomes. This is the first study to quantify the impact of self-esteem on subsequent key metrics of career achievement in academic medicine.

METHODS: The National Faculty Study (NFS) is a longitudinal survey of career outcomes among female and male faculty, across the United States. The first National Faculty Survey was conducted in 1995 among a stratified random sample of 1814 faculty members, oversampling among women and minority faculty, at 24 medical schools. In 
2012-13, we conducted a longitudinal follow-up among the sub-sample willing to participate in follow up studies. In the original survey, self-esteem was measured by the 4 item Rosenberg self-esteem scale, which was normalized to a mean of 0 and standard deviation (SD) of 1.0 for these analyses. The follow up survey inquired about four major domains of career achievement: 1) number of peer-reviewed publications as a measure academic productivity, 2) academic rank of full Professor, 3) senior leadership including dean and department chair and 4) retention in an academic career. For non-respondents to the follow up survey, we supplemented our database from publically available sources. We assessed the impact of self-esteem in 1995 on career outcomes in 2012-13 in bivariate analyses, and then in multivariate regression models. We utilized logistic regression for all analyses except that of refereed publications, for which a negative binomial model was utilized. All multivariate models included gender and race/ethnicity (dichotomized as white versus minority, both underrepresented and non-underrepresented), and used backward selection for the following additional potential covariates: specialty, effort distribution across domains of teaching, clinical, research and administration, and years since first appointment. Because we hypothesized that gender differences in outcomes may be related to self- esteem, we repeated the analyses with interaction terms between gender and self-esteem

RESULTS: The analytic sample was 1235 , of whom $50 \%$ were women and $71 \%$ were white. The mean self-esteem score in 1995 was significantly lower for women (-.119) than men $(+.118, p<.0001)$. In unadjusted models, higher self-esteem in 1995 was positively associated with attaining the rank of Professor (odds ratio (OR) 1.36; $95 \%$ confidence interval (CI, 1.20, 1.55), senior leadership (OR 1.28; CI 1.09, 1.50), number of peer-reviewed publications (OR 1.17; CI 1.10, 1.25), and retention (OR 1.29; CI 1.11, $1.50)$ in academic medicine. In multivariate regression models, female gender and minority race / ethnicity were negatively associated with all outcomes. In the multivariate models adjusted for gender and race/ethnicity, self-esteem retained its independent positive association with rank (OR 1.22; CI 1.05, 1.55), leadership (OR 1.22; CI 1.04, 1.43), refereed publications (RR 1.11; CI 1.04, 1.18), and retention (OR 1.31; CI 1.12, 1.53). There were no significant interactions between gender and self- esteem for any of the career outcomes.

CONCLUSIONS: Women reported lower self-esteem as compared to men in 1995. While higher self-esteem had a positive association with subsequent outcomes of retention, referred publications, rank and leadership, there was no confounding of this association by gender or race/ ethnicity and there was no significant interaction between selfesteem and gender. This indicates that the impact of self-esteem on career advancement was similar for women and men and for majority and underrepresented minority faculty, even though membership in these groups was associated with decreased odds of career advancement as measured by the metrics above. While self-esteem appears to be important for career advancement, it does not explain gaps in career advancement for women or minority faculty.

THE IMPACT OF THE MEDICARE ELIGIBILITY AGE ON HEALTH CARE SPENDING, VOLUME, AND PRICES: IMPLICATIONS FOR FEDERAL POLICY Zirui Song ${ }^{2,3}$; Jacob Wallace ${ }^{1} .{ }^{1}$ Harvard University, Cambridge, MA; ${ }^{2}$ Massachusetts General Hospital, Boston, MA; ${ }^{3}$ Harvard Medical School, Boston, MA. (Control ID \#2466810)

BACKGROUND: As the nation's economy recovers and increasing numbers of Americans gain insurance, health care spending is expected to accelerate. Amidst mounting pressure to control Medicare spending, some lawmakers have proposed raising the Medicare eligibility age. Proponents point out that people are living longer and retiring later than they did when Medicare was enacted in 1965. Thus, raising the eligibility age from 65 to 67 , for example, would bring fiscal relief without significant disruptions to most seniors. Opponents contend that raising the Medicare eligibility age would not generate significant savings, as federal spending on Medicaid and exchange subsidies would increase for seniors waiting longer to enter Medicare. Moreover, total health care spending could increase due to more individuals retaining commercial coverage after age 65. To date, little is known about the impact of the Medicare eligibility age on spending, volume, and prices for U.S. adults, the majority of whom transition into Medicare from private insurance.

METHODS: To assess the impact of changing Medicare's eligibility age, we examined the effect of entry into Medicare on spending, volume, and prices for beneficiaries who transitioned into the program from employer-sponsored private insurance. We identified 1,341,854 individuals who transitioned from commercial insurance to Medicare at age 65 using 2007-2013 Truven commercial and Medicare claims data. We excluded individuals who did not have at least 1 year of continuous enrollment before and after entering Medicare, as well as individuals with Social Security Disability Insurance (SSDI) or end stage renal disease as they are eligible for Medicare prior to age 65 . We restricted the sample to retired individuals, since active workers often retain employersponsored insurance after age 65. Our final sample comprised 200,870 beneficiaries. Using a regression-discontinuity design, we evaluated changes in spending, volume, and prices attributable to entry into Medicare at age 65, adjusted for age trends, quarter, year, and individual fixed effects. We focused on outpatient imaging and procedures, as these services are consistently reported in our commercial and Medicare data. We performed multiple sensitivity analyses.

RESULTS: Unadjusted medical spending fell sharply after entry into Medicare, while spending trends were similar before and after age 65. After spending on average $\$ 119.12$ per quarter in their last year of commercial insurance, new Medicare beneficiaries spent $\$ 89.28$ per quarter in their first year in Medicare. In adjusted analysis, spending was $\$ 38.56$ or $32.3 \%$ lower after transitioning into Medicare $(p<0.001)$. Spending fell by $\$ 33.75(40.2 \%)$ for imaging $(P<0.001)$ and $\$ 4.82(13.8 \%)$ for procedures $(P<0.001)$. The decrease in spending was driven by lower Medicare prices relative to commercial. Average prices were $\$ 56.48$ (29.2\%) lower in Medicare-\$63.20 (37.8\%) lower for imaging $(P<0.001)$ and $\$ 37.07(13.3 \%)$ lower for procedures $(P<0.001)$. Volume did not significantly change after entering Medicare. Sensitivity analyses were consistent with our findings. Results were similar across alterations in the model's bandwidth, functional form, and control variables, including the removal of individual, year, or quarter fixed effects. In addition, Medicare beneficiaries who were SSDI recipients, and therefore experienced no change in coverage at age 65 , exhibited no change in spending at age 65. This strengthens our inference that the sharp decrease in spending for the main sample of non-SSDI individuals at age 65 is attributable to entry into Medicare.

CONCLUSIONS: Entry into Medicare decreased medical spending by $32 \%$ for newly enrolled beneficiaries with prior commercial insurance. Lower Medicare prices, rather than changes in utilization, drove the decrease in spending, consistent with Medicare's considerable market power as a large insurer. Our estimates suggest that raising the Medicare eligibility age would at first reduce Medicare spending by removing Medicare coverage for the youngest beneficiaries. However, spending for these beneficiaries is about $30 \%$ lower in Medicare than it would be if they were to enroll in private insurance, implying total spending would rise. The full effect of such a policy on government spending must account for increased federal subsidies to privately insured individuals in health insurance exchanges and increased spending for those impacted who qualify for Medicaid and dual-eligible beneficiaries. If younger beneficiaries are healthier, the decreased risk pooling would also likely increase premiums for the remaining Medicare beneficiaries. To our knowledge, this is the first attempt to measure differences in spending, utilization, and prices between commercial insurance and Traditional Medicare using claims data from a national sample of continuously enrolled adults within a 
regression-discontinuity approach to estimate causal effects. Our findings have two main policy applications. First, it provides evidence on how spending and access differ between Medicare and private insurance, thereby helping policymakers forecast the impact of proposed changes in the Medicare eligibility age. Second, and more broadly, these findings provide new insight into the effect of Medicare's market power on prices and access to care. If these findings generalize to other services, they suggest that insurers with market power can pay lower rates without losing access to providers.

\section{THE IMPACT OF U.S. CLINICAL TRAINING ON INTERNATIONAL MEDI-} CAL GRADUATE'S CAREER FROM JAPAN: A QUALITATIVE STUDY Hirotaka Kato $^{1}$; Alfred Burger ${ }^{1}$; Robert Yanagisawa ${ }^{2}$; Jenny J. Lin ${ }^{3} .{ }^{1}$ Mount Sinai Beth Israel, New York, NY; ${ }^{2}$ Icahn School of Medicine at Mount Sinai, New York, NY; ${ }^{3}$ Icahn School of Medicine, New York, NY. (Control ID \#2465988)

BACKGROUND: International medical graduates (IMGs) consist of about onefourth of the physician workforce in the United States. The number of U.S medical graduates is surpassing the available residency positions, however losing IMGs completely from the U.S. clinical training poses a risk in diversity and care quality for diverse population. The previous articles have discussed the significance of IMGs as a contributor to academic medicine, brain gain/brain drain, but there is little data about the longitudinal impact of U.S. clinical training on IMG's career and international healthcare system. In order to shed light on academic contribution of IMGs, we sought to identify the reasons pursuing U.S. clinical training from a high-income country, and its career impact among IMGs from Japan who train or have trained in both Japan and selected residency programs in New York City, known as "N program scholars." METHODS: We used the Japanese IMGs' network, known as "N program scholars," consisting of current residents and graduates from selected New York City residency programs. A cross-sectional online survey was conducted from July 2015 to September 2015. The questionnaire included characteristics, current positions, reasons of US clinical training and its impacts on their careers. Grounded theory was used to analyze narrative scripts and identify themes.

RESULTS: One hundred eighteen out of 152 (77.6 \%) completed the survey. Respondents consisted of 20 current residents (17\%), 95 graduates $(81 \%)$ and 3 no answers ( $2 \%$ ). Of the graduates, $26 \%$ graduated within the past 5 years, $30 \%$ between 6 and 10 years ago, $24 \%>10$ years ago. At the start of their US residency program, 45 (38\%) were PGY 3 or less in Japan, 92 (78 \%) were PGY 6 or less. Twenty-nine (33\%) have or are currently pursuing an advanced degree. The most common residencies included internal medicine $(67 \%)$ and pediatrics $(18 \%)$. The most common fellowships included infectious disease (13\%), hematology/oncology $(11 \%)$, cardiology $(8 \%)$, and pulmonary/critical care $(7 \%)$. One hundred respondents $(85 \%)$ currently work in academic settings including university hospitals (52\%) and tertiary medical centers (33\%), either in the United States $(67 \%$ ) or in Japan (30\%). Among the 64 attending physicians, $15(23 \%)$ are currently either at the professor level or hold higher administrative titles (division chief, department chair, or president), 7 (11\%) are associate professors, and $26(40 \%)$ are assistant professors. Common themes with regard to reasons for US training included standardized clinical/academic training, pursuing fellowship not available in Japan, broader career options, and diverse experience. In addition to those themes, inspiring professional networking was identified as advantages of US training in their careers, whereas estrangement from Japan, additional overlapping residency, financial burden, and limited procedural training were often seen as the drawback. As for career impact of US clinical training, many Japanese IMGs reported that their US residency was the game changer for their careers, and they were recognized as a leader and/or a pioneer in Japan. US residency also gave them the foundation for clinical, academic skills and broad perspectives, as well as inspiring professional networks. In exchange, reverse culture shock was identified as a drawback for graduates returning to Japan.

CONCLUSIONS: Many Japanese IMGs sought US residency for subspecialty training not available in Japan, and they have been academically successful in both the US and Japan over the long term. They report that training in the US gave them a wealth of experience, skills, broader views and expanded professional networks. N program graduates demonstrate significant academic commitment and contributions, both in the US and Japan.

THE 1 YEAR TREATMENT COURSE OF NEW OPIOID RECIPIENTS IN VETERANS HEALTH ADMINISTRATION Hilary Mosher ${ }^{1,2}$; Kelly Richardson ${ }^{2,3}$; Brian C. Lund ${ }^{2,3}$. ${ }^{1}$ University of Iowa Hospitals and Clinics, Iowa City, IA; ${ }^{2}$ Iowa City VA Healthcare System, Iowa City, Iowa, IA; ${ }^{3}$ University of Iowa, Iowa City, IA. (Control ID \#2469127)

BACKGROUND: Recent research on patients who are on long term opioid therapy (LTOT) demonstrates that, once established, LTOT tends to be persistent. Little is known about patterns of new opioid prescription leading to long-term therapy. Short term opioid therapy (e.g. for acute pain) is common; it is not known what proportion of patients newly prescribed opioids subsequently has a pattern of ongoing opioid receipt. An understanding of the patterns of opioid receipt leading to long term therapy may improve clinical decision-making regarding repeat prescribing and inform policy decisions aimed a curtailing excess or inappropriate opioid use. Therefore our objectives were to describe the 1 -year treatment course of new opioid recipients, including the proportion that subsequently received opioids long-term; contrast initial prescription characteristics over time (e.g. dose, days supplied); and discern variation in proportion of long-term therapy initiation across Veterans Health Administration (VHA) medical centers.

METHODS: We included VHA pharmacy users in 2004 and 2011 who received a new opioid prescription preceded by 365 days in which no opioid was prescribed (i.e., new recipients). Short-term therapy was defined as an index treatment episode lasting no longer than 30 days and no additional opioid prescriptions in the year following initiation. Long-term therapy was defined as a treatment episode of $>90$ days that occurred within the year following initiation. All other patterns were considered intermediate.

RESULTS: In 2004, $43.2 \%(161,247$ / 373,276) of new opioid recipients met the definition of short term therapy, compared to $42.7 \%(224,939 / 526,499)$ in 2011. The proportion who met the definition for long-term therapy decreased from $20.4 \%(76,280)$ in 2004 to $18.3 \%(96,166)$ in 2011 . The remaining proportion, patients with intermediate patterns of receipt, increased from $36.4 \%$ $(135,821)$ in 2004 to $39.1 \%(205,394)$ in 2011 . Although initial dose remained stable between 2004 and 2011, median days supplied in the index prescription decreased from 30 to 20 days, and a greater percentage of new opioid recipients received initial prescriptions of 7 days or fewer $(20.9 \%$ in $2004 ; 27.9 \%$ in 2011). The proportion of new recipients whose therapy continued long-term use ranged from 10.5 to $38.5 \%$ across medical centers in 2011 .

CONCLUSIONS: While the overall number of new opioid recipients increased, the proportion who had subsequent long-term opioid therapy declined slightly between 2004 and 2011. This decline may reflect a growing reluctance to initiate long-term opioid therapy or the saturation of potential candidates for long-term opioids. The rising proportion of intermediate users warrants further investigation. Recent initiatives aim to curtail opioid prescribing. Our method of characterizing opioid prescribing distinguishes clinically different short- and long-term patterns, and can be applied to assessing outcomes of efforts such as the national VHA Opioid Safety Initiative. 


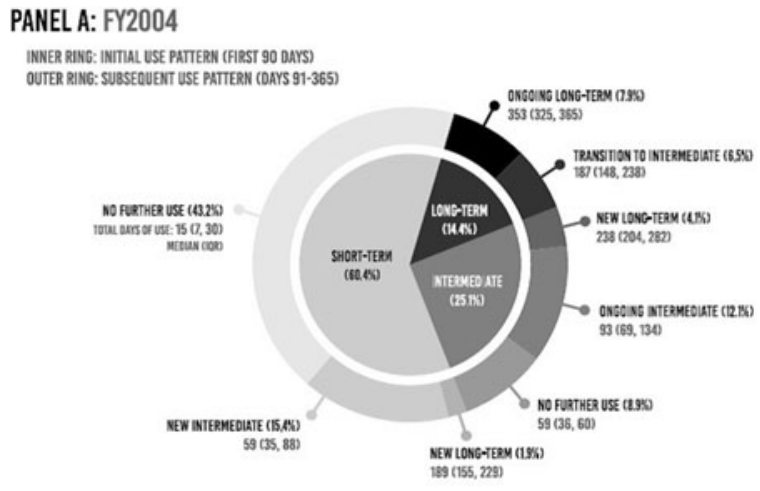

\section{PANEL B: FY2011}

IWNER RING: IMITAL USE PATTERN (FIRST 9O DAYS) OUTER RING: SUBSEOUEIT USE PATTERN (DAYY 91-365)

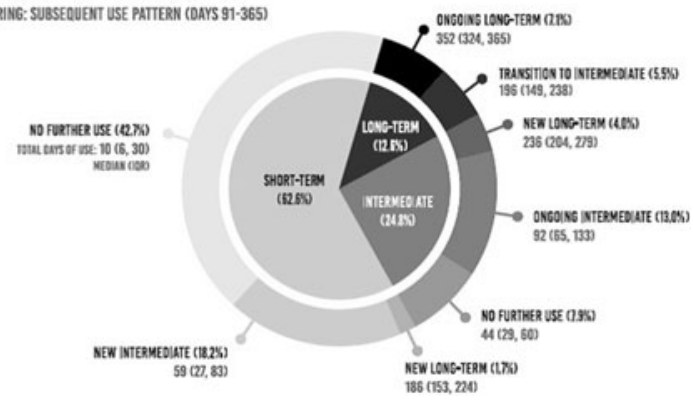

One-year pattern of opioid prescription following incident receipt in Veterans in 2004 and 2011

THE PAUSE PROCEDURE ENHANCES RECALL IN CONTINUING MEDICAL EDUCATION PRESENTATIONS: A RANDOMIZED CONTROLLED INTERVENTION STUDY Lukas W. Richards ${ }^{1}$; Amy T. Wang ${ }^{2}$; Saswati Mahapatra ${ }^{1}$; Sarah Jenkins ${ }^{1}$; Nerissa Collins ${ }^{1}$; Thomas J. Beckman ${ }^{1}$; Christopher M. Wittich ${ }^{1}$. ${ }^{1}$ Mayo Clinic, Rochester, MN; ${ }^{2}$ Harbor-UCLA Medical Center, Torrance, CA. (Control ID \#2433243)

BACKGROUND: Continuing medical education (CME) is a common method for physicians to maintain competency, though previous studies have demonstrated that CME has only a modest impact on physician behaviors and patient outcomes. Active learning strategies have been shown to improve knowledge recall and educational outcomes in CME. A "pause procedure," where students take periodic breaks during lectures to collaborate on and discuss the curricular content, has been shown to increase knowledge recall and test performance in undergraduate education. However, we are unaware of pause procedure studies in CME. Our goals were to 1) implement a pause procedure during a large CME course, 2) determine if presentations with pause procedures are evaluated more favorably based on a validated presentation assessment tool, and 3) determine if pause procedures improve knowledge recall.

METHODS: This was a randomized controlled intervention study of all participants ( $N=$ 214 ) at the 22nd Annual Mayo Clinic Internal Medicine Board Review (IMBR) course. The 48 large group lectures were randomized to either intervention group or control groups. Intervention lectures included the pause procedure, consisting of a 1-min pause every $15 \mathrm{~min}$ during 30 or $45 \mathrm{~min}$ presentations. During the pause, participants were instructed to review notes independently or with others seated nearby. Control lectures consisted of a traditional lecture with no break. At the end of each half-day participants performed rapid recalls, which required writing down as many recalled facts as possible in $5 \mathrm{~min}$. Study outcomes included 1) scores from a validated 7-item presentation evaluation instrument and 2) the number of recalled items from each lecture. Presentation evaluation scores (average percentage "strong agreement" to 7 evaluation items) and number of recalled items per presentation were compared between intervention and control groups using paired t-tests.

RESULTS: Two hundred fourteen participants returned 145 surveys (response rate $=$ $68 \%$ ). Mean presentation evaluation scores were significantly higher for the pause procedure presentations than for the traditional control presentations (70.9 vs $65.8 \%$ in strong agreement; $95 \% \mathrm{CI}$ for the difference, 3.5 to $6.7 \% ; p<0.0001)$. The number of rapid recall items per presentation was significantly higher for the pause procedure presentations than for the traditional control presentations ( 0.68 vs 0.59 ; $95 \% \mathrm{CI}$ for the difference, 0.02 to $0.14 ; P=0.01$ ).

CONCLUSIONS: We describe the implementation of a feasible pause procedure in a large, Internal Medicine CME board review course. Presentations that included a pause procedure had higher presentation evaluation scores and increased knowledge recall. Active learning methods such as the pause procedure should enhance the educational effectiveness of CME, which is a crucial venue for maintaining competence for physicians. The pause procedure arms CME presenters with an easy, effective technique to improve the delivery of their educational content. More research will be needed to determine lasting effects of the pause procedure on medical knowledge retention and patient care.

THE PREVALENCE OF OSA AMONG AN ADULT POPULATION WITH DOWN SYNDROME Janis L. Sethness ${ }^{1}$; Michelle Cornacchia ; Cynthia Peacock ${ }^{2}$; Philip Alapat ${ }^{1}$; Yu-Hsiang Lin $^{3} .{ }^{1}$ Baylor College of Medicine, Houston, TX; ${ }^{2}$ baylor college of medicine, Houston, TX; ${ }^{3}$ University of California, Los Angeles, Los Angeles, CA. (Control ID \#2455326)

BACKGROUND: Obstructive sleep apnea (OSA) is a common sleep disorder affecting $2-4 \%$ of adults, and untreated can cause significant cardiopulmonary complications. Among adults with OSA, studies involving individuals with Down syndrome (DS) are rare. One study by Trois et al. (J Clin Sleep Med, 2009) showed that individuals with DS have a high prevalence of OSA, although it was a limited study size of 16 participants. Given the lack of evidence of the association of OSA and DS in adults in the current literature, there are no universal guidelines for screening. However, the American Academy of Pediatrics (AAP) does recommend obtaining polysomnography (PSG) for all children with DS by the age of 4 . Unfortunately, many children are not screened and are left undiagnosed and untreated. Therefore, it is important to further evaluate the disease burden in this population, and if appropriate, recommend universal guidelines for OSA screening.

METHODS: A retrospective chart review study of adults with DS was performed at the Baylor College of Medicine (BCM) Transition Clinic to yield the following information: history of PSG; results of PSG; and if no sleep study completed, reason why not. The prevalence of OSA in adult patients with DS was then determined. The study was approved by the BCM Institutional Review Board.

RESULTS: Of the 125 patients with DS seen at Transition Medicine Clinic, 82 (65.6\%) had undergone PSG testing. Records of PSG testing were available for 69 of those patients. Sixty of the patients for whom records were available were adults $(\geq 18 \mathrm{yo})$ at the time of the PSG. Of those 60, 56 (93.3\%) of participants were diagnosed with OSA. For those patients who did not have PSG testing done, the most common reasons cited in clinic documentation were (1) referral made for PSG but not completed (34.9\%) and (2) patient was screened for symptoms of OSA and was asymptomatic (25.6\%).

CONCLUSIONS: The purpose of this study was to evaluate the prevalence of OSA in the adult DS population. The AAP recommends that all children with DS be screened for OSA; however, no such recommendations exist for the adult DS population. Given that adults with DS have even more predisposing factors for OSA, including obesity and 
hypothyroidism, and that the prevalence of OSA tends to increase with age across all populations, it is particularly important to understand the prevalence of OSA in this population in order to make appropriate screening recommendations. Our study shows that adult DS patients who undergo PSG testing have high rates of OSA. Because of the high prevalence of OSA among our study population, we recommend that all adults with DS should be screened for OSA with PSG.

THE PROFESSIONALISM DIVIDE: RESIDENTS' PERCEPTIONS OF PROFESSIONALISM IN THE LEARNING ENVIRONMENT Preston Reynolds; Casey White; James Martindale; Nicholas Kiefer. University of Virginia, Charlottesville, VA. (Control ID \#2469284)

BACKGROUND: Residency training occurs alongside patient care in environments that are complex. The LCME, ACGME, and Joint Commission now mandate learning environments that promote professionalism. In concert with this movement, the ACGME implemented the Clinical Learning Environment Review (C.L.E.R.) program for institutions hosting graduate medical education. The ACGME's C.L.E.R. program requires institutions and residency programs create professionalism curricula, periodically assess the culture of professionalism, and continuously improve to meet new review standards Between 2010 and 12, researchers interviewed 11 of 13 residency program directors to determine the scope of residency curricula, faculty development programs, and assessment methods focused on professionalism. Qualitative analysis of the interviews revealed a lack of structured professionalism curricula for residents and faculty, and a near universal reliance on role modeling for teaching ethics and professionalism. Evaluation methods predominantly comprised 360 assessments. One program used a professionalism assessment tool in a self-assessment exercise. The study was to assess the institution's culture of professionalism from the perspective of residents. It was hypothesized that residents would rate their commitment to professionalism greater than that of their residency program and the institution.

METHODS: A survey was constructed around 13 professionalism behaviors considered core to professionalism by the ACGME and the literature. Pilot testing with senior residents and fourth year students was conducted; the final instrument was modified based on feedback. The project was an anonymous on-line survey to be completed by all residents. Three mailings were sent out, and with endorsement from the House Staff Council, the two presidents asked residents to complete the survey honestly and notified them of a \$5 coffee card upon completion. The project received IRB approval. (\#20130110)

RESULTS: More than $47 \%$ of residents (365/771) completed the survey. Residents reported they were significantly more committed to demonstrating the set of 13 professionalism behaviors than the institution was. $(p<.001)$ Residents also perceived their residency program significantly more committed to professionalism than the institution. $(p<.001)$ Residents reported the most common reason they did not attend workshops on professionalism is that they were not offered (54\%). Respondents noted about $25 \%$ of their faculty modeled professionalism all the time. More than $50 \%$ of respondents admitted their perception of the importance of professionalism was influenced when their attending did not model professionalism. The lack of time and support services, and the number of patient admissions were cited as barriers to professionalism.

CONCLUSIONS: Residents at one teaching hospital report they work in a learning environment they perceive is not as committed to demonstrating professionalism as they are, and the difference in commitment is significant from their perspective. Moving from advanced beginner to competent professional requires residents have the right learning environment for this development to occur. Professional behaviors have to be taught, modeled by faculty and other residents, and reinforced through assessment methods. These data reveal the learning environment should remain a focus of ACGME activities, and that these requirements be expanded, if we are to train the next generation of professionals in professionalism.

THE QUALITY OF HEALTH CARE DELIVERED TO ADULTS IN THE UNITED STATES, 2002-2013 David M. Levine ${ }^{1}$; Jeffrey A. Linder ${ }^{1}$; Bruce E. Landon ${ }^{2}$. ${ }^{1}$ Brigham and Women's Hospital, Boston, MA; ${ }^{2}$ Harvard Medical School, Boston, MA. (Control ID \#2467966)

BACKGROUND: Medical care in the United States has been criticized as low-quality and high-cost. Research from over a decade ago demonstrated widespread deficits in adherence to basic recommended practices. Over the past decade, national efforts to improve the value of health care in the United States have included expanded quality measurement and public reporting programs; educational efforts such as Choosing Wisely; adoption of pay-for-performance and bundled payments; improved coverage for recommended services; and the expansion of patient-centered medical homes. To determine whether these major efforts to improve quality over the past decade have been successful, we measured the quality of care delivered to adults in the United States in the areas of high-value care, low-value care, access to care, and patient experience.

METHODS: We analyzed 2002 to 2013 data from the Medical Expenditure Panel Survey (MEPS), a nationally representative survey of patients that also collects data from their clinicians, hospitals, pharmacies, and insurance companies (annual sample size ranged from 21,915 to 26,509 respondents). We evaluated performance over time on 42 clinical quality measures including 28 high-value care measures and 14 low-value care measures. From these measures, we constructed 10 clinically meaningful composites (e.g., "highvalue diabetes care" included 5 individual metrics: hemoglobin A1c measurement, cholesterol measurement, foot exam, eye exam, and receipt of the influenza vaccine). We report performance on the clinical quality measures and composites as the proportion of eligible people who received the care. We also examined 8 measures of self-reported access to care and 10 measures of patient experience, aggregated into 2 composites. To examine temporal trends, we compared composites in 2002 and 2013 with weighted regression. In all analyses we accounted for the complex survey design of MEPS.

RESULTS: From 2002 to 2013, 3 clinical quality composites improved, 3 worsened, and 4 were unchanged. The improved clinical quality composites were high-value cardiovascular care (from $35 \%$ [95 \% confidence interval $\{\mathrm{CI}\}, 34 \%$ to $36 \%$ ] in 2002 to $42 \%$ [95\% CI, $41 \%$ to $43 \%$ ] in 2013; $p<0.01$ ), high-value counseling (from $43 \%$ [95 \% CI, $42 \%$ to $44 \%$ ] to $49 \%$ [95\% CI, $47 \%$ to $50 \%$ ]; $p<0.01$ ), and low-value cancer screening (from $37 \%$ [95 \% CI, $36 \%$ to $39 \%$ ] to $28 \%$ [95\% CI, $27 \%$ to $30 \%$ ]; $p<0.01$ ). The worsened clinical quality composites were high-value pulmonary care (from $53 \%$ [95 \% CI, $48 \%$ to $57 \%$ ] in 2002 to $43 \%$ [ $95 \%$ CI, $38 \%$ to $48 \%$ ] in 2013; $p<0.01$ ), low-value medication prescribing (from $8.1 \%$ [95 \% CI, $7.5 \%$ to $8.7 \%$ ] to $10.7 \%$ [95 \% CI, $10.0 \%$ to $11.4 \%$ ]; $p<0.01$ ), and low-value antibiotic use (from $50 \%$ [95 \% CI, $47 \%$ to $52 \%$ ] to $56 \%$ [ $95 \% \mathrm{CI}, 53 \%$ to $60 \%$ ]; $p<0.01$ ). There were no significant changes in the delivery of high-value cancer screening (from $76 \%$ [95 \% CI, $75 \%$ to $77 \%$ ] in 2002 to $77 \%$ [ $95 \%$ CI, $76 \%$ to $78 \%$ ] in 2013; $p=0.05)$, high-value diabetes care $(76 \%$ [ $95 \%$ CI, $75 \%$ to $78 \%$ ] to $76 \%$ [95\% CI, $75 \%$ to $77 \%$ ]; $p=0.9$ ), high-value diagnostic and preventive testing ( $76 \%$ [95\% CI, $75 \%$ to $76 \%$ ] to $77 \%$ [95 \% CI, $76 \%$ to $77 \%$ ]; $p=$ 0.05), and low-value imaging ( $13 \%$ [95 \% CI, $12 \%$ to $15 \%$ ] to $13 \%$ [95 \% CI, $11 \%$ to $15 \%] ; p=0.7)$. The proportion of Americans with a positive patient experience improved slightly from $87.2 \%$ ( $95 \% \mathrm{CI}, 86.9 \%$ to $87.6 \%$ ) in 2012 to $89.8 \%$ (95\% CI, $89.4 \%$ to $90.1 \%)$ in $2013(p<0.01)$. The proportion with good or very good self-reported access to care improved marginally from $84.0 \%(95 \%$ CI, $83.7 \%$ to $84.4 \%)$ in 2002 to $84.9 \%$ $(84.4 \%$ to $85.3 \%)$ in $2013(p<0.01)$

CONCLUSIONS: Despite over a decade of effort to improve the value of health care in the United States, the quality of care delivered to adults has not consistently improved. 
There have been only modest improvements in patient experience and self-reported access to care. Current deficits in the provision of most care continue to pose serious hazards to the health of the American public.

THE RELATIONSHIP BETWEEN DEPRESSION AND HEALTH CARE UTILIZATION IN PATIENTS WITH TYPE 2 DIABETES Caroline Presley ${ }^{4}$; Richard O. White $^{1}$; Ken Wallston ${ }^{3}$; Sunil Kripalani ${ }^{4}$; Russell L. Rothman ${ }^{2} .{ }^{1}$ Mayo Clinic, Jacksonville, FL; ${ }^{2}$ Vanderbilt, Nashville, TN; ${ }^{3}$ Vanderbilt University Medical Center, Pisgah Forest, NC; ${ }^{4}$ Vanderbilt University Medical Center, Nashville, TN. (Control ID \#2467811)

BACKGROUND: Depression is a common and important comorbid condition among patients with Type 2 Diabetes (T2DM) that has been associated with decreased adherence to self-care and treatment, and worse glycemic control. The relationship between depression and health care utilization in patients with T2DM is not well understood. The aim of this study was to investigate the relationship of depressive symptoms and self-reported health care utilization (clinic visits, ED visits, and hospitalizations) in a racially, ethnically diverse group of low-income patients with T2DM.

METHODS: English and Spanish-speaking patients with T2DM were enrolled in a cluster randomized trial evaluating a health literacy intervention for diabetes care at 10 Tennessee Department of Health safety net clinics. For this current presentation, we performed cross-sectional analysis of data from time of enrollment. Data on patient characteristics and clinical information were collected via self-report and from the medical record. Depressive symptoms were assessed with the Center for Epidemiological Studies Depression Scale (CES-D 20, range $0-60)$, with a score of $\geq 16$ indicating a positive depression screen. Patients were asked to report the number of encounters they had in the past 3 months for each of the following: clinic visits, ED visits, and hospital admissions. Descriptive statistics were used to characterize the sample. Negative binomial regression analysis was used to evaluate the relationship between positive depression screen (CES-D $\geq 16$ ) and number of encounters (clinic visits, ED and hospitalization, and total number of encounters), adjusted for age, gender, race, ethnicity, duration of diabetes, BMI, and current A1C.

RESULTS: Of 411 patients recruited into the study, complete data were available for 409 $(99.5 \%)$ of patients. On average, the sample was 51 years old (SD 9.6), $61 \%$ were female, $18 \%$ were African American, and $24 \%$ reported Latino ethnicity. Mean education was 11.1 years (SD 3.4). The majority ( $88 \%$ ) reported no health insurance and $83 \%$ had annual household incomes of less than $\$ 20,000$. Mean A1C was 9.2 (SD 2.1). The majority $(53 \%)$ of the sample screened positive for depression (CES-D score $\geq 16$ ). Of this group, $64 \%$ were female. There was a similar racial and ethnic distribution among those with CES-D scores $\geq 16$ and $<16$. The mean number of clinic visits was 2.16 for those with CES-D $\geq 16$ compared to 1.68 for those with CES-D $<16(p=0.032)$. The mean number of ED visits and/or hospitalizations was 0.60 for those with CES-D $\geq 16$ compared to 0.36 for those with CES-D $<16(p=0.041)$. The incidence rate ratios for health care encounters comparing those with CES-D $\geq 16$ to those with CES-D $<16$ are displayed in Table 1.

CONCLUSIONS: We found higher rates of recent health care utilization among patients with a positive depression screen in this racially, ethnically diverse sample of low-income patients with T2DM. This indicates that depression may be a risk factor for increased utilization of health care services in patients with T2DM. Given the cross-sectional nature of this study, we are unable to make inferences about causality. Future work in this area with longitudinal data would be valuable to gain a better understanding of this relationship.
Table 1: Increased rates of health care utilization for patients with positive depression screen

\begin{tabular}{lll}
\hline \hline & Incidence Rate & Incidence Rate \\
& Ratios, Unadjusted & Ratios, Adjusted* \\
Clinic Visits & $1.29(1.05,1.58)$, & $1.30(1.06,1.59)$, \\
& $p=0.013$ & $p=0.01$ \\
ED Visits and Hospitalizations & $1.64(0.99,2.68)$, & $1.54(0.93,2.56)$, \\
& $p=0.051$ & $p=0.094$ \\
All Encounters & $1.24(1.11,1.63)$, & $1.36(1.12,1.64)$, \\
& $p=0.003$ & $p=0.002$ \\
\hline
\end{tabular}

* Adjusted for age, gender, race, ethnicity, duration of diabetes, BMI, current A1C

THE RELATIONSHIP BETWEEN DIET FOOD ADVERTISEMENT AND DIET BEHAVIOR OVER A 13-YEAR PERIOD Ashley K. Haddad ${ }^{2}$; Marshall Chin²; Deborah L. Burnet ${ }^{2}$; Michael T. Quinn ${ }^{2}$; Monica E. Peek ${ }^{1} .{ }^{1}$ The University of Chicagoa, Chicago, IL; ${ }^{2}$ University of Chicago, Chicago, IL. (Control ID \#2464094)

BACKGROUND: Obesity represents a significant population health concern, with more than seventy percent of U.S. adults classified as overweight or obese. Obesity causes extensive morbidity and mortality, and disproportionately affects African-Americans and Latinos. Many attribute the rise in obesity to environmental factors such as fast-food restaurants and unhealthy advertising; in 2012, $\$ 4.6$ billion was spent on fast-food advertising alone. Numerous studies document the targeting of unhealthy food advertisements toward racial minorities and low-income communities. However, evidence is inconclusive regarding the effect of food advertising on food-related behavior, especially amongst subsets of the population at risk for health disparities. To better understand this complex relationship, we evaluated trends in diet food advertisements and self-reported diet behavior over time, stratified by body mass index (BMI) and sociodemographic factors. We hypothesized that: 1) diet food advertising would be positively associated with self-reported diet behavior, and 2) increased diet food advertising would temporally precede an increase in diet behavior for those subsets of the population with greatest potential ad exposure.

METHODS: We utilized Federal Trade Commission (FTC) data from 1985 to 1997 that catalogued all food advertisements in 5 leading women's magazines (Better Homes and Gardens, Good Housekeeping, Ladies Home Journal, McCall's, and Women's Day) and 3 general readership magazines (Reader's Digest, Time, and Newsweek). To our knowledge, this dataset is the most recent and comprehensive characterization of food advertisements available $(n=11,647)$. In these advertisements, all comments on calories, weight, weight reduction, or dieting were classified as diet claims. The percentage of food advertisements with diet claims was calculated over the total number of food advertisements. Using a Spearman correlation coefficient $(\rho)$, we compared the trend in diet food advertisements to trends in diet behavior reported in the Behavioral Risk Factor Surveillance System (BRFSS). We categorized diet behavior by the percentage of people who responded positively to the questions "are you now trying to lose weight?" and "are you eating fewer calories to lose weight?" We stratified results by age, gender, race, education, income, and body mass index of respondents.

RESULTS: The percentage of food advertisements with diet claims increased from 1985 to 1990 , and subsequently declined. In 1989 and 1990, diet content in food advertisements peeked, while self-reported diet behavior was at its lowest. Throughout the study period, we noted an inverse relationship between diet food advertisements and diet behavior (See Figure), though results were not statistically significant $(\rho=-0.42, p=0.16)$. This inverse 
relationship held for all demographic stratifications. Steepest declines in diet behavior were noted amongst individuals with annual income $<\$ 10,000(\rho=-0.37, p=0.22)$ and educational attainment $<8$ th grade $(\rho=-0.53, p=0.06)$.

CONCLUSIONS: Our hypotheses that 1 ) diet food advertising would be positively associated with diet behavior, and 2) increased diet food advertising would temporally precede an increase in diet behavior were not supported. In fact, trends suggested the opposite. The observed inverse relationship between diet ads and diet behavior may potentially be explained by one of three social psychological concepts: Self-Perception Theory, which describes the discordance between an individual's perception of himself and others when the environment changes (Bem, 1972); Reactance Theory, which describes an individual's response to the

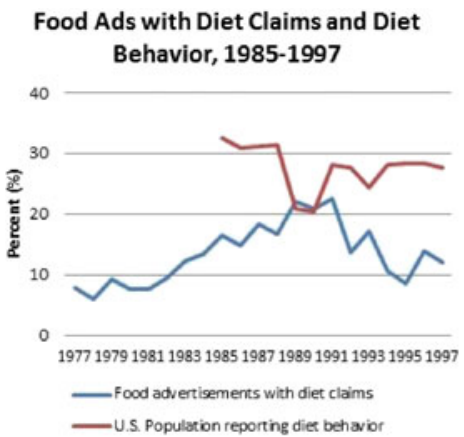

\section{Food Ads with Diet Claims and Diet Behavior, stratified by Income}

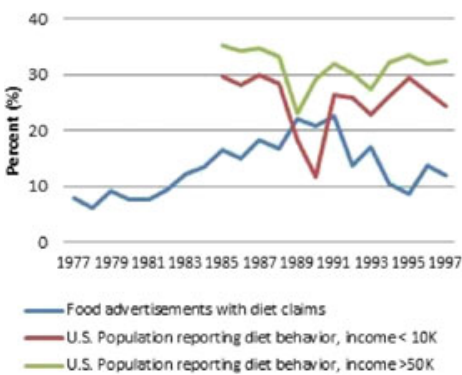

THE RELATIONSHIP BETWEEN PATIENT EXPERIENCE, CARE QUALITY, AND NO SHOW RATES IN THE OUTPATIENT PRIMARY CARE SETTING Jaya Aysola ${ }^{1}$; Marilyn M. Schapira ${ }^{1}$; Hairong Huo ${ }^{1}$; Rachel M. Werner ${ }^{2} .{ }^{1}$ University of Pennsylvania, Philadelphia, PA; ${ }^{2}$ University of Pennsylvania and Philadelphia VA, Philadelphia, PA. (Control ID \#2469427)

BACKGROUND: There is increasing emphasis on the use of patient-reported experience data in the evaluations of outpatient primary care practice and provider performance. Studies have examined the relationship between patient experience and inpatient clinical outcomes with mixed results; however, less is known about the relationship between patient experiences and primary care clinical outcomes and process measures. In addition, the relationship between patient experience and no show rates is unknown. Therefore, the objective of our study was to evaluate the associations between patient-reported experiences with care and clinical outcomes, process measures, and no show rates in the outpatient primary care setting. METHODS: We analyzed visit data from patients $(n=8355)$ between January 2012 through July 2014, at all University of Pennsylvania Health System affiliated adult perception of lost autonomy (Brehm, 1966); or Mere Exposure Effect, which describes the mechanism by which a previously neutral stimulus becomes caustic with overexposure (Zajonc, 1968). Our study is the first to compare food advertising with self-reported food behavior on a national level. There are limitations to our analysis including restricted data availability and inability to rule out confounders that may contribute to the relationship observed between diet food advertisements and self-reported diet behavior. We are currently attempting to obtain additional quarterly data that will assist in a more sensitive analysis of the relationship described above. Further research is warranted to understand the effect of food advertising on food-related behaviors, particularly amongst at-risk populations (e.g. lowincome minorities).

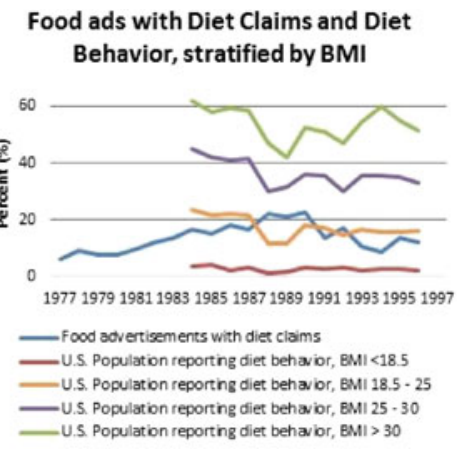

Food Ads with Diet Claims and Diet Behavior, stratified by Education

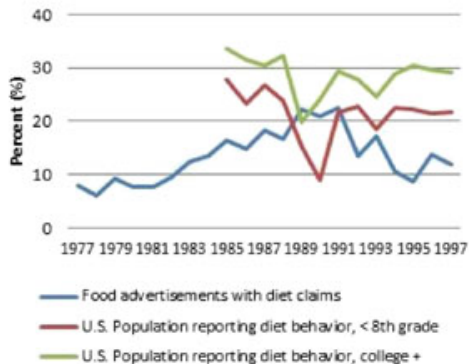

primary care practices with three or more providers $(n=22)$. Our predictors were patient-reported experiences with care in the following four domains: access, visit, nurse/staff, and care provider. Our clinical outcomes included elevated BMI $(>25)$, smoking status, elevated HbAlc (>8), and cholesterol control (LDL). Our process measures included smoking cessation discussed, diabetes eye exam referral, mammography, and colonoscopy screening. Other covariates included patient characteristics, such as race/ethnicity, age, gender, insurance type, place of residence, clinical comorbidities (Charlson Comorbidity Index (CCI)) and practice characteristics, such as location and number of providers, and patient volume. We employed generalized estimating equations (GEE) with an exchangeable correlation structure to account for patient clustering by practice. In multivariate regression models we estimated the relationship between our patient experience predictors and binary process and outcome measures, adjusting for the covariates listed above. With multivariate negative binomial models we estimated the relationship between our patient experience measures and visit no show rates, adjusting for the covariates above, while accounting for clustering of patients at the same clinical site. 
RESULTS: Of the 8355 patients in the study, $64 \%$ were female, $24 \%$ were Black, $5.3 \%$ had Medicaid, $21.2 \%$ Medicare, $5.3 \%$ had multiple chronic conditions (CCI $>2)$. The mean age (SD) was 57 (17.5). The mean patient experience scores (SD) for the four domains were as follows: 85.8 (16.0) for experience with access; 81.6 (18.4) for visit experience; 88.5 (16.7) for nurse/staff experience; and 91.5 (14.8) for care provider experience. We found no discernable patterns in associations between our patient experience measures and our clinical process and outcome measures. We did find a significant association between care provider experience and no show rates, where a one point increase in care provider experience scores was associated with a one percent decrease in no show rates $(p<0.001)$.

CONCLUSIONS: Our findings suggest that patient experience while an important standalone metric of care quality does not necessarily relate to clinical outcomes or process measures in the outpatient setting. However, patient experience with their primary care provider did appear to influence no show rates and therefore, practices would benefit from initiatives that focus on improving the provider-patient relationship.

\section{THE RELATIONSHIP BETWEEN PRIMARY CARE SPENDING, PATIENT} RISK, QUALITY, AND TOTAL COST OF CARE Amol S. Navathe ${ }^{1,2}$; Andrea B. Troxel $^{1}$; Kristen Caldarella ${ }^{1}$; Amanda T. Hodlofski ${ }^{1}$; Ezekiel J. Emanuel ${ }^{1}$; Kevin G. Volpp ${ }^{1,2}$. ${ }^{1}$ University of Pennsylvania, Philadelphia, PA; ${ }^{2} \mathrm{CMC}$ VA Medical Center, Philadelphia, PA. (Control ID \#2468907)

BACKGROUND: Recent policy reforms, such as Medicare Accountable Care Organizations (ACOs), have targeted Primary Care Physicians (PCPs) to improve quality and reduce total health care spending, without changing the underlying fee-for-service (FFS) payment system. While primary care services generally account for a small percentage (8$10 \%$ ) of total spending, the relationship between primary care expenditures and quality of care, patient risk, and total healthcare spending is not well-described.

METHODS: Our objective was to examine the relationship between spending on primary care services and quality of care, patient risk, and total cost of care at the PCP level. Using administrative data from Hawaii Medical Services Association (HMSA), the Blue Cross Blue Shield insurance plan in the state of Hawaii, we analyzed data on 435 PCPs paid FFS across the commercial, Managed Medicaid, and Medicare Advantage lines of business (LOBs) over the years 2013-2014. Primary care spending was computed by aggregating all FFS billings for associated services per Medicare definitions. Quality of care was measured by incentive payments earned by PCPs through HMSA's Pay-for-Quality (P4Q) program that was based on HEDIS measure performance versus national benchmarks. Total cost of care was calculated by summing payments for all health care claims per patient, excluding drug costs. Patient risk was measured by categories of the Episode Risk Group (ERG) metric from Optum, Inc to allow for non-linear relationships. Patients were attributed to their elected PCPs in managed care lines of business and to PCPs with the plurality of their visits when elections were not available. We computed unadjusted associations and then developed multivariate models using ordinary least squares to adjust for additional factors such as visit intensity, specialty, geographic indicators, and group size for each LOB and in aggregate. We tested alternate model specifications, including negative binomial models, to account for distributional characteristics in health care spending and used Huber-white standard errors to account for heteroscedasticity, clustering standard errors at the physician organization level.

RESULTS: The relationship between primary care spending and quality of care, patient risk, and total cost of care varied substantially by LOB. The unadjusted relationship between primary care spending and quality showed a statistically significant small negative relationship for all LOBs together $(p<0.001)$, between primary care spending and patient risk showed a positive relationship across all LOBs $(P<0.001)$, and between primary care spending and total cost of care showed a positive relationship across all LOBs $(p<0.001)$. In multivariate analysis including risk adjustment, each dollar of primary care spending was associated with 40 cent lower quality bonuses for Medicare Advantage $(p=0.008)$ and 80 cent lower quality bonuses for Managed Medicaid ( $p=0.03$ ), while associated with an additional 20 cents of quality bonus for patients with Commercial insurance. Total cost of care showed variation by LOB as well, with each dollar of primary care services associated with 18 dollar higher total cost of care for Medicare Advantage $(p=0.01), 14$ dollar lower total cost of care for Commercial $(p=0.07)$, and an insignificant change in Managed Medicaid ( $p=0.45$ ) (Figure 1).

CONCLUSIONS: The relationship between primary care spending and quality of care, patient risk and total cost of care varies significantly by line of business within a sample of PCPs representing primary care in Hawaii. The differences in the direction of the relationship were striking, indicating that PCPs who provide more services do not systematically provide higher quality of care or reduce the overall cost of care, nor do they systematically spend more if their patient panels are of higher risk. While these results may not be generalizable outside of Hawaii, they suggest the importance of understanding these relationships in other markets. Alternate payment models like ACOs may be used to create greater alignment between primary care spending, higher quality, higher patient risk, and reductions in total cost of care.

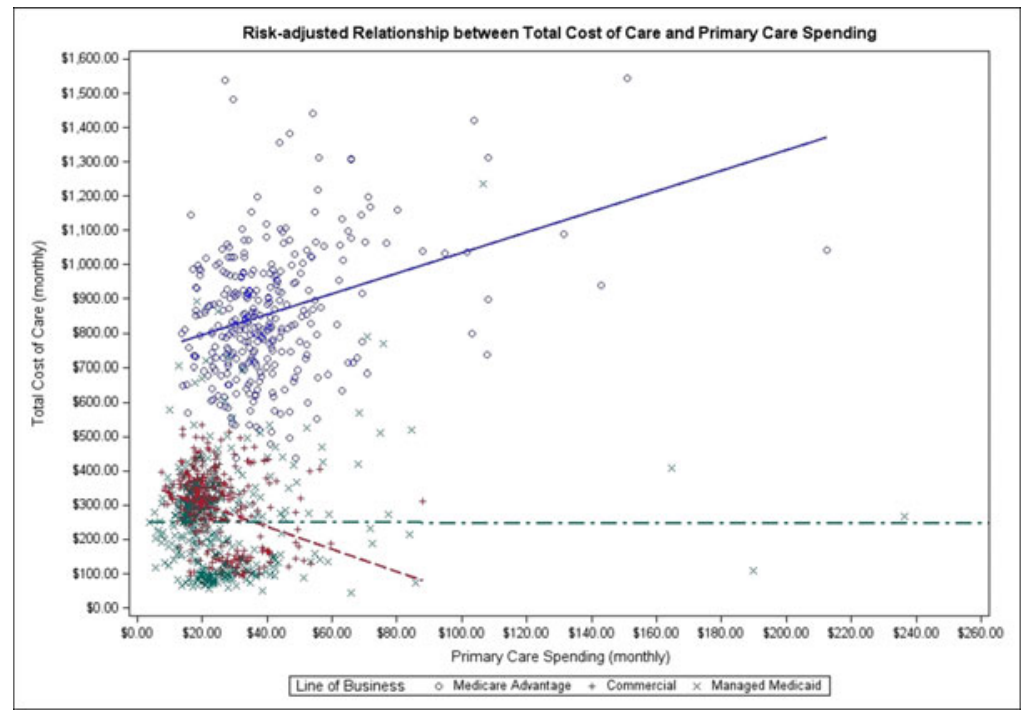

Figure 1: Risk-adjusted Relationship between Total Cost of Care and Primary Care Spending 
THE RELATIONSHIP BETWEEN RESPIRATORY DYSFUNCTION AND VISCERAL FAT ACCUMULATION. Yuichi Takahashi ${ }^{2}$; Toshio Naito ${ }^{1}$; Hirohide Yokokawa ${ }^{2}$; Kazutoshi Fujibayashi ${ }^{3} .{ }^{1}$ Juntendo University, Tokyo, Japan; ${ }^{2}$ Juntendo University School of Medicine, Tokyo, Japan; ${ }^{3}$ School of Medicine,Juntendo University, Tokyo, Japan. (Control ID \#2467085)

BACKGROUND: The aim of this study is to evaluate the association between respiratory dysfunction and visceral fat accumulation.

METHODS: A total of 385 people visited our hospital for a medical checkup from May 2015 to August 2015. We excluded 57 subjects who met the exclusion criteria and analyzed the data for 188 males (mean age: 62 years old) and 140 females (mean age: 60 years old). We measured the amount of visceral fat tissue (VFT) at the umbilical level using computed tomography. Respiratory function was evaluated using the \% vital capacity $(\% \mathrm{VC})$ and the forced expiratory volume in $1 \mathrm{~s}$ (FEV1). We estimated the correlation between $\% \mathrm{VC}$ and VFT using Spearman's rank correlation coefficient.

RESULTS: A negative correlation was detected between $\% \mathrm{VC}$ and VFT in males ( $\mathrm{rs}=-0.24$, $p<0.01)$. No significant correlation was detected between these parameters in females. CONCLUSIONS: Our results suggest that visceral fat accumulation might be associated with reductions in \% VC in Japanese males.

THE RELATIONSHIP OF HEALTH BELIEFS WITH HOSPITAL UTILIZATION Himali Weerahandi ${ }^{1}$; Juan Wisnivesky ${ }^{1}$; Minal Kale ${ }^{1}$; Rachel O'Conor ${ }^{2}$; Melissa Martynenko ${ }^{1}$; Michael S. Wolf ${ }^{2}$; Alex Federman ${ }^{1} .{ }^{1}$ Icahn School of Medicine at Mount Sinai, New York, NY; ${ }^{2}$ Northwestern University, Chicago, IL. (Control ID \#2464414)

BACKGROUND: Health beliefs are thought to affect self-management of chronic disease, which in turn has been demonstrated to affect disease flare and hospitalizations. Studies on the relationship of health related beliefs on COPD outcomes such as hospitalization are limited. We sought to investigate whether hospital utilization may be mediated by health beliefs.

METHODS: Data are from a cohort of English- and Spanish-speaking adults with COPD enrolled from outpatient practices in New York, NY and Chicago, IL $(n=391)$. Health beliefs were assessed with the Brief Illness Perceptions questionnaire. Hospital utilization and race were self-reported and obtained through chart review. COPD severity was assessed by spirometry (percent predicted $\mathrm{FEV}_{1}$ ). We modeled the associations between hospitalization and the independent variables using multivariate logistic regression, adjusting for age, gender, race, percent predicted $\mathrm{FEV}_{1}$ and chronic co-morbidities.

RESULTS: The mean age was 68.0 years, $58 \%$ were women and $44 \%$ identified as black. Hospitalization for COPD was reported by $49 \%$ of this cohort. Differences between patients with and without history of hospitalization were statistically significant in 3 of the 5 components of the cognitive illness representations of COPD. In this domain, patients who reported a history of hospitalization for their COPD were more likely to believe COPD more severely affects their life ( $p<0.01$, Cohen's $d=0.62)$, more likely to believe in treatment control of COPD ( $p<0.01$, Cohen's $d=0.33$ ), and more likely to experience symptoms of COPD ( $p<0.01$, Cohen's $d=$ 0.53). However, patients with and without history of hospitalization for their COPD were equally likely to believe they will always have their $\operatorname{COPD}(p=0.19$, Cohen's $d=0.23)$ and believe in their personal control on the disease ( $p=0.88$, Cohen's $d=0.05$ ). Among the emotional representations, patients who reported a history of hospitalization for their COPD were more likely to express concern about their COPD $(p<0.01$, Cohen's $d=$ $0.46)$ and report their COPD affects their emotions ( $p<0.01$, Cohen's $d=0.35)$. In illness comprehension, patients who reported a history of hospitalization for their COPD were more likely to believe they understood their COPD ( $p=0.02$, Cohen's $d=0.22)$. In a multivariate logistic regression controlling for age, gender, race and co-morbidities, and predicted percent FEV1, severity belief (OR 1.16, $95 \%$ CI 1.05-1.27, $p=0.02$ ), belief in treatment control (OR 1.11, $95 \%$ CI 1.01-1.22, $p=0.03$ ), experience of symptoms (OR $1.10,95 \%$ CI 1.05-1.27, $p=0.02$ ), level of concern (OR 1.09, $95 \%$ CI 1.01-1.20, $p=$ 0.04 ), and level of understanding (OR 1.11, $95 \%$ CI 1.02-1.21, $p=0.02$ ) remained significant predictors for COPD hospitalization. The belief that COPD affects emotions was not significant in the multivariate model (OR 1.01, $95 \%$ CI $0.94-1.08, p=0.89$ ).

CONCLUSIONS: In a cohort of patients with COPD, we found several health beliefs were associated with a history of COPD hospitalization. These illness beliefs reflect that patients with a history of COPD hospitalization do recognize their illness is serious and can be aided by treatment. These findings highlight potential targets for educational interventions by providers to prevent future hospitalizations. Additional work is needed to define a casual association between beliefs and hospitalization.

\section{THE ROLE OF VA PRIMARY CARE IN COORDINATING NON-VA GYNECO-} LOGIC ONCOLOGY CARE Kristina M. Cordasco ${ }^{2,5}$; Jessica L. Zuchowski ${ }^{2}$; Joya G. Chrystal $^{4}$; Laurie C. Zephyrin ${ }^{1}$; Alison Hamilton ${ }^{3}$. Department of Veterans Affairs, Washington, DC; ${ }^{2}$ US Dept of Veterans Affairs, North Hills, CA; ${ }^{3} \mathrm{VA}$ Greater Los Angeles, Los Angeles, CA; ${ }^{4} \mathrm{VA}$ Greater Los Angeles Healthcare System, Los Angeles, CA; ${ }^{5}$ University of California, Los Angeles, Los Angeles, CA. (Control ID \#2466358)

BACKGROUND: The Veterans Access, Choice and Accountably Act of 2014 ('Choice Act') is likely to result in Veterans Health Administration (VA) patients more frequently using non-VA care concurrent to using VA care. This may result in increased care coordination work for VA primary care who will need to coordinate care with additional providers outside of VA. Little is known about the potential impact of increased use of non-VA care on the care coordination workload of VA primary care providers (PCPs) and their teams, or how that work might vary across VA settings. We investigated roles of PCPs and their teams related to care coordination with non-VA providers for gynecologic oncology care.

METHODS: Between February and April, 2015, we interviewed 21 key informants at 15 VA facilities of varying size, availability of VA gynecology services, and geographic characteristics. Informants included VA Women's Health Medical Directors (many of whom were also PCPs), gynecologists, and nurse care coordinators. Interviews were semistructured; participants described the resources and processes used at their facilities for the coordination of gynecologic cancer care. We recorded, professionally transcribed, and summarized each interview, and then utilized a team-based hybrid deductive/inductive thematic analytic process to generate results.

RESULTS: The role of PCPs and their teams in coordinating care of patients with gynecologic malignancies varies substantially across VA facilities, depending on whether, and to what extent, the VA facility, or a nearby VA facility, has one or more VA gynecologists on staff. If a VA gynecologist is available, the PCP usually refers the patient to this person, who assumes responsibility for care coordination. In facilities without an available VA gynecologist, which is more common in rural areas, PCPs refer the patient directly to non-VA specialists (e.g., gynecologic oncologists) in the community, and assume most of the care coordination responsibilities. Interviewees reported that coordinating care with non-VA providers is more difficult, compared to coordinating with VA providers, due to lacking a shared electronic medical record with non-VA providers. Care coordination tasks include submitting authorization requests for VA to purchase the needed non-VA care; communicating directly with non-VA care providers; facilitating the exchange of medical records with non-VA providers; placing orders for diagnostic tests that are requested by non-VA providers and performed within VA; prescribing medications recommended by non-VA providers and filled within VA; tracking patient progress through all phases of cancer care, within and outside of VA; communicating with patients about appointments and questions; communicating with VA specialty providers who may be delivering portions of the cancer care or caring for comorbidities; and facilitating transitioning to hospice care, if needed. 
CONCLUSIONS: There are multiple tasks associated with VA PCPs and their teams coordinating care with non-VA providers for gynecologic oncology care. As Veterans begin to more frequently use non-VA specialty care providers in tandem with VA-based care, these tasks may result in a significant increase in workload for VA primary care teams, not only for gynecologic malignancies, but potentially for other complex conditions as well. Primary care teams in facilities with less specialty care services, such as those in rural areas, may be particularly affected. VA PCPs and their teams need support to help meet the increased care coordination needs of VA patients also receiving care outside of VA.

THE SKILLS OF AN EFFECTIVE PHYSICIAN LEADER: A SURVEY OF RISING CHIEF MEDICAL RESIDENTS AS A ROADMAP FOR FUTURE CURRICULAR DEVELOPMENT Sarah B. Merriam² ${ }^{2}$ Jennifer Corbelli ${ }^{2}$; Shanta M. Zimmer ${ }^{1}{ }^{1}$ University of Pittsburgh, Pittsburgh, PA; ${ }^{2}$ University of Pittsburgh Medical Center, Pittsburgh, PA. (Control ID \#2461716)

BACKGROUND: Physicians frequently occupy leadership roles in clinical, research, administrative, academic and community settings; however the majority receives no leadership training. Given increasing reliance on interdisciplinary teams to provide care and greater emphasis on cost control and quality improvement, the need to develop effective clinical leadership is paramount. Leadership development programs have been demonstrated to improve leadership ability. Rising chief medical residents (CMRs), chosen based upon their perceived leadership ability, are an ideal sample through which to identify both current best practices and gaps in leadership training during residency. The goal of this study is to assess the current state of CMR leadership training and to inform the development of curricula at the residency level. The specific objectives are to 1. Quantify the extent of current resident leadership curricula nationwide, 2 . Identify the characteristics of an effective physician leader as perceived by a nationally representative sample of CMRs, and 3. Assess CMR comfort with specific leadership skills.

METHODS: This is a cross-sectional descriptive study of 169 rising CMRs attending the 2015 Association of Program Directors in Internal Medicine CMR conference. With IRB approval, we developed a unique survey including check-box, free-response, and Likertscale questions assessing sociodemographic characteristics, exposure to leadership training, and perceived comfort with a wide variety of leadership domains. These domains were adapted existing from validated leadership development curricula, including the Stanford Leadership Development Program.

RESULTS: Fifty-one percent of the sample was male, $59 \%$ were US medical graduates, $65 \%$ were from university-affiliated residency programs, and $27 \%$ reported work experience prior to residency. The majority ( $72 \%$ ) of respondents had not received formal training in leadership skills during residency. If such training had been available, $97 \%$ would have willingly participated. Of those reporting prior leadership training, two-thirds felt that additional skill development was needed prior to the start of the chief year. Several domains emerged in which CMRs were found to have greater comfort, while in other domains CMRs reported greater discomfort (Table 1). Respondents reported a mixed comfort levels with specific skills in three domains: team leadership, communication, and acceptance of the demands of leadership (Table 2). Female respondents reported less comfort with the skills 'finding balance between personal and professional goals" $(p=0.05)$ and being "at peace with the isolation that leadership may bring" ( $p=0.01)$ than male respondents. US medical graduates were less comfortable than international medical graduates (IMGs) with skills contained in the "self-awareness" domain $(p=0.007)$, e.g. ability to identify leadership strengths and deficits. CMRs with prior work experience endorsed more comfort speaking in front of groups $(p=0.01)$ than those without work experience.
CONCLUSIONS: The reported lack of exposure to leadership training and desire for additional training suggest that rising CMRs do not feel adequately prepared for leadership roles at the end of residency. Rising CMRs report comfort with many leadership skills likely developed while leading hospital ward teams but discomfort with advanced leadership skills involving mentorship or conflict management.. Female CMRs are less comfortable with leadership skills involving intrapersonal conflict. Compared to IMGs, who have engaged in the competitive process of matching into US residency programs, US medical graduates report greater discomfort with self-awareness. These sub-groups may benefit from more targeted leadership skills development. Next steps of this study include a repeat survey of CMRs at the end of their chief year, to identify how perceived comfort with leadership skills evolve over the year as a result of practice, exposure to various leadership styles, and increasing experience. The results of this study can be used to provide an important guide the development of future leadership training curricula for residents nationally.

Table 2. Leadership domains in which CMRs report both comfort and discomfort

\begin{tabular}{ll}
\hline \hline Leadership Domain & $\%$ \\
TEAM LEADERSHIP & 88 (C) \\
- Works effectively with diverse groups & 13 (U) \\
- Time management & \\
COMMUNICATION & 80 (C) \\
- Public speaking & 11 (U) \\
- Tailors communication style to audience & 79 (C) \\
ACCEPTANCE OF THE DEMANDS OF LEADERSHIP & 32 (U) \\
- Willing to take responsibility for decisions & 34 (U) \\
- Making unpopular decisions & \\
- At peace with the isolation that leadership can bring &
\end{tabular}

(C) $=\%$ comfortable

(U) $=\%$ uncomfortable

Table 1. CMR reported level of comfort within leadership domains

\begin{tabular}{lr}
\hline \hline Respondents reporting comfort & \\
( $\mathbf{7 5} \%$ of respondents reporting Likert of 4 "somewhat comfortable" & \\
or 5 "very comfortable") & $\%$ \\
Leadership Domain & 98 \\
Initiative & 92 \\
Empathy & 91 \\
Professionalism & 85 \\
Volunteerism & 81 \\
Giving/receiving feedback & \\
Respondents reporting discomfort & \\
( $\mathbf{1 0} \%$ of respondents reporting Likert 1 "very uncomfortable" or 2 \\
"somewhat uncomfortable") & 11 \\
Leadership Domain & 14 \\
Mentoring others & 15 \\
Conflict Management & 18 \\
Fostering Creativity & \\
Self-awareness &
\end{tabular}

THE SPARSE EVIDENCE BASE FOR PRIMARY CARE INITIATIVES IN U.S. MEDICAL SCHOOLS Ryan Graddy ${ }^{1,2}$; Colleen Christmas ${ }^{2} .{ }^{1}$ Johns Hopkins Bayview Medical Center, Baltimore, MD; ${ }^{2}$ Johns Hopkins University, Baltimore, MD. (Control ID \#2469343)

BACKGROUND: There is widespread recognition that medical educators should implement interventions that promote interest and skills in primary care careers to avert a crisis in healthcare for the U.S. Indeed, according to the 2014 report from the Association of American Medical Colleges, over $70 \%$ of U.S. medical schools have or will soon have programs to do just that. Despite this sweeping support and wealth of efforts, the characteristics that render initiatives most effective are unclear. We completed a 
comprehensive literature review to identify best practices in stimulating interest in careers in primary care and teaching skills necessary to practice effective primary care among medical students.

METHODS: We searched Pubmed for references using the MeSH as a Major Topic using the term, "Medical Education, Undergraduate [MeSH]" and "Primary Care," and limited the results to those published in English over the past 10 years. Titles and abstracts were manually reviewed to identify those identifying a unique program or intervention; remaining publications were reviewed in full by both authors to identify those that described outcomes. Additionally, bibliographies of each paper were reviewed to ensure we had not missed important publications in our search methods. Descriptions of the interventions and outcomes from each paper were abstracted and collated. Because of widely disparate study designs and outcomes evaluated, meta-analysis could not be employed and content analysis was performed to identify emerging themes, using an "editing organizing" style. RESULTS: We identified 19 publications highlighting 14 unique primary care programs across the country. The results from the programs can be reported along four general themes: location (rural vs. urban), educational format, special populations, and health systems teaching. Many papers discussed several of these four main themes. A total of 11 publications describing 7 unique programs engaging students in rural primary care practice found that these programs had high rates of retention after medical school graduation in rural practice sites. A single publication described very high rates of residency placement in urban underserved communities among students completing a structured learning experience in a similar setting. The length of all educational interventions reviewed ranged from 4 weeks to more than 4 years. Some were required experiences for all students, while others were elective experiences; none were assigned randomly. Primary care tracks at 6 institutions were described in 8 publications; graduation and practice data available generally showed increased rates of rural practice and primary care practice among individuals who completed these programs. While emphases on special populations and health systems teaching were described as important focuses of many programs, there was little published about the impact of these interventions.

CONCLUSIONS: In pursuit of addressing the worsening shortage of primary care physicians across the country, many medical schools over the past decade have developed dedicated curricular interventions to engage students in primary care experiences during their undergraduate medical education and encourage them to pursue primary care careers Published outcomes from these interventions are uncommon, however. While most American medical schools will soon have interventions designed to improve interest and skill in primary care practice amongst medical students, the evidence base on which to build these interventions is stark. Limited evidence suggests the setting in which these educational interventions are delivered may influence practice location and that longer interventions may be more impactful. Outcomes from the myriad of other interventions at medical schools across the country are critical to developing an effective evidence-based primary care curriculum and to understand how best to identify and recruit appropriate students for such interventions.

THE SYNDEMIC EFFECT OF SUBSTANCE ABUSE, INTIMATE PARTNER VIOLENCE, AND MENTAL HEALTH, ON HIV RISK AMONG WOMEN ATTENDING AN URBAN STD CLINIC Kristen A. Lee ${ }^{1}$; Heidi Hutton ${ }^{3}$; Catherine R. Lesko ${ }^{3}$; Anne Monroe $^{3}$; Anika A. Alvanzo ${ }^{3}$; Mary E. McCaul ${ }^{3}$; Geetanjali Chander ${ }^{2} .{ }^{1}$ Johns Hopkins School of Medicine, Baltimore, MD; ${ }^{2}$ Johns Hopkins University, Baltimore, MD; ${ }^{3}$ Johns Hopkins University School of Medicine, Baltimore, MD. (Control ID \#2463840)

BACKGROUND: Substance abuse, intimate partner violence (IPV), HIV/AIDS, and mental health disorders are complex, co-occurring epidemics that particularly burden urban, low-income women. Together these variables are called substance abuse, violence, and HIV/AIDS (SAVA). Few studies have examined how syndemic factors influence women's HIV risk. This study investigates SAVA and risky sexual behavior among women at risk for HIV. Such understanding could better characterize the specific needs of these patients and offer more tailored HIV/ AIDs prevention. We hypothesized that women with more SAVA factors would report engaging in more risky sexual behaviors.

METHODS: This was a cross-sectional study of baseline data of women recruited at an urban STI clinic to participate in an RCT of an alcohol and sexual risk reduction intervention. Women were eligible if they were $\geq 18$ years old and either consumed $>7$ standard drinks per week or had $\geq 2$ binge drinking episodes ( $>3$ drinks on one occasion) in the past 3 months. At baseline, patients completed an audio computer administered self interview assessing substance abuse, physical or sexual IPV, and depression. Participants reporting cocaine, heroin, narcotics, or benzodiazepine use in the prior 6 months were scored positive for substance abuse. IPV was defined as physical or sexual abuse in the past year. Respondents scored positive for depression if they reported moderate to severe depressive symptoms on a 9-item measure evaluating symptoms over the prior 2 weeks (PHQ-9). A categorical SAVA variable (Table 1) was created from these dichotomous (absence/presence) variables to examine every combination of the presence/absence of each of the SAVA components. Using logistic and negative binomial regression adjusted for age, education, and housing status, we examined the relationship between the SAVA variable and three outcomes: 1) unprotected vaginal/anal sex under the influence of alcohol 2) having sex for drugs or money 3) number of lifetime sexual partners. We assessed the relative excess risk due to interaction (RERI) for each combination of SAVA factors; the RERI is interpretable as the change in absolute risk (rate) of the outcome expressed as a proportion of the risk (rate) in the triply unexposed group.

RESULTS: Fifty-eight percent of participants ( $N=438,99 \%$ African American, mean age 31 ) reported at least one SAVA factor and $23 \%$ had two or more SAVA factors (Table 1). $24 \%$ ( $n=$ 104) of participants reported unprotected sex and $40.55 \%(n=178)$ reported sex for drugs or money. Median (IQR) number of lifetime sexual partners reported was $18(10,36)$. In multivariable analyses (Table 1), respondents with one or more SAVA factors were more likely to have sex for drugs or money and have greater lifetime sexual partners compared to women with none. The RERI was 0.75 for the association between substance abuse and violence with unprotected sex; 2.85 for their association with sex for drugs or money; and 1.39 for their association with number of lifetime sexual partners. The RERI was 3.01 for the association between depression and violence with sex for drugs or money and 0.62 for the association between all three SAVA factors and lifetime number of sexual partners. All other RERIs were negative.

CONCLUSIONS: Women attending this urban STI clinic reported experiences of SAVA: violence, and co-occurring problems of substance abuse and depressive symptoms. The positive RERIs for substance abuse and violence suggest that these two SAVA factors increase the probability of reporting risky sex and greater number of sex partners more than would be expected based on their individual associations, and women who report substance abuse and violence may experience a greater benefit from intervention. Depression and IPV interact to increase the risk of having sex for drugs or money. All three SAVA factors we considered interact to increase the number of lifetime sexual partners. The study findings suggest that the burdens of SAVA may worsen sexual risk behavior and outcomes. This study underscores how using a SAVA framework can inform preventive care for patients with substance abuse, IPV, and mental health vulnerabilities around sexual risk taking.Table 1. Multivariable analysis of association between SAVA factors and risky sexual behaviors* $(N=438)$ 


\begin{tabular}{|c|c|c|c|c|c|c|}
\hline Depression & $\begin{array}{l}\text { Substance } \\
\text { Abuse }\end{array}$ & Violence & $\begin{array}{l}\text { Unprotected Sex } \\
\text { (OR } 95 \% \text { CI) }\end{array}$ & $\begin{array}{l}\text { Sex for Drugs or Money } \\
\text { (OR } 95 \% \text { CI) }\end{array}$ & $\begin{array}{l}\text { Lifetime Sexual Partners** } \\
\text { (IRR } 95 \% \text { CI) }\end{array}$ & Frequency (\%) \\
\hline- & - & - & Reference & Reference & Reference & $183(41.78)$ \\
\hline+ & - & - & $1.80(.81,4.01)$ & $2.95(1.35,6.46)$ & $2.15(1.52,3.04)$ & $43(9.82)$ \\
\hline - & + & - & $2.42(1.28,4.56)$ & $3.97(2.11,7.47)$ & $1.26(.96,1.66)$ & $84(19.18)$ \\
\hline - & - & + & $1.73(.67,4.46)$ & $2.55(1.00,6.46)$ & $1.00(.66,1.51)$ & $29(6.62)$ \\
\hline+ & + & - & $1.83(.79,4.26)$ & $5.59(2.42,12.94)$ & $1.76(1.21,2.56)$ & 41 (9.36) \\
\hline+ & - & + & $2.28(.65,7.98)$ & $7.51(2.04,27.62)$ & $1.75(.98,3.12)$ & $13(2.97)$ \\
\hline - & + & + & $3.90(1.51,10.08)$ & $8.37(2.94,23.77)$ & $2.65(1.67,4.20)$ & $23(5.25)$ \\
\hline+ & + & + & $3.17(1.17,8.58)$ & $7.16(2.40,21.38)$ & $3.03(1.90,4.83)$ & $22(5.02)$ \\
\hline
\end{tabular}

*Adjusted for age, education, and housing status. **Negative binomial regression.

THE TAILORED STUDY: A RANDOMIZED CONTROLLED TRIAL TO IMPROVE SURROGATE DECISION MAKING Mark Hughes ${ }^{1,3}$; Daniel Sulmasy ${ }^{2}$; Gayane Yenokyan ${ }^{4}$; Joan Kub ${ }^{5}$; Peter Terry ${ }^{1,3}$; Alan B. Astrow ${ }^{6}$; Julie Johnson ${ }^{2}$; Grace $\mathrm{Ho}^{5}$; Marie Nolan ${ }^{5,3}$. ${ }^{1}$ Johns Hopkins University School of Medicine, Baltimore, MD; ${ }^{2}$ University of Chicago, Chicago, IL; ${ }^{3}$ Johns Hopkins Berman Institute of Bioethics, Baltimore, MD; ${ }^{4}$ Johns Hopkins University, Baltimore, MD; ${ }^{5}$ Johns Hopkins University School of Nursing, Baltimore, MD; ${ }^{6}$ Maimonides Medical Center, New York, NY. (Control ID \#2469861)

BACKGROUND: Patients with terminal illnesses often require surrogate decision makers. Prior research has demonstrated that acting as a surrogate is highly stressful, and that despite legal and ethical standards that focus on autonomy, most patients do not want their surrogates to make pure substituted judgments for them. It is not known how best to help loved ones fulfill the surrogate decision making role.

METHODS: The study was conducted at two academic medical centers with diverse patient populations. Study subjects were dyads of adult patients with ALS or incurable gastrointestinal malignancies and adult family members whom they might involve in health care decision making (surrogates). Patients or surrogates with cognitive impairment were excluded. Dyads were randomized into intervention (TAILORED) and control groups (CONTROL). The TAILORED Intervention is a brief nurse-facilitated patient-family discussion involving assessment of patient preference for surrogate involvement in health care decisions, support for surrogate involvement in proxy decision making, and a take-home handout reflecting the patient's decision control preferences - having the surrogate perform a substituted judgment of the patient's autonomous wishes, sharing decision making with the surrogate, or deferring to the surrogate to decide what is best. The patient and surrogate were encouraged to talk further about these preferences. The CONTROL group received the standard of care with respect to advance care planning and an attention-control discussion about nutrition. Four weeks after baseline, TAILORED surrogates were given a telephone "booster," while CONTROL surrogates were only asked about satisfaction with care and if they had any questions about the study. Eight weeks $( \pm 2)$ after baseline, patients and surrogate-subjects were again asked to complete study surveys plus a measure of satisfaction with decision involvement. The main outcomes were congruence between patient and surrogate in Decision Control Preferences (DCP), decision making stress (measured with 22-item, 5-point Likert Impact of Event Scale (IES)), caregiver burden (measured with 12 item, 5-point Likert Zarit scale), decision making self-efficacy (measured with 7-item, 5point Likert Family Decision-Making Self-Efficacy Scale), and satisfaction with decision involvement. Descriptive statistics, such as means/medians or proportions, were used to summarize the demographic characteristics of the sample. Distributions of baseline variables were compared between groups. Outcomes were compared between study arms at baseline and at 8 weeks with t-tests or $\chi 2$ tests. Patient-surrogate agreement on DCPs was assessed using the kappa statistic at each time point. Generalized linear regression models with generalized estimating equations (GEE) and exchangeable working correlation structure were fitted to compare the above outcomes by group as well as over time for each group, adjusting for study site, patient's age, gender, surrogate's relationship to the patient and importance of religion.
RESULTS: The sample consisted of 163 patients (41\% with ALS and $59 \%$ with incurable gastrointestinal malignancies) and their designated surrogates. Decision Control Preference congruence between surrogate and patient improved non-significantly for both groups. The percent agreement between patients and surrogates went from $36 \%$ at baseline to $46 \%$ at follow up for CONTROL and went from 44 to $54 \%$ for TAILORED (follow-up kappa $=.23$ for CONTROL and .28 for TAILORED). Surrogates' perceptions of the patients' Decision Control Preferences shifted significantly to emphasize more shared decision making after the intervention for TAILORED patients (adjusted OR = 2.05 compared with baseline, $p=0.03$ ), but not for CONTROL (adjusted OR $=1.44$ compared with baseline, $p=0.28$ ). Stress levels decreased for TAILORED surrogates (IES $=23.1 \pm 14.6$ baseline, $20.8 \pm 15.3$ follow up, $p=0.046$ ), but not for CONTROL (IES $=23.8 \pm 15.3$ baseline, $22.8 \pm 15.8, p=.85$ ), and post-intervention stress was lower for TAILORED than CONTROL $(p=.04)$. Surrogate confidence was uniformly high at baseline and did not change. Surrogate caregiver burden increased from $12.5 \pm 6.5$ to 14.7 \pm 8.1 for TAILORED $(p<.01)$ while not changing for CONTROL $(14.1 \pm 7.2$ to $14.9 \pm$ $8.5, p=0.14)$. Satisfaction with involvement in decision making was higher at follow-up for TAILORED (71\% completely satisfied) than for CONTROL (52\% completely satisfied) $(p=.03)$.

CONCLUSIONS: TAILORED surrogates adopted more of a shared decision making style that included both patients' autonomous wishes and their own views about what would be best for the patient. Surrogates receiving the intervention demonstrated greater congruence with patient decision control preference and had less stress and more satisfaction with involvement in decision making. Confidence in surrogate decision making was uniformly high in both groups at baseline and did not change. The improvements seen with the intervention were accompanied by a modest increase in caregiver burden. These findings suggest that interventions like TAILORED can positively impact surrogate decision making.

THE USE OF EVIDENCE-BASED PROGRAMS FOR OLDER ADULTS IN UNDER-RESOURCED COMMUNITIES IN LOS ANGELES COUNTY Arleen F. Brown $^{2}$; Teresa Seeman ${ }^{4}$; Katherine Ward ${ }^{3}$; Tony Kuo ${ }^{1}$; Karina Ramirez ${ }^{4}$; Lynn Phan $\mathrm{Vo}^{2}$; Stefanie D. Vassar ${ }^{2}$; Ibrahima Sankare ${ }^{2}$; Arturo Martinez ${ }^{2}$; Sonya Pritzker ${ }^{5} .{ }^{1}$ Division of Chronic Disease and Injury Prevention, Los Angeles, CA; ${ }^{2} \mathrm{UCLA}$, Los Angeles, CA; ${ }^{3}$ Los Angeles County Department of Health Services, Los Angeles, CA; ${ }^{4}$ Division of Geriatrics, UCLA, Los Angeles, CA; ${ }^{5}$ University of Alabama, Tuscaloosa, AL. (Control ID \#2468740)

BACKGROUND: The population of older adults is growing rapidly in Los Angeles County, and the growth is most rapid among minority elders: between 2000 and 2008 the Latino and Asian/Pacific Islander population increased at a rate of $40 \%$. Minority elders have higher rates of preventable or modifiable chronic conditions, poorer control of those conditions, and lower access to evidence-based care and high quality preventive care. Although many evidence-based programs (EBPs) address common chronic conditions and geriatric syndromes, most of these 
programs were developed and studied under conditions approaching optimal efficiency. Very little information exists on their implementation in "real world" settings accounting for racial/ethnic, cultural, and language diversity among participants. The degree of reduced effectiveness of these programs in low income and minority communities and approaches for enhancing their effectiveness has not been well documented.

METHODS: We conducted semi-structured interviews with stakeholders from 25 organizations across Los Angeles County that implement EBPs for older adults in four ethnically diverse geographic areas with high levels of need. Interviews included characteristics of the organization, type and geographic areas of EBPs provided, and EBP evaluation and implementation issues. Interviews were recorded and transcribed, and data were imported into Atlas.ti. Data were analyzed by four independent reviewers using grounded theory approaches to identify key themes. A subsequent panel meeting of 17 stakeholders representing 15 organizations was held to establish preliminary priorities, metrics and implementation strategies for EBPs in diverse communities in Los Angeles County. Recommendations from this panel were incorporated into the summary.

RESULTS: Interviewed organizations were currently implementing EBPs in 8 domains: Falls Prevention (68 \%), Mental Health (64 \%), Caregiver Health (48 \%), Chronic Disease Management (48\%), Diabetes Management (36\%), Arthritis Management (28\%), Physical Activity (24\%), and Multiple Conditions Management (8\%). Three themes were identified from qualitative analyses: 1) barriers to implementation, 2) challenges in evaluation and 3) program fidelity and tailoring/modifications. Barriers to implementation of EBPs included community trust, physician or health-system buy-in and referrals, client transportation and scheduling, and long-term maintenance and program funding. Fidelity was a major concern, and several agencies and organizations expressed a need for additional program modifications to make the program culturally appropriate while maintaining fidelity to the main program goals. Additionally, most programs either had not been translated into needed languages, or the translations were suboptimal. A lack of adequate cultural tailoring of programs was often a barrier to implementation. Challenges with evaluation included the perception that the organizations and agencies were restricted to the evaluation required by funders, limited access to their own data and/or comparison to comparable local or national data, and difficulty with high attrition for post-program follow-up data collection (6 to 12 months). The stakeholder panel identified EBPs in the domains of Chronic Disease and Diabetes Management as highest priority for their organizations and client population. The pane discussed the difficulties with fidelity in low-income and minority populations, and pointed to the need for studies of equivalence of programs that had been adapted or modified. Organizations' reliance on volunteers to staff EBPs and high staff turnover resulted in a lack of trained program leaders. Evaluation metrics suggested included: 1) Demographics of program participants including home environment, transportation challenges, etc. 2) Completion rates of programs, 3) Overall health and disease diagnoses, and 4) local outcomes. The panel emphasized the need for standardized approaches to evaluation across programs, and the need for assistance with data collections and management.

CONCLUSIONS: With a projected increase in minority elders, and potential expansion of reimbursement for selected EBPs, there is need for programs that are culturally and linguistically appropriate and flexible to implement, while maintaining adequate fidelity to guarantee reimbursement. Evaluation of modified programs for several populations is necessary to ensure EBPs reach communities most in need of assistance with chronic condition management and geriatric syndromes. Strategies are needed to assist programs with data management locally to fulfill reimbursement requirements and improve program quality, and at the macro-level, to apply data to inform sensible health policies that will improve access to and sustain EBPs in Los Angeles, regionally, and nationally.
THE USE OF SOCIAL MEDIA IN GRADUATE MEDICAL EDUCATION (GME): A SYSTEMATIC REVIEW Madeline R. Sterling ${ }^{1}$; Peggy Leung ${ }^{1}$; Drew Wright $^{2}$; Tara F. Bishop ${ }^{2} .{ }^{1}$ New York Presbyterian - Weill Cornell Medical College, New York, NY; ${ }^{2}$ Weill Cornell Medical College, New York, NY. (Control ID \#2465166)

BACKGROUND: Social media platforms, which include web-based and mobile technologies, are increasingly being used in undergraduate medical education. Yet, little is known about their use in Graduate Medical Education (GME) and their effects on education, recruitment, and professionalism. We conducted a systematic review of the peer-reviewed literature to understand the use of social media platforms in GME. We sought to explore how social media platforms influence (1) residency education and learning, (2) resident recruitment, and (3) resident professionalism.

METHODS: We conducted systematic literature searches in Medline, Embase and Cochrane from their inception through November 2015. Search strategies were developed under the guidance of a research librarian. Major search terms included Social Media, Medical Education, and Residency. In addition, reference and related article searches were conducted in Scopus and PubMed respectively. We included English-language manuscripts that pertained to (1) residents in any year of training and from any specialty, and (2) social media platforms including Blogs, Microblogs (e.g., Twitter), Social networking sites (e.g., Facebook, Yammer), podcasts, video-sharing sites (YouTube) and Wiki platforms. Only full text articles were included in the review and duplicates were excluded. Two researchers independently reviewed all retrieved articles to identify studies meeting eligibility criteria and abstract pertinent data. The Medical Education Research Study Quality Instrument (MERSQI), a validated instrument which assesses the quality of medical education research on a scale of 6 to 18 , was used to evaluate study quality.

RESULTS: Of the 319 articles identified, 29 met our inclusion criteria. Regarding selection criteria, interrater reliability was high $(\kappa=0.86)$. The majority of the studies were published between 2012 and 2014 and $70 \%$ were published in sub-specialty journals. Studies were either descriptive $(n=20)$ (cross-sectional, survey designs or case studies) or evaluated interventions $(n=9)$. There were no randomized control trials (RCTs). Among the platforms studied, Facebook $(n=10)$, Blogs $(n=3)$, Twitter $(n=3)$, and podcasts $(n=3)$ were the four most common. The mean MERSQI score was 9.41 and ranged from 6.5 to 14.5 . Of the 29 studies, 13 (44.8\%) attempted to use social media to enhance resident education and knowledge with Twitter, Podcasts and Blogs were most frequently used to do so. The majority of studies that used YouTube, Wikis and Blogs were associated with improved knowledge/ technical skills and self-efficacy among residents. Twitter, which was used by several studies to promote evidence based medicine and conference material, had mixed results on residents' education value, however appears to be effective in distributing information. In addition to enhancing education, six ( $20.7 \%)$ studies explored how platforms are being used as vehicles for residency recruitment and applicant screening. Across GME, programs are transitioning residency information to social media platforms, most commonly Facebook, to attract applicants. Several studies ( $n=10,34.5 \%$ ) explored the effects of social media on professionalism in residency. Most of these studies were exploratory in nature and highlighted issues of patient and resident privacy, particularly with respect to Facebook. Four of these (13.8 \%) studies surveyed residents about their social network behavior with respect to their patients (searching for patients or accepting friend requests) while the other six (20.7\%) explored the use of social network sites by residency programs who wish to monitor and censor unprofessional behavior while aiming to develop potential professionalism guidelines in an ever evolving sector

CONCLUSIONS: The use of social media in GME is increasing, although to date, its effects remain unclear. Despite the existence of these platforms for more than a decade, the majority of studies in the literature are single-center and of modest quality. For this generation of trainees, who are both comfortable and versatile with technology, educators are challenged with how best to integrate these platforms into traditional models of 
residency education. Although there appears to be significant interest in social media across GME programs, our results suggest a lack of evidence on how to effectively use platforms to enhance education or promote recruitment. In addition, social media in GME raises concerns about professionalism and privacy, both of which merit further study.

THE UTILITY OF ULTRASOUND IN ACUTE KIDNEY INJURY Arham $\underline{\text { Barakzai }^{2}}$; Nisha Deol ${ }^{2}$; Avneil Yashpal ${ }^{2}$; Syem Barakzai ${ }^{3}$; Sarwan Kumar ${ }^{1} .{ }^{1}$ Crittenton Hospital/Wayne state university, Rochester, MI; ${ }^{2}$ Wayne State University / Crittenton Hospital, Rochester, MI; ${ }^{3}$ Tulane University School of Medicine, New Orleans, LA. (Control ID \#2456931)

BACKGROUND: Acute Kidney Injury (AKI) is one of the most common diagnoses resulting in admission to the hospital. The incidence of AKI in the developed world is 2-3/ 1000 and increases for the geriatric population to 49/1000 for MEDICARE patients above the age of 85+. Ultrasound (US) abdomen is a commonly used tool to exclude obstructive uropathy in patients admitted with AKI. An ultrasound abdomen can cost from $\$ 200$ to $\$ 1000$. We conducted a retrospective cohort study to investigate what proportion of patients with AKI who have an ultrasound, have obstructive uropathy.

METHODS: We examined medical records from patients admitted to Crittenton Hospital Medical Center with AKI between September 2014 and August 2015. We only included patients who were admitted with diagnosis of AKI (ICD-9 code 584.9 to 584.9) and had an ultrasound abdomen specifically to exclude obstructive uropathy. Out of over 200 patient charts that were reviewed at random (of patients admitted with AKI), the first 50 patients who had ultrasound to exclude obstructive uropathy were included in the study.

RESULTS: Out of 50 patients only 3 had obstructive uropathy (6\%). All 3 of these patients had signs and symptoms of obstructive uropathy: 1 patient had history of BPH and 2 patients had flank pain.

CONCLUSIONS: In this pilot study only $6 \%$ of patients with AKI who had US abdomen were diagnosed with obstructive uropathy on US scan. Ninety-four percent of patients who had an ultrasound did not have obstructive uropathy. Of the 3 patients with obstructive uropathy, $100 \%$ had elements of history and physical examination to suggest obstruction. Therefore the internist should not routinely order US abdomen in patients admitted with AKI. US abdomen should only be ordered if there is a finding on history or physical examination suggestive of renal tract obstruction.

THE VALUE OF INTEGRATED MENTAL HEALTH SERVICES IN VA PRIMARY CARE MEDICAL HOMES Lucinda B. Leung ${ }^{9}$, ; Jean Yoon $^{2}$; Jose J. Escarce ${ }^{6}$; Edward P. Post ${ }^{5,}{ }^{10}$; Maureen E. Metzger ${ }^{8}$; Kenneth B. Wells ${ }^{3}$; Catherine Sugar $^{7}{ }^{3}$; Lisa V. Rubenstein ${ }^{1} .{ }^{1}$ GLA VA, North Hills, CA; ${ }^{2}$ Palo Alto VA, Menlo Park, CA; ${ }^{3}$ UCLA, Los Angeles, $\mathrm{CA} ;{ }^{4}$ University of California Los Angeles, Department of Veterans Affairs, Los Angeles, CA $;{ }^{5}$ VA Ann Arbor Healthcare System and University of Michigan, Ann Arbor, MI; ${ }^{6}$ University of California Los Angeles, Los Angeles, CA; ${ }^{7}$ UCLA School of Public Health, Los Angeles, CA ${ }^{8}$ VA Center for Clinical Management Research and Serious Mental Illness Treatment Resource and Evaluation Center, Ann Arbor, MI; ${ }^{9}$ VA, Los Angeles, CA; ${ }^{10}$ VA, Ann Arbor, MI. (Control ID \#2465097)

BACKGROUND: Integrating mental health resources into primary care settings is widely viewed as desirable, especially among patients with complex chronic illnesses. Recognizing that nearly a third of veterans are burdened with mental health disorders, the VA introduced two major national changes: Primary Care-Mental Health Integration (PCMHI) and Patient Aligned Care Teams (PACT), i.e., patient-centered medical homes. Observed decreases in specialty mental health visits and modest cost reductions have been attributed to PACT, but, to date, no one has examined whether PC-MHI affects healthcare utilization and medical spending in the VA. Since PC-MHI aims to improve access to and quality of mental health services in primary care, we use VA data to determine whether PC-MHI use is similarly cost-saving, through increased access to outpatient care (i.e., primary care, specialty mental health care) without increasing acute care use (i.e., potentially avoidable emergency department (ED) visits, hospitalizations).

METHODS: We examined 66,638 patients with mental health diagnoses who used VA primary care in one of 29 clinics in Southern California from October 1, 2008 to September 30, 2013. To measure the level of PC-MHI use in each clinic, we calculated the proportion of primary care patients who received PC-MHI services over the last 12 months. Then, we compared healthcare utilization and medical spending in each year for patients in clinics with varying levels of PC-MHI use. We controlled for time and clinic fixed-effects, other practice redesign efforts (e.g., PACT implementation support), and patient-level factors (e.g., age, gender, chronic comorbidities)

RESULTS: Over the 5-year study period, the proportion of patients receiving PC-MHI services increased by 9.3 percentage points on average across clinics. After adjusting for patient- and clinic-level factors, we found a significant $0.5 \%$ increase in specialty mental health visits per year for each percentage point increase in the proportion of patients receiving PC-MHI services at a clinic site $(p=0.003)$. However there was no evidence of a PC-MHI effect on other utilization outcomes: primary care visits $(\operatorname{IRR}=0.81[\mathrm{SE}=0.21]$, total $\mathrm{ED}$ visits $(\mathrm{IRR}=1.58[0.43])$, ambulatory care sensitive condition $\mathrm{ED}$ visits $(\mathrm{IRR}=$ $0.59[0.44])$ ), or hospitalizations ( $\operatorname{IRR}=1.10[0.29])$. Finally, we observed a small reduction in total costs per year associated with the PC-MHI variable, but this effect was not significant $(\beta=-0.23[0.21])$.

CONCLUSIONS: PC-MHI services aim to improve management of patients with coexisting mental and physical illnesses, who are often high utilizers of medical care. In our study, patients in clinics with greater PC-MHI use tended to use more outpatient specialty mental healthcare, indicating that integrated services successfully facilitated referrals for higher-level outpatient care. Furthermore, it did not appear that patients in those clinics experienced worse outcomes in acute care use (i.e., more ED visits or hospitalizations) or had increased medical spending, compared to patients in clinics with less PC-MHI use. Further research on patient clinical outcomes is necessary to fully understand how the proportion of PC-MHI use affects healthcare value and whether it may be used as a quality metric for integrated mental health care in medical homes.

THE VETERANS AFFAIRS CARDIAC RISK SCORE: RECALIBRATING THE ASCVD SCORE FOR APPLIED USE Jeremy Sussman ${ }^{3,5}$; Wyndy L. Wiitala ${ }^{5}$; Matthew Zawitowski ${ }^{5}$; TImothy Hofer ${ }^{4}$, ; Douglas Bentley ${ }^{5}$; Sandeep Vijan ${ }^{1}$; Rodney A. Hayward 2,6 . ${ }^{1}$ Ann Arbor VA HSR\&D, Ann Arbor, MI; ${ }^{2}$ U. Michigan, Ann Arbor, MI; ${ }^{3}$ University of MIchigan, Ann Arbor, MI; ${ }^{4}$ University of Michigan, Ann Arbor, MI; ${ }^{5} \mathrm{VA}$ Medical Center, Ann Arbor, MI; ${ }^{6}$ VA Ann Arbor Healthcare System, Ann Arbor, MI. (Control ID \#2470564)

BACKGROUND: Since the publication of new statin guidelines in 2013, cardiovascular disease (CVD) risk prediction is now central to statin use. This has been controversial, in part because the risk score used in the guidelines (the Atherosclerotic CVD score, or ASCVD) has had inconsistent results in different populations. This problem could be correct with electronic health records that correct risk scores to the populations that will use them. For US Veterans, this can already be done. Here, we created risk scores that are based on the ASCVD risk score, but that are calibrated to VA and that use only information available in the VA EHR. We use three calibration techniques, each designed for a different potential user and compare how well they predict CVD in VA patients compared to each other and the original ASCVD score.

METHODS: Data: Linked national data from the VA, Medicare, and the National Death Index Population: All active VA patients ages 45-80 in 2006. We excluded patients with a 
history of CVD, heart failure, use of loop diuretics, or those with a non-CVD cause of death during 2007 . We then used $2 / 3$ of the participants in a derivation and $1 / 3$ in a validation cohort. Risk predictors Traditional CVD risk factors, including age, smoking status, gender, non-HDL cholesterol, race, systolic blood pressure, diabetes status, and blood pressure medication use. Outcome variables: "Hard" CVD events, defined as first myocardial infarction, CVD death, and fatal or nonfatal stroke, modeled on the 2013 ACC/AHA cholesterol guideline for 5 years of follow-up. Prediction models: We used 4 prediction models, designed to estimate types of recalibration available to different participants. Models used logistic regression with interaction effects for age and gender with all other predictors. 1 . The risk score reported by the ACC/AHA ASCVD risk score 2. "Population recalibration:" Adjust the overall event rate and apply to every patient. 3 "Regression recalibration:" Regress ASCVD predicted score against observed outcomes in our dataset. 4. "Refit model:" Recalibrate the entire model Analysis: We assessed model fit with the C-statistic, the Hosmer-Lemeshow Goodness of Fit, and the Brier score. RESULTS: Our full sample, with 1.5 million participants, was much larger than any previous American risk prediction tool. Our sample was also older $(61.7+/-8.6$ years), more male ( $95 \%$ ), and had more medication use than previous scores (37\% on statins). Discrimination was quite similar across all four models, for men the C-statistic was near 0.66 and for women it was near 0.73 . Calibration varied dramatically, with the standard ASCVD score estimating 0.091 events per 5 patient-years vs. 0.056 observed in men. In the 1.4 million men all 3 recalibration techniques had far better calibration, with the Refit Model having the best results, with a GOF statistic of 17.4, compared to 8318 for the ASCVD score. The results for the 76,155 women in the full sample were less dramatic. The ASCVD score only slightly overestimated event rates $(0.023$ average predicted events vs. 0.021 observed per 5 years). The Population Recalibration technique had a minimal effect on the discrimination and made calibration substantially worse, especially at the higher values (GoF increased from 49.0 to 199). The other recalibration techniques substantially outperformed the ASCVD score.

CONCLUSIONS: We found that a large managed care organization can use pre-existing electronic health record data and well-established risk factors to create a risk prediction score that has major advantages over those developed in traditional cohort studies. Our score points towards a potential solution to this problem of risk prediction in different populations - loca risk scores created from EHRs using clinical data. We have shown that people can develop risk scores within an organization's EHR that are well-calibrated, easy to integrate with local decision support tools, require no data-input, and can be easily updated with new information We used three different recalibration techniques, each designed to approximate a differen real-world situation. Population recalibration resembles how a health-system leader, with easy access to basic descriptive information about their patients but without detailed, patientlevel data, could roughly approximate the risk of his or her population. Regression recalibration could be performed by someone with minimal statistical skills using a regular spreadsheet program. Full recalibration ("refit") is the recommended, technique that recalibrates the entire model. We used clinical data, so some information was missing or inaccurate, just like in clinical practice. We think this is partly why our study's discrimination was no better than previous scores. This work has many policy and clinical implications. We have shown that clinical risk scores can be made efficiently within a single EHR, like that available in many managed care and accountable care organizations. As EHRs improve and "Big Data" becomes central to patient self-management, work like this can point the way towards how these tools can be used in clinical practice.

THINK ALOUD AND NEAR LIVE USABILITY TESTING OF TWO PRIMARY CARE CLINICAL DECISION SUPPORT TOOLS Safiya Richardson ${ }^{4}$; Rebecca G. Mishuris ${ }^{2}$; Lauren McCullagh ${ }^{3}$; Devin Mann ${ }^{1} .{ }^{1}$ Boston University, Boston, MA; ${ }^{2}$ Boston University School of Medicine, Boston, MA; ${ }^{3}$ Hofstra North Shore LIJ School of Medicine, Manhasset, NY; ${ }^{4}$ Northwell Health, Manhasset, NY. (Control ID \#2470366)
BACKGROUND: Clinical prediction rules (CPRs) integrated into electronic CDS tools have demonstrated the ability to shape health care provider behavior towards more evidence based clinical practice. However, low provider adoption, reported at 10-20\%, continues to be a significant barrier to widespread use of CDS. We conduct "Think Aloud" and "Near Live" usability testing on two electronic health record based CDS tools. Each was composed of a CPR for either strep pharyngitis or pneumonia and an automatic order set based on CPR score. The objective of this study was to further understandings of the mediators of usability by finding generalizable themes and to compare findings from these two types of usability testing. This study was done as a part of a larger, ongoing randomized controlled trial evaluating these tools effect on antibiotic ordering.

METHODS: This was a qualitative observational study analyzing video data recorded during "Think Aloud" and "Near Live" usability testing performed at the University of Wisconsin and the University of Utah. Testing was done with 12 primary care providers in the fall and early winter of 2015. During "Think Aloud" usability testing participants are provided with written clinical scenarios and asked to verbalize their thought process while interacting with the CDS tool. During "Near Live" usability testing participants interact with a standardized patient. The interaction between participant and the CDS tool was recorded using Morae, which records full screen capture and all audio. Based on a coding scheme developed for these two CDS tools in a previous study, all participant comments were coded under usability, visibility, workflow, content, understand-ability, practical usefulness, medical usefulness or navigation and analyzed for generalizable themes.

RESULTS: "Think Aloud" and "Near Live" usability testing generated similar generalizable themes under these usability codes: VISIBILITY - Visibility improves when important text is colored, larger, underlined and appropriately located. WORKFLOW Passive alerts that trigger at the time of decision making allow providers to use CDS tools without disturbing their natural workflow. CONTENT - All elements of the CDS tool should incorporate up-to-date and specialty specific medical knowledge. UNDERSTAND-ABILITY - Using common medical terminology, providing clear instructions and using simple language improves understand-ability. NAVIGATION - CDS tool components should include obvious indications of next steps. Generalizable themes differed under these codes: USABILITY - (Think Aloud) Usability increases when CDS tools correctly anticipate user needs by providing what they want, when and where they want it. (Near Live) Users perceive ease of use when CDS tools auto-populate with required clinical information and alerts can easily be permanently silenced. MEDICAL USEFULNESS - (Think Aloud) CDS that provides information that is perceived as relevant and medically accurate is helpful for decision making. (Near Live) CDS must provide unknown information or understanding to users to be perceived as medically useful. Tools that facilitate patient education are perceived as medically useful. PRACTICAL USEFULNESS - (Think Aloud) Automatically generated documentation and communication with nurses and patients decrease workload and saves time. (Near Live) To be perceived as practically useful CDS tools must generate visit aids, like smartsets, documentation and patient instructions that are complete. Additionally, "Near Live" testing provided clinical workflow maps as providers interacted with the CDS tool as they would during a real clinical encounter. All providers first completed the history and physical exam, then used the CDS tool calculator, then accessed results if applicable and then proceeded to the smartset and lastly counseled the patient.

CONCLUSIONS: "Think Aloud" and "Near Live" usability testing provide CDS tool designers with supportive and complementary understandings of the key mediators of usability. In particular "Near Live" testing, which mimics an actual clinical encounter, provided more information about workflow integration. These complementary types of usability testing generate unique and generalizable insights about provider perspective which will serve to improve ease of use of CDS, increase provider adoption and help CDS reach its potential to improve quality of care and health outcomes. 


\section{THINKING PAST THE PAP: AN EXPLORATION OF SELF-ADMINISTERED HPV TESTING AS A CERVICAL CANCER SCREENING OPTION FOR UNDERSCREENED WOMEN Elizabeth O. Kidder. George Washington University,} DC, WA. (Control ID \#2466622)

BACKGROUND: Though significant gains have been made in preventing cervical cancer over the past 30 years, it continues to cause morbidity and mortality among women in the United States, particularly among those women who are screened infrequently or not at all. More than half of cervical cancer deaths in the United States are among immigrants, and the incidence and mortality from cervical cancer is increasing among foreign-born women. Arab and Hispanic women living in the United States continue to have cervical cancer screening rates that are lower than the general population. Understanding what factors influence their cervical cancer screening practices and what new screening options may overcome their barriers to preventive screening may be effective in reducing disparities in the disease burden of cervical cancer. HPV DNA testing has taken on a larger role in cervical cancer screening, and there is increasing evidence and support for the use of HPV testing alone as a primary cervical cancer screening test. Novel health screening devices have been developed that allow women to self-screen for HPV, which may offer opportunity to simplify the cervical cancer screening protocol and reach women who are not receiving recommended cervical cancer screening services. Because selfadministered screening devices are not yet available and most women have not had exposure to them, there are limited quantitative and qualitative assessments of women's attitudes towards and likelihood to use such devices, particularly in the U.S. This study informs the development of culturally appropriate interventions and policies intended to improve cervical cancer screening rates among Arab and Hispanic women in the United States, and discusses implementation challenges and policy implications associated with incorporating self-administered HPV testing into the cervical cancer screening protocol in the United States.

METHODS: A paper-based cross-sectional survey $(n=476)$ and individual interviews $(n=31)$ were used to explore Arab and Hispanic participants' screening behaviors, their likelihood to use HPV self-administered tests to screen for cervical cancer, their perceived self-efficacy in using self-screening tests, and the major concerns they have about selfscreening. Arab and Hispanic women living in the United States were selected as the research populations of interest as their uptake of cervical cancer screening services has continually been lower than the general population of women in the United States. Women surveyed and interviewed were between the ages of 30 and 65 , as this is the age range for which HPV screening is currently recommended. The Health Belief Model was the conceptual framework used to define the survey and interview questions.

RESULTS: Approximately half of uninsured (52.7\%) and underscreened (47.1\%) participants reported they would be more likely to get screened for cervical cancer if an at-home self-screening test were available. Participants who were 1) uninsured, 2) knowledgeable about HPV and cervical cancer, 3) had high self-efficacy in their ability to use a self-screening test; and 4) had no concerns about the self-screening test were significantly more likely to use a self-screening test. Hispanic participants $(74.0 \%)$ were significantly more likely than Arab participants (43.8 \%) to report they would be likely to use a selfadministered cervical cancer screening test if it were available. Qualitative interviews revealed multiple cultural and socioeconomic factors that impact participants' likelihood to get screened for cervical cancer, and explored how the use of a self-screening test may overcome such barriers.

CONCLUSIONS: A majority of participants, many of them uninsured or underscreened, responded positively to the option for HPV self-testing as a cervical cancer screening option, suggesting that it may an effective screening modality to reach women who are not accessing routine screening. More research is needed on implementing a self-screening option, particularly among underscreened populations. A new screening modality that is patient-centered and easy to use may have significant implications on reducing morbidity and mortality from cervical cancer. Further, this new screening modality spurs practitioners to consider how it may impact the practice of women's health and the promotion of preventive health, as we move away from the "annual visit" model that revolves around the Pap test and has for decades defined why and with what frequency women seek health care.

THYROID DYSFUNCTION AND THE RISK OF DEMENTIA AND COGNITIVE DECLINE: SYSTEMATIC REVIEW, META-ANALYSIS AND CLINICAL IMPLICATIONS Carole Rieben ${ }^{4}$; Carole E. Aubert ${ }^{1}$; Daniel Segna ${ }^{1}$; Bruno Da Costa ${ }^{6}$; Christine Baumgartner'; Tinh-Hai Collet ${ }^{3}$; Layal $\mathrm{Chaker}^{5}$; Osvaldo P. Almeida'; Eef Hogervorst $^{8}$; Stella Trompet ${ }^{2}$; Kamal Masaki ${ }^{9}$; Simon P. Mooijaart ${ }^{2}$, Jacobijn Gussekloo ${ }^{2}$; Robin P. Peeters ${ }^{5}$; Douglas Bauer ${ }^{10}$; Drahomir Aujesky ${ }^{1}$; Nicolas Rodondi ${ }^{1} .{ }^{1}$ Inselspital Bern, University Hospital Bern, Switzerland, Bern, Switzerland; ${ }^{2}$ Leiden University Medical Center, Leiden, Netherlands; ${ }^{3}$ Service of Endocrinology, Diabetes and Metabolism, Lausanne, Switzerland; ${ }^{4}$ Inselspital Bern, University Hospital Bern, Bern, Switzerland; ${ }^{5}$ Erasmus Medical Center, Rotterdam, Netherlands; ${ }^{6}$ University of Bern, Bern, Switzerland; ${ }^{7}$ University of Western Australia, Perth, WA, Australia; ${ }^{8}$ Loughborough University, Leicestershire, United Kingdom; ${ }^{9}$ John A. Burns School of Medicine, University of Hawaii, Honolulu, HI; ${ }^{10}$ Univeristiy of California, San Francisco, CA. (Control ID \#2448775)

BACKGROUND: The prevalence of subclinical thyroid dysfunction (SCTD), defined as abnormal thyroid stimulating hormone (TSH) with normal free thyroxin levels, reaches $10 \%$ for subclinical hypothyroidism (SHypo) and up to $4 \%$ for subclinical hyperthyroidism (SHyper) in the elderly. Mild cognitive impairment is also a highly prevalent condition in the elderly ( 5 to $8 \%$ after 60 years, up to $22 \%$ after 71 years of age) with an annual progression rate to dementia of $15-41 \%$. While both overt hyper- and hypothyroidism are known to contribute to cognitive impairment, and clinical guidelines recommend screening for thyroid diseases among patients with cognitive disorders, data on the association between SCTD and cognitive function remain conflicting. Therefore, we performed a meta-analysis to determine whether SCTD was associated with an increased risk of both dementia and cognitive decline in prospective cohorts.

METHODS: Two physicians conducted a systematic literature search in MEDLINE and EMBASE databases to identify all prospective cohort studies among participants aged $\geq 18$ years that included thyroid function tests at baseline, a SCTD and an euthyroid control group, assessment of cognitive function (dementia and/or screening tests, such as Mini-Mental State Examination [MMSE]) during follow-up, and published risk estimates or sufficient information to calculate them. Both reviewers independently assessed eligibility and methodological quality of included studies. We expressed the estimates of the association between SCTD and outcomes as risk ratios (RR) for dementia or as changes from baseline for MMSE scores (standardized as mean MMSE differences per 1 year unit). We used an inverse variance randomeffects meta-analysis to pool estimates across studies, Q statistic to evaluate heterogeneity across studies and $\mathrm{I}^{2}$ statistic to assess the variability in estimates of effects across studies. We visually evaluated publication bias through funnel plots and statistically by the Egger's test. To explore the sources of heterogeneity, we conducted several sensitivity analyses.

RESULTS: Eleven prospective cohorts followed a total of 16,805 participants during a median time of 44.4 months. Mean age was 70 years or higher, except for one study (mean age 68.8 years). Five studies analyzed the risk of dementia in SHyper ( $n=6410,329$ cases of dementia, mean follow-up 68.3 months), and six studies in SHypo ( $n=7401,416$ cases of dementia, mean follow-up 64.6 months). Five studies analyzed MMSE decline in SHyper ( $n=7895$, mean follow-up 33.6 months) and seven studies in SHypo $(n=8960$, mean follow-up 32.2 months). In random-effects models, the pooled adjusted RR for dementia was 1.67 (95\% confidence interval [CI] 1.04-2.69) in SHyper versus 
euthyroidism, and 1.14 (95\%CI 0.84-1.55) in SHypo versus euthyroidism, both without statistical heterogeneity $\left(\mathrm{I}^{2}=0.0 \%\right)$. Sensitivity analyses pooling only studies with formal outcome adjudication or population-based studies yielded similar results. The pooled mean MMSE decline from baseline to follow-up did not significantly differ between SHyper or SHypo versus euthyroidism. A sensitivity analysis including the two studies examining the relationship between SHyper and both MMSE and dementia also showed no larger decline of MMSE among SHyper.

CONCLUSIONS: In this meta-analysis of prospective cohort studies, we found that SHyper, but not SHypo, was associated with a slightly elevated risk for dementia when compared with euthyroidism, while decline in MMSE was not faster in SCTD. This discrepancy may be explained by the modest sensitivity (79 \%) of MMSE as a diagnostic tool for dementia, the multimodal approach needed to diagnose dementia, different population investigated or by the longer follow-up in studies that analyzed dementia Given the relatively high prevalence of both SCTD and cognitive dysfunction in the elderly, even a modestly increased rate of cognitive decline among subjects with SCTD might indeed have important public health implications, including those concerning screening for and treatment of SCTD. As available data were limited, large high-quality studies with longer follow-up are needed to clarify a possible association of SCTD with cognitive impairment

TIMING OF REFUGEE HEALTH ASSESSMENTS MAY BE ASSOCIATED WITH ACUTE HEALTHCARE UTILIZATION Wagahta Semere ${ }^{2,}{ }^{1}$; Pooja Agrawal $^{2}$; Katherine Yun ${ }^{3}$; Isha M. Di Bartolo ${ }^{2}$; Aniyizhai Annamalai ${ }^{2}$; Joseph S. Ross ${ }^{2}$. ${ }^{1}$ RWJF Clinical Scholars Program with support from U.S. Department of Veterans Affairs, New Haven, CT; ${ }^{2}$ Yale University School of Medicine, New Haven, CT; ${ }^{3}$ University of Pennsylvania Perelman School of Medicine, Philadelphia, PA. (Control ID \#2464872)

BACKGROUND: Over the past 40 years, more than 3 million refugees fleeing persecution worldwide have been accepted into the United States Refugee Resettlement Program Admission to the U.S. requires that refugees undergo medical evaluations 6 months prior to departure to screen for conditions of public health significance as well as those that will require follow up on arrival. According to Centers for Disease Control and Prevention recommendations, refugees should undergo a follow-up medical evaluation within 30 days of arriving in the U.S. to identify conditions requiring follow-up medical care and provide an introduction to local healthcare systems. Our research objective was to determine refugees' acute care use during the first 8 months after U.S. arrival in Southern Connecticut, and identify factors, including timing of follow-up medical evaluation, that were associated with differences in utilization patterns

METHODS: We partnered with a local refugee resettlement agency to conduct a retrospective cohort study of all adult refugees arriving in Southern Connecticut from 2013 to 2015 . We excluded those refugees who previously resettled elsewhere in the U.S., left the region within 90 days, or lived outside of the study hospital health service area. We abstracted demographics and baseline health characteristics from mandatory pre-departure and postarrival follow-up medical evaluations. Utilizing the EHR for the largest healthcare system in the area that serves as the primary provider for all refugees in our sample, we identified all acute care visits within 8 months of a refugee's date of arrival, including Emergency Department visits and hospital admissions, abstracting dates and affiliated primary diagnoses. We used multivariate logistic regression analyses to examine associations between any acute care use and the following variables: age, gender, Iraqi origin, arrival alone, hypertension, PTSD, depression, and post-arrival medical evaluation within 30 days of arrival.

RESULTS: There were 249 adult refugees that arrived in Southern Connecticut from 2013 to 2015 that met our study inclusion criteria. Many refugees (43.1 \%) identified Iraq as their country of origin. The median age was 30 years, but more than half $(60.1 \%)$ reported having at least one chronic health condition, and $57 \%$ had a post-arrival follow- up medical evaluation within 30 days. Among all adult refugees, $104(41.9 \%)$ had at least one emergency department visit or hospitalization within 8 months of arrival. After adjusting for demographic and baseline health characteristics, male sex (OR 2.18, $95 \%$ Confidence Interval (CI), 1.21-3.95) and prior history of hypertension (OR 2.87, 95 \% CI, 1.09-7.52) were associated with greater likelihood of an acute care visit within 8 months of arrival, while having a medical evaluation within 30 days of arrival was associated with lower likelihood (OR 0.54, $95 \%$ CI, 0.30-0.95).

CONCLUSIONS: Refugees have frequent acute care utilization early on in the arrival period and a high burden of comorbid disease. Designing systems to ensure timely evaluations of newly arrived refugees may be beneficial in reducing acute care utilization during the initial period of resettlement.

TITLE: QUALITY OF CARE ASSESSMENT IN HOSPITALIZED PATIENTS WITH UPPER GASTROINTESTINAL (UGI) BLEEDING Abdulfatah A. Issak ${ }^{2}$; Sangeeta Agrawal ${ }^{1}$; Roshni Venkatesh ${ }^{3} .{ }^{1}$ Wright State University, Dayton, $\mathrm{OH} ;{ }^{2}$ Wright State University, Kettering, OH; ${ }^{3}$ Dayton VA Medical Center, Dayton, OH. (Control ID \#2462482)

BACKGROUND: Background: There are established guidelines for the acute management of patients presenting to the hospital with upper gastrointestinal bleeding. Adherence to these guidelines is inconsistent and few studies have addressed this issue.

METHODS: Methods: Data from patients admitted to one single hospital with the principal diagnosis of upper gastrointestinal bleeding during 1 year period was collected and quality indicators consistent with currently accepted guidelines for management of patients with upper gastrointestinal bleeding were used to assess the quality of care provided.

RESULTS: Results: From January 2014 to December 2014, 36 patients were admitted with a diagnosis of upper gastrointestinal bleeding. All the patients had a large bore IV access established at the ED. Twenty two percent of the patients had signs of hypovolemia at rest and IV fluids were started on all of them. Orthostatic vital signs were checked in only $28.6 \%$ of the remaining patients. Fifty eight percent of patients had no type and cross sent but other laboratory tests (CBC, Chemistry, PT, and PTT) were obtained in all. None of the patients had risk stratification done on initial encounter. Nearly forty percent of patients were admitted to the ICU, $25 \%$ of patients to a step down unit and $36.6 \%$ of patients to the floor. Intravenous proton pump inhibitor was started in $94.4 \%$ of patients. Ten patients $(27.8 \%)$ had a previous diagnosis of cirrhosis. Intravenous antibiotics were started in $60 \%$ of patients with cirrhosis. Eight patients were suspected of variceal bleeding, but IV octreotide was started in only $62.5 \%$ of these patients. About forty two percent of patients had an EGD done within 24 h, $25 \%$ within 48 h, $11 \%$ had a recent EGD done, $8.3 \%$ were deferred for an outpatient EGD, $11 \%$ refused EGD, and one patient left AMA. One-fourth of patients needed therapeutics during EGD and homeostasis was achieved in all. PPI was continued for $48 \mathrm{~h}$ in $83 \%$ of patients. Close to seventeen percent of patients had PUD and H. Pylori testing was done in all and treated if positive. The average hospital stay was 2.2 days which was equal to the national average. CONCLUSIONS: Conclusion: Major inconsistencies with adherence to guidelines for UGI bleeding were identified in this study. Practice and implementation of established guidelines at the patient level continues to lag behind research and ideal standard of care based on recommendations of expert panels.

TITLE:EVALUATION OF IMPACT OF NEUROIMAGING IN SYNCOPE IN PREDOMINANTLY BLACK PATIENTS Delamo Bekele; Agazi Gebreselassie; Yonette Paul; Daniel Larbi; Julius Ngwa. Howard University Hospital, Washington, DC. (Control ID \#2457056) 
BACKGROUND: Background Syncope is a sudden, brief loss of consciousness associated with loss of postural tone with spontaneous recovery. Careful clinical evaluation usually leads to a diagnosis in $50-85 \%$ of patients who present with syncope. Even though a limited number of studies have shown the insignificant value of neuroimaging in the diagnosis of Syncope, many physicians still order them indiscriminately with the intention of not missing conditions such as stroke, brain tumors and sub-arachnoid hemorrhage Despite several studies and clear recommendations of guidelines by the ACC, AAN and ESC, it is not clear whether clinical practice has changed to reflect the evidence. Several studies have showed no benefit in routine neuroimaging of patients presenting with Syncope. Review of literature showed scarcity of studies of Syncope in ethnically diverse populations. The purpose of this study is to evaluate the utility of routine neuroimaging in Syncope in a predominantly black patient population.

METHODS: Methods: Patients admitted with a diagnosis of Syncope from 2011 to 2014 were identified using ICD 9 codes. Patients were excluded if the diagnosis after initial assessment was not documented by the physician as syncope even though the chart was coded as syncope.151 patients were included in analysis. A retrospective review of inpatient documentation was performed. Data collected included EKG, CT Brain, MRI Brain, MRA, EEG and orthostatic vital signs (emergency room and floor). CT Brain was considered as routine neuroimaging. Demographics included age, gender, admitting service and co-morbid conditions. Syncope was classified as follows: Cardiogenic, Orthostatic, Vasovagal, Situational and Undetermined. Categorical Variables were analyzed with chi-square and Fisher's exact testing. A Student's T test was used for continuous variables. A multivariate logistic regression analysis was done to predict factors associated with syncope. We considered $P$-value less than 0.05 statistically significant and calculated confidence intervals (CI) at the $95 \%$ level.

RESULTS: Results: Of 151 patients, the average age was $56.62 \pm 18.78$ SD and $68.2 \%$ (103) were female. $128(84.8 \%)$ of the patient population was black, $8.61 \%$ white, hispanic and other both comprised $3.3 \%$. With respect to chronic medical conditions, 92 (60.9 \%) had Hypertension, 47 (31.1 \%) had Cardiac Disease, 32 (21.19\%) had Diabetes Mellitus, 32 had Psychiatric Disorders, 31 (20.67\%) had Pulmonary Disease and 10 (6.62\%) had Seizure Disorder. With respect to diagnostic workup, 147 (98\%) of patients had an EKG, 114 (75.5\%) had a CT of the Brain, 66 (43.71 \%) had Orthostatic Vital signs done after admission, 23 (15.2\%) had Orthostatic Vital Signs done in the Emergency Room. 5 out of 114 patients had an acute abnormality on CT ( $4.4 \%) .1$ of 5 patients with an acute abnormality on CT that was related to Syncope ( $0.88 \%)$. Orthostatic Vital Sign Measurement in Emergency Room was predictive of a diagnosis of syncope (OR 4.98, P Value 0.005 ). Hypertension (OR 2.81, $\mathrm{P}$ value 0.031 ) was also found to be predictive. Orthostatic Vital Signs on floor (0.112) and CT Brain (0.978) were not found to be predictive of underlying etiology of Syncope despite high frequency of use (43.71 and $75.5 \%$ ). Significantly, despite extensive work up in most cases only $65(43.05 \%)$ patients had an underlying etiology of syncope diagnosed at time of hospital discharge

CONCLUSIONS: Conclusions: Routine neuroimaging in the form of CT Brain was not found to be predictive or useful in the workup of Syncope in a predominantly black patient population. This is in keeping with studies conducted in other populations; though replicating this study in a larger population may be beneficial. Hospitals and patients may benefit from policies and protocols to regulate the utility of neuroimaging in syncope evaluation. This would involve taking current evidence, risks versus benefits and cost conscious care into consideration.

\section{TO SOAP OR NOT TO SOAP: COMPARING INPATIENT AND OUTPATIENT} ORAL PRESENTATION STRUCTURES FOR INCOMING RESIDENTS Erin D. Snyder $^{2},{ }^{1}$; Jennifer Bares ${ }^{2}$; Carlos Estrada ${ }^{2,}{ }^{1}$; Ryan Kraemer ${ }^{2,1}{ }^{1}{ }^{1}$ The University of Alabama at Birmingham, Birmingham, AL; ${ }^{2}$ University of Alabama at Birmingham, Birmingham, AL. (Control ID \#2467142)
BACKGROUND: During medical school, students learn to present a patient's history and key clinical findings to a preceptor through Introduction to Clinical Medicine courses and clerkship rotations. Traditionally, training has focused on the admission and subsequent hospital day presentation of inpatients (i.e. SOAP: Subjective, Objective, Assessment, Plan), while less time has been spent training students to present patients in the outpatient setting. Our study aimed to evaluate the oral presentation structure taught to medical students and their comfort with presenting in various settings.

METHODS: Sixty incoming PGY-1 residents from 40 different medical schools were asked to complete an anonymous, 23-item, written survey during orientation. Residents were beginning programs at the University of Alabama at Birmingham in: Categorical Medicine, Preliminary Medicine, Anesthesiology, Neurology, Medicine/Pediatrics, and Medicine/Genetics. We asked residents if they were taught a standard oral presentation format for 4 different patient situations: initial hospital admission history and physical (H\&P), subsequent day hospital rounds, acute outpatient visit, and outpatient follow-up visit for a patient with several chronic problems. They were asked to indicate their knowledge of expectations for oral presentations of the same 4 situations using a 5point Likert scale $(1=$ strongly disagree to $5=$ strongly agree $)$. Residents were also asked to evaluate their comfort level for presenting those same patient situations to faculty attendings, using a 6 -point Likert scale $(0=$ never done to $5=$ very comfortable). Finally, participants were asked to write the headings of each section used when presenting an admission H\&P, a subsequent day inpatient, and a routine follow-up outpatient with several chronic conditions.

RESULTS: The survey response rate was $100 \%(n=60)$. One hundred percent of responders indicated they were taught a structure for hospital admission oral presentations, $98 \%$ for subsequent day follow-up inpatient oral presentations, $83 \%$ for acute outpatient presentations, and $71 \%$ reported being taught a presentation structure for a follow-up outpatient visit in a patient with several chronic conditions. Ninety-five percent of residents "agreed" or "strongly agreed" that they knew what was expected for an inpatient subsequent day presentation while only $68 \%$ of residents indicated the same for an outpatient routine follow-up presentations $(p<0.001)$. Ninety-two percent of the residents were 'comfortable' or 'very comfortable' presenting a subsequent day hospital patient, while only $53 \%$ of the residents were comfortable presenting an outpatient follow-up visit $(p<0.0001)$. When listing the headings of each section used for follow-up hospital presentations, 28 of 54 responses listed SOAP, 16 listed elements similar to SOAP, and 10 responses had no common pattern. For follow-up outpatient presentations, 10 of the 53 responses listed SOAP and 43 responses listed a mixture of common presentation elements in various orders without a discernable pattern.

CONCLUSIONS: Our survey showed that incoming PGY-1 residents perceived being taught outpatient presentation structures less often than inpatient presentation structures and were less comfortable presenting outpatients. In addition, our survey revealed one common inpatient follow-up presentation structure (SOAP), while no common structure for chronic outpatient follow-up presentations was identified. Our study of new residents from 40 different medical schools indicates that current curricula do not provide a uniform presentation structure to guide medical students' outpatient presentations. Without a uniform structure, incoming residents indicate they are less comfortable presenting patients in the ambulatory setting, which may lead to a lack of self-efficacy and may influence career choice. Further studies are needed to evaluate current teaching, or lack thereof, of outpatient presentations and uniform standards for outpatient presentations should be developed.

TOBACCO TREATMENT: IDENTIFYING DISPARITIES IN TREATMENT INVITATIONS Kristin M. Berg; Stevens S. Smith; Megan E. Piper; Michael C. Fiore; Douglas E. Jorenby. University of Wisconsin - Madison, Madison, WI. (Control ID \#2467693) 
BACKGROUND: The steady progress in reducing tobacco use in the general population is much less evident in many disparity populations. One reason for this is that these populations may lack access to health system-provided evidence-based cessation treatment. To address this, the Breaking Addiction to Tobacco for Health (BREATHE) study was designed to engage all smokers presenting to 11 primary care clinics from two Wisconsin health systems in tobacco treatment. Smokers were invited to participate via an electronic health record (EHR) prompt that was activated when a patient screened positive for smoking during their intake assessment by the medical assistant.

METHODS: Using BREATHE data, this study conducted a secondary analysis that examined whether individual variables, including markers of disparities, influenced invitation rates and thus access to treatment. Logistic regression was used to predict receipt of an invitation based on: gender, age ( $<24$ years, 25-44, 45-64, $>65)$, racial background (white, African American, other), insurance status (private, Medicare, Medicaid, none), and visit diagnosis ("high-risk" including cardiovascular and pulmonary disease, malignancy and pregnancy versus "low-risk" including all other ICD-9 categories). Interactions between the individual variables and health systems were also tested to examine invitation rates to treatment dependent upon health system.

RESULTS: A total of 95,471 patients were seen, of which $89 \%(84,668)$ were screened for smoking. Among the 15,193 identified current smokers (17.9\% of screened patients), $10,242(67 \%)$ were invited to participate in the tobacco treatment program. Those who did not receive an invitation were more likely to be younger, represent a racial minority, and to carry a high-risk diagnosis. There were also clinic- and health system-specific differences: rates of non-invitation were higher in the health system and clinics with a higher proportion of health disparities within the populations they served.

CONCLUSIONS: These results suggest that despite EHR-facilitated recruiting, incentives for recruitment, and no cost for treatment, significant disparities remain regarding recruitment for clinical tobacco dependence treatment. Furthermore, these disparities appear to worsen in areas with existing disparate populations.

TRAINING QUALIFIED PRESCRIBERS TO USE COMPUTERIZED PROVIDER ORDER ENTRY SYSTEMS IN BOTH OUTPATIENT AND INPATIENT CARE: A LITERATURE REVIEW Clare L. Brown ${ }^{6,}$; Katie Reygate $^{8}$; Ann Slee ; Jamie Coleman ${ }^{5}$; Sarah K. Pontefract ${ }^{5}$; David W. Bates ${ }^{1}$; Andrew K. Husband ${ }^{2}$; Neil W. Watson ${ }^{4}$; Sarah P. Slight ${ }^{3} .{ }^{1}$ Brigham and Women, Boston, MA; ${ }^{2}$ Durham University, Teesside, United Kingdom; ${ }^{3}$ Durham University, Stockton on Tees, United Kingdom; ${ }^{4}$ The Newcastle upon Tyne Hospitals NHS Foundation Trust, Newcastle upon Tyne, United Kingdom; ${ }^{5}$ University of Birmingham, Birmingham, United Kingdom; ${ }^{6}$ University of Durham, Sunderland, United Kingdom; ${ }^{7}$ Newcastle Upon Tyne NHS Foundation Trust, Newcastle Upon Tyne, United Kingdom; ${ }^{8}$ Health Education Kent, Sussex and Surrey, Sussex, United Kingdom; ${ }^{9}$ NHS England, UK, United Kingdom. (Control ID \#2457332)

BACKGROUND: Computerized Physician Order Entry (CPOE) with clinical decision support has been associated with benefits over paper-based systems, including improved patient safety and quality of care and reduced costs. A key element of the implementation and on-going use of a CPOE system is ensuring that users are, and remain, sufficiently trained to use the system effectively. Studies have suggested that insufficient training is associated with suboptimal use. We sought to describe the approaches used to train qualified prescribers on CPOE systems, whether online methods were used, and to identify whether the training content included the pitfalls and challenges of using these systems.

METHODS: We performed a literature review using a systematic approach. Three large databases: Cumulative Index Nursing and Allied Health Literature (CINAHL), Embase and Medline were searched for relevant English language articles in May 2015. We did not restrict the timeframe for these searches in any of the respective databases. Search terms were kept deliberately broad. Articles that explored the training of qualified prescribers (medical and non-medical practitioners) on CPOE systems in both inpatient or outpatient settings were included. Studies which explored the training of undergraduate medical students, clinical skills other than prescribing, or the use of CPOE in medical education were excluded. All duplicate articles were removed. Titles were initially reviewed, followed by abstracts and then full texts of remaining articles by one author (CLB). Queries were resolved through discussion with a second author (SPS). Reference lists of included articles were also examined. Data were abstracted onto a customized data extraction sheet by one author (CLB). A narrative synthesis of all eligible studies was undertaken.

RESULTS: Our database search returned 1179 publications of which seven met our inclusion criteria. One further article was identified from the reference list search. A variety of training methods were used such as classroom-based sessions, which included demonstrations, expertise-specific scenarios and exercises, aimed at addressing commonly encountered prescribing errors, e.g. prescribing Tazocin ${ }^{\circledR}$ (piperacillin-tazobactam an antibiotic) at non-standard times. Additional methods included ward-based training facilitated by 'super-users' (expert staff members who received additional training). Some studies incorporated assessment, which allowed users to monitor about their own progress and highlighted individuals who may need further assistance. A team at the University of Victoria in Canada developed an online portal, which housed a range of simulated versions of CPOE systems. Individuals were given access to this portal where they could prescribe for fictitious patients in a safe environment. Educating prescribers about the challenges and pitfalls of electronic systems was rarely discussed.

CONCLUSIONS: We identified few articles related to the training of prescribers on CPOE systems. Examples of approaches used include demonstrations, practical exercises and ward-based training, which are likely to appeal to the learning styles of different users. Educating prescribers about the challenges of electronic systems was rarely discussed. Online training methods were reported, and may facilitate the training of larger groups. It is possible that other training methods were employed in practice, but not identified in the small number of articles retrieved. Additional research is therefore needed to inform training approaches and to explore the best methods for training users about the pitfalls and challenges associated with electronic systems.

\section{TRAUMA EXPOSURE AND POSTTRAUMATIC STRESS SYMPTOMS IN WOMEN RECRUITED FROM BALTIMORE CITY HAIR SALONS Allison F.}

Hall; Lycinda Rodriguez; Anika A. Alvanzo. Johns Hopkins University School of Medicine, Baltimore, MD. (Control ID \#2467192)

BACKGROUND: Although the relationships between trauma exposure and posttraumatic stress disorder (PTSD) have been widely studied, many of these studies have been conducted with veteran and clinical samples. Less is known about the prevalence and severity of trauma exposure and PTSD symptoms in community-based samples. This study used data from The PERMSS Project: Promoting Education and Research on Mood, Stress, and Substances to estimate the prevalence of trauma exposure and posttraumatic stress and to examine correlations between these two variables among non-treatmentseeking women recruited from urban hair salons.

METHODS: Participants were recruited from Baltimore area hair salons. Women were eligible to participate if they spoke English and were 21-65 years old. Pregnant, acutely intoxicated, and cognitively impaired women were excluded from the study. Eligible participants completed a computerized survey administered via a tablet computer equipped with voice-enabled software. The survey consisted of demographic questions and items from previously validated instruments, including the Traumatic Life Events Questionnaire (TLEQ) to measure frequency and types of trauma exposure, and the PTSD 
Checklist for DSM-5 (PCL-5) to measure symptom clusters B (re-experiencing), C (numbing), D (avoidance), and $\mathrm{E}$ (hyperarousal and hypervigilance). Because these variables were not normally distributed, we used non-parametric tests to analyze our data. We conducted Kruskal-Wallis $\mathrm{H}$ tests to identify differences between demographic categories, and Spearman's rho correlations to examine associations between measures.

RESULTS: Women $(N=57)$ were $91.2 \%$ Black with a mean age of $41.84(S D=12.97)$. Most had completed at least 2 years of college $(75.5 \%$ ), reported an annual income of over $\$ 35,000(70.2 \%)$, and were employed for wages $(71.9 \%)$. Participants experienced a median of 5.0 trauma types, with a median frequency of 7.0. PCL-5 scores $(M d n=5.0)$ showed minimal symptoms of PTSD. Kruskal-Wallis $\mathrm{H}$ tests revealed no differences in PCL-5 scores across categories of income level, education level, or employment status. Spearman's rho showed positive correlations between the amount of trauma types reported by participants, and cluster $\mathrm{B}, r_{s}(55)=.33, p=.01$, cluster $\mathrm{C}, r_{s}(55)=.26, p=.05$, and cluster $\mathrm{E}, r_{s}(55)=.32$, $p=.02$, symptoms, and between amount of trauma types and total PCL-5 score, $r_{s}(55)=.33$, $p=.02$. We also found positive correlations between trauma exposure frequency and cluster B symptoms, $r_{s}(55)=.37, p=.004$, cluster $\mathrm{C}$ symptoms, $r_{s}(55)=.30, p=.02$, cluster $\mathrm{E}$ symptoms, $r_{s}(55)=.28, p=.04$, and the total PCL-5 score, $r_{s}(55)=.34, p=.01$.

CONCLUSIONS: Women reported significant trauma exposure with minimal symptoms of PTSD. As expected, we found several correlations between trauma exposure and posttraumatic stress. Although we found no demographic differences, our sample was relatively homogenous due to our method of recruitment, and results should be interpreted with caution. Future recommendations include sampling a more representative population to explore relationships between symptoms and socioeconomic status, and using qualitative interviewing to characterize potential risk and protective factors that may have influenced our findings. Financial Support: 5K23AA020316

TRENDS IN BENZODIAZEPINE AND OPIOID ANALGESIC ABUSE AND MORTALITY IN THE UNITED STATES. Susan L. Calcaterra ${ }^{1,2}$; Stevan Severtson ${ }^{1}$; Becki Bucher Bartelson ${ }^{1}$; Gabrielle Bau ${ }^{1}$; Zachary Margolin ${ }^{1}$; Jody Green ${ }^{1}$; Richard Dart ${ }^{1,2} .{ }^{1}$ Denver Health and Hospital Authority, Denver, CO; ${ }^{2}$ University of Colorado, Denver, CO. (Control ID \#2469880)

BACKGROUND: Opioid analgesics are responsible for a large number of unintentional overdose deaths in the United States. Benzodiazepines, misused in combination with opioid analgesics to enhance opioid intoxication, are increasingly involved in overdose deaths. We examined changes over time in the number of abuse and misuse exposures to opioid analgesics, benzodiazepines and the combination of both opioid analgesics and benzodiazepines reported to poison centers. We also examine whether the use of both benzodiazepines and opioid analgesics increased the risk of death relative to cases exposed to opioids alone. METHODS: Data were obtained from the National Poison Data System (NPDS) which collects information from calls to 55 poison centers throughout the United States and its territories. From January 2000 to December 2014, we identified all benzodiazepine and opioid analgesic intentional exposures excluding self-harm reported to the NPDS with documented medical outcomes. Opioids identified included fentanyl, hydrocodone, morphine, hydromorphone, oxymorphone, tramadol and tapentadol. Exposure types were defined as Intentional Abuse, Intentional Misuse or Intentional Unknown. Medical outcomes related to the exposure of opioids, benzodiazepines or both were categorized by their severity including 1) no effect (no symptom development), 2) minor effect (minimally bothersome symptoms), 3) moderate effect (symptoms requiring medical treatment), 4) major effect (life-threatening symptoms) and 5) death. Poisson Regression was used to compare the number of cases from each year relative to the year 2000. A multiple logistic regression model was used to determine whether cases exposed to benzodiazepines and opioid analgesics together had greater odds of death relative to cases exposed to an opioid analgesic alone. We compared exposures to a single opioid analgesic, a single benzodiazepine and the combination of a single opioid and a single benzodiazepine.

RESULTS: From 2000 to 2014, there were 104,297 intentional exposures to benzodiazepines and 67,491 intentional exposures to opioid analgesics with known medical outcomes. Of the 67,491 opioid analgesic exposures, $32.3 \%(n=21,803)$ also involved a benzodiazepine exposure. Exposures for all three groups (opioid analgesics, benzodiazepines, and opioid analgesics plus benzodiazepines) were highest in 2011. Benzodiazepine exposures increased 2.3-fold ( $95 \%$ CI: 2.2 to 2.4, $p<0.001$ ) from 3571 in 2000 to 8262 in 2014. Over the same time period, opioid exposures increased 4.2-fold (95\% CI: 4.0 to 4.5 , $p<0.001$ ) from 1354 in 2000 to 5679 in 2014. Exposures involving both an opioid analgesic and a benzodiazepine increased 4.0 -fold (95\% CI: 3.6 to $4.4, p<0.001)$ from 439 in 2000 to 1744 in 2014. Of the 67,491 opioid analgesic exposures, 801 resulted in a medical outcome of death. Of these deaths, $40.2 \%(n=322)$ involved an exposure to a benzodiazepine in addition to a opioid analgesic. After adjusting for gender and age, there was a $62 \%$ (95\% CI: 19 to $119 \%, p=0.002)$ greater odds of death among cases exposed to both an opioid and a benzodiazepine compared to those exposed to an opioid alone. CONCLUSIONS: While a variety of efforts to curb opioid analgesic abuse and diversion have been implemented with varying degrees of success, less emphasis has been placed on reducing adverse outcomes associated with benzodiazepine use. Similar efforts to curb benzodiazepine abuse and diversion may be warranted, particularly when opioid analgesics and benzodiazepines are prescribed in combination.

TRENDS IN SENIORS' USE OF DIGITAL HEALTH TECHNOLOGY IN THE UNITED STATES, 2011-2014 David M. Levine; Stuart R. Lipsitz; Jeffrey A. Linder. Brigham and Women's Hospital, Boston, MA. (Control ID \#2467920)

BACKGROUND: Seniors 65 and older are the sickest, most expensive, and fastest growing segment of the US population. Seniors account for $13 \%$ of the US population but nearly $35 \%$ of national health care spending. Digital health technology has been touted by industry and incentivized by government as a solution to improve quality, cost, safety, and outcomes. However, little is known about digital health technology use among seniors, particularly among seniors in declining health who may be the most in need of innovative interventions.

METHODS: The National Health and Aging Trends Study (NHATS) is an annuallyadministered, longitudinal nationally-representative sample of community-dwelling Medicare beneficiaries (2011-2014, $n=7,609)$. Each year, NHATS asks about four digital health technology modalities: use of the Internet to refill prescriptions, contact medical providers, address insurance matters, and research health conditions. We analyzed trends over time accounting for the complex survey design of NHATS. We examined independent predictors of digital health technology use at baseline in 2011, adjusting for demographic and medical characteristics with weighted logistic regression. We also used weighted logistic regression to examine whether physical or cognitive decline between 2011 and 2014 was associated with changes in digital health technology use, adjusting for baseline digital health technology use in 2011.

RESULTS: The proportion of seniors who used any digital health technology increased from $21 \%(95 \% \mathrm{CI}$ [confidence interval], $19 \%$ to $23 \%)$ in 2011 to $25 \%(95 \% \mathrm{CI}, 23 \%$ to $27 \%)$ in 2014 ( $\mathrm{p}$ for trend $<0.001$ ). By individual modality, use increased for prescription refills (2011 to $2014,8 \%$ to $10 \%$; p for trend $<0.001$ ), medical provider contact (2011 to $2014,7 \%$ to $12 \%$; p for trend $<0.001$ ), and health condition research (2011 to $2014,16 \%$ to $18 \%$; p for trend $=$ 0.002). Use was unchanged for handling insurance matters online (2011 to $2014,5 \%$ to $6 \%$; p for trend $=0.07$; Figure). The proportion of seniors who used all 4 modalities increased significantly from $2011(1.1 \%$ [95 \% CI, $0.8 \%$ to $1.5 \%$ to $2014(1.8 \%$ [95 \% CI, $1.3 \%$ to $2.4 \%$; ; p for trend $=0.03$ ). From 2011 to $2014,14 \%$ (95 \% CI, $13 \%$ to $15 \%$ ) of seniors increased the number of digital health technology modalities used; $10 \%(95 \% \mathrm{CI}, 9 \%$ to $11 \%)$ 
decreased their use. In 2011, independent predictors of less use of any digital health technology were older age (odds ratio [OR], 0.93 per year [ $95 \%$ CI, 0.92 to 0.94$]$ ), Black race (OR, 0.5 compared to White race [ $95 \% \mathrm{CI}, 0.36$ to 0.68$]$ ), divorce (OR, 0.71 compared to married [ $95 \%$ CI, 0.55 to 0.92$]$ ), and poor health (OR, 0.47 compared to excellent health [95\% CI, 0.22 to 0.998]). Independent predictors of greater use of any digital health technology use included college education (OR, 9.57 compared to $<$ high school [ $95 \%$ CI, 6.22 to 14.71], higher annual income (OR, 2.06 for $>\$ 60,000$ versus $<\$ 15,000$ [95 \% CI, 1.41 to 3.01]), taking medications (OR, 1.94 [95\% CI, 1.32 to 2.85], and more comorbidities (OR, 1.13 per comorbidity [95\% CI, 1.06 to 1.21]). There was no significant association between digital health technology use and gender, depression, activities of daily living, instrumental activities of daily living, grip strength, and number of hospitalizations. From 2011 to 2014, measures of physical and cognitive decline were associated with significantly decreased digital health technology use. Compared to seniors who did not have such changes, seniors who experienced decreases in digital health technology use - a loss of 1 or more digital health technology modalities - were associated with decreased activities of daily living (OR, 0.4 per activity $[95 \% \mathrm{CI}, 0.3$ to 0.5$]$ ), decreased score on the short physical performance battery (OR, 0.7 per point [ $95 \% \mathrm{CI}, 0.6$ to 0.9$]$ ), new-onset dementia (OR, 0.1 [ $95 \% \mathrm{CI}, 0$ to 0.4$]$ ), new-onset depression (OR, 0.4 [ $95 \% \mathrm{CI}, 0.3$ to 0.6$]$ ), and increased hospitalization rate (OR, 0.7 per admission [ $[95 \% \mathrm{CI}, 0.6$ to 0.9$]$ ). All seniors who relocated to a skilled nursing facility had decreased use.

CONCLUSIONS: Digital health technology has been touted as a solution to improving care for seniors, yet seniors used digital health technology at low rates with only modest increases from 2011 to 2014. Low use rates, socioeconomic disparities, and decreased use with declining health are all barriers to effective delivery innovations seeking to leverage digital health technology among seniors

\section{5}

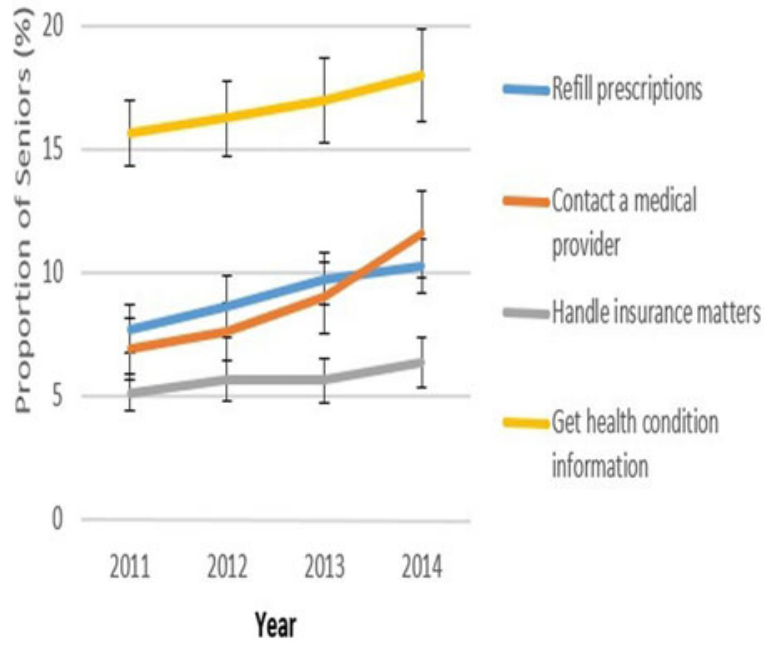

Figure. Changes in United States Seniors' Digital Health Technology Use, 2011-2014. Trends: digitally refill prescriptions ( $\mathrm{p}$ for trend $<0.001$ ), contact a medical provider $(<0.001)$, handle insurance matters $(0.07)$, and get health condition information $(0.002)$ Error bars indicate $95 \%$ confidence intervals of the weighted mean.

TRENDS IN USER RATINGS AND REVIEWS OF A POPULAR YET INACCURATE BLOOD PRESSURE SMARTPHONE APPLICATION Timothy B. Plante ${ }^{2}$; Anna C. O'Kelly ${ }^{3}$; Satish Misra ${ }^{1}$; Bruno Urrea ${ }^{1}$; Seth S. Martin ${ }^{1}$; Geetanjali Chander ${ }^{2}$. ${ }^{1}$ Division of Cardiology, Johns Hopkins University, Baltimore, MD; ${ }^{2}$ Johns Hopkins University, Baltimore, MD; ${ }^{3}$ Johns Hopkins University School of Medicine, Baltimore, MD. (Control ID \#2469742)
BACKGROUND: Instant Blood Pressure (IBP) is a popular mobile health application (mHealth; app) that was downloaded $>148,000$ times at $\$ 4.99$ per download before its removal from app stores in mid-2015. Although the app was removed from stores, it remains functional for users that previously downloaded it. IBP claims to determine blood pressure without a cuff by using only inputs from a smartphone's integrated sensors. We previously found that IBP achieved the lowest possible accuracy grade and misclassified $78 \%$ of hypertensive readings as non-hypertensive. The app and its website present disclaimers that it is for recreational use only and should not replace a standard cuff-based blood pressure machine. Whether these disclaimers are adequate in preventing potentially harmful use of the product is unclear. Users can contribute ratings and reviews of apps to most commercial app stores. Quantitative ratings range from 1 to 5 stars with a higher number indicating a more favorable user impression. Text narrative reviews often accompany ratings and document the experience of current app owners. Ratings and reviews are displayed as part of the app store listing and can help drive app uptake and use. We sought to analyze and describe IBP app store user ratings and reviews in order to better understand app usage, drivers for favorable and unfavorable impressions, and any potential harm that may come from IBP use.

METHODS: All ratings with a corresponding review for IBP were downloaded from the Apple iTunes app store. They were excluded if they were not for the most recent version of the app. Using an iterative process, the reviews were assessed for content themes and a coding scheme for these themes was developed. Additional codes for uncommon themes that were pertinent to our conceptual model for app propagation were also included. This coding scheme was then applied to all reviews. As overall star ratings on app stores are commonly presented as mean values, we calculated them as such in our analysis despite a lack of normal distribution.

RESULTS: Of the 995 ratings with reviews for IBP (June 5, 2014 - July 29, 2015), 263 were for the most recent version (v1.2.3, released May 5, 2015). The mean [SD] star rating for this version was 3.98 [1.50]. Notable review themes included convenience (mentioned in $18 \%$ of the 263 reviews; mean star rating of these was 4.72 [0.50]), ease of use ( $17 \%$; $4.71[0.72])$, inability to successfully produce a blood pressure measurement (10\%; 1.18 [0.78]), lack of Apple HealthKit integration (9\%; 3.68 [0.99]), perceived accuracy (41\%; $4.74[0.72])$, perceived inaccuracy $(10 \% ; 1.88$ [1.18]), acknowledgement of the app's disclaimer (1\%; 3.98 [1.50]), claim of being a medical professional (3\%; 4.28 [1.49]), claim of endorsement of IBP use by a medical professional known to the user ( $4 \% ; 4.9$ [0.32]), and use of the app in managing a blood pressure-related medical condition ( $8 \%$; 4.54 [0.96]). Medical conditions being managed included hypertension, hypertensive urgency, postural orthostatic tachycardia syndrome, end-stage renal disease, and postheart transplant care. Fifty-eight percent of all ratings gave 5 stars and tended to highlight convenience, ease of use, and perceived accuracy. Sixteen percent of reviews gave 1 star and tended to mention inability to successfully produce a blood pressure measurement and perceived inaccuracy.

CONCLUSIONS: User ratings and reviews for a popular, inaccurate blood pressure app demonstrate several thematic trends. The app's disclaimers were ineffective as users with serious blood pressure-related medical conditions reported using the app in their care, not just for recreational use. Furthermore, the app was highly rated in part because of its ease of use and convenience, which may drive its use as a replacement for a bulky standard device. Among patients who commented on app accuracy, a majority reported that the app was accurate despite the poor performance demonstrated in a clinical trial. Because of these findings, we think that non-validated mHealth apps with similar functionality to regulated medical devices should not be available to consumers, regardless of the disclaimers provided. For these types of apps, developers should partner with academic institutions in order to rigorously evaluate the quality of their product prior to market introduction. Finally, while it is not possible to verify if users truly were medical professionals, it is likely that at least some well-intentioned colleagues are using and promoting potentially harmful, non-validated mHealth apps. As such, professional 
organizations should educate their members about appropriate selection and utilization of mHealth apps so that they can provide the necessary counseling to their patients.

\section{UNDERSTANDING FACTORS ASSOCIATED WITH COLORECTAL CANCER} SCREENING IN THE LAO COMMUNITY IN MINNESOTA Layne Anderson ${ }^{1}$; Sunny Chanthanouvong ${ }^{2}$; Hee Yun Lee ${ }^{1}$; Elizabeth A. Rogers ${ }^{1}$; Namthip Saengsudaham ${ }^{2}$; Vilamone Tran ${ }^{2}$; Lei Zhang ${ }^{1} .{ }^{1}$ University of Minnesota, Minneapolis, MN; ${ }^{2}$ Lao Assistance Center of Minnesota, Minneapolis, MN. (Control ID \#2467709)

BACKGROUND: Colorectal Cancer (CRC) is the 2nd and 3rd most common cancer in Lao-American women and men, respectively, and is the most rapidly rising cancer found in Laotians in the U.S. CRC screening could help detect these cancers early and offer better outcomes, but CRC screening rates are significantly lower among Asian Americans than among whites and blacks. Minnesota (MN) is home to a large population of immigrants from Laos as well as one of the largest disparities in cancer screening and prevention. Several factors have been identified as barriers to CRC screening in Asian American subgroups, but the specifics of whether any is at play within the Lao-American community has not been investigated. Through a community-university partnership, we used the Anderson Model of Health Care Utilization as a framework to investigate the rates of CRC screening in the Lao-American community in MN, and to understand how predisposing characteristics, enabling resources, and perceived need were associated with screening. METHODS: We conducted a cross-sectional survey study using a non-random sample. The survey was in both English and Lao and was composed of validated measures of health literacy, self-efficacy in screening, fatalism, and family support in addition to demographics and other socioeconomic status questions. It was edited for face validity after review by Lao Assistance Center of MN (LACM) staff and 2 additional Lao speakers. Trained staff from LACM recruited 50-75-year-old men and women (recommended screening age) who spoke Lao or English. Participants completed paper surveys with support from the trained LACM staff, and responses were entered into a database. We used descriptive statistics to calculate counts and proportions, and step-wise multivariate logistic regression to identify factors associated with ever being screened.

RESULTS: One hundred eighteen participants completed surveys. Of these, only $45 \%$ reported ever receiving CRC screening. Those who had ever been screened were older (62.7 vs 59.2 years), but otherwise did not differ from those never screened by other predisposing factors (gender, years in the U.S., education, fatalism). They also did not differ by certain enabling resources (employment, income, insurance coverage, marital status, English fluency, health literacy, and family support) or by perceived need (family history of cancer). However, in univariate regression, some enabling resources (having a primary care provider, higher self-efficacy in pursuing screening) and perceived needs (knowledge that those 50 and older should be screened, higher number of chronic illnesses (average 2.6 vs 0.9 , respectively)) were associated with ever being screened. When added together in a multivariate logistic regression model, having a primary provider and higher self-efficacy were associated with screening independent of other factors. However, after adjusting for number of chronic illnesses, only primary care provider was still significant such that the odds of every being screened for CRC was 12.4 times higher for those with a primary care provider compared to those without $(p=0.045)$.

CONCLUSIONS: CRC screening rates among Lao-Americans in MN are low. Similar to other populations, having a primary care provider is important for successful completion of CRC screening. A limitation of this study is the small sample size which could mask some additional important differences between groups. Given the significant findings despite the small sample, our next steps will focus on intervention development that will include connecting community members with primary care practices as well as on education about the need for CRC screening and resources available to pursue it with the aim of promoting screening self-efficacy.

\section{UNDERSTANDING KIDNEY HEALTH AND CHRONIC KIDNEY DISEASE} (CKD) KNOWLEDGE AMONG AMERICAN INDIANS: A FOCUS GROUP STUDY Stacey E. Jolly ${ }^{1}$; Rodney C. Haring ${ }^{2}$; Marcia O'Leary ${ }^{3}$; Ashwini Sehgal ${ }^{4}$; Spero Manson ${ }^{5}$; Cheryl Killion ${ }^{4}{ }^{1}$ Cleveland Clinic, Cleveland, OH; ${ }^{2}$ Roswell Park Cancer Institute, Buffalo, NY; ${ }^{3}$ Missouri Breaks Ind Research Inc, Eagle Butte, SD; ${ }^{4}$ Case Western Reserve University, Cleveland, OH; ${ }^{5}$ University of Colorado Denver, Aurora, CO. (Control ID \#2466284)

BACKGROUND: End Stage Renal Disease (ESRD) with the need for dialysis or transplant disproportionately affects many American Indian communities. Chronic Kidney Disease (CKD) is the precursor to ESRD and people are often unaware of having CKD or its risk factors. There is limited data about the knowledge and awareness of CKD among American Indians; culturally appropriate CKD educational interventions are even rarer for this population. The aim of this study was to elicit the perspectives of participants with CKD risk factors or CKD about their disease, with a specific focus on knowledge/ awareness and their information needs related to CKD and patient education.

METHODS: Strong Heart Study and/or the Strong Heart Family Study participants were invited to contribute to talking circles (focus groups). A moderator guide with open-ended statements or questions to assess CKD knowledge/awareness and perceived information needs related to patient education was generated (Table 1); probes were used if needed to encourage discussion. The talking circles were separated by gender. Five male talking circles (19 males total) and four female talking circles (26 females total) each lasting 1 to $1 \frac{1}{1 / 2}$ hours were conducted in two American Indian communities in fall 2012. The talking circles were audio recorded and transcribed. Two independent and trained abstractors coded the talking circle (focus group) transcripts and identified major themes and subthemes.

RESULTS: From the 9 talking circles (focus groups) which included 35 participants, ranging in age from 20's to elders in their 80's, six major themes were identified: Information Seeking, Co-occurring conditions, Health Lifestyles Management, Medication, Genetics and Heredity, and Barriers to Health. Subthemes were identified and classified with the major themes and were present among both gender groups except for the subtheme want knowledge shared was only from the female transcripts. Participant quotes exemplify the themes/subthemes: "I would like to know how long does the kidney take before you know you have a bad kidney?" Information Seeking (kidney health knowledge gaps; desire for more knowledge about kidney function, kidney testing, kidney disease symptoms; trustworthy sources to get medical information), "I know about kidneys is that you need to keep your blood sugar at a low level. Because I know that diabetes attacks the organs and kidneys." Co-occurring conditions (kidney health along with other chronic conditions; awareness of risk factors for ESRD due it affecting someone in their family), "... eating the right foods one thing. Not so much carbohydrates, but more grains, and a little meat on the side of it and a lot of liquid. Those are the priority things I think, they should be. I know I swung into it, that what I'm saying. It helps. I eat a lot of oatmeal." Health Lifestyles Management (nutrition/food; physical activity/exercise), “... the hospital up here, instead of finding out, when something ails you, you go up there, instead of taking care of the thing that you went up there for, they give you all these pills, that 9 times of 10 , they don't tell people what the medicine is for, or how to take it, or how to space it; they take it home and look at, and what am I going to do with this all? And 9 times out of 10 they throw it away, because they don't know how to take it." Medication (pills; medication burden), "I don't know if his dad drank a lot or when he was younger if that was why he needed a kidney. He was one of the younger boys that gave a kidney to his dad; I know that he gets sick a lot too. I don't know what's going on with that. My oldest granddaughter lives out in the east. I don't know what is wrong with her kidney today, but she keeps landing in the hospital and she's just now 21. I 
don't know if that was hereditary or how it happened." Genetics and Heredity (importance of family history; want knowledge shared), "We have such a nice hospital but they get a good doctor, and he gets burned out and goes away because too much, too many people can't take care of them all." "You never see the same doctor twice." Barriers to Health (mistrust/ incongruent health beliefs; lack of continuity)

CONCLUSIONS: Participants identified a strong desire for further information about kidney health and CKD and its relationship with other common conditions and what nonpharmacologic or pharmacologic means can be tried to help slow the burden. Additionally keen awareness of family and how kidney health and CKD knowledge needs to be obtained from family and shared with family to help future generations. Novel educational interventions that address these factors may improve patients' knowledge and awareness of kidney health and $\mathrm{CKD}$, which in turn might help reduce the burden of kidney disease among this population.

Talking Circle (Focus Group) Moderate Guide

UNDERSTANDING MEDICAL STUDENTS' PERCEPTIONS OF MEDICAL PROFESSIONALISM AT DIFFERENT STAGES OF MEDICAL EDUCATION Danielle Reimer ${ }^{1}$; Ross Russell ${ }^{1}$; Christine Bellew ${ }^{1}$; Caridad Hernandez ${ }^{1}$; Juan Cendan ${ }^{1}$, ${ }^{2}$; Analia Castiglioni ${ }^{1,2}$. ${ }^{1}$ University of Central Florida College of Medicine, Orlando, FL; ${ }^{2}$ Orlando VA Medical Center, Orlando, FL. (Control ID \#2469607)

BACKGROUND: Medical professionalism instruction and assessment is an essential component of the medical education curriculum as professionalism is a core competency for medical students, residents and practicing physicians. Medical professionalism and associated behavior sets have been well-defined by the AAMC Professionalism Task Force. University of Central Florida College of Medicine has an integrated longitudinal curriculum that incorporates formal professionalism instruction and assessment across the continuum of medical education. The objectives of this study were to have first and second year medical students identify and cognitively organize attributes of medical professionalism in order to gain an understanding of medical students perceptions of medical professionalism at different stages of medical education. This can guide educators and curriculum advisors in assessing the effectiveness of the current medical professionalism instruction, and allow for identification of areas that could be modified or improved. METHODS: Nominal Group Technique (NGT) is a multi-step, structured group process used to elicit and prioritize answers to a specific question. We convened two NGT sessions for first year students (M1) and two sessions for second year students (M2) from UCF College of Medicine and elicited their perceptions on medical professionalism. Each group was asked the following prompt: "What is medical professionalism? Think broadly about factors which define professionalism and also factors which affect physicians' (and physicians in training) professional behavior." Participants were then asked to prioritize proposed factors with respect to their importance. Through a formal process, a group of investigators combined the generated lists of attributes from each pair of M1 and M2 NGT sessions. Similar statements were combined and repetitive statements were eliminated, resulting in one reliable and representative list of medical professionalism attributes for each class. M1 and M2 students performed an unforced card sorting task, grouping the attributes identified in the NGT sessions, based on their perceived similarity or dissimilarity. A principle component analysis (PCA) was conducted for each class

using the card sorting data. The investigators examined the factor solutions for each class, discussed possible themes and reached a consensus on a label for each factor. The labeled factors were then compared between classes and to the AAMC's defined behaviors of medical professionalism.

RESULTS: M1 students $(n=20)$ identified 51 items that contribute to medical professionalism and M2 students $(n=20)$ identified 66 items during the NGT sessions. Two principle component analyses (PCAs) were conducted using the card sorting data, separately for M1 and M2 students; for both analyses an Eigen value $>1$ was used. The PCA for M1 students derived a 7 factor solution; factor loading ranging from (.55 to .759$)$. Factors identified were: 1$)$ Self-management and Patient-centeredness, 2) Ethics and Professional reputation, 3) Dependability, 4) Selfawareness and Self-improvement, 5) Image, 6) Proficiency and 7) Lifelong learning and Integrity. The PCA for M2 students derived a 5 factor solution; factor loading ranging from (.561-.684). Factors identified were: 1) "Good Doctor" attributes, 2) Responsibility, 3) Ethics, 4) Innovation and Self-improvement and Unbiased.

CONCLUSIONS: Medical students' perceptions of medical professionalism differ from the first to second year of medical education. Both classes of students identified domains focused on the intrinsic qualities of physicians; however, second year students were able to consolidate these intrinsic professionalism attributes into a singular domain representing the personal qualities of the ideal "good doctor." Other core professionalism domains were identified by both classes of students; however their perception of medical professionalism appears to evolve throughout the first year of medical education.Overall, first year students identified domains narrower in scope, which may be consistent with a more societal view of medical professionalism. In contrast, second year students were able to identify a wider range of domains representing a more comprehensive understanding of the fundamental principles of medical professionalism as well as the role of a physician

UNDERSTANDING NEEDS AND ENGAGING COMMUNITY PARTNERS TO DEVELOP A HOSPITAL-BASED IMPROVING ADDICTION CARE TEAM (IMPACT) Honora Englander ${ }^{2}, 4$; Christine Velez Klug ${ }^{3}$; Rachel Solotaroff ${ }^{4}$; Melissa B. Weimer ${ }^{1,5}$; Alison Noice $^{5}$; Tim Hartnett ${ }^{5}$ : Ed Blackburn ${ }^{4}$; Christina Nicolaidis ${ }^{3},{ }^{1}$; Pen Barnes ${ }^{6}$; Sarann Bielavitz; Benjamin Chan ${ }^{1}$; Catherine Michalczyk ${ }^{7}$; Philip T. Korthuis ${ }^{1}{ }^{1}$ Oregon Health \& Science University, Portland, OR; ${ }^{2}$ Oregon Health \& Science University, Portand, OR; ${ }^{3}$ Portland State University, Portland, OR; ${ }^{4}$ Central City Concern, Portland, OR; ${ }^{5} \mathrm{CODA}$ Inc., Portland, OR; ${ }^{6}$ OHSU, Portland, OR; ${ }^{7}$ Coram Infusion Services, Portland, OR. (Control ID \#2469486)

BACKGROUND: Addiction is a growing national epidemic that represents a pressing need and significant burden to the healthcare system. People with substance use disorders (SUD) have high rates of hospitalization and readmission, often for medical complications of SUD. Despite this, most receive no addiction care during or after hospitalization. Models for hospital-based and post-discharge SUD care for medically complex patients are limited and face tremendous implementation barriers. We aimed to understand the needs of hospitalized adults with SUD and use findings to engage community partners to develop regional SUD improvements. METHODS: We conducted a survey of consecutive hospitalized adults at a large academic medical center who reported high-risk alcohol or any drug use (per the AUDIT-C and a single item drug screener). We excluded people reporting only marijuana and/or tobacco. We assessed social and demographic factors, healthcare utilization, substance use severity, and experience and interest in treatment for those with moderate to high-risk use on the ASSIST. We then convened a task force of leaders and front line staff from hospital, community health, and addiction agencies to develop regional improvements in SUD care. Our task force met over a series of three large group and many small group meetings to develop the Improving Addiction Care Team (IMPACT) intervention.

RESULTS: We enrolled 185 participants from September 2014 to April 2015. Participants had a mean age of 44 , were predominantly male (73\%), and identified as white (78 \%), Native American (7\%) or black (3\%). Fifty-two percent reported homelessness and $48 \%$ had at least 
some college. Seventy-three percent had Medicaid, $13 \%$ Medicare, and one $(0.5 \%)$ was uninsured. Participants had moderate or high risk alcohol (44\%), methamphetamine (40\%), and opioid (39\%) SUD. Fifty-four percent reported hospitalization, $63 \%$ ED use in the prior 6 months. Among those reporting substance use in the last 3 months, $58 \%$ of alcohol users and $67 \%$ of drug users reported interest in cutting back or quitting. Among out-of-treatment opioid users, $44.7 \%$ reported high interest in medication-assisted treatment (MAT). Participants especially those with endocarditis and osteomyelitis - had longer than expected hospital lengthsof-stay (Figure). Our task force used needs assessment findings and evidence from the literature to inform program development. Our diverse team brought unique perspectives and innovative solutions. Because many participants wanted to cut back or quit and expressed strong interest in MAT; and because community partners emphasized peers as key to engagement, our inpatient consult team includes an addiction-trained physician, social worker, and peers. Because gap time limits access to addiction care after discharge and because patients struggle to navigate a complex system, community partners suggested bringing their staff into the hospital to do intakes and facilitate rapid-access paths to care. And, learning that some patients stay in hospital for weeks to receive intravenous (IV) antibiotics for social - not medical - risk without addiction treatment, community partners suggested delivering IV antibiotics in a residential addiction setting. To do this, they would need support of infectious disease specialists, more highly trained nurses, funding for daily infusion therapy, and added support to for MAT in residential. Thus, we contracted with an infusion pharmacy, secured funding for daily infusions, and staffed our model to support complex care needs. Collectively, we developed IMPACT that includes: 1) a hospital-based addiction consult team comprised of a physician, addiction social worker, and peer mentors; 2) rapid-access pathways to community addiction care; and 3) community-based residential addiction care with integrated IV antibiotic capacity. We used utilization data to support a business case and secured hospital and Managed Medicaid Care funding. Since July 2015, we have served over 100 patients, and referred over $65 \%$ to community addiction care.

CONCLUSIONS: Our findings support that hospitalization may be a reachable moment to initiate and coordinate addiction care across a care continuum. Community and hospital partners can develop innovative care models that integrate care across hospital and community, physical and behavioral health settings. Future studies explore feasibility, acceptability, and effect on SUD, physical health, and utilization outcomes. Our experience may be relevant to other health systems facing similar challenges

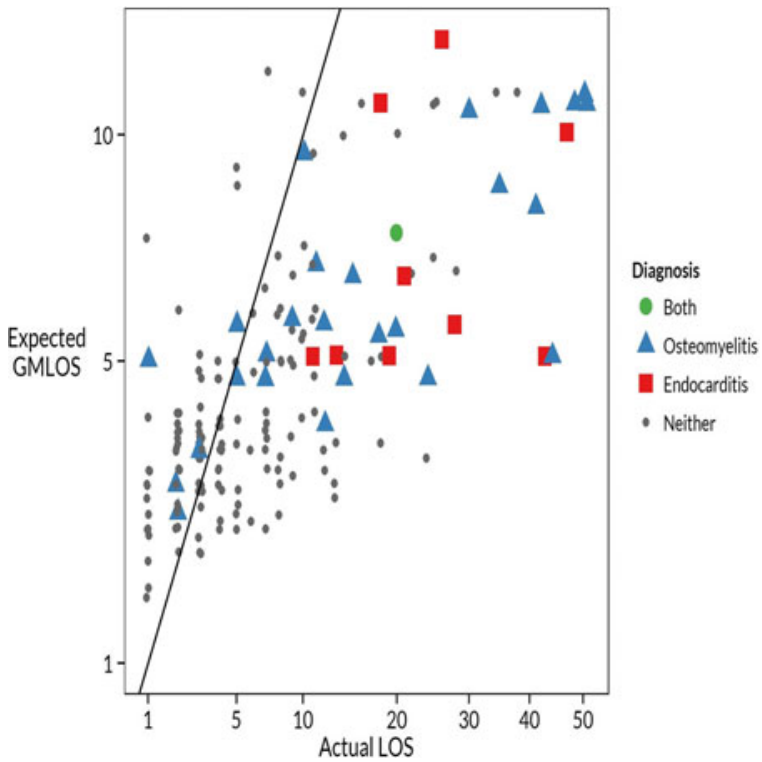

Plot compares actual vs expected hospital length of stay (LOS) in days on a logarithmic scale. The line of identity represents values where actual LOS equals expected. Diagnoses include: $\triangle$ osteomyelitis (blue), $\mathbf{n}$ endocarditis (red), $\mathrm{O}$ both (green), and neither $\bullet$ (gray)
UNDERSTANDING PREDICTORS OF REACHING SERUM URIC ACID (SUA) GOAL IN FEBUXOSTAT UTILIZERS Richard Sheer ${ }^{2}$; Keith Szymanski ${ }^{1}$; Kyle Null $^{1}$; Lavanya Sudharshan ${ }^{2}$; Margaret K. Pasquale ${ }^{2}$. ${ }^{1}$ Takeda Pharmaceuticals USA, Inc., Deerfield, IL; ${ }^{2}$ Comprehensive Health Insights, Inc., Humana Inc., Louisville, KY. (Control ID \#2467277)

BACKGROUND: Clinical guidelines recommend allopurinol or febuxostat as first-line pharmacologic urate-lowering therapy for gout patients to achieve a sUA goal of $<6 \mathrm{mg} /$ dL.(1) Previous studies have established febuxostat's effectiveness $(2,3,4)$ in achieving sUA goal, but further evidence is needed to determine the predictors of attaining sUA goal among febuxostat users. The study objective was to identify clinical characteristics of febuxostat utilizers in order to develop and validate a predictive model for achieving a goal of sUA $<6 \mathrm{mg} / \mathrm{dL}$

METHODS: This was a retrospective database analysis with a cohort design using the Humana Research Database (Feb 1, 2008 - Dec 31, 2014). The study sample consisted of commercial and Medicare members newly initiated on febuxostat (index date Feb 1, 2009 - Dec 31, 2013), with continuous enrollment 12 months pre- and post-index date, $\geq 1$ medical claim with a gout diagnosis, $\geq 1$ baseline sUA test (on or before index date), and a valid sUA test $\geq 120$ days from the index date. Descriptive statistics were used to compare baseline demographic and clinical characteristics between those that did and did not achieve sUA goal. Variables with $P<0.2$ and other key metrics were included in the predictive model. Four variations of the base model were run to finalize the input variables. Stepwise logistic regression with backward elimination, using $P<0.2$ for retention in the model, was employed to model sUA goal attainment. Sensitivity analyses included using a shorter pre-index period (180 days), using sUA goal of $<5 \mathrm{mg} / \mathrm{dL}$ as a cutpoint in the logistic model, and estimating a linear regression model with sUA as a continuous variable.

RESULTS: The eligible study sample $(n=678)$ was divided into a development (training) dataset $(n=453)$ and a validation (holdout) dataset $(n=225)$. The mean $\pm \mathrm{SD}$ age of the study sample ranged from $67.0 \pm 12.3$ years in the validation sample to $69.3 \pm$ 11.4 years in training sample. The majority of members were male ( $>60 \%)$, in the $65-$ 74 years age group $(-40 \%)$, white $(>55 \%)$, and resided in the southern region of the US $(\sim 80 \%)$. Members in the sUA $<6 \mathrm{mg} / \mathrm{dL}$ group were on febuxostat longer $(169 \pm 69 \mathrm{vs}$ $129 \pm 73$ days, $P<0.0001$ in training sample), were more adherent $(0.82 \pm 0.20$ vs $0.62 \pm$ 0.31 proportion of days covered, $P<0.0001$ in training sample), and had a lower average baseline sUA level $(8.0 \pm 2.3$ vs $9.0 \pm 2.4 \mathrm{mg} / \mathrm{dL}, P<0.0001$ in training sample) vs members in the sUA $\geq 6 \mathrm{mg} / \mathrm{dL}$ group. Comorbidities were comparable between the sUA $<6$ and $\geq 6 \mathrm{mg} / \mathrm{dL}$ groups (top 3 were hypertension [90.2 vs $93.2 \%, P=0.1599$ ], hyperlipidemia [ 82.3 vs $82.0 \%, P=0.9146$ ], and diabetes [ 43.8 vs $50.3 \%, P=0.0908$ ]). In the logistic model, febuxostat adherence increased the odds of attaining sUA goal $<6 \mathrm{mg} / \mathrm{dL}(\mathrm{OR}=1.03,95 \%$ CI: 1.02-1.04, $P<0.0001)$, and higher baseline sUA was a strong predictor of not achieving sUA goal $(\mathrm{OR}=0.84 ; 95 \% \mathrm{CI}$ : $0.77-0.92, P<0.0001)$. In the linear regression model, increase in febuxostat adherence $(P<0.0001)$ and advanced age $(P<0.05)$ were associated with reduction of sUA level; baseline sUA level $(P<0.0001)$ and congestive heart failure $(P<0.05)$ were associated with increase in sUA level. Allopurinol use in the pre-index period was retained in both models but was not as strong a predictor of attaining sUA goal $(P=0.13$ and 0.16 in the logistic and linear models, respectively). Using these results, an interactive tool was created for both models, wherein values are entered for each variable (febuxostat adherence, baseline sUA, allopurinol use, age [linear model], CHF [linear model]), and the probability of attaining sUA goal or the sUA level is calculated.

CONCLUSIONS: Among febuxostat users diagnosed with gout in a real world setting, adherence to febuxostat and baseline sUA level were the primary predictors of attaining sUA goal. These findings along with consideration of febuxostat dose may help clinicians identify appropriate patients most likely to benefit from febuxostat treatment, and 
underscore the importance of medication adherence in this challenging patient population. References: 1. Khanna D, Fitzgerald JD, Khanna PP, et al. 2012 American College of Rheumatology guidelines for management of gout. Part 1: systematic nonpharmacologic and pharmacologic therapeutic approaches to hyperuricemia. Arthritis Care Res (Hoboken). 2012;64(10):1431-1446. 2. Hatoum H, Khanna D, Lin SJ, Akhras KS, Shiozawa A, Khanna P. Achieving serum urate goal: a comparative effectiveness study between allopurinol and febuxostat. Postgrad Med. 2014;126(2):65-75. 3. Kim SC, Schmidt BM, Franklin JM, Liu J, Solomon DH, Schneeweiss S. Clinical and health care use characteristics of patients newly starting allopurinol, febuxostat, and colchicine for the treatment of gout. Arthritis Care Res (Hoboken). 2013;65(12):2008-2014. 4. Singh JA, Akhras KS, Shiozawa A. Comparative effectiveness of urate lowering with febuxostat versus allopurinol in gout: analyses from large U.S. managed care cohort. Arthritis Res Ther. 2015;17(1):120

UNDERSTANDING THE "WHY AND HOW" OF INTEGRATING COMMUNITY HEALTH WORKERS INTO PRIMARY CARE TEAMS Kathleen T. Call ${ }^{1}$; Joan Cleary $^{2}$; Eileen M. Harwood ${ }^{1}$; Sarah T. Manser ${ }^{1}$; Elizabeth A. Rogers ${ }^{1} .{ }^{1}$ University of Minnesota, Minneapolis, MN; ${ }^{2}$ Minnesota Community Health Worker Alliance, Minneapolis, MN. (Control ID \#2467760)

BACKGROUND: Health care reform has cultivated innovative care models in addressing health disparities in chronic disease management through the redesign of primary care delivery teams - called health care homes $(\mathrm{HCH})$ in Minnesota $(\mathrm{MN})$. While evidence supports the value of the newly emerging Community Health Worker (CHW) workforce in supporting patients in chronic disease self-management, in particular with populations experiencing health disparities, less than $10 \%$ of $\mathrm{HCHs}$ in $\mathrm{MN}$ have incorporated CHWs into their redesigned care teams. Little is known about the factors that impact decisions about integrating CHWs and how they are then incorporated into the structure and function of the care team. Our study aimed to: 1) identify the barriers and facilitators to integrating $\mathrm{CHW}$ in $\mathrm{HCHs}$ in $\mathrm{MN}$, and 2) define the roles played by $\mathrm{CHW}$ within $\mathrm{HCH}$ teams that employ them

METHODS: We conducted a qualitative interview study of care team key informants and clinic leaders $(n=51)$ within certified HCHs in MN. We purposefully sampled $\mathrm{HCH}$ whose patient populations included underserved, low socioeconomic and racial/ethnic minority patients with chronic health conditions. Among the recruited HCHs $(n=9), 5$ employed CHWs and 4 did not. We conducted individual, semi-structured interviews at each clinic with representatives from clinic leaders, clinicians, care coordinators, and CHWs. Respondents described decisions pertaining to team composition, patient care and team experiences, process of incorporating CHWs into the team, and roles CHWs played. Using general inductive qualitative analysis methods, we identified themes pertaining to decision-making, $\mathrm{CHW}$ integration, and $\mathrm{CHW}$ roles.

RESULTS: Participating HCH clinics were located in both rural and metropolitan areas of MN. Seven of the HCHs were embedded within a larger health system and two were independent, Federally Qualified Health Centers. Barriers to CHW integration included: 1) a lack of awareness of the CHW role, 2) perception that the current care team model is sufficient and further innovation is unnecessary, 3) concerns about supervision capacity and structure, and 4) perception of "risk" in hiring a new workforce role. Facilitators included: 1) a clinician champion for CHWs, 2) perception of diversity and psychosocial needs of the patient population, and 3) grant funding to trial the $\mathrm{CHW}$ role. Across clinics, we found high variability in team structure and member roles. CHW roles universally included patient outreach, health education and coaching, community resource linkage, and system navigation. Some $\mathrm{CHWs}$ also served in a $\mathrm{HCH}$ care coordinator role, performed home-based support, provided community health promotion, served as a community cultural liaison, and provided language-specific coordination. In clinics without CHWs, some of these functions were performed by other members of the care team, and respondents identified potential for the CHW role, particularly in the areas of community and cultural liaison and to meet home visit needs.

CONCLUSIONS: In primary care HCHs, a trial period was critical for embracing innovation and embedding the CHW role. Perceptions about new hires and supervision often rely on previous experience and perceived benefits of workforce innovation. CHWs play important roles as part of the care team. Most team members were enthusiastic about the role once the CHW was an established member of the team, and a few clinics had already expanded the role with new hires. With the increasing focus on population health in health care, new models of chronic disease care that are high-value and mitigate disparities will be essential. Clinics seeking innovative models can anticipate barriers and plan for facilitators when incorporating CHWs into their primary care teams. Our findings highlight salient issues around the integration of CHWs into the care team for consideration by health system and clinic leadership.

UNDERSTANDING THE HEALTH NEEDS TO BETTER SERVE THE GUATEMALAN-MAYA COMMUNITY OF LAKE WORTH, FLORIDA Jennifer Shiroky; Eunsung Elisa Kim; Daniel O'Shea; Lukas Gaffney; Erin Kobetz; Julia Belkowitz. University of Miami, Boca Raton, FL. (Control ID \#2469390)

BACKGROUND: The Guatemalan-Maya community is a unique, medically underserved community located in Lake Worth, Florida. Much of the population is eligible for some level of healthcare coverage through county-based health insurance programs for uninsured residents and/or via Medicaid or Florida Kid Care (state CHIP program) for qualifying adults and children. The undocumented, uninsured individuals can seek services at clinics sustained via county taxes. Despite having access, insured families do not utilize the services that are available to them. The remainder of the population does not have access to formal healthcare. Our research aims to understand the gaps in health literacy and access to healthcare for members of the Guatemalan-Maya community in Lake Worth, Florida. The ultimate goal is to develop programming that supports the needs of the community. The research goals and design have been developed in collaboration with key stakeholders and leaders in the community using the model of Community Based Participatory Research. The data will help guide the development of a culturally sensitive and appropriate health program that focuses on improving the interface between the Guatemalan-Maya community and the healthcare system, including health literacy, disease prevention and management, and access to care.

METHODS: Our research is being conducted under the tenets of Community Based Participatory Research (CBPR). CBPR methodology emphasizes conducting research that is oriented to benefit the participating community. CBPR aims to involve the community throughout every step of the research process, from development of research goals and designs to reflecting on and utilizing the results and conclusions to advance the community. Over the past year and a half students from the University of Miami have spent time at the Guatemalan-Maya Center, an integral community center, in order to develop a relationship with community leaders and members. In September 2015 we approached the Center to discuss potentially developing a health-focused project for the community. Informal interviews and informal surveys with community members and leaders were conducted in order to identify health conditions and topics community members view as most significantly affecting their community. Key stakeholders and future partners within the community were identified during this process.

RESULTS: Participation in and contributions to community-run activities and events helped students develop rapport with community members. Students were able to gain a unique, sincere impression of the values, needs, and goals of the community. Consistent attendance to and voluntary support of community happenings nurtured a trusting collaboration between the students and the Guatemalan-Maya Center, a respected and highly- 
utilized resource to the community. This allowed the Center and its community to feel comfortable with collaborating with students on future community-based interventions. The Guatemalan-Maya Center introduced us to their community partners to increase the area available for research development and programming. The rapid assessment survey was developed and administered with the assistance of community partners. The survey results and discussions with community member and leaders identified diabetes, and correspondingly obesity, diet, and exercise as health issues of great significance within the community. The surveys also suggested that community members would be receptive to educational interventions that aim to improve members' understandings of health conditions and issues that impact their community.

CONCLUSIONS: This project continues to utilize community input and collaboration throughout every step of the research process in order to gain an understanding of what the community identifies as health issues and challenges and what they believe would be the most effective ways to address them. Working together with the community fosters a trusting relationship that allows for increased creativity, flexibility, support, and appropriateness of research goals. Shared goals allow for future physicians to gain a better understanding of the lived realities of the communities they serve and to contribute to improving the health profiles of these communities in ways that maximize the benefits to the community from their research. Based on the findings and experiences gained through our relationship with the Guatemalan-Maya community thus far, we are currently developing in-depth focus groups to understand the perceptions of the community and gaps to preventing, managing, and obtaining health services in relation to diabetes. The findings of the study will then be utilized to develop a community guided and appropriate health intervention.

\section{UNDERSTANDING THE IMPACT OF TREATING LATENT TUBERCULOSIS} INFECTION IN NEW YORK CITY: A DYNAMIC MODEL Anthony T. Fojo'; Natalie Stennis ${ }^{2}$; Andrew Azman ${ }^{3}$; Shama Ahuja ${ }^{2}$; David Dowdy ${ }^{3}$. ${ }^{1}$ Johns Hopkins University School of Medicine, Baltimore, MD; ${ }^{2}$ New York City Department of Health and Mental Hygiene, New York, NY; ${ }^{3}$ Johns Hopkins Bloomberg School of Public Health, Baltimore, MD. (Control ID \#2469660)

BACKGROUND: Screening and treatment for latent tuberculosis infection (LTBI) is a key component of the U.S.'s strategy to eliminate TB, and is the focus of an upcoming recommendation from the U.S. Preventive Services Task Force. New York City has seen a steady, gradual decline in the incidence of active TB over the past decade, but the future trajectory of this decline remains uncertain, as does the potential impact of targeted testing and treatment for LTBI in high-prevalence populations such as foreign-born residents. METHODS: We constructed a dynamic transmission model of TB in New York City stratified by age and place of birth (inside or outside the U.S.). We calibrated the model using detailed epidemiological data from the New York City Department of Health and Mental Hygiene. Our primary outcome was the estimated incidence of active TB among U.S.-born and foreign-born New York City residents from 2015 to 2025, under different assumptions for rates of transmission (per person-year of active TB) and reactivation (per person-year of LTBI). We assessed the relative fit of the model to reported incidence using a likelihood ratio test. We then performed a second analysis to estimate the proportion of future TB incidence that was potentially avertable, if all foreign-born residents of New York City were to undergo screening and (when indicated) treatment for LTBI during the decade 2015-2025.

RESULTS: Under the assumptions of this model, demographic shifts plus ongoing declines in TB transmission were much more likely to fit existing data than demographic shifts alone, or demographic shifts plus declines in reactivation (likelihood ratio $>10^{9}$, $p<0.001$ for both comparisons). With no additional intervention, the pace of decline in TB incidence was projected to slow from $2.5 \%$ per year on average in 2005-2015 to
$1.3 \%$ per year in 2015-2025 under the declining transmission scenario (and to $0.8 \%$ per year under the less-likely declining reactivation scenario). Regardless of assumptions regarding transmission versus reactivation, the vast majority of TB incidence from 2015 to 2025 was potentially avertable through targeted testing and treatment of LTBI among the foreign-born population (66\% under declining transmission, versus $64 \%$ under declining reactivation)

CONCLUSIONS: Ongoing declines in transmission have likely played a major role in the downward trend of incident TB in New York City from 2005 to 2015 but have little room for further improvement. As a result, the pace of decline in TB incidence is likely to slow substantially in the next decade without further investment of resources to prevent reactivation among people with LTBI.

\section{UNDERSTANDING THE MIND-BODY CONNECTION: STATEWIDE ASSESS-} MENT OF PRIMARY CARE AND BEHAVIORAL HEALTH INTEGRATION Theodore Long ${ }^{2}{ }^{1}$; Shayla N. Durfey ${ }^{3}$; Sandra Powell ${ }^{2}$; Jeffrey Borkan ${ }^{3}$; Nicole Alexander-Scott ${ }^{2} .{ }^{1}$ Yale University School of Medicine, Providence, RI; ${ }^{2}$ Rhode Island Department of Health, Providence, RI; ${ }^{3}$ Brown University School of Medicine, Providence, RI. (Control ID \#2469565)

BACKGROUND: Thirty percent of US adults in any 12-month period are classified as having a mental health disorder. While the majority of behavioral health management is provided in the primary care setting, evidence suggests that these patients receive the highest quality care when primary and behavioral healthcare services are integrated. Integrating behavioral healthcare with primary care practices has been shown to improve the quality, outcomes, and cost of care. However, there is limited data regarding the extent of integration currently happening. We completed a statewide evaluation of the current level and characteristics of integrated practices in Rhode Island as a baseline for improvement and health policy development.

METHODS: The Rhode Island Department of Health 2015 Statewide Health Inventory Behavioral Health Survey was developed through an iterative process with stakeholder feedback. The survey was sent via email to verified administrators at licensed behavioral health clinics, outpatient psychiatrist practices, and outpatient psychologist practices. Survey questions assessed patient demographics and indicators of integration with primary care practices, including shared electronic medical records, integrated scheduling, colocation, and communication systems between providers. Practices were given 3 weeks for survey response, after which up to seven follow-up phone calls were made. In order to achieve the highest response rate, intern teams completed the survey with the remaining clinics in person.

RESULTS: The response for the primary study group, licensed behavioral health clinics, was 48 out of 52 ( $92 \%$ response rate). Among outpatient psychiatrist and psychologist practices, 171 and 187 surveys were completed, respectively. Limited integration between primary care and behavioral health was identified. Even among clinics and practices reporting some level of integration, using the same EMR for behavioral health and primary care providers as an indicator of integration, no integration was reported by $75.0 \%$, $89.1 \%$, and $100 \%$ of licensed behavioral health clinics, psychology practices, and psychiatry practices, respectively. In these same practices, only $16.7 \%, 4.3 \%$, and $0 \%$ of licensed behavioral health clinics, psychology practices, and psychiatry practices reported being in the same location as a primary care practice, respectively.

CONCLUSIONS: There was limited integration between behavioral health and primary care practices in Rhode Island. Even among those behavioral health practices reporting some level of integration, the proportion of practices reporting specific variables of integration remained very low. Policy development should support further integration of behavioral health and primary care toward the aim of improving statewide quality of care and outcomes for Rhode Islanders. 
UNEXPECTED ASSOCIATION BETWEEN PATIENT ACTIVATION AND QUESTION ASKING BEHAVIOR IN PRIMARY CARE Daniel J. Coletti ${ }^{3}$; Hara Stephanou ${ }^{1}$; Joseph Conigliaro ${ }^{2} .{ }^{1}$ Department of Medicine, New Hyde Park, NY; ${ }^{2}$ North Shore LIJ Health System, New Hyde Park, NY; ${ }^{3}$ Northwell Health, Great Neck, NY. (Control ID \#2463979)

BACKGROUND: Encouraging patient engagement is a key feature of healthcare delivery models such as the Patient Centered Medical Home (PCMH) and selfmanagement approaches to chronic illness. Patient Activation (PA) is a commonly used, well-validated proxy for patient engagement, and has been defined as "understanding one's own role in the care process and having the knowledge, skills, and confidence to take on that role." Higher levels of patient activation are associated with health-promoting patient behaviors and improved health outcomes. "Activated" patients are also assumed to communicate with their medical teams to understand what is required to manage their health. Prior research has shown that patient activation is modifiable through interventions that target the ability to ask more effective questions of medical providers. No research to date, however, has assessed the association between patientgenerated questions and PA levels in the general patient population. The objective of this investigation was to test the hypothesis that patient question asking behavior in a primary care setting would be positively correlated with PA. We also explored relations between dimensions of question formulation and sociodemographic, health-related, and psychosocial variables.

METHODS: Participants were attending an appointment at a hospital-affiliated PCMH that serves a socioeconomically diverse catchment area. All had also been prescribed at least one medication. Participants completed the 13-item Patient Activation Measure (PAM) as well as questionnaires assessing attitudes to health, medication adherence, emotional and physical functioning, and health literacy. Patients also completed a brief written exercise in which they were prompted to ask as many questions as possible about (a) their medical condition;(b) any medication they were taking, and (c) other issues concerns. Three trained raters completed a qualitative analysis of patient questions to identify common themes and also coded questions according to levels of increasing conceptual sophistication (from pre-logical, to concrete and abstract reasoning). T-tests for independent means, Pearson-r correlations, and multivariate logistic regression analyses tested the study hypothesis and identified variables associated with patient questions. RESULTS: A total of 250 patient-participants ( 160 women, 90 men, mean age $=$ 49.63 years, $\mathrm{SD}=15$ ) completed study measures. Levels of patient activation were high overall (Mean PAM score $=64.04, \mathrm{SD}=16.55)$. Over two thirds of the sample $(n=170$ or $68 \%$ ) were categorized as falling into Activation Levels 3 or 4 , suggesting proactive patient engagement and maintenance of efforts over time. Over two thirds of the sample ( $n=173$ or $69.2 \%$ ) formulated one or more questions (total frequency $=446$ questions range $=1-8$ per participant). Concerns about medications were the most frequent theme evident ( $n=146$ questions by 95 patients) —including inquiries about medication side effects, long-term complications, and required duration of treatment. In contrast to the high mean levels of patient activation, however, it was noted that a significant proportion of the respondents either had no questions $(n=77)$ or recorded simple statements such as "I'm here to get my blood pressure checked" rather than formulate actual questions. Contrary to our expectations patients who formulated questions had lower PAM scores $(\mathrm{M}=61.64$, $\mathrm{SD}=16.59)$ than patients who had no questions $(\mathrm{M}=69.42, \mathrm{SD}=16.59 ; t=3.51, \mathrm{df}=$ $248, p=.001)$. Higher PAM scores were also associated with fewer number of questions asked $(r=-.19, p=.002)$ and lower levels of conceptual sophistication $(r=-.28$, $p<.001)$. The strength of these associations remained statistically significant after controlling for insurance status (as a proxy for SES) and level of education. Modest but significant correlations were also observed between asking more questions and lower levels of self-reported medication adherence $(r=.20, p=.002)$ and poorer perceived functional status $(r=.19, p=.003)$. Controlling for demographic and the other healthrelated variables, only lower PAM scores predicted question asking behavior in the multivariate model $\left(X^{2}=12.35, \mathrm{df}=1, p<.001\right)$

CONCLUSIONS: Results depict an unexpected pattern of associations between patient questions and variables thought to represent greater self-efficacy and engagement. Patients who formulated questions within this written task in fact reported feeling less confident, knowledgeable and skilled in their healthcare. They were also more likely to report lower levels of physical functioning and more difficulties adhering to their medication regimen. We speculate that the effort to engage providers by asking questions was an attempt to address confusion about their treatment regimen and about their health in general. Therefore question asking may represent both a cognitive and a behavioral attempt to strive for higher levels of engagement rather than a sign that things are already going well. Results should be replicated using a larger sample in different treatment environments. Longitudinal research is also needed to detect changes in both patient questions and activation levels as they fluctuate over time-or as patients receive answers to their questions and apply the information to their care. These results however, highlight the importance of answering patient questions to improve engagement, and suggest a need to re-think the construct of patient activation as currently conceptualized.

USABILITY OF A NOVEL MHEALTH IPAD APPLICATION BY VULNERABLE POPUlATIONS David P. Miller ${ }^{1}$; Kathryn Weaver ${ }^{1}$; Doug Case ${ }^{1}$; Don P. Babcock $^{1}$; Donna Kronner ${ }^{1}$; Nancy M. Denizard-Thompson ${ }^{1}$; Michael Pignone ${ }^{2}$; Jennifer L. Troyer ${ }^{3}$; John G. Spangler ${ }^{4}{ }^{1}$ Wake Forest School of Medicine, Winston-Salem, NC; ${ }^{2}$ University of North Carolina, Chapel Hill, NC; ${ }^{3}$ University of North Carolina at Charlotte, Charlotte, NC; ${ }^{4}$ Wake Forest School of Medicine, Winston Salem, NC. (Control ID \#2469423)

BACKGROUND: Recent advances in mobile technologies have created new opportunities for novel health interventions. Mobile health (or mHealth) refers to the use of mobile devices such as smartphones and tablets to improve healthcare delivery. While mHealth has the potential to reach broadly into populations, it could paradoxically increase health disparities if members of vulnerable populations lack the ability to access or use mobile devices. We analyzed baseline data from an ongoing randomized controlled trial of an mHealth colorectal cancer (CRC) screening intervention to determine if patients from health disparate populations could successfully navigate and complete an mHealth patient education program.

METHODS: We designed a user-friendly mHealth iPad program for use by individuals with limited literacy and little prior technology experience. The program, call mPATH, incorporates simple language, audio narration, and large intuitive buttons to collect basic health information from patients via a selfadministered survey after which it displays a health education video. We enrolled English-speaking patients aged 50-74 years old who were due for CRC screening and were scheduled for a routine medical visit at one of six community-based primary care practices affiliated with a large academic medical center. Participants completed the mPATH iPad program immediately before their scheduled visit. After completing the self-administered survey on the iPad, the mPATH program randomly displayed either a CRC screening decision aid or a video about diet and exercise. To measure usability, the research assistant left the room while participants completed the program and recorded the number of times participants opened the door to ask for assistance. All participants also completed 5 items from the validated Systems Usability Scale.

RESULTS: All enrolled participants ( $n=351 ; 54 \%$ female) completed the mPATH iPad program. Ages ranged from 50 to 74 with a median of 57 years. Three-fourths of 
participants were members of a vulnerable population defined as either having limited health literacy (39\%), having an annual household income $<\$ 20,000(57 \%)$, or selfidentifying as black (45\%). Many participants had not used the internet in the last 30 days (40\%), but $89 \%$ owned a cell-phone. Overall, participants rated the user-friendliness of mPATH highly ( $83 \%$ "excellent", $14 \%$ "good"), and $93 \%$ of participants were able to complete the program without any assistance. Another $4 \%$ were able to complete the program with only 1 episode of assistance, $2 \%$ required 2 or more episodes of assistance, and $1 \%$ had a caregiver help them use the program. In bivariate analyses, members of vulnerable populations as defined above were more likely to need assistance to complete the program ( 9 vs $1 \%, p=0.02$ ), but even $91 \%$ of vulnerable patients completed the program without any help. The proportion requiring some assistance was similar for those who had limited health literacy, had low income, or self-identified as black $(10,9$, and $9 \%$ respectively). Other attributes associated with requiring assistance were age $>57$ years ( 11 vs $3 \%, p<0.01$ ), no internet use in last 30 days ( 14 vs $2 \%, p<0.0001$ ), and not having a cell phone ( 15 vs $6 \%, p=0.03$ ). In a multivariable logistic regression model with these factors as covariates, being a member of a vulnerable population was no longer associated with needing assistance $(p=0.18)$. Only older age $(p=.02)$ and lack of internet use $(p=.01)$ remained associated with needing assistance. The odds ratio for age over 57 was 3.1 (95\% CI 1.2-8.2) and the odds ratio for no internet use was $3.9(1.3-11.4)$

CONCLUSIONS: Over $90 \%$ of members of vulnerable populations were able to complete our iPad mHealth application without assistance. While older patients and those who do not use the internet had more difficulty with the program, a large majority were still able to successfully complete it with minimal help. These results indicate that properly designed mHealth interventions can reach vulnerable populations, including older adults and those with little technology experience. Careful consideration of the usability of newly developed applications is essential to ensure that mHealth interventions do not widen health disparities.

USABILITY TESTING OF A MOBILE-OPTIMIZED KNOWLEDGE MANAGEMENT SYSTEM TO ENHANCE LIKELIHOOD OF USE AND GENERALIZABILITY BEYOND A STUDENT-RUN CLINIC: THE EHHAPP. Kevin Hu; Mark Finkelstein; Ammar Siddiqui; Theodore Pak; Alyssa Trochtenberg; Joe-Ann Moser; Christian Pina; Nathan Lee; Chien Yi Png; David C. Thomas; Yasmin S. Meah. Icahn School of Medicine at Mount Sinai, New York, NY. (Control ID \#2470231)

BACKGROUND: The EHHapp is a mobile optimized web-based KMS (mKMS, http// :ehhapp.org) developed by students in 2013 at the East Harlem Health Outreach Partnership (EHHOP) student-run free clinic. The mKMS serves as a repository of care- coordination protocols, evidence-based guidelines and a cost-based pharmaceutical formulary. The application allows for transfer of information from year-to-year between a changing staff of students; it is mutable so that as processes and protocols become updated, users have such information readily accessible at the point of care. It serves ultimately as the memory of clinic operations and its usability had been anecdotally positive. To verify the usability of the mKMS and thus the likelihood that this mKMS would be adopted by clinics beyond the free clinic as a viable technology, we used validated survey instruments to capture user perceptions and observed live testing sessions to quantify and characterize usability barriers.

METHODS: Study participants were medical student volunteers involved in the clinical care of patients at EHHOP. Surveyed participants were recruited via email sent to a general student volunteer pool. For objective task analysis, separate emails were sent targeted to cohorts of student volunteers known to either have just started their training or to have had several months of on-the-job experience. Participants to complete two validated surveys: a 10-item System Usability Scale (SUS), and 6 items from the Technology Acceptance Model (TAM). The SUS is scored on a 100-point scale calculated from individual items' Likert scale positions. Each TAM item can be scored based on Likert scale position (1-5). A mean TAM score was then computed as the average of each of the 6 individual item scores. SUS scores were compared against a previous meta-analysis of over 500 SUS studies of a variety of different types of software, and assigned a percentile ranking. To objectively evaluate the ease of learning to use the application, inexperienced and experienced mKMS users were also compared in observed live testing sessions while completing a set of predefined tasks such as finding out locations of specialty clinics, costs of medications, and editing or updating protocols using the mKMS. Usability barriers were characterized and quantified.

RESULTS: Students gave the mKMS a SUS mean score of $79.8(n=30)$, ranking in the top $20 \%$-ile compared to previously studied software, and a mean TAM score of 4.3 ( $n=$ $30)$ suggesting moderate to high system usability in a clinical setting. Inexperienced ( $n=$ $13)$ and experienced $(n=13)$ cohorts encountered a similar number of total usability barriers (43 vs 41) that included navigation errors such as inefficiently scrolling to find information without realizing that a quick search function was available or presentation errors such as having trouble understanding how to edit or update information on a page. CONCLUSIONS: The mKMS developed at EHHOP is easy to learn and use, and is highly valuable in busy practice settings where there is a need for clinical and care coordination protocols at ready access. The application is easily searchable no matter the experience of end-users and holds promise as a versatile mKMS for clinicians, administrators and trainees in settings with rapid turnover. The EHHapp is the first student-developed mKMS for patient care, and shows high likelihood in being favorably adopted as a support tool in multiple clinical settings.

\begin{tabular}{|c|c|c|c|c|c|c|c|c|c|}
\hline EHHapp & ce en & Losocet & EHHapp & & cen & 2 noct & a & Irdilao Hother & C E⿺辶⿻二⿰丿丨土 \\
\hline Q My pationt noods.. & & (1) Card & & & (๓) & \multirow{2}{*}{\multicolumn{4}{|c|}{ This reforral roquires charity care. }} \\
\hline BENERTS & & \multicolumn{4}{|l|}{ SPECULLTY RERERRULS } & & & & \\
\hline Assistance with a bill & ( ) & \multicolumn{2}{|l|}{ Cardiology } & & 0 & \multicolumn{4}{|c|}{ Senior Clinician } \\
\hline Benefits Scroen & 0 & Cardiac Holter & & & 0 & \multicolumn{4}{|c|}{ 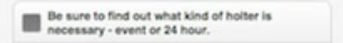 } \\
\hline Financial Assistance & 0 & Cardiac Stress Test & & & 0 & \multirow{2}{*}{\multicolumn{4}{|c|}{ 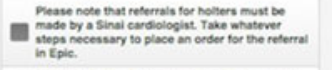 }} \\
\hline Insurance & (2) & \multicolumn{4}{|l|}{ NCCLLAY SERMCES } & & & & \\
\hline Transitioning out Patients with Insurance & 0 & EHHOP Cardiology & & & 0 & \multicolumn{4}{|c|}{ 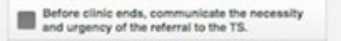 } \\
\hline maciva & & & & & & \multirow{2}{*}{\multicolumn{4}{|c|}{ 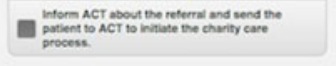 }} \\
\hline Barium Swallow & ( & & & & & & & & \\
\hline Breast Ultrasound (US) & 0 & & & & & \multicolumn{4}{|c|}{ (†) Teaching Senior } \\
\hline Carotid Ultrasound & 0 & & & & & \multicolumn{4}{|c|}{ (1) Access to Care Team } \\
\hline cr Sean & 0 & & & & & \multicolumn{4}{|c|}{$\odot$ Cost } \\
\hline O & 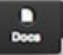 & 1 $\quad$ int & nona & $\stackrel{+}{a x}$ & $D_{0000}^{D}$ & $\underbrace{1}_{n=0}$ & $\stackrel{5}{c}$ & nowa & $D_{000}^{D}$ \\
\hline
\end{tabular}


USE AND COMPLEXITY OF NUMERIC CONCEPTS AND TERMS IN BREAST CANCER TREATMENT CONSULTATIONS Marilyn M. Schapira ${ }^{4}$; Kathlyn Fletcher ${ }^{2}$; Pamela S. Ganschow ${ }^{3}$; Elizabeth Jacobs ${ }^{5}$; Cynthia Walker ${ }^{6}$; Denisse Gil $^{3}$; Alicia J. Smallwood ${ }^{1}$; Courtney C. Okwara ${ }^{7}$; Esther Nivasch ${ }^{4}$; Joan Neuner ${ }^{1}$. ${ }^{1}$ Medical College of Wisconsin, Milwaukee, WI; ${ }^{2}$ Medical College of Wisconsin/ Milwaukee VAMC, Tbd, AL; ${ }^{3}$ Stroger Hospital/Rush University Medical Center, Chicago, IL; ${ }^{4}$ University of Pennsylvania, Philadelphia, PA; ${ }^{5}$ University of Wisconsin School of Medicine and Public Health, Madison, WI; ${ }^{6}$ University of Wisconsin-Milwaukee, Milwaukee, WI; ${ }^{7}$ Harvard University, Boston, MA. (Control ID \#2468571)

BACKGROUND: Numeric concepts are salient in cancer treatment consultations when topics such as survival, risk of recurrence, efficacy, and genomic testing are often discussed. However little is known about the frequency or level of complexity of numeric terms used in these consultations. The objective of this study was to develop a taxonomy of these terms and describe techniques utilized by clinicians to assess patient understanding of quantitative information.

METHODS: Participants included English- and Spanish-speaking women with a new diagnosis of stage $0,1,2$, or 3 breast cancer and their consulting clinicians (General Surgery, Radiation Oncology, and Medical Oncology). Consultations were audio-recorded and transcribed verbatim; Spanish transcripts were translated to English for ease of coding. A coding scheme was developed based on conceptual frameworks of health numeracy and an iterative process of transcript review. Each segment (defined as a sentence or group of related sentences) was coded by 2 independent reviewers with conflicts resolved by a 3 rd reviewer. Codes included numeric domains (number sense, tables/graphs, risk \& probability, statistics and scientific evidence), and the following categorization of terms used to convey numeric concepts; descriptive terms (i.e., high, low), nominal terms (i.e., Stage 2 breast cancer), whole numbers, percentages, decimals, fractions or proportions, measurements, mathematical operations, and indications of evidentiary or statistical uncertainty. Complexity (defined by two or more numeric categories or requiring a mathematic calculation) and use of techniques to assess patient understanding (teach back or prompting for questions) were also coded.

RESULTS: Twenty-one consultations with unique women we were recorded (4 in Spanish) and transcribed. Results are presented as percent of consultations with at least 1 use of the numeric term (\%) and number of sentences coded for each term across transcripts (n). The most commonly used numeracy domains were number sense (100\%, $n=1442)$, followed by risk and probability $(95 \%, n=412)$, statistics $(46 \%, n=26)$, and tables \& graphs $(14 \%, n=11)$. The most commonly used category for communicating numeric concepts was use of a descriptive term $(100 \%, n=1366)$, such as in the following statement, "there is a tiny difference in the chance of it coming back in the breast". Other categories used to convey numeric information were: whole numbers $(100 \%, n=826)$, time related terms $(100 \%, n=715)$, fractions or proportions $(91 \%, n=67)$, percentages $(67 \%, n=82)$, nominal terms $(57 \%, n=53)$, measurements $(52 \%, n=33)$, decimals $(19 \%, n=6)$, and mathematical operations $(5 \%, n=8)$. Only one segment was coded as conveying uncertainty. Complex numeric information, defined as 2 or more categories in one segment, was used in most consultations $(67 \%, n=41)$. For example, both risk of recurrence and time frame were conveyed in the following statement, "40 women out of 100 are going to have it come back in the next 10 years". Complex information, defined by asking patients to do mental calculations was used less frequently $(38 \%, n=16)$. For example, results of a study were summarized in the following way, "Okay? So these are studies that show about nine percent of women who take this treatment live nine percent more." The median (interquartile range) number of segments including numeric concepts per consultation was 95 (67-134), covering (median, interquartile range) $13.45 \%$ (10.9924.95) of the sentences spoken by the clinician. Prompting for questions was used in $81 \%$ ( $n=73$ sentences), and teach back techniques in $38 \%$ ( $n=18$ sentences) of consultations
For example, one doctor asked the patient, "So why don't I have you tell me why we do radiation, just so I make sure you know."

CONCLUSIONS: A wide range of terms, using both words and numbers, was used to convey numeric concepts in breast cancer treatment consultations. Although patients are prompted to ask questions in a majority of consultations, teach back techniques are less often used. This taxonomy of numeric terms developed in this study should be examined in other areas of medicine, and has the potential to inform strategies to match numeracy demand with patients and family numeracy skill.

USE OF ELECTRONIC HEALTH RECORDS FOR ADDRESSING OVERWEIGHT AND OBESITY IN RIMARY CARE: RESULTS FROM A CLUSTERRANDOMIZED CONTROLLED TRIAL Heather J. Baer ${ }^{2}$; Christina C. Wee ${ }^{1}$; Endel J. Orav ${ }^{2}$; Katerina DeVito ${ }^{2}$; Elisabeth Burdick ${ }^{2}$; Deborah H. Williams ${ }^{2}$; Adam Wright ${ }^{2}$; David W. Bates ${ }^{2}$. ${ }^{1}$ Beth Israel Deaconess Medical Center, Boston, MA; ${ }^{2}$ Brigham and Womens Hospital, Wellesley, MA. (Control ID \#2470128)

BACKGROUND: Primary care clinicians often fail to identify patients who are overweight or obese or discuss weight management with them. Electronic health record (EHR)-based tools may help clinicians with the assessment and management of overweight and obesity, but few studies have examined this.

METHODS: We developed several new features within the EHR used by primary care practices at Brigham and Women's Hospital (BWH) in Boston, Massachusetts. These features included 1) reminders to measure height and weight; 2) an alert to add overweight or obesity to the problem list for patients with body mass index (BMI) $\geq 25 \mathrm{~kg} / \mathrm{m}^{2} ; 3$ ) reminders with tailored management recommendations, based on a patient's BMI and medical problems; and 4) a Weight Management screen to help with counseling. We then conducted a pragmatic, cluster-randomized controlled trial in $12 \mathrm{BWH}$ primary care practices. We randomized 23 clinical teams (“clinics") within these practices to the intervention group $(n=11)$ or the control group $(n=12)$. The new features were activated for clinics in the intervention group and were not activated for clinics in the control group. The intervention was implemented in two phases: the height and weight reminders went live on December 15, 2011 (Phase 1), and all of the other features went live on June 11, 2012 (Phase 2). The pre-intervention period was defined as the 6-month period before Phase 1. We compared changes in documentation of BMI in the EHR from the preintervention period to Phase 1 for patients who had visits in the intervention and control clinics, using mixed-effects logistic regression models to account for the within-clinic and within-clinician correlation and to adjust for other covariates. We used a similar approach to compare changes in diagnosis and management of overweight and obesity from the preintervention period to Phase 2 for patients with $\mathrm{BMI} \geq 25 \mathrm{~kg} / \mathrm{m}^{2}$ who had visits in the intervention and control clinics. We also compared 6-month ( \pm 2 months) and 12-month ( \pm 3 months) weight change during Phase 2 for patients with $\mathrm{BMI} \geq 25 \mathrm{~kg} / \mathrm{m}^{2}$ who had visits in the intervention and control clinics, using mixed-effects linear regression models We mailed surveys to a sample of overweight and obese patients who had visits during Phase 2 to obtain more information about their experiences with weight management and about discussions of weight management with their primary care clinicians. We also assessed clinicians' attitudes about management of overweight and obesity and about the new EHR features, using web-based surveys that were administered before and after the intervention period. All statistical analyses were conducted using SAS version 9.3 (SAS Institute Inc., Cary, NC).

RESULTS: A total of 62,736 eligible patients had visits during Phase 1 of the intervention period (28,919 in the intervention group and 33,817 in the control group). From the preintervention period to Phase 1, documentation of BMI in the EHR increased from 93 to $98 \%$ among patients in the intervention group and from 94 to $98 \%$ among patients in the control group $(p=0.69)$. A total of 36,574 eligible patients with $\mathrm{BMI} \geq 25 \mathrm{~kg} / \mathrm{m}^{2}$ had visits 
during Phase 2 of the intervention period (15,669 in the intervention group and 20,905 in the control group). From the pre-intervention period to Phase 2, diagnosis of overweight or obesity on the problem list increased from 37 to $71 \%$ among patients in the intervention group, but decreased from 16 to $8 \%$ among patients in the control group $(p<0.0001)$. There were no significant differences between groups in changes in the percentages of patients who had an appointment with a registered dietitian at BWH or who were prescribed weight loss medication. During Phase 2, the mean 6-month weight change was $-0.5 \mathrm{lb}$ for patients in the intervention group and $-0.2 \mathrm{lb}$ for patients in the control group, and the mean 12-month weight change was $-1.4 \mathrm{lb}$ for patients in the intervention group and $-0.9 \mathrm{lb}$ for patients in the control group $(p<0.0001$ for the effect of the intervention over time). Among 590 patients who completed a mailed survey after their primary care visit (response rate $=25 \%$ ), $60.7 \%$ of patients in the intervention group compared to $53.9 \%$ of patients in the control group reported that their clinician recommended that they lose weight ( $p=0.03$ ), and $17.5 \%$ of patients in the intervention group compared to $13.3 \%$ of patients in the control group said that their clinician helped them set a specific weight loss goal $(p=0.05)$. On the post-intervention clinician survey $(n=86$, response rate $=49 \%$ ), most clinicians reported that they would like more help creating weight loss plans for their patients (77.6 and $89.2 \%$ in the intervention and control groups, respectively, $p=0.22)$. Among clinicians in the intervention group $(n=49), 28.6 \%$ reported that the recommendations about management of overweight and obesity were useful, and $14.6 \%$ felt that the new EHR features improved the quality of care.

CONCLUSIONS: These findings suggest that EHR-based tools can lead to substantial improvements in the diagnosis of overweight and obesity and potentially modest improvements in weight management by primary care clinicians. Although there were only small differences in weight change between the groups, even small amounts of weight loss can lead to significant health benefits.

\section{USE OF ESTROGEN-CONTAINING CONTRACEPTION AMONG REPRO-} DUCTIVE AGE WOMEN WITH MEDICAL CONTRAINDICATIONS Julianne R. Lauring ${ }^{1}$; Erik B. Lehman ${ }^{2}$; Timothy A. Deimling ${ }^{1}$; Richard Legro ${ }^{1}$; Cynthia H. Chuang ${ }^{1}$. ${ }^{1}$ Penn State College of Medicine, Hershey, PA; ${ }^{2}$ Pennsylvania State University, Hershey, PA. (Control ID \#2469704)

BACKGROUND: The Centers for Disease Control (CDC)'s U.S. Medical Eligibility Criteria for Contraceptive Use recommends that estrogen-containing contraceptives (i.e., birth control pills, contraceptive patch, vaginal ring) should be avoided in women with specific characteristics and medical conditions due to increased risk of cardiovascular events. Whether women with category 3 (theoretical or proven risk usually outweigh the advantages) or category 4 (unacceptable health risk) contraindications are appropriately avoiding estrogen-containing methods is unknown. We describe the prevalence of estrogen-containing contraceptive use among women with a medical contraindication in a sample of reproductive age women.

METHODS: Data are from the baseline survey of the MyNewOptions study, which included 987 privately-insured women residing in Pennsylvania aged 18-40, who were sexually active and not intending pregnancy in the next year. Women were surveyed about their health history, contraceptive use, and characteristics of their contraceptive prescriber Women were considered to have a contraindication to estrogen-containing hormonal contraception if they reported one of the following CDC's Medical Eligibility Criteria category 3 or category 4 contraindications - hypertension, smoker over age 35 , history of venous thromboembolism, diabetes with complications, coronary artery disease, systemic lupus erythematosus with antiphospholipid antibodies, breast cancer, or migraine headaches with aura. Chi-square tests were used to compare estrogen-containing contraceptive use and contraceptive prescriber characteristics in women with and without contraindications to estrogen-containing contraceptives.
RESULTS: The MyNewOptions baseline study sample included 987 adult women who were mostly young (46\% were 18-25 years), white (94\%), employed (70\%), and married or cohabiting (54\%). Thirteen percent $(n=130)$ of the sample had a medical contraindication to estrogen-containing contraceptive use-migraine with aura (81\%) was the most common contraindication, followed by smokers over age 35 (7\%), hypertension ( $8 \%$ ), history of venous thromboembolism (3\%), and diabetes with complications ( $2 \%$ ). High use of estrogen-containing contraceptives was reported among the women with medical contraindications to estrogen at $39 \%(n=51)$. This was not statistically different from women without a medical contraindication ( $47 \%, p=0.1)$. Among the 130 women with a contraindication, whether they did or did not use an estrogen-containing contraceptive did not vary by the woman's education level, income, or weight category. With respect to their contraceptive prescribers, there were no differences in prescriber specialty, provider type, or clinic type comparing women using and not using an estrogencontaining contraceptive.

CONCLUSIONS: Among this study sample of reproductive age women, there was a high rate of estrogen-containing contraceptive use in women with a medical contraindication. These women may be at increased risk for cardiovascular events. Processes need to be improved to ensure that women with medical contraindications to estrogen-containing contraception are being offered the safest and most effective alternatives, including longacting reversible contraceptives (LARCs), such as intrauterine devices (IUDs) and the contraceptive implant.

USE OF EXERCISE VERSUS PAIN MEDICATIONS FOR CHRONIC PAIN: ANALYSIS OF A POPULATION-BASED SAMPLE OF HISPANICS FROM FIVE STATES Barbara J. Turner ${ }^{1,1}$; Natalia Rodriguez ${ }^{1}$; Yuanyuan Liang ${ }^{1,1}$; Melissa Valerio ${ }^{2}$, ${ }^{1}$; Paula Winkler ${ }^{1,1}$; Jennifer Potter ${ }^{1,}{ }^{1}$; Kay Avant ${ }^{1,}{ }^{1}$; Jason Hill ${ }^{1} .{ }^{1}$ University of Texas Health Science Center at San Antonio, San Antonio, TX; ${ }^{2}$ University of Texas School of Public Health, Houston, TX. (Control ID \#2469965)

BACKGROUND: Minorities in the U.S. are more likely to suffer from chronic pain but little is known about their utilization of various modalities to manage pain. Prescription pain medication (PPM), especially opioid analgesics, carries risks with limited benefits for chronic pain whereas active self-management such as exercise has been linked to better outcomes. In a population-based survey of Hispanics with non-cancer chronic pain from five southwestern states, we examined factors associated with use of exercise or PPM. We hypothesized that women would be more likely to use PPM than men as in other studies and that exercise would be more likely for higher income persons who may have greater support and fewer barriers to exercise.

METHODS: From a nationally representative sample of adults in the KnowledgePanel ${ }^{\circledR}$ of GfK Custom Research used for business marketing and other research purposes, we identified Hispanic subjects, aged 35-75, reporting that they had pain affecting daily activities on most days for $>3$ months which was not due to cancer. The online survey asked about pain (location, duration, severity), impact on daily activities, approaches used to manage/treat pain, and reasons for not using other approaches. To account for the sampling design of this survey, weighted logistic regression models were used for two outcomes: 1) taking PPM and 2) exercising to manage pain. Predictors included: age, survey language preference-sex (English-men, English-women, Spanish-men, Spanish-women), employed (yes/no), household income (low $<\$ 9,999$, moderately low $\$ 10,000-34,999$, moderately high $\$ 35,000-74,999$, high $>\$ 75,000)$, and mean daily pain level on a visual scale with five levels. We adjusted for exercise in the PPM model and vice versa to account for people who reported both behaviors.

RESULTS: Among the 102 Hispanic respondents in 5 states, the mean age was 55.3 years $(\mathrm{SD}=10.1)$, mean daily pain level was $3.3(\mathrm{SD}=0.9)$, and $70.6 \%$ 
were employed. Language preference-gender groups were: $25.5 \%$ Spanishwomen; $29.4 \%$ English-women; $18.6 \%$ Spanish-men; and $26.5 \%$ Englishmen. Income distribution was: $16.7 \%$ low, $42.2 \%$ moderately low, $20.6 \%$ moderately high, and $20.6 \%$ high. Exercise was used by $33.3 \%$ alone and with PPM by $38.2 \%$ while $14.7 \%$ used PPM alone and $13.7 \%$ used neither. In the fully adjusted model for exercise with highest income category as the reference, the adjusted odds ratios (AORs) of exercising for pain were less than 0.5 for the three lower income categories but significant only for moderately high income $(0.15,95 \%$ CI $0.03-0.73, p=0.02)$. No other factors were associated with exercise but the AOR for Spanish-women was 0.28 (95\% CI 0.07-1.16, $p=0.08$ ) versus English-men. In the fully adjusted model for PPM, only income was a significant predictor with higher AORs for lower income categories compared with high income: low $(20.61,95 \%$ CI $0.95-446.14, P=0.05)$, moderately low $(6.90,95 \%$ CI $1.20-39.82, P=0.03)$, moderately high (5.03, $95 \%$ CI $0.90-28.18, P=0.07)$. Notably, mean daily pain level was not associated with either exercise or PPM.

CONCLUSIONS: To our knowledge, this is the first population-based study of Hispanics with chronic pain and self-reported use of exercise and/or PPM to manage this condition. We found that lower income groups $(<\$ 75,000$ household income) were less likely to use exercise to manage pain but markedly more likely to report using PPM than the high income group. Women preferring a Spanish language survey tended to be less likely to exercise than men preferring English suggesting both gender and acculturation effects. To reduce the risks of PPM, reasons for these differences in use need to be explored such as cultural preferences, access to exercise facilities, and limited understanding of the risk/benefits of long-term PPM for chronic pain

\section{USE OF ONLINE CROWD-SOURCING TO IDENTIFY AND STUDY PATIENTS} WITH CHRONIC CONDITIONS: IS THIS POSSIBLE AND ARE FINDINGS VALID? Veronica Yank ${ }^{1}$; Sanjhavi Agarwal ${ }^{2}$; Pooja Loftus ${ }^{2}$; Christian $\mathrm{Choe}^{2} .{ }^{1} \mathrm{UCSF}$, Menlo Park, CA; ${ }^{2}$ Stanford, Stanford, CA. (Control ID \#2466404)

BACKGROUND: Use of crowd-sourcing for health survey research may be more efficient than traditional approaches but requires validation. We focused on patients with chronic kidney disease (CKD) not yet taking recommended medications for whom we designed advertising-style medication messages (imagery and text) designed to activate emotions. Our goal was to determine whether we could validate use of crowd-sourcing to test such messages: could we identify patients and, if so, would their data be comparable to data from health system patients?

METHODS: We developed 7 advertising-style messages intended to prompt patients with CKD to discuss statin medications with healthcare providers. Using the world's largest crowd-sourcing platform, Amazon Mechanical Turk, we administered a screening survey to US-based crowd-sourced respondents. Among these we identified respondents $50+$ years old with CKD or conditions associated with increased cardiovascular disease risk: hypertension (HTN), diabetes mellitus (DM), or hyperlipidemia (HL). We also identified health system patients $50+$ years old with stages $3 \mathrm{~b}-4$ CKD (eGFR $15-44 \mathrm{~mL} / \mathrm{min}$ ) or stage 3a (eGFR 45-59) plus HTN, DM, or HL. We invited both groups to complete a message survey with validated questions on (a) 8 message characteristics (predictors) - degree of being attention grabbing, absorbing, surprising, balanced, believable, convincing, useful, and easy to understand, and (b) message impact on respondent self-efficacy for health action (outcome). Write-in boxes solicited qualitative feedback. Sample size calculations determined a target of 32/group. We confirmed comparability of each groups' evaluation of message characteristics with $\mathrm{Chi}^{2}$ analyses. We performed factor analyses to identify related message characteristics and then, for both groups, calculated Spearman correlations to determine whether identified factors predicted impact. We then confirmed correlation coefficients were similar between groups. Finally, we compared message impact for crowd-sourced vs. health system patients.

RESULTS: Among 2015 crowd-sourced respondents screened, 135 (7\%) qualified for the message survey, and 115 of those (85\%) completed it. Among 89 health system patients who confirmed receipt of invitation letters, 37 (42\%) completed surveys, whereupon we closed enrollment. Crowd-sourced patients were younger than health system patients (mean age 58 vs. 70 years, $p<.001$ ) but similar in race/ethnicity (White 89 vs. $100 \%, p=.33$; Latino 4 vs. $5 \%$, $p=.79$ ), educational attainment (some college 91 vs. $89 \%, p=.70$ ), BMI (mean $30 \mathrm{~kg} / \mathrm{m}^{2}$ vs. $30, p=.31$ ), and rate of HTN (68 vs. $70 \%, p=.78$ ). They had lower rates of HL ( 41 vs. $54 \%, p=.04)$, DM (24 vs. $38 \%, p=.09)$, and CKD ( 2 vs. $60 \%, p<.001)$. They did not differ in ratings of message characteristics, except on one message - which had significant rating differences for two characteristics. Factor analysis identified two factors: credible (balanced, believable, convincing, easy to understand, and useful) and involving (attention grabbing, absorbing, and surprising). For both groups factor ratings correlated with message impact, and correlation coefficients did not differ. Measures of self-efficacy outcome also were comparable for most messages. [Table] Among both groups two messages ("Woman with shadow" [Figure] and "Flatline") ranked high, two others had similar middle ranks ("Giant sandwich" and "Coffee mug"), and another ("Crash test dummy") received bottom ranking. But outcomes for two messages ("Bride" and "Doctor") were more disparate, significantly so $(p=.003)$ for the former. Qualitative analysis suggested the age discrepancy between groups explained these findings.

CONCLUSIONS: In general, pre-screened crowd-sourced and health system patients responded similarly to medication messages. Yet researchers seeking to build on our approach should ensure that characteristics of crowd-sourced respondents align with those of targeted patient populations. Patients who are older, from under-represented groups, or have lower educational attainment may not be reachable by crowd-sourcing. In sum, use of crowd-sourcing may be useful for survey-based health research if respondents are confirmed through pre-screening to have similar profiles to targeted patients

Message impact on patients

\begin{tabular}{|c|c|c|c|c|c|c|}
\hline Message & $\begin{array}{l}\text { Intended } \\
\text { emotional } \\
\text { activation }\end{array}$ & $\begin{array}{l}\text { Health } \\
\text { system } \\
(n=37) \\
\text { Increased } \\
\text { self- } \\
\text { efficacy, } \\
\%\end{array}$ & $\begin{array}{l}\text { Crowd- } \\
\text { sourced } \\
\text { ( } n=115) \\
\text { Message } \\
\text { rank }\end{array}$ & $\begin{array}{l}\text { Increased } \\
\text { self- } \\
\text { efficacy, } \\
\%\end{array}$ & $\begin{array}{l}\text { Message } \\
\text { rank }\end{array}$ & \\
\hline Doctor & $\begin{array}{l}\text { Trust-in } \\
\text { experts }\end{array}$ & 76 & 1 & 57 & 5 & 0.04 \\
\hline Flatline & $\begin{array}{l}\text { Fear-of } \\
\text { death }\end{array}$ & 65 & 2 & 67 & 2 & 0.82 \\
\hline $\begin{array}{l}\text { Woman } \\
\text { with } \\
\text { shadow }\end{array}$ & $\begin{array}{l}\text { Fear-of } \\
\text { vague threat }\end{array}$ & 60 & 3 & 70 & 1 & 0.26 \\
\hline $\begin{array}{l}\text { Giant } \\
\text { sandwich }\end{array}$ & Amusement & 50 & 4 & 54 & 6 & 0.40 \\
\hline $\begin{array}{l}\text { Coffee } \\
\text { mug }\end{array}$ & $\begin{array}{l}\text { Comfort-of } \\
\text { daily routine }\end{array}$ & 49 & 5 & 61 & 4 & 0.19 \\
\hline Bride & $\begin{array}{l}\text { Tenderness- } \\
\text { for family }\end{array}$ & 38 & 6 & 65 & 3 & $0.003^{*}$ \\
\hline $\begin{array}{l}\text { Crash } \\
\text { test } \\
\text { dummy }\end{array}$ & Amusement & 30 & 7 & 45 & 7 & 0.10 \\
\hline
\end{tabular}

*With Bonferroni correction, significance level is $p<.007$. 


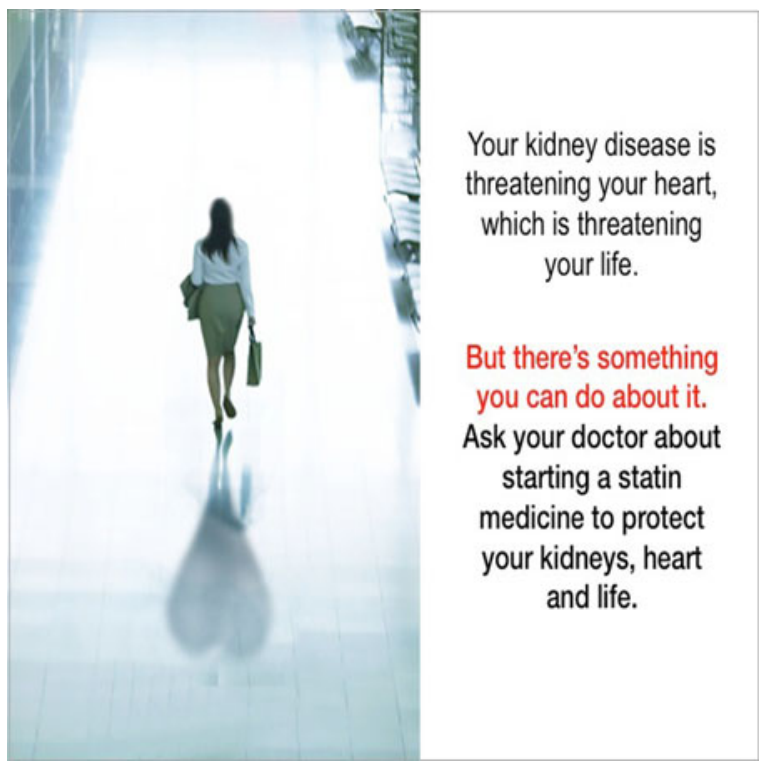

USE OF TEXTING AMONG OVERWEIGHT OR OBESE ADULTS Tracie C. Collins ${ }^{1}$; Maria Gabriela Valverde ${ }^{1,}$; Kelsey Lu ${ }^{1} .{ }^{1} \mathrm{KU}$ School of Medicine - Wichita, Wichita, MN; ${ }^{2}$ University of Kansas School of Medicine - Wichita, Wichita, KS. (Control ID \#2469790)

BACKGROUND: As part of pilot work for a behavioral intervention trial, we assessed use of text messaging among overweight/obese adults, English or Spanish-speaking. METHODS: We developed a 21-item survey that was translated from English to Spanish by a certified translator. The survey items ascertained race/ethnicity; gender; primary language spoken at home (English or Spanish); country of origin; and age. The survey also ascertained type of service (e.g., unlimited, pay per text), shared ownership of a cell phone; if shared, hours of access to the phone; access to the Internet from the cell phone; use of text messaging to send/receive messages; and frequency of texting each day. The survey was interviewer administered either by phone or during in-person interviews by a trained research assistant fluent in English and Spanish. To be eligible, survey completers were required to meet the following criteria: ownership of a cell phone and overweight/obese (BMI $25 \mathrm{~kg} / \mathrm{m}^{2}$ or higher). Survey completers were identified by study fliers distributed throughout the Wichita, KS or through a mailing list of persons who agreed to be contacted about research studies. Participants self-reported demographics, weight, and height.

RESULTS: We screened 147 adults of whom 145 were eligible based on BMI. Among the 145 survey completers, the mean age was 60.3 years (SD 6.61), 89 (61.4\%) were women, $40(27.6 \%)$ were non-Hispanic white, 61 (42.1\%) were Hispanic, and 44 (30.3\%) were African American. Among survey completers, the United States was the country of origin for 85 (58.6\%), Mexico for 25 (17.2\%), Ecuador for 13 (8.9\%), and other for $22(15.2 \%)$. Fifty eight (40\%) reported Spanish as the primary language spoken at home. One hundred thirty eight $(95.2 \%)$ survey completers reported sole ownership of their cell phones, 6 (4.1\%) reported co-ownership of a cell phone with someone else in their home, and 1 reported co-ownership with a neighbor or friend. However, all survey completers reported $24 \mathrm{~h}$ access to a cell phone. Specific to access to the Internet from their cell phones, 114 (78.6\%) survey completers reported yes, 29 (20\%) reported no, and 2 $(1.4 \%)$ did not know. For type of text messaging service, $133(91.7 \%)$ reported unlimited texting, $5(3.4 \%)$ reported pay per text, and $7(4.8 \%)$ were not sure. One hundred forty $(96.5 \%)$ of survey completers reported receiving text messages and 137 (94.5\%) reported sending text messages. Specific to frequency of text messaging, $128(88.3 \%)$ received or sent at least one message per day. Thirty seven $(25.5 \%)$ survey completers received/sent text messages in Spanish. Ninety nine (68.3\%) reported yes to an interest in receiving health tips as text messages on their cell phones, 33 (22.8\%) were not sure, and $13(8.9 \%)$ reported no.

CONCLUSIONS: Our findings highlight the common use of text messaging among an ethnically diverse group of overweight/obese adults, English or Spanish speaking. We will use the findings from this study to inform a larger trial with potential for an innovative intervention which may improve population health.

USE OF WARFARIN FOR ATRIAL FIBRILLATION RELATED STROKE PREVENTION IN AN URBAN PRIMARY CARE PRACTICE Jillian Senner²; Alia Chisty ${ }^{1} .{ }^{1}$ Temple, Philadelphia, PA; ${ }^{2}$ Temple University Hospital, Philadelphia, PA. (Control ID \#2465580)

BACKGROUND: Warfarin remains the oral anticoagulant of choice for the prevention of stroke in patients with a diagnosis of atrial fibrillation (A-fib). Initiation and management of anticoagulation is challenging in underserved populations with low health literacy. The goal of this study was to explore rates of warfarin use for A-fib and time spent in therapeutic range in an urban, underserved resident continuity clinic

METHODS: A query was performed of the outpatient electronic medical record to identify all patients who carried a diagnosis of A-fib (ICD-9: 427.31) or had received warfarin (V58.61) in our practice between January 2014 and January 2015. We excluded all patients who did not have an active diagnosis of A-fib. We recorded patient clinical characteristics, CHADS-2 scores and HASBLED scores, as well as INR values for those on warfarin therapy and calculated their respective time in therapeutic range.

RESULTS: A total of 214 patients were identified in the EMR query, of those 145 met the inclusion criteria for analysis. A total of 74 patients were treated with warfarin. Average overall CHADS2-VASc for all patients was 4.2 and average HASBLED score for all patients was 2.9, with no clinically significant difference between anticoagulated and not anticoagulated group. The average number of INRs was $17+13$ per patient per year. The average amount of time spent in the patients therapeutic range was $44 \%+26$, and no correlation was found between more frequent INR checks and increased time in therapeutic range.

CONCLUSIONS: Appropriate anticoagulation for A-fib remains challenging in the resident continuity clinic. Despite high CHADS2-VASc scores only $50 \%$ of patients received appropriate anticoagulation. The average time spent in therapeutic range is significantly lower than has been seen in prospective studies. Future studies are needed to elucidate the cause of this discrepancy and to explore future interventions.

USING ACTIVE CHOICE WITHIN THE ELECTRONIC HEALTH RECORD TO INCREASE COLORECTAL CANCER SCREENING RATES Mitesh Patel ${ }^{2,3}$; Susan C. Day ${ }^{1}$; Dylan Small ${ }^{1}$; Jingsan Zhu ${ }^{1}$; Lin Yang ${ }^{1}$; Steven Honeywell ${ }^{1}$; Kevin G. Volpp ${ }^{2,3}$. ${ }^{1}$ University of Pennsylvania, Philadelphia, PA; ${ }^{2}$ University of Pennsylvania, New York, NY; ${ }^{3}$ Philadelphia VA Medical Center, Philadelphia, PA. (Control ID \#2467616)

BACKGROUND: Despite its benefits, colorectal cancer screening rates are often low. Active choice is a method that incorporates insights from behavioral economics and has been shown to change behavior in many other settings outside of healthcare. In this study, we evaluated an electronic heath record (HER) intervention using 'active choice' to increase colorectal cancer screening rates among adults in a primary are practice. METHODS: On February 15, 2012 one of three similar academic internal medicine practices at the University of Pennsylvania implemented the intervention. Rather than the standard approach of relying on physicians to recognize the need for screening and opt into placing an order, the EHR confirmed patient eligibility during the clinic visit and used an alert 
to ask the physician and their medical assistant to actively choose to "accept" or "cancel" a colonoscopy order. A difference-in-differences approach was used to compare the change in colonoscopy order and completion rates for patients at the intervention practice compared to patients at the two control practices. Data on colonoscopy orders were obtained using Clarity, an EPIC reporting database. Data on colonoscopy completion rates were obtained from health insurance claims. Patients already screened for colon cancer were identified and excluded. Multivariate logistic regression models were fit to the binary outcome measures (colonoscopy ordered, colonoscopy completed) using the patient as the unit of analysis and adjusting for demographics (age, gender, race/ethnicity), comorbidities (using the Charlson Comorbidity Index), insurance type, whether the visit was with the primary care provider or not, and visit type (new, return, reassign provider, other). The model compared 1 year before and after the intervention, adjusting for time trends, calendar month and practice site fixed effects, and clustering on patient.

RESULTS: The sample ( $n=7,560$ patients) was $60.0 \%$ female, $50.1 \%$ white, $40.1 \%$ black with a mean age ranging by practice from 58.8 to 60.2 years. During the preintervention period, the intervention practice had a higher rate of colonoscopy orders than the control practices ( 42.4 vs. $23.4 \%$ ) but there was no difference in pre-intervention monthly trends between the two groups (Odds Ratio, 1.01; $95 \%$ Confidence Interval, $0.63-1.64 ; P=0.96)$. After the intervention, the intervention practice using active choice had a significantly greater increase in colonoscopy order rates (Adjusted difference-indifference: 11.8 percentage points; $95 \%$ CI, $8.0-15.6 ; P<0.001)$ and patient colonoscopy completion rates (Adjusted difference-in-difference: 3.5 percentage points; $95 \%$ CI, 1.1 $5.9 ; P=.04)$

CONCLUSIONS: Active choice within the EHR was associated with a significant increase in physician ordering and patient completion of colonoscopy.

USING ACTIVE CHOICE WITHIN THE ELECTRONIC HEALTH RECORD TO INCREASE INFLUENZA VACCINATION RATES Mitesh Patel ${ }^{2,3}$; Kevin G. Volpp $^{2,}{ }^{3}$; Dylan Small ${ }^{1}$; Jingsan Zhu ${ }^{1}$; Lin Yang ${ }^{1}$; Steven Honeywell ${ }^{1}$; Susan C. Day ${ }^{1}$ ${ }^{1}$ University of Pennsylvania, Philadelphia, PA; ${ }^{2}$ University of Pennsylvania, New York, NY; ${ }^{3}$ Philadelphia VA Medical Center, Philadelphia, PA. (Control ID \#2467587)

BACKGROUND: The manner in which choices are offered and displayed has influenced decisions in many contexts. Yet, there has been little evaluation of using choice architecture within the electronic health record (EHR) to change physician behavior and improve patient care. In this study, we evaluated an EHR intervention using 'active choice' to increase influenza vaccination. Rather than the standard approach of relying on physicians to recognize the need for vaccination and opt into placing an order, the EHR confirmed patient eligibility during the clinic visit and used an alert to ask the physician and their medical assistant to actively choose to "accept" or "cancel" an order for influenza vaccination.

METHODS: A difference-in-differences approach was used to compare the change in influenza vaccination order rates for patients visiting an internal medicine clinic that implemented active choice on February 15, 2012 (intervention group) to patients visiting two internal medicine clinics that did not (control group). All three clinics were academic teaching practices located within proximity at the University of Pennsylvania Health System. Data on vaccination orders were obtained using Clarity, an EPIC reporting database. Patients already vaccinated in the current influenza season were identified and excluded using data from EPIC and health insurance claims. A multivariate logistic regression model was fit to the binary outcome measure (vaccination ordered) using the patient as the unit of analysis and adjusting for demographics (age, gender, race/ethnicity), comorbidities (using the Charlson Comorbidity Index), insurance type, whether the visit was with the primary care provider or not, and visit type (new, return, reassign provider, other). The model compared the post-intervention year influenza season (September 2012 to March 2013) to influenza seasons in the prior 2 years, adjusting for calendar month, year and practice site fixed effects, and clustering on patient. The bootstrap procedure, resampling patients, was used to obtain the adjusted difference in percentage of patients with a vaccination order placed with $95 \%$ confidence intervals.

RESULTS: The sample ( $n=45,926$ patients) was $62.9 \%$ female, $35.9 \%$ white, $54.4 \%$ black with a mean age of 50.2 years. Greater than $99.9 \%$ (9938/9941) of orders placed during the study period resulted in vaccination. Between the two practice groups, preintervention influenza vaccination order rates were similar (17.7\% overall, range: 14.5 to $21.2 \%$ ). There was no significant difference in the change in order rates between groups from pre-intervention year 2 to pre-intervention year 1 (Odds Ratio, 0.98; $95 \%$ Confidence Interval, $0.87-1.10 ; P=0.70$ ). Vaccination rates increased in both groups in the post-intervention year, but the intervention practice using active choice had a significantly greater increase compared to control (Adjusted difference-in-difference: 6.6 percentage points; $95 \%$ CI, $5.1-8.1 ; P<0.001$ ), representing a $37.3 \%$ relative increase compared to the pre-intervention period.

CONCLUSIONS: Using active choice within the EHR was associated with a significant increase in influenza vaccination rates suggesting that choice architecture could be used more broadly to better optimize medical decision-making.

\section{USING HEALTH MAPS TO CAPTURE PATIENT'S PERCEPTIONS ABOUT} THEIR ILLNESS Pablo Buitron de la Vega ${ }^{1}$; Barbara G. Bokhour ${ }^{3}$; Michael PaascheOrlow2. ${ }^{1}$ Boston Medical Center / Boston University, Dorchester, MA; ${ }^{2}$ Boston University, Boston, MA; ${ }^{3}$ ENRM Veterans Affairs Medical Center, Bedford, MA. (Control ID \#2469797)

BACKGROUND: Management of chronic diseases has become one of the major challenges for the health care community. Limited health literacy is one of the important factors that has been associated with poor self-care in chronic illnesses. An individual's level of health literacy is not a fixed characteristic and could be improved by promoting productive interactions between patients and providers. In order to achieve adequate patient education and collaborative goal setting, providers need to be aware of their patient's perspectives and knowledge about their chronic illnesses (explanatory frameworks). Capturing patient's perspectives about their chronic illness continues to be a challenge due to time constraints and insufficient communication techniques to capture patient's distinct views in a systematic manner. This study used 'mind mapping', a graphic representation of ideas and thoughts, to develop a process that captures patient's perspectives about their chronic illness. We will refer to this process as "Health Mapping". We aim to explore the patient's experience using Health Mapping as a method to capture patient's explanatory frameworks systematically.

METHODS: We conducted a qualitative research study that included 20 adult ( $>18$ years old) English and Spanish speaking patients with uncontrolled (HbAlc $>7 \%$ ) type 2 diabetes. Patients were recruited from a primary care clinic of an urban safety-net hospital. Each participant met for $30 \mathrm{~min}$ with a medical assistant or research assistant who was previously trained to facilitate the process of health mapping. After the participant finished developing their health map, we conducted a semi-structured interview in which patients were asked about their experience with Health Mapping. We also asked them to describe their health map with the interviewer. The Health Mapping processes were videotaped. Interviews were recorded and transcribed. Health Mapping video and interview data were analyzed using grounded thematic analysis to identify how the process was perceived and understood by patients.

RESULTS: All patients were able to create their Health Maps during the 30-min meetings. Staff were successful in guiding patients to develop their own health maps including patient's understandings of their illness, self-care activities, challenges and goals. Figure 1, is an example of a Health Map that was developed by one of the patients. We identified several key themes regarding patient's use of the health map. Patients 
reported developing insight into their illness; reported that the process led to some 'soul searching'. They reported intention to use the health map in several ways: sharing with family members; using it to remind themselves of their goals; educating others about their illness. Patients reported feeling comfortable creating their Health Map; some patients reported that they would prefer their primary care physicians to be the facilitator of this process.

CONCLUSIONS: The health mapping process can help capture patient's understandings of their illness, self-care activities, challenges and goals. Additional research is needed to determine how to use a patient's Health Map to facilitate patient education, disease management activities, and engage patients in collaborative goal setting to improve health literacy and self-care in chronic illness.

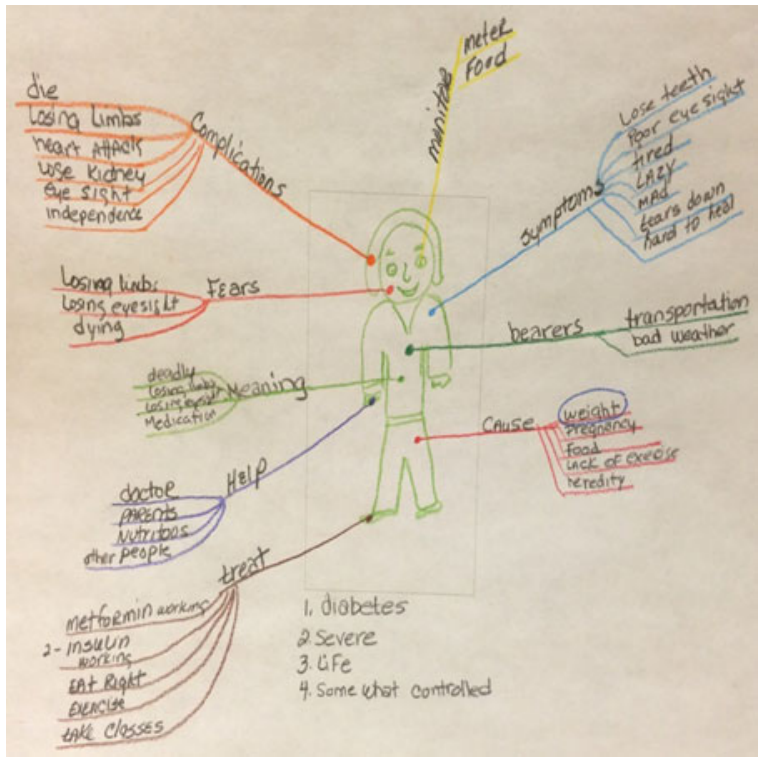

Middle aged female with morbid obesity and diabetes. Her Health Map shows fear of death. When sharing her Health Map she realized her understanding of diabetes was better than she had initially thought

USING LONGITUDINAL HBA1C DATA IN PROACTIVE DIABETES MANAGEMENT IDENTIFIES PATIENTS AT A WIDER SPECTRUM OF RISK AS COMPARED TO STATIC GOAL-BASED CUTOFFS. John A. Zambrano ${ }^{1,2}$; Pete Spanos ${ }^{2} .{ }^{1}$ Cleveland Clinic Foundation, Beachwood, OH; ${ }^{2}$ Louis Stokes Cleveland VA Medical Center, Cleveland, OH. (Control ID \#2466643)

BACKGROUND: Cross sectional HbAlc cutoffs established by quality organizations and adopted by providers to identify poorly controlled diabetes patients in population health management identify a patient at a specific point along their diabetes experience and lack the ability to characterize a trend describing actual glycemic control. An approach that characterizes the behavior of a patient's HbAlc over time may offer richer clinical understanding of risk as compared to using a static cut-off. Changes in HbAlc behavior over time including variability, upwards trends and downward trends of $\mathrm{HbAl} \mathrm{c}$ have been associated with progression to renal disease, retinopathy, and cardiovascular disease, respectively. A 2008 white paper by the NQF addresses the need for the evolution of the current diabetes quality measures including among other things, reconsidering using the use of static cutoffs and enhancing targeting of primary prevention.

METHODS: Study population included veterans with a diagnosis of type 2 diabetes mellitus currently receiving primary care at the Cleveland VA Center of Excellence in
Primary Care resident clinic. De-identified longitudinal $\mathrm{HbA} 1 \mathrm{c}$ data were obtained from CPRS for each patient from Jan 2014 to May 2015. STATA statistical software package was employed to fit regression lines of repeated $\mathrm{HbA} 1 \mathrm{c}$ measurements for each individual patient (Exhibit 1) as well as to automate the determination of the following HbA1c slope behaviors: stable, increasing and decreasing. A patient was deemed to have controlled diabetes if they demonstrated a 1 . stable $\mathrm{HbA} 1 \mathrm{c}$ slope behavior and having a mean $\mathrm{HbAlc}$ in one of the following categories: 4-6\%, 6-8\% or 2. a decreasing HbAlc slope behavior and having a mean $\mathrm{HbA} 1 \mathrm{c}$ in the categories: 6-8\%. Uncontrolled patients were identified if they demonstrated: 1 . an increasing $\mathrm{HbA} 1 \mathrm{c}$ slope regardless of average $\mathrm{HbA} 1 \mathrm{c}$ category or 2 . a stable $\mathrm{HbA} 1 \mathrm{c}$ slope and a mean $\mathrm{HbA} 1 \mathrm{c}$ in one of the following categories: 8-10\%, $10-14 \%$ or 3 . a decreasing $\mathrm{HbA} 1 \mathrm{c}$ slope and a mean $\mathrm{HbA} 1 \mathrm{c}$ in the $4-6 \%$ range. Patients with 'improving' control were only able to be identified using the LSA and were defined as those patients with a decreasing HbAlc slope and a mean HbAlc in one of the following categories: $8-10 \%, 10-14 \%$. The percentage of controlled vs. uncontrolled diabetes patients identified was compared using the LSA to those identified using static cut-offs of most recent $\mathrm{HbAlc}>9 \%$ and less than $7 \%$, drawn from nationally accepted diabetes qualtiy metrics.

RESULTS: The static goal-based approach looked at 769 diabetic veterans. The LSA approach was limited to a subgroup of the 769 diabetic veterans who had two or more $\mathrm{HbA1c}$ readings during the study time period- these included 535 of the 769 veterans. Using the above definitions for controlled and uncontrolled the static goal-based approach identified $19 \%(146 / 769)$ with uncontrolled diabetes and $65 \%$ with good diabetic control (note the different numerator definitions preclude controlled and uncontrolled percentages combining to result in $100 \%)$. The LSA approach identified $40 \%(213 / 535)$ of uncontrolled patients, $39 \%$ with good diabetic control (209/535) and $21 \%$ of diabetic veterans with improving glycemic control (113/535) (Figure 1).

CONCLUSIONS: The results of this comparative analysis suggest the use of longitudinal data over cross sectional cut-offs identifies a larger proportion of poorly controlled diabetics for those practicing proactive diabetes management. The LSA further enables identification of patients amenable to targeting for primary prevention, i.e., patients with pre-diabetes demonstrating an upward $\mathrm{HbA1c}$ slope. Current quality measures and chronic disease management approaches identify diabetics well beyond the prevention stage, missing out on a potentially important treatment window. LSA allows for identification of patients with improved glycemic control which may be used as a treatment-based and patient-centered diabetes quality metric.

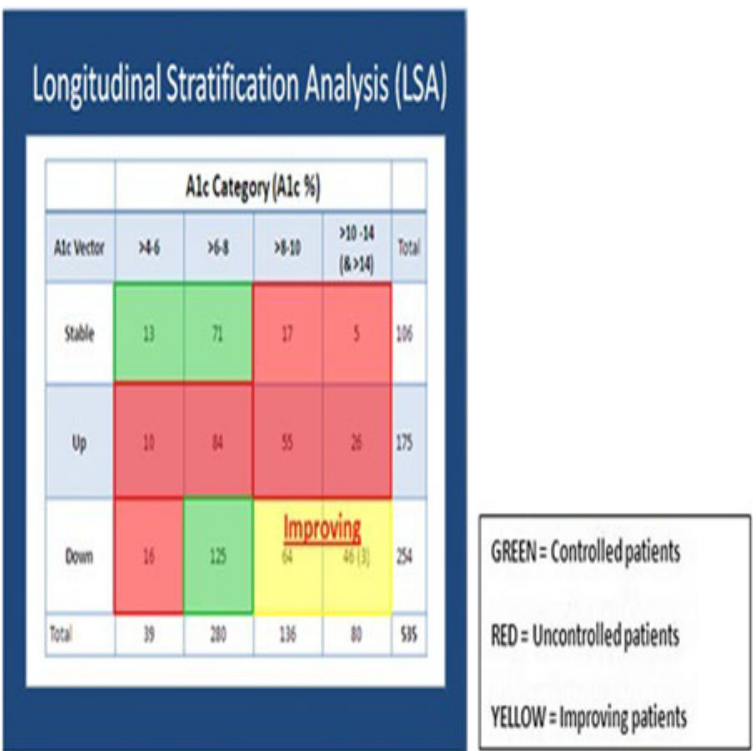

Figure 1. Results of longitudinal stratification analysis. 


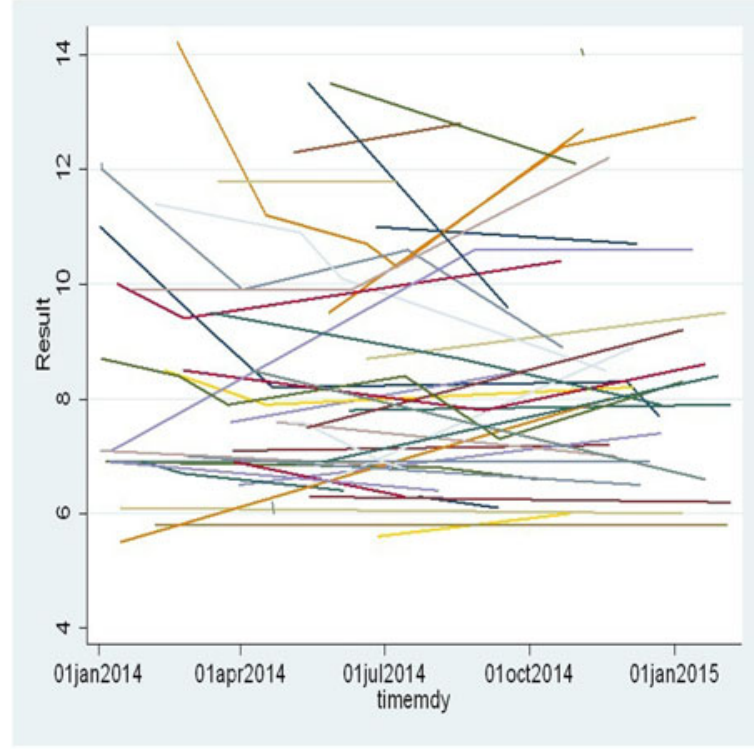

Exhibit 1. Individual patient longitudinal HbAlc curves.

USING NATURAL LANGUAGE PROCESSING TO AUTOMATE GRADING OF STUDENTS' PATIENT NOTES: PROOF OF CONCEPT Irina Gershgorin ${ }^{1}$; Marina Marin²; junchuan $\mathrm{Xu}^{2}$; So-Young $\mathrm{Oh}^{2}$; Sondra $\mathrm{Zabar}^{2}$; Ruth Crowe ${ }^{3}$; Linda Tewksbury $^{2}$; Jennifer Ogilvie ${ }^{2}$; Colleen Gillespie ${ }^{2}$; Michael Cantor ${ }^{2}$; Yindalon Aphinyanaphongs ${ }^{2}$; Adina Kalet ${ }^{4} .{ }^{1}$ NYU Langone, New York, NY; ${ }^{2}$ NYU School of Medicine, New York, NY; ${ }^{3}$ NYU School of Mediicne, New York, NY; ${ }^{4}$ New York University School of Medicine, New York, NY. (Control ID \#2468972)

BACKGROUND: Medical educators make judgments about clinical reasoning of trainees based on patient notes. This is both a rubric-driven and intuitive process, which takes a tremendous amount of effort and time. However, high stakes decisions are based on these judgments. Engineering features for inclusion in a machine learning text classification model may enable automation of this process. If we can produce a validated algorithm to score the quality of clinical reasoning in patient encounter notes produced by trainees, we may achieve automated grading of the notes. This automation will free valuable faculty time, ensure standardization and timeliness of scoring, and may have implications for assessing clinical reasoning in all clinical settings. Ultimately, it may have impact on improving healthcare quality and patient safety.

METHODS: Written and graded student notes from a high stakes OSCE held in 2013 were collected into a dataset comprising seven medical cases. The text data was cleaned, organized, analyzed and parsed using R and python. Each note was faculty rated for quality of clinical reasoning score using both a rubric and a global four-point scale. The distribution of grades was analyzed in the overall dataset and for each case. The diarrhea case was selected for deeper investigation of what constitutes a poor vs. excellent student note. Automated comparison to the rubric and manual verification of the features intended for algorithm design was performed. Each note was parsed into specific sections such as medical history, differential diagnosis (DD) and next steps (NS). DD and NS were further parsed into items rather than single words. Notes were analyzed for word and item counts as well as overlap and uniqueness of particular terms.

RESULTS: The complete dataset consists of 830 notes. The number of notes available for each case varies from 122 to 131 . Notes for all seven cases are available for 107 students. Overall, 77/830 scored 1, 401/830 scored 2, 299/830 scored 3, 53/830 scored 4. Score distributions per case varied, with some notable differences in the extreme scores $(1,4)$. Out of 118 diarrhea notes, 25 were graded as 4 and 5 as 1 . Automatic text search revealed poor agreement with the terms in the rubric both in poorest and highest-scored notes due to synonyms and spelling peculiarities. The average word count was 269 and 320 for the poorest and highest-scored notes respectively. The difference was not significant. Similarly, the average number of items for the DD and NS sections was similar, with 5 items on average in the DD and NS sections in the lowestscored notes and 6 (DD) and 7 (NS) items in the highest-scored notes. Four items appeared in both and were: 'food poisoning', 'viral gastroenteritis', 'bacterial gastroenteritis', ‘ibs'. Manual inspection showed further content similarities due to synonyms. Items such as 'malabsorption', 'hyperthyroidism' appeared only in the lowest-scored notes, while items referencing 'NSAID' and 'toxin' only in the highly-scored ones. Items in the highly-scored notes were noticeably longer and included more qualifying words such as "(un)likely", "new-onset", "possible". Comparing the distributions of item length in the DD and NS sections between the two types of notes showed significant differences (Mann-Whitney $p$-values $<0.05$ ). The average item lengths were 2 and 4 in DD and 6 and 11 in NS in poorest and highest-scored notes respectively. To increase sample sizes, this analysis was repeated by combining the notes graded as 1 and 2 vs. 3 and 4 . The highest and lowest-rated samples were 75 and 43, with average number of words per note 303 and 273 respectively. The average number of items in DD and NS was 6 for both note types. Content-wise, upon combing the 1 and 2 scored notes, "appendicitis" and "lactose" appeared as items not present in the highest-scored $(3+4)$ notes. Manual inspection revealed that appendicitis was mentioned twice in the highly-scored notes, but had "r/o" (rule-out) and "less likely" next to it. Again, item length distributions for DD and NS were significantly different in the lowest vs. highest-scored notes. The average item lengths were 2 and 5 for DD and 8 and 10 for NS for poorest and highest-scored notes respectively.

CONCLUSIONS: We engineered features that could be employed in automatic quality scoring of student free-text notes. Even though no exploitable differences can be picked up from simple note length and case-specific medical content, a thorough inspection revealed significant differences in specific properties between the highly and low-rated notes. While the number of items listed in the DD and NS sections was similar, item length distributions were significantly different. Descriptive words, often referred as semantic qualifiers which may be associated with a more sophisticated reasoning process are more prevalent in the highest-rated notes. These findings show that general features such as item length and certain word content can form the basis for an algorithm that will enable generalized non-case specific scoring of student notes.

\section{USING NATURAL LANGUAGE PROCESSING TO IMPROVE ACCURACY OF} HOSPITAL-ACQUIRED DVT AND PE MEASUREMENT Carlton R. Moore; Jamison Chang; Christine Williams; Lucy Witt; Michael Craig; Michael Langston; E. A. liles. University of North Carolina at Chapel Hill, Chapel Hill, NC. (Control ID \#2467017)

BACKGROUND: Pulmonary embolism (PE) resulting from deep vein thrombosis (DVT) - collectively referred to as venous thrombo-embolism (VTE) - is the most common preventable cause of hospital death. Patient safety indicator (PSI) \#12 is a quality measure developed by the Agency for Healthcare Research and Quality (AHRQ) that is used to assess the number of postoperative VTEs in hospitalized patients, and is reported to regulatory agencies and frequently used to provide feedback to clinicians regarding the quality of care 
they provide to patients. Tracking the total number of VTEs is currently performed at our institution by identifying relevant ICD-9 codes as secondary diagnoses. However this method, while sensitive, over-estimates true hospitalacquired VTE prevalence, making its use as a quality measure problematic. AHRQ recommends identifying VTEs cases based on results in radiology and vascular clinical reports; a process that can be time consuming and costly when numerous records need to be manually reviewed. The goal of our study is to use natural language processing (NLP) technology to develop an automated system that accurately identifies acute VTEs cases from free-text clinical reports in our institution's electronic medical record (EMR).

METHODS: We obtained the PCI \#12 reports for all perioperative VTEs occurring at UNC Hospitals from January 2014 through December $2014(n=81)$. The PCI reports were generated using VTE ICD-9 codes as secondary diagnoses. We then queried our institution's EMR and obtained all PVLs, chest CTs and VQ scans obtained on these patients during their hospitalizations. Hospitalist physicians (JC, CW, LW, EL) reviewed each of the 81 cases to adjudicate the presence or absence of hospital-acquired VTEs, this was used as the gold standard to compare NLP and ICD-9 code performance. We measured sensitivity for NLP and false-positive rates for both ICD-9 code and NLP methods. We used c-TAKES (clinical Text Analysis and Knowledge Extraction Tool), an open-source software package, to develop an NLP tool to identify the presence of VTEs in free-text electronic radiology and vascular reports (figure). We modified the c-TAKES software to negate relevant concepts not consistent with acute or new onset VTEs. For example, the 'DVT' term in the text 'stable appearance of femoral vein DVT' is negated because the DVT is not acute or new onset. The output of the program was a list of acute VTE cases.

RESULTS: Of the 81 hospital-acquired VTE cases identified using ICD-9 codes, 11 of the 81 were determined not to be hospital-acquired based on manual review ( $13.6 \%$ falsepositive rate). In the 11 false-positive cases, there was either no radiology or vascular report indicating VTE or the VTE was not a new finding found during the patient's hospitalization. The NLP tool correctly classified all 11 false-positive cases without missing cases of 'true' hospital-acquired VTEs (i.e., $100 \%$ sensitivity and $0 \%$ falsepositive rate)

CONCLUSIONS: We developed a NLP tool that accurately identifies hospitalacquired VTEs based on free-text electronic radiology and vascular report in our institution's EMR. Future directions include using the tool to prospectively review electronic reports and provide accurate real-time feedback on hospitalacquired VTE cases to clinicians and quality improvement specialists.

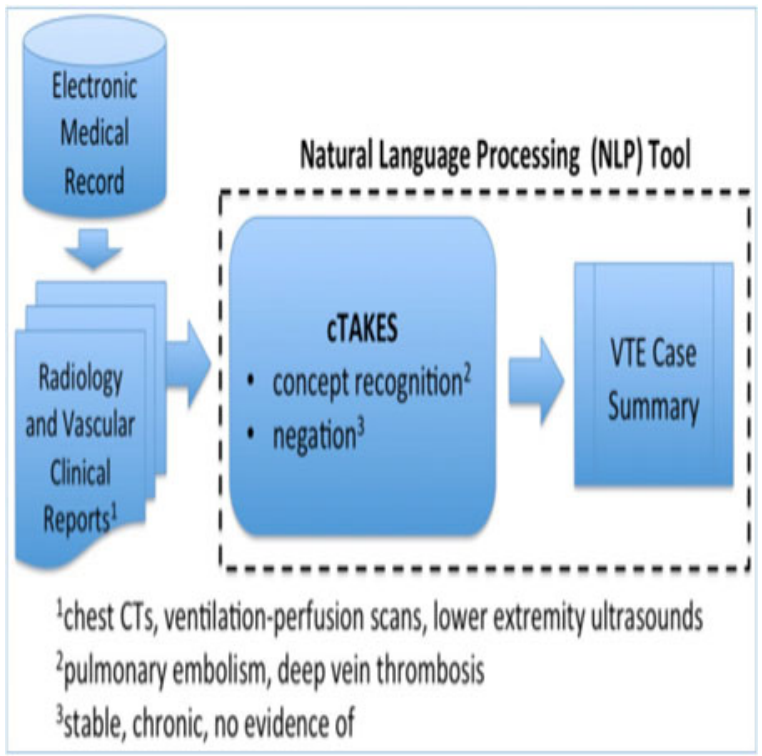

USING NON-ENGLISH SURVEY INSTRUMENT AS A MARKER FOR LIMITED ENGLISH PROFICIENCY: AN APPLICATION FOR EXAMINING NATIONAL DISPARITIES IN HYPERTENSION Eun Ji Kim ${ }^{1}$; Taekyu Kim ${ }^{4}$; Michael Paasche-Orlow ${ }^{2}$; Amresh D. Hanchate ${ }^{3} .{ }^{1}$ Boston University, Cambridge, MA; ${ }^{2}$ Boston University, Boston, MA; ${ }^{3}$ Boston University School of Medicine, Boston, MA; ${ }^{4}$ Massachusetts General Hospital, Boston, MA. (Control ID \#2468483)

BACKGROUND: Low English proficiency has been shown to be associated with poor health care status, treatment and outcomes. However, no national health and health care surveys include measures of low English proficiency. Our objective was to utilize data on the use of a non-English survey instrument as a proxy measure of limited English language proficiency and assess its applicability in understanding disparities in hypertension. We also examined the sensitivity of this measure relative to previously used indicators of acculturation: spoke English at home; US citizen; and born outside of US.

METHODS: As a nationally representative sample of all adults, we pooled data on all adults in the 2003-2012 National Health and Nutrition Examination Surveys (NHANES). We included all survey respondents 18 years or older and with valid data on hypertension status, blood pressure measurements, and anti-hypertensive medication use $(n=25,380)$. The primary survey instrument was administered in person at the respondent's residence using a Computer-Assisted Personal Interviewing (CAPI) system. Respondents were offered the choice of being interviewed in English or Spanish or a different language using an interpreter; based on this information, we defined a dichotomous indicator of the use of a non-English survey instrument and identified respondents who were interviewed in Spanish or in another language using an interpreter as having used a non-English instrument. Our main outcome was elevated blood pressure prevalence identified by $\mathrm{SBP}>140 \mathrm{mmHg}$ or DBP $>90 \mathrm{mmHg}$ on physical examination. As secondary outcomes we also examined self-report of prior diagnosis of hypertension and use of antihypertensive medication use. We compared prevalence of hypertension between those who used a non-English instrument and others who used an English instrument; using multivariate logistic regression we performed this comparison while adjusting for other potential differences across the two groups, including, in age, sex, race/ethnicity, and socioeconomic status. We performed similar analyses for the secondary outcome measures, and for subgroups based on the mode of non-English instrument: use of Spanish instrument and use of interpreter.

RESULTS: The study sample of 25,380 survey participants represented 208 million of the US population. $14.2 \%(n=3591)$ of the sample used a non-English instrument; of these, $12.0 \%(n=3055)$ used the Spanish questionnaire and $2.1 \%(n=538)$ used an interpreter to complete the survey. The prevalence of elevated blood pressure in our overall sample was $18.0 \%$. After adjustment, use of a non-English survey instrument was associated with increased odds of elevated blood pressure $(\mathrm{OR}=1.41[1.05,1.89])$ compared to those who used an English survey instrument; of the three measures of acculturation also included in the regression, two were not associated with a significant difference in elevated blood pressure (spoke English at home, and US citizen), while those born outside US had lower risk of elevated blood pressure. High risk of elevated blood pressure persisted in a group of participants with known prior diagnosis of hypertension $(\mathrm{OR}=1.50[1.05,2.15])$. Among participants with prior diagnosis of hypertension and not taking anti-hypertensive medication, non-English instrument use was associated with increased odds of having elevated blood pressure $(\mathrm{OR}=3.61[1.18,11.02])$. Use of Spanish questionnaire was not associated with increased likelihood of having elevated blood pressure on physical examination, even after looking at subgroups of participants with or without prior diagnosis of hypertension. Interpreter use was associated with increased odds of having elevated blood pressure $(\mathrm{OR}=1.55[1.10,2.18])$. This association persisted in participants with prior diagnosis of hypertension and not taking medication $(\mathrm{OR}=3.83[1.38,10.63])$ and participants with no prior diagnosis of hypertension $(\mathrm{OR}=1.57[1.01,2.43])$. There was no association between non-English instrument use 
and the likelihood of taking anti-hypertensive medications among participants with prior diagnosis of hypertension.

CONCLUSIONS: To our knowledge, this is the first study that examines the relationship between non-English instrument use and blood pressure using nationally representative data. This study suggests that non-English instrument use is associated with uncontrolled hypertension and undiagnosed hypertension, and interpreter service should be utilized to improve hypertension outcome. Future studies should examine the validity of using a nonEnglish survey instrument as a measure of limited English proficiency and the relationship between this factor and barriers to care and health literacy.

USING PHOTOVOICE AND ASSET MAPPING TO INFORM A COMMUNITYBASED DIABETES INTERVENTION Nicole M. St. Omer Roy ${ }^{1}$; Jana Florian ${ }^{1}$; Lisa M. Quintiliani ${ }^{3}$; Ve Truong ${ }^{2}$; Yi Feng ${ }^{1}$; Philippe P. Bloch ${ }^{3}$; Zlatka Russinova ${ }^{3}$; Karen E. Lasser ${ }^{2}$. ${ }^{1}$ Boston Medical Center/Boston University School of Medicine, Boston, MA; ${ }^{2}$ Boston Medical Center, Boston, MA; ${ }^{3}$ Boston University, Boston, MA. (Control ID \#2469037)

BACKGROUND: In addition to medical care, effective diabetes control requires dedicated self-care. This self-management takes place within a complex social and environmental context. A better understanding of the perceived value, awareness, and presence of community resources for diabetes management may help design a robust community-based diabetes intervention. This study utilized community asset mapping, the systematic documentation of resources in the environment, and photovoice, a participatory action research method which engages participants in identifying and reflecting on issues in their community through a specific photographic method. We utilized photovoice to explore factors that impact diabetes self-management. To our knowledge, these methodologies have not been previously combined to provide complementary data on the relationship between subjective perspectives about diabetes self-management and relevant resources in the community. The aims of this study were to (1) identify individual and community factors contributing to diabetes managemen in Boston census tracts with high numbers of patients with poorly controlled diabetes ("diabetes hot spots") and (2) document the presence and availability of resources related to the identified factors.

METHODS: Eleven patients residing in diabetes hot spots participated in a photovoice group at a public library. These participants were identified from previous geocoding work, in which we outlined diabetes hot spots by using ArcGIS software to geocode patients from Boston Medical Center with poorly controlled diabetes (hemoglobin $\mathrm{A} 1 \mathrm{C}$ levels $>9$ ). In the photovoice group, participants took photos about barriers to diabetes control, wrote accompanying narratives, and discussed photovoice pieces through a facilitated group process We audio recorded, transcribed, and coded the discussions to identify relevant themes. To further explore themes related to the environment, we conducted asset mapping in five diabetes hot spots that contained the highest numbers of patients with poorly controlled diabetes. We performed internet searches to create a preliminary list of community resources, including places of worship, community centers, libraries, community gardens, farmer's markets, food pantries, grocery stores, convenience stores, restaurants, gyms, and parks, and mapped these using ArcGIS software. Two research assistants conducted walking surveys of each census tract to verify the presence of these assets and utilized published assessment tools to evaluate physical activity resources, walking environment (e.g. presence of sidewalks and park benches), availability of "diabetic-friendly" foods in food stores, and availability of healthy food choices in restaurants.
RESULTS: Themes that emerged in the photovoice group included both barriers and facilitators of diabetes self-management. Barriers included lack of access and high cost of healthy food and exercise facilities, local restaurants serving only unhealthy foods, and family obligations, including sharing cooking and eating meals with family members. Facilitators to self-management included participation in a church community, which helped participants reduce the daily stresses of living with diabetes. Participants shared strategies for diabetes control (e.g. healthy food choices and replacements, strategies to decrease stress, and stores and community organizations that offer quality produce) and stated intentions to change behaviors. Asset mapping identified 114 community assets including 22 food stores, 22 restaurants, and five exercise facilities. Each diabetes hot spot contained at least one food store with between five and nine varieties of fruits and vegetables and $73 \%$ of food stores overall contained fresh produce. The majority of restaurants were fast food restaurants $(68 \%)$, and no restaurants served whole wheat bread or brown rice. Mapping also showed that exercise facilities had no signage regarding hours or services; memberships ranged from free to $\$ 9.95$ per month. These findings were not consistent with participants' reports in the photovoice group

CONCLUSIONS: There were two takeaways from this study. First, when used in combination, photovoice and community asset mapping provide richer data to inform chronic disease interventions than either method provides alone. We identified a mismatch between what photovoice participants thought to be available in their community and what we found to be available through asset mapping. These results support previous work that demonstrates the discordance between perceptions of the local food and amenities environment and the objective reality of that environment. Second, incorporating photovoice and community asset mapping into a communitybased diabetes intervention may help patients gain insight into their disease and motivate them to make positive lifestyle changes. In this study, the photos and narratives allowed participants to reflect at a deeper level about their personal circumstances while the group discussion facilitated knowledge sharing of different diabetes management strategies. Integrating the results of community asset mapping into a diabetes intervention may additionally bring awareness to underutilized resources in the community.

USING TEXT MESSAGES TO SUPPORT IMPROVED SELF-CARE DECISIONS IN MEDICALLY UNDERSERVED AFRICAN-AMERICANS WITH UNCONTROLLED DIABETES: BEST PRACTICES FROM THE PATIENT'S PERSPECTIVE Aniekanabasi N. Udoko; James E. Bailey; Samantha Ransone; Bonnie L. Binkley; Justin Gatwood; Mace Coday; J. Carolyn Graff. University of Tennessee Health Science Center, Memphis, TN. (Control ID \#2469838)

BACKGROUND: Text messaging (TM) is increasingly used by practices and health systems throughout the country to engage patients with chronic disease in self-care. While TM has been observed to be an effective, low cost approach for engaging patients with chronic disease self-care, little is known about the most effective TM program characteristics from the perspective of vulnerable patients in medically underserved areas. Further research is needed to direct the development of patient-centered TM approaches in primary care settings nationwide. This study sought to explore the characteristics of text messages and primary care-based TM programs most likely to be effective in supporting improved self-care decisions in medically underserved African-Americans with uncontrolled diabetes and multiple chronic conditions. In particular, we sought to determine the most desirable TM frequency, message content, message length, message interactivity, and message source for diabetes health-related text messages from the patient's perspective.

METHODS: This cross-sectional mixed methods study employed a concurrent selfadministered survey and focus group. Consistent with this method, quantitative and 
qualitative data were collected simultaneously, analyzed separately, and merged to provide a holistic view of the potential TM characteristics most likely to be effective in supporting chronic illness self-care. Quantitative data were collected using a self-administered survey, while qualitative data were collected using focus group interviews. In support of the study aims, both survey and interview questions were directed at assessing interest in a motivational TM program along with the kinds of TM that would best encourage patients to better care for themselves and their diabetes. Participants were recruited from a medically underserved area in Memphis, Tennessee. Focus group responses were uploaded into the NVivo qualitative analysis software program and main themes were identified. Standard frequencies were calculated for survey responses.

RESULTS: Three focus groups were conducted and $100 \%$ of focus group participants $(N=22)$ completed the survey. Mean participant age was 57 years old, and $86.4 \%$ of the participants were female. $86.4 \%$ were black or African American. One hundred percent of participants use a cell phone and $77.3 \%$ send and receive TM, with $50 \%$ using TM several times a day. The $22.7 \%$ that chose the "I don't text" response cited financial reasons, visual impairment, lack of interest and lack of know how as reasons for not texting. When asked about message length, $59.1 \%$ preferred short messages. Regarding message content, $77.3 \%$ indicated an interest in receiving messages about healthy eating and $86 \%$ or respondents were interested in receiving motivational messages. Main themes identified from the focus groups were: age and texting habits, TM characteristics, perceived advantages and barriers of TM, and diabetes self-care struggles. Concerning age and texting habits, participants expressed differences in the texting habits of younger and older patients. Participants also identified 'short and to the point' messages, one way messages, and inspirational messages as desirable TM characteristics. Identified TM advantages include but are not limited to: less intrusive than phone calls, avoids prolonged conversation, and convenient and quicker response. Identified TM disadvantages include but are not limited to: added phone bill expense, not conducive for visually impaired patients, and safety concerns regarding texting and driving.

CONCLUSIONS: This study suggests that health-related TM programs targeting vulnerable patients with uncontrolled diabetes should employ short and inspirational messages without a response requirement. Our results show that cellphones and TM are widely available and that age is not a deterrent to texting, suggesting that a healthrelated TM program is likely to garner a favorable response in similar underserved populations. Underserved diabetes patients are likely to participate in motivational TM programs that employ personalized TM with desirable message characteristics. Primary care practices and health systems seeking to develop patient centered TM programs for vulnerable patients with chronic diseases should strive to tailor health-related text messages to meet patients' personal health needs and interests.

USING THE HOSPITAL SETTING TO IDENTIFY AND IMPROVE VISION NEEDS OF INPATIENTS WITH DIABETES Allison J. Louis ${ }^{1}$; Vineet M. Arora ${ }^{2}$, ${ }^{1}$; Victoria I. Moreira ${ }^{3}$; Janaki Patel ${ }^{1}$; Ashley M. Snyder ${ }^{1}$; Seenu M. Hariprasad ${ }^{1}$; David Meltzer ${ }^{1}$; Valerie G. Press ${ }^{1} .{ }^{1}$ University of Chicago, Chicago, IL; ${ }^{2}$ University of Chicago Medical Center, Chicago, IL; ${ }^{3}$ Medical College of Georgia, Augusta, GA. (Control ID \#2466043)

BACKGROUND: Patients with diabetes are at higher risk for medical eye disease, such as diabetic retinopathy and macular edema as well as cataracts and glaucoma, compared to the general population. It is recommended that patients with diabetes receive outpatient preventative vision care, including retinal and dilated eye exams at least yearly. However, gaps in preventative care often occur, particularly among underserved minority patients. Therefore, to reduce health disparities for minority patients with diabetes, the hospital setting may be just the opportunity needed to identify and connect patients to vision care opportunities to address their vision care needs. Our objective was to identify the vision care needs of inpatients with diabetes and to assess the feasibility of using the hospital setting to help facilitate post-discharge vision care appointments. This is a preliminary analysis of an ongoing study. METHODS: Research assistants (RAs) at the University of Chicago Medicine enrolled participants from an ongoing quality-of-care study. Participants who were 18 years or older, English-speaking, and cognitively intact were eligible; consenting general medicine inpatients were administered the Visual Function Questionnaire (VFQ) and had their vision screened. Those with vision worse than 20/50 in both eyes were given reading glasses and had their vision rescreened to determine if vision could be corrected. Participants with diabetes were identified by medical record and self-report. A subset of eligible participants were given readers to take home. Upon discharge, participants were given information regarding their vision and how to obtain follow-up care. RAs called participants 30 days after discharge from the hospital to re-administer the VFQ and obtain follow-up vision care. Means were compared using unpaired and paired t-tests and categorical comparisons used Chi-square.

RESULTS: To date 225 inpatients were recruited for participation and had their vision screened in the hospital. One fifth (20\%) of participants had diabetes, half of whom were female (50\%), $83 \%$ were African American (83\%), and the mean age was 54 years. About $1 / 3$ of participants with diabetes had sufficient vision $(30 \%, 14 / 46)$; about $1 / 3$ of participants had insufficient vision but were wearing corrective lenses (32\%, 15/46); and the remaining participants had insufficient vision and did not have corrective lenses $(37 \%, 17 / 46)$. All participants without any lenses $(n=17)$ had their vision re-screened using non-prescription readers of whom. $2 / 3$ of paraticipants' vision corrected (11/17) just over half (9/17) went home with readers. Nearly half (49\%) reported that they see an eye doctor every year. During hospitalization, over 3/4 (176/ 225 ) of the total study population completed the VFQ. The VFQ was lower for participants with diabetes $(n=37)$ versus those without diabetes $(n=139$; mean score of 83 versus to $88 ; p<0.05$ ). Although early in the study, to date there are no significant findings at the 30-day follow-up as only 12 participants completed the post-discharge VFQ to date. Participants with diabetes $(n=7)$ had a mean VFQ score of 86 versus 90 in those without diabetes $(n=5 ; p=0.2)$. Comparing the VFQ scores of participants during hospitalization and upon 30-day follow-up also revealed no significant difference $(n=7 ; p=0.6)$. Almost $3 / 4$ (32/46) of inpatients with diabetes agreed that they needed to see an eye doctor and $89 \%$ (41/46) said that they would be very likely to see one if told to do so by a health care provider. On 30-day followup, $55 \%(6 / 11)$ endorsed that they believe they need to see an eye doctor now, however only $36 \%$ (4/11) reported that they had scheduled an appointment with an optometrist. Interestingly, on 30-day follow-up all participants (11/11) still endorsed that they would be very likely to see an eye doctor if told to do so by a health care provider.

CONCLUSIONS: Our study demonstrates that inadequate vision is a problem for inpatients with diabetes. A third of the population had insufficient vision without any corrective lenses of any kind, of whom two-thirds were able to have their vision corrected by readers commonly found at drug stores. Since this study shows that patients with diabetes have lower VRQOL in the hospital and likely after discharge as well, it is even more important to recognize and take advantage of opportunities to ensure that patients with diabetes get preventative eye care. Because hospitalization is an important transition point in care and because written materials are heavily relied upon in the hospital setting for patient discharge instructions and education materials, the hospital setting is an important opportunity to provide vision screening and intervention, especially since more than half of those with insufficient vision had their vision corrected using simple drug store readers. Hospitalization also represents an important opportunity connect patients to preventative vision care, since only half of those with diabetes report seeing an eye doctor 
every year. Although only a third of patients scheduled an appointment with an eye doctor upon 30 day follow-up, all still agreed that they would be very likely to see an eye doctor if a health care professional told them to do so, demonstrating the importance that health care professionals can play in the preventative care of their patients.

\section{UTILIZATION OF 4 T SCORE TO DETERMINE THE PRETEST PROBABIL-} ITY OF HEPARIN INDUCED THROMBOCYTOPENIA AT UNITY HOSPITAL

Yazan Samhouri; Mohammad Telfah; Ruth Kouides; John D'Souza; Timothy Woodlock. Rochester Regional Health/Unity Hospital, Rochester, NY. (Control ID \#2457653)

BACKGROUND: Thrombocytopenia is common in hospitalized patients. Heparin Induced thrombocytopenia (HIT) is a life threatening condition which can lead to extensive thrombosis. Diagnosis of HIT relies on clinical suspicion determined by $4 \mathrm{~T}$ score, and immunoassays through testing for anti PF4/Heparin antibodies. Clinical practice guidelines published by the American Society of Hematology (2013) recommended use of the $4 \mathrm{~T}$ score before ordering the immunoassays. If the score is less than or equal to 3 , a low probability for HIT is determined, and no further testing is required. If the score is more than 3, then anti PF4/Heparin ELISA should be ordered and all heparin products should be stopped. While the negative predictive value of a low probability $4 \mathrm{~T}$ score is $99.8 \%$, it has a low positive predictive value (14\%-64\%). The purpose of this study was to evaluate the utilization of $4 \mathrm{~T}$ score before ordering anti PF4/Heparin antibodies.

METHODS: We did a retrospective chart review for patients who are 18 year or older, admitted to Unity Hospital between 07/01/2013 and 12/312014, and had anti PF4/Heparin antibodies ordered. Patients who had a prior diagnosis of HIT or had ESRD on hemodialysis were excluded. The following parameters were assessed: Age, sex, length of stay, timing of platelets drop, thrombosis, other possible causes of thrombocytopenia, previous exposure to heparin, documentation of $4 \mathrm{~T}$ score, type of anticoagulation, Anti PF4/ Heparin Antibodies result, and treatment. We calculated the proportion of patients who had $4 \mathrm{~T}$ score documented prior to ELISA testing and proportion of ELISA tests which were not indicated due to a $4 \mathrm{~T}$ score less than or equal to 3 based on a retrospective calculation.

RESULTS: Review of 123 patients, with average age of 69.4 years, showed that testing was indicated in 18 patients (14.6\%). Six subjects had positive results, testing was indicated in all of them. $4 \mathrm{~T}$ score was documented in 3 patients $(2.4 \%)$. Of note, this may not reflect the rate of $4 \mathrm{~T}$ score use. Some patients with thrombocytopenia may not have the test ordered due to low $4 \mathrm{~T}$ score and would not be in our sampling frame. CONCLUSIONS: Anti PF4/Heparin antibodies are being overused in thrombocytopenia work up at Unity Hospital, with the fact that $85.4 \%$ of tests in this study were not indicated, costing $\$ 9,345$. More conferences and lectures are needed to increase awareness among health care providers in our hospital. A prompt for a $4 \mathrm{~T}$ score was added to electronic medical records before ordering the test as a step to improve high value care and reduce unnecessary health care cost

UTILIZING DIRECT ASSESSMENT TO IDENTIFY FUNCTIONAL IMPAIRMENTS IN HOMELESS ADULTS Maria Maiaroto'; Juan Rodriguez-Guzman ${ }^{4}$, 1; David DeWorsop $^{2,}{ }^{\text {; }}$, Corey Hassell ${ }^{3,1}$; Theddeus Iheanacho ${ }^{4,}{ }^{1}$; Adam P. Mecca ${ }^{4}$, ; Marcia C. Mecca ${ }^{2,1} .{ }^{1}$ VA Connecticut, West Haven, CT; ${ }^{2}$ Yale University, Hamden, CT; ${ }^{3}$ Quinnipiac University, Hamden, CT; ${ }^{4}$ Yale University, New Haven, CT. (Control ID \#2467732)

BACKGROUND: Traditionally, assessments of instrumental activities of daily living in the elderly have been based on self-reports, but concerns about underestimating impairment have led to studies of directly observed function
(Diehl et al., 1995). The Health Care for Homeless Veterans (HCHV) program at VA Connecticut aims to provide housing assistance to patients who are homeless or at risk of homelessness. Several studies have reported a high prevalence of both cognitive and functional impairment among homeless adults, even at as young as age 50 (Depp et al., 2015). This population often lacks collateral sources of information to confirm functional status. Therefore, we aim to utilize a directly observed assessment of function in a geriatric syndrome screening clinic within the HCHV at VA Connecticut, and identify relationships between directly observed functional assessment, self-report functional assessment, and cognitive assessments.

METHODS: Patients were 18 years old or older, eligible for VA benefits, and homeless or at risk for homelessness. Patients received either a full or a partial screening as part of this project. The full screening included a revised form of the Direct Assessment of Functional Status (DAFS-R; McDougall et al., 2010) to assess skills in the domains of communication, finance, shopping, and medication management. In addition, subjective measures of functional status [Modified Katz Activities of Daily Living Scale, Brief Instrumental Functional Scale (BIFS)], depression screening (Patient Health Questionnaire 9), and cognitive assessments [Montreal Cognitive Assessment (MoCA), Trail Making Test Part B (Trails B)] are performed. The DAFS-R contains 55 items that assess the skills required for communication (using a phone and mailing a letter), financial affairs (counting currency and balancing checkbooks), shopping (recalling a shopping list and choosing the shopping list items from a shelf), and medication management (identifying information on a prescription bottle, using a telephone for prescription refills, and managing medications in a pillbox.) Partial screening includes the subjective measures of functional status, depression screening, and the cognitive assessments. Here we report the results of directly observed functional testing, as well as the associations with subjective measures of function and cognitive screening.

RESULTS: A total of 17 patients received a full assessment including directly observed assessment of function. Average age was 55.8 \pm 10.2 years and $93.4 \%$ were male. Patients reported an average education level of $12.7 \pm 1.3$ years. Based on chart diagnoses, $50.0 \%$ had a mood disorder, $37.5 \%$ an anxiety disorder, $12.5 \%$ a psychotic disorder, $43.8 \%$ an alcohol use disorder, $6.3 \%$ an opioid use disorder, and $18.8 \%$ a stimulant use disorder. Four patients $(25 \%)$ had a chart diagnosis of MCI or dementia. None of the patients had a diagnosis of traumatic brain injury or stroke recorded in the chart. One patient $(6.3 \%)$ had a seizure disorder. On direct assessment of functional status (DAFS-R), the mean score was $45.6 \pm 8.2$ ( $\max$ score possible of 57$)$. DAFS-R score was significantly correlated with MoCA score $\left(r^{2}=0.2399, p<0.05\right.$, Spearman correlation, Figure 1), but not with Trails B alone. Patients that had cognitive impairment (MoCA $<26$ represented by horizontal red dotted line in Figure 1, or Trails $\mathrm{B}<-1.5$ standard deviations below age and education adjusted norms) scored significantly lower on the DAFS-R than those without cognitive impairment ( $p<0.05$, Wilcoxon rank sum test, Figure 2). Importantly, subjective assessments of function completed by asking a patient about their needs using the BIFS had no correlation with the DAFS-R, MoCA, or Trails B. CONCLUSIONS: This study demonstrates the need for directly observed assessments of function in homeless patients. Impairments are both prevalent and under-recognized by assessments based only on subjective interview. Identification and support of functional impairment may be an important intervention for patients with unstable housing whose impairment may contribute to continued housing instability or homelessness. Future goals of our work are to explore the relationship between housing instability and functional impairment and evaluate the impact of geriatric syndrome screening on housing outcomes. 


\section{Figure 1: Association between Cognition and Directly Observed Function}

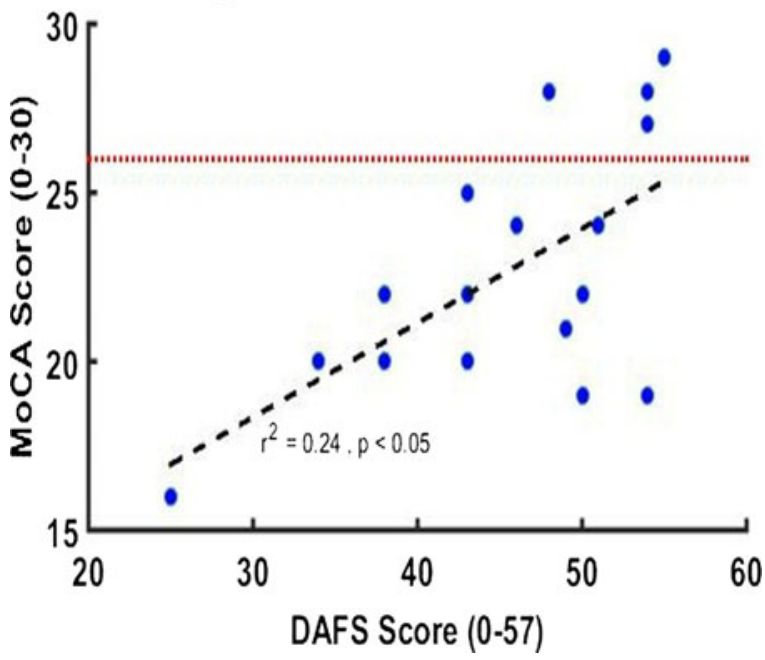

Figure 2: Patients with Cognitive Impairment have more Functional Impairment

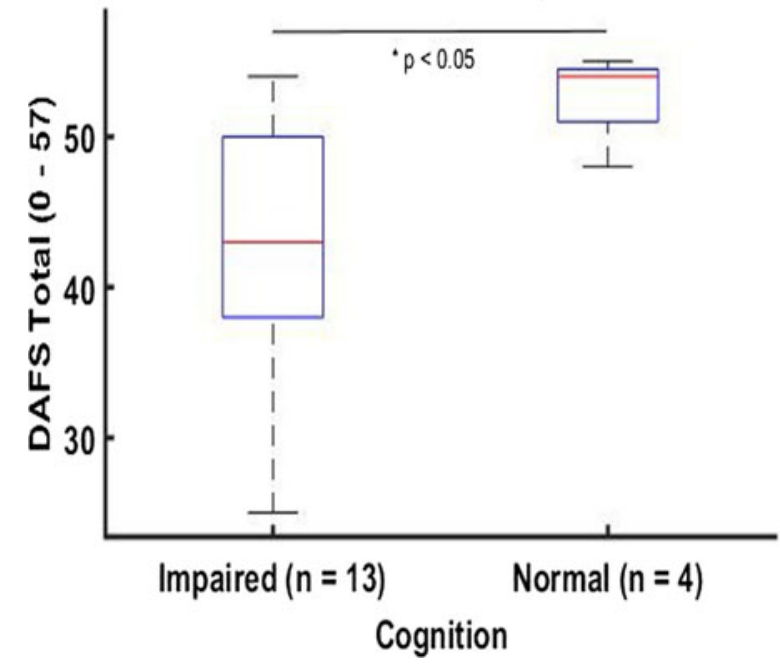

UTILIZING MEDICARE CLAIMS FOR THE ASSESSMENT OF INFLUENZA VACCINATION COVERAGE IN THE US ELDERLY Mallika Mundkur'; Clement J. McDonald ${ }^{2}$. 'Brigham and Women's Hospital, Lexington, MA; ${ }^{2}$ National Library of Medicine, Bethesda, MD. (Control ID \#2470258)

BACKGROUND: Surveys are currently the gold-standard for assessment of vaccination coverage in the population, despite criticisms of high costs of administration and issues with recall bias (Government Accountability Office, 1996; Zimmerman, 2003). Insurance claims are an underexplored and inexpensive alternative source of data that may also be used to assess vaccination (Lochner, 2015). Using influenza vaccination as a general framework, we investigated the use of Medicare claims to assess absolute rates and trends of vaccination, comparing claims-based estimates with survey-based estimates derived from the same population.
METHODS: Utilizing fee-for-service Medicare Claims representing nearly 1 billion personyears of data, we estimated year-specific rates of vaccination among adults 65 and older for years spanning 2006 through 2011 using repeated cross-sectional measurements for each year. For comparison, we derived estimates in a similar manner using responses from the Medicare Current Beneficiary Survey (MCBS). Claims-based estimates relied on HCPCS codes from Carrier and Outpatient Medicare files to identify vaccinated individuals, while MCBS-estimates used responses to a single question about receipt of influenza vaccination during the previous year. Using claims, we also characterized temporal trends in vaccination, overall, and by race and ethnicity. We also defined a novel metric that could be derived from claims data only, median time-to-vaccination, using the date of service field found within claims and using August 1st of each year as day 0.

RESULTS: For each year evaluated, rates of vaccination were estimated to be twenty percentage points higher when using survey responses rather than claims as the primary data source - in 2010-2011, rates of vaccination were 74 and $53 \%$, according to surveys and claims, respectively. Claims-based temporal trends in vaccination revealed minimal change in overall rate for the year 2006-2011, and indicated significant disparities in rates of vaccination among by race and ethnicity, with lower rates of vaccination among Blacks and Hispanics than non-Hispanic Whites. Median time-to-vaccination varied by year, with measurable population level delays in vaccination of up to 19 days when comparing vaccination seasons with and without known delays (e.g. 2004-2005 vs. 2011-2012).

CONCLUSIONS: For each year evaluated, rates of vaccination were estimated to be twenty percentage points higher when using survey responses rather than claims as the primary data source - in 2010-2011, rates of vaccination were 74 and $53 \%$, according to surveys and claims, respectively. Claims-based temporal trends in vaccination revealed minimal change in overall rate for the year 2006-2011, and indicated significant disparities in rates of vaccination among by race and ethnicity, with lower rates of vaccination among Blacks and Hispanics than non-Hispanic Whites. Median time-to-vaccination varied by year, with measurable population level delays in vaccination of up to 19 days when comparing vaccination seasons with and without known delays (e.g. 2004-2005 vs. 2011-2012). Our study is the first to directly compare trends in vaccination depicted by claims with those from traditional survey-based methods. Although we find that absolute estimates of vaccination rates differ substantially depending upon whether claims or surveys are used, temporal trends are similar, suggesting a role for claims-based approaches in longitudinal surveillance. Claims also have the unique ability to capture time-to-vaccination, and represent a novel metric that could be used to track efficiency of vaccination programs or disparities in access. In summary, we find that claims are an important source of data that may be used in conjunction with surveys.

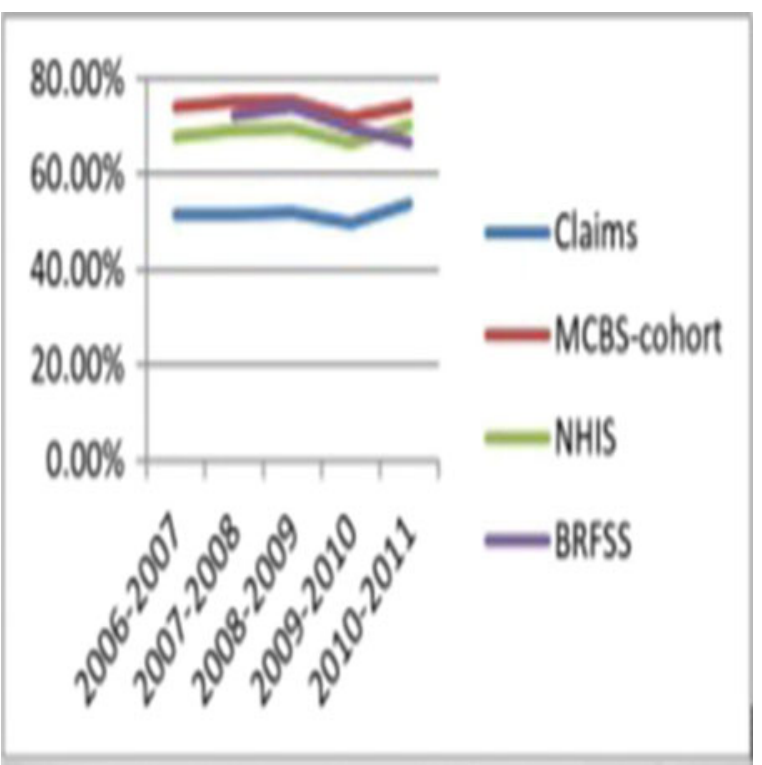


VACCINATION IN SOLID ORGAN TRANSPLANT RECIPIENTS: AN INSTITUTIONAL SURVEY Muhammad Yasser Alsafadi ${ }^{1}$; Odaliz Abreu-Lanfranco ${ }^{2}$; Linda Kaljee $^{3}$; Marwan Abouljoud ${ }^{1}$; Mayur Ramesh ${ }^{2}$; George Alangaden ${ }^{2} .{ }^{1}$ Henry Ford Hopsital, Detroit, MI; ${ }^{2}$ Henry Ford Hospital, Detroit, MI; ${ }^{3}$ Wayne State University, Detroit, MI. (Control ID \#2437312)

BACKGROUND: Immunization against vaccine-preventable diseases is recommended for solid organ transplant (SOT) patients yet vaccines remain underutilized in this vulnerable population. Prior studies at our institution estimated that completion rates of all recommended vaccinations at 1-year post liver transplantation could be as low as $5 \%$. We conducted a survey to understand the reasons for this low vaccine uptake.

METHODS: A questionnaire was developed to determine attitudes towards vaccinations, estimates of vaccine completion, potential barriers and improvement measures. The anonymous survey was distributed electronically to SOT team members. The questionnaire was based on a Likert scale format and questions could have multiple answers.

RESULTS: Twenty-six SOT team members returned the survey. Seventy-three percent agreed that vaccine administration is the shared responsibility of the primary care physician and the transplant team. Forty-six percent agreed that lack of communication between transplant teams and primary care physicians is a major barrier to vaccine uptake. Seventy-seven percent believe that easy to access web-based information about vaccine use in SOT patients will improve vaccine uptake. Seventy-three percent agreed that the availability of written materials to provide to patients and their families would improve vaccination rates. Forty-six percent agreed that vaccines not being routinely stored at the transplant clinic is a major obstacle to vaccine administration.

CONCLUSIONS: Vaccination rates in SOT recipients remain low. The low uptake appears to be the result of a combination of factors. Patients' views on vaccination, lack of ownership of the immunization process, and ineffective resource utilization were amongst the factors most often quoted. Interventions such as the use of educational materials, seminars, system-based interventions and improved communication between SOT teams and primary care physicians may improve vaccination rates.

VACCINATION RATES IN KIDNEY TRANSPLANT RECIPIENTS: ARE WE FOLLOWING THE GUIDELINES? Muhammad Yasser Alsafadi ${ }^{1}$; Shiloh Tackett ${ }^{1}$; Daryl Sudasena ${ }^{1}$; Victor Aguin Melendez ${ }^{1}$; Erica Graney ${ }^{1}$; Tommy J. Parraga $^{1}$; Odaliz Abreu-Lanfranco ${ }^{2}$; Mayur Ramesh ${ }^{2}$; George Alangaden ${ }^{2} .{ }^{1}$ Henry Ford Hopsital, Detroit, MI; ${ }^{2}$ Henry Ford Hospital, Detroit, MI. (Control ID \#2438527)

BACKGROUND: Solid organ transplant (SOT) recipients are at increased risk for vaccine preventable infections. The Infectious Diseases Society of America and The American Society of Transplantation (AST) guidelines recommend vaccination of solid organ transplant recipients 2-6 months prior to transplantation according to the CDC Advisory Comittee on Immunization Practices (ACIP) Schedule. If a vaccine is missed pre-transplantation, it is recommended that inactivated vaccines be given after the transplantation. Information on the adherence of transplant centers to these guidelines is scarce.

METHODS: We performed a retrospective chart review of 400 consecutive adult patients that received a kidney transplant between 2009 and 2012 at Henry Ford Hospital, Detroit, MI. Vaccination status, eligibility, and completion of appropriate vaccines were determined. Vaccines reviewed were hepatitis B virus, hepatitis A virus, influenza, pneumococcus, and Tdap. Eligibility for vaccination was assessed using AST and the CDC ACIP recommendations based on age, medical conditions, antibody titers, and seasons.

RESULTS: Demographics: Gender: Males $65 \%$. Average age: 52.5 years (+/- 13.5). Race: Caucasian $45 \%$, black $42 \%$, and other $12 \%$. Comorbidities: hypertension $92 \%$, diabetes $48 \%$, and malignancy $5 \%$. Completion rates of recommended vaccines pre- and post- transplantation are shown in Table 1.

CONCLUSIONS: There is a significant gap in between vaccination guidelines for SOT recipients and vaccine uptake in our kidney transplant recipients. Gap analysis to determine the challenges to implementation of vaccination guidelines are ongoing. This will help guide process improvement measures to improve vaccination rates in this vulnerable population.

Table 1: Vaccination Status in 400 Adult Kidney Transplant Recipients

\begin{tabular}{|c|c|c|c|c|}
\hline Vaccine & $\begin{array}{l}\text { Number of } \\
\text { vaccine } \\
\text { eligible } \\
\text { patients } \\
\text { pre- } \\
\text { transplant }\end{array}$ & $\begin{array}{l}\text { Number } \\
\text { vaccinated } \\
\text { pre- } \\
\text { transplant } \\
(\%)\end{array}$ & $\begin{array}{l}\text { Number of } \\
\text { vaccine } \\
\text { eligible } \\
\text { patients } \\
\text { post- } \\
\text { transplant }\end{array}$ & $\begin{array}{l}\text { Number } \\
\text { vaccinated } \\
\text { post- } \\
\text { transplant } \\
(\%)\end{array}$ \\
\hline Hepatitis B & 270 & 75 (27\%) & 195 & $9(4.6 \%)$ \\
\hline Hepatitis A & 387 & $4(1 \%)$ & 383 & $3(0.7 \%)$ \\
\hline Influenza & 308 & 107 (34\%) & 208 & 65 (31 \%) \\
\hline Pneumococcus & 386 & $82(21 \%)$ & 305 & 89 (29 \%) \\
\hline Tdap & 381 & 35 (9 \%) & 346 & $15(4 \%)$ \\
\hline
\end{tabular}

VALIDATION OF AN INTERACTIVE ADAPTIVE VIDEO MODULE EDUCATION PLATFORM TO TEACH PATIENTS EFFECTIVE RESPIRATORY INHALER TECHNIQUE Colleen A. Kelly ${ }^{2}$; Vineet M. Arora ${ }^{1}$; John Kim ${ }^{1}$; Ashley M. Snyder ${ }^{1}$; David Meltzer'; ${ }^{1}$ Steven R. White ${ }^{1}$; Valerie G. Press ${ }^{1} .{ }^{1}$ University of Chicago, Chicago, IL; ${ }^{2}$ Pritzker School of Medicine, Chicago, IL. (Control ID \#2466062)

BACKGROUND: Although effective treatments for asthma and chronic obstructive pulmonary disorder (COPD) exist, the vast majority of hospitalized patients misuse their respiratory inhalers. Efforts to develop and implement inpatient strategies for improving inhaler technique have found that an in-person "Teachto-Goal" method is initially successful at decreasing inhaler misuse. However, this strategy fails to have a long lasting effect with the majority of patients misusing their inhalers. Therefore, we sought to develop a platform for hospital use to teach inhaler technique and for educational reinforcement at home. This abstract describes the development and validation of an interactive, adaptive Video Module Education (VME) platform for inpatient and post-discharge education. Our objectives were to 1) compare pre and post knowledge, attitudes and skills using self-response to embedded questions about correct inhaler technique and 2) compare objective technique skills measured by a trained observer before and after the completed VME platform experience versus selfassessed knowledge questions corresponding to these skills embedded in the VME platform.

METHODS: Hospitalized adult patients with a diagnosis of asthma or COPD were enrolled in this quasi-experimental pre-post study. The VME is an interactive learning platform that combines up to three rounds of narrated demonstrations of correct inhaler technique with tailored self-assessment questions (7 questions pre video demonstration; up to 8 questions per round post video demonstration). Patient knowledge and attitudes of inhaler technique were selfassessed prior to and after watching the demonstration by completing responses in the VME; in addition, skill (incorrect technique video question) was assessed post-video demonstration. Patients were prompted to re-watch the demonstration 
if any questions were answered incorrectly. Participants' objective technique skill was measured by a trained research assistant prior to and after participants' completed the entire VME platform. To test for the validity and utility of the self-assessment questions, we evaluated the proportion of participants that answered each question correctly before and after the video demonstration. To test for skills versus knowledge we compared patient knowledge on VME questions with their observed ability to correctly demonstrate related skills. McNemars chi-square and t-tests were performed. Analyses were performed using Stata 14 at $p<0.05$.

RESULTS: A total of 90 participants were enrolled. Prior to VME education, around half or more of the participants answered each of the six knowledge questions correctly (49-89\% correct per question) [Table 1]. After education however, the proportion of participants answering the questions correctly improved for all of the knowledge questions (63-99\% correct with Qs 1,2,4,5 showing a significant change $[p<0.01])$. Of the four knowledge questions that tested self-assessed understanding of associated inhaler technique skills, three initially did not show congruence between knowledge and skill prior to VME (Qs 4, 5, 6 $p<0.01$ ). After VME, there was greater congruence between participant knowledge and skills (only Q6 $p<0.01$ ).

CONCLUSIONS: This study shows that the self-assessment questions are valid to test for improved knowledge, attitudes and skills as part of an interactive adaptive video module to educate patients on correct inhaler technique. Further, this study shows the importance of validating self-assessed knowledge of skills compared to objective improvement in skills. Although, for most participants, knowledge of inhaler technique improved with the use of VME, more importantly, patients' knowledge became more congruent with actual demonstration of the required skills for effective inhaler technique. As one of the first studies to compare demonstrated skills with knowledge of inhaler technique, the initial incongruence between knowledge and skill emphasizes the importance of the narrated video demonstration and elucidates that skills may be more difficult to learn or remember, even though the underlying concept is easy to grasp and recall. Further research is needed to understand the efficacy of the VME in the long-term.

Participant performance on VME knowledge and associated skills

\section{VME Question}

Pre $p$-value

Q1 T/F You should breathe in with rapid, shallow breaths...

Q2 ... Please number the steps in correct order from first to last

Q3 Which of the following is not an important step...

Q4 T/F When you are told to take " 2 puffs" of your rescue MDI (inhaler) that means pumping the inhaler twice in a row..

Q5 T/F Before you pump the MDI (inhaler) you should empty your lungs by taking a deep breath in...

Q6 T/F If you hear the spacer make a whistle sound when breathing in to $\mathrm{K}$ fill your lungs with the medicine, this means you are breathing in correctly

Q7 From the video that you just watched, please choose the step that was incorrect?

Q8 How strongly do you agree: I am confident that I know how to use my rescue inhaler correctly

$\begin{array}{llllll}\text { Domain } \dagger \text { K/A/S } & \text { Pre n/N (\%) } & \text { Post n/N (\%) } & p \text {-value } & \text { Observed Skill } & \\ \text { Post } p \text {-value } & & & & & \\ \mathrm{K} & 41 / 76(54 \%) & 50 / 64(78 \%) & <0.01 & 1.0 & 0.4 \\ \mathrm{~K} & 39 / 79(49 \%) & 56 / 78(72 \%) & <0.01 & - & - \\ \mathrm{K} & 13 / 26(50 \%) & 15 / 24(63 \%) & 0.5 & - & - \\ \mathrm{K} & 43 / 77(56 \%) & 68 / 77(88 \%) & <0.01 & <0.01 & 1.0 \\ \mathrm{~K} & 69 / 78(89 \%) & 75 / 76(99 \%) & <0.01 & <0.01 & <0.01 \\ \mathrm{~K} & 15 / 20(75 \%) & 19 / 23(83 \%) & 0.6 & <0.01 & 1.0 \\ \mathrm{~S} & - & 52 / 76(68 \%) & - & - & - \\ \mathrm{A} & & & & & -\end{array}$

$\dagger \mathrm{K} / \mathrm{A} / \mathrm{S}$ : Knowledge/Attitude/Skill

\$Confidence defined as "Agree" and "Strongly Agree" on a 5 point Likert scale

\section{VALIDITY OF IMPLEMENTING A SELF-REPORTED GLOBAL HEALTH MEASURE IN A MEDICARE ACCOUNTABLE CARE ORGANIZATION TO PREDICT HEALTHCARE UTILIZATION Karen J. Blumenthal ${ }^{1}$; Yuchiao Chang ${ }^{1}$; Timothy G. Ferris ${ }^{2}$; Jenna C. Spirt ${ }^{2}$; Christine Vogeli ${ }^{2}$; Neil W. Wagle ${ }^{2}$; Joshua Metlay ${ }^{1}$. ${ }^{1}$ Massachusetts General Hospital, Boston, MA; ${ }^{2}$ Partners HealthCare, Waban, MA. (Con- trol ID \#2467064)}

BACKGROUND: Health Care systems participating in shared savings contracts need to stratify patients to guide care coordination services. In the past systems have relied primarily on administrative and clinical data to identify those individuals at greatest risk for high healthcare utilization. Patient Reported Outcome Measures (PROMs) that assess self-reported global health may be more responsive to changes in health status and therefore better predict future healthcare needs. Partners HealthCare (Partners) recently implemented point of care collection of the PROMIS Global Health instrument (PGH) using electronic tablets at outpatient visits. We investigated the association between PGH scores and both patient demographic and clinical characteristics as well as subsequent health care utilization in our Accountable Care Organization (ACO) Medicare patients.

METHODS: We identified all patients in the Partners Medicare ACO who completed $\geq 1$ PGH between $03 / 2014$ and $09 / 2015$ at any of 54 participating ambulatory practices. The PGH includes 4 questions that generate a Global Physical Health (GPH) score and 4 questions that generate a Global Mental Health (GMH) score. Both scores are standardized for the US population with a mean score of 50 and a standard deviation of 10 . Higher scores represent higher functioning. Demographic and clinical data, including diagnostic codes for Charlson Comorbidity Index and counts of ED visits and hospitalizations, were extracted from administrative claims files. We examined the associations between GPH and GMH scores and age category, gender, race, area level poverty, and Charlson scores. A second set of analyses examined the association between GPH and GMH scores and subsequent rates of ED visits and hospitalizations limited to those subjects with a full 12 months of claims records prior to the date of PGH completion in order to adjust for past utilization as part of future analyses and at least 6 months of claims post completion. Monthly counts of visits were used to project an annual rate. As appropriate, $p$-values were generated using T-tests, Wilcoxon rank sum or ANOVA.

RESULTS: Four thousand four hundred ninety-four individuals in the Partners Medicare ACO completed $\geq 1$ PGH. Mean GPH and GMH scores were 46.5 (SD 10.7) and 52.0 (SD 11) respectively. The mean scores differed significantly across multiple demographic and clinical characteristics (Table 1). Being age 65-80, male gender, white race, living in area with lower proportion of the population living below the poverty level or having a lower Charlson score were 
all associated with significantly higher GPH scores and GMH scores (all $p<0.001$ ). Among a subgroup of 728 individuals with at least 18 months of utilization data, both the GPH and GMH were significantly associated with rates of future ED visits and hospitalizations (Table 2). Those with scores in the lowest GPH and GMH quartile had significantly higher rates of ED visits and hospitalization compared to those with scores in the highest quartile $(p<0.01)$.

CONCLUSIONS: The PGH demonstrated construct validity in that better scores were associated with patient characteristics typically associated with improved health. Moreover, the self-reported PGH was significantly associated with future healthcare utilization in this population. Ongoing analyses will assess whether this self-reported health measure provides incremental value for predicting future utilization after adjusting for administrative variables including comorbidities and past utilization. In conclusion, PROMs, such as the PGH, can be routinely collected at the point of care and may help provide unique information on patients to assist providers and health systems predict subsequent healthcare needs.

Mean PGH Scores by Demographic and Clinical Characteristics.

$* P<0.001$ for all comparisons with reference value in each category for both GPH and GMH.

\begin{tabular}{|c|c|c|}
\hline & GPH & GMH \\
\hline & Mean (SD) & Mean (SD) \\
\hline \multicolumn{3}{|l|}{ Age, Years } \\
\hline$<65 *$ & $38.1(10.1)$ & $41.6(10.8)$ \\
\hline 65-80 (reference) & $48.4(10.2)$ & $53.9(10.1)$ \\
\hline$>80^{*}$ & $44.6(9.9)$ & $51.5(10.3)$ \\
\hline \multicolumn{3}{|l|}{ Gender } \\
\hline Male (reference) & $48.2(10.7)$ & $53.4(10.8)$ \\
\hline Female* & 44.9 (10.6) & $50.6(11.0)$ \\
\hline \multicolumn{3}{|l|}{ Race } \\
\hline White (reference) & 46.8 (10.6) & $52.4(10.8)$ \\
\hline Other Race* & $42.3(11.1)$ & 47.0 (11.9) \\
\hline \multicolumn{3}{|l|}{ Area Level Poverty } \\
\hline $0-<5 \%$ (reference) & $48.0(10.1)$ & $53.3(10.2)$ \\
\hline $5-<10 \%$ & $46.3(10.8)$ & $52.3(10.6)$ \\
\hline $10-<20 \% *$ & $45.4(11.0)$ & $50.5(11.3)$ \\
\hline $20+\% *$ & $43.2(10.5)$ & $48.0(11.8)$ \\
\hline \multicolumn{3}{|l|}{ Charlson Score } \\
\hline 0-1 (reference) & $49.4(10.4)$ & $54.2(10.4)$ \\
\hline $2-3^{*}$ & $47.7(10.4)$ & $52.4(10.9)$ \\
\hline $4+$ & 43.5 (10.5) & $50.0(11.1)$ \\
\hline
\end{tabular}

Annual Rates of ED visits and Hospitalizations by PGH Scores.

$P<0.01$ for all comparisons between low and high scores for both ED visits and Hospitalizations.

\begin{tabular}{lll}
\hline \hline & ED Visits, mean (SD) & Hospitalizations, mean (SD) \\
GPH & & \\
Low (Quartile 1) & $0.9(1.7)$ & $1.0(2.0)$ \\
High (Quartile 4) & $0.6(2.7)$ & $0.1(0.4)$ \\
GMH & & $0.8(1.7)$ \\
Low (Quartile 1) & $1.2(2.0)$ & $0.2(0.7)$ \\
High (Quartile 4) & $0.5(2.2)$ & \\
\hline
\end{tabular}

VALIDITY OF VISCERAL ADIPOSITY ASSESSMENT WITH IDXA VS. CT IN RENAL DISEASE Ayesha A. Appa ${ }^{1}$; Ian de Boer ${ }^{1}$; Leila Zelnick ${ }^{1}$; Danielle Yancey ${ }^{1}$; Nicole Robinson ${ }^{1}$; Tyler Bosch ${ }^{2}$; Ellen Schur ${ }^{1} .{ }^{1}$ University of Washington, Seattle, WA; ${ }^{2}$ University of Minnesota, Minneapolis, MN. (Control ID \#2467595)

BACKGROUND: Both visceral adiposity and chronic kidney disease are independent risk factors for cardiovascular disease. Accurate and feasible quantification of visceral adipose tissue is an important component of research seeking to modify the development or persistence of visceral adipose tissue accumulation. However, validity of dual-energy x-ray absorptiometry (DXA) has yet to be demonstrated in patients with chronic kidney disease (CKD). Our objective is to evaluate validity of DXA against single-slice abdominal $\mathrm{CT}$ in visceral adipose tissue quantification in patients with and without CKD. METHODS: A cross-sectional validity study was conducted with 42 participants who were enrolled in a study of glucose and insulin metabolism. Fifteen participants had moderate to severe non-diabetic CKD $(\mathrm{eGFR}<60 \mathrm{~mL} / \mathrm{min} / 1.73 \mathrm{~m} 2$ without hemodialysis dependence), and 27 participants were controls with eGFR of 60 or greater. All participants underwent DXA (Lunar GE iDXA with CoreScan software) and single-slice CT scan at the umbilicus at the same study visit in order to measure visceral adiposity volumes $\left(\mathrm{cm}^{3}\right)$. Pearson's correlation coefficients were calculated to measure agreement between DXA and CT in the assessment of VAT. Bland-Altman analysis evaluated bias between the two methods.

RESULTS: Amongst the entire sample, the correlation coefficient for VAT measured by iDXA as compare to single slice CT was $r=0.85(P=<0.001)$. In participants with CKD, VAT volume correlation between iDXA and CT measures was nearly equivalent $r=0.86$ $(P=<0.001)$. Bland-Altman analysis revealed increasing variability across measures at higher VAT volumes.

CONCLUSIONS: DXA is is an equally valid method of VAT measurement as singleslice CT for studies comparing patients with and without CKD, though more variability may exist between modalities at higher volumes of visceral adipose tissue.

\section{VARIATION IN QUALITY OF CARE PROVIDED DURING COMMERCIAL} VIRTUAL VISITS IN URGENT CARE: A STANDARDIZED PATIENT AUDIT STUDY Adam J. Schoenfeld ${ }^{2}$; Jason Davies'; Ben Marafino²; Mitzi L. Dean²; Colette

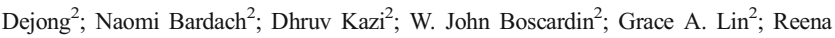
Duseja $^{2}$; John Mei ${ }^{2}$; Ateev Mehrotra ${ }^{1}$; R. Adams Dudley ${ }^{2} .{ }^{1}$ Harvard Medical School, Boston, MA; ${ }^{2}$ UCSF, San Francisco, CA. (Control ID \#2455080)

BACKGROUND: Commercial virtual visits are an increasingly popular model of care for the management of common, acute illnesses. In commercial virtual visits, patients access a website to be connected synchronously_via videoconference, telephone, or webchat - to a physician with whom they have no prior relationship. There has been no assessment of whether the care delivered through those websites is similar, or whether quality varies among the sites.

METHODS: To assess the variation in quality of care among virtual visit companies, we performed an audit study using trained standardized patients. The standardized patients presented to commercial virtual visit companies with six common, acute illnesses (ankle pain, streptococcal pharyngitis, viral pharyngitis, acute rhinosinusitis, low back pain, and recurrent urinary tract infection). The primary outcomes were completeness of histories and physical examinations, naming the correct diagnosis (versus an incorrect diagnosis or not naming any diagnosis), and adherence to guidelines of key management decisions.

RESULTS: Standardized patients completed 599 commercial virtual visits from May 2013 to July 2014. Histories and physical examinations were complete in 69.6 (95\% confidence interval [CI], 67.7-71.6\%) of virtual visits, diagnoses were correctly named in $76.5 \%$ (CI, 72.9-79.9\%), and key management decisions were adherent to guidelines in $54.3 \%$ (CI, 50.2-58.3\%). Rates of guideline-adherent care ranged from 34.4 to $66.1 \%$ across the eight websites. Variation across websites was significantly greater for viral pharyngitis and acute rhinosinusitis (12.8-82.1\%) than for streptococcal pharyngitis and low back pain (74.6-96.5\%) or ankle pain and recurrent urinary tract infection (3.4 $40.4 \%$ ). There was no statistically significant variation in guideline adherence by mode of communication (video vs. telephone vs. webchat). 
CONCLUSIONS: We found significant variation in quality among companies providing virtual visits for management of common acute illnesses. There was more variation in performance for some conditions than for others, but there was no variation by mode of communication.

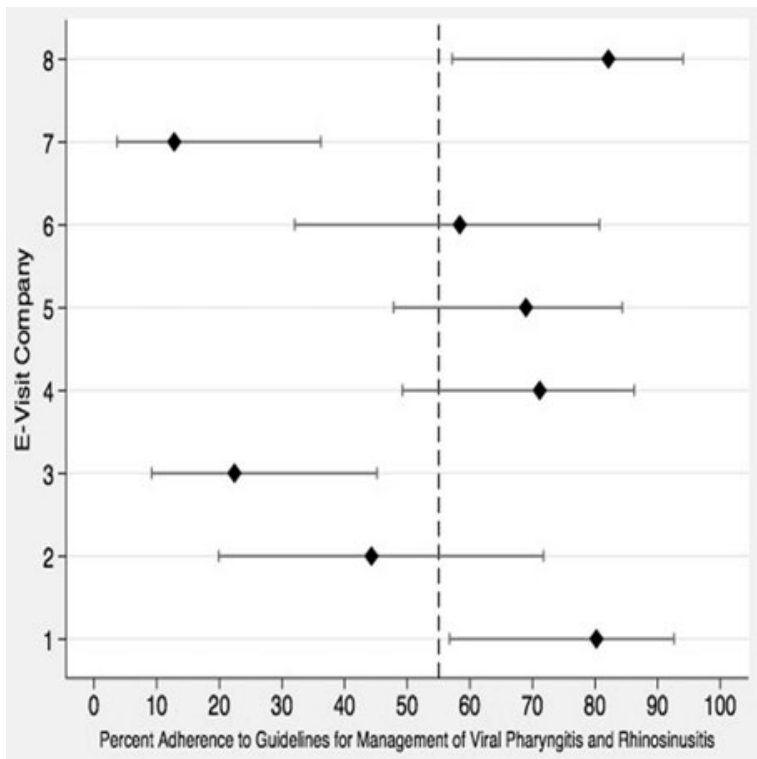

WAYS TO WRITE A MILESTONE: APPROACHES TO OPERATIONALIZING THE DEVELOPMENT OF COMPETENCE IN GRADUATE MEDICAL EDUCATION Andrea Leep Hunderfund; Rachel D. Havyer; Darcy Reed; Stephanie Starr; Tara Lang; Suzanne M. Norby. Mayo Clinic, Rochester, MN. (Control ID \#2468728)

BACKGROUND: To prepare for the Accreditation Council for Graduate Medical Education (ACGME) Next Accreditation System, specialty groups developed milestones describing the trajectory of professional development within sub-competencies relevant to their disciplines. Milestones provide a framework for describing the developmental progression of knowledge, skills, attitudes, and behaviors within a specialty and hence provide insights into how competence and its development have been defined in graduate medical education. Competence is a complex construct, and the development of competence can be operationalized in different ways. The aim of this study was to identify approaches to operationalizing the development of competence in ACGME milestones. METHODS: All "Milestone Project" documents available on the ACGME website on 9/11/2013 $(n=25)$ were reviewed to identify approaches to operationalizing the development of competence in the milestones associated with each sub-competency $(n=601)$. Agreement on a refined list of approaches was achieved via an iterative process of consensus building that extended over several months. Once consensus was achieved, the primary investigator re-reviewed the milestones of all 25 specialties to classify which approaches to operationalizing the development of competence were used in the milestones associated with each sub-competency.

RESULTS: Fifteen approaches to operationalizing the development of competence were identified. The most common approach was to infer competence by performing increasingly difficult tasks (304/601, $51 \%$ ), followed by progression from "knowing" to "applying" to “analyzing/evaluating/creating" (281/601, 47 \%) and progression from "learning" to "teaching/leading/role-modeling" (271/601, $45 \%$ ). Many sets of milestones inferred competence from the ability to perform a given task best or faster $(171 / 601,28 \%)$, in increasingly difficult or infrequently encountered situations (236/601, 29\%), or with increasing consistency (123/
$601,20 \%)$. Others inferred competence from an expanding scope of engagement (169/601, $28 \%$ ), increasing knowledge (150/601, $25 \%$ ), progression from "awareness" to "knowledge" to "ability" (141/601, $23 \%$ ), or increasing interdependence with other members of the healthcare team $(59 / 601,10 \%)$. Some inferred competence from a decreasing need for supervision or assistance $(151 / 601,25 \%)$ or progression from performing parts of a task to performing a whole task (74/601, $12 \%)$. Others included an attitudinal dimension such as progression from "unwilling" to "willing but unable" to "able" to perform a task $(57 / 601$, $9 \%$ ) or progression from extrinsic to intrinsic motivation (100/601, $17 \%)$. A minority relied on more traditional approaches to assessment such as normative comparisons $(7 / 601,1 \%)$, increasing experience or completion of special training (16/601, $3 \%$ ), or scores on a written test $(7 / 601,1 \%)$. Multiple approaches were often combined within the milestones associated with a single sub-competency (mean 4.0, SD 1.6).

CONCLUSIONS: ACGME milestones operationalize the development of competence in many different ways. Encouragingly, a number of these approaches can be mapped to established conceptual frameworks such as the Dreyfus model of skill acquisition, Bloom's taxonomy of objectives for the cognitive domain, Miller's pyramid, Ericsson's definition of expert performance, and Vygotsky's zone of proximal development. Basing milestones on conceptual frameworks contributes to their content validity and allows the education community to build upon previous work. Different approaches may offer complimentary perspectives on the development of competence. However, combining too many approaches within a single set of milestones risks confusing rather than clarifying the picture. It also complicates the assessment process for teaching faculty, clinical competency committees, and program directors. The diversity of approaches further poses a challenge to establishing the reliability and validity of milestone assessments across programs and specialties. Conversely, grounding milestones in thoughtfully selected, relevant conceptual frameworks may enhance the ease with which faculty use and understand milestones (and associated assessment instruments), hence bolstering their response process validity. Further investigation of the relative utility and acceptability of different approaches would be a useful next step.

WEIGHT GAIN IN PREGNANCY AND PREGNANCY COMPLICATIONS IN A HIGH RISK POPULATION: THE BOSTON BIRTH COHORT Zihe Zheng ${ }^{2}$; Xiaobin Wang ${ }^{3}$; Noel Mueller ${ }^{2}$; Lawrence J. Appel ${ }^{1}$; Wendy L. Bennett ${ }^{1}{ }^{1}{ }^{3}{ }^{1}$ Johns Hopkins School of Medicine, Baltimore, MD; ${ }^{2}$ Johns Hopkins University Bloomberg School of Public Health, Baltimore, MD; ${ }^{3}$ Johns Hopkins Bloomberg School of Public Health, Baltimore, MD. (Control ID \#2469724)

BACKGROUND: Pregnancy provides a unique opportunity to intervene to improve women's long-term health behaviors, especially around weight management. More than $50 \%$ of women gain an excessive amount of weight in pregnancy, according to weight gain ranges recommended by the Institute of Medicine (IOM). Understanding how weight gain in pregnancy affects pregnancy outcomes has implications for developing behavioral interventions in pregnancy, as well as for long-term maternal health. We used the Boston Birth Cohort to assess the risk of pregnancy complications associated with weight gain in pregnancy. METHODS: We conducted a retrospective analysis using the ongoing Boston Birth Cohort, an ethnically diverse population of over 8000 women, with oversampling for preterm birth. We excluded women missing pre-pregnancy BMI, gestational age at delivery, pregnancy weight gain (recorded either through the electronic medical record or through self-report at the time of delivery) or outcome data (preeclampsia, gestational diabetes and cesarean section). We defined the total weight gain goals according to the IOM guideline with individualized limits calculated from pre-pregnancy BMI and gestational age at delivery. We used multivariate logistic regression models to examine the association of pregnancy weight gain and pregnancy complications.

RESULTS: We included 6368 women in the sample. $51.0 \%$ were African American or Haitian, $24.3 \%$ were Hispanic and $11.9 \%$ were white. About one quarter had pre-term birth. 
Overall, $19.9 \%$ had adequate pregnancy weight gain, $21.4 \%$ did not gain an adequate amount of weight, while $58.7 \%$ had excessive weight gain. Overweight women had the highest percentage of excessive gain $(67.6 \%)$, followed by women with obesity class I (63.1\%) and normal pre-pregnancy BMI (56.4\%). Compared to women with adequate pregnancy weight gain, excessive weight gain was associated with $75 \%$ higher odds of experiencing preeclampsia $(\mathrm{OR}=1.75,95 \%$ confidence interval [CI], 1.38-2.23), $49 \%$ greater odds of gestational diabetes $(\mathrm{OR}=1.49,95 \% \mathrm{CI}, 1.12-1.98)$, and $30 \%$ greater odds of cesarean delivery $(\mathrm{OR}=$ $1.30,95 \% \mathrm{CI}, 1.12-1.49)$. After stratifying by pre-pregnancy BMI or pre-term birth, excessive weight gain was consistently associated with higher probability of experiencing these three pregnancy complications

CONCLUSIONS: In a multi-ethnic and high risk population, excessive gestational weight gain according to the IOM recommended ranges was associated with risks of preeclampsia, gestational diabetes and cesarean section, regardless of pre-pregnancy BMI or pre-term birth status. Our findings provide strong evidence of the applicability of the IOM recommended ranges in this high risk populations, and for the importance of targeting pregnancy weight gain to improve birth outcomes and future health risks for women.

WELLNESS AND WORK LIFE: PRESERVING THE TEACHERS OF THE FUTURE OF MEDICINE Katherine G. Romp ${ }^{4}$; Carlos Estrada ${ }^{3}$; Kay Ovington ${ }^{2}$; Sara Poplau ${ }^{1}$; Mark Linzer ${ }^{1}{ }^{1}$ Hennepin County Medical Center, Minneapolis, MN; ${ }^{2}$ Society of General Internal Medicine, Alexandria, VA; ${ }^{3}$ The University of Alabama at Birmingham, Birmingham, AL; ${ }^{4} \mathrm{UAB}$, Birmingham, AL. (Control ID \#2437338)

BACKGROUND: Physicians are under increased pressure from government agencies, insurance companies, and employers to redesign healthcare delivery that is in compliance with unfunded mandates to use electronic health records (EHR). Furthermore, against the well-established principle that every professional should provide care at the "top of their license,' more clerical functions are being imposed on physicians. We examined personal and system factors that impact wellness and work life among academic general internal medicine faculty.

METHODS: Design: cross-sectional study (2015). Setting/ Participants: General internal medicine faculty at US academic institutions. Inclusion: Division directors were invited to enroll faculty from their respective division to complete the survey. Measures: Online survey included: a) faculty characteristics; b) personal factors (satisfaction, professional values, stress, workload, burnout; 5 validated questions); and, c) system factors (work-efficiency, EHR proficiency and documentation time, work atmosphere) (5-point Likert scale). Analysis: We compared responses from a single institution to national benchmarks for top Likert scale categories (ex: agree, strongly agree) using the Chi-square test.

RESULTS: Faculty from 15 divisions of academic general internal medicine in the US participated (47 \% response rate; 579/1,235); 21 of whom were from a 'best practice' institution ( $84 \%$ response rate). Faculty from the institution scored all personal factors more favorably than their peers (Figure); however, a third had significant amounts of stress, 2 out of 10 perceived poor control over workload, and 1 out of 10 had significant burnout. Similarly, faculty rated system factors more favorably than their national counterparts. More faculty at the single "best practice' institution rated their team working as efficient ( 95 vs. $74 \%, p=0.03$ ), fewer best practice faculty noted excess documentation time at home ( 33 vs. $57 \%, p=0.03$ ), fewer noted time pressure for documentation at work ( 29 vs. $62 \%, p<0.01$ ), and fewer noted a hectic or chaotic work atmosphere ( 29 vs. $58 \%, p<0.01)$

CONCLUSIONS: In this national survey of academic general internal medicine faculty showing high stress and unfavorably work conditions among many general internists, one division had very low burnout scores and favorable work conditions. With effective strategies that address teamwork, documentation time at work and home, and chaotic workplaces, academic general internal medicine will hopefully be able to retain its talented teachers and clinicians.

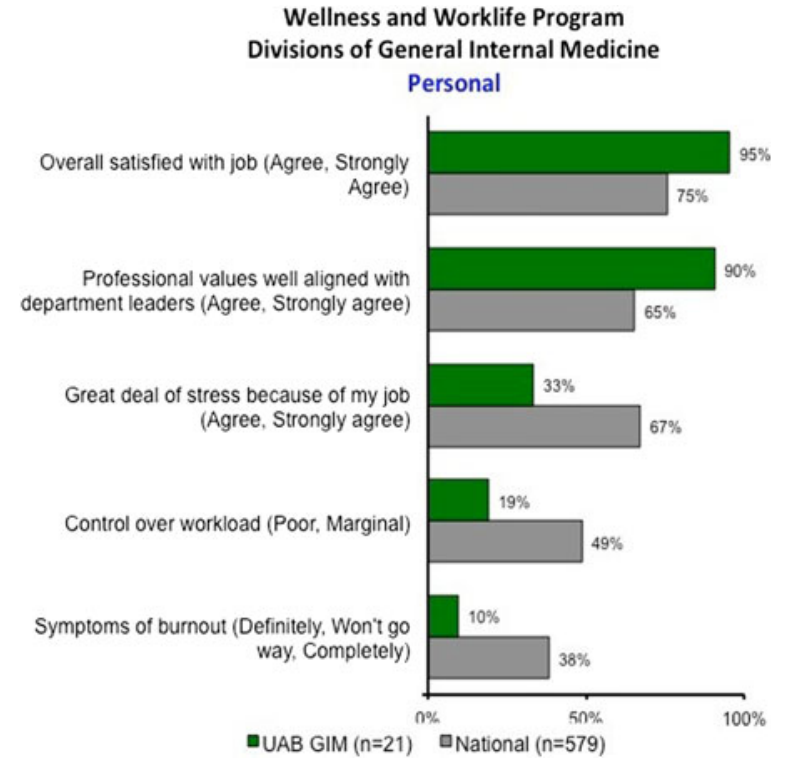

WHAT BRINGS AN OLDER VETERAN TO AN URGENT VISIT (UV)? A REVIEW OF THE CHIEF CONCERNS BY VETERANS AGED 65 AND OLDER WHO PRESENTED FOR UV DURING A 6-MONTH PERIOD AT THE WEST HAVEN VETERANS AFFAIRS CENTER OF EXCELLENCE IN PRIMARY CARE EDUCATION (VA COEPCE), AN INTERPROFESSIONAL ACADEMIC PATIENT ALIGNED CARE TEAM (PACT) Tao Liu ${ }^{1,2}$; Rebecca Brienza ${ }^{1} .{ }^{1}$ VA Connecticut Healthcare System, West Haven, CT; ${ }^{2}$ Yale-New Haven Hospital, New Haven, CT. (Control ID \#2467097)

BACKGROUND: In recent years, increasing focus has been devoted towards reducing emergency department (ED) visits for nonemergency causes. As a result, urgent care clinics and retail clinics were developed as alternative sites (1). In addition, there has been a special interest on ED usage by older adults who have been found to present to the ED more frequently than younger adults (2). At the VA, $40 \%$ of the Veterans who present to the Veterans Affairs Medical Centers emergency departments (VAMC ED) are aged 65 and older (3). Urgent visit clinics embedded within primary care clinics have the potential to play a crucial role in caring for older Veterans with urgent and unexpected medical needs, thereby alleviating some of the burdens imposed on the VAMC ED. In 2010, the Veterans Health Administration implemented Patient Aligned Care Team (PACT), which is an adoption of the patient-centered medical home (4). The VA CoEPCE is an innovative model that seeks to establish an academic PACT with emphasis on inter-professional collaboration and team-based care (5). The COE embedded UV clinics within the PACT team model to improve access and continuity of care and to reduce inappropriate ED use. This model is relatively new and allows for access for patients within a collaborative team care model. There is limited data describing the characteristics and pattern of use of UV within academic PACT settings. Learning more about UV use by older Veterans within a PACT may help to identify ways to improve care provided to older Veterans by designing innovative models of care. METHODS: UV by Veterans aged 65 and older within West Haven VA CoEPCE from April 1, 2014 to September 30, 2014 were reviewed. For each visit, we collected demographics, chief concerns, diagnostics, referrals and disposition.

RESULTS: A total of 388 visits were reviewed. Thirty-one percent of the visits were made by Veterans aged 80 and older and $2 \%$ were made by women Veterans. The most frequent chief concerns were musculoskeletal pain (29\%) with back pain and knee pain being the most common, followed by respiratory symptoms ( $15 \%)$, dermatologic concerns $(6 \%)$, dizziness $(5 \%)$, urinary symptoms $(3 \%)$ and hospital follow-up (3\%). Diagnostic testing related to the chief concerns including EKG, labs, urine studies, 
cultures, imaging and procedures were obtained during $43 \%$ of the visits. Referrals related to the chief concerns were made during $22 \%$ of the visits with Physical Therapy being the most common referral. For $3 \%$ of the UV, the Veterans were sent to the ED and for $1 \%$ of the UV, the Veterans were admitted.

CONCLUSIONS: UV can have the potential to reduce unnecessary emergency department visits particularly among older patients and primary care providers can significantly impact patient care by also taking on the role of urgent care providers. Our study shows that the majority of Veterans aged 65 and older who present for UV do not require ED visits or hospitalization. Integrating innovative models of interprofessional shared team care such as this UV model, may offer improved access and team continuity for patients needing UV. Future curriculum for making UV more effective may focus on developing teaching modules for the most common chief concerns and ways to identify and better manage common geriatric syndromes during those visits. Future work may also focus on assessment of patient satisfaction with the team care model. A major limitation of our study is the small percentage of UV by women Veterans. Future study may also assess UV by older women Veterans. References 1. Weinick RM, Burns RM, Mehrotra A. Many emergency department visits could be managed at urgent care centers and retail clinics. Health Aff (Millwood). 2010 Sep; 29(9):1630-6. 2. Samaras N, Chevalley T, Samaras D, Gold G. Older patients in the emergency department: a review. Ann Emerg Med. 2010 Sep; 56(3):261-9. 3. Hastings SN, Smith VA, Weinberger M, Oddone EZ, Olsen MK, Schmader KE. Health services use of older veterans treated and released from veterans affairs medical center emergency departments. J Am Geriatr Soc. 2013 Sep; 61(9):1515-21. 4. Nelson KM, Helfrich C, Sun H, Hebert PL, Liu CF, Dolan E, Taylor L, Wong E, Maynard C, Hernandez SE, Sanders W, Randall I, Curtis I, Schectman G, Stark R, Fihn SD. Implementation of the patient-centered medical home in the Veterans Health Administration: associations with patient satisfaction, quality of care, staff burnout, and hospital and emergency department use. JAMA Intern Med. 2014 Aug. 174(8):1350-8. 5.
Long T, Dann S, Wolff ML, Brienza RS. Moving from silos to teamwork: integration of interprofessional trainees into a medical home model. J Interprof Care. 2014 Sep;28(5):473-4.

\section{WHAT CAN FIVE HIGH COST PATIENTS TEACH US ABOUT HEALTH} CARE SPENDING? Ishani Ganguli ${ }^{2}$; Ryan Thompson ${ }^{1}$; Timothy G. Ferris ${ }^{1} .{ }^{1}$ Massachusetts General Hospital, Boston, MA; ${ }^{2}$ Massachusetts General Hospital, Cambridge, MA. (Control ID \#2470346)

BACKGROUND: The highest cost patients account for a disproportionately large share of American healthcare costs and are increasingly the focus of research and policy efforts to stem the rapid growth of these costs. These patients tend to be medically complex and frail, but we know little about how such characteristics influence healthcare spending from the perspectives of the patients and their caregivers.

METHODS: We chose five of the highest cost patients at an academic medical center based on Medicare claims data and archetype-demonstrating features. We performed claims analysis and chart review as well as semi-structured interviews with the patients, their family members, and their clinicians. We used a modified Delphi process to assess how eight prespecified patient and health system characteristics influenced costs in these interviews.

RESULTS: We found that complex medical issues, physical disability/frailty, and mental illness/substance use were consistently linked with increased costs. However, socioeconomic status, social network, activation, and trust in clinicians and the health system appeared to increase or decrease costs depending on context, challenging population-based research. We found that trust modified the interaction between patient activation and cost.

CONCLUSIONS: Our findings suggest factors mediating costs in high-cost patients may be more heterogenous than previously described, reinforcing the importance of customized interventions to address their needs. Patient trust and activation are important, potentially modifiable drivers of these costs.

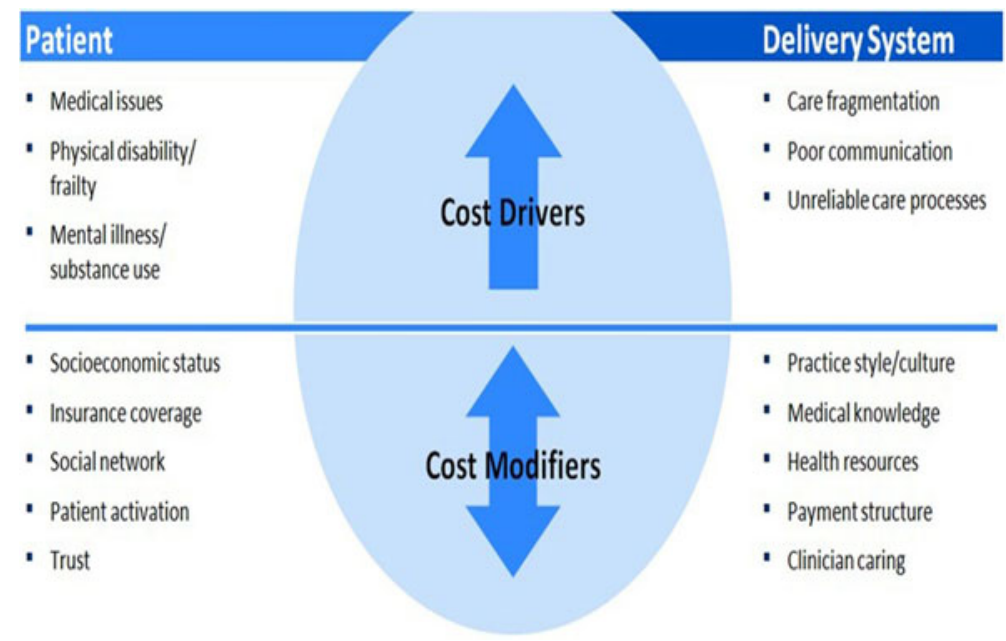

\section{WHAT DO PHYSICIANS WANT TO KNOW ABOUT OPIOID PRESCRIBING?} A QUALITATIVE ANALYSIS OF THEIR QUESTIONS Phoebe A. Cushman ${ }^{3}$; Joseph G. Hodgkin ${ }^{2}$; Christopher W. Shanahan ${ }^{3}$; Julie L. White ${ }^{1}$; Ilana Hardesty ${ }^{1}$; Jane M. Liebschutz ${ }^{3}$; Daniel P. Alford ${ }^{3,}{ }^{1}$. ${ }^{1}$ Boston University School of Medicine, Boston, MA; ${ }^{2}$ Boston University School of Medicine, Chestnut Hill, MA; ${ }^{3}$ Boston University School of Medicine and Boston Medical Center, Boston, MA. (Control ID \#2467983)
BACKGROUND: In the US, chronic pain and prescription opioid misuse are major problems, yet physicians report lack of expertise in addressing them. In an effort to improve opioid prescribing for chronic pain, in 2012 the FDA approved a single shared Risk Evaluation and Mitigation Strategy (REMS) that required all manufacturers of extended-release and long-acting opioids to jointly fund accredited continuing medical education based on the "FDA Blueprint for 
Prescriber Education." The main topics in the blueprint are I: Assessing Patients for Treatment, II: Initiating Therapy, Modifying Dosing, and Discontinuing Use, III: Managing Therapy, IV: Counseling Patients and Caregivers about Safe Use, and V: General Drug Information (characteristics, toxicities, and drug interactions). Based on the FDA Blueprint, we created the "Safe and Competent Opioid Prescribing" (SCOPE of Pain) program for physicians and other prescribers. Beginning in 2013, we have been presenting a series of live SCOPE of Pain trainings around the country. During each training, participants submit written questions on index cards about both the content of the workshop and about their clinical experiences managing chronic pain; questions are answered by faculty at the end of the training. Our study consists of qualitative analysis of the 915 written questions submitted by 2786 participants in 29 SCOPE of Pain workshops conducted in 16 states over 2 years (May 2013 to May 2015). We compare the themes that arise from the questions with the topics outlined in the FDA Blueprint. Given the large number of questions and the geographical distribution of the participants, our study offers a unique opportunity to evaluate whether the FDA blueprint addresses the needs of prescribers. Moreover, our study offers insight into physicians' current fears and frustrations as they confront the "opioid epidemic."

METHODS: We coded the question cards with conventional content analysis, using Nvivo for Mac v. 11 to organize our data. First, two team members (PAC, JGH) developed a list of preliminary codes based on an initial reading of the first 100 question cards. Second, these two team members individually coded all observations and met regularly with the other team members to review any areas of ambiguity between codes and, when appropriate, merge or divide codes. The two team members created code definitions to develop a complete code list. We finished coding and reached saturation when no new codes were generated from the data. Finally, the primary coding team grouped codes into themes, which were refined by all authors into a final list. After coding was complete, we compared the themes to the content of the FDA Blueprint

RESULTS: Most of the themes fell into the categories outlined in the FDA Blueprint: (I) Assessing Patients for Treatment ("Should tobacco abuse be a risk factor for opioid misuse risk?"), (II) Initiating Therapy, Modifying Dosing, and Discontinuing Use ("Do short-acting opioids need to be tapered in any way? "), (III) Managing Therapy ("Do you recommend contracts for patients on tramadol?"), (IV) Counseling Patients and Caregivers about Safe Use ("What about risk of driving while on opioids?"), and (V) General Drug Information (characteristics, toxicities, and drug interactions) ("Are you saying someone on a benzo should never receive opioids?'). Four main themes diverged from the topics addressed in the FDA Blueprint: (1) Participants sought information on safe pharmacologic and non-pharmacologic alternatives to opioids for treatment of chronic pain. ("In an opioid naive patient who has chronic severe low back pain, please discuss the medicines/therapies you could use before starting opioid therapy.") (2) Participants identified multiple barriers to ideal prescribing practices. ("What do you do when your pain clinics only want to treat patients with procedures but don't want to manage medical therapies?") (3) Participants voiced uncertainty about the role of government in opioid prescribing. ("What is the legal medical process for reporting a patient for suspected diversion behavior practice vs. HIPAA regulations?") (4) Participants raised concerns about how to navigate the provider-patient relationship while prescribing opioids. ("When taking over a difficult panel how do you discuss with the patient that you want to change the regimen but not harm the therapeutic relationship you are trying to form?")

CONCLUSIONS: In addition to learning the mechanics of prescribing, providers are eager to learn about the complex realities of opioids, including the role of government and the intricacies of effective patient communication. Aware of the limitations of opioids for chronic pain, providers also actively seek advice on alternatives therapies for their patients. Future updates to the FDA Blueprint and other educational guidelines on opioid prescribing should address providers' additional specific concerns.
WHAT DO WOMEN'S HEALTH PRIMARY CARE PROVIDERS NEED AND WANT TO KNOW?: LEARNING INTERESTS AND CONFIDENCE OF VA WOMEN'S HEALTH PCPS Jessica L. Zuchowski ${ }^{3}$; Alison B. Hamilton ${ }^{4}$; Donna L. Washington ${ }^{5}$; Arthur G. Gomez ${ }^{2}$; Laure Veet ${ }^{1,7}$; Kristina M. Cordasco ${ }^{6} .{ }^{1}$ Department of Veterans Affairs, Washington, DC; ${ }^{2}$ David Geffen School of Medicine at UCLA, Sepulveda, CA; ${ }^{3} \mathrm{US}$ Dept of Veterans Affairs, North Hills, CA; ${ }^{4} \mathrm{VA}$ Greater Los Angeles Healthcare System, Los Angeles, CA; ${ }^{5}$ VA Greater Los Angeles Healthcare, UCLA, Los Angeles, CA; ${ }^{6}$ VA Greater Los Angles Healthcare System/ UCLA, Los Angeles, CA; ${ }^{7}$ University of Pennsylvania School of Medicine, Philadelphia, PA. (Control ID \#2465560)

BACKGROUND: Women Veterans, comprising $<10 \%$ of the patient population in the Veterans Health Administration (VA), are increasingly using VA services for their health care. They are preferentially assigned for primary care to the panels of designated women's health primary care providers (WHPCPs). WHPCPs are faced with the task of maintaining their knowledge and skills in women's health topics in addition to general medicine topics. VA has invested substantially in providing women's health continuing education (WHCE) for WHPCPs. However, little is known about VA WHPCPs' relative confidence and learning interests, or drivers of their learning interests. As part of an ongoing needs assessment, we explored VA WHPCPs' relative confidence and learning interest in clinical topics that are specific to or more prevalent in women, and assessed the motivations behind those learning interests.

METHODS: Using mixed methods, we surveyed and interviewed VA WHPCPs (MDs, DOs, PAs, and NPs) across six VA healthcare systems. The survey asked about relative confidence and learning interest in 30 women's health topics comprised of gender-specific conditions as well as topics from previous VA WHCE programming. We asked, "How confident are you evaluating or managing the following?" (rated on a 5-point scale), and "How interested would you be in getting additional education on the following?" (rated on a 4-point scale). We evenly categorized topics into higher/lower confidence and higher/lower interest, based on the relationship among each topic's mean scores. We asked interviewees about WHCE topics of interest, and reasons for choosing those topics. All interviews were audio recorded, professionally transcribed, and coded in ATLAS.ti. Results were synthesized by grouping relevant coded sections of text to form emergent themes.

RESULTS: Twenty-six of 100 WHPCPs (26\%) completed the survey, and 31 of $111(28 \%)$ completed an interview. Survey respondents reported relative higher interest in 10 of 15 comparatively higher confidence topics (including topics related to cervical and breast cancer, sexually transmitted infections, contraception, osteoporosis, and menopause), while they reported relative higher interest in 5 of the 15 comparatively lower confidence topics (including topics related to urinary incontinence, pelvic pain, and polycystic ovarian syndrome). Ten topics in which survey respondents reported having comparative lower confidence and relative lower learning interest included topics related to dyspareunia, military sexual trauma, infertility, pregnancy pharmacology and tests, intimate partner violence, fibromyalgia, post-partum depression, and transgender medicine. Interviewees expressed greater interest in learning about topics related to conditions they more frequently encountered in their clinics, or that they anticipated seeing more frequently in the future based on patient panel demographics. Interviewees were less interested in learning about topics for conditions that they perceived as being less appropriate for management in primary care.

CONCLUSIONS: Assessing the intersection of relative confidence and interest of VA WHPCPs can guide WHCE program development. Relative lower 
confidence/higher interest topics represent a target for new continuing education programs, while relative higher confidence/higher interest topics may be appropriate for more advanced level programming. The topics in which WHPCPs reported both relative lower confidence and relative lower interest may first require understanding why WHPCPs regard them with lesser interest. Understanding the drivers of WHPCPs continuing education learning priorities lays a foundation for further development of WHCE programming that will be of interest to WHPCPs both in and outside of VA. These findings may also have applicability beyond women's health, to suggest a general approach for continuing education programming that prioritizes high-volume, in-scope topics for the target providers.

WHAT IS THE BEST WAY TO COMMUNICATE THE BENEFITS AND HARMS OF LUNG CANCER SCREENING? Tanner Caverly ${ }^{2,3}$; Angela Fagerlin 4, 5; Knoll Larkin ${ }^{6,}$ 7; Brian Zikmund-Fisher ${ }^{1}$. ${ }^{1}$ University of Michigan, Ann Arbor, MI; ${ }^{2}$ University of Michigan Medical School, Ann Arbor, MI; ${ }^{3}$ Ann Arbor VA Center for Clinical Management Research, Ann Arbor, MI; ${ }^{4}$ University of Utah, Salt Lake City, UT; ${ }^{5}$ Salt Lake City VA, Salt Lake City, UT; ${ }^{6}$ Wayne State University School of Medicine, Detroit, MI; ${ }^{7}$ Karmanos Cancer Center, Detroit, MI. (Control ID \#2468842)

BACKGROUND: Low-dose computed tomography lung cancer screening (LCS) is widely considered in guidelines to be a preference-sensitive decision context. Clinical practice guidelines explicitly recommend shared decision making (SDM) between clinician and patient prior to initiating LCS and Medicare requires it for payment. Most conceptions of SDM involve communicating quantitative information to patients so that they can understand the trade-offs between important risks and benefits. However, there are multiple important outcomes to communicate regarding the downstream consequences of LCS. Communicating this complex epidemiologic information within busy primary care clinics is quite challenging. Thus, we aimed to determine effective ways of communicating this risk information to patients by conducting a randomized survey experiment.
We compared comprehension and perception of lung cancer screening's benefits and harms when the information is presented using 4 different evidence-based formats.

METHODS: This online randomized survey experiment was conducted on December 2014 with 1612 adults aged 18 years or older recruited from Amazon Mechanical TURK who reported smoking daily or almost daily. Participants imagined they were 60 years old and eligible for lung cancer screening and then viewed the benefits and harms of screening presented in one of four different formats: 1) a screening facts box with absolute percentages (Facts Box); 2) multiple side-by-side pictographs (100 person) presenting the benefit and each harm separately (Multiple Pictographs); 3) a single combined pictograph (1000 person) depicting both benefits and harms in multiple colors (combined pictograph); and 4) a screening facts box with multiple pop-out pictographs (Facts Box + Pop-out). We measured comprehension using 11 knowledge questions (primary outcome) and perceptions of screening benefit and harm.

RESULTS: Participants viewing the combined pictograph had a harder time identifying false-positives as more likely to happen than other outcomes (67\% correct vs. $78-84 \%$ for the other formats, $P<0.001$ ). However, those viewing the combined pictograph had an easier time both a) identifying that the chance of a major complication from screening was similar to the chance of avoiding a cancer screening death (64\% correct vs. $52-58 \%, P<0.001)$ and b) identifying that the majority of people who would have died from lung cancer without screening will still die from lung cancer even with screening ( $68 \%$ correct vs. $54-57 \%, P<0.001$ ). Those viewing the combined pictograph also perceived screening as less effective $(P=0.02)$, less likely to help them avoid a lung cancer death $(P=0.02)$, and less of a good choice $(P=$ $0.002)$ than those who viewed the other formats.

CONCLUSIONS: Participants who viewed a combined pictograph (depicting both the benefits and harms of lung cancer screening in multiple colors within a single pictograph) had improved knowledge about the magnitude of the benefit while perceiving screening as less effective compared to those randomized to other formats. This study provides evidence for how to best communicate the multiple benefits and harms of lung cancer screening to patients.

Proportion of Participants Who Correctly Answered Knowledge Questions (only knowledge questions with significant response differences are presented)

\begin{tabular}{|c|c|c|c|c|c|}
\hline Question & $\begin{array}{l}\text { Correct } \\
\text { Answer }\end{array}$ & $\begin{array}{l}\text { Facts Box } \\
(n=396), \%\end{array}$ & $\begin{array}{l}\text { Multiple Pictographs } \\
(n=407), \%\end{array}$ & $\begin{array}{l}\text { Combined } \\
\text { Pictograph }(n=410), \%\end{array}$ & $\begin{array}{l}\text { Facts Box }+ \text { Pop-out } \\
(n=399), \%\end{array}$ \\
\hline \multicolumn{6}{|l|}{ Verbatim Knowledge } \\
\hline What percentage of people are overtreated? $\dagger$ & $0.4 \%, 4$ in 1,000 & 88 & $80 \ddagger$ & 88 & 90 \\
\hline $\begin{array}{l}\text { What percentage of people who are NOT screened die from } \\
\text { cancer in the next } 6 \text { years? } \dagger \\
\text { Gist Knowledge }\end{array}$ & $2.1 \%, 21$ in 1,000 & 88 & $73 \S$ & 86 & 86 \\
\hline $\begin{array}{l}\text { For people getting screened, which of the following is the } \\
\text { most likely thing to happen? (false positive, } \\
\text { invasive procedure, overtreated, avoid a lung cancer death, } \\
\text { I can }\end{array}$ & They & 84 & 78 & $67 \S$ & 84 \\
\hline $\begin{array}{l}\text { People getting screened are much more likely to have a } \\
\text { false positive (a suspicious finding that turns out not to } \\
\text { be cancer) than to avoid a cancer death. }\end{array}$ & True & 89 & 87 & $79 \S$ & 90 \\
\hline $\begin{array}{l}\text { The chance of having a major complication from screening } \\
\text { is similar to the chance of avoiding a cancer death because } \\
\text { of screening. }\end{array}$ & True & 52 & 58 & $64 \ddagger$ & 58 \\
\hline $\begin{array}{l}\text { The majority of people who would have died from lung } \\
\text { cancer without screening will avoid a lung cancer } \\
\text { death if they get screened. }\end{array}$ & False & 54 & 55 & $68 \S$ & 57 \\
\hline
\end{tabular}

$\dagger$ Format specific question in which the numerical format in the presentation matched the numerical format in the answer choices.

$\$ P<=0.01$ compared with the facts box group

$\S P<=0.001$ compared with the facts box group 
Combined Pictograph Presenting Multiple Benefits and Harms Within a Single Pictograph

Using Multiple Colors

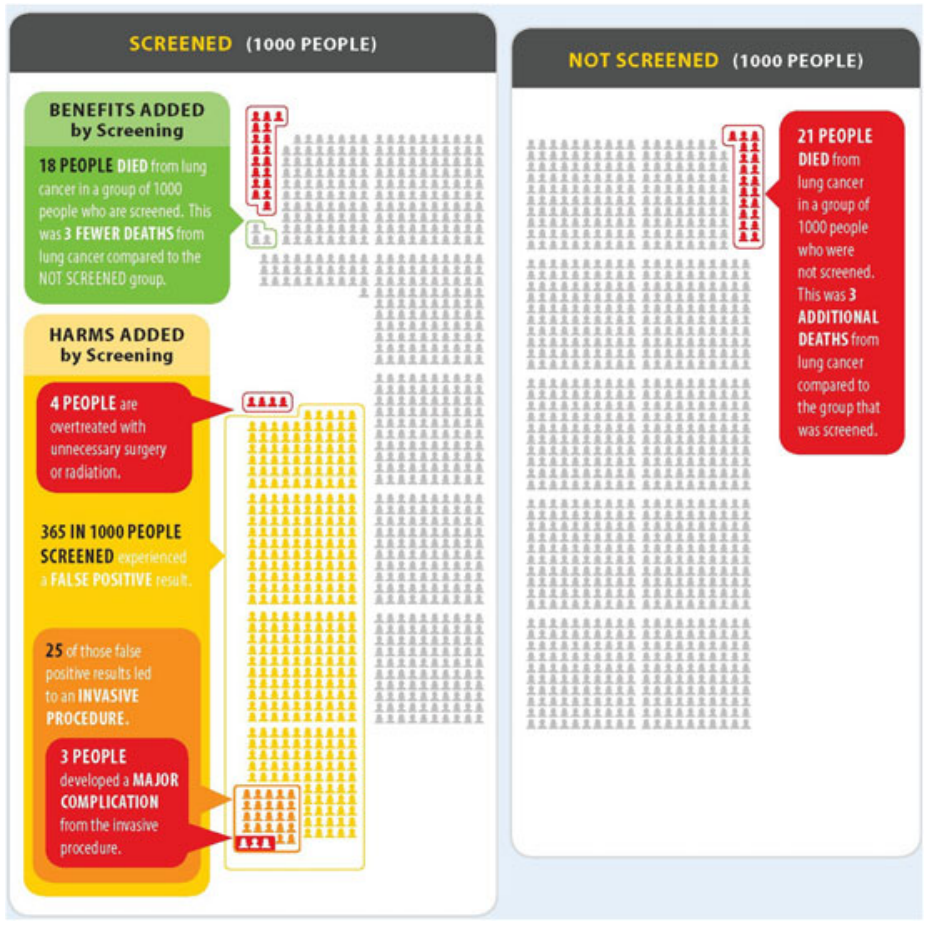

WHAT IS THE IMPACT OF AMBULATORY PRACTICE SETTING ON THE USE OF LOW VALUE HEALTH SERVICES IN THE UNITED STATES? John N. Mafi ${ }^{1,}$

2; Christina C. Wee ${ }^{3}$; Bruce E. Landon ${ }^{4}{ }^{1}$ David Geffen School of Medicine at UCLA, Los Angeles, CA; ${ }^{2}$ RAND Corporation, Santa Monica, CA; ${ }^{3}$ Beth Israel Deaconess Medical Center, Boston, MA; ${ }^{4}$ Harvard Medical School, Boston, MA. (Control ID \#2466727)

BACKGROUND: In an era of consolidation in healthcare, outpatient care is shifting away from private office practices and increasingly towards integrated hospital systems. While critics argue that consolidation will raise prices and increase incentives to overuse health services, proponents point to the potential for more coordinated, efficient, and high value care. Yet few studies have rigorously compared the value and efficiency of care among outpatient hospital based practices and other ambulatory practice settings over time.

METHODS: Using nationally representative data from the National Ambulatory Medical Care Survey and National Hospital Ambulatory Medical Care Survey we examined ambulatory visits for three common conditions: upper respiratory infections (e.g., URIs or acute bronchitis/pharyngitis), back pain, and headache from 1997 to 2011. We compared primary care practice patterns at private solo or group office practices (reference group) with hospital based outpatient departments (primary exposure of interest), freestanding urgent care clinics, health maintenance organizations (HMOs), and other community-based clinics (e.g. community health centers). We excluded the presence of clinical red flags (e.g., complaints/diagnoses of trauma for headache, or COPD for URI, etc.) in accordance with HEDIS quality measures and other national guidelines. Outcomes included use of antibiotics (URIs), CT/MRI (back pain and headache), x-rays (URI and back pain), and referrals to other physicians (all conditions). We used logistic regression models and adjusted for demographic, clinical (including case complexity), provider characteristics and year, and weighted results to reflect national estimates. We hypothesized that compared to private office practices, more integrated outpatient hospital departments would provide lower value care because of greater incentive to overuse tests and easier access to specialists, while HMOs would provide fewer low value services given their capitated budgets. We also stratified results by time, acute vs. non-acute presentations, and primary care provider (PCP) vs. non- $\mathrm{PCP}$ visits.

RESULTS: We identified 28,949 ambulatory visits (mean patient age 48.4 years; $62.9 \%$ female), representing an estimated 681 million visits during the study period. Clinicians practicing in outpatient hospital departments saw younger patients (mean age 44.5 vs. 49.1 years, $p<0.001$ ), were less likely to identify as the patient's PCP ( 74.9 vs. $81.7 \%$, $p<0.001$ ), and less likely to practice in an urban setting ( 74.9 vs. $81.7 \%, p=0.033$ ) when compared to clinicians practicing in private office practices. While there was no difference in antibiotic use, outpatient hospital-based clinicians ordered more CT/MRI studies ( 8.3 vs. $6.3 \%, p=0.012)$, $\mathrm{x}$-rays $(12.8$ vs. $9.9 \%, p<0.001)$, and referrals to other physicians ( 19.0 vs. $7.6 \%, p<0.001)$ compared to clinicians in private office-based practices. While clinicians in HMOs ordered fewer antibiotics ( 33.3 vs. $49.1 \%, p<0.001)$, they ordered similar proportions of imaging tests, and referred to other physicians more frequently than clinicians at private office practices $(15.6$ vs. $7.6 \%, p<0.001)$. Results remained similar after multivariable adjustment (see Table). Stratification revealed that practice variation between outpatient hospital department care and private office based care increased over time $(p<0.001$ for interaction for CT/MRI), was primarily driven by non-acute visits ( $p=$ 0.021 for interaction for CT/MRI, $p=0.008$ for interaction for $\mathrm{x}$-rays, and $p=0.019$ for interaction for referrals), and by non-PCP or episodic-type visits $(p<0.001$ for interaction for referrals).

CONCLUSIONS: Clinicians at outpatient hospital departments ordered more low value $\mathrm{CT} / \mathrm{MRI}$ and $\mathrm{x}$-ray studies and made over twice as many referrals to specialists compared to clinicians at private office-based practices, possibly due to greater availability of imaging scanners and specialty services. Differences in CT/MRI use widened over time and were also driven by non-acute visits, while differences in referrals were driven by clinicians who did not identify as the PCP, suggesting that prioritizing greater continuity of care may play an important role in reducing practice variation among different primary care settings. Payers and policymakers who have a stake in greater consolidation among large hospital-based health systems should take these findings into consideration when determining policy. 


\begin{tabular}{|c|c|c|c|c|c|c|c|c|c|}
\hline & $\begin{array}{l}\text { Private Solo or } \\
\text { Group Ofioe } \\
\text { Practices } \\
(n=9,785)(\text { (rel) }\end{array}$ & $\begin{array}{c}\text { Oatpatent } \\
\text { Hospial } \\
\text { Depatnerts } \\
(n=16,306)\end{array}$ & $\begin{array}{l}\text { OOR } \mid 95 \% \\
\text { C] }\end{array}$ & $\begin{array}{c}\text { Fires } \\
\text { staning } \\
\text { Ungert Care } \\
\text { Centers } \\
(n=788)\end{array}$ & $\begin{array}{c}20 R \mid 95 \% \\
\text { CI }\end{array}$ & $\begin{array}{l}\text { Heath } \\
\text { Mainterance } \\
\text { Organizatons } \\
\left(\begin{array}{l}\text { HNOS) } \\
(n=280)\end{array}\right.\end{array}$ & $\begin{array}{l}\text { OOR/P5\% } \\
\text { CI] }\end{array}$ & $\begin{array}{l}\text { Other } \\
\text { Types d } \\
\text { Contruty } \\
\text { Crics } \\
(n=1,792)\end{array}$ & $\begin{array}{c}\text { 20R } 105 \% \\
\text { Ci] }\end{array}$ \\
\hline Antbiotics & 49.4 & 497 & $\begin{array}{c}1.0[0.83- \\
1.23]\end{array}$ & 56.1 & $\begin{array}{c}1.31 \text { 10.96- } \\
1.795\end{array}$ & 37.8 & $\begin{array}{c}0.62[0.39 \\
0.99]\end{array}$ & 41.5 & $\begin{array}{c}0.73[0.47 . \\
1.12]\end{array}$ \\
\hline CTNRI & 57 & 79 & $\begin{array}{c}1.41[1.10 . \\
1.81]\end{array}$ & 5.1 & $\begin{array}{l}0.88[0.45 \\
1.73]\end{array}$ & 78 & $\begin{array}{c}1.40[0.70- \\
2800]\end{array}$ & 43 & $\begin{array}{c}0.75[0.49- \\
1.14]\end{array}$ \\
\hline Bask Pain & 53 & 7.6 & $\begin{array}{c}1.48[1.10- \\
2.00]\end{array}$ & 48 & $\begin{array}{c}0.90[0.44 \\
1.85]\end{array}$ & 7.4 & $\begin{array}{c}1.44[0.71 . \\
292]\end{array}$ & 38 & $\begin{array}{c}0.72[0.41- \\
1.26]\end{array}$ \\
\hline Headacte & 6.6 & 79 & $\begin{array}{c}1.21[0.87 . \\
1.69]\end{array}$ & 78 & $\begin{array}{c}120[0.58- \\
2.47]\end{array}$ & 89 & $\begin{array}{c}1.37[0.48- \\
3.89]\end{array}$ & 5.7 & $\begin{array}{c}0.84[0.41- \\
1.75]\end{array}$ \\
\hline$X \cdot R a y$ & 9.4 & 132 & $\begin{array}{c}1.46[1.20 . \\
1.78]\end{array}$ & 10.6 & $\begin{array}{l}1.1310 .85- \\
1.52]\end{array}$ & 14.4 & $\begin{array}{c}1.62[0.65 . \\
275]\end{array}$ & 110 & $\begin{array}{l}1.19 p 808 . \\
1.70]\end{array}$ \\
\hline UPI & 5.1 & 7.4 & $\begin{array}{c}1.50[1.09 . \\
2.06]\end{array}$ & 62 & $\begin{array}{c}124 p 727 . \\
2.13]\end{array}$ & 62 & $\begin{array}{c}1.2410 .51- \\
3000\end{array}$ & 72 & $\begin{array}{l}1.45[0.58- \\
3.61]\end{array}$ \\
\hline Back Pain & 9.4 & 18.8 & $\begin{array}{l}2.22[1800- \\
2.73]\end{array}$ & 10.5 & $\begin{array}{c}1.13 p 07 . \\
1.606\end{array}$ & 13.8 & $\begin{array}{c}1.53[0.78- \\
3.02]\end{array}$ & 142 & $\begin{array}{c}159[1.08 . \\
2.33]\end{array}$ \\
\hline $\begin{array}{l}\text { Refer to Other } \\
\text { Physiclan }\end{array}$ & 7.1 & 17.2 & $\begin{array}{c}2.71[220- \\
3.33]\end{array}$ & 86 & $\begin{array}{c}123[.088- \\
1.72]\end{array}$ & 128 & $\begin{array}{c}1.91[1.20- \\
2.10]\end{array}$ & 110 & $\begin{array}{c}1.61[1.21 . \\
2.16]\end{array}$ \\
\hline UVI & 34 & 13.4 & $\begin{array}{c}4.45[3.12 . \\
6.36]\end{array}$ & 44 & $\begin{array}{c}131[10.74 \\
233]\end{array}$ & 69 & $\begin{array}{c}2.12[0.86- \\
5.19]\end{array}$ & 6.5 & $\begin{array}{c}1.99[1.07 . \\
3.69]\end{array}$ \\
\hline Back Pain & 12.1 & 169 & $\begin{array}{l}1.49[1.19- \\
1.85]\end{array}$ & 13.4 & $\begin{array}{l}1.13 p .080 . \\
1.599\end{array}$ & 17.6 & $\begin{array}{l}1.56[0.84 \\
291]\end{array}$ & 125 & $\begin{array}{c}1.0407071- \\
1.52]\end{array}$ \\
\hline Headacte & 79 & 150 & $\begin{array}{c}2.06[1.54 \\
2.75]\end{array}$ & 125 & $\begin{array}{c}16660.097- \\
2.85]\end{array}$ & 15.4 & $\begin{array}{c}2.12[0.80- \\
5.40\end{array}$ & 8.1 & $\begin{array}{c}1.0310 .53- \\
2011\end{array}$ \\
\hline
\end{tabular}

WHAT MAKES A MEDICAL PATIENT COMPLEX IN THE HOSPITAL SETTING Baptiste Crelier $^{2}$; Drahomir Aujesky ${ }^{1}$; Jacques Donze ${ }^{1,3}$. ${ }^{1}$ Bern University Hospital, Bern, Switzerland; ${ }^{2}$ Bern University, Bern, Switzerland; ${ }^{3}$ Brigham and Women's Hospital, Boston, MA. (Control ID \#2457239)

BACKGROUND: Care of complex patients are a daily challenge in the hospital setting, with a direct impact on the health care costs. Although there is no standard definition of a "complex" patient, we can argue that they are patients who need more time and resources during their care process. Age and comorbidities are probably important factors contributing to the patient complexity, but little is known about the characteristics of complex patients, in particularly in the hospital setting. Therefore, we aim to identify the characteristics of patient's complexity in the hospital setting.

METHODS: We conducted a cross-sectional study in which we surveyed the physicians from the medical wards of 3 large Swiss hospitals between July and October 2015. All physicians involved in patient care (residents, attending, faculties) were invited to participate to the survey via Email, with a reminder 2 weeks later. All physicians working in each medical ward were eligible to participate only once, and the survey was anonymous. The survey instrument was developed based on a literature review, and clinical experience. The survey contained 21 closeended questions on characteristics that might influence patient complexity with answer on a scale from 1 (no influence) to 4 (great influence). The questions were grouped into 6 categories of characteristics: provider demographics, patient characteristics, patient diagnoses and comorbidities, treatment care, hospital structure and process, communication.

RESULTS: Among the 111 responders (response rate $53 \%), 55.5 \%(n=61)$ were female, $65.5 \%(n=72)$ were resident, $25.5 \%(n=28)$ attending, and $9 \%(n=10)$ faculty. The median number of years since MD was 5 (IQR 3-7). Comorbidity was the most overall frequently rated characteristics that was considered to influence patient complexity, with $95 \%(n=106)$ of the responders rating this characteristics as having either a moderate $(26 \%, n=29)$, or a high $(69 \%$, $n=77$ ) influence on patient complexity. The 9 other most frequently characteristics rated as high influence were the number of medication changes $(47 \%, n=52)$, a psychiatric disease ( $46 \%$, $n=51)$, difficulties in the communication with the patient ( $46 \%, n=51)$, aggressiveness ( $47 \%$, $n=52)$, drug or alcohol abuse ( $41 \%, n=46)$, interdisciplinary communication $(45 \%, n=50)$, number of drug prescription ( $41 \%, n=46)$, coordination of care $(43 \%, n=47)$. On the other hand, patient characteristics such as gender $(1 \%, n=1)$, social support $(4 \%, n=4)$, living alone ( $2 \%, n=2)$, or patient education $(9 \%, n=10)$, were not rated as having a high influence on patient complexity. Between the 6 categories of characteristics, patient diagnoses and comorbidities was rated as the most important factors. The reported mean proportion of complex patients was equally distributed between 20 and $80 \%$.
CONCLUSIONS: Most important factors identified as influence the patient complexity in the hospital setting were patient comorbidities, in particularly psychiatric disease, communication difficulties (with the patient, and also interdisciplinary), number and changes of medication, drug or alcohol abuse, and coordination, and patient aggressiveness and limited mobility. The proportion of complex patients reported ranged widely, between 20 and $80 \%$, and may suggest interpersonal differences in considering the threshold of patient complexity.

WHAT PEOPLE WITH AFRICAN ANCESTRY HAVE TO SAY ABOUT GENOMICS, DISPARITIES AND CHRONIC DISEASE Carol R. Horowitz ${ }^{3}$; Noura S. AbulHusn ${ }^{1}$; Erwin Bottinger ${ }^{1}$; Michelle A. Ramos ${ }^{3}$; Kezhen Fei ${ }^{2} .{ }^{1}$ Icahn School of Medicine at Mount Sinai, New York, NY; ${ }^{2}$ Mount Sinai School of Medical, New York, NY; ${ }^{3}$ Mount Sinai School of Medicine, New York, NY. (Control ID \#2469798)

BACKGROUND: Emergent research has uncovered genetic variants that increase risk of common chronic diseases, particularly chronic kidney disease (CKD). Specifically, APOL1 genetic variants, nearly unique to people with African ancestry, increase CKD and kidney failure risk by 5 to 10 fold among adults with hypertension. Yet, little is known about Black/ African American patients' understanding of genomics, beliefs regarding genes' contribution to chronic disease and their contribution to health disparities. This information is needed to effectively translate genomic discoveries into routine clinical practice.

METHODS: An academic-clinical-community team team recruited patients from 6 neighborhood health centers, and several primary care practices affiliated with an academic medical center in NYC. We surveyed adults with hypertension, self-reported African ancestry and normal kidney function as part of a randomized trial testing them for APOL1 variants, using validated scales and questions developed from qualitative interviews. With clinician permission, we reached 3342 patients; $38 \%$ were ineligible, $21 \%$ declined and $41 \%$ (1434) were interested, of whom to date, 1100 have enrolled, and for whom we have survey and measured blood pressure data.

RESULTS: Participants have a mean age of 53 (range 18-70), $67 \%$ are female, $20 \%$ have inadequate health literacy, $53 \%$ have household incomes under $\$ 30,000 /$ year, $48 \%$ are not adherent to their hypertensive medications and $47 \%$ have uncontrolled blood pressure. Few (11\%) had a previous genetic test or report they understand genetic testing (12\%). Yet, $93 \%$ think genes affect their risk for common diseases. Most believe hypertension is inherited (65\%), some think CKD is inherited (39\%). Most ( $85 \%$ ) also think it is a good idea to get genetic testing to assess chronic disease risk, and $78 \%$ want their children tested. Few have 
concerns: $18 \%$ are worried about the privacy of their results, $5 \%$ think doctors would discriminate against people with a genetic chronic disease risk. In terms of disparities, only $33 \%$ report that doctors treat Black and White patients equally. Most (88\%) state that Blacks are more likely to have chronic diseases than Whites, and attribute this difference to their built environments (60\%), stress (53\%), behavior/culture (45\%), and genes (41\%). Interestingly, $58 \%$ do not think providers would view Black people any differently if they think their patient's chronic disease risk is due to genetics, as opposed to other factors. Among participants who think this would change providers' views, twice as many think providers will view them more positively, rather than more negatively ( 28 vs $14 \%$ ).

CONCLUSIONS: These data highlight that many adults with hypertension and African Ancestry agree to participate in genetics research, believe that genetic factors contribute to their chronic disease risk, are interested in genetic testing, and have limited concerns about testing. Despite keen awareness of disparities and beliefs that providers do not treat Blacks equally, most believe this genetic information will either positively impact their providers' views of Black patients, or not impact these views at all. It is important to consider patient perspectives when studying the complex interplay between ancestry, race, genomics, social determinants and health

WHEN IS RANDOM GLUCOSE ABNORMAL? DETERMINING THE UPPER LIMIT OF THE RANDOM GLUCOSE REFERENCE RANGE USING NATIONALLY REPRESENTATIVE DATA Michael E. Bowen ${ }^{1}$; Lei Xuan ${ }^{1}$; Ildiko Lingvay ${ }^{1}$; Eric Mortensen ${ }^{2}$; Ethan Halm ${ }^{1} .{ }^{1}$ University of Texas Southwestern Medical Center at Dallas, Dallas, TX; ${ }^{2}$ VANTHCS, Dallas, TX. (Control ID \#2467240)

BACKGROUND: Clinical laboratories flag most laboratory values as being out of range using reference ranges derived from population norms. However, glucose values are flagged as abnormal based on diagnostic cutpoints extrapolated from diabetes diagnostic criteria. Random blood glucose (RBG) values $\geq 140 \mathrm{mg} / \mathrm{dL}$ are flagged as abnormal using criteria extrapolated from the $2 \mathrm{~h}$ Oral Glucose Tolerance Test (OGTT). However, this cutpoint is derived in a fasting population and applied to non-fasting values. Recent literature indicates that $\mathrm{RBG}$ values $<140 \mathrm{mg} / \mathrm{dL}$ are associated with diabetes risk. Thus, we sought to define the range of normal random glucose values in a non-fasting, nationally representative population without diagnosed diabetes and prediabetes and describe the frequency with which the RBG $140 \mathrm{mg} / \mathrm{dL}$ threshold identifies individuals as abnormal and in need of gold standard diabetes testing.

METHODS: Using data from the 2007-2014 National Health and Nutrition Examination Surveys, we defined a reference population of non-fasting, non-pregnant US adults. Participants with known diabetes and prediabetes were excluded. We describe the mean (+2 Standard Deviation (SD)) RBG values in the total reference population. The population was then stratified using $\mathrm{A} 1 \mathrm{C}$ as the gold standard into normal glycemia, undiagnosed prediabetes, undiagnosed, diabetes, and undiagnosed dysglycemia (undiagnosed diabetes + undiagnosed prediabetes). The sensitivity, specificity, and predictive values of the current RBG $140 \mathrm{mg} / \mathrm{dL}$ cutpoint are described.

RESULTS: The reference population $(N=9522)$ had a mean age of 45 years, $51 \%$ were female, and $31 \%$ were non-white. Mean BMI was $28 \mathrm{~kg} / \mathrm{m}^{2}$ and $25 \%$ had hypertension. The mean (+2SD) RBG value in the total reference population $(N=9522)$ was $93.3(142.1) \mathrm{mg} / \mathrm{dL}$. Using A1C to classify glycemic status, mean (+2SD) RBG values by group were: normal $(N=6656) 89.8$ (132.6) $\mathrm{mg} / \mathrm{dL}$, undiagnosed prediabetes $(N=2555) 98.4(153.0) \mathrm{mg} / \mathrm{dL}$, undiagnosed diabetes $(N=311) 159.1$ (339.0) $\mathrm{mg} / \mathrm{dl}$, and undiagnosed dysglycemia $(N=2866) 103.9$ (189) mg/dL. The current RBG cutpoint of $140 \mathrm{mg} / \mathrm{dL}$, which is used by clinicians to identify individuals with abnormal glucose in need of gold-standard diabetes testing, has low sensitivity to detect undiagnosed diabetes and prediabetes in the population (Table 1). By area-under the receiver operator curve (AROC) criteria, the $140 \mathrm{mg} / \mathrm{dL}$ cutpoint is of no value in identifying undiagnosed prediabetes $(\mathrm{AROC}=0.50)$.

CONCLUSIONS: The upper limit of the RBG reference range $(142 \mathrm{mg} / \mathrm{dL})$ in a nationally representative sample of non-fasting, non-pregnant adults without diagnosed diabetes or prediabetes is similar to the commonly used $140 \mathrm{mg} / \mathrm{dL}$ cutpoint extrapolated from the $2 \mathrm{~h}$ OGTT. However, this cutpoint fails to flag the vast majority of individuals with undiagnosed prediabetes and dysglycemia as abnormal. Re-evaluation of RBG cutpoints based on their ability to identify individuals with undiagnosed diabetes and prediabetes is needed and may improve case detection.

Sensitivity, Specificity, and Predictive Values for the Traditional $140 \mathrm{mg} / \mathrm{dL}$ Cutpoint for Flagging RBG Values as Abnormal

\begin{tabular}{|c|c|c|c|c|c|}
\hline$\overline{\text { Glycemic State }}$ & Sensitivity (95\% CI) & Specificity (95 \% CI) & PPV (95\% CI) & NPV (95\% CI) & $\overline{\text { AROC }(95 \% \mathrm{CI})}$ \\
\hline Undiagnosed Diabetes & 46.9 (39.4-54.4) & 97.9 (97.6-98.3) & 33.7 (26.4-41.0) & $98.8(98.6-99.0)$ & $0.71(0.68-0.74)$ \\
\hline Undiagnosed Prediabetes & $4.0(3.1-4.9)$ & $97.2(96.7-97.7)$ & $29.0(22.7-35.3)$ & $77.9(76.7-79.2)$ & $0.50(0.50-0.51)$ \\
\hline Undiagnosed Dysglycemia & $7.9(6.6-9.2)$ & 98.5 (98.1-98.9) & $62.7(55.1-70.4)$ & 76.7 (75.4-78.0) & $0.53(0.53-0.54)$ \\
\hline
\end{tabular}

PPV: positive predictive value

NPV: negative predictive value

AROC: area-under then receiver operator curve

Undiagnosed Diabetes: A1C $\geq 6.5 \%$; Undiagnosed Prediabetes: A1C 5.7-6.4 \%; Undi-

agnosed Dysglycemia: A1C $\geq 5.7 \%$

WHO ARE THE HEART FAILURE "FREQUENT FLIERS"? UNDERSTANDING THE CHARACTERISTICS OF HEART FAILURE PATIENTS WITH MULTIPLE READMISSIONS Madeline R. Sterling ${ }^{1}$; Parag Goyal ${ }^{1}$; Ashley Beecy ${ }^{1}$; Carla Boutin-Foster ${ }^{2}$; Erica C. Jones ${ }^{1} .{ }^{1}$ New York Presbyterian - Weill Cornell Medical College, New York, NY; ${ }^{2}$ Weill Cornell Medical College, New York, NY. (Control ID \#2465182)

BACKGROUND: While much research has focused on factors related to 30-day heart failure (HF) readmissions, less is known about patients who are readmitted multiple times for HF. Multiple readmissions result in significant morbidity and mortality for patients and are costly for the health care system. Here, we aim to examine the characteristics of patients readmitted with HF multiple times and determine the significance of those predictors on readmission risk.

METHODS: This is a single-center retrospective study of adult patients $(\geq 18$ years of age) admitted with a principal diagnosis of HF who were discharged from an urban, academic hospital from January 2013 to December 2014. Demographic, clinical and heart failure characteristics, as well as echocardiographic indices and administrative data, were 
collected via chart review. Multi-readmissions ( $\geq 2$ readmissions) was the primary outcome of interest. Patients discharged to hospice were excluded. Descriptive statistics of the study population were performed. Bivariate analyses were conducted using Chi-square and Fisher's Exact Test. Covariates which were found to be significant at the bivariate level were included in the model. Weight of Evidence (WOE) and Information Value (IV) of the qualifying covariates were used to build the final multivariable logistic regression model. RESULTS: During a period of 2 years, 658 patients were admitted for HF. The mean age of the cohort was 71 years with the majority comprising of men (57\%), non-white (67\%), and recipients of Medicare (71 \%). NYHA Class III (63\%) and NYHA Class IV (30\%) comprised the majority of the population. A total of $79(12 \%)$ patients were readmitted multiple times during the study period. Among these 79 "frequent fliers", 46 (58\%) patients were readmitted twice, $21(27 \%)$ three times and $5(6 \%)$ four times. One patient was readmitted with HF 12 times during the study period. Heart failure with preserved ejection fraction (HFpEF) $(p=0.001)$, peripheral vascular disease (PVD) $(p=0.002)$, a heart rate $(\mathrm{HR})$ greater than 70 on admission $(p=0.04)$ and severe mitral regurgitation $(p=0.01)$ were associated with multiple readmissions. In addition to the number of cardiac co-morbidities ( $p=0.003$ ) being associated with multiple readmissions, the number of non-cardiac co-morbidities $(p=0.002)$ that patients had were as well. Of the noncardiac comorbidities, End-Stage Renal Disease (ESRD) $(p=0.003)$ and a prior gastrointestinal bleeding $(p=0.003)$ were significantly associated with multiple HF readmissions. In the final regression model, $\mathrm{HFpEF}(\mathrm{OR}=2.099$, CI: 1.289-3.41), the number of cardiac co-morbidities (OR 1.264, CI: 0.981-1.628) and the number of noncardiac co-morbidities (OR 1.341, CI: 1.048-1.415) were associated with multiple readmissions. The model was significant $(p=0.0001)$ with $65.1 \%$ convergence. In neither the bivariate or regression analyses were age, gender, race/ethnicity, insurance type or social support at home associated with multiple readmissions.

CONCLUSIONS: Our study suggests that "frequent fliers", patients who are readmitted multiple times with $\mathrm{HF}$, are more likely to have $\mathrm{HFpEF}$ and a higher burden of cardiac and non-cardiac co-morbidities. Although 30-day readmissions are an important quality metric, "frequent fliers" represent a unique sub-group that merits further study. Interventions to reduce readmissions may require that comorbid conditions, as well as $\mathrm{HF}$, be addressed. Although the sociodemographic characteristics we studied were not associated with multiple readmissions, future study might explore the effect of socioeconomic status, health literacy and medication adherance on multiple readmissions given that the management of HF, particularly HFpEF, has been shown to be affected by these factors.

\section{WHO IS THE "HOSPITAL-DEPENDENT" PATIENT? MEDICAL, FUNCTION-} AL, AND SOCIAL CHARACTERISTICS Grant M. Smith ${ }^{2}$; Irena Stijacic Cenzer ${ }^{1}$; Alexander Smith ${ }^{1} .{ }^{1}$ UCSF, San Francisco, CA; ${ }^{2}$ University of San Francisco California, San Francisco, CA. (Control ID \#2469293)

BACKGROUND: As medical advances have increased the survival of older adults, there is an emerging population that does not thrive outside of the hospital, depends on the hospital to return to an acceptable quality of life, and ultimately requires frequent hospitalizations. Patients in this population have been labeled "hospital-dependent." As incentives to reduce costs expand beyond preventing 30 day readmissions, understanding the factors that differentiate hospital-dependent patients from similarly-sick peers will be important in developing targeted interventions. This study aims to identify the medical conditions, functional limitations, and social supports that differentiate hospital-dependent patients from patients who have a similar prognosis but require fewer hospitalizations.

METHODS: This is a case-control study using survey data from the Health and Retirement Study (HRS) between 2000 and 2012. The HRS follows a cohort of adults age 50 years and older with comprehensive interviews conducted every 2 years. Hospitaldependent patients (cases) were identified as participants who reported having 5 or more hospitalizations over the last 2 years on at least one HRS survey during the study period. If participants reported 5 of more hospitalizations in the last 2 years on multiple HRS surveys, data from the first survey with frequent hospitalizations was used. Controls included participants who never reported 5 or more hospitalizations. Controls were matched to cases by age, sex, and Lee index score. The Lee index is a validated prognostic tool that was developed to estimate 4-year mortality using age, sex, medical conditions, and functional measures. Each control could only be matched to a single case. Descriptive statistics were used to describe cases and controls. Two-tailed t-tests and chi-squared tests were used as appropriate to compare medical conditions, functional limitations, and social supports. Cases and controls were compared across the components that comprise the Lee index as well as hours of help with ADLs and having a child living within 10 miles.

RESULTS: Of the 31,437 participants in HRS between 2000 and 2012, 1464 (4.6 \%) reported being admitted to the hospital 5 or more times within 2 years. Their mean age was 70.7 years (SD 11.8 years). Fifty-eight percent were men. Their mean Lee index score was 11.0 (SD 4.2), which is associated with a $45 \%$ risk of mortality in 4 years and a median life expectancy of 5.9 years. Hospital-dependent patients reported a mean of 7.6 hospitalizations over 2 years (SD 5.9, range 5-94). Controls had a mean of 0.8 hospitalizations (SD 1.1 , range $0-4)$. Of cases who reported 5 or more hospitalizations in 2 years, 1450 (99\%) were able to be matched to a control based on age, gender, and Lee index score. Hospitaldependent patients were significantly more likely to have diabetes (41 vs. $34 \%$, $p<0.001$ ), heart failure (63 vs. $50 \%, p<0.001$ ), and have difficulty bathing (37 vs. $33 \%, p=0.02$ ), walking several blocks ( 79 vs. $75 \%, p=0.006$ ) and pushing or pulling heavy objects ( 73 vs. $69 \%, p=0.01$ ). They were significantly less likely to have cancer ( 27 vs. $32 \%, p=0.003$ ), be a current smoker ( 15 vs. $30 \%, p<0.001$ ) or be married ( 49 vs. $54 \%, p=0.006$ ). Hospital-dependent patients and their less frequently hospitalized peers with similar prognosis had similar ethnicities, BMIs, and they received a similar number of hours of help with ADLs and were equally likely to have an adult child who lived within 10 miles.

CONCLUSIONS: Hospital-dependent patients are more likely to have diabetes, heart disease, disability, mobility impairment, and be single compared to older adults with a similar prognosis who are not frequently hospitalized. Interventions to support hospitaldependent patients should target medical, functional and social vulnerability.

WHO SAYS NO? PATIENT CHARACTERISTICS ASSOCIATED WITH REFUSAL OF HIV SCREENING IN A PRIMARY CARE SETTING Sara Turbow ${ }^{2}$; Heather Freiman ${ }^{1}$; Bijal Shah ${ }^{1} .{ }^{1}$ Emory University, Atlanta, GA; ${ }^{2}$ Emory University School of Medicine, Atlanta, GA. (Control ID \#2456617)

BACKGROUND: Despite efforts to routinize and de-stigmatize HIV screening, many patients still decline testing. Past studies indicate that patients who refuse testing may have low self-perceived HIV risk. ${ }^{1,2}$ However, research investigating the association between patient characteristics and HIV test refusal, especially in primary care settings, is limited. Studies performed in the emergency department find that older patients are more likely to decline testing than younger patients, ${ }^{3}$ and that African American patients are the most likely to accept the test. ${ }^{4}$ Here, we assess which demographic characteristics are associated with refusal of routine HIV testing in an urban primary care clinic.

METHODS: As part of a program to implement routine HIV testing in the primary care center (PCC) of a large, urban, safety-net hospital in the Southeastern US, all patients at least 18 years old, who were not known to be HIV positive, did not have an HIV test in the past 6 months, and were able to consent were offered HIV testing during triage using optout language. Medical record data were extracted for all patients in the PCC who were offered an HIV test between October 1, 2013 and September 30, 2015. Data included age 
at first test offer, sex, and race. Patients were categorized as "declined HIV testing" if they declined testing at every visit. Patients were categorized as "ever accepted testing" if they ever accepted an HIV test, even if they declined the test offer at other visits. Patients who ever accepted testing were further categorized into "accepted first offer" and "accepted after previous decline." Chi-square tests were used to assess associations between patient characteristics and response to routine HIV test offer. T-tests were used to compare mean ages and the Z-test for two proportions was used to compare specific age category frequencies.

RESULTS: This analysis included 24,000 patients with 53,312 test offers. Eleven thousand five hundred fifty-four patients always declined $(47 \%)$ and 12,746 patients ever accepted (53\%): 10,579 accepted the first offer and 2167 accepted after previous decline. There was no difference in sex distribution ( $p=$ 0.50) when comparing patients who always declined to: 1) patients who ever accepted and 2) patients who accepted first offer and accepted after previous decline. However, there was a significant difference in race distribution when comparing these same groups; African American patients least frequently declined testing $(p<0.0001)$. Age distribution was also significantly different $(p<0.0001)$ among groups, and older patients were more likely to decline testing. However, when comparing patients who accepted first test offer and those who accepted after previous decline, a higher percentage of patients aged 61-75 accepted after a previous decline than accepted the first test offer $(p<0.0001)$.

CONCLUSIONS: While routine HIV testing should be offered to every patient, it is still important to identify factors that may decrease a patient's chances of accepting. This knowledge can help tailor messaging about HIV testing and improve provider awareness when discussing HIV testing with patients. Here, we find that patients who always decline testing are more frequently older or non-African American compared to patients who ever accept. Older patients may decline more frequently because of lower perceived HIV risk When comparing test acceptance across age groups, the oldest group of patients more frequently declined testing, regardless of number of test offers. While patients 30 years and younger most frequently accepted the first test offer (11\%), they least frequently accepted after a previous decline $(3 \%)$. When comparing frequencies of patients who always declined and accepted after previous decline, there was no significant difference in patients aged 61-75 years. The reasons for this finding are unclear, although they may be related to how the test is offered or to evolving understanding of the need for HIV screening in this age group. Further investigation into more detailed characteristics among this population is warranted, as well as more complex analyses of different patient response patterns to test offer. These analyses will provide valuable information about who declines routine HIV testing and will allow providers, social workers, and counselors to focus their efforts accordingly.

WISDOM AND BURNOUT IN MEDICAL STUDENTS Margaret L. Plews-Ogan; Justine E. Owens; Walker Redd; danielle oliver; Rachel H. Kon; Tabor E. Flickinger; John Schorling. University of Virginia, Charlottesville, VA. (Control ID \#2468762)

BACKGROUND: The ABIM chose to name their campaign to reduce unnecessary testing and treatment the "Choosing Wisely" campaign, implying it takes wisdom to provide truly high value care. Researcher Monika Ardelt describes characteristics of wise persons as: understanding the deeper meaning of things, knowing the limits of knowledge, tolerating ambiguity, engaging in reflective and self-reflective thinking, showing compassion and sympathy toward others, and the capacity to be other-centered. ${ }^{1}$ To "choose wisely" among diagnostic and treatment options for patients, a clinician must not only have expert knowledge, but must be able to apply that knowledge within the complex circumstances and goals for an individual patient, must accept (and help their patients accept) the uncertainties and ambiguities in life, and must do all this with compassion, in the most trying of circumstances. The capacity for self-reflection is essential to both preventing mistakes and learning from experience. In addition, the wisdom capacities (capacities for reflection, finding meaning, tolerating ambiguity, and compassion) may be critical to fostering professionalism and preventing burnout in physicians. As William Branch suggests, "wisdom is what we should be striving for in our development as clinicians" and that "seeking wisdom should be embedded in our culture" ${ }^{2}$, yet wisdom has never been measured in medical students or practicing physicians. The wisdom project sought to measure wisdom and burnout in medical students, in order to better understand the relationship between these factors, and to begin to explore ways in which wisdom capacities might be fostered in medical students.

METHODS: All medical students at the University of Virginia Medical School were sent an online 128 item anonymous survey that included demographic information, wellbeing measures, and validated questionnaires for anxiety, depression, burnout, resilience and wisdom. This report will focus on results on the results on Ardelt's 3-Dimensional Wisdom Scale ${ }^{1}$, and the relationship between high and less high scores on the $3 \mathrm{D}$ wisdom scale to scores on the Maslach Burnout Inventory (MBI). The 3-D Wisdom scale contains subscales of the three elements of wisdom: affective (compassion), reflective, and cognitive. The MBI contains three subscales of Emotional Exhaustion (EE), Depersonalization (DP) and Personal Accomplishment. Using cut offs consistent with other studies, we considered a score of 4 or higher on the $3 \mathrm{D}$ wisdom scale as high wisdom, scores of 27 or greater for $\mathrm{EE}$ or 10 or greater for DP as high burnout. We considered combined burnout to be a high score on either the EE or DP scales. Differences in 3D wisdom scale across 4 years, differences between students scoring high in wisdom and less high in wisdom on the three subscales of the MBI, and differences between high and low burnout students on the three dimensions of 3D Wisdom Scale were tested with ANOVA

RESULTS: Six hundred thirty students were eligible to participate, and 378 responded to at least part of the questionnaire ( $60 \%$ response rate) and 260 to the entire questionnaire (40\% response rate). Mean age 25 with $51.3 \%$ female and $48.7 \%$ male respondents. Three hundred seven medical students completed the MBI, and 260 completed the 3D wisdom scale. Mean overall scores for wisdom were $3.78(\mathrm{SD}=.28), 3.84(\mathrm{SD}=.34)$, $3.70(\mathrm{SD}=.39)$ and $3.84(\mathrm{SD}=.33)$ for years 1 through 4 respectively $(\mathrm{F}=2.63, p=0.05)$ Scores on the $3 \mathrm{D}$ wisdom scale ranged from 2.49 to 4.92 with an average score of 3.79 $(\mathrm{SD}=.34)$. Twenty-five percent of students who completed the 3D Wisdom scale scored in the high wisdom category (score $>4.0)$. High wisdom students $(n=64)$ vs less wisdom students $(n=196)$ scored as follows on the MBI: $16.9(\mathrm{SD}=8.8)$ vs $23(\mathrm{SD}=11.2)$ on emotional exhaustion $(\mathrm{F}=16.0, p<0.001), 4.8(\mathrm{SD}=4.6)$ vs $8.4(\mathrm{SD}=6.5)$ on depersonalization, $(\mathrm{F}=p<0.001)$ and $39.2(\mathrm{SD}=6.6)$ vs $36(\mathrm{SD}=6.5)$ on personal accomplish ment $(\mathrm{F}=11.5, p<0.001)$. Students scoring high on the combined burnout measure $(n=$ 115 ) scored lower on all three dimensions of 3D Wisdom Scale, compared to students scoring low on burnout $(n=154)$, with affective scores of $3.48(\mathrm{SD}=.5)$ vs $3.77(\mathrm{SD}=$ $.42)(\mathrm{F}=28.5, p<.000)$, reflective scores of $3.9(\mathrm{SD}=.45)$ vs $4.1(\mathrm{SD}=.37)(\mathrm{F}=20.8$, $p<.000)$, and cognitive scores of $3.7(\mathrm{SD}=.45)$ vs $3.8(\mathrm{SD}=.36)(\mathrm{F}=8.2, p<.001)$

CONCLUSIONS: Wisdom is a complex phenomenon which brings together many of the components critical to being a good doctor. To our knowledge this is the first measure of wisdom in medical students. Twenty-five persent of medical students scored as high wisdom. There was a trend toward differences between years, with the lowest mean wisdom scores in the third year. High wisdom students had significantly lower scores on emotional exhaustion, and depersonalization, and significantly higher scores on personal accomplishment on the MBI, suggesting that high wisdom students were less likely to suffer from burnout. Wisdom capacities include compassion, reflection, and cognitive capacities to tolerate ambiguity, complexity, and discerning meaning. These capacities may be protective of the negative influences that face physicians that contribute to burnout. Fostering wisdom capacities in young physicians may be a more durable 
approach to fostering professionalism and meaning, and reducing burnout in this challenging profession. ${ }^{1}$ Ardelt M. Empirical assessment of a three-dimensional wisdom scale. Research on Aging 2003; 25(3):275-324 ${ }^{2}$ Branch W, Mitchell G. Wisdom in medicine. The Pharos. 2011; Summer: 12-17.

\begin{tabular}{|c|c|c|c|c|}
\hline & \multirow{2}{*}{$\begin{array}{l}\text { Always } \\
\text { Declined } \\
\text { Testing } \\
N=11.254 \\
46.9 \% \text { of } \\
\text { unique pts }\end{array}$} & \multicolumn{2}{|c|}{$\begin{array}{l}\text { Accepted Testing } \\
\quad \mathrm{N}=12,746 \\
53.1 \% \text { of unique pts }\end{array}$} & \multirow[b]{2}{*}{$\begin{array}{c}\text { Aways } \\
\text { Declined vs. } \\
\text { Accepted after } \\
\text { previous } \\
\text { decline }\end{array}$} \\
\hline & & $\begin{array}{c}\text { Accepted 1st } \\
\text { Offer } \\
N=10,579 \\
44.1 \% \text { of unique } \\
\text { pts }\end{array}$ & $\begin{array}{c}\text { Accepted after } \\
\text { previous decline } \\
N=2,167 \\
9.0 \% \text { of unique } \\
\text { pts }\end{array}$ & \\
\hline \multicolumn{5}{|l|}{ Age } \\
\hline $\operatorname{Mean}(S D)$ & $55.5(14.1)$ & $49.7(13.2)$ & $55.7(10.9)$ & $p=0.22$ \\
\hline Median (IQR) & $56(47.64)$ & $53(41.59)$ & $56(50.63)$ & \\
\hline \multicolumn{5}{|l|}{ Categones } \\
\hline $18 \cdot 30$ & $671(6.0 \%)$ & $1176(11.1 \%)$ & $58(2.7 \%)$ & pro.01 \\
\hline 31.45 & $1763(15.7 \%)$ & $2287(21.6 \%)$ & $267(12.3 \%)$ & $p<0.01$ \\
\hline 46.60 & $4735(42.1 \%)$ & $4981(47.1 \%)$ & 1125 (51.9\%) & $p<0.01$ \\
\hline 61.75 & $3249(28.9 \%)$ & $1978(18.7 \%)$ & $649(30.0 \%)$ & $p=0.31$ \\
\hline $76+$ & $836(7.4 \%)$ & $157(1.5 \%)$ & $68(3.1 \%)$ & $p<0.01$ \\
\hline
\end{tabular}

WOMEN'S PERCEPTIONS OF DENSE BREAST NOTIFICATIONS: "SO WHAT IS THAT SUPPOSED TO MEAN?" Christine M. Gunn ${ }^{1,3}$; Tracy A. Battaglia $^{1}$; Amanda K. West ${ }^{1}$; Nancy R. Kressin ${ }^{2} .{ }^{1}$ Boston University Medical Center, Boston, MA; ${ }^{2}$ Dept of Veterans Affairs and Boston University, West Roxbury, MA; ${ }^{3}$ Boston University, Boston, MA. (Control ID \#2465992)

BACKGROUND: Patients and advocacy groups have influenced the adoption of legislation requiring that women be notified of their breast density in 24 states. Breast density laws have been advocated on the premise that notification will increase knowledge and empower women to make informed decisions about breast cancer screening, yet there is no evidence that this goal has been attained. Legislating the content of breast density notification letters has potential negative effects on patient-centered care, particularly if the letters are not appropriate for all health literacy levels. If certain populations do not understand notifications and therefore do not receive equitable supplemental screening services, existing disparities in breast cancer outcomes may be exacerbated. This study sought to understand women's awareness and understanding of breast density, perceptions of breast cancer risk, and planned actions following the receipt of a dense breast notification, among women receiving care at a safety-net hospital.

METHODS: We assessed women's awareness and perceptions regarding breast density notification in Massachusetts following its implementation in January 2015. Through medical records, we identified women ages 40-75 who had a mammogram (1/1/15-6/30/

15 ) with a normal mammogram result with a finding of dense breasts. Women were sent a letter from the radiology department, inviting them to participate in a qualitative interview study. Research assistants called the women, further screening them to identify those who spoke English and recalled receiving the dense breast notification. Thirty qualitative telephone interviews were conducted, audio recorded, and transcribed verbatim. A grounded content analysis identified women's perceptions and actions taken following receipt of this letter.

RESULTS: Two hundred fifty women were sent invitations to participate and received at least one phone call. Of these, $49(20 \%)$ were ineligible due to language barriers. Sixty-six women were fully screened for participation, with the remainder not reached or refused. Sixteen women $(24 \%)$ did not recall receiving a dense breast notification and were deemed ineligible. Of the 40 eligible women, 30 women agreed and participated in a telephone interview. Participants ranged in age from 42 to 69 (mean $=55.3)$. About half $(53 \%)$ were Black, $13 \%$ White, $13 \%$ Hispanic, and $17 \%$ Other. Participants were evenly split in insurance type: $50 \%$ private, $47 \%$ public, and $3 \%$ unknown. Regarding knowledge about breast density, onethird of women had never heard the term before receiving the notification. One woman said, "I don't understand what it really is...[Is it] something we had and it's going to go away, or something that was already there, or a stage?". Women struggled to understand what it meant to have dense breasts, and most developed their own explanations as to why they have them, including: breastfeeding/children, weight, having "active breasts", age, physical changes, and lifestyle. Many also relied upon physical signs and feelings to interpret how serious it might be to have dense breasts: "my breasts have always felt thick and full". Although the Massachusetts letter states that breast density can increase breast cancer risk, a minority of women recalled this aspect. One woman even stated, "I think maybe if the letter said you have dense breasts and you have a higher risk of getting breast cancer, that would trigger it more than just talk to your doctor." Emotionally, women described a range of reactions to receiving a dense breast notification: Many expressed concern, often related to family histories of breast cancer. Others felt confused, suggesting the notification was vague, they weren't provided enough information, and were unsure about what to do. About a third of women interviewed sought in-person communication to get an explanation: "I talked to my doctor, he said 'your mammogram is fine"'. Women had to balance the notification with messages from medical professionals telling them they were fine, conflicting information which led to uncertainty. Alternatively, some women found the lack of explanation in the notification made it easy to put the letter off and not follow through on concerns. A minority of women brought up insurance as being a barrier to seeking further screening or care related to breast density.

CONCLUSIONS: Among a sample of women sent breast density notifications in Boston, three-quarters recalled receiving such notifications. Those who recalled the notification expressed a lack of understanding about breast density as a concept, and many created their own interpretations about what caused their dense breasts. Regardless of the intention of this legislation to encourage informed decision-making, the language used in the notification did not have the desired effect on these women. Instead, women used their own experiences to create explanations for having dense breasts and justify their actions.

WORK LIFE AND WELLNESS IN ACADEMIC GENERAL INTERNAL MEDICINE: RESULTS FROM A NATIONAL SURVEY Stewart Babbott ${ }^{4}$; Mark Linzer ${ }^{1}$; Sara Poplau ${ }^{1}$; Tracie C. Collins ${ }^{2}$; Laura M. Guzman-Corrales ${ }^{1}$; Jeremiah Menk ${ }^{5}$; Mary Lou L. Murphy ${ }^{6} ;$ Kay Ovington ${ }^{3}$. ${ }^{1}$ Hennepin County Medical Center, Minneapolis, MN; ${ }^{2} \mathrm{KU}$ School of Medicine - Wichita, Wichita, MN; ${ }^{3}$ Society of General Internal Medicine, Alexandria, VA; ${ }^{4}$ University of Kansas, Kansas City, KS; ${ }^{5}$ University of Minnesota, Minneapolis, MN; ${ }^{6}$ Stanford, Palo Alto, CA. (Control ID \#2465026)

BACKGROUND: Careers in primary care, particularly general internal medicine (GIM), are less attractive to medical students. Insight into GIM work conditions could help address this problem. Our objective was to assess work life in academic GIM and determine remediable predictors of stress and burnout.

METHODS: Physicians, nurse practitioners, and physician assistants in 15 GIM divisions in the United States were sent a survey by email. The 10 item instrument queried stress, burnout, and work conditions such as electronic medical record (EMR) challenges. An open-ended question assessed stressors and solutions. Results were categorized into 
burnout, high stress, high control, chaos, good teamwork, high values alignment, documentation time pressure, and excess EMR use at home. Frequencies were displayed for national data, VA vs. civilian, and hospitalist vs. ambulatory roles. A General Linear Mixed Model (GLMM) assessed correlates of burnout. Content analysis of open-ended question responses produced a conceptual framework of GIM work life.

RESULTS: Five hundred seventy-nine of 1235 eligible clinicians responded (47\%). High stress was present in $67 \%$, with $38 \%$ burned out (burnout range $10-56 \%$ by division). Half had low work control, with high documentation time pressure in $60 \%$ and half describing too much home EMR time. Most respondents reported chaotic workplaces. Two-thirds felt aligned with departmental leaders' values, and three-quarters were satisfied with teamwork. Burnout was associated with high stress, low work control, and low values alignment with leaders (all $p<0.001$ ). VA faculty had less burnout than civilian counterparts (17 vs. $40 \%$ burned out, disjunct CI). Hospitalists described better teamwork and less home EMR time (both disjunct CI). Key themes from the qualitative analysis included short visits, insufficient support staff, a Relative Value Unit (RVU) mentality, documentation time pressure, and undervaluing education

CONCLUSIONS: While GIM divisions overall demonstrate high stress and burnout, division rates vary widely. Sustainability efforts within GIM could focus on visit length, staff support, schedule control, clinic chaos, and EMR stress

YIELD OF TESTING AND MANAGEMENT OF HYPERTENSIVE URGENCY IN PATIENTS REFERRED TO THE EMERGENCY DEPARTMENT FROM AN OFFICE VISIT Krishna Patel; Laura Young; Erik H. Howell; Bo Hu; Gregory W. Rutecki; George Thomas; Michael B. Rothberg. Cleveland Clinic, Cleveland, OH. (Control ID \#2469185)

BACKGROUND: Hypertensive urgency is a commonly encountered outpatient problem. When blood pressures are very high, patients sometimes are referred to the emergency department (ED) for management of their high blood pressures even in the absence of symptoms. For this population, the yield of testing in the ED, prevalence of end organ damage and treatment administered are unknown. We aim (i) To describe management of patients with asymptomatic hypertensive urgency in the emergency department. (ii) To describe the yield of testing ordered to detect end-organ damage.

METHODS: All patients who were referred to the ED after presenting with systolic blood pressure $(\mathrm{SBP}) \geq 180 \mathrm{mmHg}$ and/or diastolic blood pressure $(\mathrm{DBP}) \geq 110 \mathrm{mmHg}$ to an office visit in the Cleveland Clinic Health system from 2008 to 2013 were included. We excluded pregnant women and patients referred to the ED for symptoms or for treatment of other conditions. The details of their management in the ED were collected by chart review.

RESULTS: In our study population, 387 patients were referred to the ED. On arrival to the ED, mean SBP was $197 \pm 28 \mathrm{mmHg}$ and mean DBP was $103 \pm 19 \mathrm{mmHg}$. On discharge, mean SBP had fallen to $166 \pm 24 \mathrm{mmHg}$ and DBP to $87 \pm 15 \mathrm{mmHg}$. Seven patients $(1.8 \%)$ had evidence of target organ damage (4 had acute kidney injury, 2 had pulmonary edema and 1 had elevated cardiac biomarkers). The etiology of hypertensive urgency was unknown in $60 \%$ of patients, $25 \%$ had it attributed to non-adherence (includes running out of medications), $11 \%$ of patients had new diagnoses of hypertension and $5 \%$ of patients were referred because they were new patients who presented with elevated blood pressure readings. Treatment consisted of a one-time dose of antihypertensive medication for $59 \%$ patients ( $29 \%$ were delivered intravenously and $71 \%$ orally) while the rest received no intervention. Labetalol (11\%) was the most commonly used intravenous drug and clonidine ( $15 \%$ ) was the most commonly used oral drug. In the ED, $41 / 748$ tests ordered ( $5.5 \%$ of tests, $10.5 \%$ of patients, mean 1.9 test /patient) had an abnormal result. Of the ED patients, $17 \%$ were admitted to an inpatient service and the rest were discharged home. Of the patients admitted from the ED, $85 \%$ were admitted for observation and mean length of stay was 3.45 days. On discharge from the ED, a new BP medication was added to the home regimen for $21 \%$ of patients, the home dose of BP medication was increased for $6 \%$, and no change was made for $66 \%$ of the patients.

CONCLUSIONS: For patients referred to ED for management of asymptomatic hypertensive urgency, diagnostic testing had a low yield and only one-third of the patients had a change to their medical regimen. ED referral may represent low value care for this population.

*CLINICAL VIGNETTES ...AND THAT IS WHY I DON'T DRINK FIREBALL ANYMORE! Sarah Smith; Michael P. Smith. University of Nebraska Medical Center, Omaha, NE. (Control ID \#2435398)

LEARNING OBJECTIVE \#1: Recognize the presentation of anion gap metabolic acidosis due to propylene glycol toxicity from Fireball brand whiskey

LEARNING OBJECTIVE \#2: Develop a systematic approach to evaluate an anion gap metabolic acidosis, that can be used in both the inpatient and outpatient setting CASE: A 49 year-old woman presented for an annual exam with complaint of mild fatigue. Her physical exam was unremarkable. Lab work revealed a bicarbonate of 16 with an anion gap of 16. The remainder of the comprehensive metabolic profile, including renal function, blood glucose, and hepatic function, was within normal range. Complete blood count, thyroid stimulating hormone, and lipid profile were all unremarkable as well. On further questioning, she denied any use of salicylates or acetaminophen and had no known exposure to ethylene-glycol or methanol containing products. There had been no evidence of hypo-perfusion on exam. She did reveal that she had been drinking large amounts of Fireball brand whiskey daily, including the night prior to her lab draw. The patient was advised to discontinue her consumption of whiskey and subsequent lab work returned to normal values.

DISCUSSION: An elevated anion gap metabolic acidosis is frequently encountered by the internist, though not often in an otherwise well appearing patient. It is important to have an approach to an abnormal acid-base status in the outpatient setting. Common causes of elevated anion gap acidosis include endogenous production or accumulation of acids ( $e g$, lactic acidosis, renal failure), ketoacidosis (eg, diabetic, starvation), and toxins. Thorough history and physical, with accompanying lab work as indicated, can help one identify an etiology. An arterial blood gas, to evaluate $\mathrm{pH}, \mathrm{pCO} 2$, and $\mathrm{pO} 2$, helps delineate acid-base disturbances. Data can be used to estimate appropriate compensation and to detect mixed abnormalities. The comparison of measured osmolality to a calculated value to determine an osmol gap is also part of the evaluation. A discrepancy between measured and calculated osmolality of ten or greater suggests the presence of additional unmeasured solutes. Other laboratory to consider include lactic acid level, ethanol level, salicylate and acetaminophen levels. This patient had no evidence of type A lactic acidosis as a result of tissue hypo-perfusion and had a normal serum creatinine and glomerular filtration rate. Blood sugar was normal, she was well-nourished and had not been fasting at the visit; diabetic and starvation ketoacidosis were each considered unlikely. Thorough review of medications was performed and negative for salicylates and acetaminophen, both of which are known to cause an anion gap acidosis. She did endorse drinking several shots of whiskey every night. Fireball Whisky is a popular brand of American whiskey known to contain propylene glycol for flavoring purposes. It has been temporarily recalled in several European countries for not meeting compliance standards, though is approved by the United States Federal Drug Association for use. Propylene glycol is metabolized to Llactic and D-lactic acid and glyoxal, thus contributing to a type B lactic acidosis and elevated osmol gap. Propylene glycol is more commonly encountered as a pharmaceutical solvent for medication infusions, including lorazepam and phenobarbital. Toxicity is observed in patients receiving such infusions for prolonged periods, generally in the 
intensive care unit setting. When such patients develop an otherwise unexplained anion gap acidosis, consideration must be given to medication as the culprit. Toxicity can also cause renal injury and acute tubular necrosis. Treatment is to discontinue the medication, with administration of fomepizole if severe. The internist must have an approach for anion gap acidosis in the ambulatory setting. This approach must take into consideration ingestion of common and popular products. One must also consider the clinical context of each individual case. In the outpatient setting, it is not always with ease that additional laboratory work and testing can be obtained. If in evaluation of a stable and reliable patient a reversible and non-life threatening cause seems most likely, it can be safe to defer extensive testing and follow-up in a timely manner. With a complete history and physical and systemic approach to abnormalities, one can be judicious in ordering tests.

\section{"SMOKE IN THE AIR": A RARE CAUSE OF STROKE IN A YOUNG ADULT} Khin Sandar Lim; Arianna E. Becker; Sameen Khalid; George Everett. FLORIDA HOSPITAL, ORLANDO, FL. (Control ID \#2466766)

LEARNING OBJECTIVE \#1: Moyamoya disease is a rare, idiopathic, progressive cerebrovascular occlusive disease characterized by irreversible stenosis of bilateral intracranial vessels, particularly middle (MCA) and anterior (ACA) cerebral arteries. Blockage of cerebral blood flow causes ischemic stroke and development of collateral circulation leads to hemorrhage. Moyamoya, which means, "puff of smoke" in Japanese, was used to describe the characteristic angiographic appearance of collateral circulation. Here, we discuss a 23-year-old Hispanic female with acute stroke symptoms.

CASE: A 23-year-old Hispanic female with history of asthma developed sudden rightsided body numbness and weakness associated with slurred speech for 1 day. On physical examination, she had mild dysarthria, flattened right nasolabial fold with decreased strength and sensation in right upper and lower extremities. Initial labs were unremarkable. CT head showed $2 \mathrm{~cm}$ hypodense lesion in left basal ganglion. CTA head revealed bilateral occlusions of the M1 segments of the MCA. MRI brain showed numerous acute punctate infarcts in bilateral ACA/MCA watershed distributions. Cerebral angiogram showed bilateral proximal MCA occlusions with dilated collaterals, which is consistent with moyamoya disease. Aspirin, statin and physical therapy was started. Her speech and sensory impairment improved except slight residual hemiparesis. Two weeks later, she underwent left extracranial to intracranial carotid artery bypass surgery. All her symptoms are resolved at 6 week follow up.

DISCUSSION: Moyamoya disease was first described in Japan in 1957. Although originally considered mainly in Asian population, recent studies suggest that moyamoya has been observed in other populations. Its incidence has been reported as 0.086 cases per 100,000 people in the United States. However, incidence -rate ratios are 0.03 for Hispanics compared to 0.28 for Asian American. Moyamoya has bimodal distribution with peaks of five and mid- forties years of age. Females have double the incidence of males. While familial occurrence is approximately $9 \%$ of moyamoya disease, majority is sporadic. RNF213 gene on chromosome 17q25.3 is important for moyamoya disease in Asian populations. Patients with the angiographic appearance of moyamoya without risk factors are considered moyamoya disease, while those with recognized associated conditions (neurofibromatosis type 1, Down syndrome) are classified as moyamoya syndrome. Conventional cerebral angiography is still the gold standard for diagnosis. Management focuses on secondary prevention of ischemic stroke by revascularization procedures. Progressive neurologic deficits were reported in 50-66 \% of untreated patients vs. $2.6 \%$ after surgery in a 5-year period. Early diagnosis followed by surgical intervention is important in prevention of further neurologic deficits. This case highlights the importance of recognizing moyamoya in young patients with stroke, even in ethnic groups where it is less common.
"FAT BURNERS MAY BURN THE HEART": A RARE CASE OF LTRYPTOPHAN RELATED EOSINOPHILIA-MYALGIA SYNDROME (EMS) WITH MYOCARDITIS: waqas nawaz; Faran Ahmad; Marilou Corpuz. Montefiore Medical Center, Albert Einstein College of Medicine, Bronx, NY. (Control ID \#2437565)

LEARNING OBJECTIVE \#1: Recognize the clinical presentation of eosinophilia-myalgia syndrome, and its association with different medications and dietary supplemements.

LEARNING OBJECTIVE \#2: Understand the historical relationship between Ltryptophan containing dietary supplements and eosinophilia-myalgia syndrome.

CASE: A 26 year old woman with past medical history of asthma and eczema presented with off and on pleuritic and positional left sided chest pain for 6 days that was associated with cramp-like muscle pains in her extremities and back for 3 weeks. She denied fever, arthralgia or rash. Examination revealed normal vital signs and tenderness in chest, extremities and lower back. Initial labs showed elevated white blood cell (WBC) count of $26,000 / \mu \mathrm{L}$ with eosinophilia of $16,000 / \mu \mathrm{L}$ that was $60 \%$ of WBC count. Chest X ray was normal. CPK was elevated at $434 \mathrm{U} / \mathrm{L}$. Troponins were elevated with 1 st set as $1.26 \mathrm{ng} / \mathrm{ml}$, 2nd set as $1.74 \mathrm{ng} / \mathrm{ml}$ and $3 \mathrm{rd}$ set as $2.10 \mathrm{ng} / \mathrm{ml}$. Further cardiac work up including electrocardiogram and echocardiogram was unremarkable. The eosinophilia was extensively worked up. Infectious etiology including parasitic infestations (isospora, cyclospora, cryptosporidium, strongyloides, cryptococcus, trypanosoma, trichinella), HIV, Human T cell lymphotropic virus (HTLV) type-1 was ruled out. Work up for allergic etiology (tryptase level) and vasculitis (ANA, c-ANCA, p-ANCA, complement factors) was negative. Upon further questioning, she reported daily use of a dietary supplement containing L-tryptophan for weight reduction during the preceding 4 weeks. Based on the muscle pains and unexplained eosinophilia $>1000$ cells $/ \mu \mathrm{L}$ with negative infectious work up, she was diagnosed with eosinophilia-myalgia syndrome which was attributed to the Ltryptophan containing supplement by exclusion. Elevated troponins were attributed to te hyper-eosinophilia. She was started on steroids and counseled to stop the the supplements. Follow up 4 months later showed improvement of symptoms with WBC count of 10,700 $/ \mu \mathrm{L}$ and eosinophil count of $20 \%$.

DISCUSSION: Eosinophilia-myalgia syndrome (EMS) is a rare entity characterized by incapacitating myalgia and eosinophilia $>1000$ cells $/ \mu \mathrm{L}$ in the absence of alternative conditions that could account for eosinophilia. It presents as abrupt onset of muscle pains and spasms involving muscles of legs, shoulders and back. Muscle pains progressively worsen and eventually become incapacitating making it difficult to walk or perform daily activities. Other organs involved include skin, lungs and nervous system. Cardiac abnormalities include myocarditis and arrhythmias which are rare and occur in later phase of syndrome. It is more commonly seen in white females in age group of 35 to 60 years. Mortality ranges from 2 to $6 \%$. Eosinophilia-myalgia syndrome has been historically related with tryptophan containing dietary supplements. Three cases of EMS were first reported in 1989 following the use of tryptophan containing products in New Mexico. Based on the evidence from subsequent case-control studies, the Food and Drug Administration (FDA) banned the sale of all tryptophan containing dietary supplements in 1990, which led to a precipitous fall in the frequency of EMS. The ban was lifted in 2005. Since then, very few cases have been reported. To our knowledge, this has been the second case report of EMS since 2005. Pathogenesis is based upon a cell mediated immune response which is triggered by exposure to certain preparations of L-tryptophan in a genetically susceptible host, leading to recruitment and degranulation of eosinophils. Moreover, excessive oral ingestion of tryptophan inhibits histamine degradation by increasing formation of formate and indolyl metabolites, which block the degradation of histamine, thereby potentiating its effects. Increased histamine activity is known to induce peripheral blood eosinophilia and myalgia. Management of EMS involves supportive treatment and cessation of L-tryptophan containing supplements. Steroids may help in few cases. Prognosis is variable. Most patients show slow improvement while some patients show 
progression of symptoms after stopping L-TRP containing supplements. This case signifies the importance of obtaining a comprehensive patient history about the use of dietary supplements and herbal products, and considering them in the differential diagnosis.

\section{ACUTE RENAL FAILURE FROM INTERSTITIAL NEPHRITIS RELATED TO PROTON PUMP INHIBITORS IN A 39-YEAR-OLD PATIENT Monoj K. Konda ${ }^{1}$;} Sandeep Patri ${ }^{1}$; Hardik Chhatrala ${ }^{1}$; Sidhu Prince ${ }^{2} .{ }^{1}$ Western Michigan University Homer Stryker M.D. School of Medicine, Kalamazoo, MI; ${ }^{2}$ Nephrology Center, Kalamazoo, MI. (Control ID \#2460382)

LEARNING OBJECTIVE \#1: This case report emphasizes the need for awareness of medications, especially proton pump inhibitors (PPIs), causing acute interstitial nephritis (AIN). Physicians should be able to recognize the non-specific presentation of renal dysfunction or uremia and obtain a good history of medications and time of initiation in cases of drug induced allergic interstitial nephritis.

CASE: A 39-year-old female presented to PCP office with 4-week history of gradually worsening generalized fatigue, excessive sleepiness, nausea, metallic taste in mouth, excessive thirst and dyspnea on exertion. Blood work showed a serum creatinine of $10.7 \mathrm{mg} / \mathrm{dl}$ with a normal value 5 months ago and was referred to ED. Other review of systems was negative. Her past medical illnesses include chronic back pain, depression, morbid obesity and severe reflux disease. Home medications include meloxicam, tramadol, citalopram, rabeprazole and omeprazole with omeprazole being the newest drug, started 4-5 months ago. Physical exam was unremarkable without signs of fluid overload; vitals were normal except for blood pressure of 152/89 $\mathrm{mmHg}$. Laboratory tests showed creatinine $11.2 \mathrm{mg} / \mathrm{dl}$, GFR $4 \mathrm{ml} / \mathrm{min}$, BUN $91 \mathrm{mg} / \mathrm{dl}$, bicarbonate $15 \mathrm{mmol} / \mathrm{L}$ with normal electrolytes and CBC. Urinalysis showed 1+ protein and 1+ hemoglobin with 1 RBC, 8 WBC and no eosinophils or casts. Fractional excretion of sodium was $12.6 \%$ and $24-\mathrm{h}$ urine protein was $1269 \mathrm{mg}$ /day. Renal ultrasound and immunologic work up were normal. Meloxicam was initially thought to be the cause for renal injury and was discontinued without improvement in renal function which prompted a renal biopsy on day 5 which showed severe AIN without evidence for immune complex-mediated glomerulonephritis. It was likely from the new medication omeprazole. PPIs were discontinued and steroids were started on day 7. Patient required 2 sessions of dialysis on day 6 and day 8 . Her kidney function improved over a week to a point where she did not require dialysis and was discharged on oral steroid taper for 3 months with nephrology follow-up.

DISCUSSION: Seventy to $75 \%$ of cases of AIN are related to drugs. Other causes include infections, tubule-interstitial nephritis and uveitis syndrome and systemic diseases (Sjogren's syndrome, SLE and others). Virtually any drug can cause AIN but commonly reported ones include NSAIDs, penicillins, cephalosporins, sulfa drugs, ciprofloxacin, PPIs, cimetidine and 5-aminosalicylates. Common indications for biopsy include uncertainty in clinical features or offending agent, patients who do not improve following cessation of offending agent, new onset renal failure (within last 3 months) and in whom glucocorticoids for AIN is being considered. There are retrospective studies which showed improvement in kidney function with glucocorticoids and also studies that reported no benefit. Due to potential benefit and relative safety of short term therapy patients who do not respond to conservative management are usually started on steroids for 2-6 months Limited literature is available for treatment of steroid dependent and steroid resistant cases which reported use of mycophenolate, cyclosporine and cyclophosphamide.

COCCIDIOIDOMYCOSIS AND CARDIAC TAMPONADE- A DEADLY COMBINATION Shruti Mony; Geraldine Casino; Jeremy P. Feldman. St.Josephs Hospital and Medical Center, PHOENIX, AZ. (Control ID \#2469914)
LEARNING OBJECTIVE \#1: Coccidioidomycosis, an endemic fungal infection, is found predominantly in the Western hemisphere especially in Arizona, southern New Mexico, western Texas and the central valley of California. It primarily affects the lungs but may disseminate to virtually any organ in the body. Cardiac Involvement in cocci is a very rare and less than five cases of cardiac tamponade have been reported. We present an unusual case of disseminated cocci to the pericardium presenting with early cardiac tamponade. A high index of suspicion for cardiac coccidioidomycosis should be maintained in patients from an endemic area presenting with acute cardio-pulmonary compromise.

CASE: A 37-year old African American female, presented to our hospital with a 5 days history of pleuritic chest pain, shortness of breath and night sweats. Her chest pain was worse on leaning forward and exertion. She had initially presented to our emergency department with similar complaints 1 week ago and underwent a CT angiogram, which showed extensive para- tracheal, and hilar soft tissue concerning for a mass or mediastinal adenopathy. Since she was and hemodynamically stable, she was supposed to follow up with a Pulmonologist for further workup. Due to insurance barriers, the patient was unable to be seen promptly in the outpatient setting and she represented to the emergency department with worsening symptoms. Physical exam revealed a moderately distressed female, tachycardic at $120 \mathrm{bpm}$, blood pressure of $90 / 70 \mathrm{~mm}$ of $\mathrm{Hg}$, saturating at $95 \%$ on room air. Cardiac examination was notable for diminished heart sounds and pulmonary examination revealed clear lung fields. A transthoracic echocardiogram done in the emergency department showed a large pericardial effusion, with signs of early tamponade. Due to her rapid deterioration, she underwent a repeat CT chest which confirmed a new large pericardial effusion with increase in the mediastinal and hilar lymphadenopathy seen the earlier scan. Urgent pericardiostomy was performed and $650 \mathrm{ml}$ tan-colored fluid was removed. Other labs were significant for a high normal LDH at 220U/L and elevated ALT at $150 \mathrm{U} / \mathrm{L}$. Due to a persisting moderate posterior pericardial effusion, seen on repeat echocardiogram,and minimal fluid draining from the pericardial drain, she underwent a pericardial window. Mediastinoscopy was also performed due to concern for lymphoma. The pericardium was noted to be thickened with significant adhesions to the right ventricle and an additional $100 \mathrm{cc}$ of posterior pericardial fluid was suction and sent for culture \& cytology. Medistinoscopy revealed fibrosis in the pre-tracheal space with necrotic right paratracheal nodes. On site frozen sections of the lymph node biopsy showed organisms consistent with coccidiomycosis. Two days later her Cocci IgM \& IgG returned positive. Pericardial fluid was negative for neoplasm but the para-tracheal lymph node mass showed granulomas and coccidiodes spherules. Tissue culture subsequently returned positive for coccidiodes immitis. Immediately after her mediastinoscopy, she was started on high dose Fluconazole. Within the next $48 \mathrm{~h}$ her clinical condition significantly improved, her drain was removed and she was discharged home. At 2 weeks of follow up she is doing very well. She has returned to work and has is tolerating her therapy without problems

DISCUSSION: Pericardial involvement with Coccidioides immitis is an infrequent occurrence with a relatively unfavorable prognosis. Our patient's initial clinical picture was suggestive of malignancy given the mass like presentation and increased LDH. Disseminated Coccidiomycosis is associated with significant mortality and morbidity. Common extra-pulmonary sites include the skin or subcutaneous soft tissue, meninges, spinal cord and the musculoskeletal system. In review of literature, cardiac coccidiomycosis is not uncommonly identified at autopsy but diagnosis premortem $\mathrm{s}$ rare. Cardiac tamponade due to coccidiomyocosis is particularly uncommon. Valley fever is a great imitator and clinicians caring for patients from endemic areas should always consider the possibility of coccidiomycosis as a potential diagnosis. 
HYPOKALEMIA WITH ALKALOSIS AS A SIGN OF AN IMPORTANT SYSTEMIC ILLNESS Salik Nazir; Anthony Donato; Stephen Melnick; Rittu V. Hingorani; Richard Alweis. Reading Health System, West Reading, PA. (Control ID \#2467511)

LEARNING OBJECTIVE \#1: Recognize clinical features of Gitelman syndrome and adverse consequences associated with chronic hypokalemia and hypomagnesaemia.

LEARNING OBJECTIVE \#2: Approach to diagnose Gitelman syndrome in patients with hypokalemia \& alkalosis.

CASE: A 30 year-old female admitted for the management of pulmonary embolism was incidentally noted to have profound hypokalemia $(2.8 \mathrm{meq} / \mathrm{L}$, normal: $3.5-5 \mathrm{meq} / \mathrm{L})$. Further historical review found that her hypokalemia had persisted for 15 years without identified cause. She was a non-drinker and was never prescribed diuretics. She also noted chronic fatigue, intermittent numbness of extremities, and arthralgia's of multiple large and small joints. Exam revealed a normotensive, euvolemic and obese female with an unremarkable exam including musculoskeletal and neurologic exams. Laboratory testing also identified low magnesium $(1.7 \mathrm{mg} / \mathrm{dl}$, normal: $1.8-2.2 \mathrm{mg} / \mathrm{dl})$ and elevated serum bicarbonate (33 meq/L, normal:22-28 meq/L). To determine the cause of her presumed metabolic alkalosis, a spot urine chloride was obtained, which was $160 \mathrm{meq} / \mathrm{L}$, confirming a saline-resistant alkalosis. Hypocalciuria (spot calcium to creatinine ratio of 0.05 ) and hyperrenenimic hyperaldosteronism were also identified, confirming the diagnosis of Gitelman syndrome. X-rays of knees identified chondrocalcinosis with narrow joint space suggestive of secondary osteoarthritis due to chronic low magnesium and potassium. She was discharged on magnesium and potassium replacement and Indomethacin to block the prostaglandin effects of the disease, and noted resolution of her symptoms and electrolyte abnormalities at 6 weeks.

DISCUSSION: Gitelman syndrome is a rare autosomal recessive cause of hypokalemia and metabolic alkalosis, usually presenting in childhood or early adolescence. While most patients seen in hospitals with hypokalemia and alkalosis are most often hypovolemic; in those that are not, a urine chloride ( $>15 \mathrm{meq} / \mathrm{L}$ ) will identify those rare patients who may be saline-resistant (including those with Gitelman, Barrter and Liddle). Normotensive patients with High renin, high aldosterone, low magnesium and low urinary calcium (a“thiazide-like pattern") may have Gitelman syndrome, which responds to NSAIDs and Amiloride. Physicians frequently encounter hypokalemia, but identifying this rare pattern of electrolyte abnormalities can prevent future complications such as secondary osteoarthritis from chronically low potassium and Magnesium levels.

\section{MYCOPHENOLATE-INDUCED DISSEMINATED TB IN A PPD-NEGATIVE}

PATIENT Megan L. Srinivas; George Kunnackal John. Johns Hopkins University, Baltimore, MD. (Control ID \#2457685)

LEARNING OBJECTIVE \#1: Individuals with underlying rheumatologic diseases such as dermatomyositis may not adequately respond to tuberculin (PPD) skin tests, creating false negative results. These illnesses are frequently treated with immunosuppressive therapy making proper identification of TB infection imperative.

CASE: A 59-year-old Filipino man was diagnosed with dermatomyositis on the basis of rash, electromyography, and muscle biopsy. He was initially treated with IVIG infusions and transitioned to oral prednisone and mycophenolate. The patient's symptoms improved on this regimen. Six months after starting mycophenolate, the patient began having fevers, night sweats, and productive cough without hemoptysis. He moved from the Philippines 5 years prior to dermatomyositis diagnosis, denied sick contacts, and was PPD negative both at immigration and immediately prior to starting mycophenolate treatment. A third PPD was negative following the onset of these new symptoms. He was treated for community-acquired pneumonia, but symptoms worsened over 10 days and he developed watery diarrhea and a growing non-tender, non-mobile mass on the left side of his neck. A chest X-ray demonstrated a cavitary lesion in right upper lobe suspicious for TB that hadn't been present 1 month earlier. Chest CT corroborated this finding also exhibiting necrotic hilar and paratracheal lymphadenopathy. Neck CT demonstrated the left-sided mass as cervical chain lymphadenopathy. Expectorated sputum and stool samples contained acidfast bacilli (AFB), cultures showing TB bacteria. Fine-needle biopsy of the neck mass (scrofula) also exhibited AFB. An MRI brain showed nodular enhancement suspected to be a tuberculoma. Mycophenolate was discontinued and dermatomyositis treatment was switched to oral prednisone with a 3-day course of IVIG. The patient's infection showed sensitivity to standard RIPE (rifampin, isoniazid, pyrazinamide, and ethambutol) treatment. Within a week of starting RIPE, the patient's diarrhea subsided, scrofula diminished, and symptoms significantly improved. By the end of treatment week 3, the patient's sputum no longer contained AFB; he was removed from isolation, and was discharged to continue RIPE at home. He was discharged on oral prednisone, which effectively addressed his dermatomyositis.

DISCUSSION: This case illustrates the unreliability of PPD tests in patients with longterm inflammatory diseases such as dermatomyositis. Other immunosuppressive therapies (adalimumab, etanercept, and infliximab) have been affiliated with conversion of latent TB to disseminated TB. Mycophenolate is another immunosuppressive agent with similar mechanistic properties. Thus, it is imperative that patients with long-term inflammatory diseases and high-risk TB factors initiating immunosuppressive therapy receive a TB blood test (such as a quantiferon gold assay) prior to the initiation of therapy to ensure that latent TB is unmasked before it can evolve into a disseminated form of the disease.

WHEN TREATMENT BECOMES THE CAUSE: TMP-SMX AND ASEPTIC MENINGITIS Sabrina N. Huq; Srikanth Puli; Shahid Tamoor; Valerie Gross. Montefiore Medical Center, Wakefield, Bronx, NY. (Control ID \#2466713)

LEARNING OBJECTIVE \#1: Consider drug induced meningitis as a differential diagnosis for aseptic meningitis

LEARNING OBJECTIVE \#2: Recognize the temporal relationship and proposed mechanisms of TMP-SMX induced aseptic meningitis

CASE: A 28 year-old woman presented with headache and fever for 1 week associated with photophobia and myalgias. She denied significant travel history, exposures to ticks, rodents or sick contacts. On further questioning, she reported taking TMP-SMX for the past 4 days for an axillary abscess status post incision and drainage. Her temperature was $102.8 \mathrm{~F}$ and heart rate was 113 beats per minute, physical examination did not reveal focal neurological deficits or skin rash; her axillary abscess was well-healed. The WBC count from the prior emergency room visit was $6400 / \mu \mathrm{L}$, on presentation at our facility, WBC count: $2600 / \mu \mathrm{L}$, lumbar puncture revealed a WBC of 12 cells $/ \mu \mathrm{L}, \mathrm{CSF}$ RBC count: 8 cells/ $\mu \mathrm{L}$ with $82 \%$ lymphocytes, $13 \%$ monocytes, $0 \%$ segmented neutrophils, CSF protein: $34 \mathrm{mg} / \mathrm{dL}$ CSF glucose: $53 \mathrm{mg} / \mathrm{dL}$, serum glucose: $93 \mathrm{mg} / \mathrm{dL}$. Gram stain, bacterial, fungal and viral cultures of CSF returned negative. HIV antibody testing was negative. Her TMP-SMX was discontinued on admission day 1 and she improved rapidly within 4 days without antibiotic therapy.

DISCUSSION: Drug-induced aseptic meningitis is a rare adverse reaction but should be considered when history, CSF analyses and cultures rule out microbial causes. Common medications implicated are nonsteroidal anti-inflammatory drugs, antibiotics (most commonly Trimethoprim-Sulfamethoxazole, TMP-SMX) and intravenous immunoglobulins. TMP-SMX is widely used since it is a low-cost, broad-spectrum antibiotic. The possibility of TMP-SMX causing aseptic meningitis has been underreported to FDA/MEDWATCH program possibly since it is also used to treat meningitis from Listeria monocytogenes. Recognition of the temporal relationship between TMP-SMX ingestion, development of symptoms, and quick recovery when the drug is stopped without antibiotic therapy is crucial. Our patient developed sudden leukopenia, headaches, photophobia, myalgia, 
within hours-days of consuming TMP-SMX and all of which have been associated with TMP-SMX use. Specific to our case were the findings of a high-normal CSF pleocytosis with lymphocytic predominance and lack of elevated protein; however other case reports have demonstrated these findings as well. Proposed mechanisms include hypersensitivity reaction or direct drug toxicity but further research is needed. Drug-induced aseptic meningitis is a difficult but important diagnosis of exclusion to make as the treatment is markedly different from that of other causes of meningitis and includes discontinuation of implicated drug. Use of TMP-SMX in such cases should be re-evaluated since repeated exposures have been linked to recurrent aseptic meningitis.

WHERE DID THE WATER GO? Yoshinori Saito ${ }^{2}$; Shadia Constantine ${ }^{1} .{ }^{1}$ Teine Keijinkai Hospital, Sapporo, Japan; ${ }^{2}$ Teine Keijinkai hospital, Teine-ku, Sapporo, Japan. (Control ID \#2468382)

LEARNING OBJECTIVE \#1: Recognize that Clostridium difficile (CD) colitis may cause Systemic Capillary Leak Syndrome.

LEARNING OBJECTIVE \#2: Recognize that delirium could be the first symptom of Clostridium difficile colitis.

CASE: A 79-year-old Japanese man presented with fever and shortness of breath. His past medical history included a lymphoma in remission, hypertension, diabetes mellitus, organizing pneumonia on steroids and recurrent pneumonia. He had no allergies. Social and family histories were unremarkable. On physical exam, his temperature was $38.6 \mathrm{C}$, blood pressure $130 / 65 \mathrm{mmHg}$, heart rate $94 / \mathrm{min}$, respiratory rate $24 / \mathrm{min}$, and $\mathrm{O}_{2}$ saturation $67 \%$ on room air. His chest exam showed crackles on the right lung. The rest of his exam revealed no abnormalities. Labs showed pancytopenia and acute kidney injury. He was diagnosed with severe pneumonia and treated with cefepime and ciprofloxacin. On day 4 , he had no fever and started to eat. However, in the evening of day 5 , he developed altered mental status. Lab tests and CT scan were unremarkable except for ascites. We broadened antibiotics to meropenem and IV vancomycin (VCM). He had an episode of loose bowel movement, which was positive for $\mathrm{CD}$ toxin. We started to treat Clostridium difficile colitis with IV metronidazole (MNZ). On day 7, his condition was getting worse as he became anuric. His pleural effusion, ascites and systemic edema worsened and his renal function and plasma albumin decreased. On day 8, we diagnosed systemic capillary leak syndrome (SCLS) and treated with fluid resuscitation and steroid pulse. On day 9 , his total balance was $+6800 \mathrm{ml}$ and his urine output was only $100 \mathrm{ml}$. His $\mathrm{O} 2$ saturation was decreasing, so we intubated him and transferred him to ICU and started continuous hemofiltration. At this point, he also required pressure support with noradrenaline. Later, he developed DIC for which he needed platelet transfusions. Despite aggressive treatment, he died.

DISCUSSION: C.difficile colitis is a common disease in hospitals usually characterized by fever and diarrhea. In this case, the patient's symptoms were anorexia and altered mental status. The lack of "diarrhea" contributed to miss the diagnosis. A few hours after his first loose bowel movement, the stool tests showed $\mathrm{CD}$ toxin positive. We began treatment with metronidazole but his condition continued to worsen. He had severe CD colitis (confirmed with colonoscopy) and we changed the treatment to vancomycin and metronidazole. SCLS is a rare disorder characterized by severe hypotension, hypoalbuminemia and hemoconcentration. The cause of SCLC is not known, although monoclonal gammopathy was found in many patients. In this case, he had 3 features of SCLC. Treatment of SCLS is not yet known and all treatment strategies are based on single case reports. Those reports suggest that most cases of SCLS will recover from hypotension in a few days. We treated him with 3 days of steroid pulse therapy, but it didn't work. He needed fluid resuscitation because of continuous hypotension, but fluid almost immediately third spaced causing worse systemic edema. The etiology of SCLS is still unclear. It could be due to infections, as in this case: CD colitis. We should consider SCLS when there is a patient with hypotension, hypoalbuminemia and hemoconcentration.
TWO MONTHS OF FEVERS? WHERE'S THE INFECTION??? ROMA MEHTA $^{2}$; Blake R. Barker ${ }^{1}$; Bethany Agusala ${ }^{1} .{ }^{1}$ UT Southwestern Medical Center at Dallas, Dallas, TX; ${ }^{2}$ UTSW, DALLAS, TX. (Control ID \#2470629)

LEARNING OBJECTIVE \#1: Identify and diagnose Still's disease from clinical and laboratory data.

LEARNING OBJECTIVE \#2: Manage both acute flares and long term treatment for Still's disease.

CASE: A 20-year-old Ethiopian woman with no past medical history presented with a 3-month history of fever to $103{ }^{\circ} \mathrm{F}$, diffuse joint pain, fatigue, odynophagia and a truncal and lower extremity rash. The fevers were nocturnal, lasting for several hours, and were almost always accompanied by a pruritic, papular rash. She denied dry mouth or eyes, but reported occasional difficulty rising from a seated position. Her joint pain was worse in the morning, and would increase to such severity that the she was unable to move. The symptoms were so debilitating that the she needed to quit soccer and take a leave of absence from college. She reported abdominal pain and a ten-pound weight loss over 2 months, but denied night sweats, alopecia, malar rash, oral or nasal ulcers, chest pain, diarrhea or dysuria. On exam, she was afebrile with a heart rate of 93 , blood pressure of $114 / 70$ and body mass index of $19 \mathrm{~kg} / \mathrm{m} 2$. Small, tender right axillary lymphadenopathy was noted with soft palatal erythema. Lung and cardiac exam was normal. The abdomen was diffusely tender to palpation with hepatosplenomegaly. No rash was noted, but her right knee was mildly swollen and tender with pain during passive wrist and elbow movement. Her laboratory evaluation was notable for WBC count 14.3 × 109/L (11.8 × 109 neutrophils), hemoglobin $9.5 \mathrm{~g} / \mathrm{dL}$, ferritin $119 \mathrm{ng} / \mathrm{mL}$, ESR $60 \mathrm{~mm} / \mathrm{h}$, CRP $5.5 \mathrm{mg} / \mathrm{dL}$, normal LFTs, ANA positive to 1:80 (speckled), RF $12 \mathrm{IU} / \mathrm{ml}$ with negative CCP, Ro, La, dsDNA, Smith and Jo antibodies. A broad infectious work up (blood and urine cultures, Gonorrhea and Chlamydia PCR, HIV antibodies and numerous viral serologies) was performed; all tests were negative. A CT of the chest and abdomen was notable for bilateral axillary lymphadenopathy and hepatosplenomegaly. Ultrasound-guided biopsy of an axillary lymph node was negative for abnormal lymphoid proliferation or malignancy. A diagnosis of adult Still's disease was made, and she was discharged on prednisone and naproxen. Methotrexate was initiated as an outpatient, but she developed severe alopecia and had recurrent symptoms. Her therapy was changed to Infliximab after which she had complete resolution of symptoms. She was able to return to studying to be a nurse and is also back on the soccer field. DISCUSSION: Discussion: Still's disease is a diagnosis of exclusion. Several different diagnostic criteria have been proposed, but the most sensitive is the Yamaguchi criteria.1 These criteria include four major (fever of at least 102.2 degreesF for at least a week, arthralgias or arthritis for at least 2 weeks, a non-pruritic macular or maculopapular rash and leukocytosis defined as 10,000/microL with at least $80 \%$ granulocytes) and five minor (sore throat, lymphadenopathy, hepato- or splenomegaly, abnormal liver function levels and negative ANA and RF) criteria with reported positive and negative predictive values of 70.3 and $95.2 \%$ if two major and three minor criteria are met. 2 This patient had all 4 major criteria with 3 minor criteria. While the etiology of this disease is unknown, it has been suggested that infectious agents are responsible for the initial symptomatic presentation. Classically, hyperferritinemia is observed in patients with this disease, frequently five times the normal serum level. The ferritin level has limited diagnostic utility because as an acute phase reactant, many other diseases can present with an elevated level. It is, therefore, not included in the Yamaguchi criteria. Treatment typically involves anti-inflammatory drugs and steroids, or in more severe instances, methotrexate and tumor necrosis factor inhibitors. With appropriate treatment, many patients experience complete resolution of symptoms.

A 36-YEAR OLD WOMAN WITH RECURRENT SKIN ABSCESSES: SOMETHING IN THE WATER? Utibe R. Essien ${ }^{1}$; Chika Nwachuckwu ${ }^{1}$; William Lewis ${ }^{2}$. ${ }^{1}$ Massachusetts General Hospital, Boston, MA; ${ }^{2}$ Harvard Medical School, Boston, MA. (Control ID \#2456568) 
LEARNING OBJECTIVE \#1: Recognize the broad differential diagnosis for recurrent abscesses, particularly in a patient with a complex medical and social history.

LEARNING OBJECTIVE \#2: Identify common clinical presentations along with preventive and supportive management of patients with $\mathrm{C} 2$ deficiency.

CASE: A 36-year-old Caucasian woman with a past medical history of untreated hepatitis C, remote intravenous (IV) drug use complicated by endocarditis and recurrent skin and soft tissue infections presented to the emergency department (ED) with a right lateral thigh abscess. The patient stated that over the past 10 years she has had multiple pyogenic abscesses over all four of her extremities, armpits and inguinal area after a flooding took place in her apartment. On review of her medical record, many of these abscesses were confirmed Methicillin-Resistant Staphylococcus Aureus for which she was treated with several courses of vancomycin, incision and drainage and surgical debridement. The patient reported recent subjective fevers and chills along with a new erythematous, fluid-filled lesion on her right upper thigh. She attempted to drain the lesion at home but with no success and due to persistent pain she presented to our ED. The patient had become increasingly concerned about her recurrent skin infections, which thus far had been attributed to ongoing drug use. While the patient reported previous use of both IV heroin and injected drugs subcutaneously, she denied using illicit substances over the past 2 years. The patient was afebrile and non-toxic appearing on arrival to the ED where she underwent incision and drainage of the right thigh abscess and was started on IV vancomycin. She was subsequently admitted to the inpatient medical service for further management. Physical examination demonstrated a tearful woman without significant lymphadenopathy or detectable cardiac murmur. Initial laboratory workup included a normal complete blood cell count and negative HIV-1 RNA PCR along with normal CD3 and CD4 counts. Serum and urine tests for drug toxicology were also negative. Hepatitis serologies confirmed active Hepatitis C with a viral load of 400,000 while Hepatitis B serologies were negative. She underwent transthoracic echocardiogram which was negative for vegetations and initial blood cultures were unrevealing. Wound culture of the thigh abscess eventually grew Streptococcus anginosus, sensitive to the administered antibiotics. On hospital day 3 , while the initial abscess was healing, the patient developed new tender, pruritic, lesions in the web spaces of her hands bilaterally, concerning for an as yet undiagnosed infectious process or active subcutaneous drug abuse. However blood cultures remained negative as did serum and urine toxicology. Dermatology was consulted for a skin biopsy which revealed concern for a drug hypersensitivity reaction, thought to be in response to antibiotics. Given her history of recurrent abscesses and puzzling clinical picture Allergy was also consulted who recommended testing for autoimmune or immune deficient states. While results of an autoimmune work-up were unrevealing including ANA and ANCA, the patient's results demonstrated a total complement level less than 13 $\mathrm{U} / \mathrm{ml}$, with specifically a $\mathrm{C} 2$ level less than $10 \mathrm{U} / \mathrm{ml}$, laboratory findings consistent with homozygous $\mathrm{C} 2$ deficiency.

DISCUSSION: Homozygous C2 deficiency is a rare disorder, estimated at 1 in 20,000 persons in western populations though the most common of all complement deficiencies. Typically, the disorder is associated with recurrent infections by encapsulated organisms including Neisseria menigitidis, Streptococcus pneumoniae and Haemophilus influenza type $B$ early in the life of an affected individual. Additionally, $\mathrm{C} 2$ deficiency is associated with the development of systemic lupus erythematosus and other autoimmune diseases such as Sjogren's and thus skin manifestations such as in this patient can be expected in the disorder. $\mathrm{C} 2$ is a key part of the classical pathway of complement, the primary immune defense against pyogenic organisms such as those mentioned above. As such it is important to consider deficiencies in the complement system, including $\mathrm{C} 2$, in patients with recurrent infections. At this time replacement of $\mathrm{C} 2$ is not available and management of C2 deficiency is largely supportive, including prevention and treatment of infections early along with vaccinations against the above encapsulated organisms.
A BANANA A DAY, KEEPS THE DOCTOR AWAY Ali A. Cheema; Katherine Salciccioli; Anna Kolpakchi; Lee Lu. Baylor College of Medicine, Houston, TX. (Control ID \#2469737)

LEARNING OBJECTIVE \#1: Recognize steroid injection as a precipitant of hypokalemic periodic paralysis

LEARNING OBJECTIVE \#2: Review steroid induced hypokalemic periodic paralysis

CASE: A previously healthy 29-year-old man presented to the emergency room with progressive weakness after receiving a steroid injection. On the morning of admission, the patient presented to his primary care physician complaining of a diffuse urticarial rash and was given an intramuscular steroid injection for a presumed allergic reaction. About one and half hours after receiving the injection, he developed progressive weakness which started initially in bilateral upper extremities and progressed to bilateral lower extremities. The weakness continued to worsen over the next few hours to the point where he fell while attempting to walk. He had no previous personal or family history of similar symptoms and denied myalgia, back pain, decreased sensation, and loss of bowel or bladder continence. Physical exam was notable for $4 / 5$ strength in bilateral upper extremities and $1 / 5$ strength in bilateral lower extremities, $2+$ symmetric reflexes, and normal proprioception and sensory exams. His rash had resolved. CT head was unremarkable. His labs were significant for K $2.7 \mathrm{meq} / \mathrm{L}$ with normal renal function and blood glucose of $144 \mathrm{mg} / \mathrm{dL}$. His thyroid panel was normal. ECG was normal with normal QT. Patient was started on aggressive potassium repletion with close telemetry monitoring. Approximately $2 \mathrm{~h}$ after oral and intravenous repletion of potassium, he regained his normal motor strength of $5 / 5$ in all extremities, and the repeat $\mathrm{K}$ was $4.5 \mathrm{meq} / \mathrm{L}$. He was diagnosed with hypokalemic periodic paralysis due to steroid injection.

DISCUSSION: Hypokalemic periodic paralysis (HKPP) is rare with an estimated prevalence of 1 in 100,000. HKPP may be familial with autosomal dominant inheritance or may be acquired in patients with thyrotoxicosis. The disorder is three to four times more common in men. A mutation in the gene that codes for the alpha-1 subunit of the dihydropyridine-sensitive calcium channel in skeletal muscle is the most common genetic abnormality in HKPP and is found in about $70 \%$ of symptomatic patients with the familial form. For the rest of $39 \%$, a mutation in the skeletal muscle sodium channel, SCN4A, has been identified. This SCN4A gene mutation demonstrates more complete clinical penetrance, affecting men and women equally. Initial presentation of HKPP typically occurs in 1st or 2nd decade of life, with attacks occurring a few times per year lasting hours to days. Typical precipitants include high carbohydrate load, stress, and exercise. These events are often associated with an increased release of epinephrine or insulin, both of which cause movement of potassium into cells and low potassium blood levels. Although rare, steroids have been reported to cause HKPP especially in patients with underlying thyroid disease. However, Arzel-Herzode et al. reported a case review of 12 patients with steroid induced HKPP. Out of the 12 patients between the age of 15 to 36,11 patients were male and only one had thyroid disease. All patients received either oral or IV steroid prior to the onset of the symptoms of paralysis and fully recovered after receiving potassium supplement. Genetic testing was performed in all; seven were found to have gene mutation. The mechanism of steroid induced HKPP has been hypothesized to be due to the direct effect on the $\mathrm{Na}^{+} / \mathrm{K}^{+}$ATPase resulting in intracellular potassium shift, leading to hypokalemia and weakness. The implication of this case is significant due to extensive use of steroids in clinical practice such as for asthma, COPD, allergic reaction, rheumatologic diseases, etc. While rare, primary care physicians must be aware of steroid induced HKPP as a potential adverse effect especially in young male patients.

A BIZARRE CASE OF WATER INDUCED BLEEDING Yazan Samhouri; ming zhao; Mahesh Krishnamurthy. Rochester Regional Health/Unity Hospital, Rochester, NY. (Control ID \#2457602) 
LEARNING OBJECTIVE \#1: Recognize the importance of detailed medication history, including over the counter medications, for diagnosis of thrombocytopenia.

LEARNING OBJECTIVE \#2: Manage drug induced immune thrombocytopenia CASE: A 69-year-old man with medical history of Diabetes, Hypertension and varicose veins, presented to emergency room with hemoptysis, easy bruising and ecchymosis for 1 week. His daily medications included Amlodipine, Losartan, Insulin Lispro and Glargine. Patient denied any recent history of viral illness. On examination, he had diffuse ecchymosis on his abdomen, lower back and left thigh. Laboratory tests showed platelet count of $1000 / \mu \mathrm{l}$, WBC $9700 / \mu \mathrm{l}$ and hemoglobin of $13.6 \mathrm{~g} / \mathrm{dl}$ with normal chemistries. HCV antibody and HIV screening were negative. Peripheral blood smear showed sparse platelets with normal WBCs and RBCs. The etiology of his thrombocytopenia was unclear on admission. However, upon further questioning, patient admitted that he had been drinking tonic water containing quinine, intermittently for the past 10 years for lower extremity cramps. He increased intake to $500 \mathrm{ml}$ daily over the past 2 weeks. Patient was diagnosed as quinine induced thrombocytopenia, treated with Methylprednisolone $125 \mathrm{mg}$ twice daily given his severe thrombocytopenia. He was given intravenous immune globulin (IVIG) on day 3 for persistent thrombocytopenia (less than 1000/ $\mu$ l). Patient was discharged on day 7 with Platelet count of $72,000 / \mu 1$ and no evidence of bleeding.

DISCUSSION: The development of drug induced immune thrombocytopenia is a wellrecognized side effect of many drugs. Quinine is a classic example of drugs that cause severe immune thrombocytopenia. Tonic water is popularly used for leg cramps. Quinine stimulates IgG antibodies causing destruction of platelets only when the drug is present. The diagnosis is made by documenting prompt resolution of thrombocytopenia after discontinuation of the suspected drug and excluding other causes. Bone marrow biopsy can be used to exclude blood dyscarcias if suspected. Laboratory testing for drug dependent antibodies (DDAbs) is not required to make the diagnosis. Testing is technically demanding and not widely available, hence, not useful in the immediate care of a patient. Most patients with DIIT require no specific treatment, as their platelet counts will recover promptly following withdrawal of the offending agent. However, treatment in addition to drug withdrawal may be required when thrombocytopenia is severe or bleeding is present. Corticosteroids, IVIG and plasma exchange have been used successfully to improve platelet count, as in the present case, although the benefit of these treatments is uncertain. In conclusion, DIIT should be suspected in any patient who presents with acute thrombocytopenia of unknown cause. A detailed, careful history of drug exposure is essential.

\section{A BLUE FINGER AND HYPONATREMIA LEADING TO A DIAGNOSIS OF MALIGNANCY-ASSOCIATED MARANTIC ENDOCARDITIS. Abhishek Maiti ${ }^{1}$; Nicholas J. Short ${ }^{2}$. ${ }^{1}$ University of Texas Health Science Center at Houston, Houston, TX; ${ }^{2}$ The University of Texas MD Anderson Cancer Center, Houston, TX. (Control ID \#2469592)}

LEARNING OBJECTIVE \#1: Malignancies often present with paraneoplastic syndrome of inappropriate diuretic hormone secretion (SIADH).

LEARNING OBJECTIVE \#2: Embolic phenomenon in a patient with suspected cancer should raise concern for non-bacterial thrombotic endocarditis (NBTE).

CASE: A 62-year-old woman with history of heavy tobacco use presented with pain and discoloration in her left index finger for 1 week, which eventually became blue and cool to touch. She denied any fevers, chills, chest pain, palpitations, shortness of breath, or dyspnea on exertion. Physical examination showed a cool and blue-appearing left index finger with normal Allen's test bilaterally. Cardiac examination was unremarkable, and she appeared euvolemic on examination. Laboratory evaluation was notable only for a serum sodium of $122 \mathrm{mEq} / \mathrm{L}$. Further laboratory work-up confirmed diagnosis of SIADH
A chest radiograph ordered subsequently revealed a right hilar opacity. Chest computed tomogram confirmed a $4.5 \times 4.4 \mathrm{~cm}$ right hilar mass with hilar and mediastinal lymphadenopathy. A diagnosis of possible lung cancer with paraneoplastic SIADH was made. A transthoracic echocardiogram (TTE) performed to evaluate for embolic phenomenon showed no evidence of valvular vegetations. A transbronchial biopsy of the lung mass showed small-cell lung cancer (SCLC). A transesophageal echocardiogram (TEE) done at the same time showed marked thickening of the anterior mitral valve leaflet tip suspicious for marantic endocarditis. A unifying diagnosis of SCLC causing SIADH and marantic endocarditis with embolic manifestations was established. Staging work-up showed metastasis to the humerus, consistent with extended stage SCLC. The patient was treated with indefinite therapeutic dose enoxaparin for the marantic endocarditis. She received multiple lines of chemotherapy including a phase I trial regimen and eventually passed away in hospice 20 months after the initial presentation.

DISCUSSION: Hyponatremia is the most common electrolyte abnormality in patients with advanced cancer. Paraneoplastic SIADH is seen in around $15 \%$ patients with SCLC, and often precedes the diagnosis of cancer. Also any lung pathology can precipitate SIADH. Hence, unexplained SIADH warrants chest imaging. Marantic endocarditis, also called NBTE is caused by deposition of thrombi on undamaged heart valves in the absence of bacteremia, and is a manifestation of the prothrombotic state of cancer. It commonly occurs in patients with adenocarcinoma, and its incidence in SCLC is unknown. A high index of suspicion is needed to diagnosis NBTE as murmurs are audible in a minority of patients, and the valvular lesions are often too small to be identified with TTE. TEE has significantly better sensitivity for diagnosing NBTE and should be considered if TTE is negative and the suspicion for NBTE is high. Early identification of NBTE is crucial as it can cause substantial morbidity from embolic phenomenon, including ischemic episodes in cerebral, mesenteric, coronary or extremity arteries, which occur in roughly $40 \%$ of patients. Treatment of NBTE involves therapeutic anticoagulation to prevent further thromboembolic episodes and treatment of the underlying malignancy, if possible. In our patient the seemingly disparate clinical phenomena of SIADH and digital ischemia ultimately led to the unifying diagnosis of SCLC-associated NBTE

A BLURRY DIAGNOSIS BECOMES CLEAR Bonnie Wang. University of Michigan, Ann Arbor, MI. (Control ID \#2470440)

LEARNING OBJECTIVE \#1: Metastatic infiltration of the optic nerves is a rare but important consideration in patients with visual deficits. A thorough evaluation for extraocular malignancy must precede biopsy of the ocular lesion. The evaluation may include lymph node palpation, dermatologic survey, mammography, CXR, CT chest/ abdomen, and PET scan. Metastatic involvement of the optic nerves portends a poor prognosis.

CASE: Sixty-five year old woman with a history of tobacco use presented with a 10 day duration of progressive vision changes. She reported seeing flashing lights in the temporal portion of her left eye which progressed to blurry vision throughout the entire eye. This was associated with pain with ocular movements. Two days prior to admission, she reported right-sided blurry vision. An outpatient ophthalmic exam was unremarkable. In the Emergency Department, she could only visualize hand motions with her left eye. Visual acuity of the right eye was $20 / 20$, but she had difficulty with color vision. MRI of the orbits demonstrated bilateral optic nerve sheath enhancement extending into the prechiasmatic optic nerve. MRI of the spine and brain demonstrated leptomeningeal involvement of the seventh and eighth complex within the right internal auditory canal. High dose steroids were initiated in an attempt to preserve vision. In addition, she reported progressive dyspnea during the past 2 months. Upon admission, she was mildly hypoxic and a CXR showed a right pleural effusion. Chest CT demonstrated a $1 \mathrm{~cm}$ right upper 
lobe mass and mediastinal lymphadenopathy. Pleural fluid cytology was consistent with adenocarcinoma. A cervical lymph node excisional biopsy was performed and revealed metastatic poorly differentiated adenocarcinoma of pulmonary origin. A PET scan detailed extensive hypermetabolic lesions within the neck, mediastinal, and intraabdominal lymph nodes. The final diagnosis was stage IV non-small cell lung cancer with multi-focal leptomeningeal disease including spread to the optic nerves.

DISCUSSION: The differential diagnosis in patients with visual deficits and a cancer diagnosis include metastatic spread (breast, lung, GU, GI in order of prevalence), leukemia, lymphoma, or paraneoplastic syndromes. Most patients who develop metastatic cancer to the eye have a known prior malignancy or other sites of metastases. However, approximately $25 \%$ of individuals found to have ocular metastasis present with symptoms that precede detection of the primary malignancy. Signs and symptoms of optic nerve metastases include blurry vision, reduced visual acuity, pain, bitemporal hemianopsia, and exophthalmos. Depending on the level of involvement, visual deficits may be present in one or both eyes. When the ophthalmic exam is normal, an MRI of the orbits/brain is necessary to investigate a posterior optic nerve or cortical cause of vision loss.

A BONE TO PICK WITH DYSPHAGIA Jordan D. Becerril; Rachel Oreck. Tulane University Health Sciences Center, New Orleans, LA. (Control ID \#2464500)

LEARNING OBJECTIVE \#1: Recognize the clinical features of Forestier's syndrome LEARNING OBJECTIVE \#2: Assess treatment options for symptomatic Forestier's syndrome

CASE: A 55 year-old woman with a history of seizures and submandibular stones, presented with dysphagia. Five days prior to presentation, she woke up with urinary incontinence, headache, and confusion consistent with prior nocturnal seizures. However the morning of presentation she had difficulty swallowing solids, accompanied by nausea and vomiting. Over the next few days, her symptoms worsened to include mild dysphagia to liquids, shortness of breath, and voice changes. On physical exam, she was hypertensive at 164/97 with oxygen saturation at $97 \%$ on room air. She had submandibular fullness bilaterally with a clear oropharynx. No focal neurologic deficits were appreciated. Labs were significant for hypokalemia of $3.3 \mathrm{mmol} / \mathrm{L}$ but were otherwise unremarkable. Flexible fiber optic laryngoscopy found the posterior pharyngeal wall significantly pushed anteriorly. Modified barium swallow was performed which demonstrated a narrow pharynx due to mass versus swelling of tissue on posterior pharyngeal wall at $\mathrm{C} 3-\mathrm{C} 4$, leading to decreased laryngeal elevation and poor epiglottic inversion. CT neck with IV contrast demonstrated exuberant osteophytes with preserved intervertebral disk height located at $\mathrm{C} 3, \mathrm{C} 4, \mathrm{C} 5, \mathrm{C} 6$, and $\mathrm{C} 7$ with $\mathrm{C} 3-\mathrm{C} 4$ osteophytes displacing the prevertebral soft tissues anteriorly at the level of the epiglottis, consistent with symptomatic Forestier's syndrome. A PEG tube was placed for nutrition requirements and neurosurgery recommended outpatient surgical decompression.

DISCUSSION: Forestier's syndrome, also known as DISH (diffuse idiopathic skeletal hyperostiosis), was first described in 1950 by Forestier and Routes-Querol. While it is not rare, it is often under-diagnosed. In the United States the prevalence of DISH is approximately $25 \%$ of men and $15 \%$ of women older than 50 years. DISH is usually asymptomatic, though it can lead to compression of local tissues, manifesting symptomatically as dysphagia, dysphonia, stridor, and dyspnea. Dysphagia is most frequently reported, occurring in up to $28 \%$ of cases and is usually progressive and more severe for solids than liquids. The etiology remains unclear though risk factors include excessive mechanical stress, dyslipidemia, and hyperuricemia. Diagnosis is dependent on radiograph evaluation that can include plain radiography of the spinal column, computed tomography (CT) or magnetic resonance imaging (MRI). Diagnostic criteria include calcification and ossification along the anterolateral paravertebral ligaments, contiguously involving at least four vertebral bodies with or without specific bony outgrowths projecting into the intervertebral space; relative preservation of intervertebral disc height in involved areas; absence of apophyseal ankyloses or erosion/sclerosis/sacroiliac fusion. The treatment of DISH depends on disease severity with a conservative approach in mild to moderate cases, surgical treatment in severe or refractory cases. Muscle relaxants and anti-inflammatory drugs are recommended for initial treatment. Surgical treatment is indicated in patients with dysphagia refractory to conservative treatment or with airway impairment. The procedure is a simple osteophyte excision (osteophytectomy), performed by neurosurgery. There is a risk for recurrence of osteophytes after excision (in one case series all patients developed radiological recurrence), though few actually reported recurrence of symptoms. While the differential for dysphagia is broad, Forestier's syndrome is an underreported etiology that should be considered.

\section{A BURNING QUESTION: ERYTHROMELALGIA IN PRIMARY CARE} Alexandra M. Ristow. University of California, San Francisco, San Francisco, CA. (Control ID \#2467857)

LEARNING OBJECTIVE \#1: Describe the pattern of symptoms that distinguish erythromelalgia from more common entities, such as peripheral neuropathy or Raynaud's disease.

LEARNING OBJECTIVE \#2: Outline a first-pass diagnostic approach for a patient presenting with erythromelalgia

CASE: A 38 year old woman with a history of chronic right-sided back pain presented to clinic complaining of worsening chronic pain of her extremities. She described the pain as intermittent redness and burning in her bilateral hands and feet. The paroxysms of pain and redness were triggered by walking and prolonged dependent positioning of her limbs, and both the skin changes and pain resolved with elevation of the affected limbs. The symptoms had become progressively more debilitating since they appeared 10 months ago, now limiting ambulation to $10 \mathrm{~min}$ at a time. Her past medical history included only chronic right-sided back pain and pelvic pain, which began following a chiropractic accident 2 years ago. Her medications included baclofen $10 \mathrm{mg}$ BID, pregabalin $75 \mathrm{mg}$ BID, and duloxetine $20 \mathrm{mg}$ daily. Exam was notable for cool, pale feet and hands with intact sensation, normal nail folds, and normal pulses. Her feet were notable for a lacey pink reticular rash. She presented pictures from her cell phone of her feet before and after 10 min of slow walking in $65^{\circ}$ Fahrenheit weather showing remarkable erythema following ambulation.

DISCUSSION: Erythromelalgia is an uncommon clinical entity characterized by red, painful, extremities. It affects women more commonly than men, with a mean age of diagnosis in the fourth decade of life. The pain is generally described as burning and bilateral; unlike typical neuropathic pain, however, it is paroxysmal and accompanied by transient erythema. The pain and erythema of erythromelalgia are often triggered by exertion, dependent positioning of the affected limbs, or warm temperature. This is in contrast to Raynaud's disease, which is triggered by cold temperatures and classically presents with a triphasic pattern of white, blue, and then red discoloration of the skin. Various causes of erythromelalgia have been identified. Primary erythromelalgia can be familial or sporadic, and it is caused by a mutation of the voltage-gated sodium channel alpha subunit gene SCN9A. Secondary erythromelalgia is found in conjunction with various classes of systemic diseases: hematologic disorders such as polycythemia vera and essential thrombocytosis; connective tissue disorders; paraneoplastic syndromes; infections such as HIV; medications including calcium channel blockers; and musculoskeletal disorders including back trauma or surgery. Thus, the first-pass workup to exclude major secondary causes of this condition includes a careful history, a complete blood count, Janus Kinase 2 (JAK2) mutation testing, an HIV test, age-appropriate cancer screening, and, if the rheumatologic review of systems or exam is positive, anti-nuclear antibody testing. This case highlights an unusual pattern of symptoms that should be 
recognized by general medicine physicians as erythromelalgia, a clinically diagnosed condition that could potentially be confused with more common entities such as Raynaud's disease or peripheral neuropathy. There is no definitive treatment for this often debilitating condition, and referral to a dermatologist for monitoring and therapy is generally indicated.

A CASE OF ACUTE ADRENAL INSUFFICIENCY UNCOVERING UNDERLYING AUTOIMMUNE POLYGLANDULAR SYNDROME TYPE II Victoria Haddad $^{2}$; Hrudya Abraham ${ }^{1}$; AnnMarie Canelas ${ }^{1} .{ }^{1}$ MacNeal Hospital, Berwyn, IL; ${ }^{2}$ Ross University School of Medicine, Chicago, IL. (Control ID \#2466864)

LEARNING OBJECTIVE \#1: Patients diagnosed with primary adrenal insufficiency are at higher risk for developing further autoimmunity. Adrenal insufficiency is the initial manifestation of Autoimmune polyglandular syndrome type 2 (APS2) in about $50 \%$ of patients. In about $20 \%$ of patients, it can occur simultaneously with autoimmune thyroid disease or Type 1 diabetes mellitus

LEARNING OBJECTIVE \#2: Evaluation of primary adrenal insufficiency before levothyroxine replacement is important to prevent precipitation of Adrenal crisis in patients with Schmidt's syndrome.

CASE: Fifty four-year-old Hispanic male with no significant past medical history presented with 6 months of generalized weakness, fatigue, and decreased appetite On admission, he was found to be hypotensive and physical exam was significant for generalized tanning and hyperpigmentation of perioral mucosa, knuckles and palmar creases. Laboratory investigation was significant for hyponatremia hyperkalemia, normal renal function, A1c of $6.3 \%$, normal fasting blood glucose, elevated TSH (11.3mIU/L), positive anti-thyroid peroxidase antibodies, low free $\mathrm{T} 4$ and $8 \mathrm{AM}$ cortisol of $2.6 \mathrm{mcg} / \mathrm{dL}$. An adrenocorticotropic hormone (ACTH) level of $>2000 \mathrm{pg} / \mathrm{mL}$ and positive anti-adrenal antibodies along with low AM cortisol confirmed diagnosis of Addison's disease. Presence of autoimmune hypothyroidism in addition to diagnosis of Addison's disease cumulatively make up a diagnosis of APS2 in our patient. He was immediately started on intravenous hydrocortisone followed by levothyroxine (LT4) replacement and was late discharged on oral hydrocortisone and LT4. He followed up in our clinic 2 weeks later with significant improvement in energy level and hyperpigmentation.

DISCUSSION: APS2 forms different cluster of autoimmune disorders predominantly seen in females and is defined by the occurrence of Addison's disease with autoimmune thyroid disease and/or type 1 Diabetes Mellitus (DM1). Morning serum cortisol values below $5 \mathrm{mcg} / \mathrm{dL}$ is $100 \%$ specific for making this diagnosis. Approximately 50 to $65 \%$ of patients with autoimmune adrenal insufficiency have one or more additional autoimmune endocrine disorders and $75 \%$ of these cases are diagnosed in women. APS2 is more prevalent than APS1 syndrome with primary adrenal insufficiency being principal manifestation. They are at increased risk of developing DM1, vitiligo, alopecia, pernicious anemia, celiac disease, myasthenia gravis and primary hypogonadism. APS2 with primary adrenal insufficiency and autoimmune thyroid disease is referred to as Schmidt's syndrome. Evaluation of primary adrenal insufficiency before levothyroxine replacement is important to prevent precipitation of Adrenal crisis in patients with Schmidt's syndrome. The diagnosis of polyglandular insufficiency is often delayed due to non-specific symptoms at early disease stages. Progression may be rapid, culminating in Addisonian crisis under physical stress or infection requiring immediate high dose hormone replacement therapy. Hence careful re-examination is mandatory to ensure adequate treatment before life threatening complications occur. Organ specific autoantibody screening in patients with monoglandular autoimmune endocrinopathies undoubtedly facilitates the identification of those at risk of developing a future APS.
A CASE OF ACUTE BACK PAIN: ABIOTROPHIA DEFECTIVA THORACIC VERTEBRAL OSTEOMYELITIS. Divya Akshintala ${ }^{1}$; Lindsay O’Rourke ${ }^{3}$; John J. Farrell ${ }^{2}$. ${ }^{1}$ University of Illinois College of Medicine at Peoria, Peoria, IL; ${ }^{2}$ University of Illinois college of Medicine at Peoria, Peoria, IL; ${ }^{3}$ OSF Saint Francis Hospital, Peoria, IL. (Control ID \#2469736)

LEARNING OBJECTIVE \#1: A high degree of suspicion for discitis is warranted in bacteremic patients with back pain.

LEARNING OBJECTIVE \#2: Abiotrophia defectiva as a causative agent is rare for discitis, this could be secondary to its fastidious nature. However, with increasing use of better culture media and mass spectrometry, it is likely that the incidence rate will increase, and it is important to identify this organism as a causative agent of discitis and vertebral osteomyelitis.

CASE: A 62 year old male with no significant past medical history was transferred from an outlying hospital for bilateral lower extremity weakness. He had presented to the outlying hospital's emergency deparment (ED) a few days earlier with symptoms of low back pain. Lumbar Xrays were normal and he was treated symptomatically. However, within $24 \mathrm{~h}$ he developed urinary incontinence and later developed 3/4 signs of systemic inflamatory response syndrome (SIRS) and bilateral lower extremity weakness, prompting return to the ED. Magnetic resonance imaging of the thoracic and lumbar spine revealed a T6 burst fracture and an epidural fluid collection with compression of the thecal sac. There was clinical concern for spinal cord compression, and he was life-flighted to our neurosurgical intensive care unit for emergent laminectomy for decompression of spinal cord and spinal stabilization. Vital signs on arrival were temperature $=37.4 \mathrm{C}(99.4 \mathrm{~F})$; HR $=$ $120 ; \mathrm{RR}=22 ; \mathrm{BP}=128 / 90$. Labs revealed leukocytosis of 18,000 with $71 \%$ neutrophils. Pre-operative blood cultures grew Abiotrophia defectiva in $2 / 2$ bottles (one set of blood cultures also grew Strepcoccus mitis). Epidural abscess fluid was collected in the operating room and submitted to the microbiology lab for Gram stain and culture which were notable for Gram positive cocci and Abiotrophia defectiva, respectively. The identification was confirmed by both Vitek 2 (Biomérieux, Marcy-l'Étoile, France) and Matrix Assisted Laser Desorption/Ionization - time of flight (MALDI-tof), The inital post-operative antimicrobial treatment included combination intravenous ampicillin and clindamycin A transesophageal echocardiogram was performed which did not reveal any vegetations or valvular abnormalities, and follow up blood cultures were negative. Our patient did wel post-operatively and he had begun to regain motor function in both his lower extremities before transfer to a rehabilitation facility.

DISCUSSION: This case highlights two important points: although back pain is among the most common presenting complaints in the ED and the primary care office, a high degree of suspicion is warranted for discitis as the etiology of back pain when SIRS signs are present and timely evaluation with blood cultures and MRI are critical to prevent progression to spinal cord compression. Secondly, although Abiotrophia defectiva is a rare cause of vertebral osteomyelitis and discitis, the incidence of Abiotrophia bacteremia and endocarditis is increasing. These organisms are generally a part of normal oral flora, gastrointestinal and urogenital tracts. Bacteremia is not unusual, and may result in disc space or vertebral body infection via hematogenous dissemination. Abiotrophia species were formerly referred to as nutritionally variant Streptococcus sp. due to their fastidious nutritional growth requirements. These organisms grow poorly in conventional media and require a pyridoxine group supplementation as well as prolonged incubation. In this case, the organism was identified by MALDI-tof, which uses the principle of mass spectrometry for identification of organisms, and is now commonly employed in most diagnostic microbiology laboratories. It is likely that Abiotrophia species have been underestimated as causative organisms of vertebral osteomyelitis and discitis due to their slow growth and fastidious growth requirements, but with the increased use of improved culture media and the proliferation of MALDI-tof in clinical microbiology laboratires, we anticiapte that the incidence of Abiotrophia defectiva discitis will increase. 
A CASE OF ACUTE MYOCARDIAL INFARCTION DISGUISED AS TRANSIENT ISCHEMIC ATTACK David Cassel ${ }^{1,2}$; Jeffrey Bates ${ }^{2} .{ }^{1}$ Baylor College of Medicine, Houston, TX; ${ }^{2}$ Michael E. DeBakey Veterans Affairs Medical Center, Houston, TX. (Control ID \#2469874)

LEARNING OBJECTIVE \#1: Recognize that acute myocardial infarction can manifest with painless anginal-equivalent symptoms

LEARNING OBJECTIVE \#2: Recognize the significant overlap between anginalequivalent and Transient Ischemic Attack (TIA) symptoms

CASE: A 64-year-old gentleman with hypertension, hyperlipidemia, and TIAs in 2012 and 2013 was admitted with transient left arm and face numbness. He noticed sudden left arm numbness that radiated to his left neck and face; he was confused, diaphoretic, suddenly weak, and immediately collapsed into his seat. The symptoms lasted approximately $3 \mathrm{~min}$ before he returned to baseline; he denied chest pain, palpitations, dyspnea, or complete loss of consciousness. He was transported to a community hospital, where a computed tomography (CT) head was negative for acute intracranial hemorrhage or infarct and a troponin-I was normal; he was treated with aspirin and transferred to our hospital for further neurologic evaluation. Upon arrival approximately $7 \mathrm{~h}$ after the index event, the patient's troponin was $0.18 \mathrm{ng} / \mathrm{ml}$; his electrocardiogram $(\mathrm{EKG})$ was negative for ischemic changes. He was started on therapeutic enoxaparin ( $1 \mathrm{mg} / \mathrm{kg}$ twice daily); his troponin-I peaked at $0.32 \mathrm{ng} / \mathrm{ml}$ approximately $16 \mathrm{~h}$ after the index event. Magnetic resonance imaging (MRI) brain confirmed lack of acute infarct, and magnetic resonance angiography (MRA) head and neck demonstrated no significant carotid disease or other acute vascular abnormality. Transthoracic echocardiogram noted inferior wall hypokinesis. Coronary angiogram demonstrated a $90 \%$ stenotic lesion of the mid-left circumflex artery with extension to the second obtuse marginal artery. A single drug-eluting stent was placed and he was started on dual anti-platelet therapy with aspirin and clopidogrel, atorvastatin, and losartan; beta-blockers were held due to bradycardia

DISCUSSION: Clinicians frequently classify symptoms for acute coronary syndrome (ACS) into typical anginal chest pain and atypical symptoms; typical anginal chest pain is described as retrosternal, left-sided, crushing, worsened with exertion, and relieved by rest. This classification system is misleading as myocardial infarction (MI) frequently manifests with painless anginal-equivalents: dyspnea, diaphoresis, light-headedness, acute mental status changes, generalized weakness, nausea, or shoulder/arm/jaw discomfort. A study from a large public hospital reported that $47 \%$ of 721 consecutive patients with myocardial infarction presented with symptoms other than chest pain; and several anginalequivalent symptom complexes overlap with TIA symptoms. For example, a multicenter study examining the frequency and character of TIA diagnosis noted that 9 out of 23 patients presenting with dizziness, which had been mistakenly attributed to TIA at initial evaluation, were subsequently diagnosed with MI. The reliability of TIA diagnosis can be poor even among experienced physicians; a study examining the inter-observer variability in the diagnosis of stroke and TIA noted that agreement for the diagnosis of TIA amongst six stroke specialists was nearly as low as chance with a Kappa of 0.11 . The study and several others noted that changes in limb or face sensation, an anginal-equivalent symptom, had a wide degree of precision for diagnosing stroke or TIA (Kappa of 0.27-0.89). Additionally, ACS presentations other than chest pain occur more frequently in racial minorities, the elderly, diabetics, women, and patients with psychiatric disease or altered mental status. Patients with acute MI who present without chest pain are prone to worse outcomes due to delays in diagnosis and treatment, and they have an in-hospital mortality rate more than twice that of patients whose acute MI presents with chest pain. Clinicians should recognize the significant overlap between anginal-equivalent and TIA symptoms, and consider myocardial infarction in patients presenting with TIA-like symptoms even in the absence of chest pain.
A CASE OF ACUTE TRANSIENT FLACCID PARALYSIS WITH ANAPLASMOSIS Amanda Fernandes. Baystate Medical Center, Springfield, MA. (Control ID \#2470175)

LEARNING OBJECTIVE \#1: Review the clinical features of Human Granulocytic Anaplasmosis (HGA)

LEARNING OBJECTIVE \#2: Identify diagnostic challenges in identifying HGA CASE: A 56-year-old female patient with a past medical history of non-ischemic cardiomyopathy, chronic low back pain on narcotics presented with a 4-day history of generalized aches, weakness with periodic episodes of inability to move her legs, fever and lethargy. She had no recent sick contacts or travel and has resided in Western Massachusetts for the last year. She denied any tick bites but noted that her cat had ticks. In addition, she has had several dead birds in her yard but was under the impression that the birds were killed by her housecat. On admission, she was febrile to $102.1 \mathrm{~F}$ with laboratory workup notable for pancytopenia and mild transaminitis. Peripheral smear was normal. CT of her chest, abdomen and spine did not reveal any abnormalities. She was admitted for sepsis of unclear etiology at the time of admission and started on broad spectrum antibiotics. Following admission, she continued to spike intermittent fevers and developed two episodes of transient flaccid paralysis in both her lower extremities associated with mild confusion. CT head as well as EEG were negative. Given pancytopenia, a complete tick borne panel was sent which came back positive for Anaplasma Phagocytophyllia. She was started on doxycycline with improvement in her symptoms and no recurring episodes of flaccid paralysis.

DISCUSSION: HGA is a tick borne illness that is transmitted by the Ixodes tick in the New England region. Its counterpart from the same genus, Ehrlichia chaffeensis causes Human Granulocytic Erhlichiosis (HGE) and is transmitted by the Lone Star tick endemic in the southeastern and south central regions of the US. Both have similar clinical presentations including fevers, myalgias and headaches that begin approximately 9 days after the tick bite. Central neurological manifestations have been described in approximately $20 \%$ of patients with HGE and include meningitis or meningoencephalitis, cranial nerve palsy and seizures. In contrast, neurological involvement has been described in less than $1 \%$ of cases involving HGA. There have been case reports noting peripheral nervous system manifestations including brachial plexopathy but none reporting bilateral acute flaccid paralysis. Etiology from prior studies including EMG and muscle biopsy suggests possibly inflammatory cell infiltrates with resulting axonal or demyelinating disease that may explain our patient's presentation (Horowitz et al., 1996). PCR amplification remains the mainstay in diagnosis of HGA and HGE. Peripheral smears note morula in less than $3 \%$ of patients with HGE but are more useful in diagnosis of HGA (25-50 \%) (Ismail et al., 2010). If clinical suspicion for HGA, HGE are not high, they can often go missed resulting in progression of symptomatology. Empiric treatment with doxycycline is key to preventing progression while pending workup. References Horowitz HW, Marks SJ, Weintraub M, et al. Brachial plexopathy associated with human granulocytic ehrlichiosis. Neurology. 1996;46(4):1026-9. Ismail N, Bloch KC, McBride JW. Human Ehrlichiosis and Anaplasmosis.Clinics in laboratory medicine. 2010; 30(1):261-292.

\section{A CASE OF AN UNCOMMON SILENT KILLER: RAPIDLY PROGRESSIVE CRESCENTIC IGA NEPHROPATHY Arvind Reddy Devanabanda; Neelesh Rastogi;} Matthew Dallos. New York University, New York, NY. (Control ID \#2466340)

LEARNING OBJECTIVE \#1: To diagnose rapidly progressive crescentic IgA nephropathy and recognize the unique pathophysiology of an uncommon diagnosis

LEARNING OBJECTIVE \#2: To assess risks and benefits of timely treatments options in rapidly progressive crescentic IgA nephropathy 
CASE: A 26 year old Hispanic male presented to the ER with periumbilical abdominal pain, nausea and vomiting for 1 week. The patient was feeling well until 1 week ago when he developed abdominal pain that was sharp, constant, non-radiating and exacerbated by movement. Three days prior to admission the patient developed nausea and non-bloody non-bilious emesis, which prompted him to come to the hospital. On review of systems he also reported white foamy urine, lower extremity swelling, and sore throat over the last week. His past medical history is notable for hypertension, with no significant surgical history. He has no significant family history. He was previously prescribed an ACE inhibitor and calcium channel blocker (CCB), but at the time of admission reports noncompliance for 2 months. His significant exam findings were notable for a BP of 170/110, mild distress with peri-umbilical tenderness, clear urine, and no leg swelling. His basic metabolic panel was notable for a BUN/ Cr ratio of 69/8.6, potassium of 5.3, and bicarbonate of 18 . His $\mathrm{CBC}$, liver function tests and lipase were normal. His urine microscopy showed packed red blood cells and a $24 \mathrm{~h}$ urine protein of $6 \mathrm{~g}$. His lipid panel was notable for an HDL of 25. Additional lab work including IgG subclasses, dsDNA, anti-GBM, ANA, ANCA, HIV, and hepatitis B was negative. His complement levels and CK were normal. A renal ultrasound showed normal size kidneys with no evidence of hydronephrosis. A CT abdomen and pelvis revealed pancreatic inflammation. The patient was dually diagnosed with acute pancreatitis and non oliguric acute renal failure. His abdominal pain, nausea and vomiting were thought to be primarily related to his acute pancreatitis and resolved with supportive care. He subsequently underwent a renal biopsy which revealed severe crescentic IgA nephropathy, mesangial proliferation and $50 \%$ interstitial fibrosis and tubular atrophy. He was initially treated with a 3 day course of pulse dose methylprednisolone and then started on high dose oral prednisone daily. The patient's renal function modestly improved and upon discharge from hospital, the patient was considering starting immunosuppressive therapy with mycophenolate mofetil (MMF) or cyclophosphamide followed by azathioprine as an outpatient.

DISCUSSION: IgA nephropathy (IgAN) is the most common primary glomerulonephritis in the western world. Typically patients present with hypertension and proteinuria, followed by either microscopic or gross hematuria, after an upper respiratory infection. The pathophysiologic mechanism is thought to involve an environmental exposure that causes over stimulation of B cells that produce IgA1 with deficient galactose residues. This induces the synthesis of autoantibodies and the formation of circulating immune complexes that deposit in renal mesangium leading to local inflammation and eventually proteinuria and hematuria. IgAN progression is very variable, but after 25 years $25-30 \%$ will develop end stage renal disease. The presence of minimal $<500 \mathrm{mg}$ /day protein favors a good long term prognosis. However, our patient had $6 \mathrm{~g} /$ protein excretion /day. For patients with $>1 \mathrm{~g} /$ day of proteinuria, KIDGO (Kidney Disease: Improving Global Outcomes) guidelines recommend starting an $\mathrm{ACE}$ inhibitor (class 1B recommendation) A particularly rapid progression to ESRD has been shown in a minority $(<10 \%)$ of patients with IgAN. The treatment challenge in these patients is the paucity of evidence for immunosuppressive therapy. One Italian trial, showed that a 6 month course of corticosteroids led to better clinical disease remission and long term outcomes than no steroids But subjects with IgAN and GFR $<50$ were excluded from this trial. KIDGO recommends treating crescentic IgAN with corticosteroids combined with cyclophosphamide. For simple IgAN, a randomized control trial showed decreased proteinuria and increased kidney survival time with corticosteroids and cyclophosphamide. A Chinese study suggests the use of MMF decreases proteinuria and increases kidney survival time at 6 year follow up. To date there are no randomized control trials on the treatment of cresecentic IgAN and most of the treatment guidelines from KIDGO are based on small observational studies. Choosing an appropriate treatment regimen was especially challenging in our patient given the serious potential adverse effects of immunosuppressive drugs. Although cyclophosphamide could potential benefit our patient, he remained concerned about the risk of infertility and could not afford the cost of sperm banking. Thus, the need for newer immunosuppressive agents like rituximab, complement cascade targets and monoclonal antibodies needs to be further studied. References: Coppo R, D'Amico G. Factors predicting progression of IgA nephropathy. J Nephrology 2005; 18: 503-512 Rocatello D, Ferro M, Coppo R et al. Treatment of rapidly progressive IgA nephropathy. In: Clarkson A, Woodroffe A (eds). IgA Nephropathy: Pathogenesis and Treatment. Basel: Karger, 1995, vol 111, pp. 177-1483 Kidney Disease: Improving Global Outcomes (KDIGO). Chapter 10 Immunoglobulin A Nephropathy. Pozzi C et al. Corticosteroids in IgA nephropathy. Lancet 1999; 353: 883-887

\section{A CASE OF CHEST PAIN AND "PSEUDONORMAL" CENTRAL LINE TIP LOCALIZATION Alicia Stapleton ${ }^{1,2}$; Philip $\mathrm{Ng}^{2}$; Mark Ault ${ }^{2} .{ }^{1} \mathrm{UCLA}$, Los Angeles, CA; ${ }^{2}$ Cedars-Siani Medical Center, Los Angeles, CA. (Control ID \#2470011)}

LEARNING OBJECTIVE \#1: Recognize aberrantly-placed central venous catheters can present with nonspecific complaints

LEARNING OBJECTIVE \#2: Identify the benefits and pitfalls of various types of modalities used to assess central line placement

CASE: The patient was a 17-year-old previously health female with newly diagnosed embryonal rhabdomyosarcoma of the cervix. She was referred to our institution's Procedure Center for a port-a-cath placement to be performed by the Internal Medicine Proceduralist team. It was placed under fluoroscopy without complication and the patient was sent home. She began treatment with chemotherapy that afternoon, with cyclophosphamide, vincristine and dactinomycin. The following day, the patient called her primary physician complaining of intermittent chest and shoulder pain. She was brought into the emergency department for further evaluation. A d-dimer was found to be elevated. She then had a CT angiogram of the chest that revealed the line had migrated to the right internal mammary vein. The line was repositioned and the patient's pain resolved.

DISCUSSION: When optimally place a peripherally inserted central catheter (PICC) or PAS Port device will have the catheter tip lying at the junction of the superior vena cava and the right atrium (SVC/RA junction). While clinically there is often a fair amount of latitude in this positioning when the anticipated use of the line is for total parenteral nutrition (TPN), dessicating chemotherapy or antibiotics optimal tip location becomes crucial. We report a case of "pseudo normal" "catheter tip localization" and discuss some of the difficulties that can be encountered in confirming the true location of the catheter tip. Accurate and effective demonstration of adequate line placement is paramount prior to use of the line for infusion. Complications from failure to ensure appropriate intravascula location of the line prior to usage are well documented making this a fundamenta responsibility when placing an indwelling vascular device. This simplest and most basic technique for any line placement includes demonstration of the ability to appropriately flush saline through the line and to withdraw a blood sample. Failure to accomplish this should immediately alert the operator to a technical difficulty warranting further investigation. In this case this was accomplished and in fact the patient had received a significant infusion prior to discover of the line placement difficulty. Conventionally, a chest radiograph (CXR) is obtained post procedure to document the location of the catheter prior to usage. The CXR in this case demonstrated what could be assumed to be optimal tip location in the distal SVC. While practically CXR confirmation typically involves a single AP of PA image, it should be remembered that this is a 2 dimensional image and optimally requires an accompanying lateral image to confidently localize the course of the line. This case demonstrated the difficulty in relying on this technique, however, when the lateral film is of suboptimal penetration and the false sense of security provided by the AP view. Fluoroscopy, while not readily available to all operators placing central lines can be an effective adjunct for line placement, localization and repositioning. This case does however demonstrate the limitation of this technique when 2 dimensional assessment is utilized again providing misleading assurance of the tip location. Blood gas analysis of blood withdrawn from the catheter or wave form analysis are well reported techniques to 
discern whether a catheter has been inadvertently placed in an artery instead of a vein. These techniques would have been of little value in this circumstance as the aberrant placement was in a venous structure. Other available techniques to assure optimal tip location during insertion include placement of the catheter utilizing tip localizing devices such as the Sherlock system (Bard Access Systems) which uses a magnetic stylette to track the needle tip during catheter placement or the Arrow VPS device (Teleflex) which uses Doppler based technology for catheter guidance and tip location. Both devices have demonstrated efficacy in tip localization effectively replacing the need for CXR confirmation, and may have yielded a different outcome in this situation as the discordance between the anatomic location and the EKG may have alerted the operator of the abnormal location. Computerized tomography which ultimately confirmed the exact location of the catheter in the mammary vein can be and invaluable technique for either intended or incidental catheter localization but is clearly too costly and exposes the patient to too much radiation to be used on a routine basis. It is a good reminder that when a patient with an indwelling vascular device has a chest $\mathrm{CT}$ for any reason that attention be given to the location of the lines. Ultimately, careful assessment of the patient's symptomatic complaints may, as in this case, be the useful modality for revealing the presence of an aberrantly place line. While it may be tempting to dismiss vague or possibly unrelated complaints in an otherwise well placed and functioning line, complaints of palpitations, chest discomfort, musculoskeletal symptoms (shoulder pain in this case) in the hours to days after line placement may well be cause to consider the possibility of a pseudo normal line position.

\section{A CASE OF DERMATOMYOSITIS INITIALLY MISTAKEN FOR ANGIOEDE-}

MA Alejandra Bustillo; Danielle Jones. Emory University School of Medicine, Atlanta, GA. (Control ID \#2469644)

LEARNING OBJECTIVE \#1: Dermatomyositis (DM) is a rare, idiopathic, inflammatory myopathy with characteristic skin manifestations that can be diagnosed mainly on the basis of the clinical presentation, elevated muscle enzymes and presence of specific autoantibodies and can be associated with an underlying malignancy. Not all patients present with the full constellation of symptoms as some patients have been known to present with skin findings without weakness or abnormal muscle enzymes. Therefore, it is important to recognize the key cutaneous manifestations as these can lead to making the correct diagnosis more promptly.

LEARNING OBJECTIVE \#2: The hallmark finding of dermatomyositis is proximal weakness but because of its gradual onset, the clinician may first identify only the skin manifestations and consider primary skin or other systemic conditions. A delay in diagnosis can lead to progressive, debilitating weakness that can delay treatment and ultimately increase the morbidity associated with this disease.

CASE: A 50 year-old African American woman presented to Grady Memorial Hospital with a 2 week history of generalized edema, weakness, orbital swelling, dyspnea, and a rash on her face, chest and upper and lower extremities. She initially sought care at an urgent care center after developing the facial edema and rash and was treated with steroids for a presumed allergic reaction. Her condition worsened and on re-presentation she was treated with cephalexin for cellulitis, however her symptoms continued to progress. Four weeks prior to presentation, she had been admitted at an outside hospital and diagnosed with invasive lobular carcinoma of the left breast, but had not had further workup. On admission to Grady, her vital signs were BP 96/50, HR 120, 99 F, SpO2 $89 \%$ on room air. Her exam was concerning for periorbital edema, tongue swelling, generalized non-pitting edema, periungual erythema, an erythematous diffuse rash involving her forehead and cheeks as well as a dry, scaly rash involving the nape of the neck, chest, arms and lateral aspect of both thighs. She had generalized proximal weakness in both upper and lower extremities. Labs were significant for elevated $\mathrm{CPK}>3000$, AST $>900, \mathrm{LDH}>800$ and lactic acid $>3$. Other labs included WBC count of 22.7 ( $87 \%$ neutrophils, $7 \%$ lymphocytes and $6 \%$ monocytes). CT angiogram of the chest revealed no evidence of pulmonary embolism but did show a moderate sized right pleural effusion. Both the rheumatology and dermatology services evaluated the patient and agreed that her characteristic photodistributed erythema, poikiloderma and proximal muscle weakness were characteristic of dermatomyositis. Ultimately, she had a skin biopsy and an EMG which confirmed the diagnosis despite negative myositis-specific autoantibodies. The patient was started on prednisone with gradual improvement in her CPK, LDH and AST but only mild improvement of her weakness despite continuous physical and occupational therapy. The patient's hospital course was further complicated by HCAP, deconditioning and worsening dysphagia eventually requiring peg tube placement. Unfortunately, because of the patient's poor functional status, further management of her underlying malignancy was not possible during her hospitalization, and she was discharged to a rehabilitation facility with plans for outpatient management of her breast cancer.

DISCUSSION: This patient was diagnosed with dermatomyositis based mostly on the constellation of her clinical presentation, most notably her cutaneous findings and proximal muscle weakness. Several factors worsen prognosis, such as: greater weakness at presentation, presence of dysphagia, delayed initiation of treatment for $>6$ months, and association with a malignancy. This patient had at least three of these factors, and her diagnosis was delayed due to her physical appearance almost characterizing angioedema. Clinicians are often taught to recognize a heliotrope rash in association with dermatomyositis, but a photodistributed poikiloderma along the upper back (shawl sign) and the V of the neck is common as well. Generalized erythroderma may be confused for cellulitis as was also the case with this patient. Furthermore, not all patients may exhibit Gottron's papules, but they may have periungual erythema and a generalized non-pitting edema. Holster sign is another cutaneous manifestation of DM which appears as a dry, scaly rash on the lateral aspects of the thighs. Sometimes, the cutaneous manifestations may be more easily and readily recognized before the onset of the characteristic proximal muscle weakness. Knowing how to recognize these findings is crucial to making the diagnosis in order to initiate adequate treatment. Steroids are the mainstay of treatment for improving inflammation, and extensive rehabilitation is required to regain muscle strength. In cases associated with an underlying cancer, treating the malignancy may also provide relief from the morbidity associated with dermatomyositis. However, if the diagnosis is delayed, dermatomyositis can be debilitating enough that primary management of the cancer is not possible initially. It is an important paraneoplastic syndrome to consider especially in patients presenting with weakness and a recently diagnosed malignancy. Its characteristic photodistributed erythema as well holster, shawl and V signs helped to seal this diagnosis.

\section{A CASE OF DISSEMINATED CRYPTOCOCCOCIS IN A PATIENT WITH} CIRRHOSIS Shravana Bheemanathi; Azam Mohammad. St. Barnabas Hospital, Bronx, NY. (Control ID \#2469785)

LEARNING OBJECTIVE \#1: To consider cryptococcal infections as part of differential diagnosis in evaluating patients with peritonitis to prevent morbidity and mortality. CASE: A 54-year-old caucasian male with a history of cirrhosis due to hepatitis $\mathrm{C}$ and alcohol, stage III chronic kidney disease was admitted to the hospital for symptoms of worsening abdominal pain and swelling for 3 days. He also reported low grade fever along with a skin rash. He has history of decompensated cirrhosis with esophageal varices but no history of spontaneous bacterial peritonitis. He was afebrile on admission with blood pressure of 98/50. His abdomen was tense with positive fluid wave and he also had extremity edema on bilateral lower extremities. He also had an erythematous, macular, non-blanchable, non-pruritic, painful rash over his thighs. He had diagnostic paracentesis which showed significant neutrophilia and he was started on treatment of spontaneous bacterial peritonitis with ceftriaxone. His blood cultures from admission grew yeast and 
the patient was started on Micafungin. Later the blood cultures were reported as Cryptococcus neoformans. Given the positive blood cultures and erythematous rash, we considered him to have disseminated Cryptococcosis and his antibiotics were switched to Amphotericin B and Flucytosine. . Although he was aggressively treated with Octreotide, Midodrine, and albumin for his preexisting hepato-renal syndrome, the addition of Amphotericin B put him at further risk for nephrotoxicity and his creatinine increased from 2.76 to 5.75 in a couple of days. At this time he opted to start hemodialysis. His hospital course was complicated by worsening renal failure and respiratory distress and patient expired the next day.

DISCUSSION: Cryptococcus infections have been associated with immunocompromised individuals, $80-90 \%$ of whom have been HIV-positive patients. Increasingly, cryptococcal infections have been reported in cirrhotic patients who are HIV-negative. This case illustrates the importance of considering Cryptococcal infections in cirrhosis patients, as this fungal organism may be a more common culprit of infections in these patients than previously known. Impaired phagocyte function, increased use of antibacterial antibiotics, previous paracentesis and hypocomplementemia are thought to be possible etiologies for increased risk of cryptococcal infection in cirrhotic patients. Cryptococcal infections in this population have been associated with substantial morbidity and mortality, with some sources citing a mortality rate of $81 \%$. Early diagnosis and aggressive antibiotic therapy is vital to improve outcomes in these patients. Hence, cryptococcal infection should be considered in the differential diagnosis whenever evaluating a patient with an advanced liver disease and possible peritonitis.

\section{A CASE OF EMBOLIC MYOCARDIAL INFARCTION IN THE SETTING OF} ATRIAL ARRHYTHMIA Chioma Nwagbara ${ }^{1}$; Thomas Wool ${ }^{2}$; Kannan Thanikachalam ${ }^{1} .{ }^{1} \mathrm{UAB}$ Montgomery Health Center, Montgomery, AL; ${ }^{2}$ Southeastern Cardiology, Montgomery, AL. (Control ID \#2470212)

LEARNING OBJECTIVE \#1: Recognize embolic myocardial infarction as a potential complication of atrial arrhythmia.

LEARNING OBJECTIVE \#2: Emphasize the importance of appropriate risk stratification for anticoagulation in patients with atrial arrhythmia.

CASE: A 60 year old man with a history of hypertension and paroxysmal SVT presented with chest pain of a few hours. He described ongoing substernal chest pain, radiating to the jaw. On physical examination, temperature was $98^{\circ}$ Fahrenheit, heart rate was 87 beats per minute, respiratory rate was 22 breaths per minute, oxygen saturation was $100 \%$ on $\mathrm{O} 2$ via nasal cannula, blood pressure was 146/ 106. He was anxious. Heart sounds, S1 and 2 were heard, irregular and no murmur was present. Laboratory data include creatine kinase of 406 unit/L, CKMB of $19.8 \mathrm{ng} / \mathrm{mL}$, CPK isoindex was 4.9 and troponin was $19.4 \mathrm{ng} / \mathrm{mL}$. His EKG showed atrial flutter and ST segment elevation in Lead 1, AVL and V2-5. He underwent immediate left heart catheterization which showed totally occluded mid LAD and mild to moderate plaque with less than $50 \%$ stenosis with intravascular ultrasound. A large red thrombus was recovered via suction thrombectomy. The LAD was completely recanalized. Left ventricular ejection fraction was estimated at $25 \%$. He had a TEE after which showed a left atrial thrombus. He was treated with IV heparin and was discharged home in stable condition after 12 days.

DISCUSSION: Coronary artery embolism (CE) is a non-atherosclerotic cause of acute myocardial infarction (AMI). It often occurs in the setting of septic emboli from infective endocarditis, non-infected thrombi on prosthetic valves, calcific degeneration of the aortic valve, atrial fibrillation, coronary angiography, paradoxical emboli due to atrial septal defect and dilated cardiomyopathy as reported in literature. A recent study proposed criteria for determining the probability of coronary embolism in the setting of acute myocardial infarction. Atrial fibrillation was found to be the most prevalent risk factor among patients enrolled in that study. The major criteria included angiographic evidence of coronary artery embolism and thrombosis without atherosclerotic components, concomitant multisite $\mathrm{CE}$, and concomitant systemic embolization excluding left ventricular thrombus attributable to AMI. The 3 minor criteria includes coronary angiographic evidence of $<25 \%$ stenosis, except for the culprit lesion; evidence of an embolic source detected by any imaging modality; and coexistence of a potential for thromboembolic disease, that is, atrial fibrillation, cardiomyopathy, rheumatic valvular disease, infective myocarditis, prosthetic valve implantation, recent cardiac surgery, hypercoagulable state or atrial septal defect. They proposed that the diagnosis of definite $\mathrm{CE}$ be based on the presence of greater than or equal to 2 major criteria, 1 major criterion plus greater than or equal to 2 minor criteria, or 3 minor criteria. The patient had one major risk factor and two minor risk factors based on the above criteria. Treatment of coronary embolization entails treatment of acute MI and controlling the underlying embolic risk factors. Some studies suggest suction thrombectomy as a preferred modality during a left heart catheterization. Institution of anticoagulation is also indicated in both the acute setting and prevention of future embolic events. Atrial fibrillation remains one of common cause of systemic embolism and early diagnosis, risk stratification using CHADs-VASC score and institution of anticoagulation is helpful in preventing CE.

\section{A CASE OF GIANT CELL ARTERITIS PRECEDED BY VARICELLA ZOSTER} Jonathan J. Lee ${ }^{1}$; Barbra Blair ${ }^{2}$; John Perry ${ }^{2}$; Shiv T. Sehra ${ }^{2} .{ }^{1}$ Mount Auburn Hospital, Harvard Medical School, Boston, MA; ${ }^{2}$ Mount Auburn Hospital, Harvard Medical School, Cambridge, MA. (Control ID \#2468566)

LEARNING OBJECTIVE \#1: Recognize the clinical presentation of giant cell arteritis LEARNING OBJECTIVE \#2: Understand the possible pathogenic link between varicella zoster and giant cell arteritis

CASE: CC: Fatigue HPI: An 83-year-old female presented to our hospital with a chief complaint of persistent fatigue for 4 weeks. She had been diagnosed with shingles 7 weeks prior to presentation, based on left scalp and ear pain as well as hypersensitivity. She was treated with acyclovir for 1 week with complete resolution of these symptoms. Two weeks after finishing treatment, however, she developed progressive fatigue, malaise, weakness, as well as $10 \mathrm{lb}$ weight loss. She also endorsed intermittent headache, over the left temporal area, different from the shingles pain in location and quality. She denied any visual disturbances, muscle pain or stiffness, joint pain, or rashes. She presented to the emergency room given the progressive worsening of her symptoms, and was noted to have a fever, leukocytosis, an elevated ESR, and was admitted to the hospital for further work-up. PMHx: Hypertension, gastroesophageal reflux disease, and a remote history of breast cancer, which was treated with mastectomy in the 1980s. SHx: Retired banker with no history of smoking or alcohol abuse. She had no notable travel history. FHx: Positive for breast cancer in multiple female siblings, but negative for autoimmune disease. MEDICATIONS: Lisinopril $20 \mathrm{mg}$ daily, amlodipine $5 \mathrm{mg}$ daily, omeprazole $20 \mathrm{mg}$ daily, aspirin $81 \mathrm{mg}$ daily. PHYSICAL EXAM: Temperature 100.9, heart rate 84, blood pressure $127 / 64$, respiratory rate 20 , oxygen saturation $96 \%$ on room air. Head \& neck exam revealed tenderness in the region of the left temporal artery and diffuse scalp tenderness. Visual fields were full and intact to confrontation bilaterally. The remainder of the systemic exam showed no abnormalities. Laboratory studies showed a WBC count of 12.8, hemoglobin and hematocrit of 11 and 33, respectively, and platelet count of 617 . The ESR was 135 and the CRP 335. The basic metabolic panel and liver function tests were within normal limits. Peripheral smear was negative for intracellular parasites. The 
TSH was normal. The Monospot test was negative. Blood cultures were pending at time of initial evaluation but showed no growth after $48 \mathrm{~h}$. CLINICAL COURSE: The patient was empirically started on prednisone $60 \mathrm{mg}$ daily for possible giant cell arteritis. She underwent a left temporal artery biopsy. Pathologic examination showed granulomatous inflammation of the arterial wall with scattered neutrophils, consistent with arteritis. Given the patient's recent episode of zoster, immunohistochemical stains for varicella zoster antigen were performed but were negative. The patient's symptoms continued to improve while on prednisone. Given the above findings, a diagnosis of giant cell arteritis was made. She had a marked improvement within 2 days of starting prednisone. She has been successfully tapered to $20 \mathrm{mg}$ a day of prednisone and continues to do well.

DISCUSSION: Giant Cell Arteritis (GCA) is a type of large vessel vasculitis predominantly affecting Caucasians, older than 50 years of age with a peak incidence in the 70 's. It is a rare diseases with an incidence of close to 1 in 100,000. A growing body of evidence suggests a possible pathogenic link between varicella zoster virus (VZV) infection and giant cell arteritis (GCA). Preliminary data linking VZV infection and GCA first emerged in the form of three seminal case reports published in the neurology literature that had uncovered the presence of VZV antigen within the wall of the temporal artery (TA) in patients being evaluated for ischemic optic neuropathy due to VZV infection, with or without additional GCA symptoms (see Salazar et al. Arch of Neurol 2011; Nagel et al. Neurology 2013). Prior to these reports, the role of VZV infection in central nervous system vasculopathies (causing stroke) had already been established (Martin et al. Brain Pathol 1990). Recent analyses further implicate VZV infection in the pathogenesis of GCA. Gilden et al. (Neurology, 2015) very recently found that, among patients with GCA, VZV antigen was present (as detected through immunohistochemistry) in $74 \%$ (61 of 82) of GCA-positive TAs compared to in only $8 \%$ ( 1 of 13) normal, post-mortem controls (without clinical history suggestive of GCA). Though we did not find VZV immunoreactivity in the vessel wall in our patient, the timeline and existing literature does suggest some role of VZV in the pathogenesis of GCA, which should be explored further. This case highlights that clinicians should consider the diagnosis of GCA in elderly Caucasian patients with non-specific symptoms of new onset fatigue, fever, and headaches. Moreover, given the existing preliminary data, a recent diagnosis of VZV involving the head and neck region in such adults should prompt the clinician to exclude GCA as a cause of the symptoms.

A CASE OF HYPOKALEMIC PARALYSIS IN THE SETTING OF RENAL TUBULAR ACIDOSIS FROM SJöGREN'S SYNDROME Neha Sathe; John Hwang. NYU School of Medicine, NEW YORK, NY. (Control ID \#2469350)

LEARNING OBJECTIVE \#1: Recognize hypokalemic paralysis as a rare presentation of Sjögren's Syndrome.

LEARNING OBJECTIVE \#2: Distinguish the clinical features of proximal versus distal renal tubular acidosis.

CASE: A 36 year-old Chinese female was brought to our hospital with 2 days of diffuse, progressive weakness. Her history was notable for an unclear diagnosis of rheumatologic disease for which she had not sought further evaluation or treatment. On presentation, the patient was febrile and septic-appearing, with a urinalysis positive for nitrates, leukocyte esterase, many bacteria, $5-10$ white blood cells per high power field, and $2-5$ red cells per high power field. She exhibited diffuse hyporeflexia and reduced (4/5) strength in all extremities. Laboratory testing was remarkable for an arterial $\mathrm{pH}$ of 6.9 ; reduced serum levels of potassium $(1.9 \mathrm{mmol} / \mathrm{L})$, bicarbonate $(<10 \mathrm{mmol} / \mathrm{L})$, phosphate $(1.9 \mathrm{mmol} / \mathrm{L})$, and uric acid $(1.9 \mathrm{mmol} / \mathrm{L})$; and elevated blood urea nitrogen $(21 \mathrm{mmol} / \mathrm{L})$ and creatinine $(2.2 \mathrm{mg} / \mathrm{dL})$ levels. Other urine studies were notable for a $\mathrm{pH}$ of 6.5 , an anion gap of $+5 \mathrm{mEq} / \mathrm{L}$, and an osmolal gap of $-4 \mathrm{mOsm} / \mathrm{kg}$. Electrocardiogram showed prominent $\mathrm{U}$ waves. The patient was admitted to the ICU with presumed hypokalemic weakness in the context of urosepsis. Despite initial treatment with sodium bicarbonate and potassium chloride, she remained persistently hypokalemic below $2 \mathrm{mmol} / \mathrm{L}$, and within $8 \mathrm{~h}$ developed hypoxic respiratory failure from progressive flaccid paralysis that required emergent intubation. Concurrent proximal and renal tubular acidosis was suspected; treatment was changed to potassium bicarbonate-citrate. Over the next $24 \mathrm{~h}$, her serum potassium normalized, her weakness and electrocardiogram abnormalities resolved, and she was successfully extubated. The patient subsequently acknowledged a history of xerostomia and extensive dental caries; xerophthalmia was noted on formal exam. Autoantibody testing was positive for anti-Ro/SSA ( $>8$ units), anti-La/SSB ( $>8$ units), and ANA (1:1280 speckled). Renal biopsy showed membranous lupus glomerulopathy, as well as interstitial lymphoplasmacytic infiltration with fibrosis and tubular atrophy, suggestive of extraglandular Sjögren's syndrome. An overlap diagnosis of Sjögren's syndrome and systemic lupus erythematous was made, and she was discharged on potassium citrate, hydroxychloroquine, and high-dose prednisone.

DISCUSSION: We present a case of hypokalemic paralysis in the context of distal (type I) and proximal (type II) renal tubular acidosis (RTA) secondary to Sjögren's syndrome (SS). Distal and proximal RTA both present as a normal anion gap metabolic acidosis often accompanied by hypokalemia, but have distinctive clinical and laboratory features. In distal RTA, impaired acid secretion in the distal tubule leads to abnormally low urinary concentrations of hydrogen and ammonium ions, resulting in an inappropriately elevated urinary $\mathrm{pH}(>5.3)$, a positive urine anion gap, and a low urine osmolal gap. Depending on the degree of impairment, the ensuing acidosis can vary from imperceptible to severe $(\mathrm{HCO} 3<10 \mathrm{mEq} / \mathrm{L})$. Since sodium reabsorption in the distal tubule requires secretion of a cation to maintain electroneutrality, defective acid secretion enhances kaliuresis, often causing hypokalemia that improves with alkali therapy. In comparison, proximal RTA results from impaired reabsorption of filtered bicarbonate in the proximal tubule. The resulting acidosis is self-limited (typically $12-18 \mathrm{mEq} / \mathrm{L}$ ) because of compensatory bicarbonate reabsorption in the distal nephron, but is often accompanied by generalized wasting of other solutes such as phosphate, uric acid, magnesium (Fanconi syndrome), as seen in this case. Urinary acidification is intact and the serum potassium is often initially normal; however, alkali therapy characteristically worsens hypokalemia and increases urinary $\mathrm{pH}$, because of increased distal delivery of unreclaimed sodium and bicarbonate in the context of proximal tubular dysfunction. Both types of RTA can occur in patients with Sjögren's syndrome but are often subclinical, and therefore underrecognized in case series. For unclear reasons, the prevalence appears to be higher among Chinese as opposed to European and American patients. The pathogenesis of SS-associated RTA is poorly understood, but in the case of distal RTA may involve autoantibodies against acid efflux pumps. Hypokalemic paralysis appears to be a relatively rare presentation of Sjögren's syndrome, and seems to rarely occur before the onset of sicca symptoms. However, it is a well-documented consequence of distal RTA from other causes. Clinicians should be aware of this rare but potentially lethal complication of Sjögren's syndrome, and periodic monitoring of serum and urine electrolytes for evidence of subclinical renal involvement should be considered in patients with this condition.

\section{A CASE OF INVASIVE MENINGOCOCCAL DISEASE IN A MSM PATIENT}

Ryan Guinness. Kaiser Permanente, San Francisco, CA. (Control ID \#2469895)

LEARNING OBJECTIVE \#1: Recognize the initial clinical features of patients with invasive meningococcal disease.

LEARNING OBJECTIVE \#2: Identify when chemoprophylaxis is indicated for the prevention of meningococcal infection.

CASE: A 31 year-old man presented with sudden onset of fever, nausea, vomiting, myalgias, and diffuse purpura for 1 day. The patient denied any headaches, neck stiffness, chest pain, shortness of breath, constipation, diarrhea, or dysuria. The patient is sexually 
active with men, and is on HIV Pre-exposure Prophylaxis (PrEP) therapy. He lives in San Francisco where he works as a voice actor, and he traveled to Chicago 1 week ago. He had one sexual partner while in Chicago and has had two other sexual partners since coming back to San Francisco, all of whom he met through a GPS-based mobile application focused on meeting potential sexual partners. He reports adherence to PrEP, endorses occasional alcohol and marijuana use, but otherwise denies any other illicit drug use. On exam, the patient had a heart rate of 115 and blood pressure of $72 / 49$, with diffuse purpuric macules scattered throughout the trunk as well as upper and lower extremities. Laboratory tests were notable for a lactic acid of 5.1, platelets of 116, and creatinine of 1.74 (more than double his baseline). Lumbar puncture was performed, which did not show any organisms on gram stain. Blood and CSF cultures were sent. The patient was immediately transferred to the ICU where he was placed on droplet precautions for $24 \mathrm{~h}$ and started on IV fluid resuscitation, vasopressors, and empiric antibiotic therapy with Ceftriaxone and Vancomycin for suspected bacterial meningitis. His hemodynamics eventually stabilized and his functional status began to improve. Blood and CSF cultures eventually grew Neisseria meningitidis, serotype $C$; the same serotype involved with an invasive meningococcal outbreak among Chicago men who have sex with men (MSM) during this time. The patient's recent sexual contacts were all notified about potential exposure by the local Public Health Department so that they could receive chemoprophylaxis. He was continued on Ceftriaxone for a 7-day total course and received the meningococcal polysaccharide vaccine after discharge.

DISCUSSION: This case demonstrates some of the challenges associated with the recognition and management of meningococcal disease, particularly in the setting of an outbreak. Three clusters of the serotype $\mathrm{C}$ meningococcal disease among MSM have been reported in the United States since 2012, with the most recent outbreak occurring in Chicago during the summer and fall of 2015. The clinical expression of this infection ranges widely, and, thus a high index of suspicion and careful search for clues of disease are required to make a diagnosis. The typical initial presentation consists of sudden onset of fever, nausea, vomiting, headache, and myalgias in an otherwise healthy patient Although initial clinical features are similar to many common, self-limiting viral illnesses seen in primary care, signs of early sepsis should differentiate the patient who merits clinical monitoring. Chemoprophylaxis is typically given with a single intramuscular dose of Ceftriaxone $250 \mathrm{mg}$ for close contacts, including sexual partners, of patients with meningococcal infection and should be given as early as possible following the exposure Secondary disease usually occurs within 10 days of the primary care, though longer intervals have been described. In this case, all of the patient's recent sexual contacts were notified of exposure by the San Francisco and Chicago Department of Public Health and administered prophylaxis. Improved understanding of the epidemiology, clinical manifestation, treatment, and chemoprophylaxis modalities for invasive meningococcal disease will be necessary to prevent future outbreaks and tend to those at risk.

\section{A CASE OF LOSARTAN INDUCED IMMUNE THROMBOCYTOPENIC PUR-} PURA (ITP) PRESENTING WITH A PLATELET COUNT OF 0/UL. farhan ashraf $^{1}$; Iole Ribbizi-Akhtar ${ }^{2} .{ }^{1}$ Memorial Hospital of Rhode Island/ Alpert Medical School of Brown University, Providence, RI; ${ }^{2}$ Memorial Hospital of Rhode Island/ Alpert Medical School of Brown University, Pawtucket, RI. (Control ID \#2465407)

LEARNING OBJECTIVE \#1: Identify losartan as a provoking factor for ITP LEARNING OBJECTIVE \#2: Manage ITP resistant to multiple lines of treatment.

CASE: A 78 year old women with history of hypertension, diabetes mellitus, and hyperlipidemia presented with a rash for 3 days. She was started on losartan by her PCP 2 weeks prior to presentation. She denied fever, abdominal pain, or tiredness. Except losartan she didn't report any other change in medications in the last year. On arrival she was slightly hypertensive, otherwise vital signs were stable. Examination was remarkable for diffuse petechial rash on her upper and lower extremities, abdomen and chest, and a small hematoma on the inner aspect of her lip. Laboratory results showed a platelet count of $0 / \mathrm{uL}$, normal hemoglobin, white cell count, liver function test, prothrombin time, HIV and hepatitis panel. Serum protein electrophoresis and immunofixation were also normal. Hematology was consulted and established a diagnosis of ITP, most likely induced by losartan considering it was the only identifiable trigger. All her medications including losartan were stopped and she was started on intravenous steroids and immunoglobulin, with minimal increase of the platelets count to $2000 / \mathrm{uL}$. A bone marrow biopsy was then obtained, which showed increased megakaryocytes, supporting a diagnosis of ITP. Rituximab was then administered as next line therapy but also failed to improve her platelets count. Splenectomy was considered as a treatment option but the patient wanted to avoid surgery. Rho (D) immunoglobulin was then administered but her platelet count did not improve and concurrently she developed hematuria. She finally agreed to undergo splenectomy. Post-surgery her platelets count improved rapidly with resolution of the symptoms. She was discharged with a platelet count of $76,000 / \mathrm{uL}$, on a steroid taper Within a month her platelet count was in the normal range $(300,000 / \mathrm{uL})$ and persisted in the following 6 months.

DISCUSSION: ITP is a diagnosis of exclusion. It tends to present with severe thrombocytopenia, however a count of $0 / \mathrm{ul}$ at presentation is infrequent. In adults it usually responds to first line treatment in $2 / 3$ of cases. In our case, ITP was resistant to multiple lines of medical management and a surgical intervention in the form of splenectomy was needed. Losartan was believed to be the triggering factor as it was stared recently and there were no other identifiable causes. Per our literature search this is the only second reported case of losartan induced ITP.

\section{A CASE OF MALIGNANT PARAGANGLIOMA WITH INTESTINAL PSEUDO-} OBSTRUCTION AND HEART FAILURE. Masahiro Sakai; Shunsuke Kojima; Toru Yamada; Eiji Hiraoka. Tokyo-bay Urayasu Ichikawa medical center, Urayasu, Japan. (Control ID \#2466764)

LEARNING OBJECTIVE \#1: At the end of this presentation, the participant will demonstrate the ability to recognize paraganglioma as the rare cause of intestinal pseudo-obstruction with heart failure and new onset of hypertension and be able to manage the situation like this case.

CASE: A 57 year-old Japanese male without significant past medical history presented to the previous hospital with new-onset of heart failure with reduced ejection fraction, severe hypertension 4 months before admission. He was diagnosed with viral myocarditis based on the time course, elevated CRP and BNP level and diffuse hypokinesis detected with echocardiography. It was treated with bisoprolol, torsemide and digitalis with improvement of symptoms. One month before admission, he developed severe constipation and sweating. The constipation was gradually getting worse and was associated with appetite loss and weight loss. He was referred to our hospital. His vital signs were as follows: blood pressure, 181/93 $\mathrm{mmHg}$; pulse, 121 beats/min (regular); temperature, $37 \mathrm{C}$; respiratory rate, 22 breaths/min; and oxygen saturation while breathing room air, $94 \%$. He had elevated jugular venous distension and positive hepatojugular reflux. His heart sound was regular rate rhythm with S3 and S4. He had mild edema in bilateral legs. He was noted to have left axillary lymphadenopathy. His abdomen was distended with mild tenderness with no bowel sound auscultated. Laboratory data showed azotemia and elevated BNP level. Chest X-ray showed pulmonary edema. Echocardiogram demonstrated diffuse hypokinesis with EF $31 \%$. Computed tomography scan of the chest and abdomen identified left axillary lymphadenopathy, dilatation of the colon with no evidence of obstruction and multiple para-aortic tumors. Colonoscopy was normal. We diagnosed him with congestive heart failure and intestinal pseudo-obstruction. Laxative was not 
effective for his constipation. After treating congestion by diuresis, he was treated with bisoprolol for his heart failure and hypertension. His blood pressure did not improve at all. The biopsy of left axillary lymphadenopathy showed paraganglioma. Urinary metanephrine and normetanephrine as well as serum catecholamine levels were highly elevated. The 123I-MIBG scintigraphy demonstrated accumulation in the para-aortic lesions and didn't demonstrate accumulation in the left axillary region because of having removed the lymph node for biopsy. He was diagnosed with malignant paraganglioma. Treatment with doxazosin improved his blood pressure dramatically. He was transferred to a tertiary care center for further treatment.

DISCUSSION: We herein reported a case of intestinal pseudo-obstruction with severe constipation and heart failure with reduced EF, which was finally found to be due to paraganglioma. Paraganglioma are rare neuroendocrine tumors that arise from the extraadrenal autonomic paraganglias, which secret catecholamine. It can be associated with inhibition of the peristaltic activity of the gastrointestinal tract by direct stimulation of catecholamine on its alpha1, 2, and beta2 receptor. It can be manifested by constipation, intestinal pseudo-obstruction and perforation. Although pseudo-obstruction is often encountered in a case of post-surgery, trauma, and serious infection, paraganglioma should be considered when it is complicated with heart failure and new onset of hypertension. We found out only 35 cases of paragangliomas/phenochromocytomas with intestinal pseudoobstruction like our case from PubMed, Embase, Web of Knowledge and Scholar Google and so we think that this case is rare. As treatment with alphal and alpha 2-adrenergic receptor antagonists are often effective for intestinal pseudo-obstruction, hypertension and heart failure caused by paraganglioma, physicians should correctly diagnose it as early as possible.

\section{A CASE OF MONTELUKAST-INDUCED LIVER INJURY IN A BONE MAR-} ROW TRANSPLANT RECIPIENT Devin Loewenstein ${ }^{1}$; Trinh Khong ${ }^{2} .{ }^{1}$ UCLA Medical Center, Los Angeles, CA; ${ }^{2}$ UCLA David Geffen School of Medicine, Los Angeles, CA. (Control ID \#2469703)

LEARNING OBJECTIVE \#1: Recognize drug-induced liver injury as an adverse effect of montelukast

LEARNING OBJECTIVE \#2: Review the mechanism of drug-induced liver injury CASE: A 45 year-old female was admitted for acute on subacute transaminitis (AST 900) with complaints of nausea, lethargy, and recurrent fevers. Her medical history included end-stage polycystic kidney disease status post renal transplant complicated by rejection, post-transplant lymphoproliferative disorder, and mycosis fungoides treated with allogeneic stem cell transplant achieving remission. She had no personal history of alcohol consumption, risk factors for exposure to parenterally transmitted viruses, and no family history of liver disease including polycystic liver. Her temperature was $38 \mathrm{C}$, heart rate low $100 \mathrm{~s}$, oxygen saturation mid-90s on two liters nasal cannula, and respiratory rate low 20 s. She was chronically ill though non-toxic in appearance, her skin jaundiced, flushed, and excoriated, and her abdomen mildly tender throughout. She underwent extensive workup, including wide-ranging infectious and autoimmune panels. She was found to have a slightly elevated anti-smooth muscle antibody (1:80) with a negative ANA in the setting of immunosuppression. A trial of steroids for possible autoimmune hepatitis did not improve her transaminitis. Imaging was unrevealing with no intrahepatic thrombosis detected. Liver biopsy demonstrated portal hepatitis with periportal hepatocyte dropout, consistent with drug-induced liver injury (DILI). Her medications were reviewed, and montelukast, nifedipine, and ketoconazole were cross-referenced as having hepatotoxic potential. Upon further review, it was noted that 1 month after initiating montelukast she developed an AST-predominant transaminitis (500's-1100's), elevated alkaline phosphatase (200's-500's), and elevated conjugated bilirubin (3-4), with an R-value of 1.8 , suggestive of a cholestatic hepatic injury. Of the three potential culprit medications, the timing of her liver injury was consistent only with montelukast (nifedipine and ketoconazole had been started months earlier), which had been initiated for off-label treatment of chronic urticaria and pruritus. Montelukast was promptly discontinued and her fevers, lethargy, and transaminitis improved gradually over the remainder of her hospital stay. During her recovery, her lab test for hepatitis E IgM returned positive. Hepatitis E IgG was negative along with the remainder of her extensive infectious workup. The positive IgM was initially concerning for an acute hepatitis $\mathrm{E}$ infection. However, further confirmatory testing (including repeat antibody panel and PCR) was negative indicating a likely false positive initial result. Hepatitis $\mathrm{C}$ and $\mathrm{E}$ have been known to be masquerade as DILI, with up to $3 \%$ of individuals with suspected DILI testing positive for anti-hepatitis E IgM but with negative confirmatory testing. ${ }^{1}$

DISCUSSION: DILI is a poorly understood multifactorial process involving a complex interplay between genetic, environmental, and pharmacologic factors. ${ }^{2}$ It is further subdivided into intrinsic and idiosyncratic forms, with intrinsic DILI being caused by hepatotoxic medications in a dose-dependent manner. Idiosyncratic DILI, on the other hand, is characterized by hepatotoxicity in susceptible individuals, with some medications causing a hepatocellular, cholestatic, or mixed hepatic injury oft resembling autoimmune hepatitis. ${ }^{3}$ Proposed mechanisms of DILI include direct cell stress resulting in reactive hepatotoxic metabolites, induction of signaling pathways, and immune reactions induced by formation of neoantigens (haptens). ${ }^{2,4}$ Montelukast is a substrate and inhibitor of the hepatocyte cytochrome p450 (CYP450) enzyme system, leading to the proposed mechanism that hepatotoxic injury from montelukast is caused by an accumulation of toxic metabolites. ${ }^{46}$ This case demonstrates a rarely reported presentation of drug-induced liver injury caused by montelukast. While approximately $2 \%$ of patients taking montelukast will develop elevated AST, ${ }^{6}$ serious hepatotoxicity is even more rare with only a handful of case reports found in our literature search. ${ }^{7-10}$ It is important for physicians to be aware of this adverse effect for this commonly prescribed medication.

A CASE OF PITUITARY FAILURE FOLLOWING TREATMENT WITH IPILIMUMAB Patrick M. Gill ${ }^{1}$; Ellen S. Story ${ }^{2,1} .{ }^{1}$ Carolinas Medical Center, Charlotte, NC; ${ }^{2}$ Levine Cancer Institute, Charlotte, NC. (Control ID \#2469583)

LEARNING OBJECTIVE \#1: Recognize lymphocytic hypophysitis as a toxicity from immune checkpoint therapy.

LEARNING OBJECTIVE \#2: Describe the diagnostic criteria and management of the initial presentation of lymphocytic hypophysitis.

CASE: RL is a 59 year old male with a past medical history of stage IV metastatic melanoma without evidence of intracranial metastasis, newly diagnosed hypothyroidism, and coronary artery disease who presented following a syncopal event. He denied any orthostatic or preceding symptoms but his wife did endorse a low blood pressure following the event. RL's metastatic melanoma was undergoing treatment with dabrafenib and trametinib. He was then started on ipilimumab in addition to his other treatments 10 weeks prior to the event. Over time, he started to endorse fatigue, constipation, and a persistent frontal headache. RL was hypotensive on arrival to the emergency room and despite aggressive fluid resuscitation, he remained hemodynamically unstable. His exam was unremarkable for infectious etiology and his neurologic exam was intact. A thorough infectious workup, including a lumbar puncture, was performed and unrevealing. He was started on hydrocortisone with rapid improvement in his hemodynamics. MRI brain with gadolinium and thin pituitary cuts was performed which showed enlargement of the pituitary gland consistent with hypophysitis. Subsequent studies identified central hypothyroidism and hypogonadotropic hypogonadism. He was started on hormone replacement therapies with improvement of his symptoms. 
DISCUSSION: Lymphocytic hypophysitis is an autoimmune process resulting in inflammation of the pituitary gland that can lead to hypopituitarism. Extreme cases, if left untreated, can result in death due to adrenal crisis, characterized by electrolyte abnormalities and severe hypotension. Classic cases of lymphocytic hypophysitis will present with fatigue and a frontal headache, which can be debilitating. Common associated symptoms also include nausea and visual field defects. Given the nonspecific nature of the majority of symptoms, they may be missed unless careful vigilance is undertaken. Chart review for RL showed that he had complained of his headache and fatigue for approximately 2 months prior to presenting with adrenal insufficiency. Typical cases of lymphocytic hypophysitis result in central hypothyroidism and secondary adrenal insufficiency. It is essential to obtain a free T4 as TSH may be within normal limits in cases of central hypothyroidism. ACTH stimulation testing may be normal in the setting of acute pituitary failure as the adrenal gland has not had time to atrophy. MRI with and without contrast will further support the diagnosis by demonstrating enlargement of the pituitary gland; however, it is essential to obtain thin cuts through the pituitary as it could be missed with routine MRI brain protocols. All of these provide barriers to diagnosis, demonstrating how a strong clinical suspicion is important for prompt diagnosis. RL was diagnosed with lymphocytic hypophysitis secondary to his ipilimumab therapy. This disorder, which was once almost exclusive to young pregnant females, is increasing in prevalence with the usage of immune checkpoint therapies (i.e. anti-CTLA-4 and anti-PD-1 agents). Most studies report a 1-2\% occurrence of hypophysitis with these treatments, however, this is likely underreported due to a lack of recognition of more indolent presentations. Some studies suggest that the true prevalence may be has high as $10 \%$ following these treatments. Immune checkpoint therapy is growing in prevalence for the treatment of metastatic melanoma, small cell lung cancer, and renal cell carcinoma, providing patients with another treatment option with promising results. These drugs, however, have a unique set of toxicities and adverse effects. General internists will often be the first to hear about these symptoms as they seem nonspecific and potentially unrelated to their immunotherapy. Prompt recognition can promote an improved quality of life and preven a life threatening adrenal crisis. There are no studied treatment regimens at this time; however, there are suggestions available based on expert opinion. Initial treatments include withholding the offending agent and starting high dose steroids, which treat both hypophysitis and hemodynamic compromise, if it is present. A prolonged steroid taper is performed with close monitoring for return of symptoms, primarily headache and visua field defects, as recurrence of hypophyitis is common during the taper. With adequate treatment, enlargement of the pituitary gland will typically resolve, while return of pituitary function is much less likely. Case series estimate return of function in only 37 $50 \%$ of patients. Therefore, while glucocorticoid replacement is initiated, a full investigation of the hypothalamic pituitary axis should be performed, adequately replaced, and followed longitudinally.

A CASE OF PROGRESSIVE ISCHEMIC STROKE DUE TO THYROID CRISIS ASSOCIATED CEREBRAL VENOUS THROMBOSIS. Natsumi Tanabe ${ }^{2,3}$; Toru Yamada $^{2}$; Kuniyasu Saigusa ${ }^{2}$; Yasuhiro Norisue ${ }^{1}$; Eiji Hiraoka ${ }^{2}{ }^{1}$ Tokyo Bay Urayasu Ichikawa Medical Center, Chiba, Japan; ${ }^{2}$ Tokyo Bay Urayasu-Ichikawa Medical center, Chiba, Japan; ${ }^{3}$ Tokiwa foundation Johban hospital, Fukushima, Japan. (Control ID \#2468263)

LEARNING OBJECTIVE \#1: Diagnose cerebral venous thrombosis when there is a progressive ischemic stroke with underlying disease.

CASE: A 49-year-old Japanese female with an unremarkable medical history presented to the previous hospital with 1 month history of headache, palpitation, sweating and weight loss. She was diagnosed with hyperthyroidism based on TSH $<0.01 \mathrm{mU} / \mathrm{L}$, FT3 $7.82 \mathrm{pg} /$ dl. Treatment with methymazole was started. She also started to have weakness in her left arm. MRI of the head showed cerebral infarction in right precentral and postcentral gyruses. She was started on intravenous argatroban. During the next 6 days, she developed weakness in her legs bilaterally. The second MRI showed infarction in the left frontal lobe, posterior lobe and right parietal lobe. In addition, she was also noted to have confusion, tachypnea, tachycardia, hyperthermia and hypertension and she was transferred to our hospital for further treatment. On admission, she was diagnosed with Graves disease based on positive TSH receptor antibody. She was started on glucocorticoids, beta blockers, potassium iodide-iodine, and methimazole for the thyroid storm. Three days after admission, her MR venography showed thrombosis in left transverse sinus, sigmoid sinus, and internal jugular vein. In addition to anticoagulation, catheter thrombectomy was performed with gradual clinical improvement. On day 85 , the patient was transferred to the rehabilitation hospital.

DISCUSSION: Although hyperthyroidism is not a widely acknowledged risk factor for venous thrombosis, there are several reports confirming their association. It has been reported that thyrotoxicosis shifts the haemostatic balance toward hypercoagulable and hypofibrinolytic state with a rise in coagulation factors. We need to consider arterial stroke as well as venous sinus thrombosis when a hyperthyroid patient has stroke. To prevent progression of the thrombosis, the key to success in treatment is adequate anticoagulation and initiation of anti-thyroid agents. The pathophysiology of arterial and venous stroke is different. Arterial infarction leads to cell membrane damage and cause cytotoxic brain edema that always demonstrates hypointensity in ADC map of brain MRI. In contrast, venous sinus thrombosis increases venous and capillary pressure and induces vasogenic edema, which is shown as hyperintensity in ADC map. Later, when the increased venous pressure results in malperfusion of the artery, arterial infarction will occur, which is usually shown as hyperintensity mixed with hypointensity in ADC map. In our case, looking at the MRI retrospectively, ADC map showed hyperintensity in day 5, which most likely suggested venous sinus thrombosis. Although anticoagulation treatment in acute phase of arterial stroke is usually not recommended because of its hemorrhagic risk, it is strongly recommended in a case of venous sinus thromboembolism even in acute phase of ischemic stroke. In addition to anticoagulation therapy, it is important to detect and treat the underlying cause of the thrombosis. The Factor VIII activity usually returns to normal after a few weeks of antithyroid therapy in hyperthyroid patient. Thus, the correction of the hyperthyroid state is an important part of the treatment strategy for cerebral venous thrombosis. Therefore, the early suspicion of venous sinus thrombosis and detecting the underlying cause of the thrombosis are very important for the adequate treatment. The ADC map should be evaluated carefully in a case of ischemic stroke.

A CASE OF PURPURA FULMINANS (PF) IN A BACKGROUND OF SEPTIC SHOCK CAUSED BY STREPTOCOCCUS PNEUMONIAE Sierra Jin ${ }^{1}$; Pinky Jha ${ }^{2}$. ${ }^{1}$ Medical College of Wisconsin, Wauwatosa, WI; ${ }^{2}$ medical college of wisconsin, Milwaukee, WI. (Control ID \#2464686)

LEARNING OBJECTIVE \#1: To report a case of PF due to Streptococcus pneumoniae septic shock.

LEARNING OBJECTIVE \#2: To discuss the diagnostic approach and management of PF.

CASE: A 60-year-old female with a history of COPD, tobacco use, and childhood spinal meningitis presented with progressive shortness of breath and cough for $24-48 \mathrm{~h}$. She was admitted to the medical ICU (MICU) for acute respiratory failure and septic shock with multi-organ failure. In the MICU, the patient was intubated and was started on empiric broad-spectrum IV antibiotics and fluids. The patient also required continuous venovenous hemofiltration (CVVH) for renal failure. She was stabilized in the MICU and was extubated on day 3 of hospitalization. Labs on admission later confirmed Streptococcus pneumoniae pneumonia and bacteremia, which were successfully treated empirically with 
vancomycin, moxifloxacin, and aztreonam. On day 5 , the patient's extremities were noted to be cool and then rapidly developed well-demarcated dark mottling purpura to bilateral forearms and legs. Vascular and plastic surgery teams were consulted for management of dry gangrene of the limbs. The hematology team was also consulted to assess potential disseminated intravascular coagulopathy (DIC)/thrombocytopenic thrombotic purpura (TTP)/heparin induced thrombocytopenia (HIT). Due to high clinical suspicion of TTP, the patient was started on methylprednisolone and was transferred to the MICU of another facility for a 5-day course of plasmaphoresis. DIC and HIT were ruled out subsequently with normal fibrinogen level and negative PF4, and heparin was reinitiated for anticoagulation. TTP was also ruled out with normal haptoglobin levels and mildly diminished ADAMTS13 levels. Therefore, plasmaphoresis was stopped and tapering of methylprednisolone was initiated. The patient became hypoxic on day 6 of hospitalization and was re-intubated and received CVVT and plasmaphoresis due to concerns for cerebrovascular occlusion. She was extubated on day 9. Stevens-Johnson Syndrome/ Toxic Epidermal Necrolysis and angioinvasive organisms were subsequently ruled out by a punch biopsy, and the rheumatology and dermatology teams made a clinical diagnosis of purpura fulminans. Meanwhile, the skin lesions were protected via loose gauze, lamb's wool boots, and petroleum jelly. On day 11, the patient became hypotensive with hemoglobin decreasing from 7 to 4 in 1 day. An abdominal CT showed a rectus sheath hematoma, a right psoas hematoma, and left lower lung lobe necrosis. The patient received a transfusion and anticoagulation treatment was discontinued. The patient was intubated a third time on day 12 with respiratory distress secondary to fluid overload and was extubated on day 15 . Hemodialysis was initiated on day 17 with completion of CVVH. At this time, the patient was transferred to the medical floor with no respiratory distress and a stable hemoglobin level. The patient remained stable hemodynamically and did not need any additional antibiotic treatments. Liver function and renal function also improved. Over the following weeks, the patient continued to receive hemodialysis and began working with physical therapy as she prepared to undergo further rehabilitation at a long-term acute care facility.

DISCUSSION: PF is a life threatening and rare complication caused by microvascular occlusion followed by hemorrhagic infarction of the dermis resulting in large purpura and bullae. It is classically seen in severe infections in children but can occur in adults. Rates of mortality ranging from 33 to $90 \%$ have been reported. It is believed that bacterial endotoxininduced endothelial damage and disturbance in pro- versus anticoagulation factors are key elements leading to acute sepsis-related PF. While PF can affect any body region, it is most often seen in the extremities. Perfusion is often compromised as a result of thromboembolic lesions, and the rates of amputation have been reported ranging from 90 to $100 \%$. The management of limb ischemia differs from case to case, ranging from emergent radical debridement to more conservative measures. The reported patient tolerated protectionoriented management with non-progressing bullae and stable dry gangrenous limb ischemia. The vascular and plastic surgery teams were closely involved in the day-to-day assessment of the lesions and recommended no immediate surgical management due to the patient's medical instability. However, she will likely require various degrees of amputation to all of her extremities in the future. The majority of pneumococcal-related PF has been seen in asplenic patients, as the spleen is crucial in the clearing of encapsulated organisms. This patient has no history of splenic trauma, sickle cell disease, prior splenectomy or other known hyposplenism, and her spleen was morphologically normal on abdominal CT scan. However, functional hyposplenism was suspected due to the patient's current presentation and her past medical history of spinal meningitis. Thus, she received the appropriate pneumococcal and meningococcal vaccinations as a part of her management.

A CASE OF RECALCITRANT HYPOKALEMIA AND HYPOPHOSPHATEMIA IN A PATIENT TREATED WITH ABIRATERONE ACETATE Cynthia Woods. Methodist Dallas Medical Center, Dallas, TX. (Control ID \#2466253)
LEARNING OBJECTIVE \#1: Recognize electrolyte disturbances in patients treated with abiraterone acetate

LEARNING OBJECTIVE \#2: Manage electrolyte abnormalities caused by mineralocorticoid excess and proximal tubular dysfunction

CASE: An 86 year-old man presented to the hospital with the complaint of right ankle pain and swelling. He had a past medical history of hypertension controlled with amlodipine, and metastatic prostate cancer treated with abiraterone acetate. He was admitted for treatment of septic arthritis and noted to have alarmingly low serum potassium and phosphate levels. During his hospitalization, the patient continued requiring a significant amount of potassium chloride and phosphorus to correct the severe electrolyte abnormalities. Lab testing revealed elevated urine potassium and phosphate levels, as well as significant aminoaciduria. An initial test to detect 24-h fractional excretion of phosphorus was obtained and noted to be elevated at $35 \%$. Accordingly, testing for fibroblast growth factor 23 (FGF 23) was done, resulting in normal levels. Given the hypokalemia and a history of hypertension, serum renin and aldosterone levels were tested to rule out primary hyperaldosteronism, and were within normal limits. Abiraterone acetate was identified as the likely causative agent for the patient's hypokalemia. Abiraterone acetate was held, and spironolactone was initiated. Lab testing after the addition of spironolactone revealed an elevated ACTH level of $71 \mathrm{pg} / \mathrm{mL}$. The cortisol level was decreased at $2.24 \mathrm{mcg} / \mathrm{dL}$. Corticosterone was markedly elevated at $7140 \mathrm{ng} / \mathrm{dL}$. The patient's hypokalemia normalized with the addition of spironolactone. He continued to get oral potassium phosphate replacement, which improved the phosphate level. He was scheduled to see his oncologist and a decision to continue abiraterone acetate would be made at that visit.

DISCUSSION: This case illustrates the potential for severe electrolyte disturbances in patients treated with abiraterone acetate for metastatic castration resistant prostate cancer (CRPC). While the etiology may be the same, it is important to look at the two electrolyte abnormalities separately. Regarding the hypokalemia with significant kaliuresis, inhibition of the cytochrome P450 enzyme 17 $\alpha$-hydroxylase (CYP17A1) with abiraterone acetate results in suppression of androgen and cortisol synthesis. The decrease in cortisol synthesis causes an increase in the ACTH level. This induction of diminished negative feedback on ACTH increases manufacturing of intermediates upstream from CYP17A1 such as 11deoxycortisocosterone, corticosterone and $18-\mathrm{OH}-$ corticosterone. These intermediary products have mineralocorticoid and some glucocorticoid effects. As mentioned above, the patient also had significant phosphaturia. Metastatic prostate cancer has been associated with elevated FGF 23 production and tissue induced osteomalacia (TIO). Although the FGF 23 level was not elevated, a negative or normal FGF 23 level does not completely rule out the diagnosis of TIO because there is not a way to measure the other two phosphatonins currently. Though TIO is still in the differential, the finding of significant aminoaciduria in the setting of phosphaturia was concerning for a proximal tubular dysfunction. Abiraterone has been associated with Fanconi syndrome, and though the patient had phosphaturia and aminoaciduria, he did not have glucosuria or non-anion gap metabolic acidosis. There could be an interesting interplay of multiple influences on the ammonia antiporter in the proximal tubule, which accounts for the normal bicarbonate levels. Mineralocorticoid excess causes hypokalemia, which stimulates this antiporter and enhances bicarbonate reabsorption. Proximal tubular dysfunction, as seen in Fanconi syndrome, results in malfunction of this antiporter and hence leads to increased sodium bicarbonate losses and intravascular volume depletion. These changes lead to an increase in the renin and aldosterone level and increased potassium loses in the collecting tubule (hypokalemia in proximal RTA). Despite the potential for electrolyte abnormalities, abiraterone acetate may be the only recourse for patients with metastatic CRPC. Therefore, it seems reasonable to consider other mechanisms to abate the effects of mineralocorticoid excess. Prescribing spironolactone or epleronone usually helps prevent the severe hypokalemia. Alternatively, prescribing a small dose of glucocorticoid can suppress the ACTH production. There is evidence that addition of dexamethasone to abiraterone acetate can 
further suppress androgens by preventing a rise in substrates of backdoor androgen synthesis. Replacement of electrolyte losses in the urine as in the treatment of Fanconi syndrome may stabilize patients who exhibit signs of proximal tubular dysfunction.

\section{A CASE OF SERONEGATIVE RHEUMATOID ARTHRITIS IN THE SETTING OF HEPATITIS C Sarah Dobro. Emory School of Medicine, Atlanta, GA. (Control ID \#2470327)}

LEARNING OBJECTIVE \#1: 1. Learn to recognize the presentation of seronegative rheumatoid arthritis

LEARNING OBJECTIVE \#2: 2. Understand the management of RA in the setting of chronic active hepatitis C. 3. Reflect on the interplay between homelessness and chronic medical illness

CASE: Mr. K.J. is a 49 year-old African American gentleman who presented with acute on chronic joint pain. He reported symptoms of 15 years duration, with pain and swelling of numerous joints, morning stiffness and severe fatigue. He had been diagnosed in the past with rheumatoid arthritis, and recalled treatment with prednisone, plaquenil and methotrexate with moderate benefit. Of note, he had a history of homelessness, and reported a history of chronic active hepatitis C acquired via IV drug use, discovered recently when he was tested by a mobile health van. He first presented at our institution with a chief complaint of pain "all over", worst in his hands and knees. Physical exam was significant for bilateral ulnar deviation with evidence of synovitis at the $2 \mathrm{nd}$ and $3 \mathrm{rd}$ MCPs, bilateral wrists with moderate synovitis and severely limited range of motion, contractures of the bilateral elbows, and bilateral knees with small effusions and limited extension. Labs and imaging were ordered, and he was discharged with prednisone $5 \mathrm{mg}$ BID and close follow up with Rheumatology. Unfortunately, he was robbed of all his belongings (including his prednisone) while staying on the street. He was lost to follow up until he presented as a walk-in to Rheumatology clinic 6 months later. At this visit, he complained of severe polyarticular pain and swelling, worst in his hands and knees. Physical exam was unchanged except for increased size of his bilateral knee effusions. Labs ordered at the past visit were significant for negative RF and CCP, positive ANA with positive SSA, elevated ESR, and positive HCV Ab with elevated viral load. The effusions were aspirated and his knees injected with solumedrol. Synovial fluid studies were significant for WBC 775, no crystals, and negative culture. X-rays showed severe erosions of his MCPs and elbows, as well as moderate tricompartmental knee osteoarthritis. He was also referred to Hepatology Clinic for treatment of his hepatitis C.

DISCUSSION: The ACR Criteria for diagnosis of RA include number of joints involved, positive serology (rheumatoid factor or anti-citrullinated peptide), elevated acute phase reactants (ESR or CRP), and duration of symptoms ( $>6$ weeks). This patient met criteria but had negative serologies, as do 10 $15 \%$ of all patients with RA. The differential diagnosis in this case also includes hepatitis C-related arthritis. Approximately two thirds of cases occur in the setting of mixed cryoglobulinemia - these tend to mimic RA, but usually have positive serologies and present with mild, non-erosive disease. The other one third are not related to cryoglobulins, but are oligoarticular rather than polyarticular. One might also consider polyarticular gout, but the absence of crystals and relatively low WBC count in the synovial fluid essentially rules this out. Since most treatments for RA involve some degree of immunosuppression, our team wondered whether there are any special considerations for the treatment of RA in the setting of active hepatitis C. There is scant direct evidence on this point, but the ACR Guidelines recommend using DMARDs other than methotrexate due to concern for hepatitis, and collaborating with a hepatologist when managing patients on antiviral therapy. Otherwise, patients with hepatitis $\mathrm{C}$ should be treated identically to other patients. There is also an important point to be made in this case about the interplay between socioeconomic hardship, substance abuse, and chronic medical illness. This patient contracted hepatitis C due to IV drug use, and both homelessness and IV drug use are traditional risk factors for tuberculosis. Furthermore, his interrupted access to care has caused permanent morbidity in the form of severe erosive joint disease.

\section{A CASE OF SUSPECTED HENOCH-SCHONLEIN PURPURA IN AN ADULT}

MALE Jarred P. Reed ${ }^{1}$; Edward $\mathrm{Ha}^{2} .{ }^{1}$ UCLA, Los Angeles, CA; ${ }^{2}$ UCLA School of Medicine, Los Angeles, CA. (Control ID \#2469814)

LEARNING OBJECTIVE \#1: Recognize the clinical manifestations of HenochSchonlein purpura in the adult patient.

LEARNING OBJECTIVE \#2: Apply the American College of Rheumatology criteria as a sensitive and specific means for the diagnosis of Henoch-Schonlein purpura.

CASE: The patient is a 35-year-old male with no significant past medical history who presented with bloody stools and purpuric rash. He was in his usual state of health until 3 weeks prior to presentation, when he developed persistent sore throat which was treated empirically with azithromycin and corticosteroid taper. Several days after completing treatment, the patient presented to a local hospital with five episodes of bloody stools and associated cramping abdominal pain, nausea, and vomiting. Computed tomography of the abdomen showed focal thickening of the wall of the ileum and ulceration of the lesser curvature of the gastric antrum. He was seen by Gastroenterology, who were concerned for infectious colitis and recommended initiation of levofloxacin and metronidazole for antimicrobial coverage. Stool culture and c. diff PCR eventually returned negative and the patient's symptoms did not improve. Two days later, he developed a purpuric rash on his bilateral palms, shins, and soles of the feet. He also complained of new pain in his right knee and bilateral ankles. He received two doses of methylprednisolone $62.5 \mathrm{mg}$ IV due to concern for an autoimmune process. Doxycycline was also added for coverage of possible arthropod-borne infection. The next day, the patient was transferred to our hospital for higher level of care. On arrival, the patient had innumerable scattered purpuric macules on his palms, bilateral knees, dorsal feet, and soles. His right knee was warm without swelling, bilateral ankles were cool to touch with mild swelling. Oropharynx was clear. Abdomen was soft, non-tender, and non-distended with normoactive bowel sounds. His only medications were acetaminophen-hydrocodone and cyclobenzaprine for low back pain. He denied drug use and reported that he was in a monogamous relationship with a longtime female partner. Admission labs were notable for hemoglobin $12.3 \mathrm{~g} / \mathrm{dL}$, white count $16.9 \times 10 \mathrm{E} 3 / \mathrm{uL}$, ESR $32 \mathrm{~mm} / \mathrm{h}$, CRP $11.9 \mathrm{mg} / \mathrm{dL}$. Basic metabolic panel, urinalysis, and $\mathrm{C} 3 / \mathrm{C} 4$ were within normal limits. Extensive infectious studies were negative, as were numerous rheumatologic serologies including ANA, ANCAs, MPO Ab, Proteinase-3 Ab, and Cryocrit. Upper endoscopy and colonoscopy revealed multiple ulcerations in the antrum and terminal ileum, and scattered macules in the colon. Biopsy specimens of skin over the right ankle showed leukocytoclastic vasculitis on light microscopy. Direct immunofluorescence showed 1+ granular deposition of $\mathrm{C} 3$ in vessels of the superficial dermis but was negative for antibody deposition. The Rheumatology service felt that despite the lack of IgA staining on skin biopsy, the patient's presentation was most consistent with $\operatorname{IgA}$ vasculitis. Antibiotics were stopped and the patient received methylprednisolone $1 \mathrm{~g}$ IV daily for 2 days, followed by methylprednisolone $48 \mathrm{mg}$ po daily. His symptoms improved with this regimen and he was discharged after a 9-day hospital course. Within several days of 
discharge, he returned with worsening abdominal pain and rash. His course was complicated by an episode of hematemesis of $500-1000 \mathrm{~mL}$. He was treated with intravenous corticosteroids and eventually transitioned back to an oral regimen prior to discharge. Since that time his disease has been wellcontrolled, and he is working with his Rheumatologist to taper his steroid regimen.

DISCUSSION: Henoch-Schonlein purpura (HSP) is an IgA-mediated small-vessel vasculitis, predominantly involving the skin, glomeruli, and gastrointestinal tract. Although HSP is the most common form of systemic vasculitis in children, its annual incidence in adults is approximately $0.8-1.8 / 100,000$. The classic tetrad of clinical manifestations in HSP includes palpable purpura, abdominal pain, arthritis/arthralgia, and renal injury, however the presentation can be variable and one or more of these symptoms may be absent. The American College of Rheumatology classification criteria for IgA vasculitis requires at least two of the following four items: age less than 20 years, palpable purpura, abdominal pain, and vessel wall granulocytes on biopsy. These criteria have shown a sensitivity of $87.1 \%$ sensitivity and a specificity of $87.7 \%$. Biopsy evidence of leukocytoclastic vasculitis or proliferative glomerulonephritis, with predominant $\operatorname{IgA}$ deposition, is confirmatory. While there was wide agreement that this patient's presentation was most likely secondary to HSP, it was unclear why vascular IgA deposition was not seen on skin biopsy. We theorized that the several day delay between the onset of the patient's rash and the time of biopsy, along with receipt of pulse steroid therapy, may have reduced the yield of our patient's biopsy specimen. Although outcomes are favorable in most patients with HSP, adults tend to have more severe renal involvement which can cause significant morbidity. One retrospective study found that $85 \%$ of adult patients developed nephropathy versus $25 \%$ of pediatric patients, and that $13 \%$ of the adults developed renal insufficiency compared to none of the children.

\section{A CASE OF TAKOTSUBO CARDIOMYOPATHY IN A PATIENT WITH ANA- PHYLAXIS TREATED WITH EPINEPHRINE Guramrinder S. Thind. Western} Michigan University School of Medicine, Kalamazoo, MI. (Control ID \#2469635)

LEARNING OBJECTIVE \#1: Recognizing high-dose epinephrine therapy as a possible precipitant of Takotsubo cardiomyopathy.

LEARNING OBJECTIVE \#2: Understanding the limitations of nuclear stress test for evaluating a patient with suspected Takotsubo cardiomyopathy.

CASE: A 58-year old female presented to a nearby hospital with shortness of breath, tightness in the throat, and vomiting after being stung by a wasp. She was diagnosed with anaphylaxis, and received 0.3 milligram (mg) epinephrine intramuscularly and $1 \mathrm{mg}$ epinephrine intravenously. Electrocardiogram (EKG) done after the second dose showed some ST elevations in anterior leads. Upon first contact after her transfer to our hospital, she was found to be hypotensive and was complaining of chest pain. She had to be intubated in view of impending airway obstruction. She received steroids, antihistamines, and was started on epinephrine infusion. She was found to have elevated troponins that were trending up. Her EKG showed persistent ST elevation in anterior leads. An echocardiogram (ECHO) was performed that revealed reduced systolic function (left ventricular ejection fraction $(\mathrm{LVEF})=25 \%$ ) and apical hypokinesis suggestive of TC. Troponins eventually began trending down. An elective cardiac catheterization was contemplated but patient had a history of dye allergy. She underwent a nuclear stress test instead, which revealed reversible ischemia in the antero-septal regions. This finding raised the concern of coronary artery disease and she eventually underwent cardiac catheterization with premedication for dye allergy. It revealed a largely normal coronary circulation and apical hypokineses, thereby confirming Takotsubo cardiomyopathy. A repeat ECHO done 6 weeks after discharge revealed return of LVEF to normal (65\%).
DISCUSSION: Takotsubo (stress) cardiomyopathy is now a well-established clinical entity; however, its pathophysiological basis is still being investigated. Some well characterized risk factors have been identified including postmenopausal status and stress. Although stress cardiomyopathy has classically been associated with emotional stress, it is also seen after events that cause significant physical stress e.g. surgery, sepsis, and intracranial bleed. Of the various emerging facets of the pathophysiology, catecholamine (especially epinephrine) surge during the stress event seems to be the central mechanism. In a case series published recently by Abraham et al., iatrogenic stress cardiomyopathy was observed in patients who received epinephrine $(n=6)$ or dobutamine $(n=3)$. Other cases of stress cardiomyopathy have been reported in pheochomocytoma patients, as well as in patients with anaphylaxis treated with vigorous epinephrine therapy. Most of these cases have been associated with high doses of epinephrine. The American Heart Association guidelines recommends a $0.3 \mathrm{mg}$ intramuscular or $0.1 \mathrm{mg}$ intravenous dose of epinephrine for anaphylaxis. In our case and in most other case reports, doses in excess of $1 \mathrm{mg}$ were administered. It has been shown that pre-hospital use of epinephrine is associated with a dose-dependent decrease in chance of survival in resuscitated patients. It has also been postulated that this could at least in part be due to increased incidence of undiagnosed TC in these patients. The central role of epinephrine in stress cardiomyopathy was reinforced by recent elucidation of the phenomenon 'biased agonism'. It has been established that, at critically high epinephrine concentrations, beta- 2 adrenergic receptor ( $\beta 2 \mathrm{AR}$ ) switches its coupling from Gs protein to an inhibitory Gi protein - that results in negative inotropic effect. This switch appears to be dependent on the level of $\beta 2 \mathrm{AR}$ phosphorylation, which in turn is facilitated by $\beta 1 \mathrm{AR}$ stimulation by epinephrine. The apical myocardium has been found to have a higher $\beta 2 \mathrm{AR}: \beta 1 \mathrm{AR}$ ratio and that could explain the selective negative inotropic effects of catecholamine surge in the cardiac apex. Another facet of the pathophysiology of stress cardiomyopathy is microvascular impairment. Studies measuring myocardial perfusion via TIMI myocardial perfusion grade (TMPG) as well as nuclear imaging have demonstrated coronary microcirculatory disturbances. It has been hypothesized that these microvascular abnormalities may result from increased mechanical wall stress as a consequence of apical ballooning. These abnormalities may manifest as abnormal nuclear stress tests, as in our case, and stress tests should be interpreted with caution in patients with stress cardiomyopathy.

\section{A CASE OF THE UNSEEN: KLEBSIELLA PNEUMONIAE PYOGENIC LIVER ABSCESS WITH PNEUMONIA AND SEPTIC ENDOPHTHALMITIS IN A NON-ASIAN U.S. RESIDENT Xuan Gao; Patricia Dharapak. Mount Sinai Beth Israel, New York, NY. (Control ID \#2467887)}

LEARNING OBJECTIVE \#1: Prompt awareness on the metastatic complications of liver abscesses.

LEARNING OBJECTIVE \#2: Recognize Klebsiella pneumoniae as a growing source for pyogenic liver abscess in the United States.

CASE: A 53 year old Russian man with no significant past medical history initially presented to an ophthalmology clinic with 2 days of progressively worsening, near-total left visual field loss associated with a 1-week duration of fever, chills, and cough. He was diagnosed with bacterial endophthalmitis. His vitreous fluid was sampled, and he was referred to our institution for further evaluation. On presentation, patient appeared visibly toxic with diffuse rigors. Vital signs were Tmax $104.6^{\circ} \mathrm{F}, \mathrm{BP} 135 / 88, \mathrm{HR} 127, \mathrm{SaO} 291 \%$ on room air. Patient reported that he was born in Russia and immigrated to the United States 11 years ago; his recent travel history was limited to within the United States. Lab work performed on admission showed WBC $12.7 \mathrm{~K} / \mathrm{UL}$, AST $58 \mathrm{U} / \mathrm{L}$, ALT $92 \mathrm{U} / \mathrm{L}$, Alkaline Phosphatase $246 \mathrm{U} / \mathrm{L}$, Albumin $2.1 \mathrm{~g} / \mathrm{dL}$. Glucose was normal. HIV was nonreactive. CXR showed right middle and right lower lobe pneumonia. Urine culture was negative. His vitreous fluid and peripheral blood cultures grew Klebsiella 
pneumoniae. Pyogenic liver abscess was a concern given evidence of metastatic septic foci. An abdominal CT revealed a $6 \times 7.5 \mathrm{~cm}$ multi-septated hypodense right hepatic lobe collection. Ceftriaxone was initiated and the patient underwent percutaneous hepatic drainage, which also recovered $K$. pneumoniae. He was ultimately discharged home to complete a 4-week total course of antibiotics. Repeat abdominal CT performed at 6 weeks post-discharge showed markedly reduced abscess size.

DISCUSSION: K. pneumoniae is a well-known opportunistic nosocomial pathogen Most community acquired $K$. pneumoniae can cause pneumonia or urinary infections; however, K. pneumoniae serotype $\mathrm{K} 1$ is a major cause of community acquired pyogenic liver abscess, particularly in East Asia. The K1 strain appears to be highly invasive, affecting relatively healthy hosts like our patient, and causing metastatic complications such as endophthalmitis, necrotizing fasciitis, meningitis, and visceral abscesses. Multilocus sequence typing on our patient's isolate shows that it was sequence type 23 , a genotype associated with the K1 serotype. An infection with this serotype has a slight male predominance, and patients can present with fever, chills, right upper quadrant pain, leukocytosis, low albumin, and an elevated alkaline phosphatase. Right sided and solitary hepatic lesions are also typical. It is unclear how our patient acquired this strain as he lacked the typical epidemiologic association with East Asia travel or descent. Our case adds to the already growing evidence that community acquired liver abscess with K. pneumoniae $\mathrm{K} 1$ strain is expanding beyond Asia as a global disease. Review of literature demonstrated only a handful of cases featuring disseminated $K$. pneumoniae K1 pyogenic liver abscesses in the United States. As such, the diagnosis of $K$. pneumoniae should be considered in all cases of liver abscess, and clinicians should be prompted to closely evaluate these patients for metastatic complications to improve clinical outcomes.

\section{A CASE OF VASOMOTOR DISTURBANCES IN ANAPHYLAXIS Gloria Shan;} Omar Hadzipasic; Meghna C. Trivedi. UMass Memorial Medical Center, Worcester, MA (Control ID \#2467989)

LEARNING OBJECTIVE \#1: Recognize Kounis syndrome affecting the cardiovascular system during anaphylaxis

LEARNING OBJECTIVE \#2: Recognize reversible cerebral vasoconstriction syndrome after administration of epinephrine in anaphylaxis

CASE: A 24 year-old female with past medical history of migraine, fibromyalgia, and nicotine dependence presented to the emergency room post elective termination of pregnancy at 7 weeks of gestation. Thirty minutes after the procedure, she developed facial swelling, generalized urticaria, shortness of breath, and chest pain. She has history of allergy to iodinated contrast and morphine. She had received intravenous doses of $100 \mathrm{mcg}$ fentanyl and $2 \mathrm{mg}$ midazolam for anesthesia as well as azithromycin, ibuprofen and Rhogam. Intrauterine copper was implanted. On initial evaluation, heart rate was 115/min and blood pressure was $94 / 51 \mathrm{mmHg}$. Laboratory data was significant for a lactic acid of 2.7, Troponin I of $0.06 \mathrm{ng} / \mathrm{ml}$ and D-Dimer of $16 \mathrm{mg} / \mathrm{L}$. Complete blood count and chemistry were normal. Electrocardiogram showed diffuse T-wave inversions in inferior and lateral leads. Benadryl, epinephrine, famotidine, solumedrol and 41 of saline were administered in the ER. While cutaneous symptoms resolved, she continued to experience intermittent substernal chest pain. CTA of chest ruled out pulmonary embolism. Troponin peaked at 0.06 and EKG normalized on hospital day 2. Echocardiography was normal. Patient subsequently developed thunderclap headaches with word finding difficulty and right sided paraesthesia. The quality of headache was different than her previous migraine attacks. CT head, MRI brain and MRV were normal. MRA showed bilateral irregularity of A1 segments of anterior cerebral arteries and proximal MCA with multifocal narrowing consistent with reversible cerebral vasoconstriction syndrome (RCVS). She was started on a calcium channel blocker for RCVS Gradually her neurological symptoms and chest pain resolved. She was discharged with recommendations to repeat neuroimaging and follow up with an allergist.
DISCUSSION: Kounis and Zavras first described Kounis syndrome, also known as "allergic angina" or "allergic myocardial infarction" in 1991. It refers to concurrent occurrence of acute coronary syndrome and allergic reactions. During anaphylaxis, mast cells infiltrate myocardium and release histamine upon degranulation, which in turn causes coronary arterial vasospasm, plaque erosions, and rupture. Three variants of the Kounis syndrome have been described. In Type I, patients with no pre-existing coronary artery disease have vasospasm with or without progression to myocardial infarction secondary to inflammatory mediator release. Type II variant includes patients with pre-existing atherosclerotic disease experiencing coronary vasospasm alone or in combination with plaque erosion/rupture leading to myocardial infarction. Type III variant is seen in patients who died from stent thrombosis where histological exam of coronary vessel shows infiltration of mast cells and eosinophils. Based on observations and objective evidences, presented case could be classified as Type I Kounis syndrome. Incidence of peri-operative anaphylaxis is reported to be around 1 in 13,000 procedures. The most common triggers are neuromuscular blocking agents, antibiotics, namely penicillins and cephalosporins, and latex. However, she received fentanyl, which although uncommon, could cause IgE mediated inflammatory reaction. A case report of a young woman with confirmed fentanyl induced intra-operative anaphylaxis describes lactic acidosis and coronary vasospasm similar to our patient. Midazolam and azithromycin can rarely cause anaphylaxis. Ibuprofen usually causes pseudo-allergic reaction mimicking anaphylaxis in patients with asthma. Only three cases of anaphylactoid reaction after injection of anti-rhesus-D-immunoglobulin have been reported. Copper is a weak allergen hence IUD is the least suspected culprit. A skin patch test will help us determine the trigger for peri-operative anaphylaxis in our patient. Except IUD, she had been exposed to all described agents in the past. For treatment of anaphylaxis, patient received $0.3 \mathrm{mg}$ (1:1000) epinephrine intramuscularly. Within $24 \mathrm{~h}$ of administration, she developed thunderclap headache and acute neurological symptoms. MRA brain revealed findings consistent with reversible cerebral vasoconstriction syndrome (RCVS). Interestingly, a case report of RCVS secondary to epinephrine administration in setting of anaphylaxis has been described. It is attributed to transient disturbance of regulation of cerebral arterial tone. RCVS is characterized by acute thunderclap headaches, with or without other neurological symptoms and evidence of diffuse segmental constriction of cerebral arteries. The condition resolves spontaneously within 3 months. The headache is severe, peaks in seconds and occurs intermittently for 1-2 weeks. Ischemic and hemorrhagic strokes are the major complications of RCVS. Pregnancy, post-partum state, vasoactive agents, catecholamine- secreting tumors, blood products, neurosurgeries etc. are known precipitating agents of RCVS. Female sex preponderance is noted. Removal of offending agent and initiation of a calcium channel blocker is the mainstay of treatment. Nimodipine $60 \mathrm{mg}$ orally three times a day for $8-12$ weeks is recommended. A repeat neuroimaging in few weeks helps to assess the cerebral vessels.

A CASE SERIES ON THE USE OF LEFT VENTRICULAR ASSIST DEVICES (LVADS) IN INFLUENZA-INDUCED CARDIOMYOPATHY AND FULMINANT HEART FAILURE Jalal Vargha ${ }^{1}$; Joy Iskandar ${ }^{1}$; Sunil Pauwaa ${ }^{1,2} .{ }^{1}$ Advocate Christ Medical Center, Oak Lawn, IL, Oak Lawn, IL; ${ }^{2}$ Advocate Christ Medical Center, Oak Lawn, IL. (Control ID \#2467916)

LEARNING OBJECTIVE \#1: To describe the use of left ventricular assist devices (LVADs) in persistent cardiogenic shock from influenza induced cardiomyopathy as a bridge to transplantation or recovery. Fulminant acute myocarditis presents with sudden 
onset of cardiac symptoms, which manifest after nonspecific flu-like symptoms and rapidly progress to severe hemodynamic instability with severe heart failure or cardiogenic shock. This case series shows the use of LVADs as a viable treatment to allow time for clinical improvement or transplantation. Temporary support with LVADs has been shown to improve survival, but literature review yielded no cases in regards to influenza-induced cardiomyopathy and resulting fulminant heart failure. This case series identifies two cases of LVAD utilization which led to patient survival.

LEARNING OBJECTIVE \#2: To show the utility of LVADs when other forms of hemodynamic support have failed. In both cases, extracorporeal membrane oxygenation (ECMO), inotropes, and vasopressors did not significantly improve clinical status or cardiac status. Clinical improvement was only met when patients had LVADs implanted which reduced strain on the cardiopulmonary system and assisted the heart as it regained its ability to function properly.

CASE: The first patient is a 55 year old male with no significant past medical history that presented with worsening shortness of breath. The patient had been healthy until 2 months prior to admission, when he began to have viral-like symptoms. His primary care physician treated him with antibiotics for a presumed upper respiratory tract infection. The patient continued to have fevers, cough, and worsening shortness of breath. He was evaluated in an emergency department, diagnosed with pneumonia, and again treated with antibiotics. Patient continued to have worsening symptoms and returned to the emergency room with respiratory distress requiring emergent intubation. An echocardiogram showed a new diffuse, global hypokinesia and an ejection fraction of $30 \%$ while the chest $\mathrm{x}$-ray revealed diffuse pulmonary edema. Patient was also found to have Influenza A infection. The patient was diagnosed with severe decompensated systolic heart failure with multisystem organ failure likely secondary to influenza myocarditis. Extracorporeal membrane oxygenation (ECMO) was initiated at an outside hospital and the patient was transferred to our facility for LVAD placement for hemodynamic support. Patient was started on inotropes and vasopressors with minimal clinical improvement. Following LVAD placement, vasopressors, inotropes, and ECMO were weaned as cardiogenic shock improved. Repeat echocardiogram revealed full recovery of systolic function with ejection fraction $55-60 \%$. As cardiac function had returned to baseline, the LVAD was removed within a few weeks. The patient clinical status improved, and he was discharged with close follow up in the heart failure clinic. The second patient is a 33 year old female with a history of rheumatoid arthritis who presented to an outside hospital with 3 days of flu-like symptoms including fever, cough, and shortness of breath along with new-onset syncope. The patient was diagnosed with influenza and found to be in cardiogenic shock with elevated troponins and an ejection fraction of $15 \%$. She was stabilized with pressors, an intraaortic balloon pump (IABP) and inotropes. Right and left heart catheterization revealed new onset cardiomyopathy without coronary artery disease, strongly suggesting influenza as the cause of cardiomyopathy and the fulminant heart failure. The patient was transferred to our facility for further management. Patient continued to be in cardiogenic shock despite IABP and ECMO. Patient was also treated with Tamiflu. An LVAD was placed with end goal of heart transplant. Patient cardiac status improved following LVAD placement with repeat cardiac echocardiogram exhibiting an improvement in systolic function. The patient was eventually discharged home in stable condition. Patient continues to follow up in the LVAD clinic and is awaiting heart transplant.

DISCUSSION: Cardiovascular involvement in acute influenza infection can occur through direct effects of the virus on the myocardium or through exacerbation of existing cardiovascular disease. Our patients did not have any previous history of cardiovascular disease. In at least $10 \%$ of patients with viral infection, the virus may replicate in the heart. Most cases of acute myocarditis will be mild and will improve without intervention, but those cases that develop into fulminant acute myocarditis may be fatal. Patients with fulminant influenza myocarditis with cardiogenic shock are given hemodynamic support with vasopressors and inotropes. However, despite intensive treatment, the mortality rate has been reported to be $39 \%$ in patients with fulminant myocarditis from the H1N1 influenza virus. For those patients who did not receive mechanical circulatory support, despite failure to respond to medical management, the mortality rate has been reported to be $83 \%$. Complete recovery is possible if patients are successfully supported. In fulminant myocarditis with refractory cardiogenic shock, the use of LVAD therapy is a life saving and should be considered as an option early on in the disease before end organ failure. LVADs can be used as a temporary therapy to stabilize patients, yet are able to be used long-term for patients who will eventually need a transplant.

A CHALLENGING CASE OF ASCITIES : CHEMOTHERAPY INDUCED NODULAR REGENERATIVE HYPERPLASIA Orighomisan Pessu; Deepa R. Nandiwada. University of Pittsburgh Medical Center, Pittsburgh, PA. (Control ID \#2466515)

LEARNING OBJECTIVE \#1: Recognize Nodular Regenerative Hyperplasia (NRH) as a cause of portal hypertension

LEARNING OBJECTIVE \#2: Understand the pathophysiology, causes, and management of NRH

CASE: Case presentation: A 54 year old gentleman with past medical history of glomerulonephritis requiring two renal transplants, Hodgkin's lymphoma stage 3B on Adriamycin, Bleomycin, Vinblastine, Dacarbazine (ABVD) cycle 6 who presented with new ascites. He was diagnosed with Hodgkin's lymphoma stage 3B due to fevers, night sweats and unintentional weight loss 6 months prior to admission. He was then started on ABVD cycle 1 and had his last cycle 4 weeks prior to admission. He was in his usual state of health until 3 weeks prior to admission, when he started to notice progressive abdominal swelling. He also had bilateral lower extremity swelling which he stated was chronic for several decades and was unchanged at time of presentation. He complained of abdominal pressure and tension near his ribs and sore back at the time of presentation. He had new, progressive shortness of breath with exertion. He denied any cardiac history, chest pain, paroxysmal nocturnal dyspnea, orthopnea, nausea, vomiting, diarrhea, or change in urinary habits. The rest of his review of systems was negative for findings. Initial exam: On initial physical exam, vital signs were normal and the patient was in no acute distress. No scleral icterus was noted, mucous membranes were moist, no lymphadenopathy was present. Cardiovascular exam was remarkable for $2+$ lower extremity edema to his hips and scrotum. Abdominal exam was notable for a very distended abdomen with fluid wave and shifting dullness. He had minimal abdominal discomfort with no rebound or guarding. There were no spider angiomatas, palmar erythema, caput medusa present. Initial labs Initial labs include sodium of $133 \mathrm{mmol} / \mathrm{L}$, potassium of $4.3 \mathrm{mmol} / \mathrm{L}, \mathrm{BUN}$ of $19 \mathrm{mg} / \mathrm{dl}$, creatinine of $2.1 \mathrm{mg} / \mathrm{dl}$, Total bill of 0.9 , ALT of $15 \mathrm{IU} / \mathrm{L}$, AST of $25 \mathrm{IU} / \mathrm{L}$, ALP of $157 \mathrm{IU} /$ $\mathrm{L}$, albumin of $3.0 \mathrm{~g} / \mathrm{dl}$. White blood cell count of 7.7, hemoglobin of $9.8 \mathrm{~g} / \mathrm{dl}$. Infectious hepatitis workup was negative. Ferritin was elevated to $967 \mathrm{IU} / \mathrm{L}$. Further hospital course For progressive ascites, he had pigtail catheter placed and underwent large volume paracentesis with drainage of up to $14 \mathrm{~L}$ with appropriate albumin replacement. Ascitic fluid revealed a high serum albumin ascities gradient and low total protein consistent with portal hypertension. Fluid was sent for cytology, which was unremarkable. He underwent abdominal ultrasound, which showed unremarkable liver morphology, and patent hepatic vessels with antegrade flow of the portal vein. Transjugular liver biopsy was performed and porto-systemic pressure gradient was assessed which revealed a pressure gradient of $23 \mathrm{~mm} / \mathrm{hg}$ consistent with portal hypertension. Echocardiogram was also done to try to elucidate cause of ascites, however showed normal systolic function, grade I diastolic dysfunction and unremarkable valves. Liver biopsy revealed portal venopathy and moderate nodular regenerative hyperplasia.

DISCUSSION: Nodular regenerative hyperplasia (NRH) of the liver is an uncommon cause of non cirrhotic portal hypertension. Autopsy studies indicate an overall incidence ranging between 0.72 and $2.6 \%$. NRH is characterized by the diffuse transformation of 
normal hepatic parenchyma into small, regenerative nodules with little to no fibrosis. It has been associated with systemic diseases including autoimmune disorders, systemic malignancies, systemic drugs including chemotherapy drugs and immunosuppressants. The most widely accepted and described mechanism is micro thrombosis and decreased blood flow in the hepatic sinusoids and portal microvasculature leading to apoptosis and reactive hyperplasia of neighboring hepatocytes that are not affected, causing formation of nodules. Nodule formation cause diffuse compression of sinusoids leading to portal hypertension. On presentation, liver functions tests are typically normal and liver synthetic function remains intact as evidenced by normal INR and protein. NRH should be suspected in any patient with clinical features of portal hypertension and no hormona or synthetic manifestations of cirrhosis. Imaging methods in general have poor sensitivity and specificity for NRH. On ultrasound, regenerative nodules are usually not visible and if they are may be indistinguishable from metastases. MRI may be a better diagnostic modality than CT and ultrasound however use is still controversial. Liver biopsy is the gold standard. Once diagnosed, management options include first removing the offending agent if able, and managing the complications of portal hypertension. The natural progression is uncertain, in some cases, removing the offending agent may lead to reversal of NRH.

\section{A CLUE TO THE DIAGNOSIS: HEMOPHAGOCYTIC SYNDROME PRESENT-} ING WITH HYPERFERRITINEMIA Sujoy Phookan; Schuyler D. Livingston. Emory University School of Medicine, Decatur, GA. (Control ID \#2456158)

LEARNING OBJECTIVE \#1: Recognize the differential diagnosis of severe hyperferritinemia

LEARNING OBJECTIVE \#2: Review the causes and key clinical features of hemophagocytic lymphohistiocytosis (HLH)

CASE: A 55-year-old male with untreated HIV/AIDS presented to the emergency department with altered mental status, fever, and tachypnea. Initial evaluation revealed acute renal failure and hepatic injury. The patient's clinical course was marked by rapid decline and circulatory collapse, with development of multi-organ dysfunction. He became obtunded, anuric, and developed respiratory failure consistent with adult respiratory distress syndrome (ARDS). Urine sediment revealed acute tubular necrosis, which required hemodialysis. Though the admission blood counts were relatively preserved, he later developed pancytopenia and disseminated intravascular coagulation (DIC). Despite extensive investigation, including cultures from blood, urine, broncho-alveolar lavage, and stool, no bacterial etiology was found. Viral polymerase chain reaction studies from blood and cerebrospinal fluid were negative with the exception of serum Epstein-Barr virus (EBV). Labs for mycobacterial and fungal etiologies, including urine histoplasma antigen, were also negative. Iron studies revealed a serum ferritin level of $>15,000 \mu \mathrm{g} / \mathrm{L}$ (normal range 12-300). This, in conjunction with the patient's severe pancytopenia, raised suspicion for hemophagocytic lymphohistiocytosis (HLH), possibly secondary to EBV or HIV. A subsequent bone marrow biopsy revealed scattered hemophagocytes. Testing for soluble CD25 returned positive, thus confirming the diagnosis of HLH. The patient's multi-organ dysfunction largely resolved over several weeks with supportive care, and he was initiated on antiretroviral therapy prior to discharge.

DISCUSSION: Hyperferritinemia is a nonspecific finding often present in acute or chronic infection, rheumatologic disorders, malignancy, chronic iron overload, and severe hepatic or renal dysfunction. In acutely ill patients with underlying AIDS, it is common to find a serum ferritin $>1000$, even in the setting of concurrent iron deficiency. Extreme hyperferritinemia ( $>3000$ to $>10,000$ ), raises concern for diseases such as HLH, disseminated histoplasmosis, and adult-onset Still's disease. In children, hyperferritinemia, especially at levels $>10,000$, is highly sensitive $(90 \%)$ and specific for $(98 \%)$ for HLH. In adult patients, however, even severe ferritin elevation is a relatively nonspecific marker, and may only be helpful in certain clinical contexts such as this case. Hemophagocytic lymphohistiocytosis is a rare syndrome characterized by immune dysregulation and overproduction of inflammatory cytokines. The primary immune defect is reduced or absent function of natural killer cells, which normally function to destroy activated histiocytes and lymphocytes. Elevation of inflammatory cytokines may lead to multiorgan failure and death. Clinically, patients typically present with fever, pancytopenia, lymphadenopathy, and hepatosplenomegaly. This presentation should prompt further evaluation with biopsy of the bone marrow or an excised lymph node. Other common findings include hepatic injury, hypertriglyceridemia, low fibrinogen levels, and hyperferritinemia. Pathologic analysis in HLH reveals activated macrophages with engulfed blood cells. In some cases, additional testing for natural killer cell activity and soluble CD25 are necessary to confirm the diagnosis. Acquired HLH may be secondary to underlying infection or malignancy. Cases of HLH secondary to underlying EBV infection are associated with higher cytokine levels and increased mortality. Treatment with etoposide is recommended for HLH, but was not necessary in this case given the patient's clinical improvement while he awaited definitive diagnosis

A COMMON PRESENTATION OF A RARE DISEASE: SPORADIC CJD Kaylee J. Shepherd ${ }^{1}$; Blake R. Barker ${ }^{2} .{ }^{1}$ UT Southwestern, Dallas, TX; ${ }^{2}$ UT Southwestern Medical Center at Dallas, Dallas, TX. (Control ID \#2469679)

LEARNING OBJECTIVE \#1: Recognize clinical features that should lead to prompt evaluation for Creutzfeldt-Jakob Disease (CJD).

LEARNING OBJECTIVE \#2: Identify key differences between sporadic, inherited, and infectious forms of CJD

CASE: A 62-year-old male with a history of hypothyroidism and extensive psychiatric family history was admitted for further evaluation of progressive neurologic changes. $\mathrm{He}$ was at his baseline until 1-month prior when he became increasingly paranoid of losing his job and began working at least $20 \mathrm{~h}$ per day. A couple of weeks later, he had an episode of acute agitation and aggression (destroying his home office). Since then, he progressively declined with a variety of neurologic symptoms: he would wander around the house aimlessly, not follow simple commands, and be unable to identify or use common objects. He soon declined to the point of behaving childlike and losing the ability to use his right hand. He was admitted to an outside facility where he underwent extensive workup, including $24 \mathrm{~h}$ EEG, brain MRI, cerebrospinal fluid (CSF) analysis, and brain PET scan. MRI brain was worrisome for global anoxic injury and PET demonstrated hypometabolic activity. The patient was transferred to our facility for further investigation. Upon transfer, there were multiple episodes of aggression, and he soon developed myoclonus and pseudobulbar affect. Physical exam revealed primitive reflexes. EEG showed periodic sharp wave complexes (PSWC) and diffusion weighted imaging (DWI) on MRI of brain showed cortical ribboning concerning for CJD. Spinal fluid specimen from lumbar puncture was sent to National Prion Center at Case Western, which returned positive for real time quaking induced conversion (RT-QUIC) protein with more than $98 \%$ probability of having CJD.

DISCUSSION: CJD has sporadic, inherited, and infectious forms. Sporadic CJD constitutes $85 \%$ of cases and classically presents with rapidly progressive dementia (RPD) between the 6th and 8th decades of life accompanied by cerebellar, psychiatric, or extrapyramidal symptoms (particularly myoclonus). Progressive aphasia, apraxia, and visual symptoms may occur. These may be preceded by a prodrome of headache, fatigue, anorexia, anxiety, and vertigo/dizziness; as well as changes in mood, behavior, and sleep pattern. Median survival is 5 months, with $85 \%$ dying within 1 year. Inherited CJD constitutes $15 \%$ of cases and is caused by an autosomal dominant PRNP mutation. Variant CJD is rare, distinguished by a median age of onset of 29 , and associated with bovine spongiform encephalopathy. Iatrogenic transmission has been recorded via corneal transplants, human growth hormone 
treatments, and contaminated blood products. While the diagnostic gold standard is brain biopsy demonstrating spongiform change, this presents the risk of iatrogenic transmission. Alternatively, CDC clinical criteria for probable CJD include RPD accompanied by $\geq 2$ of the following: myoclonus, visual or cerebellar signs, pyramidal/extrapyramidal signs, or akinetic mutism AND $\geq 1$ of: PSWCs, CSF 14-3-3 protein, or characteristic hyperintensities on MRI Our patient demonstrated RPD, myoclonus, PSWCs, characteristic MRI, and CSF 14-3-3 and tau protein, which reflexed to a RT-QuIC analysis to detect the abnormal prion protein itself. The National Prion Center performed the RT-QuIC analysis and offers CSF, DNA, and tissue testing. They report results to the CDC and state health departments.

\section{A COMPACT REVIEW OF NONCOMPACTION CARDIOMYOPATHY Marga-} ret Lynch. University of California San Francisco, San Francisco, CA. (Control ID \#2466121)

LEARNING OBJECTIVE \#1: 1) Recognize isolated left ventricular non-compaction as an uncommon but high-risk cause of syncope.

LEARNING OBJECTIVE \#2: 2) Describe the classic symptom triad of noncompaction cardiomyopathy and key management principles.

CASE: A 51-year-old man with history of gastric bypass and sensorimotor neuropathy due to copper deficiency presented to transfer care to a new provider. Ten weeks prior, he had lost consciousness in his kitchen and sustained a vertebral fracture for which he was treated at an outside emergency room. He described bending over to pick up an object and losing consciousness on returning to stand. He noted the room was warm, he felt lightheaded earlier in the day, and he had skipped breakfast. He denied chest pain, shortness of breath, palpitations, or similar prior episodes. Family history was notable for stroke in both parents, with no history of sudden death. Medications were noncontributory. Cardiac exam was normal, without murmurs, rubs, gallops, or signs of volume overload. Labs were notable for low fasting blood glucose (58) and serum copper (50). Electrocardiogram (ECG) and Ziopatch were normal. Over the next 4 months, his hypoglycemia normalized with small frequent meals, he resumed copper supplementation, and had no interval presyncope or syncope. Two months later, he returned to clinic after repeat syncope with vertebral fracture, and endorsed two additional interval syncopal events, each with a prodrome of warmth and vertigo. Exam was unchanged. Echocardiogram revealed normal ejection fraction with prominent trabeculations, concerning for isolated left ventricular (LV) noncompaction. He was urgently referred to cardiology where repeat Ziopatch revealed a ten-second run of asymptomatic ventricular tachycardia, and cardiac magnetic resonance imaging (MRI) confirmed an increased ratio of noncompacted to compacted LV apical myocardium with preserved LV ejection fraction. Evaluation for inducible ventricular arrhythmia is ongoing; if present, an implantable cardioverter defibrillator (ICD) will be placed. His first degree relatives are undergoing screening echocardiogram. Given his multiple fractures, a bone densitometry scan was performed; he also has osteopenia.

DISCUSSION: Isolated LV noncompaction cardiomyopathy (LVNC) is a rare genetic cardiomyopathy which is increasingly being recognized in the era of improved echocardiographic technologies, although overall population prevalence is still less than $1 \%$. It is characterized by prominent trabeculation of the left ventricular wall in the absence of other congenital cardiac abnormalities. A number of potential candidate genes have been identified, and there is some genetic overlap with other cardiomyopathies such as hypertrophic cardiomyopathy (HOCM). The abnormalities are thought to be due to intrauterine arrest of compaction of the fetal myocardial primordium due to ischemia or other insult, and consequent persistence of deep trabecular recesses. The classic clinical presentation is a triad of heart failure (the most common presenting complaint), arrhythmia, including sudden cardiac death, and thrombotic events. Approximately $5 \%$ of patients with LVNC present with syncope. Unlike our patient, most patients have an abnormal resting ECG, with left bundle branch block the most common pattern. A delayed time to diagnosis is typical, with a mean of 3.5 years from symptom onset. Transthoracic echocardiogram is the diagnostic study of choice, and transesophageal echocardiogram or cardiac MRI can be done to confirm the diagnosis. Treatment is focused on (1) evidence-based treatment of LV systolic dysfunction, when applicable, (2) arrythmia prevention with ICD placement indicated for patients with a history of ventricular tachycardia, cardiac arrest, or who meet standard criteria for primary prevention in the setting of heart failure, and (3) anticoagulation for patients with a history of VTE. Some authors suggest a broader subset of patients with LVNC may benefit from ICD and/or anticoagulation, although current data is limited. The overall prognosis is quite poor, with cardiovascular event rates as high as $8 \%$. Sudden cardiac arrest is the most common cause of death. This case highlights an atypical but increasingly recognized etiology of cardiogenic syncope, as well as the important role of echocardiography in the workup of syncope concerning for cardiogenic etiology, such as recurrent syncope or syncope with signficant injury.

\section{A CRYPTIC PRESENTATION OF CRYPTOCOCCUS NEOFORMANS Mazie} Tsang; Sundsted Karna. Mayo Clinic, Rochester, MN. (Control ID \#2469071)

LEARNING OBJECTIVE \#1: Identify possible causes of spells in an immunocompromised patient. Spells can be a diagnostic enigma requiring consideration of a wide differential, including neurologic, cardiogenic, autonomic, infectious, and psychogenic etiologies.

LEARNING OBJECTIVE \#2: Describe the clinical presentation of Cryptococcal meningitis and recognize that it can mimic CNS vasculitis.

CASE: A 73 year old man-with a history of pulmonary sarcoidosis on chronic prednisone, stroke, and recent knee arthroplasty from which he was recovering at a swing bed-was admitted for spells, delirium, mental status changes, headaches, fevers, and cough. His delirium began shortly after being transferred to a swing bed facility, where he was noted to have a spell both at the facility and at an outpatient clinic visit. During his spells, his family noted that his upper extremities stiffen up prior to him losing consciousness. Upon admission, he was found to be febrile and coughing, so he was initiated on broad-spectrum antibiotics. He was placed on continuous electrocardiogram monitoring with no arrhythmia noted. Neurology was consulted and witnessed a spell, which was deemed to be consistent with a seizure, so he was started on levetiracetam. Magnetic resonance imaging and angiogram (MRI/A) of the brain showed new multi-territory strokes and vascular changes concerning for vasculitis; he was nearly started on high dose methylprednisolone before his cerebrospinal fluid and blood cultures grew Cryptococcus neoformans. Infectious Disease was consulted-he was started on Amphotericin B and flucytosine, and his broadspectrum antibiotics were discontinued. Repeat MRI suggested that his vasculitic-like changes and multiple strokes were thought to be secondary to his central nervous system (CNS) infection. He was eventually transitioned to fluconazole and flucytosine prior to discharge to his swing bed facility.

DISCUSSION: Cryptococcal meningitis typically is an indolent process that occurs over a period of weeks, with patients most commonly presenting with fever, malaise, confusion, and headache. Our patient who presented with spells was found to have multiple strokes that mimicked CNS vasculitis, which is a very unusual presentation of cryptococcal meningitis. One of the largest retrospective studies identified 28 patients with cerebral infarctions due to chronic meningitis, of which $39 \%$ had cryptococcal meningitis. Although there is a high index of suspicion for cryptococcal meningitis in patients with HIV and low CD4 count, it and other opportunistic CNS infections should be considered in the differential in patients on chronic immunosuppression who present with 
CNS vasculitis and stroke, especially if they are not improving despite other appropriate therapies. In conclusion, Cryptococcus neoformans is uncommonly a mimicker of CNS vasculitis, and our case emphasizes its unusual presentation and the importance of considering CNS infections in immunosuppressed patients who present with spells or stroke.

A CURIOUS CASE OF HYPOKALEMIA: MCKITTRICK-WHEELOCK SYNDROME Jennifer S. Mulliken; David Altszuler; Natalie K. Levy. NYU School of Medicine, Bellevue Hospital, New York, NY. (Control ID \#2469585)

LEARNING OBJECTIVE \#1: Review the manifestations of hypokalemia and an approach to diagnostic work-up.

LEARNING OBJECTIVE \#2: Understand the clinical features and management of an unusual cause of hypokalemia, McKittrick-Wheelock syndrome.

CASE: A 67 year-old man with a history of mild intermittent asthma was admitted to our hospital with bilateral hand paresthesias for 2 days and a serum potassium of $2.1 \mathrm{mmol} / \mathrm{L}$. Ten months prior to admission the patient began having 6 to 8 bowel movements daily associated with profuse mucous discharge. He also reported a 30-lb weight loss over the same time interval. Bowel movements were not associated with abdominal pain or tenesmus, and there were no relieving or inciting factors. He denied any recent history of fevers, hematochezia, melena, or change in dietary habits. He took no medications. He had never undergone screening colonoscopy and there was no known history of cancer in his family. On presentation vital signs were stable. Digital rectal exam was notable for a large and soft anterior rectal mass palpable at the tip of the finger. Physical exam was otherwise unremarkable. Electrocardiogram showed normal sinus rhythm with a prolonged QTc interval (490 ms), diffuse T wave flattening, and $\mathrm{U}$ waves. In addition to low serum potassium, labs were otherwise notable for a serum chloride of $97 \mathrm{mmol} / \mathrm{L}$ and bicarbonate of $36 \mathrm{mmol} / \mathrm{L}$. Calcium and magnesium levels were normal. Creatinine was $0.9 \mathrm{mg} / \mathrm{dL}$. Spot urine potassium was less than $3 \mathrm{mmol} / \mathrm{L}$, while fecal potassium was $67 \mathrm{mEq} / \mathrm{L}$. The fecal osmotic gap was $24 \mathrm{mOsm} / \mathrm{kg}$, consistent with a secretory process. Computed tomography of the abdomen and pelvis showed a 6-cm lobulated mass in the rectum without evidence of bowel obstruction or metastasis. Colonoscopy revealed a large rectosigmoid mass extending 10 to $17 \mathrm{~cm}$ from the anal verge, partially occluding the lumen. Biopsy of the mass was consistent with tubulovillous adenoma. The patient subsequently underwent surgical resection of the adenoma with resolution of his hypokalemia.

DISCUSSION: Hypokalemia can be caused by increased losses of potassium, increased uptake into cells, or decreased intake of potassium. The initial management of hypokalemia includes electrocardiography and assessment of muscle strength, as cardiac arrhythmias and muscle weakness (including diaphragmatic weakness) are the most common fatal effects. These complications most frequently occur when serum potassium is less than $2.5 \mathrm{mEq} / \mathrm{L}$. Our patient had a critically low serum potassium level on presentation. $\mathrm{He}$ had prominent $\mathrm{U}$ waves on electrocardiogram but did not have any muscular weakness. He was aggressively treated with both oral and intravenous potassium supplementation. By measuring his urine and fecal potassium levels, we determined that his hypokalemia was due to increased losses from the gastrointestinal tract. We suspected that his secretory diarrhea and hypokalemia were related to his large tubulovillous adenoma. Adenomas of the colon are common. Large studies estimate an incidence of $25-35 \%$, and prevalence increases with age (plateaus after age 65). Rarely, these adenomas can have hyperactive secretory glands and cause severe diarrhea. In 1954 Drs. McKittrick and Wheelock described 48 cases of patients with large colonic adenomas and secretory diarrhea with massive fluid losses leading to severe electrolyte abnormalities and renal failure. Patients with McKittrick-Wheelock syndrome classically present with hypokalemia and a complex acid-base disorder (metabolic acidosis and contraction alkalosis) from increased secretion of potassium and bicarbonate from the tumor. Presentations can be variable, and adenomas occasionally secrete chloride (as $\mathrm{HCl}$ ) rather than bicarbonate, which may explain our patient's alkalosis. Surgical resection of these adenomas is curative. McKittrick-Wheelock syndrome should remain on the differential for patients presenting with severe electrolyte derangements, especially hypokalemia.

A CURIOUS CASE OF JOINT PAIN Rachel Clark; Philip J. Putnam. Tulane University Health Sciences Center, New Orleans, LA. (Control ID \#2467211)

LEARNING OBJECTIVE \#1: Identify the criteria necessary to diagnose acute rheumatic fever (ARF)

LEARNING OBJECTIVE \#2: Describe the short-term and long-term sequela of ARF and current prevention methods

CASE: A 25-year-old woman presented to the emergency room with an intense burning pain in her ankles, erythema overlying her ankles that spread upwards towards her knee, and chest pain. Four weeks prior, she was diagnosed with streptococcal pharyngitis and was treated with a 10-day course of Amoxicillin. Two days following completion, she noticed circular erythematous areas on her bilateral lower shins, which spread to involve her ankles and knees. She presented to an urgent care facility and was given another 10day course of amoxicillin. She completed 7 days but noted worsening symptoms and presented to the emergency room. Her physical exam was significant for edema and erythema of the bilateral lower extremities to the knees, swollen and erythematous ankles and knees bilaterally with exquisite tenderness to palpation, and reproducible chest pain. There were no subcutaneous nodules present. Electrocardiogram showed an incomplete right bundle branch block (no previous EKG to compare). CT angiography was negative for pulmonary embolism. Lab findings were significant for elevated ESR/CRP, negative gonorrhea/chlamydia, ASO titer significantly elevated, and an autoimmune panel negative. Transthoracic echocardiogram was normal. The patient's symptoms progressed to include bilateral upper extremity joint pain with appearance of subcutaneous nodules around her elbows. During hospitalization, she also developed debilitating involuntary spasms of her extremities and experienced significant emotional lability. Based on the revised Jones' Criteria, this patient was diagnosed with Acute Rheumatic Fever (ARF) and started on appropriate therapy. Her symptoms improved over the next 5-7 days.

DISCUSSION: Acute Rheumatic Fever (ARF) is a disease resulting from an autoimmune reaction to infection with Group A Streptococcus (GAS). ARF is most commonly diagnosed in childhood, and the infrequency in which it is encountered in the adult population makes it a diagnostic challenge. The most common age group affected by ARF is 6-20. Worldwide, rheumatic heart disease, a potential sequela of ARF, accounts for as high as $50 \%$ of all cardiac admissions in certain areas. ARF is diagnosed using the Jones Criteria, which is subdivided into Major and Minor criteria. The Major criteria include polyarthritis, carditis, subcutaneous nodules, erythema marginatum, and Sydenham Chorea. The Minor criteria include fever, arthralgia, elevated acute phase reactants, and prolonged PR interval. Along with a documented recent GAS infection, $\mathrm{ARF}$ is diagnosed if either 2 of the major criteria are met or 1 major and 2 minor criteria. Current streptococcal testing has a specificity of $90-100 \%$ and a sensitivity of $70-90 \%$. The primary treatment goals include elimination of the GAS infection, symptom alleviation, and prophylaxis to prevent recurrence. GAS infections are treated preferentially with penicillin, while fevers and arthritis are treated with aspirin or other NSAIDs. Penicillin prophylaxis is necessary to reduce risk of recurrent episodes and Rheumatic Heart Disease. Long term consequences from ARF makes its recognition and treatment essential. Patients who develop an initial ARF secondary to GAS are at risk for recurrence of rheumatic fever with subsequent GAS infection. ARF incidence has decreased steadily in large part due to the introduction of antibiotics and improved sanitation practices throughout the developed world. However, internationally, outbreaks are still a common 
occurrence, likely secondary to the introduction of new virulent streptococcal strains. Although the use of antibiotics have led to the decrease of long term consequences in developed countries, ARF is still associated with the development of life threatening carditis, rheumatic heart disease, and post-streptococcal glomerulonephritis. ARF is primarily a clinical diagnosis, and requires thorough history, careful examination, and a broad differential diagnosis by the primary care physician to ensure that this disease and the potential sequelae are not overlooked.

\section{A CURIOUS CASE OF NOSEBLEEDS IN THE NIGHT-TIME: PROFOUND IRON DEFICIENCY ANEMIA AND PLUMMER-VINSON SYNDROME SEC- ONDARY TO HEREDITARY HEMORRHAGIC TELANGIECTASIA}

Mohammad-Ali Jazayeri; Douglas J. Rausch. Hennepin County Medical Center, Minneapolis, MN. (Control ID \#2469728)

LEARNING OBJECTIVE \#1: Recognize the characteristic features of hereditary hemorrhagic telangiectasia (HHT) and Plummer-Vinson syndrome.

LEARNING OBJECTIVE \#2: Avoid the pitfall of accepting the clinical reasoning of prior providers when new information comes to light.

CASE: A 34-year-old Mexican male with a history of reportedly chronic, low-level epistaxis presented with fatigue, shortness of breath, and pedal edema for 3 days. He noted that over the prior 2 weeks family members had told him he was more "yellow." Denied any other bleeding symptoms, changes in bowel habits. He did note that he had been having some difficulty with swallowing in the preceding weeks but denied weight loss and other constitutional symptoms. He also endorsed craving consumption of crayons, plastic, paper, paint, and liquid soap for years. He had previously been seen nearly 5 years prior when he was hospitalized with posterior reversible encephalopathy syndrome in the setting of microcytic anemia and hemoglobin of $5.3 \mathrm{~g} / \mathrm{dL}$ with ferritin of $3.8 \mathrm{ng} / \mathrm{mL}$. At that time he underwent upper and lower endoscopy which were wholly unremarkable aside from a single red spot detected in the lesser curvature of the stomach with no active bleeding or stigmata of recent bleeding. He received appropriate iron replacement, and his hemoglobin improved to $10.2 \mathrm{~g} / \mathrm{dL} 1$ year later. Aside from a history of nasal injury requiring surgery in 1998 , he had no other significant medical history to note. He had been lost to follow-up for approximately 4 years. On this presentation, he was afebrile on arrival, and other vital signs were in the normal range. Examination was remarkable for severe conjunctival pallor, evidence of glossitis and cheilosis, Mild bibasilar crackles were present along with $2+$ pedal edema. Jugular venous pressure was not markedly elevated. There was no significant hepatosplenomegaly or ascites. Laboratory evaluation was remarkable for hemoglobin of $2.1 \mathrm{~g} / \mathrm{dL}$, platelet count of $557 \mathrm{k} / \mathrm{cmm}$, ALT 465, AST 336, normal total and direct bilirubin. PT was prolonged at $20.7 \mathrm{~s}$, and INR was 1.9 , while PTT was normal. Iron studies were notable for ferritin of $3.7 \mathrm{ng} / \mathrm{mL}$ with low serum iron of $10 \mathrm{mcg} / \mathrm{dL}$, normal iron binding capacity of $460 \mathrm{mcg} / \mathrm{dL}$, and depressed reticulocyte count of $0.2 \%$. B12 and folate levels were normal. Peripheral blood smear revealed profound hypochromic, microcytic anemia without evidence of sideroblasts. Hepatitis serologies and HIV testing were negative. Imaging studies included an admission chest $\mathrm{x}$-ray with evidence of increased central vascular congestion and cardiomegaly. A transthoracic echocardiogram showed left ventricular ejection fraction at the lower limits of normal, mild mitral insufficiency with left atrial enlargement and borderline elevated pulmonary artery pressure. He was referred for endoscopic evaluation of his nasopharynx, which was remarkable for diffuse telangiectasias of the nasal mucosa. Further imaging with MRI Brain, CT Chest, and abdominal MRI showed no evidence of intracranial arteriovenous malformations (AVM), one right lower lobe AVM measuring $3 \mathrm{~cm}$ in longest dimension, and no hepatic AVMs.

DISCUSSION: We present the case of a 34-year-old male with a history of microcytic anemia secondary to iron deficiency who presented after nearly 5 years with severe microcytic anemia in the setting of ongoing near-daily epistaxis and a presenting $\mathrm{Hgb}$ of $2.1 \mathrm{~g} / \mathrm{dL}$. He improved following transfusion of 3-4U PRBC with small doses of diuretic administered in the setting of high output heart failure and was scheduled for serial iron transfusions in the outpatient setting. His case has tremendous learning value in demonstrating some features of the rarely seen Plummer-Vinson syndrome, namely dysphagia and weakness along with glossitis and cheilosis in the setting of profound iron deficiency anemia. Moreover, despite the underwhelming epistaxis complaint, which had previously been treated supportively while electing for an extensive endoscopic evaluation of the gastrointestinal tract, further questioning on this subsequent admission for a recurrent problem revealed a family history of nosebleeds. Despite the absence of classic oral telangiectasias on physical exam, endoscopic nasal evaluation demonstrated diffuse telangiectasias concerning for hereditary hemorrhagic telangiectasia, a rare (approximate incidence 1 in 5000), autosomal dominant disorder characterized by the following criteria: (1) spontaneous and recurrent nosebleeds, (2) multiple mucocutaneous telangiectasias, (3) visceral involvement, and (4) first degree relative with HHT. In summary, this case illustrates the features of two rare clinical syndromes and, more importantly, highlights the importance of a thorough history and physical examination, particularly in the setting of a recurrent and unsolved problem.

A DEAD STOP: VASOVAGAL MEDIATED ASYSTOLE Kristopher P. Kline; Garrett Ruth; Michael Kaufmann; Matthew McKillop. University of Florida, Gainesville, FL. (Control ID \#2469319)

LEARNING OBJECTIVE \#1: Recognize that vasovagal syncope is a diagnosis primarily based on a thorough history

LEARNING OBJECTIVE \#2: Recognize vasovagal syncope can cause profound vasodiliatory and cardioinihibitory responses including complete sinus arrest.

CASE: A 58-year-old female with medical history of prior syncope and breast carcinoma now in remission presented for elective breast reconstruction surgery. Post-operative day \#2 she was working with physical therapy and developed extreme shoulder and chest wall pain at surgical site followed by nausea, lightheadedness and diaphoresis. Continuous telemetry monitoring during the event showed sinus rhythm transition to junctional bradycardia followed by $20.7 \mathrm{~s}$ of asystole associated with loss of consciousness. After regaining consciousness she complained of fatigue and malaise with gradual recovery over several minutes. Cardiac evaluation with ECG, TTE and LHC 2 months prior were all normal. Past workup for prior episodes of syncope included multiple ambulatory ECG monitors without evidence of causative arrhythmia. The patient noted symptoms of lightheadedness, nausea, diaphoresis occurring every few months, all associated with moments of severe emotional distress or pain. Occasionally, if severe, they will progress to transient loss of consciousness. She has always been able to feel the event coming on, lies down and never been injured due to these episodes. She had no personal or family history of heart disease and no family history of sudden death.

DISCUSSION: Syncope is the transient loss of consciousness and remains a common diagnosis, with over $20 \%$ of cases considered to be vasovagally mediated. A thorough history will elicit a diagnosis in nearly $50 \%$ of syncope events. In the setting of an appropriate history and negative cardiac workup, a diagnosis of neurally-mediated vasovagal syncope was made. Vasovagal syncope occurs classically in setting of orthostatic or emotional stress resulting in inappropriate autonomic reflexes causing either a significant cardioinhibitory, vasodilatory process, or both. The cardioinhibitory response can result in sinus slowing or arrest, AV delay or block, or both. In our case the patient had a long asystolic pause due to sinus slowing/arrest and transient atrioventricular block, likely from extrinsic autonomic changes rather than intrinsic conduction disease. It is important to include all forms of syncope in the differential diagnosis. Forms of neurally mediated syncope include vasovagal, situational, carotid sinus hypersensitivity and postexertional, 
which account for up to two-thirds of etiologies behind syncope. These are largely considered benign. Orthostatic hypotension account for approximately $10 \%$ of cases. Cardiogenic syncope accounts for an additional 10-20\% of cases and occurs in the setting of structural heart disease, cardiomyopathy, outlet obstruction, and arrhythmias. Cardiogenic syncope is unlikely in the setting of a normal ECG and a structurally normal heart on echocardiography, as abnormal findings on ECG are seen in up to $90 \%$ of patients with cardiac syncope. Other important etiologies include medication effect, seizure, hypovolemia, hemorrhage, hypoglycemia, aortic dissection, pulmonary embolism, cardiac tamponade and psychogenic. Vasovagal syncope has not been associated with an increase risk of cardiovascular morbidity or mortality. Conservative management principles revolve around physical maneuvers to help increase peripheral vascular resistance and venous return including lying supine with legs raised above the heart, leg-crossing and arm gripping with muscle tension, or squatting. Remaining well hydrated for volume expansion or increasing dietary salt as tolerated is recommended. Thigh-high compression stockings can also be used. There has been no clear evidence in favor of pharmacologic therapies. While once thought to be theoretically beneficial, beta-blockers have fallen out of favor and are no longer recommended. Fludrocortisone, a mineralocorticoid to promote volume expansion, and midodrine, a vasoconstrictor, are the most commonly prescribed but have not shown consistent evidence of benefit. Other agents have been proposed but have indeterminate evidence to support their use. In our case, the prologed cessation of electrical cardiac activity was of concern. Cardiac pacing can correct the cardioinhibitory process but does not correct the vasodilatory process, leaving many patients prone to recurrent events despite an invasive procedure. A 2009 meta-anaylsis on pacemaker implantation in vasovagal syncope showed no overall benefit but did suggest an "expectation effect" on patients after pacing. A more recent trial of pacing in neurally mediated syncope and asystolic pauses showed a borderline statistically significant reduction in syncope recurrence at two years. Options were presented to our patient who elected against further testing or permanent pacemaker implantation. She had an otherwise uncomplicated hospital course without further arrhythmias or syncopal events.

A DEATHLY SPASM Jason Dinsmoor; Michael P. Smith. University of Nebraska Medical Center, Omaha, NE. (Control ID \#2469941)

LEARNING OBJECTIVE \#1: Recognize the clinical presentation of vasospastic angina in the setting of non-obstructive coronary artery disease

LEARNING OBJECTIVE \#2: Understand the pathophysiology of coronary vasospasm.

CASE: A 66 year-old man with history of periodic chest pain and prostate cancer was admitted for cystoprostatectomy and developed cardiac arrest in the post-operative setting. He was resuscitated and underwent cardiac catherization which revealed non-obstructive coronary artery disease. He continued to have intermittent chest pain during his admission. Cardiovascular, pulmonary, and abdominal exams were unrevealing. Cardiac enzymes were trended and remained negative. Echocardiogram was without regional wall abnormality. Cardiac catheterization revealed $50 \%$ stenosis in the right coronary artery and $30 \%$ stenosis in the left anterior descending artery. Telemetry revealed ST segment elevations in the inferior leads that correlated to chest pain consistent with vasospastic or Prinzmetal angina.

DISCUSSION: Chest pain is a common problem encountered by the general internist. Vasospastic, or Printzmetal, angina has a unique presentation with transient intermittent chest pain. Coronary artery spasm results in a temporary reduction in luminal diameter leading to myocardial ischemia. This ischemia is manifested on electrocardiogram with transient ST segment elevation. The vascular smooth muscle hyper-reactivity resulting in vasospasm can be reversed by nitrates and calcium channel blockers. These are first line therapies as they prevent vasoconstriction and promote vasodilation in the coronary vasculature. Vasospastic angina in the setting of pre-existing coronary artery disease can lead to complete coronary artery occlusion, infarction, and in this case arrest. Often with coronary artery disease standard management would include beta-blockade as it has shown to decrease mortality; however, nonselective beta blockers can exacerbate vasospasm and should be avoided. Aspirin is a prostacyclin inhibitor and should be used with caution with vasospastic angina; however it is recommend in vasospastic angina with underlying atherosclerotic disease. It is essential for the general internist to differentiate vasospastic angina from other forms of angina as the treatment for coronary artery disease can worsen vasospastic angina. Our patient had both leading to severe symptoms post operatively, and it is possible that if his vasospasm was treated aggressively prior to surgery, it could have prevented his cardiac arrest.

\section{A DIAGNOSTIC HEADACHE: PRIMARY CENTRAL NERVOUS SYSTEM} LYMPHOMA IN A HUMAN IMMUNODEFICIENCY VIRUS-NEGATIVE PA-

TIENT Evie A. Kalmar. University of California, San Francisco, San Francisco, CA. (Control ID \#2469768)

LEARNING OBJECTIVE \#1: Review common causes of headaches LEARNING OBJECTIVE \#2: Diagnose primary central nervous system lymphoma CASE: A 74-year-old man with a history of chronic kidney disease (CKD) stage 4 with nephrotic syndrome and polycystic kidney disease, hypertension, type 2 diabetes mellitus, and previous cerebrovascular accident of unknown etiology with mild residual left-sided deficit presented for evaluation of 4-week history of a new headache. He described the headaches as dull, frontal, associated with blurry vision, lightheadedness, and unsteadiness on his feet, and lasting 60 to $90 \mathrm{~min}$. He had a history of chronic headaches, but noted that these headaches were different in quality. He could not identify triggers or palliation, though noted they were most frequent at night and generally resolved by morning. Exam was remarkable for blood pressure of 141/64 and mild left-sided pronator drift with slowing of coordination in left upper extremity. Labs were notable for stable mild normocytic anemia and rising creatinine of 3.9 (estimated glomerular filtration rate 14), from 2.7 one-month prior. Magnetic resonance imaging (MRI) with angiogram without contrast (given CKD) showed severe periventricular microvascular disease without aneurysm, mass, or evidence of acute infarct. He continued to suffer from intermitten headaches for the following weeks, and presented to primary care and urgent care clinics during this time. Ultimately, he fell and presented to the emergency department. Computed tomography $(\mathrm{CT})$ non-contrast of the head showed a $2.1 \times 2.5 \mathrm{~cm}$ right temporal occipital mass with surrounding vasogenic edema causing a $7.8 \mathrm{~mm}$ right-to-left midline shift and right ventricular compression. Neurosurgery was consulted, and a biopsy showed central nervous system (CNS) lymphoma. Positron emission tomography and CT showed no alternate primary source, and he was diagnosed with diffuse large B cell CNS lymphoma. He was evaluated by oncology, but his renal function limited his treatment options. After a complicated 8-week course following diagnosis, he was transitioned to comfort care and passed away.

DISCUSSION: Headache is a common presenting symptom of CNS tumors, but CNS tumors are not a common cause of headache. The majority of headaches have a benign etiology, and are due to a primary cause such as migraines, tension headaches, or cluster headaches. In some patients however, such as the one in this case, a headache can be the first symptom of a more serious disease, such as vascular thrombosis or malformation, malignancy, or infection. The diagnostic approach to headache varies based on the quality of symptoms, co-existing medical issues, and neurologic exam. It is important to screen for red flag signs and symptoms, including focal neurologic signs, immunocompromised state, sudden onset of severe headache, headache worse with supine position or Valsalva, 
nuchal rigidity, or history of head trauma. Any of these red flags warrant diagnostic work up, which can include neuroimaging, lumbar puncture, and/or labs depending on the suspected underlying diagnosis. In this patient, his age and complex medical history warranted work up, but initial imaging and lab testing were unrevealing. Primary CNS lymphoma (PCNSL) accounts for less than five percent of primary CNS tumors. The vast majority of cases occur in HIV-positive patients; in fact, the only established risk factor is immunodeficiency. Consequently, a new diagnosis of PCNSL should prompt testing for HIV status. PCNSL can manifest in any of the following locations: brain, meninges, eyes or spinal cord. Clinical manifestation depends on location of the malignancy, and ranges from focal neurologic deficits, signs of increased intracranial pressure, or neuropsychiatric symptoms in cerebral disease to ocular symptoms in ocular disease or myelopathy or systemic symptoms in spinal disease. These potential sites of disease guide the initial diagnostic work up in patients with suspected PCNSL, which includes 1) imaging of CNS (MRI with contrast, preferred), 2) cerebrospinal fluid (CSF) evaluation, 3) slit lamp examination, and 4) testicular examination. Ultimately, diagnosis requires pathologic confirmation with CSF or with cerebral or vitreal tissue. This vignette reviews common causes of headache and highlights the importance of continued evaluation and work up in cases where there is concern for secondary causes of headache.

A DIFFICULT ROUTE FOR C. DIFFICILE Hili Rosen; Julia P. Brockway; Thida Aye. Mount Sinai Beth Israel, New York, NY. (Control ID \#2467644)

LEARNING OBJECTIVE \#1: Recall that fistula formation is a potential complication of diverticulitis.

LEARNING OBJECTIVE \#2: Recognize that fistulae may provide a route for Clostridium difficile entry to the bladder.

CASE: A 97-year-old female with history of CAD, atrial fibrillation, history of previous vancomycin resistant enterococcus (VRE) urinary tract infection (UTI), and an admission 1 month prior for sigmoid diverticulitis treated with a 2 week course of ciprofloxacin and metronidazole, was sent from her nursing home to the emergency department with 3 days of diarrhea and dysuria. While at the nursing home, the patient reported abdominal pain associated with multiple episodes of watery stools, as well as burning with urination. The patient's initial vital signs were T 97.6, BP 156/72, PR 70, RR 16. Labs were significant for stable anemia, a normal white blood cell count and a normal lactate. Urinalysis showed large leukocyte esterase, > $182 \mathrm{WBC} / \mathrm{HPF}$, many bacteria and white blood cell clumps. Physical exam was notable for mid-epigastric tenderness. Given her history of VRE, the patient was started on linezolid and admitted to the hospital. On hospital day two, the patient was found to have urinary retention and a foley catheter was placed, with return of feculent-appearing material. Stool cultures that had been collected at the nursing home returned positive for Clostridium difficile ( $C$. difficile), and the patient was started on oral vancomycin. The initial urine culture grew mixed organisms which, in the setting of feculent foley drainage, raised strong suspicion for a colovesical fistula. Renal ultrasound revealed a distended bladder containing a large amount of debris. On hospital day four, the patient developed a leukocytosis and a bandemia of $25 \%$. Culture of the feculent debris found in the foley bag returned positive for $C$. difficile toxin. The patient was continued on oral vancomycin, but leukocytosis worsened and she continued to have large volume stools and copious feculent drainage into the foley. The patient's fistula was deemed inoperable, and she and her family ultimately decided on a palliative care approach.

DISCUSSION: Fistula formation is a complication that can be seen after diverticulitis, especially when the sigmoid colon is involved, as was in this patient. It has been estimated that colovesical fistula may develop in $2-4 \%$ of cases of diverticulitis, and diverticulitis is the most common cause of colovesical fistula. Symptoms include pneumaturia, fecaluria and dysuria as well as frequent urinary tract infections. Diagnosis can be made through various imaging techniques, but is often obvious clinically. Definitive treatment of the fistula involves surgical repair. This patient had multiple risk factors for $C$. difficile colitis, including recent hospitalization, nursing home stay, antibiotics use and age. Her fistula provided a route for the feces to enter her bladder. To our knowledge, this is the first documented case of $C$. difficile infection in the urine via a fistulous tract. Providers should also be aware that, while metronidazole is often a treatment for $C$. difficile, it can be a causative agent.

A DYSFUNCTIONAL RELATIONSHIP - EPISODIC DYSPNEA DUE TO VOCAL CORD DYSFUNCTION Nihan K. Cannon. Cambridge Health Alliance, Cambridge, MA. (Control ID \#2469490)

LEARNING OBJECTIVE \#1: Delineate between vocal cord dysfunction (VCD) and asthma

LEARNING OBJECTIVE \#2: Manage VCD and consider the role for CPAP in an acute episode

CASE: A 20 year old female with a history of irritable bowel syndrome and nut/sesame seed allergies, requiring multiple admissions for anaphylaxis, presented with the chief complaints of dyspnea, itchiness, and throat tightness soon after a presumed peanut exposure. She explained that while dusting at work, she may have noticed a peanut shell. Soon after, she felt dyspneic and experienced worsening throat tightness and itchiness, for which she self-administered her EpiPen before calling an ambulance. En route, EMS administered a second EpiPen, IV Benadryl, Solumedrol, and Duo-nebs. On arrival, her pruritis and dyspnea persisted. Her vitals were significant for tachycardia to the $150 \mathrm{~s}$, tachypnea to the low 30 s, oxygen saturations in the upper 90 s, SBPs $120 \mathrm{~s}-130 \mathrm{~s}$; she had swollen lips and a large uvula, as well as diffuse wheezes and reduced breath sounds in all lung fields. Labs, consisting of $\mathrm{CBC}$ with differential and BMP, were within normal limits. She received additional intramuscular epinephrine, Benadryl, Solumedrol, and Duo-nebs. Her itching resolved and her dyspnea improved, though her voice remained raspy. She was admitted to the ICU for close monitoring for airway compromise. The following morning, the patient began to frantically gesture at her throat, while taking rapid, shallow breaths and making tongue-clicking noises. Her heart rate peaked at 150 and she struggled to speak due to shortness of breath, though her exam was significant for improved wheezes and lack of stridor. She received another round of albuterol, antihistamines, Solumedrol and epinephrine with little relief. Flexible laryngoscopy during this episode revealed paradoxical vocal cord movement, but a widely patent airway without evidence of edema of her epiglottis or vocal cords. She received $1 \mathrm{mg}$ Ativan, followed by $2 \mathrm{mg}$ Ativan, with partial improvement of her now diagnosed vocal cord dysfunction, likely worsened by her panic attack. She experienced a second episode several hours later, without improvement on Ativan - she began to request intubation. Instead, the patient was trialed on CPAP (PEEP 5), with full resolution of symptoms. She was discharged the following day, with plans to start speech and psychotherapy and to present for outpatient pulmonary function testing.

DISCUSSION: The patient in this vignette never desaturated or developed true, persistent stridor, although she had brief inspiratory and expiratory upper airway wheezes at times. This case thus highlights the importance of having a low threshold of suspicion for vocal cord dysfunction. Given her associated anxiety symptoms and lack of improvement with bronchodilator treatments, laryngoscopy was obtained and cinched the diagnosis by demonstrating abnormal adduction of true vocal cords. Her dysphonia outside these acute episodes likely reflected her vocal cord hyperfunction resulting in anterior-superior laryngeal compression during speech. Her VCD was likely related to both her history of anaphylaxis and 
resultant anxiety, although she may also have undiagnosed asthma. Given her severe symptoms that were only mildly responsive to Ativan, she was trialed on CPAP with good effect. Historically, patients who presented with stridor and paroxysms of wheezing and dyspnea in the face of aggressive therapy were thought to have bronchial asthma, often resulting in intubations. Interestingly, vocal cord dysfunction and asthma may co-occur $40 \%$ of the time, though it's not uncommon for patients to receive the misdiagnosis of only asthma, resulting in higher medical utilization and exposure to unnecessary medication. Case reports even allude to patients who become Cushingoid due to repeat exposure to steroids. The most effective diagnostic strategy remains laryngoscopy during an acute episode — otherwise, patients may undergo numerous tests and studies, without identification of VCD. Benzodiazepines remain the mainstay of treatment for acute attacks, with few case reports discussing CPAP. Goldman and Muers previously suggested that CPAP works by slowing expiratory flow, to result in a more open glottis, which then alleviates airway tightness. Such an approach may be especially efficacious when there is closure of the glottis on inspiration, as was visualized on laryngoscopy for this patient. A multidisciplinary approach with speech therapy and psychiatry remain crucial for reducing VCD attacks in the future and helping the patient navigate through the anxiety provoking nature of VCD. Only by identification of VCD can patients be set on the path for treatment and recovery.

\section{A FORGOTTEN CAUSE OF ANION GAP METABOLIC ACIDOSIS WITH AN} OSMOLAR GAP Marc Heincelman. Medical University of South Carolina, Charleston, SC. (Control ID \#2467439)

LEARNING OBJECTIVE \#1: Recognize the clinical presentation of alcoholic ketoacidosis with emphasis on onset occurring after cessation of alcohol ingestion, leading to undetectable ethanol levels on admission.

LEARNING OBJECTIVE \#2: Demonstrate that alcoholic ketoacidosis presents with an anion gap metabolic acidosis with an increased osmolar gap, and understand the importance of obtaining a serum beta-hydroxybutyrate for diagnosis.

CASE: A 62-year-old woman with a history of anxiety disorder and alcohol abuse presented with somnolence and slurred speech. Two weeks prior to presentation, the patient started consuming two liters of wine per day. Over the following days, she developed general weakness, nausea, nonbloody nonbilious emesis, and decreased oral intake but continued to drink alcohol. Her weakness limited ambulation to around the house and she ran out of wine 3 days prior to admission. On presentation, vitals revealed temperature 36.9C, HR $97 \mathrm{bpm}$, BP 105/72 mmHg, RR $20 \mathrm{bpm}$, and O2 saturation of $96 \%$ on room air. Examination revealed a woman appearing older than stated age with dry mucous membranes; she otherwise had a nonfocal exam. Basic metabolic panel revealed serum sodium $117 \mathrm{mmol} / \mathrm{L}$, potassium $3.9 \mathrm{mmol} / \mathrm{L}$, chloride $84 \mathrm{mmol} / \mathrm{L}$, bicarbonate $17 \mathrm{mmol} / \mathrm{L}$, anion gap 16, BUN $39 \mathrm{mg} / \mathrm{dl}$, creatinine $3.8 \mathrm{mg} / \mathrm{dl}$, and glucose $85 \mathrm{mmol} / \mathrm{L}$. Ethanol level was undetectable. Urine drug screen was positive for benzodiazepines, which she took daily for her anxiety. After receiving one liter of normal saline, she became more alert and deemed stable for admission to the general medicine teaching service. Her hyponatremia was deemed hypovolemic hyponatremia in the setting of decreased oral intake with a serum osmolality of $273 \mathrm{mosm} / \mathrm{kg}$ and positive orthostatic vital signs. Her acute kidney injury was deemed prerenal after urine sodium returned less than $10 \mathrm{mmol} / \mathrm{L}$ and fractional excretion of sodium returned $0.4 \%$. The etiology of the anion gap metabolic acidosis was unclear. Venous blood gas demonstrated $\mathrm{pH} 7.32, \mathrm{CO} 234$, and bicarbonate 17. Lactate was normal at $1.44 \mathrm{mmol} / 1$ and urinalysis did not demonstrate ketones. Given her psychiatric history with alcohol abuse, an osmolar gap was calculated and found to be elevated at 17 concerning for ingestion. Volatile solvents revealed undetectable levels of ethylene glycol, methanol, propylene glycol, and isopropanol. Interestingly, her acetone was elevated at $7 \mathrm{mg} / \mathrm{dl}$. A serum beta-hydroxybutyrate was obtained and returned elevated at $3.3 \mathrm{mmol} / \mathrm{l}$. She was diagnosed with alcoholic ketoacidosis and treated with thiamine and dextrose-containing intravenous fluids. On discharge, labs revealed serum sodium $136 \mathrm{mmol} / \mathrm{L}$, bicarbonate $23 \mathrm{mmol} / \mathrm{L}$, and creatinine $1.1 \mathrm{mg} / \mathrm{dl}$.

DISCUSSION: Alcoholic ketoacidosis is a common disease encountered by hospitalists. It presents in the setting of chronic alcoholism with a recent episode of binge drinking. Often the patient is no longer actively drinking secondary to nausea and vomiting, leading to undetectable ethanol levels on presentation. Ketoacidosis develops 1 to 2 days after ethanol ingestion has ceased, creating an anion gap metabolic acidosis. Compared to diabetic ketoacidosis, alcoholic ketoacidosis is associated with a more significant conversion of acetoacetic acid to beta-hydroxybutyrate and acetone. As the urine nitroprusside test to detect ketone bodies (part of the urinalysis) detects acetoacetate, alcoholic ketoacidosis often leads to a negative urinalysis for ketones. As such, alcoholic ketoacidosis often requires a serum beta-hydroxybutyrate for diagnosis. Further, the excessive conversion of acetoacetic acid to acetone in alcoholic ketoacidosis is responsible for the osmolar gap, which is not seen in diabetic ketoacidosis.

A HEAD TURNING CASE OF MENINGOENCEPHALITIS Muznay Khawaja ${ }^{3}$; Syed Imran Jafri ${ }^{1}$; Faizan Malik ${ }^{1}$; Yub Raj Sedhai ${ }^{2}$; IRFAN AHSAN ${ }^{1}$; Srikanth Mukkera ${ }^{4}$; Roberto Carrichio ${ }^{4} .{ }^{1}$ Abington Jefferson Health, Horsham, PA; ${ }^{2}$ Mercy Catholic Medical Center, Yeadon, PA; ${ }^{3}$ Mercy Catholic Medical Centre, Secane, PA; ${ }^{4}$ Temple University, Philadelphia, PA. (Control ID \#2469811)

LEARNING OBJECTIVE \#1: Recognize that relapsing polychondritis can present with CNS complications like meningitis and/or encephalitis.

LEARNING OBJECTIVE \#2: Treat relapsing polychondritis complications with steroids and Azathioprine.

CASE: Relapsing Polychondritis (RP) is a systemic disease involving cartilaginous structures throughout the body. It often starts with cartilage involvement, but a multiorgan damage is possible.The prognosis is poor for patients displaying respiratory tract involvement and CNS involvement. Twenty-nine years old Hispanic male presented with persistent high grade fevers $>101 \mathrm{~F}$ and pulsatile headache with poor response to Acetaminophen and Ibuprofen. He had photophobia and altered mental status. On exam, he was noted to have neck stiffness. He was started on empiric broad spectrum antibiotics (vancomycin and ceftriaxone) and antivirals (acyclovir) for meningitis treatment. CSF was drawn 3 times with no evidence of infection. There was no response to antibiotics and he continued to have high grade fevers and headache. He then developed red eyes, double vision, nystagmus and bilateral sensorineural hearing loss. Patient was then seen by ENT, Ophthalmology and Rheumatology. Inflammation of conjunctiva, episclera and sclera was noticed on slit lamp exam. Physical examination revealed VI th nerve palsy with lateral rectus weakness, boggy and enlarged, cauliflower shaped ears, leading to suspicion for relapsing polychondritis. Relevant labwork demonstrated negative RPR, negative CSF for fungal culture, HSV 1/2 PCR, HIV 1/2 and Lyme Ab were all negative. Nevertheless patient had elevated ESR/CRP and WBC with neutrophilia. ANA, ANCA, ENA, RF, Cryoglobulinemia and Complement were negative. Cogan syndrome was in the differential, but patient fulfilled criteria for RP. MR brain findings were consistent with meningitis and associated cerebritis. This pattern of involvement was concerning for subacute form of Herpes, but not for bacterial meningitis. He was given Methylprednisolone 80 IV daily and was started on Dapsone therapy, but could not tolerate the latter due to nausea. Gradually, his symptoms started to improve. Fever subsided, double vision and hearing loss also improved gradually. Antibiotics and Acyclovir were stopped. He was discharged on prednisone $60 \mathrm{mg}$ daily and Azathioprine $150 \mathrm{mg}$ daily. Follow up MR brain showed treatment response for meningoencephalitis.

DISCUSSION: There are 28 cases reported in the literature with RP and meningoencephalitis. A minority of patients with RP develop neurological 
involvement ( $3 \%$ ). The reported mortality of RP-associated meningitis is $12 \%$. Diagnosis of meningoencephalitis secondary to RP is made clinically after exclusion of other etiologies as infection, paraneoplastic syndrome, etc. A careful physical exam and a high index of suspicion is essential to make a timely diagnosis, and prevent mortality and morbidity. There are no controlled studies for treatment options for RP with CNS complications. The few cases reported show that patients generally respond well with pulse steroids but in several cases, Cyclophosphamide was also effective. Other case reports mention treatment with TNF blockers, Abatacept and Rituximab with success. Our patient was diagnosed relatively early and was started on treatment. This case reiterates that combination treatment with steroids and Azathioprine is effective treatment for CNS complications of RP.

A HEART THAT KEEPS BREAKING Gaurav Rao; Jason Salamon; Mario Garcia. Montefiore Medical Center, Bronx, NY. (Control ID \#2469912)

LEARNING OBJECTIVE \#1: Identify the clinical features of Takotsubo Cardiomyopathy (TCM) including its variable presentations

LEARNING OBJECTIVE \#2: Recognize its ability to recur and the proposed mechanisms of pathophysiology

CASE: A 52-year-old woman presented to our hospital with dyspnea and chest pain, without a preceding physical or emotional stressor. She had a medical history significant for hypertension with multiple recent admissions for shortness of breath at various metropolitan hospitals. In the emergency department, she was hypoxic to $70 \%$, had distended neck veins and diffuse crackles on lung exam. Chest radiography was significant for bilateral pulmonary edema. An electrocardiogram showed ST depressions in V4V6 leads with a laboratory testing significant for a Troponin-T of $1.18 \mathrm{ng} / \mathrm{ml}$. Echocardiogram showed basal to mid-ventricle hypokinesis and apical hyperkinesis with a left ventricle ejection fraction (LVEF) of $25 \%$. Medical therapy was initiated with furosemide and nitroglycerin infusion for acute decompensated heart failure. Cardiac catheterization was performed the following day, which revealed no obstructive coronary anatomy and a preserved left ventricular function without any wall motion abnormalities. The patient's symptoms resolved and the diagnosis of Takotsubo Cardiomyopathy (TCM) was made. Of note, the patient had a similar presentation of acute heart failure 2 years prior with similar symptoms and vitals. An electrocardiogram showed T wave inversions in V5-6 leads. Troponin-T peaked at $15 \mathrm{ng} / \mathrm{ml}$. Echocardiogram showed apical akinesis and basal hyperkinesis with LVEF of $25 \%$. Cardiac catheterization the following day revealed nonobstructive coronaries and normal LVEF of $60 \%$. Similarly, her symptoms resolved and a diagnosis of TCM was made, but this time, it was the more typical apical ballooning subtype as opposed to her later presentation.

DISCUSSION: Takotsubo cardiomyopathy (TCM) is characterized by acute, reversible left ventricular dysfunction in the absence of explanatory coronary artery stenosis. The most common presenting symptoms are chest pain or dyspnea, often preceded by an emotional or physical stressor, although one is not always present. Classically, it was thought to involve the apex, and thus termed "apical ballooning syndrome," however midventricular manifestations have been increasingly reported in patients who have apical function preserved. Despite published favorable prognosis and infrequent recurrence with TCM, our patient continued to have recurrent events. The reoccurrence rate of TCM is approximately $11 \%$ over 5 years. In addition, she had a variability in her left ventricle ballooning on echocardiography from her first episode to her second episode was which was difficult to explain using current concepts of pathophysiology in TCM. This further perplexed the mechanism responsible. Several mechanisms have been proposed to explain the pathogenesis of TCM including catecholamine cardiotoxicity, microvascular disease and neurogenically stunned myocardium. Catecholamines can exhibit a direct toxic effect on cardiac myocytes through multiple mechanisms including cAMP-mediated calcium overload. In addition, high levels of epinephrine can switch $G$ protein signaling from stimulatory to inhibitory, causing a negative inotropic effect preferentially within the myocardium. This may be more pronounced at the apex where there is higher catecholamine sensitivity secondary to increased beta-adrenergic receptor density in order to compensate for less sympathetic innervation compared to the base. The alternations in adrenoreceptor sensitivity regionally, influenced by unknown external factors, may be the cause of variant morphologies during recurrent TCM presentations. Given these theories as well as studies suggesting cardiac medications such as beta-blockers may further induce cardiodepression in TCM, our team felt unsure about optimal management. In small observational studies, there has been no difference shown in the rate of TCM recurrence in patients on aspirin, angiotensin receptor inhibitor/blocker, beta-blocker, or statin. The preferred therapeutic medication regimen to prevent these events in patients with recurrent TCM remains unknown. However, medical professionals should recognize that TCM could in fact reoccur and keep it in the differential when a patient presents with suggestive symptoms.

A HOLE-Y CASE: SARCOIDOSIS COMPLICATED BY CHRONIC PULMONARY ASPERGILLOSIS (CPA) Arfa Faiz ${ }^{2}{ }^{1}$; Hammad Bhatti ${ }^{1}$; Sayed K. Ali ${ }^{1}$. ${ }^{1}$ Orlando VA Medical Center, Orlando, FL; ${ }^{2}$ University of Central Florida, College of Medicine, Orlando, FL. (Control ID \#2466998)

LEARNING OBJECTIVE \#1: 1. Recognizing and diagnosing chronic pulmonary aspergillosis; an insidious disease process.

LEARNING OBJECTIVE \#2: 2. Realizing that chronic pulmonary aspergillosis is found in patient with pre-exsisting pulmonary disorders and often confounds the burden of disease.

CASE: Chronic pulmonary aspergillosis (CPA), an insidious and difficult to diagnose entity, has been shown to complicate the burden of disease in patients with existing pulmonary disorders. Patients usually present with dyspnea, weight loss, and sometimes life threatening hemoptysis. Untreated CPA carries a high mortality rate, yet clinical guidelines until recently were unavailable. We present one such intricate case. A 51-year-old African American male with a past medical history of sarcoidosis, diagnosed 6 years ago, on chronic low dose prednisone, presented to our clinic with complaints of fatigues and intermittent, but progressive hemoptysis for 6 months. On presentation, he had stable vital signs with an oxygen saturation of $94 \%$ on room air. His lung exam revealed coarse breath sounds bilaterally. A chest x-ray showed bilateral upper lobe parenchymal opacities. A comparison of past and his most recent chest CT revealed, new development of extensive fibro-cavitary changes in areas of previous bilateral upper lobe mass-like consolidations. His WBC was reported at $3.7 \mathrm{~K} / \mathrm{cmm}$ and his hemoglobin measured $13.9 \mathrm{~g} / \mathrm{dL}$. His INR, PT/PTT, HIV antibody, quantiferon levels, ACE levels and platelet counts were all within normal range. An ESR was measured at $33 \mathrm{~mm} / \mathrm{h}$ and a Fungitell assay (detecting 1-3- $\beta$-d-glucan) was reported over $500 \mathrm{pg} / \mathrm{mL}$. A diagnostic bronchoscopy was unsuccessful in localizing the bleeding site. The BAL fluid analysis was negative for acid-fast bacilli, but eventually grew Aspergillus fumigatus. His low dose prednisone was continued and oral voriconazole therapy was initiated. The patient showed modest improvement after 6 months of therapy, however his hemoptysis, even though improved, continued intermittently. A repeat bronchoscopy, again failed to localize the site of bleeding. A bilateral bronchial artery angiogram with embolization of two right and three left intercostal arteries was pursued. Success was achieved in temporarily 
halting his hemoptysis. Surgical excision was considered, but deferred due to the presence of bilateral lung disease. He continued to slowly improve on long term antifungal therapy with close monitoring by our pulmonary and infectious disease departments

DISCUSSION: The presence of CPA complicates many pulmonary diseases including sarcoidosis. Untreated CPA carries a high mortality rate and thus timely recognition and treatment remains crucial. Long term steroid use is the mainstay for treatment of advanced sarcoidosis. However, immunosuppression puts many patients at risk for aspergillosis invasion and dissemination. Long term antifungals use has been shown to reduce the overall mortality associated with CPA. Bronchial arterial embolization, performed by an expert physician, is usually reserved for life threatening hemoptysis. Surgical options may be considered in patients with adequate lung function and severe hemoptysis. This case highlight the insidious, often protracted nature of CPA and the need for prompt diagnosis and treatment to improve overall mortality.

A LETHAL CASE OF CHOLANGITIS IN A BURMES IMMIGRANT TO ARIZONA Shruti Mony ${ }^{1}$; Mohit Pahuja ${ }^{2}$; Abdul Nadir ${ }^{3} .{ }^{1}$ St. Joseph's Hospital and Medical Center, Phoenix, AZ; ${ }^{2}$ St. Josephs Hospital and Medical Center, Phoenix, AZ; ${ }^{3}$ Maricopa Integrated Health System, Phoenix, AZ. (Control ID \#2469989)

LEARNING OBJECTIVE \#1: Recurrent pyogenic cholangitis is exclusively seen in patients who have lived in Southeast Asia but is increasing in the West due to immigration of people from endemic countries. It is a poorly understood syndrome characterized by the intra-hepatic biliary calculi along with strictures and obstruction of the biliary tree. This results in recurrent cholangitis and transient portal bacteremia. This introduction of bacteria into the portal tract initiates cycles of infection and secondary stone formation which lead to further obstruction and infection, resulting in chronic inflammation and scarring. Clinical presentation is typical of Charcot's triad with epigastric or right upper quadrant pain, fever and jaundice. Diagnosis is made only in $15-30 \%$ of the cases after initial presentation. It is important to diagnose recurrent pyogenic cholangitis early due to severe complications in delayed cases. Here in we report a case of pyogenic cholangitis in a young Burmese gentleman who migrated to USA 7 years earlier.

CASE: A 32-year-old male without any underlying co-morbid conditions presented with epigastric pain, nausea, vomiting, anorexia and generalized weakness for 2 days. $\mathrm{He}$ complained of recurrent spells of these symptoms in the past. He denied any recent travel history; however he immigrated from Burma to USA few years back. He smoked half pack/day for 10 years and consumed 3 beers/week for the last 10 years. On examination he was tachypneic and tachycardic with epigastric tenderness, but no peritoneal signs. His labs showed normal WBC count with $90 \%$ neutrophils and hypokalemia. His total bilirubin was elevated to $2.8 \mathrm{mg} / \mathrm{dl}$. His blood cultures 2 of 2 bottles gram negative bacteria which was pan sensitive. His CT abdomen with contrast showed an ill-defined $3.3 \times 2.5 \mathrm{~cm}$ region of abnormal enhancement at the confluence of right and left intrahepatic bile ducts. Both ERCP and MRCP showed multiple intrahepatic calculi with stricture in the common hepatic duct. A guidewire was passed through the common hepatic duct, but could not be passed selectively to the left intrahepatic duct because of a stone completely occluding it. After biliary sphincterotomy, a stent was placed deep in the right hepatic system; however, it did not drain adequately. He underwent left hepatic resection, following which he improved and was discharged home in good condition. Eight weeks later, during his follow up, his stent was removed. He was doing well, with no recurrence of symptoms since surgery.

DISCUSSION: A diagnosis of recurrent pyogenic cholangitis was made based on his demograophic background, clinical presentation and radiological findings. This condition was first described in Chinese population in 1930 and has been described to be associated with infections including Clinorchis, Ascaris,liver flukes like Fascicola and bacteria like
E.coli. The diagnosis of this condition is typically delayed and many clinical presentations including sepsis, fistula formation, cirrhosis, portal hypertension and cholangiocarcinoma have been described and high morbidity and mortality has been reported. This case highlights that this condition can be seen in USA among the immigrant population. A high index of suspicion among patients with typical symptoms should lead to prompt treatment including consideration of curative surgery as was accomplished in the described case.

A MASKED CASE OF VANCOMYCIN-INDUCED IMMUNE THROMBOCYTOPENIA Samuel A. Schueler; Nilima S. Shet; Nicholas F. Stienstra; Daniel Chen. Boston University School of Medicine, Boston, MA. (Control ID \#2468406)

LEARNING OBJECTIVE \#1: Recognize the accelerated course of thrombocytopenia in a patient who may have circulating drug-dependent platelet-reactive antibodies LEARNING OBJECTIVE \#2: Recognize that confirming a diagnosis of vancomycininduced immune thrombocytopenia by testing for vancomycin-dependent platelet-reactive antibodies may improve patient safety, particularly in a patient with multiple comorbidities that can independently cause thrombocytopenia and/or predispose to infection. CASE: Mr. C is a 55-year-old gentleman with chronic hepatitis $\mathrm{C}$, a previous diagnosis of primary idiopathic thrombocytopenic purpura (ITP), prior intravenous (IV) heroin use in remission, distant history of tricuspid valve endocarditis with subsequent tricuspid valve repair, chronic obstructive pulmonary disease (COPD), and gastroesophageal reflux disease (GERD), who presented to the emergency room with altered mental status, fever, and cough. The patient met systemic inflammatory response syndrome (SIRS) criteria with temperature of 102.3 and heart rate of 100 . Serum, urine, and cerebrospinal fluid (CSF) testing was performed, and the patient was given 1 dose each of vancomycin 1 gram (g) IV, cefepime $1 \mathrm{~g}$ IV, metronidazole 500 milligrams (mg) IV, and 21 of normal saline IV. Admission labs showed a normal white blood cell count and hemoglobin level, and a platelet count of 150,000 per microliter. Serum chemistry testing was unremarkable except for an elevated lactate level of 3.3 millimoles per liter, which normalized after IV normal saline was given. CSF tests for cryptococcal antigen, herpes simplex virus, and Lyme disease were negative, and the CSF cell count and chemistry were normal. Serum and urine toxicology screens were negative. Urine testing was unremarkable. On hospital day 2, the platelet count was 2000. Physical exam, which was largely unremarkable on admission, was now significant for diffuse lower extremity petechiae. Antibiotics were held. Serum tests for human immunodeficiency virus (HIV) antigen, Lyme antibodies, heparin induced platelet antibody, unfractionated heparin serotonin release assay, platelet antibody screening (anti-glycoprotein 1ib/1iia, anti-glycoprotein 1a/1ia, anti-glycoprotein 1b/1x, and anti-human-leukocyte-antibody class 1), and direct and indirect Coombs tests were negative. On hospital day 3 , the platelet count was 1000 , and remained $<4000$ through hospital day 5 despite platelet transfusions, prednisone $80 \mathrm{mg}$ IV given on hospital day 3, and intravenous immunoglobulin (IVIG) $30 \mathrm{~g}$ given on hospital day 4. The platelet count was 40,000 on hospital day $6,69,000$ on day 7 , and 150,000 on day 8 . The patient's serum tested strongly positive for vancomycin-dependent platelet-reactive Immunoglobulin $\mathrm{G}(\mathrm{IgG})^{1}$, confirming a diagnosis of vancomycin-induced immune thrombocytopenia. We later learned that our patient was given vancomycin during another hospitalization for pneumonia 3 months prior. During that admission his platelet count was initially 161,000 , and then steadily trended down to a nadir of 10008 days later despite platelet transfusions, prednisone, and intravenous immunoglobulin administration. We are unaware of any other previous exposure to vancomycin.

DISCUSSION: As shown by Von Drygalski et $\mathrm{al}^{1}$, the usual course of vancomycin-induced immune thrombocytopenia involves an average of a $93 \%$ drop in platelets with the average nadir platelet count on day 8 after vancomycin is first administered, with a return to greater than or equal to 150,000 platelets an average of 7.5 days after vancomycin is discontinued. 
However, in this same case series, 5 patients developed acute thrombocytopenia within $24 \mathrm{~h}$ after initiation of vancomycin. Two of the 5 patients had known previous exposures to vancomycin, and it was hypothesized that all 5 of the patients may have had circulating vancomycin-dependent antibodies. Kenney and Tormey described an additional case of a male patient who experienced a drop in platelet count from 635,000 to 3000 within $15 \mathrm{~h}$ after exposure to vancomycin, with serum studies subsequently showing vancomycin-induced platelet antibodies, though it was unclear if the patient had previous exposure to vancomy$\mathrm{cin}^{2}$ In our case, we were not initially aware of the patient's previous exposure to vancomycin. Further complicating matters were that our patient carried diagnoses of chronic hepatitis C, primary ITP, and also presented meeting SIRS criteria and with altered mental status concerning for infection. Given the life-threatening thrombocytopenia associated with vancomycin-induced immune thrombocytopenia and treatment implications, we find it important to: 1) Recognize the accelerated course of thrombocytopenia in a patient who may have circulating drug-dependent platelet-reactive antibodies; and 2) Recognize that confirming a diagnosis of vancomycin-induced immune thrombocytopenia by testing for vancomycin-dependent platelet-reactive antibodies may improve patient safety, particularly in a patient with multiple comorbidities that can independently cause thrombocytopenia and or predispose to infection.

\section{A MASQUERADING PRESENTATION OF ACUTE FIBRINOUS PERICARDI-} TIS Mohit Pahuja ${ }^{2}$; Priya Radhakrishnan ${ }^{1}$; Youngsoo Cho ${ }^{3} .{ }^{1}$ St Joseph, Phoenix, AZ; ${ }^{2}$ St. Josephs Hospital and Medical Center, Phoenix, AZ; ${ }^{3}$ St. Joseph's Hospital and Medical Center, Phoenix, AZ. (Control ID \#2470021)

LEARNING OBJECTIVE \#1: Acute Fibrinous pericarditis is an inflammatory process of the visceral pericardium with the development of the fibrinous exudates. Multimodality imaging is emerging as a cornerstone in the management of pericardial disease. Echocardiogram is often the initial test performed, but CT/MRI can play a robust role in diagnosis. CASE: A 65 year old female with history of pulmonary embolism on warfarin and with an inferior vena cava (IVC) filter came with worsening shortness of breath, chest pain and cough 3 days prior to admission. She was admitted with similar symptoms 2 weeks back and was diagnosed with an acute pericarditis with an echocardiogram revealing mild pericardial effusion. She was started on aspirin and colchicine. On this admission her physical exam showed muffled heart sounds with decreased breath sounds bilaterally. Her EKG showed sinus tachycardia. Laboratory findings for HIV serology, tuberculin skin test, anti-nuclear antibody and other test were negative. A repeat echocardiogram was performed which was consistent with worsening pericardial effusion. She underwent a pericardiocentesis but it was unsuccessful with only minimal fluid removed. To further delineate the pericardial effusion, a transesophageal echocardiogram (TEE) was done which was consistent with loculated pleural effusion with multiple fibrinous strands. To confirm, a CT chest with contrast was done which in addition to massive pericardial effusion also showed a hyper dense mass in the right ventricle (RV). These findings along with her worsening condition led us to perform a sternotomy with pericardial stripping that to our surprise revealed multiple gelatinous masses within the pericardium. Biopsy of the mass confirmed an acute fibrinous pericarditis. For further evaluation, an EKG gated CT chest was performed which was consistent with the previous hyper dense mass in the right ventricle with concern for a foreign body. Considering her history of IVC filter, CT abdomen with contrast was done to look for a broken IVC filter. Abdominal CT revealed prongs of IVC extending outside the vena cava and the right ventricle hyper density consistent with fractured piece of IVC prong. Upon follow-up in clinic, her echocardiogram still showed minimal fluid. She was started on prednisone and within 3 weeks there was no fluid found on repeat echocardiogram.

DISCUSSION: Acute pericarditis is usually based on history of pleuritic chest pain, and is confirmed if pericardial friction rub is present. Multimodality imaging is an integral part in the modern management of pericardial disease. Echocardiogram remains the initial test of choice for diagnosis but CT scan and MRI are performed in cases where an echocardiogram is unrevealing with a high suspicion of the disease. EKG gated CT scan are done to minimize the cardiac motion artifact to better visualize the underlying pathology. Common complications secondary to IVC filter includes IVC filter thrombosis or stenosis. Other less common complications include filter migration, filter erosion through the filter wall, filter misplacement and rarely filter fracture and embolization. Migration of the broken strut of the filters to the heart is a very rare complication. Our case demonstrates a unique presentation of an acute fibrinous pericarditis due to a broken IVC filter. Presentation of a gelatinous mass within the pericardium is unknown. In our review of literature, we found only one case report presenting as a gelatinous pericardial mass secondary to pericardial synovial sarcoma. This is the first case report of a gelatinous pericardial mass mimicking a pericardial effusion secondary to acute fibrinous pericarditis. Both TEE and EKG-gated CT scan was the key to diagnosis in our case and further validates the power of advanced imaging in the evaluation of pericardial disease. It demonstrates the significance of utilizing multiple modalities of cardiovascular imaging in cases with an unknown cause of pericardial effusion.

A MISSED DIAGNOSIS OF AORTIC DISSECTION DUE TO ATYPICAL PRESENTATION AND FALSE NEGATIVE CT ANGIOGRAM XIAOMEI MENG. Ohio state university medical center, Dublin, OH. (Control ID \#2469775)

LEARNING OBJECTIVE \#1: CT aorta protocol is not acurrate to role out pulmonary emboliam

LEARNING OBJECTIVE \#2: family history is very important to get during routine patient's encounter.

CASE: A 47 year-old African-American woman presented to Emergency Department (ED) with sudden onset 'indigestion-like' abdominal discomfort, accompanied by severe nausea, vomiting, diarrhea and one episode of syncopal attack. In the ED her vitals were stable. After an episode of vomiting and large volume diarrhea, she developed a second syncopal attack with profound hypotension and hypoxia which resolved after fluid resuscitation. Chest CT angiogram (CTA) with pulmonary artery (PA) protocol was performed due to D-dimer elevation and hypoxia, which showed no evidence of pulmonary embolism (PE) or aortic dissection, but did reveal moderately complex pericardial effusion (fluid had a high CT attenuation). To further evaluate the pericardial effusion, transthoracic echocardiogram was performed which demonstrated small effusion without evidence of tamponade. Patient was managed conservatively and discharged 3 days later. Three days after discharge, patient presented to ED with similar complains of severe nausea, vomiting and abdominal pain along with labile blood pressure. Patient then developed pulseless electrical activity and expired after $50 \mathrm{~min}$ of resuscitation. Pericardiocentesis was performed during resuscitation but failed. Autopsy demonstrated dilated aortic root, with aortic dissection starting from $2 \mathrm{~cm}$ above sinus of Valsalva and extending up to $4 \mathrm{~cm}$ above origin of renal arteries. Additionally, pericardial sac contained $500 \mathrm{ml}$ blood and blood clots, and cause of death was assigned to cardiac tamponade. Further questioning of patient's family revealed that four related family members died due to aortic dissection.

DISCUSSION: Aortic dissection clinically manifests as 'tearing' chest pain or abdominal pain which is seen in $90 \%$ of patients. Our case had atypical presentation with mostly gastroenterological symptoms, possibly due to the dissection interfering with aortic baroreceptors axons that travel within the vagus nerve. Patient likely had some degree of cardiac tamponade secondary to aortic dissection during first ED presentation which would have explained the hypotension and syncopal attacks. The diagnosis was missed due to a combination of atypical presentation, inadequate history, and a false negative CTA. It needs to be noted that CTA protocol required for diagnosis of PE differs from that 
required to optimally visualize aortic dissection because time-to-peak enhancement differs for different target vessels (PA-coronary-aorta), and hence triggering mark of starting scan will be set at different locations. In our case the commonly used CTA with PA protocol was ordered. If clinicians suspect both PE and aortic dissection, a proper CTA protocol for double rule out (timing bolus or modified bolus tracking) needs to be discussed with radiologist. Although MRA or transesophageal echocardiogram might be better options, they require additional time and technical expertise which limit their use in ED.

A MISTAKEN CASE OF GLIOMA Nitya Ramreddy ${ }^{1}$; Aviva C. Hopkins ${ }^{2}$; Carlos J. Lozada ${ }^{3}$. ${ }^{1}$ University of Miami Miller School of Medicine/Holy Cross Hospital, Fort Lauderdale, FL; ${ }^{2}$ University of Miami/Holy Cross Hospital, Fort Lauderdale, FL; ${ }^{3}$ University of Miami Miller School of Medicine, Miami, FL. (Control ID \#2469495)

LEARNING OBJECTIVE \#1: Systemic lupus erythematosus (SLE) is a chronic autoimmune inflammatory disease with a relapsing and remitting course, often affecting women of child-bearing age. Central nervous (CNS) involvement can be the presenting clinical feature in SLE. We discuss the importance of recognizing lupus cerebritis.

CASE: A 45 year old female with no significant past medical history was brought by EMS to the ER after being seen "shaking" uncontrollably. She subsequently had two witnessed generalized seizures in the ER. Additional history from the family noted lowgrade fever, poor appetite, and mild headache. No changes in vision, confusion, weakness, slurred speech, or any other prodromal features reported. A few days prior, the patient had visited an urgent care facility and where elevated blood pressure, "abnormal liver tests" and proteinuria were recorded. The patient was not on any prescription medications but was taking a fish oil supplement. She denied any history of smoking, illicit drug or alcohol use. ROS was significant for some vaguely described arthralgias over the past few months She was afebrile and vital signs were stable. On examination, she was lethargic, responded to vigorous stimuli but could not answer any questions or cooperate with the exam. There was no neck stiffness and she was able to passively flex her knees without discomfort. The rest of the examination was unremarkable. Keppra was started for management of the seizures. Initial blood work showed $\mathrm{Hb}$ of $8.8 \mathrm{~g} / \mathrm{dL}$, Wbc- $5.9 \mathrm{~K} / \mathrm{mm}^{3}$ platelets $81 \mathrm{~K} / \mathrm{mm}^{3}$, AST 954 U/L, ALT 394 U/L, ALP 127 U/L, Creatinine 1.94 mg/dL, BUN 39 mg/dL, UA showed $3+$ protein and hyaline casts. Toxicology screen and hepatitis panel were negative Non-contrast CT brain was unremarkable; MRI of the brain with and without contrast demonstrated an anterior left frontal lesion within the superior frontal gyrus approximately $2 \times 2.5 \mathrm{~cm}$. Although initially read as a hemorrhagic stroke, the opinion of neurosurgery, neurology, and the tumor board, was that the lesion was more concerning for low-grade glioma. The patient underwent left frontal craniotomy and then resection after the frozen pathology was read as gliosis consistent with a low-grade glioma. However, because during the surgery the neurosurgeon noted abnormal appearing vessels, which raised the possibility of vasculitis, he started IV steroids and consulted rheumatology postoperatively. Rheumatology initiated pulse dose steroids $1 \mathrm{~g}$ IV daily for 3 days followed by solumedrol $1 \mathrm{mg} / \mathrm{kg} /$ day. Pathology reviewed by a neuropathologist at Emory University demonstrated findings consistent with CNS vasculitis and associated reactive gliosis and hypoxic ischemic changes secondary to the patient's autoimmune disease. With the diagnosis of CNS vasculitis, IV cyclophosphamide (CYC) $500 \mathrm{mg} / \mathrm{m}^{2}$ was added. Serologies ultimately revealed positive ANA 1:1280 titer (homogenous pattern), antidouble stranded DNA andanti-Smith Ab's, low complement levels (C3 and C4), anticardiolipin Ab's. CNS vasculitis secondary to SLE was diagnosed. Despite the pulse steroids and IV CYC, the hospital course was complicated by worsening renal function related to presumed nephritis requiring dialysis, pancreatitis, and a chylous pleural effusion bilaterally. With manifestations including seizures, hepatitis, renal failure, thrombocytopenia, hemolytic anemia, pericardial and pleural effusions, and pancreatitis, it was concluded that the patient had aggressive systemic lupus erythematosus with multi-system involvement, with initial presenting symptom of neuropsychiatric SLE. Over 2 weeks, patient improved neurologically, was slowly able to tolerate oral intake, and renal function improved. The patient was transferred to inpatient rehabilitation.

DISCUSSION: Considering the wide gamut of manifestations of neuropsychiatric lupus (NPSLE), presenting features of lupus cerebritis can be misleading and diagnostically challenging. The range of symptoms varies greatly, from non-specific features like depression, anxiety, headaches, and seizures, to severe symptoms such as major seizures, visual problems, dizziness, behavior changes, stroke, or psychosis that can occur in about $15 \%$ of the cases. Seizures, generalized or focal, tend to occur early in SLE, especially in those with African ethnicity. Assessment of clinical features and neurological signs, along with detection of antibodies in serum and cerebrospinal fluid are helpful in making a diagnosis. CT scans and MRI imaging can provide additional information, but are often non- diagnostic. In our patient, initial consideration of low grade glioma as the cause of seizures, based on a lesion seen on MRI, led to a frontal craniotomy with resection. This ultimately provided the histopathology to make a diagnosis of CNS vasculitis. Generally, only a minority of NPSLE patients have evidence of frank vasculitis on imaging or histopathology; however a small vessel thrombotic-vasculopathy has been the predominant histopathological abnormality in brains of NPSLE patients at autopsy. Lupus cerebritis should be included in the differential diagnosis of a young female patient who presents with complicated neurological manifestations and with no clear-cut clinical, pathological, or imaging test findings.

A MYSTERIOUS PLEURAL EFFUSION Dianne L. Goede ${ }^{1}$; Margaret C. Lo $^{2}$; Mohammad Abu-Farsakh ${ }^{1} .{ }^{1}$ University of Florida, Gainesville, FL; ${ }^{2}$ University of Florida College of Medicine, Gainesville, FL. (Control ID \#2469478)

LEARNING OBJECTIVE \#1: Recognize the clinical features and risk factors for primary amyloidosis in association with plasma cell dyscrasias

LEARNING OBJECTIVE \#2: Know the atypical causes of recurrent transudative pleural effusions

CASE: A 81 years-old female presents for worsening fatigue, dyspnea and syncope. This has resulted in 4 recent admissions with discharge diagnoses of orthostasis, moderate aortic stenosis and bilateral transudative pleural effusions secondary to heart failure Despite diuretics and thoracentesis, her symptoms persisted. Medical history was significant for monoclonal gammopathy of undetermined significance (MGUS), Sjogren's syndrome, moderate aortic stenosis, chronic kidney disease, and paroxysmal supraventricular tachycardia. Exam revealed mild respiratory distress with tachypnea, decreased breath sounds with dullness to percussion in left posterior lung, crescendo-decrescendo systolic murmur at right upper sternal border and $2+$ pitting edema of the bilateral lower legs. Admission labs showed: baseline creatinine $(1.32 \mathrm{mg} / \mathrm{dL})$, total protein of $6.2 \mathrm{~g} / \mathrm{dl}$, low albumin of $2.5 \mathrm{~g} / \mathrm{dl}, 30 \mathrm{mg} / \mathrm{dl}$ protein on urinalysis. Additionally, AST/ALT, bilirubin, INR, and TFTs were normal. CXR exhibited a loculated left pleural effusion and persistent right pleural effusion. Fluid analysis from repeat thoracentesis confirmed a transudative effusion; cytology was negative for malignancy. Twenty-four-hour urine collection revealed non nephrotic range proteinuria. EKG showed sinus rhythm with an old left bundle branch block. Echocardiogram (TTE) revealed moderate aortic stenosis, mild aortic regurgitation, borderline left ventricular systolic function (EF 50-55\%), and mildly elevated right ventricular pressure $(50-55 \mathrm{mmHg})$. Yet PCWP on right heart catheterization (RHC) was only $14 \mathrm{mmHg}$. Given the negative workup for heart failure and malignancy, this case was re-evaluated with Cardiology and Hematology consult. A diagnosis of amyloidosis was considered given her multi-organ dysfunction and MGUS history. An abdominal fat pad biopsy with Congo red staining ultimately confirmed the diagnosis of amyloidosis. Diuretics were stopped and patient was discharged with plans for outpatient pleurodesis. 
DISCUSSION: This case illustrates the propensity for clinicians to fall into known diagnostic errors of anchoring and pattern recognition with such common conditions as pleural effusions. Often transudative pleural effusions are attributed to typical etiologies of heart failure, hepatic hydrothorax, or nephrotic syndrome. Yet none of these causes fit the clinical context of our patient. When confronted by such diagnostic quandary, clinicians must perform diagnostic timeouts to reflect on the evolving case and avoid diagnostic heuristics. Thus, atypical causes of transudative effusions had to be considered in this case including sarcoidosis, trapped lung, malignancy, hypothyroidism and amyloidosis. Diagnostic clues for atypical causes include multi-organ involvement and persistent symptoms despite proper conventional therapy. The TTE and RHC remain important tools to disprove heart failure as the cause of transudative effusion in our patient. Other diagnostic consideration must include risk factors, notably our patient's history of MGUS and the association of amyloidosis with plasma cell dyscrasias. Literature cites the development of MGUS into AL amyloidosis, multiple myeloma, or Waldenstrom macroglobulinemia at $\sim 1 \%$ per year. AL amyloidosis is rare, mostly affecting adults $>65$ years-old. Clinical features reflect the organs affected but can be nonspecific (e.g. fatigue, weakness, anorexia) and involve multi-organ dysfunction i.e. cardiomyopathy, renal failure, macroglossia, peripheral and autonomic neuropathy, purpura and hepatomegaly. Our patient suffered from cardiac conduction abnormalities, autonomic neuropathy, and renal and pulmonary involvement. Pulmonary manifestations of amyloidosis include tracheobronchitis, pleural effusions, parenchymal nodules and pulmonary hypertension. Persistent pleural effusions develop in 1-2\% of cases and are typically exudative due to pleural infiltration with amyloid deposits. Yet, our patient's transudative effusions should not fool clinicians. Up to $64 \%$ of pleural effusions can be transudative per a retrospective case analysis of AL amyloidosis with refractory pleural effusions. Persistent pleural effusions signify poor prognosis and limited treatment response, although pleurodesis has been useful in some cases. Knowledge of amyloidosis through case reports like ours is essential to primary care clinicians who commonly manage patients with ongoing dyspnea from pleural effusions. Of particular importance is the recognition of certain diagnostic clues (i.e. multi-organ dysfunction), assessment of associated risk factors (i.e. MGUS) and timely tissue histopathology for the proper diagnosis of amyloidosis as an atypical cause of recurrent transudative pleural effusions

A NEUROLOGICAL CONUNDRUM SOLVED BY A RADIOLOGICAL DIAGNOSIS Paige Castelino; Jeffrey Genda; Sahi Rajinder; Pramil Cheriyath. Pinnacle Health, Harrisburg, PA. (Control ID \#2470220)

LEARNING OBJECTIVE \#1: To understand the various toxicities of Metronidazole. LEARNING OBJECTIVE \#2: Treatment for an intracranial abscess includes a prolonged course of antimicrobial therapy and close follow-up. Here we describe a rare medication complication that posed as a diagnostic challenge.

CASE: A 22-year-old healthy male presented with new-onset seizures. MRI showed $<2 \mathrm{~cm}$ cystic lesion in the olfactory cortex, along with concurrent sinusitis. Cultures revealed gram-positive cocci. Treatment included ethmoidectomy and maxillary sinusotomy and a prolonged medication regimen of levetiracetam $500 \mathrm{mg}$ PO BID, ceftriaxone $2 \mathrm{mg}$ IV BID, metronidazole PO $750 \mathrm{mg}$ TID, and linezolid $50 \mathrm{mg}$ PO BID. Repeat MRI showed stable lesion with no surrounding edema. At follow up visits, the patient complained of decreased hearing, gait instability and peripheral neuropathy. His peripheral neuropathy was attributed to Linezolid and it was switched to Vancomycin. MRI 7 weeks into treatment exhibited a stable lesion. However, despite broad-spectrum antibiotic therapy for 11 weeks, his condition deteriorated and he was admitted for acute cerebritis. On admission patient's vitals were stable and neurological exam was benign. MRI showed stable lesion unchanged from prior. There was also bilateral and symmetric T2-enhancement of the midbrain tectum, dorsal aspect of the pons, dentate nuclei, putamen, as well as posterior and anterolateral aspects of the medulla. Non-infectious pathology including white matter disease was considered, but symmetrical lesions pointed against the diagnosis. Radiologist reported findings likely represented metronidazole toxicity.

DISCUSSION: Metronidazole-induced encephalopathy (MIEP) is a rare toxic encephalopathy with characteristic radiographic findings of bilateral dentate nuclei T2enhancement. In 2011 a systematic review re-counted sixty-four cases of MIEP between the years 1965-2011. Only five of the sixty-four cases were being treated for an intracranial infection and radiological findings associated with MIEP were noted as early as 20 days. This is an interesting case because it was a challenge to decipher between nonspecific symptoms that could be attributed to intracranial infectious pathology versus drug-induced neurotoxicity. Moreover, the patient's onset of symptoms of gait instability and decreased hearing presented as early as 3 weeks into treatment, but MIEP MRI findings were only noted 11 weeks later. Based on our knowledge, this is the first case to demonstrate the MIEP symptomatology prior to the validation from radiological findings.

A NEW "HIGHWAY TO HEALTH:" INTEGRATED CARE MANAGEMENT OF A PATIENT WITH ILLNESS ANXIETY DISORDER AND FREQUENT EMERGENCY DEPARTMENT UTILIZATION Daniel J. Coletti; Leslie Rosenberg; Darleen Rourke; Jennifer Verbsky; Joseph Conigliaro. Northwell Health, Great Neck, NY. (Control ID \#2464065)

LEARNING OBJECTIVE \#1: To structure effective interdisciplinary care management of patients with non-medical reasons for high levels of emergency department utilization. LEARNING OBJECTIVE \#2: To identify ways psychopharmacological treatment, psychological intervention, and care management can work together to reduce symptoms that influence ED utilization and prevent relapse.

CASE: "Mary" (name changed) is a 52 year old Latina woman who presented to establish care in our hospital-affiliated resident practice in March of 2012. She had recently been hospitalized for evaluation of chest pain of unknown origin. At initial presentation Mary had no emergent complaints but described a 2 year pattern of lower extremity weakness and discomfort, bilateral eye pain, dizziness, intermittent shortness of breath (SOB), generalized fatigue and anxiety. Recent neurological and rheumatologic workups were negative; exercise stress test only showed poor exercise capacity for age and gender, and CXR, EKG and cardiac enzymes were normal during the hospitalization. Mary subsequently returned for frequent follow-up appointments after visits to the hospital's ED for acute treatment of SOB and chest pain. At outpatient visits she typically presented in no acute distress but would describe recent onset of SOB, chest tightness, and dyspnea on exertion (DOE). She had obtained independent cardiac consultation and was placed on indomethacin with no change in symptom profile. A thyroid/parathyroid ultrasound in the Fall of 2012 showed a $1 \mathrm{~cm}$ ill-defined thyroid nodule, and it was noted that Mary's mother was in treatment for cancer with thyroid involvement. Mary's ED visit frequency accelerated, with additional symptoms of tightness in her throat, difficulty swallowing and a perceived lump in her throat. Thyroid function tests and cardiac workups continued to be normal. Repeated ED visits and subsequent outpatient follow-up assessments identified no medical reason for Mary's symptoms. Given the increasingly apparent role illness-related anxiety was playing in her pattern of ED utilization, Mary was placed on citalopram $20 \mathrm{mg}$ daily in April of 2013 which was titrated to $40 \mathrm{mg}$. She was also seen by the practice's health psychologist for cognitive behavioral anxiety management and behavior planning around ED utilization. During this treatment Mary acknowledged (a) the presence of sudden, severe attacks of distress resulting from somatic sensations in multiple parts of her body, (b) that she had recently experienced the loss of a family member to a sudden MI, and (c) that particularly when alone she felt an uncontrollable impulse to pursue 
emergency evaluation for reassurance. In fact, the ED facility itself served as a discriminative cue for the behavior, as Mary described that while passing the exit sign on the highway for the medical center she would reverse course and seek evaluation "just to make sure" she was in adequate health. Combination SSRI+ psychological intervention ameliorated subjective anxiety and reduced the frequency of ED visits from 16 in the 12 months prior to beginning treatment to 4 visits in the subsequent 4 months, and then no visits after March of 2014. Then in June of 2015 Mary decided to discontinue the SSRI unilaterally. A resumption in ED utilization was subsequently observed within 4 weeks of discontinuation, and in September a new care management program identified this change in behavior pattern. An interdisciplinary meeting was held with Mary and her husband, in which both medication and psychological treatments were re-instated and in which ongoing care management was put in place. Family-based interventions and graphic representations of her behavior pattern have supplemented existing treatments, and ED visit frequency has been reduced to a single visit since the new plans were put into place 90 days ago.

DISCUSSION: The case describes the common primary care presentation of illnessrelated anxiety associated with excessive use of acute emergency services. Mary's symptom presentation was consistent with the DSM-5 diagnosis of Illness Anxiety Disorder, and the treatments deployed were broadly consistent with the Coordinated Anxiety Learning and Management (CALM) model of integrated anxiety disorder management (Roy-Byrne et al., JAMA, 2010; Joesch et al., Psych Medicine, 2011) In this model SSRI treatment and/or cognitive behavioral interventions are used according to patient preference and care managers coordinate treatment and track response over time. Mary's course was noteworthy for the potent impact of combination SSRI and psychological intervention on the patient's anxiety level as well as her subsequent use of healthcare resources. The patient's course was also a clear "AB-A-B" demonstration of treatment effectiveness- in which a treatment was effective when given, withdrawn (by the patient) with relapse in symptoms, and then reinstated to positive effect. Typical anxiety reduction training provided in psycholog ical treatment was supplemented by family-based intervention and functional analysis to help Mary and her husband identify and address contingencies associated with her feelings and behavioral responses. Finally, the patient's treatment clearly benefitted from integrated medical and behavioral health care management, in which high resource utilization triggered outreach to the patient, the resumption of treatment, and implementation of the current follow-up protocol to monitor Mary's adherence to treatment and prevent relapse.

A NOT SO MINIMAL CHANGE DISEASE Daniel H. Kil; John Allison; Michelle Udayamurthy. Baylor College of Medicine, Houston, TX. (Control ID \#2464664)

LEARNING OBJECTIVE \#1: Distinguish nephrotic syndrome in an otherwise healthy adult patient as a red flag for possible malignancy

LEARNING OBJECTIVE \#2: Recognize that thymomas are known to cause minimal change disease as a paraneoplastic syndrome.

CASE: A 63 year old African-American male with a medical history of well-controlled hypertension and hyperlipidemia presented with a 4 day history of new-onset lower extremity and abdominal swelling. He denied shortness of breath, chest pain, orthopnea, and constitutional symptoms, but did report occasional non-drenching night sweats. $\mathrm{He}$ reported being otherwise healthy with no known history of baseline heart, liver or kidney disease, and sought evaluation at the suggestion of his spouse who noticed the swelling. Vital signs were within normal limits. Physical exam was significant for abdominal distension with mild ascites and shifting dullness, 3+ pitting edema in the lower extremities to the level of the knees, decreased breath sounds in the left anterior lung fields, and mild jugular venous distention. There was no lymphadenopathy or testicular masses and neurologic examination was within normal limits. Laboratory values were indicative of renal failure with nephrotic range proteinuria. A 13x9x10 $\mathrm{cm}$ anterior mediastinal mass was found incidentally on chest $\mathrm{x}$-ray. Subsequent $\mathrm{CT}$ and MRI detailed extension of the mass from the left brachiocephalic vein to the left ventricle, abutting the aorta. Fine needle aspiration confirmed a large thymoma. Renal biopsy was consistent with minimal change disease likely secondary to the malignancy. Despite aggressive diuresis and high dose steroids, renal function continued to decline leading to initiation of hemodialysis. The hospital course was further complicated by recurrent left sided pleural effusions, twice requiring thoracentesis and chest tube placement. He ultimately underwent an uncomplicated median sternotomy and total thymectomy, however, his renal function failed to improve despite tumor resection and he continued to require outpatient dialysis.

DISCUSSION: This case depicts a rare presentation of thymoma-associated paraneoplastic glomerulonephritis, specifically manifesting as minimal change disease (MCD). Although an uncommon occurrence, MCD is the most frequent thymomaassociated glomerular lesion and its pathogenesis is linked to an altered immune response of T-cells. MCD associated with lymphocyte-predominant thymoma typically responds well to steroids, however, this patient did not respond. It is important to note that even with thymectomy, patients can continue to exhibit nephrotic syndrome with progressive renal decline, an association that is related to persistent T-cell dysfunction after tumor removal. Additional studies have shown that thymomas are chemosensitive diseases, therefore this case highlights the need for further investigation of chemotherapy in the management of paraneoplastic glomerulonephritis. Of importance, an emphasis on early detection of nephrotic syndrome, initiation of appropriate therapy, and pursuit of a multidisciplinary approach may prevent the progression to end-stage kidney disease.

A NOT SO RESTFUL NIGHT: AN UNUSUAL SIDE EFFECT OF A COMMONLY USED DRUG Ashley Husebye; Rachel Sandler. Hennepin County Medical Center, Minneapolis, MN. (Control ID \#2457368)

LEARNING OBJECTIVE \#1: Posterior shoulder dislocations are rare $(2-4 \%$ of shoulder dislocations) and $78 \%$ of bilateral shoulder dislocations are attributed to seizures. LEARNING OBJECTIVE \#2: Doxylamine toxicity is associated with seizures and rhabdomyolysis and can occur at any doxylamine plasma level.

CASE: A 63 year old man with well-controlled hypertension was admitted to the hospital after his neighbor found him slumped over in the hallway of their apartment building. He had awoken with intense bilateral shoulder pain. His physical exam revealed encephalopathy and tenderness to palpation to the bilateral shoulders with inability to passively abduct the bilateral upper extremities. He was also found to have leukocytosis and an elevated lactate level. Computer tomography of the head was without abnormalities. Shoulder x-rays showed a comminuted fracture of the right humeral head, including a mildly displaced fracture of the greater tuberosity and a comminuted displaced fracture of the left humeral head involving the lesser tuberosity. He was found to have bilateral posterior shoulder dislocations, which were reduced in the emergency department. The patient's encephalopathy resolved after a few hours and was consistent with a post-ictal state. Upon further questioning, he recalled waking in the middle of the night with excruciating bilateral shoulder pain and then going into the apartment hallway to seek help. He denied any prior history of seizures, family history of seizures, recent trauma, illicit drug use, or alcohol use. His only new medication was doxylamine, which he had taken the previous night for insomnia. He had taken this medication once previously and woke up the next day with muscle cramps.

DISCUSSION: Posterior shoulder dislocations are rare and are responsible for less than $5 \%$ of all shoulder dislocations. The most common causes of posterior shoulder dislocations are seizure, trauma, and electrocution. Seizures are the cause of $78 \%$ of bilateral 
posterior shoulder dislocations that have been reported. Doxylamine (Unisom) is a first generation $\mathrm{H} 1$ antagonist and a common medication used for insomnia. Doxylamine toxicity is associated with seizures and rhabdomyolysis. Previous studies suggest there is no correlation between the toxic effects of doxylamine and the plasma drug level. While the patient only took one tablet of doxylamine, his history suggests that he is sensitive to this medication. The first time he took doxylamine, he woke up with muscle cramps concerning for rhabdomyolysis or a possible seizure. The second time he took the medication, his combination of findings was highly suggestive of seizures. He was not taking any other medications that have known interactions with doxylamine. The patient's history and presentation is most suggestive of a doxylamine-induced seizure, which caused bilateral posterior shoulder dislocations and subsequent bilateral humeral fractures.

A NUANCE IN THE CONTAMINANT CONDUNDRUM Cory J. Rohlfsen; Christopher J. Smith. University of Nebraska Medical Center, Omaha, NE. (Control ID \#2433664)

LEARNING OBJECTIVE \#1: Discuss the microbiology and clinical relevance of Staphylococcus lugdunensis.

LEARNING OBJECTIVE \#2: Differentiate the management of Staphylococcus lugdunensis bacteremia from that of other coagulase negative staphylococci species.

CASE: A 74 year-old man presented with 2 weeks of worsening fatigue and dyspnea on exertion. He reported an 8 month history of melena with recently performed "upper and lower" scopes, both of which were negative for a bleeding source. His past medical history was significant for end-stage alcoholic cirrhosis with grade I esophageal varices, coronary artery disease with a drug-eluting stent placed 8 months prior to presentation, and sick sinus syndrome requiring a pacemaker. He had normal vital signs, icteric sclera, abdominal distension with a fluid wave, and $2+$ non-pitting lower extremity edema. His mental status was at baseline and there was no asterixis. Labs revealed a white blood cell count of 11,000 cells $/ \mathrm{cm} 3$, hemoglobin of $8 \mathrm{~g} / \mathrm{dL}$ (near baseline), and creatinine of $2.8 \mathrm{mg} / \mathrm{dL}$ (baseline $2.0 \mathrm{mg} / \mathrm{dL}$ ). A diagnostic paracentesis was negative for spontaneous bacterial peritonitis. Chest $\mathrm{x}$-ray revealed bibasilar atelectasis without any sign of hepatohydrothorax. Interrogation of the pacemaker demonstrated adequate function. Blood cultures turned positive for coagulase negative staphylococci (CoNS) within 6 hours of being drawn. Vancomycin was started after the second set became positive. The following day, the organism was identified as Staphylococcus lugdunensis. An echocardiogram revealed a new $1 \mathrm{~cm}$ vegetative lesion on the tricuspid valve which was adjacent to a pacemaker wire. An interventional pacemaker exchange was considered, however the risk-to-benefit ratio was felt to be unacceptably high given the patient's co-morbid conditions. Despite intravenous antibiotics, the patient continued to decline and eventually elected to pursue hospice care.

DISCUSSION: Interpretation of blood culture results is a skill required of all internists. Because CoNS is a normal constituent of skin flora, it commonly contaminates microbiologic samples and complicates result interpretation. In general, one of two blood cultures positive for CoNS is regarded as contaminant and should not be treated. Growing evidence suggests $\mathrm{S}$. lugdunensis is an exception to this widely accepted rule. S. lugdunensis is a catalase positive, facultative anaeroabe that lacks the MecA gene found in S. aureus. Although it only accounts for $\sim 3 \%$ of skin flora and is generally susceptible to a wide range of antibiotics, its virulence is much higher than that of other CoNS with mortality that approaches that of S. aureus. As this case demonstrates, S. lugdunensis has a well-documented proclivity for implantable devices, such as pacemakers, with tendency to form biofilms. As such, echocardiogram is recommended to evaluate for endocarditis in patients with S. lugdunensis bacteremia. Given S. lugdunensis endocarditis is cited to have an $18 \%$ risk of mortality, failing to recognize valvular vegetations could result in significant patient harm
A NUTTY CAUSE OF CHRONIC COUGH Juan N. Lessing ${ }^{1}$; Nicholas M. Mark ${ }^{2}$. ${ }^{1}$ University of Colorado, Denver, CO; ${ }^{2}$ University of Washington, Seattle, WA. (Control ID \#2467265)

LEARNING OBJECTIVE \#1: Understand common causes of chronic cough and initial steps for diagnosis and treatment.

LEARNING OBJECTIVE \#2: Recognize when higher level referral and advanced diagnostics are necessary.

CASE: A 64-year-old man came to clinic with 6 years of cough. History was notable for several bouts of pneumonia with multiple hospital admissions. His cough was occasionally productive of mucous though never hemoptysis. He denied fevers, chills, weight loss, orthopnea, dyspnea or heartburn. Trials of acid suppressants, bronchodilators, and inhaled steroids did not improve his symptoms. He previously smoked cigars. On examination, his vital signs were normal and he appeared well, with few audible expiratory wheezes on auscultation. The remainder of his exam was normal. All basic lab work, including complete blood counts, electrolytes, creatinine, and liver function tests were normal. Pulmonary function tests showed an approximately $20 \%$ volume loss. Later, on further questioning, he recalled a specific day in 2009 when his cough began. A chest radiograph and then CT scan of the chest revealed collapse of the right middle lobe (RML) with irregularities within the airway leading to it. Bronchoscopy demonstrated inflamed tissue surrounding a foreign body in the os of the RML . The object was removed and determined to be a pistachio shell. Bronchial wall biopsies returned as squamous metaplasia without evidence of malignancy. Asking again specifically about the day his cough began, he now recalled choking while eating pistachios.

DISCUSSION: Cough is one of the most common reasons for presenting to clinicaccounting for nearly 30 million clinic visits in the US alone-and is commonly encountered by general internists and specialists alike. Chronic cough is defined as cough lasting longer than 8 weeks. The differential for chronic cough includes hundreds of etiologies, however the majority of cases will be explained by the three most common diagnoses: postnasal drip, asthma and gastroesophageal reflux disease. Complete history often points to a most likely culprit. Recent initiation of angiotensin-converting-enzyme inhibitor, for example, raises suspicion for a medication-induced cause of cough, and should prompt a trial of medication discontinuation. Patients with typical presentations of chronic cough in the absence of "red flag" warning signs (e.g., weight loss or hemoptysis) can be safely followed and trialed on lifestyle modifications (such as elevation of head of bed) as well as acid suppressive and antitussive medication. A trial of short-acting beta agonist for obstructive lung disease is also usually appropriate. In the absence of a clear underlying cause, pulmonary function tests and chest radiography are reasonable first steps. Patients with significant risk factors for malignancy or those with warning symptoms require expedited work-up. Lack of symptom improvement or the presence of "red flags" or radiographic abnormalities merit further work-up including consideration of computerized tomography. If abnormalities are seen on imaging, or if work-up has been unrevealing, bronchoscopy should be considered. Conclusion In this case, a patient aspirated a pistachio shell that caused chronic airway irritation and inflammation for over half a decade, causing chronic cough. Failure to recognize that lack of symptom improvement merits broadened diagnostic consideration and further evaluation led to significant diagnostic delay and potential for more grave consequences. Squamous metaplasia from chronic irritation is a risk factor for malignant transformation, and merits follow-up to assess for progression or resolution. Fortunately for our patient, shell removal led to rapid resolution of his cough and repeat bronchoscopy with bronchial biopsy 6 months later confirmed resolution of the airway inflammation. 
A PAINFUL RENDITION OF THE NUTCRACKER Douglas Lim; Michael P. Smith. University of Nebraska Medical Center, Omaha, NE. (Control ID \#2469921)

LEARNING OBJECTIVE \#1: Recognize abdominal vascular abnormalities and potential complications

LEARNING OBJECTIVE \#2: Recognize the risk factors and presentation of SMA syndrome and Nutcracker Syndrome.

CASE: A 20 year-old man presented with diarrhea and abdominal pain over the past week that progressively worsened. The pain was non-radiating, sharp, constant, and occurred all over his abdomen. His diarrhea was watery and without blood. He reported vomiting after eating as well as fever and chills. He denied voiding problems and does not drink alcohol. He had no previous medical or surgical history. The abdomen was diffusely tender on exam without distension. His creatinine was $1.7 \mathrm{mg} / \mathrm{dL}$ and he had white blood cells of $20 \times 103 / \mathrm{uL}$. Urinalysis revealed large blood. Upon further questions, he reported a $50-\mathrm{lb}$ weight loss over a period of 1 year attributed by poor diet. A CT scan of the abdomen revealed a narrow angle between his superior mesenteric artery and abdominal aorta, commonly referred to as Superior Mesenteric Artery Syndrome; additionally, the left renal vein was compressed between those vessels, commonly referred to as Nutcracker Syndrome

DISCUSSION: Abdominal pain is a common complaint for a general internist and finding a diagnosis may be challenging. This patient was found to have superior mesenteric artery (SMA) syndrome as well as left renal vein (LRV) entrapment. These are uncommon disorders and require a high index of suspicion. SMA syndrome can cause compression of the duodenum between the aorta and the SMA resulting in abdominal pain. SMA syndrome is also a risk factor for left renal vein entrapment between the two vessels as well. Both conditions can present with non-specific symptoms and can have significant morbidity. Various medical subspecialties may encounter these patients depending on the specific presentation, as it can be varied. The most common cause of this syndrome is significant weight loss often associated with bariatric surgery and malignancy. This leads to a decrease in fatty tissue around the SMA causing the syndrome. SMA syndrome can present insidiously or acutely like our patient. Our patient's presentation was somewhat atypical as it usually occurs following rapid weight loss, however, our patient presented with chronic weight loss over a period of a year. His weight loss was due to poor caloric intake and not due to severe illness or malabsorption. The general internist must have a high index of suspicion to consider SMA syndrome and LRV compression in patients presenting with abdominal pain and acute kidney injury to ensure the correct course of treatment.

A PARTICULARLY VIRULENT CASE OF STREPTOCOCCUS BOVIS ENDOCARDITIS Timothy S. Krill ${ }^{2}$; Shivani Desai ${ }^{1}$; Blake R. Barker ${ }^{1} .{ }^{1}$ UT Southwestern Medical Center at Dallas, Dallas, TX; ${ }^{2}$ UT Southwestern Medical School, Sugar Land, TX. (Control ID \#2462467)

LEARNING OBJECTIVE \#1: 1. Recognize the virulence of Streptococcus bovis bacteremia

LEARNING OBJECTIVE \#2: 2. Recognize the sequelae associated with $S$. bovis bacteremia 3. Identify risk factors for development of $S$. bovis bacteremia

CASE: A 57 year old African American female with a history of chronic hepatitis C and prior IV drug use presented with a 1 week history of subjective fever, chills, back pain, and generalized malaise. On admission, the patient was afebrile, tachycardic to 130, and hypotensive to the 80 's systolic. Physical exam revealed a 3/6 systolic murmur over the apex radiating into the axilla, elevated JVD, and tenderness over the lumbosacral spine. The remainder of the physical exam was unremarkable. Laboratory data on admission revealed a normal basic metabolic panel, INR, and slightly elevated transaminases. Blood cultures drawn on admission revealed $4 / 4$ bottles positive for $S$. bovis. MRI of the Lumbar spine without IV contrast was unremarkable. Broad spectrum antibiotics were promptly initiated. On the 4th day of hospitalization, the patient became febrile and increasingly hypoxic, which necessitated transfer to the ICU. Transthoracic echocardiogram revealed a mobile vegetation on the anterior mitral valve leaflet measuring $1.2 \times 0.6 \mathrm{~cm}$, flail of the anterior mitral leaflet, and severe mitral regurgitation. Chest $\mathrm{x}$-ray revealed marked pulmonary edema. Due to the severity of the valvular infection and cardiogenic shock, the patient underwent urgent mitral valve replacement with a mechanical prosthesis. Subsequent colonoscopy later performed revealed a $3 \mathrm{~mm}$ sessile tubular adenoma. Two months after the acute episode, the patient presented with persistent back pain. MRI of the lumbar spine with contrast revealed L4-L5 discitis and osteomyelitis with epidural extension and crowding of the cauda equina. Additionally, a prevertebral phlegmon extending inferiorly to the L5-S1 disc was also found. She underwent L4-L5 decompressive laminectomy for cauda equina syndrome, was treated with 6 weeks of IV vancomycin, PO ciprofloxacin, and metronidazole. Fortunately, she managed to regain most of her strength in the lower extremities.

DISCUSSION: $S$. bovis is implicated as the agent in up to $5-12 \%$ of all infectious endocarditis cases. S. bovis is a normal inhabitant of the colon in a small percentage of healthy individuals; however, the prevalence is increased in patients with colonic neoplasia. In recent years, there has been an increased interest in the association between chronic liver disease and S. bovis endocarditis, and several mechanisms have been postulated to explain this association. Additionally, diskitis, if present, has typically been found to be the first sign of disease. This patient experienced several stigmata and complications from the S. bovis infection. Classically, S. bovis bacteremia is associated with colonic neoplasia. One study reported that $60 \%$ of those with $S$. bovis bacteremia who underwent colonic evaluation were found to have neoplasia (30\% carcinoma, $30 \%$ adenoma). Our patient was found to have a sessile tubular adenoma. One study evaluating echocardiographic features of $S$. bovis endocarditis found an association between this bacteria and vegetations $>10 \mathrm{~mm}$ in size. Our patient indeed had a large vegetation measuring $12 \mathrm{~mm}$ in size which resulted in significant destruction of the valve Our patient also had underlying chronic liver disease (from hepatitis c), which has also been seen in association with S. bovis bacteremia and endocarditis. One of the proposed mechanisms involves alterations in hepatic secretion of bile salts and secretory immunoglobulins, which can facilitate translocation of bacteria from the intestinal lumen to the portal system. Finally, S. bovis is now recognized as having a propensity to seed the vertebrae and cause vertebral diskitis/osteomyelitis. One study found of those with endocarditis due to $S$. bovis, the incidence of diskitis/osteomyelitis was 23.3 vs. $0.6 \%$ from endocarditis due to other causes. Our patient initially presented with back pain, but initial lumbar MRI withou contrast was negative. Subsequent MRI with contrast done at a later date for persistent back pain revealed osteomyelitis. While the bone culture was negative, this was done after antibiotic administration. Conclusion This case illustrates the virulence of $S$. bovis and the various sequelae it can cause. The individual in this case suffered from a large sized vegetation, colonic pathology, chronic liver disease, and diskitis/osteomyelitis, all of which have been shown to be associated with $S$. bovis infection. It is important for the clinician to keep in mind the aggressive nature of this organism, its association with colon cancer, and its propensity for vertebral osteomyelitis.

\section{A PENNY FOR YOUR THOUGHTS? ACUTE LIVER FAILURE DUE TO WILSON'S DISEASE Ravina Patel; Leslie Cler; Brad Pirtle. Methodist Dallas Medical Center, Dallas, TX. (Control ID \#2466106)}

LEARNING OBJECTIVE \#1: Diagnose acute liver failure due to Wilson's disease LEARNING OBJECTIVE \#2: Assess treatment options based on severity of illness CASE: Wilson's disease is an autosomal recessive condition of copper metabolism. Patients present with hepatic, neurologic, and psychiatric abnormalities due to copper 
deposition in these vital organs. The most devastating manifestation is acute liver failure. Due to the infrequency of Wilson's disease and the high fatality associated with liver failure; prompt recognition and treatment is essential. A 19 year old female with no past medical history presented with the chief complaint of "I'm turning yellow." Since starting college a few months prior the patient began experiencing lower extremity edema, abdominal discomfort, bloating, and weight gain. She attributed these symptoms to increased fast food and cafeteria food. Patient tried Tums for abdominal pains, but with no improvement. Patient dealt with the fatigue and lower extremity swelling for approximately 6 weeks. Patient ultimately presented to the hospital for unbearable fatigue, persistent abdominal discomfort and her roommate noting she was "turning yellow." The patient denied any positive family or surgical history. Patient was Guatemalan, she was a freshman at the University of Texas at Dallas, she smoked cigarettes once a week, she consumed 4-5 beers a week, and she used marijuana 4-5 times a week. She denied intravenous drugs, intra-nasal drugs, mushrooms, PCP, and amphetamines. She did not have any tattoos, transfusions, or any recent travel. Her home medications included a multivitamin. Physical exam: Patient was afebrile, hemodynamically stable and saturating well on room air. Patient was in no apparent distress, she was fatigued and skin was jaundiced. Patient had scleral icterus. She did not have any mucosal bleeding, heart was regular, there was trace to $1+$ pitting edema of the lower extremities bilaterally, no jugular venous distention, and lungs were clear to auscultation with faint crackles at the bases bilaterally. Her abdomen was soft with tenderness to palpation of the right upper quadrant, mild distention, and hepatomegaly. There was no splenomegaly, rebound, guarding, or fluid wave. Patient was alert and oriented times four with no focal deficits or altered mental status. Patient had appropriate mood and affect. Labs: The patient had a mild leukocytosis and hemolytic anemia. She had relatively normal liver function tests, however her INR was elevated to 5.8 and her total bilirubin was elevated to 35.2. She had a renal tubular acidosis due to Fanconi's syndrome with low phosphorus, low sodium, low potassium and low uric acid. Her viral panel for hepatitis and HIV was completely negative. Patient's urine drug screen was negative for acetaminophen, salicylates, and other drugs. Her serum blood alcohol was undetectable. Her abdominal sonogram demonstrated ascites and splenomegaly. The patient was diagnosed with acute liver failure. The most common causes of acute liver failure are acetaminophen toxicity and viral infections which were both ruled out. It was possible the patient was undergoing a drug reaction or had Amanita mushroom poisoning; however patient denied exposure to both. Her autoimmune panel was negative. At this point other less common etiologies of acute liver failure were discussed such as Wilson's disease. Her many other lab abnormalities like hemolytic anemia, renal tubular acidosis due to Fanconi's syndrome, and her young age could all be explained by Wilson's disease. Further work up included a slit lamp examination by ophthalmology which verified Kayser Fleischer rings. Lab work showed a low ceruloplasmin, low serum copper, and elevated urinary excretion of copper. Liver biopsy confirmed copper accumulation and a high hepatic copper content. Interventions: Initially the patient was treated with n-acetylcysteine for possible acetaminophen toxicity. As further confirmatory lab work came back, Wilson's disease became the main diagnosis despite the lack of neurologic or psychiatric changes. Patient continued to deteriorate over the next two to three days and was placed on the liver transplant list. Patient received a liver due to fulminant hepatic failure with an elevated MELD. Patient is currently status post successful orthotopic liver transplant and on chronic immunosuppresion.

DISCUSSION: Acute liver failure typically presents with encephalopathy and coagulopathy in a previously healthy individual. In the United States the most common causes are acetaminophen toxicity, Hepatitis B, or other drug reactions. However, less common etiologies such as Wilson's disease must be kept in the differential. Wilson's disease is diagnosed based on serum ceruloplasmin, copper, urine excretion of copper, and ultimately hepatic copper concentration on liver biopsy or $A T P 7 B$ mutation detection. Wilson's disease should be considered in those with unexplained hepatic, neurologic, and/or psychiatric manifestations. Other lab values associated with Wilson's disease include Fanconi's syndrome and hemolytic anemia. Patients can present at any stage of severity of illness. Some can be treated with lifelong chelation therapy, however many will present with acute decompensation of the liver which will require urgent liver transplant.

A POETRY BY INFLAMMATORY CYTOKINES! Sreelakshmi Panginikkod ${ }^{1}$; Venu Pararath Gopalakrishnan ${ }^{1}$; Niyati Gupta ${ }^{2}$. Presence Saint Francis Hospital, Evanston, IL; ${ }^{2}$ Grant Medical College and Sir JJ Group of Hospitals, Mumbai, India. (Control ID \#2469227)

LEARNING OBJECTIVE \#1: Recognize the clinical manifestations of POEMS syndrome.

LEARNING OBJECTIVE \#2: Manage a case of POEMS syndrome.

CASE: A 52 year old male presented with insidious onset of abdominal distention for 2 months prior to presentation. His past medical history was significant for hypothyroidism and sensory motor polyneuropathy diagnosed 5 years ago. Physical examination revealed a cachectic male with hyperpigmentation, sclerodermoid skin changes, significant ascites and mild pedal edema. Laboratory studies revealed normal liver function tests with albumin of $4.3 \mathrm{mg} / \mathrm{dl}$ and international normalized ratio (INR) of 1.1. Hepatitis and HIV serologies were non-reactive. CA-125 and CEA levels were within normal limits. Ascitic fluid analysis revealed a serum ascites albumin gradient of $0.9 \mathrm{~g} / \mathrm{dl}$ and ascitic fluid protein of $3.2 \mathrm{~g} / \mathrm{dl}$. Chest X-ray showed a normal sized heart with mild right pleural effusion. Transthoracic echocardiogram showed normal right and left ventricular function with ejection fraction of $62 \%$. Computerized tomography scan of the abdomen showed normal liver, massive amount of ascites, splenomegaly and retroperitoneal lymphadenopathy. Serum protein electrophoresis showed an M spike and serum Immunofixation was consistent with IgA lambda monoclonal gammopathy. Serum VEGF and IL-6 levels were elevated. Based on the overall clinical picture and investigations, the patient was diagnosed with POEMS syndrome. The patient was started on melphalan and dexamethasone and showed significant clinical improvement in the follow up period.

DISCUSSION: POEMS syndrome is a rare paraneoplastic syndrome which is caused by an underlying plasma cell disorder and is thought to involve elevations in VEGF, IL-6, Tumor Necrosis Factor-alpha and Interleukin-1beta. The acronym represents several of the defining features which includes Polyradiculoneuropathy, Organomegaly, Endocrinopathy, Monoclonal plasma cell disorder and Skin changes. Patients also have extravascular fluid overload which most commonly manifests as peripheral edema, but pleural effusion and ascites are also common. Patients have a low serum-ascites albumin gradient in the ascitic fluid, consistent with an exudative process rather than a portal hypertension process. There is no standard treatment for POEMS syndrome and no randomized controlled trials of treatment exist in the available literature. Systemic chemotherapy with the use of melphalan plus dexamethasone has demonstrated good response rates with an acceptable toxicity profile. Ascites often responds to the administration of diuretics, but may require drainage with paracentesis. Median survival in patients of POEMS syndrome with extravascular fluid overload was determined to be 6.6 years. Early diagnosis and a prompt multidisciplinary approach increase the likelihood of reduced long-term morbidity and mortality. Therefore, each case is interesting to report in order to improve the diagnosis and management of this uncommon syndrome.

\section{A POTENTIALLY FATAL PAIN IN THE NECK: REMEMBERING THE FOR- GOTTEN DISEASE OF LEMIERRE'S SYNDROME Caitlin G. Waters; Oluchi Dike; Arooj Hyat. Salem Hospital, Salem, MA. (Control ID \#2469470)}

LEARNING OBJECTIVE \#1: Recognize Lemierre's syndrome as a potential complication of sore throat. 
LEARNING OBJECTIVE \#2: Outline early management of Lemierre's to prevent rapid progression to fatal septicemia.

CASE: Thirty-one year old Cambodian female with no significant past medical history presented with a 6 days history of fever, headache, and sore throat. The sore throat lasted 2 days associated with left sided, tender swelling in the front of her neck. She had mild, non-bloody, non-painful diarrhea. The patient also endorsed nausea, poor oral intake, and dysuria a few days prior. Her urine had become very brown. She was not taking any medications and denied recent travel. She was febrile to 101.4, tachycardic at a rate of 221, with a blood pressure of 95/50, saturating $96 \%$ on room air. Exam revealed a young female with malaise, tender left anterior cervical chain lymphadenopathy, normal heart and lung sounds, and otherwise unremarkable exam. She had a white count of 13,000, $40 \%$ bands, and acute kidney injury with a creatinine of 1.71 , above her baseline of 0.8 . Urinalysis showed hematuria with $2+$ protein, $3+$ blood, $2+$ urobilinogen. Chest $\mathrm{x}$ ray was unremarkable. A renal ultrasound suggested possible pyelonephritis and appropriate antibiotic treatment was initiated; however, a urine culture came back as negative. Three days later, the patient developed a dry cough and a repeat chest $\mathrm{x}$ ray showed extensive bilateral airspace disease, most prominent in the right lung base. It was reported as multifocal bilateral pneumonia. Blood cultures from day of admission were positive for Streptococcus anginosus and Fusobacterium necrophorum. Contrast CT scan of the neck was negative for evidence of thrombophlebitis of the jugular veins, and no tonsillar or peritonsillar abscess. A new cavitation was seen in the partially imaged airspace disease of the superior segment of the left lower lobe, suggestive of septic emboli. With history of pharyngitis, tender lymph nodes, pulmonary emboli, and blood cultures with Fusobacterium necrophorum, a diagnosis of Lemierre's syndrome was made. TEE showed no evidence of endocarditis. Lower extremity ultrasound was negative for DVT. It was postulated that the patient's left-sided neck pain may have been a jugular venous thrombus that subsequently migrated to the lungs prior to the CT of the neck. Antibiotic treatment was continued for 4 weeks and the patient followed up with infectious disease as an outpatient.

DISCUSSION: Lemierre's syndrome, also known as postanginal sepsis, is a rare but potentially fatal complication of oropharyngeal infections in young, previously healthy persons. In the 1936, Andre Lemierre published a case series of 20 patients with Fusobacterium pharyngitis complicated by sepsis. Prior to penicillin, the prognosis was poor, and 18 of his 20 patients died. Penicillin eventually became more available and the condition was so rarely seen that it was termed the 'forgotten disease'. In the past few decades, it has presented more frequently and uniquely. It has been suggested that emphasis on antibiotic stewardship, with restricted uses of antibiotics, has potentially unveiled a 'reappearance' of Lemierre's syndrome. Fusobacterium necrophorum, a nonmotile, sporulating gram-negative anaerobe found in the pharynx, gastrointestinal tract, and female genital tract, is the most common causative organism. Within less than 7 days from the development of pharyngitis, the bacteria invade the lateral pharyngeal space, and subsequently, the neighboring peritonsillar blood vessels which spread to the internal jugular vein. The first sign of Lemierre's syndrome is usually a persistent fever, followed by acute pharyngitis and subsequent neck tenderness or swelling along the sternocleidomastoid. Typically, the infection progresses from the lateral pharyngeal space, due to tonsillitis, pharyngitis, mastoiditis or tooth infection, and can progress to septicemia and subsequent thrombosis. Septic emboli can lead to complications including metastatic abscesses, most commonly in the lungs and joints, if diagnosis is not made in a timely fashion. Diagnosis is based on the clinical presentation with fever, sore throat, malaise, and subsequent blood cultures positive for Fusobacterium. Imaging, such as high resolution contrast CT scan of the neck, can reveal parapharyngeal stranding, rim enhancing loculations, and occasionally, a thrombus in the jugular vein. It is imperative to suspect Lemierre's in the patient with antecedent pharyngitis, septic pulmonary emboli, and persistent fever despite antimicrobial therapy. Treatment is focused on early removal of the focus of infection and prompt administration of intravenous antibiotics. Surgical intervention, such as ligation and excision of the jugular vein or incision and drainage of abscesses, should be considered in patients with ongoing sepsis despite medical therapy. Early diagnosis of Lemierre's syndrome decreases morbidity and mortality by preventing progression to fatal septicemia through prompt medical treatment.

A PRES-ING CASE OF LUPUS NEPHRITIS Jason C. George; Jamie A. Green. Geisinger Medical Center, Danville, PA. (Control ID \#2463155)

LEARNING OBJECTIVE \#1: 1 . To diagnose posterior reversible encephalopathy syndrome (PRES) in a patient with newly-diagnosed lupus nephritis

LEARNING OBJECTIVE \#2: 2. Recognize PRES as a potential acute manifestation of previously undiagnosed lupus nephritis

CASE: A 24 year-old female with no significant past medical history presented with 6 weeks of progressive fatigue, dyspnea on exertion and lower extremity edema. She presented to an outpatient clinic, where urinalysis showed significant proteinuria. She was admitted after further lab investigation showed evidence of acute kidney injury with creatinine 7.2, nephrotic-range proteinuria (protein/creatinine ratio 12.1) and pancytopenia. She also had ANA titer of 1:10,240 with decreased complement levels and elevated anti-double stranded DNA. She underwent renal biopsy that showed active, diffuse proliferative lupus nephritis (ISN/RPS class IV) with extensive capillary microthrombi, obliterative vascular injury consistent with thrombotic microangiopathy. Biopsy also showed tubular red cell casts and only mild interstitial fibrosis, suggesting minimal chronicity. Immunofluorescence showed granular glomerular and mesangial deposition of IgA, IgM, IgG, C3 and C1q. She acutely developed severe hypertension with systolic blood pressure greater than 200 and subsequently developed seizure activity. MRI brain showed abnormal signal enhancement of the posterior aspect of both cerebral hemispheres consistent with posterior reversible encephalopathy syndrome (PRES). She was followed by Neurology and started on anti-epileptic medication. She also required escalating doses of several anti-hypertensive medications. She was started on high-dose prednisone and mycophenolate mofetil for lupus nephritis. She also required hemodialysis during admission. She began to make urine and showed some signs of renal recovery but remained hemodialysis-dependent. Her neurological symptoms completely resolved prior to discharge with repeat MRI 2 months later showing normal posterior cerebral hemispheres. Of note, the patient had an uncomplicated pregnancy 15 months prior. She developed joint pain several months after delivery and was found to have a positive rheumatoid factor. She was diagnosed with rheumatoid arthritis and was briefly treated with prednisone and methotrexate with remission of her joint pain. She did not have any further pertinent rheumatologic findings at that time.

DISCUSSION: Posterior Reversible Encephalopathy Syndrome (PRES) is an increasingly-recognized neurological disorder that typically presents acutely with a variety of clinical manifestations, including headache, seizures, confusion and transient vision loss. These symptoms are accompanied by the disorder's characteristic imaging findings, particularly vasogenic edema and white matter changes of the parieto-occipital regions. PRES has been associated with a variety of clinical conditions, including pre-eclampsia/ eclampsia, hypertensive encephalopathy, organ transplantation, prior immunosuppressant therapy and connective tissue disease. The pathophysiology of PRES is not completely understood but is thought to be related to severe hypertension leading to autoregulatory dysfunction, vasodilation and subsequent vasogenic edema. Treatment should be targeted to the underlying cause. Neurological symptoms can be completely reversible, making early and aggressive intervention essential in order to avoid rapid clinical deterioration/ permanent neurological injury. Systemic Lupus Erythematosus (SLE) is a well-known systemic autoimmune disorder that can cause a wide variety of clinical manifestations, including extensive renal and neurological involvement. PRES is a well-known but rare complication of SLE. It has previously been reported mostly in SLE patients with known 
chronic lupus nephritis. Its true incidence in the general population is not well-known and incidence as an acute manifestation of previously undiagnosed SLE has not been frequently reported. Previous case series have found the most common precipitants of PRES in SLE were acute hypertensive episodes, renal decompensation and fluid retention. In a previous systematic review of the literature from 1966 to 2007, only 22 cases of PRES associated with SLE have been reported, with a mean age of 13-40 years. Of note, most of these patients developed the syndrome in the setting of chronic SLE and treatment with immunosuppression (often in high doses), not as part of their initial presentation of lupus nephritis. A more recent case series from 2006 to 2010 investigated PRES associated with SLE and found only 6 patients had PRES as part of their initial presentation. In conclusion, PRES is an important potential neurological complication of connective tissue disease and should be considered as part of the initial manifestation of previously undiagnosed lupus nephritis.

A RARE AND FATAL CASE OF VIRAL ENCEPHALITIS IN AN IMMUNOCOMPETENT HOST Prathik Krishnan; Poornima Ramadas; RUMON CHAKRAVARTHY; Birendra Sah. SUNY UPSTATE MEDICAL UNIVERSITY, SYRACUSE, NY. (Control ID \#2442817)

LEARNING OBJECTIVE \#1: HHV-6 is a ubiquitous virus commonly causing exanthem subitum in children but can also cause meningoencephalitis in immunocompromised individuals especially after BMT. We present a case of HHV-6 encephalitis in an immunocompetent adult. CASE: A 55 y/o F with PMH of Hypertension, hyperlipidemia, depression, Hypothyroidism and type $2 \mathrm{DM}$ presented to an outside hospital with complaints of fatigue and lethargy for a days. She also complained of low-grade fever, abdominal pain and diarrhea. Over the past day the patient had become very agitated and hence was taken to the emergency room. In the ER she was found to be extremely agitated and febrile. She was sedated and intubated for airway protection. A spinal tap was done which revealed protein of 60 , glucose of 108,0 WBCs and 11 RBCs. The patient was started on acyclovir, vancomycin, ceftriaxone, ampicillin for broad coverage of infectious meningoencephalitis. A U.tox screen was also done which was negative. Since there was minimal improvement after 5 days of antibiotics, she was transferred to our facility for higher level of care. Spinal tap cultures were pending at the time of transfer. The patient underwent MRI brain which did not show any acute findings. TSH, pCO2, Lactate and anion gap all normal. EEG was done which showed no epileptiform activity but generalized slowing and semiperiodic triphasic waves. A repeat lumbar puncture was done which showed glucose 82 , protein 25, RBC 11, WBC 3. Auto-immune work up significant for elevated ESR, CRP, and speckled pattern ANA. RF and dsDNA were negative. Rheumatology was consulted who recommended starting patient on $1 \mathrm{~g}$ solumedrol daily for possible lupus Cerebritis until serology work up is complete. Further history from the family revealed patient had photosensitivity, malar rash, mouth ulcers, history of depression, and joint swelling which satisfied criteria for SLE and the patient had a positive family history of SLE in her sister. She was started on Plasmapheresis therapy every other day for six sessions and solumedrol was eventually decreased to $40 \mathrm{mg}$ daily after 5 days of $1 \mathrm{~g}$ daily. NMDA Ab's, VGKC Ab's, prion panel, CMV EBV IgM, tau protein, Toxoplasma IgM and IgG and paraneoplastic panel were all negative. New York State encephalitis panel revealed positive HHV-6 antigen but did not quantify the Viral Load. The patient was started on foscarnet and infectious disease were consulted. The patient completed 22 days of foscarnet but didn't have any improvement in her mental status. MR Spect completed with fairly heterogeneous activity throughout the brain, with a specific area of focally decreased uptake in the left frontal lobe. A repeat lumbar puncture was again done with fluid analysis revealing HHV-6 DNA PCR at 6200copies/ml. Due to the prolonged hospital stay and in light of minimal improvement of mental status after antiviral therapy comfort care measures were instituted as per family request and patient passed away.
DISCUSSION: HHV-6 is a double standard envelope DNA virus discovered in 1986. It was demonstrated later to be the causative agent of exanthem subitum in infants. The virus has two variants, A and B. Similar to other herpes viruses; HHV6 remains latent in Lymphocytes, salivary glands and brain. When the host becomes immunocompromised reactivation can occur. HHV-6 Variant B has been reported to occur in 40 to $60 \%$ of BMT and solid organ transplant. HHV-6 can also cause encephalitis of variable severity in immunocompetent hosts. There has been debate regarding the clinical relevance of HHV6 in the CSF of immunocompetent adults even in the presence of Encephalomyelitis. The virus has a tropism for glial cells and PBMCs and remains latent in all infected individuals. Therefore, the presence of HHV-6 does not necessarily establish a pathogenic role. However, in several autopsy specimens from immunocompetent patients with encephalitis, either HHV-6 early protein was localized by immunohistochemistry or HHV-6 nucleic acid was localized by in situ hybridization, specifically in involved portions of the brain. This suggests HHV-6 as the etiology in these cases. There have been no in vivo studies - except for case reports - that have addressed treatment of HHV-6 infection. Ganciclovir resistant HHV-6 has also been reported but in our case after completion of 3 weeks of foscarnet no response was seen. In conclusion we present a rare case of HHV-6 encephalitis in an immunocompetent adult who failed to show significant clinical improvement even after 3 weeks of foscarnet therapy.

\section{A RARE ASSOCIATION OF ACUTE PANCREATITIS WITH ALENDRONATE} USE Khushdeep S. Chahal ${ }^{1}$; Alexandria Kraus ${ }^{2} .{ }^{1}$ University of Alabama Birmingham, Huntsville Regional Campus, Huntsville, AL; ${ }^{2} \mathrm{UAB}$ School of Medicine, Birmingham, AL. (Control ID \#2469767)

LEARNING OBJECTIVE \#1: Recognize that Alendronate use can cause pancreatitis LEARNING OBJECTIVE \#2: Recognize that common manifestations of a drug reaction (e.g. rash, fever, and eosinophilia) may be absent in drug-induced pancreatitis. CASE: We present a rare case of Alendronate induced pancreatitis in a 60-year-old Caucasian female with history of SLE, anemia, hypertension and osteoporosis. The patient presented to the emergency department with acute onset abdominal pain of 1-day duration. She reported sharp pain in her upper abdomen associated with nausea and exacerbated by movement. The patient denied any alcohol use or history of gallstones. Home medications included fosinopril, hydrochlorthiazide (HCTZ) and low dose prednisone, all of which she had been taking for several years without any recent dose changes. She was recently started on weekly Alendronate $70 \mathrm{mg} 4$ weeks prior, with the last dose 5 days before pain onset. Patient was tachycardic and slightly tachypneic on presentation; physical exam was unremarkable except for hypoactive bowel sounds and epigastric tenderness without rebound or guarding. Labs revealed leukocytosis (WBC 17), hemoconcentration (Hct 44.7), markedly elevated lipase (3290 U/L), and elevated CRP (10.4) consistent with acute pancreatitis. The severity of pancreatitis was categorized as mild with low mortality risk based on Bedside index for severity in acute pancreatitis (BISAP) score of two. Patient did not have any rash, fever or eosinophilia. Lipid panel and calcium levels were normal, and abdominal USG and CT abdomen/pelvis showed no gallstones and normal biliary anatomy. Patient had no symptoms of an SLE flare-up, but since pancreatitis is rarely associated with such flareups, $\mathrm{C} 3$ and $\mathrm{C} 4$ complement levels were obtained, and were normal. Patient responded well to supportive treatment with intravenous fluids and pain control, and was pain free on day three while tolerating meals. Leukocytosis and hemoconcentration resolved, and CRP and lipase trended down. The patient was discharged home on all home medications except Alendronate. At 6 weeks follow-up, the patient had no recurrence of symptoms while continuing to take fosinopril, HCTZ and low dose prednisone. Alternative pharmacological options for osteoporosis management are being considered for her.

DISCUSSION: Acute pancreatitis is a leading gastrointestinal cause of hospitalization. Reported incidence ranges from 4.9 to 35 per 100,000 with an overall mortality of $5 \%$. 
Gallstones and alcohol abuse account for more than two-thirds of cases. Drug induced pancreatitis is a rare condition occurring in $0.3 \%$ to $1.4 \%$ of cases, with more than 120 drugs implicated. These drugs are classified based on reported number of cases, consistent latency periods (from drug initiation to pancreatitis), and recurrence on re-challenge. Hypothesized mechanisms include: direct idiosyncratic reaction to the drug, either due to hypersensitivity (characterized by a latency period of 1-6 weeks) or accumulation of toxic metabolites (characterized by a latency period of months). Our patient scenario had a latency period of 4 weeks and would be more consistent with hypersensitivity reaction. Our patient scored +7 on Naranjo probability scale for drug causality assessment suggesting a 'probable' association. Though fosinopril, HCTZ and prednisone have been associated with pancreatitis, our patient had been on these medications for several years and had had no recent dose modifications. In addition, prednisone induced pancreatitis is reported with use of much higher dosages. Though pancreatitis recurrence on re-challenge with alendronate would be definitive evidence, the absence of recurrence in spite of continuation all other home medications supports the suspicion for Alendronate induced pancreatitis. Rechallenge would only be ethical if Alendronate was a medical necessity and there were no other options available for managing osteoporosis. There have been two reported cases of pancreatitis related to pamidronate infusions, and only one such report with Alendronate. Although Alendronate induced esophagitis, dysphagia, and esophageal and gastric ulcers are well-described entities, acute onset of abdominal pain with recent use of the medication should prompt workup for acute pancreatitis. Considering that only a couple of case reports are available without enough rechallenge data, Alendronate would belong to Class 4 of drugs implicated for inducing pancreatitis

\section{A RARE CASE OF ACQUIRED HEMOPHILIA A IN A PATIENT WITH CHRONIC HEPATITIS C TREATED WITH PEGINTERFERON ALPHA} $\underline{\text { Safeera Javed }}{ }^{1}$; emrullah yilmaz ${ }^{2}$; Swati Goel ${ }^{2}$; Bennal Perkins ${ }^{3} .{ }^{1}$ Montefiore medical center, NYC, NY; ${ }^{2}$ Montefiore medical center, Bronx, NY; ${ }^{3}$ Montefiore Medical Center, New York, NY. (Control ID \#2460335)

LEARNING OBJECTIVE \#1: Recognize Peginterferon alpha as a potential cause of acquired hemophilia $\mathrm{A}$ in patients receiving treatment for $\mathrm{HCV}$ infection.

LEARNING OBJECTIVE \#2: Recognize the value of mixing study as an important diagnostic test, when evaluating patients with unexplained isolated prolongation of partial thromboplastin time (PTT)

CASE: Fifty-seven year old male with history of intravenous drug abuse and chronic hepatitis $\mathrm{C}$ infection, treated with peginterferon alpha and ribavarin for 6 months, completed 1 month prior to hospitalization, presented with an 8 day history of pain, swelling and bruising of hands, forearms, upper arms, knees, calves and thighs bilaterally. He also reported on and off bleeding from gums. He denied personal or family history of bleeding disorder. On examination, there was ecchymosis, swelling and tenderness in muscles of forearms, upper arms, calves, thighs, multiple joints of the fingers and toes bilaterally, with inability to extend some of the fingers. Initial laboratory tests showed $\mathrm{Hb} 10 \mathrm{~g} / \mathrm{dl}$, WBC $9.1 \mathrm{k} / \mathrm{ul}$ and platelet count of $349 \mathrm{k} / \mathrm{ul}$. He had a prothrombin time (PT) of $10 \mathrm{~s}$ and partia thromboplastin time (PTT) of $53 \mathrm{~s}$, which was not corrected by mixing with norma plasma (mixing study), indicating presence of an inhibitor. Factor VIII, IX, XI, LDH, Fibrinogen, Von Willebrand panel and lupus anticoagulant were tested. The Factor VIII activity was $1 \%$ (50-150), Factor XI $99 \%$; Factor IX $85 \%$; VWF activity $236 \%$ and Fibrinogen $567 \mathrm{mg} / \mathrm{dl}$. Lupus anticoagulant and rest of the autoimmune workup was negative. Factor VIII Inhibitor was detected in a titer of 12 Bethesda Units. Patient was diagnosed with acquired hemophilia $\mathrm{A}$ and was started on prednisone $1 \mathrm{mg} / \mathrm{kg} / \mathrm{day}$ and intravenous recombinant human coagulation factor VIIa (NovoSeven) $90 \mathrm{mcg} / \mathrm{kg}$. He developed new ecchymoses while on treatment and was started on Cyclophosphamide and intravenous immunoglobulins IVIG. PTT trended down to 33 s, Factor VIII level gradually increased to $25 \%$ and Factor VIII inhibitor level was undetectable after 10 12 days of treatment. Patient had complete resolution of his symptoms and has been asymptomatic to date.

DISCUSSION: Acquired hemophilia A is a rare life threatening autoimmune hemorrhagic disorder caused by production of autoantibodies against coagulation Factor VIII. These autoantibodies produced in acquired hemophilia A are different from alloantibodies that are produced in some individuals with congenital hemophilia, following treatment with recombinant or plasma-derived FVIII. The diagnosis of acquired hemophilia $\mathrm{A}$ is based on the demonstration of an isolated prolongation of partial thromboplastin time (PTT), not corrected by mixing with normal plasma (mixing study), in association with reduced factor VIII and presence of factor VIII antibodies. Acquired Hemophilia A has been described in association with malignancies, lymphoproliferative disorders, autoimmune diseases, pregnancy and drug reactions. Several drugs such as penicillin, sulfa drugs and anticonvulsants have been known to have a well established association with acquired hemophilia A. Recently acquired hemophilia A has been reported to occur in association with peginterferon alpha, when used for the treatment of various malignant and nonmalignant conditions. However, most of these patients had additional risk factors for the development of Factor VIII inhibitors such as hematological malignancies or congenital hemophilia, and it had been argued whether the underlying malignancy and not the interferon therapy had predisposed to the development of autoantibodies. Our case is unique as the patient developed autoantibodies against factor VIII without having an underlying malignancy, suggesting that either hepatitis $\mathrm{C}$ infection or peginterferon was the triggering factor for the development of factor VIII inhibitors. The development of acquired hemophilia $\mathrm{A}$ in association with chronic hepatitis $\mathrm{C}$ virus infection and interferon therapy has rarely been reported in literature. To my knowledge there have been five other cases, which reported acquired hemophilia $\mathrm{A}$ in patients receiving interferon therapy for chronic HCV infection and two cases which reported development of factor VIII inhibitors in patients with hepatitis $\mathrm{C}$ infection without exposure to Interferon therapy. Interferon alpha has been known to have marked immunomodulatory effects and can induce the development of autoimmune disorders, aggravate preexisting autoimmunity or unmask silent autoimmune processes. Hepatitis $\mathrm{C}$ virus infection has also been associated with a variety of autoimmune disease. When treated with Interferon alpha, it may have synergistic effect for the development of Factor VIII autoantibodies. Although acquired hemophilia A is a potentially life threatening disorder with significant mortality, prompt recognition and discontinuation of the involved drug can be life saving. Some patients with low antibody titers resolve spontaneously after removal of the triggering drug. The mainstay of treatment involves achieving hemostasis and eradication of autoantibody. Acute bleeding episodes can be treated with human factor VIII concentrates, desmopressin, activated prothrombin complex concentrates (APCC) and recombinant activated factor VII (rFVIIa). Immunosuppressive agents used for eradication of inhibitors include steroids and cytotoxic drugs. Our patient responded to treatment with recombinant human factor VIIa and immunosupressive drugs with complete eradication of factor VIII inhibitor.

A RARE CASE OF AXITINIB-INDUCED POSTERIOR REVERSIBLE ENCEPHALOPATHY SYNDROME (PRES) khalid baosman ${ }^{1}$; Gary Malakoff ${ }^{2}$; abdelazim sirelkhatim ${ }^{3}$; Ahmad U. Janjua ${ }^{4}$. University of Tennessee, East Ridge, TN; ${ }^{2}$ University of Tennessee College of Medicine, Chattanooga, Chattanooga, TN; ${ }^{3}$ University of Tennessee, Chattanooga, TN; ${ }^{4}$ Ross SOM, Miramar, FL. (Control ID \#2462702)

LEARNING OBJECTIVE \#1: Recognize the relationship between axitinib and posterior reversible encephalopathy syndrome

CASE: A 73-year-old Caucasian male with a past medical history of metastatic renal cell carcinoma, status post right nephrectomy, stage 3-chronic kidney disease, and 
hypothyroidism was found by EMS unresponsive, face-down with a right-sided gaze deviation and tonic-clonic seizure activity lasting $20 \mathrm{~s}$. He was intubated at the scene and rushed to the emergency department. He was afebrile, and had blood pressure of 253/ $140 \mathrm{mmHg}$, and heart rate of $93 \mathrm{bpm}$. CT imaging of the head revealed vasogenic edema in the bilateral frontal and parietal lobes. Laboratory studies were normal except for white cell count of 14,000 cells $/ \mathrm{mm}^{3}$ and a creatinine of $1.7 \mathrm{mg} / \mathrm{dL}$. The patient was started on a nicardipine hydrochloride and levetiracetam intravenous infusion and then transferred to the ICU. Neurology was consulted. MRI of the brain revealed diffuse white matter signal abnormalities suggestive of severe hypertensive encephalopathy and posterior reversible encephalopathy syndrome (PRES). The patient's home medications included axitinib for the past 6 months for advanced metastatic renal cell carcinoma and synthroid. The patient was extubated the next day. Improved vital signs showed a blood pressure of 152/ $76 \mathrm{mmHg}$. The patient was found to have decreased strength in the bilateral lower extremities (3/5) and the bilateral upper extremities (0/5). Gaze deviation had resolved. Patient became responsive to verbal commands. He was weaned off the nicardipine hydrochloride infusion and started on amlodipine and metoprolol to maintain a blood pressure less than $140 / 70 \mathrm{mmHg}$. Axitinib was discontinued to rule out medicationinduced malignant hypertension. Subsequent blood pressure readings were less than 140/70 $\mathrm{mmHg}$. Upper and lower extremities strength improved, 3/5 and 4/5 respectively. Three months after discharge, a repeat MRI showed near resolution of the PRES findings and his strength was back to normal in both the upper and lower extremities.

DISCUSSION: Posterior reversible encephalopathy syndrome (PRES) is a unique clinicoradiological diagnosis that is associated with clinical findings of headache, altered mental status, hypertension, visual disturbances, and characteristic MRI findings. Axitinib is a FDA-approved anti-neoplastic agent that is commonly used for the treatment of advanced, renal cell carcinoma. It is a tyrosine kinase inhibitor and a potent inhibitor of VEGFR-1, -2 , and -3 . Interestingly, axitinib has been rarely associated with the induction of malignant hypertension due to its inhibitory effect on VEGFR-2. In the case of our patient axitinib use was the likely etiology of his malignant hypertension. Our case highlights the relationship between axitinib and PRES. Clinicians should monitor regular blood pressure readings on patients who are being treated with axitinib. It is unclear if axitinib-induced malignant hypertension is related to duration of use or idiosyncratic factors. More research is needed in this area.

\section{A RARE CASE OF FULMINANT LYMPHOCYTIC MYOCARDITIS IN A PRE- VIOUSLY HEALTHY ADULT Benjamin A. Bier; Andrew Hale. Beth Israel Deaconess Medical Center, Boston, MA. (Control ID \#2431938)}

LEARNING OBJECTIVE \#1: Recognize the clinical presentation and treatment of fulminant lymphocytic myocarditis

LEARNING OBJECTIVE \#2: Identify the differential diagnosis of cardiogenic shock CASE: A 57 year-old woman presented with 2 days of chest pain, myalgias, fever, shortness of breath, and malaise. Her symptoms were sudden in onset. Her past medical history was notable for hypothyroidism, for which she took levothyroxine. She did not smoke, use drugs or have significant alcohol intake. Family history was notable for coronary artery disease in sister. On initial evaluation she was noted to have an elevated JVP to $20 \mathrm{~cm}$, and pitting edema of both ankles. Initial ECG suggested infero-lateral ST segment elevations. Troponin T was initially 5.42 . She urgently underwent right and left heart catheterization, which showed only minor luminal irregularities with no occlusive coronary disease, but significantly elevated pressures in all chambers of the heart. Transthoracic echocardiogram showed reduced ejection fraction of $45 \%$ and global dyskinesis. Over several hours the troponin $\mathrm{T}$ trended upwards to 15.96. The patient became progressively more hypotensive. Because of high-suspicion for fulminant myocarditis, she was empirically placed on venous-arterial Extracorporeal Membrane Oxygenation (ECMO) within $24 \mathrm{~h}$ of hospitalization. Repeat transthoracic echocardiogram on hospital day 2 showed no wall motion (completely akinetic ventricles), and repeat ECG showed no electrical activity (asystole). She was thus completely dependent on ECMO for perfusion for greater than $24 \mathrm{~h}$. Cardiac biopsy was obtained, which showed active lymphocytic myocarditis. She developed acute renal and hepatic failure, felt related to initial ischemic shock, and was initiated on renal replacement therapy. High dose steroids were initiated (methylprednisolone $1000 \mathrm{mg}$ daily). Given bleeding from femoral ECMO, the patient was transitioned to bi-ventricular mechanical support. Within $24 \mathrm{~h}$ of the initiation of steroids, ECG demonstrated a return of electrical function (initially a slow, wide-complex rhythm), and echocardiogram showed increased ventricular contractility. Five days after initiation of steroids, the ejection fraction and electrical activity of the heart had completely normalized, and biventricular support was removed. She was awake during this period and able to speak with her family. Unfortunately, despite improved cardiac function, the patient eventually succumbed to acute liver and kidney failure, felt secondary to her initial presentation, and expired after 26 days.

DISCUSSION: Chest pain and dyspnea are problems commonly encountered by the internist. Accurate history with an ECG can help triage more emergent causes of chest pain and dyspnea from benign diagnoses. In our patient, physical exam was consistent with acute onset of cardiogenic shock. In a patient with no significant past medical history, the most likely cause is myocardial infarction. Our patient's initial ECG showed inferolateral ST elevations, but cardiac catheterization showing no coronary disease. In the setting of her highly elevated troponin and no structural heart disease, fulminant myocarditis became the most likely explanation. The most common cause of acute fulminant myocarditis is infectious, with viruses such as Coxsackie B, adenovirus, and parvovirus B19 being the most common ${ }^{1}$. Other causes include drug-induced hypersensitivity myocarditis, often caused by anticonvulsants, antibiotics, and antipsychotics. Eosinophilic and giant-cell myocarditis are two idiopathic conditions known to cause a fulminant presentation. Lymphocytic myocarditis is a histologic diagnosis and does not refer to a specific disease entity; it is frequently the result of viral or idiopathic etiologies. In our patient's case, biopsy confirmed lymphocytic myocarditis, but no specific underlying cause was identified. In lymphocytic myocarditis, supportive care including heart failure therapy and mechanical ventricular support if necessary, are the mainstays of treatment. ${ }^{2}$ Glucocorticoids do not improve patient mortality or functional status, but have been shown to improve left ventricular ejection fraction. ${ }^{3}$ Our patient's heart function did respond to steroid therapy, however she ultimately expired of fulminant liver and renal failure. Our case highlights the need to maintain a broad differential in patients presenting with chest pain, and underscores the importance of timely mechanical ventricular support in patients with fulminant myocarditis. References 1. Cooper, L. Myocarditis. N Engl J Med 2009;360:1526-38 2. Chen, JMet al. Improved survival in patients with acute myocarditis using external pulsatile mechanical ventricular assistance. J Heart Lung Transplant:351357. 3. Caldeira, Det al. Cochrane Corner: Corticosteroids for Viral Myocarditis. Revista Portuguesa de Cardiologia. 34(1):65-67.2015 Jan.

\section{A RARE CASE OF IRON REFRACTORY IRON DEFICIENCY ANEMIA} (IRIDA). Sreelakshmi Panginikkod ${ }^{1}$; Venu Pararath Gopalakrishnan ${ }^{2}$; Niyati Gupta ${ }^{3}$. ${ }^{1}$ Presence Saint Francis Hospital, Evanston, IL; ${ }^{2}$ Saint Francis Hospital, Evanston, IL; ${ }^{3}$ Grant Medical College and Sir JJ Group of Hospitals, Mumbai, India. (Control ID \#2469244)

LEARNING OBJECTIVE \#1: Recognize the clinical presentation and laboratory findings of Iron Refractory Iron Deficiency Anemia (IRIDA)

LEARNING OBJECTIVE \#2: Recognize the role of serum hepcidin in evaluation of patients with iron deficinecy anemia unresponisve to oral iron therapy. 
CASE: A 47 year old female was referred for evaluation of chronic anemia of unknown etiology. Patient was anemic since childhood and was on iron supplements but got worsened in last 1.5 years warranting hospitalizations and blood transfusions. Patient endorsed to have dizziness, fatigue, poor exercise tolerance, dyspnea and palpitation associated with exertion. Review of system was negative for any active bleeding, epigastric pain or melena. Her menstrual cycles were regular with normal flow. Family history was significant for anemia in her niece. Laboratory values showed severe microcytic hypochromic anemia (Hemoglobin-4.5, Mean Corpuscular Volume-70, Mean Corpuscular Hemoglobin Concentration-28, Red cell Distribution Width-24) and iron panel revealed low serum iron and transferrin saturation with normal serum transferrin and unexpectedly normal serum ferritin. White cell count was normal (12.1) and platelet count was increased (443). There was no clinical or lab evidence of chronic inflammatory state or hemolysis and hemoglobin electrophoresis did not detect any hemoglobinopathy. Stool occult blood and Helicobacter Pylori antigen were negative. Esophagoduodenoscopy and Colonoscopy were unremarkable. Serum vitamin B12 and folic acid were normal. Serum anti transglutaminase antibody, parietal cell and castles intrinsic factor antibody were negative. Serum hepcidin was found to be inappropriately high. Presence of high hepcidin suggested a diagnosis of iron-refractory iron deficiency anemia (IRIDA), which was eventually confirmed by identification of a novel heterozygous TMPRSS6 mutation. Patient was treated with blood transfusion and parenteral iron therapy which she may require in future as well

DISCUSSION: Iron-refractory iron-deficiency anemia (IRIDA) is an autosomal recessive disorder caused by mutations in TMPRSS6 gene characterized by iron deficiency anemia (IDA) unresponsive to treatment with oral iron, but partially responsive to parenteral iron therapy. In patients referred for hematologic evaluation with refractory IDA, screening for celiac disease, autoimmune gastritis, Helicobacter pylori, and hereditary forms of IDA is recommended. Although iron deficiency anemia is relatively common, the prevalence of the iron-refractory form of the disease is unknown. The typical features of IRIDA are hypochromic, microcytic anemia with a very low mean corpuscular volume of erythrocytes, low transferrin saturation, no (or inadequate) response to oral iron, and only a partial response to parenteral iron. In contrast to classic iron deficiency anemia, serum ferritin levels are usually low-normal, and serum or urinary hepcidin levels are inappropriately high for the degree of anemia. Serum hepcidin should be routinely measured for differential diagnosis when patients with IDA are unresponsive to oral iron or have unusual clinical features. The current treatment of IRIDA is based on parenteral iron administration; in the future, manipulation of the hepcidin pathway might become an alternative therapeutic approach.

\section{A RARE CASE OF LYMPHOMATOID GRANULOMATOSIS OCCURRING WITH ULCERATIVE COLITIS SAMAH NASSEREDDINE MD, TIMOTHY DOUGHERTY JR MF, MARIE L.BORUM MD,EDD,MPH Samah Nassereddine; Marie Borum; Timothy Dougherty. George Washington University Hospital, WASHING-} TON, DC. (Control ID \#2467695)

LEARNING OBJECTIVE \#1: Acknowledge the potential risk of lymphoma in patients with IBD on immunomodulator therapy

LEARNING OBJECTIVE \#2: Recognize Lymphomatoid Granulomatosis as a rare distinctive type of lymphoma

CASE: A 36 year old man with longstanding ulcerative colitis, well controlled with 6-MP, presented with left eye pain associated with increased tearing and crusting . Ophthalmologic evaluation revealed no evidence of uveitis or episleritis. He was treated with antibiotics and acyclovir for dacrocystitis following evidence of methicillin resistant staphylococcus aureus (MRSA) and herpes simplex virus (HSV) on a lacrimal duct swab. While on therapy, he developed bilateral blurred vision and diplopia for which he was referred to the university hospital for further evaluation. Upon transfer he noted a $6 \mathrm{lb}$ unintentional weight loss during the past month. His examination was remarkable for a right-sided lateral gaze palsy and a large left-sided cervical lymphadenopathy. Laboratory studies were only remarkable for a white count of 2000 and a C-reactive protein of 45 . Extensive infectious disease workup included negative lyme serology, cryptococcal antigen, HSV, acid fast stain and rapid plasma regain (RPR). Cerebral spinal fluid (CSF) analysis showed a white count of 16 (100\% lymphocytic), glucose 35 , with a total protein of 178 and negative gram stain and culture. A subsequent MRA / MRV of the brain revealed multifocal supratentorial and infratentorial hyperintense lesions with enhancement of the 5th and 6th cranial nerves. His cervical lymph node biopsy revealed CD4+, atypical lymphocytic infiltrates by flow cytometry and was EBV RNA positive consistent with lymphomatoid granulomatosis. \{figure 1 \}

DISCUSSION: Lymphomatoid granulomatosis has rarely been associated with inflammatory bowel disease. The previously reported individuals with IBD were noted to be in patients with Crohn's disease, all of of whom were maintained on 6-Mercaptopurine and one was maintained on Azathioprine. Abdominal pain associated with B symptoms and respiratory symptoms was the common presentation. Diffuse systemic involvement including neurological lesions on imaging was also noted. Our patient is the first patient with ulcerative colitis who is diagnosed with lymphomatoid granulomatosis who's initial presentation was ocular manifestation with dacryocystitis, ptosis and six nerve palsy. The patient was started on systemic corticosteroids in addition to Chemotherapy with R-CHOP regimen (Rituximab,cyclophosphamide, vincristine, doxorubicin). Although orbital involvement has been described in 21 reported cases however only six patients had ocular manifestations as the initial presentation, four patients were noted to have ocular manifestation secondary to central nervous system involvement rather than direct orbital infiltration. Our case is unusual because of its atypical presentation with dacrocystitis and orbital involvement . Further work up showed diffuse disease with lung, pancreatic, prostate and neurologic involvement and rare lymphadenopathies which is unusual site for LYG. He was at increased risk for this malignancy because of the immunosuppression from 6-MP and previously unrecognized EBV infection. Discontinuation of thiopurine can result in remission in individuals with low grade disease. However, aggressive malignancies similar to our patient, may require multidrug chemotherapy. While lymphomatoid granulomatosis is an unusual complication in IBD, it is important that clinicians are aware of the possible occurrence to ensure appropriate diagnostic evaluation and intervention. The increased emergence of neoplastic process in the setting of immunomodulators use should be taken in consideration by prescribing physicians and risk should be discussed with patients . Treatment guidelines should be revised taking into account the medications side effect profile. Immunomodulators should therefore be handled by experienced and dedicated clinicians who are aware of the potential, although low, cancer risk associated with their use in patients with IBD.

\section{A RARE CASE OF PULMONARY VEIN THROMBOSIS ASSOCIATED WITH TREATMENT OF OMALIZUMAB. Sandhya Narukonda; Nagadarshini Ramagiri U. Vinod; Jahnavi Sagi; Medha Joshi; Boutros Zeidan. Conemaugh Memorial Medical Center, Johnstown, PA. (Control ID \#2467945)}

LEARNING OBJECTIVE \#1: Thrombosis of pulmonary vein is a rare entity and the diagnosis is challenging secondary to the nonspecific clinical presentation. Various recognized causes for pulmonary vein thrombosis are pulmonary tumors, trauma, lung resection and radiofrequency ablation for atrial fibrillation. We report a case of pulmonary vein thrombosis without a clear etiology.

CASE: A 53-year-old male with past medical history significant for unprovoked pulmonary embolism at the age of 38 , multiple sclerosis, COPD, and asthma presented with shortness of breath and cough. He is being treated with omalizumab for the last 2 years for 
underlying allergic asthma with significant improvement in the symptoms. No history of recent surgical interventions on the lungs and negative family history of hereditary thrombophilia. On admission vital signs were normal and physical examination was unremarkable except for diffuse wheezing. Chest computed tomography (CT) with pulmonary embolism (PE) protocol revealed thrombosis of the right inferior pulmonary vein and the contributing tributaries, extending to the left atrium, but not into the left atrium. This finding was new compared to a CT scan obtained 3 years ago. There was no evidence of intrapulmonary neoplasm. Echocardiogram was obtained which showed RV ejection fraction of $55 \%$, grade I diastolic dysfunction, no intracardiac thrombus and mild pulmonary vascular hypertension. Patient was started on IV heparin based on CT evidence of pulmonary vein thrombosis. Hypercoaguable work up was negative for ANA, ANCA, anti cardiolipin, anti phospholipid antibodies, and factor $\mathrm{V}$ Leiden. The rheumatoid factor was mildly elevated and lupus anticoagulant was positive which could be attributed to anticoagulant therapy with heparin. Patient's medical treatment was transitioned from heparin to rivaroxaban (xarelto) and omalizumab was discontinued.

DISCUSSION: Omalizumab (xolair) is an IgE monoclonal antibody that has been used over a decade for the treatment of chronic severe persistent allergic asthma, not controlled with conventional treatment. Studies have shown that it not only decreases the rate of asthma exacerbations but also reduces the use of inhaled corticosteroids. Our patient presented with isolated pulmonary vein thrombosis, after being on omalizumab for 2 years without any other identifiable causes and negative hypercoagulable work up. Data from the studies suggest that patients receiving omalizumab are at a higher risk of pulmonary embolism and deep vein thrombosis. However, there have been no reported cases of pulmonary vein thrombosis associated with omalizumab treatment in the current literature search, which should be considered as an adverse effect in patients treated with this medication on a long-term basis.

A RARE CASE OF PULMONARY-RENAL SYNDROME INDUCED BY BACTERIAL ENDOCARDITIS Iliya P. Amaza ${ }^{1,2}$; Arinzechukwu Uzoechina ${ }^{1,2}$; Jiwan Thapa ${ }^{1,2} .{ }^{1}$ Marshfield Clinic, Marshfield, WI; ${ }^{2}$ Ministry Saint Joseph's Hospital, Marshfield, WI. (Control ID \#2466052)

LEARNING OBJECTIVE \#1: Recognize bacterial endocarditis as a cause of pulmonary-renal syndrome

CASE: A 70-year-old male with a history of diet controlled diabetes mellitus and a remote history of prostate carcinoma, now in remission, was admitted to an outside hospital with complaints of progressive fatigue, renal failure, unintentional $20-1 \mathrm{~b}$ weight loss and a worsening rash over a 4-month period. A nonblanching purpuric rash had started on medial thighs bilaterally but spread diffusely involving the palms, soles as well as oral mucosa. There was no fever and no history of insect bites. Initial skin biopsy showed perivascular dermatitis. Serum creatinine ( $\mathrm{SCr}$ ) increased from $1 \mathrm{mg} / \mathrm{dL}$ to $3.7 \mathrm{mg} / \mathrm{dL}$ over 2 months. Extensive work up revealed worsening anemia (from a hemoglobin level of $13.6 \mathrm{~g} / \mathrm{dL}$ to $8.2 \mathrm{~g} / \mathrm{dL}$ ), thrombocytopenia (from a platelet count of $103,000 / \mu \mathrm{L}$ to 53,000 / $\mu \mathrm{L}$ ), negative hemolytic panel, negative vasculitis studies (antinuclear antibody, antineutrophil cytoplasmic antibody [ANCA], complements 3 and 4, anti-glomerular basement membrane, antibody antiphospholipid antibody), negative hepatitis panel, and negative serology for tick-borne diseases. Serum immunofixation, bone marrow biopsy and flow cytometry were unremarkable. Urinalysis showed dense microscopic hematuria (greater than 100 red blood cells per high-power field) and a urine protein/creatinine ratio of 630. Patient was subsequently transferred to our facility due to a difficulty in obtaining a renal biopsy with thrombocytopenia. Further workup here showed worsening $\mathrm{SCr}$ $(4.3 \mathrm{mg} / \mathrm{dL})$, a weakly positive cryoglobulin with cryocrit of $0.44 \%$, and a positive rheumatoid factor (1:640). A repeat skin biopsy showed leukocytoclastic vasculitis with perivascular immunoglobulin A deposition and he was empirically started on intravenous methylprednisolone. He, however, decompensated rapidly with altered mental status and respiratory failure requiring intubation. A computed tomography scan (CT) of the chest and a bronchoscopy confirmed diffuse pulmonary hemorrhage. A stat bedside echocardiogram showed densities on both mitral valve leaflets confirmed as vegetations on transesophageal echocardiogram. A renal biopsy performed same day revealed diffuse proliferative necrotizing glomerulonephritis with crescents and immune complex deposition in subendothelial, paramesangial \& mesangial location. Blood vessels showed no thrombi and there was no organized substructure on electron microscopy. CT and magnetic resonance imaging of the head showed a small subarachnoid hemorrhage and multiple lacunar infarcts. He was started empirically on Vancomycin and Cefepime. Blood cultures subsequently came back positive for Streptococcus viridans and his antibiotic regimen was adjusted.

DISCUSSION: Our case demonstrates how a chronic infection with low-pathogenicity organism can elicit a strong immunogenic response and present as an apparent immune disorder leading to delay in diagnosis of the underlying infection. A vasculitis-like presentation of subacute bacterial endocarditis ultimately led to the rare complication of pulmonary-renal syndrome in our patient. The pulmonary-renal syndrome is described as a combination of acute glomerulonephritis and pulmonary hemorrhage. It is commonly caused by ANCA-positive vasculitis and Goodpasture's syndrome. ${ }^{1}$ Glomerulonephritis is a well-recognized complication of bacterial endocarditis. Circulating immune complexes, occurring in $63-75 \%$ of patients, play a causative role. ${ }^{2}$ Pulmonary hemorrhage, on the other hand, is a rare finding in bacterial endocarditis. To our knowledge, only two cases reported have been reported in the literature. ${ }^{3,4}$ Our case highlights the importance of excluding infection as a cause of pulmonary-renal syndrome, because treatment with immunosuppressive agents, as is common with other etiologies, may be hazardous in an underlying infection. References 1. Hebert LA, Parikh SV. Differential diagnosis and evaluation of glomerular disease. In: UpToDate, Glassock RJ (Ed), UpToDate, Waltham, MA. (Accessed on January 12, 2016.) 2. Kauffmann RH, Thompson J, Valentijn RM, Daha MR, Van Es LA. The clinical implications and the pathogenetic significance of circulating immune complexes in infective endocarditis. Am J Med. 1981;71(1):17-25. 3. Griffin KA, Schwartz MM, Korbet SM. Pulmonary-renal syndrome of bacterial endocarditis mimicking Goodpasture's syndrome. Am J Kidney Dis. 1989;14(4):329-332. 4. Wu HC, Wen YK, Chen ML, Fan CS. Pulmonary-renal syndrome in a patient with bacterial endocarditis. J Formos Med Assoc. 2005;104(8):588-592.

A RARE CASE OF SUDDEN MALE DYSPAREUNIA Pranoti Hiremath; Meredith Barnes. University of Washington, Seattle, WA. (Control ID \#2469848)

LEARNING OBJECTIVE \#1: Recognize the variable presentations and importance of urgent treatment of pancreatic pseudoaneurysm

LEARNING OBJECTIVE \#2: Describe the complications of pancreatic cysts in the setting of chronic pancreatitis

CASE: A 70 year old man with a PMH of ETOH use presented to the ED after severe pain in his abdomen, legs, and back during orgasm, as well as persistent mild diffuse abdominal pain. He denied dysuria, hematuria, nausea, neurologic symptoms, or fevers. He had no history of acute pancreatitis and took no medications. VS included T 36.8, HR 95, BP 160/ 100, RR 18. Exam was notable for mild distress, abdomen diffusely tender without rebound/guarding, no hepatosplenomegaly, no abdominal or inguinal masses, no spider angiomata, and $2+$ pedal pulses. Labs showed WBC 5, lipase 444, Tbili 0.8. CT abdomen showed evidence of mild edematous pancreatitis with pseudoaneurysm of the inferior pancreaticoduodenal artery, peripancreatic fluid collection suggestive of slow hemorrhagic leak without active extravasation, and a $9 \mathrm{~mm}$ pancreatic cyst. Interventional radiology performed mesenteric angiography, leading to auto-spasm of vessel and no requirement 
for embolization. Symptoms had resolved following the procedure, and he was discharged with recommended CT scan in 3-6 months for pseudocyst monitoring. Five months later, he re-presented to the ED with Tbili 9 and direct bilirubin elevation. CT showed dilation of the intra- and extra- hepatic bile ducts, and $9 \mathrm{~mm}$ pancreatic cyst. ERCP/EUS demonstrated stricture at the neck of the pancreas and lower third of the main bile duct. Stents were placed in the bile duct and main pancreatic duct. ERCP/EUS also revealed evidence of chronic pancreatitis and a $2 \mathrm{~cm}$ mass in the location of the cyst. Biopsies diagnosed pancreatic adenocarcinoma. He subsequently underwent staging and a Whipple procedure, and is considering adjuvant chemotherapy.

DISCUSSION: Pancreatic pseudoaneurysm is a rare complication of pancreatitis and may present with unusual symptoms. Pathophysiology is thought to involve direct damage to arterial vessel walls from pancreatic enzymes. Indirect damage may also induce pseudoaneurysm via long-standing pancreatic pseudocysts, which cause direct compression, ischemia, or erosion of enzymes within the pseudocyst. Pancreatic pseudocyts have been seen in $41 \%$ of pancreatic pseudoaneurysm cases. Unlike splenic artery aneurysms, pseudoaneurysms almost always present with symptoms of abdominal pain and, more rarely, with active bleeding. Our patient's acute ejaculatory pain may have been a herald symptom of pseudoaneurysm formation. Risk of pseudoaneurysm rupture is as high as $37 \%$, and mortality rate approaches $90 \%$ when untreated. Mesenteric angiography allows definitive diagnosis as well as treatment with transcatheter embolization. Pancreatic cysts are associated with both biliary obstruction and adenocarcinoma in the setting of chronic pancreatitis. Cyst formation in the pancreatic head causes pancreatic ductal dilatation, thereby creating biliary strictures. Furthermore, cysts may represent either pseudocyst or neoplasm. Premalignant lesions comprise $20-42 \%$ of pancreatic cysts. Current guidelines recommend 6-month and 1-year CT/MRI surveillance of asymptomatic pancreatic cysts less than $3 \mathrm{~cm}$ without a solid component or dilated pancreatic ducts. This case emphasizes the importance of maintaining high clinical suspicion for pancreatic pseudoaneurysm, particularly when addressing unusual symptoms concurrent with common diagnoses such as pancreatitis. Pseudoaneurysms are easily treated to prevent additional complications The case further underscores the importance of appropriate follow-up in diagnosing and treating complications of pancreatitis, including pancreatic cyst formation, bile duct obstruction, and malignancy.

A RARE CASE OF SYSTEMC LUPUS ERYTHEMATOSUS AND NONVEGETATIVE MITRAL REGURGITATION Shruti Mony ${ }^{1}$; Manraj Khosla ${ }^{2}$; Taaran Cariappa Ballachanda Subbaiah ${ }^{1}$; Gabriel Colceriu ${ }^{2} .{ }^{1}$ St. Joseph's Hospital and Medical Center, Phoenix, AZ; ${ }^{2}$ St.Josephs Hospital and Medical Center, Phoenix, AZ. (Control ID \#2470027)

LEARNING OBJECTIVE \#1: Systemic lupus erythematosus (SLE) is an autoimmune disease exhibiting great diversity in presentation. Pericarditis and endocarditis are the most common cardiac manifestations secondary to SLE while isolated valvular disease is very rare. Although cardiovascular disease in SLE patients occurs earlier than in the general population and is the major cause of death, cardiac involvement is rarely the presenting manifestation of SLE. We present a unique case of a young male with SLE causing mutliorgan dysfunction and severe mitral regurgitation.

CASE: A 34 year old Hispanic male with a past medical history of SLE, Antiphospholipid syndrome (APS) and stroke presented with symptoms of recurrent hemoptysis, shortness of breath and fatigue for 3 months. He had multiple outside hospital admissions for shortness of breath of breath wherein he was found to have massive pulmonary hemorrhage confirmed by bronchoscopy and acute kidney injury requiring temporary hemodialysis. He also underwent a kidney biopsy which confirmed crescentic lupus nephritis. $\mathrm{He}$ failed therapy with high dose IV Steroids, Rituximab, Cytoxan, and 1 month of Plamsapharesis. Due to his refractory status and respiratory decline he required intubation, following which he was transferred to the critical care unit at our hospital for further evaluation. On admission, he appeared in acute distress and was ventilator dependent. His laboratory data revealed Hemoglobin/Hematocrit-6.6/19 g/dl, platelet count 50,000/uL, and Creatinine $4.68 \mathrm{mg} / \mathrm{dl}$ (from $2.45 \mathrm{mg} / \mathrm{dl} 1$ month ago). His chest X-ray was consistent with pulmonary hemorrhage showing diffuse bilateral interstitial opacities. For further evaluation, he underwent a transthoracic and transesophageal echocardiogram which showed severe Mitral regurgitation and moderate pulmonary hypertension, no vegetations were noted. His angiogram was negative for coronary artery disease. Unfortunately, he was deemed a poor candidate for cardiac surgery and was managed medically. In addition to daily high dose IV steroids, and hemodialysis he received mycophenolate mofotil. But his hospital course was further complicated with thrombocytopenic purpura (TTP) requiring a repeat cycle of plasmapheresis. Despite all aggressive measures patient failed to improve and his family decided to withdraw care, following which patient expired. DISCUSSION: Among the various cardiac manifestations of SLE, fibrinous pericarditis and verrucous endocarditis are the most recognized, but lesions of the myocardium and coronary vessels may also occur. The prevalence of SLE associated valvular disease, mainly endocarditis, is $18-74 \%$, of which mitral and aortic valves are more commonly involved Our case is exceptional as the patient was diagnosed with severe mitral regurgitation without any prior vegetation or associated cardiomyopathy. In reviewing literature, there has been only one case reported of SLE causing isolated mitral regurgitation. Treatment options for valve disease include surgical replacement and outcomes are varied depending on multiple prognostic factors. Since cardiac involvement is an increasing cause of mortality and morbidity, one should be aware of the varied manifestations of Lupus. A high index of suspicion needs to be maintained, as early diagnosis may avoid devastating consequences.

A RARE CASE OF TRANSIENT CORTICAL BLINDNESS FROM MESENTERIC ANGIOGRAM. CONTRAST INDUCED CORTICAL BLINDNESS IS AN UNCOMMON COMPLICATION. THE INCIDENCE OF TRANSIENT CORTICAL BLINDNESS VARIES FROM 0.3 TO $1.0 \%$ BUT THE RATE GOES UP TO $4 \%$ WHEN HYPEROSMOLAR IODINATED CONTRAST AGENTS ARE USED. THE INCIDENCE IS MORE WITH CEREBRAL AND VERTEBRAL ANGIOGRAMS AS COMPARED TO CARDIAC ANGIOGRAMS. THERE WERE ONLY 2 CASES THAT HAVE BEEN REPORTED INVOLVING ARTERIOGRAPHY OF THE ABDOMINAL AORTA OR ANY OF ITS BRANCHES. Neeli Thati; Sachin Goyal. Wayne State University, Detroit, MI. (Control ID \#2457174)

LEARNING OBJECTIVE \#1: Objectives 1-Recognize contrast-induced blindness LEARNING OBJECTIVE \#2: Objectives 2-Manage contrast-induced blindness CASE: We present a rare case of a 52-year-old female with a history of uroepithelial carcinoma with liver metastasis with no other significant past medical history. Patient underwent elective embolization of liver metastasis with iodinated contrast. The following day the patient developed progressive loss of vision of both eyes, and by the end of the day patient was completely blind in both eyes. On fundoscopy there was no intraocular pathology. MRI brain was done that showed areas of hyper intensity and diffusion restriction in the bilateral parieto-temporal and temporo-occipital area, consistent with contrast medium leakage through blood brain barrier. In addition a Long Term Recording (LTR) Electroencephalogram (EEG) showed epileptic activity in the bilateral occipital areas of the brain. The patient was started on anti-epileptics after which the seizures came under control. Initially the patient reported only minimal perception to light, however, over the hospital course the symptoms progressively resolved on its own. By the 5 th day the patient could percept $20 / 70$ on a snellen's chart.

DISCUSSION: Angiography is a commonly used investigative procedure and thus it is important to understand the pathophysiology behind transient cortical blindness. The contributing risk factors can be chronic hypertension, diabetes, hyperlipidemia, impaired 
renal function and/or use of hyperosmolar iodinated contrast. It is known that the integrity of the blood brain barrier is uneven, and in the occipital region it is easily penetrated. As seen in our patient, leakage of contrast can have diverse manifestations including transient cortical blindness, hemiparesis, aphasia, confusion, seizures and coma. Initial signs mimic a more serious intracranial pathology like Sub-Arachnoid Hemorrhage- which needs to be ruled out using CT scan, MRI and/or MRA. The cortical blindness is self-limiting as the effect is primarily from contrast neurotoxicity and usually resolves in 3-5 days. Treatment is mainly supportive, including adequate hydration with IV crystalloids and anti-epileptics for seizures. In a few cases, patients have been treated with intravenous steroids and mannitol with no adverse consequences.

A RARE CAUSE OF SUDDEN ONSET SEVERE ABDOMINAL PAIN: A CASE OF IDIOPATHIC RENAL INFARCTION. Hind Hadid. Hurley Medical Center, West Bloomfield, MI. (Control ID \#2468362)

LEARNING OBJECTIVE \#1: 1 . Although rare, idiopathic renal infarction should be considered as a cause of acute severe abdominal pain after more common causes of abdominal pain are ruled out

LEARNING OBJECTIVE \#2: 2. A careful search for a cause of renal infarction is crucial to guide treatment and prevent recurrence. There are no specific guidelines for the treatment of idiopathic renal infarction.

CASE: We report a case of a 35-year-old female with history of congenital complete heart block with pacemaker insertion 2 years ago, who presented to the ER with acute severe left flank pain associated with vomiting and chills of $8 \mathrm{~h}$ duration. Upon her initial assessment, she was afebrile with mildly elevated blood pressure and tachycardia. She was particularly tender to palpation in the left upper quadrant and the left costovertebral angle. Her urinalysis showed $2+$ proteinuria without hematuria, and her complete blood count, basic metabolic panel, lipase and hepatic panel were unremarkable. She had minimal relief with analgesics and anti-acid treatment. She then underwent contrasted CT abdomen and pelvis, revealing a sharply-demarcated hypodensity in the left kidney consistent with acute renal infarction. She was started on IV unfractionated Heparin and admitted to the general medical floor for further management. She underwent extensive workup for an underlying cause for her renal infarction. She denied hormonal contraceptive use, recurrent miscarriages, or family history of hypercoagulapathy or autoimmune diseases. A CT angiography of the aorta and renal arteries, and transesophageal echocardiography were unremarkable. Pacemaker interrogation and telemetry monitoring during her hospital stay were unrevealing. Her hypercoagulapathy screen, including Factor V Leiden, Prothrombin gene mutation, Lupus Anticoagulant, Beta-2 Glycoproteins, and Cardiolipin Antibodies were was essentially negative, except for inconclusive IgM Cardiolipin Antibody level. Despite the lack of an identifiable etiology, a decision was made to bridge to Warfarin and continue anticoagulation for 6 months, with a plan to repeat hypercoagulapathy workup in 6 months.

DISCUSSION: Renal infarction is an uncommon cause of acute severe abdominal pain that is often delayed in diagnosis or underdiagnosed. Atrial fibrillation and hypercoagulapathy are among the commonest identified causal mechanisms involved in renal infarction occurrence. Other causes include renal artery dissection, suprarenal plaque embolization, vasculitis, and cocaine abuse. Idiopathic renal infarction is even less common, and therefore, a high index of suspicion is required to make the correct diagnosis. The treatment of renal infarction is based on the underlying etiology involved. Renal infarction in the setting of a hypercoagulable state or atrial fibrillation has traditionally been treated with anticoagulation. Anticoagulation is not recommended in the setting of fat emboli, atheroemboli or renal artery dissection. Renal infarction due to vasospasm secondary to cocaine use is further prevented by avoiding the inciting drug. Therefore, a vigorous search for the causal mechanism of renal infarction is extremely valuable in guiding the treatment plan and preventing recurrence. Idiopathic renal infarction, as presented in our case report, poses a treatment dilemma to clinicians, since no guidelines are available to direct clinical decisions. Most clinicians have traditionally treated idiopathic renal infarction with anticoagulation for variable duration of times. In many cases, a causal mechanism was eventually found, despite the negative initial work-up. Therefore, patients with apparently idiopathic renal infarction on initial assessment need to be followed closely for any new signs or symptoms that may shed light on the underlying etiology of the renal infarction.

A RARE CO-EXISTENCE OF CHYLOUS ACCUMULATION IN CLL Elizabeth S. John ${ }^{1}$; Ramy Sedhom ${ }^{2}$; Daniel Schaar ${ }^{1} .{ }^{1}$ Rutgers Robert Wood Johnson, Montville, NJ; ${ }^{2}$ Rutgers- Robert Wood Johnson, Staten Island, NY. (Control ID \#2469885)

LEARNING OBJECTIVE \#1: Determining etiologies of chylothorax and chylous ascites and exploring the pathophysiology of these two processes.

LEARNING OBJECTIVE \#2: Briefly discussing diagnostic strategies to help differentiate etiologies.

CASE: Chylothorax and chylous ascites are lymphatic complications characterized by leakage of triglyceride-rich fluid secondary to damage of the thoracic duct. Few cases in the literature exist regarding chylous complications from chronic lymphocytic leukemia (CLL). We report a rare co-existence of chylothorax and chylous ascites in a patient with CLL. A 60-year old non-smoker, non-drinker was admitted with dyspnea. Medical history included CLL diagnosed 5 years prior. He tolerated his first cycle of chemotherapy. On presentation, the patient complained of dyspnea and fatigue. He denied fevers, chest pain, pleurisy, cough, hemoptysis or recent travel. Physical examination revealed diminished breath sounds at the right lung base and a distended, non-tense abdomen. No stigmata of liver disease were present. Admission vitals included hypotension and tachycardia. Laboratory studies were significant for WBC 11.8, creatinine 1.4. ABG: $\mathrm{pH} 7.38, \mathrm{pCO} 2$ 26, pO2 108, O2 99.2 \%. Chest x-ray showed large right-sided pleural effusion without mediastinal adenopathy. Thoracentesis drained $1600 \mathrm{cc}$ of milky fluid from the right lung. Fluid studies revealed: RBCs 10455ug/L, 1256 nucleated cells (39 \% lymphocytes, $10 \%$ monocytes). An exudative effusion was noted based on Light's criteria. Pleural fluid triglycerides, $233 \mathrm{mg} / \mathrm{dl}$, confirmed suspicion of chylothorax. After, the patient was found to have new abdominal distention. Imaging showed new cirrhosis not seen on imaging just 2 months prior, and a large amount of ascites along with significant lymphadenopathy. A diagnostic paracentesis was performed, and the aspirate yielded $400 \mathrm{cc}$ of turbid fluid with triglyceride level of $213 \mathrm{mg} / \mathrm{dL}$, consistent with chyloperitoneum. Ascitic fluid cultures were sterile and cytology demonstrated no malignant cells or neutrophils. A communication between the thorax and the peritoneum was presumed, and later confirmed by the consistent accumulation of chyle into both the left and right pleural space. A decision was made to proceed with video-assisted thorascopic surgery (VATS) and mechanical pleurodesis. The patient improved following conservative dietary management and discharged to a rehabilitation facility.

DISCUSSION: This case is unique from multiple aspects. First, chylous ascites is caused by an accumulation of lymph in the abdominal cavity, and is extremely rare, with a reported incidence of approximately 1 of 20,000 admissions at a large university-based hospital over a two decade period. In the case of our patient, he had two risk factors for developing this rare entity - cirrhosis, which accounts for two thirds of all cases, and lymphoma which accounts for one third of cases. Secondly, while CLL is often associated with pleural effusion, chylothorax is rare because CLL seldomly causes adenopathy significant enough to obstruct the thoracic duct. Understanding the causes of chylous ascites and chylothorax in the setting of malignancy can guide future care. Our patient improved with VATS and mechanical pleurodesis, but as both are extremely rare conditions, there is scant literature on their management. 
A RARE CORONARY ARTERY ANOMALY PRESENTING AS ACUTE CORONARY SYNDROME. Chandana Shekar ${ }^{1}$; William Perucki ${ }^{2}$; Charudatta Wankhade ${ }^{3}$; Nikolas Krishna ${ }^{4}$. ${ }^{1}$ University of Connecticut School of Medicine, Farmington, CT; ${ }^{2}$ University of Connecticut, Farmington, CT; ${ }^{3}$ Lincoln Medical and Mental Health Center, Bronx, NY; ${ }^{4}$ Hartford Hospital, Hartford, CT. (Control ID \#2470006)

LEARNING OBJECTIVE \#1: Early recognition of coronary artery ectasia as a cause of an acute ischemic presentation.

CASE: A 74 year old male patient who had recently moved from Turkey, with past medical history of coronary artery disease with no further details available and hypertension presented to our hospital with sudden onset severe substernal chest pain, radiating to left shoulder and relieved with nitroglycerine. Pertinent positive history included shortness of breath, nausea and headache. Pertinent negative history included no diaphoresis, syncope, palpitations or back pain. He had multiple episodes of similar chest pain, resolved with nitroglycerine over the past 4 years and had not sought medical attention. He was being treated with aspirin, clopidogrel, beta blocker and statin since the past 4 years. No recent illness or medication changes were present. No alcohol or tobacco use was present either. Family history was negative for cardiac diseases. On arrival to our hospital, he was hemodynamically stable with a normal physical examination. Initial blood work revealed negative cardiac enzymes when tested thrice; a normal complete blood count, basic metabolic panel and coagulation panel. B-type Natriuretic Peptide was elevated at $453 \mathrm{pg} / \mathrm{mL}$. Electrocardiogram showed normal sinus rhythm with a heart rate of 58 beats per minute, left axis deviation, ST segment elevation in $\mathrm{I}, \mathrm{aVL}$ and $\mathrm{V} 2$ of less than $1 \mathrm{~mm}$, ST segment depression in III and aVF. Echocardiogram showed Left ventricular Ejection Fraction of $39 \%$, basal inferior dyskinesia with moderate left ventricular hypertrophy. Chest $\mathrm{X}$ ray did not show any acute cardiopulmonary process Computed Tomography of the chest showed a small saccular aneurysm over the left side of the aortic arch, but no dissection. He was being treated with dual anti platelet therapy, statin, heparin and beta blocker in the meanwhile. On further imaging, vasodilator stress test with myocardial perfusion scan showed a medium size, severe scar in the Right Coronary Artery (RCA)/Left Circumflex artery (Lcx) territory. No evidence of ischemia was noted. Cardiac Positron Emission Tomography Viability study showed viable myocardium in the Left Anterior Descending Artery (LAD) Territory and significant hibernating scar in the Lcx/RCA territory scar. On cardiac catheterization, luminal irregularities were noted in a short $0.5 \mathrm{~cm}$ left main artery, along with diffuse ectasia of the proximal, mid and distal circumflex trunk of the Lcx and diffuse ectasia of the proximal, mid and distal RCA. He was hence diagnosed with Type I coronary artery ectasia according to Markis classification. Etiology for the ectasia in our patient was not able to be identified. Surgical revascularisation was deferred as he did not have stenotic disease. Dual anti platelet therapy with aspirin and clopidogrel was continued and anticoagulation with warfarin was initiated. The patient made a full recovery and was discharged home. $\mathrm{He}$ follows up as an outpatient and has not had a recurrence of chest pain when seen at the last follow-up.

DISCUSSION: Coronary Artery Ectasia (CAE) is a rare abnormality of the coronary anatomy. It is an abnormal, irregular, saccular dilatation of more than a third segment of the coronary artery to more than 1.5 times the diameter of the normal segment. The incidence ranges from 1.2 to $4.9 \%$. It is most commonly associated with atherosclerosis. It has also been associated with aortic aneurysms. Isolated CAE or dilated coronaropathy which excludes atherosclerosis, connective tissue diseases, and other cardiac defects, is very rare with an angiographic frequency of 0.1 to $0.32 \%$. CAE is far from benign and can potentially cause ischemia as well as acute coronary events due to coronary spasm, slow flow and thrombus formation. Optimum treatment guidelines and role of revascularization is yet to be established. However, prophylactic use of warfarin in the treatment regimen to reduce sequelae of thrombosis has been supported by literature. Hence, early identification and treatment of CAE is vital to decrease morbidity and mortality in such patients.

A RARE ETIOLOGY OF HEMOPTYSIS IN AN ELDERLY MALE Shravana Bheemanathi $^{3}$; Karthik Ragunathan ${ }^{2}$; Azam Mohammad ${ }^{1} .{ }^{1}$ Bronx, Bronx, NY; ${ }^{2}$ OSF St.Francis medical center, Peoria, IL; ${ }^{3}$ St. Barnabas Hospital, Bronx, NY. (Control ID \#2469813)

LEARNING OBJECTIVE \#1: Understand the increased risk of aortobronchial fistula in patients with history of thoracic aortic surgeries

CASE: A 86 year-old male with past medical history significant for thoracic aortic aneurysm s/p graft repair and chronic kidney disease (CKD) presented to our institution because of intermittent hemoptysis for 3 months duration. He first noticed the symptoms when he had some cough and congestion and went to a nearby emergency department where the physician suspected community acquired pneumonia as the cause of his symptoms. Patient was prescribed oral antibiotics and sent home. One week later the patient again had hemoptysis. Given his history of chronic kidney disease, a non contrast computed tomography (CT) of the chest was done and showed left pulmonary opacity. He was seen by pulmonologist and a bronchoscopy showed gelatinous mucous plugs and no bleeding lesion. Patient was discharged home as his bleeding resolved. Two months later, hemoptysis recurred and were more significant and he was transferred to our institution for further evaluation. Bronchoscopy was repeated and showed large amount of blood in lef upper lobe and lingula but no actively bleeding lesion was diagnosed. With patient's history of thoracic aortic repair with graft, we were suspicious of aortobronchial fistula. Risk and benefits of giving IV contrast for CT of chest were discussed with the patient and he agreed to proceed with the imaging study, given high suspicion for aortobronchial fistula. CT angiography found a focal $8 \mathrm{~mm}$ ulcerated plaque in the distal aortic arch with adjacent pulmonary parenchymal opacity possibly representing blood clot. This was suspicious for aortobronchial fistula so cardiothoracic surgery was consulted. Decision was made to perform endovascular aortic repair with synthetic graft. Patient's symptoms resolved post operatively and was discharged home on a stable condition.

DISCUSSION: Here we present a case of aortobronchial fistula. Literature review reveals that $75 \%$ of cases occurred in patients with history of previous thoracic aortic surgery. Presumed pathophysiology is that a foreign body can elicit chronic inflammation, leading to formation of a fistula. The bleeding is intermittent because lung tissue is thrombotic in nature. Unenhanced chest CTs and bronchoscopies may not be adequate to make diagnosis. computed tomography of the chest with intravenous contrast must be performed to diagnose and treat this potentially fatal condition. Once diagnosed with this condition, patients needs urgent surgical repair and the procedure has high risk of morbidity and mortality with some studies predicting as much as 25 to $40 \%$ mortality rate. A high index of suspicion is needed to diagnose this condition, especially in patients with history of previous thoracic aortic surgery.

A RARE PAIN IN A COMMON SCENARIO Aradhna Seth ${ }^{2}$; Minal A. Shah ${ }^{1} .{ }^{1}$ Baylor College Of Medicine, Houston, TX; ${ }^{2}$ Baylor College of Medicine, Houston, TX. (Control ID \#2457589)

LEARNING OBJECTIVE \#1: Recognize pylephlebitis as a rare etiology of abdominal pain 
LEARNING OBJECTIVE \#2: Identify the indications for anticoagulation

CASE: A 62 year old Caucasian male with history of hypertension and remote cholecystectomy presented with 1 week of malaise, night sweats, fevers, and anorexia after consuming fast food. Three days prior to admission, he developed non- bloody, non-bilious emesis and non-radiating, cramping, epigastric pain not associated with meals. He denied preceding weight loss, changes in stool frequency, color, or caliber, dysuria, joint pains, or skin rashes. He had no recent sick contacts, travel, or family history of malignancy. Upon arrival, vital signs demonstrated temperature $101^{\circ} \mathrm{Fahr}-$ enheit, blood pressure 74/53 mmHg, heart rate 105 beats/min, and respiratory rate 18 breaths/min. The patient had pallor, dry mucus membranes, diffuse abdominal tenderness with no rebound or guarding, splenomegaly without hepatomegaly, and cool extremities. Pertinent labs included leukocytosis of $14.2 \mathrm{~K} / \mathrm{ul}$, platelets $51 \mathrm{~K} / \mathrm{ul}$, creatinine $3.59 \mathrm{mg} / \mathrm{dL}$, bicarbonate $20 \mathrm{mmol} / \mathrm{L}$, total bilirubin $6.9 \mathrm{mg} / \mathrm{dL}$, direct bilirubin $5.3 \mathrm{mg} / \mathrm{dL}$, alkaline phosphatase $562 \mathrm{U} / \mathrm{L}$, alanine transaminase $128 \mathrm{U} / \mathrm{L}$, aspartate transaminase $152 \mathrm{U} / \mathrm{L}$, venous lactate $46 \mathrm{mmol} / \mathrm{L}$, and normal coagulation factors. Blood cultures grew Escherichia coli and Klebsiella pneumoniae. Abdominal computed tomography demonstrated a thrombus in the portal vein confluence and adjacent superior mesenteric vein with small bowel wall thickening. The presence of a thrombus prompted evaluation for malignancy and hypercoagulability disorder, respectively. No malignancy was found; however, he tested positive for antiphospholipid antibody syndrome (APLS). Ultimately, he was diagnosed with pylephlebitis secondary to small bowel enteritis and underlying APLS. Despite antibiotics and heparin, the patient developed recurrent abdominal pain and fever requiring partial resection of ischemic small bowel.

DISCUSSION: Septic thrombophlebitis of the portal vein, or pylephlebitis, is a rare clinical entity. In the past, pylephlebitis was frequently associated with appendicitis; however, common etiologies today are biliary infections, colonic infections and hypercoagulable conditions. Symptoms are non-specific including fevers, chills, malaise, and abdominal pain. Bacteremia often occurs, particularly due to Escherichia coli, Klebsiella pneumoniae, Peptostreptococcus, and Bacteroides fragilis. Splenomegaly in the absence of hepatomegaly or chronic liver disease should prompt consideration of portal vein thrombosis. Splenomegaly is present in $10-25 \%$ of patients with pylephlebitis. The cornerstone of treatment is antibiotics. Some patients may require surgical intervention if complications of pylephlebitis occur, which include hepatic abscess formation and small bowel ischemia. Patients with pylephlebitis should be evaluated for underlying malignancy, hypercoagulability disorders, inflammatory bowel disease, and human immunodeficiency virus. The indications for anticoagulation are controversial but include hypercoagulable state (i.e. APLS), persistent fever despite antibiotics, and thrombus involving the superior mesenteric vein due to risk of small bowel ischemia. Internists should have a high index of suspicion for pylephlebitis in patients presenting with abdominal pain and septic shock given the potential morbidity and mortality associated with this under-recognized condition. Additionally, physicians should consider the use of anticoagulation in patients with thrombus involving the superior mesenteric vein as this can lead to small bowel ischemia, as was the case in our patient. All cases of pylephlebitis should warrant work up for an underlying hypercoagulable state.

A RARE PIN CUSHING Nicole $\mathrm{Ng}^{1}$; Julie Lorton ${ }^{2}$; Eric Epstein ${ }^{2}$; Tulay Aksoy ${ }^{2}$. ${ }^{1}$ Albert Einstein College of Medicine, Bronx, NY; ${ }^{2}$ Montefiore Medical Center, Bronx, NY. (Control ID \#2469216)

LEARNING OBJECTIVE \#1: Recognize the potential effects of Epidural Steroid Injections on the Hypothalamic-Pituitary-Adrenal axis of patients on ritonavir

LEARNING OBJECTIVE \#2: Manage Iatrogenic Cushing's Syndrome and Secondary Adrenal Insufficiency due to the interaction between epidural steroids and ritonavir
CASE: A 49 year-old woman with HIV presented with 4 months of progressive facial and extremity swelling, and generalized weakness. During this time she was also found to have an episode of CD4 lymphopenia and thrush, despite adherence to her HIV regimen: tenofovir, emtricitabine, lopinavir and ritonavir. On review of systems, she reported a five kilogram weight gain despite decreased appetite. She was hypertensive with moon facies, a buffalo hump, central obesity, abdominal striae, and bilateral upper and lower extremity edema. On further questioning, she reported two recent epidural triamcinolone injections for her chronic sciatica, at 4 and 2 months prior to her presentation. Symptoms began 1 week following the initial injection. Laboratory data revealed hypokalemia to $3.1 \mathrm{mEq} /$ $\mathrm{L}$, ACTH level $<5 \mathrm{ug} / \mathrm{dL}$, morning cortisol level of $0.4 \mathrm{ug} / \mathrm{dl}$, cortisol level of $10 \mathrm{ug} / \mathrm{dL}$ at $1 \mathrm{~h}$ after Cosyntropin stimulation, and triamcinolone level of $0.3 \mathrm{mcg} / \mathrm{dL}$. Considering this dramatic and prolonged presentation, ritonavir (and lopinavir) was discontinued and dolutegravir was initiated. The patient was monitored in the hospital for an additional 2 days for possible iatrogenic adrenal crisis and she was started on maintenance hydrocortisone preventatively. Hospital course was subsequently uneventful and by day of discharge a repeat triamcinolone level showed total clearance of the drug. She was followed outpatient and adrenal axis had normalized by 1 month post-hospitalization.

DISCUSSION: Cushing's Syndrome is defined as a clinical constellation of signs and symptoms of hypercortisolism - central obesity, facial plethora, glucose intolerance, generalized weakness, hypertension, etc. The most common cause of hypercortisolism is exogenous intake of glucocorticoids, including oral, injected, topical, or inhaled routes of administration. The differential diagnosis should also include various endogenous endocrine-associated pathologies such as pituitary adenoma, adrenal gland tumor, or less commonly, the ectopic ACTH syndrome. Evaluation should be guided by history, physical exam, and laboratory values including 24-h urine free cortisol, dexamethasone suppression testing, basal and suppressed ACTH values, and additional adrenal and pituitary laboratory evaluation. Epidural Steroid Injections have been used for pain control in patients with low back pain, radiculopathy, and spinal stenosis since the 1950 s. However, the safety and efficacy of Epidural Steroid Injections have not been established and the US FDA actually does not approve of corticosteroids for this use. Furthermore, these injections have been associated with rare, but serious side effects from infection and bleeding to nerve damage. Nonetheless, it is believed that epidural administration of glucocorticoids allows for direct deposition at the site of inflammation rendering a more pronounced therapeutic effect and longer duration of action at the injection site. However, a known aspect of the pharmacokinetic profile of epidural medications, triamcinolone and other glucocorticoids included, is the diffusion of medication across spinal meninges which allows for access to the epidural venous plexus and systemic circulation. Although systemic absorption is typically negligible, when the metabolism of triamcinolone is significantly decreased, as occurs with concomitant use of ritonavir, a protease inhibitor with inhibitory effects on cytochrome $\mathrm{P} 4503 \mathrm{~A} 4$, systemic circulation can be dramatically increased. One study reported that the elimination half-life of triamcinolone was prolonged 170 -fold with co-administration of ritonavir. This increased bioavailability of epidural triamcinolone can lead to iatrogenic Cushing's Syndrome with suppression of the Hypothalamic-Pituitary axis resulting in Secondary Adrenal Insufficiency. Due to ritonavir's potent inhibition of cytochrome P450, abrupt withdrawal carries risk for sharp decline in glucocorticoid levels and subsequent adrenal crisis. In patients with less severe cases of Iatrogenic Cushing's Syndrome, an alternative management strategy is to continue ritonavir with no further steroid injections and allow self-resolution of Cushing's as triamcinolone is slowly metabolized. Increased awareness of the clinical implications and potential adverse effects of Epidural Steroid Injections in conjunction with ritonavir should lead us to: 1) Perform thorough medication reconciliation for all patients to identify drug-drug interactions 2) Consider alternative steroids for epidural injection, e.g. methylprednisolone, which to date has no reported adverse interactions with ritonavir 3) Increase monitoring for signs and symptoms of Iatrogenic Cushing's Syndrome after epidural steroid 
injection in patients on ritonavir 4) Monitor Cushing's Syndrome patients for Secondary Adrenal Insufficiency as exogenous steroids are naturally metabolized

\section{A RARE PRESENTATION OF PARVOVIRUS INFECTION WITH EDEMA,} JOINT PAIN AND PROTEINURIA Stephen Hudak ${ }^{1}$; Deepa R. Nandiwada ${ }^{2}$; Matthew Camiolo ${ }^{1} .{ }^{1}$ UPMC, Pittsburgh, PA; ${ }^{2}$ University of Pittsburgh, Pittsburgh, PA. (Control ID \#2467917)

LEARNING OBJECTIVE \#1: Recognize the clinical presentation of viral arthralgia LEARNING OBJECTIVE \#2: Understand the epidemiology of viral arthralgia CASE: A 44 year old woman without significant medical history presented with increasing fatigue, joint pain and anasarca over the preceding 10 days. Her symptoms began with swelling and tenderness of the metacarpal phalangeal joints and proximal intraphalangeal joints bilaterally. She initially sought treatment at another facility where x-rays revealed joint space narrowing and soft tissue swelling without evidence of erosive disease. She was given 5 days of oral prednisone $60 \mathrm{mg}$ out of concern for mixed connective tissue disease and instructed to follow-up with Rheumatology. Upon evaluation in clinic, she noted improvement in her swelling but continued to experience significant arthralgia Hepatitis serologies were found negative. Anti-cyclic citrullinated peptide antibody, rheumatoid factor and anti-nuclear antibodies were all within normal limits. Erythrocyte sedimentation rate and C-reactive protein were unremarkable. She was subsequently continued on steroids without clear diagnosis. She later returned to the emergency department with worsening fatigue, pain and edema that began in her distal lower extremities and extended cranially to the level of her abdomen. Throughout this time she denied fevers, chills, nausea, vomiting, anorexia, rash, focal neurologic deficit, weakness, numbness or tingling. Her exam was benign except for bilateral lower and upper extremity pitting edema to the level of the thigh, with edema of the hands as well Labs at admission were notable for a serum potassium of $6.4 \mathrm{mmol} / \mathrm{L}$ without concerning EKG changes, a creatinine of $1.3 \mathrm{mg} / \mathrm{dl}$, a bicarbonate of $19 \mathrm{mmol} / \mathrm{L}$ with an anion gap of 4. Urinalysis revealed $1+$ blood with red blood cells, trace protein, granular and cellular casts. She was admitted to the floor for further management Further Hospital Course Rheumatology was asked to evaluate the patient and felt that she was likely experiencing a viral versus reactive arthritis. They recommended discontinuing prednisone in the absence of a definitive diagnosis. Her extensive autoimmune workup was unremarkable except for low complement levels, positive EBV IgG and nuclear antigen, positive cytomegalovirus IgM with negative IgG, and positive parvovirus B19 IgM and IgG serologies. Transthoracic echo revealed normal ejection fraction. A urinalysis came back positive for moderate protein and $1+$ blod, so nephrology was asked to evaluate for possibility of a glomerular disease. One month after discharge she was evaluated by Rheumatology as an outpatient. She noted improvement in her edema mild residual joint pain. Given her positive viral serologies and the constellation of symptoms she had experienced, it was felt that her illness was most consistent with viral-related polyarthralgia secondary to Parvovirus B19. She was continued on NSAIDs for symptomatic relief.

DISCUSSION: Parvovirus is an extremely common infection, with $50-80 \%$ of adults being seropositive for parvovirus B19-specific IgG antibodies2. Women of child bearing age serocomvert at a rate of around $1.5 \%$ per year 3 . Viral related arthalgias are a common cause of self limited arthalgias, in the adult patient. Specifically, parvovirus B-19 associated arthalgias are often preceded by 7-10 days of febrile illness. They often affect the hands, feet and knees, usually symmetrically. Rash is present in approximately $75 \%$ of cases, however the characteristic "Slapped cheek," rash is only seen in approximately $20 \%$ of patients. Pain is usually the dominant characteristic, but it can be associated with swelling 1 . There have been some case reports with anasarca as a presenting finding in parvovirus infections mediated through glomerular disease. This is a possible pathophysiological pathway in our patient as well, although complement levels were normal. This phenomonen is also postulated to be due to capillary leak syndrome from systemic inflammation of a viral infection. 1. Anderson LJ. Role of parvovirus B19 in human disease. Pediatr Infect Dis J. 1987;6:711-8. 2. Koch WC, Adler SP. Human parvovirus B19 infections in women of childbearing age and within families. Pediatr Infect Dis J. 1989;8:83-7. 3. Brooks G. Parvoviruses. In: Brooks GF, Carroll KC, Butel JS, Morse SA, Mietzner TA, editors. Jawetz, Melnick, \& Adelberg's Medical Microbiology. 26th edn. New York: McGraw-Hill; 2013 4. Krishnan JK, Cinti SK. Case Report: An Unusual Presentation of Generalized Edema in an Adult with Parvovirus Infection. Infect Dis Clin Pract 2013;21: 145 Y146

\section{A RARE RUSE BY A COMMON CONDITION: MENINGITIS RETENTION} SYNDROME James S. Lang. Cambridge Health Alliance, Cambridge, MA; Harvard Medical School, Boston, MA. (Control ID \#2469770)

LEARNING OBJECTIVE \#1: Recognize acute urinary retention as a potential complication of aseptic meningitis

CASE: A previously healthy 32-year-old man presented to an outside ED with complaints of headache for 5 days and fever for 3 days, and was diagnosed with a viral syndrome. He had returned from a Caribbean cruise 5 weeks prior. He had no sick contacts. One week later, he arrived to our ED complaining of a fever of $104 \mathrm{~F}$, chills, diplopia, unsteady gait, and headache. His wife reported he had been increasingly confused. He also reported difficulty urinating over the past 5 days and was unable to void in the ED. Urinary catheter placement revealed $700 \mathrm{cc}$ of urine. On review of systems, he reported mild nasal congestion and subtle left hand numbness. On exam, his vitals were normal except for a fever of 101.4 F. He appeared exhausted and was not oriented to the day. He was mildly ataxic upon testing gait. He had $2+$ reflexes with 1 beat clonus on right ankle and 2-3 beats on the left. He had mild dysmetria on cerebellar testing, but with preserved sensation and 5/5 strength throughout. His exam was otherwise normal, notably without meningeal signs. CT and CXR were both unremarkable. CBC and CMP were normal. Lumbar Puncture demonstrated pleocytosis with lymphocytic predominance, low glucose $(36 \mathrm{mg} / \mathrm{dL})$, elevated protein $(203 \mathrm{mg} / \mathrm{dL})$, and elevated WBC (210/mm3). He was started on Vancomycin, Ceftriaxone and Acyclovir and admitted for further work-up. A brain MRI demonstrated minimal leptomeningeal enhancement with proteinaceous material in the subarachnoid space and prepontine cistern consistent with meningitis, but no intraparenchymal lesions. Spine MRI revealed a C6-7 disc herniation without compression and enhancement of the meninges with scattered inflammatory changes in the thoracic and lumbar regions of his spinal cord, consistent with spinal meningitis. EEG showed nonspecific findings suggesting an encephalopathic process. CSF cultures (bacterial and fungal) and gram stain, as well as blood and urine cultures were negative. CSF IgG, TB smear, Cryptococcal Ag, Histoplasmosis Ag, Coccidiomycoses Ab, Lyme Ab western blot and PCR, India Ink, HSV and VDRL were all negative. Oligoclonal bands in serum and CSF were negative. $\mathrm{HIVAb} / \mathrm{Ag}$ and Treponema $\mathrm{Ab}$ were both negative. Dengue Fever Ab/Ag was negative. Blood parasite smear was negative. ESR and CRP were both normal. RF and ANA were negative. ACE level was also normal. Intravenous antibiotics were discontinued after $48 \mathrm{~h}$, given negative cultures. Acyclovir was discontinued on hospital day 5 when CSF was negative for HSV. The patient failed a voiding trial on hospital day 4 so his urinary catheter was replaced. At the time of discharge on hospital day 5, the mild ataxia and confusion had fully resolved. A voiding trial on day 22 was unsuccessful, and the urinary catheter was replaced. A subsequent voiding trial on day 36 was successful. He later reported erectile dysfunction and diminished libido, though both slowly improved by 1-year follow-up. Six months from presentation, he was running 13 miles in preparation for a half-marathon with no residual ataxia.

DISCUSSION: Aseptic meningitis resulting in urinary retention has been described in case reports as Meningitis Retention Syndrome (MRS). Little is known about the pathogenesis of this entity and treatment options remain limited, often requiring urinary 
catheterization for many weeks. It is thought that MRS may be a mild form of Acute Disseminated Encephalomyelopathy (ADEM) that selectively affects the lower urinary tract innervation, since often MRS includes a mild or partial constellation of symptoms usually seen in ADEM (signs of encephalitis, myelitis, and lower urinary tract symptoms). The available literature identifies time to recovery of bladder function between 2 and 10 weeks. In the patient described above, bladder function slowly returned to normal by week 5. MRS is rare, and may often be confused with transverse myelitis. While several of the patient's symptoms overlap with this diagnosis (ataxia, urinary retention, erectile dysfunction and decreased libido), he did not have sensation loss, which would be expected. Additionally, he had only mild ataxia, which completely resolved within days of presentation. Another syndrome described in the literature and often associated with MRS is Elsberg Syndrome, which is a sacral myeloradiculitis causing urinary retention and sacral dermatome sensation loss, usually associated with genital herpes. This is not consistent with our patient's presentation, as he did not have any sensation loss or signs of genital herpes. Most investigators believe the process by which meningitis causes MRS is through inflammation of the spinal cord, or myeloradiculitis. Treatment is supportive, though some have reported the use of corticosteroids, despite a lack of data. In summary, MRS is rare, but is an important condition to identify since urinary retention is a urological emergency and may be a complication of aseptic meningitis. Prompt catheter placement should be initiated and urology follow-up arranged due to frequent need for long-term catheterization. Additionally, male patients should be counseled on the possibility of resultant erectile dysfunction and decreased libido.

A ROUTINE EXAM WITH NOT SO ROUTINE COMPLICATIONS: A CASE REPORT OF STREPTOCOCCAL PERITONITIS AND TOXIC SHOCK SYNDROME AFTER A PAP Yusuke Ohashi; Fumihiro Kodama; Shadia Constantine. Teine Keijinkai Hospital, Sapporo, Japan. (Control ID \#2442543)

LEARNING OBJECTIVE \#1: To list toxic shock syndrome among the causes of unexplained shock vital signs

LEARNING OBJECTIVE \#2: To list the vaginal mucosa among one of the potential ports of entry of Streptococcus species

CASE: A 57-year-old Asian woman presents with sudden onset of generalized, severe abdominal pain, which began hours after undergoing a routine gynecological examination and screening for cervical cancer. She reports associated vaginal discharge, nausea, vomiting, and dizziness. Other review of system is within normal limits. The patient's past medical history includes rheumatoid arthritis, and osteoporosis; she takes lansoprazole, prednisolone $4 \mathrm{mg} /$ day, methotrexate, folic acid, and alendronate. She does not smoke or use recreational drugs, but does drink alcohol occasionally. She has no known drug or food allergies. She is monogamous, not sexually active, menopause at 52 years-old, and has no history of sexually transmitted diseases. On physical exam, vital signs include temperature $37.3{ }^{\circ} \mathrm{C}$, blood pressure $65 / 49 \mathrm{mmHg}$, heart rate 120 beats per minute, respiratory rate 30 breaths per minutes, and oxygen saturation $98 \%$ on room air. She is alert and oriented to person, place, and time. Abdominal exam reveals slight distention, decreased bowel sounds, positive rigidity and rebound tenderness. Gynecological exam only shows brown discharge. Laboratory tests show increased white blood cells $12,000 / \mu \mathrm{g}$, alkaline phosphatase $327 \mathrm{U} / \mathrm{L}$, alanine transaminase $81 \mathrm{U} / \mathrm{L}$, aspartate aminotransferase $220 \mathrm{U} / \mathrm{L}$, creatine phosphokinase $591 \mathrm{U} / \mathrm{L}$, blood urea nitrogen $33.6 \mathrm{mg}$ / $\mathrm{dL}$, creatinine $2.59 \mathrm{mg} / \mathrm{dL}$, C-reactive protein $15.69 \mathrm{mg} / \mathrm{dL}$, prothrombin time $40.2 \%$, activated partial thromboplastine time $77.2 \mathrm{~s}$, fibrinogen $459 \mathrm{mg} / \mathrm{dL}$, and FDP $33 \mu \mathrm{g} / \mathrm{dL}$. Transvaginal ultrasound shows a small uterine leiomyoma without signs of pyometra. Abdominal CT scan shows inflammatory changes in the intestines without abscess formation. The patient is treated empirically for septic shock with meropenem, hydration, hydrocortisone, and vasopressors, but continues to be hemodynamically unstable. Given the suspicion of an intraabdominal source, she underwent emergent exploratory laparoscopy, which only revealed inflammatory changes in the intestines, without abscess or appendicitis. The origin of the shock remained elusive... On the next day, blood cultures, peritoneal fluid culture, and vaginal discharge culture were all reported as growing Gram positive cocci, finally identified as Streptococcus pyogenes. We diagnosed Streptococcal Toxic Shock Syndrome (TSS) given isolation of S. pyogenes from blood, hypotension, renal dysfunction, coagulopathy, and liver dysfunction. We changed antibiotics from meropenem to ampicillin and clindamycin with satisfactory clinical response. The vasopressor and hydrocortisone were tapered and discontinued at day 5. We continued antibiotics for 14 days, and the patient was discharged hospital in Day 15 in good condition.

DISCUSSION: A confirmed case of Streptococcal TSS is characterized by hypotension, group A Streptococcus isolated from a normally sterile site, and 2 or more organ dysfunctions. The usual port of entry of invasive streptococcal infection is a skin wound or surgical site, however, vaginal mucosa could be the potential entry in as much as $10 \%$ of the cases. Group B streptococcus is a well-known "colonizer" of the vaginal mucosa; in the other hand, an investigation detected vaginal colonization with group A Streptococcus in only $0.03 \%$ of the patients studied. Risk factors for TSS are minor trauma, recent surgery, viral infection, and postpartum state. If the patient has a skin wound, proper and prompt debridement is very important. CT and MRI may be helpful to locate the primary site of infection, but because the group A streptococci do not form gas or frank abscess, radiologic interpretations are frequently not definitive. Regarding antibiotic choices, penicillin monotherapy is associated with high mortality and extensive morbidity in the setting of aggressive infections associated with TSS. It is essential to add clindamycin which can affect any stage of bacterial growth. Clindamycin also reduce the production of bacterial toxins, and penicillin binding proteins which is associated with cell wall synthesis and degeneration. In addition to this, it has longer half-time than penicillin alone. Even procedures usually considered simple and routine, can be dangerous in the setting of immunosuppression.

A SECOND LOOK AT SECONDARY SYPHILIS Jaime Deseda; Tina Wang; JonEmile Kenny. Mount Sinai Beth Israel, New york, NY. (Control ID \#2469416)

LEARNING OBJECTIVE \#1: Identify the clinical manifestations of secondary syphilis LEARNING OBJECTIVE \#2: Recognize rapid progression of syphillis in an acute retroviral syndrome

CASE: A 28-year-old man with no significant past medical history presented to the emergency department for worsening pruritic rash for the past 3 days. He was in his usual state of health until 1 week prior when he developed fevers, chills, myalgias, fatigue, and poor appetite. He was initially evaluated at an urgent care center and treated empirically for influenza with oseltamivir. However, shortly after taking oseltamivir, the patient noted the development of a pruritic rash on his back. This progressed centrifugally until the patient presented for further evaluation. The history was also significant for the patient being a homosexual man who, 2 weeks prior to the initial onset of symptoms, endorsed an unprotected anal-receptive sexual encounter. Patient denies additional unprotected sexual encounters within 6 months. The HIV status of his partner was unknown. One week prior, he had also traveled to Pennsylvania and come into contact with multiple farm animals but did not recall any tick or other insect bites. On initial evaluation, the patient was afebrile with normal vital signs. Physical exam was significant for a diffuse maculopapular targetlesion rash involving anterior and posterior trunk, extensor and flexor surfaces of extremities, and the palms and soles. No oral or genital lesions were appreciated. Laboratory analysis revealed elevated aminotransferases, AST $211 \mathrm{U} / \mathrm{L}$ and ALT $175 \mathrm{U} / \mathrm{L}$. Basic metabolic panel, complete blood counts, and urinalysis were all within normal limits. Initial HIV antigen/antibody test was negative. The patient also tested negative for 
chlamydia, gonorrhea, CMV, and acute hepatitis. RPR and testing for lyme and rickettsial diseases were also sent. The patient was initially treated empirically with doxycycline for tick-borne illnesses. His rash was biopsied and he was also given solu-medrol for erythema multiforme. However, shortly after admission, RPR testing returned reactive. This was confirmed with a positive FTA-ABS test. RPR titer was 1:128. The patient was diagnosed with secondary syphilis and subsequently treated with Benzathine penicillin G. A HIV viral load was sent and later revealed greater than 1.8 million copies $/ \mathrm{mL}$. The patient was discharged with improvement in symptoms shortly thereafter.

DISCUSSION: Although significant progress has been made in the overall rates of reported primary and secondary syphilis in the United States over the past few decades, it is a disease that continues to affect 5.3 in 100,000 people. In the 2014 report by the Center for Disease Control detailing primary and secondary syphilis in the United States from 2005 to 2013, analysis showed that rates were again on the rise. These increases were seen primarily in men, particularly in men who have sex with men (MSM). We present here a case of a 28-year-old MSM who presented with an atypical time course of secondary syphilis in the setting of acute HIV infection. In the natural history of syphilis, primary syphilis presents as a chancre at the site of inoculation approximately 2 to 3 weeks after sexual contact. Around $25 \%$ of patients will then progress to secondary syphilis, which typically manifests 3 to 6 weeks after the conclusion of the primary stage and can include nonspecific constitutional symptoms, rash, condylomata lata, alopecia, and generalized lymphadenopathy. In our patient, however, constitutional symptoms and rash occurred just 2 weeks after reported unprotected sexual contact. Our patient was not entirely aware of any preceding genital ulcer, as is often the case, and none was seen on examination during his initial evaluation. It has long been known that concurrent HIV can significantly impact the presentation of syphilis including greater organ involvement, a protracted course of secondary syphilis, and more rapid progression to neurosyphilis. One study showed that men infected with HIV were more likely to have overlapping secondary syphilis with genital ulcer disease than non-infected men. However, most cases of secondary syphilis present weeks to months after the primary stage. There is little research on the effects of concurrent HIV infection on shortening the time to development of primary and secondary syphilis after inoculation. Our patient presents a case of early manifestation of secondary syphilis in which coinfection with HIV may have modulated the disease course. In high risk populations, it is important to recognize the signs and symptoms of secondary syphilis and consider simultaneous HIV infection in the case of atypical presentation.

A SHOCKING PRESENTATION OF ACUTE ISCHEMIC STROKE Daniela $\underline{\text { DiMarco }}{ }^{1}$; Rupal Panchal ${ }^{2}$. ${ }^{1}$ University of Connecticut Health Center, Farmington, CT; ${ }^{2}$ The Hospital of Central Connecticut, New Britain, CT. (Control ID \#2467819)

LEARNING OBJECTIVE \#1: Recognize systemic electrical injury as a rare cause of focal cerebral ischemia and infarct.

LEARNING OBJECTIVE \#2: Differentiate the etiology of acute stroke in a patient with electrical injury and some typical stroke risk factors.

CASE: A 47 year old male smoker with no significant past medical history presented with worsening blurred vision and right frontal headache. At his job site 1 day prior, he stepped into a puddle of water in contact with a $110 \mathrm{~V}$ electrical cable. $\mathrm{He}$ experienced full body shaking and acute vision loss, which improved to blurred vision after about $30 \mathrm{~min}$. After the event, he had a transient right frontal headache that was partially improved with ibuprofen. The exposure was believed to be only seconds, and he had no personal or family history of stroke. On presentation he was found to have a blood pressure of 166/109 (normotensive at physical 6 months prior), mild left peripheral visual field cut, with the remainder of the exam unremarkable. In cardiac workup he had right bundle branch block and left anterior fascicular block of unclear onset, and slight elevation in troponin. Non-contrast CT of the head showed a hypodensity in right occipital lobe, confirmed as acute right PCA distribution infarct on follow up MRI of the brain. The patient was treated with aspirin and statin. Further workup included CTA of the head and neck, which did not reveal dissection, occlusion, or significant stenosis of the vasculature. TTE showed reduced ejection fraction and inferior wall akinesis. The visual symptoms persisted throughout hospitalization, and the patient was discharged on aspirin and statin therapy. Follow up TTE 2 months later revealed normalized ejection fraction, and no wall motion abnormalities.

DISCUSSION: This case demonstrates a very rare cause of acute ischemic stroke in a person with some typical stroke risk factors. Although the patient did not have a highvoltage or extended duration exposure, the onset of visual symptoms coincides with the electrocution event. In addition to CNS injury, his TTE findings were seemingly consistent with stunned myocardium from electric shock. The mechanism of stroke in this patient was thought to be secondary to acute cerebral vasospasm, rather than thermal vascular injury. The electrical current can cause overstimulation of the sympathetic nervous system and subsequent vasospasm, however this has not yet been well described or understood, and requires further investigation. Treatment of stroke under such conditions has followed the traditional ischemic stroke pathway. As preventive therapy, the benefits of aspirin and statin are unclear in patients who suffered stroke from electrical shock-induced vasospasm without the typical stroke risk factors. As both medication classes have side effects, and this type of injury is more common in a younger population, further consideration of their use may be warranted.

\section{A SINGLE PLAIN ABDOMINAL RADIOGRAPHY MIGHT HAVE SAVED A} PATIENT WITH INTRAABDOMINAL INFECTION Dongkyung Seo ${ }^{1}$; Toshiaki Wakai $^{2}$; Masaji Saijo ${ }^{1}$; Keitaro Takeda ${ }^{1}$; Dongje Seo ${ }^{3}$; Yasushi Tanabe ${ }^{1}$; Urara Nakagawa ${ }^{1}{ }^{1}$ Sapporo Tokushukai Hospital, Sapporo, Japan; ${ }^{2}$ Ohasa clinic, Ebetsu, Japan; ${ }^{3}$ Dankook university, Cheonan, Korea (the Republic of). (Control ID \#2468490)

LEARNING OBJECTIVE \#1: Plain chest radiography is routinely performed for patients with an unknown focus of infection to exclude pneumonia, however, plain abdominal radiography is not. It is not recommended to perform abdominal radiography routinely for patients without abdominal symptoms, however, blindly following this recommendation may cause missing the right diagnosis.

CASE: A 79-year-old female was brought to the emergency department (ED) of Sapporo Tokushukai Hospital for generalized weakness and poor oral intake for 4 days. Her neighbor found her in this state, with evidence of vomiting around her. On arrival, her mental status was clear, and her vital signs revealed a temperature of $35.9{ }^{\circ} \mathrm{C}$, blood pressure 103/63 mmHg, heart rate of 90 beats per minute, $\mathrm{SpO} 297 \%$ in room air, and respiratory rate of $20 / \mathrm{min}$. Physical examination revealed normal lungs sounds bilaterally, slightly dry mucous membranes, no abdominal tenderness or distension, and a $5 \mathrm{~cm}$ right groin soft mass without tenderness, warmth or redness. Laboratory data showed significant leukocytosis with left shift (WBC 347,000; Bands 17.5), BUN/Cr 83.7/1.05, and normal liver function tests. Urinalysis revealed mild bacteriuria without pyuria. Chest radiography showed no infiltrates. She did not complain of any other symptoms besides mild generalized weakness, she was not ill-appearing and her vital signs were stable. However, her laboratory data suggested severe bacterial infection but the focus was not readily identified. She was admitted to the hospital for close observation and was given empiric treatment with ceftriaxone. On hospital day 2 , she vomited once so an abdominal radiography was ordered surprisingly showing signs of distended small bowel loops. The following abdominal computed tomography (CT) revealed small bowel obstruction (SBO) with an incarcerated femoral hernia. She underwent emergent surgery which found a necrotic ileum and intestinal perforation. The day after the surgery, she developed acute 
change in mental status and severe respiratory distress, requiring intubation and transfer to the intensive care unit. Her clinical condition continued to deteriorate, she did not respond to resuscitation efforts and she passed away on the same day. Autopsy was not performed. Retrospectively, the chest radiography performed on the day of admission did revealed a small part of distended small bowel loops, which should have prompted further abdominal imaging.

DISCUSSION: A single plain abdominal radiography could have shown the evidence of SBO, possibly followed by intraabdominal CT to find the source of infection and earlier intervention. The two reasons why it was not performed include: 1) According to RCR guidelines for the use of plain abdominal radiography, which includes acute abdominal pain (Smith et al., 2008), this patient unfortunately did not meet any of the criteria. 2) A small part of distended small bowel loops in chest radiography was missed due to "faulty detection or perception" in the category of Faulty Synthesis. (Graber et al., 2005) Abdominal radiography and focusing on extra-pulmonary cites of chest radiography are crucial in certain circumstances even without meeting the criteria.

\section{A SLEEPING GIANT AWAKENS: A CASE OF PHEOCHROMOCYTOMA DI-} AGNOSED AFTER ANTIHISTAMINE INDUCED ADRENERGIC CRISIS Serena M. Okoronkwo ${ }^{1}$; ALi Mehr ${ }^{2}$. ${ }^{1}$ University of Texas Health Science Center San Antonio, San Antonio, TX; ${ }^{2}$ South Texas Veteran Health Care System, San Antonio, TX. (Control ID \#2470321)

LEARNING OBJECTIVE \#1: Pheochromocytoma is a rare treatable cause of hypertension. It can be triggered by certain "innocent medications". A high clinical suspicion along with focused, detailed history taking can lead to successful diagnosis.

LEARNING OBJECTIVE \#2: Patients awaiting surgical resection should be started on alpha blockade followed by beta blockade to prevent intraoperative adrenergic crisis. After surgical resection, the patient and their family should be carefully monitored and have biochemical and genetic testing to screen for recurrent disease and familial syndromes associated with pheochromocytoma such as Multiple Endocrine Neoplasia type 2, Neurofibromatosis type $1 \mathrm{~F}$ and Von Hippel Lindau.

CASE: A 45 year old woman with history of anxiety and migraines presented to the emergency room with nausea and headache. The initial workup in the emergency room was unremarkable, and the patient was given metoclopramide and diphenhydramine for symptom relief. Shortly after these medications were administered the patient became tachycardic, hypertensive, and hypoxemic. A stat chest x-ray revealed flash pulmonary edema and the patient was intubated for respiratory distress. A complete metabolic panel and complete blood count were all within normal limits. EKG showed sinus bradycardia and shortened PR interval. Upon review of outside records, it was noted that the patient had a history of cardiac arrest during a prior surgery. She also had a history of transient depressed systolic function postoperatively -subsequent echocardiograms showed a return of normal systolic function. Based on the patient's history, additional laboratory studies were collected which were significant for elevated plasma metanephrines and normetaneprhines. Adrenal CT scan revealed a $3.1 \mathrm{~cm}$ right adrenal mass with $67 \%$ enhancement washout. The patient was started on phenoxybenzamine and metoprolol therapy and later underwent successful surgical resection of the mass.

DISCUSSION: Pheochromocytoma is a rare adrenal tumor affecting 1 to 2 patients per million population. Patients may not always have the classic triad of symptoms. As our case demonstrates, patients may present with adrenergic crisis after induction of anesthesia and after administration of certain antihistamine medications. When pheochoromytoma is suspected, initial testing should include urine or plasma metanephrine levels. Elevated levels should prompt imaging with $\mathrm{CT}$ or MRI to localize the tumor.
A SPONTANEOUSLY REGRESSED SEMINOMA PRESENTING AS A UNILATERAL INGUINAL MASS John S. Fleming ${ }^{2}$; Christopher Di Felice ${ }^{1} .{ }^{1}$ Western Michigan Universiry Homer Stryker M.D. School of Medicine, Portage, MI; ${ }^{2}$ Western Michigan University Homer Stryker MD School of Medicine, Kalamazoo, MI. (Control ID \#2435585)

LEARNING OBJECTIVE \#1: Identify factors that may affect the site of metastasis in testicular cancer

LEARNING OBJECTIVE \#2: Recognize the importance of a thorough history and physical exam in shaping a differential diagnosis

CASE: A 42 year old man presented to the clinic with the chief complaint of a groin mass. He had no constitutional symptoms. Pertinent historical findings included hydrocelectomy at age sixteen, and family history of large cell lymphoma. The physical exam was remarkable for a large right-sided inguinal mass that was firm, fixed, non-tender and non-reducible. No overlying skin changes were apparent. No additional masses or lymphadenopathy were found. CT scan of the abdomen and pelvis showed right internal iliac and inguinal adenopathy. An open biopsy of the right inguinal node was performed; results were consistent with metastatic seminoma. Testicular ultrasound revealed a smaller right testis with mild diffuse heterogenous echogenicity suggesting atrophy and fibrosis. Radical orchiectomy of the right testis revealed extensive atrophy and scarring within multiple sections of the testicular parenchyma. The patient was referred to oncology to begin systemic chemotherapy with cisplatin and etoposide. He completed four cycles with follow-up CT showing resolution of all lymphadenopathy.

DISCUSSION: Testicular cancer is relatively rare, accounting for approximately $1-2 \%$ of all male cancers. Germ cell tumors (GCTs) account for $95 \%$ of testicular cancers with seminomas being the most common histologic subtype. Metastatic disease at presentation is an unusual occurrence seen in less than fifteen percent of patients. The retroperitoneal lymph nodes are the typical site of spread while inguinal node involvement is far less common. Testicular metastasis typically occurs via efferent lymphatics draining alongside the gonadal vessels to the retroperitoneal para-aortic lymph nodes. Involvement of the iliac and inguinal lymph nodes in testicular cancer is unusual; however, it can be related to local invasion of the tumor into the tunica vaginalis or scrotal skin, bulky retroperitoneal lymphadenopathy, and previous surgical manipulation of the inguinoscrotal region. The latter is the most likely mechanism of spread in our patient who had a history of hydrocelectomy. Another unique feature to our case is the absence of a testicular mass on exam. This can likely be explained by spontaneous regression of the primary tumor. This is a well-recognized phenomenon marked by a distinct constellation of findings on biopsy that usually permits its recognition. In a large case series of patients who were found to have metastatic disease at presentation without a palpable testicular mass, biopsy specimens following orchiectomy showed irregular foci of scarring, distinct from the adjacent parenchyma, in association with widespread testicular atrophy. This case poses a diagnostic challenge for the internist who may not suspect inguinal adenopathy arising from testicular cancer. A thorough history to include previous inguinal surgery may aid in uncovering the diagnosis.

A STRONG CASE OF GRAM NEGATIVE SEPSIS Stephen Pickett; Ryle Przybylowicz; June Kampangkaew. Baylor College of Medicine, Houston, TX. (Control ID \#2467737)

LEARNING OBJECTIVE \#1: Strongyloides is an endemic disease in tropical and subtropical climates, and is less common in developed countries. However, Strongyloides hyperinfection should be on the differential when a patient presents with recurrent sepsis without conspicuous cause. Here we describe a case of chronic strongyloides leading to hyperinfection. 
CASE: An 83 year old male Veteran presented with 1 day of fevers, chills, nausea, and generalized weakness. His medical history was significant for DM type 2 (A1c 8.5) and two previous episodes of gram negative sepsis over the last decade. On further history, he denied any urinary or GI symptoms, pulmonary symptoms, neurological deficits, and had no travel outside of his home state since serving in Vietnam in the 1970's. He had never had an EGD or colonoscopy. Initial blood cultures were positive for both E. Coli and Citrobacter, urine cultures were negative, and he was started on a cephalosporin. Repeat blood cultures grew parabacteroides and pseudomonas. Throughout hospitalization, the patient had persistent leukocytosis without eosinophilia. CT abdomen/pelvis was unrevealing for source of infection. Whole-body tagged WBC scan did not demonstrate any focal uptake. Colonoscopy showed no evidence of malignancy or other possible source. Three stool examinations were negative for ova or parasites. Strongyloides IgG was strongly positive suggestive of strongyloides hyperinfection. Patient was transitioned from broad spectrum IV antibiotics to ciprofloxacin and metronidazole in addition to a 7 day course of Ivermectin. EGD was discussed for duodenal biopsies but was deferred because the patient was already improving under the current treatment plan. At a 2-month follow up, the patient was asymptomatic.

DISCUSSION: S. stercoralis is unique for its ability to autoinfect hosts: infective filariform larvae can penetrate small intestine mucosa, travel hematogenously to other organ and later migrate back to the intestine where they become adults. This may explain the possibility of persistent infection for many years in this patient despite him not having been to an endemic region in nearly 40 years. Strongyloides may lead to recurrent and lifethreatening hyperinfection, as first described in 1970. Common presenting features include fever with respiratory or gastrointestinal symptoms. Peripheral hypereosinophilia is present in only about a third of patients but is a poor prognostic indicator. Strongyloides is a rare diagnosis in immunocompetent patients, but in immunocompromised patients the rate of bacterial co-infection is approximately $38 \%$. In the ICU, mortality rates for strongyloides hyperinfection approach $60 \%$. Ivermectin is the standard of care and has been shown to be more effective than albendazole in the treatment of chronic strongyloides. A single dose of ivermectin is recommended for immunocompromised patients or those on chronic steroids visiting endemic areas.

A SURPRISE SEIZURE Lynn Zaremski; Rebecca Koransky; Daniel Wong; Yelena Averbukh. Montefiore Medical Center, New York, NY. (Control ID \#2469192)

LEARNING OBJECTIVE \#1: Understand management of zolpidem withdrawal LEARNING OBJECTIVE \#2: Recognize irregular voice volume as a possible seizure symptom

CASE: A 38 year-old woman with history of anxiety was admitted for altered mental status after being found at home in bed, lethargic, not following commands. On admission, patient would not rouse to voice or noxious stimuli, pupils showed sluggish response to light, and right arm shaking was noted. There was no nuchal rigidity. Vitals were stable, alcohol, acetaminophen, salicylate, urine toxicology were negative. Laboratory values, including CBC, BMP, LFTs were all normal. Due to suspicion of seizure activity, the patient was loaded with fosphenytoin. The next morning, neurologic exam was normal apart from patient speaking in an extremely loud, monotonic voice. The patient was not aware of this alteration in volume and her hearing was intact. During the next few days EEG showed bilateral temporal, left worse than right, elipeptogenic spike activity with a background of focal cerebral dysfunction as well as episodes of seizure activity which correlated clinically with right arm shaking and decreased sensation in right hemibody. Further history was obtained and patient reported that she had been ingesting $50 \mathrm{mg}$ of zolpidem nightly that she illegally bought from the street, but had recently run out. She reported a history of blackouts following previous episodes of abrupt zolpidem cessation. Given patient's seizure activity, she was treated with benzodiazepines and she returned to her normal cognitive baseline after 3 days of treatment. Voice returned to normal quality with normal intonation and volume. Patient expressed interest in zolpidem cessation, and planned to follow up in outpatient addiction clinic.

DISCUSSION: Zolpidem is an imidazopyridine, which selectively antagonizes the GABA receptors at the omega-1 site. Benzodiazepines work similarly, but at the alpha site of the GABA receptors. Zolpidem is typically dosed in the range of 5 to $10 \mathrm{mg}$ qhs for insomnia. Although zolpidem is much less frequently abused when compared to benzodiazepines, abuse, dependence, and withdrawal have been reported to occur. According to package insert, withdrawal typically results in fatigue, nausea, flushing, panic attacks, abdominal discomfort, uncontrolled crying, emesis, and delirium. Seizures are rare, but are known to occur. Previous case reports of seizures were shown in patients abusing zolpidem and ingesting 1000-160 mg, making this the lowest known dose to result in seizures upon cessation. For this patient, once zolpidem use was confirmed from the patient's history, a long-acting benzodiazepine taper was initiated with successful clinical outcome. Other methods of treating zolpidem withdrawal or dependence have been used including zolpidem taper, initiation of antiepileptics like levetiracetam, detoxification with flumazenil followed by benzodiazepine taper, and quietiapine. Characteristic symptoms of temporal lobe seizures include aura, motionless stare, dilated pupils, automatisms including lip smacking, chewing, or swallowing, picking or patting, or unilateral repetitive extremity movement. Voice disorders including automatisms are common results of seizure activity, and there have even been case reports of more complex vocalizations occurring such as singing, whistling, or barking. However, our patient was able to speak fluently and carry out appropriate conversation in an extremely loud and monotone voice during EEG-proven seizure activity. Seizure activity in the left temporal lobe may be able to explain these symptoms due to location in auditory centers. This vignette describes a case of zolpidem withdrawal and highlights new onset seizures as a possible symptom of zolpidem misuse. Speaking in loud and monotonic voice in appropriate patient should be further investigated as a symptom of focal partial seizure activity. Physicians should be aware that zolpidem withdrawal can present as seizures, and benzodiazepines can be successful treatment

\section{A SURPRISING CASE OF COLORECTAL CANCER WITH METASTASIS TO} THE TOE Nandita Sriram. UCLA Medical Center, Los Angeles, CA. (Control ID \#2469930)

LEARNING OBJECTIVE \#1: Recognize metastasis as a cause of bone pain in patients with a history of colorectal cancer

LEARNING OBJECTIVE \#2: Recognize the importance of post-treatment surveillance in colorectal cancer survivors

CASE: A 49-year-old female with a history of rectal adenocarcinoma presented to her primary care provider with pain in her right great toe for 3 weeks. The pain began after she accidentally stubbed her toe on pavement; it then became progressively swollen and painful without warmth or drainage, to the point that she could no longer wear her shoes. On review of systems, she denied fevers, chills, shortness of breath, and night sweats, but endorsed a dry cough that had been present for 8 months and an unintentional $30-\mathrm{lb}$ weight loss over the previous year. She denied any family history of malignancy and any history of smoking, alcohol use, or illicit drug use. She had been taking no medications and had no known allergies. Her oncologic history was notable for stage T3N1 rectal cancer diagnosed 9 years prior at an outside hospital. She underwent neoadjuvant chemotherapy with capecitabine as well as radiation therapy. Two years later, a surveillance whole body PETCT showed activity in the left lateral wall of the rectum, and biopsies showed stage T3N0 rectal adenocarcinoma with no lymph node involvement. The patient underwent abdominoperineal resection with adjuvant chemotherapy and radiation therapy, and she was then lost to follow-up until presenting with toe pain as above. Physical exam revealed 
a healthy appearing female in no acute distress, breathing comfortably on room air with markedly diminished left lung sounds throughout as well as a firm, swollen, and a multicolored mass at the distal aspect of the right great toe, with total destruction of the nail bed. Labs were notable for an elevated serum CEA at $15.8 \mathrm{ng} / \mathrm{mL}$ (normal value: $<3.1 \mathrm{ng} / \mathrm{mL}$ ). Chest x-ray showed complete opacification of the left hemithorax, concerning for left mainstem bronchus occlusion with lung collapse. CT Chest with contrast showed pleural carcinomatosis with multiple left lung masses and nodules obliterating all associated airways. CT of the right foot showed a mixed lytic and sclerotic expansile mass of the first distal phalanx. CT of the abdomen and pelvis showed no evidence of metastatic disease. Whole body PET/CT showed FDG uptake in the lungs and pleura, lymph nodes of the lower neck and chest, and in the first distal phalanx of the right foot. Biopsies of the toe and of the lung mass confirmed metastatic colorectal adenocarcinoma. Unfortunately, 6 weeks later, while awaiting initiation of chemotherapy, the patient developed acute hypoxic respiratory failure, was found to have new right mainstem bronchus occlusion due to progression of her lung metastases, and expired in the ICU.

DISCUSSION: Skeletal metastases from colorectal cancer are rare, with an estimated incidence of $4.7-10.9 \%$. When present, they are usually found in association with liver or lung metastases. The incidence of isolated bone metastases has been reported to be as low as $1-2 \%$. Autopsy studies have shown that rectal cancers are more frequently associated with bone metastases than other parts of the colon, and the route of spread is thought to be primarily hematogenous, through the Batson venous plexus. The most common sites of metastases are the vertebral column, pelvis, and long bones, and the lesions are predominantly osteolytic. The presence of bone metastases portends a grave prognosis, with an estimated 5-year survival rate of $8.1 \%$. A thorough review of the literature suggests that this may be one of the first reported cases of a skeletal metastasis from colorectal cancer to the great toe. Although rare, clinicians should consider metastasis when evaluating bony pain in patients with a history of colorectal cancer. They should also consider pursuing a biopsy of any new bony lesion as the type of malignancy (new primary malignancy vs. metastasis) can affect both prognosis and management. Finally, clinicians must educate patients about the importance of post-treatment surveillance as a means of identifying potentially treatable recurrent disease as early as possible.

A SWEet diagnosis IN A PERITONEAL DiALYSIS PATIENT Brittany K. Gary; Darlene LeFrancois. Montefiore Medical Center, New York, NY. (Control ID \#2469611)

LEARNING OBJECTIVE \#1: Diagnose acute hydrothorax in a peritoneal dialysis patient.

LEARNING OBJECTIVE \#2: Manage pleural-peritoneal communication.

CASE: A 46 year-old man who has been on automated peritoneal dialysis (PD) for approximately 1 year presented after waking with diffuse abdominal pain which radiated to his back. The abdominal pain was associated with nausea and many episodes of clear emesis. He had no dyspnea. He was afebrile with mild tachycardia. He had diffuse abdominal tenderness without rebound or guarding, and there were reduced breath sounds and dullness to percussion $2 / 3$ of the way up the right posterior chest. Patient reported infusing dialysate before going to bed, but no fluid was able to be withdrawn from the PD catheter to evaluate for peritonitis. No intraabdominal pathology was seen on CT of the abdomen and pelvis, and while only a small amount of fluid was present in the abdomen, a large right sided pleural effusion was noted in the thorax. Thoracentesis was performed and $2000 \mathrm{ml}$ of fluid was removed with immediate improvement in abdominal pain. Pleural fluid protein was $<1 \mathrm{~g} / \mathrm{dL}$ and glucose was $129 \mathrm{mg} / \mathrm{dL}$. Blood capillary glucose measured at the time of thoracentesis was $99 \mathrm{mg} / \mathrm{L}$. Based on the low protein, high glucose profile, fluid analysis was diagnostic of pleural-peritoneal communication and PD was temporarily held. A hemodialysis (HD) catheter was placed and patient was transitioned to
HD. During the admission, the patient did have some re-accumulation of pleural effusion, however he remained asymptomatic and did not require repeat thoracentesis. Imaging performed approximately 4 weeks after thoracentesis showed no evidence of pleural effusion, and after 6 weeks the patient was able to restart PD.

DISCUSSION: Acute hydrothorax is an uncommon complication of PD occurring in 1$2 \%$ of PD patients. With less than 30,000 patients in the US receiving peritoneal dialysis, general internal medicine practitioners infrequently encounter this complication. It most commonly presents with shortness of breath, but as many as $25 \%$ of patients are asymptomatic. Presentation with abdominal pain is less common, and PD patients with acute onset abdominal pain should be evaluated for secondary bacterial peritonitis. Abdominal pain that radiates to the back also warrants concern for pancreatitis, which is more common in PD patients than the general population. In the case of pleural-peritoneal communication, the diaphragmatic defect usually occurs on the right side possibly a result of increased porosity and lower abdominal pressure in the right upper quadrant. There are several proposed underlying etiologies. Cases that occur shortly after initiation of PD are more likely due to congenital diaphragmatic defects, whereas cases that develop some time later may be a result of an acquired defect caused by a sudden increase in abdominal pressure. Diagnosis can be made by analysis of thoracentesis fluid that shows low protein and high glucose. No formal cut-offs for pleural fluid glucose level, or pleural fluid to serum glucose ratio, have been validated, but it has been suggested that any elevation of pleural fluid glucose above serum glucose is specific for pleural-peritoneal communication. When the fluid analysis is not conclusive, or in cases where other causes of pleural effusion are equally likely, other diagnostic tests can be useful. Technetium- $99 \mathrm{~m}$ scintigraphy can identify a communication as can video-assisted thoracoscopic surgery (VATS). Injection of intraperitoneal methylene blue dye prior to thoracentesis is not recommended, as it is insensitive and is associated with a risk of peritonitis. Management of these communications is typically conservative. Reducing dwell volumes and performing exchanges in the semi-seated position, or temporarily transitioning the patient to hemodialysis, can allow the defect to heal. A 4-6 week period is typically tried, and if unsuccessful may be attempted again if pleural effusion recurs with dialysate infusion. Alternatively, some patients choose to continue with hemodialysis. If conservative measures fail and the patient strongly prefers $\mathrm{PD}$, repair of the defect can be attempted with more invasive techniques including talc pleurodesis and VATS repair. However, in many cases of PD-related pleural-peritoneal fistula, both diagnosis and management can be accomplished with a conservative approach, exposing the patient to less risk from invasive procedures.

\section{A TETANIC PROBLEM: A CASE OF MULTIPLE SIMULTANEOUS ENDO-} CRINE DYSFUNCTION Emily Schapira ${ }^{2}$ J Jeff $\mathrm{Min}^{2}$; Denisa Gace ${ }^{1} .{ }^{1}$ Mass General Hospital, Boston, MA; ${ }^{2}$ Massachusetts General Hospital, Cambridge, MA. (Control ID \#2456477)

LEARNING OBJECTIVE \#1: Manage patients on tyrosine kinase inhibitors in a primary care or hospitalist setting

LEARNING OBJECTIVE \#2: Recognize the potential endocrine side effects of tyrosine kinase inhibitors

CASE: An 82-year old man with renal cell carcinoma metastatic to the lungs, on axitinib for the past 2 months, was found to be in metabolic disarray with severe hypocalcemia at a routine nephrology clinic visit. His past medical history is significant for stage III-IV chronic kidney disease, type II diabetes on insulin, heart failure (EF $43 \%$ ) and hypertension. His oncologic history is significant for 6 months of treatment with pazopanib prior to axitinib, stopped after he developed pancreatitis thought to be related to pazopanib. At clinic, he was ill-appearing and tachypneic. Laboratory data showed a metabolic acidosis with $\mathrm{CO}_{2}$ of $11 \mathrm{mmol} / \mathrm{L}$, anion gap of 23 , acute-on-chronic kidney injury with a creatinine 
of $3.9 \mathrm{mg} / \mathrm{dL}$, BUN of $87 \mathrm{mg} / \mathrm{dL}$, hypocalcemia to $5.4 \mathrm{mg} / \mathrm{dL}$ (albumin $3.5 \mathrm{~g} / \mathrm{dL}$ ) and hypoglycemia to $59 \mathrm{mg} / \mathrm{dL}$. He was sent to the Emergency Department and admitted to the medicine service. On examination he appeared in mild distress, dyspneic at rest and unable to speak in full sentences. He had perioral paresthesias and positive Chvostek sign. Additional labs showed a phosphate of $5.2 \mathrm{mg} / \mathrm{dL}$, magnesium of $1.2 \mathrm{mg} / \mathrm{dL}$ and an ionized calcium of $0.7 \mathrm{mmol} / \mathrm{L}$. He had elevated PTH, low 25-OH vitamin D, and normal 1,25-OH vitamin D. An arterial blood gas showed a $\mathrm{pH}$ of $7.34, \mathrm{PaCO}_{2}$ of $19, \mathrm{PaO}_{2}$ of 115 . ECG was significant for a prolonged QTc of $525 \mathrm{~ms}$. Aggressive electrolyte repletion was started with oral calcium, calcitriol, and ergocalciferol as well as IV calcium gluconate, magnesium sulfate, and sodium bicarbonate. He continued to have episodes of hypoglycemia despite pushes of dextrose and was started on a dextrose infusion. His presentation was suspicious for endocrine disorders secondary to axitinib, which was held with consultation from his oncologist. Nephrology and Endocrinology services were also consulted for further assistance with management. The patient's calcium slowly improved and his QTc normalized. By day 3, he no longer required IV supplementation. Similarly, his glycemic control improved and he was restarted on an insulin regimen. His dyspnea resolved following correction of his acidosis. DISCUSSION: Imatinib, an inhibitor of BCR-ABL tyrosine kinase, was the first of a generation of small molecule inhibitors and monoclonal antibodies which target and inhibit the pro-growth effects of tyrosine kinases. Since its approval in 2003, a host of tyrosine kinase inhibitors (TKIs) have been developed for the treatment of cancer. Pazopanib and axitinib are TKIs that inhibit the activity of vascular endothelial growth factor (VEGF). Axitinib was approved in 2012 as a secondline agent for the treatment of advanced renal cell carcinoma. As the number of TKIs increase and the indications for their use expand, a growing population of patients now exist who are maintained on these medications. As a result, physicians in primary care and hospitalist settings should develop an awareness of TKI side effects. While some side effects mirror those of traditional chemotherapeutics (e.g., fatigue, rash, myelosuppresion, and diarrhea), TKIs are uniquely associated with diverse and potentially severe endocrine effects, notably disruptions in calcium and glucose metabolism, thyroid function, and adrenal function ${ }^{[1]}$. These effects are not rare; clinical trial data for axitinib, for example, shows that $39 \%$ of patients developed hypocalcemia, and $19 \%$ developed hypothyroidism ${ }^{[2]}$. Our patient experienced a combination of these alterations resulting in his hospitalization. On presentation, he had severe hypocalcemia with secondary hyperparathyroidism, with perioral paresthesias, Chvostek sign, and prolonged QTc. Furthermore, he had hypoglycemia refractory to pushes of IV dextrose, attributed to both decreased renal clearance of insulin from kidney injury as well as TKI side effect In fact, TKIs are being explored as a treatment for diabetes with the potential to correct insulin resistance and beta cell dysfunction ${ }^{[3]}$. In conclusion, physicians of all specialties will increasingly encounter patients on TKIs. While no definitive guidelines exist for the management of TKI side effects, providers should be cognizant of the most common effects on thyroid function, calcium metabolism, and blood glucose. A baseline TSH, free T4, and thyroid antibodies should be obtained, with regular TSH monitoring. Regular monitoring of bone density, PTH, and 25-hydroxyvitamin D levels are also recommended. Finally, hemoglobin A1c and blood glucose monitoring is suggested for all patients. ${ }^{[3]}$ This clinical vignette emphasizes the importance of considering the side effects of TKIs as potential etiologies of endocrine dysfunction in this patient population. Fountas A, et al. Tyrosine kinase inhibitors and diabetes: A novel treatment paradigm? Trends in Endo \& Metab. 2015; 26(11): 643-56. Lodish, M. Kinase inhibitors: adverse effects related to the endocrine system. J. Endo. Metab. 2013; 98(4): 1333-42. Van Geel R, et al. Concise Drug Review: Pazopanib and Axitinib. The Oncologist. 2012; 17: 1081-89.
A TOTAL LOSS: ALOPECIA UNIVERSALIS IN ASSOCIATION WITH ADDISON'S DISEASE John Webb; Vinod E. Nambudiri. Grand Strand Medical Center, Myrtle Beach, SC. (Control ID \#2470289)

LEARNING OBJECTIVE \#1: Recognize the clinical manifestations and distinctions between alopecia areata, alopecia totalis, and alopecia universalis.

LEARNING OBJECTIVE \#2: Understand the association of autoimmune and antibody-associated diseases seen in conjunction with Addison's disease.

CASE: An 80 year old Caucasian male with a history of hypertension, atrial fibrillation, and Addison's disease presented to establish care at our primary care clinic after a recent hospitalization for presumed viral gastroenteritis. His recent gastrointestinal symptoms including nausea and vomiting had resulted in his discontinuation of all medical treatment for his Addison's disease and presentation to the hospital with hypotension and renal insufficiency. Following fluid repletion and initiation of $50 \mathrm{mg}$ of intravenous hydrocortisone every $8 \mathrm{~h}$ during hospitalization, he returned to his normal baseline and was restarted on oral hydrocortisone to continue as an outpatient. In addition to Addison's disease, his past medical history was notable for atrial fibrillation, hypertension, generalized xerosis, and vitamin B12 deficiency. His medications included hydrocortisone, metoprolol, diltiazem, and rivaroxaban. His family history did not include any individuals with other autoimmune diseases. He was a never smoker. On presentation, he appeared comfortable with vital signs were within normal limits. His physical examination was notable for the total loss of hair on the scalp, which had occurred at an early age. He also had a total lack of eyebrows, eyelashes, facial hair, and axillary hair. Body hair on the arms was very sparse; hair on the legs was absent. A diagnosis of alopecia universalis was made. The patient was not interested in any treatment specifically directed at the hair loss at the time of his visit.

DISCUSSION: Alopecia universalis is a rare, extreme phenotypic variant of alopecia areata, an immunologically-mediated form of hair loss. Alopecia areata is known to be a Tlymphocyte driven process, with T-cell inflammation targeted at the hair bulb resulting in the destruction of the growing hair shaft. Given its autoimmune nature, it can be seen in association with other autoimmune conditions such as Addison's disease, which most commonly results from autoimmune-mediated destruction of the adrenal glands, resulting in a state of endogenous cortisol deficiency. Clinically, alopecia areata is typically marked by round patches of non-scarring hair loss. Individuals from children to the elderly may be affected by alopecia areata. The most commonly affected site is on the scalp, though round isolated patches of hair loss in the facial hair are also routinely observed. Several variants of alopecia areata have been described, including the ophiasis pattern which occurs with a loss of hair in a ring-like distribution around the scalp. When the entirety of the scalp hair is lost due to alopecia areata, the condition is termed alopecia totalis. When all body hair including that of the eyebrows, lashes, facial hair, axillary hair, pubic hair, and body hair is lost, the condition is termed alopecia universalis. Alopecia totalis and alopecia universalis constitute fewer than $10 \%$ of individuals with alopecia areata and represent progressively more severe variants of the disease and are generally harder to treat or reverse. Autoimmune comorbidities are common in alopecia areata. The most commonly associated disorders include vitiligo and autoimmune thyroiditis, both of which are also thought to be primary T-lymphocytic mediated disorders. Rarely, alopecia universalis can be a manifestation of an autoimmune polyendocrinopathy syndrome if present since early childhood. Of the autoimmune diseases associated with alopecia areata, Addison's disease is infrequently reported. The treatment for alopecia areata, when desired, often begins with topical or intralesional steroids. For larger areas of involvement such as alopecia totalis or universalis, systemic immunosuppression is often necessary to attempt to achieve hair regrowth. Addison's disease has itself been linked with several other autoimmune phenomena. The most common autoimmune associations are with other endocrine glands such as autoimmune thyroiditis as well as autoimmune-mediated type 1 diabetes. Our patient did not have any other associated endocrinopathies. Interestingly, he had 
previously suffered from vitamin B12 deficiency requiring supplementation, likely due to pernicious anemia — another autoimmune disease associated with these entities. Addison's disease clinically presents with features of electrolyte disturbance, hypotension, weakness, and may lead to cutaneous hyperpigmentation. Repletion of endogenous cortisol through lifelong use of corticosteroids can prevent disease flares or crises. We present this case to highlight the association of a severe phenotype of alopecia areata, alopecia universalis, occurring in conjunction with Addison's disease as a reminder of the spectrum of autoimmune diseases and their associations.

\section{A TRIPLE THREAT: LUPUS, MYOCARDITIS AND PREGNANCY IN A YOUNG} WOMAN Swati Vishwanathan ${ }^{1}$; Indu Poornima ${ }^{2} .{ }^{1}$ Allegheny general hospital, Pittsburgh, PA; ${ }^{2}$ Allegheny General Hospital, Pittsburgh, PA. (Control ID \#2470123)

LEARNING OBJECTIVE \#1: Learning Objective 1: Diagnose Myocarditis in the setting of a lupus flare.

LEARNING OBJECTIVE \#2: Learning Objective 2: Understand the challenges in managing this condition during pregnancy

CASE: A 22 years old female who was 18 weeks pregnant and had a past history of Lupus, presented with complaints of chest pain, shortness of breath and orthopnea that started a week prior to admission. On presentation to the emergency room she had an elevated BP of 190/100 mmHg, was hypoxic, tachycardic and tachypneic. Respiratory exam revealed bilateral crackles, elevated JVD and bilateral pedal edema . Chest Xray showed significant bilateral pleural effusions, pulmonary edema and cardiomegaly. A bedside Echocardiogram showed left ventricular (LV) global hypokinesis with an ejection fraction $(\mathrm{EF})$ of $25 \%$, no valvular disease and a moderate to large pericardial effusion without tamponade physiology. Labs were significant for moderate leukocytosis with WBC of 13,000, elevated ESR and CRP, hypocomplementemia and elevated troponin. EKG showed sinus tachycardia with low voltage QRS. She was treated for hypertensive emergency and acute pulmonary edema with IV nitroglycerine, and furosemide drip. Hypoxemia and BP improved. Two weeks prior to this presentation she was noted to have a fever associated with malar rash and proteinuria and she was started on Prednisone and Imuran for lupus flare with possible nephritis. Since her current presentation was suggestive of worsening lupus flare with possible myocarditis, she was started on methylprednisolone $250 \mathrm{mg} \mathrm{q} 6 \mathrm{~h}$ and the dose of Imuran was increased. Despite aggressive treatment for the lupus flare and hypertension, repeat Echocardiogram showed decrease in LVEF to $15 \%$. Cardiac MRI with T2 imaging showed markedly abnormal hyperenhancement pattern in the apex and septum and acute intramyocardial edematous process, consistent with lupus carditis. A multidisciplinary team including Cardiology, Rheumatology, and Maternal-fetal medicine discussed the risk-benefit ratio of continuing the pregnancy in the setting of untreated myocarditis and worsening LV function since definitive treatment with Cyclophosphamide is contraindicated in pregnancy. After consideration of all options patient proceeded with medical termination of pregnancy. Monthly Cyclophosphamide infusions were initiated and high dose steroids and Imuran were continued. Repeat Echo and Cardiac MRI, 2 months after discharge, showed marked improvement in her LVEF to $50 \%$. BP medications have been gradually reduced and diuretics and steroids have been discontinued.

DISCUSSION: Pregnancy is associated with a high rate of lupus flare (60\%) and lupus nephritis during pregnancy is an adverse maternal mortality predictor. It is also a predictor of fetal intrauterine growth retardation, preterm birth and fetal complete heart block. Myocarditis itself has been reported only in $10 \%$ of patients with lupus but there are only a few case reports in pregnancy. Its presentation can mimic peripartum cardiomyopathy or lupus related nephritis with hypertension. The latter can also be mistaken for preeclampsia but associated lab abnormalities help with the diagnosis. A high index of suspicion and an echocardiogram showing LV systolic dysfunction points to the diagnosis. Management is more challenging since all cytotoxics and antimetabolites such as cyclophosphamide and Mycophenolate are contraindicated in pregnancy and termination of pregnancy may be necessary to avoid maternal mortality.

A TWIST OF FATE Minnsun Park; Vinh Nguyen; Jessica DeBord. Tulane University Health Sciences Center, New Orleans, LA. (Control ID \#2468877)

LEARNING OBJECTIVE \#1: Consider Torsades de Pointe as a cause of cardiac arrest in a patient on QT prolonging medications

LEARNING OBJECTIVE \#2: Recognize the importance of medication reconciliation and patient education on side effects and interactions

CASE: A 45-year-old man with a history of depression and chronic pain lost consciousness at home. Upon emergency medical technician arrival, he was found to be pulseless. Rhythm analysis revealed polymorphic ventricular tachycardia (VT), and he was successfully defibrillated in the field. Upon ED arrival, his heart rate was $66 \mathrm{bpm}$ and blood pressure was $88 / 45 \mathrm{mmHg}$. The neurological exam revealed dilated, unresponsive pupils, and the patient withdrew from painful stimuli, but was unable to follow commands. Electrolytes were within normal ranges with the exception of low magnesium, at $1.5 \mathrm{mg} / \mathrm{dL}$. Troponin was undetectable. The patient's rhythm strip showed torsades de point. Shortly after arrival, the patient experienced another polymorphic VT arrest requiring cardioversion. An electrocardiogram revealed ST changes, and he underwent emergent coronary angiography, which found no coronary artery disease. A subsequent electrocardiogram revealed a prolonged corrected QT interval of 711 milliseconds. A magnesium infusion was initiated, QT prolonging agents were discontinued, and the QT interval was monitored closely. The patient's girlfriend explained that he had decided to wean himself off methadone several weeks prior to the event. He had reported fever, malaise, myalgia, headaches, and nausea for the preceding 2 weeks. He was prescribed two courses of azithromycin and ondansetron by his primary care physician. In addition, he was taking diphenhydramine, doubling his prescribed dose of fluoxetine, and also taking his girlfriend's sertraline to treat what he presumed was depression associated with methadone withdrawal.

DISCUSSION: Internists frequently prescribe medications that can prolong the QT interval. Torsades de Pointe, a rare form of polymorphic VT, results from QT prolongation. If not recognized and addressed promptly, it can result in sudden cardiac death. Its precise incidence is not known, but it is predicted to cause less than $5 \%$ of all sudden cardiac deaths. Torsades de Pointe occurs as a consequence of either congenital or acquired QT prolongation; acquired cases commonly involve electrolyte abnormalities and/or medications. Several classes of antibiotics, anti-depressants, and anti-arrhythmics are known to block myocardial delayed rectifier potassium $\left(\mathrm{I}_{k r}\right)$ channels. This results in prolongation of the cardiac action potential, leading to QT prolongation. In addition to DC cardioversion, as in other VT cases, the mainstay of treatment is magnesium. This electrolyte helps to terminate the arrhythmia by decreasing the influx of calcium into the myocardium and results in suppression of early afterdepolarizations (EADs). ${ }^{1}$ In addition to terminating the arrhythmia, it is important to identify any reversible causes of QT prolongation. In this case, the patient took multiple medications which are known to prolong the QT interval, including methadone, fluoxetine, sertraline, azithromycin, ondansetron, and diphenhydramine. ${ }^{2}$ Together, they potentiate each other's effects on $\mathrm{I}_{k r}$ inhibition and increase susceptibility to ventricular arrhythmia by causing electrolyte abnormalities. They may also inhibit hepatic cytochrome p450, further increasing medication levels. Adverse drug reactions (ADRs) as described in this case are common in the 
United States. Over 2 million ADRs occur annually, and result in over 100,000 deaths yearly. ${ }^{3}$ In our patient's case, a neurologically devastating cardiac arrest may have been prevented through closer medication reconciliation and patient education. Had the patient understood the symptoms of methadone withdrawal, the side effects, and potential interactions of his medications, he may have been more prudent in his self-medication. Internists should consider the potential for QT prolongation when prescribing medication, and ensure they review drug interactions and side effects with their patients to avoid similar ADRs.

A UNIQUE OCCURRENCE OF POLYCYTHEMIA VERA AND HEYDE SYNDROME IN THE SAME PATIENT Hardik Chhatrala ${ }^{1}$; Monoj Konda ${ }^{1}$; Shreya Ghetiya $^{3}$; Lokesh Yadav ${ }^{1}$; Mark Loehrke ${ }^{2} .{ }^{1}$ Homer Stryker MD WMU School of Medicine, Kalamazoo, MI; ${ }^{2}$ Western Michigan University School of Medicine, Kalamazoo, MI; ${ }^{3}$ Jersey Shore Medical Center, Neptune, NJ. (Control ID \#2466892)

LEARNING OBJECTIVE \#1: It is important to recognize extra-intestinal causes for gastrointestinal bleeding.

LEARNING OBJECTIVE \#2: Simultaneous occurrence of polycythemia vera, hypersplenism and uremic coagulopathy accentuates the challenge in diagnosis and management of symptomatic gastrointestinal bleeding in Heyde's syndrome.

CASE: A 68-year old male presented with progressively worsening angina for 2 weeks in the setting of intermittent melena for about 4 weeks. Past medical history included endstage renal disease (ESRD) on dialysis, diabetes mellitus, coronary artery disease status post triple vessel bypass grafting, hypertension, JAK2 positive polycythemia vera (PV) with massive splenomegaly. Physical exam findings included skin pallor, sinus tachycardia with a 3 out of 6 systolic murmur radiating to carotids, massive nontender splenomegaly till umbilicus, and trace pedal edema. Laboratory finding: Hemoglobin 7.8 g/dL platelets 189,000 cells/dL, normal haptoglobin, INR 1.1, serial troponin elevation, positive fecal occult blood. Aspirin and heparin were not started due to dropping hemoglobin which was attributed to gastrointestinal bleeding. CT scan of chest/abdomen/pelvis ruled out hematoma. Angina eventually resolved upon blood transfusion. Cardiac echo confirmed severe aortic stenosis and severe left ventricular hypertrophy. Esophagogastroduodenoscopy found portal hypertensive gastropathy but no active bleeding. Colonoscopy as well as capsule endoscopy at a later date failed to find active bleeding source although subsequent fecal occult blood testing was positive. Bone marrow showed hypercellular with trilineage hyperplasia, mild myelofibrosis, marked microcytic hypochromic anemia. Patient underwent aortic valve repair due to high suspicion for Heyde's syndrome as underlying cause of his gastrointestinal bleed. No further episodes of angina or melena were noted. Unfortunately, 3 weeks after the surgery, the patient passed away from sudden cardiac death at home.

DISCUSSION: There is well known correlation between aortic stenosis and gastrointestinal bleed in the setting of arterio-venous malformations (AVMs) known as Heyde's syndrome. The sheer stress across the stenotic valve leads to breakdown of high molecular weight multimers (HMWMs) of von Willibrand Factor (vWF) rendering them ineffective in aiding platelet aggregation. This leads to an acquired type $2 \mathrm{~A}$ von Willibrand disease (vWD). Patient's bleeding tendency was worsened by ESRD induced uremic coagulopathy resulting from qualitative platelet defect. In the past, our patient had a history of bleeding from fistula site during dialysis while on aspirin. This is typical of von Willibrand disease and should raise clinical suspicion. Curiously, in our case, erythrocytosis from PV was probably balanced by chronic gastrointestinal blood loss while hypersplenism could have been masking his elevated cell counts. It was unclear if portal hypertensive gastropathy secondary to massive splenomegaly was contributing to gastrointestinal blood loss. Regardless, AVMs were highly suspected even though endoscopies were negative, as it requires repeated testing in many patients for highest yield, especially with small bowel
AVMs. For the definitive treatment for gastrointestinal bleeding in Heyde's syndrome, trans-catheter aortic valve replacement was being planned and a splenectomy if bleeding was refractory. Multi-organ system involvement raised our patient's overall risk of mortality from Heyde's syndrome.

A WIN FOR EMR - PROVIDING EFFECTIVE, TIMELY AND WELL COMMUNICATED TREATMENT FOR AN INDIVIDUAL WITH A RARE MEDICAL CONDITION Ryan Quarles ${ }^{2}$; Raquel K. Belforti ${ }^{1}$. ${ }^{1}$ Baystate Medical Center, Springfield, MA; ${ }^{2}$ Baystate Medical Center, Enfield, CT. (Control ID \#2463261)

LEARNING OBJECTIVE \#1: To present a case of effective use of EMR care plans for patients with rare and difficult to treat disorders

LEARNING OBJECTIVE \#2: To prompt discussion of other novel ways to effectively communicate to improve the care we give our patients

CASE: A 32 year-old male with the rare genetic disorder known as familial dysautonomia presented to a community academic hospital for another episode of cyclical vomiting. The patient had been vomiting at least once a minute since he woke up. He denied any sick contacts, toxic ingestion or history suggestive of food poisoning. The patient has been seen in the ED and admitted to the hospital 7 times in the past year for supportive treatment. Opening the patient's chart provided every member of the healthcare team with a pop-up window with a clearly outlined summary of this patient's diagnosis, an explanation of the symptoms, triggers for acute flares and a treatment plan. This "ED Care Plan" was also sent to the user's inbox on the EMR for future reference. This system, typically used for narcotic seeking behavior, clearly communicated the patient's unfortunate situation. Specifically it states that his symptoms would be made worse by giving typical antiemetics and other common medications and suggested atypical treatment of his nausea with high dose benzodiazepines, thereby validating the patient's requests to staff when he arrived. It also warned that these patients can have decreased pain sensation and unable to communicate, all of which could be critical information for the ED and hospital staff.

DISCUSSION: Not much has been written about ED care plans being implemented to electronic medical records. Past descriptions of individualized ED care plans have shown a decrease in ED utilization. ED case management trials for high utilizers of the ED have also shown a decrease in ED use and cost while simultaneously improving socia outcomes for the patients, but their cost savings were offset by the program costs. Much has also been written about sickle cell crisis patients, which represent a distinctive group of high utilizers and found that individualized plans and protocols alike were able to decrease the number of ED visits and increased utilization of outpatient resources. Initially implemented to identify and manage narcotic seeking patients, our ED care plans provided the healthcare team an aligned and institutional approved treatment plan for these complicated and frustrating patients. Patients are identified as high utilizers by reviewing ED visit counts as other studies have done and a team of healthcare providers draft and implement a plan meant to provide efficient, safe and aligned care to these patients. Our method provides an effective way of communicating between providers and aligns the care for these patients because when fragmented care occurs with these patients, no one benefits. The only cost is the time input from the team to draft and approve these care plans. Using this same means of communication for a patient with a rare genetic disorder provided incredible benefit for the patient and staff alike. First, the patient was not given medications that had proven to make his condition worse in the past, medications that any practitioner would use to treat extreme nausea. Second, since the patient sometimes presents with somnolence and may not be able to communicate, this care plan provided effective communication to all team members. Third, by outlining a very specific treatment plan, it validated the patient's request to be treated with high dose IV benzodiazepines for vomiting, a very atypical request and one that would raise red flags for any practitioner. Fourth, the care plan was written with the patient's input, thereby providing 
patient centered, efficient and high value for the patient and health care system. Finally, should there be any concerns or something was not presenting as a normal "flair" practitioners had consolidated contact information for family members, primary care doctors and a specialist for this condition. Patients with rare disorders will undoubtedly have an incredible amount of information in their electronic medical record and the most important information and treatment plans can often become lost and easily lost at the most critical times. A simple pop-up message provides focused care plan to anyone that opens the cart provides effective, timely and well communicated treatment was disseminated to those that cared for the patient. Using these simple and cost effective systems for communication between practitioners should be considered for patients who have complex medical conditions that require aligned care in our fragmented medical system.

\section{ABNORMAL SCREENING LIPIDS AS A DIAGNOSTIC TOOL FOR RENAL} DISEASE Allison Glaser; Athanasia Vasiliadis. Mount Sinai Hospital, New York, New York, NY. (Control ID \#2467591)

LEARNING OBJECTIVE \#1: Recognize the abnormal lipid panel as a means of diagnosing renal disease in the outpatient setting

LEARNING OBJECTIVE \#2: Identify the abnormal lipid pattern in nephrotic syndrome and describe the laboratory features and treatment options

CASE: A 39-year-old African American female presented to the outpatient clinic to establish primary care. Her past medical history was significant for hypertension and superimposed pre-eclampsia during a recent pregnancy 5 months prior. The patient did not have any complaints at the time of the visit but was concerned about her weight gain. She reported compliance with her blood pressure medication of labetalol $200 \mathrm{mg}$ PO twice daily; however, she forgot to take it the morning of the visit. She denied any headache, blurry vision, chest pain or shortness of breath. The patient had no history of tobacco, alcohol or illicit drug use. She reported a strong family history of diabetes and hypertension. On exam, the patient was 5'2", weighing $265 \mathrm{lbs}$., with a BMI of 48 . Her vital signs noted an elevated blood pressure of 179/98 $\mathrm{mmHg}$, a pulse of 85 beats per minute, and a normal temperature, respiratory rate and oxygenation saturation. The patient was a well appearing obese female in no acute distress with a normal neurologic, cardiac, lung, and abdominal exam. Her musculoskeletal exam was within normal limits, and she did not have any peripheral edema. Review of the patient's medical chart revealed a transient transaminitis during her recent pregnancy. The workup revealed a positive hepatitis $\mathrm{C}$ antibody with a negative RNA; platelets had remained within normal limits, thereby ruling out HELLP syndrome. During the visit, the patient was counseled on healthy lifestyle interventions and the importance of medication compliance. Laboratory testing included a complete blood count, comprehensive metabolic panel and also included a screening lipid panel and hemoglobin A1c given her risk factors and strong family history. The results of the lipid panel revealed marked elevation of total cholesterol 433, triglycerides 209 , and LDL 317. The HDL cholesterol was normal at 74 . The labs were significant for a low albumin at 2.1, low total protein 4.8. Creatinine, electrolytes, LFTs, CBC and hemoglobin A1c were within normal limits. The patient was recalled for further studies and work up of the noted abnormal lab tests. Further testing included a urinalysis with $>500$ protein, no red cells or casts, and a spot urine protein $701 \mathrm{mg} / \mathrm{dL}$, urine creatinine $60.9 \mathrm{mg} / \mathrm{dL}$, with an elevated ratio of $11.51 \mathrm{mg} / \mathrm{mg}$. The normal urine protein to creatinine ratio is less than $0.2 \mathrm{mg} / \mathrm{mg}$. Ratios greater than $3.5 \mathrm{mg} / \mathrm{mg}$ are in the nephrotic range for proteinuria. Renal ultrasound noted echogenic kidneys with medical renal disease and no obstruction. The urine studies and lab results were concerning for nephrotic syndrome, and the patient was referred to Nephrology.

DISCUSSION: This case highlights the importance of routine lipid panel screening in eligible patients and the use of the lipid panel as a diagnostic tool for renal disease. Marked lipid abnormalities with significant elevation of total and LDL cholesterol and triglycerides are common in patients with the nephrotic syndrome. HDL chol maybe normal or reduced. Hyperlipidemia is triggered by the reduction in plasma oncotic pressure which stimulates hepatic apoprotein B gene transcription. Resolution of the nephrotic syndrome reverses the lipid abnormalities. Treatment options include dietary modification, use of angiotensin-converting enzyme (ACE) inhibitors or angiotensin receptor blockers (ARBs) to reduce proteinuria and statins. ${ }^{1}$ Nephrotic syndrome is defined by heavy proteinuria $(>3.5 \mathrm{~g} / 24 \mathrm{~h})$, hypoalbuminemia $(<3 \mathrm{~g} / \mathrm{dL})$, and peripheral edema. Hyperlipidemia and thrombotic disease are frequently observed. ${ }^{2}$ Our patient did not exhibit edema but her clinical and laboratory features are consistent with nephrotic syndrome. Serologic studies to rule out an underlying cause of disease include complement, ANA, serum free light chains and urine protein electrophoresis and immunofixation, syphilis serology, hepatitis B and hepatitis C serologies, and the measurement of cryoglobulins. ${ }^{3}$ Thus far, these studies have been negative in the patient. Renal biopsy is the gold standard for evaluating the etiology of proteinuria and is currently pending. Complications of nephrotic syndrome include protein malnutrition, symptomatic hypovolemia, acute kidney injury, urinary loss of hormones, hyperlipidemia and the potential for accelerated atherosclerosis, venous or arterial thrombosis, and increased risk of infection. ${ }^{4}$ References: ${ }^{1}$ Kronenberg, F. Dyslipidemia and Nephrotic Syndrome: Recent Advances. Journal of Renal Nutrition 2005;15:195-203 ${ }^{2}$ Stephan R.Orth, M.D., and Eberhard Ritz, M.D. The Nephrotic Syndrome. N Engl J Med 1998; 338:1202-1211 ${ }^{3}$ Howard AD, Moore J Jr, Gouge SF, et al. Routine serologic tests in the differential diagnosis of the adult nephrotic syndrome. Am J Kidney Dis 1990; 15:24. ${ }^{4}$ Crew RJ, Radhakrishnan J, Appel G. Complications of the nephrotic syndrome and their treatment. Clin Nephrol 2004; 62:245.

ACALCULOUS CHOLECYSTITIS AND EPSTEIN BARR VIRUS: A RARE COMPLICATION OF A COMMON DISEASE Omar K. Jilani ${ }^{1}$; Abubaker Jilani ${ }^{3}$; Karin Chen ${ }^{1}$; Iqbal Jilani ${ }^{2}$; Svetlana Chernyavsky ${ }^{1} .{ }^{1}$ Mt Sinai Beth Israel, New York, NY; ${ }^{2}$ Hamdard Medical University, Karachi, Pakistan; ${ }^{3}$ Mercy Hospital System, Conshohocken, PA. (Control ID \#2470214)

LEARNING OBJECTIVE \#1: Diagnose a rare complication of Epstein Barr Virusassociated Infectious mononucleosis (IM)

LEARNING OBJECTIVE \#2: Recognize the clinical presentation, radiologic features and laboratory markers in Infectious mononucleosis-associated acalculous cholecystitis CASE: A previously healthy 22-year old female with a past medical history of asthma presented to the emergency department with 2 weeks of worsening malaise and 4 days of intermittent fevers, myalgias, headaches, right neck swelling and abdominal pain. On exam patient was febrile to $103 \mathrm{~F}$ and tachycardic to 102 beats per minute. She had scleral icterus and tender mobile right cervical and left inguinal lymphadenopathy. She also had palpable splenomegaly and right upper quadrant abdominal tenderness with negative Murphy's sign. On labs patient had leukopenia of $2.8 \mathrm{~K} / \mathrm{uL}$, thrombocytopenia of $69 \mathrm{~K} /$ $\mathrm{uL}$, direct hyperbilirubinemia of $2.9 \mathrm{mg} / \mathrm{dL}$, elevated alkaline phosphatase of $488 \mathrm{U} / \mathrm{L}$ and transaminitis with AST of $215 \mathrm{U} / \mathrm{L}$ and ALT of $316 \mathrm{U} / \mathrm{L}$. A peripheral smear revealed atypical lymphocytes. Initial blood cultures showed no growth and serology for hepatitis, Lyme disease, cytomegalovirus, parvovirus B19 and HIV was negative. Epstein Barr IgM antibody was positive. On abdominal ultrasound, there was a markedly thickened and edematous gallbladder with negative Murphy's sign, absent cholelithiasis, non-dilated biliary tree and moderate splenomegaly, findings consistent with acalculous cholecystitis. Patient was empirically started on ciprofloxacin and metronidazole and infectious disease, surgery and gastroenterology were consulted. Patient was planned for cholecystectomy however this was postponed given marked clinical improvement and resolution of lab abnormalities with supportive care. Patient had resolution of ultrasound findings and was discharged with directions to avoid contact sports and return if symptoms recur. 
DISCUSSION: Epstein Barr virus (EBV)- associated Infectious mononucleosis (IM) usually presents with prolonged fever, fatigue, hepatosplenomegaly, lymphadenopathy and malaise. Lab findings include atypical lymphocytes, mild transaminitis, hyperbilirubinemia, low-grade neutropenia and thrombocytopenia - all of which were present in our patient. Acute acalculous cholecystitis (AAC) is frequently described in critically ill patients however has been documented in only 17 case reports as a very rare complication in EBV-associated Infectious mononucleosis. AAC involves inflammation of the gallbladder in the absence of cholelithiasis and clinically presents with persistent jaundice and right upper quadrant abdominal pain. The exact mechanism of AAC in EBV infection is unknown. It is thought increased bile viscosity from dehydration, hyperthermia or ileus in the setting of EBV infection can lead to cholestasis and have a direct toxic effect on the gallbladder wall, leading to AAC. The diagnosis of AAC is based on clinical suspicion however the ultimate diagnosis rests on imaging. Ultrasound criteria include major and minor criteria. Major criteria include gallbladder wall thickening greater $3.5 \mathrm{~mm}$, pericholecystic fluid or edema, intramural gas or sloughed mucosa. Minor criteria include echogenic bile or hydrops. The combination of 2 major or 1 major and 2 minor criteria are considered diagnostic. In our case, the ultrasound showed moderate splenomegaly, contracted gallbladder with a thickened wall $(10 \mathrm{~mm})$ and surrounding edema, with no evidence of biliary duct dilatation or cholelithiasis, findings consistent with AAC. Complications such as gangrene, perforation and empyema have been reported in AAC however not in EBV-associated AAC. This disease entity is usually treated with intravenous rehydration therapy and conservative management, not requiring emergent surgery as seen in our case. Clinicians should be aware of AAC complicating EBV infection in order to avoid unnecessary antibiotic treatment and surgical intervention. Acute acalculous cholecystitis is a rare but serious complication of EBV infection. Case reports show full resolution with conservative management. Patients should be monitored for clinical improvement given the significant morbidity and mortality associated with AAC.

\section{ACQUIRED HEMOPHILIA A: CASE AND DISCUSSION OF A RARE COAG-} ULOPATHY Xiaoming Jia; Jasmine Kalsi; Farrah Kheradmand. Baylor College of Medicine, Houston, TX. (Control ID \#2466680)

LEARNING OBJECTIVE \#1: Diagnose acquired hemophilia A based on clinical presentation and coagulation studies.

LEARNING OBJECTIVE \#2: Manage acute bleeding caused by acquired hemophilia A CASE: A 50 year old African American female with human immunodeficiency virus on highly active antiretroviral therapy, presented with 1 week of progressively worsening left thigh pain and swelling accompanied by fatigue and dizziness. She noticed bruising at the affected area several days prior to admission but denied any trauma. She does not have any previous history of easy bruising or bleeding and denies any family history of bleeding disorders. On exam, the lateral aspect of the left thigh was asymmetrically swollen and tense, which was confirmed by computer tomography to be a large hematoma. However, no evidence of petechiae, bruising, mucosal bleeding or hemarthrosis was noted. Complete blood count showed a hemoglobin of $5.4 \mathrm{~g} /$ deciliter with normal platelet count. Coagulation labs demonstrated an isolated elevation of activated partial thromboplastin time (aPTT) of $78.1 \mathrm{~s}$. Factor VIII level was markedly reduced with normal factor IX and XI concentrations. Mixing study was performed and resulted in an incomplete correction, suggesting the presence of an inhibitor. The patient was admitted to the medical intensive care unit for close monitoring. Repeat hemoglobin had decreased to $3.9 \mathrm{~g} /$ deciliter and she was initiated on recombinant activated factor VII and prednisone. After red blood cell transfusion, her hemoglobin stabilized and the patient was eventually discharged. A sendout lab for inhibitor titer was 11.5 Bethesda Units (BU), confirming the presence of high levels of factor VIII inhibitors. Subsequent work-up for malignancy, autoimmune disease and viral hepatitis were negative
DISCUSSION: Acquired hemophilia A, though very rare, is a potentially life threatening coagulopathy caused by the production of autoantibodies against factor VIII. Because of its increased mortality, as high as $22 \%$, acquired hemophilia A requires expedient initiation of diagnosis and management (1). Acquired hemophilia often presents with hemorrhage into the soft tissue and muscle as well as severe mucosal bleeding. However, unlike in hereditary hemophilia, hemarthrosis is usually not found. Diagnosis is based on coagulation studies, which reveal isolated elevation in (aPTT), suggesting a disturbance in the intrinsic pathway. This is followed by a mixing study to distinguish low factor levels versus presence of inhibitors. Cases where mixing study results are suggestive of inhibitors warrant confirmation by measurement of factor levels and autoantibody titers (1). The treatment strategy for acquired hemophilia A can be divided into the initial control of acute bleeds and long-term suppression of autoantibody production. Establishment of hemostasis can further be categorized into agents that bypass factor VIII in the coagulation pathway and therapies that replace depleted factor levels. Bypassing agents are currently the first line treatment for acute bleeds in patients with acquired hemophilia A. Due to the rarity of the disease, these therapies have not been studied in randomized prospective trials. However, both recombinant activated factor VII (rFVIIa) and activated prothrombin complex concentrate $(\mathrm{aPCC})$ have been shown in various retrospective studies to be highly effective $(2,3)$. RFVIIa, in particular, was found to have up to $100 \%$ response rate as first line treatment and up to $75 \%$ as a salvage therapy (4). Treatments to increase circulating levels of FVIII, meanwhile, have been found to be limited in efficacy and should be used only in disease with low inhibitor titers, $<5$ BU (2). Patients diagnosed with hemophilia A typically also undergo elimination of autoantibody in order to prevent recurrent bleeding episodes. Again, a majority of studies on therapy have been retrospective. However, at least one prospective trial has been conducted, demonstrating significant autoantibody suppression in patients treated with either prednisone alone or steroid plus cyclophosphamide (5). Other strategies that have been documented in the literature include cyclosporine, intravenous immunoglobulin and rituximab. In conclusion, the severity of bleeding in acquired hemophilia A necessitates expedient diagnosis and management. In patients with no previous personal or family history of bleeding, the presence of inhibitors against factor VIII can quickly be recognized via isolated prolongation of aPTT with incomplete correction in mixing studies. First line treatment consists of the use of a bypassing agent, such as rFVIIa, to achieve hemostasis along with suppressive therapies such as prednisone to eradicate autoantibodies. References: 1) Franchini M, Giuseppe L. Acquired factor VIII inhibitors. Blood. 2008;112:250-255. 2) Holme PA, Brosstad F, Tjonnfjord GE. Acquired hemophilia: management of bleeds and immune therapy to eradicate autoantibodies. Haemophilia. 2005;11:510-515. 3) Roberts HR, Monroe DM, White GC. The use of recombinant factor VIIa in the treatment of bleeding disorders. Blood. 2004;104:3858-3864. 4) Hay CRM, Negrier C, Ludlam CA. The treatment of bleeding in acquired hemophilia with recombinant factor VIIa: a multicenter study. Thromb Haemost. 1997;78:3-7. 5) Green D, Rademaker AW, Briet E. A prospective, randomized trial of prednisone and cyclophos- phamide in the treatment of patients with factor VIII autoantibodies. Thromb Haemost. 1993;70: 753-757.

\section{ACTINOMYCES, AN UNUSUAL SUSPECT FOR NON RESOLVING PNEUMO-}

NIA Ignacio A. De Cicco; Abhishek Maiti; Gabriel M. Aisenberg. The University of Texas Health Science Center, Houston, TX. (Control ID \#2468764)

LEARNING OBJECTIVE \#1: Many times physicians fall into the challenges of managing cases of non resolving pneumonia. Pulmonary actinomycosis is rare, but must be part of our differential diagnosis and there has to be high level of suspicion. Undiagnosed in a timely manner can signify a threat to patient's health and inappropriate use of hospital resources such as imaging, antibiotics, surgical interventions and extended hospital stay. 
CASE: Actinomyces are Gram positive branching filamentous commensals. Fifteen to fifty percent of actinomycosis cases have pulmonary involvement. The diagnosis is rarely suspected at initial presentation and commonly confused with chronic suppurative chest disease or malignancy. Often 6 months lapse before a definitive diagnosis is made. A 54 year-old man who is a nonsmoker, presented to the hospital with increasing cough for 6 months. He was producing a cup of thin whitish non-purulent, non-bloody sputum per day. He noted having exertional dyspnea, fever, malaise and weight loss. He denied any history of ill-contacts or exposure to tuberculosis. Three months prior to presentation, he was prescribed 7 days of clarithromycin for similar symptoms and a right paracardiac opacity on chest radiography. The treatment resulted in no clinical improvement. Physical exam revealed clubbing and coarse crackles with decreased breath sounds over the right sub-scapular region. Initial workup revealed persistent right paracardiac opacity, leukocytosis and elevated erythrocyte sedimentation rate. Sputum examination and cultures were unrevealing. The patient was treated empirically with 7 days of parenteral levofloxacin without significant change. Computerized tomography of the chest showed consolidation and atelectasis of right lower lobe (RLL) with scarring. There was a calcified endobronchial opacity in the RLL bronchus with right paratracheal and hilar enlarged lymph nodes. Bronchoscopy revealed erythematous mucosa in the RLL with whitish flecks and a plug of whitish friable material obstructing its bronchus. Staining, culture and cytology of bronchoalveolar lavage fluid were unrevealing. Histopathology of the biopsy demonstrated metaplasic squamous epithelium with lymphocytic and eosinophilic infiltrate in the stroma underneath. Clumps of delicate basophilic filamentous bacillary structures with Splendore-hoeppli phenomenon and focal calcifications yielded the diagnosis of actinomycosis. The patient received 4 weeks of parenteral penicillin followed by oral penicillin for 4 months with significant clinical and radiographic improvement.

DISCUSSION: Pulmonary actinomycosis is a rare, chronic infection. Presentation is usually nonspecific including productive cough, chest pain, shortness of breath, hemoptysis or localized chest swelling. Systemic features may include weight loss, malaise, night sweats, fever and generalized weakness. Advanced pulmonary disease may present with extensive cavitary consolidation, pleural effusion, empyema or draining sinuses. Early diagnosis is difficult as the presentation commonly raises suspicion for pulmonary tuberculosis, chronic suppurative chest disease, fungal infection or malignancy. To confirm the diagnosis invasive procedures are required such as bronchoscopy or biopsy. Inability to diagnose it in a timely manner, it possesses a threat to patient's health and inappropriate use of hospital resources such as imaging, antibiotics, surgical interventions and extended hospital stay. High index of suspicion is needed and actinomycosis should always be considered in the differential diagnosis

\section{ACUTE BACK PAIN IN A YOUNG WOMAN DUE TO INFECTIOUS} SACROILIITIS Dan Reynolds; Adam P. Sawatsky. Mayo Clinic, Rochester, MN. (Control ID \#2469595)

LEARNING OBJECTIVE \#1: Recognize the clinical presentation, diagnosis, and treatment for infectious sacroiliitis

CASE: A 23-year-old female with a history of chronic low back pain presented to the Emergency Department with pain in her left hip, gluteal region and lower back that had progressively worsened over 3 days. She described pain mainly located in her gluteal region, with radiation down her leg and into her back. Aggravating factors included any movement of the leg itself, and the pain had progressed to the point where she was no longer able to bear weight. Additionally, she reported 3 days of fevers and chills without evidence of rash or other signs of infection. She denied any history of drug use. Laboratory evaluation revealed: white blood cell count 7.8, hemoglobin 11.1, platelets 153,000, and an elevated CRP of 201.2. An MRI of the lumbar spine performed in the ED did not reveal any abnormalities. The patient was admitted for management of her acute on chronic back pain. A CT scan of the pelvis revealed a focus of contrast enhancement near the left sacroiliac joint. Blood cultures became positive for methicillin-susceptible staphylococcus aureus (MSSA). An indium scan showed septic arthritis of the left sacroiliac joint. A repeat MRI of the pelvis identified a loculated abscess in the left sacroiliac joint. She was treated with a CT-guided aspiration of the abscess and a 6-week course of intravenous cefazolin, which helped improve her pain.

DISCUSSION: Infectious sacroiliitis (ISI) is a rare condition affecting the sacroiliac (SI) joint, representing 1-2\% of all cases of septic arthritis (1). The diagnosis is made difficult by its rare nature, low clinical suspicion and a nonspecific presentation that is easily confused with other common causes of low back pain. A retrospective study found that the mean time to diagnosis was 43.3 days (2). Known risk factors for ISI include immunosuppression, pregnancy, trauma, drug abuse, and known infection. The most common symptom is lumbogluteal pain; radicular symptoms are also common (2). Physical examination often will often reveal pain with manipulation of the SI joint. Fever is a common symptom with some suggestion that it is present in $75 \%$ of cases (3). Laboratory evaluation typically reveals an elevated C-reactive protein and sedimentation rate; however, leukocytosis is not consistently elevated (2). The diagnosis of ISI requires a high level of suspicion in a patient with complaints of lumbogluteal pain, fever, and concern for systemic infection. The most common organisms identified in cases of ISI are MSSA, coagulase-negative staphylococcus, and streptococcus. The preferred diagnostic method is MRI of the pelvis (3). In our case, MRI of the lumbar spine initially did not reveal the diagnosis and further imaging with $\mathrm{CT}$ and eventual dedicated Pelvic MRI was required. Antibiotic therapy is recommended based on culture-specific therapy directed at specific organisms. If a specific organism cannot be identified, therapy should be aimed at MSSA. Antibiotic therapy typically lasts for 2 to 6 weeks. Untreated ISI can lead to increased morbidity such as abscess formation, osteomyelitis and fusion of the SI joint (4), making early diagnosis essential.

\section{ACUTE ENCEPHALOPATHY WITH HYPERCALCEMIA MASKING NEUROSYPHILIS MASKING CERVICAL CANCER Andrew Kelly; Vivek Alaigh.} University of Connecticut, Hartford, CT. (Control ID \#2469628)

LEARNING OBJECTIVE \#1: Recognize the importance of a thorough and complete work up for acute encephalopathy to uncover the etiology of altered mental status, especially when there are multiple organic causes that could be contributing.

CASE: A 94-year-old female with a past medical history of hypertension and a prior stroke presented to the emergency department with acute encephalopathy and 3 weeks of progressive fatigue. She had decreasing appetite over that time and ultimately stopped eating altogether. Vitals on admission included a blood pressure of $160 / 80$, heart rate of 79 , a respiratory rate of 16 , a temperature of $94.9^{\circ} \mathrm{F}$, and oxygen saturation of $100 \%$ on room air. Initial laboratory values were sodium $146 \mathrm{mmol} / \mathrm{L}$, potassium $4.7 \mathrm{mmol} / \mathrm{L}$, chloride $105 \mathrm{mmol} / \mathrm{L}$, bicarbonate $27 \mathrm{mmol} / \mathrm{L}$, blood urea nitrogen $50 \mathrm{mg} / \mathrm{dL}$, creatinine $3.5 \mathrm{mg} /$ dL. Total calcium was 16.2 and ionized calcium was 1.81 . Total protein was 8.4 and albumin was 3.2. Urinalysis revealed pyuria with bacteruria. Head CT showed chronic small vessel changes with lacunar infarcts but no masses. TSH was normal and vitamin B12 level was elevated. HIV was negative. A rapid plasma reagin (RPR) was elevated > 8.0 and the Treponema pallidum agglutination assay was reactive. The patient was originally treated for neurosyphilis and her hypercalcemia was treated with intravenous fluid hydration, which also improved her acute kidney injury. Her mental status improved but calcium levels remained elevated. $1,25 \mathrm{OH}_{2}$ Vitamin D levels were low and intact PTH was normal. Further investigations for causes of her hypercalcemia revealed elevated beta macroglobulins and bone lesions. The lambda and kappa chains were elevated but inconsistent with multiple myeloma. Further investigations for causes of hypercalcemia uncovered a cervical mass which proved to be metastatic. 
DISCUSSION: This case highlights the value of a thorough work up for acute encephalopathy. This patient had innumerable contributors to her altered mental status and the etiology could have only been uncovered through completing a thorough evaluation. It seemed that every differential being investigated was confirmed by lab or imaging. Her UTI was confirmed with urinalysis, her tertiary syphilis was confirmed with RPR but was felt to be burnt-out syphilis. Because she had bone lesions as well as elevated kappa and lamba chains in her serum and urine, there was also suspicion for hypercalcemia secondary to a paraneoplastic syndrome from malignancy, particularly multiple myeloma; however, the levels were felt to be inconsistent with that diagnosis. She was ultimately diagnosed with a pelvic mass found to be cervical cancer that could have easily been missed if the workup was stopped prematurely.

ACUTE FLANK PAIN: THINKING BEYOND THE BOX Raju Khanal ${ }^{1}$; Daniel Forman ${ }^{2} .{ }^{1}$ Reading Health System, Wyomissing, PA; ${ }^{2}$ Reading Hospital, Reading, PA. (Control ID \#2454801)

LEARNING OBJECTIVE \#1: To diagnose renal infarction in otherwise healthy patients presnted with renal colic

LEARNING OBJECTIVE \#2: To understand the management of idiopathic renal infarction

CASE: A 42 year old male with history of hypertension and 20 pack year of smoking, presented to emergency initially for sudden onset of left flank pain for half hour duration. Non-contrast CT abdomen revealed a $1 \times 1 \mathrm{~mm}$ non-obstructing stone in the lower pole of left kidney. Urinalysis, $\mathrm{CBC}$, and BMP were unremarkable. The pain was thought to be due to renal stone and discharged from emergency with analgesic. However, he returned to ED after 9 days of the discharge with worsening pain. This time, enhanced CT abdomen showed a long segment thromboembolism in the left mid to distal renal artery with renal infarct (Fig 1). He was started on intravenous heparin infusion, followed by warfarin with Enoxaparin bridge. Further work up including hypercoagulable states, echocardiogram, and telemetry monitoring were unremarkable. Warfarin was discontinued in 6 months, and started on aspirin as he did not have any other evidence of thromboembolism.

DISCUSSION: Classic presentation of renal infarction is a triad of persistent abdominal and/or flank pain, elevated serum LDH and/or hematuria and risk of thromboembolic event. However, absence of those factors does not rule out idiopathic renal infarction, like in our case. Although, non-contrast CT is the diagnostic choice for renal colic, it cannot easily detect renal arterial or venous thromboembolism. Contrast enhanced CT not only detects renal infarction/ thromboembolism but also helps to rule out other differential diagnosis of acute abdominal pain including abscess, cyst, tumor and extra-renal causes of abdominal pain. In majority of cases, the source of renal emboli with normal cardiac evaluation remains unknown. It may originate from the proximal aortic wall rather than from the cardiac chambers. Its excellent outcomes with timely diagnosis and treatment emphasize the need to consider renal artery thrombosis in differential diagnosis of acute abdomen. This can avoid surgical intervention, such as autotransplantation or nephrectomy. Most patients without cardiac disease are initially treated with anticoagulants similar to atrial fibrillation-induced embolism. However, the role of newer anticoagulants, duration of anticoagulation, benefit of anticoagulation over antiplatelet agents, and role of statin in setting of idiopathic renal infarction are unknown. Thrombolytic can also be considered in cases that are diagnosed within $6 \mathrm{~h}$ of symptoms onset. In conclusion, acute renal infarction is extremely rare disease and often misdiagnosed as its presentation is similar to other common diseases including renal stones. Contrast-enhanced CT should be considered in patient with renal colic without significant lithiasis even in previously healthy individuals without thromboembolic
ACUTE HYPERSENSITIVITY PNEUMONIA IN THE DISGUISE OF COMMUNITY ACQUIRED PNEUMONIA IN A YOUNG ADULT WORKING AS EXTERMINATOR Monoj K. Konda ${ }^{2}$; Yashwant Agrawal ${ }^{2}$; Hardik Chhatrala ${ }^{1}$; Jason K. Lam². ${ }^{1}$ Homer Stryker MD WMU School of Medicine, Kalamazoo, MI; ${ }^{2}$ Western Michigan University Homer Stryker M.D. School of Medicine, Kalamazoo, MI. (Control ID \#2467788)

LEARNING OBJECTIVE \#1: Acute hypersensitivity pneumonia can mimic community acquired pneumonia in clinical presentation. A detailed occupational history and history of exposure to various inhaled chemicals can give important clues to the diagnosis. LEARNING OBJECTIVE \#2: Removal of offending agent is all that is needed in treatment of most cases.

CASE: 39 -year-old male with no past medical illnesses presented to emergency department with 4-day history of worsening shortness of breath and productive cough with black colored sputum. He had associated features of subjective fevers, chills and decreased oral intake for past 3 days. Occupational history was significant for extermination with exposure to multiple chemicals and was working without mask recently. Physical examination was positive for fever of $102.8 \mathrm{~F}, 87 \%$ oxygen saturation on 5-1 nasal cannula, diffuse crackles and diminished breath sounds over right lower lobe. Laboratory work including $\mathrm{CBC}, \mathrm{CMP}$, lactic acid and procalcitonin were unremarkable. A chest x-ray showed bilateral peri-hilar streaky multinodular opacities. He was admitted to general medical floor with a presumable diagnosis of community acquired pneumonia and initially treated with ceftriaxone and azithromycin. The patient clinically deteriorated in $24 \mathrm{~h}$ requiring endotracheal intubation and mechanical ventilation. A high resolution CT chest showed nonspecific multifocal groundglass pulmonary opacities suggestive of hypersensitivity pneumonitis. A bronchoscopy showed normal appearing bronchial mucosa and testing of bronchoalveolar lavage (BAL) suggested the diagnosis of acute hypersensitivity pneumonitis (HP) most likely related to his occupation as exterminator (exposed to Maxxthor/Suspend SC/Tempo dust/Termidor/Gentrol and other chemicals). There were $5596 / \mathrm{cmm}$ nucleated cells with $87 \%$ polymorphonuclear cells and $13 \%$ mononuclear cells and 3954/cmm RBCs. Blood cultures and cultures of BAL were negative. Mycoplasma, streptococcus pneumoniae and legionella testing were also negative. Patient improved clinically with elimination of offending agent and ventilator support and was extubated in $72 \mathrm{~h}$. The combination of negative cultures and other infectious markers, clinical improvement with removal of offending agent and absence of other explanatory findings made acute HP most likely diagnosis and a lung biopsy was not performed. DISCUSSION: Epidemiology of acute HP is mostly unknown and varies considerably depending on case definitions, inciting agents, geographical distribution. Farmers lung is most commonly studied form of HP and prevalence per 100,000 varies from 420 to 3000 . It is commonly mistaken for viral or bacterial infection as occurred in our patient. Most classic form occurs after heavy exposure to inciting agent. Removal from exposure to the inciting agent results in resolution of symptoms within $12 \mathrm{~h}$ to several days and radiologic recovery takes several weeks. Scattered multinodular, interstitial opacities in lower and middle lung zones on chest $\mathrm{x}$ ray and presence of ground glass opacities on high resolution CT confirms the presence of pneumonitis in the right clinical scenario. The cell counts and differential on BAL is helpful in suggesting the diagnosis. Histopathology confirms the diagnosis and can differentiate between acute versus subacute or intermittent HP. Systemic glucocorticoids are only occasionally required as all categories of HP improve with removal from exposure to inciting agent. Repeated exposures can result in chronic fibrosing lung disease. Our patient was discharged with close pulmonology follow up and advised to avoid future occupational exposure or use effective protection equipment.

ACUTE LIVER INJURY IN A PATIENT WITH METASTATIC PHEOCHRO-

MOCYTOMA Dana Zalkin; Matthew McNeill. New York University, New York, NY. (Control ID \#2467303) 
LEARNING OBJECTIVE \#1: Recognize the epidemiology and clinical presentation of metastatic pheochromocytoma

LEARNING OBJECTIVE \#2: Assess various etiologies of acute liver injury in patients with metastatic pheochromocytoma

CASE: A 63 year-old woman presented with 3 days of altered mental status, fatigue, and jaundice. The patient had been diagnosed with predominantly norepinephrine-secreting pheochromocytoma at age 21 and underwent a left adrenalectomy at that time. Nine years ago, she had recurrence of disease with metastatic involvement of the lumbosacral spine and underwent palliative treatment with radiotherapy, targeted chemotherapy, and surgical resection. Blood pressure was well-controlled with phenoxybenzamine and labetalol. Vital signs on admission were unremarkable. Labs were notable for aspartate transaminase (AST) $1861 \mathrm{U} / \mathrm{L}$, alanine transaminase (ALT) $1231 \mathrm{U} / \mathrm{L}$, total bilirubin $7.7 \mathrm{mg} / \mathrm{dL}$, International Normalized Ratio (INR) 2.4 , and white blood cell count of 13,000 with $14 \%$ bands. Tests for viral hepatitis and toxins, including acetaminophen were negative. Computed tomography scans of the head and abdomen revealed numerous new osteolytic lesions involving the calvarium and skull base without any acute liver pathology. The patient was treated with broad spectrum intravenous antibiotics for presumed sepsis and intravenous $\mathrm{N}$-acetylcysteine for acute liver injury of unknown etiology. All cultures showed no growth. Her amino transaminases improved, however her INR, bilinubin, creatinine, and white blood cell count continued to rise during the hospitalization. The patient continued to decline clinically and she expired on hospital day 14 .

DISCUSSION: Pheochromocytoma is a rare catecholamine-secreting tumor arising from the chromaffin cells of the adrenal medulla. The most common presentation of pheochromocytoma is sustained or paroxysmal hypertension, however other presentations include headache, generalized sweating, or incidental findings on imaging. Diagnosis requires elevated plasma or urinary metanephrines and catecholamines. Surgical resection is recommended if anatomically feasible following appropriate pre-operative medical management. Approximately $10 \%$ of pheochromocytomas are malignant, however there are no histopathological methods to distinguish benign versus malignant tumors at presentation. The only widely accepted indicator of malignancy in pheochromocytomas is the presence of metastases. Metastatic disease may be present at the time of initial diagnosis or it may occur decades after initial presentation. The most common sites of metastasis are the lymph nodes, bone, liver, and lungs, with better survival rates in those with metastatic lesions in the bone and worse survival rates in those with metastases to the liver and lungs. Regular biochemical screening is recommended after tumor resection to detect recurrence. There is no established cure for metastatic pheochromocytoma. Treatment is aimed at controlling symptoms of excess circulating catecholamines as well as reducing tumor burden with surgery or chemotherapeutic agents. The potential etiology of acute liver injury in a patient with known metastatic pheochromocytoma includes metastatic liver infiltration, drug-induced liver injury, and hepatic ischemia. Amino transaminases levels exceeding $1000 \mathrm{U} / \mathrm{L}$, as in this patient, are usually found only in ischemic hepatitis, acute drug or toxin induced liver injury, or acute viral hepatitis. Resolution of amino transaminases elevation concomitantly occurring with worsening liver synthetic function (INR, total bilirubin) are indicative of ischemic hepatitis. Evidence of other end-organ hypoperfusion (rising creatinine) further suggests sepsis or hypotensive episodes as the likely etiology of acute liver injury. Although usually associated with hypertension, there are reports of symptomatic hypotension and fluctuating blood pressures in patients with pheochromocytoma. It has been postulated that catecholamine-associated vasoconstriction causes tumor necrosis, leading to a relative catecholamine insufficiency. The degree of hypotension is complicated further by often aggressive anti-hypertensive medication regimens. Hypotension is more commonly associated with predominantly epinephrinesecreting tumors, however episodes of pheochromocytoma-induced hypotension in predominantly norepinephrine-secreting tumors have been described. In our patient, the concern for sepsis-induced hypotension and liver ischemia was high, however no source was ever elucidated. With known metastatic pheochromocytoma, the differential for acute liver injury must include tumor-related hypotension as well.
ACUTE MESENTERIC ISCHEMIA IN PATIENTS WITH DIABETES MELLITUS: BEYOND THE CLASSIC TEACHING OF "ABDOMINAL PAIN OUT OF PROPORTION TO THE PHYSICAL EXAM" Elissa Szalkiewicz ${ }^{1}$; Lauren Shapiro ${ }^{2} .{ }^{1}$ Montefiore Medical Center, NYC, NY; ${ }^{2}$ Montefiore Medical Center, Bronx, NY, NY. (Control ID \#2470223)

LEARNING OBJECTIVE \#1: Recognition of the early presentation of acute on chronic mesenteric ischemia in the setting of critical illness

LEARNING OBJECTIVE \#2: Understand the prothrombotic state of Type II Diabetes Mellitus

CASE: A 51 year-old man with Type II Diabetes Mellitus presented with 2 weeks of polyuria and polydipsia in addition to 3 days of shortness of breath, productive cough, nausea, and emesis. He denied abdominal pain. He was obese, febrile to 102.5 and tachycardic to the $120 \mathrm{~s}$. His abdomen was soft, distended, and tympamnic. He had with mild discomfort in the bilateral lower quadrants with palpation. Initial labs revealed: anion gap 40, glucose $731 \mathrm{mg} / \mathrm{dL}$, potassium $6.7 \mathrm{mg} / \mathrm{dL}$, bicarbonate $12 \mathrm{mg} / \mathrm{dL}$, urine ketones (acetone) $>80 \mathrm{mg} / \mathrm{dL}, \mathrm{pH} 7.17$, white blood cell count $18.9 \mathrm{k} / \mathrm{uL}$, and lactic acid $3.7 \mathrm{mmol} /$ L. After 4 days of treatment for diabetic ketoacidosis (DKA) and antibiotics for a pneumonia which was thought to have precipitated the DKA, his anion gap closed to 13 and his lactic acid decreased to $1.7 \mathrm{mmol} / \mathrm{L}$; however, he continued to have nausea, emesis, abdominal discomfort and distention with an absence of abdominal pain. He also had not had a bowel movement. CT scan revealed dilated jejunum with mural thickening involving loops with areas of pneumatosis and small foci of extraluminal gas in the small bowel mesentery. MRI/MRA revealed an occlusive thrombus within the mid superior mesenteric artery (SMA), a small nonocclusive thrombus in the proximal SMA with patent collaterals and an occlusive thrombus in the superior mesenteric vein (SMV) with patent collaterals. There were multiple dilated loops of bowel with wall thickening including one loop in the left mid-abdomen with markedly decreased enhancement. Four days after presentation to the ICU, he was taken emergently to the operating room for extensive small bowel resection and underwent subsequent small bowel resections and abdominal wound wash-outs. Pathology revealed organizing thrombi in the SMA and SMV, other blood vessel thrombi, acute ischemic colitis and complete transmural necrosis. His postoperative course was complicated by septic shock and multi-organ failure. Unfortunately, the patient expired.

DISCUSSION: The classic teaching for diagnosing acute mesenteric ischemia is "abdominal pain out of proportion to the physical exam" with lactic acidosis on labs. It is important to recognize that this may not always be the case. Early acute mesenteric ischemia can present with nonspecific symptoms of abdominal discomfort, nausea, emesis, or unexplained abdominal distention. Acute ischemic episodes can be superimposed on chronic mesenteric ischemia as was likely the case for this patient given the collaterals noted on the MRI/MRA. Patients with chronic mesenteric ischemia often do not exhibit symptoms because of collaterals or they develop intestinal angina presenting as postprandial dull abdominal pain and later develop weight loss. Acute SMA thrombosis and chronic mesenteric ischemia are typically caused by thrombosis in areas of severe mesenteric atherosclerosis whereas mesenteric venous thrombosis is usually secondary to thrombophilias, local intra-abdominal processes, systemic hypercoagulable states, or idiopathic causes. Our patient's Diabetes Mellitus, Type II put him at risk of having accelerated atherosclerosis and a prothrombotic state. Increased glucose levels promote the production of free radicals with resultant oxidative stress and increase the expression of inflammatory genes resulting in endothelial cell dysfunction and vascular injury. In addition, there is an association between Diabetes Mellitus and a prothrombotic state as there is impairment of fibrinolysis by increased plasminogen activator inhibitor-1 (PAI-1) generated by insulin resistant adipocytes, promotion of platelet aggregation via glycation of platelet surface proteins and impaired calcium homeostasis, and decreased sensitivity of platelets to anti-aggregates (prostacyclin and nitric oxide). With the prevalence of Type II 
Diabetes Mellitus, physicians should have a suspicion for acute mesenteric ischemia in a critically ill patient with nausea, emesis and a distended abdomen even in the absence of "abdominal pain out of proportion to the physical exam" and lactic acidosis on labs.

ACUTE OCCLUDED LEFT ANTERIOR DESCENDING ARTERY IN A 21 YEAR-OLD MALE, NEVER TOO YOUNG TO HAVE ACUTE CORONARY SYNDROME Andrew Kelly; William Perucki. University of Connecticut, Hartford, CT. (Control ID \#2469749)

LEARNING OBJECTIVE \#1: Considering the risks associated with hypercoagulable states and even early cardiovascular disease which was demonstrated in Vietnam War autopsies of young soldiers, it is imperative to recognize the importance of thorough evaluation of chest pain irrespective of patient age.

CASE: A 21-year-old male with a past medical history of anxiety and depression presented to the emergency department with sharp chest pain that started at rest, radiated to the left shoulder and did not resolve spontaneously, He had a similar episode a week earlier that he did not seek medical attention for. He was a smoker and was obese with a BMI of 34. His initial ECG was unremarkable and troponin was 0.4. His pain resolved with Motrin but a subsequent troponin was 4.1 with creatinine kinase 506 . He had slight PR depressions on ECG and was treated for pericarditis until continuing to trend cardiac enzymes revealed a troponoin $>25$ and creatinine kinase of 1435 . At this time his ECG did show some mild ST and T wave changes but he remained chest pain free. He was taken to the cath lab to evaluate his coronary anatomy. He was found to have severe hypokinesis and akinesis in the distribution of a stenosed LAD artery. He was treated with heparin, integrilin, aspirin and ticagrelor as the stenosis was thought to be due to a thrombotic occlusion. After 3 days of medical therapy and no additional chest pain his coronary anatomy was re-evaluated with a coronary angiogram which redemonstrated $70 \%$ stenosis of the LAD which was stented. Laboratory evaluation did not confirm a hypercoagulable state.

DISCUSSION: Autopsy data from the Vietnam and Korean wars demonstrated the development of coronary atherosclerosis in young males. Furthermore it has been shown that plaques more prone to rupture are smaller and less stable. This is in contrast to larger plaques occupying a greater space in the coronary lumens that have had time to develop stability from the fibrous cap that forms over them. In our patient it was essential to evaluate the coronary anatomy when the history and laboratory data suggested acute ischemia. Even after a diagnosis of pericarditis was made, a thorough evaluation for acute coronary syndrome was able to reveal cardiac enzymes that continued to trend upwards Despite the patient being chest pain free, he had a significant lesion needing intervention If his work-up was completed prematurely it is highly probable that his lesion would have been missed putting him at risk of further ischemia and cardiac events

\section{ACUTE ONSET OF HEMIPARESIS AND DYSARTHRIA IN AN ADOLESCENT} MALE Karen A. Clarke. Emory University, Newnan, GA. (Control ID \#2470329)

LEARNING OBJECTIVE \#1: 1. Recognize acute disseminated encephalomyelitis as a cause of severe neurologic deficits in adolescents.

LEARNING OBJECTIVE \#2: 2. Understand the appropriate treatment for patients with acute disseminated encephalomyelitis, and their expected outcomes.

CASE: A 20 year old male with no past medical history experienced the acute onset of nausea and vomiting, and 3 days later he had seizure activity. Subsequently, he was admitted to the local hospital, and his initial vital signs and routine labs were unremarkable. During the course of the next 4 days, he had two more seizures, and was therefore started on levetiracetam. He experienced further clinical deterioration, including fluctuating mental status changes and right-sided weakness. The patient was transferred to a tertiary medical center for further management, and right hemiparesis, dysarthria, and encephalopathy were noted upon his arrival there. His head CT scan showed no acute intracranial abnormality. However, progressive multifocal FLAIR signal abnormality, consistent with demyelination, in the cortex/subcortical white matter in both hemispheres as well as the deep gray nuclei, was noted on his brain MRI. A lumbar puncture was performed and the cerebrospinal fluid (CSF) contained ninety (90) nucleated cells, seventy (70) per cent of which were lymphocytes. His CSF total protein was $60 \mathrm{mg} / \mathrm{dL}$. No oligoclonal bands were noted. His electroencephalogram showed diffuse theta to delta slowing, but no evidence of seizure activity. Intravenous acyclovir was started shortly after completion of the lumbar puncture, but it was discontinued after negative CSF virology PCR results were obtained. Acute disseminated encephalomyelitis (ADEM) was diagnosed, and intravenous methylprednisolone $1000 \mathrm{mg}$ daily was started. Within 3 days after the initiation of methylprednisolone, the encephalopathy and right hemiparesis resolved; however, mild dysarthria persisted.

DISCUSSION: The incidence of ADEM is uncertain, but it is not a common diagnosis. In adults, ADEM is often preceded by either a viral or bacterial infection, or less frequently, immunization. Patients with ADEM usually experience the acute onset of multifocal neurologic deficits. Motor deficits affect most patients with ADEM, and these may include paraparesis, hemiparesis, or tetraparesis. Sensory deficits and altered mental status also frequently occur. Findings that support a diagnosis of ADEM include (i) brain MRI finding of demyelinating lesions, (ii) CSF with mildly elevated protein and lymphocytic pleocytosis, and (iii) a history of recent infection or immunization. Immunosuppressive therapy is essential for the treatment of ADEM, and glucocorticoids should be used first. If glucocorticoids are ineffective, then other treatment options include intravenous immunoglobulin, plasma exchange, or cyclophosphamide. For most patients with ADEM who receive treatment, partial resolution of their neurologic deficits will occur. Complete recovery is typically seen in less than fifty percent of adults. Conclusion: ADEM should be considered in patients who experience the acute onset of unexplained multifocal neurologic deficits. Once the diagnosis of ADEM has been established, then the prompt initiation of immunosuppressive therapy will be beneficial for most patients.

\section{ACUTE PANCREATITIS AS PRESENTATION OF THROMBOTIC THROM- BOCYTOPENIC PURPURA IN A PATIENT WITH SLE Sonaina Imtiaz. North} Shore Medical Center, Salem, MA. (Control ID \#2469430)

LEARNING OBJECTIVE \#1: Complications of TTP such as pancreatitis can present as the initial presenting symptoms and a low index of suspicion is required for prompt treatment to save lives. TTP is a rare disease but $90 \%$ of the cases can be saved if recognised early and treated with intravenous steroids and plasma exchange in a timely manner.

LEARNING OBJECTIVE \#2: Microangiopathic hemolytic anemia and thrombocytopenia without an apparent obvious cause even in patient with autoimmune disease like lups should lead to a high index of suspicion for TTP.

CASE: 42 year old female with history of cutaneous SLE (biopsy proven, not treated), hypertension, diabetes mellitus and bipolar disorder presented with nausea and acute onset of epigastric pain radiating to the back 2 days prior to admission. She denied any associated diarrhea, vomiting, alcohol abuse, confusion but complained of mild yellowing of her skin over the past few days. On further inquiry she admitted to minor bleeding while brushing her teeth but no overt bleeding from any other site, no recent weight changes Subsequent CT scan without contrast of abdomen revealed heterogenous enhancement with extensive peripancreatic fat stranding of the body, head, uncinate process and to a lesser extent of the tail of the pancreas consistent with acute pancreatitis. Patient was a non 
alcoholic, denied any history of gallstones or prior history of pancreatitis. Her SLE was quiescent for many years and she was not only taking Risperidone and Ibuprofen at home. She was noticed to be mildly icteric without any rash. She was cooperative, able to answer all questions with appropriate affect, no motor or sensory deficits on admission. She was noticed to have mild petechiae over her shoulders, reproducible epigastric pain on palpation and no palpable masses. Labs revealed pancytopenia with Hemoglobin 7.1, Hct 21.4 (baseline Hct 36), WBC $3.9 \mathrm{~K} / \mathrm{microL}$, platelets $11 \mathrm{~K} / \mathrm{microL}$, bands $14 \%$, Retic count $4.4 \%$,INR was 1.2 , BMP was normal with BUN 22, creatininne 0.9 , low potassium of 3.4, LFTs revealing total protein 6.4, albumin 4.1, total bilirubin 3.2, direct bilirubin 0.7, Alkaline Phosphatase 871, ASt 30, ALT 23, Lipase 144. Peripheral smear revealed schistocytes with basophilic stippling, decreased platelets with large forms. She was started on intravenous steroids for autoimmune hemolytic anemia and autoimmune thrombocytopenia. Ultrasound of the right upper quadrant revealed biliary sludge but no stones. Over the next 4-5 days, the cause of her autoimmune anemia remained unclear. Her renal function remained intact, her WBC normalized the following day but she continued to have anemia and thrombocytopenia. Her fibrinogen levels were normal and direct and indirect Coombs test were negative. Her primary complaint remained epigastric pain with some nausea. Initially it was felt that she might have autoimmune anemia perhaps related to SLE but the schistocytes in the smear raised the concern for TTP and after a transient episode of aphasia with preceding parasthesias suspicion for TTP was increased. She was started on plasma exchange. During MRI brain was non revealing for stroke. Eventually patients condition improved with plasma exchange and she was started on Rituxan. Her aphasia completely resolved. ADAMSTS13 returned to be low $(<5 \%)$ consistent with TTP. She continues to receive Rituxan and plasma exchange and her platelets are improving. The exact etiology of TTP remains unclear and is still undergoing evaluation. A strong index of suspicion at the beginning would have hastened her treatment process

DISCUSSION: TTP is a life threatening emergency requiring a low index of suspicion. It may present as one of the complications of TTP as in this case it presented with the symptoms of Acute Pancreatitis. TTP is caused by severly low activity of ADAMTS13, von Willebrand cleaving factor. Microangiopathic Hemolytic anemia, Thrombocytopenia and signs of end organ damage are key to diagnosis. Often renal function or altered mental status are considered signs of end organ damage but it should be kept in mind any other organ be effected. In fact in one study, $69 \%$ patients with TTP presented with Gastrointestinal symptoms, $63 \%$ with weakness, $35 \%$ with major neurologic findings, $31 \%$ with minor neurologic findings, renal insufficiency may be seen but acute kidney injury and anuria are rare. Most patients have normal or only transiently elevated creatinine.TTP itself is a rare condition with 3 cases per 1 million adults per year. Not all patients are critically ill at presentation. In one post mortem study 30 out of 51 cases of TTP had pancreatic involvement. Vascular damage from thrombotic occulusion of the vessels serves as causing ischemia and triggering pancreatitis.

ACUTE PROMYELOCYTIC LEUKEMIA IN A TWIN PREGNANCY Catherine Wei ${ }^{1}$; Elan Gorshein ${ }^{2}$; Beatrice Wong ${ }^{1}$; Mecide Gharibo ${ }^{2} .{ }^{1}$ Rutgers Robert Wood Johnson, New Brunswick, NJ; ${ }^{2}$ Rutgers - Robert Wood Johnson, New Brunswick, NJ. (Control ID \#2470407)

LEARNING OBJECTIVE \#1: Acute Promyelocytic Leukemia is a clinical emergency and requires immediate diagnosis, treatment with ATRA and attention to coagulopathy disorders.

LEARNING OBJECTIVE \#2: Pregnancy presents potential complications with fetal toxicity. Management requires multidisciplinary effort between internists, oncologists and obstetricians.

CASE: Acute promyelocytic leukemia (APL) is a subtype of acute myeloid leukemia characterized by a balanced reciprocal translocation $\mathrm{t}(15 ; 17)$, a distinctive morphology of blast cells, and life threatening coagulopathy [1] All-trans retinoic acid (ATRA) and anthracycline-based chemotherapy can achieve complete remission in $95 \%$ of patients and a cure in $80 \%$ of patients $[2,3]$. Nonetheless, APL portends a 1-month death rate of $17.3 \%$ predominantly attributable to life-threatening coagulopathy [4]. Therefore, prompt recognition and therapy by internists and specialists alike is critical. A therapeutic dilemma arises, however, in a pregnant patient. Although the prevalence of leukemia in this population is approximately 1 in 10,000 [5], the benefits of treatment must be balanced against potential teratogenicity. We present a case of a new diagnosis of APL in the setting of a twin pregnancy. A 35 year-old pregnant female (G2 P1001) with twins presented at 24 weeks gestation following routine lab work revealing pancytopenia with peripheral blasts. She was asymptomatic with no signs of bleeding. Prior pregnancy was uncomplicated but current obstetrics course was significant for a short cervix and gestational diabetes. Admission labs were as follows: WBC $11.2 \mathrm{k} / \mathrm{uL}$ (n: $4-10 \mathrm{k} / \mathrm{uL}$ ) with $24 \%$ blasts, hemoglobin $10.7 \mathrm{~g} / \mathrm{dL}$ (n: $11.9-15.1 \mathrm{~g} / \mathrm{dL}$ ), and platelets $63 \mathrm{k} / \mathrm{uL}$ (n: 140-440 k/ $\mathrm{uL}$ ). Initial labs were also consistent with acute disseminated intravascular coagulation: $d-$ dimer $14306 \mathrm{ng} / \mathrm{mL}$ (n: 0-243 ng/mL), fibrinogen $118 \mathrm{IU} / \mathrm{L}$ (n: 125-220 IU/L), fibrin degradation products $>20(n<5)$, and prolonged prothrombin time $13.4 \mathrm{~s}(\mathrm{n}: 8.8-12.8 \mathrm{~s})$. Bone marrow biopsy with flow cytometry and FISH demonstrated translocation $\mathrm{t}(15: 17)$ with $72 \%$ blasts and promyelocytes, confirming high-risk APL. Induction therapy with ATRA and daunorubicin was started. Patient was treated with dexamethasone as prophylaxis for ATRA syndrome. Hospital course was complicated by neutropenia. Meropenem was started empirically for neutropenic fever. Patient also required multiple cryoprecipitate (15 units), platelets ( 2 units) and packed red blood cell transfusions (5 units). Hematologic remission was achieved and patient was discharged home. One week after discharge, patient was re-admitted for pre-term premature rupture of membrane at 28 weeks gestation, requiring urgent caesarian section delivery. Repeat bone marrow evaluation 30 days after induction demonstrated complete remission with no residual leukemia and PCR testing negative for $\mathrm{t}(15: 17)$. Patient is currently on arsenic consolidation therapy.

DISCUSSION: APL is a clinical emergency that requires immediate recognition by internists and specialists, initiation of ATRA and addressing potential coagulopathy $[1,2,3,6]$. Prompt therapy minimizes potential coagulopathy and treats the underlying disease but is problematic in pregnancy. Following the first trimester, ATRA administration is associated with less fetal toxicity [5]. Daunorubicin must be balanced with the increased risk in abortion, prematurity, low birth weight and neonatal neutropenia and sepsis [5]. Thus, recognition and management of APL requires efficient interdisciplinary care for the patient with obstetrics and leukemia expertise [6]. REFERENCES 1. Grimwade D, Hills RK, Moorman AV, Walker H, Chatters S, Goldstone AH, Wheatley K, Harrison CJ, Burnett AK, National Cancer Research Institute Adult Leukaemia Working G: Refinement of cytogenetic classification in acute myeloid leukemia: determination of prognostic significance of rare recurring chromosomal abnormalities among 5876 younger adult patients treated in the United Kingdom Medical Research Council trials. Blood 2010, 116(3):354-365. 2. Lo-Coco F, Giuseppe A, Vignetti M, Thiede C, Orlando SM, Lacobelli S, Ferrara, Felicetto, Fazi P: Retinoic Acid and Arsenic Trioxide for Acute Promyelocytic Leukemia. New England Journal of Medicine 2013, 369:111121. 3. Shen Z-X, Chen Guo-Qian, Ni J-H, Li X-S, Xiong S-M, Qiu Q-Y: Use of Arsenic Trioxide in the Treatment of Acute Promyelocytic Leukemia: Clinical Efficacy and Pharmacokinetics in Relapsed Patients. Blood 1997, 89(9):3354-3360. 4. Park JH, Qiao B, Panageas KS, Schymura MJ, Jurcic JG, Rosenblat TL, Altman JK, Douer D, Rowe JM, Tallman MS: Early death rate in acute promyelocytic leukemia remains high despite alltrans retinoic acid. Blood 2011, 118(5):1248-1254. 5. Milojkovic D, Apperley JF: How I Treat Leukemia During Pregnancy. Blood 2014, 123:974-984. 6. Rego E, Kim HT, ReuizArguelles G: Improving the Treatment Outcome of Acute Promyelocytic Leukemia in Developing Counries Through International Cooperative Network. Report on the International Consortium on Acute Promyelocytic Leukemia Study Group. Blood 2009, 22(114). 


\section{ACUTE SEVERE THROMBOCYTOPENIA INDUCED BY EPTIFIBATIDE JOSE}

B. Cruz Rodriguez; Leslie Cler. Methodist Dallas Medical Center, Dallas, TX. (Control ID \#2456802)

LEARNING OBJECTIVE \#1: Describe a case of acute severe thrombocytopenia induced by eptifibatide.

LEARNING OBJECTIVE \#2: Compare and contrast thrombocytopenia induced by eptifibatide versus other etiologies of thrombocytopenia.

CASE: CASE PRESENTATION Our patient was a 59-year-old female with history of gastroesophageal reflux, type II diabetes mellitus, hypertension, chronic left bundle branch block and coronary artery disease (CAD) status post coronary artery bypass graft in 2003 (left internal mammary to left anterior descending and saphenous vein to first obtuse marginal [OM] artery), stenting in 2004 (native mid-circumflex artery) and 2010 (ostial $70 \%$ left main). She presented with several days of burning and crushing sensation in her left chest that was not responsive to her usual treatment with esomeprazole. Sublingual nitroglycerin provided some relief and walking made the pain worse. Home medications were aspirin, carvedilol, clopidogrel, hydrochlorothiazide, insulin levemir, esomeprazole, simvastatin and valsartan. She was allergic to ACEI and losartan. The physical exam was within normal limits. EKG showed normal sinus rhythm with left bundle branch block and left axis deviation, unchanged from previous ones. Serial troponins were negative. Initial labs showed hemoglobin of $12 \mathrm{mg} / \mathrm{dL}$, platelets 181,000/microL, no leukocytosis, normal coagulation profile, kidney and liver function. She was admitted for evaluation of unstable angina. Her cardiologist was consulted and decision was made to take her to coronary angiogram 2 days after admission. A focal high-grade stenosis was found in the first $\mathrm{OM}$ just distal to the previous stent, which was treated with a new drug-eluting stent. During the procedure, the patient was placed on heparin drip per protocol, bivalirudin $500 \mathrm{mg}$, and eptifibatide $80,000 \mathrm{mcg}$. She was also loaded with $300 \mathrm{mg}$ of clopidogrel. She had received heparin 7500 units for DVT prophylaxis. Her cardiac symptoms resolved completely after the procedure. The next morning, while still asymptomatic, her platele count dropped to 21,000/microL, which was confirmed by repeating the blood count and by peripheral smear. There was no platelet clumping in the smear. TREATMENT He 4 T's score was 4 (14\% pre-test probability of heparin-induced thrombocytopenia [HIT]), so HIT-ELISA antibody was sent, prophylactic heparin was stopped and she was started on argatroban. She never developed any bleeding nor thrombotic events. After $24 \mathrm{~h}$, the HIT-ELISA came back negative, and argatroban was stopped. Medications were reviewe and it was felt that the best explanation for the severe acute drop in platelets was related to eptifibitide. The platelet count improved daily without further therapy. OUTCOME AND FOLLOW UP At the time of discharge, her platelet count was $85,000 /$ microL. She was discharged with optimal therapy for her $\mathrm{CAD}$ and other comorbidities. Follow up in clinic 3 weeks after showed a platelet count of 198,000 , with no change in the other hematologic lines.

DISCUSSION: Epitifibatide and the other glycoprotein IIb/IIIa inhibitors (GPI), commonly used in patients with CAD, can be associated with thrombocytopenia (TCP), often within minutes to hours of the initial administration. Although heparin is often coadministered with these agents, case reports and clinical trial data suggest that GPI are most commonly the etiology of TCP when both agents are used. Furthermore, heparin rarely causes severe TCP (platelet count $<20,000$ ). Randomized controlled studies have shown significantly increased rates of TCP for patients treated with eptifibatide. The TCP caused by GP IIb/IIIa inhibitors is clinically distinct from HIT. TCP caused by GP Ilb/IIIa inhibitors is sudden and severe, while TCP in HIT often occurs more gradually and platelet counts are rarely less than $30,000 /$ microL. Thrombosis is a common occurrence in HIT. On the contrary, paradoxical thrombosis is very rare with GP IIb/IIIa inhibitors although it has been reported. In addition to acute TCP, GP IIb/IIIa inhibitors can also cause pseudothrombocytopenia, a laboratory artifact when EDTA anticoagulant present in blood collection tubes causes platelet clumping. This possibility was ruled out in our patient by peripheral smear. A unique feature of GP IIb/IIIa inhibitor-induced TCP is its severity and acuity, typically occurring within $24 \mathrm{~h}$ and occasionally within $30 \mathrm{~min}$ to several hours of initial administration. This is distinct from other drugs that cause TCP mediated by drugdependent anti-platelet antibodies, which can take up to 14-21 days. It has been hypothesized that the rapid occurrence of TCP following new exposure to GP IIb/IIIa inhibitors is because of natural preformed antibodies against platelet neoepitopes, exposed during normal platelet aggregation, possibly during the process of aging. If TCP due to a GP $\mathrm{IIb} / \mathrm{III}$ inhibitor is suspected, the drug should be discontinued as well as heparin products. Pseudothrombocytopenia needs to be excluded with a blood smear and the $4 \mathrm{~T}$ score calculated to evaluate for HIT. In contrast to HIT, there is no need to anticoagulate. The use of IV gamma globulin has not been proven to be beneficial. Platelet transfusion is only recommended if the platelet count is $<20,000$, if there is overt bleeding or if an emergency invasive procedure is required. Readministration of the drug that caused TCP is not encouraged because of the risk for more severe TCP if rechallenged; furthermore, there is a $15 \%$ cross-reactivity with the other GP IIb/IIIa inhibitors.

ADRENAL INSUFFICIENCY IN A YOUNG MALE Sahar Sherf; Dorothy Martinez. UCLA Ronald Reagan Medical Center, Los Angeles, CA. (Control ID \#2469712)

LEARNING OBJECTIVE \#1: Recognize adrenal leukodystrophy as a rare cause of idiopathic adrenal insufficiency, particularly in young males

CASE: A 40 year old male initially presented to the neurology clinic for headaches, bilateral foot and thigh paresthesias, diffuse twitching, and gait instability. Neurological exam was notable for impaired attention and recall, hyperreflexia, and diminished vibratory sense. He exhibited diffuse hyperpigmentation of skin and loss of hair in his bilateral lower extremities. Cranial MRI showed increased signal in the cortex, internal capsule, and brain. Of note, his 42 year old brother had died of a motor neuron disease diagnosed on autopsy. Given his family history an Amyotrophic Lateral Sclerosis gene study was performed and was negative. Exome sequencing then identified a hemizygous c. $231 \mathrm{G}>\mathrm{A}$ nonsense variant for the $\mathrm{ABCD} 1$ gene associated with $\mathrm{X}$-linked recessive adrenoleukodystrophy (ALD)/adult-onset adrenomyeloneuropathy (AMN). Very long chain fatty acids were found to be elevated (hexacosanoic acid (C26:0) 3.1 umol/L, ratio of hexacosanoic acid to tetracosanoic acid (C26:0/C24:0), 1.69, and to docosanoic acid (C26:0/C22:0), 0.05). Adrenocorticotropic hormone (ACTH) was then found to be elevated at $1351 \mathrm{pg} / \mathrm{mL}$ (normal 6-59 pg/mL); evening cortisol level was $8 \mathrm{mcg} / \mathrm{dL}$. He was referred to the endocrinology clinic for adrenal insufficiency secondary to ALD. The patient noted generalized fatigue/weakness, lightheadedness, mild positional dizziness, salt cravings, and anorexia on presentation. He was hypotensive to $95 / 69$ on initial visit and started on daily fludrocortisone and prednisone with significant improvement in symptoms.

DISCUSSION: Adrenal insufficiency is most commonly due to autoimmune disease, infection, metastatic disease, or drugs. Less commonly, it can result from X-linked adrenoleukodystrophy (ADL)/adrenomyeloneuropathy (AMN), a rare peroxisomal disorder of beta-oxidation caused by mutations in the ATP-Binding Cassette gene at Xq28. This disorder results in accumulation of very long chain fatty acids (VLCFAs) in tissues, particularly the central nervous system, Leydig cells of the testes, and the adrenal cortex. ALD/AMN affects 1 in 16,800 and accounts for ten percent of adrenal insufficiency cases. This rare $\mathrm{X}$ linked disorder has two main phenotypes: ALD typically begins in childhood and rapidly progresses to quadriparesis; $\mathrm{AMN}$, more likely in our patient given later onset and milder form with slower progression, typically presents between 20 and 40 years of age with weakness, spastic parapresis, and polyneuropathy. While there is no correlation between the duration or severity of endocrine dysfunction and the severity of the myeloneuropathy, approximately $66 \%$ of male AMN patients have resultant Addison disease. We present a case of a young male with adrenal insufficiency, not previously on 
glucorticoids, due to likely AMN subtype of ALD. It is important to consider ALD/AMN in a young male with idiopathic adrenal insufficiency. While suspicion is higher in those with vague neurologic symptoms, up to $60 \%$ of young men with ALD have no or few neurologic abnormalities at the time of diagnosis of adrenal insufficiency. More so, biochemical evidence of adrenal insufficiency can be present for up to 2 years before the development of clinical signs. Rapid initiation of corticosteroid replacement therapy is essential.

ADULT ONSET STILL'S DISEASE : SOLVING THE JIGSAW PUZZLE Rajesh Gopalarathinam $^{1}$; Pavithra Indramohan ${ }^{2,}{ }^{1}$; Eric Orlowsky ${ }^{1} .{ }^{1}$ Allegheny General Hospital, Pittsburgh, PA; ${ }^{2}$ West Penn Allegheny Health Network, Pittsburgh, PA. (Control ID \#2456975)

LEARNING OBJECTIVE \#1: Diagnose Adult Onset Still's disease in the appropriate clinical scenario based on the Yamaguchi criteria

LEARNING OBJECTIVE \#2: Treat Adult Onset Still's Disease appropriately with corticosteroids and immunosuppressants.

CASE: A 33 old African-American male with no significant past medical history presented to the emergency department with a 4 week history of high-grade fever $(>39 \mathrm{C})$, sore throat and dry cough. His fever was accompanied by non pruritic skin rash on his trunk, arthralgia of bilateral ankles and knees, myalgias and night sweats. He denied the presence of joint stiffness in the mornings, blurry vision, eye pain, headache, back pain, burning urination, recent travel, decreased appetite or weight loss. The rest of the review of the systems was negative. He was not on any medications previously and had no significant family history. His vital signs were within normal limits. Physical exam was normal except for maculopapular skin rash on the trunk and an inflamed throat without any exudates. He was given azithromycin tablets in the ER for his symptoms. Following a week, his symptoms did not improve despite treatment. He presented to the ED again and was admitted to the hospital for further workup. His initial workup revealed elevated acute phase reactants (ESR:

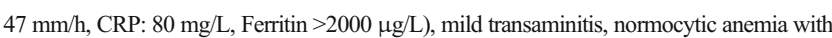
normal white cell and normal platelet count. His HIV and hepatitis panel were negtive. Chest $\mathrm{X}$-ray was normal. Subsequently, infectious diseases was consulted for his fever of unknown origin (FUO). An extensive ID workup was ordered which included fungal serologies and gallium scan, all of which turned out to be normal. Hematology was consulted for the patient's FUO and normocytic anemia. Hematological conditions such as leukemias, lymphomas and hemophagocytic lymphohistiocytosis were ruled out due to the absence of physical and laboratory findings. A 2-D echo, which was ordered to rule out endocarditis, came back as a normal study. As several investigations including SPEP and UPEP were nondiagnostic, a bone marrow biopsy with flow cytometry was done which showed no evidence of fibrosis or lymphoproliferative disorder. Finally, rheumatology was consulted for his FUO in relation to arthralgias and myalgias. His ANA, RF, CCP, ANCA panel, lyme serology and HLA-B27 tests were all negative. After ruling out sickle cell anemia, tuberculosis and other infectious causes, a diagnosis of Adult onset Still's disease was made based on the yamaguchi criteria. Subsequently, the patient was started initially on high dose prednisone $60 \mathrm{mg}$ PO daily. Over the next few days, his symptoms improved significantly following which methotrexate $10 \mathrm{mg}$ once weekly was added for steroid sparing effect. The patient was discharged home in a stable condition. During the next clinic visit in 2 weeks, his fever, rash and arthralgias subsided completely and his steroid dose was tapered appropriately. He did clinically well on methotrexate $20 \mathrm{mg}$ once weekly.

DISCUSSION: AOSD is a rare systemic inflammatory disease of unknown etiopathogenesis. It occurs worldwide and has bimodal age distribution with two peaks at 15-25 and 36-46 years of age. Clinically, the most classic manifestations of AOSD are a high spiking quotidian fever $\left(\geq 39^{\circ} \mathrm{C}\right)$, salmon colored rash in the trunk or proximal extremities, sore throat, and arthralgia involving the knees, wrists, ankles and elbows. The most common laboratory abnormalities include elevated ESR, neutrophilic leukocytosis, thrombocytosis and elevated ferritin levels. AOSD is diagnosed based on the Yamaguchi criteria which has major and minor criteria. The major criteria includes fever of at least $39^{\circ} \mathrm{C}$ for at least a week, arthralgia for atleast 2 weeks, non pruritic salmon colored rash on trunk/extremities and granuloctic leukocytosis (10,000/microL or greater). The minor criteria includes sore throat, lymphadenopathy, hepatomegaly or splenomagaly, transaminitis and negative tests for rheumatoid factor (RF) and antinuclear antibodies (ANA). Diagnosis requires at least 5 features, with at least two of these being major diagnostic criteria. AOSD is primarily a diagnosis of exclusion which should be considered only after excluding several other differential diagnoses including Infections (Infective endocarditis, Lyme disease, TB, viral infections such as HIV, hepatitis B and C, Parvovirus, Rubella), malignancies (leukemias and lymphomas), connective tissue disorders (SLE, MCTD, PAN, Wegener's granulomatosis) and granulomatous disorders (Sarcoidosis, Crohn's disease). Corticosteroids remain the first- line treatment for AOSD. Disease modifying antirheumatic drugs (DMARDs) such as methotrexate and azathioprine are often used for maintenance therapy of AOSD. Biologic agents such as TNF inhibitors (Infliximab, etanercept, adalimumab) or IL-1 antagonists (anakinra) can be used in refractory AOSD patients not responding to steroids and DMARD's.

AIR IN THE GASTRIC WALL-A RARE CASE OF EMPHYSEMATOUS GASTRITIS Thomas Chin; Mini Hariharan; Abid Shah; Peters Okonoboh. Baystate Medical Center, Springfield, MA. (Control ID \#2464367)

LEARNING OBJECTIVE \#1: Recognize the clinical presentation of emphysematous gastritis and the importance of early intervention

LEARNING OBJECTIVE \#2: Differentiate emphysematous gastritis from gastric emphysema

CASE: A 52 year-old male with a background of chronic kidney disease, type 2 diabetes mellitus on insulin, hypertension, hypothyroidism and prior DVT on warfarin presented as a transfer from an outside hospital. He had experienced 5 days of generalized, progressively severe abdominal pain associated with subjective chills, loose bowel motions, nausea and vomiting. He was afebrile but tachycardic (130 bpm) with stable BP. Examination revealed a distressed, morbidly obese gentleman with abdominal distention and tenderness to the upper abdomen without rebound or guarding. Rectal exam demonstrated melanotic stool. Laboratory studies were significant for $\mathrm{Hb}$ 6.3, WCC 12, INR 9.1, lactate 3.7, BUN of 52, Cr of 1.9, HCO3 17 and anion gap 13. Computed tomography (CT) of the abdomen was performed which showed distention of the stomach with foci of gas within the gastric wall along the greater curvature. There were also linear foci of gas extending to the periphery of the liver consistent with portal venous gas. A diagnosis of emphysematous gastritis was made. The patient was admitted to the intensive care unit. He received FFP and vitamin $\mathrm{K}$ for reversal of his INR. He was given IV fluid and packed red blood cells for resuscitation, and commenced on insulin for his hyperglycemia. Meropenem was commenced as a broad spectrum antibiotic. Surgery were consulted and felt that given his unstable clinical picture he should be managed conservatively. EGD on day 4 of presentation demonstrated Barrett's esophagus and normal appearing stomach mucosa but findings of 'chronic gastritis with focal activity' on biopsy. Blood cultures remained negative and antibiotics were eventually changed to metronidazole and levofloxacin which were continued to complete a 10 day course. The patient gradually improved and was discharged home with visiting nurse services on day 7 of admission.

DISCUSSION: Emphysematous gastritis is a rare condition, characterized by the presence of air in the gastric wall secondary to infection with gas-forming bacteria. Patients typically present with severe abdominal pain, nausea, vomiting, diarrhea, evidence of gastro-intestinal bleeding, and signs of systemic toxicity. Pre-disposing factors seem to include alcohol abuse, ingestion of corrosive substances, gastroenteritis, diabetes, NSAID 
use, abdominal surgery, gastric infarction, pancreatitis, renal failure and an immunocompromised state. Cultured organisms from stomach aspirates, blood and peritoneal fluid have included Streptococci, Escherichia coli, Pseudomonas aeruginosa, Clostridium perfringens and Staphylococcus aureus. CT is the imaging modality of choice and classically shows gas within the stomach wall and, in some cases, gas in the portal vein. This condition carries a poor prognosis with mortality rates reported at around $60 \%$. Mechanical complications of gastric strictures, ischemia and perforation are not uncommon. Management includes aggressive fluid resuscitation, early commencement of broadspectrum antibiotics, appropriate nutrition and, in select cases, surgery. Emphysematous gastritis should be differentiated from gastric emphysema which is another condition in which gas is seen within the stomach wall. Gastric emphysema is felt to be as a result of barotrauma in the absence of bacterial infection. Typically patients present relatively asymptomatically or with mild symptoms of abdominal pain. They are non-toxic appearing and hemodynamically stable. Radiologically, the pattern of gas in gastric emphysema seems to be more linear, whereas in emphysematous gastritis the gas appears in more focal collections. Gas in the portal vein is more suggestive of emphysematous gastritis. The distinction between the two entities is important as gastric emphysema tends to have a much more benign clinical course. Our patient's risk factors for developing emphysematous gastritis included his uncontrolled diabetes mellitus and renal failure. His presentation with severe abdominal pain and tachycardia fit with the expected clinica picture of emphysematous gastritis. The radiological pattern of gas was also more suggestive of emphysematous gastritis than gastric emphysema. Fortunately, he responded well to aggressive initial management and did not require surgery. A causative organism was not identified in this case. Blood cultures remained negative and gastric sampling did not reveal any bacterial species. This may have been due to the delay in performing EGD until day 4 of his hospital stay when he was more clinically stable.

ALL MYXED UP Allison Selby; Reem Taha; Sherri Sandel. Lenox Hill Hospital, New York, NY. (Control ID \#2465444)

LEARNING OBJECTIVE \#1: Atrial myxoma should be considered on the differential of patients presenting with TIA symptoms

CASE: A 73 year-old female with PMH of Depression, HLD, Breast CA status post lumpectomy 5 years ago who presented with diplopia and confusion. On the morning of admission, patient began driving to work and experienced double vision. Diplopia resolved when she covered either eye. She continued to drive despite double vision. After bumping into the car in front of her, the patient realized she had driven to an unknown location. She could not recall what had happened and called EMS. Patient never had symptoms like this before. She denied vertigo, tinnitus, headache, slurred speech, chest pain, or palpitations. Double vision and confusion resolved while patient was in the ED, where she remained hemodynamically stable. Glucose on arrival was 109. Other laboratory studies were within normal limits. CT head was negative for acute intracranial hemorrhage or infarction. CT Angiogram of the head and neck was also performed, which was negative. Patient was admitted to the regional medical floor for further work-up. An transthoracic echocardiogram was ordered as part of TIA work-up and was performed on hospital day two. TTE revealed a large $(1.8 \times 1.6 \mathrm{~cm})$, irregular, mobile clot seen in the left atrium attached to the postero-lateral wall. Patient was started on low-molecular weight heparin initially due to concern of LA thrombus. Transesophageal echocardiogram was then performed to better characterize the presumed clot. TEE revealed a $2 \mathrm{~cm}$ shaggy, mobile mass attached to the left atrial side of the base of the posterior mitral leaflet. Contrast injection was suggestive of a vascular mass, most likely a neoplasm. Patient was transferred to the cardiothoracic surgery service. Patient underwent surgery to remove mass. Biopsy of the lesion revealed atrial myxoma. Patient was discharged following surgery with no residual effects.
DISCUSSION: Myxomas are the most common primary cardiac tumors. They tend to occur in the 3rd-6th decades of life. They commonly occur in the atria, and occurrence in the left atrium is about $75 \%$. Clinical manifestations of myxoma are dependent on location, size, and mobility. Left atrial myxomas most commonly present with central nervous system manifestations. Embolism from tumor particles or thrombotic material covered with tumor cells occurs in $30-40 \%$ of patients with left atrial myxoma. Cerebral and retinal arteries are most frequently involved, manifesting as confusion, visual changes, gait imbalance, dysarthria, or weakness. Therefore, myxoma should be included in the differential of TIA presenting with confusion and diplopia. Similar cases of myxomatous embolism have been described. However, in the medical literature, a mean duration of 36 months have been described from the time of neurologic symptoms to diagnosis of atrial myxoma. For our patient, echocardiography was ordered in a timely fashion following her TIA symptoms, allowing for a speedy diagnosis and early surgical intervention.

ALL SHOOK UP WITH HYPERGLYCEMIA. Uzair Mushtaq; Rutwij K. Joshi; Rushit Kanakia. UTHSCSA, SAN ANTONIO, TX. (Control ID \#2454840)

LEARNING OBJECTIVE \#1: Recognize ballismus, chorea and Facial dyskinesias as rare clinical manifestations of common metabolic disorders like uncontrolled Diabetes Mellitus type 2 .

LEARNING OBJECTIVE \#2: Treat hyperglycemia for a prompt neurological response and recovery.

CASE: 71 year old woman presented with bizarre movements of her arms, legs and face. These symptoms began 1 week prior to the presentation. She denied headaches, vision or hearing changes, loss of consciousness and focal weakness. Physical examination revealed an alert, oriented lady with no cognitive defects who had sudden repetitive movements of oro-bucco-lingual musculature along with ballistic and choreiform movements of bilateral upper and lower extremities, more pronounced on the right side. These movements were reduced with sleep and exaggerated with activity or exertion. Patient had been diagnosed with diabetes mellitus type 24 weeks ago and was non-compliant with her medications. Laboratory studies were significant for a serum glucose of $>600 \mathrm{mg} / \mathrm{dl}$ and glycated hemoglobin of $>16 \%$ in the absence of ketones. Drug screen was negative and patient denied being on any neuroleptic medications. Neurology consult was obtained and a computerized axial tomography of brain demonstrated homogeneous hyper dense appearance of bilateral basal ganglia mainly in the caudate and putamen suggestive of a metabolic disturbance. Having ruled out other possible etiologies, the diagnosis of movement disorder secondary to non-ketotic hyperglycemia was made. Aggressive glycemic control resulted in a drastic improvement, with a complete resolution of her symptoms in 13 days. A magnetic resonance imaging done after resolution of symptoms was normal.

DISCUSSION: Ballismus and chorea are manifestations of metabolic, inflammatory or ischemic diseases of basal ganglia. Oro-bucco-lingual dyskinesia is seen most commonly with Huntington's disease and tardive dyskinea. However, these symptoms can also rarely present in non ketotic hyperglycemia. The pathophysiology of movement disorders in hyperglycemia is poorly understood. Hypotheses include direct alterations of dopaminergic activity in striatum, disruption of blood brain barrier secondary to hyper viscosity resulting in intracellular acidosis and inactivation of Krebs' cycle. Computerized axial tomography shows areas of hyper density in the striatum corresponding to the territory of the lateral lenticulostriate arteries and magnetic resonance shows high T1-weighted signal. In a majority of patients, strict glycemic control results in a rapid improvement and resolution of symptoms. Use of neuroleptics and benzodiazepines has been reported in persistent cases. 
ALPHABET SOUP: IMPORTANCE OF DISTINGUISHING AHUS AND TTP

Nina Thomas. Tulane University Health Sciences Center, New Orleans, LA. (Control ID \#2469125)

LEARNING OBJECTIVE \#1: Recognize and diagnose the different thrombotic microangiopathic syndromes

LEARNING OBJECTIVE \#2: Differentiate underlying pathophysiology of TTP and atypical HUS

CASE: A 57 year-old woman presented with several weeks of progressive shortness of breath, cough, and progressive altered mental status. The patient had been evaluated 5 weeks prior for worsening dyspnea and weakness with persistent bibasilar interstitial infiltrates on chest CT, consistent with interstitial lung disease. She had clinical signs suggesting an autoimmune process, including Raynaud's phenomenon, dysphagia, and skin hardening. She was started on rituximab infusions weekly, her last dose being 4 days prior to presentation. Upon presentation, she had rhonchi with bibasilar crackles. She was tachycardic with a heart rate of 130, blood pressure was 105/74 and tachypneic with a respiratory rate of 24 with normal oxygen saturations. Labs revealed thrombocytopenia, hemolytic anemia with schistocytes on the peripheral smear, and acute renal failure. Her chest $\mathrm{x}$-ray showed bibasilar airspace disease. She underwent emergent plasma exchange for presumed TTP. Although her mental status improved, her platelet count and renal function continued to decline leading to initiation of hemodialysis. The ADAMTS13 results showed normal activity with negative antibodies, suggesting she had aHUS, not TTP. She was started on weekly infusions of eculizumab for atypical HUS. Her platelets and anemia improved quickly, however, she still required hemodialysis. Renal biopsy revealed fibrin thrombi in the glomerular capillaries, consistent with a thrombotic microangiopathy.

DISCUSSION: Thrombotic thrombocytopenic purpura (TTP) and atypical hemolytic uremic syndrome (aHUS) are both uncommon and often clinically indistinguishable diseases, but differentiating them is vital to treatment. TTP results from autoantibodies to ADAMTS13, a von willebrand factor cleaving protein. This deficiency leads to platelet aggregation in the microvasculature. HUS, on the other hand, results from over-activation of the complement cascade, resulting in endothelial disruption, which in turn leads to thrombus formation in the microvasculature. Both result in blood cell destruction and multisystem end-organ damage. Though TTP and aHUS both present with microangiopathic hemolytic anemia, acute renal failure, thrombocytopenia and neurologic disturbance, the pathogenesis, prognosis and treatment differ making distinguishing between the two imperative. While the initial therapy for both TTP and aHUS is plasma exchange, if the patient has refractory disease then other agents are considered. Eculizumab, a monoclonal antibody against the $\mathrm{C} 5$ in the terminal portion of the complement cascade, is a newly FDA-approved therapy for refractory atypical HUS. A recent 2011 prospective trial showed significant hematologic improvement and an $80 \%$ rate of discontinuation of dialysis. After eculizumab therapy, this patient had significant hematologic improvement, but had irreversible renal failure. This case raises the question whether early recognition of the difference between TTP and aHUS could prevent such outcomes.

ALTERING MENTATION ABOUT ALTERED MENTAION Daniel Hart'ㄹ SAAD EMHMED ALI ${ }^{1}$; Marianna Sargsyan ${ }^{2} .{ }^{1}$ PRESENCE SAINT FRANCIS HOSPITAL, Chicago, IL; ${ }^{2}$ Saint Francis Hospital, Evanston, IL. (Control ID \#2469824)

LEARNING OBJECTIVE \#1: To recognize an uncommon cause of altered mental status in a critically ill patient

CASE: 25 year old male was found by his roommate to be displaying odd behavior. He was opening his eyes but not responding when spoken to. He had not left his room for a few days and had six empty half gallon bottles of vodka scattered around him in his room.
Pt was brought to the ED by EMS and was found to be jaundiced and in septic shock with multiple sources of infection being UTI, aspiration and Staph Aureus pneumonia, perirectal abscess, HSV2 perirectal infection and oral candidiasis. The pt has a past medical history of acute HIV, alcoholic hepatitis and alcoholic gastritis. During his ICU stay he required two sympathomimetic drugs to maintain his blood pressure, developed pulmonary edema, acute respiratory failure requiring intubation, ARDS, non-oliguric acute kidney injury, compensated anion gap metabolic acidosis, hypernatremia, congestive heart failure with ejection fraction of $40 \%$, heparin induced thrombocytopenia and hemolytic anemia. Pt was extubated after 9 days and was able to communicate but his mentation would wax and wane and he had significant trouble following commands and ambulating. He was alert but would not orient to time or place. He would also display intermittent burst of agitation. He was transferred out of the ICU after 14 days and each organ system recovered back to his baseline except for persistently elevated LFTs, elevated ammonia, hemolytic anemia, and his altered mentation. A lumbar puncture was preformed but came back negative for meningitis. MRI of the brain was negative for ischemia, mass effect or midline shift but was limited due to patient non compliance. At this point Wilson's disease was considered as a potential diagnosis. Further investigation revealed decreased ceruloplasmin levels and elevated $24 \mathrm{~h}$ urinary copper levels consistent with the diagnosis

DISCUSSION: Many factors could have caused this patient to have altered mental status. Septic shock and multi system organ failure are both well known causes of metabolic encephalopathy. ICU delirium is a common phenomenon and cognitive impairment is a sequele of ARDS. Alcohol withdrawal and Wernicke-Korsakoff Syndrome were also likely given this patient's history of substance abuse. All of the previously listed conditions should resolve over time and mentation should return to baseline, something which was not seen in this patient. HIV enecephalopathy was also strongly considered. This patient showed dramatic improvement in all organ systems aside from abnormal liver function tests, persistent AMS and incoordination. Given his young age the only diagnosis which truly fit his constellation of symptoms was Wilson's Disease

AN AGE-OLD DIAGNOSIS WITH A TWIST Ryan I. Grattan ${ }^{1}$; Kevin Hauck ${ }^{2} .{ }^{1} \mathrm{New}$ York University School of Medicine, New York, NY; ${ }^{2}$ NYU Langone Medical Center, New York, NY. (Control ID \#2469413)

LEARNING OBJECTIVE \#1: Recognize the clinical signs and symptoms of vitamin C deficiency

LEARNING OBJECTIVE \#2: Identify comorbid conditions that can lead to vitamin C deficiency

CASE: The patient is a 26 year-old man who presented with 3 days of moderately severe knee pain. The patient had been in excellent health until 6 weeks prior to admission, when he developed worsening fatigue and a new rash. In the days prior to admission, he noted new bruises across his arms and lower extremities, and bleeding from his gums while brushing his teeth. These symptoms began approximately 3 months after the patient moved away from his parents' house. Always a picky eater, the patient restricted his meals almost entirely to fried chicken and pasta and was unable to recall the last vegetable he had eaten. His medications consisted of lamotrigine which he had been taking chronically for a mood disorder. The physical exam was notable for large, tender bruises and perifollicular hemorrhage across the lower extremities. There was full passive range of motion in the knees and ankles with some tenderness and minimal joint swelling. Examination of the mouth revealed normal teeth, with scattered petechiae over the gums. Laboratory evaluation revealed hemoglobin of $7 \mathrm{mg} / \mathrm{dL}$ with a decreased mean corpuscular volume. Prothrombin time, activated partial thromboplastin time, and platelet count were within normal limits. Serum vitamin C level was undetectable. The patient was started on high dose vitamin $\mathrm{C}$ supplementation with resolution of his symptoms over the next 2 weeks. 
DISCUSSION: Scurvy is a disease that results from severe dietary deficiency of vitamin $\mathrm{C}$, an essential nutrient found in fruits and vegetables. Symptoms typically manifest after a number of months of poor vitamin $\mathrm{C}$ intake, but may be seen as early as after 1 month of deficient diet. These early symptoms are non-specific and include weakness, fatigue, myalgias, and sluggishness. The more classically documented skin and oral findings may be seen after 1 to 3 months of poor diet. These include hyperkeratotic perifollicular hemorrhage, corkscrew hairs, hematomas, and hyperplastic hemorrhagic gingivitis. Hematologic and vascular signs and symptoms can also be seen, including anemia, arthralgias secondary to hemarthrosis, and edema. Our patient's presentation with fatigue, arthralgias, spontaneous hematomas, gingival petechiae, anemia, and the classical perifollicular hemorrhage in the context of a diet that was absent of any fruits and vegetables was consistent with a diagnosis of scurvy. The diagnosis of scurvy is clinical. The presence of perifollicular hemorrhage with corkscrew hairs is considered by some to be pathognomonic for vitamin $\mathrm{C}$ deficiency. In practice, the presence of these signs and symptoms is variable, complicating the identification of this clinical diagnosis. Laboratory testing can help confirm the diagnosis. A skin biopsy of an affected follicle can reveal erythrocyte extravasation and torsion of the hair shaft, helping identify corkscrew hairs that may not be visible to the naked eye. Serum levels of vitamin $C$ can be tested and will confirm the diagnosis if below $0.1-0.2 \mathrm{mg} / \mathrm{dL}$. A complete blood count should also be ordered to evaluate for anemia. Treatment is straightforward and involves supplying high doses of vitamin C. No standardized dosing regimen exists, but the literature suggests a dose of 100-300 mg daily for children and 500-1,000 mg daily for adults. Treatment should be provided for 1 month or until symptoms resolve. Reports indicate symptomatic improvement can be seen within a few days to a few weeks. Scurvy is an unusual diagnosis in the present day due to high availability of fresh fruits and vegetables and foods fortified with vitamin C. Still, certain at-risk populations are unable to obtain foods rich in vitamin C. Patients with alcoholism or the elderly with poor social support are classic examples, neither of which applied to our patient. In a subsequent interview, he revealed that he did not like the peel of fruits and refused to peel them on his own. If peeled for him, he enjoyed the taste of these fruits. This inflexible behavior around fruit spurred a second review of the literature which revealed a number of case reports where vitamin C deficiency was seen in the context of schizophrenia, autism, or obsessive compulsive disorder. A heightened food selectivity was exhibited in these cases that lead to patients severely restricting their intake of fruits and vegetables, behavior very similar to what our patient exhibited. His lack of obvious psychiatric symptoms led to added history gathering around his mood disorder. While the patient denied the diagnosis, his mother stated that he had at one time been diagnosed with obsessive compulsive disorder. The diagnosis of scurvy is rarely entertained in the present day, especially in patients with apparent social supports and high levels of social function. However, patients with subclinical mental illness may develop scurvy secondary to unique food preferences and selectivity. The suspicious clinician should recognize the classical presenting symptoms of vitamin $\mathrm{C}$ deficiency and redouble her efforts to gather a detailed dietary and psychiatric history that may provide an atypical explanation for the development of scurvy.

AN ATYPICAL CASE OF APPENDICITIS Jenny S. Placido-Disla ${ }^{2}$; Tina Wang ${ }^{1}$; Faraj Faour ${ }^{1} .{ }^{1}$ Mount Sinai Beth Israel, New York, NY; ${ }^{2}$ Mount Sinai Beth Israel, New York, NY. (Control ID \#2462950)

LEARNING OBJECTIVE \#1: Recognize the effects of chronic steroid use on gastrointestinal disease

LEARNING OBJECTIVE \#2: Consideration of alternate diagnoses in patients with non-resolving nausea and vomiting.

CASE: A 55-year-old female with a history of asthma, systemic lupus erythematosus, rheumatoid arthritis, and anxiety presented with a 2-day history of nausea, vomiting, and diarrhea. She also noted diffuse abdominal discomfort during episodes of vomiting and diarrhea. She described her symptoms being similar to those which led to an admission 1 month prior where she had presented with nausea, vomiting, and productive cough, and during which time she was diagnosed with community acquired pneumonia. On initial presentation of this admission, the patient was found to be afebrile, but tachycardic. Her vital signs were otherwise stable. Her initial physical exam was within normal limits, without abdominal tenderness. Laboratory evaluation was significant for leukocytosis WBC $23.8 \mathrm{~K} / \mathrm{UL}$ with neutrophilic predominance. The rest of her labs including basic metabolic panel, hepatic function tests, and urinalysis were unremarkable. Given recent antibiotic use, an acute infection, including Clostridium difficile was suspected. Stool studies were sent and she was started empirically on antibiotics. On hospital day 2, patient became febrile to 100.5. Repeat physical examination showed mild right lower quadrant tenderness, however Rovsing, Spoas, or Obturator signs were negative and no rebound or guarding was noted. CT scan of the abdomen and pelvis was done and showed a dilated appendix to $0.8 \mathrm{~cm}$, consistent with acute appendicitis, with no perforation or abscess formation noted. The patient's atypical presentation of acute appendicitis was felt secondary to chronic steroids use masking the signs and symptoms of intra-abdominal inflammation. The surgical team was consulted for management. However, given that the patient was hemodynamically stable and clinically improving, she was treated conservatively rather than surgically. Leukocytosis subsequently resolved and patient had no further fevers over her course. Patient was discharged on hospital day 5 on oral cefpodoxime and metronidazole.

DISCUSSION: Glucocorticoids are known to cause a number of adverse effects in various organ systems when used in long durations or at high doses. Within the gastrointestinal system, the anti-inflammatory effects of glucocorticoids may mask the manifestations of serious disease resulting in delays in diagnosis and treatment. This complication has been previously described most commonly in patients who present with colonic perforations - in particular diverticular disease. In these patients, the time from initia presentation to definitive diagnosis and treatment was significantly prolonged due to the reduction in or lack of premonitory symptoms. The majority of these patients were transplant patients or patients with rheumatologic diseases on long-term steroid use for immunosuppression. Similarly, we describe a case of acute appendicitis in a patient on chronic steroids for systemic lupus erythematosus in which the presentation was very atypical. Although early symptoms of acute appendicitis may be subtle, abdominal pain is eventually reported in nearly all patients with confirmed appendicitis. Our patient, however, presented with only nausea and vomiting over her course. Her chronic steroid use may have resulted in an inability to mount the typical inflammatory response and subsequent milder symptoms. Delays in appropriate treatment of intra-abdominal disease can have serious implications on morbidity and mortality. Additional workup and more serious diagnoses should be considered in patients on long-term steroid use who present with gastrointestinal symptoms of otherwise unclear etiology. Resources: 1. Canter JW, Shorb PE. "Acute perforation of colonic diverticula associated with prolonged adrenocorticosteroid therapy.” Am J Surg. 1971; 121 (1): 46-51. 2. Candelas G, Jover JA, et al. "Perforation of the sigmoid colon in a rheumatoid arthritis patient treated with methylprednisolone pulses." Scand J Rheumatol. 1998; 27 (2): 152-53. 3. ReMine SG, Mcllrath DC. "Bowel perforation in steroid-treated patients." Ann Surg. 1980; 192 (4): 581-86. 4. Morris CR, Harvey IM, et al. "Epidemiology of perforated colonic diverticular disease." Postgrad Med J. 2002; 78: 654-58. 5. Hara T, Akutsu H, et al. "Cushing's disease presenting with gastrointestinal perforation: a case report." Endorinol Diabetes Metab Case Rep. 2013.

AN ATYPICAL CASE OF ATYPICAL HEMOLYTIC-UREMIC SYNDROME Ekta Kakkar. Baylor College of Medicine, Houston, TX. (Control ID \#2467242) 
LEARNING OBJECTIVE \#1: Multiple etiologies of thrombotic microangiopathy (TMA) exist, with the most prevalent being Shiga-like toxin-producing Escherichia coli hemolytic-uremic syndrome (STEC-HUS) followed by thrombotic thrombocytopenic purpura (TTP). Atypical hemolytic-uremic syndrome (aHUS), a rare genetic disorder of uncontrolled activation of the complement system, is another mechanism. Since each etiology of TMA presents with hemolytic anemia, thrombocytopenia, and acute renal failure, making a prompt diagnosis is difficult yet essential.

CASE: A 74-year-old woman with well controlled benign hypertension was in her usual state of health until 2 weeks prior to presentation when she noted fatigue and lower extremity and facial swelling. She denied a preceding illness or diarrhea. Upon presentation to her primary care physician, her blood pressure was noted to be 196/112 $\mathrm{mmHg}$. At that time, antihypertensive medications, including a diuretic, were added without resolution of symptoms. She subsequently presented to the emergency room with identical complaints. Initial examination was remarkable for hypertension to $162 / 93 \mathrm{mmHg}$ and $1+$ bilateral pitting pedal edema without other signs of anasarca, hemodynamic instability, or hypoxia. Initial laboratory studies suggested a new normocytic anemia with hemoglobin $9.1 \mathrm{~g} / \mathrm{dL}$, new thrombocytopenia with platelets of $73,000 \mathrm{mcL}$, and acute kidney injury with creatinine $2.79 \mathrm{mg} / \mathrm{dL}$ from a normal baseline. Further work-up was consistent with a microangiopathic hemolytic anemia with nephrotic range proteinuria without secondary causes and a negative autoimmune work up, viral hepatitis panel, and syphilis screen. Renal biopsy was pursued after ADAMTS13 activity was normal and clinical presentation did not indicate the presence of a Shiga or Shiga-like toxin. Treatment with eculizumab was initiated to prevent persistent complement activation. Renal biopsy pathology was consistent with aHUS with a component of membranoproliferative glomerulonephritis secondary to gammopathy which was seen on protein electrophoresis. Genetic testing of complement factor $\mathrm{H}$, complement factor $\mathrm{I}$, and $\mathrm{CD} 46$ were all negative in this patient. After receiving three treatments with eculizumab the patient was discharged with improving blood counts, renal function, and blood pressure control.

DISCUSSION: This is a case of aHUS that was systematically managed and treated with favorable outcomes. The presentation was typical for TMA; however, a normal ADAMTS13 activity and a clinical history without diarrhea/toxin excluded TTP and STEC-HUS. This differentiation is imperative since plasmapheresis, if used in aHUS, does not address the underlying issue of uncontrolled complement activation and treatment delays can result in renal failure. Eculizumab, a monoclonal antibody directed against the terminal complement system, has improved outcomes in aHUS and is not indicated in the treatment of STEC-HUS or TTP. Clinicians must follow a systematic diagnostic strategy in cases of TMA to avoid misdiagnosis and delays leading to suboptimal outcomes

AN ATYPICAL CASE OF SEPSIS AND MULTIPLE ANOGENITAL LESIONS IN SYPHILIS Nichole A. Smith; Sonu Dhillon; John G. Cotter; Zohair Ahmed. University of Illinois College of Medicine at Peoria, Peoria, IL. (Control ID \#2467937)

LEARNING OBJECTIVE \#1: Recognize the atypical clinical presentations of Primary \& Secondary Syphilis.

LEARNING OBJECTIVE \#2: Recognize the increasing incidence of Syphilis in the United States.

CASE: A 22-year-old male with no significant past medical history presented with fevers, chills, sore throat, diaphoresis and diarrhea. On presentation, he was febrile (T: 102.6 F) and tachycardic with hypotension which was unresponsive to fluid resuscitation requiring short term vasopressor therapy. Physical exam revealed diffuse lymphadenopathy, lower extremity macular rash involving the soles of feet, papular non-pustular lesions on the scrotum, and a $0.5 \mathrm{~cm}$ non-tender irregular, healing lesion on the shaft of the penis. Laboratory analysis was significant for leukocytosis and elevated creatinine. Serum screening RPR was positive, and further testing revealed a titer of 1:32, with confirmation via FTABS. Further serological evaluation included: Monospot, Hepatitis C, HIV RNA, antibody \& antigen testing, Q Fever serology, Lyme serology, HSV serology, CMV serology, Gonorrhea and Chlamydial testing, EBV serology, and blood cultures, all of which were negative. The etiology of the sepsis was determined to be Secondary Syphilis. The patient was treated with Penicillin G Benzathine 2.4 million units $\mathrm{IM} \times 1$ and supportive management, with improvement of symptoms. The patient eventually admitted to high risk sexual behaviors including prior unprotected sexual contact with males. RPR screening had been negative 5 months prior to diagnosis. Anticipated follow up for patient includes RPR with titer at 6 weeks, 3 months, 6 months and 1 year post treatment.

DISCUSSION: The classic presentation of Syphilis is the single, painless anogenital ulcer in an otherwise healthy individual, or the mucocutaneous eruption with mild constitutional symptoms in the secondary stage. However, as many as $1 / 3$ of individuals with Syphilis may present with multiple anogenital lesions or painful primary lesions. This case illustrates the atypical way in which Secondary Syphilis may present with a persistent chancre and multiple anogenital lesions. It also demonstrates a unique presentation of septic shock from Syphilis infection in an immunocompetent host. The incidence of Syphilis in the United States has been steadily rising since 2000, with a $40 \%$ increase since 2010 alone. As such, it is important that physicians recognize the multitude of ways in which syphilis may present and maintain a high level of clinical suspicion in the setting of high risk behaviors, especially Men who have Sex with Men (MSM).

AN ATYPICAL PRESENTATION AND RARE COMPLICATION OF TERMINAL ILEITIS Shalini Sri Kumaran. Singapore General Hospital, Singapore, Singapore. (Control ID \#2467984)

LEARNING OBJECTIVE \#1: Recognize the varied clinical presentations of terminal ileitis

LEARNING OBJECTIVE \#2: Recognize mesenteric vein thrombosis as a complication of intra-abdominal infections

CASE: A 74 year old male with a history of hypertension presents with 3 days of fevers and chills with no other associated symptoms. He was febrile but was otherwise non-toxic in appearance. Physical examination was largely unremarkable except for an enlarged non-tender prostate on digital rectal examination. Investigations revealed leukocytosis $11.6 \times 10^{9} / \mathrm{L}$, thrombocytopenia $105 \times 10^{9} / \mathrm{L}$ and elevated inflammatory markers with a C-reactive protein of $80.6 \mathrm{mg} / \mathrm{L}$ and Procalcitonin of $26.2 \mathrm{UG} / \mathrm{L}$. Urinalysis showed microscopic hematuria and leukocytosis of 40 and 55 cells respectively thus a presumed diagnosis of urosepsis was made and intravenous Ceftriaxone was commenced. Though he remained hemodynamically stable, he continued spiking high temperatures after $72 \mathrm{~h}$ of admission hence a CT intravenous pyelography was done to exclude possible renal abscesses or other genitourinary collections. The scan unexpectedly showed a grossly thickened and edematous terminal ileum, marked perienteric fat stranding and lymphadenopathy suspicious for severe acute terminal ileitis. Following this the history was revisited several times and the patient did recall having had some diarrhea for 2 days a fortnight ago which he did not think was significant. His blood cultures subsequently grew polymicrobial bacteria consisting of E.coli, Strep.constellatus and Clostridium species. A surgical consult concluded that no intervention was needed given he was completely asymptomatic but he was closely monitored with serial abdominal examinations. He was screened for both HIV and TB which were negative and subsequent stool studies sent for culture and acid fast bacilli grew Salmonella species. Antibiotics were switched to Ciprofloxacin and Clindamycin as per microbiological sensitivities. A repeat CT a week later to monitor progress showed marked improvement with only minimal stranding visible however a new long segment complete superior mesenteric vein/ileocolic vein thrombosis was now seen. A thrombophilia and JAK-2 mutation screen done was 
negative, thus the etiology was attributed to the ileitis. He was commenced on warfarin and a repeat CT 6 weeks later showed stable mild fat stranding around the terminal ileum with improvement of the thrombosis. He is to complete 6 months of anticoagulation therapy but is otherwise doing well.

DISCUSSION: Classically associated with Crohn's disease, terminal ileitis is also associated with a myriad of other causes such as infectious diseases, spondyloarthropathies, vasculitides, medication induced and neoplasms. It can be a great mimicker as it can present as acute appendicitis, acute gastroenteritis, acute intestinal obstruction or have a more subclinical presentation for example when associated with spondyloarthropathies such as ankylosing spondylitis. In our patient, the relative lack of symptoms coupled with an abnormal urinalysis which was likely the result of the underlying inflammation causing renal tract irritation led us to believe this was urosepsis. Yersinia, non-typhoidal Salmonella, Clostridium difficile and mycobacterium tuberculosis are among the commonest infectious pathogens associated with bacterial ileitis but with extremely variable clinical syndromes. Nontyphoidal Salmonella is one of the commonest foodborne illnesses worldwide and usually causes a short self-limited gastroenteritis episode. It may be complicated with bacteremia from gut translocation and affect mesenteric lymph nodes leading to adenitis and ileitis which is what likely occurred in our patient. Blood, stool and sometimes even tissue cultures via endoscopy are essential to establish the microbiological diagnosis and institution of appropriate antibiotics. Imaging modalities and mucosal biopsies help aid with the presence, severity and progress of the terminal ileitis. Mesenteric vein thrombosis although a rare complication of intra-abdominal infection has increased in prevalence mainly due to incidental findings on CT scans as with our patient. It is classically associated with thrombophilia's and hypercoagulable states and more common in the elderly population. The goal of management for mesenteric vein thrombosis would be to prevent bowel infarction and recurrent thrombosis. Anticoagulation and close serial abdominal examinations are the mainstay of treatment with a low threshold for surgery should there be suspicion for ischemic bowel. The duration of anticoagulation should be for at least 6 months or longer if a thrombophilic state has been identified. In summary, a multi-disciplinary collaboration of imaging and microbiological services are needed by the clinician in diagnosing and managing the great mimicker that terminal ileitis can be and to be aware of the rare occurrence of mesenteric vein thrombosis predominantly in the geriatric population with intra-abdominal infections.

AN ATYPICAL PRESENTATION OF BACTERIAL PNEUMONIA IN A PATIENT WITH HIV/AIDS Noor Ali; Jane Andrews. Tulane University Health Sciences Center, New Orleans, LA. (Control ID \#2467697)

LEARNING OBJECTIVE \#1: Review pathogenic causes of respiratory compromise in AIDS patients

LEARNING OBJECTIVE \#2: Recognize the importance of a broad differential diagnosis in immunocompromised patients

CASE: A 33 year-old woman with untreated HIV and a CD4 count of 24 cells $/ \mathrm{mm}^{3}$ presented with a 2-week history of shortness of breath and cough with bloodytinged sputum production. Admit labs were pertinent for an LDH level of 353 and an A-a gradient of 42 . She was a cachectic woman with labored breathing and right lung base rales. CT imaging of the chest demonstrated tree-in-bud opacities within the right lower lobe. She was empirically started on Bactrim and steroids for concern for Pneumocystis jirovecii pneumonia (PJP). Induced-sputum smears demonstrated normal oral flora. Pre-test probability for fungal and atypical mycobacterial organisms remained high in the setting of low CD4 count and immunocompromised status; therefore, she underwent bronchoalveolar lavage (BAL). Smears from the BAL returned negative for PJP. Respiratory cultures ultimately grew Klebsiella pneumoniae with susceptibility to Bactrim.
DISCUSSION: The etiology of respiratory compromise in HIV-seropositive individuals may be secondary to various disease processes, including viral, fungal, bacterial, and noninfectious. Although decreased with the availability of anti-retroviral therapy, opportunistic infections (OIs) continue to be a cause of considerable morbidity and mortality in HIV-infected individuals; therefore, prevention, detection, and treatment are crucial elements in providing quality care to patients with AIDS. When treating a patient with AIDS, CD4 counts are often used as a guidance tool to direct the clinician to the most likely OI as well as with choosing prophylactic medication. For instance, PJP infections become more common when CD4 cell counts reach less than $200 \mathrm{cells} / \mathrm{mm}^{3}$, cytomegalovirus and toxoplasmosis infections when counts reach less than $100 \mathrm{cells} / \mathrm{mm}^{3}$, and Mycobacterial avium complex infections when counts reach less than 50 cells $/ \mathrm{mm}^{3}$. However, it is crucial to note that persons infected with HIV remain susceptible to those pulmonary pathogens that also infect HIV-seronegative individuals. In particular, bacterial respiratory infections are considered common AIDS-defining illness and are associated with increased morbidity in this population. Therefore, in an untreated patient with advanced disease, suspicion for a wide-range of pathogens must be given considerable value. Bacterial pneumonias may occur at any point throughout the course of infection and with increased frequency and severity as CD4 cell counts decline. The clinical presentation of bacterial pneumonias and those caused by PJP may be similar, with fevers, dyspnea, and pleuritic pain often seen in both. However, hypoxemia and elevated LDH levels are more classical manifestations of PJP, and productive cough is more characteristic of bacterial infection. In this particular patient, presentation of bacterial pneumonia presented similarly to PJP. After further examination, the patient's bloody-tinged sputum was thought to be the currant-jelly sputum, which is associated with Klebsiella pneumoniae infection. The characteristic thick, mucoid, bloody-tinged sputum is secondary to the marked inflammation and necrosis of lung parenchyma associated with this bacterial species; hence, oxygen desaturation and elevated LDH levels may be associated laboratory abnormalities. This case plays crucial importance in both the outpatient and inpatient setting for treatment of respiratory compromise in an HIV-infected individual, with emphasis on the importance of a broad differential diagnosis without disregard for common causes of pulmonary infections despite atypical presentations.

AN ATYPICAL PRESENTATION OF CHRONIC MYELOGENOUS LEUKEMIA (CML) Farhan Ashraf ${ }^{1}$; Iole Ribbizi-Akhtar ${ }^{2} .{ }^{1}$ Memorial Hospital of Rhode Island/ Alpert Medical School of Brown University, Providence, RI; ${ }^{2}$ Memorial Hospital of Rhode Island/ Alpert Medical School of Brown University, Pawtucket, RI. (Control ID \#2465396)

LEARNING OBJECTIVE \#1: Recognize atypical presentation of Chronic Myelogenous Leukemia.

CASE: A 49 year old women with only positive past medical history of back pain went to the emergency room complaining of abdominal pain and epistaxis for 3 days. Beside 30 packs smoking history, she denied any other drug use. Her vital signs were stable and on physical examination the only positive finding was epigastric tenderness without rebound or organomegaly. Her white cell count was $13,900 / \mathrm{uL}$, of which $8600 / \mathrm{uL}$ neutrophils and $1400 / \mathrm{uL}$ basophils, the platelet count was $1,945,000 / \mathrm{uL}$. Her last known platelets count 3 months prior to this presentation was $332,000 / \mathrm{uL}$. A peripheral smear showed $1+$ larger platelets and rare giant platelets. Rest of her bloodwork including liver function tests and coagulation panel were normal. Janus kinase 2 (JAK2) and BCR-ABL analysis were sent. Abdominal ultrasound was unremarkable and head CT did not show any hemorrhage. She was initially started on aspirin $325 \mathrm{mg}$ daily and, in order to control the progressively increasing platelets count, hydroxyurea was also added. Her platelet count went down to $1,600,000 / \mathrm{ul}$ in the following 3 days. An upper endoscopy was obtained which showed gastritis. Within a week from her initial presentation JAK2 came back negative, however 
qualitative PCR for BCR-ABL was positive. A confirmatory bone marrow biopsy was obtained and was consistent with CML in chronic phase, with $1 \%$ blast cells and presence of dwarf megakaryocytes. Hydroxyurea was stopped and treatment with Imatinib $400 \mathrm{mg}$ daily was initiated. Within 2 weeks of therapy her platelet count returned to normal range, and subsequent blood work showed that she was in hematological remission within 1 month.

DISCUSSION: CML is predominately a disease of elderly. It is often diagnosed based on a routine blood work showing an abnormally elevated white count. In up to $72 \%$ of the cases the white count is greater than $100,000 / \mathrm{uL}$, and only in $15-30 \%$ of CML patients the platelets count is higher than $600,000 / \mathrm{uL}$. Our case is unusual as it presented with a minimally elevated white count, and an extremely high platelets count. There are few case reports which have described CML presenting with extreme thrombocytosis only. CML has excellent prognosis since the tyrosine kinase inhibitors were introduced, but often requires lifelong treatment. Therefore it is important to make an appropriate diagnosis and initiate treatment as soon as possible. As illustrate above, the lack of greatly elevated white count or absence of enlarged spleen should not distract from working up the thrombocytosis from all angles. Labelling it "essential " can result in missing a diagnosis of CML.

AN AUTOIMMUNE PAIN IN THE GUT Grant Jester ${ }^{1}$; Henrique E. Kallas ${ }^{2}$; Margaret C. $\mathrm{Lo}^{2} .{ }^{1}$ University of Florida, Gainesville, FL; ${ }^{2}$ University of Florida College of Medicine, Gainesville, FL. (Control ID \#2469594)

LEARNING OBJECTIVE \#1: Recognize the atypical presentations and risk factors for autoimmune hepatitis in patients with other autoimmune conditions

LEARNING OBJECTIVE \#2: Distinguish between the clinical, diagnostic and therapeutic features of autoimmune hepatitis vs. drug-induced hepatitis

CASE: A 27 years-old female presented with 7 days of diffuse abdominal pain, flank pain, arthralgias and high fevers $\left(103.7^{\circ} \mathrm{F}\right)$. Symptoms were preceded by dark urine and yellowing of the eyes. Exam revealed jaundice, scleral icterus and right-sided abdominal tenderness but stable vital signs with no fever, hepatomegaly, ascites or rebound. Labs showed severe LFT elevation (AST $1522 \mathrm{U} / \mathrm{L}$, ALT $1154 \mathrm{U} / \mathrm{L}$, total bilirubin $11.3 \mathrm{mg} / \mathrm{dl}$, direct bilirubin $8.2 \mathrm{mg} / \mathrm{dl}$, alkaline phosphatase $224 \mathrm{U} / \mathrm{L}$ ) but normal INR, lipase and WBC. Viral hepatitis panel was negative. Abdominal ultrasound showed signs of cirrhosis and portal hypertension but normal gallbladder and no ductal dilatation. Cefepime, Vancomycin and Flagyl were empirically started for acute cholangitis. Subsequent IgG level was elevated $(2523 \mathrm{mg} / \mathrm{dl})$ but IgM and IgA levels were normal. Medical history included diabetes, hypothyroidism, Crohn's disease on Humira and vague history of "hepatitis" on no current therapy. Outside records later confirmed ASMA-positive, biopsy-proven autoimmune hepatitis with associated cirrhosis. In consultation with Hepatology, the patient was diagnosed with an acute flare of autoimmune hepatitis. Antibiotics were stopped and methylprednisolone was started (due to oral prednisolone intolerance). This regimen was effective in resolving her symptoms and normalizing her LFTs. Patient was discharged with azathioprine and a prolonged 6-month steroid course with close follow-up in Liver clinic.

DISCUSSION: This case adds to the growing literature of autoimmune Hepatitis (AIH) as a rare immune-mediated condition often mistaken for infectious viral hepatitis or cholangitis The diagnostic dilemma stems from its clinical variability dependent on ethnicity and risk factors. Our patient fits the classic epidemiology of AIH as a disease of young Caucasian women with 70-80\% incidence in females. However, AIH can afflict $20 \%$ over 60 yearsold, predominantly Asians and Pacific Islanders. Known inciting events include infection (i.e. EBV), toxin (i.e. trichloroethylene), drugs (i.e. nitrofurantoin), or genetic predisposition. Our case supports the additional risk factor of pre-existing autoimmune conditions, specifically inflammatory bowel disease (IBD). Literature indeed cites a strong association of AIH to
Ulcerative Colitis with up to $16 \%$ prevalence. Crohn's disease association is much rarer with $0.4 \%$ prevalence. Other reported autoimmune conditions include autoimmune thyroiditis and synovitis. This case teaches us further about the role of IBD in altering the classic manifestations and management of AIH. Classic AIH symptomology resembles that of other types of chronic hepatitis and includes fatigue, arthralgia, right upper abdominal pain and jaundice. A major diagnostic clue for AIH is its close association with other extrahepatic autoimmune conditions. Our patient had 3 specific immune-mediated conditions - hypothyroidism, diabetes and IBD. Transaminase levels in AIH are typically $<500$ units/L, but can be exacerbated to $500-1000$ units/L in those with underlying IBD, as seen in our patient. Patients with IBD also present with $\mathrm{AIH}$ at younger ages and are less responsive to typical treatment regimens. IBD may not only increase the progression of $\mathrm{AIH}$ but also induce recurrent flares of AIH. Some hepatology experts even advocate for the IBD$\mathrm{AIH}$ combination to be a distinct subgroup of AIH. Despite diagnostic criteria by the International Association for the Study of the Liver, AIH remains a diagnosis of exclusion. Of importance is to rule out drug-induced hepatitis (DIH) given the implications for management. Common culprits of DIH include atorvastatin, diclofenac, infliximab, isoniazid, minocycline and nitrofurantoin. Our patient was not taking any such offending drugs. DIH has similar symptoms to AIH but symptoms and autoantibodies of DIH vanish rapidly after removal of the offending medication. On the other hand, the foundation of therapy for $\mathrm{AIH}$ requires prednisone monotherapy or prednisone-azathioprine combination for severe cases. The presence of cirrhosis with AIH is important to confirm given its ramifications on management. Prednisolone is the steroid of choice for our patient with known cirrhosis. Studies have shown that prednisolone has superior activity over prednisone in patients with liver cirrhosis given the liver's inability to convert the synthetic prednisone to the active prednisolone. Recognition of any associated autoimmune conditions and cirrhosis is vital to dictate management options for AIH. Early consultation with Hepatology expertise is warranted for timely diagnostic evaluation and proper therapy regimen of $\mathrm{AIH}$.

AN EASY WAY TO GET ROCK HARD ABS! Rishi Kumar. PInnacle Health, Harrisburg, PA. (Control ID \#2470216)

LEARNING OBJECTIVE \#1: Identify and manage Tetanus Recognize pathologies that share similar clinical symptoms

CASE: Although vaccination has decreased the incidence of fatal diseases globally, many of these illnesses are still prevalent in developed countries. One such examples is Tetanus, a disorder of the nervous system caused by the bacterium Clostridium tetani. The anaerobe can be inoculated into the body through a penetrating injury and with an average incubation period of 8 days. This fatal disease commonly manifests with severe generalized symptoms including the classic lockjaw presentation, also known as trismus. The prognosis of a patient with tetanus depends on early diagnosis and treatment of the disease. The following report outlines the presentation and treatment of a case of Tetanus in a patient with acute COPD. A 50 year old male, with a past medical history of COPD is transferred from a remote hospital for worsening respiratory distress secondary to an acute COPD exacerbation. The patient reported having a 1-week history of dyspnea and productive cough that prompted an ER visit. The patient was started on intravenous steroids and inhaled nebulizer treatments but showed no signs of improvement. The patient began developing respiratory acidosis requiring non-invasive positive pressure ventilation and was ultimately transferred to this hospital for further management. Upon arrival, the patient was exhibited worsening respiratory distress, complained of abdominal pain and had a diffuse macular rash. Unfortunately the patient required intubation and mechanical ventilation for acute respiratory failure. Immediately after ventilation, the patient began experiencing periods of intense abdominal muscle rigidity and periods of apnea resulting in profound hypoxia. Further examination revealed hyperreflexia and 
clonus, however the patient remained afebrile. Despite many of the patient's clinical symptoms coinciding with the diagnosis of serotonin syndrome, the patient was not on any medications that might trigger this condition. The patient's symptoms were suspicious of tetanus despite no obvious site of skin inoculation upon examination. Treatment was initiated with intramuscular tetanus immune globulin, metronidazole and active immunization with the Tdap vaccine. Muscle spasms were treated with diazepam and propofol which reduced the episodes of apnea related to thoracic muscle contractions. The patient was also treated for sepsis related to his respiratory infection present upon admission and topical antihistamine for dermatitis. After 7 days of mechanical ventilation the patient was successfully extubated and safely discharged home a few days later.

DISCUSSION: Tetanus is a rare disease with only a few hundred cases reported in the United States over the last 15 years based on literature search. This disease shares many clinical symptoms more commonly seen in diseases such as serotonin syndrome and malignant neuroleptic syndrome, which should be safely ruled out before considering a diagnosis like tetanus. Finally, this case emphasizes the importance of early recognition, appropriate treatment and supportive care to prevent death and allow for timely recovery. Despite the rarity of this disease, this case exemplifies the clinical presentation and standard of care approach to treat the disease. It is important for physicians to recognize these signs in order to potentially treat for Tetanus in the future.

AN EXACERBATION OF PAROXYSMAL NOCTURNAL HEMOGLOBINURIA IN A PATIENT WITH COBALT METALLOSIS Joseph Maciuba; Joan B. Ritter. Walter Reed National Military Medical Center, Arlington, VA. (Control ID \#2467019)

LEARNING OBJECTIVE \#1: The clinical manifestations of heavy metal toxicity are protean.

LEARNING OBJECTIVE \#2: Clinicians should consider metallosis in patients who experience a change in clinical status after prosthesis placement.

CASE: Metallosis from cobalt is a documented complication of total hip arthroplasty with certain types of prostheses. Paroxysmal nocturnal hemoglobinuria (PNH) is a hematologic disorder caused by an acquired mutation in the PIGA gene that results in complementmediated destruction of red blood cells. A 66-year-old Caucasian male with stable PNH on warfarin and dipyramidole underwent a right total hip arthroplasty with a Stryker metalon-metal hip prosthesis in April 2010, complicated by post-operative anemia requiring transfusion. In July 2010, he was noted to have anemia and thrombocytopenia and required multiple transfusions. He began oral steroids for presumed worsening of his $\mathrm{PNH}$. One month later, the patient developed gross hematuria. Bone marrow biopsy revealed $60 \%$ PNH clones in the background of hypercellular bone marrow, ruling out aplastic anemia. He stopped steroid therapy in October 2010 and received eculizumab biweekly with stabilization of his blood counts. He developed a fever of unknown origin and a steroid-responsive interstitial lung disease in March 2012. In September 2013, he was diagnosed with node-positive melanoma that was excised with clear margins. Follow up PET imaging showed no recurrence of melanoma but revealed hypermetabolism surrounding his right hip prosthesis. In November 2013, he reported worsening pain and limited movement of his right hip. Aspiration was unremarkable; inflammatory markers were elevated. Cobalt levels were $6.9 \mathrm{mcg} / \mathrm{L}$ and confirmed metallosis. Imaging revealed a fluid collection with magnetic susceptibility artifact around his implant, and a hip revision was performed in February 2014. Three months later, his cobalt level was $1.4 \mathrm{mcg} / \mathrm{L}$. In June 2014, his eculizumab treatments were spaced to 3 and then 4 weeks without decline in cell counts. In June 2015, repeat imaging was concerning for a metallosis-associated pseudotumor and his cobalt increased to $2.8 \mathrm{mcg} / \mathrm{L}$. This pseudotumor was excised in July 2015. Two months later, his anemia and thrombocytopenia have improved.
DISCUSSION: Given the temporal relationship between our patient's symptoms and his initial surgery and revisions, metallosis is a potential cause of his worsening PNH. There is a case report documenting an autoimmune hemolytic anemia in a patient with titanium metallosis that one could extrapolate to cobalt[1][1]. A hypothetical mechanism is that the PIGA mutation found in PNH clones is protective against an autoimmune hemolytic anemia exacerbated by metallosis. The chronic inflammatory state from his metallosis is likely contributing to his thrombocytopenia. This case not only shows another complication of metallosis from total hip arthroplasty, but it also illustrates the protean manifestations of heavy metal toxicity. Clinicians should consider metallosis in patients who experience a change in clinical status after prosthesis placement. [1][1] Duarte, Joana, Lurdes Correia, Adelia Simao, Antonio Figueiredo, and Armando Carvalho. "Metallosis: A Rare Cause of Autoimmune Hemolytic Anemia.” Acta Medica Portuguesa 28.3 (2015): n. pag. Web.

AN ODD CASE OF HEADACHE Kashiti Long. Emory University, Atlanta, GA. (Control ID \#2469385)

LEARNING OBJECTIVE \#1: Review the presentation of acute calcification tendinitis of the longus colli

LEARNING OBJECTIVE \#2: Recognize acute calcification tendinitis as a non-life threatening cause of severe headache

CASE: A 67 year old male presents with severe headache, neck stiffness and elevated blood pressure. He reports 3 days ago he developed pain and tightness in the right posterior neck which progressed to include his entire head. The pain was severe and constant for 2 days. He endorsed light and sound sensitivity, insomnia, and reduced appetite. Past medical history was notable for hypertension, diabetes and prostate cancer. Initial blood pressure was 200/100. Exam revealed no focal neurological deficits in motor or strength in extremities. Range of motion of the neck was limited and he was unable to flex his neck forward. CT of the head and cervical spine found no acute findings. Laboratory studies were normal including chemistry and blood counts. Lumbar puncture was performed and showed 3 WBC, 0 RBCs, and 54 total protein. Morphine and then dilaudid provided only mild relief of headache but his blood pressure did improve somewhat to $170 / 65$. Interestingly, he reported more relief with Percocet than dilaudid. MRI of his C-spine was notable for retropharyngeal effusion with calcifications anterior to $\mathrm{C} 1$ compatible with acute calcific tendinitis of the longus coli muscle.

DISCUSSION: Acute calcification tendinitis of the longus colli is an inflammatory response due to deposition of amorphous calcium hydroxyapatite crystals in the superior oblique tendons of the longus colli muscles at the C1-C2 level. Symptoms include headache, neck pain, difficulty or painfully swallowing, and neck stiffness. Imaging via computed tomography (CT) and/or magnetic resonance imaging (MRI) is necessary for definitive diagnosis. Treatment includes non-steroidal anti-inflammatory drugs and severe cases may also benefit from corticosteroids. It is a self-limited process and symptoms usually resolve with in a week. Resolution of radiographic findings may take weeks to months. Presentation can be suggestive of other more serious conditions such as retropharyngeal abscess, meningitis, bleeding, trauma, and cervical disk herniation. Evaluation is often focused on assessing for other conditions. In this case, there was concern for serious pathology due to infection or elevated blood pressure and the patient underwent lumbar puncture and CT imaging to narrow the differential diagnosis. MRI provided a definitive diagnosis. Overall acute calcific tendinitis of the longus colli muscle is an uncommon but benign cause of neck pain and headache. Physicians should be aware of the presentation of calcific tendinitis of the longus colli muscle. Although rare, awareness of the condition may be helpful in differentiating this entity from other more serious conditions it may mimic. 
AN OMINOUS CAUSE OF GUM PAIN AND FEVER IN A YOUNG MALE. A CASE OF BURKITT'S LEUKEMIA ... IE. BURKITT'S LYMPHOMA INVOLVING THE BONE MARROW (BM). Manpreet Kaur; Olga Laur; Kimberly Farrell; Barry Boyd; Irem Nasir. Greenwich Hospital, Greenwich, CT. (Control ID \#2465591)

LEARNING OBJECTIVE \#1: To identify gum pain and testicular pain as symptoms of Burkitt's leukemia.

LEARNING OBJECTIVE \#2: To recognize spontaneous tumor lysis syndrome (TLS) in a patient with a new diagnosis of Burkitt's Leukemia.

CASE: An 18 years old male who has braces for 2 years presented with mandibular gum pain and chin numbness $\times 1$ month. He was seen 1 day prior by his orthodontist and had a normal dental exam. Three weeks prior, he had testicular pain with a normal scrotal ultrasound and a urinalysis with hematuria. He was empirically given a 10d doxycycline course for possible epididymitis, but his testes pain did not resolve. He endorsed a $10 \mathrm{lb}$ weight loss over 1 month but denied rigors, night sweats, sore throat, dyspnea, chest, back, abdominal, or joint pains or rashes. He had occasional alcohol and marijuana use and was sexually active with a female partner in a monogamous relationship for 1 year. He denied any history of STDs. He had a fever $103 \mathrm{~F}$ and paresthesias in the distribution of the V3 mental nerve, mucobuccal fold edema, and pain with gum palpation, but no periodontal fluctuance or tooth decay. There was palpable cervical lymphadenopathy, but no splenomegaly. On labs, wbc was 7 (53\% segs, $22 \%$ lymph), hemoglobin and platelets were low at 11 and 50's, respectively. Potassium, calcium, phosphorus, and creatinine were all normal. The initial blood smear was read as few atypical lymphocytes and no schistocytes. HIV, CMV, and hepatitis serologies were negative. HIV RT PCR, HHV6 PCR, and CMV PCR were negative. Mononucleosis screen, Rapid Strep antigen, throat cultures, and blood cultures were negative. EBV serology indicated an old infection. ANA was negative. LDH was trending up from 1200 to 2100 over $24 \mathrm{~h}$ and uric acid was elevated at 10 . The hematology lab reevaluated the blood smear and atypical lymphocytes were reread as rare blasts. Panorex XR had no lytic or destructive lesions. CT soft tissue neck had multiple periapical lucencies involving multiple mandibular teeth. The ultrasound of the scrotum now revealed diffusely heterogenous testicles with multiple foci and illdefined intratesticular lesions bilaterally. Given the fever, nonlocalizing gum and testicular pain, peripheral blasts, and possible early TLS, a leukemia/lymphoma workup was initiated. Aggressive IV hydration and rasburicase were started. Flow cytometry and BM biopsy had $>95 \%$ cellularity (mostly medium sized lymphoid cells associated with macrophages, scattered mitotic, and apoptotic figures, resulting in a "starry sky" pattern). $30 \%$ cells were clonal B-cells, expressing CD19, CD20, CD10, CD38, FMC7, HLA-DR, and monotypic surface kappa light chain with a germinal center immunophenotype (IP). Molecular analysis revealed a cmyc-immunoglobulin (Ig) rearrangement (t8;14), characteristic of Burkitt's leukemia.MRI brain was negative, but CSF had WBC6, $49 \%$ atypical lymphs with blastoid features. The nuclear whole body PET/CT revealed intense uptake in the cervical and mediastinal lymph nodes, spleen, B testes, renal parenchyma, CSF, and diffusely involved bones. The entire spine was hyperintense on MRI consistent with diffuse BM infiltration by lymphoma. He was diagnosed with stage 4 Burkitt's leukemia, CNS positive disease and enrolled in a clinical trial that included the R-EPOCH regimen, intrathecal methotrexate and cytarabine. He had an excellent response to treatment with improvement from $>90 \% \mathrm{BM}$ involvement to $1 \%$ involvement on repeat BM biopsy after 1 month.

DISCUSSION: Burkitt's lymphoma (BL), a highly aggressive form of B cell NHL, accounts for $40-50 \%$ of pediatric NHL and a small percentage of patients present as if they had acute leukemia with extensive BM and peripheral blood involvement. BL and Burkitt's leukemia are classified as different manifestations of the same disease, with $>25 \%$ BM involvement in Burkitt's leukemia. It accounts for 1-2 \% of cases of ALL. There are 3 variants of BL (sporadic, endemic, seen mostly during childhood in Africa, and immunodeficiency-associated). All are treated the same way. Our patient fits the sporadic BL, found in the USA and Western Europe with 3:1 male to female ratio, with a peak age 11-30 years. He has the more common $\mathrm{t} 8 ; 14$ (80\%), where c-myc oncogene translocates to the Ig heavy or light chain gene and becomes deregulated. Apoptosis is frequent and phagocytic histiocytes, that have engulfed the nuclear debris left by dead cells, create a typical "starry-sky" pattern with a background of blue stained lymphocytes on staining. EBV is highly associated only with endemic BL. In sporadic Burkitt's leukemia, there is involvement of the jaw or facial bones (25\%), BM (30\%), and CNS $(15 \%)$.Most patients have tumor involving the stomach, distal ileum, cecum, mesentery, kidney, testis, ovary, and breast. These tumors are rapidly growing, with doubling time $\sim 25 \mathrm{~h}$ and have a high incidence of spontaneous TLS, a hematologic emergency, resulting in acute renal failure, arrhythmias, and seizures. Treatment for Burkitt's leukemia involves chemotherapy within $48 \mathrm{~h}$, with no role for radiation or surgery. Our patient's gum pain and chin numbness was likely due to extensive BM involvement by tumor causing compression of the mental nerve and dental roots. Our case illustrates that in the age of modern molecular studies, the blood smear remains an important diagnostic tool. And, a young male with gum or testes pain and fever, clinicians need to keep a high index of suspicion for Burkitt's leukemia, due to its highly aggressive nature and risk of developing TLS.

AN OUT-OF-BALANCE AXIS Deborah N. Lee ${ }^{2}$; Vassiliki Pravodelov ${ }^{1} .{ }^{1}$ Boston University Medical Center, Boston, MA; ${ }^{2}$ Boston University, Boston, MA. (Control ID \#2468053)

LEARNING OBJECTIVE \#1: Recognize hypopituitarism as a cause of hyponatremia and syncope.

LEARNING OBJECTIVE \#2: Discuss the initial diagnostic workup and treatment of hypopituitarism.

CASE: An 87-year-old Haitian woman with hypertension, chronic kidney disease, and chronic anemia presented to the hospital after a witnessed syncopal episode. She had intermittent syncopal episodes for a few months prior to presentation, typically while seated, with no prodromal symptoms, no seizure-like activity, no post-ictal findings, and no falls. Prior presentations for the same symptoms were attributed to her anemia and hypovolemia. Review of systems on this presentation was only positive for weakness, dizziness, and headache without red flags. On admission, she was afebrile and hemodynamically stable with negative orthostatic vital signs. The exam revealed pale conjunctivae, a normal thyroid, and a III/VI systolic murmur heard throughout the precordium with regular rate and rhythm. She appeared euvolemic with moist mucous membranes, no jugular venous distention, and no peripheral edema. She had an antalgic gait but no other focal neurologic findings. Lab results were significant for a low hemoglobin of $6.4 \mathrm{~g} / \mathrm{dL}$ (her baseline), hypotonic hyponatremia with a sodium of $123 \mathrm{mEq} / \mathrm{L}$ and a serum osmolality of $272 \mathrm{mOsm} / \mathrm{kg} \mathrm{H} 2 \mathrm{O}$, a urine osmolality of $215 \mathrm{mOsm} / \mathrm{kg} \mathrm{H} 2 \mathrm{O}$, a urine sodium of $47 \mathrm{mEq} / \mathrm{L}$, and a creatinine of $1.89 \mathrm{mg} / \mathrm{dL}$ (down from $2.28 \mathrm{mg} / \mathrm{dL}$ several days prior to admission). Her ECG showed normal sinus rhythm, normal intervals, and no ischemic changes. A transthoracic echo a few weeks prior to admission was unremarkable. Her medications were reviewed and none could explain her symptoms. She was started on intravenous normal saline. As a Jehovah's Witness, she declined blood transfusions. On admission, given her weakness and hyponatremia, thyroid function and cortisol tests were sent, revealing a low thyroid-stimulating hormone (TSH) of $0.09 \mathrm{uIU} / \mathrm{mL}$, a low free thyroxine (T4) of $0.49 \mathrm{ng} / \mathrm{dL}$, a low total triiodothyronine (T3) of $52 \mathrm{ng} / \mathrm{dL}$, and a borderline low morning cortisol of $9.9 \mathrm{ug} / \mathrm{dL}$. Thyroid function and cortisol tests were repeated with similar results. Further workup revealed a low prolactin of $4.3 \mathrm{ng} / \mathrm{mL}$ and a low post-menopausal follicle-stimulating hormone (FSH) of $11.5 \mathrm{mIU} / \mathrm{mL}$. A cosyntropin stimulation test revealed an inadequate cortisol response to cosyntropin. Cortisol was $9.7 \mathrm{ug} / \mathrm{dL}$ at baseline, $14.6 \mathrm{ug} / \mathrm{dL} 30 \mathrm{~min}$ after cosyntropin administration, and $16.1 \mathrm{ug} / \mathrm{dL}$ 
after another $30 \mathrm{~min}$. Adrenocorticotropic hormone (ACTH) was low at $12 \mathrm{pg} / \mathrm{mL}$, likely in the setting of adrenal insufficiency. A pituitary MRI showed a small pituitary gland (thought to be appropriate for her age) with severe white matter disease, an old basal ganglia infarct, and no acute infarctions. The patient was started on hydrocortisone for adrenal insufficiency. Three days later, she was started on levothyroxine for central hypothyroidism. On discharge, her sodium had increased to $133 \mathrm{mEq} / \mathrm{L}$, and her weakness had improved.

DISCUSSION: This case illustrates the importance of having a broad differential for common signs and symptoms. Our patient's hyponatremia was initially attributed to hypovolemia, whereas her weakness and syncope were separately attributed to her anemia. Given that her hyponatremia was partially refractory to volume resuscitation, and that she had recurrent syncopal episodes despite stable hemoglobin levels, it was suspected that there were other unaddressed contributing factors to her symptoms. The connection between thyroid dysfunction and hyponatremia is not fully understood, but appears to involve impaired water excretion. Adrenal insufficiency can cause both syncope and hyponatremia. Cortisol deficiency results in a lack of negative feedback on antidiuretic hormone (ADH) and on corticotropin releasing hormone, an $\mathrm{ADH}$ secretagogue. Recognition of these possible explanations for her presentation led to a diagnosis of central hypothyroidism, which upon further workup revealed panhypopituitarism. Central hypothyroidism is much less common than primary thyroid dysfunction and can be difficult to recognize, especially because TSH levels are normal in the majority of hypopituitary cases. Once hypopituitarism is suspected, initial workup should include assessment of multiple hypothalamic-pituitary axes by checking levels of TSH, free T4, total T3, prolactin, FSH, ACTH, and cortisol. If the morning cortisol is borderline low, a cosyntropin test should be performed, as described in this case. After repeating labs for verification, any abnormalities should prompt pituitary imaging. If, as in this case, no tumors are found, other causes of hypopituitarism may be investigated depending on the goals of the patient and provider. It is important to note that treatment of hypothyroidism prior to correction of hypoadrenalism (if present) can worsen the symptoms of both deficiencies, as the thyroid replacement therapy may increase clearance of endogenous cortisol. It is thus recommended that adrenal function be assessed and corrected prior to initiating thyroid replacement in patients with central hypothyroidism, as was done for our patient. This case demonstrates the importance of avoiding premature closure, as it can result in misdiagnosis and potentially adverse events. Keeping a broad differential, especially for elderly patients with non-specific and refractory symptoms, can lead to unexpected diagnoses and thus the choice of appropriate treatment and possible recovery.

AN UNCOMMON CULPRIT THAT IS FREQUENTLY MISSED-CEFEPIME NEUROTOXICITY. Pratyusha Bollimunta ${ }^{2}$; Venu M. Ganipisetti ${ }^{1}$; Malav Parikh ${ }^{3}$; Harvey J. Friedman ${ }^{4} .{ }^{1}$ Miami Valley Hospital, Evanston, IL; ${ }^{2}$ Presence Saint Francis Hospital, Evanston, IL; ${ }^{3}$ Cleaveland Clinic, Cleaveland, OH; ${ }^{4}$ Presence Saint Francis Hospital, Wilmette, IL. (Control ID \#2456980)

LEARNING OBJECTIVE \#1: Recognize that Cefepime neurotoxicity is a reversible and preventable cause of severe neurologic symptoms.

LEARNING OBJECTIVE \#2: Timely identification and discontinuation of the medication is a simple therapeutic trial which can establish the diagnosis.

CASE: A 87-year-old female presented to our hospital with repeated episodes of fevers at home. She has history of stage IIIB chronic kidney disease and type 2 Diabetes mellitus. On presentation, she had a non-healing large necrotic wound on her left leg. She was already treated with antibiotics and a revascularization procedure few weeks prior to admission. Superficial cultures taken prior to admission were growing MRSA, enterococcus and Corynebacterium. She was admitted to our hospital and started on Daptomycin and Cefepime $(1 \mathrm{~g}$ every $8 \mathrm{~h})$. On initial exam, she appeared alert, oriented $\times 3$ and completely cooperative. No focal deficits were noted. However, by day 3 , patient became confused with garbled speech along with shivering of both her arms. Continuous video EEG was performed, which showed slow encephalopathic background with triphasic waves. No epileptiform discharges or seizures were noted. Creatinine clearance was noted to be in the range of $50-60 \mathrm{~mL} / \mathrm{min}$. Due to the suspicion of Cefepime neurotoxicity, Cefepime was switched to meropenem. Patient became increasingly alert and returned to her baseline by day 5 . EEG showed improvement as well. Patient showed improvement in her fever curve with ongoing antibiotics and wound care. After careful exclusion of other potential causes, patient was diagnosed with Cefepime Neurotoxicity as the cause of her altered mental status. She was later discharged to home in a stable condition.

DISCUSSION: Cefepime is a broad-spectrum antibiotic predominantly excreted through kidneys ( $85 \%$ unchanged). Consequently, patients with renal failure (as our patient), are at increased risk of neurotoxicity as the drug can reach toxic levels by slowly accumulating in blood and CSF. This condition is a particularly under-appreciated phenomenon. Neurological symptoms may include seizures, hallucinations, myoclonus, confusion, and coma. The most common EEG findings noted are diffuse background slowing, triphasic waves, and multifocal sharp waves. Diagnosis can be challenging in hospitalized patients because of numerous possible confounding causes of 'altered-mental-status'. It can be established with careful exclusion of other etiologies. Diagnosis should be suspected if patient meets these criteria; 1.) Neurologic symptoms consisting of encephalopathy, including decreased level of alertness, seizures, myoclonus, or any combination of these with no alternative cause for neurological deterioration and 2. Clear temporal relationship between neurologic symptoms and cefepime administration (symptoms had to begin following initiation of cefepime, persist or worsen during cefepime administration, and improve or resolve when the medication was discontinued). Stopping the medication is a simple therapeutic trial which can establish diagnosis in most of the cases. Careful renal adjustment of Cefepime dosing may prevent this complication, but can occur despite dose adjustments in minority of patients. Patients usually improve dramatically after stopping medication. Conclusion: Cefepime neurotoxicity is a reversible (and preventable) cause of severe neurologic symptoms, which should be identified promptly and treated. Pre-existing kidney disease is an important risk factor for this condition and careful renal dose adjustment should be done when possible.

\section{AN UNEXPECTED COLLISION BETWEEN UNCOMMON CONDITIONS: ADULT-ONSET STILL'S DISEASE COMPLICATED BY MACROPHAGE AC- TIVATION SYNDROME Ashley Saito; Jason P. Williams. UCLA, Los Angeles, CA. (Control ID \#2438444)}

LEARNING OBJECTIVE \#1: Identify the clinical features of macrophage activation syndrome and the rheumatologic conditions with which it is associated

LEARNING OBJECTIVE \#2: Recognize the distinct differences in treatment of macrophage activation syndrome (MAS) vs. primary hemophagocytic lymphohistiocytosis (HLH)

CASE: A 31 year old previously healthy female presents with 9 months of fever, arthralgia, and painful lymphadenopathy. Her fevers were episodic, with temperatures reaching as high as $105 \mathrm{~F}$. Her joint pain was most prominent in the ankles, hips, and small joints of her hands. She also reported worsening fatigue and an evanescent rash involving her face, chest, arms, and legs. Extensive workup at outside institutions was done to rule out TB, HIV, and endocarditis. She also underwent 3 lymph node biopsies, a bone marrow biopsy, and splenectomy for massive splenomegaly, all of which were unrevealing. Upon arrival in our ED, the patient was febrile, lethargic, and reporting diffuse joint pain. Initial examination was remarkable for tender cervical and inguinal lymphadenopathy, hepatomegaly, and arthritis of the hips and ankles. Her initial laboratory workup was notable for a leukocytosis with neutrophilic predominance and transaminitis. This clinical presentation 
fulfilled criteria for Adult Still's disease. However, her low fibrinogen and markedly elevated ferritin prompted further workup, which revealed a low NK cell activity and high IL2 receptor level. These studies met criteria for a diagnosis of HLH. The patient was started on prednisone $1 \mathrm{mg} / \mathrm{kg}$ with rapid improvement in her symptoms. She is now undergoing evaluation for treatment with Anakinra, a recombinant IL-1 receptor antagonist that may provide more targeted immune-modulating therapy.

DISCUSSION: Adult Still's disease is a rare inflammatory condition that often masquerades as a fever of unknown origin. Our patient's symptoms followed a classic, polycylic pattern and met Yamaguchi's criteria for diagnosing Still's disease. However, not all of her lab abnormalities could be explained by Still's disease alone. Macrophage activation syndrome (MAS) is a potentially life-threatening condition that falls along the same spectrum of disease as HLH. It is important to distinguish MAS from primary HLH due to differences in their management. Treatment of MAS involves aggressive immunosuppression with high dose steroids, but not chemotherapy as with primary HLH. The rarity of MAS and its variable clinical presentation often makes it a difficult diagnosis to establish. However, early recognition and prompt initiation of treatment may result in rapid clinical improvement, as was the case with our patient.

AN UNFORTUNATE CASE OF HICKAM'S DICTUM Randy McCreery; Michael P. Smith. University of Nebraska Medical Center, Omaha, NE. (Control ID \#2469870)

LEARNING OBJECTIVE \#1: Identify the considerations for non-resolving pneumonia LEARNING OBJECTIVE \#2: Understand the pitfalls in the diagnosis and treatment of Actinomycosis

CASE: A 56 year-old woman presented with 3 months of productive cough, fevers and night sweats after failing multiple rounds of outpatient antibiotics. She had improved slightly with each course before her symptoms would again return. She had a significant smoking history of $>30$ pack years, reported a $70 \mathrm{lb}$ weight loss over the last 6 months, and denied any new exposures or travel. Her lungs were clear to auscultation, and she was in no distress. CT scan of the chest revealed a $1.8 \mathrm{~cm}$ mass with distal consolidation in the right upper lobe and the patient was taken for bronchoscopy. Biopsies from the area were negative for malignancy and bronchial alveolar lavage revealed $\sim 2000$ white blood cells, $97 \%$ polymorphonuclear leukocytes but no organisms. PET scan revealed a hypermetabolic lymph node in the right neck and retromolar trigone. Tongue biopsy revealed a well differentiated invasive squamous cell carcinoma with subsequent resection confirming metastasis to adjacent lymph nodes. Patient also underwent lobectomy for the lung mass. Pathology of the mass was without evidence of malignancy but did identify actinomycotic sulfur granules within bronchial lumina.

DISCUSSION: A non-resolving pneumonia is a presentation that the general internist will inevitably encounter. When this presentation is accompanied by the appearance of a mass, one should then include the infectious causes such as mycobacteria, fungi, nocardia and actinomyces in their differential in addition to malignancy. In our case, there was a mass found on imaging associated with a history of a pneumonia that had waxed and waned with short courses of antibiotics. This is a common presentation for actinomycotic infections as the empiric treatment duration for community acquired pneumonia is much shorter than that which is required for appropriate treatment of actinomyces. The partial treatment and thus chronicity of the infection may lead to fibrosis and the development of a mass on imaging. In addition, symptoms such as fevers, night sweats and weight loss may further sway the clinician toward suspicion of malignancy. The diagnosis may again be obscured by antecedent antibiotics when tissue samples are obtained. Therefore, it is not surprising that only a small minority of cases are diagnosed pre-operatively. When a concomitant malignancy is diagnosed with the associated infectious mass, as was the case with our patient, the likelihood of identifying actinomyces pre-operatively is even smaller. The general internist must know when to evaluate a non-resolving pneumonia further and must have a high index of suspicion for actinomyces in order to prevent unnecessary surgeries or interventions prior to appropriate treatment.

AN UNFORTUNATE STORM-A CASE OF A RARE ASSOCIATION WITH SLE Evan Nadler ${ }^{1}$; Stefanie D. Wade ${ }^{2} .{ }^{1}$ University of Connecticut, West Hartford, CT; ${ }^{2}$ University of Connecticut Health Center, West Hartford, CT. (Control ID \#2470239)

LEARNING OBJECTIVE \#1: Recognize the diagnosis of macrophage activating syndrome and its relationship to other autoimmune diseases

LEARNING OBJECTIVE \#2: Highlight the importance of prompt diagnosis of autoimmune diseases in the setting of fever of unknown origin

CASE: Macrophage activation syndrome (MAS) results from over-activation of Tlymphocytes and macrophages leading to a cytokine storm, which manifests as fevers, cytopenias, and elevated ferritin - a syndrome characterized by high mortality. MAS is an acquired form of Hemophagocytic lymphohistiocytosis (HLH) that is well described in pediatric populations with autoimmune conditions. MAS is infrequently described in adult onset Still's disease and other connective tissue disorders. We present a rare case of a young, previously healthy, adult male with MAS as a complicating process in the initial presentation of Systemic Lupus Erythematosus (SLE). A 19-year-old African American male presented from the department of corrections for altered mental status and fevers. Exam showed temperature $102 \mathrm{~F}$, pulse $122 \mathrm{bpm}$, respirations 30/min, with normal oxygen saturation. He was obtunded, with Glasgow coma score of 8 , cervical lymphadenopathy, diminished basilar breath sounds, symmetrical extremity weakness, and an erythematous maculopapular rash on his chest and back. Initial labs revealed pancytopenia, sodium $124 \mathrm{meq} / \mathrm{L}$, creatinine $1.2 \mathrm{mg} / \mathrm{dl}$, AST $152 \mathrm{IU} / \mathrm{L}$, ALT $19 \mathrm{IU} / \mathrm{L}$, and elevated ESR and CRP. Head CT was non revealing. Lumbar puncture was not suggestive of infection. MRI and EEG were obtained and unrevealing. Chest radiography showed cardiomegaly. On admission to the intensive care unit he remained febrile and encephalopathic despite receiving supportive care and broad-spectrum antibiotics. Extensive infectious work-up was negative. His presentation raised concern for autoimmune disease. Further testing revealed positive anti-nuclear antibody titer $>1: 5120$, speckled pattern, elevated anti-smith antibody titers, and low $\mathrm{C} 3 / \mathrm{C} 4$. Further testing showed ferritin $>40,000 \mathrm{ng} / \mathrm{ml}$, triglycerides $250 \mathrm{mg} / \mathrm{dL}$, and worsening transaminitis and pancytopenia. Fibrinogen was normal and bone marrow biopsy did not reveal hemophagocytic activity. Echocardiogram showed an enlarging pericardial effusion and the patient ultimately required a pericardial window. A new diagnosis of SLE was made (based on pancytopenia, serositis, positive ANA, antismith antibodies and nervous system involvement). Pulse dose steroids and Hydroxychloroquine were initiated. Anakinra was later added for treatment of presumed MAS. He responded quickly with improving encephalopathy, downtrending ferritin, and resolving cytopenias. His course was complicated by development of seizures likely due to neuropsychiatric SLE, and he ultimately died from septic shock secondary to invasive pulmonary aspergillosis. Histopathology on autopsy showed accumulation of macrophages in the cerebellum, a foci of inflammation in the spleen and confirmed the diagnosis of invasive pulmonary aspergillosis.

DISCUSSION: MAS as the initial presentation of SLE is a rare phenomenon. Our case demonstrates the importance of early recognition and diagnosis of SLE and increased awareness of MAS as a complication that can increase mortality. Profound ferritin elevation is the hallmark of MAS and should prompt further investigation based on MAS and HLH criteria. High dose intravenous corticosteroids are the standard of care and various immunomodulators have shown to be efficacious; however, the increased risk of opportunistic infection must be considered. 
AN UNUSUAL CASE OF BACK PAIN IN ADULT PRIMARY CARE CLINIC Eric

J. Tanenbaum. University of Washington, Seattle, WA. (Control ID \#2462501)

LEARNING OBJECTIVE \#1: Diagnose spinal epidural abscess (SEA)

CASE: Mr. R is a 65 year old man with no significant past medical history who presented to a primary care clinic with back pain. He described the gradual onset of razor-like bilateral upper back pain, 10/10 in intensity, starting adjacent to his spine and radiating to the chest in a band-like distribution. The pain started 6 weeks ago while he was on a crosscountry bike ride. The pain was made worse by inhalation but not exercise or movement. Two weeks prior to the onset of pain, Mr. R experienced fevers, lethargy, night sweats, headache, and dysuria; all of these symptoms resolved over a 2-week period. On review of systems, the patient denied recent skin changes. He endorsed being bit on his leg by a brown tick 1-2 years ago. His travel history over the past year includes riding his bike through the American southwest, northeast, northwest, and south eastern Canada. He endorsed minimal consumption of alcohol and denied use of tobacco and illicit drugs. Five weeks ago he had presented to a different primary care clinic. A PA-L chest radiograph revealed a possible left-sided pleural effusion. He was prescribed NSAIDs. He presented to an emergency department 4 weeks ago. His vital signs were normal. A workup for acute coronary syndrome was negative. $\mathrm{CBC}$ revealed a mild normocytic anemia ( $\mathrm{Hb} 12.9$ ). Basic chemistries and liver function tests were within normal limits. A PA-L chest radiograph was normal, and a thoracic $\mathrm{CT}$ angiogram revealed a $7 \mathrm{~mm}$ pulmonary nodule in the left lower lobe and no pulmonary embolism. Two weeks ago, he presented to another primary care office with persistent back pain. He was prescribed gabapentin. At this time the patient began taking oxycodone obtained from a friend. His pain was still poorly controlled. At the current visit, vital signs were: BP 105/74, Pulse 90, T 37.6C Physical exam revealed a healthy-appearing man who appeared younger than his stated age. Heart and lung exams were normal. Neurological exam revealed: CN II-XII intact, strength $5 / 5$ throughout, reflexes $2+$ throughout. No sensory deficits. No spinal or paraspinal tenderness and ROM in his spine was normal. Digital rectal exam revealed normal rectal tone and normal prostate. No rashes were seen. Lab testing revealed normocytic anemia (Hb 12.0), HIV neg, UA normal, Hep C neg, Quantiferon Gold neg, ESR 62. His gabapentin dose was increased. Mr. R was called 1 week after this visit at our clinic to evaluate pain control. He reported that the pain was worsening. Nortriptyline and naproxen were prescribed, and a thoracic MRI was ordered. The MRI revealed discitis/ osteomyelitis of T4-T5 with erosive changes and associated epidural abscesses. Cord compression was noted without signal abnormality. The patient was contacted and admitted to the spine service. Culture from spinal biopsy revealed MSSA. T3-6 laminectomy and T4-T5 corprectomy were performed with surgical stabilization and hardware placement. Broad spectrum antibiotics were begun and later narrowed to nafcillin. His hospital course was complicated by meningitis. He was discharged with levofloxacin and rifampin to complete a 6 week course. He was neurologically intact upon discharge.

DISCUSSION: Spinal Epidural Abscess (SEA) is a rare cause of back pain. The rate of spontaneous SEA is believed to be 0.88 cases $/ 100,000$ person years. Major risk factors for SEA in the absence of spinal surgery or instrumentation include concurrent infection, diabetes mellitus, immune system compromise, and intravenous drug use. Other risk factors include alcohol abuse, recent spinal fracture, indwelling catheter, cancer, and chronic renal failure. The vast majority of patients have at least one risk factor. This patient demonstrates the difficulty in early diagnosis of SEA. He presented to a primary care clinic with the common complaint of persistent back pain with normal vital signs and prior negative imaging. His red flags were new back pain at an older age and the nonmechanical nature of the pain. The sharp nature of the pain and apparent dermatomal pattern raised the concern for a neurologic source. The primary diagnoses considered included infectious causes (bilateral herpes zoster, West Nile Virus, Lyme disease), structural causes (malignancy, compressive mass, spinal fracture), and musculoskeletal causes. Multiple PA-L chest radiographs did not reveal signs of SEA nor did thoracic CT. This patient had no known risk factors for SEA and did not present with the classic triad of spinal pain, fever, and neurologic deficits which led to missed diagnosis. SEA is often missed on initial presentation because the classic triad described above is rare ( $8 \%$ of patients on initial visit). Back pain is the most common complaint with SEA, with $95 \%$ of patients describing this at their initial visit, compared to $41 \%$ reporting a neurologic deficit and $33 \%$ reporting fevers. MRI with gadolinium contrast is the diagnostic study of choice for SEA, with a sensitivity approaching $100 \%$. X-ray of the spine can often suggest an abnormality and the need for further imaging. The exact sensitivities of plain radiography and CT for SEA are unknown. Back pain is a common complaint in primary care and new non-mechanical back pain in an elderly patient must be taken seriously. One should not rely on the classic triad of symptoms to suspect SEA, as most patients present with isolated spinal pain. MRI with contrast should be obtained when SEA is suspected.

AN UNUSUAL CASE OF PROPTOSIS: IGG4-RELATED DISEASE IN THE SPOTLIGHT Shannon Ruzycki; Jay L. Patel; Margaret M. Kelly. University of Calgary, Calgary, AB, Canada. (Control ID \#2457672)

LEARNING OBJECTIVE \#1: Recognize the common manifestations of systemic IgG4-related disease

\section{LEARNING OBJECTIVE \#2: Diagnose IgG4-related disease}

CASE: A 66-year old male without significant past medical history presented to the Urgent Eye Clinic with a 1 week history of painless right eye proptosis and epiphora. Computed tomography of the head demonstrated a homogenous, $3.7 \times 1.7 \mathrm{~cm}$ enhancing mass arising from the right lacrimal gland that displaced the lateral rectus muscle. Urgent surgical biopsy was performed. Pathology demonstrated a lymphoplasmacytic infiltrate with storiform fibrosis and occasional eosinophils. Immunohistochemistry showed in creased number of IgG4-positive plasma cells, constiting $75 \%$ of total plasma cells. No granulomas or malignant cells were seen. Flow cytometry for lymphoma performed on the tissue was negative. Serum calcium, c-reactive protein (CRP), rheumatoid factor (RF), angiotensin converting enzyme (ACE), anti-nuclear antibody (ANA), and anti-neutrophi cytoplasmic antibody (ANCA) were negative. Liver and renal function were within normal limits. Serum immunoglobulin subclass analysis revealed an IgG4 level of $3.50 \mathrm{~g} / \mathrm{L}$ (normal range $0.01-1.40 \mathrm{~g} / \mathrm{L}$ ). A diagnosis of IgG4-related disease (IgG4-RD) was made, and treatment was initiated with high-dose prednisone. Follow-up CT of the orbits demonstrated significant reduction in size of the lacrimal gland mass at 6 month assessment. Prednisone was tapered over 3 months with ongoing improvement of the patient's symptoms. At 1 year follow up, the patient had not relapsed or developed symptomatic involvement of other organ systems.

DISCUSSION: IgG4-related disease is a recently described, systemic fibroinflammatory disease defined by characteristic pathologic lesions. Its discovery linked many singleorgan diseases, including autoimmune pancreatitis and idiopathic salivary gland fibrosis, under a single disease spectrum by a common histopathology. Since the first description of the disease-defining IgG4-positive plasma cell infiltrate in 2003, IgG4-RD has been found to affect nearly every organ. Thus, physicians from any speciality may encounter IgG4RD. Diagnosis of IgG4-RD has implications for treatment and prognosis, and should be sought in patients with classic clinical features for this illness. End organ damage in IgG4RD occurs due to infiltration of tissues by lymphocytes leading to organ enlargement, reactive inflammation, and eventual fibrosis. The pancreas and salivary glands are the most common affected. The most common presenting symptom of IgG4-RD is due to mass effect of the enlarging organ; for example, IgG4-related pancreatitis may present as obstructive jaundice secondary to pancreatitic head enlargement. Often, IgG4-RD is discovered as a mass on imaging studies performed for other indications. Rarely, patients present with symptoms of subacute organ failure. A retrospective review series suggested 
that IgG4-RD of the orbit may account for up to $28 \%$ of cases initially given a diagnosis of idiopathic orbital inflammation or orbital benign lymphoid hyperplasia, two idiopathic conditions that cause non-neoplastic orbital soft tissue enlargement. A recent consensus statement by an expert, international panel of specialists emphasizes the importance of histopathology in diagnosis of IgG4-RD. The number of IgG4-positive plasma cells must be elevated, with the threshold for diagnosis dependent on the organ. The proportion of IgG4-positive plasma cells must be greater than $40 \%$ of total plasma cells. Further, there must be increased lymphocytes and evidence of fibrosis. Finally, IgG4-RD is a diagnosis of exclusion; other disorders associated with elevated tissue IgG4-positive plasma cells, such as ANCA-associated vasculitidies, rheumatoid arthritis, pernicious anemia, lowgrade B-cell lymphomas, and other malignancies must be ruled out. Flow cytometry for lymphoma, and careful examination for granulomatous disease or malignancy should be performed by an experienced pathologist. Elevated serum IgG4 levels (greater than $1.35 \mathrm{~g}$ / 1) are supportive of, but not required for, a diagnosis of IgG4-RD. Many disorders, including B-cell lymphomas, Sjogren's syndrome, and minimal change disease, are also associated with elevated serum IgG4 levels. The sensitivity and specificity of an elevated serum IgG4 level for IgG4-RD is 97.0 and $79.6 \%$ respectively. Without compatible histopathology, an isolated elevated serum IgG4 level is non-specific. IgG4-RD should be suspected in a patient presenting with organ enlargement, fibrosis, or dysfunction of unknown etiology. In general, expert opinion is that tissue biopsy with immunohistochemical staining for IgG4-RD should be considered in patients with pancreatitis, retroperitoneal fibrosis, or lymphadenopathy of unknown etiology, any inflammatory pseudotumor, salivary gland enlargement, or sclerosing cholangitis. Management of patients with IgG4-RD is based on expert opinion. Treatment is usually initiated in symptomatic patients or those with critical organ involvement to prevent progression to organ fibrosis and failure. Small case series suggest that greater than $90 \%$ of patients improve on initial course of glucocorticoids; however, rates of relapse and requirement of long-term immunosuppression are reported in $20-70 \%$ of patients as glucocorticoids are tapered. Azathioprine, methotrexate, rituximab, and cyclophosphamide have all been trialed as steroid-sparing agents in IgG4-RD.

AN UNUSUAL CASE OF SEVERE, COOMBS NEGATIVE HEMOLYTIC ANEMIA Jeffrey A. Shrensel ${ }^{2}$; Aaron M. Etra ${ }^{3}$; Veenu Gill ${ }^{1} .{ }^{1}$ Mount Sinai Beth Israel, Brooklyn, NY; ${ }^{2}$ Mount Sinai Beth Israel, New York, NY; ${ }^{3}$ The Mount Sinai Hospital, New York, NY. (Control ID \#2467871)

LEARNING OBJECTIVE \#1: Recognize an emerging cause of Coombs negative hemolytic anemia in patients receiving blood transfusions

LEARNING OBJECTIVE \#2: Recognize the additional risks of infection via blood transfusion in asplenic and immunocompromised patients

CASE: DM, a 47 year old man with a medical history of type II diabetes mellitus, pancreatic adenocarcinoma status post Whipple procedure and splenectomy complicated by blood loss anemia requiring a transfusion of 1 unit of PRBC, presented 6 weeks after surgery with a chief complaint of 2 weeks of bilateral leg pain and swelling. In the emergency room, the patient was found to have bilateral deep vein thromboses and was admitted to the surgical service. Initial laboratory values were significant for moderate hyponatremia and anemia, with a hemoglobin level of $9.8 \mathrm{~g} / \mathrm{dl}$, similar to his discharge hemoglobin of $9.9 \mathrm{~g} / \mathrm{dl}$. A hematology consult was placed and the patient was started on a heparin drip and then transitioned to rivaroxaban. A CT angiogram of the chest performed the following morning revealed bilateral pulmonary emboli. Over the next 2 days, the patient became increasingly lethargic and confused, during which time he developed a Coombs' negative hemolytic anemia, with hemoglobin dropping to $5.6 \mathrm{~g} / \mathrm{dl}$, bilirubin rising to 8.2 , and an undetectable haptoglobin. The patient was transferred to the stepdown unit and a peripheral smear was prepared. The patient was then transfused two units of blood. The smear revealed numerous intracellular ring forms present in approximately $80 \%$ of the red cells. An infectious disease consult was placed as the initial smear was read as likely Plasmodium falciparum. The patient was then transferred to the medical ICU to initiate IV quinidine, and a call was placed with the CDC for possible release of artusenate. A review of the smear in the morning revealed the presence of extracellular forms, and given the high parasite burden, it was felt much more likely to be due to be Babesia microti infection, which was later confirmed by PCR assay at the New York City Department of Health. The patient was also started on IV clindamycin Q8H, and over the next $12 \mathrm{~h}$ experienced improvement in his mental status. He was able to provide more history at this point, and denied any recent travel out of his home neighborhood in Brooklyn, or travel out of New York State since moving from Albania some 18 years prior. He did live in Staten Island for 6 years before moving to Brooklyn. A repeat smear $24 \mathrm{~h}$ after initiating therapy showed parasitemia of greater than $40 \%$, and the patient received an exchange transfusion of 8 units of PRBC. As his mental status continued to improve, he was transitioned to oral quinine and clindamycin, with eventual clearance of his parasitemia over 2 weeks time. As the patient had received blood approximately 6 weeks prior to presentation, the city department of health and the division of epidemiology at the CDC were notified. Testing of the donor blood serology conducted by the department of health was positive for B. microti.

DISCUSSION: Babesia microti is a blood-borne parasite transmitted via Ixodes scapularis, commonly known as the deer tick, the same vector responsible for transmission of Lyme Disease. The nymph form of the tick acquires the babesia parasite when they feed on mice, which is passed on to human hosts during feeding after the tick molts into the adult form. Symptoms of infection in healthy, immunocompetent adults are often nonspecific, including fever, chills, body aches, and fatigue. As the parasite inhabits red blood cells, it can cause hemolytic anemia, particularly in immunocompromised or asplenic hosts, where infected cells are unable to be cleared effectively by the reticuloendothelial system. Infected deer ticks can commonly be encountered the United States Northeast and Upper Midwest, particularly in New York, New Jersey, and New England. Detection of B. microti is commonly via blood smear, where the parasite can be seen as both intracellular and extracellular forms. The intracellular form can appear as the pathognomonic merozoite "maltese cross," or the less specific trophozoite "ring form," which led to the initial lab read of P. falciparum. It should be noted that an $80 \%$ parasitemia of $\mathrm{P}$ falciparum is nearly unheard of, and in addition, extracellular parasites are generally not visualized on the smear in the case of $P$. falciparum, which can help differentiate the two. This asplenic patient almost certainly contracted babesiosis through contaminated blood, since he presented for this admission in March, and his surgery and blood transfusion occurred in mid February, an uncommon window for contact with deer ticks in an urban setting,. Recently, a number of articles have argued for the routine testing of donor blood for babesia infection, and this case illustrates the catastrophic risks that such infection poses for asplenic or immunocompromised patients.

\section{AN UNUSUAL CAUSE OF ACUTE KIDNEY INJURY IN A PATIENT WITH ALLOPURINOL-INDUCED DRESS SYNDROME Anthony J. Esposito; Ryan C.} Murphy; Joseph Merrill. University of Washington School of Medicine, Seattle, WA. (Control ID \#2469763)

LEARNING OBJECTIVE \#1: Recognize the rare but potentially fatal complication of drug reaction with eosinophilia and systemic symptoms (DRESS) with allopurinol.

LEARNING OBJECTIVE \#2: Distinguish common renal complications of DRESS from a rare case of necrotizing vasculitis.

CASE: A 59-year-old male patient with past medical history significant for coronary artery disease and chronic kidney disease (baseline creatinine $1.6 \mathrm{mg} / \mathrm{dL}$ ) who was admitted with 1 week of progressive diffuse, desquamating, morbilliform rash, fever, 
hemoptysis, malaise, and weight loss. One month earlier, he was prescribed allopurinol for asymptomatic hyperuricemia. Admission examination revealed scaly papules and plaques with areas of desquamation diffusely distributed across his face, neck, trunk, and upper extremities with involvement of the mucosal membranes of his oropharynx in addition to lymphadenopathy. Findings of leukocytosis with a predominant eosinophilia (absolute value of $4.05 \mathrm{k} / \mathrm{mcL}$ ), hepatitis (ALT $360 \mathrm{U} / \mathrm{L}$ ), and acute anuric renal failure (creatinine $15 \mathrm{mg} / \mathrm{dL}$ ) revealed a presentation consistent with DRESS syndrome. Hemodialysis was initiated, and a kidney biopsy was performed to determine the underlying cause of his renal failure. The biopsy was remarkable for tubulointerstitial nephritis with frequent plasma cells and eosinophils as well as necrotizing vasculitis of the interlobular arteries without glomerular necrosis or crescents. Immunofluorescence did not reveal immune complex deposition or linear glomerular basement membrane (GBM) staining. As necrotizing vasculitis is unusual in the setting of DRESS alone, an extensive evaluation was performed to identify a separate primary cause of the vasculitis. This included antineutrophil cytoplasmic antibodies, anti-nuclear antibodies, and complement levels, all of which were within normal limits; however, he had a strongly positive anti-glomerular basement membrane titer and a mildly elevated anti-PR3 titer that returned to normal limits on recheck 2 weeks later. Bronchoscopy revealed desquamating bronchi/ bronchioles, but no evidence of alveolar hemorrhage to correlate with anti-GBM disease. Therefore, it was concluded that the necrotizing vasculitis was likely due to DRESS. He was given cyclophosphamide and pulse corticosteroids with improvement of his rash, hepatitis, and acute kidney injury, no longer requiring hemodialysis.

DISCUSSION: DRESS is a rare but potentially life-threatening, drug-induced hypersensitivity reaction. It is characterized by a characteristic skin eruption, eosinophilia, lymphadenopathy, and end-organ damage, mainly including the liver, kidney, and lung. Allopurinol and anticonvulsants are the most common agents associated with this entity, and the onset is usually 3 to 8 weeks after initiation of the drug. The course is usually prolonged and frequently relapses despite discontinuation of the offending agent. Renal involvement occurs in about $10-40 \%$ of cases and is more common in cases induced by allopurinol (up to $68 \%$ in one case series). Kidney biopsy characteristically demonstrates tubulointerstitial nephritis with invasion of lymphocytes and eosinophils into the rena interstitium. In addition to this, our patient had interlobular artery necrotizing vasculitis. We are only aware of one case of kidney-limited vasculitis secondary to DRESS described in the literature. Hemodialysis is rarely required but is more common in patients with underlying chronic kidney disease. The mainstay of treatment is discontinuation of the offending agent and supportive therapy. In patients with severe interstitial nephritis or interstitial pneumonia, high-dose corticosteroids are used with a prolonged taper, usually over 8 to 12 weeks.

AN UNUSUAL CAUSE OF ACUTE LIVER FAILURE Sheryl K. Ramdass; Salwa Khedr; Rony Ghaoui. Baystate Medical Center, Springfield, MA. (Control ID \#2464501)

LEARNING OBJECTIVE \#1: Identify acute liver failure as a rare presentation of acute B cell lymphoblastic leukemia (B-ALL)

LEARNING OBJECTIVE \#2: Discuss the treatment limitations and prognosis of BALL in the setting of acute liver failure

CASE: A 70 year old man presented to the emergency department after a syncopal episode following a 1 week history of nausea, fatigue and weight loss. His past medical history was significant for hypertension, treated with hydrochlorothiazide. He was also on glucosamine, gingko biloba and saw palmetto. He was employed as a correctional officer and rarely consumed alcohol. On presentation, vitals were stable and physical examination was remarkable for sclera icterus without any other stigmata of chronic liver disease. Admission laboratory revealed AST $3949 \mathrm{u} / \mathrm{L}$ (normal <38 u/L), ALT $4518 \mathrm{u} / \mathrm{L}$ (normal $<41 \mathrm{u} / \mathrm{L}$ ), ALP 213 units/L (normal <129 units/L), bilirubin $13.3 \mathrm{mg} / \mathrm{dL}$ (normal $<1.2 \mathrm{mg} /$
$\mathrm{dL}$ ), direct bilirubin $10.7 \mathrm{mg} / \mathrm{dL}$ (normal $<0.3 \mathrm{mg} / \mathrm{dL}$ ), INR 1.6 , measured drug levels within normal, pancytopenia with hemoglobin $12.5 \mathrm{~g} / \mathrm{dl}$, platelet count $33.000 / \mathrm{mm}^{3}$ and white blood cell count $2500 / \mathrm{mm}^{3}$ A CT scan of the abdomen showed low attenuation of the liver consistent with hepatic steatosis without intra or extra hepatic biliary dilatation. A subsequent work-up was negative for autoimmune markers as well as viral serology for hepatitis A,B,C, E, Cytomegalovirus and Epstein-Barr virus. He was empirically treated with $\mathrm{n}$-acetyl cysteine. Bilirubin continued to increase up to $18.6 \mathrm{mg} / \mathrm{dL}$ and therefore a trans-jugular liver biopsy was pursued which revealed lymphoblasts invading the liver parenchyma and on staining with PAX-5. The diagnosis of B cell acute lymphoblastic leukemia was later on also confirmed on bone marrow biopsy. The patient was started on high dose steroids with the addition of induction chemotherapy regimen of rituximab, cyclophosphamide and daunorubicin in keeping with the modified CALGB 8811 protocol for his hyperbilirubinemia. He unfortunately decompensated with altered mental status and development of neutropenic septic shock. He expired 19 days after his admission. DISCUSSION: Acute liver failure (ALF) carries a high morbidity and mortality rate and the etiology of ALF provides one of the best indicators of prognosis as well as dictataes specific management options. ALF is most often caused by toxins and hepatitis viruses however rarer causes must be entertained when these are outruled. Acute B cell lymphoblastic leukemia (B-ALL) is a hematological malignancy characterized by abnormal clonal proliferation of lymphoblasts within the bone barrow and extra medullary involvement is unusual. ${ }^{1}$ Furthermore, it is exceedingly rare for acute liver failure to be the initial presentation with the majority of case reports limited to the pediatric population. A case series by Anderson el al in children highlighted the rarity of acute liver failure as the initial manifestation of acute leukemia and the poor prognostic associated with this diagnosis. ${ }^{2}$ B-ALL causes intrahepatic cholestasis with an infiltration of the liver sinusoids by leukemic blast cells. The prognosis is guarded with limited effectiveness and challenges of chemotherapy in presence of hepatic dysfunction as well as the clear contraindication for liver transplantation. This is corroborated by a multicenter study of 1910 patients with acute liver failure with only 27 cases (1.4\%) attributed to malignancy from which a third were due to lymphoma or leukemia and 24 patients ( $89 \%$ ) died within 3 weeks of onset of symptoms. ${ }^{3}$ In conclusion, discerning clinicians must be recognise B-ALL is a rare cause of ALF and carries a very poor prognosis.

\section{AN UNUSUAL CAUSE OF PRODUCTIVE COUGH AND HEADACHES Ana I.}

Velazquez; Mariana Mercader; Michael P. Bergman; Samuel Acquah. Mount Sinai Beth Israel, New York, NY. (Control ID \#2469933)

LEARNING OBJECTIVE \#1: Recognize the diverse clinical manifestations of lung adenocarcinoma.

LEARNING OBJECTIVE \#2: Describe the epidemiology of lung adenocarcinoma. CASE: A 30 year-old male presented to his PMD with 1 month of progressive dyspnea on exertion, wheezing, and cough productive of white sputum. He reported chills, night sweats and a 10-lb unintentional weight loss for 1 month. The patient also complained of development of worsening frontal headaches, photophobia, phonophobia, and right hand clumsiness associated with his respiratory symptoms. He was treated with oral azithromycin and amoxicillin-clavulanate for suspected community acquired pneumonia with minimal improvement. His neurologic symptoms prompted his PMD to order a brain MRI that showed supra- and infratentorial intra-axial enhancing masses with vasogenic edema and left to right midline shift. He was instructed by his PMD to go to the hospital for work up of his brain MRI findings. Regarding his medical history, the patient reported occasional alcohol use but no tobacco exposure or other toxic habits. He denied environmental exposures or history of positive PPD. Family history was significant for mother with breast cancer, lung cancer in a smoker uncle, and skin cancer in grandfather. Physical exam revealed a young male in no acute distress, breathing comfortably on room air. 
Rhonchi were appreciated over the right middle lung but neurological exam was intact. A chest CT scan was performed to evaluate the auscultatory findings, which showed a $6 \mathrm{~cm}$ necrotic and spiculated right hilar mass. CT abdomen-pelvis was unremarkable. Labs were negative for HIV and cultures for bacteria, fungi, and AFB in both blood and sputum. Bronchoscopy with endobronchial ultrasound (EBUS) demonstrated large subcarinal mediastinal lymphadenopathy with nodal heterogeneity and central necrosis. The mass visible in the CT scan appeared to be a conglomerate of necrotic mediastinal and subcarinal lymph nodes. EBUS-guided biopsy of hilar mass confirmed the diagnosis of metastatic non-small cell lung adenocarcinoma (NSCLC). Molecular DNA analysis was negative for mutations in epidermal growth factor receptor (EGFR), anaplastic lymphoma kinase (ALK), and KRAS. Systemic steroids were started for his brain metastases with improvement in headaches. Targeted palliative chemotherapy in combination with whole brain radiotherapy was recommended.

DISCUSSION: NSCLC is a rare disease with an incidence of $0.6 \%$ among adults less than 40 years of age. Adenocarcinoma is the most common cell type, however it only occurs in $0.3 / 100,000$ individuals in this age group. Lung adenocarcinoma more frequently affects females and non-smokers. In the age of targeted molecular therapy, analysis of lung adenocarcinoma has led to the development of targeted therapies to the most common mutations, at present EGFR and ALK-mutated cell lines. Mutations in the EGFR tyrosine kinase are observed in $15 \%$ of NSCLC adenocarcinoma patients in the US and occur more frequently in nonsmokers. EGFR mutations confer a more favorable prognosis and predict high sensitivity to EGFR tyrosine kinase inhibitors (TKIs) such as erlotinib. Translocations in ALK tyrosine kinase are present in only $4 \%$ of NSCLC adenocarcinoma patients, also occurring more frequently in nonsmokers and younger patients. The presence of an ALK translocation predicts sensitivity to ALK TKIs, as crizotinib, that significantly prolong progression-free survival. Given the high sensitivity of mutated tumors to TKIs, it is vital to pursue molecular testing to identify those patients who may benefit from these therapies. Our patient is a rare case of a young man with advanced local and metastatic disease without a dominant lung mass at time of diagnosis. NSCLC is more commonly seen in older men cancer presenting with cough and constitutional symptoms. Unfortunately, our patient presented at a younger age and he did not have any mutations identified, which portends a worse overall prognosis.

\section{AN UNUSUAL CAUSE OF RHINORRHEA: SPONTANEOUS CSF LEAK Victo-} ria Poplin ${ }^{2}$; James E. Kim ${ }^{3}$; Christopher L. Sweeney ${ }^{1} .{ }^{1}$ University of Missouri Kansas City School of Medicine 2. Children's Mercy Hospital, Kansas City, MO; ${ }^{2}$ University of Missouri Kansas City, Kansas city, MO; ${ }^{3}$ University of Missouri Kansas City School of Medicine, Kansas city, MO. (Control ID \#2467396)

LEARNING OBJECTIVE \#1: Acknowledge the importance of inclusion of spontaneous CSF leak in the differential for any patient presenting with persistent or unilateral rhinorrhea due to the high morbidity and mortality

LEARNING OBJECTIVE \#2: Recognize the connection between spontaneous CSF leak and idiopathic intracranial hypertension and the potential implications for diagnosis and management

CASE: Patient is a $56 \mathrm{y} / \mathrm{o}$ obese female with a past medical history significant for seasonal allergic rhinitis who first presented to her primary care physician complaining of clear rhinorrhea. Initial management included treatment with a nasal steroid and loratadine for suspected allergic rhinitis. Due to lack of improvement, CT head without contrast was obtained, which showed apparent osseous dehiscence of the posterior wall of the sphenoid sinuses and a mildly expanded partially empty sella concerning for cerebrospinal fluid leak and idiopathic intracranial hypertension. Nasal drainage was positive for Beta-2-transferrin and a glucose concentration of $124 \mathrm{mg} / \mathrm{dL}$, confirming CSF rhinorrhea. Endoscopy performed by ENT showed an area concerning for encephalocele of the cribriform plate in the left nasal cavity, and lumbar puncture (LP) prior to treatment had a non-elevated opening pressure of $24 \mathrm{~cm} \mathrm{H} 20$. These findings were consistent with left cribriform encephalocele with CSF leak and likely idiopathic intracranial hypertension. The patient underwent simultaneous endoscopic repair of her encephalocele and CSF leak with lumboperitoneal shunt placement with resolution of her rhinorrhea.

DISCUSSION: Spontaneous cerebrospinal fluid (CSF) leaks, also referred as idiopathic CSF leaks, are the inappropriate drainage of cerebrospinal fluid from the dura without an identifiable etiology. They contribute to $14-46 \%$ of all CSF leaks. The most common presenting symptom of spontaneous CSF leaks is rhinorrhea which is nonspecific to the diagnosis. In fact, spontaneous CSF leaks are often misdiagnosed as allergic rhinitis, nonallergic rhinitis or chronic sinusitis and lead to a subsequent diagnostic difficulty and delay. Delay in the diagnosis increases the potential for complications such as meningitis, intracranial abscess and pneumocephalus. A recently identified association with idiopathic intracranial hypertension (IIH) may provide an additional tool to aid in early diagnosis. Recent literature proposes an association between spontaneous CSF leaks and IIH. The association is suggested by similar patient demographics, symptoms of increased intracranial pressure (ICP) before or after repair, overlap of CT findings between the two, increased ICP in patients with spontaneous CSF leak after repair, and a higher rate of leak recurrence in patients with raised ICP. Patients with spontaneous CSF leak and IIH are often both middle aged and obese females. The most common finding shared between IIH and spontaneous CSF leaks is an empty or partially empty sella tursica on CT scan. The critical finding strengthening the association between IIH and spontaneous CSF leaks is elevated ICP. In a study by Schlosser et al., 10 out of 16 patients had a post-operative LP and all had an elevated opening pressure, average 26.5 (range 17.3-34 cm H2O, normal $0-15 \mathrm{~cm} \mathrm{H} 2 \mathrm{O}$ ). Illing et al. found $95 \%$ of their patients to have an elevated opening pressure, with a mean of 27.7 (range 9-50). The full diagnostic criteria for IIH is the modified Dandy criteria. One study found that $72 \%$ of spontaneous CSF leaks or patients with an encephalocele met the modified Dandy criteria. Additionally, IIH may also be associated with an increased risk of recurrence. Currently the risk of recurrence of CSF leak after surgical repair ranges from 25 to $87 \%$. When elevated ICP was identified and treated, the recurrence rate was less than $10 \%$. The persistent pressure exerted on the skull base at sites of inherent structural weakness may result in bony erosion leading to the initial CSF leak and subsequent poor healing after repair. Without appropriate treatment of the elevated pressure, the patient will continue to be at risk of new or recurrent bony erosion. The gold standard treatment of spontaneous CSF leak is endoscopic repair. If elevated ICP is suggested or diagnosed, elevated ICP should be managed prior to surgical repair with weight loss, medical management or surgical management. First line medical management is acetazolamide. Surgical treatment can be achieved via intracranial venous shunt, ventriculoperitoneal shunt, lumboperitoneal shunt and bariatric surgery. In conclusion, it is important to include spontaneous CSF leak in the differential diagnosis of persistent rhinorrhea. The association with IIH has already impacted treatment and will likely improve diagnosis, but more studies are required to determine if this association represents the underlying etiology or a separate disease entity.

AN UNUSUAL COMPLICATION OF FEVER Migdalia Feliciano ${ }^{2}$; Sunny-Skye Keppel ${ }^{1}$. ${ }^{1}$ Mount Sinai Beth Israel, Elmont, NY; ${ }^{2}$ Mount Sinai Beth Israel, New York, NY. (Control ID \#2469663)

LEARNING OBJECTIVE \#1: Identify unusual triggers of Brugada Syndrome LEARNING OBJECTIVE \#2: Manage patients with fever induced Brugada Syndrome CASE: A 40-year-old man presents to the emergency room with fever of $101^{\circ} \mathrm{F}$ that began 1 week prior to admission. Associated symptoms are fatigue, chills, weakness, lightheadedness, palpitations and a near-syncopal event. He denies syncope, chest pain, shortness of breath, cough, sore throat, skin rashes, nausea, vomiting, abdominal pain, 
edema or any other complaints. At home, he has been taking ibuprofen as needed for fever. His history was notable for atrial flutter, which was treated with radiofrequency ablation 10 years ago. In the past, he was also told that his heart rhythm had pauses but did not require treatment. He was not on any home medications other than ibuprofen. There was no family history of premature or unexplained death or arrhythmia. He denies alcohol, cocaine or any other toxic habits. On physical exam patient was afebrile and heart exam was remarkable for irregular rhythm and occasional pauses. Troponin was negative. Complete Blood Count (CBC) was significant for leukopenia (WBC $2.7 \mathrm{~K} / \mathrm{uL}$ ) and thrombocytopenia (platelets $104 \mathrm{~K} / \mathrm{uL}$ ) concerning for viral etiology. Comprehensive Metabolic Panel (CMP) was normal. Chest X-ray did not reveal any pathology. Electrocardiogram (ECG) had coved ST segment elevations greater than $2 \mathrm{~mm}$ in V1 and V2 followed by $T$ wave inversions, characteristic of Brugada Type I. Telemetry was significant for frequent sinus pauses of 4 to $5 \mathrm{~s}$. Patient was admitted to the Coronary Care Unit (CCU) unit for closer monitoring and further workup. He was given acetaminophen every $6 \mathrm{~h}$ to prevent fevers. He remained afebrile and infectious workup including Parvovirus titers was negative. Transthoracic echo (TTE) showed normal left and right ventricular size and systolic function and no significant valvular disease. Telemetry monitor in the $\mathrm{CCU}$ continued to show intermittent pauses of 2 to $3 \mathrm{~s}$ with sinus exit block and sinus bradycardia but patient remained asymptomatic. Electrophysiology (EP) service was consulted. Invasive EP study was performed and revealed prolonged sinus node recovery time, which in conjunction with sinus bradycardia and pauses noted on telemetry was consistent with sinus node disease. Implantable cardiac monitor (LINQ) was implanted after the EP study. During hospitalization, patient's leukopenia and thrombocytopenia improved. Repeat EKG showed improving Brugada type pattern. Patient was medically stable upon discharge and was given follow up appointment with EP.

DISCUSSION: The Brugada syndrome is an autosomal dominant genetic disorder characterized by abnormal ECG findings in association with an increased risk of ventricular tachyarrhythmias and sudden cardiac death. ECG findings consist of persistent ST segment elevation in leads V1 to V3 and pseudo-right bundle branch block. If patients have these typical ECG findings but are asymptomatic this is a Brugada pattern, whereas those with typical ECG findings who have experienced sudden cardiac death or sustained ventricular tachyarrhythmia have Brugada syndrome. Typically these ECG changes are hidden and become apparent in the presence of a trigger such as: use of a sodium channe blocker (flecainide, procainamide), pacing, vagal maneuvers, increased alpha-adrenergic tone, beta blockers, tricyclic or tetracyclic antidepressants, lithium, local anesthetics, fever, hypokalemia, hyperkalemia, hypercalcemia, and alcohol and cocaine toxicity. Type Brugada pattern is more common among patients with fever. Approximately $25 \%$ of cases of Brugada syndrome are caused by mutations in the cardiac sodium channel gene SCN5A which result in temperature dependent ionic changes that cause characteristic Brugada ECG patterns during fever. In general, treatment for patients diagnosed with the Brugada syndrome is focused around termination of any ventricular arrhythmias with an implantable cardioverter-defibrillator (ICD). However, with fever-induced Brugada, prompt control of fever with antipyretics is vital in preventing malignant arrhythmias. In conclusion, it is important for the clinician to be aware of the various triggers of Brugada syndrome, as making the diagnosis can be difficult as the ECG patterns are often hidden

AN UNUSUAL ETIOLOGY OF MENINGITIS IN A LUPUS PATIENT Saika Sharmeen. Mount Sinai St. Luke's, Mt Sinai West Hospital Center, NY, NY. (Control ID \#2468369)

LEARNING OBJECTIVE \#1: To recognize primary Varicella Zoster Virus as a cause of meningitis in patients with Systemic Lupus Erythematosus (SLE)

LEARNING OBJECTIVE \#2: To recognize that SLE patients are considered immunocompromised and are prone to opportunistic infections
CASE: A 27-year-old female with Lupus nephritis on peritoneal dialysis and history of lupus cerebritis, was admitted with 1-week of high fever, vomiting, headache, and rash. The headache was bi-frontal, associated with rigors and photophobia. She described the rash as pruritic and first noticed it a few days prior to admission. She had no other comorbidities and denied any allergies or toxic habits. Her home medications included hydroxychloroquine $200 \mathrm{mg}$ daily and Prednisone $10 \mathrm{mg}$ daily. The patient denied any recent travel or any known sick contacts. Vitals at presentation were remarkable for recurrent fevers of $103 \mathrm{~F}$ and tachycardia to $100 \mathrm{~s}$. Her physical exam was remarkable for nuchal rigidity and vesiculopapular rash that began on her left buttocks advancing to bilateral upper thighs without purulence or drainage. A detailed neurological examination demonstrated no focal motor or sensory deficits. Cranial nerve testing results were normal. Diagnostic evaluation revealed normal complete blood cell count and chemistry panel except for Hemoglobin of $11 \mathrm{~g} / \mathrm{dL}$ and Creatinine of $7.10 \mathrm{mg} / \mathrm{dL}$. Computed tomography of the head, chest X-ray, and coagulation studies were unremarkable. Despite the rash, the initial concern was bacterial meningitis. Cultures were sent for analysis including blood and peritoneal fluid. A lumbar puncture was also performed prior to initiation of antibiotics. The patient's fever persisted and her condition worsened despite being on broad spectrum antibiotics. Cerebrospinal fluid (CSF) analysis revealed normal opening pressure, marked lymphocytic pleocytosis, WBC 557/uL with $89 \%$ lymphocytes, protein of $205 \mathrm{mg} / \mathrm{dL}$, and glucose of $38 \mathrm{mg} / \mathrm{dL}$. Due to the lymphocytic predominance, the patient was promptly started on IV acyclovir. CSF polymerase chain reaction (PCR) was subsequently positive for Varicella Zoster Virus (VZV). All other CSF workup was negative. Viral culture from scraping of the skin lesion was also positive for VZV. Serum VZV IgG was negative and IgM was positive. Blood cultures and peritoneal fluid evaluation were unremarkable. Her condition markedly improved with 10-day course of IV acyclovir DISCUSSION: Varicella Zoster meningitis, either primary or latent, is an uncommon cause of meningitis in SLE patients. Primary VZV infection (chicken pox) is not common in immunocompetent adults and even less common in SLE. Moreover, while Herpes Zoster (shingles) have been reported in SLE patients, primary VZV has not been as frequently reported. This case demonstrates the severity of primary VZV in this patient population and the importance of recognizing that SLE patients are considered immunocompromised even when they are not on strong immunosuppressive agents. Hence, it is advisable for SLE patients to avoid contact with any host with such an infection. Furthermore, the diagnosis of meningitis can be challenging because SLE itself can present with wide complexity of CNS symptoms. This patient already had a history of lupus cerebritis which further complicated the diagnosis. A CSF study is crucial to identifying the etiology. The presence of pleocytosis with a cell count typically between 100 and 300 cells per mm3, with predominance of lymphocytes can indicate the possibility of lupus cerebritis, with positive neuron-reactive autoantibodies or lymphocytotoxic antibodies. Viral meningitis can be identified by the CSF cell count with predominance of lymphocytes and viral PCR. The concomitant skin lesion and viral culture of the lesion further facilitated the diagnosis. Both the viral culture from her rash and the lumbar puncture were essential in differentiating infectious causes of her symptoms from those due to SLE. Prompt diagnosis and treatment led to her successful recovery.

AN UNUSUAL PRESENTATION OF SECONDARY SYPHILIS Joseph Delio ${ }^{2}$; Heather Hopkins ${ }^{3}$; Jessica Logan ${ }^{1} .{ }^{1}$ DC VA Medical Center, Washington, DC; ${ }^{2}$ The George Washington University, Washington, DC; ${ }^{3}$ Georgetown University, Washington, DC. (Control ID \#2437811)

LEARNING OBJECTIVE \#1: Recognize an unusual presentation of syphilis LEARNING OBJECTIVE \#2: Manage secondary syphilis

CASE: A 66-year-old male with chronic systolic heart failure and hypertension presented with 2 weeks of painless pustular lesions on his face and scrotum. HIV antibody was 
negative. He was prescribed Clindamycin and referred to Dermatology. A biopsy of the left cheek revealed "subacute dermatitis with reactive epithelial hyperplasia and spirochetes" with a serum RPR of 1:64 and a positive FTA-abs consistent with pustular secondary syphilis. Given concern for possible tertiary syphilis, he was referred for hospital admission. At that time, he endorsed visual floaters, but denied any mental status changes, numbness, tingling, photophobia, headache, fever or chills. His exam was significant for multiple hyperpigmented, erythematous papules and pustules, some with central umbilication and a yellow discharge on his cheeks, glabella, nose, right postauricular scalp and occipital scalp. Similar appearing lesions were also noted on his bilateral medial thighs and scrotum. A lumbar puncture was significant for a WBC count of 7, but was not reactive for VDRL. Ophthalmologic exam showed no ocular involvement. Given his CSF leukocytosis, the infectious disease team opted for treatment with IV Penicillin for 14 days with near complete resolution of his symptoms.

DISCUSSION: In recent years, syphilis has been on the rise in the United States. In 2014, 19,999 new cases of primary and secondary syphilis were reported. This translates to 6.3 cases per 100,000 in 2014 as compared to 5.5 cases per 100,000 in 2013 [7]. Despite disease prevalence, syphilis often goes untreated for 20 or more years and approximately $15 \%$ of untreated cases will advance to late stage syphilis. Therefore, recognizing and adequately treating syphilis is paramount $[2,3]$. The primary stage of syphilis presents as a single, painless chancre which lasts between 3 and 6 weeks and heals irrespective of treatment. If primary syphilis is not recognized, it will progress to secondary syphilis. This stage is characterized by a rough, reddish brown rash involving the palms and soles and mild constitutional symptoms. Pustular syphilis is rare, with less than $2 \%$ of all secondary syphilis cases presenting this way $[1,4]$. It is characterized by pustules which may be "follicular, perifollicular or associated with other forms of secondary syphilis such as framboesiform, papulosquamous and ulcerated [4]." According to Aggarwal, et al., the pustules can further be divided based on appearance into several categories including accuminate pustular syphilid and pustuloulcerative syphiloderm [4]. Given the diverse appearance of the pustules, the initial differential diagnosis remains broad and includes HSV, folliculitis, disseminated gonococcemia, hidradenitis suppurativa, psoriasis, and medication side effects. The diagnosis of secondary syphilis can be challenging, but is vital, because if left untreated, tertiary syphilis may develop. The symptoms of tertiary syphilis vary widely, but most commonly involve the central nervous and ocular systems, which can have dire consequences. Currently, there are no comparative trials have been adequately conducted to guide the selection of an optimal penicillin regimen for secondary syphilis. Treatment is largely based upon expert opinion and uncontrolled trials. The CDC recommends Penicillin (PCN) G as the first-line treatment for all stages of syphilis, including pustular syphilis [5]. Specifically, secondary syphilis is treated with a single dose of PCN G. However, in one study by Fiumara, 165 patients with secondary syphilis were treated with PCN and 39 were alternatively treated with Tetracycline due to a PCN allergy. Within 24 months, all patients were cured clinically with seronegativity [8]. Late latent syphilis (or that of unknown duration) is treated with 3 doses of Penicillin G, each dose administered a week apart. If the CNS or ocular systems are involved, treatment is recommended with 18-24 million units/day for 10-14 days [5, 6]. Syphilis is being encountered with increasing frequency in health care settings, but the myriad of presentations, including pustular syphilis, often makes diagnosis difficult. However, given the progressive nature of syphilis, it is imperative to promptly recognize and effectively treat the infection.

\section{AN UNUSUAL SUSPECT FOR ACUTE KIDNEY INJURY IN AN END-STAGE} LIVER DISEASE PATIENT: CRYOGLOBULINEMIC GLOMERULONEPHRI-

TIS Bharat Rao; Megan Franzo-Romain; Kevin S. Fay; Kevin Whitlow; Zarina Alam. Henry Ford Hospital, Detroit, MI. (Control ID \#2466628)
LEARNING OBJECTIVE \#1: Show how a less common etiology for acute kidney injury in an end-stage liver patient may be overlooked

LEARNING OBJECTIVE \#2: Diagnosis of membranoproliferative glomerulonephritis secondary to mixed cryoglobulinemia.

CASE: A 63-year-old Caucasian male presented with a 2 day history of swelling, pain, and redness of his left upper extremity after an intravenous access was placed in preparation for a liver transplant. Surgery was aborted after the donor liver was not suitable for transplantation. He had end-stage liver disease (ESLD) from hepatitis C virus (HCV) along with a 10-year history of non-insulin dependent diabetes. In addition, he had baseline chronic kidney disease (creatinine of $1.8 \mathrm{mg} / \mathrm{dL}$ with estimate glomerular filtration rate of $31 \mathrm{~mL} / \mathrm{min}$ ). However, he did not undergo extensive work-up for a definitive etiology prior to transplant. On examination, the patient had stable vital signs. He had grade I encephalopathy. He was jaundiced with ascites and lower extremity edema. His hand through forearm was erythematous, warm, tender, and swollen. His Model for End-stage Liver Disease (MELD) score was 31 (creatinine $1.96 \mathrm{mg} / \mathrm{dL}$, bilirubin $7.4 \mathrm{mg} /$ $\mathrm{dL}$, and INR 2.48). Other laboratory values revealed a white blood cell count of $1.0 \mathrm{~K} / \mathrm{uL}$ (absolute neutrophil count of $0.58 \mathrm{~K} / \mathrm{uL}$ ), hemoglobin of $7.9 \mathrm{~g} / \mathrm{dL}$, and platelet count of $23 \mathrm{~K} / \mathrm{uL}$. Peritoneal fluid revealed a 49 polymorphonuclear cell count. He was treated for cellulitis with intravenous vancomycin with stable trough levels. His transplant listing was held until further neutropenia evaluation and treatment of infection. Bone marrow biopsy showed normal trilineage hematopoiesis. His pancytopenia was predominantly attributed to $\mathrm{HCV}$ and liver disease with splenomegaly. Moreover, he soon developed acute kidney injury (AKI) with oliguria. He was presumed to have either pre-renal disease or hepatorenal syndrome (HRS). Despite fluid challenges and initiation of albumin, midodrine, and octreotide there was no improvement. Further evaluation revealed microhematuria ( 75 red blood cells per high powered field) on UA and proteinuria (protein to creatine ratio $2.7 \mathrm{~g} / \mathrm{dL}$; albumin to creatinine ratio $1074.5 \mathrm{mg} / \mathrm{g}$ ). Urine sediment revealed dysmorphic red blood cells and casts. Cryoglobulinemic work-up was pursued given his history of HCV. He had an elevated rheumatoid factor (RF) of $339 \mathrm{IU} / \mathrm{mL}$, low complements (C3 was $32 \mathrm{mg} / \mathrm{d}$ and $5 \mathrm{mg} / \mathrm{dL}$ ), $\mathrm{HCV}$ titre $(47,177 \mathrm{IU} / \mathrm{mL}$ ). Cryoglobulinemia screen showed biclonal IgG kappa and IgM lambda. These findings were concerning for membranoproliferative glomerulonephritis (MPGN) from mixed cryoglobulinemia secondary to HCV. His renal function rapidly declined (creatinine $4.39 \mathrm{mg} / \mathrm{dL}$ on day $21 \mathrm{of}$ admission) and he remained oliguric. Given the risk for infection prior to transplant, solumedrol and rituximab were not administered. He was ultimately deemed a suitable candidate for simultaneous liver and kidney transplant and was initiated on dialysis days prior to transplantation. He underwent successful transplant on day 25 of admission. His kidney biopsy revealed fibrillary glomerulonephritis with mesangial and subendothelial deposits consistent with cryoglobulinemic glomerulonephritis. Fivemonths post-transplant his renal function has remained stable and has initiated treatment with ledipasvir/sofosbuvir for treatment of HCV (genotype 1a).

DISCUSSION: Common etiologies for AKI in the ESLD population include pre-renal azotemia, acute tubular necrosis, and HRS. Infections may precipitate any of these etiologies. The patient did have a soft tissue infection, however he did not exhibit significant systemic signs on presentation. In addition, he had ascitic fluid that was not suggestive of SBP. Nonetheless, HRS was presumed as he developed a rapid decline in renal function without improvement after fluid and albumin challenges. Only after further investigation did HRS become less likely as the cause for worsening renal function. His UA and urine protein studies highlight major findings that argued against HRS. In HRS, red blood cells in the urine should be less than 50 per high powered field along with less than $500 \mathrm{mg} /$ day of protein. Moreover, a glomerulonephritis should be excluded in an ESLD patient, particularly those with HCV, prior to making a diagnosis of HRS. MPGN can occur from mixed cryoglobulinemic syndrome secondary HCV. Diagnosis is typically made from clinical findings in combination with hypocomplementemia with low $\mathrm{C} 4$ levels, positive RF, positive HCV serology, and characteristic findings on biopsy. It is 
important to mention that although this syndrome is generally associated with arthralgias, purpura, and neuropathy, this patient did not exhibit any of these features. He did though exhibit the classic renal manifestations of microhematuria, proteinuria, and chronic renal insufficiency. In some instances, as in this patient, a rapid progressive glomerulonephritis may develop. Furthermore, a rapid renal decline is an indication to initiate immunosuppressive therapy along with treatment of the underlying etiology (HCV in this case). However, his case posed a difficult scenario in that he was recovering from an infection, already had neutropenia, and was therefore at high risk for infection prior to transplant. It is important to mention that antiviral therapy was delayed in this case in part due to insurance costs. However, renal function and cryoglobubilinemia was monitored closely while awaiting approval for treatment.

ANCHORS AWEIGH: A CLINICAL EXAMPLE OF ANCHORING BIAS William P. Nichols; Jessica S. Lavender; Yanglin Guo; Denise L. Harkins. University of Mississippi Medical Center, Jackson, MS. (Control ID \#2457417)

LEARNING OBJECTIVE \#1: Recognize situations in which anchoring may impede a physician's ability to properly diagnose and treat a patient

LEARNING OBJECTIVE \#2: Diagnose Infective Endocarditis despite highly variable presentations

CASE: Anchoring is a cognitive bias that effects physicians on a very regular basis. It refers to the tendency to focus too heavily on the initial information presented, thus obscuring one's ability to consider alternative possibilities. This clinical vignette discusses a patient who presented with what seemed like an obvious diagnosis, but ultimately turned out to be much more. A 45 year-old man who has avoided doctors his whole life is found by his wife to be completely unresponsive. Upon arrival to the emergency room, physicians learn of the patient's history of heavy alcohol abuse. His wife also reports that he has been complaining of headaches and self-medicating with dangerously excessive amount of acetaminophen and aspirin while continuing to drink alcohol. On exam, He is obtunded but responsive to noxious stimuli. He is also jaundiced and has an obvious systolic murmur which is loudest at the apex. His initial labs reveal an indirect hyperbilirubinemia and mildly elevated transaminases. Toxicology is consulted for high suspicion of acet aminophen and aspirin toxicity. He receives $\mathrm{N}$-acetylcysteine while in the emergency room. Computed tomography (CT) of the abdomen is surprisingly normal except for a renal infarct of indeterminate age. His renal function appears unaffected. He is admitted to a medicine service with the diagnosis of drug-induced liver injury with hepatic encephalopathy. Not long after admission, his acetaminophen, salicylate, and ammonia levels all return as normal. This revelation prompts a drastic redirection of diagnostic approach. A repeat physical exam reveals possible antecubital "track-marks", but the patient's family avidly denies any history of intravenous drug use. No signs of embolic phenomena are appreciated. An urgent CT of the head reveals a large stroke in the left middle cerebral artery territory. Suspicions of an embolic source of the stroke are confirmed when blood cultures grow Streptococcus pneumoniae and an echocardiogram displays an impressively large $2 \mathrm{~cm} \times 3 \mathrm{~cm}$ mitral vegetation.

DISCUSSION: Infective Endocarditis (IE) is a very common infectious process seen in the United States, and its prevalence is steadily rising. Risk factors such as heart disease and intravenous drug use make IE a particularly frequent diagnosis in Mississippi. Despite this disease being well-studied and usually straightforward to detect, presentations are highly variable which can easily distort the clinical picture and delay a diagnosis. As with all diseases, there are difficulties associated with diagnosis; however, occasionally a physician's own thought process can create the most obstacles. With every patient encounter, physicians are tasked with determining the correct diagnosis while sifting through a myriad of information that can easily lead a doctor astray. The apparent simplicity of a patient's presentation can oftentimes cause a physician to inappropriately trust his or her initial diagnosis without fully evaluating other possibilities. While hepatic toxicity could have explained plenty of the findings in this case, the murmur, the indirect hyperbilirubinemia, and the renal infarct should have raised concern for IE earlier. The lessons learned from this case will hopefully lead to more rapid identification of IE in patients with unusual presentations while also serving as a reminder to avoid a myopic approach to patient assessments.

\section{ANOMIC APHASIA: AN UNCOMMON PRESENTATION OF OTOGENIC} TEMPORAL LOBE ABSCESS Mridula Krishnan ${ }^{2}$; Felicia Ratnaraj ${ }^{2}$; Manasa Velagapudi ${ }^{1}$; Renuga Vivekanandan ${ }^{1} .{ }^{1}$ Creighton Medical center, Omaha, NE; ${ }^{2}$ Creighton University, Omaha, NE. (Control ID \#2469457)

LEARNING OBJECTIVE \#1: Temporal lobe abscess is a rare, life threatening complication of inflammation of the middle ear. Immediate surgical intervention is mandated. In $55-100 \%$ of the cases, it is preceded by chronic middle ear infection.

CASE: A 73 year old male with no significant past medical history presented with drainage and swelling in the left ear canal concerning for otitis externa. He was treated with ofloxacin ear drops and oral ciprofloxacin and was informed to remove his hearing aids. Few days later, he presented with sudden onset anomic aphasia and amnesia. MRI brain with contrast showed a $3.3 \mathrm{~cm}$ left temporal lobe abscess with mastoidits. An emergent neurosurgical drainage of the abscess and craniotomy was performed. The following day, he had left myringotomy and tube placement as the left ear mastoiditis was the most likely source contributing to the brain abscess. Cultures from the abscess yielded Pseudomonas aeruginosa and coagulase negative Staphylococcus aureus. He was discharged on an 8 week course of Vancomycin and Cefepime with improvement of his neurological symptoms during the course of his treatment.

DISCUSSION: Intracranial complications (ICC) such as meningitis and temporal lobe abscess from chronic and acute otitis media are life threatening. Due to the recent development in antibiotics for otitis media, incidence of ICC's have significantly reduced. Some patients with temporal abscess may have symptoms of raised intracranial tension or focal neurological deficits at the time of presentation, whereas others may be relatively asymptomatic. Due to the possibility of otogenic complications of brain abscess such as loss of hearing, management includes a multidisciplinary approach with surgery including aspiration through burr hole or formal craniotomy, open drainage, or total excision along with parenteral antibiotic treatment. In conclusion, even subtle neurological presentations such as amnesia in patients with chronic otitis media should raise suspicion for an otogenic brain abscess.

ANTIBIOTICS OVER STEROIDS Rehaan Shaffie; Yelena Averbukh; Sachin Jain. Montefiore Medical Center, Bronx, NY. (Control ID \#2469437)

LEARNING OBJECTIVE \#1: Expand understanding of erythrocyte sedimentation rate in the setting of decreased fibrinogen.

LEARNING OBJECTIVE \#2: Interpret common autoimmune serologies in the context of infectious endocarditis.

CASE: A 72 year old man was recently hospitalized for peripheral arterial disease requiring bilateral popliteal endarterectomies. Following discharge to a nursing home, he developed a urinary tract infection and was treated with trimethoprim/sulfamethoxazole. Soon after, lab results showed worsening renal function and rising leukocytosis, so he was readmitted. He quickly decompensated with a deep vein thrombosis, acute stroke, and splenic infarct. His labs showed renal failure $(2.9 \mathrm{mg} / \mathrm{dL})$, anemia $(8.3 \mathrm{~g} / \mathrm{dL})$, thrombocytopenia $(38,000$ / microL), leukocytosis (18,700/microL), elevated INR (5.9), and low fibrinogen ( $<70 \mathrm{mg}$ / dL). Given his disseminated intravascular coagulation (DIC) and evidence of arterial and 
venous clotting, a hematologic workup was pursued with concern for catastrophic antiphospholipid antibody syndrome (CAPS). He had a positive antinuclear antibody (ANA), positive lupus anticoagulant, and negative antiphospholipid antibody. His erythrocyte sedimentation rate (ESR) was $4 \mathrm{~mm} / \mathrm{h}$ (normal) and c-reactive protein (CRP) was $4.4 \mathrm{mg} / \mathrm{dL}$ (high). His blood cultures and transthoracic echocardiogram were negative. The differential included CAPS, non-infectious endocarditis and culture-negative infectious endocarditis. Given the autoimmune findings and negative infectious workup, the primary and Rheumatologic teams discussed using steroids to treat CAPS. However, based on further collaboration with the Infectious Disease team and based on strong clinical suspicion for endocarditis with embolic complications, the patient was continued on broad spectrum antibiotics. Further workup with a transesophageal echocardiogram showed two large vegetations on the mitral valve leaflets and a smaller vegetation on the aortic leaflet. The patient's treatment was focused on culture-negative infectious endocarditis, and he was treated with an extended course of antibiotics.

DISCUSSION: In the western world, the incidence of infectious endocarditis is between 1.7 and 6.2 cases per 100,000 individuals. Of these cases, between 2 and $7 \%$ will be culture negative, with about $50 \%$ of all cases receiving antibiotics prior to blood cultures. Interestingly, in patients with endocarditis and DIC, ESR levels may be decreased due to low levels of fibrinogen, which is responsible for red cell agglutination. Non-agglutinated erythrocytes settle more slowly, decreasing the ESR. In addition, endocarditis causes an elevation in autoimmune markers. Though literature review shows this phenomenon is well-documented, a more specific pathophysiology than widespread immune activation remains unclear. Patients without any history of autoimmune or rheumatologic disease with infective endocarditis may have: positive ANA, anti-neutrophil cytoplasmic antibody (ANCA), cardiolipin, lupus anticoagulant, and/or rheumatoid factor (RF), among others. However, though these markers are present during active infection, they resolve after successful treatment of infectious endocarditis. At best, these markers should be considered clinically insignificant, and, at worst, can be misleading diagnostically and therapeutically, with potentially life-threatening consequences. However, there is some evidence to support the idea that antiphospholipid antibodies promote endothelial cell activation and thrombin generation, leading to increased embolic events. In summary, understanding these irregularities in laboratory findings such as negative blood cultures, low ESR and positive serologies for common rheumatologic diseases can help lead to the faster and safer diagnosis and therapy of culture negative infectious endocarditis.

AORTIC RUPTURE AS A RARE COMPLICATION OF MANUAL CARDIOPULMONARY RESUSCITATION Prashanth Venkatesh; Edward Schenck. New YorkPresbyterian Hospital/ Weill Cornell Medical Center, New York, NY. (Control ID \#2469684)

LEARNING OBJECTIVE \#1: Recognize aortic rupture as a rare but potentially lethal complication of manual cardiopulmonary resuscitation

LEARNING OBJECTIVE \#2: Diagnose acute aortic rupture clinically in a hemodynamically unstable patient in the immediate aftermath of return of spontaneous circulation after cardopulmonary resuscitation

CASE: A 78-year-old woman with history of coronary artery disease, hypertension and a chronic smoking presented with acute on chronic intermittent left sided chest pain and spitting up small amounts of blood for 1 week. She also had a history of gastro-esophageal reflux, CREST syndrome and Barrett's esophagus, with periodic chest pain occurring during worsening of reflux disease. A chest radiograph revealed an enlarged aortic knob and subsequent CT angiogram of the chest showed a penetrating atherosclerotic ulcer (PAU) with associated pseudoaneurysm arising from the aortic arch, a PAU in the descending thoracic aorta and several aortic calcifications. Also found was a dilated patulous esophagus with air-fluid levels suggestive of obstruction at the gastro- esophageal junction. An esophagoduodenoscopy showed retained food and highly friable mucosa, thought to be the likely cause of her spitting up blood. She was discharged with instructions for strict outpatient blood pressure control and cardiothoracic surgery followup in case her chest pain worsened. She returned 1 week later with fatigue, fevers, chest pain and hemoptysis, and was found on exam to be hypotensive, tachycardic and febrile. Workup revealed unchanged mediastinal width and marked pyuria with Escherichia coli in blood and urine cultures. She was started on antibiotics and given successful isotonic fluid resuscitation. Approximately $24 \mathrm{~h}$ into admission, she had a witnessed aspiration event when eating and promptly went into hypoxic respiratory failure with subsequent loss of pulse. A total of four rounds of high-quality manual cardiopulmonary resuscitation (CPR) were performed with subsequent return of spontaneous circulation (ROSC). Esophageal intubation was performed during CPR and was promptly rectified. Immediately after ROSC the patient was hypotensive to $50 / 30 \mathrm{mmHg}$ with new large ecchymosis in the upper chest and neck with associated crepitus. Considerations at this point included catastrophic pseudoaneurysm and/or myocardial rupture, pneumothorax, mediastinal hemorrhage and esophageal rupture from intubation or chest wall trauma. Chest X-ray showed interval widening of the mediastinum since admission. An emergent thoracic CT angiogram revealed acute rupture of the previously seen pseudoaneurysm and a large mediastinal hematoma. Due to the high mortality associated with attempted repair of this lesion given co-morbidities of sepsis, coronary disease, age, poor functional status and renal insufficiency, the patient was made comfort care in consultation with the family and supported with vasopressors and blood transfusions. Terminal extubation was subsequently performed, resulting in rapid respiratory arrest and death likely due to tracheal compression from the surrounding hematoma.

DISCUSSION: Closed chest cardiac massage is well known to cause a multitude of iatrogenic injuries, with skeletal injury being by far the most common. Direct injury to the myocardium and great vessels is exceedingly rare and has been reported sporadically with incidence rates up to $1.7 \%$. The spectrum ranges from aortic dissection and intramural hematoma to full thickness atrial and ventricular perforation. Rupture of the thoracic aorta has been reported in large series with prevalence of up to $1 \%$. Very few case reports have described this complication in detail; the ones that do report it only as a finding on autopsy. All these cases have involved multiple concomitant rib and sternal fractures thought to have caused penetrating trauma. Some of these occurred with the use of mechanical compression devices, which have been shown to increase the risk of CPR-associated injury. Importantly, none of the cases reported in the literature to our knowledge had any evidence of pre-existing aortopathy at the site of rupture. Our case is unique in that it illustrates a diagnosis of aortic rupture made in the clinical setting immediately after CPR, with a distinct aortic lesion serving as a nidus for rupture. Mechanical compression was not utilized, nor was there any evidence of sternal or rib fracture on imaging. The rupture likely occurred due to sudden, explosive increase in velocity of blood flowing through the aorta and the consequent increase in shear stress against an already weakened aortic wall at the site of the PAU and pseudoaneurysm. Given that the patient's chest pain was a chronic complaint related to esophagitis, the decision to not intervene on the pseudoaneurysm and to instead consider elective operative management with tight blood pressure control in the interim was in accordance with current practice guidelines. The cardiac arrest and need for CPR was an unforeseen event and in this case the sentinel event of aspiration was ultimately unavoidable. This case also highlights the need to maintain a high index of suspicion for potentially lethal intrathoracic sequelae in the face of clinical instability immediately after CPR. In suitable surgical candidates unlike our patient, early diagnosis of such complications as aortic rupture could lead to lifesaving emergent surgical intervention.

AS CLEAN AS THE COW THAT CALVES: A CASE OF LEUKOCYTOSIS

Rachel Hammer; Holly Gonzales; Deepa Bhatnagar. Tulane University Health Sciences Center, New Orleans, LA. (Control ID \#2468890) 
LEARNING OBJECTIVE \#1: Discuss campylobacter as a cause of diarrhea. LEARNING OBJECTIVE \#2: Learn treatment options for campylobacter bacteremia

CASE: A 26 year-old man with past medical history of chronic myelogenous leukemia (CML) and autologous stem cell transplant 1 year ago was referred from an outside hospital for leukocytosis. He endorsed 2 weeks of fever, chills, nausea, sore throat, and mild episodic diarrhea. Review of systems revealed decreased appetite and a $20 \mathrm{lb}$. weight loss over 2 months. History was significant for CML diagnosed 3 years ago during a blast crisis and he was thought to be in remission while on dasatinib. He lives on a farm in a Southern rural area, has a dog, and tends livestock including cattle. One of his cows recently birthed. The patient was afebrile with normal vital signs. Physical exam revealed a slender man with splenomegaly. Labs showed a white count of 30.2 with $53 \%$ neutrophils, $18 \%$ immature neutrophils, $11 \%$ lymphocytes, $2 \%$ blasts, and $11 \%$ basophils. He otherwise had an anemia with hemoglobin 9.7 and thrombocytopenia with platelets of 63 . Initial infectious work-up was non-revealing and he was admitted with concern for an evolving blast crisis. On hospital day 4, while awaiting bone marrow biopsy results, the patient spiked a fever. Blood cultures obtained at that time grew spiral shaped rods and stool culture grew Campylobacter jejuni. The patient was started on ciprofloxacin and completed 2 weeks with resolution of his symptoms and leukocytosis. DISCUSSION: Campylobacter is one of the most common causes of infectious diarrhea in the United States typically contracted from ingestion of unpasteurized dairy products, contaminated water, poultry, and produce. As in the case of our patient, people in close contact with farm animals are at higher risk. Bacteremia due to Campylobacter species is rare. Less than $1 \%$ of those with campylobacter gastroenteritis contract blood stream infection; however there is increased prevalence during summer months and patients are at greater risk if they are immunocompromised. Interestingly, most cases of bacteremia do not present with gastrointestinal symptoms. Campylobacter bacteremia has been associated with fatal outcomes and mortality has been cited as high as $16 \%$. Risk of fatality appears to vary by the species, with Campylobacter fetus being the most virulent. Most Campylobacter diarrheal illnesses, unless severe, resolve spontaneously. For severe cases, or for immunocompromised patients, standard treatment is 2 weeks of azithromycin or fluoroquinolone. If the source is likely to be a farm animal, note the growing prevalence of fluoroquinolone resistance due to the use of quinolones in livestock. In patients with leukemia, bacteremia can cause a leukocytosis that mimics early blast crises. Thorough infectious work-up is required to determine the cause of leukocytosis in these cases. Though rare, Campylobacter jejuni bacteremia can cause minimally symptomatic infections in immunocompromised patients, leading to high mortality rates if not recognized and treated.

ASCITES IN A PATIENT WITH GRAVES' DISEASE Mahreen Arshad. The Baptist Health System, Homewood, AL. (Control ID \#2470335)

LEARNING OBJECTIVE \#1: Assess patients with ascites for thyroid dysfunction LEARNING OBJECTIVE \#2: Recognize the need for close follow-up for patients with thyroid suppression therapy

CASE: A 46 year-old African American female with a past medical history of hypertension and Graves' disease presented to the hospital for a 3 week history of worsening abdominal distention. She had a similar episode in April 2015, during which time she was admitted to an outside hospital and treated with Lasix and prednisone. At this presentation, approximately 1 month after her initial episode, patient reported that her episode of abdominal distention was significantly worse than her previous episode. Two weeks before her presentation to the hospital the patient had been seen by her endocrinologist and was told that her thyroid stimulating hormone level was low. Patient had been on methimazole therapy for many years for her Graves' disease and her dose was decreased due to her low thyroid function test levels. Patient's physical exam was normal except for severe tense abdominal distention, a positive fluid wave, and flank dullness to percussion. Laboratory studies showed an elevated thyroid stimulating hormone test with a low free thyroxine. CT of the abdomen showed a large amount of ascites but was otherwise negative. Ultrasound doppler of the abdomen was also negative for any thrombus. With the exception of a thickened endometrium, a pelvic ultrasound, ordered to rule out any ovarian abnormalities, was negative. A PPD test was placed on the patient but was negative. During her hospital stay the patient required two paracenteses. The first paracentesis removed three liters of straw yellow fluid and the patient reported a lot of relief of her abdominal pressure. The fluid quickly re-accumulated and patient required paracentesis again the following day. Cytology was negative for malignancy on both fluid samples. A serum ascites albumin gradient was calculated and indicated a non-portal hypertension related etiology for her ascites. After reviewing the work-up, the cause for patient's ascites was determined to be secondary to her hypothyroidism from methimazole. The patient was started on a low dose of levothyroxine and her symptoms resolved. She was seen briefly 1 week later and was doing well with no reoccurrence of ascites.

DISCUSSION: Ascites can be a complication of various diseases including multiple cardiac, hepatic, and renal processes but is seen less so in endocrinology. It is well known that ascites can be a manifestation of hypothyroidism but this is usually not the presenting symptom. Frank ascites secondary to hypothyroidism is a rare phenomenon. In most of the cases of fluid retention with thyroid disease patients develop accumulations in the pleural cavity and ascites is a less common presentation. Many case reports have noted ascites due to myxedema but ascites due to a short duration of hypothyroidism is a rare finding. The pathophysiology of the mechanism of ascites caused by hypothyroidism is not well understood but there are a few hypotheses. One of the hypothesis is that hypothyroidism causes an abnormally high levels of capillary leak with a lack of compensatory mechanisms that increase the occurrence of ascites. Another theory attributes it to obstruction of lymphatic flow by hyaluronidase-albumin complexes. Lastly, some also believe that there is a hygroscopic swelling effect due to the accumulation of hyaluronic acid. Although hypothyroidism is a rare cause of frank ascites it is important to include it in the working differential diagnosis, especially if patients have known thyroid dysfunction. For patients on thyroid suppressive medication and/or ablation therapy it is also necessary to have close follow-up in order to identify those who need replacement therapy. All patients that present with ascites should have blood testing to identify thyroid hormone level abnormalities.

ASPERGILLUS AORTITIS OF THE ASCENDING AORTA PRESENTING AS FUNGAL ENDOPHTHALMITIS Joseph M. Rocco ${ }^{1}$; Maggie K. Benson ${ }^{2} .{ }^{1}$ University of Pittsburgh, Pittsburgh, PA; ${ }^{2}$ University of Pittsburgh Medical Center, Pittsburgh, PA. (Control ID \#2462409)

LEARNING OBJECTIVE \#1: Evaluate for endogenous infection in the setting of endophthalmitis with no recent corneal trauma or surgery

LEARNING OBJECTIVE \#2: Suspect aspergillosis aortitis in patients with fever of unknown origin within 1-year post-cardiac surgery in the absence of positive blood cultures

CASE: A 76-year old woman with a bioprosthetic aortic valve replacement (AVR) 3months prior, diabetes and remote cataract removal was referred to the ED by her optometrist with acute right eye pain and decreased visual acuity. She denied having fevers, night sweats, dyspnea or chest discomfort, and did not report a history of chemical exposure or trauma to the eye. On exam, she was afebrile, HR 60 and BP 120/59. Her Reye had diffuse conjunctival injection, corneal opacification and a dense vitreous with loss of red reflex. Visual acuity was intact only to hand waving. Ophthalmology evaluated the 
patient in the ED and administered intravitreal antibiotics for a diagnosis of acute endophthalmitis. The patient was admitted due to concern for a systemic source of infection. Her cardiac exam was benign with no murmur. Lungs were clear. There were no stigmata of endocarditis. Initial labs showed a normal WBC count. Blood cultures had a negative gram stain and no immediate growth. ATTE revealed a small mobile vegetation near the aortic valve and thickening of the posterior aortic root. Subsequently, a CT scan revealed a $3.4 \mathrm{~cm} \mathrm{x} 2.25 \mathrm{~cm}$ pseudoaneurysm of the ascending aorta with extensive thrombus extending from the AV to the mid-descending thoracic aorta. The patient was taken for emergent surgery which revealed disruption of the aortotomy suture line with a large thrombus outside and within the aorta. The aortic valve was unaffected and a patch was used to close the opening. Grocott staining of the aorta revealed invasive mycoses and in situ hybridization confirmed the diagnosis of invasive aspergillosis. Systemic and intravitreal Amphotericin-B was administered and IV therapy was continued with voriconazole. Symptoms began to resolve but repeat CT chest was concerning for a new peri-aortic abscess. Patient did not wish to pursue further surgical intervention and was discharged on systemic anti-fungal therapy. Her vision did not return.

DISCUSSION: Both fungal endophthalmitis and aortitis are rare diseases that can be difficult to recognize and are associated with high morbidity and mortality. In our case, the patient presented with an acute red-eye, a common presentation for general internists, for which there is a large differential including benign and emergent etiologies. Identifying red flag signs, such as severe pain, decreased visual acuity and pupil abnormalities can indicate ophthalmologic emergencies such as keratitis, uveitis, angle-closure glaucoma or endophthalmitis which require emergent evaluation. A detailed review of systems can identify underlying systemic diseases, while other findings have classic associations, such as hypopyon with endophthalmitis. Our patient had several red flag symptoms which led to prompt referral and rapid diagnosis. Endophthalmitis is an intraocular infection affecting the inner coats of the eye leading to progressive vitreous inflammation. It classically occurs in two forms, exogenous or endogenous. Endogenous endophthalmitis is rare, occurring in only $6-12 \%$ of cases, with fungal etiologies being the most common worldwide. In developed countries, the most common organisms are bacteria such as Staph and Strep, but GNRs can also be identified. Evaluation for systemic infection is vital, with the most common etiologies including endocarditis and liver abscess. Recognizing risk factors such as prosthetic heart valves, diabetes, IV drug use and indwelling catheters is important. Fungal infections are often associated with immunocompromised states. Identifying the causative organism is crucial as intravitreal and systemic therapies are often merited. Vitrectomy has been shown to have a higher diagnostic yield compared to aspiration and can be therapeutic. PCR is highly effective but cannot provide antibiotic sensitivities. Without a clear causative organism, empiric intravitreal therapy should be administered with vancomycin/ceftazidime for bacteria and amphotericin-B when fungal etiologies are suspected. Unfortunately for our patient, a causative organism was not rapidly identified. A search for systemic infection revealed an aortic pseudoaneurysm with evidence of aspergillus on pathology. Disseminated aspergillosis often occurs in the setting of immunosuppression, however there are several case reports identifying aspergillus aortitis in immunocompetent patients after cardiac surgery. One series, including 188 cases of post-cardiac surgery aspergillosis, identified the highest proportion of infections after aortic valve surgery. The most common presentation was nonspecific fever or signs of embolic disease, usually presenting 3-months post-surgery. Blood cultures were often low yield and an ante-mortem diagnosis was made in less-than $50 \%$ of cases. TEE was useful in confirming the diagnosis and provides better visualization of the aortic root than TTE if it can be performed rapidly. However, some cases noted normal TEEs, thus CT angiography is considered the gold standard. The mortality was exceptionally high, ranging from 93 to $100 \%$ and emergent intervention with surgical repair and systemic anti-fungal therapy is required. Due to the high mortality, the optimal duration of therapy is unknown and should be continued indefinitely.
ATYPICAL HUS IN THE HIV PATIENT: A DIAGNOSTIC AND THERAPEUTIC CHALlenge Kara Dupuy ${ }^{2}$, Levent Sipahi ${ }^{1}$; Jailan Elayoubi ${ }^{2}$; Cecilia Big ${ }^{2}$. ${ }^{1}$ Wayne State University School of Medicine, Detroit, MI; ${ }^{2}$ Beaumont Hospital Dearborn, Dearborn, MI. (Control ID \#2470446)

LEARNING OBJECTIVE \#1: To describe the complexities of diagnosis and treatment of aHUS in a patient concurrently being treated for HIV.

LEARNING OBJECTIVE \#2: 1) To provide a case of aHUS treated with eculizumab. CASE: A 31-year-old HIV-positive woman with a history of prior disseminated histoplasmosis presented with hemolytic anemia, thrombocytopenia, and acute kidney injury. Workup showed elevated LDH, decreased haptoglobin and schistocytes on peripheral smear, which led to a diagnosis of TTP. Histoplasma urine antigen was elevated so liposomal amphotericin B was started as treatment for reactivated histoplasma infection. The patient had 10 sessions of plasma exchange with slow improvement of platelet count, but no improvement in kidney function. In that time the ADAMTS13 test had returned and was in the normal range. Given the incomplete response to plasma exchange and normal ADAMTS13, TTP was ruled out as the cause of the renal injury and greater consideration was given to other diagnoses including aHUS, HIV-associated nephropathy, histoplasmaassociated granulomatous nephropathy, and amphotericin-induced nephrotoxicity. Since these entities are indistinguishable clinically, and in light of the patient's acutely worsening renal function, kidney biopsy was performed and demonstrated thrombotic microangiopathy consistent with aHUS/TTP. Since the response to plasma exchange was inadequate and the ADAMTS13 level was normal, TTP was effectively ruled out and we diagnosed the patient with atypical HUS and commenced therapy with the terminal complement inhibitor, eculizumab. After the first infusion, the patient's kidney function began to improve.

DISCUSSION: Atypical hemolytic uremic syndrome (aHUS) is a rare disease characterized by microangiopathic hemolytic anemia, thrombocytopenia, and acute kidney injury, which is related to complement dysfunction and carries a $50 \%$ risk of progression to endstage renal failure (ESRF). The link between HIV and microangiopathic hemolytic anemia including Thrombotic Thrombocytopenic Purpura (TTP) and aHUS has been clearly established but distinguishing between both entities as a cause of renal failure can be challenging due to overlapping clinical and laboratory findings. Furthermore, in HIV patients with multiple comorbidities, it is challenging to elucidate the underlying cause of any kidney injury based on clinical presentation and without a kidney biopsy. We focus here on the diagnosis and treatment of aHUS in the HIV patient and provide specific take-home points: 1 . The algorithm of diagnosis of thrombotic microangiopathy and importance of having a high index of suspicion of aHUS in case of inadequate response to plasma exchange, 2. The importance of obtaining a kidney biopsy in a timely manner in HIV patients with multiple potential causes of nephropathy to further guide therapy, 3 . The effect of eculizumab on immunity in the setting of an HIV patient with active fungal infection, which is a unique situation that we did not encounter any reports of in the literature.

ATYPICAL HUS, AN ATYPICAL CAUSE OF HUS Angeline J. Sabol. Mayo Clinic, Rochester, MN. (Control ID \#2468825)

LEARNING OBJECTIVE \#1: Evaluation of atypical hemolytic uremic syndrome (HUS).

LEARNING OBJECTIVE \#2: Assess treatment options for atypical HUS

CASE: A 29 year-old female was admitted for combined liver-kidney transplantation for end stage kidney disease secondary to atypical (aHUS) due to a complement factor $\mathrm{H}$ splice variant. Approximately 7 years prior to her presentation, she had an unremarkable medical history until she developed a flu-like illness with no associated diarrhea that progressed to renal failure and presumed thrombotic thrombocytopenic purpura. She developed end stage renal disease (ESRD) and remained on dialysis until 3 years later 
when she received a living related donor allograft from her sister. Her allograft remained well-functioning for 2 years until she again developed upper respiratory tract symptoms and was found to be in acute renal failure. Renal biopsy demonstrated thrombotic microangiopathy and transplant glomerulopathy and she was re-initiated on hemodialysis. She was referred to a tertiary center for consideration of a repeat renal transplant. As there was confusion regarding the exact cause of the graft failure, she was evaluated by hematology for concern of aHUS. Further work up revealed a low-positive hemolytic assay on functional testing as well as a single allele splice site variant in the $\mathrm{CFH}$ (complement factor $\mathrm{H}$ ) gene. The patient's particular splice site variant is predicted to lead to skipping of exon 22 which encodes SCR20 and is therefore predicted to be pathogenic. Due to these findings, her risk of graft failure was predicted to be in the 60 $80 \%$ range. Her options included treatment with eculizumab versus combined liver and kidney transplant. The decision was made for combined transplant as lifelong therapy with eculizumab would lead to a high risk of infection with her history of splenectomy. On postoperative day 1 , an alternative pathway test was obtained and was abnormally low at $21 \%$. This was concerning for continued activation and dysfunction. Repeat testing was obtained a few days after surgery. This result is currently pending and the patient was discharged no longer requiring dialysis.

DISCUSSION: HUS is characterized by renal failure caused by platelet thrombi in the microcirculation of the kidney, hemolytic anemia, and thrombocytopenia (3). Typical HUS is associated with a shiga-toxin producing e. coli, and aHUS is complement mediated and can be genetic, acquired, or idiopathic. Diagnosis is made when two or more family members are affected and an exposure to a common triggering infectious agent is excluded, or one of the ten genes known to be associated with aHUS is identified (3). Evidence demonstrates that $50-60 \%$ of cases are associated with genetic alterations of the complement system (3). The complement system's excessive activation leads to direct damage of cell membranes (2). Of these, the most common alteration is that of the CHF gene, which is involved in the alternative complement pathway (3). In normal endothelial cells, $\mathrm{CFH}$ binds to the surface and to $\mathrm{C} 3 \mathrm{~b}$, and together with membrane cofactor protein, acts as a cofactor for $\mathrm{C} 3 \mathrm{~b}$ cleavage (2). $\mathrm{CFH}$ also dissociates $\mathrm{C} 3$ convertase. Therefore, the genetic alteration leads to insufficien C3b degradation and uncontrolled complement activation. When a case of aHUS is suspected, a panel approach is used to screen for a complement disorder, using a combination of functional and antigenic testing. Both the classic and alternative pathways can reveal if complement activation has consumed certain factors. It is important to note that consumption can also be present in the setting of infection. Therefore quantification of the components and their split products allows one to determine if the factor is reduced because of increased consumption or reduced production (4). After a positive screen, identification of a genetic mutation is needed to confirm the diagnosis. Recently methodologies have advanced to high output methods, such as next generation sequencing. Despite this, Sanger sequencing is still the mainstay of analysis in most laboratories, and is still used on a supplemental basis due to the presence of highly homologous regions which can lead to both false-positive and falsenegative variant results (5). When the diagnosis is made, eculizumab, an anti-C5 monoclonal antibody, is recommended (1). However, eculizumab is very expensive and leads to high incidence of infection. Liver transplant has been known to be curative when a mutation is identified, as the complement factors are produced in the liver. In cases of aHUS, diagnosis is imperative, leading to multiple therapeutic options. References: 1. Coppo, Rosanna, et al., Liver Transplantation for aHUS: Still Needed in the Eculizumab Era? Pediatr Nephrol. Nov 24, 2015. 2. Franchini, Massimo. A typical hemolytic uremic syndrome: from diagnosis to treatment. Clin Chem Lab Med 2015; 53 (11):1679-1688. 3. Noris, Marina, PhD., et al. Atypical Hemolytic-Uremic Syndrome. GeneReviews. Nov 16, 2007 4. Prohaszka, Z, et al., The use of 'real-time' complement analysis to differentiate atypical haemolytic uraemic syndrome from other forms of thrombotic microangiopathies. British journal of haematology. 2012;158:424 425. 5. Rehm, HL, et al., ACMG clinical laboratory standards for next-generation sequencing. Genetics in medicine : Official journal of the American College of Medical Genetics. 2013;15:733-747.
AUTOIMMUNE HEPATITIS TRIGGERED BY HEPATITIS A VIRUS INFECTION Takehiro Miyazaki ${ }^{1,2}$; Akinori Sasaki ${ }^{1}$; Junji Kinoshita ${ }^{1}$;oru Yamada ${ }^{2}{ }^{1}$; Eiji Hiraoka ${ }^{2}{ }^{1}$ Tokyo Bay Medical center, Urayasu, Japan; ${ }^{2}$ Tokyo Bay Urayasu Ichikawa Medical Center, Urayasu, Japan. (Control ID \#2466102)

LEARNING OBJECTIVE \#1: Autoimmune hepatitis should be taken into consideration in differential diagnosis of recurrence of liver dysfunction after hepatitis A virus infection.

LEARNING OBJECTIVE \#2: Type 1 autoimmune hepatitis with negative antinuclear antibodies (ANA) is not rare. Therefore, it is important to measure not only ANA but also anti-smooth muscle antibody (SMA).

CASE: A 31-year-old Japanese female was in her usual state of good health until 4 months before visiting our hospital, when she felt anorexia with vomiting a few weeks after she ate raw shellfish at a sushi restaurant in Japan. It was not associated with fever, abdominal pain or diarrhea. She visited a local clinic. On examination, skin and conjunctiva were icteric. There was no angioma spider, asterixis or abdominal tenderness. Laboratory test showed AST 411 U/l, ALT 316 U/1, LDH 342 U/1, ALP 381 U/l, $\gamma$ GTP154 U/1, totalbilirubin $4.3 \mathrm{mg} / \mathrm{dl}$, direct-bilirubin $2.7 \mathrm{mg} / \mathrm{dl}$, prothrombin time $40 \%$, ammonia $108 \mu \mathrm{g} /$ $\mathrm{dl}$, and immunoglobulin $\mathrm{G}(\mathrm{IgG}) 2772 \mathrm{mg} / \mathrm{dl}$. Antinuclear antibodies (ANA) and antimitochondrial antibodies (AMA) were negative. She was admitted to a local hospital for further treatment. Then, she was diagnosed with hepatitis A by presence of anti-hepatitis A virus (HAV) IgM antibody. Serology tests for hepatitis B virus (HBV), hepatitis C virus (HCV), Epstein-Barr virus (EBV) and cytomegalovirus virus were negative. A few weeks later, her transaminase levels spontaneously became normal and anti-HAV IgM converted to anti-HAV IgG. Three days after that, her transaminase levels increased again. Then, she was referred to our hospital for further evaluation. She had no symptom at the time of visit. Physical examination revealed normal except icterus in her skin and conjunctiva. Laboratory test showed AST 101 U/1, ALT 88 U/1, LDH 278 U/1, ALP 461 U/1, $\gamma$ GTP118 U/1, total-bilirubin $2.28 \mathrm{mg} / \mathrm{dl}$, and prothrombin time $51 \%$. ANA and AMA were negative. Anti-smooth muscle antibody (SMA) was $\geq 1: 40$. Serum IgG was $3216 \mathrm{mg} / \mathrm{dl}$. Ultrasound showed a hypoechoic liver, no hepatomegaly and normal spleen. A liver biopsy specimen revealed interface hepatitis, a portal lymphoplasmacytic cell infiltrate surrounding the portal triad and percolates into the surrounding lobule, as well as rosettes of hepatocytes. Her simplified diagnosis criteria for autoimmune hepatitis (AIH) were calculated to 7 , which confirmed the diagnosis of definite type $1 \mathrm{AIH}$. We started the prednisone $50 \mathrm{mg}$ daily. She had dramatical improvement in serum aminotransferases, bilirubin, and IgG within a few weeks

DISCUSSION: We reported a case with type $1 \mathrm{AIH}$, developing after HAV infection. On the first transaminase elevation, she was diagnosed with HAV infection based on positive anti-HAV IgM and development of the symptoms a few weeks after eating raw shellfish, which is typical clinical history for hepatitis A. On the second hepatic transaminase elevation, her laboratory data showed positive SMA. She was diagnosed with definite AIH by the simplified diagnosis criteria for autoimmune hepatitis. It was suggested that virus infection, such as $\mathrm{HAV}, \mathrm{HBV}, \mathrm{HCV}$, or EBV infection, may trigger $\mathrm{AIH}$. Clinically, association of HAV infection and $\mathrm{AIH}$ has been observed in case reports and case series. The first case was reported in 1991. In one case series, 58 healthy relatives of 13 patients with type $1 \mathrm{AIH}$ were observed prospectively for 4 years. Three patients developed subclinical hepatitis A, and two of them subsequently developed type $1 \mathrm{AIH}$. It was hypothesized that HAV infection triggers $\mathrm{AIH}$. In our case, the differential diagnosis included not only AIH triggered by acute hepatitis A, but also acute hepatitis A in a patient of previously undiagnosed chronic AIH. Abnormal liver test had never been recognized in annual physical check-up for several years. It is less likely that she had an undiagnosed chronic $\mathrm{AIH}$ previously. Considering the fact that she had recurrence of liver dysfunction and met the simplified diagnosis criteria after recovery from hepatitis $\mathrm{A}$, we believe she developed AIH after HAV infection. In any case, when liver dysfunction recurs 
after hepatitis A infection, AIH should be considered. A case of type 1 AIH with negative ANA is not rare. It has been reported that $15 \%$ of type $1 \mathrm{AIH}$ patients have positive ANA with negative SMA. $35 \%$ of them have positive SMA with negative ANA and $60 \%$ have positive for both SMA and ANA. Both antibodies are negative among $5 \%$ of them. Another study reported that the sensitivity and specificity for ANA was 65 and $75 \%$, respectively. For SMA, the values were 59 and $92 \%$, respectively. Therefore, negative ANA cannot rule out AIH. SMA needs to be checked as well, especially when AIH is suspected and ANA is negative, like our case. In conclusion, AIH should be taken into consideration in a case of recurrence of liver function after HAV infection. Physicians should be alert that ANA negative of type $1 \mathrm{AIH}$ is not rare and checking SMA is important.

BACTERIA IN A HALF SHELL Chellse Gazda; Poppy Markwell; John Moscona. Tulane University Health Sciences Center, New Orleans, LA. (Control ID \#2467665)

LEARNING OBJECTIVE \#1: Recognize the risk factors associated with Vibrio vulnificus infection

LEARNING OBJECTIVE \#2: Diagnose and treat Vibrio Vulnificus infection

CASE: A 51 year-old man, who was unaware of his diagnosis of hepatitis $\mathrm{C}$ or cirrhosis, presented with 4 days of progressive bilateral lower extremity swelling, and new onset of erythema and bullous lesions after doing demolition work. He endorsed associated vomiting and diarrhea, but denied trauma or exposure to oysters, seafood, ocean water, or animals. He was hypotensive, tachycardic, and tachypnic. He had a painless ecchymotic rash on his bilateral legs extending up to the mid-thigh. His left leg had bullae. His extremities were cool, but peripheral pulses were present with dopplar. Initial tests revealed disseminated intravascular coagulation and a metabolic acidosis with a lactate of 18. X-rays and lower extremity ultrasound showed soft tissue edema, but no gas or crepitus. Broad-spectrum antibiotics were started, and blood products were given to temporize the coagulopathy. The patient was taken for emergent surgery due to clinical concern for necrotizing fasciitis. He was found to have extensive soft tissue necrosis from his bilateral feet to mid-thigh. Bilateral above the knee amputations were performed to gain source control. Blood cultures grew Vibrio vulnificus. Despite numerous antibiotics, surgeries, vasopressors, transfusions, the necrotizing fasciitis continued to spread and the rash progressed to his arms. He also developed fulminate hepatic, respiratory, cardiac, and kidney failure and care was withdrawn.

DISCUSSION: Vibrio vulnificus infections can present as wound infections (45\%), primary sepsis $(43 \%)$, or gastroenteritis $(5 \%)$. These infections can occur by eating contaminated seafood or through exposure of a wound to seawater. Vibrio infections are rare but are crucial to recognize because of the high case fatality rate. Patients with chronic liver or kidney disease, diabetes, and cancer are at increased risk of mortality from vibrio infections because of low complement levels, reduced phagocytic and chemotaxis abilities, and portal hypertension which can cause the organism to bypass the reticuloendothelial clearance system. Epidemiological studies have shown that cases are prevalent in the summer months on the Gulf Coast. Symptoms of non-foodborne vibrio can include cellulitis, fever, bullae, muscle pain, nausea, vomiting, and diarrhea. Retrospective analysis of patients with non-foodborne vibrio infections revealed that $12 \%$ had no wound. History of exposure to seafood or seawater can aid in making the diagnosis of vibrio, but this case illustrates that demolition work is also a risk factor. Tetracycline and third generation cephalosporin antibiotics can decrease mortality and the need for debridement. It is important to diagnose necrotizing fasciitis quickly, but this can be extremely challenging if fever, erythema, or bullous skin lesions are not present. The Laboratory Risk Indicator for Necrotizing Fasciitis (LRINEC) was developed in 2004 to help identify patients with necrotizing fasciitis. Serum creatinine, white blood cell count, hemoglobin, sodium, glucose, and C-reactive protein levels can be converted to a LRINEC score. A number greater than or equal to six, has a positive predictive value of $92 \%$ and a negative predictive value of $96 \%$ for necrotizing fasciitis. suggests that the patient should be carefully evaluated for the presence of necrotizing fasciitis. Any scoring system has its limitations and should not replace clinical judgment. Follow up studies, specifically looking at patients with necrotizing fasciitis from vibrio have shown that this model is poorly predictive. The LRINEC score was not predictive of necrotizing fasciitis in our patient. This case reminds the internist to be mindful of Vibrio vulnificus as a cause of wound infections, sepsis, or gastroenteritis. Early identification is essential to assure appropriate antibiotic coverage and possible need for surgical debridement. Vibrio infections are typically associated with exposure to oysters or seawater but may be encountered during demolition or construction work as well. Vibrio is prevalent in the gulf coast region and is associated with a high mortality. Immunocompromised patients should be educated to not consume and to wear gloves when handling raw or undercooked shellfish, to avoid seawater with open wounds or cuts, and to avoid high risk occupations such as demolition work.

BACTRIM MAY DRESS TO KILL Kyrstin Eklund; Salman Raheem; Leslie Cler. Methodist Dallas Medical Center, Dallas, TX. (Control ID \#2437271)

LEARNING OBJECTIVE \#1: Identify the Drug Reaction with Eosinophilia and Systemic Symptoms (DRESS) syndrome as a rare cause of a potentially fatal drug reaction that can be treated through discontinuation of the offending agent or underlying cause. Be able to differentiate DRESS syndrome from other causes of systemic inflammation such as Steven Johnson Syndrome, acute generalized exanthematous pustulosis (AGEP), hypereosinophilic syndromes, Sézary syndrome, and acute cutaneous lupus erythematosus.

\section{LEARNING OBJECTIVE \#2: Manage the treatment of DRESS syndrome.}

CASE: A 44 year old African male with history of hypertension and hyperlipidemia was in his third week of treatment with trimethoprim/sulfamethoxazole (TMP/SMX) (Bactrim) for recent prostatitis when he developed fever and rash over his face, arms and back. TMP/SMX was discontinued and Levaquin started for 1 week, but symptoms persisted. He then transferred to our facility and was found to have mild thrombocytopenia, leukocytosis, acute kidney injury, and elevated liver function tests with unrevealing MRCP and serological workup for liver injury etiologies. Sepsis workup was unrevealing. Bone marrow biopsy revealed $80 \%$ cellular marrow with erythroid and eosinophilic hyperplasia and no evidence of hematolymphoid malignancy. SPEP and UPEP were normal. DRESS syndrome, secondary to TMP/SMX, was diagnosed. TMP/SMX had already been discontinued and steroids were given. Symptoms were improving or resolved at discharge.

DISCUSSION: The Drug Reaction with Eosinophilia and Systemic Symptoms (DRESS) syndrome is a rare, severe drug reaction that is potentially fatal. Early recognition is critical, but a delay in diagnosis often occurs due to a variety of cutaneous presentations and organ involvement. DRESS must be differentiated from such diseases as Steven Johnson Syndrome, acute generalized exanthematous pustulosis (AGEP), hypereosinophilic syndromes, Sézary syndrome, and acute cutaneous lupus erythematosus. DRESS syndrome can be caused by EBV, HHV 6, HHV 7 or certain drugs such as phenobarbital, carbamezapine, phenytoin, allopurinol, vancomycin, and sulfamethaxazole. It typically causes a rash, fever, inflammation of internal organs, lymphadenopathy and characteristic hematologic abnormalities such as eosinophilia, thrombocytopenia, and atypical lymphocytosis. This case illustrates a fairly typical presentation of DRESS syndrome with onset of reaction 2 to 6 weeks after initiation of the offending drug. Fever, malaise, rash, eosinophilia and organ involvement such as liver (most common), kidney and lung are well documented. The extent of visceral organ involvement dictates the risk of death. The mortality rate is about $10 \%$. Treatment includes stopping offending drugs and providing supportive care. The efficacy of systemic steroids has not been validated with controlled trials but they are commonly used. 
BAD BLOOD Meghan T. O’Brien. UCSF, San Francisco, CA. (Control ID \#2469498)

LEARNING OBJECTIVE \#1: Distinguish septic thrombophlebitis from thrombosis LEARNING OBJECTIVE \#2: Reduce iatrogenesis by appropriately using and interpreting blood cultures

CASE: A 42-year old male smoker with a history of HIV (CD4 = 600 s), active intravenous drug use, currently undergoing treatment for septic thrombophlebitis presented with persistent fevers. During a hospitalization 2 weeks prior, he was diagnosed with an occlusive thrombus of his right internal jugular vein. He was discharged to a skilled nursing facility (SNF) for IV vancomycin treatment for a diagnosis of septic thrombophlebitis after one of two blood cultures was positive for $p$. Acnes. He was started on rivaroxiban for anticoagulation. His antiretrovirals were switched to Triumeq from Truvada/darunivir due to drug interaction with vancomycin. The patient had persistent fevers since the time of discharge. On two occasions at the SNF, single blood cultures was drawn off the PICC. One grew Viridans strep, VRE (faecium), and lactobacillis spp. Ceftriaxone and flagyl were added to the patient's regimen. A separate blood culture grew candida tropicalis. When the patient re-presented for persistent fevers, his vital signs were $\mathrm{T}=39.1, \mathrm{HR}=119, \mathrm{RR}=20, \mathrm{O} 2$ sat $90 \%$ on room air. His BP was $90 / 60$, but normalized with $2 \mathrm{~L}$ normal saline. He had scattered wheezes and pitting edema of his right arm, and no signs of endocarditis. CXR revealed a patchy consolidation of the right heart border. Labs were within the patient's baseline. CTA showed a stable occlusive thrombus of left IJ. CT chest revealed multiple bi-apical nodular opacities (up to $1.3 \mathrm{~cm}$ ), possibly early septic emboli, as well as bilateral patchy ground glass opacity, peribronchovascular thickening, and septal thickening, related to underlying pulmonary edema or infection. RUE ultrasound revealed a DVT at the site of the PICC. Antibiotics were broadened to include Vancomycin, meropenem, and Caspofungin. The PICC was removed. Heparin was started to treat both thrombi. Albuterol improved his wheezing and $\mathrm{O} 2$ sats. Two sets of repeat blood cultures were negative, however the patient's fevers persisted. A rapid respiratory panel was positive for rhinovirus, which was felt to explain his initial hypoxia and CT findings. Out of concern for drug fever due to recently started Triumeq (Abacavir/ dolutegravir/lamivudine), the patient was switched back to Dolutegravir, Truvada (tenofivir/emtricitabine). His fevers resolved. He was discharged on fluconazole and rivaroxiban.

DISCUSSION: Suppurative thrombophlebitis is an endovascular infection hallmarked by venous thrombosis, inflammation and persistent bacteremia despite $72 \mathrm{~h}$ of appropriate antibiotics. Clinically, it presents with high-grade fever, erythema, tenderness, and purulence at the site of the involved vessel. Often it is complicated by septic pulmonary emboli or secondary pneumonia, which may be the presenting signs. Antibiotics are targeted at the offending organism (usually staph, strep, or enterobacteriacae). Evidence for anticoagulation is equivocal, and surgical debreidment may be required. Given that this patient's blood cultures were incomplete and grew different organisms, it is unlikely that he ever had septic thrombophlebitis. The harms he suffered as a result of misdiagnosis underscore the importance of appropriate use and interpretation of blood cultures. Detection of bacteremia and interpretability of blood cultures depends on three factors: 1) technique; 2) number of cultures; 3) volume of blood drawn. Detection of bacteremia increases $3 \%$ per $\mathrm{ml}$ of blood drawn. The optimal number of culture sets varies with the clinical condition, pre-test probability, and volume of blood obtained with each set (set $=1$ aerobic and 1 anaerobic culture). One set is never sufficient and leads to unnecessary intervention and testing. For a patient with suspected persistent bacteremia (i.e. endovascular infection), two sets are sufficient. Three sets should be drawn to detect bacteremia not due to common contaminants ( 2 in succession, the $3 \mathrm{rd} 6 \mathrm{~h}$ later). Ideally, 4 sets of cultures should be drawn for bacteremia related to contaminant organisms, or if the patient recently received antibiotics. False-positives increase as more blood cultures are obtained. Certain organisms are always considered pathogens (i.e. candida). The likelihood that an organism that is usually a contaminant is a pathogen increases if repeat cultures demonstrate continued positivity. For this patient, only one of two cultures was positive for an organism that is commonly a contaminant and that was never redemonstrated on subsequent cultures, making endovascular infection unlikely. The poly-microbial second single culture is un-interpretable, especially since it grew contaminant organisms. While candida also grew off a single culture, candida is always considered a pathogen that warrants treatment, underscoring the importance of considering both pre-test probability and pathogen profile when interpreting and acting on results. The inappropriate use and misinterpretation of this patient's blood cultures resulted in misdiagnosis and iatrogenic harms including a 2-week stay in SNF for unnecessary vancomycin treatment, re-hospitalization for drug fever related to switch in his antiretroviral regimen to avoid interaction with vancomycin, and placement of a PICC line that was complicated by a second thrombus.

BAD BLOOD: RASBURICASE-INDUCED HEMOLYTIC ANEMIA CAUSING SPECTROPHOTOMETER ERROR Clark A. Veet; Amar Kohli. UPMC, Pittsburgh, PA. (Control ID \#2432385)

LEARNING OBJECTIVE \#1: Identify two serious side effects of rasburicase, a medication used in lowering uric acid levels.

LEARNING OBJECTIVE \#2: Successfully evaluate and list potential etiologies for discrepancies in pulse oximetry and blood gas analysis

CASE: An 85 year old African American man with ischemic cardiomyopathy, hypertension, chronic kidney disease, and heart block with pacemaker presented with progressive volume overload. He was admitted for elective pacemaker upgrade after cardiac evaluation suggested pacemaker dyssynchrony. Preoperative testing showed creatinine elevated to 5.7 with BUN of 129 (baseline Cr 2.0, BUN 60). Abdominal CT showed a large right perinephric mass with a necrotic center. Additional labs showed elevated uric acid to 16.1 and LDH of $865(<171)$. On days 4 and 5 of admission the patient received rasburicase for suspected tumor lysis syndrome. On day 6 , the patient became acutely short of breath with pulse oximetry showing $70 \%$ saturation. Chest x-ray was negative. Arterial blood was chocolate in color with $\mathrm{pH} 7.35, \mathrm{PaCO} 2$ of 25 and $\mathrm{PaO} 2$ of 167 on $15 \mathrm{~L}$ nasal oxygen but uninterpretable saturated hemoglobin $(\mathrm{SaO} 2)$, methemoglobin, and carboxyhemoglobin. Additional labs showed a hemoglobin of 6.7 (from 10.7), LDH of 2352, haptoglobin of $<5.8$ (36-195), total bilirubin of $15.8(0.3-1.5)$ and direct bilirubin of $9.0(0.1-0.5)$ suggesting severe hemolytic anemia. While hemolysis was identified, questions remained regarding the etiology and an apparent disconnect between blood and hemoglobin $\mathrm{PaO} 2$ and pulse oximetry readings.

DISCUSSION: In this case, the patient experienced severe hemolytic anemia due to rasburicase which caused spectrophotometer error in both pulse oximetry and arterial blood gas studies. Rasburicase (recombinant urate oxidase) catalyzes oxidation of uric acid to non-toxic allantoin. It is indicated in tumor lysis syndrome for rapid breakdown of uric acid, which likely caused the patient's acute kidney injury. While it is generally well tolerated, it does carry a black box warning for methemoglobinemia and hemolysis, both of which occur predominantly in those with glucose-6-phosphate deficiency (G6PD), an $\mathrm{X}$-linked enzymatic disorder of red blood cells. G6PD deficiency testing was performed with blood smear showing absence of bite cells and confirmatory testing with red cell hemolysate assay showing normal NADPH generation of $8.7 \mathrm{u} / \mathrm{g} \mathrm{Hgb}$ (4.6 to 13.5), making G6PD deficiency unlikely. The patient nonetheless experienced severe hemolysis in response to this agent. It is suspected that the severe hemolytic burden caused multiple automated lab and monitoring errors delaying diagnosis. Both ABGs and pulse oximetry rely on spectrophotometric analysis of hemoglobin wavelength to generate data. ABG analyzers in our institution use whole blood to detect wavelengths of molecules that correlate with $\mathrm{SaO} 2$, methemoglobin, and carboxyhemoglobin. Seven ABG and VBGs 
were drawn during hospitalization and run on different samplers, but all were unable to process $\mathrm{SaO} 2$, methemoglobin, or carboxyhemoglobin. Samples were subsequently submitted to an outside lab where spectrophotometer testing was performed on whole blood and centrifuged blood samples. When plasma was separated from red cells, isolated red cell pellet was analyzed with the spectrophotometer able to detect all wavelengths without disturbance. This suggested that the interference was not caused by hemoglobinopathy or red blood cell issue. Confirmatory hemoglobin electrophoresis showed no methemoglobin or additional hemoglobinopathy. Additional testing showed high concentrations of plasma bilirubin and hemosiderin, which is normally absent from plasma. This confirmed severe red cell hemolysis and leakage of breakdown product into whole blood. Quantitative plasma hemoglobin was $142.8 \mathrm{mg} / \mathrm{dl}(<6)$. Consultation with the hospital lab affirmed that all blood gases are run on whole blood without centrifugation, differing from the external lab where the blood was centrifuged. These findings support the hypothesis that serum pigmentation prevented accurate interpretation of $A B G$ and pulse oximetry by means of optically obscuring detection of normal hemoglobin wavelengths in whole blood. Identifying discrepancies between arterial blood gas values and pulse oximetry should prompt rapid evaluation into cause. First, ensure correct site of phlebotomy (arterial vs venous) and exclude transportation delays which can cause erroneous values. Next, consider hemoglobinopathies (methemoglobinemia, carboxyhemoglobinemia, or sulfhemoglobinemia) or pigmentation causing lab error, as in this case. Rasburicase induced hemolytic anemia is a rare but potentially life threatening side effect of a commonly used medication for tumor lysis syndrome. Severe hemolysis of any etiology, however, can cause similar errors in common laboratory techniques and management delays. While these laboratory errors were the result of an unusual inciting factor, the lab errors seen can occur as the result of any severe hemolytic anemia and thus should prompt novel means to obtain appropriate data for patient care. A possible solution in future cases is centrifugation of whole blood prior to testing by eliminating pigment interference with the spectrophotometer.

BAROREFLEX FAILURE FOLLOWING RADIATION THERAPY TO THE NECK Jonathan Arend. Mount Sinai, New York, NY. (Control ID \#2469971)

LEARNING OBJECTIVE \#1: Describe the clinical features of orthostatic hypotension secondary to baroreflex failure

LEARNING OBJECTIVE \#2: Recognize the potential significance of prior neck irradiation in the evaluation of patients with orthostatic hypotension

CASE: HPI: RE is a 62 year-old man with a history of hypertension who presented to our primary care practice with the chief complaint of episodic dizziness for 2 years. The episodes occur exclusively and reliably when rising from a seated or supine position. On 2 occasions, the dizziness was followed by loss of consciousness, resulting in falls without significant trauma. The episodes are not related to eating, exercise, urination or defecation, emotional stress, or coughing. They are not associated with flushing, palpitations, diaphoresis, headache, or anxiety. The patient has no known history of diabetes and denies polyuria, polydipsia, polyphagia, nausea, or vomiting. He has had no memory changes, visual disturbances, speech or swallowing difficulty, focal numbness or weakness, imbalance, gait disturbance, clumsiness, or involuntary shaking. He has had hearing loss, requiring augmentation, since age 40 , without recent changes. He has a history of thyroid and tonsillar cancer, both of which are in remission. Radiation therapy (RT) for the latter has resulted in some dysphagia. He has a significant smoking history but denies chronic cough, hemoptysis, abdominal pain, and hematuria. He has a moderate exercise routine and maintains adequate hydration. He consumes 1 alcoholic drink per week on average. There were no medication changes at the time of symptom onset. He had tolerated valsartan (320 mg) since he was diagnosed with hypertension in 2000, but since 2011, his blood pressure regimen has been adjusted repeatedly due to low blood pressure when standing up. Past Medical History: Hypertension, treated with amlodipine 2.5, previously with losartan 25, valsartan 320, lisinopril 10 Hyperlipidemia Hypothyroidism, s/p total thyroidectomy and RAI in 2006 for incidentally discovered $1 \mathrm{~mm}$ papillary thyroid carcinoma PUD (duodenal ulcer), complicated by hemorrhage requiring emergency surgery (anatomy unknown) and vagotomy in 2007 Tonsillar squamous cell carcinoma, stage IV1 (T1 N2b M0), s/p excision, chemotherapy and RT 2010, in remission Former smoker (25 pack-years), quit 1985 Medications: Amlodipine $2.5 \mathrm{mg}$ qd, Levothyroxine $175 \mathrm{mcg}$ qd, Pravastatin $40 \mathrm{mg}$ qd. No known allergies. Social history: Health care administrator. Married, with stable female partner for 30 years, no h/o STDs. 2 children. Family History: Mother with coronary artery disease (CABG in 60's, died of MI in 80's), father with coronary artery disease (MI in 40's, died of MI at 50) Physical Exam: Supine: BP 150/103, HR 71; Standing (2 min): BP 73/55, HR 79 RR: 12, T $97.2^{\circ} \mathrm{F}$, W $178 \mathrm{lb}$, BMI $23 \mathrm{~kg} / \mathrm{m}^{2}$, SpO2 $98 \%$ Well-appearing. HEENT unremarkable. Normal s1, s2, regular rate and rhythm, no murmurs. Lungs clear to auscultation. Abdomen with midline scar, otherwise normal. No peripheral edema. Normal pulses. Skin normal. Mildly diminished hearing bilaterally, neurologic exam otherwise normal. Diagnostic Testing: BMP, CBC, TSH, LFTs, RPR, B12 were normal. EKG was normal. Tilt table testing was consistent with office readings. 24-h ambulatory blood pressure monitoring revealed wide fluctuations in blood pressure, with a mean systolic blood pressure of 136 and a mean diastolic blood pressure of 92, and a mean heart rate of 79. The etiology of the patient's orthostatic hypotension was presumed to be baroreflex failure secondary to radiation injury to the carotid sinus. Interventions: The patient was referred to a hypertension specialist. A number of medication adjustments were made but failed to control his hypertension without exacerbating his orthostatic symptoms. Antihypertensive treatment was eventually discontinued, resulting in symptom improvement. Aggressive cardiovascular risk factor modification was otherwise pursued.

DISCUSSION: Orthostatic hypotension associated with a moderate rise in the heart rate (approximately $10 \mathrm{bpm}$ ), often in the setting of labile hypertension, is consistent with baroreflex failure. To date, there have been 8 case reports, and 2 case control studies of patients exposed to RT to the neck who subsequently developed symptomatic barofeflex failure, as suggested by tilt table and autonomic testing (1-10). Eliciting a history of neck irradiation may facilitate diagnosis in patients with otherwise unexplained autonomic dysfunction. Treatment of such patients is challenging and often involves weighing the risks of uncontrolled hypertension against the risks of symptomatic hypotension. 1. Robertson et al. Am J Med 1983;74:193-200. 2. Robertson et al. NEJM 1993;329:1449-55. 3. Timmers et al. Clin Auton Res 1999;9:317-324. 4. Sharabi et al. Hypertension 2003;42:110-116. 5. Heusser et al. Hypertension 2005;45:834-839. 6. Park et al. Int J Card 2006;115:117-119. 7. Hutchinson et al. Age and Aging 2008;37:602-604. 8. Goodman et al. The Neurologist 2009;15:102-104. 9. Timmers et al. Int J Radiation Oncology Biol Phys 2002;53:1203-1210. 10. Huang et al. Muscle and Nerve 2012; doi:10.1002/mus.23530.

\section{BE IMPRESSED WITH PRES: KEEP IT IN THE DIFFERENTIAL EVEN WITH LOW GRADE HYPERTENSION Devi Sampat; Patricia Dharapak. Mount Sinai Beth} Israel, New York, NY. (Control ID \#2468932)

LEARNING OBJECTIVE \#1: Recognize Posterior Reversible Encephalopathy Syndrome (PRES) can occur in the absence of severe blood pressure elevations, and providers should be prompted to consider it in patients presenting with neurologic complaints and any degree of hypertension.

CASE: A 33-year-old female with hypertension diagnosed at age 14, prior preeclampsia, and a family history of early onset hypertension presented with 4 days of sudden nausea, vomiting, and abdominal pain. Home medications 
included Labetalol and Amlodipine, which were intermittently taken. On exam she was afebrile, BP 137/88, and there was diffuse abdominal tenderness. An abdominal CT scan was unremarkable, and she was treated for a presumed gastroenteritis with fluids, anti-emetics, opioid analgesics and then discharged. Two days later, the patient returned with persistent nausea and vomiting in addition to headaches and intermittent blurred/double vision. She was admitted inpatient and reported headaches and vision changes that began prior to her initial visit 2 days earlier. Her exam was further significant for T 101.3, BP $183 / 125$, and intermittent drowsiness without focal deficits on neurologic exam. A basic metabolic profile and complete blood count were unremarkable. Urine toxicology was positive for opiates. Due to continued nausea, a Clonidine patch was administered for severe hypertension. An infectious workup was negative and the fevers gradually resolved, coinciding with blood pressure control. Given the persistence of headaches, nausea and vomiting, a brain MRI was obtained which revealed extensive bilateral hemispheric subcortical white matter changes without restricted diffusion. In consultation with Neurology, posterior reversible encephalopathy syndrome (PRES) was likely the unifying diagnosis of our patient's constellation of symptoms. With maintenance of normotension, her symptoms gradually resolved. She was referred for further outpatient work-up but was lost to follow-up.

DISCUSSION: PRES is a neurotoxic state associated with a unique pattern of vasogenic edema on brain CT or MRI. The edema is often widespread but predominates in the parietal and occipital regions. Clinical symptoms may develop over several days as in our case or acutely and can include headache, vision change, paresis, nausea and altered mentation. Seizures and coma may occur. In $70-80 \%$ of patients, moderate to severe hypertension is noted, but normotension doesn't exclude the diagnosis. As initially seen in our case, hypertension can be absent in $20 \%$. PRES can occur in association with specific conditions including pre-eclampsia/eclampsia, sepsis, shock and autoimmune disease. Its cause is not yet understood, but hypertension with failed auto-regulation and hyperperfusion is a possible etiology. The diagnosis is typically corroborated when neurologic recovery is accompanied by resolution of imaging abnormalities within a few weeks. It is important to note that the radiographic MRI findings are often transient in relation to symptoms - therefore clinicians must be aware that patients can still exhibit clinica findings of PRES without MRI confirmation. This is yet another reason why regardless of imaging or blood pressure, providers should be prompted to consider PRES in patients presenting with neurologic complaints and any degree of hypertension. It is important to keep this diagnosis in our differentials in order to prevent worsening manifestations of the disease, such as seizures or a coma.

BEATING INSIDE AN EFFUSIVE CONSTRICTIVE JACKET Deirdre Lewis; Sotiris Stamou; John Rousou; Jaime Hernandez. Baystate Medical Center, Springfield, MA. (Control ID \#2469449)

LEARNING OBJECTIVE \#1: Recognize the signs and symptoms of constrictive pericarditis

LEARNING OBJECTIVE \#2: Describe the diagnosis and management of constrictive pericarditis

CASE: A 51-year-old male with rheumatoid arthritis recently off disease modifying agents for spinal surgery presented to his PCP with complaints of lack of energy, nausea, chills, and subjective fevers. He received a CXR and was diagnosed with pneumonia, and received a course of levofloxacin without improvement. He was subsequently referred to pulmonology who performed a CT scan without contrast demonstrating a right pleural effusion and a moderate pericardial effusion, and was admitted to the hospital for further management. Physical examination at that time revealed distant heart sounds, elevated
JVD with respiratory variation, abdominal distension and pitting edema of the lower extremities. An echocardiogram demonstrated a large pericardial effusion, preserved LVEF, and septal bounce consistent with RV-LV interdependence. The patient underwent diagnostic and therapeutic $\mathrm{CT}$ guided thoracentesis to relieve the pericardial exudative effusion and was discharged on steroid therapy with close rheumatology and cardiology follow-up. The patient subsequently represented to our cardiology clinic for worsening exercise intolerance. Findings in the cardiology office were significant for notable weight gain, JVD elevated to the angle of the jaw, and a repeat echocardiogram once again demonstrating a constrictive pattern but now with only a trivial pericardial effusion. The patient was readmitted to the hospital where he underwent a cardiac MRI relevant for circumferential thickening of the pericardium without delayed gadolinium enhancement. Simultaneous cardiac catheterization showed dynamic respiratory changes with discordant RV/LV pressure curve area during expiration with near equalization of right and left right ventricular end diastolic pressures, non-elevated mean pulmonary artery pressure and lack of respiratory variation in the mean RA. The constellation of symptoms with multimodality imaging and hemodynamics suggested chronic constrictive pericarditis. He subsequently underwent pericardiectomy of the parietal pericardium with no major changes in hemodynamics (elevated RA pressure) followed by visceral pericardium (epicardium) dissection of thickened fibrous looking tissue. Post operatively he had normalization of RA pressure and resolution of cardiopulmonary symptoms. Patient was discharged with close follow up with cardiology and rheumatology.

DISCUSSION: Constrictive pericarditis is an inflammatory condition of the pericardium which can present as either acute or subacute diastolic heart failure. A high clinical suspicion for this condition should be maintained in all patients with risk factors such as rheumatoid arthritis. The pericardium is the most common cardiac structure affected in patients with rheumatoid arthritis. Constrictive pericarditis leads to impaired diastolic filling, with subsequent clinical manifestations of diastolic heart failure. Diagnosis of constrictive pericarditis is made via comparative multi-modality imaging and hemodynamic measurement. In our case further evaluation of the visceral pericardium (epicardium) was prompted by persistently elevated intraoperative right atrial pressures. Constrictive pericarditis may be either transient or chronic, and treatment varies as such. Transient constrictive pericarditis may resolve spontaneously or with anti-inflammatory treatment. Chronic constrictive pericarditis requires definitive surgical management with pericardiectomy. In our patient more than 3 months of anti-inflammatory agents were utilized with no improvement in hemodynamics whereas hemodynamic response was obtained after surgical removal of the pericardium. Timing from medical to surgical intervention is important in order to avoid end-organ dysfunction that could potentially increase eventual surgical risk. Therefore in patients with constrictive pericarditis, a trial of anti-inflammatory therapy may be attempted to see if the condition remits, however if symptoms persist beyond several months surgical intervention needs to be considered.

BEER POTOMANIA: TOO MUCH BEER, TOO FEW PRETZELS Yaniuska Lescaille; Jason A. Korcak. Montefiore Medical Center, Bronx, NY. (Control ID \#2468658)

LEARNING OBJECTIVE \#1: Recognize beer potomania as an uncommon cause of hyponatremia.

LEARNING OBJECTIVE \#2: Describe the pathophysiology, diagnosis and treatment of beer potomania.

CASE: A 64-year-old man with a history of longstanding alcohol abuse, hypertension, and diabetes mellitus type 2 presented with confusion and disorientation. His family members noted strange behavior over the prior 2 weeks, such as attempting to use a credit card to change television channels. The patient 
reported drinking at least twenty-four beers and one gallon of vodka daily. On presentation, he was hemodynamically stable and afebrile. He appeared chronically ill, but in no acute distress. There was a bilateral hand tremor with arms extended, but no asterixis, nystagmus, or tongue fasciculations. Labs were significant for serum sodium $118 \mathrm{mEq} / \mathrm{L}$, urine osmolality $93 \mathrm{mOsm} / \mathrm{Kg}$, urine sodium $<20 \mathrm{mEq} / \mathrm{L}$, and urine specific gravity $<1.005$. Thyroid stimulating hormone (TSH) was normal. A head CT revealed generalized prominence of the cerebral sulci and ventricles compatible with volume loss and microvascular ischemic changes. Given the presence of euvolemic hypoosmolar hyponatremia with a history of excessive beer intake, low urine sodium, and low urine specific gravity, the patient was diagnosed with beer potomania. His serum sodium steadily improved with water restriction, and the patient was discharged 4 days later with a normal mental status.

DISCUSSION: Beer potomania is a rare form of hyponatremia caused by excessive beer consumption without adequate solute intake. The condition was first described in 1971 as "hyponatremic coma" in patients with marked beer intake and serum sodium ranging from 98 to $107 \mathrm{mEq} / \mathrm{L}$. Hyponatremia due to beer potomania results from the dilution of serum sodium after the consumption of a large volume of hypotonic solution, such as beer, combined with a low solute state from poor dietary intake. In other instances of low solute intake, ketone formation provides effective osmoles. However, the metabolism of carbohydrate-rich beer prevents ketone formation and aggravates sodium depletion. Low plasma sodium leads to a low renal solute load, which impairs the normal diluting capacity of the kidneys. This results in decreased free water clearance and dilutional hyponatremia. Even with heavy beer consumption, beer potomania is uncommon, as most individuals continue to eat enough to maintain adequate amounts of solute. The pathophysiology of beer potomania is similar to other causes of euvolemic hypoosmolar hyponatremia with low urine sodium, such as psychogenic polydipsia and the low solute "tea and toast" diet. The management of beer potomania presents a challenge as diuresis can begin spontaneously after the introduction of solutes, likely due to an underlying low antidiuretic hormone state. This brisk diuresis may cause a rapid increase in sodium concentration and increased risk of complications, such as central pontine myelinolysis. Treatment for concurrent alcohol withdrawal is also important given the overlap with the sequelae of hyponatremia. Moreover, individuals with alcohol withdrawal and beer potomania are at a particularly high risk of seizures from the combined neurological effects. Beer potomania represents an uncommon, but clinically significant form of hyponatremia with a classic presentation of water intoxication and reversible mental status changes. Clinicians should be mindful of beer potomania when they encounter patients with too much beer and too little salt.

BEYOND SPELUNKING: DISSEMINATED HISTOPLASMOSIS COMPLICATED BY POLYMICROBIAL PERITONITIS IN AN EL SALVADORIAN FARMER WITH AIDS Jyothi Ravindra; Amy Pasternack; Julia Braza; Jonathan Opraseuth; Rebecca Osgood; Eirini Iliaki. Cambridge Health Alliance, Cambridge, MA. (Control ID \#2469593)

LEARNING OBJECTIVE \#1: Rapidly diagnose disseminated histoplasmosis in a critically ill patient after initial standard testing is negative.

LEARNING OBJECTIVE \#2: Recognize the possibility of managing polymicrobial peritonitis nonsurgically in a severely immunocompromised patient.

CASE: A 24-year-old man from El Salvador presented with subacute onset of fevers, weight loss, hemoptysis, and dyspnea. Medical history included an episode of shingles 9 months prior for which he had no follow up. He was febrile, tachycardic, mildly hypotensive, and tachypneic with an oxygen saturation of $91 \%$ on room air. He was cachectic and ill-appearing, with clear lungs and benign abdomen. Labs were significant for hemoglobin $8.6 \mathrm{~g} / \mathrm{dL}$, white count 6,700 cells/uL ( $82 \%$ neutrophils), albumin $2 \mathrm{~g} / \mathrm{dL}$, and lactate dehydrogenase $729 \mathrm{IU} / \mathrm{L}$. Chest x-ray showed diffuse tiny nodules with a miliary distribution. A rapid HIV test was positive and CD4 count was 11 cells/uL. Within hours of admission, the patient developed severe abdominal pain and peritoneal signs. $\mathrm{He}$ became increasingly hypotensive and hypoxic, requiring pressors and intubation. Abdominal CT showed retroperitoneal lymphadenopathy, mild hepatosplenomegaly, and ascites but no free air. Blood cultures from admission remained negative, but blood cultures drawn after this decompensation grew bacteroides. Peritoneal fluid cultures grew E. coli, S. anginosus, P. aeruginosa, corynebacterium, g-hemolytic streptococcus, and C. albicans. The fluid also contained debris consistent with fecal matter, and microperforation was suspected. Surgical consultants recommended that risks of surgical washout outweighed benefits for this patient, and therefore, a drain was placed by interventional radiology. On hospital day 2 , despite the positive cultures related to the peritonitis, microbiology samples remained unrevealing with regard to the patient's initial subacute systemic and respiratory symptoms. Therefore, the patient underwent a bone marrow biopsy to attempt rapid diagnosis of tuberculosis, atypical mycobacteria, dimorphic fungi, or lymphoma. The biopsy showed granulomas and budding yeast identified as histoplasma, which facilitated timely administration of liposomal amphotericin B. The culture was confirmed positive for histoplasma at 5 weeks, despite serum and urine antigens returning falsely negative. Eventually, all testing for P. jiroveci was negative, as were all blood and sputum cultures for acid fast bacteria. Over the 6 week hospital course, multiple loculations developed in the peritoneal fluid, necessitating a total of six procedures to place and move drains or aspirate fluid collections, but the patient steadily improved. On hospital day 36 , once he could tolerate oral intake, antiretroviral therapy was started. 10 months later, his CD4 count is 377 cells/uL. He is doing well-working and playing sports.

DISCUSSION: This case highlights the importance of maintaining a high index of suspicion for disseminated histoplasmosis in immunocompromised patients. Though in the United States, histoplasma is commonly associated with the Mississippi and Ohio River Valleys, it is also endemic to many regions of Central America, where farmers are exposed through contact with soil. As evidenced in this patient, manifestations of disseminated histoplasmosis can include fever, weight loss, lymphadenopathy, hepatosplenomegaly, pulmonary involvement, bone marrow infiltration, and gastrointestinal lesions. Other commonly involved organs include the skin, central nervous system, and adrenal glands. Given that delays in diagnosis and treatment can lead to death in critically ill patients, prompt diagnosis of disseminated histoplasmosis is important. While histoplasma antigen detection is highly sensitive $(\sim 90 \%)$, the false negative result in this case reminds us that no single test can rule out histoplasmosis when the clinical suspicion is high. Culture (of blood, bone marrow, or other body tissue) is the gold standard but typically takes 4 to 6 weeks to result (up to $85 \%$ sensitive, but with sensitivity dependent upon disease burden and collection of multiple specimens). Direct microscopy of blood or buffy coat and histopathology of bone marrow are less sensitive $(<10 \%$ and $10-34 \%$ respectively) but play a crucial diagnostic role by yielding more timely results. With regard to the patient's peritonitis, bowel wall perforation is a rare but well-documented complication of disseminated histoplasmosis. Although other infections could have caused the suspected microperforation, histoplasma likely played a central role, given improvement without specific treatment of other opportunistic pathogens. Endoscopy to rule out such pathogens was considered but carried too great a risk of frank bowel wall perforation. Gastrointestinal involvement is common in disseminated histoplasmosis (70-90\% on autopsy), as are symptoms (up to $37 \%$ diarrhea and $45 \%$ nausea/vomiting), though exact correlation between lesions and symptoms is not definitively known. Although surgical washout is standard treatment for peritonitis, it was avoided in this case due to the high mortality rate of emergency laparotomy in critically ill patients with AIDS. With close collaboration among the medicine, surgery, pathology, and interventional radiology teams, the patient's peritonitis was successfully managed nonsurgically, with percutaneous drainage and appropriate antimicrobials 
BEYOND THE PALE: THE ASSOCIATION BETWEEN SEVERE ANEMIA AND RENAL CELL CARCINOMA Karma-Jaya Parbhu ${ }^{1}$; Christopher J. Smith ${ }^{2}$. ${ }^{1}$ University of Nebraska, Omaha, NE; ${ }^{2}$ University of Nebraska Medical Center, Omaha, NE. (Control ID \#2434809)

LEARNING OBJECTIVE \#1: Recognize the association between severe anemia and renal cell carcinoma

CASE: A 68 year-old woman presented with increasing shortness of breath for 3 days Associated symptoms included a 2-month history of a dry "hacking" cough, fatigue, anorexia, unintentional weight loss of $15 \mathrm{lb}$, and skin pallor forcing her to purchase different makeup. She had long-standing dark-colored stools related to iron supplementation. The remainder of her review-of-systems was normal. Her past medical history was notable for iron deficiency anemia with a normal colonoscopy 2 years prior. The patient was in no acute distress, but had 4-word dyspnea. Her vital signs were normal. She had generalized pallor. Her heart demonstrated a regular rate and rhythm with no murmurs. She had diminished breath sounds in the lower left lung field, but the other lung fields were clear. Her abdomen was tender in the right lower quadrant with no organomegaly or palpable masses. Her rectal exam was normal with no frank blood. $\mathrm{CBC}$ revealed the following: hemoglobin $4.7 \mathrm{~g} / \mathrm{dL}$, MCV $75.7 \mathrm{fL}$, WBC 15,200/uL, RDW $20.1 \%$, and platelets 535,000/ul. Blood chemistries were normal, except for elevated alkaline phosphatase of $194 \mathrm{U} / \mathrm{L}$. Iron studies showed: iron $15 \mathrm{mcg} / \mathrm{dl}$, transferrin $141 \mathrm{mg} / \mathrm{dl}$, iron saturation $8 \%$, and ferritin $518 \mathrm{ng} / \mathrm{ml}$. Hemolysis studies were negative with normal LDH and elevated haptoglobin. Reticulocytes were $4 \%$ and calculated reticulocyte index was 0.6. ESR was $109 \mathrm{~mm} / \mathrm{h}$ and the CRP was $19.7 \mathrm{mg} / \mathrm{dl}$. Guaiac-based fecal occult blood test was negative. Chest $\mathrm{x}$-ray revealed a large left pleural effusion. Thoracentesis was performed and pleural fluid was exudative by Lights criteria. Gram stain and culture were negative for infection. Cytology was negative for malignancy. CT of chest, abdomen, and pelvis revealed a renal mass invading the IVC with suspected metastasis in the left lower lung. A biopsy of the renal mass demonstrated renal cell carcinoma, clear cell type. DISCUSSION: With 62,000 new cases diagnosed annually in the U.S., renal cell carcinoma $(\mathrm{RCC})$ is a disease frequently encountered by general internists. RCC can present with a wide array of symptoms, with $<10 \%$ of patients having the classic triad of hematuria, flank pain, and abdominal mass. Anemia can preceded the diagnosis of RCC and has been reported in $29-88 \%$ of patients with advanced disease. Anemia is typically normocytic or microcytic and associated with iron studies similar to anemia of chronic inflammation. Researchers have found an association between the anemia found in metastatic RCC and IL-6. IL-6 is a strong inducer of acute phase proteins: CRP, haptoglobin, and hepcidin. Hepcidin is the principal iron-regulatory hormone and key mediator of anemia of chronic inflammation. Anemia is an important prognostic factor in RCC, as patients with anemia at presentation are more likely to die from RCC that those with normal/high hemoglobin. In conclusion, anemia is commonly associated with RCC and is associated with worse outcomes. Internists should consider RCC in the evaluation of a patient with otherwise unexplained anemia of chronic disease.

BILATERAL GIANT CELL ARTERITIS PRESENTING AS BILATERAL SUDDEN VISION LOSS Dhruv K. Modi ${ }^{1}$; Khushboo Sheth ${ }^{2}$; Sonam Puri ${ }^{3}$; Stefanie D Wade $^{2}$; Sukrut Nanavaty ${ }^{4}$; Syed Salman Ali ${ }^{5}$. ${ }^{1}$ Jamaica Hospital Medical Centre, Jamaica, NY; ${ }^{2}$ University of Connecticut, Harftord, CT; ${ }^{3}$ University of Connecticut Health Center, Hartford, CT; ${ }^{4}$ Wright Center for graduate medical education, Scanton, PA; ${ }^{5}$ St Francis Hospital, Hartford, CT. (Control ID \#2469187)

LEARNING OBJECTIVE \#1: Consider giant cell arteritis (GCA) in the differential diagnosis of patients with sudden bilateral vision loss
LEARNING OBJECTIVE \#2: Early diagnosis and prompt treatment of patients with GCA can prevent permanent vision loss

CASE: We present the case of a 77-year-old Caucasian female with history of diabetes, hypertension, and bilateral cataract surgery who presented with sudden onset of bilateral vision loss. Review of systems was positive for neck stiffness and pain radiating to shoulder and diplopia about 2 weeks prior to presentation. Patient denied any headaches, jaw claudication, tongue pain, scalp tenderness, fever, fatigue or other neurological symptoms. At presentation, vital signs were normal. Physical exam revealed tenderness over temporal arteries with decreased pulses. She had no light perception, and pupils were trace reactive with normal intraocular pressures. Ophthalmoscopy exam showed pale nerves with poor vascular flow, positive cherry red spot and no retinal detachment, consistent with anterior ischemic optic neuropathy. Laboratory data was significant for Erythrocyte Sedimentation Rate (ESR) of $71 \mathrm{~mm} / \mathrm{h}$ and C-Reactive Protein (CRP) $5.1 \mathrm{mg} /$ dl. Computerized Tomography (CT) angiogram of the head and Magnetic Resonance Imaging (MRI) brain did not reveal any acute pathology. Bilateral temporal artery biopsies were done, which showed chronic inflammatory cell infiltration without any granulomatous infiltration or medial necrosis. CT angiogram of chest was also done which was negative for involvement of aorta or its major branches. Patient was initially treated with pulse doses of steroids $1 \mathrm{~g}$ for 3 days without any improvement of visual symptoms, and later maintained on prednisone $1 \mathrm{mg} / \mathrm{kg}$ daily which also failed to improve symptoms DISCUSSION: GCA is an inflammatory vasculitis of medium to large vessels and mostly involves the external carotid branches (e.g., temporal and occipital arteries), the ophthalmic, vertebral, distal subclavian, and axillary arteries, and the thoracic aorta. Visual disturbances are frequently present at initial presentation and can cause permanent vision loss in some patients. Anterior ischemic neuropathy (AION) secondary to luminal occlusion is the most common cause of blindness in these patients. Although vision loss is unilateral in majority of the cases, GCA can rarely present as bilateral vision loss as in our patient. AION is a true emergency and immediate therapy with high dose steroids is warranted to prevent permanent vision loss. Temporal artery biopsy with assessment of vascular segment of 1.5-2 cm length has very high yield in confirming diagnosis of GCA (85-95\%) and is considered the gold standard of diagnosis. Treatment consists of initial pulse dose steroids, followed by a slow taper and steroid sparing drugs, if required. Treatment with steroids should not be delayed, as the yield of biopsy remains high up to 2 weeks after initiation of steroids. Several vasculitis symptoms may mimic conditions which are more commonly seen and the diagnosis of vasculitis is often delayed or missed due to ambiguous presentations. Advances are made in diagnosing GCA and other form of vasculitis but the role of detailed history and physical exam are still vital in early diagnosis of GCA. GCA has to be considered in differential diagnosis of bilateral vision loss as early diagnosis and prompt treatment initiation are imperative to prevent dreaded complications.

BLACK ESOPHAGUS Michelle Simonait ${ }^{1}$; Nitya Ramreddy ${ }^{1}$; Charles Hotte ${ }^{2}$. ${ }^{1}$ University of Miami Miller School of Medicine/Holy Cross Hospital, Fort Lauderdale, FL; ${ }^{2}$ University of Miami/Holy Cross Hospital, Fort Lauderdale, FL. (Control ID \#2469681)

LEARNING OBJECTIVE \#1: Esophageal necrosis is an uncommon complication of gastrointestinal tract. It is associated with a two hit theory leading to the development of esophageal necrosis, a low flow vascular state, and gastric outlet obstruction. CASE: Patient is an 83 year old female with past medical history of atrial fibrillation, hypertension, diabetes mellitus type II, end stage renal disease on hemodialysis admitted for hip pain and difficulty walking for 3 days. She was found to have a non-traumatic fracture of the left hip. She underwent a bipolar left hip replacement and was recovering well until post-operative day two. Rapid response was called. Patient was found to be in acute distress with nausea with multiple episodes of coffee ground emesis. On examination, vitals showed tachycardia and hypotension, chest auscultation revealed bibasilar 
crackles bilaterally, abdominal tenderness in the epigastric area, and the remainder of the examination was unremarkable. There was high suspicion for a GI bleed. A nasogastric tube (NGT) was placed with over 11 of black tarry fluid removed, and she was started on a proton pump inhibitor (PPI) drip, ondansetron, intravenous fluids, and pain control. $\mathrm{Hb}$ dropped $2 \mathrm{~g} / \mathrm{dl}$ from her baseline. She was transferred to the critical care unit. Patient underwent endoscopy the next day, which revealed a black esophagus in the lower $2 / 3$ of the distal esophagus with a sharp demarcation at the gastro-esophageal junction with superficial ulceration with no active bleeding seen. The gastric mucosa was normal and there was a superficial duodenal ulcer. Gastroenterologist made the diagnosis of esophageal necrosis. Patient was kept NPO for next 24 h, the NGT was removed, PPI drip continued, and sucralfate was added the following day. Patient was then advanced to a clear liquid diet, which was not tolerated, she complained of chest pain while drinking liquids. Chest XRAY was done which showed bilateral pleural effusions and cardiomegaly. Subsequently, a CT chest with contrast showed compressive bilateral pleural effusions, air in the anterior mediastinum, and subcutaneous emphysema in the neck. Patient decompensated with Pseudomonas Aeruginosa bacteremia, and treated with IV piperacillin sodium-tazobactam. She became septic and was no longer tolerating hemodialysis despite pressors. Her family made the decision to change her code status to DNR and she passed away shortly after.

DISCUSSION: Acute esophageal necrosis (AEN) commonly referred to as "black esophagus" or "acute necrotizing esophagitis", is a rare clinical condition seen with upper GI bleed cases. Clinical presentation of esophageal necrosis ranges from hematemesis to coffee ground emesis to melena. It is thought to arise from a combination of ischemic insult to the esophagus, impaired mucosal barrier systems, and a backflow injury from chemical contents of gastric secretions. Low-flow state related to sepsis, arrhythmias, CHF, acute blood loss, trauma and shock may lead to ischemic compromise of the esophagus. It may also be associated with diabetes mellitus, atherosclerosis, cardiovascular, and renal diseases due to vasculopathy. It is characterized by diffuse black appearing distal esophageal mucosa on EGD that stops abruptly at gastroesophageal junction. The endoscopic appearance makes the diagnosis. Esophageal biopsy or brushings of the affected esophagus are supportive but not required to make the diagnosis. The most serious complication is perforation of the esophagus, which can occur in severe cases that result in full-thickness necrosis of the esophageal tissue. Complications should be suspected in rapidly decompensating patients. Perforation may lead to mediastinitis, empyema, sepsis, and rapid clinical deterioration. Prompt recognition, intravenous antibiotics, and surgical intervention may be life-saving. Acute esophageal necrosis generally has a poor prognosis and the goal of therapy is to treat the coexisting medical conditions. Overall mortality from AEN is up to $31 \%$. Intravenous hydration, transfusion of packed red blood cells, and keeping the patient NPO should be instituted. Insertion of the nasogastric tube is contraindicated to avoid perforation. Medical management is aggressive intravenous PPI until clinical condition improves, and then should be switched to oral therapy for a few months to avoid esophageal stricture formation. Surgical intervention is reserved for patients with perforated esophagus with mediastinitis and abscess formation.

BLAST FROM THE PAST: RADIATION INDUCED OSTEOSARCOMA 35 YEARS LATER IN A CHILDHOOD EWING CANCER SURVIVOR Andrew Trifan; Amar Kohli. UPMC, Mars, PA. (Control ID \#2469158)

LEARNING OBJECTIVE \#1: Identify red flags for limb pain

LEARNING OBJECTIVE \#2: To describe long term complications in Ewing sarcoma patients

CASE: A 51-year-old woman with a past history of Ewing sarcoma of her right proximal fibula, severe peripheral vascular disease status post fem-pop bypass in 2005, presented to her PCP with worsening right lower leg pain. She was diagnosed with Ewing sarcoma at age 16 and was treated with 1.5 years of chemotherapy (vincristine, Adriamycin, cyclophosphamide) followed by partial resection of the fibula and 6 weeks of radiation. Shortly before her PCP visit, she started having right leg pain after she stepped "wrongly" off a sidewalk curb. Her exam showed an atrophic right lower leg, tenderness to palpation on the posterolateral side, difficult to appreciate distal pulses, motor/sensation intact and normal temperature to touch. She smoked 1 pack/day for 30 years and had a remote history of heavy alcohol use. Her PCP diagnosed her with shin splints and managed the symptoms conservatively. However, the pain became more severe and persistent, even throughout the night or when non-weight bearing. X-rays were obtained, showing nonspecific bony changes in the tibia. Imaging for a vascular etiology, given her history, was negative. A CT scan showed a $3.8 \mathrm{~cm}$ lytic lesion in her anterior right tibial meta-diaphysis suspicious for radiation induced osteosarcoma or metastatic Ewing sarcoma along with radiation osteonecrosis of the lateral tibia and fibula. Bone biopsy revealed high grade undifferentiated spindle cell sarcoma. A knee amputation was performed with final pathology showing poorly differentiated high grade spindle cell sarcoma of the metaphysis with extension in to soft tissue, no lympho-vascular invasion and resection margins free of tumor. CT of the chest did not show metastatic disease. She was referred to medical oncology for further workup.

DISCUSSION: Up to $60 \%$ patients with cancer receive radiotherapy. The diagnosis of radiation-induced sarcoma is clinical, based off criteria from 1948, including prior history radiation exposure, occurrence of sarcoma in or near field of radiation and pathologic confirmation. The time interval is one criterion still subject to debate as it is necessary to differentiate radiation induced from second primary, and varies from several months to $10+$ years. Current studies suggest radiation sarcoma accounts for $0.5-5 \%$ of all sarcomas with median development time of 10 years. Osteosarcoma is the second most frequent primary cancer occurring during the first 20 years following radiation for solid cancer in childhood, and when seen in adults is considered to be transformation of Paget disease or from irradiated bone. Median age of diagnosis is 55-65. Typical symptoms include pain that is worse at night and occasional swelling with or without tender soft tissue adherent mass. One of the most common locations for metastatic spread is the lungs which needs to be evaluated with a new diagnosis. Musculoskeletal pain is a very common encounter in the primary care setting, and it is important to distinguish "red flag" symptoms. Identification of these red flag symptoms will help the generalist determine when additional work up is necessary versus watchful waiting. In regards to malignant bone pain, a hallmark feature is intense unremitting pain, particularly at night, that is not relieved with aspirin or NSAIDs, as well as having a prior malignancy. In fact, the source of pain in patients with a history of cancer can be elusive, with a broad differential including inflammatory lesions (arthritis, bursitis, gout), metastasis, stress fraction, radiation osteitis, etc. In this case, her history of severe peripheral vascular disease also put a vascular etiology on differential. Finally, survival of Ewing patients has improved with patients at risk for future health complications that a PCP should be aware of, including: second malignancy, radiation complications (wound, pulmonary fibrosis, neuropathy, limb leg discrepancy, femoral head necrosis) and chemotherapy related (renal insufficiency, bowel toxicity, cardiomyopathy, neuropathy).

BOERHAAVE'S SYNDROME: AN EARLY RECOGNITION HAMPERS MORTALITY Mary A. Esho; Kusuma A. Kurmayagari. St. Vincent Charity Medical Center, Cleveland, OH. (Control ID \#2469624)

LEARNING OBJECTIVE \#1: To underscore the magnitude of early diagnosis and treatment in preventing mortality.

CASE: A 51-year-old male presented with sudden onset of sharp retrosternal chest pain, which started immediately after the inability to swallow his breakfast. Pain was rated $8 / 10$, non-radiating, worse with deep breathing and followed by vomiting of bright red blood. 
He denied cough, SOB, fever and chills. He had a history of type 2 DM, alcohol abuse with 8 cans of beer daily, arthritis with long-term use of ibuprofen and tobacco smoking for 12 years. Ten years prior he had dysphagia, was diagnosed with peptic stricture and underwent repeated mechanical dilations. On examination, BP was 172/97, pulse 71, temperature 37 , RR 20 , pulse oximetry $96 \%$ on room air. He was awake, alert but in moderate painful distress. There was subcutaneous crepitus in the bilateral neck. He had epigastric tenderness without rigidity, guarding or rebound tenderness. Review of other systems was negative. Chest x-ray showed streaky opacities in the right lung base with air in the soft tissue of the lower neck. CT chest confirmed a right pneumothorax and pneumomediastinum tracking around the subcutaneous soft tissues of the neck and all the way down the lesser curvature of the stomach. Also noted was a right pleural effusion. He was intubated, admitted to the ICU and started on antibiotics. Subsequently he underwent a Gastrografin fluoroscopy showing an esophageal tear with fibrosis and a false lumen. Due to extensive esophageal damage, he underwent a video assisted thoracoscopic surgery (VATS) esophagectomy, esophagostomy, gastrostomy and jejunostomy tube placements. He was later discharged home.

DISCUSSION: Risk factors for Boerhaave's syndrome include medications (NSAIDS, bisphosphonates), alcohol, Barrett's and eosinophilic esophagitis. Patients present with severe neck, retrosternal chest and epigastric pain (for cervical, intrathoracic and intraabdominal ruptures respectively). Mortality rate is $14 \%$ for treatment onset within $24 \mathrm{~h}$ of incident versus $27 \%$ after $24 \mathrm{~h}$ and is heralded by complications like mediastinitis and sepsis. Hence early institution of management is imperative. Diagnosis is established with contrast esophagram or chest CT scan. Medical management includes antibiotics, IV fluids, PPI and chest tube placement for air or fluid removal. Surgery may include primary repair/ resection of the defect, diversion or esophagectomy. Other measures include metal or plastic stents via EGD. A single important life saving measure here was the early recognition and management of the condition. The patient's previously fibrosed esophagus from chronic NSAID use, chronic dysphagia and inflammation made salvaging the esophagus impossible.

\section{BOWEL PERFORATION SECONDARY TO APPENDICEAL METASTASIS OF} TESTICULAR SEMINOMA. Briana E. DiSilvio; Josebelo Chong; Mark Lega. Allegheny Health Network, Pittsburgh, PA. (Control ID \#2469854)

LEARNING OBJECTIVE \#1: In $90 \%$ of cases, testicular seminoma presents as localized disease. Unfortunately, in $10 \%$ of cases, the cancer has already metastasized by the time of diagnosis. This, however, should not deter physicians and patients from pursuing aggressive chemotherapy considering the remarkably high response rate (over $90 \%$ ) even in advanced stages.

LEARNING OBJECTIVE \#2: Gastrointestinal metastasis of testicular seminoma is rare, but can have fatal sequelae if not identified and intervened upon in a timely manner.

CASE: A 32 year old caucasian male without significant medical history presented with severe, sudden onset abdominal pain, nausea and vomiting. He stated 4 months of progressive abdominal distention, leg edema, and a $50 \mathrm{lb}$ weight loss. He further reported a painless right testicular mass which had steadily increased in size over the previous 13 years, for which he had never sought medical attention on account of being embarrassed. Physical examination revealed cachexia, a distended and diffusely tender abdomen, a firmly enlarged scrotum, and 3 plus pitting bipedal edema up to the thighs. CT imaging of the abdomen and pelvis demonstrated a large scrotal tumor and a retroperitoneal soft tissue mass, measuring $14 \times 15 \mathrm{~cm}$, which encased the small bowel, inferior vena cava (IVC) and aorta. Serial chest radiographs revealed the development of free air under the diaphragm, and an emergent exploratory laparotomy was performed. Intraoperative findings included a large mass enveloping the bowel and IVC, multiple omental adhesions, and tumor growth from a perforated appendix. The remaining bowel was examined and no additional sites of perforation were visualized. Appendectomy was performed and histology revealed sheets and nests of cells with abundant pale cytoplasm and large nuclei with prominent nucleoli, consistent with seminoma. 10 days after admission, following a complicated perioperative course, the patient expired.

DISCUSSION: Testicular cancer is the most common solid malignancy affecting males aged 15 to 35 years. The classic presentation is a painless lump in the testis found during self-examination. In approximately $10 \%$ of patients however, the initial manifestation is attributable to metastatic disease. Above we describe the unusual case of a patient who presented with seminoma metastatic to the appendix, resulting in bowel perforation. Seminoma with metastasis to the lungs, bone, and retroperitoneum is well-documented and presents with such symptoms as dyspnea, back and bone pain, and lower extremity swelling. Conversely, gastrointestinal metastasis is rare, occurring with an estimated incidence of $5 \%$, and results mainly from direct invasion by adjacent tumor present within the retroperitoneal lymph nodes. Subsequent complications typically consist of intestinal obstruction and hemorrhage. Bowel perforation is far less frequent, especially when the appendix is the site involved. This is an extremely rare presentation of a common disease, with fatal consequences. Making this case even more unfortunate is the fact that seminoma is usually quite sensitive to chemotherapy and has a remarkably high response rate (over $90 \%$ ), even in advanced stages. Early recognition and treatment of the disease in this individual could have been life-saving.

BREAKING THE LINE: AN UNUSUAL CASE OF BACK PAIN Fiore Mastroianni Tulay Aksoy; Sarah Baron. Montefiore, New York, NY. (Control ID \#2468205)

LEARNING OBJECTIVE \#1: Recognize the potential complications of intrathecal catheter placement for long-term analgesia

LEARNING OBJECTIVE \#2: Identify new neurological exam findings important in the assessment of patients with chronic pain complaints

CASE: A 52 year-old woman presented with 3 weeks of progressively worsening back and leg pain. She had chronic non-malignant lower back pain secondary to disc disease and a fall which caused frequent hospitalizations over the past 9 years. She had an L4-L5 discectomy and fusion 7 years ago and an intrathecal hydromorphone pump was placed for management of her pain 5 years prior to presentation. Despite this, she had multiple admissions for pain control in the past year. The pump had been empty of hydromorphone for the preceding month due to lack of insurance coverage. She reported adequate analgesia with a functional pump and oral methadone. On interview, she described worsening urinary retention for at least 1 month as well as heaviness and intermittent burning sensation in her legs and perineum. She had antalgic gait and silent patellar and Achilles reflexes. Sensation over the legs was intact. There was decreased perineal sensation and decreased rectal tone. Both the anocutaneous and bulbospongiosus reflexes were diminished. CT imaging of the lumbar spine revealed that the intrathecal catheter had fractured at its point of entry into the thecal sac at the L1-L2 space. There was a free $12 \mathrm{~cm}$ segment in the thecal space extending from the level above entry to the L5 vertebra. There was also epidural and paraspinal free air. Neurosurgery and interventional radiology were consulted for management of the fragment and pump replacement, though, given risks of the procedure and ongoing insurance concerns, no intervention has been performed. We could not determine when the catheter fractured based on patient's history, and no imaging was performed between placement of the intrathecal pump and this discovery.

DISCUSSION: Patient with chronic pain are often encountered by internists in and out of the hospital. These patients take a combination of opiates, neuroleptics, and antiinflammatories as well as have implanted devices. It is important to account for all of these when assessing patients with new pain and complaints. Intrathecal catheters can be used to control pain in individuals who benefited from opiate therapy and either fail more 
conservative treatment or suffer intolerable adverse effects like constipation and concerns of opioid dependence. Patients may be able to significantly reduce their oral medication burden with intrathecal delivery. Intrathecal delivery removes the risk of systemic side effects and very low doses are needed. Unfortunately, about five percent of catheters fracture. Continued infusion of medication with a fractured catheter can lead to epidural or intramuscular delivery of those very small doses which do not produce adequate analgesia. The free intrathecal catheter can be a source of neurological complaint and deficits, though this is rare. Symptoms related to the free catheter can mimic the original pain for which the catheter was placed. Catheter removal can be considered if there is associated morbidity, however, removing the fragment is not without risk as it requires laminotomy or laminectomy. Non-fracture complications include infection and fibrosis around the catheter tip, both of which can cause pump failure and exacerbate symptoms. Physical exam signs should be used to localize neurological lesions in patients with chronic low back pain who present with new or worsening complaints. "Red flag" symptoms of urinary retention, fecal incontinence, perineal anesthesia, and any weakness merit focused evaluation of the lumbar and sacral nerve roots. Patients with chronic non-cancer pain often have other comorbidities managed by internists. Presentations of pump failure or neurologic signs and symptoms of lumbosacral dysfunctino should be familiar to internists. Hypoactive patellar and Achilles reflexes can be seen in lower motor neuron dysfunction. These can be silent in patients with significant osteoarthritis or other knee deformity and it is normal for the Achilles reflex to diminish with age, potentially limiting their usefulness. The other useful reflexes of sacral dysfunction are those mediated by nerves to the perineal area. The anocutaneous reflex is a multisynaptic reflex of sacral nerves (S2-S4) and is elicited by tactile stimulation of the perianal and perineal skin; normal response is contraction of the external anal sphincter. The bulbospongiosus reflex is a multisynaptic sacral reflex as well. It is elicited by compression of the glans penis, clitoris, or stimulation of the urethra by lightly pulling on a foley catheter, if present; normal response is contraction of the anal sphincters. Absence of these reflexes indicate sacral dysfunction and offer compelling evidence to look for structural causes of new complaints. Internists should be aware of and look for red flags, particularly in patients with chronic nonmalignant pain. The sacral reflexes are an important part of the physical exam when assessing patients with chronic back pain who present with new, severe, or urinary complaints.

BROADENING THE DIFFERENTIAL FOR SEVERE HEPATITIS IN ANOREXIA NERVOSA. Victor D. Babatunde ${ }^{1}$; Adam C. Nadelson ${ }^{2}$; Vilas R. Patwardhan ${ }^{2,1}$; Eric U. Yee ${ }^{2,}{ }^{1}$. ${ }^{1}$ Harvard Medical School, Boston, MA; ${ }^{2}$ Beth Israel Deaconess Medical Center, Boston, MA. (Control ID \#2469732)

LEARNING OBJECTIVE \#1: Recognize that abnormal and severe aminotransferase elevations in anorexia nervosa patients may serve as a marker of disease severity.

LEARNING OBJECTIVE \#2: Identify malnutrition-induced hepatitis as distinct from hepatitis from refeeding syndrome.

CASE: A 31 year old female with a past medical history notable for anxiety, bulimia from the ages of 18-28, with subsequent anorexia nervosa from the ages of 28-31, presented for evaluation of elevated aminotransferase levels, discovered during a medical evaluation for anorexia. On admission, alanine aminotransferase (ALT) was 514 IU/L; aspartate aminotransferase (AST) was $130 \mathrm{IU} / \mathrm{L}$; with normal alkaline phosphatase and total bilirubin levels. She was asymptomatic without fevers, chills, night sweats, nausea, vomiting, or abdominal pain. She denied recent alcohol consumption, use of acetaminophen or herbal supplements, high-risk sexual behavior, or intravenous drug use. Outpatient medications included a daily multivitamin and $1000 \mathrm{mg}$ per day of ascorbic acid. She had no allergies to medications. There was no family history of liver disease. Vital signs on admission were notable for a blood pressure of $93 / 62 \mathrm{mmHg}$, heart rate of 56 beats per minute. Admission weight was $35.7 \mathrm{~kg}$ (Body Mass Index (BMI): $13.5 \mathrm{~kg} / \mathrm{m}^{2}$ ). She was thin appearing, with anicteric sclera. She had no hepatosplenomegaly, palmar erythema, asterixis or altered mental status. Admission laboratory evaluation revealed a normal complete blood count, chemistry panel and liver synthetic function. Viral hepatitis serologies were negative. Serum toxicology screen was negative for acetaminophen. An abdominal ultrasound showed normal hepatic parenchyma with a smooth liver contour, patent portal vein, and no evidence of biliary ductal dilatation. Given her low BMI, she was evaluated by psychiatry and started on a strict eating disorder protocol, with daily weights, and strict meal requirements. Aminotransferase levels up-trended over the next 6 days peaking with an ALT 1368 IU/L and AST 544 IU/L. There was no clinical evidence of re-feeding syndrome and serum electrolytes remained normal, without requiring repletion. Subsequent testing for other causes of hepatitis was negative. A liver biopsy was performed on hospital day 6. At that time, ALT was $1368 \mathrm{IU} / \mathrm{L}$, AST $544 \mathrm{IU} / \mathrm{L}$ and weight was near its nadir (BMI $12.8 \mathrm{~kg} / \mathrm{m}^{2}$ ). Liver biopsy pathology revealed relatively minimal changes. There were focal portal areas with mild, mononuclear inflammation. Lobules showed no significant inflammation and only rare acidophil bodies (apoptotic hepatocytes). Centrivenular zones showed mild sinusoidal dilatation with hepatocytes that appeared slightly atrophic. Nutrition was continued and aminotransferase levels downtrended with steady weight gain. ALT was $100 \mathrm{IU} / \mathrm{L}$ and AST $47 \mathrm{IU} / \mathrm{L}$ at a peak weight of $52.2 \mathrm{~kg}$ (BMI 19.8), 84 days after her initial presentation. In summary, this patient's presentation was consistent with severe aminotransferase elevations secondary to severe malnutrition in association with anorexia nervosa.

DISCUSSION: This case demonstrates severe malnutrition to be an important cause for elevated aminotransferase levels and highlights abnormal liver chemistries as a sign of clinically severe malnutrition. "Starvation-induced autophagy," where the nutritional stress of malnourishment induces autophagocytic mechanisms without evoking an overt process of apoptosis, has been described. The initial response of autophagy to cope with nutrient depletion without cell death may be a hepatoprotective response. ${ }^{1}$ According to Kheloufi et al ${ }^{1}$, when starvation continues and BMI reaches a dangerous level of $13 \mathrm{~kg} / \mathrm{m}^{2}$ or less, excessive autophagocytic processes may no longer be protective and lead to hepatocellular death and liver failure. Maintaining this patient's weight above the critical BMI may have prevented further hepatocellular damage and averted hepatic failure. Thus, starvation-induced autophagy with resultant hepatocyte plasma membrane permeability could, at least in part, explain the increase in serum aminotransferases without extensive liver cell necrosis or inflammation on liver histology in our patient. ${ }^{2}$ Although refeedinginduced hepatic steatosis has been implicated in ALT and AST elevations in anorexia nervosa patients with malnutrition, there was no evidence of hepatic steatosis on our patient's liver biopsy specimen. In our patient, hepatitis was most severe at the patient's nadir weight with subsequent refeeding with controlled enteral supplementation eventually leading to a decline in aminotransferases, as her weight increased. These findings suggest that aminotransferase levels in anorexia nervosa patients may serve as a marker of disease severity in anorexia nervosa and help to guide monitoring and treatment. In conclusion, differentiating malnutrition-induced hepatitis from refeeding syndrome is pivotal and attention should be paid to the BMI during the course of refeeding as judicious early weight restoration ought to be pursued, not delayed, for these severely undernourished patients. References 1. Kheloufi M, Boulanger CM, Durand F, Rautou P-E. Liver autophagy in anorexia nervosa and acute liver injury. Biomed Res Int. 2014;2014:701064. 2. Rautou P, Cazals-Hatem D, Moreau R, et al. Acute Liver Cell Damage in Patients With Anorexia Nervosa: A Possible Role of Starvation-Induced Hepatocyte Autophagy. Gastroenterology. 2008;135(3):840-848.

BUDD CHIARI: A RARE INITIAL PRESENTATION OF RENAL CELL CARCINOMA Raagini Jawa; Stephanie Le; Warren Hershman. Boston University Medical School, Plaistow, NH. (Control ID \#2456830) 
LEARNING OBJECTIVE \#1: Diagnosis and consequences of Budd Chiari Syndrome from Renal Cell Carcinoma

LEARNING OBJECTIVE \#2: Recognize the complex management options and treatment challenges of Budd Chiari Syndrome

CASE: A 74-year-old woman presented with 2 weeks of night sweats, nausea, jaundice, mild abdominal pain with distension and new onset lower extremity edema. Her clinical exam revealed scleral icterus, tender hepatomegaly, shifting dullness to percussion, and $2+$ bilateral edema to the thighs. Her labs were significant for lactate $7 \mathrm{mmol} / \mathrm{L}$, Creatinine $2.5 \mathrm{mg} / \mathrm{dL}$, AST $885 \mathrm{u} / \mathrm{L}$ and ALT $548 \mathrm{u} / \mathrm{L}$, total bilirubin $2.3 \mathrm{mg} / \mathrm{dL}$. Given her clinical findings of ascites, lower extremity edema, and hepatic dysfunction, there was a concern for possible intra-abdominal pathology. Her admission CT abdomen showed a heterogeneous right renal mass with IVC occlusion with a thrombus. Decision was made to not anti-coagulate the patient since the etiology of the thrombus was unknown and additional more sensitive imaging was needed for further diagnosis and evaluation of thrombus extension. MRI confirmed the presence of a multi-lobulated, Stage 4 RCC with tumor thrombus extending into the IVC to the right atrium, left renal vein, middle and right hepatic veins confirming the clinical diagnosis of BCS. Though the patient's full metastatic workup was negative, her hospitalization was complicated by progressive hepatic failure with rising transaminases, coagulopathy, and resistant ascites due portal hypertension from BCS. Multidisciplinary management options such as surgical excision with revascularization and liver transplant, or chemotherapy were considered but because of the extent of our patient's tumor burden and her hepatic dysfunction the patient was deemed to be a high surgical risk and ineligible for most chemotherapy; thus she opted for home hospice care

DISCUSSION: RCC is often diagnosed at an advanced stage and approximately $5 \%$ of patients have IVC extension of tumor thrombus. Rarely this tumor thrombus can progress to hepatic venous obstruction leading to a primary BCS causing ischemic hepatic centrilobular necrosis. Since the etiology of BCS is usually a vascular thrombus from a hyper-coagulable state, anticoagulation or thrombolysis is usually first line to prevent further thrombus propagation. Unlike most cases of BCS, this case illustrates the need to have a high clinical suspicion for malignancy. Malignancy warrants multidisciplinary approach for aggressive surgical resection with full removal of the primary tumor and tumor thrombus thus posing as both a diagnostic and management challenge.

BUERGER'S IMITATOR: A CASE OF COCAINE INJECTION MIMICKING THROMBOANGIITIS OBLITERANS. Sarah Tietz ${ }^{2}$; Maria G. Frank ${ }^{1,2}$; Quocan Nguyen ${ }^{2} .{ }^{1}$ Denver Health Hospital Authority, Denver, CO; ${ }^{2}$ University of Colorado, Denver, CO. (Control ID \#2455221)

LEARNING OBJECTIVE \#1: To highlight the importance of excellent history taking and its importance of arrival to accurate clinical diagnosis.

LEARNING OBJECTIVE \#2: To review clinical presentation and differential diagnosis of Thromboangiitis obliterans (TAO) through discussion of a case of cocaine-induced distal ischemia masquerading as TAO.

CASE: A 37 year old male with history of multiple sclerosis, depression, and polysubstance abuse (current tobacco, remote heroin use) presented with a 5-day history of pain, numbness, and ulcerated lesions of the first three digits of left hand with positive Allen's test. He denied current illicit drug use. Diagnostic work up included ESR, CRP, anti-cardiolipin IgG, lupus anticoagulant, complement levels, ANCA IgG, anticentromere $\mathrm{IgG}$, SCL70 IgG, and serine protease $3 \mathrm{IgG}$, all of which were negative, Echocardiogram excluded embolic source. Urine toxicology was positive for cocaine metabolites. Arterial angiogram evidence showed multifocal segment occlusion of multiple digital arteries involving all digits of the left hand, sparing the right hand and left foot. Ultimately, the patient admitted to injecting cocaine into the dorsum of his left hand and wrist, but was concerned of receiving substandard care had he admitted it. Heparin drip was discontinued and patient was started on aspirin.

DISCUSSION: Thromboangiitis obliterans (TAO), formerly Buerger's Disease, is a small to medium vessel vasculitis that can affect the arteries, veins, and nerves of the extremities of young smoker males. We present a case of a patient who met all diagnostic criteria TAO in the setting of wrist cocaine injection. TAO usually presents with ischemia of the distal small arteries, frequently involving more than one limb when explored with an arteriogram. Clinical presentation includes claudication of distal extremities; necrotic ulcerations; thrombophlebitis, Raynaud's phenomenon, abnormal sensory findings, and positive Allen's test. Acute phase reactants, immunological markers and auto-antibodies are non-contributory. Despite a strong association with heavy tobacco use, etiology is still unknown. Diagnosis is made angiographically. The treatment is smoking cessation. Multiple diagnostic criteria have been proposed for Bueger's Disease, recently Olin suggested the following diagnostic criteria: (1) onset before 45 years of age, (2) current tobacco use, (3) distal extremity ischemia such as claudication, rest pain, ischemic ulcers or gangrene documented with non-invasive testing, (4) laboratory tests to exclude autoimmune or connective tissue diseases and diabetes mellitus, (5) exclusion of a proximal source of emboli with echocardiography and arteriography, and (6) demonstration of consistent angiographic findings in the involved and clinically noninvolved limbs. Cocaine injection into the radial artery has also been shown to cause similar symptoms to TAO; however, the symptoms tend to be more severe, have a faster onset and with a clear history of cocaine injection such as our patient. Case reports have shown inadvertent arterial injection of cocaine at the wrist leading to distal vascular compromise. A variety of mechanisms of injury have been proposed including vasospasm, particulate embolization, and endarteritis with resultant thrombosis. Our patient met 5/6 of Olin's criteria. Angiography result suggested an alternative diagnosis; and upon further conversation patient admitted to injecting cocaine in his wrist. This case highlights the importance of complete history taking and its clinical and angiographic correlation in the diagnosis of TAO mimics.

BUT I THOUGHT SHE WAS VACCINATED: BREAKTHROUGH HEPATITIS B INFECTION IN A YOUNG ADULT Gretchen Snoeyenbos ${ }^{2}$; Jennifer Bracey² ${ }^{2}$ Robin Klein ${ }^{1}{ }^{1}$ Emory, Atlanta, GA; ${ }^{2}$ Emory University School of Medicine, Atlanta, GA. (Control ID \#2467763)

LEARNING OBJECTIVE \#1: Understand the natural history of acute hepatitis B disease including serologic markers of infection.

LEARNING OBJECTIVE \#2: Recogonize the indications for and efficacy of hepatitis B vaccination

CASE: 22 year old African American woman presents with 2 weeks of fatigue, nausea, and abdominal pain. She reports generalized fatigue, dark urine, decreased oral intake, nausea and vomiting, subjective fevers, and intermittent abdominal pain. She travelled to Nigeria 4 months prior. Social history was significant for recent unlicensed tattoos, unprotected sex with 1 male partner, weekly alcohol use of $200 \mathrm{~mL}$ vodka. She reported being vaccinated to hepatitis B as a child and Hepatitis A and yellow fever prior to travel. She denied recent acetaminophen ingestion or IV drug use. Exam revealed scleral icterus and right upper quandrant tenderness without hepatomegaly. Initial laboratory evaluation revealed AST of 1273, ALT of 1968, total bilirubin of $8.4 \mathrm{mg} / \mathrm{dL}$ and direct bilirubin of $6.0 \mathrm{mg} / \mathrm{dL}$. Alkaline phosphatase was normal and gamma glutamyl transpepdidase was elevated at 81 units/L. A right upper quadrant ultrasound revealed no evidence of obstruction and normal echogenicity of the liver. She was admitted for evaluation of her transaminitis. HIV and HCV serologies were negative. Hepatitis A IgG was positive but 
IgM negative. Hepatitis B surface antigen was positive, surface antibody was indeterminate and core IgM was positive. Hepatitis B DNA copies were $3.37 \mathrm{IU} / \mathrm{mL}$. Hepatitis B virus $\mathrm{E}$ antigen was positive with a negative $\mathrm{E}$ antibody. Autoimmune disease markers were unremarkable. She was diagnosed with acute hepatitis B infection. With supportive care and counseling about risk behaviors, she recovered without complication.

DISCUSSION: Acute hepatitis B infection is diagnosed through serologic markers of infection. Hepatitis B surface antigen is the hallmark of infection and the presence of IgM antibody to hepatitis $\mathrm{B}$ core antigen indicates acute infection. Overtime, surface antigen and core IgG antibodies form. Hepatitis B E antigen is a marker of viral replication and increased infectivity. The natural history of hepatitis B infection is dependent on the age at which an individual in infected. For those over the age of 5 years, $>95 \%$ of cases are self limited and the virus is cleared from the body with lasting immunity within 6 months. A small percentage of patients develop chronic hepatitis B and are at risk for cirrhosis and hepatocellular carcinoma. Though there are effective treatments for chronic hepatitis B, treatment in the acute phase is largely supportive. In the US, widespread vaccination for Hepatitis B started in the 1990s. The vaccine is delivered in a 3 dose regimen and if completed, $>90 \%$ of people will develop seroprotective titers. Breakthrough acute infections have been reported in vaccinated individuals, though there are no reports of chronic HBV disease in a vaccinated individual. While current guidelines do not recommend routine assessment of titer levels, testing of high risk individuals may be warranted. High risk groups include healthcare workers, men who have sex with men, injection drug users and persons with multiple sexual partners. As those vaccinated as children grow into adulthood and engage in high risk behaviors, breakthrough infections may become more common. As this case illustrates, physicians need to be aware of the possibility of breakthrough infection in vaccinated patients and be cognizant of risk behaviors.

CALCIPHYLAXIS IN NORMAL RENAL FUNCTION Molly Wolf ${ }^{1}$; Utibe R. Essien ${ }^{2} .{ }^{1} \mathrm{MGH}$, Boston, MA; ${ }^{2}$ Massachusetts General Hospital, Boston, MA. (Control ID \#2469525)

LEARNING OBJECTIVE \#1: Recognize risk factors for calciphylaxis in non-renal patients.

LEARNING OBJECTIVE \#2: Describe diagnostic techniques and initial management for calciphylaxis.

CASE: A 57-year-old Egyptian female, with a history of atrial fibrillation on warfarin, hypoparathyroidism on calcium supplementation, diabetes type 2 , and asthma presented with 6 months of painful indurated nodules involving the abdomen, legs, and breasts. The nodules ulcerated through her skin surface, eventually forming large black echars. The patient was initially hospitalized in Egypt where her lower extremity wounds were debrided and biopsied, but a diagnosis was not made. She then traveled to the U.S. where she was admitted for expedited workup and pain management. On presentation, exam was notable for stable vital signs, with painful bilateral lower extremity symmetrical wounds, as well as tender palpable abdominal nodules and ulcerations on her breast areolas. Additionally she had bilateral great toes with dry gangrene, and scattered purpuric patches over her dorsal feet. Laboratory data revealed a normal complete metabolic panel including serum calcium, phosphorus, and creatinine. Additionally, she had a negative rheumatoid factor and ANCA titers. CT-scan of the abdomen revealed calcific atherosclerotic plaques of the abdominal aorta, with scattered small vessel irregularities. Biopsy of the skin wounds revealed skin and subcutis with fat necrosis, and calcifications within the fibroadipose tissue and the arteriolar walls, consistent with calciphylaxis, A radionuclide bone scan showed soft tissue calcifications on the sites of her palpable nodules, consistent with the diagnosis. The patient was started on sodium thiosulfate (STS) therapy for treatment under the guidance of dermatology, and had notable reductions in pain after 2 weeks of therapy. A wound culture grew Pseudomonas for which she completed a course of cefepime. Her anticoagulation management was switched to rivaroxaban. Her calcium supplementation was carefully titrated to avoid hypercalcemia. Patient was discharged to a rehabilitation facility with plans to continue STS therapy.

DISCUSSION: Calciphylaxis is a dermal arteriolar calcification disorder most commonly diagnosed in dialysis-dependent, end-stage renal disease (ESRD) patients, though also reported in patients with $\mathrm{CKD}$, in renal transplant patients, and in those with normal renal function $^{1,2}$. As diagnosis requires a high index of suspicion, physicians should be aware of common risk factors including ESRD, primary hyperparathyroidism, warfarin (vitamin K antagonist) therapy, malignancy, liver disease, female gender, autoimmune diseases, and hypercoagulable states ${ }^{1}$. Presenting symptoms generally include painful skin nodules, livedo reticularis, and ulcerations with black eschars in adipose-rich areas ${ }^{1}$. Diagnosis requires a skin biopsy to look for calcification of the tunica media and elastic membrane of small to medium sized arterioles ${ }^{1}$. Calcium stains, such as von Kossa, are frequently required to detect tissue calcium deposits. Treatment requires a multi-disciplinary team, and using STS to increase calcium solubility ${ }^{2}$. The high mortality rate is due to sepsis complications ${ }^{1}$. While calciphylaxis was originally thought to be due to deranged metabolism with an elevated calcium-phosphorus product resulting in precipitation, literature review demonstrates that mineral parameters may be within normal limits ${ }^{1}$. One theory is that patients who have pre-existing risk factors (most commonly renal disease) have a trigger (most commonly skin trauma), which precipitates calciphylaxis. This patient had calciphylaxis lesions at the sites of skin trauma including the points of contact between her two legs. In non-renal calciphylaxis, the dose and duration of STS in a non-dialysis patient is not standardized, but is titrated based on side effect tolerance and efficacy ${ }^{2,3}$. If possible, warfarin should be discontinued and alternative anticoagulation pursued ${ }^{3}$. Follow-Up: The patient completed 16 weeks of STS therapy. At follow up, her wounds were healing. She continues to suffer from toe pain due to dry gangrene. References: Nigwekar SU, Wolf M, Sterns RH, Hix JK. Calciphylaxis from nonuremic causes: a systematic review. Clinical journal of the American Society of Nephrology 3 (2008): 1139-43. Nigwekar SU, Brunelli SM, Meade D, Wang W, Hymes J, Lacson E, Jr. Sodium thiosulfate therapy for calcific uremic arteriolopathy. Clinical journal of the American Society of Nephrology 8 (2013): 1162-70. Ross EA. Evolution of treatment strategies for calciphylaxis. American journal of nephrology 34 (2011): 460-7.

CARDIAC ARREST IN A YOUNG MAN FROM THYROID STORM Weijia Wang. Tufts Medical Center, Boston, MA. (Control ID \#2463015)

LEARNING OBJECTIVE \#1: Recognize the symptoms and signs of thyroid storm. CASE: A 34-year-old man with no past medical history presented to the emergency room with 3 months of progressive dyspnea on exertion, fatigue, palpitation, and weight loss of $10 \mathrm{lb}$. He also complained of subjective fever and chills. Family history was significant for thyroid diseases of his mother. He denied taking medications and illicit drugs. On physical examination, he appeared restless. He was febrile to $38.3^{\circ}$ of Celsius with heart rate at 157 beats per minute in atrial fibrillation and blood pressure of $150 / 60 \mathrm{mmHg}$. He was breathing at 25 times per minute with $\mathrm{SaO} 2$ of $95 \%$ on room air. Fullness of the neck, hyperdynamic precordium, bilateral crackles and brisk reflexes were noted. No proptosis or pretibial edema was observed. In the emergency room, he quickly deteriorated into pulseless electrical activity from hypoxia and was resuscitated with ROSC achieved in $5 \mathrm{~min}$. TSH came back as undetectable. Total T3 was $116 \mathrm{ng} / \mathrm{dL}$ and free T4 was $>8 \mathrm{ng} /$ dL. He was started on Propylthiouracil and Propranolol for thyroid storm. Iodide was added for Wolff-Chaikoff effect. Thyroid ultrasound next morning showed diffuse enlargement of the thyroid gland with increasing vascularity consistent with Grave's disease. Transthoracic echocardiography revealed a diffusely hypokinetic left ventricle with ejection fraction of $30 \%$. His hospital course was complicated by pneumonia and GI bleeding. He recovered well despite of lengthy ICU stay and was discharged home on Propylthiouracil and standard heart failure regimen with follow up in the clinics. 
DISCUSSION: Thyroid storm, an endocrine emergency first described in 1926 remains to be a diagnostic challenge. Its diagnosis is based on clinical findings. High fever (above $40^{\circ}$ Celsius) is very common. Cardiac manifestations include tachycardia out of proportion to the underlying illness, dyspnea on exertion, widened pulse pressure, cardiac ischemia and atrial fibrillation. The increased cardiac output and accompanying tachyarrhythmia can progress into cardiovascular collapse and shock. CNS manifestations are almost invariably seen and range from agitation, delirium, confusion and coma. Gastrointestinal symptoms include nausea, vomiting and severe diarrhea. Laboratory abnormalities can only aid in the diagnosis and there is no definite serum T4 or T3 cutoff that differentiates uncomplicated thyrotoxicosis from crisis. This case demonstrated a typical manifestation of thyroid storm which deteriorated rapidly into cardiac arrest unfortunately. It is important that primary care physicians and hospitalists be able to recognize the signs and symptoms of impending failure as well as start the therapy as soon as possible. The most teachable moment here is that thyroid storm is a clinical diagnosis and we should not let laboratory results delay the treatment if the clinical suspicion is high.

CAREFUL HISTORY AND PHYSICAL EXAM REMAINS THE CORE OF ACCURATE DIAGNOSIS Joydeep Chakraborty; Christopher E. Forsmark. University of Florida, Gainesville, FL. (Control ID \#2469082)

LEARNING OBJECTIVE \#1: Recognize pertinent history and physical exam findings required to diagnose Hereditary Hemorrhagic Telangiectasia (HHT).

LEARNING OBJECTIVE \#2: Manage various clinical presentations of HHT and perform appropriate screening and preventive treatment to avoid catastrophic consequences.

CASE: A 60 year old male presented to our Gastroenterology clinic to establish care for history of recurrent upper gastrointestinal (GI) bleeding over the past 12 years. He had moved to our city 10 months ago. The initial episode manifested as progressive fatigue due to anemia. He was evaluated and underwent an upper endoscopy, which revealed several bleeding arteriovenous malformations (AVMs) in the stomach and these were successfully cauterized. Subsequently, he required repeat endoscopy approximately every 18 to 24 months for recurrent severe symptomatic anemia (with a drop in $\mathrm{Hb}$ to $7 \mathrm{~g} / \mathrm{dl}$ from his baseline of 9 to $10 \mathrm{~g} / \mathrm{dl}$ ). Each recurrence was treated with local endoscopic therapy of the bleeding gastric AVMs. After this therapy, his hemoglobin $(\mathrm{Hb})$ improved to baseline with iron supplementation and monthly Epoetin alfa shots for his associated Chronic Kidney Disease (CKD). He had received blood transfusions on a few occasions. He denied a history of hematemesis but did report intermittent black stools. Colonoscopy was normal 18 months ago. He had not undergone enteroscopy or capsule endoscopy. On further probing into additional history, he reported having recurrent self-limiting epistaxis since the age of 3 years that occurred 2 to 4 times a week in recent years. He denied any history of stroke, hemoptysis or shortness of breath. His family history was notable for recurrent frequent epistaxis in his 20 year old son. His past medical history was significant for Hypertension, Type 1 Diabetes Mellitus with CKD stage 4 (not on dialysis) and recent NSTEMI. He denied tobacco, alcohol or recreational drug use. His physical examination was unremarkable other than large telangectasias on his tongue, with multiple smaller ones on the inner margin of his lips and on periungual fingertips. Laboratory testing revealed anemia, renal dysfunction (stable) and normal liver chemistries. Multiple gastric AVMs are exceedingly unusual. Based on his history, he met clinical criteria for a diagnosis of HHT. Despite multiple previous endoscopic procedures and clinic visits, this was the first time he had been told he might have a genetic syndrome causing his illness. He was scheduled for deep enteroscopy to look for AVMs in small intestine. He was referred to otolaryngology for management of recurrent epistaxis. He was scheduled for screening with a brain MRA and Transthoracic contrast echocardiography (TTCE). He was offered genetic testing for both him and his son
DISCUSSION: HHT, also known as Osler-Weber Rendu disease, is an uncommon autosomal dominant disease with a prevalence of one in 5000 persons. It is caused by abnormal blood vessel development which manifests as AVMs and telangiectasia on mucocutaneous surfaces (lips, tongue, chest or hands) and visceral organs including GI, lung, brain, liver and spinal cord. Recurrent spontaneous epistaxis is the most common symptom and often leads to iron deficiency anemia (IDA). GI telangiectasias (mainly gastric and small intestinal) can cause slow intermittent GI bleed. This commonly occurs after the 5 th decade of life and in up to $25-30 \%$ patients, and presents as symptomatic anemia. Pulmonary AVMs (PAVMs) are seen in $15-50 \%$ and can have life threatening manifestations in the form of paradoxical embolization (stroke, cerebral abscess) or massive hemoptysis. Cerebrovascular malformations (CVMs) can present as headaches, seizures or bleeding which can lead to long term disability. HHT is a clinical diagnosis or can also be diagnosed by identification of causative mutation. As per the Curaçao criteria, we can diagnose 'definite' HHT if 3 or more of the 4 clinical criteria (epistaxis, telangiectasias, visceral AVMs or family history of HHT) are present. The goal of genetic testing is to find the specific HHT mutation (of endoglin gene or activin A receptor type II like 1 gene or SMAD4 gene). Identification of the specific gene allows for effective screening of family members who do not meet the clinical criteria. Screening with brain MRA (for CVMs) and TTCE (for PAVMs) should be performed in all patients with HHT. Repeated epistaxis can be managed with humidification of indoor air and by endonasal coagulation as first line therapy. Repeated GI bleeds is managed by endoscopic therapy (argon plasma coagulation is preferred), treatment of IDA and systemic hormonal or antifibrinolytic therapy in selected patients. PAVMs are treated with transcatheter embolotherapy. Patients with documented PAVMs and those not yet screened must get antibiotic prophylaxis for dental procedures, avoid SCUBA diving and air filters should be used on all intravenous lines. Asymptomatic children of an affected parent should be considered to have HHT unless it is excluded by genetic testing. This case exemplifies Sir William Osler's best known saying, "Listen to your patient, he (she) is telling you the diagnosis". HHT is often unrecognized and physicians may be unaware of current screening and treatment guidelines leading to unnecessary stroke and life threatening hemorrhage in children and adults. A thorough history and exam will lead to a timely and accurate diagnosis of HHT, treatment of acutely symptomatic disease and prevention of complications.

CAROTID PSEUDOANEURYSM: A RARE COMPLICATION OF 'THE FORGOTTEN DISEASE'. Audrey Tran; Gursimran Chandhoke; Christopher A. Smith. Queen's University, Kingston, ON, Canada. (Control ID \#2468549)

LEARNING OBJECTIVE \#1: Identify the key clinical features and principles of management in Lemierre's syndrome.

LEARNING OBJECTIVE \#2: Recognize the indications for surgical management of Lemierre's syndrome.

CASE: A 37 year-old previously healthy man presents with right sided pleuritic pain and shortness of breath on a background of a 4 day history of fever, neck pain and odynophagia. He was not taking any medications prior to hospitalization and denied illicit drug use. Physical examination showed left neck tenderness and decreased air entry to the right lung base. He had poor dentition but no evidence of abscess formation. A CT neck confirmed the presence of a $4 \mathrm{~cm}$ left internal jugular thrombus extending into the facial vein, septic arteritis of the left common and internal carotid arteries and an associated carotid mural thrombus. Furthermore, CT chest revealed multifocal cavitary pneumonia associated with a loculated right-sided pleural effusion. Thoracentesis was performed and revealed a parapneumonic effusion. With antibiotic treatment, the effusion resolved. Blood cultures were positive for Streptococcus pharynges which was pansensitive. Due to the extent of the necrotizing pneumonia, he was treated with piperacillin/tazobactam for a total of 8 weeks as per Infectious Diseases. Due to worsening septic emboli in the lung 
despite appropriate antibiotic therapy and evidence of a carotid mural thrombus, a decision was made to initiate anticoagulation. This was continued for 3 months total therapy. Finally, repeat imaging within 1 week of his initial presentation demonstrated the development of a carotid pseudoaneurysm at the level of the carotid bulb with underlying thrombus. This was not repairable and the patient underwent a carotid bulb resection with a saphenous vein bypass graft.

DISCUSSION: Internal jugular vein suppurative thrombophlebitis, otherwise known as Lemierre's syndrome and 'the forgotten disease', is a rare clinical entity characterized by persistent fever, neck pain and septic emboli—particularly pulmonary abscesses and septic arthritis. This is often preceded by pharyngeal infections and occurs in young, healthy adults. Classically, it is caused most commonly by Fusobacterium necrophorum, a gram-negative pharyngeal anaerobe. Diagnosis is confirmed by CT of the neck. The mainstay of treatment is prolonged antibiotics. The use of anticoagulation is controversial. In the setting of ongoing clot extension and embolic phenomenon, anticoagulation should be considered. In our case, ongoing embolic phenomenon (pulmonary) and the presence of a carotid mural thrombus despite appropriate therapy provided reasoning for anticoagulation as per Hematology. Persistent sepsis despite adequate therapy is an indication for surgical referral. Classically this will involve, ligation or excision of the internal jugular vein. The development of an aneurysm in Lemierre's is a rare occurrence. We make note of a single case of mycotic aneurysm involving the vertebral artery. Our patient represents the first case of Lemierre's that has led to the development of a carotid pseudoaneurysm. Surgical repair is indicated in extracranial carotid pseudoaneurysms that are symptomatic, greater than $2 \mathrm{~cm}$ in diameter or in the presence of an intraluminal thrombus. In an infected pseudoaneurysm, open repair is preferred over endovascular repair. Carotid resection for mycotic pseudoaneurysm represents a rarely performed procedure in the setting of Lemierre's syndrome not previously found in the literature. Though the mainstay of treatment is antibiotics, vascular surgery involvement should be considered in the setting of persistent infection and mycotic aneurysm.

CASES OF GRAM NEGATIVE RODS BACTEREMIA SECONDARY TO RUPTURED NON-ANEURYSMAL INFECTIOUS AORTITIS AND UNRUPTURED ANEURYSMAL INFECTIOUS AORTITIS WITH INTRAMURAL ABSCESS Masaji Saijo ${ }^{1}$; Toshiaki Wakai ${ }^{2}$; Dongkyung Seo ${ }^{1}$; Keitaro Takeda ${ }^{1}$; Urara Nakagawa ${ }^{1}$; Yasushi Tanabe ${ }^{1} .{ }^{1}$ Sapporo Tokushukai Hospital, Sapporo, Japan; ${ }^{2}$ Ohasa Clinic, Ebetsu, Japan. (Control ID \#2468498)

LEARNING OBJECTIVE \#1: Infectious aortitis (IA) is one of the essential differential diagnosis for persistent bacteremia even by the atypical causative organism including Gram Negative Rods (GNR) because of its high mortality.

CASE: Case1: An 82-year-old male with a history of bladder cancer, recurrent urinary tract infection (UTI), diabetes mellitus, and cerebral infarction was brought to the Emergency Department (ED) of Sapporo Tokushukai Hospital from skilled nursing facility (SNF), presenting with fever and chills. On arrival, he was febrile with other normal vital signs, and pyuria and leukocytosis were present. Pyelonephritis was suspected and Piperacillin/Tazobactam was initiated. Blood culture showed ended-spectrum betalactamases (ESBL)-producing Escherichia coli on hospital day 3, and the antibiotics was switched into Meropenem. His clinical condition was improved, and it was discontinued on hospital day 21 . He was stable enough to be prepared for the transfer back to SNF. However, he was found to be a status of sudden cardiac arrest by a registered nurse and passed away despite resuscitation efforts. Autopsy reported GNR induced IA, ruptured into bilateral thoracic cavities without aneurysm formation. Case 2: An 82-yearold male with a history of chronic obstructive pulmonary disease (COPD) and thoracic aneurysm was brought to the ED, presenting with shortness of breath for 1 day. On arrival, he was severely respiratory distressed with desaturation of $88 \%$ on room air and has severe bilateral wheezing, and COPD exacerbation was suspected. Short-acting beta agonist inhaler, intravenous (IV), Methylprednisolone and IV Ceftriaxone with oral clarithromycin were initiated. His clinical condition was improved and those treatments were discontinued on hospital day 21 . His respiratory status suddenly exacerbated on hospital day 30 , cefepime was initiated for possible hospital-acquired pneumonia. However, his urine output had been decreased, and he became hypotensive and severely respiratory distressed with acute renal failure on hospital day 47, requiring intubation and transfer to intensive care unit. Cefepime and meropenem were initiated for positive blood cultures of carbapenem-resistant Pseudomonas aeruginosa and ESBL-producing Klebsiella pneumonia, possibly secondary to UTI or catheter-related blood stream infection (CRBSI). His clinical condition continued to deteriorate, he did not respond to resuscitation efforts and he passed away due to multiple organ dysfunction syndrome (MODS) on hospital day 79. Autopsy reported unruptured thoracic aneurysmal IA complicated by intramural abscess, whose culture revealed carbapenem-resistant P. aeruginosa and ESBL-producing K. pneumonia.

DISCUSSION: IA is a comparatively rare disease, remains a significant cause of mortality, which is ranging from 21 to $44 \%$. (Foote et al., 2005). Staphylococcus species and Salmonella species are reported as a major offending organism of IA as opposed to GNR which is rare. Furthermore, AI with GNR has a higher rate of rupture and fatality than that caused by gram-positive organisms (72\% vs $25 \%$ ). (Johnstone et al., 2012) In general, IA requires surgical debridement and revascularization followed by parental or oral antibiotics for at least 6 weeks for the treatment. Even with the invasive surgery, the overall mortality rates range from 40 to $45 \%$, whereas mortality from IV antibiotics alone approaches close to $100 \%$. (Rondina et al., 2006)

CAUSE OF URINARY RETENTION? THINK OUTSIDE OF THE BLADDER Kazusa Saegusa $^{2}$; Satoshi Kobayashi ${ }^{2}$; Sandra Y. Moody ${ }^{1}$; Makito Yaegashi ${ }^{2} .{ }^{1}$ Kameda Medica Center \& UCSF/SFVAMC, Kamogawa City, Japan; ${ }^{2}$ Kameda Medical Center, Kamogawa City, Japan. (Control ID \#2448689)

LEARNING OBJECTIVE \#1: Recognize a possible differential diagnosis and relevant management of urinary retention.

CASE: A 54-year-old woman presented with urinary hesitancy for 10 days, fever and severe headache for 5 days, and urinary retention for 2 days. She saw her primary care physician 2 days before admission and was treated for cystitis with levofloxacin $500 \mathrm{mg}$ orally for 2 days. Since her symptoms did not improve, she was admitted for further evaluation and treatment. Her physical examination was unremarkable. Although we did not find bacteria on urine gram stain or culture, leukocyte esterase and nitrates were positive. We treated her with a $2 \mathrm{~g}$ dose of cefotiam, a second-generation cephalosporin, intravenously three times daily for 10 days. Though her fever and headache improved, she still had trouble with urination. Abdominal CT with and without contrast showed no evidence of urethral stenosis or obstruction. Thoracic and lumber MRI scans revealed bulging discs at L4/L5 and L5/S1, slightly compressing the dural sac, but no signs of spinal cord compression. Brain MRI was unremarkable. The imaging tests did not reveal a primary cause of her urinary retention. Thus, we performed a lumber puncture that showed a lymphocytic pleocytosis with 181 cells, $91 \%$ of which were mononuclear cells. The CSF protein level was slightly elevated at $86 \mathrm{mg} / \mathrm{dL}$, and the glucose level was normal at $63 \mathrm{mg} / \mathrm{dL}$. These findings were consistent with aseptic meningitis. Intermittent selfcatheterization was initiated; however, at discharge, her urinary retention had completely resolved without medications or further interventions.

DISCUSSION: Meningitis-retention syndrome (MRS) is a clinical entity presenting with aseptic meningitis and urinary retention without other significant neurological abnormality except for mild pyramidal signs. There are several differential diagnoses of aseptic meningitis with urinary retention, including sacral herpes, acute disseminated 
encephalomyelopathy (ADEM), acute multiple sclerosis (MS), neuromyelitis optics (NMO), Guillain-Barre syndrome, and other common causes of neurogenic urinary retention such as diabetic neuropathy and lumbar spondylosis. Although the pathological mechanism of MRS is incompletely understood, it appears to be a very mild form of $\mathrm{ADEM}$, one of the autoimmune demyelinating diseases of the central nervous system, which is triggered by infection. While acute MS, ADEM, and NMO require therapy with corticosteroids or immunosuppressive agents, MRS has a benign and self-remitting course with a duration of 2 to 10 weeks. Elsberg syndrome, another similar condition to MRS, is nonspecific, but possibly encompasses MRS or ADEM, sacral myeloradiculitis of undetermined etiology, or sacral herpes. Some experts define Elsberg syndrome as urinary retention with aseptic meningitis and abnormalities on imaging studies, and others think it is due to varicella-zoster virus (VZV). Sacral herpes, however, often accompanies skin rashes and elevated viral titers, while MRS does not. Though the etiologies of MRS have not been determined, several previous reports suggest viral and bacterial infections (HSV2, VZV, Campylobacter etc.) as well as herbal medication. Specific localization of lesions in MRS also remains unclear. Prospective studies are needed to uncover the etiologies, establish diagnostic processes and determine appropriate management of MRS. This case illustrates one possible differential diagnosis of urinary retention with aseptic meningitis, and the need for lumber puncture in such cases. Physicians need to be aware of these conditions, since urinary retention is a urologic urgency. By diagnosing MRS by lumbar puncture, physicians may be able to avoid medications (e.g., alpha-blockers), intermittent catheterization, and indwelling bladder catheterization, since uneventful recovery is expected.

CAVITARY RHIZOPUS INFECTION Hannah Oakland; Jane Andrews. Tulane University Health Sciences Center, New Orleans, LA. (Control ID \#2467547)

LEARNING OBJECTIVE \#1: Develop a differential diagnosis for cavitary lesions in renal transplant patients.

LEARNING OBJECTIVE \#2: Manage pharmacologic immunosuppression in renal transplant patients with pulmonary mucormycosis.

CASE: This patient is an 18 year-old man with a past medical history of insulin-dependent diabetes and renal agenesis who presented to our hospital with fever, hemoptysis, and back pain. On admission he was found to have a 5-6 cm cavitation in his left lower lobe with surrounding consolidation. The patient had undergone his first renal transplant 4 months prior and was immunosuppressed with cyclosporine and prednisone. His initial workup was significant for a fever to $103 \mathrm{~F}$ and $\mathrm{WBC}$ of 21.9. He was started on fluconazole, piperacillin/tazobactam, and vancomycin. On the 7th day of admission, he underwent thoracotomy with left lower lobe lung resection. Sputum cultures from admission and lobectomy tissue cultures would eventually reveal Rhizopus, so the patient was started on liposomal amphotericin B, the first-line therapy for Rhizopus. It was replaced by isavuconazonium after 4 days of treatment due to concern for a rising creatinine. His hospital course was also complicated by mycoplasma hominis infection, treated with 14 days of levofloxacin. Over the course of admission, cyclosporine dose was gradually reduced as the patient continued to have fevers and chills despite surgical resection of the cavitary lesion. As his creatinine rose, a renal biopsy was performed which was suspicious for T-cell mediated rejection as well as moderate to severe tubuloepithelial injury consistent with acute tubular necrosis. Therefore, cyclosporine was stopped entirely. He was discharged almost 2 months after admission with a creatinine which had risen to 4.5 but not yet requiring dialysis.

DISCUSSION: Although mucormycosis infections are becoming more common, the incidence of rhizopus infections is likely far below $1 \%$ in renal transplant patients. This case demonstrates several important teaching points. The differential diagnosis of a cavitary lung lesion is broad, including bacterial infections (anaerobes, S. aureus, S. pneumo, klebsiella, P. aeruginosa), mycobacteria, and fungi. Non-infectious causes must also be considered, including vasculitides, neoplasm, bullae with air fluid levels, or pulmonary sequestration. Pulmonary mucormycosis infections are rare and occur mostly in immunosuppressed patients, including transplant patients and diabetics. The mortality of these infections is not well established but is certainly high; one extensive review calculated $76 \%$ in pulmonary infections and $96 \%$ in disseminated infections as opposed to $45 \%$ in rhino-orbital infections. Rhizopus species generally account for one third to one half of mucomycosis infections. Early surgery including debridement and lobectomy has been retrospectively linked to lower mortality. Although no randomized controlled trials exist establishing it as first line treatment of Rhizopus, liposomal amphotericin B has long been used and has also been retrospectively shown to be associated with lower mortality rates. The decision to use this drug in a life-threatening infection was complicated by the patient's very recent renal transplant. This transplant had significantly improved the quality of life of this young man, and he and his family initially strongly advocated for treatment approaches that would preserve its function. Eventually, the severity of the infection forced the patient and team to decide upon the cessation of cyclosporine and prednisone with concomitant treatment with intravenous amphotericin B.

CEFTAROLINE INDUCED AGRANULOCYTOSIS Najmus Sahar ${ }^{1}$; Theresa Ratajczak $^{1}$; Nicholas Conger ${ }^{2}$. ${ }^{1}$ Wright State University, Beavercreek, OH; ${ }^{2}$ WPAFB, Beavercreek, OH. (Control ID \#2456673)

LEARNING OBJECTIVE \#1: Ceftaroline fosamile is a new fifth generation cephalosporin with broad spectrum bactericidal activity. It is currently approved for acute bacterial skin and skin structure infections (ABSSSI) and community-acquired bacterial pneumonia. We report a case of agranulocytosis as a side effect of ceftaroline use, of which the internist needs to be aware.

CASE: A 24-year-old male, intravenous (IV) drug user, presented with right sided chest pain of 3 days duration, associated with productive cough, dyspnea, fatigue, and abdominal pain. Physical exam revealed a heart rate of $143 \mathrm{bpm}$, and a holosystolic murmur along the left sternal border. Admission complete blood count (CBC) showed a leukocyte count (WBC) of $11.7 \mathrm{~K} / \mathrm{MM} 3$ with $81 \%$ neutrophils. Chest computer tomography revealed multiple septic pulmonary emboli. Acute coronary syndrome was ruled out with normal cardiac enzymes and non-specific ST changes on EKG. The diagnosis of infective endocarditis was clinched when transthoracic echocardiogram demonstrated tricuspid valve vegetation with severe tricuspid regurgitation and blood cultures grew methicillin resistant staphylococcus aureus (MRSA). The patient's empiric antimicrobials were narrowed to ceftaroline $600 \mathrm{mg}$ IV every $8 \mathrm{~h}$ for a planned 6 week course because of cost effectiveness. On ceftaroline day 15 , WBC was $8.2 \mathrm{~K} / \mathrm{MM} 3$ with $82 \%$ neutrophils and on day 41 , WBC was $0.5 \mathrm{~K} / \mathrm{MM} 3$ with an absolute neutrophil count of 0 . The agranulocytosis was felt to be a side effect of the antibiotic, which was stopped at day 41 . The patient's WBC gradually trended upward to $3.3 \mathrm{~K} / \mathrm{MM} 35$ days later and $11.2 \mathrm{~K} / \mathrm{MM} 3$ 8 days after stopping ceftaroline therapy.

DISCUSSION: Agranulocytosis is defined as a neutrophil count of $<100 /$ microL. It is a rare but serious side effect associated with beta lactam antibiotics use in large doses for longer than 10 days. Cephalosporins have been used $>40 \%$ of the time as outpatient IV therapy with associated incidence of leukopenia of $<1 \%$ but has been historically reported as high as $5-15 \%$ with large doses and prolong course. Complete recovery within 1 week after discontinuation of therapy has been observed in most cases. The mechanism is thought to be either from immune mediated cell destruction, or the direct toxic effects on bone marrow. Ceftaroline is recommended for MRSA infections, multidrug resistant strains of streptococcus pneumoniae as well as many gram-negative organisms. It's general side effects include positive coombs test without hemolysis, hypersensitivity and gastrointestinal manifestations. Agranulocytosis has been reported in $<1 \%$ of users. 
In our patient, the high dose, prolong treatment course and the timeline are consistent with ceftaroline associated agranulocytosis. Severe neutropenia and agranulocytosis are rare but reversible side effects of ceftaroline. Monitoring weekly CBC and patient's education about neutropenia symptoms are important aspects of management.

\section{CHALLENGES IN DIAGNOSING AND TREATING IGG4-RELATED DISEASE}

Dawn Zhao; Ifeoma Aduba; David Schmit. UTHSCSA, San Antonio, TX. (Control ID \#2469757)

LEARNING OBJECTIVE \#1: Recognize the differential diagnosis of peripheral eosinophilia in a patient presenting with pneumonia

LEARNING OBJECTIVE \#2: Differentiate the first and second line therapies in the treatment of IgG4-Related Disease

CASE: A 67-year-old male with a history of presumed pancreatic cancer, thyroid cancer, lacrimal gland mass, cryptogenic cirrhosis, and recently diagnosed IgG4-related disease presented from clinic with hypoxia. He had been recently discharged on a 10-day course of antibiotics after a hospitalization for multi-lobar pneumonia. However, his symptoms of cough, shortness of breath, and hypoxia did not improve despite therapy. A chest x-ray performed during his next admission showed worsening left upper lobe infiltrates/mass as well as a right lower lobe consolidative mass. Suspicion for recurrent pneumonia was low, given that he was afebrile. He had a normal white blood cell count significant for peripheral eosinophilia that was unimproved with antibiotics. Given eosinophilia, persistent infiltrates, and history of IgG4-related disease, the work up for his current symptoms was broadened to include autoimmune, IgG4-related disease, malignancy, atypical pneumonias, and eosinophilic etiologies. Fungal serologies, p-ANCA serology, and stool ova and parasite returned negative. He then underwent bronchoscopy with biopsy. Pathology results came back as "features suggestive of IgG4-related disease," with an IgG4 ratio $>40 \%$. He started a course of high-dose prednisolone. Post bronchoscopy, he developed acute hypoxic respiratory failure that required intubation and mechanical ventilation. He developed hemoptysis, and repeat bronchoscopy showed diffuse alveolar hemorrhage. Anti-glomerular basement membrane serology for Goodpasture's disease work up returned negative. Given lack of clinical improvement, he was transitioned to rituximab therapy. His condition further deteriorated, and the patient expired 1 month after admission. A CT scan of the head showed massive intracranial and parenchymal bleeds with midline shift and herniation. An autopsy revealed IgG-4 fibrosing pulmonary disease and intracerebral bleed as a complication of liver cirrhosis and coagulopathy as the causes of death.

DISCUSSION: This case illustrates the diffuse nature of IgG4-related disease and the multiorgan involvement that can be seen. It can be the underlying etiology behind a collection of disorders that initially seem unrelated. Initially viewed as a "zebra" condition, IgG4-related disease is now increasingly recognized as the etiology behind isolated organ pathologies. In this particular case, clinical suspicion for IgG4-related disease should have been higher given this patient's multi-organ involvement and history of pancreatic mass. Unfortunately, by the time his IgG4-related disease diagnosis was certain and glucocorticoid and rituximab therapy was initiated, the patient showed symptoms of multi-organ failure. This case highlights the need for disseminated guidelines to facilitate early IgG4-related disease diagnosis and more comprehensive therapeutic options for managing it. Current management relies on early immunosuppression to prevent disease progression with limited established options for latestage disease and acute exacerbations. Perhaps future studies should examine the effectiveness of screening criteria or treatments that further decrease plasma cell burden.

CHEST PAIN AND A CROOKED FINGER: RECURRENT PERICARDITIS AS THE PRIMARY MANIFESTATION OF A CONNECTIVE TISSUE DISORDER Jennifer P. Weintraub. Icahn School of Medicine at Mount Sinai, New York, NY. (Control ID \#2469903)
LEARNING OBJECTIVE \#1: Identify autoimmune disease as an uncommon cause of pericarditis

LEARNING OBJECTIVE \#2: Diagnose jaccoud's arthropathy and recognize its relationship to autoimmune disease

CASE: A 57-year-old male with a history of diet controlled diabetes and asthma presented to our clinic with 2 days of chest pain. His pain was difficult to characterize but was described as constant and exacerbated only by deep breaths and leaning back. His chest pain was not worsened with exertion, not associated with food, and not accompanied by symptoms of chest pressure, cough, fever, or rash. He denied a history of oral lesions, rashes, arthralgias, and a family history of autoimmune disease. Vital signs were within normal limits and the cardiopulmonary exam was unremarkable. An electrocardiogram done in the office showed concave ST elevations in I, II, V4-V6 with concurrent PR depression in these leads. Labs were notable for an elevated ESR $(46 \mathrm{~mm} / \mathrm{h})$ and CRP $(78.3 \mathrm{mg} / \mathrm{L})$ and a negative troponin. Blood counts as well as liver and renal function were within normal limits. Tuberculosis, hepatitis $\mathrm{C}$, and HIV testing were also negative. Initial transthoracic echocardiogram (TTE) showed no pericardial effusion, and the patient was promptly started on indomethacin and colchicine for a diagnosis of acute pericarditis. Despite adherence to these medications, his symptoms worsened. He was admitted to the hospital with an enlarging pericardial effusion and rising inflammatory markers. An extensive work-up revealed only a positive ANA (1:160), and was notably negative for antibodies to dsDNA, Smith, Ro, La, and Histone-DNA IgG. His symptoms improved on systemic steroids and he was discharged home on colchicine and a steroid taper. Four weeks later, his symptoms recurred. He was then was noted to have a mild jaccoud's arthropathy on exam. These physical exam findings in conjunction with a positive ANA were highly suggestive of undifferentiated connective tissue disease. The patient has now had sustained improvement on his current regimen of azathioprine, prednisone, and colchicine

DISCUSSION: Though the majority of pericarditis cases diagnosed in developed countries are idiopathic, as many as $5-15 \%$ of cases may be secondary to an underlying systemic autoimmune disease. Pericardial involvement is rarely the initial manifestation of these diseases and there is little published data to help discern the etiology of pericarditis. In our case, a positive ANA along with the unusual physical exam finding of jaccoud's arthropathy led us to the etiology of his recurrent pericarditis. Jaccoud's arthropathy is a non-erosive joint disorder characterized by ulnar deviation of the digits and subluxation of the metacarpalphalangeal joints. The arthopathy is reversible with physical manipulation and thought to be secondary to soft tissue abnormalities rather than joint destruction. It has classically been described as a sequelae of rheumatic fever, but has been increasingly associated with systemic lupus erythematosus and other connective tissue diseases.

CHEW ON THE DIAGNOSIS: GRADENIGO'S SYNDROME KoKo Aung ${ }^{2}$; John DeMis $^{2}$; Thwe Htay ${ }^{1}$. ${ }^{1}$ University of Texas Health Science Center at San Antonio, Shavano Park, TX; ${ }^{2}$ University of Texas Health Science Center at San Antonio, San Antonio, TX. (Control ID \#2470272)

LEARNING OBJECTIVE \#1: To consider skull base osteomyelitis in patients with persistent unilateral facial pain and neurological signs after dental extraction LEARNING OBJECTIVE \#2: To recognize the limitations of empirical antibiotic therapy in skull base osteomyelitis without microbiological confirmation. CASE: A 76-year-old man with uncontrolled diabetes mellitus presented with a 2-month history of worsening right-sided headache and facial pain, right facial weakness, dysphagia, hearing loss, and right otalgia with intermittent otorrhea following a right upper molar extraction. CT scan done 1 week prior to presentation showed only right sphenoid sinusitis and lucency in the molar extraction region. Physical exam findings were confined to the right side: mastoid tenderness, purulent discharge from the external auditory canal, 
profound mixed sensorineural hearing loss, lower motor neuron-type facial palsy, and rightward gaze diplopia. An MRI and CT showed extensive inflammatory changes throughout the right petrous temporal bone, sphenoid sinus, right carotid space, right pterygopalatine fossa, and clivus. The 3-phase technetium scan of temporal bone showed increased focal uptake in the right mastoid region and petrous temporal bone consistent with right mastoiditis and petrous apicitis. The patient underwent a wide field myringotomy and was discharged after several days of antibiotic treatment with ciprofloxacin-dexamethasone eardrops and a 5 to 6-week course of IV cefepime and vancomycin. Six weeks later, repeated imaging studies showed worsening osteomyelitis now involving the right occipital condyle and left occipital bone. His right-sided frontalis weakness had resolved, dysphagia had improved, but the headache and otorrhea had become worse. He was readmitted and underwent right mastoidectomy, sphenoidotomy and ethmoidectomy. Purulent fluid was cultured from the right sphenoid sinus and mastoid sinus, which showed growth of Enterococcus spp. and Aspergillus fumigatus. Currently, he is being treated with IV vancomycin, metronidazole, cefepime, voriconazole, along with ciprofloxacin-dexamethasone eardrops.

DISCUSSION: In our case, the ascending infection plausibly spread from the dental operation through the sinuses, middle ear to petrous temporal bone and then adjacent bones in the base of the skull. Previously reported cases of aspergillus petrous apicitis in immunocompetent hosts primarily involved older adults. Our patient's final outcome is yet to be determined, but the overall mortality of aspergillus osteomyelitis of skull base is about $27 \%$. Persistent unilateral facial pain and neurological signs after dental extraction should prompt a clinician to consider petrous apicitis, especially in the setting of uncontrolled diabetes. Our case underscores the limitations of empirical antibiotic therapy in skull base osteomyelitis without microbiological confirmation. Surgical debridement of devitalized tissues assists in source control as well as providing intra-operative specimens for microbiological diagnosis that would be crucial in deciding appropriate antimicrobial therapy. Invasive fungal infection should be considered when there is lack of response to empirical therapy.

CHLAMYDIA PROCTOCOLITIS MASQUERADING AS AN ULCERATIVE COLITIS FLARE Patrick B. Wu; Mila Ortigoza; Daniel Eiras. New York University School of Medicine, New York, NY. (Control ID \#2464569)

LEARNING OBJECTIVE \#1: Recognize the role of sexually transmitted infections as a possible etiology for new symptoms in high-risk patients with chronic clinical conditions LEARNING OBJECTIVE \#2: Recognize the clinical features of and treatment options for lymphogranuloma venereum

CASE: A 41-year-old man was seen in the Emergency Department with 2 weeks of fever, night sweats, profuse diarrhea, and hematochezia. His past medical history was significant for HIV well-controlled on HAART as well as ulcerative colitis (UC) diagnosed 15 years ago. Notably, he reported similarities between his current presentation and several past "UC flares." However, he could not recall biopsy evidence for his UC diagnosis, nor was he taking UC medication. He reported being sexually active with his male partner, but specifically denied engaging in anal intercourse within the month prior to presentation. The patient's physical examination was notable for tenderness upon digital rectal exam, and he was started on empiric intravenous ceftriaxone and metronidazole out of concern for a prostatic abscess. He was admitted to an inpatient medical ward, and CT imaging revealed rectal wall thickening with associated fat stranding and local reactive lymphadenopathy. By the second day of the patient's admission, he was noted to be tachycardic and febrile to $102.3^{\circ} \mathrm{F}$. His empiric Gram-negative antibiotic coverage was broadened to piperacillin-tazobactam, and doxycycline was added for suspicion of possible anorectal chlamydia. Infectious workup revealed negative blood and stool cultures and a positive Chlamydia rectal swab by nucleotide amplification. Serum immunofluorescence chlamydial antibody panel revealed evidence of a chronic Chlamydia trachomatis serovar L2 infection suggesting lymphogranuloma venereum (LGV). Flexible sigmoidoscopy was performed, revealing histopathologic evidence of infectious proctitis with no evidence of underlying UC or acute UC flare. Piperacillin-tazobactam was discontinued, and the patient clinically improved on doxycycline. The patient was discharged with a 21-day course of oral doxycycline to treat LGV. He ultimately admitted to having intercourse with multiple partners, all of whom were advised to seek testing and treatment.

DISCUSSION: Sexually transmitted infections (STIs) are caused by a wide range of organisms, many of which are highly prevalent, causing an estimated one million infections per day worldwide. In particular, chlamydia can manifest with dysuria, dyspareunia, and discharge at the site of infection. Complications of untreated chlamydia include trachoma and blindness, pelvic inflammatory disease, infertility, and lymphogranuloma venereum (LGV). Caused primarily by Chlamydia trachomatis serovars L1, L2, and L3, LGV is an infection of the lymph nodes, presenting in three stages: 1) a self-limiting painless mucosal genital ulcer; 2) local lymphangitis and lymphadenitis which may progress to inguinal or femoral buboes; and 3) fibrotic changes causing lymphatic obstruction, chronic edema, and local fistulas. LGV can also arise from a rectal musocal infection, and manifests as proctitis or proctocolitis with associated anorectal pain and diarrhea concurrently with lymphatic inflammation, matching this patient's presenting symptoms and CT scan results. Beyond usual risk factors for contracting STIs, risk factors for chlamydial LGV include unprotected anal sex, concurrent ulcerative disease, and a history of STIs, in particular concurrent HIV, all of which were reported by this patient. Standard treatment for LGV is $100 \mathrm{mg}$ oral doxycycline twice daily or $500 \mathrm{mg}$ oral erythromycin four times daily for 21 days, with possible incision and drainage of large buboes as well as testing and treatment for HIV and other STIs. This case illustrates a diagnostic dilemma, specifically LGV versus a UC flare, in a high-risk patient with a history of a chronic inflammatory disease. Obtaining a detailed sexual history and taking into account this patient's HIV status led to appropriate treatment for secondary-stage LGV. By adding STIs to the differential for higher-risk patients, more patients may benefit from earlier identification and treatment, preventing long-term complications of undiagnosed or misdiagnosed STIs.

CHOLECYSTOENTERIC FISTULA RESULTING IN BOUVERET'S SYNDROME Eric Huynh; Roger D. Smalligan; Abdel Rahman A. Omer. Texas Tech Univ Health Sc Center - Amarillo, Amarillo, TX. (Control ID \#2469213)

LEARNING OBJECTIVE \#1: Determine the possiblity of a cholecystoenteric fistula early.

LEARNING OBJECTIVE \#2: Understand the appropriate treatment of a cholecystoenteric fistula and Bouveret's syndrome.

CASE: A 61-year-old man with a PMH of HTN, diverticulitis, hepatitis C, osteoarthritis and chronic pain presented to the emergency room after 1 week of nausea, intermittent vomiting with coffee-ground emesis and black tarry diarrhea. He had increasing epigastric and RUQ pain with occasional chills but no fever. He denied hematemesis, recent travel, sick contacts and new foods. Home medications included hydrocodone-acetaminophen, lisinopril and sulindac. Surgical history: sigmoid colectomy for diverticulitis, spinal cord stimulator placement via RUQ, colostomy creation and takedown, abdominal herniorrhaphy and left total knee replacement. He is a current smoker and drinks occasional alcohol and uses marijuana. On physical exam he was alert, BP 126/90, P 74/min, R 16/min, O2 sats $97 \%$ on RA, clear lungs, regular heart without murmurs, mild tenderness in the RUQ of the abdomen. There were no peritoneal signs and digital rectal exam did not reveal melena. Laboratory: WBC 12.8 k, Hgb 10.7 and normal platelets, PT and INR counts. CMP was largely unremarkable with normal transaminases and bilirubin. Stool was positive for lactoferrin, fecal occult blood and cryptosporidium and negative for 
C. difficile, Giardia and campylobacter. HIV antibody was negative as were blood cultures. RUQ ultrasound revealed normal pancreas and liver, with contracted gallbladder and possible air in the fossa. CT of the abdomen and pelvis showed chronic cholecystitis and suspicion of biliary-enteric fistula due to large amount of air identified within the gallbladder fossa, but no free intraperitoneal air. There was a large calcified gallstone in the duodenal bulb.

DISCUSSION: This patient demonstrated a potential serious complication that can follow episodes of acute or chronic cholecystitis. The gallbladder with a large stone can adhere to an adjacent organ and eventually erode through the wall and cause a biliaryenteric fistula. While the cause of these fistulas can vary from iatrogenic, peptic ulcer associated, malignancy and Crohn's disease, over $90 \%$ of them are associated with chronic cholelithiasis. Our patient exhibited both a chronic inflammatory state as well as previous surgery in the region from the spinal stimulator placement. The most frequently reported cholecystoenteric fistula locations are duodenum, colon and stomach. However, identifying these fistulas radiologically is often challenging, with the only hint being pneumobilia. Furthermore, with neither fever nor significant elevations in liver function tests in our patient, there was not clear evidence of acute or emphysematous cholecystitis. Infusion of dye by interventional radiology can be used to visualize such a fistula. One of the complications of such a fistula can be the passage of air from the bowel into the gallbladder leading to distention and discomfort. Gallstones have also been reported to pass through the fistula into the bowels and cause a classic gallstone ileus as the small intestine narrows into the ileum. If a gallstone is large enough to obstruct and become impacted in the duodenum, proximal to the pylorus, the condition is called Bouveret's syndrome. Traditionally, this syndrome has been managed with open cholecystectomy and closure of the fistula however increasingly, laparoscopic tools and techniques are being used instead. Because Bouveret's syndrome typically occurs in older patients with multiple comorbidities and thus higher surgical risk, attempts can be made to remove the stone by EGD. The disadvantage is that the fistula cannot be addressed. In one study this was only successful $9 \%$ of the time. In our patient, there was an unsuccessful attempt at endoscopic removal of the duodenal stone followed by a successful open laparotomy with duodenotomy to evacuate the large stone in the duodenum and repair of the fistula. The patient recovered well and was discharged home.

CHOOSING THE RIGHT NEEDLE FOR THE RIGHT HAYSTACK: USING A DIAGNOSTIC ALGORITHM TO DIAGNOSE AN UNCOMMON CAUSE OF UNIFOCAL LYMPHADENOPATHY Jenny K. Cohen. UCSF, San Francisco, CA. (Control ID \#2466650)

LEARNING OBJECTIVE \#1: Identify the limits and merits of fine needle aspiration (FNA) and core needle biopsy (CNB) for further characterization of lymphadenopathy LEARNING OBJECTIVE \#2: Understand the typical presentations of Castleman's disease

CASE: A previously healthy 32 year old woman presented to re-establish primary care. Three years prior, she underwent fine needle aspiration (FNA) of an enlarged lymph node on the lateral side of her neck. FNA showed reactive pattern lymphadenopathy without significant increase of eosinophils or the presence of granulomas, abscess, or ReedSternberg/Hodgkin cells. Flow cytometry was normal. Serologic testing for Bartonella, Toxoplamosis, Epstein-Barr Virus, and Human Immunodeficiency Virus was negative. Cytomegalovirus IgG was positive, but thought not to be the cause of her adenopathy. Despite unclear pathology, the patient was prescribed a course of antibiotics and noted improvement in the size of the lesion but not full resolution. An excisional biopsy had been planned, but she was lost to follow-up. Upon re-presentation to care, she noted the mass was stable over the last 3 years, but over the last few weeks it was larger, painful, and "pushing the jaw out". She denied any change in saliva production, stridor, or difficulty swallowing. She had no surgical history and no family history of lymphoma or other malignancies. Her only medication was a daily multivitamin. The patient's vital signs were normal and her exam was only notable for a $2 \mathrm{~cm} \times 3 \mathrm{~cm}$ rubbery, fixed mass below the angle of the mandible on the left lateral neck. There was no axillary or inguinal lymphadenopathy. She subsequently underwent excisional biopsy, which revealed hyalinevascular Castleman's disease. Complete blood count, lactate dehydrogenase, interleukin6 (IL-6), C-reactive protein, serum free light chains, and quantitative immunoglobulins were all normal. Given her presentation was thought to be consistent with unicentric disease, no further treatment after excisional biopsy was required.

DISCUSSION: Lymphadenopathy is defined as an abnormal number of palpable lymph nodes, abnormal nodal texture, or a node greater than $1 \mathrm{~cm}$. While lymphadenopathy is a common complaint in the primary care setting, it usually does not represent malignancy. Much of the diagnostic work-up hinges on history and exam, and if the patient does not have risk factors for malignancy or serious infection, it is reasonable to observe for 3 to 4 weeks. In the presence of risk factors, or if the lymphadenopathy does not resolve with observation, biopsy is indicated. Historically, fine needle aspiration (FNA) and core needle biopsy $(\mathrm{CNB})$ were thought to be prone to sampling error and to offer incomplete information for diagnosing lymphoma, as architectural information is needed to use the WHO Classification of Tumors of Haematopoietic and Lymphoid Tissues. However, newer research shows that CNB and FNA are highly accurate, with a sensitivity and specificity both above $96 \%$ (1), particularly in combination with technologies such as immunohistochemical staining. As excisional biopsy is still the reference standard, some authors have argued that perhaps patients with certain risk factors should skip FNA or CNB and go straight to excisional biopsy. These risk factors include age above 40 years, lack of tenderness of the lymph node, lymph node size, generalized pruritus, supraclavicular location, or hard texture of the lymph node. This patient appropriately received initial FNA with flow cytometry and immunohistochemical staining, and then was referred for excisional biopsy when the initial diagnostic testing was inconclusive. Although this patient had a very typical presentation, she was diagnosed with Castleman's disease, which is an uncommon lymphoproliferative disorder that can either be unicentric or multicentric. The disease is caused by a proliferation of B- and T-cells with an overproduction of cytokines, most commonly IL-6, though its exact pathogenesis is not well-understood. Other etiologies such as infections, autoimmune disorders, and malignancies must be ruled out, as they were in this case, before the diagnosis of Castleman's can be made. While it is not a type of cancer, it is similar to lymphoma and some patients do go onto develop lymphoma. In the case of multicentric Castleman's, all patients should be tested for Human Herpes Virus 8 (HHV-8) and if positive, treated with ganciclovir and rituximab. This patient's disease was thought to be unicentric, in which case surgery represents definitive treatment. 1. Amador-Ortiz et al. Combined Core Needle Biopsy and Fine-Needle Aspiration With Ancillary Studies Correlate Highly With Traditional Techniques in the Diagnosis of Nodal-Based Lymphoma. Am J Clin Pathol 2011;135:516-524

CHRONIC EOSINOPHILIC LEUKEMIA IN AN AFRICAN AMERICAN MALE Onyekachi H. Ogbonna; Stanley M. Nwabudike. Howard University Hospital, Hyattsville, MD. (Control ID \#2454692) 
LEARNING OBJECTIVE \#1: Recognize the clinical features of Chronic Eosinophilic Leukemia (CEL), and identify diagnostic modalities and treatment options.

LEARNING OBJECTIVE \#2: Report a case of CEL, a rare hematologic malignancy. CASE: A 54-year-old African-American male with history of asthma presented with sudden onset pleuritic chest pain and dyspnea. His chest x-ray was normal and cardiac work up was negative. He was treated for acute asthma exacerbation with steroids and nebulizers with improvement in his symptoms. Interestingly, he was found to have significant eosinophilia of $11,070 \mathrm{eos} / \mathrm{mm}^{3}$ (45\% of leucocytes) on admission, which had been longstanding on review of his medical record. On evaluation by the hematology team, he denied constitutional symptoms, travel abroad, camping and exposure to unsanitary water or food. He denied easy bruising or bleeding, allergic reactions or history of allergy to medication. His family history was significant for Asthma in his mother who died of asthma related complications. His home medications were albuterol and Advair inhalers only. Physical examination revealed diffuse inspiratory and expiratory wheezing in all lung fields and moderate splenomegaly. He underwent a bone marrow biopsy, which revealed a markedly hypercellular marrow with predominant eosinophilia (60\% of total marrow cellularity). Flow cytometry showed myeloid predominance with increased CD52+ eosinoforms; and florescent in-situ hybridization (FISH) was positive for the FIP1L1-PDGFRA (F/P) mutation, consistent with chronic eosinophilic leukemia (CEL). Cytogenetic analysis revealed normal karyotype. Given that patients with F/P mutated CEL have virtually universal response to Imatinib, we encouraged the patient repeatedly to begin treatment with Imatinib. Unfortunately, he declined.

DISCUSSION: Hypereosinophilic syndrome (HES) refers to a rare group of disorders characterized by a persistent, marked proliferation of eosinophils ( $>1500$ eos/ $\mathrm{mm}^{3}$ ) with end-organ involvement, in the absence of secondary causes of eosinophilia. Chronic eosinophilic leukemia (CEL) is a very rare myeloproliferative variant of HES characterized by clonal eosinophilia. It discriminately affects men and can present with anemia, thrombocytopenia, hepatosplenomegaly, and bone marrow dysplasia/fibrosis among other findings. A patient suspected of having HES/CEL owing to prolonged profound eosinophilia should first undergo evaluation to rule out secondary causes of eosinophilia. Failure to identify a secondary cause for the eosinophilia should then lead to a comprehensive work-up to identify possible endorgan damage from HES and a possible clonal population of eosinophils, as is the case with CEL. Screening of the peripheral blood for the F/P mutation by FISH or reverse transcription polymerase chain reaction and bone marrow biopsy with cytogenetics is crucial in identifying clonal eosinophilia. Patients with F/P positive CEL or PDGFR-associated CEL should be treated with Imatinib given their almost universal response to Imatinib. Second-line agents include corticosteroids and interferon alpha. Novel therapies in the management of HES and/or CEL include Alemtuzumab and Mepolizumab, both of which are humanized monoclonal antibodies that target CD 52 and IL-5 respectively. Given the rarity of CEL, these cases should be reported if identified to increase our understanding of the disease.

CHRONIC HEART FAILURE WITH PRESERVED EJECTION FRACTION (HFPEF): AN UNAPPRECIATED CONTRIBUTOR Raju Khanal ${ }^{1}$; Ronald Herb ${ }^{2}$. ${ }^{1}$ Reading Health System, Wyomissing, PA; ${ }^{2}$ reading health system, Reading, PA. (Control ID \#2454850)

LEARNING OBJECTIVE \#1: To know when to suspect restricive cardiomyopathy in heart failure with preserved ejection fraction.
LEARNING OBJECTIVE \#2: To understand senile cardiac amylodosis as a contibutor of heart failure

CASE: A 69-year old male, presented with progressive worsening dyspnea on exertion for several months. He had a history of CAD status post CABG and multiple stentings, type 2 diabetes mellitus, obstructive sleep apnea, chronic atrial fibrillation, and HTN. . Electrocardiogram showed atrial fibrillation with controlled ventricular rate without evidence of left ventricular hypertrophy. Echocardiogram revealed normal size left ventricle, with normal ejection fraction, moderate left ventricular hypertrophy, moderate biatrial enlargement and dilated right ventricle. Initially he was treated for decompensated diastolic heart failure. Because of worsening symptoms, he underwent right heart catheterization, which revealed restrictive physiology. Subsequently, left ventricular myocardial biopsy was positive for transthyretin amyloid deposition (ATTR), consistent with wild type or senile amyloidosis. As there was no effective treatment for the condition, he was referred to tertiary center for phase 3 clinical trial with tafamidis on progression of TTR amyloidosis. After a year on the study, his symptoms were controlled on diuretics

DISCUSSION: More than $50 \%$ of chronic heart failure patients are older adults with preserved ejection fraction (HFpEF). Effective therapy for this condition is still not well defined. There are two major types of cardiac amyloidosis; cardiac amyloid light-chain (AL), and transthyretin-related cardiac amyloidosis (ATTR). ATTR has two types, familial (mutant) TTR amyloidosis and wild types (WT) or senile TTR amyloidosis. The early recognition of cardiac amyloidosis and differentiating the types is very important for treatment and prognosis Restrictive cardiomyopathy is the dominant manifestation of wild type ATTR amyloidosis. Cardiac amyloidosis should be considered in any adult with unexplained heart failure with left ventricular hypertrophy and a normal left ventricular cavity on echocardiogram. Cardiovascular MRI may support the diagnosis of all forms of cardiac amyloidosis. However, radionuclides imaging using $99 \mathrm{mTc}$-phosphate derivatives may differentiate subtypes. A definitive diagnosis requires myocardial biopsy. Several novel therapies including TTR protein stabilizers (tafamidis and diflunisal) and RNA inhibitors are in clinical trial for ATTR amyloidosis, which may prevent progression of ATTR amyloidosis. In conclusion, the understanding of underlying process is crucial for the treatment and prognosis of heart failure with preserved ejection fraction $(\mathrm{HFpEF})$. Wild type ATTR cardiac amyloidosis is an under-recognized contributing factor to HFpEF. Its recognition, and its treatment with developing novel therapies might prevent progression of heart failure in these populations.

CLARIFYING ALTERED MENTAL STATUS: A BLACK, WHITE, OR GREY MATTER? Sushma Kola ${ }^{1}$; Siddharth Sheth $^{2}$; Joseph M. Rocco ${ }^{2}$; Amar Kohli ${ }^{2}$; David McAdams ${ }^{2} .{ }^{1}$ University of Pittsburgh, Pittsburgh, PA; ${ }^{2}$ University of Pittsburgh Medical Center, Pittsburgh, PA. (Control ID \#2466158)

LEARNING OBJECTIVE \#1: List at least three common causes of AMS in elderly patients.

LEARNING OBJECTIVE \#2: Explain that multiple etiologies of AMS may exist concurrently.

CASE: An 80-year-old African American female with history of hypertension presented to the ED with altered mental status (AMS). As reported by her family, the patient became increasingly confused over several weeks and concomitantly developed worsening anorexia, weight loss, and generalized weakness. Three days prior to presentation, she complained of dysuria and constipation with abdominal discomfort. Due to worsening mental status and inability to 
care for herself, she was brought to the ED for further evaluation. On physical exam, the patient was awake but only responded to questions with persistent repetition of phrases. She was afebrile and hemodynamically stable. Pulmonary exam was notable for decreased breath sounds and dullness throughout the left hemithorax. Her abdomen was distended without focal tenderness. Neurologically, the patient was fully disoriented without other focal deficits. Labs were unremarkable except for WBC 11,000 and UA with positive nitrite, LE 1+ and WBC 9. Urine culture grew $>100,000 \mathrm{col} / \mathrm{mL}$ of E. coli. Blood cultures yielded no growth. Imaging included a negative non-contrast head CT. Chest X-ray revealed a large left pleural effusion with complete compressive atelectasis of the left lung. CT surgery placed a chest tube, which drained serosanguinous fluid. Pleural studies were exudative based on elevated pleural LDH, however cytology and cultures were negative for malignancy and infection, respectively. Repeat CXR demonstrated appropriate lung re-expansion. Initially, AMS was felt to be secondary to UTI and large pleural effusion, although treatment of both offered no improvement in cognition. Due to persistent abdominal distention despite normalization of bowel movements, an abdominal CT was obtained and found multiple large $(10 \times 10 \mathrm{~cm})$ bilateral adnexal masses with omental nodules in the right lower quadrant suspicious for malignancy. Serum CA-125 levels were elevated $(749 \mathrm{U} / \mathrm{mL})$ and subsequent omental nodule biopsy confirmed stage IIIC ovarian adenocarcinoma. Despite a new diagnosis of malignancy, we still could not adequately explain the patient's perseverating speech. On the 6th day of admission, a contrasted brain MRI showed an acute right middle cerebral artery ischemic stroke. Due to poor performance status and inability to tolerate physical therapy, our oncology consultants deemed the patient a poor candidate for chemotherapy or surgery. She was ultimately transitioned to hospice care and passed away 3 weeks later.

DISCUSSION: We present an elderly patient who had four different reasons for acute altered mental status: UTI, pleural effusion, malignancy, and stroke. AMS is noted in $10 \%$ of elderly patients who present to the ED. Infectious, metabolic, or neurologic causes are typical; however, the differential is vast. Initially, we focused on UTI, which was appropriately treated. Diagnosis of UTI in older patients can be complicated by a lack of typical symptoms. More than one-third of elderly patients present without pyrexia and a decline in general status is often the only indication. We also identified a large pleural effusion based on abnormal lung exam and radiographic evidence. Large pleural effusions can cause impaired gas exchange and lead to hypoxia, hypercapnia, and AMS. Exudative effusions occupying greater than two-thirds of the hemithorax are strongly associated with malignancy, tuberculosis, or parapneumonic effusions. Diagnostic sensitivity of cytology for malignant effusions is $<76 \%$ with $40 \%$ of cancer patients undiagnosed by cytology alone. Additionally, weight loss, anorexia, and persistent abdominal distention led to an eventual diagnosis of ovarian malignancy. Neurologic complications occur in $20 \%$ of all patients with cancer. Brain metastases causing AMS arise predominantly in lung and breast tumors, with $<5 \%$ occurring in genitourinary tumors. While paraneoplastic syndromes such as encephalitides are rare in gynecologic malignancies, cerebellar degeneration has the highest incidence in ovarian and breast cancers and can lead to impaired cognition, ataxia, and dysarthria. Cerebrovascular disease is more common in cancer patients, however, and can be caused by direct tumor invasion, coagulation disorders, or marantic endocarditis. Gynecologic neoplasms have the highest potential for developing ischemic stroke.
In our patient, both advanced age and underlying hypertension further increased her stroke risk. In conclusion, acute cognitive changes are commonly precipitated by underlying medical conditions that can be life-threatening with a multitude of adverse outcomes. Therefore, evaluation should focus on early diagnosis with awareness that multiple etiologies may exist concurrently.

CLOPIDOGREL-INDUCED THROMBOTIC THROMBOCYTOPENIC PURPURA PRESENTING AS COLITIS IJEOMA NNODIM; ASHLY DUCHARME. WAYNE STATE UNIVERSITY-DETROIT MEDICAL CENTER, DETROIT, MI. Ashly Ducharme. Detroit Medical Center/Wayne State University, Brownstown Township, MI. (Control ID \#2469256)

LEARNING OBJECTIVE \#1: Identify atypical presentations of thrombotic thrombocytopenic purpura

LEARNING OBJECTIVE \#2: Management of non-ST elevation myocardial infarction in acute thrombocytopenia

CASE: A 68 year old woman with history of hypertension, coronary artery disease, and chronic kidney disease presented with a 1 day history of right lower quadrant abdominal pain, nausea, vomiting, and bloody diarrhea. She had a drug-eluting stent 11 days prior to admission and was initiated on clopidogrel 3 days prior to admission. On admission, vitals showed temperature 36.2 , heart rate 104 , blood pressure $134 / 98 \mathrm{mmHg}$, and respiratory rate of 16 . Laboratory results were remarkable for leukocytosis at $20.6 \mathrm{~K} /$ cumm, hemoglobin $10.6 \mathrm{~g} / \mathrm{dL}$, platelet count of $123 \mathrm{~K} / \mathrm{cumm}$, carbon dioxide $18 \mathrm{mMol} / \mathrm{L}$, and creatinine $2.28 \mathrm{mg} / \mathrm{dL}$. Lactate was normal at $1.0 \mathrm{mMol} / \mathrm{L}$. CT abdomen and pelvis without contrast revealed abnormal thickening of transverse and right colon consistent with nonspecific colitis. Platelets trended down to $7 \mathrm{~K} / \mathrm{cumm}$ over the next 4 days with a drop in hemoglobin to $6.8 \mathrm{~g} / \mathrm{dL}$. ADAMTS13 level was low at $13 \%$ with an elevated ADAMTS13 Inhibitor of $32 \%$. Schistocytes were seen on peripheral smear. She was diagnosed with TTP and initiated on plasmapheresis with improvement in platelet count, anemia, and LDH. Hospital course was complicated by NSTEMI with new basal and mid inferior segments appearing hypokinetic to akinetic on transthoracic echo, for which the patient was initiated on argatroban.

DISCUSSION: Thrombotic thrombocytopenic purpura (TTP) is a microangiopathic hemolytic process characterized by development of microthrombi in small vessels and capillaries. TTP has a high mortality rate without prompt emergency treatment. Pathophysiology involves deficiency of ADAMTS13, a metalloprotease that cleaves von Willebrand factor, leading to VWF and platelet aggregates in arterioles and capillaries. Our patient presented with symptoms of abdominal pain, vomiting, and bloody diarrhea and CT findings suggestive of colitis. This presentation could be secondary to development of microthrombi in the arterioles and capillaries of the colon and mesentery leading to the development of abdominal pain and bloody diarrhea. Given her history of CAD and presentation, there was initial concern for ischemic colitis. She was also initiated on cefoxitin and metronidazole for possible infectious etiology as well; however, stool culture for Salmonella, Shigella, and Campylobacter, Shiga toxin 1 and 2, and Clostridium difficile toxin were negative. Further evaluation for ischemic colitis with angiography was not obtained due to improvement in symptoms and chronic kidney disease. She developed a non-ST elevation myocardial infarction during her hospitalization with troponin elevation and new basal to inferior wall motion abnormalities seen on echocardiography on the day after admission. She was initiated on argatroban for the NSTEMI rather than a heparin-based product as there was concern for heparin-induced 
thrombocytopenia (HIT). HIT was subsequently excluded with negative serotonin release assay and platelet factor 4 . The possibility of disseminated intravascular coagulation was also considered given elevated prothrombin (PT), activated partial thromboplastin time (aPTT), and D-dimer but was thought less likely as she had an elevated fibrinogen level. The elevations in PT and aPTT could be secondary to argatroban therapy. The development of TTP was thought to be secondary to clopidogrel. Initiation of clopidogrel leading to TTP has been often seen within 2 weeks of initiation and has been reported with as little as one dose. The decision was made to avoid dual antiplatelet therapy following DES due to her risk of TTP with both clopidogrel and ticlopidine and due to previous adverse reaction to ticagrelor.

CLOSTRIDIUM DIFFICILE AND OPIOIDS: A TOXIC MIX Jasmine Dukandar; Kristina Chacko. Montefiore Medical Center, Bronx, NY. (Control ID \#2469471)

LEARNING OBJECTIVE \#1: Understand the medical and surgical management of toxic megacolon.

LEARNING OBJECTIVE \#2: Understand the effects of opioid analgesia on bowel dysfunction.

CASE: A 67 year-old man with Hepatitis C cirrhosis and hepatocellular carcinoma who underwent orthotopic liver transplantation presented to the hospital on post-operative day 20 with abdominal pain. At the time of transplant, he received antibiotics and standard immunosuppressive drugs. His post-operative course was complicated by an excess need for narcotic medications, out of proportion to his pain. The patient, an addiction social worker at an outside hospital, had been found to be abusing narcotics and recently enrolled in a methadone maintenance program. Despite methadone, he required increasing doses of opiates. Throughout his post-operative hospital stay he complained of constipation, requiring an aggressive bowel regimen. One week after hospital discharge he presented to the Emergency Department (ED) with 4 days of abdominal pain and diarrhea. He had severe lactic acidosis (lactate $9.6 \mathrm{mmol} / \mathrm{L}$ ), renal failure (creatinine $3.4 \mathrm{mg} / \mathrm{dL}$ ), and worsened leukocytosis (WBC $27 \mathrm{k} / \mathrm{uL}$ ). Abdominal radiograph revealed distended airfilled loops of small bowel and a thickened colon. He became hypotensive in the ED, requiring intravenous fluids, norepinephrine, and vasopressin. He developed abdominal compartment syndrome. His transplant incision was initially opened in the ED, to find dilated loops of bowel, but no ischemia. He was taken emergently to the operating room. The proximal half of the small bowel, cecum, and transverse colon were markedly dilated. Intra-operative stool sample was positive for clostridium difficile. Despite all aggressive life-saving measures, he developed ischemic bowel requiring extensive resections of the small bowel and colon, and died on hospital day 3 .

DISCUSSION: Clostridium difficile infection (CDI) is one of the leading causes of hospital-associated gastrointestinal illness, placing an increasingly higher burden on the healthcare system. Recent figures estimate annual costs of 3.2 billion dollars. In adults, toxic megacolon is a relatively uncommon but potentially lethal complication of infectious colitis. Mortality rate exceeds $60 \%$. While diarrhea is a common presentation of CDI, this is not reliable in toxic megacolon. Patients will have signs of sepsis, such as fever, hypotension, decreased level of consciousness, and electrolyte imbalances. The diagnosis is made based on colonic distension exceeding $60 \mathrm{~mm}$, clinical evidence of toxicity, and positive stool assay for clostridium difficile toxin. Medical management of this condition includes bowel rest and decompression, fluid volume and electrolyte replacement, antibiotic therapy, and close monitoring. Surgical management is necessary if medical therapy is not successful during the first $72 \mathrm{~h}$ or when there are signs of deterioration, perforation, hemorrhage or sepsis. Known risk factors of toxic megacolon are medications that decrease gastrointestinal motility. Prolonged opiate use increases the risk of developing "opioid-induced bowel dysfunction" (OBD), of which the sequelae include constipation, delayed gastric emptying, and decreased motility. The basis of OBD is $\mu$-receptor activation, decreasing the peristaltic contraction. Opioid analgesics are increasingly being used for the treatment of many types of pain, including post-operative pain. Patients with a history of narcotic abuse have tolerance, requiring higher doses of opiates for pain. In this unfortunate case of an early post-transplant patient on immunosuppressive therapy, the overuse of opioids masked early symptoms of CDI such as diarrhea. It is important to recognize that patients who use opiates or any other medication that slows gastrointestinal motility are at an increased risk of the feared complication of toxic megacolon.

CO-INFECTION OF MYCOBACTERIUM AVIUM COMPLEX AND MYCOBACTERIUM ABSCESSUS - NONTUBERCULOUS MYCOBACTERIA IN AN IMMUNOCOMPETENT HOST. Sonal Pruthi; Amit Sharma; Rumon Chakravarty; Ambika Eranki. SUNYUpstate Medical University, Syracuse, NY. (Control ID \#2470228)

LEARNING OBJECTIVE \#1: 1. Clinical and radiological presentation of Nontuberculous mycobacteria infection

CASE: A 63 year old gentleman with a past medical history significant for stroke, CAD, OSA and Scatzki ring presented to us with complaints of nasal congestion, post nasal drip, stuffiness and headaches for 4 months, unresponsive to three courses of antibiotics. He also developed progressive cough with white, mucoid sputum and a $55 \mathrm{lb}$ weight loss over 6 months. This was not associated with shortness of breath, fever or chills. He did have a 40 pack year history of smoking cigarettes and had quit smoking 26 years ago. He was a truck driver and had history of travel to regions endemic for NTM including Colorado. The patient did not have any history of diabetes mellitus, recent/long term steroid use or HIV. A CT scan of the thorax, revealed multiple bilateral nodules, some with tree in bud appearance. There were two more distinct nodules noted - one in the left lower lobe, other in the right lower lobe with a cavity. A bronchoscopy with bronchioalveolar lavage (BAL) was negative for Pneumocystis jiroveci. It was positive only for Mycobacterium abscessus and MAC. The BAL culture grew Mycobacterium abscessus, ERM 41, suggesting inducible clarithromycin resistance and was confirmed with DNA PCR. The patient was started on intravenous amikacin, cefoxitin for Mycobacterium abscessus; azithromycin, ethambutol and rifampin for MAC. He developed a diffuse rash secondary to cefoxitin use needing hospitalization, and was switched over to imipenem/cilastatin therapy. After 2 months into the therapy the sputum was negative for AFB and the patient is currently undergoing oral treatment with azithromycin, ethambutol and rifampin, for a year.

DISCUSSION: There is an increase in incidence of diseases secondary to Nontuberculous mycobacteria (NTM). From over 150 species known, the commonly implicated species in pulmonary diseases are Mycobacterium avium complex (MAC), Mycobacterium kansasii, and Mycobacterium abscessus. It is difficult to accurately characterize the incidence and prevalence of NTM, as isolation of the organism does not necessarily indicate a disease. NTM are identified as colonizers of pulmonary tract and disseminated disease is seen in association with profound immunosuppression or in patients who have undergone lung transplantation. Clinical symptoms of disease includes chronic cough, purulent sputum, fatigue, and weight loss. Thin walled cavities, clusters of small nodules less than $0.5 \mathrm{~mm}$ (called tree-in-bud sign) are seen on imaging. Our patient had a similar clinical and radiological picture and the most likely cause in him was repeated silent aspiration of contaminated drinking water when travelling, secondary to 
a Schatzki ring. This case highlights the fact that NTM are not as benign as they were once thought to be and can cause significant diseases in immunocompetent individuals.

COCAINE INDUCED ACUTE HEMOPERITONEUM RESULTING FROM RUPTURE OF SURFACE UTERINE VESSELS: A CASE REPORT Tatvam T. Choksi ${ }^{1}$; Nicole Vital ${ }^{2}$; Michael F. McCollum ${ }^{3} .{ }^{1}$ Mercy Catholic Medical Center, Lansdowne, PA; ${ }^{2}$ Philadelphia University, Sugar Land, TX; ${ }^{3}$ Mercy Catholic Medical Center, Darby, PA. (Control ID \#2458417)

LEARNING OBJECTIVE \#1: Cocaine abuse has long been associated with a variety of hemorrhagic events including gastrointestinal, pulmonary and intracranial bleeding. But from the review of literature, it has not been well associated with uterine bleeding in the absence of uterine rupture. We describe a case of acute massive hemoperitoneum due to rupture of the surface vessels of a fibroid uterus resulting secondary to cocaine abuse. CASE: 42 year old African-American female with history of uterine fibroids presented with acute onset diffuse abdominal pain. She reported snorting 'crack' cocaine $3 \mathrm{~h}$ ago. On examination, she was found to be hypotensive with blood pressure of $70 / 40 \mathrm{mmHg}$ and tachypneic with respiratory rate of $25-30 / \mathrm{min}$. She was also noted to have moderate diffuse abdominal and flank tenderness without guarding or rigidity. An emergent bedside ultrasound of abdomen and pelvis showed free intra-peritoneal fluid. Aggressive resuscitation with intravenous fluids was initiated. But soon her mental status deteriorated and she was intubated for airway protection. Lab studies showed hemoglobin of $6.8 \mathrm{~g} / \mathrm{dl}$ (down from baseline of $10 \mathrm{~g} / \mathrm{dl} 2$ months ago). Coagulation profile was normal. Central venous access was obtained; resuscitation with intravenous fluids and packed red blood cells was continued. Soon, CT scan of abdomen and pelvis was obtained which showed massive hemoperitoneum and severely enlarged uterus with multiple uterine fibroids. Emergent surgical and gynecological consultation was obtained and the patient was taken to operating room immediately for exploratory laparotomy. Hemoperitoneum was evacuated. It was noted that 2 large vessels on the surface of anterior fundal uterine myoma were actively oozing. They were cauterized to stop bleeding. After making sure that there was no other intra-abdominal bleeding source contributing, supra-cervical hysterectomy was performed and the patient was transferred to ICU for close monitoring. She received total of 6 units of packed red blood cells and 2 units of fresh frozen plasma. She was extubated the next day. The patient gradually improved and finally, discharged in a stable condition.

DISCUSSION: Internal bleeding from leiomyoma has been reported in the event of trauma or torsion. However, there is a lack of association between uterine vessel rupture and substance abuse. The use of 'crack' cocaine has been reported to cause hemoperitoneum from uterine rupture in pregnancy secondary to forceful uterine contractions along with focal and systemic vasoconstriction induced by cocaine. But in the absence of scar tissue or gravid uterus; cocaine use itself causing hemoperitoneum from sudden rupture of surface uterine vessels has not been reported. Cocaine has direct toxicity on capillary endothelium. It induces hypertensive surges on the vessel wall with resultant acute shearing stress on the vasculature. It can inhibit the reuptake of norepinephrine at pre-synaptic junction resulting in increased and perhaps, sustained effects of norepinephrine on alpha-adrenergic receptors with significant vasoconstriction. In addition, cocaine has sympathomimetic effects on uterus, which can result in repetitive forceful uterine contractions. These effects can last longer in those with chronic abuse, as the half-life of cocaine and its metabolites is relatively prolonged among them. Vascular network feeding sub-serosal fibroids, due to lack of connective tissue support and lack of myometrial base, is significantly affected by these mechanisms and may in-turn rupture causing potentially massive hemoperitoneum. Interestingly, despite significant hemoperitoneum, hypotensive response may be blunted with concomitant cocaine use. Therefore, hypotension in this setting may be suggestive of a delayed presentation requiring immediate attention, as in our patient. In conclusion, though hemoperitoneum from rupture of surface vessels of uterine fibroid is extremely rare, the association should not be overlooked especially when history of substance (especially cocaine) abuse is present.

COCAINE-INDUCED MESENTERIC ISCHEMIA: THE RISK OF PROFILING PATIENTS SUSPECTED OF DRUG-SEEKING BEHAVIOR Ethan Y. Zhao; Anna M. Ledgerwood. Wayne State University, Detroit, MI. (Control ID \#2454177)

LEARNING OBJECTIVE \#1: Recognize that the clinical picture of cocaine-induced mesenteric ischemia can be confused for drug-seeking behavior

LEARNING OBJECTIVE \#2: Treat cases of severe abdominal pain in the context of a positive cocaine screen with high clinical suspicion for mesenteric ischemia CASE: A 63 year old Caucasian male presented with severe abdominal pain. The patient was struck in the abdomen during a construction incident 5 days prior resulting in intermittent mild abdominal pain that subsequently developed into sharp tearing abdominal pain. The patient stated the pain was $15 / 10$ and was in visible distress. Vomitus was non-bloody. The abdominal pain was diffuse and non-radiating. The patient denied fever, chills, chest pain, shortness of breath, syncope, dizziness and focal neurological deficits. The patient had not passed flatus or a bowel movement since the morning. Bowel sounds were normal and fecal occult blood was negative. Physical exam was unremarkable except for diffuse abdominal tenderness that was out of proportion in relation to physical findings. Peritoneal signs were not present. The patient; however, admitted to alcohol, marijuana and occasional cocaine use. Urine drug screen was positive for cannabinoids, cocaine metabolites, and opiates. Basic metabolic panel, liver enzymes (ALT: 19 U/L; AST: 17 U/ L; Alkaline Phosphatase: $77 \mathrm{U} / \mathrm{L})$, troponin level $(<0.017)$ were all within normal range. Vital signs were within normal limits. White blood cell count was elevated $\left(14.6 \mathrm{~K} / \mathrm{mm}^{3}\right)$. Chest X-ray revealed no acute cardiopulmonary process. Multiple dilated loops of small bowel and air-fluid levels were seen on abdominal x-ray with minimal air and stool in the colon, which suggested distal small bowel obstruction. Computed tomography of the abdomen without contrast revealed inflammatory stranding in the mesentery adjacent to the dilated loops of small bowel. No bowel wall thickening, air within the small bowel walls, free air and fluid collection were present. The initial presentation and toxicology results led to the suspicion of drug-seeking behavior. The patient was kept overnight but continued to be symptomatic. The next afternoon, the patient was tachycardic (HR: 101113), white blood cell count elevated to $17.7 \mathrm{~K} / \mathrm{mm}^{3}$ and his abdomen was found to be rigid and exquisitely tender prompting the suspicion of mesenteric ischemia. The patient was subsequently consented for an emergency laparotomy. In the OR, findings suggestive of ischemic changes were noted and $4.5 \mathrm{ft}$ of necrotic small bowel was resected. DISCUSSION: Mesenteric ischemia is a life threatening vascular emergency with overall mortality of $60-80 \%$ and comprises a group of pathophysiological processes that result in bowel necrosis ${ }^{1}$. Early diagnosis and intervention are required to adequately restore mesenteric blood flow and prevent subsequent bowel infarction and patient death. Mesenteric ischemia remains a diagnostic challenge for clinicians due to the difficulty in recognizing the condition before bowel infarction occurs. Clinical presentation is nonspecific in most cases and is characterized by an initial discrepancy between severe abdominal pain and minimal findings on physical exam ${ }^{2}$. A delay in diagnosis contributes to the high 
mortality rate. Thus, early diagnosis and prompt effective treatment are essential to improve clinical outcome. This patient sustained blunt abdominal trauma from a workrelated incident. Many patients often self-medicate with over-the-counter drugs, sedatives, opiates and psycho-stimulants such as cocaine to address their chronic pain states, mental distress, and anxiety ${ }^{3}$. In this case, the patient was found to have a positive toxicology screen for cannabinoids, opiates and cocaine metabolites. An ischemic event precipitated from cocaine-induced vasospasm is a very likely possibility with regards to this patient ${ }^{4}$. Given the positive drug screen and a physical presentation of abdominal pain out of proportion to physical findings, this scenario can falsely paint a clinical picture of drugseeking behavior. Being cognizant of this scenario in the future will allow for more timely treatment to a medical emergency.

COMA THE PRESENTING SYMPTOM OF A BILATERAL THALAMIC STROKE INVOLVING THE ARTERY OF PERCHERON Kushal A. Shah; Shahniwaz Labana; Jandark Yuseif; Alaeddin Maeza. Wayne State University, Rochester, MI. (Control ID \#2469998)

LEARNING OBJECTIVE \#1: To assess a possibility of a stroke with a negative CT head without contrast in an unresponsive patient. To recognize the symptoms associated with a stroke involving Artery of Percheron which is a rare variant of thalamic strokes.

LEARNING OBJECTIVE \#2: To remember that imaging modalities and interventions which can facilitate diagnosis and treatment of a stroke of the Artery of Percheron.

CASE: This is a 56-year-old right-handed Caucasian female with a history significant for right hemispheric cerebrovascular accident manifested as left-sided weakness, history of grand mal seizures, IDDM with peripheral neuropathy, hypertension and hyperlipidemia She was poorly compliant with her medications, which included Clopidogrel. She was found unconscious in her bed. Blood glucose at that time was 229. The patient was an alert, oriented and fully functioning individual prior to this episode. There was no evidence of a suicide attempt. On examination, her pulse rate was 60 beats $/ \mathrm{min}$, blood pressure 178 $104 \mathrm{mmHg}$, respiratory rate 28 breaths/min and oxygen saturation $92 \%$ on room air. The Glasgow coma-scale score was 7 . She did not respond to any verbal stimuli, but she was able to localize painful stimuli. Pupils were $4 \mathrm{~mm}$ bilaterally and the pupillary reflex was nonreactive in both eyes. No extra ocular movements were noted. She was flaccid in all four extremities. Her EKG was within normal limits. She was intubated for airway protection. A CT of the head without contrast done $90 \mathrm{~min}$ after the incident showed no gross intracranial hemorrhage or mass effect. There was presence of a left fronto-parietal chronic white matter ischemic changes. A stat EEG showed slow background rhythm, excessive slow frequency along with low amplitude and asymmetry, suggestive of an underlying diffuse cerebral dysfunction. There were no metabolic abnormalities noted. Her clinical course did not change over the next few days, so a repeat CT $72 \mathrm{~h}$ later was obtained and demonstrated symmetric acute infarcts in the medial thalami bilaterally, related to occlusion of the Artery of Percheron and an old infarct in the left occipital lobe and right cerebellum. The patient was not a candidate for IPA and the patient was managed conservatively. An MRI showed increased signal in the medial thalami bilaterally, consistent with a sub-acute infarct in the artery of Percheron and small infarct in the right cerebellar hemisphere. The patient's neurological examination did improve over the course of the next couple weeks. She regained motor and neurological function and was eventually extubated. A PEG tube was placed due to persistent dysphagia and she was eventually transferred to an extended care facility.
DISCUSSION: Bilateral thalamic infarcts are a rare presentation of a stroke, accounting for only $22-35 \%$ of all thalamic infarcts. The Artery of Percheron is a rare vascular variant in which a single dominant thalamo-perforating artery arises from the P1 segment and bifurcates to supply the paramedian thalamic regions. Occlusion of this artery typically results in characteristic ischemic radiologic findings involving the paramedian thalami, with symptoms ranging from confusion and visual impairment to full blown coma. Our case illustrates the more severe presentation of this rare type of infarction and elucidates the necessity for vigilance when considering differential diagnoses for a comatose patient. Diagnosis can be made with CT Head without contrast or an MRI. Initial imaging can be negative when it has been performed too close to the initial insult. If clinical suspicion is high for a stroke, hypo attenuation on CT or hyper intense T2 or FLAIR signal intensity on MRI with or without restricted diffusion in the distribution of the artery can suggest a stroke. We also suggest that an MRI or CT scan be performed again within $48 \mathrm{~h}$ if the initial imaging is normal and clinical suspicion is high. After diagnosis, treatment is consistent with that of an ischemic stroke. Thrombolysis is a treatment option if occlusive phenomenon is observed, otherwise conservative treatment with IV Heparin is also an option. Early recognition and initiation of treatment is imperative as it directly effects long term neurological outcome. References: G. Raphaeli, A. Liberman, J. M. Gomori, and I. Steiner, "Acute bilateral paramedian thalamic infarcts after occlusion of the artery of Percheron," Neurology, vol. 66, no. 1, p. E7, 2006. G. Percheron, "The anatomy of the arterial supply of the human thalamus and its use for the interpretation of the thalamic vascular pathology,” Journal of Neurology, vol. 205, no. 1, pp. 1-13, 1973. Guillaume Cassourret, Bertrand Prunet, Fabrice Sbardella, Julien Bordes, Olga Maurin, and Henry Boret, "Ischemic Stroke of the Artery of Percheron with Normal Initial MRI: A Case Report,” Case Reports in Medicine, vol. 2010, Article ID 425734, 4 pages, 2010. doi:10. 1155/2010/425734 Rodriguez EG, Lee JA. Bilateral thalamic infarcts due to occlusion of the Artery of Percheron and discussion of the differential diagnosis of bilateral thalamic lesions. Journal of Radiology Case Reports. 2013;7(7):7-14. doi:10.3941/jrcr.v7i7.961.

\section{COMMUNITY ACQUIRED LEGIONNAIRE'S DISEASE FROM AN INDOOR} SPRAY BOTTLE Andrew Herman ${ }^{1}$; Vishisht Mehta ${ }^{1}$; Karishma Bhatia ${ }^{1}$; Felicia Ratnaraj ${ }^{2}$; Lee Morrow ${ }^{1} .{ }^{1}$ Creighton University, Omaha, NE; ${ }^{2}$ Creighton University, Iowa, Cabo Verde. (Control ID \#2469693)

LEARNING OBJECTIVE \#1: Recognize that Legionnaire's Disease can be sporadic and community aquired \& is not always associated with air conditioning systems and larger outbreaks.

CASE: A 57 year old man was admitted for shortness of breath, fever and cough since 2 weeks. He had no recent travel nor exposure to sick contacts. Initial vitals included temperature a of $104.8 \mathrm{~F}$, blood pressure of $136 / 69 \mathrm{mmHg}$, heart rate of 140 beats/min, respirations of 30 breaths/min and oxygen saturation of $85 \%$ on room air. The patient was alert $\&$ in moderate respiratory distress, using his accessory muscles of respiration. Auscultation revealed bibasilar dullness with increased tactile and vocal fremitus throughout the lungs. The rest of his exam was unrevealing. Laboratory studies included white blood cell (WBC) count of $11,100 / \mathrm{mcl}$, platelet count of $143,000 / \mathrm{mcl}$, hemoglobin of $11.6 \mathrm{~g} / \mathrm{dL}$, low serum sodium at $134 \mathrm{mEq} / \mathrm{L}$ and elevated creatinine at $2 \mathrm{mg} / \mathrm{dL}$. Computed tomography imaging of the chest showed multifocal bilateral opacities, with near complete consolidation of the left lung. The sputum Gram stain was negative. Given the severity of his illness, a history of alcohol abuse and hyponatremia, Legionella urine antigen was obtained. Despite empiric ceftriaxone and levofloxacin the patient became 
increasingly unstable and required mechanical ventilator support. The Legionella urine antigen subsequently was positive. Azithromycin was added due to clinical deterioration and a rising WBC count. The patient subsequently improved, was extubated and discharged home on oral levofloxacin and azithromycin.

DISCUSSION: A detailed source investigation was carried out by the medical team in collaboration with the county health departments' epidemiology division. The patient denied Legionella risk factors including recent travel, hotel stay, long term care exposure, occupational exposure, contact with saunas or mines and recent plumbing work. No other individuals in his household were ill and there were no local clusters of Legionella. The county health department reported one confirmed case in the 5 months preceding ours. The source was traced back to a spray bottle the patient used to water his indoor plants. He reported using the bottle as a mister, regularly storing water in the vessel and topping it off with tap water daily. He was the only person in his household who used the mister bottle to water the plants. He was advised to replace the contaminated bottle, clean it regularly with bleach and avoid storage of tap water. Although water distribution and air conditioning systems are classically thought of as culprit sources for Legionella outbreaks, other sources are responsible for sporadic, community acquired Legionnaire's disease. Likelihood of infection is influenced by host susceptibility and inoculum size. In turn inoculum size is driven by the degree of water reservoir contamination. Man-made reservoirs have larger bacterial loads than natural reservoirs: infections have resulted from contaminated water fountains, potable water, compost and cooling towers. This case highlights the need for a high index of suspicion for Legionella in the appropriate clinical setting, even in the absence of an obvious source.

COMPLETE HEART BLOCK AFTER STEMI William Perucki ${ }^{1}$; Chandana Shekar ${ }^{2}$. ${ }^{1}$ University of Connecticut, Farmington, CT; ${ }^{2}$ University of Connecticut School of Medicine, West Hartford, CT. (Control ID \#2467943)

LEARNING OBJECTIVE \#1: Discuss complete heart block as an acute complication of MI

CASE: This is a case of an 83-year-old female with past medical history significant for hypertension, coronary artery disease, diverticulosis, migraine, Raynaud phenomenon, and GERD who presented initially to an outside facility with a chief complaint of substernal chest pain precipitated by minimal exertion. She was found to have STsegment elevations in leads II, III, and aVF with ST-segment depressions in leads V2 and V3. She was transferred to a facility capable of coronary angioplasty, and prior to catheterization was found to have hematochezia. She was unable to undergo angioplasty secondary to this GI bleed and the high risk of further bleeding with antiplatelet agents. She was also not a candidate for anticoagulation for the same reason so she was managed medically. Initial EKGs showed sinus rhythm with frequent premature atrial contractions, which subsequently converted to a ventricular rhythm with retrograde $\mathrm{P}$-wave conduction. Echocardiogram was performed which showed a left ventricular ejection fraction of 25 $30 \%$ with severe hypokinesis to akinesis of the inerior, inferolateral, and inferoseptal segments of the left ventricle. Her troponin remained elevated. She subsequently became bradycardic, initially in third degree heart block and subsequently with an atrial flutter rhythm though an intrinsic ventricular rhythm of 30-40. A trans-venous pacemaker was placed in the EP lab and the patient was kept in the cardiac intensive care unit. DISCUSSION: In the current age of revascularization, complications of myocardial infarctions are seen far less frequently. Complications of MI include mechanical, arrhythmic, ischemic, embolic, and inflammatory. With respect to arrhythmic complications, atrial and ventricular arrhythmias are often seen, with the later at times being a fatal presentation of an acute MI. Less often seen are sinus or atrioventricular node dysfunctions. This is a case of atrioventricular node dysfunction due to myocardial ischemia. The atrioventricular node is located at the superior aspect of the interventricular septum and normally supplied by the right coronary artery but may also be supplied by the left anterior descending artery. Infarction involving either of these arteries, depending on coronary dominance, can lead to dysfunction of the AV node as seen in this patient, resulting in complete heart block. Studies have shown that the incidence of complete heart block has decreased over time. Further, complete heart block has been shown to be reversible if revascularization occurs, but the same is not necessarily true of the patient is given thrombolytics. Thus, it appears as though early revascularization is one of the factors contributing to decreased incidence of complete heart block as an acute complication of myocardial infarction as well as improved survival overall.

\section{COMPLICATED CO-INFECTION: MANAGING HIV AND HCV INFECTION} Trenton C. Elliott ${ }^{2}$; Rebecca Glassman ${ }^{1} .{ }^{1}$ Beth Israel Deaconess Medical Center, Boston, MA; ${ }^{2}$ Beth Israel Deaconess Medical Center, Brookline, MA. (Control ID \#2470389)

LEARNING OBJECTIVE \#1: Recognize the natural progression of HIV/HCV coinfection

LEARNING OBJECTIVE \#2: Identify medical and psychosocial considerations to treatment in $\mathrm{HIV} / \mathrm{HCV}$ co-infected patients

CASE: A 47 year old man with a history of hepatitis C (HCV) cirrhosis (failed interferon therapy in 2007 due to side effects), HIV (never on anti-retrovirals), and anxiety presents for routine follow-up. He was diagnosed with HIV in 2012 on routine screening. His current CD4 count is 410 (24\%) and HIV viral load 5,020 copies/mL. Other current notable labs include platelets 51 , creatinine 0.8 , alanine transaminase 113 , aspartate transaminase 119 and total bilirubin 1.0. He has genotype 1a HCV with a viral load of $2,730,000 \mathrm{IU} / \mathrm{mL}$. Liver ultrasound shows a coarsened, nodular liver without focal liver lesions, and FibroScan Score is $35.8 \mathrm{kPa}$ consistent with Metavir Stage 4 cirrhosis. The patient is interested in initiating a medication to cure his HCV. He has been hesitant to start highly active anti-retrovirals (HAART). For the past 3 years, the patient has been compliant with regular medical visits and laboratory monitoring, but consistently declines HAART. He was initially living in a group home and was concerned about other tenants knowing his status. He currently lives with his girlfriend and fears her children discovering his status. He has never had an AIDS defining illness and his CD4 count has never been less than 200 . He has intermittently started and stopped medications for anxiety, and is routinely followed by a therapist.

DISCUSSION: Approximately $20-30 \%$ of HIV-infected persons are co-infected with Hepatitis C, with rates as high as $75-90 \%$ in high-risk groups such as intravenous drug users. HIV/HCV-coinfected patients have a three fold greater risk of progression to cirrhosis or decompensated liver disease than HCV-monoinfected patients. The risk of progression is even greater in co-infected patients with CD4 counts below 350. In one prospective cohort, co-infected patients receiving HAART were $66 \%$ less likely to experience end-stage liver disease, $\mathrm{HCC}$, and fatal hepatic failure than patients not receiving HAART. Great strides have been made in treatment options for HCV, with single pill, once daily, 12-week regimens now available. Multiple clinical trials (Phase II ERADICATE, ION-4, ALLY-2) have shown these medications to have equivalent efficacy in achieving sustained virologic response (SVR) in HIV/HCV co-infection compared to HCV-monoinfection. Despite high sustained virologic response rates, survival benefits 
and quality of life improvements have yet to be demonstrated in co-infected patients undergoing $\mathrm{HCV}$ treatment. When considering initiation of $\mathrm{HCV}$ treatment, particularly in patients with HIV co-infection, it is important to account for psychosocial factors, drugdrug interactions, and treatment adherence capabilities. Adherence is vital for maximizing SVR and preventing class-wide resistance mutations. In addition, given the high cost of these medications, most insurers will only authorize one lifetime course of the therapy. Despite these concerns, multiple studies have shown equivalent rates of medication adherence in HCV mono-infected compared to HCV/HIV co-infected patients, regardless of HAART use. With advances in HCV medication regimens, treatment of co-infected patients should be considered in order to prevent progression of liver disease. Medication adherence capabilities and drug-drug interactions should be evaluated in advance of initiation of $\mathrm{HCV}$ treatment. In the appropriate patient, where compliance is not a concern, treatment of $\mathrm{HCV}$ without concurrent HAART may be reasonable.

COMPLICATED PANCREATITIS - AN UNUSUALLY SYMPTOMATIC PSEUDOCYST Harsha Mudrakola ${ }^{1}$; Lubna Khawaja ${ }^{2} .{ }^{1}$ Baylor College of Medicine, Houston, TX; ${ }^{2}$ Baylor College of Medicine, Sugar Land, TX. (Control ID \#2469787)

LEARNING OBJECTIVE \#1: Recognize that pseudocysts may develop at sites distant from the pancreas and may cause symptoms independent of the pancreatic inflammation LEARNING OBJECTIVE \#2: Identify situations appropriate for more invasive strategies for pseudocyst management

CASE: A 35 year-old man with a history of alcohol abuse presented with abdominal pain. The patient had been abstinent from alcohol for several years, but 4 weeks prior to presentation he had relapsed and imbibed daily for nearly a week. Abdominal pain started shortly afterwards. He reported sharp, burning, epigastric pain that worsened considerably with eating. It was associated with nausea, but not vomiting. The pain progressed to the point where the patient could only tolerate clear liquids. Patient also noted early satiety; he denied diarrhea, steatorrhea, melena, and hematochezia. Physical examination revealed a soft abdomen with marked tenderness in the epigastric and right upper quadrant areas with voluntary guarding but without rigidity or other peritoneal signs. Laboratory abnormalities included a lipase of 595 and BUN of 2; all other lab values were in the normal range. An abdominal and pelvic computed tomography (CT) was done, which revealed marked peripancreatic fat stranding and nodularity consistent with pancreatitis. It also showed a fluid collection measuring $4.1 \mathrm{~cm} \times 4.5 \mathrm{~cm} \times 7 \mathrm{~cm}$ with a formed wall located anterior the stomach with no apparent communication with the pancreas, thought to be a pseudocyst At first the patient was treated with supportive measures including IV hydration and pain medications. Due to continuation of symptoms and continued intolerance of PO intake, it was thought that the pseudocyst was contributing significantly to the patient's symptoms. Hence gastroenterology was consulted for intervention. An esophagogastroduodenoscopy (EGD) was performed, which showed a large extrinsic bulge on the anterior gastric body wall. Endoscopic ultrasound (EUS) was used to confirm that said bulge was due to the pseudocyst seen on CT. The cyst was endoscopically aspirated and drained, producing $200 \mathrm{cc}$ of dark fluid. Cultures of the fluid were negative and amylase level in the fluid was $27,683 \mathrm{U} / \mathrm{L}$ consistent with a pseudocyst. By the next day the patient's symptoms greatly improved. Two days after the procedure he was able to tolerate a regular diet and was discharged.

DISCUSSION: Pancreatic pseudocysts are well known complications of acute pancreatitis, occurring in approximately $10 \%$ of the cases. This particular case was notable for the unusual location of the pseudocyst, its lack of direct contact with the pancreas and also for the symptoms it was causing. Previous studies on the natural history of pseudocysts show that cysts $6 \mathrm{~cm}$ or smaller can be managed expectantly as the majority resolve on their own. However, indications for intervention include symptomatic pseudocysts regardless of the size. Historically, these were drained surgically. If anatomically feasible, endoscopic drainage is preferred due to the relative non-invasive nature compared to surgical drainage. However, studies report no difference between endoscopic versus surgical among rates of resolution and complications - including hemorrhage, infection, and fistula formation.

CONGENITAL CARDIAC DIVERTICULUM-A RARE CAUSE OF THROMBOEMBOLIC DISEASE Rehan Mujahid ${ }^{1}$; Fahd A. Almohid ${ }^{2}$; Shakil Shaikh ${ }^{1}$. ${ }^{1}$ Baystate Medical Center, Springfield, MA; ${ }^{2}$ Baystate medical center/ Tufts medical school, West Springfield, MA. (Control ID \#2469952)

LEARNING OBJECTIVE \#1: Recognize congenital cardiac diverticulum as a rare cause of transient ischemic attack and embolic cerebrovascular accident

LEARNING OBJECTIVE \#2: Distinguish between cardiac diverticulum, aneurysm, and pseudoaneurysm and their clinical implications

CASE: A 74-year-old female with diabetes and hypertension presented to our hospital with acute onset vertigo and left upper extremity weakness. Her symptoms resolved spontaneously and a work up for posterior circulation transient ischemic attack (TIA) was initiated. Transthoracic echocardiogram revealed an area of akinesis in the left ventricle periapical wall with an out-pouching in the endocardium. She was diagnosed with a cardiac diverticulum however it remained unclear whether symptoms were related to this diverticulum. No other causes of TIA were identified on electrocardiogram, laboratory data, or head imaging. She was started on antiplatelet therapy and discharge from the hospital.

DISCUSSION: Cardiac Diverticulum is a rare congenital malformation with diverse clinical manifestations. Although it is more common in pediatric populations, the prevalence in adults is $0.4 \%$ of the general population. Most patients are asymptomatic and the anomaly is usually found incidentally. However, severe hemodynamic instability and cardiovascular collapse have been reported. Other potential complications include thromboembolism, arrhythmias, ventricle rupture, heart failure, and valvular heart disease. Development of these complications depends on the size and location of the diverticulum. Once diagnosed, it is important to differentiate a cardiac diverticulum from aneurysm or pseudoaneurysm. Although they may appear similar on echocardiogram, the etiology and clinical manifestations are different. The latter two are generally due to infectious or ischemic events and the former is a congenital anomaly thought to develop during early embryonic life. Small differences in appearance may aid with the diagnosis. Aneurysms appear as a bulge during systole with or without paradoxical movement. Diverticulum is usually seen as an appendix or finger like structure out-pouching from the ventricular wall. Treatment is undefined and is mainly dictated by clinical presentation. Most patients can be treated conservatively while others may require surgical correction. For patients presenting with thromboembolism, such as our patient, some authors would suggest systemic anticoagulation to prevent recurrent events but this remains an area of dispute among experts. We preferred antiplatelet therapy over systemic anticoagulation since the diverticulum was small with no evidence of thrombus or turbulent flow. The rare nature of cardiac diverticulum and lack of substantial literature brings a unique challenge for practitioners. A high index of suspicion is required to make the diagnosis when other causes of TIA are ruled out. More research is needed to fully understand its pathophysiology and the role of anticoagulation in management of patients with cardiac diverticulum. 
CONNECTING THE DOTS OF SEEMINGLY UNRELATED COMPLAINTS: A CASE OF NEUROSARCOIDOSIS Theotonius J. Gomes ${ }^{2}$; Jason K. Lam ${ }^{1} .{ }^{1}$ Western Michigan University Home Stryker M.D. School of Medicine, Kalamazoo, MI; ${ }^{2}$ Western Michigan University Homer Stryker MD School of Medicine, Kalamazoo, MI. (Control ID \#2469817)

LEARNING OBJECTIVE \#1: Recognize neurosarcoidosis as a clinical entity presenting with multiple different clinical features

LEARNING OBJECTIVE \#2: Distinguish neurosarcoidosis as a clinically distinct diagnosis from Multiple Sclerosis

CASE: A 31 year old male presented with multiple symptoms over 7 months worsening over 3-4 weeks with increased neck stiffness, dizziness with episodes of vertigo, blurry vision, near syncope, holocephalic headaches, increased pressure behind eyes, muffled hearing, constant numbness in his tips of his fingers, thigh weakness with difficulty climbing stairs and frequent lancinating pain radiating down his right leg from the hip. Physical exam showed bilateral inguinal lymphadenopathy, 4/5 muscle strength with bilateral hip flexion and paresthesias at his fingertips with normal sensory exam to light touch, pinprick and cold. Initial laboratory workup including complete blood count, complete metabolic panel, lactate dehydrogenase, creactive protein, and thyroid stimulating hormone were normal. Lyme, HIV, Serum and urine protein electrophoresis were also negative. Cerebrospinal fluid (CSF) was negative for oligoclonal bands, acid-fast bacillus testing, cryptococcus, lyme and syphilis. CSF bacteria and fungal cultures were also negative. CSF analysis showed: glucose $39 \mathrm{mg} / \mathrm{dl}$, Protein $368 \mathrm{mg} / \mathrm{dl}$, and angiotensin converting enzyme level (ACE) 6 units/L (Reference range 0-2.5 units/L). Serum ACE was mildly elevated at 57 units/L. ANA was elevated to 1:160- Speckled pattern. A chest $\mathrm{x}$ ray showed right hilar prominence. MRI Brain and Cervical Spine showed enhancement in the basal cisterns and spinal cord at T1 with edema at T7-8. There was leptomeningeal enhancement over the occipital lobes into the parietal regions, right frontal lobe, optic chiasm, cerebellar hemispheres, medulla and pons. Extensive T2 hyperintensity was noted through the base of the pons, medulla and cervical cord. A biopsy of a subcarinal lymph node showed non-necrotizing granulomatous lymphadenitis. Clinical and radiographic data were most consistent with a diagnosis of Neurosarcoid. The patient was started on prednisone with resolution of his symptoms and improved areas of enhancement in his MRI on follow up.

DISCUSSION: Although multiple unrelated neurological symptoms can be indicative of a primary nervous system disorder such as multiple sclerosis, clinicians should recognize that it can also be the presenting symptoms of sarcoidosis with neurologic involvement. Sarcoidosis is characterized by non-necrotizing granulomatous inflammation with varying degrees of inflammation in multiple organs especially in the lung, eyes and skin. The cause of sarcoidosis is unknown. However, sarcoidosis has been linked to autoimmune processes related to molecular mimicry from infectious causes (viral, bacterial, fungal) as well as non-infectious causes (dust, metals). Classically it has been described in young to middle aged African American women with prolonged cough and shortness of breath Neurological complications can occur in $5-10 \%$ of those with sarcoid. Literature reviewed show that $50 \%$ of patients with neurosarcoidosis present with neurological symptoms as their initial presentation. Any portion of the central or peripheral nervous system can be affected leading to a multitude of common symptoms based on the location of the lesions. If a diagnosis of neurosarcoid is suspected a biopsy of non-neural tissue should be attempted first. There is no neurodiagnostic test specific for Neurosarcoidosis. Therefore, studies should be performed to rule out other causes. First line therapy for Neurosarcoid is corticosteroids dosed between 0.5 and $1.5 \mathrm{mg} / \mathrm{kg} / \mathrm{day}$ for 2-4 weeks based on area of neurological involvement. Immunomodulators, radiation and surgery have also been utilized for symptomatic control. Disease course may be monophasic, progressive or relapsing/remitting.

CONTRACEPTIVE HORMONE THERAPY INDUCED SPONTANEOUS CORONARY ARTERY DISSECTION Chandana Shekar ${ }^{1}$; William Perucki ${ }^{2}$; Charudatta Wankhade ${ }^{3}$; Nikolas Krishna ${ }^{4}{ }^{1}$ University of Connecticut School of Medicine, Farmington, CT; ${ }^{2}$ University of Connecticut, Farmington, CT; ${ }^{3}$ Lincoln Medical and Mental Health Center, Bronx, NY; ${ }^{4}$ Hartford Hospital, Hartford, CT. (Control ID \#2469925)

LEARNING OBJECTIVE \#1: Early recognition of spontaneous coronary artery dissection as a cause of acute coronary event in a patient without traditional cardiac risk factors

LEARNING OBJECTIVE \#2: Early recognition of hormone therapy as a cause of spontaneous coronary artery dissection.

CASE: A 47 year old caucasian female with past medical history of bipolar disorder, anxiety, depression, with etonogestrel-ethinyl estradiol vaginal ring for contraception presented to our hospital with severe substernal chest pain of $4 \mathrm{~h}$ duration, radiating to both arms and neck which woke her up from sleep. Pertinent positive history included nausea and generalized myalgia. Pertinent negative history included no diaphoresis, palpitations, shortness of breath or back pain. No recent illness or medication changes were present. Occasional use of alcohol was present but no tobacco use. Family history was negative for cardiac disorders. On arrival to our hospital, she was hemodynamically stable with a normal physical examination. Electrocardiogram (EKG) done immediately, showed normal sinus rhythm with a heart rate of 90 beats per minute, $\mathrm{T}$ wave inversions in lead III which were similar to previous EKG from 6 years ago and ST segment flattening in V2 which was a new finding when compared to previous study. No ST elevations were seen even on serial EKGs or lateral EKG. Initial blood investigations revealed a normal complete blood count, basic metabolic panel and coagulation panel. Troponin was elevated at $1.47 \mathrm{ng} / \mathrm{ml}$ and increased to $12.46 \mathrm{ng} / \mathrm{ml}$. Creatine Kinase was elevated at $168 \mathrm{U} / \mathrm{L}$ and later increased to $519 \mathrm{U} / \mathrm{L}$. Creatine Kinase-MB (CK-MB) was $15.2 \mathrm{ng} / \mathrm{ml}$ increasing to 78.0 and CK-MB relative index was $9 \%$, increasing to $15 \%$. Chest $\mathrm{X}$ ray was negative for acute cardiopulmonary process. Chest Pain was controlled with sublingual nitroglycerin. Our differential diagnosis then included Non ST elevation Myocardial Infarction and Myocarditis and she underwent medical management for the same. On cardiac catheterization, she was found to have no culprit occlusion of any coronary vessel. However, the circumflex provided a large marginal branch in its mid portion, which bifurcated into superior and inferior branch. The superior branch, before further bifurcation into two divisions had a severe focal narrowing, which extended into both divisions. This was concerning for spontaneous coronary artery dissection. There was evidence of stagnant contrast in the area supporting the diagnosis of spontaneous coronary dissection. As the patient was pain-free and marginal vessels involved in the dissection did not appear to be large in caliber, no coronary intervention was done and she underwent medical management. Echocardiogram showed a left ventricular ejection fraction of $50 \%$ and apical and mid-inferolateral hypokinesis. On further workup for identifying etiology of the condition, inflammatory markers were elevated with Erythrocyte Sedimentation Rate at $28 \mathrm{~mm} / \mathrm{h}$ and C-Reactive Protein at $2.68 \mathrm{mg} / \mathrm{dl}$. Rheumatologic workup for possible vasculitis or fibromuscular dysplasia including Anti Nuclear Antibody, Cyclic Citrullinated Peptide Antibody, Rheumatoid Factor, Double stranded DNA were negative. As she had no other risk factors and rheumatologic work-up was negative, it was concluded that combined hormone therapy in the form of estrogen-progesterone vaginal ring for contraception was the etiology of the spontaneous 
coronary artery dissection. She had the hormonal vaginal ring removed and has not had cardiac symptoms ever since.

DISCUSSION: Spontaneous Coronary Artery Dissection (SCAD) is defined as a tear in the coronary arterial wall that is not related to trauma or medical instrumentation. The build up of blood inside the arterial wall compresses the lumen, blocking the blood flow to the heart. Incidence of SCAD has been estimated to be $0.07-1.1 \%$, leading to underdiagnosis as well. Since it's first description in 1931, over 440 cases have been reported and the strongest link is with peri-partum period and with fibro-muscular dysplasia (70$80 \%$ ). Oral contraceptive use is attributed to $5.8 \%$ of SCAD cases. Incidence with topical and other forms of hormonal therapy, like in our patient is much lesser. Hormonally (particularly progesterone) induced degeneration of collagen, fragmentation of reticulin fibres along with decreased mucopolysaccharide content of the arterial media and hemodynamic changes are thought to play a role in hormone-associated SCAD. High degree of suspicion for SCAD is thus warranted when a patient on any form of hormonal therapy presents with an ischemic presentation without traditional cardiac risk factors.

\section{CORYNEBACTERIUM, A CORNY CONTAMINANT OR A REAL} PATHOGEN? Erica Altschul; Delphine Tang; Sherri Sandel. Lenox Hill Hospital, New York, NY. (Control ID \#2447486)

LEARNING OBJECTIVE \#1: To understand Corynebacterium striatum as a pathogen in endocarditis

LEARNING OBJECTIVE \#2: To understand which patients are at risk for infection by Corynebacterium

CASE: 58 year old male with bipolar disorder on diazepam, hypothyroidism, and lithium induced chronic kidney disease stage $\mathrm{V}$ on hemodialysis was brought to Lenox Hill Hospital for altered mental status (AMS) for 3 weeks. The patient was originally evaluated by an outpatient neurologist who performed head CT and MRI, both of which showed no acute pathology. Subsequently, low grade fevers developed at dialysis and he was sent to the hospital for further workup. The patient's mental status worsened and he became nonverbal, lethargic and was unable to follow commands. Initially symptoms were thought to be secondary to benzodiazepines, however symptoms persisted despite adjustment of medications. Vital signs in the ER were normal, physical exam findings significant for a 3/6 holosystolic murmur heard at the left sternal border with no appreciable radiation, bilateral pitting edema, and a left great toe ulcer with mild purulent drainage. Laboratory workup demonstrated chronic anemia unchanged from baseline, chronic renal failure, and an elevated TSH of $26 \mathrm{mcIU} / \mathrm{mL}$ with a decreased thyroxine level of $4.0 \mathrm{mcg} /$ dL, an elevated ESR of $62 \mathrm{~mm} / \mathrm{h}$. He was admitted to the hospital for EEG and LP. Bedside debridement of the left toe ulcer was performed by podiatry. Within $24 \mathrm{~h}$ of admission, the patient developed high fever and had respiratory distress. He was started on vancomycin, ceftazidine, and gentamicin and transferred to the ICU requiring intubation. Two sets of blood cultures returned positive for Corynebacterium striatum and a TEE was performed given the presence of fevers and murmur on exam. There was a large vegetation seen on the aortic valve mainly on the non-coronary cusp, however appeared to be involving the whole valve causing obstruction across the valve with a mean gradient of $28 \mathrm{mmHg}$. Also noted was a possible abscess formation around the valve. The patient was taken for emergent aortic root debridement and valve and root replacement. Due to the extensive destruction from the endocarditis, the patient required reconstruction of the left ventricular outflow tract and anterior mitral leaflet. After a prolonged hospital course, the patient was discharged to subacute rehabilitation facility to complete 6 weeks of antibiotic therapy. His mental status and functional status improved to baseline.

DISCUSSION: Corynebacterium species (CS) are gram positive bacilli considered to be a normal part of the flora of skin and mucous membranes; when present in clinical specimens, they are commonly thought to be contaminants. Developing data has demonstrated that CS is capable of causing serious infections in humans, occurring in any age group as well as immunocompromised and immunocompetent hosts. In a study that assessed Corynebacterium endocarditis risk factors and outcomes, $28 \%$ of patients required valve replacement and $43.5 \%$ died. The source of corynebacterium striatum likely results from cutaneous or mucosal colonization, even in superficial wounds such as the ulcer debrided in our patient. Another possible risk factor for the development of CS endocarditis in a native valve is the presence of a fistula which our patient possessed. The most common indications for valvular surgery include congestive heart failure, refractory sepsis, embolic complications, and vegetation size. The extensive destruction of the valve was not as evident on imaging - the presence of a large vegetation just inferior to the destroyed aortic leaflet prevented what would have been severe aortic insufficiency and likely cardiovascular demise. The severity of disease was not fully appreciated until the patient underwent a surgical procedure. While prosthetic valve endocarditis due to corynebacterium is considered well known and native valve endocarditis is considered rare, the diagnosis of endocarditis must be considered in all bacteremic patients. The presence of a murmur on exam and positive blood cultures, even if the species is usually considered contaminant, should prompt immediate investigation to rule out endocarditis.

COXSACKIEVIRUS B2 INDUCED HAND-FOOT-AND-MOUTH DISEASE COMPLICATED WITH HENOCH-SCHÖNLEIN PURPURA: AN UNUSUAL CLINICAL PICTURE. Tomoya Shiba ${ }^{1}$; Tadahiro Suzuki ${ }^{1}$; Taro Okabe ${ }^{1}$; Tomohiro Ogawa $^{2}$; Takuya Murakami ${ }^{3}$; Yu Yamamoto ${ }^{1}$; Shuji Hatakeyama ${ }^{1}$; Masami Matsumura ${ }^{1}$. ${ }^{1}$ Jichi Medical University Hospital, Shimotsuke, Japan; ${ }^{2}$ Department of Dermatology, Shimotsuke, Japan; ${ }^{3}$ Division of Nephrology, Shimotsuke, Japan. (Control ID \#2468201)

LEARNING OBJECTIVE \#1: Recognize the unusual clinical presentation of handfoot-and-mouth-disease complicated with Henoch-Schönlein purpura.

CASE: A 62-year-old immunocompetent woman was referred to our hospital because of painful oral vesicles and rashes in the hands and feet. She had close contact with her grandchild who had common cold-like symptoms few days previously. On day 1 of illness, her body temperature was $39.7^{\circ}$ Celsius, and she had a sore throat and chills. On day 3, she was diagnosed with group A streptococcal pharyngitis by a positive rapid antigen test. Anti-streptolysin-O (ASO) titer was $539 \mathrm{IU} / \mathrm{mL}$ (reference range, < $186 \mathrm{IU} /$ $\mathrm{mL}$ ). Oral cephalosporin antibiotics were administered for 10 days. The fever subsided in a few days. On day 7 of illness, however, small papules and vesicles appeared on palms and feet. The rashes gradually progressed to extensive hemorrhagic bullae $(10-30 \mathrm{~mm})$ and pastules. Small vesicles also appeared on the lips, tongue, throat, and buccal mucosa. Erythema with exfoliation and small purpura were observed on the lower legs and the lateral region of the abdomen. The patient was diagnosed with hand-foot-and-mouth disease (HFMD), and coxsackievirus B2 was isolated from the bloody fluid in a bulla on the hand. By day 32, the skin lesion began to reduce without specific treatment. However, on day 40 of illness, pitting edema on the lower legs, hypoalbuminemia $(1.6 \mathrm{~g} / \mathrm{dL})$, and proteinuria ( $8.6 \mathrm{~g} /$ day) appeared and the patient was diagnosed with nephrotic syndrome. Renal biopsies revealed proliferation of the mesangium with immunoglobulin A deposition, which is compatible with Henoch-Schönlein purpura nephritis. Her ASO titer significantly decreased to $61 \mathrm{IU} / \mathrm{mL}$ after 8 weeks.

DISCUSSION: HFMD commonly affects children and outbreak of the disease usually occurs during summer and autumn. Coxsackievirus A4, A6, A9, A10, and A16 and enterovirus 71 are known to be the major causative viruses. During the summer of 2015, a large outbreak of coxsackievirus A6 and A16-associated HFMD occurred in Japan. HFMD caused by coxsackievirus B2 is rare, but clinical presentation is considered to be similar to that in HFMD caused by other enteroviruses. Typical skin lesions are 1-2 mm vesicular exanthema in the hands, feet, and mouth and last for 3-7 days. However, in this 
patient who simultaneously suffered from HFMD caused by coxsackievirus B2 and Henoch-Schönlein purpura caused by group A Streptococcus, large (10-30 mm) hemorrhagic bullae and small purpura were observed and lasted for about 1 month. HFMD occurring simultaneously with Henoch-Schönlein purpura can cause unusual, severe skin lesions involving the hands, feet, and the mouth.

CRACKING THE CASE Joseph Delio ${ }^{1}$; Kendall Stone ${ }^{3}$; Anne Lesburg ${ }^{2} .{ }^{1}$ GWU, Washington, DC; ${ }^{2}$ George Washington University Hospital, Washington, DC; ${ }^{3} \mathrm{GWU}$ School of Medicine \& Health Sciences, Washington, DC. (Control ID \#2468258)

LEARNING OBJECTIVE \#1: Recognize that chest pain in a patient without cardiac risk factors may be due to an anterior mediastinal mass.

LEARNING OBJECTIVE \#2: Create a broad differential diagnosis for sternal fractures including lymphoma.

CASE: A 37 year-old woman without any past medical history presented with 5 days of constant, progressive, central pleuritic chest pain despite non-steroidal anti-inflammatory use and an associated non-productive cough. She denied trauma, fevers, chills or weight loss. She had no significant family history and denied tobacco use. She had exquisite tenderness to palpation from the mid sternum to the xiphisternal junction with an otherwise normal cardiopulmonary exam. She also had left axillary lymphadenopathy with adequate peripheral pulses and no peripheral edema. Thyroid exam was normal. She was afebrile and without leukocytosis. There was a widened mediastinum on chest x-ray and commuted tomography of the chest, abdomen and pelvis revealed an anterior mediastinal mass with local involvement of the sternum, suggesting a pathologic fracture. There was also surrounding mediastinal, left axillary and left subpectoral lymphadenopathy as well as two left upper lobe pulmonary nodules. Her sternal biopsy was inconclusive, but biopsy of the anterior mediastinal mass revealed classic bi-nucleated ReedSternberg cells, confirming Hodgkin's Lymphoma, nodular sclerosing subtype.

DISCUSSION: Chest pain is one of the most common chief complaints encountered by internists. However, in a young healthy patient without cardiac risk factors or associated gastrointestinal or concerning pulmonary symptoms, less common etiologies must be explored. In this patient's case, imaging first revealed a widened mediastinum. Although we are taught to immediately associate a widened mediastinum on chest $\mathrm{x}$-ray with an ascending aortic dissection, in this patient who had no alarming symptoms to suggest this diagnosis, an alternative diagnosis needed to be found. This case reminds clinicians that it is important to develop a broad differential in patients with a widened mediastinum as treatment and outcome varies drastically. Items on the differential can be categorized into one of the following: traumatic, infectious, inflammatory/autoimmune, neoplastic, anatomic and vascular. In this patient who denied trauma and was afebrile without a leukocytosis, the differential narrowed fairly quickly. When further imaging revealed the anterior mediastinal mass, only a few items remained on the differential and the biopsy result provided the answer. While the bulky anterior mediastinal mass contributed to this patient's pain, the sternal fracture no doubt was the main cause. While sternal fractures are most commonly due to trauma, the differential diagnosis also includes secondary malignancy, myeloma and rarely osteoporosis, frequently in the setting of glucocorticoid use. In this patient's case, the fracture was caused by contiguous spread of Hodgkin's disease In Hodgkin's disease, osseous involvement occurs in 5-20\% of patients but is only observed in $1-4 \%$ of cases at initial presentation. When osseous involvement is in fact present at diagnosis, the sternum is the most common site affected, postulated to be due to its superficial location and proximity to thoracic lymph ducts. A sternal mass can develop over a variable time period, ranging from days to months and is usually solitary in nature. Unlike other skeletal masses, it is often associated with subtypes of Hodgkin's disease with good prognoses, such as the nodular sclerosing subtype as in this patient. This case highlights the importance of creating a broad differential diagnosis for chest pain including anterior mediastinal mass in the appropriate setting. While sternal fracture without trauma is a rare cause of chest pain, clinicians should consider it especially in the presence of an anterior mediastinal mass. Doing so may be the key to cracking the case.

\section{CUTANEOUS PRESENTATION OF METASTATIC THYROID CANCER Derek}

Nguyen ${ }^{2}$; Yvonne N. Covin ${ }^{2}$; Blake R. Barker ${ }^{1} .{ }^{1}$ UT Southwestern Medical Center at Dallas, Dallas, TX; ${ }^{2}$ University of Texas Southwestern Medical Center, Arlington, TX. (Control ID \#2465826)

LEARNING OBJECTIVE \#1: Expand the differential diagnosis of scalp lesions to include cutaneous metastasis

LEARNING OBJECTIVE \#2: Recognize atypical metastatic manifestations of papillary thyroid carcinoma

CASE: A 74 year-old male with a past medical history of hypertension and type 2 diabetes presented with three prominent growing skin lesions on his face within the past year: an asymptomatic scalp lesion and intermittently bleeding lower lip and right helix lesions. He denied any fevers, weight changes, night sweats, dyspnea, palpitations, or fatigue. He is a former smoker of 20 pack-years who quit 49 years ago. He worked outside and did not wear sunscreen. He denied any history of skin cancer. On presentation, he was afebrile with skin findings consisting of a $7 \mathrm{~mm} \times 7 \mathrm{~mm}$ pearly telangiectatic papule on midline scalp, a $9 \mathrm{~mm} \times 4 \mathrm{~mm}$ eroded thin papule with induration on lower left lip, and a $12 \mathrm{~mm} \times$ $14 \mathrm{~mm}$ erythematous, eroded, and friable plaque on right helix. Neck showed no tracheal deviation, thyromegaly, or cervical adenopathy. Excisional skin biopsy was performed for each lesion. The lip and helix lesions' pathology were consistent with basal cell carcinoma and squamous cell carcinoma, respectively. The scalp lesion, however, showed welldifferentiated adenocarcinoma. Subsequent immunohistochemistry suggested thyroid metastasis and PET scan revealed FDG-avid lesions in the right thyroid lobe and right cervical lymph nodes. Neck ultrasound showed a suspicious $2.8 \mathrm{~cm}$ right thyroid mass as well as abnormal lymph nodes in the central and right lateral neck. FNA findings from the right thyroid mass and level 3 right cervical node were consistent with papillary thyroid carcinoma. He then underwent a total thyroidectomy followed by remnant ablation with radioactive iodine for additional metastatic disease in lungs and liver. Post-ablation, he began maintenance levothyroxine with TSH suppression goal of $<0.10$.

DISCUSSION: Given his heavy unprotected sun exposure and characteristic location of the lesions, an initial evaluation for skin cancer was warranted. When the scalp biopsy revealed an unexpected adenocarcinoma, the differential was broadened to include metastatic disease. An array of immunohistochemical markers and a PET scan aided in identifying the primary origin. The incidental discovery of an asymptomatic thyroid nodule prompted suspicion for thyroid carcinoma - ultimately confirmed to be papillary thyroid carcinoma. Papillary thyroid carcinoma (PTC) is the most common type of thyroid malignancy. PTC usually presents as an asymptomatic, palpable thyroid nodule. Approximately $40 \%$ of PTC cases involve local metastasis to cervical lymph nodes. Distant metastasis to the lungs, liver, bones or brain occurs in $4 \%$ of patients. PTC manifesting as a cutaneous metastasis has an infrequent incidence ranging from 0.06 to $0.82 \%$. Interestingly, the scalp is the most common site. The scalp possesses extensive dermal capillary networks where embolic tumor cells from the systemic circulation can become trapped within the skin. This nutrient-rich vascularity provides the optimal environment for formation of metastatic foci. An excisional skin biopsy with subsequent immunohistochemistry staining of thyroid transcription factor-1 (TTF-1), thyroglobulin, or cytokeratin 7 serves to distinguish thyroid carcinoma from other primary carcinomas. Due to concurrent presentation of cutaneous and disseminated metastasis, average survival time of PTC is 19 months. This case was unusual as the patient had no previous history or sign 
of a palpable thyroid mass despite a sizable thyroid nodule on PET scan identified months after the initial skin presentation. Our case illustrates that internists and dermopathologists should be cognizant of this atypical clinical manifestation, despite its rarity. Thus, we ought to consider metastatic thyroid cancer on the differential diagnosis of scalp lesions and to perform excisional skin biopsy on all atypical cutaneous lesions.

\section{CYCLICAL THROMBOCYTOPENIA: A RARE BUT FATAL IMITATOR OF} IDIOPATHIC THROMBOCYTOPENIA Shazia Samanani; Rehan Mujahid; Shrinkhala Khanna. Baystate Medical Center, Springfield, MA. (Control ID \#2468558)

LEARNING OBJECTIVE \#1: Recognize the patterns needed for diagnosing cyclical thrombocytopenia

LEARNING OBJECTIVE \#2: Distinguish the potentially life threatening features of cyclical thrombocytopenia as compared to idiopathic thrombocytopenia

CASE: A 42 year old female of Eastern European origin, with a past medical history of systemic lupus erythematosis (SLE), was referred to outpatient hematology for management of her presumed diagnosis of idiopathic thrombocytopenia (ITP). Previous treatments including Aspirin, Eltrombopag, Azathioprine, Prednisone, Rituximab, Intravenous Immunoglobulin (IVIG) and splenectomy had been unsuccessful in raising her platelet count. The patient experienced symptoms of thrombocytopenia which included diffuse petechiae, mucosal petechiae, self-limiting epistaxis and significant menorrhagia which required hysterectomy. Symptoms were intermittent and occurred approximately once a month. Upon investigating the patient's previous lab work, a cyclic pattern of thrombocytopenia alternating with rebound thrombocytosis was revealed. A nadir of platelet count as low as $1000 / \mathrm{mm} 3$ with a peak count of as high as $2,000,000 / \mathrm{mm} 3$ was observed occurring approximately every 28 days. Interestingly, a similar pattern was noticed in the white blood cell levels. A correlation was also seen with her reproductive hormone levels, with luteinizing hormone running synchronously and estradiol cycling inversely to the platelet trends. This laboratory pattern, coupled with her lack of response to the aforementioned therapies, allowed us to make a diagnosis of cyclical thrombocytopenia (CT). Although the patient did not always experience symptoms during periods of rebound thrombocytosis, over the years, thrombotic events included lower extremity deep venous thrombosis and a myocardial infarction thought to be a result of peaked platelet levels. Treatment since the diagnosis of CT was made included Eltrombopag, Romiplostim, and Prednisone with dose adjustment at 2-week intervals depending on her most recent platelet levels. Hormone therapy was trialed using Premarin, a conjugated estrogen therapy. Concurrent management of SLE with Belimumab was also held for a period of time. A combination of these interventions decreased the life threatening peaks but failed to inhibit platelet nadirs.

DISCUSSION: Cyclical thrombocytopenia is a rare but potentially life threatening condition. A recent literature review identified only 51 reported cases. Correlation with cyclical leukocytosis is found in a small subset of CT patients making it a more uncommon variant. Diagnosis is challenging and most patients are misdiagnosed with ITP. Differentiating CT from ITP is crucial to prevent life threatening thrombotic events. Differentiating factors include rebound cyclical thrombocytosis alternating with cyclical thrombocytopenia. These cycles typically occur every 28 days. CT patients may have a history of thrombotic events in addition to typical ITP symptoms and may fail to respond to conventional ITP therapies. CT predominantly affects pre-menopausal females, however, post menopausal females and men may also be affected. The pathophysiology of CT is poorly understood. Multiple theories exist including intermittent autoimmune destruction of platelets, cyclical fluctuations of megakaryocytes, and abnormal biochemical signaling between cytokines and hematopoietic cells. An association with female reproductive hormones and a positive response to hormone therapy has also been documented in the literature. More research is needed to fully understand the pathogenesis of CT. Such an understanding may increase identification of the disease, provide better treatment modalities, and prevent poor outcomes in these patients.

DAPTOMYCIN INDUCED EOSINOPHILIC PNEUMONIA Alex Katz ${ }^{2}$; Adrian P. Umpierrez De Reguero ${ }^{1}$; Pinky $\mathrm{Jha}^{3} .{ }^{1}$ Froedtert \& the Medical College of Wisconsin, Milwaukee, WI; ${ }^{2}$ Medical College of Wisconsin, Wauwatosa, WI; ${ }^{3}$ Medical College of Wisconsin, Milwaukee, WI. (Control ID \#2467712)

LEARNING OBJECTIVE \#1: Recognize the clinical presentation of daptomycin induced eosinophilic pneumonia.

LEARNING OBJECTIVE \#2: Understand the hypothesized pathophysiology of daptomycin induced lung injury.

CASE: A 60 year-old woman presented with 2 days of severe shortness of breath associated with low oxygen saturation and lower extremity swelling. She had jugular venous distension, bilateral crackles, and significant bilateral lower extremity edema. Laboratory results were remarkable for elevated creatinine kinase, leukocytosis and abnormal creatinine. Chest X-ray revealed new bilateral patchy opacities and bilateral pleural effusions. Two weeks prior to admission, she had been started on a 6 week course of I.V ertopenem and daptomycin for cervical discitis. A CT chest revealed multifocal consolidations with surrounding ground glass opacities and adenopathy. Pulmonary and Infectious diseases were consulted. Further labs revealed abnormal peripheral eosinophilia and elevated serum IgE. These results, coupled with an unremarkable infectious work up, allowed us to broaden our differential to autoimmune and other hypersensitivity disorders. A bronchial-alveolar lavage revealed marked eosinophilia. A diagnosis of daptomycin induced eosinophilic pneumonia was made once other causes were ruled out. Fortunately, daptomycin had already been switched to linezolid on admission. A chest x-ray was repeated 1 week after admission and showed complete clearing of the bilateral interstitial thickening.

DISCUSSION: Eosinophilic pneumonia is a rare lung pathology associated with a broad differential. It includes drug interactions, infectious causes and vasculitis. It can be acute or chronic and usually presents with fever, hypoxia, new lung infiltrates and either peripheral eosinophilia or pulmonary eosinophilia. Here we present a case of eosinophilic pneumonia induced by daptomycin administration in the setting of diastolic heart failure exacerbation. Hospitalists commonly encounter patients presenting with edema and pulmonary infiltrates who are taking antibiotics. While daptomycin does not have an increased incidence of adverse events when compared with other anti-biotics, eosinophilic pneumonia is a known, if rare, side effect. We found 8 cases of definite daptomycin induced eosinophilic pneumonia in the literature. Diagnostic criteria included were 1) bilateral pulmonary infiltrates, 2) hypoxia 3) bronchial-alveolar lavage with $25 \%$ eosinophils, 4) exposure to drug or toxin 5) no other cause of pulmonary eosinophilia, 6) clinical improvement after cessation of drug and finally, 7) recurrence of symptoms after re-introduction of toxin or drug. While bronchial-alveolar lavage revealed only $19 \%$ eosinophils, our case still met 5 of 7 requirements for diagnosis. Daptomycin causes injury by forming a complex with pulmonary surfactant, activating alveolar macrophages and leading to IL-5 release by Th2 cells, leading to eosinophilic release and inflammation. In our patient, this damage led to a CHF exacerbation. Once the offending agent was removed and fluid taken off, our patient rapidly improved. Daptomycin is an increasingly common second line antibiotic prescribed due to the emerging incidence of multi-drug resistant organisms. It is important for hospitalists to recognize the clinical symptoms of possible drug induced lung injury so as to discontinue the medication promptly.

DE NOVO SPLENIC CD5+ DLBCL Zaid Abdelrahman; Yaser Alkhatib; Vrushali Dabak. Henry Ford Health System, Detroit, MI. (Control ID \#2457400) 
LEARNING OBJECTIVE \#1: Describe a rare case of an aggressive de novo splenic CD5+ DLBCL

LEARNING OBJECTIVE \#2: Managing tumor lysis syndrome in the setting of an aggressive lymphoma might require treating the underlying lymphoma

CASE: A 63 year-old female patient presented with worsening abdominal pain for 1 month. This was associated with nausea, vomiting, early satiety and a $40 \mathrm{lb}$ weight loss. She denied fever, night sweats, or changes in bowel movement, hematochezia or melena. Patient was evaluated before for these symptoms with esophagogastroduodenoscopy and colonoscopy which were unremarkable. Initial blood workup was significant for a Sodium level at $125 \mathrm{mmol} / \mathrm{L}(135-145 \mathrm{mmol} / \mathrm{L})$, a potassium at $3.9 \mathrm{mmol} / \mathrm{L}(3.5-5.0 \mathrm{mmol} / \mathrm{L})$, a Creatinine at 1.58 from a baseline of $1.1 \mathrm{mg} / \mathrm{dL}$, a hemoglobin at $8.9 \mathrm{~g} / \mathrm{dl}$ and a platelet count at $94 \mathrm{~K} / \mathrm{uL}$ $(150-450 \mathrm{~K} / \mathrm{uL})$ with normal white blood count. Further workup showed elevated LDH at $3300 \mathrm{IU} / \mathrm{L}(100-220 \mathrm{IU} / \mathrm{L})$, uric acid at $18 \mathrm{mg} / \mathrm{dL}(3.5-7.2 \mathrm{mg} / \mathrm{dL})$, serum calcium at $6.9 \mathrm{mg}$ $\mathrm{dL}(8.2-10.2 \mathrm{mg} / \mathrm{dL})$ and a phosphorus at $3.9 \mathrm{mg} / \mathrm{L}(2.5-4.5 \mathrm{mg} / \mathrm{dL})$. A CT of the abdomen and pelvis to rule out acute abdomen was negative for any acute process but showed significant splenomegaly at $21.1 \mathrm{~cm}$. Patient was diagnosed with spontaneous tumor lysis syndrome (TLS) secondary to a likely underlying lymphoproliferative disorder. Aggressive IV hydration was initiated and rasburicase was given resulting in transient improvement of her electrolytes and uric acid. A bone marrow biopsy showed atypical large B-cell infiltrates Immunohistochemistry stains revealed scattered occasional intrasinusoidal or interstitial infiltrates of large B-cells (CD20+) that aberrantly co-expressed CD5, CD10 and Bcl-6, highly suspicious, however, morphologically insufficient to establish a diagnosis of lymphoproliferative disorder. Subsequently a diagnostic and therapeutic splenectomy was performed; pathology was consistent with diffuse large B-cell lymphoma. The immunohistochemistry stains were positive for CD5, CD20 and BCL-2. C-Myc was positive in less than $40 \%$ and MIB-1 was positive in $70 \%$. Following the surgery, her functional status declined (Eastern Cooperative Oncology Group performance scale of 3) and developed anasarca due to poor nutritional status and hypoalbuminemia. Patient continued to be in clinical TLS despite the first rasburicase dose and removing the bulk of her disease (the spleen). Chemotherapy was held initially due to the recent surgery and declined functional status, however, in light of persisten clinical TLS, which was attributed to the bone marrow involvement, a decision was made to start chemotherapy with R-CHOP 2 weeks after surgery. Aggressive hydration and an additional dose of rasburicase were given to control the worsening TLS. One week following chemotherapy a response was demonstrated by halting the tumor lysis and improving counts Her nutritional and functional status continued to improve and she was discharged to subacute rehabilitation, where she regained her strength and successfully completed a total 6 cycles of chemotherapy. She is now in complete remission, and is back to her normal daily activities. DISCUSSION: De novo CD5-positive diffuse large B-cell lymphoma (CD5+ DLBCL) is rare and accounts for less than $10 \%$ of all DLBCL. Isolated de novo splenic DLBCL is even rarer and it is known to be associated with a more aggressive clinical course and worse outcomes. Spontaneous TLS can be seen with aggressive lymphoid malignancies, even though it is more commonly encountered after initiation of chemotherapy. TLS is a medical emergency where early detection and management are essential to avoid serious, life threatening complications including kidney failure, cardiac arrhythmias, seizures and even death. Even though IV hydration, anti-hyperuricemic drugs and correction of electrolytes abnormalities can transiently decrease the risks associated with TLS; the ultimate management is treating the underlying malignancy. DLBCL is very sensitive to chemotherapy; which can be lifesaving and it should not be delayed due to declined functional status, especially if this decline was secondary to the underlying lymphoma.

DEBILITATING MUSCLE PAIN IN A DIABETIC PATIENT: A DIAGNOSIS OF DIABETIC MYONECROSIS Sophie M. Hapak ${ }^{1}$; Orighomisan Pessu ${ }^{2}$; Courtney Mechling ${ }^{2}$; Deepa R. Nandiwada ${ }^{1}$. ${ }^{1}$ University of Pittsburgh, Pittsburgh, PA; ${ }^{2}$ University of Pittsburgh Medical Center, Pittsburgh, PA. (Control ID \#2467172)
LEARNING OBJECTIVE \#1: Recognize the clinical presentation and differential of diabetic myonecrosis

LEARNING OBJECTIVE \#2: Understand the management and clinical course of diabetic myonecrosis

CASE: A 50-year-old man presented from an outside hospital with 4 weeks of increasing left anterior thigh pain and swelling. His past medical history was significant for type II diabetes complicated by end stage renal disease now on dialysis, coronary artery disease with an automatic implantable cardioverter defibrillator (AICD), and deep vein thrombosis. Of note, he had a $50 \mathrm{lb}$ unintentional weight loss over the past 6 months allowing his diabetes to be controlled by diet alone. He described the pain as constant, aching, and worse with movement and palpation. The pain prevented him from bearing weight, prior to this he was able to ambulate with a cane. On physical exam, the patient appeared thin and was in moderate distress due to pain. Vital signs were normal. Cardiac exam was significant for a $2+$ systolic ejection murmur best heard at the left upper sternal border. His left anterior thigh was swollen, warm, and exquisitely tender to light palpation without evidence of trauma or erythema. Strength was limited due to pain. There was no erythema or edema below the left knee. His right leg was normal on exam. He had palpable dorsalis pedis pulses bilaterally; his femoral pulse was diminished on the left. No inguinal lymphadenopathy was noted. Abdominal exam did not reveal any significant masses or organomegaly. Laboratory exam was significant for an erythrocyte sedimentation rate of $118 \mathrm{~mm} / \mathrm{h}$ and a high sensitivity CRP of $5.284 \mathrm{mg} / \mathrm{dL}$. He had a white blood cell count of $6.3 \mathrm{k} / \mathrm{mm}^{2}$, hemoglobin of $9.4 \mathrm{~g} / \mathrm{dL}$, INR of $1.2, \mathrm{CPK}$ of $42 \mathrm{IU} / \mathrm{L}$, and blood glucose of 84 . Alkaline phosphatase of $371 \mathrm{IU} / \mathrm{L}$, total bilibrubin of $1.8 \mathrm{mg} / \mathrm{dL}$, with a normal AST and ALT. IgG4-subclass was elevated to $132 \mathrm{mg} / \mathrm{dL}$. Rheumatoid factor, anti-nuclear antibody, and anti-neutrophil cytoplasmic antibody were within normal limits. Venous Doppler was negative for deep vein thrombosis. EMG of the anterior left thigh muscle from an outside hospital demonstrated severe left lumbosacral polyradiculopathy, advanced peripheral polyneuropathy, and fibrosis of the left vastus medialis and vastus intermedialis muscles concerning for myconecrosis, vasculitis, plexopathy, and amyotrophy. Computed tomography angiogram of the abdomen, pelvis, and left lower extremity was significant for diffuse low attenuation and enlargement of the left anterior thigh musculature. There was no evidence of malignancy or thrombi. Magnetic resonance imaging was unable to be performed due to the patient's AICD. Open biopsy of the left anterior thigh muscle demonstrated abnormal muscle with neurogenic changes and fibrosis, patchy necrosis, and focal perivascular inflammation. All test results were consistent with a diagnosis of diabetic myonecrosis with amyotrophy. The patient was started on aspirin $325 \mathrm{mg}$ and 2 days of methylprednisolone $500 \mathrm{mg}$ intravenously. Oxycodone, oxycontin, and hydromorphone were required to manage his pain. After steroid administration, he experienced significant improvement in pain, decreased swelling, and increased range of motion. He was discharged to a skilled nursing facility.

DISCUSSION: Diabetic myonecrosis is a rare complication of long-standing diabetes. It has been reported mostly in patients with poorly controlled type I diabetes with multiple associated complications (retinopathy, renal disease, and neuropathy). Our patient was unique in that his diabetes was well controlled for months prior to disease onset. Additionally, he had type II diabetes, although he suffered from multiple diabetic complications. Diabetic myonecrosis is challenging to diagnose given that its presentation can resemble that of other diseases, including myositis, infection, vasculitis, and thrombosis. The classic presentation is in a poorly controlled diabetic patient with acute progressive onset of severe thigh or calf muscle pain. Currently, contrast-enhanced MRI is recommended for diagnosis. An MRI was contraindicated in our patient given his AICD. The combination of an EMG, CT angiogram, and muscle biopsy were sufficient to diagnosis this patient, suggesting that these modalities may be useful in patients in which an MRI is contraindicated. Current recommendations for treating diabetic myonecrosis include antiinflammatory therapy (including high dose methylprednisolone) and anti-platelet agents. Our patient experienced significant improvement in his pain and range of motion after 
2 days of methylprednisolone $500 \mathrm{mg}$ intravenously. Of note, there is evidence to indicate that physical therapy and muscle biopsies may prolong a patient's disease course, although the disease has been shown to be self-limited to weeks or months. As our differential was so broad in this patient, we opted for a muscle biopsy to confirm the diagnosis prior to initiating high dose steroids. The prognosis for these patients is generally poor, with the majority experiencing a second episode involving the contralateral leg and most suffering death from a major vascular event within a few years.

\section{DEHYDRATION AND DOXYCYCLINE PRECIPITATING LITHIUM}

TOXICITY Melissa Itsara. Kaiser Foundation Hospital - San Francisco, San Francisco, CA. (Control ID \#2468007)

LEARNING OBJECTIVE \#1: Assess for signs and symptoms of lithium toxicity LEARNING OBJECTIVE \#2: Recognize potential precipitants of lithium toxicity CASE: A 47 year old Caucasian female with a history of bipolar disorder presented to the emergency department with 2 days of tremors and altered mental status. Five days prior to admission, she was seen in clinic for cough and fever, and was prescribed doxycycline for presumed pneumonia. At that time, the husband noticed his wife appeared more unsteady on her feet and had difficulty with manual dexterity, such as holding a cup. The patient's home medications included lithium (for 10 years), aripiprazole, escitalopram, and klonopin. No recent changes had been made to her medications except the addition of doxycycline. On examination in the emergency department, her vitals were within normal limits. She had dry mucus membranes. Cardiac, pulmonary, and abdominal exams were unremarkable. She was awake and alert, with a normal cranial nerve exam, full strength, and normal sensation. She had no rigidity or clonus. She had an expressive aphasia limiting her ability to answer questions and a resting tremor of her bilateral hands and feet. Non-contrast CT of the head was unremarkable. Chest x-ray showed a resolving pneumonia. EKG was unremarkable. Laboratory studies revealed a creatinine of 1.46 (last known of 0.97 ), white blood cell count of 18.6, and a negative urine drug screen. Lithium level was noted to be 1.2 when she initially presented to clinic. At time of admission, repeat lithium level was elevated at 2.6. Aggressive intravenous fluids were given for treatment of lithium toxicity. By the following day, her lithium level came down to $<0.1$. Her hospital course was complicated by profound urine output and development of hypernatremia to the $150 \mathrm{~s}$, which corrected with D5W. Her altered mental status resolved. The patient later reported having chronic polydipsia necessitating her to drink approximately five liters of water every day, which she was unable to continue once she began feeling unwell. It was suspected the patient had developed nephrogenic diabetes insipidus from chronic lithium use and she was treated with amiloride. By time of discharge, her creatinine was also improving.

DISCUSSION: Due to its narrow therapeutic index (0.8-1.2), lithium toxicity can occur frequently. Acute toxicity occurs with ingestions of large amounts of lithium, while chronic toxicity usually occurs in conjunction with impaired renal function or excretion. Predisposing factors that can contribute to lithium toxicity include dehydration, fever, infection, and medications (commonly diuretics, ACE inhibitors, and NSAIDs). Clinical manifestations of chronic toxicity may include neurological symptoms such as ataxia, confusion/agitation, coarse tremors, fasciculations, myoclonic jerks, or even seizures. With chronic lithium use, nephrogenic diabetes insipidus (DI) can develop, manifesting with polyuria, polydipsia, and hyponatremia. Lithium accumulates in the collecting ducts, leading to resistance to $\mathrm{ADH}$ and preventing its ability to increase water permeability (ADH normally regulates aquaporin-2 channels to fuse to the luminal membrane, thereby allowing water reabsorption). Nephrogenic DI is treated with a potassium-sparing diuretic, such as amiloride. In this patient, it was suspected that the combination of her recent infection, decreased fluid intake, as well as the addition of doxycycline (which is known to increase lithium levels), likely led to renal impairment and accumulation of lithium. In summary, this case is a reminder to be aware of the narrow therapeutic index of lithium and the predisposing factors to toxicity.

DELAYED DIAGNOSIS OF DENGUE FEVER Gina Maki ${ }^{1}$; Alexis C. Haftka ${ }^{2}$; Milind Patel ${ }^{1}$; Indira Brar ${ }^{1} .{ }^{1}$ Henry Ford Health System, Royal Oak, MI; ${ }^{2}$ Henry Ford Hospital, Detroit, MI. (Control ID \#2457583)

LEARNING OBJECTIVE \#1: Diagnose Dengue Fever. LEARNING OBJECTIVE \#2: Recognize Anchoring Bias.

CASE: The patient is a 34 year old Filipino male with no significant past medical history who presents with a 4 day history of fever, chills, headache and malaise. He returned from a trip in Vietnam 2 days prior to presentation. A work up was initiated in the emergency department. A rapid HIV screen was performed which was positive. He denied any known exposure to HIV, unprotected intercourse, or intravenous drug use. A CT of the head and lumbar puncture were performed, which were both negative. Patient was admitted to the medical ward where he continued to have cyclic fevers, night sweats and headache. Lab work showed leukopenia, lymphopenia, thrombocytopenia, and elevation of transaminases. On day two of admission patient developed a faint petechial rash. Patient's rapid HIV screen was determined to be a false positive after an HIV viral load returned negative. Patient's transaminases continued to worsen, as did his petechial rash. Further laboratory testing was performed to determine a diagnosis. RPR, HSV, West Nile, Malaria smear, as well as fungal and blood cultures remained negative. Based on patient's history, physical examination, laboratory abnormalities, and recent travel history a concern for Dengue fever was present. On day four of admission patient remained afebrile with normalization of his white blood cell count and platelets. His liver enzymes improved, as well as his petechial rash. Shortly after discharge, patient's Dengue IgG and IgM were found to be positive.

DISCUSSION: Dengue is the most prevalent mosquito-borne viral illness disease. It can present with a wide range of clinical manifestations, from mild febrile illness to a lifethreatening shock syndrome or organ dysfunction. The classic presentation of Dengue fever is an acute febrile illness accompanied by headache, retro-orbital pain, myalgias and arthalgias. Symptoms typically develop 4 to 7 days after the bite of an infected mosquito. Dengue is mainly a clinical diagnosis, which can be confirmed using serology. Treatment is supportive care. It is important to rule out other treatable diseases which may have a similar presentation such as malaria and typhoid fever. This case also exemplifies the need to avoid anchoring bias. It is easy to narrow ones' differential diagnosis once a test result returns positive. However, this can be dangerous when the test result returns prior to the initial expansion of the differential diagnosis. When our patient was found to have a positive HIV test in the emergency department the differential diagnosis was narrowed immediately, which contributed to a delayed diagnosis of Dengue fever.

DENTAL ANTIBIOTIC PROPHYLAXIS: ARE WE DOING ENOUGH? Nikhil $\underline{\text { Shah; }}$ Jorge Lascano. University of Florida, Gainesville, FL. (Control ID \#2469659)

LEARNING OBJECTIVE \#1: Review antibiotic prophylaxis guidelines for infective endocarditis before dental procedures

LEARNING OBJECTIVE \#2: Review evidence surrounding infective endocarditis antibiotic prophylaxis.

CASE: A 53 year old previously healthy male presented to an outside hospital with 3 weeks of abulia noted by family, right-sided weakness with a constant, pill-rolling tremor, decreased vision, fevers and night sweats. He had a dental extraction without antibiotics 4 weeks prior. He was transferred to our facility with strokes, pneumonia, splenic abscess and possible infarct, atrial fibrillation, moderate aortic regurgitation (AR), 
and reduced ejection fraction. He had a history of hypertension, bicuspid aortic valve (BAV), viral myocarditis with heart failure in 2011 (since resolved) and a heavy smoking history. He had minimal verbal output but spontaneously opened his eyes and was oriented to self. His lungs were clear, heart rhythm was irregular with a 3/6 systolic murmur, legs had 2+ edema, skin had no lesions, and right facial droop and hemiparesis were present with bilateral plantar extensor reflexes. Blood cultures on admission grew Streptococcus viridans. A brain MRI revealed a subacute right PCA infarct and trans-esophageal echo showed a normal ejection fraction with akinesis of the basal inferior wall, moderate aortic stenosis and AR, and severe mitral regurgitation with infective endocarditis (IE) and small mobile mitral leaflet vegetations. His hospital course had multiple, life-threatening complications. He had 12 min of pulseless ventricular tachycardia and torsades de pointes on back-to-back days. Subsequent left heart catheterization showed mild coronary disease unlikely to have caused his arrhythmias. He also had alternating tachycardia and bradycardia, and hypotension requiring pressors intermittently for weeks. Renal failure and hypervolemia required $\mathrm{CVVH}$ as tolerated by blood pressure. He had hemorrhagic conversion of his stroke with new deficits. He was anemic and often lost blood iatrogenically, requiring multiple transfusions. Given his mental status and episodes of flash pulmonary edema from $\mathrm{AR}$, he was intubated twice, ultimately requiring tracheostomy. His medical therapy included ACLS, several antimicrobials, pressors, anti-arrhythmics, and diuretics. After several weeks of tenuous hemodynamics with waxing and waning mental status, he was fully alert, oriented and able to move his extremities again. He was transferred to a long-term acute care facility after 47 days in our facility.

DISCUSSION: Seemingly benign dental procedures may result in IE with life-altering complications. It is commonly diagnosed at a rate of $11-15$ per 100,000 in the US. Given the relatively low risk of IE in patients, and weighing the risk of antibiotic resistance and overuse, the guidelines for antibiotic prophylaxis were narrowed in 2007 for only certain invasive dental procedures in patients at high risk for IE due to prosthetic cardiac valves or valve repair materials, previous IE, certain congenital heart diseases, and transplanted hearts with valvulopathy. In these patients, a single dose 30 $60 \mathrm{~min}$ before the procedure is recommended with either amoxicillin orally, or cephalexin, ceftriaxone, clindamycin or azithromycin if allergic or unable to take oral antibiotics. The patient presented above, at low risk for IE by current guidelines, underwent an unknown dental procedure and later developed IE with multiple, severe complications, with lasting implications on his lifestyle, health and finances. He was hospitalized for at least 47 days. Assuming an average cost of nearly $\$ 4000$ per day in the ICU, adjusting for inflation, and excluding procedures or long-term acute care, this patient's hospital bill alone is estimated to be over $\$ 280,000$. This high degree of morbidity and cost associated with IE from a dental procedure without antibiotic prophylaxis begs the question: is our prophylaxis enough after the 2007 guidelines narrowed the indications for antibiotics? Several investigators examined whether the rates of IE increased after 2007. In Minnesota, no significant change was noted in the incidence of S. viridans IE, but other investigators showed a national increase in streptococcus IE rates. Other studies researched whether dental procedures and IE rates were associated, or if prophylaxis was even necessary. A study in Europe showed that only $5.3 \%$ of IE was due to dental procedures, and 5 out of 20 patients with IE had received antibiotics, indicating that even appropriate prophylaxis may not prevent IE. In Taiwan, there was no association between dental procedures and IE rates, but in England, guidelines were updated in 2008 to cease all prophylaxis for dental procedures, after which IE incidence rose. Furthermore, the 1997 criteria placed patients with BAVs at moderate risk, but there is no mention of BAVs in the 2007 criteria. Based on the evidence above, it is unclear if prophylaxis would have prevented IE and its complications in this patient. He may have had other unknown predisposing risk factors, or he could have developed idiopathic IE even if his dental procedure had not occurred. More research is needed to know how much prophylaxis is enough. It is therefore at the discretion of providers, weighing the risks and benefits of each patient's predisposing factors, to appropriately prescribe prophylaxis with the knowledge that though appropriate and guideline-based, prophylaxis may not always prevent IE.

DERANGEMENTS OF HEROIN WITHDRAWAL IN PRISON Nina Thomas. Tulane University Health Sciences Center, New Orleans, LA. (Control ID \#2467544)

LEARNING OBJECTIVE \#1: Recognize symptoms and complications of acute heroin withdrawal

LEARNING OBJECTIVE \#2: Understand appropriate evidence-based detoxification methods

CASE: A 63 year-old man with a history of heroin abuse presented with lethargy, weakness and altered mental status. He had been arrested 4 days prior to presentation and had 4 days of nausea, vomiting, chills, and profuse diarrhea that was untreated. He was tachycardic with heart rate in the $120 \mathrm{~s}$. Blood pressure was 93/52 and he was tachypneic. On exam, he was lethargic and somnolent, arousable only to painful stimuli. He had dry mucous membranes and a benign abdominal exam. Labs revealed acute renal failure with a BUN of 225 and creatinine of 8.5. He was hyponatremic with a sodium of 127 and hypokalemic with a potassium of 2.5 . His chloride was 82 and bicarbonate was 15. He had a metabolic anion gap acidosis with an anion gap of 35 , a metabolic alkalosis with a concurrent respiratory alkalosis. He was treated supportively with aggressive hydration, symptomatic control and electrolyte replacement. His sodium corrected to 138 , potassium to 3.5 and creatinine improved to 1.0 . His acidosis resolved completely. He was discharged back into custody.

DISCUSSION: Heroin withdrawal has been considered to cause low mortality or morbidity and is often undertreated or not treated at all. However, there are some dangerous complications associated with heroin withdrawal. Most US jails do not provide appropriate treatment for opioid detoxification upon arrest, which can potentially endanger prisoners addicted to heroin. The lack of opiate detoxification protocol in most US prisons has caused an increase in complications and even death due to opiate withdrawal while incarcerated. Opiate withdrawal is associated with severe muscle cramps, diarrhea, abdominal cramps, lacrimation, rhinorrhea, tachycardia, piloerection, and chills. However, if prisoners, with opiate withdrawal, are left untreated with appropriate detoxification and supportive care, they can develop life-threatening conditions. Complicated opiate withdrawal has been associated with delirium and generalized tonic-clonic seizures. In this case, the patient developed multiple electrolyte abnormalities and acute renal failure due to severe dehydration and profuse diarrhea that was left untreated. He also had elements of delirium, which could either be attributed to the electrolyte abnormalities or the opiate withdrawal itself. Common methods used for opiate detoxification include partial opiate agonists, like methadone or buprenorphine or autonomic control, using alpha-2 agonists. In a Cochrane review, both methadone and buprenorphine had better efficacy than alpha- 2 agonists. In 2001, the Vermont Department of Corrections (VDOC) refused to administer methadone to inmates, despite recommendations from the medical community that methadone is effective in treatment of heroin withdrawal. This was after two cases, in which current methadone users after being arrested went into abrupt withdrawal after VDOC withheld methadone treatment. If the patient in this case had been left untreated for a longer period of time in prison, it may have resulted in death.

DERMATOMYOSITIS: WHEN MUSCLE WEAKNESS SIGNALS MORE THAN FATIGUE Leah M. Bruno. Tulane University Health Sciences Center, New Orleans, LA. (Control ID \#2467694)

LEARNING OBJECTIVE \#1: Recognize the clinical presentation of dermatomyositis. LEARNING OBJECTIVE \#2: Understand the pathophysiology of dermatomyositis. 
CASE: A 52 year old African American woman, with no prior medical history, was admitted directly from clinic for failure to thrive, 3 months of progressive weakness, hair loss, $20 \mathrm{lb}$ weight loss, difficulty swallowing and diffuse joint pains. She reported a decline over 3 months from full functionality to wheelchair bound and unable to perform activities of daily living without assistance. Her weakness had progressed from difficulty standing to complete loss of muscle strength. On physical exam, she exhibited dysphonia, but no dysarthria. She was noted to have significant bilateral periorbital edema and bilateral lower extremity edema. She could not keep her eyes open and had nearly complete hair loss, which her family noted was thick and full prior to this illness. Her neurologic exam demonstrated proximal greater than distal weakness with $3 / 5$ strength in upper extremities bilaterally and $2 / 5$ strength in lower extremities bilaterally, $2 / 5$ grip strength and inability to walk on presentation. She had a heliotrope rash on her face and hyperpigmented, itchy rash on her back, arms and lower extremities. Kidney biopsy revealed minimal change disease versus acute tubular necrosis secondary to severe proteinuria (8-12 g), spot protein/creatinine ratio: 16,207. Labs were significant for acute kidney injury with a creatinine of 3.5 (.7 baseline). Other notable labs included an ESR 94, CRP 2.2, CK 454, LDH 630, and an eleveated aldolase at 14.3. MRI revealed diffuse myositis throughout thighs and hips. Muscle biopsy remains pending. She was discharged with a diagnosis of dermatomyositis and minimal change disease, etiology concerning for underlying malignancy. Initial gynecology visit has revealed cervical mass with benign biopsy. However, age appropriate cancer screenings as an outpatient remain pending.

DISCUSSION: General internists frequently encounter complaints of weakness in both outpatient and inpatient settings. One method of evaluating weakness is to begin with a stepwise approach to differentiate muscle weakness from overlapping complaints of fatigue or a sense of exhaustion in the absence of muscle fatigue. This patient presented to multiple emergency departments. Yet, each time an isolated subjective complaint was elicited, it was attributed to a benign cause such as a virus and she was discharged home without further workup. Her primary care physician ultimately directly admitted her due to complete loss of functional status. The differential diagnosis for this presentation includes ALS, myasthenia gravis, Guillian Barre, systemic rheumatologic disease such as SLE or scleroderma, polymyalgia rheumatica, rhabdomyolysis, hypothyroidism, or other toxic myopathies (statin, alcohol, steroid induced). Because the differential is vast, muscle weakness should be evaluated by determining its' pattern and associated symptoms. DM can mimic inclusion body and polymyositis. All may present with gradual onset of respiratory, pharyngeal and proximal muscle weakness. However, gottron papules and heliotrope rash, if present, are telling of DM. Skin rash presents concurrently with muscle symptoms in $60 \%$ of cases. Initial diagnostic tests include muscle enzymes (CK and aldolase) EMG, and MRI. Muscle biopsy should be considered only after preceding workup remains inconclusive. Additionally, there are a number of autoantibodies present in myositis, associated with distinct clinical features. DM is thought to be the result of an unclear immune mediated attack against the arterioles and muscle capillaries. As the disease progresses, perifascicular atrophy progresses to necrosis and eventual degeneration of muscle fibers. Dysphagia, heliotrope eye rash and shortness of breath are key presenting symptoms, with scalp involvement that sometimes mimics psoriasis. Rash may be the only manifestation in 10-30\% and muscle involvement is notably bilateral and proximal. Formal diagnosis is made when at least 1 skin symptom +4 criteria are met such as: systemic signs of inflammation, muscle weakness, elevated CK or aldolase, myogenic changes on emg, nondestructive arthritis, + anti Jo-1, MRI findings compatible with inflammatory myositis. If criteria are met in absence of skin findings, the diagnosis is polymyositis. Associated with an underlying malignancy in 1 of 4 cases (ovarian being the most common), it is important to catch DM early. Peak age of onset is 50 years old and course is often chronic and progressive. Strong risk factors for the development of malignancy in this population include advanced age, elevated ESR and dysphagia at presentation. It is most commonly misdiagnosed in low income and African American populations. Physicians should be adept at identifying dermatomyositis as a cause of muscle weakness and ruling out other etiologies of acute weakness.

DEVELOPMENT OF PLATYPNEA-ORTHODEOXIA SYNDROME IN AN ELDERLY PATIENT AFTER NISSEN FUNDOPLICATION Michael A. Harms; Beth S. Zha; David G. Rosenthal; Gabrielle Berger. University of Washington, Seattle, WA. (Control ID \#2469048)

LEARNING OBJECTIVE \#1: Recognize platypnea-orthodeoxia syndrome, and define three major etiologies to guide evaluation.

LEARNING OBJECTIVE \#2: Emphasize the importance of physical examination in evaluation of patients with hypoxemia.

CASE: An 80 year-old woman presented to the emergency department with acutely worsening dyspnea 10 days after Nissen fundoplication. The patient had a history of a remote motor vehicle accident with resultant tracheal fracture and paraesophageal hernia. Two years prior to presentation, she developed new hypoxia requiring two liters/min of supplemental oxygen at home. Workup of hypoxia did not reveal a specific etiology, however there was concern for recurrent aspiration, and after expert consultation, the patient underwent Nissen fundoplication. Her postoperative course was complicated by acute respiratory failure due to subglottic edema requiring a short stay in the intensive care unit (ICU). She improved with glucocorticoids and was discharged on her previous home supplemental oxygen. Four days after discharge, the patient represented with worsening dyspnea and hypoxia. On evaluation, she was normotensive with a heart rate of 80 but required 6 1/min of supplemental oxygen to maintain a saturation of $91 \%$. On physical exam, there were no signs of respiratory distress, good airflow throughout the lungs fields and faint bibasilar crackles. She had an otherwise unremarkable exam. Laboratory studies were significant for a mild leukocytosis of 13 and an arterial blood gas that demonstrated an elevated arterial-alveolar gradient, with arterial oxygen saturation of $51 \%$ on $61 / \mathrm{min}$ of oxygen. A chest computed tomography (CT) with contrast demonstrated a small subsegmental pulmonary embolism, slight evidence of chronic aspiration, and esophageal swelling from her recent fundoplication. Her hypoxemia was attributed to a combination of postoperative swelling and pulmonary embolism in the context of baseline chronic lung disease, and she was admitted to our hospital with initiation of anticoagulation. Soon after admission, it was noted that hypoxemia present while upright resolved in the recumbent position, and her oxygen saturation reached $95 \%$ on room air. This was diagnostic of platypnea-orthodeoxia syndrome (POS), and further workup ensued. Repeat CT scans were notable for a small posterior esophageal seroma and a large epicardial fat pat causing a mild compressive effect on the tricuspid annulus. Transthoracic echocardiography (TTE) demonstrated right-to-left shunting through a patent foramen ovale (PFO). A right heart catheterization was not consistent with pulmonary arterial hypertension. She was unable to perform spirometry testing due to fatigue and dyspnea. Based on these findings, the patient's hypoxemia was attributed to worsening right-to-left shunting through a PFO that resulted from external compression of the right atrium and stretching of the interatrial septum due to a postoperative esophageal seroma. Due to the progression of symptoms, she ultimately underwent successful PFO closure and was discharged without supplemental oxygen.

DISCUSSION: Postsurgical hypoxemia is a frequent phenomenon, often attributed to common perioperative complications such as pulmonary edema, pneumonia, and pulmonary embolism. POS is an uncommonly recognized clinical entity that can narrow the differential diagnosis of a patient with hypoxemia. The profound positional effects on oxygenation in POS with a largely otherwise unremarkable examination undersores the importane of careful history and physical exam to elucidate precipitating factors which can help determine the cuase. The underlying etiologies of POS can be categorized into intracardiac shunt, intrapulmonary shunt, or ventilation-perfusion mismatch. As such, 
initial appropriate workup includes diagnostic imaging with contrast-enhanced chest CT to evaluate the pulmonary vasculature, a TTE with bubble study, pulmonary function testing, and right heart catheterization. The most common cause of POS is an intracardiac shunt due to a PFO. Since physiologic intracardiac pressures favor a left-to-right shunt in patients with a PFO, the etiology of shunt reversal in patients with POS must be investigated. If pulmonary arterial hypertension is excluded, alternative causes to consider are decreased right atrial compliance, anatomic alterations, or extracardiac compression. POS is one of only a few clinically indicated reasons for PFO closure, and should be strongly considered to eliminate positional hypoxia and the need for supplemental oxygen.

\section{DIABETIC AMYOTROPHY, MORE THAN JUST STOCKINGS AND GLOVES}

David N. Brennan; Adam P. Sawatsky. Mayo Clinic, Rochester, MN. (Control ID \#2469184)

LEARNING OBJECTIVE \#1: Recognize diabetic amyotrophy as a distinct form of diabetic neuropathy

LEARNING OBJECTIVE \#2: Differentiate diabetic amyotrophy from the more common distal symmetrical polyneuropathy with regard to risk factors, presentation, treatment and clinical course

CASE: A 55 year old man with a recent diagnosis of type II diabetes mellitus developed severe burning pain over the lateral aspect of his left hip. Over weeks, the pain spread to the lower back and down to the knee, and eventually affected the same distribution in the contralateral leg. Workup with his local providers included a normal lumbar spine MRI, abdominal CT and head CT. An EMG was inconclusive The patient was started on gabapentin with little relief. Over the next 2 months, the patient lost $50 \mathrm{lb}$, required narcotics for pain control, and developed severe proximal lower extremity weakness. After falling over in the bathroom one morning, he decided to travel to an academic tertiary care hospital for further workup. On admission, exam showed a well-appearing man with significant proximal muscle atrophy in the lower extremities, left greater than right, with intact reflexes, sensory function and dista strength. Standing from a seated position was difficult. Back exam was normal, and there was no rash or lymphadenopathy. Blood tests were normal except for a hemoglobin A1C of 7.9. CSF analysis showed elevated protein. MRI of the lumbar plexuses showed increased $\mathrm{T} 2$ signal and enlargement of the bilateral lumbosacral plexuses and peripheral nerves extending to the level of the knees. A repeat EMG showed a bilateral lumbar radiculoplexus neuropathy affecting the L3, 4, and 5 myotomes. After ruling out infectious, neoplastic and auto-immune etiologies, we made the diagnosis of diabetic amyotrophy. We discussed treatment options with the patient and initiated a 12 week course of pulse corticosteroids. Four weeks after discharge, the patient's pain and weakness were gradually improving.

DISCUSSION: Diabetic amyotrophy is a distinct but uncommon form of diabetic neuropathy. It is not associated with prolonged hyperglycemia, but rather thought to be caused by axonal ischemia and microvasculitis. It typically arises in older and middle age men with recent-onset and well-controlled type II diabetes. Onset is relatively abrupt and characterized by severe pain and weakness in the proximal lower extremities. Massive weight loss is also common. Symptoms reach maximal severity within 1 or 2 years, with many patients requiring wheelchair assistance. This is usually followed by a spontaneous gradual recovery. Corticosteroids and/or IVIG are often used to hasten recovery, and there are several case reports describing dramatic results, but no randomized controlled trials have been published to date.

DIAGNOSING A PULMONARY EMBOLISM AFTER A NEGATIVE CT PULMONARY ANGIOGRAM Stephanie Chiao. University of Texas Southwestern, Dallas, TX. (Control ID \#2470051)
LEARNING OBJECTIVE \#1: Evaluate a patient with a high clinical suspicion for a PE but a negative CTA.

LEARNING OBJECTIVE \#2: Evaluate a patient without lower extremity signs or symptoms for a DVT.

CASE: The patient is a 71-year-old man with a family history of deep vein thromboses (DVTs) and a personal history of a left lower extremity DVT who presented with 1 day of left-sided pleuritic pain. The patient recently arrived from Mexico via an 18-h truck ride. He was feeling well until the day prior to admission when he acutely developed sharp chest pain while at rest, without associated fevers, chills, diaphoresis, nausea, emesis, cough, dyspnea, lower extremity edema or pain. His past medical history was notable for a DVT 2 years ago for which the patient had been on rivaroxaban until 2 months ago due to inability to afford the medication. Initial evaluation was notable for mild tachypnea and hypoxia, requiring 2-3 1 of oxygen. Physical exam was notable only for bibasilar crackles and lower extremity varicose veins. An arterial blood gas showed an alveolar-arterial gradient of 31 with an expected gradient of 17 . The patient underwent multidetector computed tomography angiography (CTA) which showed bilateral lower lobe atelectasis, left greater than right; a small left pleural effusion; and consolidation of the posterior basal segment of the left lower lobe favoring atelectasis or pneumonia over a pulmonary infarct, as no pulmonary emboli (PE) were identified. Pleural fluid from a thoracentesis had 2425 nucleated cells with monocytic predominance and 7900 red blood cells. Given the high clinical suspicion for PE and the relative lack of signs and symptoms consistent with pneumonia, lower extremity ultrasonography (LE US) was completed, revealing extensive bilateral lower extremity DVTs. Consequently, the patient's presentation was thought to be most consistent with a PE. The patient was treated with an enoxaparin bridge to warfarin therapy. His chest pain gradually improved, and he was weaned off oxygen. The patient also received 5 days of antibiotics for empiric pneumonia treatment.

DISCUSSION: This case highlights the importance of clinical probability assessment in the diagnostic algorithm of a PE for the subset of patients with a false negative CTA. For the vast majority of patients, a negative CTA alone is sufficient for ruling out a PE. Multiple studies have shown that in patients with a negative CTA, the incidence of subsequent thromboembolic events is very low (less than $2 \%$ at 3 months) $[1,2]$. However, there is a fraction of patients who have a false negative CTA, and that fraction is larger in those with a high clinical probability of a PE [3, 4]. Therefore, in patients with discordant imaging and clinical findings, it is important to consider further evaluation, such as LE US or CT venography to evaluate for DVT, or a ventilation-perfusion scan. This case also demonstrates the value of using formal prediction scores when evaluating for DVTs, rather than relying solely on lower extremity signs and symptoms. Prediction scores such as the Wells or Geneva score not only include DVT signs and symptoms, but also include DVT risk factors, such as recent travel or a history of DVTs, which can contribute to a moderate or high risk score, prompting further evaluation by LE US [5]. Furthermore, studies have shown that any single lower extremity sign or symptom is of little value in diagnosing or excluding a DVT [6]. Therefore, it is important to consider prediction scores and the various DVT risk factors that are incorporated into these scores to ensure that patients with grossly normal lower extremities are appropriately screened. References 1. Perrier A, Roy PM, Sanchez O, et al. Multidetector-row computed tomography in suspected pulmonary embolism. N Engl J Med 2005; 352:1760-8. 2. van Belle A, Büller HR, Huisman MV, et al. Effectiveness of managing suspected pulmonary embolism using an algorithm combining clinical probability, D-dimer testing, and computed tomography. JAMA 2006; 295:172-9. 3. Stein PD, Fowler SE, Goodman LR, e al. Multidetector computed tomography for acute pulmonary embolism. N Engl J Med 2006; 354:2317-27. 4. Musset D, Parent F, Meyer G, et al. Diagnostic strategy for patients with suspected pulmonary embolism: a prospective multicentre outcome study. Lancet 2002; 360:1914. 5. Wells PS, Hirsh J, Anderson DR, Lensing AW, Foster G, Kearon C, et al. Accuracy of clinical assessment of deep-vein thrombosis. Lancet. 1995; 345:1326-30. 6. 
Goodacre S, Sutton AJ, Sampson FC. Meta-analysis: The value of clinical assessment in the diagnosis of deep venous thrombosis. Ann Intern Med 2005; 143:129.

\section{DIAGNOSING ESOPHAGEAL DYSPHAGIA WITH A HISTORY-BASED}

WORK UP Micah Eades; Kerry B. Dunbar. University of Texas Southwestern, Dallas, TX. (Control ID \#2464652)

LEARNING OBJECTIVE \#1: Identify the correct study for esophageal dysphagia workup using specific components of a patient's history.

LEARNING OBJECTIVE \#2: Recognize the diagnosis of esophagogastric junction outflow obstruction.

CASE: Ms. B was a 67 year old African-American female with a history of diabetes mellitus, hypertension, and osteoarthritis who presented with several weeks of difficulty swallowing. She initially described a "hiccup-like sensation" with both liquids and solids. Over the course of 14 months, she began to also have difficulty swallowing both liquids and solids. Pills particularly would become stuck in her throat several seconds after initiating a swallow. When asked about the location that food would become stuck, she gestured to her mid sternum. She denied having a burning sensation in her throat, or a sour taste in her mouth. Ms. B had a history of heavy smoking using 3 packs per day for 39 years but quit 17 years ago. She would have a glass of wine only on special occasions. Social, family, and surgical history were otherwise non-contributory. Medications included a 70/30 combination of aspart protamine and aspart insulin, metformin, amlodipine, losartan, hydrochlorothiazide, rosuvastatin, and aspirin. Physical exam revealed an obese African-American woman with pink, moist oral mucosa, no lymphadenopathy, an unremarkable thyroid, normal heart sounds, lungs clear to auscultation, and a soft, nontender abdomen with normal bowel sounds and no masses. Because her history was more consistent with esophageal dysphagia and she had no history of esophageal surgery or structural abnormalities, we ordered an upper endoscopy. This revealed a normal esophagus, stomach, and duodenum. Because she was experiencing dysphagia to both liquids and solids, we then ordered a high resolution manometry study. This revealed an elevated lower esophageal sphincter basal pressure at $54.2 \mathrm{mmHg}$ (normal is $13-43 \mathrm{mmHg}$ ), and incomplete relaxation with a high residual pressure of $32.8 \mathrm{mmHg}$ (normal is less than $15 \mathrm{mmHg}$ ). Interestingly, peristalsis was slightly weak but otherwise normal. These findings were consistent with a rare motility disorder subtype called esophagogastric junction (EGJ) outflow obstruction which is distinct from achalasia in that while integrated relaxation pressure is elevated, some peristalsis is preserved. Because EGJ outflow obstruction can be caused by gastroesophageal junction strictures, a barium esophagram was next ordered. This revealed no obstruction, stricture, intrinsic or extrinsic filling defect, diverticulum, signs of esophagitis, or gastroesophageal reflux, but confirmed disruption of peristaltic wave primarily due to frequent and persistent tertiary contractions in the caudal thoracic esophagus. Finally, because EGJ outflow obstruction has been reported to occur in benign and malignant infiltrative disorders, endoscopic ultrasound was performed to rule out submucosal neoplasia. No signs of endosonographic abnormality were found, so 100 units of botulinum toxin were successfully injected in the four quadrants of the lower esophageal sphincter. Ms. B subsequently reported a significant improvement in swallowing function.

DISCUSSION: Because the availability of evidence on dysphagia work up is limited, study selection should be based on a carefully collected history guided by key components of classic dysphagia disorders. Dysphagia evaluation begins with determining whether symptoms are due to oropharyngeal or esophageal dysphagia. Oropharyngeal dysphagia is characterized by difficulty initiating a swallow, localized in the cervical region, and associated with drooling, dysarthria, coughing, or choking. Esophageal dysphagia, however, is characterized by difficulty in swallowing several seconds after initiating a swallow, and is localized superior to or behind the sternum. An upper endoscopy is an appropriate first diagnostic test to determine the etiology of esophageal dysphagia. In a study of 1649 patients with dysphagia, upper endoscopy found a pathological diagnosis in $70 \%$ of patients (Varadarajulu 2005). If, however, the patient has a history of recent proximal esophageal surgery, radiation therapy, Zenker's diverticulum, or known stricture, they should first receive a barium swallow study in order to avoid the risk of perforation during a blind upper endoscopy (Fass 2015). Finally, the types of foods which elicit symptoms, solids or liquids, should be determined. Dysphagia to liquids can be suggestive of an esophageal motility disorder, while dysphagia to solids alone is suggestive of a mechanical obstruction. If solids alone, barium swallow study may be performed to evaluate for lower esophageal rings or extrinsic compression which may be otherwise missed (Ott 1990). If dysphagia is present with both solids and liquids, the next appropriate choice is a manometry study. In summary, dysphagia work up should be guided by a careful history including assessment for oropharyngeal or esophageal characteristics, history of procedures or structural abnormalities, and finally whether symptoms are present with solids or solids and liquids. Identifying the correct history-based dysphagia workup can eliminate waste, reduce costs, streamline patient care, and minimize risk.

\section{DIAGNOSTIC DILEMMA BETWEEN TUBERCULOUS PERITONITIS AND} LYMPHOMA WITH ASCITES Ayako Kumabe; Shuji Hatakeyama; Koki Kosami; Tadahiro Suzuki; Yu Yamamoto; Masami Matsumura. Jichi Medical University Hospital, Shimotsuke, Japan. (Control ID \#2467980)

LEARNING OBJECTIVE \#1: Recognize elevated adenosine deaminase (ADA) levels in ascitic fluid is a sensitive diagnostic marker for tuberculous peritonitis.

LEARNING OBJECTIVE \#2: Rule out lymphoma in cases where ascitic ADA levels are significantly elevated.

CASE: Herein, 46 patients who underwent ascitic fluid adenosine deaminase (ADA) level examination at the Division of General Internal Medicine at Jichi Medical University Hospital between 2006 and 2015 were included in the study. Of these, 9 were diagnosed with hypoalbuminemia, 6 with tuberculous peritonitis, 6 with peritoneal carcinomatosis, 5 with lymphoma, 5 with liver cirrhosis, and 11 with other diseases. Four patients were undiagnosed. Elevated ascitic ADA levels, which was defined as $>39 \mathrm{IU} / \mathrm{L}$ according to a previous study, were observed in 9 patients (median, $87.4 \mathrm{IU} / \mathrm{L}$; range, 44.0-176.1 IU/L). Among the 9 patients with high ascitic ADA levels, 6 had tuberculous peritonitis and 3 had lymphoma-related ascites. Therefore, in our cohort, elevated ascitic fluid ADA levels (>39 IU/L) showed $100 \%$ sensitivity and $92.5 \%$ specificity for the diagnosis of peritoneal tuberculosis. The diagnoses of tuberculous peritonitis were established on the basis of bacteriologic and/or histopathologic results (4 patients) or clinically ( 2 patients), although no patient showed positive results for acid-fast stain, culture, or polymerase chain reaction for Mycobacterium tuberculosis using ascitic fluid samples. Among the cases of lymphoma with high ascitic ADA levels, 2 involved natural killer/T-cell lymphoma (including 1 case of extranodal lymphoma) and 1 involved B-cell lymphoma. Furthermore, 2 patients with tuberculosis showed clinical manifestations mimicking lymphoma.

DISCUSSION: Tuberculous peritonitis has been difficult to diagnose owing to its varying clinical features, in addition to the low yield on bacterial culture or polymerase chain reaction using ascitic fluid samples. Common symptoms of tuberculous peritonitis include abdominal pain, fever, and weight loss, which are not specific for tuberculosis. The sensitivity of acid-fast stain and mycobacterial culture using ascitic fluid samples for the diagnosis of tuberculous peritonitis has been reported as $0-6$ and $20 \%$, respectively. Furthermore, mycobacterial culture is not appropriate for a prompt diagnosis of tuberculous peritonitis because it typically requires more than 2 weeks of incubation. Surgical peritoneal biopsy may yield high culture positivity and pathology for a definitive diagnosis. However, this surgical procedure is sometimes too invasive to perform in the clinical setting. ADA levels in ascitic fluid have been reported as a potential marker for the 
diagnosis of tuberculous peritonitis, with a sensitivity of $100 \%$ and specificity of $97 \%$ in a previous study and sensitivity of $100 \%$ and specificity of $93 \%$ in this study. It is important for clinicians to understand the cause of this relatively low specificity. ADA activity is related primarily to the number, maturation, and level of stimulation of lymphocytes. Therefore, ADA levels can theoretically increase in cases of effusion (including pleural, ascitic fluid, and cerebrospinal fluid effusion) due to infection, rheumatic diseases, and lymphoproliferative disorders. In our series, significant elevation of ascitic fluid ADA levels was observed in only 2 diseases: tuberculous peritonitis and lymphoma. Ascitic fluid ADA levels $>39$ IU/L show an excellent sensitivity, despite a relatively low specificity, for the diagnosis of tuberculous peritonitis. This series highlights the important mimics of tuberculous peritonitis. However, clinicians should be aware that both tuberculosis and lymphoma can result in high ascitic fluid ADA levels with similar clinical manifestations.

DIAGNOSTIC DILEMMA OF NON-RESOLVING PNEUMONIA: A CHALLENGING CASE OF HISTOPLASMOSIS IN AN IMMUNOCOMPETENT PATIENT Neal Spada; Kristian Feterik; Scott Curry; Ghady Haidar. University of Pittsburgh Medical Center, Pittsburgh, PA. (Control ID \#2469358)

LEARNING OBJECTIVE \#1: Diagnose histoplasmosis in an immunocompetent patient

LEARNING OBJECTIVE \#2: Recognize the need to rule out infection before starting immunosuppression for pulmonary granulomatous disease

CASE: A previously healthy, 41-year-old male plasterer and resident of rural Pennsylvania presented with 3 weeks of fevers, malaise, weight loss, night sweats, dyspnea, and cough. He did not respond to multiple courses of antibiotics for community-acquired pneumonia at an outside hospital. He was then transferred to our center due to persistent fevers, bilateral pulmonary nodules, and hilar lymphadenopathy. Transbronchial biopsies showed noncaseating granulomas. Grocott and acid fast stains for micro-organisms were reported as negative. Preliminary bronchoalveolar lavage (BAL) bacterial, fungal, and mycobacterial cultures showed no growth. Methylprednisolone $100 \mathrm{mg}$ IV Q8H was given over $48 \mathrm{~h}$ for presumed sarcoidosis with profound clinical improvement. He was transitioned to oral prednisone and discharged home in good condition on PCP prophylaxis and prednisone $40 \mathrm{mg}$ daily without a taper. BAL cultures had not yet been finalized. Two weeks later, he was readmitted with recurrent fevers and worsening dyspnea. He had new pancytopenia, severe hypoxia, and extensive consolidations on chest CT. HIV testing was negative. He deteriorated despite broad spectrum antibiotics and pulse-dose steroids, necessitating intubation. An infectious disease consultation was obtained. Urine and serum Histoplasma capsulatum antigens were above the limit of detection. Sectioning of deeper portions of the previous transbronchial biopsies revealed oval yeasts suggestive of Histoplasma. The original BAL fungal culture from the first admission grew yeast after 13 days that was identified as $H$. capsulatum after re-admission. Bone marrow biopsy showed involvement by yeast forms consistent with Histoplasma and grew the same on fungal culture. The patient completed a 2-week course of liposomal amphotericin B with good clinical response and a decrease in the Histoplasma antigen level. His corticosteroids were stopped, and he was discharged on long-term itraconazole. He is doing well at 6 months of follow-up. The initial diagnosis of sarcoidosis was rejected in favor of acute pulmonary histoplasmosis with subsequent respiratory failure and dissemination due to iatrogenic immunosuppression. DISCUSSION: This case of histoplasmosis in a previously healthy man demonstrates the challenges associated with diagnosing the illness in immunocompetent patients. Histoplasmosis shares clinical, pathologic, and radiographic features with many diseases, including tuberculosis, other endemic mycoses, malignancy, and sarcoidosis. Because of their similarity, some experts recommend evaluation for histoplasmosis prior to starting immunosuppression for sarcoidosis. The performance of tests available for the diagnosis of histoplasmosis is variable. Histopathology is about $20 \%$ sensitive for acute pulmonary histoplasmosis and $76 \%$ sensitive for disseminated disease. ${ }^{1}$ Similarly, the sensitivities of culture are 42 and $74 \%$ for acute pulmonary and disseminated disease respectively, and cultures can take up 6 weeks to turn positive. ${ }^{1}$ During the patient's initial presentation, Grocott stains of the transbronchial biopsies were negative for micro-organisms, and Histoplasma yeast forms were only seen upon retrospective re-evaluation of deeper sections of the original sample. Further, because H. capsulatum grew 13 days after the initial BAL was obtained, the diagnosis was delayed, and the presumptive treatment for sarcoidosis likely resulted in dissemination of what may otherwise have been a self-limited infection. Non-culture-based diagnostics, such as antigen testing, are extremely useful in these evaluations. This patient may have benefitted from antigen testing during his first admission, as it is $83 \%$ sensitive in acute pulmonary disease. ${ }^{1}$ However, immunocompetent patients are less likely to have a positive antigen than immunosuppressed patients, and the level of positivity may also be lower. ${ }^{2}$ As such, the yield of these tests during his initial presentation may have been low. Further consideration of his profession may have also pointed to the diagnosis, as two case reports have linked histoplasmosis to plasterers, and he was likely exposed to construction materials from areas where histoplasmosis is more highly endemic. ${ }^{3,4}$ Infectious disease consultation on initial presentation would have likely identified the need for non-culture-based testing. Ultimately, careful re-evaluation of the differential diagnosis led to more detailed workup and the rapid initiation of life-saving therapy. Diagnosing histoplasmosis is challenging and requires a high index of suspicion and understanding of the available diagnostics. References 1. Hage CA, et al. "Histoplasmosis: Up-to-Date Evidence-Based Approach to Diagnosis and Management." Semin Respir Crit Care Med 36.5 (2015) 729-45. 2. Hage CA, et al. "A multicenter evaluation of tests for diagnosis of histoplasmosis." Clin Infect Dis 53.5 (2011) 448-54. 3. Weaver DK, et al. "Histoplasma Endocarditis" Arch Surg 96.1 (1968) 158-62. 4. Schochet SS Jr, et al. "Symptomatic cerebral histoplasmoma. Case report." $J$ Neurosurg 52.2 (1980) 273-5

DIARRHEA WITH PERIPHERAL EOSINOPHILIA: ANOTHER CASE OF INTESTINAL PARASITES? Leigh Cagino ${ }^{1}$; Margaret C. Lo $^{2} .{ }^{1}$ University of Florida, Gainesville, FL; ${ }^{2}$ University of Florida College of Medicine, Gainesville, FL. (Control ID \#2466656)

LEARNING OBJECTIVE \#1: Diagnose and manage eosinophilic gastroenteritis in the setting of recurrent GI symptoms and peripheral eosinophilia

LEARNING OBJECTIVE \#2: Identify human and clinical factors leading to difficulties in diagnosing eosinophilic gastroenteritis

CASE: A 55 year-old female presented with 4-months of recurrent diffuse abdominal pain with watery diarrhea and anorexia, unresponsive to dietary changes. This had led to two outside hospitalizations with no resolution of her symptoms, first treated with levofloxacin and flagyl empirically for gastroenteritis and second with protonix for gastritis and duodenitis per endoscopy. Medical history includes diabetes and hypertension but no prior atopy or GI conditions. Exam revealed diffuse abdominal tenderness but stable vital signs and no rebound, organomegaly, ascites or jaundice. Labs were notable for severe leukocytosis $(24500 \mu \mathrm{L})$ with $2 \%$ bands and $39 \%$ eosinophils. Eosinophilia was also seen on outside records (55\%). Stool cultures and ova/parasites were repeatedly negative. $\mathrm{CT}$ abdomen was unremarkable. Repeat upper endoscopy found an esophageal stricture and patchy erythematous, nonbleeding mucosa in gastric body, antrum, duodenal bulb and 1st to 2 nd part of duodenum. Colonoscopy showed normal mucosa of ileum and entire colon. Pathology revealed a patchy increase in the lamina propria of the terminal ileum, right and left colon and a marked increase in intraepithelial eosinophils in the duodenum. Patient was diagnosed with eosinophilic gastroenteritis (EosGE) and responded well to 
enteric azathioprine per GI consult. Enteric budesonide was added upon discharge with GI clinic follow-up arranged.

DISCUSSION: This case demonstrates the common diagnostic errors of framing and availability heuristics. Very often peripheral eosinophilia with GI symptoms is attributed to intestinal parasitic infections or allergic processes. This results in repeated GI evaluations including stool ova/parasite tests and food diaries, as seen in our case. EosGE is a rare condition with variable clinical manifestations depending on the depth of mucosal involvement. This results in its underdiagnosis. Index of suspicion must be high in those with recurrent GI symptoms and peripheral eosinophilia unexplained by drug reactions, parasitic infections or malignancies. History of asthma, atopy or food allergies are other diagnostic clues. Our patient did not have such risk factors. Yet, her persistent eosinophilia remained a crucial finding and was overlooked once parasitic infections were excluded and gastritis/ duodenitis was diagnosed on endoscopy. Given its vast clinical presentation, endoscopy with biopsy is vital in the diagnosis of EosGE. EosGE in the mucosa layer manifests as vague abdominal symptoms i.e. nausea, vomiting, anemia, anorexia. Those within the muscle layer present with gastric outlet or bowel obstruction. Subserosal EosGE develops ascites and eosinophilia which is responsive to steroids. The diagnosis of EosGE requires GI symptoms, biopsy-proven eosinophilic infiltration of one or more areas of the GI tract and no other extraintestinal or parasitic involvement. Our patient met these diagnostic criteria. Eosinophilic infiltrates are often patchy in distribution so multiple biopsies must be obtained from both affected and unaffected areas. Patchy lesions can be active during asymptomatic times and may reflect the persistence in peripheral eosinophilia and risk for relapse. Such fragmented distribution may explain our patient's recurrent course as well as the equivocal pathology on the first endoscopy. This case further supports the current recommendation for repeat endoscopic biopsy if initial pathology is undiagnostic and GI symptoms and eosinophilia persist. Elimination diets are the first line therapy for EosGS but relapses are high. Topical enteric steroids are effective for those refractory to dietary therapy or with relapses. Medications such as antihistamines and cromolyn to target allergy control have had equivocal benefits. Monoclonal antibodies and/or nonbiologic immunosuppressants are reserved for severe cases or associated complications, such as in our patient. Close endoscopic surveillance is needed for those with esophageal strictures or change in symptoms. The rare and diverse presentation of EosGE has led to multiple reports of misdiagnosis, in favor of well-known GI conditions with eosinophilia such as intestinal parasitic infections. When faced with such diagnostic dilemma, clinicians must execute diagnostic time-outs and reflect on their own clinical thought process. This practice in meta-cognition will avert diagnostic errors of pattern recognition and framing as seen in this case. Case reports like ours are needed to disseminate knowledge of this rare condition. Of significance are its associated risk factors and need for multiple, repeated endoscopic biopsies. Early diagnosis of EosGE may not alter prognosis but will surely prevent unnecessary interventions and potential complications.

DIFFUSE RASH IN A PATIENT WITH AIDS Chetaj A. Mahabir. University of Nebraska Medical Center, Omaha, NE. (Control ID \#2465551)

LEARNING OBJECTIVE \#1: Develop a differential diagnosis for rash in a patient with HIV/AIDS.

LEARNING OBJECTIVE \#2: Discuss the diagnosis and management of DRESS

CASE: A 50 year-old man with HIV/AIDS (CD4 count 92 cells/mcL) was admitted to the hospital with a diffuse rash. Beginning 2 weeks prior, he noted a rash on his forearms that spread to his trunk, legs, face, and hands. Associated symptoms included a sore throat and swelling of his hands and face. He denied sick contacts, new sexual partners, and recent travel. A thorough medication history revealed that dolutegravir (an HIV integrase inhibitor) and sulfamethoxazole were started 6 weeks prior to presentation. At the time of admission, the patient was afebrile and tachycardic with a heart rate of 103 . His skin exam demonstrated confluent erythema of the face with crusted areas extending to the scalp, swelling of the ears and confluent maculopapular lesions of his trunk, arms, back and legs. He did not have any lesions on his mucosal membranes. His palms and soles were not involved. The reminder of his exam was within normal limits. His white cell count was 15400 cells/mm3 with $16 \%$ eosinophils. His AST was 43 , ALT 70 alkaline phosphatase 103, creatinine 1.4. Parvovirus, herpes viral panel, syphilis, and Cryptococcus Ag were within normal limits. He underwent a skin biopsy of the left thigh which showed perivascular mononuclear inflammatory cell infiltrate with occasional red blood cells and eosinophils, consistent with a drug reaction. Based on the drug history, skin biopsy, and evidence of end-organ damage, a diagnosis of drug reaction with eosinophilia and systemic symptoms (DRESS) was made. Dolutegravir and sulfamethoxazole were discontinued, and he was started on hydroxycine and triamcinolone ointment for his skin lesions. At follow up 2 weeks later, his rash and lab values were improved.

DISCUSSION: This case demonstrates the importance of taking a thorough medication history in evaluating a patient with a diffuse rash. DRESS usually manifests 2 to 6 weeks after drug exposure. It is characterized by fever, lymphadenopathy, skin eruption, facial edema, and internal organ involvement (typically the liver, kidneys and lungs). In a patient with HIV, DRESS needs to be distinguished from other causes of fever and rash, including staphylococcus, bacillary angiomatosis, secondary syphilis, HSV, parvovirus, endemic fungi, and Kaposi sarcoma. A skin biopsy is often helpful to make the diagnosis. Common culprit medications for DRESS include antiepileptic and sulfur containing drugs. While not commonly associated, there are case reports of integrase inhibitors causing DRESS. The cornerstone of managing DRESS is withdrawal of the offending agent. Patients without significant organ involvement can be treated symptomatically, including topical steroids for rash. Corticosteroids are usually reserved for patients with severe end-organ involvement.

DISSECTION OF THE CORONARY ARTERY WITH STRESS AND EXERTION. Hamza Rayes ${ }^{2}$; Aiman M. Smer ${ }^{2}$; Haitam Buaisha ${ }^{2}$; Yazeid Alshebani ${ }^{1}$. ${ }^{1}$ Creighton University, Omaha, NE; ${ }^{2}$ Creighton University Medical Center, Omaha, NE. (Control ID \#2456920)

LEARNING OBJECTIVE \#1: Recognize coronary artery dissection as a cause of ischemic heart disease in patients with no risk factors of atherosclerosis.

LEARNING OBJECTIVE \#2: Managing spontaneous coronary artery dissection and recognizing the risk of recurrence.

CASE: An otherwise healthy 43-years-old male presented with new onset chest pain after a $30 \mathrm{~min}$ intense exertion. He described his pain as a pressure like sensation which lasted 10 min radiating to the left shoulder and left arm and relieved gradually by rest but recurred intermittently the days after. Past medical history was only significant for a remote 5 pack-year smoking history. Patient denied illicit drug use, family history of sudden death or premature coronary artery disease (CAD) or aortic aneurysm nor any family history of connective tissue diseases. Cardiopulmonary examination was normal and no evidence of Marfanoid habitus. Laboratory testing was significant for mild troponin elevation with a peak at $0.57 \mathrm{ng} / \mathrm{ml}$. Electrocardiogram showed sinus rhythm with non specific ST-T changes. Transthoracic echocardiography showed mildly hypokinetic apex. Aortic root was normal and no valvular abnormalities noted. Patient underwent coronary angiography which revealed abrupt narrowing of the distal LAD with very limited coronary blood flow due to intraluminal hematoma. This finding was consistent with SCAD at distal LAD. The lesion was not amenable to percutaneous intervention so patient was managed medically with dual antiplatelet therapy, beta blocker and high intensity statin. Connective tissue disease work up panel was negative. CT scan of the chest, abdomen and pelvis which didn't show any vascular abnormalities. He then 
was discharged in a stable condition. However, 2 weeks after discharge he presented to the emergency room with recurrent chest pain of similar character precipitated by emotional stress. He had repeat workup including EKG and three sets of cardiac biomarkers which were all negative. The patient was discharged on Isosorbide mononitrate and asked to refrain from strenuous exercise and avoid emotional stress.

DISCUSSION: SCAD is a very rare entity with an estimated incidence of $0.2 \%$ among patients who undergo diagnostic coronary angiography (1). In 1931, Pretty described the first case of SCAD, since then few case reports have been published about this rare disease (2). SCAD usually affects middle age women and it has a characteristic association with peripartum state. Nonetheless, when found in men it is typically associated with an underlying atherosclerotic coronary artery disease, connective tissue diseases or blunt trauma but still can happen in the absence of the above risk factors like in our case (4). SCAD is defined as a non traumatic separation between the layers of a coronary artery wall creating an intimal flap, false lumen and subsequently obstructing blood flow. It can occur as an isolated phenomenon or in association with coronary atherosclerosis. The left anterior descending coronary artery is the most commonly involved vessel. Unfortunately, the pathogenesis of SCAD remains poorly understood. Clinically, SCAD can not be differentiated from acute coronary syndrome. Coronary angiogram is the gold standard diagnostic test. However, CT coronary angiogram and novel intravascular ultrasound technique can be valuable both in confirming the diagnosis and in guiding treatment decisions. There is no consensus on the optimal management for patients with SCAD. In general, most patients with stable SCAD can be managed medically. However, in patients with ongoing ischemia, hemodynamic instability, percutaneous coronary intervention or coronary bypass graft is recommended. These patients should avoid emotional and physical stress to prevent recurrence. In a case series with extended follow up of 13 patients approximately $50 \%$ of the patients developed recurrent dissection within 1 month involving a different coronary artery (3). This supports the need of clinical surveillance of such patient and brings the question of how invasive we should be on patient presenting with recurrent symptoms. Reference: (1) Idiopathic spontaneous coronary artery dissection: incidence, diagnosis and treatment. Maeder M1, Ammann P, Angehrn W, Rickli H. (2) Dissecting aneurysms of coronary artery in woman aged 42 Pretty H. (3) DeMaio SJ, Kinsella SH, Silverman ME. Clinical course and longterm prognosis of spontaneous coronary artery dissection. Am J Cardiol 1989; 64:471-474. (4) Onset during exercise of spontaneous coronary artery dissection and sudden death. Occurrence in a trained athlete: case report and review of prior cases. Chest 1995; 108:284-287.

\section{DISSEMINATED CRYPTOCOCCOSIS WITH ENDOBRONCHIAL LESIONS} Kusha Davar ${ }^{1}$; Arun Jose ${ }^{1}$; Anne Catherine Miller Cramer ${ }^{1}$; Alexander E. Aldrich ${ }^{1}$; Jillian S. Catalanotti ${ }^{2}$. ${ }^{1}$ George Washington University, Washington, DC; ${ }^{2}$ The George Washington University, Washington, DC. (Control ID \#2469668)

LEARNING OBJECTIVE \#1: 1) Discuss the clinical presentation of disseminated Cryptococcosis

LEARNING OBJECTIVE \#2: 2) Discuss the appropriate treatment of disseminated Cryptococcosis

CASE: A 52-year-old man with HIV/AIDS and recent CD4+ T-cell count of 11 (CD4+ percent of $3 \%$ ) presented with shortness of breath and non-productive cough for 3 months. He was otherwise asymptomatic, denying headaches, photophobia, fevers, chills, night sweats, and hemoptysis. He had no concerning environmental exposures. Medications included darunavir/cobicistat and emtricitabine/tenofovir. Vital signs and physical examination were normal with the exception of several $1 \mathrm{~mm}$ umbilicated flesh-toned nontender papules spread across his face. Chest X-ray on admission showed a diffuse reticular pattern. Computed tomography of the thorax without contrast showed a tree-in-bud nodularity pattern with peribronchovascular thickening in addition to a large cavitary lesion within the right lower lobe. Laboratory analysis revealed a positive serum Cryptococcal serum antigen titer of $1: 16,000$ and CSF antigen titer of 1:32,000. Bronchoscopy showed ulcerated erythematous endobronchial plaques within the right segmental bronchi. The lesions were biopsied and mucicarmine stain revealed diffuse encapsulated yeast forms. Cryptococcus neoformans was cultured from bronchioalveolar lavage fluid, cerebrospinal fluid, and blood. The patient was treated with a combination of amphotericin B and flucytosine with improvement in his facial rash, symptoms, and Cryptococcal antigen titer.

DISCUSSION: Disseminated Cryptococcosis occurs in immunocompromised patients, often those with AIDS. This case demonstrates a patient with disseminated Cryptococcosis who presented with pulmonary and meningeal involvement manifested by positive cultures and notably high Cryptococcal angiten titers. The patient was also found to have endocbronchial lesions harboring Cryptococcus neoformans as well as cutaneous umbilicated papules across his face. Although these findings have been described in prior literature, disseminated Cryptococcosis was more common in the preHAART era, and this case serves as an important reminder and an educational tool for today's physicians to correctly identify disseminated Cryptococcosis. Cryptococcal infections primarily affect the lungs, however the most common extrapulmonary site of infection is the central nervous system. In patients with disseminated Cryptococcosis and AIDS, management includes long-term treatment with anti-fungal medications, beginning with induction therapy with amphotericin B and flucytosine for at least 2 weeks of treatment or until repeat lumbar puncture demonstrates negative CSF cultures. Treatment then shifts to a consolidation phase with oral fluconazole for 10 weeks, followed by maintenance therapy with a lower dose of oral fluconazole until the CD4+ T-cell count is above 200. Anti-retroviral medications are typically held for 5 five weeks after initiation of anti-fungal therapy to decrease the risk of immune reconstitution inflammatory syndrome (IRIS). Interestingly, our patient had started anti-retroviral therapy for several weeks prior to admission, which may have precipitated an immune reconstitution phenomenon, causing his presenting symptom.

DISSEMINATED HERPES ZOSTER AFTER IPILIMUMAB TREATMENT FOR METASTATIC MELANOMA John P. Russo ${ }^{1}$; Armand V. Russo ${ }^{2} .{ }^{1}$ University of Connecticut, Newington, CT; ${ }^{2}$ Yale School of Medicine, New Haven, CT. (Control ID \#2470303)

LEARNING OBJECTIVE \#1: To review side effects in new immunoligc targeted therapy for cancer and discuss their mechanisms of action.

CASE: A 67 year-old Caucasian woman, diagnosed with stage IV cutaneous melanoma, was treated with anti-CTLA-4 antibody (ipilimumab) every 3 weeks for 12 weeks. An episode of bilateral conjunctivitis occurred after the second dose of ipilimumab and resolved with steroid eye drops. It was believed to be an immune-mediated adverse event. Six weeks after the final dose of ipilimumab, the patient developed an acute, painful, vesicular necrotic skin rash of the right upper back along the T2 dermatome that was accompanied by a widespread cluster of erythematous vesicular lesions on the trunk and extremities (Figure 1). A clinical diagnosis of disseminated herpes zoster infection (DHZ) was confirmed by a positive direct fluorescent antibody test of skin lesion scrapings leading to its successful treatment with a 21-day course of intravenous acyclovir. A whole body positron emission tomography performed approximately after 4 months of ipilimumab treatment confirmed a partial response to treatment

DISCUSSION: Ipilimumab is a humanized monoclonal antibody of IGG1k specificity, directed against the inhibitory CTLA-4 molecule on effector T lymphocytes, that imparts a survival advantage to patients with metastatic melanoma $(1,2)$. A number of autoimmune 
side effects following ipilimumab treatment have been described (3). Here we note the development of DHZ after ipilimumab treatment, which has been reported only incidentally in a study on rare side-effects (4). The precise immune defects leading to herpes zoster are not clear. Available evidence indicates VZV specific CD4+ T lymphocytes are responsible for maintaining the virus in its dormant state in the sensory dorsal root ganglia $(5,6)$. The immune augmentation resulting from ipilimumab treatment is reflected by the non-specific $\mathrm{T}$ lymphocyte activation of central memory lymphocytes as evidenced by their expression of induced co-stimulatory molecules and HLA-DR molecules (7). Salutary effects are also seen on regulatory $\mathrm{T}$ lymphocytes by the increased ratio of effector to regulatory $\mathrm{T}$ lymphocytes in the regressing tumors (8). Our patient's manifestation of autoimmune conjunctivitis and objective tumor regression following ipilimumab treatment conform to its immune stimulatory effects. However, the diagnosis of DHZ makes this effect of ipilimumab paradoxical. It appears to be reminiscent of an acute immune reconstitution syndrome familiar to physicians treating severely ill patients diagnosed with human immunodeficiency virus who develop new or exaggerated opportunistic infection following initiation of highly active retroviral therapy $(9,10)$. In the emerging era of immune enhancing anti-cancer treatment, consequences of acute immune reconstitution will merit the attention of clinicians.

DISSEMINATED NOCARDIOSIS - AN OPPORTUNISTIC INFECTION IN A POST LIVER TRANSPLANTED PATIENT Evan Nadler'; Andrew P. Scatola ${ }^{2}$.

${ }^{1}$ University of Connecticut, West Hartford, CT; ${ }^{2}$ University of Connecticut School of Medicine, Farmington, CT. (Control ID \#2470284)

LEARNING OBJECTIVE \#1: Recognize nocardiosis as an opportunistic infection in post liver transplant patients

LEARNING OBJECTIVE \#2: Understand the workup and management of disseminated nocardiosis

CASE: We present a case of a 68 year old male with history of alcoholic cirrhosis who received a Hepatitis B core antigen positive orthotopic liver transplant 4 months prior to this admission. His immunosuppressive regimen consisted of prednisone, mycophenolate mofetil and tacrolimus. He was on atovaquone for PCP prophylaxis, valganciclovir for CMV prophylaxis and tenofovir for Hepatitis B. In addition he had a history of type 2 diabetes and atrial fibrillation for which he is on warfarin. The patient presented with 3 days of worsening cough associated with moderate hemoptysis. Additionally, 1 month prior to presentation, he had noticed a large skin lesion on his right upper chest wall which had been spontaneously draining purulent material. He was afebrile on exam with mild tachypnea and $\mathrm{SpO} 2$ of $88 \%$ on room air. Lab results showed acute on chronic anemia with a hemoglobin of $7 \mathrm{~g} / \mathrm{dL}$, platelets of $164,000 \mathrm{u} / \mathrm{L}$ and a WBC count of $30,000 \mathrm{u} / \mathrm{L}$. Chest X-ray showed a pulmonary mass in the right upper lobe, right lower lobe and right middle lobe with a small right pleural effusion. The patient was placed on oxygen via nasal cannula, started on azithromycin and ceftriaxone to cover for community acquired pneumonia, he was placed on airborne precautions and his immunosuppressive therapy was held. His sputum culture grew gram-positive branching rods suspicious for nocardia. The patient was given a single dose of amikacin and was started on meropenem and linezolid. CTA of the chest showed multiple focal nodules and masses, including two rounded lesions in the right lung apex and upper lobe measuring up to $3 \mathrm{~cm}$ by $4 \mathrm{~cm}$, bulky mediastinal and right hilar lymphadenopathy and a moderate right pleural effusion with a large rind of loculation and pleural thickening, consistent with pulmonary nocardiosis. Given concern for possible disseminated disease, an MRI of the brain was performed which showed a single, ring enhancing lesion in the left parietal lobe, consistent with nocardia abscess. He later underwent a diagnostic and therapeutic thoracentesis. Pleural fluid revealed an uncomplicated exudative effusion with predominantly polynucleated cells. His sputum and pleural cultures both grew Nocardia farcinica. He was continued on meropenem and was able to be weaned to room air with repeat chest $\mathrm{X}$ ray showing significant improvement in his pulmonary disease. His immunosuppressive regimen was then resumed. He was treated with long-term meropenem and repeat CT of the chest and MRI of the brain 3 months later showed resolution of pulmonary, skin and CNS disease. DISCUSSION: Nocardiosis is a bacterial infection caused by gram positive, aerobic filamentous actinomycetes of the Nocardia species occurring most commonly in immunocompromised hosts, including those with malignancy, HIV and history of transplantation. The most common sites of infection include the lungs, involving more than twothirds of cases and often with parapneumonic effusions, the CNS and the skin. About onethird will have disseminated disease involving at least two organ systems, secondary to hematogenous spread. One case control study found that out of 5126 organ transplant recipients, 35 patients were found to have Nocaria spp. infections. Of the 1840 liver transplant recipients two $(0.1 \%)$ were found to have Nocardiosis. In this report 5 out of the 35 patients died $(11 \%)$ which was directly attributable to their Nocardia infection. In patients who have received organ transplantation, the use of steroid sparing regimens has significantly decreased rates of Nocardia infection from 2.6 to $0.7 \%$ in post-renal transplant patients. In transplant recipients, the risk for Nocardiosis tends to be greatest in the first year post transplant due to greater amounts of immunosuppressive therapy. Nocardia farcinica has been implicated as a source of infection in liver transplant recipients causing isolated pulmonary infection, isolated brain abscesses, cutaneous manifestations and occasionally disseminated disease. Mortality in these patients is about $25 \%$ and approaches $50 \%$ in the presence of brain involvement. The mainstay of treatment for Nocardiosis is trimethoprim-sulfamethoxazole (TMP-SMX) although there are significant discrepancies in susceptibility patterns amongst different reports and for different species of Nocardia. While N. farcinica has been reported as susceptible to TMP-SMX, one 10 year review noted a resistance rate as high as $80 \%$ while all isolates were susceptible to linezolid. Optimal duration of therapy is not defined, however treatment for at least 1 year in immunocompromised patients and those with CNS infections has been described. All patients with Nocardiosis should have identification performed and antimicrobial therapy guided based on antimicrobial testing for optimal outcomes.

DISSEMINATED VARICELLA ZOSTER PRESENTING AS MYOPERICARDITIS IN AN IMMUNOCOMPETENT MALE Srinidhi Jayadevappa Meera; Dharmarajan S. Thiruvinvamalai. Montefiore Medical Center, Mamaroneck, NY. (Control ID \#2469600)

LEARNING OBJECTIVE \#1: Varicella zoster can cause serious primary infection with multi organ involvement in immunocompetent individuals and may not necessarily begin with the onset of skin rashes . Low threshold of suspicion, early diagnosis and therapy should be provided

LEARNING OBJECTIVE \#2: Myopericarditis is a rare manifestation of disseminated varicella zoster and can rarely occur even in immunocompetent adults

CASE: 44 year old male with no significant past history presented to the ED with dyspnea, dry cough, unintentional weight loss over 3 months, and diffuse muscle soreness, no fever and chills. In the ED: BP 136/86, heart rate $95 / \mathrm{min}$, respiratory rate $22 / \mathrm{min}$, afebrile. EKG: T wave inversion in lead III and $1 \mathrm{~mm}$ ST elevation in V2, V3. Troponin was $0.89 \mathrm{ng} / \mathrm{ml}(\mathrm{N}: 0.00-0.10)$ and CPK $5165 \mathrm{U} / \mathrm{L}$. SGOT was $96 \mathrm{U} / \mathrm{L}(\mathrm{N}: 13-50 \mathrm{U} / \mathrm{L})$ and SGPT 113 U/L. He had pleurtic pain and bilateral diffuse crackles. CT Angiography thorax showed opacities and pulmonary vascular congestion. Initiated IV antibiotics for community-acquired pneumonia. Troponin trend was 0.89 -à 0.78 à.0.71, Echocardiogram: normal ventricular wall motion with left ventricular ejection fraction of $65 \%$. Myocardial perfusion scan normal. Myocarditis associated with pericarditis was suspected. ANA, Rheumatoid Factor, Strep pneumo, legionella, amylase, lipase, HIV, 
hepatitis panel, CRP, ANCA, Anti Jo, anti Mi 2 antibodies were negative. He became better in days. With suspicion for autoimmune disorder, outpatient evaluation was planned but he was lost to follow up. He returned in 2 weeks with worsening symptoms and elevated troponin, CPK of 4845 and diffuse ST elevations on EKG. He had worsening muscle weakness with inability to turn in bed. Biopsy of new onset skin eruptions confirmed multinucleated giant cells (Tzank smear) suspicious for varicella. He developed hypoxic respiratory failure with ARDS requiring mechanical ventilation. CT thorax showed new bilateral lung nodules suggesting varicella pneumonitis. Varicella IgM and IgG were positive. EMG with muscle biopsy confirmed inflammatory myopathy. Acyclovir with steroids helped improvement and discharge.

DISCUSSION: Varicella Zoster Virus (VZV) causes two clinically distinct diseases Primary infection (Varicella/ chicken Pox) is characterized by a generalized vesicular rash. Following primary infection, VZV establishes latency in the sensory ganglia and reactivates in immunocompromised states / elderly leading to more localized cutaneous eruption associated with neuralgic pain . Varicella zoster (VZ) infection seldom presents with life-threatening complications in immune-competent adults. However atypical presentations and uncommon complications in immunocompetent individuals can pose diagnostic and therapeutic challenges. Recent studies have shown that rashes are present in only $45 \%$ of the cases. In this case, there was a late onset of skin rashes following development of multi organ involvement . Our patient had developed pneumonitis, elevated transaminases, myopericarditis, myositis leading to diffuse muscle weakness Cardiac involvement in VZ is rare in an immunocompetent patient. Reported cardiac manifestations of varicella include pericarditis, myocarditis, endocarditis, pericardial effusion, cardiac tamponade, arrhythmias, and heart block . Features of myopericarditis or pericarditis can mimic acute coronary syndrome, with the distinction subtle. Our patient had elevated troponin and diffuse ST segment elevations, with negative stress test. Once coronary artery occlusion is excluded, management of myopericarditis involves therapy with nonsteroidal anti-inflammatory drugs, monitoring myocardial function, and addressing LV dysfunction or arrhythmias. There may be a role for antiviral agents, immunoglobulins or pacemaker.

DIVERTICULITIS WITHOUT DIVERTICULA Mehtap Canastar; Darlene LeFrancois. Montefiore Medical Center, Bronx, NY. (Control ID \#2469485)

LEARNING OBJECTIVE \#1: Recognize the atypical presentation of recurrent diverticulitis in the form of a colonic stricture

LEARNING OBJECTIVE \#2: Identify the indications for surgical resection of diverticular disease

CASE: A 52 year-old woman with chronic constipation presented with 1 day of left lower quadrant (LLQ) abdominal pain associated with chills, and one episode of vomiting. In addition to years of constipation, the patient noted the development of pencil thin stools for several months. She had experienced approximately three similar pain episodes per year over the last 5 years, frequently necessitating inpatient stays in which she was often treated with fluids and antibiotics. Colonoscopy done 1 month prior to presentation revealed congested mucosa of the distal descending colon, possibly suggesting ischemia, but no other abnormalities. Additional history was notable for a remote history of a medically treated prolactinoma and postmenopausal status. On exam she was afebrile but had a very tender $\sim 10 \mathrm{~cm}$ tubular mass palpable in the LLQ. There were focal but no generalized peritoneal signs and the abdomen was soft, nondistended, Carnett's sign was absent, and McBurney's point was nontender. On pelvic exam the patient had tenderness of the left adnexa. Laboratory testing was remarkable for an absence of leukocytosis and normal hemoglobin. CT of the abdomen with contrast revealed a long segment of marked circumferential bowel wall thickening extending from the proximal sigmoid colon to the mid descending colon, with surrounding inflammatory stranding and mild diverticulosis. CT scan findings of this visit was without significant change from her previous two admissions spanning 9 months at our institution. Flexible sigmoidoscopy revealed gross colonic spasm at the involved segment. During the current hospitalization patient had a partial laparoscopic colectomy. Pathologic examination of the resected bowel showed acute and chronic diverticulitis with associated abscess formation, mural scarring, and mucosal ischemic changes.

DISCUSSION: Diverticulitis is a complication of diverticulosis commonly encountered by internists, affecting $4-15 \%$ of patients with diverticulosis. Accurate diagnosis of diverticulitis and its complications in the absence of classical presenting symptoms and signs is dependent on provider familiarity with the spectrum of disease observed with this disorder. Our patient had left lower quadrant pain with vomiting and chills but she did not have leukocytosis, fever nor diverticula on colonoscopy. Differential diagnosis of LLQ pain include diverticulitis, ischemic colitis, irritable bowel syndrome, inflammatory bowel disease, ovarian pathology, malignancy. In this case suspicion for complicated chronic diverticular disease remained high given her history of previous intermittent pain episodes with chronic constipation. We continued to consider ischemic colitis and malignancy in our differential. The persistent colonic wall thickening of the distal segment of descending colon on CT imaging was consistent with stricture formation, a surgical indication. Other indications for surgical management of diverticular disease include sepsis, fistula formation, abscess that failed percutaneous drainage, failure to respond to medical management, and recurrent disease. In patients with low preoperative risk and recurrent attacks of diverticulitis surgical resection of the diverticular disease can remove diseased colon, prevent obstruction and alleviate chronic pain with a low risk of morbidity. Our patient had recurrent attacks of diverticulitis that led to progressive fibrosis of the colonic wall causing stricture. Chronic recurrent diverticulitis complicated by abscess formation and fibrosis likely caused microvascular insufficiency and resulted in ischemic changes seen on the surgical specimen of the resected colon. Closing off of diverticular necks during recurrent inflammatory episodes will make diverticula unapparent on colonoscopy. If this stricture was not surgically removed recurrent symptoms and hospitalizations would likely continue and the patient would be at high risk for obstruction. As the incidence of diverticular disease continue to rise, prompt identification of patients who would benefit from surgical resection can decrease future hospital admissions, prevent chronic pain, and other complications of diverticular disease including strictures which can lead to bowel obstruction.

DOC, I SWALLOWED THE PACK: WHEN TO RETRIEVE INGESTED FOREIGN BODIES Danica S. Carew ${ }^{1}$; Kristopher P. Kline ${ }^{1}$; Maryam Sattari ${ }^{3}$; Ryan Nall $^{2}$. ${ }^{1}$ University of Florida, Gainesville, FL; ${ }^{2}$ University of Florida, Gainseville, FL; ${ }^{3}$ University of Florida College of Medicine, Gainesville, FL. (Control ID \#2468722)

LEARNING OBJECTIVE \#1: Describe the diagnostic approach after blister pack ingestion

LEARNING OBJECTIVE \#2: Recognize the indications for retrieval of ingested foreign objects

CASE: A 74-year-old man with past medical history of dementia, GERD, and Parkinson's disease called his primary care physician with symptoms of odynophagia and abdominal pain after unintentionally swallowing an omeprazole blister pack that morning. The incident occurred while taking a handful of his morning medications and he felt immediate and continuous sharp pains in the throat unresponsive to drinking water. He also complained of positional upper abdominal pains but denied nausea or vomiting. He was instructed to present to the Emergency Department for evaluation. On exam his abdomen was soft, non-tender and non-distended. Plain abdominal films showed no evidence of radiopaque foreign bodies. The patient was subsequently admitted for EGD with possible removal. EGD the following morning revealed several small non-bleeding erosions throughout the esophagus. An omeprazole 
blister pack was visualized in the gastric antrum and removed with a Roth net and foreign body hood protector. He tolerated the procedure well and was discharged the following day. The patient is doing well to-date, is tolerating a regular diet and reports complete resolution of his symptoms.

DISCUSSION: Acute onset dysphagia, inability to swallow saliva, cough, chest/ abdominal pain and choking are common presenting symptoms after foreign body ingestion. Foreign body ingestion is most common in adults with mental health disorders, dementia, and alcoholism. Blister pack ingestion is also frequently seen in older adults with visual impairment. Initial workup of known or suspected foreign body ingestion should include plain AP and lateral radiography in order to determine the location. Blister pack material is often difficult to detect on plain radiograph directly because of its radiolucency, however the air trapped in the pack might make the drug tablet visible. If radiography is negative and patient is symptomatic or clinical suspicion is high endoscopy is indicated. The timing of endoscopy in retrieval of foreign bodies is dependent on patient's symptoms, the characteristics of the object and its location. As in our case, emergent endoscopy is indicated if high-risk objects such as disc batteries or sharp/pointed substances are located in the esophagus due to increased risk of perforation, bleeding and obstruction. It is important to note that blister packs should be categorized as sharp along with more typical objects such as toothpicks, paperclips and fish or chicken bones. In fact, there have been several published reports of perforation secondary to blister pack ingestions that were not immediately recognized. Our patient was fortunate to have a favorable outcome due to early recognition of the need for foreign body retrieval with endoscopy. Primary care physicians can reduce risk of this complication by educating patient and caregivers about the risk. Recognition of perforation risk associated with blister pack ingestion and timely referral for retrieval would reduce risk of perforation.

DOC, MY URINE IS PURPLE! Kannan Thanikachalam ${ }^{1}$; Chioma Nwagbara ${ }^{3}$; Krishna Venkata ${ }^{2} .{ }^{1} \mathrm{UAB}$ Health Center Montgomery, Montgomery, AL; ${ }^{2} \mathrm{UAB}$ Montgomery, Montgomery, AL; ${ }^{3} \mathrm{UAB}$ Montgomery Health Center, Montgomery, AL. (Control ID \#2470326)

LEARNING OBJECTIVE \#1: Caregivers should be aware of purple urine bag syndrome in patients with chronic indwelling catheters and the association of urinary tract infections along with it.

EARNING OBJECTIVE \#2: Early treatment should be initiated if warranted, to prevent further complications.

CASE: The patient is a 74-year-old African American male with a past medical history of cerebrovascular accident, diabetes mellitus, sacral decubitus ulcers and chronic indwelling Foley's catheter since 1999 due to neurogenic bladder after a gun shot injury to the spine and was admitted with the chief complaints of discoloration of urine. The patient's home health had noticed purplish discoloration of urine and was sent to the ER. On physical examination, patient was alert, oriented and not in any distress. Abdominal examination did not reveal any significant abnormalities. While the color of urine from the plastic urine bag was purple in color, the color of the urine in the glass container attached to the bag before emptying into the plastic bag was yellow in color. When the urine from the glass container was emptied into the plastic bag, the color changed from yellow to purple in color. Patient also denied having fever or chills or lower abdominal pain. Patient had a normal WBC count and the lactic acid level was normal. Urinalysis revealed red-purple urine with a positive leukocyte esterase, positive nitrite, $4+$ bacteria, moderate triple phosphate crystals but normal urine WBC count. Urine cultures grew Escherichia coli, Enterococcus faecalis and Proteus mirabilis. Patient did not seem to have any signs or symptoms of urinary tract infection and the bacteriuria was attributed to colonization and we chose not to treat with antibiotics.

DISCUSSION: Purple urine bag syndrome is described as purple discoloration of urine which commonly occurs in cases of catheter associated urinary tract infection. Risk factors include female sex, chronic indwelling foley, alkaline urine, constipation, polyvinyl chloride catheter use, and a diet high in tryptophan. Discoloration is due to the metabolism of indole sulfate in urine by bacterial indoxyl phosphatase into indirubin and indigo. Indole is a product of the bacterial metabolism of tryptophan in the gastrointestinal tract. Indole is absorbed and converted to indoxyl sulfate which is excreted in the urine. The permeation of the indirubin and indigo into the plastic tubing of the catheter and urine bag also contribute to the distinct color observed. Providencia stuartii is the bacterium most commonly isolated from persons with purple urine bag syndrome, followed by Klebsiella pneumoniae, Enterobacter agglomerans, Providencia rettgeri, Proteus mirabilis, Escherichia coli, Morganella morganii, and Pseudomonas aeruginosa. Studies have shown that a several-log higher mean urinary bacterial count being the most strongly predictive factor, rather than the presence of one particular organism. Management includes recognizing it, testing and treating urinary tract infection and changing the urinary catheter. PUBS is a rare disorder which can be alarming to caregivers and attempts should be made at reducing duration of catheterization and improved catheter care remain vital in preventing the disorder in at-risk patients.

DOES HE HAVE DIABETES MELLITUS? Raju Khanal ${ }^{1}$; Ronald Herb ${ }^{2} .{ }^{1}$ Reading Health System, Wyomissing, PA; ${ }^{2}$ reading health system, Reading, PA. (Control ID \#2457913)

LEARNING OBJECTIVE \#1: Not all HbA1c in diabetic range suggests diabetes mellitus

LEARNING OBJECTIVE \#2: To recognize the false elevation of HbA1c in iron deficiency

CASE: 72 year old Caucasian male, presented to the primary care doctor for regular follow up for Iron deficiency anemia, hyperlipidemia, and impaired fasting glucose. He had been doing well. Initial iron deficiency was attributed to the bleeding esophageal ulcer documented by EGD. Subsequent EGD and colonoscopy were unremarkable. Celiac antibody panel was also negative. He had been donating whole blood three or four times in a year and was not taking any iron supplementation, which led to the persistent iron deficiency state. He was doing regular exercise and watching diet for his impaired fasting glucose and hyperlipidemia. Physical examination including vitals were unremarkable. Hemoglobin was stable at around $13 \mathrm{~g} / \mathrm{dl}$ with $\mathrm{RBC}$ of $4.37 \times 10^{12} / \mathrm{L}$. Ferritin was $17 \mathrm{ng} /$ $\mathrm{ml}$. His fasting blood sugars was $85 \mathrm{mg} / \mathrm{dl}$. HbAlc was $6.9 \%$. Considering $\mathrm{HbAlc}$ in diabetic range but fasting sugar within normal range, $2 \mathrm{~h}$ post prandial blood sugar was done, which was $111 \mathrm{mg} / \mathrm{dl}$. Considering nondiabetic range of both blood sugars in fasting as well as in post-prandial state, elevated $\mathrm{HbAl} \mathrm{c}$ was considered to be spurious secondary to iron deficiency. So it was recommended to take iron supplements, and repeat his ferritin and $\mathrm{HbAlc}$ after correction of iron deficiency.

DISCUSSION: According to the American Diabetes Association (ADA), diabetes mellitus is considered if $\mathrm{HbAlc}$ is $6.5 \%$ and prediabetes if it is $5.7-6.4 \%$. The $\mathrm{HbAlc}$ level depends not only with glycemic control but also the life span of RBCs. Nutritional deficiencies including iron are a major factor affecting RBC survival, which limits the diagnostic value of $\mathrm{HbAlc}$ in some situations. It may be misleadingly high in iron deficiency because of increase in RBC survival. It is also proposed that iron deficiency may alter the quaternary structure of hemoglobin molecule and facilitate glycation of the B-globin chain. A fall in $\mathrm{HbAlc}$ levels after iron supplementation supports the causal role of iron deficiency in elevating $\mathrm{HbAlc}$ concentration. It may be falsely high or low in patients with hemoglobin variants. Additionally, patients with low hemoglobin or recent blood transfusion or hemolytic anemia may have a false low $\mathrm{HbA} 1 \mathrm{c}$. In conclusion, the significance of $\mathrm{HbAlc}$ in screening, diagnosis and monitoring of treatment response may be limited in some situations including Iron deficiency, which may give a false higher 
value. As an astute physician, we need to aware of this situation to avoid unnecessary investigations, treatment, and also need to confirm the causal relation with repeat $\mathrm{HbAlc}$ after correction of iron deficiency to avoid misdiagnosis.

DON'T BE FOOLED BY A NEGATIVE PPD, POTT'S DISEASE MAY STILL BE PERCOLATING Brendan Gilmore; Toug M. Tanavin. Baylor College of Medicine, Houston, TX. (Control ID \#2469407)

LEARNING OBJECTIVE \#1: Recognize the risk factors and clinical symptoms of disseminated tuberculosis

LEARNING OBJECTIVE \#2: Diagnose disseminated tuberculosis when the tuberculin purified protein derivative skin test is negative

CASE: This is a case of an immunocompetent patient with a recent negative tuberculin purified protein derivative (PPD) skin test who presents with physical exam findings concerning for cord compression and is found to have an epidural abscess secondary to tuberculosis mycobacterium (TB). Diagnosing disseminated tuberculosis is challenging because of its often non-specific symptoms. Moreover, TB is paucibacillary disease which further complicates making a microbiological diagnosis. A 55 year-old African-American male with a history of homelessness and incarceration was in his normal state of health until 2 months prior to presentation when he went to his primary care provider with complaint of 1 week of shortness of breath, productive cough and poor appetite. A chest $\mathrm{X}$ ray $(\mathrm{CXR})$ showed a right sided pleural effusion. He was treated with azithromycin for community acquired pneumonia in the outpatient setting. At this time, a human immunodeficiency virus (HIV) antibody test and a tuberculin PPD skin test were negative. Two months after presentation to his primary care provider, the patient was admitted for continued respiratory symptoms, back pain and new onset lower extremity weakness. Vitals were notable for low grade fever and tachycardia. Lung exam revealed diminished breath sounds in the right lower lobe. Neurological exam was remarkable for diffuse lower extremity weakness, bilateral patellar and achilles hyperreflexia, and clonus. Labs showed a mild leukocytosis. As a result of upper motor neuron exam findings, an MRI was obtained. It showed extensive osteomyelitis with an epidural abscess and cord compression extending from the eighth to the eleventh thoracic vertebrae. MRI also revealed a chronic small rim-enhancing right pleural effusion. The patient was taken for laminectomy, lateral decompression, and incision and drainage of an epidural abscess. Sputum acid fast bacilli smear and culture were negative. Thoracic abscess culture had no growth. However, pathology returned from the vertebral biopsies with acid fast bacilli and necrotizing granulomas. The patient was started on treatment for disseminated TB with rifampin, isoniazid, pyrazinamide, and ethambutol.

DISCUSSION: The patient's presentation demonstrates the challenges in diagnosing disseminated TB. At first, it often presents with non-specific symptoms. Most common symptoms include anorexia, fever, weight loss, productive cough and night sweats. There symptoms overlap with many disease presentations, which can lead to a misdiagnosis. In this patient, his initial respiratory symptoms and CXR findings were also consistent with a much more common diagnosis of community acquired pneumonia. Despite the patient's TB risk factors, which included a history of homelessness and incarceration, the negative PPD skin test was misleading. PPD skin tests have been shown to have a false negative rate of approximately ten percent in active pulmonary TB cases. The false negative rate actually increases if pleurisy is present and as the infection disseminates. Moreover, TB is paucibacillary disease, which makes obtaining a microbiological diagnosis difficult. As a result, if TB is suspected or risk factors exist, further diagnostic testing should be pursued via advanced imaging and fluid or tissue cultures as appropriate. Multiple sputum, blood, urine or CSF samples need to be obtained as all have diagnostic yields of less than fifty percent in cases of disseminated TB. Notably, if active disease exists in lymph node or liver tissue, tissue biopsies have been shown to have the highest diagnostic yields of over eighty percent. Active pulmonary TB can progress to disseminated TB via massive hematogenous spread. However, it is less commonly seen in adult immunocompetent patients. When it does spread, it can involve the spine (Pott's disease). In these cases, it often presents with symptoms of cord compression, which require emergent surgical evaluation. Unfortunately, as a result of non-specific symptoms and a false negative PPD, the often challenging diagnosis of disseminated TB was initially overlooked. It is critical to understand TB risk factors, clinical symptoms and diagnostic yields to diagnose and treat this high mortality disease in a timely manner.

\section{DON'T IGNORE THE YOUNG MALE WITH RECURRENT PNEUMONIA} Hyejo $\mathrm{Jun}^{2}$; Judy $\mathrm{Vu}^{2}$; Amar Kohli ${ }^{1} .{ }^{1} \mathrm{UPMC}$, Pittsburgh, PA; ${ }^{2}$ University of Pittsburgh Medical Center, Pittsburgh, PA. (Control ID \#2467094)

LEARNING OBJECTIVE \#1: Discuss the epidemiology and pathophysiology of chronic granulomatous disease (CGD)

LEARNING OBJECTIVE \#2: Name the four clinical manifestations of Aspergillus infections and describe findings of pulmonary aspergillosis Outline the general management principles of CGD

CASE: The patient is a 29-year-old male with history of CGD manifested by recurrent pneumonias as a child and active recurrent perirectal abscesses who presented with 1 week of fevers, chills, dyspnea, and non-bloody, non-bilious emesis. He admitted to longstanding noncompliance with prophylactic antimicrobials as prescribed by his infectious disease team. He initially visited a different hospital where chest radiography showed no infiltrates. The patient was hydrated intravenously and discharged home. He became progressively fatigued, dyspneic, and developed a cough intermittently productive of sputum and presented to our hospital. Upon arrival the patient was afebrile, tachycardic to the $120 \mathrm{~s}$, and tachypneic to the $20 \mathrm{~s}$. Blood pressure was $118 / 64$ and the patient was saturating $96 \%$ on 31 by nasal cannula. He was diaphoretic and using accessory respiratory muscles. Auscultation of the posterior lung fields was notable for diffuse, bilateral rales with some wheezing audible in the bibasilar spaces. Arterial blood gas was 7.45/37/79/25. Bloodwork revealed a white count of 10.5, Hgb 13, Plts 320, Cr 0.7, and ESR 67. Chest radiography now demonstrated widespread lung opacities without focal consolidation and reactive hilar adenopathy. An azithromycin allergy limited typical community-acquired pneumonia coverage, therefore the patient was given a dose of intravenous levofloxacin. Due to clinical deterioration over the subsequent $12 \mathrm{~h}$, the patient was transferred to the intensive care unit and intubated. After intubation, the patient underwent bronchoscopy with bronchoalveolar lavage. Antibiotics were broadened and antifungal coverage with voriconazole and caspofungin were added. Eventually sputum culture and fungal culture grew Aspergillus fumigatus. Serum galactomannen was also negative. Antimicrobials were narrowed to voriconazole. The patient improved and was extubated 5 days after intubation. He was discharged home 3 days later off of any supplemental oxygen. The importance of adherence to prophylactic antimicrobials was stressed. At the 3-month follow up, the patient was doing well and compliant with his medications, and discussing with Hematology/Oncology about the possibility of an allogeneic stem cell transplant.

DISCUSSION: This case offers a window into a rare, genetic cause of immunodeficiency and its complications. CGD occurs in about 1:200,000 live births in the United States and is caused by mutations to any of the five proteins that form nicotinamide adenine dinucleotide phosphate (NADPH) oxidase in phagocytes. Men are more affected than women as the mutation that causes $65-70 \%$ of the cases is X-linked. Phagocytes use NADPH oxidase to help generate reactive oxygen species necessary for defense against invading pathogens. Organisms that produce catalase are able to protect themselves against reactive oxygen species. Thus the most common pathogens seen in CGD are Aspergillus species, Staphylococcus aureus, Burkholderia cepacia, Serratia marcescens, 
and Nocardia species, respectively. Pneumonia and abscesses are the two most frequent types of infections. Aspergillus favors the respiratory tract and causes four main clinical syndromes: allergic bronchopulmonary aspergillosis, chronic necrotizing Aspergillus pneumonia, aspergilloma, and invasive pulmonary aspergillosis. Our patient suffered from pulmonary aspergillosis, confirmed by sputum culture data. He did not present with the classic triad of fever, pleuritic chest pain, and hemoptysis, although this was mostly described in studies of neutropenic patients. Although computed tomography (CT) of the chest was deferred in our patient, it remains a useful diagnostic tool. Typical findings on CT chest include nodules with or without cavitation, patchy or segmental consolidation, or peribronchial infiltrates. Again in neutropenic patients, the initial findings are that of nodules with surrounding ground-glass opacities from hemorrhage into the area, dubbed the halo sign. The progression of disease is then often marked by cavitation of the nodules, producing air-crescent signs. The foundations of CGD management are antimicrobial prophylaxis, early diagnosis of infections, and aggressive management of infectious complications. Recommended prophylactic medications are trimethoprimsulfamethoxazole and itraconazole. Our patient was also discussing the possibility of allogeneic stem cell transplant; unfortunately there is little literature around transplantation in CGD patients. A recent prospective study published by Güngör et al. studied fifty-six patients considered high-risk due to therapy-refractory infections or autoinflammation. They were recruited from 16 centers worldwide and underwent allogeneic stem cell transplantation with reduced-intensity conditioning. The 2-year probability of overall survival was $96 \%$ and the events-free survival was $91 \%$. Although this study shows promise, there remains limited evidence and availability of centers with adequate experience in transplantation of CGD patients

DON'T LEAVE ME BROKEN-HEARTED: A CLASSIC CASE OF TAKOTSUBO CARDIOMYOPATHY Andy Y. Cheng; David C. Demoise; Amar Kohli. University of Pittsburgh Medical Center, Pittsburgh, PA. (Control ID \#2450789)

LEARNING OBJECTIVE \#1: Differentiate Takotsubo Cardiomyopathy from STElevation Myocardial Infarction

LEARNING OBJECTIVE \#2: Describe key epidemiological features of Takotsubo Cardiomyopathy

CASE: A 71-year-old Caucasian female, past medical history significant for severe malnutrition, failure to thrive, and CT chest findings concerning for chronic Mycobacterium avium-intracellulare infection presented 1 day after bronchoscopy with acute shortness of breath. She repeatedly stated that she could not breathe, but denied any chest pain or other discomforts. Her baseline nasal cannula oxygen requirement of $4 \mathrm{~L}$ had increased to $6 \mathrm{~L}$ to maintain an oxygen saturation of $92 \%$, and her baseline tachycardia had worsened from a rate in the 110's to the 130's. However, her blood pressure remained stable at 111/79. Cardiac exam did not reveal new rubs, murmurs, gallops, or changes in heart sounds, but pulmonary exam was significant for new extensive crackles throughout all lung fields. Immediate workup including basic metabolic panel, complete blood count, troponin, brain natriuretic peptide, electrocardiogram, and chest $\mathrm{x}$-ray were obtained. Basic metabolic panel was within normal limits, but complete blood count showed a worsening leukocytosis from 18.9/mcL to 21.6/mcL. Brain natriuretic peptide was elevated at $1552 \mathrm{pg} / \mathrm{mL}$, compared to $115 \mathrm{pg} / \mathrm{mL}$ upon admission, and chest x-ray was suggestive of newly developed vascular congestion. Electrocardiogram, when compared to prior, showed ST elevations in leads I, II, and V2 through V6, alongside ST depressions in aVR and V1. Troponin returned at $0.98 \mathrm{ng} / \mathrm{mL}$. Cardiology was consulted, and after echocardiogram showed transient apical ballooning with regional hypokinesis and cardiac catheterization showed clean coronary arteries, the patient was diagnosed with Takotsubo Cardiomyopathy.

DISCUSSION: Takotsubo Cardiomyopathy and ST-Elevation Myocardial Infarction closely mimic each other in several aspects of patient presentation and diagnostic work- up, but closer examination of the Mayo Clinic Criteria for diagnosing Takotsubo Cardiomyopathy elicits several key differences. Firstly, new ECG abnormalities or modest elevation in cardiac troponin should be present. This patient demonstrated a modest troponin elevation of $0.98 \mathrm{ng} / \mathrm{mL}$, alongside marked EKG changes from baseline. It has been documented that the combination of absent ST elevation in V1 and present ST depression in aVR carries a $91 \%$ sensitivity and $96 \%$ specificity for Takotsubo Cardiomyopathy, and this patient's electrocardiogram depicted such a combination. Furthermore, one study showed an average BNP:Troponin ratio of 1089.4 in Takotsubo versus 97.4 in STEMI. This patient had a ratio of 1581.6, thus strongly suggestive of Takotsubo Cardiomyopathy. Secondly, there must be an absence of coronary disease or angiographic evidence of acute plaque rupture, and this patient's cardiac catheterization showed clean coronary arteries. Thirdly, there should be regional wall motion abnormalities beyond a single coronary vascular distribution. This patient's echocardiogram showed hypokinesis in the entire anterior septum, mid and distal anterior wall, and apical lateral wall - regions that certainly extend beyond a single vascular distribution. Furthermore, four-chamber views on echocardiogram also demonstrated transient apical ballooning, or classic “octopus trap" Takotsubo appearance, of the left ventricle. Fourthly, pheochromocytoma and myocarditis have to be clinically ruled out. This patient did not have classic pheochromocytoma symptoms of sustained or paroxysmal hypertension, headache, and sweating at any point in her history or during her inpatient stay. This patient also did not demonstrate symptoms such as chest pain, acute decompensation of documented heart failure, and flu-like syndrome to strongly point towards myocarditis. Thus, this patient fulfilled all four of the Mayo Clinic Criteria for Takotsubo Cardiomyopathy. Furthermore, she also exemplified classic epidemiological features of the disease. She matched the disease's predominant occurrence in elderly women. While she did not report the typical symptom of chest pain, she did report the second-most common symptom of dyspnea. Lastly, she had several possible preceding stressors, including very recent bronchoscopy, long-standing generalized anxiety disorder, and a worsening infection that was later identified as Pseudomonas pneumonia. In summary, this case encompasses classical aspects of Takotsubo Cardiomyopathy and highlights clinical features differentiating Takotsubo Cardiomyopathy from STElevation Myocardial Infarction.

DON'T TAKE THE BAIT: FISHING FOR A DIAGNOSIS IN A MAN WITH RECURRENT FEVERS AND HALLUCINATIONS Nicholas Duca; Swati Shroff; Amar Kohli. UPMC Presbyterian, Pittsburgh, PA. (Control ID \#2433923)

LEARNING OBJECTIVE \#1: Identify three potential biases that may occur during the evaluation of a patient with fever of unknown origin

LEARNING OBJECTIVE \#2: Describe the utility of inflammatory markers in distinguishing infectious and non-infectious causes of fever of unknown origin

CASE: A 54 year old man with history of toe osteomyelitis presented with fever and altered mental status. One month prior, the patient began having fevers to $106^{\circ} \mathrm{F}$, every 2 to 3 days. During the last month, his wife noted worsening memory, somnolence, occasional hallucinations, and a $15 \mathrm{lb}$ weight loss. The patient was admitted due to persistent fevers and worsening hallucinations. Medical history included osteomyelitis of the toes 8 months prior, complicated by sepsis, status post amputation of the right second and third toes and left third toe, peripheral vascular disease and chronic lower extremity pain. Medications included pregabalin $150 \mathrm{mg}$ every $8 \mathrm{~h}$, tizanadine $4 \mathrm{mg}$ as needed for muscle spasm, and trazodone $100 \mathrm{mg}$ nightly. The patient was hemodynamically stable and afebrile upon admission. The patient was oriented but somnolent. Amputation sites were clean, dry and intact. White blood cell count was $5.9 \times 10^{9} / \mathrm{L}$ with normal differential, hemoglobin $8.5 \mathrm{~g} / \mathrm{dl}$, ferritin $3656 \mathrm{ng} / \mathrm{ml}$, C-reactive protein $23.1 \mathrm{mg} /$ 
$\mathrm{dl}$, and erythrocyte sedimentation rate $>120 \mathrm{~mm} / \mathrm{h}$. Blood cultures were negative. Brain imaging was normal. X-rays of the feet were negative for osteomyelitis. Lumbar puncture revealed normal cerebrospinal fluid and negative Lyme, herpes simplex virus, and varicella-zoster PCR, and cryptococcal antigen. After decreasing the dose of pregabalin and discontinuing tizanadine and trazodone, the patient's mental status improved. He remained afebrile throughout the admission. The patient was discharged and referred for colonoscopy to evaluate for occult gastrointestinal malignancy, given anemia and elevated inflammatory markers. One month after discharge, the patient was readmitted with persistent fevers. A CT scan with IV contrast of the chest, abdomen and pelvis revealed multiple, enlarged retroperitoneal lymph nodes measuring up to $4 \times 3 \mathrm{~cm}$. Lymph node biopsy demonstrated a lymphoproliferative process. Bone marrow biopsy confirmed Hodgkin lymphoma. PET scan showed extensive lymphadenopathy with osseous involvement of the spine and ribs. The patient was diagnosed with stage IV Hodgkin lymphoma, and referred to oncology for treatment.

DISCUSSION: Despite advances in laboratory and imaging modalities, the etiology of fever of unknown origin (FUO) is undiagnosed in up to $51 \%$ of cases. During evaluation, physicians may be susceptible to cognitive biases while attempting to synthesize nonspecific historical and laboratory data. These biases can lead to a delay in diagnosis or inappropriate testing and treatment. This vignette identifies three biases which occurred during the evaluation of a patient with FUO and examines important laboratory studies which aid in distinguishing between infectious and non-infectious causes of FUO. Early in this patient's illness, cognitive biases led to an incomplete diagnostic evaluation. The patient's presenting complaints included altered mental status and fever, triggering an infectious work-up. Upon learning that the patient had cyclical fevers and weight loss over the prior month, the team did not aggressively explore potential non-infectious etiologies, such as malignancy, due to anchoring bias. Anchoring led to confirmation bias; the team sought diagnostic studies which supported their number one diagnosis rather than other key tests, such as a CT scan of the chest, abdomen, and pelvis which eventually diagnosed Hodgkin lymphoma. Finally, when the patient's mental status improved with medication changes, the team failed to consider alternative diagnoses, an example of premature closure. Inflammatory markers including ESR, CRP, and ferritin were significantly elevated in this patient, which may have aided in differentiating infection from malignancy. In one study of patients with FUO, CRP levels greater than $6 \mathrm{mg} / \mathrm{dl}$ and concurrent ferritin levels less than $500 \mathrm{ng} / \mathrm{mL}$ were independently associated with infection. In another study, the optimal cutoff for serum ferritin levels to predict non-infectious cases of FUO was $>561 \mathrm{ng} / \mathrm{mL}$. In a third study, all FUO patients with malignancy had an ESR $>40$. Taking these findings together, our patient had significantly elevated CRP $(23.1 \mathrm{mg} / \mathrm{dl})$, ferritin $(3656 \mathrm{ng} / \mathrm{ml})$ and ESR ( $>120 \mathrm{~mm} / \mathrm{h}$ ) which suggested a non-infectious, and likely malignant etiology. In summary, reaching a diagnosis in patients with FUO is complex. To avoid inappropriate testing or treatments, physicians must be aware of cognitive biases, such as anchoring, confirmation, and early closure. Furthermore, utilization of key inflammatory markers such as ESR, CRP, and ferritin may be of benefit in differentiating infectious and non-infectious causes of FUO and should be obtained in all patients presenting with FUO.

\section{DON'T TURN A BLIND EYE TO THE SERUM SODIUM: AN UNUSUAL CASE OF MONOCULAR VISION AND SIADH IN AN ALCOHOLIC PATIENT Ernie L.} $\underline{\text { Esquivel }^{1}}{ }^{1}$ Esther Chung ${ }^{1}$; Alejandro Pino ${ }^{1}$; Harpreet S. Bhatia ${ }^{2} .{ }^{1}$ Weill Cornell Medical College, New York, NY; ${ }^{2}$ Weill-Cornell Medical Center, Stamford, CT. (Control ID \#2470260)

LEARNING OBJECTIVE \#1: Recognize the historical and physical exam findings of a cavernous sinus-carotid fistula as a cause of monocular vision loss.

LEARNING OBJECTIVE \#2: Appreciate the need for vigilant search for an underlying cause of hyponatremia in patients with unexplained SIADH.
CASE: A 64-year-old undomiciled man with unknown past medical history presented with nausea and vomiting, and incidentally mentioned left eye blindness. A poor historian, he described a vague bitemporal headache with no associated aura, and painless loss of vision in his left eye sometime in the prior 3 weeks. Physical exam was notable for a $10 \mathrm{~cm}$ linear scar on the left temple and a cast of the left wrist, neither of which he could explain. The temporal artery was not inflamed and no scalp tenderness or jaw claudication were elicited. His left eye showed chemosis and conjunctival injection, no light perception and an afferent pupillary defect. Funduscopic exam showed diffuse pallor of the optic nerve. No neurologic deficits were observed and his right eye was normal. Laboratory data were suggestive of recent alcohol intake and ESR and CRP were unremarkable. Noncontrast head CT showed evidence of subacute traumatic brain injury and radiographs demonstrated a left wrist fracture. While attempting to obtain an MRI/MRA of the brain and head to explain the vision loss and headaches, the patient's hospital course was complicated by persistent headache requiring intravenous narcotics and a dramatic drop in his serum Na to $120 \mathrm{mmol} / \mathrm{L}$ over 4 days. The patient was noted to be euvolemic, and urine studies revealed a urine sodium of $116 \mathrm{mmol} / \mathrm{L}$, urine osmolality of $348 \mathrm{mOsm} / \mathrm{kg}$ and a low serum uric acid of $2.8 \mathrm{mg} / \mathrm{dL}$, all consistent with syndrome of inappropriate anti-diuretic hormone (SIADH). Because of worsening confusion and an inability to perform the MRI due to agitation, treatment of SIADH was initiated with fluid restriction and high dose sodium chloride tablets, but ultimately was refractory and required intravenous furosemide and hypertonic saline. MRA and MRI were eventually performed under general anesthesia and revealed a high-flow direct cavernous sinus-internal carotid fistula (CCF), likely due to prior head trauma. Indeed, brain imaging also showed subacute to chronic bilateral hemorrhagic contusions, bilateral frontoparietal subdural collections, thin focal scarring/hematoma along the left orbital roof, and left optic nerve edema/ gliosis at the optic canal. This prompted us to re-examine the patient's left eye, which revealed mild proptosis and a left orbital bruit which are consistent with CCF. Interventional neuroradiology performed an uncomplicated parent vessel occlusion of the left internal carotid artery and coil embolization of the left CCF. The patient's hyponatremia and headaches subsequently improved and he was discharged to a homeless shelter

DISCUSSION: Monocular vision loss and headache in our patient was attributed to a posttraumatic cavernous sinus-internal carotid fistula (CCF), classified as a high-flow, direct Barrow type A. The cavernous sinus is a paired dural venous sinus that receives drainage from the inferior and superior ophthalmic veins and the sphenoparietal sinus, and contains the cavernous segment of the internal carotid artery. This type of CCF typically causes high cavernous sinus pressure refluxed to the ophthalmic veins, resulting in vein engorgement and elevated intraocular pressure. Elevated intraocular pressure decreases retinal perfusion compromising visual acuity and ultimately resulting in vision loss. Our patient demonstrated several classic signs and symptoms of CCF including monocular vision loss, severe headache, conjunctival injection, chemosis, proptosis, and ocular bruit . The development of worsening hyponatremia in our patient due to unexplained SIADH served as a warning sign for us of potential CNS pathology. In patients with a history of alcoholism and homelessness vague complaints may easily be dismissed and this case is a reminder of the importance of understanding the potential for attribution bias. Identification and treatment of CCF in this patient led to a resolution of his headache and hyponatremia, although vision loss was not restored. Although CCFs are rare, monocular vision loss, headache, chemosis, proptosis, and ocular bruit with evidence of trauma should prompt physicians to consider CCF as an etiology. Immediate treatment of $\mathrm{CCF}$ can reduce risk of vision loss and potentially life threatening complications such as cerebral hemorrhage. Physicians are also reminded to evaluate undomiciled patients for a history of trauma and traumatic brain injury, as substance abuse is common among this population and increases risks of falls.

DON'T BYPASS YOUR LIFE: POST-BARIATRIC STARVATION Michael Gillette; Naree Whang; Ahmed G. Mohiuddin. Tulane University Health Sciences Center, New Orleans, LA. (Control ID \#2468920) 
LEARNING OBJECTIVE \#1: Recognize protein-energy malnutrition (Kwashiorkor) LEARNING OBJECTIVE \#2: Recognize medical side-effects of bariatric surgery CASE: A 47 year-old woman presented to the emergency department with new onset dyspnea on exertion, anasarca, and a hyperpigmented truncal rash. She had a past medical history notable for a Roux-en-Y gastric bypass performed 10 years earlier. After the bypass, she achieved significant weight loss and developed a high functional capacity. However, the patient's course was complicated by early satiety that lasted for many years. In the months prior to admission, she developed increasing exercise intolerance progressing to dyspnea on exertion. She further complained of worsening memory, intermittent confusion, and peripheral neuropathy. In the weeks prior to admission, the patient experienced an un-provoked DVT and subsequent pulmonary embolus. Vital sings at triage: T $36.8{ }^{\circ} \mathrm{C}$, HR 107, BP 135/82, RR 14, WT $72 \mathrm{~kg}$, BMI 30. Physical exam revealed cachexia, temporal wasting, and frank anasarca with thinning hair, and a dry, hyperpigmented, macular rash limited to the trunk. Laboratory work showed a total protein of $5 \mathrm{~g} / \mathrm{dL}$, albumin of $1.6 \mathrm{~g} / \mathrm{dL}$, pre-albumin of 7, thiamine (Vit. B1) of $97 \mu \mathrm{g} /$ $\mathrm{dL}$, niacin (Vit. B3) $<3 \mu \mathrm{g} / \mathrm{dL}$, and hemogolbin A1c $<3.5 \%$. Renal function, transaminases, and urine studies were normal. Cardiac testing with an EKG, transthoracic echocardiogram, left and right heart catheterization did not reveal any significant findings. An abdominal ultrasound and contrasted abdominal CT showed hepatosteatosis but no other derangements. Esophagogastroduodenoscopy (EGD) showed post-surgical changes without significant changes on pathology. The patient was placed on escalating doses of peripheral parenteral nutrition (PPN), vitamin supplementation, and underwent a partial reversal of her Roux-en-Y. One year after the reversal, the patient has a steadily increasing functional status and a normal nutritional status.

DISCUSSION: Severe malnutrition is a well-recognized problem in resource-poor settings, but may be under-recognized in developed countries. In developed countries, severe malnutrition is often a consequence of chronic disease states (e.g. alcoholism, AIDS, and cancer). Iatrogenic protein-energy malnutrition after bypass surgery is noted in several previous case reports. Bariatric surgery is an increasingly common treatment for morbid obesity with approximately 113,000 cases performed in the US a year and may become an under-recognized cause of malnutritioned states. Our patient presented with several hallmarks of Kwashiorkor, namely: (1) anorexia driven by insufficient caloric intake, (2) profound edema due to a combination of low serum oncotic pressure and decreased hepatic clearance of antidiruetic hormone, (3) dermatosis, depigmented thinning hair, and cognitive changes resulting from amino acid metabolism derangements and concomitant vitamin deficits. The history of recent DVT and PE was likely driven by a pro-inflammatory and hypercoagulable state similar to nephrotic diseases with albumin levels of $<2 \mathrm{~g} / \mathrm{dL}$. Laboratory findings were also consistent with Kwashiorkor: (1) low total protein, albumin and pre-albumin from a global lack of amino acids, (2) hepatosteatosis due to lack of beta-lipoproteins, (3) concomitant water-soluble vitamin deficiencies. Management of acute malnutrition requires reversal of both the nutritional state and the iatrogenic cause. Resuscitation is begun slowly with vitamin and micronutrient supplementation while slowly titrating up caloric intake to $100 \%$ of caloric needs over a week. This gradually shifts metabolism from a catabolic to anabolic state, avoiding re-feeding syndrome - a dangerous complication resulting from sudden metabolic shifts with insufficient total body phosphorus, potassium, and macronutrients needed to maintain homeostasis.

DOUBLE TROUBLE: SIMULTANEOUS PRESENTATION OF CHRONIC LYMPHOCYTIC LEUKEMIA AND CHRONIC MYELOGENOUS LEUKEMIA Asha Kovelamudi; Roger D. Smalligan; Sue Nadesan. Texas Tech Univ Health Sc Center-Amarillo, Amarillo, TX. (Control ID \#2466851)

LEARNING OBJECTIVE \#1: Diagnose concurrent CLL and CML in a patient. LEARNING OBJECTIVE \#2: Understand the possible origens of a rare combination of CLL and CML in a patient.
CASE: An 88-year-old Caucasian male who was able to care for himself, exercised three times a week, and played golf weekly presented to his primary care doctor with an unexplained 20-lb weight loss over 6 months. He denied anorexia, nausea, vomiting, diarrhea, fatigue, fever and night sweats. He did have shortness of breath with exertion and some urinary frequency as well as back stiffness and cold intolerance. PMH: Coronary artery disease, COPD, GERD, Osteoarthritis, BPH and Hematuria Medications: Carvedilol 6.25 mg daily. PSH: Tonsillectomy, Vasectomy, Cataract Surgery, TURP, Right Hip Replacement and Pacemaker Placement. PE: BP 124/63, HR 59, RR 18, T 98 deg F, O2 sat $100 \%$ on RA. Alert and in no distress. HEENT, pulmonary, cardiac, abdominal, skin and neurological exam was normal. He had no lymphadenopathy. Labs: WBC 21,000, $62 \%$ segs, $29 \%$ lymphs, $4 \%$ monos, $1 \%$ eos; Hgb 15.4 g/dL, HCT $42.6 \%$, MCV $92.6 \mathrm{fL}$, platelets 256,000/uL, ESR $6 \mathrm{~mm} / \mathrm{h}, \mathrm{CRP}<0.2$, vitamin B12 $2210 \mathrm{pg} / \mathrm{mL}$, folate $11.4 \mathrm{ng} / \mathrm{mL}$ Course: The patient was referred to oncology due to an unexplained leukocytosis. The peripheral smear showed hypersegmented neutrophils and some atypical lymphocytes. ANA and RA tests were negative. SPEP was normal. Flow cytometry showed a clonal CD5-positive B-cell population that was ZAP-70, and CD38 positive which was consistent with chronic lymphocytic leukemia (CLL). In addition, he had aberrant CD-56 granulocytes with $1 \%$ blasts and a quantitative RT-PCR assay for $\mathrm{BCR} / \mathrm{ABL} 1$ that was positive at $45 \%$ which was consistent with chronic myelogenous leukemia (CML). A hypercellular bone marrow showed CML in chronic phase and B-cell CLL. Cytogenetics showed a 9;22 translocation (Philadelphia chromosome) and a FISH profile showed a 13q deletion. A diagnosis of simultaneous CLL and CML was confirmed. The patient was started on imatinib which he took intermittently for a 2-month period but stopped due to an extensive rash. He was then given dasatinib which he tolerated better. Flow cytometry was done at intervals to follow blast numbers; CD5, CD38 cell numbers followed for CLL and periodic FISH to follow the 13q deletion. He is currently having a good response and maintaining his functional status on this therapy. DISCUSSION: Primary care internists and hospitalists are often the first to detect the possibility of a hematologic malignancy. Most patients with both CLL and CML are asymptomatic at the time of diagnosis, with their first sign being the presence of an unexplained leukocytosis. Our patient was more advanced in that he had symptoms consistent with either diagnosis such as weight loss and fatigue although he lacked other classic symptoms of fevers and night sweats and lymphadenopathy in the case of CLL. The incidence of CLL is 4.5 per 100,000 with a lifetime risk of $6 \%$. CML occurs in 1.7 per 100,000 and a lifetime risk of $2 \%$. Coexistence of CLL and CML is a rare event with only eight documented case reports. There are several other case reports showing one disease developing after the other months to years later (i.e. CML develops in a patient with CLL or vice versa). Research has shown that BCR/ABL-transformed cells from CML patients secrete TNF- $\alpha$, IL-1 $\alpha$, IL-1RA, IL-3, IL-6, and GM-CSF. However, the cytokine of special importance is interleukin-3 (IL3). IL3 has been shown to increase B-lymphoid production from cord blood and bone marrow CD34+/CD 38- cells. The effect of IL-3 was highly reproducible in cell cultures with a significant increase in B-cells numbers in both total cell output and in the purity of CD19 positive B-cells. This evidence suggests a mechanism by which IL-3 might influence the emergence of CLL in a patient with CML. Another possible mechanism is the fact that hematologic stem cells are capable of differentiating into two or more different hematologic cell lines. One study demonstrated that there were two distinct clonal proliferations in a patient with both CML and CLL, one was a population of clonal $\mathrm{B}$ cells with no $\mathrm{BCR} / \mathrm{ABL}$ fusion and other was the $\mathrm{BCR} / \mathrm{ABL}$ fusion CML clone providing evidence for a separate clonal origin for each disorder. Other studies have also used gene probe analysis to show the two independent origins of CLL and CML that have occurred simultaneously. Further investigation needs to be done on the present case to determine the origin of the CLL and CML that seemingly arose simultaneously in this patient. Utilization of CBC analyzers, flow cytometry, and FISH can help diagnose the two separate hematological entities in an individual patient. Regardless, internists need to be alert to early laboratory and clinical signs and symptoms of 
hematologic malignancies to facilitate early diagnosis and treatment to preserve the functional status of older adults as was achieved in this case.

DRAMATIC IMPACT OF MILD QTC PROLONGATION Stephanie Windish Creighton University, Omaha, NE. (Control ID \#2469639)

LEARNING OBJECTIVE \#1: Recognize patient specific risk factors for development of ventricular arrhythmias.

LEARNING OBJECTIVE \#2: Manage patients with high risk QTc prolongation. CASE: A 25-year-old female presented to the emergency department with a 1 day history of intractable nausea and vomiting. She has no known past medical history and review of systems was otherwise negative. In the ED, electrocardiogram (ECG) showed a rhythm of sinus bradycardia with a QTc of 462. Laboratory data showed potassium of 3.3. She was given a single dose of $4 \mathrm{mg}$ ondansetron intravenously. She was later found to be unresponsive. Telemetry showed ventricular fibrillation (VFib.) Advanced Cardiac Life Support protocol was initiated and a $200 \mathrm{~J}$ biphasic shock was administered with immediate return to spontaneous circulation and ECG showed return to sinus bradycardia Given the cardiac arrest in a previously healthy 25 year old, with no family history of sudden cardiac death or arrhythmias, an echocardiogram was done to rule out structural abnormalities. Subsequently the electrophysiology team was consulted. The assessment was that the emesis induced electrolyte abnormalities in combination with her prolonged QTc and the administration of a QT prolonging medication led to development of Torsades de Pointes and VFib. The plan was for aggressive electrolyte monitoring and replacement and to avoid any QT prolonging medications. After successful treatment of her underlying infection the patient was discharged to home with a LifeVest and plan for follow up, including genetic testing.

DISCUSSION: Prolonged QTc is a known risk factor for development of ventricular arrhythmias. In previously asymptomatic patients, the right clinical settings and compounding risk factors can lead to life threatening arrhythmias. If QTc prolongation is found, even in the absence of confirmed congenital long QT syndrome, risks and benefits of administration of QT prolonging medications should be carefully weighed After diagnosis of QTc prolongation is made by ECG further work up depends on the specific clinical course. Although our patient had no family history, the VFib arrhythmia and sudden cardiac arrest warranted consideration for ICD placement, as well as genetic testing for congenital prolonged QT syndrome.

DRESS SYNDROME IN A PATIENT RECEIVING LEVOFLOXACIN Brian J. Ferrara; Kathryn A. Jobbins. Baystate Medical Center, Springfield, MA. (Control ID \#2463680)

LEARNING OBJECTIVE \#1: Recognize the common features of Drug Reaction with Eosinophilia and Systemic Symptoms (DRESS) syndrome and the potential for atypical agents to act as a trigger.

LEARNING OBJECTIVE \#2: Identify Levofloxacin as a rare trigger of DRESS.

CASE: An 86-year-old man with past medical history of bronchogenic carcinoma status post resection, hypertension, chronic kidney disease, and hyperlipidemia presented to the Emergency Department after referral by his PCP. Following routine labs, he received a call from his PCP that his labs were abnormal, including an elevated potassium, alkaline phosphatase, AST, and ALT. On presentation, the patient and his wife reported a pruritic rash that had become more prominent and itchy over the past 5 days. The rash had not improved with application of topical hydrocortisone cream. Notably, the patient had been diagnosed with community-acquired pneumonia about 2 months earlier. He had completed a 10-day course of levofloxacin and had resolution of his pneumonia. Due to an abnormal chest x-ray in the ED, he received an additional dose of levofloxacin there. He had no other recent medication changes. On admission, the patient was found to be afebrile and had occasional tachycardia into the 90 s. He had a morbilliform rash developing on the chest, upper back, and proximal upper and lower extremities. The rest of his examination was within normal limits. Relevant labs on presentation included WBC 11.3 with $17.1 \%$ eosinophils ( 1900 cells $\mathrm{mm}^{-3}$ ), alkaline phosphatase 277, AST 67, and ALT 73. Eosinophils peaked at $23.4 \%\left(2200\right.$ cells $\left.\mathrm{mm}^{-3}\right)$. Ultrasound of the abdomen did not reveal any acute biliary process. Levofloxacin was stopped upon admission. The patient initially received topical betamethasone, but due to the persistent skin symptoms, Prednisone $40 \mathrm{mg}$ daily was added. Peripheral smear was notable only for eosinophils. A skin biopsy revealed diffuse mild epidermal spongiosis with a perivascular infiltrate composed of intense eosinophils and lymphocytes. This pattern is most consistent with DRESS syndrome. Following initiation of the prednisone, the rash improved, and AST, ALT, and eosinophils normalized. The patient steadily improved and was discharged to home with plan to complete a total of 5 days of prednisone. He was advised to avoid any future exposure to levofloxacin.

DISCUSSION: DRESS syndrome is a rare, drug-induced hypersensitivity reaction. It is notable for a prolonged latency time of 2 to 8 weeks from start of the culprit medication and presents with a variety of appearances, most commonly with the diffuse, pruritic morbilliform rash seen in our patient. Though our patient did not have a fever on presentation, he did have leukocytosis and eosinophilia consistent with the expected presentation of DRESS. The condition can result in injury to internal organs, the most commonly affected visceral organ being the liver. In our patient, there was a relatively mild degree of hepatic injury. (1) Identification and appropriate management of DRESS syndrome is critical, as the condition has a mortality of $10 \%$, most often secondary to fulminant liver failure. The most common offending drugs include allopurinol, sulfonamides, and aromatic anti-epileptic drugs. (2) However, review of the literature reveals just a single case of DRESS syndrome following levofloxacin, which did not involve eosinophilia. (3) Given our patient's history and physical, this case highlights how essential it is to ask about previous medication exposure to ensure an appropriate diagnosis can be reached. As with most drug reactions, the mainstay of management of DRESS is discontinuation of the causative drug, with many also requiring systemic corticosteroids. (4) Consideration of an unexpected trigger such as levofloxacin and subsequent discontinuation are key pieces of appropriate care. Reference: 1. Husain Z, et al. DRESS syndrome: Part I. Clinical Perspectives. J Am Acad Dermatol. 2013 May;68(5):693.e1-14 2. Cacoub P, et al. The DRESS Syndrome: a literature review. Am J Med. 2011 Jul;124(7):588-97 3. Charfi O, et al. DRESS Syndrome Following Levofloxacin Exposure With Positive Patch-Testing. Therapie. 2015 Aug 3. [epub ahead of print] 4. Husain Z, et al. DRESS syndrome: Part II. Management and therapeutics. J Am Acad Dermatol. 2013 May;68(5):709.e1-9

DRUG-INDUCED CATATONIA, A CASE FOR EARLY DETECTION Tse-Shuen

J. Ku; Hannah Oakland; Meredith M. Barr. Tulane University Health Sciences Center, New Orleans, LA. (Control ID \#2466099)

LEARNING OBJECTIVE \#1: Review symptoms of drug-induced catatonia and its relationship to neuroleptic malignant syndrome.

LEARNING OBJECTIVE \#2: Describe the manifestations of catatonia in a patient with pre-existing dementia.

CASE: A 70 year-old man with history of advanced microvascular dementia, hypertension, and alcohol abuse in remission presented with a 4-day history of decreased verbal responsiveness, urinary incontinence, and inability to ambulate. At baseline patient is dependent for most activities of daily living, but is able to reply with three-word phrases, feed himself, and walk a few feet with assistance. He previously took quetiapine, haloperidol, and sertraline for treatment of aggressive features. Outpatient records reveal 
that haloperidol had been exchanged for risperidone with rapid increase in risperidone dose over the prior week, however he continued to receive high doses of both antipsychotics due to misunderstanding of physician instructions. At presentation, vital signs were within normal limits. Physical exam was significant for decreased attention and tracking, increased rigidity, hypertonia, hyporeflexia, cogwheel rigidity in right arm and resting tremor in both hands. He was reactive to verbal and painful stimuli. Laboratory results including TSH and basic chemistry were within normal limits. Infective work up was negative. Creatinine Kinase was 1903. CT brain showed only chronic microvascular changes consistent with previous imaging. EEG indicated generalized slowing without seizure activity. MRI brain was deferred as the patient was unable to cooperate without sedation. Patient slowly improved over the next week with supportive treatment and withholding of all home psychotropic medications. He remained afebrile with down trending of CK without indication for treatment with benzodiazepines or dantrolene. At discharge, he continued to have some mild cogwheel rigidity but was verbally responsive and improving in strength with physical therapy.

DISCUSSION: The mechanism of drug-induced catatonia is unclear, but likely involves dopamine, gamma-aminonutyric acid, and glutamate neurotransmitters. Clinical diagnosis can be made using rating scales such as the Bush-Francis Catatonia Screening Instrument. Such scales help in the identification of drug-induced catatonia, especially in patients with poor baseline functioning or multiple risk factors for altered mental status who may not have clear fever, rigidity, or rhabodmyolysis as malignant signs of catatonia. Patients with dementia may have agitation requiring treatment with antipsychotics or antidepressants. Unlike NMS and serotonin syndrome, drug-induced catatonia has variable presentation, including dysautonomia and hyper or hyporeactivity. It is also associated with both neuroleptic and non-neuroleptic medications, including alcohol, aspirin, metoclopramide, lithium, morphine, and steroids. Early recognition prevents unnecessary interventions and imaging, as standard treatment is the withholding of the offending agent. The existence of catatonia in patients with dementia has been rarely reported and can be confused with symptoms of the underlying disease, causing delay in diagnosis and adverse outcomes. Early detection and initiation of supportive treatment such as intravenous fluids and withholding of the offending medication can result in complete resolution and prevention of worsening to NMS, suggested to be on the same continuum as drug-induced catatonia.

\section{EARLY BREAST CANCER DETECTION - NOT ALWAYS AN EASY TASK} Dietlind L. Wahner-Roedler. Mayo Clinic, Rochester, MN. (Control ID \#2443049)

LEARNING OBJECTIVE \#1: Recognize the importance of mammographic breast density

LEARNING OBJECTIVE \#2: Recognize the difficulty in detecting invasive lobular carcinoma of the breast

CASE: In January 2015, a 56-year-old nurse noted some asymmetry and skin retraction of her right lower breast. Under the care of her local provider, she underwent a screening mammogram which was unremarkable. Although reassured by this report, she was concerned about the self-detected changes in her breast and referred herself to our Diagnostic Breast Clinic. On breast examination, the right breast was smaller than the left. There was significant dimpling at the 6 o'clock position of the right breast, and in the area of the dimpling, a $3 \mathrm{~cm}$ palpable thickening was noted. The patient's outside mammogram was reviewed, her breast density was noted to be D3, and the mammogram was read as negative. She then underwent an ultrasound which showed a $3.3 \mathrm{~cm}$ region encompassing 2 hypoechoic shadowing areas which were ill defined. A unilateral right diagnostic mammogram demonstrated a $4 \times 3.5 \times 2.4 \mathrm{~cm}$ region of architectural distortion in the subareolar breast, corresponding to the sonographic findings. An ultrasound-guided core biopsy revealed an invasive lobular carcinoma (ILC), Nottingham grade I (of III). The tumor was ER, PR positive, HER 2 negative, and proliferation index Ki-67 1.7 \%.
Axillary ultrasound showed mildly prominent lymph nodes, subsequent FNA was negative. MRI showed a $7.4 \times 7 \times 3.5 \mathrm{~cm}$ non mass enhancement of the right breast. In early March 2015, the patient underwent a right-sided skin-sparing mastectomy and axillary lymph node dissection. Pathology showed a grade I, ILC $12 \times 8.2 \times 6 \mathrm{~cm}$, surgical margins were negative, $2 / 2$ sentinel lymph nodes were positive. Overall, 16 lymph nodes were positive for metastasis. The patient completed postmastectomy radiotherapy to the reconstructed chest wall and regional lymph nodes to a total dose of 50 Gy delivered in 25 fractions in May 2015. An Oncotype DX testing revealed a low score (7) consistent with her grade I, ILC and therapy with Letrozole was initiated.

DISCUSSION: This case is unfortunate in that the patient's breast cancer was only detected at a locally advanced stage although the patient had undergone regular annual screening. There are 2 factors contributing to the late detection: 1 ) the patient's breast density (D3) and 2) the histologic type of the tumor (infiltrating lobular). Breast density refers to the relative proportion of fat versus connective and epithelial tissues as depicted on mammography. It is most commonly placed into one of the following categories: D1-almost entirely fatty; D2scattered areas of fibroglandular density; D3-heterogeneously dense; D4-extremely dense. Increased breast density has been shown to increase the risk of breast cancer from 2.2 to fivefold when breasts with densest grades are compared with fatty breasts and decreases the sensitivity of mammography to detect small lesions with as few as $30-48 \%$ of cancers depicted in extremely dense breasts. As of January 2015, 21 states in the US have adopted dense breast legislation. These laws require that patients be informed of their breast density, that dense breast tissue may be a risk factor for breast cancer, and that dense tissue may interfere with cancer detection. Supplemental screening for patients with dense breasts includes digital breast tomosynthesis and molecular breast imaging (MBI). Digital breast tomosynthesis also known as "3-D mammography" has been approved by the FDA for routine clinical use as an adjunct to standard mammography. It is a modification of digital mammography and uses a moving x-ray source and digital detector. A three dimensional volume of data is acquired and reconstructed using computer algorithms to generate thin sections of images. The use of low-radiation-dose MBI for supplemental screening of women with mammographically dense breasts has been very helpful. Adding MBI to mammography increases the cancer yield from 3.2 per 1000 with mammography alone to 12.0 per 1000 . Breast MRI, the most sensitive screening modality for breast cancer and by far the most expensive one, is not indicated as a screening test for patients with dense breasts and no other risk factors. Besides breast density, tumor pathology significantly impacts mammographic breast cancer detection. Infiltrating ductal carcinoma is the most common type of invasive breast cancer, accounting for 70 to $80 \%$ of invasive lesions; ILCs are the second most common type of invasive breast cancer, accounting for about 5 to $10 \%$ of invasive lesions. ILC is an important cause of false negative mammograms. ILC tends to be mammographically subtle if it is seen at all. In one series, more than one of three mammographically depicted ILCs was seen as a vague asymmetry, poorly defined opacity, or area of possible architectural distortion. The poor visibility of ILCs may be partly related to their low density (mass/unit volume), which may also reduce the palpability of the tumor. ILCs may, however, be excellent absorbers and attenuators of ultrasound, causing significant shadowing in a high percentage of cases. The incidence of ILC is rising, and knowledge of its special features is therefore relevant. In summary, the combination of having dense breasts and the development of an invasive lobular rather than a ductal carcinoma led to the delayed diagnosis of breast cancer in the patient presented.

\section{EBV-ASSOCIATED SMOOTH MUSCLE TUMOR IN A PATIENT WITH AIDS}

Kirk E. Cahill; Amrish Deshmukh; Shannon Martin. University of Chicago, Chicago, IL. (Control ID \#2456864)

LEARNING OBJECTIVE \#1: Define Epstein-Barr virus-associated smooth muscle tumors (EBV-SMTs) 
LEARNING OBJECTIVE \#2: Describe the differential diagnosis of a soft-tissue mass in a patient with HIV/AIDS.

CASE: A 33-year old man with HIV/AIDS presented to the emergency department with right lower leg pain, swelling, and superficial ulceration secondary to scratching over the previous week. For the last year, he had a mass on his left upper arm with some tenderness. He was re-started on highly active antiretroviral therapy (HAART) 3 weeks prior with CD 4 count 1 cell $/ \mu \mathrm{L}$ and viral load 74,000 copies/mL. Diagnosed with HIV 8 years ago, he has been mostly nonadherent with medications. On exam, there was a $2 \mathrm{~cm}$ ulcer on the dorsum of the right foot with swelling and tenderness, but no erythema. At the right groin there was a $2.5 \mathrm{~cm}$ ulcer with purulent drainage and tender inguinal lymphadenopathy. On the lateral aspect of his left upper arm overlying the deltoid, there was a firm, tender, mobile mass $(3 \mathrm{~cm})$ without ulceration or warmth. Wound cultures from his foot grew methicillin-resistant Staphylococcus aureus (MRSA) sensitive to vancomycin and tetracycline. His groin ulceration and induration was suspicious for lymphogranuloma venereum (LGV), and he was empirically treated with a 21-day course of doxycycline to cover both LGV and MRSA. Bacterial and fungal blood cultures were negative. Blastomyces, coccidioides, histoplasma, RPR, and hepatitis serologies were negative. Ultrasound of the mass on his left arm showed vascularity, and MRI with contrast demonstrated a lobulated irregular mass $(4.2 \mathrm{~cm} \times 1.4 \mathrm{~cm} \times 3.8 \mathrm{~cm})$ along the lateral deltoid with heterogeneous T1 and T2 enhancement. Excisional biopsy revealed neoplastic cells staining positive for desmin and smooth muscle actin, and negative for $\mathrm{S} 100$ and CD31, stains evaluating for schwannoma or Kaposi sarcoma. In-situ hybridization for Epstein-Barr virus-encoded small RNA (EBER) was strongly positive and consistent with EBV-associated smooth muscle tumor (EBV-SMT). He was continued on HAART and 2 months later his CD4 count improved to 10 cells $/ \mu \mathrm{L}$ with a viral load of 30,000 copies $/ \mathrm{mL}$. After removal of the mass, he no longer had pain in his arm.

DISCUSSION: EBV-SMTs are rare neoplasms that occur in patients with AIDS or solid organ transplants. As more cases have been reported, EBV-SMTs are recognized as a class different from other SMTs given the association with immunosuppression, occurrence anywhere in the body at multiple sites, and low mortality from the tumor. While the mechanism of oncogenesis is unclear, EBV-SMTs are hypothesized to derive from smooth muscle cells of small vessel walls that become transformed after EBV infection. Interestingly, EBV-SMTs most commonly occur in the central nervous system where there is little smooth muscle. The next most common locations include soft tissue, lung, and liver. The majority of cases involve multi-focal lesions, which are considered separate infectious transformations rather than metastasis from a single tumor. While other foca EBV-SMTs in soft-tissue have been reported, few presented on the arm as in this case Patients typically present with pain related to the site of involvement or symptoms related to dysfunction of the organ involved. In this case, the patient presented with an unrelated MRSA infection of the leg and was found to have an EBV-SMT after noting the chronic painful mass on his arm. In addition to SMTs, the differential for a chronic soft tissue mass in the setting of immunosuppression is broad and includes: Kaposi sarcoma, bacillary angiomatosis, mycobacterium or fungal infection, lymphoma, leiomyoma, leiomyosarcoma, schwannoma, neurofibroma, lipoma, hemangioma, and osteosarcoma. Ultrasound and MRI narrow the diagnosis, but biopsy is required. For any SMT, testing for $\mathrm{EBV}$ is imperative given that EBV-SMTs vary in appearance ranging from leiomyoma or leiomyosarcoma to angioleiomyoma or myopericytoma. Despite the sarcoma-like histopathologic appearance and multi-focal nature, EBV-SMTs are slow growing with low tumor-related mortality. In contrast to SMTs in immunocompetent patients, mortality from the tumor is low at less than $10 \%$. Survival largely depends on the status of the underlying HIV infection. Surgical excision and HAART are the treatment of choice for EBV-SMTs. The benefit of complete versus partial removal is unknown, but complete removal is preferred when possible. This can be difficult in the setting of multi-focal disease. Surgical removal and adjuvant radiotherapy or chemotherapy have not been compared in the context of EBV-SMTs and must be decided on an individual basis.
Once diagnosed, a clinician should be suspicious of signs or symptoms suggestive of other lesions given their multiplicity. If resection is not possible, improving the CD4 count and reducing viral load with HAART alone may also reduce tumor burden. 1. Deyrup AT, Lee VK, Hill CE, Cheuk W, Toh HC, Kesavan S, et al. Epstein-Barr Virus-Associated Smooth Muscle Tumors Are Distinctive Mesenchymal Tumors Reflecting Multiple Infection Events. Am J Surg Path. 2006; 30(1): 75-82. 2. Hussein K, Rath B, Ludewig B, Kreipe H, Jonigk D. Clinico-pathological Characteristics of Different Types of Immunodeficiency-associated Smooth Muscle Tumours. Eur J Cancer. 2014; 50 (14): 2417-24. 3. Purgina B, Rao UN, Miettinen M, Pantanowitz L. AIDS-Related EBV-Associated Smooth Muscle Tumors: A Review of 64 Published Cases. Patholog Res Int. 2011: 1-10.

\section{EGGS TRAPPED IN THE LIVER: A MORBID CASE OF SCHISTOSOMIASIS}

Tatiana Teslova ${ }^{2}$; Arthur O. Omondi ${ }^{2}$; Sheira Schlair ${ }^{1} .{ }^{1}$ Albert Einstein College of Medicine/Montefiore Medical Center, Bronx, NY; ${ }^{2}$ Montefiore Medical Center, Bronx, NY. (Control ID \#2469729)

LEARNING OBJECTIVE \#1: Recognize pathophysiology and epidemiology of schistosomiasis

LEARNING OBJECTIVE \#2: Diagnose hepatosplenic schistosomiasis and recognize treatment options

CASE: A 54 year old man from Yemen presented with intermittent episodes of small volume hematemesis for 3 months. Medical history included schistosomiasis treated with praziquantel 4 years prior. Upper endoscopy revealed esophageal varices which were banded. Five days later he developed dizziness and had one episode of large volume hematemesis. He also reported abdominal distension and burning chest pain. Initial vital signs were stable. He appeared diaphoretic. Labs included hemoglobin $9.5 \mathrm{~g} / \mathrm{dL}$, hematocrit $34.1 \%$, platelets $188 \mathrm{k} / \mathrm{uL}$, total bilirubin $1.4 \mathrm{mg} / \mathrm{dL}$, AST $21 \mathrm{U} / \mathrm{L}$, ALT $11 \mathrm{U} / \mathrm{L}$, alkaline phosphatase $110 \mathrm{U} / \mathrm{L}$. Hepatitis $\mathrm{B}$ and $\mathrm{C}$ serologies were negative. Schistosomiasis serology was positive. Emergent EGD revealed active bleeding from prior banded varices, which was successfully controlled with additional banding and sclerotherapy. Abdominal CT showed hepatic cirrhosis, massive splenomegaly, paraesophageal varices and ascites, all suggestive of portal hypertension. Liver MRI revealed periportal fibrosis. On Hospital day \# 7, the patient had several more episodes of hematemesis with precipitous hemoglobin drop (nadir of $7.1 \mathrm{~g} / \mathrm{dL}$ ) and hemorrhagic shock. Four more endoscopies and multiple transfusions of blood products were required to control variceal bleeding. Because of the high risk of re-bleeding, he underwent emergent TIPS procedure. He had no further episodes of hematemesis and was discharged home 10 days later. Diagnosis of hepatosplenic schistosomiasis was made based on his constellation of symptoms and supporting imaging with serologic diagnosis of schistosomiasis.

DISCUSSION: Schistosomiasis is a prevalent disease worldwide but is infrequently seen by internists in the United States, except those who practice in immigrant rich areas. Affected regions in the world include Sub-saharan Africa, the Middle East, western South America and South Caribbean islands. The larvae of Schistosoma mansoni, the most common schistosome species, penetrate the skin of humans in contact with infested water. Most infected individuals do not develop systemic illness; however the repeated exposure to schistosome species can lead to chronic infection. Liver, spleen, intestinal tract, genitourinary tract, lungs and central nervous system are organs with potential involvement. About $5-10 \%$ of patients infected with schistosomiasis progress to the most severe form, hepatosplenic schistosomiasis. Clinical manifestations include periportal fibrosis, portal hypertension, splenomegaly and frequent episodes of gastrointestinal bleeding. Liver function is often preserved. Pathogenesis of hepatosplenic schistosomiasis involves induction of chronic inflammation by the eggs leading to tissue fibrosis. Within few weeks 
of infection, parasites migrate to the portal and mesenteric veins of their host, where they mature into male or egg-laying female worms. Most eggs pass into the intestines, but some are taken by the portal vein blood flow into the liver, where they get deposited in small vessels, causing inflammatory granuloma. The granuloma causes cell-mediated immune response with subsequent collagen deposition and periportal fibrosis, also known as "Symmers fibrosis". Splenomegaly often develops due to fibrosis, egg obstruction and from hyperplasia of cells of the reticuloendothelial system, induced by immunological stimulation due to antigens released by the worms and eggs. As our patient's case demonstrated, variceal bleeding is the most morbid complication and the primary cause of death in hepatosplenic schistosomiasis with rates approaching 10-20\%. Beta blocker prophylaxis, endoscopic banding or sclerotherapy are shown to reduce the bleeding risk. In patients with refractory bleeding effective treatment options include splenectomy with esophagogastric devascularization. Interventional therapy such as TIPS or splenorenal shunts should only be considered for patients with failed endoscopic therapy because of the high risk of encephalopathy. Liver function is usually preserved in patient with schistosomiasis; therefore shunting of the blood away from the liver may cause encephalopathy. Antihelminthic therapy with praziquantel may reverse early stages of periportal fibrosis and portal hypertension. However, as in our patient's case, antihelminthic therapy is not beneficial for reversing late-stage fibrosis, as he had previously taken praziquantel however was noted to have significant fibrosis on advanced imaging. This case of advanced hepatosplenic schistosomiasis illustrates a few central learning points for the general internist: Schistosomiasis should always be considered in the differential diagnosis of non-cirrhotic portal hypertension (particularly in appropriate ethnic populations), the most morbid presentation is that of profound variceal bleeding (which may require emergent intervention e.g. TIPS), and early treatment of schistosomiasis is paramount in preventing late stage fibrosis and other non-cirrhotic portal hypertensive complications.

\section{EHLERS DANLOS SYNDROME, NOT SIMPLY A JOINT PROBLEM Ashley M}

Castillo; Robin Klein. Emory University, Atlanta, GA. (Control ID \#2469386)

LEARNING OBJECTIVE \#1: Review the clinical features of Ehlers Danlos Syndrome LEARNING OBJECTIVE \#2: Discuss the diagnosis of Ehlers Danlos Syndrome

CASE: 28 year old female presents with weight loss, weakness and fatigue. She reported dysphagia to both solids and liquids and decreased taste with poor appetite for the last several years. She started to lose weight ultimately losing 80 pounds over 2 years. As her weight loss progressed, she began to have generalized weakness with difficulty walking and multiple falls. Ultimately severe malnutrition required gastric tube placement for supplemental nutrition. She also reported longstanding constipation alternating with diarrhea and chronic joint hypermobility and joint dislocation. Past medical history was notable for gastroparesis, abdominal hernia, and mild cognitive impairment. Family history included hypermobile joints in her grandmother, mother, and brother. She had an extensive work-up at multiple outside institutions over the last 2 years including unremarkable EMG, MRI brain/spinal cord, HIDA scan, lumbar puncture, infectious disease evaluation, autoimmune panel, EGD and colonoscopy evaluation without a clear diagnosis. In the past her symptoms were attributed to anorexia nervosa or psychosomatic disorders as a cause for her significant weight loss and failure to thrive. Exam was notable for an extremely cachectic appearing female. She had decreased strength in her upper and lower extremity with atrophy of her extremities with normal tone. She had impaired sensation in the feet and hands to light touch and pinprick. She had hypermobility of multiple joints without evidence of skin hyperextensibility. Laboratory studies were unremarkable. Based on the clinical history and exam findings, she was diagnosed with Ehlers Danlos Syndrome Type III (Hypermobility Type).

DISCUSSION: Ehlers Danlos Syndrome is an autosomal dominant connective tissue disease than affects as many as one in five thousand individuals. Clinical history may include complaints of joint pain affecting multiple joints and recurrent dislocation. A majority of patients experience chronic fatigue and chronic pain which can cause significant disability and may be the most bothersome complaint to the patient. Other common manifestations include poor wound healing with frequent bruising. Neurological complaints may also include neuropathies, impaired motor development, gait instability, headache, mild cognitive impairment and myalgias with accompanying weakness. Gastrointestinal manifestations are common and can incorporate abdominal hernia, functional bowel disorders such as dysphagia, decreased taste, reflux, chronic constipation, IBS, and poor nutritional status. Diagnosis is largely based on clinical history with family history being a key component, as well as physical examination. Genetic markers have been identified for some subtypes but are not required for diagnosis. Diagnosis for Ehlers Danlos Syndrome is differentiated by subtype but includes both major and minor criteria. Major criteria include skin involvement with hyperextensibility, generalized joint hypermobility, and tissue fragility (widened atrophic scars). Minor criteria can include recurrent joint dislocation, subcutaneous spheroids, chronic joint/limb pain, molluscoid pseudotumors, and a positive family history for joint complaints. Other reported associated symptoms may include functional bowel disorders, gastrointestinal dysautonomia, easy bruising, muscle hypotonia, hernias, mitral valve prolapse, or aortic dilation. This case highlights the constellation of systemic manifestations seen in Ehlers Danlos Syndrome. Due to variability and nonspecific nature of many of the systemic findings, diagnosis often being missed, delayed or symptoms incorrectly attributed to psychosomatic illness. Physicians need to be aware of the range of clinical manifestations of Ehlers Danlos Syndrome. In this case, a comprehensive history rather than extensive tests was key to making the diagnosis.

EIK! AN UNUSUAL CAUSE OF ABSCESS Savan Shah; Deepa Bhatnagar. Tulane University Health Sciences Center, New Orleans, LA. (Control ID \#2467561)

LEARNING OBJECTIVE \#1: Recognize uncommon organisms as causes of abscess in IV drug users

LEARNING OBJECTIVE \#2: Illustrate need for obtaining history in patients suspected of IV drug use

CASE: A 33-year-old man presented to the ED with a left antecubital arm wound. He reported that during the week prior to presentation he was helping his mother in her attic when he punctured his arm on a nail. He did not seek immediate medical attention. In the interval period, his arm became red and painful. The wound developed purulent drainage, prompting him to seek medical attention. He reported a fever of 100.6 at home. At the time of admission, he endorsed using marijuana and denied using intravenous drugs. His past medical history included a diagnosis of chronic SVT controlled with propafenone. On presentation, the patient was afebrile, with a BP of $97 / 54$, pulse of 69 and a respiratory rate of 16. Examination of the left antecubital fossa revealed a $1 \mathrm{~cm} \times 1 \mathrm{~cm}$ puncture wound with yellow pus actively draining with surrounding erythema and tenderness. No other cutaneous findings were noted. Initial labs showed a mild leukocytosis with WBC of 12.6. $\mathrm{X}$-rays did not demonstrate the presence of a foreign body or bony erosions. Initial gram stain of the cutaneous wound showed numerous gram positive cocci and gram variable rods. Given progression of the wound, he was hospitalized and was treated with intravenous clindamycin and ciprofloxacin for an antecubital fossa abscess and surrounding cellulitis. For pain management he was initially started on ibuprofen and oxycodone with morphine for breakthrough pain. After stating that he was not opioid-naïve and that his pain was not controlled, he asked for hydromorphone for pain management. Given the location of the abscess and the likely need for incision and drainage, surgery was 
consulted. In the operating room, surgery performed an I\&D. During drainage of the abscess, a puncture wound was seen over the cephalic vein and the vein appeared to be necrotic with a thrombus. Post-operatively, the patient recovered well. Two days later, on the day of discharge, the patient's aerobic cultures returned, demonstrating heavy Eikenella corrodens and moderate Haemophilus influenzae. The patient was sent home on oral clindamycin and ciprofloxacin.

DISCUSSION: General internists frequently encounter intravenous drug users in the hospital. These patients are at risk for cutaneous complications and are particularly susceptible to formation of cellulitis and abscesses. The risk factors for abscess formation in IV drug users include unsanitary equipment, the lack of skin asepsis, and even the drugs themselves have shown to be sources of pathogens. The antecubital fossa is a common site of these abscesses due to involvement of the cephalic vein. While Staph aureus and gram positive organisms are commonly considered causes of abscesses, gram negative flora may also be pathogens in IV drug users. Eikenella corrodens and Haemophilus parainfluenzae are members of the HACEK group and are common human oral gram negative flora. In the literature, and as seen with our patient, the cause of the infection seems to be from a "needle-lick." Though the patient was appropriately covered for gram positive cocci and gram negative rods, an earlier recognition of possible IV drug use as a cause of his abscess could have furthered this patient's care. This patient had contextual clues indicating IVDU, including cephalic vein involvement and asking for hydromorphone by name. He also changed his story multiple times when talking to different providers. Despite the difficulty obtaining history from this patient, the burden to obtain proper history in order to provide proper care is on the providers. Important techniques when interviewing patients suspect of IVDU include ensuring privacy, approaching the patient with a non-judgmental line of questioning, understanding the patient's social context and obtaining collateral history if necessary. Due to the increased prevalence in IV drug users, correct identification of these patients can help further screen patients for HIV, and Hepatitis B and C as part of our duty as physicians to improving public health.

ELDERLY ONSET OF DIABETES MELLITUS TYPE 1 Ryosuke Minami ${ }^{2}$; Shadia Constantine ${ }^{1}$. ${ }^{1}$ Teine Keijinkai Hospital, Sapporo, Japan; ${ }^{2}$ Teine Kejinkai Hospital, Sapporo, Japan. (Control ID \#2468288)

LEARNING OBJECTIVE \#1: Recognize that the elderly can develop type 1 DM LEARNING OBJECTIVE \#2: Describe characteristic of the elderly that should influence the choice of treatment of their DM type 1

CASE: A 78-year-old Japanese woman arrived to the emergency room via ambulance with chest pain, confusion and generalized weakness of 1-day duration. The patient admitted malaise, headache and cervical pain for 5-days before admission. The patient's past medical history included an episode of acute pancreatitis 1-month prior. In addition, she suffered from chronic kidney disease, anemia of chronic disease, rheumatoid arthritis, liver cirrhosis, hypertension and gastric cancer treated with endoscopic removal. She did not drink alcohol, smoke or use recreational drugs. On physical exam, her vital signs showed hypotension $(80 / 60 \mathrm{mmHg})$, hypothermia ( $\mathrm{T} \mathrm{35.6)}$ bradycardia (50/min) and tachypnea (30/min). Her oxygen saturation was normal on room air. The patient looked sick, she was obtunded. Her oral mucosa was very dry. Respiratory sounds were clear bilaterally. Her abdomen was soft, flat and appeared non-tender. Her lower extremities showed pitting edema. Her GCS was E1 V4 M5. Cranial nerves were intact. Her IVC was collapsed according to a bedside US examination. Labs showed leukocytosis $(16790 / \mathrm{mcL}$, ) anemia $(8.9 \mathrm{mg} / \mathrm{dL}$,) prolonged INR $(6.86$,) elevated amylase (503 IU/L) BUN $(66.3 \mathrm{mg} / \mathrm{dL}$,) and potassium $(8.4 \mathrm{mEq} / \mathrm{L})$ and decreased sodium (124 mEq/L.) Her blood glucose was 1127 and interestingly, her HbAlc was 5.8 .
Urinalysis showed $(+)$ protein, $(4+)$ glucose, $(2+)$ ketone and $(+)$ occult blood. She received fluid resuscitation but still required pressure support with norepinephrine. Her hyperkalemia was treated with calcium gluconate infusion and continuous drip of insulin; however, she still developed bradycardia and then atrial fibrillation. Hyperkalemia and hyperglycemia were eventually controlled with continuous drip of insulin. Three days after admission, it was found that her C-peptide was very low $(0.3 \mathrm{ng} / \mathrm{ml})$ and her anti-GAD-antibody was positive $(3.3 \mathrm{U} / \mathrm{ml})$. At this point, she was diagnosed with immune mediated diabetes (Diabetes mellitus Type 1). It was difficult to obtain stability of her glucose levels even with the insulin drip and we were concerned about her ability to use insulin injections. She developed several episodes of nocturnal hypoglycemia, which were thought to be associated with her liver cirrhosis and renal failure. Our patient was unable to continue living by herself due to this new diagnosis. Fortunately, her daughter was able to move with her to help with the injections of insulin. Nocturnal hypoglycemia was prevented with the introduction of nighttime snacks.

DISCUSSION: Immune-mediated diabetes commonly develops in childhood and adolescence, but it can occur at any age, even in the 8th and 9th decades of life, though few cases of such late onset have been reported in the literature. Patients with autoimmune destruction of beta cells are also susceptible to other autoimmune disorders because of multiple genetic predispositions and environmental factors that are still poorly defined. This patient had RA before developing DM, and during her hospitalization, she was also diagnosed with Sjogren's syndrome. Given that she had multiple autoimmune diseases, she most likely had a predisposition to immune mediated diabetes. In adult patients with DM type 1 , intensive insulin therapy is strongly recommended. Intensive insulin therapy is defined as the use of basal insulin once a day + nutritional insulin three times a day. However, introduction of insulin therapy in the elderly is difficult not only because they seem to be more sensitive to its effects, but also because of the multiple comorbidities they usually have that alter the metabolism of insulin. They also might not have the cognitive ability or manual dexterity to apply the injections by themselves. This is why family support is critical since they can administer the insulin and monitor for the development of hypoglycemia and extreme hyperglycemia. According to Up-to-date, the target A1C for adults with DM is $7 \%$ (using an assay aligned to the Diabetes Control and Complications Trial $[\mathrm{DCCT}]$ in which the upper limit of normal is $6.0 \%$ ) or lower in patients in whom the benefits outweigh the risks. Glycemic targets are generally set somewhat higher (eg, $<8 \%$ ) for older patients and for those with comorbidities or a limited life expectancy and little likelihood of benefit from intensive therapy. In our patient, the target fasting and postprandial blood glucose levels were set around $200 \mathrm{mg} / \mathrm{dL}$ $250 \mathrm{mg} / \mathrm{dL}$ with insulin therapy. According to a certain study, HbAlc is predicted to be $8-9 \%$ or over $9 \%$ with this blood glucose range. It is thought that by achieving tight glucose control with insulin, we can prevent complications including, retinopathy, renal failure and neuropathy. But those complications usually develop over a decade. Also, close insulin control is necessary to prevent overdose and subsequent hypoglycemia, which sometimes leads to death. Given that her capacity of glucose storage is very low and that the metabolism of insulin is prolonged due to liver cirrhosis and renal failure, a high glucose target seemed appropriate for her.

ELECTRICAL STORM IN A MAN WITH A PRIOR MYOCARDIAL INFARCTION Christopher J. Westley ${ }^{1}$; Thomas Middour ${ }^{2} .{ }^{1}$ Tulane University Medical Center, New Orleans, LA; ${ }^{2}$ Tulane University School of Medicine, New Orleans, LA. (Control ID \#2470479)

LEARNING OBJECTIVE \#1: Define electrical storm, list the causes, and recognize the need for urgent workup and treatment 
LEARNING OBJECTIVE \#2: Manage electrical storm using a multi-tiered approach including ACLS guidelines, current pharmacologic regimens, implantable cardiac defibrillators (ICDs), and catheter ablation

CASE: A 67 year old gentleman with a history of coronary artery disease presented to the hospital after an episode of acute shortness of breath for ten minutes. His initial rhythm strip from EMS showed monomorphic ventricular tachycardia that spontaneously converted to atrial fibrillation. Upon arrival, vital signs were stable and he was loaded with amiodarone $150 \mathrm{mg}$ IV. He was sent to the cardiac care unit for monitoring and was started on an additional intravenous amiodarone load of $900 \mathrm{mg}$ over $24 \mathrm{~h}$ and an oral load of $400 \mathrm{mg}$ four times daily. A work up for new ventricular tachycardia was unrevealing. Serial troponins were negative as were blood and urine cultures and drug screens. Electrolytes and thyroid function tests were normal. An echocardiogram showed an ejection fraction of $45 \%$, unchanged from previous. Less than 1 day after admission, the patient developed sustained monomorphic ventricular tachycardia with stable vital signs. An additional load of amiodarone $150 \mathrm{mg}$ IV over $10 \mathrm{~min}$ and then metoprolol $5 \mathrm{mg}$ IV over five minutes failed to convert the arrhythmia. The patient's mental status waned prompting a DC shock of $100 \mathrm{~J}$, which did not affect the rhythm. Lidocaine $100 \mathrm{mg}$ IV converted the patient to atrial fibrillation and his mental status improved. One further episode of ventricular tachycardia resolved with an additional intravenous infusion of lidocaine. The patient returned to sinus rhythm and was transitioned to amiodarone $400 \mathrm{mg}$ and metoprolol succinate $50 \mathrm{mg}$ orally once per day. He was sent home after receiving a single chamber automated implantable cardioverter defibrillator.

DISCUSSION: Electrical storm has been defined as frequent and recurrent episodes of ventricular tachycardia or ventricular fibrillation within $24 \mathrm{~h}$, which may result in significant symptoms or hemodynamic compromise. Its poor prognosis mandates immediate investigation of the cause. Work up should include a history and physical including possible offending medications, a work up for myocardial infarction (MI) and new or worsening heart failure, serum electrolyte and thyroid function tests, and drug screen and infectious workup if warranted. In our case, this workup was unrevealing and old scar from a prior MI caused electrical storm. Management of electrical storm requires a multitiered approach. Addressing any underlying etiology, if found, will often solve the arrhythmia. This includes urgent coronary revascularization if there is suspicion of new or ongoing MI. Hemodynamically unstable patients with ventricular arrhythmias should initially undergo cardioversion or defibrillation per advanced cardiac life support protocol Because of the adrenergic surge associated with recurrent ventricular arrhythmias and shocks, hemodynamically stable patients with electric storm should receive beta blocker and antiarrhythmic medications in accordance with ACC/AHA/ESC guidelines. Amiodarone, a class III antiarrhythmic drug, has been shown to reduce mortality in this setting and is the first line antiarrhythmic of choice. Lidocaine, a class Ib antiarrhythmic drug, can help suppress refractory arrhythmias, as with our patient. While ICD placement does not resolve electrical storm, consultation with an electrophysiologist is important to determine if the patient will benefit. In patients with electrical storm that persists despite medical therapy, catheter ablation is recommended.

ELECTRO CONVULSIVE THERAPY TRIGGERED VARIANT OF TAKOTSUBO CARDIOMYOPATHY Charudatta Wankhade ${ }^{2}$; Chandana Shekar ${ }^{1}$. ${ }^{1}$ University of Connecticut School of Medicine, Farmington, CT; ${ }^{2}$ Lincoln Medical and Mental Health Center, Bronx, NY. (Control ID \#2470132)

LEARNING OBJECTIVE \#1: Early recognition of Electroconvulsive therapy as a precipitating factor for Stress-Induced Cardiomyopathy

LEARNING OBJECTIVE \#2: Recognizing echocardiogram findings of variants of stress induced cardiomyopathy
CASE: A 59 year old female patient, with past medical history of depression, chronic obstructive pulmonary disease and hyperlipidemia was brought to our hospital after an episode of syncope. She was admitted to the psychiatric hospital 3 days ago for treatment of severe depression and underwent Electro Convulsive Therapy (ECT) for the same. She was noted to have a syncopal episode the following day and was transferred to our hospital for further evaluation. She did not have any symptoms on presentation, including chest pain, shortness of breath or dizziness. She was noted to be hemodynamically stable with a normal physical examination. Initial blood investigations revealed a normal complete blood count, basic metabolic panel, coagulation panel and cardiac markers. B-type natriuretic peptide was elevated at $331 \mathrm{pg} / \mathrm{ml}$. Chest $\mathrm{X}$ ray did not show any acute cardiopulmonary process. Electrocardiogram (EKG) showed normal sinus rhythm with new T wave inversions in the precordial leads and QT prolongation. Given the new EKG changes and syncope, an echocardiogram was performed for further evaluation. It showed severe left ventricular systolic dysfunction with an ejection fraction of $30 \%$. There was hypokinesis of the mid ventricle, but normal kinesis of basal myocardium as well as distal apex. She underwent cardiac catheterization which showed normal coronary arteries with a normal left ventricular end diastolic pressure. Left Ventriculography performed at that time showed mid ventricle akinesis, which was consistent with a variant of stress-induced cardiomyopathy, similar to inverted-takotsubo cardiomyopathy. She was started on low dose beta blockers and angiotensin converting enzyme inhibitors, and she did fairly well. A correlation between the stress cardiomyopathy and ECT was suspected given the timeline of events and she was advised against ECT for the future. She was doing fairly well from a cardiac and psychiatric standpoint and was discharged home. Repeat echocardiogram on follow up visit after 2 months showed a left ventricular ejection fraction of $60-65 \%$ and no wall motion abnormalities. Hence, it was concluded that the stress induced cardiomyopathy was trigerred by ECT.

DISCUSSION: Stress-induced cardiomyopathy is an enigmatic entity characterized by acute, reversible and transient left ventricular systolic dysfunction that resembles acute coronary syndrome but does not show significant stenosis on coronary angiography. There are a few variants to this condition, including Takotsubo Cardiomyopathy which is characterised by regional or global hypokinesis of the left ventricle with a relatively hypercontractile base. An Inverted-Takotsubo pattern is noted when there is midventricular hypokinesis, like our patient with sparing or hyperkinesis of apex. It may rarely occur as a complication of ECT. There are many factors that make patients who get ECT susceptible to stress induced cardiomyopathy. The coexistence of emotional distress, pharmacological therapy that modifies the cellular metabolism of catecholamines and associated surge of central nervous system and systemic catecholamines during the grand mal seizure triggered by ECT treatments places these patients at increased risk for stress cardiomyopathy. The clinical observation of extreme emotional trauma, syncope, female sex and subtle EKG changes are non-specific indicators currently available to indicate susceptibility to this rare complication of ECT. ECT at that point is discontinued and the patient closely followed up for reversal of the cardiomyopathy. Hence, it is vital that the risk factors are identified early so as to prevent significant morbidity and mortality in these patients.

ELEVATED CARDIAC TROPONINS IN MULTIPLE MYELOMA Lindsey Aurora; Alexander Michaels. Henry Ford Hospital, Detroit, MI. (Control ID \#2465441)

LEARNING OBJECTIVE \#1: Cardiac troponins represent a biochemical marker in the detection of myocardial damage. However, recognize that elevated troponins do not always indicate a diagnosis related to acute coronary syndrome.

LEARNING OBJECTIVE \#2: Identify etiologies of hyponatremia in asymptomatic patients before institution of therapy. 
CASE: 54-year-old male with a past medical history significant for multiple myeloma on treatment presented to his cardiologist for a 3 month history of dyspnea on exertion and exertional chest pain, which was relieved by rest. He had a recent left heart catherization with no obstructive disease and cardiac stress test, which was negative for ischemia - though at submaximal stress. The patient was on Metoprolol and Isosorbide Dinitrate with no change in symptoms. While at the cardiology clinic, he experienced chest discomfort while walking from the clerk's desk to the clinic room. EKG showed sinus tachycardia with a new $2 \mathrm{~mm}$ ST depression on lateral leads. Patient was thereafter admitted to the telemetry floor. Troponin levels were elevated initially to 1.1 with a slow rise to 2.2 over $24 \mathrm{~h}$ with no dynamic EKG changes and resolved chest pain. Cardiology consult was obtained and a 2D echocardiogram showed preserved ejection fraction with no wall motion abnormalities. Cardiac magnetic resonance imaging showed no evidence of infiltrative disease. The patient remained free of chest pain during the hospitalization and medical management was continued. Of note, patient had a history of IgG lambda multiple myeloma, stage IIIA, diagnosed 5 years prior, status post multiple bouts of chemotherapy and autologous transplant with partial remission. The patient had known prior lytic lesions of the lower extremities and despite resumption of chemotherapy, had evidence of disease progression. There were new findings on recent magnetic resonance imaging revealing lesions on the left chest wall, ribs, and spine. He was on a trial of Daratumumab at presentation. During the admission, globulin levels peaked at 9.1 - total protein of 12.9 - and he was also found to have asymptomatic hyponatremia as low as 128 from prior of 140 . Hematology was consulted for management of multiple myeloma and pulse Decadron was administered for 4 days with plan at discharge for weekly Procrit. Close outpatient Hematology follow-up was scheduled.

DISCUSSION: Cardiac troponins serve as the most sensitive test for myocardial damage. ${ }^{1}$ Elevations of this biochemical marker do not only occur in acute coronary syndrome but also in various other settings such as pulmonary embolism, chronic kidney disease, sepsis, and cardiomyopathy. This case illustrates elevated troponin concentrations associated with progression of multiple myeloma and elevated globulin levels. The mechanism is hypothesized to be cardiac troponins being sequestered as a high molecular mass enzyme complex with serum IgG in patients with mutiple myeloma. ${ }^{2}$ Therefore, it is important to differentiate true troponin elevations due to myocardial injury and proteins such as IgG leading to false-positive results in the clinical setting. Review of the literature leads us to believe this is only the third documented case of troponin elevation due to high serum globulins. The alternative rise of cardiac troponins in myeloma is well documented and due to myocyte compression injury and infiltrative cardiomyopathy by amyloid proteins. ${ }^{3}$ Cantwell et al. presented a case of multiple myeloma and elevated troponins with myocardial ischemia resulting from the toxic effects of amyloid proteins. ${ }^{3}$ Cardiac function can be subsequently compromised due to potential injury. However, it is important to keep in mind that elevations in troponin can also be secondary to microvascular coronary ischemia, explained by the presenting anginal symptoms in our case report. Elevations in serum proteins can also have implications on concentrations of electrolytes. In this case, laboratory abnormalities included elevated globulins and hyponatremia. Patient remained asymptomatic throughout the admission. Multiple myeloma is defined as uncontrolled proliferation of plasma cells resulting in secretion of Ig or paraproteins. Increased proteins in the serum can cause pseudohyponatremia due to increased intravascular oncotic pressure. ${ }^{4}$ Non-aqueous components such as triglycerides or proteins usually constitute $7 \%$ of normal plasma volume. ${ }^{4}$ Elevations in paraproteins such as in multiple myeloma displace plasma water, subsequently resulting in a falsely low sodium. Appropriate assessment of electrolyte abnormalities requires further characterization based on the patient's medical history before instituting therapy. References 1 . Zabernigg A, Schranzhofer R, Kreczy A, Gattringer K. Continuously elevated cardiac troponin I in two patients with multiple myeloma and fatal cardiac amyloidosis. Ann Oncol. 2003;14(12):1791. 2. Domanski O, Maréchaux S, Forzy G, Lemahieu JM. An unexpected rise in cardiac troponin I in a patient with multiple myeloma. BMJ Case Rep.
2012;2012. 3. Cantwell RV, Aviles RJ, Bjornsson J, et al. Cardiac amyloidosis presenting with elevations of cardiac troponin I and angina pectoris. Clin Cardiol. 2002;25(1):33-7. 4. Mirvis E, De-silva D, Mehta A. Hyponatraemia in patients with multiple myeloma. BMJ Case Rep. 2015;2015.

ELEVATED CPK FOR AN INTERNIST: A CASE OF IDIOPATHIC INFLAMMATORY MYOPATHY Hina N. Khan ${ }^{1}$; Usman K. Jilani ${ }^{2}$; Shitij Arora ${ }^{1}$. ${ }^{1}$ Albert Einstein- Montefiore Medical Center, Bloomfield, NJ; ${ }^{2} \mathrm{New}$ York Institute of Technology College of Osteopathic Medicine, Long Island, NY. (Control ID \#2462333)

LEARNING OBJECTIVE \#1: Recognize idiopathic inflammatory myopathy as a potential cause of myasthenia

LEARNING OBJECTIVE \#2: Describe the current treatment and treatment response in polymyositis.

CASE: A 64-year-old man presented with 3 weeks of progressive proximal muscle weakness causing him difficulty ambulating, combing his hair, and raising himself from a seated position. He also reported dysphagia to solids over the past week. The patient was recently admitted twice to an outside hospital for an elevated CPK, and diagnosed with "rhabdomyolysis." Past medical history was significant for poorly controlled diabetes mellitus. There was proximal muscle weakness to $4 / 5$ bilaterally in the shoulders and hips. Hemoglobin was $10.5 \mathrm{~g} / \mathrm{dL}$ with a normal MCV, and CPK was $2705 \mathrm{U} / \mathrm{L}$. ESR and CRP were within normal limits and RF, anti-Ro/La, anti-Jo1, anti-RNP, anti-centromere, antiScl-70, and anti-dsDNA were all negative. An MRI of the right arm revealed prominent edema involving the deltoids and an MRI of the bilateral thighs revealed myositis with no discrete collections. An EMG showed evidence of irritative myopathy affecting the bilateral deltoids. A muscle biopsy of the right deltoid was negative for inflammation but presumed to not be representative. He was started on Prednisone $60 \mathrm{mg}$ with resolution of symptoms within 1 month of starting therapy.

DISCUSSION: Myasthenia, or simply muscle weakness, is a common admitting diagnosis in the inpatient setting with a broad differential. However, in the setting of an elevated CPK the differential diagnosis for myasthenia is narrowed to rhabdomyolysis or a myopathy. While acute exertional rhabdomyolysis can be diagnosed by history, the syndrome of idiopathic inflammatory myopathies must be differentiated from myopathies caused by infections, toxins, paraneoplastic syndrome, and endocrinopathies. Idiopathic inflammatory myopathy encompasses systemic rheumatic diseases including polymyositis and dermatomyositis. Our patient met three out of the four criteria for polymyositis per the American College of Rheumatology including symmetric proximal muscle weakness, elevation of skeletal muscle enzymes, and an abnormal EMG showing polyphasic, short, small motor unit potentials, fibrillation potentials, positive sharp waves, and repetitive high-frequency discharges, suggesting a probable diagnosis of polymyositis. The final criteria needed for definitive diagnosis is an abnormal muscle biopsy with histopathologic findings of degeneration, regeneration, necrosis, and interstitial mononuclear infiltrates; in this case, muscle biopsy was presumed not representative. However, several epidemiologic features make this a particularly difficult diagnosis in this patient. Foremost, polymyositis is a rare disease with incidence rates occurring in about 1 per 100,000 people annually. Additionally, polymyositis is seen twice as commonly in women than in men. Furthermore, although polymyositis can occur at any age, it typically peaks in the 30 50 year age range, and our patient had a later age of onset. This case also highlights the low sensitivity of serologic tests including anti-Jo-1, anti-Scl-70, and anti-RNP which are prevalent in 21,6 , and $5 \%$ of patients with polymyositis, respectively. However, when considering a case of myasthenia with an elevated CPK, some key features can be used to differentiate polymyositis from other diagnoses. These include chronicity of symptoms, presentation of symmetric proximal muscle weakness, and presence of bulbar features, which can be seen in polymyositis, dermatomyositis, or inclusion body disease. Other 
systemic signs including rash seen in dermatomyositis, or Raynaud Phenomenon seen in scleroderma or CREST can also aid in correctly arriving at a diagnosis. Steroids remain the cornerstone of treatment in polymyositis although lack of response as high as $40 \%$ has been reported. Such a high non-response rate may partly be due to misdiagnosis of inclusion body myositis as polymyositis. Studies are ongoing to identify specific mechanisms for steroid resistance with higher expression of proinflammatory molecule "granulysin" in CD8+ T cells and glucocorticoid receptor polymorphisms as possible explanations. In conclusion, polymyositis is a rare disease with several epidemiologic features and serologic markers which are neither sensitive nor specific, but with key history and physical exam findings that can help arrive at a diagnosis of polymyositis when confronted with a case of myasthenia and an elevated CPK, and thus aid in promptly initiating appropriate therapy.

EMPHASIZING EMPYEMA：THE GROWING CONCERN OF STREPTOCOCCUS CONSTELLATUS Vincent Chau; Jason A. Korcak. Montefiore Medical Center, New York, NY. (Control ID \#2469520)

LEARNING OBJECTIVE \#1: Identify the factors that impede resolution of pneumonia. LEARNING OBJECTIVE \#2: Recognize the growing significance of streptococcal, non-pneumococcal empyemas.

CASE: A 58-year-old man presented with 1 month of productive cough, dyspnea, leftsided pleuritic chest pain, malaise, and recent weight loss. The patient had a history of poorly-controlled diabetes mellitus. Physical exam, as well as chest x-ray and computed tomography were consistent with pulmonary consolidation. The patient was discharged and completed a 10-day course of levofloxacin. He presented 17 days later with persistent symptoms. The patient was febrile to $100.1^{\circ} \mathrm{F}$ with a heart rate of $123 \mathrm{bpm}$. He had decreased breath sounds, egophony, and dullness to percussion at the left lower lung field. White blood cell (WBC) count was 19,400 cells/uL. Chest radiograph revealed a left lower lobe airspace opacity and pleural effusion. He was treated with piperacillin tazobactam, but WBC count continued to rise. Computed tomography of the chest performed 4 days later demonstrated a left pleural fluid collection concerning for empyema. Tube thoracostomy removed $250 \mathrm{~mL}$ of pus with $\mathrm{pH} 6$, LDH $21,540 \mathrm{U} / \mathrm{L}$, WBC count 445,800 cells/uL with $94 \%$ polymorphonuclear (PMN) cells, and Gram-positive cocci in pairs. Pleural fluid culture subsequently grew Streptococcus constellatus.

DISCUSSION: Resolution of community-acquired pneumonia typically follows a timeline, including an initial improvement in hemodynamics, followed by the resolution of cough and fatigue within several weeks, and chest radiograph normalization after approximately 1 month. Non-resolution or slow resolution is defined as persistence of radiographic evidence beyond the expected time course. There are multiple factors that contribute to delayed resolution, including comorbid conditions like diabetes mellitus and immunosuppression, lifestyle factors such as alcohol dependence, and characteristics of the infectious organism. In addition, parapneumonic effusions develop in $20-40 \%$ of patients with acute bacterial pneumonia. While most of these effusions resolve with antibiotic therapy, empyema formation occurs in a subset of patients. The rate of parapneumonic empyema-related hospitalization increased from 2.04 per 100,000 in 1996 to 5.98 per 100,000 in 2008 , while the rate of parapneumonic empyema causing hospital death increased from 0.24 per 100,000 in 1996 to 0.43 per 100,000 in 2008 . Reasons for this increase are unclear, but may be related to the spread of antibioticresistant bacteria or more frequent diagnosis from increased use of computed tomography. The causative organisms for empyema include aerobic bacteria in $64 \%$ of patients, $13 \%$ anaerobic, and $23 \%$ mixed aerobic and anaerobic. In addition, streptococcal, nonpneumococcal empyema rates have increased 1.9-fold between 1996 and 2008 with the greatest absolute increase in those greater than age 65. S. constellatus is a nonpneumococcal streptococcus that is part of the normal oral flora, but also a clinically- important cause of empyema. Classically, S. constellatus is associated with polymicrobial abscesses that present in the mouth, throat, neck, brain, and perirectal/perianal regions. $S$. constellatus is often isolated along with other oral anaerobes. In mice models, S. constellatus synergizes with the anaerobe Prevotella intermedia to produce a $60 \%$ mortality rate compared to $10 \%$ with each organism alone. The presence of both organisms also delays microbial clearance. In patients with persistent symptoms despite treatment of pneumonia, clinicians must always consider empyema formation, as well as the increasing presence of streptococcal, non-pneumococcal pathogens such as S. constellatus.

EMPHYSEMATOUS PYELONEPHRITIS, A RARE DISEASE WITH A COMMON PRESENTATION Somwail Rasla ${ }^{1,3}$; Marina Garas ${ }^{2}$; Saira Imran ${ }^{1,3}$; Patrick KOO ${ }^{1,3}$. ${ }^{1}$ Memorial Hospital, Pawtucket, RI; ${ }^{2}$ Roger Williams Medical Center, Providence, RI; ${ }^{3}$ Brown University, Providence, RI. (Control ID \#2455525)

LEARNING OBJECTIVE \#1: 1- Recognize that DKA by itself may be a symptom of underlying catastrophe

LEARNING OBJECTIVE \#2: 2- This case demonstrates the presentation, diagnosis, and management of Emphysematous Pyelonephritis.

CASE: A 55-year-old Caucasian female with a past medical history of insulin-dependent DM, presented with nausea, non-bloody vomiting, and left upper quadrant pain over a 1 day period. Due to these symptoms and loss of appetite, she stopped taking insulin 3 days prior to presentation. She was afebrile, tachycardic, tachypneic, and toxicappearing. Her physical exam was remarkable for Kussmaul breathing, dry mucous membranes, and severe abdominal tenderness to palpation. Laboratory investigations revealed an elevated white blood cell count of 36,000 with immature forms, anion gap metabolic acidosis, elevated serum glucose of $679 \mathrm{mg} / \mathrm{dl}$, elevated beta-hydroxybutyrate of $7.77 \mathrm{mmol}$, and acute kidney injury. Urinalysis and chest x-ray did not reveal an acute process. In the emergency department, the patient had blood cultures drawn and was started on broad-spectrum antibiotics. She was admitted to the intensive care unit for management of DKA. She received aggressive fluid resuscitation and continuous insulin delivery until her anion gap was normalized. Dietary and medication non-adherence and infection were considered to be the causes of her developing DKA. Since the abdominal pain was out-of-proportion to the typical symptoms of DKA and to the ultrasound findings, an abdominal and pelvis computed tomography with IV contrast was obtained. It revealed grade $3 \mathrm{~A}$ (presence of gas in the perinephric space) emphysematous pyelonephritis with an obstructing stone at the ureteropelvic junction. Treatment with antibiotics and percutaneous drainage or emergent nephrectomy was considered based on the extent of the disease. The decision was made to transfer the patient to the operating room for emergent nephrectomy given the severity of medullary necrosis. She recovered uneventfully after nephrectomy and antibiotic therapy.

DISCUSSION: Emphysematous Pyelonephritis (EPN) is a rare urologic complication caused by gas forming bacteria affecting the renal parenchyma, collecting system, or perinephric tissue. Patients with an immunodeficiency syndrome or diabetes mellitus (DM) have increased risk of EPN. EPN has a high mortality rate, and early recognition is paramount. This case demonstrates the presentation, diagnosis, and management of EPN. EPN is a rapidly progressing disease that if left untreated is associated with high mortality due to overwhelming sepsis. Although early diagnosis is important, presenting symptoms and laboratory findings, as with our case, may not be helpful in guiding the clinician to the diagnosis of EPN. If the patient were only treated for DKA and not infection given a bland urinalysis, the outcome would have been catastrophic. Therefore, in patients with immunodeficiency syndrome or poorly controlled diabetes who present with severe abdominal pain and signs of sepsis, an acute 
intra-abdominal process should not be overlooked. Antibiotics should be initiated followed by abdominal imaging to exclude highly morbid diseases such as EPN.

ENDOCARDITIS AN UNUSUAL CAUSE FOR A SKY HIGH BNP! Tiba Alwardi ${ }^{4}$; Arham Barakzai ${ }^{2}$; Reem Mahdawi ${ }^{3}$; Zain Kulairi ${ }^{1}$. ${ }^{1}$ Wayne State University, West Bloomfield, MI; ${ }^{2}$ Wayne State University / Crittenton Hospital, Rochester, MI; ${ }^{3}$ Wayne state University, Rochester, MI; ${ }^{4}$ Wayne state university, Rochester Hills, MI. (Control ID \#2454513)

LEARNING OBJECTIVE \#1: Identify unuasal causes of significantly elevated B-type natriuretic peptide (BNP)

LEARNING OBJECTIVE \#2: discuss BNP significance directing the treatment and prognosis

CASE: An 80 year old female with no significant past medical history presented with altered mental status and generalized weakness of few hours duration. She denied chest pain, palpitations, shortness of breath or cough. On presentation she was hypotensive, febrile and tachycardic, labs were significant for leukocytosis and high lactic acid. As part of sepsis workup CXR was done and reported possible right lobe pneumonia versus pulmonary congestion. Considering CXR findings BNP was ordered and returned significantly high at 62000 . Interestingly ECHO reported nondilated left ventricle, estimated ejection fraction of $60 \%$, normal left atrium and sever mitral regurgitation complicated by mobile mass at the mitral valve annulus. Blood cultures grew MSSA confirming the diagnosis of Infective Endocarditis. Despite starting appropriate antibiotics she developed multifocal embolic stroke leading to severe debility and death shortly after.

DISCUSSION: Atrial myocytes secrete natriuretic hormones that play a major role in fluid homeostasis and blood pressure regulation. In ventricular diseases ventricular myocytes secrete B-type natriuretic peptide (BNP) further increasing their serum concentrations. Acute mitral regurgitation increases volume into a normally compliant left atrium resulting in a marked increase in atrial pressure and commonly ends with pulmonary edema, in rare instances regurgitant flow is directed preferentially to a single pulmonary vein with edema seen mostly in that lung segment. This finding is easily confused with pneumonia particularly if the patient has endocarditis. BNP helps to distinguish cardiac from non-cardiac causes of shock, This is particularly true in those presenting with unilateral pulmonary edema. BNP activation in acute MR is an emerging biomarker of severity of MR consequences and of poor outcome. with further investigation BNP level may be employed in directing the management of acute MR regurge.

EOSINOPHILIC GASTROENTERITIS - A RARE CASE IN THE SETTING OF TOXACARA INFECTION Elizabeth S. John ${ }^{1}$; Richard Tangel ${ }^{2}$; Betty Chung ${ }^{2}$; James R. Penn ${ }^{1}$. ${ }^{1}$ Rutgers Robert Wood Johnson, Montville, NJ; ${ }^{2}$ RWJ, New Brunswick, NJ. (Control ID \#2469869)

LEARNING OBJECTIVE \#1: To understand the presentation and differential diagnosis of eosinophilic gastroenteritis.

LEARNING OBJECTIVE \#2: To review the diagnostic workup and treatment of eosinophilic gastroenteritis.

CASE: Eosinophilic gastroenteritis (EGE) is an extremely rare inflammatory condition marked by eosinophilic infiltrates limited to the gastrointestinal (GI) tract. We present a unique case of a patient with eosinophilic gastroenteritis in the setting of a Toxacara infection, which can also elevate the eosinophilic count, making the diagnosis more nebulous. The patient is a 32-year old female born in the Dominican Republic (DR) with a history of iron deficiency anemia who presented with an 8 day history of crampy epigastric and right upper quadrant abdominal pain. Associated symptoms included non-bilious, non- bloody emesis and multiple episodes of diarrhea. The patient denied any relation of the pain to food intake or bowel movements, fevers, or chills. Notably, the patient was seen 5 days prior to admission, at which point abdominal imaging revealed enteritis. She was treated with antibiotics without improvement. On exam, the patient had tenderness in the epigastrium and RUQ without rebound, guarding, or organomegaly. Labs revealed leukocytosis with $31.5 \%$ eosinophils (Absolute Eosinophil Count 4060). Her initial infectious work up including stool ova and parasites and Strongyloides Ab were negative. The patient underwent upper endoscopy that showed erosive gastropathy with gastritis. Biopsies for $\mathrm{H}$. pylori were negative. Subsequently, her Toxocara antibody was found to be positive, but treatment with albendazole resulted in no clinical improvement or changes in imaging. Push enteroscopy 5 days later showed chronic gastritis with minimally erythematous proximal jejunum. Biopsies of the Stomach/Duodenum showed eosinophils in the proximal jejunum, duodenum, stomach, and throughout the esophagus (Figure 1). The patient was started on a restrictive diet and discharged home. On outpatient follow up, the patient was also started on glucocorticoids, which further minimized her pain.

DISCUSSION: EGE's rarity and non-specific presentation make the condition a diagnostic challenge. As it is a diagnosis of exclusion, a detailed interview to review all drugs, as well as family history, recent travel, and past malignancies, should be conducted to rule out other etiologies that can result in eosinophilia (1). Interestingly, our patient was born in the DR and likely had a positive Toxocara antibody from prior exposure. Ultimately, diagnosis is made with endoscopic biopsies that reveal eosinophilia in one or more parts of the GI tract. Eosinophils may penetrate any layer of GI tract including the serosa, which means they may not be seen on mucosal biopsies. Also, eosinophils are normally present in the rest of the GI tract, and pathologists will often use any level of eosinophils between 20 and 80 per hpf in one or more hpf (3) because there is no definition of pathological eosinophilia. Most importantly, the biopsy results should be correlated with the overall clinical picture and response to therapy. Guidelines are sparse in regards to treatment as well (2). Oral steroids are the most effective treatment in both the short and long term management, and our patient's pain improved drastically with steroids. Some case reports show that PPI and mast cell stablilizers are also effective. Still, some patients can often have spontaneous remission, either from the medications or spontaneous remission (4). Lastly, amino-based elemental diets and elimination diets in which milk and wheat are the most common foods excluded, have been highly debated (5). Our patient improved with a low residue, lactose free diet and small frequent meals (4-6 times/day). References: 1. Cianferoni A, Spergel JM. Eosinophilic Esophagitis and gastroenteritis. Current Allergy Asthma Rep. 2015; 15:58. 2. Straumann A. Idiopathic eosinophilic gastrointestinal diseases in adults. Best Pract Res Clin Gastroenterol. 2008;22:481-96. 3. DeBrosse CW, Rothenberg ME. Allergy and eosinophil associated gastrointestinal disorders (EGID). Curr Opin Immunol. 2008;20:703-8. 4. Pineton de Chambrun G, Gonzalez F, Canva JY, et al. Natural history of eosinophilic gastroenteritis. Clin Gastroenterol Hepatol. 2011;9:950-956 e951. 5. Lucendo, AJ, Serrano-Montalbán B, Arias A, Redondo O, Tenias JM. Efficacy of Dietary Treatment for Inducing Disease Remission in Eosinophilic Gastroenteritis. Journal of Pediatric Gastroenterology and Nutrition. 2015; 61 (1):56-64.

\section{EOSINOPHILIC GRANULOMATOSIS WITH POLYANGIITIS : NOT FOR THE} LIGHTHEARTED Rajesh Gopalarathinam ${ }^{1}$; Pavithra Indramohan ${ }^{2}$; Swathi Vishwanathan ${ }^{1}$; Aarthi Madhanakumar ${ }^{1}$ Tarun Sharma ${ }^{1} .{ }^{1}$ Allegheny General Hospital, Pittsburgh, PA; ${ }^{2}$ West Penn Allegheny Health Network, Pittsburgh, PA. (Control ID \#2465596)

LEARNING OBJECTIVE \#1: Diagnose Eosinophilic Granulomatosis With Polyangiitis in patients presenting with pericardial effusion.

LEARNING OBJECTIVE \#2: Treat Eosinophilic Granulomatosis With Polyangiitis with corticosteroids and immunosuppressants. 
CASE: A 50 year old caucasian male with past medical history of asthma and chronic sinusitis presented with a 1-week history of gradual onset worsening exertional dyspnea. He denied orthopnea, paroxysmal nocturnal dyspnea, chest pain, palpitations, syncopal episode, fever, cough with sputum, upper respiratory tract symptoms, sick contacts, recent travel and lower extremity swelling with pain. Remainder of the history was unremarkable. On physical exam, patient was found to be afebrile, tachycardic and tachypneic with mild wheezes in bilateral lung bases. Complete blood count revealed significant eosinophilic leukocytosis with a white count of 19,700 and $28 \%$ eosinophils. EKG showed sinus tachycardia. Chest x-ray revealed mild cardiomegaly without any pulmonary infiltrates. Subsequently, an echocardiogram was done which revealed an EF of $40 \%$ with moderate to severe pericardial effusion. He underwent pericardiocentesis and his dyspnea improved significantly. Analysis of the pericardial fluid revealed a transudative effusion. However, the etiology for his pericardial effusion remained unclear. Over the next $48 \mathrm{~h}$, he developed a new non-blanching purpura on the forehead and chest wall. Biopsy of the skin lesions showed eosinophils and leukocytoclastic vasculitis. His ANCA panel returned negative. Further review of his outpatient records revealed an elevated IgE level of $893 \mathrm{kU} / \mathrm{L}$ (reference range: $0-100 \mathrm{kU} / \mathrm{L}$ ). Based on the presence of peripheral blood eosinophilia, evidence of vasculitis with extravascular eosinophils on skin biopsy and a history of asthma with chronic sinusitis, a diagnosis of EGPA was made. Prompt initiation of pulse IV corticosteroids and oral cyclophosphamide led to rapid improvement of his skin lesions over the next 2 days. He also had a repeat echocardiogram during his hospitalization which revealed an improved EF of $55 \%$ with no evidence of pericardial effusion.

DISCUSSION: Eosinophilic granulomatosis with polyangiitis (EGPA) is a rare form of necrotizing vasculitis that affects small to medium sized vessels and is characterized by asthma, chronic rhinosinusitis, peripheral eosinophilia and necrotizing granulomas in the affected tissues. Although EGPA affects multiple organ systems, cardiac involvement is noted to be the most serious complication of the disease and it occurs in $50 \%$ of affected patients. Cardiac involvement in the form of myocardial infarction and arrythmias accounts for $50 \%$ of the deaths in EGPA patients and is more commonly seen in patients who are ANCA negative with a high eosinophil count. The cardiac manifestations are variable and include pericarditis, myocarditis, cardiomyopathy, myocardial infarction, pericardial effusion, heart failure, arrythmias and valvular insufficiency. The differential diagnoses that should be excluded are other forms of vasculitides such as Granulomatosis with Polyangiitis, Microscopic polyangiitis and Hypereosinophilic syndrome. It is important for internists to be cognizant of the fact that pericardial effusion and other cardiac manifestations can be the first presentation in patients with EGPA and there should be a high degree of clinical suspicion for EGPA in an appropriate clinical setting. Early diagnosis and aggressive treatment with corticosteroids and cyclophosphamide can result in improved cardiac function and survival in these patients.

\section{EOSINOPHILIC GRANULOMATOSIS WITH POLYANGIITIS PRESENATING} AS CNS VASCULITIS Mosumi Majumder. New York Methodist Hospital, Brooklyn, NY. (Control ID \#2470230)

LEARNING OBJECTIVE \#1: Dx of EGPA CNS vasculitis and manifestation LEARNING OBJECTIVE \#2: Management of EGPA involing CNS

CASE: Eosinophilic granulomatosis with polyangiitis (EGPA)A is a multisystem entity that often involves the pulmonary and the renal systems but at times nervous system involvement is also seen. When it does affect the nervous systems, it generally causes peripheral neuropathy such as foot drop however CNS involvement is generally rare. We present a case of CNS vasculitis secondary to EGPA in an elderly patient who was previously diagnosed with EGPA 20 years ago. 70 year old man who was initially brought in for evaluation after a non traumatic fall. His family also reported what they thought were changes in his general behavior as well as weakness of the right upper and lower extremity first noted about 2 weeks prior to the fall. Patient has an established history of Lewy Body dementia diagnosed 2 years ago and Eosinophilic Granulomatosis with Polyangiitis (EGPA), diagnosed 20 years ago with nerve biopsy when he presented to another facility with acute right foot drop. At the time of diagnosis he was given pulse dose steroids, which improved his symptoms. He has since then been in his usual state of health and able to carry about most of his activities of daily living with minimal help from family. He had been ambulatory with a walker at baseline because of mild residual weakness of the right lower extremity. On presentation, patient had a physical exam notable for decreased strength in the right lower extremity. He had a normal WBC count but had significant peripheral eosinophilia at $>50 \%$, a normal complete metabolic profile. ESR and CRP were both elevated and complement levels were normal. A CT brain showed ventriculomegaly associated with supratentorial volume loss and mild microvascular ischemic changes but no infarcts nor gross deformities. Subsequent evaluation with an MRI showed multiple small foci of peripheral and cortical enhancement along the convexity of the right cerebral hemisphere. A lumbar puncture showed elevated protein of 69, a normal glucose level and no WBCs. CSF fluid was negative for HSV and bacterial gram stain and cultures were negative. MPO antibody was elevated at $>8$ with a negative proteinase 3 antibody. An EEG showed left and right cerebral dysfunction. EGPA remained the primary differential and in light of the MRI and serologic findings he was initiated on $1 \mathrm{~g}$ intravenous (IV) Methylprednisolone for 5 days followed by IV dose Cyclophosphamide with partial improvement in mental status and muscle strength. The significant eosinophilia noted on admission also quickly resolved during his management. DISCUSSION: Eosniphilic Granulomatosis with Polyangiitis is a multisystem disorder usually characterized by allergic rhinitis, asthma and peripheral eosinophilia $>10 \%$. Isolated CNS manifestation of EGPA is rare and especially rare to manifest after recovering from peripheral neuropathy due to EGPA many years prior. Our patient was positive for MPO antibody, which is positive in 40-60\% of EGPA patients making this an ANCA positive vaculitis. Literature for isolated CNS EGPA such as our patient is scarce however; management with steroids and Cyclophosphamide have change the course of the disease and lead to symtomatic improvement.

EVALUATING PULMONARY EMBOLISM IN PATIENTS WITH FONTAN PHYSIOLOGY Joel D. Beachey; Jacob C. Jentzer; R. J. Widmer. Mayo Clinic, Rochester, MN. (Control ID \#2466120)

LEARNING OBJECTIVE \#1: Identify the appropriate diagnostic work-up of pulmonary embolism in patients with Fontan physiology.

CASE: A 42-year-old male smoker presented to a local emergency department with recent onset dyspnea and pleuritic chest pain. His past medical history was significant for tricuspid atresia status-post Waterston shunt at 16 months of age, Fontan procedure at 5 years, and redo-Fontan at 14 years. He developed complications with massive right atrial dilation and dysrhythmias, prompting conversion of his Fontan from a right atriopulmonary anastomosis to an intra-atrial conduit at age 29 . At the time of current presentation, he had lapsed in follow-up for several years and had self-discontinued all of his medications including warfarin. On physical exam, he was normotensive with a normal heart rate and regular rhythm. Jugular venous pressure and perfusion were normal. Respiratory rate was normal and oxygen saturation was $92 \%$ on room air. He had a pleural rub over the left posterior chest and significant pain with deep inspiration. The remainder of his exam was unremarkable. CT angiography of the chest with contrast injection into the left antecubital fossa was performed and interpreted as positive for a massive pulmonary embolism (PE) with near complete occlusion of the left pulmonary artery and extension through the right atrium and inferior vena cava (IVC). He was initiated on IV heparin and transferred to our medical center, where he was admitted to the Cardiac 
Intensive Care Unit. Upon arrival to our hospital, he remained surprisingly hemodynamic stabile, given the degree of his apparent PE. This diagnosis was thus called into question. Echocardiogram demonstrated a widely patent IVC and inferior segment of the intra-atrial conduit, inconsistent with prior CT images. A thrombus could not be excluded at the superior vena cava anastomosis on echocardiogram. A repeat CTA was performed with dual upper and lower extremity contrast injection. This demonstrated a widely patent Fontan anastomosis with no evidence of PE. Heparin was discontinued and naproxen was initiated for pleurisy. The patient's home medications were restarted, and smoking cessation counseling was provided. At follow-up 3 weeks later, his symptoms had nearly completely resolved.

DISCUSSION: This case illustrates the ease of misdiagnosis of pulmonary embolism in patients with Fontan physiology. The Fontan procedure results in complex altered anatomy with diversion of venous blood directly from the right atrium or vena cavae into the pulmonary arteries, avoiding the morphologic right ventricle. This is accomplished via creation of a direct atriopulmonary or cavopulmonary anastomosis. This connection can be either intra- or extra-cardiac. The finished result significantly alters pulmonary blood supply. In some instances, as with the patient described in this case, venous blood flows preferentially from the superior vena cava through the right pulmonary artery, while blood from the inferior vena cava travels into the left pulmonary artery. This discrepancy significantly complicates interpretation of CT angiography (CTA) of the pulmonary tree. With traditional single-injection CTA involving injection into the upper extremity, contras preferentially flows directly from the super vena cava through the right pulmonary artery, creating the appearance of an occluded left pulmonary artery. To improve diagnostic evaluation in these patients, it is critical to utilize dual-site power injection into the upper and lower extremities. A modified CTA protocol is thus essential to avoid false-positive results from incomplete contrast mixing. Accurate evaluation is highly important in this population, given a significant increase in the rate of pulmonary embolism in Fontan patients. Utilization of dual site injection into upper and lower extremity veins improves diagnostic accuracy, reducing the rate of misdiagnosis and avoiding unnecessary use of therapeutic anticoagulation.

EVERYTHING IN MOODERATION: A CASE OF HYPERCALCEMIA Karthik J. Kota. UPMC, Pittsburgh, PA. (Control ID \#2469803)

LEARNING OBJECTIVE \#1: Describe the work-up and treatment for hypercalcemia LEARNING OBJECTIVE \#2: Describe the pathophysiology of milk-alkali syndrome CASE: JS is a 72 year old accountant with history of stage IV melanoma of the left toe (metastatic to mediastinal lymph nodes; on pembrolizumab and peg-interferon) who presented with dry mouth, generalized weakness, and word-finding difficulties. Symptoms began 4 days prior to admission, starting with word-finding difficulty and progressing to generalized weakness. Associated symptoms included frequency and constipation; patient denied fevers, sudden weakness/mental status change, dysuria/ urgency/nocturia, chest pain, dyspnea, and bleeding JS worked as an accountant, denied smoking, alcohol, and drug use, and was independent at baseline. Family history was not significant. Physical exam on presentation was significant for slight word-finding issues only (otherwise, normal neurologic exam). Lab data showed a bicarbonate of 32, BUN of 31, Cr of 1.8 (b/1 1), normal CBC, normal LFTs (albumin 4), and calcium of 13.8 with phosphorus of 4 (previous calcium 1 month prior 10). Chest x-ray, CT head, and MRI head were negative Upon admission, patient was given calcitonin and started on IV fluids, w/Q6H calcium checks; although calcium initially improved to 10.8 , it slowly climbed back to the upper $11 \mathrm{~s} /$ low $12 \mathrm{~s}$. Work-up showed suppressed PTH, normal PTHrP, normal TSH, normal VitD25 (slightly elevated VitD1, 25), free kappa light chain of 38.3 with free lambda of 19.2 (ratio 1.92), and negative SPEP. Bone scan showed no metastases. Repeat history taking on day of admission revealed patient drank 4 pints of milk per day; repeat history on third hospital day revealed patient continued drinking milk. After restricting dairy intake, patient's calcium dropped below 11 in $24 \mathrm{~h}$, and was at 10 on discharge. Repeat labs 2 weeks after discharge (with continued dairy restriction) showed calcium staying at 10

DISCUSSION: This case demonstrates a slightly uncommon manifestation of milk-alkali syndrome, insofar as most current cases are from antacid overuse. The three most common etiologies of hypercalcemia are hyperparathyroidism, hypercalcemia of malignancy, and milk-alkali syndrome. Clearly, then, the first step (after ruling out medications) is to distinguish whether hypercalcemia is PTH-mediated or not. If PTH is elevated, a 24-h urine calcium will distinguish between familial hypocalciuric hypercalcemia (benign) vs primary/tertiary hyperparathyroidsim requiring surgery. If not PTH-mediated, the next step involves checking for granulomatous disease (vit D metabolites) versus malignancy (PTHrP). If none of these are elevated, then it is time to look for rarer causes, such as hyperthyroidism, adrenal insufficiency, and acromegaly Treatment of hypercalcemia boils down to levels and symptoms. Asymptomatic hypercalcemia or hypercalcemia $<12$ can be treated with avoiding factors that elevate serum calcium (e.g., diet, medication) and keeping up adequate PO intake. Asymptomatic moderate hypercalcemia (12-14) is monitored closer, with initiation of supplemental IV fluids and treatment of underlying causes (steroids for granulomatous disease, bisphosphonates for malignancy) started when patients begin having more symptoms. Severe hypercalcemia $(>14)$ requires calcitonin to quickly reduce calcium, IV fluids for re-expansion (goal of urine output of 100-150 mL/ h), and concurrent treatment of underlying causes as above. Of note, hypercalcemia of malignancy refractory to bisphosphonates used to be treated with gallium (mechanism involving, among others, arresting osteoclasts and stimulating bone growth), but fell from favor due to common side effects of ATN and hypophosphatemia; since 2014, denosumab (a RANK-ligand inhibitor, preventing osteoclast maturation) has taken this role Milkalkali syndrome (commonly called calcium-alkali syndrome due to the prevalence of antacids, rather than dairy, causing the condition) is, at its core, a problem with calcium buffering and excretion. Bone resorption/deposition moves 200-500 mg of calcium/day out of a pool of 3-6 $\mathrm{g}$ of calcium in the bones; as patients age, more is resorbed and less deposited, both increasing calcium in serum and reducing the total capacity to store it (in part by elevated calcium levels decreasing PTH, which activates bone remodeling). Calcium is excreted primarily via the kidneys, with a three-fold difference in calcium excretion in low PTH states (IE, with hypercalcemia) versus high PTH states (IE, with hypocalcemia). In hypercalcemic states, GFR is reduced (secondary to a direct vasoconstrictive), decreasing calcium filtering and excretion; as well, hypercalcemia activates the calcium-sensing receptor of the thick ascending loop of Henle, leading to natriuresis and diuresis (further decreasing GFR and causing a metabolic alkalosis). In turn, alkalotic urine increases calcium reabsorption. Of note, all these renal effects are worsened in chronic kidney disease, often found in the elderly For JS, then, his hypercalcemia was less a result of his lifelong 4 pints of milk per day habit, and more due to his body no longer being able to handle calcium loads as it once could

EXOGENOUS LIPOID PNEUMONIA Kevin K. Manocha; David Snipelisky; Dante Schiavo. Mayo Clinic, Rochester, MN. (Control ID \#2462117)

LEARNING OBJECTIVE \#1: The importance of taking a good history. A good clinical history can obviate unnecessary invasive testing.

LEARNING OBJECTIVE \#2: Ground glass opacities on Chest Computed Tomography imaging have a broad differential including lipid.

CASE: A 61-year-old man with a past medical history of head and neck squamous-cell carcinoma treated with neck dissection and radiation presented to our clinic with an 
abnormal surveillance computed tomogram (CT) of the chest. The patient denied dyspnea, cough, sputum production, chest pain, fevers, and rigors. He did however complain of significant oropharyngeal dryness, particularly night. He used gastric tube for nutrition and was otherwise not eating or drinking anything by mouth due. To moisten his oropharynx, however, he had been coating his mouth with coconut oil every night before going to bed. Review of the CT revealed geographic ground glass opacities in both lower lobes medially with interspersed small centrilobular micronodules. The distribution was predominantly in dependent segments of lung, suggestive of aspiration. The findings were present and had worsened compared to a prior scan despite symptomatic stability. Because of the history and CT findings, we deduced that the patient was aspirating coconut oil at night which he was using to moisten his oropharynx resulting in an exogenous lipoid pneumonia. Given his satisfactory clinical status, we did not introduce any pharmacotherapy and recommended aspiration precautions, alternative oropharyngeal moistening strategies, and termination of coconut oil to treat our patient. Repeat CT scans after termination of his coconut use revealed significant improvement of the GGOs. He will be followed with serial imaging until resolution.

DISCUSSION: Lipoid Pneumonia is an uncommon and often unrecognized condition. Lipoid pneumonia refers to the presence of oil or fat like substance in the lung parenchyma. It can be exogenous or endogenous. Endogenous refers to cholesterol escaping into the lung parenchyma because of an underlying malignant, inflammatory or suppurative process. Exogenous lipoid pneumonia refers to lipid from an external source. It was relatively common in the 1930s on autopsies of children because of the use of oils in inhalants and widespread popularity of mineral oil for constipation, nosebleeds, etc. An important concept is the oil/fat can evade mucociliary clearance given its texture allowing for easy entry into the lung parenchyma. Once in the lungs, it can cause either an aspiration pneumonitis or if chronic, may be asymptomatic- as was the case in our patient. Because of unfamiliarity, often times the diagnosis is missed and when found, can incite unnecessary testing and costs. However a careful history and characteristic imaging of paraffin like appearance of ground glass opacities in the dependent positions of the lung can make the diagnosis. In asymptomatic individuals, the treatment is to remove the offending agent and monitor for resolution

EXTRA ADRENAL PARAGANGLIOMA IN A YOUNG FEMALE, WITH THE CLASSIC “3 P'S” Zain Kassam ${ }^{1}$; David DiCaprio ${ }^{3}$; Rebecca A. Mazurkiewicz ${ }^{2}$; Robert E. Graham ${ }^{1} .{ }^{1}$ Lenox Hill Hospital, New York, NY; ${ }^{2}$ Lenox Hill Hospital Medicine, New York, NY; ${ }^{3}$ Lenox Hill NSLIJ Hospital System, New York, NY. (Control ID \#2469499)

LEARNING OBJECTIVE \#1: 1. Identify the classic presentation of pheochromocytoma despite the age of the patient.

LEARNING OBJECTIVE \#2: 2. Pheochromocytoma as an important cause of secondary hypertension in young adults, in the appropriate clinical setting. 3. Discuss different presenting clinical profiles of the tumor.

CASE: A 25-year-old female who was in her usual state of health started experiencing sudden episodes of headaches, excessive sweating and palpitations a couple of weeks prior to her presentation to the ER. Her initial episode was self-limited. Her second episode occurred at work, which prompted her to present to the ER. Patient denied any other symptoms or complains and states she is relatively healthy exercising about 4 to 5 times a week. She denied significant past medical, past surgical, or family history. She took no prescribed or over the counter medications, and used no herbal supplements. She denied use of tobacco, alcohol, oral contraceptive pills and recreational drugs. On exam, the patient's blood pressure was 200/100 and her heart rate was 110 . The remainder of her vital signs were normal and she was in no distress though she periodically experienced flushing. Her exam was negative for papilledema, JVD, murmurs, abdominal masses, organomegaly, bruits of any kind, and edema. Her labs including a CBC, CMP, TSH, UA, urine toxicology screen, and pregnancy test were all negative for pathology. A retroperitoneal ultrasound showed a well-circumscribed mass adjacent to the left kidney comprising of mixed cystic/solid features with mild vascularity in the mass on Doppler. A MRI abdomen with and without contrast revealed a $8.5 \mathrm{~cm}$ mass in the left abdomen lateral to the left adrenal gland and anterior to the left kidney. $24 \mathrm{~h}$ fractionated metanephrines, normetanephrines and total metanephrines, were extremely elevated at 1753, 23688, 25441, respectively. Her CT head was negative for any acute intracranial pathology. A thyroid ultrasound was remarkable for sub-centimeter colloid cyst in each lobe of the thyroid gland and a MIBG scan was negative for metastatic lesions. The patient's blood pressure was controlled with alpha-blockers perioperatively and she underwent an exploratory laparotomy and resection of a paraganglioma. Surgical pathology showed an abdominal paraganglioma measuring $9.2 \times 8.1 \times$ $6.3 \mathrm{~cm}$, without lymphovascular invasion and clear margins. Her subsequent course was unremarkable with complete resolution of her symptoms.

DISCUSSION: Catecholamine-secreting tumors are rare neoplasms, occurring in less than $0.2 \%$ of patients with hypertension. It is estimated that the annual incidence of pheochromocytoma is approximately 0.8 per 100,000 person years. If the diagnosis of a pheochromocytoma is overlooked, the consequences can be disastrous, even fatal; however, if a pheochromocytoma is found, it is potentially curable. Pheochromocytomas are most common in the fourth to fifth decade, but may occur at any age and have no gender preference. Approximately $10 \%$ of pheochromocytomas and $35 \%$ of extra-adrenal pheochromocytomas are malignant (i.e. have metastases). The classic triad of symptoms in patients with a pheochromocytoma consists of episodic headache, sweating, and tachycardia; however, most patients do not exhibit these symptoms. $90 \%$ of patients will develop headaches. While about half have paroxysmal hypertension; most of the rest have what appears to be primary hypertension or normal blood pressure. Other signs and symptoms that may occur include orthostatic hypotension, visual blurring, papilledema, weight loss, polyuria, polydipsia, constipation, increased ESR, hyperglycemia, leukocytosis, and psychiatric disorders. Rarely, patients with pheochromocytomas can develop secondary erythrocytosis due to overproduction of erythropoietin and even reversible cardiomyopathy. These catecholamine-secreting tumors are almost always treated surgically with preoperative blood pressure control with alpha-blockers. In sum, pheochromocytoma should be suspected if there are paroxysmal elevations in blood pressure (which may be superimposed upon stable chronic hypertension), particularly if associated with the triad of headache (usually pounding), palpitations, and sweating. Our case is one of the rare situations where the triad was evident, it was a young female and we were able to quickly identify and manage the patient without complications.

\section{EXTRAPULMONARY SARCOIDOSIS WITH PANCYTOPENIA AND COEXISTENCE OF SJOGRENS'S SYNDROME Tuncay Taskesen; Bara} Alzghoul; Adnan Elghezewi; Monika Tripathi; Ashley Wang; Ira Oliff. Saint Francis Hospital, Evanston, IL. (Control ID \#2466350)

LEARNING OBJECTIVE \#1: Atypical presentation of systemic diseases should be in differential diagnosis of patients with unexplained medical conditions.

LEARNING OBJECTIVE \#2: Bone marrow biopsy is a gold standard for the diagnoses of pancytopenia etiology, after ruled out obvious, reversible causes.

CASE: A 72-year-old female presented with generalized weakness and bilateral leg swelling for the past 2 months. Her review of system was positive for exercise intolerance, dry eyes, photophobia and ecchymosis over her legs. She denied cough, chest pain, shortness of breath, or weight loss. Her primary care physician noticed new developed pancytopenia and referred her to our hospital for further investigation. Her past medical history include CAD, HTN, HLD, stage 4 CKD, insulin dependent DM type 2, and hypothyroidism. Her physical examination revealed normal vital signs, with dry eyes, 
splenomegaly, bilateral $2+$ leg edema and diffuse ecchymosis over both lower legs. Her CBC showed pancytopenia (WBC: 2.4 k/uL, Hb: 8.9 g/dl, PLT: 60.000); CMP showed increased serum $\mathrm{Cr}(3.2 \mathrm{mg} / \mathrm{dl})$ and Ca level $(13.3 \mathrm{mg} / \mathrm{dl})$. Abdominal US showed splenomegaly $(21.3 \mathrm{~cm})$. Reversible causes of pancytopenia were ruled out. Serum and urine protein electrophoresis were normal; light chain and Immunoglobulin levels also came out normal. Bone marrow biopsy showed noncaseating granulomatous infiltration of bone marrow, which was consistent with sarcoidosis. Bone marrow aspirate (BMA) culture was negative for mycobacterial and fungal infections. Flow cytometry from BMA identified small monoclonal B-cell population, peripheral blood flow cytometry showed reactive inflammatory response. The chest and abdominal CT showed several small lymphadenopathy and splenomegaly without lung involvement. Her chest X-ray also did not show any infiltration or granulomatous changes. The patient declined lymph node biopsy. Tumor and infectious granulomatous diseases such as tuberculosis and fungal infections were ruled out with appropriate lab studies. Serum ACE-I and Vit D 1-25 HI-OH level were high, PTH level was low, which was explained by resistant hypercalcemia and deteriorated kidney function. Patient was diagnosed with extrapulmonary sarcoidosis with bone marrow and most likely spleen infiltration. Autoimmune serologic tests revealed a positive ANA, anti Ro antibody and increased ESR and CRP. She was diagnosed with Sjogrens's disease. She started oral prednisone $40 \mathrm{mg}$ daily. She improved clinically, her hematologic parameters and high calcium level improved with steroid therapy, her kidney function stabilized. She was discharged to subacute rehabilitation center on tapering dose of oral steroids with follow up plan with nephrology and hematology.

DISCUSSION: Sarcoidosis is a systemic disorder characterized by granulomatous infiltration of various organs. The lungs are involved in $90 \%$ of patients. The skin, eyes, and heart are affected in significant number of patients. Although, disease remits within 3 year in most patients, $10 \%$ to $30 \%$ of patients need ongoing treatment. This case is an unusua presentation of sarcoidosis with bone marrow infiltration, pancytopenia, and also comorbid Sjogrens's disease. A detailed evaluation for pancytopenia did not show any infectious or primary hematological disorder. The bone marrow biopsy showed noncaseating granulomas, and lab results supported the diagnosis of sarcoidosis. The pancytopenia manifested in this patient was most likely a combination of splenic sequestration and bone marrow infiltration by sarcoidosis. Sjogrens's disease was considered less likely to cause the pancytopenia as the reticulocyte count was normal and bone marrow was normocellular without fibrotic changes. The splenomegaly may be a result of sarcoid involvement or extramedullary hematopoiesis. The subacute presentation of the patient makes the last explanation less likely. Sarcoidosis and Sjogrens's syndrome are chronic, inflammatory, and multisystem diseases of unknown etiology. The coexistence of both diseases has been reported in only $1 \%$ of patients with Sjogrens's syndrome. In the presented case high ANA, anti- Ro antibody titer, elevated inflammatory markers, and clinical features were consistence with Sjogrens's syndrome. Relatively acute presentation of the patient resulted in overlap of clinical features from both diseases. The bone marrow biopsy clarified etiology of pancytopenia by showing histopathologic feature of sarcoidosis. Primary renal manifestations of sarcoidosis are nephrolithiasis, nephrocalcinosis, and acute interstitial nephritis with or without granuloma formation. In this case, the renal CT scan did not show nephrocalcinosis or nephrolithiasis, could be attributed to subacute presentation. It has been shown that glucocorticoids can improve calcium metabolism and kidney function in patients with sarcoidosis. However, despite early initiation of steroid therapy, the patient's kidney function did not improve. Patient had underlying chronic kidney disease due to HTN, DM, CHF, and prior contrast medium exposure. Prognosis of acute kidney injury in sarcoidosis depends on baseline kidney function, severity, and longevity of hypercalcemia. We present a rare case of extrapulmonary manifestation of sarcoidosis with pancytopenia, acute kidney injury, and coexistent Sjogrens's syndrome. Awareness of atypical presentations of sarcoidosis will prompt for early diagnosis and targeted therapy to prevent long-term consequence of this progressive disease.
FALLING ON OUTSTRETCHED HANDS: THINKING OUTSIDE THE SNUFFBOX Helen $\mathrm{Ma}^{1}$; David Cheng ${ }^{1}$; Patrick M. Cocks ${ }^{2} .{ }^{1} \mathrm{NYU}$ School of Medicine, New York, NY; ${ }^{2}$ NYU School of medicine, New York, NY. (Control ID \#2465179)

LEARNING OBJECTIVE \#1: Recognize a rare cause of syncope in a patient with cardiac risk factors

LEARNING OBJECTIVE \#2: Recall the epidemiology, pathophysiology and clinical presentation of carotid body tumors

CASE: An 86-year-old woman is admitted for loss of consciousness for 1 day while waiting to cross the street. Her history begins 1 year ago when she reports an unexplained fall in the street resulting in a fractured left wrist. The patient was unable to provide more detail. Three months prior to admission, she fell when she slipped on an icy sidewalk causing a right wrist fracture. On the day of admission, patient was waiting at an intersection waiting to cross the street when she lost consciousness and fell. She denies prodromal symptoms. Review of symptoms was notable for right-sided neck fullness, treated unsuccessfully with topical herbal medication. Her medical history consists of hypertension, diabetes, and osteoporosis. Her medications include metoprolol, amlodipine, olmesartan, hydralazine, glipizide and raloxifene. On exam, the patient was normotensive, found to have a scalp laceration and a painless right neck swelling without a bruit. ECG revealed a wandering pacemaker. Telemetry was notable for intermittent bradycardia to the 50s. The only finding on head and neck CT was a lobular neck mass along the right carotid artery concerning for neoplasm. MRI of the neck confirmed a $2.3 \times$ $2.7 \times 2.8 \mathrm{~cm}$ carotid body tumor at the bifurcation of the right carotid artery. Given the suspicion that the mass was contributing to her falls, carotid massage was preformed, which slowed her heart rate from the 70 s to 50 s. Upon discussion of findings, the patient declined diagnostic biopsy or surgery because of her age and acceptable quality of life. Metoprolol and hydralazine were discontinued and she was discharged to home with follow up.

DISCUSSION: The differential for elderly patients with unexplained syncope is quite broad. Common etiologies such as vasovagal or hypovolemia are often realized with history and physical alone. In our patient, imaging preformed as part of a trauma revealed the culprit etiology: carotid body tumor. Carotid body tumors are a rare cause of syncope. The carotid body is a paraganglion and acts as a chemoreceptor by registering oxygen, carbon dioxide and protons. Paragangliomas are tumors that arise from chief cells within the carotid glomus, vagus nerve, middle ear or jugular foramen; carotid body tumors are most common. Carotid body tumors are associated with chronic hypoxia as seen in people living in high altitudes or chronic heart and lung disease. The pathophysiology of tumor genesis is due to hypoxia leading to hypertrophy and hyperplasia of chief cells and eventual neoplasia. There is also familial association with the mutation of mitochondrial SDH genes. Clinical findings include neck mass, cranial nerve palsies and cardiovascular effects. The mechanism of syncope is the hypersensitivity of the carotid body, leading to increased vagal tone, bradycardia and hypotension. It is likely that the patient turned her head to cross the street, causing compression of the tumor resulting in an exaggerated parasympathetic response. While in the majority of cases patients are cured with surgical resections, taking into consideration the 4-year doubling time and reported recurrence in these tumors, our patient declined biopsy and surgery, opting for watchful waiting. Interestingly our patient was only symptomatic from her tumor when crossing the street and turning her head calling into question the old adage "look both ways before crossing the street".

FALSE POSITIVE CEREBROSPINAL FLUID CRYPTOCOCCUS ANTIGEN IN LIBMAN SACKS ENDOCARDITIS Iyad Isseh; Kassem Bourgi; Asaad Nakhle; Mahmoud Ali; Marcus Zervos. Henry Ford Hospital, Detroit, MI. (Control ID \#2466776) 
LEARNING OBJECTIVE \#1: Recognize false positive cerebrospinal fluid cryptococcal antigen of low titers in the setting of systemic lupus erythematous.

LEARNING OBJECTIVE \#2: Distinguish the clinical features of cryptococcal meningoencephalitis.

CASE: 58 year old lady who presented with acute and suddent onset of altered mental status. It was described to be lethargy with slurring of the speech and inability to recognize her husband. Notably, our patient had a long history of SLE with secondary immune thrombocytopenic purpura. She was also formerly diagnosed with cerebral vasculitis and stable cerebral aneurysmal dilatation. For the past year she had been maintained on mycophenolate mofetil and hydrochloroquine. During evaluation, $16 \mathrm{~h}$ after the initial symptom onset, the patient was back to her normal state of health. By that time no intervention had been done yet. On evaluation she was afebrile with stable vital signs and unremarkable findings on physical examination. She was awake, alert and fully oriented. She had no neck stiffness and had intact sensation and motor power in all four extremities. Reflexes were symmetric and cranial nerve exam was grossly intact. Her recent and remote memory were appropriate and she had fluent speech with no dysarthria. The initial work-up revealed a slightly elevated white blood cell count of $11.6 \mathrm{~K} / \mathrm{uL}$ and a platelet count of $450 \mathrm{~K} / \mathrm{uL}$. Erythrocyte sedimentation rate and antinuclear antibody were at $72 \mathrm{~mm} / \mathrm{h}$ and 1:320 Titer, respectively. Angiographic computed tomography, to evaluate for aneurysmal rupture, revealed a stable small $2.5-3.0 \mathrm{~mm}$ carotid terminus aneurysm with no evidence of intra-cranial hemorrhage. CSF fluid sample was then obtained. The opening pressure, glucose and protein were all within normal limits, however there was evidence of mild pleocytosis with a total of 24 leukocytes $/ \mathrm{mm} 3$. Few hours later CSF Cryptococcus antigen testing resulted as 1-2 Titer. At that time she was started on antifungal therapy with intravenous liposomal amphotericin B along with oral flucytosine. Given the paucity of congruent infectious signs along with the acuity of symptom onset and resolution, a repeat lumbar puncture was done 3 days later. On repeat analysis the leukocyte count dropped to $16 / \mathrm{mm} 3$ and the Cryptococcus antigen titer was "negative". Results of India-ink examinations were negative and CSF cultures showed no growth on both occasions and therefore anti-fungal therapy was discontinued. Further assessment with magnetic resonance imaging showed signal abnormality within the cortex of the superior left frontal lobe with cortical enhancement favored to represent subacute ischemia of unknown etiology. A trans-esophageal echocardiogram, done to investigate for an embolic source, showed thickening and calcification of posterior mitral leaflet suggestive of SLE-associated valvular abnormalities. The patient was diagnosed with having thrombo-embolic cerebrovascular transient ischemia, for which she was started on long term anticoagulation.

DISCUSSION: Cryptococcus neoformans meningoencephalitis usually presents in immuno-compromised patients. Typically, it presents as subacute to chronic meningitis with headache, lethargy, personality and memory change. In our patient, her presentation was more acute in both onset and resolution. Definitive diagnosis of Cryptococcus meningoencephalitis in HIV-seronegative patients requires a lumbar puncture with opening pressures and careful evaluation of the CSF with India-ink, antigen testing and cultures. While culturing the organism from the CSF is the preferred confirmatory test with a positive result in $90 \%$ of non-AIDS patients, cultures can take 3-7 days to grow in the laboratory. As prompt initiation of therapy is crucial, CSF Cryptococcus antigen testing has been established as an indispensable adjunct test with results available more rapidly. These assays possess a high degree of sensitivity and specificity, ranging 93$100 \%$ and $93-98 \%$, respectively. However, false positive results have been previously reported in the literature, and usually take place at extremely low titers (1:8 or under). While initial CSF evaluation revealed a positive Cryptococcus antigen assay, patient's symptoms were inconsistent with Cryptococcus meningoencephalitis, and a repeat CSF evaluation, done 3 days later, revealed a negative CSF Cryptococcus antigen assay. A significant proportion of serological studies in patients with SLE may have cross reactivity or non-specific interference from circulating autoantibodies causing false-positive results.
Our case is the first reported false positive CSF Cryptococcus antigen in the setting of active SLE disease. Our patient was thought to have Cryptococcus meningitis based on isolated positive CSF Cryptococcus antigen titers, when in fact she had experienced a cardiac thrombo-embolic cerebrovascular transient ischemic event as a result of occult Libman-Sacks Endocarditis. While CSF serologic testing is very useful in ensuring prompt diagnosis and management, it's imperative to remember that false-positive results can occur even in highly specific tests, such as the CSF Cryptococcus antigen assay. Any isolated positive CSF Cryptococcus antigen test result has an unclear clinical value and should be interpreted with a grain of salt, especially in the setting of low titers. In our case we believe that the false-positive result was attributed to test interference by autoantibodies in the setting of SLE and high ANA titers.

FALSE POSITIVE HIV RESULT AND LOW CD4 IN BABESIOSIS Nabil Mesiha ${ }^{2}$; Arthur Ablayev $^{1}$; Ekaterina Vypritskaya ${ }^{1}$; Marc Whitman ${ }^{3}$; Saba A. Hasan ${ }^{1}{ }^{1}$ Capital Health Regional Medical Center, Trenton, NJ; ${ }^{2}$ Capital health Regional Medical Center, Trenton, NJ; ${ }^{3}$ Capital Health Medical Regional Center, Trenton, NJ. (Control ID \#2456708)

LEARNING OBJECTIVE \#1: To demonstrate that babesiosis, is a condition where we need to be aware of the possibility of false positive EIA result for HIV.

LEARNING OBJECTIVE \#2: To demonstrate that CD4 count should not be used as a surrogate test for HIV diagnosis, especially in patients with acute immunologic response to infection.

CASE: Background: Babesiosis is a disease, prevalent in the Northeast during warm months, May through September. The majority of those infected with Babesia microti are asymptomatic. However, the elderly, immunocompromised and asplenic patients can develop life-threatening complications including intravascular hemolysis. Patients with HIV are known to have high morbidity and mortality, as $\mathrm{CD}^{+} \mathrm{T}$ helper cells are the subpopulation chiefly responsible for protection against Babesia microti. Therefore, all patients with symptomatic babesiosis are usually screened for underlying HIV. EIA (enzyme immunoassay) is the screening test with high sensitivity. Some infections, such as malaria, and autoimmune diseases, such as systemic lupus erythematosis, are known to cause false positive results on HIV screening. We report two consecutive cases of symptomatic babesiosis with false positive EIA. Case Presentation: Case \#1. A 29 years male, who works as a landscaper, presented with 1 week history of fever, chills, night sweats, myalgia, headache and dizziness. On admission temperature was 101,5 and vital signs were otherwise stable. Hb was normal and wbc was 4.7 with $16 \%$ bandemia. His peripheral smear confirmed babesiosis parasitemia of $1.1 \%$. Atovaquone and azithromycin were started and we observed steady improvement of his symptoms. HIV screening was performed on admission and EIA came back positive. On further questioning, we identified no risks factors for HIV. A western blot confirmation was pending and CD4 count was ordered. Western blot was negative, but absolute CD4 count was 20 and $\mathrm{Cd} 4 / \mathrm{Cd} 8$ ratio was 0,08 At his 2 week outpatient visit, he was asymptomatic. The CD4 count and CD4/CD8 ratio normalized. Case \#2. An afebrile 62 years old male presented with 1 week history of generalized fatigue and dizziness. He was diagnosed with severe hemolytic anemia secondary to babesiosis with $11.5 \%$ parasitemia. He was started on atovaquone and azithromycin. HIV EIA was positive with total CD4 suppressed at 412. The CD4/CD8 ratio was normal. The western blot was negative. On follow up visit in the outpatient clinic patient the patient was asymptomatic and his CD4 normalized.

DISCUSSION: Discussion: We are well aware that some infectious and autoimmune diseases are associated with false-positive EIA results for HIV. Malaria, for example, may cause this false positive result due to hypergammaglobulinemia from immunological stimulation. We postulate that babesiosis, similar to malaria, is another condition where we need to be aware of the possibility of false positive EIA result for HIV. Also, our cases 
demonstrated that CD4 count should not be used as a surrogate test for HIV diagnosis, especially in patients with acute immunologic response to infection.

FEMORAL AVASCULAR NECROSIS AS A COMPLICATION OF PRIMARY MYELOFIBROSIS Prashanth Venkatesh. New York-Presbyterian Hospital/ Weill Cornell Medical Center, New York, NY. (Control ID \#2470209)

LEARNING OBJECTIVE \#1: Recognize femoral avascular necrosis as a potential complication of primary myelofibrosis

CASE: A 73-year-old woman presented to our hospital with acute, severe, intermittent, throbbing pain of the groin and both thighs of 5 days' duration. She was diagnosed with primary myelofibrosis 2 weeks prior, after presenting with night sweats, weight loss and massive splenomegaly. Workup included peripheral flow cytometry, which was positive for the JAK2 exon 14p V716F mutation, and bone marrow biopsy that confirmed the diagnosis of primary myelofibrosis. She was started on ruxolitinib $500 \mathrm{mg}$ daily which was uptitrated to $1000 \mathrm{mg}$ daily. On current presentation she had intermittent pain in the left thigh, worse with movement, radiating to the buttock and occasionally to the left big toe. She had had similar symptoms on the right side 4 days prior, which had spontaneously resolved. Physical examination was significant for tenderness to palpation over the left thigh and restriction in range of motion of the left hip with severe pain on flexion or extension of the left hip and 3/5 strength on left hip flexion. Laboratory tests showed leukocytosis to $75,700 / \mathrm{mm} 3$ with $85.9 \%$ polymorphonuclear cells, hemoglobin of $7.5 \mathrm{~g}$ $\mathrm{dL}$ and platelet count of $159,000 / \mathrm{mm} 3$. Magnestic resonance imaging of the left hip revealed diffusely decreased $\mathrm{T} 1$ and increased $\mathrm{T} 2$ signal throughout the bone marrow, with bone marrow edema pattern throughout the femoral head and neck (Fig 1,2). Post-contrast sequence showed non-enhancement in these regions (Fig 3). A small region of T2 hyperintensity with lack of central enhancement was found within the subtrochanteric femur likely representing infarct. Overall, the findings were consistent with extensive ischemia and developing femoral avascular necrosis. Also found was a moderate reactive left hip joint effusion that was subsequently aspirated under imaging guidance, following which the patient's pain and hip function remarkably improved and she was able to weight bear on the left leg hours afterwards. The aspirate revealed 4222 erythrocytes/uL and 6,200 leukocytes /uL with $99 \%$ PMNs, but Gram stain and culture were negative. The patient's history was negative for prior thrombotic events, recurrent miscarriages, chronic steroid use or other risk factors for avascular necrosis. A comprehensive inherited thrombophilia screen was negative. The patient received daily physical therapy and was eventually able to ambulate with decreasing analgesic requirements. Bone decompression surgery, an initial consideration, was hence deferred and the patient was discharged with orthopedic follow-up and plan for subsequent elective hip replacement. $<$ !- Copyright (c) 2006 Microsoft Corporation. All rights reserved. $-><$ ! - OwaPage $=$ ASP.webreadyviewbody aspx $-><$ !-Copyright (c) 2006 Microsoft Corporation. All rights reserved. $->$

DISCUSSION: Myeloproliferative neoplasms have been well documented to give rise to a hypercoagulable state. PMF has been well associated with a plethora of thrombotic complications, including fatal and non-fatal conditions such as acute myocardial infarction, venous thromboembolism, ischemic stroke and peripheral arterial thrombosis. In addition, the risk of recurrence of thrombosis in patients presenting with thrombosis on diagnosis or a history of thrombosis is as high as $9 \%$. Risk factors found to confer the highest risk of thrombosis in PMF include age $>65$ years, leukocytosis $>15,000 / \mathrm{uL}$ and the presence of the JAK2 V617F mutation. Femoral avascular necrosis is a profoundly debilitating, progressive and often irreversible condition with limited joint-sparing treatment options available. The value of core decompression in advanced or chronic disease is questionable, and the safety of such a procedure in patients with bone marrow fibrosis and resulting thrombocytopenia that is often difficult to correct, is always an issue. The definitive treatment for advanced disease is total hip replacement, a procedure frought with severe complications including cardiovascular morbidity/mortality, venous thromboembolism and sepsis - conditions that a PMF patient with poor bone marrow reserve is ill-equipped to face. This is to the best of our knowledge the first reported case of femoral avascular necrosis in a patient with PMF and no other risk factors. Extensive thrombophilia workup was unrevealing. We feel that formation of microthombi in the skeletal vasculature due to the hypercoagulable milieu created by PMF was the likely underlying mechanism. Our report sheds light on a possible new thrombotic complication of PMF and urges clinicians to consider avascular necrosis in the differential diagnosis of hip pain in patients with myeloproliferative neoplasia.

FEVER, MYALGIA, NEUROPATHIC PAIN AND LUNG NODULES IN A PATIENT WITH POOR ORAL HYGIENE: RARE CASE OF DISSEMINATED INFECTION WITH PARVIMONAS MICRA Naoko Kunami; Kazumi Motomura; Rika Yamaguchi; Yuka Naito; Motoshi Fujiwara; Akihiko Ogushi; Motosuke Tomonaga; Masaki Hyakutake; Itaru Kyoraku; Shu-ichi Yamashita. Saga University Hospital, Saga, Japan. (Control ID \#2454937)

LEARNING OBJECTIVE \#1: Parvimonas micra (P. micra) is a rare but increasingly important pathogen of sepsis and disseminated infections, especially immunecompromised patients including poor controlled DM with poor oral hygiene. A high clinical suspicion and careful identification of anaerobic bacteria appear to be the key factor for the diagnosis of $P$. micra infection.

CASE: A 43-year-old man was admitted to our hospital with a 1-month history of fever, cough and right side of neck pain. He also suffered from poor controlled diabetes mellitus (DM). On admission, temperature was $37.7^{\circ} \mathrm{C}$, blood pressure $130 / 77 \mathrm{mmHg}$, heart rate $118 / \mathrm{min}$, respiratory rate $22 / \mathrm{min}$, and oxygen saturation was $98 \%$ while the patient was breathing ambient air. His right shoulder motion was limited and palpations of right neck and shoulder were painful. The patients also complained tingling sensation in the right arm. The deep tendon reflexes were normal except those in the right arm, which were completely absent. Oral hygiene was extremely poor with severe periodontal disease and multiple decayed teeth. The white-cell count was $2.08 \times 10^{10} / 1$, blood levels of glucose was $246 \mathrm{mg} / \mathrm{dl}$, CRP was $26.6 \mathrm{mg} / \mathrm{dl}$ and $\mathrm{HbAlc}$ was $9.3 \%$. The cervical T2-weighted and diffusion-weighted MRI images revealed some increased signal-intensities in the right side of scalene muscles, C3-7 spinal nerves, brachial plexus and C3/4 intervertebral foramens. The chest $\mathrm{CT}$ scan with enhancement showed many pulmonary nodules located along the running direction of pulmonary arteries. The cervical echo and transesophageal echocardiography were normal. We diagnosed his illness as sepsis from severe odontitis with disseminated infections including scalene myositis, C3-7 spinal nerves radiculitis, brachial plexitis, and septic pulmonary embolisms. We empirically started Ceftriaxone ( $2 \mathrm{~g}$ daily) and Metronidazole ( $1.5 \mathrm{~g}$ daily), mainly targeting oral anaerobes. In addition, we controlled his blood sugar level at least always below $200 \mathrm{mg} / \mathrm{dl}$. His periodontal disease was treated with strict oral hygiene, resection of poor granulation tissues and decayed teeth, and removal of dental calculi. According to the positive result of blood cultures of anaerobic bacteria, P. micra, obtained 1 week later, we changed antibiotics to intravenous ampicillin-sulbactam (12 g daily), which was given for another week. The successful treatments with intravenous antibiotics were followed by 8 weeks of oral minocycline (200 mg daily) on the outpatient basis. After the cessation of antibiotics, the patient didn't show any signs or symptoms of recurrence.

DISCUSSION: $P$. micra is a nonspore-forming anaerobic gram-positive coccus widely distributed as commensal flora in the oral cavity that, under immunosuppressed conditions including poor controlled diabetes mellitus or traumatic conditions, can become pathogenic and cause sepsis, brain, liver, and thoracic infections, as well as generalized necrotizing soft tissue infections. Its strong proteolytic activity may 
be significant in the development of mixed extraoral anaerobic abscesses. Although P. micra was considered to be a relatively rare cause of disseminated infection, it has increasingly been recognized as an important oral pathogen.

FIBRILLARY GLOMERULONEPHRITIS: TIME TO LOOK BEYOND AMYLOID! ZAIN UL ABIDEEN ASAD ${ }^{1}$; Amna Mohyud Din Chaudhary ${ }^{3}$; Usman Z. Bhutta ${ }^{2} .{ }^{1}$ UNIVERSITY OF OKLAHOMA HEALTH SCIENCES CENTRE, Oklahoma City, OK; ${ }^{2}$ University of Oklahoma Health Sciences Centeriversity of Oklahoma Health Sciences Center, Oklahoma City, OK; ${ }^{3}$ Nishtar Medical College \& Hospital, Multan, Pakistan. (Control ID \#2469929)

LEARNING OBJECTIVE \#1: Recognize fibrillary glomerulonephritis as a cause of proteinuria and renal failure to increase awareness among health care professionals about this rare condition.

LEARNING OBJECTIVE \#2: Recognize Focal Segmental Glomerulosclerosis as an uncommon morphology in fibrillary glomerulonephritis.

CASE: Introduction: Fibrillary glomerulonephritis is a rare disorder with a prevalence of $1 \%$ in renal biopsies. The mean age of presentation is 50 years, proteinurea in $100 \%$, nephrotic syndrome in $70-75 \%$, renal insufficiency $(\mathrm{Cr} \geq 1.5)$ in $50-55 \%$, hypertension $70 \%$ and hematuria in $70 \%$ cases. Etiology is unknown and diagnosis is established by pathognomonic electron microscopy findings. This case illustrates the presentation, workup and diagnosis of fibrillary glomerulonephritis (FGN). Case Description: A 49 year-oldfemale with history of hypertension, obstructive sleep apnea, non-steroidal anti inflammatory drug use for chronic back pain presented with shortness of breath on exertion and bilateral lower extremity swelling. Vital signs showed temperature $35.6 \mathrm{C}$, heart rate 75 / $\mathrm{min}$, respiratory rate $21 / \mathrm{min}$ and blood pressure $112 / 68 \mathrm{mmHg}$. She had mild respiratory distress, bilateral crackles at lung bases, normal heart sounds, no murmurs and bilateral +2 pedal edema on physical examination. Complete blood count showed hemoglobin $10.7 \mathrm{~g} /$ $\mathrm{dl}$ and normal white cell count. Complete metabolic panel showed creatinine $1.1 \mathrm{mg} / \mathrm{dl}$, blood urea nitrogen $21 \mathrm{mg} / \mathrm{dl}$, albumin $2.6 \mathrm{~g} / \mathrm{dl}$ and normal electrolytes. Urinalysis showed $\mathrm{pH} 6.0$, specific gravity $1.028,+4$ proteinuria, +2 hematuria and no casts. 24 h urine collection showed $4.3 \mathrm{~g} /$ day nephrotic range proteinuria. Lipid profile showed cholesterol $308 \mathrm{mg} / \mathrm{dl}$ and non-HDL cholesterol $246 \mathrm{mg} / \mathrm{dl}$. Hepatitis C, HIV, Goodpasture Disease, Cryoglobulinemia, Systemic Lupus Erythematosus and other autoimmune diseases were ruled out by appropriate tests. Serum and urine protein electrophoresis were without Mspike but with high free Lambda $(29.7 \mathrm{mg} / \mathrm{dl})$ and Kappa chains $(23.6 \mathrm{mg} / \mathrm{dl})$. A diagnosis of nephrotic syndrome was made due to the presence of edema, proteinuria, hypoalbuminemia and hyperlipidemia. A renal biopsy was done to evaluate the etiology of nephrotic syndrome. Biopsy showed focal segmental and global glomerulosclerosis with mild to moderate interstitial fibrosis and tubular atrophy. Immunofluorescence showed staining of glomerular capillary walls and mesangium for IgG, kappa and lambda. Electron microscopy showed linear, non- branching fibrils in the mesangium that averaged $20 \mathrm{~nm}$ in width (11.7-28.6 nm). Congo red staining was negative. A diagnosis of nephrotic syndrome with FGN was made due to characteristic electron microscopy apparence.

DISCUSSION: This case illustrates the clinical presentation, workup and diagnosis of fibrillary glomerulonephritis (FGN). This patient presented with edema, hypertension, shortness of breath, nephrotic proteinuria and the electron microscopy findings on biopsy established the diagnosis. A study of 66 cases identified most common histologic patterns as mesangial, membranoproliferative, endocapillary proliferative, crescentic and necrotizing, membranous and diffuse sclerosing in order of likelihood. This case had focal segmental and global glomerulosclerosis that is a rare finding. In the same study immunofluorescence staining was positive for IgG in $100 \%, \mathrm{C} 3$ in $92 \%$ and both kappa and lambda in $84 \%$ cases. This case also demonstrated positive staining for IgG, kappa and lambda. The pathognomonic finding is presence of fibrillary deposits in the mesangium and glomerular capillary walls distinct from amyloidosis. The size of fibrils ranges from 8 to $15 \mathrm{~nm}$ in Amyloid and 12-24 nm in FGN. IgG is usually monoclonal in AL Amyloid as compared to polyclonal in FGN. The characteristic difference from amyloid is absence of reaction to histochemical dyes like Congo Red and Thioflavin T in FGN. One third of FGN cases are associated with malignancy, monoclonal gammopathy and autoimmune disorders. In our case an extensive workup was negative for all these conditions. Angiotensin inhibitors (ACEI) are used if the glomerular filtration rate is normal and proteinuria is present, to control blood pressure and reduce disease progression. Evidence for use of steroids and immunosuppressants is based on uncontrolled studies with variable success. In our patient a limited trial of corticosteroids with mycophenolate resulted in some improvement in proteinuria along with ACEI.

FLASH PULMONARY EDEMA-NO, IT'S NOT MY HEART! Vishal K. Patel ${ }^{2}$; Nway Koko ${ }^{2}$; Maryam Qadir ${ }^{1,2}$; Sayed K. Ali ${ }^{1,2}$. ${ }^{1}$ Orlando VA Medical Center, Orlando, FL; ${ }^{2}$ University of Central Florida, College of Medicine, Orlandi, FL. (Control ID \#2468503)

LEARNING OBJECTIVE \#1: A. Recognizing flash pulmonary edema as a cause of renal artery stenosis

LEARNING OBJECTIVE \#2: B. A unique scenario where revascularization might be superior to medical management of renal artery stenosis.

CASE: Flash pulmonary edema most often occurs when there has been an insult to the pumping function of the cardiac system. However, flash pulmonary edema due to renal artery stenosis remains rare. We present a intricate case of recurrent flash pulmonary edema in a patient with uncontrolled hypertension and renal artery stenosis. A non- tanned 68-year-old male with a history of coronary artery disease and uncontrolled hypertension, presented to our clinic complaining of monthly episodes of shortness of breath, due to flash pulmonary edema over the past 6 months. These episodes occurred suddenly, and repeatedly required diuresis in an inpatient setting. Upon evaluation, the patient was found to have a history of difficult to control hypertension, despite a treatment regimen consisting of four antihypertensive agents, including a diuretic. His physical exam revealed an obese male with a mid-pitched systolic murmur and bilateral lower extremity trace edema. A review of his past medical records showed a normal sleep study and a 2D echo showing a normal EF of $65 \%$ with moderate aortic stenosis. His creatinine was reported at $2.1 \mathrm{mg} / \mathrm{dL}$, renin $27.47 \mathrm{ng} / \mathrm{mL} / \mathrm{h}$ and an aldosterone of $2 \mathrm{ng} / \mathrm{dL}$. His urinalysis was normal with no hematuria or proteinuria. Further workup including an ANA, SPEP, UPEP and complement level analysis was performed, and returned normal. A renal ultrasound showed a left kidney measuring $9.8 \times 4.5 \times 4.6 \mathrm{~cm}$ while the right kidney measured $11.6 \times 4.6 \times 5 \mathrm{~cm}$ without any evidence of hydrouretronephrosis; however mild prostatic hypertrophy was noted. Due to his elevated creatinine and recurrent flash pulmonary edema, an abdominal MRA without contrast was pursued, which showed significant stenosis of his bilateral renal arteries. A CO2 angiogram of both renal arteries confirmed the diagnosis; high-grade renal artery stenosis due to atherosclerotic plaque, which responded well to bilateral balloon angioplasty and primary stenting. The patient did well post operatively, and was later discharged home. At time of follow up examination, his creatinine had improved to $1.5 \mathrm{mg} / \mathrm{dL}$. His blood pressure had gradually improved as well with home readings ranging from $130-140 / 70-80 \mathrm{mmHg}$. He was followed in our clinic at months 1,3 and 6 following his stenting, and has remained asymptomatic since without any recurrence of flash pulmonary edema.

DISCUSSION: Atherosclerotic renal artery stenosis is defined as a $>50 \%$ stenosis of the proximal one third of the renal artery. It has been found to be associated with renal failure, uncontrolled hypertension, and sudden onset fluid overload with resultant flash pulmonary edema. The exact mechanism of flash pulmonary edema is not fully understood but is 
most likely related to the renin-angiotensin-aldosterone system. The management of renal artery stenosis has yielded much debate with recent studies suggesting no added benefit to stenting over medical therapy. The role of revascularization in alleviating flash pulmonary edema has been studied in small prospective and observational trials, showing improvement in renal function and symptoms. Individualized therapies should be considered based on the clinical scenario, after weighing the risks and benefits of each treatment modality. In this particular patient, due to his frequent episodes of flash pulmonary edema, revascularization was pursued and proved to be successful overall. This case highlights one of the few scenarios in which revascularization was found to be superior to medical management in this unique presentation of renal artery stenosis.

FLUSHING OUT VIBRIO PARAHAEMOLYTICUS INFECTIONS: NOT JUST A CAUSE OF GI DISTRESS Amir Kazerouninia ${ }^{2}$; Nicolas Cortes-Penfield ${ }^{2}$; Barbara Trautner ${ }^{1} .{ }^{1} \mathrm{BCM} / \mathrm{VA}$ Hospital, Houston, TX; ${ }^{2}$ Baylor College of Medicine, Houston, TX. (Control ID \#2469475)

LEARNING OBJECTIVE \#1: Identify patients susceptible to more severe manifestations of Vibrio parahaemolyticus infection.

CASE: A 70-year-old gentleman with morbid obesity, uncontrolled diabetes mellitus, heart failure with preserved left ventricular function, and atrial fibrillation on warfarin presented to the emergency room following collapse at home. In the preceding days, he had experienced non-bloody diarrhea with progressive lower extremity swelling and erythema. Although he denied fever and chills, his wife stated he had appeared sweaty and ill. Review of systems was positive for progressive complaints of shortness of breath orthopnea, and paroxysmal nocturnal dyspnea. He denied focal weakness, loss of consciousness, and palpitations prior to collapse. Social history, which was not elicited in the emergency room or initially upon admission, indicated that the patient enjoyed fresh water fishing and wading in Gulf waters (but had done neither recently), and that he had eaten seafood gumbo from a restaurant 5 days prior to his collapse. Vitals at presentation were temperature of $98.2^{\circ} \mathrm{F}$, heart rate of 90 , blood pressure of $109 / 62$, and respiratory rate of 18. Examination revealed a sallow gentleman with bilateral $2+$ pitting edema in the lower extremities with erythema, warmth, tenderness to palpation, and several tense, purple bullae. Electrolytes, blood count, liver profile, and coagulation studies were notable for leukocytosis of 14,800 cells $/ \mathrm{ml}$ with a neutrophil predominance of $85 \%$, INR of 7.79 , and total bilirubin of 1.8. The emergency room physician held warfarin then started furosemide, vancomycin, and cefepime, and admitted the patient to acute care where antibiotics were changed to clindamycin. The patient deteriorated within $24 \mathrm{~h}$ of admission with worsening skin findings and the development of sepsis. Ertapenem was started and later changed to cefepime. Admission blood cultures returned Vibrio parahaemolyticus sensitive to levofloxacin. Of note, only at this point was the patient's ingestion of seafood elicited. Following change in antibiotics, he improved and was discharged on hospital day seven to a skilled nursing facility, to complete 10 days of Gram-negative coverage on oral levofloxacin and for routine management of acute on chronic heart failure.

DISCUSSION: This case illustrates the importance of a thorough social history upon admission; the patient's ingestion of seafood was an obvious risk factor for V. parahaemolyticus. More importantly, however, this case presents an unusually severe illness given the organism involved and the routine mechanism of infection. $V$. parahaemolyticus is a halophilic gram-negative bacillus found in warm coastal waters; infection is acquired by consumption of undercooked seafood or wound exposure to contaminated water. Annually, it causes 4,500 infections, with 215 culture-confirmed cases, 30 hospitalizations, and 1-2 deaths. V. parahaemolyticus typically causes gastroenteritis after ingestion or cellulitis after wound exposure, while the syndrome of bullae, sepsis, and bacteremia as seen here is more commonly caused by ingestion of $V$. vulnificus. In a study by Hou et al., $59.8 \%$ of patients with vibrio infection (non Vibrio cholera) presented with acute gastroenteritis, with $V$. parahaemolyticus responsible for $92.1 \%$ of those cases. In contrast, $V$. vulnificus, the prototypic invasive human pathogen in this genus, was responsible for all cases of bacteremia in the aforementioned study. Even though it is extremely rare, $V$. parahaemolyticus sepsis can occur and can be fatal if not rapidly diagnosed, as the organism usually has skin manifestations suggestive of skin and soft tissue infections, but is not susceptible to empiric antibiotics routinely used to treat these infections. A case report by Ahmad et al. identified a patient who had fatal necrotizing fasciitis and sepsis from a wound infection by $V$. parahaemolyticus after sustaining a water skiing injury in brackish water. What makes our patient unique is that he did not have any wound exposure; his exposure was most likely through consumption of undercooked seafood. Given that the majority of V. parahaemolyticus infections cause only mild infection, it is important to identify the subset of patients at increased risk for severe, life-threatening disease. In a study of vibrio infections, of patients with V. parahaemolyticus infection, those who presented with sepsis had a greater incidence of diabetes, alcoholism, and liver disorders than patients who presented with gastroenteritis alone. In our case, diabetes may have been the predisposing factor for invasive disease and the elevated INR and bilirubin may have been indicative of underlying liver dysfunction. Of note, the patient from the Ahmad et al. case report was similarly morbidly obese and had diabetes mellitus.

FOLLOWING THE CLUES IN THE DISTRIBUTION: FEVER AND PHOTODISTRIBUTED RASH IN A PATIENT WITH AIDS AND LATELATENT SYPHILIS Alan H. Baik; Madhavi Dandu. University of California, San Francisco, San Francisco, CA. (Control ID \#2469840)

LEARNING OBJECTIVE \#1: Recognize the diagnostic value of the distribution of an unidentified rash in a patient co-infected with HIV and syphilis

LEARNING OBJECTIVE \#2: Describe the manifestations of HIV photodermatitis

CASE: A 47 year-old Samoan man presented to the emergency room with 6 months of pruritic rash on the dorsal aspects of his bilateral hands, anterior lower extremities, and posterior neck. He reported fevers, chills, night sweats, and 50-pound weight loss. He also endorsed 2 weeks of bilateral thigh myalgias. He did not take any medications or supplements. He reported unprotected sex only with his wife, with whom he was in a monogamous relationship. He denied use of injection drugs. He had tattoos from Samoa that were made using sticks from a lime tree, which were not shared with others. $\mathrm{He}$ reported inconsistent use of protective clothing while working in construction as a pipe layer. On examination, the patient was febrile to $39.4^{\circ}$ Celsius, blood pressure was 148 $85 \mathrm{mmHg}$, and heart rate was 115 beats per minute. He had decreased breath sounds in bilateral upper lung fields. Skin examination revealed hyperpigmented thick plaques on his posterior neck, scaly hyperpigmented violaceous papules coalescing into lichenified plaques on the dorsal aspects of his hands and forearms, and scaly papular nodules with areas of hyper- and hypo-pigmentation on his anterior lower extremities. His trunk, palms, soles, and mucocutaneous membranes were spared. There was no cervical, axillary, or inguinal lymphadenopathy. Admission labs included WBC $2.5 \times 10^{9} / \mathrm{L}$ with ANC $1.28 \times$ $10^{9} / \mathrm{L}, \mathrm{Cr} 1.62 \mathrm{mg} / \mathrm{dL}, \mathrm{LDH} 472 \mathrm{U} / \mathrm{L}$, lactate $1.2 \mathrm{mmol} / \mathrm{L}$. ANA, dsDNA, anti-Smith, and complement levels were negative. Subsequent lab results included positive HIV antibody with CD4 count $57 \times 10^{6} / \mathrm{L}$ and viral load 5771 copies $/ \mathrm{mL}$. Rapid plasma reagin was positive with a 1:1 titer for treponemal antibody. Skin biopsy from his posterior neck prior to exposure to penicillin showed lichenoid interface dermatitis with plasma cells, and was negative for fungi, bacteria, and mycobacteria. Treponema pallidum immunoperoxidase stain was negative. Histopathology was consistent with lichenoid photoeruption of HIV. He was initiated on Emtricitabine/Tenofovir and Dalutegravir. He was discharged with plans to complete treatment for late-latent syphilis.

DISCUSSION: This case highlights the clinical dilemma of fever and rash in a patient coinfected with HIV and syphilis. The photodistributed pattern of the rash narrowed the 
differential into four broad categories: medication side effect, autoimmune, inherited, and infectious. The presence of fever and absence of medication use made autoimmune and infectious causes more likely; his autoimmune work up was negative. His fever was ultimately attributed to a concomitant infection with Pneumocystis pneumonia, rather than related to the rash. Skin biopsy was notable for lichenoid interface dermatitis with predominance of plasma cells, and negative immunoperoxidase stain for Treponema pallidum, most consistent with HIV lichenoid photodermatitis. Rashes in sun exposed areas are often secondary to medications or due to photoexacerbated dermatoses, most commonly cutaneous lupus erythematosus and dermatomyositis. Approximately $5 \%$ of HIV-seropositive patients have HIV photodermatitis, which includes actinic prurigo, porphyria cutanea tarda, and lichenoid photoeruption. Many of these rashes appear similar to each other, often with hyperpigmented and lichenified plaques. Porphyria cutanea tarda usually causes a blistering rash. Chronic lichenoid actinic photodermatitis is characterized by hyperpigmented, pruritic, and scaly lesions on the neck, upper chest, and dorsal surfaces of the hands and forearms. AIDS interface dermatitis causes a lichenoid reaction pattern rash that can resemble erythema multiforme or a fixed drug eruption. It is often preceded by new medication use, which was not present in this case. Histopathology is important in the diagnosis of HIV photodermatitis due to non-specific skin findings. In this case, it was unclear if the patient's rash was a manifestation of syphilis or HIV, especially since syphilitic rashes can be altered in the setting of HIV co-infection and are typically characterized by plasma cell predominance on pathology. Lichenoid reactions can develop in late stages of secondary syphilis, and cutaneous lesions can re-appear in latent syphilis after the disappearance of lesions in secondary syphilis even without treatment. However, it is unusual for syphilitic rashes to have a photodistributed pattern. Negative immunochemistry and silver stains do not exclude the diagnosis of cutaneous syphilis, as their sensitivities are $70-90 \%$ and $40 \%$, respectively. In conclusion, this patient's rash was thought to be HIV lichenoid photodermatitis based on appearance and histopathology findings. It was unlikely to be secondary to syphilis due to its photodistributed pattern, as well as the patient's low RPR titer and negative T. pallidum immunoperoxidase stain.

FROM GASTROENTERITIS TO BILATERAL THALAMIC HEMORRHAGE: A CASE OF THIAMINE DEFICIENCY IN A NON-ALCOHOLIC PATIENT Brennan S. Mosch; Natasha Kassim; Jill Zupetic; Dayakar Kancherla. University of Pittsburgh Medical Center, Pittsburgh, PA. (Control ID \#2466294)

LEARNING OBJECTIVE \#1: Recognize bilateral thalamic hemorrhage as a rare, but recognized complication in patients with thiamine deficiency

LEARNING OBJECTIVE \#2: Identify risk factors for development of thiamine deficiency

CASE: A 48 year-old white male with a past medical history of morbid obesity, hypertension, and coronary artery disease was admitted to the ICU after he was found unresponsive and incontinent of urine at home. Four weeks prior to presentation, family members reported acute onset of abdominal cramping, nausea, vomiting, diarrhea, anorexia, and poor oral intake. A week later, he developed unsteady gait and worsening fatigue when he confined himself to bed for most of the day. Over the following weeks, his fatigue worsened and his oral intake remained poor. Initial work-up was largely negative. Patient was afebrile with stable vitals. Neurologic exam was limited on account of patient's lethargy and confusion, but notable for absence of nystagmus, presence of spontaneous movement of all extremities, normal deep tendon reflexes bilaterally of upper and lower extremities, and downgoing plantar reflexes bilaterally. Labs were remarkable for mildly elevated creatinine, normoglycemia, and negative toxicology screen. Initial computerized tomography (CT) scan of the head, electroencephalogram, and cerebrospinal fluid analysis were unremarkable. Per his family members, he maintained a poorly nutritious diet consisting strictly of "pizza and chips." He was not known to consume alcohol. Intravenous infusion of normal saline resulted in transient improvement in his level of alertness. Within $24 \mathrm{~h}$ the patient became more obtunded and was intubated for airway protection. Subsequent magnetic resonance imaging study of his brain demonstrated bilateral thalamic and intraventricular hemorrhages. CT venogram and cerebral angiogram ruled out cerebral venous thrombosis and arteriovenous malformation, respectively. An external ventricular drain was placed for treatment of the cerebral hemorrhage. A blood thiamine level was noted to be markedly low at $37 \mathrm{nmol} / \mathrm{L}$ (normal reference range: 78 to 185), confirming the diagnosis of severe thiamine deficiency. Thiamine was repleted intravenously with gradual improvement in alertness over the following weeks. However, expressive aphasia and unsteady gait were among persistent remaining deficits. He was discharged to a long-term acute rehabilitation facility hoping for further recovery. DISCUSSION: We present a case of severe thiamine deficiency in a non-alcoholic presenting with bilateral thalamic hemorrhage, a complication of thiamine deficiency that has been reported very rarely in the literature. Thiamine (or vitamin B1) acts as a coenzyme in a number of cellular processes, most notably, processes involving carbohydrate metabolism and glucose generation. Thiamine is ubiquitous in most foods and adequate stores are easily obtained with a typical western diet. Due to its short half-life of 9-18 days, deficiency can result from poor nutrition and/or high catabolic states (e.g. hyperthyroid, infection, etc.). Thiamine deficiency, also referred to as beriberi, presents in two forms: dry beriberi when the nervous system is affected and wet beriberi when the cardiovascular system is affected. Classically, thiamine deficiency is associated with alcoholics who present with Wernicke's encephalopathy, which is characterized by a triad of confusion, ophthalmoplegia, and ataxia. In our case, the patient likely had a poor reserve of thiamine due to the lack of diversity in his diet, which consisted of mostly processed foods ("pizza and chips"). A bout of gastroenteritis likely further precipitated acute thiamine deficiency from poor oral intake and rapid depletion of previously compromised reserves during the illness. His initial clinical signs of thiamine deficiency were new onset gait disturbance, severe fatigue, and persistent anorexia that had further exacerbated the deficiency. This ultimately led to encephalopathy and stupor. Our case highlights the development of bilateral thalamic hemorrhages as a rare complication of severe thiamine deficiency. Interestingly, a common gastroenteritis infection precipitated this near-fatal complication. Our case illustrates how some symptoms of thiamine deficiency, such as anorexia and fatigue, can perpetuate those very symptoms into an everworsening deficiency. Due to the short half-life of thiamine (9-18 days), clinicians should consider the possibility of deficiency when evaluating patients with a history of poor oral intake over a period of weeks, regardless of history of alcohol use.

FROM PNEUMONIA TO CABG- A CALL FOR CALCIUM REPORTING ON IMAGING Teresa Ratajczak ${ }^{1}$; Ameesh Vora $^{2}$; Arpan Patel ${ }^{1}$; Timothy G. Petrie ${ }^{1}$; Mukul Chandra ${ }^{1,2}$. ${ }^{1}$ Wright State University, Beavercreek, OH; ${ }^{2}$ Wright State University, Dayton, OH. (Control ID \#2431778)

LEARNING OBJECTIVE \#1: Coronary artery calcium can be identified on nongated chest computer tomography, and should be considered suggestive of coronary artery disease. It is an underreported, noninvasive finding that could have great impact on patient morbidity.

LEARNING OBJECTIVE \#2: With the increased use of CT scans of the chest in the emergency department and for lung cancer screening, a more uniform reporting of vessel calcifications can lead to secondary prevention of coronary artery disease.

CASE: 56 year old Caucasian female with past medical history significant for diabetes mellitus type II, hypertension, hyperlipidemia and family history of cardiac disease was found to have a pulmonary irregularity on standard chest xray done to rule out pneumonia. She subsequently underwent a CT chest to evaluate the nodule which showed a benign calcified lesion. The radiologist commented on the moderate to severe calcific plaques in 
the left anterior descending, circumflex and right coronary arteries. She was referred to Cardiology where she described a 1-year history consistent with angina pectoris, endorsed substernal chest pain occurring on exertion and relieved with rest. Lexiscan stress test showed moderate size reversible perfusion defect and a moderate scar on the posteriorlateral wall of the heart. Cardiac catheterization revealed multivessel disease and a coronary artery bypass graft surgery was recommended. Patient was started on beta-blocker and continued on statin and aspirin. She underwent a triple bypass surgery and continued on optimal medical therapy for risk and lifestyle modification.

DISCUSSION: Coronary artery calcium scanning (CACS) is a method of noninvasively evaluating the coronary arteries in order to check for the presence of atherosclerosis. It is used to risk stratify patients in the intermediate ASCVD risk group of 5-7.5 \%. The technique uses ECG gated CT imaging synchronized to diastole, to minimize motion artifacts. A score of 0 means the risk of coronary artery disease is very low, 101 to 400 suggests mild disease, and $>400$ suggests a high likelihood of at least one significant coronary narrowing However, most chest CT scans that are done on a daily basis in inpatient and outpatient settings are nongated. It is estimated that about 13 million nongated CT scans are performed annually, in contrast to 0.7 million gated exams Several studies have shown a favorable correlation between coronary calcium assessment using nongated and gated images. With the increased use of chest CT scan for lung cancer screening, one study found that $>40 \%$ of patients had a CAC of $>1000$, and were not taking the appropriate statin or antihypertensive medications. Our patient was diagnosed with coronary disease using standard nongated $\mathrm{CT}$ imaging that was done due to suspicion of pneumonia Conclusion: Reporting of coronary artery calcification on nongated chest imaging represents a great opportunity to implement strategies for primary and secondary prevention in order to reduce morbidity of patients

FROM SEAWATER TO BLOODSTREAM Amy J. Sheer ${ }^{1}$; Kristopher P. Kline ${ }^{1}$; Dhaval K. Naik ${ }^{2}$; Sherri Gampel ${ }^{2}$; Margaret C. Lo ${ }^{2}{ }^{1}$ University of Florida, Gainesville, FL; ${ }^{2}$ University of Florida College of Medicine, Gainesville, FL. (Control ID \#2469666)

LEARNING OBJECTIVE \#1: Compare and contrast the clinical and therapeutic features of Vibrio vulnificus vs. Vibrio cholera infection

LEARNING OBJECTIVE \#2: Identify the risk factors and poor prognostic indicators for Vibrio vulnificus sepsis

CASE: A 77 years-old male with history of chronic renal insufficiency (creatinine $1.4 \mathrm{mg}$ / $\mathrm{dL}$ ) and provoked DVT on warfarin presented with an acute onset of a blistering rash on his right arm with severe pain, vomiting, chills and dizziness. He further reported crabbing the day prior in the Gulf of Mexico and being stuck multiple times by crab claws. Exam revealed hypotension $(82 / 51 \mathrm{mmHg})$, confusion and severely tender ecchymosis with hemorrhagic bullae of right forearm. Initial labs showed leukocytosis $(13200 / \mathrm{uL})$ with $81 \%$ PMN, acute on chronic renal dysfunction (creatinine $2 \mathrm{mg} / \mathrm{dL}$ ) and lactic acidosis (5.4 mmol/L) but normal CPK, D-dimer and LFTs. Right arm x-ray showed soft tissue edema of superficial and deep tissues but no gas or foreign body. Patient was admitted to the MICU in septic shock, requiring vasopressor support. Vancomycin, doxycycline and ceftriaxone were empirically started. Surgery was consulted for concerns of compartment syndrome or necrotizing fasciitis but felt no surgical intervention was required. Aggressive wound care was continued. Over the next $24 \mathrm{~h}$ patient developed profound bandemia ( $25 \%$ ) and mild CPK elevation ( $240 \mathrm{U} / \mathrm{L}$ ) but was successfully weaned off vasopressors. Blood cultures grew out pan-sensitive Vibrio vulnificus. Antibiotic regimen was narrowed to ceftriaxone and doxycycline. Patient improved on this regimen and was discharged on a 6-week course of IV antibiotics with close follow-up with PCP, surgery, and wound care. Over the next 3 months, he continued to suffer from ongoing right arm pain, requiring 2
ED visits and 2 readmissions for cellulitis with additional antibiotic regimen of cefepime then keflex.

DISCUSSION: This case illustrates the growing incidence of Vibrio vulnificus infection in the Gulf Coast and its associated rapid morbidity. It further adds to the literature on the important distinction between the rare Vibrio vulnificus infection and the well-known Vibrio cholera infection. Vibrio illnesses are classified into 2 categories - cholera from $V$. cholera and vibriosis from $V$. vulnificus. Both are gram negative rod bacteria in estuarine and marine settings. Ingestion of contaminated seafood is the primary mode of infection. $V$. cholera either causes no symptoms or acute diarrhea which is usually mild and seldom life-threatening ( $\sim-10 \%)$. Infection is rare in the US but common in third-world nations. Antibiotics are hardly indicated and prompt high-volume rehydration results in full recovery, even in severe cases. On the other hand, V. vulnificus is found worldwide in any warm coastal waters, peaking during the summer months and in the Gulf Coast region. The increased incidence in the Gulf Coast is likely due to exposure of skin and soft-tissue injuries to contaminated waters. This is surely the mode of infection for our patient. Social history of such exposure and associated risk factors is of utmost importance. Typically, $V$. vulnificus causes three severe syndromes: primary bacteremia, wound infection and GI illness but pneumonia, osteomyelitis, eye infections, meningitis, and endocarditis have also been reported. As true for our patient, $V$. vulnificus septicemia presents as abrupt fever and/or chills followed by hypotension and metastatic cutaneous lesions, which rapidly evolve into hemorrhagic bullae and necrotic cutaneous ulcers. The presence of hemorrhagic cutaneous bullae or necrotizing fasciitis is an important predictor of mortality. The presence of lesions involving two or more limbs and septic shock increases mortality risk. Over $50 \%$ have a median survival of $\sim 2$ days. These 3 poor prognostic features may explain our patient's ongoing morbidity. The most severe $V$. vulnificus cases have been reported in certain high-risk conditions, including chronic hepatitis, immunodeficiency states, iron-storage disorders, ESRD and diabetes. Those with chronic liver diseases are at highest risk with over 80-times higher likelihood of $V$. vulnificus septicemia. Yet our patient's immunocompetent state should not mislead clinicians as severe cases have been reported in healthy individuals. Early surgical intervention (e.g. incision/drainage, debridement, fasciotomy, or amputation) is essential in treating soft tissue infections from V. vulnificus and significantly decreases mortality. This is the reason for our prompt surgical consultation. Immunocompromised people should be advised to avoid consuming raw seafood and exposing wounds to seawater; all must wear gloves while handling seafood. In addition to prevention, a detailed history of exposure, assessment of risk factors and identification of prognostic indicators are vital to expedite proper treatment and prevent the rapid morbidity and mortality of $V$. vulnificus infection.

FROZEN: A RARE CASE OF PARALYSIS IN A 29 YEAR OLD MAN Li-Chi Hung; Jennifer Mandal. Kaiser Permanente, San Francisco, CA. (Control ID \#2469896)

LEARNING OBJECTIVE \#1: Diagnose and recognize the clinical presentation of periodic paralysis

LEARNING OBJECTIVE \#2: Treat severe hypokalemia caused by thyrotoxic periodic paralysis

CASE: A 29 year old otherwise healthy Asian man presented to the ED with 3 weeks of intermittent bilateral leg and arm weakness, and 1 day of repeated falls. He denied pain in his extremities or head trauma. On review of systems, he endorsed palpitations, heat intolerance, and frequent bowel movements. His only medication was an over-the-counter antihistamine as needed for allergic rhinitis. He denied illicit drug use or excessive alcohol. On physical exam, temperature was 97.7 , blood pressure was $167 / 76 \mathrm{mmHg}$, and heart rate was 101/min. Cardiac exam was notable for tachycardia and a soft systolic murmur. On neuro exam, he was alert and oriented, but he was unable to move against gravity in all four extremities. Deep tendon reflexes were absent, and sensation was grossly intact. 
Laboratory tests revealed severe hypokalemia at $1.3 \mathrm{mEq} / \mathrm{L}$ (normal 3.5-5.3) without evidence of potassium wasting in urine. Subsequent testing showed thyroid stimulating hormone $<0.01 \mathrm{uIU} / \mathrm{mL}$ (normal $0.10-5.50$ ) and free T4 at $4.3 \mathrm{ng} / \mathrm{dL}$ (normal $0.8-1.7$ ). EKG showed a junctional rhythm and presence of $U$ waves, consistent with hypokalemia. The patient was admitted to ICU for close monitoring and treatment. He received potassium repletion and had regained some strength in his extremities within hours. Nerve conduction study and electromyography showed findings most consistent with non-irritative myopathic process. The patient's strength normalized to baseline after potassium repletion. His course was complicated by asymptomatic rebound hyperkalemia. He was discharged home on propranolol and methimazole with advice to avoid excessive carbohydrate. Office thyroid ultrasound showed small symmetric vascular heterogeneous goiter, consistent with Graves' disease. Ultimately, the patient was diagnosed with hypokalemia periodic paralysis secondary to hyperthyroidism from Graves' disease. He subsequently underwent thyroidectomy and was recovering well.

DISCUSSION: Hypokalemic periodic paralysis (PP), characterized by episodes of painless muscle weakness, is a rare neuromuscular disorder. This condition can be either hereditary, or acquired, in which case it is commonly in association with thyrotoxicosis. The clinical features of thyrotoxic PP are symptoms of hyperthyroidism and infrequent weakness attack lasting hours to days, usually affecting proximal muscles more than distal, and the legs more than the arms, together with hyporeflexia. Bulbar and respiratory muscles may be mildly affected, but consciousness is preserved. Symptoms are precipitated by exercise, carbohydrate load, or stress. The highest incidence is seen among Asian men. The mechanism by which hyperthyroidism induces hypokalemic PP is not well understood. It has been proposed that thyroid hormone increases tissue sensitivity to betaadrenergic stimulation, which increase sodium-potassium ATPase activity on the skeletal muscle membrane, thus driving potassium into cells. This leads to hyperpolarization of membrane and relative inexcitability of muscle fibers, resulting in muscle weakness and hyporeflexia. Treatment for acute thyrotoxic PP consists of cardiac monitoring and incremental replacement of potassium chloride (10-60 $\mathrm{mEq}$ per hour) until potassium normalized with careful monitoring for $24 \mathrm{~h}$, as posttreatment hyperkalemia may occur due to intracellular shift. Dextrose should be avoided. The definitive treatment is to reach euthyroid state with standard workup and treatment for hyperthyroidism. To prevent attacks, low carbohydrate diet and refraining from alcohol and vigorous exercise are advised. Symptomatic potassium supplementation may be used. In contrast to familial hypokalemic PP, carbonic anhydrase inhibitors have not been shown to be beneficial in thyrotoxic PP. This case illustrates the importance of recognizing thyrotoxic PP as a rare but treatable cause of severe muscle weakness. With careful correction of severe hypokalemia in the acute phase and long-term management for hyperthyroidism, these patients can have excellent prognoses.

GALLOPING GAMMOPATHIES John L. Elliott; Jennifer Bracey. Emory University, Decatur, GA. (Control ID \#2469422)

LEARNING OBJECTIVE \#1: Recognize how macroglobulinemia can mimic CHF exacerbations

LEARNING OBJECTIVE \#2: Discuss the complications of treatment in advanced cases of macroglobulinemias

CASE: Mrs. H was a 64 year old African American woman who presented with shortness of breath, cough, orthopnea, and lower extremity swelling that began several months ago. Her symptoms had become much worse over the past week and prevented her from performing some activities of daily living. Her past medical history was significant for hypertension, coronary artery disease, and GERD. All were being treated appropriately. Patient's pressure was $98 / 65$, pulse 115 , and respiratory rate 18 with $96 \% \mathrm{O}_{2}$ saturation on room air. Her physical exam revealed a cachectic appearing woman with $3+$ edema to her umbilicus, jugular venous distention of the neck, several $0.5 \mathrm{~cm}$ papules on her forehead, tachycardia with a prominent $\mathrm{S} 3$, prominent inspiratory crackles bilaterally with decreased breath sounds, dullness to percussion at the lung bases, and hepatomegaly. Labs showed a hemoglobin of 7.1, white blood cell count of 6.5 , sodium of 128 , potassium of 3.9 , chloride of 91, anion gap of $6, \mathrm{BUN}$ of 18 , and Creatinine of 0.7 . A chest $\mathrm{x}$-ray revealed bilateral pleural effusions. Mrs. H was admitted for a suspected CHF exacerbation. As standard CHF treatment did not provide relief a diagnostic/therapeutic thoracentisis revealed a triglyceride count of 256 , which is diagnostic of a chylothorax, and a previous $\mathrm{CBC}$ was found to have rouloux. Further examination of the papules were shown to be BCell CD 20+ Lymphoma infiltrating the dermis. Patient also admitted to substantial weight loss in the last year. A lymph node biopsy was performed and patient was initiated on treatment for Waldenström's macroglobulinemia. After one round of chemotherapy, patient decided to forgo treatment.

DISCUSSION: Monoclonal gammopathies are categorized as light chain, heavy chain, or whole immunoglobins, although many diseases may produce a combination of chain types. Bence-Jones protiens of Multiple Myeloma and AL amyloidosis are the most common light chains. Heavy chain diseases are less common, with alpha chain disease (Seligmann's disease) and gamma chain disease (Franklin's disease) being the most commonly occurring out of this group. Whole immunoglobin gammopathies are also seen with Multiple Myeloma and Waldenström's macroglobulinemia. Waldenström's is a rare disease with less than 1,500 cases occurring every year in the US. The paraprotiens of Waldenström's are known to aggregate and increase blood viscosity which often leads to CHF symptoms from expanded blood volume. It can also lead to neuropathies, skin papules, and chylothoracies from destruction of lymphatic vessels. Hepatomegaly is present in about $20 \%$ of cases. Advanced cases can prove difficult to treat as the insidious nature of the disease can deplete a patient's physiologic reserves, as was the case in our patient. Initiation of chemotherapy in patients with a high disease burden can lead to tumor lysis syndrome which must be prepared for. Destruction of lymphatic vessels leading to chylothoracies are rare but must be treated rapidly. A dietitian is needed to assess the patient's nutritional status and create a diet low in triglycerides to reduce the buildup of chylous fluid. In advanced cases such as this, the 5 year survival rate is $<36 \%$.

\section{GASTRIC LYMPHOMA AS A PRIMARY PRESENTATION OF NEWLY} DIAGNOSED HIV Ayesha S. Ali ${ }^{1}$; Fazel Dinary ${ }^{1}$; Kulsoom Fatima ${ }^{2}$. ${ }^{1}$ Saint Vincent Charity Medical Center, Cleveland, OH; ${ }^{2}$ St.Vincent Charity Medical Center, Cleveland, OH. (Control ID \#2467520)

LEARNING OBJECTIVE \#1: Overall, lymphomas of the gastrointestinal (GI) tract are rare, although they are the most frequent extranodal location. The incidence of primary GI lymphoma, above all in the non-Hodgkin variant, is clearly higher in the HIV positive population, especially in subjects with AIDS. We present a case of lymphoma in newly diagnosed HIV patient in whom the only symptom was abdominal pain.

CASE: A 52-year-old African American female with history of chronic obstructive pulmonary disease and multiple sexual partners presented with sharp epigastric abdominal pain radiating to the back, melena and constipation. Physical Exam was unremarkable except mild epigastic abdominal tenderness on palpation. Laboratory findings showed hemoglobin $11.4 \mathrm{~g} / \mathrm{dl}$, hematocrit $36.2 \%$, platelets 243,000/mm3, BUN $26 \mathrm{mg} / \mathrm{dl}$, Creatinine $2.0 \mathrm{mg} / \mathrm{dl}$, Alkaline phosphatase $94 \mathrm{U} / \mathrm{L}$ (normal 90-240 U/L). Transaminase levels were normal. Upper endoscopy revealed $3 \mathrm{~cm}$ ulcerated hard friable mass, biopsy was consistent with ulcer and acute on chronic inflammation and necrosis. CT scan of the abdomen showed mural thickening of the posterior stomach wall at the antrum extending to duodenal, and multiple prominent mesenteric lymph nodes involving celiac chain. Patient underwent exploratory laparotomy with roux-en-Y bypass and preliminary 
intraoperative stomach, lymph node biopsy was consistent with GI lymphoma. HIV test was performed while waiting for final biopsy result; which revealed a positive HIV result, with absolute CD 4 counts of 111 cells/uL. Patient started HAART therapy and later begins Chemotherapy.

DISCUSSION: The incidence of non-Hodgkin's lymphoma (NHL) has greatly increased in the AIDS population. AIDS-associated NHLs are classified according to their anatomic site of location into three classes: (1) systemic (both nodal as well as extranodal), (2) primary central nervous system, and (3) body cavity-based lymphomas. Virtually any area of the GI tract may be involved, but the most common sites are the stomach, duodenum, perianal/anal area, and the oropharynx. The major presenting features are abdominal, fever, diarrhea, and/or weight loss; life-threatening complications such as bleeding, perforation and obstruction are not uncommon. When GI involvement is suspected, the evaluation includes imaging studies, upper and lower endoscopy with evaluation of the small bowel, and biopsy. This case underlines the following points: 1 . In the evaluation of acute abdominal disease in a patient with high risk factor for HIV clinicians should think about, AIDS-related infections as well as malignancies should be sought in the differential diagnosis. 2. Computed tomographic scanning of the abdomen and biopsy are the modalities of choice for characterization of disease as well as direction of appropriate therapy. 3. AIDS-related NHL remains an important biologic model for investigating the development and progression of lymphomas in the immune-deficient population.

GIANT LIVER CYST CAUSING OBSTRUCTIVE JAUNDICE; A COMPLICATION RARELY SEEN. GEORGE KHOUDARI ${ }^{1}$; Yazan Samhouri ${ }^{2}$; Tarun Kothari ${ }^{1} .{ }^{1}$ ROCHESTER REGIONAL HEALTH/UNITY HOSPITAL, ROCHESTER, NY; ${ }^{2}$ Rochester Regional Health/Unity Hospital, Rochester, NY. (Control ID \#2457596)

LEARNING OBJECTIVE \#1: Recognize liver cyst as a rare cause of obstructive Jaundice.

LEARNING OBJECTIVE \#2: Manage a large symptomatic liver cyst causing obstructive jaundice.

CASE: A 67 year old female with a history of diabetes and hypertension. Presented to the hospital with jaundice, associated with dull aching right upper quadrant (RUQ) pain, nausea, and fever for 3 weeks. $\mathrm{CBC}$ and chemistries showed $\mathrm{WBC} 11.8 / \mu \mathrm{l}$, alanine aminotransferase $167 \mathrm{u} / \mathrm{l}$, aspartate aminotransferase $114 \mathrm{u} / \mathrm{l}$, alkaline phosphatase $625 \mathrm{u} / \mathrm{l}$, total bilirubin $2.3 \mathrm{mg} / \mathrm{dL}$, and conjugated bilirubin $1.6 \mathrm{mg} / \mathrm{dL}$. Physical exam was remarkable for icterus, epigastric and RUQ tenderness, no ascites, hepatomegaly, or palpable masses. Abdominal US showed changes of acute cholecystitis and new $14.5 \mathrm{~cm}$ cyst in the right hepatic lobe. She was started on antibiotics (Ciprofloxacin and Metronidazole). MRCP done to better characterize the cyst, It showed a large cyst within the central liver cephalic to the gallbladder, with thickened gallbladder wall, no distention, or gallstones. Gallbladder was drained and culture of the fluid showed no growth, thus antibiotics were stopped. Follow up liver function tests showed worsening obstructive pattern. So, ERCP was done which revealed a patent cystic duct, and marked narrowing and irregularity of the right hepatic duct. Surgical team was consulted and diagnostic laparoscopic fenestration and cholecystectomy was done and revealed the diagnosis of simple cyst by frozen section. She was seen frequently by her primary care physician for the next 6 months after the procedure, no complains re-occurred, and all her liver function tests were within normal range.

DISCUSSION: Hepatic cysts are classified as benign non-parasitic liver cyst (NLC), parasitic cyst, and neoplastic cyst. NLCs are cystic formations containing clear fluid that do not communicate with the intrahepatic biliary tree. NLCs are becoming more frequently detected and have been found in approximately $5 \%$ of the population. Presentation can varies and mainly depends on the size of the cyst and the location. A majority of cases are found incidentally on imaging studies and tend to have a benign course. A minority can cause symptoms like abdominal discomfort, pain, or nausea. Jaundice is considered a rare presentation and can happen if cysts are larger than $10 \mathrm{~cm}$ in diameter as in the present case. A review by Ishiwika, et al. reported only 19 cases of solitary cysts causing obstructive jaundice. Diagnosis of symptomatic benign liver cysts necessitates intervention, laparoscopic fenestration has been favored compared to open surgery. In conclusion, this case adds to the sparse literature about NLC and highlights their ability to cause obstructive jaundice.

\section{GOING SKIN DEEP: A CASE OF NODULO-PUSTULAR LESIONS IN ACUTE} MYELOID LEUKEMIA Adrienne N. Poon ${ }^{1}$; Minh-Hai Ngo ${ }^{2}$; William B. Ershler ${ }^{3}$; Jennifer Primeggia ${ }^{4} .{ }^{1}$ The George Washington School of Medicine and Health Sciences, Washington, DC; ${ }^{2}$ Virginia Commonwealth University \& Fairfax Family Practice, Fairfax, VA; ${ }^{3}$ Institute for Advanced Studies in Aging, Falls Church, VA; ${ }^{4}$ Inova Fairfax Hospital, Falls Church, VA. (Control ID \#2457578)

LEARNING OBJECTIVE \#1: Recognize the need for skin biopsy of lesions in the setting of acute myeloid leukemia

LEARNING OBJECTIVE \#2: Determine appropriate treatment of atypical skin lesions in the setting of acute myeloid leukemia

CASE: A 59 year old female with acute myeloid leukemia M7 variant presented with 1 week of progressive facial, head, and neck lesions preceded by a small right thigh macular lesion that had developed into a larger papulo-pustular lesion. Subsequently, a number of similar papular lesions erupted across her forehead and grew into painful nodulo-pustular lesions. Associated right sided periorbital edema prompted her hospitalization. She has a history of recurrent upper respiratory infections, pulmonary aspergillosis, staphylococcus epidermidis vertebral osteomyelitis, diabetes mellitus, and genital herpes. For AML, she had received two remission induction cycles of idarubicin/cytarabine, then high dose ara-C 5 months prior to admission. For persistent neutropenia, she required daily granulocytecolony stimulating factor. Other medications included carvedilol, lisinopril, gabapentin, lorazepam, oxycodone, and valacyclovir. Admission vital signs were unremarkable. Examination revealed cervical lymphadenopathy and multiple nodulo-pustular lesions scattered across the forehead and neck, with a similar lesion on her right thigh. Lesions ranged from papules with surrounding erythema to $2 \mathrm{~cm}$ indurated erythematous nodules with central ulcerations and drainage. Right sided periorbital edema was present without visual field deficits, conjunctival injection, or extraocular movement pain. Labs included: WBC 3,720 with ANC of 2,976, hemoglobin $9.4 \mathrm{~g} / \mathrm{dL}$, platelets of 80,000, $\mathrm{HCO}_{3} 21 \mathrm{mEQ} /$ L without anion gap, glucose $115 \mathrm{mg} / \mathrm{dL}$, and lactate $1.5 \mathrm{mEq} / \mathrm{L}$. A skin biopsy was obtained, and IV vancomycin, acyclovir and voriconazole were initiated due to concern for superinfection. Lesions continued to progress to pustules that eventually crusted. Biopsy revealed acute neutrophilic dermatitis. Antibiotics were discontinued and prednisone was initiated with further improvement. Two months later, she was rehospitalized for bilateral pneumonia with skin lesion recurrence, now on her chest and left wrist. Skin biopsy again confirmed acute neutrophilic dermatitis.

DISCUSSION: Sweet Syndrome is associated with acute myeloid leukemia. Though typically presenting as well-demarcated papules and plaques with surrounding erythematous base, this presentation was atypical, as lesions were predominantly nodulo-pustular. She had multiple risk factors for Sweet Syndrome that may have contributed including chronic intermittent upper respiratory infections. Even after she steroid treatment at initial presentation, she had recurrence in the setting of pneumonia, suggesting an infectious precipitant. She also received daily tbo-filgrastim, a growth factor associated with Sweet Syndrome when neutrophils rapidly increase. The patient's M7 variant of AML also provides a unique aberration that may have made her more susceptible. In patients with a 
combination of risk factors including URIs, AML, and G-CSF use, Sweet Syndrome may present atypically. Skin biopsy should be considered in such patients to confirm diagnosis and initiate appropriate treatment with corticosteroids.

\section{GRANULOMATOSIS WITH POLYANGIITIS : A STORM IN THE BRAIN} Rajesh Gopalarathinam ${ }^{1}$; Pavithra Indramohan ${ }^{2}$; Tarun Sharma ${ }^{1} .{ }^{1}$ Allegheny General Hospital, Pittsburgh, PA; ${ }^{2}$ West Penn Allegheny Health Network, Pittsburgh, PA. (Control ID \#2465612)

LEARNING OBJECTIVE \#1: Diagnose Granulomatosis With Polyangiitis in patients presenting with acute ischemic stroke.

LEARNING OBJECTIVE \#2: Treat Granulomatosis With Polyangiitis with corticosteroids and immunosuppressants

CASE: A 62 year old Caucasian female with past medical history of hypertension, diabetes, hyperlipidemia and 40 pack year smoking presented to the emergency room with sudden onset aphasia. On physical exam, she was found to have tachycardia, altered mental status and severe expressive aphasia. A CT head without contrast revealed an acute left middle cerebral artery infarct. Unfortunately, she was outside the window period for tPA administration. MRA of the head was not done as she had a metal screw in her left shoulder joint from a previous injury. Upon admission, her labs showed acute kidney injury with a creatinine of 4.3 (Baseline Creatinine was 1.2). Review of her previous medical records revealed worsening kidney function over the past few months without a clear etiology. Urinalysis showed hematuria and dysmorphic RBC's. Renal ultrasound was unremarkable. ESR and CRP were significantly elevated at $140 \mathrm{~mm} / \mathrm{h}$ and $3.5 \mathrm{mg} / \mathrm{dl}$ respectively. Workup for glomerulonephritis revealed abnormally elevated c- ANCA and PR3 antibody titers. ANA, cryoglobulins, lupus anticoagulant tests were negative. C3, C4 and anti-GBM antibodies were within normal limits. A kidney biopsy was performed and it revealed necrotizing pauci-immune crescentric glomerulonephritis confirming the diagnosis of GPA presenting as acute stroke leading to Broca's aphasia and acute kidney injury. Subsequently, she was started on pulse IV corticosteroids and Rituximab infusion. Over the next few days, her aphasia and renal function gradually improved during the hospital stay. She was discharged on oral prednisone and weekly rituximab infusions.

DISCUSSION: Graulomatosis with Polyangiitis (GPA) is a systemic necrotising granulomatous vasculitis, typically affecting small vessels. Although GPA characteristically involves the kidneys, upper and lower respiratory tract, it may also affect the nervous system. About $50 \%$ of GPA patients have some form of neurological involvement. Peripheral neuropathies and cranial neuropathies are more common than involvement of the central nervous system (CNS) which is rare. Manifestations of the CNS are extremely variable and can include seizures, cerebritis, focal motor or sensory complaints and stroke syndromes. Diagnosis involves a combination of history, physical exam findings, elevated c-ANCA with PR3 antibody titres, imaging such as MRA of the head and neck and tissue biopsy showing characteristic necrotizing granulomas. Treatment involves immunosuppresants such as rituximab with high dose steroids. It is important for internists to be cognizant of the fact that acute stroke and other neurological manifestations can occur in patients with GPA and there should be a high degree of clinical suspicion for GPA in an appropriate clinical setting. Early diagnosis and treatment is critical as it can improve survival and prevent complications of pulmonary and renal vasculitis.

GRANULOMATOUS LIVER DISEASE: A DIAGNOSTIC AND THERAPEUTIC DILEMMA Muznay Khawaja ${ }^{1}$; Faizan Malik $^{3}$; Syed Imran Jafri ${ }^{3}$; Vivek R. Mehta ${ }^{1}$; Tawseef A. Dar ${ }^{2}$; Yub Raj Sedhai ${ }^{1}$; Jayamohan Nair ${ }^{1} .{ }^{1}$ Mercy Catholic Medical Center, Yeadon, PA; ${ }^{2}$ Mercy Catholic Medical center, Drexel Hill, PA; ${ }^{3}$ Abington Memorial Hospital, Abington, PA. (Control ID \#2457650)
LEARNING OBJECTIVE \#1: Distinguish tuberculosis from sarcoidosis in a patient with granulomatous liver disease.

LEARNING OBJECTIVE \#2: Recognize persistently elevated alkaline phosphatase with normal ALT and AST, recurrent transudative ascites could be from tuberculosis of liver.

CASE: Leading causes of hepatic granulomas in US are sarcoidosis and tuberculosis. Sarcoidosis and tuberculosis share many clinical and pathological features and the differentiation can be quite challenging. Treatment strategies are entirely different; immunosuppression is the main treatment for sarcoidosis, however it could be harmful in tuberculosis. Our case report illustrates the diagnostic and therapeutic challenges in differentiating sarcoidosis from tuberculosis. High index of clinical suspicion for TB is important to prevent morbidity and mortality. A 53 year old gentleman from Haiti with coronary artery disease, systolic congestive heart failure, diabetes mellitus and end stage renal failure presented with fatigue, fever and intractable nausea and vomiting for 5 days. He had multiple prior hospital admissions with vague abdominal symptoms. On examination, he had fever of 101.2; liver was palpable and firm in consistency. Laboratory tests were remarkable for elevated alkaline phosphatase (947) and gamma glutamyltranspeptidase (904). ALT and AST were within normal range. CT abdomen showed hepatomegaly, and moderate ascites. Paracentesis revealed SAAG of 0.6. Previous labs also showed persistently elevated alkaline phosphatase and low SAAG. The main differentials with hepatomegaly, low SAAG and persistently elevated alkaline phosphatase were peritoneal carcinomatosis, pancreatitis, sarcoidosis, tuberculosis and serositis. Imaging, biochemistry and cytology were not suggestive of cancer or pancreatitis. Sputum AFB was negative. Gallium scan was not convincing for sarcoidosis. Quantiferon gold test was positive. Liver biopsy showed non-caseating granulomata. DISCUSSION: The finding of non-caseating granuloma in biopsy makes sarcoidosis the most likely diagnosis. However, for diagnosing sarcoidosis, more than one organ system has to be involved. As the patient was from an endemic area, and was immunocompromised (ESRD), the rare possibility of TB causing non-caseating granuloma was considered. Caseating granuloma is hall mark of tuberculosis, however caseating and noncaseating granulomas can co-exist in TB and in rare cases, biopsy may yield only noncaseating granuloma. Positive quantiferon also led to suspicion of TB, and patient was started on empiric anti-tuberculosis therapy. His clinical improvement was marked after initiating anti TB treatment. It is already established that liver involvement is particularly frequent in patients with disseminated miliary tuberculosis. However, the local form of hepatic tuberculosis, with minimal extrahepatic manifestations, is much less common. In cases of suspected granulomatous disease of liver of unknown etiology, a trial of antituberculosis therapy is worth considering prior to immunosuppressive therapy, especially if suspicion for TB is high.

GROUP B STREPTOCOCCAL SEPTIC ARTHRITIS OF THE STERNOCLAVICULAR JOINT Sarah Chung; Jennifer Lyden. University of Washington, Seattle, WA. (Control ID \#2464019)

LEARNING OBJECTIVE \#1: Diagnose community-acquired pneumonia (CAP) using suggestive radiographic features in the appropriate clinical scenario.

LEARNING OBJECTIVE \#2: Recognize that infections with Group B streptococcus (GBS) are increasingly common in older adults with chronic medical conditions.

CASE: A 61-year-old diabetic African-American man presented to the emergency department with reports of increasing weakness and falls over the last 3 weeks. The patient was a poor historian and the initial history was obtained primarily from his family. At presentation, he did not have any other specific complaints. He had a history of hypertension and right thalamic stroke in the distant past, with residual left hemiparesis, allodynia, and dysphagia. Review of systems was negative for fevers, shortness of breath, 
or productive cough. On examination, vital signs were all within normal limits, other than a mild tachycardia, and he demonstrated normal oxygen saturation on room air. $\mathrm{He}$ appeared older than his stated age. Head and neck exam was within normal limits aside from poor dentition. Cardiac exam revealed no murmurs. There were no signs of respiratory distress and lungs were clear to auscultation without wheezes or rhonchi. Neurology exam affirmed his left-sided upper and lower extremity weakness, but no other significant deficits. Basic labs revealed a leukocytosis of $17.82 \mathrm{k} / 1000 \mathrm{ul}$ with bandemia $(0.23 \mathrm{k} / 1000 \mathrm{ul})$ and a mildly elevated creatinine. A chest $\mathrm{x}$-ray revealed a consolidation in the right lung apex and the patient was started on ceftriaxone and azithromycin in the emergency room for community-acquired pneumonia (CAP) and admitted to the acute care medicine floor. On arrival to the floor, he continued to deny shortness of breath, recent cough, increased sputum production or fevers. He did, however, report 1-month duration of dull and achy pain over the right shoulder exacerbated by movement without known trauma. Focused exam revealed a $2 \mathrm{~cm}$ by $4 \mathrm{~cm}$ soft tissue swelling at the right sternoclavicular joint with mild erythema, warmth and tenderness to palpation. A CT chest revealed an extrapulmonary abscess at the right chest wall with extension into the sternoclavicular joint and clavicular head. Using ultrasound guidance, cultures were obtained and returned positive for beta-hemolytic group B streptococcus (GBS) Intravenous ceftriaxone was continued and Thoracic Surgery was consulted to assist with source control. The patient underwent several incision and drainage procedures and ultimate resection of the head of the clavicle. He completed a 6-week course of antibiotics for osteomyelitis and septic arthritis

DISCUSSION: This case illustrates the importance of interpreting radiographic findings within the context of the clinical scenario. Between 5 and 10 million Americans are diagnosed with CAP annually, with 350,000 to 620,000 cases among the elderly requiring hospitalization. Despite the high incidence, patients are frequently misdiagnosed with CAP based on chest radiograph findings alone. In the absence of supportive physical exam findings, such as fever, cough, hypoxia, or abnormal breath sounds, alternative diagnoses should be sought. Secondly, this case highlights the rising trend of invasive GBS infections among non-pregnant adults, specifically the elderly and those with a high burden of immunosuppressive comorbidities, particularly diabetes and obesity. GBS is understood to be a colonizer of the intestinal and vaginal flora, yet this organism has highly invasive potential, causing sepsis and death, most commonly via the genitourinary tract or by skin and soft tissue infections. Cases outside the perinatal risk period account for nearly $90 \%$ of the national burden of disease, and patients in older age groups are more likely to die from infection than infants. In our patient, we identified GBS septic arthritis of the sternoclavicular (SC) joint and clavicular osteomyelitis, presumably from an oropharyngeal source. Septic arthritis of the SC joint carries the potential for significant complications if left untreated, including retrosternal abscesses, mediastinitis, superior vena cava syndrome, and osteomyelitis. Symptoms of GBS septic arthritis are generally mild or non-specific and thus require a high index of suspicion for appropriate diagnosis and management, particularly in the elderly diabetic patient population who are at increased risk for skin and soft tissue infection.

GUILLANE-BARRE SYNDROME IN THE SETTING OF SALMONELLA INFECTION AND INFLUENZA VACCINE OBJECTIVES: Hadeel Zainah $^{1}$; Fadi samaan ${ }^{2}$; Sarah Freeman ${ }^{1} .{ }^{1}$ The Miriam Hospital, Providence, RI; ${ }^{2}$ Rhode Island Hospital, Providence, RI. (Control ID \#2469881)

LEARNING OBJECTIVE \#1: Recognize the etiology and diagnosis of Guillane-Barre Syndrome

LEARNING OBJECTIVE \#2: Identify the significance of neurological exam in patients with generalized weakness
CASE: A 77-year-old woman with chronic kidney disease stage III and hypothyroidism presented with falls from generalized weakness in the setting of a diarrheal illness. Patient endorsed non-bloody watery diarrhea twice a day for the past 9 days, without vomiting, pain, or fever. She developed lower extremities (LE) weakness and numbness 1 day prior to presentation and upper extremities (UE) weakness and difficulty with silverware on day of presentation. Examination revealed normal vital signs, non-tender abdomen, decreased extremity sensation, decreased strength (plantarflexion R 3/5, L 2/5; dorsiflexion R 1/5, L $0 / 5$; straight leg raise both $2-3 / 5$; hand grip both $2-3 / 5$; triceps both $2 / 5$; and biceps both $3 / 5$ ), and areflexia. Laboratory results showed leukocytes 6.4,000/ $\mu \mathrm{L}$, hemoglobin $10.3 \mathrm{~g} /$ $\mathrm{dL}$, platelets $475,000 / \mu \mathrm{L}$, creatinine $1.23 \mathrm{mg} / \mathrm{dL}$, and normal TSH, B12 and lyme reflex. Stool cultures grew Salmonella group D. Spine magnetic resonance imaging showed degenerative changes without compression. Further questioning revealed influenza vaccination 4 weeks prior. Lumbar puncture was performed given suspicion for Guillane-Barre syndrome (GBS). Cerebral spinal fluid (CSF) analysis revealed normal results with protein $36 \mathrm{mg} / \mathrm{dL}$ and nucleated cells $1 / \mu \mathrm{L}$. Antibiotics were held as diarrhea resolved shortly after admission. Five days of empiric IVIG treatment led to improvement in neurologic exam (3/5 in LE, $4 / 5$ in UE). CSF eventually returned positive for oligoclonal bands, consistent with GBS. Nerve conduction studies (NCS) were scheduled as an outpatient. DISCUSSION: GBS is an acute self-limiting immune-mediated polyradiculopathy presenting with ascending paralysis and areflexia. Two-thirds of GBS follow a gastrointestinal (GI) or respiratory infection. Of the associated GI pathogens, Campylobacter jejuni is most common (30\% of all GBS cases) but Salmonella typhi has been reported. Seasonal influenza vaccination, within the past 6 weeks, is also associated with GBS (Relative Risk $(\mathrm{RR})=1.2)$, however, the risk pales in comparison to recent $C$. jejuni infection $(\mathrm{RR}=48)$ CSF analysis typically reveals albuminocytologic dissociation, however CSF protein levels will be normal in approximately $1 / 3$ of patients if checked within a week of symptoms onset. NCS can confirm the diagnosis and provide prognostic information. IVIG or plasmapheresis should be initiated immediately upon diagnosis. Physicians should perform a neurological exam on all patients presenting with generalized weakness and diarrheal illness. It is also important to recognize the non-classic CSF results during early GBS. Although the risk of GBS with influenza vaccines is statistically significant, the absolute risk is only 2 in a million. The annual morbidity and mortality associated with influenza infections far outweighs the risk of vaccine induced GBS. In our case, the trigger for her GBS was likely her antecedent Salmonella infection.

\section{GUT VERSUS HOST DISEASE IN POST LUNG TRANSPLANTATION Peter}

Nguyen; Betty La; Mohamed Othman. Baylor College of Medicine, Houston, TX. (Control ID \#2465453)

LEARNING OBJECTIVE \#1: To recognize and diagnose graft versus host disease in patients with solid organ transplant.

LEARNING OBJECTIVE \#2: To manage and treat graft versus host disease in patients with solid organ transplant.

CASE: Introduction: Graft Versus Host Disease (GVHD) is a known complication of hematopoietic stem cell transplants, but it can also present in patients who receive solid organ transplants. Solid organs contain lymphoid tissue and immunologically competent cells that can migrate from the transplanted organ into both lymphoid and nonlymphoid tissues of the host, resulting in chimerism. The disease can then affect different organ systems with high morbidity and mortality. We present an unusual case of gastrointestinal GVHD in a post lung transplant patient. Case Presentation: A 50 year old man with pulmonary sarcoidosis, end stage lung disease, and bilateral lung transplant (CMV+ donor/CMV- recipient) who presented 3 months post-transplant with acute profuse watery diarrhea for 3 days. After successful lung transplant, the patient was managed on 
immunosuppressive therapy with tacrolimus and mycophenolate mofetil and prophylactic antibiotics with bactrim, voriconazole, and valganciclovir. His diarrhea was described as watery, mucoid, and greenish in color. Vitals, physical exam, and initial labs were unremarkable. Abdominal CT showed evidence of thickening in the right colon. He later developed a maculopapular rash that was pruritic and painful. Infectious disease was consulted and studies including stool culture, ova/parasite, protozoan, fungal, C. difficile, and strongyloides were negative. Serology studies for viral causes of diarrhea including CMV were all negative. Colonoscopy revealed erythema, edema, severe ulcerations, and pseudomembranes in the right colon. Biopsies were obtained and histopathology revealed severe active colitis with ulceration and granulation, severe cryptitis, blunted villi, and focal apoptosis consistent with grade III GVHD of the colon. Chimerism study was significant for two genetically distinct lymphocyte population. The patient was treated with high dose steroids of solumedrol with resolution of symptoms, but was eventually readmitted for reoccurrence. He was again given high dose steroids and also TNF-alpha inhibitor infliximab with some clinical improvement. He was later discharged to M.D Anderson to receive extracorporeal photopheresis therapy.

DISCUSSION: Gastrointestinal GVHD can be difficult to diagnose in transplant patients as it typically presents with non-specific symptoms such fever, rash, and diarrhea that can be misdiagnosed as infection, CMV colitis, cellcept induced colitis, or inflammatory bowel disease. It is important to rule out infectious causes as it can affect management. Since GVHD is a potentially lethal disease, recognition of histological features are crucial for accurate diagnosis and need for aggressive treatment. First line therapy includes high dose steroids; second line therapy include TNF-alpha inhibitors; and third line therapy would involve extracorporeal photophoresis. As more and more patients are receiving solid organ transplants, it is important to recognize that GVHD as a potential complication.

HARD PRESED TO STOP BENZODIAZEPINES? A CASE OF POSTERIOR REVERSIBLE ENCEPHALOPATHY FROM BENZODIAZEPINE WITHDRAWAL James Higgs ${ }^{1}$; Gregory Braden ${ }^{2} .{ }^{1}$ Baystate Medical Center, Enfield, CT; ${ }^{2}$ Baystate Medical Center, Springfield, MA. (Control ID \#2469620)

LEARNING OBJECTIVE \#1: Identify the clinical and radiological features of Posterior Reversible Encephalopathy (PRES)

LEARNING OBJECTIVE \#2: Describe possible pathophysiologic explanations for PRES in benzodiazepine withdrawal

CASE: A 61-year-old female with history of chronic back pain on narcotics and chronic anxiety on clonazepam presented after being found unresponsive at home by her family. On arrival of ambulance crew, patient was minimally responsive to pain with an oxygen saturation of $79 \%$ and respiration rate of 4 . Her mentation improved after naloxone administration. Laboratory workup was notable for WBC $9.3 \mathrm{k} / \mathrm{mm} 3$, creatinine kinase 1120 units/L, creatinine $2.49 \mathrm{mg} / \mathrm{dl}$ (baseline 0.9 ), lactate $1.3 \mathrm{mmol} / \mathrm{L}$. CT head and MRI showed subacute bilateral basal ganglia infarcts. She was fluid resuscitated for her kidney injury and gradually had improvement in her mental status. Her clonazepam $2 \mathrm{mg}$ three times/day was held on admission. On day 5, patient became febrile to $102.3 \mathrm{~F}$ and hypertensive (170/80) with two generalized tonic-clonic seizures. This was accompanied by visual impairment and left lower extremity weakness. A lumbar puncture was performed which showed 14-23 WBCs, glucose of 57 and protein of 37. Blood and CSF cultures had no growth. MRI was performed which demonstrated T2 signal abnormalities in the posterior cortical hemispheres, posterior frontal lobes, parietal occipital regions and posterior right temporal lobe consistent which given her clinical picture was felt secondary to posterior reversible encephalopathy syndrome (PRES). EEG showed evidence of severe encephalopathy but no epileptiform discharge. Patient was briefly commenced on antiepileptic therapy. She had gradual improvement back to her baseline neurologic function over the next 3 days prior to be discharged.

DISCUSSION: PRES is a clinico-radiological syndrome characterized by headaches, visual changes, encephalopathy, and seizures in addition to areas of focal reversible vasogenic edema best seen on MRI. The syndrome has mostly been described in cases of severe hypertension, immunosuppressive medications, preeclampsia and autoimmune diseases. This case illustrates PRES in the setting of benzodiazepine withdrawal. Benzodiazepines act as positive allosteric modulators at GABA receptors. Sudden withdrawal can result in unbalanced glutamate and catecholaminergic function resulting not only in hypertensive states but potential neurotoxicity. During this patient's admission, her clonazepam was held and despite complete initial recovery from her narcotic overdose developed seizures with MRI findings supportive of PRES. The timing of her symptoms corresponds to the half-life of clonazepam which is approximately $8-50 \mathrm{~h}$. While cases associated with benzodiazepines have yet to be reported in the literature, a handful of cases of PRES have been seen in those with severe alcohol withdrawal. Similar to benzodiazepines, alcohol exerts its activity through GABA channels with potential for glutamate toxicity in setting of withdrawal. This neurotoxicity could affect neurovascular permeability, resulting in vasogenic edema seen in imaging of those with PRES. As this case illustrates, those on chronic benzodiazepines should be gradually tapered off their medications to avoid potential serious neurologic complications of PRES.

HEADACHE IN A PATIENT WITH HIV? LOOK WEST! Zahra Rahman; Anna Marie Lewarchik. University of Pittsburgh Medical Center, Pittsburgh, PA. (Control ID \#2468225)

LEARNING OBJECTIVE \#1: To review the differential and workup for headache in patients with HIV.

LEARNING OBJECTIVE \#2: To review high value imaging in patients presenting with headache.

CASE: The patient is a 50 year old African- American man with uncontrolled HIV, history of herpes zoster who presented with a 3 day history of right- sided posterior scalp pain, bilateral intermittent blurred vision and photophobia. He did not endorse unilateral vision loss, temporal artery tenderness, neck stiffness, fevers or chills. The patient presented to the ER with similar complaints 2 months ago, which improved with a migraine cocktail of metoclopramide and diphenhydramine, and the patient was discharged. On his current physical exam, the patient had normal vital signs and no focal findings. In the ER he was given acetaminophen, morphine, methylprednisolone with some pain improvement. A head CT done in the ER showed no acute abnormalities. Initial labs showed ESR of 50 (normal $<20$ ), urine positive for cocaine and barbiturates. CMP and $\mathrm{CBC}$ were normal. Pain was initially thought secondary to herpes zoster, and the patient was started on valacyclovir. Initially there was some concern for temporal arteritis given his visual complaints, ESR and possible improvement with steroids. Biopsy was deferred given the bilateral visual complaint and lack of temporal artery tenderness. Neurology and Rheumatology were consulted and in agreement. On day 2 the patient's headache improved with oxycodone and valacyclovir, however the next day he developed a fever of $38.5^{\circ} \mathrm{C}$ and his CD4 count resulted at 150 . Sulfamethoxazole/ Trimethoprim (TMP-SMX) was started, a lumbar puncture (LP) was ordered and Infectious Disease was consulted. Lumbar puncture showed protein 56 , glucose 45 , WBC $25, \mathrm{RBC}<25$, lymphocytes 29 , neutrophils 26 . The patient was started on IV fluconazole for empiric cryptococcal coverage. On day 5 , the patient's headache and visual changes had resolved, and the cryptococcal Ag in serum and CSF was negative. The patient was discharged on valacyclovir and TMP-SMX. Post-discharge LP results showed West Nile IgM Ab of 2.79 and IgG 18.6 confirming West Nile. Post- discharge the patient has experienced no further headache or visual symptoms. 
DISCUSSION: This case encourages the importance of considering a broad differential for headache in patients with HIV. Our evaluation and differential were greatly affected by our patient's CD4 count and subsequent fever. Whilst imaging was performed in the ER, the infectious differential continued to be broad. In the patient with HIV and CNS symptoms, the differential is vastly different compared to an immunocompetent host. The leading diagnostic considerations in HIV patients are toxoplasmosis, primary CNS lymphoma, PML, HIV encephalopathy and CMV encephalitis. Patients with high viral loads and low CD4 counts often present with their first opportunistic infection in the CNS Our patient had not previously been on HAART thus further predisposing him to infection. In evaluating HIV patients with potential CNS lesions, imaging has limited utility unless considering advanced imaging modailities such as thallium SPECT scans, perfusion MRI, MR spectroscopy or PET. Many of these tests also have decreased specificity. Instead, evaluation should start with the CSF. Cell counts and cytology] differentiate infection from lymphoma, while PCR isolates specific etiologies. Whilst the final diagnosis in our patient was West Nile Virus (WNV), this case provided the opportunity to review the differential for CNS lesions in a patient with HIV while recognizing that immunocompromised patients present with a varied etiology. Although WNV is uncommon it is worth noting it has increased incidence in patients with age $>50$, malignancy, transplant or immunocompromised state. It is important to address the prudence of imaging when evaluating headaches as MRI and CT are often first line. Our case warranted imaging in the ER given the patient's complaint of fever, visual changes and immunocompromised state, however imaging is often unnecessary. As physicians our history and comprehensive neurologic exam can provide a valuable differential while assuring cost conscious-care. The Choosing Wisely Guidelines, an initiative of the American Board of Internal Medicine, corroborate the minimization of imaging. The Guidelines recommend against routine imaging in headaches, as they provide little diagnostically and are often an unnecessary cost and radiation exposure Symptoms prompting imaging include headaches that are sudden or explosive, distinctive, prompted by exertion, or accompanied by fever, seizure, vomiting, loss of coordination, change in vision, speech or alertness. The case of a patient with HIV and a headache helped cement that imaging in the evaluation of headaches, whilst warranted in our patient, is not always necessary. Rather a lumbar puncture is essential in the workup of an immunocompromised patient with a headache and fever, as illustrated by our patient's final diagnosis of WNV.

HEADACHE/HEARTACHE: STEMI, BRADYCARDIA, AND SHOCK FOLLOWING MIGRAINE ATTACK HeeEun Kang; Girish L. Kalra. Emory University School of Medicine, Atlanta, GA. (Control ID \#2470238)

LEARNING OBJECTIVE \#1: Recognize potential cardiovascular complications arising from triptan therapy.

LEARNING OBJECTIVE \#2: Identify contraindications to starting triptans.

CASE: A 64-year-old woman with hypertension, hyperlipidemia, type 2 diabetes mellitus, and COPD presented to the emergency department with headache and hypertensive urgency. She had been out of all medications for 3 weeks due to financial hardship. The headache started 2 days ago, was severe, left-sided, throbbing, and associated with photophobia, nausea, visual disturbance, and intermittent left-sided weakness and numbness. Symptoms were characteristic of headaches that she had experienced intermittently since childhood; episodes would occur 3-4 times annually and typically abate with overthe-counter analgesics. She denied chest pain or shortness of breath. She admitted to smoking cannabis, but quit cigarettes and alcohol. On presentation, she was hypertensive to $246 / 127 \mathrm{mmHg}$ and appeared uncomfortable, though her exam was otherwise unremarkable. Diagnostic evaluation, which included chemistry, CBC, cardiac enzymes, EKG, chest radiograph, and head $\mathrm{CT}$, was also unrevealing. She was admitted for hypertensive urgency, reinitiated on oral antihypertensives, and her blood pressure gradually improved. For her headache, she was diagnosed with atypical migraine and treated with IV dexamethasone, ketorolac, and metoclopramide. The headache improved initially, but it recurred later that evening, whereupon she was initiated on oral sumatriptan with good effect. Later that night, she experienced chest pain; this was poorly described, and resolved within one hour without intervention. Troponin and EKG checked the next morning were negative. She had a negative stress test documented 4 years previously. She was discharged home with a short supply of her home medications and as-needed sumatriptan. Two days later, while at home, the patient developed new-onset severe, retrosternal chest pain that radiated to the back, lasted for hours, and was associated with nausea, vomiting, and presyncope. She called 911 and was found by paramedics to have an acute inferior ST-elevation myocardial infarction. On arrival, she was hypotensive to $62 / 23 \mathrm{mmHg}$ and experienced occasional bradycardia to $30 \mathrm{bpm}$ in the setting of intermittent sinus arrest with junctional escape rhythm. Emergent left heart catheterization revealed $100 \%$ occlusion of the mid-circumflex artery, which was co-dominant, as well as $100 \%$ occlusion of the right coronary artery, which was believed to represent chronic occlusion. She underwent percutaneous intervention of the circumflex lesion with stent placement, and she was briefly treated with a transvenous pacemaker. She was monitored in the ICU, and her bradycardia, hypotension, and chest pain resolved. Echocardiogram showed preserved contractility. She recovered quickly and was medically optimized for her coronary heart disease. She was counseled on medication adherence and cannabis cessation, and she was instructed to discontinue sumatriptan.

DISCUSSION: Triptans are serotonin $1 \mathrm{~b} / 1 \mathrm{~d}$ agonists used for treatment of acute migraine headache. They work by producing cerebral vasoconstriction, reducing vasoactive peptide secretion, and reducing the conduction of pain sensation. Outside the central nervous system, triptans have been shown to produce vasoconstriction in systemic, pulmonary, and coronary vasculature. Coronary vasospasm following sumatriptan administration is welldescribed, especially with injectable forms, and there have been many documented cases of myocardial infarction following triptan administration in patients with and withou underlying coronary disease. While observational analyses of migraine patients on triptan therapy have not demonstrated an association with stroke, heart disease, or death, these analyses are limited by prescribing practices that favor triptan therapy in lower-risk patients. Contraindications to triptan therapy include history of ischemic stroke, coronary artery disease, coronary vasospasm, uncontrolled hypertension, peripheral vascular disease, pregnancy, hemiplegic migraine, and basilar migraine. Our patient developed transient chest pain- and later, acute ST-elevation MI - following initiation of triptan therapy for migraines, indicating coronary vasospasm superimposed upon coronary atherosclerotic disease. Though she lacked a clear contraindication to triptan therapy such as known cardiovascular disease, she had multiple coronary risk factors, including hypertension, smoking history, hyperlipidemia, and diabetes. Moreover, her uncontrolled hypertension and atypical migraine features should have prompted caution when starting sumatriptan, as triptans can transiently increase blood pressure, and they may be associated with stroke in patients with hemiplegic or basilar migraine syndromes. It should be noted that in lower-risk patients, triptans have an excellent cardiovascular safety profile that is considered superior to ergotamine preparations. Nonetheless, the internist should take care to review cardiac risk factors before starting triptans.

\section{HEMODYNAMIC INSTABILITY SECONDARY TO PROFOUND BRADYCARDIA AND COMPLETE HEART BLOCK FROM CARDIAC INVOLVEMENT OF LYMPHOMA Rana Z. Tariq; Kumar S. Lal; Tochukwu Okwuosa. Rush University Medical Center, Chicago, IL. (Control ID \#2457570)}

LEARNING OBJECTIVE \#1: •Recognize cardiac arrhythmias, including symptomatic bradycardia and/or complete heart block as presenting symptoms of cardiac lymphoma. 
LEARNING OBJECTIVE \#2: •Recognize transthoracic echocardiogram (TTE) and cardiac computed tomography (CT) findings suggestive of cardiac involvement of lymphoma.

CASE: A 68 year-old male with diffuse large B-cell lymphoma (DLBCL), coronary artery disease status post coronary artery bypass graft, and atrial fibrillation was admitted for mediastinal lymph node biopsy due to disease progression of lymphoma, as seen on staging CT scan. On day 1 of admission, the patient developed new-onset atrial flutter with intermittent asymptomatic bradycardia. The patient proceeded to the operating room for the planned mediastinal lymph node biopsy on day 2 of admission. Prior to surgical incision the patient developed severe bradycardia (heart rate of $23 \mathrm{bpm}$ ) and hypotension $(55 / 30 \mathrm{mmHg})$ requiring cardiac resuscitation. The patient was subsequently emergently transferred to the Cardiac Intensive Care Unit with temporary pacemaker placement. Based on TTE findings of a left atrial mass with mitral valve involvement and moderate mitral regurgitation, there was concern for lymphomatous infiltration of the heart as the cause of the patient's acute decompensation. An MRI could not be completed given the patient's hemodynamic instability. A cardiac CT scan showed diffuse pericardial, myocardial, bypass graft, and aortic involvement, presumably from his previously diagnosed lymphoma. High dose IV corticosteroid therapy was consequently initiated to reduce inflammation and edema around the cardiac mass and led to initial improvement in heart block. The pacemaker dependence subsequently resolved after radiation therapy. The patient eventually converted to normal sinus rhythm, and was discharged home with a plan for continued chemotherapy after receiving one cycle of ESHAP (Etoposide, Methylprednisolone, Cytarabine, and Cisplatin) chemotherapy regimen during his hospitalization.

DISCUSSION: While primary cardiac neoplasms are rare, the incidence of cardiac metastases in the literature ranges from 2.3 to $18.3 \%{ }^{[1]}$ Postmortem studies have shown that cardiac involvement is present in $16 \%$ of patients with Hodgkin lymphoma and $18 \%$ of patients with non-Hodgkin lymphoma. ${ }^{[2]}$ Our patient's history of DLBCL is therefore a strong predisposing factor. Although the clinical presentation varies depending on the neoplasm's location, size, growth rate, degree of invasion, and friability, complete heart block, as seen in our patient, is an uncommon presentation. ${ }^{[2],[3]}$ Metastasis to the pericardium commonly results in hemorrhagic pericardial effusions while myocardial infiltration can present as arrhythmias (e.g. atrial flutter, atrial fibrillation, or premature atrial/ventricular complexes), conduction disturbances, and congestive heart failure with systolic or diastolic dysfunction. Thus, on exam the signs and symptoms can include chest pain, dyspnea, hypotension, or superior vena cava syndrome. Notably metastasis to the valves is uncommon, possibly due to the absence of vessels in the valvular stroma and constant cusp motion. ${ }^{[1]}$ However, as noted earlier, our patient had evidence of mitral valve involvement on TTE. The variability in these presenting symptoms leads to these neoplasms often going unrecognized until autopsy. ${ }^{2,3}$ Therefore, a high index of suspicion is necessary to identify cardiac involvement in these patients. While endocardial metastases usually localize to the right atrium and ventricle, myocardial metastases can involve any of the heart chambers. ${ }^{1}$ Diagnosis of these cardiac metastases requires leveraging multiple imaging techniques, including echocardiography, cardiac computed tomography (CT), cardiac magnetic resonance imaging (MRI), and fluorodeoxyglucose positron emission tomography (FDG PET). Pathologic and immunohistochemical characterization may also be helpful in guiding chemotherapy and can be performed with thoracotomy or less invasively by pericardiocentesis with cytology. ${ }^{1-3}$ Our case illustrates the need for high index of suspicion for cardiac involvement in a patient with known (current or past) history of lymphoma presenting with brady or tachyarrhythmias, with or without hemodynamic instability. In addition to aiding immediate management decisions and possibly saving the patient's life, such recognition could be the initial pathway to diagnosing recurrence of past lymphoma. Other tumors that metastasize to the myocardium and could therefore present similarly include lung, breast, other hematologic malignancies (i.e. leukemias and lymphomas), and melanoma. ${ }^{[4],[5]}$ References: [1] Bussani R, De-Giorgio
F, Abbate A, Silvestri F. Cardiac Metastases. J Clin Pathol. 2007; 60: 27-34. [2] O'Mahony D, Piekarz RL, Bandettini WP, et al. Cardiac Involvement with Lymphoma: A Review of the Literature. Clin Lymphoma Myeloma. 2008; 8(4): 249-252. [3] Jorge VJ, Bernardino V, Araujo AC, et al. Complete heart block as a complicating feature of a mediastinal lymphoma. BMJ Case Reports. 2012: published online 30 April 2012. [4] Bruce CJ. Cardiac tumours: diagnosis and management. Heart. 2011; 97: 151-160. [5] Goldberg AD, Blankstein R, Padera RF. Tumors Metastatic to the Heart. Circulation. 2013; 128: 1790-1794.

HEMORRHAGIC CHOLECYSTITIS MASQUERADING AS PERSISTENT ACUTE LIVER DYSFUNCTION Rebecca Haberman ${ }^{3}$; Alicia Cowley ${ }^{2}$; Monica Gupta ${ }^{1}{ }^{1}$ NYU School of Medicine, New York, NY; ${ }^{2}$ New York University, New York, NY; ${ }^{3}$ New York University School of Medicine, New York City, NY. (Control ID \#2466458)

LEARNING OBJECTIVE \#1: Recognize hemorrhagic cholecystitis in a patient without known risk factors

LEARNING OBJECTIVE \#2: Diagnose hemorrhagic cholecystitis

CASE: A 49 year-old man with known alcohol abuse, type 2 diabetes mellitus on insulin, and chronic pancreatitis was admitted with 1 day of hematemesis and abdominal pain. The patient reported that he had not used his insulin for 15 days. He was hemodynamically stable upon admission. Initial physical exam was only pertinent for mild right upper quadrant abdominal tenderness. Admission laboratory values were significant for a glucose of $378 \mathrm{mg} / \mathrm{dL}$ and an anion gap of 18 with positive ketones in the urine. The patient's hepatic panel showed an alanine aminotransferase (ALT) of 1828 units/L, an aspartate aminotransferase (AST) of 279 units/L, and an alkaline phosphatase of 720 units/ L with normal bilirubins and coagulation panel. He was treated with fluids and insulin with resolution of his diabetic ketoacidosis. However the patient's AST, ALT, and alkaline phosphatase all continued to trend up dramatically, as did his bilirubins and markers of synthetic function. ALT and AST peaked at 2196 units/L and 618 units/L respectively, alkaline phosphatase at 1891 units/L, and total bilirubin increased from normal to $4.1 \mathrm{mg} /$ dL. Hepatitis serologies and an autoimmune hepatitis workup were unrevealing. Out of concern for impending liver failure, emergent right upper quadrant ultrasound with doppler was performed and showed a dilated common bile duct and a distended gallbladder containing a large amount of avascular heterogeneous material suspicious for hemorrhagic cholecystitis. Abdominal computed tomography (CT) and abdominal magnetic resonance imaging (MRI) were consistent with this diagnosis. The patient's hemoglobin continued to downtrend and surgery was consulted for possible urgent cholecystectomy. A $\mathrm{CT}$ angiogram did not reveal an active bleed and the patient's hemoglobin stabilized. Given the stabilization of hemoglobin and downtrend of his transaminases, a joint decision was made with surgery to manage the patient conservatively with plan for elective cholecystectomy after improvement of his hepatitis.

DISCUSSION: Hemorrhagic cholecystitis is a rare and challenging diagnosis. It was first described in the literature in 1892 by Naunyn, with the first set of case reports published in the New England Journal of Medicine in 1946. The hemorrhage is hypothesized to occur when transmural inflammation of the organ walls causes erosion into the veins supplying the gallbladder, leading to hemorrhage within the lumen. Risk factors for major hemorrhage include trauma, underlying malignancy, and coagulopathies due to anticoagulants or conditions such as cirrhosis or renal failure. In the 14 most recent case reports reviewed, patients ranged from 17 to 91 years old. In this group, four patients had a history of abdominal trauma, two had underlying malignancies, five had coagulopathies (three were on anticoagulants and two were considered uremic), and one had a gallbladder ulcer, but in the remaining two cases, no underlying causes were discovered. The diagnosis is particularly difficult as it often masquerades as more common and less dangerous diagnoses. 
Patients often present with symptoms similar to acute calculous cholecystitis, including right upper quadrant pain, fever, and leukocytosis. Additionally, they may demonstrate hallmarks of gastrointestinal bleeding such as hematemesis, melena, and hematobilia when blood refluxes into the bowel tract from the biliary tree. Clot formation from the hemorrhage can also lead to biliary obstruction and subsequent hepatic enzyme elevation. This case is unique in its presentation as this patient presented with mild right upper quadrant pain, hematemesis and a transaminitis much higher than has previously been recorded in the literature (prior case reports have described peak transaminase levels of $700-800$ units/L). Our initial clinical suspicion did not include hemorrhagic cholecystitis as common risk factors were all absent. Due to the varied clinical manifestations of hemorrhagic cholecystitis, the definitive diagnosis must be made with imaging. Ultrasound remains the first-line imaging modality, though both CT and MRI can be used to confirm the diagnosis. Practitioners should have a high index of suspicion for hemorrhagic cholecystitis if patients present with evidence of both gallbladder disease and gastrointestinal bleeding. Early diagnosis is critical as it can have high morbidity and mortality rates, particularly when complicated by gallbladder perforation and intraabdominal hemorrhage. Treatment is typically surgical or via angiography with embolization, but is ultimately dictated by the patient's overall clinical status.

\section{HENOCH SCHöNLEIN PURPURA: IT'S NOT JUST FOR KIDS Kelly} Pennington $^{1}$; Mithulan Jegapragasan ${ }^{1}$; Anjali Bhagra ${ }^{2} .{ }^{1}$ Mayo Clinic School of Graduate Medical Education, Rochesster, MN; ${ }^{2}$ Mayo Clinic School of Graduate Medical Education, Rochester, MN. (Control ID \#2466534)

LEARNING OBJECTIVE \#1: Be able to recognize key differences between adult and pediatric Henoch Schönlein Purpura.

LEARNING OBJECTIVE \#2: Be able to identify possible inciting events for adult Henoch Schönlein Purpura.

CASE: Henoch Schönlein Purpura (HSP), otherwise known as IgA vasculitis, is a wellrecognized, self-limited disease process in the pediatric population characterized by nonthrombocytopenic purpura, tenosynovitis, gastrointestinal angina, and rarely renal insufficiency. Over the past 20 years, HSP has become increasingly recognized in the adult population and has been strongly associated with gastrointestinal and/or pulmonary malignancies. Unfortunately, the clinical manifestations and natural progression of the disease in the adult population are poorly understood and do not mirror that of the pediatric population. In our case, we have a patient who presented with cutaneous manifestations that progressed to end-stage renal disease despite immunosuppressive therapy, consistent with previous case reports of the aggressive nature of adult-onset HSP. A 61-year-old woman with a past medical history of poorly controlled diabetes (hemoglobin Alc of $11 \%$ ) and nicotine dependence presented to the Emergency Department with a 1 week history of a painful, erythematous rash over her bilateral lower extremities initially starting on her tibia and spreading up her legs to include her thighs, lower back, forearms, and face. She had an upper respiratory infection approximately 3 weeks prior, which had completely resolved upon presentation. She denied any abdominal pain, melena, hematochezia, or hematuria. Physical examination revealed an afebrile Caucasian woman who was in obvious pain. Diffuse palpable purpura was noted over her bilateral lower extremities and forearms, along with petechiae over her low back and buttocks with small pustules starting to appear on her face. Other systemic examination was normal. Initial laboratory evaluation was non-revealing including a complete blood count, complete metabolic profile, and urinalysis. C-reactive protein was mildly elevated with a normal erythrocyte sedimentation rate. Work-up for infectious and autoimmune etiologies was negative. Skin biopsy revealed strong IgA deposition in superficial blood vessels suggestive of an IgA predominant vasculitis. Without systemic manifestations, the patient was started on Prednisone $60 \mathrm{mg}$ daily for cutaneous small vessel vasculitis without specific subtype.
Over the next 2 weeks, her cutaneous lesions continued to progress, and she developed clawing of her left ring and small finger concerning for mononeuritis multiplex. Concurrently, she developed acute renal failure and worsening of her inflammatory markers. Repeat urinalysis revealed significant hematuria with nephrotic range proteinuria and granular casts. Renal biopsy revealed a proliferative, necrotizing, and crescentic glomerulonephritis with IgA-dominant deposits consistent with HSP. Further work-up to identify respiratory or gastrointestinal malignancy was negative. She was initiated on cyclophosphamide in addition to prednisone with continued progression of her renal and cutaneous manifestations. The decision was made to trial Plasma Exchange Therapy. Unfortunately, she did not regain renal function and was started on hemodialysis with possible future consideration for renal transplant. With aggressive wound care, her cutaneous lesions gradually improved.

DISCUSSION: This case illustrates that the presentation of HSP differs significantly with age. Adult patients have a much more aggressive and severe course with over half developing renal insufficiency versus five to fifteen percent in pediatric patients. Likewise, common manifestations in children, such as tenosynovitis and gastrointestinal angina, are frequently absent in the adult population. Adult-onset HSP is also commonly associated with upper respiratory and gastrointestinal cancers; although, gastrointestinal and respiratory infection can still be the inciting event.

HEPARIN-INDUCED THROMBOCYTOPENIA: A HIT AND A MISS Ayushi Chauhan $^{1}$; John P. Russo ${ }^{2}$. ${ }^{1}$ University of Connecticut, Hartford, CT; ${ }^{2}$ University of Connecticut, Newington, CT. (Control ID \#2470252)

LEARNING OBJECTIVE \#1: Recognize heparin-induced thrombocytopenia (HIT) in ICU setting in presence of confounders

LEARNING OBJECTIVE \#2: Treat with therapeutic, not prophylactic anti-coagulation in critically ill patients with suspected HIT

CASE: 51 year old male was admitted for an elective open aorto-bifemoral bypass. The patient had a past medical history significant for severe peripheral vascular disease, ischemic cardiomyopathy, hyperthyroidism and single-vessel coronary artery disease. developed acute hypoxic respiratory failure on post-operative day (POD) 1, requirIng transfer to Intensive Care Unit for rescue BIPAP and subsequent intubation. The patient was found to be in severe sepsis associated with non-ST elevation myocardial infarction (NSTEMI) secondary to increased demand. CTA chest on POD 1 revealed bilateral aspiration pneumonia which, on respiratory cultures grew Klebsiella and Pneumonia. The patient was started on Vancomycin and Piperacillin-Tazobactam (Zosyn) on POD 1 along with Heparin infusion (for $48 \mathrm{~h}$ ) for the NSTEMI. The pre-operative platelet count of 193 steadily decreased to a nadir of 95 on POD 4 , a greater than $50 \%$ decline from preoperative values. There were no bleeding or thrombotic manifestations. A 4 T score, although not calculated at the time, showed an intermediate HIT probability $(14 \%$, for a score of 4). The thrombocytopenia was attributed primarily to Zosyn adverse effect. A HIT antibody panel was however sent. At this time, both subcutaneous (sc) Heparin and Zosyn were discontinued. PAS stockings were started for deep venous thrombosis (DVT) prophylaxis. Successful extubation was achieved on POD 4. On POD 6, the HIT antibody panel returned positive and Fondaparinux was initiated at prophylactic doses of $2.5 \mathrm{mg} \mathrm{sc}$ per day (Patient's weight was $99 \mathrm{~kg}$ ). After transfer to the floors, the patient continued to require supplemental oxygen at 3-41 nasal cannula (NC). On ambulatory, he exhibited significant exertional hypoxemia to $80 \%$ with tachypnea to $40 \mathrm{~s}$. This was attributed to worsening of previously existent ischemic cardiomyopathy as well as deconditioning. He was continued on prophylactic doses till POD 10 when the Serotonin release assay returned strongly positive. At this time, the haematology—oncology team were consulted and Fondaparinux initiated at anti-coagulant doses of $7.5 \mathrm{mg} s \mathrm{sc}$ per day. An ultrasound of bilateral upper and lower extremities exhibited significant DVT in all 4 extremities. A 
CTA chest on POD 11 showed multiple pulmonary emboli with possible extension to right atrium at the confluence with inferior vena cava. Pulmonary team was collaborated with at this juncture who recommended an IVC filter considering the constellation of comorbidities and clot burden. The patient was finally discharged on POD 14 on Fondaparinux $7.5 \mathrm{mg} \mathrm{sc}$ per day for 6 months, IVC filter and home oxygen.

DISCUSSION: HIT is considered an uncommon cause of thrombocytopenia in critically ill patients with an estimated incidence in ICU patients varying from 0.39 to $0.48 \%$. However, The diagnosis is often confounded by concomitant HIT-mimicking disorders such as septicaemia, thrombocytopenia of critical illness and adverse effects of broadspectrum antibiotic therapies. Recognising and treating HIT in this population becomes imperative considering the morbidity inflicted by under-diagnosis and unde-treatment as illustrated above. The $4 \mathrm{~T}$ scoring in such patients usually reveals an intermediate probability secondary to other possible causes of thrombocytopenia usually existent. However the compete clinical picture needs to be taken into consideration. The patient above was status post an open aorto-bifemoral bypass. There would be reasonable suspicion of the first exposure to heparin intraoperatively in vascular surgeries. Also, HIT develops more frequently in patients who have had a major surgery. The platelet count had a $50 \%$ fall consistent with Day 5 of first exposure with repeated exposures via the heparin infusion and sc heparin. The $4 \mathrm{~T}$ score was not calculated at this time which would have shown an intermediate probability of HIT. Guidelines recommend sending a heparin antibody panel at this time and awaiting results which was performed. However, therapeutic anticoagulation (AC) was not initiated despite the initial screen being positive. The trifecta of clinical suspicion, intermediate $4 \mathrm{~T}$ score and positive screen would be sufficient evidence for initiating therapeutic $\mathrm{AC}$ without awaiting the platelet function assay. Early testing with venous dopplers also should have been performed to detect thrombosis. The choice of anticoagulation should also be tailored to the appropriate scenario. Coumadin use is not recommended till HIT has resolved with a platelet count is above 150,000 secondary to risk of ischemic limb gangrene due to elevated protein $\mathrm{C}$ levels. Per Greinacher, two drugs- Argatroban and Danaparoid have been approved for use in HIT by FDA. Fondaparinux as used here however has shown efficacy in multiple clinical studies but pending FDA approval. Ease of use and lack of monitoring however drove the choice in this scenario. In conclusion, ICU patients are more vulnerable to under detection of HIT and any suspicion should prompt investigation and early initiation of therapeutic anti coagulation even in intermediate probability.

HEPATIC MANIFESTATIONS OF A PSYCHIATRIC ILLNESS Thomas Starnes ${ }^{2}$; Nila Radhakrishnan ${ }^{1} .{ }^{1}$ University of Florida, Gainesville, FL; ${ }^{2}$ University of Florida College of Medicine, Gainesville, FL. (Control ID \#2469464)

LEARNING OBJECTIVE \#1: List a differential diagnosis for transaminits in patients with anorexia nervosa

LEARNING OBJECTIVE \#2: Recognize starvation-induced hepatic autophagy as a cause of severe transaminitis in a patient with anorexia nervosa.

CASE: A 30-year-old female with an 18-year history of severe anorexia nervosa presented with profound weight loss, hypoglycemic episodes, and severe electrolyte disturbances. She weighed $27.2 \mathrm{~kg}$ and stood $1.63 \mathrm{~m}$ tall, resulting in a body mass index of $10.29 \mathrm{~kg} / \mathrm{m}^{2}$. She was mildly hypotensive at $81 / 59 \mathrm{mmHg}$, but her heart rate was $68 / \mathrm{min}$, respiratory rate was $14 / \mathrm{min}$ breathing ambient room air, and temperature was $36.6{ }^{\circ} \mathrm{C}$. She was noted to be severely cachectic in appearance and displayed facial lanugo but no scleral icterus, jaundice, or angiomata. She displayed no signs of decompensated heart failure. Admission laboratory work was significant for near-pancytopenia with a white blood cell count of 1.3 thousand $/ \mathrm{mm}^{3}$, hemoglobin/hematocrit of $8.3 \mathrm{~g} / \mathrm{dL} / 23.6 \%$, and a platelet count of 159 thousand $/ \mathrm{mm}^{3}$. Her initial potassium was measured at $2.0 \mathrm{mmol} / \mathrm{L}$. The initial hepatic function panel revealed a total bilirubin of $0.7 \mathrm{mg} / \mathrm{dL}$, alkaline phosphatase of $150 \mathrm{U} / \mathrm{L}$, aspartate transaminase (AST) of $99 \mathrm{U} / \mathrm{L}$, and alanine transaminase (ALT) of $110 \mathrm{U} / \mathrm{L}$. Her caloric intake remained poor in the hospital due to a refusal of most food, and 10 days after admission her hepatic function panel was found to be grossly abnormal. AST peaked at $817 \mathrm{U} / \mathrm{L}, \mathrm{ALT}$ at $1066 \mathrm{U} / \mathrm{L}$, and alkaline phosphatase at $457 \mathrm{U} / \mathrm{L} 11$ days after admission. At this time, the patient agreed to enteric feeding via a small-bore feeding tube. She was evaluated for a cause of her worsening transaminitis. Abdominal ultrasonography of the right upper quadrant was unrevealing and showed a normal hepatopedal flow of the portal vein. Laboratory evaluation with an acute hepatitis panel, ceruloplasmin, iron studies, and immunoglobulin levels did not reveal an etiology of the liver injury. The patient and her mother declined a recommended liver biopsy. Liver function lab values trended down slowly after the initiation of the enteric feeding, suggesting that the transaminitis was due to severe malnutrition in the setting of anorexia nervosa. As feeding was continued, electrolyte abnormalities slowly corrected and hypoglycemic episodes became more infrequent. After being seen to be medically stable, she was transferred to an inpatient psychiatric unit of the hospital for continued treatment of her anorexia nervosa.

DISCUSSION: Transaminitis is a well-known complication of eating disorders, having been described in the medical literature previously ${ }^{1}$, but significantly elevated transaminases are infrequently reported. A 2014 article in the Turkish Journal of Gastroenterology mentioned that "transaminase levels over $1000 \mathrm{u} / \mathrm{L}$ is a very rare complication." ${ }^{2}$ In one study, hepatic biopsies were taken in 12 patients with anorexia nervosa and hepatic failure. Of the 12 biopsies, 4 were examined by electron microscopy and found to have autophagosomes present. ${ }^{3}$ These findings, along with the absence of hepatocyte necrosis or alterations in hepatic blood flow, suggest that autophagocytosis is a significant cause of transaminitis in severely malnourished patients. Clinicians need to recognize starvation-induced autophagocytosis as a cause of significant acute transaminitis. However, alternative etiologies need to be ruled out because of the morbidity and mortality rates associated with diagnoses such as acute viral hepatitis, autoimmune hepatitis, Wilson's disease, hemochromatosis, or acetaminophen toxicity. Clinicians also should be aware of the constellation of problems associated with severely elevated transaminase levels. Tomita et al. found that patients with ALT values $>840 \mathrm{U} / \mathrm{L}$ were more likely to display hypoglycemia, bradycardia, hypothermia, and thrombocytopenia, ${ }^{4}$ findings that our patient displayed at one point or another in her hospital stay. Previous case reports and series report resolution of transaminitis with gradual reintroduction of caloric intake, often via feeding tubes. Our patient demonstrated this same response, further confirming that her liver injury was secondary to severe malnutrition. References 1. Tsukamoto M, Tanaka A, Arai M, et al. Hepatocellular injuries observed in patients with an eating disorder prior to nutritional treatment. Intern Med. Vol 47. Japan2008:1447-1450. 2. Bridet L, Martin JJ, Nuno JL. Acute liver damage and anorexia nervosa: a case report. Turk $J$ Gastroenterol. Apr 2014;25(2):205-208. 3. Rautou PE, Cazals-Hatem D, Moreau R, et al. Acute liver cell damage in patients with anorexia nervosa: a possible role of starvation-induced hepatocyte autophagy. 4. Tomita K, Haga H, Ishii G, et al. Clinical manifestations of liver injury in patients with anorexia nervosa Hepatol Res. Oct 2014;44(10):E26-31

HEPATITIS C SHOCKS LIVER: A CASE OF ACUTE HEPATITIS C INFECTION CAUSING TRANSAMINITIS IN HIV PATIENT. Waqas Nawaz; Tehseen Haider; Elizabeth Clain; Bennal Perkins. Montefiore Medical Center, Albert Einstein College of Medicine, Bronx, NY. (Control ID \#2433857)

LEARNING OBJECTIVE \#1: Consider acute hepatitis C viral infection as a cause of transaminitis especially in HIV infected patients.

LEARNING OBJECTIVE \#2: Recognize importance of diagnosing co-infections in HIV, as they affect the disease course and response to treatment. 
CASE: A 25 year old African American male with HIV, with CD4 count of 482/microL and HIV viral load of $437 \mathrm{copies} / \mathrm{ml}$, not adherent to antiretroviral therapy, presented with right upper quadrant abdominal pain and dark urine. He had right upper quadrant tenderness to palpation with elevated aspartate aminotransferase (AST) of $703 \mathrm{U} / \mathrm{L}$, alanine aminotransferase (ALT) of $695 \mathrm{U} / \mathrm{L}$, alkaline phosphatase of $157 \mathrm{U} / \mathrm{L}$, and Direct bilirubin of $1.0 \mathrm{mg} / \mathrm{dL}$. Urinalysis showed urobilinogen of $4.0 \mathrm{EU} / \mathrm{dl}$. He had leukopenia to 4100 /microL and negative antinuclear, anti-mitochondrial and anti-liver and kidney microsomal antibodies. Monospot test was negative, but Epstein Barr Virus DNA viral load was $1500 \mathrm{IU} / \mathrm{ml}$. Hepatitis C antibody was positive with viral load of 47,007,341 IU/mL. Abdominal ultrasound revealed normal liver texture and size. His HCV screen 6 months prior was negative suggesting a diagnosis of acute HCV. Syphilis screen was positive. He clinically improved and was started on ledispravir and sofosbuvir as an outpatient for treatment of HCV. He planned to follow up with infectious disease to start treatment for syphilis when his transaminitis resolved.

DISCUSSION: There is growing awareness of sexual transmission of HCV among HIV positive homosexual and bisexual men. Risk factors for spread of HCV include direct blood to blood contact for e.g. sharing syringes for drug injection, accidental needle sticks among health workers, unsafe reuse of medical equipments or tattooing with non sterile equipment. Acute $\mathrm{HCV}$ is diagnosed when patients present with newly detectable $\mathrm{HCV}$ RNA and anti-HCV antibodies within 6 months of a negative HCV antibody test. While $\mathrm{HCV}$ infection is estimated to account for $15 \%$ of symptomatic cases of acute hepatitis in the United States, the majority of patients with acute HCV are asymptomatic. When symptomatic, it presents as anorexia, abdominal pain, darkening of urine and jaundice. It can cause transaminitis that can be as high as 10-20 times upper limit of ALT and AST. The aminotransferases start to increase shortly before the onset of clinical symptoms and usually before anti-HCV antibodies are detectable but levels may fluctuate. Co-infection with HIV and HCV is common. Thirty percent of HIV patients are co-infected with HCV. These patients usually have higher aminotransferase levels and HCV viral loads than people with $\mathrm{HCV}$ alone. HIV hastens the progression towards fibrosis and liver cirrhosis, especially if the CD4 count is less than 200. HIV positive patients still have good virologic response to $\mathrm{HCV}$ treatment. However, $\mathrm{HCV}$ increases risk of progression to AIDS and decreases response to ART for treatment of HIV, while also increasing the risk of hepatotoxicity from ART. Clinicians typically monitor non-HIV acute HCV patients for 12 weeks, anticipating spontaneous clearance of the virus, prior to initiating $\mathrm{HCV}$ treatment. HIV positive patients are less likely to spontaneously clear HCV infection. Early treatment is associated with high sustained virologic response (SVR). Treatment of acute HCV in HIV patients involves an interferon-based regimen with ribavirin for 12 24 weeks depending on the genotype. Recent trials with novel direct acting antivirals (DAA) in patients with HIV/HCV co infection showed promising SVR at 12 weeks (61\% in DAA plus interferon ribavirin regimen vs $27 \%$ in interferon ribavirin group alone) When considering treatment of $\mathrm{HCV}$, it is imperative to test and treat for other sexually transmitted infections. Syphilis and EBV may contribute to a slow virologic response to the initial treatment of HCV. This is supported by studies revealing HCV SVR improvement after treatment of syphilis infection. This case illustrates the importance of considering acute $\mathrm{HCV}$ in the differential for transaminitis in HIV-positive patients, as well as testing for other infections that may interfere with the recovery.

HEPATOCELLULAR CARCINOMA PRESENTING WITH SCROTAL SWELLING AND CLINICAL FEATURES OF HEART FAILURE WITH RIGHT ATRIAL METASTASIS AT THE TIME OF DIAGNOSIS Monoj K. Konda $^{2}$; Christopher Di Felice ${ }^{1}$; Sandeep Patri ${ }^{2}$; Hardik Chhatrala ${ }^{2} .{ }^{1}$ Western Michigan Universiry Homer Stryker M.D. School of Medicine, Portage, MI; ${ }^{2}$ Western Michigan University Homer Stryker M.D. School of Medicine, Kalamazoo, MI. (Control ID \#2460673)
LEARNING OBJECTIVE \#1: Hepatocellular carcinomas (HCC) can metastasize to heart at the time of diagnosis and can pose a diagnostic dilemma by mimicking cardiac disease.

LEARNING OBJECTIVE \#2: Detailed history and high clinical suspicion is required to make an accurate diagnosis. We describe an unusual presentation of $\mathrm{HCC}$ with metastasis to right atrium causing features of right heart failure

CASE: An 83-year-old gentleman presented to the emergency department with shortness of breath, fatigue with worsening scrotal and bilateral lower extremity swelling for 1-month duration. Patient also reported $40 \mathrm{lbs}$ of unintentional weight loss over past year despite his edema. He had diabetes mellitus and essential hypertension. He was previously evaluated with an exercise stress test which was negative. Laboratory results included: Hemoglobin $9.4 \mathrm{mg} / \mathrm{dl}$, MCV 72.9 FL, Sodium 132 mmol/L, AST 70 IU/L, ALT 28 IU/L, alkaline phosphatase $515 \mathrm{IU} / \mathrm{L}$, total bilirubin $1.6 \mathrm{mg} / \mathrm{dl}$, albumin $2.7 \mathrm{~g} / \mathrm{dl}$, prothrombin time $15.1 \mathrm{~s}$, pro-BNP $1446 \mathrm{pg} / \mathrm{ml}$ and troponin $<0.03 \mathrm{ng} / \mathrm{ml}$. Physical exam revealed bibasilar crackles, grade 2/6 holo-systolic murmur, 1+ bilateral lower extremity edema extending to thighs and scrotum and a positive abdomino-jugular reflux. A chest x-ray demonstrated mild cardiomegaly with increased pulmonary vascular markings. A CT of abdomen revealed hepatic lesion concerning for malignancy and patient was transferred to tertiary-care facility for further management Vitals were stable with exception of mild hypoxia supplemental oxygen. A transthoracic echocardiogram showed a $7.0 \times 5.3 \mathrm{~cm}$ mass in the right atrium suggestive of metastatic tumor in transit. Follow-up CT of the chest, abdomen and pelvis with contrast revealed $19 \mathrm{~cm}$ hepatic mass replacing the entire right hepatic lobe with bilateral pulmonary emboli. Alpha-fetoprotein level was $2,669 \mathrm{ng} / \mathrm{ml}$. A CT guided biopsy confirmed poorly differentiated HCC. Cardiothoracic surgery deemed patient as a poor operative candidate while hematology oncology felt he would be intolerant of systemic therapy. The patient was transitioned to hospice care and passed away 2 months later.

DISCUSSION: HCC is the fifth most common neoplasm with $5.5-14.9 \%$ incidence. It is an aggressive tumor with median survival of 6-20 months following diagnosis Extrahepatic metastases at the time of diagnosis is seen in $5-15 \%$ of cases occurring more commonly when tumor size is $>5 \mathrm{~cm}$ and common sites are lung, intra-abdomina lymph nodes, bone and adrenal glands in that order. Metastasis to heart is relatively rare with an incidence of $0.67-3 \%$. In cases of cardiac metastasis cancer spreads through hepatic veins, inferior vena to the right atrium and can involve other chambers of heart. A right atrial mass can cause significant hemodynamic instability, pulmonary emboli from the friable tumor, systolic murmur due to tricuspid valve dysfunction and can lead to sudden death in severe cases with large obstruction or pulmonary embolism. Standard treatment is resection of primary tumor and intra-cardiac mass; However, prognosis was reported to be poor with only $12-39 \% 5$-year survival even with surgical treatment. It is important to recognize the possibility of HCC when a patient with history of chronic hepatic disease presents with symptoms of right heart failure.

\section{HEREDITARY ANGIOEDEMA ATTACK PRESENTING AS URINARY TRACT} OBSTRUCTION: CASE REPORT Asaad Nakhle; Rimma Polevoy; Iyad Isseh; Mahmoud Ali; Jian Li. Henry Ford Hospital, Detroit, MI. (Control ID \#2466722)

LEARNING OBJECTIVE \#1: Recognize renal manifestations in patients with hereditary angioedema.

CASE: The patient was a 20-year-old African American female with a past medical history of hereditary angioedema (HAE) who had undergone multiple admissions with respiratory distress requiring intubations and was maintained on Danazol as an outpatient. She presented to the emergency department with an acute onset of bilateral flank discomfort, abdominal pain, and nausea. At the time of presentation, she was afebrile, and her vital signs were within normal limits. Physical examination was significant for bilateral costovertebral angle tenderness, and fine miliary rash over the right scapula. Her 
laboratory studies showed an elevation in her serum creatinine to $3.4 \mathrm{mg} / \mathrm{dL}$ from a previous value of $0.6 \mathrm{mg} / \mathrm{dL}$. Given her known history of HAE, she was given a dose of Ecallantide to block the activity of plasma kallikrein which offered mild relief of her symptoms, and she was subsequently admitted to the hospital for work-up of acute kidney injury (AKI). A CT scan of the abdomen and pelvis showed mild calyectasis with bilateral perinephric, parenchymal, and peripelvic edema. Slight urothelial thickening was also noted in the renal collecting systems and proximal ureters, with additional periserosal stranding of the bladder. Such bilateral findings may be typically seen with a distal obstruction or an infectious process. Obstruction due to a calculus was ruled out with renal protocol CT showing no evidence of stones. The patient's urinalysis was benign, revealing small leukocyte esterase but no nitrites, no bacteria, no proteinuria, and RBC and WBC levels within normal limits. A urine culture additionally showed no bacterial growth, effectively ruling out an ascending urinary tract infection. Her urine sediment was bland. While the patient showed no signs of fluid depletion, the patient was fluid challenged with continuous intravenous fluids for the first $48 \mathrm{~h}$ of her hospitalization without any improvement in her creatinine, ruling out hypovolemic etiology. Allergy service was involved in the patient's care, and the patient was given a dose of $\mathrm{C} 1$ - inhibitor (Berinert) on day 1 and continued on Danazol. While the patient's abdominal pain improved and resolved by day 4 , she continued to show no change in her renal function (Cr 3.8-4.0) or flank pain, and she was given an additional dose of Berinert on day 4. Due to her continued symptoms and renal impairment, she underwent a kidney biopsy on day 5. Pathology was consistent with mild interstitial edema, and this was able to effectively rule out acute tubular necrosis (ATN) or interstitial nephritis as potential causes of her AKI. On day 6, the patient was noted to have an increased urine output ( $3 \mathrm{~L}$ from $1.8 \mathrm{~L}$ ), a decrease in serum creatinine $(2.9 \mathrm{mg} / \mathrm{dL}$ from $3.9 \mathrm{mg} / \mathrm{dL})$, and resolving flank pain. She was discharged on Danazol, and arrangements were made for her to follow up with both Nephrology and Allergy services. She was seen 19 days after her discharge and found to have near complete recovery of her kidney function, with a serum creatinine of $0.86 \mathrm{mg}$ / $\mathrm{dL}$. This remained normalized an additional 2 months later

DISCUSSION: Although rare, HAE has previously been shown to manifest as renal impairment. HAE is most commonly associated with membranous glomerulonephritis, membranoproliferative glomerulonephritis or IgA nephropathy, but can also cause reduced glomerular perfusion pressure leading to AKI. The patient in this case had a prolonged history of HAE with multiple typical presentations including abdominal pain and respiratory distress, but this was her first documented instance of renal involvement. An obstructing calculus was excluded by imaging and urinalysis, hypovolemic etiology was excluded with fluid challenge, an infectious process was excluded with urinalysis and urine culture, and additional intra-renal pathologies such as glomerulonephritis were excluded by urine sediment and renal biopsy. After HAE-appropriate treatment, the patient's urine output increased in conjunction with improving serum creatinine levels. She eventually regained full kidney function with a return of her creatinine to previous baseline value, which was consistent with a post-obstructive picture. In conclusion, due to the time connection and exclusion of other possible etiological factors, the reported case of acute kidney injury was most likely secondary to a transient swelling in the endothelium of the urinary tract due to HAE attack.

HEREDITARY ANGIOEDEMA: IMPLICATIONS OF DIAGNOSIS AND MANAGEMENT Mohini Pathria; Juan C. Guarderas. University of Florida, Gainesville, FL. (Control ID \#2455530)

LEARNING OBJECTIVE \#1: Describe the presentation, diagnosis, and management of hereditary angioedema

LEARNING OBJECTIVE \#2: Recognize limitations of implementing available therapy
CASE: A 38-year-old woman with anxiety disorder presents for evaluation of recurrent angioedema. At the age of 7, she had severe abdominal pain and underwent laparotomy with the presumed diagnosis of appendicitis; however, her appendix was normal. At age 13, she started to experience episodes of lip, hand, and foot swelling associated with menses. Since adolescence, she has 2-7 episodes of angioedema per month. During these episodes, she visits the emergency room, usually treated with antihistamines, and has limited symptom improvement. Her mother had similar attacks. She has no allergies and no skin manifestations during attacks. She smokes tobacco and drinks alcohol occasionally. She takes escitalopram and hydrocodone-acetaminophen for pain during her attacks, reporting dependency on narcotics. She has a normal physical exam. Her laboratory studies show a total complement of $<13$ (normal 31-60), C4 level of $<3$ (normal 1647), and $\mathrm{C} 1$ inhibitor level of 3 (normal 21-39), confirming the diagnosis of hereditary angioedema. While initiating therapy with $\mathrm{C} 1$ inhibitor, neither medication nor protocols were available in the facility pharmacy. Ultimately, pharmaceutical companies were directly contacted and assisted in handling insurance and administration services. The process approximated 2 months from diagnosis to medication acquisition

DISCUSSION: Hereditary angioedema is an autosomal dominant condition characterized by recurrent episodes of edema of the subcutaneous tissue, gastrointestinal tract, and airway. The prevalence of hereditary angioedema is approximately 1 in 50,000 people. Affected individuals have decreased production or dysfunction of the $\mathrm{C} 1$ inhibitor, a serine protease inhibitor that plays a role in contact, complement, and fibrinolytic systems. Symptoms begin in childhood with most patients diagnosed during adolescence as attack frequency generally increases during puberty. Mechanical trauma, mental stress, airway infection and menses are triggers for an attack. Attacks occur on average every 1 to 2 weeks. Swelling occurs most commonly in the extremities, genitalia, and face, and can also involve the intestines and airway. Angioedema worsens over the first $24 \mathrm{~h}$ and subsides over 48-72 h. Patients can also have laryngeal attacks and abdominal pain without peripheral swelling. One-third of patients with abdominal pain due to hereditary angioedema have received an unnecessary operation due to misdiagnosis. Notably, hereditary angioedema is not associated with food, environmental allergies, or pruritis. The condition does not respond to therapies targeted toward allergic angioedema, such as antihistamines, epinephrine, or corticosteroids. Patients have reported depression and anxiety and should be screened for co-morbid mood disorders at time of diagnosis. Patients can also develop narcotic dependence due to the pain associated with acute symptoms. Diagnosis can be difficult given clinical mimickers and unfamiliarity with the condition. Presentation, family history, and decreased $\mathrm{C} 1$ inhibitor levels contribute to the diagnosis; however one-fourth of patients have a spontaneous mutation resulting in the condition, so a lacking family history should not preclude the diagnosis. Tests to screen for hereditary angioedema include CH50 (total complement), C4 level, and C1 inhibitor level and function. The combination of low $\mathrm{C} 4$ and $\mathrm{C} 1$ inhibitor function has a reported $98 \%$ specificity and $96 \%$ negative predictive value. Plasma derived $\mathrm{C} 1$ inhibitor replacement has been approved in the United States for treatment of acute attacks, with the highest efficacy occurring when the medication is administered early. $\mathrm{C} 1$ inhibitor provides relief in the first 30 to $120 \mathrm{~min}$, with full resolution in $24 \mathrm{~h}$. Recombinant $\mathrm{Cl}$ inhibitor is effective within $3 \mathrm{~h}$. Icatibant, a synthetic bradykinin receptor antagonist, is administered subcutaneously and the time of onset to relief occurs after approximately $1 \mathrm{~h}$. Ecallantide is an inhibitor of kallikrein that has recently been approved for use in the United States. Fresh frozen plasma contains $\mathrm{C} 1$ inhibitor and is second line treatment as other substrates in plasma are thought to potentially worsen an attack. For long-term prophlyaxis, antifibrinolytics such as epsilon aminocaproic acid and transexaminc acid have been used, with the presumed mechanism of reducing peripheral consumption of $\mathrm{C} 1$ inhibitor. Attenuated androgens like danazol have also been used when antifibrinolytics are contraindicated, such as with a history of thromboemoblism or hemophilia, but have side effects such as virilization. Medications for acute attacks average $\$ 5,000$ to $\$ 11,000$ per dose and require coordination with insurance companies. The World Allergy Organization has 
recommended self-administration at home providing the earliest intervention at the first sign of attack. This requires amenable patients to be trained in intravenous adminstration. In addition to reduced attack severity, patients report decreased interference in family and social life, sleep habits, and missed days at school or work. Hereditary angioedema is a rare condition and delays in diagnosis lead to a significantly decreased quality of life. Difficulty implementing appropriate treatment is caused by unfamiliarity of current therapies, expense, and administrative protocols.

\section{HISTORY AND PHYSICAL IS THE BACKBONE OF CLINICAL DIAGNOSIS} Yelena Kreymer. Mount Sinai Beth Israel, New York, NY. (Control ID \#2469571)

LEARNING OBJECTIVE \#1: Recognize signs of cord compression by physical exam CASE: A 72-year-old male, with PMH of large B-cell lymphoma status post R-CHOP chemotherapy, presents for an outpatient 2 week follow-up for leg edema. The swelling is symmetric, without erythema or pain. He denies any shortness of breath, orthopnea, or chest pain. He had a normal TTE 2 years ago. The swelling presented acutely after taking ibuprofen for back pain after a fall 1 month ago. Edema was thought to be a side effect of ibuprofen and he was switched to Percocet at previous visit. He notes that the swelling has decreased. He does not complain of any other symptoms. As he walks over to the exam table, I note he has an ataxic gait and inquire further history. Previously to a fall 1 month ago, he used to be very active, walking six miles a day, but now mostly works from home Over the past month he had two falls. The first fall was accidental due to a heavy backpack. The following fall 2 weeks ago was due to his right knee buckling. He denies leg weakness, but has mild leg numbness. He does not seem concerned about his unstable gait. He minimizes most symptoms and attributes it to the swelling. On further review of systems, he reports that he has persistent left sided rib pain, which radiates in a band-like distribution around his back, but is well controlled with pain medications, although he takes them daily. He denies urinary retention, bowel or bladder incontinence, fever, chills, night sweats or weight loss. On physical exam, his pupils are equal and reactive, extraocular movements are intact. There is no lymphadenopathy. Lungs are clear to auscultation, no crackles. Heart is with regular rate and rhythm, no murmurs. Abdomen is soft, non-tender, no hepatosplenomegaly. Lower extremities have trace edema bilaterally, no calf tenderness. Neuro exam reveals ataxic gait, mildly decreased sensation in lower extremities to light palpation, strength $5 / 5$ in all extremities, reflexes - right patellar 0 , left patellar 2+, normal nose-to-finger, normal rapid alternating movement, Romberg negative. MRI of the thoracic spine shows marked focal spinal cord compression with cord edema at primarily $\mathrm{T} 7$ level due to a bulky epidural mass.

DISCUSSION: This case highlights the importance of a physical exam. This was my initial encounter with the patient. He did not present complaining of neurologic symptoms, or gait instability, which was clearly evident on physical exam. That forced me to ask more probing questions about his gait and any neurologic symptoms that he did not find alarming. My concern, especially in a patient with a history of lymphoma, back pain and gait instability was cord compression. We referred him for emergent MRI of the spine and brain, which confirmed cord compression and the patient immediately started radiation therapy and further treatment. Biopsy of the epidural mass later confirmed B-cell lymphoma. There have only been a handful of studies that looked at the importance of history and physical exam in clinical diagnosis. The data, although limited, consistently shows that the majority of final diagnoses can be made with history and physical alone (up to $90 \%$ of cases), without further lab tests or imaging. ${ }^{1,2}$ However, as medical technology continues to advance, physicians are more likely to rely on lab tests and imaging, especially if clinical exam skills are deemphasized or lacking. Not only could this lead to more misdiagnosis, but also harm to the patient and increased cost. ${ }^{3}$ In the past decade there has been more interest in reviving the fundamentals of medicine- history and physical exam. And as health care costs continue to rise, this might prove to be a solution to an ever growing problem. References: 1. M.C. Peterson, J.H. Holbrook, D.V. Hales, et al. Contributions of the history, physical examination, and laboratory investigation in making medical diagnoses. West J Med, 156 (1992), pp. 163-165. 2. Hampton JR, Harrison MJ, Mitchell JR, Prichard JS, Seymour C. Relative contributions of historytaking, physical examination, and laboratory investigation to diagnosis and management of medical outpatients. Br Med J. 1975;2(5969):486-489. 3. Feddock CA. The lost art of clinical skills. Am J Med 2007; 120(4):374-8.

HIV PRESENTING AS WEAKNESS Jenny Cunningham; Catherine Jones. Tulane University Health Sciences Center, New Orleans, LA. (Control ID \#2468656)

LEARNING OBJECTIVE \#1: Diagnose an unusual late stage presentation of AIDS LEARNING OBJECTIVE \#2: Recognize the evidence to incorporate routine HIV screening into clinical practice

CASE: A 51-year-old male with no past medical history presented initially to the emergency department with a 3-week history of worsening left hand weakness. On review of social history, he was divorced and in a monogamous sexual relationship with his girlfriend of 5 years. He denied history of IVDU. As part of the routine practice of the emergency department, the patient was offered opt-in HIV testing. HIV-1 antibody test came back positive. MRI of brain showed multiple bilateral T2 flair abnormalities without diffusion restriction or enhancement. Lumbar puncture was preformed with testing for fungal, viral and bacterial studies, including toxoplasma antibody and crytococcus antigen. These studies were all negative. With MRI findings and HIV status in mind, JC virus qualitative PCR was also sent and returned positive and the patient was diagnosed with Progressive Multifocal Leukoenceophalopathy. He was started on ART. At the time of discharge from the hospital his exam revealed only mild grip strength weakness in his left hand. Two months later, he again presented to the emergency department complaining of rapidly progressive weakness over the last week involving his entire left arm, left leg, and face. The patient reported careful adherence to ART regimen. Physical exam revealed overall decreased strength, right-sided nasolabial fold flattening, and mild decreased sensation in the right hand. Repeat Viral Panel demonstrated CD4 count of 293 with undetectable viral load. Repeat MRI showed worsening edema and mass effect suggestive of inflammation consistent with IRIS rather than direct progression of PML. The patient was treated with $1 \mathrm{~g}$ Solumedrol daily for 5 days for treatment of PML-IRIS, followed by $4-6$ weeks taper. ART medications were also continued. After approximately 1 week, modest gains were noted in lower extremity strength, with patient regaining ability to ambulate with a foot drop. His hand remained weak, but significant gains were seen at the elbow and shoulder. Patient was eventually discharged with physical therapy.

DISCUSSION: CDC recommendations for HIV testing in health care settings were published in September 2003 that called for HIV testing to become part of routine medical services, without regard to risk factors. Like this patient, $48 \%$ of those in the study who received a new diagnosis would not have qualified for testing by risk factor assessment alone. Many of these patients did not present for complaints related to HIV, but were able to be diagnosed and connected with treatment services. In the case of this patient, routine screening also led to speedy diagnosis and treatment of an unusual late-stage manifestation of HIV. PML-IRIS is a syndrome in patients infected with HIV who display marked improvements in CD4 $\mathrm{T}$ cell counts and a decrease in viral load which leads to an inflammatory reaction of previous PML lesions as a manifestation of the immune reconstitution syndrome. In addition to a known diagnosis of PML by brain biopsy or PCR testing of CSF, IRIS can be suggested by contrast enhancement of typical lesions on brain MRI. While this condition is too rare for treatment recommendations based on large scale RCTs, expert opinion recommends high dose glucocorticoids for severe neurologic 
deterioration or mass effect noted on imaging. This patient demonstrates that early diagnosis and implementation of steroids in PML-IRIS can lead to significant neurologic improvements, though he will continue to live with significant morbidity. However, median survival for those diagnosed with PML even in the era of ART is only 1.8 years. Wide adaptation of routine HIV screening in emergency departments and in other health care settings and novel approaches to community based screening are needed to prevent tragic and preventable cases that present when the initiation of ART may come too late to avoid serious morbidity and mortality.

HOME VISITS: POPUlATION HEALTH ONE PATIENT AT A TIME Nora Hutchinson; Carol Landau. Brown University, Providence, RI. (Control ID \#2469333)

LEARNING OBJECTIVE \#1: Describe the educational value of a home visit and narrative experience from the provider perspective.

LEARNING OBJECTIVE \#2: Recognize the impact of a home visit on how lowincome women with psychiatric diagnoses are perceived by their medical providers.

CASE: In our General Internal Medicine Program, 4-6 residents, a psychologist and a psychiatrist conduct a home visit each year. The goal of the visit is to provide an integrative psychosocial approach to care for patients, while also creating an in-depth experience for residents. After a 1-h visit, residents write a reflective piece and discuss it with the group. We present one case, with excerpts from resident narratives, to highlight the impact of these visits. Ms $\mathrm{H}$ was a 30-year-old woman who had been recently diagnosed with major depressive disorder and moderate alcohol use disorder. Initial interventions had included the initiation of an SSRI, behavioral health counseling by the clinic social worker, and a referral to a community mental health center for depression and substance use. Her apartment was located in a notoriously dangerous neighborhood. Ms H greeted us warmly in her living room, where an outline of a family tree had been stenciled onto the wall, with nameplates left blank. The living area itself was freshly vacuumed with the vacuum cleaner still sitting in plain view. It was that familiar feeling of a woman preparing her home for guests (R. Pyngolil PGY-2, 2015). She told us the story of her relationship with her partner who was busy working 2 full-time jobs. She expressed concern about her eldest son who had been diagnosed with ADHD and Obsessive Compulsive Disorder, and had been suspended from school. She had taken him out of football due to his school performance and agonized over this decision. She described selfmedication with daily drinking and new frequent marijuana use, both of which were having a significant negative impact on her life. She missed several shifts of work each week and when she was at work she frequently cried. All were concerned that her depression and substance use would lead to the loss of her job and an income that was needed. After finishing her overnight shift she returned home to care for her 2 youngest children. It became clear why a prior attempt to address her disturbed sleep with trazodone had failed-Ms H could not take this medication because she needed to stay alert during the day to care for her sons. She downplayed the severity of her alcohol use, coming back to her low mood as her main concern. She had good insight, but did not want to think of herself as a depressed person, and admitted that it was difficult to accept help. We concluded the visit with a plan to integrate onsite medical visits with behavioral counseling visits, and with a cautious optimism that $\mathrm{Ms} \mathrm{H}$ would accept the help she needed. I'm left wondering what this fall will bring. My hope is that she adds photos to her family tree and in doing so has some sense of what an insightful mother she is (M. Geary PGY-2, 2015).

DISCUSSION: This case highlights the enormous value of home visits as an educational tool. Home visits allow housing, mobility status, nutrition, medications and spiritual health to be evaluated in a comprehensive manner (3). They can also alter the way in which a provider practices medicine, fostering empathy and the development of a stronger patientphysician relationship. $84 \%$ of medical graduates from the Weill Cornell Medical
College, who had taken part in a half day of house calls, reported a continued positive impact on their approach to patient care (4). Home visits also create the possibility of narrative engagement with patients. Rita Charon described the impact of this, both for the patient and the physician, in which "deep and therapeutically consequential understandings of the persons who bear symptoms are made possible in the course of hearing the narratives told of illness" (1). In all of our visits, it was through listening to the life stories of our patients that their struggles were better understood. In our program a greater understanding of the patient is achieved through writing. Charon has highlighted this second aspect of narrative medicine that allows physicians to "reveal transcendent truths, exposed in the course of illness, about ordinary human life" (1). Narrative writing reinforces the memorable experience, allows it to be retained, considered and applied to new settings. Her obstacles are insurmountable in the span of a doctors appointment ... I've concluded that for now, the thing I can do is tell her story ... to help others understand the injustice of poverty (M. Garg PGY-2, 2013). Our case also reveals the close relationship between poverty, hardship and depression (2). Ms $\mathrm{H}$ has since served to represent and inform interactions with many other women who have been seen in our clinic. In this way, a single home visit and the sharing of narratives can inspire practitioners to take a step back and remember the individual stories and patients that compose a population. References: 1. Charon R. Narrative and medicine. NEJM. 2004;350(9):862-864. 2. Heflin, CM, Iceland J. Poverty, material hardship, and depression. Soc Sci Q. 2009;90(5):10511071. 3. Unwin BK, Tatum PE. House calls.Am Fam Physician. 2011;83(8):925-931. 4. Yuen JK, Breckman R, Adelman RD, Capello CF, LoFaso V, Reid MC. Reflections of medical students on visiting chronically ill older patients in the home. J Am Geriatr Soc.2006;54:1778-1783.

HOW A CBC AND PERIPHERAL BLOOD SMEAR CAN BE LIFESAVING Ian Cormier ${ }^{1}$; Meltiady Issa ${ }^{2} .{ }^{1}$ University of Queensland, Albuquerque, NM; ${ }^{2}$ Mayo Clinic, Rochester, MN. (Control ID \#2460467)

LEARNING OBJECTIVE \#1: Identify the early signs and symptoms of thrombotic thrombocytopenic purpura (TTP) Prompt diagnosis of TTP is crucial for initiating plasma exchange therapy, which drastically decreases patient morbidity and mortality. However, the variability in the presentation of this disease and the lack of concrete criteria can make the diagnosis a challenge. The diagnosis of TTP should be made clinically since, though helpful, an ADAMTS13 assay may take days to obtain results. The most common presenting symptoms in patients with TTP are gastrointestinal symptoms (pain, nausea, vomiting and diarrhea), neurologic symptoms (headache, confusion, seizures, stroke and coma), fatigue, weakness, purpura, bleeding and hematuria. Fever and acute renal failure are uncommon. The classic pentad of fever, MAHA, thrombocytopenia, neurologic symptoms and renal failure occurs in roughly $5 \%$ of patients and thus, is not useful in diagnosing TTP. Once a history and physical exam have been performed, an initial complete blood count (CBC) can confirm anemia and thrombocytopenia. These abnormalities should trigger reflex hemolysis labs, including haptoglobin and LDH, as well as a peripheral smear. Peripheral smears demonstrating schistocytes are vital to the workup of TTP. Thus, a CBC and peripheral smear can be quick, cheap and effective screening tools for this disease.

LEARNING OBJECTIVE \#2: Assess severity and appropriately triage patients with a thrombotic microangiopathy (TMA) As TTP is a life threatening pathology requiring immediate treatment, this disease must be differentiated from other forms of TMAs quickly in order to guide proper therapy. The presence of schistocytes on a peripheral smear is an ominous sign that should incite an immediate workup, including renal and liver function tests, coagulation studies and fibrinogen levels. The severity of TMAs is sometimes difficult to quantify, but may clinically be appreciable by the extent of bleeding, renal failure, neurologic symptoms and laboratory abnormalities. IfTTP is at all suspected, 
plasma exchange should begin immediately. First line treatment for TTP is daily plasma exchange until laboratory values have returned to baseline and 2 days after symptoms have resolved. Hematology/Oncology should be consulted for continued outpatient treatment with long term therapies, which include corticosteroids and rituximab. Other TMAs generally do not require as urgent intervention as patients with suspected TTP.

CASE: A 53 year old woman with no significant past medical history presented to her primary care physician with the complaint of "purple dots on the skin". Physical exam revealed petechial rash on the lower extremitites and soft palate with no other abnormalities. The patient was reassured and sent home with conservative measures. Several days later, she began experiencing fevers, chills, night sweats and a severe unilateral headache associated with photophobia. The patient returned to her primary care physician, who thought that these symptoms could be due to a viral illness. However, the patient's symptoms became progressively worse and a few days later, her urine became dark red. She was transported to the emergency department for further evaluation. Initial laboratory workup revealed anemia and thrombocytopenia. Serum LDH and bilirubin were elevated and her haptoglobin was low. Coagulation studies were within normal range. Peripheral blood smear demonstrated schistocytes. Urinalysis demonstrated hemoglobinuria. The presence of thrombocytopenia, hemolytic anemia and schistocytes raised suspicion for microangiopathic hemolytic anemia (MAHA) and the patient was transferred to the hematology/oncology unit. She received multiple units of fresh frozen plasma and was immediately started on daily plasma exchange (PEX). Subsequent investigations revealed an ADAMTS13 activity level of $<5 \%$ and a positive ADAMTS13 inhibitor study. The patient was diagnosed with TTP.

DISCUSSION: TTP is a rare, life threatening disease characterized by MAHA and thrombocytopenia leading to the formation of widespread microvascular thrombi with variable signs and symptoms of ischemic organ damage. It was originally characterized by the pentad of hemolytic anemia, thrombocytopenia, fever, neurologic symptoms and rena failure. However, less than $5 \%$ of patients with TTP present with all five of these findings, making the classic pentad obsolete in clinical medicine. A common feature of TTP is decreased levels of ADAMTS13, a metalloprotease responsible for cleaving ultra large multimers of von Willebrand factor, which in turn sequester platelets out of the circulation, creating platelet microthrombi, hemolytic anemia and ischemic organ damage. This case illustrates a classic presentation of a relatively rare but potentially life-threatening TMA. The wide range of non-specific presenting symptoms in patients with TTP can make this disease a challenge to diagnose. A good history and physical exam is helpful in raising TTP suspicion, in which case, a simple complete blood count with a peripheral blood smear are excellent screening tools. This will help to triage those patients appropriately, as any delay in treatment is associated with a higher morbidity and mortality. TTP requires urgent intervention with plasma exchange therapy to prevent serious or potentially life-threatening complications. Other causes of MAHA do not necessitate such emergent intervention.

\section{HOW PULMONARY MYCOBACTERIUM AVIUM COMPLEX INFECTION MASKED INVASIVE MUCINOUS ADENOCARCINOMA Natalia Lipin; John} McConville; Aliya N. Husain; Valerie G. Press. University of Chicago, Chicago, IL (Control ID \#2432561)

LEARNING OBJECTIVE \#1: Learning Point 1: Maintain a broad differential for nonresolving pneumonia Learning Point 2: Understand the typical presentation and treatment of mycobacterium avium complex infection Learning Point 3: Recognize anchoring heuristic and its deterrence to timely recognition of the proper diagnosis Learning Point 4: Identify diagnostic difficulties of invasive mucinous adenocarcinoma due to its bland cytology

CASE: The patient is a 68-year-old man with an 8-month history of progressive dyspnea who was transferred to a tertiary facility for progressive hypoxia, after initially being treated for community acquired pneumonia (CAP) without resolution of symptoms. At that time, the CT scan showed bilateral cavitary lung lesions and the bronchoalveolar lavage (BAL) grew mycobacterium avium complex (MAC) infection, though transbronchial biopsies were negative for malignancy and granuloma formation. Despite 4 months of MAC treatment, he became progressively hypoxic, with daily fevers and a 40lb weight loss. At admission, he had respiratory failure due to acute respiratory distress syndrome with blood cultures, repeat BAL, and rheumatologic workup negative for etiology. Open lung biopsy demonstrated invasive mucinous adenocarcinoma (IMA). Despite maximal pulmonary support, the patient's clinical status deteriorated and he was transitioned to comfort care.

DISCUSSION: This case highlights several important learning points: Learning Point 1: Importance of maintaining a broad differential in non-resolving pneumonia, particularly when a patient experiences treatment failure for a suspected etiology. The British Thoracic Society guidelines provide an approach to non-resolving pneumonia where considerations of both infectious and non-infectious causes for symptoms offer a comprehensive approach. Local or distant spread of infectious pathogens may limit efficacy of oral antibiotics or require source control. Alternately, non-infectious vasculitic, inflammatory, or oncologic conditions may mimic CAP. Workup for non-resolving pneumonia includes serologic testing, radiography, and bronchoscopy with biopsy. For this patient, with a significant smoking history and multiple occupational toxin exposures, malignancy was certainly on the differential. While bronchoscopy is standard of care for workup of accessible pulmonary lesions, the yield is less-than perfect even with ultrasound guidance ( $\sim 88 \%$ for central lesions, $70 \%$ for peripheral lesions). Therefore, if suspicion for malignancy is high despite negative transbronchial biopsies, patients should proceed to video-assisted thoracoscopic surgery (VATS), which has sensitivities of $99 \%$. Learning Point 2: Understanding the typical presentation and treatment of mycobacterium avium complex infection. Nontuberculous mycobacterial diseases represent a diverse group of clinical presentations described by the national clinical guidelines. Cavitary and interstitial forms of MAC are diagnosed by presence of all three criteria: (1) Clinical pulmonary symptoms, (2) Radiographic evidence of cavitation or interstitial pattern with bronchiectasis and small nodules, and (3) Minimum two sputum specimens (expectorated or bronchoscopic samples) demonstrating MAC. Therapeutics depend on severity of disease and require monthly monitoring of sputum specimens to determine success of treatment. Specifically, patients without clinical improvement after 6 months of treatment or persistently positive cultures represent treatment failure. For the patient in question, his symptom progression despite appropriate treatment should have been identified as treatment failure and been further evaluated. Learning Point 3: Recognition of anchoring heuristic and its deterrence to timely recognition of proper diagnosis. In this case, understanding the typical presentation of MAC and its response to treatment was essential to identifying that the patient's case was either extremely atypical MAC, or, more likely, that an alternative diagnosis was present. Acknowledgement of anchoring and maintaining a broad differential perhaps could have led to additional tissue earlier in this patient's course. Learning Point 4: Identification of diagnostic difficulties of invasive mucinous adenocarcinoma due to its bland cytology. IMA is characterized by its invasiveness, quick progression, bilateral, multi-centric involvement, and likely aerogenous spread. Cells often lack cytologic atypia, which may contribute to frequent misdiagnosis as an inflammatory process. In 2011, the International Association for the Study of Lung Cancer et al. (IASLC/ATS/ERS) revised the former 2004 non-small cell lung cancer (NSCLC) guidelines to reflect further understanding of the clinical course and genetics of its various subtypes. Sensitivities of $20 \%$ for fine needle and $60 \%$ for core needle transthoracic biopsies have been described. Given the bland pathology of IMA, it is not surprising that this patient's initial transbronchial biopsies lacked evidence of malignancy. This case highlights a rare form of NSCLC, invasive mucinous adenocarcinoma, which is known for its aggressive nature and poor prognosis. Its abundance of mucin makes needle biopsy diagnosis challenging. When there is high clinical suspicion of malignancy, more invasive tissue sampling is necessary for early diagnosis and treatment. 
HOW SWEET IT IS John Anderson; Jason Dinsmoor; Michael P. Smith. University of Nebraska Medical Center, Omaha, NE. (Control ID \#2469934)

LEARNING OBJECTIVE \#1: Recognize the clinical presentation and the clinical criteria required to make the diagnosis of acute febrile neutropenic dermatosis (Sweet Syndrome)

LEARNING OBJECTIVE \#2: Understand the underlying factors in the pathophysiology in order to appropriately treat acute febrile neutropenic dermatosis

CASE: A 54 year-old man with a recent diagnosis of mantel cell lymphoma returned to the hospital with hypotension, fever, and leukocytosis. The patient was admitted a week prior with similar complaints in addition to headache, diffuse myalgia, and arthralgia. A thorough infectious work up had been performed during his hospitalization; however, no infectious agent was identified. He was discharge on oral antibiotics with only moderate improvement. During that hospitalization, he abruptly developed a diffuse rash consisting of painful erythematous patches and plaques. Upon returning to the hospital, the patient was febrile with a temperature of $103^{\circ}$ Fahrenheit and systolic blood pressure in the eighties. He had bilateral conjunctival injection and his erythematous patches and plaques were unchanged. Heart, lung, and abdomen exam had no abnormalities. He had a leukocytosis of 13.6 with $96 \%$ neutrophils, erythrocyte sedimentation rate of 50, a positive c-reactive protein, and increased liver enzymes. A skin biopsy of an erythematous plaque showed superficial neutrophilic inflammation consistent with febrile neutropenic dermatosis, or Sweet Syndrome.

DISCUSSION: Fever and leukocytosis are commonly encountered by the general internist. Acute febrile neutropenic dermatosis often presents with sudden onset of fever. Often this can be associated with leukocytosis as well as a diffuse rash composed of tender, well-demarcated papules and plaques. Major criteria use to diagnose sweet syndrome include histological evidence of a dense neutrophilic infiltrate without evidence of leukocytoclastic vasculitis and abrupt onset of painful erythematous plaques or nodules. Minor criteria that assist in the diagnosis include fever; an associated process including: malignancy, inflammatory disease, pregnancy, or preceding infection; excellent response to treatment; and laboratory evaluation with erythrocyte sedimentation rate $>20 \mathrm{~mm} / \mathrm{h}$, positive C-reactive protein, and greater than 8000 leukocytes with greater than $70 \%$ neutrophils. The pathophysiology of Sweet Syndrome is thought to be related to hypersensitivity reactions, cytokine dysregulation, and genetic susceptibility. A patient presenting with acute febrile neutropenic dermatosis can appear critically ill and can be confused with alternative diagnosis's including severe sepsis. It is the internist role to be able to use diagnostic criteria in the evaluation the patient in order to make the correct diagnosis. Accordingly, understanding that the underlying causative factors result in a reactive process, provides the internist insight in how to treat the patient. This is a very satisfying diagnosis, as the patient will have an excellent response to oral or intravenous steroids.

HOW WOULD YOU DESCRIBE THAT MURMUR? Meghan Combs; Micah Beachy; Michael P. Smith. University of Nebraska Medical Center, Omaha, NE. (Control ID \#2469837)

LEARNING OBJECTIVE \#1: Recognize the variable presentation of endocarditis. LEARNING OBJECTIVE \#2: Develop an approach to hemoptysis.

CASE: A 25 year-old man presented with 2 months of bilateral lower extremity edema and hemoptysis. One month prior, he was hospitalized for a pneumonia but left against medical advice despite persistent symptoms. Over the next month, his condition worsened with an increase in hemoptysis and a decrease in urine output. He was breathing 27 times per minute, had bilateral inspiratory and expiratory rhonchi with late expiratory wheezes, and had decreased breath sounds at the right base. Sinus tachycardia with $2 / 6$ pan-systolic heart murmur across a hyperdynamic precordium was heard. His abdomen was soft, edematous with noted hepatomegaly. Multiple large joint effusions were present including the left shoulder and bilateral knees. Pain was elicited with any joint movement. Lower extremity $2+$ pitting edema was present. No petechial rashes or lesions were noted. Laboratory evaluation revealed an elevated serum creatinine with severe acidosis. Multiple peripheral pulmonary infiltrates were demonstrated on computed tomography. He quickly decompensated, was intubated, admitted to the intensive care unit, and required intermittent hemodialysis. Multiple blood cultures grew methicillin-resistant Staphylococcus aureus. Further evaluation revealed vegetation of four native valves with aortopulmonic paravalvular abscess. The patient admitted to 7 months of intravenous methamphetamine use. Ultimately, he received triple mechanical valve placement with debridement of the paravalvular abscess. Less than $24 \mathrm{~h}$ after surgery, he self-extubated without complications

DISCUSSION: Hemoptysis is a problem commonly encountered by the general internist that can be due to benign, self-limiting pathologies or can be due to pathologies with significant morbidity and mortality. When evaluating hemoptysis, it is important to consider the vascular anatomy of the lung broken up into the low pressure pulmonary circulation and the bronchial circulation that branch from the aorta and are at systemic pressure. Hemoptysis is due to a breakdown in the structure of the normal lung anatomy and the most common causes are related to common infections, such as pneumonia, or inflammation, as in bronchitis. Other considerations include severe infections that distort the anatomy of the lung as in tuberculosis, lung abscess, or fungal infections within an already structurally distorted lung. Structural issues related to inflammation such as malignancy and autoimmune disorders must be considered as well. The structure of the heart-lung circuit along with the contents of the blood should also be considered, specifically mitral stenosis, emboli, and coagulopathies. Our patient presented with active hemoptysis and grossly abnormal exam. In this situation, flexible bronchoscopy and/or computed tomography is warranted to evaluate for the structural abnormalities at the root of his hemoptysis. Bronchoscopy and/or transthoracic needle aspiration is helpful for obtaining material for culture when multiple infiltrates are present on imaging or infection is suspected. The presence of edema with hemoptysis may increase suspicion for structural issues within the heart and an echocardiogram is useful. The presentation of endocarditis varies by each patient. Most patients present acutely; however, this is not always true. It is essential to evaluate for new murmurs, signs of acute heart failure, and neurologic, joint, skin, pulmonary, renal and splenic sequelae. All or none of these can be present as our patient demonstrated at his various presentations with limited evidence of sequelae at the outside hospitals but severe sequelae and all four heart valves involved upon presentation at our facility. Given his risk factors, a more aggressive investigation at a prior presentation perhaps could have prevented the worsening and spread of his infective endocarditis With intravenous drug abuse a common problem along with hemoptysis a common presenting compliant, the general internist should be aware of the various presentations of endocarditis and a structural approach to hemoptysis can aid in making an accurate diagnosis to ensure timely, appropriate treatment.

\section{HYDRALAZINE ASSOCIATED CRESCENTIC GLOMERULONEPHRITIS}

David Gessert; Adrienne Lenhart; Vallari K. Kothari; Lenar Yessayan. Henry Ford Hospital, Detroit, MI. (Control ID \#2466679)

LEARNING OBJECTIVE \#1: Diagnose hydralazine-induced vasculitis LEARNING OBJECTIVE \#2: Manage hydrlazine-induced vasculitis aggressively CASE: A 67 year old Caucasian male with history of diabetes and hypertension, on hydralazine, was admitted secondary to a several day history of shortness of breath and lower extremity edema after recently traveling to Aruba. The patient had been started on hydralazine therapy 18 months prior to presentation and his dose had been increased from 25 to $50 \mathrm{mg}$ three times daily 12 months ago. Initial workup revealed a serum creatinine of $3 \mathrm{mg} / \mathrm{dL}$, which had increased from $0.78 \mathrm{mg} / \mathrm{dL}$ just 3 weeks prior. Urinalysis was 
significant for a large amount of blood and proteinuria. The patient denied any recent contrast exposure, antibiotic use, or nephrotoxic medications. His family history was only significant for a relative with scleroderma. Laboratory results were as follows: Urine protein/creatinine ratio was 1.51 , consistent with non-nephrotic range proteinuria. Urine protein electrophoresis suggested glomerular proteinuria. Urine sediment showed many non-dysmorphic red blood cells without evidence of cellular or granular casts. Serological tests for hepatitis B and HIV were negative. Serum C3 and C4 levels were normal. Rheumatoid factor, anti-glomerular basement membrane antibody, anti-topoisomerase 1 antibody (Scl-70) and anti-double stranded DNA antibody were negative. Anti-nuclear antibody (ANA; titer 1:320), anti-histone antibody (1.2 units) and anti-neutrophil cytoplasmic antibodies (both P-ANCA and C-ANCA) were positive. Anti-myeloperoxidase (anti-MPO) and anti-proteinase 3 (anti-PR3) titers were elevated at 92 and 28, respectively (normal $\leq 20$ ). Renal ultrasound showed echogenic kidneys without evidence of hydronephrosis. Hydralazine was subsequently discontinued. He underwent renal biopsy, which revealed glomerulonephritis with immune complex deposition and scattered necrotizing lesions and crescents. Based on his pathology and positive ANCA status, he was diagnosed with hydralazine-induced vasculitis. Despite discontinuation of hydralazine, the patient's renal function continued to worsen rapidly and hemodialysis was initiated. The patient was additionally started on high dose corticosteroids, cyclophosphamide and plasmapheresis therapy. He was ultimately discharged on hemodialysis, with plans to continue immunosuppressive medications, dialysis and plasmapheresis treatment in the outpatient setting.

DISCUSSION: ANCA associated vasculitis has been linked with multiple drugs including hydralazine. It is a rare disease and can cause significant multi organ damage, but the mechanism by which hydralazine leads to an autoimmune response is unclear. It is not uncommon for renal involvement to be the only manifestation of the disease and it is estimated that only $5-10 \%$ of cases of hydralazine-induced kidney disease are crescentic glomerulonephritis as seen in our patient. Risk factors include advanced age, Caucasian race, and female sex. Hydralazine-induced vasculitis is usually associated with doses greater than $200 \mathrm{mg}$ per day as opposed to our patient, who was only taking a total of $150 \mathrm{mg}$ per day. It is important to note that the patient had been taking the drug for 18 months, and in almost all cases of the disease, length of therapy had been greater than 1 year and usually longer. Diagnosis is often challenging as physicians may gloss over medications that have been used safely for a long time. Furthermore, there is no single test to confirm the disease. Anti-histone antibody and ANA are the most common laboratory abnormalities seen in patients with hydralazine-induced vasculitis but positive ANCA studies can be seen as well. Anti-double stranded DNA is unusual in the disease but has been reported in the past. Our patient had positive ANCA studies and high titer anti-MPO. This is often associated with more severe disease. The glomerular crescents with necrotizing lesions confirmed his severe and rapidly progressing disease. This case was unique in that his anti-histone antibody was only weakly positive when usually it is the most strongly positive lab study. It was also unusual that our patient had immune complex deposition on biopsy as rapid progressive glomerulonephritis is pauci-immune. Our patient was quickly started on immunosuppression with corticosteroids, cyclophosphamide and plasmapheresis in an attempt to prevent significant kidney damage or death. In an era where hydralazine is very commonly used, it is important for internists and society to be aware of hydralazine induced vasculitis for early recognition and treatment

HYPERSENSITIVITY PNEUMONITIS PRESENTING AS A CARDIAC EMERGENCY Oluwaseun Shogbesan; Priya Rajagopalan; Jessica Young; Eugene York. Reading Health System, West Reading, PA. (Control ID \#2469699)

LEARNING OBJECTIVE \#1: Recognizing pericardial effusion as a presentation of Hypersensitivity Pneumonitis
CASE: A 31-year-old, nonsmoker, male presented with a history of bilateral leg swelling, progressive shortness of breath and cough. He denied orthopnea, PND, fever, weight loss, hemoptysis or recent travels. Physical examination revealed $3+$ pitting pedal edema, tachycardia and tachypnea. Cardiac examination was negative for jugular venous distension and crackles. Brain natriuretic peptide was 6; TSH, CBC, electrolytes and liver function were within normal limits. Chest radiograph showed diffuse bilateral nodular infiltrates. Computed tomography of chest was remarkable for diffuse alveolar opacity with sparing of the lung periphery, moderate bilateral pleural and pericardial effusion. Echocardiogram was remarkable for pericardial effusion with early tamponade, serial echo cardiogram demonstrated a progressive increase in size of pericardial effusion requiring emergent pericardiocentesis. Pericardial fluid analysis showed a sterile exudate with $87 \%$ predominance of lymphocytes. Post pericardiocentesis echocardiogram showed trace pericardial effusion and was otherwise normal. Additional work up was negative. On further questioning, he divulges moving to a new house with exposure to birds perching in the loft and to molds. He had noticed resolution of respiratory symptoms when away from the house. Tested bird serum precipitins were negative. Transbronchial biopsy revealed patchy chronic peribronchial inflammation predominantly lymphocytic with a component of organizing pneumonia suggestive of HP. He was started on high dose steroids with dramatic clinical and radiographic improvement. He was discharged with a plan for steroid taper over 4 weeks and counselled on need for relocating to a new apartment. Follow-up chest radiograph showed normal lungs with complete resolution of nodular opacities. Two months after discharge, patient represented with progressive shortness of breath, leg swelling which started 2 days after steroid taper. He was tachycardic and tachypneic. Admission chest x-ray and chest $\mathrm{CT}$ again showed diffuse bilateral nodular airspace disease similar to prior. Patient was still living in his old apartment. Echo showed large pericardial effusion with concerns for tamponade and a pericardial window was placed. Histology of pericardium was consistent with acute on chronic pericarditis with predominantly lymphocytic infiltrate without malignant cells. He was again restarted on steroids with resolution of pulmonary symptoms. He was discharged on long term low dose steroids given continued exposure to inciting antigen in present residence.

DISCUSSION: Cardiac tamponade is a dreaded cardiac emergency with grave sequelae if missed. Hypersensitivity pneumonitis (extrinsic allergic alveolitis), a diverse collection of diseases with varied and nonspecific clinical, radiologic and histopathologic findings, presents primarily with respiratory symptoms however pericardial effusion with cardiac tamponade is an uncommon presentation of HP and likely represents inflammation extending to the adjacent pericardium. Our patient presented with a regional, subacute cardiac tamponade hence the absence of the classical signs of tachycardia, jugular venous distension, distant heart sounds or pulsus paradoxus. Predominance of lymphocytosis in pericardial effusion with negative cultures or malignant cells mirrors the finding of predominant lymphocytosis seen in bronchoalveolar lavage in HP. Detailed exposure history, compatible clinical and radiographic finding, and histopathology all play a role in diagnosis of HP. Early diagnosis, identification and removal from allergen prior to lung fibrosis are key. Awareness of, diagnosis and prompt management of uncommon but fatal presentations such as cardiac tamponade is crucial.

HYPERTENSIVE EMERGENCY INDUCING THROMBOTIC MICROANGIOPATHY Jonathan P. Salud. Emory University School of Medicine, Atlanta, GA. (Control ID \#2469601)

LEARNING OBJECTIVE \#1: Identify thrombotic microangiopathy (TMA) and its associated conditions

LEARNING OBJECTIVE \#2: Recognize hypertensive crisis as a cause of thrombotic microangiopathy (TMA) 
CASE: A 40 year-old male presents with abdominal pain. He reports severe, diffuse abdominal pain and anuria for the past 2 days. Review of systems notable for 2 weeks of generalized weakness. He reported a distant history of hypertension but did not regularly take antihypertensive medications. He denied use of alcohol, tobacco, and recreational drugs. On admission, his blood pressure was 261/144 mmHg. Exam revealed scattered pulmonary crackles, increased work of breathing, and bilateral lower extremity edema. Initial labs were significant for serum creatinine greater than $25 \mathrm{mg} / \mathrm{dL}$, BUN of $160 \mathrm{mg}$ / $\mathrm{dL}$, and total bilirubin of $2.2 \mathrm{mg} / \mathrm{dL}$. Hemoglobin was $10.6 \mathrm{~g} / \mathrm{dL}$, platelet count was $74,000 / \mu \mathrm{L}$, with minimal schistocytes. Urinalysis revealed hematuria and proteinuria. $\mathrm{X}-$ ray showed marked pulmonary edema. He developed worsening respiratory distress requiring intubation and underwent urgent hemodialysis. His respiratory status improved and he was extubated. Blood pressure was controlled with nifedipine and hydralazine. Even with continued hemodialysis, his $\mathrm{Cr}$ remained elevated with scant urine output. His pattern of findings raised suspicion for thrombotic microangiopathy. Plasmapharesis was initiated. HIV, hepatitis B and C serologies, and serum and urine protein electrophoresis were negative. ADAMTS13 activity was $58 \%$ (normal >67\%). Complement, antidsDNA, anti-GBM antibodies, and ANCA were normal. Renal biopsy revealed acute and chronic changes consistent with thrombotic microangiopathy, glomerulosclerosis, tubular injury, interstitial fibrosis, and tubular atrophy.

DISCUSSION: Thrombotic microangiopathy (TMA) is a pathologic finding of thrombosis in capillaries and arterioles. In TMA, endothelial injury leads to thrombosis and hemolysis causing thrombocytopenia and anemia. Classically, TMA is seen in hemolytic uremic syndrome (HUS) and thrombotic thrombocytopenic purpura (TTP), so called primary TMA syndromes along with drug-induced and complement-mediated. TMA is also seen in a variety of other conditions including infection, malignancy, HELLP syndrome, disseminated intravascular coagulation, systemic lupus erythematosus, and hypertensive crisis. Findings concerning for TMA should prompt evaluation to determine its etiology and guide management. Identifying the presence of TTP is of particular importance. It demonstrates a higher mortality compared to other primary TMA syndromes, and the pentad of TTP (microangiopathic hemolytic anemia, thrombocytopenia, renal failure, fever, neurologic deficit) is not uniformly present. Evaluation for TTP anchors on testing for severe deficiency (activity $<10 \%$ ) of ADAMTS13, a zinc metalloprotease that cleaves von Willebrand factor. However, ADAMTS13 testing should not preclude initiation of plasmapheresis. Demonstration of ADAMTS13 activity is not required to initiate plasmapheresis if TTP is suspected, and severe deficiencies typically persist even after the patient undergoes plasmapheresis. It is important to remain cognizant of the wide range of conditions associated with TMA. In our case, TMA was associated with hypertensive crisis. Intervention hinges on appropriate and timely blood pressure management.

HYPOPARATHYROIDISM MASQUERADING AS NEUROPSYCHIATRIC ILLNESS: UNMASKING THE IMPORTANCE OF COGNITIVE BIAS IN CLINICAL DECISION-MAKING Danielle Fine; Vasudev Mandyam. Beth Israel Deaconess Medical Center, Brookline, MA. (Control ID \#2463797)

LEARNING OBJECTIVE \#1: Recognize the overlapping clinical presentations of hypoparathyroidism and neuropsychiatric illness

LEARNING OBJECTIVE \#2: Illustrate how cognitive biases can negatively affect diagnostic and therapeutic clinical decision-making

CASE: A 21-year-old female with a past medical history of hypothyroidism, depression, and anxiety presented to an outpatient practice to establish primary care with self-reported worsening anxiety. She was recently diagnosed with a panic attack in the emergency department (ED), where she presented with hyperventilation, perioral numbness, hand tingling, and a sensation that her hands were "clenching up". She reported a 3-year history of anxiety manifested by similar symptoms, but noted that the episodes were becoming more frequent and severe. On review of systems, she noted occasional non-exertional chest tightness, headaches, and persistent anhedonia. On physical exam, the patient was well-groomed, anxious appearing, and intermittently tearful. She was afebrile with normal vital signs. Neck was supple with no thyroid enlargement or nodules. Her heart, lung, and abdominal exams were normal. An initial laboratory evaluation to rule out secondary causes of depression and anxiety revealed a normal TSH and a mild normocytic anemia. The EKG obtained from her recent ED visit revealed a prolonged QT interval ( $470 \mathrm{msec})$, which was confirmed with a follow-up outpatient EKG obtained in consideration of starting an SSRI for her symptoms of anxiety and depression. She was started on low dose fluoxetine with a plan to recheck her EKG in 1 month to assess for further QT prolongation induced by the SSRI. The patient presented for a follow-up appointment 8 weeks later with progressive drowsiness and self-reported anxiety attacks characterized by whole body numbness and the inability to use her hands because they "clenched up". A repeat EKG revealed a QT interval of $500 \mathrm{msec}$. A laboratory evaluation to assess for reversible causes of QT prolongation revealed critically severe hypocalcemia ( $4.7 \mathrm{mg} / \mathrm{dl})$ and hyperphosphatemia $(6.2 \mathrm{mg} / \mathrm{dl})$, with a normal serum albumin $(4.3 \mathrm{~g} / \mathrm{dl})$. She was triaged to the ED for diagnostic work-up and urgent therapy. Further laboratory evaluation revealed a parathyroid hormone of $<6 \mathrm{pg} / \mathrm{ml}$ (normal 15-65 pg/ml), 25-OH-Vitamin D of $14 \mathrm{ng} / \mathrm{ml}$ (normal 30-60 ng/ml), with normal TSH and morning cortisol levels. She was treated with IV calcium and oral calcitriol, calcium carbonate, and Vitamin D with complete resolution of her symptoms. She was diagnosed with primary hypoparathyroidism of presumed autoimmune etiology.

DISCUSSION: This patient presented with an uncommon condition, primary autoimmune hypoparathyroidism, which was initially misdiagnosed as depression and anxiety disorder, illustrating how distinguishing between a primary neuropsychiatric illness and the manifestations of hypoparathyroidism can be extremely difficult. Many of the symptoms observed in anxiety and hypoparathyroidism result from a common end pathway of neuromuscular excitability caused by hypocalcemia. Whereas hypoparathyroidism results in hypocalcemia from decreased bone resorption, decreased calcium absorption from the gastrointestinal tract, and increased kidney excretion of calcium, anxiety results in hypocalcemia by means of hyperventilation with resultant respiratory alkalosis leading to a decrease in serum ionized calcium levels. Neuromuscular excitability from hypocalcemia can have both sensory and motor manifestations. The most common sensory symptoms of hypocalcemia are peri-oral, acral, and symmetrical distal extremity paresthesias. In fact, the hyperventilation syndrome associated with anxiety is one of the most common causes of symmetrical upper extremity paresthesias. The most common motor symptoms are stiffness, clumsiness, myalgias, muscle spasms, and cramps. In addition to anxiety and hypoparathyroidism having similar sensory and motor symptoms, neuropsychiatric symptoms are also very common in hypoparathyroidism. The major neuropsychiatric symptoms observed in this condition are depression, anxiety, delirium, psychosis, lethargy, stupor, and coma. This case illustrates the importance of recognizing how two common forms of cognitive bias, diagnosis momentum and premature closure, can negatively affect clinical decision-making. Diagnosis momentum occurs when a new physician carries forward a prior physician's diagnosis. In this case, the patient presented to a new physician with established diagnoses of depression and anxiety, which were presumed to be accurate without confirmation or consideration of a broader differential diagnosis. Premature closure occurs when a physician relies to heavily on initial information and makes a diagnosis prematurely, without full consideration of all potential diagnostic information. For this patient, the physician focused on a cluster of symptoms commonly associated with depression and anxiety and did not consider the full range of diagnoses potentially responsible for her symptoms. To ensure accurate and timely diagnosis and treatment, it is extremely important to recognize and eliminate such cognitive biases in clinical decisionmaking. 
HYPOTHERMIA CAUSED BY QUETIAPINE: A CASE LEFT UNDIAGNOSED FOR 1 YEAR LEADING TO MULTIPLE ADMISSIONS- A CASE REPORT Waqas Nawaz; Prerna Kumar; Barry Fomberstein. Montefiore Medical Centre, YONKERS, NY. (Control ID \#2465171)

LEARNING OBJECTIVE \#1: Consider medications in the differential diagnosis of hypothermia besides the more commonly expected causes such as sepsis, malnutrition and endocrine disorders.

LEARNING OBJECTIVE \#2: Recognize quetiapine as a cause of hypothermia, especially at high doses in patients with central nervous system disorders.

CASE: A 59 year old woman with a history of hypothyroidism, mental retardation, seizure disorder and bipolar disorder, who had been taking quetiapine, among other medications, presented with altered mental status and hypothermia of $91 \mathrm{~F}$. Routine laboratory testing, including white blood cell count, thyroid function tests and cortisol levels, was normal. She was initially treated for sepsis of unknown origin and was started on broad spectrum antibiotics. The patient had four episodes of hypothermia on the antibiotics during the hospital stay, which occurred around $10 \mathrm{PM}$ after she had taken the 3 daily doses of quetiapine. The previous record showed that she was initially started on quetiapine $200 \mathrm{mg}$ daily without any episodes of hypothermia noted. Subsequently the dose was increased to $200 \mathrm{mg}$ TID and this was followed by 3 subsequent admissions for hypothermia in the following year. The antibiotics and quetiapine were stopped, and no further episodes of hypothermia were noted Divalproex sodium was continued as treatment for seizure disorder. The hypothermia was attributed to quetiapine. The mystery of her hypothermia was solved and she did not have further admissions for this reason.

DISCUSSION: Hypothermia is defined as core body temperature below $95 \mathrm{~F}$ Differentials include sepsis, malnutrition, hypothyroidism, hypoadrenalism, hypopituitarism, opioids and barbiturates overdose, head trauma and medications. Hypothermia in patients on atypical antipsychotics is a serious, unpredictable, idiosyncratic adverse reaction that frequently leads to hospital and intensive care unit admission and sometimes even to death. Among atypical antipsychotics, hypothermia is attributed more often to olanzapine and resperidone, due to their antagonistic actions on D2 and 5HT2 receptors However, there are a few case reports which described quetiapine-induced hypothermia Quetiapine may cause hypothermia by both peripheral and central mechanisms. It has antagonistic action on D2, $5 \mathrm{H} 2-\mathrm{A}$ and alpha-2 receptors with the highest affinity for alpha-2 receptors. The alpha 2 receptor blocking inhibits the peripheral vasoconstriction and shivering, and thus may play a role in inducing the hypothermia. The high 5 HT-2/D2 affinity ratio also contributes to this adverse effect by centrally disrupting the thermoregulation. Quetiapine also contributes to hypothermia by affecting the complex reactions related to activation of orexinergic system. Interestingly, the hypothermia caused by quetiapine is dose dependent and is observed at high doses. Moreover, it is more marked in patients with CNS disorders (as in our case). This case signifies the importance of considering broad differentials for hypothermia including medications.

\section{IATROGENIC CUSHING SYNDROME DUE TO RITONAVIR-FLUTICASONE} INTERACTION Fasil Tiruneh ${ }^{1}$; Doshi Saumil ${ }^{2}$; Ankit Mahajan ${ }^{1} .{ }^{1}$ Howard University, Washington DC, DC; ${ }^{2}$ Howard University hospital, WASHINGTON DC, DC. (Control ID \#2467843)

LEARNING OBJECTIVE \#1: High clinical suspicion is needed for early diagnosis and prevention of iatrogenic cushing's syndrome

LEARNING OBJECTIVE \#2: Avoid Fluticasone in patients who are treated with Ritonavir

CASE: A 50-year-old African American male patient with a past medical history of HIV on HAART for 15 years and long standing asthma that has been treated with inhaled fluticasone $200 \mathrm{mcg} /$ day and as neded albuterol for more than 15 years presented with sharp lower back pain of 3 days' duration that started while he was lifting heavy weight. For his HIV, he had been initially treated with abacavir and Efavirenz which was later changed to truvada one tablet, atazanavir $300 \mathrm{mg}$, and ritonavir $100 \mathrm{mg}$ PO daily because of virologic failure. He has recent onset type two diabetes mellitus and hypertension. On examination, Blood pressure was $168 / 98 \mathrm{mmHg}$ and pulse rate was 90 per min. He had mild pallor and oral trush. Central obesity with prominent abdominal striae and wasted extremities were noted. Localized tenderness was noted at the lower lumbar vertebral body. A CT of the spine showed compression fracture of inferior endplate of L3 vertebra, diffuse osteoporosis, and aseptic necrosis of bilateral femoral head. Laboratory tests showed low morning serum cortisol level $(0.2 \mathrm{ug} / 1$ [normal $5-25 \mathrm{nmol} / \mathrm{L})$ and suboptimal cosyntropin stimulation test consistent with adrenal suppression $(8.14,12.65$, and 13.25 at 0,30 , and 60 min respectively). HbAlc was elevated at 7.8. Luteinizing and folicular stimulating hormones were 3.7 and $7.9 \mathrm{mIU} / \mathrm{mL}$ respectively (Normal FSH 1.5 to $12.4 \mathrm{IU} /$ L and LH 1.8 to $8.6 \mathrm{IU} / \mathrm{L}$ ). Celiac screening with tissue transglutaminase antibody and gliadin antibodies were negative. His thyroid function test was normal. Patient was started on hydrocortisone to prevent adrenal crises. Decompressive lumbar laminectomy with bilateral facetectomies and foraminotomies and arthrodesis at L2-L3 and L3-L4 were done. Fluticasone was discontinued and he was given bronchodilator. Patient was also started on bisphosphonate therapy with calcium and vitamin D supplementation.On subsequent clinic visit he showed marked symptomatic improvement and good glycemic control.

DISCUSSION: Ritonavir, a potent inhibitor of the hepatic cytochrome P450, is commonly used in low doses to boost plasma levels of other protease inhibitors in patients with HIV. Intranasal and inhaled corticosteroids are widely used for the treatment of allergic rhinitis and asthma. Inhaled steroids do not usually lead to systemic adverse events, since their plasma concentrations are low due to extensive first-pass metabolism and clearance by CYP3A4. However, the coadministration of ritonavir with inhaled (or intranasal) corticosteroids may result in an increase in the plasma corticosteroid levels due to the potent CYP3A4 inhibition by ritonavir. This may cause iatrogenic Cushing's syndrome with adrenal suppression. More than 10 million Americans receive pharmacologic doses of glucocorticoids each year making Iatrogenic Cushing's syndrome resulting from long-term use of exogenous glucocorticoids the most common cause of Cushing's syndrome. Drugs that have been reported to result in hypercortisolism are glucocorticoids, megestrol acetate, and herbal preparations that contain glucocorticoids. We suggest that high clinical suspicion is needed for early diagnosis and prevention of Addisonian crises. Fluticasone treatment should be avoided in patients who are treated with Ritonavir. Other inhaled glucocorticoids, such as beclomethasone and budesonide, appear to be safer options because of their lower binding affinity for glucocorticoid receptors and shorter elimination half-life. However, they are also CYP3A4 substrates, and similar cases have been described. Caution should be used when any inhaled glucocorticoid is combined with ritonavir. Alternative therapeutic options for asthma control such as oral Montelukast or bronchodilators alone should be considered.

IFLUTTER: THE USE OF WEARABLE TECHNOLOGY IN THE MANAGEMENT OF CARDIAC ARRHYTHMIAS Becky Lou; Matthew Schaikewitz; Nina Nguyen; Joseph Mermelstein. Mount Sinai Beth Israel Medical Center, New York, NY. (Control ID \#2468214)

LEARNING OBJECTIVE \#1: To become familiar with new and developing technology and apply it to patient care

LEARNING OBJECTIVE \#2: To empower patients by teaching them how to interpret data from mobile apps and wearable technology 
CASE: Mr. H is a 71 year-old man, who presented to the Emergency Department with a chief complaint of "my Apple Watch shows a fast pulse." The patient had a past medical history of paroxysmal atrial fibrillation and atrial flutter for which he was taking metoprolol $100 \mathrm{mg}$ daily and apixaban $5 \mathrm{mg}$ twice a day for anticoagulation. Prior to arrival, the patient noted a rapid carotid pulse. His Apple Watch, which recorded his heart rate through a mobile app, confirmed that his heart rate at that time was approximately 150 beats per minute. The recordings further showed that the episode began between 12:00 AM1:00 AM. He denied chest pain, shortness of breath, dyspnea on exertion, heart palpitations, dizziness, and syncope. Physical exam revealed tachycardia but was otherwise unremarkable. Troponin-I was elevated to $0.036 \mathrm{ng} / \mathrm{mL}$ (normal $<0.035 \mathrm{ng} / \mathrm{mL}$ ). EKG showed atrial flutter with 2:1 AV conduction and a ventricular rate of 150. Three doses of metoprolol $2.5 \mathrm{mg}$ IV and two doses of diltiazem $10 \mathrm{mg}$ IV were given but failed to slow the heart rate. Cardiology was consulted, and the patient underwent cardioversion, which successfully resolved the tachycardia.

DISCUSSION: Within the past decade there have been advances in the use of mobile technology. Apps on smart phones and watches have become prevalent in today's society, and most patients use them on a daily basis, whether it is to keep track of their day, communicate via social media, or even as a pedometer. Now mobile apps and smart watches are being applied to the field of medicine - programs are being developed to identify ST changes in the detection of myocardial infarction, to distinguish between atrial fibrillation and other irregular rhythms, and to screen healthy patients for irregular rhythms. The benefits and potentials of this developing area of medicine are numerous. As seen in our case, this patient had an asymptomatic exacerbation of his atrial flutter. By checking his Apple Watch and mobile app he was able to both confirm his elevated heart rate and pinpoint exactly when it started. This case highlights the promising use of mobile technology, especially in monitoring patients with known arrhythmia for asymptomatic exacerbation, which led to a more rapid diagnosis and treatment. Logs kept by apps and wearable mobile technology, such as the Apple Watch, can act as a surrogate to an event recorder and show the timing of an arrhythmia. Our patient was able to self-diagnose, and he sought attention when necessary. Similar to home blood pressure monitoring and glucometers, these devices can aid physicians, who review the data and make adjustments in medication regimens or properly space outpatient appointments. In the inpatient setting, hospitalists are often the first physicians to discover and teach patients about newly diagnosed diseases, they can use this technology as they transition patients to the outpatient setting. Mobile devices and apps also empower patients and encourage them to participate in their own medical care as seen in this case. With the proliferation of such advances in the future, we expect such cases to increase. Thus it is important for physicians to become familiar with new mobile technology as it applies to medicine and become leaders in designing and using these technological advances to their patients.

IGD MYELOMA: A RARE DIAGNOSIS Medhavi Gupta ${ }^{2}$; Aditya Kotecha ${ }^{3}$; Abbinaya Elangovan ${ }^{2}$; Gaurav Goyal ${ }^{1} .{ }^{1}$ Creighton university medical center, Omha, NE; ${ }^{2}$ Mount Sinai St. Luke's Roosevelt Hospital, New York, NY; ${ }^{3}$ Wayne State University/ Detroit Medical Center, Farmington Hills, MI. (Control ID \#2468275)

LEARNING OBJECTIVE \#1: Recognize the rare entity of IgD myeloma.

LEARNING OBJECTIVE \#2: Understand the importance of prompt recognition and management of this aggressive subtype of multiple myeloma.

CASE: A 53-year-old Asian male with a known history of hyperlipidemia presented with central chest pain along with fatigue, anorexia and polyuria for 15 days. Physical examination was insignificant except for tenderness over the manubrium. Initial work up showed elevated serum creatinine $(2.57 \mathrm{mg} / \mathrm{dl})$, hypercalcemia (total calcium $15.4 \mathrm{mg}$ / $\mathrm{dl}$ ), elevated phosphorus ( $4.9 \mathrm{mg} / \mathrm{dl}$ ), leukocytosis (WBCs $13.7 / \mathrm{mcl}$ ), normal total protein $(6.7 \mathrm{~g} / \mathrm{dl})$, normal albumin $(4.2 \mathrm{~g} / \mathrm{dl})$, and normal hemoglobin $(13.8 \mathrm{~g} / \mathrm{dl})$. Further work up revealed low parathyroid hormone ( $<3 \mathrm{pg} / \mathrm{ml})$, low 25-hydroxy Vitamin D ( $20 \mathrm{pg} / \mathrm{ml})$, low 1,25 vitamin D $(<8 \mathrm{pg} / \mathrm{ml})$, normal PTH related protein $(16 \mathrm{pg} / \mathrm{ml})$, an elevated lactate dehydrogenase (368 U/L) and elevated beta-2 microglobulin levels $(5.775 \mathrm{mg} / \mathrm{L})$. The hypercalcemia was managed with intravenous normal saline and pamidronate leading to reduction of total calcium levels to $8.5 \mathrm{mg} / \mathrm{dl}$. Chest x-ray revealed a linear lucency suspicious for a non displaced right lateral eighth rib fracture. CT scan of chest showed an expansile lytic osseous lesion centered at the sternal manubrium. Skeletal survey did not show any osteolytic lesions. A bone scan was subsequently performed and demonstrated foci of mildly increased tracer uptake within the skull, along the manubrium, ribs and the left femoral neck. Twenty-four hour urine studies revealed a wide discrepancy in the degree of proteinuria ( $3.94 \mathrm{~g}$ ) and albuminuria $(0.127 \mathrm{~g})$, which was highly suspicious for paraproteinemic cast nephropathy. Serum and urine protein electrophoresis showed an abnormal band in the gamma and beta globulin regions respectively with immunofixation showing a free lambda M protein. Serum measurements of light chains revealed normal kappa chains $(8.6 \mathrm{mg} / \mathrm{L})$ and extremely high lambda chains (6669 mg/L), with kappa/ lambda ratio being nearly zero. A definitive diagnosis of multiple myeloma was established with bone marrow biopsy which showed a markedly hypercellular bone marrow with plasma cells contributing greater than $80 \%$ of the total cellularity. Immunochemistry showed the cells bearing surface markers of CD138/CD38/Lambda. Since the more common serum immunoglobulins were low (IgG $405 \mathrm{mg} / \mathrm{dl}$, IgA $17 \mathrm{mg} /$ $\mathrm{dl}$, IgM $7 \mathrm{mg} / \mathrm{dl}$ ), IgD levels were tested and found to be high at $11600 \mathrm{mg} / \mathrm{l}$. Thus the diagnosis of IgD myeloma was established and the patient was started on dexamethasone as a part of initial therapy.

DISCUSSION: Multiple myeloma is characterized by the neoplastic proliferation of a single clone of plasma cells producing a serum and/or urine monoclonal (M) protein. The gammopathy leads to end organ damage evidenced by one or more of the myeloma defining events such as hypercalcemia, renal insufficiency, anemia and osteolytic bone lesions (commonly depicted by the acronym CRAB). Immunoglobulins found in myeloma in decreasing frequency are $\operatorname{IgG}$, IgA and light chains. IgD myeloma is a rare variant found in approximately $1-2 \%$ of the myeloma patients 1 . IgD myeloma is more common in men and the patients present at a relatively younger age (median age of 52 years in $\operatorname{IgD}$ myeloma versus 58 years in other isotypes). Although IgD myeloma has many resembling clinical features as compared to other forms of myeloma, it has a higher prevalence of hypercalcemia, renal failure, Bence Jones proteinuria, anemia, bony lesions, extra osseous disease, extramedullary plasmacytomas, hepatosplenomegaly, lymphadenopathy, amyloidosis, a higher occurrence of cytogenetic abnormalities and a lambda chain bias. Studies have suggested that $\operatorname{IgD}$ myeloma usually presents at a more advanced stage and have a poor prognosis than other variants of myeloma. In one series, the median overall survival of patients with $\operatorname{IgD}$ myeloma was around 18 months, compared to 50 months in other myeloma patients 2 . IgD myeloma is treated in the same manner as other forms of myeloma. High dose chemotherapy along with autologous stem cell transplantation seems to have a better prognosis over the standard chemotherapy, with an added survival advantage with the use of newer drugs such as immunomodulatory agents (lenalidomide) and proteasome inhibitors (bortezomib).Due to the aggressive clinical behavior of IgD myeloma, its prompt diagnosis and treatment is imperative to maximize patient survival. One should have a high index of suspicion for myeloma if the initial presentation includes unexplained bone pain, hypercalcemia, acute renal failure, anemia. As IgD levels in the serum are generally very low, IgD myeloma may lead to a small or absent M-protein spike on electrophoresis. Therefore, testing for IgD should be done when they do not have an IgG or IgA M protein but have free light chains to prevent a false diagnosis of light chain or non-secretory myeloma. References: (1) Pandey, S. and R. A. Kyle (2013). "Unusual myelomas: a review of $\operatorname{IgD}$ and $\operatorname{IgE}$ variants." Oncology (Williston Park) 27(8): 798-803. (2) Kim, M. K., et al. (2011). "Immunoglobulin D multiple myeloma: response to therapy, survival, and prognostic factors in 75 patients." Ann Oncol22(2): 411-416 
IGG-4 RELATED DISEASE PRESENTING AS LACRIMAL GLAND ENLARGEMENT IN A PATIENT WITH ESTABLISHED SJOGREN'S SYNDROME Maaman Bashir ${ }^{1}$; Abdullah Ali Ahmed ${ }^{1}$; Daniel Hart'; Manish Jain ${ }^{2}$. ${ }^{1}$ Presence Saint Francis Hospital, Evanston, IL; ${ }^{2}$ St Francis Hospital, Evanston, IL. (Control ID \#2465887)

LEARNING OBJECTIVE \#1: To recognize the unusual presentation of IgG-4 Related Disease in a patient with established Sjogren's Syndrome

CASE: An 88 year old female was being treated for SS-A positive Sjogren's syndrome complicated by sicca symptoms and inflammatory joint symptoms for many years. Her past medical history was significant for untreated hepatitis $\mathrm{C}$ and interstitial lung disease (ILD) presumed related to Sjogren's syndrome. Her Sjogren's was treated with lubricant eye drops and hydroxychloroquine. She presented to her ophthalmologist with 6 months of worsening bilateral ptosis and swelling of her eyelids. Physical examination revealed enlarged lacrimal glands. Blood count, metabolic panel, erythrocyte sedimentation rate (ESR) and C-reactive protein (CRP) were all normal. Anti-nuclear antibody (ANA) was positive at 1:80 and SS-A was positive again. Magnetic resonance imaging (MRI) of her orbits revealed bilateral symmetrically enlarged lacrimal glands with suggestion of lymphoid infiltration. Given particular concern for lymphoma, a tissue diagnosis via lacrimal gland biopsy was quickly pursued. Biopsy showed large areas of fibrous band cicatrization with intervening areas of chronic inflammation associated with follicular lymphoid hyperplasia and a lymphoplasmacytic infiltrate. The biopsy showed no morphologic or immunohistochemical evidence of malignant lymphoma. Staining for $\operatorname{IgG}$ and $\operatorname{IgG} 4$ demonstrated a predominance of IgG4 positive plasma cells with an IgG4/IgG ratio of greater than $75 \%$. A subsequent serum IgG4 level was elevated at $>300 \mathrm{mg} / \mathrm{dL}$ (norma range 4-86). The histology along with elevated serum IgG4 level pointed towards the diagnosis of IgG4 related disease (IgG4-RD). On further questioning, she did have a pas episode of pancreatitis decades prior without clear etiology. The patient was started on prednisone $40 \mathrm{mg} /$ day with marked improvement in lacrimal enlargement, eyelid swelling, and ptosis just 2 weeks later. Steroid sparing agents such as methotrexate or azathioprine were deferred in the setting of her hepatitis $\mathrm{C}$ and ILD. The patient continued to do well on a tapering dose of steroid.

DISCUSSION: Autoimmune syndromes can share clinical manifestations but usually have distinct pathogenic, diagnostic and treatment modalities. Sjogren's syndrome and IgG4 related disease are two such syndromes that can present a diagnostic challenge. IgG4-RD is a relatively newly described immune-mediated condition with a ubiquitous set of disease manifestations ranging from local glandular enlargement to retroperitoneal fibrosis and autoimmune pancreatitis. Here we describe an unusual presentation of IgG4$\mathrm{RD}$ in a patient with established Sjogren's syndrome with a high clinical concern for lymphoma. While elevated IgG4 levels have been described in patients with Sjogren's, to our knowledge this is the first case of biopsy-proven IgG4-RD in a Sjogren's patient.

IGG4-RELATED SYSTEMIC DISEASE MANIFESTED AS AUTOIMMUNE HEPATITIS: IT DOESN'T JUST AFFECT THE PANCREAS Anirudha Rathnam²; Ayush Motwani ${ }^{1}$; Dilip Moonka ${ }^{1}$; Heidi Gunderson ${ }^{1} .{ }^{1}$ Henry Ford Health Systems, Detroit, MI; ${ }^{2}$ Wayne State University School of Medicine, Detroit, MI. (Control ID \#2470122)

LEARNING OBJECTIVE \#1: Recognize the unusual presentation of $\operatorname{IgG}_{4}$-associated autoimmune hepatitis.

LEARNING OBJECTIVE \#2: To the expansion of the HISORt criteria used to diagnose autoimmune pancreatitis to $\mathrm{IgG}_{4}$-related systemic disease.

CASE: A 67 year old former male smoker male with a history of Crohn's disease, anemia, and coronary artery disease presented with jaundice, severe fatigue, dyspnea, and unintentional weight loss over 9 months with persistently elevated liver enzymes. The patient worked as a pastor and had no history of alcohol or illicit or IV drug use. Physical exam revealed a thin but fully oriented and conversant male with a Karnofsky score of 80 and icterus on eye exam. His exam was otherwise negative for any sequelae of cirrhosis or encephalopathy. Laboratory data was significant for the patient's liver enzymes, which were elevated on admission and continued to increase: AST 203 to 448, ALT 161 to 293, alkaline phosphatase (AlkP) 1155 to 1474 , and total bilirubin 7.3 to 7.9. Alpha-1 antitrypsin, ceruloplasmin, ANA, antimitochondrial and anti-smooth muscle antibodies, and hepatitis panel were all negative. Amylase and lipase were within normal limits and the patient did not exhibit clinical signs of pancreatitis. $\mathrm{His} \mathrm{IgG}_{4}$ levels were found to be significantly elevated at 6020 . Imaging was generally nonspecific with MRI abdomen showing nonspecific periportal enhancement likely secondary to underlying hepatitis/ cholangitis, and CT abdomen/pelvis showing borderline enlarged porta hepatis. A liver biopsy was pursued which revealed dense plasma cell infiltrate centered on the bile ducts with diffusely positive IgG and $35-40 \%$ of the plasma cells staining positive for $\operatorname{IgG}_{4}$. The biopsy also showed venules surrounded by fibrous tissue consistent with obliterative phlebitis. The patient was diagnosed with IgG4-associated autoimmune hepatitis and initiated on prednisone. After several weeks, his liver enzyme panel improved remarkably, with AlkP trending down to 800 , AST to 95 , and ALT to 184 . Impressively, total bilirubin decreased 7.9 to 1.6 and the patient was no longer icteric. Ursodiol was added to his regimen and mycophenolate mofetil was added to help with the prednisone taper. One month later, his liver enzymes were near normal. At present, the patient is maintained on the above three medications. He continues to improve clinically

DISCUSSION: $\mathrm{IgG}_{4}$-associated autoimmune hepatitis (IAAH) can often be confused with primary sclerosing cholangitis (PSC), especially in patients with preexisting risk factors like Crohn's disease. It is important to notice the subtle differences in this patient's presentation that do not favor PSC, such as abrupt increase in liver enzymes, very high bilirubin and AlkP, rapid weight loss, and unimpressive MRI and MRCP. Such as in this case, patients with IAAH often present at the time of diagnosis with an abrupt onset of obstructive jaundice that responds to corticosteroids. In contrast PSC is often diagnosed in asymptomatic patients with abnormal liver function tests and does not respond to corticosteroids ${ }^{1}$. Previously considered autoimmune pancreatitis (AIP) with extrapancreatic presentations, $\mathrm{IgG}_{4}$-related systemic disease (ISD) is a more recently proposed, broader clinicopathologic disease process that does not consistently involve the pancreas ${ }^{2}$. This case highlights the importance of recognizing ISD in the context of hepatitis with or without concomitant pancreatitis. Hepatic manifestations are less well described within the literature $^{3}$. The HISORt criteria was initially developed by Mayo Clinic for diagnosing AIP and later adapted for extrapancreatic $\mathrm{IgG}_{4}$ diseases like $\mathrm{IgG}_{4}$-related sclerosing cholangitis ${ }^{4}$. The criteria includes $(\mathrm{H})$ histology such as lymphoplasmocytic infiltrate with $>10 \mathrm{IgG}_{4}+$ cells under high power field, storiform fibrosis, and obliterative fibrosis, (I) imaging including intrahepatic strictures or irregular pancreatic ducts, (S) serology indicating elevated $\mathrm{IgG}_{4}$ levels, (O) other organ involvement, and (Rt) response to corticosteroids, which is to say normalization of liver enzymes or resolution of strictures ${ }^{3}$. Our patient had findings consistent with these criteria. In summary, ISD is an important cause of autoimmune hepatitis, which may often go misdiagnosed due to its resemblance to more common conditions like PSC. Broadening the HISORt criteria to involve organs other than the pancreas and gallbladder may improve diagnosis. 1. Kamisawa T, Tu Y, Nakajima H, Egawa N, Tsuruta K and Okamoto A. Usefulness of biopsying the major duodenal papilla to diagnose autoimmune pancreatitis: a prospective study using $\operatorname{IgG}_{4}$ immunostaining. World J Gastroenterol 2006; 12:2031-2033. 2. Zhang L, Smyrk T. Autoimmune pancreatitis and $\mathrm{IgG}_{4}$-related systemic diseases. int $\mathrm{J}$ Clin Exp Pathol 2010; 3(5):491-504 3. Joshi D, Webster JM. Review article: biliary and hepatic involvement in $\mathrm{IgG}_{4}$-related disease. Alimentary Pharmacology and Therapeutics 2014; 40:12511261. 4. Chari ST. Diagnosis of autoimmune pancreatitis using its five cardinal features: introducing the Mayo Clinic's HISORt criteria. J Gastroenterol 2007; 42 Suppl 18:39-41. 
IMMUNE COMPLEX-MEDIATED RAPIDLY PROGRESSIVE GLOMERULONEPHRITIS IN HIV Allison Guttmann; Galen Leung; Alejandro Gomez-Viso; Taiye Odedosu. New York University School of Medicine, New York, NY. (Control ID \#2469294)

LEARNING OBJECTIVE \#1: Recognize the differential diagnosis of rapidly progressive renal failure in patients with underlying HIV.

LEARNING OBJECTIVE \#2: Recognize the importance of early renal biopsy to determine the etiology of rapidly progressive renal failure in patients with HIV.

CASE: A 53 year-old man with a history of HIV on anti-retroviral therapy (ART) with a CD4 count of $470 / \mu \mathrm{L}$ and undetectable viral load, and stage III chronic kidney disease presented with 2 weeks of progressive shortness of breath, scrotal and leg edema. Initial laboratory evaluation was notable for a creatinine of $4.1 \mathrm{mg} / \mathrm{dL}$ (baseline $2 \mathrm{mg} / \mathrm{dL}$ ), albumin of $2.4 \mathrm{~g} / \mathrm{dL}$, urine microscopy showing RBCs without casts, spot urine protein of $1851 \mathrm{mg} / \mathrm{dL}$ and spot urine creatinine of $94.5 \mathrm{mg} / \mathrm{dL}$. Renal ultrasound demonstrated enlarged, hyperechoic kidneys without evidence of hydronephrosis or renal vein thrombosis. Left-sided renal biopsy revealed glomeruli with crescent formation and necrotizing lesions by light microscopy, irregular capillary loop staining by immunofluorescence, and scattered immune complex deposits by electron microscopy. Immune and rheumatologic work-up included ANA, ANCA, immunoglobulins, SS-A, SS-B, Scl-70, rheumatoid factor, $\mathrm{C} 3 / \mathrm{C} 4$, anti-double stranded DNA antibody, anti-smooth muscle antibody, antihistone antibody, anti-cardiolipin antibody, antiphospholipid panel, and glomerular basement membrane antibody, which returned negative or within normal limits. Hepatitis serology was also negative. In the setting of the patient's HIV as well as his negative rheumatologic and immune work-up, the diagnosis of HIV immune complex kidney disease (HIVICK) was suspected. The patient was aggressively diuresed with intravenous furosemide with improvement in his hypervolemia. His renal function continued to worsen with serum creatinine rising to $5.7 \mathrm{mg} / \mathrm{dL}$. Given concern for rapidly progressive glomerulonephritis, intravenous, then oral steroids were started to decrease the inflammatory reaction to immune complex nephritis. ART was also held due to development of acute pancreatitis of unclear etiology, with a working diagnosis that the pancreatitis was related to his initial high dose steroids. The patient's serum creatinine eventually downtrended after the initiation of steroids. The patient was discharged on oral steroids with plans to follow-up with nephrology and his virologist for continued treatment of his HIV.

DISCUSSION: HIVICK is composed of immune complex related diseases affecting HIV-positive patients and include post-infectious glomerulonephritis, IgA nephropathy, membranous nephropathy, and membranoproliferative glomerulonephritis. Patients may present with worsening kidney injury, dependent edema, and proteinuria/hematuria. Patients usually have normal or slightly increased C3 and C4 complement levels. Interestingly, IgA and IgG immune complexes to HIV antigens have also been found in serum and in higher amounts in biopsy specimens, and HIV-antigens in glomeruli, suggesting the role of HIV-antigen specific immune complex deposition. Pro-viral HIV DNA has been detected in tubular and glomerular epithelial cells. Histopathology would demonstrate HIV antigen-antibody complexes and renal tissue appearance of immune complex-mediated glomerulonephritis. The proposed mechanism behind HIVICK involves polyclonal B-cell activation leading to increased immunoglobulin production in response to HIV infection. HIV p24 antigens are bound to either IgA or IgG antibodies or HIV gp120 bound to IgG. The high level of HIV-related immune complexes, irrespective of the stage of HIV, can deposit causing glomerulonephritis. Due to the presence of HIV DNA in glomerular and tubular cells, another proposed mechanism is that direct HIV infection or incorporation of the HIV genome into renal cells leads to expression of antigen and subsequently, the formation of immune complexes against renal cells. Compared to HIV Associated Nephropathy cases, patients with HIVICK were more likely to be on ART, have lower HIV RNA copies and higher CD4 counts ${ }^{1}$. They also have less advanced HIV infection ${ }^{2}$, milder proteinuria, and were at lower risk of progression to endstage renal disease (ESRD) ${ }^{1}$. The use of ART and the copies of HIV RNA did not influence the progression to ESRD among HIVICK patients. Despite the lack of randomized controlled trials in the use of ART in treating HIVICK, it has suggested that ART should be implemented and that corticosteroids may attenuate interstitial inflammation. ART has also been associated with regression of immune complex deposits histological$1 y^{3}$. Our patient presented with a rapidly progressive acute kidney injury despite being on ART, having an undetectable HIV viral load and high CD4 count. His rheumatologic workup and hepatitis serology were negative. HIVICK patients have a lower risk of progressing to ESRD, and fortunately, the patient's kidney function eventually improved. The optimal management of HIVICK is unclear and more studies are needed on treatment. References Foy MC, Estrella MM, Lucas GM, et al. Comparison of risk factors and outcomes in HIV Immune Complex Kidney Disease and HIV-Associated Nephropathy. Clin J Am SocNephrol. 2013. 8: 1524-1532. Humphreys MH. Human immunodeficiency virus-associated glomerulosclerosis. Kidney Int 48: 311-320. 1995. Fabian J, Naicker S, Goetsch S, et al. The clinical and histological response of HIV-associated kidney disease to antiretroviral therapy in South Africans. Nephrol Dial Transplant. 2013. 28: 15431554

IMMUNOGLOBULIN FREE LIGHT CHAINS AS A HARBINGER FOR LUPUS MYOSITIS Christina A. Mosher ${ }^{1}$; Rebecca F. Yarborough ${ }^{2}$; Salahuddin Kazi ${ }^{3} .{ }^{1} \mathrm{UT}$ Southwestern Medical Center, Dallas, TX; ${ }^{2}$ University of Texas Southwestern Medical Center, Dallas, TX; ${ }^{3}$ Ut Southwestern Medical Center, Dallas, TX. (Control ID \#2437274)

LEARNING OBJECTIVE \#1: 1. Suspect systemic lupus erythematosus (SLE) as the underlying etiology in the setting of systemic symptoms and myositis, and the additional presence of oral ulcers and/or pulmonary disease are associated with a worse prognosis. LEARNING OBJECTIVE \#2: 2. Obtain immunoglobulin free light chain levels in SLE patients both during and between disease flares to assess treatment efficacy and predict relapses, respectively.

CASE: A 44 year old African American female with no significant past medical history presented with progressive muscle pain and weakness. Six months prior to presentation, she developed diffuse muscle tightness, followed by myalgias and symmetric arthralgias with prolonged morning stiffness. On presentation, she was unable to lift her arms or thighs against gravity. Associated symptoms include a sixteen-kilogram weight loss, oral ulcers, dysphagia to solids and liquids, and severe constipation. Vital signs on arrival were within normal limits. Physical exam was most remarkable for severe, proximal (2/5) greater than distal (3/5) muscle weakness with associated tenderness in bilateral arms and legs. Oral exam revealed a left lateral tongue ulceration. Cardiovascular examination was notable for a loud P2 and bilateral pitting edema to the knees. Abdominal and respiratory examination was unremarkable. The skin was notable for a discoid rash in the concha bowl of both ears. Laboratory data revealed an elevated creatine kinase. Electromyography showed an irritative myopathy, and a muscle biopsy revealed perifascicular atrophy and frequent tubuloreticular inclusions. Serum protein electrophoresis revealed elevated FLC with a globulin gap and an elevated kappa to lambda light chain ratio. Urine studies revealed non-nephrotic proteinuria likely due to increased FLC, an elevated FLC excretion rate, and a urine immunoglobulin M spike. Additional studies revealed decreased complements, and positive speckled antinuclear, anti-Smith, antiribonucleoprotein, and U2 snRNP antibodies. Double stranded DNA antibodies were negative. Chest imaging was notable for an enlarged pulmonary artery, a small pericardial effusion, and space-occupying ground glass opacities. The patient was diagnosed with SLE with serositis, an oral ulcer, arthritis, anemia and thrombocytopenia, positive ANA, positive anti-Smith antibodies, and a discoid rash. She was started on high dose solumedrol before transition to a prednisone taper, mycophenolate mofetil, and plaquenil. 
DISCUSSION: Systemic lupus erythematosus is an autoimmune disease that can affect any organ system and is marked by flares alternating with remission. ${ }^{1,2}$ Flares are treated with immunosuppressants only after active disease becomes clinically apparent, when irreversible tissue damage may have already occurred. ${ }^{2,3}$ Skeletal muscle involvement is most commonly a subclinical myopathy, but rare cases of myositis demonstrate significant inflammatory damage on muscle biopsy and electromyography, and patients have a similar or worse overall SLE prognosis. ${ }^{4,5,6}$ Especially in severe manifestations such as SLE myositis, biomarker screening could allow early treatment initiation that curtails the extent of inflammatory damage. Recent work has attempted to correlate SLE disease activity with serum levels of various immunologic parameters, and thus find a biomarker predictive of flares. ${ }^{2,7,8,9}$ Polyclonal free light chains have been shown to correlate with SLE disease activity, and relapses can be preceded by 4 to 8 weeks of elevated urinary FLC. ${ }^{2,7,8}$ The literature has not previously documented whether myositis in SLE is independently associated with elevated FLC or another immunologic parameter. The severity of this case also provides the impetus to conduct larger trials to assess the relationship between SLE myositis and free light chain concentrations to mitigate future disease flares. This opens the possibility for a paradigm shift in SLE disease management from a reactive to a proactive treatment strategy. 1. Hochberg MC. Systemic lupus erythematosus. Rheum Dis Clin North Am 1990; 16: 617-639. 2. Aggarwal R, Sequeira W, Kokeibie R, et al. Serum free light chains as biomarkers for systemic lupus erythematosus disease activity. Arthritis Care Res 2011; 63(6): 891-898. 3. Mirzayan MJ, Schmidt RE, Witte T. Prognostic parameters for flare in systemic lupus erythematosus. Rheumatol 2000; 39: 1316-1319. 4. Dayal NA, Isenberg DA. SLE/myositis overlap: are the manifestations of SLE different in overlap disease? Lupus 2002; 11: 293-298. 5. Maazoun F, Frikha F, Snoussi M, et al. Systemic lupus erythematosus-myositis overlap syndrome: report of 6 cases. Clin Pract 2011;1(89) 189-192. 6. Garton MJ, Isenberg DA. Clinical features of lupus myositis versus idiopathic myositis: review of 30 cases. Br J Rheumatol 1997; 36: 1067-1074. 7. Draborg AH, Lydolph MC, Westergaard M, et al. Elevated serum immunoglobulin free light chains in systemic lupus erythematosus patients in relation to disease activity, inflammatory status, B cell activity and Epstein-Barr virus antibodies PLOS One 2015; 10(9): e0138753. 8. Brebner J, Stockley R. Polyclonal free light chains: a biomarker of inflammatory disease or treatment target? F1000 Medicine Reports 2013; 5(4): 1-6. 9. Chiche L, Cournac JM, Mancini J, et al. Normalization of serum-free light chains in patient with systemic lupus erythematosus upon rituximab treatment and correction with biological disease activity. Clin Rheumatol 2011; 30: 685-689.

IN DENIAL UNTIL THE LAST MOMENT. Weijia Wang; Patrick Abt; Laura K. Snydman. Tufts Medical Center, Boston, MA. (Control ID \#2470140)

LEARNING OBJECTIVE \#1: Recognize the symptoms and signs of alcoholism and alcoholic withdrawal.

CASE: A 46-year-old Cambodian woman with past medical history of bicuspid aortic valve complicated by aortic root aneurysm and hypertension who presented to the emergency room with 3 days of chest tightness and decreased appetite along with nonbilious and non-bloody vomiting for the last 1.5 days. She described the chest discomfort as substernal and radiating to her back and both shoulders and it had been associated with trembling. Her medications were Hydrochlorothiazide, Amlodipine, Diltiazem, Losartan and Sertraline (which was started 2 weeks ago). Social history was significant for her losing her job recently. She denied using alcohol or illicit drugs. She reports that she immigrated from Cambodia when she was 10 years old. On physical exam, she was afebrile with a heart rate of 99 beats/min and blood pressure 133/79 $\mathrm{mmHg}$. Respiratory rate and oxygenation were normal. She appeared anxious and had pronounced trembling in her right arm along with mild diffuse abdominal tenderness without guarding. EKG revealed what was initially thought to be new T wave inversions in V4-V6 so she was admitted for a myocardial infarction rule out. Laboratory data on admission were significant for mild pancytopenia (WBC $2 \mathrm{~K} / \mathrm{uL}, \mathrm{Hgb} 10 \mathrm{~g} / \mathrm{dL}$ and platelets $130 \mathrm{~K} / \mathrm{uL}$ ), a transient anion gap that resolved quickly after 11 of normal saline, an elevated gamma glutamyl transferase level of $117 \mathrm{U} / \mathrm{L}$, and mild transaminitis (AST $81 \mathrm{U} / \mathrm{L}$, ALT $63 \mathrm{U} / \mathrm{L}$ ). Right upper quadrant ultrasound revealed fatty infiltration of the liver. On hospital day 2 , she developed severe hypokalemia $(2.6 \mathrm{mEq} / \mathrm{L})$ and severe hypophosphatemia $(0.4 \mathrm{mg} /$ dL). Her folic acid was found to be low at $3 \mathrm{ng} / \mathrm{mL}$ and she had severe vitamin $\mathrm{D}$ deficiency (25-hydroxy vitamin D $12 \mathrm{ng} / \mathrm{mL}$ ). On hospital day 3, she reported visual hallucinations of seeing her mother cleaning the floor and black caterpillars on the bed sheet. At this point, she was tachycardiac to 120 beats $/ \mathrm{min}$. She continued to adamantly deny alcohol use. CIWA protocol was initiated and lorazepam was administered liberally given our high suspicion for alcohol withdrawal delirium. We were able to reach her husband on hospital day 3 and he confirmed that she was an alcoholic. Her symptoms resolved gradually over the next few days and she was discharged on hospital day 5 . The patient only admitted to heavy drinking on the date of discharge.

DISCUSSION: Alcoholic withdrawal is commonly encountered in general hospital settings. The acute withdrawal syndrome is often completely unexpected and occurs when the patient has been hospitalized for an unrelated problem. Keeping a vigilant eye on signs and symptoms of alcohol withdrawal is the key to a timely diagnosis. Symptoms of mild withdrawal, including tremor, elevated heart rate, and anxiety may begin within $8 \mathrm{~h}$ of the patient's last drink. Delirium tremens is an acute organic psychosis accompanied by autonomic hyperactivity, which usually manifests within $72 \mathrm{~h}$ after the last drink. Electrolyte abnormalities such as unexplained anion gap acidosis, hypokalemia and hypophosphatemia can be clues as well. Alcoholism can be elusive and challenging to diagnose in the primary care physician's office. Unexplained work absences can be one of the subtle signs. Pancytopenia, severe vitamin D deficiency, folic acid deficiency and elevation of gamma glutamyl transferase should raise suspicion for alcohol abuse. Our patient underwent extensive rheumatologic workup for her pancytopenia previously in the outpatient setting but it took the development of delirium tremens for her alcohol abuse to be brought to light.

\section{IN T CELL LYMPHOMA, AN ELEVATED FERRITIN DOES NOT BODE} WELL Adrienne D. Workman. Methodist Dallas Medical Center, Dallas, TX. (Control ID \#2431928)

LEARNING OBJECTIVE \#1: Early recognition of the vague and diagnostically challenging presentation of Hemophagocytic Lymphohistiocytosis is imperative for initiation of treatment and improvement of patient survival.

CASE: A 79-year-old female with a history of hypertension and hyperlipidemia presented to the Emergency Department complaining of "tea colored urine". She had a 1 month history of decreased appetite, poor oral intake and dizziness followed by a 1 week history of tea colored urine and not "feeling like herself". The patient had no prior surgeries, significant family history or toxic habits. Physical exam was significant for a temperature of 101.6 F, blood pressure of 174/78, HR 98, scleral icterus, jaundice and trace lower extremity swelling. Labs were significant for pancytopenia, hyponatremia, elevated creatinine, normal lactic acid with elevated transaminases and coagulopathy. Further imaging revealed diffuse lymphadenopathy in her chest, abdomen and pelvis with fasting triglycerides of 275 , low \% saturation of 17, Iron 38 and ferritin greater than 10,000 ng/ $\mathrm{mL}$. Due to the elevated unconjugated bilirubin and conjugated bilirubin both hematology/oncology and hepatology were consulted. Biopsies of both an iliac lymph node and BM aspiration with biopsy were positive for T cell lymphoma. Due to her ongoing B symptoms she was diagnosed with Grade $4 \mathrm{~T}$ cell lymphoma. HLH was diagnosed based on the modified HLH-2004 criteria requiring three of four clinical criteria (including fever $>101.3 \mathrm{~F}$, cytopenia, hepatitis or splenomegaly) plus one of four possible 
immune markers (including increased ferritin, hemophagocytosis, hypofibrinogenemia or absent or very decreased NK cell function). The patient continued to deteriorate, with worsening of her liver function, increased coagulopathy, oliguria progressing to the need for hemodialysis and development of spontaneous tumor lysis syndrome. Given her precarious hemodynamic state and multiorgan failure, she was transferred to an academic center with a leading program for the treatment of hematologic malignancies allowing her full access to potential treatment for her TCL. Despite rapid diagnosis (within $48 \mathrm{~h}$ of presentation) and though initially her clinical picture improved with high dose steroids 2 weeks following her transfer she expired due to complications from acute blood loss following a liver biopsy. Laboratory analysis confirmed HLH with an interleukin 2 receptor (IL2R) level of 9,240 pg/mL.

DISCUSSION: A ferritin greater than $10,000 \mathrm{ng} / \mathrm{mL}$ should always warrant suspicion for HLH. Diagnosis is often delayed secondary to its vague presentation: generalized symptoms of fever, rash and neurological findings, as well as a clinical picture consistent with underlying infection and sepsis. Two sets of clinical criteria, HLH 2004 and modified HLH 2004, or the presence of pathologic mutations of PRF1, UNC13D, MUNC13E, MUNC18-2, RAB27A, STX11, SH2D1A or BIRC4 establish diagnosis. Early diagnosis is imperative, enabling immediate treatment as the mortality rate is reported anywhere from 50 to $90 \%$. Treatment is guided via the HLH-2004 treatment protocol and varies depending on neurological involvement and underlying autoimmune, malignancy or infection as the cause of HLH.

IN THE EYE OF THE BEHOLDER: A CASE OF ENDOGENOUS CANDIDAL ENDOPHTHALMITIS DUE TO INTRAVENOUS DRUG ABUSE Sue Gu'; Hermione Hurley ${ }^{1}$; Sansrita Nepal ${ }^{2} .{ }^{1}$ University of Colorado School of Medicine, Aurora, CO; ${ }^{2}$ Denver Health Medical Center, Denver, CO. (Control ID \#2469918)

LEARNING OBJECTIVE \#1: To describe the clinical presentation, epidemiology, and natural course of endogenous endopthalmitis.

LEARNING OBJECTIVE \#2: To raise awareness of the rare clinical entity endogenous endophthalmitis among internal medicine providers to allow prompt recognition, referral for consultation, and treatment.

CASE: A 20 year-old male presented with sudden onset progressive unilateral vision loss. He reported small dark spots that began in his left eye and progressed over a few days to complete blurring of vision, associated with redness, pain and photophobia. He denied fevers, chills or other symptoms. He had a history of asthma, cigarette and marijuana smoking, and IV heroin use two to three times weekly with accidental injection of his arm after a "missing a vein" a few weeks prior. On exam, his temperature was 36.5 , heart rate 91 , respiratory rate 16 , blood pressure $129 / 78,98 \%$ saturation on room air. He was a thin male in no acute distress with a firm induration in his right forearm without erythema, warmth or fluctuance, no cardiac murmurs, lungs clear to auscultation, and no stigmata of endocarditis. On eye exam, he had injected sclera on the left, cloudy appearance to the anterior chamber, normal lids and lashes, pupils equal round and reactive to light, extra ocular movements intact and no hypopion. Visual acuity was 20/20 in the right and 20/400 in the left eye, with visual fields full to confrontation but inability to count fingers. Ophthalmology consultant saw him urgently and on fundoscopic exam, he had hazy vitreous on the left with three fluffy white retinal lesions extending into the vitreous concerning for endogenous endophthalmitis from presumed hematogenous spread from his IV drug use. A vitreous sample was sent for aerobic, anaerobic and fungal cultures. He underwent intravitreal injections of vancomycin, ceftazadime and voriconazole. He was started on IV vancomycin, levofloxacin, fluconazole, and ceftazadime as well as moxifloxacin, cyclopentolate, and prednisolone eye drops. Vitreal cultures, blood cultures on the day of admission and subsequent surveillance blood cultures were negative for bacterial or fungal growth. CT of the orbits was unremarkable. Ultrasound of the forearm lesion showed no abscess. TTE was negative for vegetations. HIV and RPR were negative. His pain improved but his visual acuity remained diminished at 20/400. Serial direct fundoscopic exams showed continued but slightly improved vitritis with unchanged fluffy white lesions. His systemic antibiotics were narrowed to levofloxacin and fluconazole prior to discharge due to negative blood cultures. He was continued on the eye drops. Upon follow up in clinic, he had no clinical improvement. He underwent core vitrectomy followed by pars para lensectomy. The vitreal biopsy grew candida albicans susceptible to azoles. He was put on an increased dose of fluconazole $800 \mathrm{mg}$ PO daily. He continues to have poor eyesight and can see hand movements only, but has not proceeded to enucleation.

DISCUSSION: While most cases of endophthalmitis develop after surgery, trauma, or an infected cornea and are termed exogenous endophthalmitis, endogenous endophthalimitis from metastatic spread in the setting of bacteremia or fungemia is rare and accounts for only $2-8 \%$ of all cases of endophthalmitis. Intravenous drug abuse is an independent risk factor for endogenous endophthalimitis with a high proportion of pathogens being fungal. Many cases of fungal endophthalmitis occur without fungemia or endocarditis, as a transient fungemia is likely sufficient to cause seeding of this protected space. The diagnosis of endogenous endophthalmitis is difficult to make due to a variability in clinical signs and lack of sensitivity of blood and vitreous cultures ( $44 \%$ ) and thus requires a high index of suspicion. Diagnostic vitrectomy has higher diagnostic yield for culture (92\%). Case series have shown improved outcomes with prompt vitrectomy with intravitreal injection of amphotericin or voriconazole and systemic antifungals for a minimum of 6 weeks. Endogenous endophthalmitis is a serious disease with potentially devastating results including complete vision loss or in systemic disease, death. A high index of suspicion should be maintained in patients with immunocompromise or history of intravenous drug abuse presenting with decreased vision and vitreoretinal changes on examination. An early consultation by an ophthalmologist is critical to prevent mortality or result in better visual outcomes.

INCIDENCE AND RECURRENCE OF ATRIAL FIBRILLATION IN A YOUNG ADULT FOLLOWING HEAVY MARIJUANA SMOKING: IS IT MORE THAN A TEMPORAL ASSOCIATION. Prakrity Urja ${ }^{2}$; Arjun K. Theertham ${ }^{2}$; Abhilash Akinapelli ${ }^{2}$; Muhammad S. Azzouz ${ }^{2}$; Hussam Abuissa ${ }^{1} .{ }^{1}$ Creighton University, Omaha, NE; ${ }^{2}$ Creighton University Medical centre, Omaha, NE. (Control ID \#2469336)

LEARNING OBJECTIVE \#1: Learn cardiovascular effects of Marijuana CASE: Background: Marijuana is the most common illicit drug in the USA. It has been reported that marijuana smoking is associated with adverse cardiovascular effects. We report a case of young African American patient presenting with symptomatic Atrial Fibrillation (AF) twice after heavy marijuana smoking. Case report: An apparently healthy 22 year old African-American male presented to emergency room with sudden onset of chest pressure, palpitations, shortness of breath and anxiety. He reported smoking large quantity of the marijuana approximately one hour prior to the onset of symptoms. He has no significant past medical history. He was vaccinated appropriately as a child. He has two kids, lives with his girlfriend. He had been smoking 20 cigarettes a week for last 2 years. He was smoking large amount of marijuana 3-4 times a week for about 4 years. He had not used alcohol and any other recreational drugs except marijuana. He is not taking any medications. Physical examination revealed patient to be in distress, heart rate of 200 per minute, blood pressure of $90 / 60 \mathrm{~mm}$ of $\mathrm{Hg}$, and respiratory rate of 25 per minute. Cardiovascular examination revealed irregularly irregular rhythm, variable S1, normal S2, tachycardia, no rubs, gallops or murmurs. He had clear lung sounds. All peripheral pulses were palpable and equal bilaterally with low volume. Twelve lead Electrocardiogram showed AF with rapid ventricular response. The patient underwent 
successful emergent synchronized cardioversion with $120 \mathrm{~J}$. He converted to sinus rhythm and his vital signs and symptoms improved. His laboratory work up revealed no elevation in cardiac enzymes, normal complete blood count, electrolytes, renal function tests, liver function tests and thyroid stimulating hormone. His urine drug screen was positive for tetrahydrocannabinol, negative for amphetamines, cocaine, methadone, opioids, and benzodiazepines. His blood alcohol level was undetectable. His chest X-ray was normal. Patient refused hospital admission and medications. Appropriate counselling was provided to quit smoking. A follow up appointment was made with a cardiologist. One month later patient again presented to the emergency room with similar and more severe complaints and hemodynamic instability requiring repeated emergent cardioversion. Patient admitted to smoking large amount of marijuana $1-2 \mathrm{~h}$ prior to the onset of symptoms. He was admitted to the hospital. A transthoracic echocardiogram revealed no abnormalities. $24 \mathrm{~h}$ rhythm monitoring showed no recurrence of AF. Patient refused to take antiarrhythmic medications, decline further investigations and procedures. He wanted to quit smoking marijuana before considering further treatment. He was discharged on short term anticoagulation.

DISCUSSION: Discussion: Cardiovascular adverse effects of marijuana have been studied. The mechanism of AF associated with marijuana use is not well understood. Increased adrenergic activity combined with microvascular changes and ischemia caused by the marijuana smoking might contribute to the development of atrial automaticity and AF. Marijuana smoking leads to an increase in sympathetic and a reduction in parasympathetic activity. The most consistent finding is the rapid increase in heart rate secondary to enhanced sinus node automaticity that lasts $2-3 \mathrm{~h}$ after cessation of Marijuana smoking Beaconsfield et al. showed that marijuana induced tachycardia could be prevented with propranolol, supporting an adrenergic mediated sinus tachycardia in humans. Miller et al showed that administration of intravenous THC resulted in significant decrease in the A-H interval and in the atrioventricular refractoriness. Adrenergic stimulation reduces action potential duration and alters the electrophysiological properties of myocardium favoring automaticity, triggered activity and micro-reentry. A subset of healthy individuals sensitive to the catecholamine surge may be prone to AF development. Rezkalla et al. reported that a 34-year-old man presenting with syncope and ventricular tachycardia after marijuana use. In the electrophysiology laboratory, ventricular tachycardia was inducible. Coronary angiography showed normal coronary arteries with a significant reduction in coronary flow. After cessation of marijuana, coronary flow reverted to normal, and the patient was no longer inducible in the electrophysiology laboratory. Marijuana use is also associated with myocardial ischemia. In 2001 Mittleman et al. interviewed 3,882 patients with acute myocardial infarctions about marijuana use and found that the risk for developing myocardial infarction was 4.8 times higher than average in the hour immediately after marijuana use. In a study involving 1,913 adults after hospitalization for myocardial infarction, Mukamal et al. found a 4.2-fold increased risk for mortality in marijuana users compared with nonusers. Recurrence of AF after the heavy use of marijuana in our patient may represent strong (or causative) association rather than temporal association. More studies with long term ambulatory rhythm monitoring of the marijuana users is required to further confirm the causal association of marijuana and AF.

\section{INDUCING HYPOTHYROIDISM-AN UNUSUAL DRUG INTERACTION}

Lima Lawrence; Aleida Rodriguez; Natasha Shah; Armand Krikorian; Tahira Yasmeen. UIC - Advocate Christ Medical Center, Chicago, IL. (Control ID \#2431977)

LEARNING OBJECTIVE \#1: Recognize the effect of rifampin on thyroid function CASE: Mycobacterium avium intracellulare (MAI) is a nontuberculous mycobacterial infection that is treated with multi-drug therapy including rifampin. Rifampin is a potent inducer of hepatic enzymes with multiple drug interactions and hormone derangements. Rifampin induced hypothyroidism in euthyroid patients with Hashimoto's thyroiditis is well described. We present a case where thyroid stimulating hormone (TSH) levels escalate due to rifampin therapy in a patient with previously controlled hypothyroidism. Our aim is to increase awareness of the effect of rifampin on thyroid function. A 71 yearold female with a past medical history of hypothyroidism, chronic obstructive pulmonary disease and recently diagnosed MAI infection presented with recurrent pneumonia. She was started on standard therapy for MAI including rifampin $450 \mathrm{mg}$ daily, ethambutol $800 \mathrm{mg}$ daily and azithromycin $500 \mathrm{mg}$ on Monday, Wednesday and Friday. Patient was diagnosed with hypothyroidism over 20 years ago and maintained on levothyroxine (LT4). Her thyroid hormone replacement regimen consisted of LT4 $50 \mathrm{mcg} 5$ days a week and $75 \mathrm{mcg} 2$ days a week. A few weeks after initiation of rifampin, the patient reported symptoms of constipation, cold intolerance and severe fatigue. On physical exam, there was no thyromegaly or proptosis. Laboratory evaluation demonstrated a progressive increase in TSH over 2 weeks from a normal value of $1.557 \mathrm{mU} / \mathrm{L}(0.4$ and $4.0 \mathrm{mU} / \mathrm{L}$ ) before initiation of rifampin, to $14.889 \mathrm{mU} / \mathrm{L}$. Free thyroxine level was normal at $1.0 \mathrm{ng} / \mathrm{dL}(0.7$ to $1.9 \mathrm{ng} / \mathrm{dL})$. Subsequently, LT4 dose was increased to $75 \mathrm{mcg} 6$ days a week with instructions to repeat a TSH level in 6 weeks.

DISCUSSION: Rifampin has been well-documented to cause clinically significant drug interactions and alterations in hormone levels. Case reports of rifampin-induced hypothyroidism during tuberculosis (TB) treatment exist in the literature. We report a rare case of rifampin-induced hypothyroidism during MAI treatment. A Japanese team reported a case series of three euthyroid patients with Hashimoto's thyroiditis with mycobacterium tuberculosis infection who developed hypothyroidism with elevated TSH that resolved after rifampin was discontinued. Our patient's progressive rise in TSH and transition from clinical euthyroidism to symptomatic hypothyroidism is likely related to increased thyroid hormone requirement due to enhanced hepatic metabolism and biliary excretion mediated by rifampin. Rifampin is a well-known inducer of cytochrome $\mathrm{P} 450$ hepatic enzymes and may result in increased T4 requirement. Physicians should be aware that patients with underlying thyroid disorders on rifampin may be vulnerable to developing worsening hypothyroidism. Thyroid function should be monitored after initiation and termination of therapy with rifampin.

INEVITABLE EXTRAPONTINE MYELINOLYSIS Ellen Wang ${ }^{1}$; Ali Hakim Shoushtari ${ }^{1}$; Nabil Mesiha ${ }^{1}$; Olga Tarasova ${ }^{1}$; Stephen Tieku ${ }^{2}{ }^{1}$ Capital Health Regional Medical Center, Yardville, NJ; ${ }^{2}$ Capital Health, Trenton, NJ. (Control ID \#2470267)

LEARNING OBJECTIVE \#1: Hypernatremia is most commonly due to free water loss, therefore resulting in elevated plasma osmolality. It becomes pathologic when the body fails to adequately compensate the volume and fluid deficits through thirst mechanism or prompt rehydration. In a critical setting of symptomatic hypernatremia, careful fluid replacement and closely monitored sodium level are warranted to slowly lower the serum sodium to avoid pontine demyelination. In practice, correction of hypernatremia is often clinically challenging despite careful fluid replacement. We report a case of extrapontine myelinolysis occurred in a setting of very gradual correction of hypernatremia.

CASE: A 44 years old female was found in the woods after being reported missing for 6 days by family. She was tachycardic, hypertensive, tachypneic, febrile of 101.4, and appeared dry, warmth, with moderate distress. Initially lethargic, but overall she was awake, oriented and followed commands. Physical exam is significant for multiple bruises, excoriations, and moderate epigastric tenderness. The laboratory workup revealed sodium of 191 , osmolality of 431 , potassium of 5.4 , chloride of 145 , bicarbonate of 16 , creatinine of 5.06 , BUN of 149 , calcium of 10.4 , white blood cell of 14.4 with $30 \%$ bandemia, and creatine kinase of 7558. Trauma workup was negative. After receiving resuscitation with IV fluids and antibiotics in emergency room, she was admitted to intensive care unit for severe hypernatremia, acute renal injury and rhabdomyolysis. Intravenous normal saline was initiated with the goal to correct the hypernatremia set at 
$1 \mathrm{meq} / \mathrm{L}$ per hour or $10 \mathrm{meq} / \mathrm{L}$ in $24 \mathrm{~h}$. We frequently followed the sodium level at interval of 4-6 h, and adjusted rate of infusion per recalculation by the formula. On day 3 with sodium of $177 \mathrm{meq} / \mathrm{L}$, she became more lethargic with left proximal drifting, hypoactive deep tendon reflex, downward plantar responses, and bilateral leg weakness with strength $2 / 5$. Therefore she was intubated for respiratory distress and airway protection. The MRI revealed symmetric restricted diffusion in the posterior limbs of the internal capsules and brachium pontis but spared globus pallidus which was not typical for osmotic demyelination syndrome. The intravenous fluid treatment continued along with very frequent sodium level check. Gradually the patient became normonatremic, however she remained unresponsive mentally. With a repeat MRI which revealed increasing diffusion restriction in brachium pontis, internal capsules, cerebellum, crura of fornices and persistent mental changes, it was concluded the patient had developed extrapontine myelinolysis in setting of adequate sodium correction. Her mental status slowly improved with supportive management. She was finally extubated on day 14 and discharged to rehabilitation on the day 22. She was alert, awake, and oriented time three at time of discharge.

DISCUSSION: The recommended rate of correction for hypernatremia is $10 \mathrm{meq} / \mathrm{day}$, however data have shown most cases have slower rate at $<0.25 \mathrm{meq} / \mathrm{h}$. Furthermore, most reported cases of pontine demyelination are among pediatric population. We speculate the adequate fluid replacement therapy in adult population, especially in cases of severe hypernatremia, can be further reduced to even $5 \mathrm{meq} / \mathrm{day}$, give no significant focal neurologic deficits or seizures occurred.

INFECTIVE ENDOCARDITIS: REIGNITING THE QUESTION OF ANTIBIOTIC PROPHYLAXIS Amit Gupta ${ }^{1}$; Melanie M. Holtrop ${ }^{2}$; Jamie A. Sutton ${ }^{1}$. ${ }^{1}$ University of Michigan Health Systems, Ann Arbor, MI; ${ }^{2}$ University of Michigan Medical School, Ann Arbor, MI. (Control ID \#2448222)

LEARNING OBJECTIVE \#1: Identify patient populations that may benefit from antibiotic prophylaxis for infective endocarditis

LEARNING OBJECTIVE \#2: Recognize complications of infective endocarditis

CASE: A 67 year-old gentleman with a history of moderate-to-severe aortic stenosis presented with three days of fevers and altered mental status. History was notable for a dental procedure 2 weeks prior without prophylactic antibiotics. Vital signs and physical exam were otherwise unremarkable. Initial laboratory workup was only notable for a slightly elevated white blood cell count of $12.5 \mathrm{~K} / \mathrm{uL}$. Lumbar puncture did not support bacterial meningitis. He was treated empirically with vancomycin, piperacillintazobactam, azithromycin, and acyclovir for broad empiric coverage of healthcareassociated pneumonia, infective endocarditis, and HSV-meningitis. On day three, multiple sets of blood cultures grew gram-positive cocci, and he developed an Osler's node, several Janeway lesions, and splinter hemorrhages, raising concern for infective endocarditis (IE). Subsequent transesophageal echocardiography (TEE) demonstrated a tiny vegetation on the aortic valve, confirmed his known history of aortic stenosis, and did not show any significant insufficiency, paravalvular abscess, or fistula. Brain MRI showed numerous septic emboli. Cultures speciated to methicillin-sensitive staphylococcus aureus prompting antibiotics to be narrowed to nafcillin. He remained afebrile on nafcillin for an additional day and was discharged with home nursing, intravenous (IV) antibiotics, and follow-up labs. Three days later he returned with fevers. Electrocardiogram demonstrated first-degree heart block raising suspicion for complication of IE. Repeat TEE showed possible abscess. Surgery was consulted and recommended urgent surgery. He was taken to the operating room for surgical intervention and aortic valve replacement. He tolerated the surgery well and was discharged on a 4 week course of IV nafcillin.

DISCUSSION: Infective endocarditis (IE), an infection of the inner lining of your heart and/ or heart valves, is a rare condition that often presents with nonspecific findings. Diagnosis requires astute history taking, physical examination, laboratory testing, and imaging. The cost of missing a diagnosis of IE is significant, as untreated IE can cause a wide variety of short and long-term complications affecting several organ systems and possibly leading to death. In this case we reported IE in a man with congenital bicuspid aortic valve and severe aortic stenosis, recent dental procedure without antibiotic prophylaxis, nonspecific presentation, and the development of an arrhythmia raising concern for the possibility of an abscess. On presentation he had two minor criteria (predisposition and fever), which we considered clinically sufficient to warrant empiric coverage for IE. ${ }^{1}$ The diagnosis was then confirmed by day three, given the development of Janeway lesions and presence of two major criteria (typical microorganism for IE from two separate blood cultures and evidence of endocardial involvement on TEE). ${ }^{1}$ The subsequent development of an arrhythmia was an important clue to a potential complication prompting repeat TEE, diagnosis of a possible abscess, and ultimate decision to urgently proceed to surgery. This case also reignites the debated question regarding appropriate populations within which to use antibiotic prophylaxis for IE. Had this patient presented prior to 2007, the American Heart Association (AHA) guidelines would have recommended antibiotic prophylaxis given his moderate risk for acquiring IE due to his known bicuspid aortic valve and aortic stenosis. ${ }^{2}$ The 2007 guidelines, however, do not advise the use of antibiotics in our patient, as these guidelines were modified to limit antibiotic prophylaxis to only the highest risk groups: those with prosthetic cardiac valves, previous IE, certain types of congenital heart disease (not including bicuspid aortic valve), and cardiac transplantation recipients who develop valvulopathy. ${ }^{3}$ The decision to decrease the scope for antibiotic prophylaxis was made given increased recognition of adverse effects of antibiotic use and realization that prior recommendations were largely based on consensus opinion as opposed to prospective data. This case, however, raises questions as to whether the benefits of antibiotic prophylaxis in moderate risk groups may actually outweigh the risks, and stresses the need for randomized controlled-trials to better answer this question. 1. Li JS, Sexton DJ, Mick N. Proposed modifications to the Duke criteria for the diagnosis of infective endocarditis. Clinical infectious diseases : an official publication of the Infectious Diseases Society of America. 30(4):633-8. 2000 2. Dajani AS, Taubert KA, Wilson W et al. Prevention of Bacterial Endocarditis : Recommendations by the American Heart Association. Circulation. 96(1):358366. 1997. 3. Wilson W, Taubert KA, Gewitz M et al. Prevention of Infective Endocarditis: Guidelines From the American Heart Association: A Guideline From the American Heart Association Rheumatic Fever, Endocarditis, and Kawasaki Disease Committee, Council on Cardiovascular Disease in the Young, and the Council on Clinical Cardiology, Council on Cardiovascular Surgery and Anesthesia, and the Quality of Care and Outcomes Research Interdisciplinary Working Group. Circulation. 116(15):1736-1754. 2007.

INFLAMED BRAIN: AN ATYPICAL PRESENTATION OF CEREBRAL EDEMA SECONDARY TO LUPUS CEREBRITIS Mira John; Aimee Aysenne. Tulane University, New Orleans, LA. (Control ID \#2465609)

LEARNING OBJECTIVE \#1: Diagnose new-onset lupus cerebritis based on atypical clinical findings and MRI scans.

LEARNING OBJECTIVE \#2: Recognize the rapid progression of lupus cerebritis's systemic manifestations.

CASE: A 41 year-old African American lady with a recent diagnosis of heart failure, newonset allergies, and 4 days of malaise presented with new-onset seizures. She was hypotensive and tachypnic with Kussmaul respirations and required intubation. Neurological exam revealed intact brainstem reflexes with right gaze deviation, no blink to threat, and withdrawal to noxious stimuli. Non-contrast head computer tomography (CT) revealed bilateral sulcal effacement with loss of the grey-white junction. MRI showed bilateral cortical, thalamic, and cerebellar hyperintensities. MRA and MRV were 
unremarkable. EEG was isoelectric on all leads. Chest x-ray showed left lower lobar consolidation with bilateral pulmonary opacities. Admit labs included 16,5000 WBC with $21 \%$ bandemia and abnormal red cell morphology, elevated ESR at 84 , and urinalysis which revealed $100 \mathrm{~g} / \mathrm{dL}$ protein, $20 \mathrm{mg} / \mathrm{L}$ ketones, and $50 \mathrm{mg} / \mathrm{dL}$ glucose. She was started on levetiracetam and Ativan for seizure control and broad-spectrum antibiotics, azithromycin, and acyclovir for concern of pneumonia and encephalitis. Her gaze deviation and altered mental status persisted despite therapy. Lumbar puncture revealed $140 \mathrm{mg}$ / $\mathrm{dL}$ protein and $75 \mathrm{mg} / \mathrm{dL}$ glucose. MS panel had an elevated myelin basic protein of $2.3 \mathrm{ng} / \mathrm{mL}$, serum IgG of $171 \mathrm{~g} \mathrm{mg} / \mathrm{dL}$, and CSF $\operatorname{IgG}$ of $30.0 \mathrm{mg} / \mathrm{dL}$, along with decreased serum albumin of $3.0 \mathrm{~g} / \mathrm{dL}$, and CSF albumin of $68 \mathrm{mg} / \mathrm{dL}$; these results suggested a demyelinating process consistent with earlier MRI findings. Antibody testing reveled antiSjogren syndrome antibody $\mathrm{A}>9.0$, anti-Sjogren syndrome antibody $\mathrm{B}>1.1$, 1:640 anti-nuclear antibody (ANA) titers with speckled pattern, suggesting SLE or some other autoimmune process. Her lupus panel had elevated prothrombin of $13.5 \mathrm{~s}$; her International Normalized Ratio (INR) and activated partial thromboplastin time (aPTT) values were within normal range. Factor 8 activity was elevated at $611 \%$. Her blood and CSF cultures, HIV, RPR, hepatitis panels, and West Nile CSF tests were all negative. She was eventually extubated and was hemodynamically stable but displayed intermitted agitation. The diagnoses of lupus cerebritis and nephritis were made based off her seizures, proteinuria, and abnormal ANA and antibody titers. A repeat MRI revealed a new ischemic insult in the left occipital area on T2 flare, but she began to receive high dose steroids for her presumed diagnosis of SLE. The next day she was found to be nonverbal with livedo reticularis over lower extremities along with her right eye held in adduction. Despite careful management, she declined over the next $24 \mathrm{~h}$ and went into respiratory distress and cardiac arrest. The diagnosis based on autopsy was CNS Lupus, stroke, and cerebral edema which caused cerebellar tonsillar herniation.

DISCUSSION: Common neurologic manifestations of SLE include cognitive dysfunction, stroke, mood disorders and psychosis, peripheral neuropathy, and seizures. Less common neurologic presentations include cranial neuropathies such as eye movement abnormalities, optic neuritis or sensorineural hearing deficits, meningitis, and posterior reversible encephalopathy syndrome (PRES). MRI findings for SLE are varied and include infarcts, hyperintense focal white matter lesions, and demyelinating lesions. It is extremely unusual for a patient to have coma and diffuse cerebral edema that progresses to death. Standard treatment of lupus cerebritis is high dose cortical steroids. Given that this patient presented without a previous diagnosis of lupus and with unusual clinical findings, there was a delay to insure the diagnosis and exclude infectious etiologies. Her systemic manifestations were in rapid progression from the onset of any symptoms of the disease, and her neurologic manifestations were sudden and unexpected. While this is an unusual case, the general internist should be aware of neurologic presentations and MRI findings of SLE in order to more quickly reach a diagnosis, begin treatment, and monitor for the development of cerebral edema and other potential life-threatening complications associated with the disease.

INTERNISTS DIAGNOSING A SURGICAL EMERGENCY: AN UNEXPECTED ETIOLOGY OF A NON-ANION GAP METABOLIC ACIDOSIS Neelroop Parikshak; Sara-Megumi L. Naylor. UCLA Medical Center, Los Angeles, CA. (Control ID \#2465384)

LEARNING OBJECTIVE \#1: Diagnose rare causes of acid-base disorders by applying a systematic approach and type 2 (rational) clinical reasoning.

LEARNING OBJECTIVE \#2: Recognize the importance of care coordination between multiple consulting teams for optimal management of a complicated clinical case.
CASE: A 73 year old male with castration-resistant metastatic prostate cancer, hypertension, and obesity presented to the emergency department with left lower extremity pain, swelling, and erythema consistent with cellulitis. On further questioning, he complained of incomplete voiding of his bladder for 3 weeks, and gross hematuria for 5 days. The cellulitis was treated with a standard course of antibiotics and resolved. His history and admission labs suggested an obstructive nephropathy, presumably from prostatic enlargement, metastatic cancer lesions, and blood clots from bleeding malignant lesions. A urinary catheter was placed with immediate return of a large volume of gross hematuria and blood clots. Urology was consulted, and recommended serial bladder irrigations with normal saline over the next few days. However, new blood clots continued to form and obstruct the catheter. On day five of admission, continuous bladder irrigation (CBI) with normal saline was initiated to prevent further obstruction from new clots. Cystoscopy revealed a large clot burden as well as a friable mass extending from the prostate. Fulguration of the prostate was performed. However, gross hematuria and clot formation persisted and the patient had to be kept on CBI with normal saline. Over the subsequent 2 days on daily labs, his bicarbonate was noted to downtrend. He developed a progressive, non-anion gap metabolic acidosis (NAGMA) of unclear etiology. The patient was not receiving any IV fluids, had no diarrhea or other intestinal losses of bicarbonate, his renal function remained stable over this course, and he did not have labs suggestive of a renal tubular acidosis (RTA). Nephrology was consulted. Sodium bicarbonate was initiated, but the NAGMA continued to worsen. At this point, the patient had developed new abdominal distension, worsening bilateral lower extremity swelling, and dyspnea. Possible etiologies of a NAGMA were explored, and standard causes did not fit. Given the patient's known metastatic prostate cancer with invasion to the bladder, friable mucosa, recent instrumentation, CBI, and new hyperchloremia, we hypothesized that normal saline may be leaking into the peritoneal space through a bladder wall tear and subsequently being absorbed into the intravascular space. Given the lack of alternative explanations and the patient's worsening condition, emergent CT cystography was pursued. The tip of the urinary catheter was found to be in the perivisceral fat, and contrast injected into the bladder passed into the intraperitoneal space, mixing with existing free intraperitoneal fluid. The patient had a bladder wall rupture. The case was discussed with Urology and Interventional Radiology for optimal management. Bladder irrigation with normal saline was stopped despite the risk of obstruction. Bilateral nephrostomy tubes were placed to preserve renal function while definitive management of the bladder rupture was decided upon. The patient's NAGMA resolved over the next day.

DISCUSSION: This vignette illustrates the importance of using a systematic approach and type 2 (rational) clinical reasoning to solve challenging clinical cases. While there is a crucial role for type 1 clinical reasoning, which is fast and instinctive, type 2 clinical reasoning that is slow and analytical is crucial to solve unfamiliar problems. This case highlights how a list of memorized causes for a NAGMA would miss the underlying cause of this specific patient's NAGMA and the persistence to find a rational explanation helped solve the clinical problem. There are multiple recommendations on how to approach acidbase disorders, and we support methods that use a systematic approach. The case also emphasized the importance of working with consultants in an organized, coordinated fashion. The urology team was focused on the urinary catheter and hematuria, while the nephrology team had no clear reason for the NAGMA but attempted to address it with sodium bicarbonate. Overcoming heuristics, having a primary team that interpreted all aspects of the clinical case and took ownership to address the unfamiliar problem was vital in unraveling this case.

INTESTINAL SPIROCHETES AS AN UNUSUAL CAUSE OF DIARRHEA IN AN HIV POSITIVE PATIENT Dima Dandachi ${ }^{2}$; Abhinav Dhakal ${ }^{1}$; Roshanak Habibi ${ }^{1}$. ${ }^{1}$ Presence Saint Francis Hospital, Evanston, IL; ${ }^{2}$ Saint Francis Hospital, Evanston, IL. (Control ID \#2466651) 
LEARNING OBJECTIVE \#1: To recognize a rare cause of acute diarrhea in a patient with HIV

CASE: A 29 year old male presented to emergency department with episodes of watery stools and abdominal pain of 1 week duration. The pain was crampy and generalized with 6 out of 10 severity. He had no complaints of fever, weight loss, bloody stools or vomiting. He was sexually active with men and had multiple partners in the past. He was tested negative for HIV and viral hepatitis 6 months prior to his admission. He was not taking any medication. Physical exam showed periumbilical tenderness with inguinal lymphadenopathy and guaiac negative stool. In laboratory work up he was found to have reactive ELISA for HIV with CD4 of 650 cells $/ \mathrm{mm} 3$. Blood counts and basic metabolic panel were normal. Fungal panel and Stool cultures for common germs were negative. In abdominal CT diffuse dilatation of distal small bowel and colon plus multiple enlarged lymph nodes in abdominal and pelvic area were detected. Colonoscopy showed segmental mild inflammation and erythema of right ascending colon with biopsy results positive for intestinal spirochetes. The patient was started on Metronidazole and HAART for newly diagnosed HIV. His symptoms were significantly improved after few days.

DISCUSSION: Human intestinal spirochetosis (IS) is a condition defined histologically by the presence of spirochetal microorganisms attached to the apical cell membrane of the colorectal epithelium. It can manifest as a very rare cause of diarrhea in HIV positive patients. Clinical significance in individual cases has remained unclear up to now Spirochetes comprise a heterogeneous group of bacteria. In humans, Brachyspira aalborgi and Brachyspira pilosicoli predominate. A review of the literature assumes that invasion of spirochetes beyond the surface epithelium may be associated with gastrointestinal symptoms which respond to antibiotic treatment (Metronidazole), whereas individuals lacking this feature may be mostly asymptomatic. Of unknown reason, homosexual and HIVpositive men as well as children are more likely to be symptomatic irrespective of invasion. Many patients are with CD4 count of more than 200 cells $/ \mathrm{ml}$. Pathophysiology is still unclear. Symptoms happens after invasion of spirochetes beyond the surface epithelium or apical membrane. Colonoscopy can be normal or showing erythematous inflammation. Diagnosis is made by seeing false brush border pattern of spirochetes in $\mathrm{H}$ and $\mathrm{E}$ stain of biopsy samples from colon. Worthen starry stain is gold standard to show the microorganism. Treatment with metronidazole leads to symptomatic remission and histologic clearance. As IS is more consistently recognized with better identification techniques, it is hoped that the clinical significance of this condition, particularly that of different strains and their potential of invasiveness, will soon become evident.

ION MEMBRANE CHANNELOPATHIES: A CONNECTED GATEWAY: BRUGADA PATTERN IN A PATIENT WITH UNDERLYING CHARCOT MARIE TOOTH SYNDROME Shakil Shaikh; Khawar Maqsood. Baystate Medical Center, Princeton, NJ. (Control ID \#2470155)

LEARNING OBJECTIVE \#1: Recognize the link in genetic mutations of Brugada Syndrome and Charcot Marie Tooth Syndrome

LEARNING OBJECTIVE \#2: Identify instance of fever-induced Brugada Syndrome, and importance of rapid anti-pyretic therapy to prevent fatal arrhythmias.

CASE: A 52-year old male with a past medical history of Charcot Marie-Tooth disease presented to our facility for evaluation of acute dyspnea. He presented for respiratory distress, mentioning progressive generalized weakness and symptoms of an upper respiratory tract infection. On arrival, the patient was found to be febrile to 101.8, along with associated tachypnea (respiratory rate of 24), and tachycardia (heart rate: 112). He required BiPAP therapy for respiratory distress secondary to accessory muscle use. The patient was admitted for a diagnosis of pneumonia, and started on antibiotics. Of note, an EKG done on arrival was consistent with ST segment elevations in leads V1 and V2, characteristic of
Brugada pattern. Per collateral history, the patient never had any underlying diagnosis of Brugada syndrome. He was diagnosed with Charcot Marie Tooth Syndrome as a child, but did not have any family history of sudden cardiac death. His cardiac enzymes were trended during his hospitalization, and were always within normal limits $(<0.01$ and $<40$ for troponin, and CPK, respectively). The patient received $72 \mathrm{~h}$ of antibiotics with respiratory improvement, and resolution of his fevers. Cardiac rhythm strips showed resolution of the patients ST segment changes after the patients fevers had resolved.

DISCUSSION: Brugada Syndrome is an autosomal dominant genetic disorder seen with ECG findings of ST segment elevations in leads V1 to V3. These patterns are thought to occur from multiple causes, including mutations in cardiac sodium channel SCN5A and SCN10A genes, significant febrile illness, or psychotropic medications. Due to ECG findings, Brugada pattern can be misconstrued as an ST-elevation myocardial infarction; as a result, it becomes prudent for the clinician to identify the appropriate illness, as both can cause sudden cardiac death. Charcot Marie Tooth Syndrome consists of a group of hereditary motor sensory neuropathies caused by specific mutations in myelin genes that result in defects in myelin structure and function. Gene mutations commonly link various diseases, however research connecting Brugada Syndrome and Charcot Marie Tooth Syndrome is sparse. Recent reports have shown a new lamin $\mathrm{A} / \mathrm{C}$ gene mutation that has connected spinal muscular atrophy, as seen in Charcot-Marie-Tooth disease with cardiomyopathies, dysrhythmias, and sudden cardiac death. The lamin gene mutations do result in ion channel defects, as seen with the genetic mutation of Brugada syndrome. Due to the rarity of simultaneous presentation of Brugada and Charcot-Marie-Tooth Syndrome, further molecular genetic research is needed to identify underlying mechanisms, thereby allowing for therapeutic strategies to treat affected individuals.

IRON DEFICIENCY ANEMIA：THROMBOCYTOSIS TO THROMBOCYTOPENIA. Dipen B. Khanapara; Nurilign A. Bulcha; David Fernandes; Sofia Terner. Montefiore Medical Center, Bronx, NY. (Control ID \#2470276)

LEARNING OBJECTIVE \#1: Recognize the spectrum of quantitative platelets derangements associated with variable severity of Iron Deficiency anemia.

CASE: A 48-year-old female was admitted for loss of consciousness following the recurrence of heavy vaginal bleeding. She complained of menorrhagia for the past 3 years. Family history was negative for any bleeding diathesis. A review of systems was negative for any epistaxis, bleeding gums or melena. She was not on any medication or herbal supplements. On physical exam, she had anicteric sclerae, dry mucous membranes without evidence of bleeding, a grade 2/6 systolic murmur, cool skin without petechiae or ecchymoses, and a firm non-tender mass in the suprapubic area. Her initial laboratory results were significant for hemoglobin of $3.2 \mathrm{mg} / \mathrm{dl}$, hematocrit $12.3 \%$, mean corpuscular volume (MCV) $56.5 \mathrm{FL}$, red-cell distribution width (RDW) $27.8 \%$ and platelets 24,000 cell/ul. Peripheral Smear showed very severe hypochromic red blood cells ( $>70 \%$ central pallor), no schistocytes and a severely decreased platelet number per high power field without obvious clumping. Transvaginal Ultrasound revealed a heterogeneous intramural mass, likely a uterine fibroid. Five units of packed red blood cell (PRBC) and two units of platelets were transfused. The patient had an optimal response with hemoglobin and platelet count rising to $8.2 \mathrm{mg} / \mathrm{dl}$ and $60,000 \mathrm{cell} / \mathrm{ul}$ respectively. Initial workup was negative for other causes of thrombocytopenia. Her platelet count was well maintained after transfusion making Immune Thrombocytopenia (ITP) unlikely. She underwent hysteroscopy guided transvaginal myomectomy. She was also started on oral iron and folic acid supplements that led a rise in platelet count to $1,66,000$ cells/ul in 2 days.

DISCUSSION: Iron deficiency is the most common cause of anemia worldwide. The incidence of Iron deficiency anemia (IDA) is significantly high in both low-income and developed countries, affecting about $30 \%$ of premenopausal females. Thrombocytosis is 
commonly seen with IDA but thrombocytopenia is very rare and is associated with severe IDA. Platelet derangements are found in about $15 \%$ of patients with IDA. Thrombocytosis is very common, seen in about $13 \%$ of cases. In less severe IDA, thrombocytosis is likely reactive. However, thrombocytopenia is rare. Most documented cases of IDA associated thrombocytopenia occur in the setting of heavy vaginal bleeding or chronic prolonged dietary iron deficiency. Thrombocytopenia is seen towards the more severe spectrum of disease when the body stores of iron are severely deficient. Studies have linked the relationship between iron levels and the maintenance of platelet count as a "two-compartment" model (Figure 1). In the "Inhibitor compartment" of the model, sufficient iron quantity inhibits platelet production above steady state levels. In the "essential compartment", iron is necessary for the synthesis of platelets. With bleeding, the loss of iron initially only affects the inhibitor compartment, and so platelet quantity loses regulatory control by iron and this leads to reactive thrombocytosis. With continued blood loss, a severe or prolonged iron deficiency develops which affects the essential compartment, and in turn suppresses platelet production leading to thrombocytopenia. Our patient presented at the extreme end of the spectrum of IDA with very severe anemia and thrombocytopenia. After blood transfusion, surgery and iron replacement therapy, the platelet count went up immediately. It should be noted that Iron replacement therapy can also lead to both thrombocytosis as well as thrombocytopenia depending on the severity of iron deficiency. In mild cases, Iron replacement therapy cause thrombocytopenia via the inhibitor compartment and in severe cases; it can lead to thrombocytosis as seen in our case. It is very important to recognize IDA as a cause of thrombocytopenia. Any patien who presents with severe IDA and thrombocytopenia needs an initial basic workup to rule out life-threatening conditions. Appropriate treatment of IDA can reverse thrombocytopenia in a matter of days without exposing the patient to invasive procedures such as bone marrow biopsy.

IS FLATBUSH, NY, IN FLORIDA?-A CASE OF KETOSIS PRONE DIABETES Adam Levine; Sweny Gulati; Maryam Sattari. University of Florida, Gainesville, FL. (Control ID \#2467696)

LEARNING OBJECTIVE \#1: Become familiar with diagnosis, classification, and treatment of Ketosis-Prone Diabetes

LEARNING OBJECTIVE \#2: Recognize similarities and differences in immediate and long-term treatment of Ketosis-Prone Diabetes, type 1 diabetes, and type 2 diabetes

CASE: A 52-year-old African American man presented with 1 month of polyuria, polydipsia, progressive fatigue, and 30-lbs weight loss. While his HgbA1C had been only 6.46 months prior to presentation, lab results on presentation revealed a blood glucose of $388 \mathrm{mg} / \mathrm{dL}$, anion gap of 32, B-hydroxybutyrate of $7.5 \mathrm{mmol} / \mathrm{L}$, urine and serum ketones, and hemoglobin $\mathrm{AlC}(\mathrm{HbA1c})$ of 16.6. The patient was admitted for treatment of diabetic ketoacidosis (DKA) and discharged on metformin $1 \mathrm{~g}$ BID, glipizide $10 \mathrm{mg}$ BID, and glargine insulin 40 units daily. At 2-month follow-up, the patient reported hypoglycemic episodes and HgbA1C was 5.9. Glipizide was discontinued and insulin dose was decreased. The patient has since further reduced his insulin dose and continues to do well with $\mathrm{HgbA1C}$ of 5.6 approximately 10 months after initial presentation. We are awaiting the results of the following labs at this time: autoantibodies to glutamic acid decarboxylase 65 (GAD65), islet cell antibodies (IA-2), and C-peptide.

DISCUSSION: Our patient presented with DKA in the absence of type I diabetes and was diagnosed with "ketosis-prone diabetes" (KPD). This condition, also known as "atypical diabetes," "Flatbush diabetes," or "diabetes type 1B" is being increasingly recognized and is believed to account for $25-50 \%$ of new onset DKAs in African-American and Hispanic patients. These patients are typically obese, middle-aged men with a family history of type 2 diabetes. While the exact pathophysiology is unknown, glucose desensitization, insulin insensitivity, and impaired insulin release are thought to play a role in the DKA development. Four subgroups have been defined based on the presence or absence of autoantibodies and pancreatic beta-cell functional reserve. At initial presentation, KPD can be difficult to distinguish from type 1 diabetes. However, the long term outcomes and insulin requirements of these patients are variable and depend on presence of autoantibodies and beta-cell reserve. The initial treatment of patients with suspected KPD who present with DKA is similar to that of type 1 diabetes and all patients should also be discharged on insulin. For patients presenting in an atypical fashion (older, overweight, non-Caucasian ethnicity), assessment of beta-cell secretory reserve, by measurement of Cpeptide, and testing for autoantibodies (GAD65, IA-2) can aid in making the accurate diagnosis and classification as well as provide guidance on long-term management. These measurements are best done 4-8 weeks after DKA resolution to minimize effects of glucose toxicity or desensitization on beta-cell function. Insulin can be titrated down and eventually discontinued under careful supervision and close follow up in patients with present beta-cell function and without autoimmunity, whereas patients without beta-cell reserve generally remain insulin-dependent and require long-term insulin therapy to prevent recurrent ketoacidosis. This case highlights the importance of prompt diagnosis and treatment of DKA in patients without classical risk factors for type 1 diabetes. After successful treatment of the initial DKA episode, awareness of KPD and its natural history can lead to appropriate testing, identification and accurate classification of KPD, and safe discontinuation of insulin therapy in some cases.

\section{ISCHEMIC STROKE AS AN UNUSUAL PRESENTING SYMPTOM OF SYSTEMIC LUPUS ERYTHEMATOSUS IN A YOUNG PATIENT Pooja} Agrawal; Ana I. Velazquez; Jeffrey Hollis; Heather Siedenburg; Erin Patton; Alfredo J. Astua. Mount Sinai Beth Israel, New York, NY. (Control ID \#2465165)

LEARNING OBJECTIVE \#1: Recognize the possibility of an underlying rheumatologic disorder in young patients presenting with acute strokes.

CASE: A 33-year old female with no medical history presented after witnessed collapse with subsequent right-sided weakness and inability to speak. At time of presentation, physical exam was remarkable for global aphasia, right facial droop, a flaccid right upper and lower extremity not responsive to pain, sustained right ankle clonus, and an inability to respond to simple commands. A stroke code was called and although CT head was negative, given a high degree of suspicion, she received tissue plasminogen activator (tPA). Subsequent CT angiogram of the head and neck showed complete occlusion of the left distal-third middle cerebral artery. Clot retrieval was performed with improvement of her hemiplegia. Initial labs were significant for C-reactive protein $1.2 \mathrm{mg} / \mathrm{dL}$, lymphopenia $700 \mathrm{~mm}^{3}$, and negative toxicology. Hospital course was complicated by hemorrhagic stroke transformation over the left hemisphere with intraventricular extension that remained stable. Magnetic resonance angiography (MRA) of the neck and bubble echocardiogram were normal. A comprehensive hypercoagulable workup was negative for antiphospholipid $\mathrm{Ab}$, anti-cardiolipin $\mathrm{Ab}, \beta 2$-glycoproteins, sickle cells, homocysteine, proteins C/S deficiency, antithrombin III deficiency, SSA Ab, SSB Ab, and factor V Leiden mutation among others. Autoimmune workup showed positive ANA 1:640, dsDNA $45 \mathrm{IU} / \mathrm{mL}$, and low C3 $71 \mathrm{mg} / \mathrm{dL}$ consistent with systemic lupus erythematosus (SLE). Further history later provided by the family revealed oral contraceptive (OCP) use and complaints of Raynaud's phenomenon. There was no history of joint pains, stiffness, rashes, oral ulcers, or dermal photosensitivity. The patient was transferred to acute rehabilitation where she made marked improvement. On outpatient follow-up, the diagnosis of SLE was confirmed by AVISE SLE panel and treatment with hydroxychloroquine was started.

DISCUSSION: The prevalence of ischemic strokes in SLE ranges between 2 and $15 \%$ with the highest risk among individuals younger than age 50. Pathogenesis has been 
attributed to a higher incidence of hypertension, earlier development of atherosclerosis, presence of lupus anticoagulant, propensity for hyperviscosity, and steroid administration. Despite this known correlation between SLE and cerebrovascular diseases, our case illustrates three unique concepts given it is one of few reported in which the ischemic event preceded the diagnosis of SLE. Firstly, given our patient's young age and otherwise absent traditional risk factors for stroke, it became vital to explore unconventional etiologies which may otherwise not be pursued in other populations. She fulfilled 4 of 17 SLICC classification criteria for diagnosis of SLE, confirmed by AVISE SLE panel. This emerging diagnostic assay has $78 \%$ sensitivity and $87 \%$ specificity for SLE. Thus, when combined with traditional biomarkers including ANA and anti-dsDNA, the diagnostic accuracy for SLE is improved by approximately $30 \%$. Secondly, the patient's use of OCPs may have synergized her underlying prothrombotic state, contributing to unmasking her disease. Yet lack of knowledge regarding her OCP use became beneficial to the eventual diagnosis, as this knowledge may often deter autoimmune workup when still clinically warranted. Lastly, as in our case, patients with some degree of SLE activity are generally treated with hydroxychloroquine. This therapy has added benefit of being effective for stroke prevention by mechanistically decreasing low-density lipoproteins and increasing platelet inhibition.

\section{ISCHEMIC STROKE AS THE PRESENTING SYMPTOM OF LEFT} VENTRICULAR NON-COMPACTION William Perucki ${ }^{1}$; Chandana Shekar ${ }^{2}$. ${ }^{1}$ University of Connecticut, Farmington, CT; ${ }^{2}$ University of Connecticut School of Medicine, West Hartford, CT. (Control ID \#2469465)

LEARNING OBJECTIVE \#1: Describe the pathophysiology of ventricular noncompaction as one of the cardiomyopathies.

LEARNING OBJECTIVE \#2: Identify the major subtypes of left ventricular noncompaction and discuss their management

CASE: This is a case of a 20 -year-old female with a past medical history including psoriasis and is currently on Humira therapy. She presented to our hospital with the acute onset of blurriness of vision. On neurologic testing she was found to have left-sided hemianopsia. She was previously very active- working at an outdoor adventure park and often hiking several miles without any complaints of dyspnea. A CT scan of her head showed a large acute infarct in the right posterior cerebral artery territory and a small additional acute infarct in the cortex of the left occipital lobe. As she was out of the time window, the patient did not receive tPA, and was admitted to the neurology service for stroke workup. During the standard workup for ischemic stroke, echocardiogram indicated a left ventricular ejection fraction of $15 \%$ with global hypokinesis, elevated right ventricular systolic pressure, and a normal right ventricle. Cardiology was consulted and recommended further imaging in the form of Cardiac MRI for her severe cardiomyopathy, with plans for cardiac catheterization shortly thereafter should MRI be inconclusive. Cardiac MRI revealed left ventricular noncompaction, most serious along the lateral wall.

DISCUSSION: Left ventricular noncompaction was first described in 1926 and formally classified as a cardiomyopathy by the American Heart Association in 2006. It is a congenital cardiomyopathy characterized by failure of the myocardium to condense and compact. The early embryonic myocardium is a loose network of muscle fibers, and during embryonic development the myocardium compacts to form a structure that is efficient in contracting and working as needed for effective pumping of the heart. Non-compacted myocardium is characterized by deep trabeculae which are in communication with the lumen of the left ventricle. On histologic cross section, these trabeculae are lined by endothelium. There is often fibrous and/or elastic tissue noted in these trabeculations. Patients can present with symptoms of heart failure or arrhythmia, or may be diagnosed on the basis of echocardiography ordered for other symptoms such as a murmur. LVNC is divided into the following subcategories: Benign LVNC, LVNC with arrhythmias, Dilated LVNC, Hypertrophic LVNC, Hypertrophic Dilated LVNC, Restrictive LVNC, Right ventricular or biventricular LVNC, and LVNC with congenital disease. Imaging studies of choice include echocardiography or cardiac MRI. Either of these tests show a ratio of non-compacted myocardium to compacted myocardium at end diastole of $2: 1$. Because this disease may be congenital one, at-risk first degree relatives of the patient are often screened. Treatment of patients with such a diagnosis is similar to the treatment of heart failure, with inclusion of ACE-inhibitors, beta blockers, and aldosterone antagonists to assist with left ventricular remodeling. Loop diuretics are used for patients with signs and symptoms of congestion. Aspirin or other anticoagulation, as needed, is used to mitigate the risk of thrombosis. Implantible cardioverter-defibrilatiors may also be indicated. As with any form of cardiomyopathy, a patient may require advanced therapies or even transplant for full restoration of function.

IT'S COMPLICATED: AN UNUSUAL CASE OF AN ESCHERICHIA COLI SKIN \& SOFT TISSUE INFECTION David Kline ${ }^{1}$; Taimur H. Khan ${ }^{1}$; Laila Castellino ${ }^{1,2}$. ${ }^{1}$ Wright State University, Kettering, OH; ${ }^{2}$ Wright State University, Dayton, OH. (Control ID \#2469695)

LEARNING OBJECTIVE \#1: Identifying \& managing complicated soft tissue infections, while maintaining a broad differential for possible microbial etiologies in immunocompetent patients to include unlikely pathogens.

CASE: An 82 year old man was admitted to the VA Medical Center in Ohio presenting with a 2 day history of left lower extremity pain and swelling. The patient had a history of coronary artery disease, chronic kidney disease, heart failure, and chronic venous stasis in his lower extremities. He had no history of malignancy, diabetes or liver disease. The patient denied local trauma, fever, chills, diarrhea, sick contacts, or history of intravenous drug abuse or any immunosuppressive agents. He was an ex-smoker and rarely consumed alcohol. On exam patient was afebrile, non-tachycardic, mildly tachypneic, borderline hypotensive with a systolic blood pressure in the $90 \mathrm{~s} \mathrm{mmHg}$ and obese. Patient endorsed baseline dark discoloration (purplish hues) around his calves bilaterally from his chronic co-morbidities as well as a non-purulent non-healing ulcer present on the medial aspect of his left ankle for several weeks. The ulcer was not significantly erythematous or tender. An erythematous, warm, and indurated area with appreciable skin tightening was present on the left lateral calf, measuring $8 \mathrm{~cm} \times 12 \mathrm{~cm}$, not contiguous with the ankle ulcer. Peripheral pulses were intact. Labs revealed a leucocytosis of $21.15 \mathrm{~K}(91 \%$ granulocytes), ESR of $20 \mathrm{~mm} / \mathrm{h}, \mathrm{CRP}$ of $124 \mathrm{mg} / \mathrm{L}$, lactate of $2.2 \mathrm{mmol} / \mathrm{L}$, and creatinine was elevated at $3.0 \mathrm{mg} / \mathrm{dL}$ (baseline $1.8 \mathrm{mg} / \mathrm{dL}$ ). Others labs were within normal limits. Vancomycin and intravenous fluids were initiated for management of sepsis secondary to cellulitis. Urine studies/cultures were unrevealing; however one out of two blood culture bottles from admission grew gram negative rods therefore antibiotic coverage was expanded with piperacillin-tazobactam. The left ankle ulcer was easily managed with topical wound care per podiatry. Final speciation of the blood culture grew pan-susceptible Escherichia coli. Given the uncommon bacteria within the context of cellulitis, computerized tomography (CT) of the abdomen and pelvis were obtained and were unrevealing for a source for the bacteremia. Despite initial clinical improvement, the patient developed worsening left calf cellulitis erythema, pain, and fluctuance. An abscess spontaneously drained purulent fluid also growing pan-susceptible E. coli. On hospital day 5, CT of the left lower leg was without abscess, significant soft tissue swelling or subcutaneous air. However due the patient's worsening clinical status, surgeons performed bedside incision and drainage (I\&D) revealing multiple small subcutaneous abscesses. Despite I\&D, the 
patient's pain worsened and the surgeons felt he would benefit from further surgical exploration. Per their report, multiple microabscesses were noted within the subcutaneous fat which itself appeared to be necrotic and liquefied. Surgical exploration revealed what appeared to be necrotizing fasciitis for which a four compartment fasciotomy was performed on hospital day 9 and 11. The pathology report supported skin and soft tissue demonstrating necrosis and abscess formation. Further discussion with the pathologist revealed panniculitis and soft tissue necrosis/inflammation; fasciitis was not excluded. Surgical specimen cultures continued to grow pan-susceptible E. coli without other bacteria present. The patient improved with routine wound care, wound vac placement, and IV antibiotics (piperacillin-tazobactam with de-escalation to ampicillin-sulbactam). Blood cultures and wound cultures were negative for bacterial growth prior to discharge. The patient was discharged to a rehabilitation facility in stable condition with wound vac and oral amoxicillin-clavulanate therapy with plans for definitive closure and skin graft at a later date.

DISCUSSION: Our case represents a rare event. Non-GAS/staphylococcal infections are sparse, but those documented previously in medical literature are almost exclusively limited to those with immunodeficiency or malignancy. In 2002, Gach, Charles-Holmes, and Ghose noted in Clinical and Experimental Dermatology that their case of suspected E. coli cellulitis was the first documented case in an immunocompetent patient They restated that renal/hepatic failure, haematological malignancy, diabetes, alcoholism and corticosteroid treatment are all risk factors for cellulitis as they may be associated with leukocyte dysfunction. Their source was likely enteritis which evolved in septicemia and cellulitis. Our patient had neither gastrointestinal disease, hepatic failure, malignancy, diabetes, history of alcoholism or chronic steroid treatment. He was obese, had a history of saphenectomy with chronic venous stasis, and mild chronic kidney disease as well as a noncontiguous ankle ulcer. No source for infection was found. Additionally, the presentation of our patient's E. coli cellulitis was atypical. In the limited literature, E. coli cellulitis was often hemorrhagic or bullous. Our patient presented with a fairly classic cellulitis presentation which later developed into deeper panniculitis requiring surgical debridement. Fasciitis could not be excluded but was not present on admission as noted on CT imaging, labs, or physical exam. Despite the lack of classic risk factors, E. coli SSTIs are possible and given the poor diagnostic yield of cultures obtained for cellulitis, may be more common than previously thought.

IT'S MORE THAN JUST A NECROTIZING PNEUMONIA Poornima Ramadas'; Prathik Krishnan ${ }^{1}$; Viveksandeep Thoguluva Chandrasekar ${ }^{2}$; Christy Ann Gilman ${ }^{2}$; Jivan Lamichhane ${ }^{1} .{ }^{1}$ SUNY UPSTATE MEDICAL UNIVERSITY, SYRACUSE, NY; ${ }^{2}$ SUNY Upstate Medical University, Syracuse, NY. (Control ID \#2462669)

LEARNING OBJECTIVE \#1: To identify potentially disastrous aortic pathology which may be missed as an underlying diagnosis of pneumonia

LEARNING OBJECTIVE \#2: To identify and manage anaerobic bacteremia CASE: 59 year old male with history of hypertension, who presented with substernal chest pain, vomiting, chills and one episode of hemoptysis. Patient was admitted a week ago for similar chest pain and stress ECHO was negative for ischemia. He had presented to the Emergency room 4 days ago, was diagnosed with pneumonia and was discharged home on oral levofloxacin. The new onset of hemoptysis and the persistent symptoms prompted him to come to the Emergency room again. He was a smoker and had 10 pack year smoking history, consumed alcohol every day and used marijuana. He was tachycardic, otherwise hemodynamically stable and was saturating well on room air. Lung exam revealed decreased breath sounds in the left anterior lung. He was not noted to have any further episodes of hemoptysis after the initial episode. Labs revealed hemoglobin of $9.6 \mathrm{~g} / \mathrm{dl}$, which was lower than his baseline of around $12 \mathrm{~g} / \mathrm{dl}$, leukocytosis of $18.5 \times 10^{3} / \mathrm{mm}^{3}$ and new onset acute kidney injury with blood urea nitrogen of $53 \mathrm{mg}$ / $\mathrm{dl}$ and creatinine of $4.92 \mathrm{mg} / \mathrm{dl}$. Chest X-ray done showed interval increase in mass like border of the upper mediastinum compared to the previous X-ray, suspicious of aortic aneurysm. CT chest without contrast was done and showed a cavitating left upper lobe mass in contact with the aortic arch, likely necrotizing pneumonia, however limited exam without contrast. Patient was diagnosed with severe sepsis secondary to necrotizing pneumonia, blood cultures were drawn and he was started on intravenous vancomycin and piperacillin/tazobactam. With the concern for aortic involvement, CT angiogram of thorax was done despite the risk with contrast and showed inflammatory or infectious process involving the proximal ascending aorta, with a saccular aneurysm, indicating a contained rupture of the thoracic aorta with surrounding necrotic lung tissue with gas formation. Vascular surgery consult was done and he underwent emergency endovascular stent graft in thoracic aorta. Blood cultures were later found to grow Fusobacterium mortiferum. Infectious diseases consult was done and antibiotic was switched to ampicillin/sulbactam. Patient remained hemodynamically stable and his renal function slowly recovered to baseline. Repeat blood cultures were negative. Plan is to discharge the patient on a total of 6 weeks of intravenous ertapenem from negative cultures and chronic suppression with penicillin as the stent was inserted when the blood cultures were positive.

DISCUSSION: This rare case describes a possible mycotic aneurysm with contained aortic rupture, which initially presented as necrotizing pneumonia. This has not been described in any recent case reports and demonstrates the importance of ruling out an aortic pathology which could be disastrous if missed. The source of infection was presumed to be the oral cavity, given his poor dental hygiene and the risk of aspiration given his alcohol use. Anaerobic bacteremia is also rare and Fusobacterium accounts for $0.9 \%$ of patients with bacteremia.

JAMAICAN STONE: IS THE STRENGTH WORTH THE RISK? Pooja Agrawal; Ana I. Velazquez; Heather Siedenburg; Jennifer Park; Tamara Brodsky; Jason Rubinov; Erica Bang. Mount Sinai Beth Israel, New York, NY. (Control ID \#2467904)

LEARNING OBJECTIVE \#1: Recognize the clinical course of a potentially lethal, nonFDA approved product so as to facilitate timely management of its toxicity.

CASE: A 49 year old male presented with sudden onset nausea, non-bloody, non-bilious vomiting, periumbilical abdominal pain, and tongue numbness two hours after ingesting a one centimeter piece of "Jamaican Stone." He denied chest pain, palpitations, dyspnea, headaches, or fevers. He was not taking any medications. Vital signs were remarkable for a heart rate of $27-40$ beats per minute, but blood pressure was stable. Physical exam was remarkable for $8 \mathrm{~mm}$ dilated pupils bilaterally, bradycardia, hypoactive bowel sounds, and preserved mental status. Laboratory values were significant for potassium $6.1 \mathrm{mmol} / \mathrm{L}$ and creatinine $1.40 \mathrm{mg} / \mathrm{dL}$. Complete blood count, liver function tests, troponin levels, coagulation studies, and urine toxicology were all normal. An initial electrocardiogram showed sinus bradycardia with inverted $\mathrm{T}$ waves in the precordial leads. Given these findings, the patient was admitted to the medical intensive care unit for life-threatening Jamaican Stone toxicity. Following treatment with three doses of intravenous digoxinspecific antibody fragments, the patient's hyperkalemia and bradycardia resolved. Activated charcoal was initiated for possible delayed-release bezoar effects. The patient remained asymptomatic for 2 days and was discharged home on hospital day four with outpatient follow-up.

DISCUSSION: Jamaican stone, otherwise marketed as Black Stone, Love Stone, or Piedra among others, is an illegal topical aphrodisiac used to prevent premature ejaculation. Derived from West Indian tree sap and dried toad venom, Jamaican stone's active ingredients are termed bufadienolides. These naturally occurring cardioactive steroids 
have a surprisingly similar chemical structure to digoxin. As such, these toxic substances inhibit the sodium-potassium ATPase and increase calcium influx into cells, mimicking the classic symptoms of digoxin poisoning in the nervous, cardiac, and gastrointestinal systems. Given that the Food and Drug Administration placed a ban on import of Jamaican Stone following four reported fatalities between 1993 and 1995 in the United States, encounters with this substance are now rare, limiting clinical knowledge. Interestingly, gas chromatography mass spectrometry (GC/MS) of obtained samples has confirmed the absence of pure digoxin substrate in Jamaican Stone, but does demonstrate digoxin immunoassay cross-reactivity. Thus, digoxin levels are expected to be elevated on laboratory testing. Research has also shown digoxin-specific antibody fragments to be effective in treating overdose. Per the Poison Control Center's recommendations, if either the ingested amount of digoxin-like substance or the digoxin laboratory level is unknown, empiric treatment with 10 vials of digoxin-specific antibody fragments, equating to $400 \mathrm{mg}$, should be initiated. Given toxicity effects often last 2 to 3 days, doses may be repeated until heart rate stabilizes to greater than 40 beats per minute and potassium levels stabilize to less than $5.5 \mathrm{mmol} / \mathrm{L}$.

JAUNDICE, PRURITUS, AND A 'NAGGing FEeling': A CASE OF KLATSKIN TUMOR Charles Frost ${ }^{2}$; Navya Nambudiri ${ }^{1}$; Andrew Pearson ${ }^{2}$; Andrew F. Glover ${ }^{2}$; Dipali R. Sahoo ${ }^{2} .{ }^{1}$ Cochin Medical College, Myrtle Beach, SC; ${ }^{2}$ Grand Strand Regional Medical Center, Myrtle Beach, SC. (Control ID \#2467759)

LEARNING OBJECTIVE \#1: Recognize the clinical presentation of jaundice and abdominal pain as worrisome for potential biliary tract cancer.

LEARNING OBJECTIVE \#2: Understand the features of Klatskin tumors and their management.

CASE: A 55 year old African American male with a history of hypertension, type 2 diabetes, and bipolar disorder presented for evaluation with 1 week of worsening abdominal pain, dark urine, and pruritus. The abdominal pain was diffuse and described as a "nagging feeling". It had been present for 1 week, and acutely worsened 2 days prior to presentation. Over-the-counter antacids and bismuth had not relieved the pain. Additionally, he noted the presence of dark urine and pruritus that had begun 4 days prior to presentation. He endorsed fatigue and decreased appetite, but denied any associated nausea, vomiting, diarrhea, constipation, chest pain, fever, recent weight loss or other symptoms. His medications on admission included lisinopril, hydrochlorothiazide, metformin, haloperidol, and benztropine. He had no known drug allergies. He did not smoke or use illicit drugs; he consumed 2 alcoholic beverages per week. He worked as a cook at a local restaurant. He had no recent travel. His family history included a brother who suffered a stroke at age 64 and another brother who had passed away from pancreatic cancer at age 53 . On presentation, he appeared comfortable with vital signs within normal limits. His examination was notable for scleral icterus, and abdominal pain with tenderness to palpation. Labs on presentation revealed an elevated total bilirubin of $8.7 \mathrm{mg} / \mathrm{dL}$, elevated alanine aminotransferase of $111 \mathrm{U} / \mathrm{L}$, elevated aspartate aminotransferase of $95 \mathrm{U} / \mathrm{L}$, and elevated alkaline phosphatase of $201 \mathrm{U} / \mathrm{L}$. An abdominal ultrasound was performed on admission, which demonstrated gallbladder wall thickening up to $9 \mathrm{~mm}$ and a dilated common bile duct. Subsequently, magnetic resonance cholangiopancreatography (MRCP) was performed and showed intrahepatic duct dilatation with a focal bile duct defect and a caliber change at the level of the porta hepatis, suspicious for a stricture, mass, or intraluminal obstruction. To follow up on this defect, the patient underwent endoscopic retrograde cholangiopancreatography (ERCP) which showed pancreas divisum, as well as marked tortuosity and firmness in the region of the ampulla of Vater, suggestive of a mass effect. An abdominal CT confirmed intrahepatic biliary dilatation with the level of obstruction just below the confluence of the bile ducts, concerning for a Klatskin tumor. No masses within the pancreas or liver were noted. The patient declined placement of a percutaneous biliary drain. He was referred to the hepatobiliary surgeon for consideration of operative management

DISCUSSION: "Klatskin tumors" are cholangiocarcinomas arising at the bifurcation of the common hepatic duct into the right and left hepatic ducts. They are a rare subtype of gastrointestinal malignancy and carry a relatively poor prognosis. Cholangiocarcinomas are rare malignancies arising from the cells of the intrahepatic, perihilar, or extrahepatic portions of the biliary tract that account for fewer than $3 \%$ of all gastrointestinal malignancies. Individuals with these tumors generally develop symptoms secondary to obstruction of the biliary tract. There is an increased risk in individuals with primary sclerosing cholangitis. The most common symptoms of cholangiocarcinoma are abdominal pain, weight loss, fevers, malaise, and pruritus. The obstruction of the biliary tract results in jaundice in the vast majority of affected individuals, and will also result in dark colored urine, light colored stools, and icterus as seen in our patient. The typical laboratory abnormalities that are seen with Klatskin tumors are a result of blockage of the biliary system as well. Most patients present with elevations in their bilirubin, alkaline phosphatase, and liver transaminases, as was the case in our patient. The clinical picture is primarily cholestatic, but hepatocellular injury can result from longstanding biliary obstruction in these patients. Additionally, serum tumor markers such as CA-19-9 and CEA may be elevated in patients with cholangiocarcinoma. Imaging tests are helpful in evaluating individuals with a suspected cholangiocarcinoma and localizing the lesions. Abdominal ultrasound is often obtained in jaundiced individuals but is not specific enough to make the diagnosis. MRCP, ERCP, and CT can all be used in further imaging of the biliary system. Imaging findings suggestive of a perihilar cholangiocarcinoma such as a Klatskin tumor include dilatation of the intrahepatic bile ducts with normal extrahepatic ducts as seen in our patient. The definitive diagnosis of Klatskin tumors and all forms of cholangiocarcinoma is based on histopathology, which may be obtained via brushings of the bile tract, fine needle aspiration, biopsy, or at the time of surgical resection of the mass. Surgery is the definitive treatment for cholangiocarcinomas that have not metastasized or invaded local structures. However, many such tumors present with lymph node or metastatic spread at the time of diagnosis. Adjuvant therapy with radiation and chemotherapy is often given. For individuals with non-surgically removable disease, radiation, chemotherapy, hepatic embolization, and liver transplant have been tried. The prognosis of all cholangiocarcinomas is poor, with a 5 year survival of $15-20 \%$.

KEEP AN "EYE OF THE TIGER" OR BETTER, AN "EYE ON THE SINUS"? Hiroko Taguchi; Shadia Constantine. Teine Keijinkai Hospital, Sapporo, Japan. (Control ID \#2468146)

LEARNING OBJECTIVE \#1: Include cavernous sinus thrombosis in your differential diagnosis for sinusitis with eye complaints.

LEARNING OBJECTIVE \#2: Is a "delay in diagnosis" a diagnostic error? Describe the main finding of the recent Institute of Medicine Report on Diagnostic Error.

CASE: 60-year-old Japanese previously healthy woman complains of 1-week history of productive cough, gradual onset of fever, left-sided headache, and facial pain. She did not have significant past medical history and this was the first time she experienced these symptoms. She did not use oral contraceptives, she did not smoked and there was no family history of thrombosis. The examination revealed normal vital signs, one left cervical lymphadenopathy, left ptosis, but no facial palsy. A contrast-enhanced computed tomography (CT) scan showed fluid in the left maxillary sinus with ring enhancement. She was initially diagnosed with acute sinusitis. However, a few days later, her blood cultures became positive for Streptococcus intermedius. She was admitted and treated with ampicillin-sulbactam. After the admission, she complained of double vision; however, her external ocular movements were reported as intact so no further workup was done at the time. However, the neurological compromise was fully evident 3 days later and it was 
characterized by ptosis (left oculomotor nerve paralysis,) double vision (left abducens nerve paralysis,) and deviation of the tongue (paralysis of the left hypoglossal nerve.) A contrast-enhanced CT scan of the head showed bilateral thrombosis of the internal jugular veins and thrombosis of the cavernous sinus. The overall clinical course suggested septic thrombosis of the cavernous sinus and internal jugular veins secondary to maxillary sinusitis. Anticoagulation was added to the management. She successfully completed treatment and at her 6-month follow-up, the radiological and clinical examinations had significantly improved.

DISCUSSION: In the old days, when there were not enough antibacterial drugs, this diagnosis certainly would have been fatal. Today, septic thrombosis of the cavernous sinus is rare, and risk factors include: trauma or fractures, surgery, oral contraceptives and hormone replacement therapy, pregnancy and puerperium, hypercoagulability, previous venous thromboembolism, age, bed rest ( $>3$ days), prolonged travel, metabolic syndrome and air pollution. Early diagnosis is crucial to decrease morbidity and mortality; however, it requires a high level of suspicion. In our patient, the complaint of double vision should have prompted imaging earlier than the 3 days it took. In retrospect, we believe this delay can be categorized as a diagnostic error. This year, the Institute of Medicine released the Report on Diagnostic Error and they define diagnostic error as "the failure to (a) establish an accurate and timely explanation of the patient's health problem(s) or (b) communicate that explanation to the patient." This case illustrates an example in which the diagnosis was neither accurate nor timely.

KLEBSIELLA OXYTOCA (KO) ENDOCARDITIS PRESENTING WITH COMPLETE HEART BLOCK $\underline{\mathrm{Saad} \mathrm{Ullah}^{2}}{ }^{2}$ Omar Elbita ${ }^{2}$; Puneet Tuli ${ }^{2}$; Joshan Suri ${ }^{1} .{ }^{1}$ Conemaugh Memorial Medical Center, Jeannette, PA; ${ }^{2}$ Conemaugh Valley Memorial Hospital, Johnstown, PA. (Control ID \#2467738)

LEARNING OBJECTIVE \#1: A rare cause of Infective endocarditis presenting as variable degree of conduction abnormalities requiring transvenous pacing.

CASE: A 76 years old caucasian male was admitted with a right middle cerebral artery ischemic stroke. The patient had a dense left hemiplegia with respiratory failure that required a tracheostomy on the 12th day of his admission. On his 14th hospital day, he developed symptoms and signs of septic shock with fever of $39^{\circ}$ celcius and hypotension. Cultures were obtained and empiric antibiotics were started for a suspicion of Ventilator Associated Pneumonia. In addition, he also underwent percutaneous gall bladder (GB) drainage and an ERCP based on an abdominal CT finding of a dilated common bile duct, concerns of cholecystitis and a clinical suspicion of cholangitis. His two blood cultures grew KO the same day. Antibiotics were tailored to Ceftriaxone based on the sensitivity results. None of his following blood cultures grew any organism. However, he continued to require vasopressors until a new onset intermittent complete heart block developed on the 22nd day. On physical examination, the patient was afebrile while on minimal ventilator settings. His blood pressure was $100 / 40 \mathrm{mmHg}$ on a low dose of norepinephrine. The cardiac monitor showed sinus rhythm most of the time. However, intermittent periods of variable atrioventricular blocks ranging from first degree to complete heart block with few occasions when the patient developed pauses up to $10 \mathrm{~s}$. An emergent temporary transvenous pacemaker was inserted. Repeat echocardiography showed aortic incompetence as compared to a 2nd day echocardiography, with a large vegetation measuring $1.7 \mathrm{~cm}$ in length and $0.6 \mathrm{~cm}$ in width. A diagnosis of Infective Endocarditis (IE) was confirmed at that point. The patient was transferred to a tertiary center for cardiac surgery evaluation. He succumbed to his illness 3 days later because of septic shock. The diagnosis of Klebsiella Oxytoca Infective Endocarditis was based on the two positive blood cultures and the presence of the valvular vegetation.

DISCUSSION: Klebsiella species account for $1.2 \%$ of gram negative native valve endocarditis and $4.1 \%$ of prosthetic valve endocarditis and is associated with a higher rate of complications and mortality than other gram-negative endocarditis. The organism is under the non-HACEK group of gram negative bacteria. The two most common pathogens in the non-HACEK group are e-coli and pseudomonas. Klebsiella species, which comes third, only accounted for $10 \%$ of cases. Gram negative bacteremia is widely seen in the inpatient setting. However, it rarely infects native heart valves owing to less adherent potential to the endocardium in contrast to higher virulent organisms such as staphylococci. This patient has the host risk factors for developing gram negative IE including age, severe GI infection, prolonged hospital stay, and the gram negative bacteremia. KO bacteremia is commonly seen in the setting of infections of the hepatobiliary tract, urinary tract, an indwelling urinary catheter, skin and soft tissue and the peritoneum. One study that looked at KO bacteremia records in one community hospital over 16 years found that in more than half of the cases biliary tract infection was the source. However, none of these cases were screened for IE. Therefore, we believe that the port of entry in this patient was the cholangitis/cholecystitis episode. Diagnosis of gram negative IE in our patient was based on one major (oscillating intracardiac mass on the aortic valve) and three minor (predisposing aortic valvular regurgitation, fever $>38.0$ $\mathrm{C}$, and two positive KO blood cultures) modified Duke's criteria for infective endocarditis. To our knowledge, this is the second reported case of $\mathrm{KO}$ endocarditis in an adult that completely meets the modified Duke criteria after J.-Y. Chen et al. reported the first one in 2005. Our patient has an interval development of a severe AV incompetence with a large vegetation on TTE that accompanied the new intermittent heart block. Based on these findings, we believe that he had a peri-valvular abscess that affected the conduction system of his heart. The pathogenesis is postulated to be from the direct spread from of the infected valve ring to the conduction system or micro emboli through coronary arteries. TEE was the next step to the diagnosis. However he was transferred to a tertiary care hospital for surgical intervention and succumbed to his sepsis 3 days later. The multiple co-morbidities, the type of organism involved and the presence of the alternative source may lead to the delay in diagnosis to the point that the infection affected the conductive system of the heart. The main potential clue for IE in our case was the variable cardiac conduction defects. Persistent gram negative bacteremia should always raise the suspicion of endocarditis when the primary source is controlled. None of the following blood cultures were positive in this patient. We treated him according to the sensitivity results. His sepsis continued to progress which indicates that the organism may have developed resistance. According to American Heart Association Guidelines, the recommended antibiotic treatment for gram negative hospital acquired IE is a combination therapy because these organisms rapidly develop resistance.

\section{KLEBSIELLA PNEUMONIAE HEPATIC ABSCESS IN A WOMAN FROM} CHINA Sumona Bhattacharya ${ }^{1}$; Gabriela Ferreira ${ }^{3}$; Ali Abbas Saifuddin ${ }^{2} .{ }^{1}$ Robert Wood Johnson University Hospital, Plainsboro, NJ; ${ }^{2}$ Rutgers, Robert Wood Johnson Medical School, Kendall Park, NJ; ${ }^{3}$ Rutgers-Robert Wood Johnson Medical School, New Brunswick, NJ. (Control ID \#2470002)

LEARNING OBJECTIVE \#1: To learn the different microbiology of a hepatic abscess. CASE: We introduce the case of a 68 year-old Chinese woman visiting the United States who presented with 2 weeks of fevers to $104 \mathrm{~F}$, dyspnea, nausea, and vomiting. Her temperature was $102.4 \mathrm{~F}$, pulse 117 , blood pressure $133 / 89$, respiratory rate 18 and shallow, and oxygen saturation $90 \%$ breathing room air. She was ill-appearing and looked dehydrated, with scleral icterus, clear lungs, and a distended and tender upper abdomen. Her white blood cell count was 12.6, alkaline phosphatase 424, total bilirubin 3.4, direct bilirubin 2.7, and lactate 5.4. An abdominal CT revealed a $10 \mathrm{~cm}$ unilocular abscess with an adjacent $8 \mathrm{~cm}$ multilocular abscess. Blood cultures and amebic serology were drawn 
and we empirically started piperacillin-tazobactam and metronidazole to provide coverage for enteric flora and Entamoeba histolytica, respectively. A percutaneous drain inserted into the larger abscess released $200 \mathrm{~mL}$ of purulent bloody fluid. This fluid culture revealed pan-sensitive Klebsiella pneumoniae, and, as the amebic serology was subsequently negative, metronidazole was discontinued and piperacillin-tazobactam was replaced with ceftriaxone. The patient improved clinically and defervesced. A repeat abdominal CT at 2 weeks showed near-complete resolution of the larger collection and a notable reduction of the smaller collection. The patient was discharged home to complete 3 weeks of intravenous ceftriaxone. A final abdominal CT at 4 weeks showed almost complete resolution of both abscesses.

DISCUSSION: Hepatic abscesses are categorized as either amebic or pyogenic in origin. Amebic abscesses, which are caused by Entamoeba histolytica, are more common in developing countries. Pyogenic abscesses, which have a mortality rate of up to $30 \%$, are more common in developed countries and are typically polymicrobial, composed of gut flora such as Escherichia coli, Enterobacter cloacae, and Bacteroides species. Recently, mono-microbial Klebsiella pneumoniae liver abscesses, which are frequent in Asia, are becoming more common in the United States. It is important to be aware of the microbiology of liver abscesses because Klebsiella abscesses may develop extra-hepatic ocular and nervous system involvement. Over the past few decades, pyogenic liver abscesses have increased in incidence from 13 to 20 per 100,000 hospitalizations, while at the same time overall mortality has decreased. The microbiology of these abscesses has also shifted: the incidence of E. coli has decreased while the incidence of Klebsiella, Staphylococcus, Streptococcus, and Pseudomonas have increased. While in the Western hemisphere E. coli is the most common pathogen, in the Eastern hemisphere Klebsiella is recognized as very common. In fact, it is the most common pathogen in Hong Kong, Taiwan, Singapore, and South Korea. We choose to highlight this case as an example of the atypical microbiology that can present as the cause of pyogenic hepatic abscesses in the United States

KLIPPEL-FEIL SYNDROME: AN UNUSUAL CASE OF NECK PAIN Thwe Htay $^{2}$; Bryan S. Brockman ${ }^{1}$; Cristin Harper ${ }^{1}$; Michelle V. Conde ${ }^{1}$; KoKo Aung ${ }^{2}$. ${ }^{1}$ UTHSCSA, San Antonio, TX; ${ }^{2}$ University of Texas Health Science Center at San Antonio, Shavano Park, TX. (Control ID \#2470188)

LEARNING OBJECTIVE \#1: To recognize the clinical features and radiological findings in Klippel-Feil syndrome

LEARNING OBJECTIVE \#2: To heighten sense of awareness of childhood disorders in adults

CASE: Introduction: Klippel-Feil syndrome describes a heterogeneous group of patients unified by the presence of congenital defects in the formation or segmentation of the cervical spine. Diagnosis predominates in the pediatric population but does occur infrequently in adults. Once cervical spinal defects consistent with Klippel-Feil are identified, a thorough head-to-toe work-up must be conducted to evaluate for the numerous and variable anomalies associated with this syndrome. Case Report: A 40 year-old male presents to the clinic with worsening midline neck pain. Symptoms have been progressing for 7 years and now include left temporal headache with sharp shooting pain and photophobia. He also reports left upper extremity weakness and numbness which intermittently involves his hand, the lateral aspect of his forearm, arm and shoulder. He denies any bowel or bladder incontinence but does endorse a history of chronic lower back pain with radiation down to the right lateral thigh and knee and difficulty ambulating. Physical Exam: Physical exam is remarkable for short neck and restricted range of flexion and extension of cervical spine. Motor strength in right upper extremity and bilateral lower extremities are $5 / 5$ throughout. Motor strength in left upper extremity is $4 / 5$ throughout with intact sensation. Deep tendon reflexes are diminished in bilateral lower extremities. Imaging: Imaging reveals moderate S-shaped scoliotic curvature of the cervicothoracic spine. There is partial fusion of the skull base, $\mathrm{C} 1, \mathrm{C} 2$ and $\mathrm{C} 3$. C4 demonstrates a right hemivertebra. Congenital fusion of $\mathrm{C} 6, \mathrm{C} 7$ and $\mathrm{T} 1$ is seen. $\mathrm{T} 1$ also demonstrates a hemivertebra. $\mathrm{C} 3 / \mathrm{C} 4$ shows severe central canal stenosis with moderate right/severe left neuroforaminal narrowing. A cervical rib is also present. Clinical course: Patient is scheduled to undergo a cervical 3-4 decompressive laminectomy due to severe spinal canal stenosis.

DISCUSSION: Klippel-Feil syndrome is a rare condition estimated to occur in approximately 1 in 40,000-42,000 births. Mutations in the GDF6, GDF3, or MEOX1 gene, which are involved in bone development, can cause Klippel-Feil syndrome. The defining feature of this condition is the congenital fusion of 2 or more cervical vertebrae, however patients can present with a wide range of other anatomic anomalies. The most common anomalies in decreasing order of incidence are congenital scoliosis, rib abnormalities, deafness, genitourinary abnormalities, Sprengel's deformity, synkinesia, cervical ribs and cardiovascular abnormalities. Treatment for this condition is guided by symptoms. Some patients will be asymptomatic and if discovered incidentally and cervical fusion are stable, no treatment may be needed. However, if patients complain of neurologic symptoms, decompression to relieve spinal stenosis, correction of scoliosis and stabilization of unstable fusions through procedures such as occipitocervical arthropodesis may be warranted. Conclusion: Klippel-Feil syndrome is typically diagnosed in the pediatric population, however, primary care physicians should maintain a high suspicion in adult patients presenting with a short neck, decrease range of motion of neck extension and or flexion, in the presence of neck pain and neurologic symptoms. Prompt identification and intervention may minimize potential for more serious and permanent neurologic injury and improve patient quality of life.

LAMBERT EATON MYASTHENIC SYNDROME ASSOCIATED WITH SMALL CELL LUNG CANCER-IS IT GOOD TO FEEL WEAK? Lindita Shehu $^{1}$; Arian Majko ${ }^{1}$; Majlinda Xhikola ${ }^{1}$; Emily Chen ${ }^{1}$; Izabella Zathureczky ${ }^{2}$. ${ }^{1}$ Capital Health Regional Medical Center, Trenton, NJ; ${ }^{2}$ Capital Health Regional Medical Center, Lawrencewille, NJ. (Control ID \#2466151)

LEARNING OBJECTIVE \#1: Recognize myasthenic syndromes associated with small cell lung cancer and their prognostic significance.

CASE: A 55 year old male with extensive tobacco history presented with 1 month of progressive bilateral upper extremity proximal weakness, cough, hemoptysis, pleuritic chest pain, shortness of breath and 1 week of progressive face and neck swelling. Vital signs included blood pressure 131/ $83 \mathrm{mmHg}$; heart rate $108 \mathrm{bpm}$; respiration rate 25/min; temperature $97.9 \mathrm{~F}$; oxygen saturation $97 \%$ on ambient air. Physical examination was significant for symmetrical face, neck and upper extremity swelling, jugular venous distention, chest wall tenderness, decreased breath sounds on the right and 1+ deep tendon reflexes. Laboratory data was unremarkable. Chest x-ray showed a large heterogenous mass in the right upper lobe extending to the right hilum and paratracheal space. CT chest showed a paratracheal, retrocaval mass measuring $7.6 \times 7.2 \mathrm{~cm}$ in the right mediastinum and SVC obstruction. The patient underwent endovascular stenting of SVC and thrombolysis with rapid relief of symptoms. Tissue from transbronchial biopsy was inadequate and nondiagnostic. A thoracentesis drained 21 of turbid fluid, and pleural fluid cytology was consistent with SCLC. Because of proximal upper extremity weakness, serum for antibodies directed against VGCC was sent to a reference laboratory and later returned positive. Patient received multiple cycles of Cisplatin and etoposide chemotherapy and radiation therapy. The initial lung mass was significantly reduced and at one year of diagnosis, is resolved. Patient gradually regained his weight, fatigue improved and proximal limb weakness resolved. Patient continues to receive chemotherapy and anticoagulation.

DISCUSSION: Clinically, LEMS patients suffer from characteristic proximal muscle weakness that is caused by the presence of antibodies directed against their voltage-gated 
calcium channels (VGCC). These channels are localized in the presynaptic membrane of their motor nerve terminals. Binding of autoimmune antibodies to the VGCCs leads to reduced neuromuscular transmission. Typically, muscle weakness is in the lower extremities, however can be observed in the upper or all extremities. Atypical presentations should not preclude testing. Better understanding of the pathophysiological mechanisms of LEMS has helped with the development of new diagnostic approaches and has led to targeted symptomatic and immunosuppressive therapy. For LEMS patients with an underlying malignancy, specific tumor therapy is the first choice. Patients with SCLC who develop LEMS have been suggested to have a more effective immunologic response to the cancer, which may result in improved survival.

\section{LANCE-ADAMS SYNDROME: THE CONTROVERSY OF TREATING POST-} HYPOXIC MYOCLONUS IN A DELIRIOUS PATIENT Katie Kaput ${ }^{1}$; Robert M. Garcia $^{2} .{ }^{1}$ Exempla Saint Joseph Hospital, Denver, CO; ${ }^{2}$ University of Colorado, Denver, CO. (Control ID \#2465550)

LEARNING OBJECTIVE \#1: Utilizing an individualized approach including a combination of clinical judgment, risk-benefit analysis and compassion when treating LanceAdams Syndrome (a.k.a post-hypoxic myoclonus) in the setting of delirium.

CASE: Introduction: Lance-Adams syndrome (LAS) is characterized by generalized myoclonus with onset days to weeks after anoxic brain injury. It generally has a better prognosis than its more acute counterpart, myoclonic status epilepticus. Treatment typically includes levetiracetam, benzodiazepines or valproic acid. We examine a case of LAS in a patient with prolonged hospitalization already complicated by impaired cognition and episodic delirium. Despite concerns that treatment with benzodiazepines might worsen the cognitive disorder, we concluded that the LAS was potentially contributing to the delirium via secondary mechanisms of pain and sleep disturbances. After careful clinical consideration, we initiated a trial of clonazepam which resulted in marked symptomatic improvement. Case Report: A 65-year-old male with a recent prolonged ICU hospitalization for necrotizing pancreatitis, complicated by a cardiopulmonary arrest requiring ACLS intervention and approximately eight minutes of CPR, presented for volume overload. On physical exam, he was noted to have anasarca, cognitive impairment with SLUMS 21/30 and quick, irregular, involuntary myoclonic jerks with symmetrical hyperreflexia. Per the patient's wife, he began to experience the jerk-like movements approximately 2 weeks after his hospital stay. At first the symptoms were mild, but then they became more frequent and occurred during sleep. They also caused the patient significant distress due to the inability to eat and tongue pain due to recurrent bite wounds. An EEG showed diffuse slowing, but no epileptiform activity. His medication list was reviewed and metabolic disturbances were corrected, but the myoclonus persisted. Based on clinical, imaging and physical exam findings, his myoclonic jerks were felt to be due to multifocal cortical irritability following post anoxic brain injury. After numerous discussions of the risk and benefits of treatment, he and his wife agreed to a trial of clonazepam $(0.25 \mathrm{mg}$ TID). Three days later his symptoms improved without further impairment in his cognition.

DISCUSSION: As detailed in the case above, the persistent nature of LAS can cause significant distress via complications such as tongue-biting, sleep disorder and muscle exhaustion. However, there remains a paucity of randomized controlled trial data to define the proper treatment of this condition. Typical treatment with either levetiracetam, benzodiazepines or valproic acid is most commonly used. Doses are started low and titrated upward for symptom relief. Due to the possible side effects of these treatments, this can become extremely challenging for patients with concomitant LAS and delirium. When faced with this clinical situation, treatment must be weighed against the possibility of exacerbating a cognitive deficit, particularly one that could prove dangerous in the hospital setting. However, the risk of undertreatment of the condition is not small and may prove more bothersome to the patient than is realized. In this case, the myoclonic jerks were preventing restful sleep, impairing the patient's PO intake and the tongue-biting was constant enough to cause excessive pain and discomfort which was likely contributing to his cognitive dysfunction. Using an individualized approach including a combination of clinical judgment, risk-benefit analysis, and compassion we suspected that these secondary complications of LAS were likely contributing to risk factors for delirium and that the benefits of the proposed treatments would outweigh the risks. Thus, a trial of clonazepam was deemed appropriate and resulted in a desirable clinical outcome. Conclusion: Treatment of LAS may be clinically challenging in the face of worsening delirium, especially in elderly patients who have experienced a prolonged hospitalization and previous signs of cognitive impairment. This complicated clinical picture can be navigated by careful investigation into the direct causes and potentiators of confusion in the hospital setting which in our case was Lance-Adams syndrome.

LEAVE NO STONES UNTURNED Lisa Hirahara; Ryuichi Sada; Mitsuya Katayama. Kameda Medical Center, Kamogawa-shi,Chiba, Japan. (Control ID \#2468294)

LEARNING OBJECTIVE \#1: Close discussion with pathologist and provision of pertinent clinical information to pathologist improve the accuracy of pathologic diagnosis LEARNING OBJECTIVE \#2: Enteropathy associated T cell lymphoma (EATL) usually associated with celiac disease but EATL without celiac disease exist.

CASE: A 67-year-old man with past medical history of left urolithiasis presented to our hospital with chronic diarrhea and fatigue. He was well until one and a half years before admission, when nonbloody loose stools developed which were three times per day. Six months prior to the admission, the consistency of his stools became watery and the frequency increased to four to five times per day. He started having a 7 day history of mild abdominal cramping associated with fatigue. Review of systems revealed weight loss of $12 \mathrm{~kg}$ in the last 10 months. He had no fever, chills, oral ulcers, arthralgia, rash, night sweats, food intolerance, and travel history. He took no regular medications. On examination, he was afebrile, blood pressure was $103 / 70 \mathrm{mmHg}$, and pulse rate was 120 beats per minutes. Physical examination was unremarkable except for increased bowel sounds No peripheral lymph nodes was palpable. The laboratory data showed elevated inflammatory markers and renal failure of serum creatinine of 3.1 with elevated anion gap. Fecal leukocyte was positive. The fecal osmotic gap was normal of $6 \mathrm{mOsmol} / \mathrm{kg}$. A computed tomography of abdomen revealed diffuse thickening of the intestinal walls. He was kept nothing per oral since admission, however diarrhea was persistent. We evaluated these results were consistent with inflammatory or secretory diarrhea. Results of anti-neutrophil antibody, blood and stool cultures, and ova and parasites were all negative. A colonoscopy showed erosion of the ascending colon, multiple ulcers of the sigmoid colon and rectum which biopsies were done. Pathological findings of the biopsy revealed medium sized lymphocyte infiltration to the colon gland which was consistent with nonspecific colitis. The stains for amyloidosis and cytomegalovirus, and tuberculosis PCR were all negative. The patient also had esophagogastroduodenoscopy done which showed gastric ulcer and two depressed areas. Pathological findings of those biopsies presented the medium sized lymphocyte infiltration to the gastric gland which reportedly suggested Mucosa associated lymphoid tissue (MALT) lymphoma. The diagnosis of MALT lymphoma, however, could not explain his clinical presentation, and we thought both changes in his stomach and colon had the same etiology. Thereby, we provided pathologists the patient's clinical information and asked them to reassess of colon biopsies including histological staining for the work up of the lymphoma. Consequently, the histologic result showed natural killer $(\mathrm{NK}) / \mathrm{T}$ cell invasion of the stomach and the colon mucosa. The $\mathrm{T}$ cell receptor gene rearrangement are identical in both tissue, that is, clonal proliferation of $\mathrm{NK} / \mathrm{T}$ cell was found. Finally, the diagnosis of enteropathy associated T cell lymphoma (EATL) type 2 was made.

DISCUSSION: EATL is a rare primary gastrointestinal lymphoma which is known to be strongly related to celiac disease. The current WHO classification divided EATL into two 
subtypes based on the existence of celiac disease and the histology. Type 1 is a classic form of EATL with celiac disease and type 2, histological variant, is less common and often occurs without celiac disease. In Asia, type 1 is extremely rare because celiac disease is also uncommon. The early diagnosis of type 2 EATL is quite difficult because of the nonspecific symptoms and a very low index of clinical suspicion due to its low incidence. Therefore, type 2 EATL is often found in an advanced disease such as intestinal perforation, bowel obstruction or hemorrhage, and many EATL are diagnosed with the pathology findings from the surgical specimen. In our diagnostic management, there are two learning points. First, we should not exclude EATL just because the patient does not have celiac disease especially in Asia. Although the presentation of this case resembled to celiac disease, chronic diarrhea was occurred from EATL because symptoms were not improved while he was under gluten-free diet and anti-gliadin antibody was negative. Second, a close communication between clinician and pathologist is essential in the diagnostic management. EATL has no specific symptoms and he pathological findings guide us to the appropriate diagnosis. Type 2 EATL is characterized by monomorphic small-medium sized lymphocyte infiltration which is easily confused with benign lymphocyte infiltration or low-grade B cell lymphomas. Thus, the histologic examination of intestinal biopsies is crucial in the diagnostic workup of EATL. In this case, the histologic stains for colon biopsies were not performed because the initial pathology report showed a benign colitis. As the evaluation progressed, the likelihood of gastrointestinal lymphoma was increased which led us to discuss with pathologists again and could reach the final diagnosis. When the clinical suspicion of gastrointestinal lymphoma arises, it is crucial for clinicians to discuss closely with pathologists during evaluation of chronic diarrhea to make a diagnosis of this rare disease. In fact, some observational studies reported that the provision of pertinent clinical information to pathologist improve the accuracy of pathologic diagnosis.

\section{LEFT VENTRICULAR NON-COMPACTION - A DANGEROUS TRIAD OF} HEART FAILURE, ARRHYTHMIAS AND THROMBOEMBOLISM IN A YOUNG ADULT Joydeep Chakraborty ${ }^{2}$; Nimesh Patel ${ }^{2}$; Maryam Sattari ${ }^{1}$; Juan M. Aranda ${ }^{2} .{ }^{1}$ University of Florida College of Medicine, Gainesville, FL; ${ }^{2}$ University of Florida, Gainesville, FL. (Control ID \#2467926)

LEARNING OBJECTIVE \#1: Recognize Left Ventricular Non Compaction cardiomyopathy (LVNC) as an underdiagnosed cause of heart failure in young adults without preexisting risk factors.

LEARNING OBJECTIVE \#2: Manage complications of LVNC, identify the indications for anticoagulation in LVNC patients to prevent thromboembolic events and screen for silent arrhythmias.

CASE: An 18-year-old man with no known pre-existing medical problems presented to an outside hospital with 1 week of worsening non-productive cough and shortness of breath. He denied fever, chest pain, palpitation, leg swelling, viral prodrome or family history of heart disease, but endorsed marijuana use. Physical exam revealed tachycardia with normal systemic exam. Lab results revealed normal troponins, renal and thyroid function, leukocytosis, elevated BNP, and negative urine drug screen. Chest X-ray demonstrated bilateral interstitial infiltrates. Despite antibiotic coverage for possible community acquired pneumonia, the patient had worsening respiratory failure and eventually required intubation and mechanical ventilation. Echocardiogram showed left ventricular ejection fraction (LVEF) of 5-10\% and he was started on dobutamine infusion for cardiogenic shock and transferred to our medical center. At our facility, we added dopamine and intravenous diuretics to the dobutamine. He responded well and was weaned off inotropic support and extubated within $72 \mathrm{~h}$. Repeat echocardiogram demonstrated severely reduced left ventricular systolic function (LVEF $15 \%$ ) and prominent left ventricular trabeculae with deep intertrabecular recesses in the apical walls suggestive of LVNC. Cardiac MRI confirmed the diagnosis of LVNC. The patient was discharged on a medical regimen that contained an ACE inhibitor, beta blocker and oral diuretics. The patient missed his first follow-up appointment and in the subsequent clinic visit, reported an interval cerebrovascular accident without residual focal deficits. Subsequently, he has been placed on a Life vest, oral anticoagulation, and home dobutamine infusion for worsening heart failure. Currently he is undergoing evaluation for Implantable Cardioverter Defibrillator (ICD) placement and cardiac transplant.

DISCUSSION: LVNC can present as an isolated finding (as in our patient) or in association with neuromuscular or mitochondrial disorders or congenital heart defects. The pathology is thought to be due to arrest of normal left ventricular (LV) myocardial compaction during embryogenesis, which leads to a two layered ventricular wall with prominent trabeculations in LV cavity. The clinical presentation can vary from being asymptomatic to presenting with clinical features at any age. The Triad of heart failure (50\%), ventricular arrhythmia and sudden cardiac death (41\%) and systemic thromboembolic events (5-38\%) comprise the typical symptoms and complications of LVNC. Prognosis of LVNC varies widely. Presentation with heart failure or arrhythmia is associated with worse clinical prognosis compared to patients with incidental diagnosis or familial forms of LVNC. Various criteria for diagnosis of LVNC have been proposed. Echocardiography is the first line imaging modality of choice. The characteristic finding is "spongy myocardium," which refers to a bilayered myocardium composed of a thinner compact epicardial layer and an inner non-compacted endocardial layer with prominent trabeculations and deep recesses which are in continuity with the LV cavity. However, correct diagnosis can pose a challenge since prominent trabeculations can also be found in healthy subjects (athletes, transiently in pregnancy) and lead to over diagnosis. Cardiac MRI is considered the best modality for diagnostic confirmation and a diastolic noncompacted to compacted ratio $>2.3$ diagnoses pathologic LVNC with high specificity. Genetic testing (for mutation in various myocardial proteins like tafazzins, $\alpha$ dystrobrevin) is of limited utility for diagnosis due to genetic heterogeneity and lack of specific genotype-phenotype associations. The main treatment goals in LVNC are prevention and management of the triad of complications. Asymptomatic patients should be evaluated every 2-3 years with physical exam, echocardiography and Holter monitoring (to detect silent arrhythmias). Arrhythmia should be managed according to the current arrhythmia management guidelines. Treatment for heart failure in LVNC is similar to that of dilated cardiomyopathy and consists of pharmacologic therapy, ICD placement and evaluation for heart transplant. Routine anticoagulation remains controversial and merits consideration in patients with LVNC and atrial fibrillation, significant LV dysfunction, known ventricular thrombus or prior history of thromboembolic phenomenon. Decreased awareness about LVNC among physicians has presumably led to its underdiagnoses. This case highlights the importance of considering LVNC in young patients without classic risk factors presenting with heart failure. Holter monitoring every 2-3 years and anticoagulation in patients with significant LV systolic dysfunction merits special consideration in these patients to prevent thromboembolic events and subsequent morbidity and mortality.

LEFT-SIDED ENDOCARDITIS AS A COMPLICATION OF RIGHT ATRIAL CENTRAL VENOUS CATHETER-ASSOCIATED THROMBUS Kainat Khalid; Shira Sachs; Arunima Misra. Baylor College of Medicine, Houston, TX. (Control ID \#2466075)

LEARNING OBJECTIVE \#1: Recognize left-sided endocarditis as a rare complication after right atrial central venous catheter-associated thrombus

LEARNING OBJECTIVE \#2: Recognize that anticoagulation strategy should be individualized based on clinical scenario

CASE: A 49 year old male with history of HIV (CD4 544, on HAART), hemodialysisdependent end-stage renal disease (ESRD), untreated hepatitis $\mathrm{C}$ infection, controlled type II diabetes mellitus, and non-obstructive coronary artery disease presented with 2 months 
of watery, non-bloody diarrhea. He was admitted for similar symptoms 1 month prior with a negative infectious work up. At that time he was diagnosed with ESRD and was started on dialysis via a right internal jugular dialysis catheter. During this admission, he was diagnosed with line-associated methicillin-sensitive Staphylococcus aureus bacteremia, prompting removal of the dialysis catheter. Physical exam was unremarkable without stigmata of subacute endocarditis. Labs were significant for WBC of $15.8 \times 10^{9} / \mathrm{L}$ with $85 \%$ neutrophils and baseline BUN and creatinine of $73 \mathrm{mg} / \mathrm{dL}$ and $8.5 \mathrm{mg} / \mathrm{dL}$. A transthoracic echocardiogram (TTE) was performed and demonstrated a mobile, filamentous mass $(2.2 \times 0.8 \mathrm{~cm})$ on the atrial side of the posterior leaflet with mild mitral regurgitation and normal left ventricular systolic function (LVEF $60-65 \%$ ). The patient subsequently developed isolated left lower extremity weakness and brain MRA showed a recent small left posterior cerebral artery infarct, concerning for septic embolism. Transesophageal echocardiogram (TEE) revealed a large, mobile filamentous vegetation $(1.8 \times 1.9 \mathrm{~cm})$ on the posterior mitral valve leaflet with valve perforation at the base of the posterior leaflet. The TEE also showed a thrombus in the right atrium and superior vena cava, continuing to the right internal jugular. This was attributed to the prior right internal jugular HD catheter. The patient was treated with IV nafcillin for endocarditis. However, given the recent embolic stroke, a decision was made not to start the patient on anticoagulants for the thrombus. Additionally, based on the echocardiographic appearance, the thrombus had characteristics of fibrosis and thus was believed less likely to cause further embolic phenomena.

DISCUSSION: In patients requiring dialysis, central venous catheters are temporarily used until arterio-venous shunts mature. Hemodialysis catheters have been associated with risk for right atrial thrombus formation, particularly in the chemotherapy pediatric population. The mechanism is thought to be the catheter tip causing irritation of atrial walls. Although uncommon, it has also previously been suggested that catheter associated thrombi can become infected. However, left-sided endocarditis is a rare presentation for an infected right-sided central venous catheter-associated thrombus. In patients with hemodialysis catheters, it is important to have high clinical suspicion not only for line-associated thrombosis but also for infectious complications such as endocarditis. Literature suggests that TEE is superior to TTE for evaluation of right atrial thrombosis. Consideration should also be given to prophylactic anticoagulation for prevention of embolic complications from both the thrombus as well as the endocarditis vegetation. However, such therapy must be individualized based on the clinical situation and based on the risks and benefits to the patient

LEGIONNAIRE'S DISEASE TREATED WITH LEVOFLOXACIN AND TIGECYCLINE Johanna Hase; Kevin Hauck. New York University School of Medicine, New York, NY. (Control ID \#2467901)

LEARNING OBJECTIVE \#1: Diagnose Legionnaire's disease

LEARNING OBJECTIVE \#2: Compare antibiotic choices for Legionnaire's disease

CASE: The patient is an 84 year-old man who presented with 6 days of fevers, chills, and productive cough. He last felt well 7 days prior to presentation and did not endorse any sick contacts or recent travel. Associated symptoms included decreased appetite and two episodes of non-bloody diarrhea. Vital signs were notable for tachycardia, and a blood pressure of $86 / 46 \mathrm{mmHg}$. The patient was tachypneic, and hypoxic to an oxygen saturation of $93 \%$ on room air. On exam he was lethargic and had coarse rhonchorous breath sounds bilaterally. Initial laboratory tests revealed leukocytosis $(17,000 / \mathrm{mcL}$ with left shift), elevated creatinine ( $4.0 \mathrm{mg} / \mathrm{dL}$ ), as well as elevated transaminases (AST $78 \mathrm{U} / \mathrm{L}$, ALT $44 \mathrm{U} / \mathrm{L})$ and total bilirubin $(3.2 \mathrm{mg} / \mathrm{dL})$. Serum procalcitonin was $33 \mathrm{ng} / \mathrm{mL}$. Computed tomography of the chest showed dense consolidation of the right upper lobe, right middle lobe and posterior right lower lobe, with small bilateral pleural effusions. The patient was treated for severe community-acquired pneumonia and started on broadspectrum antibiotics with vancomycin, cefepime and azithromycin. On hospital day two, the urine legionella antigen returned as positive and the antibiotics were changed to high-dose levofloxacin mono-therapy for treatment of Legionnaire's disease. The patient continued to be intermittently febrile and hypoxic, requiring high flow nasal cannula. His leukocytosis worsened to $45,000 / \mathrm{mcL}$ and his procalcitonin increased to $43 \mathrm{ng} / \mathrm{mL}$. Given his overall worsening clinical status and no new identified source of infection, tigecycline was added to his antibiotic regimen. Hypoxia slowly improved, and leukocytosis and procalcitonin downtrended. He completed a total of 21 days of levofloxacin and 7 days of tigecycline, and was discharged to an acute rehabilitation facility, where he continued to improve.

DISCUSSION: Rapid diagnosis of Legionnaire's disease improves clinical outcomes and may lead to recognition or prevention of other cases. The gold standard for diagnosis is positive culture, but this requires selective media (buffered charcoal yeast extract plates) and prolonged incubation, which may delay diagnosis. A four-fold increase in serum antibody is another method for diagnosis, but can only be used to make the diagnosis retrospectively. The legionella urinary antigen test is a rapid, inexepensive, and widely used test, but only detects Legionella pneumophila serogroup 1 and has a sensitivity of $77 \%$. Polymerase chain reaction (PCR) is a new tool that can lead to rapid diagnosis of Legionella infection. Sputum PCR has a sensitivity of $94.5 \%$ and specificity of $99 \%$, but is expensive and requires dedicated laboratory equipment. PCR can be especially helpful in patients with severe pneumonia in which the urinary antigen test is negative but suspicion for Legionnaire's disease remains. First line therapy for Legionnaire's disease includes macrolides or high-dose quinolones. Legionella species are intracellular bacteria and are therefore unaffected by beta-lactam antibiotics. There have been no randomized, controlled studies comparing antimicrobials in the treatment of Legionnaire's disease but several small observational studies have shown that treatment with levofloxacin compared with macrolides had no mortality benefit but produced shorter time to defervescence, shorter time to stabilization, and shorter hospital stay. In vitro studies confirm quinolones have superior activity when compared to macrolides, but quinolones are concentrationdependent drugs, so correct dosing is important. Tigecycline is a second line option for treatment of Legionnaire's disease. Intracellularly, tigecycline is superior to both quinolones and macrolides in kill-time however there are only several case reports in the medical literature of patients being treated with tigecycline for Legionnaire's disease. Tigecycline is generally well tolerated with minimal drug interactions, and does not need to be dose-adjusted for renal impairment or mild to moderate hepatic impairment, making it an excellent option for mono-therapy or combination therapy for Legionella pneumonia. Internists should be aware of the different methods available for diagnosing Legionella pneumonia as well as their limitations. Swift diagnosis and appropriate treatment are crucial in improving clinical outcomes for patients with Legionnaire's disease.

LETHARGY AS THE SOLE PRESENTING SYMPTOM OF ACUTE CORONARY SYNDROME -AN OFTEN UNDERDIAGNOSED ENTITY LEADING TO INCREASED IN-HOSPITAL MORTALITY . Srinidhi Jayadevappa Meera ${ }^{1}$; Sofia Terner ${ }^{3}$; Safeera Javed ${ }^{2} .{ }^{1}$ Montefiore Medical Center, Mamaroneck, NY; ${ }^{2}$ Montefiore medical center, NYC, NY; ${ }^{3}$ Montefiore Medical Center, Bronx, NY. (Control ID \#2469387)

LEARNING OBJECTIVE \#1: Identify individuals with high risk for cardiovascular events who have non specific symptoms on presentation

LEARNING OBJECTIVE \#2: Early diagnosis and treatment is imperative to prevent catastrophic cardiac sequelae and sudden cardiac death

CASE: 57-year-old man with DM1 and cocaine and marijuana abuse presented with 1 day of generalized weakness. He denied chest pain, dyspnea, diaphoresis, or palpitations. Initial blood pressure was $81 / 49 \mathrm{mmHg}$, heart rate was 106 beats/min, respiratory rate was 16 breaths/min and oxygen saturation was $100 \%$ on room air. Physical examination was 
otherwise unremarkable. Initial EKG and troponins were normal. He had no ECHO or cardiac stress testing done in the past. Urine drug screen was negative. Labs on presentation were consistent with diabetic ketoacidosis (DKA), was treated with fluids and insulin drip with improvement in blood glucose and anion gap. However patient complained of persistent generalized weakness. Vitals and Repeat electrolytes were normal . A repeat 12 lead EKG revealed new deep T wave inversions in anterior leads and next set of troponin began to rise, reached a peak level of $1.48 \mathrm{ng} / \mathrm{mL}$. Diagnosis of non-ST elevation myocardial infarction (NSTEMI) was made and he received Clopidogrel, Aspirin, Atorvastatin and Heparin drip. Echocardiogram revealed severe mid-septal hypokinesis and mild apical hypokinesis with preserved ejection fraction. He underwent urgent coronary angiography, showing 70-80\% stenosis of the left anterior descending coronary artery for which drug eluting stent was placed

DISCUSSION: Acute coronary syndrome (ACS) presents without chest pain in a significant proportion of patients,particularly females, diabetics, and elderly and atypical symptoms are usually dyspnea, diaphoresis, nausea, vomiting, and syncope. ACS patients presenting without chest pain often have adverse outcomes secondary to misdiagnoses and under-treatment. These patients may have delays in seeking medical care, less aggressive therapies, and increased in-hospital mortality. Atypical presentation of acute and chronic ischemic heart disease in diabetic patients is under-investigated despite extensive research into coronary artery disease.. In Diabetics, Atherosclerotic plaques develop earlier, advance faster and are more diffuse .These factors contribute to a two to four-fold increased risk of cardiovascular events. Diabetic patients with silent myocardial ischemia have evidence of diffuse abnormality in metaiodobenzylguanidine (MIBG) uptake, suggesting that sympathetic denervation may be linked to abnormalities in pain perception. In a retrospective Multicenter study conducted in 2013, STEMI Patients presenting with chest pain were compared with patients presenting with non specific symptoms and outcomes were analysed . Compared with those who presented with chest pain, patients admitted for other reasons waited longer before going to the hospital (prehospital delay $<12 \mathrm{~h}: 32.0 \%$ vs $73.3 \% ; P<0.001$ ), presented with more severe clinical symptoms (Killip score $\geq$ III: $28.0 \%$ vs $10.5 \% ; P=0.001$ ), waited longer to be examined in the hospital (waiting time > $1 \mathrm{~h}: 36.0 \%$ vs $11.4 \% ; P<0.001$ ), were less likely to receive reperfusion therapy ( $40.7 \%$ vs $77.1 \% ; P<0.001)$ and had a higher mortality rate at 1 month $(42.7 \%$ vs $21.0 \%$; $P<0.001)$. This case demonstrates that ACS can present with atypical and nonspecific symptoms in diabetic patients and clinicians must have a high degree of suspicion to accurately diagnose and provide urgent treatment to improve outcomes

LEVAMISOLE ADULTERATED COCAINE INDUCED TOXICITY Thierry N. Ngansop $^{1}$; Lenar Yessayan ${ }^{3}$; Daryl Sudasena ${ }^{2} .{ }^{1}$ Henry Ford Health System, Madison Heights, MI; ${ }^{2}$ Henry Ford Hospital, Detroit, MI; ${ }^{3}$ Henry Ford Health System, Detroit, MI. (Control ID \#2466582)

LEARNING OBJECTIVE \#1: To educate healthcare professionals on the systemic manifestations of levamisole contaminated cocaine for early recognition and intervention.

LEARNING OBJECTIVE \#2: To heighten awareness of adulterant (levamisole) use in cocaine.

CASE: 49 year old African American male with a history of cocaine use, hypertension, hepatitis $\mathrm{C}$ and $\mathrm{CKD}$ presented with acute shortness of breath and hemoptysis. He was admitted to the ICU, intubated and started on broad spectrum antibiotics. Physical exam was remarkable for diffuse adventitious breath sounds and bluish discoloration of his fingertips. Laboratory results were as follows: hemoglobin $5.8 \mathrm{~g} / \mathrm{dL}$; leukocyte count $5.5 \mathrm{~K} / \mathrm{uL}$; platelet count $463 \mathrm{~K} / \mathrm{uL}$; serum creatinine $3.2 \mathrm{mg} / \mathrm{dL}$. ANA, anti-MPO, antiPR3 and anti-cardiolipin antibodies were positive. Serum C3 and C4 levels were low; rheumatoid factor and serum cryoglobulins were undetectable. Anti-centromere, Anti-Scl70, anti-Ro/SSA, anti-La/SSB, anti-GBM, anti-ENA antibodies were all negative.
Hepatitis C viral load was 623149 copies/mL. Serological test for hepatitis B and HIV were negative. Urine microscopy revealed many RBCs, few hyaline and granular casts. Urine drug screen was positive for cocaine. Blood and respiratory cultures were negative for bacteria, viruses and fungi. Chest radiography showed diffuse bilateral opacities. Chest tomography revealed multiple nodular opacities. Bronchoalveolar lavage showed progressively bloody lavage fluid over serial aliquots consistent with diffuse alveolar hemorrhage. On the fourth day of admission his fingers, toes, ears and nasal fold started to develop necrotic skin changes. In lieu of the presentation and the presence of both antiMPO and anti-PR3, he was diagnosed with levamisole/cocaine induced systemic vasculitis. He received 3 doses of pulse intravenous steroids followed by daily oral prednisone and completed 11 sessions of plasmapheresis. Skin punch biopsy showed epidermal necrosis and widespread vascular thrombosis consistent with levamisole induced vasculopathy. Kidney biopsy showed global glomerulosclerosis, interstitial fibrosis, chronic interstitial inflammation, no active crescents or necrotizing lesions and partly reabsorbed deposits suggestive of a pauci-immune type glomerulonephritis in the past. Despite treatment, his skin lesions continued to become more necrotic and the hemoptysis persisted. Due to lack of improvement, patient was terminally extubated on day 23 from admission.

DISCUSSION: Levamisole is a veterinary anti-helminthic medication which has immunomodulatory properties. It had been used for the treatment of colorectal cancer, however it was removed from the United States market in 1999 due to significant side effects. It has been increasingly used to adulterate cocaine due to its psychotropic effects and morphological properties. Levamisole-induced vasculitis is characterized by neutropenia/ agranulocytosis, purpuric lesions involving the face, especially the ear lobes, with varying frequencies of glomerulonephritis and lung hemorrhage. Serologic abnormalities include unusually high titers of p-ANCA, combined positivity for MPO and PR3 antibodies, positivity for antiphospholipid antibodies as well as antinuclear and anti-double-stranded DNA antibodies and hypocomplementemia. Beside discontinuation of offending agents, there is no proven medical treatment. Therapy with immunosuppressive medications may be prohibitive in those with agranulocytosis and severe wounds. Resolution of the vasculitic process after cessation of cocaine has been described. Our patient had fulminant form of vasculitis and had a fatal outcome. Pulse steroids and plasmapheresis could not reverse the vasculitic process. In an era where cocaine use is common, it is important for physicians to be aware of the signs of levamisole induced toxicity for early recognition and withdrawal of the agent. More research needs to be done on treatment options.

\section{LINEZOLID-INDUCED LACTIC ACIDOSIS AND THROMBOCYTOPENIA IN} A PATIENT WITH OSTEOMYELITIS: A CASE REPORT Takaaki Kobayashi ${ }^{1}$; Michael J. Lau ${ }^{1}$; Akihiro Kobayashi ${ }^{2}$; Aloke Chakravarti ${ }^{1}$; Alfredo J. Astua ${ }^{1}$; Jose A. Cortes ${ }^{1} .{ }^{1}$ Mount Sinai Beth Israel, New York, NY; ${ }^{2}$ Mount Sinai Beth Israel, New York, NY. (Control ID \#2469540)

LEARNING OBJECTIVE \#1: Describe differential diagnosis of lactic acidosis LEARNING OBJECTIVE \#2: Identify the potential side effects of linezolid

CASE: A 77 year-old male with a past medical history of coronary artery disease, systolic heart failure, lymphoma, type 2 diabetes mellitus, with a recent admission for right toe osteomyelitis actively on treatment with linezolid and cefepime, presented with a 2-day history of shortness of breath and several episodes of hypoglycemia. He had a metatarsal head resection for acute osteomyelitis of his first toe 6 weeks prior. The pathology of the metatarsal head proximal margin was initially negative for osteomyelitis, but the surgical site eventually became infected. A repeat biopsy 1 week later was positive for acute osteomyelitis, and the bone culture grew vancomycin resistant enterococci and proteus mirabilis. The patient was subsequently discharged to a nursing home to complete a 6week regimen consisting of linezolid and cefepime. During his treatment he became 
increasingly lethargic and developed shortness of breath 2 days prior to admission and was subsequently brought to the hospital. His initial vital signs showed a blood pressure of $115 / 80 \mathrm{mmHg}$, pulse rate of $72 / \mathrm{min}$, respiratory rate of $18 / \mathrm{min}$, oxygen saturation of $99 \%$ on room air and a body temperature of $98^{\circ} \mathrm{F}$. On physical examination, the surgical site of the right foot was clean, with no erythema, discharge or tenderness noted. Blood work revealed a white blood cell count of $5.9 \mathrm{~K} / \mathrm{uL}$, platelet count of $17 \mathrm{~K} / \mathrm{uL}$, lactic acid of $15 \mathrm{mmol} / \mathrm{L}$, and an elevated anion gap of 29 . A chest $\mathrm{x}$-ray showed a mildly worsening pleural effusion. A critical care medicine consult was requested from the ED, and he was admitted to the MICU for sepsis, and severe lactic acidosis. Ongoing linezolid administrations were continued empirically, and an infectious disease consult was requested. The lactate failed to clear with adequate supportive care, and a Type B lactic acidosis secondary to Linezolid was suspected. After discontinuing linezolid, the lactic acid gradually decreased to $0.6 \mathrm{mmol} / \mathrm{L}$, and his platelet count increased to $166 \mathrm{~K} / \mathrm{uL}$ by hospital day 17. Additional bone resection was performed, however, the proximal margin was positive again. Another bone culture grew pseudomonas resistant to cefepime, and he was discharged with oral ciprofloxacin.

DISCUSSION: Lactic acidosis is a condition characterized by $>4-5 \mathrm{mmol} / \mathrm{L}$ serum lactic acid and is the most common cause of metabolic acidosis in hospitalized patients. It is often associated with poor clinical prognosis, and sepsis is a well-known primary cause of lactic acidosis. Type A lactic acidosis occurs by decreased end-organ perfusion or oxygenation secondary to hypovolemia, cardiac failure, and sepsis. Type B lactic acidosis occurs with no clinical evidence of hypoperfusion or oxygenation. Type B1 occurs by systemic diseases such as diabetes, renal failure and malignancy. Type B2 is caused by drug intoxication. Type B3 is caused by inborn errors of metabolism. Some common causes of Type B lactic acidosis include DKA, ethanol intoxication, drug use, malignancy, alcoholism, HIV, thiamine deficiency, mitochondrial myopathy, trauma, genetic diseases, among other causes of which the mechanism is not entirely clear. Drugs such as linezolid, antiretroviral drugs, and propofol that can impair mitochondrial protein synthesis and reproduction can lead to type B lactic acidosis. Linezolid is an antibiotic of the oxazolidinone class, and is often used to manage drug-resistant gram-positive bacterial infections. This inhibits bacterial protein synthesis by binding to residues within the $23 \mathrm{~S}$ ribosomal RNA of the 50S large subunit of bacterial ribosomes. Linezolid use can be associated with some adverse effect including thrombocytopenia, neuropathy, serotonin syndrome, and lactic acidosis. A longer duration of linezolid leads to greater exposure to mitochondrial damage, therefore we expect that the duration of linezolid use is an important risk factor for those adverse reaction. The primary treatment for linezolidinduced lactic acidosis is discontinuation of linezolid, and most of previous reports demonstrated the recovery within 1-15 days. Although some other treatments such as hemodialysis or bicarbonate have been reported, there is still limited evidence to support their clinical utility. The prognosis of linezolid-induced lactic acidosis is poor, and one case series revealed $26 \%$ of mortality. Sepsis is often considered a major cause of lactic acidosis. However, less common causes of lactic acidosis, such as drug adverse effects, should also be considered. Clinicians should consider these causes when lactate trends are inconsistent with other clinical data

LONGITUDINALLY EXTENSIVE TRANSVERSE MYELITIS: A RARE COMPLICATION OF SLE Pranav D. Patel ${ }^{2}$; Srinidhi Jayadevappa Meera ${ }^{1}$; Eric J. Mariuma $^{3}$; Keron Lezama ${ }^{2} .{ }^{1}$ Montefiore Medical Center, Mamaroneck, NY; ${ }^{2}$ Montefiore Medical Center Wakefield Campus, Bronx, NY; ${ }^{3}$ Montefiore Medical Center, Bronx, NY. (Control ID \#2469615)

LEARNING OBJECTIVE \#1: Recognize the rare but potentially debilitating neurological manifestation associated with systemic lupus erythematosus (SLE) and early treatment to prevent permanent disability.
LEARNING OBJECTIVE \#2: Longitudinally extensive transverse myelitis (LETM) has been known to occur with NMO (neuromyelitis Optica) but can sometimes occur with other autoimmune conditions even when they are on adequate treatment.

CASE: A young 22 year old black female presented with bilateral leg tingling and numbness for 3 days. Tingling sensation was in both legs below knee. Patient didn't have any fall, weakness or imbalance. There was no fever, headache, nausea, vomiting, blurry vision, loss of bladder or bowel control. Physical examination revealed bilateral decreased sensation to temperature in stocking distribution, near-absent vibration in the toes and feet, and very poor proprioception in toes. Hyperreflexia was noted in bilateral lower and upper extremities with positive Hoffman's sign. Hypertonia was noted with wide based gait. There were no significant motor deficits. Patient's past medical history was significant of systemic lupus erythematosus (SLE) diagnosed 3 years ago. She was on immunosuppression with Methylprednisolone and Mycophenolate started after complication of SLE nephropathy with WHO class 3 which was proven by kidney biopsy a year ago. She had stable chronic kidney disease stage 3. SLE flare up with neurological complication was suspected but inflammatory markers including $\mathrm{C}$ reactive protein, complements $(\mathrm{C} 3$, C4) were normal. There were no other clinical findings like skin rash, arthralgia which are commonly associated with acute flare up of SLE. Cerebrospinal fluid (CSF) analysis was negative for herpes virus, cytomegalovirus, west nile virus, toxoplasma and histoplasma. There were normal CSF cell count, total protein and low lactate dehydrogenase. MRI of brain was unremarkable. MRI of spine was consistent with extensive signal abnormality within the spinal cord from level $\mathrm{C} 1$ through at least $\mathrm{T} 9$ which was most prominent in the central cord and dorsal columns representing a demyelinating process. There was no evidence of spinal cord compression, tumor or epidural abscess. Neuromyelitis optica was ruled out with negative Anti NMO serology. So diagnosis of transverse myelitis secondary to SLE was made. Patient was treated with high dose of parenteral glucocorticoids which improved patient's clinical symptoms. Patient was discharged with prophylactic long term immunosuppression and close follow up with Rheumatology and Neurology.

DISCUSSION: This patient had longitudinally extensive transverse myelitis (LETM) with a lesion on MRI that extends sixteen vertebral segments. LETM is defined as a spinal cord lesion that extends over three or more vertebrae which usually leads to complete or incomplete spinal cord dysfunction. LETM usually manifests with sudden onset of lower extremity weakness and/or sensory loss, plus loss of rectal and urinary bladder sphincter control. But this patient had only lower extremity sensory disturbances which could be confusing with SLE peripheral neuropathy. It is important to know that SLE transverse myelitis is thought to be due to an arteritis, with resultant ischemic necrosis of the spinal cord. But there are normal inflammatory markers in this patients which are usually associated with vasculitis. CSF analysis in SLE transverse myelitis is usually consistent with elevated protein level and a moderate lymphocytic pleocytosis but this patient's CSF analysis showed normal lymphocytes and protein. It is debatable to rely on serological inflammatory markers in patients who are already on immunosuppressive therapy. It is prudent to assess for rare causes of CNS inflammation before making definitive diagnosis of SLE transverse myelitis in immunosuppressed patients. Very few cases of LETM in association with SLE have been reported in the literature. LETM is most commonly described as a feature of neuromyelitis optica (NMO), but can be seen in other autoimmune diseases such as SLE, as in this case.

LOST TO FOLLOW UP Douglas Lim; Michael P. Smith. University of Nebraska Medical Center, Omaha, NE. (Control ID \#2469916)

LEARNING OBJECTIVE \#1: Recognize the severe clinical features of IgA Nephropathy.

LEARNING OBJECTIVE \#2: Recognize that early pre-eclampsia can be an indication of primary renal disease. 
CASE: A 25 year-old woman presents with 3 months of worsening dyspnea, weakness, and chest pain. Her systolic blood pressure was 300 . She had diffuse lung crackles with lower extremity edema. Urinalysis revealed significant blood and protein. Chest x-ray revealed cardiomegaly, pulmonary edema with bilateral effusions. Small hypo-echoic kidneys were found on abdominal ultrasound. Her parathyroid hormone level was 500 . A renal biopsy revealed IgA nephropathy with severe atrophy and fibrosis. On further questioning, she has a history of pre-eclampsia early in her previous pregnancy several years ago that was never followed post partum.

DISCUSSION: Chronic kidney disease commonly presents with hypertension and edema; these symptoms are frequently faced by general internists. IgA nephropathy is the most common glomerulonephritis in the world. Most patients are diagnosed with hematuria or proteinuria detected on routine tests. If undetected longterm, a minority of patients present with reduced GFR, hypertension, and persistent proteinuria which are all markers for worse prognosis. Severe hypertension can lead to cardiac failure such as in our patient. Pulmonary hemorrhage in the setting of IgA nephropathy is also a rare but poor prognostic maker when other causes of hemorrhage are excluded. IgA nephropathy is commonly associated with early onset pre-eclampsia. It is difficult to differentiate undiagnosed glomerulonephritis during pregnancy from pre-eclampsia induced kidney injury. This is an important distinction because management is different for each condition. A renal biopsy is the definitive way to diagnose $\operatorname{IgA}$ nephropathy although this has been controversial during pregnancy. However, women who have continued hematuria and proteinuria postpartum should get a biopsy. Our patient experienced proteinuria and hypertension at 22 weeks gestation thought to be due to pre-eclampsia. She had an underlying primary renal disease that was undiscovered until she had severe symptoms several years later. Complications from progressive IgA nephropathy can be life threatening. Pre-eclampsia can mask underlying primary renal disease and the general internist can prevent these complications if a high index of suspicion is maintained.

LOVING HAART BUT LOSING HIP! Sreelakshmi Panginikkod ${ }^{1}$; Venu Pararath Gopalakrishnan ${ }^{1}$; Niyati Gupta ${ }^{2}$; Bara Alzghoul ${ }^{1} .{ }^{1}$ Presence Saint Francis Hospital, Evanston, IL; ${ }^{2}$ Grant Medical College and Sir JJ Group of Hospitals, Mumbai, India. (Control ID \#2469377)

LEARNING OBJECTIVE \#1: Recognize Avascular Necrosis (AVN) as an increasingly identified complication of protease inhibitor-based Highly Active Anti-Retroviral Therapy (HAART)

LEARNING OBJECTIVE \#2: Recognize Avascular Necrosis (AVN) as a cause of hip pain in HIV patients

CASE: A 57 year old gentleman with past medical history significant for HIV presented with bilateral hip pain of 2 weeks duration. Pain was more on the left side, progressively worsening and aggrevated with movement and ambulation. Patient denied any joint stiffness or involvement of other joints. Review of system was negative for fever, chills, urinary symptoms or change in bowel habits. Patient denied any trauma or chronic steroid use. He quit smoking 7 years ago and drinks about $1-2$ cans of beer occasionally. He was diagnosed with HIV 10 years ago and was started on emtricitabine-tenofovir and fosamprenavir 5 years ago. On physical examination, he was moderately built and nourished with stable vitals. Hip examination did not show any warmth, swelling or joint tenderness, but was remarkable for painful range of motion bilaterally, especially on passive internal rotation. Initial lab evaluation showed normal Complete Blood Count, Comprehensive Metabolic Panel and Urinalysis were normal. Inflammatory biomarkers and Autoimmune panel were negative. Serum cortisol level and Thyroid Function Test were normal . His CD4 count was 450 and HIV viral load was undetectable. X-ray of the pelvis showed bilateral femoral head avascular necrosis and mild articular surface collapse of the left femoral head. Magnetic Resonance Imaging (MRI) revealed avascular necrosis
(AVN) of bilateral femoral head and an anterosuperior subchondral crescentic fracture with suggestion of a collapse of left femoral head. Since patient did not have any other risk factors, the AVN was attributed to his HIV and Protease Inhibitor-based HAART therapy The patient underwent total hip arthroplasty of left hip and HAART therapy was modified to elvitegravir, cobicistat, emtricitabine and tenofovir alafenamide. The patient was doing well on 1 month follow up visit.

DISCUSSION: Avascular Necrosis (AVN) indicates ischemic death of the bone as a result of insufficient arterial blood supply. The incidence rate of AVN in HIV infected patients is greater than in general population. Though it is unclear whether this is the result of the HIV virus or the HAART treatment, the incidence has increased in the HAART era. The protease inhibitor drugs have been specifically reported to lead to AVN. Clinicians need to be aware of the subtle yet frequent complication of AVN in HIV patients on HAART therapy especially those patients on protease inhibitors. Preventive strategies should focus on avoiding risk factors like corticosteroid use, smoking, alcohol, intravenous drug use, hyperlipidemia and radiation exposure.

\section{LOWER EXTREMITY ULCER IN AN AFRICAN AMERICAN MALE} Muhammad A. Saeed; Bennal Perkins. Montefiore Medical Center, New York, NY. (Control ID \#2469295)

LEARNING OBJECTIVE \#1: Recognize squamous cell carcinoma as a differential in chronic leg ulcers and long standing lymphedema.

LEARNING OBJECTIVE \#2: Identify paraneoplastic syndrome as an etiology for hypercalcemia presenting with anemia, weight loss and venous thrombosis.

CASE: A 58 year old African American male with medical history of chronic lymphedema presented with chronic nonhealing left lower leg ulcer which had recently become foul smelling. On admission, he was febrile to 101.3; labs revealed leukocytosis, lactic acidosis, elevated creatinine, severe anemia, and severe hypercalcemia to 16.5. Imaging revealed bone loss in the distal left tibia and fibula. Considering the clinical picture, there was high suspicion for osteomyelitis and he was started on antibiotics. Aggressive Intravenous hydration was done initially. He was given Calcitonin $30 \mathrm{mg}$ and Pamidronate in several doses, totally $150 \mathrm{mg}$. As the patient was severely anemic, he was initially administered 4 units of packed red blood cells (he received a total of 6 units during hospital stay). Flow cytometry was normal. Serum Protein Electrophoresis revealed elevated kappa and lambda chains raising suspicion for multiple myeloma; however, his skeletal survey and bone biopsy were normal. We also evaluated his Parathyroid hormone (PTH), which was low, but Parathyroid Hormone related Peptide (PTHrP) was high. He underwent left below the knee amputation (BKA) and biopsy was sent to pathology. Preliminary biopsy obtained during BKA reported invasive squamous cell carcinoma (SCC). Upon discharge, an outpatient PET scan and Dermatology appointment was recommended to further evaluate surrounding scar tissue for SCC. He developed nonocclusive thrombi in the right common iliac and right internal iliac veins and later in the left basilic vein. The need for life long anticoagulation was strengthened as he developed two venous thrombus emboli in the setting of malignancy.

DISCUSSION: Squamous cell carcinoma has various etiologies and diagnosing it in chronic ulcers poses a challenge to the physician. SCC commonly occurs on sun exposed areas; often in the neck and on the back of fair skin people. Compared to Caucasians, SCC in the African American population is not as common. Females tend to be more affected than males, and lower extremities being the most common site. No study has been done discussing etiologies of lower extremity SCC; however, it is suggested that lower extremity SCC are distinct and may exhibit a pathogenesis less reliant on actinic damage. Two unique factors were involved in our case: chronic lymphedema and chronic ulcers. Only a few case reports support SCC developing secondary to chronic lymphedema, which historically is related to lymphangiosarcoma not SCC. Incidence of SCC in chronic ulcers 
has been reported to be approximately $1 \%$ in a small retrospective study. Another challenge in our case was hypercalcemia. Our patient was initially believed to have osteomyelitis or multiple myeloma based on clinical presentation of sepsis in setting of chronic ulceration and hypercalcemia, respectively. Of note, hypercalcemia is not an uncommon finding in both multiple myeloma and squamous cell carcinoma. Maintaining a broad differential diagnosis is the cornerstone of managing similar cases. The diagnosis of SCC in the setting of low PTH and high PTHrP explained paraneoplastic syndrome of hypercalcemia secondary to malignancy and anemia. Internists should consider Squamous cell carcinoma as a differential in chronic leg ulcers and long standing lymphedema; and maintain a broad differential for hypercalcemia presenting with anemia, weight loss and venous thrombosis.

\section{LUPUS, AN UNLIKELY SUSPECT FOR DYSPNEA IN A YOUNG MALE} Michele Yeung; Marcelo Mendez; Faraj Faour. Mount Sinai Beth Israel, New York, NY. (Control ID \#2468939)

LEARNING OBJECTIVE \#1: Recognize that systemic lupus erythematous (SLE) has varying manifestations and that pulmonary complaints can be the sole presenting symptom

LEARNING OBJECTIVE \#2: Understand the American College of Rheumatology (ACR) diagnostic criteria for SLE

CASE: A 21-year-old male with no past medical history presented to the hospital with 2 days of shortness of breath. He had a routine physical by his primary care physician 1 week prior to admission, at which time the only abnormal finding was a blood pressure of 149/95. He described dyspnea exacerbated with exertion and lying flat; which was associated with a nonproductive cough and intermittent pleuritic chest pain. He denied fevers, chills, hematuria, dysuria, rhinitis, epistaxis, or hemoptysis. He further denied a history of prolonged immobilization, sinus infections, or asthma. The remainder of his review of systems was negative, including lack of edema, arthralgias, myalgias, rashes, or photosensitivity. The physical exam was only significant for hypertension (153-173/106125 ) and sinus tachycardia (95-105). Lungs were clear and there was no rashes, oral ulcers, joint abnormalities, peripheral edema, elevated JVD, or chest wall tenderness Initial labs revealed Cr 3.24 (0.66-1.25 mg/dL), BUN $58(8-24 \mathrm{mg} / \mathrm{dL})$, total protein 5.1 (6.3-8.2 g/dL), albumin 2.3 (3.5-5 g/dL), and B-type natriuretic peptide 393.8 (0-100 pg/ $\mathrm{ml})$. Serial cardiac enzymes were negative. Chest radiograph was normal. Ventilation perfusion scan reported low probability for a pulmonary embolism and transesophageal echocardiography without signs of heart failure. Further workup with urinalysis revealed nephrotic range proteinuria, microscopic hematuria, along with dysmorphic red blood cells and red blood cell casts. Immunologic studies showed an elevated ANA with a 1:320 titer, positive anti-dsDNA antibody, and low C3 /C4 levels. Given the suspicion for intrinsic renal disease our patient underwent a renal biopsy. Pathology was consistent with rapidly progressive glomerulonephritis (RPGN) due to lupus nephritis and the patient was started on methylprednisolone and cyclophosphamide. Patient's initial complaints of dyspnea were deemed to be secondary to pulmonary involvement of his newly diagnosed SLE.

DISCUSSION: We present an unusual case of Systemic lupus erythematous (SLE) in a male patient where the presenting symptom was a pulmonary complaint. It is well established that SLE is substantially more common in females of child-bearing age, where the reported female:male ratio is $8-15: 1$. In addition, SLE is typically thought of as an autoimmune disease comprised mainly of musculoskeletal and dermatologic symptoms. In fact, approximately $73 \%$ of patients will present with cutaneous findings and $65 \%$ with musculoskeletal manifestations. In contrast, less than $12 \%$ of patients present with pulmonary complaints. Of those $12 \%$ of patients presenting with solely pulmonary complaints such as pleurisy and dyspnea, clinicians are more likely to consider other diagnoses such pulmonary embolism, pneumonia, and pneumothorax particularly in the young, otherwise healthy population. While this patient did have an elevated BNP, there was no clinical evidence for volume overload such as crackles or elevated JVP, and chest radiography confirmed clear lungs. Further, imaging studies and lab work also excluded pneumonia, pulmonary embolism, or pneumothorax. Early diagnosis and treatment of SLE is the key to better outcomes. However, the clinical heterogeneity of SLE and the lack of pathognomonic features or tests pose a diagnostic challenge for the clinician. Many use the classification criteria proposed by American College of Rheumatology (ACR) to document key disease features. A minimum of 4 of the 11 ACR criteria should be met to diagnose SLE. The 11 ACR criteria are broken into systems: cutaneous, musculosketetal nonerosive arthritis, cardiopulmonary pleuritis or pericarditis, renal, neurological disorder with seizures or psychosis due to unknown causes, and positive immunologic antibodies. We demonstrate with this case that SLE can present with acute dyspnea and that SLE should be included in the wide range of differential diagnoses of dyspnea particularly in a young patient where common etiologies have been ruled out. This case reminds clinicians to be cognizant of atypical presentations of SLE.

LYMPHOGRANULOMA VENEREUM PROCTITIS Madalina Ilioaia. Emory, Atlanta, GA. (Control ID \#2469599)

LEARNING OBJECTIVE \#1: Review the presentation of LGV

LEARNING OBJECTIVE \#2: Discuss the importance of diagnosis and treatment of patient and sexual partners

CASE: 28 year old male with PMH of HIV/AIDS (CD4 101 VL 209,462) who had been off antiretrovirals since September 2014 presenting due to abdominal pain and bloody diarrhea for 3 weeks. He reported 15 watery bowel movements per day. Also reported fever and chills. He denied sick contacts or recent travel. He did engage in unprotected anal intercourse 3 months prior to onset of symptoms. On exam his abdomen was diffusely tender to palpation. He had small perirectal ulcerations and had inguinal lymphadenopathy as well. Work up included CT abdomen/pelvis, which showed rectal wall thickening and adjacent fat stranding and adenopathy. Stool cultures were negative for cryptosporidium, isospora, and cyclospora. Lymphadenopathy, hemorrhagic diarrhea and proctitis on imaging in the setting of HIV/AIDS was concerning for GC proctitis. GC PCR was positive and he was treated with 21 day course of Doxycycline.

DISCUSSION: Lymphogranuloma venereum (LGV) is a genital ulcer disease caused by Chlamydia trachomatis. It was initially described in heterosexual males in tropical and subtropical areas and in the early 2000 s became increasingly prevalent in temperate climates in men who have sex with men. In MSM HIV infection was a risk factor for acquiring LGV. LGV presents in multiple stages following infection. With primary infection patients usually experience genital ulcer that can be small in size and often missed. Two to six week following primary infection the secondary stage appears which is caused by the direct spread of the infection to nearby lymph nodes. This stage is characterized by inguinal lymphadenopathy that can form buboes. This stage can also present with anorectal symptoms/ proctocolitis with rectal discharge, anal pain fever as well as hemorrhagic proctocolitis. If left untreated Diagnosis can be difficult as clinical presentations vary and testing modalities are not standardized however NAAT testing has shown to have high sensitivity/specificity for detection of Chlamydia trachomatis. Physicians should have a high index of suspicion for potential LGV infection in patient with underlying HIV infection presenting with symptoms of proctocolitis. It is important to consider LGV as a potential cause of hemorrhagic proctocolitis as untreated LGV can result in late complications including genital elephantiasis, anal fistulas, strictures, infertility. During proctocolitis episode there is also enhanced shedding of HIV that could increase the risk of HIV to uninfected partners. Partners of LGV positive patients should be screened and treated in order to prevent further spread and long term complications of untreated disease. Asymptomatic partners should be empirically treated as well. 
MAC 'N' CHEESE 'N' PNEUMONITIS, IF YOU PLEASE? Poppy Markwell; Lawrence Purpura; John Moscona. Tulane University Health Sciences Center, New Orleans, LA. (Control ID \#2468853)

LEARNING OBJECTIVE \#1: Recognize the clinical presentation of hypersensitivity pneumonitis

LEARNING OBJECTIVE \#2: Recognize the importance of considering environmental exposures for repetitive exacerbations of respiratory failure

CASE: A 28 year-old healthy woman presented with 4 days of progressive dyspnea. She had associated fever, chills, malaise, cough with productive sputum, congestion, and myalgias. She had been hospitalized 2 weeks prior with similar symptoms. Her symptoms had resolved prior to discharge without any treatments and her work-up was negative for pulmonary embolism. Her vital signs were notable for a heart rate of 120 beats per minute, blood pressure of 126/95, respiratory rate of 38 breaths per minute, with a $97 \%$ oxygen saturation on $21 \% \mathrm{FiO} 2$. She was diaphoretic with respiratory distress, accessory muscle use, and had fine bibasilar crackles. Labs revealed hypoxia and leukocytosis but were negative for influenza and HIV. The chest X-ray revealed diffuse opacities in all lobes with small bilateral pleural effusions. The patient was treated for presumed atypical bacterial pneumonia and improved to baseline within $24 \mathrm{~h}$ of hospitalization. She was discharged but returned 5 days later with worsening tachypnea, hypoxia, tachycardia, and leukocytosis. She was admitted to the intensive care unit for hypoxic respiratory distress and was treated with Bipap and antibiotics. CT scan revealed innumerable centrilobular nodules. This time the patient did not respond to antibiotics. Blood and respiratory cultures, influenza PCR, and PCP and induced AFB sputum smears were negative. The immunodeficiency panel was non-revealing. The patient was treated empirically for PCP pneumonia with bactrim and steroids and in 4 days, she returned to baseline and was discharged again without oxygen. She developed respiratory distress for a fourth time, 2 days after finishing the steroid taper and was readmitted to the intensive care unit. She quickly responded to prednisone. Sputum AFB cultures became positive on day eight for a rapid grower. A bronchoscopic alveolar lavage and transbronchial biopsy revealed a moderate lymphocytosis and ill defined non-caseating granulomas with clusters of acid-fast bacilli consistent with Mycobacterium Avium Complex. It was determined that she had developed hypersensitivity pneumonitis with underlying MAC after using a new humidifier at home. She was able to stop steroids after removing the humidifier from her home

DISCUSSION: If left undiagnosed, extrinsic allergic alveolitis can be a life-threatening emergency and can lead to irreversible lung damage. The disease is commonly misdiagnosed as asthma, virus, or reaction to inhaled molds. Unlike Organic Dust Toxic Syndrome, hypersensitivitiy pneumonitis requires a prolonged exposure to an inhaled antigen. Respiratory symptoms, reticulo-nodular opacities on chest imaging, BAL with lymphocytosis, histology showing non-caseating granulomas or mononuclear cell infiltrate, and positive inhalation challenge testing all support the diagnosis. There is no definitive laboratory test, and serum precipitins are typically useless. The serum precipitant profile tests for lifetime exposure to common molds, grain, and fungi. Oftentimes, the test will be falsely negative or pan-positive and the antigen identified is not the cause of disease. Hundreds of antigens have been implicated in this disease, and a careful history must be taken to identify the inciting agent. The key to treatment is preventing the exposure. In this patient with mycobacterium avium complex induced hypersensitivity pneumonitis, her symptoms spontaneously resolved during hospitalization because she was separated from the exposure. On discharge, she was re-exposed to her humidifier and each acute episode was worse than the previous. Only after identifying and removing the exposure did she get better. Hypersensitivity pneumonitis can occur from many exposures including contaminated humidifiers, unventilated showers, hot tubs, saunas, and basements. Mycobacterium avium complex grows well in warm water and can be aerosolized and inhaled in hot tubs but is not often considered in humidifier exposures. Routine disinfection of humidifiers can be helpful in reducing overgrowth of common pathogens such as thermoactinomyces, klebsiella, naegleria, and acanthamoeba. However, it is also important to avoid prolonged exposure to the disinfectant, as this can lead to lung injury and pulmonary fibrosis in a dose-response manner.

MACROPHAGE ACTIVATION SYNDROME IN A 38 YEAR-OLD WOMAN WITH ULCERATIVE COLITIS RECEIVING INFLIXIMAB Megan L. Krause²; Eric L. Matteson ${ }^{2}$; Alexandra Messerli ${ }^{1} .{ }^{1}$ Mayo Clinic School of Graduate Medical Education, Rochester, MN; ${ }^{2}$ Mayo Clinic, Rochester, MN. (Control ID \#2462385)

LEARNING OBJECTIVE \#1: Recognize clinical presentation of macrophage activation syndrome and identify appropriate diagnostic evaluation

CASE: A 38 year-old woman with past medical history of asthma and ulcerative colitis presented with fever, nausea/ vomiting, abdominal pain and elevated liver enzyme tests 2 weeks after a second infusion of infliximab. The day after the infusion she developed headache, dizziness, nausea, dry heaves and fever to $103{ }^{\circ} \mathrm{F}$. Her symptoms progressed to include epistaxis, cough productive of blood-tinged sputum and tea-colored urine. On initial evaluation at our facility, temperature was $39.4^{\circ} \mathrm{C}$, with tachycardia, tachypnea and oxygen saturation of $94 \%$ on room air. Physical exam was notable for mild scleral icterus, right upper quadrant tenderness with smooth hepatomegaly nearly to the umbilicus and scattered lower extremity petechial rash. Complete blood count revealed mild leukocytosis with lymphocytic predominance and normal hemoglobin. Alkaline phosphatase (931 U/L, nl 37-98 U/L), AST (559 U/L, nl 8-43 U/L), ALT (409 U/L, nl 7-45 U/L), and total and direct bilirubin ( 2.9 and $2.5 \mathrm{mg} / \mathrm{dL}, \mathrm{nl} 0.1-1.0 \mathrm{mg} / \mathrm{dL}$ and $0.0-0.3 \mathrm{mg} / \mathrm{dL}$, respectively) were all elevated; prothrombin time was normal. Computed tomography (CT) and ultrasonography of the abdomen revealed hepatomegaly without evidence of acute cholecystitis, and she was admitted with a diagnosis of acute hepatitis. The patient remained febrile over the next 10 days. Liver test abnormalities worsened with development of frank jaundice on day 4. Dyspnea and hypoxia worsened with progressive patchy pulmonary opacities on chest radiograph. Initial infectious evaluation, including blood cultures and serologies/PCR for hepatitis A, B, C, and E, Epstein-Barr virus, cytomegalovirus, HIV, HSV, VZV and syphilis, was negative. Clinical and serologic evaluation for autoimmune hepatitis or lupus was also negative. Ferritin was markedly elevated at $17,810 \mathrm{mcg} / \mathrm{L}$ (nl 11-307 mcg/L). The erythrocyte sedimentation rate (ESR) was $3 \mathrm{~mm} / 1 \mathrm{~h}(\mathrm{nl} 0-29 \mathrm{~mm} /$ $1 \mathrm{~h})$, with C-reactive protein (CRP) elevated at $104.6 \mathrm{mg} / \mathrm{L}(\mathrm{nl}<8 \mathrm{mg} / \mathrm{L})$. Triglycerides were elevated at $389 \mathrm{mg} / \mathrm{dL}(\mathrm{nl}<150 \mathrm{mg} / \mathrm{dL})$. The unremitting fever with hyperferritinemia and hypertriglyceridemia were suggestive of macrophage activation syndrome (MAS), with progressive anemia, thrombocytopenia and low fibrinogen (124 mg/dL, nl 200-430 mg/dL) concerning for an incipient coagulopathy. Methylprednisolone was initiated on day 5 , however on day 6 , she had an episode of syncope with an acute drop in hemoglobin. Abdominal CT showed new retroperitoneal hematoma. Supportive transfusions were initiated. Subsequently, the serum histoplasma antibody screen returned positive; confirmatory testing with urine histoplasma antigen, and BAL PCR was also positive as eventually were blood and BAL cultures. Amphotericin B was initiated. By day 11, the patient had remained afebrile for $24 \mathrm{~h}$ for the first time since admission, with improvement of clinical status and laboratory parameters. She was transitioned to oral prednisone with plan for gradual taper. Amphotericin was continued for 6 weeks, followed by transition to itraconazole for a prolonged course. Additional testing was significant for hemophagocytosis on bone marrow biopsy, decreased Natural Killer (NK) cell cytotoxicity with normal degranulation and soluble IL-2 receptor of 4359 units $/ \mathrm{mL}$ (nl 4-1105 units $/ \mathrm{mL}$ ). The final diagnosis was macrophage activation syndrome secondary to disseminated Histoplasmosis caused by reactivation in the setting of anti-TNF therapy.

DISCUSSION: Macrophage activation syndrome (MAS), which overlaps with hemophagocytic lymphohistiocytosis (HLH), typically presents with acute to subacute 
(1-4 weeks) onset of nonspecific symptoms, including high fever $\left(>38.5^{\circ} \mathrm{C}\right)$, lymphadenopathy, hepatosplenomegaly and petechia/purpura. Nearly $80 \%$ of patients have abnormal liver enzyme tests with coagulopathy evident in almost $60 \%$. Pulmonary, neurologic, skin, gastrointestinal and renal involvement is common. The pathophysiology of HLH is related to uncontrolled activation of antigen presenting cells and $\mathrm{T}$ cells leading to hypersecretion of proinflammatory cytokines (interferon gamma, TNF-alpha and interleukins 1, 4, 6, 10 and 18) which contributes to tissue damage and progressive systemic organ failure. The diagnosis can be made if there is a molecular diagnosis consistent with HLH or five out of eight of the following clinical criteria are met: fever, splenomegaly, cytopenia affecting at least two lineages (hemoglobin $<90 \mathrm{~g} / \mathrm{L}$, platelets $<100 \times 109 / \mathrm{L}$ or neutrophils $<1.09 / \mathrm{L}$ ), hypertriglyceridemia and/or hypofibrinogenemia (fasting triglycerides $>265 \mathrm{mg} / \mathrm{dL}$ or fibrinogen $<1.5 \mathrm{~g} / \mathrm{L}$ ), hemophagocytosis in the bone marrow, spleen or lymph nodes, low or absent NK cell activity, ferritin $>500 \mathrm{mcg} / \mathrm{L}$ and soluble CD25 (i.e., soluble IL-2 receptor) $>2400 \mathrm{U} / \mathrm{mL}$. Management consists of supportive measures, elimination of the inflammatory trigger and suppression of inflammation by immunosuppressive and/or cytotoxic drugs. As this case demonstrates, the cornerstone of treatment is identification and treatment of the underlying cause, including evaluation for opportunistic infections in immunosuppressed patients. Prompt recognition and treatment is required, as estimated mortality for this condition approaches $40 \%$. Reference: Ramos-Casals M, Brito-Zerón P, López-Guillermo A, et al. Adult haemophagocytic syndrome. The Lancet; 383: 1503-1516. DOI: 10.1016/s0140-6736(13)61048-x

\section{MALALIGNED - METFORMIN ASSOCIATED LACTIC ACIDOSIS AND MEDICATION RECONCILLIATION ERROR Angela J. Kang; Aydin Pinar. Yale New Haven Hospital, New Haven, CT. (Control ID \#2467919)}

LEARNING OBJECTIVE \#1: Recognize metformin associated lactic acidosis LEARNING OBJECTIVE \#2: Improve medication reconciliation at hospital discharge to avoid adverse medical events

CASE: A 68-year-old man with Diabetes Mellitus, Peripheral Vascular Disease, and End Stage Renal Disease (ESRD) on hemodialysis for 1.5 years presented with lethargy and back pain for 1 week. In the Emergency Room, he was found to have temperature: $86^{\circ} \mathrm{F}$, blood pressure: $82 / 54 \mathrm{mmHg}$, heart rate: $30 \mathrm{bpm}$. Finger stick glucose was $7 \mathrm{mmol} / \mathrm{L}$. His point of care venous blood gas showed: pH: 6.72, pCO2: $22 \mathrm{mmHg}$, lactate: $>20.0 \mathrm{mmol} / \mathrm{L}$. His labs were also significant for potassium: $5.7 \mathrm{mEq} / \mathrm{L}$, creatinine: $7.6 \mathrm{mg} / \mathrm{dL}$, white blood cell count: $32.8 \times 10^{3} / \mathrm{mm}^{3}$, and thyroid stimulating hormone: $4.45 \mu \mathrm{U} / \mathrm{mL}$. His anion gap was too high to determine on admission, but was measured to be 49 the next day. He was intubated, and started on broad-spectrum antibiotics and vasopressors. The differential diagnosis included: septic shock, myxedema coma, ischemic cardiac event, and hemorrhagic shock from patient's past history of repaired abdominal aortic aneurysm (AAA). However, a CT chest/abdomen/pelvis was negative for evidence of acute inflammatory process, a trans-thoracic echocardiogram was negative for focal wall motion abnormalities, and an abdominal ultrasound was negative for ruptured AAA. Blood cultures showed no growth. He was on norepinephrine and dopamine drips at this time, and was maintaining adequate blood pressure with mean arterial pressures $>65 \mathrm{mmHg}$. Considering his low vasopressor requirement and high lactic acidosis, the team began to consider Type B lactic acidosis as the primary etiology causing the patient's hemodynamic instability. Upon further chart review it was determined that the patient had been admitted 1 month prior with colitis, treated with antibiotics, and discharged with instructions to restart metformin, which had previously been discontinued secondary to his severe renal disease. Metformin associated lactic acidosis became the most likely diagnosis. Continues veno-venous hemodialysis was emergently started and his lactic acid downtrended immediately and normalized after 3 days. He was transitioned to conventional hemodialysis and extubated. His metformin level was found to be $20 \mathrm{mcg} / \mathrm{mL}$ (therapeutic range $1-2 \mathrm{mcg} / \mathrm{mL}$ ). The patient improved and was discharged after 2 weeks.

DISCUSSION: Lactic acidosis is an anion-gap metabolic acidosis with a plasma lactate level greater than $5 \mathrm{mmol} / \mathrm{L}$ and $\mathrm{pH}$ less than 7.35. It can be associated with tissue hypoperfusion, hypoxia, and multi-organ failure including neurologic and cardiovascular dysfunction, as was seen in this patient. The FDA has estimated the rate of fatal or nonfatal lactic acidosis associated with metformin use to be 5 cases per 100,000 persons treated over 1 year, although the true incidence is not known. A 2010 Cochrane Review found no evidence that metformin was associated with an increased risk for lactic acidosis when prescribed under study conditions (Salpeter 2010). However, there are no randomized controlled trials to test safety in patients with significantly impaired kidney function. In this case, a very high anion gap with profound lactic acidosis and hemodynamic instability that rapidly corrected with renal replacement therapy was a clue to metformin associated lactic acidosis. Despite findings that lactate levels have not been significantly impacted in patients with mild to moderate kidney disease that are taking metformin, this patient had severe renal impairment and was on hemodialysis. His metformin consumption proved nearly fatal. The transition home following hospital admission is a vulnerable period for patients. Nearly half of hospitalized patients experience at least 1 medical error upon discharge. In one case study, post discharge injuries ranged from laboratory abnormalities to permanent disabilities. Many of the preventable or ameliorable injuries were results of ineffective communication, including errors reviewing medication regimens (Forster 2003). Adverse drug events, or injury due to medication, affect 11 to $17 \%$ of patients during the first weeks after hospital discharge. In this case, the patient received erroneous instructions to restart metformin after his prior hospital discharge, which resulted in his admission for metformin associated lactic acidosis. REFERENCES Forster AJ, Murff HJ, Peterson JF, Gandhi TK, Bates DW. The Incidence and Severity of Adverse Events Affecting Patients after Discharge from the Hospital. Ann Intern Med. 2003;138:161-167. doi:10.7326/0003-4819-138-3-200302040-00007 Inzucchi SE Lipsaky KJ, et al. Metformin in Patients with Type 2 Diabetes and Kidney Disease: A Systemic Reivew. JAMA. 2014; page, 2668-2675.

MALE ANOREXIA NERVOSA: A UNIFYING DIAGNOSIS FOR MULTISYTEMIC SYMPTOMS Sinny Wang ${ }^{1}$; Margaret Park ${ }^{1,2}$. ${ }^{1}$ UCLA Medical Center, Los Angeles, CA; ${ }^{2}$ Kaiser Permanente-Oakland Medical Center, Oakland, CA. (Control ID \#2470085)

LEARNING OBJECTIVE \#1: Identify the presenting features, risk factors and prevalence of anorexia nervosa in a male patient

LEARNING OBJECTIVE \#2: Manage eating disorders as a general internist

CASE: 25-year-old male with history of unexplained hypogonadism, hypothyroidism, pancytopenia, and asymptomatic sinus bradycardia presents to hepatology clinic for evaluation of transaminitis than began 4 years ago. He had undergone extensive prior work up including liver biopsy (lobular hepatitis with minimal activity), bone marrow biopsy (hypocellular marrow with serous fat atrophy), fat pad biopsy (depletion of fatty tissue, negative stain for amyloidosis), transthoracic echocardiogram (aortic root dilatation without other dysfunction), and whole body PET CT (no foci of increased metabolism). Despite negative serologies, he was presumed to have autoimmune hepatitis and was empirically treated with steroids with some improvements. Prior to his illness, he was reportedly healthy and was a competitive athlete in an elite college program. He had been taking testosterone and levothyroxine, and had stopped steroids due to psychiatric side effects. He does not smoke and does not use any illicit drugs. Review of systems is remarkable for fatigue, frequent infections. He also had unintentional weight loss of $45 \mathrm{lb}$ in 4 years, despite rigidly adhering to a regimented diet of $>5000 \mathrm{kcal}$ daily. To cope with his depression and anxiety, he exercises 6-7 h daily. On exam he appeared cachectic with body-mass index (BMI) of 
14.8, significant bitemporal and total body muscle wasting with trace lower extremity edema. He did not exhibit signs of chronic liver disease. Patient had a withdrawn affect despite constant fidgeting. Labs showed hyponatremia (127 mmol/dL); non-obstructive transaminitis (AST $171 \mathrm{U} / \mathrm{L}$, ALT $123 \mathrm{U} / \mathrm{L})$ with elevated total bilirubin $(3.2 \mathrm{mg} / \mathrm{dL}$ ); pancytopenia with normocytic anemia (hemoglobin $11.8 \mathrm{~g} / \mathrm{dL}$ ). He underwent repeat liver biopsy, showing nodular regenerative hyperplasia without features of autoimmune or infectious liver disease. Upon recognition of his underlying anorexia nervosa (restrictive type with compulsive exercise) complicated by multisystem medical issues, patient was transferred to inpatient Psychiatry/Eating Disorder Unit (EDU) for further care. His course was complicated by refeeding syndrome while in EDU. He also was also diagnosed with comorbid psychiatric conditions including major depressive disorder and obsessivecompulsive disorder. He eventually refused the recommended transfer to higher level of care at a dedicated male eating disorder facility. He transitioned to outpatient internal medicine clinic for close medical supervision and refused further inpatient psychiatric hospitalization.

DISCUSSION: Diagnostic and Statistical Manual of Mental Disorders, 5th edition (DSM-5) defines feeding and eating disorders (ED) are a group of disorders characterized by persistent disturbance in eating behaviors. Despite some common features, these heterogeneous diagnoses differ significantly in their outcomes and treatments. Specifically, Anorexia Nervosa (AN) is a disorder of "restriction of energy intake relative to requirements," with "persistent behavior that interferes with weight gain... lack of recognition of the seriousness of the current low body weight." AN is generally thought of as predominantly affecting young females, with an estimated 12-month prevalence of $0.4 \%$. In comparison, minimal data exist for its prevalence among males, but clinic populations estimate approximately 10:1 female-to-male ratio. Studies focusing on male patients diagnosed with ED have been limited by sample size and study methods. In general, the majority of male patients presenting for care had very low BMI percentile for age $(<10$ th percentile), frequently engaged in compulsive exercise $(40-$ $82 \%)$ and had significant long-term medical ramifications such as osteopenia or osteoporosis on presentation (30-40\%). Although similar data is not available in men, compulsive exercise has been identified in female AN patients as being associated with lower BMI, as well as higher level of anxiety and obsessive-compulsive disorder symptom traits. It is also a predictor of poor treatment outcome in female patients. Medically, eating disorders can concurrently affect multiple organ systems, and patients may seek medical attention for a wide variety of presenting complaints and lab abnormalities. Depending on severity of malnutrition, major physiologic complications of AN can involve all organ systems, including reproductive (amenorrhea, hypogonadism), endocrine (hypoglycemia, hypothyroxinemia, osteoporosis), cardiac (bradycardia, arrhythmias, systolic heart failure), gastrointestinal (hepatitis, delayed transit time), renal (electrolyte abnormalities, renal injury, refeeding syndrome), pulmonary (pneumothorax, respiratory muscle failure), and neurologic (seizure, peripheral neuropathy). Because of these complications and difficulty treating underlying psychopathology, patients with $\mathrm{AN}$ have one of the highest mortality rates of all psychiatric disorders, estimated to be 5.1 deaths per 1000 person-years. As illustrated in the case, general internists play a unique role in diagnosing eating disorder-related complications when patients present with multisystemic abnormalities. Especially in the rare cases of male patients with eating disorders, interdisciplinary management is paramount to its successful treatment.

MALIGNANT PLEURAL MESOTHELIOMA AS A RARE CAUSE OF HEMORRHAGIC PLEURAL EFFUSIONS: A CASE REPORT Tiffany Seto ${ }^{3}$; Daniel I. Kim ${ }^{2}$; Ramiz Fargo ${ }^{1}$. ${ }^{1}$ Riverside County Regional Medical Center, Moreno Valley, CA $;{ }^{2} \mathrm{UC}$ RIverside, Moreno Valley, CA; ${ }^{3} \mathrm{UC}$ Riverside, San Mateo, CA. (Control ID \#2467762)
LEARNING OBJECTIVE \#1: Recognize the signs and symptoms of malignant pleural mesothelioma as a rare cause of hemorrhagic pleural effusions.

CASE: A 56 year old male presented with a recent history over the last month of recurrent left hemorrhagic pleural effusion of unclear etiology. He was admitted to our hospital with a leaking chest tube in place after hospitalization in Tijuana. The patient's past medical history included COPD, atrial fibrillation, hypertension, Type II diabetes mellitus and latent TB status post INH therapy. Social history was significant for current 5 year employment as a machine worker in an aluminum factory and history of an 11-year employment in construction work. The patient was a light smoker for 2 years and quit 10 years prior. The patient initially presented 1 month prior to another hospital for acute respiratory failure secondary to a left pleural effusion; 21 of hemorrhagic fluid were drained via thoracentesis and the patient was discharged home. Approximately 2 weeks later, while vacationing in Tijuana, Mexico, the patient again developed severe dyspnea and was admitted for recurrent left pleural effusion. Tube thoracostomy was performed and 41 of hemorrhagic fluid were drained. The patient was discharged from the hospital in Tijuana with a water-sealed chest tube in place and was promptly brought to our hospital. Upon presentation, the patient was admitted for chest tube evaluation for leakage and found to have a pneumothorax with subcutaneous emphysema of the left chest and serosanguinous fluid draining from the chest tube. Physical exam was significant for crepitus localized at the tube insertion site, decreased breath sounds and a pleural rub in the left posterior lung field and an irregularly irregular heart rhythm. Lab findings demonstrated normal electrolytes, renal and liver function with the exception of reduced serum albumin $(2.6 \mathrm{~g} / \mathrm{dL})$. Serum LDH $237 \mathrm{U} / \mathrm{L}$ and protein total $6.6 \mathrm{~g} / \mathrm{dL}$. Pleural fluid analysis revealed glucose $88 \mathrm{mg} / \mathrm{dL}$, LDH $251 \mathrm{U} / \mathrm{L}$, and protein $2.9 \mathrm{~g} / \mathrm{dL}$, while fluid cytology revealed brown turbid fluid with few eosinophils, elevated WBC 846 with $68 \%$ lymphocytes and macrophages, and RBC count of 26076; no malignant cells were identified. Blood and pleural fluid cultures were negative. A chest radiograph and CT with contrast demonstrated left basilar infiltrates with left-sided pleural effusion, suggestive of hemothorax in lower left base, a small left-sided pneumothorax $10 \%$, and nonspecific thickening along pleural surface abutting the mediastinum. The patient was taken into the operating room for VATS with lung biopsy, which was suggestive of mesothelioma vs adenocarcinoma. Final pathology revealed epithelial malignant mesothelioma, tubulopapillary subtype. Oncology was consulted and the patient began chemotherapy with pemetrexed and carboplatin.

DISCUSSION: Malignant pleural mesothelioma (MPM), most commonly associated with long term exposure to asbestos, is epithelial in origin $50-70 \%$ of the time. Histologically, MPM may be divided into three variants: epithelial (with tubulopapillary being the most common subtype), sarcomatoid and biphasic. Epithelioid MPM carries a slightly better prognosis than the other variants of MPM with a median survival time about a year after diagnosis. Our patient presented at a much earlier age than the median age of diagnosis in Western countries (56 years vs 72-74 years). The average interval between symptom onset and diagnosis is 23 months. Although patients with MPM typically present with an insidious onset of dypsnea, non-pleuritic chest pain, weight loss and right-sided disease, our patient presented with acute respiratory failure secondary to left-sided effusion and did not have a history of recent weight loss. MPM spreads rapidly through pleural surfaces and by local invasion. Early diagnosis is key to improving prognosis by several months to potentially years, but early diagnosis can be challenging as patients are usually asymptomatic in early stages and tubulopapillary MPM may be mistaken for benign inflammatory conditions or metastatic adenocarcinoma. Fluid cytology is often unrevealing (documented sensitivity between 32 and $76 \%$ ) and VATS biopsy with immunohistochemical analysis is required for definitive diagnosis. The mainstay of treatment for unresectable MPM and for poor surgical candidates is palliative chemotherapy with pemetrexed and cisplatin. This case demonstrates the difficulty of diagnosis and the need for early recognition of this rare and deadly cancer. 
MALIGNANT SKIN LESION WITHOUT MALIGNANCY: CALCIUM CHLORIDE EXTRAVASATION. OMAR A. MAHASNEH ${ }^{1}$; Abdelrazig Suliman ${ }^{2}$; Roger D. Smalligan ${ }^{4}$; Bharat Khandheria ${ }^{3} .{ }^{1}$ TEXAS TECH UNIVERSITY HSC, Amarillo, TX; ${ }^{2}$ TTUHSC, Amarillo, TX; ${ }^{3}$ Texas Tech, Amarillo, TX; ${ }^{4}$ Texas Tech Univ Health Sc Center - Amarillo, Amarillo, TX. (Control ID \#2462633)

LEARNING OBJECTIVE \#1: Understand the proper technique of administrating IV calcium salts.

LEARNING OBJECTIVE \#2: Assess the need for IV calcium supplementation and manage incidental calcium salt extravasation properly to minimize complications. CASE: A 70-year-old Hispanic male patient was transferred to us from the ICU. He had presented with severe sepsis secondary severe complicated $C$. difficile colitis with acute kidney injury due to acute tubular necrosis. Upon noticing a developing ulcerated area on his arm, further investigation revealed that he had been resuscitated appropriately in the ICU but was found to have asymptomatic, severe hypocalcemia with a calcium of $6.1 \mathrm{mg} /$ $\mathrm{dl}$ (albumin $1.1 \mathrm{mg} / \mathrm{dl}$ ) and an ionized calcium of 0.83 (normal 1.1-1.3), phosphorus of $10.6 \mathrm{mg} / \mathrm{dl}$ (normal $2.5-4.5 \mathrm{mg} / \mathrm{dl}$ ), a magnesium of $2.7 \mathrm{mg} / \mathrm{dl}$ (normal $1.6-2.5 \mathrm{mg} / \mathrm{dl}$ ) and a creatinine of $4.6 \mathrm{mg} / \mathrm{dl}$. The patient was given $1 \mathrm{~g}$ of calcium chloride through a peripheral IV line. During the administration, the nurse noted a significant drop in the flow rate that led her to find that the IV line had infiltrated. The IV was pulled out and a new one inserted. A day later the patient developed an erythematous nodular skin lesion that worsened over the following days. Skin necrosis of the affected area ensued that ultimately required surgical debridement and engraftment of a $15 \times 18 \mathrm{~cm}$ skin flap to save the arm.

DISCUSSION: Hypocalcemia is a common finding in critically ill patients. Calculated correction of the observed serum calcium level is necessary if the serum albumin is significantly below $4 \mathrm{mg} / \mathrm{dl}$. Measuring ionized serum calcium, as was done in this case, is accurate and is not affected by the total albumin level. The standard of care is to replace calcium aggressively if the patient is symptomatic (carpopedal spasms, tetany, seizures or a prolongted QT interval) or if the ionized calcium is $<0.8$ (or corrected total serum calcium $<7.5 \mathrm{mg} / \mathrm{dl}$ ). Mild symptoms like paresthesias are not an indication for IV replacement. Due to the known highly toxic effects of calcium salts on subcutaneous tissues, they should ideally be administered via a large, deep vein or via a central line to avoid the risk of extravasation. Some hospitals have a standard policy to give calcium chloride only through a central line, while calcium gluconate can be given through a deep peripheral large vein if diluted appropriately as it is less destructive. Hyperphosphatemia in the setting of renal failure, as in this case, would normally be treated with phosphate binders first. Hypomagnesemia is another issue that should be corrected first, before addressing a very low calcium levels in most cases. In the case of accidental extravasation of a calcium salt like calcium chloride, the following management protocol should be implemented to decrease the extent of the injury: 1) Stop the infusion immediately. 2) Disconnect the IV line but leave the needle/cannula in place. 3) Try to aspirate as much of the infiltrated fluid as possible. 4) Inject hyaluronidase enzyme subcutaneously (15 units/ $\mathrm{ml}$ ) in a clockwise fashion at 5 different sites with $0.2 \mathrm{ml}$ injected in each area. 5) Remove the needle. 6) Apply cold dry compresses to the affected area. 7) Keep tha arm elevated. This case reminds physicians of the indications for IV calcium replacement with emphasis on safe administration techniques as well as how to best address complications should accidental extravasation of a calcium salt occur.

MALNUTRITION OR SOMETHING MORE SERIOUS? FRAMING THE CASE TO ARRIVE AT THE CORRECT DIAGNOSIS Alex J. Sommerfeld ${ }^{2}$; Tala Achkar ${ }^{1}$; Thomas R. Radomski ${ }^{3}$. ${ }^{1}$ UPMC, Pittsburgh, PA; ${ }^{2}$ University of Pittsburgh Medical Center, Pittsburgh, PA; ${ }^{3}$ University of Pittsburgh School of Medicine, Pittsburgh, PA. (Control ID \#2467205)
LEARNING OBJECTIVE \#1: To describe an appropriate expansion of the differential diagnosis of anasarca when common causes have been ruled out.

LEARNING OBJECTIVE \#2: To illustrate how framing bias can lead to errors in diagnostic reasoning and delay arriving at a definitive diagnosis

CASE: A 63 year-old African-American woman with a history of pancytopenia attributed to a B-cell lymphoproliferative disorder was admitted to the inpatient oncology service with worsening edema and 40-lb weight gain over the past 6 months. She had multiple prior hospitalizations for edema and once for syncope, with multiple 24-h urine collections exhibiting sub-nephrotic proteinuria (250-750 mg/day) but otherwise unremarkable workups. She was previously diagnosed with malnutrition and deconditioning and was prescribed furosemide and instructed to consume a high protein diet. On physical exam, the patient was normotensive though tachycardic to 150 beats per minute with movement. She exhibited significant bi-temporal wasting, decreased breath sounds bilaterally with dullness to percussion up to the right middle lung zone. Abdominal distention and a fluid wave were both appreciated. She had $2-3+$ tense but pitting total body edema. There was no jugular venous distension, periorbital edema, rashes, jaundice, or stigmata of chronic liver disease. An electrocardiogram revealed sinus tachycardia with low-voltage $\mathrm{QRS}$ and no ischemic changes. A chest $\mathrm{x}$-ray was notable for bilateral pleural effusions. Her electrolytes were normal and her serum creatinine was $1.1 \mathrm{mg} / \mathrm{dL}$. The patient had a stable anemia and leukopenia (Hemoglobin $10.6 \mathrm{~g} / \mathrm{dL}$ and white blood cell count $3.4 \times 10^{\wedge} 3 / \mathrm{uL}$, respectively). She was hypoalbuminemic, with a serum albumin of $2.1 \mathrm{~g} / \mathrm{dL}$ and a total protein of $7.9 \mathrm{mg} / \mathrm{dL}$. Her erythrocyte sedimentation rate was markedly elevated to $143 \mathrm{~mm} / \mathrm{h}$. The patient underwent an extensive workup, which included an unremarkable CT scan of the chest, abdomen, and pelvis, SPEP/UPEP, bone marrow biopsy, and EGD with biopsies. Her ascitic fluid was exudative with a serum-ascites albumin gradient of 0.7 , and her pleural fluid also was noted to be exudative by Light's Criteria given pleural protein/serum protein ratio of $>0.5$. Given evidence of serosal involvement (exudative ascites and pleural fluid), constitutional symptoms, anemia, leukopenia, and persistent subnephrotic proteinuria, we were suspicious of systemic lupus erythematosus (SLE). Further rheumatologic work-up revealed a + ANA 1:80 (speckled pattern), low complements (C3 of $27, \mathrm{C} 4$ of $<8$ ) with + Anti-Sm Ab, +Anti-RNP, and + DS-DNA $(>300 \mathrm{IU} / \mathrm{mL})$. She was ultimately started on pulse dose steroids by rheumatology prior to discharge. She has continued to follow up with outpatient rheumatology for which she is currently on hydroxychloroquine $400 \mathrm{mg}$ daily and prednisone $15 \mathrm{mg}$ daily. Her anasarca has completely resolved. Nephrology is continuing to follow the patient and is planning to perform a renal biopsy to asses for Class III/IV Lupus nephritis given up-trending urine protein/cr ratio, most recently measured at $2.8 \mathrm{mg} / \mathrm{mg}$. On follow up with hematology, her B-cell lymphoproliferative disorder was believed to be a manifestation of autoimmune disease rather than a true lymphoma.

DISCUSSION: Generalized edema is a common complaint encountered by general internists. Classically, the initial approach to edema/anasarca is to consider cardiac causes such as congestive heart failure, nephrotic syndrome, and cirrhosis. However, this case illustrates that the workup should not end once these most common causes are excluded. In this case, the patient's presentation was presumed to be secondary to malnutrition despite normal calorie/ protein counts. In addition to lupus, neoplastic causes such as lymphoma with lymphatic obstruction were at the top of our differential given the patient's history of a lymphoproliferative disorder. Protein wasting enteropathies — including intestinal amyloid - were also in our differential given the patients significant hypoalbuminemia. We also considered multiple myeloma, due to her elevated protein gap and family history. Additionally, despite normal $\mathrm{EF}$ and diastolic function, we considered right sided heart failure due to pulmonary hypertension. We believe our case was affected by framing bias as her presentation was framed as a female with an indolent B-cell lymphoproliferative disorder with concern for developing lymphoma. Prior to her admission, she even received rituximab, further framing our approach to her work up. As such, etiologies such as lymphatic obstruction, multiple myeloma, and amyloidosis were highest in our differential. The presence of exudative ascites and pleural effusions were initially considered to be further evidence of malignancy rather than serositis. However, when re-framed as a patient with marked anasarca, hypoalbuminemia, anemia, 
leukopenia, serosal involvement, and persistent sub-nephrotic proteinuria, our differential diagnosis expanded to include lupus.

MARKEDLY ELEVATED FERRITIN WITH FEVER AND LYMPHADENOPATHY: A COMMON PRESENTATION OF A RARE DISEASE, KIKUCHI'S Julie S. Nusbaum. New York Presbyterian Cornell, New York, NY. (Control ID \#2470347)

LEARNING OBJECTIVE \#1: Learn about the typical features of a rare disease, Kikuchi's Disease, and how to distinguish it from similar diseases.

LEARNING OBJECTIVE \#2: Learn the differential diagnosis for a ferritin level $>10,000$.

CASE: A 41-year-old previously healthy man presented with fevers, chills, and lymphadenopathy for 2 weeks. Had recently had a sore throat and cough which had resolved a few weeks ago. He had no recent travel, sick contacts, and review of systems was negative for other infectious symptoms. He was found to be hypotensive to $80 / 40$, tachycardic to 120 , tachypneic to 30 , and febrile to $102^{\circ}$ farenheit. His physical exam was notable for an ill appearance, nontender cervical lymphadenopathy, diaphoresis, and was otherwise negative. He was admitted to the ICU for presumed sepsis and responded well to aggressive IV Fluid resuscitation. Labs showed WBC 20, BUN elevated to 24, Creatinine elevated to 2.2 from a baseline of 0.7 . No anemia, thrombocytopenia, or liver chemistry abnormalities. Chest XRay showed atelectasis versus consolidation at the bases bilaterally. Patient was admitted to the ICU for SIRS and received aggressive IV fluid resuscitation and empiric antibiotics with vancomycin and piperacillin/tazobacterim. Infectious studies all came back negative including rapid strep test, urine culture, blood culture, and respiratory viral panel. The patient continued to have intermittent fevers, chills, tachycardia, and hypotension in spite of antibiotics and fluids. He was briefly started on pressors. The differential diagnosis at this time included lymphoma and viral illnesses like EBV, CMV, HIV, and adenovirus. CT Chest/Abd/Pelvis was done which showed impressive necrotizing lymphadenopathy of the cervical and mediastinal lymph nodes. LDH was elevated to 400. PCR for EBV, CMV, and HIV were all negative. Ferritin was sent which came back at 14,000 adding to the differential HLH, Adult Onset Still's Disease, and Kikuchi's Disease. A mediastinal lymph node biopsy was done which showed necrosis w/ histiocytic infiltration consistent with Kikuchi's. The patient was started on $40 \mathrm{mg}$ prednisone on hospital day 6 with good results. He quickly defervesced. His vital signs gradually normalized and his lymphadenopathy diminished. He was treated with a 4 week course of steroids as an outpatient.

DISCUSSION: There is a short list of diseases that cause a ferritin level greater than 10,000. They include HLH, Adult Onset Still's Disease, and Kikuchi's disease. Kikuchi's disease is a necrotizing, histolytic lymphadenitis associated with fever and cervical lymphadenopathy that more commonly affects women than men. Although usually benign and self limited, cases of Kikuchi's can be quite severe as in the young man in this case. The pathogenesis remains unclear but is speculated to be a deranged $\mathrm{T}$ cell and histiocyte response to a viral illness, with EBV being implicated in a number of case studies. Most patients are under 40 years of age with a mean age at presentation of 30 , but the disease has been rarely reported in patients up to age 80 . As a relatively rare disease confirmed only on histology, the incidence of the disease is likely underreported. Extranodal manifestations of the disease reported in case studies have included diarrhrea, night sweats, weight loss, aseptic meningitis, autoimmune hepatits, and a transient maculopapular rash. The symptoms of Kikuchi's can be easily confused for SLE, lymphoma, and Adult Onset Still's Disease. The diagnosis is primarily made by pathology with biopsies showing foci of necrosis and hystiocytic infiltrate. The disease is usually self limited but severe cases can be treated with steroids. Patients with Kikuchi's develop SLE at a higher incidence than the general population and should subsequently be closely observed for symptoms of SLE. Physicians should have a low threshold to check ferritin in patients with a fever that persists in spite of antibiotics. A ferritin above 10,000 should prompt consideration for Kikuchi's disease.

MASSIVE LOWER GASTROINTESTINAL BLEEDING : A RARE PRESENTATION OF CARCINOID TUMOR Khushdeep S. Chahal. University of Alabama Birmingham, Huntsville Regional Campus, Huntsville, AL. (Control ID \#2469809)

LEARNING OBJECTIVE \#1: Recognize that carcinoid tumors though usually asymptomatic, can present with lower gastrointestinal bleeding.

LEARNING OBJECTIVE \#2: Recognize that though obscure gastrointestinal bleeding can be associated with carcinoid tumor, but very rarely massive and potentially fatal bleeding can occur

CASE: We present a rare case of massive lower gastrointestinal bleeding (GI) in a 75 year old healthy caucasian female with past history of hypothyroidism. Patient presented with transient loss of consciousness early in the morning. She reported vague abdominal pain for the last 4 days and episodes of bright red blood per rectum in the morning. Patient reported a colonoscopy in 2014 that was normal. On physical exam patient was found to be hypotensive $90 / 56$ and tachycardic to 107 , had mild tenderness in right lower quadrant but no rebound or guarding. Initial $\mathrm{Hb}$ of 11.1 trended down to 7.1 on repeat and 5.7 on the next day. Patient was aggressively fluid resuscitated and admitted to ICU, had episodes of bright red bleed per rectum in the ICU. Emergent colonoscopy was done which showed blood in the entire colon but source couldnt be localised. A red cell scan localised to the sigmoid colon, a follow up angiography and CT angiogram were non diagnostic. Patient then re-bled and had another red cell scan done which localised to the terminal ileum. Surgery was consulted, patient underwent laparotomy which revealed a small carcinoid in proximal ileum that was resected to the terminal ileum and a right hemicolectomy was performed. Pathology was positive for single carcinoid tumor without lymph node involvement. Patient required 18 units of packed red blood cells during the hospitalisation. She did well post surgery except for post operative ileus which resolved in a week.

DISCUSSION: Carcinoid tumors are rare, incidence in the United States has increased significantly, due to increased detection on imaging and endoscopy. In 2000, carcinoid tumors surpassed adenocarcinomas as the most common small bowel tumor reported to the National Cancer Data Base. Within the GI tract, most carcinoids arose in the small intestine (45\%), most commonly in the ileum within $60 \mathrm{~cm}$ of the ileocecal valve, More commonly present in African Americans and in males. In general, midgut carcinoid tumors produce serotonin and other vasoactive substances that give rise to the typical carcinoid syndrome of flushing and diarrhea and occurs predominantly in patients with metastatic carcinoid tumors originating in the small intestine. Our patient with carcinoid localised to the terminal ileum did'nt have any of these features. Commonly intestinal carcinoid presents asymptomatically or with diffuse abdominal pain, intermittent obstruction can occur in $25 \%$,only rarely it can cause obscure GI bleeding but massive GI bleed is extremely rare. There are only a couple of cases with massive GI bleed reported. The fact that colonoscopy didnt reveal a bleeding source calls for high index of suspicion for carcinoid tumor and use of angiography and Red cell scans to aid in timely diagnosis and treatment in patients presenting with massive GI bleeds to avoid morbidity and mortality.

MASTER OF DISGUISE: IGG4-RELATED DISEASE Simeng Sun; Michael P. Bergman; Geeta Varghese. Mount Sinai Beth Israel, New York, NY. (Control ID \#2467542)

LEARNING OBJECTIVE \#1: Recognize IgG4-related disease. 
CASE: A 55-year-old male with no known past medical history presented with shortness of breath, nonproductive cough and subjective fevers for 1 week. Review of systems revealed a 20-lb unintentional weight loss over the past year. He was admitted to the MICU for sepsis and hypoxic respiratory failure secondary to $S$. pneumoniae multifocal pneumonia, empyema and bacteremia, which required noninvasive mechanical ventilation. Initial labs showed leukocytosis (WBC $20.6 \mathrm{~K} / \mu \mathrm{L}$ with $16 \%$ bands), troponin $0.122 \mathrm{ng} / \mathrm{mL}, \mathrm{Hgb} 11.5 \mathrm{~g} / \mathrm{dL}$, total protein $10.5 \mathrm{~g} / \mathrm{dL}$ and lactate $1.4 \mathrm{mmol} / \mathrm{L}$. CT chest demonstrated mediastinal, hilar, retroperitoneal and mesenteric lymphadenopathy in addition to pneumonia. The patient clinically improved after chest tube drainage, aided by intrapleural fibrinolytics, along with a prolonged course of antibiotics. At outpatient follow up, non-tender cervical and submandibular adenopathy along with lacrimal gland enlargement were noted. Fine needle aspiration was performed; cytology and flow cytometry results were only suggestive of reactive lymphocyte populations. Repeat CT chest 6 weeks later showed persistent consolidations and diffuse lymphadenopathy; subsequently, endobronchial ultrasound with trans-bronchial needle aspiration failed to show lymphoma or granulomas. A trans-bronchial biopsy in an area of persistent right middle lobe consolidation showed a mixed inflammatory infiltrate with plasma cells, lymphocytes and eosinophils with fibrosis. Given these inconclusive results, he was referred to otolaryngology for excisional cervical lymph node biopsy. A pre-operative stress echo showed severe pulmonary hypertension with right ventricular systolic pressure of $83 \mathrm{mmHg}$ that increased to $104 \mathrm{mmHg}$ post-exercise. Left and right heart catherizations were subsequently performed, which showed single-vessel (right coronary artery) nonobstructive disease and pulmonary artery systolic pressure of $82 \mathrm{mmHg}$. The patient was readmitted to the hospital for worsening dyspnea on exertion. At that time, the peripheral blood smear revealed rouleaux, plasmacytoid cells, plasmablasts, plasma cells and eosinophilia concerning for plasma cell dyscrasia. Cervical lymph node excisional biopsy showed atypical lymph nodes with sclerosis, vascular proliferation and plasmacytosis There was a significant increase in $\mathrm{IgG} 4$ to total $\mathrm{IgG}$ ratio with the association of sclerosis, which raised the possibility of IgG4 sclerosing disease. IgG4 serum level was also elevated to $>300 \mathrm{mg} / \mathrm{dL}$. Whole body PET scan revealed hypermetabolic activity in the following organs: bilateral lacrimal glands, retrobulbar right orbital apex and ocular muscles, extensive cervical, thoracic, abdominal and pelvic lymphadenopathy, soft tissue in bilateral renal pelvises and right middle lobe in the lung. Patient was started on prednisone $40 \mathrm{mg}$ daily for treatment of IgG4-related disease. The patient was also incidentally found to have rectal adenocarcinoma and underwent transanal excision of the rectal polyp.

DISCUSSION: IgG4-related sclerosing disease is a fibroinflammatory condition that is characterized by dense lymphoplasmacytic infiltration consisting of IgG4-positive plasma cells organized in a storiform pattern, obliterative phlebitis, eosinophil infiltration, and at times, elevated IgG4 serum concentrations. The disease process has been described in almost every organ system, linked by the same histopathological characteristics, and usually presents subclinically. Clinical pathology can include but is not limited to lymphadenopathy, autoimmune pancreatitis, chronic sclerosing dacryoadenitis and sialadenitis. About $40 \%$ of patients also have symptoms of asthma or allergies. Given this varied presentation and little information on this entity, it was often not placed on the differential. Diagnosis is made through tissue biopsy and the presence of defining pathological characteristics. No optimal treatment has been established, while the international consensus among experts agrees that glucocorticoids are the first line treatment. Most patients have been shown to improve clinically within weeks. B-cell depletion therapy with rituximab has been effective in cases refractory to glucocorticoids in case series, but has not been studied in randomized trials. Interestingly, some studies have shown increased risk of malignancy in patients with IgG4-related disease, especially in the year after diagnosis. IgG4-related sclerosing disease is an increasingly recognized disorder, which affects multiple organ systems. Due to its non-specific presenting symptoms, diagnosis is often challenging, leading to possible disease progression and a delay in initiating effective therapies. A high degree of clinical suspicion is therefore needed to make the correct diagnosis and begin treatment.

MAY-THURNER SYNDROME; AN UNDER-RECOGNIZED CAUSE OF VENOUS THROMBOEMBOLISM Yub Raj Sedhai ${ }^{1}$; Shoaib A. Sheikh ${ }^{1}$; Tawseef A. Dar ${ }^{2}$; Muznay Khawaja ${ }^{1}$; James C. McMaster ${ }^{1}{ }^{1}$ Mercy Catholic Medical Center, Yeadon, PA; ${ }^{2}$ Mercy Catholic Medical center, Drexel Hill, PA. (Control ID \#2457652)

LEARNING OBJECTIVE \#1: To recognise May-Thurner syndrome as one of the treatable causes of recurrent left lower extremity deep vein thrombosis.

LEARNING OBJECTIVE \#2: To recognise the role of thrombolytic and endovascular interventional therapies as well as maintenance anticoagulation therapy in patients with acute extensive ileofemoral deep vein thrombosis.

CASE: A 50-year old African-American female with history of chronic smoking and COPD presented to emergency with complaints of pain, swelling of left leg and dyspnea on exertion for 3 days. On examination she had sinus tachycardia with heart rate of $110 / \mathrm{min}$, blood pressure was $100 / 70 \mathrm{mmHg}$ and saturated at $87 \%$ on room air. Left lower extremity was swollen, tender and edematous. Blood count, metabolic panel \& coagulation profile were normal. Lower extremity doppler ultrasound showed deep vein thrombosis (DVT) extending from left common femoral to popliteal vein. Computed Tomographic (CT) angiography of chest showed right upper and lower lobe pulmonary embolism (PE). A subsequent thrombophilia work up was negative. She was admitted and anticoagulated with heparin. During the hospital course she developed abdominal pain. CT scan of the abdomen was done. It showed extensive left iliofemoral thrombosis extending to infra renal inferior vena cava (IVC). It also revealed right common iliac artery compressing the left common iliac vein; a feature consistent with May-Thurner Syndrome (MTS). An IVC filter was immediately deployed to prevent further embolism. Catheter directed thrombolysis (CDT) was done with tissue plasminogen activator (tPA). After complete thrombolysis a metallic stent was deployed in Left common and external iliac vein to correct the anatomical defect. She had uneventful recovery and was discharged home on coumadin for 6 months.

DISCUSSION: May-Thurner syndrome (MTS) is an anatomical defect described as external compression of left common iliac vein by right common iliac artery against fifth lumbar vertebrae. Long standing compression leads to intimal fibrosis with venous spur formation resulting in venous stasis \& thrombosing tendency as per Virchow's triad. MTS has a reported prevalence of $22-34 \%$ in cadaveric studies, however, only $2-3 \%$ of overall left lower extremity DVTs are reported as MTS related. A reason for such under diagnosis is probably because patients often have easily identifiable risk factors and work up is generally stopped once the diagnosis of DVT is confirmed. MTS in our case too was a completely unanticipated incidental diagnosis. MTS related DVTs are commonly associated with excessive clot burden which increases the risk of emolism, compartmental syndrome and post-thrombotic syndrome. Because of excesive clot burden and associated risks treatment of MTS related DVTs require catheter directed thrombolysis (CDT) along with anticoagulation. After completion of thrombolysis anatomical defect i.e. luminal narrowing caused by venous spur and external compression by artery should be recanalized with venous stenting. This is followed by maintenance anticoagulantion for 6 months like provoked DVTs. CDT and Venoplasty has good immediate and intermediate term outcome. Venous stent patency rate is reported as high as $83 \%$ at the end of 1 year. Failure to correct anatomical defect predisposes recurrent DVTs, PE and even iliac vein rupture. Given the prevalence and promising outcome of endovascular treatment, May-Thurner syndrome should be sought for in unprovoked left lower extremity DVTs especially in the absence of thrombophilia and risk factors. 
MAY-THURNER SYNDROME: A RARE CAUSE OF DEEP VENOUS THROMBOSIS Nabeel Siddiqui; Ubaid Sherwani. Presence Saint Francis Hospital, Evanston, IL. (Control ID \#2469926)

LEARNING OBJECTIVE \#1: To consider rare causes of Deep Venous Thrombosis such as May-Thurner Syndrome in young patients with no identifiable etiology

LEARNING OBJECTIVE \#2: To recognize the role of stent placement as a means of treating May-Thurner Syndrome

CASE: A 20-year-old female presented to the hospital with left lower extremity pain and swelling for 1 week. The swelling was initially in her foot and traveled up to her thigh, and the pain made her unable to ambulate. She denied any recent immobilization, travel or limb trauma. She reported oral contraceptive use and history of smoking one cigarette daily each for the past 1 year. She denied any history of blood clots and her family history was insignificant. She had stable vital signs. On exam, she had unilateral pitting edema in her left lower extremity up to the thigh, and tenderness to palpation in left posterior leg. DDimer was elevated at 7.2. Her coagulation profile and other tests for hereditary thrombotic disorders were normal. Ultrasound venous duplex identified a blood clot from the calf extending cephalad proximal to the femoral head, confirming the presence of DVT. She was started on anticoagulation with therapeutic enoxaparin. To prevent clot embolization, inferior vena cava filter (IVC) was placed. Moreover, tissue plasminogen activator was infused through a catheter in the left iliac vein. However, her pain persisted. CT angiogram showed extensive thrombus within the left common iliac vein, left external iliac vein and left femoral vein. There was also found to be narrowing in the distal aspect of left common iliac vein secondary to compression from a crossing left common iliac artery. This suggested the diagnosis of a variant of May-Thurner Syndrome. She underwent placement of stents in left common iliac vein and left iliac vein at level of pelvic rim. Eventually, her symptoms of pain and swelling resolved with the preceding interventions. Her IVC filter was removed, and she was started on warfarin. Once cinically stable, she was discharged with follow up arranged with her hematologist.

DISCUSSION: May-Thurner syndrome is a rare condition usually involving compression of the left common iliac vein by the overlying right common iliac artery, however, other variants like the one in our patient also exist. This can result in stasis of blood, leading to thrombosis. Vascular ultrasound or CT angiogram are used to establish the diagnosis. Treatment is based on angioplasty and stenting of iliac vein. Our case demonstrates that one should consider rare causes of DVT such as May-Thurner syndrome in young patients with no other identifiable causes. Thorough investigation and imaging can help identify the condition, and hence, prevent re-thrombosis in such patients.

MEGACOLON AND REFRACTORY HYPOGLYCEMIA Krishna Venkata; Ashish Manne; Hardik Satashia; Ashwini Sharma. UAB Montgomery, Montgomery, AL. (Control ID \#2470337)

LEARNING OBJECTIVE \#1: We present a case of pheochromocytoma who presented with megacolon, resistant hypoglycemia and was managed successfully, novel strategy with use of chemotherapeutic drugs.

CASE: A 42 year old male with no significant past medical history presented with 6 months history of gradual worsening of right upper quadrant abdominal pain. He was unable to tolerate food for past 2 days and had lost $10 \mathrm{lbs}$ in past 2 weeks. On physical exam he had abdominal tenderness with guarding but no rigidity, heart rate of 120 , rest of vitals were normal. Radiograph of the abdomen showed megacolon. Computed tomography revealed a right adrenal mass measuring $15 \times 13 \times 19 \mathrm{~cm}$ with necrosis and cystic changes, necrotic retroperitoneal lymphadenopathy and hepatic hypodensities. Biochemical tests showed elevated levels of plasma normetanephrine, metanephrine and chromagnin A. Patient also had persistent hypoglycemia and required continuous infusion of dextrose during hospitalization. Biochemical tests to evaluate hypoglycemia showed low insulin, low C-peptide, low IGF-I and normal cortisol levels at time of hypoglycemia which was consistent with consumptive mechanism. He was started on alpha-adrenergic blockade with doxazosin; hydrocodone for pain control and bowel regimen was maximized with inclusion of polyethylene glycol, docusate, lactulose and magnesium citrate. Despite maximal medical treatment the catecholamine excess resulted in worsening of hypoglycemia, ileus and abdominal pain. His symptoms necessitated urgent chemotherapy, surgery was not indicated due to hyper-vascularity of the tumor. He received cyclophosphamide, vincristine, dacarbazine. Two days after initiation of chemotherapy patient was able to pass flatus. After completion of 3 cycles, ileus improved and patient had a bowel movements. Even though the patient improved symptomatically, considering the poor prognosis of his malignant pheochromocytoma, he opted for palliative care and had a PEG tube placed for nutrition and hypoglycemia.

DISCUSSION: Malignant pheochromocytoma is a rare disease. Paralytic ileus, megacolon and refractory hypoglycemia are it's rare and life threatening complications. Typical presenting symptoms of pheochromocytoma include hypertension, anxiety attacks and palpitations. Ileus and megacolon are unusual presentations of pheochromocytoma. The physiology behind this complication is proposed to be secondary to excess amount of catecholamine secretion by the tumor. The elevated catecholamines in pheochromocytoma can lead to constipation affecting up to $13 \%$ of the patients. Improvement of gastrointestinal manifestations of pheochromocytoma by use of an alpha blockers indicates catecholamines may indeed be involved to cause constipation, paralytic ileus or megacolon. Catecholamines affect glucose homeostasis through both alpha and beta receptors. Habra et al. proposed that the cause of hypoglycemia might be related to rapid consumption of glucose. The findings in this case with low insulin, $\mathrm{C}$ peptide are consistent with this hypothesis. Malignant pheochromocytoma should be considered into differential diagnosis of unusual cases of megacolon or refractory hypoglycemia as illustrated in this case.

\section{METABOLIC ENCEPHALOPATHY DUE TO SEVERE HYPERNATREMIA} Harsha Mudrakola. Baylor College of Medicine, Houston, TX. (Control ID \#2470015)

LEARNING OBJECTIVE \#1: Recognize the risk of electrolyte abnormalities and their sequelae in patients dependent on tube feedings

LEARNING OBJECTIVE \#2: Recognize the risk of patient morbidity with lack of proper communication with patients and their families

CASE: A 64 year old female with recently diagnosed tonsillar squamous cell carcinoma was brought in by her family due to altered mentation. The patient had been diagnosed 3 months prior and was subsequently started on chemotherapy with cisplatin and concomitant external radiation therapy (XRT). 1 month prior to presentation, the patient developed mucositis and severe dysphagia. A percutaneous endoscopic gastrostomy (PEG) tube was placed and the patient was placed exclusively on tube feeds. Over the following month, the patient's family reported that she became more lethargic, confused, and weaker. On examination, the patient was lethargic and somnolent. She was afebrile, but had dry mucous membranes and was tachycardic. Neurological exam revealed intact cranial nerves and global weakness, but it was without focal deficits. Laboratory abnormalities included a sodium of 181 , chloride of 134 , bicarbonate of 33 , blood urea nitrogen (BUN) of 53, creatinine of 1.2, and glucose of 182 . Urine osmolality was $\mathrm{mOsm} / \mathrm{kg}$. Computed tomography (CT) of the brain did not reveal any abnormalities. The patient's symptoms were attributed to her severe hypernatremia. The etiology was lack of sufficient free water wit her tube feeds. During the previous hospitalization, the patient was prescribed $150 \mathrm{cc}$ of Nutren 2.0 every $4 \mathrm{~h}$ along with free water flushes of $200 \mathrm{cc}$ every four hours. The patient's husband had never received explicit tube feeding instructions and had been giving the patient feeds three times a day without any free water. Her calculated free water deficit was 11.51 . The hypernatremia was corrected with intravenous infusion of D5W and free water via the PEG tube at a 
rate of 8-10 meq/L per day. The patient's symptoms resolved completely upon correction of her serum sodium.

DISCUSSION: Encephalopathy due to metabolic disturbances is common in patients with cancer. Hypernatremia typically does not cause symptoms, but in this patient it was profound enough to do so. Correcting the hypernatremia should be done gradually — no more than 10-12 meq/L per day - to avoid cerebral edema and the resulting sequelae. The free water deficit can be easily calculated to find out exactly how much extra water the patient requires. The most common etiology of hypernatremia is the inability to replace free water losses from the skin, gastrointestinal tract and kidneys. This inability arises from a pre-existing condition precluding enough water intake - dementia, delirium, intubation/ sedation in the ICU setting etc. It may also be as simple as lack of proper communication with patients and their families as in this case. This case demonstrates the importance of properly educating patients in addition to properly managing their illnesses.

METASTATIC NEUROENDOCRINE TUMOR MASQUERADING AS CUSHING'S SYNDROME Andrew P. Scatola ${ }^{2}$; Virginia O. Volpe ${ }^{1}$; Amy Kost ${ }^{2}$. ${ }^{1}$ University of Connecticut, Farmington, CT; ${ }^{2}$ University of Connecticut School of Medicine, Farmington, CT. (Control ID \#2467858)

LEARNING OBJECTIVE \#1: Recognize and treat neuroendocrine tumors presenting as Cushing's Syndrome

LEARNING OBJECTIVE \#2: Appreciate that pancreatic neuroendocrine tumors can transform hormone production

CASE: A 37 year old male presents to clinic with new onset proximal weakness and hypertension. The patient was diagnosed 18 months ago with metastatic neuroendocrine tumor (NET) of the head of the pancreas following a work-up for unexplained jaundice Biopsy revealed that the NET produced. proinsulin, gastrin and chromagranin A. Initial symptoms were minimal, except for pain from liver metastases. He had a positive octreotide scan and was started on long-acting octreotide depot and everolimus, which lead to normalization of his fasting gastrin, proinsulin and chromagranin A. Cortisol and ACTH levels were normal at that time. A surveillance octreotide scan was suspicious for increased uptake in his liver metastases, however there were no new metastatic lesions. A month after the repeat scan, he was found to have the aforementioned findings, new hyperglycemia and a serum potassium of $2.5 \mathrm{mEq} / \mathrm{L}$. An evening cortisol and ACTH were elevated at $44 \mathrm{mcg} / \mathrm{dL}(3-12 \mathrm{mcg} / \mathrm{dL})$ and $225 \mathrm{pg} / \mathrm{mL}(7-69 \mathrm{pg} / \mathrm{mL})$ respectively. Due to concern for Cushing's Syndrome, he underwent an $8 \mathrm{mg}$ dexamethasone suppression test, with cortisol and ACTH remaining unchanged. MRI of the brain revealed no pituitary lesions, and MRI of the abdomen revealed new liver metastases with no changes in his prior lesions. Given this and the non-suppression of the dexamethasone test, it was felt these new metastatic lesions were responsible for his ACTH production and Cushing's Syndrome. He was started on metyrapone in order to prevent further symptom development and he experienced normalization of his serum cortisol.

DISCUSSION: Pancreatic NETs are rare, with an incidence of $1-5$ cases per million population. The most common types are insulinomas, gastrinomas and non-functional tumors, while primary ectopic ACTH producing NETs are exceedingly rare. The term nonfunctional is misleading since most of these tumors produce peptides but not a clinical syndrome. Symptoms typically only arise as a physical consequence of the tumor itself. Due to severe symptoms, functional tumors usually present early with a small primary tumor, while nonfunctional NETs present later with invasive tumors and metastatic lesions. NETs may produce one or more hormones and their clinical course may be unpredictable. Reports exist of new metastatic lesions with de novo ACTH secretion, which have even occurred years after initial resection of a non-ACTH secreting, nonmetastatic primary tumor. This suggests that metastatic lesions can dedifferentiate and are principally responsible for ectopic ACTH secretion and development of Cushing's
Syndrome. Treatment starts with controlling the hormone-excess state to attenuate clinical symptoms. Treatment must also target the tumor itself, and can include traditional chemotherapy, somatostatin analogs, mTOR and tyrosine kinase inhibitors, and hepatic arterial chemoembolization. Given the unpredictable nature of NETs, as in our patient's case, it is important for clinicians to anticipate that new symptoms in a NET patient may herald new hormone production or worsening metastatic disease.

\section{METASTATIC PROSTATE CARCINOMA MASQUERADING AS CIRRHOSIS} Osman Bhatty; Karishma Bhatia. Creighton University, Omaha, NE. (Control ID \#2467856)

LEARNING OBJECTIVE \#1: Recognize a rare radiological manifestation of prostate cancer metastases

CASE: A 76 year old male with past medial history of hypertension, gastroesophageal reflux disease, colonic tubular adenoma and chronic low back pain presented to the emergency department with new onset dyspnea on exertion for 3 day's duration. He denied any associated cough, fevers, chills, lower extremity swelling and prior respiratory or cardiac problems. His other complaint was worsening of chronic low back pain that he was experiencing some relief of with acetominophen. This was an ongoing issue but at the time he felt it had acutely worsened over the past few weeks. Other history was significant for alcohol use (one drink a day) and chewing tobacco. Physical exam was noncontributory and review of systems was unimpressive. Patient's workup revealed D dimer grossly elevated to about 1100 along with a marked elevation in alkaline phosphatase as well as gamma glutamyl transferase. Mild elevation in aspartate transferase, alanine transferase and total bilirubin was also noted. He was admitted for workup of his hypoxia of unknown etiology and elevated liver enzymes. CT-Angiogram revealed no thromboembolic disease. The liver was visualized and had an appearance consistent with chronic liver disease. A right upper quadrant ultrasound of the abdomen showed findings that corresponded to fatty liver disease. Due to the elevation of gamma glutamyl transferase liver specific etiology was higher in the differential and thus a hepatitis panel, acetaminophen and alcohol level were ordered which were all negative. Gastroenterology service was then consulted and a MRCP was done which showed innumerable hepatic metastases and multifocal retraction of the capsule consistent with pseudocirrhosis/carcinomatous cirrhosis appearance of the liver. PSA was then checked which was markedly elevated and a bone scan was ordered considering his history of worsening back pain. His results showed multiple regions of uptake (ribs, hips, femur, pelvis, spine). A diagnosis of metastatic prostate cancer was thus made.

DISCUSSION: Pseudocirrhosis or metastatic carcinomatous cirrhosis refers to a radiographic finding that resembles macronodular cirrhosis, but in which histopathological specimens fail to show the typical findings of cirrhosis. This condition has been observed in patients with cancer metastatic to the liver, both in those who have undergone prior systemic chemotherapy and those who have not. Pseudocirrhosis has been most widely described with metastatic breast cancer, however, cirrhotic changes have also been reported in hepatic metastasis of a variety of cancers, including pancreatic cancer, esophageal cancer, small-cell lung cancer, and thyroid cancer. Here we describe, to the best of our knowledge the first reported case of prostate cancer with metastasis to the liver with radiographic appearance of pseudocirrhosis. The radiographic features of pseudocirrhosis are similar to liver cirrhosis: nodularity of the liver contour with capsular retraction, decreased size of the liver, diffuse heterogeneity associated confluent fibrosis, and enlargement of the caudate lobe. In our patient ultrasound of the right upper quadrant revealed cirrhotic appearance of the liver, computed tomography suggested abnormal liver morphology representing chronic liver disease and magnetic resonance cholangiopancreatography demonstrated markedly heterogeneous liver with surface nodularity and multifocal retraction of the capsule compatible with pseudocirrhosis secondary to metastatic disease. The precise mechanism 
underlying development of pseudocirrhosis remains unclear. Based on previous studies, pseudocirrhosis occurs in cases in which hepatic histopathology shows evidence of extensive fibrosis representing a profound desmoplastic response to the infiltrating tumor, which is potentially related to an altered expression pattern of adhesion molecules. Despite its name, 'pseudocirrhosis', its clinical significance is equivalent to that of 'true' liver cirrhosis as it can cause hepatic decompensation and complications of portal hypertension, such as hepatic encephalopathy and variceal bleeding and have an effect on prognosis. Therefore, these patients should be monitored carefully for progression of hepatic failure.

\section{METHAMPHETAMINES AND DANCE CARNIVAL ON A HOT DAY IN} VEGAS: A DEADLY COMBINATION! Simran Kaur B. Matta. University of Nevada School of Medicine, Las Vegas, NV. (Control ID \#2470259)

LEARNING OBJECTIVE \#1: To understand the effects of methamphetamines and hyperthermia on cardiovascular system

LEARNING OBJECTIVE \#2: To identify that the two may potentiate the effect of each other and worsen the outcome

CASE: A 20-year-old male with no known past medical history was brought in from Electric Daisy Carnival in Las Vegas. He had seizures for $15 \mathrm{~min}$ and had to be intubated at the field for airway protection. His core temperature was as high as $108.7^{\circ}$ Fahrenheit. The EKG obtained in the ER showed diffuse ST-T depressions. He had been tachycardic in 140-160 s but maintained a stable BP initially. Pertinent labs: Urine drug screen was positive for amphetamines. The initial troponin was 0.33 which continued to trend up to $50.00 \mathrm{ng} / \mathrm{ml}$. CK levels were elevated and quickly increased to $>40800 \mathrm{U} / \mathrm{L}$. He had a potassium level of $6.6 \mathrm{mmol} / \mathrm{L}$, bicarbonate of $15 \mathrm{mmol} / \mathrm{L}$ and lactic acid of $7.5 \mathrm{mmol} / \mathrm{L}$ The echocardiogram showed global left ventricular dysfunction with an EF of 20-25\%. Pulmonary catheterization revealed low PCWP, low SVR and a high cardiac index. The patient also suffered DIC, shock liver and renal failure. He also developed severe sepsis and was on antibiotics for much of his hospital stay. He was on multiple vasopressors. We continued to provide supportive care and cooling measures to bring the temperature down. The patient persistently remained in respiratory failure requiring high $\mathrm{FiO} 2$ and PEEP. Unfortunately he developed cardiac arrest on Day 23 and expired

DISCUSSION: Reports of methamphetamine-related emergency room visits suggest that elevated body temperature is a universal presenting symptom, with lethal overdoses generally associated with extreme hyperthermia. The mechanisms through which methamphetamine affects body temperature include both central and peripheral targets. It stimulates the HPA axis, increases metabolic activity, pyrogen formation and release, and promotes heat generation and retention while suppressing responses that would facilitate heat dissipation. Stimulated alpha- and beta-adrenergic receptors produce hypertension, tachycardia, hyperthermia, and vasospasm. High catecholamine levels are also known to be cardiotoxic. Resulting coronary artery vasospasm and/or atherosclerotic plaque rupture can cause ACS. Myocardial necrosis and recurrent vasospasm from chronic use have been also proposed to result in methamphetamine-induced cardiomyopathy ${ }^{1,2,3}$. Hyperthermia, on its own, has been known to cause cardiac injury. In animal studies, elevation of body temperature to $40^{\circ}$ Celcius was associated with increased portal and systemic arterial lipopolysaccharide (endotoxin) concentration. Such high levels have been identified to cause pulmonary and systemic edema and cardiac dysfunction ${ }^{4}$. The exact mechanisms, however, of myocardial injury from hyperthermia remain unclear. The degree of troponin elevation has been shown to reflect the severity of heat stroke in rat models $^{5}$. It has been suggested that simple cooling techniques alone may abate the EKG changes and lower the troponins since the changes are not always related to a coronary lesion ${ }^{6}$. However, concurrent amphetamine overdose complicates the case and the possibility of true AMI in such situations cannot be excluded. Our patient had classic hypercirculatory hemodynamic profile on pulmonary catheterization as has been reported previously in cases of heat strokes. This made acute myocardial infarction a less likely cause of shock, and cardiac catheterization was not deemed necessary especially in the face of ongoing sepsis and multi-organ failure. While both hyperthermia and high doses of amphetamines are cardio-toxic, their combination may have a synergistic effect on myocardial injury. More studies are needed to fully elucidate mechanisms and potential cardiovascular effects of the combination of amphetamines and hyperthermia. This case highlights that methamphetamines and hyperthermia can be a fatal association and more awareness is needed among the people to prevent future episodes. 1. Darke S, et al. Major physical and psychological harms of methamphetamine use. Drug Alcohol Rev. 2008, 27 : 253-262. 2. Turdi S, et al. Acute methamphetamine exposure inhibits cardiac contractile function. Toxicol Lett. 2009, 189: 152-1528. 3. Volkow ND, et al. Distribution and pharmacokinetics of methamphetamine in the human body: Clinical implications. PLoS One. 2010, 5 (12): e15269-10.137. 4. Suffredini A, et al. The cardiovascular response of normal humans to the administration of endotoxin. N Engl J Med 1989; 321:280-87 5. Hausfater $\mathrm{P}$, et al. Elevation of cardiac troponin I during non-exertional heat-related illnesses in the context of a heatwave. Crit Care. 2010;14(3):R99. 6. Wakino S, et al. A case of severe heat stroke with abnormal cardiac findings. Int Heart J. 2005 May;46(3):543-50

MIGRATING BELL'S PALSY: A CASE OF NEUROSARCOIDOSIS Crysta $\mathrm{Iv}^{2}$; Michelle L. Kraslow ${ }^{2}$; Ryan Nall ${ }^{1} .{ }^{1}$ University of Florida, Gainseville, FL; ${ }^{2}$ University of Florida College of Medicine, Gainesville, FL. (Control ID \#2468985)

LEARNING OBJECTIVE \#1: Recognize the neurological clinical manifestations of sarcoidosis.

LEARNING OBJECTIVE \#2: Describe the diagnostic approach to neurosarcoidosis. CASE: A 31 year old African American male with no significant past medical history presented with a 2 month history of constant headache and a migrating Bell's palsy. Two months prior to evaluation, patient experienced a facial palsy of his right lower face associated with dysarthria of complex words. One week later, he developed a persistent headache (8/10 in severity). Additional symptoms included a dry cough, swollen right preauricular lymph node, right eye blurred vision, and photosensitivity. Three weeks later, his left lower face became paralyzed, associated with left eye blurred vision, photosensitivity, and lagophthalmos. As he developed left-sided palsy, he regained motor function in the right lower face, but noted persistent tingling and numbness. Headache, tinnitus, dizziness, and left-sided facial palsy continued to presentation. Prior to admission, the patient took prednisone to reduce his facial palsy symptoms. He denied seizures, memory loss, coordination impairments, skin rashes, joint pain, fever, fatigue, shortness of breath, wheezing, or hemoptysis. On physical exam, patient was alert and oriented. Cranial nerve (CN) examination revealed normal bilateral sensation to light touch. Unable to smile on left, normal movement on right. Right cheek could puff air, but it seeped out of the left side of the mouth. Left eye could be pried open. All other cranial nerves intact. Babinski sign positive in left foot. Exam otherwise unremarkable except for minimal expiratory wheezing in the right lower lobe. Contrast MRI of the brain revealed a cyst in the corpus callosum and enhancement around right Meckel's cave and right CN VII extending into the proximal right mandibular nerve. Chest $\mathrm{CT}$ showed bilateral centrilobular pulmonary nodules with mediastinal and hilar lymphadenopathy. A fine needle biopsy of mediastinal lymph nodes indicated epithelioid granulomas consistent with sarcoidosis. CSF analysis revealed elevated protein and leukocytosis (95\% lymphocytes) with normal glucose. Infectious workup was negative. Patient was started on $60 \mathrm{mg}$ oral prednisone once daily to be tapered every 2 weeks. Given no improvement at 6 weeks, methotrexate was added for long-term immunosuppression.

DISCUSSION: Sarcoidosis is an idiopathic multisystemic non-infectious inflammatory disorder characterized by noncaseating granulomas. Only $5-10 \%$ of patients with systemic 
sarcoidosis have neurological complications resulting in neurosarcoidosis (NS). However post-mortem studies indicate central nervous system (CNS) involvement in up to $25 \%$, suggesting the underdiagnosis of NS. Clinical diagnosis relies on the Zajicek criteria, which defines the likelihood of a NS diagnosis as definitive, probable, or possible, based on the amount of evidence. Noncaseating granulomas in the CNS are the histopathological hallmark of NS and are the only definitive diagnostic marker, however biopsy of brain parenchyma is highly invasive. Neurosarcoidosis is probable when neurological inflammation is concurrent with systemic disease and possible as a diagnosis of exclusion when patients present with typical neurological symptoms. Neurosarcoidosis most commonly manifests as cranial nerve abnormalities, especially optic (CN II) and facial (CN VII) neuropathies. Vestibulocochlear (CN VIII) and trigeminal (CN V) neuropathies may also present, resulting in facial sensory loss, hearing loss, and dizziness. Aseptic meningitis is the next most common neurological sign of sarcoidosis. Patients may less commonly display hypothalamic-pituitary dysfunction, encephalopathies, seizures, focal deficits due to mass effect of cerebral lesions, cerebral vasculopathy, and peripheral neuropathies or myopathies. Since no individual non-invasive test is specific for NS, practitioners should utilize a wide scope of diagnostic tools to confidently differentiate NS from neoplasms, inflammatory diseases, and infections. When NS is suspected, contrast enhanced T1-weighted MRI is the most sensitive and preferred diagnostic imaging technique. Basilar leptomeningeal enhancement is most often observed on MRI, but other common findings include focal lesions, diffuse dural thickening, hypothalamic-pituitary enhancement, and cranial nerve enhancement. Cerebrovascular findings, hydrocephalus, and spinal cord lesions are less common. CSF analysis may reveal elevated protein or white blood cells, decreased glucose, and increased IgG; however, increased sIL2-receptor alpha chain levels $>150 \mathrm{pg} / \mathrm{mL}$ and elevated ACE are the most specific findings in the CSF for NS. Electromyography may be used to determine peripheral nervous system involvement. Malignancy and infectious meningitis can result in MRI and CSF findings similar to NS. Therefore, cytology, flow cytometry, and cultures are used to rule out these processes. Lymphadenopathy and nodules on chest, abdomen, and pelvis CT can be indicative of systemic sarcoidosis and add further evidence to the overall clinical and diagnostic picture for neurosarcoidosis.

MINDING THE ANION GAP Meaghan S. Roche. Boston University Medical Center, Boston, MA. (Control ID \#2454741)

LEARNING OBJECTIVE \#1: Identify euglycemic diabetic ketoacidosis in an appropriate clinical context

LEARNING OBJECTIVE \#2: Recognize common cognitive biases seen in clinical practice

CASE: A 47-year-old woman with a history of insulin-dependent diabetes, Graves' disease, prior spinal fusion surgery with indwelling hardware, and fibromyalgia presented to the ED with complaints of severe low back pain, myalgias, headache, nausea, and vomiting of 2 days' duration. She smoked one-half pack of cigarettes daily and denied alcohol or illicit drug use. She had not had any recent illnesses or sick contacts. Of note, her diabetes was poorly controlled despite insulin therapy for the past 8 years, and she had started canagliflozin, a novel SGLT2 inhibitor, 2 weeks prior to her presentation. She was initially seen at an outside ED and transferred for MRI of the lumbar spine given concern for epidural abscess. On arrival to the ED, the patient was afebrile, and her vital signs were stable. Physical exam was remarkable for an ill-appearing woman without localized pain, tenderness, or focal neurologic deficits. Initial labs were notable for serum bicarbonate of 16 , creatinine 0.76 , glucose 152 , lactate 0.9 , and anion gap of 17 . Serum and urine toxicology screens were negative. Venous blood gas revealed pH 7.20 and pCO2 43, and urinalysis showed moderate ketones and large glucose. The patient underwent lumbar puncture and lumbar spine MRI, and she was treated with broad-spectrum antibiotics, anti-emetics, and aggressive fluid resuscitation with normal saline. Neither lumbar puncture nor MRI showed any acute pathology. Repeat labs on arrival to the medicine floor revealed worsening combined anion gap and non-gap metabolic acidosis with serum bicarbonate 10, chloride 111 , and serum glucose of 78 . Venous blood gas showed pH 7.14 and pCO2 51.6, and the patient was visibly distressed, vomiting and diaphoretic. She was treated overnight with intravenous fluid boluses and anti-emetics. Arterial blood gas obtained a few hours later showed $\mathrm{pH} 7.05, \mathrm{pCO} 226.9$, bicarbonate 7 , and lactate 1.1. The patient was started on a bicarbonate infusion and transferred to the MICU, where an insulin infusion was initiated. Repeat labs and arterial blood gas showed resolving metabolic acidosis, and the patient's nausea and vomiting improved. Her anion gap closed within $24 \mathrm{~h}$, and she was started on weight-based insulin glargine with mealtime lispro. Notably, review of outside records, where the patient received her diabetes care, suggested a diagnosis of latent autoimmune diabetes of adults, or LADA. This form of diabetes is sometimes referred to as late-onset type 1 diabetes due to its common pathogenesis of pancreatic beta cell destruction versus the insulin resistance that characterizes type 2 diabetes.

DISCUSSION: This case illustrates an important cause of euglycemic diabetic ketoacidosis (DKA) associated with a novel class of oral antidiabetic medications, the SGLT2 inhibitors. Their mechanism of action is blockade of the sodium-dependent glucose cotransporter in the renal proximal tubules, which is responsible for urinary glucose reabsorption. This leads to marked glycosuria and polyuria due to osmotic diuresis, and it also promotes weight loss without a significant risk of hypoglycemia. Canagliflozin, the first SGLT2 inhibitor to be released, has been on the market in the United States since 2013, and post-marketing surveillance has led to multiple case reports of euglycemic ketoacidosis, mostly in type 1 diabetics. The exact mechanism by which SGLT2 inhibitors are linked to DKA has yet to be fully elucidated, but the number and severity of case reports that have arisen prompted the FDA to issue a warning in May 2015. Furthermore, this case depicts a clinical scenario in which common heuristics were employed, particularly the framing and availability biases. Upon transfer to the ED, the patient was initially framed as a diabetic with an unknown source of infection; her headache and back pain were major presenting symptoms because she had been transferred specifically for an MRI to rule out epidural abscess. Later, the patient's elevated anion gap, ketonuria, and worsening acidosis with concomitant nausea and vomiting strongly suggested DKA, but her blood glucose was normal. The leading differential diagnosis was starvation ketoacidosis in the setting of a viral illness, which was based on review of available common etiologies of elevated anion gap metabolic acidosis. As the patient's acidosis worsened, her new medication was investigated further, and the link between SGLT2 inhibitors and euglycemic DKA was discovered. The patient's brisk response to insulin and bicarbonate infusions supported this diagnosis. While this patient had a very good outcome, her diagnosis was not obvious or a well-described phenomenon. It is important to recognize common clinical reasoning "shortcuts" in cases like this one and to re-evaluate both the patient and the clinical data frequently in order to ensure timely diagnosis and correct management.

\section{MODERN-DAY AWARENESS OF ZOONOTIC DISEASES AND CLINICAL} IMPLICATION IN THEIR EARLY DIAGNOSIS Md U. Ali ${ }^{1}$; Mohammed ALJumayli ${ }^{1}$; Mohamed Hassanein ${ }^{2}$; Bipinpreet Nagra ${ }^{3}{ }^{1}$ Capital Health Regional Medical Center, Plainsboro, NJ; ${ }^{2}$ Capital health regional medical center, Plainsboro, NJ; ${ }^{3}$ Capital Health Medical Center, Trenton, NJ. (Control ID \#2470334)

LEARNING OBJECTIVE \#1: Staphylococcus intermedius associated infections are very rare in human beings but should always be considered among the differential diagnosis in serious invasive infections, especially among patients who are in close contact with dogs LEARNING OBJECTIVE \#2: Canine associated zoonotic disease should always be considered under differential diagnosis especially in patients who are immunocompromised and having intimate contact with dogs 
CASE: A 60 year old male with a history of diabetes, hypertension, pituitary mass, hypothyroidism and end stage renal disease on dialysis presented with complaints of fever, transient confusion and lethargy. The patient denied headache, neck pain, change in vision, nausea, vomiting, shortness of breath, recent fall, chest pain or palpitations. Patient has been on long term peritoneal dialysis and recently switched to hemodialysis for which he had tunneled dialysis catheter. There were reports of patient maintaining an intimate contact with his dog, where dog was licking him on several occasion. Patient had temperature of $102^{\circ} \mathrm{F}$ and tachycardia on arrival. Laboratory evaluation showed leukocytosis of 18.4 with $16 \%$ bandemia. CT of head showed no change in pre-existing pituitary mass and chest X-ray was normal. Blood cultures returned positive for $S$. intermedius and therefore transesophageal echocardiogram was done which showed a mobile vegetation which was attached to the posterior mitral leaflet measuring approximately $18 \mathrm{~mm}$. The patient was initially started on nafcillin resulting in a significant improvement in his condition. The patient was eventually discharged on cefazolin during which time he was asymptomatic and repeat blood cultures were negative for the microorganism

DISCUSSION: Zoonotic diseases are those that can be transferred between animals and humans. The CDC estimates that more than 6 out of every 10 infectious diseases in humans are spread from animals; fortunately the numbers are low in North America. The risk in humans becomes greater with immunosupression. Skin and oral flora of dogs harbor various pathogenic microorganisms including Staphylococcus intermedius. Case reports of human infections from this microorganism are relatively rare, but the true incidence is unknown because the pathogen is frequently misidentified as Staphylococcus aureus. There are only 16 cases in the literature that have described $S$. intermedius as a cause of infection in humans ranging from soft tissue infections to bacterial endocarditis. Most of these cases have been described in association with exposure to animals, mostly dogs. Above we report a rare case of $S$. intermedius causing bacterial endocarditis in a dialysis patient. The presumed source of infection was the patient's dog. Although very rare in human beings, disease caused by $S$. intermedius should always be considered among the differential diagnosis of serious invasive infections, especially among patients who are in close contact with dogs

MORAXELLA BACTEREMIA, A FORGOTTEN INFECTION Hadeel Zainah ${ }^{1}$; Seungjun $\mathrm{Kim}^{2} .{ }^{1}$ The Miriam Hospital, Providence, RI; ${ }^{2}$ Brown University, Providence, RI. (Control ID \#2469818)

LEARNING OBJECTIVE \#1: Recognize the clinical manifestations and treatment of Moraxella bacteremia

CASE: A 62-year-old man with a history of chronic obstructive pulmonary disease (COPD) on home oxygen $(3 \mathrm{~L} / \mathrm{min})$, coronary artery disease, diabetes, congestive heart failure and hypertension presented with dyspnea on exertion, orthopnea, chills, legs edema and productive cough with whitish sputum. He also had painful right shin lesions after a local trauma. He was found to have a temperature of $101.9^{\circ} \mathrm{F}$, pulse of $125 / \mathrm{min}$, blood pressure of $192 / 79$ and oxygen saturation of $92 \%$ on $6 \mathrm{~L} / \mathrm{min}$ of supplemental oxygen. Exam showed wheezing and bibasilar lung crackles, regular heart rhythm, and right leg pus-filled pustules $(2 \mathrm{~cm})$ with 2 smaller ulcers. Laboratory work revealed leukocytosis of $17.400 / \mu \mathrm{L}$. Chest radiography showed mild edema. He was started on vancomycin and piperacillin/tazobactam, and underwent surgical debridement of the leg lesions. Additionally he was treated for COPD and CHF exacerbations. Blood cultures grew Moraxella osloensis and Micrococcus species. The latter was considered a contaminant, while M. osloensis was treated with ceftriaxone for 14 days, and other antibiotics were discontinued. Transthoracic echocardiogram showed no vegetations but suspected severe mitral regurgitation. He refused transesophageal echocardiogram as well as spine imaging despite briefly worsening chronic neck pain at the site of old hardware. He was discharged after improvement.
DISCUSSION: Moraxella osloensis is a Gram-negative coccobacilli which exists on skin and in environment. It can rarely cause invasive infections in pediatric, adult, immunecompetent, or immune-compromised patients. It has been reported to cause bacteremia, osteomyelitis, endophthalmitis, meningitis, endocarditis and septic arthritis. Bacteremia has also been reported in patients with reactive airway disease or with impetigo. It is usually susceptible to penicillin, cephalosporins and aminoglycosides. Our patient's likely source was the upper respiratory tract or leg impetigo. He was treated with ceftriaxone given the reported resistance to penicillin. We could not rule out endocarditis or cervical osteomyelitis, which were still suspected given the clinical findings. Moraxella osloensis bacteremia could be serious. The source should be investigated extensively since M. osloensis could cause many infections. It might be associated with upper respiratory or skin infections as seen in this case. Physicians should be aware of this organism as a cause of bacteremia in order to seek the appropriate consultation.

MORE THAN A CASE OF RESISTANT HYPERTENSION: CUSHING SYNDROME SECONDARY TO ADRENAL CORTICAL CARCINOMA Nicoletta Machin; Rahul A. Parikh. UPMC, Pittsburgh, PA. (Control ID \#2466523)

LEARNING OBJECTIVE \#1: Hormonal evaluation to determine etiology of Cushing syndrome

LEARNING OBJECTIVE \#2: Diagnosis and management of secretory adreno-cortical carcinoma

CASE: A 55 year-old male with a history of previously well controlled hypertension and pre-diabetes on metformin presented to his PCP with uncontrolled hypertension and blood glucoses $>500 \mathrm{mg} / \mathrm{dl}$. The patient also noted increasing fatigue, weakness and rapid weight gain. He was started on a four drug anti-hypertensive regiment and basal/bolus insulin. The patient subsequently presented to the ED with worsening symptoms and new onset chest. In the ED the patient was afebrile, hypertensive 165/101 on amlodipine, carvedilol, furosemide, hydrochlorothiazide and valsartan. No abnormal physical exam findings were noted on this encounter. Laboratory examination revealed a potassium of $2.9 \mathrm{mEq} / \mathrm{l}, \mathrm{CO} 235 \mathrm{mEq} / \mathrm{l}$ and serum glucose of $263 \mathrm{mg} / \mathrm{dL}$. During his evaluation a CT chest angiogram identified a right $(10.6 \mathrm{~cm} \times 11.1 \mathrm{~cm})$ adrenal mass. The patient was evaluated by Endocrinology and Endocrine Surgery and on repeat examination was noted to have facial plethora, moon facies, truncal obesity, atrophic skin over the hands and shins with multiple ecchymosis and violaceous abdominal striae. A hormonal evaluation was conducted revealing $24 \mathrm{~h}$ cortisol elevated at $675 \mathrm{ug} / \mathrm{dl}$ (range 4-50). Followed by an afternoon ACTH level, which was suppressed at $<5$. The patient's DHEA-S level, urine catecholamines and urine metanephrines were unremarkable. His laboratory evaluation was consistent with ACTH independent disease and given his CT findings the most likely diagnosis was a cortisol producing adrenal tumor. He underwent open adrenalectomy and was found to have a pT2Nx Stage II, $12 \mathrm{~cm} \times 7 \mathrm{~cm}$ adrenal cortical carcinoma with large vessel venous invasion. The patient was subsequently started on adjuvant mitotane therapy to reduce the risk of developing distant metastatic disease.

DISCUSSION: Cushing's syndrome is a constellation of clinical and laboratory abnormalities secondary to excessive glucocorticoid ingestion or production. For a primary care provider, the principle challenge is to determine when to consider it, given the large number of patients with diabetes and hypertension who are seen in clinic daily. The Endocrine Society's clinical practice guidelines recommend after the exclusion of exogenous glucocorticoid, cortisol testing is recommended for patients with multiple, progressive discriminating features of the disease including, exam findings of easy bruising, facial plethora, proximal myopathy and striae. Combined with new onset or worsening of previously controlled hypertension, new or worsening glucose intolerance and rapid weight gain. Additionally, any patient with an incidental adrenal mass and one of the above findings should undergo screening for Cushing's syndrome. Recommended initial 
tests include urine free cortisol, late-night salivary cortisol, 1-mg overnight dexamethasone suppression test or low does dexamethasone suppression testing over $48 \mathrm{~h}$. Our patient's progressive hypertension, worsening glycemic control, weight gain and physical findings meet criteria for diagnostic evaluation. For patients found to have hormonally confirmed Cushing's syndrome the next step is determination of source i.e. pituitary vs ectopic. Our patient presented with a new adrenal mass, elevated cortisol and suppressed ACTH consistent with ectopic cortisol production secondary to an adrenal cortical carcinoma. Adrenal cortical carcinomas (ACC) are rare tumors with an incidence of 0.7-2 per million and only a subset of these tumors are hormonally active or secretory. Overall, ACC have a poor prognosis secondary to late diagnosis (Stages III, IV). Management of these tumors is based on whether surgical resection is possible, if so open resection is currently recommended. Tumors less than $10 \mathrm{cms}$ in size, which are resected with negative surgical margins or tumor spillage portend the best prognosis. Unfortunately, our patient's surgical margins were $<1 \mathrm{~mm}$ and he went on to require adjuvant mitotane. Mitotane is an adrenolytic agent, which causes adrenal cortical atrophy, it also decreases the production of cortisol and the metabolism of peripheral steroids. The role of adjunctive mitotane has mixed results and there is a lack of randomized clinical data given the rarity of this disease. It is currently the first line therapy for non-operative ACC or resectable disease with high risk of recurrence. Overall the goal of our presentation is to alert primary care physicians to closely monitor patients who have had worsening hypertensive disease or glucose intolerance for secondary causes. Treatment failure should raise the specter of the development of secondary hypertension including the diagnosis of Cushing's syndrome and appropriate work-up.

MOYAMOYA SYNDROME: A RARE PRESENTATION OF VZV VASCULOPATHY. Safeera Javed ${ }^{1}$; Srinidhi Jayadevappa Meera $^{2}$; Marilou Corpuz ${ }^{3}$; Andrea Porrovecchio ${ }^{4}$; Deepa Bhupali ${ }^{3}$; Bennal Perkins ${ }^{5} .{ }^{1}$ Montefiore medical center, NYC, NY; ${ }^{2}$ Montefiore Medical Center, Mamaroneck, NY; ${ }^{3}$ Montefiore Medical Center, Bronx, NY; ${ }^{4}$ Montefiore Medical Center, Bronx, NY; ${ }^{5}$ Montefiore Medical Center, New York, NY. (Control ID \#2457584)

LEARNING OBJECTIVE \#1: Understand that the complete work up moyamoya spectrum vasculopathy should comprise of autoimmune workup, hematologic work up and cerebrospinal fluid analysis including VZV serology, to look for acquired and potentially treatable etiologies.

LEARNING OBJECTIVE \#2: Obtain CSF samples for both VZV DNA and VZV IgG in order to make the diagnosis of VZV vasculitis, as the diagnostic value of detecting VZV IgG antibody in CSF is greater than that of detecting VZV DNA.

CASE: 23 year old female with diabetes mellitus presented with 2 weeks of twitching of the left side of the face, associated with slurred speech and weakness of left upper extremity. Examination was remarkable for mild nystagmus on lateral gaze and decreased strength in left upper extremity. MRI revealed right frontal cortical lesion, suggestive of infarct. MRA head revealed proximal occlusion of both left and right middle cerebral arteries MCAs, with areas of narrowing throughout the anterior and posterior circulations. Cerebral angiogram confirmed narrowing and extensive collateral vessels in the anterior and posterior circulations; features most consistent with moyamoya spectrum vasculopathy. A complete autoimmune, hematologic and infectious work up was conducted. Antinuclear antibody (ANA), Anti-neutrophilic cytoplasmic antibody (ANCA), C3/C4 and Antiphospholipid antibody were negative. Cerebrospinal fluid (CSF) analysis was negative for VZV PCR, but positive for VZV IgG, consistent with a diagnosis of VZV vasculitis. Patient was started on a 3 weeks course of acyclovir for VZV vasculitis.

DISCUSSION: Moyamoya cerebral angiopathy is characterized by progressive stenoocclusion of arteries around the circle of Willis, such as terminal portion of internal carotid arteries and proximal portion of middle and anterior cerebral arteries, with compensatory development of extensive collateral circulation. This collateral network of vessels gives a characteristic "puff of smoke" appearance on angiography, giving the disease its name, "moyamoya" in Japanese. This characteristic angiopathic pattern is referred to as moyamoya phenomenon, which either exists as an isolated idiopathic disease entity called moyamoya disease, or it can be associated with a variety of conditions, including infections, autoimmune, hematological disorders and connective tissue disorders, thus giving rise to the concept of moyamoya syndrome. Diagnosis of moyamoya angiopathy is based on radiological visualization of steno-occlusive disease in the branches of circle of Willis as well as visualization of an abnormal arterial collateral circulation in the vicinity of the steno-occlusive lesions. Infections are a wellrecognized cause of intracranial vasculopathy with progression to moyamoya syndrome. The inflammation associated with infections can lead to a progressive angiopathy which may represent a pathophysiologic mechanism for the arterial occlusion seen in moyamoya syndrome. Chronic brain hypoxemia results in secretion of pro-angiogenic factors, which results in development of collateral circulation as a compensatoy mechanism. Moyamoya syndrome has been reported after many viral and bacterial infections such as bacterial meningitis, neurosyphilis, leptospirosis, tuberculosis and HIV. We report a case of moyamoya syndrome in a patient diagnosed with VZV vasculitis. To our knowledge this is the first case of moyamoya syndrome that has been reported in an adult patient with VZV vasculitis. Over the last several decades, there has been a reported increase in the number of vasculopathies following VZV reactivation. VZV vaculopathies can be seen in both immunocompetent and immunocompromised individuals and can occur after a primary infection (chicken pox) vs reactivation of VZV in ganglions (zoster). These vasculopathies involve both small and large vessels, can be unifocal or mutifocal. The clinical spectrum of VZV vasculopathy has expanded to include TIA, ischemic and hemorrhagic stroke, temporal artery infection, aneurysm and arterial dissection. It is important to recognize that all of these variants can present without rash. While vasculopathy is a well-recognized complication of VZV infection, VZV as a cause of moyamoya syndrome has never been reported. Our patient presented with simple partial seizures and was found to have frontal infarct, along with VZV vasculitis. Cerebral angiogram showed severe narrowing of MCAs in conjunction with extensive collateral vessels, features most consistent with a moyamoya spectrum vasculopathy. The diagnosis of VZV vasculitis, however, is not always straightforward. Rash and CSF pleocytosis are absent in about one-third of the cases. Furthermore, CSF VZV PCR is not a sensitive test for diagnosis of VZV vasculitis. Compared to the common clinical practice of testing for VZV DNA in CSF alone, testing for VZV IgG antibody in CSF identifies more cases of VZV vasculopathy. This is in contrast to HSV encephalitis where PCR is very sensitive and specific for diagnosis. This is likely due to the chronic and protracted clinical course of VZV mediated neurologic disease. In VZV vasculopathy, the diagnostic value of detecting VZV IgG antibody in CSF is greater than that of detecting VZV DNA. Thus new guidelines recommend that where VZV vasculopathy is suspected, cerebrospinal fluid (CSF) should be analyzed for polymerase chain reaction (PCR) for VZV DNA as well as VZV IgG in CSF.

\section{MULTIPLE ACQUIRED PLASMA COAGULATION FACTOR DEFICIENCIES IN AN HIV-INFECTED PATIENT: A CASE OF ACQUIRED HEMOPHILIA}

Daryl Sudasena; Jennifer Brescoll; Nichole Miller; Vijayalakshmi Donthireddy. Henry Ford Hospital, Detroit, MI. (Control ID \#2467880)

LEARNING OBJECTIVE \#1: Recognize risk factors attributed to the development of acquired hemophilia which could potentially result to earlier diagnosis and decreased morbidity and mortality.

LEARNING OBJECTIVE \#2: Describe current available treatments including novel agents and their respective indications. 
CASE: A 66-year old African American woman with a past medical history significant for HIV on HAART with recent CD4 count of 159 and viral load of 212, multiple deep venous thromboses on lifelong warfarin, was admitted to our hospital initially with suspicion for severe sepsis with a lactate of 12.3 and likely source thought to be a breast abscess. Initial lab work showed an INR of 2.95, PT of 30.6, PTT > 200, D-dimer of 1280 and fibrinogen of 284 . She had an incision and drainage of the breast mass by the surgery team and the fluid collection was found to be a hematoma. After this procedure, her hemoglobin continued to drop to 4.7, she was intubated and required vasopressor support. She was taken back to the operating room twice more for washout and cauterization due to continued bleeding from the wound. Hematology was consulted and mixing studies showed failure of PTT correction and she was found to have factor VIII, IX and XI inhibitor proteins. Her quantitative inhibitor level initially was 702. Autoimmune work-up including ANA, rheumatoid factor and lupus anticoagulant were negative. Her CD4 was 18 and her viral load was 175 . She was treated with intravenous Solumedrol $500 \mathrm{mg}$ twice daily for 3 days followed by Prednisone $60 \mathrm{mg}$ daily and Cyclophosphamide $100 \mathrm{mg}$ daily. She received three doses of Human Prothrombin Complex Concentrate (Kcentra) which was later switched to Recombinant Human Coagulation Factor VIIa (Novoseven) at $30 \mathrm{mcg} / \mathrm{kg}$ every $4 \mathrm{~h}$. Attempts at weaning off Novoseven were complicated by recurrent bleeding from previous intravascular line sites and at the breast hematoma pocket itself. PT monitoring had shown a decreasing trend hence frequent Novoseven dosing was stopped as thrombosis was of concern. She was switched to Factor 8 Inhibitor Bypassing Activity (FEIBA) twice daily with complete cessation of bleeding allowing her to be completely taken off treatments. The patient was discharged to a rehabilitation facility on Prednisone and Cyclophosphamide. Her quantitative inhibitor level decreased from 702 to 19.5 by the time of her discharge and further declined to zero within 2 months as an outpatient. Throughout her hospitalization, she was transfused a total of 24 units of PRBC, 11 units of FFP, 3 units of cryoprecipitate and 2 units of platelets. It was determined that this persistent left breast hematoma was secondary to acquired hemophilia from HIV.

DISCUSSION: Acquired hemophilia is a rare but life-threatening bleeding disorder caused by the development of autoantibodies (inhibitors) targeting plasma coagulation factors. Characteristically, there is spontaneous hemorrhaging in soft tissue and within intramuscular planes in a patient with no previous personal or family history of bleeding as opposed to inherited hemophilia wherein males are more affected and bleeding most commonly occurs intra-articularly. Several risk factors have been linked to this coagulopathy including malignancy, autoimmune disease, infection (HBV, HCV, HHV, HIV) and drugs, however, the majority are thought to be idiopathic. Goals of management are to control bleeding and decrease factor inhibitor levels. Our patient is the first case to be reported of multiple acquired factor deficiencies (FVIII, FIX, FXI) occurring in a female presenting with left breast hematoma and known controlled HIV on ART. Our patient's presentation is unique in scope due to the severity of the multiple factor inhibition exhibited in a setting of controlled HIV resulting to clinically significant refractory hemorrhaging. Other potential concomitant causes of acquired hemophilia, which may have contributed to this patient's disease severity, were effectively ruled out. The tendency for certain patients to develop acquired hemophilia is thought to be due to the presence of certain gene polymorphisms or autoreactive CD4+ T-lymphocytes. It is presumed that high physiologic stress states involving humoral overactivity such as during immune reconstitution, parturition, exposure to an immunomodulatory agent, autoimmunity and active malignancy lead to increased antibody production in a milieu of T-cell imbalance and dysregulation of cytokine, plasma cell and antibody formation. While the generation of autoantibodies are controlled with immunosuppression, minor bleeding with low inhibitor levels are treated with Desmopressin or high purity factor VIII concentrate whereas more fulminant cases with high inhibitor levels call for the use of bypassing agents or recombinant porcine factor VIII concentrate. It is important to be aware of this rare although potentially lethal entity especially when a patient presents with known risk factors. Improved familiarity and recognition would likely decrease morbidity and mortality although more studies are indicated for risk stratification of patients identified as being prone to develop factor inhibitors which would determine appropriate treatment regimens.

\section{MULTIPLE MYELOMA WITH AMYLOIDOSIS: A CLINICAL CONUNDRUM} Lesley Jackson; Vimal Patel; Daniel Ely. UT Graduate School of Medicine, Knoxville, TN. (Control ID \#2466257)

LEARNING OBJECTIVE \#1: Diagnose amyloidosis when initial diagnostic tests are negative

LEARNING OBJECTIVE \#2: List the clinical signs that help diagnose amyloidosis in the presence of an associated underlying plasma cell dyscrasia

CASE: We present a case of a 68 year old African American male with a history of chronic diastolic heart failure, type II diabetes, hypertension, generalized weakness, dysphagia with weight loss, and normocytic anemia. He presented with progressive dyspnea of 6 months duration. Previously he had been admitted for a heart failure exacerbation and treated with aggressive diuresis. He endorsed orthopnea, exercise intolerance, weight loss, and fatigue. On exam, he had diminished breath sounds in the bases and 1+ pitting leg edema. Labs revealed mild anemia (HGB $10.2 \mathrm{~g} / \mathrm{dL}$ ). BMP was significant for a sodium of $130 \mathrm{mEq} / \mathrm{L}$, albumin $3.3 \mathrm{~g} / \mathrm{dL}$, and creatinine $0.8 \mathrm{mg} / \mathrm{dL}$; BNP was elevated at $329 \mathrm{pg} / \mathrm{mL}$. Chest X-ray showed bilateral pleural effusions. A prior chest CT noted hilar and mediastinal adenopathy and granulomatous calcifications in the right hilar region. Transthoracic echocardiography showed a normal ejection fraction (EF) of 55-60\% with left ventricular hypertrophy and slight "speckling" which was considered compatible with either hypertrophic or infiltrative cardiomyopathy. Pulmonary function tests revealed a restrictive pattern with FVC and FEV1 between 40 and $50 \%$. Due to progressive neurological symptoms despite therapy, our differential included some neuromuscular disorders and amyloidosis. We obtained a repeat echocardiogram. This revealed restrictive cardiomyopathy with progressive left ventricular dysfunction showing a reduced $\mathrm{EF}$ of $40-45 \%$. An abdominal fat pad biopsy was obtained to evaluate for amyloidosis but this was negative for amyloid in routine and Congo red stains. Amyloidosis remained high on our differential diagnosis, thus we pursued endomyocardial biopsy. This showed subendocardial fibrosis and positive staining with Congo red for amyloid. A cardiac MRI showed diffuse subendocardial enhancement pattern and other findings very suggestive of cardiac amyloidosis. Serum protein electrophoresis was suggestive of paraproteinemia, with immunofixation studies noting elevated IgA and lambda light chains. Urine free light chains and urine/lambda light chains were also elevated. Bone marrow biopsy revealed a monoclonal lambda restricted plasma cell neoplasm and negative Congo red stain. Nerve conduction studies showed presence of a severe symmetrical sensorimotor axonal neuropathy and electromyographic examination showed presence of a severe proximal myopathic process. A diagnosis of multiple myeloma with amyloidosis was made at that time. He received weekly dexamethasone. His symptoms stabilized, and he was discharged to a rehabilitation center, where Revlimid was later initiated for further treatment of the myeloma. Unfortunately, the patient did succumb to his illness and passed away at home from respiratory failure.

DISCUSSION: This case demonstrates that diagnosing amyloidosis can be elusive, and often multiple diagnostic techniques may be necessary. Amyloidosis is a condition in which a variety of serum proteins arranged in an abnormal configuration of fibrils deposit in extracellular matrix. The serum proteins are often derived from either monoclonal immunoglobulin light chain fragments (as in AL amyloidosis) or from the acute phase reactant serum amyloid A (as seen in chronic inflammatory disorders). Deposition occurs in multiple organs, including the heart, liver, lung, kidney, and nervous system, and often results in significant morbidity and mortality. It is suspected in patients that present with any of the following: restrictive 
cardiomyopathy, nephrotic range proteinuria, unexplained edema, increased NT-proBNP without known primary heart disease, or macroglossia. Congo red stained tissue showing the presence of apple-green birefringence when subjected to polarized light microscopy is pathognomonic of the disease. Diagnosis is confirmed only by biopsy, often initially by sampling an abdominal fat pad biopsy, as it is generally an uninvolved site with less risk of bleeding. Alternatively, biopsies can be obtained from other noninvolved sites such as minor salivary glands or rectal mucosa, or from dysfunctional organs like the kidney, nerve, or heart. Amyloidosis is in itself a type of monoclonal plasma cell proliferative disease as manifested by the presence of serum or urine monoclonal proteins ( $M$ protein), clonal plasma cells in the bone marrow, or an atypical serum free light chain ratio. It can occur alone or in the presence of an underlying plasma cell disorder like MGUS, multiple myeloma, or Waldenstrom macroglobulinemia, which makes diagnosis problematic. Amyloidosis should be suspected in patients with known underlying plasma cell dyscrasia that also have heavy proteinuria, edema, peripheral neuropathy, hepatosplenomegaly, or carpal tunnel syndrome. However, the high prevalence of MGUS in the general aging population means that the presence of M protein along with deposition of amyloid is not diagnostic of amyloidosis. One should examine the biopsy of amyloid directly to verify the presence of light chains using spectrometry-based proteomic analysis or immunoelectron microscopy.

MUSCULOSKELETAL CHEST PAIN- NOT JUST A DIAGNOSIS OF EXCLUSION Daniel J. Howell; Matthew Shaines. Montefiore Medical Center, Bronx, NY. (Control ID \#2469436)

LEARNING OBJECTIVE \#1: Recognize the presenting features of multiple myeloma LEARNING OBJECTIVE \#2: Identify appropriate imaging studies in myeloma and chest pain

CASE: This 65 year-old woman with a history of hypertension presented with central chest pain. The pain had been present at low intensity throughout the year with occasional random exacerbations for which she had had repeated non-diagnostic workups with EKG cardiac markers and chest radiographs. Other than chest wall tenderness her vitals and exam were unremarkable. On labs hemoglobin was $13.4 \mathrm{~g} / \mathrm{dl}$, WBC $3.9 \times 1000 / \mu \mathrm{L}$, creatinine $1.0 \mathrm{mg} / \mathrm{dl}$, albumin $3.8 \mathrm{~g} / \mathrm{dL}$, total protein $8.5 \mathrm{~g} / \mathrm{dL}$, calcium $9.9 \mathrm{mg} / \mathrm{dL}$, troponin T undetectable. A CT Thorax to better evaluate her persistent chest pain revealed several expansible lytic lesions with associated cortical destruction and soft tissue extension in the body of the sternum. Subsequent biopsy revealed a sheet of atypical plasma cell infiltrate expressing CD138 and lambda restricted consistent with plasma cell myeloma. SPEP was positive for an IgG-lambda monoclonal protein and her bone marrow revealed $0.5 \%$ polytypic plasma cells. A skeletal survey showed no lytic lesions. She commenced Lenalidomide and Dexamethasone and is currently doing well.

DISCUSSION: Chest pain is a commonly encountered complaint in primary care and it is the second most common chief complaint in the ED. $36.2 \%$ of chest pain presentations in primary care are due to musculoskeletal disease, the vast majority of which is self-limiting. While more serious causes of musculoskeletal chest pain are uncommon, bone pain as a feature of multiple myeloma is not. It is in fact present in $58 \%$ of individuals at presentation. In this patient the location of the bone pain in the chest and presentation on multiple occasions to the ED rather than to primary care may have distracted from suspicion of the diagnosis. However in patients with myeloma causing only a bone lesion around $20 \%$ will involve the sternum, rib, scapula or clavicle and therefore it is a reasonable diagnosis to consider in chronic chest pain. While additional findings of anemia, renal failure and hypercalcemia are often associated with myeloma, they are absent in 26,52 and $62 \%$ of patients respectively who present with myeloma. Thus, the lack of these hallmark clinical features should not dissuade one from pursuing a diagnostic workup. While almost $80 \%$ of patients with multiple myeloma will have radiological evidence of skeletal involvement on the skeletal survey, one of the major disadvantages of plain radiography is its high false-negative rate of $30-70 \%$, leading to significant underestimation in diagnosis. MRI is the most sensitive modality for imaging lesions in the spine but it misses skull and rib lesions so should be used in conjunction with plain radiographs. CT is the preferred imaging modality in detecting the osteolytic lesions of multiple myeloma and has a higher sensitivity than plain radiography and outperforms MRI. Further imaging is indicated either when there is uncertainty about the extent of disease or if bone pain is present without any skeletal survey abnormality. When bone pain is the only symptom there is added prognostic value in detailed imaging given that a lesion of $>5 \mathrm{~mm}$ or multiple lesions are predictive of progression to end-organ damage in $80 \%$ of patients. When to image a patient in primary care presenting with chest pain is more nuanced as there is little evidence or guidelines to support decision making. The persistence of symptoms without a cause in this case justified further imaging and future research may focus on when and how to image. Physicians should recall that the time course of symptoms is a key aspect of the history and to not prematurely close on a diagnosis that does not fit the chronicity of illness but rather widen the differential diagnosis and consider more uncommon diagnoses.

MYCOBACTERIA ARUPENSE OLECRANON BURSITIS IN AN IMMUNOCOMPETENT KAYAKER John D. Herlihy; James Horton. Carolinas Medical Center, Charlotte, NC. (Control ID \#2466036)

LEARNING OBJECTIVE \#1: Recognize exposure risk factors and presentation of atypical Mycobacteria infection

CASE: 68 year old male without significant medical who presents with recurrent right elbow pain and swelling over the past 8 months. The patient had been previously healthy and was an active kayaker and rock climber. Symptoms started shortly after a kayaking trip to Utah. He denied fever, chills, nausea, vomiting, shortness of breath, or diarrhea. He did complain of a nonproductive cough which had been going on for approximately 3 weeks. Aspirate from the elbow showed no organisms, but acid fast bacilli in blood culture after incubation was grown out. Synovial fluid showed 20,000 RBC, 3,500 nucleated cells, $84 \%$ sets, $16 \%$ lymphocytes. The patient was taken for debridement and cultures were sent for regular and cooler temperature cultures to look for atypical mycobacterium. Post op chest x-ray showed a $10 \mathrm{~mm}$ pulmonary nodule in the lower lateral right lung and a $13 \mathrm{~mm}$ nodule in the left costophrenic angle. The patient was started on Doxycycline and Levaquin while culture results grew out. The surgical culture results eventually grew out Mycobacteria arupense approximately 2 months after being drawn. At that time antibiotics were changed to Clarithromycin, Ethambutol, and Rifabutin for planned 6 month course. The patient's cough resolved shortly after starting antibiotics. His elbow pain and swelling resolved with debridement and antibiotic treatment. He had no further joint pain or stiffness. Chest CT was done to further evaluate the pulmonary nodules and it showed a $1 \mathrm{~cm}$ discrete round nodule in the right lower lobe and a similar $1 \mathrm{~cm}$ nodule laterally within the left lower lobe. A follow up chest CT 6 months later showed the pulmonary nodules to be unchanged in size or appearance. The patient is to have scheduled CT scans every 6 months for continued monitoring of these pulmonary nodules.

DISCUSSION: Mycobacteria arupense was first described in 2006 by Cloud et al. (2) and since that time there have been numerous case reports of infections caused by the bacteria. On review of the literature only one case report describes large joint infection caused by $M$. arupense. Seidl and Lindeque in 2014 described a patient with a history of traumatic knee arthrotomy in the past who presented with knee swelling and inability to bear weight. The patient was found to have osteoarticular infection secondary to $M$. arupense which responded well to debridement and appropriate antibiotics. Beam et al. (1) presented a case report of an otherwise healthy farmer who developed flexor tenosynovitis of his fingers and swelling of his entire right hand with the only pertinent history being a remote history of blunt trauma to his 
hand. Beam et al. divided the presentation of $M$. arupense into 3 clinical syndromes with the first being tenosynovitis/extremity infection after traumatic injury and environmental contamination, the second being pulmonary infection, and finally disseminated infection in immunocompromised host. Antibiotic susceptibility testing by Beam et al. (1) showed that $97.5-100 \%$ of isolates to be susceptible to clarithromycin, ethambutol, and rifabutin. Greater than $78 \%$ of these isolates were resistant to ciprofloxacin, moxifloxacin, rifampin, and doxycycline. Most reported cases show rapid improvement with initiation of antibiotics except for the cases in immunocompromised patients with disseminated disease. Based on a review of the available literature, most described peripheral infections from $M$. arupense tend to be tenosynovitis of the hand. The case described above is the second reported case of large joint infection. The patient has history of kayaking with trauma to the elbows suggesting a water source for the infection. The bacteria has been found to be prevalent in the water supply as shown by Castillo-Rosal et al. in 2012 (3) where they analyzed the water distribution system from the Mexico City Metropolitan Area and found M. arupense to be prevalent in the water supply. Given the prevalence of $M$. arapense in water supplies and the time and difficulty to culture many cases likely go underreported. In conclusion this case of $M$. arupense is unique in that it involves a large joint infection and also has associated pulmonary nodules which responded well to a 6 month course of clarithromycin, ethambutol, and rifabutin. This case highlights the importance of evaluating patients for environmental exposure risks and identifying the specific species of Mycobacterium in order to initiate prompt and appropriate antibiotic therapy and to outline follow-up procedures as needed. References Beam E, Vasoo S, Simner PJ, Rizzo M, Mason EL, Walker RC, Deml SM, Brown-Elliott BA, Wallace RJ, Wengenack NL, Sia IG. Mycobacterium arupense flexor tenosynovitisL case report and review of antimicrobial susceptibility profiles for 40 clinical isolate. Journal of Clinical Microbiology. 52(7): 27062708. Cloud JL, Meyer JJ, Pounder JI, Jost KC, Sweeney A, Carroll KC, Woods GJ. Mycobacterium arupense sp. nov., a non-chromogenic bacterium isolated from clinical specimens. Int. J. Syst. Evol. Microbiol. 56:1413-1418. 10.1099/ijs.0.6419-0 Castillo-Rosal AI, Mazari-Hiriart M, Lloret-Sanchez LT, Sachman-Ruiz B, Vinuesa P, Lopez- Vidal Y. 2012 Potentially pathogenic nontuberculous mycobacteria found in aquatic systems. Analysis from a reclaimed water and water distribution system in Mexico City. European Journal of Clinical Microbiology and Infectious Disease. 31:683-694

MYCOPHENOLATE-INDUCED POSTERIOR REVERSIBLE ENCEPHALOPATHY SYNDROME Victoria Menashy; Amanda Levine; Kara Stoudt; Rebecca Mazurkiewicz; Ira Wagner. Lenox Hill Hospital, New York, NY. (Control ID \#2468626)

LEARNING OBJECTIVE \#1: Posterior Reversible Encephalopathy Syndrome (PRES) is a neurological disease characterized by seizures, headaches, focal neurological deficits, changes in mental status. Hyperperfusion, probably related to endothelial dysfunction and increased capillary pressure in the brain, plays a key role in the pathogenesis. Common etiologies of PRES include hypertension, encephalopathy, chemotherapy or calcineurininhibitor drug toxicity and lupus among others. The neurotoxic effects of many immunosuppressive therapies are well-known but poorly understood.

CASE: An 18-year-old female with chronic history of WHO class IV lupus glomerulonephritis treated with prednisone, mycophenolate and hemodialysis, presented after having three witnessed generalized tonic-clonic seizures. Past medical history significant for seizure disorder, hypertension, lupus complicated by lupus nephritis and cerebritis, endstage renal disease (ESRD) on hemodialysis and iron-deficiency anemia. Home medications include levetiracetem, mycophenolate, metoprolol, nifedipine, folic acid and epoetin alfa. The patient has a history of marijuana and amphetamine drug abuse. No allergies. Review of systems otherwise negative. In the emergency department, she was noted to be hypertensive with a blood pressure of $239 / 118 \mathrm{mmHg}$, temperature of 98.3 , a heart rate of 110 , respiratory rate of 28 and SPO $100 \%$ on room air. Shortly after, the patient had another generalized tonic-clonic seizure. Examination revealed no focal neurological deficits, there was no neck stiffness or papilledema. A head CT revealed no mass lesion or ischemia. Her symptoms included seizures, headache and confusion. Her blood work was negative for any significant metabolic abnormalities and her urine toxicology was positive for opiates. Her blood pressure was treated acutely with hydralazine intravenously and clonidine orally. She had another witnessed seizure for which she received lorazepam and levetiracetam intravenously. She was admitted to the medical intensive care unit to begin nicardipine intravenous drip and to continue intravenous levetiracetam. Once stabilized, an electroencephalogram (EEG) showed frequent left temporal sharp waves, with no epileptiform activity. Cerebral MRI showed symmetric signal abnormality involving the cortex and subcortex of the occipital lobes. The MRI did not show a signal abnormality of the medial temporal lobes to correspond with the EEG findings. Based on the clinical presentation and imaging abnormalities, a diagnosis of Posterior Reversible Encephalopathy Syndrome (PRES) was made. Further testing revealed a homogeneous ANA at a titer of 1:1280, markedly elevated dsDNA antibody level of $146[<30 \mathrm{IU} / \mathrm{mL}]$, C3 $98 \mathrm{mg} / \mathrm{dL}$ [80-180 mg/dL], C4 $23 \mathrm{mg} / \mathrm{dL}$ [10-45 mg/dL]. She continued to receive hemodialysis throughout her hospital stay and a weaning regimen of oral prednisone along. She was transitioning to oral levetiracetam for seizure control. With this treatment, her mental status improved and patient was discharged from the hospital.

DISCUSSION: The patient may have concomitantly had a lupus flare with PRES, as her DsDNA and ANA were elevated from baseline. This thereby leads to conflicted opinions on management of PRES with continued use of current immunosuppressants for patients who have lupus and present with a flare up, as the medications needed to treat can be potentially causing the neurotoxic effects of PRES. There are currently no clear guidelines on further management of immunosuppressants in this context once the diagnosis of PRES is made. Early diagnosis of PRES is very important to prevent irreversible neurological sequelae. The diagnosis of PRES is often unsuspected by clinicians, and is often diagnosed by neuro imaging. PRES is reversible with cessation of the offending agent, rapid control of hypertension, and treatment of the underlying disease. This case illustrates severe PRES syndrome with use of cellcept as well as the value of a complete clinical history and picture. Although PRES is commonly associated with certain immunosuppressants, there have only been two case reports documenting cellcept as a potential agent in causing with PRES in lupus patients, one of these reports was in the setting of a renal transplantation where discontinuation of cellcept resulted in resolution of symptoms. Recognition of this syndrome and identification of agents that potentiate the development of PRES in lupus patients is critical for determining appropriate therapy and prevention of complications

\section{MYOPERICARDITIS: A DIAGNOSTIC CHALLENGE IN A PATIENT WITH} RECURRENT CHEST PAIN Maaman Bashir; Nabeel Siddiqui. St Francis Hospital, Evanston, IL. (Control ID \#2469845)

LEARNING OBJECTIVE \#1: To identify myopericarditis as an important differential in patients with recurrent chest pain and elevated biomarkers

LEARNING OBJECTIVE \#2: To recognize the importance of cardiac magnetic resonance (CMR) in diagnosing myopericarditis

CASE: The patient is a 45 year old male, with no significant past medical history, who presented with recurrent left sided chest pain. The first episode occurred the night before admission, lasted about $4 \mathrm{~h}$ and resolved without intervention. The second episode occurred the morning of the admission and prompted him to come to the emergency department. The pain was 'pressure-like', non-radiating, accompanied by mild shortness of breath and not related to positional change. A review of systems was negative. Physical examination was unremarkable. Work up revealed elevated troponins and a normal electrocardiogram. Complete blood count and basic metabolic panel were unremarkable. 
An angiogram the next day revealed normal coronary arteries. The echocardiogram was negative for structural, valvular or wall motion abnormailities. An elevated erythrocyte sedimentation rate prompted inflammatory work up for vasculitis including anti-nuclear antibodies, anti-neutrophil cytoplasmic antibodies and complement levels, which were also negative. At this point, vasospasm was considered as a possible cause of his symptoms and he was started on nifedipine. However, over next few days of admission, the patient continued to have bouts of severe, recurrent chest pain, usually lasting for hours. These episodes were accompanied by an increase in troponin (up to 6.6) and did not respond to nitroglycerin. Serial electrocardiograms revealed ST elevation in the precordial leads along with PR depression. Myocarditis viral panel was negative. The patient subsequently underwent a cardiac magnetic resonance (CMR). The CMR showed epicardial, subepicardial and myocardial enhancement within the infero-lateral wall and cardiac apex, consistent with myopericarditis. The patient was started on ibuprofen and colchicine. His chest pains resolved completely and he was discharged in stable condition.

DISCUSSION: Myopericarditis has a variable presentation; from being clinically asymptomatic with a diagnosis being made at autopsy, to intractable ventricular arrhythmias, syncope, congestive heart failure and even sudden cardiac death. Therefore, relying entirely on presenting signs and symptoms and an initial work up may not be sufficient. It is imperative to rule out other causes of persistent chest pain after ruling out acute coronary syndrome. Although no test definitively confirms the diagnosis of myopericarditis, CMR holds promise for an effective non-invasive diagnostic tool. It could be a valuable addition to other methods particularly in diagnostically challenging patients with troponin-positive, recurrent chest pain and normal coronary arteries. If the diagnosis remains uncertain after performing CMR, an endomycoardial biopsy can help confirm the diagnosis. Diagnostic challenges may occasionally arise, especially in the setting of unusual presentations as in our patient. The application of timely diagnostic measures and appropriate treatment remains the most effective strategy to ensure the correct diagnosis and treatment plan.

\section{NAPROXEN INDUCED PHENYTOIN TOXICITY IN A YOUNG AND} HEALTHY PATIENT. Oleksandra Lupak; Kassem Bourgi. Henry Ford Hospital, Detroit, MI. (Control ID \#2462332)

LEARNING OBJECTIVE \#1: To recognize and diagnose phenytoin toxicity secondary to phenytoin interaction with non-steroidal anti-inflammatory medications (NSAIDs) in patients with normal albumin levels and without major comorbidities.

CASE: Phenytoin is widely used for the treatment of major types of seizure disorders. Its mechanism of action involves inhibition of voltage-gated sodium channels that slows action potential propagation in the neurons of the central nervous system. However, its narrow therapeutic index and complex pharmacokinetics may lead to drug toxicity. Many case reports exist in the current literature regarding phenytoin toxicity in elderly patients, critically ill patients, and in patients with low albumin levels. This report presents a case of phenytoin toxicity secondary to phenytoin interaction with the naproxen in a relatively young patient without any major comorbidities and with a normal albumin level. Our patient is a 58-years-old African-American male with a known past medical history of a grand mal seizure secondary to head trauma during his early adulthood who presented to the Emergency Department with the chief complaint of bilateral blurred vision for one hour and dizziness for the past 2 days. He denied weakness or numbness of his extremities. Review of systems was negative for fever, chills, nausea, vomiting, or abdominal discomfort. He was under the care of neurologist and had been on a stable dose of phenytoin $200 \mathrm{mg}$ twice daily for seizure control, with normal phenytoin levels measured over the last 10 years. Upon further questioning, he reported low back pain that was recently treated by his primary care physician with naproxen. No other changes in medications were identified. Patient's social history was negative for any recreational drug use or excessive alcohol use. Physical examination revealed stable vital signs, was positive for horizontal nystagmus of both eyes and negative for any focal neurological deficits. Ocular motor abnormalities were not identified. Computer tomography of the head without contrast was negative for acute intracranial processes. The urine toxicology screen was also negative. Electrolytes and liver profile were within normal limits with the normal albumin level. Total phenytoin level was $50.3 \mathrm{mcg} / \mathrm{ml}$ and free phenytoin level was $3.6 \mathrm{mcg} / \mathrm{ml}$. According to the review of the patient's medical record, his phenytoin level 2 months prior to presentation was $1.9 \mathrm{mcg} / \mathrm{ml}$. The patient was diagnosed with phenytoin toxicity secondary to presumed phenytoin-naproxen interaction as no other causes of phenytoin toxicity were identified. The phenytoin was temporarily discontinued. The patient's vertigo and the nystagmus improved within $24 \mathrm{~h}$ of observation and he was discharged home. Patient's repeated free phenytoin level 3 days after the discharge was $0.7 \mathrm{mcg} / \mathrm{ml}$. His regular dose of $200 \mathrm{mg}$ of phenytoin twice daily was restarted and he was instructed to stop using naproxen for pain management.

DISCUSSION: We present a case of phenytoin toxicity secondary to phenytoin-naproxen interaction. Although displacement of phenytoin that is $90 \%$ albumin-bound by other highly albumin-bound agents, such as salicylate, has been documented in vitro, not much data exist regarding phenytoin-NSAID interaction in vivo. Ibuprofen and naproxen are partially albumin-bound and can potentially displace phenytoin resulting in an elevated free phenytoin level. Some studies report an increase in free phenytoin concentrations in the uremic pool of patients with normal serum albumin level that were concurrently treated with NSAID. However, limited data exist regarding phenytoin toxicity secondary to phenytoin-NSAID interaction in relatively young patients without major comorbidities and with normal albumin levels. Phenytoin has complex pharmacokinetics and concentration-dependent metabolism that can result in an unpredictable increase in serum concentration even after a small change in its dosing. Enzymes of the cytochrome P450 catalyze phenytoin metabolism. The kidneys subsequently excrete its active metabolites. Concurrent administration of substances that can potentially inhibit cytochrome P450 or cause liver and renal damage can cause phenytoin toxicity in susceptible patients. Symptoms of phenytoin toxicity include nausea, vomiting, nystagmus, ataxia, seizure, and, in rare cases, coma. Coma and seizure are usually associated with a phenytoin level greater than $200 \mathrm{mmol} / \mathrm{L}$. In the central nervous system (CNS) phenytoin potentiates inhibitory synapses in the vestibule-oculomotor pathway that uses gamma-aminobutyric acid resulting in nystagmus and ocular motor abnormalities. Phenytoin also increases discharging of the cerebellar Purkinji cells, causing ataxia and cerebellar dysfunction. To date, phenytoin toxicity is managed by supportive care as there is no known reversible agent. As our report demonstrates, in most cases it resolves uneventfully. Rarely prolonged intoxication can cause permanent cerebellar damage and residual neurological dysfunction. This case report recognizes clinically significant interaction between NSAIDs and phenytoin. Bringing awareness of phenytoin toxicity secondary to its interaction with common co-prescription medications, such as NSAIDs, can prevent potentially serious side effects and decrease the cost of hospitalizations. It can also lay down a platform for further investigation of new methods that can decrease toxic medication interactions

\section{NASAL CAVITY CHONDROSARCOMA PRESENTING AS RECURRENT} SINUSITIS Yuko Shirouchi ${ }^{1}$; Shadia Constantine ${ }^{2} .{ }^{1}$ Teine Keijinkai Hospital, Hokkaido, Japan; ${ }^{2}$ Teine Keijinkai Hospital, Sapporo, Japan. (Control ID \#2468169)

LEARNING OBJECTIVE \#1: Recognize that a nasal-cavity tumor can present as a treatment-resistant sinusitis.

LEARNING OBJECTIVE \#2: Recognize the radiographic characteristics of a nasa cavity chondrosarcoma.

CASE: This is a case of a 59 year-old woman with history of chronic sinusitis, chronic cough and chronic dyspnea of unclear etiology, which presented to the hospital with 3 days history of worsening of these symptoms. Her story began 6 months ago, when she 
developed persistent nasal congestion with inability to breath through her nose and muffled voice. She then developed progressive fatigue for 2 months. In the previous 3 days, her cough had turned productive of yellow sputum with streaks of blood and her dyspnea had also worsened. She has been unable to sleep because of coughing. She also mentioned that she had been sleeping on her right side because sleeping on her left would make her dizzy when getting up. Moreover, she reported new onset of low-grade fever also for the past 3 days. The review of system was positive for night sweats, weight loss ( $2 \mathrm{~kg}$ in 4 months) and appetite loss. The patient's past medical history included chronic sinusitis, diabetes mellitus, Klebsiella pneumonia and renal cell carcinoma for which she underwent a right partial nephrectomy. She had no history of tuberculosis and she had no sick contacts. She had no known drug allergy, she did not smoke, nor did she drink any alcohol. She had two dogs but no exposure to birds or recent travel history. On examination, her vital signs revealed a blood pressure of 137/86 $\mathrm{mmHg}$, heart rate 119 beats per minute and regular, temperature $37.6{ }^{\circ} \mathrm{C}, \mathrm{O}_{2}$ saturation $94 \%$ room air and respiratory rate 30 per minute. She was laying on her right. Other significant findings included crackles and squawks on the right back and decreased air entry on the right lung field. No wheezing was heard. The laboratory data revealed leukocytosis (WBC 15640/ $\mu \mathrm{L})$ and elevated C-reactive protein $(10.20 \mathrm{mg} / \mathrm{dL})$ but her procalcitonin level was low at $0.11 \mathrm{ng} / \mathrm{mL}$. Her sputum was negative for acid-fast bacilli. Her chest X-ray showed a consolidation on the right lung so she was started on ceftriaxone $1 \mathrm{~g} /$ day to treat community-acquired pneumonia. The fever disappeared on day 6 and ceftriaxone was stopped after day 9. Meanwhile, a computed tomography (CT) of the chest showed brochodilation and mucoid impaction in the right lung. We hypothesized that the mucus was coming from her upper airway. Imaging of her sinuses revealed complete bilateral opacification of her maxillary sinuses. This prompted a nasal examination revealing an easy bleeding tumor in the right common nasal meatus, which was causing the displacement of the nasal septum to the left, and both cavities were completely blocked. The CT scan confirmed a tumor occupying the nasal cavity. It also showed lytic lesions of the palatine bone, sphenoid bone and cranial base.The biopsy of the tumor confirmed chondrosarcoma.

DISCUSSION: Chondrosarcomas of the nasal cavity and sinuses are uncommon. Tumors tend to remain asymptomatic and therefore, have often reached a considerable size by the time they are discovered. Our patient had been diagnosed with chronic sinusitis on multiple occasions and had been treated for over a year with numerous rounds of antibiotics. On plain radiography, calcifications are seen in about the half of the cases. However, computed tomography is needed to examine the extent of tumor. Surgical removal is the first-line of treatment. When sinusitis symptoms persist despite proper treatment, underlying causes such as nasal tumors should be considered. When a large mass with calcifications and bone expansion are seen in an otherwise asymptomatic patient, chondrosarcoma should be considered as one of the differential diagnoses.

NEUROCYSTICERCOSIS: AN UNUSUAL CAUSE OF HEADACHES Ana I. Velazquez; Alan Tso; Geeta Varghese. Mount Sinai Beth Israel, First Avenue at 16th Street, NY. (Control ID \#2469849)

LEARNING OBJECTIVE \#1: Recognize the clinical features of neurocysticercosis. LEARNING OBJECTIVE \#2: Discuss the diagnosis and treatment of a patient with neurocysticercosis.

CASE: A 46 year-old Spanish-speaking immigrant female from Ecuador developed episodes of fainting spells associated with frontal headaches, nausea and vomiting. The patient had no prior medical history or toxic habits. Vitals and physical examination were normal. Laboratory evaluation including HIV, tuberculosis, and strongyloidiasis testing were also unremarkable. Radiologic studies revealed multiple parenchymal and extraparenchymal cysts with scolex on head CT diagnostic for neurocysticercosis. The patient was treated with antiparasitic therapy, corticosteroids, and endoscopic debulking with resolution of headaches. 8 months later, she developed worsening frontal headaches associated with intractable nausea and vomiting. Repeat brain imaging showed development of obstructive hydrocephalus secondary to entrapment of calcified cysticerci in the interventricular foramina. An emergent ventriculo-peritoneal shunt was placed for cerebrospinal fluid diversion with improvement in symptoms. 6 months after ventriculoperitoneal shunt placement, the patient's course was complicated by development of dizziness, nausea, vomiting, and drop attacks - characterized by sudden sensation of being pulled to the ground without loss of consciousness. Evaluation with brain imaging revealed two calcified cysticerci involving the left vestibular system. Corticosteroid therapy was initiated with resolution of dizziness, vomiting, and drop attacks. At 3month follow up patient remained asymptomatic while on corticosteroid therapy. Surveillance imaging at that time showed stable disease.

DISCUSSION: Cysticercosis is a parasitic infection caused by ingestion of the pork tapeworm Taenia solium. Transmission is via ingestion of Taenia solium eggs that are shed in the stool of humans or pigs harboring the intestinal tapeworm. Infection caused by this parasite is more prevalent in endemic areas including Latin America, India, Asia, and Africa. The diagnosis can be challenging as clinical manifestations are nonspecific and vary significantly depending on involvement of the central nervous system (CNS) or extraneural systems. Brain parenchymal involvement of neurocysticercosis commonly presents with headaches and seizures. Extraparenchymal CNS disease can present with ocular symptoms, spinal involvement, and hydrocephalus from ventricular system involvement. Neurocysticercosis can be diagnosed based on clinical findings and neuroimaging with CT or MRI showing cystic lesions with the scolex. Serology and CSF analysis can be helpful in equivocal cases, as well as brain biopsy, but is rarely necessary. Treatment depends on the location, number, and type of cysts. It is focused on management of symptoms, treatment of increased intracranial pressure if present and seizure control. In patients with multiple lesions, antiparasitic therapy should be initiated with albendazole or praziquantel combined with corticosteroid use. Concomitant treatment with corticosteroids reduces inflammation and decreases the risk of seizures and mass effect. As in our case, patients with ventricular involvement leading to increased intracranial pressure require emergent cerebrospinal fluid diversion with ventriculo-peritoneal shunt in addition to endoscopic removal or debulking of intraventricular cysts, if available. Following treatment, surveillance imaging may be performed at 1 to 2 months and 6 months post-treatment. Given the rising number of immigrants from diverse backgrounds to the US, it is important to consider and become familiar with the diagnosis of management of infections as cysticercosis that are rare in the US but endemic in other countries.

NEW DIAGNOSIS OF MARFAN SYNDROME PRESENTING AS ACUTE HEART FAILURE Nicholas Hendren ${ }^{1}$; Purav Mody ${ }^{2}$; Michael Luna ${ }^{2} .{ }^{1}$ University of Texas-Southwestern, Irving, TX; ${ }^{2}$ University of Texas-Southwestern, Dallas, TX. (Control ID \#2463691)

LEARNING OBJECTIVE \#1: Manage an evidence based medical and screening regimen for a patient with Marfan syndrome

LEARNING OBJECTIVE \#2: Identify patients with high risk clinical features for Marfan syndrome

CASE: A 28 year old woman visiting from Honduras with a history of atrial fibrillation and mitral valve repair presented to an emergency room with 1 day of shortness of breath, orthopnea, lower extremity edema and palpitations. Her medical history included severe mitral valve regurgitation and a mitral valve ring repair in 2014 with a cardiac surgeon on a medical mission. Her family history was negative for cardiac disease, connective tissue diseases or other genetic conditions. A transesophageal echocardiogram was notable for a 
left ventricular ejection fraction of $56 \%$, a dilated aortic root $(3.9 \mathrm{~cm})$, prior mitral valve repair, severe mitral valve prolapse and two mitral valve perforations with resultant severe mitral regurgitation. She subsequently underwent an uncomplicated mechanical mitral valve replacement and returned to Honduras. Given her mitral valve prolapse, aortic root dilation, arachnodactyly and scoliosis on X-ray, she was evaluated for Marfan syndrome (MFS) using the modified Ghent nosology. Her aortic root dilation per the Marfan.org calculator resulted in a Z-score of 3.9 (Z-score $>2$ is positive) and her systemic score was 9 ( $>6$ is positive). An FBN1 gene sequencing test revealed a mutation in one of two gene copies resulting in a premature stop codon causing premature peptide truncation, confirming the MFS diagnosis.

DISCUSSION: MFS is an autosomal dominant disease involving the fibrillin-1 (FBN1) gene with an estimated prevalence of $1.5-15$ per 100,000 people. Twenty-five percent of these mutations are likely de novo and the disease occurs in all populations without significant differences in genetic penetrance or incidence. When considering evaluating a patient for MFS, findings associated with a higher likelihood of MFS diagnosis include: a diagnosis of MFS in childhood ( $88 \%$ ), eye manifestations ( $83 \%$ ), family history of MFS (52\%), aortic dissection (50\%), pneumothorax (43\%), skeletal features (30\%) and joint hypermobility (14\%). Recognition of MFS in a patient is imperative to avoid the mortality associated with cardiovascular complications. Prior to prophylatic aortic root replacement, the mean age of death in patients with MFS was 32 years old with approximately $90 \%$ of deaths related to cardiac complications, most commonly from aortic valve insufficiency, aortic aneurysm rupture and congestive heart failure. The discovery of the FBN1 gene association with MFS in 1991 represented a new frontier for diagnosis. Despite the known genetic connection between the FBN1 gene and MFS, a genetic defect without clinical symptoms consistent with MFS is not diagnostic of the disease. Despite an improved understanding of FBN1 mutations, consistently predicting which mutations produce a Marfan phenotype remains challenging and no consistent correlation between FBN1 mutations and a phenotype has been determined. Since MFS represents a constellation of clinical findings without a clear reference standard, a true sensitivity and specificity cannot be determined solely by genomic testing. The modified Ghent nosology remains the gold standard for diagnosis. Once MFS has been diagnosed, appropriate monitoring, medical therapy, genetic counseling and prophylactic surgery improve the length and quality of life for patients. MFS patients should undergo at least yearly echocardiograms to monitor for aortic root dilation that could require aortic root replacement. Patients should be referred for aortic root replacement if dilation progresses at more than $0.5 \mathrm{~cm}$ per year or the aortic root dilates to $5.0 \mathrm{~cm}$ at the sinus of Valsalva, although some advocate replacement at $4.5 \mathrm{~cm}$. Additional recommendations include exercise, weight lifting, pregnancy, vision exams, musculoskeletal exams and medical management. Betablockers are a staple of therapy for MFS based on clinical trials despite an unclear impact on survival as they reduce wall stress by reducing inotropy and chronotropy. Developments in the pathophysiology of MFS suggest an abnormality in the transforming growth factor (TGF- $\beta$ ) pathway and matrix metalloproteinase (MMP) may play a role in aortic enlargement. MMP pathogenesis is an evolving concept with animal models suggesting altered MMP gene expression resulting in inappropriate extracellular membrane remodeling. In addition, over activity of TGF- $\beta$ signaling with increased serum levels of TGF- $\beta$ has been positively associated with progressive aortic root enlargement in MFS as well as other genetic aortopathies. Subsequent animal studies have suggested angiotensin receptor blockers (ARBs) attenuate the TGF- $\beta$ pathway by lowering TGF- $\beta$ ligands, receptors and activators. Several small studies suggest ARBs reduce the rate of aortic dilation both as a monotherapy and in combination with beta-blockers; however, robust efficacy in large randomized studies is lacking. Statins, doxycycline and peroxisome-proliferator-activated receptor (PPAR) gamma agonists have also been theorized to have a role in treatment as they have both anti-MMP and TGF- $\beta$ compound effects, but clinical efficacy remains unclear. Overall, MFS is a clinical syndrome with a genetic causative mutation diagnosed with the modified Ghent nosology criteria. With appropriate screenings, medical management and surgical intervention, the life expectancy of MFS patients approaches the population average.

NO RENAL DISEASE HERE: A CASE OF GOODPASTURE'S DISEASE PRESENTING AS ISOLATED ALVEOLAR HEMORRHAGE Allison C. Heacock. The Ohio State University Wexner Medical Center, Columbus, OH. (Control ID \#2469519)

LEARNING OBJECTIVE \#1: Recognize the clinical signs and symptoms of isolated alveolar hemorrhage in Goodpasture's (anti-GBM) disease

LEARNING OBJECTIVE \#2: Diagnose and treat patients with Goodpasture's (antiGBM) Disease and isolated alveolar hemorrhage

CASE: 29 year old man presented with 1 week of progressive pleuritic midsternal chest pain and dyspnea on exertion. The symptoms were associated with two episodes of "quarter size, cherry-red" hemoptysis the week prior. He did not have fever, chills, or night sweats. No signs of bleeding from bowel or bladder and no change in urine output. Past medical history included anxiety and alcohol abuse. Family history was significant for rheumatoid arthritis. He smoked a cigar a week. He did not have exposure to other inhalants. He denied intravenous drug abuse and had only had one sexual partner in the last year. He had no sick contacts and recent travel. He had one cat and no other animal exposures. Initial physical exam was notable for a heart rate of 120 , respiratory rate of 18 and oxygen saturation of $84 \%$ on RA initially. Patient looked in mild distress but had no increased work of breathing. Lungs auscultation revealed course breathe sounds but no wheezes or rhonchi. Labs on admission were significant for a white blood count of 12.6 (74 \% neutrophils), Hemoglobin of $6.1, \mathrm{MCV}$ of 95 and retic count of $10.8 \%$. Chemistry including creatinine was normal Urinalysis showed trace blood with bacteria present. Electrocardiogram showed sinus tachycardia. Chest Xray revealed diffuse bilateral airspace opacification with a nodula appearance. Follow up Chest Tomography Pulmonary Embolism Study had diffuse bilateral nodular ground glass opacities and centrilobular nodules greater at the bases. The patient was admitted to the hospital and had worsening hypoxia requiring 151 of oxygen to maintain saturations. Bronchoscopy was performed and the findings were consistent with alveolar hemorrhage. Workup was begun but patient had persistent hypoxia so underwent video assisted thoracotomy for lung biopsy which was complicated by worsening hypoxia and inability to come off the ventilator. At that time his anti-glomerular basement membrane antibodies came back positive, while anti-nuclear antibody and anti-neutrophil cytoplasmic antibody were negative. He was started on methylprednisolone and plasmapheresis 6 days after presentation. He was extubated 2 days later and quickly weaned to room air. $\mathrm{He}$ completed 3 days of pulse steroids and a total of nine plasmapheresis treatments. He was discharged on oral prednisone. On follow up 2 weeks later he had a normal chest xray. Two months later he continues to have no lung function deficit by report and has maintained an normal creatinine. He will be continued on a long term steroid taper potentially followed by cyclophosphamide.

DISCUSSION: Our patient was diagnosed with anti-glomerular basement membrane disease (Anti-GBM) or Goodpasture's disease. A rare disease which occurs in less than one million with only $5 \%$ presenting with isolated aveolar hemorrhage such as our patient. Diagnosis of anti-GBM disease peaks in second and seventh decade of life. Alveolar hemorrhage should be diagnosed by broncho-alveolar lavage after suspicion from clinical symptoms such as dyspnea, cough, hemoptysis and anemia. Chest xray and chest tomography may show bilateral alveolar opacities including ground glass to consolidations. Some chest imaging may be normal so suspicion should be high for alveolar hemorrhage. While most patients have both alveolar hemorrhage and glomulonephritis, a small minority of patients with severe alveolar hemorrhage have normal creatinine. As in our patient, most of these patients have minor urinary abnormalities usually trace hematuria. Diagnosis requires presence of Anti-GBM antibodies which can be detected through 
serologic testing or renal biopsy. It is uncertain at this time if the subset of patients that have isolated alveolar hemorrhage without detectable renal disease are early in their disease process or a variant of the disease. Initial renal function has been shown to be the strongest determinant of long term renal function in anti-GBM disease. Treatment with high dose glucocorticoids, immunosuppressive agents and plasma exchange has been shown to stop auto-antibody production and induce remission especially if started early in disease course. Therefore, early detection of alveolar hemorrhage by broncho-alveolar lavage with systematic diagnosis of Anti-GBM disease in these rare cases may lead to improved outcomes especially a higher chance of renal survival.

NON-TYPE 1 OR TYPE 2 DIABETES MELLITUS: JUST AS SWEET Elisabeth Askin. UCSF, San Francisco, CA. (Control ID \#2466329)

LEARNING OBJECTIVE \#1: Review Mature Onset Diabetes of the Young (MODY) LEARNING OBJECTIVE \#2: Recognize when to refer diabetics for genetic evaluation CASE: A healthy 31 year old male presented to the emergency department with a 2 week history of insatiable thirst and fatigue. He took no medications. Family history included type 1 diabetes in his mother and pre-diabetes in his sister. On exam, he had a thin body habitus. Laboratory studies were remarkable for blood glucose of 576, normal anion gap, elevated beta-hydroxybutyrate, and hemoglobin A1c of 14.5. Bloodwork was negative for autoantibodies including islet cell, GAD 65, insulin, islet cell antigen 512, and zinc transporter 8 . Review of the mother's medical records indicated she was diagnosed around age 35 but was still producing c-peptide into age $60 \mathrm{~s}$. The patient was started on a basalbolus insulin regimen during hospitalization, but, after discharge, his insulin requirements rapidly decreased. Given frequent hypoglycemia, his insulin was switched to short-acting mealtime coverage only. Repeat A1c was 8.2 then 6.9. The endocrinologists suspected Mature Onset Diabetes of the Young (MODY), but an initial panel of MODY testing returned negative. Further genetic testing was deferred due to cost

DISCUSSION: While this patient's disease initially appeared consistent with Type 1 diabetes, there are elements which stand out. First, despite his thin body habitus, relatively young age, and presentation in a catabolic state (consistent with Type 1), he never exhibited diabetic ketoacidosis (more consistent with Type 2). Second, his autoantibody testing was negative (nearly excluding Type 1). Third, after his initial presentation, his insulin requirements were low, without need for basal insulin (not consistent with Type 1). Fourth, there was a strong family history of diabetes (more consistent with Type 2 or genetic types of diabetes), with a mother still producing c-peptide (inconsistent with Type 1). In such a case, with a mixture of "types," particularly with lean body habitus, and negative autoantibody testing, it is appropriate to suspect a non-Type 1 or Type 2 diagnosis and to refer to endocrinology. There are many diabetes syndromes. One can classify these as involving either (a) destruction of beta cells or (b) genetic changes affecting the activity of insulin production or uptake. MODY falls into the latter category and makes up 2-5\% of diabetics. Thirteen autosomal dominant genes have been implicated, though only three - hepatocyte nuclear factor 1-alpha (HNF1a), glucokinase (GCK), and hepatocyte nuclear factor-4-alpha (HNF4a) - make up roughly $90 \%$ of the disease. These genes all affect glucose sensing or insulin production by beta cells rather than insulin activity. Clinically, MODY is characterized by early onset (before age 25), normal or lean body habitus, a family history of early onset diabetes, and negative autoantibodies. Insulin resistance is not consistent with MODY. MODY is diagnosed through genetic testing. Distinguishing MODY from Type 1 and Type 2 diabetes as well as determining the specific MODY gene has implications for treatment. No MODY types require insulin. Of the three most common, HNF1a and HNF4a are treated with sulfonylureas, and GCK with lifestyle modification. Further, genetic testing can also alert other family members to the diagnosis and need for testing themselves. However, genetic testing is limited. First, by cost. Even with insurance coverage, out-of-pocket costs may be hundreds of dollars. Second, most laboratories only test for HNF1a, HNF4a, and GCK. Thus, in the case of our patient, testing can be non-diagnostic. Treatment in these cases may follow the presumptive diagnosis. This case describes a young adult patient with new onset diabetes where testing was not consistent with Type 1 or Type 2 diabetes, and demonstrates the appropriate diagnostic and therapeutic approach when MODY is suspected.

NORMOKALEMIC THYROTOXIC PERIODIC PARALYSIS: A CASE REPORT OF A PAINFUL, EPISODIC MUSCLE PARALYSIS AS INITIAL MANIFESTATION OF GRAVES' DISEASE IN 15-YEAR-OLD ADOLESCENT. Kanako Horibe $^{1}$; Shadia Constantine ${ }^{1}$; Hotaka Kamasaki ${ }^{2}$; Noeru Miyake ${ }^{1}$; Shuji Sai ${ }^{1}$; Takako Takeuchi ${ }^{2}$; Hiroyuki Tsutsumi ${ }^{2} .{ }^{1}$ Teine Keijinkai Hospital, Sapporo, Japan; ${ }^{2}$ Sapporo Medical University Hospital, Sapporo, Japan. (Control ID \#2468342)

LEARNING OBJECTIVE \#1: List thyrotoxic periodic paralysis in the differential diagnosis of muscle weakness and/or acute paralysis.

LEARNING OBJECTIVE \#2: Recognize that normal potassium level during paralytic attacks does not exclude the diagnosis of thyrotoxic periodic paralysis.

CASE: A 15-year-old Japanese adolescent male presented to our hospital with a 2-week history of intermittent, proximal femoral weakness. These episodes were associated with femoral pain, worsened with movement and occurred only at night to early morning. During the episode, he was unable to stand up or walk. The symptoms usually resolved spontaneously without any intervention. The rest of the review of systems was negative. The patient had no significant past medical history. Nobody in his family had history of similar paralytic episodes or other diseases. He had been nervous and irritable which he attributed to lack of sleep and stress for the upcoming high school entrance exams. His vital signs revealed mild hypertension $(135 / 79 \mathrm{mmHg})$ and tachycardia $(104 / \mathrm{min})$. The musculoskeletal exam was significant for slightly decreased iliopsoas muscle strength (5on the right and $4+$ on the left.) Deep tendon reflexes were normal. Initial blood work was normal including creatinine phosphokinase $(126 \mathrm{U} / \mathrm{L})$ and potassium levels $(3.6 \mathrm{mEq} / \mathrm{L})$. The electrocardiogram and spine X-ray showed no abnormal findings. We were unable to make a specific diagnosis at that time so he was discharged the next day with a referral to the outpatient clinic. As an outpatient, periodic paralysis was considered, despite the absence of other typical symptoms of hyperthyroidism, by the characteristics of paralytic episodes. Then additional blood testing was ordered, and findings revealed hyperthyroidism; serum free thyroxine (FT4) was greater than $7.70 \mathrm{ng} / \mathrm{dL}$ (range, 1.00-1.80), free triiodothyronine (FT3) was $30.10 \mathrm{pg} / \mathrm{mL}(2.20-4.40)$ and thyroid-stimulating hormone (TSH) was lower than $0.01 \mu \mathrm{IU} / \mathrm{mL}(0.27-4.20)$. High levels of thyroid stimulating antibodies were detected at $2822 \%(0-120)$. Thyroid ultrasonography showed a mild diffusely enlarged thyroid gland with hypervascularity. He was diagnosed with Graves' disease with secondary normokalemic thyrotoxic periodic paralysis (TPP). He began treatment with methimazole, potassium iodine and beta-blocker, and shortly after his thyroid function became controlled and his TPP gradually resolved.

DISCUSSION: Graves' disease (GD) is very rare in adolescent males, and it often presents with behavioral disturbances such as decreased attention span, difficulty concentrating, hyperactivity and difficulty sleeping instead of the typical thyrotoxic symptoms. TPP is a rare complication of hyperthyroidism and it occurs mostly in Asian male. Its incidence among Asian patients with hyperthyroidism is approximately $2 \%$, on the other hand is $0.1 \%$ in non-Asian populations, and seldom seen in adolescence. TPP is typically characterized by acute paralytic attacks with hypokalemia, and its etiology remains unclear. TPP can be a life threatening condition since it can lead to cardiac arrhythmias and respiratory failure. It is challenging to diagnose TPP during the initial presentation as it is a rare condition, and moreover, because the clinical features of hyperthyroidism tend to be subtle in patients with TPP. Although hypokalemia is said to be a typical factor of TPP, there are a few reported cases in the literature with preserved serum potassium levels called 'normokalemic' TPP. The presentation resembles other conditions such as Guillain-Barre 
syndrome, myasthenia gravis, multiple sclerosis, transverse myelitis, botulism and psychogenic paralysis. In our case, we reached the diagnosis of normokalemic TPP secondary to GD based on his characteristic clinical history of episodic paralytic attacks. This is a first report of normokalemic TPP seen in an adolescent. In conclusion, diagnosis of GD in adolescence is often delayed due to their atypical symptoms and it can present as normokalemic TPP. TPP is a highly treatable disorder and early diagnosis could prevent lethal complications. Therefore, clinicians should consider TPP in the differential diagnosis of muscle weakness or acute paralysis, even with normal potassium levels.

NOT A PNEUMONIA: A CASE OF STREPTOCOCCUS PNEUMONIAE SEPTIC ARTHRITIS AND AN UNDERLYING IMMUNODEFICIENCY Hyejo $\underline{\text { Jun }}^{2}$; Roger W. Sands ${ }^{2}$; Amar Kohli ${ }^{1} .{ }^{1}$ UPMC, Pittsburgh, PA; ${ }^{2}$ University of Pittsburgh Medical Center, Pittsburgh, PA. (Control ID \#2465959)

LEARNING OBJECTIVE \#1: Describe the epidemiology and clinical manifestations of Strepococcus pneumoniae infections

LEARNING OBJECTIVE \#2: List important pathogens and risk factors for septic arthritis Review the diagnostic criteria for multiple myeloma

CASE: The patient is a 66-year-old Caucasian male with a history of tobacco use, hypertension, poorly controlled diabetes mellitus, and medication non-compliance who presented to the hospital with malaise, nausea, progressive right knee pain, swelling, and decreased ambulation in the week prior to presentation. Upon arrival to the hospital, the patient was tachycardic to the $110 \mathrm{~s}$ but was otherwise hemodynamically stable. Physical exam was notable for right knee anterior erythema and palpable effusion. His bloodwork demonstrated a white count of 25.1 ( $88 \%$ neutrophils, $32 \%$ bands), elevated CRP 35.3 and ESR 76, Cr 1.57 from baseline 1.0, Ca 8.7, albumin 2.4, and total protein 6.4 Immediate joint aspiration drained turbid fluid containing WBC of 560000 (95\% neutrophils) concerning for a septic joint. He was started on broad-spectrum antibiotics and underwent two irrigation and debridement procedures. Gram stain and cultures from the synovial fluid and blood cultures all subsequently grew penicillin-resistant $S$. pneumoniae. Antibiotics were narrowed to vancomycin and a 4-week course of treatment was planned. His hospital course was complicated by a right lower extremity DVT, the treatment of which was made difficult by a transfusion-dependent anemia without source and epistaxis requiring an IVC filter, and significant delirium. Extensive testing was performed to determine a possible source of the pathogen, including a CT head/sinuses, CT chest/ abdomen/pelvis, and an echocardiogram, all of which were unremarkable. Lumbar puncture was deferred due to low suspicion for meningitis. After discussing with our Infectious Disease team, testing for an immunodeficiency was performed. Serum and urine electrophoresis (SPEP, UPEP) testing revealed elevated gamma globulin fractions, and immunofixation (IF) confirmed an IgG-kappa gammopathy. The patient also displayed an elevated kappa:lambda ratio of 14.8 and an elevated beta-2 microglobulin at $6.56 \mathrm{mg} / \mathrm{L}$, the latter portending a poor prognosis. Skeletal survey revealed a $1 \mathrm{~cm}$ osteolytic lesion in the left proximal humerus. Even with strong clinical suspicion, the Hematology/Oncology team recommended bone marrow biopsy to confirm multiple myeloma (MM). However, due to stress of the acute illness, the patient and his family deferred the procedure as an outpatient and this still remains pending.

DISCUSSION: S. pneumoniae is familiar to physicians as one of the infectious agents in community-acquired pneumonia. If $S$. pneumoniae is isolated from the blood or CSF, normally sterile sites unlike the sputum, then the disease is termed invasive pneumococcal disease. Incidence is highest in patients greater than 65 years old or less than 2 years old or in those with underlying risk factors such as rheumatoid arthritis, alcoholism, degenerative joint disease or prosthetic joints, steroid use, HIV, malignancy, and other chronic disease states. The most frequent complication of invasive pneumococcal disease is meningitis, which has a high mortality rate of $20-30 \%$. Pneumococcal endocarditis is another more typical clinical manifestation of invasive disease. Together, pneumococcal meningitis, endocarditis, and pneumonia form a clinical triad called Austrian's syndrome originally described by William Osler, now rare in the penicillin era. Septic arthritis is a relatively uncommon manifestation of invasive pneumococcal disease. However, in case reviews of septic arthritis by Ispahani et al. and Ross et al., S. pneumoniae is the second most common pathogen occurring between 6 and $8 \%$. Staphylococcus aureus comprises the majority of cases of septic arthritis. Although multiple myeloma was the underlying risk factor in $6 \%$ of patients, it preceded or prompted the diagnosis in multiple cases. In our patient, this diagnosis was considered in light of transfusion-dependent anemia and persistent renal dysfunction. An abnormal SPEP, UPEP, and IF and a lytic bone lesion increased clinical suspicion. S. pneumoniae is one of the most common pathogens in multiple myeloma. As an encapsulated organism, it takes advantage of the immunocompromised state in MM due to polyclonal hypogammaglobulinemia and defects in the complement system, amongst other deficiencies. Hypogammaglobulinemia stems from the neoplastic proliferation of plasma cells in MM, one of the diagnostic criteria in MM. Specifically, MM is diagnosed by the following according to the International Myeloma Working Group: clonal bone marrow plasma cells $\geq$ $10 \%$ or biopsy-proven plasmacytoma, evidence of end-organ damage (hypercalcemia, renal insufficiency, anemia, or osteolytic bone lesions) and/or positive biomarkers such as an involved:uninvolved serum free light chain ratio $\geq 100$. Monoclonal gammopathy of undetermined significance and smoldering multiple myeloma are asymptomatic plasma cell dyscrasias that are at risk of progressing to MM. This case represents an interesting unmasking of an underlying immunodeficiency in a patient initially with invasive pneumococcal disease.

NOT ALL SCLERODERMA IS THE SAME SCLERODERMA John S. Fleming ${ }^{2}$; Christopher Di Felice ${ }^{1}$; MARK SCHAUER ${ }^{2}$. ${ }^{1}$ Western Michigan Universiry Homer Stryker M.D. School of Medicine, Portage, MI; ${ }^{2}$ Western Michigan University Homer Stryker MD School of Medicine, Kalamazoo, MI. (Control ID \#2435414)

LEARNING OBJECTIVE \#1: Recognize the differences between types of scleroderma LEARNING OBJECTIVE \#2: Recognize the clinical features and serologies of scleroderma

CASE: A 75 year old white male initially noticed finger swelling and polyarthralgias 8 months prior to admission. Subsequently he developed worsening skin thickening advancing proximally to mid-forearm and mid-thigh. He was diagnosed with scleroderma. The skin thickening progressed until his walking was impaired by the reduced range of motion at his knees and ankles. He could not flex his fingers into a fist and exhibited facial involvement with decreased mouth opening at $40 \mathrm{~mm}$. Intermittent skin pruritis occurred over the affected areas. He complained of progressively worsening dyspnea on exertion and underwent HRCT which confirmed interstitial pulmonary fibrosis. He was initially treated with diltiazem, losartan, methotrexate, and prednisone. His hypertension worsened and continued to be difficult to control. The addition of mycophenolate mofetil increased his range of motion somewhat. However, his renal function progressively declined during the 4 months after diagnosis. He underwent renal biopsy which demonstrated ATN and findings consistent with scleroderma renal crisis. While awaiting the results of this biopsy his dyspnea and fatigue acutely worsened and he was admitted to the inpatient medical floor. Hemodialysis was initiated. Captopril was used to control his hypertension. Serology revealed that he was PM-SCL negative but he was RNA Polymerase III strongly positive at $>150$

DISCUSSION: Scleroderma also called systemic sclerosis is actually a group of disorders characterized by abnormal inflammation and subsequent overproduction of collagen resulting in skin thickening and hardening, along with multi-organ system involvement, and vascular dysfunction. Women are affected four to nine times more often than men and are generally diagnosed between the ages of 20 and 50 years old. Although clinical 
presentation is the foundation of diagnosis, there are numerous serologies that help support a diagnosis of scleroderma for example: ANA, Anti-Scl-70, Anticentromere antibodies, anti-RNA polymerase III. This case is unusual for several reasons. First, the patient was an elderly male. Scleroderma usually presents in younger women. His disease, RNA polymerase III positive scleroderma, is rapidly progressive. Typically other sclerodermas demonstrate a more indolent course. In addition he was Scl-70 negative and yet had severe systemic disease including skin, lung, and renal manifestations, which is a hallmark of RNA polymerase III positive scleroderma. RNA polymerase III positive scleroderma is a significant risk factor for scleroderma renal crisis which this patient developed. In addition, scleroderma renal crisis can be precipitated by corticosteroids which he received. It should be kept in mind that not all scleroderma is the same and that there are significant differences in disease manifestations and progression which can in part be predicted by serology.

\section{NOT JUST A SORE THROAT: A CASE OF LARYNGEAL TUBERCULOSIS} Mohamad Firas Barbour ${ }^{1}$; Jaleh Fallah ${ }^{1}$; Claire Thomson ${ }^{2}$; Valeria Fabre ${ }^{2}$; Taro Minami ${ }^{2}$. ${ }^{1}$ Memorial Hospital Of Rhode Island, Pawtucket, RI; ${ }^{2}$ Memorial Hospital of Rhode Island, Pawtucket, RI. (Control ID \#2463988)

LEARNING OBJECTIVE \#1: Maintain a high clinical suspicion for tuberculosis in this vulnerable population.

CASE: Introduction: Despite advances in therapy, tuberculosis represents a major burden of disease worldwide with 9 million cases and 1.5 million deaths annually. Laryngeal tuberculosis, once a common presentation of Mycobacterium tuberculosis infection, has become increasingly rare since the advent of effective antitubercular therapy. The following describes a case of laryngeal tuberculosis presenting as acute epiglottitis. Case report: A 48-year-old man with a history of binge drinking and a positive PPD 20 years prior presented to the emergency department complaining of worsening sore throat, dysphagia and hoarseness for 1 week. Review of systems revealed a 15-lb weight loss and productive cough over the previous 6 months. He migrated to the United States from Guatemala 22 years prior to presentation and denied any exposure to tuberculosis. Physical examination was remarkable for hoarseness and oropharyngeal thrush. Computed tomography (CT) of the neck revealed epiglottitis and CT of the chest showed a large cavitary lesion in the right upper lobe. Flexible laryngoscopy revealed supraglottic edema as well as granular, superficial ulceration on the laryngeal surface of the epiglottis. Laboratory findings were remarkable for a lymphocyte count of 300 cells $/ \mu$ l. Anti-HIV antibody, respiratory viral panel, legionella and Streptococcus pneumoniae urine antigens, and blood cultures were negative. Sputum samples showed numerous acid fast bacilli and nucleic acid testing confirmed the presence of Mycobacterium tuberculosis organisms. His oropharyngeal symptoms improved with fluconazole and dexamethasone. He was started on isoniazid, rifampin, ethambutol, and pyrazinamide for laryngeal and pulmonary tuberculosis and was eventually discharged home with isolation precautions after coordination with the State Department of Health.

DISCUSSION: Discussion: Laryngeal tuberculosis (LT) is an increasingly rare manifestation of tuberculosis, now seen in only $1 \%$ of cases. Diagnosis requires a high index of suspicion, particularly in US natives or migrants who have resided in the US for a long time, as LT is frequently confused with laryngeal carcinoma in these populations. On laryngoscopy, lesions vary in both appearance and location, and may appear ulcerative, ulcerofungative, or polypoid. While LT is typically associated with concomitant pulmonary tuberculosis, as seen in our case, up to $20 \%$ of LT cases present as primary disease. Typically LT presents in men in their forties or fifties and the most frequent complaints are dysphonia and weight loss. Risk factors include lack of prior vaccination with BCG, tobacco or alcohol use, acquired immunosuppression or malnutrition. Our patient serves as a reminder to maintain a high clinical suspicion for tuberculosis in this vulnerable population.
NOT JUST ANOTHER CASE OF BACK PAIN Micah Yost; Andrew Greenlund. Mayo Clinic, Rochester, MN. (Control ID \#2464352)

LEARNING OBJECTIVE \#1: Recognize red flag symptoms in a patient with back pain and systematically work through a case to detect the underlying cause

LEARNING OBJECTIVE \#2: Identify the importance of not anchoring on a premature diagnosis

CASE: A 62-year-old male presented for progressive lower back pain with bilateral lower extremity weakness and numbness. His medical history was significant for post-polio syndrome characterized by chronic weakness of the proximal right lower extremity as well as chronic back pain. Symptoms began 6 weeks earlier with weakness and numbness in both lower extremities, which progressed to difficulty ambulating. The back pain was located in the lumbar area midline and radiated to the abdomen. Also of note, he had a diarrheal illness 2 weeks before symptoms started. He worked a desk job and denied tobacco, alcohol, illicit and prescription drug abuse. He informed us that he was MSM with approximately 50 different partners dating back over 20 years. He used protection only intermittently and had previously been treated for genital herpes. On initial presentation his pain and weakness had worsened and the numbness had spread proximally up his legs and also involved his hands and forearms bilaterally. Exam showed bilateral proximal lower extremity weakness, right worse than left, as well as left triceps weakness. Muscle wasting was noted over the medial and lateral quadriceps, right worse than left. He was areflexic throughout with flexor plantar response bilaterally. Decreased superficial pain sensation was also noted in the left lateral leg and foot without a clear sensory level. Lab studies including TSH, CK, aldolase, B12, thiamine, ceruloplasmin, monoclonal protein, ANA, ENA's, tickborne disease panel, and HIV did not reveal a cause for his symptoms. EMG was complicated due to his history of polio as well as compressive polyradiculopathy. It suggested an acute on chronic process, likely a polyradiculoneuropathy with mixed axonal and demyelinating features. Motor neuron disease could not be excluded due to prominent fibrillation potentials. Lumbar puncture was performed and showed protein of 111 , total nucleated cells 27 with $93 \%$ lymphocytes glucose 71 with blood glucose 143 and cytology negative for malignancy. All viral studies performed on the CSF were negative. VDRL was ordered on the CSF and RPR was ordered on serum along with syphilis $\operatorname{IgG} \mathrm{Ab}$. At this point a tentative diagnosis of inflammatory demyelinating polyradiculoneuropathy was made. Treatment with plasma exchange was initiated and five treatments were performed. The patient's symptoms did not change significantly. At this point the VDRL, RPR, and syphilis IgG Ab all returned positive and a diagnosis of neurosyphilis was made. Treatment with 5 million units IV Penicillin G was initiated followed by continuous infusion of 24 million units of Penicillin $\mathrm{G}$ daily for 14 days. Retinal photo was obtained, and he was noted to have some mild optic disc edema. MRI head and orbits showed left optic disc edema. He was discharged to a skilled nursing facility with plans to recheck the CSF every 6 months until the VDRL, cell count, and protein return to normal. These labs will also be used as a measure of the need for retreatment. Follow-up with neurology and ophthalmology was planned.

DISCUSSION: Syphilis is an infection with Treponema pallidum and can affect many systems including the integumentary, musculoskeletal, cardiovascular, and central nervous systems. Currently, neurosyphilis is most frequently seen in HIV infected patients, though it is not known if this is due to a difference in patient-to-patient susceptibility, or it simply reflects patient lifestyle and common risk factors. Presentations vary based on the site of infection and can be anywhere from asymptomatic to potentially fatal. Infection begins with invasion of the CSF and spreads to the meninges, blood vessels, spinal cord, brain, or nerve roots. The presentation we observed in our patient was a polyradiculopathy presenting with back pain, weakness, sensory symptoms, and muscle wasting. Neurosyphilis is diagnosed with lumbar puncture and both treponemal and non-treponemal testing. Non-treponemal testing is primarily helpful as treponemal studies may be negative in late stage syphilis. Treatment typically involves IV penicillin by continuous infusion for 10-14 days. In our 
patient the primary risk factor was unprotected sexual intercourse. His situation was complicated by his history of polio as well as his spine disease. Inflammatory demyelinating polyradiculoneuropathy was initially suspected due to his history of recent diarrheal illness, areflexia, and lower extremity predominant symptoms. Elevated CSF protein was noted, although he did not have the classic albuminocytologic dissociation as his total nucleated cells were 27. By not anchoring on the diagnosis of inflammatory demyelinating polyradiculoneuropathy we were able to determine the true cause of our patient's symptoms. In his classic syphilis text Modern Clinical Syphilology, John Stokes wrote "The frequency of neurosyphilis in general medical practice depends to a large extent on the thoroughness of the search for signs of neuraxis involvement and the frequency with which the spinal fluid examination is employed." This case is a good reminder that this remains true today, and testing for "the great imitator" should be considered when investigating neurologic disease.

NOT RECOMMENDED FOR HUMAN CONSUMPTION- LEVAMISOLEADULTERATED COCAINE Megan Jagosky; Kristi M. Moore; Leslie Ranken. Carolinas Medical Center, Charlotte, NC. (Control ID \#2467771)

LEARNING OBJECTIVE \#1: Recognize the potential complications of ingesting levamisole-adulterated cocaine.

LEARNING OBJECTIVE \#2: Treat both the common and rarer complications of levamisole-mediated vasculitis.

CASE: A 47-year-old woman with a history of active cocaine abuse, prior carotid artery dissection and deep venous thrombosis presented to the emergency department with encephalopathy, joint pains, and palpable purpura. The purpuric lesions were initially on extremities but progressed to involve the tip of the nose, cheeks and helices of the ears Initial laboratory results demonstrated anemia, neutropenia, and an acute kidney injury with a creatinine of $2.5 \mathrm{mg} / \mathrm{dL}$ (from a baseline of $1.4 \mathrm{mg} / \mathrm{dL}$ ). She was positive for ANA pANCA, ds-DNA, myeloperoxidase (MPO), anti-SSA, rheumatoid factor, and lupus anticoagulant. Based on this positive panel, it was presumed that this was a case of cocaine-mediated vasculitis, specifically from levamisole adulterated cocaine. Initially, steroids were avoided since it is not the primary treatment for this condition and supportive care was provided. Unfortunately, over the course of a week she developed progressive renal failure and hematuria prompting further investigation. Renal biopsy was performed showing pauci-immune crescentric glomerulonephritis (GN). She also developed hemoptysis and hypoxemic respiratory failure from diffuse alveolar hemorrhage (DAH) which required intubation. In light of her worsened clinical status and the development of DAH and GN, management was escalated and high dose steroids were initiated. Plasmapheresis was also performed with the plan to escalate immunosuppression if need be. Unfortunately, 2 weeks later, cavitary lesions were incidentally seen on an abdominal CT scan with blood cultures positive for Klebsiella pneumoniae; therefore further immunosuppression was held. After infection resolution she received two doses of rituximab, 2 weeks apart, leading to improvements in renal and respiratory function. She was placed on a long steroid taper and the skin necrosis had healed by the time of discharge. DISCUSSION: In 2013, approximately 1.5 million Americans used cocaine with an estimated $70 \%$ adulterated with levamisole. It is sold as an antihelminthic agent in veterinary medicine and is added to cocaine for its bulking and psychotropic effects. When ingested by humans, it has a variety of effects largely mitigated via a transient production of autoantibodies including p-ANCA, c-ANCA, ANA, and anticardiolipin antibodies. Levamisole-induced vasculitis has a characteristic dermatologic presentation with a purpuric rash that typically involves the ears and can extend to the cheeks, nose, and extremities. It can also lead to skin necrosis, agranulocytosis, and less commonly systemic organ dysfunction. A handful of cases of ANCA-positive GN caused by levamisole have been reported, with an even smaller occurrence of DAH. The best treatment of levamisolemediated vasculitis has not been fully expounded however cessation of the offending drug is recommended since the majority of effects are self-limited. When there is end-organ involvement, as in our patient, immunosuppression is warranted. Corticosteroids, azathioprine, and cyclophosphamide have been used successfully. In addition, rituximab has proven to be non-inferior to cyclophosphamide for ANCA-associated vasculitis therefore it is also a therapeutic option.

NOT YOUR AVERAGE BABY BUMP: PREGNANCY AND THROMBOCYTOPENIC PURPURA (TTP) Jillian Edmunds; Kathryn Jobbins. Baystate Medical Center, Enfield, CT. (Control ID \#2467249)

LEARNING OBJECTIVE \#1: Recognize thrombotic microangiopathy (TMA) syndromes and distinguish between acquired and hereditary thrombocytopenic purpura (TTP) LEARNING OBJECTIVE \#2: Understand the management of hereditary TTP in pregnancy

CASE: Sister 1 A 34 year-old female at 25 weeks gestation with past medical history of psoriasis presented with headache and epistaxis. Physical exam was notable for petechial rash on abdomen and lower legs. She had marked thrombocytopenia with platelets of $17,000 \mathrm{~mm}^{3}$ and hemolytic anemia with hemoglobin of $8.7 \mathrm{~g} / \mathrm{dL}$ and hematocrit of $24.3 \%$, LDH of 840 units $/ \mathrm{L}$ and haptoglobin $<10 \mathrm{mg} / \mathrm{dL}$. She also had proteinuria and mild transaminitis. Her peripheral smear showed schistocytes. Given concern for HELLP syndrome, she underwent a C-section and delivered a healthy baby. In the post-partum period her platelets continued to decline and she had an ADAMTS13 level of $<5 \%$ with negative ADAMTS13 inhibitor level. Her platelets dropped below $30,000 \mathrm{~mm}^{3}$ and she was started on plasmapharesis for TTP and after 6 treatments platelets improved to $160,000 \mathrm{~mm}^{3}$ and she was discharged home. Sister 2 A 31 year-old female with no known past medical history but family history of sister with TTP admitted for acute appendicitis and underwent appendectomy without complications. On postoperative day 3 , she had thrombocytopenia with platelets dropping from 170,000 to $27,000 \mathrm{~mm}^{3}$. Physical exam was remarkable for right lower quadrant pain and well healing surgical site. She also had hemoglobin of $10.4 \mathrm{~g} / \mathrm{dL}$, decreased from $13.9 \mathrm{~g} / \mathrm{dL}$, and hematocrit of $30.8 \%$, decreased from $40.9 \%$. Peripheral smear did not show signs of hemolysis. She was started on prednisone for possible ITP and platelets improved to $107,000 \mathrm{~mm}^{3}$. She later followed up in hematology clinic given her family history of TTP to plan for potential pregnancy. Labs at that time showed ADAMTS13 activity level of $<5 \%$ without presence of detectable inhibitor. Follow up genetic testing showed ADAMTS13 gene mutation confirming the diagnosis of hereditary TTP.

DISCUSSION: Thrombotic thrombocytopenic purpura (TTP) is a type of thrombotic microangiopathy (TMA) syndrome. It is also known as ADAMTS13 deficiency mediated TMA. It is caused by an acquired autoantibody inhibitor of the ADAMTS13 enzyme or an autosomal recessive ADAMTS13 gene mutation. The ADAMTS13 protease is the enzyme that cleaves von Willebrand factor. Deficiency in this enzymes allows for the accumulation of von Willebrand multimers and platelet thrombi leading to vascular injury causing thrombocytopenia, hemolytic anemia and end organ damage ${ }^{1}$. The hereditary form is also known as Upshaw-Schulman Syndrome. It is a rare disorder that is estimated to affect only 150 families and comprises less than $5 \%$ of TTP cases. Diagnosis of TTP may be challenging if it is not considered as part of the differential for thrombocytopenia. One case series reports that $29(79 \%)$ of 37 patients diagnosed with hereditary TTP based on genetic testing had episodes of neonatal thrombocytopenia diagnosed as idiopathic thrombocytopenia at the time. ${ }^{3}$ Diagnosis of TTP is made by presence of thrombocytopenia and microangiopathic hemolytic anemia with schistocytes on a peripheral smear, increased indirect bilirubin, and increased lactate dehydrogenase. Patient will often have neurologic symptoms, like headache. Unlike the other TMA syndromes, TTP is associated with only minimal acute kidney injury ${ }^{2}$. In acquired TTP there is a deficiency of ADAMTS13 activity with an ADAMTS13 autoantibody inhibitor present. In hereditary TTP patients will have deficiency 
of ADAMTS13 activity in the absence of ADAMTS13 autoantibody inhibitor. Diagnosis of hereditary TTP is confirmed with molecular testing for the ADAMTS13 gene mutation. The management of acute episodes of hereditary TTP involves plasma infusion to provide the deficient ADAMTS13 protease, which will result in an increase in platelet count in 1 to 2 days. In contrast, acquired TTP requires plasmapharesis to remove the offending ADAMTS13 autoantibody. Prophylactic management is indicated during pregnancy. Von Willebrand factor increases physiologically during pregnancy and patients with TTP are unable to cleave these multimers given ineffective or absent ADAMTS13 enzyme resulting in thrombosis ${ }^{1}$. Often pregnancy can be the initial precipitant of $\mathrm{TTP}^{3}$. Patients are at highest risk in the second and third trimester. To prevent this pregnant patients with hereditary TTP require prophylactic plasma infusion at 10 to $15 \mathrm{ml} / \mathrm{kg}$ weekly or every other week throughout the pregnancy and 6 weeks post-partum. Platelet counts must be closely watched to guide dosing of plasma infusion. The frequency of the dosing is based on the half-life of ADAMTS13, which is 3 to 5 days. One case series describes fetal outcomes of mothers with hereditary TTP and found that out of 15 pregnancies (one twin pregnancy), 8 babies were still born or died shortly after birth, 7 were born premature, and only one mother who received plasma infusion delivered at full term without complications. ${ }^{3}$ TTP is a form of TMA presenting with thrombocytopenia, hemolytic anemia, and end organ damage with varying degrees of severity. The diagnosis may be overlooked if it is not considered and fatal if not managed appropriately. It is important to remember both acquired and hereditary TTP as part of the differential of thrombocytopenia and TMA and begin prophylactic treatment to prevent morbidity and mortality.

NOT YOUR AVERAGE SYNCOPE Lily L. Yung; Caren Waintraub; Matthew Harrington. Mount Sinai Beth Israel, New York, NY. (Control ID \#2465931)

LEARNING OBJECTIVE \#1: Identify Multiple Myeloma as a cause for recurrent syncope

LEARNING OBJECTIVE \#2: Describe the autonomic dysfunction associated with Multiple Myeloma

CASE: An 84 year-old male with a history of recurrent epistaxis is admitted to the hospital with syncope. This is his fifth episode in 3 months. Prior to each episode, he relates a prodrome of dizziness followed by a loss of consciousness lasting seconds. He also relates frequent epistaxis without trauma and unintentional weight loss. Review of systems is negative for vertigo, palpitations, chest pain, jerking movements, tongue biting, and incontinence. At each prior admission, he had postural hypotension, anemia, and kidney injury. Previous workup included normal Head CT, Brain MRI, EKG, loop recorder interrogation, and echocardiogram. Hematologic workup revealed normocytic anemia with normal B12 and iron levels. Endoscopy and colonoscopy were also normal. The patient was discharged with a diagnosis of hypovolemia related orthostatic hypotension. Labs on current admission show BUN/Creatinine 38/1.7, hemoglobin $7.9 \mathrm{~g} / \mathrm{dL}$, corrected calcium $11.3 \mathrm{mg} / \mathrm{dL}$, and total protein of $9 \mathrm{~g} / \mathrm{dL}$. Physical exam is notable for positional orthostasis and decreased plantar proprioception. SPEP/UPEP reveals IgG kappa monoclonal M protein spike and elevated free kappa/lambda ratio. Notably, the monoclonal protein is not IgM. Skeletal survey shows diffuse lytic lesions. Bone marrow biopsy reveals increased plasma cells (immunohistochemistry with CD138 $>25 \%$ plasma cells), confirming diagnosis of Multiple Myeloma (MM). The patient is treated with IV fluids and a bisphosphonate. After resolution of symptoms, he is discharged with Hematology/ Oncology follow up. This patient's recurrent syncope is likely due to MM related autonomic dysfunction, causing orthostatic hypotension. The constellation of recurrent epistaxis and syncope in a patient with plasma cell dyscrasia raised suspicion for hyperviscosity syndrome, an entity most commonly associated with Waldenstrom's Macroglobulinemia. Due to the patient's improvement with conservative measures, nontoxic clinical appearance, and diagnostic workup consistent with Multiple Myeloma, hyperviscosity was deemed unlikely, and plasmapharesis not required for acute management.

DISCUSSION: Multiple Myeloma is often difficult to recognize, as many of the signs and symptoms of the disease are commonly seen in hospitalized patients. When present, the classic combination of anemia, renal injury, hypercalcemia, and bone pain raises suspicion for the diagnosis. MM is a neoplastic plasma cell proliferative disorder which produces a monoclonal immunoglobulin. Infiltration of plasma cells and light chains into bones and organs causes end-organ damage, manifested as bone pain from osteolytic lesions, anemia, hypercalcemia, and renal injury. Damage to sensory, motor, and autonomic nerve fibers cause peripheral neuropathy and autonomic dysfunction. Additional testing to confirm diagnosis of MM includes bone marrow biopsy and imaging such as skeletal survey or whole body PET/CT. Patients with hypercalcemia should receive IV fluid resuscitation and a bisphosphonate. Potentially nephrotoxic medications including NSAIDs and IV contrast should be avoided. This patient's presentation was initially concerning for Waldenstrom's macroglobulinemia, which causes hyperviscosity syndrome more frequently than MM due to the large size of the IgM pentamer. Symptoms of hyperviscosity include syncope, as well as oronasal bleeding, and dizziness. Hyperviscosity syndrome is due to positively charged, large globulins binding to red cells, causing aggregation and increased viscosity. It is an oncologic emergency requiring swift recognition and treatment. While laboratory testing should include serum viscosity, this test frequently takes days to be resulted and in a scenario of high clinical suspicion, plasmapharesis should be initiated under the consultation of hematology

NOT YOUR AVERAGE UTI Cathleen Kenary; Meghana Bhatta; Suzanne Chang. George Washington University Hospital, Washington, DC. (Control ID \#2435510)

LEARNING OBJECTIVE \#1: Diagnose and treat urinary tract infections in a timely manner

LEARNING OBJECTIVE \#2: Recognize risk factors for complications of recurrent urinary tract infections

CASE: A 60-year-old obese female with diabetes, coronary artery disease, and recurrent urinary tract infections presented to the hospital with 2 weeks of severe abdominal pain, confusion, weakness, and unintentional weight loss. Three months earlier, a left perirenal mass measuring $14 \times 13 \times 17 \mathrm{~cm}$ was discovered on CT scan. At the time, further workup of the mass was delayed due to urosepsis. One month after detection of the mass, renal biopsy showed collections of foamy macrophages with mixed inflammatory cells and fibroadipose tissue. Management was again deferred. On arrival to our institution, physical exam was notable for tachycardia, hypotension and a tender leftsided palpable abdominal mass. CT scan demonstrated that the mass had grown to $15 \times$ $16 \times 23 \mathrm{~cm}$, now with extensive local invasion and adherence to surrounding structures. The patient was started on meropenem for presumed sepsis. She underwent an emergent radical nephrectomy, exploratory laparotomy and drainage of a large retroperitoneal abscess. Per the operative report, the kidney appeared grossly purulent with neoangiogenesis, and extended from the spleen to the pelvis. A large amount of retroperitoneal pus and necrotic tissue was debrided. Surgical pathology showed extensive inflammation composed of lipid-laden macrophages and foamy giant cells, with areas of suppuration, sclerosis, and fibrosis throughout the mass, consistent with Xanthogranulomatous Pyelonephritis. Urine culture grew Klebsiella pneumoniae. Post-operatively, the patient became profoundly anemic due to hemorrhage. She required three subsequent operations for re-packing the wound, drain placement and closure. In the intensive care unit, the patient required pressor support and mechanical ventilation. After a month-long hospital course, the patient was stable and successfully extubated. She was discharged to a rehab facility. 
DISCUSSION: Our patient was diagnosed with Xanthogranulomatous Pyelonephritis (XGP), a rare complication of recurrent urinary tract infections. This case illustrates the importance of timely treatment and vigilance for urinary tract infections, a common occurrence in the primary care setting. It also highlights the importance of a thorough workup for recurrent urinary tract infections, including imaging, prompt biopsy and possible resection if a mass is found. Unfortunately, because of the obscurity and limited understanding of XGP, our patient was shuffled between different medical facilities for months before receiving definitive diagnosis and treatment. By the time she reached the operating room, she was septic and her mass had grown to such a large size that it had begun to infiltrate surrounding structures. A more thorough and timely workup of our patient's recurrent UTI may have prevented delays in treatment, which ultimately led to her complicated perioperative course. Our middle-aged female patient, with history of metabolic syndrome and recurrent urinary tract infections had multiple risk factors for XGP and warranted a more comprehensive workup of her condition to determine effective treatment. Definitive treatment of XGP is radical nephrectomy, a technically difficult procedure. Most cases require open nephrectomy as there are reportedly high conversion rates from laparoscopic to open procedure. As this case demonstrates, early intervention is paramount for reducing surgical complications. Furthermore, early tissue characterization and the appropriate imaging studies can inform a pre-operative diagnosis and thereby guide operative planning. In conclusion, this case highlights the importance of clinical curiosity and awareness. The potentially devastating consequences of XGP, though rare, warrant closer observation in patients with recurrent urinary tract infections.

NOT YOUR TYPICAL SICKNESS Rasheen Imtiaz ${ }^{1}$; Beenish Ahmed ${ }^{1}$; Zeeshan Mirza $^{3}$; Barry J. Zeluff ${ }^{2} .{ }^{1}$ Baylor College of Medicine, Houston, TX; ${ }^{2} \mathrm{CHI}$ St. Luke's Health, Houston, TX; ${ }^{3}$ Jiangsu University, Zhenjiang, China. (Control ID \#2470244)

LEARNING OBJECTIVE \#1: Recognize the entity of serum sickness and its common clinical features

LEARNING OBJECTIVE \#2: Recognize etiologies of serum sickness that have been reported in literature

CASE: A 46 year-old man with history of hypertension and end stage non-ischemic cardiomyopathy status post orthotropic heart transplant 8 months ago presented to the emergency room (ER) with subjective fevers, joint pains and rash. In the 1 to 2 months preceding this, routine cardiac biopsies had revealed acute cellular rejection for which he received pulse dose corticosteroids on two occasions with no response. He was admitted a third time for acute rejection 10 days prior to his current presentation and was treated with steroids and 3 days of rabbit anti-thymocyte globulin (r-ATG). This time, he responded with subsequent cardiac biopsy showing no acute rejection. Of note, throughout the period since transplantation, he was adherent to his immunosuppressant agents including mycophenolate, tacrolimus and prednisone. On the day of presentation he started experiencing bilateral shoulder pain that then progressed to involve several large and small joints including his elbows, hips, knees and phalangeal joints. He developed diffuse myalgias and also noticed swelling of his face, lips, tongue, neck, hands and feet. Additionally, he developed red bumps over several areas of his abdomen and thighs. He began having lowgrade fevers and subsequently presented to the ER. He denied dyspnea, headaches, blurry vision, abdominal pain, nausea, vomiting, diarrhea, and hematuria. He had no unusual exposures including sick contacts. The only new drug he had taken was ATG for transplant rejection. He did report having eaten lunch at a Mexican restaurant earlier in the day but this was his routine lunch stop. On evaluation, the patient had a temperature of $100.6 \mathrm{~F}$, heart rate of 110 beats per minute, blood pressure of $147 / 93 \mathrm{mmHg}$ and oxygen saturation of $100 \%$ on room air. His physical exam was significant for angioedema involving his lower face, neck and tongue, bilateral swelling of his hands and feet and a faint maculopapular rash on his anterior thighs. He oral mucosa was normal. There was no erythema or warmth of the joints but they were tender to touch and with movement. The remainder of his examination was normal. On laboratory evaluation, his c-reactive protein (CRP) and erythrocyte sedimentation rate (ESR) were markedly elevated. He had a lownormal complement 4 and normal complement 3 . He had mild elevation in creatinine but urinalysis did not reveal any proteinuria or casts. The remainder of his metabolic panel and complete blood counts were normal. His blood cultures showed no growth and cytomegalovirus viral load was undetectable. The patient was diagnosed with serum sickness secondary to rabbit anti-thymocyte globulin. He was treated with diphenhydramine, antihistamines and corticosteroids and supported with fluids for his kidney injury. He began feeling better soon and his symptoms including joint pains and swelling significantly improved by the next day.

DISCUSSION: Serum sickness is a syndrome characterized by malaise, rash, fevers, polyarthralgias and myagias, occurring in response to a foreign antigen such as r-ATG. Rash is usually the earliest feature and can be urticarial, macular and papular or even manifest as palpable purpura. Arthalgias occur in two-thirds of the patients and can involve large and small joints. Less common features include facial and peripheral edema, lymphadenopathy, headache and uveitis though these have been reported in literature. The onset of symptoms is typically $1-2$ weeks after first exposure to a drug. If a patient has been exposed to the offending drug before, the syndrome can start earlier within 1-7 days of re-exposure and may have a more severe, sudden onset. Serum sickness is a type III hypersensitivity reaction and is mediated by antigen-antibody immune complexes (ICs). It takes 1-2 weeks for the body to mount an immune response. When there is excess antigen, ICs form and preferentially deposit in blood vessels (vasculitis), joints (arthritis and athralgias) and glomeruli (glomerulonephritis) precipitating an inflammatory response with activation of the complement cascade. Glomerulonephritis is not a common feature of serum sickness. Of note, medical conditions which are also type III hypersensitivity reactions include systemic lupus erythematosus, rheumatoid arthritis and hypersensitivity pneumonitis among others. Laboratory findings may reveal elevated ESR and CRP along with low complements although the latter finding is not always seen. Proteinuria, eosinophilia and thrombocytopenia may also be seen but none are diagnostic. Serum sickness remains a clinical diagnosis. Some etiologies of serum sickness that have been reported in literature include equine ATG (more common than rabbit ATG), monoclonal antibodies such as rituximab and infliximab, venom anti-toxins, rabies vaccine, penicillins and trimethoprim-sulfamethoxazole. Patients are treated by withdrawing the offending agent and often with corticosteroids. The prognosis is good with resolution of symptoms usually within days. Conclusion: Serum sickness is an uncommonly encountered entity and, therefore, may prove to be a difficult diagnosis for internists. This case highlights the important clinical features, pathophysiology and some rare but well established etiologies associated with serum sickness.

\section{NOTHING WILL KEEP ME DOWN: A CASE OF DESCENDING WEAKNESS IN A PATIENT WITH A PURE MOTOR GUILLAIN-BARRE SYNDROME VARIANT Peggy Leung. Weill Cornell/ NewYork Presbyterian, New York City, NY. (Control ID \#2466844)}

LEARNING OBJECTIVE \#1: To recognize the presentation of a motor variant of Guillain-Barre Syndrome with atypical features

LEARNING OBJECTIVE \#2: To manage a patient with an acute motor axonal neuropathy

CASE: 66 year old woman with no significant past medical history presents with descending weakness and pain. Four days prior to admission, she developed bilateral proximal upper extremity dull, aching pain followed by difficulty raising her arms above her head. She went to an emergency department and was discharged with pain medicines. Two days prior to presentation, she had difficulty ambulating due to lower extremity 
weakness and pain. On arrival, she was unable to stand. She denied any confusion, vision changes, jaw pain, chest pain, shortness of breath, abdominal pain, bowel/bladder incontinence, or other sensation changes. One week prior to hospitalization, she hiked effortlessly on a well-paved trail in the Mid-Atlantic and did not recall any bug bites. She also described a brief diarrheal illness around the same time. She denied recent travel, recent influenza vaccination, ingestion of canned foods, infected wounds, and history of intravenous drug use. Her physical exam is notable for pain with any movement of her arms and legs. Her muscles are tender on palpation. No rashes are appreciated. Her speech is intact. She has intact cranial nerves. She has $3 / 5$ strength at bilateral deltoids and hips, $4 / 5$ at bilateral wrists and knees, and 5/5 with dorsiflexion and plantarflexion. She is intact to light touch and hypo-reflexive throughout. She had down-going toes. Her CBC, BMP, and creatine kinase were all within normal limits. MRI brain did not reveal any acute intracranial pathology. MRI cervical spine only showed C3-C4 disc protrusion without spinal cord impingement. Lumbar puncture showed 1 wbc, 1 rbc, negative gram stain, glucose 55 , and protein 69 , consistent with cytoalbuminologic dissociation. Her negative inspiratory force (NIF) was at -30 . Given rapid progression in symptoms and picture most consistent with Guillian-Barre Syndrome (GBS), plasma exchange (PLEX) was initiated. Electromyography (EMG) was consistent with pure motor axonal GBS. She was also ganglioside antibody positive. Infectious work up was largely negative. Despite receiving plasma exchanges, her NIF progressively worsened to -18 and she was intubated for airway protection. Subsequently, she was given intravenous immunoglobulin (IVIG) and later required a tracheostomy. Repeat EMG 1 month after admission showed minimal improvement in motor function. Ultimately, she was discharged to acute rehabilitation.

DISCUSSION: GBS is an immune-mediated polyradiculoneuropathy with a mean age of onset of over 40 and with a male predominance. Worldwide incidence of GBS is quoted at 0.6 to $4 / 100,000$ people. Typically, GBS presents with hyporeflexia and neuropathic pain in a symmetric ascending pattern. Our patient's descending presentation (less commonly seen in GBS) led us to consider botulism. However, this idea was quickly dismissed given lack of typical exposures to botulinum toxin. Her notable proximal muscles weakness also triggered concerns over possible acute myopathy/myositis. However with such a quick onset and a normal creatine kinase, the likelihood was low. This patient's clinical pattern is most consistent with acute motor axonal neuropathy (AMAN), a pure motor axonal subtype of GBS rarely seen in North America (3-5\% of all GBS cases in North America). It is frequently associated with Campylobacter jejuni enteritis and the molecular mimicry of human gangliosides by $C$. jejuni lipo-oligosaccharides. Compared to the most common GBS subtype, acute inflammatory demyelinating polyradiculopathy (AIDP), AMAN typically has preserved reflexes (unlike this patient), less cranial nerve involvement, less autonomic dysfunction, and a more rapid progression of disease. Interestingly, despite having a pure motor GBS, this patient had significant pain on arrival. Unlike typical neuropathic pain that results from the degeneration of sensory nerve fibers as seen in AIDP, the pain she experienced in the acute phase of her disease was likely of nociceptive origin secondary to inflammation or inflammatory mediators. Another notable difference between more common forms of GBS is that recovery from axonal degradation (as in AMAN) takes longer than recovery from demyelination (as in AIDP). Regaining the ability to walk, if possible, may range from months to years. Supportive care plays a large role in AMAN as roughly $30 \%$ of GBS cases progress to respiratory failure. Frequent telemetry and respiratory monitoring is critical. PLEX (to remove autoantibodies, immune complexes, and other inflammatory mediators) and IVIG (to inhibit costimulatory molecules involved in antigen presentation and attenuate autoantibody-initiated inflammation) had both been shown in large randomized control trials to be effective therapies. However, patients in these studies were predominantly diagnosed with AIDP rather than AMAN. It is debated whether combined PLEX and IVIG (which our patient received) offer improved outcomes over monotherapy with PLEX or IVIG. Corticosteroids have no role in the treatment of GBS. Despite usage of these immunemodulating treatments, approximately $20 \%$ of patients are left with severe disability.
NOW SERVING PALM OIL Kara B. Ruder ${ }^{1}$; Joshua Wong ${ }^{1}$; Sweny Gulati ${ }^{2}$; Margaret C. Lo ${ }^{3,2}$; Dianne L. Goede ${ }^{3}$; Juan C. Guarderas ${ }^{3} .{ }^{1}$ University of Florida, Gainesville, FL; ${ }^{2}$ University of Florida, Lady Lake, FL; ${ }^{3}$ University of Florida College of Medicine, Gainesville, FL. (Control ID \#2456313)

LEARNING OBJECTIVE \#1: Diagnose and manage atypical presentations of anaphylaxis due to food allergies.

LEARNING OBJECTIVE \#2: Identify and improve system flaws for optimal management of anaphylaxis.

CASE: A 25-years-old female presented with sepsis from worsening periodontal abscess despite outpatient clindamycin. Medical history includes gastroparesis, asthma, and multiple allergies including anaphylaxis to coconut, ibuprofen, naproxen and hives to latex and codeine. On admission, resuscitative IV fluid and cefepime, vancomycin and flagyl were started. Oromaxillofacial surgery extracted teeth \#7-10 and drained an abscess pocket. The next day the patient was doing well until she developed acute pruritus and throat irritation after eating chocolate pudding. These symptoms mirrored her prior anaphylactic reactions. The primary team found her in acute distress with tachycardia and tachypnea. She was able to speak in full sentences and had normal blood pressure and oxygen saturation. Exam revealed diffuse wheals and stridor but no angioedema. IM epinephrine (EPI) was ordered but not readily available. Her clinical status rapidly deteriorated to hypoxia and inability to speak. With staff inertia and continual delay in IM EPI, the primary team had to give IV diphenhydramine, IV methylprednisolone, and racemic EPI followed by IV EPI as last resort. Code BLUE was called; patient was intubated for airway protection and stabilized in MICU. The next day, she was extubated and transferred to the floor on room air. A patient safety report (PSR) was filed.

DISCUSSION: This near-miss case illustrates the continual misconception of clinicians on the diagnostic management of anaphylaxis and the need to teach to these knowledge gaps. Anaphylaxis typically presents with urticaria, hypotension and angioedema. However, in the inpatient setting, active medical issues and existing comorbidities can cause atypical presentations and lead to diagnostic confusion. Our patient's active infection and extensive allergies were such risk factors. Contrary to popular belief, neither hypotension nor angioedema is required in the diagnosis of anaphylaxis, as true in our case. Per 2011 World Allergy Organization guidelines, our patient met 1 of 3 diagnostic criteria of anaphylaxis - specifically, sudden skin and respiratory signs after allergen exposure. Treatment of anaphylaxis involves removal of the trigger and immediate administration of IM EPI. IM EPI is the only effective treatment and should be given at $0.2-0.5 \mathrm{~mL}$ of $1 \mathrm{mg} / \mathrm{mL}(1: 1000)$ solution every $5 \mathrm{~min}$ as needed. IV EPI may be given for hypotension refractory to IM EPI but must be more dilute (1:10,000 solution) than IM concentration. Telemetry monitoring is needed given its risk of arrhythmias. Second line agents for anaphylaxis are antihistamines and steroids for symptom relief and prevention of a biphasic reaction, respectively. Hospitalization is required for management of any biphasic reaction. In addition to uncovering provider inertia and misconceptions about anaphylaxis, a root cause analysis (RCA) of this case discovered that IM EPI was not in the unit's Omnicell and was not considered a standard stock item. In response to this RCA, an anaphylaxis kit was created as a standard stock item on every unit. The kit includes IM EPI at $0.3 \mathrm{~mL}$ of 1:1000 solution as well as IV methylprednisolone and IV diphenhydramine. Additionally, the RCA found that food allergies were not routinely reported to dietary staff. It further found that the chocolate pudding trigger contained palm oil which may cross-react with coconut per 3 case reports. With such rare events, our allergist did caution against adding palm oil allergy to all patients with coconut allergy. Clinicians must still be cognizant of cross-reactivity between certain allergy groups (i.e. tropical fruits and latex) and certain foods, in this case coconut and palm oil. In patients with history of anaphylaxis, proper food and allergy labeling are vital to dieticians. In response to this RCA, food allergies 
are now fully disclosed to our dieticians and staff must verify any reaction in the meal checklist. Our near-miss case demonstrates that a well-known condition as anaphylaxis can cause management dilemmas when atypical presentations and systems flaws come into play. Near-miss events are often regarded as "no harm, no foul" situations. Yet our case supports the need for reporting near-miss events to address hazards in need of system redesign and patient safety initiatives. With a keen ability to identify critical system gaps, residents play a frontline role in improving health systems and must be encouraged to file PSRs on all safety events. The RCA in this case led to implementation of specific patient safety measures including Medicine Grand Rounds on anaphylaxis education, disclosure of food allergies to dietary staff, and stocked anaphylaxis kits on every unit. Such quality initiatives for early recognition and proper treatment of anaphylaxis will prevent future patient harm.

\section{NOW THAT I THINK ABOUT IT, YOUR HEART IS ACTUALLY FINE Kathleen} McKillip; Scott Lundgren; Michael P. Smith. University of Nebraska Medical Center, Omaha, NE. (Control ID \#2469904)

LEARNING OBJECTIVE \#1: Recognize the alternative causes of lower extremity edema and the role of oncotic pressure.

LEARNING OBJECTIVE \#2: Understand the typical presentation and laboratory findings in nephrotic syndrome.

CASE: A 65 year-old man presented with 3 months of progressively worsening lower extremity edema and dyspnea on exertion. He had previously been able to walk more than a block, but now could not walk from the parking lot without dyspnea. He had a history of chronic systolic heart failure, atrial fibrillation, and hypertension. He was treated over the past few months by his primary care provider and cardiologist as worsening systolic heart failure without improvement. He had no orthopnea and no paroxysmal nocturnal dyspnea. He was noted to have chronic appearing non-pitting edema present up to his thighs, mild abdominal distension, no evidence of jugular venous distension, and heart was irregularly irregular without murmurs or gallops. A chest X-ray showed mild cardiomegaly, but without evidence of overt heart failure Repeat echocardiogram was done which showed improvement in his overall heart function with his ejection fraction now at 50-55\%. Laboratory evaluation showed an albumin of $1.7 \mathrm{~g} / \mathrm{dL}$, total protein of $4.1 \mathrm{~g} / \mathrm{dL}$, total cholesterol of $358 \mathrm{mg} / \mathrm{dL}$, LDL of $177 \mathrm{mg} / \mathrm{dL}$, and creatinine of $0.8 \mathrm{mg} / \mathrm{dL}$. A $24 \mathrm{~h}$ urine collection showed $13 \mathrm{~g}$ of proteinuria in the $24 \mathrm{~h}$ period. A renal biopsy was done which showed randomly arranged fibrils consistent with fibrillary glomerulonephritis. Patient was discharged on prednisone $60 \mathrm{mg}$ daily, furosemide $80 \mathrm{mg}$ three times daily, and prophylactic trimethoprim/sulfamethoxazole with nephrology follow up to discuss further immunosuppressive therapy.

DISCUSSION: Edema and dyspnea are problems commonly encountered by the general internist. An important part in evaluating edema and dyspnea in patients with a prior diagnosis of heart failure or other cardiac problems is looking at all of the information available, ordering tests in a methodical approach, and referring on to sub-specialists when help is needed. Looking back on his course prior to being admitted to the hospital, the patient continually had his diuretic dosing increased for complaints of dyspnea on exertion and lower extremity edema without objective evidence of heart failure exacerbation or worsening renal function. While fibrillary glomerulonephritis is an uncommon cause of edema, renal causes including nephrotic syndromes should be in the differential, especially given our patient's history of proteinuria and hypoalbuminemia. Recognition of his low oncotic pressure due to his proteinuria could have led to an earlier diagnosis and appropriate management. With edema and dyspnea likely to be encountered frequently by the general internist, a thorough differential needs to be considered in order to make the appropriate diagnosis in a timely manner.
NSAID USE WITH CAPNOCYTOPHAGA OCHRACEA: THE PERFECT STORM? Nina Suda ${ }^{2}$; Vidya Atluri ${ }^{2}$; Richard J. Silvera ${ }^{2}$; Tulay Aksoy ${ }^{1} .{ }^{1}$ Montefiore Medical Center, Bronx, NY; ${ }^{2}$ Montefiore Medical Center, New York, NY. (Control ID \#2470248)

LEARNING OBJECTIVE \#1: Identifying Capnocytophaga ochracea (C. ochracea) as a rare cause of severe sepsis in an immunocompetent host with no significant comorbidities. LEARNING OBJECTIVE \#2: Recognizing that nonsteroidal anti-inflammatory drugs (NSAIDs) may affect the presentation of sepsis and blunt the systemic response in immunocompetent patients.

CASE: A 63 year-old woman presented with 2 months of progressively worsening shortness of breath. She also complained about intermittent chest pain radiating to her left shoulder, anorexia and significant dyspnea on exertion forcing her to leave her job as a home health aid. She had a temperature of 100.4 and was tachycardic to 118 . She had normal blood pressure of 119/75. Breath sounds were decreased on the left side of her chest. Jugular venous pressure was not elevated. A large palpable mass was noted six $\mathrm{cm}$ above the umbilicus. She had bilateral lower extremity edema with chronic venous stasis ulcers on her right leg. Laboratory examination was remarkable for a leukocytosis of $20 \mathrm{k} /$ $\mathrm{uL}$ and a serum creatinine of $1.6 \mathrm{mg} / \mathrm{dl}$. HIV testing was negative. Computerized Tomography of the chest and abdomen revealed several findings including a large loculated pleural collection in the left lung with complete lung collapse and right shift of the mediastinum, large rim-enhancing collections of gas and fluid around the spleen and liver, and a $10 \mathrm{~cm}$ pelvic mass consistent with a lipoleiomyoma. A chest tube was placed and culture of the drained pleural effusion grew Capnocytophaga ochracea. Dental examination revealed no likely source of infection or dental destruction. Of note, the patient reported taking $1320 \mathrm{mg}$ naproxen daily for the past 1.5 years to treat pain from a chronic venous ulcer, which itself was likely from compression of the iliac vein/artery by her undetected pelvic mass. Our patient was treated with intravenous Ampicillin/ Sulbactam and responded well with almost complete resolution of her symptoms within the first 5 days of her hospitalization.

DISCUSSION: Capnocytophaga ochracea is a gram negative bacillus most commonly found as a constituent of the normal human oral flora and may occasionally be found in the vagina and the gastrointestinal tract. It typically causes localized periodontal disease that through disruption of the oral mucosa can induce bacteremia and severe sepsis, particularly in the setting of an immunocompromised host. Severe infection has been reported in the setting of splenectomy, neutropenia, malignancy and immunosuppressive therapy. Reports of Capnocytophaga ochracea infection in immunocompetent patients tend to be localized and of lesser severity. The present case is remarkable in that it illustrates a rare cause of sepsis in an immunocompetent patient with intraperitoneal abscesses without evidence of a recent nosocomial exposure, underlying predisposing condition, or oral lesion. Although the portal of entry of this infection could have been vaginal or oral despite no concomitant dental pathology, the degree of sepsis that our patient presented should still be explored, and her nonsteroidal anti-inflammatory drug (NSAID) use should not be overlooked. NSAIDs have numerous effects on neutrophil function. Prior use ameliorates symptoms of infection, which may lead to delays in presentation, diagnosis, and treatment. In the absence of other risk factors for infection, long term, daily use of NSAIDs may be sufficient to induce substantial immune dysfunction and mask the underlying process to allow infection with an otherwise benign organism and a diagnostic delay. While infection and sepsis with Capnocytophaga ochracea has been identified in literature with less reported cases in immunocompetent, this case demonstrates that seemingly benign factors can blunt a systemic immune response, delaying presentation to the clinic, and leading to an unexpected outcome. Given the widespread use of daily NSAIDs, clinicians should keep these factors in mind and recall that such patients constitute a vulnerable population to infection from uncommon organisms even in the absence of other risk factors. 
NUMB CHIN SYNDROME: AN UNUSUAL YET OMINOUS SIGN Jie Qu ${ }^{2}$; Adam P. Sawatsky ${ }^{1} .{ }^{1}$ Mayo Clinic, Rochester, MN; ${ }^{2}$ Mayo Clinic- Rochester, MN, Rochester, MN. (Control ID \#2469947)

LEARNING OBJECTIVE \#1: Recognize numb chin syndrome (NCS) as a possible presenting symptom of an underlying malignancy

LEARNING OBJECTIVE \#2: Understand that the erythema migrans rash in potential Lyme disease is often misleading, and inthe setting of worsening symptomatology, laboratory values, and lack of response to antibiotics, careful re-evaluation is necessary to avoid premature closure

CASE: A previously healthy 38 year-old male presented with diffuse myalgias, night sweats, nausea, and fevers. He removed a tick after a hunting trip and described a rash consistent with erythema migrans. Patient was given a clinical diagnosis of Lyme disease and was started on doxycycline. After 4 doses, he presented to the ED for lower lip and bilateral jaw numbness, pain, and swelling. Due to concerns for doxycycline allergy, he was switched to amoxicillin. His symptoms persisted and he presented to the following day. He was diagnosed with Rocky Mountain Spotted Fever and doxycycline was restarted. Several days later, he returned to the ED with persistent numbness and pain in the lower lip and chin, and severe headaches. He experienced conjunctival hemorrhage after an episode of emesis. Patient was febrile and tachycardic, and was admitted for severe tick-borne illness. Basic labs noted a leukocytosis of 26.9 with lymphocyte predominance (46\%), severe thrombocytopenia with platelets 18 , a drop in Hgb from 14.5 to 12.6 , transaminitis, and lactate 4.3 . Tick-borne disease antibody panel was negative for Anaplasma, Ehrlichia, Lyme, and Babesia. A head CT was unremarkable. Quinine and clindamycin were added to empirically for Babesiosis. Patient received 1 unit of platelets and was transferred to our institution for further evaluation and management. A peripheral smear for Babesia was negative but showed highly atypical lymphocytes, prompting a leukemia/lymphoma flow cytometry study that was consistent with Burkitt's lymphoma. Bone marrow biopsy showed extensive involvement by Burkitt's lymphoma ( $>90 \%$ of cellularity). An initial PET scan demonstrated diffuse skeletal and splenic involvement. Patient was initiated on appropriate therapy and treated for tumor lysis syndrome. Patient tolerated chemotherapy well, and on dismissal, pain and numbness of his lower lip and chin had improved.

DISCUSSION: This case describes NCS in the setting of a complex set of symptoms, as the first presentation of Burkitt's Lymphoma. Numb chin syndrome (NCS) is characterized by pain, numbness, and paresthesias of the lower lip and chin, restricted to the distribution of the mandibular branch of the trigeminal nerve. NCS is rare presentation and has been reported not only in various hematologic malignancies such as acute leukemia, Burkitt lymphoma, multiple myeloma but also solid tumors such as breast cancer, prostate cancer, and esophageal cancer. It is most commonly associated with breast cancer and lymphoma, and is generally thought to be the consequence of bone metastases or leptomeningeal seeding. Although a very rare neuropathy, the clinical significance of this syndrome lies in its frequent association with both the first presentation of an underlying malignancy or signs of recurrence or metastasis in patients with history of malignancy. For our patient, initial diagnosis of Burkitt's lymphoma was delayed as his symptoms were initially attributed to a severe tick-borne illness. Further workup, however, revealed lack of response to antibiotic treatment, worsening symptomatology and laboratory findings. This case not only demonstrates the importance of recognizing NCS as a prompt for further evaluation of an underlying malignancy, but also encourages developing a differential diagnosis throughout a patient's clinical course to avoid premature closure in the diagnostic process.

NUMBNESS AND TINGLING: A CASE OF METFORMIN-INDUCED ELECTROLYTE ABNORMALITIES Ankit Bhargava; Pamela Vohra-Khullar. Emory, Atlanta, GA. (Control ID \#2465927)
LEARNING OBJECTIVE \#1: Recognize that even common side effects of widely prescribed medications can lead to severe symptomatic manifestations.

LEARNING OBJECTIVE \#2: Recognize that diabetics are a particularly vulnerable population for hypomagnesemia, which can be worsened by the effects of metformininduced diarrhea and lead to subsequent electrolyte abnormalities such as hypokalemia and hypocalcemia.

CASE: A 47 year old female with a recent diagnosis of type 2 diabetes mellitus $(\mathrm{HbAlc}=$ $12.3 \%$ ) approximately 3 weeks prior presents to primary care clinic with complaints of nausea, diarrhea, and a "tingling" sensation throughout her body. She was recently started on metformin $500 \mathrm{mg}$ twice daily 2 weeks prior, with subsequent symptoms of nausea with occasional emesis and increasing bouts of diarrhea, approximately 2-3 loose stools per day. A week prior to presentation to clinic, she started to have symptoms of perioral numbness and tingling, tingling in her lower extremities that began in her feet and radiated up to her thighs, and accompanying lower extremity weakness and dizziness. The patient denied having any fever, nightsweats, or abdominal pain. Further history revealed that the patient had mistaken her instructions for metformin $500 \mathrm{mg}$ twice daily as $1000 \mathrm{mg}$ twice daily. At this point it was felt that her symptoms were medication-induced, and a basic metabolic panel was drawn revealing a potassium level of $2.7 \mathrm{mmol} / \mathrm{L}$, calcium of $6.3 \mathrm{mg} /$ $\mathrm{dL}$, and a magnesium of $0.4 \mathrm{mg} / \mathrm{dL}$. Notably, her calcium level was $9.9 \mathrm{mg} / \mathrm{dL}$ just 3 weeks before her clinic appointment. The patient was advised to have the labs repeated immediately; repeat labs confirmed the severity of her hypomagnesemia $(<0.04)$, hypokalemia (2.6) and hypocalcemia (6.4). Ionized calcium was low at 0.9, PTH was appropriately elevated given hypocalcemia. She was admitted to the hospital for treatment of severe electrolyte abnormalities. Treatment was initiated with magnesium and potassium replacement protocol, IV calcium gluconate, and IV fluids. Metformin was discontinued for the duration of her admission and insulin therapy was instituted. By the next day, her labs began to normalize with a calcium of 7.1, potassium 3.5, and magnesium of 1.8 . The patient had improved clinically, having improved strength, decreased nausea, and tolerating PO intake. Numbness and tingling sensation also improved. She was discharged on oral supplements for potassium, magnesium and calcium.

DISCUSSION: For patients initially diagnosed with type 2 diabetes, metformin is the preferred initial pharmacologic therapy. Its most common side effects relate to the gastrointestinal system - diarrhea, flatulence, and abdominal discomfort-and are dose dependent. Usually these symptoms can be ameliorated by slow titration of the initial dosage, and generally will resolve within 2 weeks of treatment. Diarrhea can cause magnesium deficiency. Diabetics are particularly predisposed to hypomagnesemia, as seen in increased urinary magnesium excretion in connection with glycosuria. In this case, metformin-induced diarrhea likely led to further magnesium depletion, and this in turn could have led worsening hypokalemia and hypocalcemia . Additionally, this patient had been taking proton-pump inhibitor for years for gastroesophageal reflux symptoms; proton pump inhibitor medications can also frequently cause hypomagnesemia. This case further illustrates that even the common side effects of a widely prescribed drug can lead to severe clinical manifestations.

NUTRIENT VARIENT STREPTOCOCCAL ENDOCARDITIS-A DIAGNOSTIC AND THERAPEUTIC CHALLENGE Sandeep Patri ${ }^{1}$; Monoj K. $\mathrm{Konda}^{1}$; Hardik Chhatrala ${ }^{1}$; Yashwant Agrawal ${ }^{2}$. ${ }^{1}$ Western Michigan University Homer Stryker M.D. School of Medicine, Kalamazoo, MI; ${ }^{2}$ Western Michigan University Homer Stryker M.D. school of medicine, Kalamazoo, MI. (Control ID \#2468017)

LEARNING OBJECTIVE \#1: Granulicatella elegans (G. elegans) is a rare cause for infective endocarditis (IE) and a likely culprit in culture negative endocarditis. Clinicians should be aware of the high rates of complications associated with it and consider early surgical management when warranted. 
LEARNING OBJECTIVE \#2: Irrespective of antimicrobial sensitivities, G. elegans endocarditis should be treated with a combination of ampicillin or penicillin and an aminoglycoside. Infectious disease consultation should be sought to determine length of therapy.

CASE: A 63 year old Caucasian man with history of non-ischemic cardiomyopathy presented to the emergency room with worsening shortness of breath, fatigue and malaise of 10 days duration. His other comorbidities included hypertension, paroxysmal atrial fibrillation and stage 3 chronic kidney disease and a bi-ventricular implanted cardioverter defibrillator implantation for the cardiomyopathy. Physical exam in the emergency room was significant for a new onset early diastolic decrescendo murmur, signs of congestive heart failure and poor dentition. Soon after admission he was transferred to the intensive care unit for acute respiratory failure and septic shock. Vancomycin and ceftriaxone was started as empiric antibiotic therapy. He had to be started on pressor support, was mechanically ventilated and underwent continuous venovenous hemofiltration $(\mathrm{CVVH})$ for worsening acute kidney injury (AKI). A trans-esophageal echo was done in view of the new murmur which showed a large aortic valve vegetation and associated new onset moderate to severe aortic incompetence. Blood cultures grew G. elegans for which antimicrobial susceptibilities could not be done in our hospital. Antibiotics were changed to ampicillin and ceftriaxone per infectious disease recommendations. Cardiothoracic surgery was consulted but the patient was too unstable for valve replacement surgery. Despite initial improvement patient became progressively hypotensive with worsening congestive heart failure. He expired on day 17 of hospital stay.

DISCUSSION: G.elegans,previuosly grouped under nutrient varient streptococci (NVS) is an extremely fastidious pleomorphic gram-positive bacteria and is often considered a main culprit in culture negative endocarditis. NVS has been reported to cause between 5 and $6 \%$ of all cases of streptococcal endocarditis with G. elegans constituting about $8 \%$ of NVS. It is estimated to be present in about $10 \%$ of adult dental plaques which suggest that the likely source of infection in our patient was his poor dentition. Isolation and correct identification of the organism is often an arduous task because of the fastidious growth requirements and pleomorphism. Identification of G. elegans is best done based on a combination of its special phenotypic characteristics and 16S rRNA gene sequencing. A recent review of 29 cases of Granulicatella species endocarditis by Adam et al. reported aortic valve to be the most commonly involved valve. Unlike the Viridans group, endocarditis caused by this organism has a very poor outcome and higher mortality rate. It is associated with a high rate of complications that include embolic cerebral infarcts, atrial vegetations, need for early surgical intervention, heart failure and multi-valvular involvement. IE caused by the NVS group as a whole has been reported to have treatment failure rates of $41 \%$, despite the organisms being sensitive to the antibiotics used. The high treatment failure rates might be due to the phenomenon of penicillin tolerance or a delay in isolating and identifying the organism and also initiation of appropriate antibiotics. American Heart Association guidelines specifically recommend that Granulicatella endocarditis should be treated with a penicillin derivative and an aminoglycoside for 4 6 weeks, similar to Enterococcal endocarditis. Aminoglycosides could not be added in our patient due to advanced renal failure. Infectious disease consultation should be sought to determine length of therapy. Early detection of the organism and appropriate antibiotics with surgical management when indicated is the key to management.

OBSTRUCTING DESTRUCTION: SEPTAL AND VALVULAR INFECTIVE ENDOCARDITIS IN HOCM AND MITRAL REGURGITATION Ryan Barry;

Kevin Lazo; Rebecca A. Mazurkiewicz. Lenox Hill Hospital Medicine, New York, NY. (Control ID \#2469598)

LEARNING OBJECTIVE \#1: Manage a complicated case of sepsis caused by infective endocarditis.
LEARNING OBJECTIVE \#2: Understand how structural heart disease impacts sepsis management.

CASE: A 46-year old man with HOCM and an AICD placement 12 years prior presented to the ED with a chief complaint of intermittent fever for 10 days. His maximum temperature at home was $103.0 \mathrm{~F}$. The fevers were associated with chills and sweats. The patient denied any recent travel history, sick contacts, dental procedures, or prior history of injection drug use. On physical exam, the patient had a temperature of 103.3, a $4 / 6$ holosystolic murmur on the left parasternal border of the 5 th intercostal space, clear lungs, two erythematous and warm nodules on his left elbow and one on his left 3rd metacarpal joint, and appeared diaphoretic with tachypnea. Initial labs showed CRP 17.6, ESR 20 and WBC $13.9 \times 103 / \mu$ L. Patient had adequate fluid resuscitation which subsequently led to acute pulmonary edema. He received non-invasive positive airway pressure support but did not need intubation. Patient was transferred to the ICU. He was initially started on Cefepime and Vancomycin IV, to treat for presumed infective endocarditis. On TTE, patient was found to have an LVOT gradient of $105 \mathrm{mmHg}$, systolic anterior motion (SAM) of mitral valve and severe mitral regurgitation. One day later the patient's blood cultures were found to have Staphylococcus lugdunensis in two different sets. A Transesophageal echocardiogram contained hemodynamic and anatomical findings significant for HOCM with thickened irregular mitral valve leaflets. No definite vegetations were seen on the AICD leads. The patient was maintained on Vancomycin and Rifampin. Repeat blood cultures on days 3 and 5 continued to be positive for Staphylococcus lugdunensis. The patient underwent a mitral valve replacement with a $31 \mathrm{~mm}$ bioprosthetic valve, a laser lead extraction of his AICD, and removal of visualized vegetations of the interventricular septum adjacent to the anterior mitral valve leaflets. Intraoperative cultures were positive for Staphylococcus lugdunensis on the ventricular septum, and on the anterior leaflet of the mitral valve. The defibrillator capsule, leads, and intraoperative blood cultures were negative. The patient had no post-operative complications, and was discharged with Rifampin and Nafcillin IV for 6 weeks.

DISCUSSION: The estimated incidence of Endocarditis in Hypertrophic Cardiomyopathy is $1.4 / 1000$ people per year and increases to $3.8 / 1000$ people per year with a concomitant left ventricular outflow obstruction. A literature review of 67 patients with Staphylococcus lugdunensis endocarditis revealed an approximately $40 \%$ mortality rate, when medical therapy was used alone. Given this patient had both HOCM and severe mitral regurgitation, it was difficult managing hemodynamics. HOCM patients are preload dependent, especially in the setting of sepsis, which is a relative decrease in blood volume. Management of acute mitral regurgitation often necessitates use of vasodilators and diuretics to reduce both preload and afterload, to reduce the risk of pulmonary edema. Because of the aggressive nature of this pathogen, surgical intervention in combination with antibiotics may be considered. Surgery with prosthetic valves is technically difficult but necessary and often leads to lower mortality rates. Surgery is indicated for patients with $\mathrm{CHF}$, persistent bacteremia and aortic and mitral regurgitation. Our patient also had an AICD, which was removed despite evidence of infection related to the device. There are no exact guidelines regarding the removal of a device in this situation. Post surgical antibiotic treatment for Methicillin-Sensitive Coagulase Negative Staph consists of Nafcillin or Cefazolin IV for a total of 6 weeks, as well. This is the first reported case to our knowledge of Staphylococcus lugdunensis endocarditis in a patient with HOCM and an AICD.

OMEPRAZOLE-INDUCED ACUTE INTERSTITIAL NEPHRITIS-A CASE REPORT Fang Liu. Metrowest Medical Center, NORTHBOROUGH, MA. (Control ID \#2463804)

LEARNING OBJECTIVE \#1: To know how to identify early symptoms of omeprazole-induced acute interstitial nephritis 
LEARNING OBJECTIVE \#2: To know how to manage omeprazole-induced acute interstitial nephritis

CASE: An 81-year-old female with multiple comorbidities including, non-insulindependent diabetes mellitus, hyperlipidemia, Barrett's esophagus, monoclonal gammopathy of uncertain significance, chronic kidney disease with bilateral hydronephrosis of unknown reason, status post ureteral stent placement 1 month ago, who was referred to the Emergency Department by her primary urologist due to worsening renal function. Her creatinine was found to be 7.11 , with a baseline of 2.5 to 3 . Patient was asymptomatic. No shortness of breath, peripheral edema, nausea, vomiting, headache, or altered mental status. She had been producing the same amount of urine as before. No change with her medications except that patient was restarted on omeprazole $40 \mathrm{mg}$ daily about 3 weeks ago for heartburn. On physical exam, patient was alert and oriented, afebrile, euvolemic and hemodynamically stable. Lungs were clear to auscultation bilaterally. Regular heart rate and rhythm, normal S1 and S2, no murmur, rubs, or gallops. Abdomen was soft, nontender and nondistended. No peripheral pitting edema. Laboratory data was significant for a creatinine of 7.11. Urinalysis was remarkable for urine white blood cells more than 100 , urine glucose 300 , and many urine bacteria. Retroperitoneal ultrasound showed resolution of the previously noted bilateral hydronephrosis. Patient had a chronic foley catheter, and there was no evidence of obstruction. It was noted that patient had severe acute interstitial nephritis (AIN) 8 months ago which was diagnosed by renal biopsy and attributed most likely to high dose of proton pump inhibitor (PPI). Patient responded to PPI discontinuation and steroid. Resumption of PPI use 3 weeks ago correlated with the timing of worsening renal function, suggesting AIN as idiosyncratic reaction to re-exposure to PPI. We discontinued PPI indefinitely, and gave patient methylprednisolone $500 \mathrm{mg}$ intravenous daily for 3 days, followed by steroid taper by mouth for 2 weeks. Her renal function started to recover. Patient was discharged to rehab facility, and followed up with nephrologist

DISCUSSION: AIN is characterized by an inflammatory infiltrate in the kidney interstitium. The mechanism of AIN is unclear but probably involves immunemediated hypersensitivity reaction. AIN can be caused by drugs ( $70-75 \%)$, infections (4-10\%), tubulointerstitial nephritis and uveitis syndrome (5-10\%), or systemic diseases $(5-10 \%)$. A large number of medicines are reported to have caused various forms of AIN, including but not limited to, nonsteroidal anti-inflammatory agents, penicillin and cephalosporins, rifampin, antimicrobial sulfonamides, ciprofloxacin, diuretics, and allopurinol. Virtually any drug can cause AIN. Omeprazole is a proton pump inhibitor that is used commonly in the treatment of acid-peptic disorders. Although omeprazole is generally well tolerated, serious adverse effects such as renal failure have been reported. Thus far, 13 cases of AIN secondary to omeprazole have been described. Patients commonly present with malaise, fever, nausea, lethargy and weight loss. The classic triad of immunological reaction, fever, rash, and eosinophilia, was uncommon. The time of onset varies considerably, from 2 weeks to over 1 year's use of omeprazole. Doses have been within the recommended range of 20 or $40 \mathrm{mg}$ daily. Key laboratory features include raised creatinine and reduced GFR, hematuria, proteinuria, and pyuria. Urine microscopy may show white cells including eosinophils, white cell casts and few red cells, but may be unremarkable. Urinary eosinophils are only rarely found and require special stains for their identification. Renal biopsy is required to confirm the diagnosis. Management consisted of withdrawal of omeprazole and corticosteroid therapy in some patients. Patients usually respond rapidly to discontinuation of omeprazole, but full recovery of renal function may take 2-3 months, or, occasionally, even longer. All but one patient recovered normal renal function. Recurrence was universal on re-exposure. This case demonstrates a typical manifestation of omeprazole-induced interstitial nephritis on re-exposure. The most teachable moment here is that recognition of interstitial nephritis may be challenging because the symptoms of renal impairment are non-specific. Patients taking omeprazole, or any of the medicines listed above, who present with malaise, fever, nausea, lethargy or nonspecifically unwell should have urine microscopy and an assessment of renal function. It is important for health professionals to be aware of this adverse drug reaction, because an accurate and timely diagnosis and withdrawal of the offending drug can prevent potentially life-threatening renal failure.

ONE IS THE LONELIEST NUMBER: HIV STIGMA AND MEDICAL DECISION MAKING Richard J. Silvera ${ }^{2}$; Tulay Aksoy ${ }^{1} .{ }^{1}$ Montefiore Medical Center, Bronx, NY; ${ }^{2}$ Montefiore Medical Center, New York, NY. (Control ID \#2469939)

LEARNING OBJECTIVE \#1: Recognize that HIV stigma remains a powerful cultural phenomenon and some patients may prioritize non-disclosure over care, even to a mortal outcome

LEARNING OBJECTIVE \#2: Recognize that the presence of family and friends is not synonymous with social support nor can a patient always access the support available to them

CASE: 42 year-old man presented with 1 week progressively worsening abdominal distension and jaundice. He was diagnosed with HIV infection over 20 years ago without any history of opportunistic infection. He had never revealed his HIV status to his family, despite being deeply involved with them, out of fear of a hostile reaction. To avoid being identified as HIV positive he had also avoided all medical care. At presentation, he was jaundiced with scleral icterus; his abdomen was distended, diffusely tender, with shifting dullness. Abdominal ultrasound revealed enlarged cirrhotic liver with multiple small echogenic foci, splenomegaly, and ascites. He rapidly declined with acute kidney failure due to Hepatorenal Syndrome type I. Etiology of his liver failure remained undetermined but was not thought to be HIV related given his excellent CD4 count and undetectable HIV viral load without therapy. Transplant Committee concluded he was not a transplant candidate due to his lack of social support and concerns about adherence. With the encouragement of medical teams he disclosed his HIV status to a single family member whom he assigned as his Health Care Proxy. The family and he were informed about his poor prognosis. Hospice care was offered as there was no treatment option other than liver transplant. He is now engaging in end of life planning with no further disclosure of his HIV status.

DISCUSSION: HIV-stigma influences people with HIV/AIDS (PWHA), and those at risk, in many ways. Persons at risk may avoid testing out of fear of joining a stigmatized group. Surveys have found that up to $33 \%$ of participants would avoid an HIV-positive grocer, $12 \%$ would avoid an HIV-positive co-worker, and $10 \%$ would prohibit HIV-positive children from schools. PWHA may fear being identified in the community and may avoid, or limit their interactions with, not just HIV care but care in general. Lifelong exposure to HIV stigma may cause these patients to internalize these messages and make them a part of their self-identity. Research has shown PWHA with higher ratings of internalized stigma had more severe HIV symptoms, less accepting families, less social support, more fear of transmitting HIV to others, and significantly lower rates of healthcare access. This case illustrates that patients with internalized HIV stigma may prioritize non-disclosure over even lifeprolonging interventions. A patient's social context is complicated, and simplifying that interaction can obscure factors critical to medical decision making. A survey of PWHA found that as many as $21 \%$ had not disclosed their HIV status to family and $5 \%$ had not disclosed to friends. Those who had not disclosed their status had significantly lower perceptions of social support. It is important to remember that social interactions are a negotiation of social, cultural, and economic relationships that may vary in utility for various medical scenarios. The social, political, and cultural worlds in which patients' make decisions is complex and varies by individual. Caregivers cannot assume what an individual's priorities will be and must be ready to address the whole person when making medical decisions. 


\section{ONE PATIENT, FOUR CARDIAC ARRESTS AND AN UNLIKELY CULPRIT}

Maaman Bashir; Nabeel Siddiqui; Harvey J. Friedman. St Francis Hospital, Evanston, IL. (Control ID \#2465884)

LEARNING OBJECTIVE \#1: To recognize the life-threatening potential of autonomic dysfunction

LEARNING OBJECTIVE \#2: To identify simple life-style modification techniques that can be life-saving in patients with dysautonomia

CASE: The patient is a 68-year-old male who presented with syncope. At admission, he had a blood pressure of 249/128. After acute management in the emergency department for hypertensive urgency, his blood pressure normalized. He had a history of Shy-Drager syndrome, mitral valve replacement, sarcoidosis, and atrial fibrillation. His blood pressure over subsequent days was labile and upon postural change, profound and symptomatic hypotension was identified. On one such occasion, while measuring his orthostatic vitals, his blood pressure readings were 170/80 (supine), which dropped to 64/40 (standing). Soon after, he collapsed in his chair, became unresponsive, and his cardiac monitor showed pulseless electrical activity. He was successfully resuscitated. An angiogram performed the next day revealed normal coronary arteries. An echocardiogram did not show structural or valvular abnormalities. His hospital course was complicated by three additional witnessed cardiac arrests, each leading to a prolonged stay in the critical care unit. Precipitating events seemed to be large meals, straining for defecation or changing posture. During arrests, he remained normothermic and had no metabolic or electrolyte derangements, ruling out other etiologies. Serum and urine protein electrophoresis were normal. There was no myocardial uptake of gallium. An endocardial biopsy showed nonspecific myocardial inflammation. Congo red staining for amyloidosis was negative Tilt-table testing, paraneoplastic antibodies, skin biopsy for peripheral nerve evaluation, and quantitative pseudomotor axon reflex test, were all negative, suggesting that his dysautonomia was of central rather than peripheral etiology. After the extensive work up, it was concluded that the patient had severe autonomic dysfunction, whereby certain events such as consuming large meals, straining during defecation, or changing posture, triggered profound hypotension. This led to myocardial hypo-perfusion resulting in spontaneous arrhythmias and cardiac arrests. Hence, an automated implantable cardioverter-defibrillator was placed. He was discharged to a rehabilitation center on fludrocortisone and midodrine to maintain his blood pressure along with thigh high compression stockings and instructions for lifestyle modification.

DISCUSSION: This patient's fascinating case demonstrates that evaluation and management of syncope can be far more complex than is usually appreciated. Autonomic dysfunction is a commonly overlooked cause of syncope that is rarely considered serious but that can be life threatening, as demonstrated here. It is important to tread cautiously when managing these patients. Close cardiac monitoring and education are required for these patients, especially those with severe dysautonomia. Medications to increase blood pressure, such as fludrocortisone and midodrine, are recommended but carry the risk of rebound hypertension. Simple lifestyle modification techniques, such as arising slowly from supine to standing, avoiding straining, maintaining hydration, consuming high salt diet, eating small meals, and using pressure stockings, may be life-saving.

ORANGE TO RED: A POOR PROGNOSTIC SIGN Cory J. Rohlfsen; Michael P. Smith. University of Nebraska Medical Center, Omaha, NE. (Control ID \#2435673)

LEARNING OBJECTIVE \#1: Recognize the diseases associated with Agent Orange exposure

LEARNING OBJECTIVE \#2: Develop an approach to chronic pruritus

CASE: A 73 year-old man presented with acute on chronic pruritus for the past 3 years. He described monthly flares of severe pruritus which responded only to high dose steroids
The pruritus had been moderately well controlled on doxepin and atarax in between flares. He had been adherant to avoidance of known allergans and no medications were found to be related to his symptoms. He admitted to Agent Orange exposure during his military service in Vietnam but had no other chemical exposures. His past medical history was significant for chronic paronychia, chronic dermatitis, and multiple skin allergies including silicone, adhesive tape, and propylene glycol preps. He had xerostomia, hypertrophic nails, and non-blanching, diffusely erythematous skin from head to toe. Volar surfaces were atrophic with multiple fissures. His skin was otherwise warm, dry, and non-tender without ecchymosis or petechiae. Multiple skin biopsies were obtained during this particular flare which revealed small lymphocytes in the superficial dermis with irregular nuclear contours. A peripheral blood smear revealed lymphocytes with cerebriform nuclei. Flow cytometry demonstrated that the T-cell clone in skin specimen was identical to the monoclonal T-cells found in the peripheral blood. The patient was diagnosed with Sezary Syndrome and underwent phototherapy and chemotherapy.

DISCUSSION: As Vietnam war veterans age, the manifestations of Agent Orange exposure are becoming more prevalent. It is essential that general Internists are able to recognize the classic presentations of the rare conditions associated with Agent Orange exposure. Diseases commonly associated with Agent Orange exposure include Parkinson's Disease, Multiple myeloma, Porphyria Cutanea Tarde, Hodgkin's disease, Non-Hodgkin's Lymphoma, Prostate cancer, Lung cancer, Diabetes Mellitus, Ischemic Heart Disease, and T-cell lymphoma. Without a high degree of clinical suspicion, these diseases could easily be missed. Chronic pruritus in an otherwise healthy patient can be difficult to diagnose without associated symptoms and is a problem frequently encountered by the general Internist. Ruling out uremia, biliary cirrhosis, iron deficiency anemia, HIV, and Hepatitis C are often the first steps. However, if chronic pruritus is associated with a rash, then skin biopsy should also be included in the diagnostic work up. Moreover, chronic pruritus should be considered as a potential paraneoplastic sign. This is especially true in patients with a history of Agent Orange exposure, who are at increased risk of several malignancies. Chronic pruritus with dermatitis that is diffuse should raise suspicion for Cutaneous T-cell lymphoma (Mycosis Fungoides). Once a patient develops leukemia of T-cells (involvement in the blood), the diagnosis is upstaged to Sezary Syndrome which is associated with poorer prognosis. A definitive diagnosis is obtained when clonality of T-cells is confirmed amongst skin biopsy, peripheral blood smear, and flow cytometry results. Chronic pruritus is a common problem with a wide scope of underlying causes, ranging from the benign to the extremely malignant. The general Internist must have an approach to this symptom to accurately diagnose and treat appropriately.

\section{OSSEOUS SARCOIDOSIS MASQUERADING AS MULTIPLE MYELOMA}

Sherif Shoucri; Robert Freilich. NYU, New York, NY. (Control ID \#2469908)

LEARNING OBJECTIVE \#1: Demonstrate that osseous sarcoidosis can present as lytic bone lesions

LEARNING OBJECTIVE \#2: Consider osseous sarcoidosis in the differential diagnosis for asymptomatic patients presenting with multiple lytic lesions

CASE: A 57-year-old male with a history of lytic lesions presented with refractory bone pain. He was diagnosed with a monoclonal gammopathy of undetermined significance 1 year prior, after presenting with a mechanical fall. At that time, numerous lytic lesions were discovered on computed tomography (CT) scans of his skull and spine. Follow-up blood work revealed elevated lambda light chains. A subsequent bone marrow biopsy was positive for a plasma cell neoplasm replacing $8 \%$ of his marrow cellularity. He underwent two bone biopsies; one showed granulomatous disease and the other showed plasma cells. In light of these findings he was treated with cyclophosphamide, bortezomib, and dexamethasone for eight cycles. His total light chains decreased with chemotherapy. However, his lytic 
lesions worsened. One week prior to his admission, a positron emission tomography (PET) scan was obtained and showed new uptake throughout his lungs, as well as hilar lymphadenopathy that had increased in size. He was admitted to the hospital for pain control and further work-up of his lung disease. The patient described worsening bone pain limiting his ability to ambulate. He denied having shortness of breath, cough, weight loss or fatigue. His exam demonstrated gait instability but was otherwise unremarkable. He was neither anemic nor hypercalcemic and his creatinine clearance was normal. Initial differential diagnosis of his lung lesions included metastatic multiple myeloma, secondary malignancy, or atypical infection. A bronchoscopy was performed and a bronchial lymph node biopsy revealed noncaseating granulomatous inflammation and fibrosis consistent with pulmonary sarcoidosis. In view of this diagnosis, combined with the fact that his prior bone biopsy showed granulomatous disease, it was determined that his lytic lesions were a manifestation of osseous sarcoidosis. The patient was started on prednisone $50 \mathrm{mg}$ daily and after 1 month of therapy his bone pain had significantly improved.

DISCUSSION: Sarcoidosis is a systemic granulomatous disease that classically involves the lungs, skin, lymph nodes, and eyes. Osseous sarcoidosis is only reported in 1-13\% of patients. The small bones of the hands and feet are most commonly affected. However, there are case reports of osseous lesions involving the skull and axial skeleton. These lesions are described as a cystoid osteitis when seen in the hands and feet. They can also appear as multiple lytic lesions, permeative lesions with bone remodeling, or destructive lesions resulting in pathologic fractures. Although patients can present with bone pain, many are often asymptomatic. This can lead to difficulty in diagnosing patients with osseous sarcoidosis. Especially in cases where many lytic lesions are present, one must be cautious in differentiating between osseous sarcoidosis and multiple myeloma.

OTOGENIC BRAIN ABSCESS COMPLICATING OTITIS MEDIA: A CASE REPORT Oluchukwu Oluoha ${ }^{1}$; Olufolahan Carrena ${ }^{2}$; Ali Salem ${ }^{1}{ }^{1}$ John H. Stroger, Jr. Hospital of Cook County, Chicago, IL, Chicago, IL; ${ }^{2}$ Meharry Medical College, Brentwood, TN. (Control ID \#2470310)

LEARNING OBJECTIVE \#1: Recognize the need for serial neuroimaging studies in patients with suspected intracranial complication of otitis media.

LEARNING OBJECTIVE \#2: Recognize the intracranial complications of otitis media CASE: A 41-year-old man presented to the emergency department with a 5-day history of headache, fever and chills. In the preceding week, he was seen at a private clinic for similar complaints including left otorrhea, and was treated with antibiotics for presumed otitis media. Ibuprofen prescribed for headache provided no significant relief prompting his presentation to emergency department for worsening headache. On examination, his vital signs were normal but he was ill-appearing. Otologic examination revealed brownish left otorrhea with associated ruptured tympanic membrane and mastoid tenderness. Neurological examination revealed no focal deficits or evidence of meningitis except for equivocal nuchal rigidity. Laboratory studies showed blood leukocyte count of $11.7 \mathrm{k} / \mathrm{uL}$, otherwise unremarkable. Initial CT scan was remarkable for complete opacification of left mastoid air cells and edema of left middle ear cavity. No bony erosive changes or intracranial extension of middle ear disease was noted. Patient was subsequently admitted to the medicine floor. Due to initial concerns of intracranial complications of otitis media in the form of meningitis, he was started on empiric antibiotics. Lumbar puncture attempted prior to initiation of antibiotic was unsuccessful but was successfully obtained the next day. Cerebrospinal fluid (CSF) analysis revealed mildly elevated protein and leukocyte count. CSF gram stain was negative. Headache worsened even after initiation of antibiotics therapy and pain management. On day 2 of hospitalization, patient was noted with new-onset generalized tonic-clonic seizure. CT scan of head and temporal bone was repeated and it revealed loss of left mastoid trabeculae and focal dehiscence of the tegmen tympani with empyema involving the left temporal lobe and left middle cranial fossa. MRI re-demonstrated similar findings but also revealed partial thrombosis of left cavernous sinus. Otorhinolaryngology surgery service was consulted and prompt otosurgical intervention including left tympanomastoidectomy, middle ear exploration and temporal bur hole aspiration of abscess was performed. Intraoperative culture later grew Peptostreptococcus and pathology of ear debri was consistent with cholesteatoma. Sequelae of his infection include left conductive hearing loss and mild aphasia. Aphasia and headache subsequently improved and he was discharged to a nursing home to complete 6 weeks of culture-directed antibiotics therapy. At 3-month follow-up after discharge, the patient's condition has improved. Repeated MRI showed resolution of left temporal lobe empyema. Future follow-up was scheduled with Otorhinolaryngology surgery service where audiogram is planned for hearing loss

DISCUSSION: Otogenic brain abscess (OBA) is the most serious complication of otitis media (OM). Its incidence has decreased following the advent of antibiotics, however, it remains a potentially fatal complication. It typically arises following spread of infection beyond the mucoperiosteal lining of the middle ear and mastoid that extend via the tegmen tympani and trautmann's triangle to involve the temporal lobe (more commonly) and cerebellum respectively. Streptococcus pneumonia is the most common offending bacteria but Peptostreptococcus species are often isolated. Symptoms of OBA largely depends on location and extent of the abscess, however, as seen in this case and as described by Derić D et al., headache is the most common presentation and may or may not be associated with fever, nausea and vomiting. Neurologic deficits including seizure, aphasia, altered mental status and motor dysfunction may also occur. Diagnosis of OBA is made by neuroimaging studies with CT scan or MRI. This case highlights the importance of a high index of suspicion for intracranial complication of otitis media in patients with OM presenting with severe headache. It also shows the importance of serial imaging studies if clinically indicated even if initial studies were negative. This would be the case with persistence or worsening of symptoms despite appropriate therapy, and development of new complications. Treatment of OBA requires prompt antibiotic therapy and otosurgical management to reduce associated morbidity and mortality.

PAIN IN THE NECK Moira Scaperotti; Lisa N. Glass; Yelena Averbukh. Albert Einstein College of Medicine, Montefiore Medical Center, Bronx, NY. (Control ID \#2467747)

LEARNING OBJECTIVE \#1: Recognize the causes of autonomic dysreflexia LEARNING OBJECTIVE \#2: Highlight the role of the autonomic nervous system in glucose regulation

CASE: The patient is a 64-year-old woman with multilevel degenerative disc disease, including large herniations at $\mathrm{C} 3-\mathrm{C} 4$ and $\mathrm{C} 4-\mathrm{C} 5$, and associated spinal cord contusion. She presented with facial pain and swelling after receiving a steroid injection in her cervical spine the day prior. The patient was in no distress, and all of her symptoms had resolved. However, her systolic blood pressure ranged $80-150 \mathrm{~s} \mathrm{mmHg}$, while her heart rate ranged $40-80 \mathrm{~s} \mathrm{bpm}$. She was found to be hypoglycemic to $50 \mathrm{mg} / \mathrm{dl}$, and was admitted. A physical examination revealed tenderness of the posterior neck. Laboratory tests were unrevealing. She had been experiencing labile blood pressures and erratic blood sugar for approximately 1 year prior to presentation. Over the course of a day, her systolic blood pressure could range from the $70 \mathrm{~s}$ to $190 \mathrm{~s} \mathrm{bpm}$. She also experienced intermittent, symptomatic hypoglycemia. An extensive work-up, including a cardiac catheterization, stress test, tilt-table testing and endocrinology blood work, was unrevealing. Medicine, neurology and surgical services attributed her symptoms to autonomic dysfunction as a result of cervical sympathetic ganglia compression in the setting of degenerative joint disease. The patient has since undergone cervical decompression surgery, with some improvement in her symptoms.

DISCUSSION: The patient's labile blood pressures and bradycardia can be attributed to autonomic dysreflexia caused by the spinal cord contusion. Autonomic dysreflexia is 
characterized by acute hypertension with simultaneous bradycardia, and can be found in spinal cord injury (SCI). Blood pressure is normally controlled by supraspinal sympathetic preganglionic neurons. However, in SCI, reflex processes play a larger role in blood pressure regulation than they typically do. The mechanism is not well understood. However, when a somatic or visceral stimulus below the SCI travels proximally, it becomes interrupted at the lesion. Subsequently, there is thought to be a sympatheticmediated vasoconstrictive response, leading to hypertension. There are several welldescribed physiologic mechanisms that combat hypoglycemia in non-diabetics. The role of the autonomic nervous system (ANS) is specifically pertinent to this case. Once the blood glucose reaches $50 \mathrm{mg} / \mathrm{dl}$, the sympathetic chain is activated. This sympathetic contribution descends to ganglia in the celiac and superior mesenteric plexi, and terminates in the pancreatic islet. Insulin secretion decreases, while glucagon secretion increases. The preganglionic fibers in the chain also directly innervate the adrenal gland, and release adrenaline. Subsequently, insulin secretion decreases, glucagon secretion increases, gluconeogenesis increases and glucose uptake decreases in skeletal muscle. Given the sympathetic chain's close proximity to the cervical and thoracic vertebral columns, it is not unlikely that disc disease could impinge on it, potentially disrupting glucose control. This patient's cervical disc disease created generalized ANS malfunctioning, manifesting as improper glucose control, labile blood pressure and bradycardia. The patient was educated about symptomatic hypoglycemia and encouraged to be proactive with regard to self-administration of glucose. She was also counseled about hypotension and bradycardia, and asked to ensure adequate salt and fluid intake.

PAIN OUT OF PROPORTION IN A YOUNG MAN, UNUSUAL PRESENTATION OF THROMBOPHILIA. Uzma Imran ${ }^{1}$; Phaniram Sumanam ${ }^{1}$; Arian Majko ${ }^{1}$; Mohammed AL-Jumayli ${ }^{2}$; Ali M. Usman ${ }^{2}$; Supriya Sekhar ${ }^{2}$; Mohamed Hassanein ${ }^{3}$; Anubha Tewary ${ }^{2} .{ }^{1}$ Capital Health RMC Trenton NJ, Hamilton, NJ; ${ }^{2}$ Capital Health Regional Medical Center, Trenton, NJ; ${ }^{3}$ Capital health regional medical center, Plainsboro, NJ. (Control ID \#2455403)

LEARNING OBJECTIVE \#1: Consider mesenteric ischemia in younger patients with non-resolving and out of proportion abdominal pain.

CASE: A 25-year-old African American gentleman with family history of Lupus presented with moderate, intermittent right upper quadrant pain for 2 weeks which was worse after meals, associated with non bloody emesis, occasional dark stools and constipation. On exam, he was in moderate distress secondary to abdominal pain, afebrile with tachycardia, soft abdomen with mild epigastric and RUQ tenderness and positive bowel sounds. He was admitted for mild gastroenteritis as initial abdominal CAT scan showed mild thickening of small bowel. Patient's condition worsened with interval development of leukocytosis of 26 without lactic acidosis and worsening abdominal pain. Gall bladder ultrasound revealed no flow in the portal vein, prompting a repeat $\mathrm{CT}$ abdomen on 3rd day of hospitalization showing a filling defect in a distended superior mesenteric vein and confirmed thrombosis of the portal vein leading to small bowel ischemia. Heparin infusion was started and he was taken to OR for small bowel resection next day. He was discharged home in stable condition after 19 days, on coumadin therapy. Hypercoagulability work up before heparin therapy revealed decreased protein S activity.

DISCUSSION: Mesenteric venous thrombosis, though less common than arterial thrombosis, remains an important cause of mesenteric ischemia. There is often a considerable delay in the diagnosis because of a low degree of suspicion and the nonspecific clinical presentation. The broad and atypical list of etiologies to consider in those under age 40 years include congenital aortic anomalies, hypercoagulable states, inflammatory conditions, collagen vascular disorders, and environmental agents such as cocaine use, smoking, or trauma. Presentation of mesenteric venous thrombosis can be more insidious, with sub acute symptoms that can be present for days to weeks before diagnosis.
Nonspecific abdominal pain may be the only feature as was the case with our patient. Acidosis, acute renal failure, and septic shock/sepsis can develop in roughly half of patients with more extensive liver or bowel ischemia or necrosis. According to one study overall postoperative complication and overall mortality rates were 61.5 and $26.9 \%$, respectively. Early recognition of mesenteric ischemia in young adults and children is essential to prevent bowel loss and other serious consequences associated with this rare condition.

PANCREATIC AND HEPATIC ABSCESSES IN A DIABETIC PATIENT SIMULATING A MALIGNANCY Nurilign A. Bulcha; Dipen B. Khanapara; Olena Slinchenkova. Montefiore Medical Center, Bronx, NY. (Control ID \#2470301)

LEARNING OBJECTIVE \#1: Identify Streptococcus Aginosus as an emerging cause of pyogenic liver and pancreatic abscesses.

LEARNING OBJECTIVE \#2: Recognize that uncontrolled DM is a risk factor for pancreatic and hepatic abscesses.

CASE: A 72 year old man presented with 6 months of progressively worsening lethargy and epigastric discomfort. He had a history of DM and an episode of acute pancreatitis 2 years prior. On presentation, he was febrile and had diffuse abdominal tenderness. He had a white count of 14,700. His liver function tests and pancreatic enzymes were normal. His hemoglobin A1C was $8.8 \%$. A CT scan of the abdomen revealed a $3.5 \mathrm{~cm}$ necrotic mass in the uncinate process and numerous hepatic masses. Blood culture grew Streptococcus Aginosus. EUS guided FNA of the pancreatic mass and a CT guided FNA of a sentinel hepatic lesion revealed inflammatory cells and was negative for malignant cells. The patient received 4 weeks of IV antibiotics and was discharged on a 2 week course of oral antibiotics. Three months later, a repeat CT scan revealed complete resolution of the pancreatic and hepatic lesions.

DISCUSSION: A suspicious abdominal mass on imaging is a common finding encountered by the internist. As illustrated in our case, pyogenic intra-abdominal abscesses can present as mass lesions with vague symptoms and laboratory investigation results. The liver is the most common site for pyogenic intra-abdominal abscesses with a prevalence rate of 8-16 cases per 100,000 among hospitalized patients and an estimated 2.3 cases per 100,000 in the population. Pancreatic abscesses are rare but are associated with greater mortality. The underlying mechanisms of pancreatic and liver abscess formation are variable. In the liver, about $60 \%$ of abscesses can be attributed to biliary tract obstruction. Other frequent causes of liver abscess include infections of the portal bed infections and direct extension from local infections. Most cases of pancreatic abscess occur following an episode of acute pancreatitis and infection of a pancreatic cyst. Penetrating abdominal trauma, surgery and bowel perforation due to foreign body, inflammatory bowel disease and ischemic colitis have been implicated in both pancreatic and liver abscesses, but are less common. Escherichia Coli is the most frequently identified pathogen from intraabdominal abscesses. Among gram positives, Streptococcus Millieri is an important cause of pyogenic bacterial abscess. This group of bacteria is present in $12 \%$ of liver abscesses and has been identified in up to $20-40 \%$ of isolates from healthy appendices. Streptococcus Millieri are also known as the streptococcus Aginosus group after the most commonly encountered bacteria in the group. Streptococcus Aginosus has been found in $64 \%$ of isolates from healthy individuals containing streptococcus Millieri species. Streptococcus Aginosus also has the highest predilection to cause intra-abdominal abscess formation. The other members of this group are Streptococcus Intermedius and Streptococcus Constellatus. All members of this group are normal flora, found in the oral cavity, the GI and the GU tracts. Infectious abscesses due to these organisms usually occur in patients with cancer and impaired immunity or following surgery and abdominal trauma Diabetes Mellitus is also a risk factor for infectious abscesses, particularly in the liver. Patients with DM can have a 3.6 fold increased risk of developing pyogenic liver 
abscesses. This risk is attributable to the hyperglycemic environment in DM which leads to neutrophil dysfunction and promotes the growth of pathogenic bacteria. Patients with DM have decreased chemo taxis, phagocytosis and oxidative action of phagocytes. Other features of inflammation such as protein leakage and edema formation in response to inflammatory mediators are also impaired in diabetic patients. One mechanism for neutrophilic dysfunction in hyperglycemia is thought to be the result of accumulation of advanced glycation end products. (AGEs), formed due to the interaction of glucose with proteins and lipids. Advanced glycation products modify several receptors on endothelial cells, smooth cells and mononuclear phagocytes, altering membrane structure and function. The interaction between AGEs and phagocyte cell membranes leads to decreased fluidity and leukocyte migration. Hyperglycemia also causes increased levels of sorbitol in phagocytic cells via activation of the polyol pathway which further impairs leukocyte and endothelial cell interactions. The mechanisms leading to neutrophilic dysfunction are related to the degree of hyperglycemia and can be reversed with improved glycemic control. In our patient, his uncontrolled DM was a likely predisposing factor for his liver and pancreatic abscesses. Good glycemic control can thus prevent intraabdominal infections and complement antibiotic therapy. Streptococcus Aginosus is sensitive to commonly used antibiotics but there is data suggesting emerging resistance to antibiotics which could pose a significant problem in the management of infections caused by this organism. Further studies are warranted to better understand resistance patterns and guide antibiotic therapy.

PARAGANGLIOMA OF THE URINARY BLADDER: A RARE AND UNSUSPECTED CAUSE OF SEVERE HYPERTENSION AND RECURRENT URINARY TRACT INFECTIONS Gerard Chaaya; Jorge A. Morales; Noman Subhani; Analia Castiglioni. University of Central Florida College of Medicine Internal Medicine Residency Program, Orlando, FL. (Control ID \#2469487)

LEARNING OBJECTIVE \#1: Review the pathophysiology, clinical presentation and localization of pheochromocytomas.

LEARNING OBJECTIVE \#2: Recognize bladder paraganglioma as a rare type of extraadrenal pheochromoyctoma, its clinical presentation and management.

CASE: We present a case of a 28 -year-old Hispanic woman, with a past medical history significant for ulcerative colitis, who at age 23 developed episodic headaches, palpitations and sweating, along with recurrent urinary tract infections (UTIs) and pelvic pain. She was subsequently diagnosed with hypertension at age of 28 . Because of recurrent lower abdominal pain, a pelvic ultrasound was performed and showed a $5 \mathrm{~cm}$ adnexal mass with papular structures and septations; Ca- 125 was negative. Laparoscopy revealed a $5 \mathrm{~cm}$ cystic-solid peritoneal wall mass that extends from the midline to the left anterior pelvic wall, up to the lower uterine segment and upper third of the cervix. An abdominal and pelvic computer tomography (CT) scanner revealed an irregular thick-walled left adnexal cystic mass $(4 \times 6 \mathrm{~cm})$ abutting the urinary bladder, a left pelvic lymphadenopathy $(1.7 \mathrm{~cm})$, along with a T12 and sacral lucencies. Cystoscopy was performed showing a growing mass thru the left bladder wall, which prompted a transurethral resection of the bladder tumor (TURBT) with a histological diagnosis of paraganglioma. Physical exam and laboratory evaluation were normal except for elevated $24 \mathrm{~h}$ urinary cathecolamines (norepinephrine), vanillymandelic acid (VMA) and metanephrines. A decision for surgical excision was made and patient was started on perioperative alpha blockage (phenoxybenzamine) followed by beta-blockage (propranolol). The patient underwent a partial cystectomy with lymph node dissection. The histopathological exam revealed proliferation of neuroendocrine cells arranged in balls and nests with round to ovoid nuclei, nucleoli and fibrillar cytoplasm, evidence of bladder muscular and vascular walls invasions, along with positive left iliac and obturator lymph nodes; all consistent with a diagnosis of paraganglioma of the bladder with lympho-vascular invasion (T3aN2M1).
The patient did well and was discharged in order to follow-up with urology and oncology. A PET and bone scans still pending at this time.

DISCUSSION: Pheochromocytomas are catecholamine-producing tumors that originate from the chromaffin cells derived from the sympathetic or parasympathetic paraganglia. Although the etiology of sporadic pheochromocytomas is unknown, there is a recognized genetic component seen in $25-33 \%$ of patients, including those found in multiple endocrine neoplasia type 2 (MEN2) and von Hippel-Lindau disease. The classic triad of symptoms includes headache, sweating, and palpitations/tachycardia. Although the majority of patients do not have all three of these classic symptoms, the presence of all three in association with hypertension makes pheochromocytoma a likely diagnosis. Diagnosis is made using urinary and plasma fractionated metanephrines and catecholamines mesasurement. Elevated urinary metanephrines is both sensitive and specific for pheochromocytoma, 96 and $98 \%$ respectively. Pheochromocytomas can be located extramedullary in $10 \%$ of the cases, usually located within the abdomen in association with the celiac, superior mesenteric, inferior mesenteric ganglia and Organ of Zuckerkandl. Approximately $10 \%$ of all extra-adrenal pheochromocytomas are malignant. In 2004, the World Health Organization differentiated pheochromocytoma, a catecholamineproducing tumor that originates within the adrenal medulla, from paraganglioma, a catecholamine-producing tumor found outside the adrenal glands. Paragangliomas of the bladder are exceedingly rare tumors, comprising less than $0.05 \%$ of all bladder tumors. Although less than $1 \%$ of all pheochromocytomas are located within the urinary bladder, it is still the most common location of origin within the genitourinary system $(79.2 \%)$. Less common sites include the urethra (12.7\%), the pelvis (4.9\%), and the ureter $(3.2 \%)$. The most common associated symptoms are hypertension $(54.7 \%)$, headache (48.1\%), hematuria (47.2\%) and syncope/palpitations (43.4\%). Imaging plays an ancillary role in the detection of paragangliomas of the bladder, but is vital in the staging and follow-up of urological disease. CT is the most commonly used imaging modality for initial evaluation of patients with suspected bladder tumors, although magnetic resonance imaging offers better soft tissue contrast for more accurate staging. The most commonly reported treatment for patients with paraganglioma of the urinary bladder is partial cystectomy. Other treatment modalities include trans-urethral resection of bladder tumor (TURBT) $(19.8 \%$ ) and radical cystectomy (11.3\%). Regimens including cyclophosphamide, vincristine and dacarbazine (Averbuch protocol) have been shown to be effective against malignant pheochromocytomas. This case illustrates a case of malignant paraganglioma of the bladder presenting as recurrent UTIs and hypertension in a young female. A reminder is to consider pheochromocytomas in the differential diagnosis of bladder tumors and to recognize their malignant potential when in extraadrenal locations.

PARANEOPLASTIC PEMPHIGUS CAUSING BRONCHIOLITIS OBLITERANS David Pineles ${ }^{2}$; Grace Huang ${ }^{2}$; Natalie K. Levy ${ }^{1} .{ }^{1}$ NYU School of Medicine, Bellevue Hospital, New York, NY; ${ }^{2}$ New York University School of Medicine, New York, NY. (Control ID \#2465578)

LEARNING OBJECTIVE \#1: Recognize the clinical presentation of paraneoplastic pemphigus.

LEARNING OBJECTIVE \#2: Understand that with bronchiolitis obliterans, severe pulmonary disease can be present in the absence of significant CT chest findings. Understand that paraneoplastic pemphigus (also known as paraneoplastic autoimmune multiorgan syndrome) is a clinical syndrome that should still be considered in cases where direct and indirect immunofluorescence are negative.

CASE: A 51 year-old man who presents with progressively worsening shortness of breath and cough for a month. He had been diagnosed with mucosa-associated lymphoid tissue (MALT) lymphoma about 2 months prior to admission, and had undergone 1 cycle of 
rituximab, cyclophosphamide, doxorubicin, vincristine and prednisone (R-CHOP). He then developed painful erosive oral lesions and worsening dyspnea. Biopsy of the oral lesions showed lichen planus, and the patient was given oral prednisolone as well as oral pain relievers with some improvement in oral symptoms. However, his shortness of breath continued to worsen and he presented to the hospital. On physical exam, the patient was tachypneic, and pulmonary exam was notable for inspiratory wheezes, especially in the upper lung fields. A CT scan of the chest was significant only for bilateral cylindrical bronchiectasis. However, an arterial blood gas showed respiratory acidosis, despite the patient's tachypnea. Pulmonary function tests were performed which were consistent with severe obstructive pulmonary disease. Paraneoplastic pemphigus was suspected, and biopsy specimens were sent for direct and indirect immunofluorescence. These did not show any abnormal deposit, although studies have shown the sensitivity for direct and indirect immunofluorescence to be approximately $41 \%$ and $74-86 \%$, respectively. DISCUSSION: The patient's constellation of symptoms likely represents paraneoplastic pemphigus in the setting of his primary hematologic neoplasms. Paraneoplastic pemphigus (PNP) is a paraneoplastic mucocutaneous blistering disease that is most commonly associated with lymphoproliferative disorders. Another name for this entity is paraneoplastic autoimmune multiorgan syndrome (PAMS) which encompasses the heterogeneous signs and symptoms associated with this paraneoplastic process: erosive mucositis, variable cutaneous lesions (pemphigus-like, bullous pemphigoid-like, erythema multiforme-like, and lichen planus-like), and pulmonary disease. The patient was treated with intravenous steroids, rituximab, and IVIG with moderate improvement in respiratory symptoms. Studies have shown that when PNP is associated with Castleman's disease or thymoma, resection of these underlying tumors often results in disease remission. However, when PNP is associated with other malignancies, the response by treating these underlying neoplastic disorders is not as beneficial. Given this syndrome's extremely high mortality rate, it is important for practitioners to consider PNP in patients with a primary neoplasm, mucocutaneous lesions, and respiratory symptoms, and to promptly provide appropriate treatment.

PARINAUD OCULOGLANDULAR SYNDROME: RARE BUT TREATABLE CAUSE OF NEURO RETINITIS Krishna Venkata ${ }^{2}$; Caitlin Jones ${ }^{2}$; Chioma Nwagbara $^{3}$; Sanam Anwer ${ }^{2}$; Kannan Thanikachalam ${ }^{1}$; Ashwini Sharma ${ }^{2} .{ }^{1}$ UAB Health Center Montgomery, Montgomery, AL; ${ }^{2} \mathrm{UAB}$ Montgomery, Montgomery, AL; ${ }^{3} \mathrm{UAB}$ Montgomery Health Center, Montgomery, AL. (Control ID \#2470265)

LEARNING OBJECTIVE \#1: To recognize the clinical symptoms, various manifestations, diagnosis, management and complications of Bartonella henselae infection.

LEARNING OBJECTIVE \#2: To emphasize importance of cat scratch disease manifesting as Parinaud Oculoglandular syndrome, a rare but potentially treatable cause of neuroretinitis and need to be considered as one of the possible differential diagnosis.

CASE: A 27 year old Caucasian male presented with a history of acute painful loss of left eye vision, 4 weeks prior to the admission. History was negative for purulent or serous eye discharge, red eye, fever or sore throat or any medical comorbidities. He had a remote history of exposure to cats, almost 6-7 months ago. On exam, his vital signs were normal. His corrected visual acuity was $20 / 20$ in the right eye and 20/200 in the left eye. Right pupil was $3 \mathrm{~mm}$ in size, round and reactive to light whereas the left was $5 \mathrm{~mm}$ and had Marcus Gunn defect. Color vision was also impaired in the left eye. Confrontation visual field testing revealed superior altitudinal field defect in left eye. The anterior segment exam was normal in both the eyes. The posterior segment exam in the right eye showed mild edema in the optic nerve with streaking exudates towards the fovea and intact retina. The exam on the left showed significant swelling of the optic nerve with extensive fluids and exudates forming a macular exudate on the left. No signs of retinal artery or vein occlusion were visualized. No lymphadenopathy and no cutaneous manifestations were appreciated. Several tests were ordered including: human immunodeficiency virus, antineutrophilic antibody, cytomegalovirus antibody, neuromyelitis optica (NMO)-specific autoantibodies, Rocky Mountain spotted fever, West Nile antibody, Lyme antibody, angiotensin-converting enzyme, syphilis IgG with rapid plasma reagin, $B$. henselae IgG and IgM levels in the blood and CSF oligoclonal bands, cerebrospinal fluid (CSF) cryptococcal antigen, CSF Ebstein Barr virus, CSF Herpes simplex virus, CSF Lyme disease, CSF myelin basic protein, CSF acid fast bacilli, CSF culture and Gram stain, Epstein-Barr virus, CSF toxoplasma by PCR. All the laboratory data came back negative except $B$. henselae antibody levels. MRI of brain was normal while MRI of orbit showed normal right optic nerve and enhancement of left optic nerve with very subtle enhancement of retina as well concerning for optic neuritis. The patient was treated empirically with high dose intravenous steroids. Eventually diagnosis of cat scratch- optic neuroretinitis was made as the $B$. henselae $\operatorname{IgG}$ titer was elevated (1:1024). The patient was started on trimethoprim- sulphamethoxazole therapy for a month. On outpatient follow up after 1 week his optic nerve swelling improved with disappearance of the macular exudates over time, but the vision in the OS failed to improve most likely secondary to ischemic damage to optic nerve.

DISCUSSION: Cat Scratch Disease (CSD) is a fairly common infectious disease caused by intracellular gram negative bacterium Bartonella henselae with around 24,000 cases yearly in United States (US). Humans get infected through cat clawing, biting and bite of cat flea (Ctenocephalides felis).The ocular signs and symptoms are seen in 5-10\% of patients. Around $1-2 \%$ of cases have neuroretinitis which manifests as acute unilateral vision field loss secondary to optic nerve edema and stellate macular exudate. We present a case of Bartonella infection involving bilateral retina and left optic nerve. Early antibiotic treatment has been suggested to shorten course of infection and improve outcome. Doxycycline has been reported to achieve good intraocular concentration. Other antibiotics include azithromycin, ciprofloxacin, clarithromycin, gentamicin, rifampin and trimethoprim sulfamethoxazole. Immunocompetent patients are usually treated for 2 4 weeks and immunocompromised patients are treated for 4 weeks to treat the infection. Prompt diagnosis and treatment of neuroretinitis due Bartonella henselae is required, as delay in treatment can cause permanent visual impairment as with our patient.

\section{PAROTID GLAND SWELLING IN A PATIENT WITH CLINICAL FEATURES OF SYSTEMIC LUPUS ERYTHEMATOSUS (SLE) Lucas Bruton; Aliyah Sadaf.} Rush University Medical Center, Chicago, IL. (Control ID \#2469689)

LEARNING OBJECTIVE \#1: Recognize bilateral parotid gland swelling as a presenting feature of Systemic Lupus Erythematosus (SLE)

CASE: A 38-year-old woman with a past medical history of gastroesophageal reflux disease (GERD), goiter and a family history of systemic lupus erythematosus (SLE) presented with a 3 week history of a persistent sore throat that was followed by progressive swelling of her hands and feet, dyspnea with exertion, nausea with occasional vomiting, diffuse arthralgias, and fatigue. She was seen at an outside hospital multiple times for these complaints and was prescribed oral antibiotics for coverage of a possible upper respiratory infection without relief. Vital signs were unremarkable. Initial physical examination showed bilateral scleral injection, left anterior cervical lymphadenopathy, mild tenderness to palpation of shoulders bilaterally, warmth and tenderness to palpation of bilateral PIPs, and diffuse non-pitting edema of the upper and lower extremities bilaterally. Initial laboratory evaluation was significant for WBC of $2,550 / \mu \mathrm{L}$ (ANC of 0.82 ), hemoglobin of $10.5 \mathrm{~g} / \mathrm{L}, \mathrm{MCV}$ of $86.3 \mathrm{fL}$, platelets of $148 \mathrm{~K} / \mu \mathrm{L}, \mathrm{BUN}$ of $17 \mathrm{mg} / \mathrm{dL}$, creatinine of $0.74 \mathrm{mg} / \mathrm{dL}$, and an albumin of $2.6 \mathrm{~g} / \mathrm{dL}$ with a total protein of $6.5 \mathrm{~g} / \mathrm{dL}$. CXR was notable for new enlargement of the cardiac silhouette with changes of volume overload and associated left retrocardiac atelectasis and small bilateral pleural effusions. Physical exam on second day of hospitalization was notable for tender, bilateral parotid gland enlargement and bilateral submandibular 
adenopathy. Due to concern for parotitis on exam, mumps serologies were obtained. Mumps IgG was positive ( $>5.00)$, but mumps IgM was not detected $(<1: 20)$. She was also found to have elevated SS-A titers of 3.37 U; however, SS-B titers were negative. She did not exhibit or report any dryness of her eyes or mouth, and did not meet the clinical criteria for Sjögren's Syndrome. Subsequent laboratory evaluation showed decreased C3 of $40 \mathrm{mg} / \mathrm{dL}$ and C4 of $4 \mathrm{mg} / \mathrm{dL}$, elevated ESR of $35 \mathrm{~mm} / \mathrm{h}$, and elevated ANA of 1:640 and anti-dsDNA of $>180 \mathrm{IU} / \mathrm{mL}$. Rheumatoid factor, TSH, monotest, CMV DNA, EBV DNA, Parvovirus B19, CCP, Anti-DNAse B and Anti-Streptolysin O titers were negative. The patient was diagnosed with SLE. Prednisone $20 \mathrm{mg}$ PO daily was started, and her bilateral parotid gland swelling diminished. Following discharge, the patient followed with rheumatology and was started on Plaquenil.

DISCUSSION: This case demonstrates a rare presentation of bilateral parotid gland swelling in a patient with SLE, which is not often observed in patients presenting with pure SLE (not compounded with other diseases). While there was initial concern for mumps infection in this patient given her symptoms, mumps serologies were consistent with prior immunization or exposure and did not suggest acute infection. The most likely cause of her bilateral parotid gland enlargement was, therefore, SLE. Though salivary gland involvement is extremely rare in SLE, it is more often seen in females affected with the disease. Previously diagnosed cases involving parotid and submandibular gland enlargement in SLE patients have shown lymphoplasmacytic infiltration of perisalivary areas or gland lobules, with occasional fibrosis and mucoid degeneration also having been observed. In addition, standard treatment with steroids appeared to reduce inflammation and decrease swelling in the patient presented above. SLE is a common disease that can present in a variety of uncommon ways. Physician understanding of unusual presentations of common illnesses such as SLE is important to promote prompt diagnosis and administer appropriate treatments for these conditions. It also remains essential to exclude infection in patients with suspected or confirmed SLE who present with parotid gland swelling.

PINKY PROMISE- AN ATYPICAL ANATOMICAL SITE OF PRIMARY CUTANEOUS MUCINOUS CARCINOMA. Sree V. pilla ${ }^{2}$; Siwadon Pitukweerakul ${ }^{1}$; Harvey J. Friedman ${ }^{3}$; Shahriar Dadhkhah ${ }^{1}$. ${ }^{1}$ Presence St. Francis Hospital, Evanston, IL; ${ }^{2}$ St Francis Hospital, EVANSTON, IL; ${ }^{3}$ St Francis Hospital, Evanston, IL. (Control ID \#2470306)

LEARNING OBJECTIVE \#1: Primary Cutaneous Mucinous Carcinoma is a rare cancer of sweat gland origin. Only 200 cases of PCMC have been reported to date in literature. Our case is the first to report a nail bed in upper extremity as a potential site of origin for this rare tumor.

CASE: An 82 y/o gentleman presented to our clinic complaining of a subcutaneous lesion measuring about $1 \mathrm{~cm}$ on the nail plate matrix on the right 5 th finger growing steadily in size over several years which was occasionally painful. He never sought medical attention about this finger lesion before. However, this time the patient also had a 4 × $3 \mathrm{~cm}$ cyst that had broken down to form a chronic ulcer that had recently started draining on his lower back which prompted family members to bring him to the clinic. The patient is a retired businessman and he denied any injury or pricks at the finger site prior to development of the lesion, denied noticing any bleeding from the finger nail lesion. He denied any recent weight loss or loss of appetite. No lumps or bumps in his neck or axilla. He had no history of bloody stools or melena. No history of gastritis or hematemesis. He had a remote smoking history of five pack years and drinks alcohol socially. He did not have family history of cancer. On physical examination in our office the finger lesion was pink in color, glossy in appearance and soft in consistency and fixed to nail matrix area. No drainage was noted. It was mildly tender. The chronic ulcer on upper right back had an exophytic round granulating mass which was friable. He had neither lymphadenopathy nor organomegaly. Rest of the examination was unremarkable. Patient underwent excisional biopsy with wide margins. The back lesion was confirmed as Basal cell carcinoma. The finger mass histopathology revealed that it was a mucinous adenocarcinoma. Special immunohistochemical stains including Cam 5.2 (positive), AE1/3 (positive), p63 (negative) and EMA (negative) were done. Since a mucinous carcinoma of skin is more frequently a metastatic lesion we did a thorough work up to look for a primary tumor. A CT scan of Chest/ Abdomen/Pelvis was unremarkable. EGD and Colonoscopy with biopsies were also unremarkable. Combining the immunohistochemical findings and the lack of a primary tumor we concluded this was a Primary Cutaneous Mucinous Carcinoma (PCMC). The lesion was excised with negative margins and patient was scheduled for close follow up for monitoring recurrence and metastasis.

DISCUSSION: Mucinous Adenocarcinoma in the skin is commonly a result of metastasis and should always prompt us to evaluate for a primary site of malignancy, which is usually an adenocarcinoma. Most common primary sites are from the Breast, GI Tract, ovary and rarely lungs. PET-CT scans are often found helpful in finding the primary. Due to the mucoid material, they appear glossy and trans-illuminate with light. However, Mucinous Adenocarcinoma originating from skin as a Primary malignancy is quite rare. Approximately 200 case reports have been published to date. Based on literature review, PCMC has a predilection to occur in the head and neck area, arising from lachrymal and salivary glands, followed by vulva and great toe. Our case is unique in that the site of lesion is a finger nail bed and this has not been reported before. IHC stains such as P63, CK5/6, CAM5.2, EMA, AE1/3 help in distinguishing primary versus secondary lesions. Presence of myoepithelial cells has been proposed to help distinguish primary from secondary lesions, although they may not always been seen in preparations. Conclusions: Primary Cutaneous Mucinous Carcinoma is a rare cancer of sweat gland origin. Only 200 cases of PCMC have been reported to date in literature. Our case is the first to report a nail bed in upper extremity as a potential site of origin for this rare tumor. The significance of this case is to understand the pathology and implications of finding mucinous adenocarcinoma in skin. This case highlights that distinguishing primary and secondary mucinous adenocarcinoma is crucial and prompt intervention can alter the course of disease. While the primary variety is slow growing with a benign course and is managed conservatively, a metastatic lesion warrants a more urgent and thorough screening to find a primary tumor to initiate treatment in a timely manner.

PLANTING THE SEED: A CASE OF HEMOPTYSIS GROWS INTO A SURPRISE DIAGNOSIS Karen E. Schmitz; Ashley Ullrich. University of Colorado, Denver, GA. (Control ID \#2469469)

LEARNING OBJECTIVE \#1: To report foreign body aspiration as an unusual, but important cause of hemoptysis

LEARNING OBJECTIVE \#2: To delineate the diagnostic challenges of foreign body aspiration in the adult population

CASE: A $43 \mathrm{M}$ with no significant past medical history presented to an emergency department with an 18 month history of non-productive cough and 3 weeks of moderatevolume hemoptysis. Prior to presentation, he was evaluated several times by his primary care physician for the non-productive cough. He was given a presumptive diagnosis of reactive airways disease and started on a steroid taper and inhaled beta-agonist without improvement in symptoms. On presentation, the patient reported producing quarter-sized blood clots in his sputum, which were increasing in severity over the past 3 weeks. He denied any associated dyspnea, fevers, night sweats or weight loss. He denied any sick contacts or tobacco use history. He had no history of incarceration, homelessness, or occupational exposures. Travel history was limited to California and Arizona, last foreign travel was to Saudi Arabia and Mexico 20 years prior. On physical exam he was non-toxic appearing with stable vital signs; oxygen saturation was $>95 \%$ on ambient air. Pulmonary exam was notable for ronchi in the right lower lobe; physical exam was otherwise 
unremarkable. Initial laboratory work including comprehensive metabolic panel and complete blood count were within normal limits. Chest radiograph was without abnormality, however subsequent chest CT scan revealed a $3-4 \mathrm{~cm}$ cavitary lesion in the basilar anterior segment of the right lower lobe. He was admitted and underwent work-up for both infectious and non-infectious causes of hemoptysis and cavitary lung lesions. On hospital day 3 , he underwent bronchoscopy which yielded a foreign body lodged at the bifurcation of the right lower lobe with surrounding inflammation and nodular protrusions. The foreign body was easily removed and proved to be a full, intact, sunflower seed. On review of the diagnosis with the patient, he was able to recall an episode approximately 18 months prior in which aspirated a sunflower seed. He was discharged with a short course of amoxicillin-clavulanic acid and scheduled for interval repeat CT scan to ensure resolution of the cavitary lesion.

DISCUSSION: Foreign body aspiration is a rare occurrence in the adult population and requires a high index of suspicion to make the diagnosis. Foreign body aspiration occurs in less than 1 per 100,000 person years (LIMPER 1990), but are higher among the elderly and those with neuromuscular disorders. Unlike in pediatrics where patients often present acutely with life-threatening tracheal or upper airway obstruction, adults often present with chronic symptoms due to lower airway obstruction. Frequently, there is no memory of the inciting aspiration event. Presenting symptoms often mimic infection and include chronic cough in $80 \%$ of patients. Other symptoms include hemoptysis, fever and pleuritic chest pain. Symptoms are due to both airway obstruction by the foreign body as well as parenchymal inflammation from the irritant properties of the foreign body. The lack of sensitive, non-invasive diagnositic testing adds further challenge to making the diagnosis of foreign body aspiration. The majority of aspirated items are radiolucent and not visible on chest radiographs. CT scans of the chest have less than $85 \%$ sensitivity for detecting endobronchial foreign bodies (ZISSEN 2001). When present, CT findings are variable and include cavitary lesions, bronchiectasis, volume loss and hyperluciency. While invasive, bronchoscopy with direct visualization of the foreign body is the gold standard for diagnosis. Fortunately, bronchoscopy is often simultaneously diagnostic and therapeutic, with success rates for initial bronchoscopy approaching $75 \%$ (CHEN 1997). Due to the subtle nature of presentation, it is paramount that the clinician consider foreign body aspiration on the differential diagnosis for a variety of pulmonary conditions including chronic cough, recurrent pneumonia, hemoptysis and abrupt onset obstructive lung disease. Early broncoscopy is both diagnostic and therapeutic and is key to management of foreign body aspiration. REFERENCES: Chen, C. H., C. L. Lai, T. T. Tsai, Y. C. Lee, and R. P. Perng. "Foreign Body Aspiration Into the Lower Airway in Chinese Adults." CHEST Journal CHEST 112.1 (1997): 129. Limper, A. H., and U. B. Prakash. "Tracheobronchial Foreign Bodies in Adults." Annals Of Internal Medicine 112 (1990): 604-09. Zissin, R., Myra Shapiro-Feinberg, Judit Rozenman, Sara Apter, Jehoshua Smorjik, and Marjorie Hertz. "CT Findings of the Chest in Adults with Aspirated Foreign Bodies.” European Radiology 11.4 (2001): 606-11.

\section{PLASMAPHERESIS AS AN EFFECTIVE MODALITY FOR TREATMENT OF} HYPERTRIGLYCERIDEMIA INDUCED PANCREATITIS. Nabil Mesiha ${ }^{2}$; Mohamed Hassanein ${ }^{3}$; Majlinda Xhikola ${ }^{2}$; Daniel Goldsmith ${ }^{1}$; Tania Calzada ${ }^{1}$; Emily Chen ${ }^{4}$; Manish Gugnani ${ }^{4} .{ }^{1}$ Capital Health Regional Medical Center, Trenton, NJ; ${ }^{2}$ Capital health Regional Medical Center, Trenton, NJ; ${ }^{3}$ Capital health regional medical center, Plainsboro, NJ; ${ }^{4}$ Capital Health Medical Regional Center, Trenton, NJ. (Control ID \#2456720)

LEARNING OBJECTIVE \#1: To demonstrate that Plasmapheresis as an effective modality for treatment of hypertriglyceridemia induced pancreatitis.

CASE: Background Hypertriglyceridemia is the third most common cause of acute pancreatitis, accounting for 1 to $4 \%$ of cases presenting to the hospital. A serum triglyceride (TG) level of approximately $1000 \mathrm{mg} / \mathrm{dL}$ or greater increases the risk of acute pancreatitis (AP), although some patients have developed AP at lower levels. The risk of developing acute pancreatitis is approximately $5 \%$ with TG $>1000 \mathrm{mg} / \mathrm{dL}$ and 10 to $20 \%$ with TG $>2000 \mathrm{mg} / \mathrm{dL}$. The optimal approach to hypertriglyceridemia induced pancreatitis has not been established in will designed clinical trials. Case Report A 48 Hispanic female presented with epigastric pain, radiated to the back, associated with nausea and 3 episodes of non-bloody vomiting. There was no fever, change of bowel habits or urinary symptoms. Her past medical history was significant for alcohol induced pancreatitis 10 years prior. She took no medication, and last alcohol drink was a few weeks previously. There was a positive family history of dyslipidemia. On physical examination T 97.8, BP 140/87, Heart rate 118, RR 22 and BMI 32. Mucous membranes were dry. There was significant epigastric tenderness with no rebound tenderness. Laboratory findings: Lipase of 1682 Units/L, amylase 504 Units/L, Total Bilirubin $0.8 \mathrm{mg} / \mathrm{dl}$, WBC 21.2 cells/mm3. $\mathrm{Hb} 12.2$, lactic acid $3.7 \mathrm{mg} / \mathrm{dl}$, Triglyceride $3321 \mathrm{mg} / \mathrm{dL}$. The blood sample was extremely lipophilic and needed to be tested multiple times to obtain accurate results. Computed tomography showed severe inflammatory stranding abutting the pancreas and duodenum, compatible with pancreatitis. Ultrasound of the gall bladder showed no cholelithiasis or cholecystitis. Conservative treatment of AP was started including bowel rest, aggressive fluid resuscitation, and parenteral analgesia. The patient was still severely symptomatic so plasmapheresis was initiated. After 2 sessions of plasmapheresis, the patient improved significantly and she was able to start oral intake and required less parenteral analgesics. By the fifth day of hospitalization, TGs decreased to $347 \mathrm{mg} / \mathrm{dL}$, and she was able to tolerate a full liquid diet and oral analgesics. Statin therapy and finofibrates were initiated. The pancreatitis resolved and she was discharged home on the seventh day of hospitalization.

DISCUSSION: Discussion: This case demonstrates plasmapheresis was an effective modality in treating HTG-induced acute pancreatitis. Medical therapy of severe hypertriglyceridemia is effective but may take time to achieve a result that allows resolution of AP. Intravenous insulin is frequently used for rapid correction of severe hypertriglyceridemia, however use of plasmapharesis in the acute setting is recognized as a viable alternative. Our experience suggests that plasmapharesis is rapid and effective not only for the hypertriglyceridemia but for the AP as well. A large case control analysis or prospective clinical trial is warranted to establish the best approach to this challenging condition.

\section{PNEUMOCOCCAL SEPTIC ARTHRITIS AND PURULENT PERICARDITIS} IN AN IMMUNOCOMPROMISED HOST Rina Mauricio; Jennifer S. Mulliken; Ronald Goldenberg. New York University Langone Medical Center, New York, NY. (Control ID \#2469496)

LEARNING OBJECTIVE \#1: Review the risk factors for purulent pericarditis and the common pathogens involved.

LEARNING OBJECTIVE \#2: Recognize the clinical features of purulent pericarditis and when to have a high index of suspicion for infection.

CASE: A 69 year-old man with a history of advanced gastric adenocarcinoma undergoing chemotherapy presented with progressively worsening right knee pain and swelling for 1 month. He denied preceding knee trauma or surgery, though prior to the onset of symptoms he had received a course of ciprofloxacin for a right lower extremity cellulitis. His knee pain progressed to the point of immobility, and he subsequently developed pleuritic chest pain and shortness of breath. On arrival to our hospital he was tachycardic, tachypneic, and hypotensive. He had distant heart sounds on exam with elevated jugular venous pressure. The right knee was erythematous, warm, and swollen with severe pain on small movements. Labs were notable for a leukocytosis to $40.8 / \mathrm{mm}^{3}$ ( $75 \%$ neutrophils) and an elevated lactate. Knee arthrocentesis revealed purulent synovial fluid with a white

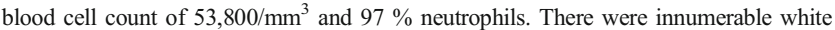


blood cells on gram stain. A transthoracic echocardiogram showed a large pericardial effusion with tamponade physiology. The patient received intravenous fluids and broadspectrum antibiotics and was admitted to the intensive care unit. Synovial fluid culture later speciated to Streptococcus pneumoniae. On hospital day two, the patient underwent a pericardial window with $300 \mathrm{cc}$ of purulent fluid drained from the pericardium. Gram stain showed innumerable white blood cells, but cultures of the pericardial fluid and of the excised pericardium were sterile. Blood cultures throughout the admission were negative, however none of these was collected before the initiation of antibiotics. On hospital day three, the patient developed worsening septic shock on maximum dose vasopressors. The decision was made to stop escalating care, and the patient expired on hospital day four DISCUSSION: Purulent pericarditis is characterized by frank pus in the pericardial sac or by microscopic purulence. Most cases are caused by gram-positive organisms, with Staphylococcus aureus and Streptococcus pneumoniae accounting for the majority of infections. Gram-negative and fungal organisms are less common pathogens. Infection usually spreads hematogenously from another site but can also arise from an intrathoracic or intracardiac source. Primary bacterial infection of the pericardium is rare. Purulent pericarditis requires prompt identification, but diagnosis can be challenging. Patients typically present with fever, chest pain, and cough, however the index of suspicion is typically low in the absence of recent cardiothoracic surgery or a known pulmonary or cardiac infection. In addition, patients may not have the classic symptoms of pericarditis including chest pain, a pericardial friction rub, or electrocardiographic abnormalities. As a result, purulent pericarditis often goes unrecognized until the late stages of infection. Once purulent pericarditis is suspected, empiric intravenous antibiotics should be promptly administered and pericardial drainage should be performed. Without treatment the infection is universally fatal. Death usually occurs secondary to septic shock or obstructive shock from either tamponade or constrictive pericarditis. Even with appropriate treatment, the mortality rate ranges from $20 \%$ to as high as $77 \%$. Often the diagnosis is made postmortem. In conclusion, Streptococcus pneumoniae is an uncommon cause of septic arthritis, but it is a well-known cause of bacterial pericarditis. While blood cultures were repeatedly negative, the organism presumably spread hematogenously to his pericardial sac. His immunocompromised state likely predisposed him to such widespread infection. To our knowledge this is the first reported case of pneumococcal septic arthritis leading to purulent pericarditis

POST-TRANSFUSION PURPURA INDUCED THROMBOCYTOPENIA - A RARE CAUSE OF A COMMON CONDITION IN PATIENTS WITH MALIGNANCY Kartik Sehgal ${ }^{2}$; Aakriti Gupta ${ }^{1}$; Catherine $\mathrm{Gao}^{2} .{ }^{1}$ Yale University, New Haven, CT; ${ }^{2}$ Yale University School of Medicine, New Haven, CT. (Control ID \#2469627)

LEARNING OBJECTIVE \#1: To learn about the clinical presentation of posttransfusion purpura in the setting of malignancy

LEARNING OBJECTIVE \#2: Review the differential of thrombocytopenia in a patient with malignancy in the inpatient setting

CASE: A 59-year-old lady with widely metastatic renal cell carcinoma s/p cytoreductive surgery and chemotherapy (temsirolimus, sunitinib, bevacizumab with interferon and finally pazopanib) was admitted to the hospital with vaginal bleeding and anemia (hemoglobin $(\mathrm{Hb})$ of $7.8 \mathrm{~g} / \mathrm{dl})$. On day 4 of hospitalization, she developed an acute vaginal hemorrhage that prompted transfusion with 4 units of packed red blood cell (PRBC) followed by arterial embolization of her vaginal metastases. On Day 9 , she was treated for presumed sepsis with broad spectrum antibiotics and additional PRBC transfusions. She received 1 unit of leukocyte-reduced PRBC administered each day on days 1, 3, 8 and 9 of hospitalization; and 4 units of PRBC on day 4 of hospitalization. Of note, she had a history of 2 uncomplicated pregnancies and was not known to have had any prior transfusions. On day 12 , her platelet counts abruptly declined to $17,000 / \mathrm{mm}^{3}$ from $211,000 / \mathrm{mm}^{3}$ the previous day. Physical examination was only significant for petechiae on the buccal mucosa. Within a span of $8 \mathrm{~h}$, platelet counts reached a nadir of $4,000 / \mathrm{mm}^{3}$ despite transfusion with 2 units of platelets. Examination of the peripheral smear for suspected microangiopathic coagulopathies showed no evidence of schistocytes, and pseudothrombocytopenia was excluded based upon the absence of platelet clumping. Blood urea nitrogen, creatinine, fibrinogen, prothrombin time and partial thromboplastin time were within normal ranges, thus ruling out disseminated intravascular coagulation (DIC). Blood cultures showed no growth. The absence of recent exposure to unfractionated or low molecular weight heparin excluded the possibility of heparin induced thrombocytopenia (HIT). The patient was given a presumptive clinical diagnosis of post-transfusion purpura (PTP) and empirically started on intravenous Immune globulin (IVIG) at $1 \mathrm{~g} / \mathrm{kg}$ for 2 consecutive days in addition to prednisone $40 \mathrm{mg}$ orally twice a day for 7 days. Antibiotics were discontinued and patient's blood sample was sent to the Blood Center of Wisconsin for platelet serology and genotyping. Six days after IVIG infusion, the patient's platelet count reached $56,000 / \mathrm{mm}^{3}$ and she was discharged. Platelet genotyping results showed human platelet antigen (HPA)-1b/1b and $3 b / 3 b$ alleles and serological studies indicated the presence of anti-HPA-1a and probable anti-HPA-3a platelet reactive antibodies, confirming the diagnosis of PTP. She was discharged in a stable condition and has had no further episodes of thrombocytopenia.

DISCUSSION: Differential diagnoses of thrombocytopenia entertained in this case included pseudothrombocytopenia, DIC, idiopathic thrombocytopenia (ITP), antibioticinduced thrombocytopenia and HIT, each of which was considered less likely as described above. In patients who have previously received blood transfusions with mismatched HPA, subsequent exposure to PRBC, platelet concentrates or plasma leads to an increase in HPA antibody levels, resulting in the characteristic severe thrombocytopenia $(<10,000$ / $\mathrm{mm}^{3}$ ) in post-transfusion purpura. It usually occurs within 5-12 days of immune challenge. The majority of cases occur in patients with HPA- $1 \mathrm{~b} / \mathrm{b}$ genotype, leading to PTP in which anti-HPA-1a antibodies are produced after transfusion of HPA -1a platelets. Serology confirmed the presence of these antibodies in our patient. While the pathophysiology of PTP has yet to be established, the anamnestic response that boosts HPA antibodies is clearly a key to clinical presentation and several hypotheses have been proposed to account for the observed HPA antibody reaction to self and donor platelets. During the acute thrombocytopenic phase, HPA alloantibodies become auto-reactive and bind autologous HPA with broad reactivity against platelet glycoproteins receptors. Although the extent to which renal cell carcinoma may have predisposed our patient to PTP is unclear, the detection of IgM and IgG anti-HPA antibodies clearly indicate a potent immune response to platelet antigens. Management with IVIG and steroids aids in suppressing this robust immune reactivity that leads to thrombocytopenia, and rapid and prompt responses to IVIG are seen in $85 \%$ of cases. Platelets are generally ineffective and not warranted unless life-threatening hemorrhage occurs and transfused platelets lack the causative antigen. In conclusion, this presentation illustrates the value of formulating a thorough differential of thrombocytopenia in patients with malignancy. Once more common causes are ruled out, PTP should be considered in this subset of patients. Early diagnosis and management of PTP can prevent a detrimental outcome of this rare transfusion-related phenomenon.

POSTPARTUM WOMAN WITH CHEST PAIN HEADING YOUR WAY Teresa Ratajczak; ameesh Vora; Arpan Patel; Amy Jiang; Mukul Chandra. Wright State University, Dayton, OH. (Control ID \#2431782)

LEARNING OBJECTIVE \#1: Spontaneous coronary artery dissection is a very rare cause of acute coronary syndrome. It can occur during the postpartum period and is a serious complication that the internist needs to be aware of. 
LEARNING OBJECTIVE \#2: Recognize the presentation and learn about the workup and management for a coronary artery dissection.

CASE: 29 year old female presented to the emergency department with substernal chest pain of 1 week duration, increasing in severity over past several hours. She denied any prior episodes, relation to changes in position or exertion. Past medical history was significant for an uncomplicated vaginal delivery 1 week prior. Medications consisted of prenatal vitamins. Admission labs were significant for troponin of 0.92 (baseline 0.070.09) and ECG with acute ST segment elevations in the inferior leads II, III, AVF with reciprocal depression in the lateral leads. Echocardiogram revealed ejection fraction of $45 \%$ and moderate inferior wall hypokinesis. She underwent an urgent cardiac catheterization that showed an extensive dissection of the right coronary artery extending from the os to its distal aspect. Chest pain resolved with nitroglycerin, medical management was pursued with plans for a follow up angiography the following week. She was started on nitroglycerin, heparin and a beta blocker. Unfortunately, the patient developed recurrence of chest pain the following day, evidence of heart failure and ventricular fibrillation requiring defibrillation. She was taken to the catheterization lab. Stenting of the vessel was attempted however was unsuccessful due to extent of the dissection. The decision was to pursue a single vessel bypass surgery. Patient was discharge home with appropriate medications; she has been seen in follow up clinic with continued improvement.

DISCUSSION: Case discussion: Spontaneous coronary dissection is a rare disease that should be considered in young female patients presenting with an acute coronary syndrome. The frequency is 1 in 100,000 pregnancies with most occurring within 2 weeks post-partum. Proposed mechanism consist of hormonal alterations causing structural changes in the medial wall during pregnancy, sheer mechanical stress on the coronary arteries during labor and the use of an uterine constrictor. Dissection separates the layers of the arterial wall, creating a false lumen. This can impair blood flow, resulting in acute coronary syndrome. The initial diagnosis includes an emergent catheterization Hemodynamic status is usually related to extent of the dissection. In stable patients, with adequate blood flow without persistent ischemia medical management is pursued. In ongoing signs of ischemia the options are stenting or surgery. Indications for bypass include involvement of the left main coronary artery, multi-vessel dissection or failure of stenting. Conclusion: Postpartum coronary dissection is a rare but serious cause of acute coronary syndrome. Urgent diagnosis by angiography is required with treatment based on hemodynamics and vessel involvement

\section{PRECIPITATION OF PRIMARY ADRENAL INSUFFICIENCY BY THYROID SUPPLEMENTATION IN A PATIENT WITH UNDIAGNOSED AUTOIMMUNE POLYGLANDULAR SYNDROME TYPE II Daniel Restrepo; George Alba. Massachusetts General Hospital, Boston, MA. (Control ID \#2432093)}

LEARNING OBJECTIVE \#1: Identify thyroid hormone supplementation as a potential precipitation of primary adrenal insufficiency in patients with multiple endocrinopathies LEARNING OBJECTIVE \#2: Discuss the common clinical features of the autoimmune polyglandular syndrome type II

CASE: A 42 year-old woman with a history of hypothyroidism, remote type II diabetes mellitus, and premature ovarian failure (POF) presented to the Emergency Department of an academic community hospital with the 1 day of diffuse myalgias, nausea, and vomiting. Her only medication changes were notable for the recent initiation of levothyroxine for hypothyroidism. On physical exam, she was noted to be hypotensive and had palmar hyperpigmentation. Initial laboratory values were notable for hyperkalemia of $9.3 \mathrm{mmol} /$ L, hypoglycemia, hyponatremia, non-anion gap metabolic acidosis, mild peripheral eosinophilia, and random cortisol level of 0.2 micrograms/dL. Adrenocorticotropin stimulation had no effect on serum cortisol values. A preliminary diagnosis of primary adrenal insufficiency (AI) was made based on clinical and laboratory data, and the patient was started on empiric stress-dose intravenous hydrocortisone with marked improvement in her symptoms and metabolic disarray. Additional testing revealed an adrenocorticotropic hormone $(\mathrm{ACTH})$ of $1120 \mathrm{pg} / \mathrm{mL}$ (upper limit of normal $=60 \mathrm{pg} / \mathrm{mL}$ ), undetectable aldosterone levels, and an anti-21 $\alpha$ hydroxylase antibody level of 85 units $/ \mathrm{mL}$ (upper limit of normal $<1 \mathrm{unit} / \mathrm{mL}$ ). Subsequent testing for other causes of adrenal insufficiency such as human immunodeficiency virus (HIV), tuberculosis, and bilateral adrenal hemorrhage were unremarkable. Given her autoimmune adrenalitis, history of hypothyroidism and premature ovarian failure, a diagnosis of autoimmune polyglandular syndrome type II was made.

DISCUSSION: Autoimmune polyglandular syndromes are a heterogenous group of diseases that involve immune-mediated destruction of endocrine glands. Autoimmune polyglandular syndrome type II (APS2) is characterized by autoimmune thyroid disease, adrenal insufficiency, and oftentimes type I diabetes mellitus. Although almost half of APS2 cases are familial, the majority are sporadic. Interestingly, although precipitation of adrenal insufficiency by initiation of thyroid supplementation is a known physiologic phenomenon, it is a rather uncommon presentation of primary AI, reported sparingly in the literature. The mechanism of thyroxin-induced $\mathrm{AI}$ is thought to be secondary to increased metabolic demand in addition to enhanced glucocorticoid clearance. A history of multiple endocrinopathies such as autoimmune thyroiditis, type I diabetes mellitus, vitiligo and POF should alert the clinician that the patient is at high-risk for adrenal insufficiency, given that this is the hallmark of the autoimmune polyglandular syndromes. Although patients with polyglandular endocrinopathies will almost all eventually develop autoimmune adrenalitis, it is important that adrenal insufficiency is noted at presentation in only 19$50 \%$ of cases. Symptoms inconsistent with iatrogenic thyrotoxicosis, such as fatigue, nausea and abdominal pain, should serve as warning signs that the patient may develop adrenal insufficiency in the setting of thyroid replacement therapy and should prompt further investigation.

PREDNISONE CAUSING DRUG-INDUCED LIVER INJURY LEADING TO FULMINANT LIVER FAILURE: A CASE REPORT Muna Sheikh ${ }^{2}$; Sumeet S. Pawar $^{1}$; Craig Noronha ${ }^{1} .{ }^{1}$ Boston Medical Center, Boston, MA; ${ }^{2}$ Boston University School of Medicine, North Andover, MA. (Control ID \#2469747)

LEARNING OBJECTIVE \#1: Recognize the clinical and histologic features of druginduced liver injury

LEARNING OBJECTIVE \#2: Diagnose acute fulminant liver failure based on clinical and laboratory findings and escalate care appropriately

CASE: A 62-year-old Caucasian male with a past medical history notable only for hypertension was admitted with nausea, vomiting, and jaundice, and was found to have markedly deranged liver function tests. One month prior to presentation, the patient was treated with prednisone (100 mg on the first day, followed by a 10-day taper) for a diffuse rash consistent with contact dermatitis. The rash subsequently resolved, and the patient took no other hepatotoxic medications in the weeks preceding his admission. On the first hospital day, the biochemical tests showed total bilirubin of $13.1 \mathrm{mg} / \mathrm{dl}$; direct bilirubin, $9.0 \mathrm{mg} / \mathrm{dl}$; ALT, $931 \mathrm{IU} / \mathrm{L}$ (normal value $<40 \mathrm{U} / \mathrm{L}$ ); AST, $1315 \mathrm{IU} / \mathrm{L}$ (normal value = $40 \mathrm{U} / \mathrm{L}$ ); ALP, $202 \mathrm{IU} / \mathrm{L}$ (normal value $=20-130 \mathrm{U} / \mathrm{L}$ ), and INR 1.31. Ultrasound and CT scan of the upper abdomen revealed no hepatobiliary pathology. Serologic tests for hepatitis and hepatotropic viruses and antinuclear, antimitochondrial, and smooth muscle antibodies were all negative. Liver biopsy ultimately revealed necrosis, portal tract inflammation, fibrosis, and microvesicular hepatic steatosis, suggesting drug-induced liver injury as the most likely etiology. The patient received $\mathrm{N}$-acetyl cysteine and supportive care. He was discharged on day 8 with stable-to-improving AST, ALT, and INR, and given instructions to avoid the offending drugs with close monitoring of his biochemical tests. One week later, the patient returned from his primary care physician's office with new altered mental status concerning for hepatic encephalopathy. Biochemical tests showed an 
increased INR to 2.16, as well as continued elevations of other markers: AST 399 IU/L, ALT $382 \mathrm{IU} / \mathrm{L}$, total bilirubin $22.4 \mathrm{mg} / \mathrm{dl}$, direct bilirubin $12.9 \mathrm{mg} / \mathrm{dl}$, and ALP 197. Due to concern for fulminant hepatic failure, the patient was transferred to a liver transplant center for urgent evaluation. In the week following transfer, his symptoms resolved, and he was discharged from the facility in stable condition without liver transplantation. Given the pattern of his liver histology, as well as the resolution of his symptoms after discontinuation of drugs, prednisone-induced injury was thought to be the most likely cause of his acute steatohepatitis.

DISCUSSION: Acute hepatitis with microvesicular steatosis is relatively rare and associated with acute fatty liver of pregnancy, Reye's syndrome, and drug-induced liver injury (DILI). While there are no specific diagnostic tests that establish DILI, a wide array of medications has been associated with this pattern of injury. Corticosteroids, in particular, have been associated with microvesicular steatosis that may lead to fulminant liver failure. This case illustrates the importance of retaining a high index of suspicion for DILI in the setting of recent corticosteroid use. Recognition of DILI is critical not only for withdrawal of potential offending medications, but also for close monitoring and the provision of supportive care. In rare cases, chronic or progressive DILI may persist after discontinuation of the offending medications. This case highlights that patients with drug-induced liver injury must be observed closely for the development of acute fulminant liver failure. The diagnosis of acute fulminant liver failure is made with the presence of elevated aminotransferases, hepatic encephalopathy and INR greater than 1.5. As patients may decompensate rapidly, transfer to an intensive care facility that is capable of liver transplantation should be performed in a timely manner.

PREPPING THE INTERNIST FOR PREP Paul M. Lu ${ }^{1}$; Lee S. Shearer ${ }^{2} .{ }^{1}$ New York Presbyterian-Weill Cornell, New York, NY; ${ }^{2}$ Weill Cornell Medical College, New York, NY. (Control ID \#2464087)

LEARNING OBJECTIVE \#1: To understand the importance of the primary care provider to identify candidates and prescribe HIV Pre-Exposure Prophylaxis (PrEP). LEARNING OBJECTIVE \#2: To recognize candidates at high risk for HIV infection and to know how to prescribe and manage patients on PrEP.

CASE: A 28 year old MSM patient with a history of gonorrhea and syphilis presented to our outpatient clinic requesting STD testing. The patient reported multiple male sex partners in the past 6 months and had engaged in oral and insertive/receptive anal sex with inconsistent condom use. His medical history was limited to an episode of gonorrhea (2014) and early syphilis (2015) which were both treated. Social history included occasional episodes of binge drinking and rare marijuana use. No history of intravenous drug use (IVDU). He worked as a professional singer in a traveling choir. His vital signs and physical exam were within normal limits. STD testing, including HIV were negative and his RPR titer remained at baseline. The patient returned to the our clinic 3 months later again requested STD testing. His sexual behavior was unchanged. He tested negative for all STDs including HIV and his RPR was stable. 5 months later, the patient returned with a sore throat and for repeat STD screening. His HIV antibody test returned positive.

DISCUSSION: This case demonstrates a missed opportunity to have prescribed HIV PrEP. It also shows the need for the internist to recognize their role as first-line PrEP prescribers and to be learn to identify at-risk patients, how to prescribe PrEP, and how to manage the long-term care of these patients. Internists as first line prescribers: The incidence of new HIV infections have remained stable over the past 2 decades with an estimated 50,000 new cases per year. Despite this, the incidence of HIV infection has actually increased among particular groups, including men who have sex with men (MSM) and certain minorities. In 2010, the Centers for Disease Control and Prevention (CDC) reported that nearly 2 out of every 3 cases of newly diagnosed HIV occurred in MSM patients, with Black and Hispanic men disproportionately affected the most. One method to reduce new HIV infection is pre-exposure prophylaxis (PrEP). Multiple randomized control studies including the Pre-exposure Prophylaxis Initiative (iPrEx) and the Intervention Préventive de l'Exposition aux Risques avec et pour les Gays (IPERGAY) have demonstrated that daily or on-demand Tenofovir and Emtricitabine (Truvada) reduce the rate of HIV seroconversion by $44-86 \%$. Despite the clear evidence, a recent study on provider knowledge and utilization of PrEP demonstrated that though a majority of providers were aware of PrEP, only a minority had prescribed PrEP in the past. This study showed that for non-HIV providers, $77 \%$ were aware of PrEP as a tool for HIV prevention, $38 \%$ were comfortable determining candidacy for PrEP, and only $9 \%$ had previously prescribed PrEP. Identifying candidates: The CDC provides 3 groups of patients to consider: MSM, heterosexual adults, and intravenous drug users (IVDU). MSM: HIV negative men not in a monogamous relationship with male sex partner(s) in the past 6 months who have either: participated in unprotected anal sex (receptive or insertive), had any sexually transmitted disease in the past 6 months, or is in a sexual relationship with an HIV-positive male partner. Heterosexual men or women: Any HIV negative adult not in a monogamous relationship who is sexually active in the past 6 months who either: engages in unprotected sex with partner(s) of unknown HIV status who are known to be at substantial risk of HIV infection (i.e. IVDU or bisexual men) or in a sexual relationship with an HIV-positive partner. IVDU: Any HIV negative adult that has either shared injection drugs or equipment or enrolled in a drug abuse treatment program (i.e. methadone, buprenorphine, or suboxone) in the past 6 months. Prescribing and management: Prior to initiating PrEP, it is necessary to ascertain medication compliance and to perform specific laboratory testing, including HIV, hepatitis B, renal function, and pregnancy testing. If compliance is satisfactory and laboratory values show a negative HIV, hepatitis B, and pregnancy test with a creatinine clearance of $>60 \mathrm{ml} /$ min, then you may proceed to prescribe PrEP. Specifically, Tenofovir $300 \mathrm{mg}$-Emtricitabine $200 \mathrm{mg}$ (Truvada 1 tablet) daily should be prescribed at a maximum of 3 month intervals. The CDC recommends close routine follow up at least every 3 months. At each follow up visit, assessment of medication side effects should be elicited in addition to repeat HIV, renal function, and pregnancy testing. STD testing should be conducted at least every 6 months. Conclusions: 1) Primary care providers must learn and be comfortable with HIV preexposure prophylaxis. 2) PrEP should be offered to high risk groups, especially MSM, serodiscordant couples, and IVDU. 3) Medication compliance and specific laboratory testing must be checked before initiating PrEP. Once started, patients should follow up at least every 3 months.

PREVALENCE AND DIAGNOSIS OF MULTIPLE DRUG RESISTANT TUBERCULOSIS Abd almonem Abdelrahman ${ }^{2}$; Vishisht Mehta ${ }^{2}$; Amanda Krantz ${ }^{2}$; Stephen Cavalieri ${ }^{2}$; Renuga Vivekanandan ${ }^{1}$. ${ }^{1}$ Creighton Medical center, Omaha, NE; ${ }^{2}$ Creighton University, Omaha, NE. (Control ID \#2457666)

LEARNING OBJECTIVE \#1: Recognize the support and the tools offered by the Center for Disease Control and Prevention (CDC) that can be used in the diagnosis of MDR-TB cases.

LEARNING OBJECTIVE \#2: Identify the conditions and risk factors when a case of MDR-TB infection should be considered.

CASE: A 43-year-old Chinese male, who is visiting US, without any significant medical history presented to the emergency department complaining of hemoptysis of 10 days duration. This was associated with mild right-sided pleuritic chest pain and night sweats. He denied any shortness of breath, fever or weight loss. On admission, the patient had normal physical examination and no significant laboratory abnormalities. Chest CT scan showed a cavitary lesion with air crescent sign in the apical segment of his right lower lobe, suggestive of fungal infection. AFB and fungal cultures were performed on 3 early morning sputum specimens, all were positive for AFB but were negative for fungi. M. tuberculosis infection was confirmed by DNA probe method. The patient was discharged home on first line therapy with isoniazid (INH), rifampin (RIF), pyrazinamide (PZA) and 
ethambutol (EMB), with pyridoxine. Since, the patient's country of origin was China, there was concern for MDR-TB. However, a molecular test for the rpoB gene coding for rifampin resistance was negative. Two weeks later, susceptibility testing of the M. tuberculosis isolate showed resistance to INH, PZA and EMB. Therapy was subsequently changed to amikacin, linezolid, moxifloxacin and rifampin. The isolate was sent to Center for Disease Control (CDC) for molecular testing for resistance genes. Over the following 2 weeks, patient symptoms were stable. Results from the CDC were negative for resistance genes. Repeat susceptibility testing showed that INH, EMB, and PZA were susceptible. As a result, patient was restarted on his initial anti-tuberculosis regimen and was able to return to China. Most likely the initial susceptibility test was contaminated with a respiratory tract organism that was resistant to INH, EMB, and PZA.

DISCUSSION: Tuberculosis (TB) is caused by strains of Mycobacterium tuberculosis complex. The main goals for the treatment of TB are to treat the individual patient and to minimize transmission. Directly observed therapy (DOT) has been a key component of keeping resistance rates low. The development and spread of drug-resistant strains of $\mathrm{M}$. tuberculosis greatly jeopardize TB control efforts. In the U.S., 91 cases of multiple drug resistant tuberculosis (MDR-TB) were reported in 2014. It is estimated that preventing a single case of MDR-TB would save the U.S. health care system more than \$250,000. MDRTB is resistant to at least INH and RIF. Extremely drug resistant TB (XDR-TB) is also resistant to quinolones and at least one of the injectable drugs (e.g. amikacin, kanamycin, or capreomycin). TB is endemic in China, with 1.4 million cases and 130,000 deaths annually. Rates of MDR-TB (approximately $10 \%$ of cases) and XDR-TB (approximately $0.8 \%$ of cases) are epidemic. When treating patients with TB from high resistance area it is important to consider MDR-TB and XDR-TB. Clues in the history may point to the possibility of drug resistant TB. A previous history of TB, inadequate or short course treatment and unsupervised therapy are all red flags, as are contact with a patient with MDR TB, origin from an endemic region or failure of therapy with documented supervision. It is very important to verify lab results if there are questionable results. As in our case repeat susceptibility testing and molecular tests for resistance genes helped aid in making the right diagnosis. The molecular tests offered by the $\mathrm{CDC}$ were instrumental in this regard and served to aid in the diagnosis, treatment, and control in this case of TB. TB could be successfully treated if the right antibiotics are administered for the correct length of time. MDR-TB is significantly more difficult and expensive to treat than drug-susceptible TB. Therefore, the timely detection and confirmation of MDR-TB is crucial. References: 1- CDC. Reported Tuberculosis in the United States, 2014. Atlanta, GA: U.S. Department of Health and Human Services, CDC, October 2015. 2- Zhao, Y., et al. (2012). National survey of drugresistant tuberculosis in China. N. Engl. J. Med. 366, 2161-2170. 3- Günther, G. (2014) Multidrug-resistant and extensively drug-resistant tuberculosis: a review of current concepts and future challenges. Clin Med 14, 279-285. 4- Blumberg, H.M., et al. (2003). American Thoracic Society/Centers for Disease Control and Prevention/Infectious Diseases Society of America: treatment of tuberculosis. Am. J. Respir. Crit. Care Med. 167, 603-662.

PRIMARY CERVICAL SQUAMOUS CELL CARCINOMA PRESENTING WITH A CARDAC MASS AND PULMONARY EMBOLISM. Crystal A. Shaw ${ }^{1}$; Wilmer F. Davila ${ }^{2}$; Sandeep Banga ${ }^{1}$; Kavitha Kalvakuri ${ }^{3}$. ${ }^{1}$ OSF Peoria, UICOMP, Peoria, IL; ${ }^{2}$ UICOMP, Peoria, IL; ${ }^{3}$ OSF, Peoria, IL. (Control ID \#2467432)

LEARNING OBJECTIVE \#1: Metastatic cardiac tumors from gynecological malignancies are uncommon, and metastasis originating from cervical carcinomas is extremely rare, with fewer than 44 cases reported.

LEARNING OBJECTIVE \#2: When a diagnosis of PE and treatment plan seem obvious, it is essential to conduct a thorough and prompt investigation to assess for additional factors. A multimodality approach is necessary for early diagnosis, prompt action and improved prognosis.
CASE: A 49-year-old female smoker, on estrogen supplements and a history of recent air travel presented to the ED complaining of pleuritic chest pain and dyspnea. Two years prior she had a TAH-BSO for refractory menometrorrhagia. On exam her HR (113) BP (152/106) SpO2 (97 \%) RR (28) BMI (28) and bilateral pedal edema. Initial labs showed an elevated BMP (308). The electrocardiogram showed T-wave inversion in inferior leads, prolonged QT interval. CT angiography showed a PE in the right lower lobe pulmonary artery, multiple nodules and a mass within the right ventricle extending into the pulmonary artery. The patient was started on a UFH infusion, to treat PE. Cardiology was consulted. ECHO showed EF (50 \%) RVSP (51) and a mass in the RV. Cardiac MRI confirmed the presence a mobile mass extending to the pulmonary outflow tract. Metastasis become a likely diagnoses and a PET CT confirmed our suspicion. Inguinal node pathology revealed poorly differentiated carcinoma most likely SCC. Patient went into PEA and did not survive. Autopsy showed squamous cell carcinoma of the cervix.

DISCUSSION: In our patient, the diagnosis of PE was not surprising, considering her risk factors of prolonged immobility, hormone replacement therapy, smoking and evidence of a PE on CT angiography and EKG showing right heart strain. Her symptoms can be explained by the propagation of the cervical cancer along the inferior vena cava to the heart where it formed an intracavitary mass with extension into the pulmonary arteries. Review of 44 cases, indicates that $66 \%$ (29 of the 44$)$ of the patients with metastatic squamous cell carcinoma of the heart, presented with dyspnea and $34 \%$ had documented pulmonary emboli due to tumor embolization. It also showed that CT has been the most useful tool for identifying metastasis and MRI the most valuable in confirming the diagnosis

PRIMARY EFFUSION LYMPHOMA IN A HIV POSITIVE MALE WITH A HISTORY OF KAPOSI SARCOMA HoanVu N. Nguyen ${ }^{2}$; Ashley Ullrich ${ }^{2}$; Penny $\mathrm{Li}^{2}$; Noelle Northcutt ${ }^{1}{ }^{1}$ Denver Health, Denver, $\mathrm{CO} ;{ }^{2}$ University of Colorado Anschutz Medical Campus, Aurora, CO. (Control ID \#2467663)

LEARNING OBJECTIVE \#1: Diagnose pericardial effusion in patients with HIV LEARNING OBJECTIVE \#2: Recognize clinical features of primary effusion lymphoma

CASE: A 44 year old male with a history of HIV infection on HAART, malignant Kaposi sarcoma post doxorubicin chemotherapy, and hepatitis $\mathrm{C}$ post antiviral treatment presented to Denver Health Medical Center complaining of constipation for 2 weeks. Review of systems was positive for abdominal discomfort, dyspnea on exertion, and lower extremity swelling. Pertinent exam findings included distant but normal heart sounds without murmurs, rubs or gallops. Lungs were clear to auscultation bilaterally. Abdomen was mildly distended and diffusely tender to palpation. Bilateral lower extremities were swollen with $2+$ pitting edema. Chest x-ray (A) showed an enlarged cardiac silhouette, confirmed to be a large circumferential pericardial effusion on CT (B) and transthoracic echocardiography $(\mathrm{C})$. He underwent a pericardiocentesis which found an opening pressure of 8-9 mmHg and drained $1500 \mathrm{cc}$ of cloudy, bloody fluid. A pericardial drain was left in place for the next $24-48 \mathrm{~h}$ until drainage ceased. A repeat TTE showed only trace effusion. Cytology from the pericardial fluid confirmed the diagnosis of Primary Effusion Lymphoma (PEL). Throughout his hospitalization, the patient did not exhibit tamponade physiology. It was recommended to him that he start inpatient treatment, but he requested to be discharged as he was unsure about pursing therapy. He was followed in Oncology clinic, and ultimately began treatment after developing a pleural effusion.

DISCUSSION: Primary effusion lymphoma (PEL) is a monoclonal B-cell malignancy that accounts for $1-4 \%$ of all HIV-associated lymphomas. Involved in its pathogenesis is HHV-8, the virus that also causes Kaposi's sarcoma, although the exact mechanism of oncogenesis is uncertain. PEL begins on serosal surfaces and has a predilection for body cavities including the pleural space (60-90\%), peritoneum (30-60\%), pericardium 
$(<30 \%)$, and rarely joint spaces and meninges. The clinical manifestations are variable. Most patients present with symptoms of serous effusion — dyspnea, abdominal distension and/or joint swelling. These effusions are exudative, often bloody, and contain high-grade malignant lymphocytes. Of note, solid tumor variants occur predominantly in the gastrointestinal tract. Current treatments are not optimal. Prognosis remains very poor. Treatment of PEL requires chemotherapy and ART (survival benefit when compared to chemotherapy alone). Suggested chemotherapy regimens include CHOP (cyclophosphamide, doxorubicin, vincristine, prednisone) or dose-adjusted EPOCH (CHOP + etoposide) which can extend median survival from 2 to 3 months to 5-6 months. If a patient has failed ART + chemotherapy, local radiation to the body cavity involved has been used and may provide palliation for up to 1 year. HIV viral loads should be measured at 4 and 12 weeks after starting treatment. If viral load is still detected at 12 weeks, changing the treatment regimen must be considered. Many patients with PEL often show response to treatment, but remissions are often short in duration. Death typically results from progression of the malignancy, opportunistic infections and other HIV-associated complications.

PRIMARY HYPERALDOSTERONISM AS AN UNCOMMON CAUSE OF RHABDOMYOLYSIS: A CASE REPORT Tiffany Seto ${ }^{1}$; Iqbal Munir ${ }^{2} .{ }^{1} \mathrm{UC}$ Riverside, San Mateo, CA; ${ }^{2}$ Riverside University Health System, Moreno Valley, CA. (Control ID \#2466320)

LEARNING OBJECTIVE \#1: Recognize the risk of hypokalemia-induced rhabdomyolysis in patients with primary hyperaldosteronism

CASE: A 34 year old female with early onset resistant hypertension, hypokalemia and primary hyperaldosteronism was admitted for acute metabolic encephalopathy, rhabdomyolysis and proximal myopathy secondary to severe hypokalemia. The patient has a past medical history of severe hypokalemia with rhabdomyolysis requiring multiple hospitalizations in the past 2 years and hypertension for greater than 10 years. Surgical history included uncomplicated surgical excision of left breast mass. Social history was significant only for former use of marijuana. Prior to presentation, the patient had been on antihypertensive medications, spironolactone and potassium supplements, but had run out of spironolactone and potassium supplements for the previous 4 days. The patient had begun experiencing fatigue, weakness, dizziness, bradycardia, hypotension and progressive somnolence before being brought into the ER with an altered level of consciousness. Aside from hypertension, the patient's vital signs were stable upon presentation. EKG demonstrated non-specific ST-T changes. Physical exam was significant for proximal muscle weakness. Lab findings demonstrated profound hypokalemia $1.5 \mathrm{mEq} / \mathrm{L}$ and elevated total creatine phosphokinase $6897 \mathrm{U} / \mathrm{L}$. Plasma renin activity was $0.36 \mathrm{ng} / \mathrm{ml} / \mathrm{h}$ and plasma aldosterone $15 \mathrm{ng} / \mathrm{mL}$ (ARR 514). AM cortisol was $19.7 \mathrm{ug} / \mathrm{dL}$. A partial workup had been started for primary hyperaldosteronism during a previous admission, but the patient was lost to follow up. Previous lab results include $24 \mathrm{~h}$ urine metanephrine 159 $\mathrm{mcg}$ and noremetanephrine $546 \mathrm{mcg}$ (in normal range). Twenty four hour serum total catecholamine, dopamine, epinephrine and norepinephrine were within normal limits, ruling out pheochromocytoma as the cause of the patient's hypertension and as a rarer secondary cause of rhabdomyolysis. Adrenal venous sampling was not performed after discussion with the patient at length after reviewing the advantages and risks of the procedure. CT of the abdomen revealed a cyst in the left adrenal gland and an MRI of the abdomen confirmed a $2.6 \times 1.5 \times 2.7 \mathrm{~cm}$ mass that was suggestive of a lipid rich adenoma. The patient was treated appropriately and subsequently admitted for an elective laparoscopic left adrenalectomy at a later date. On pre-surgical admission, the patient was found to be hypertensive (BP 207/106) and again, severely hypokalemic (serum K $1.7 \mathrm{mEq} / \mathrm{L}$ ), but relatively asymptomatic. Post-adrenalectomy, the patient's serum potassium was $3.5 \mathrm{mEq} / \mathrm{L}$ and ARR dropped to 7.14. Final pathology revealed adrenal cortical adenoma $3.0 \mathrm{~cm}$ in diameter. The patient was discharged with scheduled follow up at the endocrinology clinic.

DISCUSSION: Rhabdomyolysis is the necrosis of striated muscle leading to release of myocyte contents and intracellular potassium into the bloodstream, leading to hyperkalemia. In patients with primary hyperaldosteronism (PA), mineralocorticoid excess leads to hypokalemia, which can be severe enough to cause rhabdomyolysis. Because rhabdomyolysis is usually associated with trauma, crush injuries, seizures, overexertion of muscle, hereditary muscle enzyme defects and renal disease, rhabdomyolysis caused by hypokalemia secondary to PA is usually missed. Notably, only 16 cases of rhabdomyolysis as the first manifestation of PA has been reported in literature so far. Other endocrine causes of rhabdomyolysis include diabetic ketoacidosis, non-ketotic hyperosmotic hyperglycemia, hypo- and hyperthyroidism as well as pheochromocytoma. Primary hyperaldosteronism is a common cause of secondary hypertension resistent to medication. Before surgical resection of a hypersecreting adrenal adenoma, confirmation of the diagnosis and laterality of the adrenal lesion should be done by adrenal venous sampling (AVS); however, this procedure was not performed for our patient due to younger age of onset of the disease and CT/MRI imaging indicating the possible culprit lesion. For patients of advanced age, AVS would be necessary to localize the source of excess aldosterone since non-functioning adrenal incidentalomas are more prevalent in this population and current imaging modalities can only detect adrenal masses $\geq 5 \mathrm{~mm}$. Although CT imaging is most widely used to visualize the adrenal glands, CT proved to be inadequate in our case; multiple CT scans suggested an adrenal cyst. An MRI was required to provide concrete morphological evidence of a lipid-rich adrenal adenoma. In conclusion, it is important to bear in mind that (1) patients with PA are at risk of developing rhabdomyolysis secondary to hypokalemia, (2) AVS is not warranted for all patients, and (3) although CT of adrenal glands is adequate for most cases, occasionally MRI could be necessary to confirm an adenoma.

PRION DISEASE: AN UNEXPECTED DIAGOSIS IN A PATIENT PRESENTING WITH DKA John L. Ebrahim; Alexandra Sowa; Arnab K. Ghosh; Amit Uppal. NYU, New York, NY. (Control ID \#2469622)

LEARNING OBJECTIVE \#1: Recognize that prion disease is a rare but fatal cause of progressive neurological decline.

LEARNING OBJECTIVE \#2: Recognize when to consider prion disease in the evaluation of a persistently comatose patient.

CASE: A 64 year old male with Type 1 diabetes mellitus and squamous cell carcinoma of the tongue was found unresponsive. He was found to have diabetic ketoacidosis (DKA) and was treated appropriately. His mental status did not improve with resolution of DKA, prompting further evaluation. Computed tomography $(\mathrm{CT})$ of the brain was unremarkable, lumbar puncture (LP) was not consistent with meningitis, and electroencephalogram was not consistent with subclinical seizures. Magnetic Resonance Imaging (MRI) of the brain revealed abnormal T2/FLAIR intensity throughout bilateral cerebral hemispheres, predominantly cortical in distribution. Further history revealed that he had been a highly functional person until 1 year prior to admission, when he suffered a subdural hematoma requiring craniotomy. Over the subsequent months, family noticed a decline in his level of organization, initially manifest as a decreased attention to personal hygiene. This progressed to forgetfulness, impaired judgment, and disorganized thinking. The differential diagnosis at that time included paraneoplastic limbic encephalitis and prion-related disease. Repeat LP revealed no evidence of paraneoplastic antibodies, but marked elevations of 14-3-3 and Tau proteins. Given the compatible history, imaging, and CSF findings, the patient was diagnosed with Creutzfeldt-Jacob disease (CJD). Given the advanced nature of his disease, the goals of his care were transitioned to symptomtargeted therapy. 
DISCUSSION: Prion diseases are a unique class of neuro-degenerative disorders that are rapidly progressive and universally fatal. They are caused by misfolded proteins that cause neuronal cell death. CJD is the most common form. The majority of cases are acquired through spontaneous mutations, though person-to-person transmission has been documented. In our patient, CJD may have been acquired during the neurosurgical procedure that preceded the onset of symptoms, and this also represents a risk to subsequent patients treated in that institution. The case was reported to the Department of Health for further investigation. In conclusion, prion disease, while rare, should be considered in the differential diagnosis of a patient with an otherwise unexplained progressive neurological decline. Brain MRI findings of abnormal T2/FLAIR intensity in a bilateral cortical distribution and elevated CSF levels of 14-3-3 and Tau proteins are highly suggestive of CJD.

PROLONGED HYPERCALCEMIA CAUSED BY ADULT-T CELL LEUKEMIA/ LYMPHOMA IN A PATIENT INITIALLY SUSPECTED AS VITAMIN D INTOXICATION Iori Motohashi; Yoko Kanamitsu; Chieko Sumiya; Taro Shimizu; Yasuharu Tokuda. JCHO (Japan Community Health care Organization) Tokyo Joto Hospital, TOKYO, Japan. (Control ID \#2468132)

LEARNING OBJECTIVE \#1: Design a new diagnostic strategy for helping to perform accurate and cost effective differential diagnosis of hypercalcemia based on recent epidemiologic background indicating high prevalence of vitamin D supplementation CASE: A 60-year-old woman with osteoporosis and lumbar spondylolisthesis presented with a 1-month history of appetite loss. Review of systems revealed she had low back pain and low-grade fever. She denied nausea, vomiting, diarrhea, polyuria, polydipsia, weight loss, shaking chill or night sweat. On examination, she was alert and the vital signs were normal. She appeared dehydrated but there were no other abnormal physical findings including no palpable lymphadenopathy and no rash. Laboratory data revealed serum calcium concentration of $15.4 \mathrm{mg} / \mathrm{dL}$ with serum albumin of $4.5 \mathrm{~g} / \mathrm{mL}$, phosphate of $3.7 \mathrm{mg} / \mathrm{dL}$, creatinine of $1.48 \mathrm{mg} / \mathrm{dL}$, urea nitrogen of $31.3 \mathrm{mg} / \mathrm{dL}$ and lactate dehydrogenase of $317 \mathrm{IU} / \mathrm{L}$. Her baseline medication list included oral activated vitamin D (eldecalcitol, $0.75 \mathrm{mcg} / \mathrm{day}$ ) and non-steroidal anti-inflammatory drug (NSAID, diclofenac), which had been prescribed by her home doctor. Under the tentative diagnosis of hypercalcemia due to vitamin D intoxication and renal dysfunction due to NSAID these drugs were discontinued along with intravenous infusion of normal saline. However, even after the medication discontinuation and fluid replacement during several days of the hospitalization, serum calcium was not normalized. In careful clinical reevaluation, numerous lymphocytes with abnormal morphology of their nuclei were identified in the peripheral blood smear. Additional test results revealed positive human T-cell lymphotropic virus type 1 (HTLV-1) antibody, elevated levels of serum parathyroid hormone related protein (PTHrP) and serum soluble interleukin-2 receptor. Bone marrow examination showed predominant CD4-positive cells in flow cytometry. Accordingly, a diagnosis of hypercalcemia due to adult T-cell leukemia/lymphoma (ATLL) was made. While the patient developed double vision and worsening back pain which were considered to be symptoms of ATLL invasion into brain and bone, she was transferred to another hospital for a plan of chemotherapy.

DISCUSSION: Vitamin D intoxication has recently become an important cause for hypercalcemia in addition to primary hyperparathyroidism and malignancy in Japan because there has been the widespread recommendation for vitamin D supplementation as preventive medication for osteoporosis. However, hypercalcemic patients on vitamin D supplementation may have other causes for hypercalcemia, including humoral hypercalcemia of malignancy (HHM). Despite the increasing incidence of vitamin D intoxication in Japan, another cause for hypercalcemia should be considered especially in patients on vitamin D but with prolonged hypercalcemia several days after discontinuation of vitamin D. We will propose a novel diagnostic algorithm for helping to perform accurate and cost effective differential diagnosis of hypercalcemia based on recent epidemiologic background indicating high prevalence of vitamin D supplementation. The algorithm provides consequential approaches; firstly in patients on vitamin D to rule out drug-induced hypercalcemia by discontinuing the medication and as a next step for patients with prolonged hypercalcemia to consider HHM or local osteolytic hypercalcemia as malignancy-associated conditions in addition to primary hyperparathyroidism.

PROTEINURIA IN ELDERLY: A SIGN OF SOMETHING SINISTER IRFAN AHSAN $^{1}$; Faizan Malik ${ }^{1}$; Syed I. Jafri ${ }^{1}$; Muznay N. Khawaja ${ }^{2}$; Hamid Rashid ${ }^{1}$; Rabia Naheed ${ }^{1}$. ${ }^{1}$ Abington Jefferson Health, Abington, PA; ${ }^{2} \mathrm{MCMC}$, DAby, PA. (Control ID \#2469577)

LEARNING OBJECTIVE \#1: Recognize proteinuria in elderly patients.

CASE: Systemic lupus erythematosus (SLE) is an autoimmune disorder usually affecting women aged 20 to 40 years. The incidence of nephropathy in Lupus is 11 to $18 \%$. Clinical features range from proteinuria (nephritic and nephrotic range), normal or slightly elevated serum creatinine, microscopic hematuria, nephrotic syndrome (with hypoalbuminemia and edema) and hypertension. These usually occur concurrently with other clinical feature of SLE. Isolated proteinuria as the sole initial presentation of Lupus Nephritis in elderly has not been reported in the literature. We report a case of an elderly male who was found to have membranous glomerulonephritis on renal biopsy consistent with Lupus nephritis. A 73 year-old-male presented with 6 months history of ongoing dyspnea on exertion. He has a pertinent medical history of hypertension and coronary artery disease status post Coronary Artery Bypass Graft 10 years ago. He had dyspnea on exertion about 6 months ago when he was diagnosed with pericardial effusion of unclear etiology, underwent pericardial window and had normal coronary arteries on cardiac catheterization. His workup at that time including ANA, RA, ACE level, complements (C3/C4/CH50), ANCA, Anti-MPO \& Antiproteinase was negative. His dyspnea did not completely resolve after pericardial window and now he can only walk one block before getting dyspneic. He denies dyspnea at rest, orthopnea and in fact, feels better lying down. His review of the systems was pertinent for 2 weeks symptoms of swollen hands, some mild lower extremity edema, and approximately ten pounds weight gain. He denied chest pain, palpitations, lightheadedness, paroxysmal nocturnal dyspnea, and claudication. His examination was pertinent for hypertension of $181 / 87 \mathrm{mmHg}$, mild periorbital swelling, and trivial bilateral pitting pedal edema. Laboratory data revealed normal complete blood count, renal and liver function with an albumin level of $2.1 \mathrm{~g} / \mathrm{dl}$ and total proteins of $5.0 \mathrm{~g} /$ $\mathrm{dl}$, cardiac BNP of $430 \mathrm{pg} / \mathrm{ml}$, urine analysis with proteinuria $500 \mathrm{mg} / \mathrm{dl}$ and hematuria of $27 \mathrm{RBCs} / \mathrm{HPF}$. Electrocardiogram was normal, a chest x-ray was unremarkable and an echocardiogram showed normal ejection fraction, borderline left ventricular hypertrophy, no regional wall motion abnormalities, no pericardial effusion or constriction and no valvular abnormalities. His lower extremity venous ultrasound was negative for deep vein thrombosis. In light of low albumin, periorbital edema, and dyspnea a random urine protein-creatinine ratio check was $860 \mathrm{mg} / \mathrm{dl}$ and $24 \mathrm{~h}$ urine proteins were $14.3 \mathrm{~g} / \mathrm{day}$ consistent with nephrotic syndrome ( 2 week ago random urine protein-creatinine ratio was $185 \mathrm{mg} / \mathrm{dl}$ ). Lipid panel revealed total cholesterol $159 \mathrm{mg} / \mathrm{dl}$, LDL $114 \mathrm{mg} / \mathrm{dl}$, HDL $27 \mathrm{mg} / \mathrm{dl}$ and triglycerides $75 \mathrm{mg} / \mathrm{dl}$. Rheumatological workup including ANA was positive with homogenous pattern and dsDNA positive at $25 \mathrm{IU} / \mathrm{ml}(0-9 \mathrm{IU} / \mathrm{ml})$. He had normal RF, ASO titer, ANCA panel, complements (C3, C4, and total hemolytic complement), serum free light chains and urine protein electrophoresis and immunofixation, HIV, Syphilis, hepatitis B, C serology, and cryoglobulins. Renal biopsy revealed membranous glomerulonephritis with focal endocapillary proliferative features, highly suggestive of membranous and focal proliferative lupus nephritis (lupus nephritis class V and IIIA) along with tubular degenerative changes and arterio-arteriolosclerosis with hyalinosis. The patient was treated with antihypertensive, diuretics, pulse dose 
steroids and cyclosporine along with Bactrim for PCP Prophylaxis resulting in resolution of proteinuria on follow-up.

DISCUSSION: Nephrotic syndrome patients present with marked edema, proteinuria, hypoalbuminemia, and often hyperlipidemia. Proteinuria should not be ignored in elderly patients presenting with unexplained dyspnea. This could be the sole initial diagnostic clue to the underlying nephropathy secondary to underlying SLE.

PULMONARY NODULES MADE CRYSTAL CLEAR Claire A. Evans; Douglas Haden. Carolinas Medical Center, Charlotte, NC. (Control ID \#2470254)

LEARNING OBJECTIVE \#1: Recognize uncommon causes of pulmonary nodules. LEARNING OBJECTIVE \#2: Identify populations at high risk for adverse sequelae from sodium polystyrene sulfonate (SPS).

CASE: A 66-year-old African American man with history of end stage renal disease status post renal transplant from deceased donor and now on chronic immunosuppression presented with shortness of breath and generalized weakness. He reported increasing exercise intolerance over a 1 month period but denied associated cough, hemoptysis, or chest pain. On admission, patient was well-appearing, afebrile, and had normal vital signs. Lung exam revealed clear breath sounds without crackles, wheezes, or rhonchi. Oxygen saturation was $99 \%$ on room air. Serologic testing revealed leukocytosis of 12,500 with $77 \%$ neutrophils. Given his complaint of shortness of breath and finding of leukocytosis, computed tomography of the chest with contrast was obtained. Imaging revealed multiple pulmonary nodules in the bilateral lungs, the largest of which was estimated to be $15 \mathrm{~mm}$. Notably, these findings were not present on CT of the chest obtained 5 months prior. He underwent bronchoscopy with broncheoalveolar lavage as well as transbronchial biopsies of two nodules in the right lung. Bacterial and fungal cultures were negative. Biopsies revealed foreign body giant cell reaction with basophilic crystals consistent with sodium polystyrene sulfate (Kayexalate) aspiration. On chart review, it was noted that patient was suffering from severe constipation during a hospitalization several months prior. At that time, he received an aggressive bowel regimen including SPS, magnesium citrate, bisacodyl suppositories, and tap water enemas. Shortly thereafter, patient suffered nausea and emesis leading to aspiration which in retrospect was identified as the inciting event

DISCUSSION: The etiology of multiple pulmonary nodules should include both malignant and benign conditions. Certain characteristics of the nodules may help distinguish between the two. For example, multiple nodules greater than $1 \mathrm{~cm}$ in diameter or those detected on chest radiography are more likely due to metastatic disease. Conversely, multiple nodules that are less than $5 \mathrm{~mm}$ in diameter or those found incidentally on computed tomography are more likely benign lesions. Further narrowing of the differential can be accomplished by careful history. For example, in our immunocompromised patient we included opportunistic fungal and bacterial infections in the differential such as invasive aspergillosis, Cryptococcus, and tuberculosis or other mycobacteria. Other benign processes considered included inflammatory diseases such as granulomatosis with polyangiitis, sarcoidosis, and rheumatoid arthritis. The patient's medication list was also reviewed for common culprits such as methotrexate, amiodarone, or bleomycin. An overlooked cause of pulmonary nodules is foreign body giant cell reaction. While commonly associated with intravenous injection of pulverized pharmaceutical tablets, the inhalation or aspiration of certain agents can also cause giant cell reaction. The introduction of foreign material into pulmonary interstitial tissue causes influx of neutrophils leading to inflammation. The foreign particles are then phagocytized by macrophages and giant cells, leading to the gradual replacement of normal lung tissue with mononuclear cells and multinucleated giant cells which are identifiable on pathology. These nodules may remain relatively benign, but in some cases can progress to significant fibrosis with associated dyspnea and hypoxia. Interestingly, the crystals of common agents are also distinguishable by properties including light polarization and staining characteristics. For example, SPS material is identified as polygonal or amorphous basophilic crystals. The identification of these crystals on transbronchial biopsy sealed the diagnosis for our patient. SPS has been associated with rare but serious and sometimes fatal lower gastrointestinal adverse events including colonic necrosis and perforation. In one case report of 12 patients with bowel necrosis after treatment with SPS, five had fatal outcomes within 6 weeks. In an effort to decrease incidence, populations have been identified as high risk for complication. These groups include patients within the 2 week post-operative period, those with obstructive bowel disease, and those with end-stage renal disease. Conversely, while adverse aspiration and upper gastrointestinal complications are also being described in literature, identification of high risk patients is lacking. For example, a retrospective study of 59 cases of pulmonary complications from aspiration revealed two cases of SPS crystals on biopsy. Another report described 11 cases of upper gastrointestinal sequelae including mucosal ulceration or erosion in the esophagus, stomach, and duodenum. However, neither of these articles addressed potential risk factors. Our case presentation of an adverse event associated with aspiration demonstrates that there may be other unidentified populations at high risk such as patients with nausea, altered mental status, or dysphagia.

PULMONARY VEIN THROMBOSIS: NOT THE GARDEN VARIETY LUNG CLOT Mojun Zhu; Ashley Woods; Antonios Charokopos; Abhay J. Dhond. Yale New Haven Hospital, New Haven, CT. (Control ID \#2469442)

LEARNING OBJECTIVE \#1: Management of Pulmonary Vein Thrombosis in Hemodynamically Stable Patients

CASE: A 90+ year old female patient with hypertension presented to the emergency department (ED) with left rib pain that started 2 days prior to admission. The pain was sharp in quality and localized to the area under her left breast. It was moderate, constant, and independent of position and activity; however, it was exacerbated by deep inspiration and occasionally radiating to her left flank. She initially thought that her undergarments irritated her skin and took acetaminophen but the pain persisted. She denied recent falls, lightheadedness, shortness of breath, dyspnea on exertion, cough, hemoptysis or palpitations. Her medical history is significant for colorectal cancer status-post resection 40 years prior, recent falls and multiple ED visits for hypertensive urgency likely due to medication non-adherence. She is a never smoker and her home medications include daily aspirin, olmesartan, labetalol, and vitamin D. At the ED, she was found to be hypertensive with systolic blood pressure in $200 \mathrm{~s}$ that quickly improved after IV labetalol. Other vital signs were within normal limits. On physical exam, she had mild tenderness to percussion under her left breast and diminished breath sounds bilaterally. The remainder of her physical exam, including cardiac exam, was normal. CT angiography (CTA) was ordered to rule out pulmonary embolism (PE), which showed a 1.7 x $0.3 \mathrm{~cm}$ filling defect within the left inferior pulmonary vein. Also noted were a few scattered nonspecific small pulmonary nodules measuring up to $0.4 \mathrm{~cm}$. Venous duplex ultrasonography did not reveal any deep vein thrombosis (DVT) in her lower extremities. Echocardiogram showed a normal appearing left atrium (LA) with no evidence of thrombus and left ventricular ejection fraction of $72 \%$. She was then admitted to the geriatric medicine service and remained hemodynamically stable. Her pain resolved with topical lidocaine application. Anticoagulation was not initiated considering lack of symptoms, her advanced age, and high risk of fall. Family was in agreement and patient was discharged with close outpatient follow up.

DISCUSSION: Pulmonary vein thrombosis (PVT) is an extremely rare phenomenon. It is associated with lung and metastatic malignancy, radiofrequency ablation for atrial fibrillation, chest trauma, and surgical procedures such as lobectomy and lung transplantation. Direct tumor invasion, pulmonary vein stenosis with turbulent blood flow, endothelial injury due to mechanical manipulation, as well as hypercoagulability can all lead to thrombosis $^{1-7}$. A few cases of idiopathic PVT have also been reported, such as with our 
patient. However, malignancy as an alternative precipitant in this patient cannot be excluded taking into account of the presence of pulmonary nodules and her remote history of colorectal cancer. Clinical manifestations of PVT vary widely from pleuritic chest pain to massive hemoptysis but most patients presented with respiratory distress ${ }^{1,2,4,6}$. Due to nonspecific symptoms and rarity of this condition, diagnosis is often made as a result of incidental imaging. Transthoracic echocardiogram followed by transesophageal echocardiogram appears to be the preferred test in patients with known cardiac conditions or in the post-operative period ${ }^{2,5}$. CTA or CT with IV contrast identifies filling defects effectively but its specificity is limited by the presence of artifacts. In our case, after discussion with radiology, her finding was felt to be a true finding. There is no consensus on management of PVT. Lobectomy and thrombectomy were only performed in patients with hemodynamic instability with evidence of active hemorrhage ${ }^{2,6}$. Systemic heparinization was attempted in patients with PVT after lung transplantation but the treatment was complicated by increased risk of bleeding ${ }^{5}$. One infrequent but important consequence of PVT is distal embolization, Anticoagulation with warfarin seems to be the default medical therapy for most stable patients. However, there is no data on the length of treatment with warfarin $^{1,2}$. Additionally, patients with small PVT's $(0.6-0.7 \mathrm{~cm})$ were managed conservatively without anti-coagulation and subsequent resolution of the thrombus without any clinical sequelae $e^{5}$. Interestingly, prophylactic therapeutic anticoagulation with warfarin did not prevent occurrence of PVT in one patient ${ }^{4}$. In conclusion, treatment of PVT depends on the hemodynamic stability of the patient, size of the PVT and risk of bleeding with the patient. References: 1. Selvidge SD, Gavant ML. Idiopathic Pulmonary Vein Thrombosis Detection by CT and MR Imaging. AJR Am J Roentgenol. 1999;172(6); 1639-41. 2 Garcia MJ, Rodriguez L, Vandervoort P. Pulmonary Vein Thrombosis and Peripheral Embolization. Chest. 1996;109(3):846-47. 3. Kim NH, Roldan CA, Shively BK Pulmonary Vein Thrombosis. Chest. 1993;104:624-26. 4. Yataco J, Stoller JK. Pulmonary Vein Thrombosis and Infarction Complicating Venous Stenosis Following Radiofrequency Ablation. Respir Care. 2004 Dec;49(12):1525. 5. Schulman LL, et al Four-Year Prospective Study of Pulmonary Venous Thrombosis After Lung Transplantation. J Am Soc Echocardio. 2001 Aug;14(8); 806-12. 6. Alexander GR, Reddi A, Reddy D. Idiopathic Pulmonary Vein Thrombosis: A Rare Cause of Massive Hemoptysis. Ann Thorac Surg. 2009 Jul; 88(1) 281-3. 7. Tamizifar B, Zadeh MR, Foroghi E. Pulmonary Vein Thrombosis After Metastatic Liposarcoma. Medicinski arhiv 2012;66:68-9.

\section{PURPURA FULMINANS DUE TO STREPTOCOCCUS PNEUMONIA Shunsuke} Kojima $^{1}$; Jun Ehara ${ }^{4}$; Takaki Naito ${ }^{2}$; Toru Yamada ${ }^{5}$; Hidetaka Kitazono ${ }^{3}$; Eiji Hiraoka ${ }^{5}$ ${ }^{1}$ Tokyo Bay Urayasu Ichikawa Medical Center, Urayasu, Japan; ${ }^{2}$ Tokyo Bay Urayasu/ Ichikawa Medical Center, Urayasu, Japan; ${ }^{3}$ Tokyo-Bay Urayasu/Ichikawa Medical Center, Urayasu-shi, Japan; ${ }^{4}$ Tokyobay Urayasu Ichikawa Medical Center, Urayasu, Japan; ${ }^{5}$ Tokyo Bay Urayasu Ichikawa Medical Center, Chiba, Japan. (Control ID \#2470033)

LEARNING OBJECTIVE \#1: Identify the features of Purpura Fulminans LEARNING OBJECTIVE \#2: Recognize the importance of early diagnosis and treatment of purpura fulminans

CASE: A 57-year-old previously healthy man was brought to the emergency department with 3 days of generalized malaise, muscle sore and fever followed by witnessed syncopal episode and a diffuse macular rash noted on the arms, chest, abdomen, and pretibial areas. On admission, he was oriented. Vital signs were blood pressure 73/ $47 \mathrm{mmHg}$, pulse rate 132 beats/min, respiratory rate 24 breaths/min, temperature of $38.7^{\circ} \mathrm{Celsius}$. Initial laboratory tests showed thrombocytopenia and coagulopathy, being consistent with DIC. Liver and renal insufficiency was also noted. Level of troponin I was normal on admission, followed by elevation within a few hours.
Arterial blood gas analysis detected severe lactic acidosis. Computerized tomography showed no pathological findings, except hypoplasty of spleen. Howell-Jolly bodies were not observed on peripheral blood smear. An electrocardiogram demonstrated sinus tachycardia with ischemia-like ECG changes including ST-segment elevation in leads V1, 2, and aVR, along with ST-segment depression in leads II, III and aVF. On echocardiogram, his cardiac contractility was diffusely reduced to $18 \%$ of the ejection fraction, without any valvular diseases or vegetations. Septic shock and cardiogenic shock were considered possble, although no infectious source was detected on admission. Rapid fluid resuscitation with intravenous empirical antibiotics with vancomycin and meropenem was started immediately followed by mechanical ventilation. In view of his unstable status and multiple organ failure, treatment with an intra-aortic balloon pumping (IABP) and continuous renal replacement therapy was started. Cardiac catheterization confirmed normal coronary disease. The initial resuscitative management resulted in a reduction of his serum lactate level and hemodynamical stabilization. Two days after the admission, Streptococcus pneumonia was identified by blood culture and he was diagnosed with invasive pneumococcal disease. IABP and mechanical ventilation was weaned off on the second and the seventh day, respectively. However, he developed painful purpura on his tip of his fingers, which gradually spread to his forearms and calves, consistent with purport fulminans (PF) due to Streptococcus pneumonia. Seven weeks after his admission, he required amputation of all extremities. After the 4-month hospital stay, he was transferred to another hospital for further rehabilitation without any other sequela.

DISCUSSION: We experienced a case of pneumococcal sepsis-induced PF. PF is a lifethreatening condition of symmetric peripheral gangrene, fever, hypotension and DIC that requires early diagnosis and management. Its mortality is up to $40 \%$. It is usually occurs in the setting of severe sepsis which our patient suffered from. The most common pathogens are Meningococcus, followed by Varicella, Pneumococcus, and measles. Recent studies reported that the protein $\mathrm{S}$ or $\mathrm{C}$ deficiency can be a risk factor of this disease. Currently, there is no standard treatment for PF. Intensive care with aggressive fluid resuscitation for adequate peripheral perfusion and antibiotics play the main role in management. Because the pathophysiology of PF is intravascular thrombosis, heparin is empirically administered to prevent further thrombus formation in spite of lack of evidence. Administration of protein $\mathrm{C}$ product might contribute to restore peripheral perfusion by its repletion reportedly. Once peripheral gangrene occurs, surgical intervention including fasciotomy and debridement might be needed to save their lives. Our patient was in shock state associated with severe cardiac systolic dysfunction and normal coronary artery. Our initial differential diagnosis of shock with low EF and fever includes septic cardiomyopathy, myocarditis, and septic shock occurring to cardiomyopathy patients. Retrospectively, the cardiac dysfunction was most likely to be sepsis cardiomyopathy based on normal coronary artery and full cardiac recovery. Given the fact that it is difficult to differentiate between sepsis cardiomyopathy and severe myocarditis and the high mortality $(70 \%)$ with sepsis cardiomyopathy, empirical treatment with broadspectrum antibiotics should be initiated for possible sepsis cardiomyopathy, encountering a case with low EF with fever even if no other signs and symptoms exist like our case. Asplenic patients and those with reduced splenic function are at the risk of sepsis especially due to encapsulated bacteria, such as streptococcus pneumonia. The incidence of sepsis is $3.2 \%$ and its mortality risk is up to 50 to $70 \%$ among splenic patients. Our patient may have splenic dysfunction based on the small size of spleen though spleen scintigraphy, the most reliable method to assess its function, was not performed. The Centers for Disease Control and Prevention recommends asplenic patients receive pneumococcal vaccine. Our patient received pneumococcal vaccine after discharge. In conclusion, we herein reported a rare case of $\mathrm{PF}$ associated with pneumococcal infection in adult. Early diagnosis and aggressive interventions including fluid resuscitation, broadspectrum antibiotics are necessary to save life and extremities. Prompt surgical consultation should also be considered. 
PYODERMA GANGRENOSUM AN EXPLODING PUSTULE OFTEN MISDIAGNOSED Daniel Puneky ${ }^{3}$; Samit Datta ${ }^{1}$; Sherri Sandel ${ }^{2} .{ }^{1}$ Lenox HIll Hospital, New York, NY; ${ }^{2}$ Lenox Hill Hospital, New York, NY; ${ }^{3}$ North well Health/ Lenox Hill Hospital, New York, NY. (Control ID \#2469121)

LEARNING OBJECTIVE \#1: Diagnose pyoderma gangrenosum early in its course using clinical features and comorbidities

CASE: Our patient is a 34-year-old obese, African American female, who presented with a purulent, exquisitely painful right lower extremity ulcer. Our patient also has a history of hidradenitis suppurativa (HS), inactive at presentation. Two and a half weeks earlier, the patient noticed a small pustule that rapidly developed into an ulcer, which became progressively more painful. Prior to presentation, she was treated with 10 days of doxycycline without improvement. On exam, she was afebrile with no abnormal vital signs or systemic symptoms. She had a $7 \times 7 \mathrm{~cm}$ purulent ulcer with undermined, violaceous borders and surrounding erythema. Exam was otherwise benign. Laboratory studies, including chemistries and complete blood count, were within normal limits with the exception of a known iron deficiency anemia secondary to dysfunctional uterine bleeding. CT with contrast of the affected extremity showed shallow ulceration with infiltration of the underlying subcutaneous tissues and no drainable fluid collection or abscess. Duplex ultrasound of the affected extremity was negative for DVT. The patient was started on vancomycin and Plastic Surgery was consulted for debridement of the wound. She was taken to the OR where surgical debridement was performed, wound vacuum was placed, and both wound cultures and biopsy of the lesion were collected. Cultures failed to grow any bacterial species. Throughout her course, the patient's only complaint was pain at the site without systemic symptoms. She was found to have an ANA titer of 1:80, but other labs were negative including rheumatoid factor, cANCA, pANCA, serum protein electrophoresis, Hepatitis A, B and C, and HIV. Chest $\mathrm{X}$-ray was negative for hilar lymphadenopathy. On day four of her course, the patient returned to the OR for autologous mesh skin grafting. On hospital day five, histopathology of the wound biopsy showed a dense, diffuse neutrophilic infiltration of the dermis and subcutaneous fat consistent with a diagnosis of pyoderma gangrenosum (PG). Antibiotic therapy was discontinued and the patient was started on immunosuppressive therapy with $60 \mathrm{mg}$ of oral prednisone daily. The patient improved, and on hospital day seven the wound vacuum was removed. She was discharged to home on oral prednisone with outpatient dermatology follow up.

DISCUSSION: Owing to its rarity (3-10 people per million per year), many physicians are unaware of PG. This leads to late diagnosis and significant iatrogenically induced morbidity. PG is a rapidly progressive ulcerative skin disorder characterized by neutrophilic infiltration of the dermis and subcutaneous fat. While it was first described in 1930, the pathogenesis is still largely unclear due to a lack of prospective research on the subject. PG has a predominance in women, a preference for the lower extremities, and an association with inflammatory bowel diseases, hematologic disorders (leukemias, myelofibrosis and monoclonal gammopathy), and arthritis (rheumatoid arthritis, seronegative arthritis). Our patient had a coexistent diagnosis of HS. To our knowledge, there is only one case series documenting the association of HS with PG in 11 patients. In that case series, 10 of the 11 patients were women and 9 were obese, characteristics shared by our patient. Patients typically present with an infectious appearing follicular pustule or papule that rapidly develops into a painful ulcerative lesion, which may be mucopurulent or hemorrhagic. While infectious in appearance, the ulcer is sterile with an undermined violaceous border, surrounding area of erythema and edematous infiltration of surrounding tissue. Clinical and laboratory findings are nonspecific. Histopathologic evidence of sterile neutrophilic infiltration of the dermis, when present, is only suggestive of the disease. There are no universally accepted or validated diagnostic criteria, so recognition requires a high level of suspicion from the astute physician. Clues to the diagnosis include comorbid conditions. In our case, we present a patient with a PG ulcer classic in appearance with violaceous, undermined borders, surrounding erythema and histopathology consistent with PG. While our patient had minimal symptomatic improvement after surgical debridement, it is important to note that many patients experience significant worsening of the disease following surgical manipulation. This is poorly understood pathophysiologically, but the proposed mechanisms are pathergy or an Arthus-like reaction. Surgical biopsy is a useful tool in diagnosis, but further manipulation should be held until after the initiation of immunosuppresive therapy. Pyoderma gangrenosum is a rare disease, easily overlooked, and a late or misdiagnosis results in significant morbidity. Our case serves as a reminder that even apparently straightforward dermatologic cases deserve rigorous scrutiny for underlying pathology that may significantly change management. Ruocco E, Sangiuliano S, Gravina AG, Miranda A, Nicoletti. Pyoderma gangrenosum: an updated review. J Eur Acad Dermatol Venereol. 2009 Sep;23(9):1008-17. doi: 10.1111/j.1468-3083.2009.03199.x. Epub 2009 Mar 11. Hsiao JL, Antaya RJ, Berger T, Maurer T, Shinkai K, Leslie KS. Hidradenitis Suppurativa and Concomitant Pyoderma Gangrenosum: A Case Series and Literature Review. Arch Dermatol. 2010;146(11):1265-1270. doi:10.1001/archdermatol.2010.328.

\section{PYOGENIC SPONDYLITIS INITIALLY UNDIAGNOSED BY IMAGING} STUDIES Reiko Sakama; Naoto Matsuda; Kenji Murai; Masahiro Fujioka; Yuki Uehara; Toshio Naito. Juntendo University School of Medicine, Bunkyoku, Japan. (Control ID \#2466999)

LEARNING OBJECTIVE \#1: Understand the significance of clinical diagnosis over imaging study results in patients with acute neck pain and fever in the early stage of disease

LEARNING OBJECTIVE \#2: Recognize the importance of blood culture for patients presenting with fever and neck pain

CASE: A 63-year old man with a history of left cervical spondylotic radiculopathy, prostate cancer, and impaired glucose tolerance was admitted to our hospital because of fever, headache, neck pain, and bilateral shoulder pain. Six days prior to admission, he visited another hospital due to a fever of $39.9^{\circ} \mathrm{C}$, shivering, headache, and joint pain. A rapid influenza test result was negative; hence, he was diagnosed with a viral infection and sent home. One day before admission, he visited his orthopedist with worsening symptoms, but plain radiography of the neck and shoulders did not reveal any possible causes for the pain. The day of admission, fever, headache, and neck pain persisted and he reported a rating of 8 out of 10 points on the pain scale and returned to our hospital for further evaluation. His body temperature was $38.8^{\circ} \mathrm{C}$. He could barely turn his neck to the right or left and had difficulty elevating his upper extremities. He did not complain of numbness in the fingers, dyspnea, or difficulty swallowing while eating. Blood testing revealed a white blood cell count of $11,300 / \mu \mathrm{L}$ and a C-reactive protein level of $19.8 \mathrm{mg} /$ dL. Enhanced computed tomography (CT) of the neck to pelvis was performed, revealing no significant findings. He was admitted to the department of general medicine. Two sets of blood cultures were obtained, and sulbactam/ampicillin were administered. The next day, a blood culture was positive for group B streptococcus after $12 \mathrm{~h}$ of incubation. For further analysis, enhanced magnetic resonance imaging (MRI) of the neck was performed. MRI showed an inhomogeneous contrast effect of fluid retention in front of the cervical vertebrae. Based on the MRI findings, the orthopedic surgeon could not deny a retropharyngeal abscess, but the otolaryngologists diagnosed the patient with calcific retropharyngeal tendinitis based on the MRI and previous enhanced CT findings of possible calcification in front of the $\mathrm{C} 1$ vertebra. On day 4 of hospitalization, enhanced $\mathrm{CT}$ of the cervical spine was repeated, but it revealed neither findings of abscess formation nor definitive calcification in front of the $\mathrm{C} 1$ vertebra due to halation of an implanted tooth. Two weeks later, enhanced MRI of the neck was repeated for a follow-up evaluation. It showed a high-intensity area on the body of the C6 and C7 vertebrae on Short-T1 Inversion Recovery (STIR), and a final diagnosis was reached of pyogenic spondylitis. 
We continued administering him sulbactam/ampicillin for 6 weeks overall, followed by amoxicillin/clavulanate for 4 weeks. The patient was discharged without any complications.

DISCUSSION: We experienced a diagnostic challenge in determining the cause of neck pain and fever, and this case showed the importance of obtaining blood cultures as well as of the clinical diagnosis over imaging studies in the early stage of disease. Differential diagnosis for acute neck pain with fever includes retropharyngeal abscess, pyogenic spondylitis, calcific retropharyngeal tendinitis, and crowned dens syndrome. It is crucial to distinguish retropharyngeal abscess and pyogenic spondylitis from others since they could lead to severe complications, such as airway stenosis and quadriplegia. We learned, however, that it was not always easy to differentiate between those with only imaging studies, especially in the early stage. Enhanced CT and MRI taken about 1 week after the beginning of symptoms showed no evidence of abscess formation or spondylitis. The diagnosis of pyogenic spondylitis was made about 2 weeks after admission. One of the biggest reasons for diagnostic confusion could have been that imaging was performed too early to show meaningful results. The diagnosis could have been calcific retropharyngeal tendinitis with calcification in front of the $\mathrm{C} 1$ vertebra, but the positive blood culture result was inconsistent with this diagnosis. Initially a route of entry was not clear in spite of careful examination and our diagnosis did not include pyogenic spondylitis. Fortunately, we initiated the treatment based on physical examination and the results of blood cultures In recent years, imaging modalities, such as CT and MRI, are often used to make a diagnosis. However, physicians must be mindful that diagnostic imaging does not always reflect the conditions of the disease in the early stages, and it is important to give weight to clinical diagnosis through careful physical examination.

RAPIDLY ENLARGING LUNG NODULE: YES, IT WAS FROM ASPIRATION! Dipti Baral $^{1}$; Bhishma Pokhrel ${ }^{3}$; Rhisti Shrestha ${ }^{4}$; Birendra Sah ${ }^{2} .{ }^{1}$ SUNY Upstate Medical University, Syracuse, NY; ${ }^{2}$ Upstate Medical University, Syracuse, NY; ${ }^{3}$ Patan Academy of Health Sciences, Patan, Nepal; ${ }^{4}$ Institute of Medicine, Kathmandu, Nepal. (Control ID \#2469698)

LEARNING OBJECTIVE \#1: Identify aspiration as a possible cause for any rapidly enlarging lung nodule in a patient with acid reflux disease.

LEARNING OBJECTIVE \#2: Positron Emission Tomography (PET) scans can be positive in metabolically active infectious or inflammatory lesions. Lung nodules with surrounding glass opacities could suggest inflammatory or hemorrhagic process.

CASE: 78 year-old-male presented with 1 episode of moderate amount of hemoptysis. He reported subjective fever with no chills for last few weeks, occasional dry cough and reflux symptoms. He had chronic sinusitis, barrett's esophagus and coronary artery disease with drug eluting stent placed 2 months ago. He had smoked 1.5 pack-per-day for last 50 years. His mother was treated for tuberculosis (TB) twice. On exam, vitals were stable except for scattered wheezes on auscultation. Electrocardiogram showed no new changes. Chest Xray showed $1.6 \mathrm{~cm}(\mathrm{~cm})$ right upper lobe (RUL) mass. Computed Tomography $(\mathrm{CT})$ revealed $1.9 \mathrm{~cm}$ RUL lung mass with surrounding ground glass halo with $1-1.5 \mathrm{~cm}$ partially calcified mediastinal lymph nodes. Galactomannan test for aspergillus, immunologic studies, sputum cytology and quantiferon test for TB came back negative. Patient was empirically treated with levofloxacin for 10 days. Repeat CT in 6 weeks showed that the nodule had increased to $2.5 \mathrm{~cm}$ cavitary lesion and also showed a $2 \mathrm{~cm}$ right paratracheal lymph node. The RUL mass and right paratracheal lymph node had increased metabolic activity in PET scan (Standardized uptake value, SUV of 3.8 and 4.4 respectively). Patient opted for diagnostic mediastinoscopy and right thoracotomy and if needed, therapeutic resection of a part of lung in the same setting to reduce his time off plavix. During thoracotomy, $1 \mathrm{~cm}$ nodule was found medial to the large cavitary nodule. Hence, right posterior segmentectomy was done with lymph node biopsy. Pathologic exam of the large cavitary mass revealed fibroblast plugs within air spaces, interstitial chronic inflammation and remnants of degenerated vegetable particles, which suggested organizing aspiration pneumonia. The enlarged mediastinal lymph node was reactive and the smaller $1 \mathrm{~cm}$ nodule was old calcified granuloma.

DISCUSSION: Lung disease due to aspiration is very common but is often misdiagnosed. Aspiration to lungs commonly present as aspiration pneumonia, diffuse aspiration bronchiolitis and aspiration pneumonitis. Presentation of aspiration as a lung mass is extremely rare. Most of the cases described in literature are associated with reflux disease. One of the key features looked to characterize lung cancer is the size of the nodule and it's doubling time. It is important to remember that benign lung lesions tend to grow either very fast or very slow. Lung nodules with surrounding ground glass opacities could suggest inflammatory or hemorrhagic process. Increased 18 F-2-deoxy-2-fluoro-D-glucose (FDG) uptake in PET scans can be seen in active infective or inflammatory lesions like active tuberculosis, acute and chronic pneumonia, abscess, sarcoidosis, pneumoconiosis, vasculitic lung lesion and organizing pneumonia.

\section{RAPIDLY PROGRESSIVE NEUROLOGIC DECLINE AND PAPULAR RASH} PRESENTING IN A PATIENT WITH LYMPHOMA Dean Ehrlich; Jennifer Phan; Alexandra Drakaki. University of California, Los Angeles, Los Angeles, CA. (Control ID \#2458120)

LEARNING OBJECTIVE \#1: Recognize the risk factors and presentation of neuroinvasive West Nile Virus

LEARNING OBJECTIVE \#2: Counsel patients and families on the prognosis of neuroinvasive West Nile Virus

CASE: Mr. B is a 67 year-old male with past medical history of mantle cell lymphoma who presented with papular rash followed by an acute onset of bilateral lower extremity weakness. On the day of presentation his family found him down and unable to stand, and he was brought to the local hospital. In the emergency department, he presented with fever to $103^{\circ}$, mild confusion, and bilateral lower extremity weakness without meningismus. He had an erythematous papular rash on his trunk, back, and lower extremities. Labs were notable for mild leukocytosis with $80 \%$ neutrophils. He denied travel history outside of California, bug bites, and history of stroke or seizure. Empiric broad-spectrum antibiotics were started, but over the course of the next 3 days the patient's mental status deteriorated. Upon transfer to our tertiary center, the patient had a GCS of 6 . He had diffuse flaccid paralysis, absent upper and lower extremity reflexes, aphasia and dysphagia. Lumbar puncture demonstrated elevated white blood cells with neutrophilic predominance and negative gram stain. One week after admission, CSF West Nile Virus (WNV) IgM became positive. Once the diagnosis of neuroinvasive WNV was made, all antibiotics and antiviral medications were discontinued. The patient made significant neurologic progress over his 1-month hospital course, regaining his ability to speak and follow complex commands. On discharge, however, there were persistent deficits from baseline, including significant lower extremity weakness.

DISCUSSION: West Nile Virus encephalitis is the most common viral encephalitis in the United States, yet less than $1 \%$ of those infected with WNV develop neuroinvasive disease. The best described risk factors for neuroinvasive WNV include advanced age, malignancy and organ transplantation. This case demonstrates a particularly profound presentation of neuroinvasive $\mathrm{WNV}$, which classically presents with fever, flaccid paralysis, and signs of meningoencephalitis, often accompanied by a maculopapular or morbilliform rash. Interestingly, the neutrophilic CSF with negative gram stain found in our patient can be a clue to the diagnosis because it is a well described phenomenon in neuroinvasive WNV, despite its viral etiology. Prognostication with the patient's family becomes important once the diagnosis is made. Neuroinvasive WNV is estimated to carry a $2-12 \%$ overall mortality rate. Age $(>50)$ is the most important predictor of lasting 
complications. In the Houston West Nile Cohort followed since 2002, $86 \%$ of patients and $43 \%$ of patients with WNV encephalitis had neurologic deficits $1-3$ years and 8-11 years post-infection, respectively. Wide variability in the literature between individual studies makes prognostication challenging, but based on a recent review of all known WNV studies, persistent long-term sequelae are likely. The most frequently observed sequelae are muscle weakness, fatigue, myalgia, memory loss, depression and difficulty concentrating. Patients and families must be counseled that recovery will be slow and that patients may continue to make progress even past 1 year.

\section{RARE CASE OF VIRULENT NATIVE VALVE ENDOCARDITIS AND} PERIVALVULAR ABSCESS DUE TO COAGULASE NEGATIVE STAPHYLOCOCCUS Khushdeep S. Chahal; Esmeralda B. Gutierrez Asis. University of Alabama Birmingham, Huntsville Regional Campus, Huntsville, AL. (Control ID \#2469786)

LEARNING OBJECTIVE \#1: Recognize Staphylococcus lugdunensis as a unique coagulase negative Staphylococcus (CONS) that causes virulent native valve endocarditis. LEARNING OBJECTIVE \#2: Recognize bradycardia and conduction abnormailities as signs of perivalvular abscess.

CASE: We present a rare case of native valve endocarditis due to Staph. Lugdunensis (SL) in a 21-year-old Caucasian male with history of bicuspid aortic valve (BAV). The patient had visited an outside hospital twice in the past 2 weeks with fevers, nausea and undue fatigue and was discharged after receiving antiemetics and intravenous fluids .On presentation to our emergency department (ED)patient complained of nausea, fevers and fatigue, was tachycardic and febrile to $103.1 \mathrm{~F}$. Exam was within normal with good dentition, except for blowing diastolic murmur over the aortic area. No history of iv drug use or high risk sexual behavior. He worked for a tree company and had a minor scalp laceration from a wooden plank for which he didn't seek medical attention. Labs revealed leukocytosis (WBC $12 \mathrm{~K}$ ) and elevated C Reactive protein (14). EKG showed sinus tachycardia with 1st degree AV block. 2 sets of blood cultures were drawn a few hours apart, Vancomycin initiated for endocarditis and infectious disease consultation sought. A transthoracic echocardiogram revealed aortic valve vegetation with preserved ejection fraction and moderate aortic insufficiency (AI). Follow up transesophageal echocardiogram (TEE) confirmed Aortic valve endocarditis. Meanwhile patient continued to be febrile and Gentamicin was added. Blood cultures grew methicillin sensitive S. Lugdunensis, vancomycin was switched to nafcillin. Patient's fever defervesced, tachycardia resolved, bacteremia resolved and a PICC line placed to continue antibiotics for 6 weeks. Next day patient was noted to have bradycardic episodes and EKG showed persistence of AV block, cardiology was consulted due to concern for perivalvular abscess. Since the patient was asymptomatic and bacteremia resolved, an echo wasn't repeated. Bradycardia was ascribed to gentamicin use which was discontinued with some improvement in heart rate, patient was discharged next day. Patient returned to the hospital within 2 weeks with severe dyspnea, productive cough, in decompensated heart failure with tachycardia, tachypnea, wide pulse pressure, elevated JVD, rales on lung exam, proBNP of 14000 and elevated troponin but no acute ST changes on EKG. TEE revealed Ejection fraction of $35 \%$ with posterior aortic valve abscess and severe AI. The patient was started on Nitroprusside drip and transferred to University Medical center for aortic valve replacement surgery and a metallic valve was placed. On clinic follow up, patient had been doing fine off antibiotics, was on Coumadin with therapeutic INR of 3.7.

DISCUSSION: Infective Endocarditis (IE) incidence has increased in the past decade from $10 / 100000$ to $15 / 100000$ cases. Major risk factors are age $>60$, male sex, injection drug use, poor dentition, structural heart disease, Intravascular devices, prosthetic heart valves, hemodialysis and HIV. Our patient had a bicuspid aortic valve which increases risk of IE. Most common cause is Staph. Aureus, CONS accounts for $10 \%$ cases. Usual presentation is of acute onset of fever, chills, nausea, fatigue with exam findings of heart failure, new murmur and stigmata of IE from vascular embolic or immunological phenomenon mainly affecting fundi, conjunctivae, skin and digits with petechiae being a common finding. Diagnosis requires careful history and exam, blood cultures, echocardiographic findings and modified Duke criteria are the accepted diagnostic criteria.SL is a unique CONS in its propensity to affect native valves and susceptibility to wide range of antibiotics. Incidence is underreported as most labs don't speciate CONS. In a clinical study of 495 CONS endocarditis cases, SL was the cause in $3 \%$ cases. It shares virulence factors of Staph aureus (SA) thus morbidity and mortality that rivals SA .SL has higher rate of embolic phenomenon ( $30 \%$ ) which was absent in our patient and in a review it was seen to cause heart failure in $45 \%$ with perivalvular abscess in $19 \%$. Detection of CONS in a clinically sick patient warrants speciation of organism.. Perivalvular abscess is reported in $30-40 \%$ cases of IE and has higher mortality. It is more common in aortic valves and bicuspid aortic valves are more prone. Involvement of conducting system is common, rarely causes acute coronary syndrome due to extrinsic compression of coronaries. Abscess should be suspected in patients with conduction blocks or persistent bacteremia and fever, TEE is more sensitive for diagnosis and diagnosis should prompt timely cardiovascular surgery evaluation. In our patient bacteremia had resolved, AV block was present at baseline but bradycardia was a new finding and a repeat TEE might have avoided associated morbidity.

READING BETWEEN THE EKG LINES Savannah R. Pena; Joshua Wong; Sweny Gulati; Margaret C. Lo. University of Florida College of Medicine, Gainesville, FL. (Control ID \#2455844)

LEARNING OBJECTIVE \#1: Recognize the classic symptoms and risk factors for pericarditis in patients with end stage renal disease (ESRD)

LEARNING OBJECTIVE \#2: Compare and contrast the etiology and treatment of acute pericarditis vs. recurrent pericarditis in setting of ESRD

CASE: A 55 years-old male presented with 5-days of dyspnea and pleuritic chest pain. Medical history includes ESRD from polycystic kidney disease, coronary artery disease on dual antiplatelet therapy (DAPT) for multiple stents and recent tamponade from hemorrhagic pericardial effusions treated with NSAIDS, colchicine and pericardial drainage. He had no history of autoimmune disease, recent infections or noncompliance with dialysis. Exam revealed stable vital signs and distant heart sounds but no rubs, gallops, JVD or pulsus paradoxus. Initial EKG showed sinus tachycardia with no segment changes. CTA chest found no pulmonary embolism but did reveal a moderate pericardial effusion unchanged from 3-months ago. A 2Dechocardiogram (TTE) showed a small pericardial effusion with no hemodynamic compromise. On hospital day 2, patient reported sudden sharp chest pain of 10/10 severity in the subxiphoid with no radiation elsewhere. The pain worsened with supine position but was not relieved by leaning forward. EKG now showed ST elevations in leads V2-V4 and diffuse PR depression. Troponins were stable at baseline values. Repeat TTE revealed unchanged pericardial effusion with no tamponade. In consult with Cardiology, patient was diagnosed with recurrent pericarditis and treated with colchicine every $72 \mathrm{~h}$ and prednisone $40 \mathrm{mg}$ daily. His dialysis was switched to a heparin-free protocol. Patient responded well to this regimen with complete symptom resolution. He was discharged on 6 months of colchicine and 3 weeks of prednisone taper for recurrent pericarditis.

DISCUSSION: This case demonstrates that severe chest pain syndromes do not always represent acute coronary syndrome and can result from common non-ischemic processes such as pericarditis. Indeed, literature cites at least 12 cases of acute pericarditis misdiagnosed as acute myocardial infarction, some receiving thrombolysis. Key diagnostic clues lie in the symptomology, risk factors and EKG findings. Acute pericarditis 
typically presents as sharp, pleuritic chest pain, worsened with lying down and relieved with leaning forward. EKG showing diffuse ST elevation with PR depression is pathognomonic. Our patient had these classic symptoms of pericarditis. His initial atypical EKG should not mislead clinicians. One case series report $43 \%$ of pericarditis with atypical EKGs, mostly seen in ESRD and hypothyroidism. If so, a TTE is indicated for further evaluation and over $50 \%$ show pericardial effusion, as true in this case. Our case further emphasizes the need to distinguish between acute pericarditis vs. recurrent pericarditis given differing etiologies and therapies. While acute pericarditis is mostly idiopathic or infectious, recurrent pericarditis is often autoimmune in etiology. This case suggests additional risk factors for recurrent pericarditis, namely ESRD and DAPT. ESRD patients with no typical risk factors of autoimmune conditions, repeat infections, or metabolic abnormalities can develop recurrent dialysisassociated pericarditis. Incidence can be as high as $20 \%$. The predisposition for uremic bleeding from platelet dysfunction and the use of heparin during hemodialysis elevates this risk to the hemorrhagic form of pericarditis. These risk factors along with active DAPT resulted in our patient's recurrent hemorrhagic pericarditis. Poor prognostic indicators include worsening anemia, volume overload state, and rapid accumulation of pericardial effusion. Close monitoring with serial CBCs and TTEs is recommended. Treatment of acute pericarditis involves NSAIDS as first line therapy and colchicine as adjunct therapy to improve symptoms and decrease recurrence. For recurrent pericarditis, the NSAIDs-colchicine combination can be effective as well. However, those refractory to NSAID therapy may warrant a short course of high dose glucocorticoids to be slowly tapered in conjunction with a prolonged course of colchicine Glucocorticoids are not recommended in one episode of acute pericarditis due to an increase recurrence rate of pericarditis. Additionally, ESRD patients on active DAPT should avoid NSAIDS and be switched to a heparin-free dialysis protocol to prevent the hemorrhagic type of recurrent pericarditis. Colchicine should also be encouraged as it reduces recurrences of uremic pericarditis. Timely recognition of pericarditis in ESRD patients may not aid in prevention but will certainly reduce costly and harmful interventions. Of importance is the clinical history with consideration of patients' risk factors and assessment of diagnostic needs, including EKG and TTE. Further distinction between acute pericarditis and recurrent pericarditis in ESRD patients is essential in the proper management of this potentially fatal condition.

\section{RECCURENT THROMBOTIC THROMBOCYTOPENIC PURPURA IN A POST SPLENECTOMY PATIENT Preeti Patel. UICOMP, Peoria, IL. (Control ID \#2466016)}

LEARNING OBJECTIVE \#1: A high index of suspicion for recurrence of thrombotic thrombocytopenic purpura should be maintained in any patient with a previous history of TTP, including those who are status post splenectomy.

LEARNING OBJECTIVE \#2: Patients may present with thrombotic thrombocytopenic purpura early in the disease course with only minor signs and symptoms such as gingival bleeding. These patients frequently present to their primary care physician's office. Physicians should be aware that early recognition and prompt imitation of steroids and plasmapheresis will decrease mortality.

CASE: A 54-year-old female presented to the emergency department with gingival bleeding, hematuria and bilateral lower extremity petechial rash. A few days prior, she presented to a prompt care with concerns of prolonged gingival bleeding after brushing her teeth. She was offered supportive care and a follow up appointment in 1 week if she had no improvement. Within $24 \mathrm{~h}$, she developed gross hematuria followed by a petechial lower extremity rash prompting her to visit the emergency department. She denied any headaches, recent changes in mental status or any recent infections. Her past medical history was significant for idiopathic thrombocytopenic purpura diagnosed in 1995, thrombotic thrombocytopenic purpura (TTP) and acute cerebrovascular attack diagnosed in 1997, and a recurrent attack of thrombotic thrombocytopenic purpura which resulted in a splenectomy in 1999. She also has a history of hyperthyroidism, and hyperlipidemia. Patient has not had any issues with platelets or symptoms since her splenectomy in 1999. Family history was not significant. Physical exam at the time of admission revealed a well appearing, alert and oriented female. She had a fine petechial rash on the bilateral lower extremities and neurological exam was intact without focal deficits. Initial laboratory studies showed elevated reticulocyte count, lactate dehydrogenase, indirect bilirubin and fibrinogen. Initial platelet count was 17 and she had decreased haptoglobin prompting urgent treatment with steroids while awaiting further testing. Peripheral smear revealed multiple schistocytes. Imaging of her abdomen revealed evidence of her previous splenectomy and lack of accessory spleen formation. Hematology/oncology as well as nephrology were consulted, who agreed the patient was likely experiencing a repeat occurrence of thrombotic thrombocytopenic purpura. Interventional Radiology was then consulted for temporary dialysis catheter placement and the patient began plasmapheresis. In the meantime, the patient's ADAMTS13 activity returned low. Patient improved and platelets increased to 127 at time of discharge. Her steroids were continued on discharge as a prolonged taper. Patient was followed closely by Hematology and Nephrology.

DISCUSSION: This case illustrates the potential for recurrence of TTP in patients with a history of TTP, including those who have previously undergone splenectomy. Although post splenectomy patients were previously presumed to have a lower risk of recurrence, new studies show this may not be the case. Prompt initiation of plasmapheresis and steroids are the cornerstones of therapy to decrease morbidity and mortality. There is no consensus regarding the benefit of splenectomy in patients with recurrent TTP and risks outweigh potential benefits. These include overwhelming infection with encapsulated organisms and abdominal vein thrombosis, which prompt the need for postoperative prophylactic vaccination, thromboembolism prophylaxis, and patient education. This case also provides insight on the spectrum of presentation of TTP. Thrombotic thrombocytopenic purpura is characterized classically by the presence of fever, anemia, thrombocytopenia, renal insufficiency, and neurological changes ranging from headache to coma However, it exists on a spectrum from mild signs and symptoms to fulminant TTP with coma. Many patients with milder initial manifestations may present to their primary care physician's office or prompt care where the diagnosis may be missed. Clinicians in ambulatory care facilities should also keep a high index of suspicion they may be seeing a patient present with early signs and symptoms of TTP, and obtain platelet counts and a peripheral smear.

RECURRENT DELAYED TRALI Andrei Yankovich ${ }^{1}$; Tatiana McKenna ${ }^{2}$; Stephen Tieku ${ }^{3}$. ${ }^{1}$ Capital Health Regional Medical Center, Levittown, PA; ${ }^{2}$ Capital Helath Regional Medical Center, Hopewell, NJ; ${ }^{3}$ Capital Health, TRENTON, NJ. (Control ID \#2449575)

LEARNING OBJECTIVE \#1: Clinicians need to recognize this condition, as it has high prevalence in critically ill patients and carries a high mortality risk.

LEARNING OBJECTIVE \#2: A patient with history of TRALI requiring blood transfusion needs special attention; blood products should have minimal length of storage, and transfusion should be performed using leukocyte filters. Conservative transfusion practices, such as using the cut off for $\mathrm{Hb}$ of less than $7 \mathrm{mg} / \mathrm{dl}$ for non-cardiac patients, may decrease transfusion reaction rates

CASE: A 48 year old Caucasian male with history of Type 2 Diabetes, ESRD, testicular cancer, and polysubstance abuse was admitted to the hospital with sepsis secondary to legs cellulitis. On day 2, he was transfused with 2 Units of PRBC's for a hemoglobin of $7.0 \mathrm{mg} /$ dL. Patient's condition was relatively stable, with no leukocytosis or fever, but 5 days later, he developed acute respiratory failure with picture of ARDS. There was no cardiomegaly 
on $\mathrm{CXR}$ and $\mathrm{PO} 2 / \mathrm{FiO} 2122$, and patient required mechanical ventilation with PEEP of 8 . Echocardiogram showed EF $55 \%$ with impaired diastolic relaxation. The patient received supportive treatment including IV methylprednisolone. On day 3 after intubation the patient showed significant improvement and on a day 4 was successfully extubated. The patient was discharged with diagnosis of noncardiogenic pulmonary edema. Two months later, the patient was readmitted to the hospital with left foot gangrene and leg cellulitis. He was treated with vancomycin, aztreonam, and metronidazole and had great toe amputation. After the surgery, the patient required PRBC transfusion. He was relatively stable without fever. Three days later, the patient developed respiratory failure and ARDS picture without cardiomegaly on CXR, PO2/FiO2 112, and NT-proBNP 6191. Initially he received trial of diuretics but later required mechanical ventilation with PEEP of 8 . The patient responded well to supportive treatment including IV steroids, and on a day three after intubation was successfully weaned from ventilator.

DISCUSSION: The classic TRALI syndrome is a rare condition characterized by the acute onset of respiratory failure within hours of the transfusion of a blood product. The transfusion of blood products in critically ill patients increases the risk for the development of lung injury 6-72 $\mathrm{h}$ after the transfusion. The syndrome known as a "Delayed TRALI" is occurs in up to $25 \%$ of critically ill patients receiving a blood transfusion, and is associated with a mortality of up to $40 \%$. "Delayed TRALI syndrome" is more common than we thought. The risk increases as the number of transfused blood products increase Leukodepletion may reduce the incidence of delayed TRALI as leukocyte reduction has been demonstrated to reduce the accumulation of biologically active mediators in stored blood. Recipient's antiplatelet antibody and the length of blood storage before transfusion may increase the risk of developing delayed TRALI

RECURRENT HYPOGLYCEMIA NEEDS TO BE EVALUATED FOR INSULINOMA Md U. Ali ${ }^{2}$; Mohammed AL-Jumayli ${ }^{2}$; Daniel Eichorn ${ }^{1}$; Agustin Busta ${ }^{3}$; Phaniram Sumanam ${ }^{1} .{ }^{1}$ Capital Health Regional, Hamilton, NJ; ${ }^{2}$ Capital Health Regional Medical Center, Plainsboro, NJ; ${ }^{3}$ Mount Sinai Beth Israel, New York, NY. (Control ID \#2468867)

LEARNING OBJECTIVE \#1: Recurrent hypoglycemia presenting as a seizure in a patient underscores the need to assay insulin, $\mathrm{C}$ peptide, and proinsulin

LEARNING OBJECTIVE \#2: The essential aspect of the diagnosis of insulinoma is a high index of clinical suspicion in the setting of unexplained recurrent hypoglycemia, and it is associated symptoms

CASE: A 65-year-old man with a history of ulcerative colitis and hypertension presented with seizures in the setting of severe hypoglycemia. Workup included a CT abdomen that showed multiple liver masses in both lobes of the liver. Blood sugar, Proinsulin, Insulin and C- peptide were 32, 83.2 (normal <18), 32.6 (normal <29), 12 (normal <3.1) U/ml respectively. Liver biopsy showed moderately differentiated carcinoma with polygonal cells growing in nests, suggestive of neuroendocrine origin. Immunohistostaining for keratin 7, CD56, synaptophysin, and chromogranin were positive suggesting a diagnosis of malignant insulinoma. Chemotherapy treatment with everolimus was considered; however, given the burden of disease patient underwent immediate hepatic artery embolization. The patient eventually had subsequent resolution of the hypoglycemia, and it is related symptoms

DISCUSSION: Most episodes of recurrent hypoglycemia occur in patients with diabetes mellitus and can be prevented by changes in medication, diet, or activity. However, persistent, unexplained hypoglycemia in the absence of diabetes can indicate a potentially grave, often treatable, underlying disorder, such as insulinoma. It is a rare pancreatic islet cell tumors, with an incidence of 4 cases per million person-years. Most insulinomas are benign whereas multiple insulinomas are usually associated with MEN1. Malignancy is rare with the liver and regional lymph nodes being the most common sites of metastasis.
Although Malignant insulinoma, a very rare variant, needs to be identified early and differentiated from more common benign variant as the management of both the entity is entirely different. Most patients present with characteristic symptoms such as hypoglycemia, weakness, blurred vision, palpitations though the loss of consciousness and seizures is described. The fasting plasma levels of insulin, C-peptide, and, to a lesser degree, proinsulin are elevated. Imaging techniques are then used to localize the tumor, most frequently transabdominal or endoscopic ultrasound, CT, MRI, or somatostatin receptor scintigraphy. Medical treatments of insulinomas include diazoxide and octreotide. Resection of insulinoma is often curative in benign cases, but metastasis reduces survival rates by $60 \%$. Hepatic artery embolization, radiofrequency ablation, and hepatic resection can be tried in hepatic metastasis.

RECURRENT SINUSITIS MAY NOT ACTUALLY BE SINUSITIS Mohammad Jawish $^{2}$; Robert Asbury ${ }^{1}$; Yazan Samhouri ${ }^{1} .{ }^{1}$ Rochester Regional Health/Unity Hospital, Rochester, NY; ${ }^{2}$ Unity Hospital, Rochester, NY. (Control ID \#2469688)

LEARNING OBJECTIVE \#1: To recognize the need to broaden the differential after failed therapy for sinusitis in a patient with prior Non Hodgkin lymphoma (NHL). CASE: A 74 years old female with the past medical history of Chronic Lymphocytic Lymphoma (CLL) was treated with two cycles of Bendamustine 2 years ago. She presented to the ED with nasal congestion, sinus headache, and post-nasal drip for the last 3 weeks. Patient had multiple similar attacks in the last couple months, and she was treated with Cephalexin, Amoxicillin, and Prednisone without complete relief of her symptoms. Her physical exam showed a low grade fever of $37.5 \mathrm{C}$, sinus tachycardia, cervical lymphadenopathy and right deviation of her intranasal septum. Her CBC and chemistry were unremarkable. Patient was started on intravenous (IV) fluid, Methylprednisolone, and IV Moxifloxacin. A sinus CT showed moderate to severe mucosal thickening of the bilateral ethmoid, maxillary, and sphenoid sinuses. Patient was symptomatically improved with the steroid, so she was discharged home on oral Cetirizine and nasal fluticasone with instructions to follow up with an Otolaryngologist. She had an outpatient nasal diagnostic endoscopy that showed a lobulated soft tissue filling the nasopharynx. A biopsy was performed and the patient was diagnosed with low grade B-cell lymphoma with positive CD20, CD19, and CD5, and negative for CD23. Patient had a CT scan of chest/abdomen/pelvic that revealed mildly enlarged axillary lymph nodes and interval progression of mesenteric, retroperitoneal, and iliac chain lymphadenopathy. Treatment with Bendamustine and Rituxan was instituted.

DISCUSSION: Lymphoma is a malignant neoplasm of lymphocytes. The two major classes of lymphoma are Hodgkin disease which typically arises in the lymph node, and Non-Hodgkin lymphoma (NHL) which presents as extranodal disease. NHL may be a B-cell lymphoma or a T-cell Lymphoma. The most common B-cell lymphomas are diffuse large B-cell lymphoma, follicuar lymphoma, CLL/small lymphocytic lymphoma, mantle cell lymphoma and marginal zone lymphoma. NHL can involve any organ, however the gastrointestinal tract is the most frequent extranodal site. Sinonasal tract involvement is rare in the Western World, comprising only $1.5 \%$ of all cases, and the lymphoma of primary paranasal sinus made up only $0.17 \%$ of all lymphomas in the Kiel Lymph Node Registry. Sinonasal tract involvement with NHL typically presents with nasal obstruction, epistaxis, nasal discharge and recurrent sinusitis. Radiologically, NHL appears like a mass, cloudy, or opaque sinus due to infiltrative growth and may cause prominent bony erosion of the sinus wall. Treatment options vary from radiation therapy, chemotherapy, or combination therapy. Surgical resection is a possibility in bulky disease for symptom control. This case shows the importance of identifying NHL as a possible cause for persistent sinusitis in patients with a previous history of CLL. Our patient suffered from her symptoms for a few months before the cause of her problems was recognized. 
REFUGEES FROM ERITREA TO SWITZERLAND: COMMON SKIN INFECTIONS DUE TO PANTON-VALENTINE LEUCOCIDIN-PRODUCING STAPHYLOCOCCUS AUREUS STRAINS. Laure Jaton ${ }^{2}$; Trestan Pillonel ${ }^{1}$; Katia Jaton $^{1}$; Elodie Dory ${ }^{2}$; Guy Prod'hom ${ }^{1}$; Dominique Blanc ${ }^{1}$; Frédéric Tissot ${ }^{1}$; Gilbert Greub $^{1}$; Patrick Bodenmann ${ }^{2} .{ }^{1}$ University hospital of Lausanne, Lausanne, Switzerland; ${ }^{2}$ Department of Ambulatory Care and Community Medicine, University of Lausanne, Lausanne, Switzerland. (Control ID \#2461072)

LEARNING OBJECTIVE \#1: Europe is facing the largest number of refugees since World War II. Between January and October 2015, 427'000 refugees, mainly Syrians, reached Greece and $132^{\prime} 000$, mainly Eritrean, reached Italy. Eritrea is the main country of origin of refugees arrived in Switzerland since 2012. During 2014 and 2015, the University Hospital of Lausanne faced an increasing number of cases of dermatological infections in the Eritrean refugee population. The hospital is located in an area named "Canton of Vaud", which is in charge of $10.5 \%$ of the refugees in Switzerland. Infections were due to $S$. aureus producing the Panton-Valentine leucocidin. The lesions were often multiple and recurrent abscesses or boils appearing after scabies infections. Most of them required drainage and some of them the administration of different antibiotics. PVLproducing $S$. aureus strains are known to be responsible for severe abscedizing skin infections, necrotizing pneumonia and severe osteomyelitis. Instead of the USA were the majority of community-associated methicillin-resistant Staphylococcus aureus (CAMRSA) are PVL positive, in Europe, less than $5 \%$ of the recovered $S$. aureus strains are PVL producers and most of them are susceptible to methicillin (MSSA). The spread of many epidemic infectious diseases is influenced by immigration (tuberculosis, HIV, relapsing fever due to $B$. recurrentis, scabies), but to our knowledge, such common occurrence of PVL-producing MSSA has not been yet described in migrant populations The aim of the present study was to investigate the diversity and origin of isolated PVL positive S. aureus strains, and to determine by whole genome sequencing (WGS) whether this high prevalence of $S$. aureus skin infections results from an outbreak.

CASE: The study population consisted in all the patients from whom a $S$. aureus PVL producer strain was isolated from January 2014 to July 2015 (15 cases). We used WGS to identify if all strains were identical or not and we checked for the presence of different kind of toxins and adhesins encoding genes; in addition, a phylogenic tree was reconstructed based on core genome single nucleotide polymorphisms (SNPs). There was 14 MSSA and 1 MRSA infections. 14/15 PVL-positive patients exhibited a skin infection (abscess, boil, adenitis or cellulitis), and 1 presented a necrotising pneumonia. Five patients needed to be hospitalized, and drainage of the lesions was required in 13 cases. In 9/15 cases, amoxicillin and clavulanate (co-amoxicillin) have been used as empirical treatment and in 4 of those cases, clindamycin had to be introduced because of an unfavourable evolution with co-amoxicillin alone. SNPs and phylogenetic analyses based on whole genome sequences showed that 8 cases (all MSSA strains) were due to MLST Sequence Type (ST) 152, 3 from ST15 and 4 from ST 121, 72, 80 and 5 . The 8 cases infected by ST152 strains include 4 Eritreans men, one Ethiopian woman, one Swiss man living with his Helveticoethiopian daughter, one Swiss female working with migrant people and married to a Rwandan man and one Portuguese child with a Cape Verdean mother. The 8 ST152 strains and 3 ST15 strains exhibited a few hundred of SNPs, indicating that those strains did not originate from a single clone.

DISCUSSION: Informations about prevalence and type of S. aureus endemic in Africa are scarce but the studies of Ruimy et al. in Mali and Breurec et al. in Cameroon, Niger and Senegal showed that ST 152 and ST 15 are frequent in West Africa. This work suggests that these 2 subtypes are also widespread in East Africa and that our patients have been infected by different strains of the 152 and 15 subtypes, respectively. We can not completely exclude some transmission between refugees or between refugees and the Swiss population because we did not analysed all this large population but only those consulting in our hospital. Nevertheless, the present whole genomic sequences analysis showed that we are not actually facing an outbreak of infection due to PVL-producing S. aureus strains. Moreover, the difficult travelling and living conditions of the refugees, the promiscuity as well as concomitant skin infections such as scabies make them at high risk of S. aureus PVL-producer infection due to the damage to the skin. Thus, in front of an unusual dermatological infection in African refugees, and before searching for an exotic infection such as leishmaniasis, rickettsiosis or even mycobacteria, clinicians must be aware that it could be a $S$. aureus PVL-producer. The clinicians also need to remember that PVL might be produced by a MSSA, especially in Europe or in Africa. Because betalactam antibiotics at sub-inhibitory concentrations can enhance PVL levels whereas antibiotics targeting protein synthesis have been shown to reduce PVL release, the better treatment seems to be an antibiotic acting against the toxin, for example clindamycin or linezolid. Finally, this study showed that whole genomic sequencing is a good promising tool to rapidly assess the risk of facing an epidemic.

\section{RELAPSING THYROTOXICOSIS AFTER TOTAL THYROIDECTOMY IN A} PATIENT WITH GRAVES' DISEASE Charlotte Clark; Colette Knight. Montefiore Medical Center, Wakefield Campus, Bronx, NY. (Control ID \#2469573)

LEARNING OBJECTIVE \#1: Recurrence of hyperthyroidism post-total thyroidectomy is possible in patients with Graves' disease.

LEARNING OBJECTIVE \#2: Close medical follow-up is recommended to ensure sustained resolution of thyrotoxicosis post-total thyroidectomy and should include periodic thyroid function tests and assessment of symptoms and signs that may be suggestive of hyperthyroidism.

CASE: A 60-year-old-woman with Graves' disease complicated by exophthalmos and dermopathy presented for treatment of hyperthyroidism. Her hyperthyroidism remained refractory to methimazole after 1 year. Fine-needle aspiration of a left cold thyroid nodule was negative for malignancy and cytology findings were in accordance with a nodular goiter. Total thyroidectomy was advised for definitive management. The patient was treated with levothyroxine $50 \mathrm{mcg}$ daily for post-surgical hypothyroidism. Initial Thyroid Function Tests (TFTs) were normal. Several months after surgery the patient had a decrease in Thyroid Stimulating Hormone (TSH) levels that persisted despite decreasing dosing of levothyroxine. Nine-months post-thyroidectomy the patient presented in endocrine clinic for routine follow-up, where she described worsening palpitations associated with episodic dyspnea and anxiety. She also reported progressive bilateral foot and ankle swelling over 2-months. Her vitals revealed blood pressure of 151/101 and pulse rate of 96 . Mild-asymmetric orbitopathy was noted with proptosis, bilateral lid retraction with left-eye predominance, and mild peri-orbital fullness. A persistent keloid scar was noted over incision site on the anterior neck. A low-amplitude tremor was observed upon extension of hands. Lower extremity examination was significant for bilateral diffuse lymphedema (dorsal toes, dorsal feet, and anterior shins), with extensive nodular and indurated plaques, and peau d'orange appearance over the bilateral pretibial areas. Levothyroxine was discontinued and repeat TFTs after 1 month showed persistent TSH suppression, raising concern for recurrent thyrotoxicosis from a thyroid remnant or ectopic thyroid source. Thyroid whole body uptake and nuclear imaging revealed an intense thyroid remnant and $24 \mathrm{~h} 41 \%$-RAIU, without evidence of ectopic thyroid tissue. The patient was treated with $29 \mathrm{mCI} \mathrm{I-131}$ for remnant-thyroid ablation, resulting in hypothyroidism post-ablation (TSH: 94, FT4: 0.15). Thyroxine replacement was initiated and well tolerated. Dermopathy and ophthalmopathy improved after resolution of thyrotoxicosis.

DISCUSSION: Relapsing hyperthyroidism after total thyroidectomy is rare. However, recurrence after subtotal thyroidectomy and Radioactive Iodine (RAI)-ablation has been described. We present a case of recurrent-thyrotoxicosis requiring RAI-ablation 1 year after total thyroidectomy. Total thyroidectomy is reserved for specific circumstances, and 
is usually recommended for those with longstanding Graves', a diffusely enlarged thyroid, and ophthalmopathy. In such cases, RAI ablation is not recommended as first line treatment due to potential worsening ophthalmopathy and frequent recurrences necessitating multiple treatment sessions. In addition to the adrenergic symptoms, cutaneous and ocular symptoms can worsen in the setting of persistent Graves' thyrotoxicosis. Due to the heterogeneous treatment response of Graves' hyperthyroidism, we conclude that close follow-up following surgical and RAI ablation therapy is essential to avoid a potentially life-threatening recurrence of thyrotoxicosis.

REMEMBERING A FORGOTTEN DISEASE Justin S. Louie. Kaiser Permanente San Francisco, Berkeley, CA. (Control ID \#2469943)

LEARNING OBJECTIVE \#1: Recognizing Lemierre's syndrome as an uncommon, but increasingly prevalent disease with significant morbidity and mortality, necessitating high clinical suspicion and rapid diagnosis

LEARNING OBJECTIVE \#2: Discussing the core treatment principles and management of these patients

CASE: A 23 year old man with no significant medical history presented with 1 week of body aches, chills, cough, sore throat, and fevers. Initial labs were notable for a WBC count of 14.9 and lactate of 3.5. His chest x-ray revealed extensive infiltrates, and follow up CT scan showed bilateral opacities. He was started on IV vancomycin and piperacillin/ tazobactam for suspected multi-lobar pneumonia. Blood cultures from admission grew anaerobic gram negative rods, with speciation to Fusobacterium necrophorum, and his antibiotics were changed to IV ampicillin/sulbactam. Neck ultrasound and subsequent neck CT revealed non-occlusive thrombophlebitis of the right internal jugular vein (IJV) and right external jugular vein, leading to the diagnosis of Lemierre's Syndrome. His care was managed by a multidisciplinary team including infectious disease, head and neck surgery, and vascular surgery. Anticoagulation was deferred in lieu of monitoring with daily ultrasounds to track clot length. His course was complicated by hypoxic respiratory failure, requiring continuous high flow oxygen, and septic myelosuppression; both of which resolved after 1 week of IV antibiotics and supportive care. He was discharged with oral metronidazole to complete a 6 week total antibiotic course. During his follow up appointment 2 weeks later, he showed clinical improvement, with only occasional neck pain, and marked improvement on his chest x-ray.

DISCUSSION: Lemierre's syndrome, also known as jugular vein suppurative thrombophlebitis, is a rare condition. It often affects previously healthy young adults and is characterized by a primary oropharyngeal infection, followed by metastatic spread and suppurative thrombophlebitis of the carotid sheath vessels, classically the IJV. Though once relatively common, the number of cases has declined since the discovery of antibiotics. However, there are now concerns about a reemergence of this condition, thought mostly due to antibiotic resistance and decreased antibiotic prescription for minor oropharyngeal conditions. This increasing prevalence, along with an unfamiliarity of many clinicians with the classical features of this "forgotten disease", risks misdiagnosis or delay in identifying this potentially fatal illness. Lemierre's syndrome carries a mortality of over $90 \%$ if untreated; thus, early diagnosis and prompt treatment initiation is vital. Patients typically present with acute fever, rigors, respiratory distress and localized neck and/or throat pain. Septic emboli to the lungs, as seen in our patient, occur in up to $97 \%$ of cases. Metastatic infectious seeding of the organs, soft tissue, joints, and bones is also possible. CNS involvement is uncommon, but not unheard of, and carries an even worse prognosis. Causative organisms are usually normal oropharyngeal flora, most commonly Fusobacterium necrophorum. Other etiologies include primary dental infection, infectious mononucleosis, and IV catheter insertion. The diagnosis of this condition is made with consistent microbiology and identification of jugular vein thrombophlebitis on CT scan or ultrasound. Unfortunately, given the rarity of this condition, there have been no randomized trials done to help guide management. That being said, current treatment principles include prompt IV antibiotic administration and consideration for surgical intervention and/or anticoagulation. Empiric antibiotic therapy includes a beta-lactamase resistant antibiotic for at least 4 weeks. Although ligation or resection of the IJV was common before antibiotics, surgical intervention is now generally only favored with refractory sepsis, abscess formation, rapid clot extension, or ongoing septic emboli despite antibiotic therapy. The role of anticoagulation in patients with Lemierre's syndrome remains controversial. Proponents argue that anticoagulation prevents clot extension, increases penetration of antibiotics, and hastens resolution of the thrombus. Arguments against anticoagulation cite the inherent risks of therapy, especially as sepsis related thrombocytopenia is not uncommon, as well as a potential for worsening metastatic spread of septic emboli. This case is a classic presentation of Lemierre's syndrome. It illustrates the rapidity of clinical decline seen in these patients, and therefore highlights the need for high clinical suspicion from physicians, as well as aggressive and multidisciplinary management. Lemierre's should be suspected in patients with antecedent pharyngitis, septic pulmonary emboli, and persistent fever despite antimicrobial therapy. Appropriate and prompt institution of antibiotics remains the mainstay of therapy, with surgical drainage where indicated. Though there are no clear guidelines, a review of the literature notes that there is an unofficial consensus that anticoagulation is indicated in certain circumstances, such as a lack of response despite adequate antimicrobial therapy, underlying thrombophilia, or potential for retrograde progression to the cavernous sinus. Regardless, during therapy, close observation for signs of continued sepsis, propagation of thrombus, or septic emboli is vital.

REMISSION OF ADULT ONSET TYPE 1 DiABETES IN A PATIENT PRESCRIBED IMMUNE MODULATING THERAPY Scott J. Pilla ${ }^{1}$; Amy Q. Quan $^{3}$; Emily L. Germain-Lee ${ }^{2}$; David B. Hellmann ${ }^{4}$; Nestoras N. Mathioudakis ${ }^{4}$. ${ }^{1}$ Johns Hopkins University, Baltimore, MD; ${ }^{2}$ Johns Hopkins University School of Medicine/Kennedy Krieger Institute, Baltimore, MD; ${ }^{3}$ Johns Hopkins School of Medicine, Baltimore, MD; ${ }^{4}$ Johns Hopkins Medicine, Baltimore, MD. (Control ID \#2467783)

LEARNING OBJECTIVE \#1: Recognize type 1 diabetes as a relapsing remitting disease with changes in disease activity that could lead to hypoglycemia.

LEARNING OBJECTIVE \#2: Define common medications used to treat autoimmune disease that can cause changes in glycemic control.

CASE: The patient is a 55 year old woman with adult onset diabetes mellitus type 1 who presented to the hospital with vomiting and hypoglycemia. She is a pediatric endocrinologist who has a 30-year history of systemic lupus erythematosus (SLE) which was well controlled on hydroxychloroquine until it was discontinued 4 years ago due to retinal toxicity. She then suffered an SLE flare requiring multiple therapy adjustments until achieving control on a regimen of mycophenolate mofetil and prednisone. During the SLE flare, the patient was diagnosed with type 1 diabetes after a rapid rise in blood glucose and positive levels of anti-GAD65 and anti-insulin antibodies. She was transitioned to therapy with an insulin pump 1 year prior to admission. In the weeks preceding admission, the patient noted her insulin needs were steadily decreasing to less than half her usual dose. The night prior to admission she awoke at midnight to her hypoglycemia alarm: her glucose was $65 \mathrm{mg} / \mathrm{dL}$. She tried drinking juice, taking glucose tabs, and eating, but was not able to increase her glucose. Shortly thereafter she became nauseated and had several episodes of vomiting food particles. She called her providers who advised she come to the hospital. On evaluation, the patient's heart rate was $108 \mathrm{bpm}$, blood pressure 168/ $87 \mathrm{mmHg}$, temperature $37.6{ }^{\circ} \mathrm{C}$, respiratory rate $18 \mathrm{bpm}$. She was alert, oriented, and appeared uncomfortable due to nausea. Her abdomen was soft, non-tender, with active bowel sounds and no rebound tenderness. The remainder of the exam was unremarkable, 
and her insulin pump was functioning normally. Initial laboratory tests were notable for mild neutrophilic leukocytosis, blood glucose $195 \mathrm{mg} / \mathrm{dL}$, and no urine ketones. Fasting C-peptide was $2.78 \mathrm{ng} / \mathrm{mL}$ (range $0.80-3.10 \mathrm{ng} / \mathrm{mL}$ ), GAD65 antibody was $4.3 \mathrm{U} / \mathrm{mL}$ from a previous value of $19.6 \mathrm{U} / \mathrm{mL}$ (range $\leq 1.0 \mathrm{U} / \mathrm{mL}$ ), and insulin autoantibody was undetectable from a previous value of $0.4 \mathrm{U} / \mathrm{mL}$ (range $<0.4 \mathrm{U} / \mathrm{mL}$ ). The patient was treated with antiemetics, IV fluids, and a single dose of hydrocortisone given concern for adrenal suppression. Nausea and vomiting resolved by morning. She was observed for $24 \mathrm{~h}$ off all insulin and remained normoglycemic without developing ketosis. The diagnosis was acute gastroenteritis and remission of type 1 diabetes. Over the next 4 weeks her insulin requirements gradually returned to near pre-remission levels.

DISCUSSION: This case demonstrates the importance of recognizing the distinct natural history of type 1 diabetes. Incident type 1 diabetes can occur at any age with approximately one fourth of patients diagnosed as adults. ${ }^{1}$ Unlike type 2 diabetes which is usually relentlessly progressive, the course of type 1 diabetes is more fluid. After diagnosis, half of patients experience a temporary decrease in insulin needs called the honeymoon phase. This usually occurs within 6 months of starting insulin, but may happen years later. The patient in this report presented with a normal C-peptide level and absent ketosis demonstrating a return of pancreatic function, which is characteristic of the honeymoon phase. It is possible that this patient's remission was related to insulin initiation months prior, but also to the change in her therapy for SLE. Medications used to treat autoimmune disease can change the course of type 1 diabetes. This patient developed type 1 diabetes after stopping hydroxychloroquine, then entered a remission while on corticosteroids and mycophenolate mofetil. Corticosteroids can have paradoxical effects: they worsen insulin resistance, but may induce remission of type 1 diabetes, as can other immunosuppressive agents. $^{2}$ The immunomodulators hydroxychloroquine and etanercept are unique in that they have a strong antihyperglycemic effect in type 1 diabetes as well as type $2 .^{3}$ It is important to note that as many as $12 \%$ of adults diagnosed with type 2 diabetes may have an autoimmune component to their disease, and it is not known how immunomodulatory agents will affect this subgroup. Therefore, providers should recognize changes in a patient's immune modulating regimen as a high risk period for diabetes care for all diabetic patients at risk for hypoglycemia. The patient in this report had many resources to prevent hypoglycemia including a continuous glucose monitor and her experience as a pediatric endocrinologist. Nevertheless she found insulin management challenging during the remission. This highlights the importance of educating patients to notify providers of falling insulin requirements which could herald a coming remission and need for more active management. References 1. Maahs DM, West NA, Lawrence JM, Mayer-Davis EJ. Epidemiology of type 1 diabetes. Endocrinol Metab Clin North Am. 2010; 39(3):481-97. 2. Silverstein J, Maclaren N, Riley W, Spillar R, Radjenovic D, Johnson S, Immunosuppression with azathioprine and prednisone in recent-onset insulin-dependent diabetes mellitus. N Engl J Med. 1988; 319(10):599-604. 3. Gerstein HC, Thorpe KE, Taylor DW, Haynes RB. The effectiveness of hydroxychloroquine in patients with type 2 diabetes mellitus who are refractory to sulfonylureas-a randomized trial. Diabetes Res Clin Pract. 2002; 55(3):209-19.

RESPIRATORY DISTRESS? DON'T FORGET DRESS! Christopher Su; Jason A Korcak. Montefiore Medical Center, New York, NY. (Control ID \#2469232)

LEARNING OBJECTIVE \#1: Describe the pulmonary presentation of DRESS syndrome.

LEARNING OBJECTIVE \#2: Recognize DRESS syndrome as an important consequence of drug allergy.

CASE: A 43-year-old morbidly obese woman with a past medical history of mild intermittent asthma presented with severe shortness of breath at rest for 1 day. She also noted a significant decrease in exercise tolerance, chest tightness, and a non-productive cough. The patient was discharged 2 days prior following a complicated hospitalization for breast abscess. Four weeks prior, the patient began a course of vancomycin for treatment of the staphylococcal abscess. Three weeks after initiation of the antibiotic, she developed diffuse macular erythema over her entire body complicated by hypotension and fever to $39.5^{\circ} \mathrm{C}$. Vancomycin was immediately discontinued and the patient was discharged 3 days later with topical steroid cream and outpatient dermatology and allergy follow-up for presumed drug rash. On this presentation, she was readmitted with a respiratory rate of $33 \mathrm{bpm}$ and an arterial blood gas demonstrating respiratory acidosis with $\mathrm{pH} 7.13$ and pCO2 $52 \mathrm{mmHg}$. Skin exam revealed diffuse macular erythema with desquamation, and lung exam was notable for bilateral crackles. WBC was $20,000 /$ microL (4700/microL lymphocytes and 500/microL eosinophils), kidney and liver function tests were normal, and blood cultures were negative. A chest CT demonstrated moderate bilateral pleural effusions, interstitial infiltrates, and enlarged bilateral axillary lymph nodes. The patient was initially treated for asthma exacerbation and possible pneumonia. However, given the patient's recent constellation of findings, including fever, rash, hypotension, lymphocytosis, and eosinophilia, as well as new respiratory distress with lymphadenopathy, the diagnosis of drug reaction with eosinophila and systemic symptoms (DRESS) was made. The patient was started on a prednisone taper with progressive improvement in symptoms.

DISCUSSION: DRESS syndrome is an uncommon, but serious complication of drug allergy. Clinical diagnosis of DRESS syndrome requires three of the following major criteria: acute rash, fever above $38^{\circ} \mathrm{C}$, lymphadenopathy, internal organ involvement, and hematologic abnormalities including eosinophilia. Symptoms typically begin 2 to 6 weeks following drug initiation; in our patient, symptoms started 3 weeks after exposure. Major organ systems affected include hepatic (60-80\% of total cases), renal (10-30\%), and pulmonary (5-25\%). Diagnostic testing for DRESS is limited, but patch testing may be helpful to identify the culprit drug. Medications most commonly implicated in DRESS include allopurinol, aromatic anticonvulsants, and dapsone. However, vancomycin has been reported in several studies to be a precipitant of DRESS syndrome. Current treatment is supportive with immediate cessation of the offending medication. A course of systemic corticosteroids is recommended for patients with severe pulmonary and/or renal involvement. Mortality rate of DRESS is reported at approximately 5-10\%, most often due to acute liver failure or multiorgan failure. In this case, the patient presented with respiratory distress suggesting significant pulmonary involvement of DRESS syndrome. Although hepatic and renal presentations of DRESS are more common, clinicians should consider DRESS when pulmonary distress develops in the setting of recent drug reaction.

\section{REVIEWING THE EXPANDING NATURE OF ACHROMOBACTER INFECTIONS: A CASE REPORT OF BACTEREMIC ACHROMOBACTER XYLOSOXIDANS CELLULITIS IN AN IMMUNOCOMPETENT HOST Tatvam T. Choksi ${ }^{1}$; William B. McNamee ${ }^{2}$; Ted Martynowicz ${ }^{3}$. ${ }^{1}$ Mercy Catholic Medical Center, Lansdowne, PA; ${ }^{2}$ Mercy Catholic Medical Center, Darby, PA; ${ }^{3}$ Coatesville VA Medical Center, Coatesville, PA. (Control ID \#2457004)}

LEARNING OBJECTIVE \#1: Achromobacter xylosoxidans has generally been considered as an organism with low virulence potential. Traditionally, it has been associated with hospital acquired infections related to infected solutions, or a pathogen associated with bacteremia in immunocompromised patients. It has recently been isolated from sputum of cystic fibrosis patients. The organism also has been reported as a cause of soft tissue infection involving the existing wounds. But it has been rarely, if ever, associated with primary skin and soft tissue infections in immunocompetent patients. Here we describe a unique case of acute bacterial cellulitis caused by A. xylosoxidans in a previously healthy male patient.

CASE: 46 year old morbidly obese male with chronic venous insufficiency presented with acute onset left lower extremity pain and swelling. The patient was in his usual state 
of health the night before; but woke up in the morning with severe pain in left lower extremity associated with subjective fevers and chills. His leg became very red and warm over several hours. He was not able to bear weight on it. Vital signs were noticeable for temperature of $39.1^{\circ}$ Celsius, and heart rate of 110 beats per minute. Physical exam of his left lower extremity (LLE) revealed $2+$ pitting edema with erythema, warmth and moderate tenderness. Right lower extremity examination revealed $1+$ pitting edema and chronic venous stasis changes without any tenderness. He had inter-triginous disease in bilateral lower extremities. Laboratory data was remarkable for white blood cell count of 18,000 . Ultrasound lower extremity was negative for any deep venous thrombosis. Given patient's presentation was suggestive of acute bacterial cellulitis, he was started initially on intravenous Vancomycin, which was later changed to intravenous Ceftriaxone. But high grade fevers continued and there was minimal improvement in LLE signs. Blood culture from aerobic bottles grew gram negative rods which later on were identified as 'Achromobacter xylosoxidans' (VITEK 2 GN Card/ BioMerieux, Durham, NC)! The organism was sensitive only to Trimethoprim-sulfamethoxazole and Piperacillintazobactum. Antibiotic was changed to intravenous Piperacillin-tazobactum. The patient rapidly became afebrile with improvement in LLE signs. Follow up visit showed consistent improvement and the patient returned back to his baseline level of activities.

DISCUSSION: This case represents the first case of community-acquired bacteremic cellulitis in a non-immunocompromised host due to Achromobacter xylosoxidans. A recent review, from Spain, of skin and soft tissue infections associated with Achromobacter found that most of their cases were related to previous trauma, surgery or vascular disease. In addition, most of these patients had severe underlying diseases and in around $50 \%$ of cases, the infection was polymicrobial. In a prior large scale review of the cases of Achromobacter bacteremia, only 1 of 77 cases attributed the bacteremia to a soft tissue infection and the suffering patient had severe systemic lupus erythematosus. Interestingly, many of these cases were associated with secondary metastatic skin infections. Our patient was an uncommon host as he had no underlying disease or existing wound and presented with the typical presentation of cellulitis with fever and local signs of inflammation in his leg. Blood cultures grew Achromobacter xylosoxidans and he didn't clinically respond until antibiotics active against this organism were administered. This case further contributes to the expanding spectrum of infections due to Achromobacter xylosoxidans

RHABDOMYOLYSIS AND COMPARTMENT SYNDROME IN AN OPIATE OVERDOSE Daniel Edwards. Tulane University Health Sciences Center, New Orleans, LA. (Control ID \#2467677)

LEARNING OBJECTIVE \#1: Recognize rhabdomyolysis as an important cause of renal failure in opiate overdose cases.

LEARNING OBJECTIVE \#2: Appreciate the importance of rapidly identifying compartment syndrome in obtunded patients.

CASE: A previously healthy 21 -year old man was found unresponsive and cyanotic in a hotel room after reportedly participating in recreational intravenous drug use. Naloxone was administered with marginal results. He was transported to a local emergency department where he was vomiting and subsequently intubated for airway protection. Admission labs were significant for hyperkalemia, lactic acidosis, cardiac troponemia, transaminitis, elevated creatinine, and creatinine kinase above the level of detection. Urine drug screen was positive for opiates and barbiturates. Chest $\mathrm{x}$-ray was significant for right lower lobe airspace opacities consistent with aspiration pneumonia. EKG showed peaked T-waves but no ST-segment elevations. Echocardiography was significant for decreased leftventricular ejection fraction, as well as global hypokinesis without regional wall motion abnormalities. Cardiorespiratory stability was achieved in the ICU setting and his hyperkalemia was treated medically with calcium gluconate, albuterol, and insulin/ dextrose. Soon after admission, the patient complained of right forearm pain despite sedation with propofol and pain control with IV fentanyl. His right upper extremity rapidly developed tense, non-pitting edema, though remained neurovascularly intact. Surgery was consulted for compartment syndrome and the patient underwent emergent fasciotomy of his forearm. After fasciotomy, the patient improved and was extubated on hospital day three. His cardiac enzymes down-trended and his cardiac function improved to normal. Blood, urine, and sputum cultures remained negative. His hyperkalemia initially improved, but the patient became anuric and required hemodialysis for hypervolemia and hyperkalemia. As of hospital day 14, the patient remained anuric and dependent on hemodialysis with a diagnosis of acute tubular necrosis

DISCUSSION: Opiate use and dependence has been elevated to crisis status in the United States. As such, opiate overdoses have become much more common in the community setting necessitating a need for rapid identification and management of possible complications arising in these patients. While the initial obtundation is often reversible with agents such as naloxone, other acute medical complications must often be addressed. As with any patient that is found unresponsive for an unknown period, rhabdomyolysis should be considered in overdose patients. Rhabdomyolysis represents the syndrome that occurs with myonecrosis and the release of intracellular components of skeletal muscle into circulation. Hallmarks of the disease include elevated creatinine kinase as well as myoglobinuria. As noted above, rhabdomyolysis can importantly lead to critical hyperkalemia and risk for arrhythmia. Other important manifestations include hyperphosphatemia, hypocalcemia, and metabolic acidosis. However, perhaps the most important complication of this disease is acute renal failure, which occurs in up to fifty percent of cases. Depending on the severity of renal injury, this may result in long-term, if not permanent reliance on hemodialysis. Equally important in those who are obtunded is the identification of evolving compartment syndrome. Compartment syndrome is caused by increased pressure from swelling secondary to injury within the finite space defined by fascial compartments of the limb. If this pressure reaches critical levels, the neurovasculature of the limb may be compromised resulting in limb ischemia. While $75 \%$ of compartment syndrome cases involved long bone fracture, almost one quarter are the result of soft-tissue injury without fracture, such the crush injury noted above. Rapid identification and treatment is the initial step in preventing limb ischemia. This is especially important in patients such as ours who cannot reliably report pain, either due to recreational drug use or therapeutic sedation.

\section{RISKS OF ALTERNATIVE MEDICINE: A CASE REPORT OF SEPSIS DUE TO MULTIPLE MSSA SUBCUTANEOUS ABSCESSES CAUSED BY INFECTED ACUPUNCTURE NEEDLES. Kanako Horibe; Shadia Constantine. Teine Keijinkai Hospital, Sapporo, Japan. (Control ID \#2467021)}

LEARNING OBJECTIVE \#1: Recognize that alternative medicine, including acupuncture, may cause harm.

LEARNING OBJECTIVE \#2: List the potential harms of acupuncture, including the risk of infections, in your shared-decision-making discussion.

CASE: A 40-year-old previously-healthy Japanese woman was referred to our hospital due to fever, progressive edema in her lower extremities and lower back pain. She mentioned that she had developed lower back pain 1 month prior to her admission and underwent acupuncture treatments along her back, hip and the posterior side of her lower extremities. Over the following 3-4 weeks, while continuing with the acupuncture treatments, she developed intermittent high grade fevers and chills, progressive edema, and focal areas of pain, redness and induration in her lower extremities. Her back pain progressed, then she visited a local orthopedic clinic and they found one abscess in her left calf. The culture of abscess grew methicillin-sensitive Staphylococcus aureus. She received initial treatment with cefazolin and cefcapene; however, the infection severity progressed so she was referred to our hospital. Her past medical history 
included left partial nephrectomy due to traumatic kidney injury. She drank alcohol, 1 bottle of wine or 1000-1500 $\mathrm{ml}$ of beer daily, and denied smoking or recreational drug use. On physical examination, her vital signs showed blood pressure of $135 / 80 \mathrm{mmHg}$, heart rate of 90 beats $/ \mathrm{min}$, temperature of $37^{\circ}$ Celsius, respiratory rate of 12 times $/ \mathrm{min}$, and $\mathrm{O} 2$ saturation at room air of $98 \%$. The physical findings were significant for diffuse pitting edema in both lower extremities with livedo reticularis, a 3/6 systolic murmur on left sternal border and slightly anemic eye conjunctiva. The biopsy site in her left calf was visible. Interestingly, no other abscesses were reported in the physical exam. Her laboratory studies showed elevated white blood cells (10310/ $\mu \mathrm{L})$ and $\mathrm{C}$-reactive protein, normocytic normochromic anemia $(9.4 \mathrm{mg} / \mathrm{dL})$, slightly elevated liver enzymes, and coagulation abnormalities suggesting disseminated intravascular coagulation. Blood cultures taken on admission turned back negative. Computed tomography (CT) scan of her lower extremities confirmed eight bilateral subcutaneous abscesses in her hip and lower extremities, corresponding exactly with the sites where acupuncture needles were placed. Magnetic resonance imaging (MRI) of her lumbar spine also showed L4 pyogenic spondylitis. She underwent surgical drainage and completed treatment with cefazolin for 6 weeks. She was discharged home in good condition.

DISCUSSION: Acupuncture is a complementary and alternative medicine (CAM) therapy derived from China, and the techniques most frequently used are manipulation and/or electrical stimulation of thin, rigid, metallic needles inserted into the skin. Nowadays, acupuncture has been studied for many conditions including chronic and acute pain and postoperative and chemotherapy induced nausea, and it is estimated that in the US alone, 8.2 million adults receive acupuncture treatments at some point. Acupuncture is probably one of the CAM therapies with the most evidence supporting its benefits. Even though acupuncture is generally considered very safe as long as appropriate sterile techniques are followed, there are certainly several adverse effects reported, including pneumothorax, central/peripheral nervous system injury, organ injury, infection and hemorrhage/hematoma. Our case is unique in that the patient had multiple subcutaneous abscesses corresponding exactly with the sites of acupuncture, which was strongly suggestive of an adverse event by infected acupuncture needles, and that is rarely reported in the literature. Moreover, acupuncture associated infection is said to be severe and sometimes life-threatening as was seen in this case and therefore, worth reporting as a reminder of severe complications of CAM therapy.

SARCOID EVERYWHERE!!. A CASE OF SYSTEMIC SARCOIDOSIS WITH ASSOCIATED SEVERE SARCOID MYOPATHY. Pratap Reddy Tetali ${ }^{1}$; Marc Bernstein $^{3}$; Richard Brasington ${ }^{2} .{ }^{1}$ Mercy Hospital, St. Louis, MO; ${ }^{2}$ Washington university, St. Louis, MO; ${ }^{3}$ Mercy Hospital, Creve Couer, MO. (Control ID \#2466911)

LEARNING OBJECTIVE \#1: Sarcoid myopathy is a very rare condition but it should be considered in the differential diagnosis, in patients with sarcoidosis, who have elevated creatinine kinase or aldolase.

LEARNING OBJECTIVE \#2: Golimumab could be a good alternative treatment option for patients who develop side effects from other tumor necrosis factor inhibitors like infliximab.

CASE: A 28 year-old Africian-American female with a past medical history of sarcoidosis diagnosed by a skin biopsy at age 17 , was seen in the hepatology clinic for elevated Aspartate aminotransferase (AST) and Alanine transaminase (ALT). She had negative work up which included viral hepatitis serology, autoimmune hepatitis, celiac disease and hemochromatosis. Subsequently, she had a liver biopsy as part of the work up which showed granulomatous hepatitis. Since then, she had multiple visits to the emergency department (ED) for various complaints including blurry vison, redness of eyes, pain in bilateral eyes and paratoid gland swelling. Biopsy of lower eyelid at that time was positive for sarcoidosis. She was again seen in the ED for pain in bilateral thighs, swelling of bilateral thighs and dark urine. Work up at that time showed an elevated creatinine kinase value of $44,000 \mathrm{mg} / \mathrm{dl}$. Muscle biopsy was negative for granulomas but was consistent with sarcoid myopathy. She was treated appropriately with intravenous normal saline and was discharged to home on prednisone and infliximab infusions. Three months after that episode, she had another visit to the ED for chest pain, orthopnea and dyspnea on exertion. Echocardiography showed an ejection fraction of $35 \%$. She had a normal cardiac catheterization. Infliximab was discontinued. Cardiac MRI 1 month later showed a normal EF of $67 \%$ with no cardiac sarcoidosis features. She is currently being treated with Golimumab and is in remission with a good clinical response.

DISCUSSION: This case illustrates the an interesting presentation of sarcoidosis involving liver, skin and muscle but sparing lungs. Sarcoid myopathy is a very uncommon presentation of sarcoidosis but it should be considered in the differential diagnosis in patients with systemic sarcoidosis with elevated muscle enzymes. We assume that the patient developed severe congestive heart failure due to infliximab, as the ejection fraction improved to normal value when she was taken off the infliximab. Golimumab could be considered as an alternative in patients with severe sarcoid myopathy with systemic sarcoidosis to achieve remission

SARCOID VS. LYMPHOMA: ELEVATED ACE LEVEL AND CLINICAL OVERLAP Daniel Restrepo; Alyssa Sclafani; Puig Alberto. Massachusetts General Hospital, Boston, MA. (Control ID \#2432094)

LEARNING OBJECTIVE \#1: Identify similar clinical, radiographic and laboratory features of Sarcoidosis and Lymphoma

LEARNING OBJECTIVE \#2: Identify common causes of elevated Angiotensin Converting Enzyme levels outside of Sarcoidosis

CASE: A 33-year-old man presented to our emergency department with a chief complaint of 1 week of fatigue and hypersomnolence. Additionally, he reported night sweats, subjective fevers, a $6.8-\mathrm{kg}$ (15-lb) unintentional weight loss, and progressive left-sided abdominal discomfort of several weeks duration. Physical exam was notable for cervical, axillary, and inguinal lymphadenopathy, as well as tender palpable splenomegaly. Initia laboratory values demonstrated hypercalcemia, pancytopenia, and an ACE level of 225 units/l (upper limit of normal $53 \mathrm{U} / \mathrm{L}$ ). Sputum cultures for acid fast bacilli and HIV antibody and PCR were negative. Chest x-ray showed prominent bilateral hilar shadows concerning for a granulomatous process. In the setting of an elevated ACE level and hilar adenopathy, initial concern on admission to the general medicine floor was a diagnosis of sarcoidosis. However, computed tomography of the chest, abdomen and pelvis demonstrated diffuse, extensive bulky lymphadenopathy of the supraclavicular, mediastinal, hilar, axillary, abdominal, retroperitoneal, and pelvic areas, concerning for a lymphoproliferative disorder. Additionally, serum 1,25-OH vitamin D level returned within normal limits, which was inconsistent with sarcoidosis as the presumed cause of the patient's hypercalcemia on presentation. The final diagnosis of Diffuse Large B-Cell Lymphoma was confirmed on pathology from excisional cervical lymph node biopsy. The patient was transferred to the oncology unit and completed the first cycle of intravenous chemotherapy with cyclophosphamide, doxorubicin, rituximab, vincristine, and oral prednisone. Calcium levels normalized following intravenous fluids and bisphosphonate infusion. He was discharged from the hospital with a plan to complete further cycles of chemotherapy as an outpatient.

DISCUSSION: An elevated serum Angiotensin Converting Enzyme (ACE) level is clinically most commonly associated with sarcoidosis. However, elevations in this enzyme are also seen in a host of granulomatous, infectious, pulmonary and malignant processes, including lymphoma. This case illustrates the complexity of differentiating between sarcoidosis and lymphoma. Both disorders can present with similar features including lymphadenopathy, B symptoms, splenomegaly, hypercalcemia, pancytopenia, and elevated ACE level. Although serum ACE levels are elevated in approximately $60 \%$ of patients with sarcoidosis, this test is not specific for sarcoid as elevated ACE can be seen 
in other macrophage-driven processes such as IBD, Gaucher's Disease, Tuberculosis and Leprosy, as well as both Hodgkin and Non-Hodgkin Lymphoma. Sarcoidosis and Lymphoma can present with similar clinical, laboratory, and radiographic features, differentiation between the two disorders is often mired in diagnostic uncertainty. As demonstrated in this case, even when patients present with symptoms of sarcoidosis and elevated ACE levels it is essential for clinicians to avoid the errors of premature closure and diagnostic anchoring. Only by maintaining a broad differential diagnosis and seeking histologic confirmation will physicians ensure that malignancies such as lymphoma are not overlooked.

SCLERITIS, HYPERTENSION, AND RENAL FAILURE Nancy Haff; Jason P. Cooper; Lee Park. Massachusetts General Hospital, Boston, MA. (Control ID \#2466718)

LEARNING OBJECTIVE \#1: Recognize the clinical presentation of scleritis and its association with systemic disease.

LEARNING OBJECTIVE \#2: Describe the features of IgA nephropathy and the clinical characteristics associated with prognosis.

CASE: A 32-year-old woman who had immigrated from India in 2011, with a history of a spinal cyst resected in 2009 and epidermoid tumor resected in 2014 presented with 1 week of right eye pain and erythema, fever to 101 , and headache. She noted right eye pain primarily with movement and blinking. The erythema and pain did not improve with saline drops. She noted a similar episode several years ago that resolved spontaneously. She denied changes in her vision. On exam, she was hypertensive to 178/118 and had injection of the right lateral sclera. She did not have any evidence of joint disease or rash. Dilated eye exam was consistent with scleritis. Labs were notable for creatinine elevation to $1.67 \mathrm{mg} / \mathrm{dL}$ ( 1.29 nine months prior) and a urinalysis with $2+$ blood and protein. Urine microalbumin to creatinine ratio was $1.6 \mathrm{~g}$. ESR was $28 \mathrm{~mm} / \mathrm{h}$ and CRP $2.2 \mathrm{mg} / \mathrm{L}$. Tests for HIV and T-spot were negative. Hepatitis serologies were negative for hepatitis $\mathrm{C}$ and consistent with prior cleared hepatitis B infection. Cryoproteins were not present. ANA, ANCA, anti-dsDNA, anti-Ro, anti-La, APL antibody, rheumatoid factor, and anti-CCP were all negative. Serum protein electrophoresis and complement levels were normal. The patient underwent renal biopsy which showed IgA nephropathy (IgAN).

DISCUSSION: While scleritis is uncommon, it is important for the generalist to be able to recognize scleritis as it is often associated with systemic disease. Scleritis is characterized by inflammation of the outer white external coating of the eye with accompanying engorged vasculature. It often presents with erythema, usually confined to the anterior eye. It is very painful which distinguishes it from conjunctivitis as conjunctivitis is usually painless. About $25-50 \%$ of patients with scleritis have an associated systemic disease ${ }^{1}$. About half of these patients have a nodular, seropositive rheumatoid arthritis. Other associated systemic diseases include Wegener's granulomatosis, systemic lupus erythematosis, inflammatory bowel disease, the seronegative spondyloarthropathies, and polyarteritis nodosa ${ }^{1}$. We initially evaluated our patient for rheumatologic disease but all work up was negative. Her creatinine did not improve with blood pressure control. The patient was diagnosed with IgAN by renal biopsy. While less common, scleritis has also been associated with $\operatorname{IgAN}^{2}$. $\operatorname{IgAN}$ is the most common lesion associated with primary glomerulonephritis. IgAN most commonly occurs in Asians and Caucasians and generally presents in patients aged 20 to 40 years. It is pathologically characterized by the presence of mesangial IgA deposits, and most often presents with macroscopic hematuria that occurs during or 1 to 2 days after an upper respiratory or gastrointestinal illness ${ }^{3}$ (distinguishing it from post-streptococcal glomerular injury which generally occurs 1 to 3 weeks after a URI). However, the presentation can also include proteinuria, hypertension, and acute kidney injury ${ }^{3}$, all of which were seen in our patient. The degrees of renal impairment, proteinuria, and hypertension at diagnosis are strong initial clinical predictors of unfavorable outcomes, and the continued presence of proteinuria during follow-up is an even stronger predictor ${ }^{4}$. Our patient was started on prednisone and mycophenolate mofetil. At follow-up 2 months after presentation the patient continued to have proteinuria $>1.3$ g per day. 1. Smith JR, Mackensen F, and Rosenbaum JT. Therapeutic Insight: Scleritis and its relationship to systemic autoimmune disease. Nat Clin Proc Rheumatol 2007;3: 219-226. 2. Nomoto Y, Sakai H, Endoh M, et al. Scleritis and IgA Nephropathy. Arch Intern Med 1980;140(6): 783-785. 3. Wyatt RJ, Julian BA. IgA Nephropathy. New Engl J Med 2013;368: 2402-2014. 4. D'Amico G. Natural history of idiopathic IgA nephropathy and factors predictive of disease outcome. Semin Nephrol 2004;24(3): 179-196.

SCROTAL EXTRAMAMMARY PAGET'S DISEASE WITH SEVERE HYPERCALCEMIA AOI YOGO ${ }^{2}$; Sandra Y. Moody ${ }^{1}$; Takahiro Mori ${ }^{2} .{ }^{1}$ Kameda Medica Center \& UCSF/SFVAMC, Kamogawa City, Japan; ${ }^{2}$ Kameda Medical Center, Kamogawa, Japan. (Control ID \#2466205)

LEARNING OBJECTIVE \#1: Recognize cancer-related hypercalcemia as the most common cause of hypercalcemia in hospitalized patients.

LEARNING OBJECTIVE \#2: Perform a thorough physical examination in a hospitalized patient with hypercalcemia of unknown etiology to look for signs of undiagnosed cancer.

CASE: A 72-year-old Japanese man with a history of acute myocardial infarction, hypertension, and hyperlipidemia presented with low back pain exacerbated by standing for 3 weeks. He also had unintentional weight loss of $3-\mathrm{kg}$ (from 53 to $50 \mathrm{~kg}$ ) over 3 weeks, increased thirst, anorexia, and constipation. Initial physical examination was thought to be unremarkable, but repeat physical examination later in the hospital course was notable for a painless erythematous ulceration on the scrotum and right inguinal lymphadenopathy. Laboratory data revealed calcium of $16.2 \mathrm{mg} / \mathrm{dL}$ (reference range: 8.5 10.2), albumin $3.6 \mathrm{mg} / \mathrm{dL}$, phosphorus $4.9 \mathrm{mg} / \mathrm{dL}$, intact parathyroid hormone (PTH) $8 \mathrm{pg} / \mathrm{ml}$ (reference range: 10-65), elevated parathyroid hormone-related protein (PTHrP) 16.5 Pmol/L (reference range: 0-1.1), 1,25-dihydroxyvitamin D $19.2 \mathrm{pg} / \mathrm{ml}$ (reference range: 20-60), and creatinine $3.22 \mathrm{mg} / \mathrm{dL}$. A 25-hydroxy vitamin D level was not measured, as it is not commercially available in Japan. Whole body computed tomography showed multiple osteolytic changes in the lumber spine without evidence of overt malignancy. We performed a skin biopsy of the scrotal lesion and bone biopsy of the lumber spine, leading to a diagnosis of extramammary Paget's disease (EMPD) with bone metastasis.

DISCUSSION: Hypercalcemia has been reported to occur in up to 20 to $30 \%$ of patients with cancer. Cancer-related hypercalcemia is the leading cause of hypercalcemia in hospitalized patients, and cancer has often already been diagnosed by the time it causes hypercalcemia. In this case, two mechanisms contributed to the patient's severe hypercalcemia: systemic secretion of PTHrP and marked increase in osteoclastic bone resorption in carcinomatous metastasis. The level of 1,25-dihydroxyvitamin D was not elevated. Cancer-related hypercalcemia usually suggests a very poor prognosis. EMPD is a rare form of cancer, and is more common in women of lighter skin. It is an adenocarcinoma of apocrine gland-bearing skin (i.e., vulval, perineal, perianal, scrotal, or penile), and usually presents as a slowly expanding well-defined patch that is often pruritic. Although EMPD rarely invades the dermis, it is helpful to make an early diagnosis because dermal invasion is associated with regional lymph node involvement and systemic metastasis, and possibly a high mortality rate. Cancer-related hypercalcemia usually suggests a very poor prognosis. In this case, two mechanisms contributed to the patient's severe hypercalcemia: systemic secretion of PTHrP and marked increase in osteoclastic bone resorption in carcinomatous metastasis. The level of 1,25-dihydroxyvitamin D was not elevated. The oncology service was consulted and determined that the patient was not a candidate for chemotherapy, given his poor prognosis and low performance status. Palliative care was provided, and he died on hospital day 41. The cutaneous lesion was 
missed because detailed genitourinary examination and lymph node survey were not performed on the initial examination, which delayed skin biopsy of the scrotal lesion until the tenth day of hospitalization. When a patient presents with severe hypercalcemia of unknown etiology, an initial evaluation should include a thorough physical examination including perianal and genital regions, which may be easily omitted, to look for signs of underlying malignancy. Severe hypercalcemia can be a clue for undiagnosed malignancy.

\section{SCRUB TYPHUS MIMICKING INFECTIOUS MONONUCLEOSIS Hirom}

Sekiguchi; Koki Kosami; Jun Suzuki; Kozue Murayama; Yu Yamamoto; Shuji Hatakeyama; Masami Matsumura. Jichi Medical University Hospital, Shimotsuke, Japan. (Control ID \#2465714)

LEARNING OBJECTIVE \#1: Diagnose scrub typhus correctly based on detailed history and physical examination.

LEARNING OBJECTIVE \#2: Recognize that scrub typhus can mimic infectious mononucleosis and present as aseptic meningitis.

CASE: A 34-year-old previously healthy Japanese man presented with a 2-day history of fever, headache, and malaise. Six days prior to admission, the patient visited a nearby hospital, where acetaminophen was prescribed without improvement. Four days before, he was admitted to another hospital because of fever of $39^{\circ}$ Celsius Erythematous papules 5 to $10 \mathrm{~mm}$ in diameter on the whole body and bilateral posterior neck lymphadenopathy were noted. Examination of the cerebrospinal fluid (CSF) showed 16 white blood cells/ $\mu \mathrm{L}$ (mononuclear cells 16), glucose of $57 \mathrm{mg} / \mathrm{dl}$, and protein of $68.7 \mathrm{mg} / \mathrm{dl}$. He was diagnosed with meningitis and transferred to our hospital. On examination, his temperature was $37.2^{\circ}$ Celsius, blood pressure 114 $66 \mathrm{mmHg}$, and pulse rate 52 per minute. He was oriented. Nuchal rigidity was noted. Oropharynx examination showed no abnormality. Bilateral cervical and inguinal lymph nodes were enlarged, 10 to $20 \mathrm{~mm}$ in diameter. No erythematous rash was observed, but an eschar on the posterior aspect of the right lower leg was noted Leukocyte count was $6,600 / \mu \mathrm{L}$ with $1,000 / \mu \mathrm{L}$ of atypical lymphocytes, hemoglobin $14.4 \mathrm{~g} / \mathrm{dL}$, platelets $193,000 / \mu \mathrm{L}$, aspartate aminotransferase (AST) $107 \mathrm{IU} / \mathrm{L}$, alanine aminotransferase (ALT) $174 \mathrm{IU} / \mathrm{L}$, lactate dehydrogenase $447 \mathrm{IU} / \mathrm{L}$, and creatinine $0.8 \mathrm{mg} / \mathrm{dL}$. At this point, infectious mononucleosis was suspected because of lymphadenopathy, atypical lymphocytosis, and liver dysfunction. However, Epstein-Barr virus viral capsid antigen IgM was negative, and Epstein-Barr virus nuclear antigen was positive. Cytomegalovirus IgM antibodies were negative. Antibodies and antigen to human immunodeficiency virus were also negative. Second CSF examination showed white blood cells $58 / \mu \mathrm{L}$ (mononuclear cells 58), glucose $52 \mathrm{mg} / \mathrm{dl}$, and protein $83 \mathrm{mg} / \mathrm{dl}$. He was started on $4 \mathrm{~g}$ per day of ceftriaxon, $2 \mathrm{~g}$ per day of vancomycin, and 2,250 mg per day of acyclovir intravenously for meningitis. On hospital day 5, results of CSF, blood, and urine cultures turned out to be sterile. Although the patient's place of residence were not an endemic area of scrub typhus, scrub typhus was suspected because of an eschar on his right leg. Repeated history taking clarified that 3 weeks before he had gone to Chiba prefecture, an endemic area of scrub typhus in Japan, for orange picking. His serum IgM antibodies to Orienta tsutsugamushi (O. tsutsugamushi) were positive at a titer of 1: 640 for Gilliam, 1: 320 for Karp, and 1: 320 for Kato. Scrub typhus was diagnosed, and symptomatic improvement was followed by administration of $200 \mathrm{mg}$ per day of intravenous minocycline. On hospital day 7 , he made a full recovery.

DISCUSSION: Scrub typhus, called "Tsutsugamushi disease" in Japan, is a chiggerborne rickettsial infection caused by $O$. tsutsugamushi. In Japanese, "tsutusuga" and "mushi" mean "illness" and "mite", respectively. The prevalence of this infection differs in each district in Japan. Commonly there is a sudden onset of chills, fever, headache, and myalgia, after which a maculopapular rash appears. Characteristic eschar by chigger bite is observed. Lymphadenopathy and elevated AST and ALT levels are common. Atypical lymphocytosis can be observed in some cases. Therefore, we might misdiagnose infectious mononucleosis based on the combination of observed manifestations and laboratory data. 2-6\% of patients with scrub typhus have associated meningitis. Meningitis in scrub typhus is generally mild with quick and complete recovery and 7.7 times more frequent in patients with pneumonitis. CSF findings of mild leukocytosis, increased protein level, and normal glucose level resemble viral meningitis or tuberculous meningitis. Though we did not consider the possibility of scrub typhus in the early clinical course, a characteristic eschar on the right leg and repeated history taking led to the correct diagnosis. Detailed history and precise physical examination are the basis for achieving the correct diagnosis. Physicians should keep in mind that scrub typhus can mimic infectious mononucleosis and present as aseptic meningitis.

SCURVY: A FORGOTTEN SYNDROME Najmus Sahar ${ }^{1}$; Asghar Ali ${ }^{1}$; Theresa Ratajczak $^{2}$; Steve Burdette ${ }^{3}$. ${ }^{1}$ Wright State University, Beavercreek, OH; ${ }^{2}$ Wright State University, Dayton, OH; ${ }^{3}$ Wright State University, Dayton, OH. (Control ID \#2470353)

LEARNING OBJECTIVE \#1: Scurvy is a clinical syndrome caused by vitamin C (Ascorbic acid) deficiency. Population at risk includes the elderly, alcoholics, smokers, and low socioeconomic class, patients on restricted diets and after gastrointestinal surgeries. A survey in the USA in 2003/2004 showed that $7.1 \%$ of healthy middle class participants were vitamin $\mathrm{C}$ deficient, and $13.7 \%$ were depleted. We present a case of scurvy after gastric bypass surgery.

CASE: A 66-year-old Caucasian female presented with right lower extremity (RLE) pain, swelling and rash after blunt trauma to her leg. Her past medical history was significant for bariatric Roux-en-Y gastric bypass surgery and end stage renal disease (ESRD) status post renal transplantation, on immunosuppressive therapy including tacrolimus and mycophenolic acid. Physical examination revealed an afebrile, underweight patient with patchy mottling of the skin of RLE extending from knee to ankle. RLE was tender, swollen and had black leathery eschar in multiple small painful ulcers. Pulses were intact in both lowe limbs. Admission laboratory workup showed leukopenia of 3.3 K/MM3 and normocytic anemia. Comprehensive metabolic panel showed metabolic acidosis with elevated blood urea nitrogen and creatinine, low total protein and low albumin levels. The rash was initially attributed to ecthyma gangrenosum in the presence of Pseudomonas bacteremia, but skin biopsy revealed calciphylasix associated with ESRD. Patient developed bilateral lower extremity deep vein thrombosis (DVTs) due to persistent pain and immobility. Magnetic resonance imaging (MRI) of her legs revealed edema of the subcutaneous tissue and underlying muscles. Being malnourished with poor wound healing, a serum vitamin C level was checked and found to be deficient, at levels less than $0.12 \mathrm{mg} / \mathrm{dl}$ (reference range $0.20-1.90 \mathrm{mg} / \mathrm{dl}$ ) confirming the diagnosis of scurvy. Started on a daily $500 \mathrm{mg}$ tablet of vitamin $\mathrm{C}$, the patient experienced significant improvement in her pain and rash within $24 \mathrm{~h}$.

DISCUSSION: Patients following bariatric gastric bypass surgery are at risk for development of micronutrient deficiencies. The incidence of vitamin $\mathrm{C}$ deficiency is not uncommon if surgery is followed by poor diet. Symptoms of scurvy generally occur when plasma levels of ascorbic acid are less than $0.2 \mathrm{mg} / \mathrm{dL}$, and include ecchymosis, bleeding gums, petechiae, coiled hairs, hyperkeratosis, arthralgia, impaired wound healing (due to impaired collagen synthesis) and impaired iron metabolism causing anemia. Although scurvy is uncommon in developed nations with advanced dietary knowledge and widespread availability of over-the-counter (OTC) multi-vitamins and sufficient food supply, clinicians should have a high index of suspicion in patients at risk for developing scurvy. Early recognition and initiation of treatment provides a better outcome and good prognosis in these patients. It can prevent development of comorbidities, shorten hospital stay; saving time and money, precluding unnecessary tests. 
SEA ANEMONE ON A MITRAL VALVE! Sreelakshmi Panginikkod ${ }^{1}$; Venu Pararath Gopalakrishnan $^{1}$; Niyati Gupta ${ }^{2}$; Bara Alzghoul ${ }^{1}$; Pratyusha Bollimunta ${ }^{1}$. ${ }^{1}$ Presence Saint Francis Hospital, Evanston, IL; ${ }^{2}$ Grant Medical College and Sir JJ Group of Hospitals, Mumbai, India. (Control ID \#2469217)

LEARNING OBJECTIVE \#1: Recognize the clinical presentation of Cardiac Papillary Fibroelastomas (CPF)

LEARNING OBJECTIVE \#2: Recognize the management a patient with Cardiac Papillary Fibroelastoma.

CASE: A 44 year old male presented to our hospital for evaluation of mild chest discomfort associated with nausea and dizziness that lasted for about $2 \mathrm{~h}$. The discomfort was compressive and unrelated to activity. He also endorsed to have excessive sweating and occasional palpitation for last 3 months. Review of systems was negative for any syncopal episodes, fever or weight loss. Cardiovascular examination was unremarkable. Cardiac enzymes, chest radiograph, electrocardiogram and 24-h Holter monitoring were normal. Two-dimensional echocardiographic study showed a highly mobile $1 \times 1 \mathrm{~cm}$ mass attached to the posterior leaflet of mitral valve. Valve function was preserved without significant mitral regurgitation or stenosis. Blood cultures were negative and chemistry analyses revealed no elevation of inflammatory markers. Autoimmune work up was also negative. A preoperative coronary angiogram was performed and demonstrated normal coronaries. The patient underwent surgical resection of the mass lesion including a small portion of the endocardium and the leaflet under cardiopulmonary bypass. The postoperative echocardiogram confirmed absence of residual tumor and normal mitral valve function. Histopathologic examination of the mass confirmed the diagnosis of a cardiac papillary fibroelastoma. The postoperative course was uneventful and the patient was discharged on day 7 after the surgery.

DISCUSSION: Primary tumors of the heart are rare, with a reported frequency of $0.02 \%$ according to large autopsy series. Cardiac papillary fibroelastoma (CPF) of the mitral valve does not occur frequently and therefore each case is interesting to report in order to improve the diagnosis and management of this uncommon tumor. CPFs resemble 'a sea anemone', with multiple papillary fronds attached to the endocardium by a short pedicle. Historically, fibroelastomas have been incidental findings at autopsy. With routine use of echocardiography, clinicians are able to detect these tumors readily in living patients. The clinical presentation of cardiac papillary fibroelastoma varies from no symptoms to severe embolic sequelae and may be associated with myocardial infarction, transient ischemic attacks, strokes, or sudden death attributed to occlusion of the coronary ostia. A review of the literature suggests that the cardiac papillary fibroelastoma is a rare but potentially treatable cause of embolic, vascular and neurological complications, therefore, a strong suspicion; appropriate use of imaging modality, preoperative anticoagulation and urgent surgical resection is warranted.

\section{SECONDARY AORTOENTERIC FISTULA AFTER THE PRESERVATION OF INFECTED ABDOMINAL AORTIC ENDOVASCULAR GRAFTS AOI YOGO ${ }^{2}$;} Ryota Hase $^{3}$; Mitsuhisa Kotani ${ }^{2}$; Sandra Y. Moody ${ }^{1}$; Akiyuki Sato ${ }^{2}$. ${ }^{1}$ Kameda Medica Center \& UCSF/SFVAMC, Kamogawa City, Japan; ${ }^{2}$ Kameda Medical Center, Kamogawa, Japan; ${ }^{3}$ Japanese Red Cross Narita Hospital, Narita, Japan. (Control ID \#2468139)

LEARNING OBJECTIVE \#1: Recognize secondary aortoenteric fistula (AEF) is a fatal infectious complication of endovascular aneurysm repair (EVAR).

LEARNING OBJECTIVE \#2: Suspect a secondary AEF in patients with gastrointestinal (GI) bleeding after EVAR.

CASE: A 67-year-old bedridden man with a history of radiation-treated laryngeal carcinoma with subsequent radiation myelopathy and ruptured aortic artery aneurysm status post endovascular aneurysm repair (EVAR) and left iliopsoas abscess due to abdominal aortic endograft infection, presented with a 4-h history of epigastralgia and hematemesis. Contrast abdominal computed tomography (CT) revealed a bleeding defect of the duodenal wall near the ligament of Treitz. Emergent gastroscopy confirmed a secondary AEF of the third portion of the duodenum as the source of bleeding. Blood clots and an exposed abdominal aortic stent graft were observed in the adjacent anatomical location near the distal duodenum. Under general anesthesia, EVAR was performed via the left femoral artery as a lifesaving, temporizing option to control the bleeding, because the patient was too high risk for aortic reconstruction given his significant comorbidities and hemodynamic instability. Endovascular stent grafts (PXA260300 and PXA230300; Gore ${ }^{\circledR}$ Excluder $^{\circledR}$, Gore) were placed proximal and distal to the infected graft to extend coverage into the uninfected aorta. There was no further hemorrhaging from the AEF. He received a 4-week course of intravenous antibiotics, and lifetime chronic oral antimicrobial suppression was initiated. Despite 5 weeks of oral antimicrobial suppression, massive melena developed 75 days after his second EVAR. He refused surgery as well as upper endoscopy. He died of massive gastrointestinal (GI) bleeding despite receiving multiple transfusions, including packed red blood cells and fresh frozen plasma, over a 7-day period. Autopsy revealed clotted blood between the weak aortic wall under the stent graft and the third portion of the duodenum. A fistula in the adjacent anatomical location was found to be the source of bleeding.

DISCUSSION: Secondary AEF, which results from infection, and may cause fatal GI bleeding, is a rare, but well-known complication of EVAR, and is a surgical emergency. The incidence of abdominal endovascular graft infection after EVAR is estimated to be $<1 \%$, and secondary AEF is extremely rare. The third and fourth portions of the duodenum are the most frequent sites of fistulae. The standard treatment is wide resection of the aorta around the duodenal erosion. However, it is a demanding operation that several patients may not tolerate owing to significant comorbidities. Thus, in high-risk patients, endovascular graft preservation and conservative treatment remain viable options. Based on the literature review, there were only seven patients in whom endovascular graft infections were treated with graft preservation followed by secondary AEF. None of the seven patients survived, however, during approximately 1-year follow-up. Most of the patients with an AEF had GI bleeding, and a contrast CT confirmed the diagnosis of an endovascular graft infection, whereas patients with suspected AEF, endoscopy was required. In patients with GI bleeding after EVAR, a secondary AEF must be suspected and emergently treated to prevent a poor outcome.

SEEING RED EVERYWHERE: AMYLOIDOSIS ASSOCIATED GASTROINTESTINAL BLEEDING IN A PATIENT WITH MULTIPLE MYELOMA Woody Chang ${ }^{2}$; Bing Ren ${ }^{1}$; David McAdams ${ }^{1} .{ }^{1}$ University of Pittsburgh Medical Center, Pittsburgh, PA; ${ }^{2}$ University of Pittsburgh Medical Center, PresbyterianMontefiore, Pittsburgh, PA. (Control ID \#2470040)

LEARNING OBJECTIVE \#1: To recognize the association between AL amyloidosis and multiple myeloma

LEARNING OBJECTIVE \#2: To define the treatment options for AL amyloidosis

CASE: A 64-year-old woman, recently diagnosed with multiple myeloma following the repair of a spontaneous hip fracture, was admitted to the outside hospital from a skilled nursing facility for presumed post-operative ileus complicated by nausea and vomiting. During that stay, she developed significant hematochezia and a hemoglobin drop from 10.2 to $7.9 \mathrm{~g} / \mathrm{dL}$. CT showed dilatation of small bowel without bowel wall thickening. The patient was transferred to our facility due to concern for gastrointestinal bleed. The patient was tachycardic but normotensive and afebrile on arrival. Her exam was positive for gross blood per rectum with infrequent bowel sounds, but otherwise unremarkable. Pertinent labs include a hemoglobin of $8.3 \mathrm{~g} / \mathrm{dL}$, hematocrit of $25.4 \%$, creatinine of $1.7 \mathrm{mg} / \mathrm{dL}$, and 
a corrected calcium of $10.1 \mathrm{mg} / \mathrm{dL}$. The colonoscopy showed maroon colored stools, without an obvious source of bleeding. Endoscopy showed friable, congested mucosa of the second part of the duodenum with oozing but no ulcers or visible vessels. The duodenum was subsequently biopsied. These procedures were complicated by hypotension and further blood loss with a hemoglobin of $6.3 \mathrm{~g} / \mathrm{dL}$, necessitating multiple transfusions and transfer to the ICU. After the patient was stabilized, she was transferred to the medicine service. She was placed on a proton pump inhibitor and showed no further signs of gastrointestinal bleeding. The biopsy from the duodenum stained positive for Congo red in both the mucosal lamina propria and submucosal vessel walls, consistent with amyloidosis. She was discharged to follow up with the oncology service for discussion of outpatient chemotherapy treatment.

DISCUSSION: This case encourages the physician to think about amyloidosis as part of the differential for any patient with a plasma cell dyscrasia that presents with GI bleeding. In particular, $\mathrm{AL}$ amyloidosis is a known association with multiple myeloma, less commonly with Waldenstrom's macroglobulinemia or non-Hodgkin's lymphoma. It is estimated that $10-15 \% \mathrm{AL}$ amyloidosis cases present with multiple myeloma. This disease commonly presents with renal or cardiac symptoms, though virtually all patients have multisystem amyloid deposition. Gastrointestinal involvement is seen in only $1-8 \%$ of AL amyloidosis cases with $25-45 \%$ of these presenting with bleeding. Other complications include malabsorption, dysmotlility, pseudo-obstruction, and mesenteric ischemia Diagnosis of gastrointestinal amyloidosis requires a tissue biopsy with positive staining with Congo red or the presence of amyloid fibrils found on electron microscopy. Typically, the work up also includes an endoscopy and/or colonoscopy to rule out other etiologies of the bleeding. Such was done with this patient, as the endoscopy and colonoscopy were done to exclude other sources of bleeding such as peptic ulcer disease, diverticular disease, or angiodysplasia. Initial management involves controlling the source of the bleeding and stabilizing the patient. The next step is to treat the underlying disease that is causing the deposition of amyloid. In the case of AL amyloidosis, the deposition is caused by amyloidogenic light chains produced by underlying clonal plasma cells. Hence the objective of any regimen is to target these plasma cells. The first-line treatment that this patient could undergo is a course of melphalan followed by autologous hematopoietic cell transplantation, assuming she is transplant-eligible. A case series of patients treated with high dose melphalan followed by autologous hematopoietic cell transplant showed complete response in $34 \%$ of patients. Those with a complete response have a median event-free survival of 8.3 years versus 2 years for those that do not have a complete response. For patients that do not qualify for an autologous hematopoietic cell transplantation because of advanced age, renal insufficiency, advanced heart failure, or multiorgan involvement, treatments of melphalan and dexamethasone, as well as bortezomib-based regimens have shown promise in smaller studies. Supportive care should be provided to maintain quality of life and delay organ failure in order to give time for any of the above treatments to take effect. In conclusion, in patients with multiple myeloma and new onset gastrointestinal bleeding, amyloidosis should be considered as part of the differential diagnosis. In this case, definitive diagnosis of the amyloidosis was achieved with a tissue biopsy that was positive for Congo red staining. Treatment involves controlling the underlying plasma cell dyscrasia. If the patient qualifies for a hematopoietic cell transplantation, the likely treatment involves melphalan followed by autologous hematopoietic cell transplantation. However, if the patient does not qualify for a hematopoietic cell transplantation, there are other chemotherapies available that show some success at slowing disease progression.

SEPTIC SHOCK SECONDARY TO CAPNOCYTOPHAGA GINGIVALES BACTEREMIA IN AN IMMUNOCOMPETENT HOST. Kelley L. Coffman ${ }^{1}$; Ronald Goldenberg ${ }^{2}$. ${ }^{1}$ New York University School of Medicine, New York, NY; ${ }^{2}$ New York University Langone Medical Center, New York, NY. (Control ID \#2470290)
LEARNING OBJECTIVE \#1: Recognize a rare cause of septic shock from Capnocytophaga gingivales bacteremia in an immunocompetent host.

CASE: This is a 58 year-old man with a history of hypertension, hyperlipidemia, and mild COPD who presented with acute-onset of fevers, mild headache, and generalized myalgias for 1 day. He had recently vacationed in a wooded area of upstate New York; however, he denied any animal or insect bites. He denied any other travel, toxic ingestions, or sick contacts. Review of systems was negative for neck stiffness, photophobia, nausea or vomiting, diarrhea or abdominal pain, cough, dyspnea, or diarrhea. He had been in his usual state of good health except for exertional dizziness for the past month that had been diagnosed as benign paroxysmal positional vertigo after a negative cardiac work-up with echocardiography and EKG. His medical history was otherwise notable for active smoking and heavy alcohol use. Upon presentation, he was febrile $(103.8 \mathrm{~F})$, tachycardic (110 s), and hypotensive (100 s/50s). He appeared diaphoretic but was alert and oriented, and his exam was non-focal. His initial labs showed a leukocytosis (19) and an elevation of creatinine (1.2), troponin (0.112, subsequently trended down), CK (568), BNP (1020), and procalcitonin (10). His chest $\mathrm{X}$-ray, urinalysis, hepatic panel, and lactate were within normal limits. In the emergency department, he was fluid resuscitated with two liters of normal saline and started on broad-spectrum antibiotics with intravenous vancomycin and cefepime. His blood pressures dropped to $70 \mathrm{~s} / 40$ s, so he was initially admitted to the step down unit. His antibiotics were broadened to cover atypical organisms with azithromycin and endotoxin-releasing bacteria with clindamycin. Despite three additional liters of fluids, he remained hypotensive, so he was started on norepinephrine and transferred to the intensive care unit. Given his deterioration and no identifiable source, his antibiotics were again broadened to include coverage of tick-borne infections with doxycycline. Azithromycin was changed to levofloxacin when his serum antibody titer for Legionella returned weakly positive (1:256). Overall, he appeared to be improving clinically and was titrated off vasopressors after approximately $48 \mathrm{~h}$. His initial blood cultures returned with gram-negative rods (GNR) on gram stain. Overall, he appeared to be improving clinically and was titrated off vasopressors after approximately $48 \mathrm{~h}$. Blood cultures did not speciate prompting concern for a fastidious organism. During initial cardiac evaluation to rule out endocarditis, his TTE showed global hypokinesis with a depressed ejection fraction (EF) but no valvular disease. In addition to bacterial endocarditis including $H A C E K$ organisms, Legionella myocarditis was on the differential diagnosis given evidence of acute heart failure, mildly elevated troponin, and weakly positive Legionella antibody. His antibiotics were transitioned to piperacililn-tazobactam for broad gram-negative coverage and azithromycin for possible extrapulmonary legionellosis. Cardiac MRI showed no evidence of late gadolinium enhancement or myocardial involvement. Off pressors, TEE showed normalization of his EF and mild thickening of the anterior mitral valve with possible attachment of a very small $(\sim 1.5 \mathrm{~mm})$ fibrin strand, but no obvious vegetation. Upon further discussion of his prior exposures, he eventually revealed that within the last month, his dentures had begun to fit improperly, causing mild pain and bleeding. He continued to wear the ill-fitting dentures and had not yet sought dental evaluation. Closer inspection of his oral cavity showed gingival irritation but no obvious fluid collections or evidence of infection. He was ultimately discharged home with 4 weeks of intravenous ceftriaxone for presumed GNR endocarditis. During follow-up in an infectious disease clinic approximately 2 months after his initial presentation, the culture was reported by the Department of Health to have grown an organism with an appearance most closely resembling Capnocytophaga gingivales but was unable to be confirmed by DNA analysis. DISCUSSION: Capnocytophaga is a genus of fastidious, slow-growing Gram-negative rods, found typically in the gingiva of humans, canines, and felines. Transmission usually occurs through animal bites but has also been reported with dental surgical procedures. This bacteria is considered a low-virulence organism in healthy individuals; however, it can cause severe infections in immunocompromised hosts, specifically cancer, transplant, and HIV-positive patients. In the literature, most case reports identify C. canimorus, transmitted through dog and cat bites, as the causative organism in bacteremia, 
pneumonia, and lung abscesses. It is a rare cause of septic shock, and the species C. gingivales is an even more exceedingly rare etiology of human infections. In this case report, an immunocompetent middle-aged male developed septic shock with multi-organ failure from an unusual bacterial infection though an uncommon portal of entry. His illfitting dentures likely created a pathway of transmission for this commensal bacterium of the oral cavity, and his heavy alcohol use may have weakened his immune response, predisposing him to its pathogenicity. In conclusion, this case vignette depicted a rare cause of septic shock from bacteremia with Capnocytophagia gingivales likely originating from ill-fitted dentures.

\section{SERRATIA MARCESCENS CAUSING SUBACUTE CONSTRICTIVE} PERICARDITIS : A RARE CASE Amruta Panwala. University of Connecticut, Hartford, CT. (Control ID \#2470235)

LEARNING OBJECTIVE \#1: Recognize Serratia marcescens infection as one of the possible etiology for subacute constrictive pericarditis with pericardial effusion.

LEARNING OBJECTIVE \#2: Recognize various predisposing risk factors for Serratia marcescens infection along with knowing various infections caused by this rare organism. CASE: A 94 year old male with pertinent past medical history of atrial fibrillation but not on anticoagulation, coronary artery disease managed medically without any history of percutaneous intervention, hypertension, diabetes type 2 well controlled, stage 3 chronic kidney disease and history of chronic DVT presented from a nursing home with complain of progressive shortness of breath and orthopnea. Physical examination reveled jugular venous dilation. Initial evaluation with chest $\mathrm{x}$-ray showed only moderate enlargement of cardiac silhouettes without any air space disease. On 2nd day of hospitalization, patient had an acute episode of severe substernal chest pain radiating to back, increased shortness of breath with hypoxia along with hemodynamic decompensation with hypotension and tachycardia though EKG showed sinus tachycardia without any acute ST-T changes. CT angiography of chest was performed which was negative for PE or aortic dissection but demonstrated incidental finding of large pericardial effusion. Transthorasic echocardiography was done which showed constrictive physiology with large pericardial effusion. Patient underwent pericardiocentesis with pericardial window formation. $1.4 \mathrm{~L}$ of hemorrhagic pericardial fluid was drained during the procedure and pericardial fluid was sent for appropriate testing including cytology, cell count and microbiological evaluation with staining and culture. To our surprise, culture of fluid grew Serratia marcescense. Cytological evaluation was negative for any malignant cells. Patient was started on appropriate antibibacterial therapy with Meropenam. Second set of culture of pericardial fluid drained from pericardial window also grew Serratia marcescens. Through out the treatment blood culture remained negative. Patient showed marked improvement in symptoms within next 3 days and remained hemodynamically stable. Further radiological scan of abdomen/pelvis to rule out possible malignancy was also negative. Unfortunately, patient went into cardiac arrest with ventricular tachycardia as an underlying rhythm.

DISCUSSION: Serratia marcescens is a ubiquitous, saprophytic, motile, gram negative bacillus belonging to the division of Klebsiella Enterobacter- Serratia within the family of enterobacteriaceae. In recent years, this organism has assumed increasing importance as an opportunistic pathogen with many reported hospital based outbreaks of Serratia infection with high morbidity and mortality. It has been implicated in all kinds of organ system involvement including pulmonary, cardiovascular, urinary tract infections and eye along with Serratia bacteremia. Here we describe a case with subacute constrictive pericarditis with large pericardial effusion caused by Serratia marcescens. Serratia marcescens is a unique microorganism which has a romantic history dating to antiquity, when historically, because of production of a red pigment, it masqueraded as blood. Serratia marcescens infection has been reported to occur in mainly 2 types of patients, 1) IV drug users, 2) old debilitated as well as immune-compromised hosts. The predisposing factors for infection with this unique pathogen are cardiovascular catheterization, intravenous alimentation, peritoneal dialysis or hemo-dialysis and most commonly genitourinary catheterization. To date reported heart Infection by this organism have constituted only endocarditis especially involvement of valvular prosthesis. In our extensive literature search, we found only one case report of subacute constrictive pericarditis with Serratia marcescens bacteremia reported by Khan MY et al. To our best knowledge, this is only the second case reported for Serratia marcescens infection in form of constrictive pericarditis. This case is unique as first of all, our patient did not have any risk factors as mentioned above for Serratia infection so the source of Serratia infection remained unclear. Our patient was elderly 94 year old gentleman without any other risk factor for immune-compromisation except his age. Second, three sets of blood culture which remained negative indicated that pericardium was likely the primary site of infection rather than spread of Serratia from any other infected organ through bacteremia. Third, Involvement of heart with Serratia infection in form of constrictive pericarditis causing large pericardial effusion rather than endocarditis was unique in itself. In a nutshell, this case prompts us to consider Serratia as a very rare but distinctive cause of subacute constrictive pericarditis with pericardial effusion even in the absence of well established risk factors for Serratia infection

SEVERE ANION GAP METABOLIC ACIDOSIS ASSOCIATED WITH INITIATION OF A VERY LOW-CARBOHYDRATE DIET Asad Jehangir ${ }^{2}$; Ahmed Salman ${ }^{1}$; Sachin Gupta ${ }^{1}$; Anthony Donato ${ }^{1}$. ${ }^{1}$ Reading Health System, Wyomissing, PA; ${ }^{2}$ Reading Health System, West Reading, PA. (Control ID \#2434600)

LEARNING OBJECTIVE \#1: Recognize severe anion-gap acidosis as a complication of very-low carbohydrate diets

LEARNING OBJECTIVE \#2: List differential and initial evaluation steps in patients with severe acidosis

CASE: A 31-year-old male presented to the emergency department with shortness of breath and palpitations 1 week after starting a very-low carbohydrate diet without pharmacologic agents. He denied any history of diabetes, alcohol abuse, intensive exercise or illicit drug use. On examination, his heart rate was $136 \mathrm{bpm}$, respiratory rate 30, $\mathrm{SpO} 2$ $100 \%$ on room air and BMI $37.6 \mathrm{~kg} / \mathrm{m}^{2}$. Laboratory tests showed bicarbonate of $9.2 \mathrm{mEq} /$ L (normal: $24.0-31.0 \mathrm{mEq} / \mathrm{L}$ ) anion gap of $20 \mathrm{mEq} / \mathrm{L}$ (normal: 4-12 mEq/L), blood glucose of $86 \mathrm{mg} / \mathrm{dL}, 2+$ serum ketones and a creatinine of $1.43 \mathrm{mg} / \mathrm{dL}$ (baseline $1.0 \mathrm{mg}$ / dL). Arterial blood gas showed a pH of 7.35 (normal 7.36-7.46), pCO2 of $10 \mathrm{mmHg}$ (normal 34-46 mmHg), pO2 of $120 \mathrm{mmHg}$ (normal $85.0-95.0 \mathrm{mmHg}$ ) and calculated bicarbonate of $5.7 \mathrm{mEq} / \mathrm{L}$ (normal: $22.0-27.0 \mathrm{mEq} / \mathrm{L}$ ). Serum lactate, acetaminophen, salicylate and all alcohol levels were normal and osmolar gap was borderline high $(11 \mathrm{mOsm} / \mathrm{kg})$. He received IV sodium bicarbonate and dextrose $5 \%$ in water for $12 \mathrm{~h}$, however his anion-gap metabolic acidosis persisted, requiring acute hemodialysis. Following hemodialysis his tachypnea, acidosis and acute kidney injury resolved.

DISCUSSION: Very-low carbohydrate/ketogenic diets (generally less than $50 \mathrm{~g}$ of carbohydrates per day) have been used since 1920s for the treatment of epilepsy, and from 1960s onwards have been utilized for management of obesity as well. However, low carbohydrate diets have not been shown to have better long-term outcomes and safety profiles compared to other diets, hence there is not sufficient evidence for or against their use. Nonetheless, low-carbohydrate diets (e.g. Atkins diet) are becoming increasingly popular as they have been shown to induce ketosis-promoting rapid short-term weightloss. Moreover, these dietary plans are easily accessible on the internet and media, are often utilized without medical advice. Severely ketotic states from low carbohydrate diets may be a potential health hazard in patients predisposed to this condition, and can result in a protracted acidosis as was seen in the case presented here. Given that very low carbohydrate diets have appeared frequently throughout time and have been touted by 
some to be a treatment for many diseases, physicians caring for patients with protracted acidosis should consider dietary manipulation in the differential of protracted unexplained acidosis.

SEVERE CLOSTRIDIUM DIFFICILE INFECTION PRESENTING AS UNEXPLAINED LEUKOCYTOSIS. Kartik Kumar ${ }^{1}$; Samran Haider ${ }^{1}$; Nicholas Rickert $^{2}$; Tomas Murphy ${ }^{2}$; Jefferey Van Hooser ${ }^{2}$; Diane L. Levine ${ }^{1} .{ }^{1}$ Wayne State University, Detroit, MI; ${ }^{2}$ Wayne State University/Detroit Medical Center, Detroit, MI. (Control ID \#2469035)

LEARNING OBJECTIVE \#1: Recognize Clostridium Difficile Infection (CDI) can occur without traditional risk factors.

LEARNING OBJECTIVE \#2: Avoid and recognize premature closure, a common physician cognitive error

CASE: An 87 year old female with a past medical history significant for HTN, CHF, and lower extremity edema secondary to CHF, presented to our ED with left lower quadrant abdominal pain, nausea, and loss of appetite for 7 days. She describes the pain as sharp, intermittent, $8 / 10$ in severity, with radiation to groin as well as associated dysuria. Review of systems revealed history of diarrhea 2 months ago which occurred during a previous hospitalization. The cause of the diarrhea was not known and had subsequently resolved after taking medication she was discharged with. The diarrhea had restarted 7 days ago for which her PCP prescribed loperamide with resolution of symptoms. She denied subjective fevers, chills or back pain. Vitals signs were 144/66, HR 91, RR 16, Temperature 36.2, and $100 \% \mathrm{O} 2$ saturation on room air. On examination she was in no acute distress except for mild left lower quadrant tenderness without costovertebral angle tenderness. Urinalysis revealed WBC $>100$, Blood 2+, pH 5.0, Protein 1+, Nitrite Negative, 2+ Leukocyte Esterase, 2+ Bacteria, 0-2 squamous epithelial cells. CBC showed WBC count of $29.3 \mathrm{k} /$ $\mathrm{mm} 3$ and $\mathrm{Hb} 10.8 \mathrm{~g} / \mathrm{dl}$. Na $+140 \mathrm{mMol} / \mathrm{L}, \mathrm{K}+3.3 \mathrm{mMol} / \mathrm{L}, \mathrm{Cl}-105 \mathrm{mMol} / \mathrm{L}, \mathrm{CO} 2$ $21 \mathrm{mMol} / \mathrm{L}$, BUN $29 \mathrm{mMol} / \mathrm{L}$, and Creatinine of $1.78 \mathrm{mg} / \mathrm{dl}$. Abdominal X-ray did not show obstruction. Blood cultures were drawn. The patient was admitted with a diagnosis of acute pyelonephritis with acute kidney injury likely pre-renal given her decreased ora intake. She was started on IV ceftriaxone. On the following morning the patient complained of 3-4 loose bowel movements. On admission day 1; Leukocyte count increased to $34.5 \mathrm{k} /$ cumm while creatinine decreased to $1.32 \mathrm{mg} / \mathrm{dl}$ with $1 \mathrm{~L} 0.9 \%$ $\mathrm{NaCl}$. Given her recent history of diarrhea with increased white count, abdominal pain and increased creatinine, stool sample was sent for C. Diff PCR with initiation of empirical treatment with PO Vancomycin $500 \mathrm{mg}$ PO Q6H. PCR returned positive for CDI. Her blood pressure subsequently decreased to $82 / 46$. She was given normal saline boluses (>30 ml/kg body weight), with minimal improvement of BP to $90 / 50$. Metronidazole IV $500 \mathrm{mg}$ Q8H was added as she was assessed to have Severe Clostridium Difficile with assumed first recurrence. She was transferred to MICU where pressors were started immediately. The patient's ICU course was complicated by worsening of sepsis with a peak WBC count of $116 \mathrm{k} / \mathrm{cumm}$. Patient developed worsening abdominal distension and abdominal X-ray revealing ileus with possible distal obstruction. On her second ICU night the patient was intubated. She expired on Day 4 of her admission due to septic shock secondary to severe CDI after withdrawal of care decision was made with family. DISCUSSION: The Institute of Medicine (IOM) recently published a report highlighting diagnostic errors and their prevalence in medical practice. Amongst the various diagnostic errors is premature closure, a common diagnostic error that is defined as making a diagnosis based on classical symptoms that fit a pattern of a particular diagnosis. This prompts cessation of further data collection and prevents consideration of other diagnosis. The IOM outlined eight goals in their report to improve diagnostic accuracy and reduce diagnostic errors which generally highlight team work, recognition of errors and teaching diagnostic processes. [1]. Importantly, recognition of the existence of such errors is crucial in order for a system to be developed in order to gather data and learn from these errors. It is important to note the committee also concluded that diagnostic error is just as prevalent with common diseases as it is with rare diseases. Our patient had worsening leukocytosis which appeared to be unexplained given that she was on appropriate antibiotics for pyelonephritis. Unexplained leukocytosis can be a clue towards CDI and a recent study found that in 60 prospectively followed patients with unexplained leukocytosis 35 (58 \%) had CD Toxin present as compared to 3 (12\%) of hospitalized patients without leukocytosis [2]. Health-care associated infections are a fast growing problem in the USA with a large 2014 study involving 11,282 patients found 452 had 1 or more health-care associated infections. Clostridium difficile was the number one most isolated pathogen. [3]. CDI has been steadily increasing in both incidence as well as mortality with 30-40 cases per 100,000 in mid/late 1990 's to 84 per 100,000 in 2005 and a 4 fold increase in mortality from 1999 to 2004. [4, 5]. Early detection and recognition of the severity of disease is important across all hospital settings despite other admitted diagnosis. 1. National Academies of Sciences, Engineering, and Medicine. 2015. Improving diagnosis in health care. Washington, DC: The National Academies Press 2. Wanahita, A., et al., Clostridium difficile infection in patients with unexplained leukocytosis. Am J Med, 2003. 115(7): p. 543-6. 3. Magill, S.S., et al., Multistate point-prevalence survey of health care-associated infections. N Engl J Med, 2014. 370(13): p. 1198-208. 4. Kelly, C.P. and J.T. LaMont, Clostridium difficile-more difficult than ever. N Engl J Med, 2008. 359(18): p. 1932-40. 5. Redelings, M.D., F. Sorvillo, and L. Mascola, Increase in Clostridium difficile-related mortality rates, United States, 1999-2004. Emerg Infect Dis, 2007. 13(9): p. 1417-9.

\section{SHARED DECISION MAKING AND THE USE OF DEEP BREATHING EXERCISES IN A PATIENT WITH DEPRESSION AND ANXIETY Brett J.} Ehrmann. NewYork-Presbyterian Hospital, Weill Cornell Medical Center, New York, NY. (Control ID \#2466059)

LEARNING OBJECTIVE \#1: Recognize the importance and technique of using a shared decision making model in the development of treatment plans, particularly when physicians and patients have discordant preferences

LEARNING OBJECTIVE \#2: Recognize the role of breathing exercises as a potentially effective, simple, and free component of therapy in patients with depression and anxiety CASE: The patient is a 41 year old female with history of depression, anxiety, and PTSD (from childhood trauma) transferring care to our clinic due to a change in her insurance. The change also caused her to terminate her relationship with her psychiatrist. On questioning, she cites sleep disturbances, anhedonia, guilt, poor energy, lack of concentration and attention, but no suicidal nor homicidal ideation. She was previously taking Paroxetine with improvement, but self-discontinued it after feeling it was no longer effective. She was also previously taking Alprazolam as needed, but discontinued it 18 months prior to her visit. For the past 12 months, she attributes the above symptoms, as well as occasional dysphagia, hyperacusis, and abdominal pain, to a prolonged ongoing Alprazolam withdrawal syndrome. Past medical and surgical history, medications, allergies, and family history were all otherwise unremarkable. She denies tobacco, alcohol, or illicit substances, and has a MSW degree. On exam, vitals were within normal limits. The exam was unremarkable except for a guarded and depressed affect, appearing distant during the interview. On laboratory analysis, $\mathrm{CBC}$, Comprehensive Metabolic Panel, and TSH were within normal limits. She was determined to have poorly controlled major depression and generalized anxiety, with a possible somatic symptom disorder. The patient was unwilling to be restarted on pharmacotherapy, as she felt it may disturb or delay recovery from what she considers as her Alprazolam withdrawal symptoms. She was also unwilling to establish care with a new psychiatrist, stating she had no energy to do so. 
Using a shared decision making model as a tool for patient engagement and negotiation, mutual agreement was reached to focus on home relaxation therapy with breathing exercises, frequent visits with her PCP, and continuous reassessment for her willingness to re-start pharmacotherapy and psychiatric care. Instruction and handouts for the breathing exercises were provided in the office. In 4 months after the initial visit, patient notes mild improvement in her presenting symptoms and asks for help in re-establishing with a psychiatrist.

DISCUSSION: Treatment decision tool: Shared Decision Making Model In a shared decision making model, clinicians and patients share the best available evidence for a medical decision, and patients are supported to consider options and achieve informed preferences. This is in contrast with a paternalistic model, where the physician is viewed as capable of discerning and implementing patient preferences, therefore bearing sole responsibility for making treatment decisions. Compared to a paternalistic model, shared decision making has been demonstrated to enhance patients' sense of control, lower levels of concern about their disease, increase satisfaction with treatment, and improve outcomes. It can be implemented in three key steps: 1 . Choice talk - making sure that patients know that reasonable options are available. 2. Option talk - providing more detailed information about options, and 3. Decision talk — supporting the work of considering preferences and deciding what is best. This was the framework used in negotiating the treatment plan for our patient. Negotiated Treatment Plan: Home Relaxation and Breathing Exercises Breathing exercises (such as Sudarshan Kriya Yoga) have been associated with improved depression and anxiety symptoms, independent of the use of pharmacotherapy. It involves cycles of easy-to-learn alternate nostril breathing, fast diaphragmatic breathing, rapid exhalation, and cyclical breathing. The patient was able to learn and perform these exercises at home. Take-Home Points Using a shared decision making model is critical when there are discordant treatment preferences between physician and patient. In this case, the differences were attributed primarily to a psychiatric diagnosis, but the principle applies generally. Breathing exercises can be a free and effective component of therapy in patients with depression and anxiety, including in those unwilling or unable to be treated with medications or CBT. References J Gen Intern Med 2012; 27(10):1361-7; Am J Prev Med 1999;17(4):285-294; J Affect Disord 184(2015): 310-317

SHOULD WE PERFORM ECHOCARDIOGRAPHY IN PATIENTS WITH FEVER AND HEADACHE? Takuma Yamada ${ }^{1}$; Some Furukawa ${ }^{1}$; Takashi Okajima ${ }^{1}$; Atsushi Kawabata ${ }^{1}$; Yasuhiro Ito ${ }^{1}$; Mitsunori Iwase $^{2} .{ }^{1}$ TOYOYA Memorial Hospital, Toyota city, Japan; ${ }^{2}$ TOYOYA Memorial Hospital, Toyota, Japan. (Control ID \#2469968)

LEARNING OBJECTIVE \#1: Recognize how to determine infective endocarditis as a cause of the symptoms of brain abscess, such as fever and headache.

LEARNING OBJECTIVE \#2: Manage follow-up for patients with infective endocarditis with attention to symptoms of systolic embolism.

CASE: A 37-year-old woman was admitted to our hospital for headache and drowsiness. Two months before this admission, she experienced severe headache followed by anorexia resulting in weight loss. On examination, the patient was alert and oriented. But she appeared somewhat drowsy. Vital signs revealed temperature of $38.8^{\circ}$, blood pressure of $103 / 58 \mathrm{mmHg}$, pulse of 80 beats/min, respiratory rate of 18 breaths/min with her oxygen saturation of $97 \%$ on room air. White blood cell count was $9500 / \mu \mathrm{L}$, and C-reactive protein level was $3.2 \mathrm{mg} / \mathrm{dL}$. Neck stiffness was noted, so the lumbar puncture was performed suspecting of meningitis, which showed cell counts of 755 cells/ $\mu \mathrm{L}$ ( $92 \%$ polynuclear cells). Magnetic resonance imaging showed multiple lesions in the right cerebral hemisphere. Based on the above findings, the presence of multiple brain abscesses were highly suspected. On physical examination, both bleeding from the palms and digits of the hands were noted. The Barré test of the left side was positive. Systolic murmur was present at the cardiac apex. Therefore infective endocarditis was suspected as a cause of multiple brain abscesses. Accordingly echocardiography was performed. The vegetations in the mitral valve and mitral valve prolapse were noted. We initiated treatment with empiric antibiotic therapy. Blood cultures on admission grew streptococci from the $\gamma$ viridans group. This finding combined with the observations from echocardiography did confirm the diagnosis of infective endocarditis. A few days after the initiation of treatment, the contrast abdominal CT revealed embolisms noted in the spleen as well as the left kidney, except in the brain. Although the patient had multiple embolisms, surgery was not performed because of her poor condition. After 6 weeks of antibiotic therapy, the patient improved clinical. The palsy of left hand wasn't getting worse, and the abscess of the brain reduced. She didn't need the surgical treatment because heart failure didn't develop, and she discharged from our hospital after 6 weeks of therapy.

DISCUSSION: According to some case reports, hematogenous spread, such as infective endocarditis, causes $20-35 \%$ of brain abscesses. During the active phases of infective endocarditis, neurological complications occur in $20-40 \%$ of patients, and brain abscesses occur in $2-4 \%$ and have been associated with a poor outcome. Physicians examining patients with brain abscesses, especially those with multiple embolisms, should consider the possibility of the infective endocarditis as a potential cause and should perform echocardiography. In this case, the patient complained of fever, headache, anorexia, weight loss, and drowsiness. The symptoms of infective endocarditis can present in various ways. For example, some patients complain of stomachache, back pain, or nausea. Because of the variable symptoms, there are several pitfalls. Above of the all, it is important to suspect it when patients present with those various symptoms. A number of factors predispose to the onset of infective endocarditis, such as injection drug use, dental infection, valvular disease, congenital heart disease, HIV infection and others. The oral environment of this patient was clean, and her HIV antibody levels were negative. Mitral valve prolapse was the likely cause in this case. Risk factors for multiple embolisms in infective endocarditis include the presence of left-sided vegetations, the larger size of vegetation, microbiology itself, age, the presence of diabetes, and atrial fibrillation. In this case, we focus on embolization prior to the initiation of antibiotics. The patient had experienced headache for 2 months. The delay in diagnosis may have induced development of multiple embolisms. We have learned from the interesting case how to define infective endocarditis as a cause of the symptoms of brain abscess, such as fever and headache.

SKIN RASH AS THE ONLY MANIFESTATION OF A METASTATIC CANCER! Pratyusha Bollimunta ${ }^{3}$; Venu M. Ganipisetti ${ }^{1}$; Malav P. Parikh ${ }^{4}$; Harvey J. Friedman ${ }^{4}$; Sreelakshmi Panginikkod ${ }^{2} .{ }^{1}$ Miami Valley Hospital, Evanston, IL; ${ }^{2}$ Presence Saint Francis Hospital, Evanston, IL; ${ }^{3}$ Presence saint francis hospital, Evanston, IL; ${ }^{4}$ Saint Francis Hospital, Evanston, IL. (Control ID \#2470222)

LEARNING OBJECTIVE \#1: Recognize that skin rash should have a broader differential of diagnosis as it may be part of a serious underlying condition.

LEARNING OBJECTIVE \#2: Realize that as there are no clear guidelines for screening of ovarian malignancies in Dermatomyositis patients; a thorough physical exam and close follow up is required.

CASE: We are reporting a case of an unfortunate patient who presented to our hospital seeking medical care for a simple skin rash, which was later found out to be a manifestation of paraneoplastic Dermatomyositis. By the time of presentation, she was already harboring an advanced underlying malignancy. We take this as an opportunity to present the case and discuss the existing literature linked with the condition. A 73-year-old female who has not seen a physician for several years presented to our emergency room with chief complaint of rash on her face, upper chest and arms. Patient mentioned that symptoms started a week prior to presentation with no associated fevers or chills. There was some reported crusting and weeping of the lesions. She did not have any previous rashes, myalgias or muscle weakness. Her initial diagnosis was a probable photosensitive rash with secondary cellulitis. On physical exam, her vitals were temperature 97.7 F, BP - 159/ 
$85 \mathrm{~mm} / \mathrm{hg}$, pulse $95 / \mathrm{min}$, respiratory rate $-18 / \mathrm{min}$, oxygen saturation $97 \%$ on room air Skin rash was prominent in the sun exposed areas of the chest, arms and face. She also had some scaling on the forehead. No heliotrope rash or gottron's papules were noted. Her neurological exam was nonfocal. On abdominal palpation, a mass like periumbilical fullness was felt. Initial labs showed elevated troponins without any EKG changes. Further cardiac workup was negative for any ischemia. She had abnormal liver function tests with elevated AST- 591 and ALT-90. Her CK level was noted to be 10,690 (normal 0-200 units per liter) . Her aldolase level was elevated to 54.4 (normal less than 8.1). She also had elevated TSH-29.1, T4 level-0.24. CA-125 was elevated to 1445 (normal range less than 35). CT scan done to evaluate for abdominal fullness showed extensive omental caking suggestive of peritoneal carcinomatosis. Subsequent PET scan showed extensive peritoneal carcinomatosis throughout abdomen and pelvis and abnormal uptake in the left adnexa and surrounding uterus. Biopsy was consistent with metastatic adenocarcinoma with a ovarian primary. She was empirically started on antibiotics along with local wound care on admission. However, by day 3 of admission, she started developing weakness of her extremities. Exam revealed symmetric, proximal, bilateral, muscle weakness with depressed reflexes throughout. By this time, we had our preliminary workup indicating myopathy. ANA was positive with speckled pattern. She was empirically started on steroids at $1 \mathrm{mg}$ per KG per day. Patient was started on chemotherapy with carboplatin and Taxol for treatment of metastatic ovarian carcinoma, once the diagnosis was confirmed. Upon further work up, skin biopsy taken from the chest skin showed interface dermatitis. EMG showed decreased amplitude indicating myopathy. Muscle biopsy confirmed the diagnosis of dermatomyositis. She is currently receiving her 2 nd week of treatment with high dose steroids and chemotherapy. So far, she only had minimal improvement in her weakness with moderate improvement in CK levels.

DISCUSSION: Dermatomyositis is known to be associated with malignancies for a long time and its association with ovarian cancer is frequently reported. It may present as a paraneoplastic syndrome in up to $25 \%$ of cases. This condition can be a late manifestation of already advanced underlying metastatic cancer. By the time patients start having skin rash, they may already be harboring an advanced malignancy like our patient. Unfortunately, no histologic, clinical, or laboratory markers completely specific for paraneoplastic disease in dermatomyositis have been identified so far. CT scan is advised only for patients who are high risk or have lab or have physical exam abnormalities suggestive of cancer. Elevated CA-125 levels were found to have sensitivity of $50 \%$, and specificity of $100 \%$. However, no major studies have been done on using CA 125 as a tool for screening patients with dermatomyositis. Serial CA 125 levels to screen patients seems to be reasonable approach in view of poor prognosis of late stage ovarian cancer. Usually, paraneoplastic dematomyositis presents as amyopathic dermatomyositis (without muscle involvement). Patients with this condition may not have all the classic signs such as Gottron's papules and heliotrope rash and these are usually late manifestations of the condition. Diagnosis can be a challenge in patients who present only with a photo distributed rash. Physician should have Dermatomyositis in the differential while evaluating photo-distributed rash. Existing literature suggests ovarian cancers are diagnosed within few months of presentation with rash and in most cases, time of diagnosis ovarian cancer is in advanced stages. By the time patients are reevaluated for rash, ovarian cancers will have progressed and it's a missed opportunity to catch the cancer early. Hence a close follow up for resolution of rash is important. This case emphazises the fact that skin rashes can be a manifestation of a serious systemic condition and a thorough physical exam is necessary.

SKIN ULCER AND LEUKEMOID REACTION AS A PRESENTING FEATURE OF NON-SMALL CELL LUNG CANCER (NSCLC) Osama Elsallabi ${ }^{2}$; Haitam Buaisha ${ }^{1}$; Vijaya R. Bhatt ${ }^{3}$; Peter Silberstein ${ }^{4} .{ }^{1}$ Creighton University, Omaha, NE; ${ }^{2}$ Creighton University Medical Center, Omaha, NE; ${ }^{3}$ University of Nebraska Medical Center, Omaha, NE; ${ }^{4}$ Creighton, Omaha, NE. (Control ID \#2466567)
LEARNING OBJECTIVE \#1: Skin metastasis and leukemoid reaction are uncommon manifestations of NSCLC.

LEARNING OBJECTIVE \#2: Skin metastasis may be difficult to diagnose, particularly without a high index of suspicion.

CASE: A 68-year-old man with a history of chronic obstructive lung disease and heavy smoking presented with infected right buttock ulcer refractory to trimethoprimsulfamethoxazole and incision and drainage. He also had 40-lb weight loss, productive cough and dyspnea empirically treated with levofloxacin. Examination revealed $4 \times 5 \mathrm{~cm}$ right inguinal lymph node. Laboratory evaluation showed leukocytosis of $25,000 / \mu \mathrm{L}$, predominantly neutrophils $(87 \%)$. Wound culture did not grow any organism. Further evaluation demonstrated $13 \mathrm{~cm} \times 8.4 \mathrm{~cm} \times 14.4 \mathrm{~cm}$ right lung mass and diffuse lymphadenopathy. Bronchoscopic biopsy of right hilar lymph node demonstrated poorly differentiated carcinoma with large cells showing prominent nucleoli, which was positive for cytokeratin AE1/AE3; however, p40 and TTF-1 were negative. Biopsy of right inguinal lymph node and right gluteal ulcer was also consistent with metastatic carcinoma. With a clinicopathological diagnosis of NSCLC, the patient was treated with 2 cycles of carboplatin and paclitaxel with progressive disease requiring switch to pemetrexed. After 3 cycles of pemetrexed, the patient presented with increased dyspnea and mild hypoxia. The patient denied any headache, double or blurry vision, paresthesia, bleeding or chest pain. Laboratory evaluation showed leukocytosis of $137,700 / \mu \mathrm{L}$, predominantly neutrophils (94\%) but also immature granulocytes $(\sim 4 \%)$. Neutrophils had toxic granulation and inflammatory parameters such as $C$ reactive protein $(13 \mathrm{mg} / \mathrm{dl})$ and erythrocyte sedimentation rate $(50 \mathrm{~mm} / \mathrm{h})$ were chronically elevated. Based on his chest xray finding of right middle zone opacity, he was treated with piperacillin-tazobactam and vancomycin for presumptive healthcare associate pneumonia. Blood and sputum cultures were negative. Polymerase chain reaction for BCRABL rearrangement and JAK2V617F mutation were negative. A computed tomography scan of chest and abdomen showed increasing lymphadenopathy including massively enlarged mediastinal and abdominal lymphadenopathy up to $14 \mathrm{~cm}$ and right gluteal masses up to $4.5 \mathrm{~cm}$. The patient subsequently developed progressive hypoxia, hypercarbia and respiratory distress, and was transitioned to comfort cares.

DISCUSSION: Leukemoid reaction should trigger investigations to rule out the possibility of acute leukemia, infection, hyperleukocytosis, tumor lysis syndrome and disseminated intravascular coagulation. However, as illustrated by this case, advanced NSCLC or other malignancy may also be an etiology due to the production of granulocyte-colony stimulating factor. In the absence of leukemia and its associated complications, stable patients without symptoms of hyperleukocytosis may be managed conservatively with treatment of infection or other causes. Our case also highlights that malignant ulcer should be considered in patients with unexplained skin ulcers refractory to appropriate antibiotics and incision and drainage. Understanding of such uncommon manifestations may expedite accurate diagnosis and avoid inappropriate tests.

\section{SMA SYNDROME IN A PATIENT WITH IBS AND PTSD: A CASE REPORT}

Mona Lalehzari; Hao Cheng; Pinky Jha. Medical College of Wisconsin, Milwaukee, WI. (Control ID \#2467319)

LEARNING OBJECTIVE \#1: Recognize biases we have in working with psychiatric patients and avoid premature closure as healthcare providers.

LEARNING OBJECTIVE \#2: Distinguish organic from non-organic etiologies of abdominal pain

CASE: A 24-year-old woman presented to the hospital with "5 weeks of hell" from a sharp right upper quadrant and rib pain, nausea, and daily episodes of diarrhea and bilious emesis. She lost $16 \mathrm{lbs}$ over this time, putting her BMI at 17.4. Initially, her labs and imaging from multiple visits to the ED showed no acute process. Pregnancy test, UA, CBC, CMP, liver/gallbladder/pancreas U/S, and CXR were all normal. Her symptoms 
were attributed to an exacerbation of her IBS potentially secondary to her anxiety and PTSD, which are due to childhood sexual abuse. Her pain continued and she sought out a gastroenterologist who ordered a right upper quadrant U/S, HIDA scan, and abdomen/ pelvic CT. All were normal. The gastroenterologist initially concurred with the working diagnosis of IBS exacerbation. The patient returned a few days later for a UGI series which showed dilation of the third portion of her duodenum and proximally. During an EGD, the gastroenterologist found it difficult to cannulate the patient's jejunum due to duodenal tortuosity. Gastric biopsy showed mild chronic gastritis and negative H. pylori. A CTA showed possible $70 \%$ celiac artery occlusion with dissection. She then came to our hospital for potential emergency surgery. Vascular surgery and radiology at the tertiary care center reviewed her CTA and ruled the celiac artery dissection finding as motion artifact. A repeat CT showed compression of the third portion of her duodenum by the SMA with loss of her fat pad and the patient was diagnosed with SMA syndrome. The patient was made NPO and was started on NJ feedings and IV analgesics. The patient's pain resolved and she was able to avoid surgical intervention by slowly gaining proper weight via NJ feeds. She was discharged home with appointments for follow-up care.

DISCUSSION: SMA syndrome is a condition where the SMA obstructs the third portion of the duodenum secondary to diminished fat pad between the SMA and duodenum, often seen in anorexics. As it is exceedingly rare, with an estimated incidence of $0.1-0.3 \%$, it is frequently a diagnosis of exclusion. This case recognizes an organic etiology for abdominal pain in a patient who might otherwise have been misdiagnosed due to her other psychiatric and GI comorbidities, thus highlighting the importance of avoiding premature closure. Data in the literature as well as observation of clinical practice supports that physicians experience difficulty and bias in separating organic from non-organic etiologies of symptoms in patients with psychiatric comorbidities. Past studies have found that up to half of physical illnesses amongst psychiatric patients go undiagnosed and are instead labeled as "psychosomatic." Further, studies show that morbidity in psychiatric clinic patients also far surpasses the expected rate found in the general population, partly attributable to inadequate diagnostic workup for physical complaints and misdiagnoses. This dynamic is illustrated here with our patient with preexisting IBS, PTSD, and anxiety disorder. She was a psychiatric patient that presented with non-specific symptoms and underwent multiple appointments with a number of providers before getting the right diagnosis. Although the SMA syndrome seen in this patient is rare, it was a predictable etiology of her GI symptoms given her low BMI and change in symptoms from baseline. Recognizing our biases in working with psychiatric patients in the healthcare setting can help in avoiding early write off of symptoms as merely psychological manifestations and thus dismissal without further diagnostic workup. Avoidance of biases and thus early recognition of this syndrome is essential for timely therapy and avoidance of dehydration and malnutrition. This principle can apply to all patients with psychiatric comorbidities.

SMALL RODENTS, BIG APPLE: AN ATYPICAL SETTING FOR WEIL'S DISEASE Jaime Deseda ${ }^{1}$; James Kaus ${ }^{1}$; Tina Wang ${ }^{1}$; Alfred Burger ${ }^{2} .{ }^{1}$ Mount Sinai Beth Israel, New York, NY; ${ }^{2}$ Mount Sinai Beth Israel, New York, NY. (Control ID \#2469864)

LEARNING OBJECTIVE \#1: Recognize clinical characteristics of leptospirosis

CASE: 60 year old male with no history of any medical illnesses presented with complaints of subjective fevers, worsening proximal bilateral leg pains and general weakness for 4 days. He was in his normal state of health prior to this and denies any additional symptoms. Social history was significant for recent travel to his home country, Uzbekistan, where he had emigrated to the United States from 5 years prior. He stayed only in urban environments and denied any livestock contact. He was currently employed as a masseuse but had worked as an athletic coach prior to his emigration. He had no history of toxic habits or sexual promiscuity. He took no medications or over the counter supplements. Vital signs included T 98.9 F HR 87 BPM BP 118/79 mmHg RR 18 BPM at $100 \% \mathrm{SaO} 2$ on room air. Physical exam significant for scleral icterus without conjuctival suffusion, diffuse, blanching raised pink rash on trunk and extensor extremity surface independent of sun exposed areas, and inability to ambulate independently due to profound weakness. On presentation, patient was oliguric. Laboratory values were significant for Na $129 \mathrm{mmol} / \mathrm{L}, \mathrm{K} 3.2 \mathrm{mmol} / \mathrm{L}$, BUN $57 \mathrm{mg} / \mathrm{dL}$ and Cr $7.59 \mathrm{mg} / \mathrm{dL}$, elevated aminotransferases of AST $208 \mathrm{U} / \mathrm{L}$, ALT $189 \mathrm{U} / \mathrm{L}$, total bilirubin $9.3 \mathrm{mg} / \mathrm{dL}$ and direct of $7.9 \mathrm{mg} / \mathrm{dL}$. Complete blood counts showed WBC was $19.0 \mathrm{~K} / \mathrm{uL}, 94 \%$ neutrophils with 4 bands and lactic acid $1.0 \mathrm{mmol} / \mathrm{L}, \mathrm{Hgb} 12.5 \mathrm{~g} / \mathrm{dL}$, PLT $66 \mathrm{~K} / \mathrm{uL}$. CPK $2331 \mathrm{U} / \mathrm{L}, \mathrm{LDH}$ $1003 \mathrm{U} / \mathrm{L}, \mathrm{CRP} 18.0 \mathrm{mg} / \mathrm{dL}$, ESR $86 \mathrm{~mm} / \mathrm{h}$. UA significant for protein 300, glucose $150 \mathrm{mg} / \mathrm{dL}$, WBC $9 \mathrm{HPF}, 5 \mathrm{RBC}$ HPF with muddy brown casts. Urine toxicology for basic illicit substances negative. Acute hepatitis panel negative and HIV Ab screen negative. Peripheral smear significant for anisocytosis. Initial assessment included systemic infection, malignancy, rheumatological process or vasculitis. Several services were consulted, including nephrology, hematology/oncology, gastroenterology and dermatology. Several liters of isotonic fluids were given. Hematology/oncology recommendations did not believe systemic malignancy was at play. Dermatology obtained one biopsy which showed nonspecific inflammation. GI workup included sonogram and MRI of the liver, which was remarkable for gallstones, multiple gallbladder polyps up to $1 \mathrm{~cm}$ and $15 \mathrm{~mm}$ hepatic mass, considered to be hemangioma. Sonography of kidneys unremarkable for acute pathology. Initial imaging included only chest x-ray which was unremarkable. Renal workup was significant for late presentation of acute tubular necrosis likely from rhabdomyolysis secondary to unknown cause. The oliguria improved with isotonic fluids, electrolyte repletion and trials of diuretics. Hemodialysis was not initiated. Subsequently, hyponatremia was managed and improved with salt tablets. Initially, mild improvement was seen with supportive management. However, over the course, bilateral conjunctival suffusion was observed, thus zoonotic workup was performed. Zoonotic panel revealed positive screening leptospirosis antibody, prompting oral doxycycline and an infectious disease consult. Our patient improved significantly, including return of strength and ability to ambulate independently. Was discharged with antibiotic regimen on outpatient. No complications occurred during hospitalization.

DISCUSSION: We present a case of multi-organ failure of unknown etiology later diagnosed as leptospirosis, specifically Weil's disease, in a patient with limited animal exposures. Leptospirosis, a zoonotic disease caused by a spirochete, common in rodents, dogs, horses, and domesticated farm animals, is widespread and underreported, with a worldwide mortality of $5.5 \%$. Humans are often infected after exposure to urinecontaminated waters or contaminated soil, particularly at risk if they are barefoot. In cities, rodents are the main reservoir, transmitted with exposure to animal urine contaminating water sources and soil. From 2006 to 2013, only 116 diagnosed cases were reported in New York City. Recent epidemiological data in NYC found $93 \%$ of human infections in the city related with exposure to the leptospirosis subtype associated with rodents, which can be transmitted to dogs. Our patient had severe leptospirosis, which can manifest as acute kidney failure, jaundice, pulmonary hemorrhage, acute respiratory distress syndrome, myocarditis, rhabdomyolysis and uveitis. This potentially life-threatening condition is called Weil's disease, which requires a high clinical suspicion based on physical exam findings and significant exposures. Currently there are no specific laboratory abnormalities that increases suspicion for leptospirosis, except serologic testing. Our case highlights the necessity of a greater clinical suspicion despite low risk exposures, particularly in the physical exam. Conjunctival suffusion is a distinguishing element in Weil's disease, and its presence should lead to high clinical suspicion for leptospirosis as it is rarely present in our systemic infections. Our initial workup was approached on a systemby-system basis. Multiple consulting teams were involved in the initial workup. The result being that each organ dysfunction was evaluated independently rather than searching for a unifying diagnosis. As the primary team, consideration of the broad picture and overarching diagnosis should always be crucial. 
SPECIAL K AND UN-KOMMON BILE DUCT DILATATION Paul Leis; Gregory Haber; Steven Shamah. Lenox Hill Hospital, Brooklyn, NY. (Control ID \#2470293)

LEARNING OBJECTIVE \#1: Recognize inhalational ketamine as a cause of common bile duct dilatation.

LEARNING OBJECTIVE \#2: Identify the pathogenesis of ketamine induced common bile duct dilatation and urinary tract infections.

CASE: A 33 year old Asian woman with a history of choledocholithiasis with cholecystectomy 1 year prior to admission, and frequent urinary tract infections (UTIs) presented to our institution with 3 days of epigastric pain associated with mild dysuria. The patient stated the pain began suddenly and was not associated with any fevers, chills, or recent travel. Physical exam of the abdomen did not reveal any tenderness to palpation, with a negative Murphy's sign. Initial labs were significant for a total bilirubin of $0.4 \mathrm{mg} / \mathrm{dL}$, ALT of $83 \mathrm{U} / \mathrm{L}, \mathrm{AST}$ of $30 \mathrm{U} / \mathrm{L}, \mathrm{ALP}$ of $222 \mathrm{U} / \mathrm{L}$, and a urinalysis with $>10 \mathrm{WBC}$, and positive leukocyte esterase. A CT scan of the abdomen and pelvis with contrast displayed an enlarged common bile duct (CBD) of approximately $1.9 \mathrm{~cm}$ with mild intrahepatic dilatation and no evidence of gallstones. Abdominal MRI confirmed a CBD of $1.9 \mathrm{~cm}$ without filling defects, and a normal pancreatic head. On further history, the patient reported inhalational ketamine use about 3-5 times/week over the past 2 years with most recent use 2 days prior to admission. An ERCP was recommended to further evaluate the $\mathrm{CBD}$, however the patient's symptoms and liver tests trended down with etiology thought to be secondary to inhalational ketamine use.

DISCUSSION: Ketamine was initially developed as an anesthetic, acting as an antagonist on the NMDA receptor. Today ketamine is a commonly abused street drug producing short term effects of euphoria, hallucinations, and out of body experiences. However recently many adverse effects have been reported including detrimental effects on the hepatobiliary and urinary tract systems. In our literature review there have been up to twenty cases of reported dilated common bile ducts at times mimicking choledochal cysts, as well as frequent urinary tract infections amongst ketamine users. The mechanism for CBD dilatation and frequent UTIs is postulated to be caused by direct toxic metabolites on biliary and uroepithelial cells since the drug is metabolized by the cytochrome P450 system and excreted in the urine and bile. Another mechanism suggests that ketamine functions as an NMDA antagonist and causes relaxation of smooth muscle at the biliary tree and ureter which can result in cholestasis and hydronephrosis, respectively. Cessation of the drug should strongly be encouraged since studies have shown normalization of the CBD on follow up imaging. Although uncommon, as the use of ketamine rises, it is important to recognize its adverse effects, including CBD dilatation, in an effort to prevent unnecessary imaging, endoscopic, or surgical intervention.

\section{SPILLING THE BEANS: AN UNUSUAL CASE OF RECALCITRANT ASCITES}

Chadwick S. Richard. University of Pittsburgh Medical Center, Pittsburgh, PA. (Control ID \#2467749)

LEARNING OBJECTIVE \#1: List the common causes of high-SAAG ascites. LEARNING OBJECTIVE \#2: Recognize nephrogenic ascites as an uncommon and difficult-to-treat cause of ascites in long-term dialysis patients.

CASE: The patient is a 39 year old Caucasian woman with end-stage renal disease from uncontrolled hypertension who has required renal replacement therapy with hemodialysis for the past decade. She is poorly adherent to hemodialysis and misses four to six sessions monthly. She was seen on the general medical service after initially presenting with profound hypertension and a generalized seizure secondary to the Posterior Reversible Encephalopathy Syndrome and transferred out of the ICU once her blood pressure was well controlled. During her course, the patient noted progressing abdominal pain and distention with exam consistent with large-volume ascites and without peripheral edema.
Her ascites began suddenly, 6 months prior to admission, and became tense within $48 \mathrm{~h}$ of onset. She had two previous large-volume paracenteses with less than 2 days before complete re-accumulation. This pattern continued during the subsequent three drainages during her stay. Her initial fluid studies revealed clear yellow free-flowing fluid with a serum-ascites albumin gradient of 1.3 and normal white blood cell count. Abdominal ultrasound with dopplers, CT of the abdomen and pelvis, and transthoracic echocardiography were unrevealing. Additional testing including fluid glucose, amylase, triglyceride, lactate dehydrogenase, adenosine deaminase, acid-fast bacilli stain and culture, fungal culture, two sets of bacterial culture, two sets of cytology, serum liver function tests, hepatitis $\mathrm{B}$ and $\mathrm{C}$ serologies, and ammonia level were negative. Further history taking revealed the diagnosis of cervical cancer and unknown ovarian pathology in the patient's mother. Transvaginal ultrasound revealed a right ovarian abnormality later demonstrated as benign hydrosalpinx by pelvic MRI. The patient's ascites and abdominal pain became tolerable after a week of serial paracenteses. After reviewing her extensive negative evaluation in consultation with the nephrology service, the diagnosis of nephrogenic ascites was made. She was discharged and strongly encouraged to continue hemodialysis, undergo scheduled therapeutic paracenteses, and transition to peritoneal dialysis.

DISCUSSION: This case demonstrates a diagnostic challenge in the context of the common clinical problem of ascites. The most sensitive test for focusing the differential diagnosis of ascites is the serum-ascites albumin gradient (SAAG). Her initial evaluation revealed a high SAAG, leading to evaluation for typical causes of portal hypertension. However, no evidence of underlying cirrhosis, systolic or diastolic heart failure, pericardial or valvular disease, acute hepatitis, liver mass, or porto-hepatic venous thrombosis was found. After her subsequent workup also returned negative, a compelling diagnosis of nephrogenic ascites was reached only after consultation with the senior nephrology faculty who were familiar with the condition. Nephrogenic (or dialysis-associated) ascites is a well-described clinical entity in the nephrology literature but is relatively unknown to generalists. This case highlights many of the commonly described features. The condition is characterized by long-standing renal replacement therapy with either peritoneal or hemodialysis and the development of ascites with no identifiable cause. The incidence is likely $<1 \%$ of dialysis patients but is cited as high as $20 \%$ in older case series. Patients often have hypertension and minimal signs of total body volume overload. Multiple case reports describe rapid onset and re-accumulation of ascites which is often refractory to serial paracenteses and aggressive ultrafiltration. Per one literature review, the SAAG in nephrogenic ascites is often low, but several published cases also report a high SAAG. The underlying cause is not well described, particularly in hemodialysis patients, but may be due to altered peritoneal membrane permeability or impaired lymphatic drainage. Treatment of nephrogenic ascites is often difficult. Trials of salt and water restriction may be effective but are often limited by hypovolemia and hypotension. Peritoneovenous shunt placement is durable and effective but complicated by malpositioning or infection in onehalf of cases. Transitioning from hemodialysis to continuous ambulatory peritoneal dialysis often improves symptoms, but requires patients to tolerate iatrogenic ascites. The most effective treatment is kidney transplantation, which often results in complete resolution of ascites within weeks of graft implantation, but recurrence of the ascites after graft failure is very common. Regardless of treatment, limited mortality data shows life-expectancy is between 7 and 18 months after developing the condition. Why patients die rapidly after developing nephrogenic ascites is poorly understood.

SPINAL ARACHNOIDITIS AND CAUDA EQUINA SYNDROME - A RARE MIX FOR A PRIMARY CARE PHYSICIAN Sisir Akkineni ${ }^{1}$; Lisa C. Martinez ${ }^{2}$. ${ }^{1}$ University of Miami/HolyCross Hospital, Ft Lauderdale, FL; ${ }^{2}$ University of Miami, Miami, FL. (Control ID \#2467204)

LEARNING OBJECTIVE \#1: Recognize post-operative lumbar spinal arachnoiditis as a rare cause of cauda equina syndrome 
LEARNING OBJECTIVE \#2: Management of chronic adhesive lumbar spinal arachnoiditis with cauda equina syndrome in a primary care setting

CASE: Our patient is a 63 year old woman who presented in a primary care clinic with a 3 month history of severe pain refractory to analgesics in the low back and perineal region, urinary retention, and constipation. She had undergone lumbar spinal laminectomies from L2-L3 to L4-L5 for spinal canal stenosis 3 months prior to presentation. Her present symptoms started 2 weeks after the surgery and progressively worsened. She experienced intermittent episodes of urinary incontinence but urinary retention was predominant. She had significant $9 / 10$ pain in the lower back and perineal region refractory to Percocet and NSAIDs. She had a bowel movement once a week. There was difficulty evacuating her bowel despite use of multiple stool softeners. Prior to presenting to us, she visited an Emergency Room for similar complaints. CT abdomen done there revealed significant stool impaction and "fullness" of renal system. She was prescribed docusate for constipation with little improvement of her symptoms. She denied pain or weakness in the lower extremities, and she was able to ambulate well without support. She denied history of stumbling while walking or recent falls. She also reported subjective fever. On examination, she was afebrile. There was suprapubic tenderness with a distended bladder. There was mild lumbar spinal tenderness. She had good motor strength in lower limbs, equal bilaterally without asymmetry. Tactile stimulation demonstrated a decreased light touch and pinprick sensation in the buttock/perianal region (S 3, 4, 5) bilaterally. Rectal examination revealed reduced anal sphincter tone. Given her presentation, there was concern for cord compression. We emergently referred her for Lumbar spine MRI without contrast which revealed clumping of the nerve roots of the lower thecal sac consistent with arachnoiditis. There was no evidence of epidural collection or discitis, and no mass effect on the conus. Lumbar spinal arachnoiditis was the likely cause of her cauda equina syndrome. She was conservatively managed with NSAIDs, stool softeners for chronic constipation, and intermittent self-catheterization. This provided significant pain relief to the patient.

DISCUSSION: Chronic adhesive spinal arachnoiditis is a rare, non-specific inflammation of the leptomeninges whose incidence with Cauda Equina Syndrome (CES) is not well known. A patient presenting to the primary care clinic with history of spinal insult such as spinal infection, spinal surgery, intraspinal injections, spinal anesthesia or oil myelography should receive a thorough physical examination and symptom review for CES. As in the case of our patient, symptoms such as constipation, urinary retention or chronic pain can be misleading. There is high risk if symptoms of CES are present and emergent MRI is not performed to rule out reversible causes. The treatment of chronic adhesive lumbar spinal arachnoiditis with CES is primarily conservative. There is little evidence to support surgical intervention. Supportive care includes management of chronic urinary retention, chronic constipation, treatment of recurrent urinary tract infections, appropriate pain management, psychotherapy, and physical therapy. Individualized goals of care discussion must be had with every patient and their caregivers.

SPLENIC AND HEPATIC VEIN THROMBOSIS: AN ATYPICAL PRESENTATION OF POORLY DIFFERENTIATED COLON ADENOCARCINOMA Jehan Feroz. University of Connecticut, Hartford, CT. (Control ID \#2470036)

LEARNING OBJECTIVE \#1: Recognize the clinical features and atypical radiographic findings of poorly differentiated adenocarcinoma of the colon.

CASE: Splenic and Hepatic Vein Thrombosis: An Atypical Presentation of Poorly Differentiated Colon Adenocarcinoma A 52 year old female with no significant past medical history presented to the emergency room with abdominal fullness, nausea, and vomiting for a week. She also had been having subjective fevers and chills for 3 weeks. She had a family history of ovarian cancer. Her initial laboratory workup revealed microcytic anemia with a hemoglobin in the low $7 \mathrm{~s}$. CT scan of abdomen and pelvis with contrast demonstrated a thrombus in the main portal and splenic veins and also in the left portal vein. There was also a large splenic infarct and a small perisplenic fluid collection, left adnexal cyst and fibroids. She was found to bacteremic with Bacteroides fragilis and also found to have a poorly differentiated colon adenocarcinoma in the splenic flexure of the colon. She had her splenic abscess drained which grew Bacteroides caccae. She also had surgery with left sided colectomy, splenectomy, and partial resection of the pancreas. She was placed on intravenous ceftriaxone and metronidazole for her bacteremia, and low molecular weight heparin for her thromboses.

DISCUSSION: Splenic and hepatic vein thromboses are a less common manifestation of colon cancer. Generally, they are more associated with pancreatic cancer. Poorly differentiated adenocarcinoma of the colon was found in the above patient. Clinical suspicion should be high of a malignancy in such a case as above. The etiology of splenic and hepatic vein thrombosis in this case was likely secondary to the fact that there was compression effect by the mass in the splenic flexure.

\section{SPONTANEOUS CORONARY ARTERY DISSECTION : AN UNCCOMMON} CAUSE OF CHEST PAIN THAT SHOULD NOT BE OVERLOOKED. Siwadon Pitukweerakul $^{2}$; Sai Prasad Gadapa ${ }^{1}$; Sree V. Pilla ${ }^{3}$; Harvey J. Friedman ${ }^{1} .{ }^{1}$ Presence Saint Francis Hospital, Evanston, IL; ${ }^{2}$ Presence St. Francis Hospital, Evanston, IL; ${ }^{3} \mathrm{St}$ Francis Hospital, EVANSTON, IL. (Control ID \#2469177)

LEARNING OBJECTIVE \#1: To present the case of spontaneous coronary artery dissection which is a rare cause of chest pain. This clinical vignette would be helpful for clinicians to recognize SCAD and create a reasonable differential diagnosis list for chest pain which is a commom problem in general medical practice.

CASE: A 48-year-old woman presented to the emergency department of severe chest pain. It began $2 \mathrm{~h}$ prior to presentation while walking down the street and was described as severe pulling and dull pain. The pain radiating to the right arm and associated with profound sweating. Her medical history included hypertension and diabetes. She had no surgical history. She had no recent prolonged traveling. She denied smoking and drinking alcohol. In the emergency department, she was hemodynamically stable. Physical examination was normal. The initial electrocardiogram (ECG) at presentation revealed T-wave inversions in the inferior/lateral leads (II, III, aVF, V5 and V6 leads). Laboratory tests were normal except for an elevated serum troponin level of $19 \mathrm{ng} / \mathrm{ml}$. Chest radiograph and computed tomographic (CT) angiography were normal. Echocardiography revealed an ejection fraction of $55 \%$ and relatively hypokinetic area of the apex. Aspirin, metoprolol, atorvastatin, nitroglycerin, and heparin infusion were given for non-ST-segment elevation myocardial infarction (NSTEMI) Coronary angiography was performed after initial management which revealed spontaneous dissection of the second marginal branch of left circumflex artery (LCX). Percutaneous coronary intervention (PCI) was successfully performed without complications. She was discharged on aspirin, ticagrelor, warfarin, metoprolol, atorvastatin and nitroglycerin. She did well on follow-up 1 and 3 months after hospitalization.

DISCUSSION: Spontaneous coronary artery dissection (SCAD) is a rare, but lifethreatening cause of chest pain. It is also an important cause of acute coronary syndrome and sudden cardiac death. Clinical manifestations vary from mild chest pain to myocardial infarction and sudden cardiac death. SCAD is more common in women than in men. It often affects younger women in the peripartum period. Diagnosis of SCAD is mostly made by coronary angiography. The most common site of dissection is the Left anterior 
descending artery (LAD). LCX involvement like our case is likely the least common site of dissection. As the appropriate treatment of SCAD has not been established. Treatment should be based on findings and severity of the disease. Medical treatment, percutaneous coronary intervention, and surgery are considered mainstay of therapeutic management. Medications such as anticoagulants, aspirin, clopidogrel, beta blockers and nitrates are usually recommended for SCAD medical management. Surgery or percutaneous coronary intervention are usually preserved for patients with extensive dissections resulting in persistent ischemia. Clinicians should be aware of SCAD as a rare cause of chest pain because early recognition and treatment of SCAD is crucial given the high mortality rate of the condition.

SPONTANEOUS MULTIVESSEL CORONARY ARTERY DISSECTIONS CAUSING MYOCARDIAL INFARCTION IN A MULTIPAROUS PREGNANT WOMAN. Pratap Reddy Tetali ${ }^{1}$; Anthony Sonn ${ }^{1}$; Preethi Chiluveru ${ }^{2} .{ }^{1}$ Mercy Hospital, St. Louis, MO; ${ }^{2}$ SSM health St. Mary's Hospital, Richmond Heights, MO. (Control ID \#2466783)

LEARNING OBJECTIVE \#1: Spontaneous coronary artery dissection should be considered as one of the differentials in young multiparous, pregnant patients presenting with chest pain. Emergent cardiac cath and prompt treat in those patient can decrease mortality.

LEARNING OBJECTIVE \#2: Auto immune disorders and connective tissue disease should be considered as one of the risk factors for spontaneous coronary artery dissection. CASE: A 44 year female Gravida 8, Para 7 at 33 weeks gestational age presented to the emergency department complaining of chest pain. She denied any significant past medical history. Family history was negative for coronary artery disease. EKG showed ST elevations in lead V1,V2,V3 consistent with myocardial infarction. She was taken to emergent cardiac cath which showed a mid spiral dissection of the left anterior descending coronary artery (LAD) and a linear dissection in the diagonal artery with TIMI grade III flow. She received two bare metal stents to LAD. Diagonal artery was not stented as the patient was chest pain free and was treated medically. Echo showed an ejection fraction of $25 \%$. She was subsequently discharged to home with a life vest, aspirin $81 \mathrm{mg}$, and plavix for a month. Three months after her discharge, she presented to the hospital complaining of intermittent exertional chest pain and had another cardiac cath which showed right coronary artery dissection with second injection of dye which could have been catheterinduced. She received three bare metal stents to the right coronary artery. Genetic testing and autoimmune work-up is pending.

DISCUSSION: Spontaneous coronary artery dissection (SCAD) is a rare cause of acute coronary syndrome. The exact etiology is unclear but literature suggests multiparity, female gender, peripartum state and connective tissue diseases are common risk factors. It is important to consider SCAD in the differential diagnosis in mulliparous women presenting with chest pain. Emergent cardiac cath and promt treatment can be life saving. Consider ruling out other risk factors like connective diseases and auto immune disease in patients with SCAD.

SPOTTED FEVER WITH NO SPOTS: CHALLENGES IN DIAGNOSING ROCKY MOUNTAIN SPOTTED FEVER IN NON ENDEMIC AREAS Sheena Mathew. NYP Cornell, New York, NY. (Control ID \#2462647)

LEARNING OBJECTIVE \#1: Recognize the nonspecific presenting symptoms of RMSF and importance of having high clinical suspicion in establishing the diagnosis LEARNING OBJECTIVE \#2: Diagnose Rocky Mountain Spotted Fever in patients without a rash
CASE: 45 year old female with hypothyroidism presents with 4 days of severe headache, chills, night sweats, and fatigue this past July. Her headache was frontal and associated with nausea and vomiting. Her pain was $9 / 10$ and partially improved with naproxen. She has no history of migraines. She reported a red, circular rash that developed over the dorsal surface of the left wrist that resolved within $24 \mathrm{~h}$. She denied a sore throat, cough, dysuria, abdominal pain, diarrhea, sick contacts or recent travel history. Her home meds are Synthroid and Naproxen. She reported poor appetite on review of systems. She lives in Staten Island with family and denies drug, alcohol use and has been smoking for 1 year. She has no pets, denies tick bites but lives in a wooded area. On presentation, she was febrile to $38.1 \mathrm{C} \mathrm{HR} \mathrm{84,} \mathrm{BP}$ 94/58, RR 18 Sat $98 \%$ on RA. She was an ill appearing, somnolent but arousable to voice. Her oropharynx was clear, with no lymphadenopathy or neck stiffness. Kernig's sign was positive, but she had a negative Brudzinski's sign and no cranial nerve abnormalities or focal neurologic deficits. Heart, lung and abdominal exam were benign. No rashes were appreciated. Labs found leukopenia to 2.2 and thrombocytopenia to 122 with a mild trasaminitis. Her metabolic panel, blood cultures, urine culture, respiratory viral swab, HIV, and Hepatitis panel were negative. CT head was negative for acute bleed or mass and lumbar puncture was attempted but unsuccessful. Patient was started empirically on antibiotics for meningitis, and admitted to neurology for further workup. The next day, LP found 0 WBC, 1 RBC, Glucose of 58, Protein of 15 and negative gram stain, and HSV PCR from the CSF. Her antibiotics were stopped and she was transferred medicine. Her leukopenia progressed to 1.4 with $60 \%$ neutrophils and $27 \%$ lymphocytes and ANC of 800 . Her thrombocytopenia dropped to 96 and her transaminitis peaked at 369 for AST and ALT of 308. We suspected a tick borne illness, specifically Anaplasma given her lab abnormalities, the season and her location. We started the patient on doxycycline. Blood smears for Anaplasma, Erhlichea were sent along with titers for RMSF. Her RMSF titers were negative. Three smears were negative along with anaplasma PCR but the patient clinically improved along with her labs on doxycycline She was continued on doxycycline for presumed RMSF. One week post discharge repeat RMSF antibodies were drawn, and were positive with a titer of 1:128.

DISCUSSION: RMSF is caused by Rickettsia rickettsii, which transmitted primarily through the American Dog Tick. RMSF is primarily seen in North Carolina, Oklahoma, Arkansas, Tennessee, and Missouri but has been reported in most of the 50 states including 4 cases in the Bronx, NY in 1987. The incidence of RMSF varies per year, was 1791 in 2009 , with a mortality rate less than $1 \%$ of cases since 2001 . The diagnosis requires a high clinical suspicion as the initial symptoms are nonspecific and could be seen in many other viral infections or tick borne illnesses. Patients will present with fever, abdominal pain, severe headache, nausea, vomiting, arthralgia and weakness. The typical rash is macular, erythematous and non-blanching and usually will start distally on ankles and wrist and then spread towards the trunk within 3 to 5 days. A sign of worsening disease is when the rash becomes petechial. $90 \%$ of patients will have a rash, however $10 \%$ will never develop a rash, further complicating the diagnosis. Typical lab abnormalities include leukopenia, thrombocytopenia with a mild transaminitis which can be seen in Anaplasmosis and Erlichia. Hyponatremia is seen in severe disease in RMSF. Treatment within the first 5 days has been shown to improve mortality, and typical diagnostic testing takes weeks to confirm so the initial treatment decision is purely clinical. If a patient has a rash the diagnosis can be made based on skin biopsy. However, the biopsy yield decreases if patient is already on treatment for 2 days. In patients' sans rash, the diagnosis is confirmed by serologic testing for IGG during the acute illness and 2 weeks post, and observing a four fold increase in titers. The diagnosis of RMSF can be challenging unless the physician has high clinical suspicion given the similar presenting symptoms to viral syndromes and other tick borne illnesses. It is important for clinicians regardless of geographic location to keep RMSF in the differential for patients with consistent symptoms and lab abnormalities to avoid a delay in treatment. References 1 . Centers for Disease Control and Prevention. Diagnosis and management of tickborne rickettsial diseases: Rocky Mountain spotted fever, ehrlichiosis, and anaplasmosis-U.S.: A practical guide for physicians and other health-care and public health professionals. MMWR 2006; 55 (No.RR-4) 2. Salgo MP, et. Al. A focus of Rocky Mountain spotted 
fever within New York City. N Engl J Med. 1988;318(21):1345. 3. Kirkland KB, et al. Therapeutic delay and mortality in cases of Rocky Mountain spotted fever. Clin Infect Dis. 1995;20(5):1118. 4. Kaplan JE, Schonberger LB. The sensitivity of various serologic tests in the diagnosis of Rocky Mountain spotted fever. Am J Trop Med Hyg. 1986;35(4):840.

\section{ST ELEVATION IN EKG CONFOUNDING THE DIAGNOSIS OF} PANCREATITIS WITH LITERATURE REVIEW XIAOMEI MENG. Ohio state university medical center, Dublin, OH. (Control ID \#2469740)

LEARNING OBJECTIVE \#1: to identify pancreatitis in case of epigastric pain with ST elevation

LEARNING OBJECTIVE \#2: inferior ST elevation is most common in pancreatitis with ST elevation

CASE: A 59 year old male presented to emergency department (ED) with abdominal pain and persistent dry heaves after consuming alcohol. The patient's past history was significant for pancreatitis related to alcohol abuse, pancreatic pseudocyst, and myocardial infarction (MI) 10 years ago treated with right coronary artery stent. Patient was free of any angina like symptoms since stent placement. Initial blood tests showed mildly elevated lipase and CT scan confirmed the presence of pancreatic pseudocyst. EKG in ED demonstrated ST elevation in leads II, III and aVF, which raised the possibility of an inferior wall MI. Nitroglycerine was administered, but the patient reported no relief of abdominal pain. EKG $2 \mathrm{~h}$ later showed no progression of ST elevation. Given patient's history of recurrent pancreatitis with pseudocyst, alcohol consumption, elevated lipase, no EKG progression after $2 \mathrm{~h}$, the diagnosis of recurrent pancreatitis was considered to be more likely. Accordingly patient was monitored conservatively and coronary angiography was not performed. Subsequently, echocardiogram did not reveal any cardiac wall motion abnormalities, and serial blood tests showed no troponin I elevation confirming the absence of MI. With conservative management, the next day patient reported symptomatic improvement. Repeat EKG showed resolution of ST changes and patient was discharged 4 days later.

DISCUSSION: In the ED, detection of ST segment elevation in EKG starts a rapid sequence of events which usually ends with interventional or thrombolytic treatment. Therefore, in patients with abdominal pain with ST elevation the diagnosis of pancreatitis can be missed. Acute pancreatitis with ST elevation could be real or pseudo MI. We reviewed 17 case reports about acute pancreatitis with ST elevation and found that in patients without evidence of MI, inferior lead ST elevation is the most common territory involved. 11 out of 17 cases were proved negative for MI, and 7/11 non-MI cases presented with inferior leads ST elevation with similar pattern. The diagnosis of acute pancreatitis was initially missed in 10 of these 11 non-MI patients. Multiple hypotheses have been presented to explain the non-MI ST elevation in acute pancreatitis including the effect of pancreatic proteolytic enzymes on the myocardium, vagal reflex, cardiobiliary reflex or electrolyte disturbances. In our case, the time concordance between ST elevation and persistent severe dry heaves raises the hypotheses of diaphragm stimulation as an explanation. Additionally, we found that thrombolytic therapy might be detrimental in this population. $4 / 17$ cases received thrombolytic therapy, leading to death by retroperitoneal hematoma in one patient with MI, and development of DIC and sepsis in one non-MI patient. Clinicians should be mindful about the possibility of acute pancreatitis when dealing with inferior lead ST elevation.

STATIN-INDUCED NECROTIZING AUTOIMMUNE MYOPATHY: A NOVEL DIAGNOSIS REQUIRING A MORE WIDESPREAD RECOGNITION Lillian Gonzales $^{1}$; Mahrukh Ali ${ }^{1}$; Ramon Rodriguez-Cruz ${ }^{2},{ }^{1}$; Aunali $\mathrm{Khaku}^{2},{ }^{1} .{ }^{1} \mathrm{UCF}$, Orlando, FL; ${ }^{2}$ Orlando VA Medical Center, Orlando, FL. (Control ID \#2467896)
LEARNING OBJECTIVE \#1: Recognize clinical and diagnostic features of statininduced necrotizing autoimmune myopathy.

CASE: A 33-year-old African American man, with history significant for hypertension and hyperlipidemia, presented at our neurology clinic for evaluation of a long-standing bilateral upper and lower extremity weakness. He reported a 2-year history of progressive extremity weakness, initially involving the lower limbs with gradual progression to both upper limbs. He was initially seen in a primary care clinic where laboratory studies were done, including a CPK level that was significantly elevated at 25,257 U/L. His renal functions were within normal range, and LFTs were mildly elevated. At that time, as he was on a chronic statin therapy (Simvastatin $40 \mathrm{mg}$ daily), his symptoms and abnormal laboratory work up were attributed to drug-induced myositis. He was advised to stop taking Simvastatin and was followed closely, with serial CPK levels noted to have eventually improved to $7,800 \mathrm{U} / \mathrm{L}$. Upon evaluation in our clinic, he reports minimal improvement of weakness despite being off of simvastatin for over 6 months. Medication reconciliation did not reveal any other cause for his symptoms. Neurologic examination was significant for profound proximal muscle weakness at both upper and lower extremities. As myopathy was suspected, initial work up included a CPK level, noted to be elevated at $7640 \mathrm{U} / \mathrm{L}$. Other investigations as TSH, HIV and hepatitis serologies, B12, folate, ANA, ESR, CRP were all negative. Antibody panels for paraneoplastic and inflammatory myopathy were all unremarkable. Nerve conduction studies (NCS) and electromyography (EMG) were consistent with a myopathic process, worse at proximal extremities. Muscle biopsy revealed evidence of necrotizing myopathy without active inflammation. Recently recognized antibodies relating to a statin-induced autoimmune myopahty (anti-3-hydroxy-3-methyglutaryl-coenzyme A/HMGCoA and anti-signal recognition particle/SRP) were negative. On the basis of long-term statin therapy and after exclusion of all other causes of necrotizing myopathy, the patient was diagnosed with statin-induced necrotizing autoimmune myopathy (NAM). He was started on steroid therapy at $1 \mathrm{mg} / \mathrm{kg}$ daily dose, followed by intravenous immune globulin (IVIG) therapy. He was monitored closely and at 2 months follow-up, he reported minimal subjective improvement with only mild decrease in CPK level. He was then started on Rituximab, which eventually improved his symptoms, accompanied by normalization of his CK levels.

DISCUSSION: Necrotizing autoimmune myopathy (NAM) is a recently defined subcategory of the acquired idiopathic myopathies. It is a severe immune-mediated myopathy presenting as proximal muscle weakness and mostly associated with identifiable risk factors, the most common of which is statin exposure. The prevalence and annual incidence of NAM are not known. Although considered to be relatively rare, it may be more frequent than postulated, owing to the extensive and growing use of statins. As widely encountered in the primary care setting, the spectrum of statin side effects including myalgia, myositis and rhabdomyolysis are mostly due to the direct toxic effect of the offending drug and typically resolves with drug discontinuation. Statin-induced NAM should be suspected in patients who develop progressive muscle weakness and persistently elevated CPK levels despite discontinuation of statins, as in our patient. Though the pathophysiology for statin-induced NAM has not been clearly established, it has been suggested that statins are capable of trigerring an immune-mediated reaction causing myopathy, that is then perpetuated even if the drug is discontinued. Diagnosis is made with muscle biopsy showing necrotizing myopathy without significant inflammation and EMG consistent with myopathy. Other entities as inflammatory myopathy, connective tissue disease, neoplasm or viral diseases as hepatitis or HIV should also be excluded. More recently, novel autoantibodies (anti- SRP and anti-HMGCoA) are now available commercially to aid in diagnosis. However, normal range of antibody titers, as in our patient, does not exclude the diagnosis. While treatment regimens for NAM have not been evaluated prospectively, multi-agent immunosuppressive therapy is usually indicated. Based on most recent observations, excellent outcomes were seen with use of IVIG, corticosteroids and a steroid-sparing immunosuppressant for at least 3 months. For refractory patients, agents as rituximab, methotrexate, cyclosporine and mycophenolate mofetil may be 
considered. Thus, our case emphasizes on the need for general awareness and early recognition of this rare disease entity. Diagnosis of NAM is critical given the potential for improved outcomes with early aggressive therapy and hence prevention of lifelong disability.

\section{STEVENS JOHNSON SYNDROME (SJS): WHEN IT IS NOT EXPLAINED BY} MEDICATIONS Meredith R. Sherrill ${ }^{2}$; Dustyn E. Williams ${ }^{1}$; Virginia Reeder ${ }^{2}$. ${ }^{1}$ Baton Rouge General Medical Center, Baton Rouge, LA; ${ }^{2}$ Tulane University Health Sciences Center, New Orleans, LA. (Control ID \#2469309)

LEARNING OBJECTIVE \#1: Learn to quickly recognize Stevens Johnson Syndrome LEARNING OBJECTIVE \#2: Suspect HIV when Stevens Johnson Syndrome is present in the absence of medications

CASE: A 49 year-old man complains of 2 weeks of a painful rash. He is unable to note where the rash first started, only that it is painful, covers the majority of his body, and includes his scrotum and penis. He endorses a thirty pound weight loss over 6 months with progressive malaise. He denies night sweats, pruritis, or purulence. He has no medical history and takes no medications. Vitals are temperature $99.8 \mathrm{~F}, 115$ beats per minute, $155 / 99 \mathrm{mmHg}, 18$ respirations per minute, with $100 \%$ saturation on room air. He is cachectic. There are no mucosal lesions, but there are hemorrhagic crusts on his lips, and it is painful to open his mouth. There are ulcers with smooth borders on the scrotum and urethral meatus. The skin is dusky with violaceaous macules covering $75 \%$ of his body. These rashes are tender to palpation, involve the palms and soles, and demonstrate full thickness epidermal desquamation on less than $10 \%$ of the body surface area. Absoe-Hanson sign and Nikolsky sign are positive. Laboratories reveal a white count of $4.0^{3}$ cells/dL, hemoglobin of $8.1 \mathrm{mg} / \mathrm{dL}$, and a creatinine of $12.61 \mathrm{mg}$ dL. ELISA for HIV is positive. The CD4 count is 19. Biopsy of the lesions reveal ulcerative dermatitis with superficial fungal infection, lymphohistiocytic and neutrophilic vasculitis with thrombosed superficial small vessels and eosinophils. Stains were positive for yeast and pseudohyphae. No herpetic cells were seen. The patient is started on emergent hemodialysis and intravenous immunoglobulin, methylprednisolone, fluconazole, and acyclovir. After 3 days he is transitioned to oral medications with a prednisone taper. The rash resolves after 4 weeks. DISCUSSION: Steven Johnson's Syndrome (SJS) is a dermatologic emergency, thus al practitioners should be able to quickly identify and treat. The history often includes medications known to cause SJS, such as antiepileptics, sulfonamide and B-lactam antibiotics or allopurinol ${ }^{1}$. The skin reaction usually occurs within weeks of drug initiation and results in the sloughing of skin on up to $10 \%$ of the body surface area, with at least two mucosal surfaces usually involved. It is crucial to differentiate between Erythema Multiforme (EM) and SJS, as the latter is more likely to progress to TEN and thus requires intensive care monitoring until improved. The internist must maintain a high degree of suspicion when the dermatologic exam is inconsistent with any given diagnosis. This patient's initial clinical presentation was complicated by his previously unknown AIDS. His skin exam suggested old herpetic lesions that could not be confirmed with Tzanck smear, though the desquamative lesions were more suggestive of the SJS/TEN spectrum. As he had no acknowledged history of any medication use or previous medical history to predispose him to SJS/TEN, testing for HIV was crucial. HIV is an independent risk factor, associated with a 100 fold increased risk of SJS/TEN ${ }^{2}$. Once an immunocompromised status is established, infections, including viral, bacterial or fungal, must be considered as additional precipitating factors to development of SJS and warrant treatment if present. In this case, there was clinical evidence of herpetic lesions, biopsy evidence of fungal infection, and coexisting SJS.

STOP THE BLEEDING! A NOVEL APPROACH TO REVERSING FACTOR XA INHIBITORS USING PLASMA EXCHANGE Varun Kumar; Alice J. Chen; Arthur W. Bracey; Wilson W. Lam. Baylor College of Medicine, Houston, TX. (Control ID \#2469062)
LEARNING OBJECTIVE \#1: To report the use of therapeutic plasma exchange (TPE) to remove the anticoagulant effect of rivaroxaban.

CASE: A 65-year-old male with paroxysmal atrial fibrillation and CKD stage 5 with GFR 13 secondary to hypertension and diabetes was admitted for renal transplantation. The patient had been compliant with rivaroxaban for anticoagulation. In the setting of impaired clearance from his kidney disease, there was significant concern that an appreciable anticoagulant effect would remain in the bloodstream. Anti-Xa levels were monitored to determine anticoagulant effect and the anti-Xa Lovenox level was $0.49 \mathrm{IU} /$ $\mathrm{ml}$ (therapeutic range $0.5-1.2$ ) approximately $12 \mathrm{~h}$ after the patient's last dose of rivaroxaban. The patient was evaluated by clinical pathology and scheduled for therapeutic plasma exchange (TPE) as an experimental approach to decreasing the anticoagulant effect of rivaroxaban prior to transplant. A repeat anti-Xa level $5 \mathrm{~h}$ after the first level, just prior to TPE, was $0.40 \mathrm{IU} / \mathrm{ml}$. The patient urgently underwent $90 \mathrm{~min}$ of TPE with $3 \mathrm{~L}$ fresh frozen plasma via arteriovenous fistula. Anti-Xa level immediately after TPE was $0.21 \mathrm{IU} / \mathrm{ml}$. Patient was taken immediately for transplant thereafter, which was successfully completed without complication. There was no evidence of bleeding diathesis during the perioperative or postoperative periods. A final anti-Xa level was checked the next morning $(\sim 17 \mathrm{~h}$ after TPE) and the anti-Xa level was $<0.05$. Anti-Xa level of waste fluid from TPE was $0.22 \mathrm{IU} / \mathrm{ml}$.

DISCUSSION: Factor Xa inhibitors are increasingly replacing older agents like warfarin for patients in need of anticoagulation. Patients on warfarin are plagued by supratherapeutic and infratherapeutic levels, and centers considered to have wellcontrolled levels remain within the therapeutic range only approximately two-thirds of the time. The advent of factor Xa inhibitors provided a more consistent anticoagulant effect with significantly decreased logistical costs. Rivaroxaban, apixaban and edoxaban all showed decreases in major bleeding episodes when used for atrial fibrillation instead of warfarin. When used for venous thromboembolism, apixaban and edoxaban also showed decreased bleeding episodes compared to conventional therapy. However, the lack of antidote has posed a major challenge to the use of factor Xa inhibitors. Recently, andexanet alpha, a recombinant factor Xa molecule, has been proposed as an antidote to rivaroxaban and apixaban. The drug showed effective suppression of anticoagulant therapy during infusion, but patients showed rebound anticoagulation soon after the discontinuation of the antidote. This poses significant risks, especially if permanent reversal of anticoagulation is desired. Furthermore, the tenuous pharmacokinetics in healthy volunteers raises the question of whether the outcomes may significantly vary in patients undergoing active major bleeding events or surgical procedures. Since rivaroxaban has been shown to remain $95 \%$ protein-bound in the serum, we considered TPE as an option for removal of serum rivaroxaban. In the setting of an emergent transplant, the patient underwent a single shortened course of plasma exchange with $3 \mathrm{~L}$ FFP over $1.5 \mathrm{~h}$ showing a precipitous, non-physiologic decrease in anticoagulant effect followed by a successful renal transplant. The elevated anti-Xa level in the TPE waste fluid further corroborates the removal of rivaroxaban from the serum. Patients requiring more complete removal of anticoagulant effect can undergo further TPE. In addition, follow up studies the next morning showed no rebound anticoagulant effect. In 2015, Lam et al. showed a similar effect of TPE in removing the anticoagulant effect of apixaban (1). We suggest that this first report of TPE to acutely reverse the effects of rivaroxaban in addition to prior studies should prompt clinical trials to evaluate therapeutic plasma exchange as a method for reversal of factor Xa inhibitors. References: 1. Lam, W. W., Reyes, M. A., \& Seger, J. J. (2015). Plasma Exchange for Urgent Apixaban Reversal in a Case of Hemorrhagic Tamponade after Pacemaker Implantation. Tex Heart Inst J, 42(4), $377-380$.

STOP THE MEDICATION, STATIN! (AND START AN IMMUNOMODULATOR)

Austin T. LeMaster; Justin Rodriguez; Michael Shoffeitt. UTHSCSA, San Antonio, TX. (Control ID \#2469833) 
LEARNING OBJECTIVE \#1: Learning objectives: 1. Be familiar with a rare but serious adverse effect of a commonly prescribed medication.

LEARNING OBJECTIVE \#2: 2. Distinguish between statin-associated myopathy and immune-mediated necrotizing myopathy (IMNM) associated with a statin, and understand the differences in management

CASE: A 52 year-old man presents with shortness of breath and a cough that has been worsening for the past 2 weeks. He was in his usual state of health until about a month ago. At first, he felt like he did not have his usual amount of energy. He would easily fatigue while riding horses and would have difficulty staying seated in the saddle. Over the last 2 weeks, he is experiencing worsening fatigue, as well as cough, dyspnea on exertion, orthopnea, and paroxysmal nocturnal dyspnea. He has a past medical history significant for hypothyroidism, diabetes mellitus type II, and hyperlipidemia treated with levothyroxine, metformin, and atorvastatin. He denies smoking and his family history is noncontributory. His lungs are clear to auscultation, and he has no peripheral edema or elevation of his jugular venous pressure. Strength is four out of five in his bilateral upper and lower extremities. Further history is obtained, which reveals he has some difficulty standing up and recently has to pull himself with his arms to step into his pickup truck. Laboratory testing reveals a creatine kinase level of 13,000 International Units per liter and an arterial blood gas with a partial $\mathrm{CO} 2$ pressure of $49 \mathrm{~mm} \mathrm{Hg}$. B-type natriuretic peptide is normal. Chest radiograph reveals low lung volumes and an elevated right diaphragm, and is otherwise normal. Bedside negative inspiratory force is twenty centimeters of water. Pulmonary function tests are notable for a total lung capacity that is $50 \%$ of predicted value with a preserved corrected diffusing capacity. Aldolase level is elevated, and anti-Jo-1 antibody is normal. On magnetic resonance imaging, there are patch T2 hyperintense signals within multiple proximal muscles of the lower extremities. On electromyography of affected muscle there is early recruited short duration motor units with membrane instability and pseudomyotonic discharges, consistent with a generalized necrotizing myopathic disorder. Muscle biopsy reveals myriad necrotic and regenerating fibers indicating a necrotizing myopathy. The anti-HMGCR antibody test—sent out previouslysubsequently returns positive.

DISCUSSION: Dyspnea is a chief complaint commonly encountered by the internist. It is important to approach the complaint with a broad differential diagnosis, and to consider neuromuscular weakness in the appropriate setting. Statins are frequently prescribed due to their clear benefits in cardiovascular disease. While it is well known that statins can cause myopathies and that these can be severe, it is important to know of the unique clinical entity of statin-associated immune-mediated necrotizing myopathy (IMNM). The anti-HMGCR antibody) lab test is supportive of this diagnosis. Patients who test positive have a specific genetic background (HLA-DRB1*11:01)[i] and on biopsy demonstrate a characteristic pattern which is now called statin-associated IMNM with anti-HMGCR antibodies.[ii] Performing a biopsy and identifying the anti-HMGCR antibody is important because unlike in statin-associated myalgia, terminating statin administration is usually inadequate for clinical response. In statin-associated IMNM, muscle damage initiated in the presence of statins may be sustained even after the statin is discontinued. Immunosuppressive therapy is usually required, and corticosteroids alone are often inadequate. Our patient discontinued atorvastatin, and he was started on prednisone and azathioprine. He has not yet been seen in follow up while taking azathioprine in order to monitor his treatment response. Interestingly, the anti-HMGCR antibody test is not specific for IMNM due to statins as $30-50 \%$ of patients with anti-HMGCR antibodies and IMNM have never received statins.[iii] [i] Drouot L, Allenbach Y, Jouen F, et al. Exploring necrotizing autoimmune myopathies with a novel immunoassay for anti-3hydroxy-3-methyl-glutaryl-CoA reductase autoantibodies. Arthritis Res Ther. 2014;16:R39. [ii] Labrador-Horrillo M, Martínez MA, Selva-O’Callaghan A, et al. Identification of a novel myositis-associated antibody directed against cortactin. Autoimmun Rev. 2014;13:1008-1012. [iii] Ramanathan S, Langguth D, Hardy TA, et al. Clinical course and treatment of anti-HMGCR antibody-associated necrotizing autoimmune myopathy. Neurol Neuroimmunol Neuroinflamm. 2015;2:e96.
STRAWBERRY MILKSHAKE HYPERTRIGLYCERIDEMIA MASQUERADING AS HEMOLYSIS Jeb W. Rodgers ${ }^{2}$; Kevin Means ${ }^{3}$; Jenell Stewart ${ }^{3}$; Kathryn Christofersen ${ }^{1}$; Genji Terasaki ${ }^{1} .{ }^{1}$ University of Washington, Seattle, WA; ${ }^{2}$ University of Washington, Missoula, MT; ${ }^{3}$ University of Washington School of Medicine, Seattle, WA. (Control ID \#2469857)

LEARNING OBJECTIVE \#1: Demonstrate that severe hypertriglyceridemia is a possible explanation of spurious hemolysis.

LEARNING OBJECTIVE \#2: Describe gross lipemia as a "strawberry milkshake"appearing layer in centrifuged blood.

CASE: A 31 year-old man with a history of poorly controlled diabetes mellitus type II and intermittent alcohol abuse presented to the emergency department with new-onset nonvertiginous dizziness and muscle cramps following a night of heavy drinking. His vital signs and physical exam were normal including an unremarkable neurologic exam. Initial chemistry panel had sodium of $123 \mathrm{meq} / \mathrm{L}$ and unreportable potassium and calcium due to hemolysis. Five subsequent metabolic panels remained unreportable due to hemolysis before whole blood labs were drawn. Following centrifugation, the phlebotomized blood had a pink, viscous lipemia layer described as "strawberry milkshake" in appearance. ${ }^{[1]}$ This prompted lab personnel to contact the medical team and suggest measuring a lipid panel. A non-fasting lipid panel returned with triglycerides greater than $5,000 \mathrm{mg} / \mathrm{dL}$. The complete blood count and liver function tests were within normal limits. Hemoglobin A1c was $10.7 \%$. Total bilirubin was elevated at $2.2 \mathrm{mg} / \mathrm{dL}$. Further investigation of the hemolysis revealed a low haptoglobin of $38 \mathrm{mg} / \mathrm{dL}$ and an elevated lactate dehydrogenase of $531 \mathrm{U} / \mathrm{L}$, consistent with hemolysis. Peripheral smear showed abnormal asymmetric red cells without schistocytes and was notable for small translucent droplets visible on some RBC membranes. Whole Blood Lab Draw (WBLD) measurement returned a potassium of $4.7 \mathrm{mEq} / \mathrm{L}$. Serial CBCs remained stable.

DISCUSSION: Despite repeatedly critical laboratory abnormalities, patient remained clinically well. After stabilization with fluids and gemfibrozil, a fasting lipid panel on hospital day five revealed a triglyceride level of $1,541 \mathrm{mg} / \mathrm{dL}$. After continuing those therapies and maintaining sobriety his triglycerides were within normal limits at $140 \mathrm{mg} / \mathrm{dL} 1$ week later at outpatient follow up. Follow up labs and clinical presentation were without signs or symptoms of hemolysis. The initial concern was for in vivo hemolysis given the automated report of hemolysis with abnormal haptoglobin and LDH. However, the hemolysis was occurring in vitro, which preserved hematocrit and hemoglobin levels. The potassium and calcium were accurately measured on the blood gas instrument, which directly measures the ions, by requesting a Whole Blood Lab Draw. The usual chemistry instrument uses a colorimetric assay which is disrupted by the color of free hemoglobin. It is important to note that whole blood labs are not centrifuged. ${ }^{[2]}$ Severe hypertriglyceridemia was presumably to have driven the in vitro hemolysis and was secondary to recent alcohol abuse, uncontrolled diabetes mellitus, and familial hypertriglyceridemia. Hypertriglyceridemia affecting red blood cells has been observed before but no mechanism of in vitro hemolysis has been identified. ${ }^{[3]}$ Excess triglycerides may have incorporated into the erythrocyte membrane, disrupting the phospholipid bilayer and increasing membrane fragility. ${ }^{[1]}$ Metabolic stress resulting from cessation of Sodium-Potassium pumps contributes to cellular swelling of ex-vivo erythrocytes. This effect may have been magnified in the setting of fragile membranes. Centrifugation of these fragile RBCs may have contributed to the in vitro hemolysis. The whole blood sample measurement yielded a potassium level of $4.7 \mathrm{mEq} / \mathrm{L}$. Knowing that even a $1 \%$ hemolysis rate will increase the potassium measurement by $55 \%$, the rate of hemolysis in the whole blood specimen (not centrifuged) was very low. ${ }^{[4]}$ Finally, the excess lipid may have interfered with tube additives such as EDTA . Further investigation of the hemolysis mechanism may be useful in preventing further unnecessary hemolysis workup in the future. 
STREPTOCOCCUS AGALACTIAE BACTEREMIA PRESENTING AS TOXIC SHOCK-LIKE SYNDROME AND INFECTIVE ENDOCARDITIS: A CASE REPORT Rimma Polevoy ${ }^{1}$; Adrienne Lenhart ${ }^{1}$; Asaad Nakhle ${ }^{1}$; William J. Kane ${ }^{2}$; Jeffrey Jennings ${ }^{3}$. ${ }^{1}$ Henry Ford Health System, Oak Park, MI; ${ }^{2}$ Wayne State University, Detroit, MI; ${ }^{3}$ Henry Ford Health System, Detroit, MI. (Control ID \#2468039)

LEARNING OBJECTIVE \#1: Recognize the clinical features of toxic shock syndrome LEARNING OBJECTIVE \#2: Recognize an atypical cause of infective endocarditis

CASE: This is the case of a 44-year-old woman with a history of alcoholic liver cirrhosis, who presented as a transfer from an outside facility with sepsis and altered mental status. According to her husband, about a week prior to admission she had an episode of confusion, fatigue, and headache. Her mental status worsened over the course of the next week so she was brought to the hospital for evaluation. At the outside hospital she was found to be hypothermic to $34.7^{\circ} \mathrm{C}$ with a leukocytosis to $40,000 / \mathrm{uL}$ and lactate of $10.9 \mathrm{mmol} / \mathrm{L}$. Chest $\mathrm{x}$-ray and urinalysis were negative for infection and CT head was unremarkable. She was transferred to our facility for escalation of care. On arrival, she was unresponsive, but withdrew to pain, and was intubated for airway protection. Of note, while cleaning the patient, nursing staff found a retained tampon intravaginally, which was removed at that time. Her husband noted that she had recently started her menstrual cycle. On admission she was afebrile, but hypotensive, tachycardic, and tachypneic. Labs revealed a leukocytosis to $48,000 / \mathrm{uL}$, lactate $5.3 \mathrm{mmol} / \mathrm{L}$, and creatinine elevation to $2.34 \mathrm{mg} / \mathrm{dL}$ from a normal baseline. She was started on vancomycin, cefepime, and metronidazole for empiric coverage of infection. Blood cultures subsequently grew pansusceptible Streptococcus agalactiae, so her antibiotics were deescalated to ceftriaxone. Given her initial presentation of septic shock, group B streptococcal (GBS) bacteremia, and retained tampon, there was concern for toxic shock syndrome. Clindamycin was added for potential suppression of bacterial toxin production. Echocardiogram was ordered and revealed a $7 \times 2 \mathrm{~mm}$ vegetation on the aortic valve with severe aortic regurgitation. Due to ongoing encephalopathy, MRI brain was obtained and showed bilateral frontal empyemas with findings in the adjacent frontal lobes related to meningitis and cerebritis. Patient's condition initially improved with antibiotics, however her hospital course was complicated by ventilator associated pneumonia and renal failure. Per cardiothoracic surgery, she was not a candidate for valve repair or replacement because of her cirrhosis. Due to continued deterioration and worsening sepsis with renal failure, the decision was made by her family to withdraw life sustaining measures, and the patient expired.

DISCUSSION: $S$. agalactiae is a beta-hemolytic GBS organism commonly known to colonize the genital and gastrointestinal tracts of adults. GBS carriage rates are highest in women of reproductive age and are known to cause infections in pregnant women. It less frequently presents as a pathogen in non-pregnant adults, with a yearly incidence around 4 to 8 per 100,000 . Risk factors for GBS infection in non-pregnant patients include diabetes, HIV, malignancy, and advanced hepatic or renal disease. Our patient was of reproductive age with a history of alcoholic cirrhosis, putting her at increased risk for infection. Toxic shock syndrome (TSS) is a clinical entity typically caused by Staphylococcal aureus, classically associated with tampon use. Though rare, GBS is an increasingly recognized cause of toxic shock-like syndrome (TSLS). Various GBS strains have been found to produce a pyrogenic toxin or superantigen that causes TSS-like symptoms, however there have been only several reported cases in the literature. Clinical manifestations of TSLS include shock with fever or hypothermia, altered mental status, and multisystem organ involvement, all exhibited by our patient. Diagnosis is made by isolation of the organism from a normally sterile site (eg, blood) with hypotension and two of the following: renal or liver dysfunction, coagulopathy, ARDS, macular rash, and soft tissue necrosis. Reports of GBS TSLS to date have primarily been attributed to skin and soft tissue infections, however our patient appears to one of few reported cases of GBS TSLS associated with tampon use. This case was unique in that the patient's $S$. agalactiae bacteremia not only presented as toxic shock-like syndrome, but also resulted in infective endocarditis (IE). GBS rarely causes IE, accounting for only about $1.7 \%$ of cases. Endocarditis caused by S. agalactiae is characterized by acute onset, valvular destruction, and large vegetations. Systemic emboli are common, occurring in about $50 \%$ of cases. In our patient, GBS endocarditis resulted in septic embolization to the brain with frontal lobe empyemas and cerebritis. Treatment of IE involves antibiotics for 4 to 6 weeks, depending on the causative organism. Surgical intervention is indicated for patients with valvular dysfunction leading to heart failure, paravalvular extension of infection, infection due to fungal or resistant organisms, or persistent infection despite adequate medical therapy. American Heart Association criteria for early surgery to prevent embolization include recurrent emboli and persistent or enlarging vegetations despite appropriate antibiotic therapy, or severe valve regurgitation with mobile vegetations $>10 \mathrm{~mm}$. Our patient did not meet these criteria, therefore did not receive valve repair or replacement during her hospitalization. In conclusion, we present a rare case of GBS bacteremia due to tampon use leading to toxic shock-like syndrome and infective endocarditis, with resulting septic emboli to the brain.

\section{STRONGYLOIDIASIS WITH COEXISTING SARCOIDOSIS: STEROIDS CAN} BE TRICKY Xuan Gao ${ }^{2}$; Vijan Joshi ${ }^{2}$; Sarun V. Thomas ${ }^{1}$; Erica Grabscheid ${ }^{2} .{ }^{1}$ Mount Sinai Beth Israel, New York, NY; ${ }^{2}$ Beth Israel Medical Center, New York, New York, NY. (Control ID \#2467847)

LEARNING OBJECTIVE \#1: Learn the clinical features of Strongyloides-induced pneumonitis, and how it can be mistaken for other pulmonary disorders.

LEARNING OBJECTIVE \#2: Recognize that steroid treatments used in the setting of Sarcoidosis and/or COPD can have dire effects in Strongyloidiasis.

CASE: A 77 year old male with COPD and 25 pack year tobacco use presented with dyspnea associated with a productive white cough for 3 days. Patient denied any recent travel, but was born in Puerto Rico and moved to New York at age 19. He was admitted 2 months prior for similar complaints and was found to have newly diagnosed chronic obstructive pulmonary disease (COPD) complicated by community acquired pneumonia, for which the patient received Levofloxacin and a steroid taper. On this admission, vital signs: T $97^{\circ} \mathrm{F}$, HR 99, BP 147/79, SaO2 98 \% RA. Physical exam showed diffuse inspiratory and expiratory wheezing. Initial labs: WBC $10.0 \mathrm{~K} / \mathrm{uL}$ with marked eosinophilia of $25 \%$. CXR demonstrated bilateral perihilar fullness. CT Chest showed perihilar calcified lymphadenopathy and scattered pulmonary granulomas. There was clinical suspicion for sarcoidosis, however, bronchoscopy was postponed as per patient's request. Stool ova and parasite studies returned negative, but IgE levels were elevated (5947 KU/L) and ELISA for Strongyloides IgG antibody was positive. Steroids (for possible sarcoidosis) were held due to concern for parasitic infection. Patient was diagnosed with Strongyloides-induced pneumonitis and discharged after Ivermectin treatment. 1 month follow-up labs showed: $\operatorname{IgE} 15,564 \mathrm{KU} / \mathrm{L}$ and $\mathrm{WBC} 8.0 \mathrm{~K} / \mathrm{uL}$ with uptrending eosinophilia of $31.4 \%$; repeat ova and parasite was again negative. Given the uptrending $\operatorname{IgE}$, patient received a second dose of Ivermectin. 2 month follow-up labs showed IgE 4479 $\mathrm{KU} / \mathrm{L}$ and WBC $6.8 \mathrm{~K} / \mathrm{uL}$ with eosinophils of $6.2 \%$. Outpatient bronchoscopy later showed cytology findings consistent with sarcoidosis. Patient underwent monitored pulse steroid doses with tapering, and, thereafter, achieved resolution of respiratory symptoms. DISCUSSION: Strongyloidiasis is an infection caused by the parasitic nematode Strongyloides. This parasite has the distinctive ability to replicate within a host for decades, producing minimal symptoms. Because of its subclinical course in immunocompetent individuals, the incidence of Strongyloidiasis is often underestimated. Our case illustrates a misdiagnosis of COPD, diagnosing a coinfection of Stronglyoides and sarcoidosis, successfully treating the Stronglyoides first and, then, treating the sarcoidosis. Additionally, it highlights the need for broadened differential in treating at-risk patients for 
Strongyloidiasis, especially in the setting of sarcoidosis. The case also serves to remind providers not to over-prescribe systemic corticosteroids if there is a risk of disseminated parasitic infection. Careful physical exam with social history intake must be performed when treating such patients.

STRUCK TWICE: AN UNUSUAL CASE OF GRAVES' DISEASE WITH THYROTOXICOSIS AND DIABETES INSIPIDUS IN A PREVIOUSLY HEALTHY MAN Annalise O. Abiodun; Binh Nguyen; Muddassir Salyani. Greater Baltimore Medical Center, Towson, MD. (Control ID \#2468556)

LEARNING OBJECTIVE \#1: To recognize that polyuria in a patient with thyrotoxicosis may have a separate etiology other than hyperthyroidism.

LEARNING OBJECTIVE \#2: To discuss the importance of early diagnosis and treatment of Graves' disease.

CASE: A 35 year-old previously healthy African American man was admitted for further evaluation of a 4 month history of intractable nausea, vomiting, abdominal pain and decreased oral intake. He had undergone an outpatient upper endoscopy which revealed reflux esophagitis and possible Barrett's esophagus. Despite the use of various antiemetics and antacids, he continued to have persistent nausea and vomiting. The patient also reported a $13 \mathrm{lb}$ unintentional weight loss over a period of 3 weeks, as well as occasional migraine headaches which started approximately 1 year prior to his presentation. Two days after his admission, he suddenly became febrile with a temperature of $104.4^{\circ} \mathrm{F}$. His vital signs were also notable for a pulse of 150 , blood pressure of $150 / 58$ and respirations of 28. The patient appeared to be anxious and was sweating profusely. His physical examination was also significant for smooth, nontender thyroid gland which was not grossly enlarged, tachycardia, fine tremors, and soft abdomen which was slightly tender to palpation. There was no exophthalmos, lid lag, thyroid bruit, pretibial myxedema, or neurological deficits on exam. An electrocardiogram showed sinus tachycardia. He was admitted to the Intensive care unit (ICU) for management of thyroid storm. Thyroid function tests were ordered and showed severe thyrotoxicosis with undetectable TSH and free T4 of $3.49 \mathrm{mcg} / \mathrm{dL}$. A diagnosis of Graves' disease was made on the basis of high titres of thyroid antibodies (thyroglobulin antibody of $5 \mathrm{IU} / \mathrm{mL}$, thyrotropin receptor antibody of $22 \mathrm{mIU} / \mathrm{L}$, and thyroid stimulating immunoglobulin of $220 \%$ of basal activity). With the initiation of propranolol, potassium iodide, methimazole and hydrocortisone, his symptoms resolved and his thyroid indices gradually improved. In the ICU, the patient was noted to have profound polydipsia, polyuria, and nocturia. On average, he was voiding 71 of dilute urine each day. The patient reported that his symptoms started insidiously over a 1 year period but had worsened 3 to 4 months prior to his admission. With fluid restriction, he continued to have significant diuresis and serum sodium increased to 154 from 144 . A desmopressin stimulation test was highly suggestive of central diabetes insipidus, and further laboratory investigation revealed panhypopituitarism. An MRI of his brain was obtained and showed a $1.5 \mathrm{~cm}$ rimenhancing complex lesion with mixed cystic and solid components in the suprasellar region. He subsequently underwent resection of the suprasellar mass via endonasal approach. The pathology results showed Rathke's cleft cyst. The patient tolerated the procedure well and was discharged home in stable condition on hormone replacement therapy. He was scheduled for close follow up with Endocrinology, Otolaryngology and Ophthalmology.

DISCUSSION: Hyperthyroidism may be associated with polyuria due to a variety of factors caused by an abundance of thyroid hormone. These factors include the downregulation of renal water channels (aquaporin 1 and 2) and increased solute excretion, increased blood pressure, increased cardiac output and increased renal blood flow. Though thyrotoxicosis due to Graves' disease has been well described in the literature as a known cause of polyuria, this case highlights the importance of ruling out other causes of polyuria such as hyperglycemia, diuretic use and diabetes insipidus. This case is particularly interesting because the patient did not have the classic triad associated with Graves' disease: goiter, exophthalmos and pretibial myxedema. It is important to obtain a thorough history to identify risk factors for Graves' disease including family history of hyperthyroidism or various autoimmune disorders, excess dietary iodine, increased stress, smoking and the use of gonadal steroids. Early diagnosis of Graves' disease is crucial as delay of appropriate treatment has severe clinical implications such as cardiomyopathy, progression of ophthalmopathy and subsequent vision loss, myopathy, development of affective disorders and increased risk of neonatal hyperthyroidism in pregnant women. Ultimately, untreated Graves' disease may lead to thyroid storm which may be lifethreatening.

SUBACUTE DIFFUSE LARGE JOINT OSTEONECROSIS IN AN HIV PATIENT ON HAART Nicolas Cal. Baystate Medical Center, Middletown, CT. (Control ID \#2466224)

LEARNING OBJECTIVE \#1: To discuss protease inhibitor-induced AVN as a common etiology for osteonecrosis in patients with HAART.

LEARNING OBJECTIVE \#2: To make internists aware of the increasing incidence of AVN in HIV patients since HAART therapy and to consider osteonecrosis as a possible etiology for chronic arthralgias in any HIV patient

CASE: 49 year old female with a past medical history of HIV on HAART since 2007, CKD stage 2 and diffuse chronic large and small joints arthralgia who presented with subacute worsening of her chronic pain in her left shoulder, right hip and bilateral knees in a 6 month period. Patient had multiple urgent care and emergency room visits for the same complaint over the past 6 months with unremarkable physical exam findings and negative X-Ray studies. She had reported intermittent swelling of her knees and was treated symptomatically with acetaminophen and ice therapy. She had also received short term prednisone regimen without marked improvement of symptoms. Given the chronicity and progression of her symptoms, patient underwent a comprehensive rheumatologic work up (ESR, CRP, ACE, C2, C50, RF, anti-CCP, c-ANCA, p-ANCA, atypical ANCA, Chlamydia Trach, GC, CMV IgG, viral hepatitis panels, lyme serologies, Parvovirus B-19, Mycoplasma, and syphillis) which was negative. CD4 count had always been appropriate and HIV load was undetectable since the beginning of her HAART 8 years ago. She also tested negative for STD-associated intra articular processes. We proceeded with MRI studies of the different painful joints which demonstrated avascular necrosis type $2(\mathrm{AVN})$ of the right hip, right knee and left shoulder.

DISCUSSION: HIV is known to be associated with induction of a hypercoagulable state due to the activation of lupus anticoagulant, anti-protein $\mathrm{S}$ and anti-protein $\mathrm{C}$ gene expression. However, the incidence of HIV-associated AVN is reported at $0.3-3$ per 1000 patients and it has been increasing since the development of HAART therapy. There is new strong evidence that suggests that protease inhibitors are directly associated with osteonecrosis side effect. However, patients should also be evaluated for more common etiologies for osteonecrosis such as chronic corticosteroid therapy, chronic alcohol intake, trauma or hemoglobinopathies. Treatment for AVN is based on its severity: AVN type 1 and 2, are considered milder cases and observation with or without bisphosphonate therapy is indicated. AVN type 3 or 4 , are associated with trabecular or medullary necrosis, in this cases surgical intervention is indicated. In cases with appropriate viral suppression, careful discussion regarding risks and benefits of altering HAART regimen should take place. Given her excellent compliance to HAART and good antiviral therapy response, out patient's regimen was not modified. She was referred to orthopedic surgery for evaluation for possible knee replacement. Given that she had AVN type 2 she was started on bisphosphonate therapy with improvement of chronic pain for the past 8 months. She continues to closely follow up with her rheumatology, orthopedics and internist. 
SUBARACHNOID FAT EMBOLI: A RARE CAUSE OF FALL-RELATED DELIRIUM Asad Jehangir ${ }^{3}$; Shoaib B. Fareedy ${ }^{4}$; Qasim Jehangir ${ }^{1}$; Anthony Donato ${ }^{2}$. ${ }^{1}$ Rawalpindi Medical College, Rawalpindi, Pakistan, Lahore, Pakistan; ${ }^{2}$ Reading Health System, W. Reading, PA; ${ }^{3}$ Reading Health System, West Reading, PA; ${ }^{4}$ Reading Hospital, Wyomissing, PA. (Control ID \#2434605)

LEARNING OBJECTIVE \#1: Fat in the subarachnoid space suggests an intraspinal fatcontaining mass such as a desmoid cyst or teratoma

LEARNING OBJECTIVE \#2: Recognize vertebral fractures following trauma as a rare cause of fat in the subarachnoid space

CASE: An 86-year-old male presented to the emergency with increasing lethargy preceeding a mechanical fall. The examination revealed left-sided ptosis and ataxia. No rash or pulmonary complaints were noted. A computerized tomography of the head was unremarkable. A magnetic resonance imaging (MRI) of the brain revealed findings consistent with acute left basal ganglia lacunar infarct. Additional note was made of numerous new bilateral nodular foci of increased signal intensity on T1 imaging throughout the subarachnoid space at the base of the brain suggestive of subarachnoid fat or blood. Given the distribution of the findings were atypical for hemorrhage, these were considered subarachnoid fat and the absence of subdural CNS masses made an embolic source most likely. These foci were not visualized on the prior MRI performed 2 years ago. MRI of spine revealed acute/subacute fractures at T6 and T11, with several foci of subarachnoid fat within the lower thoracic region, leading us to conclude that these fractures were the source of the fat in the subarachnoid space. The patient's neurological deficits resolved completely with medical management.

DISCUSSION: Fat in the subarachnoid space is an unusual finding that has been infrequently reported in the literature. Subarachnoid fat most commonly arises from the rupture of intracranial or extra-spinal desmoid cysts. Other reported causes include rupture of teratomas, following foramen magnum meningioma resection and traumatic sacral pseudomeningocele in a patient with spina bifida occulta. While fat emboli to the brain may be seen in trauma patients, particularly with fractures of the long bones, post-traumatic subarachnoid fat emboli is extremely rare. Subarachnoid fat emboli on MRI brain in the setting of a recent fall in the elderly, especially if diffuse, should raise suspicion of hematogenous migration of fat from a fracture of long bones or spine. In trauma patients, this finding warrants prompt recognition of an underlying spinal injury.

\section{SUBTLE PERSONALITY CHANGES AS A PRESENTING SIGN OF SEIZURE} Jason K. Lam; Sumaiya Ansari; Monoj Konda; Theotonius J. Gomes. Western Michigan University Home Stryker M.D. School of Medicine, Kalamazoo, MI. (Control ID \#2468010)

LEARNING OBJECTIVE \#1: Recognize status epilepticus may be non-convulsive and have nonspecific, pleomorphic signs and symptoms. These findings may be subtle and difficult to distinguish from normal behaviors, leading to under-recognition and diagnosis. LEARNING OBJECTIVE \#2: Diagnose Non-convulsive Status Epilepticus

CASE: A 63 year old male with a history of viral meningitis, idiopathic epilepsy, and multiple myeloma on experimental antibody infusion (daratumumab) was brought to the emergency department by his wife for acute confusion and subtle personality change. Patient was typically interactive, joyful, and routinely asked about his farm animals. On the day of presentation, patient did not ask about his animals, was reserved, had forgotten how to use the microwave, and was observed staring blankly at his computer screen. His wife indicated he was normal the day before presentation, had no observed seizure activity, and believed he was compliant with levetiracetam. His oncology clinical investigator did not deem his symptoms were related to daratumumab infusion he received for multiple myeloma. Neurological exam revealed mild deficits with short and long term memory. Complete blood count, complete metabolic panel, thyroid studies, urinalysis, and urine drug screen were unremarkable. CT and MRI of the brain revealed no acute abnormalities. Continuous video Electroencephalogram (EEG) was obtained. Patient was awake, alert, answering questions, and following commands appropriately without any obvious behavioral changes or alteration of consciousness during the study even though his wife reported he was not "himself." EEG revealed findings consistent with nonconvulsive status epilepticus (NCSE). Continuous epileptiform activities gradually attenuated with the administration of intravenous Lorazepam and were accompanied by a gradual return of patient to his baseline. Levetiracetam dose was increased. Patient had no further episodes of confusion or personality change at follow up visit.

DISCUSSION: NCSE is defined as a persistent change in behavior or mental processes compared to baseline that is associated with continuous epileptiform discharges on EEG. Clinical manifestations vary significantly, ranging from subtle changes that are only appreciated by family members to coma. Presentations reported in the literature include mild cognitive disturbances (e.g. impaired attention, mild disorientation), amnesia, mood disturbance, speech disturbance (e.g. reduced verbal fluency, muteness, aphasia), and bizarre behaviors (laughing, dancing, singling inappropriately). Motor activity is usually normal but facial twitching and automatism (licking, chewing, or picking) have been reported. These findings may be subtle and difficult to distinguish from normal behaviors, leading to under recognition and diagnosis. Therefore, a high index of suspicion is required. NCSE should be considered in any patient who has unexplained changes in behavior or mental status, especially in those with a history of seizure. NCSE is typically diagnosed by EEG patterns showing frequent or continuous non-convulsive seizures. A trial of intravenous anti-epileptic drug can be combined with EEG when the diagnosis is uncertain and is diagnostic when there is an improvement in clinical state or a return of normal EEG pattern.

\section{SUCCESSFUL TREATMENT OF GI INVOLVEMENT IN AN ADULT WITH} IGA VASCULITIS Alexander L. Cho; Aneesha Hossain; Sam W. Serafi; Jehan ElBayoumi. George Washington University, Washington DC, DC. (Control ID \#2469105)

LEARNING OBJECTIVE \#1: Treat severe GI manifestations of IgA vasculitis in adults with pulse methylprednisolone.

LEARNING OBJECTIVE \#2: Recognize clinical features of GI involvement in the context of $\operatorname{IgA}$ vasculitis in adults.

CASE: A healthy 27-year-old female presented to the hospital with hematochezia. Ten days prior, she noticed worsening purpura on her trunk and extremities with concomitant arthralgias and abdominal discomfort. Past history was significant for presumed persistent streptococcal pharyngitis for which she completed amoxicillin and azithromycin therapy 1 month before presentation. Outpatient work-up revealed elevated ASO titers and normal inflammatory markers while skin biopsy showed leukocytoclastic vasculitis with extensive hemorrhage and necrosis. She was prescribed a low-dose prednisone; however, 3 days after initiating treatment, she presented with hematochezia. On admission, she remained hemodynamically stable and did not require transfusion. Routine lab findings, infectious disease and rheumatologic work-up were normal. Endoscopic evaluation demonstrated diffuse duodenal, ileal, colonic ulcers (Fig. 1) with hemorrhage and neutrophilic infiltration of the lamina propria, consistent with mesenteric vasculitis. She was started on a 3-day course of pulse IV methylprednisolone ( $250 \mathrm{mg} \mathrm{q} 12 \mathrm{~h}$ ) with resolution of her abdominal pain and hematochezia and was discharged with a high-dose oral prednisone taper. DISCUSSION: IgA vasculitis classically manifests as a triad involving cutaneous purpura, arthralgias and abdominal symptoms. Though rare in adults, roughly 1.4 cases per 100,000, they present with more severe disease and poorer prognosis. While $65 \%$ of adult patients present with abdominal pain, $13 \%$ present with hematochezia, requiring transfusion and surgery secondary to intussusception, bowel perforation and infarction. Given no established guidelines to treat severe GI manifestations of $\operatorname{IgA}$ vasculitis, this case illustrates effective treatment with pulse methylprednisolone, frequently studied to specifically treat renal complications. The decision for treatment extended from a review paper by Audemard-Verger et al., 
who proposed an algorithm recommending 1 g-pulse methylprednisolone for severe GI involvement (Fig. 2). Upon literature review, two cases of IgA vasculitis with GI bleeds were successfully treated with $1 \mathrm{~g}$ pulses and subsequent oral taper. We decided to lower the dose due to the lack of massive overt hemorrhage. Worsening symptoms may predicate the use of immunosuppressants; however, most trials study their effects on renal complications. One prospective open-label study comparing the use of corticosteroids with and without cyclophosphamide showed resolution of visceral involvement in both groups at 6-month follow-up, inferring the significance of steroid use alone. Also, up to $25 \%$ of cases may present with GI symptoms 2-21 days prior to the onset of characteristic purpuric lesions, which may be mistaken for an acute abdomen. Therefore, GI involvement of IgA vasculitis should be considered in a patient with previous history of upper respiratory infection as adults typically have a longer hospital length of stay due to delayed diagnosis and require more aggressive treatment

\section{SUCCESSFUL TREATMENT OF HIGH-LEVEL AMINOGLYCOSIDE RESISTANCE ENTEROCOCCUS FAECALIS ENDOCARDITIS IN PENICILLIN-ALLERGIC PATIENT Reem Mahdawi; Sarwan Kumar; Tiba} Alwardi; MHD Hussam Al-Jandali. Wayne State University, Crittenton Hospital, Rochester, MI. (Control ID \#2466137)

LEARNING OBJECTIVE \#1: Enterococci are isolated from 10 to $15 \%$ of patients with endocarditis and rank as the third commonest cause of endocarditis, behind viridans streptococci and Staphylococcus aureus Enterococcal endocarditis is usually caused by S. faecalis and rarely by S. faecium or S. durans. Enterococcal IE requires bactericidal therapy for an optimal outcome. For decades, cell-wall-active antimicrobial agents (penicillins or vancomycin) in combination with aminoglycosides were the cornerstone of the treatment . Endocarditis caused by penicillin-resistant, aminoglycoside-resistant enterococci was reported frequently. In the event of such a case, antimicrobial options include linezolid, or uinupristin/dalfopristin, but emergence of antibiotic resistance has significantly reduced the efficacy of these regimens. We here discuss a case of enterococcus faecalis IE with aminoglycoside resistance along with known penicillin allergy (anaphylaxis), successfully desensitized to penicillin in ICU and was subsequently treated with ampicillin and ceftriaxone for 6 weeks.

CASE: 81 year-old lady with significant past medical history of Diabetes II, CAD Hypertension, PAD, COPD was brought to ED by her daughter for worsening SOB and productive cough, was not reversed by breathing treatment at home, vitals in the ED showed SBP in 80 s, along with hypoxia. On physical exam, patient was alert but looked in distress, with audible wheezes, EKG showed normal sinus rythm with Q waves in V1, V2. Chest radiograph showed cardiomegaly and finding consistent with pulmonary edema, blood work including $\mathrm{CBC}$ revealed low hemoglobin, BMP and urinalysis were both within normal, BNP was very high . Blood culture grew enterococcus faecalis sensitive to ampicillin and vancomycin, resistant to gentamycin and streptomycin synergy . Patient was initially started on Vancomycin for bacteremia, TEE was done and ascertained the presence of $1.5 \mathrm{~cm}$ mobile echogenic filamentous mass attached the chordae tendineae of anterior mitral leaflet, suspected vegetations. Diagnosis of endocarditis was made, given the aminoglycoside and anaphylactic reaction to penicillin patient was started on vancomycin, but after persistent bacteremia decision was made to desensitize the patient to penicillin she was transferred to ICU and had penicillin desensitization which was tolerated without any complications, patient was started on Ceftriaxone 2 g q12 and Ampicillin $2 \mathrm{~g}$ q6 for total 6 weeks with significant improvement.

DISCUSSION: Approximately $97 \%$ of cases of enterococcal IE are caused by E faecalis, and the majority of these remain susceptible to $\beta$-lactams and vancomycin, but aminoglycoside resistance is increasing in frequency. First, in an in vitro study, Mainardi et al. observed that a double $\beta$-lactam combination was synergistic through partial saturation of
PBPs 4 and 5 due to amoxicillin and total saturation of PBPs 2 and 3 due to cefotaxime. A observational, open-label, non-randomized, multi- center clinical trial Gavaldà et al., the same group evaluated the efficacy of a 6-week course of ampicillin ( 2 g every 4 h) plus high- dose ceftriaxone ( $2 \mathrm{~g}$ every $12 \mathrm{~h}$ ) for the treatment of IE due to E. faecalis. Cure rates at the end of treatment and at 3 months in the HLAR (High-level aminoglycoside resistance) and non-HLAR groups were 71.4 versus 72.7 and 71.4 versus $63.6 \%$, respectively. Recently, in an another observational, non-randomized comparative multicenter cohort study, Fernández-Hidalgo et al. compared the effectiveness of the ampicillin plus ceftriaxone and ampicillin plus gentamicin combinations for treating IE caused by E. Faecalis. Mortality rates at the end of treatment and at 3 months in the ampicillin/ ceftriaxone and ampicillin/gentamicin groups were 22 versus 21 and 8 versus and $7 \%$, respectively. However, the rate of adverse events requiring treatment withdrawal, mainly due to kidney failure, was significantly higher in the aminoglycoside based regimen compared to the experimental regimen. Double- $\beta$-lactam has become a widely accepted therapy for the management of HLAR E. faecalis in Spain with a significant increase of its use over time and without affecting the clinical outcome compared to ampicillin/ gentamicin regimen. American Heart Association recommends 6 weeks of therapy if ampicillin plus ceftriaxone to be selected as the treatment regimen, regardless of symptom duration (Class IIa; Level of Evidence B). Given the above rationale, we decided to proceed with penicillin desensitization with escalating doses of IV penicillin $\mathrm{G}$ along with close monitoring in the ICU, patient was successfully desensitized in $24 \mathrm{~h}$, and she was switched to Ceftriaxone $2 \mathrm{~g} \mathrm{q} 12$ and Ampicillin 2 g q6 for total 6 weeks. This case illustrates that penicillin desensitization should be considered if serious penicillin allergy prevents penicillin therapy in infective endocarditis caused by Enterococcus Faecalis because of high success rate and less side effect.

SUPRAVENTRICULAR TACHYCARDIA WITH RIGHT ATRIAL MASS: AN UNUSUAL PRESENTATION OF A COMMON TUMOR Melissa Stein; Irene J. Soloway. Montefiore Medical Center, Bronx, NY. (Control ID \#2469743)

LEARNING OBJECTIVE \#1: Identify an uncommon presentation of hepatocellular carcinoma (HCC)

LEARNING OBJECTIVE \#2: Recognize limitations of ultrasound in screening for $\mathrm{HCC}$

CASE: A 58 year old African American woman with chronic hepatitis $\mathrm{C}$ infection and opiate dependence on treatment with sofosbuvir/ribavirin presented to her primary care provider for routine treatment monitoring. At age 51 she was diagnosed with chronic active HCV (genotype 4). Two years after diagnosis she underwent treatment with interferon and ribavirin but did not achieve sustained viral response. Five years after her initial treatment, reevaluation with fibrosure blood test revealed progression of liver disease to cirrhosis. MRI was scheduled to screen for hepatocellular carcinoma (HCC) but was aborted secondary to patient discomfort. The MRI was not conclusive and could not rule out a space occupying lesion. The patient refused to attempt another MRI but ultimately underwent abdominal ultrasound at a community radiology clinic which revealed hepatosplenomegaly, cholelithiasis and a widened common bile duct. No masses were noted and the IVC was unremarkable. Retreatment with sofosbuvir/ribavirin was initiated. On the day of presentation, she had no acute complaints but reported intermittent palpitations on ROS. Physical exam was significant for heart rate of 160 beats/min and hypotension. EKG revealed SVT and the patient was transferred to the ED. In the ED the patient received $6 \mathrm{mg}$ of adenosine and returned to normal sinus rhythm. She was admitted to the hospital for evaluation. Echocardiogram showed large mass in right atrium which was believed to be an atrial myxoma. Cardiothoracic surgery was consulted to evaluate for resection. Pre-operative cardiac catheterization showed normal coronary arteries. The hepatology service was consulted and ordered a CT scan which revealed a $9.5 \times 4.5 \mathrm{~cm}$ 
hypervascular hepatic mass growing into the right hepatic vein and through the IVC to the right atrium. The patient remained in sinus rhythm and was discharged with a diagnosis of (inoperable) hepatocellular carcinoma. She received home hospice care and died a month following discharge.

DISCUSSION: Hepatocellular carcinoma is the fifth most common malignancy and the third leading cause of cancer-related mortalities in the world. Risk factors for HCC include cirrhosis, alcoholic liver disease, nonalcoholic fatty liver disease, chronic hepatitis B infection, and chronic hepatitis $\mathrm{C}$ infection. Hepatitis $\mathrm{C}$ affects approximately 4 million people in the U.S. Chronic hepatitis $\mathrm{C}(\mathrm{HCV})$ has become the leading cause of liver transplantation in the United States. HCV is most common among former or current IV drug users, $65-95 \%$ of whom are $\mathrm{HCV}$ antibody positive. The rising prevalence of cirrhosis due to HCV has resulted in an increase in the prevalence of hepatocellular carcinoma. Current guidelines recommend using imaging and alpha-fetoprotein testing every 6 to 12 months to screen for HCC in patients with a history of chronic HCV infection and advanced fibrosis or cirrhosis. CT scan and MRI are more sensitive than ultrasound in identifying $\mathrm{HCC}$ but obtaining these tests can be challenging Most patients with HCC are identified through screening or identification of a primary liver lesion. However, HCC can present with symptoms caused by advanced local tumor spread or metastatic disease. This case illustrates a rare presentation of HCC with tumor thrombus extending through hepatic vasculature up the IVC into the right atrium. As HCC becomes increasingly common, we must be aware of the range of possible presentations.

TAKE YOUR PICC... INTRA-CAVITARY EKG OR CXR Michelle O. Forson; Abdullah Ali Ahmed. Presence St Francis Hospital, Evanston, IL. (Control ID \#2463462)

LEARNING OBJECTIVE \#1: To recognize the risk of central venous catheter malpositioning despite electrocardiographic guidance.

CASE: A 35 year old female presented with progressive fatigue and drowsiness of 3 days duration. Her past medical history (PMH) included type 1 diabetes mellitus, Clostridium difficile colitis, left vena caval stenosis, and ESRD (on hemodialysis via a right internal jugular tunneled catheter). She reported diarrhea, decreased appetite and malaise but denied fever or chills. On examination she was somnolent, hypotensive (blood pressure was $83 / 29$ ), with tachycardia and in circulatory shock. Her lab results were significant for hyperglycemia (326), hyperkalemia, and metabolic acidosis. She received two liters of normal saline ( $>30 \mathrm{mls} / \mathrm{Kg}$ ) but remained hypotensive (72/30). After consideration of her current clinical features, $\mathrm{PMH}$, and urgent dialysis requirement, the decision was made to place a Peripherally Inserted Central Catheter (PICC) line following consultation with her nephrologist. The vascular nurse placed a right sided PICC under ultrasound guidance. Placement -in the lower superior vena cava- was confirmed by Sherlock 3CG intracavitary EKG which showed peaking $P$ waves. The patient was started on norepinephrine and IV fluids. A chest X-ray obtained a few hours later showed the right sided PICC crossing the midline terminating in the brachiocephalic artery at the junction with the aorta. All infusions were stopped immediately. Blood gas performed on the blood sample from the PICC had PO2 of 136 confirming an intra-arterial placement. On examination, her peripheral pulses were symmetric, extremities warm, pink and no localizing features on neurological exam. The vascular surgeon evaluated the patient, removed the PICC and applied pressure to the insertion site. Frequent neurovascular checks did not reveal any further complications. Over the next $24 \mathrm{~h}$ the patient's mental status improved significantly and she was eventually discharged home.

DISCUSSION: Peripherally Inserted Central Catheters (PICCs) are increasingly utilized in modern medicine and there is a recent trend towards the use of intra-cavitary/ endovascular electrocardiography (EKG) for placement confirmation. In September 2015 guidelines regarding CVCs were released-The Michigan Appropriateness Guide for Intravenous Catheters (MAGIC). MAGIC guidelines state that "routine radiographic verification of PICC tip position is inappropriate provided that proficiency with this technology had been demonstrated and adequate tracings (such as P wave deflections) were observed". However as this case shows catheter tip malposition should not be dismissed entirely. The overall incidence of primary PICC malposition is $2-30 \%$. Post procedural diagnosis of malposition is associated with logistical problems and increased costs; therefore a method of confirming placement during the procedure is preferable. The three options available are; fluoroscopy, echocardiography and EKG. The EKG method is easier to use, more promptly accessible and cost effective. Our institution had adopted the EKG method for a few years with the vascular team trained and certified in its use. The Sherlock 3CG is the EKG verification device used by our institution for PICC line insertion (in combination with ultrasound guidance). The Sherlock $3 \mathrm{CG}$ uses the catheter tip as the endovascular electrode and internal EKG measurements to confirm the position of the catheter tip relative to the heart using the amplitude of the $\mathrm{P}$ waves compared to the QRS complex. The maximum $P$ wave height indicates the catheter tip is at the entrance of the atrium and retraction to the point at which the $\mathrm{P}$ wave height is half the maximum corresponds with the lower third of the superior vena cava. The placement accuracy of PICCs using Sherlock 3CG ranges from 79.5 to $100 \%$. However both intra- arterial and intra-venous catheters will have increasing $\mathrm{P}$ waves as the tip approaches the pericardial fold. Therefore identification of inadvertent intra-arterial cannulation would be difficult when using the EKG verification alone. Considering the significant complications associated with PICC malposition such as-vasospasm, arterial thrombosis, pseudoaneurysms and AV fistulas - it would appear that additional radiographic confirmation would be beneficial.

TAKOTSUBO CARDIOMYOPATHY IN THE PERIPARTUM PERIOD: A CLINICAL VIGNETTE Mahesh A. Chandrasekhar; Zuyue Wang. Medstar Washington Hospital Center, Washington, DC. (Control ID \#2469967)

LEARNING OBJECTIVE \#1: Identify the distinction between Takotsubo cardiomyopathy and peripartum cardiomyopathy in the peripartum period in regard to the prognosis, time to recovery, and the planning of future pregnancies.

LEARNING OBJECTIVE \#2: Recognized that Takotsubo cardiomyopathy can lead to cardiac arrest.

CASE: A 29-year-old multiparous Caucasian female at 40 weeks gestation by 18 week ultrasound presented to an obstetrics and gynecology service to rule out spontaneous rupture of membranes. At presentation, the patient denied chest pain, shortness of breath, or new exertional difficulty, but noted new bilateral lower extremity swelling first noted 5 days prior to admission. Medical history was otherwise unremarkable. Social history was relevant for tobacco use stopped 3 months prior and intermittent marijuana use; patient mentioned she recently traveled several hours to visit family for personal reasons, but did not discuss further psychosocial stressors. Her only medications included prenatal vitamins; patient had little prenatal care outside of the 18 week ultrasound. Physical exam was unremarkable on presentation; vital signs were normal except a pulse of 59 beats per minute. Overnight on the day of admission, fetal heart monitoring showed variable decelerations, for which the patient was turned on her side. An epidural was administered prior to a planned delivery for spontaneous rupture of membranes. Within $15 \mathrm{~min}$ of administration, the patient became hypoxic, requiring usage of a face mask for oxygenation. The patient was soon after found to be somnolent and unresponsive, with a thready pulse. Pulseless electrical activity was noted and the patient received $2 \mathrm{~min}$ of chest compressions and a total of $2 \mathrm{mg} / \mathrm{mL}$ of epinephrine. Return of spontaneous circulation was reach and the patient was intubated and placed norepinephrine. Vaginal delivery was performed, and the infant was delivered by vacuum assisted delivery without complication. Electrocardiogram (ECG) post resuscitation showed no elements of current injury, myocardial infarction, or left ventricular strain. The patient was transferred to the intensive care unit (ICU) for further evaluation. Patient was quickly stabilized within the ICU, with 
removal of vasopressors upon arrival and extubated soon after. A brain natriuretic peptide level of $5600 \mathrm{pg} / \mathrm{mL}$ was obtained. The patient was treated with supportive care including furosemide diuresis and initiation of both beta blockade and an angiotensin-converting enzyme inhibitor for afterload reduction. The initial echocardiogram in the ICU demonstrated mild left ventricular dilation, severe reduction of left ventricular systolic function with an ejection fraction of $25 \%$. Regional wall motion abnormalities included severe anterior wall, inferior wall and infero-lateral wall hypokinesis, were present, along with ballooning of the cardiac apex. As part of an ischemic work up, a chest and cardiac computed tomography scan was performed, showing neither any evidence of pulmonary embolism nor abnormal coronary artery origins or ostia. No coronary atherosclerosis, stenosis or other pathology in the coronary arteries was noted. Patient was soon discharged and asked to follow up with the outpatient cardiology service. At 1 month follow up, patient was symptom free. Echocardiogram at that time showed demonstrated left ventricular concentric hypertrophy, near normal left ventricular systolic function with an ejection fraction of $55 \%$. No regional wall motion abnormalities were noted at follow up. DISCUSSION: Takotsubo cardiomyopathy has been described in the peripartum period. Onset of the symptoms in the perioperative/delivery period is found less in the literature; to date only four cases were found that exhibited severe cardiac dysfunction in pregnant patients post placement of an epidural anesthesia while also documenting lack of coronary artery or pulmonary thromboembolic disease. In those described cases, none progressed to cardiac arrest. There is no consensus on the mechanism of action for Takotsubo cardiomyopathy, the leading theories focus on cardiac dysfunction secondary to acute catecholamine surge. Urgent Caesarean delivery could be a reasonably stressful event for our patient. In addition, epidural anesthesia, thought to be the trigger for this patient's cardiomyopathy, may not have been expected, given our patient's relative lack of prenatal care. This stress response, coupled with variability in myocardial adrenoceptors may explain why, rather than the characteristic apical ballooning seen in Takotsubo cardiomyopathy, our patient had a variety of notable wall motion abnormalities. It is important to make distinctions between Takotsubo cardiomyopathy and peripartum cardiomyopathy. Less than $10 \%$ of peripartum cardiomyopathy cases occur in the prepartum period, and there is a paucity of cases presenting in the perioperative state prior to delivery. Furthermore, the time course of recovery is significantly different; while Takotsubo cardiomyopathy may see recovery by 4 week follow up, most peripartum cases require at a minimum 3 to 6 months for recovery of ventricular function. Ultimately, the importance of distinguishing the etiology of heart failure becomes important in the planning of future pregnancies. While Takotsubo cardiomyopathy is relatively rare to recur, often reported less than $5 \%$ recurrence, the risk of peripartum cardiomyopathy with subsequent pregnancies is substantially higher.

\section{TB OR NOT TB: CAUDA EQUINA SYNDROME SECONDARY TO INVASIVE MYCOBACTERIUM INFECTION CAUSING LUMBAR POLYRADICULITIS} Jennifer Phan ${ }^{2}$; Iram Dubin ${ }^{1}$; Neal Shah ${ }^{1} .{ }^{1}$ Olive View Medical Center, Sylmar, CA; ${ }^{2}$ University of California, Los Angeles, Los Angeles, CA. (Control ID \#2456820)

LEARNING OBJECTIVE \#1: Identify infectious and non-infectious etiologies resulting in acute cauda equine syndrome.

LEARNING OBJECTIVE \#2: Evaluation of an uncommon presentation of extrapulmonary TB infection.

CASE: We present the case of a 50 year-old immigrant Filipino male with history of polyarthritic gout and stage IV squamous cell carcinoma of the neck status post-resection who presented initially to the hospital for hyponatremia to 109 , but developed acute onset of left lower extremity hemiparesis and bowel incontinence. Patient was initially treated in the intensive care unit for proper serum sodium correction, and found to have a new large calvarial mass with biopsy confirmed moderately differentiated keratinizing squamous cell carcinoma. He also developed an intermittent dry cough, therefore respiratory cultures were sent however chest imaging remained unimpressive. Due to history of chronic lower back pain, lumbar magnetic resonance imaging (MRI) was performed demonstrating possible leptomeningeal carcinomatosis. Lumbar puncture was significant for lymphocytosis with negative cultures and acid-fast bacilli. Two weeks into hospitalization, patient developed acute loss of sensorium in the left leg and rectal incontinence. Neurological exam was significant for peri-anal sensory loss, unilateral sensory and motor loss of the left lower extremity to T11-T12, and mute plantar reflex. Repeat MRI showed diffuse multinodular enhancement of thickened nerve roots of the cauda equina consistent with carcinomatous polyradiculitis - no masses or spinal edema identified. Patient was initiated on high dose dexamethasone and pain control for presumed SCC leptomeningeal carcinomatosis with minimal improvement in his cauda equina symptoms. The option for intrathecal chemotherapy was not available for the patient's underlying malignancy. Four weeks later, patient's respiratory culture grew Mycobacterium tuberculosis and patient was initiated on RIPE therapy. Patient was able to regain sensation in the effected limb as well as some mobility, though it was self-limited.

DISCUSSION: Cauda equina syndrome (CES) is a neurological emergency characterized by acute loss of function due to disruption of neurological elements below termination of the spinal cord. Infective pathology has been associated with acute CES, with etiologies including epidural abscess from gram-positive, fungal or tick-borne organisms, viral polyradiculoneuropathy or vertebral osteomyelitis. While tuberculosis has many unique extra-pulmonary manifestations, this is the first reported case, to our knowledge, of it causing lumbar polyradiculitis through nerve root invasion causing CES. Treatment of polyradiculitis can be delayed given organisms are slow growing, as seen in our case, and are difficult to isolate on culture. Delay in treatment with antibiotics can result in worsening neurological compromise. Cases of spinal-invasive infections are more likely identified in immunocompromised hosts, such as patients with HIV, organ transplantation, malignancy, or long-term steroid use, and therefore should be highly considered in patients with acute CES without compressive lesions on imaging. Our patient presents with a diagnostic dilemma of possible malignancy- and infectious-driven polyradiculitis causing CES

TEAR IT UP! EXERCISE INDUCED CORONARY ARTERY DISSECTION Coral Olazagasti; Carolina Bernabe; Ana I. Velazquez; Matthew Harrington. Mount Sinai Beth Israel, New York, NY. (Control ID \#2469793)

LEARNING OBJECTIVE \#1: Define the clinical presentation of coronary artery dissection

CASE: A 40 year-old female with history of pulmonary embolism during pregnancy presented with sudden onset of substernal chest pain and shortness of breath, which began an hour after she finished a rowing class. She also experienced nausea and left hand numbness and tingling. On presentation, vital signs were within normal limits and physical examination was unremarkable. EKG revealed ST elevation in V2 and ST depression in leads II, III and AVL (Fig. 1). Bedside ultrasound revealed anterior wall motion abnormality. Patient received aspirin $325 \mathrm{mg}$ PO, heparin IV bolus and drip, and clopidogrel $300 \mathrm{mg}$ PO load. She was taken for emergent cardiac catheterization, which revealed dissection of the mid left anterior descending coronary (LAD). A drug eluting stent (DES) was placed in the artery with normalization of flow. The remainder of the coronary arteries showed no obstruction. Post-catheterization EKG showed normalization of ST segments (Fig. 2), repeat echocardiogram showed normal left ventricular systolic size and function. Blood work was remarkable for normal lipid profile and negative ANA, ANCA panel, and rheumatoid factor. Urine pregnancy was negative. Troponin peaked was $7.7 \mathrm{ng} / \mathrm{ml}$. Patient was discharged on dual antiplatelet agents, low dose B-blocker and a statin. 
DISCUSSION: Spontaneous coronary artery dissection (SCAD) is a rare cause of acute coronary syndrome (ACS), accounting for less than $0.5 \%$ of total cases. SCAD is usually seen in young women in the peripartum state. The underlying mechanism is not well described; it is theorized that an intramural hematoma creates a false lumen, which compresses the true lumen of the coronary artery, resulting in myocardial ischemia. Risk factors linked to SCAD include severe exertion, postpartum status, life-threatening arrhythmias, connective tissue disorders, and inflammatory diseases. Clinical presentation varies from unstable angina to sudden cardiac death; severity of presentation correlates with the degree of dissection. Coronary angiography with possible percutaneous coronary intervention (PCI) are the diagnostic and therapeutic modalities of choice. Recurrence rate is up to $10 \%$ of having a new cardiovascular event. SCAD as an etiology of ACS should be considered in patients without traditional cardiovascular risk factors, as well as in specific populations including peripartum women, and patients with connective tissue disorders or inflammatory states. Mortality rate is as high as $75 \%$ and diagnosis is usually post-mortem. Early recognition with appropriate and rapid intervention is critical for survival.

TESTING FOR HUMAN IMMUNODEFICIENCY VIRUS (HIV) IN THE SETTING OF NEWLY DIAGNOSED PORPHYRIA CUTANAE TARDA Lauren M. Fontana; Arun K. Singavi. Medical College of Wisconsin, Wauwatosa, WI. (Control ID \#2435667)

LEARNING OBJECTIVE \#1: Recognize the importance of testing for HIV in a patient diagnosed with Porphyria Cutanae Tarda

LEARNING OBJECTIVE \#2: Recognize risk factors that increase the susceptibility for Porphyria Cutanea Tarda

CASE: A 49 year old male with a past history of type II diabetes mellitus presented to his dermatologist with worsening bullae on the dorsum of his hands and fingers that started in the spring after significant sun exposure. Physical exam was significant for tense bullae, erosions, and small ulcers on his dorsal fingers and hands. Labs revealed elevated porphyrins in the urine and blood. Direct immunofluorescence confirmed the diagnosis of PCT. Hepatitis A and C were negative, with demonstrated immunity to hepatitis B from prior infection. Three months later he presented to an urgent care with a fever and cough, was diagnosed with community acquired pneumonia, and started on levofloxacin. One week later the patient presented to the emergency department with worsening malaise, fevers, productive cough, night sweats, and a 50-lb weight loss over 3 months. Physical exam was significant for diffuse cervical lymphadenopathy, oral thrush, and crackles bilaterally. Labs revealed an elevated LDH. Chest x-ray revealed bilateral interstitial opacities. The patient had numerous clinical signs that were concerning for pneumocystis jiroveci pneumonia (PJP) so he was started on atovoquone. Trimethoprimsulfamethaxazole is contraindicated in active PCT. His PJP NAAT and HIV test were positive.

DISCUSSION: Type I Porphyria Cutanea Tarda (PCT) is an acquired cutaneous porphyria due to the deficient activity of the heme biosynthetic enzyme uroporphyrinogen decarboxylase (UROD) in the liver. This enzyme is important for the synthesis of heme. This leads to an increase in circulating iron levels. PCT typically presents with pruritic bullous and vesicular lesions in areas of sun exposure and trauma. Some factors increase the susceptibility to PCT such as liver damage from alcohol use, hepatitis C virus, HIV, and estrogen. Addressing these underlying issues is a component of PCT treatment. The incidence of PCT is reported to be higher in HIV infected individuals. Between 1987 and 1999, there were 75 reported PCT cases in HIV infected patients. Forty-percent of these patients were diagnosed with PCT prior to the detection of HIV by an average of 17 months (calculated on 64 of the 75 patients). Other factors such as a concomitant infection with hepatitis $\mathrm{C}$ may also be associated with the development of PCT. Forty-five percent of patients with HIV and PCT also had positive HCV serology (calculated on 24 of the 75 patients of patients). The exact mechanism by which HIV contributes to the development of PCT is not fully understood. Screening for HIV in appropriate patients with newly diagnosed PCT can lead to an earlier diagnosis of HIV and treatment, preventing worsening immunosuppression.

TESTS ARE NEGATIVE, BUT STILL POSITIVE Kathleen McKillip; Michael P. Smith. University of Nebraska Medical Center, Omaha, NE. (Control ID \#2469927)

LEARNING OBJECTIVE \#1: Recognize the presentation and diagnostic criteria of late neurosyphilis

LEARNING OBJECTIVE \#2: Understand the diagnostic performance of tests used to evaluate for neurosyphilis

CASE: A 56 year-old woman presented with 1 week of progressive total body pain, weakness, and confusion. She was not able to provide a reliable history. Her past medical history was significant for untreated hypothyroidism, partially treated hepatitis $\mathrm{C}$ with viral load over 4 million, alcohol and polysubstance abuse, and malnutrition. She showed slight improvement with management of these conditions, but family reported ongoing alogia and slow speech. Physical therapy noted ongoing weakness and ataxia. CT of her head was negative for acute changes. Further social history revealed high risk sexual behavior; STI screen was significant for positive serum RPR with a 1:2 titer. The patient could not recall a prior diagnosis or treatment of syphilis. Lumbar puncture revealed 2 WBC per field, elevated protein at $99 \mathrm{mg} / \mathrm{dl}$ and normal glucose. Her cerebrospinal VDRL returned negative, however treatment for neurosyphilis was initiated due to her high protein and symptoms. Her alogia improved on a 14 day course of penicillin.

DISCUSSION: The incidence of neurosyphilis has declined in recent decades likely due to widespread use of antibiotics for other reasons. While early neurosyphilis more often manifests positive titers of both nontreponemal (VDRL, RPR) and treponemal (fluorescent treponemal antibody absorption [FTA-ABS], treponema pallidum particle agglutination assay [TPPA], or syphilis enzyme immunoassay [EIA]) assays, late neurosyphilis often loses reactivity to the nontreponemal tests. This was demonstrated in the case above. As in this case, cerebrospinal VDRL has a false negative rate of up to $70 \%$ in late neurosyphilis. When considering a diagnosis of late neurosyphilis, serum treponemal tests (FTA-ABS, TPPA, or syphilis EIA) should be considered. However, expert consensus suggests that leukocytosis and elevated protein on CSF studies in the setting of a positive serum titer and high clinical suspicion is sufficient evidence for initiating treatment. It is essential that the general internist recognizes late neurosyphilis and is able to make the accurate diagnosis as the most well-known diagnostics for syphilis often are falsely negative. Without a high index of suspicion, the correct treatment will not be initiated, potentially leading to severe sequelae.

THE A, B, CS OF HEPATITIS Vassiliki Pravodelov; Bailey Y. Chang. Boston University Medical Center, Boston, MA. (Control ID \#2468095)

LEARNING OBJECTIVE \#1: Recognize the importance of Hepatitis B vaccination in patients with chronic Hepatitis C

LEARNING OBJECTIVE \#2: Review the intricacies of Hepatitis B and C co-infection CASE: A 52 year-old woman with history of remote Hepatitis A infection and chronic untreated Hepatitis C presented to the primary care clinic with a week-long history of fatigue, new diffuse hand joint pain, worst at the proximal interphalangeal (PIP) joints, bilateral shoulder pain, and worsening right knee pain. Her past medical history was significant for chronic knee and ankle pain, former intravenous heroin use now in remission on suboxone, and alcohol use disorder in remission. The patient had received 
one dose of the Hepatitis A vaccine and 2 doses of the Hepatitis B vaccine in the past, never completing either vaccination series. In clinic, her vital signs were unremarkable but due to distress on exam, she was referred to the emergency department (ED). Lyme IgG was negative, erythrocyte sedimentation rate (ESR) and basic metabolic panel were normal, while the C-reactive protein was elevated at $63.5 \mathrm{mg} / \mathrm{L}$. In the $\mathrm{ED}$, she received intravenous fluids and intramuscular ketorolac, and was sent home with ibuprofen. However, she returned to the clinic 6 days later with persistence of diffuse joint pain and interval development of hand joint swelling. Her vitals were again unremarkable though her exam revealed synovitis of the PIP joints bilaterally. Further testing revealed a positive rheumatoid factor, a positive Hepatitis B core antibody, and elevated liver transaminases with aspartate transaminase (AST) at $291 \mathrm{U} / \mathrm{L}$ and alanine transaminase (ALT) at $607 \mathrm{U} / \mathrm{L}$. Serum cryoglobulins were also detected. The patient's symptoms continued to worsen and 5 days later she was sent to the ED because of nausea, vomiting, and worsening polyarthralgias. In the ED, she had an abdominal CT scan that revealed perihepatic lymph nodes and an abdominal ultrasound that showed mild splenomegaly and was discharged home. At the follow-up clinic visit she was found to have persistent joint pain. Anti-cyclic citrullinated peptide antibody was negative. However, her Hepatitis B viral (HBV) load was found to be greater than 8 million with a positive Hepatitis B core IgM antibody and she was then admitted to the hospital for acute hepatitis B management. She received ketorolac and opiates for pain control. Her exposure to Hepatitis B was thought to be an unprotected sexual encounter with a Hepatitis B positive partner. Her AST and ALT peaked at 690 and $1022 \mathrm{U} / \mathrm{L}$ respectively. Her alkaline phosphatase peaked at $525 \mathrm{U} / \mathrm{L}$, from a baseline of about 110 . Her hepatitis C viral (HCV) load was noted to be $3355 \mathrm{IU} / \mathrm{mL}$. At discharge, her AST and ALT had normalized and the HBV viral load was noted to be $132 \mathrm{IU} / \mathrm{mL}$. Her symptoms of nausea and vomiting improved, but the arthralgias and fatigue persisted, likely due to the presence of untreated cryoglobulin positive chronic Hepatitis C.

DISCUSSION: This case serves as a reminder that immunization against the hepatitis $\mathrm{B}$ virus can be defeated in clinical practice. Our patient developed acute hepatitis B in spite of having recently begun the vaccination series, when reason would dictate that the hepatitis B surface antibody (HBsAb) response would have been most potent. However, antibody unresponsiveness, has been described in certain populations, including those with chronic liver disease, such as Hepatitis C. Our patient did not complete her vaccination series and therefore an antibody titer was not obtained prior to the onset of her symptoms. Furthermore, it is not recommended to routinely check an HBsAb titer after completing the Hepatitis B vaccination in the immunocompetent population, as the antibody response is reported to be about $95 \%$. In those at risk for non-response, however, it is recommended to give an additional $10 \mathrm{mg}$ dose and to check the antibody titer approximately 3 months after the last dose of vaccine. If the HBsAb level is then less than $10 \mathrm{IU} / \mathrm{mL}$, the four-dose vaccination series should be repeated as $50-60 \%$ of the nonresponders tend to respond to it. In light of the above, it is likely that the patient developed insufficient immunogenicity from her vaccine doses. It must be noted that several international studies have showed that individuals who respond to the HBV vaccination series are still at risk for developing an acute $\mathrm{HBV}$ infection. One issue that bears mentioning is the suppression of the HCV viral load, noticed during our patient's hospitalization. Case reports have shown that patients with underlying chronic HCV infection who developed acute HBV infection exhibited accelerated clearance of the HBV surface antigen molecule and HBV and HCV viral loads. In conclusion, the antiHBV vaccine can and should be used to mitigate the possibility of developing severe acute hepatitis in those individuals with chronic hepatitis C. However, in those individuals who are at risk for a poor immunologic response to the standard vaccine regimen, care must be taken to increase the normal vaccination dosage and routinely check for poor antibody response. As a final point, for those individuals who do go on to develop acute hepatitis B, serologic monitoring is of utmost importance given the possibility to clear both the hepatitis B and C viruses. Perhaps even more importantly, appropriate patient education regarding preventable exposures, such as sexual contacts and intravenous drug use, should be given as the efficacy of vaccination is not perfect.

THE AGONY BEHIND LACTIC ACIDOSIS Qinyun Wang; Diane Davis; Philip J. Putnam. Tulane University Health Sciences Center, New Orleans, LA. (Control ID \#2467220)

LEARNING OBJECTIVE \#1: Differentiate lactic acidosis Type A vs. Type B LEARNING OBJECTIVE \#2: Encourage consideration of reversible causes of lactic acidosis before initiating invasive work-up.

CASE: A 56 year-old woman with history of asthma presented with 3 days of shortness of breath. She was afebrile and in mild respiratory distress with an oxygen saturation of $100 \%$ on room air. Lung exam was significant for diffuse wheezing, poor air movement, and bibasilar crackles. Initial laboratory data showed leukocytosis (WCC 13.0) and elevated lactic acid $(3.2 \mathrm{mmol} / \mathrm{l})$. Chest X-ray revealed no acute airspace disease, and blood and urine cultures demonstrated no growth. After admission, the patient's lactic acid peaked at $6.9 \mathrm{mmol} / \mathrm{l}$. D-lactate was normal $(0.35 \mathrm{mmol} / \mathrm{d})$. Septic shock was ruled out on account of reassuring vitals, absence of localizing signs of infection, and otherwise normal lab values. Because of the patient's history of insulin-dependent diabetes and oral squamous cell carcinoma, the differential diagnosis also included metformin-induced lactic acidosis and malignancy-related lactic acidosis. These were deemed unlikely given the patient's longtime metformin use and close oncology follow-up. Review of her pre and inhospital treatment regimen ultimately revealed a temporal relationship between inhaled $\beta 2$-agonist administration and lactic acid levels. Prior to presentation, the patient had been using her albuterol inhaler and nebulizer 3-4 times daily for several days (compared to typically sporadic monthly use). In the ER, she was given 2 additional rounds of $7.5 \mathrm{mg}$ of albuterol sulfate $(5 \mathrm{mg} / \mathrm{ml})$. Roughly $6 \mathrm{~h}$ later, her lactic acid rose from 3.2 to $6.9 \mathrm{mmol} / \mathrm{l}$. On the floor, the patient continued to receive $3 \mathrm{ml}$ of ipratropium bromide/albuterol ( 0.5 $3 \mathrm{mg} / 3 \mathrm{ml}$ ) every $4 \mathrm{~h}$, resulting in persistently elevated lactic acid $(4 \mathrm{mmol} / \mathrm{l})$. After inhaled 32-agonist discontinuation, it decreased to 2.1 .

DISCUSSION: Lactic acidosis falls under 2 broad categories: Type A, in which lactic acid is produced due to tissue hypoxia/hypoperfusion, and Type $\mathrm{B}$, in which lactic acid is produced in the absence of such conditions. In this and other reported cases of Type B $\beta 2$ agonist-induced lactic acidosis, it is thought that stimulation of $\beta 2$ adrenergic receptors increases glycolysis and glycogenolysis, thus increasing pyruvate production. A concurrent rise in lipolysis prevents pyruvate conversion to coenzyme A, leaving an excess of pyruvate to be converted to lactic acid via anaerobic glycolysis. Additionally, concomitant steroid administration increases the sensitivity of $\beta 2$ receptors - this particular patient received $125 \mathrm{mg}$ IV methylprednisolone sodium succinate in the ER, thus setting the stage for an exaggerated $\beta 2$ adrenergic response to inhaled albuterol. Therefore, this case demonstrates that lactic acidosis can arise from reversible etiologies such as inhaled $\beta 2$ agonist use. Consideration of such causes, especially in patients with respiratory issues, may prevent unneeded testing and intervention in otherwise stable patients.

THE CASE BEHIND THE SWINGING HEART Nicole R. Zeisig; Betty La; Randy Chung; Jeffrey Bates. Baylor College of Medicine, Houston, TX. (Control ID \#2469800)

LEARNING OBJECTIVE \#1: Recognize tuberculous pericarditis as a potential complication of tuberculosis which can result in late sequelae of constrictive pericarditis

LEARNING OBJECTIVE \#2: Recognize that patients with tuberculous pericarditis should be managed with corticosteroids in addition to typical antituberculous RIPE therapy CASE: A 59-year-old man with a remote traumatic spinal cord injury, incarceration, and latent tuberculosis treated with 6 months of isoniazid was admitted with 3 months of 
worsening lower extremity edema and 5 days of shortness of breath. At baseline he is wheelchair bound and able to independently care for himself; he noted a 20-lb weight gain and is now unable to perform any activities of daily living. Physical examination was significant for pulsus paradoxus of $20 \mathrm{mmHg}$, elevated jugular venous distention, distant heart sounds and lower extremity edema. An electrocardiogram (EKG) showed low voltage with electrical alternans, and a transthoracic echocardiogram showed a very large circumferential pericardial effusion with collapse of the right atrium and ventricle. Pericardiocentesis drained $1125 \mathrm{cc}$ of serosanguinous fluid; pericardial fluid revealed more than 2 million leukocytes with $86 \%$ lymphocytes, total protein of $3.4 \mathrm{~g} / \mathrm{dL}$, elevated lactate dehydrogenase of $>27000$ units/L, adenosine deaminase of 138 units/L, negative smear for acid fast bacilli and negative polymerase chain reaction for Mycobacterium tuberculosis (MTB). A serum T-SPOT assay was positive for MTB. Computed tomography $(\mathrm{CT})$ of the chest, abdomen, and pelvis were negative for any cavitary lung lesions. The patient was diagnosed with tuberculous pericarditis and treated with RIPE therapy (rifampin $600 \mathrm{mg}$ daily, isoniazid $300 \mathrm{mg}$ daily, pyrazinamide $2 \mathrm{~g}$ daily, ethambutol $1600 \mathrm{mg}$ daily), pyridoxine $50 \mathrm{mg}$ daily, and prednisone $60 \mathrm{mg}$ daily. He is scheduled for a follow-up appointment in 1-2 months.

DISCUSSION: Although causing less than $5 \%$ of cases in advanced countries, Mycobacterium tuberculosis is the most common cause of pericarditis worldwide, accounting for more than $70 \%$ of all cases. Tuberculous pericarditis may present clinically as a pericardial effusion, constrictive pericarditis or a combination of both features. More than $80 \%$ of cases present with pericardial effusion, and progressive disease can lead to cardiac tamponade. Common manifestations of tuberculous pericarditis are identical to the non-specific symptoms seen in pulmonary tuberculosis including fever, night sweats, fatigue, and weight loss. Constrictive pericarditis is a dreaded complication of tuberculous pericarditis as it almost inevitably results in death, and it occurs in $17-60 \%$ of cases despite appropriate medical therapy. Diagnosis may be challenging but should be considered for patients with pericardial effusions who are from regions with a high prevalence of tuberculosis and for patients who have other risk factors, including incarceration. Unlike isolated pulmonary tuberculosis, tuberculous pericarditis should be treated with corticosteroids in addition to RIPE therapy; pericardiectomy is required for constrictive disease. While corticosteroids have been suggested to prevent progression to constrictive pericarditis, statistically significant evidence remains lacking. Four randomized controlled trials have studied a total of 469 patients to assess the effect of adjunctive corticosteroids on mortality and other clinical sequelae. In all four studies the intervention group had lower mortality, but the findings were not statistically significant. Other findings that were not statistically significant included faster resolution of symptoms and less frequent need for pericardiocentesis in the group treated with corticosteroids, but evidence has not supported the notion that corticosteroids prevent constrictive pericarditis. Because of some promising findings from these studies, the Infectious Diseases Society of America (IDSA) recommends adjunctive corticosteroids, and it is considered the standard of care. RIPE therapy does demonstrate statistical significance, dramatically decreasing mortality in tuberculous pericarditis from $90 \%$ without therapy to $8-17 \%$ in patients without HIV and $17-$ $40 \%$ in patients with HIV. In conclusion, providers need to consider tuberculous pericarditis in patients presenting with a pericardial effusion, as diagnosis can lead to timely medical therapy with the goal of decreasing mortality.

THE CASE OF THE FORTUITOUS FRACTURE William A. $\mathrm{Li}^{2}$; Christopher Garcia $^{2}$; Daniel Peisach ${ }^{2}$; Diane L. Levine ${ }^{1} .{ }^{1}$ Wayne State University, Detroit, MI; ${ }^{2}$ Wayne State University, Troy, MI. (Control ID \#2457044)

LEARNING OBJECTIVE \#1: Bone fractures after minor trauma in a middle-aged individual should raise the level of suspicion for multiple myeloma (MM).
LEARNING OBJECTIVE \#2: Early diagnosis and treatment of MM prolong disease free survival and decrease morbidity.

CASE: A 55-year-old incarcerated man presented for the evaluation of a swollen left jaw after a minor altercation with another inmate. Plain film x-ray of the jaw was negative. CT showed a left jaw fracture just below the condylar process. Upon close scrutiny, CT also revealed minuscule lytic bone lesions in the cervical spine. The patient denied neck or back pain. Further work up included a bone survey, which revealed multiple sub-centimeter lytic lesions in the skull, clavicles, and thoracic and lumbar spines. Serum protein electrophoresis (SPEP), and serum-free light chain assay results supported the diagnosis of MM. The bone marrow biopsy was diagnostic. Beta-2-microglobulin, an important prognostic factor for MM, was $2.14 \mathrm{mcg} / \mathrm{ml}$ in our patient at the time of diagnosis, compared to a published average value at presentation of $2.7 \mathrm{mcg} / \mathrm{ml}$. Fluorescent in situ hybridization (FISH) analysis showed a translocation of chromosome 11 and 14. Albumin was $3.39 \mathrm{~g} / \mathrm{dL}$. The patient was diagnosed with early stage multiple myeloma before the manifestation of back pain, kidney insufficiency, neurological symptoms, and other complications that usually prompt individuals to seek medical attention. Based on the revised International Staging System (R-ISS) for MM, our patient is currently at stage 2 with an estimated median overall survival of 83 months and progression-free survival of 42 months.

DISCUSSION: Multiple myeloma (MM) accounts for approximately $1 \%$ of all cancers and more than $10 \%$ of hematologic malignancies in the U.S.. MM is a malignancy characterized by monoclonal proliferation of plasma cells in the bone marrow microenvironment. MM commonly manifests as bone pain, hypercalcemia, anemia, kidney insufficiency, and neurological symptoms. Among these, the most common symptoms reported is back pain, which prompts the patient to seek medical attention. MM is frequently accompanied by a poor prognosis because it is often diagnosed during at an advanced stage. Early diagnosis and treatment, however, have been shown to significantly prolong progression-free survival and decrease morbidity, especially with new drug regimens that include lenalidomide over traditional melphalan based regimens. It is difficult to diagnose MM early. Often, MM does not manifest in symptoms or causes vague symptoms until it reaches an advanced stage. MM is rarely diagnosed early when a routine blood test shows an abnormally high protein in blood. Our case of a fortuitous fracture, which led to the early diagnosis and intervention of multiple myeloma, emphasizes the importance of having a high index of suspicion for MM. Bone fractures after minor trauma in a middle-aged individual should raise the level of suspicion for MM. Primary care physicians play an important role in diagnosing MM. This is particularly important because early treatment has the potential to prolong survival.

\section{THE CAUSE OF MY CONFUSION: AN ADULT CASE OF HAEMOPHILUS INFLUENZAE MENINGITIS AND BACTEREMIA Beenish Ahmed; Rasheen Imtiaz; Dana M. Larsen. Baylor College of Medicine, Houston, TX. (Control ID \#2469752)}

LEARNING OBJECTIVE \#1: To recognize Haemophilus influenza as a rare cause of meningitis in immunocompetent adults.

CASE: A 70-year-old Caucasian female with history of hypothyroidism and osteoarthritis was brought to the hospital with altered mental status. Two days prior to presentation, the patient started complaining of a severe headache associated with nausea and vomiting. The day prior to presentation, she also began experiencing subjective fevers. The morning of admission, her husband was unable to wake her up. She was incontinent of urine, and her face was covered with vomitus. Per her husband, the patient had been exposed to sick grandchildren. He noted that she had no recent travels or other exposures and said she was up to date with her immunizations. The patient's exam was notable for a fever of $101.2 \mathrm{~F}$ 
and tachycardia. She was lethargic and had nuchal rigidity. Laboratory studies were significant for platelets of 54 thousand, white blood cell count of 4.5 thousand with $45 \%$ bands. Computed tomography of her head was unremarkable. With her clinical presentation concerning for meningitis, a lumbar puncture was performed. She was empirically started on treatment with dexamethasone, vancomycin, ampicillin, ceftriaxone, and acyclovir. Cerebrospinal fluid culture and blood cultures subsequently grew Haemophilus influenza type B (Hib). Her treatment regimen was then narrowed to ceftriaxone. The patient remained somnolent for the next week after treatment initiation. Magnetic resonance imaging of the head was done for persistent altered mental status and showed changes consistent with meningitis and ventriculitis. A week after admission, her mental status abruptly improved. She recognized her husband and conversed appropriately. She continued to improve markedly, though still suffered from problems with shortterm memory. Her bacteremia resolved and she was successfully transferred out of the neurologic intensive care unit. She completed a 14-day course of ceftriaxone. Her strength improved with physical therapy, and she was discharged in stable condition.

DISCUSSION: Haemophilus Influenzae type b (Hib) is rare cause of meningitis in adults behind Streptococcus pneumoniae, Neisseria meningitides and Listeria monocytogenes. Its incidence has markedly decreased since the advent of immunization in mid-1980s. Predisposing factors are similar across all causes of bacterial meningitis and typically include immunosuppression, trauma, neurosurgery and other underlying diseases such as malignancy. What makes this case unique is that no such factor was identified in our patient. The most common presenting symptom in Hib meningitis has been found to be fever, but the classic triad with nuchal rigidity and altered mental status is rare. Diagnosis is confirmed with CSF culture growth, but can be presumed from CSF gram stain and cell count. Treatment includes intravenous third generation cephalosporin and conjunctive dexamethasone to reduce neurological complications. Prognosis is generally better when compared to streptococcus pneumoniae meningitis.

THE CONNECTION TO CONNECTIVE TISSUE DISEASE IN NONCIRRHOTIC PORTAL HYPERTENSION Angela K. Kleiber. Ucla, Santa Monica, CA. (Control ID \#2469898)

LEARNING OBJECTIVE \#1: Recognize Nodular Regenerative Hyperplasia as a cause of non-cirrhotic portal hypertension in patients with connective tissue diseases.

LEARNING OBJECTIVE \#2: Diagnose Nodular Regenerative Hyperplasia.

CASE: VH is a 47 year old man with a 5 -year history of recurrent ascites, $\mathrm{s} / \mathrm{p}$ liver biopsy showing congestive hepatopathy without bridging fibrosis, and a past venogram concerning for Budd-Chiari syndrome, who was admitted with acute kidney injury requiring hemodialysis. He reported increasing frequency of need for therapeutic paracentesis (from monthly to several times per week) and newly bloody ascitic fluid. He also had bilateral lower extremity edema, scrotal edema, and frequent nose bleeds. $\mathrm{He}$ denied fevers, chills, confusion, chest pain or difficulty breathing. He had no change in urination, no change in oral intake, and no recent adjustments to his diuretic medications. Medical history included hypothyroidism, chronic kidney disease and anemia requiring intermittent transfusions; family history was pertinent for brother with diabetes. He was originally from Mexico but had been living in Southern California for over 15 years; he was married with three children and worked as a handyman. He was a former smoker, but did not drink or use drugs. His medications were lasix, aldactone and synthroid. On exam his vital signs were within normal limits and he was in no acute distress. Lungs were clear to auscultation and heart was regular rate and rhythm without extra heart sounds. Abdomen was significantly distended and positive for fluid wave, but soft and nontender to palpation. He had pitting edema of bilateral lower extremities. His strength and sensation were grossly intact, he was fully alert and oriented and had no asterixis. Labs on admission were significant for BUN 70, creatinine of 3.5, INR 1.1, Albumin 3.4, Hemoglobin 9.2, platelets 152, AST 12, ALT 6, bilirubin 0.5, alk phos 141. During admission he had multiple studies done including: diagnostic paracentesis showed ascites fluid with elevated SAAG (2.2) and total protein (2.5) consistent with portal hypertension, right heart catheterization showing normal left and right heart filling pressures, renal biopsy showing evidence of thrombotic microangiopathy. Given the concern for TMA plasmaphoresis was initiated. Further lab work was significant for ANA 1:40 and presence of anticardiolipin antibody, raising concern for antiphospholipid syndrome. Liver biopsy was repeated and reticulin stain highlighted nodular regenerative changes, providing unifying diagnosis of nodular regenerative hyperplasia secondary to antiphospholipid antibody syndrome and thrombotic microangiopathy. He was anticoagulated with warfarin, and started on rituximab. Unfortunately he did not have significant improvement with this treatment, perhaps due to the extent of organ damage by the time of diagnosis, and when last seen he was being evaluated for liver transplant to address his refractory and disabling ascites.

DISCUSSION: Nodular Regenerative Hyperplasia (NRH) is an uncommon cause of noncirrhotic portal hypertension that can be seen in patients with rheumatologic disease. It is a hyperproliferative response to obstructive portal venopathy, resulting in the development of small $(1 \mathrm{~mm})$ regenerative nodules without surrounding fibrosis. It is common for AST, ALT, bilirubin and INR to be normal in these patients, as they do not have necessarily have liver dysfunction, though alkaline phosphatase can be elevated, as was seen in our patient. To make this diagnosis other etiologies of portal hypertension must be ruled out such as cirrhosis, heart failure and Budd-Chiari syndrome - all of which our patient was evaluated for but did not have. It is helpful to have a degree of suspicion for NRH prior to obtaining the liver biopsy as the diagnosis is difficult to make on histopathology alone. It may not be obvious with routine hematoxylin-eosin staining, reticulin staining is needed to show it more clearly. Also if the needle gauge is too small, the regenerative nodules may not be seen on the sample. The treatment for NRH is treatment of the underlying condition, in this case antiphospholipid syndrome (diagnosed based on TMA on kidney biopsy and presence of anticardiolipin antibody), which was treated with anticoagulation, plasmaphoresis and rituximab. References: Reshamwala, P., Kleiner, D., \& Heller, T. (n.d.). Nodular regenerative hyperplasia: Not all nodules are created equal. Hepatology, 7-14.

THE CORONARY CT: WHERE ONE DIAGNOSIS TURNED INTO THREE. Vimal Jhaveri. Massachusetts General Hospital, Cambridge, MA. (Control ID \#2470263)

LEARNING OBJECTIVE \#1: Identify the indications for coronary CT scans and its potential use in undifferentiated chest pain.

LEARNING OBJECTIVE \#2: Describe the pathophysiology of penetrating aortic ulcers.

CASE: A 74-year-old man with chronic obstructive pulmonary disease, hypertension, hyperlipidemia, cerebrovascular accident and a 60 pack-year smoking history, presented with chest pain. Four days prior, he developed squeezing chest pain with walking in the central chest with radiation to both shoulders and jaw, diaphoresis and dyspnea, but no nausea or vomiting; 1 day prior, he had 3 episodes of chest pain (two with walking, one at rest) for which he took nitroglycerin with minimal relief. He then came to the emergency department (ED). On presentation, he was afebrile, with pulse 64, blood pressure 159/86, and oxygen saturation of $100 \%$. His chest pain had resolved. He was in no acute distress, heart had regular rate and rhythm without murmurs, rubs or gallops, lungs were clear, and with trace lower-extremity edema bilaterally. His distal pulses were 2+ bilaterally; he had no bruits. Initial evaluation revealed first degree heart block with no ischemic changes on EKG; two sets of negative troponins; and chest $\mathrm{x}$-ray showing normal lung parenchyma, a calcified aortic arch, and a right hilar opacity. A chest CT was recommended. He was diagnosed with unstable angina, started on full-dose aspirin and heparin drip, and admitted for further evaluation. Instead of an early catheterization or stress test, a coronary CT (cCTA) was chosen to further evaluate for coronary artery disease (CAD) and the hilar 
opacity. The cCTA revealed: a calcium score of 627 (severe $>400$ ) predominantly affecting his left circumflex artery, a $10 \mathrm{~mm}$ spiculated nodule in the left upper lobe of the lung and multiple penetrating aortic ulcers (PAU) in the descending thoracic aorta. The right hilum contained enlarged lymph nodes within the upper limit of normal. With this one test, three new diagnoses were discovered: a potential malignancy, potentially serious aortic syndrome, and severe CAD. Following the cCTA, oncology and thoracic surgery were consulted. Oncology recommended a PET scan for nodule staging. Thoracic surgery recommended deferring coronary catheterization (and associated dual anti-platelet therapy) pending further oncology evaluation. The patient was medically optimized during his hospitalization with aspirin, high dose statin, and improved blood pressure control and smoking cessation. A few weeks later, the lung nodule was found to be PET-avid, suggestive of malignancy, and a resection is planned. His penetrating aortic ulcers will be monitored with periodic aortic CT.

DISCUSSION: Since 1999, the quality of CT has allowed for visualization of coronary calcifications. In 2010, the AHA released the Coronary Artery Calcification Score as a screening tool for coronary disease. CCTA is useful in ruling out CAD, as a zero calcium score has a very high negative predictive value (NPV) for major cardiac events within 30 days. The incidence of $>50 \%$ coronary obstruction is $<1 \%$ with a zero calcium score Furthermore, the NPV of cCTA for major adverse cardiac events is $99.3 \%$ [1]. As a result, cCTA is most useful in patients with low-to-moderate pre-test probability for CAD because negative results lead to decreased use of invasive coronary angiography, length of stay, and cumulative radiation dose. The cCTA was not the ideal test for evaluating this patient's coronary ischemia given his high pre-test probability, but proved invaluable in its' other findings. The incidence of significant incidental clinical findings is suggested to be $13 \%$ [2]. A CT with timed angiography has been proposed for use as a "triple rule out" for pulmonary embolism, CAD, and aortic dissection, but ED studies have not shown improved mortality, cost effectiveness, or time to discharge [3]. However, excluding aortic pathology in patients with acute chest pain syndromes is crucial, since stress testing may increase wall stress on a diseased aorta. Penetrating aortic ulcers are sequelae of severe atherosclerotic disease. The atherosclerotic plaque disrupts the vessel intima, penetrating into the media of the aorta. From there, it can remain stable, progress to a focal dissection or aneurysm, or evolve to aortic rupture. Data on the natural course of PAU is lacking; the incidence of progression to dissection or aneurysm varies between studies. However, the finding of PAU portends a more aggressive lesion that should be monitored and managed with a high-intensity statin and tight blood pressure and heart rate control to decrease plaque progression and aortic wall stress [4]. CCTA is likely to become more prevalent in the future as it has a high negative predictive value for coronary ischemia, but it also can have a role when multiple thoracic pathologies are suspected. [1] Takakuwa KM, et al. A meta-analysis of 64-section coronary CT angiography findings forpredicting 30-day major adverse cardiac events in patients presenting with symptoms suggestive of acute coronary syndrome. Acad Radiol. 2011 Dec;18(12):1522-8. [2] Buckens CF, et al. Unrequested findings on cardiac computed tomography: looking beyond the heart. PLoS One. 2012;7(4):e32184. [3] Hoffmann U, et al. ACR Appropriateness Criteria Acute Nonspecific Chest Pain-Low Probability of Coronary Artery Disease. J Am Coll Radiol. 2015 Dec;12(12 Pt A):1266-71 [4] Dudzinski DM, Isselbacher EM. Diagnosis and Management of Thoracic Aortic Disease. Curr Cardiol Rep. 2015 Dec;17(12):106.

THE COST OF REJECTION: UTILIZING SPECIALTY-BASED MULTIDISCIPLINARY TEAM MEMBERS TO ADDRESS UNSUSTAINABLE

MEDICATION COSTS Grant M. Smith. University of San Francisco California, San Francisco, CA. (Control ID \#2466591)

LEARNING OBJECTIVE \#1: Identify medication non-adherence due to financial burden as a common cause of acute and chronic renal transplant rejection.
LEARNING OBJECTIVE \#2: Recognize the importance of utilizing specialty-based multidisciplinary team members to reduce the financial burden of medication costs for renal transplant recipients.

CASE: A 49 year old man with a history of heart failure with preserved ejection fraction, type 2 diabetes complicated by diabetic and IgA nephropathy, status post directed donor renal transplant 4 years ago, presented to primary care clinic with 1 week of progressive chest pressure and dyspnea on exertion. His medications included tacrolimus, prednisone, mycophenolate, amlodipine, clonidine, metoprolol, lisinopril, glargine, and lispro. Exam was remarkable for tachypnea, JVP of $11 \mathrm{~cm}$, bilateral pitting edema to the knees, and bilateral crackles to the mid lung fields. Laboratory evaluation showed a creatinine of $1.66 \mathrm{mg} / \mathrm{dL}$ (from a baseline of $1.0 \mathrm{mg} / \mathrm{dL}$ ), urine protein to creatinine ratio $1.05 \mathrm{mg} / \mathrm{mg}$ (from a baseline of $0.38 \mathrm{mg} / \mathrm{mg}$ ) and a tacrolimus level of $2.2 \mathrm{ug} / \mathrm{L}$. An urgent referral was placed to the inpatient kidney transplant unit (KTU), and a renal biopsy showed chronic active antibody mediated rejection and acute cellular rejection (Type 2a). He received thymoglobulin, IVIG, methylprednisone, rituximab, and IV diuretics. Following treatment, his proteinuria returned to baseline, and his creatinine fell to a new baseline of $1.2-1.3 \mathrm{mg} / \mathrm{dL}$. Further history obtained by the patient's primary care team, primary care social worker, and KTU social worker revealed that the patient's medication costs were more than $\$ 400$ per month, with yearly costs accounting for almost $20 \%$ of the patient's income. Despite endorsing that he was "taking all of his medications," he disclosed taking partial doses and spacing his doses to make his medications "last longer." Ultimately, the KTU pharmacist, KTU socia worker, and primary care social worker were able to obtain a voucher for a 30 day supply of medications while the patient worked with his employer and the local Health and Human Services department to find an insurance plan that would provide better coverage for his medications. In addition, the KTU pharmacist worked with a loca pharmacy and several medication assistance programs to reduce the patient's costs by over $25 \%$

DISCUSSION: As of 2012, there were more than 186,000 people living with a functioning renal transplant. The limited capacity of transplant centers necessitates that patients receive post-transplant care from their primary care providers (PCPs) within 6 months of receiving a transplant. Because of this, PCPs need to be familiar with the outpatient management of patients with renal transplants including screening for rejection, monitoring for complications of immunosuppression, and knowing when to enlist the help of renal transplant specialists and other members of the multidisciplinary healthcare team. Rejection of a renal transplant should be considered when there is a greater than $50 \%$ rise in the serum creatinine or greater than $1 \mathrm{~g} /$ day of proteinuria. Evaluation of these findings should include renal biopsy coordinated by the renal transplant team. However, PCPs can plan an important role by identifying potential underlying etiologies of rejection by asking about barriers to medication compliance. In particular, PCPs should be aware of the high cost of immunosuppressant medications and be conscientious of social factors that may contribute to an inability to afford these medications, such as changes in insurance coverage or wages. While there is no nationally representative study of the burden of medication costs for renal transplant recipients, more than $70 \%$ of renal transplant programs report that their patients have an "extreme" or "serious" problem paying for their medications. Sixty-eight percent of renal transplant programs report deaths and graft losses attributable to cost-related medication non-adherence. Although Medicare covers immunosuppressant medications for 3 years following transplant, over half of renal transplant patients are unaware that their coverage will terminate and $71 \%$ do not know how long their Medicare coverage lasts. To address the cost burden of immunosuppressant medications, PCPs should enlist the assistance of specialty-based pharmacists and social workers who may have unique relationships with local pharmacies, pharmaceutical companies, and charitable foundations that can help bridge the gap for patients struggling to afford their medications. 
THE CURIOUS CASE OF THE DISAPPEARING BLOOD Sanket Borgaonkar'; Courtney Shipman ${ }^{1}$; Jaclyn M. Munoz ${ }^{1}$; Rehman Sheikh ${ }^{1}$; Sarvari Yellapragada ${ }^{2} .{ }^{1}$ Baylor College of Medicine, Houston, TX; ${ }^{2}$ Michael E. DeBakey VA Medical Center, Houston, TX. (Control ID \#2469095)

LEARNING OBJECTIVE \#1: Diagnose an acquired Factor VIII inhibitor LEARNING OBJECTIVE \#2: Understand management of bleeding in a patient with an acquired Factor VIII inhibitor

CASE: A 50 year-old female with well-controlled HIV (CD4 403), presented for further evaluation of left thigh swelling in absence of any trauma. She was in her usual state of health until 1 month prior to admission, when she experienced a mechanical fall and landed on her back and right arm. This resulted in bruising and swelling of her right arm that resolved spontaneously. One week prior to admission, she noted began to notice left thigh swelling associated with slight tenderness that did not affect her ability to ambulate. She denied fever, chest or abdominal discomfort, dyspnea, change in appetite or bowel habits, weight loss, signs of bleeding, recent travel or a family history of bleeding. On physical exam, she had noticeable pallor in absence of lymphadenopathy. Her cardiovascular and pulmonary exams were normal. Examination of her extremities revealed strong pulses bilaterally, with her left thigh circumferentially larger than her right; she also had noticeable left knee swelling, and swelling of her left forearm at the site of a blood draw. No ecchymosis or petechiae were noted. Her lab results were notable for a hemoglobin $(\mathrm{Hgb})$ of $6.0 \mathrm{~g} / \mathrm{dL}$ (reference range: 12.0-15.0) and an isolated partial thromboplastin time (PTT) elevation at 88.4 (reference range: 23.6-36.6). Iron studies indicated iron deficiency anemia and a peripheral smear did not show any evidence of hematologic malignancy or schistocytes. LDH, total bilirubin, and haptoglobin were normal. Comprehensive labwork for rheumatologic conditions, such as Sjogren's disease, rheumatoid arthritis, and lupus were negative. A CT scan of the left thigh revealed diffuse thigh soft tissue edema and a small suprapatellar knee joint effusion. Her Hgb continued to decrease to $3.9 \mathrm{~g} / \mathrm{dL}$ and she subsequently required intensive care for stabilization of her profound bleeding. A mixing study showed incomplete correction of the PTT. Factor VIII activity was severely low and factor VIII inhibitor was elevated at 11.5 Bethesda units. The patient was diagnosed with acquired factor VIII inhibitor and treated with methylprednisolone, factor VII, desmopressin, fresh frozen plasma (FFP), and packed red blood cells (pRBC). Her $\mathrm{Hgb}$ recovered to 10.4, she remained asymptomatic with no further evidence of bleeding, was started on outpatient daily prednisone therapy and discharged home with hematology follow-up.

DISCUSSION: Acquired factor VIII inhibitor, also known as acquired hemophilia A, is a rare disease with an incidence of 1 per 1 million people in the non-hemophiliac population. It is caused by the development of auto-antibodies to factor VIII leading to a bleeding diathesis that typically occurs in the muscle, soft tissue, retroperitoneal space, or the gastrointestinal tracts. However, unlike in congenital hemophilias, hemarthrosis are rare in acquired conditions. Nevertheless, bleeding can be severe, with mortality rates estimated between 8 and $22 \%$. The underlying pathophysiology is unclear with proposed mechanisms ranging from gene polymorphisms to autoreactive $\mathrm{T}$ lymphocytes. Although the majority of cases are idiopathic, associated conditions include pregnancy, autoimmune disorders, malignancy, or drugs such as penicillins or sulfonamides. Only 4 reported cases of HIV-associated Factor VIII inhibition are reported in literature. Diagnosis is based upon clinical suspicion and supported by appropriate laboratory values. Prolonged activated PTT that initially corrects but after prolonged incubation continues to remain prolonged is a diagnostic feature. Diminished Factor VIII levels and the presence of factor VIII inhibitors confirm the diagnosis. Treatment is centered around achieving hemostasis and eradication of the inhibitor. The severity of the bleeding along with the level of the inhibitor can help guide the treatment approach. Minimal bleeding with low inhibitor titers can typically be managed with observation. Minor bleeding with low titer levels can be treated with human factor VIII or desmopressin. However, for patients with severe bleeding or high inhibitor titers, aggressive measures such as recombinant factor VIIa, factor VIII bypass activity (FEIBA), therapeutic plasmapheresis, or intravenous immunoglobulin may be required. Following hemostasis, treatment approach is tailored towards eradication of the Factor VIII inhibitor. First-line treatment is an oral corticosteroid as was used in our patient. Combination therapy with cyclophosphamide can be used, and recent studies have investigated the use of rituximab when corticosteroids are contraindicated. In patients who present with bleeding without an identifiable source and laboratory values that suggest coagulation cascade disorders, the diagnosis of acquired hemophilia must be considered. Although rare, acquired Factor VIII inhibitor can result in life-threatening bleeding that can be managed effectively and successfully with hemostasis and immunosuppressive therapy if recognized promptly.

THE CURIOUS CASE OF THE VANISHING DUCTS Michael Gao; Tanping Wong. Weill Cornell Medical College/New York Presbyterian Hospital, New York, NY. (Control ID \#2469118)

LEARNING OBJECTIVE \#1: Recognize the presentation of and diagnostic criteria for Vanishing Bile Duct Syndrome (VBDS).

LEARNING OBJECTIVE \#2: Differentiate VBDS from other pathologies which affect small bile ducts without involving large ducts or hepatocytes.

CASE: Our patient is a 71 year old female who presented to the hospital with fatigue and odynophagia. She had a PMH of Graves' disease s/p radioactive iodine therapy on levothyroxine (1.5 years prior to admission) as well as ophthalmopathy for which she started prednisone (20 mg downtitrated to $5 \mathrm{mg}$ ) and prophylactic omeprazole $1 \mathrm{month}$ prior to admission. She denied any other medications, herbs, teas, imported foods, environmental exposures, drugs, or alcohol intake. The patient was generally wellappearing with only scleral icterus on physical exam. Labs demonstrated cholestasis which rapidly progressed from an alkaline phosphatase of 196 and bilirubin of 6.2 on hospital day (HD) \#1 to 1944 and 48.9 by HD\#42. A comprehensive workup was negative including hepatitis A, B, C, HIV, AMA, ANA, ANCA, MPO, Sm3, anti-LKM, soluble liver antigen, and acetaminophen level. Imaging including MRCP was negative. Her first liver biopsy on HD\#4 demonstrated cholestasis with sparse lymphocytic infiltrate in the interlobular bile ducts. There was no ductopenia or hepatitis. Due to the rate of progression we performed a second biopsy on HD\#21, which showed a loss of bile ducts with mild chronic portal inflammation and without fibrosis. There were no viral cytopathic changes and tissue cultures were negative. The patient's initial odynophagia was due to esophageal candidiasis (confirmed by EGD brushing) and resolved after an antifungal regimen with fluconazole, micafungin, and amphotericin B. The patient developed an IV-associated cellulitis which was treated with Vancomycin and Zosyn. There was no evidence of systemic infection. Serum cultures as well as EBV, CMV, VZV, and HSV were negative. Potential offending drugs were removed by discontinuing the patient's home omeprazole and by changing the antifungals (as above). Finally, we attempted empiric treatment with ursodiol and prednisone. None of our interventions slowed the progression of her cholestasis. After careful discussion the patient and her family opted against liver transplant. On our last encounter (HD\#42) she had worsened jaundice and itching but was still without liver synthetic dysfunction (albumin 2.4 but PT and PTT within normal limits) or encephalopathy. The patient died on day 69 .

DISCUSSION: Vanishing bile duct syndrome is a clinical-pathologic entity diagnosed by (1) persistent cholestatic hepatitis, (2) liver biopsy showing greater than $50 \%$ loss of interlobular bile ducts, and (3) exclusion of other potential causes. Unlike AMA-negative PBC and autoimmune cholangitis, VBDS is ANA negative and has a rapid clinical course. The disappearance of bile ducts is not accompanied by granulomatous change (also 
differentiating it from sarcoidosis), cirrhosis, or periportal fibrosis. Unlike small duct PSC, VBDS has no association with IBD and no onion-skinning or fibrosis. VBDS also progresses more quickly. VBDS can be idiopathic or associated with drugs (e.g. chlorpromazine), Hodgkin's Lymphoma (HL), or infection. Our patient's VBDS was most likely idiopathic. Although she had a history of Graves', we excluded autoimmune cholangitis. None of her medications have been reported to cause VBDS, and her inhospital medications were given only briefly (the longest antifungal was for 4 days) and only after her presenting cholestasis. Its clinical course varies from complete recovery to rapidly progressive and fatal. Recovery is more likely in cases where an etiology is identified and either treated (HL-associated) or removed (medication-induced). In progressive cases, liver transplantation is the only known therapy.

THE DARK SIDE OF CHOLESTEROL GUIDELINES: STATIN-INDUCED RHABDOMYOLYSIS WITH MULTIPLE ORGAN INVOLVEMENT Kristopher

P. Kline; Brent King; Jared Plumb. University of Florida, Gainesville, FL. (Control ID \#2469280)

LEARNING OBJECTIVE \#1: Myopathy is a common side effect of statin therapy with a wide spectrum of severity and presentation. Recognize the clinical features of statininduced myonecrosis and rhabdomyolysis.

LEARNING OBJECTIVE \#2: New cholesterol guidelines trend towards a lower threshold of starting high intensity statin medications but it is important to be cognizant of their potentially lethal side effect profile.

CASE: A 66-year-old man with non-small cell lung cancer (post chemotherapy and radiation therapy), chronic ischemic cardiomyopathy, and hypertension was brought in by family members to our emergency room with a complaint of progressive and fully debilitating muscle weakness and fatigue over 2 weeks' duration. His weakness began in the thighs and trunk, but over time began to include his back, shoulders, and neck. He stated his weakness was so profound that he was unable breath without keeping his head in full extension. He denied any pain symptoms. Initial laboratory values on presentation included CPK 4198 U/L (nl: 5-180), CKMB 102 ng/mL (nl: 0-5), Troponin T 0.12 ng $\mathrm{mL}$ (nl: <0.03), Platelets $63 \mathrm{k} / \mathrm{cmm}$ (nl: 130-440), BUN $43 \mathrm{mg} / \mathrm{dL}$ (nl: 9-20), Creatinine $7.2 \mathrm{mg} / \mathrm{dL}$ (nl: 0.5-1.2), AST $528 \mathrm{U} / \mathrm{L}$ (nl: 0-45), ALT $201 \mathrm{U} / \mathrm{L}$ (nl: 0-40), and Total Bilirubin $2.0 \mathrm{mg} / \mathrm{dL}$ (nl: 0.0-1.3). Urinalysis detected significant globulin, with follow-up confirmation of myoglobin. On gaining more information it was noted that 2 weeks prior he was seen by his primary care physician who adjusted his atorvastatin dosage from $20 \mathrm{mg}$ daily, a dose he had been on for several years, to $40 \mathrm{mg}$ daily in accordance to new cholesterol guidelines for coronary artery disease. After careful exclusion of other causes a diagnosis of statin-induced rhabdomyolysis with both hepatic dysfunction and acute renal failure was made. He was treated with aggressive yet cautious intravenous hydration in the setting of his cardiomyopathy with baseline EF of $20 \%$, paying close attention to urine output and respiratory efforts. His atorvastatin was discontinued. He had a prolonged hospital course of 13 days due to the tenuous status of balancing fluid resuscitation with his heart failure. His renal function, liver function tests and platelets all gradually returned to normal and he was seen in clinic 3 weeks after discharge with full recovery to his prior level of independence.

DISCUSSION: Statins are a widely-used class of medication for lipid-lowering therapy and have been shown to be effective in the primary and secondary prevention of coronary disease. They are felt to be safe in the majority of patients, with common side effects including hepatotoxicity and myopathy. Myopathy is a relatively common side effect of statin use with a wide spectrum of severity, ranging from mild pain and weakness to severe rhabdomyolysis as in our case. Sequelae of rhabdomyolysis include kidney injury, hepatic dysfunction, electrolyte disorders, acid/base disorders, and hematologic disturbances such as disseminated intravascular coagulation. While pain is a common complaint of severe statin-induced myositis and myonecrosis, patients may rarely present with only weakness in the absence of pain such as our case. Therapy is directed at correcting the underlying disorder, fluid support, and preventing or treating the sequelae. Mortality in patients with severe complications including acute renal failure has been observed to be $58 \%$ in the ICU setting. Statin-induced myopathy is a relatively common side effect, with observation between 10 and $25 \%$, and higher effect seen in observational studies vs. controlled trials however clinical rhabdomyolysis is rare. Occurrence and severity of myopathy due to statin use has been shown to exhibit dose-dependency, and some statins may show increased risk, with cerivastatin having been pulled from the market due to increased reported rate of fatal rhabdomyolysis. There is also increased risk when statins are combined with other lipid-lowering agents, with the greatest risk appearing to be when a statin is combined with fibrate therapy. Other risk factors include elderly patients, low body mass, underlying renal, hepatic, metabolic, or endocrine disorders, alcohol consumption, surgery, strenuous physical exertion, and CYP-450-affecting medications. In cases of frank rhabdomyolysis, statin reintroduction may be considered on a case-by-case basis, but it is recommended to discontinue therapy. Recent cholesterol guidelines such as Adult Treatment Panel IV from the ACC/AHA have trended towards a lower threshold of starting statin medications, particularly the high intensity statins atorvastatin and rosuvastatin. Guidelines aim to do the greatest benefit to the greatest population with their recommendations coming from a variety of resources ranging from double-blinded clinical trials to expert opinion. It is important to remember that even widely-used therapies which are considered to have strong positive benefit may have severe and potentially fatal complications. When complications are rare and nonspecific, clinical suspicion should be higher if there is a strong temporal association or other risk factors are present.

THE DIAGNOSTIC DILEMMA OF CATASTROPHIC ANTIPHOSPHOLIPID SYNDROME Sungwon R. Kyung; Emil Heinze. Olive View-UCLA Medical Center, Sylmar, CA. (Control ID \#2466635)

LEARNING OBJECTIVE \#1: In this case we will define Catastrophic Antiphospholipid Syndrome (CAPS), a rare but critical condition resulting in multiorgan damage secondary to microthrombi, and identify the challenges in early identification, given the multiforme presentation of this disease.

CASE: A 31 year old female followed in rheumatology clinic for possible systemic lupus erythematosus (SLE) with a history of known anti-phospholipid syndrome (positive B2 glycoprotein, anti-cardiolipin, and lupus anticoagulant antibodies, and prior miscarriages), presented to the emergency room with 1 day of chest pain and shortness of breath as well as a malar rash and splinter hemorrhages. She was noted to be persistently tachycardic and tachypneic on presentation. Laboratory studies revealed a weakly positive troponin-I, bacteruria with pyuria, renal insufficiency with new proteinuria, leukocytosis, anemia and thrombocytopenia. Peripheral smear showed occasional schistocytes and multiple tear drop cells. Workup for microangiopathic hemolytic anemia (MAHA), thrombotic thrombocytopenic purpura (TTP), and disseminated intravascular coagulation (DIC) remained negative. Patient's chest $\mathrm{x}$-ray revealed evidence of vascular congestion but no focal consolidations. Patient's CT angio chest revealed ground glass opacities, bronchial wall thickening, and centrilobular nodules, but no pulmonary embolus. At this time sepsis due to a pulmonary infection or an SLE flare (with possible pleuritis, myocarditis, and lupus nephritis) were highly considered. The patient was admitted to the intensive care unit and diagnosed with probable CAPS, likely precipitated by a pyelonephritis, based on acute multi-organ involvement, known APS serologies, and lack of strong support for a lupus flare. Patient underwent IV anticoagulation, plasmapheresis, and high dose corticosteroid therapy throughout her hospital course with improvement of her symptoms. Once the patient's acute illness resolved, the patient underwent a renal biopsy which revealed 
chronic thrombotic microangiopathy consistent with definite APS with no evidence of lupus nephritis.

DISCUSSION: CAPS is defined by a history of APS or the presence of APS antibodies with acute three or more organ involvement within a 1 week span (in this case: cardiac, pulmonary, renal, skin) as well as histopathologic tissue confirmation of microthrombi and exclusion of other causes of microthrombi. Definite CAPS is only diagnosed after biopsy tissue confirmation and thus, the decision to treat must often occur prior to the final diagnosis. Severe SLE flare was also considered in this patient with the possibility of pleuritis, myocarditis and lupus nephritis, but patient's C3 and C4 levels remained normal, and renal biopsy ultimately revealed no evidence of SLE nephritis. Because of the rarity of true CAPS cases and given that most patients are initially identified with multi-organ system involvement with end-organ damage, CAPS cases are oftentimes mistaken for severe sepsis or septic shock, as well as TTP, HUS, MAHA or DIC, as well as SLE flares. Rapid diagnosis is key as CAPS can have up to a $50 \%$ mortality rate, but treatment for CAPS (urgent anticoagulation, high dose steroids, plasmapheresis, IVIG) differs from that for a severe SLE flare (steroids with immunosuppressing agents). Normal complement levels in addition to the presence of schistocytes on peripheral smear in the setting of a patient with a known history of APS assisted in expediting the decision for urgent anticoagulation in this patient. It is crucial for clinicians to be aware of this rare condition given the high mortality associated with delayed treatment in CAPS

THE DIRTY HIGH: WHEN THE PATIENT KNOWS BETTER Yanting Wang; Molly Fisher; Jennifer Corbelli. University of Pittsburgh Medical Center, Pittsburgh, PA. (Control ID \#2467678)

LEARNING OBJECTIVE \#1: Recognize clinical features of Cotton Fever LEARNING OBJECTIVE \#2: Appreciate the importance of asking patients what they believe is causing their symptoms

CASE: AS is a 31-year-old male with a past medical history significant for active heroin use and chronic back pain, who presented with recurrent high-grade fevers (up to $106^{\circ} \mathrm{F}$ ) for 4 days. His history was also significant for unilateral knee pain, worsening of chronic low back pain, dull pleuritic chest pain, and intermittent mild diffuse abdominal pain. He denied any visual symptoms, headache, dyspnea, rashes, or urinary symptoms. He injected heroin the morning of presentation, as well as the day before, but denies sharing needles or usage of additional drugs. On arrival, initial vital signs were notable for temperature of $39.4{ }^{\circ} \mathrm{C}$, heart rate of 150 , and blood pressure of 174/100 $\mathrm{mmHg}$. His cardiac exam revealed a II/VI systolic murmur at the right upper-sternal border. Abdominal exam was soft non-tender, nondistended and negative for peritoneal signs. No focal neurological signs were noted and there was no spinal tenderness to palpation. He had numerous needle marks on his arm but no other rashes or stigmata of endocarditis. In the setting of back pain and fever, a lumbar spine MRI was obtained and showed no evidence of an epidural abscess. Blood cultures were drawn and the patient was started on vancomycin and piperacillin/tazobactam. An EKG and chest $\mathrm{x}$-ray were normal. Initial labs were significant for a sodium level of $130 \mathrm{mEq} / \mathrm{L}$, otherwise chemistries, urinalysis, and blood counts were within normal limits. Urine drug screen was positive for benzodiazepines and opiates. Additional labs including HIV, Hepatitis B and C, and inflammatory markers were all negative. His tachycardia and fever improved with intravenous fluids and antipyretics within $24 \mathrm{~h}$ of admission. A transesophageal echocardiogram showed no evidence of endocarditis and blood cultures remained negative for 2 days. With no clear diagnosis, continued clinical improvement, and the patient wanting to leave against medical advice, plans were made for discharge. On the day of discharge, it was thought that his symptoms may have been caused by cotton fever. When asked if he injected his heroin through cotton, he replied, "Do you think this was cotton fever?" The diagnosis of cotton fever was made and the patient was discharged without antibiotics in good condition.
DISCUSSION: Fever in an intravenous drug user can have many etiologies, among which sepsis, endocarditis, and an epidural abscess are the most concerning. However, there are other causes which must be considered. Originally coined in 1975, cotton fever, a benign and self-limited febrile syndrome that occurs after filtering the solubilized drug through cotton prior to injection, is well known amongst the injection drug user population but poorly described in medical literature. The pathophysiology of cotton fever is poorly understood and there is a paucity of research on it. However, multiple theories have been proposed, including the endotoxin hypothesis. Gram-negative bacilli, such as Enterobacter agglomerans, have been shown to colonize cotton plants and to produce endotoxins, causing "Mill Fever" in cotton mill workers. The toxin itself is water-soluble and heating can enhance the toxic effect, therefore the febrile syndrome may be more pronounced in intravenous drug users. Interestingly, in our patient, E. agglomerans was not found, but Onchobactrum sp. was isolated in one blood culture on day four. Although Onchobactrum has not been described in literature as a colonizer of cotton, it is a common soil bacterium and could be a contaminant in our patient's cotton filter. Onchobactrum sp. has low pathogenic potential and is rarely reported as a human pathogen. Most published cases were found in immunocompromised patients or in nosocomially acquired patients with indwelling medical devices. With this patient's clinical improvement and low pathogenicity of the bacteria, no treatment was necessary. Cotton fever is a diagnosis of exclusion and is characterized by onset of symptoms within ten to thirty minutes after injection. Symptoms include headache, malaise, myalgias, arthralgias, nausea, vomiting, chills, rigors, shortness of breath, abdominal pain, and back pain. Fever, with temperatures up to $40.3^{\circ} \mathrm{C}$, and tachycardia develop within first few hours of injection. The syndrome usually resolves after $12-24 \mathrm{~h}$ but can continue up to $24-48 \mathrm{~h}$. Our patient's initial symptoms and signs were concerning for SIRS and endocarditis, however he rapidly improved as the team ruled out major infectious causes. Therefore, a presumed diagnosis of cotton fever was made. This case highlights the disparities in cultural awareness and medical knowledge between physicians and their patients. Only after an extensive inpatient evaluation was the diagnosis of cotton fever considered. Differentiating cotton fever from more serious etiologies remains challenging; therefore every febrile, acutely ill intravenous drug user should undergo a thorough evaluation. However, taking a detailed history regarding heroin filtration methods can help alert the physician and may reduce extensive secondary evaluations as well as hospitalization lengths once serious etiologies are excluded. It is also a reminder that we as physicians should always ask the patients what they think may be the cause of their symptoms, as they often have clinically important insights into their illnesses.

THE DOWNSTREAM EFFECTS OF INACCURATE ALLERGY DOCUMENTATION Emily S. Bowen; Udayan K. Shah; Christian Wysocki. UT Southwestern, DALLAS, TX. (Control ID \#2465571)

LEARNING OBJECTIVE \#1: Recognize the importance of completely and accurately documenting allergies

CASE: A 62-year-old man with ESRD on hemodialysis, atrial fibrillation on warfarin, congenital heart block with biventricular pacemaker, heart failure with EF of $39 \%$, history of stroke, hypertension, and Child's Class B cirrhosis due to HCV presented with chest pain, elevated troponin, and AV-paced rhythm. He was given full dose aspirin and admitted for further evaluation of possible acute coronary syndrome. He then developed altered mental status and gaze deviation and was found to have an acute left temporal lobe infarction, complicated by seizure and post-ictal state. This was thought to be an embolic stroke from atrial fibrillation in the setting of a sub-therapeutic INR of 1.8. He was ineligible for TPA because he was taking warfarin. He was transferred to the MICU where his troponin and CK MB were noted to be rising. Heparin was listed as an allergy, so he was started on continuous argatroban (dosed for hepatic impairment) for management of NSTEMI. Nine hours after the 
argatroban was started, he had melena and became hypotensive to the 60 's/40's. His hemoglobin had decreased from $9.2 \mathrm{~g} / \mathrm{dL}$ earlier that day to $7 \mathrm{~g} / \mathrm{dL}$. His INR was 5.2 and PTT was 118.5 s. All anticoagulation was held, and he received a total of 4 units of blood. Upper endoscopy showed a small amount of fresh blood in the pylorus with no varices or obvious source of bleed, so his GI bleed was attributed to anticoagulation. His clinical condition stabilized, and he was transferred to the general medicine ward after 1 week in the MICU. After consultation with neurology and cardiology, there were felt to be clear indications for antiplatelet therapy and anticoagulation, given his recent NSTEMI, recent embolic stroke, and atrial fibrillation with CHADSVASC of 4 . The desired course of action was to start daily aspirin and re-initiate warfarin, but due to risk of a hypercoagulable state during warfarin initiation, bridging therapy was needed until INR was in the therapeutic range of $2-3$. The patient's cirrhosis made argatroban a sub-optimal choice for bridging therapy, and his recent GI bleed while on argatroban reinforced these concerns. His ESRD made him a poor candidate for low molecular weight heparin or fondaparinux. Heparin was the optimal choice for bridging therapy, but it was included on his allergy list. Unfortunately, the nature of the patient's reaction to heparin was not documented. The allergy had been entered by a nurse 11 months prior. Neither the patient nor his wife could recall him ever receiving heparin and both were unaware of any allergy. The electronic medical record indicated that he had received two doses of subcutaneous heparin for DVT prophylaxis after the allergy was entered, and no allergic reaction was noted after these administrations. Platelet counts showed a stable thrombocytopenia, likely secondary to cirrhosis. There were no precipitous drops in platelet count, and no heparin-platelet factor 4 antibody or serotonin release assays were ever checked to indicate suspicion for heparin induced thrombocytopenia (HIT). Outside medical records were also reviewed and did not indicate heparin allergy or history of HIT. Thus, after discussion with the patient, the decision was made, in association with our allergy and hematology staff, to cautiously challenge him with a therapeutic dose of heparin, and begin heparin for bridging therapy. The patient tolerated the heparin challenge without any signs or symptoms of an immediate hypersensitivity reaction. He was monitored closely during heparin administration with particular vigilance for HIT, but there were no adverse reactions. The rest of his admission was uncomplicated, and heparin was removed from his allergy list.

DISCUSSION: Documenting a patient's medication allergies is an important part of the medical history. Modern electronic medical records require allergies be checked during each clinical encounter by multiple members of the healthcare team. We are appropriately concerned about causing harm by administering a medication to which a patient is allergic, but this case highlights how a patient can also be harmed by incorrect or incomplete documentation of an allergy and the resultant inability to treat them with a necessary or optimal medication. If heparin had not been listed on this patient's allergy list, he likely would have received heparin for treatment of his sub-therapeutic INR and NSTEMI rather than argatroban, which is not a first line agent for patients with hepatic impairment due to its hepatic metabolism and extended duration of action in patients with hepatic impairment. Additionally, while protamine sulfate is a well-established antidote to heparin, there is currently no antidote for argatroban. It is possible that the use of heparin rather than argatroban might have prevented the patient's GI bleed and also possible that if heparin had been used he could have received protamine sulfate to limit the extent of the bleed. Finally, once he was clinically stable after his GI bleed, his anticoagulation would not have been delayed by the time consuming investigation required to minimize the suspicion of a true heparin allergy. While documenting medication allergies can prevent adverse drug reactions, it is important to thoroughly document the type of reaction so that patients are not inappropriately denied necessary medications

THE GIST OF AN OBSCURE BLEED Megan Maddox ${ }^{1,2}$; Jeffrey Bates ${ }^{1,2}$. ${ }^{1}$ Baylor College of Medicine, Houston, TX; ${ }^{2}$ Michael E DeBakey VA Medical Center, Houston, TX. (Control ID \#2469570)
LEARNING OBJECTIVE \#1: Recognize the diagnostic challenge of obscure GI bleeding.

LEARNING OBJECTIVE \#2: Identify the potential utility of CT enterography in the workup of an obscure GI bleed.

CASE: A 67-year-old man was admitted for recurrent episodes of melena. Eighteen months prior to his index admission, he presented with melena, a hemoglobin of $8.5 \mathrm{~g} / \mathrm{dL}$, and iron deficiency; he had been taking occasional NSAIDs and daily aspirin. An esophagoduodenoscopy (EGD) showed a clean based ulcer, and he was treated with daily omeprazole and cessation of NSAIDs. Over the next 18 months, he continued to have recurrent episodes of melena and persistent iron deficiency anemia. He had three EGDs, one colonoscopy, one capsule endoscopy, and a push enteroscopy, none of which revealed any significant abnormalities. He was also treated for possible Helicobacter pylori. At his index admission, he had two episodes of melena and a 10-pound weight loss. Physical examination was normal. Laboratory data were significant for a hemoglobin of $8.7 \mathrm{~g} / \mathrm{dl}$ and a gastrin level of $99 \mathrm{pg} / \mathrm{mL}$. Another EGD was normal, and biopsies were negative. A computed tomography (CT) enteroscopy identified a suspected AVM in the mid-jejunum. During an embolization procedure, a hypervascular tumor was noted. A positron emission tomography (PET)-CT revealed a $5.3 \mathrm{~cm}$ jejunal mass. An exploratory laparotomy was performed, and the patient was diagnosed with a gastrointestinal stromal tumor (GIST). One month after diagnosis, imatinib was started. Two years after diagnosis, the patient's hemoglobin level has remained normal, and there is no evidence of disease on surveillance CT scans.

DISCUSSION: This case demonstrates the classic dilemma of determining the etiology of an obscure GI bleed. Obscure GI bleeding is defined as bleeding that persists or recurs without an obvious etiology; it can be further subcategorized as obscure occult (a positive fecal occult blood test with or without iron deficiency and without obvious bleed loss) and obscure overt (clinically evident bleeding). Approximately $80 \%$ of obscure GI bleeds originate in the small bowel. Most experts on obscure GI bleeding agree that repeating an EGD can have a high diagnostic yield of 35-65\%. If the repeat EGD is negative, a capsule endoscopy is usually the next recommended test, with a diagnostic yield of 55-70\%. If these two procedures are unrevealing, although there are other tests that can be performed, the diagnostic yield decreases greatly. Also, there are different algorithms in the literature, guided by different expert opinion. However, Agrawal et al. demonstrated that CT enteroscopy can be useful in diagnosing obscure overt bleeding. In their study, after nondiagnostic EGDs and capsule endoscopies, CT enteroscopy had a diagnostic yield of $50 \%$ in these patients. CT enteroscopy, a newly-available test, successfully identified our patient's GIST, which accounts for $0.1-3 \%$ of all GI malignant tumors. CT enteroscopy can thus be an effective tool in the diagnosis of an obscure bleed.

THE GRAVITY OF STATINS William Fuller; John Ragsdale. University of Kentucky, Lexington, KY. (Control ID \#2468912)

LEARNING OBJECTIVE \#1: Identify myasthenia gravis as a rare complication of statin use

LEARNING OBJECTIVE \#2: Discuss the diagnosis of ocular myasthenia gravis

CASE: A 55 year-old woman with a history atrial fibrillation was recently started on atorvastatin after a CVA. Eleven days later, she presented to the emergency department with acute diplopia leading to falls. On exam, her pupils were equally round and reactive to light. She had significant bilateral ptosis. Her right eye had limited medial movement with some upward drift. She had no dysarthria, dysphagia, or proximal muscle weakness. Ocular myasthenia gravis was suspected. Edrophonium test was not attempted because of her history of atrial fibrillation. Instead, a bedside ice pack test was performed which attenuated the patient's ptosis. Acetylcholine receptor modulating and blocking antibodies were negative. Because of the temporal relationship with her atorvastatin and its known association with myasthenia gravis, it was held. The following morning, her ptosis and 
diplopia had resolved, confirming the diagnosis of statin-induced ocular myasthenia gravis.

DISCUSSION: Myasthenia gravis is a rare complication of statin use, restricted to case reports. Despite its rarity, clinicians should consider this diagnosis when a patient presents with signs or symptoms of myasthenia gravis who is on a statin. In this case, the patient presented with ptosis and eye muscle weakness, classic findings of myasthenia gravis. Typically, the onset occurs about a week after starting the statin, but can present as late as 10 weeks. Since statin-induced myasthenia occurs through the same mechanism as idiopathic, the testing is identical. In making a diagnosis of statininduced ocular myasthenia, the temporal relationship of when the statin was started and the weakness first appreciated is key in establishing a causal relationship. This is further confirmed when cessation of the statin therapy leads to improvement or resolution of the patient's symptoms. Bedside tests include edrophonium and the ice pack test. Edrophonium is indicated in patients with evidence of obvious ptosis or ophthalmoparesis because the effect of the test is more easily observed. Edrophonium increases the amount of acetylcholine in the neuromuscular junction, improving the strength of the small eye muscles. This test is positive in over $80 \%$ of patients with generalized myasthenia but in only approximately $60 \%$ of those with ocular myasthenia. In our patient, edrophonium was contraindicated. The icepack test involves applying a bag of ice to the patient's affected eyes and evaluating attenuation of the patient's ptosis. Neuromuscular transmission is affected by the cold in the superficial eyelid musculature not the extraocular musculature. In ocular myasthenia, this test has a sensitivity of approximately $80 \%$. Other testing includes serum and electrodiagnostic studies. Patients with generalized myasthenia are positive for acetylcholine receptor antibodies $80-90 \%$ of the time, but patients with only ocular myasthenia, have positive antibodies that are positive $40-50 \%$ of the time. Testing for muscle specific receptor tyrosine kinase antibodies can also be pursued but the sensitivity is even lower than for acetylcholine receptor antibodies. Repetitive nerve stimulation and single-fiber electromyography are positive in patients with generalized myasthenia gravis approximately 75 and $95 \%$ of the time, respectively, but in only approximately 50 and $90 \%$, respectively, for ocular myasthenia. Given the limited sensitivity of the above tests in ocular myasthenia, a single test may not be sufficient to make the diagnosis. Often multiple tests are needed and should be combined with clinical judgment.

THE GREAT PRETENDER: MORE THAN MEETS THE EYE Jamal Z. Saleh ${ }^{1}$; Chad Glisch $^{2}$; Jeremiah Stromich ${ }^{2}$; Vipal P. Durkal ${ }^{2}$; Tadeo A. Diaz Balderrama ${ }^{2}$; Pinky Jha ${ }^{1} .{ }^{1}$ Medical College of Wisconsin, Waukesha, WI; ${ }^{2}$ Medical College of Wisconsin Affiliated Hospitals, Milwaukee, WI. (Control ID \#2469517)

LEARNING OBJECTIVE \#1: 1. To recognize, diagnose, and manage ocular manifestations of syphilis

LEARNING OBJECTIVE \#2: 2. To assess for other comorbid conditions (i.e. HIV) with patients presenting with ocular syphilis

CASE: A 29-year old previously healthy bisexual African-American male presented with a 3-week history of right eye blurriness. Ophthalmology was consulted and performed a dilated fundus exam that revealed a right eye with severe vitritis, panuveitis and retinal vasculitis and a left eye with mild vitritis. Snellen vision of R 20/300 and L 20/30 was noted. Extensive workup to evaluate for Lyme disease, sarcoidosis, tuberculosis, syphilis, toxoplasmosis, and rheumatic disease was performed. Workup revealed a positive serum treponemal antibody testing (FTA-Abs) and RPR (1:256). Patient was admitted for treatment with aqueous crystalline penicillin-G 3 million units every $4 \mathrm{~h}$ for a 14-day duration. A lumbar puncture was performed and cerebrospinal fluid (CSF) analysis revealed lymphocytic pleocytosis (97\%), leukocytosis (22), and elevated protein (54), all highly characteristic findings of neurosyphilis. CSF VDRL studies were negative, however. Upon examination, patient had multiple genital chancres along the base of his glans penis. Skin examination revealed tan-brown macules and patches along the right palm and the plantar surfaces of his feet bilaterally. Neurological exam was unremarkable, other than the noted visual deficits. Subsequent workup for HIV was positive with a CD4 count of 255. With treatment, patient reported gradual improvement in right eye vision and improvement in vitreous haze on ophthalmic exam. He was referred to a local HIV/AIDS clinic for continued therapy.

DISCUSSION: Syphilis has emerged in the last decade as a formidable infectious disease in the United States, with the rates of primary and secondary syphilis doubling since the turn of the century. Ocular manifestations of syphilis present less commonly, though the true epidemiology is unclear. We present a rare case of ocular syphilis presenting as unilateral blurriness. Ocular syphilis, an insidious but potentially sightthreatening illness, has been of special concern for the CDC which has seen an alarming rise in the number of cases over the past 2 years. The rising rates of primary and secondary syphilis may be largely attributed to unprotected sex in a time period of major advances in HIV treatment. The CDC has issued a clinical advisory regarding the increasing incidence of ocular syphilis, especially in the men who have sex with men population. The condition most commonly presents as posterior uveitis or panuveitis, two conditions with many infectious, autoimmune, and rheumatic mimickers. Of note, CSF VDRL may be falsely negative in up to $70 \%$ of patients as in the case presented. Clinicians should have a high index of suspicion for a syphilitic etiology in patients presenting with the characteristic ocular complaints and a suggestive social history.

THE IDENTIFICATION OF A STEROID CELL TUMOR IN A PATIENT WITH PRESUMED MENOPAUSE Carol Rupprecht; Nancy Noel. St. Vincent Indianapolis Hospital, Carmel, IN. (Control ID \#2465572)

LEARNING OBJECTIVE \#1: Recognize when additional laboratory evaluation is indicated in a woman presenting with presumably menopausal symptoms.

CASE: A 49-year-old Spanish-speaking female presented to our clinic for follow-up of non-insulin-dependent diabetes mellitus. Despite compliance with low-dose metformin, her A1c had increased from 6.4 to 7.9 over 10 months. She had gained seven pounds, which she attributed to exercising less. She endorsed mild depression which had begun 2 years prior when her father died and had improved over time. She had new complaints of facial hair and acne. She had not menstruated in 1.5 years but had not yet experienced hot flashes. Her mother had reached menopause in her late 40s. Her medical history included essential hypertension and a postpartum ovarian cystectomy. Physical examination revealed shaved facial hair. Morning cortisol was normal; her FSH was $6.5 \mathrm{mIU} / \mathrm{mL}$, not consistent with menopause. The patient returned a month later with heartburn; pelvic examination at this visit, done to obtain a Pap smear and for further evaluation of her premenopausal amenorrhea, revealed clitoromegaly. Further blood tests included high total testosterone at $613 \mathrm{ng} / \mathrm{dL}, \mathrm{LH}$ at $4.3 \mathrm{mIU} / \mathrm{mL}$, and normal DHEA-S at $119 \mathrm{mcg} / \mathrm{dL}$. For 5 months she presented for follow-up of diabetes but delayed in obtaining a transvaginal ultrasound; hair began to grow on her shoulders. Transvaginal ultrasound later revealed a solid left adnexal mass. Its morphology score was 5 , associated with an increased risk for malignancy. Three months later, the mass was visualized on CT scan with central enhancement. At time of total abdominal hysterectomy with bilateral salpingo-oopherectomy, her tumor measured $4.2 \times 4.0 \times 3.5 \mathrm{~cm}$ with no involvement of 
sampled lymph nodes or the omentum. A pathologist characterized it as a nonspecific steroid cell tumor.

DISCUSSION: Laboratory evaluation is not often indicated for a female presenting with likely menopause. However, this patient's mild acne and hirsutism plus history of ovarian cyst prompted us to check an FSH level, primarily to differentiate between PCOS and menopause. We did not expect this would lead to a diagnosis of a sex cord-stromal ovarian neoplasm; incidence is 1 per 500,000 women. Few case reports of steroid cell tumors or Sertoli-Leydig cell tumors describe the presentation of women who are neither clearly premenopausal nor known to be postmenopausal. This case prompts us to consider a wide differential diagnosis for amenorrhea in middle-aged women, especially in those with any atypical symptoms or history.

THE INTERPLAY BETWEEN HCV AND EBV INFECTIONS IN A PRESENTATION OF ACUTE HEPATITIS. Mckenna Longacre ${ }^{1}$; Brian W. Boyle ${ }^{3}$; Denisa Gace ${ }^{2} .{ }^{1}$ Harvard Medical School, Cambridge, MA; ${ }^{2}$ Mass General Hospital, Boston, MA; ${ }^{3} \mathrm{MGH}$, Boston, MA. (Control ID \#2470377)

LEARNING OBJECTIVE \#1: Increase awareness of concurrent viral infections leading to acute hepatitis presentation

LEARNING OBJECTIVE \#2: Differentiating between new infections versus viral reactivation (EBV and $\mathrm{HCV})$

CASE: A 43yo M with a past medical history of HCV (undetectable viral loads 3 months prior to admission), remote history of alcohol and injection drug use, presented with worsening fatigue, myalgia and nausea after initially experiencing sore throat and a runny nose 2 weeks prior to coming to the hospital. His history was significant for distant EBV infection, prior gastritis and duodenitis on PPI, and recent negative PPD. Social history was significant for incarceration 2 years prior to his admission. He denied any family history of liver disease. His exam was notable for jaundice, sclera icterus and right upper quadrant tenderness with a palpable liver edge and shotty anterior cervical lymphadenopathy. His blood tests at presentation showed WBC $10.3 \mathrm{~K} / \mathrm{uL}$ with $5 \%$ atypical lymphocytes, hemoglobin $16.1 \mathrm{~g} / \mathrm{dL}$, platelets $124 \mathrm{~K} / \mathrm{uL}$; normal renal function; ALT $1649 \mathrm{U} / \mathrm{L}$; AST $966 \mathrm{U} / \mathrm{L}$; Alkaline Phosphotase266 U/L, total bilirubin7.6 mg/dL, direct bili $5.9 \mathrm{mg} / \mathrm{dL}$, INR 1.4 and albumin $4.1 \mathrm{~g} / \mathrm{dL}$. Of note, liver function tests 3 months prior to admission were within normal limits. His serum toxicology panel was negative, including an acetaminophen level. Serologies for HAV, HBV, HCV, EBV, EMV, CMV and HIV were notable for a hepatitis C viral load of 548,000 copies/ml and an EBV viral load of $2580 \mathrm{copies} / \mathrm{ml}$. Tests also showed HBV core antibody positive, surface antibody positive, surface antibody negative, and HBVe antigen negative). He was HAV immune and Influenza, HIV and CMV tests were negative. His antinuclear antibody tested was positive $(1: 20)$; smooth muscle antibody was positive $(1: 80)$; antimitochondrial antibody was negative; and ceruloplasmic were normal. Abdominal ultrasound showed a diffusely hypoechoic liver parenchyma most consistent with acute hepatitis, as well as mild gallbladder wall thickening, thought to be secondary to adjacent hepatitis rather than primary gallbladder process given no sonographic evidence of cholecystitis. During his hospitalization, the patient was managed conservatively with fluids, antiemetics and pain medications. Following conservative approach, his abnormal liver tests started to normalize. On the day of discharge, his LFTs were: AST 239 U/L, ALT 677 U/L, Alkaline Phosphatase 212 U/ $\mathrm{L}$, total bilirubin $3.8 \mathrm{mg} / \mathrm{dL}$ and direct bilirubin $2.5 \mathrm{mg} / \mathrm{dL}$. Three days following the hospital discharge, the patient was seen in the outpatient setting; his HCV viral load was down to 1200 copies (despite the absence of specific HCV therapy) and EBV viral load remained elevated to 3030 . He appeared to have clinically improved. HCV viral typing on this admission revealed serotype 1a. Patient was found to have cleared his second infection less than 3 months after infection, most recent follow up visit $\mathrm{HCV}$ viral load being $<15$

DISCUSSION: While viral hepatitis is a common presentation, coinfection with EBV and $\mathrm{HCV}$ is less common, and may help important implications for the natural history of illness. In this case, the relationship between concurrent EBV and HCV infections is unclear. It has been reported that EBV infection may be reactivated by HCV, though in this case we lack data differentiating between viral reactivation versus new infection. Of note, there have been some studies noting increased HCV viral replication in the setting of EBV infection. Another possibility is that EBV could have been reactivated by a subsequent $\mathrm{HCV}$ infection. Therefore, this raises the question of whether this patient is prone to acute presentations of $\mathrm{HCV}$ due to chronic underlying EBV. This is an important distinction given that we do not typically think of HCV as contributing to acute presentations such as this. The second interesting point is that, in addition to contributing to his acute presentation, it is possible that his concurrent EBV infection also contributed to his ability to clear $\mathrm{HCV}$ twice. Clearing multiple $\mathrm{HCV}$ infections is not in and of itself uncommon. Approximately $25 \%$ of patients clear HCV infection spontaneously, usually in within the first 6 months of infection. Individuals with previous resolution of $\mathrm{HCV}$ infection have been found to be four times less likely to develop a subsequent $\mathrm{HCV}$ infection than those infected for the first time. This may reflect acquired immunity to one strain, but not to all strains of HCV. However, the relatively rapid viral clearance of this second $\mathrm{HCV}$ infection make this patient somewhat unique. It is possible that by increasing viral replication, EBV may also increase the host immune response, and thus in fact contribute to a more rapid clearance in some patients. Therefore, this case illustrates a curious interaction between these two viruses. Further study is likely to yield a greater understanding of the pathophysiology of acute hepatitis, and may nuance our clinical insight into the natural history of a HCV.

THE KEY TO SOLVING THE MYSTERY OF A REFRACTORY PLEURAL EFFUSION IS... A PELVIC EXAM? Jennifer Mandal. Kaiser San Francisco, San Francisco, CA. (Control ID \#2469130)

LEARNING OBJECTIVE \#1: To review the differential diagnosis and workup for refractory lymphocyte-predominant pleural effusions.

LEARNING OBJECTIVE \#2: To recognize the differential diagnosis for ovarian tumor and concurrent pleural effusion, which includes metastatic ovarian carcinoma and a rare and fascinating condition known as Meigs syndrome (the triad of pleural effusions, ascites, and benign ovarian fibroma)

CASE: A 65 year-old woman presented to clinic to establish primary care. She had not seen a doctor in over 10 years. She reported a persistent dry cough that began 8 months ago. She denied fevers, nightsweats, weight loss, chest pain, or any history of asthma, acid reflux, or allergic rhinitis. She quit smoking 15 years ago. Physical examination was notable for decreased breath sounds at the right base. A chest x-ray revealed a large right pleural effusion. Thoracentesis was performed and $1.5 \mathrm{~L}$ of straw-colored pleural fluid was removed. The fluid was exudative, with 1650 white blood cells ( $96 \%$ lymphocytes). Our differential diagnosis included malignancy, tuberculosis, and autoimmune disease. Pleural fluid cytology, flow cytometry, and bacterial, fungal, and acid fast bacilli (AFB) cultures returned negative. Chest CT was unrevealing. The patient's pleural effusion rapidly reaccumulated, requiring frequent large-volume thoracenteses and eventually, placement of a chronic indwelling pleural catheter. Over the next 4 months, the patient was frequently readmitted to the hospital for complications related to her persistent pleural effusion and 
indwelling pleural catheter. Four additional samples of pleural fluid were sent for cultures, cytology, and flow cytometry, and all returned negative. Antinuclear antibody, rheumatoid factor, and anti-neutrophil cytoplasmic antibody were negative. Lung biopsy revealed chronic inflammation without evidence of malignancy or AFB. Talc pleurodesis was discussed, but the patient declined. The patient eventually presented to the gynecology clinic for a routine Pap test, and she was found to have a palpable left adnexal mass. Pelvic MRI revealed small ascites and a large heterogeneous mass in the central pelvis, concerning for possible ovarian malignancy. Serum CA 125 level was 458 (normal $<35$ ). The mass was surgically removed, and was found to be a benign ovarian fibroma. Following removal of the fibroma, the patient's pleural effusion rapidly resolved. The patient was given a final diagnosis of Meigs syndrome.

DISCUSSION: This case illustrates a rare and fascinating cause of pleural effusion. Meigs syndrome is characterized by the triad of 1) pleural effusion, 2) ascites, and 3) benign ovarian fibroma. The effusions and ascites rapidly resolve after resection of the fibroma. The clinical presentation of Meigs syndrome can closely mimic ovarian carcinoma, which can also cause pleural effusions and ascites (due to metastatic disease). Both conditions are associated with elevated CA 125 level. The diagnosis of Meigs syndrome can only be made after ovarian carcinoma is definitively ruled out via surgical pathology. Fortunately for our patient, surgical removal of her ovarian mass simultaneously ruled out ovarian carcinoma, and cured her longstanding pleural effusion.

THE MAN WHO COUGHED UP LUNG Xixi Zhao; Mark Yoder. Rush University Medical Center, Chicago, IL. (Control ID \#2457711)

LEARNING OBJECTIVE \#1: Recognize the life-threatening nature of plastic bronchitis when casts obstruct major airways, causing acute respiratory failure.

LEARNING OBJECTIVE \#2: Identify plastic bronchitis as part of the differential diagnosis of acute respiratory failure in addition to other causes

CASE: A 48-year-old man with a history of congestive heart failure (CHF) secondary to severe mitral regurgitation presents with persistent cough for 4 years. The patient described the cough as productive of a "meaty substance," resembling lung tissue coated with blood and thick secretions. The tissue would become stuck in his throat, causing him to choke, become short of breath and sometimes faint. Only after he expectorated this substance would he experience respiratory relief until he produced another one. He noticed an increased production after developing pneumonia 3 months ago. On day 4 of admission, the patient expectorated a large bronchial cast. Cytology revealed rare bronchial cells and scattered macrophages. Bronchoscopy was preformed but no additional casts were located.

DISCUSSION: Plastic bronchitis is a rare and enigmatic disease, characterized by the expectoration of bronchial casts. Case reports describe patients mistaking casts for undigested food and 1 patient in particular mistook it for squid because of its large branching pattern and rubbery consistency. It can mimic status asthmaticus and foreign body aspiration when the casts obstruct major airways, causing acute respiratory failure. Although the first report of bronchial casts dates A.D. 131, there remains a dearth of information on the subject. The pathogenesis of cast formation is unclear and therapeutic options are limited and largely based on anecdotal experience. A major advancement was made when Seer et al. categorized patients into 2 groups based on histologic examination. Type I or "inflammatory" casts consist of fibrin with eosinophilic infiltrates and are associated with inflammatory diseases of the lung (e.g. asthma and pulmonary infections). Type II "acellular" casts comprise mainly mucin and are typically found in patients with congenital heart disease following the Fontan procedure. Some experts postulate that trauma to the lymphatic channels or increased inflammatory response provokes the formation of the casts, and others attribute it to increased pulmonary venous pressure leading to mucus hypersecretion; however, most cases are idiopathic. In our patient, although stains were not performed to classify the cast, we hypothesize that his are due to a combination of factors. Increased pulmonary venous pressure in the setting of mitral regurgitation may be the underlying etiology, with worsening due to recent pneumonia. The patient is being considered for the Clinical Outcomes Assessment of the MitraClip Percutaneous Therapy (COAPT) Trial in an effort to reduce mitral regurgitation, and potentially the formation of bronchial casts. This case suggests a possible novel mechanism of this disease, reinforces its potentially lethal nature and highlights the paucity of data regarding its management.

THE MUFFLED TRAGEDY OF PSEUDOANEURYSMS Joseph H. Song; Evan Shlofmitz; Karen Friedman. Northwell Health, Manhasset, NY. (Control ID \#2469919)

LEARNING OBJECTIVE \#1: Recognize ventricular pseudoaneurysms after an acute myocardial infarction

CASE: A 79 year-old Korean female with a history of hypertension and atrial fibrillation presents with two episodes of new-onset substernal chest pain over 2 days. Electrocardiogram showed ST depressions in V1-V4. Cardiac enzymes were elevated at a creatine kinase of 1328, a creatine kinase-MB of 94, and a troponin I of 2.6. Cardiac catheterization revealed $100 \%$ occlusion of the mid left circumflex coronary artery. The patient was diagnosed with a late presentation of an inferolateral non-ST elevation myocardial infarction (NSTEMI), as she had presented to the hospital more than $12 \mathrm{~h}$ after the onset of chest pain. Thrombectomy was performed without stent placement. Transthoracic echocardiogram (TTE) showed a small to moderate pericardial effusion without evidence of cardiac tamponade, a right pleural effusion, and aneurysmal basal/ inferolateral segments. Cardiac enzymes continued to trend down. Given the absence of clinically significant coronary artery disease or risk factors and a subtherapeutic INR of 0.94 , the etiology of the coronary artery occlusion was presumed to be embolic from the patient's atrial fibrillation, a rare cause of acute myocardial infarction. Over the following $36 \mathrm{~h}$, the patient developed progressive weakness, poor appetite, and an inability to ambulate - a change from her independent and functional baseline - with a new fever of up to $101.5^{\circ}$ Fahrehneit and blood pressure down to $81 / 50 \mathrm{mmHg}$. The patient was unable to otherwise localize her symptoms, denying chest pain, shortness of breath, or other focal complaints. Pulsus paradoxus was not present. The patient was started on IV fluids and empiric broad spectrum antibiotics for severe sepsis of unclear etiology, and further imaging was ordered. CT of the chest revealed a left lateral ventricular rupture with a $2.5 \mathrm{~cm}$ pseudoaneurysm formation. Over the following several hours, the patient developed worsening respiratory distress with subsequent ventricular fibrillation and cardiac arrest. Return of spontaneous circulation was briefly achieved, and emergent bedside pericardiocentesis with drain was performed. The patient was taken to the operating room where she arrested again for $45 \mathrm{~min}$ before resuscitative efforts were discontinued.

DISCUSSION: Left ventricular free wall rupture occurs in $1-4 \%$ of all heart attacks, is the cause of $15-23 \%$ of all deaths after myocardial infarction, and is associated with greater than $80 \%$ mortality. A pseudoaneurysm is a complication of an acute MI where the ventricular free wall rupture is contained by parietal pericardium. A pseudoaneurysm has a $30-45 \%$ chance of rupturing and leading to tamponade. Unlike a true left ventricular aneurysm, a pseudoaneurysm is an indication for emergent intervention. TTE's have a low sensitivity for identifying pseudoaneurysms, and pseudoaneurysm are difficult to differentiate from true aneurysms. Pseudoanuerysms are best diagnosed with angiography, trans-esophageal echocardiogram, cardiac CT, or cardiac MRI. Risk factors for 
myocardial rupture after a myocardial infarction include: first myocardial infarction, transmural infarction, female sex, age older than 60 years, low body mass index, hypertension, use of nonsteroidal anti-inflammatory drugs or steroids during the acute phase of myocardial infarction, late thrombolysis ( $>12 \mathrm{~h}$ ), and post-infarction angina. Common symptoms of a pseudoaneurysm include chest pain and dyspnea. Common signs of pseudoaneurysms are a new "to-and-fro" murmur and signs of cardiac tamponade, including elevated jugular venous pressure, pulsus paradoxus, diminished heart sounds, and electrical alternans. Treatment of pseudoaneurysms including surgical resection and endocardial patch technique. Pseudoaneurysms are difficult to diagnose and associated with very high mortality if not aggressively managed early. It requires a high degree of suspicion for diagnosis in those with the appropriate risk factors.

THE NOSE KNOWS: A CASE OF REFRACTORY OLFACTORY HYPERTENSION Amanda Fernandes ${ }^{1}$; Anna Yousaf ${ }^{2} .{ }^{1}$ Baystate Medical Center, Springfield, MA; ${ }^{2}$ Baystate Medical Center, Northampton, MA. (Control ID \#2467700)

LEARNING OBJECTIVE \#1: Recognize the importance of a thorough physical examination looking for signs and symptoms of cocaine ingestion

LEARNING OBJECTIVE \#2: Discuss the pathophysiology and management of cocaine intoxication as it relates to hypertension (HTN).

CASE: A 53-year-old female with a past medical history of HIV and HTN presented after being found unresponsive by her husband. On arrival by ambulance crew, she was found to be in ventricular fibrillation requiring $30 \mathrm{~min}$ of resuscitation before return of spontaneous circulation was achieved. Initial lab workup was notable for mild leukocytosis, elevated creatinine kinase and cocaine in the urine. She was started on hypothermic protocol. Bedside echocardiogram noted significantly reduced left ventricular ejection fraction of 10-15\%. Despite being in cardiogenic shock, she remained hypertensive with systolic blood pressure over $160 \mathrm{mmHg}$ requiring multiple doses of anti-hypertensives including a nitroprusside infusion. On the second day of admission, she was noted to have some foaming around her nose. Aspiration of her nares revealed packed cocaine crystals. On removal of this cocaine, her blood pressure began to decrease. Unfortunately, she did not show any signs of neurological recovery after rewarming and decision was made to withdraw care

DISCUSSION: Following alcohol, cocaine is the most common cause of drug related visits to the emergency department (ED) accounting for $33 \%$ of ED visits in the US. Of these visits, $6 \%$ are related to cardiovascular events including chest pain, HTN, myocardial ischemia, ventricular arrhythmias and heart failure. These effects occur following inhibition of the re-uptake of norepinephrine at preganglionic sympathetic nerve endings resulting in stimulation of alpha and beta adrenergic receptors in both the cardiac and peripheral vasculature. Furthermore, the onset and duration of these effects are determined by route of administration with insufflation being absorbed more slowly than intravenous or inhalation routes but the effects of cocaine lasting longer (Riezzo et al. 2012). In chronic users attempting to prolong the euphoria from cocaine, nasal packing further perpetuates these effects as vasoconstriction prolongs rate of absorption. Cocaine induced hypertension results from the direct presence of cocaine in the body with removal resulting in normalization of blood pressure. In cases of mild overdose, HTN can be managed with the use of benzodiazepines given the central nervous system stimulation. In cases of severe or refractory hypertension, intravenous infusion of phentolamine is first line treatment in counteracting the alpha-adrenergic effects of cocaine (Lange and Hillis, 2001). This highlights the importance of physical examination of the nares both in the workup and treatment of cocaine induced hypertension. References Riezzo I, Fiore C, De Carlo D, et al. Side effects of cocaine abuse: multiorgan toxicity and pathological consequences.
Current Medical Chem. 2012;19:5624-5646. Lange R, Hillis D. Cardiovascular complications of cocaine use. New England Journal of Medicine. 2001;345:351-58.

THE NOTORIOUS WOLFF Zain Kassam ${ }^{1}$; Sanjeev Sharma ${ }^{1}$; Neil Bernstein ${ }^{1}$; Robert Glatter $^{3}$; Rebecca A. Mazurkiewicz $2 .{ }^{1}$ Lenox Hill Hospital, New York, NY; ${ }^{2}$ Lenox Hill Hospital Medicine, New York, NY; ${ }^{3}$ Lenox Hill Hospital, New York, NY. (Control ID \#2469460)

LEARNING OBJECTIVE \#1: Identify the subtleties between Wolff-Parkinson-White Syndrome (WPW) with atrial fibrillation (AFib) in rapid ventricular response (RVR) and supra-ventricular tachycardia (SVT) with abberancy on EKG.

LEARNING OBJECTIVE \#2: Identify the pre-excitation pattern on an EKG Select which anti-arrhythmic medications are appropriate for treatment of this fatal arrhythmia. CASE: A 46-year-old healthy male with no past medical history presented to our ER with progressively worsening palpitations over the past 2 months. The patient described his symptom as a "rapid fluttering sensation of his heart" occurring almost always on exertion, resolving with rest. He denied having any associated chest pain or pressure, syncope, orthopnea, paroxysmal nocturnal dyspnea, or history of murmurs. He denied significant past medical or cardiac history, surgical history and family history. He denied tobacco, alcohol, or drug use. He recently consulted a cardiologist for the palpitations and was found to have a normal EKG and echocardiogram. In the ED, the patient's heart rate ranged from $140 \mathrm{~s}$ to $160 \mathrm{~s}$ but his vital signs were otherwise stable. His exam was negative for carotid bruits, jugular venous distention, murmurs, gallops and rubs. His labs including a including a $\mathrm{CBC}, \mathrm{CMP}, \mathrm{TSH}, \mathrm{UA}$, urine toxicology screen were all negative for pathology. His EKG was concerning for SVT with aberrancy. After receiving adenosine, the patient developed vetricular fibrillation requiring cardioversion by defibrillation. An emergent electrophysiology consultant re-evaluated the EKG and found it be consistent with pre-excited AFib in RVR, which was then managed with a procainamide infusion. His subsequent EKG showed normal sinus rhythm (NSR) with delta waves in the antero-septal leads indicative of a pre-excitation pattern. After he underwent an uncomplicated radio-frequency ablation of the accessory pathway, the patient's symptoms resolved and his EKG no longer showed delta waves.

DISCUSSION: WPW pattern, which is seen in 0.1 to 3 per 1000 people, is a common electrocardiographic phenomenon due to an accessory conduction pathway. WPW syndrome presenting with tachycardia is rare. WPW syndrome presenting with AFib in RVR is even rarer. Most patients are asymptomatic and the diagnosis is made after a routine EKG; however, $30 \%$ of patients with WPW experience paroxysms of tachycardia and less than $10 \%$ develop atrial fibrillation. Rarely, some patients may experience frequent and prolonged episodes of tachycardia that may be disabling or life threatening. The risk of sudden death in these patients is less than $3 \%$. Judicious and careful selection of antiarrhythmics along with ablation of the accessory pathway can potentially cure the syndrome. This case highlights the stepwise clinical progression of this arrhythmia starting with a fatal, confounding rhythm to it's complete resolution post-ablation. The subtle differences between the EKG and subsequent management of SVT with aberrancy and pre-excitation in the setting of AFib with RVR makes this case an excellent teaching tool.

THE PARADOX OF AUTOIMMUNITY AND IMMUNODEFICIENCY Brandon M. Huffman; Adam P. Sawatsky. Mayo Clinic, Rochester, MN. (Control ID \#2459706)

LEARNING OBJECTIVE \#1: Review diagnostic criteria for common variable immunodeficiency (CVID). 
LEARNING OBJECTIVE \#2: Recognize autoimmune manifestations in CVID.

CASE: A 26-year-old male presented to our institution with a prior history of sacral osteomyelitis secondary to immobilizing debility on IV antibiotics and a new fluctuant mass on his right clavicle. On physical examination, he was afebrile, normotensive, but mildly tachycardic at 95-105. Diaphoretic appearing. There was an erythematous and fluctuant mass about the right clavicular head without tenderness. He had severe pain with range of motion in elbows, wrists, knees and ankles. No pain in sacroiliac joint. There was fluctuance, warmth, and severe contractures around his left elbow, right wrist, and left knee. No erythema. He had $3+$ pitting edema of his lower extremities bilaterally. Areas on his skin were notable for depigmentation and alopecia. On review of systems, he endorsed night sweats with rigors, debilitating arthralgias, alopecia, and skin depigmentation. He was previously healthy until 10 months prior to admission when he subacutely developed right knee pain and swelling. An arthroscopy was performed because he was thought to have a meniscal tear, but the scope was nondiagnostic. He developed unilateral elbow and wrist pain with swelling over the following 4 months, so he sought rheumatology evaluation. He was diagnosed with seronegative polyarthritis and methotrexate (MTX) was initiated at that time. He tried acetaminophen, ibuprofen, and hydrocodone for the pain with no symptomatic improvement. He did have mild improvement in his pain and swelling on the MTX over 3 months of therapy, though he had intermittent adherence to the medication. He trialed prednisone for 3 days, but he developed cramping in his legs, so he decided to stop the medication. Joint aspiration was attempted, but only the elbow yielded any fluid, which was yellow and cultures were negative. No antibiotics were administered. On initial evaluation by an outside rheumatologist, he was noted to have absent IgG, IgA, and IgM. He was given IV immunoglobulin therapy with adequate increase of IgG levels. At our institution, vancomycin, piperacillin-tazobactam, levofloxacin, and doxycycline were initiated for broad-spectrum coverage of potential organisms. His osteomyelitis was debrided and histopathology showed clavicular osteomyelitis with an abscess that was culture negative. Blood cultures and serum infectious PCR/serology samples were repeatedly negative. Synovial fluid of the left elbow and left knee were repeatedly analyzed without any positive PCR or growth of infectious organisms. Left elbow joint fluid showed 25,931 cells (60 \% N, $16 \%$ L, $24 \%$ Mono), no crystals. Left knee joint fluid showed 230,254 cells ( $74 \%$ N, $9 \%$ L, $15 \%$ Mono), no crystals. Flow cytometry of peripheral blood revealed $1 \%$ CD19 B cells with $90 \%$ CD3 T cells. Bruton tyrosine kinase flow cytometry showed normal Btk expression on monocytes, and full genome analysis was negative, ruling out X-linked agammaglobulinemia and CVID was diagnosed. Transesophageal echocardiogram was negative for valvular vegetations or thickening. In the setting of his sacral and right clavicular osteomyelitis, subacute culturenegative polyarthritis could not be ruled out, so broad-spectrum antibiotics were continued for 6 weeks duration. Hydroxychloroquine and NSAIDs were initiated for seronegative polyarthritis, which was a presumed autoimmune component of common variable immunodeficiency. He plans to begin etanercept therapy after discontinuation of IV antibiotics.

DISCUSSION: Common variable immunodeficiency (CVID) affects 1 in 25,000 individuals. The classic presentation of CVID includes hypogammaglobulinemia, poor response to immunizations, and the absence of other defined immunodeficiencies. Primary and secondary causes of agammaglobulinemia must be ruled out by history and laboratory evaluation in order to diagnose CVID. This patient has no notable history of recurrent infections, pulmonary or otherwise, which is unusual for primary immunodeficiencies except CVID. This young man presents in the typical age group for CVID of $20-40$ years. Additionally, he is in the $5 \%$ of patients that have a low/absent B-cell population that yields low gamma globulin. The treatment includes monthly immunoglobulin therapy and frequent revaccination. Approximately 20-26\% of patients with CVID will have associated autoimmune findings, most commonly autoimmune hemolytic anemia or immune thrombocytopenic purpura.
Between 1 and $11 \%$ of patients with autoimmunity present with seronegative inflammatory arthritis such as rheumatoid arthritis, juvenile rheumatoid arthritis, and systemic lupus erythematosus. This patient did not have a hematologic manifestation of autoimmunity. Attention to detail in the history and physical in this patient highlights his associated findings of vitiligo, alopecia, and seronegative inflammatory polyarthritis. Retrospective reviews suggest that patients with concomitant immunodeficiency and inflammatory arthritis may respond to steroids, hydroxychloroquine, anti-TNF therapy, or rituximab.

THE PERFECT STORM: FEVER, TACHYCARDIA, AND ALTERED MENTAL STATUS AFTER PTCA Minghao Liu ${ }^{2}$; Benjamin T. Galen ${ }^{1}$; Lydia Kruge ${ }^{1} .{ }^{1}$ Albert Einstein College of Medicine, Bronx, NY; ${ }^{2}$ Montefiore Medical Center, Bronx, NY. (Control ID \#2470697)

LEARNING OBJECTIVE \#1: Recognize thyroid storm as a rare but lifethreatening complication after administration of iodinated contrast media.

LEARNING OBJECTIVE \#2: Understand the treatment options for thyroid storm in relation to the pathophysiology of thyrotoxicosis.

CASE: A 68 year-old woman with history of hyperthyroidism developed tachycardia, fever and altered mental status after percutaneous transluminal cardiac angioplasty (PTCA). She initially presented to the emergency room with abdominal pain, shortness of breath, shoulder pain and vomiting for 1 day, with no fever. She appeared cachectic and had proptosis, an enlarged and smooth thyroid, and warm, moist skin, with no edema. EKG revealed ST elevations in II, III, and aVF. She underwent PTCA with placement of 3 drug-eluting stents to the right coronary artery. Six hours later, she was delirious and tachypneic, requiring intubation. Family members revealed her hyperthyroidism had been diagnosed years prior, however she was nonadherent to treatment. Heart rate was 142 beats per minute, temperature was $102.5^{\circ}$ Fahrenheit, and capillary blood glucose was $179 \mathrm{mg} / \mathrm{dL}$. There was no acute pathology on Computed Tomography of the head without contrast. White blood cell count was $15.6 \mathrm{k} / \mu \mathrm{l}$. TSH was $<.005 \mathrm{microU} / \mathrm{mL}$ and free T4 was $7.08 \mathrm{ng} / \mathrm{dL}$ (normal $0.8-1.7 \mathrm{ng} / \mathrm{dL}$ ). Thyroid stimulating immunoglobulin index (TSI) was $454 \%$ (normal $<140 \%$ of baseline). She was diagnosed with thyroid storm in the setting of longstanding, uncontrolled Graves' disease (autoimmune hyperthyroidism), and treated with hydrocortisone, metoprolol and methimazole followed by potassium iodide drops. Agitation resolved the next day and she was extubated. Two days after treatment, she briefly developed atrial fibrillation with rapid ventricular response (heart rate $150 \mathrm{~s}$ ) which ceased with uptitration of metoprolol. She regained her baseline mental status over the next 2 weeks.

DISCUSSION: Thyroid storm is a rare and life-threatening condition characterized by symptoms of severe thyrotoxicosis including tachycardia, hyperpyrexia and altered mentation. In addition to high levels of T4, T3 and low TSH, laboratory abnormalities can also include mild hypercalcemia, hyperglycemia, elevated liver tests, and high or low white blood cell count. In patients with a history of hyperthyroidism, thyroid storm may be precipitated by trauma, infection, surgery, or iodine load - such as the iodinated contrast media used during cardiac catheterization in our patient. Autonomy of thyroid function from the normal feedback loop by which high levels of T3 and T4 inhibit TSH is key to the mechanism of iodine-induced thyrotoxicosis. In Graves' disease, activating antibodies against TSH receptors on the thyroid gland make the gland independent of stimulation from native TSH. When the hyperfunctioning thyroid tissue encounters an excess of iodine, the substrate for thyroid hormone formation, the ensuing increase in production of thyroid hormone can lead to a hypermetabolic state with mortality rates of 10-30\%. Patients with thyroid storm require ICU monitoring. Treatment includes thionamides to inhibit new thyroid hormone synthesis followed by iodine to block thyroid hormone release, glucocorticoids, beta-blockers, and bile acid sequestrants. 
THE RARE CASE OF INFECTIOUS THYROIDITIS AFTER ROOT CANAL THERAPY Calvin C. Tran; Diane L. Levine. Wayne State University, Detroit, MI. (Control ID \#2466648)

LEARNING OBJECTIVE \#1: To recognize the difference between subacute thyroiditis and infectious thyroiditis

LEARNING OBJECTIVE \#2: To identify the possible etiology of infectious thyroiditis CASE: A previously healthy 23 year old man presented to the ED with chief complaint of neck pain and swelling. Approximately 1 week before admission, he has an upper respiratory infection that resolved uneventfully. 5 days before admission, the patient went to his dentist and have a root canal treatment started on the lower left molar. Over the nex several days, he experienced neck pain, swelling and discomfort with increasing odynophagia. He finished his root canal treatment on day 3 before admission. After which, he developed fever, increased neck swelling and malaise. His mother was worried and brought him in for evaluation. Physical exam reveal a tired young man with fluctuant left neck swelling and pain with no submandibular swelling or exudate. Laboratory work up reveal decreased TSH, elevated T4. He was preliminary diagnosed with subacute thyroiditis. However, CT scan revealed a cystic structure with enhancing capsule within the thyroid. Ultrasound guided needle aspiration showed purulent material. Gram stain and cultures reveals streptococcus anginosus. His diagnosis was changed to infectious thyroiditis. He underwent incision and draining and was treated with ampicillin/ sulbactam.

DISCUSSION: Infectious thyroiditis is an uncommon condition. There are only less than 100 cases reported in the literature. The distinguishing feature is that in infectious thyroiditis, radiographic evidence can suggest an abscess formation. Fever, elevated white blood count can also present. Biopsy of infectious thyroiditis in this patient shows fibrinopurulent exudate with necrosis and abscess formation. Biopsy of a subacute thyroiditis would have shown multinucleated giant cells. The etiologies of thyroid abscess are few. The most common cause is congenital esophageal or tracheal fistula that exposes the thyroid gland to a body cavity. This patient however, does not have CT or ultrasound evidence of a fistula present. The second most common etiology is hematogenous spread in an immune-compromised individual. This patient was a previously healthy male with no evidence of HIV or immunocompromised conditions. The only significant event in his HPI was a resolved URI and having a tooth treated with root canal therapy. The case of thyroid abscess from an odontogenic origin has never been reported in the literature. It is our hypothesis that during and after his root canal therapy, there is bacteremia and thus seeding the thyroid gland with streptococcus anginosus, a resident of a normal oral flora Although the current recommendation does not require antibiotic prophylaxis before root canal therapy, regimen may have to be considered if there is high risk of bacteremia.

THE RED RASH, THE RED HERRING Ashley R. Dahl. Mayo Clinic, Rochester, MN. (Control ID \#2469711)

LEARNING OBJECTIVE \#1: A simple foot abscess resulting in postinfectious glomerulonphritis can be mistaken as Henoch-Scholein Purpura due to their overlapping symptoms. This is where a clinician's history taking and data gathering skills are vital as treatments greatly differ.

LEARNING OBJECTIVE \#2: IgA deposition found on skin biopsy is a non-specific finding.

CASE: The patient is a 54 year old man with past medical history significant for diabetes mellitus type 2, hypertension, hyperlipidemia (all three resolved after gastric bypass in 2010), left Charcot foot status post multiple surgeries, chronic osteomyelitis, bilateral second toe amputations, chronic lower extremity lymphedema, bilateral neuropathy in lower extremities who was admitted the hospital from an outside institution for new onset: anemia, cough, diffuse petechial rash, bilateral upper extremity edema, acute kidney injury, and diarrhea. Three weeks prior to admission, the patient experienced discomfort in his left foot and calf. Another institution ruled out a DVT and a foot X-ray was indeterminate. He completed a 10-day Clindamycin course for question of infection. He followed up with his podiatrist and an MRI revealed chronic osteomyelitis versus new abscess. The rash, cough, and upper extremity edema started 1 week prior to admission without an obvious trigger. The non-pruritic and nonblanching rash started on the dorsum of his hands and over 1 week involved his arms, flanks, and legs. The edema also started in his hands and extended to the mid-humerus bilaterally. The cough was nonproductive with no shortness of breath or increased work of breathing. The diarrhea had persisted for 2-6 weeks. No hematochezia or melanotic stools. The only source of blood loss was the chronic seepage of serosanguineous fluid from ulcer on ventrum of left foot. On admission, pertinent physical exam findings included: $2+$ pitting edema bilateral upper extremity through mid-humerus, mild edema bilateral lower extremities, diffuse petechial rash (non-blanching, flat, discreet and coalescing lesions involving hands, palms, arms, flanks, lower extremities). Two ulcers on left foot, ventral and dorsal aspects; weeping serosanguineous and purulent matter respectively. No evidence of active synovitis. Initial complete blood count (cbc) showed a hemoglobin of 7.5 (baseline 13), platelets of 136, leukocytes of 14.0, INR of 1.5, DDimer 3460, Fibrinogen 177, PT 17.1, aptt 44, Potassium 2.7, Creatinine 3.7 (baseline 1.1), Albumin 1.5, Chloride 118, Bicarb 7, CRP 57.5, Urinalysis: hemoglobin large protein, Renal Epithelial Cells 1-3, Red Blood Cells (RBCs) 11-20, <25\% dysmorphic RBCs. ABG: pO2 104, pCO2 21, pH 7.23, HCO3 9. Peripheral smear: schistocytes and helmet cells As the laboratory data returned, thrombocytopenia, anemia, compensated disseminated intravascular coagulation (DIC), and renal failure were seen. After admission to the floor, the dorsal foot ulcer broke open in an eruption of purulent, malodorous drainage. Day 1, the diffuse petechiae started transforming into palpable purpura and a skin biopsy stained highly positive for IgA. The concern for IgA Vasculitis and/or Henoch-Schonlein Purpura (HSP) was heightened due to the skin biopsy, gastrointestinal symptoms, acute renal failure, and pulmonary involvement (new cough). A renal biopsy was scheduled and $750 \mathrm{mg}$ methylprednisolone initiated for treatment of probable HSP. Day 2, the patient remained stable and underwent renal biopsy. Initial biopsy weakly stained for IgA. HSP dropped down the differential and we investigated other causes. He continued to require transfusions secondary to DIC, his renal function continued to worsen, and we consulted orthopedic infectious disease for possibility of antibiotic initiation for his abscess. Upon further review of the history, the consultant initiated treatment for the ulcerated abscess, not the chronic osteomyelitis. The final renal biopsy revealed postinfectious glomerulonephritis (PIGN) most likely secondary to the foot abscess. Source control could only be gained via a below the knee amputation. After the amputation, his hematologic abnormalities normalized, requiring fewer transfusions and his coagulopathies also normalized. His renal function, purpuric rash, diarrhea, and cough had also improved by discharge to a rehabilitation facility. DISCUSSION: Satoskar, et al's paper, Henoch-Schonlein purpura-like presentation in IgA-dominant Staphylococcus infection-associated glomerulonephritis- a diagnostic pitfall, published January, 2013 in the Journal of Clinical Nephrology, outlined eight case studies between 2004 and 2011 with PIGN and the associated purpuric skin rash. The article illustrates the importance of distinguishing HSP and PIGN-picture as the treatments greatly differ and the incorrect treatment can cause harm. IgA staining on a skin biopsy is a nonspecific finding. In our case, the presentation was so consistent with HSP that we started treating for HSP based on biopsy and clinical picture prior to having all of the necessary information. The erupted foot ulcer was a minor part of the history rather than an obvious contributing aspect of his disease process. Upon reflection, purulent drainage from the ulcer should have played a more central role in the discussion of pathologic etiologies. As we learned in medical school, and are frequently reminded, the patient will tell you the diagnosis, just listen to the history. 
THE RIGHT HEART IN METASTATIC RENAL CELL CARCINOMA Virginia O. Volpe; Andrew P. Scatola. University of Connecticut School of Medicine, Farmington, CT. (Control ID \#2467861)

LEARNING OBJECTIVE \#1: Recognize alternative presentations of late occurring metastatic renal cell carcinoma

LEARNING OBJECTIVE \#2: Appreciate rare metastases in renal cell carcinoma CASE: A 58 year old man with no prior past medical history presented with right thigh pain to the emergency department. He was in his usual state of health until 4 months previously when this pain began to develop. Imaging of his leg on presentation revealed hyperlucent areas of his femur consistent with lytic lesions, concerning for metastatic disease, which prompted a CT of the chest, abdomen and pelvis. This showed a large left kidney mass measuring $12.3 \times 8.1 \times 13.5 \mathrm{~cm}$ and multiple nodules in his lung. IR guided biopsy of a destructive rib lesion indicated a poorly differentiated renal cell carcinoma. CT of the chest suggested cardiac metastases involving the right atrium and right ventricle, which were confirmed by echocardiography. Surprisingly, despite the intracardiac masses, there was no inferior vena cava involvement. While the right ventricular mass did show evidence of outflow tract obstruction, he was minimally symptomatic experiencing only lower extremity edema. Due to the size and location of the heart masses, they were deemed inoperable. He did undergo successful stabilization and treatment of the lytic lesions in his femur with cementing and radioablation. He received palliative radiation to multiple areas and subsequently was trialed on temsirolimus. Unfortunately, he continued to have progression of his disease with worsening bilateral malignant pleural effusions and continued to decline, at which point, he decided to transition to hospice care.

DISCUSSION: Metastatic renal cell carcinoma carries a poor prognosis with a median survival of 6-12 months and a 5-year survival of less than $10 \%$. The classic triad of hematuria, flank pain, and flank mass, occurs in only a minority of patients, especially as more cases of renal cell carcinoma are detected incidentally by imaging. Approximately $30 \%$ of patients will present with metastatic disease, of which, only $20 \%$ are bone metastases. Therefore, our patient's initial complaint of right thigh pain is an unusual presenting symptom. Cardiac metastasis is a rare complication of renal cell carcinoma, occurring in $5-15 \%$ of patients and is most commonly associated with clear cell carcinomas and rarely occurring in non-clear cell RCCs. Cardiac metastases rarely cause symptoms and when they do, they tend to be from outflow tract obstruction. Spread to the right atrium and ventricle is seen via contiguous spread of the inferior vena cava. However, there are rare reports of cardiac metastatic spread without macroscopic involvement of the IVC, like in our presented case. When this occurs, it has been theorized that there is microscopic hematogenous spread or lymphatic spread via the pulmonary and carinal nodes. Treatment is traditionally by nephrectomy, which can be limited by tumor stage and anatomic location. In metastatic RCC, three classes of systemic drugs are used: VEGF pathway inhibitors, cytokines, and mTOR inhibitors. Adjuvant therapies, thus far, have not shown significant benefit, however, further studies are being conducted. Studies of new treatments, such as immune checkpoint blocking agents and PI3K pathway inhibitors, are underway and already showing promise.

THE TELL-TALE HAND Vishnu Ganesan ${ }^{1}$; Neil Mehta ${ }^{2} .{ }^{1}$ Cleveland Clinic, Cleveland, $\mathrm{OH} ;{ }^{2} \mathrm{Cleveland}$ Clinic Lerner College of Medicine, Cleveland, OH. (Control ID \#2466770)
LEARNING OBJECTIVE \#1: Recognize typical and atypical clinical manifestations of a CNS tumor

LEARNING OBJECTIVE \#2: Identify clinical details from the history and imaging characteristics that should raise the suspicion for a CNS lymphoma.

CASE: A 73-year-old male presented to our clinic with a 1 day history of his handwriting getting smaller. A week prior, his wife had observed slowing of speech and word-finding difficulties. The patient had been seen twice in clinic in the last month for increasing lethargy and fatigue which was attributed to his depression. There was an associated slight weakness in the right arm and a complaint of morning occipital headaches . The patient denied any other concerns on review of systems. Past medical history was significant for well-controlled Type 2 Diabetes, hyperlipidemia, coronary artery disease, migraines, and ulcerative colitis. In addition, there was a distant history of low-grade bladder cancer, which had been resected. He was free of disease at most recent surveillance visit. His medications at home included infliximab, metformin, sertraline, pantoprazole, and pravastatin. There was a family history of stroke in his mother. On examination, his blood pressure was very elevated at 147/108. There was asymmetry of the smile and decreased sensation on the right face. There was weakness of the intrinsic muscles of the right hand and slowed finger-tapping but otherwise normal strength throughout. Examination of reflexes showed a positive Babinski on the right. The gait was tentative and unsteady, favoring the left side. At this point the suspicion was for a left MCA stroke and the patient was transferred to the ED for further evaluation. The patient received a CT head and a CT angiography which showed a questionable and subtle hypodensity in the left thalamocortical region. A MRI with contrast revealed an enhancing mass in the left basal ganglia extending to the cerebral peduncle with the suspicion of a lymphoma vs. glioma. A FDG whole body PET/MRI did not show other areas of focal uptake. The patient underwent two low volume lumbar punctures with cytology to evaluate for lymphoma and infections, which were unrevealing. A stereotactic biopsy of the enhancing brain mass was performed, demonstrating glioblastoma, WHO Grade IV.

DISCUSSION: This clinical vignette demonstrates how the presentation of a central nervous system (CNS) tumor in the outpatient setting can be varied making the diagnosis challenging. Typical symptoms commonly seen in patients with gliomas are headaches (53$57 \%$ ) and seizures (23-56 \%). The more atypical symptoms include memory loss (36\%), personality changes $(23 \%)$, and cognitive changes $(34 \%)$. Deep seated CNS tumors such as those near the thalamus, as in our case, are particularly challenging because they interfere with multiple neural pathways. Motor deficits are common due to the proximity to the internal capsule and while the masses are close to basal ganglia, development of tremors or other involuntary movements are infrequent. Involvement of the mammillothalamic fibers or fornices can lead to changes in cognition and memory while involvement of the optic tracts and the lateral geniculate body lead to changes in vision. Interestingly, when compared to patients with unilateral strokes in the same location as tumors, the cognitive impairments resulting from tumors tended to be milder. While the presenting complaint in our patient was difficulty with writing secondary to weakness limited to his distal upper extremity, the patient had previously reported increasing lethargy, fatigue, and cognitive slowing in the weeks to month prior. These other symptoms were initially thought to be due to depression but in retrospect they were likely the result of the expanding tumor. On the basis of the findings on CT, MRI, and PET-CT, the differential diagnosis for this patient includes both neoplastic and non-neoplastic causes. Of neoplastic causes the main diagnostic consideration was between a primary CNS lymphoma and high-grade glioma. A primary CNS lymphoma is rare, representing $4 \%$ of CNS tumors. However it was highly suspected in our case because of location, imaging characteristics, presenting symptoms, and the patient's immunocompromised state (on infliximab for IBD). The most common site for a primary CNS lymphoma is periventricular (i.e thalamus, basal ganglia, and corpus callosum) and on imaging it shows iso- to hypertense on T2 weighted MRI which enhances homogeneously with contrast. Furthermore, behavior and cognitive changes are the most common presenting symptom 
occurring in approximately two thirds of patients. Deep seated CNS tumors represent a clinical challenge given the diverse presentations, delays in diagnosis, and late treatment initiation. Primary care physicians should keep these in mind as they see patients with suspected depression and cognitive impairment. A thorough history taking and neurologic examination can lead to earlier diagnosis and initiation of appropriate treatment. Neuroimaging should be considered in patients who experience new onset of depressive symptoms without obvious cause and without a prior history of depression.

THE UGLY TRUTH OF SAGLIKER SYNDROME Kaylee J. Shepherd ${ }^{1}$; Blake R. Barker ${ }^{2}$. ${ }^{1}$ UT Southwestern, Dallas, TX; ${ }^{2}$ UT Southwestern Medical Center at Dallas, Dallas, TX. (Control ID \#2469637)

LEARNING OBJECTIVE \#1: Recognize the clinical features of Sagliker Syndrome (SS). LEARNING OBJECTIVE \#2: Recognize the importance of early diagnosis and treatment of ESRD to slow the progression of SS.

CASE: A 23-year-old female with history of end stage renal disease (ESRD) of unknown etiology on emergent dialysis since the age of 18 , secondary hyperparathyroidism, seizures, chronic respiratory failure with a tracheostomy was admitted to the ICU for pneumonia, requiring ventilator support and continuous renal replacement therapy. Her exam was particularly notable for short stature with height of $3 \mathrm{ft}$ and a very thin and distorted trunk and extremities due to frequent fractures. She was wheelchair bound and unable to ambulate. Her head comprised approximately $1 / 3$ of her body mass with severe maxillary and mandibular bony enlargement. Her oral exam revealed extensive tooth loss and bleeding gums. Her appearance was distinctly different from pictures her family shared from just 3 years prior. In addition, her lab values showed substantial abnormalities including PTH $>5000 \mathrm{pg} / \mathrm{mL}$, calcium $7 \mathrm{mg} / \mathrm{dL}$; phosphorous $2 \mathrm{mg} / \mathrm{dL}$, vitamin D-25-OH $9.8 \mathrm{ng} / \mathrm{dL}$, and alkaline phosphatase 827 units/L. This pattern of physical exam findings and extreme abnormalities was felt consistent with the unusual disorder, Sagliker Syndrome. Her secondary hyperparathyroidism was refractory to optimal medical management, and her hospital course was further complicated by endocarditis, right atrial thrombus, and inability to be weaned off of the ventilator. After nearly 90 days in the hospital, she developed acute neurologic changes, and CT head showed massive frontal lobe hemorrhage. The family ultimately decided to withdraw care. DISCUSSION: Yahya Sagliker, M.D. described Sagliker Syndrome (SS) in 2004. By 2012, only 60 cases had been recognized, however, Sagliker estimates the observed incidence to be near $0.5 \%$. Sagliker described this special population of patients with ESRD with an "uglifying" facial appearance, short stature, severe maxillary and mandibular changes, finger abnormalities, dental abnormalities, soft tissue tumors of the mouth, and neurologic and psychiatric symptoms. These patients are most often children who had a delay in treatment of renal insufficiency and are most frequently seen in underdeveloped countries. It has also seen in adults related to a severe form of renal osteodystrophy with parathyroid hyperplasia leading to lab abnormalities, as seen in our patient. These patients suffer from severe peripheral and central nervous system complications from the chaotic bone growth, including spinal cord compression, deafness, blindness, cortical deficits, as well as respiratory failure due to thoracic bone involvement. Vascular and neurologic impairment are the leading causes of death. While there is no treatment and it is unclear why certain patients develop this syndrome, a small study concluded that SS should be added to indications for total parathyroidectomy as this treatment has improved psychiatric symptoms and lab abnormalities. However, it could not reverse the skeletal malformations. Ultimately, there is no treatment beyond slowing the progression of the disease with optimal medical management.

THE UNEXPECTED HEARTBREAK: DISSECTING AN UNUSUAL CASE OF ACUTE CORONARY SYNDROME Rasheen Imtiaz; Sarathi Kalra; Nadeen Faza; Mark Pollet; Nasser Lakkis. Baylor College of Medicine, Houston, TX. (Control ID \#2456994)
LEARNING OBJECTIVE \#1: Recognize coronary artery dissection as an etiology of acute coronary syndrome

LEARNING OBJECTIVE \#2: Recognize that women with history of peripartum coronary artery dissection are at risk for recurrence and should avoid future pregnancies CASE: A 27 year-old gravida 3, para 3 African American woman with a history of delivery by cesarean section 2 weeks ago presented to the emergency department with chest pain. She was in her usual state of health when she developed severe, burning, sub-sternal chest pain at rest associated with dyspnea, diaphoresis and nausea, lasting over $30 \mathrm{~min}$. She denied pleurisy, hemoptysis, leg swelling or pain, radiation of pain to the back and symptoms of reflux disease. On evaluation, she was afebrile with a blood pressure of 109/30 $\mathrm{mmHg}$, heart rate of 105 beats/min, respiratory rate of 19 , normal oxygenation and body mass index of 29 . She was in moderate distress due to chest pain but the remainder of her examination including cardiac and pulmonary examination, was normal. An electrocardiogram showed ST segment elevation in leads V3 to V6 consistent with anterolateral ST-elevation myocardial infarction (STEMI). An initial troponin was $1.7 \mathrm{ng} / \mathrm{mL}$. She has immediately taken for cardiac catheterization which revealed significant coronary artery dissection involving the proximal and middle left anterior descending artery (LAD), the ostial left circumflex, and the first and third obtuse marginal arteries. She received multiple drug-eluting stents in the affected coronary arteries. Echocardiogram revealed an ejection fraction of $35 \%$ with wall motion depression in the anterolateral walls. She had a negative urine drug screen, antinuclear antibody and anti-neutrophil cytoplasmic antibodies. During her hospital course, her troponin peaked at $148 \mathrm{ng} / \mathrm{mL}$ and creatine kinase at $4266 \mathrm{IU} / \mathrm{mL}$. Her chest pain remained resolved after cardiac stenting and cardiac enzymes decreased at which time she was discharged with close follow-up.

DISCUSSION: Acute coronary syndrome (ACS) is a routinely encountered diagnosis for internists. However, ACS in young patients without usual risk factors can be puzzling. Spontaneous coronary artery dissection (SCAD) is defined as a non-traumatic and noniatrogenic separation of coronary arterial walls resulting in an intraluminal hematoma that compresses the true lumen of the artery, compromising blood supply. Most commonly, a single artery, usually the LAD is affected. SCAD can result in morbidities including ACS, ventricular arrhythmias and even sudden death. Risk factors include peripartum state (most common precipitating factor in women), multiple pregnancies, hormonal therapyparticularly progesterone, extreme physical activity (most common factor in men) and diseases that predispose arterial walls to injury such as fibromuscular dysplasia, connective tissue disorders and systemic inflammatory disorders like lupus or various vasculitides. Those with acute presentations including ACS, ongoing chest pain or hemodynamic instability are recommended to undergo percutaneous coronary intervention. Emergency coronary artery bypass surgery must be considered in patients with dissections of the left main coronary artery. In the long-term, there is a significant event recurrence rate of nearly $20 \%$ in once-affected individuals. Peripartum patients must be advised to avoid future pregnancies. In summary, SCAD is a rare, under-recognized but important cause of ACS. Awareness of this condition is important because the management may differ.

THE UNUSUAL FATAL PRESENTATION OF COCAINE LEVAMISOLE INDUCED VASCULITIS Ajay Kumar ${ }^{1}$; Kulsoom Fatima ${ }^{2}$; Etleva Bejko ${ }^{2} .{ }^{1}$ St. Vincent Charity Medical Center, Cleveland, OH; ${ }^{2}$ St. Vincent Charity Medical Center, Cleveland, OH. (Control ID \#2467865)

LEARNING OBJECTIVE \#1: Cocaine use have been associated with spectrum of diseases. Approximately $70 \%$ of cocaine consumed in USA is contaminated with Levamisole, an immunomodulating agent, which attributed to increase profit and volume along with enhanced euphoria and stimulatory effect of cocaine by increasing the dopamine and producing amphetamine like metabolites. Cocaine mixed with Levamisole poses 
risk of vasculitis when consumed for a long time which have a range of different clinical manifestation among which most common are arthralgias $(83 \%)$, cutaneous lesions (61\%) and Hematological (Leukopenia—-28\%). There are also multiple rare findings associated with cocaine induced vasculitis including abnormal urinalysis, glomerulonephritis and pulmonary hemorrhages ( 3 cases). We now present a rare case of Cocaine induced vasculitis resulting in intracranial pathology.

CASE: A 64 Year old morbidly obese female with PMHx of COPD, CAD, Hypertension, OSA, Hyperlipidemia and Renal cell carcinoma (s/p R Nephrectomy) brought to the ER by EMR in an unresponsive state for $>6 \mathrm{~h}$, GCS Score of $3 / 15$ with BP 180/89 $\mathrm{mmHg}$ otherwise vitals stable and non significant physical exam findings. She was admitted to ICU under critical care team with close monitoring. Blood work on admission have no significant finding except for leukocytosis (SIRS 2/4) with no identified source of infection with normal procalcitonin levels. Stat CT Scan and later MRI was ordered which showed multiple lesion mostly on watershed areas suggesting infarcts vs brain mets. Neurology and cardiology were consulted. MRA was performed which ruled out any thrombosis. Cardiac ECHO also showed no evidence of thrombus or vegetations. EEG reported continuous generalized slowing, no focal slowing, no seizure and no asymmetry. Her urine tox was + ve for Cocaine. Her initial presentation of unresponsiveness led to limited rhematological tests. Her basic rheumatological panel showed ANA positive 1:160, normal Complement $\{\mathrm{C} 3-126 \mathrm{mgs} / \mathrm{dl}(90-180 \mathrm{mg} / \mathrm{dl} \mathrm{NR})$ and C4 $-25.2 \mathrm{mgs} / \mathrm{dl}(10-40 \mathrm{mgs} / \mathrm{dl})\}$ She was also found to be + ve for pANCA $(1: 120)$. She was out of the window for levamisole testing so it was deferred. Considering the initial rheumatological panel and positive cocaine thus the diagnosis of cocaine induced vasculitis was made by the rheumatologist and other specialists. With respect to cocaine levamisole induced vasculitis she was started on IV steroid therapy which is the mainstay of the treatment She showed a favorable response with an initial neurological recovery but later deteriorated with time. Considering her guarded prognosis her code status was discussed with her family and was changed. She was extubated on day 13th and care was transferred to hospice. She died on 17 th day of event and 4 th day post-extubation DISCUSSION: Cocaine-levamisole vasculopathy is classified as small vessel vasculitis. Though the cutaneous and musculoskeletal are the most common presentation of Cocaine-Levamisole induced vasculitis but the spectrum of disease associated are still under observation. Typical cutaneous lesions include but not limited to retiform purpura of the trunk (most common, mostly involving lower extremities, $84 \%$ ), ear purpura $(73 \%)$ and lichenoid. Whereas most common musculoskeletal manifestation is arthralgias and hematological features include neutropenia and agranulocytosis. Other common non specific features which makes $72 \%$ include low grade fever, night sweats and weight loss. A case series of 4 patients also suggested association with pauci-immune vasculitis. One of the case series also suggested a female predominance of about $81 \%$. Typical lab findings suggested by multiple case reports are ANCA positivity more specifically p-ANCA $(88 \%)$ which our patient had. Other suggested findings are presence of anti-MPO and anti-PR3 (50\%) positivity. The half life of levamisole is $\sim 5.6 \mathrm{~h}$. Multiple case reports showed that test was positive in less than $50 \%$ patients, which makes positive labs a challenge. Thus multiple authors have suggested that not all rheumatogical results are not required to be positive if clinical and basic rheumatological panel are suggestive of pathophysiology related to cocaine-levamisole induced vasculopathy. The recommended treatment is steroids along with other immunomodulating agents, choice of drug is still on the discretion of physician and needs to be further studied. On the basis of the above discussion I strongly suggest our case of "Cocaine-Levamisole Induced Vasculitis".

THE USE OF HIGH DOSE INSULIN EUGLYCEMIA THERAPY IN CALCIUM CHANNEL BLOCKER AND BETA BLOCKER OVERDOSE Mohammed Qureshi ${ }^{1}$; Gerardo Catalasan ${ }^{2} .{ }^{1}$ Texas Tech University Health Science Center, Odessa, TX; ${ }^{2}$ Midland Memorial Hospital, Midland, TX. (Control ID \#2469913)
LEARNING OBJECTIVE \#1: Manage calcium channel blocker and beta blocker overdose in a critically ill patient with High Dose Insulin-Euglycemia Therapy

CASE: A 48-year-old male with history of Diabetes Mellitus type 2 and Hypertension was found unresponsive after overdosing on unknown number of Carvedilol, Amlodipine, Hydralazine, Hydrochlorothiazide, Lisinopril, and Doxazosin tablets. In ED, patient was unresponsive with BP of 53/21 and pulse of $89 \mathrm{bpm}$. Initial labs revealed only early sign of acute kidney injury. Urine drug screen was normal. Initial Troponin I was mildly elevated. EKG showed junctional tachycardia with no ST abnormalities. Chest x-ray was consistent with cardiomegaly and pulmonary edema. Lactate was elevated. Echo showed a moderately dilated left ventricle with bi-atrial enlargement but with grossly normal systolic function. Patient was intubated for airway protection and had BP of 49/29 immediately afterwards. He was given IV fluids, norepinephrine, dopamine, vasopressin and epinephrine. He received activated charcoal, Calcium Chloride, Glucagon Sodium Bicarbonate and intravenous Lipid infusion. Even with conventional resuscitation patient had profound refractory shock with bp 55/24. High dose Insulin Eugycemia Therapy (HIET) was initiated 90 min after presentation with $135 \mathrm{U}$ regular insulin bolus $(\sim 1 \mathrm{U} / \mathrm{kg})$ and continuous infusion at $300 \mathrm{U} / \mathrm{h}(2 \mathrm{u} / \mathrm{kg} / \mathrm{h})$ with dextrose to maintain euglycemia. Insulin was titrated to a maximum $900 \mathrm{U} / \mathrm{h}(\sim 6 \mathrm{U} / \mathrm{kg} / \mathrm{h})$ over $3 \mathrm{~h}$, with hemodynamic stabilization (BP 91/54) occurring thereafter. This infusion rate is larger than current HIET protocols. Cardiology was consulted and an Intra-aortic Balloon Pump (IABP) was placed. Patient further improved with bp of 100/76 within $6 \mathrm{~h}$ of initiation of HIET, enabling titration off lipid emulsion infusion, calcium chloride, vasopressin and epinephrine. HIET was discontinued after $36 \mathrm{~h}$ of infusion with no hypoglycemic episodes. Unfortunately, patient suffered from iatrogenic dissection of the left superficial femoral artery during placement of femoral central line on admission, leading to blood loss, limb ischemia, and prolonging vasopressor requirements. Calcium channel blocker (CCB) and betablocker (BB) overdose was likely not contributing to the ongoing shock as half-life of these medications is 30 and $10 \mathrm{~h}$, respectively. IABP was removed by the 8 th ICU day, all vasopressors were titrated off by the 9th ICU day, and patient self-extubated by the 10th ICU day with full neurologic recovery. By the 17th ICU day as CT abdomen showed retroperitoneal bleeding from the Left iliac artery, a sequela of femoral arterial dissection. Patient eventually expired secondary to hemorrhagic shock by the 18 th ICU day.

DISCUSSION: $\mathrm{BB}$ and $\mathrm{CCB}$, aside from their cardiovascular affects, block the secretion of insulin from the beta cells of the pancreas causing a hypoinsulinemic state. During circulatory collapse, the myocardium metabolizes glucose preferentially over free fatty acid as the primary energy source. Acute $\mathrm{CCB} / \mathrm{BB}$ intoxication causes myocardial glucose dependence and hypoinsulinema, causing ATP depletion and cardiogenic shock. Insulin allows the myocardium to uptake and use serum glucose as its primary energy source, increases cytosolic calcium within cardiac myocytes thus increasing inotropy, and promotes afterload reduction to maximize cardiac output. It increases myocyte cytosolic calcium levels by activating Ca-ATPase and allows the myocardium to uptake serum glucose to utilize aerobic respiration. Insulin activates endothelial Nitric Oxide synthase (eNOS), allowing for vasodilation of coronary arteries and systemic arterioles, reducing afterload and increasing cardiac output in cardiogenic shock. High dose insulin infusion was first tested on dogs poisoned with verapamil in the 1990s. Success in animal models led to the pioneer case study conducted by Yuan in 1999, where 5 patients with cardiogenic shock secondary to CCB overdose were succesfully treated with insulin infusion as high as $1 \mathrm{U} / \mathrm{kg} / \mathrm{h}$. Thus, the initial recommendation was " $0.5 \mathrm{U} / \mathrm{kg}$ regular insulin bolus, followed by a $0.5-1 \mathrm{U} / \mathrm{kg} / \mathrm{h}$ continuous infusion" with concern that higher doses may lead to hypoglycemia and electrolyte imbalances. Since its first introduction in 1999, multiple case reports and case series have been published using the aforementioned protocol with relative success. St-Onge in 2014 published a systematic review of the use of HIET in $\mathrm{CCB} / \mathrm{BB}$ overdose and found two observational studies five case series using Yuan's HIET protocol. Only one case series in 
2011 by Holger used infusion rates greater than the traditional $1 \mathrm{u} / \mathrm{kg} / \mathrm{h}$-with average insulin infusion $8.35 \mathrm{U} / \mathrm{kg} / \mathrm{h}$ in 12 patients admitted for $\mathrm{CCB} / \mathrm{BB}$ overdose with eleven of twelve surviving. Cases implementing higher than original protocols are exceedingly rare and the optimal dose of insulin in HIET remains unclear. Our patient received a maximum infusion rate of roughly $6 \mathrm{u} / \mathrm{kg} / \mathrm{h}$, with no significant adverse effects with excellent cardiovascular recovery prior to his demise from iatrogenic causes. We believe it is imperative to make known such case reports demonstrating higher infusion rates with minimal adverse effects to adjust protocols regarding HIET. As more data such as our case report among others become available, we anticipate a change in guidelines, as there is no universally accepted protocol for HIET, and no official position statement by the American College of Medical Toxicology (ACMT) to date.

THE WAY TO A PERSON'S HEART MAY REALLY BE THROUGH THEIR STOMACH Jessica Berman; Jane Andrews. Tulane University Health Sciences Center, New Orleans, LA. (Control ID \#2467202)

LEARNING OBJECTIVE \#1: Recognize alternative explanations for elevated troponin levels in patients with no clear cardiac etiology.

LEARNING OBJECTIVE \#2: Understand the importance of neuroendocrine tumor production of bioactive amines and effects on the heart.

CASE: A 56 year-old woman presented with a 2-day history of decreased intake and weakness. She also reported two episodes of diarrhea the day of admission and a 1-week history of abdominal pain for which she had seen her primary care physician 4 days prior to admission. Vital signs were within normal limits. On physical exam, liver edge was noted to be beyond the costal margin. She had mild tenderness to palpation in the epigastric region of her abdomen. Her troponin I was elevated to 0.180 on admission and increased to 0.441 before trending down. Cardiac workup included an EKG, which showed no acute ischemic changes, an echocardiogram that showed normal left ventricular function and ejection fraction with diastolic dysfunction. Her consequent nuclear stress test was classified as negative and a low risk study for major cardiac events. A right upper quadrant ultrasound ordered by her primary care physician and done prior to admission revealed a heterogeneous liver and a gall bladder filled with echogenic material possibly sludge. A triple phase CT was obtained while she was an inpatient to further clarify these findings. The CT revealed five heterogeneous contrast-enhancing lesions in the liver with disruption in the gallbladder wall. The largest liver lesion measured $10.1 \mathrm{~cm} \times 8.3 \mathrm{~cm}$ and there was mild intrahepatic biliary dilation. Subsequently, a liver biopsy was performed. The pathology revealed a poorly differentiated neuroendocrine carcinoma with a very high proliferative index. (Ki67 95\%). Her serum serotonin was $3039 \mathrm{ng} / \mathrm{mL}$ (normal range: 0-420 ng/mL) and chromogranin-A was $670 \mathrm{nmol} / \mathrm{L}$ (normal range: $0-5 \mathrm{nmol} / \mathrm{L}$ ).

DISCUSSION: The internist commonly encounters patients who present with nonspecific symptoms and are found to have an elevated troponin. In this case, the patient had a cardiac workup that negative for evidence of ischemia though was found to have a neuroendocrine tumor (NET). The well-established non-cardiac causes of an elevated troponin include cerebrovascular accident, critical illness, COPD, myocarditis, cardiomyopathy, congestive heart failure, sepsis, pulmonary embolism, or rhabdomyolysis. There is no recognized connection between NETs and an elevated troponin, however it is an area for future research. New tools could be useful for diagnosing NETs because they most often are found incidentally and if not, they can present with nonspecific symptoms. The incidence of NETs has been increasing over the past three decades and these patients are at a higher risk of developing additional malignancies, up to $52 \%$ of patients with NET develop a second primary tumor. The rate of incidence of NETs is estimated at about 2.47 4.48 per 100,000 people, highest incidence in African-American men. About $70 \%$ of NETs are found in the GI tract and $25 \%$ are found in the respiratory system. NETs are a very heterogeneous group of neoplasms and are typically separated into two groups: well differentiated and poorly differentiated. Those that are well differentiated are more likely to secrete vasoactive substances and are typically slow growing, while those that are poorly differentiated are less likely to secrete and tend to be more aggressive. As previously mentioned, some of these tumors, depending on their location, can produce biogenic amines, e.g. serotonin or bradykinin, both of which can result in carcinoid syndrome, which includes diarrhea, flushing, wheezing or palpitations. Less than $10 \%$ of people with a NET develop carcinoid syndrome, yet most that do have a midgut tumor. About half of those with carcinoid syndrome eventually develop carcinoid heart disease, especially when there are liver metastases. Carcinoid heart disease occurs when the bioactive substances secreted by the tumor enter the right side of the heart and cause fibrosis of the endocardium, which mostly affects the valves. The left side of the heart is protected by the inactivation of these substances in the lungs. Currently, the average time between symptom onset and diagnosis of a neuroendocrine tumor is about 9 years. We may not know exactly the utility of an elevated troponin in detecting neuroendocrine carcinomas, however a potential for improved recognition may exist.

THEOPHYLLINE: A SURPRISING USE FOR AN OLD MEDICATION Jeffrey A. $\underline{\text { Shrensel}^{2}}$; Jeffrey Ma ${ }^{2}$; Perry Fisher ${ }^{1}{ }^{1}$ Mount Sinai Beth Israel, Astoria, NY; ${ }^{2}$ Mount Sinai Beth Israel, New York, NY. (Control ID \#2467881)

LEARNING OBJECTIVE \#1: Recognize the cardiac side effects of theophylline, an infrequently used treatment for asthma

CASE: D.A., an 83 year-old man presented to the ER following two syncopal episodes at home while eating breakfast. Per patient and his wife, the syncope was preceded by a prodrome of dizziness and lightheadedness, witnessed by the patient's wife both times. Initial EKG showed complete heart block with a ventricular escape rhythm in the 30 s, which spontaneously resolved to a normal sinus rhythm in the 70s. Medical history was significant for long-standing asthma treated with a combined inhaled corticosteroid/long acting beta agonist, monteleukast and theophylline; and hypertension controlled with enalapril. The patient denied any cardiac history, chest pain or palpitations. Apart from a moderate pectus carinatum, physical exam was unremarkable, including cardiac exam. Initial electrolytes were within normal limits, with sodium of $140 \mathrm{mmol} / \mathrm{L}$, potassium of $4.3 \mathrm{mmol} / \mathrm{L}$, and magnesium of $2.2 \mathrm{mmol} / \mathrm{L}$. CBC was unremarkable, and troponin was undetectable at $<0.012 \mathrm{ng} / \mathrm{ml}$. Theophylline level was $8.6 \mathrm{mg} / \mathrm{dL}$. In the emergency room, the patient became unresponsive, with asystole seen on the monitor and loss of pulse. Chest compressions were immediately initiated; return of spontaneous circulation (ROSC) occurred after approximately $20 \mathrm{~s}$. The patient was admitted to the CCU for emergent transvenous pacing (TVP) prior to placement of a permanent pacemaker (PPM). Placement of the TVP was technically challenging, and was complicated by another episode of cardiac arrest during the procedure. The following day, the patient developed leukocytosis and fevers and was started on empiric vancomycin. PPM implantation was postponed until resolution of symptoms and completion of 7 days of antibiotics. While awaiting PPM placement, the TVP sensing wire failed, precluding synchronized pacing. Unsynchronized pacing was deemed risky and pacing was held; subsequently the patient again had episodes of complete heart block occasionally leading to asystole, which generally resolved within $2-3 \mathrm{~min}$ of CPR. Given the low theophylline level on admission, patient was started on IV theophylline, after which he was noted to maintain a sinus rhythm without further high degree block or asystole. After 7 days of vancomycin, the patient was without leukocytosis or fevers, and a permanent pacemaker was placed. The patient was discharged to rehab for reconditioning but was otherwise neurologically intact at time of discharge.

DISCUSSION: Theophylline is a methylxanthine drug with a long history of clinical use for the treatment of respiratory disease, with its first reported use occurring in 1937. Prior to the widespread availability of inhaled corticosteroids, long acting beta agonists, and leukotriene receptor antagonists, it was a mainstay of therapy, although it is now used quite infrequently. 
Its activity in pulmonary disease stems from its activity as a nonselective phosphodiesterase inhibitor, causing smooth muscle relaxation, reduced inflammation, and leukotriene synthesis. Theophylline has a narrow therapeutic window, and toxicity is known to cause sinus tachycardia, multifocal atrial tachycardia, non-sustained ventricular tachycardia, and rarely, sustained ventricular tachycardia. In addition to its traditional uses as in asthma and COPD, theophylline is known to have both inotropic and chronotropic effects. Increased chronotropy is mediated through inhibition of the A1 adenosine receptors, causing SA nodal hyperpolarization counteracting normal vagal tone. The effect of theophylline on the conducting system is less understood, but has been studied in the setting of complete heart block following recent inferior myocardial infarction (MI). In this setting, the release of endogenous adenosine in locally ischemic tissue around the AV node is thought to depress AV node conduction, and this is reversed by theophylline. In a case study by Viskin et al., administration of aminophylline, the active metabolite of theopylline, was useful in achieving ROSC in cardiac arrest following administration of epinephrine, indicating a possible synergistic effect causing increased phosphodiesterase activity or further catecholamine release. We hypothesize that in our patient, his chronic theophylline use masked his severe underlying conduction disease, which became evident as his underlying disease worsened in the setting of a sub-therapeutic theophylline level. When we administered IV theophylline, we noted cessation of his episodic complete heart block and asystole events.

THERE IS SOMETHING IN THE AIR Effie Tsomos ${ }^{1}$; Brandon Trapp ${ }^{3}$; Julie Kanevsky ${ }^{2} .{ }^{1}$ Montefiore Medical Center, Bayside, NY; ${ }^{2}$ Montefiore Medical Center, Bronx, NY; ${ }^{3}$ Albert Einstein College of Medicine, Bronx, NY. (Control ID \#2469648)

LEARNING OBJECTIVE \#1: Understand the clinical presentation of myocarditis. LEARNING OBJECTIVE \#2: Recognize that Legionella myocarditis can precede pulmonary manifestations of Legionella

CASE: A 59 year-old man with no significant past medical history and unlimited exercise tolerance had a single five-minute episode of substernal chest pain. EKG showed anterolateral T-wave inversions and he was admitted to the hospital. Troponin was 0.26 , echocardiogram revealed an ejection fraction of $20 \%$. Diagnostic cardiac catheterization showed normal coronary arteries. The patient was asymptomatic with stable vital signs and thus was discharged with cardiology follow-up. Five days later, he returned to the hospital with severe shortness of breath and diarrhea. He was tachycardic, tachypneic, and febrile to $103.8 \mathrm{~F}$. He was visibly dyspneic with bilateral rhonchi and diminished breath sounds over the left lower lobe. Chest X-ray revealed consolidation of the left lower lobe and lingula and mild pulmonary vascular congestion. Labs revealed a sodium of 122 , WBC count of 11, CPK of 149 and Troponin of 0.01 . Repeat echocardiogram was unchanged. Patient was started on antibiotics for healthcare-associated pneumonia but continued to rapidly deteriorate, desaturating to $78 \%$ on room air. The next day Legionella urine antigen was reported as positive. The patient was treated for Legionnaire's Disease with levofloxacin. EKG remained unremarkable except for prolonged QT interval. Cardiac MRI was consistent with myocarditis, thought to be a complication of Legionnaire's disease. The patient improved clinically and was discharged with a life vest. A repeat echocardiogram 3 months later showed improvement of the ejection fraction to $50 \%$. A New York City Department of Health investigation later determined that this was a community-acquired rather than a nosocomial case of Legionnaire's Disease.

DISCUSSION: Myocarditis is an "inflammation of the heart muscle" that is commonly infectious in etiology, but can also be the result of systemic diseases, drugs, and toxins. The disease received increasing recognition by clinicians after its identification in postmortem studies and the introduction of the myocardial biopsy which established an association between viral myocarditis and dilated cardiomyopathy. Animal studies have revealed that myocarditis can present with an initial non-inflammatory phase in which there is no histologic evidence of myocarditis. This is then followed by frank myocarditis.
During this phase, the myocardium is infiltrated by natural killer cells and macrophages resulting in release of pro-inflammatory cytokines, causing damage to myocytes and eventually a dilated cardiomyopathy. The spectrum of clinical presentation varies widely. This can range from completely asymptomatic patients to patients with severe heart failure and ventricular dilation. Electrocardiographic abnormalities may be present and serum levels of troponin and creatine kinase can be elevated. Occasionally, patients present with atrial or ventricular tachyarrhythmias or systemic and pulmonic emboli from intracardiac thrombi. Myocarditis is a rare complication of Legionella infection. It was first reported by Gross in 1981, occurring after the diagnosis of Legionella pneumonia. What has not been previously described is Legionella myocarditis preceding pneumonia by more than a week as in our patient. Given the proximity of his initial presentation to the pneumonia and the resolution of both myocarditis and pneumonia with antibiotics, it is likely that these events were presentations of the same disease. Endomyocardial biopsy is the gold standard for diagnosis of active myocarditis. In this patient, a myocardial biopsy was not performed due to the risk of complication without a change in management. Thus, in patients presenting with myocarditis whose initial workup does not reveal an etiology, consider testing for Legionella, as this is a treatable cause of a potentially fatal disease.

THINK ABOUT IT: ALTERED MENTAL STATUS DUE TO HASHIMOTO'S ENCEPHALOPATHY Kimberly M. LaMartin; Christopher Richardson. USF Health Morsani College of Medicine, Tampa, FL. (Control ID \#2469815)

LEARNING OBJECTIVE \#1: Recognize the key clinical manifestations of Hashimoto's Encephalopathy.

CASE: A 71-year-old man with past medical history of coronary artery disease, aortic stenosis, hypothyroidism, and chronic respiratory failure requiring tracheostomy developed altered mental status on the second day after transfer to the floor from the intensive care unit. He initially presented as code stroke alert from a long term care facility where he was undergoing weaning from mechanical ventilation. Magnetic Resonance Imaging (MRI) of brain and Computed Tomography (CT) of head on presentation were without acute abnormalities, revealing only left occipital encephalomalacia consistent with prior infarct. He was treated for hypercapneic respiratory failure and health care associated pneumonia, quickly returning to his baseline mental status, and was alert, oriented and able to communicate by mouthing words. On the second day after transfer, he developed a marked change in his mental status, with decreased responsiveness. He was afebrile, pupils were equal, reactive, eyes deviated upward and to right, moving all extremities spontaneously, and withdrawing to pain in all four extremities with symmetric grimace but not following commands or tracking. Lungs were clear to auscultation bilaterally, heart with regular rate and rhythm with $3 / 6$ systolic ejection murmur heard best at right upper sternal border. Arterial blood gas was without hypercapnia. Lorazepam was administered and levetiracetam initiated. Brain MRI and Head CT revealed no acute change from prior. Electroencephalography (EEG) with no epileptiform discharges. Four days later, neurological status continued to deteriorate, and patient exhibited multiple myoclonic movements. Repeat EEG at this time showed multiple seizures arising from right posterior quadrant and severe diffuse cerebral dysfunction, worse on the right hemisphere. MRI now revealed increased hyperintensity and edema involving cerebellar hemispheres, dorsal pons, supratentorial subcortical and periventricular white matter as well involvement of bilateral basal ganglia and thalami with relative sparing of cortical gray matter. Cerebrospinal fluid with 1 nucleated cell, total protein $92 \mathrm{mg} / \mathrm{dL}$, myelin basic protein $<2$ $\mathrm{mcg} / \mathrm{L}$, IgG $16 \mathrm{mg} / \mathrm{dL}$, oligoclonal bands and viral PCR were negative. TSH $42 \mathrm{IU} / \mathrm{mL}$, T4 $0.40 \mathrm{ng} / \mathrm{dL}$, Thyroglobulin antibody $1731 \mathrm{IU} / \mathrm{mL}$, thyroid microsomal antibody 21 . $71 \mathrm{IU} / \mathrm{mL}$. Vitals with no significant bradycardia, hypothermia or hypotension indicative of myxedema coma. He was treated with intravenous (IV) levothyroxine, and intially IV hydrocortisone which was followed by a 5-day course of $1 \mathrm{~g}$ of IV methylprednisolone 
daily and subsequent prolonged steroid taper. He responded to pulse steroids with improved alertness, and was able to track and follow simple commands with no further myoclonic jerks or seizure activity appreciated. Repeat EEG improved, still with moderate to severe background slowing, but no focal asymmetries or epileptiform discharges. MRI revealed interval improvement in hyperintensities in both cerebellar hemispheres and mild improvement in cerebral hemispheres.

DISCUSSION: This clinical vignette highlights the importance of considering Hashimoto's Encephalopathy (HE) as an etiology of altered mental status in all patients with autoimmune thyroid disease after ensuring more common causes of encephalopathy having already been excluded. Hashimoto's Encephalopathy manifests with seizures, cognitive decline, myoclonus, neuropsychiatric symptoms, and stroke like illness. High serum titers of antithyroid antibodies are supportive of the diagnosis, although these are not felt to be pathogenic and do not correlate with severity of HE. MRI findings are diverse, ranging from revealing leukoencephalopathy, remaining unremarkable, or changing abruptly. Elevated protein in the cerebrospinal fluid is found in $80 \%$ of cases. Thyroid function may vary from euthyroidism to overt hypothyroidism and rarely hyperthyroidism. HE is a relatively rare disease that is notable for its responsiveness to glucocorticoids, which supports autoimmunity as the underlying pathogenesis. Patients with Hashimoto's Encephalopathy often have a dramatic response to therapy, further emphasizing the importance of recognizing this entity

THINK OUTSIDE THE BOX TO DISCOVER POTT'S Konstantin Boroda ${ }^{1}$; Darlene LeFrancois ${ }^{2} .{ }^{1}$ Albert Einstein College of Medicine, Bronx, NY; ${ }^{2}$ Montefiore Medical Center, Bronx, NY. (Control ID \#2466796)

LEARNING OBJECTIVE \#1: Recognize the clinical features including the natural history of spinal tuberculosis.

LEARNING OBJECTIVE \#2: Identify HIV as a risk factor for the development of spinal and other extrapulmonary forms of tuberculosis.

CASE: A 47 year old woman with HIV on HAART, with a CD4 count of 150 and no prior opportunistic infections, presented with 6 weeks of worsening right sided lower thoracic back pain accompanied by subjective fever, fatigue, and headache for 1 week. The patient had lower thoracic back pain for 1 year and had undergone several therapeutic epidural steroid injections. Her last injection was 1 month prior to presentation, but since then, her back pain subsequently worsened and was now severe and disabling. On examination her temperature was $102.3^{\circ} \mathrm{F}$ and she was exquisitely tender to palpation of her right posterior lower ribs and lower thoracic spine. There were no overlying skin changes in the tender area. Neurologically she was intact without hyperreflexia or weakness of her lower extremities and with normal sphincter tone and perineal sensation. She also had several tattoos, piercings, and clubbing of her fingers. Laboratory analysis revealed a normal WBC count and differential. Chest x-ray did not reveal evidence of any bone destruction nor pulmonary infiltrate. MRI showed vertebral osteomyelitis and discitis of T9-T10, with a small right paraspinal abscess and an epidural phlegmon. Subsequent guided bone biopsy stained positive for acid-fast bacilli (AFB). Her quantiferon assay was also positive. Sputum smears for AFB were negative. Culture and sensitivities several weeks later confirmed Mycobacterium tuberculosis that was sensitive to Streptomycin, Rifampin, Isoniazid, Ethambutol, and Pyrazinamide. The patient had been born in Puerto Rico and moved to the United States at age 2, she visited Puerto Rico several times throughout her life, but had no other travel. She had no history of prior pulmonary tuberculosis (TB) and reported previous negative PPDs. She reported no history of intravenous drug abuse nor known contact with any infected individuals. Of note, during initial evaluation the patient declined HIV and CD4 testing, only disclosing her HIV positive status and that she was taking HAART several days into the hospitalization. She was initiated on therapy with Rifampin, Isoniazid, Pyrazinamide, and Ethambutol (RIPE) which she continued as an outpatient. At 3 month follow up, she had significant improvement of her back pain and had not suffered any other complications.
DISCUSSION: Typical pyogenic infection complicating epidural steroid injections occurs following 1-2 \% of all procedures. These infections can result in epidural abscess, osteomyelitis, and discitis. With the high prevalence of chronic back pain, physicians often care for patients that receive epidural steroid injections. While practitioners should be aware of this complication, they should also consider other diagnostic possibilities in patients presenting with back pain and fever. Our patient presented with fever and increasingly severe back pain subsequent to an injection 1 month prior, and a typical pyogenic infection caused by the injection was suspected. However, the bone biopsy yielded AFB on staining, exposing the protopathic bias, or reverse-causality, of our thinking. In retrospect, her spinal TB was likely the original cause of her back pain, which prompted her to seek out epidural injection therapy. Spinal TB or "Pott's Disease" most often results from reactivation of a remote primary infection. It is rare, in that it accounts for $1-2 \%$ of total TB cases worldwide. The primary TB infection is asymptomatic in $95-97 \%$ of immunocompetent hosts. When the primary TB infection is clinically apparent, the symptoms are pulmonary in nature $85 \%$ of the time, and may or may not leave chronic radiographic scars. However, due to the bacillemia that occurs during primary infection, tissues with high regional oxygen tension (vertebral bodies, adrenal glands, meninges, GI tract) are seeded with bacilli and thus harbor the organisms for potential later reactivation. The overall risk of developing reactivation TB is $10 \%$, but increases to approximately $20 \%$ in certain individuals including those with purified protein derivative (PPD) induration of more than $10 \mathrm{~mm}$, HIV infection, or evidence of old, healed tuberculosis on radiographic imaging. The mean CD4 count for HIV infected individuals with TB infection is between 200 and 300 cells $/ \mathrm{mm} 3$, earlier than that seen in other opportunistic infections. In addition, HIV-infected individuals are four times more likely to develop extrapulmonary TB than HIV-uninfected persons. Our patient had a typical presentation of Pott's disease, with back pain worsening over weeks to months. The most commonly affected part of the spine is the lower thoracic and upper lumbar vertebrae, and the typical rate of concomitant pulmonary TB is usually low, as was the case with our patient. In sum, it is important to consider spinal $\mathrm{TB}$ as a possible diagnosis in patients with increasing back pain and associated fever. In the United States, there should be particularly high clinical suspicion for this diagnostic possibility in HIV positive individuals and patients who have traveled to endemic areas.

\section{THINK OUTSIDE THE LUNG: AN UNUSUAL CASE OF SPONTANEOUS COLON PERFORATION MISSED ON ROUTINE IMAGING. Andrew Trifan;} Amar Kohli. UPMC, Mars, PA. (Control ID \#2469198)

LEARNING OBJECTIVE \#1: Illustrate the impact of cognitive errors in medical decision making

LEARNING OBJECTIVE \#2: Describe a classic finding of free air on routine imaging CASE: A 71-year-old man with a past history of diverticulitis and alcohol abuse presented to an outside hospital with three witnessed tonic-clonic seizures, presumed to be related to alcohol. He was intubated for airway protection and transferred to our hospital for further management. Work up for his seizures involved a brain MRI with findings concerning for low grade glioma in right temporal lobe, supported by EEG findings. He was extubated and kept on a withdrawal assessment protocol while in the ICU. His course was noteworthy for hypertension and tachycardia that responded well to valium, increased stool output (C.diff negative) and electrolyte abnormalities requiring aggressive repletion. He was transferred to the general medicine floor overnight with an elevated withdrawal score earlier in the day. His last drink was 2 days prior to transfer out of the ICU. On arrival to the medicine floor, he was AAOx3, lethargic but easily arousable and following commands. He was mildly diaphoretic and tremulous. Shortly thereafter his tachycardia worsened, requiring several doses of valium. He spiked a temperature to 38.3 and had worsening mental status later in the night, attributed to the excessive valium. Crackles were heard at the right base and chest $\mathrm{x}$-ray was ordered to assess for aspiration, none which were seen on the night float medicine resident's initial read. His autonomic instability was still felt to 
be delirium tremens at that time. Morning labs showed a new AKI and bandemia of $37 \%$. Repeat exam showed involuntary guarding of the abdomen (worse on the right) and increasingly tenuous vitals. A non-contrast CT abdomen was ordered showing diffuse pneumoperitoneum without obvious point of perforation or obstruction/volvulus. Surgery was called and patient underwent emergent ex-lap with right hemicolectomy for a microperforation in the cecum. He remained hospitalized for 2 weeks until stable for discharge. Review of his initial chest x-ray revealed evidence of free air and "Riggler's sign" which was missed on initial assessment.

DISCUSSION: This case highlights examples of cognitive errors in medical decision making, including anchoring/availability/representation/premature closure errors that involve faulty assessment of pre-test probability, over/under estimating disease likelihood, and failure to consider other possibilities. Awareness of such errors can help doctors recognize and avoid them. The patient was 2 days out from his last drink, so it was initially assumed that his worsening clinical picture must have been alcohol withdrawal. This anchoring prevented consideration of a larger differential at the time. There is also an assumption that patients transferred out of ICU have been "tucked away" and stabilized prior to moving, though this may not always be the case depending on the situation in the ICU. In fact, evening transfers are associated with a much higher rate of re-admission back to ICU, thus suggesting need for more careful assessment of such patients. Due to his alcohol history and being in the prime window period for delirium tremens, this patient was a set up for complications that masked his underlying condition, resulting in his transfer ultimately back to an ICU. Finally, this case demonstrates an example of free air on radiographic imaging. Although spontaneous colon perforations are rare, it is importan to use a structured process when looking at even the most routine imaging as to not miss an alternate diagnoses, such as this case where the initial differential focused on the lungs and the free air was missed. While the RUQ is the most common location for free air, Riggler's sign is the second most common finding with a prevalence of $33 \%$, where intralumina and serosal surfaces are seen due to free air. This finding, however, can be mimicked by two close contiguous loops of bowel.

THINK OUTSIDE THE LUNG: COLON CANCER PRESENTING SOLELY AS COUGH Mariana Mercader; Ana I. Velazquez; Krystle Hernandez; Nicholas Rohs; Erica Grabscheid. Mount Sinai Beth Israel, Icahn School of Medicine, New York, NY. (Control ID \#2469776)

LEARNING OBJECTIVE \#1: Identify typical and atypical presentations of colon adenocarcinoma

LEARNING OBJECTIVE \#2: Recognize the importance of investigating abnormal imaging findings.

CASE: A 55 year old Moroccan male with no past medical history presented with 3 months of a productive cough. Patient had no fevers, chills, weight loss, night sweats or changes in bowel habits. One week prior to admission, his PCP treated him with mucolytics and 5 days of azithromycin without symptom improvement which prompted him to walk in to the ER. He was previously healthy, exercised regularly and had not had a screening colonoscopy. He worked with wood, aluminum, acrylic resins and paint, but there were no toxic habits or animal exposures. He traveled to Morroco 3 weeks prior to admission and had no sick contacts. ER vitals: T $100.4{ }^{\circ}$ F, BP 130/76, P 111, R 16, Pulse Ox 93\% RA. Physical exam was remarkable for bilateral diffuse rhonchi. CXR showed bilateral diffuse ground glass infiltrates. Patient was started on IV ceftriaxone and azithromycin for presumed community acquired pneumonia. CT Chest revealed diffuse bilateral ground glass and solid opacities mostly in airway-centric distribution, interstitial/ peribronchial thickening and intrathoracic/ upper abdominal lymphadenopathy (LAN). Infectious work-up including Chlamydia, Mycoplasma, Histoplasma, Cryptococcal and HIV serologies, along with blood and sputum bacterial, AFB and fungal cultures were all negative. After 3 days of IV antibiotics, the patient had no improvement in his symptoms. Given the abdominal LAN on imaging, a CT abdomen pelvis was obtained showing extensive abdominal LAN and a circumferential sigmoid mass associated with left hydronephrosis/ hydroureter. Poorly differentiated invasive colon adenocarcinoma was found via colonoscopy. Patient was discharged with oncology referral for metastatic colon cancer treatment planning.

DISCUSSION: Colorectal Cancer (CRC) remains the third most common cancer among adults in the United States and the third most common cause of death from cancer. CRC most commonly presents with weight loss, change in stool consistency, abdominal pain, bleeding and iron deficiency anemia. While the lung is second only to liver as the most common site of metastatic disease (with approximately $15-25 \%$ of patients having disease in the lung), it is rare as a primary presentation of colon cancer. In one retrospective study only $6.3 \%(N=252)$ of patients even had lung metastasis at time of diagnosis. This case is a rare presentation of metastatic colon cancer, given the complete lack of abdominal symptoms and a productive cough being the sole complaint. Ground glass opacities on CXR may be a typical radiologic finding of lung metastases from a GI adenocarcinoma, and one's only clue. Although this appeared to be a simple case of community acquired pneumonia, thinking outside the lung led us to pursue extra-pulmonary evaluation that yielded the correct diagnosis.

THYMIC ENLARGEMENT IN GRAVES DISEASE Sara Zahedpour Anaraki; Lalit Narayan; Vafa Tabatabaie. Montefiore Medical Center, Bronx, NY. (Control ID \#2459128)

LEARNING OBJECTIVE \#1: Recognize thymic hyperplasia as a benign condition commonly associated with untreated Graves disease.

LEARNING OBJECTIVE \#2: Identify red flags that should prompt further workup at time of initial diagnosis and describe appropriate follow-up of patients with low suspicion of thymic malignancy.

CASE: A 22 year old woman with no significant medical history presented to the emergency room with palpitations, shortness of breath, fatigue, heat intolerance, menstrua changes and unintentional weight loss over the course of 2 months. Physical examination revealed resting tachycardia, mild tachypnea and a soft diffuse non-tender goiter. No lid lag, exophthalmos, hand tremors, muscle weakness or lymphadenopathy was appreciated. The examination of heart, lungs and abdomen was otherwise unremarkable. An initia chest $\mathrm{x}$-ray revealed an anterior mediastinal mass. The patient was admitted for further workup. A non-contrast computerized tomography (CT) scan revealed an enlarged lobulated thymus $(2.5 \times 5.8 \times 6.1 \mathrm{~cm})$ with surrounding paratracheal lymphadenopathy but no enlarged hilar or axillary lymph nodes. Laboratory tests revealed a thyroidstimulating hormone (TSH) level of $0.007 \mathrm{uU} / \mathrm{ml}$ (normal, $0.40-4.60 \mathrm{uU} / \mathrm{ml}$ ), free thyroxine level of $6.17 \mathrm{ng} / \mathrm{dl}$ (normal, $0.80-1.70 \mathrm{ng} / \mathrm{dl}$ ), free triiodothyronine more than $20.0 \mathrm{pg} / \mathrm{ml}$ (normal, $2.3-4.2 \mathrm{pg} / \mathrm{ml}$ ) and thyroid stimulating immunoglobulin (TSI) level at $256 \%$ of baseline (normal, $<140 \%$ baseline). The patient was diagnosed with Grave's Disease. Her enlarged thymus was determined to be most likely secondary to benign hyperplasia by endocrine and oncology specialists and further workup was deferred. She was started on methimazole ( $30 \mathrm{mg}$ daily) and atenolol (50 $\mathrm{mg}$ twice daily) with plan to follow up the thymus enlargement with repeat CT scan in 3 months.

DISCUSSION: The association between untreated Graves disease and thymic enlargement was first described about 100 years ago. Thymic enlargement in Grave's disease is secondary to benign medullary lymphoid hyperplasia and thymic parenchyma hyperplasia. These processes are thought to be responses to stimulation of the thymus by the autoimmune disease state as well as by hyperthyroidism. Younger patients are more likely to have thymus enlargement. Since an enlarged thymus is often only incidentally found, its prevalence in untreated Grave's disease has not accurately been reported. In most cases the thymus has been noted to involute with treatment of the primary process. The time to involution varies from 
8 weeks after thyroidectomy to 4 to 25 months after initiation of anti-thyroid medication. The differential diagnosis for thymic enlargement also includes rare neoplasms such as thymoma, thymic carcinoma, lymphoma and mediastinal germ cell tumors. This leads to a dilemma regarding the decision about pursuing further diagnostic workup. A review of 107 case reports revealed thymic malignancy associated with only 4 cases. While no formal guidelines exist, the current practice is to monitor the gland for involution with treatment of the primary problem. Red flags that should prompt biopsy or removal of the thymus at the time of initial diagnosis include radiological findings such as heterogeneity, calcifications, and spread into surrounding structures. Failure of the thymus to involute within the expected time with treatment of Grave's Disease should also trigger further investigation.

TIME CAN TELL WHAT EXTENSIVE WORK-UPS CANNOT Akari Nakayama ${ }^{1}$; Yoshihisa Kanamoto ${ }^{1}$; Sandra Y. Moody ${ }^{1,2}$; Makito Yaegashi ${ }^{1} .{ }^{1}$ Kameda Medecal Center, Kamogawa, Japan; ${ }^{2}$ UCSF \& SFVAMC, San Francisco, CA. (Control ID \#2465232)

LEARNING OBJECTIVE \#1: Recognize the usefulness of watchful waiting in evaluating fever of unknown origin in patients

LEARNING OBJECTIVE \#2: Recognize the potential complementary role that PET/ CT scanning can play in the etiological diagnosis of FUO

CASE: Fluorodeoxyglucose-positron emission tomography (PET) is a valuable imaging technique as part of a diagnostic evaluation in patients with fever of unknown origin (FUO), adding value to the history and physical examination. Even when extensive evaluation does not reveal the etiology of FUO, time and easy access to care may be the path to diagnosis. A 52-year-old man with a history of hypertension presented to our outpatient clinic with fever for 2 months. Three months before the visit, he had noted multiple small erythematous lesions on his upper and lower extremities, bilaterally, extending to his entire body, accompanied by finger and toe arthralgias. Two months before his visit, a fever of $38^{\circ} \mathrm{C}$ and night sweats developed. He was seen at three outside clinics, but no diagnosis was made. Because his symptoms persisted, he presented to our clinic for the first time in late August 2015 for further evaluation. At that time, all of his medications, including Chinese herbal medicine, were discontinued, as possible drug fever was considered in the differential diagnosis. Additionally, two sets of blood cultures, parvovirus B19 antibody, erythrocyte sedimentation rate, C-reactive protein, anti-nuclear antibody, rheumatoid factor, anti-citrullinated peptide antibody, and anti-neutrophil cytoplasmic antibody were ordered and were later found to be negative. Contrast computed tomography (CT) of the chest, abdomen and pelvis did not reveal any masses or other etiology of his fever. At his follow-up visit in early September, soluble interleukin-2 receptor level was found to be high at $3290 \mathrm{U} / \mathrm{mL}$ (normal range: 122 to 496). Hematology was consulted, and a PET/CT was obtained to rule out a malignant lymphoma. It revealed no obvious lymphadenopathy, but a small focus of uptake in the subcutaneous tissue of his right thigh was noted. The uptake was deemed a schwannoma or other benign tumor. The lesion was not amenable to biopsy because there was no palpable mass formation. At his third visit to our clinic, in mid-September 2015, his fever, erythema, and arthralgias had improved spontaneously. Thus, bone marrow and random skin biopsy was postponed. The patient was instructed to contact us if any new symptoms developed. In mid-October, new right cervical lymphadenopathy developed, and he contacted us immediately and was admitted for further evaluation. On physical examination, multiple rubbery, mobile, and non-tender cervical and supraclavicular lymph nodes were palpable. A palpable right thigh mass was also noted. Repeat PET/CT revealed widespread lymphadenopathy (bilateral cervical, right supraclavicular, bilateral axillary, paraaortic, left common iliac, and bilateral femoral nodes) and a right thigh mass with increased uptake. He subsequently underwent biopsy of the right cervical lymph node and right thigh mass, and a diagnosis of angioimmunoblastic T cell lymphoma (AITL), Ann Arbor classification Stage IV was made. His bone marrow aspiration was normocellular and negative for lymphoma cells.
DISCUSSION: PET/CT scanning plays a complementary role in the etiological diagnosis of FUO. Even in the absence of palpable lymphadenopathy, PET/CT is valuable for the metabolic assessment of a normal-sized lymph node, which would otherwise be dismissed as normal on conventional imaging. In one study, $15.9 \%$ of patients with lymphoma presenting with FUO were found to have lesions by PET/CT that had been overlooked by conventional imaging such as ultrasonography, CT scan and magnetic resonance imaging. Lymph node biopsy is important to establish an accurate diagnosis once suspicious lesions are found by imaging studies because of its high positive yield and low complication rate. AITL is one of the common peripheral T cell lymphomas. Patients typically present with the acute onset of a systemic illness characterized by generalized lymphadenopathy, hepatosplenomegaly, and B symptoms (i.e., fever, night sweats, and weight loss), with or without a rash. Thus, it is an important etiology of FUO. It can also be associated with immunologic abnormalities, such as autoimmune hemolytic anemia, plasmacytosis, or polyclonal hypergammaglobulinemia. The vast majority ( $90 \%$ ) of patients present with advanced (Stage III/IV) disease. AITL is generally an aggressive disease, with estimated rates of $33 \%$ for overall and $18 \%$ for progression-free survival at 5 years. In the present case, although the patient underwent extensive evaluation early in his clinical course, the definitive diagnosis was made 3 months and a half after the onset of symptoms. The diagnosis was difficult even with PET/CT. The diagnosis was finally made in a timely manner, however, after the patient contacted us immediately with a report of new symptoms. It is of paramount importance to have a good physician-patient relationship and easy access to care when necessary.

TITLE: EXFOLIATE WITH LYMPHOMA: THE PARANEOPLASTIC PEMPHIGUS Kristen Shealy; Poppy Markwell; John Moscona. Tulane University Health Sciences Center, New Orleans, LA. (Control ID \#2469091)

LEARNING OBJECTIVE \#1: Recognize the clinical presentation of paraneoplastic pemphigous

LEARNING OBJECTIVE \#2: Examine the pathophysiology of paraneoplasic pemphigous

CASE: A 58-year-old man with 1 year history of coexistent stage IV follicular lymphoma and multiple myeloma treated with Rituximab, Velcade and Bendamustine resulting in remission of both conditions, presented with 1 month history of a pruritic, painful desquamative rash. It started as a single blister on the right hand and gradually progressed to the arms, trunk, back, abdomen, legs and most recently to the periorbital area and oral cavity. He had not been able to eat due to the pain and blisters in his mouth. On physical exam, he had annular erythematous erosions and desquamating plaques of the bilateral upper and lower extremities, abdomen, trunk and upper back. He had mucositis, but no ocular involvement. Skin biopsy showed suprabasal acantholytic dermatitis suspicious for paraneoplastic pemphigus. This was confirmed with a direct immunofluorescence test showing deposition of $\operatorname{IgG}, \mathrm{C} 3$ along the basement membrane correlating with high titers of desmoglein antibodies. Repeat myeloma panel was unremarkable, however abdominal imaging showed multiple newly enlarged lymph nodes concerning for recurrence of malignancy. Bone marrow and lymph node core biopsies revealed recurrence of grade IIIa follicular lymphoma. Immunohistochemical stains were positive for CD20, PAX5, CD10, and BCL-2 with a high proliferative index. He was started on high dose steroids and broad-spectrum antibiotics along with aggressive daily dressing, but had no response to treatment. He then developed sepsis, prohibiting chemotherapy with progression of mucocutaneous, periorbital and facial disease. He died 3 weeks later.

DISCUSSION: Follicular lymphoma is the most common of the indolent Non-Hodgkin's lymphomas with a median survival of 10 years. Treatment is often not required for asymptomatic patients. Follicular lymphoma is considered incurable, however serious life threatening complications can occur requiring immediate therapy. Paraneoplastic pemphigus (PNP) is a rare fatal skin blistering disorder occurring in association with variety of 
neoplastic diseases most commonly Non-Hodgkin lymphomas. The latter induces an immune response with antibodies formation directed against epithelial antigens playing integral role in cell adhesion. The detection of antibodies against two desmoplakins (periplakin and envoplakin) are the most specific laboratory findings in PNP. Although the disease is initiated by the tumor, simply debulking the tumor or reducing tumor burden through chemotherapy will not halt disease progression. It seems that once the autoimmune disease is initiated, it progresses independently of the underlying neoplasm. Treating the underlying disease may or may not affect the disease process with variable response of skin lesions to treatment. Remission can take up to 2 years to occur and most of these patients succumb before then from complications. For unknown reasons, cases associated with NHL seem to be more difficult to treat than other diseases. The exact cause of death in PNP has been attributed to multiple factors including sepsis, gastrointestinal bleeding, multi-organ failure, and respiratory failure. Several treatment modalities have been reported that include steroids, Rituximab and immunosuppressive medications, yet limited data on treatment is available. Individual patients have widely variable responses to therapy depending on their underlying malignancy, progression of disease at the time of treatment, and preexisting comorbidities. This case teaches the internist about the clinical presentation of a rare disease. Paraneoplastic pemphigus should be suspected in patients with history of neoplasm that develop oral and cutaneous blistering.

TITLE: SEEK AND YE SHALL FIND: AN UNUSUAL CAUSE OF IRON DEFICIENCY ANEMIA AFTER BARIATRIC SURGERY Emmett A. Kistler'; Laurie Edelman ${ }^{2} .{ }^{1}$ Icahn School of Medicine at Mount Sinai, New York City, NY; ${ }^{2}$ Mount Sinai Medical Center, New York, NY. (Control ID \#2467099)

LEARNING OBJECTIVE \#1: Recognize that gastric cancer may occur in the nonalimentary limb post-bariatric surgery

LEARNING OBJECTIVE \#2: Identify clinical features that may necessitate further work up of iron deficiency anemia in patients who have undergone bariatric procedures CASE: A 72 year old woman who underwent uncomplicated Roux-en-Y gastric bypass surgery for morbid obesity 25 years prior presented to her primary care provider with a $20 \mathrm{lb}$ unintentional weight loss and fatigue for several months. She noticed a decreased exercise capacity, some dizziness and vague upper abdominal pain with decreased appetite. She denied any change in her diet, early satiety, bright red blood per rectum, melena, nausea, vomiting or changes in bowel habits. Additionally, she reported adherence with her iron, vitamin B12 and vitamin D supplements. She had routine colon cancer screening with normal colonoscopies at age 59 and 68 . Prior to these recent symptoms, she felt well and denied and other complaints. Her past medical history was remarkable for well controlled hypertension and hyperlipidemia. She had a family history of breast cancer. She did not smoke or drink alcohol and denied any illicit drug use. On exam, she appeared pale and tired. Her vital signs were normal. Her weight was $129 \mathrm{lb}$ (BMI 22.2), stable weight of $149 \mathrm{lb}$ (BMI 25.5) for the past several years prior to presentation. Pertinent clinical findings included pale conjunctivae, a soft, non-distended abdomen with active bowel sounds, and mild epigastric tenderness on deep palpation. No masses or cervical lymphadenopathy were appreciated. Her labs were notable for hemoglobin of $9 \mathrm{mg} / \mathrm{dl}$ (Baseline $11 \mathrm{mg} / \mathrm{dl}$ ) and normal AST/ALT/albumin. Iron studies revealed ferritin 8, TIBC 261, iron 25 and saturation 9\%. B12 and folate were normal. As part of her iron deficiency work up, she had a normal esophagogastroduodenoscopy and colonoscopy. A computed tomography (CT) scan of the abdomen and pelvis was performed which revealed a $2.6 \mathrm{~cm}$ rim-enhancing mass along the gastrohepatic ligament and prominent perigastric and mesenteric lymph nodes. This was new compared with CT performed 2 years prior. The patient was referred for endoscopic ultrasound with fine needle aspiration (EUS-FNA) of a perigastric lymph node which revealed benign pathology. A position emission tomography (PET) scan showed hypermetabolic wall thickening of the gastric antrum/pylorus most consistent with gastric carcinoma of possibly diffuse infiltrative type and lymph nodes consistent with metastatic disease. Repeat EUS-FNA biopsy of the peripancreatic mass was positive for poorly differentiated adenocarcinoma with focal signet cell features. Following completion of three cycles of neoadjuvent chemotherapy in accordance with the MAGIC Trial regimen, she was scheduled for laparoscopic gastrectomy, but the procedure was aborted intra-operatively upon discovery of extensive tumor invasion from the antrum to the second portion of the duodenum. She was discharged in stable condition with palliative care follow up and died several months later in hospice. DISCUSSION: The Roux-en-Y gastric bypass is a commonly utilized surgical treatment for medically severe obesity, with hundreds of thousands of operations performed since 1993. Animal models suggest that the Roux-en-Y may actually decrease the risk of gastric cancer by reducing exposure to carcinogens and bile reflux. Gastric cancer accounts for less than $5 \%$ of cancer diagnoses with approximately 25,000 cases annually; however, there is high mortality with less than $30 \% 5$-year-survival. Case reports of adenocarcinoma of the gastric remnant following gastrojejunostomy are increasing in the literature and these patients appear to offer new diagnostic challenges. Iron and other micronutrient deficiencies are common in this population further confounding the clinical picture. A review of previous case reports suggests that a common presenting feature is abdominal pain, and as, with our patient, the initial work up is often negative, making detection of malignancy in the non-alimentary limb difficult. Endoscopy, including retrograde techniques utilizing pediatric scopes, is technically difficult and unreliable in patients with Roux-en-Y anatomy. CT scanning is considered the primary screening modality. While no routine screening guidelines for gastric cancer have been validated for this population of patients, earlier investigation and diagnosis may improve prognosis. Therefore, a high suspicion for lesions affecting the gastric remnant is warranted for patients with this clinical picture.

TO B OR NOT TO B: A CASE OF CD20 ANTIGEN EXPRESSION IN SYMPTOMATIC MULTIPLE MYELOMA Monica Thim ${ }^{1}$; Shrinkhala Khanna ${ }^{2}$; Donielle F. Sliwa ${ }^{2}$; Sung-Hee Oh ${ }^{1} .{ }^{1}$ Baystate Medical Center, Broad Brook, CT; ${ }^{2}$ Baystate Medical Center, Springfield, MA. (Control ID \#2470174)

LEARNING OBJECTIVE \#1: To distinguish a rare form of multiple myeloma through discussion of its diagnostic difficulty and unique immunology

LEARNING OBJECTIVE \#2: To discuss the rising role of immunotherapy in medicine and its therapeutic implications on this rare form of multiple myeloma

CASE: 66 year old male with a history of encapsulated stage I renal cell carcinoma status post left partial nephrectomy in 2008 presented from outside hospital for concern of spinal cord compression in the setting of a pathologic T5 fracture. Four months prior to presentation, outpatient workup had been completed for progressive back pain and lower extremity weakness. Outpatient MRI of the spine revealed an extradural mass at T5 along with diffuse lytic lesions to his spine. Serum laboratories were worrisome for a gammopathy, leading to multiple myeloma workup. Unfortunately, 1 week prior to presentation to the outside hospital, he had acute worsening of his gait, leading to hospitalization. He was immediately evaluated by Neurosurgery who recommended Decadron $6 \mathrm{mg} \mathrm{q6H}$ with frequent neurochecks. CT Chest and Abdomen/Pelvis confirmed findings of pathologic compression fractures at $\mathrm{T} 5$ and $\mathrm{T} 7$. There were also osseous lesions of the right iliac crest and left ilium. Hematology was consulted. Core bone marrow biopsy of the right iliac crest, SPEP, and UPEP were obtained. Preliminary pathology on the core bone marrow biopsy revealed small, mature cells that were positive for B cell markers CD20 and PAX-5, suggestive of lymphoma. However, overall clinical picture was more concerning for multiple myeloma. Consequently, further novel immunohistochemical evaluation for CD 138, MUM-1, and cyclin D1 markers were pursued for differentiation between lymphoma and multiple myeloma. Meanwhile, SPEP was 
concerning for M spike $1.64 \mathrm{~g} / \mathrm{dL}$ with immunofixation confirming IgA lambda monoclonal protein $1131 \mathrm{mg} / \mathrm{dL}$ with corresponding suppression of IgG and IgM. Free lambda light chain level was $311 \mathrm{mg} / \mathrm{L}$ and kappa light chain level was $4.45 \mathrm{mg} / \mathrm{L}$ with kappa/ lambda ratio of 0.01 . Due to concerns of spinal cord compression in the setting of T5 and T7 fractures as well as T5 extradual mass, radiation therapy was initiated. He completed four fractions of radiation. Despite this, he had worsening bilateral lower extremity weakness. He was taken emergently for spinal cord decompression by neurosurgery and showed minimal post-operative improvement. Eventually, immunohistochemistry confirmed positivity for CD 138, MUM-1, and cyclin D1, which along with his bone marrow aspirate confirmed a $64 \%$ lambda restricted population of CD20 plasma cells, consistent with multiple myeloma. Trials of Rituxan therapy were discussed; however, he was initiated on CyBorD therapy, due to controversial evidence.

DISCUSSION: Myeloma is a neoplastic disorder that is predominantly characterized by the clonal proliferation of malignant plasma cells, whose pathogenesis is characterized by several genetic abnormalities that determine prognosis and therapy. Our case presents an uncommon form of symptomatic multiple myeloma (MM) in which CD20 antigen was detected. This is an interesting case of MM as it refutes prior studies that showed the complete absence of CD20 antigen on MM cells. More recent studies have shown that indeed $13-22 \%$ of MM patients can have partial or complete CD20 expression, which made the diagnosis of MM more difficult as CD20 antigen expression is more classically associated with lymphoma. After further investigation, more recent literature revealed an association for cyclin D1 positivity in CD20 type MM, thus allowing for pathologic finalization. Implications of cyclin D1 positivity in multiple myeloma patients is currently unclear; and, there is conflicting literature as to whether expression portends a poor or favorable effect. However, it has been useful in the identification of CD20 MM for which immunotherapy may have a role. With the advent of immunotherapy, particularly that of Rituximab, the identification of CD20 antigen on MM cells has been a very exciting finding for its favorable serotherapeutic properties, as it is not internalized, shed, or secreted into the circulation. This assists in the reduction of competitive binding of soluble antigen. Despite this, however, newer studies have shown prolonged survival of mature, malignant plasma cells even with the elimination of clonogenic "premyeloma" CD20 positive cells. Consequentially, due to lack of consensus and sparse evidence, rituximab therapy in MM remains controversial. Therefore, we opted not to treat our patient with this therapy. Continuing advances in cytogenetic and chromosomal analysis as well as immunologic techniques have resulted in an increase of possible therapeutic targets, such as that of CD 20 antigen in MM. As medicine becomes more individualized with the identification of these targets and specific cytogenetics, how we interpret this data and its prognostic and therapeutic implications must be considered in the context of our current therapeutic capacity. Much more work and research is still required for further elucidation of this antigen's potential.

TO BIOPSY OR...TO BIOPSY? THERE IS NO QUESTION. Heather Root ${ }^{1}$; Shelly-Ann Fluker ${ }^{2} .{ }^{1}$ Emory University, Atlanta, GA; ${ }^{2}$ Emory University School of Medicine, Atlanta, GA. (Control ID \#2466593)

LEARNING OBJECTIVE \#1: Describe the diagnostic criteria for chronic osteomyelitis.

LEARNING OBJECTIVE \#2: Describe the extrapulmonary manifestations of sarcoidosis.

CASE: Ms. LG is a 43 year old African American Female with no significant past medical history who presented with swelling, pain and "peeling and crusting" of the tip of her left ring finger, which started 1 year prior and had not resolved with a course of antibiotics. Exam was notable for swelling and redness of the tip of the fourth digit and atrophy and splitting of the nail. A mildly tender bump was present on the dorsum of her left hand and multiple round, mobile, subcutaneous masses were present in both arms. Labs were notable for ANA negative, CRP and ESR elevated at 0.66 and 43 respectively, and WBC normal at 5.6. An x-ray of her left hand had "findings compatible with osteomyelitis involving the distal aspect of the fourth phalanx." MRI of the left hand showed "soft tissue mass with bony destruction of the distal phalanx with differential diagnosis including chronic infection, glomus tumor, inclusion cyst, sarcoidosis and carcinoma." The patient saw Orthopedics and underwent excisional biopsy of the left finger distal tip and of the dorsal hand mass. Pathology of both specimens showed extensive dermal granulomatous focally necrotizing inflammation concerning for sarcoidosis vs tuberculous granulomas. Tissue stain showed moderate WBCs with no organisms and positive culture for staph epidermidis. Fungal and AFB stain and fungal, AFB and anaerobic culture were negative. At the patient's follow-up visit in primary care, a chest X-ray was done which showed "mild hilar adenopathy with calcified lymph nodes likely representing a granulomatous process." Of note the patient had a negative PDD done 5 months prior. The patient saw Rheumatology 1 week later where she complained of shortness of breath with exertion and tingling in her feet. Exam was notable for intention tremor of the left hand, left facial droop, and decreased sensation in hands and feet. ACE level was 62 (normal 9-67) and Quantiferon test was negative. EKG and PFTs were normal. Subsequently MRI brain with and without contrast showed "abnormal enhancement throughout the course of the left facial nerve (CN VII) likely a manifestation of neurosarcoidosis given patient history."

DISCUSSION: This case highlights the importance of isolating bacteria from a bone biopsy in order to diagnose osteomyelitis and the varying presentations and manifestations of sarcoidosis. Chronic osteomyeltitis should be suspected in patients with chronic, poorly healing, painful soft tissue wounds. Initial diagnostic testing should include $\mathrm{x}$-rays and laboratory tests including ESR, CRP, WBC count, and blood cultures. If x-ray suggests osteomyelitis or is normal with high clinical suspicion, MRI is usually the next test of choice. Evidence of osteomyelitis by MRI should prompt bone biopsy to confirm the diagnosis and guide antimicrobial therapy. Definitive diagnosis of osteomyelitis requires isolation of bacteria from the bone biopsy with histologic findings of bone necrosis. In this case, there was a high suspicion for osteomyelitis, but the biopsy showed a granulomatous process later confirmed to be sarcoidosis. This case underscores that a diagnosis of osteomyelitis should not be solely based on supportive testing such as blood tests and imaging. Sarcoidosis is a multisystem granulomatous disorder of unknown etiology that is characterized by the presence of noncaseating granulomas in involved organs. It typically affects young adults and initially presents with: bilateral hilar adenopathy, and/or pulmonary reticular opacities, and/or skin, joint and/or eye lesions. Up to $30 \%$ of patients present with extrapulmonary sarcoid. The most common sites of extrapulmonary disease include the skin, eyes, reticuloendothelial system, musculoskeletal system, exocrine glands, heart, kidney, and central nervous system. In most instances, the diagnosis of sarcoidosis requires identifying organs that may be affected, histopathology demonstrating noncaseating granulomas in at least two organ systems, and exclusion of other causes of granulomatous histopathology. In this case, the patient had bone and skin involvement with likely lung, peripheral nerve and brain involvement. Sarcoidosis of bone occurs in approximately $5 \%$ while cutaneous involvement is seen in approximately $25 \%$ of patients. This patient had both a focal bone lesion and subcutaneous involvement. Approximately 5-10\% of patients with sarcoidosis have neurologic involvement. This patient likely has a peripheral facial nerve palsy, which develops in $25-50 \%$ of patients with neurosarcoidosis and peripheral neuropathic involvement. This case highlights the importance of biopsy in the definitive diagnosis of chronic osteomyelitis. In addition it showcases the variety of extrapulmonary manifestations of sarcoidosis, a diagnosis in which biopsy is also critical.

TO OCCAM OR TO HICKAM? THAT IS THE QUESTION. Wei Han; Shadia Constantine; Hideki Takizawa. Teine Keijinkai Hospital, Sapporo, Japan. (Control ID \#2468272) 
LEARNING OBJECTIVE \#1: To recognize that amyloidosis can present as a simple gastrointestinal bleeding.

LEARNING OBJECTIVE \#2: To describe the mechanism in which amyloidosis can cause gastrointestinal bleeding.

CASE: A 53-year-old man came to the emergency department (ED) complaining of hematochezia and melena for 2 days. Since he had a hemorrhoid he assumed the bleed was from it, and decided to wait until it improved. Two days after the gastrointestinal bleed he also experienced abdominal pain, appetite loss, cold sweats and fatigue so he came to the emergency department. He denied weight change, fever, night sweats, nausea, vomiting, use of NSAIDs and excessive alcohol use. His past medical history is significant for duodenal ulcer requiring surgical repair 10 years prior. He smokes 5 cigarettes per day for 33 years and does not drink any alcohol. His family history was not relevant. His physical exam revealed tachycardia of $101 \mathrm{bpm}$, pale face and conjunctiva. His heart and lungs were normal. His abdomen was not distended but he had tenderness to palpation around the epigastric area without rebound or guarding. The rectal exam revealed a lateral hemorrhoid that was not bleeding nor inflamed. No mass was palpated, however; his stool were black with positive for fecal occult blood. His initial blood work showed a slightly elevated WBC of $9780 / \mu \mathrm{L}$ and $\mathrm{Hb} 8.8 \mathrm{~g} / \mathrm{dL}$ indicating anemia. His total protein was low at $3.1 \mathrm{~g} / \mathrm{dL}$ with a very low albumin of $1.7 \mathrm{~g} / \mathrm{dL}$. His blood urea nitrogen and creatinine were also elevated, but without any significant electrolyte abnormalities. His initial chest $\mathrm{x}$-ray showed mild pleural fluid. Our initial diagnostic impression was a gastrointestinal (GI) bleeding most likely from an upper source. He underwent an upper GI endoscopy, which revealed an ulcer in the duodenum with edema, bleeding and erosion on the body of stomach and pylorus, but no active bleeding was found anywhere. The duodenal ulcer was treated with ablation. However, the patient experienced a large amount of melena overnight after the procedure and anemia progressed requiring transfusion. A repeat ed endoscopy was done on the next day but to find no bleeding. A biopsy of the gastric lesion was reported as negative for malignancy. The initial small pleural effusion found at admission worsened significantly in both sides. We performed a thoracentesis which resulted to be a transudate without malignant cells in the cytology. For further work up on the bilateral pleural effusion, transthoracic echocardiog raphy (TTE) and urinalysis was done. Nephrotic range proteinuria was discovered and the TTE revealed increased echogenicity, left ventricular hypokinesis and concentric hypertrophy with ejection fraction of $42.9 \%$. Later, the neurological exam revealed decreased vibratory sensation in lower extremities. With the involvement of several organs, a systemic disease was suspected. Repeated GI biopsies found amyloid deposition around the small vessels and muscularis mucosa of greater curvature, posterior wall of stomach, duodenum and colon. These findings were confirmed by dual fluorescent staining. In the serum, $k / \lambda$ ratio was 0.08 indicating $\lambda$ dominant monoclonal immunoglobulins. Monoclonal free light chain protein was also found in the electrophoresis. The patient was diagnosed with systemic amyloidosis. Later, a bone marrow biopsy confirmed the existence of AL amyloidosis.

DISCUSSION: The patient came to the ED with GI bleed, which was later found to be amyloidosis. We have considered it nothing but just another gastro-duodenal ulcer because of the typical presentation and the past medical history of having an ulcer although there was no clear mechanism responsible for the lesion as he was not taking any medications and there was no sign of Helicobacter pylori. The low albumin level was thought to be from the hemorrhage. It was the worsening pleural fluid that made us wonder about nephrotic syndrome and amyloidosis. Although there were no findings on the first biopsy, the repeated GI biopsies confirmed our suspicions. Amyloid deposition in the gastrointestinal (GI) tract occurs most frequently in the small intestine and GI hemorrhage can be seen in up to $45 \%$ of the patients with amyloidosis. Gastrointestinal disease in amyloidosis results from either mucosal infiltration or neuromuscular infiltration. The organ dysfunction is thought to come from the disruption of the tissue architecture by amyloid deposits and from direct cytotoxic effect from amyloid precursor proteins. Amyloidosis gastric hemorrhage could be from the fragility of the small vessels by the amyloid infiltration, which causes ischemic changes, and from the deposition of the amyloid, which causes atrophy and destroys the mucosal structure. The AL type amyloidosis may be considered for patients with plasma cell diseases, and the AA type amyloidosis in patients with chronic inflammatory disease. However, it is difficult to distinguish between the two when no past medical history such as above is available. This case illustrates how a clinician should always keep in mind systemic diseases such as amyloidosis, even in patients with an apparently simple hematochezia. In our case, the Occam's razor philosophy, rather than Hickam's dictum, was useful to solve the mystery of this patient with gastrointestinal bleeding, nephrotic syndrome and cardiomyopathy.

TOO LITTLE, TOO LATE: WHEN LACK OF ACCESS MAKES A RARE DISEASE LESS RARE. Robert Metter; Amar Kohli. UPMC, Pittsburgh, PA. (Control ID \#2469625)

LEARNING OBJECTIVE \#1: To list the clinical and CSF features of tuberculous meningitis

LEARNING OBJECTIVE \#2: To describe the appropriate treatment regimen for tuberculous meningitis

CASE: A 39 year old undocumented male Guatemalan immigrant with history of alcohol abuse presented with nausea, emesis, headache, and lethargy. One week prior to admission he developed headache, which progressively worsened to include nausea and non-bloody, non-bilious emesis. On the day of admission, he became severely lethargic. He denied fevers, cough, diarrhea, or rash. He endorsed weight loss of $30 \mathrm{lb}$ over the preceding 6 months. Vital signs were unremarkable on admission. On exam, the patient was somnolent but arousable to voice. A neurologic exam yielded no focal deficits. Labs were remarkable for leukopenia and anemia. CT of the chest demonstrated calcified granulomas. HIV-1 testing was positive with viral load 328,000 and CD4 count of 2. Antiretroviral therapy was initiated with raltegravir, tenofovir, and emtricitabine. The patient remained somnolent, and subsequently became febrile. He was empirically started on vancomycin, ceftriaxone, ampicillin, and acyclovir. CT scan and MRI of the brain were unremarkable. Lumbar puncture demonstrated normal opening pressure, WBC 311 ( $8 \% \mathrm{~N}, 78 \% \mathrm{~L}, 14 \%$ mono), RBC 25, glucose 15, protein 447. CSF cultures returned negative and antibiotics were discontinued. Given these CSF findings, the patient was started on rifabutin, isoniazid, pyrazinamide, and levofloxacin as well as prednisone for suspected TB meningitis. However, he developed severe hyponatremia requiring ICU transfer, and quickly progressed to multi-organ failure and death. Autopsy pathology showed necrotizing granulomatous meningitis.

DISCUSSION: Tuberculous meningitis must be included in the differential when lumbar puncture results yield a lymphocytic pleiocytosis with low glucose (hypoglycorrhachia) and markedly elevated protein. Our patient displayed all three of these characteristic findings. The disease course consists of three stages: a prodromal phase lasting 2-3 weeks with malaise, headache, and low-grade fever, a meningitic phase with neurologic features including confusion, lethargy, and occasionally focal deficits, and finally, a paralytic phase with coma, seizures, and possible hemiparesis. Diagnosis is made by visualization of acid-fast bacilli in the CSF and/ or by nucleic acid testing (PCR) of CSF. Empiric therapy should not be delayed until the diagnosis has been made. Treatment of TB meningitis differs from that of pulmonary tuberculosis in two main aspects: moxifloxacin or levofloxacin is substituted for ethambutol due to higher CNS penetration, and corticosteroids are added to the antibiotic regimen. Rifabutin was chosen in this case due to interaction of rifampin with raltegravir. The four-drug regimen is continued for 2 months, after which rifampin and isoniazid are continued for an additional 9 to 12 months. 
TOO MUCH OF A GOOD THING: CALCIUM CHANNEL BLOCKER TOXICITY PRECIPITATED BY AN INTERACTION WITH RITONAVIR EMma B. $\underline{\text { Shak }}^{1,2}{ }^{2}$; Richard Ramonell ${ }^{2}$; Schuyler D. Livingston ${ }^{2} .{ }^{1}$ UCSF, San Francisco, CA; ${ }^{2}$ Emory University School of Medicine, Decatur, GA. (Control ID \#2464726)

LEARNING OBJECTIVE \#1: Recognize the clinical signs of calcium channel blocker toxicity.

LEARNING OBJECTIVE \#2: Appreciate the importance of drug-drug interactions in patients on antiretroviral therapy.

CASE: A 56 year-old female with hypertension, nonischemic cardiomyopathy with ejection fraction $40 \%$, chronic hepatitis $\mathrm{C}$, and human immunodeficiency virus (HIV) well-controlled on antiretrovirals (ARVs) presented to the emergency department for 1 week of progressive diffuse weakness and dizziness. Notably, her ARV regimen had been changed 1 month prior to a regimen that included ritonavir, a protease inhibitor (PI), because of tenofovir nephropathy. Her home medications included amlodipine, ritonavir, atazanavir, abacavir-lamivudine, and lisinopril. On initial evaluation, the patient was found to be afebrile but had orthostatic hypotension with supine blood pressure of 102/74 and was bradycardic to 38 beats per minute. Physical examination was significant only for trace pitting edema in the lower extremities bilaterally. Labs were significant for potassium of 6.0 , bicarbonate of 18 , creatinine of 2.9 , and troponin of 0.03 . Urine drug screening was negative. Cardiac monitoring showed new-onset first-degree atrioventricular block with interventricular conduction delay of left bundle morphology alternating with short periods of complete heart block. The patient was diagnosed with calcium channel blocker (CCB) toxicity secondary to interaction with ritonavir complicated by acute kidney injury. She was initially treated with discontinuation of her CCB, gentle fluid resuscitation, and intravenous calcium gluconate. External pads for transcutaneous pacing were applied but were not required as the patient gradually improved with supportive care and discontinuation of amlodipine. Within twenty-four hours of admission, the patient's EKG returned to normal sinus rhythm with heart rate at 70 beats per minute. Her first-degree atrioventricular block and interventricular conduction delay were persistent during admission but she was no longer orthostatic and her weakness had completely resolved. She was discharged without amlodipine and, at a follow-up clinic visit 2 weeks following admission, was doing well. An electrocardiogram obtained at that visit demonstrated resolution of all previous abnormalities. DISCUSSION: CCB toxicity is a medical emergency that can have serious consequences including profound hypotension and cardiac arrhythmias. CCB toxicity is usually encountered following overdose but can sometimes be seen with drug-drug interaction. Amlodipine, a dihydropyridine $\mathrm{CCB}$, is a common first-line antihypertensive that is known to have a major drug-drug interaction with protease inhibitors. This case illustrates the potential for interaction between protease inhibitors and amlodipine, a phenomenon that occurs because both medication classes are metabolized by the cytochrome P450 3A system. Protease inhibitors have been shown to clinically increase the serum concentration of amlodipine in healthy volunteers resulting in PR prolongation, symptoms of orthostasis, and hypotension. ${ }^{1}$ Given the prevalence of hypertension in the aging HIV population and the frequency with which amlodipine and protease inhibitors are used, this adverse interaction is likely to become more common. It is important for clinicians to monitor for and recognize this interaction after making any changes to patients' HIV and hypertension regimens, and choose appropriate alternative medications.

TRADING ONE FOR ANOTHER: A CASE OF DE NOVO CROHN'S DISEASE AFTER LUNG TRANSPLANT Valentine O. Millien ${ }^{2}$; Lubin A. Santana ${ }^{1}$; Ana Kolpakchi ${ }^{1}$; Lee Lu ${ }^{1} .{ }^{1}$ Baylor College of Medicine, Houston, TX; ${ }^{2}$ Baylor College of medicine, Houston, TX. (Control ID \#2469980)
LEARNING OBJECTIVE \#1: Report a case of de novo Crohn's disease in a post lung transplant patient.

LEARNING OBJECTIVE \#2: Report the association between immunosuppressive agents (mycophenolate and tacrolimus) and the development of de novo Crohn's disease following lung transplantion.

CASE: A 71- year-old -male with history of idiopathic pulmonary fibrosis status post right lung transplant presented with hematochezia. As a part of evaluation prior to lung transplant, he did have a colonoscopy which revealed a tubular adenoma polyp, and it was resected. After transplant, patient was started on mycophenolate and tacrolimus for immunosuppression, but mycophenolate was discontinued 5 months after transplant. One year after transplant, he presented with gastrointestinal bleed and was found to have ileal ulcers suggesting the possibility of graft vs. host disease. The rectal bleed recurred 6 months later and repeat colonoscopy showed 2 ulcerated malignant appearing masses in distal ileum which were biopsied. Biopsies revealed chronic ulcers without evidence of malignancy or granulomas. He was treated with budesonide and mesalamine without improvement. Subsequently, he underwent partial resection of terminal ileum and cecum with pathology showing normal mucosa adjacent to areas of chronic changes suggestive of inflammatory bowel disease consistent with Crohn's disease (CD). The patient was diagnosed with de novo IBD.

DISCUSSION: Gastrointestinal complications are common side effects observed after solid organ transplantation but are usually limited to diarrhea either from infections or medications. Interestingly, data suggest IBD is more common with an estimation of ten times more in the post organ transplant population than in the common population with ulcerative colitis more common than $\mathrm{CD}$. The so called "de novo" IBD has been mainly reported in patients with liver, heart, or renal transplant. The mechanism is thought to be due to innate immune inflammatory responses within the transplanted organ exerting their effects on the gut microbiome. Tacrolimus based immunosuppressive regimens have been associated with higher relapses in IBD patients after liver transplant with 13 and $64 \% 1$ - and 5 - year risks of IBD flares, respectively. In addition, mycophenolate has also been implicated in IBD specifically a colitis-like illness that can be sometimes confused with $\mathrm{CD}$ and may be a result of induction of release of the inflammatory cytokines tumor necrosis factor and IL-6 by a metabolite of the active form of mycophenolate. Although de novo IBD is reported following other solid organ transplantions, we could not find another case of de novo $\mathrm{CD}$ in a post lung transplant patient. It is important to recognize IBD as a serious complication after lung transplant and understand that the therapies used for immunosuppression can cause development of de novo IBD, particularly in patients on mycophenolate or tacrolimus as part of their immunosuppressive regimen.

TRUE POSTERIOR MYOCARDIAL INFARCTION Saagar Sanghvi; Edward S. Roberto; Teresa Ratajczak; Mukul Chandra. Wright State University, Dayton, OH. (Control ID \#2437823)

LEARNING OBJECTIVE \#1: Distinguish true posterior myocardial infarction from precordial ischemia or reciprocal changes in an anteroseptal myocardial infarction using a 12-lead electrocardiogram.

CASE: A 54 year-old Caucasian male presented to the ED with left chest pressure, non-radiating, associated with numbness in his left arm. The patient initially experienced symptoms while driving; he consequently took 2 aspirins and sat down to rest. The chest discomfort became worse when he stood up and drove. He did not have any shortness of breath, diaphoresis, or dizziness. Medical history was positive for hyperlipidemia, without any history of cardiac diseases or acute 
vascular events. Initial electrocardiogram (ECG) revealed ST-depression in leads V1-V4, with positive troponins within $2 \mathrm{~h}$ of presentation. The patient was treated as NSTEMI and cardiologist was consulted for further management. Diagnosis was revised to posterior myocardial infarction (PMI) after assessing ECG inverted on its long axis. Echocardiogram showed a severely hypokinetic and thinned basal mid-posterior wall, confirming a true PMI. Coronary catheterization revealed $99 \%$ stenosis of Left Anterior Descending and $100 \%$ stenosis of Left Circumflex arteries. Drug-eluting stents were placed in both arteries. Dual-antiplatelet medications, statin, beta-blocker, and ACE-inhibitor therapies were initiated. He was observed for 1 day after catheterization before discharge with no complications. DISCUSSION: Because anterior leads record the internal surface of the posterior heart, typical ST-elevation becomes ST-depression, and pathological R waves develop analogous to Q waves seen in anterior STEMI's. T-wave inversion is instead seen as an upright T wave. Clinicians must be suspicious of a PMI when ECG shows STdepression in precordial leads (V1-V4) with concurrent tall R waves. Diagnosis is aided by "flipping" the ECG on its long axis, revealing ST-elevation in the precordial leads, correlating to a posterior infarction. Utilizing 15-lead ECG's to evaluate STelevation increases specificity and sensitivity in detecting LCX artery injury. A combination of ST-depression in anterior leads with ST-elevation in posterior leads correlates to high probability of PMI. The T2 to T6 index is the voltage difference between $\mathrm{T}$ waves in $\mathrm{V} 2$ and $\mathrm{V} 6$; greater than $0.38 \mathrm{mV}$ increases probability of PMI This is most easily seen in our second ECG. Distinguishing tissue injury from tissue ischemia is critical to initiating the appropriate workup and timeline for treatment For many clinicians, ST-segment depression in precordial leads is sufficient for thrombolysis and angioplasty to establish a definitive diagnosis.

TUBERCULOSIS: A PAIN IN THE BRAIN James Higgs ${ }^{1}$; Asad H. Khan ${ }^{3}$; Puncho Gurung 2 . ${ }^{1}$ Baystate Medical Center, Enfield, CT; ${ }^{2}$ Baystate Medical Center, Springfield, MA; ${ }^{3}$ Baystate Medical Centre, Broad Brook, CT. (Control ID \#2469631)

LEARNING OBJECTIVE \#1: Identify the presenting signs and symptoms of Tuberculosis (TB) Meningitis

LEARNING OBJECTIVE \#2: Review the diagnostic modalities and complications of TB in the Central Nervous System (CNS)

CASE: 26-year-old healthy female of Nepalese origin presented for evaluation of a 1month history of headaches, anorexia and 10-lb weight loss. Her headaches were frontotemporal in location and worse upon waking. Her travel history was notable for a brief trip to Ohio just prior to the onset of symptoms. She denied any history of contact with TB patients. On arrival, she was febrile to $102.9 \mathrm{~F}$ with no leukocytosis. Lumbar puncture was performed demonstrating 558 WBCs with $88 \%$ neutrophils, 16 RBCs, protein of 217, and glucose of 10. T-spot results could not be obtained due to insufficient number of mononuclear cells. HIV was negative. She was treated with Ceftriaxone, Vancomycin, and Dexamethasone for presumed bacterial meningitis. Despite this regimen, she continued to spike intermittent fevers. CSF bacterial cultures as well as AFB and fungal smears were negative. An MRI of the brain revealed multiple ring-enhancing brain lesions and subtle leptomeningeal enhancement with basilar predominance. Chest X ray did not show any acute cardiopulmonary disease while $\mathrm{CT}$ of the chest revealed interstitial thickening with a slight reticular nodular pattern. Transthoracic echocardiogram did not demonstrate any evidence of valvular vegetations. Metronidazole was subsequently added given concern for brain abscesses. Repeat cerebral spinal fluid (CSF) studies performed 5 days after hospitalization showed continued neutrophilic pleocytosis, elevated protein, and hypoglycorrhachia. Six days into hospitalization, she became increasingly more lethargic. Repeat CT head demonstrated acute obstructive hydrocephalus for which she underwent an emergent external ventricular drain placement. Given her worsening clinical condition and persistent fever despite treatment for bacterial meningitis, she was empirically started on amphotericin B for fungal meningitis and anti-tubercular therapy with steroids for tubercular meningitis on day 7 of hospitalization. Over the next 2 days, she defervesced and began to show clinical improvement. Eventually 16 s Ribosomal RNA studies of the CSF came back positive for Mycobacterium tuberculosis.

DISCUSSION: Due to its relative rarity and variability in clinical presentations, CNS TB infection can be a formidable diagnostic challenge. With high mortality even with treatment (upwards of $50 \%$ ) and associated morbidity (30\%), early recognition is vital (1). While CNS TB infection is often associated with active pulmonary disease, upwards of $30-50 \%$ have no pulmonary involvement (1). CSF studies can initially demonstrate neutrophilic pleocytosis with elevated protein and low glucose, which often transitions to a lymphocytic pleocytosis. Diagnosis is very challenging with sensitivities of CSF studies ranging from: Culture 73-82 \%, PCR $57 \%$, Ziehl-Neelsen stain $27 \%$, and adenosine deaminase $29 \%$ (2). Imaging can demonstrate proliferative arachnoiditis (prominently in basilar regions), tuberculomas, and vasculitis-associated infarction of spinal or cerebral structures. Occurring in the majority of those with TB CNS infection, obstructive hydrocephalus is a life threatening complication that needs to be recognized promptly in those with changes in mental status. References: 1 . Rock RB, Olin M, Baker CA, Molitor TW, Peterson PK. Central nervous system tuberculosis: pathogenesis and clinical aspects. Clinical Microbiology Reviews. 2008;21(2):243-261. 2. Erdem H, Ozturk Engin D, Elaldi $\mathrm{N}$, et al. The microbiological diagnosis of tuberculous meningitis: results of Haydarpasa 1 study. Clinical Microbiology and Infection. 2014;20(10):O600-O608.

TUBERCULOUS MYOCARDITIS: AN UNUSUAL PRESENTATION. Rashi Khanna $^{2}$; Rana Wajahat ${ }^{2}$; Anil Kumar Reddy Anumandla ${ }^{1}$; Gaurav Kistangari ${ }^{3}$. ${ }^{1}$ Clevaland Clinic, Cleveland, OH; ${ }^{2}$ Fairview Hospital-Cleveland Clinic, Westlake, OH; ${ }^{3}$ Medicine, Cleveland, OH. (Control ID \#2468606)

LEARNING OBJECTIVE \#1: Recognize clinical features of rare tubercular cardiac involvement in high risk population.

LEARNING OBJECTIVE \#2: Treat with early initiation of medical therapy for a potentially reversible cause of cardiomyopathy.

CASE: Tuberculosis (TB) a common disease in developing countries, rarely manifesting as myocarditis. We present a case of a 34 year old male, a recent immigrant from India, admitted to the hospital with 2 days symptoms of left sided chest pain .Associated symptoms included shortness of breath, intermittent episodes of fever, malaise for the past 9 months, loss of appetite and $20 \mathrm{kgs}$ weight loss in 6 months. A chest x ray and computed tomography (CT) chest done in the emergency department did not reveal any pathology. However, echocardiogram and nuclear stress test done thereafter showed wall motion abnormalities along with ejection fraction (EF) of $35 \%$. No significant coronary artery disease was seen on cardiac catheterization . Cardiac Magnetic Resonance Imaging (MRI) showed basal septal thinning mainly in the anteroseptal and inferoseptal regions and multiple tiny hyper intense nodules in the right upper lobe of lung suspicious of cardiac sarcoidosis .Medical treatment was initiated for congestive heart failure . Going back to his history, he had latent TB 2 years back treated with isoniazid (INH) for 9 months with noncompliance to the regimen .Other past medical history was significant for psoriasis treated with adalimumab. Around 6 months back he travelled to India to visit his family member who was suffering from TB . Bronchoscopy was performed with a lymphocytic Broncho-alveolar lavage and necrotizing granulomatous inflammation on biopsy. A presumptive diagnosis of TB myocarditis was made and empiric treatment was started with rifampin, INH, pyrazinamide and ethambutol for 3 months, then rifampin and INH for 3 months. Adalimumab was discontinued. At 1 year follow up patient had complete clinical recovery and improvement in EF to $56 \%$ that further confirmed the diagnosis of TB myocarditis. He was able to restart his adalimumab without recurrence of $\mathrm{TB}$. 
DISCUSSION: Cardiac involvement in tuberculosis accounts for only $1 \%$ of the disease, mainly seen in the pericardium. Tubercular myocarditis, as a manifestation of reactivation of latent TB, is a rare entity as the myocardium is largely resistant to inflammation. Although it can remain clinically asymptomatic, sudden cardiac death, intractable ventricular arrhythmias, long QT syndrome, high grade heart block or congestive heart failure can be common manifestations. Although endomyocardial biopsy was not performed clinical suspicion and response to treatment with antituberculous therapy were strong pointers to make a diagnosis. Reactivation of latent tuberculous infection should be strongly suspected especially among patients from TB endemic regions who have compromised immune system. Although rare, reactivation of TB may present with myocarditis and hospitalist should have high index of clinical suspicion to promptly diagnose and isolate such patients to reduce the risk of transmission and emergence of drug resistance.

\section{TWO CASES OF AMYLOID HEART DISEASE- EARLY RECOGNITION OF A POTENTIALLY LETHAL DISEASE Namita R. Joseph; Sagger Mawri; Emmanouil} Alimpertis; Abdullah Alshammaa; John R. Schairer. Henry Ford Hospital, Detroit, MI. (Control ID \#2470339)

LEARNING OBJECTIVE \#1: Recognize the most common clinical features and objective findings of cardiac amyloidosis, and the most common causes of death.

LEARNING OBJECTIVE \#2: Recognize the progressive nature of cardiac amyloidosis, and thus the importance of early recognition.

CASE: Amyloidosis is a systemic disorder caused by extracellular deposition of insoluble protein fibrils derived from aggregation of mis-folded proteins which can affect various organs and impair tissue structure and function. Cardiac amyloidosis is a devastating and progressive disease, often diagnosed at an advanced stage due to the ambiguity of symptoms, and thus is associated with high mortality rates. Herein, we discuss two clinical cases of patients diagnosed with amyloidosis, highlighting key features for establishing diagnosis and describing the consequences of delayed diagnosis in this disease. The first case is of Mr. BN, a 64-year-old male lawyer who initially presented with a suspected infection of his left eyelid, and received antibiotic therapy without resolution. He subsequently developed progressive shortness of breath and was admitted with left-sided pleural effusion that required thoracentesis. The transudative fluid was thought to be secondary to cardiac diastolic dysfunction shown on 2D echocardiogram. He was readmitted several times over the next 9 months for recurrent heart failure exacerbation and pleural effusions. It was only after his fourth hospitalization when dermatology was consulted for his unresolved eyelid lesions that amyloidosis was considered, due to physical examination signs including red eyelid papules and macroglossia. A punch biopsy of the eyelid gave pathologic confirmation of amyloidosis as evidenced by positive Congo red staining. Cardiac MRI showed evidence of cardiac amyloid involvement. Bone marrow biopsy revealed plasma cell dyscrasia, supporting likely AL amyloidsosis type. Immunohistochemistry testing for the definitive type of amyloidosis were sent and he was discharged home to follow up with his primary care physician, cardiology, and hematology. Unfortunately, he went into cardiac arrest 3 days later and died before any further management. The second case is of Mrs. CT, a 62-year-old woman who initially presented with generalized weakness and found to be in an exacerbation of new-onset heart failure. She had a more speedy diagnosis of amyloidosis after low QRS voltage was noted on her electrocardiogram (ECG). A subsequent echocardiogram and cardiac MRI revealed biventricular and septal wall thickening, bi-atrial dilatation, diastolic dysfunction and "granular sparkling" of the myocardium; that is, increased myocardial and valvular echogenicity characteristic of amyloid deposition. Congo red stain on abdominal fat pad biopsy was positive, confirming amyloidosis. UPEP and SPEP were positive for monoclonal free kappa light chains in the gamma region. Bone marrow biopsy revealed plasma cell myeloma. Further amyloid sub-type evaluation was performed and pending (later confirmed AL-type). Meanwhile, she was discharged to subacute rehabilitation to followup within 2 weeks with hematology and cardiology. Unfortunately, she too went into cardiac arrest soon after discharge and died.

DISCUSSION: Cardiac amyloidosis is the most important predictor of survival in patients with amyloidosis and is associated with a median survival of only 4 to 6 months. Many patients have symptoms for 2 years or longer before a diagnosis of amyloidosis is established and as a result, is often at an advanced stage. This was seen with our first patient, as diagnosis was established only after multiple heart failure readmissions. Fortunately, important objective findings exist in patients with heart failure that may point towards cardiac amyloidosis. On ECG, low QRS voltage (defined as all limb leads $<5 \mathrm{~mm}$ in height) may be seen due to infiltration and replacement of the myocardium by amyloid fibrils. This was the case in our second patient, triggering suspicion for underlying infiltrative cardiomyopathy. Other ECG findings include abnormal axis and poor R wave progression. Key features on echocardiogram include concentric left and right ventricular thickening, prominent valves, and infiltration of the atrial septum. The myocardial tissue may be described as "granular sparkling," as was noted in the case of our second patient. Diastolic rather than systolic dysfunction is a hallmark of cardiac amyloidosis and may be one of the earliest signs when only minimal cardiac wall thickening has occurred. Subendocardial myocytes are most vulnerable to infiltration, leading to impairment in longitudinal contraction best seen with tissue doppler. In contrast, left ventricular ejection fraction is calculated from contraction along the short axis and is often spared in early states of amyloidosis. Because early symptoms may be nonspecific in this uncommon disease, there is often a delay in diagnosing cardiac amyloidosis. In a patient with symptoms of congestive heart failure, particularly recurrent diastolic heart failure symptoms, coupled with ECG findings such as low QRS voltage, and echocardiographic features such as thickened ventricles, diastolic dysfunction/impaired relaxation, and granular sparkling, the diagnosis of cardiac amyloidosis should be very strongly considered. As our two unfortunate cases illustrate, delay in diagnosis in this merciless infiltrative disease may cause cardiac failure or lead to arrhythmias, the most common causes of death in patients with amyloidosis.

TYPE B LACTIC ACIDOSIS DUE TO THIAMINE DEFICIENCY IN A PATIENT WITH B CELL LYMPHOMA Umair Masood; Anuj Sharma; Sonny Nijjar; Karthikeyan Sitaraman. SUNY Upstate Medical University, Syracuse, NY. (Control ID \#2466179)

LEARNING OBJECTIVE \#1: Neoplasms can be associated with type B lactic acidosis due to thiamine deficiency

LEARNING OBJECTIVE \#2: Type B lactic acidosis causes should also be considered when dealing with lactic acidosis

CASE: An 82 year old male with past medical history of depression, hypothyroidism, and hyperlipidemia presented to the hospital with a retroperitoneal mass found on physical examination in an out-patient clinic. On presentation, he reported chronic urinary difficulties with worsening in the last few weeks to the point of significant oliguria. He reported nausea, left lower extremity edema, poor appetite, and $20 \mathrm{lbs}$ weight loss over the course of 1 month. His vitals on admission were stable. His physical examination was pertinent for cachectia, left lower quadrant palpable mass with mild diffuse tenderness, and $3+$ edema of the entire left lower extremity. His laboratory findings were significant for blood urea nitrogen of $84 \mathrm{mg} / \mathrm{dL}$, creatinine of $5.7 \mathrm{mg} / \mathrm{dL}$, potassium of $5.5 \mathrm{mmol} / \mathrm{L}$, bicarbonate of $15 \mathrm{mmol} / \mathrm{L}$, anion gap of 21 , lactic acid of $6.6 \mathrm{mmol} / \mathrm{L}$, and hemoglobin of $10.0 \mathrm{~g} / \mathrm{dL}$. CT scan of the abdomen showed a $9.9 \times 8.4 \times 12.0(\mathrm{~cm})$ retroperitoneal mass and moderate right sided hydronephrosis. Patient had a nephrostomy tube placed in his right ureter with significant improvement in urinary output and creatinine. The retroperitoneal mass was biopsied and was found to be a diffuse large B cell lymphoma. Patient 
was adequately fluid resuscitated without improvement in his lactic acidosis. He was then empirically treated with intravenous thiamine ( $500 \mathrm{mg} \mathrm{IV} \mathrm{q} 8 \mathrm{~h}$ ) with normalization of lactic acid level $(1.7 \mathrm{mmol} / \mathrm{L})$ the next day. Patient was later transferred to the oncology service for chemotherapy for the lymphoma.

DISCUSSION: Type B lactic acidosis is found in the absence of tissue hypoperfusion. It rarely occurs in patients with leukemia, lymphomas and solid malignancies. While the exact mechanism is not fully understood, altered lactate metabolism due to kidney or liver dysfunction, lactate production by the tumor, glycolytic metabolism by tumor cells, riboflavin or thiamine deficiency are amongst the proposed mechanisms. It is proposed that aggressive tumors use lactate to metastasize and evade immune response and even radiation. As the most common cause of lactic acidosis is tissue hypoperfusion, it was assumed to be the guiding force in our patient in particular. Patient did not have any hypotension, hypoxia, or infection that could have been contributing to the lactic acidosis. Despite adequate hydration, his lactic acid level remained elevated. His anion gap did improve with the correction of the acute kidney injury without any improvement in the lactic acidosis. Empiric treatment with intravenous thiamine rapidly improved the lactic acid level to normal values within $24 \mathrm{~h}$ This signified that the driving force behind the patient's lactic acidosis was thiamine deficiency that can sometimes occur in tumors and has been reported in lymphomas. In conclusion, even though type A lactic acidosis is more common, a clinician must also keep type B lactic acidosis in the differentials particularly in cancer patients

UNCOMMON EKG FINDINGS: NOT ALL IS WELL IN WELLEN'S SYNDROME Osman Bhatty; Jeff Murray. Creighton University GME, Omaha, NE. (Control ID \#2466779)

LEARNING OBJECTIVE \#1: To recognize Wellen's Syndrome on EKG LEARNING OBJECTIVE \#2: To heighten awareness and emphasize the critical nature of this finding

CASE: An 81 year old female with a past medical history of gastro-esophageal reflux disease, Polymyalgia Rheumatica, Chronic Kidney Disease Stage 3, and peripheral vascular disease was transferred from an outside hospital with a chief complaint of chest pain. She had no prior cardiac investigations including stress tests, catheterizations or echocardiograms. She described that 2 days prior she felt a left-sided, $7 / 10$, non-radiating chest pain that was brought on by walking lasting $30 \mathrm{~min}$ to an hour. A second episode occurred the day after while brushing snow off her deck with minimal exertion with a similar presentation though this time associated with left arm radiation and nausea. This prompted her initial presentation to an outside facility where she was found to have an elevated troponin of 0.03 . She was given sub-lingual nitroglycerin and then transferred for further work-up and evaluation. Initial EKG showed poor R-wave progression without accompanying acute S-T segment changes. By this time chest pain had resolved and she otherwise denied shortness of breath, palpitations, nausea, vomiting, diarrhea, dizziness, or lightheadedness. She was placed on guideline directed medical therapy including administration of full dose, $325 \mathrm{mg}$ aspirin, high-intensity statin and metoprolol tartrate $12.5 \mathrm{mg}$ twice daily. She was also started on a heparin drip. Physical exam did not elucidate any further abnormalities. The only significant laboratory investigations included a troponin that was trended as $0.03,0.58,0.31$ (.04 normal) The next morning a followup EKG during a chest pain free period was significant for new deep, symmetrical T-wave inversions in leads V2, V3, V4, and V5. Given dynamic T-wave changes and the possibility of Wellen's Syndrome she was urgently consented for a cardiac catheterization via right radial approach with potential percutaneous coronary intervention. Invasive investigation was significant for $90-99 \%$ focal stenosis of proximal left anterior descending artery. She subsequently received a $3.5 \times 15 \mathrm{~mm}$ bare metal stent to the area without complications. Patient was discharged in stable condition having just avoided major occlusion and subsequent infarction.
DISCUSSION: Wellen's syndrome or "LAD Coronary T Wave syndrome" is a preinfarction syndrome characterized by certain EKG findings that imply severe stenosis of the proximal LAD artery. These have been reported to develop into acute anterior wall myocardial infarctions within several days in as high as $75 \%$ of patients. First described in 1982 by Wellen et al. he published the clinical and EKG criteria of a subgroup of patients who presented with deeply inverted T waves in the precordial leads who though initially responding to medical therapy later required revascularization. Among patient's admitted with unstable angina about $14-18 \%$ have this pattern on EKG. Mean time to infarction is 8.5 days from onset and so recognizing it to be a critical stenosis of the $\mathrm{LAD}$ is paramount. The clinical criteria for diagnosis is as follows: (1) Recent history of chest pain (2) Symmetric, deep T wave or biphasic T wave in V2-V5 or V6 during pain free period (3) No precordial Q waves or loss of R waves (4) Minimal (<1 mm) ST elevations (5) Unremarkable elevation of cardiac enzymes. Recognizing this abnormality before it progresses to infarction is critical in preventing a NSTEMI. This can prove difficult since most patients with Wellen's sign do not have chest pain during the visit or have unremarkable troponin elevations. There are 2 types; type A occurs in $75 \%$ of patients and is characterized by deeply inverted T-waves in V2 and V3. Type B occurs in $25 \%$ of cases and is characterized by biphasic T-waves in V2 and V3. The patient in our case had a type A abnormality which was recorded during a pain free period the morning after she was placed on heparin. The EKG features of Wellen's syndrome have a sensitivity, specificity, and positive predictive value of $69 \%, 89 \%$, and $86 \%$ respectively making it useful knowledge for any general practitioner. Urgent revascularization is the necessary next step to prevent possible anterior infarction and subsequent ventricular dysfunction.

UNEXPLAINED ANEMIA NOT ALWAYS IRON DEFICIENCY: A CASE OF ACQUIRED FACTOR VIII INHIBITOR Emily Miller; Tariq Horani. Palmetto Health, Columbia, SC. (Control ID \#2470213)

LEARNING OBJECTIVE \#1: Recognize presenting symptoms and characteristics of patients with Acquired Factor VIII Inhibitor

LEARNING OBJECTIVE \#2: Review diagnosis of Acquired Factor VIII Inhibitor and discuss therapeutic approach

CASE: The patient is a 68-year-old male with past medical history of Rheumatoid Arthritis, Chronic Iron deficiency anemia, and prostate cancer receiving radiotherapy who presented to the hospital with 2 week history of increased fatigue and dyspnea on exertion. Physical exam at admission was unremarkable with exception of hypotension. On laboratory evaluation, the patient was noted to have hemoglobin of $2.8 \mathrm{~g} / \mathrm{dL}$. The patient denied signs of active bleeding and no etiology could be found initially. The patient was transfused with packed red blood cells with good response in hemoglobin to $10.0 \mathrm{~g} /$ dL. Roughly 4 days after admission, the patient developed hypotension, tachycardia, and rapid decline in hemoglobin to $4.6 \mathrm{~g} / \mathrm{dL}$. CT of the abdomen and pelvis revealed developing retroperitoneal hematoma. Hematology was consulted, at which time PTT mixing and Factor studies were performed ultimately indicating development of Factor VIII inhibitor.

DISCUSSION: Acquired Factor VIII Inhibitor, also referred to as Acquired Hemophilia $\mathrm{A}$, is a rare condition characterized by development of autoantibodies against endogenous Factor VIII. Acquired Factor VIII Inhibitor has an overall incidence of roughly 1.5 per million per year with the majority of cases occurring in the elderly population and a male predominance. There is a small increase in incidence of pregnancy related cases in women of childbearing age as well as African Americans. In roughly $50 \%$ of cases there is an identifiable underlying condition associated with development of Acquired Factor VII Inhibitor. Common underlying conditions include malignancies, pregnancy, autoimmune conditions such as lupus erythematosus and rheumatoid arthritis, infections, drug-induced and dermatological conditions. Acquired Factor VIII Inhibitor should be considered in 
patients who present with acute onset of bleeding or unexplained anemia in individuals with no personal or family history of bleeding disorder. Bleeding often manifests as soft tissue hematomas, bruising, deep muscle or retroperitoneal, gastrointestinal, and urogenital bleeding. Intracranial hemorrhage has been rarely described in association with Acquired Factor VIII Inhibitor. This is sharply contrasted with congenital hemophilia A, which classically manifests with hemarthroses or muscular bleeding. Additionally, bleeding associated with Acquired Factor VIII Inhibitor is often severe and multifocal. Diagnosis of Acquired Factory VIII Inhibitor is typically delayed due to lack of clinical suspicion for this disease. However, one study noted that despite delay in initiation of treatment due to diagnostic delay overall treatment response and outcomes were unchanged. As previously noted, Acquired Factor VIII Inhibitor should be considered in patients with unexplained bleeding and no prior or family bleeding history. The differential diagnosis for anemia and unexplained bleeding is broad and includes provoked bleeding, chronic anemia as well as less common etiologies such as Lupus anticoagulant and Acquired von Willebrand disease. Initial laboratory investigation leading to diagnosis will demonstrate prolonged activated partial thromboplastin time, which is only not fully corrected by mixing study. Measurement of Factor VIII and associated antibodies confirms the diagnosis. It is important to note that in some instances, patients have presented with isolated prolonged aPTT without evidence of bleeding, which may be inappropriately attributed to lupus anticoagulant. Treatment of acute bleeding episodes consists of agents that bypass Factor VIII. The use of recombinant activated Factor VII (rFVIIa) or plasmaderived activated prothrombin complex concentrate (pd-aPCC) effectively bypass Factor VIII in the clotting cascade. While these agents have not been compared for efficacy against one another, multiple studies have proven these bypassing agents to be more effective in controlling bleeding when compared with Factor VIII replacement and desmopressin. Long-term management of Factor VIII inhibitor consists of inhibitor eradication with immunosuppressive therapy. Some regimens for immunosuppression consist of corticosteroids alone or in combination with cyclophosphamide or rituximab. One study indicated more stable remission rates in individuals treated with steroids plus cyclophosphamide compared to steroids alone and steroids combined with rituximab however survival was not significantly effected by treatment regimen. As such, no standardized treatment protocol exists at this time. Acquired Factor VIII Inhibitor is a rare but potentially life threatening condition. Diagnosis of these patients is often delayed due to lack of familiarity with the condition and its characteristics. It is important to consider this diagnosis in patients who present with unexplained anemia or obvious bleeding in order to solidify diagnosis and initiate therapy for bleeding control as quickly as possibly.

\section{UNEXPLAINED HYPERBILIRUBINEMIA IN A PATIENT WITH SICKLE} CELL DISEASE Vernisha hearn ${ }^{2}$; Aliaksandr Trusau ${ }^{1}$; Rehan Qayyum ${ }^{1}$; Mukta Panda ${ }^{1}$. ${ }^{1}$ University of Tennessee, Chattanooga, TN; ${ }^{2}$ University of Tennessee, Medical School, Memphis, TN. (Control ID \#2469194)

LEARNING OBJECTIVE \#1: Discuss and differentiate the hepatic manifestations in a patient with sickle cell disease and its management implications.

CASE: An 18 year old African American male with sickle cell disease diagnosed at birth presented with a 1 week history of diffuse abdominal pain, nausea, vomiting, dark urine and yellow discoloration. The abdominal pain was moderate, intermittent, colicky, without any aggravating or alleviating factors. He had six pain crises in the past requiring hospitalizations but received only five PRBC transfusions in his life. He was not taking any medications and denied use of tobacco, alcohol or illicit drugs. Review of system was negative for fever, chills, dyspnea, chest pain, headache, rashes, or pain in digits or back, diarrhea or constipation. On exam he looked healthy with normal vital signs. His skin and mucous membranes were jaundiced. Abdomen was soft, tender in paraumbilical and epigastric area without guarding, Murphy sign was negative, tender hepatomegaly was noted. CBC showed WBC-12.8 k/ul, Hg-9.8 g/l, reticylocyte count-9.8 \%, and platelets$527 \mathrm{k} / \mathrm{ul}$. Total bilirubin was $41.5 \mathrm{mg} / \mathrm{dL}$, direct-24.2 mg/dL, AST- $80 \mathrm{u} / 1$, ALT-79 u/l, and alkaline phosphatase-179 u/l. Patient's base levels: hemoglobin 8 to $10 \mathrm{~g} / \mathrm{L}$, total bilirubin3 to $6 \mathrm{mg} / \mathrm{dL}$ with normal level of direct bilirubin. PT, PTT, INR, BMP, UDS and acetaminophen level were normal. CXR was normal. CT abdomen and pelvis showed hepatomegaly, bilateral nephrolithiasis, and cholelithiasis. MRI abdomen showed cholelithiasis, small amount of pericholecystic fluid and mild mucosal enhancement of gallbladder, filling defect in the extrahepatic common bile duct (CBD) with possible choledocholithiasis, without significant CBD enlargement, autoinfarcted spleen. RUQ US showed signs of venoocclusive disease, nonobstructive cholelithiasis and abnormal hepatic echotexture. ERCP showed no stone in the CBD and normal filling of gallbladder. Biliary sphincterotomy was performed with stent placement to eliminate obstruction secondary to cholelithiasis. Through the hospital course patient was treated with IV hydration with normal saline and symptomatic therapy with Zofran and morphine. His symptoms waxed and waned. His bilirubin was persistently elevated around $40 \mathrm{mg} / \mathrm{dL}$ with mild transaminites. We pursued investigation of alternative diagnoses with iron studies, ANA, cerulloplasmin, $24 \mathrm{~h}$ urine cooper level- all of which were normal. Liver biopsy was performed with showed marked microvascular cholestasis and prominently dilated sinusoids with numerous sickled RBCs. Patient's benign clinical course, laboratory findings, imaging studies and results of liver biopsy were consistent with benign variant of intrahepatic cholestasis. He was discharged with outpatient follow up with his hematologist.

DISCUSSION: There appears to be a spectrum of hepatic dysfunction in patients with sickle cell disease related to disease itself and its treatment. Vascular complications from sickling process, multiple blood transfusions increasing the risk of viral hepatitis and iron overload, development of pigment gallstones may all contribute to the development of liver disease in these patients. Patterns of hepatic dysfunction in sickle cell disease are diverse and vary individually. "Sickle cell hepatopathy" represents overlapping causes of liver dysfunction. Elevated bilirubin, predominantly indirect, is universal due to chronic hemolysis. Total bilirubin concentrations are usually $<6 \mathrm{mg} / \mathrm{dL}$ and is often the only liver chemistry abnormality observed. Elevation of the serum alkaline phosphatase is common, particularly during pain crises. In sickle cell hepatic crisis, the transaminase level is usually below $300 \mathrm{mg} / \mathrm{dL}$ and total bilirubin below $15 \mathrm{mg} / \mathrm{dL}$. In rare cases transaminases may exceed $1000 \mathrm{mg} / \mathrm{dL}$, which may indicate severe hepatic hypoxia. Whereas sequestration and cholestasis have previously been regarded as separate manifestations, they actually coexist with varying degrees of predominance. Cholestasis predominates in acute presentations with bilirubin levels often exceeding $48 \mathrm{mg} / \mathrm{dL}$. Acute sequestration, which may precede cholestasis, is striking in these patients, causing clinical and radiologic hepatomegaly. Pathogenesis of sickle cell intrahepatic cholestasis is obstruction of the hepatic sinusoids by sickled RBCs and bile with resulting vascular stasis, ballooning of hepatocytes and local hypoxia. Benign hyperbilirubinemia, predominantly conjugated bilirubin of up to $57 \mathrm{mg} / \mathrm{dL}$ with only mild elevation in ALT has been described in children and adults with minimal or no symptoms and resolves spontaneously within 2-8 weeks with no subsequent recurrences. Prolongation of PT, PTT, elevation of urea, creatinine and ammonia, hypofibrinogenemia, thrombocytopenia, anemia, lactic acidosis, marked hyperbilirubinemia, severe transaminitis as well as patient's clinical course would indicate necessity of prompt investigation of underlying causes and specific therapy.

UNEXPLAINED HYPERTENSION: AN UNUSUAL CASE Christopher A. D'Avella $^{1}$; Rahul A. Parikh ${ }^{2}$; Jennifer Rodriguez ${ }^{1} \cdot{ }^{1}$ University of Pittsburgh Medical Center, Pittsburgh, PA; ${ }^{2}$ University of Pittsburgh, Pittsburgh, PA. (Control ID \#2466490)

LEARNING OBJECTIVE \#1: Recognize the Causes of Secondary Hypertension and Evaluation and Treatment of Primary Hyperaldosteronism 
LEARNING OBJECTIVE \#2: Describe the Presentation and Treatment of Adrenocortical Carcinoma

CASE: 53-year-old woman with prior history of hypothyroidism presented with palpitations and bilateral hand paresthesias for 1 month. On presentation, she was hypertensive to 170 / 110 and tachycardic to 103 . Labs showed a potassium of 2.0; all other labs including CBC, BMP, and thyroid studies were normal. EKG showed normal sinus rhythm with frequent PVCs. She had a normal chest x-ray and CT angiogram. Her potassium normalized and she was discharged with outpatient follow up. She was initially started on HCTZ but ultimately had poor blood pressure control with three anti-hypertensive agents (HCTZ, Lisinopril, Metoprolol) and persistent hypokalemia. A secondary hypertension evaluation showed an elevated aldosterone:renin ratio of 187 consistent with primary hyperaldosteronism. A CT abdomen and pelvis demonstrated a $2.3 \mathrm{~cm}$ adrenal lesion, and subsequent adrenalectomy revealed adrenocortical carcinoma. Pathology demonstrated lymphatic and adrenal vein invasion with extension into the peri-adipose fat in addition to positive surgical margins. She was staged at least T3N0, Stage III adreno-cortical carcinoma. CT chest and MRI brain showed no evidence of metastasis. Her hypokalemia and hyperaldosteronism normalized post-surgery. She was started on mitotane, hydrocortisone and completed etoposide, doxorubicin and cisplatin (EDP chemotherapy) for treatment of her high risk disease.

DISCUSSION: Hypertension is one of the most common diagnoses encountered by internists. According to the CDC, approximately 1 in 3 adults have hypertension. Essential hypertension is most prevalent, with secondary causes accounting for $5-10 \%$ of hypertensive patients. The majority of cases of secondary hypertension are linked to obstructive sleep apnea, renal parenchymal/vascular disease, and hyperaldosteronism; medications, pheochromocytoma, and endocrine disorders are less common. Evaluation is recommended for patients with clinical signs suggestive of a secondary etiology (i.e. abdominal bruit) and/or certain features of hypertension: resistance, early or late onset, or severe/accelerated course. Primary Hyperaldosteronism (PH) is the most common etiology of secondary hypertension. Aldosterone enhances the absorption of sodium and secretion of potassium by binding to mineralocorticoid receptors. $\mathrm{PH}$ is most commonly idiopathic or caused by an aldosterone producing adenoma, whereas adrenal hyperplasia/carcinoma, ovarian tumors, and familial causes are rare. Many cases do not present in textbook fashion with hypokalemia and metabolic alkalosis; normokalemia is actually a common finding Hyperaldosteronism is most known to cause hypertension. Additionally, literature links it to endothelial dysfunction, left ventricular hypertrophy, renal/myocardial/vascular fibrosis and metabolic syndrome, all independent of blood pressure. A plasma aldosterone:renin ratio $\geq 20 \mathrm{ng} / \mathrm{dl}$ and a morning plasma aldosterone $\geq 15 \mathrm{ng} / \mathrm{dl}$ indicate the diagnosis. Confirmation testing consists of oral sodium/intravenous saline loading followed by CT imaging to look for adrenal pathology. Adrenal venous sampling is recommended for all patients with $\mathrm{PH}$ who are surgical candidates to distinguish between unilateral versus bilateral disease. For adenomatous disease or unilateral hyperplasia, the treatment of choice is laparoscopic adrenalectomy; multiple studies demonstrate postoperative improvement of hypertension and hypokalemia. Idiopathic cases or non-surgical candidates are treated with mineralocorticoid antagonists (spironolactone, eplerenone). Adrenocortical carcinomas account for $14 \%$ of adrenal incidentalomas. Adrenocortical carcinoma (ACC) is a rare malignancy with an incidence of approximately $0.7-2.0$ cases per million per year. Though ACC can occur at any age, it is most commonly diagnosed in children under 10 and adults ages 40-50s. It is slightly more prevalent in females (F:M ratio 2.5:1). In adults, ACC can be associated with $\mathrm{Li}$-Fraumeni syndrome and multiple endocrine neoplasia type 1 (MEN1). Approximately $60 \%$ of ACC are functioning tumors and can present with hypercortisolism, hirsutism/virilization, feminization, precocious puberty, or hyperaldosteronism. Staging at diagnosis is the most important prognostic factor. The 5-year survival rate in patients with local disease is $60-080 \%, 35-50 \%$ in locally advanced disease and $13-28 \%$ in metastasized disease. Common sites of metastases are the lung, liver, peritoneum, bones and major veins. The only curative treatment for ACC is surgery- an option limited to resectable disease in stage I, II and III disease. In stage III-IV disease, surgery can be combined with radiation and/or chemotherapy. Chemotherapy options include mitotane, streptozotocin plus mitotane, or etoposidedoxorubicin-cisplatin plus mitotane. In cases of increased hormone production, ketoconazole and steroid receptor antagonists, such as spironolactone and mifepristone, should be considered. Our subject remains on adjuvant mitotane and completed adjuvant EDP chemotherapy. Evaluation for secondary hypertension can be an important element of the diagnostic evaluation of hypertensive patients in the right clinical setting. Adrenocortical carcinoma is a rare cause of hypertension but should be considered as a potential diagnosis in any patient with hyperaldosteronism and an adrenal nodule.

UNMASKING THE BLACK SCHWAN Brendan Gilmore; Toug M. Tanavin; Rajeev Balchandani. Baylor College of Medicine, Houston, TX. (Control ID \#2453583)

LEARNING OBJECTIVE \#1: Recognize the clinical features of a vestibular schwannoma

LEARNING OBJECTIVE \#2: Obtain a thorough history and physical to facilitate highvalue diagnostics and management

CASE: Vestibular schwannoma is a rare cause of unilateral sensorineural hearing loss. This case describes how a meticulous history and physical exam can result in the foresight to pursue additional imaging to accurately diagnosis a vestibular schwannoma and facilitate swift intervention. A 72 year-old African American male with esophageal squamous cell carcinoma in remission presented with 6 weeks of urinary incontinence, progressively worsening balance and unusual behavior. Non-contrast computerized tomography $(\mathrm{CT})$ of the head showed lateral and third ventricular enlargement out of portion to cortical atrophy, which was most consistent with normal pressure hydrocephalus (NPH). However, on assessment of vestibulocochlear cranial nerve function, the patient was found to have unilateral sensorineural hearing loss. Weber test was positive. Additional history revealed that the patient had consistent asymmetric hearing loss for the las 6 months. Audiogram confirmed unilateral sensorineural hearing loss. At this point, the decision was made to obtain a magnetic resonance imaging (MRI) brain with gadolinium. It showed a $3 \mathrm{~cm}$ by $3.5 \mathrm{~cm}$ by $3.3 \mathrm{~cm}$ heterogeneous left cerebral pontine angle mass. The mass created a partial obstruction of the fourth ventricle, which resulted in obstructive hydrocephalus. Neurosurgery was consulted and patient underwent a left retrosigmoid craniotomy. Pathology results confirmed the diagnosis of a vestibular schwannoma. Patient did well post-operatively. Mental status and balance improved. Patient was transferred to inpatient rehabilitation floor for intensive physical and occupation therapy. DISCUSSION: In the current health care environment, high value care is becoming increasingly emphasized. It is critical to avoid unnecessary tests and imaging, while still providing the best possible care to patients. Specifically, cost-consciousness often precedes consideration for an MR. This case highlights the utility of MRI when indicated by a truly thorough history and physical. Without this clinical acumen, the patient may have been confined by a diagnosis of NPH, which may have led to misguided management and persisting morbidity for the patient. This patient's diagnosis of vestibular schwannoma with associated obstructing hydrocephalus was set in motion by a well-executed history and physical. Misuse and overuse of diagnostic studies remains a pressing issue in highvalue care. However, this case illustrates the ideal allocation of clinician resources, which can help augment management and in turn, patient outcomes.

UNUSUAL RASH IN A BURMESE IMMIGRANT Mohit Pahuja ${ }^{2}$; Geraldine Casino $^{3}$; Hiral Thakrar ${ }^{2}$; Sami Hoshi ${ }^{1}$; Mirella Mircescu ${ }^{4}{ }^{1}$ St Joseph hospital and medical center, Phoenix, AZ; ${ }^{2}$ St. Josephs Hospital and Medical Center, Phoenix, AZ; ${ }^{3}$ St. Joseph's Hospital and Medical Center, PHOENIX, AZ; ${ }^{4}$ St. Joseph's Hospital and Medical Center, Phoenix, AZ. (Control ID \#2470000) 
LEARNING OBJECTIVE \#1: Cellulitis and rash are common causes of ER visits in US. Rashes not responding to the usual treatment regimen should prompt one to consider autoimmune and other atypical infections like viral, mycobacterium, fungal and parasitic. CASE: A 28 year old Burmese male came with the complaint of right forearm swelling \& painful rash 4 days prior to admission. He noted rash on both forearms which initially were described by the patient as mosquito-bites which became larger and caused bilateral forearm swelling. He also noted a similar rash on both thighs \& lower legs. It was associated with shortness of breath, fever, cough \& occasional blood tinged sputum. He worked as a gardener in Burma, lived in Malaysia for a couple of years and moved to Arizona 1 year back. He worked as a utility man on a golf course and denied exposures to toxic materials or animals. On admission his blood pressure was $99 / 40 \mathrm{~mm}$ of $\mathrm{Hg}$ and heart rate of 110/min. Physical exam revealed diffuse firm, tender nodular and urticarial rashes on bilateral forearms, anterior thighs \& shins. His right forearm was more swollen, warm \& tender. He had generalized lymphadenopathy and thickening of the left ear helix. Laboratory studies revealed WBC count of 12.9 thousand/ul with predominantly neutrophils (75.5\%). His ESR was elevated to $64 \mathrm{~mm} / \mathrm{h}, \mathrm{CRP}$ to $84.3 \mathrm{mg} / \mathrm{l}$ and procalcitonin to $1.74 \mathrm{ng} / \mathrm{ml}$. Ultrasound of right upper extremity was negative for DVT. His Chest X-ray showed a small old calcified granuloma on left middle lobe which was confirmed on a CT scan without contrast. He was empirically started on vancomycin and Zosyn for cellulitis. However, the distribution and characteristic were not typical of cellulitis. Differential diagnosis of his rash included viral, fungal or atypical bacterial infection or a vasculitis. Infectious disease was consulted for further workup. Further investigations included positive ANA \& Anti-SSB/La antibodies; HIV, coccidiomycosis, strongyloides and RPR serology were negative. AFB sputum stain was positive for 2 samples but DNA probe for mycobacterial tuberculosis were negative. A Skin biopsy from the lesion showed subcutaneous inflammation and macrophages with multiple acid fast bacilli. Additional stains for mycobacterium infection including fite stain for Mycobacterial Leprae (M. Leprae) came back negative. The skin biopsy pathology block was sent to University of Washington for further testing of all mycobacterial species and came back positive for $\mathrm{M}$. Leprae PCR. With the assistance of AZ Hansen disease Clinic patient was started on treatment for lepromatous leprosy (LL) with triple regimen of Dapsone $100 \mathrm{mg}$ daily, Rifampin $600 \mathrm{mg}$ daily and Clofazimine $50 \mathrm{mg}$ daily. He responded well to the treatment and was discharge home with 24 months of antibiotics and was registered at our local Leprosy clinic.

DISCUSSION: Leprosy also known as Hansen's disease is commonly seen in developing countries involving Asia, Africa, Latin America and the Pacific. In United States 100-200 cases are diagnosed annually. Most of the cases are seen in New York, Louisiana, Texas, Massachusetts, Hawaii, California and Florida. In 2010 only 2 cases were reported in Arizona. Early examination findings include hypopigmented or reddish skin patches, diminished sensation in involved areas and tender, enlarged peripheral nerves. Untreated patient can have worsening neuropathy resulting in severe pain, muscle weakness, deformities and paralysis. It can in also lead to ophthalmic injury causing blindness. M. Leprae is a very slow growing intracellular acid fast organism and hence difficult to culture. The diagnosis cannot be made without skin biopsy. Fite stain may be used for identification but PCR DNA has higher sensitivity and specificity. Our patient was negative for Fite stain but his PCR DNA came back positive. Leprosy can be classified on the basis of number of bacilli within the dermis into: Tuberculoid (TT), borderline tuberculoid (BT), mid-borderline (BB), borderline Lepromatous (BL) and Lepromatous leprosy (LL). Tuberculoid disease (TT, BT) are treated with dapsone, rifampin for 6 12 months, while Lepromatous disease (BB, BL, LL) is treated with additional clofazimine for total 24 months. M. Leprae can cause severe systemic inflammatory reactions. Thery are divided into 2 types: Type I Lepra reaction (TT, BT and BB) present as inflammation in previously formed skin lesions. A more severe type II lepra reaction (BL, LL) causes sudden eruption of severe painful erythematous nodules which may develop into maculopapular lesions as seen in our case. In addition to the above treatment regimen, patients with neuritis undergo treatment with prednisone. In type II lepra reaction, cases refractory to prednisone are treated with thalidomide. Patients may have false-positive ANA tests as seen in our case, which can lead to incorrect diagnosis of lupus erythematosus. Diagnosis in the US is often delayed because of its rare nature; unawareness of the disease and its symptoms. Early diagnosis and treatment can prevent neuropathies and deformities. Hansen's disease should be suspected in patients presenting with rash not responding to standard therapy especially in patients with potential exposures based on travel and social history.

UNUSUAL TOXICITIES OF SYNTHETIC MARIJUANA Angelica Nocerino; Nazish Ilyas. Northwell Health -Lenox Hill Hospital, Bronx, NY. (Control ID \#2464783)

LEARNING OBJECTIVE \#1: To recognize that synthetic cannabinoids can be associated with gastrointestinal bleeds and disseminated intravascular coagulation.

LEARNING OBJECTIVE \#2: To show that more studies are needed to identify the toxicities of synthetic cannabinoids.

CASE: Our patient is a 30 year old male with no significant past medical history who presented to our hospital with coffee ground emesis and melena. On initial presentation, vital signs were stable, with laboratory values significant for a hemoglobin of $13.9 \mathrm{~g} / \mathrm{dl}$. History was only significant for use of synthetic marijuana four times a month. The patient denied use of NSAIDs, alcohol, or other illicit subtances. Within $8 \mathrm{~h}$ of admission, melena progressed to bright red blood per rectum, and the hemoglobin dropped to $7.7 \mathrm{~g} / \mathrm{dl}$. The patient required a massive blood transfusion, including 4 units of packed red blood cells (prbcs), 6 units of platelets, 6 units of fresh frozen plasma (FFP), and 10 units of cryoprecipitate. After receiving the blood products, the patient developed acute shortness of breath and was intubated for transfusion related acute lung injury (TRALI). A mesenteric angiogram was immediately performed which demonstrated active hemorrhage from a branch of the proximal superior mesenteric artery, most consistent with the jejunal branch. Attempts of cannulation and embolization by interventional radiology were unsuccessful due to the small size of the bleeding vessel, and thus the patient was brought emergently to the operating room for exploratory laparotomy. During surgery, a massively dilated small bowel and colon filled with blood was seen. A diverticulum in the proximal jejunum was ligated. An esophagogastroduodenoscopy (EGD) with push enteroscopy was performed immediately after surgery demonstrating old blood in duodenum and stomach, with no active bleeding. During the surgery, the patient was noted to have bleeding from his nose and mouth. Laboratory values at that the time were significant for a prolonged PTT (52.7 s) and PT (17.3 s) and a low fibrinogen level of $101 \mathrm{mg} / \mathrm{dL}$. The platelet count had also decreased from 230 to $8 \mathrm{~K} / \mathrm{uL}$. Based on the clinical and laboratory findings, the patient was determined to be in disseminated intravascular coagulation (DIC). Throughout the course of admission, the patient received 2 additional units of prbc, 2 units of FFP, and 1 unit of platelets. Over the next 7 days, the patient was extubated and his hemoglobin, platelet count, PTT, and PT stabilized back to his baseline with no further bleeding. Given that our patient was a young, healthy male with no comorbidities, the etiology of his gastrointestinal hemorrhage and DIC was thought to be secondary to his SCB use. At follow up 3 months later, patient remained clinically stable and reported cessation of synthetic cannabinoids.

DISCUSSION: To our knowledge, we describe here the first case of synthetic cannabinoid associated DIC and gastrointestinal (GI) hemorrhage. Synthetic cannabinoids (SCB) is inhaled or consumed orally for its psychoaffective effects, and it has recently become a large public concern due to increased use and unpredictable toxicity. It is marketed as containing blends of herbs and is given the street names " spice", "K2", "herbal incense", "cloud 9", and "mojo". The prevalence of its use is not well documented given the inability to be detected on routine drug screen. Like natural cannabinoids or tetrahydrocannabinol (THC), synthetic cannabinoids stimulate cannabinoid receptor 1 in the brain 
and cannabinoid receptor 2 in the gastrointestinal system and immune system. There have, however, been higher rates of hospital admissions and toxicities with synthetic cannabinoids as compared to the natural cannabinoids. The reason is thought to be due to the fact that the synthetic cannabinoids act directly on the cannabinoid receptors, while the natural cannabinoids act only as a partial agonist. Furthermore, these SCBs often contain unknown contaminants. To date there have not been many studies that examine the toxicity profile of synthetic cannabinoids. According to the Poison Control Center and few case reports, the most common toxicities include the following: dry mouth, memory impairments, hallucinations, delusions, nausea, and dizziness. There are also few case reports that describe more serious complications, including myocardial infarction, acute kidney injury, hyperthermia, and rhabdomyolysis. In conclusion, it is important to recognize that SCB can be associated with GI bleeds and DIC. The mechanism of action to describe this toxicity is not completely understood. Detection of SCB use is extremely difficult since it is not picked up on routine drug screening. It is based solely on patient's history. Treatment of the toxicities associated with SCB centers around symptomatic control.

\section{UREMIC LUNG: IS IT SIMPLE PULMONARY EDEMA OR PNEUMONITIS?} Alka Lamsal Ghimire. Suny Upstate Medical University, Jamesville, NY. (Control ID \#2469829)

LEARNING OBJECTIVE \#1: \# To broaden the differential diagnosis of hemoptysis in patients with ESRD.

CASE: A 26 year-old male with ESRD due to congenital dysplastic left kidney and focal segmental glomerulosclerosis (FSGS) of right kidney presented with coughing up blood for over a month. He was initially treated as community acquired pneumonia with levofloxacin by primary care physician after a chest $\mathrm{x}$-ray showed bilateral lung infiltrate Patient denied fever, chills, night sweats or chest pain. After completing antibiotics, he continued to report hemoptysis. A CT chest was done that showed diffuse alveolar infiltrate and patient was admitted for further evaluation. On admission, patient reported missing hemodialysis for more than a month and complained of dyspnea on exertion along with coughing up blood. On examination, he was afebrile, tachycardic with HR of 117, BP 141/86, and saturating $97 \%$ on $2 \mathrm{~L}$ nasal cannula. He was in mild respiratory distress with hyper dynamic precordium and coarse rales were heard bilaterally on auscultation. There was no JVD, pericardial friction rub, asterixis or peripheral edema. Laboratory tests revealed Creatinine 27.2, BUN, 226, Na 139, K 5.4, Hb 5.0, Hct 15.3, UA showed 2+ proteins, WBC 1, RBC 4, hemoglobin 1+. CXR showed diffuse interstitial and alveolar opacities within the lung bases. CT thorax showed ground glass opacities predominantly in the lower lobe with smooth interlobular septal thickening. Echo showed normal ejection fraction with moderate mitral regurgitation. Patient received hemodialysis on day 1, 3 and 5 with total fluid removal of 81 . Hemoptysis resolved and chest $\mathrm{x}$-ray improved but the interstitial changes didn't completely resolve. A bronchoscopy with BAL was done which was consistent with diffuse alveolar hemorrhage. Cultures were negative and so were the work up for autoimmune diseases. Based on the resolution of hemoptysis and DOE but incomplete resolution of reticular infiltrate and absence of clear signs of fluid overload, we concluded that patients' symptoms were from uremic pneumonitis resulting in alveolar hemorrhage rather than just from fluid overload from missed hemodialysis.

DISCUSSION: Discussion: Pulmonary manifestation of chronic kidney disease and ESRD has been historically lumped as "uremic lung" without clear distinction between cardiogenic pulmonary edema resulting from volume overload and left ventricular dysfunction from non-cardiogenic pulmonary edema resulting from pneumonitis secondary to inflammation and epithelial injury. The first entity readily resolves with fluid removal whereas acute lung injury from pneumonitis may not completely resolve with fluid removal. This "uremic pneumonitis" lung has more inflammatory component with histological evidence of proteinaceous material and hyaline membrane formations in the lung.
This however doesn't completely correlate with degree and the duration of uremia i.e pneumonitis can develop with BUN level less than 100 and within a week of uremia symptoms. When this is coupled with platelet dysfunction from uremia, it can lead to diffuse alveolar hemorrhage which resolves after restoration of platelet function by hemodialysis but the radiological resolution lags behind the clinical resolution. With improved recognition and care of renal disease and access to dialysis, "uremic pneumonitis" which was common in the pre-dialysis era, is rare nowadays. Patients typically present with dyspnea and reversible radiological findings. Uremic pneumonitis can be protean in presentation and could be confused for other disease entities such as acute pulmonary edema, pneumonia, autoimmune, fungal or metastatic disease. It is important for clinicians to consider this rare condition as part of their differential diagnosis in the setting of hemoptysis and renal failure even when blood urea nitrogen levels are not very high

URINARY RETENTION AND SHINGLES IN A PATIENT WITH HIV/AIDS Hugo A. Torres ${ }^{1,2}$; Krupa Parikh ${ }^{1,2}$; Sarah Stoneking ${ }^{1,2}$. ${ }^{1}$ Cambridge Health Alliance, Cambridge, MA; ${ }^{2}$ Harvard Medical School, Cambridge, MA. (Control ID \#2469505)

LEARNING OBJECTIVE \#1: Diagnosis and management of Immune Reconstitution Inflammatory Syndrome (IRIS)

LEARNING OBJECTIVE \#2: Diagnosis of Varicella Zoster Virus (VZV) neurological disease

CASE: A 31-year-old woman recently diagnosed with Acquired Immunodeficiency Syndrome (AIDS) presented to our hospital with acute back pain and urinary retention. Three days prior to admission, the patient developed right lower back pain and right lower quadrant abdominal pain. The following day, she developed urinary retention and noticed a red painless rash on her upper back. At the time of diagnosis of AIDS 2 months prior to her admission, the patient's CD4 count was 14 with a CD4 percentage of 2 . Her viral load was 1.2 million and genotyping showed no evidence of resistance to anti-retrovira medications. She had sore throat and pharyngeal exudate at that time but no other evidence of opportunistic infection (OI). She was started on tenofovir-emtricitabine-efavirenz. Two weeks after starting this medication, her viral load decreased to 681, with CD4 of 19 (CD4 percentage of 3). She was taking no medication other than the anti-retrovirals. She was from Thailand and had been in the United States for 1 year, working at a restaurant. She reported being sexually active with men in the past and used condoms the majority of the time. She had no history of substance use. On admission, she was afebrile and tachycardic She had mild abdominal tenderness over the suprapubic area, a vesicular rash on the right in approximately the T6-8 distribution, diminished tendon reflexes throughout, mild weakness of the lower extremities, and bilateral up-going Babinski reflexes. Sensation, coordination, and gait were intact. Her labs were notable for a mild hyponatremia. Other electrolytes, GFR, and CBC were within normal limits. Hepatic function tests were normal, and total protein was elevated at 9.7. A foley catheter drained $1100 \mathrm{cc}$ of urine. Abdominal CT showed mild hydronephrosis of both kidneys. A contrast-enhanced MRI of the brain and spinal cord showed long segment signal abnormality throughout the thoracic cord and increased dural enhancement of the lumbar spine. There were scattered signal abnormalities in the brainstem and bilateral cortical/subcortical regions without contrast enhancement. A lumbar puncture revealed 99 RBCs and 449 WBCs with $84 \%$ lymphocytes and $15 \%$ monocytes. Glucose was 94 and protein was 689 . VZV IgG was strongly positive. The remainder of testing of the CSF was negative. These findings raised concern for VZV encephalomyelitis and IRIS. Treatment was initiated with IV Methylprednisolone and Acyclovir. On hospital days 2-3, the patient's sensation to light touch and pin prick decreased from approximately the left T5-6 level and down the left abdomen and entire left leg. Subsequently, her neurological exam remained stable, and she was discharged on hospital day eight. Four weeks after discharge, she was able to void on her own and no longer had any sensory symptoms. 
DISCUSSION: VZV neurological disease can manifest in a myriad of ways including multifocal leukoencephalitis, ventriculitis, disseminated hemorrhagic necrotizing vasculitis, myelitis, myeloradiculitis, and vasculopathy of the leptomeningeal arteries causing cerebral infarction. These processes may be associated with lymphocytic pleocytosis of the CSF and evidence of dermatomal zoster, but such findings are not always present. Symptoms are variable and depend on the anatomic site of CNS involvement. Diagnosis is made by demonstration of VZV antibody or DNA in the CSF. Our patient had evidence of dermatomal zoster, urinary retention, as well as sensory deficits on the left side of her body, strongly positive VZV IgG in the CSF, and MRI findings consistent with transverse myelitis. Urinary retention is very rarely reported as a presenting feature of VZV neurological disease. Furthermore, VZV neurological disease in the setting of IRIS is an uncommon phenomenon described in case reports. There is currently no consensus definition of IRIS, thus limiting its diagnosis and further study of appropriate therapeutic regimens. Theoretically, IRIS is a clinical manifestation of enhanced inflammatory response by CD8 T cells to an underlying OI. Most proposed definitions of IRIS include the following requirements: worsening of clinical status after the initiation of highly active anti-retroviral therapy (HAART), log fold or greater decrease in viral load in response to HAART therapy, and the presence of new symptoms not otherwise explainable by a new infection, inflammatory disease, or by the known course of a previously diagnosed disease. Patients with CNS-IRIS tend to have enhancement of the leptomeningeal spaces, spinal cord, and brain parenchyma on neuroimaging. The treatment of IRIS is controversial. Generally, HAART should be continued to maximize immune function and avoid development of new OIs. The initiation of steroids is a point of contention, and further study is needed to address this issue. Our patient improved after treatment with a short course of steroids and 2 weeks of Acyclovir, along with continuation of HAART. In conclusion, VZV-IRIS is a rare entity diagnosed with appropriate CSF studies and neuroimaging findings. The diagnosis of IRIS is clinical and based on timing of symptoms with regard to initiation of anti-retroviral therapy. Treatment consists of continuing HAART, managing the underlying OI and providing supportive care, with the possible addition of steroids dependent on patient clinical condition.

USING BEDSIDE ULTRASOUND TO DIAGNOSE AN UNUSUAL CAUSE OF DYSPNEA IN A PATIENT WITH LYMPHOMA Yifei Zhang; Martin C. Fried; Saleem Ali. NYU, Astoria, NY. (Control ID \#2469638)

LEARNING OBJECTIVE \#1: Recognize that Diffuse Large B-Cell Lymphoma can rapidly progress to cardiac involvement

LEARNING OBJECTIVE \#2: Encourage resident education in the use of bedside ultrasound as part of the evaluation of patients with dyspnea

CASE: Mr. B is a 78 years old man with history of coronary artery disease and paroxysmal atrial flutter on warfarin who presented with dry cough, fatigue, and weight loss for the past 6 weeks. His physical exam was notable for right supraclavicular lymphadenopathy and decreased breath sounds on his left lung with chest x-ray revealing a moderate-sized left pleural effusion. CT chest and abdomen showed diffuse lymphadenopathy above and below the diaphragm, as well as a loculated left pleural effusion. Mr. B underwent core biopsy of a left cervical lymph node which was consistent with diffuse large B cell lymphoma. He was subsequently discharged with plans for oncology followup. Unfortunately, soon after discharge he was readmitted with new shortness of breath at rest while on 21 of oxygen via nasal cannula. Because of the known unilateral pleural effusion, pulmonology was consulted to consider thoracentesis. Bedside ultrasound showed stable left pleural effusion as well as a new small right pleural effusion. Due to suspicion that patient's hypoxia was out of proportion to his pulmonary findings, bedside echocardiogram was done. This revealed an epicardial mass and pericardial effusion. A formal echocardiogram confirmed the presence of a large heterogeneous mass adherent to the epicardial surface of the right heart within the atrio-ventricular (AV) groove. On doppler imaging flow was noted within the mass which likely represented encasement of the right coronary artery (RCA). A moderate-sized pericardial effusion was also present without echocardiographic evidence of tamponade. The patient's dyspnea was likely caused by both the enlarging bilateral pleural effusions as well as the newly discovered epicardial mass with pericardial effusion. He was transferred to the MICU for urgent chemotherapy. A bedside thoracentesis was performed draining $1500 \mathrm{~cm}^{3}$ of exudative fluid. During the hospitalization the patient underwent two cycles of R-miniCHOP, and was discharged in stable condition. Repeat echocardiogram 1 month later showed decreased size of the epicardial mass as well as reduced pericardial effusion.

DISCUSSION: Cardiac involvement from diffuse large B-cell lymphoma is a rare clinical presentation and makes up less than $1 \%$ of the total cases. Lymphomas can metastasize to the heart through direct extension, hematogenous and lymphatic spread, or by intracavitary extension through either the inferior vena cava or the pulmonary veins. Epicardial involvement, which is seen in our patient, is hypothesized to be the result of retrograde lymphatic spread through tracheal or bronchomediastinal lymphatic channels after prior diffusion of the tumor to the pericardium. Our case also demonstrates how early detection of cardiac metastases is critical in preventing cardiac complications. Bedside echocardiogram was done in our patient because the pleural effusions seen on the lung ultrasound did not seem sufficient to explain the patients' dyspnea. This led to the accurate diagnosis and appropriate initiation of emergent chemotherapy. Although bedside ultrasound education has long been a facet of critical care and emergency medicine education, this case highlights the usefulness of bedside ultrasound education for internal medicine (IM) residents while evaluating patients with unexplained dyspnea. A 2012 survey of US IM programs found that, despite being viewed as a highly valuable tool, only $25 \%$ of the programs had formal ultrasound training with another $25 \%$ planning to initiate such a program. The programs averaged about $10 \mathrm{~h}$ on didactics and $13 \mathrm{~h}$ on hands-on training, and most of the curriculum include ultrasound-guided central venous catheter placement, paracentesis, and thoracentesis. Time required to train faculty and residents is rated to be the greatest barrier to implementation. However, in a group of emergency residents, it was found that after a 30 -min training course in thorax examination novice sonographers were able to identify B-lines with similar accuracy as an expert sonographer. Some studies have shown that with limited education novice ultrasonographers can identify abnormalities more accurately than specialists just relying on physical examination. A study done with IM residents showed that training in the usage of pocket ultrasound for two 90-min session corresponded to improved accuracy in diagnosis of dyspnea in diagnoses of COPD, pulmonary edema, pneumonia, and pleural effusion. Similarly, studies on cardiac ultrasound education show that physicians can be trained to detect cardiac abnormalities such as systolic dysfunction and pericardial effusion. Our case demonstrates the utility of bedside ultrasound in evaluating a new complaint. While ultrasound education has traditionally been organized by organ system, perhaps it is time to teach symptomfocused ultrasound education. Such a shift may lead to even more rapid diagnoses of uncommon pathologies, as was the case in our patient with an epicardial lymphomatous mass.

VALPROATE-RELATED HYPERAMMONEMIC ENCEPHALOPATHY WITHOUT UNDERLYING LIVER DISEASE Brian V. Dinh ${ }^{1}$; Kyle Ball ${ }^{1}$; Sangeeta Agrawal $^{2} .{ }^{1}$ Wright State University, Dayton, $\mathrm{OH} ;{ }^{2}$ Dayton VA Medical Center, Dayton, OH. (Control ID \#2457640)

LEARNING OBJECTIVE \#1: Valproic acid (VPA) has been used for years to treat seizure disorders, mania, mood disorders, neuropathy, dementia, and for migraine prophylaxis. Common side effects are pancreatitis, teratogenicity, hepatotoxicity, and thrombocytopenia. Less commonly, VPA has been known to cause hyperammonemia with normal liver enzyme levels 
termed valproate-related hyperammonemic encephalopathy (VHE). Symptoms vary from lethargy and confusion to coma and death. Because it is rarely reported, the diagnosis is often missed.

CASE: A 60 year-old white male with a history of depression, migraine, osteoarthritis, neuropathy, and chronic opioid use for back pain presented to the emergency department with generalized weakness, somnolence, and confusion for 1 week. His medications included fluoxetine, VPA, sumatriptan, methocarbamol, gabapentin, oxycodone-acetaminophen, and a fentanyl patch. On examination, the patient was afebrile, somnolent, and slow to respond. Strength was $4 / 5$ in upper and lower extremities with patchy decreased sensation. Basic lab work was within normal limits. Urine drug screen was positive for opiates, and ammonia was elevated at 48 umol/L. CT head and brain/spine MRI showed no acute abnormalities. Vitamin B12, folate, and TSH/T4 were within normal limits. VPA level was $58 \mathrm{mcg} / \mathrm{ml}$ (nl 50-125). Hyperammonemia was initially attributed to some underlying liver disease. Lactulose was started resulting in improvements of his symptoms. An outpatient liver biopsy 2 weeks later was normal. Four months later, his symptoms recurred, and rifaximin was added. Concurrently, VPA was discontinued due to ineffectiveness for migraines. Follow up at the gastroenterology clinic showed resolution of weakness and confusion with normalization of his hyperammonemia

DISCUSSION: VHE is a rare side effect of VPA. Therefore, it is not routinely considered in the differential diagnosis. While previous reports describe VHE with toxic VPA levels, our case stresses the importance of considering VHE despite normal drug levels and a normal liver function test. Studies have shown that VHE may occur at therapeutic levels of VPA. Because this patient underwent unnecessary liver biopsy and extensive workup before VPA was discontinued and the diagnosis was made, the case highlights the need to consider all adverse drug reactions. Diagnosis of VHE can present a challenge and lead to unnecessary testing and invasive procedures. Other causes of acute hyperammonemia include hepatic congestion, cirrhosis, acute hepatitis, drugs, and inborn errors of metabolism. VPA is suspected to induce hyperammonemia via VPA's active metabolite or through interactions related to carnitine deficiencies. Therefore, isolated hyperammonemia in a patient taking VPA should lead to a diagnosis of VHE and help eliminate unnecessary testing.

VANCOMYCIN INDUCED LINEAR IGA BULLOUS DERMATOSIS Kurtis J. Swanson. Medical College of Wisconsin Affiliated Hospitals, Milwaukee, WI. (Control ID \#2470255)

LEARNING OBJECTIVE \#1: To report a case of vancomycin induced linear IgA bullous dermatosis

LEARNING OBJECTIVE \#2: To discuss diagnostic approach and management of this disease

CASE: 86 years old male with a PMH of left knee osteoarthritis s/p total knee arthroplasty complicated by prosthetic joint infection s/p vancomycin/tobramycin spacer placement plus IV vancomycin, hypothyroidism, Raynaud's phenomenon, anemia, and CKD presents with rash. No other complaints. Vitals stable on presentation. On exam, found to have multiple eruptions including $<5 \mathrm{~mm}$ erosions with scant overlying hemorrhagic crust on the bilateral lower lip, few $<5 \mathrm{~mm}$ erosions with fibrinous base on hard/soft palate and upper lip mucosa, many $0.2 \mathrm{~cm}-5 \mathrm{~cm}$ circular urticarial papules and plaques, some vaguely targetoid on the back $>$ upper arms, axillae, and trunk, few $<1 \mathrm{~cm}$ vesicles on the scrotum and intertriginous areas of inner thighs, $1.5 \mathrm{~cm}$ bulla on the right medial thigh, few $<2 \mathrm{~cm}$ bulla on the left lateral thigh, multiple hemorrhagic vesicles and bullae on the bilateral posterior left > right thigh, many erosions with overlying hemorrhagic crusts on the left thigh and proximal lower leg, surrounding previous surgical incision and multiple pink edematous papules and scattered $1-4 \mathrm{~cm}$ tense bullae on the bilateral palmar hands and plantar feet. Also had periorbital erythema and conjunctival injection of the left eye. Bullae remarkable for hemorrhagic and serous fluid. Labs remarkable for negative ANA, negative anti-dsDNA, negative anti-SCL-70. Dermatology consulted who obtained punch biopsy remarkable for focal subepidermal blistering disease; numerous neutrophils and some eosinophils plus small collections of neutrophils in dermal papillae consistent with linear IgA bullous dermatosis, bullous lupus erythematosus, or dermatitis herpetiformis. Direct immunofluorescent stain remarkable for linear deposits of IgA at the basement membrane zone. Infectious disease was consulted and recommended changing antibiotic to daptomycin based on organism susceptibilities. Orthopedics was consulted and recommended keeping antibiotic spacer to optimize future joint mobility. Ophthalmology was consulted and recommended conservative compress management given lack of concerning eye involvement. Patient started on colchicine $0.6 \mathrm{mg}$ BID due to concerns with dapsone (anemia), prednisone (infection), and unavailability of sulfa-based agents used abroad. Patient received BID colchicine for 14 days with improvement of his rash per Dermatology follow up with discharge to subacute rehabilitation facility.

DISCUSSION: Linear IgA bullous dermatosis (LABD) is a rare idiopathic, drug-induced or immune mediated mucocutaneous dermatologic disorder $(0.25-1.45$ cases/million worldwide). It occurs in a bimodal distribution, manifesting in patients age 6 months and 10 years old and over 60. Lesions varied in nature ranging from tense serous/ hemorrhagic bullae, string of pearl "herpetiform" configurations to targetoid/erythema multiforme-like eruptions. Linear deposition of $\operatorname{IgA}$ at the dermoepidermal junction - seen both on H\&E as well as direct immunofluorescence staining- is a key feature of LABD. Diagnosis centers on the absence of gluten enteropathy/dermatitis herpetiformis or systemic lupus erythematosus as these entities can be indistinguishable from LABD grossly (pending the size and extent of vesicles or bullae) or microscopically. In our case, patient denied any history of gluten enteropathy, dermatitis herpetiformis, or similar eruption. History alone with sufficient evidence to suggest a drug related cause led us to not test for gluten intolerance. Patient did manifest 3/11 criteria for SLE (anemia, ora sores, rash) plus a history of Raynaud's phenomenon, warranting serologic testing, which was negative. While patient could theoretically have mixed connective tissue disease, which can overlap with SLE, we did not test for anti-U1 RNP given negative ANA, antidsDNA, and lack of other key historical and clinical findings (predominance in women; age of onset 20-30s; myalgias/myopathy; pulmonary HTN). Should his rash return and/or Raynaud's worsen, and/or myalgias, respiratory issues, arthralgias arise, further testing and Rheumatology referral would be indicated. Anemia mixed with more likely explanations (iron deficiency + chronic kidney disease) and sores/rash and timeframe consistent with drug induced linear IgA bullous dermatosis. Of known drug causes of LABD, Vancomycin in the most common. Other implicated medications include PCNs, cephalosporins, ACEis, NSAIDs. Rarely, furosemide, atorvastatin and ARBs can cause LABD. Accepted treatments for LABD are dapsone, topical corticosteroids, sulfonamides (sulfapyridine and sulfamethoxypridazine) and colchicine. While Red Man Syndrome, Type I hypersensitivity reactions, Drug Reaction with Eosinophilia and Systemic Symptoms (DRESS) and other drug eruptions can occur in the setting of vancomycin administration, linear IgA bullous dermatosis is another important eruption to recognize, diagnose via biopsy while ruling out gluten enteropathy and SLE, and manage medically with a patient appropriate agent

VEGGIES IN PLEURAL FLUID Kannan Thanikachalam ${ }^{1}$; Benjamin Butler ${ }^{2} .{ }^{1} \mathrm{UAB}$ Health Center Montgomery, Montgomery, AL; ${ }^{2}$ University of Alabama Birmingham, Montgomery, AL. (Control ID \#2470317)

LEARNING OBJECTIVE \#1: To recognize gastro-pleural fistula as a possible cause of empyema in patients with metastatic GI cancer

CASE: Patient is a 63 year old black female with a past medical history of metastatic colon cancer and was admitted with worsening abdominal pain and 
fatigue. Patient had refused chemotherapy and surgery in the past and was no longer a surgical candidate. On examination, patient was alert, oriented, and cardiovascular examination was normal. Patient had decreased breath sounds bilaterally and had a tense and distended abdomen. CT Abdomen and pelvis was done which showed a huge abdomino-pelvic tumor extending from just beneath the hemidiaphragms down into pelvis and was filling almost the entirety of the intraabdominal peritoneal space. Patient also had bilateral hydronephrosis and metastases to left lower lobe of lung, multiple mesenteric, peritoneal and pelvic implants. Patient underwent placement of bilateral ureteral stents. However, during the course of hospitalization, patient went into respiratory distress and chest xray revealed worsening left sided pleural effusion. Patient was intubated. A chest tube was inserted to the left pleural cavity and greenish brownish fluid was drained. The fluid was grossly exudative with a WBC count of 35,000 with $80 \%$ Monocytes and LDH of 10,000. Culture and sensitivity grew Stenotrophomonas maltophilia, Enterococcus faecium and yeast. Cytology of the pleural fluid showed acute inflammation, yeast forms and vegetable type material. Patient was on tube feeds and the colour of the pleural fluid changed to yellowish in colour. Given the findings of the pleural fluid and macroscopic appearance of the pleural fluid, a pleuro-enteral fistula was suspected. A Gastrograffin study was done which revealed contrast extravasating from the stomach into the pleural cavity and also around the spleen. Patient was started on Vancomycin, piperacillin-tazobactam and micafungin.

DISCUSSION: Gastro-pleural fistulas are uncommon connections between the stomach and pleural cavity. Gastro-pleural fistulas have been reported in literature as complications following intrathoracic perforation of stomach in hiatal hernia, traumatic diaphragmatic hernia with perforation of stomach and intraperitoneal gastric perforation with erosion of sub phrenic abscess via diaphragm, multiple chest tube insertions, esophageal rupture either spontaneously or iatrogenically, gastric malignancy such as lymphomas, trans diaphragmatic gastric penetration after an empyema thoracis, following surgical procedures. Gastro-pleural fistulas should be suspected in patients where the pleural fluid composition resembles gastric fluid. Bedside procedures such as $\mathrm{pH}$ of the pleural fluid, presence of bile salts, and appearance give us a better idea about the possibility of gastro-pleural fistulas. For a definitive diagnosis, patients may require esophagogastroduodenoscopy or diagnostic laparoscopy or administration of contrast orally and aspiration from pleural cavity. Patient's etiology for the fistula was attributed to the metastatic mesenteric implant located near the stomach. Given the severity of illness in our patient, we chose to perform upper GI series with administration of oral contrast and looked for aspiration into pleural cavity. Management involves treatment of the empyema with antibiotics, Chest tube placement, feeding either enterally or parenterally. Definitive correction by surgery is required.

VENLAFAXINE INDUCED HYPOGLYCEMIA, A RARE SIDE EFFECT OF A COMMON DRUG. Ayush Motwani; Jayna M. Gardner-Gray; Fawad Virk. Henry Ford Health System, Detroit, MI. (Control ID \#2469964)

LEARNING OBJECTIVE \#1: To identify the rare and potentially life threatening side effects associated with the use of Venlafaxine, a commonly used antidepressant and anti anxiety medication.

LEARNING OBJECTIVE \#2: To recognize the importance of dose adjustments of medications in patients with preexisting co morbidities to prevent their accumulation in the body and subsequent development of side effects.

CASE: A 39 year old Caucasian male presented to a community emergency department with chief complaint of increasing weight gain, fatigue and lower extremity edema. His past medical history was significant for morbid obesity with gastric bypass in 2006, liver cirrhosis most likely secondary to alcohol abuse and anxiety. On presentation he was found to be persistently hypoglycemic despite 12 ampules of dextrose $50 \%$ injections. The patient was subsequently started on a dextrose $10 \%$ intravenous infusion and transferred to our intensive care unit at a tertiary academic care facility for escalation of care. On arrival to the intensive care unit he was having episodes of symptomatic hypoglycemia evidenced by tremors, dizziness and tachycardia. Investigation into the etiology of his persistent hypoglycemia included an infectious evaluation. Diagnostic paracentesis, chest radiograph, blood cultures and urine analysis were all negative for infection. Endocrinology evaluation revealed an Insulin level of $3 \mathrm{uIU} / \mathrm{mL}$, Insulin like growth factor of $17 \mathrm{ng} / \mathrm{mL}$, cortisol level of $6.4 \mathrm{ug} / \mathrm{dL}$. Co syntropin test was within normal limit. Of note our patient did not have a history of diabetes and did not take any insulin products. Computed tomography of the abdomen did not reveal any pancreatic mass. Upon medication reconciliation it was discovered that our patient had been taking Venlafaxine for anxiety. This was discontinued on hospital admission. During the first 2 days of our patient's hospitalization it was observed that his blood sugars were improving. However, on second day of admission he developed anxiety and was given his home dose of venlafaxine. Within $30 \mathrm{~min}$ of receiving this medication his blood glucose level dropped to 35 . At this point the medication was discontinued and the patient's blood glucose levels subsequently normalized.

DISCUSSION: The common side effects associated with venlafaxine are nausea, dizziness, dry mouth, insomnia, diaphoresis and constipation. Drug overdose can lead to hemodynamic instability, cardiac arrhythmias, seizures, and serotonin syndrome. However a less common side effect associated with this drug is hypoglycemia which has been rarely reported. The effect of Venlafaxine on glucose metabolism has not been well understood however it has been hypothesized that it increases the uptake of glucose in the peripheral tissues. Venlafaxine is primarily metabolized in the liver mainly via the CYP2D66 isoenzyme. The rate of poor metabolizers is higher in the Caucasian population $(6-10 \%)$. The primary route of excretion of the drug and its metabolites is through the kidneys Thus in patients with preexisting liver disease and acute kidney injury, decreased excretion of the drug may lead to its accumulation leading to toxic effects at otherwise normal therapeutic doses.

VENOUS AND ARTERIAL THROMBOSES IN NEPHROTIC SYNDROME: WHERE ONLY WARFARIN HAS WALKED Hassan Kamran ${ }^{1}$; Erin Finn ${ }^{1}$; Qasim Khalil $^{2,}{ }^{1}$; Jeffrey Bates ${ }^{2,1}$; Meroe Morse ${ }^{2}$. ${ }^{1}$ Baylor College of Medicine, Houston, TX; ${ }^{2}$ Michael E. DeBakey VA Medical Center, Houston, TX. (Control ID \#2469697)

LEARNING OBJECTIVE \#1: Recognize nephrotic syndrome as a cause of both venous and arterial thromboses.

LEARNING OBJECTIVE \#2: Understand the role of anticoagulation in nephrotic syndrome.

CASE: A 34-year-old man with a history significant only for hypertension was admitted and treated at an outside hospital for a pulmonary embolism and a non-ST elevation myocardial infarction. His pulmonary embolism was unprovoked and his troponin-I peaked at $33 \mu \mathrm{g} / \mathrm{L}$. Cardiac catheterization showed only a $50 \%$ mid-LAD stenosis. Transthoracic echocardiogram demonstrated global systolic and diastolic dysfunction with a LVEF of 35-40\%. The patient was discharged on rivaroxaban but discontinued use after 1 week. Two months later he was readmitted to our hospital and found to have a left ventricular thrombus on cardiac MRI and bilateral renal infarcts. Troponins were undetectable, and a repeat transthoracic echocardiogram was unchanged from the prior exam. Laboratory data showed proteinuria $>500 \mathrm{mg} / \mathrm{dl}$, albumin of $1.4 \mathrm{~g} / \mathrm{dL}$, total cholesterol of $538 \mathrm{mg} / \mathrm{dL}$, and a protein-to-creatinine ratio of 6.9 , consistent with nephrotic syndrome. ANA, C-ANCA, P-ANCA, C3, C4, Hepatitis B/C, HIV, RPR, kappa free light chains, 
renal ultrasound with doppler, and serum/urine electrophoresis were all unrevealing for additional etiologies. Factor V Leiden mutation, homocysteine levels, anticardiolipin antibody, protein $\mathrm{C} / \mathrm{S}$ levels, and lupus anticoagulant were all sent as part of a secondary hypercoagulable workup and were negative. A kidney biopsy was obtained, and he was treated with IV heparin while being transitioned to warfarin. Two weeks later, the patient was readmitted to our hospital a second time with a subtherapeutic INR and a second nonST elevation myocardial infarction; his troponin-I peaked at $11 \mu \mathrm{g} / \mathrm{L}$. His kidney biopsy revealed membranous nephropathy, and the patient was initiated on pulse steroids followed by daily maintenance therapy. He was managed conservatively with observation and anticoagulation optimization.

DISCUSSION: Nephrotic syndromes are known to cause hypercoagulable states; however, the venous thromboembolic risk is often appreciated more than the arterial thromboembolic risk. The prothrombotic state seen in patients with nephrotic syndrome has been attributed to urinary loss of antithrombotic proteins such as antithrombin III, protein $\mathrm{C}$, or protein $\mathrm{S}$, impaired thrombolytic activity due to urinary loss of plasminogen, and increased hepatic production of prothrombotic proteins such as fibrinogen. Thrombotic events include not only the classic findings of deep venous thromboses and pulmonary emboli, but also arterial events such as acute coronary syndromes (ACS) and renal artery thromboses. Studies looking at rates of thromboembolism show high rates of both venous and arterial events in the initial 6 months of diagnosis, 9.85 and $5.52 \%$ respectively Unlike venous thromboses, arterial thromboses rates have no relationship to serum protein or albumin levels and are more difficult to predict and thus risk stratify. Prophylactic anticoagulation in asymptomatic patients is not routinely performed and the decision to anticoagulate is made on a case-by-case basis. Patients with overt thromboembolic events are treated long term with warfarin as there is currently limited data on the use of the nove oral anticoagulants in this population. The duration of treatment to prevent recurren events is unknown, but treatment is typically continued as long as the nephrotic state remains. The increased risk of arterial events in nephrotic syndrome has important implications for how we approach and risk stratify individuals presenting with classic symptoms such as chest pain for acute coronary syndrome or abdominal pain for nephrolithiasis.

VENTRICULAR SEPTAL DEFECT COMPLICATING AN ACUTE MYOCARDIAL INFARCTION: A CASE REPORT Oluchukwu Oluoha ${ }^{1}$; Olufolahan Carrena ${ }^{2}$; Ali Salem ${ }^{1}$. John H. Stroger, Jr. Hospital of Cook County, Chicago, IL, Chicago, IL; ${ }^{2}$ Meharry Medical College, Brentwood, TN. (Control ID \#2470234)

LEARNING OBJECTIVE \#1: Identify the risk factors for mechanical complications of acute myocardial infarction

LEARNING OBJECTIVE \#2: Recognize the clinical features of post myocardial infarction venticular septal defect

CASE: A 55-year-old man presented to the emergency department with a 3-week history of exertional dyspnea and bilateral lower extremity edema. Medical history is significant for hypertension. His symptoms started shortly after an episode of chest pain which he described as sudden, severe, retrosternal pain that lasted approximately $4 \mathrm{~h}$. Pain was associated with nausea, diaphoresis and palpitation. Patient did not seek medical care at the time and only took ibuprofen for pain relief. He started experiencing progressive exertional dyspnea afterwards. On initial examination, vital signs were normal. Cardiopulmonary examination revealed jugular venous distension, bilateral basilar crackles, grade 4/6 holosystolic murmur across the precordium, and bilateral pitting pedal edema. Electrocardiography showed Q waves and ST depression in leads II, III and AVF. Laboratory studies revealed an elevated serum troponin I levels and a B-type natriuretic peptide level of $1322 \mathrm{pg} / \mathrm{mL}$. Chest radiography showed pulmonary vascular congestion. Patient was subsequently admitted to the medicine floor with presumptive diagnosis of heart failure, prompting further evaluation with transthoracic echocardiography which revealed mild systolic dysfunction with dyskinesia and aneurysmal deformity of the basal inferoseptal wall. Echocardiography with color flow doppler also revealed moderate-sized defect in ventricular septum with left-to-right shunt, and total pulmonary blood flow to total systemic blood flow ratio (Qp/Qs ratio) of 1.9. These findings were consistent with ventricular septal defect (VSD) complicating an acute myocardial infarction. Patient was subsequently transferred to the coronary care unit where he underwent coronary angiography that showed severe stenosis in mid-right coronary artery. This lesion appeared to be the culprit for the recent acute myocardial infarction and consequent septal rupture. Right heart catheterization was performed to confirm the diagnosis of VSD. Cardiothoracic surgery service was consulted for VSD repair, which patient ultimately had with associated coronary artery bypass graft approximately 4 weeks after acute myocardial infarction. Unfortunately, patient died 2 days after surgery from cardiogenic shock.

DISCUSSION: Ventricular septal rupture is a rare but potentially devastating complication that can occur following acute myocardial infarction (AMI). It typically occurs 3 to 5 days after an AMI and delayed hospitalization $(\geq 24 \mathrm{~h})$ increases the risk in predisposed patients. First MI, advanced age, history of hypertension, absence of heart failure before rupture and sustained physical activity after AMI, particularly before hospital admission may increases the risk of septal rupture as described by Figueras J et al. Anatomically, rupture is usually due to anterior infarcts in the apical septum but right coronary artery infarct can causes inferior septal rupture as in the case with this patient. Rupture usually occurs at the margin of necrotic and non-necrotic myocardium and patients typically present with an abrupt onset of heart failure, new systolic murmur, and a palpable thrill. This case highlights the risk of ventricular septal rupture following AMI in patients with delayed hospital presentation. It also demonstrated the need for a high index of suspicion for this complication in patients with AMI presenting with acute heart failure, new systolic murmur with possible thrill. Diagnostically, two-dimensional transthoracic echocardiography with color flow doppler provides a rapid and reliable means of diagnosis but confirmation of diagnosis requires right heart catheterization to document the left-toright shunt. Management varies with clinical presentation but surgical repair is the mainstay of treatment, although, the timing of surgery is controversial. Urgent surgical interventional is warranted in patients with cardiogenic shock but delayed elective surgical repair is feasible in patients with heart failure without shock. Bypassing of associated coronary artery disease increases long-term survival after successful surgical repair.

VENTRICULAR STANDSTILL WITH P WAVES, HOLD THE QRS. Michael

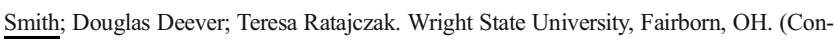
trol ID \#2470274)

LEARNING OBJECTIVE \#1: Ventricular Standstill is a form of ventricular asystole with only $\mathrm{P}$ waves present on electrocardiogram. This is a high-degree AV block that normally presents as syncope or sometimes as sudden cardiac death. We report a case of ventricular standstill that presented as multiple episodes of near syncope

LEARNING OBJECTIVE \#2: Demonstrate the importance of telemetry for presyncopal patients. Recognize a potentially fatal arrhythmia.

CASE: A 57-year-old Caucasian female presented to the air force base emergency department with complaints of pre-syncopal symptoms for the past $24 \mathrm{~h}$. She described multiple episodes of nausea, shortness of breath, lightheadedness, and blurred vision. Past medical history included hypertension treated with $25 \mathrm{mg}$ of hydrochlorothiazide and $10 \mathrm{mEq}$ of potassium chloride daily. Physical exam was unremarkable. EKG on presentation revealed less than $4 \mathrm{~s}$ of lone $\mathrm{P}$ waves, without subsequent QRS complexes, consistent with ventricular standstill that transitioned to tachycardia. Repeat EKG showed normal sinus rhythm. Patient was noted to have two subsequent episodes of ventricular standstill lasting from 4 to $9 \mathrm{~s}$ each while inpatient. These were associated with dizziness, 
presyncope, and slurred speech that resolved within two minutes of the episodes. She was transferred to a facility capable of advanced cardiac interventions where she underwent placement of a temporary pacemaker, followed by a permanent pacemaker the next day. She was monitored after pacemaker insertion and discharged home in good condition.

DISCUSSION: Discussion: Ventricular Standstill is an uncommon, but potentially fatal arrhythmia that can be seen on electrocardiography. Though $\mathrm{P}$ waves are present and occur at regular intervals, there is an absence of QRS complexes. The sinoatrial node continues to function appropriately without a ventricular response, resulting in interuption of cardiac output. Ventricular standstill can present as syncope or sudden cardiac death if occurring for a prolonged period without cardiopulmonary resuscitation. There are a few cases reported in literature which descibe this arrhythmia as a lone entity, however more commonly, complete AV block has been described as leading to ventricular standstill. The definitive treatment for high degree or complete AV block is the insertion of a permanent pacemaker. There are no definite guidelines for treatment of lone ventricular standstill. Our patient's presentation is unique in several ways. During the $4-9 \mathrm{~s}$ events of ventricular standstill captured on telemetry, she did not experience any syncopal episodes. Additonally the acute onset of her symptoms, which developed in a $24 \mathrm{~h}$ period does not fit the usual timeline of degenerative processes of the conduction system. Conclusion: Presyncope is a common complaint and a frequent cause for presentation to an emergency department. As exhibited by our patient, a normal EKG does not preclude an underlying cardiac cause of presyncope. Telemetry monitoring is imperative for patients admitted for presyncopal symptoms.

VERY HIGH FERRITIN IN AN HIV-POSITIVE PATIENT WITH FEVER OF UNKNOWN ORIGIN. John C. Ricketts; Aaron Gluth; Shea Cochi. Emory University, Atlanta, GA. (Control ID \#2467253)

LEARNING OBJECTIVE \#1: Describe the limited differential in patients with extremely elevated ferritin.

LEARNING OBJECTIVE \#2: Recognize that patients with HIV/AIDS often present with multiple acute issues that can make definitive diagnosis difficult.

CASE: A 33 year old man with a past medical history of G6PD deficiency and a very recent diagnosis of HIV presented with a 3 week history of fevers and profuse, watery diarrhea. He also reported a 2-day history of a diffuse, non-pruritic rash. His initial physical exam was notable for a temperature of $39.9^{\circ}$ Celsius, HR 101, tender axillary lymphadenopathy, and an erythematous papular rash on the face, chest, trunk, and extremities. His abdominal exam was benign. The patient's labs were notable for serum sodium $130 \mathrm{mmol} / \mathrm{L}$, bicarbonate $17 \mathrm{mmol} / \mathrm{L}$, creatinine $1.36 \mathrm{mg} / \mathrm{dL}$, ALT $318 \mathrm{u} / \mathrm{L}$, AST $1281 \mathrm{u} / \mathrm{L}$, alkaline phosphatase $111 \mathrm{u} / \mathrm{L}$, and ferritin level 70,170. His urinary Histoplasma antigen was positive, and a bone marrow biopsy revealed yeast suggestive of Histoplasma capsulatum. The patient was started on Amphotericin induction therapy and he improved clinically.

DISCUSSION: Histoplasmosis is an infection caused by the fungus Histoplasma capsulatum. The disease affects primarily the lungs. Disseminated infection can affect the gastrointestinal system, skin, adrenal glands, bone marrow, central nervous system, spleen, and cardiovascular system. As such, the clinical manifestations of histoplasmosis are myriad. Severe cases can result in shock and multi-organ failure. The mainstay of treatment in severe cases is Amphotericin B, but mortality can approach $50 \%$ even with appropriate antifungal therapy. Diagnosis of histoplasmosis can be a challenge, especially in immunocompromised patients. The differential diagnosis for diarrhea, rash, and fever in an AIDS patient is broad, and often there are multiple processes at work. In this particular case, the elevated ferritin provided a key clue. In the setting of serum ferritin levels $>10,000$, diagnostic considerations include Still's disease, systemic lupus erythematosus, disseminated histoplasmosis, disseminated mycobacterial infection, hemophagocytic lymphangiohistiocytosis (HLH), and hemochromatosis. There is considerable overlap between the clinical features of disseminated histoplasmosis and hemophagocytic lymphangiohistiocytosis (HLH). In this case, the urine histoplasmosis antigen was consistent with histoplasmosis while the soluble IL-2 receptor was consistent with HLH. Ultimately, bone marrow biopsy confirmed the diagnosis. In our case, the elevated ferritin provide a key clue. This widely available, cost effective test helped to narrow the differential and allowed for more focused evaluation and testing. With the push for more cost conscious case, physicians should remember the utility of commonplace tests such as ferritin in guiding a difficult diagnosis.

VITAMIN C DEFICIENCY AND PANCYTOPENIA- STILL AN UNDER RECOGNIZED CAUSE FOR MORBIDITY FROM LONG TERM TOTAL PARENTERAL NUTRITION IN SPITE OF SUPPLEMENTATION WITH MULTIVITAMIN FORMULATIONS Abdurahman Alloghbi ${ }^{1,3}$; Scott Huff ${ }^{2}$; Arindam Bagchi $^{3}$; Nauman Shahid ${ }^{4}$; Naga Praneeth Raja ${ }^{5}$; Veena Iyer ${ }^{6}$; Cherian Verghese ${ }^{3}$. King Khalid University, Toledo, OH; ${ }^{2}$ University of Toledo, Holland, OH; ${ }^{3}$ University of Toledo, Toledo, $\mathrm{OH} ;{ }^{4}$ University of Toledo Medical Center, Perrysburg, $\mathrm{OH} ;{ }^{5} \mathrm{University}$ of Toledo Medical center, Toledo, OH; ${ }^{6}$ University of Toledo, Toledo, OH. (Control ID \#2466623)

LEARNING OBJECTIVE \#1: Review features of Vitamin C deficiency LEARNING OBJECTIVE \#2: Discuss causes of Vitamin C deficiency during long term Parenteral Nutrition

CASE: A 25 year-old male presented with painful leg swelling and a papular rash. Venous Doppler didn't reveal any DVT's. 6 months earlier he was managed for Acute Kidney Injury and Lithium toxicity with IV hydration and replacement of Lithium with Olanzapine. He had then presented with diarrhea, abdominal pain, generalized weakness and oliguria. Physical examination showed multiple scars from skin infections, a G- tube for stomach decompression and bilateral knee ecchymosis along with follicular cutaneous bleeding. Medical history revealed Hypothyroidism, Prader-Willi Syndrome and Gastroschisis corrected by partial bowel resection. Due to Short-Gut Syndrome, he was dependent on TPN. Medications included Nystatin, Vitamin D supplements, Levothyroxine, Lorazepam, Octreotide, Oxycodone/Acetaminophen, Pantoprazole and Zolendronic acid. Laboratory studies showed Pancytopenia with WBC 0.9 L/Thou/mm3, HGB $6.3 \mathrm{~g} / \mathrm{dl}$ and Platelets of 27000/mm3. Metabolic panel showed BUN $42 \mathrm{mg} / \mathrm{dL}$, Sodium $132 \mathrm{meq} / \mathrm{L}$ and Chloride of $96 \mathrm{meq} / \mathrm{L}$. Due to peri-follicular hemorrhages a Vitamin C level was done and found to be critically low at $<5 \mathrm{umol} / \mathrm{L}$.

DISCUSSION: This case illustrates the need for early investigation of Vitamin C deficiency in patients on long term TPN. Vitamin C deficiency/Scurvy is rare in industrialized nations. It is important to have a high degree of suspicion because when missed chronic deficiency may result in recurrent infections, hemorrhages, seizures and even death. Scurvy (aka Barlow's disease; Moeller's disease; Cheadle's disease) was first described in 1753 in Sailors who didn't have access to citrus fruits/juices. Features include poor wound healing, recurrent infections, gum and skin bleeds, jaundice, mental status changes, seizures, renal failure etc. Vitamin $\mathrm{C}$ is an important micro-nutrient with antioxidant properties scavenging reactive oxygen free radical molecules. It is also essential for synthesis of Collagen and Carnitine as well as absorption of iron from the intestines. Deficiency is rare, even in patients on TPN because commercial preparations provide in excess of basal requirements. In humans, the average store of Vitamin C is $1.5 \mathrm{~g}$, of which $3-5 \%(40-60 \mathrm{mg})$ is used up daily. Based on pharmacokinetic data, doses of $100 \mathrm{mg} / \mathrm{d}$ for patients on home Parenteral Nutrition and $200 \mathrm{mg} / \mathrm{d}$ for stable adult patients receiving TPN are adequate. However in situations such as shock, trauma, burns, post-surgery, acute illness, sepsis and critical illnesses, Vitamin C requirement is increased due to abnormal losses, mal-absorptive states, utilization for wound healing, and increased oxidative stress. 
Requirements during these states can be as high as $3 \mathrm{~g} / \mathrm{d}$. In this case the patient showed features of Vitamin C deficiency even during the first admission. However due to the rarity of Vitamin C deficiency, this was not investigated further. On the second admission, the patient showed additional features of Vitamin C deficiency including pancytopenia and peri-follicular bleeding. On supplementation the peri-follicular bleeding resolved with improvement of joint and leg swelling. He was successfully discharged home on an adjusted TPN regimen with increased dosing of Vitamin C.

\section{WARFARIN IN HEMODIALYSIS PATIENTS: MORE HARM THAN GOOD?}

Meghana Bhatta. George Washington University, Arlington, VA. (Control ID \#2466761)

LEARNING OBJECTIVE \#1: To recognize the classic presentation of calciphylaxis on skin exam

LEARNING OBJECTIVE \#2: To educate about important considerations when selecting anticoagulant regimens for dialysis patients

CASE: A 57 year-old woman with end-stage renal disease on hemodialysis, mechanical mitral valve replacement due to endocarditis, hypertension, and diabetes was admitted directly from wound care clinic due to worsening chronic lower extremity ulcers. The patient's primary complaint was severe pain at the ulcer sites. Physical exam revealed three ulcers on the left thigh and bilateral calves, the largest of which measured $23 \times 13 \mathrm{~cm}$. These lesions contained large areas of eschar and slough with progressive necrosis at the margins The ulcers had doubled in size over the past 2 weeks. Numerous tense serous-filled bullae were also noted to be new over the past 2 weeks. She had undergone debridement of the ulcers 1 month prior to this admission. The pathology report showed fibrin thrombi in derma capillaries suggestive of a vasculopathy. Though no calcium deposits were visualized on histology or X-ray, calciphylaxis was strongly suspected given her comorbidities. Sodium thiosulfate had been unsuccessfully attempted for treatment of calciphylaxis. Additionally, the patient had recently completed a course of levofloxacin for growth of Stenotrophomonas maltophilia on wound culture. She had been on warfarin therapy for 1 year and had a history of very labile INR, with repeated readjustments of her warfarin dose as well as vitamin $\mathrm{K}$ administration for INR reversal. On this admission, her INR was 4.3. She was switched from warfarin to heparin drip and then given oral vitamin K. Her PTH was $126 \mathrm{pg} / \mathrm{mL}$, calcium was $8.4 \mathrm{mg} / \mathrm{dL}$, and phosphorus was $4.1 \mathrm{mg} / \mathrm{dL}$. It was strongly suspected that the patient's recent wide INR fluctuations contributed to tissue ischemia. One of the key clinical dilemmas of this case was weighing the risks and benefits of replacing warfarin with an alternative anticoagulant regimen. Options included lovenox or a novel oral agent, none of which had well-established therapeutic ranges in the setting of kidney disease or were approved for use with mechanical mitral valves. Ultimately, it was decided to keep the patient on warfarin despite the risk of wound deterioration. The patient was discharged once her wounds appeared stable. She was readmitted less than one week later for supratherapeutic INR and passed away soon after. Cause of death was suspected to be sepsis originating from the enlarging leg ulcers.

DISCUSSION: Calcific uremic arteriolopathy, also known as calciphylaxis, is a condition in which the medial layer of arterioles becomes calcified, leading to vascular occlusion and skin necrosis. It predominantly affects end-stage renal disease patients, as the etiology is thought to be due in part to abnormal calcium-phosphate balance resulting in extraskeletal calcification. The mortality of calciphylaxis may be as high as $80 \%$, usually secondary to sepsis. Warfarin use is thought to be a precipitant via inhibition of the matrix G1a protein, making anticoagulation for hemodialysis patients a risky endeavor. Frequent dose adjustments and loading dose administrations of warfarin also carry a risk of transient protein $\mathrm{C}$ depletion leading to tissue death, as in warfarin-induced skin necrosis. The ulcers are extremely painful and tend to congregate in adipose-rich tissues, such as the buttocks, thighs, and pannus. Biopsy is needed for diagnosis, but can hasten infection in these patients who have a propensity for poor wound healing. Treatment is geared towards reducing the calcium-phosphate product, for example by selecting non-calcium based phosphate binders, maintaining parathyroid hormone below 300 , and using dialysate with low calcium. Sodium thiosulfate and debridement have conflicting evidence, with the latter carrying a significant risk of superinfection. Hemodialysis patients have a higher incidence of conditions which require anticoagulation than the general population. Special precaution should be taken to avoid administering warfarin in these patients when possible - for instance, opting for bioprosthetic valves rather than mechanical. Providers must be vigilant for signs of calciphylaxis, recognizing the poor prognosis of this condition and instituting early interventions when appropriate.

WATER YOU LOOKING FOR? PAN-HYPO-PITUITARISM IN NEUROSARCOIDOSIS John Doehring; John Moscona. Tulane University Health Sciences Center, New Orleans, LA. (Control ID \#2467654)

LEARNING OBJECTIVE \#1: Identify appropriate fluid management strategies in patients with central diabetes insipidus

LEARNING OBJECTIVE \#2: Recognize the extensive and variable presentation of sarcoidosis

CASE: A 39 year-old man presented with sudden onset of altered mental status and unintelligible speech. He had associated weakness and nystagmus without loss of consciousness, postural tone, or seizure. His temperature was $92.4 \mathrm{~F}(33.5 \mathrm{C})$ and he was hypotensive. There was left-sided exotropia, ptosis, diplopia, and facial hypoesthesia; no motor deficit was appreciated. A bolus of 21 of normal saline was administered. The patient subsequently developed sinus bradycardia in the 30 s with a regular rhythm and no murmur. Laboratory studies revealed a sodium value of 174 , chloride of 133 , serum osmoles of 332, urine osmoles of 130, TSH of 0.01, and a corrected free T4 of 1.11. A free water deficit of 171 was calculated. Further chart review elicited a history of pan-hypopituitarism as a result of neurosarcoidosis in the setting of poor medication compliance (mycophenolate mofetil and prednisone). The patient's fluids were switched to $1 \frac{1}{2}$ norma saline at a rate of $250 \mathrm{cc} / \mathrm{h}$ and he was encouraged to increase free water intake. An MRI of the brain confirmed abnormal enhancement of hypothalamic regions consistent with an infiltrative process; the patient was subsequently started on desmopressin.

DISCUSSION: Altered mental status is a common initial presentation encountered by internists. In the setting of hemodynamic instability with concomitant electrolyte derangements, central diabetes insipidus is an important diagnosis to consider. The clinical presentation and laboratory patterns of central DI can vary and may be confused with alternative diagnoses. Chronic tolerance of creeping electrolyte abnormalities may be a result of an impaired thirst mechanism, as in our patient, and can lead to delayed presentation. It is important to establish the diagnosis early, however, as default fluid resuscitation with normal saline can exacerbate the problem. A judicious approach includes slow correction of hypernatremia with dilute fluid to prevent cerebral insult; a decrease of $8-10$ over $24 \mathrm{~h}$ is appropriate. The presence of other signs, symptoms, or laboratory abnormalities should alert the clinician to the possibility of pan-hypothalamicpituitary pathophysiology. Hypothermia, hypothyroidism, and hypogonadism are common signs suggestive of this etiology that were found in our patient. Associated secondary adrenal insufficiency presents with persistent hypotension, dehydration, hypoglycemic episodes, and disorientation. Moon facies and a Cushinoid appearance can be an important diagnostic clue, as exogenous steroid use either recreationally or as a result of overtreatment are common occurrences. It is imperative to initiate corticosteroid supplementation immediately to achieve hemodynamic stability, as well as replace deficient hormones in these patients to avoid permanent endocrine sequelae. Patient education is paramount to prevent repeat hospitalizations. Sarcoidosis is a common disease that will present with extrapulmonary symptoms in $30 \%$ of cases. Organs commonly affected include the skin, eyes, musculoskeletal system, heart, and central nervous system. Often the pituitary gland 
is involved, resulting in extensive endocrine manifestations including central diabetes insipidus. Identifying neurosarcoidosis as a unifying diagnosis streamlines safe fluid and electrolyte management in the acute presentation of these patients and allows transition of focus to long-term maintenance strategies.

WEAK AT THE KNEES FROM COLCHICINE Meghana Bhatta; Anne Lesburg. George Washington University, Arlington, VA. (Control ID \#2466786)

LEARNING OBJECTIVE \#1: To elucidate the multifactorial etiology of colchicine myopathy, with emphasis on drug interactions leading to toxicity

LEARNING OBJECTIVE \#2: To increase awareness of potential side effects of colchicine and encourage greater caution with initiation of colchicine therapy

CASE: A 68 year-old male was admitted for 1 month of progressive weakness in his legs, requiring the use of a wheelchair. His past medical history included gout on colchicine maintenance therapy, hypertension treated with diltiazem, chronic kidney disease, and prostate cancer in remission. He reported 1 week of diarrhea prior to onset of the symptoms. He had taken a short course of prednisone for gout flare during the past month, with resolution of the gout but no improvement in his weakness. Neurologic examination revealed decreased muscle bulk and hyporeflexia of the legs, $2 / 5$ strength of the lower extremities, and $3 / 5$ strength of the upper extremities. Laboratory examination revealed: Cr $3.0 \mathrm{mg} / \mathrm{dL}$ (baseline 1.6) with urine studies consistent with prerenal azotemia, CK $5249 \mathrm{U} / \mathrm{L}$, ESR $36 \mathrm{~mm} / \mathrm{h}$, CRP $13 \mathrm{mg} / \mathrm{L}$, aldolase $28.2 \mathrm{U} / \mathrm{L}$ (3.3-10.3), and anti-Jo <0.2. He also had a normal TSH, negative PSA, and negative HIV. MRI of the spine indicated mild stenosis of L3/L4, and MRI of the brain showed acute-subacute bilateral pontine ischemic infarcts. These focal lesions were unlikely to explain the patient's severe upper and lower extremity weakness. MRI of the legs showed mild myositis of the quadriceps. Initiation of high-dose statin for secondary stroke prevention was held due to potential for exacerbating muscle inflammation. Ultimately, colchicine myopathy was suspected, secondary to decreased clearance of the medication from acute kidney injury and drug interaction with diltiazem. Guillain-Barre Syndrome, polymyositis, paraneoplastic disease, and spinal metastases were also considered but excluded due to incongruence with the patient's clinical presentation. Therefore, colchicine and diltiazem were discontinued, and within 3 days of cessation, CK level decreased to $517 \mathrm{U} / \mathrm{L}$ and motor function gradually recovered. At 1-month follow-up, he had resumed independent ambulation.

DISCUSSION: Colchicine, whether in overdose or chronic use, can be directly myotoxic. This usually manifests as proximal muscle weakness and is thought to be related to interference with microtubule function in myocytes. Muscle biopsy would show a vacuolar myopathy and myofibrillar disorganization. Treatment consists of withdrawing the offending agents; there is no established role for steroids. Medications that inhibit CYP3A4 and P-glycoprotein transporter impede metabolism of colchicine and can lead to serum accumulation of the drug. It is well-documented that potent inhibitors of these enzymes, such as protease inhibitors and macrolide antibiotics, carry a clinically significant risk of colchicine myopathy. However, this case suggests that co-administration of diltiazem, a weaker inhibitor, may also predispose to myopathy, especially in the setting of other risk factors such as renal impairment or statin use. With this patient, dehydration from the gastrointestinal side effects of colchicine likely contributed to the kidney injury, further decreasing colchicine clearance. Thorough review of comorbidities and medications is integral in preventing colchicine toxicity. Gout patients have an especially high prevalence of concurrent metabolic syndrome, increasing the likelihood of having kidney impairment as well as being on a medication that may interact with colchicine. This case demonstrates that clinicians must maintain a broad differential for drug interactions that can result in colchicine myositis.
WEAK IN THE KNEES: A COMPLICATION OF INTRA-ARTICULAR KNEE INJECTIONS Reem M. Hanna ${ }^{2}$; Amar Kohli ${ }^{1} .{ }^{1}$ UPMC, Mars, PA; ${ }^{2}$ University of Pittsburgh Medical Center, Pittsburgh, PA. (Control ID \#2469726)

LEARNING OBJECTIVE \#1: To identify adrenal insufficiency as a cause of hypotension

LEARNING OBJECTIVE \#2: To recognize that intra-articular corticosteroid knee injections can induce adrenal insufficiency

CASE: A 48 year old woman with knee osteoarthritis presented with a 1 day history of nausea, lethargy, and lightheadedness. One day prior to presentation, the patient experienced nausea and one episode of nonbloody, nonbilious emesis. The next morning, the patient noted lethargy and lightheadedness, which was exacerbated by standing. The patient has a history of chronic knee osteoarthritis and underwent intra-articular injection of methylprednisolone acetate 2 weeks prior. Upon presentation to the hospital, her initial vital signs were remarkable for blood pressure of $55 / 32$ with a heart rate of 50 . No abnormalities were noted on physical examination. Laboratory evaluation demonstrated transaminitis with an AST of $172 \mathrm{IU} / \mathrm{L}$ and ALT of $231 \mathrm{IU} / \mathrm{L}$, consistent with shock liver. The patient was initiated on norepinephrine, normal saline, and empiric antibiotic therapy with vancomycin and piperacillin/tazobactam. Due to persistence of symptoms, an AM cortisol was obtained, which was $6 \mathrm{mcg} / \mathrm{dL}$. Intravenous hydrocortisone was started, allowing the norepinephrine to be quickly weaned off. Infectious work-up was negative and empiric antibiotics were discontinued. The patient underwent cosyntropin stimulation testing which confirmed the diagnosis of adrenal insufficiency, suspected to be iatrogenic due to chronic intra-articular steroid injections. Thus, the patient's hypotensive shock was most consistent with an adrenal crisis due to a possible viral illness. Her symptoms resolved on hydrocortisone replacement therapy.

DISCUSSION: Symptoms associated with adrenal insufficiency are often vague and variable. The most common clinical manifestations include weakness, anorexia, orthostatic hypotension, and nausea. Administration of pharmacologic doses of synthetic glucocorticoids is the most common cause of adrenal insufficiency. Intra-articular corticosteroid injection of the knee joint, which is a common clinic procedure utilized to provide pain relief in patients with knee osteoarthritis, has been known to induce adrenal insufficiency. While one of the advantages of intra-articular corticosteroid injections is to decrease systemic effects, a significant proportion of the injected steroids do reach the circulation and have been shown to suppress the hypothalamic-pituitary-adrenal axis. Recent studies have found that intra-articular corticosteroid injection of $80 \mathrm{mg}$ of methylprednisolone acetate at the knee was associated with subsequent adrenal insufficiency in approximately $25 \%$ of patients. A case series corroborated these findings, as the authors noted that, after the first intra-articular steroid injection, 9 out of 10 subjects had a blunted or completely suppressed cortisol response. Physicians should be aware of the potential for adrenal insufficiency in patients following intra-articular corticosteroid injections, and patients should be educated about signs and symptoms to anticipate.

WEAK IN THE KNEES: AN UNUSUAL PRESENTATION OF SARCOIDOSIS

Tse-Shuen J. Ku; Deepa Bhatnagar. Tulane University Health Sciences Center, New Orleans, LA. (Control ID \#2468982)

LEARNING OBJECTIVE \#1: Review common causes of weakness and workup for peripheral neuropathy.

LEARNING OBJECTIVE \#2: Recognize the neurologic manifestations of sarcoidosis and difficulties in diagnosis.

CASE: A 37 year old man with no significant past medical history presented to emergency room with complaints of 2 weeks progressive lower extremity weakness. Weakness was most noticeable with standing from sitting or walking up steps. He endorsed 
generalized arthralgias with pain worse in his lower back, knees, and ankles. He was visiting family in Jerusalem 4 weeks prior to presentation. While there, he ate unpasteurized soft cheeses, but otherwise denied changes in his diet. He had a dental procedure done 3 weeks ago, after which his dentist prescribed him a 5 day course of antibiotics, of which he took only one dose. He denied any trauma, joint swelling, fever, chills, rhinorrhea, cough, shortness of breath, abdominal pain, diarrhea, jaundice, rashes, or heat intolerance. He did note that he had 3 week course of severe arthralgias and weakness in his early twenties, similar to his presenting symptoms, that was treated with ibuprofen. Examination and findings are as follows: T 98.1, HR 72, BP 138/80, RR 12; no lymphadenopathy, no stomatitis, glossitis. Lungs were without adventitious breath sounds, abdomen soft and nontender. There was tenderness to palpation of medial malleoi bilaterally, $4 / 5$ strength in bilateral lower extremities and hyporeflexive bilateral patellar reflex. Complete blood count, comprehensive metabolic panel, and thyroid function unremarkable. Infectious workup unremarkable, with HIV, RPR, Tspot negative. Vitamin B12 low normal at $187 \mathrm{pg} / \mathrm{ml}$, Vit B6 low $3.7 \mathrm{ug} / \mathrm{L}$, methylmalonicacid (MMA) normal at $159 \mathrm{nmol} / \mathrm{L}$ ANA panel was normal. CSF studies revealed 3 oligoclonal bands not detected in serum, less than criteria of positivity consistent with multiple sclerosis. CT imaging of thoracic and lumbar spine without spinal abnormality, but significant for incidental finding of enlarged medistinal and hilar lymph nodes and right upper lobe pulmonary nodule. High resolution $\mathrm{CT}$ chest revealed multiple pulmonary nodules and extensive mediastinal lymphadenopathy without cavities or calcification. Bronchoalveolar lavage and EBUS fine needle aspiration of subcarinal lymph node was performed. Pathology significant for granulomatous lymphadenitis, multiple nonnecrotizing epithelial granulomas. AFB and fungal stains negative. Given travel history of pulmonary nodules, Brucellosis IgM also tested, along with urine histoplasmosis antigen, both of which were negative. Finally, MRI brain, thoracic, and lumbar spine were unremarkable. Patient was initially treated w parenteral B12, daily multivitamin without change in symptoms. After workup for pulmonary nodules, he was also steroids and ibuprofen with improvement of his weak ness. He was discharged after 3 days with a month-long course of prednisone.

DISCUSSION: Polyneuropathy commonly presents with bilateral weakness and hyporeflexia. The most common causes are metabolic, including diabetes and chronic kidney disease, but is also seen in vitamin deficiencies including B12 and B6 deficiencies, autoimmune diseases including SLE and sarcoidosis, infection including hepatitis B, C and HIV, and malignancy (1). Basic evaluation therefore includes CMP, CBC, TSH, HIV hepatitis B and C, and Vit B12/B6, as in this case. In this patient, we completed a rheumatologic workup for peripheral neuropathy given inconsistencies in his presentation. Although low Vit B6 can be associated with polyneuropathy, it is a less common manifestation than mucosal involvement. Additionally, his B12 level in the lower spectrum of normal, but B12 metabolite testing including MMA level was normal. Furthermore, the patient's history of a similar acute presentation a decade earlier also was not consistent with persistent vitamin deficiency. Further imaging and tissue evaluation was continued for workup for sarcoidosis, especially in light of hilar lymphadenopathy incidentally seen on CT, and neurologic complications of sarcoidosis including polyneuropathy. Neurosarcoidosis, either as initial presentation of sarcoidosis, or neurologic complications in patients with known sarcoidosis, affects $5-10 \%$ of sarcoidosis patients. Any portion of the central or peripheral nervous system can be affected in neurosarcoidosis. Symptoms, physical exam findings, and imaging can be broad and nonspecific. Common manifestations include involvement of cranial nerves, especially CN II, VII, VIII, neuro-endocrine dysfunction involving hypothalamus resulting in polyuria, granulomatous inflammation of the brain or spinal cord, meningitis or mass, hydrocephalus, peripheral neuropathy, myopathy. Although some sources cite brain biopsy as definitive diagnosis, neurosarcoidosis is generally a diagnosis of exclusion. Differential diagnosis is usually broad, including viral/aseptic meningitis, multiple sclerosis, malignancy, CNS vasculitis, meningeoma, polymyositis, toxin exposure, metabolic abnormalities, HIV, TB, syphilis. Testing includes LP, CT chest, serum ACE, contrast enhanced MRI, and biopsy. Regardless of presentation and findings, first line treatment for neurosarcoidosis is with corticosteroids, with duration of steroid taper dependent on improvement in patients' symptoms. In this case, workup for underlying causes of polyneuropathy enabled us to recognize and treat two exclusive causes of weakness in a patient with both $\mathrm{B}$ vitamin deficiency and neurosarcoidosis.

WELLENS' SYNDROME- A WARNING SIGN OF IMPENDING ANTERIOR WALL MYOCARDIAL INFARCTION Sandeep Patri; Monoj K. Konda; Hardik Chhatrala; Yashwant Agrawal. Western Michigan University Homer Stryker M.D. School of Medicine, Kalamazoo, MI. (Control ID \#2466514)

LEARNING OBJECTIVE \#1: Wellens' syndrome is a pre-infarction stage of coronary artery disease characterized by a specific EKG pattern. Recognition of this pattern allows the identification of patients who have critical proximal left anterior descending artery (LAD) stenosis and hence, are at risk for extensive anterior wall myocardial infarction.

LEARNING OBJECTIVE \#2: All clinicians should be able to recognize this syndrome early on so that prompt coronary angiogram and revascularization can be done, thereby preventing a myocardial infarction.

CASE: A 64 year old man with hypertension and no prior history of coronary artery disease presented with atypical chest pain of 1 day duration. Physical examination was significant for tachycardia and an irregularly irregular pulse. Initial EKG showed atrial fibrillation with rapid ventricular rate for which IV Diltiazem was administered. He reported complete resolution of chest pain following that. A repeat 12 lead EKG showed normal sinus rhythm with biphasic T wave inversions in V2 and V3. Troponin T and CPKMB were minimally elevated at $0.16 \mathrm{ng} / \mathrm{ml}(0.0-0.03 \mathrm{ng} / \mathrm{ml})$ and $8.6 \mathrm{ng} / \mathrm{ml}(0.0-7.0 \mathrm{ng} /$ $\mathrm{ml}$ ) respectively. Emergent coronary angiogram revealed $90 \%$ occlusion of the proximal LAD for which a drug eluting stent was placed. Left ventriculogram done at that time showed normal left ventricular function. He was discharged with dual antiplatelet therapy for 1 year and was doing well at his cardiology outpatient follow up.

DISCUSSION: Rarely are we warned by resting electrocardiographic findings well before an impending myocardial infarction (MI), but on other occasions, that may be the only indication. The immediate recognition of Wellens' pattern led to emergent coronary revascularization and prevention of MI in our patient. First decribed by Wellens' in 1982, this characteristic EKG pattern is associated with critical LAD stenosis. The criteria for the syndrome include biphasic or inverted T-wave changes in precordial leads, history of anginal chest pain, normal or minimally elevated cardiac enzyme levels, EKG without Q waves, without significant ST-segment elevation and with normal precordial Rwave progression. It has been reported to occur in 14 to $18 \%$ of patients presenting with unstable angina. The T-wave changes in the syndrome usually occur during a pain-free interval. When present, this syndrome may be even more useful to guide management in patients with atypical chest pain and in whom there is complete resolution of chest pain. These patients fare poorly with conservative therapy. This is evidenced by Wellens' initial study which showed that $75 \%$ of patients with this syndrome who did not receive coronary revascularization developed extensive anterior wall MI within a few weeks after admission. Coronary angiogram and subsequent revascularization, must be performed on an emergency basis. Stress testing is absolutely contraindicated as increasing cardiac demand with a highly stenosed LAD may lead to MI. Clinicians should be aware of this syndrome so that prompt invasive therapy can be done to avoid evolution into MI, subsequent left ventricular dysfunction and sudden death.

WERNICKE ENCEPHALOPATHY POST ACUTE PANCREATITIS Mahmoud Ali; Rimma Polevoy; Iyad Isseh; Asaad Nakhle; Laura Johnson. Henry Ford Hospital, Detroit, MI. (Control ID \#2466742) 
LEARNING OBJECTIVE \#1: Recognize Wernicke encephalopathy in patients with acute pancreatitis on TPN without vitamin B1 supplementation

LEARNING OBJECTIVE \#2: Confirm Wernicke encephalopathy in patients with altered mental status using MRI brain

CASE: This is a 44-year-old female with a past medical history of HIV/AIDS (noncompliant with antiretroviral therapy), depression, and hypothyroidism who presented to the hospital after multiple episodes of nausea and vomiting. Patient was dyspneic at that time and was found to have extensive pneumonic infiltrates. The patient was also found to have thyroid stimulating hormone level elevation to $99 \mathrm{mIU} / \mathrm{L}$ that was secondary to noncompliance with levothyroxine. The patient's CD4 count was 12 cells $/ \mathrm{mm} 3$, and she had a viral load of $860 \mathrm{~K}$ copies $/ \mathrm{mL}$. Lipase was elevated to $>800 \mathrm{U} / \mathrm{L}$ with $\mathrm{CT}$ abdomen findings of prominent pancreatitis. The patient was transferred to the medical intensive care unit (MICU) where she was treated for acute pancreatitis with intravenous (IV) hydration and bowel rest, for right middle lobe pneumonia with azithromycin and ceftriaxone, for hypothyroidism with IV levothyroxine, and, for oral candidiasis with fluconazole; during her stay in the MICU she was also started on total parenteral nutrition (TPN). The patient's mental status started to deteriorate 2 weeks after presentation. It progressively worsened to the point she was difficult to arouse and had limited response to sternal rub. She was not alert or oriented to self, place, or time, and had abnormal recent and remote memory. She had no response to light touch or pinprick in four extremities, $2 / 4$ symmetrical reflexes in upper extremities, no response visible in lower extremities, and flexor planter response bilaterally. No nystagmus was noted and gait could not be assessed. Psychiatry suggested she may have had an exacerbation of bipolar disorder for which they recommended continuation of desvenlaxafine. Neurology recommended obtaining a brain MRI and treating with IV thiamine for suspected WE after acute pancreatitis. MRI brain Flair and T2 findings confirmed the diagnosis of WE with high signal intensity in bilateral medial thalamus, both lateral walls of the third ventricle, and periaquaductal gray matter; all of which were new findings compared to her admission MRI 15 days earlier. Patient responded to IV thiamine and her mental status improved significantly in the first 3 days after treatment.

DISCUSSION: WE is a neurologic disorder caused by vitamin B1 deficiency and characterized by confusion, nystagmus and gait ataxia. It is usually associated with chronic alcoholic patients, but can also occur in non-alcoholic patients; patients receiving prolonged IV feeding without proper supplementation are at risk for this condition. The diagnosis of WE can be missed or delayed because patients tend to manifest with mostly an atypical clinical process. Urgent treatment with IV thiamine is critical and should be given without delay. MRI is useful in confirming the diagnosis of acute WE; typical findings are high-signal-intensity symmetric alterations in the thalami, mammillary bodies, tectal plate, and periaqueductal area on T2 and Flair images. Patient's mental status improved significantly in the first 3 days after treatment. Associated comorbidities like hypothyroidism, psychotic disorder and AIDS/HIV could contribute to the patient's mental status and confusion, but the patient came back to baseline according to the family. Patient was discharged home with a percutaneous endoscopic gastrostomy tube and on HIV medication. WE should be considered in every patient with malnutrition, and early recognition is critical for appropriate management and therefore better prognosis. WE should not only be considered in the differential diagnosis of alcoholic patients with neurological deficits, but also for those who are malnourished or recieving TPN.

WHAT A HEADACHE - NEURO-BEHCET'S DISEASE Monika K. Kakol; Sisir Siddamsetti. John H Stroger of Cook County Hospital, Chicago, IL. (Control ID \#2470229)

LEARNING OBJECTIVE \#1: To recognize and identify clinical findings pertaining to Neuro-Behcet's Disease
LEARNING OBJECTIVE \#2: To utilize imaging and laboratory data to support diagnosis of Neuro-Behcet's Disease

CASE: A 20 year-old African American male presented with chronic intermittent headaches with an acute onset of fever, right sided hemiparesis, diplopia, nausea and vomiting. Physical exam demonstrated temperature $102 \mathrm{~F}$, cardiac murmur, right sided hemiparesis and sensory impairment, left lower facial paresis, right abducens palsy, gait ataxia, oral aphthous ulcers and a genital ulcer. CT imaging demonstrated hypodensities involving the left temporal lobe and right frontal parietal lobe. Echo was negative for vegetations. Blood and urine cultures were negative. Ophthalmological examination did not reveal vasculitis or uveitis. RPR, VDRL, HIV, RF, ANA, c-ANCA, p-ANCA, quantiferon, lysozyme and compliments were negative. CRP $1.14(0.00-0.50)$. CSF demonstrated - clear, lymphocytic predominant leukocytosis, normal glucose, normal protein, negative PCR for HSV, West Nile and Lyme. Oligoclonal bands were present in both serum and CSF, IgG index 0.51(0.00-0.7). MRI demonstrated T2 weighed hyperintense lesions involving left temporal cerebral cortex, right frontal cerebral cortex, pons and medulla. In the setting of negative workup with supportive imaging and clinical findings the diagnosis of NeuroBehcet's Disease (NBD) was placed. Patient had multiple subsequent re-admissions for episodes of seizures, fluctuating neurologic and cognitive impairment, with most recent MRI depicting further progression of lesions involving the brainstem.

DISCUSSION: Behcet's disease (BD) has an epidemiological prevalence in ethnical groups of Asian and North African decent, with reported rates of 2.1-240 per 100,000 population. In comparison to prevalence rates of $0.3-7.5$ per 100,000 population in European and Northern American decent. International Behcet Study Group Criteria (IBSGC) is validated for diagnosis of BD. The criteria requires presence of oral ulcerations in addition to two of three findings of either ocular, genital and/or dermatological involvement. Central nervous system (CNS) involvement in BD carries the highest morbidity and mortality. NBD pathologically is a perivasculitic demyelinating inflammatory disease of the central and peripheral nervous system. Percentage of BD with neurological involvement ranges between 1.3 and $59 \%$. Usual age of onset is between 20 and 40 years of age. It is uncommon for NBD to present without systemic symptoms. NBD is divided into two groups, parenchymal and non-parenchymal involvement. Clinically, subacute meningoencephalitis presents in $75 \%$ of patients with parenchymal involvement, however meningitis alone is rare. The location of lesion involvement will give patients a multitude of neurological symptoms. Non- parenchymal CNS involvement is present in $20 \%$ of NBD, it pertains to involvement of vasculature of the CNS by means of cerebral venous thrombosis. ESR and CRP are elevated and coincide with disease activity. CSF fluid analysis typically demonstrates moderately elevated protein, absence of oligoclonal bands, leukocytosis with neutrophil predominance in early stages and lymphocytic predominant in later stages. CT imaging is of minimal value. MRI is the imaging modality of choice. Characteristic findings on T2 weighed MRI depict unilateral hyperintense lesions localized to the upper brainstem extending into the thalamus and basal ganglia. More extensive meningoencephalitis will demonstrate hyperintense lesions involving the cortices. Diffusion weighed MRI is of assistance when attempting to differentiate NBD from stroke. Headaches and cognitive-psychiatric impairment dominate patient's clinical complaints. A lack of large prospective trials limits our understanding of this complex disease; however recently there is a renewed interest in NBD.

WHAT YOU SEE IS WHAT YOU GET: VISUAL FIELD DEFICIT HERALDING CEREBRAL VENOUS THROMBOSIS. Bryce Somer ${ }^{1}$; Hugo Narvarte ${ }^{2} .{ }^{1}$ University of South Florida, Tampa, FL; ${ }^{2}$ University of South Florida Morsani College of Medicine, Tampa, FL. (Control ID \#2469782)

LEARNING OBJECTIVE \#1: Recognize transient visual disturbances or auditory symptoms as a potential clinical manifestation of cerebral venous thrombosis. 
LEARNING OBJECTIVE \#2: Recognize that chronicity of symptoms does not exclude the diagnosis of cerebral venous thrombosis.

CASE: A 40 year old man presented to clinic with complaints of 8 months of transient pulsations in his ears, lightheadedness, and changes in vision of his right eye. Symptoms started with a transient pulsating sound in both ears (worse on the right). About 2 months later he began to notice dizziness, described as light-headedness when standing which progressed from 3 times weekly to multiple times per day. About 4 months later he developed episodic decreased vision in his right eye. It initially manifested as "haziness" in the right upper temporal quadrant and progressed to a transient "brownish tint" in the lower nasal quadrant. Three days prior to presentation he temporarily lost all peripheral vision in his right eye while running, but maintained central vision. Vision gradually returned to normal by the end of his run. He denied any recent trauma, headache, weakness, difficulty breathing, or chest pain. Past medical history was significant for a tooth abscess in a right upper incisor 1 year prior to presentation requiring tooth extraction and incision and drainage of the abscess. He had never used tobacco, and drank 3-4 glasses of wine or beer per week. He took no medications and had no known allergies. Family history was significant for DVT/PE in his mother. Physical exam was normal, including a normal neurologic exam, although papilledema was noted on evaluation by ophthalmology later in the course. Given the patient's constellation of symptoms, an MRI brain was obtained, which demonstrated thrombosis of the right internal jugular vein, superior sagittal sinus and right sigmoid sinus. These findings were confirmed with MRV, which also demonstrated extensive venous collateralization, suggesting a chronic process Venous dopplers of the upper extremities were negative. A hypercoagulable evaluation at the time of diagnosis was normal, including antithrombin III, cardiolipin antibodies, lupus anticoagulant, fibrinogen, factor V mutation, protein C and S, ANA, ESR, and homocysteine. Repeat testing after the completion of warfarin therapy remained normal. Panorex scan did not reveal any acute dental abnormalities to suggest recurrent abscess. MRA showed no cerebral vascular abnormalities. The patient was treated with enoxaparin bridged to warfarin, and treated for 6 months. Due to concerns for increased intracrania pressure, he was also treated with acetazolamide. He did well until approximately 1 year later, when he developed an intermittent "whooshing" sound in his left ear. Cerebral angiogram revealed a development of an AV fistula. This was treated with embolization with subsequent improvement in his symptoms. He has remained in good health since, with no residual deficits or impairments.

DISCUSSION: Cerebral Venous Thrombosis (CVT) is a rare but important disease, which accounts for approximately $0.5-1 \%$ of strokes $(1)$. The majority of cases $(85 \%)$ are associated with at least 1 risk factor for CVT. Risk factors include prothrombotic conditions, oral contraceptives, hormone replacement, or steroid use, pregnancy and puerperium, malignancy, infection, or injury $(1,2)$. Clinical manifestations generally relate to either increased intracranial pressure or ischemia from the thrombosis (1). The most common symptom is headache. Other manifestations may include seizures, focal neurological deficits such as numbness or weakness, aphasia, or hemianopia (1). Diagnosis is generally suggested with imaging findings on MRI, and then confirmed with either CTV or MRV (1). Occasionally angiography is needed to confirm the diagnosis (1). Treatment is generally with anticoagulation, with length of treatment and additional therapies determined by the patient's underlying risk factors (1). Long term complications can include recurrent thrombosis, severe headache, visual loss, seizures, and development of a dural AV Fistula $(1,2)$. In the case described above, the chief presenting symptom was transient vision loss in addition to chronic pulsatile tinnitus and dizziness. The presentation was atypical in that headache was not present. In addition, no clear predisposing factors were found to explain a CVT in this patient other than a remote history of a dental infection. This case highlights the varied nature of manifestations of CVT. It also shows that, although the majority of patients have at least 1 risk factor for CVT, a lack of risk factors does not preclude the diagnosis. Regardless, any patient diagnosed with a CVT should undergo a thorough evaluation to identify any predisposing factors to CVT in order to determine specific treatments and long-term management. Due to its variable presentation, physicians must maintain a high index of suspicion, as the diagnosis can be easily missed. References: 1. Saposnik G, Barinagarrementeria F, Brown RD Jr, et al. Diagnosis and management of cerebral venous thrombosis: a statement for healthcare professionals from the American Heart Association/American Stroke Association. Stroke 2011; 42:1158. 2. Ferro JM, Canhão P, Stam J, et al. Prognosis of cerebral vein and dural sinus thrombosis: results of the International Study on Cerebral Vein and Dural Sinus Thrombosis (ISCVT). Stroke 2004; 35:664

\section{WHAT'S INSIDE THE FRIDGE? INTEGRATING HOME VISITS INTO THE} CARE OF PATIENTS WITH POORLY CONTROLLED DIABETES. AmY S.

Tang; Jessica Tanenbaum. NYU School of Medicine, New York, NY. (Control ID \#2469950)

LEARNING OBJECTIVE \#1: Recognize the advantage of home visits compared to usual clinic-based care of patients with poorly controlled diabetes.

LEARNING OBJECTIVE \#2: Identify social determinants of health that affect patients' adherence to diabetes management recommendations.

CASE: A 43-year old man with poorly controlled adult-onset diabetes mellitus presented to primary care clinic with symptomatic hyperglycemia. His examination was notable for normal vital signs and body mass index, normal skin without acanthoses nigricans, and an odd affect and communication style suggestive of autism spectrum disorder. Laboratory studies revealed a hemoglobin Alc greater than 17 and negative islet cell and glutamic acid decarboxylase antibodies. The patient was referred to a nutritionist, initiated on weight-based once daily detemir insulin therapy, and offered neuropsychiatric evaluation which he declined. He attended half of his follow-up appointments and reported insulin adherence, however remained hyperglycemic and without hemoglobin A1c improvement at 3 months. The patient agreed to a diabetes home visit conducted by two resident physicians including his primary care provider. An environmental assessment revealed poor lighting, cramped living quarters, and diffuse clutter including sugary beverages and processed meat and bread in the patient's bedroom where he slept on the floor. His exam was notable for a poorly healing lower extremity wound not observed previously in the clinic. A home medication reconciliation revealed unrefrigerated bottles of determir and unopened bottles of aspart insulin. Further, he was unable to locate his glucometer and reported disuse for more than a month. The patient was reeducated on the diabetic diet, emphasizing the high glycemic index foods in his home to avoid and identifying affordable produce in his neighborhood. He was instructed on proper wound care including prevention of further wounds, given a new glucometer and logbook, and educated on the proper storage and administration of detemir insulin. Close clinic follow-up appointment was made to review his glucometer log and monitor his leg wound.

DISCUSSION: Physicians gain valuable insight into social determinants of health such as housing, food scarcity, and neighborhood safety through the evaluation of patients in the context of their home. This case exemplifies the advantage of home visits compared to usual clinic-based care for assessing patients' understanding of and adherence to clinicbased diabetes recommendations. Despite attending clinic-based diabetes nutritional counseling, the patient demonstrated poor comprehension of diabetic diet recommendations. Given the patient's autism-like traits, a more concrete approach to diabetes nutritional education with identification of specific foods in his home and neighborhood to consume or avoid was more effective than usual clinic-based counseling. Studies show that home visits for patients with poorly controlled diabetes significantly decrease emergency department visits and hospitalizations. The patient's misplaced glucometer and unrefrigerated detemir insulin revealed several gaps in his knowledge of diabetes medication administration, safety, and efficacy. This discovery allowed his provider to adopt a more tailored and concrete didactic approach to help the patient achieve adequate glucose 
control. Further, the patient's living conditions likely predisposed him to leg wounds missed during his clinic visits. Such wounds may have progressed to an emergency department visit or hospitalization for diabetic ketoacidosis or sepsis if his primary care provider had not conducted a home visit to aid in the assessment of this patient with seemingly refractory diabetes.

WHAT'S UP, DOC? Minnsun Park; Brian Payne; Alvaro Alonso. Tulane University Health Sciences Center, New Orleans, LA. (Control ID \#2469132)

LEARNING OBJECTIVE \#1: To identify acute coronary syndrome as a result of medication side effects

LEARNING OBJECTIVE \#2: To recognize the importance of reviewing medication side effects and interactions prior to prescribing

CASE: A 41-year-old man presented to outside ED with priapism. He was in his usual state of health until 3 days before when he woke up with an erection that would not subside. He denied any trauma, ingestion of medications to treat erectile dysfunction, and past family history of sickle cell disease or any other hematologic disorders. In the ED, he received two doses of pseudoephedrine and terbutaline each without any improvement. A couple hours later, as he was being transferred to a tertiary center for further evaluation, he developed excruciating chest pain that radiated to his shoulders bilaterally with dyspnea and diaphoresis. He denied any chest trauma. His medical history was significant for hypertension and occasional tobacco chewing, alcohol consumption, and marijuana use. Upon arrival to our facility, the patient was tachycardic with a heart rate of 111/min and slightly hypertensive with a blood pressure of $145 / 68 \mathrm{mmHg}$. His physical exam was unremarkable except for priapism. Basic labs, including blood count, comprehensive metabolic panel, and troponin, were unremarkable. Electrocardiogram showed $2 \mathrm{~mm}$ ST-segment elevation in lead aVR and diffuse ST-segment depressions in the inferolateral leads. After confirming with the patient that he did not take any phosphodiesterase inhibitors, he was given a dose of sublingual nitroglycerin without any significant improvement. The patient was then taken emergently for a coronary angiography, which showed diffuse coronary artery vasospasm without any evidence of angiographically significant coronary artery disease. The patient was given intracoronary nitroglycerin and IV labetalol, with resolution of the vasospasm and symptoms. As for the patient's priapism, he refused surgical intervention. It was also later discovered that he had been taking trazodone, which he previously failed to report. Once this medication was discontinued, the priapism eventually resolved.

DISCUSSION: Priapism is a relatively rare and unpredictable disorder with an incidence of 1.5 cases per 100,000 person years ${ }^{1}$. When it does occur, it is considered a medical emergency. If not treated timely, it can have devastating complications, such as permanent erectile dysfunction and penile necrosis. Some medications, such as terbutaline and pseudoephedrine, have been shown to effectively achieve detumescence (65 and $74 \%$ respectively), and are considered first line therapies to treat priapism ${ }^{3}$. However, their side effects can be significant. Both terbutaline and pseudoephedrine are sympathomimetic, and the risk of cardiovascular side effects increases if both are used together. Terbutaline, a selective beta $_{2}$ agonist, can cause coronary vasoconstriction, and pseudoephedrine, an alpha and beta agonist, can increase cardiac output. Together, they can precipitate angina or acute myocardial infarction by vasoconstriction and increasing myocardium oxygen demand. Studies show that medication adverse reactions in general occur in about $6.7 \%$ of hospitalized patients ${ }^{4}$. The rate of these reactions increases exponentially if the number of medications is greater than four per patient ${ }^{5}$. However, in many patients, polypharmacy cannot be avoided. Therefore, it is important for an internist, prior to prescribing or administering the drug, not only to review a medication's known side effects, but also its potential interactions with other medications that can lead to life-threatening adverse reactions.
WHEN A CARROT A DAY DOESN'T KEEP THE DOCTOR AWAY: AN UNCOMMON CAUSE OF ABDOMINAL PAIN \& SEPSIS Ayesha A. Appa ${ }^{1}$; Lindsay Collins ${ }^{1}$; Steven Mitchell ${ }^{2} .{ }^{1}$ University of Washington, Seattle, WA; ${ }^{2}$ Harborview Medical Center, Seattle, WA. (Control ID \#2467754)

LEARNING OBJECTIVE \#1: Recognize esophageal rupture as a cause of abdominal pain, even without clear history of vomiting.

LEARNING OBJECTIVE \#2: Identify complications of esophageal rupture, such as pleural effusions, mediastinitis, and sepsis.

CASE: A 49-year-old woman with active polysubstance abuse presented with 1 day of severe left upper quadrant (LUQ) abdominal pain that radiated to the left flank and was associated with left-sided, pleuritic chest pain. She denied associated nausea/vomiting and endorsed methamphetamine use prior to arrival, though additional historical details were difficult to elicit, as she was moaning in unremitting pain. Initial exam was pertinent for tachycardia with otherwise normal vital signs, LUQ tenderness without peritoneal signs. Lab evaluation yielded mild acute kidney injury and lactate 2.5 , but no leukocytosis or elevations in serum lipase, liver enzymes, troponin, or irregularities on EKG. CT Abdomen/Pelvis failed to further elucidate the etiology of her pain and was only remarkable for small to moderate bilateral $(\mathrm{L}>\mathrm{R}$ ) pleural effusions. On hospital day 2, the patient developed fever, tachycardia, and hypoxia with respiratory distress that led to intubation for hypoxemic respiratory failure in the setting of persistent LUQ and left-sided chest pain. Pt transferred to the ICU, and upon Radiology re-review of the initial CT, distal esophageal thickening was noted with adjacent mediastinal air, concerning for esophageal rupture or Boerhaave's syndrome. Thoracentesis returned a complicated exudative effusion that eventually grew gut flora, and chest tubes were placed. Repeat EKG was also notable for new diffuse ST elevations and "knotching" of the PR segment of AVR, suggestive of irritative pericarditis. Curiously, both fluoroscopic esophagram and endoscopy did not display any esophageal mucosal defects. However, thoracoscopy revealed a piece of carrot that measured $0.7 \times 1.2 \times 0.9 \mathrm{~cm}$ adjacent to the esophagus in the posterior mediastinum with erosion into the L pleural space with L empyema. Given normal bronchial anatomy, these findings were thought consistent with relatively recent esophageal rupture, now healed, with resultant mediastinitis and empyema. Following thoracotomy and decortication as well as treatment with IV antibiotics and a proton-pump inhibitor, the patient was extubated and is now recovering at a medical respite facility. DISCUSSION: Spontaneous esophageal rupture, first described by Herman Boerhaave in 1724 , is a rare clinical entity (with incidence reported as approximately 3 per 1 million person years) and may present variably. The mechanism of injury is related to sharply increased intraesophageal pressure due to vomiting; however, between 25 and $45 \%$ patients do not report retching or vomiting. Additionally, while the classic symptom of esophageal rupture is severe, acute onset retrosternal chest pain, the location of the tear may change the localization of pain. If the tear is closer to the gastroesophageal junction, then a patient may complain of epigastric pain radiating to the shoulder. If the tear is in the cervical esophagus, a patient may present with neck pain or dysphagia. Most cases, however, are longitudinal tears that usually involve the left posterolateral aspect of the distal intrathoracic esophagus, which was consistent with symptoms in this patient. Common sequelae of esophageal rupture include fever, pleural effusion/empyema, mediastinitis, and sepsis or septic shock. Pericardial effusion and pericarditis have been reported rarely. Fungemia may also be seen in these patients, and portends poorer outcomes. Mortality on the whole is high with estimates around $20 \%$, and this increases with diagnoses delayed greater than $24 \mathrm{~h}$, as was with our patient. Given the low incidence and variable presentation, it is critical to keep esophageal rupture on the differential, as prompt diagnosis will improve patient outcomes.

WHEN A DEAD BUG CAN STILL BITE Jenny K. Nguyen; Roger D. Smalligan; Scott Milton. Texas Tech Univ Health Sc Center - Amarillo, Amarillo, TX. (Control ID \#2463628) 
LEARNING OBJECTIVE \#1: Understand that fully treated leprosy patients may develop severe immunologic reactions.

LEARNING OBJECTIVE \#2: Recognize erythema nodosum leprosum (ENL) type 2 immunologic reaction and the importance of prompt treatment.

CASE: A 20-year-old Burmese woman with a past medical history of treated leprosy (Hansen's Disease) presented to the emergency room for a worsening skin rash, fever and generalized debility. She was diagnosed 4 years prior in a Thai refugee camp where she was treated for 2 years with appropriate, curative therapy. Her father, the index case, and 2 of her 4 brothers also developed the disease although her mother was spared. The patient had a post inflammatory reaction of Hansen's disease a year prior. She reported treatment at that time with methotrexate and prednisone and that the lesions healed completely. She had recently moved to our city but had run out of prescribed medications. Gradually over the last 2 months she developed a diffuse rash that extended from her face to her lower extremities. It was not itchy but was painful. She denied loss of sensation, tingling or burning of the lesions and no loss of pigmentation. She did have generalized weakness, anorexia, fever to $102 \mathrm{~F}$ and night sweats. All of this was similar to her previous reaction. Meds: none; Allergies: NKDA; Family History: as above regarding Hansen's disease, Personal/Social: denies alcohol, tobacco, or drug use. Patient is not sexually active. Physical Exam: BP 101/69, HR 137, RR 20, T 100.9 F, SpO2 $96 \%$ on RA. The patient appears weak but alert and oriented. Lungs clear, heart had a regular rate and rhythm, abdomen was soft and nontender, extremities showed 1+ edema of lower legs, skin: on the upper and lower extremities, face, and back there are multiple blueishpurple subcutaneous indurated lesions with other erythematous superficial lesions, some with pustules. Some lesions were ulcerated and others were scabbed over. All lesions were tender Labs: chem 7 was normal except glucose $139 \mathrm{mg} / \mathrm{dl}$; liver function was normal, CBC showed a WBC $18.3 \mathrm{k}$. CXR was normal. The patient was admitted, infectious disease service was consulted and a diagnosis of erythema nodosum leprosum (ENL) type 2 immunologic reaction was made. Hypotension ensued and the patient was cultured and treated with IV fluids, vancomycin and steroids initially. A skin biopsy was done to rule out active leprosy Contact was made with the national Hansen's disease program which it turned out knew the patient well. It was recommended that antibiotics be stopped, methotrexate added and a steroid taper begun once stable. After 2 days in the ICU, she had an acceptable blood pressure, felt some better and by day 4 was discharged home on the steroids and methotrexate regimen. DISCUSSION: Hansen's Disease is caused by Mycobacterium leprae. It is highly contagious and is transmitted by contact and respiratory routes. Peripheral nerves and skin are affected and without treatment, nerve damage and physical deformity may result. Despite adequate treatment, patients can still develop immunologic reactions as seen in our patient. ENL is characterized as an immune complex mediated disease. It generally presents in patients with borderline lepromatous and lepromatous leprosy that have highly elevated levels of $M$. leprae antigens and anti-M. leprae antibodies. Deposition of these immune complexes results in the activation of the complement system, which recruits neutrophils and promote the release of inflammatory cytokines like TNF-alpha and interferon-gamma. Hence, although patients can be cured of the organisms, inflammatory reactions can still occur. It is important to note that the resulting cytokine storm look very much like sepsis. These reactions are medical emergencies and must be treated promptly to prevent further nerve damage. Clinically, ENL type 2 reaction is characterized by the rapid appearance of painful, erythematous crops of subcutaneous nodules over the extensor surfaces of extremities and the face. These nodules may ulcerate. Over time, the patient may develop fibrosis, scarring and postinflammatory hyperpigmentation. Systemic signs and symptoms including anorexia, fever, and malaise are also seen. The most common complication is a chronic, painful neuritis. The treatment of choice is thalidomide (if birth control is absolutely certain due to birth defect risk). High dose prednisone with methotrexate or clofazamine can also be used. Other alternatives include NSAIDs, pentoxifylline, TNF-alpha inhibitors, and azathioprine. Although it is prudent to treat patients who present with fever, hypotension and a rash as sepsis initially, careful history and monitoring can reveal this uncommon immunologic reaction to previously treated leprosy.
WHEN A SIMPLE CYST REQUIRES A COMPLEX SOLUTION Tahmina Begum $^{1}$; Miles Grant ${ }^{1}$; Subhasis Misra ${ }^{2}$; Nail Aydin ${ }^{2}$; Roger D. Smalligan ${ }^{1} .{ }^{1}$ Texas Tech Univ Health Sc Center - Amarillo, Amarillo, TX; ${ }^{2}$ Texas Tech Univ HSC-Amarillo, Amarillo, TX. (Control ID \#2468715)

LEARNING OBJECTIVE \#1: Distinguish a massive benign hepatic cyst from a parasitic or malignant cyst.

LEARNING OBJECTIVE \#2: Understand that surgical deroofing and alcohol sclerotherapy are recognized treatment options for symptomatic simple cysts.

CASE: A 36-year-old woman was admitted with worsening chronic abdominal pain. The pain was aching, intermittent, low grade and dull. She denied fever, sweats, chills, nausea, vomiting, diarrhea and trauma. Six months prior to admission the patient had an ultrasound during her fourth month of pregnancy which incidentally revealed a large, ill-defined, giant cystic mass in the liver. She had increasing abdominal pain as her uterus enlarged and unfortunately went in to preterm labor at 28 weeks, gave birth precipitously at home and the baby died. Due to funding issues she did not follow up with any physician until this presentation. PMH was otherwise unremarkable; she was taking no medications. Family and personal social history was unremarkable except that she immigrated to the USA from rural Mexico in her teens. Labs were unremarkable and serology for Echinococcus and cancer biomarkers were all negative. An ultrasound of the abdomen showed the cystic hepatic mass which had grown to $15 \times 20 \times 20 \mathrm{~cm}$ and was further confirmed by CT and MRI. Albendazole was given for 1 week in case the Echinococcus was a false negative result. She then underwent interventional radiology guided drainage of the cyst and infusion of hypertonic saline. Cytopathology was benign. The patient was discharged home and after a 3 month period the patient came back with similar increasing abdominal pain and repeat imaging showed recurrence and enlargement of the cyst. Surgical unroofing with omental patch was performed laparoscopically and pathology once again showed benign hepatic cyst. The patient made a full recovery and thus far has had no recurrence. DISCUSSION: Hepatic cysts are seen regularly by practicing physicians as they evaluate patients with various abdominal symptoms by CT and ultrasound. In fact, almost $5 \%$ of the population has a liver cyst (most often a simple cyst). Hepatic cysts are more common in women than men, with a female to male ratio of 1.5:1. Furthermore, liver cysts are symptomatic more often in women than in men. Liver cysts may be classified as true cysts or false cysts depending on the presence or absence of epithelial lining respectively. True cysts can be further divided into parasitic and non-parasitic types. Parasitic cysts are caused by Echinococcus granulosis and E. multilocularis tapeworms. Non-parasitic liver cysts include congenital cysts, simple cysts, neoplastic cysts (e.g., cystadenoma, cystadenocarcinoma, cyst sarcoma, squamous cell carcinoma, metastatic ovarian, pancreatic, colon, renal and neuroendocrine cancers), and biliary ductrelated cysts (Caroli's disease, bile duct duplication, and peribiliary cysts). Simple liver cysts are congenital. They are generally stable in size over time, but may slowly increase in size and occasionally become symptomatic due to mass effect, as occurred in our patient, or they can rupture, hemorrhage, or become infected. Treatment alternatives for symptomatic simple liver cysts include laparoscopic unroofing and alcohol sclerotherapy. Wide unroofing and cyst resection is associated with a low incidence of cyst recurrence and complications. Our patient's exposure during childhood to an area endemic for Echinococcus prompted us to treat her medically prior to the first aspiration. Recurrence of the cyst with significant symptoms required surgical intervention which to date has been very successful. While the vast majority of liver cysts seen on a daily basis will be benign and inconsequential, physicians must be prepared to diagnose and treat symptomatic, infected or malignant cysts promptly.

WHEN CT IS NOT ENOUGH: USING EUS TO DETECT INSULINOMAS WHEN NONINVASIVE IMAGING IS NEGATIVE Woody Chang ${ }^{2}$; Thomas J. Walk ${ }^{3}$; Thomas Painter ${ }^{1}$. ${ }^{1}$ University of Pittsburgh, Pittsburgh, PA; ${ }^{2}$ University of Pittsburgh Medical Center, Presbyterian-Montefiore, Pittsburgh, PA; ${ }^{3}$ VA Pittsburgh Healthcare System, Pittsburgh, PA. (Control ID \#2469997) 
LEARNING OBJECTIVE \#1: To describe the relative strengths and weakness of different imaging modalities for detecting insulinomas

LEARNING OBJECTIVE \#2: To define the diagnostic criteria of insulinomas CASE: A 46-year-old woman with a 4-year history of episodic symptomatic hypoglycemia was transferred from an outside hospital following an episode of altered mental status and lethargy at home. Her symptoms had resolved at the outside hospital with infusion of glucose and oral intake. She had been previously been seen by outpatient endocrinologists, but though she had lab findings that were consistent with a neuroendocrine tumor, she had a negative abdominal CT for neoplasm 6 months prior to this admission. The initial physical exam was unremarkable and vitals were within normal limits. Notable admission labs included a serum glucose of $37 \mathrm{mg} / \mathrm{dl}$, but otherwise unremarkable chemistries, as well as negative testing for sulfonylureas. Subsequent testing during a fasting challenge revealed an insulin level of $25 \mu \mathrm{IU} / \mathrm{mL}$, proinsulin of $48.9 \mathrm{pmol} / \mathrm{L}$, and a C-peptide of $3.59 \mathrm{ng} / \mathrm{mL}$ when the serum glucose was $42 \mathrm{mg} / \mathrm{dL}$, confirming the presence of an insulinoma. After consultation with the endocrinology service, the patient was referred for an outpatient endoscopic ultrasound (EUS) following stable discharge. The EUS showed a $20 \mathrm{~mm} \times 10 \mathrm{~mm}$ mass in the pancreatic head, adjacent to the superior mesenteric vein. The subsequent biopsy had stained positive for synaptophysin but did not show any invasion into the surrounding lymph nodes or soft tissue. Three weeks later, the patient underwent robot-assisted laparoscopic resection of the insulinoma. Following surgery, the patient's symptoms resolved with stabilization of blood glucose levels.

DISCUSSION: Insulinomas are rare neuroendocrine tumors, occurring in 1-4 persons per million in the general population. About $90 \%$ of insulinomas are reported as solitary, with the vast majority localized in the pancreas without invasion or metastases into other organs or lymph nodes. These tumors classically present with symptoms of Whipple's triad: hypoglycemia, neuroglycopenic symptoms, and relief of symptoms with glucose administration. The biochemical requirements for diagnosis are finding serum values for insulin greater than $3 \mu \mathrm{IU} / \mathrm{mL}$, proinsulin greater than $20 \mathrm{pmol} / \mathrm{L}$, and C-peptide greater than $0.6 \mathrm{ng} / \mathrm{mL}$ during a fasting challenge when the serum glucose is less than $45 \mathrm{mg} / \mathrm{dL}$. In addition, one must rule out the abuse of medications by testing for hypoglycemic agents such as sulfonylureas in either through a serum or urine test. Some insulinomas can be medically managed with ocetrotide. However, definitive treatment involves surgical management, either with resection or ablation. In order to maximize the chance of a curative surgical solution, the insulinoma must be localized. Prior to the widespread use of current imaging modalities, blind distal pancreatectomies for occult insulinomas had a failure rate of greater than $20 \%$. The first line of noninvasive imaging is typically CT or MRI, whereas transabdominal ultrasound is usually not recommended because of its comparatively lower detection rate. CT and MRI both can have high sensitivity in detecting insulinomas as well as metastases. CT, in particular, has been shown in one study to be able to detect about $94 \%$ of insulinomas. MRI has a similar detection rate and is typically better than $\mathrm{CT}$ in detecting extrapancreatic masses. If either of those modalities do not find the neoplasm, the next step would be an invasive imaging study, such as EUS. Such was the case in this patient, in which the EUS was able to localize the mass. It should be noted that while EUS is a highly reliable procedure for pre-operative localization of insulinomas, the sensitivity depends on the location of tumor. In particular, it is comparatively better at detecting masses in the pancreatic head than in the tail or extrapancreatic sites. In this patient, the insulinoma was found in the head of the pancreas, a site that EUS detects masses comparatively better than $\mathrm{CT}$, which has been known to miss lesions at that location. In addition, localization with EUS also allows for a concurrent biopsy of the lesion, which can be stained to confirm the presence of a neuroendocrine tumor as well as looking for invasion for tumor staging. The biopsy done showed that this patient's insulinoma did not invade the surrounding soft tissue or lymph nodes, thus it is classified as benign and hence the patient underwent surgical resection as treatment. To summarize, the diagnosis was made with the patient's symptoms, a negative screen for exogenous hypoglycemic agents, as well as elevated insulin, proinsulin, and c-peptide during a fasting challenge. Because of a prior abdominal CT that was unable to be localize a mass, an EUS was done. Once the tumor was localized, a laparoscopic excision of said lesion was curative with the patient having prompt resolution of symptoms and stabilization of blood glucose levels.

WHEN IN DOUBT, CHECK THE THYROID. Faraz Fiazuddin ${ }^{3}$; Uzair Mushtaq ${ }^{1}$; Kanapa Kornsawad ${ }^{2} .{ }^{1}$ UTHSCSA, SAN ANTONIO, TX; ${ }^{2}$ University of Texas Health Science Center, San Antonio, TX; ${ }^{3}$ University of Texas Health Science Center - San Antonio, San Antonio, TX. (Control ID \#2469476)

LEARNING OBJECTIVE \#1: Recognize altered mental status and seizures as clinical manifestations of the rare Hashimto's Encephalopathy.

LEARNING OBJECTIVE \#2: Initiate steroid therapy for a prompt clinical response and recovery.

CASE: 67 years old lady presented with confusion, poor oral intake, subjective fevers and generalized weakness for 4 weeks. Physical examination revealed a lady oriented only to person, who was experiencing visual hallucinations and had tangential speech. She had no focal motor or sensory deficits. Initial laboratory workup was unremarkable other than a toxicology screen positive for cannabinoids. Serum and CSF workup had no evidence of an active infection. Her TSH was 2.6. Patient had multiple episodes of generalized tonic clonic (GTC) movements following which she became agitated, disoriented and aggressive. Her brain MRI was normal and there was no epileptiform activity on EEG. Given the sudden deterioration in her mental status she was started on empiric therapy for bacterial and viral meningitis, which was later held after her HSV PCR and CSF cultures came back negative. Attributing her symptoms to polypharmacy, all her medications were held. She was started on levetiracetam and discharged with only partial improvement in her mental status to follow up with Neurology. A week later she presented with worsening mental status and GTC movements. EEG showed Left lateralized epileptiform discharges. Lumbar puncture showed elevated proteins. Repeat TSH came back as 13.8 and Anti-TPO was elevated to 49. A diagnosis of Hashimoto's encephalitis was made and she was promptly started on therapy with IV solumedrol and synthyroid.

DISCUSSION: Hashimoto's encephalopathy (HE) described first in 1966; is a rare disease with an estimated prevalence of 2.1/100,000 and only around 300 reported cases. The diagnosis is often missed because of nonspecific clinical presentation and laboratory findings. The mean age of onset is $40-45$ years with a female to male ratio of $4: 1$. There are two distinct clinical presentations of HE. Vasculitic type has an acute presentation with seizures, stroke-like episodes and sudden onset neuropsychiatric symptoms. Progressive type presents with gradual decline in cognition, agitation and hallucinations. Most common symptom for HE is seizures (seen in $66 \%$ of patients), followed by psychosis and stroke-like symptoms. Initial finding on Imaging, CSF and EEG are non-specific but are useful in ruling out differentials. Anti-thyroid antibodies are frequently present but most patients are euthyroid at the time of diagnosis. CSF findings of elevated protein and pleocytosis are common. Diffuse slowing of the background is the most common EEG abnormality seen in patients with HE, followed by epileptiform activity. Pathophysiology of HE continues to be unclear. Treatment of HE is centered on immunomodulation with steroids, which results in significant improvement in a majority of patients.

WHEN IT ISN'T STREPTOCOCCUS - UTILIZING THE CENTOR CRITERIA IN A YOUNG PATIENT WITH SORE THROAT Lisa Rucker; Haider Ali. Albert Einstein College of MedicineJacobi Medical Center, Bronx, NY. (Control ID \#2469340)

LEARNING OBJECTIVE \#1: Familiarize the learner with the Centor Criteria for the diagnosis of group A Streptococcal pharyngeal infections 
LEARNING OBJECTIVE \#2: Demonstrate how the Centor Criteria can help guide when an expanded clinical assessment and investigation pathway is warranted CASE: The patient is a 26 year-old obese female with asthma who walked in to our clinic with symptoms of sore throat, right ear pain, and trouble swallowing for 3 days. She had nasal discharge with thick, white sputum but denied cough. She denied fever, tinnitus, hemoptysis and sick contacts. She had not traveled recently. She felt her symptoms were different from her asthma. One month prior to her presentation she had similar symptoms. She took 5 days of amoxicillin, which she happened to have at home, with apparent improvement in symptoms. Physical examination was significant for the following: Temperature: 98.1 Pulse 57 Respirations 14 Blood Pressure 138/88 Ears: normal tympanic membranes; Nose: inflamed turbinates; Throat: enlarged, red tonsils with white exudates bilaterally; Neck: no adenopathy; Skin: no rash. Because only two of the four Centor criteria were positive, the diagnosis of group A Streptococcus was improbable so we obtained more historical information. The patient described having oral sex with her partner about 3 days prior to the onset of symptoms and recalled the same timing of symptoms after the same sexual contact 1 month prior. We performed nucleic acid amplification testing for neisseria gonorrhea and chlamydia trachomatis, and the former was positive. A single injection of ceftriaxone cured her infection.

DISCUSSION: The Centor Criteria were developed in 1981 as an aide to the correct diagnosis of group A Streptococcal sore throat. The Centor criteria are: •Tonsillar exudates $\bullet$ Tender anterior cervical adenopathy $\bullet$ Fever by history $\bullet$ Absence of cough The likelihood of having group A Streptococcal infection increases with the number of Centor criteria. Patients having two or fewer of the criteria are not likely to have the infection so testing and treatment costs can be saved. In this scenario, other causes must be entertained. The differential diagnosis of sore throat includes viruses (HIV, Herpes, Epstein-Barr), bacteria (other Streptococci, Fusobacteria, Corynebacterium, Neisseria Francisella, Mycoplasma), and non-infections (toxins, allergens, stomach acid). In this case we asked about sexual practices, HIV risks, and rashes before testing for gonorrhea and other sexually transmitted infections. Gonococcal pharyngitis is seen most often in people who practice oral sex. There are no pathognomonic findings with gonococcal pharyngitis. The infection is more likely after fellatio than cunnilingus. The prevalence of infection in patients with sore throat is $1-6 \%$. Asymptomatic infections are common Besides pharyngitis, Gonococcal infection can manifest as cervicitis, urethritis, pelvic inflammatory disease, epididymitis, proctitis, Bartholin gland infection, and disseminated disease. In this case, application of the Centor criteria appropriately broadened our differential diagnosis and helped us highlight risk factors for rarer pathogens.

\section{WHEN IT SOUNDS AND LOOKS LIKE APPENDICITIS AND IT'S...NOT?: A CASE OF APPENDICULAR ADENOCARCINOMA PRESENTING AS APPEN- DICITIS Andrew Trifan; Amar Kohli. UPMC, Mars, PA. (Control ID \#2469450)}

LEARNING OBJECTIVE \#1: To formulate a differential for abdominal pain in elderly patients

LEARNING OBJECTIVE \#2: To describe the treatment approach to adenocarcinoma of the appendix

CASE: A 67-year-old woman presented to the emergency department with diffuse bilateral lower abdominal pain for a few days. Her vitals were stable aside from a low grade temperature and labs unremarkable except for a slight leukocytosis. Her exam consisted of diffuse abdominal pain but no rebound or guarding. Given her symptoms along with nausea that co-incided with the pain, a CT scan was done showing findings concerning for appendicits. She underwent a laparoscopic appendectomy without any complications, though given the enlarged thickened appendix adherent to the cecum, it was sent off for pathology. The report came back as adenocarcinoma ex goblet cell carcinoid tumor. Invasive carcinoma was noted in proximal margins along with perineural invasion (no lympho-vascular invasion) and no lymph nodes were assessed, thus staging her as pT3Nx. CT abdomen showed hepatic steatosis but otherwise unremarkable for liver lesions and none seen on CT chest. Her chromogranin levels were normal as were other tumor markers assessing for synchronous malignancy, including CEA, CA 19-9, CA 125. She denied a prior history of unintentional weightloss. She had two colonoscopies at age 50 and 60 , both normal. No family history of carcinoid or GI cancers. Smoked 1 pack/day for 30 years but quit 10 years ago. She continues to drink 2-3 glasses wine/day and denied any carcinoid syndrome symptoms. Given that no lymph nodes were assessed during the operation, along with positive margins noted on the appendectomy, she underwent a right hemicolectomy and started on adjuvant FOLFOX therapy.

DISCUSSION: Abdominal pain is an extremely common presenting symptom in emergency rooms among all age groups. Regarding appendicitis, it is a less common cause of abdominal pain in elderly $(\sim 10 \%$ cases $)$ though there is a $>50 \%$ mortality from appendicitis is in this age group as most wait $>24 \mathrm{~h}$ until seeking help. Only $1 / 3$ of elderly patients present with classic symptoms of appendicitis. The differential also includes mesenteric ischemia, diverticulitis, bowel obstruction, AAA, malignancy and gynecologic causes in women. This case highlights a common diagnosis in a more unusual age group and should prompt the need to "think outside the box" regarding other diagnosis such as malignancy given her age and social history. Cancers of the appendix themselves are very rare, found accidentally in $1 \%$ of appendectomies and account for $0.5 \%$ all GI tumors overall. Over $80 \%$ are carcinoid whereas remainder are adenocarcinoma. Majority occur in 55-65 year-old. This case was an adenocarcinoma ex goblet cell tumor, found almost exclusively in the appendix with the most frequent presenting symptom as appendicitis (60-75\% cases), as they occur at the base compared to classic carcinoid which occurs at the tip of appendix. Classic symptoms of carcinoid are rare to begin with $(<10 \%)$ and require tumor metastasis to lung or liver as vasoactive substances are otherwise cleared. The size of the tumor dictates treatment. If $>2 \mathrm{~cm}$, a right hemicolectomy and possibly adjuvant therapy with imaging 3 months follow up is the initial route. If $<1 \mathrm{~cm}$ (75-80\% tumors) appendectomy alone is suffice. If $1-2 \mathrm{~cm}$ and evidence mesoappendiceal extension, positive margins or involving cecum/high grade, then an approach is similar to $>2 \mathrm{~cm}$ one. In general, only $5 \%$ of appendiceal cancers are metastasized at presentation, and the overall prognosis, particularly with this subtype, has an $80 \% 5$ year survival with treatment.

WHEN IT'S JUST NOT HOT FLASHES Maile Ray; Jenny J. Lin. Icahn School of Medicine at Mount Sinai, New York, NY. (Control ID \#2469310)

LEARNING OBJECTIVE \#1: Recognize side effects of hormonal therapy for breast cancer

LEARNING OBJECTIVE \#2: Understand treatment options for hormonal therapy side effects

CASE: A 46 year-old woman with a history of estrogen-receptor positive stage IIA breast cancer diagnosed 2 years ago was seen in primary care for regular follow-up. Her breast cancer had been treated with lumpectomy followed by chemotherapy and she had done well with treatment, continuing to work almost full time. Currently taking tamoxifen, she had experienced only some hot flashes a few months after starting the medication. She had no other past medical history and was taking no other medications. At this visit, she reported that she was experiencing more dyspareunia over the past several months to the point where it was starting to impact her relationship with her partner. Due to the pain with intercourse and other hormonal changes, she no longer had any libido and was becoming more depressed. She reported anhedonia, insomnia, decreased concentration and appetite. Exam revealed a tearful young woman, PHQ-9 score was 18 and gynecological exam showed moderate vaginal atrophy. Patient was recommended to start regular use of a vaginal moisturizer and referred for behavioral counseling and to consider starting anti- 
depressants. Several months later, patient returned after having started to see a therapist and psychiatrist regularly. She had been started on an anti-depressant, started to exercise regularly, and had also been referred to a sexual health psychologist. She was doing pelvic floor exercises, using vaginal moisturizers, and using vaginal lubricants during sex. She had also started using herbal remedies to help improve her sexual functioning. She was able to continue on tamoxifen.

DISCUSSION: Hormonal therapy for breast cancer (HT), including tamoxifen and aromatase inhibitors (AIs), is known for its abrupt and dramatic estrogen depletion, which often leads to menopausal symptoms often more severe than those seen in natural menopause. While hot flashes and musculoskeletal symptoms are the side effects most commonly associated with HT, sexual side effects are actually more prevalent and tend to be underreported. Estrogen helps maintain good sexual health in many ways, including objective measures (vaginal $\mathrm{pH}$, maturation index, cornification index) and subjective measures (libido, sexual satisfaction, dyspareunia). Estrogen deprivation can cause the loss of genital tissue elasticity and lubrication, as well as changes in vaginal $\mathrm{pH}$, resulting in an array of sexual symptoms such as dyspareunia, vaginal dryness, soreness, irritation, discharge, and decreased libido. Our patient presented with loss of libido, moderate vaginal atrophy, and moderate dyspareunia. Our patient also presented with depression. Mood disturbances are also common with HT yet can be stigmatized. Depression can also be attributed, in part, to other factors associated with a cancer diagnosis. Estrogen is thought to have a neuroprotective effect and also acts on multiple neurotransmitter systems, including those that regulate serotonin, dopamine, GABA, glutamate, and monoamine oxidase. Our patient presented with several classic symptoms of depression, including anhedonia, decreased appetite, and cognitive difficulties. Treatment for the sexual and mood side effects of HT can be complex because some potential treatments may interfere with HT itself. Estrogen supplementation is often used in postmenopausal women without a breast cancer predisposition as a treatment for menopausal symptoms However, because estrogen feeds estrogen receptor-positive cancers, it is contraindicated to use estrogen supplementation to alleviate HT symptoms. Instead, other treatment strategies are needed. Sexual health symptoms are best addressed by interventions that maintain or restore the natural vaginal $\mathrm{pH}$ and replenish intracellular fluids to the vaginal epithelium. The primary evidence-based strategies to accomplish this include vaginal moisturizers, such as polycarbophil gel and hyaluronic acid, and the herbal supplement, red clover. There is also mixed evidence that red clover may improve libido. Further, pelvic floor exercises and vaginal dilator therapy help women increase control over pelvic floor muscles, thereby alleviating some of the pain associated with vaginal atrophy. While there is limited evidence base for these treatments, a combination of them did alleviate symptoms substantially in our patient. Our patient used traditional strategies to treat depression, not specific to symptomatology brought on by estrogen depletion. There is a significant evidence base for the use of antidepressants and regular exercise to reduce or eliminate depressive symptoms. There are many differing views on the use of phytoestrogens, such as red clover. Some say phytoestrogens prevent side effects without significant estrogenic effect or cancer risk, while others contend phytoestrogens may increase breast cancer risk and should be avoided, at least among women with breast cancer predisposition. The interaction of phytoestrogens with the $\mathrm{ER} \alpha$ and $\mathrm{ER} \beta$ receptors appears complex and may differ according to cancer status. Our patient showed significant improvement in sexual symptoms after 2 months of treatment. Additionally, her depression lifted within a month. She will likely benefit from long-term treatment to alleviate sexual and mood symptoms so she may continue using tamoxifen to prevent a recurrence of breast cancer.

WHEN RARE BECOMES COMMON : ACUTE PANCREATITIS IN CHRONIC MARIJUANA USE Sabrina N. Huq ${ }^{2}$; Bharadwaj Ravi ${ }^{1}$; Sofia Terner ${ }^{2} .{ }^{1}$ Kasturba Medical College \& Hospital, Boston, MA; ${ }^{2}$ Montefiore Medical Center, Wakefield, Bronx, NY. (Control ID \#2467863)
LEARNING OBJECTIVE \#1: Recognize the association of chronic marijuana use with acute pancreatitis

LEARNING OBJECTIVE \#2: Identify that increasing prevalence of marijuana use could increase incidence of hitherto rare complications

CASE: A 33 year old man with no significant past medical history presented for evaluation of gradually progressive mid abdominal pain of 6 days duration. He was in his usual state of health, working as a software specialist prior to onset of symptoms. He noted severe, "punching" abdominal pain, worsened on food intake and associated with nausea. He denied fevers, sick contacts, excess alcohol intake (last intake was a month prior) or excess fatty food consumption, yellowing of skin, rashes, joint pains or right upper quadrant abdominal pain. He denied using any medications. He was found to have moderate epigastric tenderness with negative Murphy's and McBurney's signs, and no evidence of jaundice. Laboratory results revealed elevated lipase of $1200 \mathrm{U} / \mathrm{L}$ and amylase of $450 \mathrm{U} / \mathrm{L}$ which correlated with $\mathrm{CT}$ scan findings of heterogeneous and enlarged pancreatic head consistent with pancreatitis. An abdominal ultrasound did not reveal gallstones or biliary ductal dilatation. Further workup revealed normal triglyceride levels, negative alcohol level, negative hepatitis serologies and negative autoimmune serologies. On further questioning, he revealed that he had been growing and smoking marijuana daily since age 16, and had last smoked marijuana on the morning of presentation. He improved clinically and lipase levels normalized after 3 days of conservative management with intravenous fluids, pain control, and slow advancement of diet.

DISCUSSION: Marijuana is the most commonly used illicit drug in the United States, and its prevalence is increasing, with almost 20 million current users aged 12 or older ( $7.5 \%$ of the population) up from almost 15 million users $(5.8 \%)$ in 2007. Despite its current Federal classification as a Schedule I substance (having no medicinal use and high risk for abuse), marijuana has been legalized for adult recreational use in four states at present and nineteen other states (and the District of Columbia) have legalized use of marijuana for medical purposes. As its prevalence of use increases, we are uncovering rare complications seen after long term use. While alcohol and gall stones remain the most common etiologies for acute pancreatitis, drugs are a contributory cause in a small yet considerable portion of acute pancreatitis cases. Marijuana (Cannabis) has several chemical constituents, the chief among them being Tetrahydrocannabinol (THC). Cannabinoid receptors in the human body are of two known types - CB1 and CB2, both of which are expressed throughout the body. CB1 is predominantly found in the central and peripheral nervous systems whereas CB2 is found in macrophages and immune tissues such as spleen, tonsils and thymus. Both CB1 and CB2 are present in pancreatic tissue. Both are present in the Islet of Langerhans; CB1 receptors are seen in glucagon-containing $\alpha$ cells while CB2 receptors are seen in both $\alpha$ and insulin-containing $\beta$ cells. Cannabinoids have been shown to have both protective and destructive effects on different parts of the gut. They have been used in prevention and treatment of nausea and stimulation of appetite in the treatment of anorexia. In the stomach, cannabinoids act to relax the lower esophageal sphincter, reduce gastric acid secretion and delay gastric emptying. They also decrease small intestinal secretions and increase GI transit times. However, a harmful effect of cannabinoids has been observed in the small intestine where they activate the capsaicinsensitive vanilloid receptor (transient receptor potential vanilloid 1-TRPV1) and can cause or worsen ileitis. In addition, administration of a CB1 receptor antagonist (SR 141716) has been shown to reduce TNF- $\alpha$ levels and reduce ulceration of the small intestine in rodents. In the pancreas, studies have shown that cannabinoids worsen cerulein-induced pancreatitis in mice. Pre-treatment with Anandamide, an endocannabinoid, increased the severity of pancreatic tissue damage, increased serum levels of pancreatic enzymes and levels of pro-inflammatory cytokines like interleukin$1 \beta$. With increasingly prevalent use of marijuana, rare complications of its use must be considered in diagnosis. THC-induced pancreatitis has been seen in patients with excessive use of marijuana and is considered dose related. The exact mechanism is yet unknown. With discontinuation of usage, regression of symptoms has been reported in 
most cases. However, the diagnosis of marijuana-induced pancreatitis is to remain one of exclusion, only after having ruled out more common etiologies such as alcohol, cholelithiasis, infections, trauma, tumors or other medications.

WHEN WEARING A RING IS NOT SAFE: NUVARING RELATED ACUTE ISCHEMIC STROKE Tahmina Begum; Sarah Ahmed; Miles Grant; Roger D. Smalligan. Texas Tech Univ Health Sc Center - Amarillo, Amarillo, TX. (Control ID \#2468687)

LEARNING OBJECTIVE \#1: Reminder that hormonal contraceptive methods place women at risk for ischemic stroke.

LEARNING OBJECTIVE \#2: Young people may present with atypical clinical features of ischemic stroke.

CASE: A 24-year-old previously healthy young woman was admitted with sudden onset confusion, trembling and weakness of the left side of her body. At the time of the event she had just fallen off her jet ski but she denied any significant trauma as she entered the water with her life vest on. She was carried out of the water by her friends who found her confused and unable to walk. She felt numb on her left side, was nauseated and vomited in the emergency room. Past medical history was completely negative and her only medication was birth control using an etonogestrel/ethinyl estradiol vaginal ring (NuvaRing)a NuvaRing which had been using for some time. She did not smoke, use illicit drugs or drink alcohol to excess. No family history of premature vascular disease or events or coagulation disorders. On physical exam she was alert but anxious, BP 150/90, P 80, R 18 , afebrile, mild left facial droop, neck supple, lungs, heart, abdomen and extremities normal, strength $4 / 5$ in left arm and left leg. Labs including CBC, complete metabolic panel and coagulation studies were normal. Noncontrast head CT was negative but MRI showed a right middle cerebral artery ischemic infarction. She was started on aspirin and atorvastatin and showed rapid though partial recovery. She was sent to an inpatient rehabilitation unit DISCUSSION: The NuvaRing is a small, flexible vaginal ring used to prevent pregnancy. It delivers etonogestrel/ethinyl estradiol $0.12 \mathrm{mg} / 0.015 \mathrm{mg}$ daily. Although the amount of delivered hormone is very small, it still can cause significant morbidity related to thromboembolism to different organ systems including the brain, the heart, the lungs or the extremities. A recent meta analysis showed the odds ratio for first-ever ischemic stroke risk associated with current oral contraceptive pill (OCP) use compared with noncurrent OCP use was 2.47 [95 \% confidence interval (CI), 2.04-2.99]. The risk of ischemic stroke among current OCP users decreased significantly with decreasing estrogen dose: OCPs of $\geq 50 \mu \mathrm{g}$ ethinyl estradiol (EE) OR 3.28; 30-40 ug EE OR 1.75; 20 ug EE OR 1.56. All different generations of progestin only pills were associated with an elevated risk of ischemic stroke as well. Based on this data and other studies, the NuvaRing has a black box warning by the FDA regarding use in active smokers and those over age 35 due to the high risk of stroke or thromboembolism. Our case is an unfortunate example of this very risk except that she was young and had no history of smoking or other risk factors. Physicians need to continue to discuss these thromboembolic risks with all women choosing hormonal contraceptive methods.

WHEN YOUR LIVER CAN NOT BREATHE Issra Jamal. Baystate medical center Broad Brook, CT. (Control ID \#2469511)

LEARNING OBJECTIVE \#1: Recognize the signs and symptoms of chronic hypoxia as a presenting symptom of hepatopulmonary syndrome in patients with an underlying liver disease

LEARNING OBJECTIVE \#2: Recognize the importance of discerning pattern of dyspnea when assessing cirrhotic patients presenting with shortness of breath
CASE: A 53 year old Korean speaking female with a past medical history significant for cirrhosis due to chronic hepatitis B infection presented with sub-acute mild progressive shortness of breath in the setting of a recent flu-like illness and exposure to sick contacts. In the ED she was found to be hypoxic to $83 \%$ on room air. She was also found to have bilateral hand and feet clubbing on physical examination. Infectious etiologies were investigated; Influenza and other viral respiratory pathogens were negative by PCR. Mycoplasma, HIV and TB were negative too, and her CXR was unremarkable. CT pulmonary angiogram was also non-diagnostic for a pulmonary embolism. She continued to be hypoxic though clinically looked well. On further questioning, given her signs of chronic hypoxia consisting of clubbing, we were able to discern the pattern of her dyspnea, as platypnea, which lead to the consideration of hepatopulmonary syndrome. This was further corroborated by positive orthostatic desaturations or orthodeoxia. The diagnosis was confirmed with a trans-thoracic contrast induced ECHO with agitated saline which showed a moderate-to-severe right-to-left shunt with delay suggesting an intra-pulmonary shunt indicative of a hepatopulmonary syndrome. Furthermore, it also showed an elevated pulmonary artery pressure and A-a gradient which both also support a diagnosis of hepatopulmonary syndrome. A formal pulmonary angiogram was not done due the recent CT pulmonary angiogram that did not show any evidence of a collateral pulmonary pathway. She was discharged on supplemental oxygen with a follow up to arrange for a liver transplant.

DISCUSSION: The clinical features of hepatopulmonary syndrome (HPS) are the consequences of both hepatic and pulmonary dysfunction. More than $80 \%$ of patients present with symptoms of liver disease; the remainder experience dyspnea as their initial symptom . Most patients with HPS eventually develop dyspnea on exertion, a rest, or both, usually after years of liver disease. However, dyspnea is a non-specific finding in patients with liver disease because it may also be the consequence of a hepatic hydrothorax, Porto pulmonary hypertension, anemia, ascites, fluid retention, and muscle wasting. Dyspnea may be accompanied by pulmonary findings that are more specific for HPS like Platypnea which is an increase in dyspnea that is induced by moving into an upright position and relieved by recumbency. Another specific sign is Orthodeoxia which refers to a decrease in the arterial oxygen tension (by more than $4 \mathrm{mmHg}[0.5 \mathrm{kPa}]$ ) or arterial oxyhemoglobin desaturation (by more than $5 \%$ ) when the patient moves from a supine to an upright position, which is improved by returning to the recumbent position. The presence of orthodeoxia in a patient with liver disease is strongly suggestive of HPS, although it can be seen in other situations (eg, post-pneumonectomy, recurrent pulmonary emboli, atrial septal defects, and chronic lung disease). Orthodeoxia affects up to $88 \%$ of patients with HPS, compared to $5 \%$ or fewer of patients with cirrhosis alone. The diagnostic tests that can be used to detect IPVDs include contrast-enhanced echocardiography, technetium-99 m-labeled macro aggregated albumin scanning, and pulmonary arteriography . Contrast-enhanced echocardiography is generally preferred because it is more sensitive than technetium-99 m-labeled macro aggregated albumin scanning and less invasive than pulmonary arteriography, with an intrapulmonary shunt, contrast generally appears in the left heart three to six heart beats after its appearance in the right heart . In patients with liver disease, detection of an intrapulmonary rightto-left shunt is considered indicative of IPVDs.

WHERE'S WALDEN?: AN UNCOMMON CAUSE OF AUTOIMMUNE CYTOPENIAS Cameron Kemal ${ }^{1}$; Daniel M. Huck ${ }^{2}$; Viraj V. Patel ${ }^{1} .{ }^{1}$ Albert Einstein College of Medicine, Bronx, NY; ${ }^{2}$ Montefiore Medical Center, Bronx, NY. (Control ID \#2466414)

LEARNING OBJECTIVE \#1: Diagnose Waldenström Macroglobulinemia based on clinical features, laboratory values, and diagnostic studies

LEARNING OBJECTIVE \#2: Recognize common and uncommon autoimmune manifestations of Waldenström Macroglobulinemia 
CASE: An 86 year old woman presented with 2 weeks of dizziness, fatigue, several days of abdominal pain and constipation, and noted a $30 \mathrm{lb}$ weight loss over the past year. She denied having fevers, chills, night sweats, or appetite changes. She had paresthesias in her lower extremities and a 2-year history of isolated neutropenia. On exam, she had a 2/6 systolic crescendo-decrescendo murmur heard throughout the precordium and conjunctival pallor, but no splenomegaly or hepatomegaly. Initial diagnostic work up revealed pancytopenia with a white blood cell count of $4.4 \mathrm{~K} / \mathrm{uL}$, low absolute neutrophil count of $1.0 \mathrm{~K} / \mathrm{uL}$, and a normal lymphocyte count, low platelet count of $(47 \mathrm{~K} / \mathrm{uL})$, and low hemoglobin $(6.8 \mathrm{~g} / \mathrm{dL})$ and hematocrit $(21 \%)$ with a slightly elevated MCV $(97.2 \mathrm{fL})$ and reticulocyte count of $2.6 \%$. LDH was elevated (1187 U/L). A Direct Coombs test was positive for a pan-agglutinin ( $\operatorname{IgG})$ antibody, erythrocyte sedimentation rate was elevated $(140 \mathrm{~mm} / \mathrm{h})$, and an anti-nuclear antibody (ANA) test was negative. Serum immunoglobulin analysis revealed a markedly elevated IgM of $1820 \mathrm{mg} / \mathrm{dL}$ with a kappa/lambda ratio of 44 . Based on these preliminary findings, we entertained a diagnosis of malignancy versus autoimmune disease. Prednisone was initiated to treat autoimmune hemolytic anemia. On day 3 , we performed a bone marrow biopsy which revealed a lymphoplasmacytic infiltration consistent with Waldenström Macroglobulinemia. Chemotherapy was initiated with bendamustine and rituximab and a tapering steroid course was continued with improvement in hemoglobin $(11.0 \mathrm{~g} / \mathrm{dL})$, platelet count $(126 \mathrm{~K} / \mathrm{uL})$ and absolute neutrophil count $(3.9 \mathrm{~K} / \mathrm{uL})$ at 2 months after initiation.

DISCUSSION: Waldenström Macroglobulinemia (WM) is a rare malignant plasma cell dyscrasia characterized by a monoclonal IgM gammopathy and lymphoplasmacytic infiltration of the bone marrow or lymphatic tissue. The incidence of Waldenström macroglobulinemia is estimated around 2 to 5 cases per 1 million most commonly diagnosed in white males with a median age of 65 years. The most common clinical presentations of WM are fatigue and weakness, peripheral neuropathy, anemia, constitutional "B" symptoms, and abnormal bleeding. There are also unique manifestations accountable to the monoclonal gammopathy of IgM antibody, including hyperviscosity syndrome and autoimmune manifestations. Currently, several criteria exist for the diagnosis of WM including the World Health Organization Lymphoma Classification as well as the Mayo Clinic. Clinically, however, monoclonal IgM gammopathy in the presence of $10 \%$ or more lymphoplasmacytic infiltrate of the bone marrow can be considered diagnostic for WM if other lymphoproliferative diseases (including chronic lymphocytic leukemia and mantle cell lymphoma) have been excluded. This lymphocytic infiltrate must demonstrate plasmacytoid or plasma cell differentiation and one of many typical immunophenotypes. Autoimmune manifestations in WM are varied. A mutation in myeloid differentiation of factor 88 (MYD88) is found in almost all WM cases, causing malignant proliferation and likely having a role in the autoimmune manifestations in the disease. Recent studies suggest that autoimmune cytopenias are relatively common in WM with anemia most frequently the result of autoimmune hemolytic anemia; both cold (IgM) and warm (IgG) subtypes have been associated with the disease. Immune thrombocytopenia (ITP) can also occur in the setting of WM, thought to be due to a platelet associated IgM or IgG antibody. Neutropenia as seen in this patient has not been well described in the literature. A past medical history of neutropenia, persistence at the time of this admission and responsiveness to corticosteroid treatment would suggest an autoimmune causation. Peripheral neuropathy due to monoclonal $\mathrm{IgM}$ antibodies to neural antigens, and rheumatic diseases including rheumatoid arthritis and antiphospholipid syndrome are also associated with WM. In summary we present an unusual case of Waldenström Macroglobulinemia presenting as isolated neutropenia that then evolved into pancytopenia in the presence of autoimmune hemolytic anemia

WILL SHE RECOVER? A PROLONGED HOSPITALIZATION FOR A COMATOSE FEMALE WITH NEUROPSYCHIATRIC LUPUS Srikar Kumar; Christian Geier; Jillian Catalanotti. George Washington University, Arlington, VA. (Control ID \#2465977)
LEARNING OBJECTIVE \#1: Recognize symptoms and appropriate management of neuropsychiatric lupus

LEARNING OBJECTIVE \#2: Identify the typical disease course and prognosis in neuropsychiatric lupus

CASE: A 25 year old African-American woman was brought into the emergency department by EMS after she was found unresponsive in her apartment. Her mother had called 911 as she was unable to reach her daughter for a day. Police found the patient unconscious and unable to be aroused, lying in her own feces and urine, with no witnesses or medical history available. In the ED, physical exam showed an obtunded female with GCS of 6 T. Neurological exam revealed a left eye gaze with horizontal nystagmus, absent Babinski reflex, 3+ reflexes in upper extremity, and 4+ reflexes in lower extremity bilaterally. CT head did not demonstrate acute hemorrhage or masses. The patient was intubated to protect her airway and admitted to the ICU. She was started on empiric therapy for seizure with levetiracetam and for meningoencephalitis with ceftriaxone, vancomycin, and acyclovir. Lumbar puncture revealed no infection, and urine drug screen was normal. Brain MRI showed restricted diffusion along the posterior frontal, parietal and temporal cortices bilaterally with diffuse pachymeningeal enhancement concerning for an autoimmune process. Using the regional online health information exchange, we discovered that the patient had been diagnosed with SLE at a nearby hospital, 2 weeks prior to this admission. She had been started on steroids and hydroxychloroquine, and was discharged with plans for rheumatology follow-up. However, she missed that appointment, and medication compliance was unknown. Workup at the other facility revealed a mixed lupus and Sjogren's picture, with evidence of renal and hematologic involvement of her SLE. In our ICU, the patient was treated with levetiracetam, lacosamide, and pregabalin for seizures, and diagnostic EEG was performed. She was also started on pulse dose steroids and cyclophosphamide for SLE. Her neurologic status remained unimproved, and her ICU course was marked by multiple complications, including massive hemoptysis at her tracheostomy site, prolonged seizures, and anoxia. Her chance of recovery was believed to be very poor given intensity of her condition. She was transferred to the medical floor on day 26 after passing tracheostomy collar trial, but she continued to have no purposeful movements. Over the next 2 months, she slowly regained neurological function in her extremities, and was discharged to inpatient rehabilitation on oral prednisone and lacosamide. She returned home, and in the rheumatology clinic 1 month after discharge, had regained full neurological function. She began her third dose of cyclophosphamide and was continued on her seizure medications, and returned to work. She continues to follow with rheumatology and neurology. DISCUSSION: SLE affects the neurological system by affecting inflammatory, vasculitic, thromboembolic, and meningeal pathways through a series of complex mechanisms. As a result, patient may experience a range of symptoms including stroke, seizures, cognitive dysfunction, and headaches. Risk factors for neuropsychiatric SLE include the presence of antiphospholipid antibodies, nephritis, young age, and African American ethnicity. Recognizing neuropsychiatric manifestations of SLE is important as management involves treating both the neurological symptoms and the underlying SLE. Symptoms of neuropsychiatric SLE should be evaluated and treated just as in patients without lupus. Our patient presented with a likely seizure, and was treated empirically for seizure. Lupus patients with stroke should be treated as any other stroke patients. Airway, breathing, and circulation should always be assessed first when a patient presents with severe neurological symptoms, followed by an appropriate workup to rule out any common causes of neurological dysfunction. Once the patient has been stabilized and started on appropriate neurological treatments, SLE treatments such as pulse dose steroids are initiated to help recover from the underlying disease. Neurological symptoms may persist longer in SLE patients, leading to a long and difficult hospital course. Our patient was initially thought to have a very low chance of regaining neurological function. Despite these concerns, she recovered fully after 68 hospital days and an additional 16 days in inpatient rehab. Because neuropsychiatric symptoms of SLE are often indistinguishable from non-SLE neurological symptoms, diagnosis can be difficult, requiring strong clinical suspicion and accurate medical history, 
WORMS AND COMPLEMENT OVERACTIVATION Hadi Zein; Stanley Josue. New York Presbyterian Queens, Flushing, NY. (Control ID \#2468805)

LEARNING OBJECTIVE \#1: Recognize the implications of chronic corticosteroids use in patients that require lifelong maintenance on immunosuppressants

LEARNING OBJECTIVE \#2: Recognize the presentation of atypical hemolytic uremic syndrome

CASE: A 51 year old female with known polymyositis on chronic prednisone presented with 4 months of diarrhea. She denied any other symptoms. Physical examination was notable for tachycardia of 110 , temperature of $37.5 \mathrm{C}$, abdomen was non tender. Laboratory testing showed hypoalbuminemia, elevated creatinine kinase, leukocytosis and microcytic anemia. Her subsequent stool testing was positive for Strongyloides stercoralis. Despite treatment with ivermectin and albendazole, her condition worsened. She developed gram negative bacteremia with Bacteroides fragilis and Klebsiella pneumoniae. Subsequently, her platelet count and hemoglobin dropped and her kidney function worsened. A peripheral blood smear revealed the presence of schistocytes. ADAMSTS-13 activity was $77 \%$. Her deteriorating kidney function required her to be initiated on hemodialysis. She was intubated as she developed acute respiratory syndrome (ARDS) and was moved to the intensive care unit. The patient's diagnosis was atypical hemolytic uremic syndrome.

DISCUSSION: Strongloides stercolaris is a human intestinal parasite, usually treated with albendazole or ivermectin. Because of chronic corticosteroid therapy, our patient had hyperinfection syndrome characterized by cycles of autoinfection. It is often accompanied by sepsis from intestinal bacteria, hypothesized to be from the intestinal parasites migrating through the enteric mucosa. In our case, the severe sepsis triggered a thrombotic microangiopathic syndrome. This syndrome is a diverse group of disorders united by common features: microangiopathic hemolytic anemia with thrombocytopenia. Hemolyt ic uremic syndrome (HUS) is usually precipitated by Escherichia coli O157:H7 and other Shiga toxin producing bacteria. On the other side, thrombotic thrombocytopenic purpura (TTP) is caused by a deficiency of ADAMSTS-13, a protease that cleaves the von Willebrand factor multimers. As our patient had normal ADAMSTS-13 activity and no evidence of Shiga toxin producing bacteria, her diagnosis was atypical hemolytic uremic syndrome (aHUS). It is thought to be provoked by an uncontrolled activation of the complement system. The distinction between these different syndromes is not only academic but also gears physicians towards treatment. HUS and TTP are treated with plasma exchange in adults whereas aHUS is treated with eculizumab. Eculizumab is a humanized monoclonal antibody that binds C5 complement protein. Given the patient's septicemia and hyperinfection with Strongyloides, initiating an immunosuppressant would worsen the infectious process. So eculizumab therapy was not initiated. This case highlights the adverse effects of chronic corticosteroid therapy. An otherwise simple parasitic infection caused bacteremia, complicated by atypical HUS. Patients should be aware of the implication of steroids use and given alternatives when possible such as biologic therapies. Biologic agents would offer a more targeted immune suppression and fewer side effects.

XOXO: MANAGING THE MISSING X Kristi Larned; Rebecca Glassman. Beth Israel Deaconess Medical Center, Boston, MA. (Control ID \#2469405)

LEARNING OBJECTIVE \#1: Describe the signs, symptoms, and evaluation of Turner's Syndrome

LEARNING OBJECTIVE \#2: Recognize the deleterious effects of long-term hypoestrogenic states

CASE: A 49-year-old woman with no significant past medical history presented to establish care with an internist after 35 years without medical care. She had presented to the emergency department 3 weeks prior for hematuria and was found to have bladder cancer. She had undergone a transurethral resection while admitted and was awaiting further outpatient oncologic care. On review of systems, the patient reported primary amenorrhea. On further questioning, she noted that a pelvic ultrasound in her twenties had shown absence of ovaries. The patient had been informed she would likely not be able to bear children. No additional work-up was pursued. Physical exam was notable for a height of 58 in., small breasts with widely spaced nipples, and a broad chest. Labs revealed an A1c of $9.2 \%$, total cholesterol of 212, and LDL of 118 . Given her history of primary amenorrhea and physical features, the patient was counseled on the possibility of Turner's Syndrome, and agreed to a karyotype which confirmed 45, X. For additional risk stratification, a DEXA scan was performed diagnosing osteoporosis (T score PA spine -2.7 , left hip total -3.0 and femoral neck -2.9$)$. An echocardiogram did not identify any congenital cardiac abnormalities.

DISCUSSION: Turner's Syndrome (TS) results from the complete or partial absence of the second X chromosome. It affects about 1 in every 2500 live-born females. Typically a diagnosis is made prenatally or during the pediatric years based on suspicion from ultrasound findings in utero and physical exam features, with confirmation by karyotype. It is, therefore, uncommon to make an adult diagnosis of TS. Only about $10 \%$ of women with TS are diagnosed as adults. Women with TS are of short stature, typically 52-60 in.. Due to altered skeletal growth, they have upper bodies which are larger and out of proportion to the lower body, resulting in a "shield chest" appearance. Other phenotypic features may include micrognathia, cubitus valgus, webbed neck, low posterior hairline, convex nails and edema of the hands and feet. As a result of gonadal failure, most TS patients lack pubertal development and suffer from infertility. Therefore management in early adulthood often is focused on treatment of hypoestrogenic state and reproductive assistance. There are also many psychosocial ramifications of this diagnosis, prompting early intervention through social work and counseling. The workup for a woman of any age newly diagnosed with TS includes a karyotype in addition to bloodwork to screen for associated conditions. All women need fasting glucose and cholesterol testing, a yearly TSH, urinalysis, and serum creatinine. Blood pressure should be monitored closely as the risk for hypertension is increased 3-fold in women with TS. Imaging of the heart and aorta is crucial to assess for cardiac abnormalities, such as bicuspid aortic valve and aortic arch defects. Approximately one-third of TS patients have aortic enlargement putting them at risk for fatal aortic dissection or rupture. Even those without anatomic abnormalities are at twice the average risk for ischemic heart disease due to metabolic complications. The liver should be closely monitored since women with TS have a five-fold increased risk for cirrhosis. A pelvic ultrasound must be obtained in patients with $\mathrm{Y}$ chromosome mosaicism to assess for gonadal dysgenesis. If the patient has "streak ovaries," prophylactic removal is recommended to reduce the risk for gonadoblastoma. Renal ultrasounds are needed for newly diagnosed patients, as structural abnormalities are common resulting in frequent urinary tract infections as well as hydronephrosis. Hearing and vision screenings are also important. Later in life, patients are at risk of cardiovascular disease, metabolic syndrome and osteoporosis as long term side effects of low estrogen. In most cases, this is mitigated through early diagnosis and treatment with supplemental estrogen therapy. Our patient exemplifies the unfortunate result of decades without estrogen: advanced osteoporosis and metabolic syndrome. However, with a clear diagnosis these and other comorbidities will be routinely screened for, caught early and treated appropriately.

\section{*INNOVATIONS IN MEDICAL EDUCATION (IME)}

\section{FACULTY INTEGRATED TO RESIDENT SCHEDULING TEMPLATES} (FIRST) Halle G. Sobel ${ }^{6}$; Rachel Swigris ${ }^{2}$; Karen Chacko ${ }^{1}$; Alison R. Landrey ${ }^{6}$; Monica C. McNulty ${ }^{3}$; Kaitlyn Vennard ${ }^{6}$; Susan M. Nikels ${ }^{3}$; Suddarth Kathleen ${ }^{4}$; Edward N. Murphy ${ }^{4}$; Eva M. Aagaard ${ }^{5}$. ${ }^{1}$ Univeristy of Colorado Denver, Aurora, CO; ${ }^{2}$ University of Colorado, Denver, CO; ${ }^{3}$ University of Colorado Denver, Aurora, $\mathrm{CO} ;{ }^{4}$ University of 
Colorado School of Medicine, Denver, CO; ${ }^{5}$ University of Colorado School of Medicine, Aurora, CO; ${ }^{6}$ University of Vermont Medical Center, Burlington, VT. (Control ID \#2459914)

NEEDS AND OBJECTIVES: Many Internal Medicine residency programs have implemented block-scheduling templates ( $\mathrm{X}+\mathrm{Y}$ models). In an attempt to maximize continuity between supervising faculty and residents in the outpatient setting, some programs have also adjusted faculty precepting schedules to mimic those of the residents. "Intensivist" preceptors are assigned to a cohort of residents and precept this cohort during the majority of the days during their outpatient weeks. In contrast, "traditional" preceptors generally precept one half day per week. Little is known about the impact of these different types of faculty schedules on residents, faculty or the patients they serve.

SETTING AND PARTICIPANTS: Our study included two internal medicine resident continuity clinics at the University of Colorado: the Lowry Clinic and the Anshutz clinic. Our study also included the single continuity clinic at the University of Vermont Medical Center. A total of 97 internal medicine residents and 37 faculty members were included in the study. Faculty members who were part of the study did not participate in the surveys. All the clinics operate with a $4+1$ block scheduling model. In this model, residents are in their continuity clinic for 1 week followed by in-patient rotations or other electives for 4 weeks and this template repeats throughout the year. The Lowry and Vermont clinics have an intensivist preceptor model and the Anshutz clinic has a traditional precepting model

DESCRIPTION: Our study examined the impact of intensivist and traditional faculty scheduling models at two academic medical centers. We examined faculty and resident perceptions of the ability of faculty to assess resident progress, resident and faculty ability to develop interpersonal relationships with each other, faculty familiarity with residents' complex chronic patients, and their opportunity to discuss patients and their results both during and in between clinic weeks. Residents and faculty at both sites were surveyed in December of 2014 using either a web-based or paper survey. The survey included definitions of intensivist and traditional faculty preceptors, basic demographic information and likert scale questions ( $1=$ strongly disagree and $5=$ strongly agree $)$ assessing perceptions of each of the domains noted above. To analyze differences between intensivist and traditional models, we used the Wilcoxon Rank Sum test. We did not keep track of the actual number of clinic sessions each type of preceptor spent with the residents.

EVALUATION: There were 26 faculty survey responses ( $83 \%$ response rate) and 75 resident survey responses (77\% response rate). Intensivist preceptors reported feeling more familiar with the residents' complex patients $(4.00 \pm 0.76$ vs. $2.88 \pm 1.23, p=0.05)$ and more comfortable evaluating their residents' performance ( $4.38 \pm 0.52$ vs. $3.72 \pm 0.67$, $p=0.02)$ than traditional preceptors. Intensivist preceptors and traditional preceptors reported adequate opportunity to get to know the resident interpersonally $(4.25 \pm 0.71$ vs. $4.01 .06, p=0.636$ ). Both preceptor groups noted a limited opportunity to discuss results between clinic weeks (Intensivist $1.62 \pm 0.92$ vs. $2.11 \pm 1.23, p=0.352$ ) and an overall sense of being reasonably satisfied with quality of care provided (Intensivist 3.62 \pm 1.06 vs. $3.72 \pm 9.6, p=0.902$ ). Patterns similar to the faculty results were found among the residents with the exception that residents identified greater satisfaction with the ability to follow-up with intensivist preceptors between clinic compared to traditional preceptors $(3.21 \pm 1.00$ vs. $2.42 \pm 0.94, p=0.001)$

DISCUSSION / REFLECTION / LESSONS LEARNED: Enhancing continuity between residents and faculty in block-schedule outpatient clinics impacts faculty and, to a lesser extent, resident perceptions regarding faculty ability to assess residents and their familiarity with the residents' complex patients. Such models may help to deliver milestone- feedback in a way that enhances resident development especially in the age of hand-offs when some faculty evaluators may not have the oppportunity to know residents well. The lack of opportunity to discuss patients and results in between clinic weeks as noted by the residents should be further explored. In the era of increasing weight on the value on resident assessments combined with a focus on patient outcomes, research assessing the impact of new models of scheduling is needed.

IMPLEMENTATION OF A TRAINEE-LED CASE CONFERENCE TO IMPROVE INTERPROFESSIONAL, TEAM-BASED CARE FOR HIGH COMPLEXITY PATIENTS Anna Strewler ${ }^{2}$; Nathan Ewigman ${ }^{1}$; Bridget O’Brien ${ }^{1}$; Jessica A. Eng ${ }^{1}$; Rebecca L. Shunk ${ }^{3} .{ }^{1}$ SFVA/UCSF, San Francisco, CA; ${ }^{2}$ San Francisco VA Medical Center, San Bruno, CA; ${ }^{3}$ UCSF, San Francisco, CA. (Control ID \#2469960)

NEEDS AND OBJECTIVES: Team-based care and interprofessional collaboration are central tenets of the Patient-Centered Medical Home (PCMH), yet despite educational and clinical training in this setting, trainees are still greatly challenged by caring for patients with the most complex medical and psychosocial needs. To build the skills necessary to function optimally in a PCMH while simultaneously aiming to improve the care of highly complex patients, we introduced a monthly clinical case conference in July 2014 entitled PatientAligned Care Teams Interprofessional Care Update (PACT-ICU). The goals of the conference are to develop a collaborative, interprofessional treatment plan and to increase trainees' understanding of the roles each team-member can play in caring for complex patients. SETTING AND PARTICIPANTS: PACT-ICU is part of the curriculum of the San Francisco VA Center of Excellence in Primary Care Education (CoEPCE). The SFVA CoEPCE trains core internal medicine residents and nurse practitioner trainees to deliver team-based patient-centered care through Patient Aligned Care Teams (PACTs), the VA's version of PCMH. A PACT team consists of an RN, LVN, and clerk as well as the core and associated health trainees (clinical psychology fellows, clinical pharmacy residents, social work interns, and dietetic interns). PACT Intensive Management (PIM) is a case management program into which all PACT-ICU patients are referred. PIM consists of a geriatrician, RN's, social workers, and a clinical psychologist.

DESCRIPTION: Core trainees use a risk stratification tool developed by the VA called the (Care Assessment Need) CAN Score to choose patients with a high risk of hospitalization or mortality to present at PACT-ICU. Trainees write a patient narrative from the trainee and patient perspectives and present the patient during the hour-long conference. Interprofessional trainees, staff, and PACT and PIM team members discuss the case and formulate a treatment plan with assigned action items. Staff-members with relevant expertise are also invited to present a clinical teaching pearl. The patient is automatically referred into PIM, which works in tandem with the trainee and PACT team for a period of 6-12 months to support the patient's needs. To date, twenty-five patients have been presented.

EVALUATION: Evaluation of PACT-ICU includes conference evaluation forms after each session, a retrospective pre-post survey after the first session, and patient intervention outcomes. Over the course of 1 year, participants rated "participation by a variety of health professionals" and "collaborative nature of discussion during the session" very good to excellent (mean 4.6/5; sd 0.6; $n=96$ ). Learners indicated that the conference was helpful for their development of a treatment plan for the patient (mean 4.1/5, sd $1.1 ; n=17$ ). Trainees indicated that their understanding of the roles that each team member could play in hard to manage patients' care was 2.7 (SD 1.0) before the session and 4.1 (SD 0.7) after the session (scale $1=$ minimal, $3=$ adequate, $5=$ excellent; $n=17$ ). Comments from conference evaluation forms revealed that trainees found the "interdisciplinary aspect," "learning about different resources available at the VA and how to access them," and "subspecialty teaching topics" most valuable. Constructive feedback included requests to "make the session shorter" and to "explain med[ical] issues briefly to be more inclusive of non-medical providers." A qualitative list of trainee, PACT, and PIM interventions for patients following PACT-ICU conferences has been obtained by chart biopsy. Prominent examples include co-visits between trainee providers and PIM team-members, frequent motivational interviewing, and assisting with in-home support and long-term care placement. 
DISCUSSION / REFLECTION / LESSONS LEARNED: Having an assigned faculty member who mentors trainees and helps coordinate the conference has been critical. Based on feedback from trainees, the faculty made several changes to PACT-ICU. The faculty mentor started sending patient identifiers via secure email to interprofessional trainees and PACT team-members prior to the conference so they could feel more prepared and comfortable participating. They also invited experts, rather than trainees, to present clinical pearls, which made the content more accessible to non- medical trainees and teammembers. The faculty mentor needed to prepare experts for time-efficient clinical teaching in order to increase inclusiveness, while still providing satisfactory clinical content. The partnership developed between PACT-ICU and PIM has been an important component of our conference. Patients and their trainee providers and PACT teams receive case management services, often provided in the home setting, beyond the capabilities of clinicbased PACT team. The PIM team elicits the valuable perspectives of the trainee provider, PACT team, and interprofessional trainees at the conference and collaboratively formulates a treatment plan before enrolling and providing individualized services to the patient

\section{A 6-DOMAIN HISTORY-TAKING FRAMEWORK WAS USEFUL IN EFFI-} CIENTLY IDENTIFYING CLINICALLY RELEVANT BIOPSYCHOSOCIAL INFORMATION Denege A. Ward; Davoren Chick; Paula T. Ross; Patricia Mullan; Brent C. Williams. University of Michigan, Ann Arbor, MI. (Control ID \#2466473)

NEEDS AND OBJECTIVES: Clinical assessment and planning by physicians frequently omits psychosocial information necessary for mutual goal setting, facilitating patient selfmanagement, and identifying and addressing barriers to care. Physicians cite as barriers to gathering more complete biopsychosocial information: a) limited time, b) limited skills and responsibility for managing non-biomedical problems, and c) that social determinants of health require interventions other than health care. The traditional 'History and Physical' data gathering format emphasizes biomedical diseases and underemphasizes behavioral, environmental, and social determinants of health. We sought to provide physician learners a method for efficiently gathering information from patients during routine clinical care that would: a) enhance identification of clinically relevant behavioral and social factors, b) be perceived as immediately relevant to physicians' work, and c) facilitate mobilization and collaboration with non-physician health care providers.

SETTING AND PARTICIPANTS: Participants were fourth-year medical students on a 1-month rotation on an 'uncovered' (without residents) general medicine inpatient service. Fifteen students participation from May to July, 2015.

DESCRIPTION: Based on best practices in settings emphasizing comprehensive care planning - geriatrics, mental health, and care of homeless persons - we developed a 6domain framework for patient assessment. The domains are: 1) Biomedical Conditions, 2) Mental Health, 3) Behavioral Health, 4) Social Support, 5) Resources and Living Environment, and 6) Functional Status. Students underwent a 1-h orientation to the framework at the beginning of the rotation, and were assigned to use the framework to: a) complete an admission or progress note ('write-up'), and b) identify one problem to address with the health care team. At the end of the rotation students answered 10 (5-point) Likert-type questions about the usefulness of the framework and provided free-text comments (available for 10 students). Two authors (DW and BW) applied content analysis ${ }^{4}$ to the 15 write-ups to determine whether: a) information on all 6 domains was included, and b) information was misclassified by domain. Authors independently rated the write-ups for completeness and accuracy, and resolved the few differences by consensus.

EVALUATION: Student ratings of the 6-domain framework were highly positive, indicating that the framework was "helpful in identifying barriers to care and follow-up" (mean $\{\mathrm{SD}\}$ rating 4 \{1.1)), "improved my understanding of my patient and their needs" (4.2 $\{0.8\})$, that "there were resources to help with barriers" ( $4.4\{0.7\})$, and that they were "able to develop a plan to address at least one barrier to care" $(4.4\{0.8\})$. Lower but positive ratings were reported for other items including "(the framework) helped me create a comprehensive problem list" (3.8 $\{1.4\})$, and "(the team used the framework to) help utilize resources in nursing, pharmacy, and social work" (3.5 $\{1.4\})$. Comments were uniformly positive, and included "This was a great exercise and really helped my patient.", "Would be great to do on every patient...", and "Very helpful...Ensures complete H and P". Nine of the 15 write-ups included information for 4 or more domains; only 2 included all 6 domains. Domains most frequently missing were Behavioral Health (missing in 10) and Functional Status (missing in 9). Seven write-ups misclassified information by domain.

DISCUSSION / REFLECTION / LESSONS LEARNED: In this pilot study, a 6domain framework for patient assessment was useful in facilitating medical students' identification of clinically relevant issues not included in the traditional ' $\mathrm{H}$ and $\mathrm{P}$ ' without feeling burdened by the more comprehensive approach. It also appears useful in facilitating care planning involving other physician and non-physician members of the health care team. We plan to investigate the validity and feasibility of applying the 6-domain framework in other inpatient and outpatient teaching settings for students and residents. Primary barriers to implementation will be the need for faculty development and resistance in the dominant culture to expanding the scope of 'physicians' work' to include biopsychosocial and social determinants of health in patient care. 1. Engel GL. From biomedical to biopsychosocial. Psychother Psychosom 1997;66:57-62. 2. Astin JA, Sierpina VS, Forys K, Clarridge B. Integration of the biopsychosocial model: Perspectives of medical students and residents. Academic Medicine: Journal of the Association of American Medical Colleges 2008;83:20-27. 3. Morley CP, Flad JR, Arthur M, ReckerHughes $\mathrm{C}$, et al., Pilot evaluation of a piopsychosocial integrated standardized patient examination in a family medicine clerkship. Intl J Psychiatry in Medicine 2011;41:309328. 4. Hsieh H-F, Shannon SF. Three approaches to qualitative content analysis. Qualitative Health Research 2005; 15:1277-88.

A CURRICULUM TO IMPROVE RESIDENT KNOWLEDGE AND SATISFACTION IN CARING FOR HOSPITALIZED PATIENTS WITH ADDICTION Payel J. Roy $^{3}$; Zoe M. Weinstein ${ }^{1}$; David Yuh $^{4}$; Linda Neville ${ }^{4}$; Alexander Y. Walley ${ }^{2} .{ }^{1}$ Boston Medical Center, Boston, MA; ${ }^{2}$ Boston Univ, Boston, MA; ${ }^{3}$ Boston University Medical Center, Boston, MA; ${ }^{4}$ Boston University School of Medicine, Boston, MA. (Control ID \#2465573)

NEEDS AND OBJECTIVES: Residents at academic teaching hospitals are the frontline of inpatient care. They frequently care for patients with substance use, with approximately 1 out of every 6 hospital stays including a substance-related diagnosis at our institution. However, resident physicians are significantly less likely to experience satisfaction in managing addiction in comparison to other medical issues. This low satisfaction is associated with low confidence in assessment and treatment of substance use disorders (SUD). At our institution, usual inpatient practice for patients with SUD is to manage acute withdrawal symptoms and provide information for patients to self-refer to further addiction treatment and mutual support groups, without initiation of long-term pharmacotherapy or direct linkage to post-hospitalization addiction treatment. This is a lost opportunity for residents to improve the overall health of their patients and their own job satisfaction. We addressed these gaps in knowledge and satisfaction by providing a medical curriculum as part of our new Addiction Consult Service (ACS) with three objectives: increase resident ability to identify and treat addiction; improve resident satisfaction in managing patients with addiction; and provide linkage to addiction care after hospital discharge.

SETTING AND PARTICIPANTS: Setting: academic safety net hospital. Primary Curricular Participants: Internal Medicine (IM) and Family Medicine (FM) residents rotating on the ACS. Secondary: IM residents who consult the service.

DESCRIPTION: The Addiction Consult Service (ACS) is a new medical service at our hospital aimed to improve the care of hospitalized patients with SUD. Consults have 
included initiation of pharmacotherapy for alcohol and opioid use disorder, complicated benzodiazepine withdrawal, and pain management for patients on opioid agonist therapy. IM and FM residents at our hospital rotate on the service as a 1 week elective. The purpose of the curriculum is to improve resident knowledge and satisfaction in managing addiction, which occurs in several ways. Firstly, residents on the elective serve as primary consultant for 1-2 new patients per day, which includes obtaining a thorough substancerelated history and physical, presenting on rounds, developing a plan, relaying recommendations to the primary team, and managing follow-up. Residents have a rotationspecific curriculum we developed using ACGME Core Competencies. This curriculum is a 14-page document emailed to every resident before starting the rotation and includes 16 detailed learning objectives. Objectives include taking a substance use history, diagnosing substance use disorders, teaching harm reduction strategies, and understanding pharmacotherapy for alcohol and opioid use disorder. Residents also receive interdisciplinary education with staff psychiatrists to discuss difficult patient cases. Finally, residents who consult the ACS have increased exposure to SUD and its treatment from direct communication with the ACS team.

EVALUATION: A resident quality improvement project, prior to the initiation of the ACS, demonstrated a local need for more resident expertise in addiction medicine. Following this project, $40 \mathrm{IM}$ residents were asked to complete a survey regarding their attitudes towards patients with SUD; two-thirds reported dissatisfaction with the addiction treatment they provided, $87 \%$ reported the need for a change, and over $90 \%$ felt a consult service would be helpful. Since this survey, we began the consult service and our curriculum. We have had $14 \mathrm{IM}$ residents and $5 \mathrm{FM}$ residents rotate on the service. Overall the rotation and curriculum have been well received. We will evaluate rotating residents' addiction knowledge with pre- and post-elective multiple choice tests. We will also ask these residents to evaluate their comfort and satisfaction in managing patients with addiction pre- and post-elective. Finally, another 40 IM residents will be asked to complete a survey regarding their attitudes towards patients with addiction at the end of the academic year to evaluate the overall impact of the Consult Service on IM residents' satisfaction in managing patients with addiction

DISCUSSION / REFLECTION / LESSONS LEARNED: The Addiction Consul Service provides a unique opportunity for residents to expand their knowledge and comfort in managing addiction during inpatient rotations. Strengths of the service include training residents to care for an underserved population and to manage medications not always used in ambulatory training, such as opioid agonist therapy. Educational initiatives focused on improving resident ability to identify and treat addiction can improve job satisfaction and patient-provider relationships. Long-term, we anticipate this will increase patient engagement in addiction treatment, improve hospital quality metrics such as length of stay and readmission rates, and encourage residents to incorporate addiction treatment into their future practice.

\section{A CURRICULUM TO OPTIMIZE MEDICAL STUDENT EXPERIENCE WITH PATIENT-CENTERED DISCHARGE CARE Maureen D. Lyons; Adam Cifu; Amber} Pincavage. University of Chicago, Chicago, IL. (Control ID \#2445413)

NEEDS AND OBJECTIVES: Given the increased focus on effective care transitions, comprehensive patient-centered discharge care is critical but infrequently formally taught in clerkships. Our objective was to create a curriculum to address the gap in patientcentered discharge care education during clinical clerkships.

SETTING AND PARTICIPANTS: University of Chicago Pritzker School of Medicine, third year medical students

DESCRIPTION: Based on a needs-assessment demonstrating students do not frequently provide ambulatory post-hospital follow-up nor receive supervision providing discharge care, we created a curriculum. Students participated in an interactive workshop focused on patient-centered discharge care techniques that included our teaching video of one patient's flawed care transition and a reflective small group exercise. To promote direct observation, a mobile application tool was designed for attendings and residents to observe students providing discharge care. Students were required to complete one observed discharge education and encouraged to attend one post-hospital follow-up visit. For assessment, surveys were administered in the academic year before and after the curriculum.

EVALUATION: There was a pre-curriculum response rate of $84 \%(73 / 87)$ and a postcurriculum response rate of $80 \%$ (68/85). Compared to pre-curriculum, after the curriculum more students completed a post-hospitalization telephone call $(53 \%$ vs. $91 \%, p=$ $<0.001$ ), assessed medication adherence after hospitalization ( $19 \%$ vs. $61 \%, p<0.001)$, and saw a patient at a hospital follow-up visit (30\% vs. $79 \%, p<0.001)$. More students provided discharge education more than twice per clerkship ( $63 \%$ vs. $84 \%, p=0.005$ ) and students reported spending more time ( $>5 \mathrm{~min}$ ) reviewing discharge materials with patients $(81 \%$ vs. $94 \%, p=0.03)$. Students were more often directly observed completing discharge paperwork ( $52 \%$ vs. $78 \%, p=0.004)$ and at a hospital follow-up visit ( $21 \%$ vs. $43 \%, p=0.004)$. Students also were more likely to elicit patients' perspectives about hospital discharge more than twice per clerkship (78\% vs. $96 \%, p=0.006$ ). Student satisfaction with their discharge care ( $33 \%$ to $60 \%, p=<0.001$ ), discharge planning competency ( $34.3 \%$ vs. $60 \%, p=0.01$ ), and post-hospital follow-up care competency ( $25.7 \%$ vs. $51 \%, p=0.001)$ improved.

DISCUSSION / REFLECTION / LESSONS LEARNED: Our curriculum improved student participation in discharge care and direct observation of these activities. Similar to previous studies, it improved students' satisfaction and self-reported competency. However, it also increased patient-centered discharge behaviors. Next steps include adapting the curriculum for the sub-internship. This curriculum needs further evaluation but has potential to address competencies focused on patient-centeredness, system-based practice, and communication as well as the core EPA addressing care transitions.

\section{A HOSPITAL WITHOUT WALLS: ADDRESSING SOCIAL DETERMINANTS OF HEALTH THROUGH A RESIDENT PHYSICIAN-LED ADVOCACY CUR-} RICULUM Colleen Vessell; Amy S. Tang; Angela Arbach; Janine Knudsen. NYU School of Medicine, New York, NY. (Control ID \#2468260)

NEEDS AND OBJECTIVES: With an increasingly diverse patient population, physicians must understand the social determinants of health and the physician's role in advocating for health equity. In response to this need, an interdisciplinary group of resident physicians, partnered with faculty, formed the Social Justice Interest Group (SJIG) to create a curriculum that encourages trainees to: 1) Demonstrate sensitivity and responsiveness to the social determinants of health (including gender, race, economic class, religion, immigration status, and sexual orientation) that affect the diverse patient populations that residents serve 2) Work in interprofessional teams to advocate for high-quality patient care and health systems that are inclusive of patients from a broad range of socioeconomic and cultural backgrounds 3) Identify career pathways into advocacy SETTING AND PARTICIPANTS: Bellevue Hospital is the flagship tertiary safety-net hospital in New York City. It is the oldest public hospital in the United States and serves patients from a broad range of ethnic and socioeconomic backgrounds. It is also the primary academic hospital affiliated with New York University School of Medicine's residency programs. SJIG participants include internal medicine, primary care, psychiatry and emergency medicine resident physicians.

DESCRIPTION: The curriculum has multiple components, including a monthly noon conference, clinical electives, community site visits, and a quarterly newsletter. Noon conference: The core curriculum is structured around a monthly noon conference series featuring health advocates from New York City. Curriculum topics include refugee and immigrant health, substance abuse, homelessness, women's health and reproductive 
rights, legal advocacy, and health policy and systems. Speakers are invited from city agencies including Bellevue Hospital, non-profit advocacy groups, and community based organizations. In the future, the SJIG plans to organize dinner discussions with speakers to encourage residents to network and discover career pathways into advocacy. Clinical Elective: Bellevue Hospital is the designated inpatient site for inmates requiring tertiary care in New York City's correctional system. The residents' interest in correctional health led to a collaboration between the departments of medicine and psychiatry to create a 2 week elective in which trainees work in a correctional health setting and learn about the specific health needs of incarcerated individuals. Community site visits: To emphasize the relationship between community and health, the curriculum includes visits to community sites such as the Bellevue men's shelter and Riker's Island correctional facility. Newsletter. Each quarter, the SJIG distributes a newsletter that focuses on a core curricular topic and includes relevant articles, topical think pieces, and an interview to highlight a faculty member's advocacy work in this area

EVALUATION: The impact of our curriculum will be assessed through pre- and postevent surveys of the participants. The surveys will assess resident knowledge related to learning objectives, attitudes towards physician advocacy, and perceived impact on behaviors regarding patient care and choice of career path. In order to foster a multidisciplinary environment, we will track attendance from various specialties and work with other specialties to ensure that the events are accessible to all. Finally, we will solicit feedback following each event in order to improve the curriculum.

DISCUSSION / REFLECTION / LESSONS LEARNED: A curriculum addressing social determinants of health is a vital component of residency programs that seek to provide high quality care to vulnerable patients. This resident-led curricular intervention equips residents with the knowledge and skills needed to address patients' social needs and health barriers. It also offers opportunities for broader physician advocacy. To date, the program has successfully connected residents with physician-advocate role models and enhanced residents' agency in improving the care for vulnerable patient groups. The SJIG's efforts to integrate diverse disciplines, including medicine, policy, and research, have also highlighted the multidisciplinary efforts required to address community needs. Further, the SJIG's efforts to work with the city's largest correctional health facility to create a new elective may be a successful model for future community-based electives. Resident physician's discordant schedules and limited protected time for planning curricular events have been the main challenge to organizing the curriculum and engaging residents in advocacy efforts. Support from faculty mentors in scheduling and coordination across programs is essential to the success of the curriculum. Finally, the safety-net hospital's history of physician advocacy and mission to serve the city's most vulnerable patient populations provided a strong basis on which to build our curriculum and speaker series.

\section{A MULTI-FACETED EDUCATIONAL INTERVENTION FOR ADVANCE} CARE PLANNING IN RESIDENT CLINIC Jennifer Russo ${ }^{3,2}$; Irina Vovnoboy ${ }^{1,2}$; Jessica Bender ${ }^{4}, 2$; Ryan Chippendale ${ }^{1}$; Sandhya Rao ${ }^{1} .{ }^{1}$ Boston University School of Medicine, Boston, MA; ${ }^{2}$ Boston Medical Center, Boston, MA; ${ }^{3}$ Harvard Vanguard Medical Associates, Peabody, MA; ${ }^{4}$ UCSF, San Francisco, MA. (Control ID \#2470247)

NEEDS AND OBJECTIVES: Advance care planning (ACP) in primary care is increasingly important as the population ages, yet internal medicine trainees report lack of confidence and variable skills in leading these discussions in the outpatient setting. We conducted a needs assessment and multi-step educational intervention to improve levels of confidence in several specific ACP-related skills and to increase the number of ACP discussions within resident clinics.

SETTING AND PARTICIPANTS: The study was conducted in an urban academic medical center in Boston. Participants included categorical and primary care interns in an internal medicine residency program.
DESCRIPTION: We planned a multi-step educational intervention to improve the confidence and skills of IM interns in discussing ACP in the primary care setting at an urban academic medical center. A voluntary needs assessment survey was conducted to assess interns' confidence in leading ACP discussions. An educational intervention followed, consisting of 1) a practice improvement module (PIM) chart audit to determine baseline practice; 2 ) small-group educational session to address areas of lower confidence; 3) a second chart audit and post-intervention survey 3 months later.

EVALUATION: The needs assessment survey response rate was $86 \%(n=38) .74 \%$ of respondents indicated that ACP in primary care was important or very important. Yet, $45 \%$ reported that they had not facilitated any ACP discussions with their primary care patients. Interns reported high baseline confidence in discussing advance directives and naming healthcare proxies, but low confidence in estimating prognosis and discussing changes in care needs. After our intervention, the percentage of interns having ACP discussions increased from 6 to $19 \%$, based on PIM chart audits. The post-intervention survey response rate was $75 \%(n=33)$. The percentage of interns reporting high confidence in facilitating ACP discussions with primary care patients increased from 58 to $80 \%$. The percentage of interns reporting high confidence increased for all 7 specific ACP-related skills queried.

DISCUSSION / REFLECTION / LESSONS LEARNED: Although interns initially reported high levels of confidence in several specific ACP-related skills, they held few ACP discussions in the primary care setting. We theorize that interns' high level of selfreported skill is due to experiences they have had in the inpatient setting where they frequently are responsible for assisting patients in naming healthcare proxies, discussing goals of care, and leading family meetings. The interns' lower confidence in estimating prognosis in the outpatient setting likely results in an underestimation of the number of primary care patients who would benefit from advance care planning. The multi-step educational intervention was associated with increased confidence among IM interns and a greater number of ACP discussions in the primary care setting. This feasible and attainable intervention was well-received and is scalable; it has been added to the IM residency curriculum.

\section{A NOVEL APPROACH TO THE PROGRAM EVALUATION COMMITTEE} Amy R. Schwartz ${ }^{1,2}$; Mark Siegel ${ }^{2}$; Alfred Lee ${ }^{2} .{ }^{1}$ VA Connecticut Healthcare System, West Haven, CT; ${ }^{2}$ Yale University School of Medicine, New Haven, CT. (Control ID \#2469382)

NEEDS AND OBJECTIVES: The Accreditation Council for Graduate Medical Education (ACGME) requires every residency program to have a Program Evaluation Committee (PEC) to systematically evaluate the program's educational activities, curriculum, and compliance with ACGME standards. According to ACGME recommendations, the PEC should consist of at least one trainee and members of the teaching faculty who are appointed by the residency program director. There are minimal other stipulations as to how the PEC should carry out its duties. We created a novel format for the PEC, in the form of resident focus groups, guided by the mentorship of designated teaching faculty. Our goals included promoting resident leadership and providing actionable feedback to rotation Firm Chiefs.

SETTING AND PARTICIPANTS: Yale University School of Medicine, Traditional Internal Medicine Residency Program

DESCRIPTION: All interested residents and select faculty in the Yale Traditional Internal Medicine Residency Program are invited to join the PEC. One resident and one faculty member are paired and together lead a focus group for each rotation at the Yale New Haven Hospital and VA Connecticut Healthcare System campuses. Each focus group consists of all available residents who have participated in the relevant rotation and who provide verbal feedback about their experiences. The feedback is elicited in the form of a 
structured, one-hour discussion based on a survey. The survey was designed by the faculty PEC chairs and program director, and addresses general strengths, areas for improvement, educational goals, curriculum, workload, resident/faculty evaluations, and mentorship opportunities for each rotation. The resident/faculty pair summarizes the feedback in a written document and meets with the rotation Firm Chief to deliver the feedback. The resident/faculty PEC pair and Firm Chief propose an action plan, which is included in the feedback document that is shared with the residency program director, hospital leadership, and Chief of Medicine.

EVALUATION: Residents identified many strengths of their rotations, particularly in the caliber of attending precepting and teaching and in richness and diversity of patient care experiences. Suggestions for programmatic improvement included (a) optimizing the formal curriculum for each rotation, (b) addressing technology issues (e.g., phones, badges), (c) improving relationships with other services (e.g., emergency and radiology departments) and (d) streamlining workload or workflows. Faculty and resident PEC committee members found the committee to be a rewarding mentorship experience and provided a valuable opportunity to engage in quality improvement. Residents expressed satisfaction about having a forum to voice concerns and an opportunity to shape the trajectory of the program. Firm Chiefs expressed satisfaction about in-person meetings with actionable feedback to optimize their rotations.

DISCUSSION / REFLECTION / LESSONS LEARNED: We created a novel format for the PEC, consisting of resident/faculty teams that generate actionable, resident-led feedback for each rotation, based on a structured discussion. Feedback is presented formally to firm chiefs, concrete action plans created, and residency and hospital leadership systematically alerted to rotation strengths and areas for improvement. Residents, PEC faculty, and firm chiefs derived satisfaction about numerous aspects of the new PEC format. Based on the success of the new PEC, going forward we plan to expand the scope of the PEC to include perceptions of the residency program at-large and will seek quantitative data for these measures

A NOVEL CURRICULUM TO FOSTER INTERN RESILIENCY Amber-Nicole Bird $^{2}$; Amber Pincavage ${ }^{1}$. ${ }^{1}$ University of Chicago, Chicago, IL; ${ }^{2}$ University of Pennsylvania, Philadelphia, PA. (Control ID \#2458507)

NEEDS AND OBJECTIVES: Burnout amongst medical professionals is pervasive and it is a growing concern. It is associated with increased rates of depression, suicide, and poor clinical performance. Increased depressive symptoms amongst medical trainees are associated with factors encountered during residency, including perceived medical errors, increased work hours, and stressful life events. Despite increased recognition of physician burnout, little data exists on how it relates to resilience and few interventions focus on developing resilient trainees. Our objectives were to develop and pilot a novel skill-building based curriculum to foster resilience within our intern class. Through this curriculum we aimed to identify the impact of trainee burnout on clinical performance and work-life balance, as well as introduce trainees to basic concepts of resilience including managing expectations and setting realistic goals, finding gratitude, and letting go after stressful clinical events. In addition, we wanted to assess for any change in resilience scores after delivery of our curriculum.

SETTING AND PARTICIPANTS: Our resilience curriculum was piloted with the Internal Medicine interns at the University of Chicago over the 2014-2015 academic year. There were four small group sessions delivered from August of 2014 through March of 2015. DESCRIPTION: The curriculum focused on four skill-building workshops - setting realistic goals, managing expectations, letting go after stressful clinical events, and finding gratitude. All sessions were delivered by a chief resident during the outpatient portion of the interns' block schedule. Sessions consisted of 8-10 interns and combined small group discussion, reflection, didactic lecture, and simulated skill-building exercises. Interns were surveyed on their levels of resilience using the Connor-Davidsone Resilience scale (CD-
25). In addition, they were asked to respond to questions on their reported levels of stress, burnout, and involvement in medical errors using a standard Likert scale. These surveys were conducted prior to initiating the resiliency curriculum and again at the completion. EVALUATION: A total of 36 interns completed the resilience curriculum in 2014-2015. The pilot had promising results with $69 \%(25 / 36)$ of interns stating that the sessions should continue the following year. As a result of the sessions, a majority of interns are more comfortable talking about stress and burnout (64\%,23/36), are more comfortable talking about medical errors $(64 \%, 23 / 36)$, have the necessary skills to manage stress and burnout $(61 \%, 22 / 36)$, and learned new ways to approach challenges $(67 \%, 24 / 36)$. Half of interns used something they learned outside of the sessions $(51 \%, 18 / 35)$. There were no differences in resilience scores after the curriculum (low resilience pre 10/32 $31 \%$ vs. post $17 / 3647 \% p=0.22$; high resilience pre $7 / 3222 \%$ vs. post $4 / 3611 \% p=0.33$ ). Qualitative feedback from the interns indicated the sessions fostered a sense of togetherness amongst their peers and provided them with additional support systems.

DISCUSSION / REFLECTION / LESSONS LEARNED: Small group resilience workshops provided interns with new skills to approach challenges and manage burnout. While we did not demonstrate a quantitative improvement in resilience scores, sessions were well received by interns and provided an open forum for discussing medical errors, stress, and burnout. In addition, the interns' qualitative feedback suggested that these sessions fostered a sense of togetherness with their peers and provided access to additional support systems that may not have been evident at the start of their residency training. While a resilience curriculum for interns shows promise as a way to teach and foster resilience in residents, more in-depth interventions may be required.

\section{A PATIENT-CENTERED INTERPROFESSIONAL LEARNING EXPERIENCE} IN TRANSITIONS OF CARE Christopher Bruti; Lynette Richter; Irene Hossain; Jason Pagsisihan; Erika Schwelnus. Rush University Medical Center, Chicago, IL. (Control ID \#2469446)

NEEDS AND OBJECTIVES: The safe transition of patients from inpatient to less acute settings requires the concerted effort of a multidisciplinary team. The AAMC's Core EPAs for Entering Residency (CEPAER) lists "collaborate as a member of an interprofessional team" as one of the activities on which students should be taught and evaluated. CEPAER also lists "actively engages with the patient and other team members to coordinate care and provide for seamless transitions between care providers and from one setting to another" as an expectation for an entrustable learner. We sought to formalize this learning activity through an interprofessional 'transitions of care' activity in conjunction with our institution's college of nursing. Transitions of care can be a complicated process involving many disciplines and steps. We designed a 2-h activity for medical and nursing students to acquire both an appreciation for this process and knowledge of barriers to effective transitions of care. We followed the RIME framework (Pangaro, 1999) which acknowledges that a learner must be able to report and interpret a problem before managing it. The objective of the activity was to obtain and interpret information regarding a patient's home needs and risks for readmission or decompensation after their transition out of the hospital. This would be done in partnership with a nursing student, allowing for increased insight into the unique, differing, and complimentary roles of each profession's participation in the important task of safely discharging a patient.

SETTING AND PARTICIPANTS: The interactive activity was done as part of the M3 Clerkship in Internal Medicine and Term 5 of the nursing college once a month during the fall term (4 sessions to date). We used patients on a general medicine ward in a tertiary academic medical center. The patients were screened in the morning by faculty who sought patients who were willing and able to speak with the students, were planning on returning home after the hospitalization, and were thought to have risk factors complicating their transition. Preference was given to newly admitted patients. 
DESCRIPTION: Students were placed in interdisciplinary teams consisting of one or two nursing and medical students. We provided each team with a patient to interview and instructed them via a minimially prescriptive worksheet to focus on barriers to safe transition, readmission risks, and nonmedical risks to disease progression. They were given $45 \mathrm{~min}$ to complete the interview and $15 \mathrm{~min}$ to complete the worksheet that was shared with the patient's case manager. Each of the activities was followed by a large group debrief session where both medical and nursing students discussed their experiences. The debrief focused on findings from the patient interview, differences in findings and perspectives between the professions, and overall experience as part of a multidisciplinary team. Nursing and medical school faculty facilitated, helping explain any concepts when needed.

EVALUATION: The activity was evaluated through a short post-session survey. Nearly all respondents enjoyed the session and found it helpful. Many remarked that it was the first time they had worked in a multidisciplinary team and enjoyed the experience. Many requested more multidisciplinary learning sessions in the future. Constructive criticism included attempting to have the activity involve patients that the medical students were already following in order to lessen the burden of multiple providers involved in the patient's care. During the debriefings, a few themes emerged: students felt each discipline had a slightly different approach to patients' transition-related risks, and teamwork and collaboration is essential in this task. Medical students tended to focus more on the medical problems, medications, and physician follow-up. The experience gave them an appreciation for the importance of knowing a patient's social background as well as their disease process. Nursing students tended to focus more on the psychosocial stressors that affected the patients in the outpatient setting and learned more about the patient's medical risks. Both professions gained an appreciation for the value of good communication and the importance of each profession's role within the team.

DISCUSSION / REFLECTION / LESSONS LEARNED: We found this interprofessional activity on transitions of care to be highly valuable and enriching to the training of both medical and nursing students. The students learned a lot from each other and the experience, including alternative approaches to transitions and patient care, the complimentary roles of each discipline, and an the importance of good communication between providers. Future goals include shifting the time of the activity to earlier in the day so we can give the information to case managers in a timely manner making it more impactful in real-time patient care. Reference: Pangaro LN. A new vocabulary and other innovations for improving the descriptive in-training evaluations. Acad Med. 1999;74:1203-1207.

A RESIDENT-LED INITIATIVE TO ADDRESS THE IMPACT OF LAW ENFORCEMENT INTERACTIONS ON PATIENTS Jessica Bloome; David Tian; Danielle Alkov; Aisha Scherr-Williams; Lamercie Saint-Hilaire; Diana Wu. University of California, San Francisco, San Francisco, CA. (Control ID \#2469825)

NEEDS AND OBJECTIVES: Public attention has recently turned towards the epidemic of police violence in inner-city communities, encouraged by social movements such as Black Lives Matter. In clinics and hospitals, health care providers routinely care for individual survivors of violence. However, medical residents and other providers may struggle to identify ways in which they can engage social movements and create change more broadly. After attending this session, participants will be able to: --Recognize opportunities for medical residents to engage social movements as an educational tool Identify ways in which residency programs and educators can support resident-driven advocacy initiatives -Describe methods for physicians to address social determinants of health, including interactions with law enforcement

SETTING AND PARTICIPANTS: Inspired by the Black Lives Matter movement, residents at San Francisco General Hospital organized, seeking to understand police violence as a public health issue. Here, we explore how this group of residents sought to educate themselves, their peers, and their community.
DESCRIPTION: Residents from the Departments of Internal Medicine, Family and Community Medicine, and Emergency Medicine formed a multidisciplinary working group to educate ourselves on ways that police violence impacts our patients. Reflecting on our patients' traumatic encounters with law enforcement, we realized these issues were especially relevant within our own hospital, where the Sheriff's Department provides security. Through small-group self-education, we identified ways in which police interactions affect our community. We then partnered with community organizations to understand the rights of arrested and incarcerated patients within the health care setting. With faculty support, we developed a well-attended hospital-wide grand rounds panel discussion on the intersection of medicine and law enforcement. This experience led us to develop a quality improvement project to address the lack of standardization in hospital security protocols when the Sheriff is called. We created a checklist to encourage best practices in communication between health care providers and security staff, with the goal of improving patient experiences and minimizing the use of force. Recognizing our efforts, hospital management invited residents from the working group to join a multidisciplinary team of medical providers, hospital administrators, and security officers to improve handling of patient security on our campus.

EVALUATION: This educational effort harnessed small-group learning, lectures, and experiential learning through quality improvement. Process metrics included attendance at educational sessions and the successful inclusion of residents, attending physicians, nurses, administrators, and security staff in a multidisciplinary working group. Outcome metrics included the integration of a best practices security checklist into formal hospital policy. Data collection via this checklist is underway and will allow better understanding of the role of security in the hospital, as well as identify ways to decrease the use of unnecessary force.

DISCUSSION / REFLECTION / LESSONS LEARNED: While the primary focus of residency is the clinical education of residents, training programs should encourage leadership and the pursuit of social change through community engagement. Residencyled advocacy initiatives, especially with faculty support, can be an effective educational tool to foster awareness of social determinants of health and to translate these understandings into changes in clinical practice. Our experience demonstrated manners in which residents interested in a broader social issue can work together and with faculty to develop their interests. Our efforts showed how a multidisciplinary working group can identify previously unharnessed resources to teach residents. For instance, internal medicine residents can learn de-escalation techniques for agitated patients from psychiatric nursing staff who regularly use these skills. In addition, our efforts showed how experientia learning through quality improvement projects can help residents improve the sustainability of change by engaging hospital leadership. More broadly, history has shown that health care institutions can choose to perpetuate or to dismantle structural inequality. Importantly, we realized that turning a critical eye on health care institutions is key in improving communities' trust of doctors, as well as improving health care outcomes for historically marginalized communities.

A TALE OF TWO KROGERS: DEVELOPING AN INTEGRATED SOCIAL MEDICINE \& SERVICE LEARNING CURRICULUM Ayrenne Adams'; Lurit Bepo $^{3}$; Jada C. Bussey-Jones ${ }^{2}$; Maura George ${ }^{2}$; Natalie Stahl ${ }^{1} .{ }^{1}$ Emory School of Medicine, Atlanta, GA; ${ }^{2}$ Emory University, Atlanta, GA; ${ }^{3}$ Emory University School of Medicine, Atlanta, GA. (Control ID \#2463555)

NEEDS AND OBJECTIVES: During the course of medical training, it is critical that physician learners develop a depth and breadth of medical knowledge and clinical skills. However, since clinical care is estimated to have less than a $20 \%$ influence on health outcomes, it is also essential that physician learners develop an understanding of how the social determinants of health (the social, environmental, and institutional conditions in 
which people are born, grow, work, and age) influence health outcomes and disparities. Many training programs fail to adequately teach and prepare learners to engage with these broader health issues. Longitudinal opportunities to facilitate student knowledge of local communities, increase student appreciation of the social context of patients' lives, and develop student understanding of the influence of social determinants on health outcomes improves their ability to care for underserved and marginalized patients and encourages them to make patient advocacy and community engagement part of their professional practice. By the end of the course, learners should be able to: 1 . Describe the complexity and impact of community and other social determinants of health on individual patients and patient populations a. Describe how disparities in health can be conceptualized in relation to the institutions and social conditions that determine health related resources ("structural competency") 2. Discuss ways to connect structural competency and service learning with their clinical and academic work through didactic, experiential, and service activities

SETTING AND PARTICIPANTS: Students in their first year at Emory University School of Medicine became acquainted with the unique social challenges of two Atlanta communities through a series of small group sessions and activities. The two neighborhoods, known to the developers of this activity, were selected for their relevance to Emory medical students: one as a familiar and relatively wealthy area of residence for many students, and the other as a poverty-dense community home to many patients from the safety net hospitals through which all Emory medical students rotate.

DESCRIPTION: Students first independently researched two neighborhoods in Atlanta with differential access to wealth, income, and resources. They compared demographics, life expectancies, home values, educational backgrounds, graduation rates, and transportation options, among other statistics. This provided students with ample contextual knowledge to then conduct neighborhood exploration activities, which included window community surveys and a comparison of Kroger grocery stores. The window community survey allowed students to drive around the two neighborhoods and make note of the presence/absence of sidewalks, tobacco and alcohol advertisements, parks and other public spaces, grocery and liquor stores, and abandoned buildings. During the "Tale of Two Krogers" activity, students were armed with checklists assessing the presence, absence, and nature of fresh produce, sale items, unhealthy snack foods, and the general conditions and patronage of these two stores. Throughout these activities, learners had the opportunity to come together to teach each other as well as to engage in group discussions about the impacts of these social determinants of health on the communities they visited. EVALUATION: Using an electronic Likert scale survey, students completed an evaluation assessing overall feelings concerning the activity as well as strengths and areas for improvement of the program. Specific students were then contacted to provide further feedback in semi-structured interviews. At baseline, students reported having an elementary understanding of the social determinants of health and felt the activities provided a more applied understanding of those issues. The activities were generally well rated, with students expressing great support for even more interactive, less passive activities in future iterations. DISCUSSION / REFLECTION / LESSONS LEARNED: First year medical students who completed the neighborhood exploration activities were overwhelmingly in favor of bolstering their medical knowledge and clinical skills with interactive community knowledge and engagement. The students were particularly struck by the differences in access, environment, and resources available to different communities that would significantly impact their recommendations as future physicians about treatment, diet, and exercise. This knowledge lays the groundwork for the development of structural and cultural competency throughout their medical careers. Current ongoing efforts to build a longitudinal community-based service learning curriculum that expands students' functional knowledge of social medicine concepts will likely be well-received by students, particularly during the pre-clinical years. However, more structured evaluation is required to direct these efforts and to further develop activities centered on health disparities, patient advocacy, and structural and cultural competency.
A VIRTUAL LIBRARY TO PROMOTE PATIENT-CENTERED LEARNING ON INPATIENT MEDICAL SERVICES Richard E. Leiter; William Zhang; Kerri I. Aronson. Weill Cornell Medical College, New York, NY. (Control ID \#2469136)

NEEDS AND OBJECTIVES: Medical teams currently lack effective methods of sharing and discussing medical literature that answer clinical questions for daily patient care. We describe the use of a novel, web-based, collaborative reference manager and workspace on the General Medicine ward service. We show how the team's use of this program enhances both the breadth and depth of evidence-based learning at all levels of training (medical student, intern, resident, and attending) in a time-efficient and userfriendly manner.

SETTING AND PARTICIPANTS: This pilot program is currently being conducted on the PGY4 Chief Resident-led General Medicine service at a large, urban, academic medical center in the Northeastern U.S. This team consists of two medical students, one intern, one senior resident, and an attending physician (one of four Chief Residents) and is charged with caring for a diverse census and promoting the teaching skills of the senior resident as a "junior attending."

DESCRIPTION: Faculty of 1000 Workspace (F1000) is a suite of tools that assists physicians and scientists with research, writing, collaboration, and reference management. This platform was selected for use on the Medicine service because it allows for the simple addition of citations and full-text papers to a shared bibliography through the use of a webbrowser plug-in extension. References can be indexed to multiple projects or subprojects visible by different invited users allowing for logical and intuitive organization. The program also encourages collaboration between individuals with shared annotations and online space for discussion. At the beginning of each 2-week block, the attending physician introduces the team members to the online platform and ensures team members have access to the workspace (provided by the institution's medical library). Team members are then able to add articles to a shared project, highlight key portions of the text, annotate them, and comment on each other's annotations. Once a project has been created, it remains active, even after the block has finished, thus providing team members with an indexed repository of patient-centered literature.

EVALUATION: To date, three attendings and six residents have participated in the intervention. Faculty and residents have posted annotated articles based on clinical questions raised on the ward rotation. Team members have used the F1000 platform daily, and have reported high ease of use and ready integration into a demanding house staff work flow. Participants posted articles and read those posted by others, but did not discuss them online; most of the discussion has taken place on afternoon teaching rounds.

DISCUSSION / REFLECTION / LESSONS LEARNED: As the pilot progresses, we plan to track the number of papers submitted by the team as a whole and by its individual members. As objective measures of the team's utilization of the workspace and engagement with the literature, we will quantify the number of articles annotated by team members at each level and the number of virtual "conversations" between team members. We will hold focus groups with involved students and residents to ascertain the pilot's impact on their educational experience throughout their block on the Medicine service. We also hope to be able to document the discussions the articles generate so that residents and students have a centralized record of what they have learned on service. As a next step, we plan to expand this pilot to other Medicine services as other hospitalists and attending physicians become more familiar with the program. Eventually, we hope to link projects across the Medicine services to provide residents with an even larger pool of medical literature. We believe that the addition of a web-based, collaborative reference manager and workspace will increase both the quantity of literature reviewed and the quality of evidence-based discussion on a General Medicine teaching service. We expect that this program will inspire the spirit of scholarship and academic rigor in its participants, particularly the more junior trainees. 
ADDRESSING THE CHRONICALLY UNEXPLAINED: USING STANDARDIZED PATIENTS TO TEACH MEDICAL RESIDENTS PRAGMATIC ADVERSE CHILDHOOD EXPERIENCE (ACE) BASED INTERVENTIONS Martina Jelley; Julie Miller-Cribbs; Linda Oberst-Walsh; Kristin Rodriguez; Frances K. Wen; Kim Coon. University of Oklahoma School of Community Medicine, Tulsa, OK. (Control ID \#2469617)

NEEDS AND OBJECTIVES: Exponential growth has occurred in awareness of the long-term consequences of adverse childhood experiences (ACEs) on adult health risk behaviors and chronic disease. Primary care physicians need education and training in practical skills in caring for adults with high ACE scores. These physicians may be able to affect improved health outcomes by addressing ACEs through an intervention comprised of asking, listening, accepting a patient's report of an ACEs history and consideration of referral for counseling. There is significant need to develop effective and efficient training in this intervention for primary care residents. Our overall goal is to develop a longitudinal curriculum involving standardized patient (SP) encounters.

SETTING AND PARTICIPANTS: Setting - General internal medicine and family medicine residency program. Trainees - 59 IM and FP residents Faculty -IM and FP MDs, primary care based psychologist, psychiatry based counselor, social work faculty and director of simulation center

DESCRIPTION: Faculty from the IM and FM programs, along with faculty from socia work and psychiatry, created a curriculum to educate residents about ACEs and adult health effects. Fifty-nine residents attended a 3-h training session that consisted of video review of an example of a SP session, 15 min patient visit with an SP, followed by a 5 min debriefing session that included one faculty member, one fellow resident and the SP. The two cases used in this study were based on real de-identified patients, the encounters were held in a simulated exam room, and the SPs were experienced actors. In additional to their training on accurate portrayal and feedback, the SPs received $2 \mathrm{~h}$ of instruction on accurate portrayal of physical and emotional symptomology of ACEs. The cases the SPs portrayed were individuals with chronic health issues (an perceived autoimmune condition and COPD with substance abuse) who also had a previously unaddressed history of childhood trauma. The simulations began with review of the SPs "chart". The chart described the patient's medical and social history, specifically outlining ACEs including an ACE score Copies of infographics explaining ACES were made available in each room for patient education. Study team members live-viewed the residents' encounter and the faculty member annotated the sessions using Learning Space software. Immediately following each simulation, residents, SPs and the faculty mentors completed a debriefing session to help process the experience. The SPs, faculty, and residents gave qualitative and quantitative feedback on the encounters, and each resident also reviewed his/her annotated video. Once the surveys were complete, the large group met all together for a final debriefing session led by the study team and consisting largely of residents' critical evaluation of their experience. During debrief, the residents were encouraged to give candid feedback, including suggestions for improvements in the simulations. At the conclusion of all simulations the subsequent data was compiled and analyzed in order to identify trends and relationships between variables.

EVALUATION: Upon examination of individual items, the scores between the faculty and SP feedback were very similar, although the faculty consistently provided higher scores, particularly in regards to establishing rapport, efficacy in explaining the link between ACEs and current health issues, and the resident's attitude throughout the encounter. Evaluation topics were: 1. Establishes Rapport (verbal and non-verbal communication, respect with feeling and a sense of ordinariness) 2. Creates 'time tardis' effect (complete focus, unrushed appearance) 3. Recognizes and capitalizes on empathic moments 4. Considers and asks about potential ACEs background 5. Explains link between known or potential unknown ACEs and current health issues patient facing - facilitates or encourages patient's insight in to own personal links 6. Appropriately handles any disclosures by acknowledging and normalizing patients feeling and behavior responses. 7. Offers resources for more information, support, assistance — including referrals and/or warm hand-offs if available. 8. Obtain short term goals and develop shared plan for next steps on health care issues with plan for continued follow-up. 9. Student Attitude 10. Time Management - don't have to 'fix it', don't have to resolve in one visit, $15 \mathrm{~min}$ visits, agreement for regular follow-up. 11. Patient Comfort Measures 12. Overall Satisfaction DISCUSSION / REFLECTION / LESSONS LEARNED: It is clear that primary care physicians need the ability to address the effects of childhood trauma in their adult patients. There is not a standard for how this interaction is approached or addressed in medical education. Using SPs to teach this communication skill appears to be promising. We intend on continuing the project by creating a video for teaching such interventions, standardizing and validating the evaluations scales and developing training materials for distribution.

\section{ADVANCED CARE PLANNING IN THE OUTPATIENT SETTING: WHAT DO I} SAY? A WORKSHOP FOR RESIDENTS Emily Rumora; Jessamyn Blau; Michelle Rappaport. University of Washington, Seattle, WA. (Control ID \#2465579)

NEEDS AND OBJECTIVES: Residents typically do not receive formal training in advance care planning (ACP) in the outpatient setting. While conversation guides can help structure an ACP session, residents must develop their own vocabulary to use during these conversations. We created a workshop for residents to generate, share, and deliberately practice language to use in ACP discussions. Our goal was to increase resident comfort with such discussions and ultimately engage in ACP more often with their patients. Participants will be able to: Facilitate a structured participant-guided discussion to help trainees generate ideal language for advance care planning discussions Generate a unique template for documenting $\mathrm{ACP}$ conversations that incorporates participant-generated language

SETTING AND PARTICIPANTS: Internal medicine residents attending weekly didactic sessions during outpatient and consult rotations at a large academic medical center. DESCRIPTION: The workshop began with a short didactic component focused on defining key terms (e.g. living will, health care proxy) with an emphasis on encouraging residents to think of ACP as an ongoing conversation with their patients. Residents then role-played essential components of an ACP discussion including asking permission to engage in the conversation, eliciting patient understanding of disease, addressing prognosis, eliciting the patient's values, and offering the provider's opinion. After each role play, residents playing both the doctor and the patient were asked to reflect on which phrasing felt genuine and any positive or negative impacts of specific wording. We asked focused questions to build a participant-generated list of specific wording that residents found effective, which we projected on a screen and updated in real time. We subsequently disseminated a template for ACP documentation that included select participant-generated phrases to use during future conversations. To assess the effectiveness of our workshop, we administered pre- and post-tests to assess previous experience with ACP and to determine level of comfort with various aspects of ACP.

EVALUATION: Prior to our workshop, 10 of 34 residents indicated that they had never conducted an outpatient ACP conversation, and only 3 of 34 had conducted more than five. After the workshop, the percentage of residents who felt prepared to have an ACP conversation increased from 19 of 34 residents (55\%) to 25 of 28 residents ( $89 \%)$. The number of residents stating that they felt comfortable with specific components of ACP also increased after the session; specifically, they were more comfortable making a recommendation (pre $63 \%$; post $79 \%$ ) and discussing the appointment of a surrogate (pre $76 \%$; post $92 \%$ ). Several residents specifically indicated in their comments on the post-workshop survey that they were now more encouraged to engage in ACP with their patients. After the workshop, residents were more likely to indicate that they understood 
the differences between advance care planning, advance directives, health care proxy and living will (pre: $52 \%$; post: $77 \%$ ).

DISCUSSION / REFLECTION / LESSONS LEARNED: ACP conversations are inherently difficult, and the skills required are not formally taught in many residency programs. Our workshop was an effort to strengthen resident competence in ACP through deliberate practice in a structured and low-stakes environment. This approach was unique because it afforded residents a chance to not only practice difficult conversations, but to reflect in a group setting on which language was most comfortable for them. Our data demonstrate that this deliberate process made residents feel more prepared to engage their patients in ACP, though we do not yet know whether this type of workshop leads to practice change among residents. We will follow up with participants at 6 months to reevaluate their comfort with ACP and to assess how many ACP conversations they have conducted. Going forward, we anticipate refining the structure of this workshop to emphasize the goal of residents generating their own "ideal" language for ACP. A similar strategy of relying on participants to generate comfortable language may be useful in other arenas of difficult communication in medicine and could easily be adapted to other levels of training.

\section{AN EFFECTIVE QUALITY IMPROVEMENT CURRICULUM CREATED AND IMPLEMENTED BY A SINGLE FACULTY PRECEPTOR Nancy T. Kubiak;} Jennifer Koch; Charlene K. Mitchell. University of Louisville, Louisville, KY. (Control ID \#2464436)

NEEDS AND OBJECTIVES: To equip residents for their future roles in improving patient care and healthcare quality, residency programs must teach trainees skills to analyze and improve their care of patients. Due to meeting time conflicts, our residency was unable to integrate residents into existing hospital quality improvement (QI) efforts. We also faced the barrier that no available faculty member had experience or training in QI projects or teaching QI. Existing curricula could not be modified to fit the available time for QI training, and the schedule could not be adapted to existing curricula. Finally, aggregate data for each resident's patient panel could not be extracted from the electronic medical record. In order to surmount these common barriers, this curriculum was created to provide trainees with experiential learning in quality improvement via regular chart reviews and application exercises, facilitated by a single faculty member.

SETTING AND PARTICIPANTS: Each categorical internal medicine resident participated in a semi-annual, half day session in the outpatient setting. The faculty preceptor gained training by participating in Institute of Healthcare Improvement (IHI) modules, reviewing available curriculum, and attending QI workshops at national program director meetings.

DESCRIPTION: The curriculum development included identifying readings and creating checklists so residents could gather data on their patients and compare their performance to national standards. Short mini lectures and application exercises were constructed to reinforce QI skills covered in each semi-annual session. Prior to each half-day session, residents completed assigned 30-60 min readings. At the beginning of the learning session, residents collected information from a sample of the charts of their outpatient panels. Each learner used a checklist to evaluate his or her performance in quality measures for preventive care (PGY 1), diabetes (PGY 2), or hypertension (PGY 3). Mini lectures and discussions reinforced QI concepts. Using performance data from the review, residents applied those concepts to their own patient panels via exercises created for the session. Residents then created an improvement cycle for their individual panels and discussed the aim, measures, and changes with the small group and faculty member. Each resident's final plan for the improvement cycle incorporated these suggestions and was implemented. At subsequent reviews, each resident again collected data on the patient panel, assessed the results of the improvement cycle, and modified or changed the goal to further improve patient care.
EVALUATION: After two chart review sessions, residents $(n=55)$ showed statistically significant improvements over baseline in self-ratings of all twelve QI skills outlined in a previously published self-assessment ( $\mathrm{p}$ value range: $<0.001-0.04$ ). Knowledge improved significantly $(p=0.034)$ in residents who completed the baseline and follow up QI quiz after three chart reviews $(n=55)$. After 6 sessions, 17 of $21(81 \%)$ residents scored $>20$, and none scored $<10$ on the validated Quality Improvement Knowledge Application Tool (QIKAT), where they outlined plans for improvement in healthcare scenarios. Using the QIKAT-revised rubric, scores of $>20$ and $<10$ differentiate those with and without previous QI training, respectively. Attendings that completed 2 assessments $(n=4)$ over 2 years did not significantly improve in self-confidence or knowledge. Since beginning the QI curriculum, residents have completed a clinic-wide QI project that improved the rate of diabetic foot exams and are currently working to improve the percentage of diabetic patients with an $\mathrm{A} 1 \mathrm{C}<8$. Three additional resident-initiated QI projects are in progress, and two are in the planning stage for a total of twelve residents participating in optional projects. No quality improvement initiatives were started or completed prior to implementation of this curriculum.

DISCUSSION / REFLECTION / LESSONS LEARNED: A semi-annual, experiential QI curriculum facilitated by a single faculty member resulted in improved internal medicine resident knowledge, confidence, and an ability to apply QI principles to validated scenarios. It also spurred residency wide and resident driven QI projects. Faculty performance did not increase, suggesting the lack of a local trend of improvement, although a beta error cannot be excluded due to the low numbers who completed two assessments. Faculty training occurred via program director and medical education conferences, which did not incur additional monetary outlays, as well as through the freely available IHI Open School. Programs with minimal quality improvement resources can implement a meaningful QI educational endeavor for its residents. In the next phase of the curriculum, an assessment of needs and attitude of faculty will occur, with subsequent faculty QI training as needed. The lack of available faculty to mentor projects remains a barrier to expansion of QI within the residency and department.

AN INNOVATIVE SHARED DECISION MAKING CURRICULUM FOR INTERNAL MEDICINE RESIDENTS Jen Rusiecki ${ }^{2,3}{ }^{3}$ Jane Schell $^{1}$; Sarah B. Merriam²; Melissa McNeil ${ }^{2}$; Carla Spagnoletti ${ }^{1} .{ }^{1}$ University of Pittsburgh, Pittsburgh, PA; ${ }^{2}$ University of Pittsburgh Medical Center, Pittsburgh, PA; ${ }^{3}$ VA Pittsburgh Healthcare System, Pittsburgh, PA. (Control ID \#2466022)

NEEDS AND OBJECTIVES: A needs assessment revealed that internal medicine residents at the University of Pittsburgh Medical Center (UPMC) felt Shared Decision Making (SDM) was important but cited lack of training in SDM as a barrier to using this practice. The residents struggled with defining equipoise and identifying appropriate clinical situation in which to use SDM. $26 \%$ of residents indicated minimal comfort with negotiating decisions. Objectives: 1. Identify the key concepts of shared decision making through teachable communication steps and skills. 2. Demonstrate shared decision making communication steps and skills both in simulation and clinical practice. 3. Identify and address barriers towards practicing shared decision making.

SETTING AND PARTICIPANTS: The SDM curriculum takes place over two-half days per month as part of the senior ambulatory rotation for internal medicine and medicine-pediatric residents at the UPMC.

DESCRIPTION: SDM is a practice in which physicians and patients resolve an issue together both informed by medical evidence and weighted according to specific values of the patient. Patient centered care and SDM have recently drawn emphasis in health care policy, education, clinical guidelines and patient satisfaction measures. The ACGME echoes this sentiment requiring residents to be able to perform and incorporate SDM into their practice as a milestone for effective patient communication. Despite this, many US 
internal medicine residency programs lack a SDM education curriculum. We developed and implemented a 4-h curriculum, geared towards common primary care clinical situations, that uses didactics, case-based discussions, and practice with a simulated patient to teach the key concepts and skills of SDM.

EVALUATION: A total of 32 residents completed the curriculum. They were surveyed to assess perceived barriers to SDM, as well as attitudes towards and knowledge of SDM concepts. The most frequently reported barriers to practicing SDM were time constraints, lack of training in SDM, and patients' health literacy and interest in participating in SDM. Paired t-test analyses were performed to evaluate changes in attitudes and knowledge before and after the curriculum. There was significant improvement in resident knowledge of SDM elements after completing the curriculum (median pre $75 \%$, post $100 \%, p=$ $0.0001)$. Residents ranked their level of confidence with and the importance of SDM on a 4-point Likert scale $(1=$ not confident/important to $4=$ very confident/important). Residents also demonstrated significant improvement in confidence (median composite score pre 2.87, post 3.0, $p=0.001$ ) and importance of SDM (median composite score pre 3.14, post $3.5, p=0.0002$ ). There was also significant improvement in residents' understanding of the importance of SDM to the practice of high value care (median score pre 3, post 4 , $p=0.02$ ) but not to evidence based medicine (median score pre 3, post 3.5, $p=0.223$ ). To evaluate SDM skills, residents submitted audio recordings of primary care clinical decision-making in their own continuity clinics before and after participating in the curriculum. 30 clinical recordings ( 24 pre, 6 post) were assessed using the Observing Patient Involvement in Decision Making (OPTION) scale, which consists of 12 items with a total of 48 possible points. Items evaluated include: identifying the issue, explaining equipoise, assessing the patient's preferred approach to receiving information and involvement in decisions, listing options with pros and cons, exploring patient's expectations and concerns, checks patient's understanding, indicate the need for and reviewing the decision. The two raters were blinded to the pre or post status of the recording. Inter-rater reliably was high (ICC 0.87). Mixed effects modeling was performed on each of the OPTION items and total score. There was improvement in total score from pre to post with a mean difference of 4.17 (mean pre 18.6, post 22.7, $p=0.0587$ ). Residents also demonstrated trend toward improvement in discussing options and pros and cons of options with patients with a mean difference of $0.83(p=0.0853)$ and $0.80(p=0.0558)$ respectively. There was significant improvement in residents' ability to elicit a decision from the patient with a mean difference of $0.92(p=0.007)$

DISCUSSION / REFLECTION / LESSONS LEARNED: Our skill-based curricula intervention demonstrated improvement in internal medicine residents' knowledge of, attitudes towards and utilization of SDM skills. An essential element of SDM is the patient-centered nature of this practice. The improvement in residents' ability to elicit a decision from the patient suggests an improved understanding and application of this patient-centered element. While a trend toward improvement was seen in total OPTION score, we were unable to demonstrate significance likely due to the limited number of post-curriculum recordings. Overall, SDM is a teachable concept and training improves residents' understanding of, attitudes towards, and utilization of this important patientcentered skill set.

AN INTEGRATED PROGRAM TO PREPARE SECOND YEAR MEDICAL STUDENTS FOR CORE CLINICAL ROTATIONS J. H. Isaacson ${ }^{1}$; Mark Mayer ${ }^{2}$; Elham Yousef ${ }^{1}$; Giselle Velez ${ }^{1}$ 'Cleveland Clinic, Cleveland, $\mathrm{OH} ;{ }^{2}$ Cleveland Clinic Lerner College of Medicine, Cleveland, OH. (Control ID \#2468545)

NEEDS AND OBJECTIVES: Medical students traditionally learn clinical skills related to interviewing, history taking and physical examination in the first 2 years. Challenges remain in preparing students for the transition to core clinical rotations in the hospital settings where they need to apply interviewing and examination skills in acutely ill patients. In the hospital students are also exposed to a complex care system with frequent transitions of care. A common strategy to prepare students for this transition is to provide just-in-time training with an inpatient orientation or boot camp experience focused on skills needed in core clinical rotations. The objectives for our medical innovation focused on an integrated approach to prepare second year students for core clinical rotations to: 1 . Perform interviews and examinations with hospitalized patients 2 . Display the ability to do an oral presentation of a case in front of peers and faculty 3 . Analyze clinical information to generate a problem list and differential diagnosis for patients in the outpatient and hospital setting 4 . Identify components of the admission, daily care and discharge of hospitalized patients

SETTING AND PARTICIPANTS: Second year medical students in both outpatient and inpatient settings.

DESCRIPTION: Background Students learn interviewing, history and physical examination skills in small groups with standardized patients beginning in their first year. In parallel with their clinical skills training, students are assigned to a longitudinal clinic with a primary care preceptor in their first year and continue in that clinic every other week in year 1 and weekly in year 2. Education Innovation Our education innovation builds on the core clinical skills described above to prepare 2 nd year students for the transition to core clinical rotations. Students have a total of 5 half-day sessions over 5 months -2 clinica reasoning sessions and 3 acute care medicine sessions. The clinical reasoning sessions include 4 students and one faculty preceptor. Each student presents a case from his or her longitudinal clinic to the other members of the small group and the group generates a problem list and differential diagnosis. The group then goes to the inpatient setting to interview and examine an unknown patient. All patients are informed in advance and give verbal consent to participate. All students participate in at least one component of the interview or examination. After completing the interview and examination the group leaves the patient to discuss the differential diagnosis and reviews the medical record, which allows feedback regarding their clinical reasoning. Students have a clinical reasoning session early in the second semester and late in the second semester. In parallel with clinical reasoning sessions, students have 3 distinct acute care sessions. Each student is assigned a faculty preceptor a hospitalist physician in either adult or pediatric medicine. In the first session students perform a history and physical examination on a patient and practice writing admission orders. In the second session students identify components of ongoing care of a hospitalized patient and write a progress note. In the final session students are assigned a patient nearing discharge and identify components of the transition of care from hospital to home or another setting. For each of the 3 acute care sessions students and faculty have a defined skills list. Students submit assignments on line and receive feedback from faculty.

EVALUATION: A total of 282 students have participated in acute care and 191 participated in clinical reasoning sessions since 2007. Ninety-two and $94 \%$ of students were satisfied or very satisfied with their acute care experience and clinical reasoning sessions respectively. Regarding acute care sessions, $92 \%$ of students stated they were oriented to the inpatient medical record, $88 \%$ knew how to write admission orders and $94 \%$ felt improved understanding of the follow-up of hospitalized patients. Students were asked about their perception of clinical preparation for a variety of skills relevant to core rotations. Eighty two percent felt they had excellent or good skills in oral presentations, $76 \%$ in performing a history and physical examination in a hospitalized patient, $90 \%$ in developing a problem list and $77 \%$ in generating a differential diagnosis.

DISCUSSION / REFLECTION / LESSONS LEARNED: Our program in clinical reasoning and acute care medicine has allowed students to develop experience interviewing and examining patients in the hospital, practice oral presentations, differential diagnosis and identify components of care of patients in the inpatient setting. Students have responded favorably to this program. We believe components of our program are adaptable to other medical schools. 
AN INTERNAL MEDICINE DIDACTIC CURRICULUM UNDERGOES A 21ST CENTURY REDESIGN Jennifer Koch; Nancy T. Kubiak; Charlene K. Mitchell; Clayton Smith; Laura Bishop; Michael Burk. University of Louisville, Louisville, KY. (Control ID \#2467622)

NEEDS AND OBJECTIVES: The ACGME requires a core curriculum in internal medicine delivered via regularly scheduled didactic sessions. Previously, our program utilized a $3 \mathrm{~h}$ academic half-day, but found that overall attendance and resident enthusiasm were suboptimal, especially in the third hour of lecture. When a resident missed a lecture, there was no effective curricular alternative. Additionally, some didactic material might be more effectively and efficiently learned via high-quality, guided self-study. A needs assessment revealed an overwhelming majority of internal medicine residents favored changing the lecture format to two $45 \mathrm{~min}$ interactive lectures, supplemented by structured, self-directed learning. Additionally, self-directed study and conference attendance have been associated with increased In-Training Exam (ITE) scores. The curricular redesign aimed to deliver an efficient and thorough internal medicine curriculum that increased resident satisfaction and resulted in stable or improved markers of academic success (e.g. ITE scores, board pass rate).

SETTING AND PARTICIPANTS: The innovation occurred in a medium sized internal medicine residency in an academic medical center. All categorical residents $(n=67)$ were enrolled in a self-study program and experienced the change in the didactic curriculum. In addition, 20 combined medicine-pediatrics residents experienced the change in the lecture series, but had previously-established expectations for self-study.

DESCRIPTION: In a two-pronged redesign of our didactic curriculum, we established a self-study curriculum which provided for a broad medical knowledge base and allowed lecture time to be used more efficiently. First, the academic half-day was shortened to two 45-min high-yield lectures. Concomitantly, utilizing purchased board review materials and free online resources, we developed a self-study curriculum for each PGY year. Residents reported study progress using an online tracking system, viewable by their advisors. The effort was complimented by a resident-led project to record lectures and make them freely viewable on a website, LouisvilleLectures.org. Lectures that residents missed were now available on this local free online access to medicine (FOAM) website

EVALUATION: At baseline and after the first year of implementation, residents were surveyed to assess their opinions about how well the existing curriculum improved their medical knowledge and patient care, and prepared them for their board certification examination. Additional questions investigated the residents' overall satisfaction with the curriculum and with the guidance provided for self-study. Answers ranged from 1 (strongly disagree) to 5 (strongly agree) on a five point Likert scale. Initial values were used to establish the test statistic for use in the One-Sample T-test post intervention analysis. Mean scores for each of the five questions were analyzed using 2-tailed T-test. Compared to baseline, mean scores significantly improved for all five items ( $p<0.001$ for each question). Residents thought the curriculum improved medical knowledge (baseline 3.813 , post 4.27 , mean difference $0.458,95 \%$ CI $0.31-0.61$ ) and improved their patient care (baseline 3.694, post 4.24, mean difference 0.545 , CI $0.40-0.69$ ). Residents perceived that the newer curriculum better prepared them for board certification examination (baseline 3.417 , post 4.10 , mean difference 0.686 , CI $0.52-0.85$ ). Overall resident satisfaction with the didactic curriculum increased from 3.563 to 4.15 (mean difference 0.592 , CI $0.43-0.75$ ) as did their satisfaction with guidance provided for self-directed learning (3.75 pre, 4.14 post, mean difference 0.393 , CI $0.21-0.58$ ). The program's aggregate ITE score increased 2 percentage points ( 4 percentile points) after 1 year of implementation, though possible confounders cannot be excluded. After the initial year of implementation of the combined high yield didactics and self-study program, the graduating class had a $100 \%$ boards pass rate (compared to $89 \% 3$-year pass rate for the program).

DISCUSSION / REFLECTION / LESSONS LEARNED: Early outcomes of the curricular redesign show a statistically significant improvement of resident satisfaction with the combined lecture and self-study program as well as with their perception of its effect on medical knowledge, patient care, and board preparation. An initial change in the program's aggregate ITE score and board pass rate was also noted. The self-study curriculum and online lectures provide an alternative to learn material when a resident cannot attend a lecture. After the initial year of implementation, the platform for tracking progress was changed to a more accessible site, and a required number of lectures was changed to include the option of a percentage that could be viewed on the LouisvilleLectures website. The core aspects of this redesign are easy to implement, and few resources are needed.

ONLINE RESOURCE URL (OPTIONAL): www.LouisvilleLectures.org AN INTERPROFESSIONAL EDUCATION INITIATIVE TO REDUCE PHARMACEUTICAL RISK IN PRIMARY CARE Marcia C. Mecca ${ }^{1,2}$; John M. Thomas ${ }^{1,}$ ${ }^{3}$; Kristina Niehoff ${ }^{1,4}$; Anne Hyson ${ }^{1,3}$; Rebecca Brienza ${ }^{1,3}$. ' West Haven VA, Cos Cob, CT; ${ }^{2}$ Yale University, Hamden, CT; ${ }^{3}$ Yale University School of Medicine, New Haven, CT; ${ }^{4}$ Veterans Affairs Connecticut Healthcare System, West Haven, CT. (Control ID \#2469243)

NEEDS AND OBJECTIVES: Polypharmacy is common in older adults, yet there is limited geriatric medication management training within primary care education. Through this initiative, trainees identify polypharmacy, analyze complex medication regimens, and manage potentially inappropriate medications through medication dose reduction and deprescribing, and evidence-based nonpharmacologic interventions. Trainees reflect on the experience and identify strengths and areas of improvement.

SETTING AND PARTICIPANTS: An interprofessional team training program at the West Haven Center of Excellence in Primary Care Education, including residents in Internal Medicine (MD), advanced practice nursing (NP), pharmacy, and health psychology.

DESCRIPTION: The IMPROVE clinic (Initiative to Minimize Pharmaceutical Risk in Older Veterans) occurs one half-day per month and involves three sequential sessions: a pre-clinic conference, trainee-led group visit, and individual trainee visits with precepting provided by a geriatrician, geriatric pharmacist, and primary care faculty. Approximately five trainees schedule a patient from their continuity patients who meet the eligibility criteria of age 65 or older and prescribed 10 or more medications. The pre-clinic conference consists of a 20-min didactic taught by a pharmacist or geriatrician, followed by a 40-min discussion about the medication regimens which are reviewed prior to the clinic by the trainee and the faculty for potentially inappropriate or unnecessary medications and nonadherence. Next, the trainees and faculty join the patients and their caregivers for a 40-min group visit, which is led by a trainee and includes discussions about side effects, adherence strategies, and non-pharmacological treatment alternatives. Following the group visit, each patient has an individual visit with a resident (MD or NP) consisting of a medication reconciliation, geriatric assessments, and shared decision making about potential medication changes. Trainees precept with IMPROVE faculty to finalize the plan and ensure appropriate follow-up and communication with other providers.

EVALUATION: We assessed MD and NP resident knowledge with a 12 -item multiple choice polypharmacy knowledge test that was previously validated for use in primary care residents. The test was administered before participation in IMPROVE and 6 months later. A total of 16 of 21 eligible residents (13 MD and $3 \mathrm{NP}$ ) completed the initial and follow-up knowledge tests (response rate $76 \%$ ). More than two-thirds (69\%) participated in IMPROVE at least twice. The pre- and post-intervention test scores were 62 and $76 \%$, respectively $(p=0.017)$. In addition, two focus groups were conducted with 17 trainees as part of an exploratory sequential mixed methods design to characterize the perceived impact of IMPROVE on trainee skills and attitudes. Three analysts independently reviewed the transcripts to identify emerging themes, then met and discussed until they 
reached consensus. Themes included the following: 1) the interprofessional educational experience is highly valued among trainees; 2) trainees perceive improvements in their knowledge, skills, and attitudes that are generalizable to other patients; 3 ) trainees strongly prefer to see their own patients in the program so they can continue to implement the plan and observe the clinical impact over time; and 4) trainees find it valuable to lead group visits but less valuable to be observers.

DISCUSSION / REFLECTION / LESSONS LEARNED: This hands-on, team-based approach was a valuable learning experience for the trainees and increased their knowledge of polypharmacy. To our knowledge, this is the first polypharmacy intervention that involves a trainee-led group visit. Given the trainees' feedback, we will rotate trainees leading the group visit and simultaneously hold the pre-clinic conference with the rest of the trainees to increase efficiency. In conclusion, this program is an effective model of post-graduate primary care training in polypharmacy, and may be implementable in other academic settings.

BIRMINGHAM VOICES: DEVELOPING NARRATIVE COMPETENCY TO BETTER SERVE VULNERABLE POPULATIONS Amy J. Kennedy ${ }^{1}$; Gaetan Sgro ${ }^{1}$

${ }^{2}$. ${ }^{1}$ University of Pittsburgh Medical Center, Pittsburgh, PA; ${ }^{2}$ VA Pittsburgh Healthcare System, Pittsburgh, PA. (Control ID \#2449292)

NEEDS AND OBJECTIVES: Narrative medicine is an emerging competency that enables physicians to practice with greater empathy and attentiveness. Human beings have always relied on storytelling to process and to learn from their experiences. Today, physicians and patients alike need stories to cultivate meaning in their lives, to endure hardships and to locate the common ground on which the doctor-patient relationship is based. The framework of patient-centered care encourages physicians to engage in shared decision making that is informed by an understanding of each patient's unique needs and environment. While a patient-centered approach has been shown to improve health outcomes, the obstacles to practicing patient-centered care among vulnerable populations are many, including patients' lack of trust in the medical system and in individual providers. Proponents of narrative medicine have suggested that developing patientcentered narratives encourages doctors to engage their patients on a more personal level than is customary within the confines of a typical office visit, a practice that can cultivate empathy. Furthermore, the ability of physicians to view situations from their patients' perspectives has been shown both to increase patient satisfaction and to reduce burnout among providers. With these concepts in mind, we developed a creative nonfiction writing workshop to help residents better care for patients from an underserved population. SETTING AND PARTICIPANTS: The writing workshop took place over four Friday mornings during the month of October 2015, as part of the curriculum for the Global Health Seminar Series. Five trainees in University of Pittsburgh Internal Medicine Residency Program's Global Health and Underserved Populations Track participated in this curriculum, as well as two medical students from the University of Pittsburgh School of Medicine. Each session was facilitated by an internal medicine faculty member who is also a published author.

DESCRIPTION: In preparation for the workshop, trainees were provided a list of questions designed to explore the personal, social and emotional aspects of the patient experience, and asked to interview one of their patients at the Birmingham Free Clinic (a community partnership between the Program for Health Care to Underserved Populations and The Salvation Army). Each workshop session was organized around a different theme related to the craft of storytelling. Participants were asked to prepare for sessions with readings chosen to explore the week's theme. Following a period of discussion, participants were given time either to start or to continue writing narratives that featured the patients they had interviewed. Weekly prompts/themes related to: 1) channeling Hemingway to "write one true sentence;" 2) developing characters through dialogue; 3) defining a character's wants/desires through the action of the story; and 4) evoking emotion through imagery. At the end of each session, participants were given the opportunity to share their writing and to hear feedback from their peers. Following the final workshop session, participants completed a brief survey about the experience, and were given the option of continuing to develop their narratives in collaboration with the faculty mentor

EVALUATION: Of the seven trainees enrolled in this course, six completed post-course evaluations. Overall, trainees most appreciated having the opportunity to improve their writing skills, and having protected time to participate in group reflection on their clinical experiences. The parts of the course they would have changed included having more time per session and allowing their writing to be about any patient (not just ones from the Birmingham Free Clinic). Among many narrative comments, one resident wrote, "Reflecting and writing about my visit with him made me feel like I know [my patient] better." Another noted, "While I love to read, I have always thought of reflective writing as 'difficult' and 'scary,' but the structure of the sessions actually made for a very fluid writing process."

DISCUSSION / REFLECTION / LESSONS LEARNED: In general, the narrative medicine workshop was easy to implement; it cost nothing other than a modest time commitment from both the trainees and facilitators. The workshops were well attended and all trainees were active participants in the discussions and the writing exercises. The excerpts that residents shared were beautifully written and often quite moving. We plan to continue to mentor the trainees to prepare manuscripts for publication, and hope to continue this workshop in the coming years, potentially expanding it to the entire residency program.

BRIDGING THE LANGUAGE GAP: TRAINING MEDICAL STUDENTS IN THE USE OF PROFESSIONAL INTERPRETATION SERVICES Christopher Moreland $^{1}$; Todd Agan ${ }^{1}$; Diane Ferguson ${ }^{1}$; Kristy Y. Kosub ${ }^{2} .{ }^{1}$ The University of Texas HSC - San Antonio, San Antonio, TX; ${ }^{2}$ University of Texas Health Science Center at San Antonio, San Antonio, TN. (Control ID \#2457058)

NEEDS AND OBJECTIVES: People who are limited English proficient (LEP) or deaf or hard of hearing $(\mathrm{DHH})$ experience disparities in accessing health care due to communication barriers. ${ }^{1-3}$ In our program, medical students frequently encounter LEP or, less often, DHH patients. Professional interpretation can improve health outcomes, ${ }^{1}$ yet information is sparse on best practices for training students to work with interpreters. Our innovation attempts to address this educational gap, so that medical students can outline the evidence describing those disparities and effectively communicate with LEP/DHH standardized patients (SPs) using interpreters.

SETTING AND PARTICIPANTS: Within an academic medical center, we targeted our innovation to fourth-year medical students enrolled in an internal medicine boot camp elective $(n=18)$. Participants also included DHH SPs and American Sign Language (ASL) interpreters from a community college interpreter training program and bilingual, Spanish-speaking SPs as interpreters and LEP SPs.

DESCRIPTION: We developed educational content to address 1) communication barriers' impact on the health of LEP and DHH people, 2) professional interpreters' role, 3) and working effectively with professional interpreters. The course concluded with a formative five-station communication-oriented objective structured clinical examination (OSCE) with SPs. Two stations focused on obtaining informed consent with interpretation: one in Spanish and one in ASL. Students received verbal feedback after each encounter.

EVALUATION: Students completed pre- and post-tests assessing comfort level with interpreters and language fluency. -Pre-OSCE: Sixteen of 18 students reported being comfortable (14) or very comfortable (2) utilizing Spanish interpreters with patients. Nine students reported speaking Spanish well (8) or very well (1); these students reported using 
an interpreter less than $10 \%$ of the time. Fewer students reported being comfortable ( 9 ) or very comfortable (1) with ASL interpreters. Seventeen students reported that they were not or not at all proficient in ASL. -Post-OSCE: All students (18) reported being comfortable or very comfortable working with both Spanish and ASL interpreters.

DISCUSSION / REFLECTION / LESSONS LEARNED: Our curriculum introduced senior medical students to the disparities surrounding language inequality in health care and the importance of professional interpretation. The OSCE provided an opportunity to practice communication skills utilizing interpreters-in-training with LEP and DHH SPs through sustainable resources. Debriefing post-OSCE supported that this initial educational effort was central to students' understanding of working with professional health care interpreters. We will survey these students during internship to assess the intervention's impact on their interpreter use during training. References 1. Karliner LS, Jacobs EA, Chen AH, Mutha S. Do Professional Interpreters Improve Clinical Care for Patients with Limited English Proficiency? A Systematic Review of the Literature. Health Services Research. 2006;42(2):727-754. 2. McKee MM, Barnett SL, Block RC, Pearson TA. Impact of communication on preventive services among deaf American Sign Language users. Am J Prev Med. 2011;41(1):75-79. 3. O’Day BL, Killeen M, Harker H, Iezzoni LI. Communicating about health care: observations from persons who are deaf or hard of hearing. Ann Intern Med. 2004;140(5):356-362

CALling A CONSUlt: A STANDARDIZED PROCEDURE FOR A STANDARD TASK Amar Kohli ${ }^{1}$; Alda Maria Gonzaga ${ }^{2}$; Shanta M. Zimmer ${ }^{2}$; Michael Elnicki ${ }^{2} .{ }^{1}$ University of Pittsburgh, Mars, PA; ${ }^{2}$ University of Pittsburgh, Pittsburgh, PA. (Control ID \#2468789)

NEEDS AND OBJECTIVES: Consulting a colleague is an integral and germane task for internal medicine residents. Although long known to be suboptimal, the process continues to be characterized by a lack of didactic teaching in doctor-todoctor communication. Our hospital has had increasing levels of discord between medicine residents and medicine subspecialty consultants. This discord stemmed from poor transfer of information and a lack of appropriate consult questions. We sought to develop a curriculum to present a standardized way for internal medicine residents to convey information to subspecialty medicine consultants as well as hone skills in developing specific and actionable consult questions. We hypothesized that resident confidence in developing consult questions would increase as a result of our curriculum

SETTING AND PARTICIPANTS: The curriculum was implemented and evaluated at the The University of Pittsburgh Medical Center. The center is comprised of an academic teaching hospital, community based teaching hospital and affiliated Veterans Affairs hospital.

DESCRIPTION: The consultation curriculum was implemented as a set of 1-h, large group, noon conferences that were given at all three sites over a 1-week period (i.e. the same block). A pretest survey was completed by residents and collected at the beginning of the conference. The curriculum consisted of a short didactic session that first reviewed needs assessment data about which aspects of a consult call were most important to our subspecialty medicine consultants (consult question, consult urgency, whom to call back and medical record number). It was emphasized that these were the same 4 items that residents felt were most important, but in a different order. This was followed by an introduction to the $5 \mathrm{C}$ 's of consultation. The $5 \mathrm{C}$ 's consist of contact, communicate, core question, collaborate and close the loop. This method was adapted from a validated tool created for emergency medicine residents. Next, 2 five-minute videos were shown to the residents. The first video portrayed poor communication skills, while the second utilized the 5 C's correctly. The video's consisted of a phone call interaction between an internal medicine intern and cardiology fellow consultant. After each video medicine residents were asked to critique the video's and to provide specific examples on how to improve the interactions. The curriculum concluded with 3 short vignettes. Each vignette was a patient case which required the resident to call a consultant. The residents were tasked with developing specific and actionable questions for the vignettes.

EVALUATION: We analyzed pre and post curriculum survey data as well as resident attitudes to curriculum quality with descriptive and non-parametric statistics. Medicine residents were asked what information was deemed most important by subspecialty medicine consultants in a consult call. When comparing pretest and posttest data; selection of consult question increased from 88 to $98 \%(p=0.047)$. Medical record number increased from 88 to $92 \%(p=0.60)$, consult urgency increased from 6 to $84 \%$ $(p<.001)$, and whom to call with recommendations increased from 41 to $75 \%$ ( $p=$ $0.02)$. Overall, self reported confidence of residents to accurately develop a consult question decreased from $88 \%$ pretest to $77 \%$ posttest $(p=0.39)$. Residents unanimously agreed that the curriculum effectively explored good and bad communication techniques in a consult call as well as provided a standardized way to call a consult. $83 \%$ of attendee's indicated that they would use the 5 C's while on consult rotations. At the end of the curriculum resident's were asked, "No matter how much I learn about communication through consultation, consultants will always be difficult to work with." $20 \%$ of participants agreed.

DISCUSSION / REFLECTION / LESSONS LEARNED: Through disseminating our curriculum we found that residents incorrectly felt consultants valued patient data over items such as consult urgency and whom to call with recommendations. Residents are subject to overconfidence when it comes to developing consult questions. This sentiment may lead to the thought that consultants will always be difficult to work with. We learned the importance of deliberately teaching this skill and not allowing it to develop through trial and error. In the future we hope to investigate consult questions placed by medicine house staff to see if they have become more specific and actionable as a result of this curriculum.

CAN A MULTI-DISCIPLINARY APPROACH TO TEACHING INTERNAL MEDICINE RESIDENTS IMPROVE SCREENING FOR FALLS IN OLDER PRIMARY CARE PATIENTS? Jennifer Lom; Jonathan Flacker. Emory University, Atlanta, GA. (Control ID \#2435389)

NEEDS AND OBJECTIVES: Internal Medicine residents see increasing numbers of geriatric patients in their primary care clinics. Unfortunately, many do not have Geriatric Medicine either early or substantive enough to have a positive impact on the patients they care for. Thus, common, important conditions in older adults such as falls may not be appropriately screened for or evaluated. Barriers to preventing falls among resident primary care patients include a lack of knowledge and lack of self-confidences in implementing fall screening and evaluation. The purpose of the workshop is to educate learners earlier in their training on which of their primary care patients should be screened for falls, provide a framework for obtaining a falls history and targeted physical exam and finally to offer evidence based interventions they can prescribe to their patients. Learning Objectives: 1. Describe which primary care patients need to be screened for falls 2 . Demonstrate the components in a comprehensive falls history 3. Demonstrate how to do the timed up and go test (TUG) and the 4 stage balance test 4 . List 3 evidence based interventions for falls 5 . Demonstrate 3 basic exercises that would be recommended by physical therapy for your patients 6 . Prescribe the appropriate assistive device for their patient

SETTING AND PARTICIPANTS: This workshop was designed to be given in a small group setting during the residents ambulatory block month. Participants were PGY2 and PGY3 Internal Medicine residents in an academic medical setting. The workshop ran over 6 months and the average number of residents per session was around 10 . 
DESCRIPTION: This curriculum combines a flipped classroom with a multidisciplinary, interactive workshop. The residents first complete an interactive case (online, self-directed module) prior to attending the session. This case uses a mock clinic patient to provide a framework for obtaining a focused falls history/physical, and prescribing evidence based interventions. The case also provides links to key references. When they attend the workshop, they are fully prepared to participate and build upon the basic skills reviewed in the pre-work. The workshop starts with a short review of pertinent screening concepts. Following this, half of the residents complete interactive mini-CEX type cases while the other half worked with a physical therapist on evaluation of gait and demonstration of various assistive devices as well as instruction on when to choose which device. Each group had $20 \mathrm{~min}$ to complete the session and then they switched. At the end, our physical therapist demonstrated exercises they could provide their patients with to improve balance and gait. This workshop lasted for $1.5 \mathrm{~h}$.

EVALUATION: In terms of evaluation, not only was satisfaction with the teaching session analyzed, but also the proxy outcome measures of importance, confidence and attitudes as well as the clinic outcome of fall screening rates. Evaluation of the module: Residents answered a series of questions on a Likert scale of $1-5$ ( 5 being the highest). There was high satisfaction with the Relevance of the topic/information to ambulatory care $(4.57 / 5)$. The content was felt to impact patient care/practice: $(4.57 / 5)$ and the residents reported important knowledge gained from the workshop (4.57/5) Proxy outcome measures showed that before this workshop residents expressed that this was an important topic to address but had little confidence in their ability to screen. 44 residents completed a survey that used a scale of strongly disagree to strongly agree ( 1 being assigned to strongly disagree and 5 being assigned to strongly agree). Confidence in their ability to screen patients, perform a comprehensive falls assessment and treat ranged from 2.58 to $3.05 / 5$. In terms of importance of the topic, the average score was $4.58 / 5$. Residents will be surveyed again in the spring of 2016 to evaluate long term changes in confidence An analysis of fall screening rates was also performed. A chart review of 50 patients seen by PGY-2 residents prior to the workshop found that only $10 \%(5 / 50)$ of eligible patient were screening for falls. The same group of PGY-2 residents were reviewed after the workshop and the screening rates had increased to $30 \%(15 / 50)$.

DISCUSSION / REFLECTION / LESSONS LEARNED: Overall this workshop demonstrated success in substantially improving the main outcome of fall screening rates among older adults by residents in the Primary Care Clinic setting. While final analysis of some proxy outcomes of the workshop are still being evaluated, initial data suggests that it was well received. This workshop can be emulated across institutions with little faculty time commitment. The content has been published on MedEDPORTAL making dissemination easy. Lessons learned: 1 . The pre-work should be mandatory and tracked. 2 . Making the workshop interactive and multidisciplinary rather than a powerpoint lecture helped keep the residents engaged in the discussion. 3. This workshop is easy to modify and replicate for various specialties.

\section{CAN WE IMPROVE HOW MEDICAL RESIDENTS IDENTIFY AND ADDRESS SOCIOECONOMIC AND ENVIRONMENTAL RISKS THAT ADVERSELY AF-} FECT THEIR PATIENT'S HEALTH STATUS? Iman F. Hassan ${ }^{1}$; Lalit Narayan ${ }^{1}$; Mayce Mansour ${ }^{1}$; Darlene LeFrancois ${ }^{2}$; Lauren Shapiro ${ }^{2} .{ }^{1}$ Montefiore Medical Center, Bronx, NY; ${ }^{2}$ Montefiore Medical Center, Bronx, NY, NY. (Control ID \#2469546)

NEEDS AND OBJECTIVES: Recognize the link between socioeconomic and environmental factors and health. Recognize when the management of a patient's medical problems is significantly affected by socioeconomic or environmental factors. Assess and provide targeted interventions for the following socioeconomic and environmental risks: no insurance/underinsurance; food insecurity; sub-optimal housing; transportation barriers; need for supplemental income, disability and other public benefits.
SETTING AND PARTICIPANTS: The curricular intervention will be administered to 38 PGY2 internal medicine categorical residents at Montefiore Medical Center, a large, urban, academic medical center, during their ambulatory clinic blocks. All categorical residency clinics have social workers and a medico-legal partnership with LegalHealth. At each clinic, residents discuss patient encounters with a clinic preceptor. They also participate in Ambulatory Morning Rounds (AMRs), where real patient encounters are used to teach evidence-based primary care, and in lecture-based sessions on aspects of primary care medicine. We plan to integrate our curriculum into this preexisting structure.

DESCRIPTION: The proposed curriculum incorporates didactic and case-based sessions, community and clinic resource guides, and faculty training. Residents receive seminars on social determinants of health, immigration, and incarceration, and a session on identifying socioeconomic and environmental risks in the clinic and referring patients to available community and clinic resources. Residents apply this knowledge in an AMR based on their own patients. Additionally, there is a journal club session discussing a paper relevant to the link between socioeconomic status and health. In addition to resident training, there is a faculty development seminar including evidence based teaching points for clinic preceptors to use during discussion of individual patient encounters. We have created a community resource website for use by residents and preceptors that will encourage identification, assessment and intervention on patient risk factors, along with visual aids at clinics, and periodic emails to residents and preceptors

EVALUATION: A pre- and post-intervention survey is being administered to current PGY2 residents assessing confidence, self-reported behavior and knowledge about social and economic risk factors. The survey includes questions on prior training and experiences working with underserved populations, confidence levels identifying and intervening on socioeconomic and environmental risks in the clinic, current rates of addressing these issues in the clinic and perceived barriers. It also includes knowledge-based questions in the domains of access to healthcare, socioeconomic position and health, and racial and ethnic disparities from the Wieland et. al. 2010 survey instrument, and additional questions on the medical effects of incarceration. The same survey is being administered to current PGY3 residents who will serve as historical controls. Social work and medicolegal referral rates from 4 months prior to the intervention will be compared to referral rates during the intervention period and a 4-month period after completion of the curricular intervention.

DISCUSSION / REFLECTION / LESSONS LEARNED: While many family medicine and pediatric residency programs have specific health disparities curricula in place, and require demonstrating skill in determining and addressing social determinants of health, this is not the case in categorical internal medicine residency programs. A 2014 survey of categorical programs showed that $0 \%$ of respondents had social determinants of health or health disparities curriculum as part of their core curriculum. Despite this, socioeconomic and environmental factors are fundamentally related to health, and interventions on the social determinants of health can have measurable health benefits. A study by Garg et al. (2015) showed that screening for socially-based health risks at pediatric well care visits yielded increased referrals to social work and higher utilization of community resources at a 12 month follow up. Studies by Klein et al. $(2011,2014)$ demonstrated that teaching pediatric residents a screening and referral model increased self-reported knowledge and attitudes, as well as patient medico-legal referral rates. We have developed and are studying a curriculum that will teach categorical internal medicine residents to identify and address social and environmental factors affecting their patients' health within a clinic setting.

CANADA-GUYANA MEDICAL EDUCATION PROJECT: A WIN-WIN PARTNERSHIP AMONG TRAINEES Shannon Ruzycki; Victoria Weaver; William Stokes; Debra L. Isaac. University of Calgary, Calgary, AB, Canada. (Control ID \#2457698) 
NEEDS AND OBJECTIVES: Lead Calgary-based Internal Medicine residents to develop a tele-health based curriculum for medical trainees in Guyana

SETTING AND PARTICIPANTS: Core Internal Medicine (IM) residents from the University of Calgary (Calgary, Alberta, Canada) and IM trainees in Guyana.

DESCRIPTION: Opportunities for post-graduate medical education in Guyana are limited by lack of specialist-preceptors, remote training sites, and financial resources. Training is obtained in Caribbean and American institutions; however, foreign-trained Guyanese physicians often remain in these countries once trained, contributing to the paucity of specialists in Guyana. To combat this problem, the University of Maryland created a Guyana-based internal medicine (IM) program in 2013. Lack of instructors and formalized teaching sessions are barriers to the program's success. University of Calgary IM residents have created a series of teleconference teaching sessions for Guyanese residents. Core Internal Medicine (IM) residents from the University of Calgary (Calgary, Alberta, Canada) develop one hour presentations based on learning objectives from the Royal College of Physicians and Surgeons of Canada. These presentations are then delivered to medical trainees in Guyana via tele-conferencing during their academic half-day. Feedback is collected via survey and forwarded to participating IM residents who can then iteratively improve on their teaching style and presentation skills.

EVALUATION: A self-administered, cross-sectional questionnaire was created using a 5-point Likert scale to evaluate presentations on six fields; organization, pace of delivery, ease of understanding, relevance to clinical practice, involvement of participants, and the likelihood that students would recommend the session to colleagues. Guyanese trainees each complete this survey after every presentation. Calgary resident presenters completed a similar 5-point scale evaluating the usefulness of the session for the development of their own teaching skills. Both groups were asked whether sessions increased the trainees' level of comfort in interacting with physicians from other countries, as well as the desire to be involved with further international collaborative efforts. Responses from each group were pooled and averaged.

DISCUSSION / REFLECTION / LESSONS LEARNED: After seven teaching sessions, the average rating by Guyanese residents on organization, pace of delivery, ease of understanding, relevance to clinical practice, and involvement of participants was $4 / 5$. The likelihood of a Guyanese trainee to recommend each session to colleagues was consistently ranked 5/5. Canadian presenters ranked usefulness of sessions for development of their teaching skills as $4 / 5$. Both groups rated their level of comfort interacting with doctors from other countries and their desire to be involved in future international collaborative efforts as an average of $4 / 5$. These early survey results suggest that the formation of a resident led, tele-health teaching series is a mutually beneficial for Canadian and Guyanese residents. We are developing a focus-group assessment of Guyanese trainees to further guide session development.

CARING FOR FORMERLY INCARCERATED PATIENTS: A LONGITUDINAL CURRICULUM WITH OSCE Jonathan M. Giftos ${ }^{2}$; Ross Macdonald ${ }^{3}$; Melissa Stein ${ }^{2}$; Aaron D. Fox ${ }^{2,1}$. ${ }^{1}$ Montefiore Medical Center, Brooklyn, NY; ${ }^{2}$ Montefiore Medical Center, Bronx, NY; ${ }^{3}$ NYC Health + Hospitals, New York, NY. (Control ID \#2466816)

NEEDS AND OBJECTIVES: Mass incarceration represents an important public health problem in the United States. Formerly incarcerated patients have higher rates of mental illness, substance use disorders and other chronic medical conditions than those without prior incarceration. Health risks are increased during the reentry period following release from incarceration. To address this challenge, our residency program has developed a multidimensional curriculum that trains internal medicine residents to care for formerly incarcerated patients. To complement and evaluate this curriculum, we sought to design and implement an innovative
Objective Structured Clinical Examination (OSCE) assessing third year medicine residents' ability to provide evidence-based, culturally appropriate care to formerly incarcerated patients during reentry. This OSCE is unique in that it a) focuses on the reentry period, b) assesses residents as part of a primary care training program located in a community affected by high incarceration rates, and c) specifically evaluates a longitudinal and experiential curriculum dedicated to caring for formerly incarcerated patients.

SETTING AND PARTICIPANTS: Our primary care internal medicine residency program's continuity clinic is located in a federally-qualified health center (FQHC) in the South Bronx neighborhood with the highest incarceration rate in New York City (371 in 100,000)-four times the citywide rate. The FQHC houses the Bronx Transitions Clinic, which provides medical care to individuals who recently have been released from incarceration. During their first 25 years of training, Montefiore's primary care internal medicine residents receive the multidimensional curriculum dedicated to caring for formerly incarcerated patients. In September 2016, third-year primary care and categorical medicine residents will undergo the Caring for Formerly Incarcerated Patients OSCE with standardized patients (SP)

DESCRIPTION: Our innovative curriculum begins during first year, when primary care residents have the opportunity to visit the NYC jail system on Rikers Island. This experience is complemented by directed readings and case discussions regarding the effect of incarceration on health. Prior to second year, residents receive a didactic session that summarizes recommendations for providing evidence-based, culturally appropriate care to formerly incarcerated individuals. This session is informed by focus group data collected from formerly incarcerated patients regarding their experiences with the healthcare system. Key concepts include use of non-judgemental language, screening for medical conditions that are overrepresented in formerly incarcerated individuals (e.g., opioid use disorder), reentry health risks (e.g., relapse to opioid use) and interventions to reduce that risk (e.g., opioid overdose prevention with naloxone). During second and third year, primary care residents provide patient care at the Bronx Transitions Clinic. A preceptor with expertise in medical care for formerly incarcerated individuals reinforces key concepts. Patients seen during these sessions become part of residents' primary care panel providing a longitudinal patient care experience. At the beginning of third year, residents will participate in our Caring for Formerly Incarcerated Patients OSCE. During the OSCE, residents will be asked to simulate a visit with a formerly incarcerated patient seeking care during the reentry period. As part of this process, the resident must build rapport, screen for medical conditions that are overrepresented in formerly incarcerated patients and counsel patient on ways to reduce health risks during this time of transition. Categorical medicine residents who do not receive the dedicated curriculum on care for formerly incarcerated individuals, but who often care for this patient population in the hospital, will also participate in the OSCE and will serve as a comparison group.

EVALUATION: During the OSCE, a trained faculty member will use a checklist of key concepts, as outlined above, to assess the resident's ability to care for formerly incarcerated patients. For feedback, each resident will review his or her graded checklist. After data collection is complete, we will assess the relationship between participation in our longitudinal curriculum and performance on the OSCE.

DISCUSSION / REFLECTION / LESSONS LEARNED: Formerly incarcerated patients have unique healthcare needs and the reentry period following release from incarceration represents a time of substantially increased health risk. Our innovative curriculum, including the Caring for Formerly Incarcerated Patients OSCE, provides residents with the tools necessary to safely care for this high risk population during the reentry period. It also offers residents the opportunity to provide longitudinal care to formerly incarcerated patients during which ongoing medical needs can be identified and treated. Curricula such as ours may ultimately make the reentry period safer and improve health outcomes for formerly incarcerated patients. 
COACHING FACULTY FOR THE DIGITAL CLASSROOM: A TEAM-BASED APPROACH TO CREATING HIGH-QUALITY DIGITAL LEARNING CONTENT Geoffrey V. Stetson; Julia VanderMeer; Catherine R. Lucey. UCSF, San Francisco, CA. (Control ID \#2465634)

NEEDS AND OBJECTIVES: The University of California, San Francisco (UCSF) is undergoing many changes in its approach to teaching in both undergraduate (UME) and graduate medical education (GME). A large-scale curriculum redesign at the UME level is prompting the need to convert approximately one third of traditional in-class lectures to a digital format over approximately 3 years. In GME, duty hours and competing clinical demands make the need for flexible learning opportunities a priority. At UCSF, previous attempts at faculty development geared towards technology-enhanced learning, most of which had been conducted in seminar format, had been met with poor satisfaction and attendance. In response to these demands, and previous experiences, UCSF developed a unique faculty-coaching model to assist lecturers convert their materials to new, highquality, digital formats. Objectives: - Describe why an institution might feel the need to create digital learning content — Compare and contrast traditional lectures vs. video-based lectures-Identify the need for a team of experts to work closely with faculty in order to create high-quality materials - Outline the stepwise approach a multidisciplinary team uses to create digital learning content

SETTING AND PARTICIPANTS: This process is a form of faculty development that is quite individualized. Team members include instructional designers, videographers and video editors who work closely with specific lecturers and faculty in conjunction with input and feedback from course directors.

DESCRIPTION: In recent decades, the model of team-based care has been adopted in the inpatient and outpatient settings with the goal of improving not only the patient care, but also the patient and provider experience. This model stems from the idea that practitioners with varying expertise can share their knowledge through a common goal such as caring for a sick patient. Physicians continue to play a significant role in the team, but they rely on experts in fields outside of their own to enhance the care of their patient Similarly, in our digital-teaching model, the faculty member remains a central figure and serves as the content expert for the topic at hand. However, they rely on members of the team that include experts in pedagogy, and have specific knowledge in teaching using technology. These experts then support faculty members to restructure their lectures to help students learn in an asynchronous format. The team also employs professional videographers and video editors to help create the highest quality digital content. At the course director level, this team can help increase learner engagement with online videos, and grapple with the intricacies of weaving these materials into an integrated curriculum. All members of this team have a vested interest in great learning outcomes, and therefore are motivated to create an excellent product.

EVALUATION: With any curriculum innovation, evaluation is a critical component for success. To understand the impact of our video lessons on student learning, we have a number of metrics that we track. We collect information on student views of videos to confirm that our "virtual attendance" remains high. For UME courses, we can also track the overall class performance on exams and subjective feedback on the impact that the videos have had on other learning activities through surveying faculty. To date, we have seen high rates of video views and similar or improved measures of student performance across various metrics. Importantly, we also have high levels of satisfaction with the video format from both students and faculty.

DISCUSSION / REFLECTION / LESSONS LEARNED: This team-based approach has led to the creation of high-quality, and highly utilized, digital learning content. It has allowed both UME and GME curriculum the flexibility of space and time to innovate while still ensuring learners have access to core didactic materials. In addition, this has all been achieved with a group of faculty, many of whom were hesitant before they got involved with our faculty development process, because of their previous experiences.
The instructors report that for them, the difference is in the support that they receive from the team-based model. This team has been the key to all parties being satisfied with the products, as well as the process, and ultimately excellent learning outcomes.

COMING TO THE TECH TABLE TO LEARN NUTRITION COUNSELING: THE EXPERIENCE OF ONE ACADEMIC INTERNAL MEDICINE PROGRAM Brian Ricci ${ }^{2}$; Joseph A. Hardman ${ }^{1} .{ }^{1}$ OHSU, Portland, OR; ${ }^{2}$ Oregon Health and Science University, Portland, OR. (Control ID \#2455606)

NEEDS AND OBJECTIVES: 1 . Teach residents how to navigate a smartphone app to recognize the complexities of maintaining a food diary 2 . Encourage residents to analyze their own caloric intake and output as a means of applying the principles of energy balance 3. Describe an approach to nutrition counseling 4. Differentiate among several diet types in terms of efficacy for weight loss and cardiovascular health 5. Perform motivational interviewing around nutrition by recognizing a patient's preferences and then facilitating goal-setting

SETTING AND PARTICIPANTS: Internal medicine residents in an ambulatory didactic setting.

DESCRIPTION: Counseling on nutrition requires integration of basic science skills, an understanding of macronutrients, calorie balance and the interplay between disease processes, eating habits, and culture. Motivational interviewing techniques are an essentia aspect of the patient encounter, which includes a review of a patient's knowledge, comorbid conditions and facilitated goal-setting. All of this is further complicated by the demand of residency programs to offer a broad general internal medicine curriculum and the lack of time available for nutrition education. As a result of these demands, interna medicine residents lack the confidence and skill set to counsel patients in their continuity clinics about nutrition. Our residency program offers a one-hour educational intervention that provides an opportunity for general practice residents to achieve a strong foundation on which to offer nutrition-based counseling to patients. To achieve this, we have utilized an app called my fitness pal, which is free and widely available. This app utilizes the basal metabolic rate (BMR) and input of caloric intake and output to facilitate weight loss goals while also emphasizing macronutrient balance. It is a user friendly means by which to create a food diary that exhibits learning over time. Our interventional education occurred in four parts: (1) Food diary utilizing my fitness pal (2) Analysis of literature supporting common diet types (3) Reflection on the food diary experience (4) Motivational interviewing practice built from personal experience with food diary We asked internal medicine residents to utilize my fitness pal for 3 days in advance of a session focused on nutrition counseling. Participants were able to analyze their own calorie intake, which facilitated learning about energy and macronutrient balance. These concepts were reinforced in our session, in which we analyzed several diet types (Mediterranean, Paleolithic, Atkins, DASH, Weight Watchers and Plant-based). Participants were asked to find and review data supporting each of these diet types and were later provided a "pocket guide to nutrition" derived from and supporting their findings. Differentiating among these diet types allowed participants to understand how to approach nutritional counseling and also provided a review of the major trials in the medical literature. Through reflection of the food diary experience participants were able to appreciate the barriers to nutritional improvement and themes around motivation, change-talk and health literacy surfaced. This supported the final phase of the intervention, in which participants practiced motivational interviewing with concepts derived from their own personal experience.

EVALUATION: Participants were asked if the session accomplished its objectives, what worked well, what did not work well and how likely this intervention might result in a change in practice. Our hope is to survey residents 6 months following this educational intervention to assess for ongoing application and to reflect on perceived knowledge. 
DISCUSSION / REFLECTION / LESSONS LEARNED: There is limited undergraduate medical education focused on nutrition, yet obesity remains a critical public health problem. As medical educators, we are met with multiple competing demands for residents' time. A goal of our educational innovation was to provide a robust foundation in nutrition even with limited time (just one hour). Technology such as the my fitness pal app can be used in educational initiatives to make sessions time-efficient. In our experience, asking participants to create a food diary with a weight loss app facilitated introduction of concepts, which were reinforced in our session. Though my fitness pal is not designed as an educational app and rather a weight loss tool, experiential learning occurred as participants used the app. We learned that there was a wide range of knowledge in the areas of nutrition and diet counseling among participants. Use of small groups allowed for those with substantial knowledge to help others with less. We learned that specific teaching on how to later integrate the app in motivational interviewing with patients would be beneficial. We will also be assessing participant adherence to practice recommendations in a post 6-month survey to learn how we might improve the session. In the future, we would also like to do a deeper assessment of topic knowledge prior to this session.

\section{COMMUNITY BASED SERVICE LEARNING: A SHIFT FROM STUDENT} CLUBS TO AN EDUCATIONAL CURRICULUM Heather A. Archer-Dyer; Ana J. Cruz; Irene Blanco; Martha S. Grayson. Albert Einstein College of Medicine, Bronx, NY. (Control ID \#2469713)

NEEDS AND OBJECTIVES: The Liaison Committee on Medical Education (LCME) mandates opportunities for student participation in service-learning activities. Medical schools across the country are adopting programs in service-learning that incorporate meaningful service, link to the curriculum, and provide opportunities to construct collaborative partnerships. Therefore, the Community Based Service Learning Program (CBSL) was established in July, 2013 at Albert Einstein College of Medicine (Einstein) located in Bronx, NY, in order to fulfill this need in the Einstein community. The CBSL program is a community based educational program for $\mathrm{MD}, \mathrm{PhD}$ and $\mathrm{MD} / \mathrm{PhD}$ students. The program provides early career exposure via experiential education, training, workshops and seminars to develop the skills necessary for community engagement. Our mission is to provide all students with opportunities in which to engage the Bronx community and have an impact on health and social justice issues. Students learn, share, and nurture the skills needed for their roles as future physicians, physician-scientist and compassionate professionals; which are essential to work in our ever expanding communities of the Bronx. Students are encouraged and supported by engaged faculty mentors and CBSL staff. 1) Foster leadership development and community outreach by students. 2) Provide students with opportunities to learn about the health inequities and social justice issues affecting the Bronx community. 3) Offer students guidance in the development of action plans and supervision for their community outreach. 4) Develop curriculum appropriate and needed for students involved in community action projects. 5) Empower students and encourage engagement in meaningful service. 6) Offer supervision and support with evaluation and reflection

SETTING AND PARTICIPANTS: In the 2014-2015 academic year there were a total of 313 students involved in 10 projects and the Community-Student Advisory Board (CSAB). Examples of projects include: BODY—Bronx Obesity Diabetes and You, EiSci High School Mentoring Program, HEART-HIV Education and Rapid Testing, HOPE - Homeless Outreach Project at Einstein, PACt—Patient Advocate ConnecTion, and SPHERE-Space in Prisons for Health Education for Re-Entry. All CBSL groups have the opportunity to receive direct feedback from C-SAB, which consists of educators, community members, case managers, physicians, and students from our $\mathrm{MD}, \mathrm{MD} / \mathrm{PhD}$, and $\mathrm{PhD}$ programs.

DESCRIPTION: Early career exposure, through leadership training, guided reflection, and mentoring, provides greater awareness of health disparities and community health needs in our community. The training and guided reflection sessions were integrated into the service-learning curriculum allowing for active and self-directed learning. Examples of topics covered during leadership training include: HIV training for HEART, motivational interviewing, health literacy, an introduction to the IRB process, applying cultural competency training to community projects, developing research, surveys and questionnaires. Additionally, CBSL students have presented posters and participated as panelists to discuss their projects at conferences around the country.

EVALUATION: In 2014 the CBSL program was formally evaluated. The areas most valued by student leadership were logistical support by CBSL staff and mentorship received from the Director and Manager of CBSL. The evaluation of the leadership training revealed that the quality and appropriateness of the content exceeded the expectation of the students. The overall skills and knowledge shared during the sessions met expectations and students felt that their concerns were heard and questions answered. Overall, students felt that they were supported and that the service learning experience was invaluable. Additionally, C-SAB meets bimonthly to evaluate current student efforts, discuss specific community needs, and cultivate new ideas for partnerships. The CBSL program has proven to be beneficial for all students, regardless of specialty or career choice.

DISCUSSION / REFLECTION / LESSONS LEARNED: Students learn, share, and nurture the skills needed for their roles as future physicians, physician-scientists and compassionate professionals needed to work in our ever expanding communities in the Bronx. Additionally, CBSL cultivates strong leaders in service that will help to prepare all students for the clinical years and ultimately provide equitable healthcare as future physicians and physician-scientists. We believe that these invaluable lessons should ultimately be incorporated into our required curriculum for all students.

COORDINATING COMPLEX CARE - TEACHING INTERNAL MEDICINE RESIDENTS A FRAMEWORK FOR MANAGING HIGH-RISK PATIENTS Riley Lipschitz; Rebecca Davis. Hospital of the University of Pennsylvania, Philadelphia, PA. (Control ID \#2465768)

NEEDS AND OBJECTIVES: In the era of the Affordable Care Act, primary care and the patient centered medical home have taken center stage in the push to reduce health costs, improve outcomes and improve population health. Increased attention has therefore been focus on "high-risk" or "super-utilizer" patients in the primary care setting. While reducing risk and improving outcomes for these patients is complex, a growing body of evidence shows success requires risk stratification, interdisciplinary care coordination, and innovative care delivery systems. The Penn Center for Primary Care has developed the Coordinated Complex Care (C3) Clinic for high-risk patients as a novel clinical innovation to expose residents to the needs of this patient population, to utilize an inter-disciplinary team to develop integrated care plans, and to offer targeted interventions to improve health outcomes. By the end of this clinic experience: 1) Residents will identify the "high risk" patients in their continuity panel by using EMR data 2) Residents will utilize an interprofessional care team to develop individualized patient care plans 3) Residents will be able to define the role of the medical physician within the care team to reduce risk and improve outcomes for high risk patients 4) Residents will be able to develop a framework to risk stratify their panel of outpatients 5) Residents will be able to understand the interdisciplinary resources available to improve outcomes of high-risk patients

SETTING AND PARTICIPANTS: This experiential curriculum will take place within the Penn Center for Primary Care at Penn Presbyterian Medical Center with second and third year Internal Medicine/Primary Care residents.

DESCRIPTION: The C3 Team includes the resident physician, a nurse practitioner, a social worker, a clinical pharmacist, a community health worker (CHW), and an attending physician. In order to be selected for participation in the $\mathrm{C} 3$ Clinic patients had to have two 
or more poorly controlled chronic diseases and have been seen in the office within the past 12 months. Patients with serious mental illness or active substance abuse were excluded from the clinic. Each resident invited two of their primary patients to participate. For each patient, there was a "pre-clinic huddle" during which time the patient's overall health challenges were discussed and preliminary goals established. Following this, the PCP had a one-hour clinic visit with his/her patient. This visit focuses on understanding the patient's social determinants of health, evaluating functional health/well-being (SF-12), and establishing a 6-month goal to improve chronic disease. The CHW then spends an additional hour with the patient, further evaluating social, cultural and economic barriers to health. A "post-clinic huddle" allows the entire C3 team to debrief and identify areas for risk reduction. Then "next steps" are established including follow up clinic visits with NP, clinical pharmacist or PCP as well as home visits, phone calls, or virtual visits. The CHW engages with the patient weekly either in person or by phone. Additionally the $\mathrm{C} 3$ team has monthly discussions to evaluate each patient's progress. After development of the longitudinal, multi-disciplinary care plan, high-risk patients will be re-integrated into the resident continuity clinic.

EVALUATION: The evaluation of this project will primarily rely on patient and resident interviews, focus groups, surveys and multi-source feedback through the $\mathrm{C} 3$ clinic team. Prior to starting the clinic, each resident was interviewed by a medical student to evaluate why the resident chose his/her high-risk patient for the $\mathrm{C} 3$ clinic, the resident's understanding of the patient's health challenges, as well as the resident's goals for the clinic experience. After seeing their patients, residents received a post-clinic interview to assess any changes in perspective and reflections on the experience. Additionally, we plan to survey primary care residents as well as categorical internal medicine residents to evaluate their comfort level when caring for complex or high-risk patients within the outpatient setting. This will allow for more focused evaluation of the clinic experience against standard care

DISCUSSION / REFLECTION / LESSONS LEARNED: As this clinic continues to develop, several key lessons have already come to the forefront. The residents' understanding of their patients' health challenges has been greatly augmented by the presence of the CHWs as part of the interdisciplinary team. A preliminary review of the resident perspective indicates that the $\mathrm{CHW}$ involvement has helped change the way the residents approach their patients as well as their comfort level with managing high risk patients through interdisciplinary care planning. We have found that for many in our high-risk population, social obstacles have far outweighed the organic, diseased-based problems that our patient's face. This knowledge has allowed us to tailor our care planning to focus greater energy and resources on this facet of patient's overall plan.

\section{CREATING A CURRICULUM AROUND POPULATION MANAGEMENT FOR INTERNAL MEDICINE RESIDENTS Suneet Kaur; Jennifer Frazer. Carolinas} Healthcare System, Charlotte, NC. (Control ID \#2470167)

NEEDS AND OBJECTIVES: Since the Institute of Medicine (IOM) report in 2012 ${ }^{1}$, population health has been at the forefront of discussions in healthcare. Despite dollars spent on healthcare, population health outcomes fall short in comparison to other developed nations. As outlined by the IOM report, this places a need to create physician leaders that will integrate primary care and public health to improve the health of our populations. Some of the challenges faced in residency education is developing a curriculum that addresses the concerns of population health and create residents comfort with getting involved while understanding the limits on time and energy. The curriculum designed is meant to encourage physicians to become leaders; that they can help direct efforts to improve health of populations once in practice.

SETTING AND PARTICIPANTS: Carolinas Healthcare System is the 2nd largest nonprofit healthcare system in the country. The Internal Medicine Residency Program is a community-based program at Carolinas Medical Center. The hospital and clinic serve the local community as well as a large section of the southeast region, as the referral center for the system. We have a total of 42 Internal medicine residents. Six are interns completing a preliminary year. Our clinic serves a mix of underserved (25\%), Medicaid (25\%), Medicare (40\%) and some privately insured $(<10 \%)$ populations.

DESCRIPTION: Using competency principles outlined in a 2013 article from Duke University $^{2}$, we have so far created resources to educate our residents on the importance of population health and to perform routine measurement and monitoring. Using quality improvement techniques and interdisciplinary collaborative efforts, we teach residents to tackle population health issues in practice. As part of our orientation, residents are first exposed to their individual clinic population health reports. Throughout residency they receive updated reports monthly along with a quarterly performance report card that allows them to not only track their progress but to compare preventative care goals achieved amongst residents within the program. Twice a year each resident formally meets with faculty to review reports as well as select goals of improvement for the next meeting. During that time, a formal presentation takes place reviewing importance of population health, updated target preventative goals in our community, and resources within and outside of clinic. Early on, residents are assigned a primary nurse in the clinic who works directly with them to address both population and individual patient needs. We train residents on how to incorporate clinic nurse practitioners, health educators, social workers, pharmacists, dietitians, and mental health counselors to achieve population health goals. Formal training on quality improvement (QI) occurs each fall and as part of the introduction, we spend time educating residents on identifying population health concerns and focusing project goals to meet the needs of populations. Each year, every second year resident leads a QI team project. Interns on these teams learn from their peers in preparation to lead the following year, and third years mentor the team leaders along with faculty.

EVALUATION: Each year we survey residents regarding curriculum design. We have also added a survey sent to our recent graduates to assess their comfort and understanding of population health. Throughout the year we review target goals reached to determine how effective our curricular design has been.

DISCUSSION / REFLECTION / LESSONS LEARNED: In our initial approach, we have found residents have a deeper understanding of the importance, community goals, implications and ways to approach quality care goals. We have had significant improvements in reaching target goals in our clinic through quality improvement. However, we have reached plateaus just below target goals. Most recently, we added performance score cards for residents to identify, more clearly, areas that need attention and progress made. The curriculum continues to be a work in progress. We are hopeful that by adding the performance scorecard and outlining clear, achievable goals of improvement, we will see more quality targets achieved and overall improved population health for the patients we serve. Surveys of residents that have graduated recently are helping to guide us in this process of improved curricular design.

ONLINE RESOURCE URL (OPTIONAL): 1. Committee on Integrating Primary Care and Public Health; Board on Population Health and Public Health Practice. Primary Care and Public Health: Exploring Integration to Improve Population Health. Institute of Medicine Report; March 2012. 2. Kaprielian, VS, Silberberg, M, McDonald, MA, Koo, D, Hull, S, Murphy, G, Tran, AN, Sheline, BL, Halstater, B, Martinez-Bianchi, V, Weigle, NJ, Strand de Oliveira, J, Sangvai, D, Copeland, J, Tilson, HH, Scutchfield, D, Michener, JL. Teaching Population Health: A Competency Map Approach to Education. Acad Med. 2013; 88(5):626-637. [PMID: PMC3636155] doi: 10.1097/ACM.0b013e31828acf27.

CREATING A MULTIFACETED HEALTH DISPARITIES CURRICULUM IN AN INTERNAL MEDICINE RESIDENCY PROGRAM Ashley K. Haddad ${ }^{3}$; Nicole Redmond $^{2}$; William Curry ${ }^{1}$; Rebekah Weil ${ }^{1}$; Lisa L. Willett ${ }^{2}$. ${ }^{1} \mathrm{UAB}$, Birmingham, AL; ${ }^{2}$ University of Alabama at Birmingham, Birmingham, AL; ${ }^{3}$ University of Chicago, Chicago, IL. (Control ID \#2463556) 
NEEDS AND OBJECTIVES: Disparities in healthcare should be discussed during residency training; however many program directors cite barriers to implementing disparities curricula. We aim to 1) describe the development, implementation, and evaluation of a multifaceted health disparities curriculum in an urban academic medical center, and 2) discuss barriers and solutions to development of disparities curricula.

SETTING AND PARTICIPANTS: We developed the Health Disparities Track (HDT) for the Tinsley Harrison Internal Medicine Residency Program at the University of Alabama at Birmingham (UAB). UAB is a non-profit, 1400 bed quaternary care center in Birmingham, Alabama. UAB provides a university-based training program for 118 Internal Medicine (IM) residents who, during the 2014-2015 academic year, were $85 \%$ White, $6 \%$ Asian/Indian, $5 \%$ Black, and $4 \%$ Hispanic. According to recent Census data, the Birmingham community is $74 \%$ Black, $23 \%$ White, $30 \%$ impoverished, and $12 \%$ uninsured. Curricular participation was offered to all second and third-year residents in the 2014-2015 academic year.

DESCRIPTION: We began curricular planning in April 2014 and implemented the HDT in July 2014. We used Kern's six-step model for curricular design including problem identification, targeted needs assessment, definition of goals and objectives, implementation, use of varied educational strategies, evaluation and feedback. We developed goals and objectives in accordance with the Society of General Internal Medicine Health Disparities Task Force recommendation for disparities education. We surveyed residents to determine their preferred methods of instruction when learning to care for patients of cultures other than their own. Based on results, we developed didactic, clinical, and experiential learning content for our curriculum. For didactics, we used publically available resources and local expertise to create a 2-year lecture series on bias and discrimination, social and environmental determinants of health, patient-provider interactions, language and acculturation, disparities in research, special populations, and advocacy. For clinical experience, we partnered with a local clinic offering free care to uninsured patients in Birmingham, MPower Ministries Health Center. Based on clinic availability, we assigned nine residents to one-quarter of their continuity clinic time at MPower; the remaining three-quarters of their continuity clinic time was spent at a previously assigned UAB clinic site. Finally, for experiential leaning, we tasked residents with structured community exploration and reflective writing. We evaluated feasibility of curricular implementation as well as curricular outcomes at the program and resident levels. Program outcomes included number of didactic and clinic sessions delivered. Resident outcomes included reaction (compliance with assignments and interest in completing a second year of the curriculum), learning (delivery of a didactic conference on health disparities and creation of a resource guide for services available to low-income patients in the community), and behavior change (commitments to change and pre-post-test preparedness to care for patients of a different culture)

EVALUATION: Sixteen (21\%) IM residents volunteered to participate in the first year of the HDT. Feasibility was high, evidenced by successful implementation of all curricular components with no required funding. Program outcomes included delivery of 4 didactic and 84 resident clinic sessions. Overall resident reaction was positive (increased participation from 16 to 25 in the second year of the curriculum). Compliance with educational activities was higher for HDT residents assigned to the clinic experience than for those who were not ( $59 \%$ vs $33 \%, p=0.07$ ). HDT residents disseminated knowledge to their peers through a 1-h lecture on social and environmental determinants of health, bias and discrimination, and patient-provider interactions. They created a resource guide of 22 medical, dental, psychological, and social services available in the community. Fifteen HDT residents made 20 commitments to change with themes including communication, community resources, barriers to care, bias and discrimination, and patient perspective. HDT residents achieved increased preparedness to care for patients of cultures other than their own (somewhat unprepared to somewhat prepared, $p=0.025$ ).

DISCUSSION / REFLECTION / LESSONS LEARNED: A Health Disparities Track was feasible to design and implement. Residents who participated in the HDT showed positive reaction, learning, and behavior change. Facilitators to implementation included supportive program leadership, two chief resident champions, use of publically available resources and a community clinic with aligning priorities. Barriers included availability of resident time and program sustainability. In spite of these barriers, HDT expansion in the second year suggests success in implementation and overall satisfaction with the program. ONLINE RESOURCE URL (OPTIONAL): http://libguides.lhl.uab.edu/healthdisparities

CREATING AN INTERNET BASED MEDICAL EDUCATION JOURNAL CLUB Saima Chaudhry ${ }^{2}$; Alice Fornari ${ }^{1}$. ${ }^{1}$ Hofstra NSLIJ SOM, Hempstead, NY; ${ }^{2} \mathrm{Me}-$ morial Healthcare System, Hollywood, FL. (Control ID \#2469143)

NEEDS AND OBJECTIVES: Needs: Busy clinician educators need an efficient way to help develop their medical education skills. On line medical education journal clubs can provide such a forum. Running an on line journal club is substantively different than the traditional face to face small group journal clubs most clinician educators are familiar with. We will introduce participants to the nuts and bolts of running on line medical education journal clubs in their home institutions. Objectives: 1 . To list the pros and cons of an online medical education journal club for clinical educators 2 . List the steps needed to create an online medical education journal club 3 . Identify skills and resources needed to facilitate an online medical education journal club

SETTING AND PARTICIPANTS: This session will be of value to all clinician educators, program directors, associate program directors, at all levels of their careers (junior or senior) and in all settings (community, university). It would also benefit DIOs including physician and non-physician DIOs.

DESCRIPTION: Medical Educators would benefit from regular medical education journal clubs to discuss best practices and disseminate findings of importance to GME. However, attendance at such meetings, especially amongst busy clinician educators, teaching faculty, program and associate program directors can be a barrier to success. Internet based online learning has become a popular pedagogy for professional development and would be a good forum for medical education journal club. Online learning provides asynchronous discussion and allows busy clinicians access to materials anytime and anywhere the internet is available. As an alternative to face to face meetings, online journal clubs allows medical educators who are geographically isolated to participate in "virtual" forums to discuss innovations in GME. Furthermore, online learning can promote self directed learning and the concept of the flipped classroom by assigning readings and pre-work to participants who register and participate in the forum. Online journal clubs should be clear on the goals of the club whether they are to disseminate best practice in GME, improve critical appraisal of medical education research, or generate ideas for future studies. The creation of the server site, content of the site, discussion board, archiving of material, and pilot testing will be demonstrated. In addition, skills needed to facilitate the online journal club will be demonstrated with audience participation.

EVALUATION: Evaluation tools used to assess the success and utility of on line medical education journal clubs will be disseminated.

DISCUSSION / REFLECTION / LESSONS LEARNED: Our institution has engaged in an online medical education journal club for almost 1 year. We have found such a forum to be successful and popular. It has helped unify the culture of medical education at our multi-hospital health system and has brought together medical educators who would otherwise never have "met". This forum has allowed our faculty to cross collaborate on medical education programs and share resources, evaluation tools, curricula, databases etc. One line asynchronous learning is becoming an increasingly popular pedagogy. Millenial learners in particular have been noted to lean towards this type of flipped classroom instruction. Providing clinician educators and training program leadership with the skills to run an on line medical education journal club will serve to promote medical education literature and scholarship, promote cross collaboration between distant and diverse clinician educators, and promote a culture of medical education interest and buy in from diverse constituencies. 
CULTURAL DIVERSITY DAY: ENHANCING RESIDENT WELLNESS AND UNDERSTANDING Michael J. Rosenblum ${ }^{2}$; Gina Luciano ${ }^{2}$; Sudeep Aulakh ${ }^{1}$; Michael Picchioni $^{3}$; Raquel K. Belforti ${ }^{2}$; Christine L. Bryson ${ }^{2} .{ }^{1}$ Baystate Health, Springfield, MA; ${ }^{2}$ Baystate Medical Center, Springfield, MA; ${ }^{3}$ Baystate Medical Center/Tufts University School of Medicine, Springfield, MA. (Control ID \#2469075)

NEEDS AND OBJECTIVES: How do you design and implement a forum for residents to reflect upon and present their personal narratives to enhance cultural competence and promote wellness? Our objective was to design and implement a unique opportunity for residents and faculty to learn from each other in a safe environment that would encourage the sharing of life experiences with an emphasis on enhancing cultural awareness and personal connections. SETTING AND PARTICIPANTS: The setting is the Baystate/Tufts internal medicine (66 residents) and medicine-pediatrics (32 residents) programs in Springfield, MA. The participants are the residents, faculty, fellows, and office team.

DESCRIPTION: Acculturation to the complexities of the U.S. healthcare system and the New England area are often significant challenges impacting wellness for international and U.S. medical school graduates. Developing cultural competence for both national and international residents is an essential element to enhance wellness for all members of the healthcare team. Nurturing appreciation and celebration of the unique experiences of all residents is integral for wellness and resilience. Our Cultural Diversity Day is a monthly forum for residents and faculty to showcase their native culture and pre-residency experiences. Our program design: Final Friday of each block from noon to one with "catered" lunch Program Coordinator/program office organize the sessions with an individual presenter Safe setting for residents and faculty All Competing conferences cancelled "Indigenous foods" relished by attendees and prepared by our food service Presentations highlight: Discussing communication and cultural expectations in different regions of the United States and world Healthcare system, strengths and challenges as well as medical education Geography and climate of each region History, Economy and Politics Religion and Sports Tourism Fun video clips

EVALUATION: We have learned about diverse cultures and countries across the globe Food service has developed expertise in ethnic foods benefitting all Residents love the chance to "boast" about their home and their development Attendance is standing room only Residents make a point to attend demonstrating solidarity, support for each other and appreciation of cultural differences Residents see this as an opportunity to enhance their resilience by strengthening bonds with peers and faculty Illustrative resident comments: "Insight into cultural intricacies...that helps me to approach patient care with a broader perspective" "Great to learn about my fellow residents. Builds better relationships and promotes teamwork"

DISCUSSION / REFLECTION / LESSONS LEARNED: Cultural diversity day affords residents, faculty and office team opportunities to better understand and connect at a personal level which in turn helps to promote wellness and resilience Learning about a wide variety of cultures and countries has led to a broader perspective for building cultural competency and breaking down walls The sessions are cherished by our residents and faculty The sessions are effective because of the safe and supportive culture that sees diversity as strength We have "turned the table" to learn and understand about the culture of our residents This has become a remarkably important aspect of our curriculum and is incredibly beneficial for our culture of respect, acceptance and teamwork

DESIGN, IMPLEMENTATION, AND EVALUATION OF AN INTEGRATED 'CORE' ULTRASOUND AND PHYSICAL EXAMINATION FOR FIRST-YEAR MEDICAL STUDENTS Lauren Block ${ }^{2}$; Maria Barilla-Labarca ${ }^{3}$; Taranjeet Ahuja ${ }^{3}$; William Rennie ${ }^{3}$; Judith M. Brenner ${ }^{1}$. ${ }^{1}$ Hofstra NS LIJ SOM, Hempstead, NY; ${ }^{2}$ Johns Hopkins University School of Medicine, Baltimore, MD; ${ }^{3}$ Hofstra Northwell School of Medicine, Hempstead, NY. (Control ID \#2469552)
NEEDS AND OBJECTIVES: Medical educators recommend teaching physical examination to students using a 'core' checklist of comprehensive, evidence-based maneuvers to be performed at preventive care visits [1]. Ultrasound skills are increasingly taught to medical students to improve knowledge of anatomy and to supplement more traditional physical diagnosis skills. A 'core' ultrasound examination to complement traditional physical examination has not yet been proposed or evaluated. We sought to design, implement, and evaluate a core ultrasound examination to reinforce learning in anatomy and to enhance physical diagnosis. [1] Gowda D, Blatt B, Fink MJ, Kosowicz LY, Baeker A, Silvestri R. A core physical exam for medical students: Results of a national survey. Acad Med. 2014;89:436-442.

SETTING AND PARTICIPANTS: All first-year medical students at Hofstra-Northwell School of Medicine participated in six weekly sessions of supervised training by an interprofessional group of ultrasound faculty integrated into their introductory physical diagnosis course. Each week, a 'core' ultrasound maneuver was taught to complement the physical examination.

DESCRIPTION: Physical diagnosis faculty (internists, pediatricians, and ED physicians) and ultrasound faculty (radiologists, radiology techs) designed core competencies collaboratively. Core ultrasound maneuvers were chosen to complement each physical exam system set of 'core' maneuvers, including vital signs, head and neck, pulmonary, cardiac, abdominal, and musculoskeletal exams. Core ultrasound competencies included: 1. Identify the carotid artery and jugular vein 2 .Find the thyroid gland 3 . Identify diaphragmatic excursion 4. Demonstrate apical four chamber view of heart 5. Demonstrate liver and spleen tip 6. Identify patellar and quadriceps tendons The sessions were co-taught by a physical diagnosis and an ultrasound faculty member. Faculty development for physical diagnosis faculty prior to sessions insured that all faculty members were aware of al components of the examination being taught. Core maneuvers were taught to students in small groups of 2-3 students weekly over the 6-week course by the joint physical diagnosis-ultrasound faculty. Each week of the course, students practiced physical exam skills and flow for $90 \mathrm{~min}$ and ultrasound skills for $30 \mathrm{~min}$, alternating as patients and providers. One mandatory and additional optional practice sessions were offered by joint faculty. As part of clinical assessment activities, students were evaluated by standardized patients and by radiologists in the following components: 1 . comfort with the exam, and 2 . the quality of images attained. Students provided qualitative feedback on their experiences via end-of-course evaluations. This was the first in a 2-year series of clinical skills assessments to include physical exam and ultrasound image acquisition.

EVALUATION: All 100 first-year students completed the integrated physical diagnosis and ultrasound course. Following completion of the course, students were assessed in a clinical skills center by standardized patients on communication skills and patient comfort during the procedure. Ultrasound faculty evaluated the quality of images uploaded by the students. 94/102 (92\%) first-year students verbally prepared standardized patients and did at least a cursory job attending to the patient's comfort and modesty in the ultrasound exam. 85/102 (83\%) first-year students were judged to be at least somewhat comfortable working the ultrasound equipment. Time was judged by students and faculty to be a limiting factor in the performance of a full history, physical exam, and core ultrasound exam. Student comments praised the interdisciplinary nature of the PD-ultrasound integration, but asked for more consistency between faculty and homogeneity of equipment between didactic and assessment sessions.

DISCUSSION / REFLECTION / LESSONS LEARNED: An interdisciplinary group of faculty designed a 'core' ultrasound exam of six maneuvers to complement the 'core' physical exam. Faculty development was necessary to ensure physical diagnosis faculty competence in ultrasound maneuvers, to field student questions, and to assist radiology faculty by pointing out clinical correlations. This curriculum was taught in an integrated manner to early medical students with no prior training in physical examination. Student competence in the 'core' ultrasound exam was evaluated in terms of patient comfort, student communication, and image quality. The 'core' set of ultrasound maneuvers will be 
evaluated longitudinally in clinical skills assessments. Integrated education in physical diagnosis and ultrasound skill was taught seamlessly to early medical students using a 'core' exam highlighting key anatomical correlations of each physical exam, but requires additional faculty development and time allocation in clinical skills assessment.

DEVELOPING A 'COMMUNITY OF PRACTICE' AMONG VA ADVANCED FELLOWSHIPS IN WOMEN'S HEALTH Christine J. Kolehmainen ${ }^{11,}$ 7; Anne Stahr $^{9}$; Christine Pribbenow ${ }^{7}$; Carolyn B. Allard ${ }^{9}$; Lisa Altman ${ }^{10}$; Linda M. Baier ${ }^{4}$; Cynthia Brandt ${ }^{12}$; Megan R. Gerber ${ }^{1}$; Kathryn Havens ${ }^{9}$; Sally G. Haskell ${ }^{3}$; Karla Kerlikowske $^{5}$; Melissa McNeil ${ }^{6}$; Robin Moyer ${ }^{9}$; Cynthia H. Phelan ${ }^{11}$; Donna L. Washington ${ }^{10}$; Laure Veet ${ }^{4}$; Molly Carnes ${ }^{8}$; Elizabeth M. Yano ${ }^{2} .{ }^{1}$ Boston University, Jamaica Plain, MA $;{ }^{2}$ VA Greater Los Angeles HSR\&D Center, Sepulveda, CA; ${ }^{3}$ Yale University, VA CT, and VA Central Office, Madison, CT; ${ }^{4}$ Department of Veterans Affairs, Washington, DC; ${ }^{5} \mathrm{UCSF}$, San Francisco, CA; ${ }^{6}$ University of Pittsburgh Medical Center, Pittsburgh, PA; ${ }^{7}$ University of Wisconsin, Madison, WI; ${ }^{8}$ University of WisconsinMadison, Madison, WI; ${ }^{9} \mathrm{VA}$, Madison, WI; ${ }^{10} \mathrm{VA}$ Greater Los Angeles Healthcare, UCLA, Los Angeles, CA; ${ }^{11}$ William S Middleton Memorial VA Hosptial, Madison, WI; ${ }^{12}$ VA Connecticut Healthcare System, West Haven, CT. (Control ID \#2467073)

NEEDS AND OBJECTIVES: To help meet the healthcare needs of the growing number of women Veterans, the Veterans Health Administration (VHA) created Advanced Fellowships in Women's Health (AFWH) for post-residency physicians to develop academic and VA leaders in women's health. Since 1994, graduates of the program have achieved academic success with many alumni in leadership positions. In 2012, VA expanded fellowship eligibility to postdoctoral fellows from other ancillary clinical disciplines. In 2013, VHA's Office of Academic Affiliations (OAA) and VHA's Women's Health Services (WHS) partnered to inaugurate a national coordinating center (NCC) to coordinate and support the existing 8 AFWH training sites. An initial needs assessment conducted with the program directors (PD) and key site personnel identified a need to develop interdisciplinary, cross-site connections as a priority. We viewed this need for connection through the lens of Etienne Wegner's 'Community of Practice' (CoP) educational theory. We describe the NCC's efforts to achieve this goal.

SETTING AND PARTICIPANTS: There are fellowships at 8 VA medical centers who were competitively selected to host post-doctoral $\mathrm{PhD}$ and post-residency MD/DO trainees to develop leaders in women's health. Each of the 8 sites has a MD director and most have a $\mathrm{PhD}$ associate or co-director. There are currently 26 fellows in training ( 8 internal medicine MDs, 1 family medicine MD, 1 obstetrics-gynecology MD, 1 psychiatry MD, 13 psychology PhDs, 1 RN, 1 Speech, Language and Pathology PhD).

DESCRIPTION: With the intent of developing a Community of Practice (CoP), the NCC initiated the following activites: 1) regular communications via conference calls with the PDs and email distribution lists for PDs and fellows; 2) personalized business cards for each fellow; 3) a website, 4) a bimonthly eNewsletter highlighting a different site and a featured fellow each issue; 5) collaborative development of fellows' core competencies; 6) face-to-face meetings at national conferences where fellows and PDs were likely in attendance; 7) graduation certificates; 8) common advertisements in academic journals; and 9) online synchronous mentor training.

EVALUATION: As a follow-up to the needs assessment conducted 2 years ago, we interviewed a majority of PDs at the current sites (9/14), and surveyed current and former fellows. In general, the PDs describe feeling a part of a CoP. They appreciate the regular calls and other types of communication, especially when they are discussing common concerns and needs of the fellowships (e.g., core competencies). They have formed professional connections and are able to connect at meetings and conferences. The newer PDs are not as connected to the others, but believe it will develop. Some PDs expressed concern non-medical fellows are still "on the margins" and are not as included as they could be. The fellows indicated they felt a part of a larger $\mathrm{CoP}$ appreciated the eNewsletter and the mentor training. Their "mentor" was consistently the cited the best part of their experience in the fellowship. Further recommendations were for opportunities for collaborative research and networking at meetings.

DISCUSSION / REFLECTION / LESSONS LEARNED: CoPs are formed through the process of sharing information and experiences so that members of a group learn from each other and create a shared identity. Our results suggest that in its first 3 years, the VA AFWH NCC has been successful in creating a CoP among the 8 AFWH sites. We have found the sites are unique with different needs to engage in the larger community. There is further work to do to target the newer, smaller sites and bring in community members who are on the periphery.

ONLINE RESOURCE URL (OPTIONAL): www.afwh.wisc.edu

DEVELOPING A SUSTAINABLE MODEL FOR INTERPROFESSIONAL PARTNERSHIPS IN MEDICAL STUDENT-RUN CLINICS Maya Appley ${ }^{2,2}$; Alex Schmalz ${ }^{1}$; Linda Nix ${ }^{4}$; Mary K. Orsulak ${ }^{3}{ }^{1}$ Tulane, New Orleans, LA; ${ }^{2}$ Tulane University, New Orleans, LA; ${ }^{3}$ Tulane University School of Medicine, New Orleans, LA; ${ }^{4}$ Tulane University School of Medicine, School of Public Health and Tropical Medicine, New Orleans, LA. (Control ID \#2466348)

NEEDS AND OBJECTIVES: This project was initiated to address the needs of improving patient care and creating interprofessional learning opportunities for students at Tulane University School of Medicine (TUSOM) and School of Public Health (SPHTM) working in medical student-run clinics (SRCs). Our objective is to describe the development of a sustainable model for interprofessional collaboration for public health (MPH) and health administration (MHA) students working in SRCs.

SETTING AND PARTICIPANTS: Over the past two decades, TUSOM students have created, staffed, and served as administrators for numerous SRCs throughout New Orleans. The Tulane SRCs are united under the Student Clinic Council (SCC), whose medical student leaders collaborate to achieve two overarching goals: to provide quality healthcare to underserved populations and to offer students opportunities to hone their clinical skills. Wanting to expand these learning opportunities to MPH and MHA students, a group of students from the SOM and SPHTM came together in early 2015 to develop a structure for interdisciplinary collaboration in the SRCs. Key participants include medical student leaders from eight SRCs, leadership teams of MPH and MHA students, MPH and MHA volunteers, and leaders from the SCC.

DESCRIPTION: Since their inception, the Tulane SRCs have contributed significantly to the health of underserved communities in New Orleans and provided invaluable learning opportunities to countless medical students. The goals of this partnership are: to expand these opportunities to MPH and MHA students, enhance healthcare provision in the clinics and foster understanding and teamwork amongst future health professionals. The leaders endeavored to create a durable structure with support systems and accountability measures in place. First, SPHTM student groups were established for the MPH and MHA cohorts, pairing each with a faculty advisor and designating an annually elected leadership team. The leaders of the three student groups (MPH, MHA and SCC) work together to advance the partnership and to hold each other accountable. Each semester, MPH and MHA leaders work with clinic leaders to identify needs in the clinics, define discrete projects relevant to the interests and skills of their peers, and connect interested MPH and MHA students with these opportunities. The first wave of volunteer placements occurred in January 2015, placing six MPH and MHA students in quality improvement projects. In the Fall of 2015, 36 MPH and MHA students were placed in roles ranging from patient education to the development of patient follow-up and tracking systems. New volunteers attend an orientation, during which they work with clinic leaders to complete 
goal-setting exercises. Progress is monitored by monthly reports including data from the previous month, challenges, areas for improvement and goals. Through these reports, the leaders are able to monitor project development, learn how better to support the volunteers and build a repository of "lessons learned" for future leaders.

EVALUATION: Currently, the partnership is in the midst of its first leadership transition, which will be a major test of the organizational structure and sustainability. The success and sustainability of the partnership will be evaluated based on the number of projects initiated and completed each term, the number of active student group members, patient care results, and the continued evolution of the program. Additionally, each project has its own specific goals, objectives and metrics of success, established jointly by the clinic leaders and volunteers. These will be evaluated using qualitative and quantitative data from the monthly reports. We also plan to evaluate the impact of this partnership on attitudes towards interprofessional collaboration by comparing results of pre- and posttests administered to SOM and SPHTM students before and after their participation in this partnership. We will also compare the results of the participants' self-administered questionnaires with a control group of randomly selected SOM and SPHTM students who were not involved in this partnership. The questionaire is adapted from the IHI's Readiness for Interprofessional Learning Scale

DISCUSSION / REFLECTION / LESSONS LEARNED: Thus far, we have created system that is adept at identifying opportunities and matching students with volunteer positions. Moving forward, our focus is shifting towards ensuring that these projects are undertaken with the support necessary to produce the best possible results. To this end, we are working with faculty to integrate the IHI Change Action Network (I-CAN) course into TUSOM and SPHTM curricula. The I-CAN course provides leadership training and structure for approaching quality improvement projects, and allows students to receive course credit for their work, enhancing accountability and creating a strong incentive. In the coming years, we plan to continue to expand, evolve, and refine this partnership to produce maximal benefits to students and the community.

DEVELOPMENT OF A NEW ELECTIVE IN PRIMARY CARE AND TEACHING FOR SENIOR MEDICAL STUDENTS Lydia A. Flier ${ }^{3}$; Sara Fazio ${ }^{1,3}$; Barbara Gottlieb $^{2,3}$; Barbara Ogur ${ }^{4,}{ }^{3}$; Kristen Goodell ${ }^{3} .{ }^{1}$ Beth Israel Deaconess Medical Center, Boston, MA; ${ }^{2}$ Brigham and Women's Hospital, Jamaica Plain, MA; ${ }^{3}$ Harvard Medical School, Brookline, MA; ${ }^{4}$ Cambridge Health Alliance, Cambridge, MA. (Control ID \#2466710)

NEEDS AND OBJECTIVES: Learners will be able to: 1) describe learning strategies best suited to adult learners; 2) demonstrate skills of giving feedback to near-peers in the ambulatory clinical setting; 3 ) prepare and present an effective presentation on a primary care topic in a large-group setting; and 4) prepare a concept video on a primary care topic SETTING AND PARTICIPANTS: Despite a growing body of literature underscoring the value of teaching medical students to teach $[1,2]$, and the resulting increase in programs for peer-assisted learning (also called medical students-as-teachers and nearpeer instructors) $[3,4]$, most undergraduate curricula do not include teaching competencies. There is variability in student-teaching programs with respect to structure, preparation, and both student and program evaluation. At our institution, there are several informal mechanisms for participating in near-peer teaching. However, at present there is only 1 formal educational offering, and that focuses on the physical exam of standardized or hospitalized patients and does not evaluate students' teaching proficiency.

DESCRIPTION: We designed a pilot "Elective in Primary Care Medicine and Teaching" for senior students who had completed their core clerkship year and desired instruction and experience in teaching, centered on a longitudinal primary care clinic. Seven 4th-year medical students $(n=7)$ and one student in a research year $(n=1)$ enrolled in the capped 8-person pilot, out of 50 who initially expressed interest in an outpatient teaching course. We sought to provide opportunities to explore a range of teaching venues and skills. The course consists of seven 90-min didactics on medical education from expert faculty; a concurrent, part-time longitudinal primary care clinic placement as junior preceptors to MS1s in a continuity clinic; the design and delivery of a 30-min Grand Rounds presentation; and the creation of a 35 min educational "concept video" on a topic in primary care. Students receive evaluations of their work as a preceptor, their grand rounds and concept video, and in a 3-station Observed Structured Teaching Evaluation (OSTE) at the conclusion of the course. The didactics cover Adult Learning Theory, Giving Feedback, Evidence Based Medicine, Ambulatory Teaching and the 1-min Preceptor, Effective Lecturing, Designing a Concept Video, and Endings, an at least 6 clinic sessions between October and March.

EVALUATION: In addition to the multimodal evaluation of students described above, we plan to pursue a comprehensive assessment of the course, including pre/post survey selfassessment of perspectives on teaching and preparedness to teach compared to controls; OSTE outcomes; a focus group reviewing the course; and both informative interviews with faculty and surveys with MS1s about the experience of having near-peer preceptors in clinic. Though the pilot is ongoing, initial feedback is positive. After each didactic session, students take anonymous surveys assessing (a) confidence in applying the newly-taught skills, and (b) the overall effectiveness of the teaching format, on a 5-point Likert scale from not at all confident/effective (1) to very confident/effective (5). For the first 5 lectures, we identified mean confidence of 4.44 (range 4.0-5.0) and effectiveness of 4.72 (3.71-4.88).

DISCUSSION / REFLECTION / LESSONS LEARNED: The field evaluating peerassisted learning has thus far focused heavily on the efficacy of medical students-asteachers compared to faculty in delivering discreet knowledge, such as physical examination or procedural skills, with few examples of advanced students participating in an outpatient practice [5]. We describe a well-received program training and deploying advanced students as near-peer educators in an ambulatory, primary care setting with a unique multimodal approach to evaluate students on teaching performance rather than participation alone. To prepare for future iterations of this course, we intend to review the data from our evaluative process to allow for modifications based on the strengths and weaknesses identified by all participants, including near-peer preceptors, MS1s, and faculty preceptors. We are planning to expand enrollment next year. As the curriculum reform at our institution reaches students in their senior years, we see this course as an opportunity for MS1s to return to the clinic where they first learned the H\&P to begin the process of growing as teachers, preparing them for residency and beyond. References: 1 . Tolsgaard et al. 2015 2. Lockspeiser et al. 2008 3. Yu et al. 2011 4. Soriano et al. 20105. Henschen et al. 2013

DOES 4+1 RESIDENCY SCHEDULING ADD UP TO IMPROVED PATIENT OUTCOMES? Cara Foldes; Larry Laufman; Achilia Morrow; Lee Lu; David J. Hyman; Joslyn Fisher. Baylor College of Medicine, Houston, TX. (Control ID \#2464985)

NEEDS AND OBJECTIVES: Little is known about the patient's perspective of continuity and quality of care in primary care teaching clinics. New changes in residency schedules to improve resident satisfaction have led to a movement towards a block $4+1$ resident schedule. The new $4+1$ schedule is designed to create a more satisfying ambulatory experience for IM residents, but how this effects patients is unknown. The objectives for this innovation include: (1) identify patient perceived satisfaction and perception of continuity and quality of care with a change in scheduling from a traditional IM residency schedule to a newer $4+1$ schedule (2) identify ways to assess patient perceptions of quality and continuity of care.

SETTING AND PARTICIPANTS: The educational intervention and study were performed at a large inner city academic internal medicine primary care clinic where approximately $100 \mathrm{IM}$ residents and 8 GIM faculty see patients and supervise the resident clinic. In May/June 2014, before implementing the new $4+1$ block schedule, 300 clinic 
patients were randomly selected to complete the survey. This was repeated with 200 patients in May/June 2015, under 4 + 1. Participating patients were each given the choice of either the English or the Spanish survey, and the option to complete it themselves or in an interview format.

DESCRIPTION: The new $4+1$ structure attempts to eliminate the stress of caring for inpatients while away from the medical wards at continuity clinic. As less internal medicine residents pursue careers in primary care and more choose careers in subspecialties, the $4+1$ rotation structure is designed to make the ambulatory care experience a more positive experience. Residents often cite unfulfilling ambulatory internal medicine experiences as a reason why they do not chose careers in primary care. The IM resident $4+1$ block schedule consists of 4 weeks of an inpatient service followed by 1 week of continuity clinic. Prior published research on the $4+1$ block internal medicine residency scheduling is based mostly on resident perceived outcomes. In a separate study, $75 \%$ of the residents in this IM program indicated they preferred the $4+1$ schedule for a positive continuity clinic experience. This study attempts to assess the impact of the schedule change on patient satisfaction and patient perceived continuity.

EVALUATION: We developed a 53-item patient questionnaire which incorporated the 18-question Patient Satisfaction Questionnaire (PSQ-18) developed and validated by the RAND Corporation as well as questions specific to our clinic. Questions used a 5-point Likert scale. The full survey was then translated into Spanish. The questionnaire was either self-administered or administered to 300 patients under traditional scheduling in 2014 and 200 patients in 2015 under novel $4+1$ scheduling. The proportions of English and Spanish surveys collected in both years were statistically similar. Of the 300 surveys in 2014, 226 (75.3 \%) were English and 74 (24.7 \%) were Spanish. Of 200 survey in 2015, 147 (73.5 \%) were English and 53 (26.5 \%) were Spanish. Cronbach's alpha reliabilities for the 7 PSQ-18 scales were .847 , and .789 for the locally developed questions. Data were analyzed using Spearman's rho and Mann-Whitney tests for ordinal ratings, Chi-square and analysis of variance for nominal and ratio level comparisons. There were no significant differences in the 7 PSQ-18 subscales and in particular, the General Satisfaction subscale (Pre-Mean $=3.89$; Post-Mean $=3.94 ; p=.549$ ). There was significant improvement in the question "the medical care I have been receiving is just about perfect" (Pre$\mathrm{M}=4.03$, Post $\mathrm{M}=4.20 ; p=.024$ ) and "sometimes doctors make me wonder if their diagnosis is correct" (Pre-M=3.31, Post-M=3.06 $p=.021$ ). Patient perception of the doctor-patient encounter as unhurried decreased (Pre-M $=3.84$, Post-M $=3.63 ; p=.026$ ), though the overall rating of doctor interpersonal manner was high and remained unchanged (Pre-M $=4.16$, Post-M $=4.12 ; p=.456$ ). Patient perception of continuity measured as "I usually see my doctor at my clinic appointments" also remained high and unchanged (Pre-M=2.09, Post-M=2.12; $p=6.14$ ).

DISCUSSION / REFLECTION / LESSONS LEARNED: As more IM residency programs change to novel scheduling, it is reassuring that the overall patient perception of continuity, quality of care and satisfaction is either improved or unchanged with a $4+1$ scheduling model. Our study found that with the new model, patients may perceive more rushed behavior from the physicians. In a separate study evaluating the same residents who provided medical care to the patients participating in this study, the investigators found that the residents perceived they were seeing more patients under the new $4+1$ model. Such a perception may have led to the patients in this study perceiving a more rushed behavior by those physicians. If novel scheduling improves IM trainee's primary care experience and more trainees seek to pursue careers in primary care, then more programs may change to a $4+1$ or similar structure.

DUKE HOTSPOTTING INITIATIVE (DHSI): INTEGRATING MEDICAL EDUCATION WITH COMMUNITY-BASED CARE COORDINATION Jerry Lee; Morgan Hardy; Julie Rivo; Marigny M. Bratcher; Natasha T. Cunningham. Duke University Health System, Durham, NC. (Control ID \#2469647)
NEEDS AND OBJECTIVES: Amid challenges of rising costs, increasing fragmentation of care, and ongoing epidemics of preventable chronic illnesses, undergraduate medical educators recognize the tremendous need to prepare the next generation of doctors to practice cost-effective and coordinated preventive medicine. While most teaching strategies approach these topics through didactic instruction, medical students consistently attribute the difficulty in integrating these concepts into clinical care to the lack of practical training. Confronting these challenges, we seek to create a novel, hands-on approach to teaching population health and systems-based practice for first year medical students.

SETTING AND PARTICIPANTS: We enrolled a pilot group of 8 students primarily for program evaluation in 2014. As part of a "phased adoption" strategy, the program commenced the following year, with an initial enrollment of 20 students out of 36 applicants.

DESCRIPTION: Duke Hotspotting Initiative (DHSI) was developed as a new curriculum based on "hotspotting," a concept pioneered by MacArthur Genius Grant winner Dr. Jeffrey Brenner and the Camden Coalition of Healthcare Providers. Hotspotting provides comprehensive care management to the highest utilizing, medically-complex patients in order to reduce ED/hospital visits and improve care quality. Partnering with the Duke Outpatient Clinic (DOC), a hospital-based internal medicine clinic with an active care management program, DHSI incorporates medical students directly into care management services by having students work in pairs to improve the health of a high-utilizing patient. Over a 6-month period and under the supervision of a team consisting of a nurse care manager, psychiatrist, nurse practitioner and social worker, students elicit SMART selfcare goals from their patients through motivational interviewing, particularly focusing on altering modifiable risk factors for chronic disease with behavioral modification strategies. Additional responsibilities include weekly communication with their supervisor; transportation arrangements for patient primary and specialty care appointments; and notewriting using electronic medical records (EMR). Supplemental didactic sessions focus on practical skills, including how to navigate EMRs, write progress notes, give oral presentations, assess major social factors determining their patient's well being, and conduct home visits. In this way, students are able to impact the lives of some of the sickest patients at Duke, while occupying roles on the healthcare team appropriate to their level of training. EVALUATION: The overall curriculum and learning objectives were evaluated through a mixed methods approach, composed of analyses of student written reflections, and preand post-participation surveys assessing the acquisition of core competencies. Content analysis of written reflections indicated that participants felt more comfortable referring patients for social services and pain and/or drug rehabilitation, as well as obtaining a history, performing a physical examination, and communicating treatment plan options to at-risk populations than their peers, with high endorsement of the program over traditional didactic approaches. Core competencies reflecting the AAMC's Core Entrustable Professional Activities for Entering Residency were specifically examined in targeted questionnaires, and results reflected an increase in competency domains such as patient-centered care, interpersonal and communication skills, professionalism, systems-based practice, and interprofessional collaboration. These preliminary results indicate high student satisfaction, improvement in quality measures, and an increase in students' self-assessment of their knowledge in the biopsychosocial determinants of health, prevention strategies, and skills in motivational interviewing. Additionally, several patient care factors are currently being evaluated, including SMART goal adherence 6-months post-participation; improvement in Patient Activation Measure ${ }^{\circledR}$ (PAM) scores pre- and post-participation; and decreases in ED utilization and admission rates at 6-months and 1-year post-participation. DISCUSSION / REFLECTION / LESSONS LEARNED: Medical students gain a deeper understanding of the biopsychosocial determinants of health and learn about critical local health resources. While not otherwise taught in a traditional medical school curriculum, such knowledge is essential for physicians to make authentic, sustainable improvements in their patient's well-being. Concomitantly, patients receive support to meet a self-determined health goal integral to their wellbeing and assistance coordinating 
care for complex health needs. Ultimately, this program is a low-cost mechanism to simultaneously train future physicians and improve the health of the system's most vulnerable members.

ONLINE RESOURCE URL (OPTIONAL): Syllabus: bit.ly/1P0E04s

ENHANCING THE INTERPROFESSIONAL LEARNING ENVIRONMENT THROUGH A COLLABORATIVE CARE MODEL TO IMPROVE PATIENT CARE: A PILOT STUDY Temple Ratcliffe ${ }^{3}$; Kanapa Kornsawad ${ }^{4}$; Christopher Moreland $^{2}$; Meghan Crabtree ${ }^{2}$; Karthik Garapati ${ }^{2}$; Stephanie Ossowski ${ }^{2}$; Erika Bowen ${ }^{2}$; Luci Leykum ${ }^{1} .{ }^{1}$ STVHCS / UTHSCSA, San Antonio, TX; ${ }^{2}$ The University of Texas HSC - San Antonio, San Antonio, TX; ${ }^{3}$ UTHSCSA, San Antonio, TX; ${ }^{4}$ University of Texas Health Science Center, San Antonio, TX. (Control ID \#2460867)

NEEDS AND OBJECTIVES: Our objective was to evaluate the impact of delivering a collaborative care model on rounding patterns and student and resident learning experiences during an inpatient teaching service at a single academic medical center. Attendees will: - Identify potential barriers to implementing fully interprofessional approaches to rounding and patient care while optimizing the learner's experience — List components of an academic inpatient collaborative care model

SETTING AND PARTICIPANTS: Despite efforts to improve the care of hospitalized patients, adverse events remain common. Care is siloed across disparate providers and patients are not engaged in their own care plans. In a collaborative care model, patients, families and providers partner to integrate high-quality care across disciplines to best meet patients' needs. While support for delivering inter-professional, patient-centered care is growing, limited research exists examining the impact of delivering a collaborative care model, particularly with regard to learners' experiences. At an academic county hospital, we implemented a collaborative care model on a single inpatient ward team. The team included nurses, physicians (attending, residents), medical students, pharmacists, a social worker, and a physical therapist.

DESCRIPTION: Each discipline's workflow was adjusted to allow frequent interactions and bedside-rounding. Additionally, the team met daily for a 30 min reflective session. Rounds were patient-centered and focused on patients' stories and values surrounding care. Plans were scribed on a communication board and verbally summarized afterwards A standardized safety checklist was utilized during every patient discussion.

EVALUATION: Our mixed-methods approach to assessing the model's impact included direct observation; provider and patient interviews; and administrative data analysis. Conventional inpatient teams served as comparators. We engaged stakeholders to identify key elements for successful collaborative care implementation. Elements included workflow, observation, practice-simulation, geographic localization, daily goals of care, reflection, safety checklists, and patient/family engagement. Once implemented, mean time per patient discussion for traditional vs collaborative rounds was $16: 57$ vs 17:20 mins. Mean $\%$ of time spent with the patient during traditional vs collaborative rounds was 28 vs $99 \%$. Patients/families contributed to discussions $2 \%$ of the time in the traditional care model vs $17 \%$ in the collaborative model. We interviewed 22 house staff \& 16 medical students who worked on the collaborative team and coded interviews to identify themes related to learner's interprofessional and educational experiences. Respondents generally saw the benefits of the collaborative model, citing improved interprofessional communication and more patient-centered care delivery. Concerns and negative responses were also identified, of which $40 \%$ related to perceived workload and "time required" for collaborative rounds. Other concerns related to less explicit teaching time and expressing uncertainty over care plans in front of patients and other providers.

DISCUSSION / REFLECTION / LESSONS LEARNED: Implementing a collaborative care model on a medicine teaching service was feasible and did not substantially increase rounding time per patient. Improved patient and interprofessional communication was tempered with concerns over learners' educational experiences. These concerns could be addressed through improved orientation and targeted curricular objectives and content. We will continue to assess this model's impact on patients, learners, and providers to optimize its implementation in a teaching environment.

ESTABLISHMENT OF A PRIMARY CARE RURAL HEALTH FELLOWSHIP TO ADDRESS HEALTH DISPARITIES Matthew Tobey ${ }^{1}$; Devin Oller ${ }^{1}$; Omar Amir ${ }^{2}$; Tom Peteet ${ }^{3}$; Katrina Armstrong ${ }^{1}{ }^{1}$ Massachusetts General Hospital, Somerville, MA; ${ }^{2}$ Brigham and Women's Hsopital, Boston, MA; ${ }^{3}$ Boston University Medical Center, Boston, MA. (Control ID \#2466572)

NEEDS AND OBJECTIVES: Needs: -Areas of significant health need in the U.S. are often not served by nearby Academic Medical Centers -Some communities in rural areas face health outcomes on par with developing nations -There are few pathways to train primary care physicians as leaders in rural health Objectives: -To partner with rural communities with poor health outcomes in order to implement meaningful clinical and community interventions -To train leaders in rural health disparities -To innovate in the creation of curricula and career pathways to empower physicians to address rural health needs

SETTING AND PARTICIPANTS: A fellowship program has been established with a primary community partner site of Rosebud, South Dakota. Fellows are drawn from primary-care field residency programs, and two are recruited for each 2-year period The Rosebud Sioux Reservation is home to a community of 30,000 individuals stretching over an area of southern South Dakota larger than Rhode Island. The Rosebud Sioux Tribe faces a number of challenges, including a per-capita income in the five lowest counties nationally, an unemployment rate of over $80 \%$, and an age-adjusted mortality rate roughly equivalent to that of homeless individuals in Boston. Rosebud is remarkable for its strong traditions, tightly knit community, many community-led programs, and for serving as the seat of a Lakota-led university.

DESCRIPTION: The fellowship program is a 2-year program comprised of clinica service, implementation of a project, and participation in several curricula including a Master's Degree in Public Health. Service: -Fellows will spend approximately one-third time providing clinical service, primarily in Rosebud, South Dakota at the Indian Health Service center. -Clinical service in Rosebud will be split approximately $75 \%$ outpatien and $25 \%$ inpatient, and offer a chance to strengthen skills in providing care in a rural site. -As fellows adjust to the clinical needs of the rural setting, the fellowship program will offer regular phone meetings and around-the-clock support. Project Implementation: -Fellows will complete a project in a topic related to health disparities and are encouraged to do so at the primary clinical site. Possible formats include an implementation project or community-based participatory research in partnership with the community. Education: -Fellows will participate in three non-degree curricula: a novel rural health curriculum, an established community health leadership curriculum, and an established health disparities program. -Fellows will obtain a Master's Degree in Public Health through a summer-only program. The features of the program that have facilitated its development have included: -Attracting fellows through an emphasis on flexibility, the acquisition of a graduate degree, and the offer of connections with numerous peers and mentors through curricular offerings. -A stable financial model based upon compensation through the Indian Health Service, which offers flexible remuneration at its most rural sites.

EVALUATION: The fellowship will be formally evaluated by fellows, community partners, and program leadership with pre-specified instruments. The program's success will be judged by its ability to accomplish its mission of providing effective service, meaningful community partnerships, and career development.

DISCUSSION / REFLECTION / LESSONS LEARNED: Key lessons from fellowship program development include that -It is feasible to create a fellowship program in 
partnership with the Indian Health Service with a model of rotating fellows. A pediatrics department in another institution is currently exploring a similarly structured fellowship. -Recruitment of fellows for community-based domestic and international fellowships is becoming increasingly competitive given that the number of such positions is increasing. -Community needs are foremost and should guide program development. Our partner community's primary requests have been for a broadening in the availability of health services. -There is a landscape of increased focus on innovation for particularly challenged communities. Potential partners - e.g. community organizations, non-profit programs and academic institutions - are often available for collaboration. Reflections regarding next priorities for the program: - The next step in community partnership includes establishing increased reciprocity, ideally through training of community members in health fields or through pursuing other community priorities. The fellowship program plans to make a direct contribution to the tribe's programs and is exploring philanthropic and grant-based options for bidirectional training. -The fellowship's mission is to explore ways to grow to partner with other rural communities. -The fellowship recognizes the need to seek more interdisciplinary partnerships to further community and partner goals.

ONLINE RESOURCE URL (OPTIONAL): http://www.massgeneral.org/education/ fellowship.aspx?id=223

EVIDENCE BASED MEDICINE ON THE FLY: INTERDISCIPLINARY COMMUNICATION ON THE CONSULT SERVICES Deepa R. Nandiwada ${ }^{3}$; Shanta M. Zimmer $^{3}$; Megan McNamara ${ }^{1}$; Melissa McNeil ${ }^{4}$; Carla Spagnoletti ${ }^{3}$; Amar Kohli ${ }^{2}$; Kathryn Berlacher ${ }^{4}$. ${ }^{1}$ Louis Stokes Cleveland VAMC, Cleveland, OH; ${ }^{2}$ UPMC, Mars, PA; ${ }^{3}$ University of Pittsburgh, Pittsburgh, PA; ${ }^{4}$ University of Pittsburgh Medical Center, Pittsburgh, PA. (Control ID \#2468708)

NEEDS AND OBJECTIVES: 1. Utilize the interface between the generalist and specialist as a way to promote high value care through deliberate communication of Evidence Based Medicine (EBM) 2. Develop lifelong learning habits for Evidence Based Practice (EBP) on consult electives that can be transferable to other rotations 3. Apply a framework to promote and practice self-directed evidence based learning The interface between the generalist and specialist is a prime point to implement EBM that promotes high value care. EBP, or the daily practice of EBM (in clinics, on rounds), is more effective than journal clubs and group didactics. There is limited literature examining EBP curricula on medical subspecialty consult services. Our goal was to expand our small group critical appraisal EBM curriculum to promote the communication and daily practice of EBM on our subspecialty consult electives by utilizing faculty and fellows as EBP coaches. An extensive needs assessment of our consult rotations surveying medicine subspecialty consult attendings, fellows, and internal medicine residents revealed that learners wanted brief interventions for more deliberate communication of evidence, skills to write a consult note assessment and plan, and increased accountability to presenting the evidence.

SETTING AND PARTICIPANTS: The pilot curriculum was implemented to internal medicine residents and fellows at the University of Pittsburgh Medical Center on the cardiology consult elective rotation from October of 2015 to February 2016.

DESCRIPTION: A multicomponent curriculum was developed utilizing the cardiology consult faculty and fellows as EBP coaches. Parts of the curriculum are an introductory EBP module which includes a self-directed teaching and learning EBM framework that is sent out to faculty, fellows, and residents. Residents are asked to use an electronic health record based "dot phrase" which brings up a templated framework for an EBM teaching point in consult notes, present brief structured EBM summaries on rounds using a mini Clinical Evaluation Exercise (CEX), and get supervisor feedback on notes guided by a checklist. Faculty and fellows are to use the EBM teaching points as a tool for giving feedback on evidence being looked up. A face-to-face introduction session and debriefing session is held for feedback at the end of each block.
EVALUATION: To assess the pilot curriculum, a post module survey and pre and post curricular surveys were given to fellows and residents that addressed educational value, accountability, and barriers to the curriculum. As this is also a communication tool we surveyed the primary teams placing consults. 5-point agreement and frequency Likert scales were used. At this point, 9 residents and 5 fellows on the cardiology consult service have undergone the curriculum and 46 residents from primary medicine teams have been surveyed. Descriptive statistics and paired t tests were used for analysis. Post module data showed increased understanding in how to write a consult assessment and plan and knowledge of EBM resources with mean ratings going from 3.6 to $4.5(p=0.001)$ and 3.6 to $4.2(p=.04)$ respectively. In the post curriculum survey analysis $85 \%$ of residents and fellows felt the curriculum was useful. $80 \%$ of the residents felt that writing the teaching point was educationally valuable to themselves and $75 \%$ felt that it was educationally valuable to the primary team. Within the debriefing sessions, discussion points included challenges with attending agreement with the evidence, difficulty designing answerable questions, and a struggle with balancing expert opinion discordance with evidence. 46 learners were surveyed on primary medicine teams placing consults to see the impact of the EBM teaching point in the note on their learning. The residents were shown an example of the EBM teaching point template and assessed cardiology consult notes they had placed over the month. $91 \%$ of all learners liked the format of the teaching point, $98 \%$ of learners wanted other consult services to include an EBM teaching point. Ten learners had a cardiology consult with a teaching point, $100 \%$ felt that the teaching point in the note was educationally valuable.

DISCUSSION / REFLECTION / LESSONS LEARNED: We found that this EBP curriculum was useful to learners on both ends of the consult spectrum. The response from primary team placing consults was overwhelmingly positive inciting us to disseminate the curriculum more widely. As of January 2016, the curriculum is expanding to include renal, hematology/oncology, infectious disease, rheumatology, palliative care, and gastroenterology. Challenges include residents having variable amounts of time on the consult services, difficullty balancing evidence with expert opinion, and the curriculum requiring significant buy in from fellows and faculty who vary in holding residents accountable to expectations. Moving forward we are including a more robust faculty and fellow development to augment the roll-out.

ONLINE RESOURCE URL (OPTIONAL): http://vpsim.pitt.edu//player/vpplayer. aspx?caseID=7be3e993-0568-41c7-b1ce-0b6f776126bf

EXAMINING DIAGNOSTIC REASONING USING SMART PHONE-BASED CASE SIMULATION: A CASE OF CHEST PAIN DUE TO PULMONARY EMBOLUS James L. Wofford ${ }^{2}$; Shantanu Nundy ${ }^{1} .{ }^{1}$ University of Chicago, Chicag0, IL; ${ }^{2}$ Wake Forest University, Winston-Salem, NC. (Control ID \#2466477)

NEEDS AND OBJECTIVES: Better tools and strategies are needed to improve diagnostic reasoning among active clinicians and students. The advent of smart phone applications should provide a more available and more efficient way of practicing and improving the quality of differential diagnosis, but published efforts are not available.

SETTING AND PARTICIPANTS: Practicing clinicians and students have free access to this online database of clinical cases and accompanying smartphone application. Over 1000 clinicians from 47 countries have thus far contributed to the project.

DESCRIPTION: The human diagnosis project (humandx.org) offers a smart phone application for encouraging the practice of differential diagnosis by offering short, reallife cases authored by practicing clinicians. HumanDx is unique as a means of studying clinical reasoning in that (a) learners need to update their differential diagnosis with each new case finding and (b) learners enter their differentials using free text response, rather than a predefined set of responses. The resulting database of clinical cases and step-by-step diagnostic reasoning provides a means of comparing and sharing diagnostic performance. 
EVALUATION: We analyzed data from the most often attempted case in order to assess case performance and compare clinical diagnostic strategies among clinicians. The three-step case of a 50 year old man with chest pain from pulmonary embolus offered demographics and chest pain characteristics in step 1 of the case presentation, the history of prostate cancer in step 2, and recent prostatectomy in step 3.96 clinicians attempted to solve the case (33 students, 43 physicians, 20 anonymous) $55 \%$ (53/96) matched the intended diagnosis of pulmonary embolism. Students were less likely to match the intended diagnosis than physicians ( $48 \%, 16 / 33$ versus $60 \%, 26 / 43)$. Early platform users were less likely to match the intended diagnosis than experienced platform users ( $48 \%, 25 / 52$ versus $64 \%, 18 / 28$ ), and anonymous users $(63 \%, 10 / 16)$. Inclusion of the intended diagnosis on the differential occurred in an expected stepwise fashion with each step of the case (39 to $53 \%$ to $60 \%$ ), with little difference between students ( $39 \%$ to $48 \%$ to $58 \%$ ) and physicians ( $39 \%$ to $53 \%$ to $62 \%$ ). Identification of the intended diagnosis (pulmonary embolism) as the number one diagnosis increased stepwise from 10 to $25 \%$ to $36 \%$. Early users ( $8 \%$ to $19 \%$ to $31 \%$ ) were less likely to have the intended diagnosis as the number one diagnosis at each step than experienced users ( $21 \%$ to $39 \%$ to $53 \%$ ). Students ( $12 \%$ to $30 \%$ to $39 \%$ ) were as likely to have the intended diagnosis as the number one diagnosis at each step as physicians (14\% to $26 \%$ to $40 \%$ ). The case elicited an average of 3.8 items ( +2.2$)$ for the differential diagnosis with experienced users averaging more than early users $(4.3+1.8$ versus $3.3+2.2)$. More than 34 users (34/89) had more than 5 items on the differential diagnosis.

DISCUSSION / REFLECTION / LESSONS LEARNED: The smart phone application offers an innovative and enjoyable means of providing practice cases in differential diagnosis. Based on a single case from the humandx database, there is significant variability in differential diagnosis with a standard case of chest pain. Comparing and sharing stepwise progression in the diagnostic process should have value to students of diagnostic reasoning and educational institutions.

ONLINE RESOURCE URL (OPTIONAL): http://humandx.org

EXPLORING THE STRUCTURAL CAUSES OF HEALTH DISPARITIES: NOVEL, STUDENT-LED STRUCTURAL COMPETENCY AND LEADERSHIP CURRICULUM AT OREGON HEALTH \& SCIENCES UNIVERSITY Michelle A.

Beam; Brianna Muller; Justin Lee; Paul Gorman. Oregon Health \& Sciences University, Portland, OR. (Control ID \#2470633)

NEEDS AND OBJECTIVES: Structural competency is an emerging effort in medical education that strives to train current and future physicians to identify and work to improve the institutions, policies, and other social structures that shape both individual health and clinical interactions. OHSU is nationally respected as a top primary care institution with a strong social mission. As a result, top medical school applicants from around the country choose to attend OHSU because of the institution's reputation for emphasizing social medicine in its undergraduate medical curriculum. In 2014, a cohort of Class of 2017 medical students articulated a need for more rigorous and clinically integrated discussions of the effects of politics, policies, and social structures on the health of populations and individuals in the formal medical curriculum. We created a student-led component of the MS1 curriculum with the primary objective to refine students' ability to define, identify, and analyze structural issues and their influence on health and medical practice. In addition, an MS2 elective course "Leadership, Education and Structural Competency" was created as a mechanism for students to learn curriculum development as well as facilitative leadership and teaching skills.

SETTING AND PARTICIPANTS: Seventeen MS2 students participating in the "Leadership, Education, and Structural Competency" elective facilitated group sessions for all MS1 students (137), after receiving formal facilitation training. Each MS2 led/co-led the same 12-13 MS1 students over 5 sessions, which helped create a space in which students felt comfortable to engage in dialouge about nuanced and sometimes sensitive or polarizing topics.
DESCRIPTION: Student collaboration with administration resulted in five $1-1.5 \mathrm{~h}$ sessions woven into the "YourMD" OHSU first-year curriculum. The five topics were: 1. Defining Structural Competency: An Overview 2. Implicit Bias 3. Urban Health Issues 4. Rural Health Issues 5. Specialty Care and Structures The format for the session was largely interactive and discussion-based. Each session culminated with a "Clinical Pearl" to ensure that students leave each session with practical tools that they can apply as a care provider in clinical settings. Each MS2 student-facilitator developed a practical understanding of structural competency while developing leadership skills through workshops with OHSU faculty as well as Portland community leaders. Trainings in group facilitation, collective-group process, teaching sensitive topics, and leadership were required for all MS2 students who facilitated sessions. Each session lesson plan was created by a student team and collectively edited using consensus-building and group facilitation models. Each session culminated in a "Plus/Delta" exercise to allow students to contribute areas of strength and weakness in each session. MS2 student facilitators assimilated this formative feedback after each session and applied it to subsequent sessions. Over the course of our iterative curriculum development, each session came to include: -Pre-Assigned Homework -A Warm Up Exercise -1-2 Facilitated Group Exercises Created Around the Selected Topic -A Clinical Pearl to Apply Structural Analysis in Clinical Settings -Community Resources Pertinent to the Discussion -Plus/Delta -Follow-Up E-mail to students sharing any resources that were discussed in the session

EVALUATION: Summative evaluation of the effect of this 5-session curricula on prior knowledge and knowledge gained was assessed with IRB-approved Likert and descriptive evaluation adapted from cultural competence literature to assess comprehension and problem-solving surrounding structural parameters. Pre and post assessments were gathered and the data is currently being analyzed. Non-IRB descriptive feedback was gathered after final session to provide preliminary feedback to administration for curricular planning purposes.

DISCUSSION / REFLECTION / LESSONS LEARNED: The implementation of this student-facilitated curriculum addressing the effects of political and social structures on health in formal medical education in the framework of "structural competency" was the first of its kind. Preliminary analysis of measurement outcomes suggest that curriculum improved foundational knowledge, identification, and differentiation of structural vs behavioral factors influencing health and clinical practice. Participating students described improved comfort in identifying and discussing structural issues. Additionally, collaborative creation of a curriculum amongst MS2 students provided valuable process-based leadership training, $>10 \mathrm{~h}$ of facilitation training and practice, and $>20 \mathrm{~h}$ curriculum development for 17 aspiring clinician leaders. Novel lessons and tools that emerged from this formative process included: -Critical analysis of behavioral vs. structural explanations of health and disease -Participatory facilitation enhances depth of analysis and peer tolerance of diverse opinions -Value of non-hierarchical teaching for exploratory critical thinking in medical education

FACILITATING FEEDBACK ON A CLINICAL CLERKSHIP USING A SMARTPHONE APP Aditya Joshi; Jenilee Generalla; Britta M. Thompson; Paul Haidet. Penn State College of Medicine, Hershey, PA. (Control ID \#2469087)

NEEDS AND OBJECTIVES: Feedback is a crucial component of medical education, however, students often report a paucity of it. The objective of this project was to evaluate and report the effectiveness of a novel smartphone app aimed at facilitating feedback delivery on a clinical clerkship.

SETTING AND PARTICIPANTS: This program was implemented in the setting of the Psychiatry clerkship, a mandatory, 4-week long clinical rotation offered to students enrolled at the Penn State College of Medicine. In the course of an academic year, approximately 150 students complete the Psychiatry rotation 
DESCRIPTION: We used commercially available software (Yapp, Inc.) to create a smartphone app to organize student activities on the psychiatry clerkship. We created a template, using the app, to simplify and enable the feedback process between attendings and students. We created an interactive electronic feedback form, trained students in its usage, and made it available to students to use to solicit immediate, clinically-based feedback from their attending physicians. The feedback form simplifies the process for attending physicians, who can use a drop-down menu to provide real-time feedback about a variety of issues to the student.

EVALUATION: At the end of the Psychiatry clerkship, students are typically asked to evaluate various aspects of the clerkship experience. This includes questions regarding their perception of the oral and written feedback process. We compared responses to the feedback questions from students who experienced the clerkship before the introduction of the app-based feedback process to those who experienced the clerkship after the introduction of the app-based feedback process. We also compared responses to the feedback evaluation for students on the Psychiatry rotation to those on the Internal Medicine, Family Medicine, and Pediatrics rotations. All three of these rotations used standard clerkship feedback methods for the duration of our evaluation period. In addition, we added supplemental questions to the Psychiatry clerkship evaluation questionnaire for the current academic year to ascertain the ease, timeliness, quality and effectiveness of the app-based feedback method and to compare its usefulness to other feedback methods. DISCUSSION / REFLECTION / LESSONS LEARNED: In the first 3 quarters of AY 2014-15 (prior to institution of our app-based feedback method), only 74,63 , and $58 \%$ of responders reported receiving oral feedback and 30,56 , and $43 \%$ of responders reported receiving written feedback at any point during the clerkship. During the fourth quarter, when we piloted the app in limited fashion, the percentage of students reporting receiving feedback increased to $80 \%$ for both oral and written feedback. For the entire AY 2014-15, a comparison of Psychiatry to the three other clerkships in our institution (Internal Medicine, Family Medicine, and Pediatrics) showed that Psychiatry was rated lower on average by 18 percentage points for oral and 28 percentage points for written feedback. During the first 2 quarters of the current AY 2015-16 year (using a full implementation of the app), 91 and $100 \%$ of Psychiatry clerkship students reported receiving oral feedback, and 88 and $100 \%$ reported receiving written feedback. Comparison to the other three clerkships during this period showed that Psychiatry was rated on average 4 points lower for oral feedback and 14 points higher for written feedback. $89 \%$ of responders rated the ease in accessing feedback, $93 \%$ reported the timeliness of feedback, and $75 \%$ rated the quality of feedback to be good, very good, or excellent. $66 \%$ of students reported that receiving feedback via the app was helpful in improving their performance. $62 \%$ of responders indicated this method was somewhat or much better compared to other feedback methods. Qualitative comments suggested that students thought the app facilitated initiating a conversation about performance with their supervisors. Students valued the convenience of receiving feedback as well as the promptness with which feedback was provided. One weakness reported was that the use of a drop-down menu to present items for feedback from the attending physician seemed to limit the scope of conversation on certain occasions. In conclusion, our preliminary data point to the effectiveness of using an app to cue attending physicians to provide feedback to medical students. Further improvements in the process, based on student feedback, will help shape this program further.

GENDER MATTERS: ASSESSING DISPARITIES AND PROMOTING EQUITY THROUGH IMPROVED RESIDENT COMMUNICATION, LEADERSHIP, AND NEGOTIATION Mumtaz Mustapha; Elizabeth A. Rogers; Briar Duffy; Sophia Gladding. University of Minnesota, Minneapolis, MN. (Control ID \#2468036)

NEEDS AND OBJECTIVES: Gender disparities persist in academic medicine across leadership roles, promotion, and pay. Women comprise about half of US medical students, still academic medical centers have yet to close the gender gap that exists in associate and full professor ranks. Multiple studies have documented persistent gender disparities in academia: across senior level positions, promotion decisions, and salaries. Studies suggest that women who participate in leadership development curricula are more likely than their peers to attain deanships, department chairmanships, or full professorship. Despite these well documented needs, medical residents are rarely targeted for early interventions. To meet this need, we developed a resident curriculum addressing the interplay between gender and communication, leadership and negotiation. The overall curricular objective is to provide career development support to residents and promote career satisfaction and equity. By sharing our work, we hope attendees will be able to: 1) Recognize the need to address the influence of gender in GME 2) Perform an assessment for gender disparities amongst their own learners 3 ) Identify resources for building and delivering a curriculum to address needs

SETTING AND PARTICIPANTS: Residents in Internal Medicine (90), Med-Peds (40) and Med-Derm (10) at the University of Minnesota (UMN). The curriculum is delivered during mandatory educational conferences and optional conferences.

DESCRIPTION: In a needs assessment conducted among UMN medicine-pediatrics residents in spring 2015 (26 respondents of 39 total residents, 16 female), males more often than females reported positive experiences in communicating with ancillary staff ( $p=0.02$ ), and females more often than males had negative experiences in communicating with both consulting services and ancillary staff ( $p=0.03$ for each). There was a trend toward a difference in leadership, with males more often feeling their gender positively influenced opportunities for leadership and their teams' response to their own leadership style $(p=0.1)$. Overall, males felt their gender more positively affected their work experience $(p=0.001)$. From this assessment, we found that the male vs female difference was strongest for communication and leadership. Based on these data and literature review, we have developed and are implementing a resident curriculum that addresses the interplay between gender and communication, leadership, and negotiation. Our overall objective is to provide career development support to residents with a goal of promoting career satisfaction and equity. Our curriculum is innovative in two key ways: 1) designed specifically for residents instead of practicing physicians; 2) delivered to all residents - male and female- for broadening gender discussions, instead of many existing programs exclusively delivered to women. The curriculum includes interactive seminars in communication, leadership, and negotiation, focusing on knowledge acquisition, skill development, self-reflection and goal setting. Communication sessions focus on: different historical gender-based communication styles; strengths and weaknesses of these styles; and how clinical communication is affected by gender-based expectations. During leadership sessions, all residents complete a personality/skills assessment (StrengthsQuest ${ }^{\mathrm{TM}}$ ) followed by an interactive "debriefing" on their individual results., Participants then create their own learning goals to implement during their next clinical rotations. In follow-up, senior residents attend an interactive workshop focused on different leadership styles to discuss their own experiences in leading a medicine team since their first leadership assessment.

EVALUATION: The pilot phase of our curriculum focuses on the first two levels of Kirkpatrick's Four-Level Training Evaluation Model - measuring Reaction (how trainees react to the training) through individual post-session evaluations, and measuring Learning (how trainee knowledge has increased) through a pre-post curriculum knowledge assessment. Future assessments will study longer term learner outcomes.

DISCUSSION / REFLECTION / LESSONS LEARNED: Our needs assessment surprised us in a few ways. Though we expected gender disparities in communication and leadership, we were surprised that our small sample would reflect this so clearly. Additionally, several residents wrote about a lack of female role models, though the educational leadership in Medicine, Pediatrics, and Med-Peds is dominated by women. Male trainees also acknowledged disparate treatment based on gender and difficulty reconciling how to approach this disparity. The needs assessment data focused us on the topics of 
communication and leadership, but also clarified that the curriculum needed to include all genders, and it needed to include specific skill building and culture change. Initial seminar attendees have discussed intrinsic biases, ways in which both men and women can become victim to stereotype, and ways that individuals and groups can mitigate these disparities.

GLOBAL IS LOCAL: DEVELOPING A MULTIDISCIPLINARY MOBILE HEALTH EXPERIENTIAL ROTATION WORKING WITH MIGRANT AND SEASONAL FARMWORKERS IN RURAL MINNESOTA Rachel Sandler ${ }^{1,3}$; Jonathan D. Kirsch ${ }^{2}$; Oscar Garza ${ }^{2} .{ }^{1}$ Hennepin County Medical Center, Minneapolis, MN; ${ }^{2}$ University of Minnesota, Minneapolis, MN; ${ }^{3}$ University of Minnesota School of Medicine, Minneapolis, MN. (Control ID \#2457575)

NEEDS AND OBJECTIVES: 1) Describe the unique challenges of healthcare delivery for the migrant farm worker population 2) Recognize social determinants of health for a transient, migrant population in a rural context through experiential learning 3) Experience clinical medical practice in mobile units, specifically related to chronic disease management and occupational health 4) Identify a specific area of interest related to migrant farmworker health to write a scholarly publication and reflection

SETTING AND PARTICIPANTS: The clinical portion of this rotation took place in 4 rural, agricultural communities in Minnesota. Each community had a variety of work experiences for migrant workers including agricultural fieldwork, dairy work, and food processing. The didactic sessions took place at Hennepin County Medical Center and the University of Minnesota in Minneapolis, Minnesota. The participants included 4 Internal Medicine residents from the University of Minnesota and Hennepin County Medical Center, 1 pharmacy student, and 4 undergraduate pre-health students. The rotation also piloted a pipeline project incorporating 5 women ages 12 to 20 of Latino backgrounds from the rural communities interested in health careers to participate in multiple activities alongside the health professional trainees.

DESCRIPTION: In this 4-week rotation, we incorporated multiple modalities for learning about the migrant and seasonal farmworker population including didactic lectures, documentary films, guest speakers from the community, review and discussion of medical literature, and work-site tours to provide a contextual background for this unique and underserved group. Specific topics of discussion included occupational health and safety, pesticide exposure, immigration law and policy, agricultural health, and medical homes for mobile groups. Our lecturers included local, regional, and national experts and included other patient community resources, such as the Minnesota Mexican Consulate. Work site tours included farms, canneries, and dairies where residents also had opportunities to observe and participate in the work process. We also provided direct medical care to the migrant and seasonal farmworker population on a mobile medical unit through a community partner, Community Health Service, Inc. This rotation was collaboratively developed with multiple community partners including a migrant worker community organizing non-profit organization, a community health center, the Migrant Clinicians' Network, the University of Minnesota Colleges of Medicine and Pharmacy, and Hennepin County Medical Center.

EVALUATION: A pre-test and post-test assessment was developed to measure changes in knowledge-based content of the course. Evaluation also included weekly surveys via an online tool, Survey Monkey. The evaluation included Likert scale questions regarding participants' perspective on the activities and experiences within the rotation, as well as the likelihood of changing future clinical practice. Weekly dialogue sessions were also conducted to provide qualitative feedback. on the course and clinical experiences and activities.

DISCUSSION / REFLECTION / LESSONS LEARNED: Residents overall felt this was a useful clinical rotation as they gained exposure to community health in a rural setting, unlike the urban ones in which they train. They also stated that they gained benefit from the discussions about providing effective care for mobile populations and insights into specific cultural challenges faced by subsets of the Latino community. One major challenge we had was providing care to large numbers of patients throughout our time due to challenges related to the growing season and lack of community engagement prior initiating our rotation. Going forward, we plan to work with our community partners to strengthen ties with the local communities and build capacity within these organizations to provide more multidisciplinary educational opportunities in internal medicine and pharmacy along with other medical subspecialties, dentistry and public health while also improving access to care through the mobile units.

\section{HOME: HOUSE OFFICER MEDICAL EDUCATION HOME VISITING INITIA-}

TIVE J. Deanna Wilson; Katherine Shaw; Leonard S. Feldman. Johns Hopkins, Baltimore, MD. (Control ID \#2464513)

NEEDS AND OBJECTIVES: Even as the United States population continues to grow more racially and ethnically diverse, high rates of health disparities persist. Many patients of color are cared for in race-discordant relationships, which has been linked to decreased patient satisfaction and trust in providers, and decreased involvement in clincial care. There is growing evidence that patient-centered communication can lead to improved patient trust, satisfaction, and adherence. As a means to address ongoing heath disparities in vulnerable groups, current residency pedagogy should incorporate both patient-centered communication skills and cultural competency, which has been defined as "a set of congruent behaviors, attitudes and policies that enables effective work in cross-cultural situations." Home visitation programs have been shown to improve communication skills in residents and increase patient-reported satisfaction. The objective of the HOME Initiative is to improve patient-centered communication and cultural competency in interns through home visits with recently discharged patients under the intern's care. SETTING AND PARTICIPANTS: During 2014 to 2015, 28 interns (half of all first year internal medicine residents) were randomized to two of the four general medicine inpatient firms serving as the "intervention" groups. On the intervention firms, residents were paired with trained community health nurses, called transition guides (TG), to visit recently discharged patients from their team. Eligible patients had to be discharged within a week of the home visit and had to meet hospital criteria to be eligible for a TG visit. Patients eligible for a TG did not qualify for other resources through a traditional home care agency and met criteria based on their Early Screen for Discharge Planning (ESDP) score.

DESCRIPTION: The HOME Initiative is an innovative medical educational curriculum where interns participated in monthly home visits while on the intervention firms from October 2014 through April of 2015. Interns were given guidance on how to complete medication reconciliation with each patient, a guided questionnaire to probe a patient's understanding of their hospital diagnoses and disease, and modeling for Teach-back education during the home visit.

EVALUATION: We conducted baseline surveys during orientation and at the conclusion of intern year assessing self-reported patient-centered communication skills and cultural competency. We assessed feasibility and acceptability of the program for interns and patients and identified potential barriers to implementation. Twenty-three of the interns in the intervention group completed the survey (response rate $82.1 \%$ ). The majority of interns $(N=16)$ visited a patient for whom they were the primary provider in the hospital. The most common barrier to visit completion was difficulty coordinating schedules with interns and TGs and competing demands from the inpatient service. Seventeen of 23 interns reported that the experience was a somewhat or very valuable experience. Only 2 interns reported that it was not valuable. Twenty-one of 23 reported that it improved or may improve their cultural awareness. Interns in the intervention reported greater skill in "assessing the patient's understanding of their own illness" compared to those in the control group $(p=0.04)$. Those in the intervention group also reported greater confidence 
in caring for patients who have limited health literacy compared to those in the control group $(p=0.07)$. Scores showed a trend towards greater cultural competency (by reported level of skill, confidence, and familiarity in dealing with a variety of patient issues) for providers who completed the intervention, however, the differences between the intervention and control groups were often small and did not reach statistical significance. For example, providers in the intervention group reported greater confidence in their ability to care for patients who distrusted the health care system (mean score $2.65(0.16)$ versus 2.29 (0.16) on scale from 0 , not confident at all to 4 , very confident; $p=0.14$ )

DISCUSSION / REFLECTION / LESSONS LEARNED: Our data demonstrate that incorporating home visits into the traditional structure of a hospital-based rotation is feasible and acceptable to both patients and providers. Although all interns experienced gains in cultural competency and patient-centered communication skills during intern year, those who participated in the HOME Initiative were more sensitive in their evaluation of patients' understanding, an integral component in delivery of patient education, and also in addressing barriers to patient adherence and safe discharge. Although our small sample size limited our ability to see statistically significant differences between the intervention and control groups, trends show participants in the HOME Initiative had greater self-reported confidence and skill in caring for patients. Home visits are a novel way to integrate training in patient-centered communication skills and cultural competency into medical education.

HOSPITAL HORROR STORY: SITUATIONAL AWARENESS TO ASSESS INTERNS' RECOGNITION OF SAFETY AND LOW-VALUE HOSPITAL HAZARDS $\underline{\text { Kathleen M. Wiest }}{ }^{1}$; Jeanne M. Farnan ${ }^{1}$; Ellen Byrne ${ }^{1}$; Lukas Matern ${ }^{1}$; Melissa Cappaert ${ }^{1}$; Kristen Hirsch ${ }^{1}$; Vineet M. Arora ${ }^{2} .{ }^{1}$ University of Chicago, Chicago, IL; ${ }^{2}$ University of Chicago Medical Center, Chicago, IL. (Control ID \#2466845)

NEEDS AND OBJECTIVES: Patient safety training has recently become a paramount focus of academic health systems, and the ACGME's CLER (Clinical Learning Environment Review) has called for formal and hands-on patient safety training during residency. However, while many institutions train house staff to mitigate hospital hazards, few have exploited the crucial concept of situational awareness (i.e. mindfulness of the patient environment) to teach patient safety. One method to promote situational awareness is through the embedding of safety and low-value care hazards into simulation-based training exercises. The aim of this study is to assess incoming interns' ability to identify safety and low-value hazards of hospitalization in a simulation designed to promote situational awareness.

SETTING AND PARTICIPANTS: An inpatient simulation was constructed in collaboration with the University of Chicago Simulation Center as a required component of GME (Graduate Medical Education) orientation. One hundred twenty-five incoming interns (100\% of those eligible) participated in the simulation, representing thirteen specialties and sixty medical schools. All interns were eligible except those entering into pathology.

DESCRIPTION: The simulation consisted of a 55 year old male admitted for pneumonia and $C$. difficile diarrhea. Incoming interns were given ten minutes to independently review a mock patient chart and list all hazards they identified in a simulated hospital room. Eight safety hazards (e.g. penicillin allergy, wrong patient name) and four low-value hazards (e.g. unnecessary Foley catheter, unnecessary restraints) were included in the simulation based on Medicare Hospital Acquired Conditions, AHRQ Patient Safety Indicators, and Choosing Wisely recommendations. Interns completed a short survey on their prior safety training in medical school, and a follow-up survey 1 month after beginning internship. Simulation performance was measured by the percentage of total hazards identified correctly. T-tests were used to compare the identification of safety versus low-value hazards and to associate performance with prior safety training.
EVALUATION: Seventy-three percent (89/121) of participating interns had received prior safety training in medical school, and $50.0 \%(61 / 122)$ were satisfied with their prior safety training. The mean percentage of hazards correctly identified was $50.4 \%$ (median $50.0 \%$, SD $11.8 \%$ ). Interns identified significantly more safety hazards (mean $66.0 \%$, SD $16.0 \%$ ) than low-value hazards (mean $19.2 \%$, SD $18.6 \%)(P<0.001)$. There was no significant association between hazard identification and prior safety training or satisfaction with prior training. Interns entering less procedural-intensive specialties identified significantly more safety hazards (mean $69.1 \%$, SD $16.9 \%$ ) than those entering highly procedural-intensive specialties (mean $61.8 \%$, SD $13.7 \%$ ) $(P=0.012)$. Interns provided written feedback that the simulation experience was fun, engaging, and challenging enough to cultivate situational awareness, with one intern writing that they would "inherently approach every patient room looking for safety issues as a direct result of this exercise." One month after the simulation, $68.9 \%(82 / 119)$ of interns reported being more aware of how to identify hospital hazards as a result of the exercise, and $52.1 \%(62 / 119)$ had taken action during internship to reduce a hazard that was included in the simulation. DISCUSSION / REFLECTION / LESSONS LEARNED: Interns identified significantly more safety hazards than low-value hazards in the simulation. Prior safety training in medical school was not associated with interns' ability to detect hazards, and satisfaction with prior safety training was low. The simulation resulted in increased situational awareness leading to hazard mitigation 1 month into internship. These findings reinforce the ACGME's position that formal patient safety training is needed in GME, and suggest that a brief, simulation-based experience results in the retention of situational awareness and behavior change. Our results highlight the need for experiential learning and simulation-based training in medical education to improve safety and value of hospital care, and future work aims to expand the simulation to undergraduate medical education.

HOW ASSUMPTIONS AND PREFERENCES AFFECT PATIENT CARE: AN INTRODUCTION TO IMPLICIT BIAS FOR FIRST YEAR MEDICAL STUDENTS Cristina M. Gonzalez ${ }^{2}$; Alyssa R. Liguori ${ }^{1}$; Martha S. Grayson ${ }^{1} .{ }^{1}$ Albert Einstein College of Medicine, Bronx, NY; ${ }^{2}$ Albert Einstein College of Medicine/Montefiore Medical Center, Bronx, NY. (Control ID \#2466743)

NEEDS AND OBJECTIVES: Evidence suggests that individual physicians may deliver varied levels of quality of care for patients of different racial or ethnic backgrounds, contributing to health disparities. It is thought that these differences are partly due to implicit bias- the unconscious, unintentional assumptions we make about each other. Because biases are so common, and affect medical care, we are developing a comprehensive curriculum to teach medical students to recognize and manage their biases in clinical encounters. Discussions of racial and ethnic implicit bias are potentially emotionally charged. Careful measures must be taken to engage learners in a safe, respectful learning environment. Safety and a small group setting may foster true reflection and discourse, while working to counter socially desirable answers, which can hinder growth. We designed a half-day workshop introducing the concept of implicit bias and its potential clinical implications. This is another step in including bias instruction in the longitudinal, compulsory curriculum. Learning Objectives By the end of the session students will: Identify one assumption they have made about someone else. Describe in their own words the impact unconscious assumptions can have on their interactions with classmates and with future patients. List one strategy to become aware of when they may be jumping to a conclusion based on an assumption.

SETTING AND PARTICIPANTS: Participants were first year medical students ( $n=$ 187) during Orientation at Albert Einstein College of Medicine in Bronx, NY.

DESCRIPTION: We delivered an introduction to implicit bias in a large group setting highlighting its relevance to both nonclinical and clinical settings. After transitioning to small groups, content focused more on reflecting and personal strategy building. Students 
participated in a first-thought exercise looking at assumptions and stereotypes that are visually provoked using an iceberg analogy (i.e. you only see the tip of the iceberg). The ubiquitous nature of implicit bias in society was explained and further emphasized by showing a video from the technology industry on their approach to the effect of their biases about their end users in product development. The neuroscience of cognitive processing, the fast and slow thinking pathways that lead to our assumptions, was demonstrated through videos. After those efforts to normalize the concept, students were presented with the evidence of the potential influence of implicit bias in clinical decisionmaking. We reviewed the evidence from chest pain vignettes and thrombolysis, pain management, and others. The large group sessions incorporated several interactive questions using an audience-response system, allowing for anonymous participation by the students. The small groups were co-facilitated by a faculty member and a senior student, both having received facilitator training during the prior weeks. To illustrate the influence of bias in all our lives, students were asked the following three questions: 1) identify one fear that they had for the session, 2) identify one hope, and 3) identify one assumption they were worried people would make about them while in medical school, using the audience response system. Students then broke into pairs and discussed their own personal narratives, including how where they grew up might influence their current view of the world, and to consider the power/privilege/oppression of the system in which they grew up Volunteers then reported out to the entire group. Finally, students moved on to strategy building. After a short video, students again anonymously identified one strategy they could implement to become aware of when they are making an assumption that may lead them to jump to a conclusion about someone else. These strategies were shared with the group on a large screen in real time and discussed.

EVALUATION: Students took the Implicit Bias Attitude Scale as a pre-test. It will be administered as a post-test later in the curriculum.

DISCUSSION / REFLECTION / LESSONS LEARNED: We developed the first compulsory session related to implicit bias recognition and management delivered to entering students at our institution. This innovation introduced implicit bias to medical students utilizing a safe, interactive, multimedia approach. There were several lessons learned. First, in response to feedback from student focus groups and after piloting the session, we focused heavily on safety of the learning environment by normalizing the concept of bias, easing students into the concept of racial and ethnic implicit bias in clinical care, and flattening the hierarchy in small groups with student co-facilitators Second, the audience response system was a useful tool for the large group because it allowed for anonymous participation, visualization of common concerns, and student engagement. Finally, this session provides a baseline session so that all students have the same minimum knowledge base about implicit bias. We expect this will facilitate integration of implicit bias content into existing courses.

\section{LEARN BEST THIS WAY! DEVELOPMENT AND IMPLEMENTATION OF A TEAM-BASED LEARNING CURRICULUM FOR INTERNAL MEDICINE CONTINUITY CLINIC RESIDENTS David A. Williams. University of Colorado, Denver, CO; Denver VA Medical Center, Denver, CO. (Control ID \#2469219)}

NEEDS AND OBJECTIVES: Internal medicine residents at pre-continuity clinic conferences at Denver VA Medical Center (DVAMC), had been expressing dissatisfaction with the lecture-based outpatient curriculum and attendance at conferences had begun to dwindle. Additionally, continuity clinic structure had changed so that residents were in continuity clinic for a week at a time. Internal medicine clinic preceptors therefore went about revitalizing the continuity clinic curriculum at DVAMC with the following overarching objectives: 1. Develop and deliver Team-Based Learning (TBL) modules which use teams to promote learner interaction with one another and curricular content. 2 . Engage the continuity clinic residents in highly-interactive learning experiences which push the boundaries of their knowledge. 3. Deliver content comparable to or exceeding the content to the prior lecture-based outpatient curriculum

SETTING AND PARTICIPANTS: All 39 internal medicine residents in academic year 2014-2015 and 43 internal medicine residents in academic year 2015-2016 with continuity clinic at DVAMC participated.

DESCRIPTION: Prior to academic year 2014-2015, a single, 90-min faculty training session was conducted by local experts in TBL. Ambulatory Care VA faculty then developed TBL curriculum which was given to continuity clinic residents in lieu of the traditional lecture-based curriculum. As residents are in continuity clinic for one full week at a time, the curriculum was delivered over three sessions for a total of $2 \mathrm{~h}$ each week. To date, seven different TBL modules (Depression/PTSD, Diabetes, COPD, Low Back Pain, Breast Cancer Screening, GERD, and Dementia) have been taught.

EVALUATION: The curriculum was evaluated with post-session evaluations using a 5point Likert scale and free-text responses. The seven TBL modules resulted in 201 unique post-session evaluations. Overall, residents rated the new TBL curriculum highly, with average responses to the questions "I feel more confident in my knowledge of the session's topic as a result in participating in TBL," "TBL was more fun/enjoyable than the lecture format," and "TBL was an effective way to teach the pre-clinic conference material" of $4.71+/-0.45,4.86+/-0.40$, and $4.83+/-0.39$ respectively on a 5 -point Likert scale. Free-text comments included: "TBL > Lecture," "TBL is a great break from PowerPoint!," and "Improves retention, discussion, and enjoyment!"

DISCUSSION / REFLECTION / LESSONS LEARNED: TBL was a highly enjoyable and effective way to engage learners in delivering curriculum to outpatient continuity clinic residents. It should not only continue to be used to deliver formal continuity clinic content, but dissemination into additional clinic sites and other venues should be strongly considered.

IF YOU BUILD IT, THEY WILL COME: A POINT-OF-CARE ULTRASOUND CURRICULUM FOR INTERNAL MEDICINE RESIDENTS Stephanie Sun ${ }^{2,1}$; Junaid Raja ${ }^{2,}{ }^{1}$; Gourg Atteya ${ }^{2,}{ }^{1}$; David Chia ${ }^{2,1} .{ }^{1}$ Yale University, New Haven, CT; ${ }^{2}$ Yale-Waterbury Internal Medicine Residency, Waterbury, CT. (Control ID \#2435956)

NEEDS AND OBJECTIVES: Point-Of-Care Ultrasound (POC-US) has rapidly gained recognition as a useful, cost-effective tool to help guide medical decision-making. POCUS has already gained traction in emergency medicine and critical care. The ease and speed at which information can be gathered makes it a valuable augmentation of the traditional physical exam. However, it remains underutilized in general internal medicine. Objectives: 1) To measure the baseline level of understanding and training in POC-US among internal medicine residents; 2) To pilot, refine and integrate POC-US educational modules into the formal internal medicine residency curriculum; 3) To measure the effectiveness of the POC-US educational modules by utilizing pre- and postintervention testing to objectively trend changes in knowledge, confidence, and utilization of POC-US among internal medicine residents.

SETTING AND PARTICIPANTS: The POC-US curriculum was developed at an internal medicine residency program with 36 resident physicians based in an academic community hospital. In Year 1, educational modules were created by a faculty course director and resident leaders and then piloted with resident and medical student participants at an extracurricular POC-US interest group. Each of the modules were piloted twice. A total of four sessions were held over the course of the academic year. Participation was optional and open to all residents and medical students. In Year 2, the refined modules were incorporated into the formal residency curriculum for all three PGY levels as well as rotating medical students. Sessions were co-precepted by the resident leaders and the faculty course director. Participation was mandatory for all residents during their ambulatory block. A total of three sessions were held every 6 months in order to deliver the content to all residents within the program 
DESCRIPTION: Each educational module consisted of a 30 min lecture followed by a $60 \mathrm{~min}$ hands-on session practicing image acquisition on healthy peer volunteers. Two scanning stations were set up each consisting of an ultrasound machine, a cot, 1-2 trained preceptors and 4-5 participants. Topics included an overview of POC-US, inferior vena cava assessment, and basic echocardiography.

EVALUATION: Resident Attitudes and Beliefs Residents completed a 9-question selfassessment survey prior to the intervention in order to measure their baseline knowledge, prior training, current interest and confidence in POC-US. Resident Knowledge Residents completed an 8-question pre- and post-intervention knowledge assessment tool regarding ultrasound theory, image acquisition techniques and image identification.

DISCUSSION / REFLECTION / LESSONS LEARNED: Resident Attitudes and Beliefs A total of 19 residents completed the aforementioned evaluations. All residents believed that POC-US was an important skillset to acquire during their residency training. Less than half of residents (43\%) reported prior POC-US training. Only a quarter (26\%) stated that they regularly utilized POC-US in their clinical practice. Resident Knowledge Resident knowledge improved between pre- and post-intervention from $63 \%$ correctly answered questions to $81 \%(p<0.01)$. Future Directions Directions in the immediate future will involve inclusion of modules on lung ultrasound and the RUSH exam. In Year 3 , we will begin enriching each educational module with image review sessions focused on interpreting pathology and conducting "Ultrasound Rounds," i.e. integrating image acquisition with image interpretation by bringing the curriculum to the bedside and applying POC-US to actual patients. The accuracy of POC-US interpretations will be compared to known pathologies previously confirmed with the current gold standard (e.g. CT, X-Ray). Residents will then build individual portfolios of successfully acquired and interpreted images in order to become formally credentialed in POC-US by hospital standards. We will promote program sustainability by recruiting new resident leaders from each PGY year and encourage them to pursue additional formalized training through comprehensive POC-US courses and conferences. Lessons Learned Today's residents and residency applicants are eager to learn and integrate POC-US into their clinical practice. However, POC-US remains underutilized in internal medicine training. Our curriculum demonstrates one potential strategy for formally integrating POC-US into a residency program by partnering faculty with resident leaders to employ a peer-teaching model with positive results.

\section{NALOXONE PRESCRIBING WITHIN AN INTERNAL MEDICINE TRAINING PROGRAM: DOES A FOCUSED CURRICULUM CHANGE THE NUMBERS?}

Jessica Taylor; Alison B. Rapoport; Chris Rowley; Wendy Stead. Beth Israel Deaconess Medical Center, Boston, MA. (Control ID \#2468410)

NEEDS AND OBJECTIVES: In the midst of a growing opioid epidemic, concern has been raised that medical trainees do not receive adequate training in substance use disorders. There is mounting evidence that providing overdose education and naloxone kits to at-risk patients, relatives, and first responders reduces overdose deaths. However, naloxone rescue kits are still not routinely prescribed. A review of endocarditis admissions at Beth Israel Deaconess Medical Center (BIDMC), a large academic tertiary care center in Boston, MA, demonstrated that $0 / 102$ patients with endocarditis as a result of intravenous drug use were discharged with a prescription for naloxone. In addition, very few had an addiction plan explicitly outlined in their discharge summary. The BIDMC Internal Medicine Residency Program has developed an addiction medicine curriculum for its trainees. The purpose of this study is to evaluate whether a brief, targeted curricular intervention improves resident knowledge and comfort level regarding naloxone rescue and increases self-reported prescribing of naloxone rescue kits.
SETTING AND PARTICIPANTS: All current Beth Israel Deaconess Internal Medicine Residents $(n=160)$ have the academic year broken up into inpatient and outpatient rotations. For the purposes of outpatient curriculum, the academic year is divided into eight 6-week blocks. Each junior and senior resident spends 1 week out of every six in the outpatient/clinic setting. Over the course of a single 6-week block (November-December, 2015), all 50 PGY-2 residents and 47 PGY-3 residents underwent the first didactic session in this small group setting. The structure of the outpatient curriculum for interns does not lend itself to delivery of the didactic session in this setting. Therefore, the 63 interns were offered the first didactic session during their weekly "Intern Report" session. All residents then received the second session in the setting of a program-wide "Noon Conference."

DESCRIPTION: In November and December, 2015, BIDMC internal medicine residents received a series of two, one-hour didactic sessions that outline the scope of the opioid epidemic and strategies for harm reduction, including how to identify patients at risk for opioid overdose and how to prescribe intranasal naloxone overdose rescue kits. An electronic survey assessing residents' knowledge about naloxone use for opioid overdose reversal as well as attitudes regarding the management of opiate addiction was administered before these sessions and will be administered again 2 months following these sessions (February, 2016). A chart review to determine the number of naloxone prescriptions generated in the 3 months prior to the intervention compared with the 3 months following the intervention will be performed to see if the intervention contributed to a change in prescribing patterns

EVALUATION: Forty internal medicine residents (25\%) completed the pre-intervention survey, including 15 interns, 13 s-year residents, and 12 third-year residents. Twenty percent of respondents $(n=8)$ had received prior training in the use of naloxone rescue kits to reverse opioid reversal. One resident $(2.5 \%)$ had prescribed naloxone in the past. Just $12.8 \%$ of the sample $(n=5)$ agreed that they were adequately trained to prescribe naloxone rescue kits, whereas $79.5 \%(n=31)$ disagreed. One half of the sample $(n=$ 20) disagreed with the statement, "Treating patients with opiate addiction is professionally rewarding." The duration of naloxone action, approximately one hour, was correctly identified by $77.5 \%$ of respondents $(n=31)$. Eighty-five percent of the sample $(n=32)$ correctly identified that physicians in Massachusetts can prescribe intranasal naloxone to patients with opioid addiction, patients without opioid addiction who take narcotics for pain, and third parties. This varied by level of training, and senior residents were significantly more likely to answer correctly than first and second-year residents (91.2 \% vs. $33.3 \%, p=0.02)$.

DISCUSSION / REFLECTION / LESSONS LEARNED: Internal medicine trainees are often the front-line providers for patients with opioid use disorder; however, addiction medicine has historically been underrepresented in residency curricula. Prescribing intranasal naloxone for reversal of opioid overdose is a relatively new intervention that reduces mortality. Our study confirms that few of our internal medicine trainees have received formal training in how to prescribe intranasal naloxone and just $12.5 \%$ feel comfortable prescribing it currently. Half of residents do not find the treatment of addiction professionally rewarding. We are hopeful that our targeted curricular sessions will improve resident knowledge, attitudes, and prescribing practices regarding intranasal naloxone for opioid overdose reversal and we will be measuring this effect by post-intervention survey to be distributed in February, 2016.

IMPACT OF FACULTY TRAINING ON PATIENT SATISFACTION WITH PATIENT-CENTERED ELECTRONIC MEDICAL RECORD USE Obiama Ukabiala $^{3}$; Lollita Alkureishi ${ }^{3}$; J. H. Isaacson ${ }^{1}$; Mark Mayer ${ }^{2}$; Vineet M. Arora ${ }^{4}$; Wei Wei Lee ${ }^{3}$. ${ }^{1}$ Cleveland Clinic, Cleveland, $\mathrm{OH} ;{ }^{2}$ Cleveland Clinic Lerner College of Medicine, Cleveland, OH; ${ }^{3}$ University of Chicago, Chicago, IL; ${ }^{4}$ University of Chicago Medical Center, Chicago, IL. (Control ID \#2467484) 
NEEDS AND OBJECTIVES: Use of the electronic medical record (EMR) in exam rooms can impede patient-doctor communication and negatively impact the doctor-patient relationship. Despite rapid EMR adoption, few if any faculty providers receive formal training on how to incorporate patient-centered communication strategies while managing demands of the EMR. Given the widespread use of the EMR in clinical settings, it is crucial to examine how we teach best practices to attendings, and understand what impact that training has on patient perceptions of their care and patient-doctor communication. SETTING AND PARTICIPANTS: We aim to train General Internal Medicine (GIM) faculty at the University of Chicago on patient-centered EMR use and survey patients to assess the impact of our training.

DESCRIPTION: After reviewing the literature, we developed a 90-min patient-centered EMR use training targeting GIM faculty and a 12 item Likert scale patient survey to assess faculty performance. Five faculty members were randomly selected to be directly observed in clinic pre and post intervention and received a formal debriefing. Two months after the workshop, we surveyed patients seen by trained faculty. The following is a sample questions from the survey: 'During my clinic visit today, my doctor used the computer to educate me on my medical conditions or treatment.' Likert responses at the high end of the scale were grouped to dichotomize data (i.e. $4=$ agree and $5=$ strongly agree were simply categorized as "agree"). Descriptive statistics were summarized and we compared results from faculty who participated in the additional direct observation with those who did not.

EVALUATION: Nineteen GIM faculty members participated in the training. Seventy-six patient surveys were analyzed and patients from all 19 providers were included. The majority $[81 \%(57 / 70)]$ of respondents were female and the mean age was 59 (18 91 years old); $56 \%$ (38/68) were African American and $32 \%(22 / 68)$ were Caucasian. Almost all patients agreed that their doctors 'started the visit technology free and asked about my concerns, 'positioned herself so that we could talk face to face', and 'maintained good eye contact during the visit' [99\% (75/76), $99 \%(75 / 76), 96 \%(73 / 76)$ respectively] While $87 \%$ (66/76) of patients agreed that their doctor 'ensured the computer was set up so I could see the computer and physician at the same time,' only $82 \%(61 / 74)$ reported their doctors 'explained what she was doing on the computer.' Only $71 \%$ (53/75) agreed that their doctors 'encouraged me to interact with the computer (i.e. to review results) Only $69 \%$ of patients agreed that their doctors 'logged off the computer at the end of the visit,' and interestingly $23 \%$ (17/73) were unsure if their providers did so. Overall, $63 \%$ (50/73) agreed that compared to 6 months ago, 'today my doctor used the computer more effectively to communicate with me' and $77 \%$ (58/75) agreed that 'I am more satisfied with how my doctor uses the computer to communicate with me.' When comparing patient surveys from 5 faculty $(n=22)$ who participated in the direct observation to the remaining 14 faculty $(n=54)$, there was no significant difference in mean ratings on the 12 items measured $(\mathrm{p}>0.05$ for all)

DISCUSSION / REFLECTION / LESSONS LEARNED: A short faculty training on patient-centered EMR use could be associated with increased patient satisfaction with EMR-related communication. Best practices on patient-centered EMR use can be taught and positive objective behaviors (i.e. screen sharing and good eye contact) were reported by patients post training. Areas for improvement include ongoing faculty training and assessment of patient-perceptions, as well as formal training and evaluation of resident and medical student skills. Our short curricula can be easily adapted for use at other institutions. Furthermore, a potential avenue for training can include incorporating best practices into existing required EMR onboarding training at academic institutions, thereby taking advantage of available resources and champions.

IMPLEMENTATION OF A MEDICAL SPANISH CURRICULUM FOR MEDICAL STUDENTS AND RESIDENTS Colin Robinson ${ }^{1}$; Michelle Aguilar ${ }^{2}$; Kristen A. Kipps ${ }^{1} .{ }^{1}$ University of California, Los Angeles, Los Angeles, CA; ${ }^{2}$ St. John Well Child and Family Center, Los Angeles, CA. (Control ID \#2468136)
NEEDS AND OBJECTIVES: Physician ability to accurately communicate with Spanishspeaking patients has been correlated with improved outcomes and patient satisfaction. The availability of trained interpreters is often limited and, in many clinical scenarios, not readily accessible. To train our house staff and medical students to better care for this population, we have developed a novel Medical Spanish curriculum that combines classroom instruction, proctored patient encounters and independent online modules.

SETTING AND PARTICIPANTS: In our first pilot year, from January to June of 2015, we implemented a 6-month pilot course for 21 Internal Medicine and Pediatrics residents as well as 20 medical students. We expanded the course into a 9-month curriculum for the current academic year. We have currently enrolled 20 undergraduate medical students and 27 residents from the departments of Internal Medicine, Pediatrics and Emergency Medicine. All are required to have taken at least 1 year of college level Spanish or 2 years of secondary school Spanish.

DESCRIPTION: Undergraduate medical students and residents are distributed into groups of 10 to 15 based on a written pre-course evaluation of their Spanish ability. The course consists of four key components: instructor-led didactics, independent online study modules, small group role-play and patient encounters. The interactive didactics are led by an attending physician who is a native Spanish speaker; these sessions occur twice monthly and focus on organ- and problem-based history-taking and counseling. The monthly didactic topics are based around those of the Canopy Medical Spanish curriculum (www.canopyapps.com). Participants are required to complete online modules that correlate thematically with the week's instruction. Following the initial small group instruction, we hold breakout sessions in groups of 3-4 students, led by current medical students at our institution who are bilingual Spanish speakers. During these small group discussions, students act out patient scenarios that build upon the lessons covered in that week's lecture. At the midpoint of the course, we begin to incorporate patient encounters on the inpatient medicine wards also proctored by our teaching assistants. During these sessions, residents and students are observed taking a history from a Spanish-speaking patient and get realtime feedback on their history-taking in the hospital setting.

EVALUATION: In a pre-course survey of our Internal Medicine and Medicine-Pediatrics residents, $50 \%$ of residents reported "no" or "some basic" Spanish skills, and $30 \%$ reported only "moderate" Spanish skills. Meanwhile, 50 \% reported they "always" or "frequently" felt limited by their language skills, but only $31 \%$ of these residents reported "always" or "usually" utilizing Spanish translators when necessary. Course participants had completed an average of 2-3 years of high school or 1-2 years of college level Spanish. Twenty percent had participated in prior formal Medical Spanish instruction. Overall, students rated that they were "comfortable" with receptive Spanish (mean 2.7, standard deviation 0.8) on a 5-point Likert scale. Students were more "uncomfortable" with expressive Spanish (mean 2.2, standard deviation 0.89). Students were more likely to rate that they were "uncomfortable" with medical Spanish (mean 1.95, standard deviation 0.9 ). When asked to consider a variety of clinical scenarios, students were generally "somewhat likely" to use a Spanish interpreter for an initial outpatient encounter. They responded that they were "very likely" to use a Spanish interpreter to explain a new diagnosis or counseling on disease management. We will be comparing participants' performance on pre- and post-course examinations as an objective measure of progress. We will also administer post-course surveys to assess participants' comfort level with Spanish language after completing our curriculum.

DISCUSSION / REFLECTION / LESSONS LEARNED: Our Medical Spanish curriculum has been extremely well-received by both medical students and residents, with requested enrollment far exceeding our capacity. Our curriculum is innovative in that it integrates in-person instruction, online modules and simulated and real patient encounters with real-time feedback. In addition, since participants are grouped by pre-test performance rather than level of medical training, classroom participation has improved and medical students have learned how to better take patient histories at an earlier stage. Ultimately, with success in our second pilot year, we will formally study the effect of our course on the Spanish of our trainees and expand to other departments within the hospital. 
IMPROVING COMMUNICATION SKILLS: A PROSPECTIVE STUDY OF ONE-ON-ONE FEEDBACK TO IMPROVE RESIDENT COMMUNICATION. Siddhartha Kattamanchi ${ }^{1,2}$; Lisa Benson ${ }^{1,2}$; Andrew Weier ${ }^{2} .{ }^{1}$ Marshfield Clinic, Marshfield, WI; ${ }^{2}$ Ministry St Joseph's Hospital, MARSHFIELD, WI. (Control ID \#2468827)

NEEDS AND OBJECTIVES: Effective communication has been shown to influence rate of patient recovery, effective symptom control, treatment adherence, psychological stability, and satisfaction with care. ${ }^{1}$ So important is communication and interpersonal skills that the Accreditation Council for Graduate Medical Education (ACGME) has recognized and designated it as one of the six core competencies. ${ }^{2}$ Opportunity for improved patient-physician communication was identified through assessment of patient experience through HCAHPS (Hospital Consumer Assessment of Healthcare Providers and Systems) score on the general internal medicine residency service, which was around $57 \%$, well below state and national averages for physicians. To improve physician-patient communication, a prospective interventional trial was proposed to assess whether training residents in communication skills would impact patient communication scores, which could be assessed through HCAHPS score.

SETTING AND PARTICIPANTS: A total of 37 residents from internal medicine, combined medicine-pediatrics, and transitional year training programs participated in the coaching sessions while they were working on internal medicine wards during the 1-year measurement period (January-December 2013). This cohort was selected because they carried the lowest physician communication scores in the hospital.

DESCRIPTION: Beginning in January 2013, the preceptor identified each resident working on the inpatient service during that month and emailed them approximately 3 to 5 days before the start of the rotation, letting them know of the one-on-one coaching expectation. On that day, the preceptor shadowed each resident during one patient interaction (new admission, pre-rounds, follow-up visit, etc.) for approximately15$30 \mathrm{~min}$. After the patient interaction, the preceptor provided the resident with brief feedback. The feedback included objective observations on a few specific behaviors and subjective feedback on what was done well, with two to three suggestions for improving communication techniques. The resident's performance was logged. Most residents were shadowed at least 2-3 times during the study period. The HCAHPS score before the study served as the pretest data. This was compared to the HCAHPS score during the study period. The goal was to improve patient experience and communication by achieving a percent "always" response on the HCAHPS survey questions specific to physician communication of $70 \%$ in 1 year.

EVALUATION: When compared, the HCAHPS score between pre- and postintervention showed significant improvement after intervention. Overall communication improved from 61 to $81 \%$, indicating a $20 \%$ change from baseline with a p-value of 0.0019. Even the sub-group analysis showed a significant improvement in all three components of the communication. The response related to "treated with courtesy and respect" improved by $23 \%$, and the response related to "listened carefully" scored $80 \%$ in the post-intervention measurement, which was an $18 \%$ improvement compared to baseline. Performance in the response "explained things in a way you could understand" improved from 51 to $70 \%$. The p-value for all variables was $<0.05$, indicating the improvement was statistically significant.

DISCUSSION / REFLECTION / LESSONS LEARNED: Can communication skills be taught? Our study results are in line with previous studies, which showed that communication skills can be taught ${ }^{3}$. There was statistically significant improvement across all three components of the physician communication questions as well as the overall physician communication composite score. Some commonly identified and worked on skills in the group were physician positioning, engaging the patient in discussions, showing empathy, and asking the patient to teach back to ensure the patient has understood clearly. While multiple methods can be used to train communication skills, a model that used brief immediate feedback after patient interactions was found, in this study, to be an effective training method. It was also an efficient model from the resident's perspectives, because it did not require additional scheduled time and was integrated into their current workflow. By using one-on-one interaction, the feedback was personalized and customized, with a better chance of improving communication skills among individuals. An advantage of using HCAHPS was that it offered a standardized measurement tool utilized by most hospitals without requiring development of a new questionnaire.

ONLINE RESOURCE URL (OPTIONAL): 1. . Cantwell BM, Ramirez AJ. Doctorpatient communication: a study of junior house officers. Med Educ. 1997;31(1):17-21. 2. Accreditation Council for Graduate Medical Education. ACGME Outcome Project; 2007. http://www.acgme.org/outcome/comp/compFull.asp. Accessed April 14, 2014. 3. Langewitz WA, Eich P, Kiss A, Wossmer B. Improving communication skills: a randomized controlled behaviorally oriented intervention study for residents in internal medicine. Psychosom Med. 1998;60(3):268-276.

IMPROVING IUD COUNSELING AMONG INTERNAL MEDICINE RESIDENTS; A RANDOMIZED CONTROL MULTI-SITE EDUCATIONAL TRIAL. Heather D. Hirsch ${ }^{2}$; Megan McNamara ${ }^{4}$; Abby Spencer ${ }^{1}$; Pelin Batur ${ }^{2}$; Pete Spanos ${ }^{3}$. ${ }^{1}$ Cleveland Clinic, Chagrin Falls, OH; ${ }^{2}$ Cleveland Clinic, Cleveland, OH; ${ }^{3}$ Louis Stokes Cleveland VA Medical Center, Cleveland, $\mathrm{OH} ;{ }^{4}$ Louis Stokes Cleveland VAMC, Cleveland, OH. (Control ID \#2462129)

NEEDS AND OBJECTIVES: Knowledge is insufficient among practicing clinicians regarding contraceptive counseling, with particular deficit in intrauterine device (IUD) literacy. IUDs are becoming increasingly popular, and their efficacy and cost-effectiveness has been found in multiple studies to be superior to almost all other forms of birth control methods. Program directors (PDs) of internal medicine residencies are reporting that despite national guidelines endorsing women's health education, they find a negative discrepancy between what they feel residents should master and what the estimate they master, necessitating the need to re-examine how providers and users are selecting their method of birth control. The objective of this trial was to determine if exposure to a multicomponent educational intervention for internal medicine residents, which included online lectures, videos, and a "hands on" experience, as compared to the traditional lecture based curriculum, would improve provider knowledge, referral patterns, and self-reported ability to counsel on IUDs. Primary objectives included a change in IUD general knowledge, a change in self-reported comfort regarding IUD counseling, the number of referrals made for IUDs, and satisfaction with the simulation workshop.

SETTING AND PARTICIPANTS: Participation in the study was open to all first year Internal Medicine residents at both the University Hospitals (UH) Case Medical Center, and the Cleveland Clinic (CC) hospital systems. Enrollment began in March of 2015 and extends to April of 2016. Thirty-six first-year residents in the UH program and 53 firstyear residents in the CC program were eligible for enrollment. Ten residents who were coenrolled as "centers of excellence" learners, a pathway focused heavily on ambulatory medicine, were excluded from study participation. Enrollment was voluntary and participants were randomized after enrollment. Both sites were granted Institutional Review Board (IRB) approval.

DESCRIPTION: This is a multi-site, randomized, prospective, controlled study designed to assess the effectiveness and feasibility of a multi-component educational intervention on long acting contraception, as compared to a traditional lecture based education, for increasing Internal Medicine residents' knowledge about IUDs, improving attitudes and confidence regarding IUD counseling, and increasing referral rates for IUD insertions. All participants completed pre-intervention surveys assessing their general baseline knowledge of IUDs and their self-reported confidence in patient counseling. Residents in the control group received the traditional lecture-based education. Lectures were standardized so that the same content was delivered in the same way at both sites. Participants assigned 
to the intervention group received via secure email an online lecture-based video and mock physician-patient interaction to view online. Approximately 2 weeks later these residents participated in a simulation workshop, during which they will practiced inserting IUDs on plastic models. At the completion of this workshop they completed a brief survey indicating their satisfaction with the workshop. Towards study completion, all participants will complete post intervention surveys assessing their knowledge and comfort in prescribing IUDs, along with a log of IUD referrals, and complete a virtual online clinical exam which will be graded by local contraception experts.

EVALUATION: Participants' knowledge and self-reported confidence in counseling on IUDs will be assessed via completion of a pre and post intervention surveys. The knowledge survey includes 15 evidence based questions concerning IUDs. Statistics analysis will be done to asses for any change in knowledge at the completion of the study period. Participants in both groups will rate their comfort with regards to IUD counseling at the beginning and end of the study. Self-reported confidence will be rated on a 5-point Likert scale and compared between the groups' pre and post-intervention responses and change will be analyzed by a paired $t$ test. At the end of the study period, all participants will complete an assessment of their skills on IUD counseling via completion of a virtual online clinical exam. These will be scored by experts in the field and analyzed using a sample $t$ test. Provider referral patters will be based on self-reported data and analyzed using a paired $t$ test.

DISCUSSION / REFLECTION / LESSONS LEARNED: Initial survey response a the beginning of the study resulted in $70 \%$ participation rate. Most participants in the simulation workshop rated the session very highly. On a 5 point Likert scale residents overall gave the session a 4.7, and rated the clinical usefulness a 4.68 . The online virtua clinical examination will be distributed in March of 2016, with data collection to be concluded by April of 2016. Final results are expected in May of 2016. This educational research will be utilized to help build the most efficacious and useful curricular experience for future generations

IMPROVING ONLINE MEDICAL STUDENT LEARNING THROUGH INTERACTION: A RANDOMIZED CONTROLLED TRIAL Thomas D. Shiffler ${ }^{2}$; David Feldstein $^{2}$; Yuyen Chang ${ }^{1}$; Christopher B. Bundy ${ }^{3}$; Emmanuel Contreras Guzman ${ }^{3}$. ${ }^{1}$ University of Wisconsin-Madison, Madison, WI; ${ }^{2}$ University of Wisconsin School of Medicine and Public Health, Madison, WI; ${ }^{3}$ University of Wisconsin-Madison, Madison, WI. (Control ID \#2464401)

NEEDS AND OBJECTIVES: There is a need for increased opportunities for distance learning and asynchronous learning in undergraduate medical education. Students have many reasons for not being at their institution's main campus including visiting electives or rotations at other campuses. Online courses, particularly those that are asynchronous, can fill this need, but different strategies for effective instruction have not been studied extensively. Of particular interest are strategies that increase interactivity without overburdening faculty. The main objectives of this project are to: 1) Determine the feasibility of adding basic interaction to recorded lectures using a novel learning tool; and 2) Determine whether this interaction improves students' short- and intermediate-term knowledge and self-efficacy. We will determine these outcomes by performing a randomized controlled trial (RCT) comparing recorded lectures to an interactive lecture.

SETTING AND PARTICIPANTS: Clinical Therapeutics is an entirely online elective course available to all senior medical students at the University of Wisconsin School of Medicine and Public Health. The course is almost entirely asynchronous and is available from the beginning of December until the end of March to coincide with the residency interview season

DESCRIPTION: The RCT involves comparing interactive lectures that use a novel program developed at the University of Wisconsin called "Case Scenario/Critical Reader
Builder" (CSCR) to matched recorded lectures without interaction. All students taking the Clinical Therapeutics course during the 2015-2016 academic year $(n=118)$ were randomized to the recorded lecture group (control) or the interactive lecture group (intervention). Students in the control group view a recorded lecture online with accompanying PowerPoint slides roughly $45 \mathrm{~min}$ in duration. The recorded lectures have example cases mixed throughout the lecture and faculty pose questions about the cases to learners in the recording. Students in the intervention group view the same components, however, the cases and questions are presented to them via CSCR which requires them to answer questions about the cases correctly before proceeding with the lecture. CSCR provides feedback for each correct and incorrect answer chosen. We selected five learning topics taught by five faculty members for this RCT: depression, hypertension, sexually transmitted diseases, skin and soft tissue infections, and vasopressors. Students were randomized to receive all five topics in either the control or intervention format. Following the completion of each topic, students answer a five-question multiple-choice quiz querying the most important content of the lecture. Students are asked as part of the evaluation, "Overall this was an excellent session," rated on a 4 point scale $(1=$ strongly agree to $4=$ strongly disagree). In addition, the students complete a self-efficacy question, "I am confident in my ability to treat a patient with [conditions described in the lecture]", rated on a 10-point scale ( $1=$ Not at all Confident to $10=$ Extremely Confident). Students are given the same five-question quiz 2 weeks after completion of the topic to determine intermediate-term knowledge.

EVALUATION: The main outcome measure is difference in student short-term and intermediate-term knowledge, as measured by the five-question quizzes. Knowledge difference will be analyzed using repeated analysis of variance in order to account for the five matched lecture pairings. Self-efficacy in the topic area will be evaluated by the single confidence question, and learning session satisfaction will be assessed by student ratings of the sessions. Feasibility of the intervention will be evaluated by comparing the time it takes for students to complete the interactive learning session versus the recorded lecture, and the self-reported additional time it takes faculty to develop the interactive learning session. To date, intervention students have completed 89 interactive lectures with a rating of 1.28 (standard deviation 0.48). Control students have completed 76 recorded lectures with a rating of 1.44 (standard deviation 0.50 ). Faculty reported an average of 5.75 extra hours to develop the interactive content versus the lecture.

DISCUSSION / REFLECTION / LESSONS LEARNED: Data obtained from this study will help inform the use of interactive methods for online medical student education. Early results show that students rate the interactive sessions highly and additional faculty time to prepare the sessions using CSCR is manageable. If this moderate amount of interactivity improves students' short-term and/or intermediate-term knowledge, we will have identified a basic online teaching method that increases learner knowledge without requiring a large amount of faculty time. If we find no increases in student knowledge, further investigations will focus on whether increased amounts of interactivity will improve online learning, understanding that this will be increasingly burdensome for faculty.

IMPROVING PATIENT CARE AND COMMUNICATION IN A 4+1 SCHEDULE: DESIGNING A STANDARDIZED LAB FOLLOW-UP PROTOCOL Sue $\mathrm{Gu}^{2}$; Virgil Secasanu ${ }^{2}$; Charles Lundquist ${ }^{2}$; Jason Henry ${ }^{2}$; Julie Knoeckel ${ }^{2}$; Joel Womack $^{2}$; Victoria Maldonado ${ }^{2}$; Quocan Nguyen ${ }^{2}$; Vishnu Kulasekaran ${ }^{1}$; Laura P. Hurley ${ }^{1} .{ }^{1}$ Denver Health, Denver, CO; ${ }^{2}$ University of Colorado Denver, Denver, CO. (Control ID \#2469949)

NEEDS AND OBJECTIVES: An increasing focus on duty hour restrictions has led to the restructuring of many residency program schedules. A number of programs have adopted a variation on a $4+1$ schedule that alternates traditional rotations with a continuity clinic block. During absences from clinic, residents may have difficulty following up on studies ordered 
during their clinic block. Prompt follow-up of lab results is essential for quality patient care and communication, as otherwise there can be delays in diagnostic and therapeutic decisionmaking, as well as delayed patient communication resulting in erosion of the patient-provider relationship. Our objective was to create a standardized method by which studies could be reliably followed up and acted upon in a timely manner.

SETTING AND PARTICIPANTS: A quality improvement project was conducted in one continuity clinic site at an internal medicine residency program in Denver, Colorado. All residents at the clinic and faculty preceptors were involved in the design and implementation of the QI project.

DESCRIPTION: Residents and attending preceptors were assigned into "pods." A resident or attending from each pod was responsible for performing follow-ups for their pod during each week of a schedule cycle. In the first PDSA cycle, a paper spreadsheet was kept in each pod's folder. In the second PDSA cycle, an electronic form was saved on a PHI-protected encrypted network folder. Residents were asked to input patient identification number, date of lab draw, and date of complete follow-up for each study ordered. The data recorded was analyzed to determine the rate of follow-up and the percentage of labs with documented follow-up within 5 days of order. Communication about the project was maintained with a weekly e-mail update. The scope of this project was limited to basic labs and excluded imaging and other diagnostic studies. We did not include labs ordered at a visit to be drawn at a future date. Follow-up was defined as when resident attempts communication with results of all labs ordered, including 1-way communication such as leaving a voicemail or sending a letter. An audit was conducted of charts from July 2014, prior to implementation of the QI project. Residents and attendings were surveyed prior to any interventions about their perspectives on the lab follow-up system prior to intervention and surveyed again at the end of the study period. We created the following aim statement: To improve the follow-up rate on patient lab results to $100 \%$ within 5 days of lab draw. EVALUATION: To establish baseline data, an audit of 85 charts from resident clinic visits in which labs were performed conducted in July 2014 demonstrated any form of follow-up documented in 70/85 cases ( $84 \%$ ). Our average days to follow-up was 2.7 days with an average of 2.7 labs per visit. Over the study period from November 2014-March 2015, 333 encounters with lab orders were recorded. Of these encounters, 319/333 had documented follow-up within the 5 day window period (95\%). We performed a chart review of 30 lab follow up documentation encounters selected by a random number generator to ensure that our self-reported follow up data and type of documentation was concordant with the data derived from the spreadsheets. We found that $29 / 30$ or $96 \%$ of spreadsheet documentation reflected medical record documentation, which correlates well with data derived from resident self-reporting. Common reasons for delay in follow-up were 1) ambiguity in responsibility for follow-ups during a week when no "pod" resident was scheduled; 2) labs with result times that exceeded 5 days (i.e. HCV genotyping); and, 3) failure to document follow-up. A pre- and post-test survey of residents showed an improvement in percentage of residents that report always following up on labs from 60 to $100 \%$, and the percentage that felt extremely confident that their labs were being followed up from 20 to $64 \%$.

DISCUSSION / REFLECTION / LESSONS LEARNED: The design of a standardized work flow and creation of a shared document for tracking lab follow-up resulted in a $>10 \%$ increase in the rate of lab follow-up in the 4 month study period and improved resident confidence that labs were being followed up. Limitations are that this process relies on individual compliance and buy-in and is a cumbersome extra step in busy clinic settings. Additional PDSA cycles have been instituted to improve the layout of the spreadsheet to make it more user-friendly and to limit information inputed to only the most essential items. Future plans include training providers that cover follow-up responsibilities while residents are not in clinic. We plan to continue utilizing this tool and to expand it to include imaging and other studies. This tool is low-cost and requires little training, and could be implemented in multiple clinic settings where there is no automated system for forwarding lab results to covering providers and where providers are out of clinic for periods of time, such as in other residencies with a $4+1$ schedule.
IMPROVING THE PRACTICE OF PRACTICE IMPROVEMENT: RESIDENT CONTINUITY CLINIC PLAN-DO-STUDY-ACT CYCLES Stacy T. Charat, ${ }^{2}$; Simerjot K. Jassal ${ }^{1} .{ }^{1}$ UCSD \& VASD, San Diego, CA; ${ }^{2}$ University of California San Diego, Del Mar, CA; ${ }^{3}$ Veterans Health Administration, San Diego, CA. (Control ID \#2468058)

NEEDS AND OBJECTIVES: The ACGME Internal Medicine Program Requirements include provisions for practice improvement projects to be completed in the resident longitudinal clinic: "[The longitudinal resident clinic experience] must include evaluation of performance data for each resident's continuity panel of patients relating to both chronic disease management and preventive health care. Residents must receive faculty guidance for developing a data-based action plan and evaluate this plan at least twice a year." At the University of California, San Diego (UCSD), residents fulfill this requirement by completing two Plan-DoStudy-Act projects (PDSAs) in each year of residency training (total of six) as part of their continuity clinics with the support of the teaching faculty. Residents have struggled to complete these in the expected timeframe, instead frequently completing multiple PDSAs in the third year of residency in order to meet graduation requirements, thereby undermining the goal of teaching residents to incorporate quality improvement into everyday practice. The objective of this project was to improve timely completion rates for high-quality PDSAs among PGY-1 residents in our program.

SETTING AND PARTICIPANTS: This intervention took place at UCSD, where residents care for patients at ten continuity clinic sites. Participants were PGY-1 residents in the Internal Medicine Residency program with continuity clinic during the 2014-2015 academic year

DESCRIPTION: Resident Intervention: A brief educational intervention was presented at two sessions in September 2014 and was subsequently emailed to all PGY-1 residents. This included a series of short videos from the Institute for Healthcare Improvement describing the PDSA process, a chart review template, a PDSA completion worksheet with guiding questions, and a description of a specific proposed PDSA project. The proposed intervention was intentionally simple and could be completed in a single visit. The first PDSA cycle, completed in fall, 2014, involved documentation of obesity in the problem list for patients with BMIs $\geqq 30$. The second cycle, completed in spring, 2015, involved increasing the frequency of conversations about obesity in the visit plan for appropriate patients. Residents had one hour of protected clinic time in both the fall and the spring to work on their PDSAs and received a total of four email reminders (two in the fall and two in the spring.) Faculty Intervention: Site directors at each continuity clinic site reviewed the project materials (described above) via email and at a site directors meeting. They received one email reminder for each PDSA cycle (total of two.)

EVALUATION: The primary outcome was the number of PGY-1 residents who completed PDSAs at the expected rate of two per year during this intervention period compared to prior years. Graduation requirement logs are updated monthly based on New Innovations. These were reviewed along with completed submitted PDSA worksheets. In the years prior to implementation, PGY-1 completion rates by mid-year for the first PDSA cycle were 3 out of 38 (7.89 \%) in January 2013 and 2 out of 35 $(5.71 \%)$ in January 2014 , compared to a post-intervention rate of 16 out of $38(42.11 \%)$ in January 2015. In the years prior to implementation, PGY-1 completion rates by the end of the academic year for two PDSA cycles were 5 out of 38 (13.16\%) in June 2013 and 1 out of 34 (2.94 \%) in June 2014, compared to a post-intervention rate of 8 out of 37 (21.62\%) in June 2015. Select comments after the first PDSA cycles were as follows: "I was actually surprised that my patient census was a pretty accurate representation of the general US population...given how sick my patients seem to be in clinic, I expected more of them to be obese." "I also noticed that I would spend more time discussing lifestyle modification with a patient that had obesity on their problem list" "By adding this I have found that I am less likely to consider obesity as an unalterable fact about a patient and more likely to see it as a treatable problem like hypertension...This project helped me to realize that there may 
be other aspects of a patient's history that I treat in similar ways... I could have probably predicted this change before starting my PDSA however it is valuable to actually perform the act and realize how much of a difference one small change can make."

DISCUSSION / REFLECTION / LESSONS LEARNED: This relatively simple and low-resource intervention significantly increased the expected PDSA completion rate of two per year among internal medicine residents at UCSD. Completion rates were still lower than expected and desired, and the effect of the intervention waned by the second PDSA cycle. Differences in patient populations, clinic resources, faculty involvement, and electronic medical records at the different continuity clinic sites may contribute to variability in PDSA completion. Next steps include assessing the quality of PDSA projects in addition to the quantity, and assessing resident and faculty knowledge and attitudes about PDSAs.

IMPROVING TRAINEES' DIAGNOSTIC REASONING THROUGH STRUCTURED PEER FEEDBACK Kathleen Lane; Andrew Olson. University of Minnesota, Minneapolis, MN. (Control ID \#2466310)

NEEDS AND OBJECTIVES: Trainees on nightfloat rotations admit approximately $40 \%$ of the patients who are cared for by daytime ward teams. However, these trainees typically receive little formal feedback regarding their diagnostic reasoning. Instead, it is up to the nightfloat trainees' prerogative to determine how patients' disease courses evolved, why the differential diagnoses shifted, and if cognitive errors occurred. Effective feedback and calibration of medical-decision-making is critical for trainee development. Trainees perceive this lack of feedback: $40 \%$ of University of Minnesota Internal Medicine residents are dissatisfied with the feedback they receive. Training programs lack regular, standardized, formal feedback regarding diagnostic reasoning. Thus, we aimed to create and study a solution that allows for standardized, rapid, and effective feedback about diagnostic reasoning for nightfloat trainees

SETTING AND PARTICIPANTS: We developed and piloted the "Diagnostic Reasoning Feedback (DxRF) Form" at the University of Minnesota Medical Center with Internal Medicine and Internal Medicine-Pediatrics trainees on ward day and nightfloat teams. DESCRIPTION: When a patient was admitted by a nightfloat trainee, the day team accepting that patient was requested to discuss the case and fill out the feedback form, briefly explaining if, how, and why the patient's differential diagnosis changed over the first day of admission, and provide relevant clinical pearls. The form was designed to be a concise tool that would be easily incorporated into the teams' preexisting workflow while simultaneously provoking analysis of the decision-making that occurred at the time of admission. This program allowed for formative feedback over the course of that trainee's nightfloat rotation directly from his or her colleagues.

EVALUATION: A total of 25 participants completed 66 feedback forms during the 2month pilot. Trainee self-perception of diagnostic reasoning skills trended towards increased confidence. Trainees rated attending physicians as less frequently discussing why a differential diagnosis was established or prioritized after the project ( $3.95 \mathrm{vs} .3 .43, p=$ 0.01). Additionally, trainees reported increased confidence in identifying strategies to mitigate cognitive bias ( 3.05 vs. $3.5, p=0.049)$. The pilot had neutral impact on trainee feedback satisfaction. Overall, trainees viewed the project favorably.

DISCUSSION / REFLECTION / LESSONS LEARNED: After the pilot phase of this program, DxRF was made a permanent part of inpatient training at the University of Minnesota. The program will be refined and studied further at partnering institutions. In an era of heightened awareness regarding diagnostic error, feedback systems for trainees are critical. With improved feedback in training programs, all trainees will be able to hone their diagnostic reasoning skills and improve patient care. Structured peer feedback is acceptable to trainees and effective in improving self-perception of diagnostic reasoning skills by providing a forum for open communication and encouraging a culture of effective feedback for all providers
INTEGRATION OF DERMATOLOGY-FOCUSED PHYSICAL DIAGNOSIS ROUNDS AND LARGE GROUP CASE-BASED ACTIVE LEARNING WITHIN THE INTERNAL MEDICINE CORE CLERKSHIP Brian Scott ${ }^{2}$; Reenie Abraham ${ }^{1}$; $\underline{\text { Blake R. Barker }}{ }^{1}$; Heather Wickless ${ }^{3} .{ }^{1}$ UT Southwestern, Colleyville, TX; ${ }^{2}$ UT Southwestern Medical School, Dallas, TX; ${ }^{3}$ UT Southwestern Medical Center at Dallas, Dallas, TX. (Control ID \#2465850)

NEEDS AND OBJECTIVES: A cutaneous issue is the primary complaint in $5 \%$ of patient visits to internists. Despite the prevalence of these conditions, medical students only receive a median of $10 \mathrm{~h}$ of formal dermatology education. Medical students at our institution received no formal teaching regarding dermatological physical diagnosis during the third year of medical school prior to 2014. Through informal feedback, our students often expressed a lack of comfort with basic physical diagnosis skills for dermatological conditions. Our objective was to implement dermatology-focused curricula within the Internal Medicine (IM) Core Clerkship after which students should be able to perform the following tasks adapted from the American Academy of Dermatology (AAD) Basic Dermatology Curriculum: (1) Develop a systematic approach to skin examination; (2) Effectively communicate a description of common skin conditions using appropriate dermatological terms; and (3) Develop a differential diagnosis for common dermatologic manifestations.

SETTING AND PARTICIPANTS: All UTSW third-year medical students during their Internal Medicine Core Clerkship (240 per year).

DESCRIPTION: In the 2014-2015 academic year, two new dermatology-focused sessions were integrated into the IM Core Clerkship. All students were exposed to each session. Each week, a faculty dermatologist lead 5 students on "Skin Rounds", a 1-h dermatology-focused physical diagnosis rounds session. Patients are identified by the inpatient dermatology consult service or by the medical students from their genera medicine inpatient service. Students are asked to describe skin findings at the bedside and form a differential diagnosis with the assistance from the dermatologist. Students also participated in a large group $1.5 \mathrm{~h}$ case-based active learning session on common dermatologic findings called "Dermatology: What's Your Diagnosis?" A faculty dermatologist reviews 20 high-definition images of common dermatologic conditions drawn from the AAD Basic Dermatology Curriculum with a group of 20-25 students. For each slide, one student is asked to describe the condition utilizing appropriate terminology and offer a differential diagnosis.

EVALUATION: The impact of these sessions was assessed through online survey student feedback and standardized test performance. The survey was distributed to al 2014-2015 students including two questions regarding the new dermatology sessions, utilizing a 5-point Likert scale (strongly agree to strongly disagree). Response rate was $32 \%$. Nearly $98 \%$ of students strongly agreed or agreed that the activity was effective and useful. Additionally, $92 \%$ of students agreed or strongly agreed that they felt more confident describing skin lesions. Many students commented that they would like more of these sessions. Medical student performance within the "Diseases of Skin and the Nervous and Musculoskeletal Systems" content area of the National Board of Medical Examiners Internal Medicine Subject Examination was also analyzed before (2013-2014) and after (2014-2015) implementation of the dermatology teaching sessions. Specifically, we analyzed the percent of items within this content area that were answered correctly prior to and after intervention using an independent samples z-test. While not statistically significant at $p<0.05$, students improved from an average of $77 \%$ before intervention to $79 \%$ afterwards $(p=0.60)$. This increase was seen despite a national decrease from 68 to $66 \%$ over the same time frame $(p=0.51)$. Total test mean score was analyzed using an independent samples t-test. There was a 1 point increase in total test mean after the intervention; this increase also lacked statistical significance $(p=0.18)$.

DISCUSSION / REFLECTION / LESSONS LEARNED: One study at a US medical school found that 4 th year medical students were highly dissatisfied with their 
dermatology training and were able to correctly diagnose less than $50 \%$ of common dermatologic conditions. Our intervention was designed to address inadequacies in medical student dermatologic education by embedding activities within the IM clerkship. We utilized bedside teaching and case-based active learning, which have been shown to be superior to lectures at increasing medical student confidence in their ability to diagnose dermatologic disease. Additionally, these sessions were led by a faculty dermatologist, which has been shown to be superior at increasing diagnostic self-confidence relative to training from a primary care physician. Our intervention was met with high satisfaction from medical students, and they reported increased confidence describing skin lesions. We also saw a trend towards improvement in the dermatology questions on a national examination although this lacked statistical significance. Case-based and bedside teaching utilizing real patients improves medical students' confidence in approaching a dermatologic patient. This intervention can serve as a model for implementation of curricula within the IM clerkship to address specific inadequacies at other institutions.

INTERPROFESSIONAL COLLABORATIVE PRACTICE: A FORMAL CURRICULUM FOR RESIDENTS IN PRIMARY CARE CLINIC Anna Volerman ${ }^{3}$; Natalia Lipin ${ }^{1}$; Gianna Sparks ${ }^{1}$; LaToya Bradford ${ }^{3}$; George Weyer ${ }^{3}$; Julie Oyler ${ }^{2}$. ${ }^{1}$ University of Chicago, Chicago, IL; ${ }^{2}$ University of Chicago Medical Center, Chicago, IL; ${ }^{3}$ University of Chicago Medicine, Chicago, IL. (Control ID \#2468962)

NEEDS AND OBJECTIVES: In the current climate of health care reform, primary care is increasingly delivered by interprofessional (IP) teams that aim to provide high quality, high value care to individuals and populations. Prior studies have demonstrated that highly functioning IP teams improve both patient satisfaction and patient outcomes; they also improve employee satisfaction and retention. Thus, it is essential for physicians to learn the foundational skills to work within and lead IP teams. Despite this, formal training in interprofessional collaboration has traditionally been limited. The objective was to enhance interprofessional collaborative practice (IPCP) among resident physicians in a primary care clinic. SETTING AND PARTICIPANTS: First year internal medicine and medicine-pediatrics residents (PGY1) and ambulatory clinic nursing staff at one academic medical center between July 2015 and April 2016.

DESCRIPTION: Through an application of Kern's model for curriculum development, we developed and evaluated an IPCP curriculum for PGY1s residents in the ambulatory setting. A literature review and needs assessment informed the curriculum development. Clinic nursing staff, including registered nurses (RN), licensed practical nurses (LPN), and medical assistants (MA), served as educators in the curriculum. Following Kolb's learning cycle, the curriculum consists of: 1 . Seminar led jointly by an RN, LPN, MA, and a physician. 2. Clinical experiences where residents worked one-on-one with nursing staff within the setting. 3. Application in clinical care. The curriculum promoted the acquisition of core competencies of IPCP: values for interprofessional practice, roles and responsibilities, communication, and teamwork.

EVALUATION: During the pilot year, 45 PGY1 residents and 16 nursing staff members have participated in the curriculum. At the start of the curriculum, $95.6 \%$ of residents $(n=43)$ completed survey instruments focused on knowledge, skills, and attitudes for IPCP. Residents were most confident in their teamwork abilities (mean = 4.0/5.0) and less confident with responsibilities $($ mean $=3.6 / 5.0)$ and giving feedback to team members (mean 3.4/5.0). Residents rated the case-based seminar highly; $97 \%$ stated it was beneficial for understanding responsibilities, learning effective communication skills, and learning skills for working in and leading IP teams. Overall, $97 \%$ residents were satisfied with the seminar (mean 4.42, SD 0.56). To date, 21 of the 45 PGY1s (47\%) have completed clinical experiences with nursing staff. As a result of the experience, $95 \%$ of PGY1s reported they have a better understanding of responsibilities of nursing staff and $84 \%$ stated they are able to collaborate more effectively on an interprofessional team. Qualitatively, the majority of residents described it was most beneficial for learning about "what happens behind the scenes" and understanding different roles of nursing staff. Overall, $84 \%$ of residents were satisfied with the clinical experience and $74 \%$ would recommend it to future PGY1s. Clinical experiences are ongoing through spring 2016; final data will be presented at the conference.

DISCUSSION / REFLECTION / LESSONS LEARNED: The literature includes few curricula designed to provide formal training for residents physicians in interprofessional collaborative practice, particularly in the clinic setting. We successfully implemented a curriculum to provide formal training in IPCP for resident physicians by utilizing IPCP competencies and empowering nursing staff to be educators for resident physicians. Future studies will need to examine the impact of this curriculum on teamwork in the clinic and on patient-level outcomes.

INVITING THEM IN: TEACHING MEDICAL STUDENTS ABOUT THE PATIENT CENTERED MEDICAL HOME Stefani Russo ${ }^{1}$; Andrew Schreiner ${ }^{2}$; Kimberly S. Davis ${ }^{2} .{ }^{1}$ Jefferson University, Philadelphia, PA; ${ }^{2}$ Medical University of South Carolina, Charleston, SC. (Control ID \#2468913)

NEEDS AND OBJECTIVES: As the Patient Centered Medical Home model of care delivery moves to the forefront of primary care re-design nationally, it is critical that medical students understand the principles of PCMH and are given hands-on opportunities to put these concepts into practice. We agree with a joint statement from multiple primary care professional organizations citing a "remaining need" to "provide a foundation in primary care medicine and PCMH relevant for all students." To this end, we created a fourth year medical student elective to teach students the concepts of PCMH. Our objectives were: 1 . To create a PCMH curricular map, cross-walking medical student learning objectives with entrustable professional activities (EPAs) and ACGME core competencies. 2. To embed students within a $\mathrm{PCMH}$, and include multi-disciplinary members of the PCMH care team as part of the students' educational team. 3. To implement a small group curriculum to teach PCMH concepts. 4. To assess students' understanding of PCMH concepts before and after participation in our elective. 5. To identify barriers to implementation.

SETTING AND PARTICIPANTS: Fourth year medical students were given the opportunity to participate in our PCMH elective. Participating students spent one half day per week in the classroom and 4.5 days per week in our NCQA-recognized Level 3 PCMH. DESCRIPTION: The overarching goal of our 4-week elective was to teach students about the PCMH mode of care by pairing a rich hands-on clinical experience in a PCMH with a small group didactic component.

EVALUATION: Students were asked to complete a survey both before and after their participation in our elective. The survey asked students, "As of today, how well do you understand..." on a scale of 1 to 5 , with a score of 1 being "not at all" and a score of 5 being "completely." We have pre and post-elective survey results for three students who have completed our elective. All students reported an increase in their understanding of the PCMH model (pre-participation average 2.33 increased to 4.0 post-participation), personal physician (3.0 à4.33), physician-directed medical practice (1.67 à4.33), whole person orientation (2.33 à4.0), integrated and coordinated care (2.33 à4.0), quality and safety (2.0 à4.0), enhanced access (3.33 à4.0) and payment (1.0 à4.0). We are continuing ongoing data collection.

DISCUSSION / REFLECTION / LESSONS LEARNED: Clinical experience: Students spent 4 weeks working with us in our level 3 PCMH. Because we wanted students to learn about the roles and responsibilities of all of our staff, all clinical team members were included as part of the students' educational team. Students had assigned times to work 
with our LPNs, RNs, case managers, pharmacists, as well as a list of skills to practice with these team members, such as vaccine administration and insulin teaching. Students saw patients with our attending physicians, and were encouraged to follow up with patients by phone to check on their progress. Students also participated in multi-disciplinary weekly team meetings. Their clinical assignments mirrored concepts taught in our small groups, and included creating patient education hand-outs, assessing barriers to care, using motivational interviewing, and setting self-management goals. Small group: Our small group didactics focused on the following key PCMH concepts: personal physician, physician-directed medical practice, whole person orientation, integrated and coordinated care, quality and safety, patient access, and payment. These were taught in an interactive small group setting led by a faculty member with expertise in PCMH. Students received training in clinical microsystem design and population health, and were asked to design a quality improvement project during their elective that allowed for further immersion in these concepts. With our faculty leader, students discussed areas for improvement in our practice, and identified specific, measurable, and patient-centered outcomes for an ongoing quality improvement project. Lessons learned: Integrating fourth year medical students into the daily activities of LPNs, RNs, and case managers required additional education for the interdisciplinary team. With varying degrees of direct teaching experience, team members needed reinforcement of learning objectives, strategies for learner engagement, and tools to provide student feedback. Additionally, in a departure from historical faculty precepting, student education in the PCMH model required exposure to elements of care delivery outside of the exam room. As such, the amount of protected faculty time needed was greater than originally anticipated.

KICK THE BUTT! - A PILOT SMOKING CESSATION PROGRAM USING GROUP VISITS IN AN INTERNAL MEDICINE RESIDENT CLINIC Patricia $\mathrm{Ng}^{1}$; Susan Oliverio ${ }^{1}$; Mindy Sobota ${ }^{2} .{ }^{1}$ Alpert Medical School of Brown University/ Rhode Island Hospital, Providence, RI; ${ }^{2}$ Brown, Providence, RI. (Control ID \#2465031)

NEEDS AND OBJECTIVES: Tobacco cessation counseling is a grade A USPSTF recommendation. A 2012 Cochrane review showed that training health professionals in tobacco counseling led to increased discussions about quit dates and abstinence (Carson et al.). However there is limited literature about effective tobacco cessation training programs for internal medicine residents. Our objective was to develop a comprehensive tobacco education curriculum with low-literacy pictorial patient education materials and to determine the feasibility of training residents to provide tobacco counseling in group visits. Our primary outcomes were to train 30 primary care residents and provide an opportunity for them to counsel smokers using a group visit model. Our secondary outcomes were to assess the efficacy of a group program in changing patients' attitudes about smoking, readiness and confidence to quit, and quit rates.

SETTING AND PARTICIPANTS: Our smoking cessation program took place at a large academic resident clinic that serves predominantly underserved, multilingual patients in Providence, RI. Patient participants were clinic patients referred by their PCP from Sept 2014 to Feb 2015. Thirty primary care residents who were on their ambulatory block were eligible to be trained as group visit leaders.

DESCRIPTION: Our program included 3 weekly sessions that repeated each month in English or Spanish for 6 months. Sessions were approximately $2 \mathrm{~h}$ long and were led by $1-$

2 residents and a supervising attending experienced in tobacco cessation and motivational interviewing. Prior to leading the group visits, residents participated in a $1 \mathrm{~h}$ tobacco counseling training program led by the senior resident and supervising attending who created the pilot program. During the group visits, residents practiced motivational interviewing techniques to help participants reflect about their smoking behaviors and develop individualized quit plans. Patients also learned about the health and financial consequences of tobacco use, potential behavior strategies to avoid smoking and medication assistance for smoking cessation. Information was presented through an interactive PowerPoint presentation that included low-literacy pictorial education materials from ThumbsUpForHealth.org. Patients received a printed workbook to help create their individualized plan. If appropriate, patients were prescribed varenicline, bupropion, and/or nicotine replacement therapy during the group visit. Feedback and communication with patient's referring PCP and/or mental health provider was provided.

EVALUATION: All 30 eligible primary care residents participated in the $1 \mathrm{~h}$ training program. Twelve residents had schedules permitting them to lead the group visits. Informal resident feedback was collected after each group visit, which was uniformly positive. All residents reported improved knowledge about smoking cessation medications and practical aspects of prescribing, including cost and insurance coverage. Residents who participated as group visit leaders reported improved confidence in counseling patients about smoking cessation using simple motivational interviewing techniques. To assess patient outcomes, participants completed pre/post-program surveys and a 1 month follow-up telephone survey. Patients were asked about smoking history, nicotine dependence (ex. Fagerstrom Test for Nicotine Dependence [FT]), and attitudes about smoking cessation. All statistical analyses were performed with Microsoft Excel using paired t-test for difference in means for continuous variables. At 6 months, 62 patients were referred to the program but only 12 attended (19.5\%). Six patients completed the pre/post-program surveys which were included in the analysis. At post-program, $50 \%(n=3)$ quit smoking, average smoked cigarettes per day decreased from 20.7 to 9.2 cigarettes ( $p<0.05$ ), and FT scores decreased from 5.2 to $2.8(p=0.51)$. Mean readiness and confidence to quit increased from 8.8 to $9(p=0.81)$ and 6.2 to $7.5(p=0.22)$ respectively. Most participants $(85 \%, n=5)$ thought the group was very helpful and all attendees requested to repeat the program.

DISCUSSION / REFLECTION / LESSONS LEARNED: We were successful in developing a comprehensive tobacco education curriculum using low-literacy pictorial patient education materials. We found group visits to be a dedicated venue for residents to learn and to practice motivational interviewing for smoking cessation, but the experience can be inefficient due to low patient attendance. The subset of patients who completed all three sessions had improved smoking behaviors and motivation to quit. There were likely multiple factors contributing to the low patient attendance, including inconvenient program schedules, poor winter weather conditions that led to program cancellations, and referrals by PCPs when patients were pre-contemplative about quitting. In the future, we want to explore pairing residents with faculty to deliver point of care tobacco cessation counseling for patients identified by medical assistants.

ONLINE RESOURCE URL (OPTIONAL): http://www.thumbsupforhealth.org

\section{LET'S GIVE THEM SOMETHING TO TALK ABOUT: USING AN INTERPRO- FESSIONAL SMALL GROUP ACTIVITY TO TEACH STUDENTS MEDICAL ETHICS. Liam P. Howley; Laura Hinkle. Indiana University, Indianapolis, IN. (Control ID \#2468952)}

NEEDS AND OBJECTIVES: Students receive a plethora of didactic lectures throughout their medical education. And a majority of the learning during medical school is isolated from other health services students (such as nursing, dental and pharmacy). Medical ethics is a unique topic that allows for alternative and collaborative methods of learning. Previously, the ethics mini-course at Indiana University School of Medicine (IUSM) had only involved medical students discussing and analyzing ethical cases from the perspective of a medical student or physician. The medical ethics course directors for IUSM saw this mini-course as a wonderful opportunity to incorporate interprofessional education into the analysis of common healthcare ethical scenarios.

SETTING AND PARTICIPANTS: At IUSM, there is an established mini-course to teach concepts in medical ethics to third-year medical students. This time is divided into two sessions, one 3-h session at the start of their Obstetrics and Gynecology rotation and a 
2-h session at the end of the rotation (approximately 6 weeks apart). The meetings include didactic sessions on concepts such as the history of medical ethics, medical research ethics, disclosing medical errors, and interactions with the pharmaceutical industry. The interprofessional small group exercise has been incorporating into the three-hour orientation session.

DESCRIPTION: The full group (approximately 100 students from the schools of medicine, nursing and dentistry) is divided into ten or 15 smaller groups. After about ten minutes of introduction and a short ice-breaker activity, the groups are given one of five cases to read and discuss. They then are asked to analyze the ethical principles involved in the case and to respond to a specific question about the next step in resolving their case. After approximately $30 \mathrm{~min}$ in the small groups, the large group reconvenes and each case is reviewed with a representative from each of the small groups summarizing the ethical dilemma along with which principles of ethics are involved in their case and how they chose to resolve the situation. Of note, there is a facilitator assigned to each small group who is available to guide the group through the discussion.

EVALUATION: Surveys are distributed to all student participants at the end of each session to assess both the comfort of analyzing ethical situation and also to gauge the students' perceptions on the value of the interprofessional small group exercise using an adapted version of the Attitudes Towards Interprofessional Health Care Teams survey. The feedback has been very positive, with $85 \%$ of student participants agreeing with the statement "I have a better understanding of other disciplines'/health professions' perspective" and $83 \%$ agreeing with the statement "Working in a group with students from other disciplines/health professions during this seminar contributed to my learning."

DISCUSSION / REFLECTION / LESSONS LEARNED: This interprofessional small group session during the IUSM ethics mini-course has been a unique \& innovative way to enhance medical ethics learning. Our limitations are that the activity may not be translatable to other institutions who do note have other professional schools on the same medical campus, or who don't have the leadership and resources we have in Indianapolis through the Indiana University Center for Interprofessional Health Education and Practice. In the future we plan to expand the audience to include other professional students - we have had some sporadic participation from the School of Pharmacy, and plan to include other groups such as the Schools of Physical Therapy and Occupational Therapy in upcoming semesters.

\section{LEVERAGING 4 + 1 SCHEDULING FOR A PRIMARY CARE LEADERSHIP PATHWAY (PCLP) IN AN INTERNAL MEDICINE RESIDENCY PROGRAM} Nancy T. Skehan ${ }^{2}$; Allen Chang ${ }^{1}$; Sheri Keitz $2 .{ }^{1}$ UMASS, Worcester, MA; ${ }^{2}$ University of Massachusetts Medical School, Worcester, MA. (Control ID \#2467836)

NEEDS AND OBJECTIVES: The increasing need for PCPs in the United States has been well-documented, and it has been noted that residents participating in designated primary care internal medicine residency tracks are twice as likely to become general internists when compared to their categorical counterparts. Additionally, trends in healthcare delivery suggest that there is increasing need for physician leadership with the rise of population management, accountable care organizations, and advances in health information technology. The University of Massachusetts Medical School (UMMS) has long valued its role in providing primary care for the Commonwealth and has an established reputation for its excellence in primary care education. However, in the past decade, on average only $11 \%$ of the graduates from our categorical internal medicine residency have gone on to enter primary care, compared to $22 \%$ at the national level. To address these needs, we recruited a community of self-identified categorical residents interested in primary care and developed curriculum to enhance outpatient skills and provide structured leadership training. Implementation of $4+1$ scheduling in the residency program afforded opportunities for a cohesive structure and deployment of novel curriculum. Our primary goal was to increase the number of graduates from the UMMS
Internal Medicine Residency who pursue careers in primary care, and to equip these residents with skills to succeed as leaders in healthcare.

SETTING AND PARTICIPANTS: During AY14-15 and AY15-16, 19 of 110 eligible internal medicine residents at an academic tertiary medical center (17\%) self-identified as having interest in Primary Care and enrolled into the PCLP. The program provides didactic and virtual classroom content in clinical, administrative and leadership realms as well as 1:1 resident-to-faculty mentorship and social networking. In AY15-16, the residency restructured to a " $4+1$ " model allowing PCLP residents to be cohorted into a firm to create a sense of community and deliver synchronous experiences and educational content during their "+1" ambulatory weeks.

DESCRIPTION: Since its inception PCLP residents have participated in didactic sessions on topics such as resiliency and burnout, fundamentals of business in Medicine, fundamentals of medical education and LEAN quality improvement training. We provided online training done individually by the resident from resources such as the Institute for Healthcare Improvement leadership modules and MedU: High Value Care Modules. We developed clinical experiences in homeless health, endocrinology and behavioral health. We invited residents to attend the UMass Leadership Seminar Series, which include topics on "Leading Interprofessional Teams" and "Transformational Leadership." We paired residents with primary care faculty members in 1:1 mentor relationships to provide counseling in career development and work-life balance.

EVALUATION: Metrics of evaluation include the percentage of residents who chose to participate in the PCLP, along with the percentage of graduates that subsequently enter primary care. Currently, we have 15 residents involved with the PCLP (14\% of all categorical residents.) The class of 2015, which coincided with the first year of the PCLP, produced 7 residents who went on to fields in primary care ( $23 \%$ of the class), a marked increase over an average of $11 \%$ in the past 10 years.

DISCUSSION / REFLECTION / LESSONS LEARNED: Prior to the development of the PCLP, residents did not have the opportunity to explore primary care in a comprehensive and coordinated fashion beyond their mandated longitudinal clinic. The creation of the PCLP in the context of the transition to a $4+1$ block schedule allowed us to focus educational resources and mentorship on a self-identified group of residents interested in primary care. Events currently being finalized and scheduled include outpatient procedure clinics, DiSC Personal Assessment Workshop, Clifton StrengthsFinder Workshop, Crucial Conversations, Crucial Accountability and an Evidence-Based Medicine workshop. Our future focus will be to redesign 4-week subspecialty experiences to focus on the outpatient aspect of the care that they deliver. We will also develop thematic blocks that would group many specialties in one coordinated rotation such as a rotation in Metabolic Health, which would include experiences in diabetes clinic, nutrition, and cardiology. We will offer rotations in Women's Health, Dermatology, Musculoskeletal Health and other skills we identify as being critical for an excellent primary care internist. Our leadership curriculum will feature collaborative programming with our institution's Career Development and Research Office, Junior Faculty Development Program, as well as area colleges. Ultimately, this will be a comprehensive curriculum that spans the 3-year cycle of a resident's training, complemented by a set of unique clinical experiences to help prepare them for a career in primary care.

MAKING IT COUNT TWICE: COMBINING CLINICAL AND EDUCATIONAL SKILL LEARNING IN FAULTY DEVELOPMENT Anne Dembitzer ${ }^{2}$, 1; Verity Schaye $^{1}$; Lynn Bui ${ }^{1}$; Mark D. Schwartz ${ }^{2,1}$. ${ }^{1}$ NYU School of Medicine, New York, NY; ${ }^{2}$ VA NY Harbor Health Care System, New York, NY. (Control ID \#2469267)

NEEDS AND OBJECTIVES: Clinician educators (CE) are the primary teachers of medical students and residents; however most have not had any formal training in how to teach. CE want to improve their teaching skills but choose to attend clinically oriented 
CME programs. Engaging CE in faculty development programs (FDP) that focus on teaching skills is a challenge. To meet this challenge we created a FDP that combined clinical and educational skill learning. The goals of the program were to improve CE teaching skill confidence, performance and self reported behavior change.

SETTING AND PARTICIPANTS: A total of $37 \mathrm{CE}$ completed our 8 month long program. All participants teach medical students or residents, and were recruited from NYU's main 3 academic affiliate hospitals. The first cohort included 18 hospitalists and the second cohort included 19 outpatient CE. Participants completed training an average of 5 years ago (range 1 to 15 years)

DESCRIPTION: We interviewed educational and clinical leaders to identify the educational skill needs of our faculty based on student and resident feedback. We conducted an assessment of CE FDP participants regarding their clinical and educational skill needs. The teaching skills identified by the needs assessments were observation and feedback, teaching a physical examination or procedural skill, precepting, and leading ward rounds. The identified clinical skills were the dermatologic and shoulder exams, pain management, and lumbar punctures. The FDP consisted of five 3-h small group ( $8-10$ faculty) sessions. The first and last sessions were 3-station Observed Structured Teaching Examinations (OSTE) that assessed participants' educational and clinical skill performance. The workshops for the hospitalist cohort were 1. Teaching Procedures: Performing Lumbar Punctures 2. Observation and Feedback: Dermatologic Examination and 3. Ward Rounds: Pain Management. The workshops for the outpatient CEs were 1. Precepting: Pain Management 2. Observation and Feedback: Dermatologic Examination and 3. Teaching Physical Examination: Shoulder Exam. Each workshop began with an interactive discussion of the clinical topic led by local experts followed by an introduction to the educational skill. The faculty were divided in to groups of 3-4 and participated in a series of role-plays. For the workshop "Ward Rounds: Pain Management", the first hour was a discussion of pain management strategies led by a pain specialist followed by review of an educational skill - the 5 step clinical Microskills. The participants practiced using the Microskills through role-play. During the role-play the participants assumed the role of intern, student, resident or attending. The "intern" presented a case of a patient in pain to the "attending". The "attending" led a discussion using the Microskills and practiced their new pain management knowledge. Following each role-play, the participants and medical education experts provided peer to peer feedback to the "attending" on their teaching skills, and then switched roles so all participants could practice leading ward rounds. At the end a medical education expert led a discussion on how to contextualize the learning, and participants completed commitment to change statements (CTC).

EVALUATION: We measured change in teaching confidence using retrospective pre-post surveys at the conclusion of each workshop, and pre and post program surveys. Teaching performance was assessed using OSTEs. Behavior change was measured by assessing participant's ability to implement their CTC statements at the conclusion of the program. DISCUSSION / REFLECTION / LESSONS LEARNED: Participants in the program significantly increased their teaching confidence in covered teaching domains and improved their teaching performance in observation and feedback. At the end of the program $69 \%$ of participants had either fully or partially implemented their teaching behavior change goals from their commitment to change statements. The greatest barrier to implementation of the FDP was scheduling participants due to their heavy clinical responsibilities which we overcame by scheduling sessions 6 months in advance and holding each workshop three times. Conducting needs assessments with participants and educational and clinical leaders led to tremendous support for the program and facilitated support for coverage of clinical time so faculty could participate. Combining educational skills with clinical skill learning helped create interest and buy in from the participants. Although many participated in the program for the clinical skills in the end they were equally likely to make clinical and educational behavior change commitments. With future programs we plan to include a component of peer to peer observation in the participants teaching environment as participants reported challenges implementing new clinical and teaching skills in their setting.
MAKING THE CASE FOR "HIGH VALUE"-COMMUNICATING VALUEBASED DECISION MAKING TO PATIENTS Jill Bowman Peterson ${ }^{3}$; Crystal Donelan ${ }^{4}$; Craig Roth ${ }^{2,}{ }^{1}$; Peter Weissmann ${ }^{2,}{ }^{1}$; Sophia Gladding ${ }^{1}$; Alisa Duran ${ }^{1}$. ${ }^{1}$ University of Minnesota, Edina, MN; ${ }^{2}$ Minneapolis VA Medical Center, Minneapolis, MN; ${ }^{3}$ University of Minnesota, MInneapolis, MN; ${ }^{4}$ University of Minnesota Medical School, Minneapolis, MN. (Control ID \#2464429)

NEEDS AND OBJECTIVES: With health care costs soaring in the United States, physicians have a greater responsibility to assess the value of the tests and therapeutics they order for their patients and balance that with the potential harm and costs. Many residents do not understand their role in driving costs, how to integrate value into their decision-making, and communicating their reasoning to their patients. We developed an innovative high value care (HVC) communication skills intervention for our trainees, using the "Four Habits Model" as a framework. The objectives of the value-based care communication skills innovation include: 1 . Apply the 4-Habits communication model to high value care discussions with patients 2 . Practice and improve value-based communication skills with the use of standardized patients 3 . Train faculty in the communication and role modeling of value based communication skills

SETTING AND PARTICIPANTS: This study is being conducted at the University of Minnesota and involves all first postgraduate year residents (PGY-I) in Internal Medicine $(n=31)$, Medicine-Dermatology $(n=2)$ and Medicine-Pediatrics $(n=10)$ at the University of Minnesota during the 2015-2016 academic year. Participants were randomized to a standard value based care curriculum, including a HVC communication skills encounter, or the standard curriculum plus a high intensity communication skills curriculum.

DESCRIPTION: All interns $(n=43)$ participated in a single station, video-recorded, standardized patient (SP) encounter during orientation as part of the standard value-based care curriculum. This session focused on the communication of value-based decision making and avoidance of unnecessary testing on a patient presenting with back pain. Half of the interns participated in the high-intensity communication curriculum plus standard, and half in the current standard. The high intensity curriculum included a 90-min facilitated small-group review session of the video SP encounters with a communications expert facilitator, a junior faculty co-facilitator and peer participation and feedback. Two months later, interns participated in a $10 \mathrm{~min}$ SP phone encounter focused on value based prescribing, followed by a second, 60-min review of the audio encounters. Small group participants received expert feedback from the group facilitators and from their peers as well. All interns participated in a second video-recorded standardized encounter 11 months after the initial encounter; to measure an improvement in value based communication skills using a validated checklist.

EVALUATION: We evaluted the success of our intervention through the following methods: 1 . Surveys of intern and faculty satisfaction with the communication skills program as well as the overall educational value of the training program. 2. Pre/post assessment during SP encounters to identify improvement in value-based communication skills. This checklist was based upon the Four Habits model and was validated using a Delphi technique with a group of communication experts.

DISCUSSION / REFLECTION / LESSONS LEARNED: Our intervention occurred over an 11-month period. Preliminary feedback revealed that interns found participation in the SP encounters and small groups to be beneficial to their communication skills. Similarly, junior faculty serving as co-facilitators with communication experts have found this to be of educational value as well. Final data analysis will be completed prior to the abstract presentation. Our intervention provided necessary, focused training on the communication of value-based decision making, which is becoming increasing important in our health care systems. Challenges faced during our intervention period included the timing of the small group sessions and conflicts with the redesign of our continuity clinics into a block schedule. Additionally, one intern did not respond to any communication from our group and did not participate in the small group review serssions. We concluded that future interventions may function better administered as a workshop. 
MED REC: A SKILLS-BASED CURRICULUM ON MEDICATION SAFETY AND MEDICATION RECONCILIATION Katelyn Stepanyan ${ }^{2}$; Josette Rivera ${ }^{1}$; Joan Abrams $^{1}$; Michi Yukawa ${ }^{1}$; Anna Chang ${ }^{1}$; Yali Brennan ${ }^{1}$; Stephanie Rennke ${ }^{1} .{ }^{1}$ University of California San Francisco, San Francisco, CA; ${ }^{2}$ UCLA David Geffen School of Medicine, Los Angeles, CA. (Control ID \#2469074)

NEEDS AND OBJECTIVES: Medication errors are common, and more than half of hospital patients are estimated to have one or more unintended medication discrepancies at admission. Medication reconciliation can identify errors. Although there has been significant investigation into the most effective strategies for performing medicine reconciliation within the healthcare system, there is little published on teaching this skill to medical students and direct impact on medication management in the hospital. The objectives for this skills-based curriculum for students included: 1) Apply skills in patient interviews to obtain a medication history (best possible medication history or BPMH); 2) Identify medication discrepancies during transitions of care and 3) Assess for facilitators and barriers to medication safety and provide recommendations to the primary team.

SETTING AND PARTICIPANTS: The curriculum was piloted with 21 first year medical students in 2014 and 2015 at 3 hospitals affiliated with an academic medical center (tertiary care center, county hospital and veterans affairs hospital)

DESCRIPTION: Over the course of ten half-day sessions, students met with a faculty facilitator and identified patients for medication reconciliation. Using a "med rec" worksheet each student performed at least one patient interview per session completing the BPMH, including all medication discrepancies and identified facilitators and barriers to medication management (e.g. adherence, cost, polypharmacy). The students contacted the primary hospital providers using a templated communication form, including recommendations regarding patient-reported barriers.

EVALUATION: On average students identified 2.3 medication errors and one or more barriers to medication management per patient encounter. Compared to pre-test findings, there was a measurable improvement in students' knowledge and skills. Using a 5-point Likert scale after the curriculum, all students agreed or strongly agreed that they applied these skills during their clinical rotations. Students independently identified several areas involving the electronic health record and communication between providers as potential systems gaps in safe medication management during care transitions. Hospitalist faculty and residents highly rated the student communications to the primary team and identified a plan to implement at least one of the recommendations for each patient.

DISCUSSION / REFLECTION / LESSONS LEARNED: This skills-based curriculum embedded early medical students into the workplace in value-added roles for a critical patient safety activity. The curriculum provided a systematic and comprehensive approach to medication reconciliation and medication management. In learning skills such as medication reconciliation, early medical students can have direct positive impact on patient care systems and contribute to high quality care.

\section{MODIFIED NOMINAL GROUP TECHNIQUE TO GALVANIZE RESIDENT LED INCORPORATION OF PATIENT CENTERED MEDICAL HOME PRIN- CIPLES AND VALUES INTO AN AMBULATORY CURRICULUM Frank Cacace $^{1}$; Jennifer Verbsky ${ }^{3}$; Jason Ehrlich ${ }^{2} .{ }^{1}$ Hofstra North Shore LIJ, Great Neck, NY; ${ }^{2}$ North Shore LIJ, Great Neck, NY; ${ }^{3}$ North Shore LIJ Health System, Great Neck, NY. (Control ID \#2467955)}

NEEDS AND OBJECTIVES: Training residents in an ambulatory patient centered medical home should solidify exposure to patient centered team based care that is more coordinated among inter-professionals and serves patient and population based outcomes. Prior surveys delving into trainee awareness and understanding of $\mathrm{PCMH}$ principles at our institution revealed a disconnect from the site infrastructure already in place. The objective of our teaching endeavor was primarily to improve upon this disconnect by having residents identify and brainstorm improved workflows to highlight PCMH principles in their own practice sites. Through a modified nominal group technique and brainstorming session described here residents were thus placed on a path to develop their own PCMH based educational curriculum. The objectives of our innovation can serve to help other residency clinic faculty 1) recognize that nominal group technique can be used to have medical trainees identify their own unmet curricular needs, 2) perform a brainstorming session to devise curricular change encouraging the inclusion of $\mathrm{PCMH}$ principles in a trainee's ambulatory experience, and 3) list EPAs within a PCMH training site to target for curricular efforts. SETTING AND PARTICIPANTS: All interns and residents on weekly firm ambulatory assignments attended the nominal group and brainstorming session at one of our two ambulatory teaching sites over a 5 week period during our Thursday morning "academic half days", with protected time from clinical responsibilities. The session was moderated by $2-3$ teaching attendings, with one attending introducing basic $\mathrm{PCMH}$ principles in a framing discussion at the start of the session. Four medical or clerical office assistants also actively participated in the framing talk and the brainstorming session.

DESCRIPTION: A weekly series of sessions was held for 5 weeks across the internal medicine residency in ambulatory clinic during which a) PCMH principles and values were reviewed, b) a modified nominal group technique was held ascertaining which PCMH entrustable professional activities (EPAs) were not being appreciated by resident learners at their site, and c) resident led brainstorming ensued. The list of PCMH EPAs were presented to the residents via review of: Chang et al. Transforming Primary Care Training-Patient Centered Medical Home Entrustable Patient Activities for Internal Medicine Residents, JGIM, 2012. 28 (6): 801-9. Each resident then named which three EPAs they felt individually most in need of experiencial exposures. Small groups reflected on care based activities and curricular improvements to address one of three most frequently chosen EPAs and reported out to the overall group. The resulting curricular project list across five firms at two clinic sites now represents the substrate for resident led curricular design in PCMH training. Ambulatory faculty will now target the recommended educational content and PCMH minded workflow improvements to be made.

EVALUATION: Pre and post session surveys assessing PCMH principle awareness and understanding, and awareness of clinic site infrastructure and inter-professional services available for patient care, are being administered. Educational and clinical outcomes that are PCMH minded will also be tracked once curricular improvements are put in place. DISCUSSION / REFLECTION / LESSONS LEARNED: This curriculum building exercise demonstrates that nominal group technique as a way of performing trainee needs analysis followed by a brainstorming session is an effective means of galvanizing learner paticipation with curricular design. A core principle in curricular design is learner needs analysis and in this exercise, internal medicine residents identified PCMH EPAs that represented gaps in their training experience at both a PCMH certified training site and in a second site preparing its PCMH application. In particular, the EPAs most chosen among five firm teams involved 1) interrogation of a registry and use of risk stratifcation tools to better manage an entire panel, 2) activation and orchestration of community resources to meet a patient's or population's needs, and 3) performance of a root cause analysis and reflection on items such as medical error, near miss, preventable emergency room visits or readmissions, or patient complaints. Resident ideas to modify educational content and clinical workflow to serve these PCMH anchored activities will be enthusiastically cultivated and implemented. The authors believe conducting this session has focused efforts to guide trainees and faculty to fill important gaps, and to advance PCMH values and improve patient care in the residency clinic. It has proven to be a high yield innovation in curricular development for our clinic that was easy to implement

NIGHT FLOAT 101: ENTRUSTMENT OF PROFESSIONAL ACTIVITIES Deepa Bhatnagar; Ahmed G. Mohiuddin; Cathy Lazarus. Tulane University, New Orleans, LA. (Control ID \#2470320) 
NEEDS AND OBJECTIVES: In medical education today, there is a desire to enhance the value of the fourth year of medical school by clarifying its purpose and reducing its ambiguity. Both fourth year medical students and GME program directors are interested in ensuring that students are clinically rounded and prepared for residency. The AAMC recently released a framework for clinical skills expected of medical students at the start of internship - 13 core professional entrustable activities. Fourth year sub-internships provide an ideal venue to solidify these skills. However, currently at our institution and nationwide (CDIM 2014 survey), our fourth year medical students have minimal opportunities available for observation of the acute issues that arise during nighttime patient care. To prepare students for this environment, education on transitions of care and the delivery of patient care at night is needed. Through a new curriculum at our institution, we hoped to educate our fourth year students on evaluating urgent or emergent conditions and developing interpersonal communication skills with peers and nurses involved in hospital care.

SETTING AND PARTICIPANTS: We worked with 40 fourth year medical students rotating on Internal Medicine Sub-internship over the course of four 1-month blocks. We developed and implemented case-based learning to experience nighttime coverage and to understand hand-overs of care for hospitalized patients.

DESCRIPTION: The curriculum was introduced during sub-internship orientation. In the second week of the block, we conducted a classroom, small group teaching session that provided a structured template for written and verbal sign-out. The third week of the block, we utilized the Simulation Center, allowing each fourth year medical student to act as a night float intern with other students acting as the night shift nurse. We discussed data gathering and communication in time-sensitive evaluations of patients and the development of management plans on acute care patients. Each case was followed by debriefing to provide feedback on each scenario based on direct observation of each student in the scenario.

EVALUATION: To evaluate the effectiveness of this curriculum, we utilized standard ized patients in the beginning and end of the block of the Internal Medicine sub-internship Each student interacted with a nurse followed by a patient - roles both played by standardized patients. 36 of the 40 medical students completed the pre and post-tes evaluation. Using a checklist to score performance, students were evaluated on their interaction with the nurse, their medical decision to evaluate a patient based on triage of information, their interaction with the patient, and their evaluation of the patient. Following the curriculum, students improved in their communication with the nurse $(0 \%->$ $14 \%)$ and medical decision-making in an acute setting (61\% - > $81 \%)$. Students consistently introduced themselves to the patient $(80 \%->86 \%)$ but improved in clarification of their role as a night float physician $(58 \%->75 \%)$. Both pre and post curriculum, the students performed a targeted history and physical exam in the acute setting but improved in communication to the patient about possible diagnoses and next steps $(69 \%->97 \%$ ). Some representative comments about the curriculum include "I do think that the SIM center cases helped me feel a lot more prepared and comfortable when faced with the SP encounter the second time around! I also think that the sign-out part of the curriculum has improved the quality of the written list and the oral sign-out that I give to night float" and "I really like this part of the sub-I curriculum. In fact, since starting my sub-I I have been in at least one emergent situation where I was making decisions ... And I think we do NOT have adequate training thus far for triage and emergent situations." Students expressed a desire to debrief following the standardized patient encounters. While many students agreed that the scenarios were moderately or extremely believable, students were interested in having more time and/or information to evaluate the patient. DISCUSSION / REFLECTION / LESSONS LEARNED: As a national survey in 2014 demonstrates, fourth year medical students are interested in using this time to prepare for residency. This dedicated curriculum on important entrustable professional activities can improve the clinical skills of fourth year medical students. While third year rotations often focus on the attainment of medical knowledge in various subject areas, the fourth year can be used to refine certain skills necessary at the beginning of internship. Our curriculum demonstrates that students can consistently perform well on data gathering through history-taking and physical exam after third year clerkships. This curriculum, however, can be helpful in assisting students in evaluating urgent and emergent conditions effectively and improving communication skills with interdisciplinary team members.

NOVEL CHAMPIONS FOR PROFESSIONALISM: INTEGRATING A PATIENT-CENTERED EMR USE CURRICULA INTO REQUIRED EMR TRAINING Lollita Alkureishi ${ }^{1}$; Wei Wei Lee ${ }^{1}$; Sandra Webb ${ }^{1}$; Vineet M. Arora ${ }^{2}$. ${ }^{1}$ University of Chicago, Chicago, IL; ${ }^{2}$ University of Chicago Medical Center, Chicago, IL. (Control ID \#2467460)

NEEDS AND OBJECTIVES: EMR use with patients in clinical care is the new norm. Despite ACGME competencies regarding professional documentation expectations and interpersonal communication skill development in order utilize patient-centered education strategies, few institutions provide formal curricula on patient-centered EMR use. Furthermore even if such curricula exist, it is difficult to practically deliver it to all institutional trainees, thus limiting training to specific departments. Without required institutional education, trainees are left to rely on the hidden curriculum to learn EMR integration strategies and can adopt unprofessional documentation practices. Our objective was to develop and provide mandatory patient-centered EMR training to all incoming postgraduate medical trainees at the University of Chicago.

SETTING AND PARTICIPANTS: We aimed to embed training on institutional documentation expectations and patient-centered EMR use strategies for all incoming postgraduate medical trainees at the University of Chicago (UChicago) into their required EPIC onboarding training and assess the impact of our curricula

DESCRIPTION: After reviewing the literature, we developed a 10-min presentation on patient-centered EMR use best practices and issues related to documentation professionalism including "cut and paste" and authorship. UChicago EPIC trainers were instructed by the PI's on curricula content and delivered it as part of the 8-h required EMR training for all 2015 new interns, residents and fellows. Post-training, a 10-item Likert-scale survey was used to evaluate trainee self-assessed patient-centered EMR use knowledge, ability, and likelihood to change clinical practice. Likert responses at the high end of the scale were grouped to dichotomize data (i.e. $4=$ agree and $5=$ strongly agree combined as "agree").

EVALUATION: One hundred fifty-eight trainees completed evaluations (72 residents and fellows, 86 interns; 32 primary care and 126 surgical or specialty trainees in 27 specialties). Trainees reported increases in their knowledge of patient-centered EMR use barriers (Pre-training 3.1 (0.9 SD) vs Post-training 3.9 (SD 0.8), $p<0.001$ ), best practices (Pre-training 3.1 (0.9 SD) vs Post-training 3.9 (SD 0.8), $p<0.001$ ), and ability to implement best practices (Pre-training 3.1 (0.9 SD) vs Post-training 3.9 (SD 0.8), $p<0.001)$. Most felt the training was effective $(90.5 \%, n=143)$, that it should be required $(86.7 \%, n=137)$ and that it would change their future clinical practice as a result $(70.9 \%$, $n=112$ ). When comparing program type, primary care trainees were more likely to report training was effective (4.34 vs $4.09, p=0.003)$ and that it would change their future practice ( 4.13 vs $3.73, p=0.02$ )

DISCUSSION / REFLECTION / LESSONS LEARNED: Graduate Medical Education faculty partnership with EMR trainers who do routine required on-boarding is a novel, timely and effective method to facilitate training on patient-centered EMR communication strategies and professional documentation expectations across a variety of residency and post-residency training programs. Future training should aim to longitudinally reinforce best practices for trainees, train faculty to promote positive role-modeling in order to proactively shape the hidden curriculum' of EMR use, and measure patient-satisfaction with trainee communication to determine if training was effective in creating an 
institution-wide shared model of professionalism and high quality patient care in the computerized setting. Given the success of training and its reception by both trainees and trainers, the curriculum will continue yearly for all incoming trainees and also be incorporated into all new hire EMR training for attendings, nurses and support staff at UChicago. This curricula capitalizes on existing support structure available at most academic settings, involves minimal effort on part of the faculty and EMR trainers, and does not require additional cost making it extremely feasible and easily replicated at other institutions.

ORTHO TO-GO: THE UCSF MUSCULOSKELETAL PHYSICAL EXAM APP Carlin Senter ${ }^{1}$; Dylan Alegria ${ }^{2}$; Christy K. Boscardin ${ }^{1}$; Chandler Mayfield ${ }^{1}{ }^{1}$ University of California San Francisco, San Francisco, CA; ${ }^{2}$ University of California Los Angeles, Los Angeles, CA. (Control ID \#2467796)

NEEDS AND OBJECTIVES: Musculoskeletal (MSK) problems are the second most common reason that people seek medical care in the United States; however, many primary care providers lack competence and confidence in practicing MSK medicine. To address this gap in knowledge and skills, we created the UCSF Musculoskeletal Physical Exam App. The app teaches the MSK physical exam to novice-intermediate learners and serves as a resource for practicing clinicians. Based on a needs assessment using data on frequency of referrals from primary care internal medicine to orthopaedic surgery at UCSF and with faculty input, we chose seven key MSK problems on which to focus app content: meniscus tear of the knee, adhesive capsulitis and impingement syndrome of the shoulder, carpal tunnel syndrome, hip osteoarthritis, ankle sprain, and ankylosing spondylitis. Learning objectives: Upon mastery of each case in the app, the learner will be able to: 1 . Name the exam maneuvers needed, 2. Identify the abnormal exam findings, and 3 . Use case history and exam findings to make the correct diagnosis Self-assessments are embedded in each case to allow the learner to measure his or her success in achieving these objectives.

SETTING AND PARTICIPANTS: The app is recommended curriculum for UCSF School of Medicine first-year anatomy students and for UCSF primary care internal medicine residents. The app was provided to participants of a Sports Medicine continuing medical education course. The app is available for free download to the UCSF community and to the public through the Apple app store.

DESCRIPTION: The app contains two main sections. First, there are seven patient cases, each of which focuses on one of the key MSK problems. Each case contains embedded questions and a learner assessment at the end. The learner can repeat the cases as many times as they wish. Second, the app contains exam maneuver video clips with text describing the maneuver as well as its clinical utility, sensitivity, and specificity. These short video clips are meant for targeted review or on-the-fly use in clinical settings. Content was written by Technology Enhanced Learning Medical Education Fellow (DA), and edited by faculty lead (CS) with resident and faculty experts. UCSF faculty, students, and patients volunteered to participate in filming. Content was filmed by a professional crew. The app was created by a developer using an existing platform.

EVALUATION: The app has been downloaded by 345 internal (UCSF) and 509 external users in 31 countries. Learners accessed the app 138 times in the last month. The top 3 maneuvers accessed by learners since the app's inception are: AC joint crossover, Hawkins impingement test (shoulder), and knee range of motion. We are in the process of evaluating the app's efficacy in improving retention of MSK exam knowledge among primary care providers after attending a Sports Medicine continuing medical education course.

DISCUSSION / REFLECTION / LESSONS LEARNED: Improvements to be made in version 2.0 of the app: 1.) Clustering exam maneuvers according to use (not alphabetically), 2.) Including only the essential exam maneuvers for the target audience, and 3.) Porting the app to all mobile platforms (currently iOS only). Due to user feedback, we would like to develop pediatric MSK medicine and MSK procedure content. Lastly, we plan to disseminate the app more widely both in our own institution as well as in collaboration with other institutions in the future.

ONLINE RESOURCE URL (OPTIONAL): UCSF community MSK App downloading home page: http://meded.ucsf.edu/tel/msk-exam-tutor-ios-app-iphone-ipad

PHYSICIAN ADVOCATE AND HEALER ATTORNEY: A HEALTH LAW PARTNERSHIP IMPROVES HEALTH AND EDUCATION Mukta Panda ${ }^{1}$; Benjamin Danford ${ }^{2} .{ }^{1}$ University of Tennessee, Chattanooga, TN; ${ }^{2}$ Legal Aid of East Tennessee; Chattanooga, Chattanooga, TN. (Control ID \#2469114)

NEEDS AND OBJECTIVES: In order to deliver holistic patient centered care, health care trainees must demonstrate an awareness of and responsiveness to the larger context of the health care system as well as the ability to call effectively on other resources in the system to provide optimal health care. While the hospital is proficient at providing medical treatment, some of these patients have unmet legal needs that can exacerbate their medical conditions. One in six individuals in the United States live in poverty, and current research shows that each of these individuals has at least one civil legal problem that negatively affects their health. The introduction of a poverty-law attorney into the hospital provides an opportunity to identify and address legal problems as an integral part of overall patient care from the outset, instead of only providing a referral to an attorney upon discharge. This challenge led to the creation of the University of Tennessee (UT) Health Law Partnership.

SETTING AND PARTICIPANTS: The attorneys are key members of an integrated multi-disciplinary team, bringing together medical residents, students, physicians, midlevels practitioners, nurse managers, nurses, case management personnel (case managers, documentation specialists, resource utilization specialist), physical, occupational, speech and respiratory therapists, dietitians, pharmacists, legal aid staff, and chaplains

DESCRIPTION: Once a patient with a legal problem is identified, a member of the clinical team alerts the attorney. Subsequently, a meeting is arranged either at the attorney's office inside the hospital or at the patient's bedside, thus establishing an attorney-client relationship and providing much needed services without requiring the patient to ever leave the hospital. Some of the legal services include planning documents, such as, advanced care plans, durable power of attorney documents, wills, and healthcare power of attorney documents.

EVALUATION: Over the 3 years, the UT Health Law Partnership has provided assistance to individuals with more than 300 distinct legal problems and created monetary benefits for patients and clients in excess of 1.1 million US dollars. The partnership has demonstrated better health care outcomes as evidenced by improved patient care and compliance, securing reimbursement for medical expenses, reducing unnecessary readmissions, and decreased length of stay. Hospital patients benefit by meeting with an attorney in an efficient manner while in the hospital, and without having to wait or navigate the additional obstacles to obtain the necessary legal services. Other much needed services inherent to having additional in-house resources available to assist patients deal with problems outside of the traditional scope of case management, including issues related to domestic violence, housing, public benefits, planning documents, and the protection of a patient's income.

DISCUSSION / REFLECTION / LESSONS LEARNED: This partnership brings an attorney into the hospital that interacts with clinical staff as a member of the patient care team. Utilizing the services of an attorney as stated above is the important first step in eliminating one of the most persistent problems in providing legal services to indigent patients. This way, many of the obstacles that prevent patients from seeking legal services are eliminated. The traditional delivery system for legal services is a tedious one that involves a multi-step process: making 
contact with legal services over the phone, preparing an application for assistance with intake staff, scheduling an appointment to meet with an attorney or other staff, then arranging for transportation to and from the appointment. By placing an attorney in the hospital, the traditionally cumbersome procedure has been streamlined. Both patients and clinical staff benefit from the increased predictability and decreased anxiety as the result of having conveyed important decisions to the decision making authority. This interdisciplinary model is exemplary of providing not only patient centered care but developing a relationship centered care and an educational opportunity for training the future generations of healthcare providers with countless advantages for both the patient and health care system as outlined above.

\section{POINT OF CARE ULTRASOUND FACULTY DEVELOPMENT FOR GENER-} AL INTERNISTS Anna Maw; Cathy Jalali; Deanna Jannat-Khah; Kirana Gudi; Lia S. Logio; Stacy Anderson; Joshua B. Smith; Arthur Evans. Weill Cornell Medical College, New York, NY. (Control ID \#2463028)

NEEDS AND OBJECTIVES: Needs: There is growing evidence that the use point of care ultrasound (POCUS) improves patient care. Internal Medicine (IM) Residency programs have been slow to adopt the routine use of POCUS to augment the traditional physical exam. One of the identified barriers to integration of POCUS into IM residency training is lack of faculty expertise. Objectives: Following completion of training workshop, the internists were expected to 1) demonstrate skills in acquiring and interpreting images and 2) report enhanced confidence in using POCUS in patient care.

SETTING AND PARTICIPANTS: Over a 10-week period, 15 full-time academic general medicine faculty members at a single urban academic medical center participated in a 12-h (2-h online didactic by SonoSim ${ }^{\circledR}$ and $10 \mathrm{~h}$ of small-group hands-on POCUS sessions) faculty development course led by an academic hospitalist.

DESCRIPTION: Hands on sessions consisted of 1-4 faculty members performing supervised scanning of medical inpatients, who volunteered to be examined for the purposes of the course and included modules of the diagnostic characteristics and limitations of the POCUS exams most useful for an internist including: vascular, pulmonary, cardiovascular, biliary, and renal. All faculty participants completed pre and postintervention exams and surveys to assess knowledge of and attitudes toward POCUS. The questions centered on test characteristics, image acquisition, interpretation, and clinical correlation of bedside echocardiography including IVC assessment, lung examination, abdominal/aortic and renal imaging, and lower extremity DVT evaluation.

EVALUATION: Pre and post-test scores were analyzed using paired t-test. Surveys of pre and post attitudes towards POCUS were analyzed using Paired-Sample Wilcoxon Signed Rank Test. STATA software (Stata Statistical Software: Release 13) was used to analyze the data.

DISCUSSION / REFLECTION / LESSONS LEARNED: Faculty had statistically significant improvements in their ability to interpret ultrasound data (pretest mean score $=$ $40.3 \%$, post-test mean score $=73.9 \%, p<0.001)$. Prior to the training session, most faculty reported minimal to no comfort in their ability to understand and operate the ultrasound equipment (90\%). After training, they all (100\%) reported at least moderate comfort ( $p=$ 0.003). Similarly, most participants had very limited confidence with respect to understanding the capabilities and limitations of ultrasound and this attitude was also significantly improved by the training provided $(p=0.003)$. We asked the participants about perceived barriers to integrating bedside ultrasound into their practice. Pre-test survey data revealed that most identified access to training resources $(67 \%)$ and lack of time to acquire training $(73 \%)$ as the greatest obstacles to achieving this goal. This pilot study showed that a brief intensive course that included didactics and hands-on training yielded significant improvements in the ability of our faculty to obtain and interpret ultrasound data as well as change faculty attitudes regarding the utility of this new tool. While we feel that our pilot study brings to light several barriers with respect to integration of POCUS in IM Programs, our study was a small group of self-selected faculty with $N=15$. Faculty clearly benefitted from this brief intensive training course, but it remains unclear how long this higher level of skill is maintained in the absence of continued training.

\section{POST-DISCHARGE FOLLOW-UP: AN EDUCATIONAL INTERVENTION TO} ENHANCE CLINICAL REASONING Sirisha Narayana; Alvin Rajkomar; James D. Harrison; Victoria Valencia; Gurpreet Dhaliwal; Sumant Ranji. University of California San Francisco, San Francisco, CA. (Control ID \#2447394)

NEEDS AND OBJECTIVES: Feedback on patients' longitudinal clinical courses may enhance physicians' learning, improve their understanding of health systems, and reduce diagnostic error. However, few trainees have the opportunity to follow up on their patients after discharge. Barriers to longitudinal follow-up include time constraints, discontinuous training environments, and difficulty accessing patient information. We developed a novel program to teach internal medicine interns how to conduct post-discharge follow-up on their patients, with the goal of enhancing life-long learning by stimulating self-directed feedback

SETTING AND PARTICIPANTS: Interns on their 4-week patient safety rotation at an academic internal medicine residency program.

DESCRIPTION: We developed a worksheet outlining a process for structured chart review and reflection. At the beginning of their rotation, interns were given lists of hospitalized patients they cared for earlier in the year generated from the electronic health record (EHR). They collected information on patients' post-discharge courses using the EHR and completed reflection worksheets for a sample of these patients. They then met at the end of the rotation to debrief with their peers about what they learned.

EVALUATION: We thematically analyzed four debrief sessions and 104 completed patient reflection worksheets from 22 interns to assess initial perceptions of the curriculum and evaluate the content of reflections.

DISCUSSION / REFLECTION / LESSONS LEARNED: Several themes emerged from our analysis. Interns reported that post-discharge patient follow-up was extremely valuable to their professional development. It helped them appreciate the natural history of disease and the variability of illness course in patients with complex disorders. They also reported that reviewing patients' clinical courses would change their future practices by advocating for earlier end-of-life counseling, improving discharge transitions, and adjusting their clinical decision-making while the patient was still hospitalized. Sample reflection worksheet questions and interns' responses are highlighted below: What medical questions or concerns did you have at the time of this patient's discharge? $\bullet$ Would this patient adhere to insulin after multiple admissions for DKA? • Was atrial fibrillation the source of this patient's stroke? • How would this patient adjust to hospice given her functional status? What lessons did you learn from this patient's post-discharge course? $\bullet$ Anticipate patient positioning during tube feeding prior to discharge. Begin conversations about prognosis and disease severity early on. $\bullet$ Patients with advanced cancer can decompensate and die quickly. $\bullet$ Be cognizant of the realities of depression at a skilled nursing facility. What would you do differently the next time you have a similar patient or case? • Continue addressing goals of care even in short-term stays in the hospital. $\bullet ~ I$ would engage family members so all are working towards a similar goal. $\bullet$ I would do more research into the procedure I was recommending. $\bullet$ I would ensure the date and time for the PCP appointment is set prior to discharge. Future evaluation will include surveying interns before and after the intervention to determine if participation encourages habits of regular patient follow-up. In summary, a structured program coupling postdischarge follow-up data from an EHR with reflection has the potential to influence trainees' professional development and to mold their clinical reasoning. 
PREPARING MEDICINE RESIDENTS TO READ AND REVIEW THE MEDICAL LITERATURE- A PARTNERSHIP WITH JAMA INTERNAL MEDICINE Rachel J. Stern ${ }^{2}$; Blake Charlton ${ }^{2}$; Rita F. Redberg ${ }^{2,3}$; Jeff Kohlwes ${ }^{2,1}{ }^{1}{ }^{1}$ VAMC/UCSF, San Francisco, CA; ${ }^{2}$ University of California, San Francisco, San Francisco, CA; $;{ }^{3} \mathrm{JAMA}$ Internal Medicine, Chicago, IL. (Control ID \#2469835)

NEEDS AND OBJECTIVES: Many internal medicine residents aspire to academic careers. Skills in academic writing and manuscript review are needed for a successful academic career, but building them is not part of traditional residency curricula. To meet these career training needs, the UCSF Internal Medicine residency and JAMA Internal Medicine (JAMA IM) developed two mentored, longitudinal editorial experiences to be completed within senior residents' existing project/administrative time.

SETTING AND PARTICIPANTS: Sixteen second and third year internal medicine residents at the University of California, San Francisco (UCSF) who were interested in gaining manuscript review skills and writing opportunities were selected after a competitive application process

DESCRIPTION: The JAMA IM editor-in-chief, residency leadership, and two residents collaborated to create two experiences: an editorial fellowship and an editorial elective. The editorial fellowship is a 2-year apprenticeship. Two new JAMA IM editorial fellows annually participate in the editorial decision making process, scholarly writing, and manuscript review during their six outpatient months. Fellows also coordinate the elective and provide near-peer mentoring to the 12 senior resident elective participants each year. After completing a selfguided curriculum on high-quality peer review, elective residents review three manuscripts and complete a scholarly manuscript over a 4-month period. Journal editors provide feedback on residents' reviews and faculty members mentor residents' writing projects.

EVALUATION: From inception in August 2014 to the present, four housestaff have served as fellows. Six others have participated in the 2-month elective. These residents have completed 10 mentored, peer-reviews of submitted manuscripts and have published 12 peer-reviewed manuscripts themselves- five editor's notes, four commentaries, one review, one perspective and one piece of original research. These were published in JAMA IM, JAMA, BMJ, JGIM and AJIM. Initial participants report high satisfaction. The JAMA-IM editors greatly value the residents work and contributions. They comment that the trainees offer an important new perspective on many of the manuscripts, in addition to help with journal-related projects. All of the resident manuscript reviews were rated Very Good to Excellent by the editors. Qualitative comments from housestaff participants include "[the elective] built my confidence and my ability to critically evaluate research" and "made me feel like my voice mattered."

DISCUSSION / REFLECTION / LESSONS LEARNED: Academic writing and formal reviewing are discrete, teachable skills that can feasibly integrated into residency curricula. With existing project time, faculty mentoring, and a self-guided curriculum, our residents successfully improved their peer review skills and published their work. Journal editors are an underutilized source of mentoring for trainees interested in academic careers. Our elective model could be replicated at any institution where faculty members regularly review manuscripts.

PUTTING THE ACTION BACK IN ADVOCACY TRAINING: SKILLSFOCUSED TRAINING IN ADVOCACY AND LEADERSHIP FOR GRADUATE MEDICAL RESIDENTS Jane Andrews; Kris Coontz. Tulane School of Medicine, New Orleans, LA. (Control ID \#2457560)

NEEDS AND OBJECTIVES: Advocacy is regarded as a fundamental principle of professionalism for physicians in the ABIM charter, the AMA's Declaration of
Professional Responsibility, and by academic thought-leaders in the field(1,2). Social determinants are critical to improving health outcomes, and thus the responsibility of Graduate Medical Education (GME) extends beyond a commitment to providing direct care for impoverished patients and toward advocating around the conditions that underlie to health disparities (3). Given the public funding nature of residency programs, GME has a responsibility to incorporate social accountability into training.(4) In fact since 2011 the ACGME has incorporated advocacy as a curriculum requirement for pediatrics programs (5). While pediatric and family residencies have developed several models for incorporating advocacy into their curricula, internal medicine curriculum models are scarce. $(6,7)$ Objectives To empower residents to affect social determinants of health through hands-on experiences in health advocacy, providing them with the skills, confidence and contacts necessary to be an advocate and leader in their career To foster a community of changemaking individuals who can support each other in their work To elevate the role of the physician-advocate for residents in training To increase capacity for meaningful hands-on research among residents, leading to increased national reputation and increased funding SETTING AND PARTICIPANTS: Up to four interns per year will be selected for this program. Those selected will remain in the program for their entire residency, giving the program a size of approximately twelve people. Monthly advocacy track dinners are open to all members of the Tulane community, including medical students and faculty. Six faculty mentors participate in the track and are individually matched with resident mentees based on area of interest.

DESCRIPTION: Tulane's Advocacy and Leadership Track seeks to train a small group of health advocates and leaders who will receive personalized attention and increased focus on career development from institutional mentors. Residents will collaborate with an organization working on an issue of health justice during years two and three, partnering on a campaign with specific policy outcomes during two, 2-week blocks each year. By the end of the 3-year program, residents should be able to develop a strategy for a successful campaign, communicate with media around policy issues, advocate with legislators around an issue of their choice, and lay the foundations for a successful career through personal leadership development and networking. The advocacy track will hold a protected 3-week course for interns annually. All participants will begin an Advocacy Portfolio at that time, strategizing about their personal goals for this experience. Each resident will present the results of their project prior to graduation. We hope this track will create long-lasting partnerships with local and national organizations working on health advocacy campaigns. An academic objective of the track is to create a curriculum that models advocacy training as a part of internal medicine residency.

EVALUATION: We plan to use qualitative evaluation for interns after their 3-week course. The program will track resident progress through evaluation of each resident's advocacy portfolio, looking at their campaign-specific action plan and a menu of advocacy milestones. Given that activist and community-based approaches often generate unanticipated benefits, we also will track serendipitous occurrences as a result of coalitions and new partnerships formed. In the future we will survey newly-matched interns to determine whether the advocacy track contributed to their rank decision. The development of measurable competencies in social justice and advocacy training is both vital and lacking (2). By evaluating our curriculum and milestones longitudinally, we hope to contribute to this conversation.

DISCUSSION / REFLECTION / LESSONS LEARNED: One of the difficulties of any longitudinal curriculum (i.e. monthly dinners, campaign work) is that residents will give up free time to participate. We have attempted to mitigate this by having weeks set aside for campaign work that will balance this out. Additionally finding the right issue for each resident can be a challenge; some residents can find an issue they are passionate about that is also current (i.e. gun sense legislation), for others we struggle to find one that matches them personally and has a movement in progress. Using national networks is one technique that has been successful.

ONLINE RESOURCE URL (OPTIONAL): http://tulane.edu/som/advocacy-leadership-track/ 
RESIDENCY WELLNESS: CHANGING CULTURE THROUGH EXPERIENTIAL LEARNING Margaret Horlick ${ }^{1}$; Patrick M. Cocks ${ }^{3}$; Lisa Altshuler ${ }^{2}$; Colleen Gillespie ${ }^{4}$; Sondra Zabar ${ }^{2} .{ }^{1} \mathrm{NY}$ Harbor VA Healthcare Center, Brooklyn, NY; ${ }^{2} \mathrm{NYU}$ School of Medicine, New York, NY; ${ }^{3}$ NYU School of Medicine, New York, NY; ${ }^{4}$ NYU SoM, Brooklyn, NY. (Control ID \#2467148)

NEEDS AND OBJECTIVES: Medical trainees may experience stigma when seeking help for mental illness and burnout. Renewed attention has focused on maintaining wellness and supporting trainees with mental illness after the alleged deaths from suicide of two IM interns in 2014. Our objectives were to: 1) Change the culture at our institution and 2) Equip our chiefs, residents and core faculty with skills to recognize struggling residents and knowledge of resources available. SETTING AND PARTICIPANTS: Large, urban academic medical center. 16 chief residents, 74 residents and 6 faculty participated in program

DESCRIPTION: We instituted two experiential learning activities to change our residency's culture around the approach to burnout and mental illness. Over the 2014-2015 academic year we included a "struggling colleague" case in the established intern and PGY-2 OSCEs and instituted a group three-station OSCE on struggling residents for the chief residents in our IM program. The learning objectives were that learners would 1) understand their role in identifying and assisting struggling colleagues 2) recognize problem behaviors, 3) effectively discuss concerns with colleagues who may be impaired and, 4) identify available resources. The intern and PGY-2 OSCE case portrayed a colleague who struggled with depression and alcohol abuse. The station involved $10 \mathrm{~min}$ with the standardized learner, then immediate feedback and discussion with faculty. The three-station group OSCE for the chiefs was preceded by a discussion of the impaired physician and the epidemiology of substance abuse, burnout and mental illness in our profession and chief residents' role in identifying and assisting struggling trainees. Cases included standardized trainees who evidenced alcohol abuse, depression, and adjustment disorder and who had varied insight and willingness to accept help

EVALUATION: Both housestaff and chief residents noted the value of this learning experience. Of the housestaff (41 interns and 33 PGY-2 s) who participated in the OSCE, one-third felt unprepared for the case. Most struggled with roles and boundary issues discussing concerns with peers rather than patients. All 16 chief residents who completed the group OSCE valued reviewing their role and learning approaches to these conversations. Themes expressed at 1 month follow up included: support for group OSCE, importance of seeing alternate approaches to these conversations, new perspective on skills needed for their job and the relevance of available resources.

DISCUSSION / REFLECTION / LESSONS LEARNED: Instituting experiential learning activities with chiefs, residents and faculty on mental illness and burnout can generate greater awareness of the problem, promote skills in approaching these situations and effectively disseminate resources. Learners valued the opportunity to discuss resources directly with faculty and understand program's supportive environment. Moreover, it demonstrated leadership's awareness of these issues and reinforced efforts at culture change.

RESOURCE STEWARDSHIP CURRICULUM: TEACHING INTERNAL MEDICINE AND PEDIATRIC RESIDENTS TO COMMUNICATE EFFECTIVELY WITH PATIENTS TO AVOID POTENTIAL HARM FROM UNNECESSARY DIAGNOSIS TESTS Adina Weinerman ${ }^{1}$; Geetha Mukerji ${ }^{2,3}$; Sarah Schwartz ${ }^{3,4}$; Adelle R. Atkinson ${ }^{3,4}$; Lynfa Stroud ${ }^{1,3}$; Brian Wong ${ }^{1,3}$. ' Sunnybrook Health Sciences Centre, Toronto, ON, Canada; ${ }^{2}$ Women's College Hospital, Toronto, ON, Canada; ${ }^{3}$ University of Toronto, Toronto, ON, Canada; ${ }^{4}$ Hospital for Sick Children, Toronto, ON, Canada. (Control ID \#2467809)
NEEDS AND OBJECTIVES: With rising healthcare costs and focus on quality, there is a growing need to promote resource stewardship in medical education. One driver of overuse is patient request for unnecessary testing. As emphasized in the Choosing Wisely ${ }^{\circledR}$ campaign, physicians can play a critical role in ensuring delivery of high-value care. Training residents to engage with patients in shared decision making about appropriate resource utilization is one important strategy to prevent overuse. With the increased emphasis on competency based medical education, evaluating residents' ability and uptake of these learned skills is equally important. Objectives: 1) Deliver an experiential curriculum on resource stewardship to develop residents' skills to counsel patients about unnecessary testing; 2) Evaluate the effectiveness of this curriculum in improving resident knowledge of resource stewardship and communication skills with patients to reduce overuse.

SETTING AND PARTICIPANTS: Participants were third year residents in the postgraduate internal medicine program $(N=45)$ and first to third year residents in the postgraduate pediatrics program $(N=38)$ at the University of Toronto.

DESCRIPTION: The project involved 2 phases. In Phase 1, a 2-h experiential workshop on resource stewardship with a focus on developing communication skills to counsel patients about unnecessary testing was delivered to internal medicine and pediatric residents. The workshop included 4 components: 1 ) an introductory didactic mini-lecture providing an overview of resource stewardship, 2) a trigger video that introduced attendees to a communication framework with four key elements (provide clear recommendations to patients; elicit patients' beliefs and concerns; provide empathy, partnership and legitimation; and confirm agreement), 3) role play with residents in small group break-out sessions and 4) a large group facilitated interactive discussion. In Phase 2, we carried out a structured assessment of residents' communication skills with patients requesting an unnecessary diagnostic test as part of a larger formative observed structured clinical examination (OSCE) approximately 6 months after the delivery of the workshop. Standardized patients completed a multi-item scoring sheet and rated performance on a 5-point Likert scale. Residents also completed a knowledge questionnaire to assess their knowledge of resource stewardship. We compared residents' scores who attended the workshop on the knowledge assessment to those who did not using a T-test. We also summarized resident skills using descriptive statistics, and performed a mixed-effects linear regression to identify predictors of better performance on the skills assessment.

EVALUATION: This study included 83 residents, 57(69\%) of whom attended the resource stewardship workshop. On delayed knowledge testing, residents who attended the workshop scored significantly better on the knowledge questionnaire than residents who did not attend the workshop $(4.25 \pm 1.90$ vs. $3.09 \pm 1.65$ out of $8, p=0.01)$. The mean overall score on the structured assessment was $3.95 \pm 0.68$ on a 5-point Likert scale. The linear regression found that both higher training level and higher score on the delayed knowledge assessment were independent positive correlates of better overall performance on skills assessment after adjusting for gender, training program and level, workshop attendance, and selfreported prior feedback on communication skills.

DISCUSSION / REFLECTION / LESSONS LEARNED: A workshop on resource stewardship that introduced a structured approach to communicating with patients about unnecessary diagnostic tests improved residents' knowledge on a delayed knowledge assessment tool. Improved knowledge, in addition to higher training level, was independently correlated with improved performance on a structured assessment of communication skills. This study demonstrates that an easily implementable resource stewardship workshop can improve residents' knowledge in this area, which in this study correlated with improved performance on a standardized communication station. This is one step in ensuring that these necessary skills can be transferrable into actual practice, to ensure delivery of highvalue care and prevent overuse. 
RIGHTCARE ROUNDS: A CONFERENCE DESIGNED TO RAISE HEALTHCARE VALUE BY REDUCING UNNECESSARY CARE Edward N. Murphy ${ }^{4}$; Fred R. Buckhold ${ }^{1}$; Tyler M. Miller ${ }^{2,6}$; Darlene Tad-y ${ }^{3}$; Brandon Combs ${ }^{2,5}$. ${ }^{1}$ Saint Louis University School of Medicine, St. Louis, MO; ${ }^{2}$ University of Colorado, Denver, $\mathrm{CO} ;{ }^{3}$ University of Colorado Denver School of Medicine, Aurora, $\mathrm{CO} ;{ }^{4}$ University of Colorado School of Medicine, Denver, CO; ${ }^{5}$ Lown Institute, Brookline, MA; ${ }^{6} \mathrm{VA}$ Medical Center, Denver, CO. (Control ID \#2469561)

NEEDS AND OBJECTIVES: The practice of high-value, patient-centered care is a key competency for medical trainees and providers. However, there is high variability in how and to what extent these principles have been incorporated at individual institutions. We sought to implement an educational conference format that would 1) Enable recognition of medical overuse as a significant barrier to high-value care, 2) Provide a forum for participants to analyze the drivers of medical overuse and underuse, and 3) Diagnose shortcomings in care delivery to identify individual and systems-level opportunities to enhance the value of patient care

SETTING AND PARTICIPANTS: RightCare Rounds is an adaptation of the familiar case conference format and thus is appropriate for a multitude of settings including morning report and grand rounds. The intended audience is any group with need for heightened awareness of the detrimental effects of medical overuse and underuse on the delivery of high-value care. While our initial focus has been on medical trainees, RightCare Rounds is appropriate for audiences composed of trainees, faculty, private practitioners or any combination of those groups.

DESCRIPTION: RightCare Rounds is an initiative promoting a new paradigm of doing "as much as possible for the patient and as little as possible to the patient." Initially conceived by the Lown Institute, an organization determined to improve value of care through elimination of unnecessary medical treatment, RightCare Rounds transforms the familiar case presentation format to facilitate an evidence-based discussion with the goal of improving value and promoting appropriate patient care. This initiative was promoted nationally to chief residents from several specialties as part of an educational program aimed at improving awareness around the origins and harms related to low-value care. RightCare Rounds is structured to address the whole patient and the entire continuum of care providing a unique opportunity to reflect more deeply on patient preferences, social or cultural contexts and systems-level dysfunction that is often "business as usual." It also explores the many drivers of overuse and underuse that may obstruct appropriate medical care. RightCare Rounds borrows on the traditional case presentation model and uses a familiar cause and effect diagram, or "RightCare Fishbone," to identify the drivers of lowvalue care. This familiarity and adaptability allow RightCare Rounds to be tailored to each programs' need and can be used to explore these issues in both inpatient and outpatient environments. By providing a venue for discussion and tools for analysis, RightCare Rounds enables practitioners to translate lessons from the conference to their own practice. EVALUATION: RightCare Rounds has been presented to audiences at division and department Grand Rounds and as noon conference to graduate medical education trainees. Qualitative information has been obtained from surveys of the audience members. The survey was created by the Lown Institute for evaluation of RightCare Rounds and includes questions asking the respondents level of agreement with statements such as "this conference effectively discussed the drivers of overuse/underuse," and "this conference uncovered opportunities for improving quality of care." Respondents could choose one of 4 responses: agree, somewhat agree, somewhat disagree and disagree. Also respondents were asked if the information from RightCare Rounds was useful in their practice answered as yes or no.

DISCUSSION / REFLECTION / LESSONS LEARNED: In the past year, RightCare Rounds has been adopted as part of the curricula at 32 residency programs across a range of specialties including internal medicine, family medicine, emergency medicine, and general surgery. To date, survey data from three presentations at two institutions is available. An internal medicine Grand Rounds with 33 respondents was notable for
$97 \%$ favorable rating ("agree" or "somewhat agree") to the statement "this conference effectively discussed the drivers of overuse/underuse." Similarly $94 \%$ of respondents gave favorable ratings to "this conference uncovered opportunities for improving quality of care." $88 \%$ of the respondents found the information applicable to their practice. For two presentations to a total of 27 internal medicine residents, there were unanimous favorable ratings to the same statements and unanimous agreement that the information was applicable to their practice. These data suggest that the RightCare Rounds format is easily implemented and well received by medical trainees. Most trainees noted that issues pertaining to medical overuse and underuse were directly applicable to daily practice. As training programs look for innovative ways to promote competencies in high value care, the positive responses to RightCare Rounds obtained thus far indicate RightCare Rounds is an effective means to address this unmet need

ONLINE RESOURCE URL (OPTIONAL): http://lowninstitute.org/toolkit-2/

SERVICE TRANSFORMED: LESSONS IN U.S. VETERAN CENTERED CARE: DEVELOPING AN INTERPROFESSIONAL MASSIVE OPEN ONLINE COURSE (MOOC) Monica L. Lypson ${ }^{3,2}$; Paula T. Ross ${ }^{1}$; Kathryn Goldrath ${ }^{4}$. ${ }^{1}$ University of Michigan Medical School, Ann Arbor, MI; ${ }^{2}$ University of Michigan, Ann Arbor, MI; ${ }^{3}$ Ann Arbor VA Healthcare System, Ann Arbor, MI; ${ }^{4}$ University of Michigan Medical Scholl, Ann Arbor, MI. (Control ID \#2463778)

NEEDS AND OBJECTIVES: With over 200,000 veterans reintegrate into civilian life each year, it is important that training programs identify appropriate content to educate health professions on caring for veteran patients; this content is critical in the development of the necessary clinical acumen whether working in Veteran Affairs (VHA) Healthcare System or civilian clinical settings. Educating trainees on issues related to veteran centered care is critical given that $11 \%$ of US citizens have served in the military; however, only $30 \%$ of veterans seek care in the VHA. In addition, each year approximately 150,000 health professional trainees rotate in VHA facilities. We developed a Massive Open Online Course (MOOC) to introduce health professionals to critical concepts in providing veteran centered care

SETTING AND PARTICIPANTS: The MOOC format will facilitate the delivery of this much needed content in an easily accessible manner. The on-line format incorporates key domains of e-learning: lessons that communicate information and lessons that develop skills. The MOOC will allow for open global enrollment.

DESCRIPTION: The course is delivered over 7 weeks and include lecture videos, content expert commentary, perspectives from veterans, educational readings, documentary footage, and active learning techniques (e.g., photo-elicitation, reflective writing) to help learners recognize their own biases regarding veterans specifically, but for all patients in general. The active learning techniques and approach to assessment was an important valued added part of the development of the course to attempt to evaluate some aspects of the affective domain. Each week also includes a learner assessment using multiple choice questions and peer assessment. (March-April 2016)

EVALUATION: In year one of the live course $66.6 \%$ of students strongly agreed/agreed that exercises helped with my observational \& critical-thinking, as well as noting this activity heightened my awareness about providing patient-centered health care. Also $83.3 \%$ strongly agreed/agreed the photographs helped me to reflect on my own attitudes toward people from different racial groups. In year two of the live course implementation sample comments related to the role providers have in addressing disparities: "Providers need to check their own biases so they don't propagate them. Providers also need to join together to advocate for and address health disparities." "I need to check my own biases frequently so that they propagate into patient care as little as possible." Also, $100 \%$ of the students agreed or strongly agreed that the photographs helped me to reflect on my own attitudes toward veterans. 
DISCUSSION / REFLECTION / LESSONS LEARNED: The course was designed in collaboration with staff at the University of Michigan's Digital Education \& Innovation (DEI). We plan to offer the course as an on-demand enrollment session offer flexibility to learners and provide a platform for completion prior to healthcare encounters with veterans or at a VHA healthcare facility. Teaching: .The development of a quality MOOC takes the input and resources of a large university infrastructure to ensure high fidelity. Learning how to approach and evaluate learning analytics developed from peerassessment and reflections will be an important strategy for course evaluation. The views expressed in this poster are those of the authors and do not necessarily reflect the position or policy of the Department of Veterans Affairs or the U.S. government

ONLINE RESOURCE URL (OPTIONAL): http://digitaleducation.umich.edu/dei/vacentered-care/

SOCIAL JUSTICE \& COMMUNITY ADVOCACY: CREATION OF A NOVEL PATHWAY IN RESIDENCY TRAINING Utibe R. Essien; Julian Mitton. Massachusetts General Hospital, Boston, MA. (Control ID \#2469731)

NEEDS AND OBJECTIVES: The work of a physician is complementary with that of a social justice pioneer and community organizer. Those interdependent social roles can add value and meaning to practice across all clinical environments. Such efforts require unique skills and experiences that are not traditionally emphasized in medical education. As such, through this Social Justice and Community Advocacy (SJCA) pathway we aimed to: - provide Internal Medicine resident trainees and medical students with a longitudinal pathway in the interdisciplinary practice of social justice and community advocacy; - draw on opportunities at the academic medical center, Massachusetts General Hospital (MGH), Harvard Medical School, Harvard University and in the greater Boston community; - assist residents in tailoring their 3 years of residency toward the acquisition of leadership and clinical skills needed to impact change in the lives of their patients; - create and maintain a pathway that is mentored, patientand community-centered and culminates in a final project or service initiative.

SETTING AND PARTICIPANTS: The pathway includes a series of experiences aimed at skill acquisition and mentored advocacy. Pathway design is tailored to the particula interests of the resident, ideally within the context of patient care and their clinic community. There are suggested rotations and experiences in all 3 years in a graduated and longitudinal fashion, with increasing responsibility in the second and third year Activities will culminate in a senior capstone project, colloquium or service initiative. Each resident is mentored through their pathway, working with an assigned faculty member to acquire the unique skill set and experiences needed for the trainee to impact change in the lives of their patients and/or community of interest. Mentorship is also promoted in social gatherings, aimed at connecting residents with local and national leaders. Longitudinal mentorship will help residents culminate their work in a final project, research colloquium or service initiative during the senior year. It is the hope that these senior capstones will be presented at a national forum.

DESCRIPTION: Through the SJCA pathway, residents have the opportunity to tailor their pathway with required experiences and elective outpatient rotations, leadership opportunities and community experiences: Advocacy and community leadership Global Health rotation Homeless Health Prison Health Addiction Medicine Refugee/Immigrant Health Away rotations (Uganda, South Dakota/Indian Health Services, Boston Public Health Commission) Hospital administration Medical writing Research Through the pathway there are also leadership experiences through the newly created Social Justice Interest Group, developing resident report and noon conference case presentations. Community engagement is key to the pathway and involves participation in extracurricular activity programming within the Department of Medicine and other clinically-based community health staff including community health workers, population health managers, mental health and addiction medicine specialists and local community leaders.
EVALUATION: As the pathway is in its infancy, with the first cohort beginning in June 2016, the expectation is that most of the evaluation will take place over the next few years including pre and post surveys of resident trainees as well as patients. Each resident that goes through the pathway will be expected to complete: - Capstone Project, Colloquium or Service Initiative-Presentation at Regional or National Conference

DISCUSSION / REFLECTION / LESSONS LEARNED: Resident trainees have the privilege of providing care to patients of all social and ethnic backgrounds, requiring a nuanced approach that considers the social determinants and community context of health. Yet there often is little consideration to this nuance in daily education and system modeling. Addressing these considerations is increasingly important as our institutions try to engage patients in their homes and neighborhoods. Many residency programs across the country have taken on this challenge by developing social justice programming to ensure that residents and other health professional trainees are trained in the skills and afforded the opportunity to consider the social challenges that impact patient health. Through the experiences of other training programs we learned that rather than creating a track where residents were expected to follow a strict set of rotations over their training period, we would create a pathway, pairing residents with faculty mentors to tailor an interdisciplinary training experience through the preferential selection of several electives, leadership opportunities and community opportunities. We hope to share this innovative approach to increasing social justice and community advocacy training in residency while seeking opportunities for further collaboration that will help sustain this work of addressing the social determinants of health

ONLINE RESOURCE URL (OPTIONAL): http://www.altfutures.org/pubs/ leveragingSDH/IAF-CHCsLeveragingSDH.pdf

STUDENT HOTSPOTTING: CARING FOR THE SICKEST AMONG US THROUGH NARRATIVE-BASED EXPERIENTIAL INTERPROFESSIONAL EDUCATION John Marshall ${ }^{1}$; Rita K. Kuwahara ${ }^{3}$; Aziza Dang ${ }^{2} .{ }^{1}$ Emory University, Atlanta, GA; ${ }^{2}$ Virginia Commonwealth University, Richmond, VA; ${ }^{3}$ University of North Carolina at Chapel Hill School of Medicine, Durham, NC. (Control ID \#2469847)

NEEDS AND OBJECTIVES: In the US, caring for the top one percent of health care utilizers constitutes twenty percent of national health care expenditures. Our health professions education system is currently failing to prepare students for the highly interprofessional, high-touch work required to serve these patients. Our objectives included: 1 . To provide an immersive, longitudinal, patient-driven experience in interprofessional education at our major academic medical institutions 2 . To provide case-based training in skills required to care for medically and socially complex patients with recurrent hospitalizations, including motivational interviewing, barrier recognition and troubleshooting, and patient advocacy

SETTING AND PARTICIPANTS: Teams consisted of five to six members and were required to be interprofessional. Students represented a variety of fields, including medicine, nursing, public health, social work, physical and occupational therapy, divinity, and business. In the inaugural year, 2014, ten schools completed the pilot program. Ten more joined in 2015, with all twenty recently completing the wrap-up conference in Camden, NJ.

DESCRIPTION: After assembling an interprofessional team of students with at least one faculty mentor, institutions applied for a hotspotting mini-grant through the AAMC, Camden Coalition of Healthcare Providers, and Primary Care Progress. Teams attended a kick-off conference in Camden, $\mathrm{NJ}$ with training in teamwork and an introduction to high utilizing work. Teams were then given autonomy as they recruited and worked with five to six patients with multiple health and social needs. Interventions were unique to each patient but typically included home visits, accompaniment to health provider visits, care coordination, and assistance with health systems navigation. Patient education and empowerment were emphasized, culminating in having patients graduate the program and move towards more independent 
functioning. For students, relevant background information and skills were taught via monthly webinars. MacArthur Genius award winner and pioneer in high utilizing work, Dr. Jeffrey Brenner, who founded and serves as executive director of the Camden Coalition of Healthcare Providers, provided regular guidance as students presented their cases during monthly calls. At the conclusion, teams reassembled in Camden for a wrap-up conference complete with poster presentations and leadership training.

EVALUATION: Most immediately, poster presentations demonstrated both the intangibles learned by teams and their patients, as well as "utilization EKGs," which visually represented timing and costs of healthcare utilization events before and during the intervention. Evaluations completed by each team as well as a student advisory council help provide feedback on the project itself. At some institutions students were able to receive elective credit, for which the faculty mentor evaluated students per institutionspecific guidelines.

DISCUSSION / REFLECTION / LESSONS LEARNED: Numerous barriers exist to interprofessional education, including scheduling, poor avenues of communication, and lack of joint mission. However, shared experiences during training and adopting a narrative-based approach to understanding patient stories is essential to addressing the crisis of persistently poor health outcomes among our nation's most disenfranchised and frequently ill individuals. Participating students cited this program not just as an opportunity to further skills in patient communication, but also to directly learn scope of practice across health disciplines and to practice effective team communication and project management.

ONLINE RESOURCE URL (OPTIONAL): Online information regarding the minigrant: https://www.aamc.org/initiatives/hotspotter/

SWIM LANES AND CLINICAL COMPETENCY COMMITTEE NEEDS: USING QUALITY IMPROVEMENT (QI) TECHNIQUES TO ENHANCE A RESIDENCY PROGRAM'S ASSESSMENT SYSTEM James Thomascik; Colleen Colbert; Andrei Brateanu; Eric Holizna. CCF, Cleveland, OH. (Control ID \#2470147)

NEEDS AND OBJECTIVES: Clinical Competency Committee (CCC) reviews of resident progress are dependent upon quality of the data used to assess residents; faculty assessor and CCC reviewer training; and time invested in the assessment process maintenance and redesign. Analyses of the CCC assessment process and faculty evaluation completion rates per rotation were performed previously within the Cleveland Clinic Internal Medicine residency program $(N=168$ residents $)$ and identified gaps in the assessment system. Our goal was to improve the residency program assessment system using quality improvement techniques.

SETTING AND PARTICIPANTS: A hybrid of Six Sigma - DMAIC (Define, Measure, Analyze, Improve, Control) and Lean tools was chosen due to the complexity of the residency assessment system and variability of each resident's assessment path. The initial analysis identified high variability in the spectrum of potential critical assessment points for each resident, number of contributing assessors, assessment tools used, and stakeholders' knowledge of their roles in the assessment system.

DESCRIPTION: A swim lane diagram was used to model current assessment workflow, while capturing variable inputs. Horizontal swim lanes identified all assessment system stakeholders and vertical lanes identified points at which residents interacted with an assessor or assessment tool during the academic year. Next, an "Effort vs. Impact" model was used to prioritize each gap into the appropriate quadrant and identify concurrent themes. As a result, low effort/high impact or high effort /high impact processes were identified, requiring immediate adjustments, or rigorous, systematic approaches, respectively. For projects requiring controlled and cyclical improvement tools, a Lean A3 approach was selected. In addition, resetting the improvement cycle to repeatedly assess progress and successful gap closures and identify new gaps is essential to reducing variability in the resident assessment process.
EVALUATION: Redesign of resident assessment tools was identified as the highest priority project. Redesign of assessment tools is in process and includes review of content domains, revision of items, alignment with sub-competencies, and examination of frequency and timing of assessment tool distribution.

DISCUSSION / REFLECTION / LESSONS LEARNED: To our knowledge, this is the first time an educational assessment system within a residency program has been improved using QI techniques. Through systematic, iterative QI approaches, seemingly entrenched and "unfixable" problems can be ameliorated.

TAKING A DEEP DIVE IN PRIMARY CARE: USING A PRIMARY CAREFOCUSED AMBULATORY BLOCK ROTATION TO IMPROVE INTERN CONFIDENCE AND ENTHUSIASM IN OUTPATIENT GENERAL MEDICINE AND CONTINUITY CLINIC Jennifer R. Lukela. University of Michigan Medical School, Ann Arbor, MI. (Control ID \#2466819)

NEEDS AND OBJECTIVES: It has previously been reported that internal medicine residents do not value their experiences in outpatient continuity clinic as highly as they value their inpatient clinical rotations. Questions remain about the cause and significance of this finding. Not surprisingly, there is evidence that higher resident satisfaction with their continuity clinic experience positively influences residents' likelihood in choosing a career in outpatient general medicine. Therefore, innovations to improve both residents' perceived value and satisfaction in continuity clinic are important to potentially increase the number of general outpatient internists in the future. Previous work demonstrates that early immersion weeks and short term (ie, 2 day) ambulatory boot camps are helpful in improving intern confidence. We sought to show that a primary care focused month-long ambulatory block rotation could have similar impact. In the past few years, we have made changes to our continuity clinic curriculum including the introduction of week-long intern immersion weeks during elective time. For the 2015-2016 academic year, we modified the clinical content of our month-long intern ambulatory block rotations to provide a "deeper dive" into outpatient general medicine in a variety of primary care settings. With this intervention, we sought to address several questions: would a focused ambulatory block rotation in primary care improve intern confidence in managing acute complaints and chronic illness routinely seen in continuity clinic? Would a deeper dive in outpatient general medicine early in training impact resident interest in a future career in outpatient general medicine? Two additional questions we sought to address focused on interns' past experience in outpatient general medicine during medical school: what influence did outpatient general medicine exposure in medical school have on incoming intern confidence in managing common outpatient complaints and chronic illness? Did the extent or type (ie, longitudinal vs. episodic) of exposure to outpatient medicine in medical school impact interest in a future career in outpatient general medicine?

SETTING AND PARTICIPANTS: All categorical Interal Medicine interns in our program (total number 44) participate in at least one of 4 month long ambulatory block rotations during their HO1 training year. Each intern also maintains a continuity clinic at one of three clinical sites: a large hospital-based academic general medicine practice, a University-affiliated community-based ambulatory care clinic or the general medicine clinic at our affiliated Veteran's Administration Hospital.

DESCRIPTION: After our curricular re-design, each intern ambulatory block rotation included 3-4 half day sessions/week in the intern's own continuity clinic, 3 half day sessions/week in a community-based outpatient general medicine clinic and 1-4 half day sessions/month working in a clinic for underserved or high risk populations. In addition, interns participated in other aspects of our overall ambulatory curriculum including daily ambulatory morning report and a half day per week of didactic sessions throughout the month. EVALUATION: At the end of each month, all interns who have completed an ambulatory block rotation are invited to participate in a voluntary post-rotation survey seeking to 
address the questions noted above. Data collection is currently on-going. At this point, we have collected data for a total of 6 months. 19 of the 23 interns who have completed their ambulatory block rotation have completed the survey ( $83 \%$ response rate). In addition to questions about intern confidence in outpatient medicine, the survey also collects information on past experiences in outpatient medicine during their time in medical school.

DISCUSSION / REFLECTION / LESSONS LEARNED: Analysis of our survey results is ongoing. Initial results do not demonstrate a clear correlation between past medical school exposure to primary care and either confidence among incoming interns to provide care in the outpatient setting or interest in a career in general medicine. Participation in our revised intern ambulatory block rotation did appear to improve self-reported intern confidence in addressing common outpatient conditions and managing chronic illnesses often seen in continuity clinic. Initial review suggests participation in the rotation had minimal impact on interest in careers in outpatient general medicine, though the overall trend was positive. Further work will need to address whether an early deep dive in outpatient general medicine impacts overall resident competence (vs. confidence) in the skills necessary to function in the outpatient setting. Our hypothesis is that with improved confidence and competence in outpatient medicine, resident satisfaction and their overall assessment of educational value of continuity clinic will improve. Further work is needed to address this hypothesis and to identify other strategies to improve resident's perception of the value of their continuity clinic experience.

TEACHING INTERPROFESSIONAL COLLABORATIVE CARE SKILLS TO STUDENTS USING A BLENDED LEARNING APPROACH Maria A. Wamsley ${ }^{3}$; Angel $\mathrm{Chen}^{4}$; Cathi Dennehy ${ }^{4}$; Amber Fitzsimmons ${ }^{4}$; Susan $\mathrm{Hyde}^{4}$; Kirby Lee $^{4}$; Bridget C. OBrien ${ }^{2}$; Josette Rivera ${ }^{3}$; Rebecca L. Shunk ${ }^{1,5} .{ }^{1}$ UCSF, San Francisco, CA; ${ }^{2}$ Univ of California, San Francisco, San Rafael, CA; ${ }^{3}$ University of California, San Francisco, San Francisco, CA; ${ }^{4}$ University of California San Francisco, San Francisco, CA; ${ }^{5}$ VA Medical Center, San Francisco, CA. (Control ID \#2469804)

NEEDS AND OBJECTIVES: There is growing recognition of the need to teach interprofessional (IP) collaborative competencies to health professions learners. Barriers to implementing curricula include scheduling, geographical constraints and resources Asynchronous online learning may overcome these barriers. Studies support the effectiveness of online learning to teach IP concepts, but suggest that a blended learning approach may appeal more to learners. We developed a blended course to teach core IP concepts to students. Representative learning objectives for the course include the following: 1) Discuss the role of teamwork and communication in medical errors. 2) Describe the training program and potential career paths of some of the health professions. 3) Demonstrate the use of strategies and tools that facilitate effective communication and collaboration. 4) Demonstrate a three-step approach to managing conflict. 5) Identify and analyze leadership compass styles and discuss how to support team members with different styles. SETTING AND PARTICIPANTS: The curriculum was developed at a health sciences university with five health professional training programs (dentistry, medicine, nursing, pharmacy and physical therapy). All first-year learners participated in the curriculum. DESCRIPTION: We developed 5 ninety-minute online modules to teach learners core concepts in IP collaborative practice: 1) core IP concepts 2) roles and responsibilities of healthcare professionals 3) effective IP communication 4) conflict management 5) team leadership and membership. Each module includes interactive cases, demonstrations and expert interviews and is paired with a facilitated small group session for skills practice. Sessions are required and held quarterly throughout the first and second-year.

EVALUATION: Learners and faculty facilitators were surveyed after each small group session and learner focus groups were conducted at the end of the first-year of the curriculum. Six-hundred learners and 55 faculty facilitators participated in the first-year of the curriculum. Mean learner ratings of the overall value of the online modules ranged from 2.80 to 3.15 ( 1 = poor, 5 = excellent); mean learner ratings of small group sessions ranged from 3.12 to 3.57 . Most learners agreed or strongly agreed (75-91\%) that they met individual learning objectives for the sessions. Mean faculty ratings of the value of the online modules ranged from 3.75 to 4.46 and the small group sessions ranged from 4.13 to 4.38. Focus groups revealed that students perceived that the curriculum was an "add-on" to existing curriculum. Students appreciated the flexibility of the online modules, but felt that they were too lengthy and desired greater interactivity. Students desired more time to interact informally in small group sessions and commented on the challenges of creating activities that integrated all professions and were appropriate to learner skills and varying degrees of clinical experience. Students also commented that the quality of the small group sessions was dependent upon faculty facilitator skills which were variable.

DISCUSSION / REFLECTION / LESSONS LEARNED: Learners are satisfied with online materials developed to teach IP collaborative care skills using a blended learning approach. While learners value the flexibility of online content, IP small group sessions are rated more highly. Faculty rate both the online and face-to-face sessions more highly than students. Students desire better integration of IPE curriculum into their core curriculum. Course planning/development and course implementation were very resource intensive.

TEACHING POPULATION MANAGEMENT THROUGH A TEAM BASED HTN DISPARITY QI CURRICULUM Erika L. Hoffman ${ }^{4}$; Jaishree Hariharan ${ }^{3}$; Elena Lebduska ${ }^{2}$; Amar Kohli ${ }^{1} .{ }^{1}$ UPMC, Mars, PA; ${ }^{2}$ University of Pittsburgh Medical Center, Pittsburgh, PA; ${ }^{3}$ University of Pittsburgh Medical center, Pittsburgh, PA; ${ }^{4}$ VA Pittsburgh Healthcare System and The University of Pittsburgh School of Medicine, Pittsburgh, PA. (Control ID \#2469555)

NEEDS AND OBJECTIVES: In reviewing VA Pittsburgh quality metric data, a disparity in blood pressure (bp) control between white and black hypertensive patients with and without diabetes was found. We saw this as an opportunity to teach QI principles using a new curriculum developed at the University of Pittsburgh. While components of quality improvement education are infused into our residency education, hands on practice using resident-level population databases to help drive performance improvement were lacking. Residents had an opportunity to develop patient, provider and system-based interventions. SETTING AND PARTICIPANTS: The setting was a VA Primary Care Clinic serving as a training site for 56 residents. 4011 patients were cared for in medical home (PACT) teams. 7 teams were each supported by an attending, MSA, LPN, and RN along with Pharm Ds, social workers, and telehealth specialists.

DESCRIPTION: Didactic sessions were given on principles of QI. Residents were introduced to the VSSC and disparity databases. They completed worksheets for their patient panels which sought information on the percent of their patients with bp $>150 / 90$ and $>160 / 100$. They looked at the number of their patients who had not been to clinic in over 6 months. Residents drilled down to patient level data and used chart review to reflect on possible patient, provider and system-based barriers to bp control. Resident team leaders were chosen for each clinic day. Team leaders completed worksheets, collating team data along with potential solutions to pilot to overcome barriers. Teams created aim statements. They chose 3 agreed upon ideas to present as pilots. Ideas were presented to VA leadership via a QI council. At the QI council, interventions were prioritized and voted on by the team leaders. The QI nurse discussed feasibility of ideas. The QI council chose to implement several clinic-based interventions. Blood pressure technique training was standardized for the LPNs and became part of their annual competency. A free LPN-driven blood pressure clinic was created to help alleviate patient perceived financial barriers to care. This was piloted in one of seven academic PACT teams. 
Residents chose to increase home telehealth use to decrease cost associated with travel and visits for care. Residents asked for team based data to track performance improvement. The number of combination medications in clinic quick order sets was maximized to help decrease cost of drugs. Residents received patient-based outlier lists, paying attention patients who had not been to clinic in $>6$ months

EVALUATION: All residents learned to use VA databases to assist in panel management. Initially, there was a disparity in bp control between black and white patients of $7.4 \%$. For diabetics the disparity was $6.6 \%$. At the end of the project, the disparity was 7.5 and $4.2 \%$, respectively. We could not drill down into the data in order to know the impact individual providers made in closing the disparity gap. At the end of the project, $100 \%$ of the team LPNs met with a nurse educator who assessed clinical skills \& competency. For the PACT team trialing the LPN bp clinic, residents documented proposed plans of care in the medical record for the LPN to follow. Preceptors supported the LPN if the resident could not be contacted. $4 / 6$ residents in the LPN clinic pilot team saw a reduction in the percentage of patients with stage II hypertension. 1 pilot resident saw a reduction in the percent of patients with stage II hypertension that fell from 33.1 to $13.3 \% .13 / 46$ residents in teams without LPN bp clinics saw a reduction in the percentage of patients with stage II hypertension. Residents not involved in a pilot team either personally contacted patients from their outlier list or sought the help of a PACT team member and made plans to address elevated blood pressures. At the end of 6 months, LPNs blood pressure clinics were established for all resident PACT teams. Data analysis continues.

DISCUSSION / REFLECTION / LESSONS LEARNED: Resident feedback about the curriculum was positive. Residents felt they had a bigger role in QI this year, improving their ability to impact outcome and process. They said that seeing data helped them to understand how they were doing. Residents said they never thought of those patients who don't come to clinic as something they should address. They identified important process changes to help keep the momentum going, asking for time to review outlier lists and to call patients. They noted that end of year transitions of care should be evaluated in future QI phases. Designing projects that can yield significant outcomes in a short time frame can be a challenge. Some residents saw no improvement in the percentage of their patients who had uncontrolled bp and pointed out that 6 months may not be enough time for evaluation. Many asked us to continue to follow our hypertensive population. This year, we will continue to focus on bp control and will add glucose control as our next resident QI project.

TEACHING QUALITY IMPROVEMENT IN THE STUDENT-RUN FREE CLINIC Robert A. Rifkin ${ }^{3}$; Ammar Siddiqui ${ }^{3}$; Brijen Shah ${ }^{2}$; David C. Thomas ${ }^{1}$; Yasmin S. Meah ${ }^{4} .{ }^{1}$ Icahn Scholl of Medicine at Mount Sinai, New York, NY; ${ }^{2}$ Icahn School of Medicine, New York, NY; ${ }^{3}$ Icahn School of Medicine at Mount Sinai, New York, NY; ${ }^{4}$ Mount Sinai School of Medicine, New York, NY. (Control ID \#2469670)

NEEDS AND OBJECTIVES: Quality Improvement (QI) is an essential skillset for increasing efficiency and patient safety in healthcare, but most US medical students receive little or no classroom or hands-on instruction in QI.

SETTING AND PARTICIPANTS: The East Harlem Health Outreach Partnership (EHHOP) is a student-run, free clinic for the uninsured in New York City, sponsored by the Icahn School of Medicine at Mount Sinai (ISMMS). ISMMS students $(n=100)$ in their second year were participants in the described course.

DESCRIPTION: Launched in 2010, Quality Improvement Council (QIC) is a year-long, hands-on course for second-year medical students. Meetings are monthly and provide an opportunity for two-way communication between faculty and students. Between meetings, students work in groups and communicate regularly with faculty and student leaders by email. The course uses a combination of didactic and project-based learning. At the first meeting, students receive an overview of the Institute for Healthcare Improvement (IHI) Model for
Improvement, based on the "Plan, Do, Study, Act" (PDSA) cycle. Students are divided into groups of 5 and, at the second meeting, are guided through a "Define the Problem" Workshop, with the goal of clearly articulating a problem observed in the free clinic and establishing a quantitative goal. Over the next 8 months, students collect baseline data in the clinic, and implement 1-3 PDSA cycles consisting of piloted improvements and evaluations of these changes. At monthly meetings, they present their findings and receive feedback from faculty and peers. At the end of the year, students present their findings at EHHOP QIC Grand Rounds. Successful projects are incorporated into the standard operating procedures of the clinic. In total, 19 projects have been completed. In the first year, projects focused on day-today clinic operations issues, such as reducing referral completion time, reducing patient noshows, and improving physician recruitment. Most recently, projects have focused on patient experiences and outcomes, such as medication adherence and diet/exercise goal adherence; or tackled larger operational issues such as medication costs.

EVALUATION: Each year, projects pursued by students are recorded in a table and their main interventions, main outcomes, strengths and challenges are recorded. Over time, this institutional knowledge has been used to guide groups toward achievable projects. Qualitative feedback on learning experience has been solicited from each class and used to improve the class structure; for example, by limiting class size and providing peer feedback in an anonymized fashion.

DISCUSSION / REFLECTION / LESSONS LEARNED: Now in its fifth year, QIC has succeeded in establishing numerous improvements in clinic standard operating procedures, including establishment of a permanent referrals and patient navigation team known as Access to Care Team (ACT), and building a mobile knowledge management system (mKMS) app known as the EHHapp. Over time, the focus of QIC projects has shifted from internal operational problems (e.g. physician recruitment, referrals) to issues directly related to patient outcomes (e.g. medication adherence, diet/exercise goal adherence). Student presentations and feedback indicate that they gain a strong working knowledge of QI principles and the anatomy of a successful health care improvement project.

TEACHING RESIDENTS ABOUT QUALITY IMPROVEMENT: SWITCHING THE FOCUS FROM KNOWLEDGE TO PRACTICE Elena Lebduska ${ }^{3}$; Carla Spagnoletti ${ }^{2}$; Amar Kohli ${ }^{1}$; Erika L. Hoffman ${ }^{5}$; Gary Fischer ${ }^{2}$; Jaishree Hariharan ${ }^{4}$. ${ }^{1}$ UPMC, Mars, PA; ${ }^{2}$ University of Pittsburgh, Pittsburgh, PA; ${ }^{3}$ University of Pittsburgh Medical Center, Pittsburgh, PA; ${ }^{4}$ University of Pittsburgh Medical center, Pittsburgh, PA; ${ }^{5}$ VAPHS/Univeristy of Pittsburgh, Pittsburgh, PA. (Control ID \#2465365)

NEEDS AND OBJECTIVES: ACGME recognizes that internal medicine (IM) trainees must achieve competence in systems based practices. The outpatient setting is an ideal place to teach these skills. With many residency programs going to an inpatient/outpatient "block system", achieving continuity for quality improvement (QI) projects in the outpatient setting becomes a greater challenge. With this in mind, we implemented an educational initiative that aimed to: 1) Increase resident participation in QI projects in the outpatient setting by using a resident-driven team-based approach; 2) Improve patient level chronic disease outcomes measures; 3) Evaluate resident attitudes and interest in interdisciplinary QI projects.

SETTING AND PARTICIPANTS: Participants were the 140 residents affiliated with the University of Pittsburgh Medical Center. All residents followed a panel of continuity patients with faculty supervision in one of three hospital-based clinical sites: a university clinic $(n=52)$, a community clinic $(n=36)$ and a VA clinic $(n=52)$. In the new block system, residents have clinic one full day each week (Monday-Thursday) for 4 weeks, followed by no clinic for 4 weeks. Residents were placed into QI teams based on their clinic day, for a total of 8 interdisciplinary QI teams per site.

DESCRIPTION: The focus of our curriculum was on using a longitudinal, multistep, innovative approach to teach QI by focusing on single area patient level outcomes measure 
with the lowest compliance rates. At non-VA clinic sites, the focus of the resident QI project was on diabetes, with improving eye and foot exam rates as the goal for the 2014 2015 academic year, and followed these steps: Step 1) Residents received a short didactic session on principles of QI. Step 2) Using automated chart review, residents identified their diabetic patients who had not received these exams. Each resident then used a worksheet to review their patients' charts to identify key barriers and then brainstormed interventions for improvement. Step 4) Residents joined together in their QI teams and compiled their individual data using a team worksheet. They then selected interventions for implementation. Step 5) A resident "team leader" from each group, along with ancillary staff, the clinic director, and faculty clinicians, convened to form a QI council. Interventions developed by the teams were voted on and the top 3-4 were selected to be clinic-wide QI interventions. Step 6) Team leaders worked with ancillary staff to finalize protocols for the chosen interventions, and these were initiated. QI Teams met every other month to review process measures and make adjustments. The VA-based clinic residents followed a similar 7-step approach but focused their QI interventions on improving hypertension metrics.

EVALUATION: Results from the 2014-2015 pilot year are discussed here. Worksheets were developed to assist individual residents and QI teams with Steps 1-3. Data derived from the university hospital site ( 52 residents), showed that 47 of 52 completed individual worksheets (participation rate $(\mathrm{PR})=90 \%$ ) and all 8 QI teams completed worksheets ( $P R=100 \%$ ). Effectiveness of the QI curriculum was assessed with quarterly patient outcomes reports. At the university hospital site the residents had 328 diabetic patients in June 2014. From June 2014 to June 2015, resident patients' eye exam rates increased from 42 to $69 \%(p<.001)$ and in addition foot exam rates increased from 58 to $78 \%(p<.001)$ Post curriculum both eye and foot exam rates remained stable in Sept 2015. All residents were surveyed at the end of June 2015 on the QI curriculum. Over $78 \%$ of residents thought that the topic of QI was important or very important to their residency training, and over $90 \%$ believed that working in an interdisciplinary team was important or very important to a successful QI project. QI team leaders met regularly to collate successes and failures and performed a Plan Do Study Act (PDSA) cycle of the curriculum, allowing changes to be made in real time.

DISCUSSION / REFLECTION / LESSONS LEARNED: This is a team-based educational initiative to teach residents about QI and to improve patients' chronic disease management. It is unique in that it is resident-driven and it utilizes an innovative stepwise approach that can easily be extrapolated to a variety of QI interventions at different clinical sites and institutions. To date, the initiative has successfully engaged nearly 140 residents in the QI process, has fostered collaboration between residents and ancillary clinic staff, as well as led to improved patient outcomes. By switching the focus of resident QI education from knowledge to practice, we were able to see improvement in patient level outcomes in a relatively short period of time. Getting resident buy in, compiling clinic resources for a focused QI project, making quality metric data transparent, and using resident-driven QI interdisciplinary teams were keys to success. Because of this success in the 2014-2015 pilot year, our program intends on continuing this curriculum indefinitely.

TEACHING THE TEACHERS: ADAPTING A FACULTY DEVELOPMENT WORKSHOP ON PATIENT-CENTERED ELECTRONIC MEDICAL RECORDS USE FOR BUSY CLINICIANS Wei Wei Lee ${ }^{3}$; Lollita Alkureishi ${ }^{3}$;. H. Isaacson ${ }^{1}$; Mark Mayer ${ }^{2}$; Vineet M. Arora ${ }^{4} .{ }^{1}$ Cleveland Clinic, Cleveland, $\mathrm{OH} ;{ }^{2}$ Cleveland Clinic Lerner College of Medicine, Cleveland, OH; ${ }^{3}$ University of Chicago, Chicago, IL; ${ }^{4}$ University of Chicago Medical Center, Chicago, IL. (Control ID \#2467410)

NEEDS AND OBJECTIVES: Electronic medical record (EMR) use in exam rooms can distract providers from their patients. Despite rapid EMR adoption in academic institutions, few faculty receive training or feedback on how to integrate patient-centered communication skills while managing the demands of the EMR. Further, given the important function faculty serve as role-models, effective education on EMR best communication practices is critically important so they can in turn demonstrate and teach best behaviors to resident and student trainees. Our objective was to develop and evaluate a faculty workshop to improve knowledge and ability to implement best practices regarding patient-centered EMR use.

SETTING AND PARTICIPANTS: We developed a four hour patient-centered EMR use workshop for primary care faculty at the Cleveland Clinic (CC), which was then adapted for University of Chicago (UC) faculty in $90 \mathrm{~min}$. We aimed to assess 1) faculty learner satisfaction 2) post-training self-assessed knowledge, attitude, skills 3 ) efficacy of longer vs. shorter patient-centered EMR training.

DESCRIPTION: After literature review, we developed a faculty workshop to improve patient-centered EMR use which consisted of: (1) a lecture highlighting barriers and best practices for patient-centered EMR use; (2) a Group-Objective Structured Clinical Exam (GOSCE) to practice skills with a standardized patient (SP) and mock patient chart in an EPIC training environment. Faculty interacted with the SP to take a history, integrate the EMR for chart review and document a HPI. Groups consisted of 2-4 faculty, 1 facilitator and $1 \mathrm{SP}$ with immediate performance feedback. At CC, training was completed during a dedicated 4-h block routinely reserved for Continuing Medical Education (CME). At UC, because such a block did not exist, time constraints necessitated a 90 min session (1-h of lunch with 30 additional minutes of time donated by the clinic). All faculty received CME credit. The longer CC workshop included; 75 min lecture, $100 \mathrm{~min}$ GOSCE, two $20 \mathrm{~min}$ breaks, 25 min of closing feedback. The shorter UC workshop included; 20 min lecture, $60 \mathrm{~min}$ GOSCE, 10 min of feedback.

EVALUATION: 32 academic primary care faculty [Family Medicine (FM) and General Internal Medicine (GIM)] at two academic medical centers completed training [13 $\mathrm{cm}^{3}$ faculty (5 FM; 8 GIM) and 19 UC (GIM)]. All faculty completed a 23 item post-workshop evaluation assessing knowledge, attitude and skills. Responses to Likert items were dichotomized at the high end of the scale to denote agreement (i.e. agree/strongly agree). Descriptive statistics were summarized and compared between sites. We analyzed 30/32 (94\%) post-workshop evaluations. Overall, the majority $(67 \%, 20 / 30)$ of respondents were female (CC $50 \%$ vs UC $72 \%, p=0.22$ ), with mean age of 48 (range 31-65) (CC 47 years old vs UC 45 years old, $p=0.59)$. Overall, $100 \%$ (30/30) of faculty agreed it was 'important to receive training,' 'relevant to their practice,' and enabled them 'to better teach and role model patient-centered care for trainees', with no difference in mean ratings between CC and UC faculty ( 4.75 vs $4.72,4.64$ vs $4.89,4.42$ vs $4.50, p>0.05$ for all). Overall, $97 \%$ (29/30) agreed that the workshop should be 'required for all health care providers' with no difference between CC and UC ( 4.75 vs $4.56, p=0.40)$. There were significant post-workshop increases in mean scores of 'awareness of barriers' and 'best practices' at both sites with no site differences (pre vs. post; 3.7 vs 4.5 and 3.1 vs 4.3 respectively, $p<0.001$ for both). Additionally, there was a significant post workshop increase in mean ratings on ability to 'implement best practices' and 'teach trainees how to implement best practices' (3.3 vs. 4.2 and 2.9 vs 4.1 respectively, $p<0.001$ for both) with no site differences. While almost all faculty $(29 / 30,97 \%)$ agreed the GOSCE was an 'effective way to practice skills,' the CC mean ratings were significantly higher than UC (4.58 vs $4.12, p=0.04)$. However, more faculty at the UC agreed that the workshop was 'informative and effective' and that they 'gained new knowledge' ( 4.45 vs $4.83 p=0.04 ; 4.12$ vs. $4.67 p=0.21$ respectively) DISCUSSION / REFLECTION / LESSONS LEARNED: We were successful in developing faculty training on patient-centered EMR use for busy faculty at two academic medical centers, which included adapting a $4 \mathrm{~h}$ workshop into a 90 min session. Faculty reported training in patient-centered EMR use was important, relevant and should be required for all providers and no difference was found between the longer and shorter training curricular. Faculty participating in longer training reported higher GOSCE efficacy, however the shorter workshop appeared more informative and effective with higher 
reported rates of gaining new knowledge. Given busy primary care clinician schedules and demands, shorter patient-centered EMR training may be a feasible and effective way to spread this model to other institutions and other levels of trainees.

TEAM BASED LEARNING APPROACH TO RESIDENT EDUCATION IN OUTPATIENT INTERNAL MEDICINE Mirela Feurdean; Ahmad Yousaf; Neil Kothari; Ana Natale-Pereira. Rutgers NJMS, Newark, NJ. (Control ID \#2467615)

NEEDS AND OBJECTIVES: Providing adequate academic and experiential education in outpatient general internal medicine is a focus of Internal Medicine residency programs, in keeping with the societal needs for improved Primary Care for our aging population. An Academic Half Day (AHD) dedicated to education in Ambulatory Medicine was implemented in July 2013, 1 year after the introduction of an $\mathrm{x}+\mathrm{y}$ ambulatory block schedule. In our experience, it was hard to motivate residents to do the required readings, difficult to keep them engaged during the $2.5 \mathrm{~h}$ weekly sessions, and challenging to remain excited and enthusiastic as educators. For these reasons, in July 2015 the program transitioned to a Team Based Learning (TBL) format in which the focus was shifted to high yield topics that residents reported having a difficult time with in the clinic. We maintained Evidence Based Medicine sessions, journal clubs and documentation workshops, as well as the requirement for independent study using online ambulatory care modules.

SETTING AND PARTICIPANTS: Our residency program (Rutgers New Jersey Medical School) is the largest program in the state, and includes 90 categorical residents across 3 years of training. In the $\mathrm{x}+\mathrm{y}$ schedule, every 8 weeks of core rotations is followed by 2 weeks in the continuity clinic, such that all categorical residents are exposed to at least five 2-week ambulatory blocks per academic year. Every Friday of the ambulatory block residents must attend the AHD in Ambulatory Medicine.

DESCRIPTION: The AHD curriculum spans over the 2 weeks of the ambulatory block, as follows: 1st Friday : TBL1 + Clinical vignette from clinic (group develops question in PICO format) + Workshop (librarian, documentation and coding, substance use disorders) 2nd Friday: TBL $2+$ Answers to PICO based on literature review (assigned resident presents) + Journal Club Topics are organized thematically as much as possible (e.g. substance use disorder topics during 1st week are followed by HIV related skin disorders in the second.) Topics are chosen to supplement the online ambulatory care modules which residents are assigned to complete during each block. The TBL sessions include a pre-test based on the assigned reading material, followed by the group application of knowledge to clinical scenarios. We focus on high yield materials that residents report having a difficult time with in the clinic (Dermatology, Diabetes, Obesity, Preoperative evaluation, Substance Use Disorders). The Pre-Test is a short (10 min) quiz, and is followed by $10-15 \mathrm{~min}$ of collective review of answers, moderated by the faculty preceptor. The pre-test is used as a way to assess whether residents have read the materials assigned at the beginning of the ambulatory block. It is also a self-assessment of individual knowledge, hence raises interest in the discussion of correct answers. For the TBL session (45-60 min), residents are split up into groups of four or five, with residents at each PGY level represented. Complex, case based, multiple choice questions are posed and time is given for each group to discuss internally (team leaders are encouraged to involve interns in the discussion). Questions are purposely vague and often have more than one possible answer. When time for internal debate is up, the team leader raises the answer placard(s) and each team's answers are written on the board. Each answer choice is debated out loud. Points are given for correct answers and for well thought out arguments. The discussion is moderated by the faculty preceptor and the Ambulatory Chief Resident. The tone of the process is 'fun and competitive' but the focus is on knowledge application and clinical teaching points, while winning is secondary. Winners are given token prizes based on the topic at hand (e.g. Pre-Operative Evaluation TBL winners received EKG pins for their white coats.)
EVALUATION: The TBL model is engaging and fun for both faculty and the learners. Our residents stay engaged, look forward to the 'soft' competitive nature of the session and are motivated to prepare well so that they can contribute to their team's success. We will compare resident medical knowledge in general internal medicine as reflected by ITE scores in the academic year before and after implementation of the TBL sessions. We also plan to anonymously survey the residents for their satisfaction with this novel method of teaching.

DISCUSSION / REFLECTION / LESSONS LEARNED: The team based learning (TBL) model is a successful model of teaching in the undergraduate world, but sparingly used in post graduate medical education. TBL is engaging and fun for both faculty and the residents. It requires more faculty preparation before the sessions compared to the traditional models where learners present assignments. With the exception of session preparation time by faculty, there is minimal additional cost.

TEAM RETREATS FOR INTERPROFESSIONAL TRAINEES AND STAFF: GETTING TOGETHER TO GET IT TOGETHER Shalini Patel ${ }^{5,}{ }^{6}$; Bridget O’Brien $^{6,5}$; Maya H. Dulay ${ }^{3}$; Gillian Earnest ${ }^{5}$; Terry Keene ${ }^{1}$; Rebecca L. Shunk ${ }^{2}$; Denise L. Davis ${ }^{4} .{ }^{1}$ San Francisico VA Health Care System, San Francisco, CA; ${ }^{2}$ UCSF, San Francisco, CA; ${ }^{3} \mathrm{UCSF} / \mathrm{VA}$, San Francisco, CA; ${ }^{4}$ Univeristy of Califonia San Francisco, San Francisco, CA; ${ }^{5}$ San Francisco VA, San Francisco, CA; ${ }^{6}$ University of CA, San Francisco, San Francisco, CA. (Control ID \#2469846)

NEEDS AND OBJECTIVES: Primary care trainees such as internal medicine (IM) residents and nurse practitioner (NP) students need to be prepared to work in team-based medical home settings. A team-based approach is critical to managing the health care needs for patients who have part-time trainee providers. High-functioning teams require trainees and staff to learn and practice skills together, and to know each other both professionally and personally. This can be limited by irregular trainee schedules and lack of protected time for team development during a trainee clinic day. To address this challenge, we developed and delivered a team retreat curriculum with the following objectives: -Provide protected time for discussion of members' roles, team communication and panel management processes -Provide an opportunity to review team performance, discuss team goals and areas for improvement -Train faculty in group facilitation skills

SETTING AND PARTICIPANTS: At the San Francisco VA Medical Center of Excellence in Primary Care Education (COEPC), IM residents, NP students and associated health trainees practice in teams in a medical home setting. Trainees are assigned to a Patient Aligned Care Team (PACT) consisting of a Registered Nurse (RN), Licensed Vocational Nurse (LVN), and Medical Support Assistant (MSA). All 2nd year IM residents, NP students, associated health trainees and PACT teams participate in an annual half-day retreat. The retreat is delivered on two different days each fall to reduce the impact on staffing in the clinic.

DESCRIPTION: In 2011, the COEPC invited the American Academy of Communication in Healthcare to deliver and facilitate PACT retreats with trainees. In subsequent years, retreats have been led by local interprofessional (IP) faculty and are structured primarily as small group meetings with one PACT team, their affiliated trainees (5-8 participants) and two IP facilitators. These facilitators are often junior faculty who are competent but not necessarily proficient in the content. To help deliver a robust retreat each year, we developed a retreat curriculum "kit" that includes: -A standardized template with timeline of logistical items to complete starting several months prior to retreat date -A detailed facilitator guide with specific scripts -Facilitator training material in preparation for retreat to be delivered during a faculty preparation session. Also, material for case based session on tips for challenging facilitation scenarios for advanced faculty development -A description of team retreat goals and activities such as speed meeting to get to know team members personally, discussing challenging team experiences to improve 
communication, reviewing roles of team members, and identifying specific team goals for the future -A survey, completed by each team member prior to the retreat, to assess baseline team function (Team Development Measure) -An electronic folder of materials for retreat participants including communication tools and retreat evaluation forms

EVALUATION: The team retreat has been attended by $72 \mathrm{IM}$ residents, 33 NP trainees, 42 associated health trainees and 109 PACT staff since 2011. Participants completed post-activity evaluations that showed the overall high quality of the team retreat (mean $4.5 / 5$; strongly disagree $=1$ to strongly agree $=5 ; n=257$ ). Most participants strongly agreed that they know their team members better on both a personal and professional level after attending the retreat (mean $=4.6 / 5, n=181$;). Participants rated the question "I am likely to make changes in my interactions with my team as a result of this retreat" as 4.4/5 $(n=119)$. Additionally, the qualitative comments from retreat evaluations consistently reflected the benefits of protected time together and discussion of team member roles. We also identified themes related to improved communication, huddling, and team processes

DISCUSSION / REFLECTION / LESSONS LEARNED: A team retreat can be a significant curricular activity for team development among trainees and staff on primary care teams to manage a panel of primary care patients. Role clarity, communication and team processes are small group content that can be valuable for all interprofessional participants. Lessons learned over 4 years of team retreats include: -Advanced planning to coordinate schedules to maximize attendance is key. -Support from primary care leadership is critical in order to allow release from clinical duties for all participating staff. Retreats can address broader medical center population management goals as well. -Team retreats can also be a time to review team performance or panel management data -Retreats using trained primary care faculty as facilitators can be as successful as using 'expert' outside facilitators; this also has the added benefit of strengthening connections between faculty, staff, and trainees. -Future steps include dissemination of a team retreat curriculum toolkit for implementation at other sites.

THE "NO SHOW" PATIENT: HOW TO ENGAGE THE PATIENT WHO DOES NOT COME FOR RESIDENT CLINIC APPOINTMENTS Etsemaye P. Agonafer ${ }^{2}$; Wishwa N. Kapoor ${ }^{1,2}$; Peggy B. Hasley ${ }^{1,2}$. ${ }^{1}$ Univ of Pitstburgh, Pittsburgh, PA; ${ }^{2}$ University of Pittsburgh Medical Center, Pittsburgh, PA. (Control ID \#2455762)

NEEDS AND OBJECTIVES: An evaluation of resident and patient surveys was performed and identified multiple reasons for frequent missed appointments in resident continuity clinics. To specifically address the resident-patient relationship, and to improve the show rates to resident clinic, a pre-clinic conference was developed to enhanec resident understanding of patient barriers to regular attendance to clinic appointments. The preclinic conference including the following objectives: 1) Recognize that there are multiple factors that influence a person's ability to make it to their scheduled clinic appointments 2) Report clinic data about patient demographics specifically of those who miss appointments 3) Identify alternative methods to effectively communicate with patients who have difficulty coming to their clinic appointments; specifically how to conduct and document a telephone visit with a patient who does not show for their appointments

SETTING AND PARTICIPANTS: University of Pittsburgh Medical Center (UPMC) Internal Medicine Residency is comprised of an academic center and community-based residency program. Categorical residents attend continuity clinics at UPMC Montefiore, UPMC Shadyside and the VA Health System in Pittsburgh, PA

DESCRIPTION: A pre-clinic conference was developed and implemented as a one-hour faculty facilitated small group discussion centered on a case of a patient who frequently missed appointments. The case highlighted the complex factors that influence a person's ability to attend their scheduled appointments. Specific topics addressed in the conference were: clinic patient demographics, physician perception of patients who miss appointments, the impact of high no show rates on health outcomes and healthcare utilization, and methods to address patient barriers to fulfilling office visits. The use of telephone visits was suggested as an alternative method of communication with patients who do not show for appointments. Guidance on how to conduct and document a telephone visit was provided. After the session was completed, residents were assigned the task of performing a telephone visit with at least of their patients who missed an appointment.

EVALUATION: To evaluate the residents' perspective on missed appointments and to assess the impact of their telephone visit, residents were asked to reflect upon the telephone encounter using a structured reflection tool and report back to their clinic group 3 weeks later. Questions about this experience will be included in the annual resident survey.

DISCUSSION / REFLECTION / LESSONS LEARNED: Using a case about a resident clinic patient with a complex medical history and several barriers to fulfilling clinic appointments coupled with resident clinic patient demographic data, showed residents to recognize that there are several social determinants of health that limit patients' ability to attend their appointments. While recognizing the barriers is the first step and often takes time to incorporate into daily practice, the curriculum educated residents to address these barriers with clinic resources as well as external references. Because of our patients' socioeconomic status we educated residents about how to conduct a telephone visit instead of employing web-based communication. We thought a telephone visit was the most ideal form of communication that could substitute for the face-to-face clinic encounter Trainees used telephone visits to address current symptoms, chronic medical conditions, refills, and to start to work on barriers to clinic visits for those who could not routinely follow up in person. This curriculum attempted to tackle the trainees' attitudes about frequent no show patients while providing them with tools to address the complexity surrounding a patient's inability to fulfill an appointment. The pre-clinic conference permitted residents to use information about their own patients and to learn to provide care that meets the individual needs of their patients. The hope is that by reaching out to their patients via telephone, residents will deepen their relationship with their patients who do not routinely come to clinic. This may help attendance and improve the health care for no show patients.

THE DISCHARGE PROCESS AND UNEXPECTED < 30-DAY READMISSIONS: LESSONS LEARNED FROM OUR RESIDENTS - RESULTS FROM A NOVEL CURRICULUM PILOT PROJECT Mark T. Upton; Rachel K. Miller; Jennifer S. Myers; Lisa Bellini. University of Pennsylvania, Philadelphia, PA. (Control ID \#2447521)

NEEDS AND OBJECTIVES: Hospitals across the country are increasingly focusing on improving discharge transitions as the number of $<30$-day hospital readmissions is now a quality and value-based purchasing metric. The Accreditation Council for Graduate Medical Education (ACGME) includes the safe transition of patients as an internal medicine program training milestone. Given these imperatives, we created a pilot curriculum incorporating self-evaluation and continuous improvement learning in transitions of care with a focus on understanding the individual, team, and systems-based factors that can lead to preventable $<30$ day readmissions to our teaching hospitals. 1.) To create an effective educational curriculum that teaches residents to critically evaluate the factors that lead to unexpected readmissions, engages residents in self-reflection, and encourages individual practice-based learning and improvement. 2.) To identify systems issues in our residency program that, if addressed, could lead to safer transitions of care for our inpatients. 3.) To increase participation and interest in quality improvement initiatives from our residents. 
SETTING AND PARTICIPANTS: Categorical junior internal medicine residents $(n=24)$ rotating through a 2-week elective rotation block between January 2015 and May 2015. DESCRIPTION: We created a 2-week curriculum for second-year internal medicine residents that consisted of a 15-min introductory didactic session, an independent review of a readmission case utilizing an online tool, and a 1-h faculty mentored small group feedback and reflection session. Using electronic health record data, each case review involved a unique patient previously under the resident's care who had been readmitted to one of our teaching hospitals in $<30$ days. The online tool was created specifically for this curriculum utilizing components of multiple published tools. Data from completed readmission tools and post-curriculum resident evaluations were collected and analyzed.

EVALUATION: Twenty-four residents participated in this curriculum between January and May 2015. $89 \%$ of residents reported that they were not previously aware that their patient had been readmitted within 30 days of discharge. $74 \%$ of the readmissions reviewed were felt by residents to be either possibly preventable or preventable. Numerous potential opportunities for improvement were identified and included improving the content/quality of discharge instructions and medication reconciliation, better postdischarge anticipatory guidance, and improved coordination of outpatient follow up appointments. Fourteen residents (58\%) completed a post-curriculum survey. 11/14 (79\%) agreed or strongly agreed that the individual admission review was useful to their education. 14/14 (100\%) believed that the group discussion was beneficial, and 13/14 (92\%) stated that the curriculum should be continued for future residents.

DISCUSSION / REFLECTION / LESSONS LEARNED: Junior residents who participated in this novel curriculum enthusiastically identified opportunities for improvement and the majority of participants found it to be beneficial to their education and recommended that it be continued in the future. This curriculum is now part of a longitudinal transitions of care curriculum in our residency program and we are hopeful that it will lead to improved discharge documentation, medication reconciliation, communication with patients, and interdisciplinary teamwork. In addition, our hope is that the innovative improvement ideas generated by residents will become targets for QI work in our residency program and hospital system. Future directions include expanding the curriculum to include participants from nursing, pharmacy, social work, and case management to further emphasize the critical importance of interprofessional collaboration in safe transitions of care.

ONLINE RESOURCE URL (OPTIONAL): https://upenn.co1.qualtrics.com/SE/? $\mathrm{SID}=\mathrm{SV} \_$aY3hdAiPrB9Xpf 7

THE FLIPPED CLASSROOM: BASIC QI TRAINING FOR RESIDENTS Roberto O. Diaz Del Carpio; Smita Y. Bakhai; Scott H. Stewart. University at Buffalo, Buffalo, NY. (Control ID \#2468988)

NEEDS AND OBJECTIVES: The Accreditation Council for Graduate Medical Education calls for training and integration of residents in quality improvement (QI), patient safety (PS) initiatives within their clinical learning environments. Our current healthcare environment demands physicians who deliver safe, effective and high value care. The objectives for the first cycle are: to identify the key elements of an effective aim statement and to develop an effective aim statement. Objectives for the second cycle: to describe the difference between project-level measures and PDSA-level measures and to create a family of measures for their QI project.

SETTING AND PARTICIPANTS: The QI training module took place during our outpatient clinical rotation within the 4 plus 1 schedule. Our residents have a mandatory 4-h block for teaching sessions. The first training module included a 60-min workshop that was delivered to a group of 23 residents on average. This workshop was delivered five times during 5 consecutive weeks by one faculty member. We aimed to deliver the training session to our categorical internal medicine residents.
DESCRIPTION: Our first training module (1st PDSA cycle) aimed to teach the model for improvement. This training session comprised the use of massive online open courses and a hands-on workshop. Residents were asked to complete 7 selected online QI lessons from the Institute for Healthcare Improvement open school. The workshop included open discussions, role-play and open source feedback. The goal of our second module was to introduce residents to QI measurement concepts. We tailored the use of MOOC based on data from 1st PDSA. We continued using role-play, open source feedback and we added clinical experiences with feedback simulations.

EVALUATION: Learner assessment was done using multiple choice questions (MCQ), simulation and the submission of a QI aim statement. To evaluate the first training module we surveyed residents who completed the live workshop. The survey offered binary (yes/ no) and Likert scale responses (strongly agree/strongly disagree). Of 113 internal medicine residents, 107 (95\%) completed the live workshop module. 72 (67\%) completed the first 3 online QI lessons, 68 (64 \%) completed lessons 45 and, 61 (57\%) completed lessons 67. 39 residents (54\%) passed lesson 13 on the first try, 41 (60\%) for lessons 45 and 39 (64\%) for lessons $67.95 \%$ of residents reported that they feel confident on writing an effective aim statement. $91 \%$ of residents submitted a successful QI aim statement. 95 (89\%) residents identified this experience as their first formal exposure to QI. The module was rated as outstanding by $95 \%$ of the residents and more than $90 \%$ enjoyed the handson experience more than the online modules. More than $90 \%$ of residents reported that they would use what they learned in the next months. Data from our second module is being processed.

DISCUSSION / REFLECTION / LESSONS LEARNED: Initial data showed that residents enjoyed the hands-on experience and after the workshop they were confident enough to translate an improvement idea into an effective aim. The flipped classroom approach allowed more time for interactive discussions and use of QI concepts in real life scenarios. The number of online lessons reviewed by residents decreased over time. This helped us assigned a more tailored group of online lessons for our second module.

THE PHRONESIS (PRACTICAL WISDOM) PROJECT: FOSTERING CAPACITIES FOR PRACTICAL WISDOM IN MEDICAL STUDENTS Margaret L. Plews$\mathrm{Ogan}^{2}$; Walker Redd ${ }^{2}$; Natalie May ${ }^{1} .{ }^{1}$ University of Miami Miller School of Medicine, Charlottesville, VA; ${ }^{2}$ University of Virginia, Charlottesville, VA. (Control ID \#2469234)

NEEDS AND OBJECTIVES: The calls for change in health care systems have strikingly similar themes: they call for a culture of compassion, other-centeredness and selfawareness, relationality and collaboration, teamwork, embracing complexity, applying knowledge to discern the right action, and a re-focusing on the greater good. Wisdom researcher Monika Ardelt describes characteristics of wise persons as understanding the deeper meaning of things, knowing the limits of knowledge, tolerating ambiguity, engaging in reflective and self-reflective thinking, showing compassion and sympathy toward others, capacity to be other-centered and an overall focus on the greater good.. This abstract describes a longitudinal professional formation curriculum at the University of Virginia organized around the conceptual framework of wisdom, and centered on a 4 year long patient relationship, with the educational goal of fostering the capacity for wisdom development in the medical student, setting the stage for wisdom development throughout their careers.

SETTING AND PARTICIPANTS: This project, called the Phronesis project, began in 2014, with a cohort of 12 first year students at the University of Virginia medical school. The project is now in its second year, enrolling its second cohort of 12 students for a total of 24 students currently in the program. In the first year students were chosen from a group of 40 applicants based on an application essay. In year two the incoming cohort of 12 students was chosen randomly from the volunteer pool of over 40 students who wished to participate. 
DESCRIPTION: The curriculum is grounded in a longitudinal patient-student relationship and instruction is both clinical and seminar based. Students are matched with two patients longitudinally (one adult with chronic illness, one pediatric with chronic illness or multiple complex medical and social challenges) for the entire 4 years, curricular objectives and seminar-based teaching are linked with specific patient-student interactions (for example: narrative interviews, house calls, advocacy for an identified needed program or benefit on behalf of their patient, health goal setting and behavior change, re-designing a part of the health system to be more patient centered based on experience of accompanying their patient on visits). Curricular elements track with elements for wisdom articulated in wisdom researcher Monika Ardelt's 3-Dimensional Wisdom model: reflective practice, compassion, and the cognitive capacity to discern meaning, tolerate ambiguity, notice the limits of knowledge, embrace complexity, make choices in the face of conflicting values, apply expert knowledge toward right action, and focus on the greater good. Students learn habits of mindfulness, fostering positive emotion, empathy, compassion, use of narrative, and advocacy. They learn skills of narrative interviewing, relationship building, motivational interviewing, design thinking, error analysis, advocacy.

EVALUATION: Evaluation includes qualitative interviews, narratives, patientlevel outcomes of health goals, and student surveys comparing Phronesis students to their non-participating peers.(3-D wisdom scale, Connor-Davidson Resilience scale, Tolerance for Ambiguity scale, the PERMA wellbeing survey, the Maslach burnout inventory). By April 2016 researchers will have analyzed 2 years of survey data and qualitative interviews from two cohorts of Phronesis students.

DISCUSSION / REFLECTION / LESSONS LEARNED: Our first cohort of 12 students is just entering their clerkship year. Qualitative interviews suggest that the longitudinal patient interaction is a rich learning laboratory for developing capacities for wisdom, and the focus on reflective practice, compassion, and the cognitive capacities related to wisdom development are helpful as they gain experiences over time. Early results of the medical student surveys that include the 3-D wisdom scale and the Maslach Burnout Inventory suggest that wisdom may be a protective factor in reducing burnout in medical students.

THE ROLE OF DEBRIEF GROUPS IN PRESERVING EMPATHY DURING THIRD-YEAR CLINICAL ROTATIONS Laura K. Bloom² ${ }^{2}$ Madhuri Nagaraj ${ }^{2}$; Kelli Columbo $^{2}$; Alex J. Egusquiza ${ }^{2}$; Alex J. Mechaber ${ }^{1}$; Jeffrey P. Brosco ${ }^{1}$; Kenneth W. Goodman $^{3}$; Hilit F. Mechaber ${ }^{3} .{ }^{1}$ University of Miami, Miami, FL; ${ }^{2}$ University of Miami, Miami Beach, FL; ${ }^{3}$ University of Miami Miller School of Medicine, Miami, FL. (Control ID \#2469645)

NEEDS AND OBJECTIVES: Statement of Problem: Do debrief groups, focused on combatting the fatigue and stress of new roles in the clinical environment, mitigate the decline in empathy experienced by third year medical students? Objectives: 1 . Characterize the severity of the decline in empathy faced by medical students, particularly during third-year clerkship rotations. 2. Create a "safe space" for discussion of clinical experiences allowing students to express ways they are emotionally affected, increase awareness of difficult situations, and foster peer problem solving.

3. Measure and assess the extent to which participation in a debrief group aids in preserving empathy.

SETTING AND PARTICIPANTS: In 2012, University of Miami Miller School of Medicine educators and students joined together to create "Third-Year Debrief Groups" to address the erosion of empathy in medical students documented during their clinical years. All third-year students were invited to participate in a voluntary peer group facilitated by a trained faculty mentor, totalling 95 students over the last 3 years. Each summer a new cohort of third-year students are invited to participate, and currently the program is in its fourth year.
DESCRIPTION: "Third-Year Debrief Groups" were created in 2012 by the collaboration of Miller School of Medicine faculty educators and students. These groups were created with the intention of forming a "safe space" in which to discuss the emotionally difficult aspects of becoming a clinician, improving student well-being, and diminishing the erosion in empathy. All third-year medical students were voluntarily invited to participate in these small group sessions, and non-administrative faculty volunteers were trained to serve as facilitators. The facilitator's role was to foster discussion and guide students without dominating conversation while promoting problem-solving and resolution of issues commonly faced during clinical training. Sessions were held once monthly throughout the fall semester over dinner in a comfortable environment decided by the group themselves. Each session focused on a different "problem" or content area, combining selected readings with open discussion, allowing each group autonomy. Common topics have included how to communicate best with colleagues, how to approach ethical concerns, how to maintain inspiration and how to emotionally handle the stress of adjusting to a new clinical environment.

EVALUATION: Measures of Success: Over the last 3 years, survey data was collected from the 95 participants. Data included overall satisfaction with the program as well as open-ended responses to questions including whether they would recommend it to a colleague and benefits they noted from participation. This year, the Jefferson Scale of Empathy is being incorporated to create quantitative measures for the comparison of empathy between participants and non-participants. Findings to Date: Of the 95 participants over the last 3 years, $70 \%$ completed the survey. Of these responses, $83 \%$ expressed overall satisfaction with the debrief groups and noted they would recommend the program to a colleague. Qualitative responses richly demonstrated that group discussions positively influenced behaviors on clinical clerkships. Students commented specifically that the groups helped to provide support, strategies to deal with stressful situations, and a means to address burnout.

DISCUSSION / REFLECTION / LESSONS LEARNED: Key Lessons for Dissemination: Our data show that participation in "Third-Year Debrief Groups" has had positive influences on students in various aspects of their clinical training. Benefits include: obtaining advice on how to behave or react in stressful situations, creating a "safe space" in which to address ethical issues seen during clinical practice, and maintaining a support system to prevent burnout. Student feedback suggests that this faculty-facilitated peer group design is a strong model with measurable benefits. Data from multiple cohorts suggests that sharing personal experiences within these groups may confer immediate benefits in safeguarding against student distress and fatigue. In addition, sustained benefits could promote supportive peer rapport and long-term improvement in patient outcomes and physician satisfaction. The incorporation of validated empathy scales that allow for the quantitative comparison of participants to non-participants may be a useful indicator of the natural trend in empathy over third-year as well as the potential effectiveness of these groups as an intervention. In addition, this model of debrief groups may be adapted into the curriculum of other medical schools to maintain empathy among trainees and further enhance their resilience.

TIME TO AUDIT YOUR OWN PATIENTS' BILLS TO PROMOTE COSTCONSCIOUSNESS Simran Kaur B. Matta. University of Nevada School of Medicine, Las Vegas, NV. (Control ID \#2470298)

NEEDS AND OBJECTIVES: While the current system equips residents with clinical knowledge and skills, they should also be trained to become good stewards of high value care (HVC). The purpose of our project is to create a "culture" of cost-consciousness that will be embraced by this generation of physicians and next to come. We aim to assess if active participation of residents in auditing their patients' bills improves costconsciousness among residents. 
SETTING AND PARTICIPANTS: All internal medicine residents at PGY-1, PGY-2 and PGY-3 level at our institution (73 participants).

DESCRIPTION: After a baseline survey to assess internal medicine resident awareness and attitudes towards HVC, the residents on inpatient medicine rotations will do assignments to audit patient bills. At the end of every medicine rotation, each medicine team will be required to audit the bill of any one of the patients they have cared for. They will discuss strategies to deflate the bill and calculate how much money could have been saved by wisely choosing the labs and medications. They will also reflect upon if the hospital stay could have been shortened, which services were unnecessary and if any unnecessary services inflicted harm to the patient. We have dedicated High Value Care noon conferences and morning reports to disseminate the lessons learnt. These conferences are paired with brainstorming sessions including root cause analysis of over-use leading to improvement ideas that can be translated into sustainable developments in the system. This will help foster a culture of cost-consciousness and also a culture where residents feel encouraged to discuss costs during inpatient rounds.

EVALUATION: The project is currently being implemented and is due for completion in June 2016. Post intervention survey will be conducted in June and the results will be compared to the pre-intervention survey. We will assess the impact of this learning modality on resident test-ordering behaviors and their attitudes towards cost-conscious care

\section{DISCUSSION / REFLECTION / LESSONS LEARNED: Residents cannot learn how} to deliver cost-effective medicine if it is not being practiced at their institution of training. The issues are that the residents lack adequate knowledge about costs of diagnostics and medications as well as the training to incorporate cost considerations into medical practice. We are trying to develop a comprehensive approach utilizing multiple strategies that will bring high value care to our collective consciousness and create a "culture" of high value care. Formal lectures and powerpoint presentations may have minimal impact on shaping residents' behaviors. Critically analyzing the bills that the residents have generated for their patients allows them to reflect upon their own practices and test ordering behaviors The bill audit project is also being augmented by several fun games to learn the costs of various diagnostic and treatment modalities. Besides this also meets ACGME requirements for systems learning, cost of care, etc. Residents are being encouraged to choose wisely, discourage practices of 'overuse', thus shifting the culture "More is not always better".

TRAINING PHYSICIANS TO PERFORM MEDICAL EVALUATIONS OF ASYLUM SEEKERS Triveni DeFries ${ }^{2}$; Hala Borno ${ }^{2}$; Debra Keller ${ }^{3}$; Cristina Biasetto ${ }^{4}$; Sharad Jain ${ }^{1} .{ }^{1}$ UCSF, San Francisco, CA; ${ }^{2}$ University of California, San Francisco, San Francisco, CA; ${ }^{3}$ University of California San Francisco, San Francisco, CA; ${ }^{4}$ Trauma Recovery Center, San Francisco, CA. (Control ID \#2469760)

NEEDS AND OBJECTIVES: The United States grants asylum to persons who have faced persecution or have a well-founded fear of persecution in their countries of origin. Asylum is more likely to be granted in cases that include medical evaluations; however, few physicians are trained to perform these. Physicians can play an integral role in evaluating and documenting the physical and psychological sequelae of torture and human rights abuses, as well to link clients to comprehensive services including ongoing medical care. Our project aims to train resident physicians in performing medical evaluations for persons seeking asylum.

SETTING AND PARTICIPANTS: We designed and launched a 1-year curriculum for licensed Internal Medicine resident trainees at University of California, San Francisco to learn and enact the skills to perform medical evaluations for asylum seekers. Eligible clients are San Francisco residents who are adult survivors or secondary survivors of torture or gender-based violence in their countries of origin. Clients are referred to our partnering community organization, Survivors International/Trauma Recovery Center, by local attorneys. Our partner offers comprehensive services to survivors of trauma and provides logistical support for the evaluations.

DESCRIPTION: Physician trainees attended an intensive training session led by social worker and physician experts. The didactic curriculum included five modules focused on: 1) introduction to the asylum process; 2) medical evaluations using Istanbul Protocol guidelines; 3) forensic photography; 4) interviewing techniques for torture survivors; 5) provider well-being to prevent secondary trauma. The curriculum was adopted from training materials of Physicians for Human Rights and previous literature. After completing these modules, trainees are paired with an experienced evaluator to prepare and shadow an evaluation, and then participate in an established peer review process of completed affidavits.

EVALUATION: Ten physicians participated in the training program. A pre-training assessment based on a previously published survey assessed baseline knowledge, practice patterns and attitudes on immigrant patients who have suffered trauma. All participants agreed that physicians have a responsibility to advocate for victims of torture, and 6 reported prior experiences working in this realm. Few providers (7/9) were confident performing the components of medical evaluations for asylum seekers. 1/9 reported skills to support a client who is triggered or re-traumatized by telling their story of abuse. None of the participants routinely screen for torture in their practices, while $5 / 9$ screen for domestic violence. We will administer a post-training assessment after 1 year of participation to assess impact. To date, our medical team has completed 7 evaluations, 2 have been adjudicated and resulted in asylum being granted while the other 5 are at various stages of the legal process.

DISCUSSION / REFLECTION / LESSONS LEARNED: Possessing the knowledge and skills to perform medical evaluations of asylum seekers provides a powerful opportunity for physician advocacy and can enhance providers' overall care of patients who have survived torture and trauma. This active service learning program trains providers to utilize their medical skills outside of the traditional clinical setting, and offers a unique opportunity for trainees to work in a highly interdisciplinary medical-legal collaboration with attorneys, social workers and psychologists. Challenges to the program include ensuring sustainability among resident leaders and coordinating schedules of attorneys and courts with those of clinicians.

TRAINING THE NEXT GENERATION OF CLINICAL EDUCATORS: A LONGITUDINAL PRACTICE-BASED CURRICULUM FOR BEGINNING CLINICAL TEACHERS Catherine Jones. Tulane University SOM, New Orleans, LA. (Control ID \#2470399)

NEEDS AND OBJECTIVES: At the conclusion of this session, attendees will: 1) Learn the components of a successful train-the educator pilot program 2) Address the common pitfalls and challenges faced by beginning clinical educators 3) Create relevant and appropriate curriculum for the beginning clinical educator 4) Identify opportunities for longitudinal train-the-educator teaching at their home institutions

SETTING AND PARTICIPANTS: This curriculum, called the "T4 Clinical Educators," occurs longitudinally during the fourth year of medical school for students interested in teaching. Participation is voluntary and has included 50 of the 180 graduating fourth year students this pilot year. Interested students sign up to facilitate clinical teaching sessions for first and second year medical students; precept in the student run clinics; and run other educational events for underclassmen through the year. They participate in monthly workshops (also voluntary) geared toward building teaching skills in beginning clinical educators. 
DESCRIPTION: This longitudinal program grew out of a need to have a large number trained facilitators for our first year Introduction to Clinical Skills course at Tulane. At the same time, we have a strong interest among our fourth year students in gaining more clinical teaching exposure. The program combines focused skill-building workshops for the fourth year students, with real-time opportunities to practice the skills they learn. The fourth year students volunteer to participate in this program. They attend bimonthly workshops on specific teaching skills, such as "How to Facilitate a Small Group Discussion;" "How to Give Effective Feedback;" "Basic Principles of Clinical Teaching." They then are qualified to facilitate special sessions for first year students working on historytaking skills, specific physical exam maneuvers, SIM Center sessions, and sessions drilling other clinical skills such as suturing. Participating faculty in the sessions for the first year students act in a dual role, directly teaching and mentoring the first year students, while at the same time observing and providing backup for the fourth year facilitators, and then engaging in a structured debriefing of the teaching process after the sessions.

EVALUATION: At this time, evaluation is qualitative and geared toward survey data of participants' attitudes and self-assessment of their teaching skills and confidence. Future evaluations will include evaluations by first and second year medical students on components of teaching sessions; observed sessions by academic faculty with structured formal feedback; and survey data assessing knowledge and skills in addition to attitudes.

DISCUSSION / REFLECTION / LESSONS LEARNED: This is the inaugural year of this project and it has provided many lessons, most quite positive. The fourth year students come to clinical teaching with a lot of enthusiasm and more knowledge than they realize Perhaps the biggest lesson learned this year is that much of the clinical teaching workshops for the fourth year students need to be distilled down to one or two easy-to-practice topics, interspersed with confidence-building for the vast majority of participants, who do not yet understand that they already have something to teach the younger generation of physicians in training. The most effective workshop was one on "How to Give Feedback," where introduced a few simple principles and skills on effectively giving clinical feedback, and then we spent the rest of the session practicing scenarios. The remainder of this year's workshops are structured around this template. The first year students appreciate the facetime with the fourth years during their small group facilitated sessions, and the fourth years step into their role as leaders when they are actually facilitating the sessions with the first years. Both levels of students report that these sessions are less intimidating than sessions directly led by faculty, even though faculty members are present to oversee the sessions. In terms of faculty bandwidth, this is a way to allow for more small-group participation and skill-building for beginning students, without encountering the rate-limiting step of needing 6 or 7 faculty members per afternoon to work on clinical skills. As the primary faculty member overseeing the fourth years and the first years in these sessions, I found that pre-briefing and debriefing with the fourth year facilitators helped ensure that objectives were met and the session was smooth and effective for all learners (first and fourth year students) Motivated fourth year students can certainly be trained to effectively teach basic clinical skills to beginning learners. I anticipated that the voluntary nature of this program would make recruiting and retaining fourth year facilitators a challenge, however this has not been the case.

TRANSFORMING TRADITIONAL M\&M INTO AN INTERACTIVE QI CONFERENCE Thomas J. Walk ${ }^{2,}{ }^{1}$; Gaetan Sgro ${ }^{2} .{ }^{1}$ UPMC, Pittsburgh, PA; ${ }^{2}$ VA Pittsburgh Healthcare System, Pittsburgh, PA. (Control ID \#2468619)

NEEDS AND OBJECTIVES: In response to the ACGME's Next Accreditation System and CLER program, residency leaders have been working to increase trainee engagement in patient safety and quality improvement (QI). While traditional morbidity and mortality (M\&M) conference remains the dominant model for discussing medical error among physicians, the content of these conferences varies widely and often focuses on human rather than systems error. If we really want residents to appreciate how errors occur in complex systems and to improve care for our patients, traditional M\&M format needs to be re-focused. We re-engineered M\&M at our VA setting into our Quality Improvement Conference (QUIC). By the end of each session, residents will: 1. Describe how latent errors lead to adverse events and near misses 2 . Perform a contributing factors analysis on an actual case 3 . List local processes for addressing error and mitigating risks

SETTING AND PARTICIPANTS: The 60-min QUIC conference is delivered monthly to Internal Medicine trainees and medical students. The discussion is led by our Chief Resident in Quality and Patient Safety accompanied by a faculty facilitator. We plan to expand into a multidisciplinary conference with participation from other specialties and clinical areas.

DESCRIPTION: In an effort to combine an open forum for discussing adverse events with instruction on QI methodology, we overhauled M\&M conference at our VA site. Rather than selecting only the worst patient outcomes for discussion, we select cases involving serious or multiple systemic errors for our Quality Improvement Conference (QUIC). We begin by leading trainees through an interactive case presentation and an introduction to root cause analysis. Attendees then identify contributing factors in small groups before reconvening to brainstorm potential interventions. We conclude by disclosing how our local QI offices are addressing the issues raised and by reviewing the event reporting process.

EVALUATION: Preliminary feedback is generally positive. Residents are gaining a better understanding of the process for addressing incident reports as well as hands-on experience using common QI techniques with which they were previously unfamiliar. In addition, they note an increased likelihood of reporting adverse events in the future. A comparison between evaluations for traditional M\&M and QUIC format is pending with results available later this spring. Ultimately, we hope to capture increased resident engagement in our patient safety culture as measured by our annual program evaluation and a positive impact on CLER visits.

DISCUSSION / REFLECTION / LESSONS LEARNED: We restructured M\&M into a novel interactive conference that ushers the discussion of adverse events into the era of quality improvement and patient safety. Our QUIC conference engages trainees in the foundations of QI and bolsters ACGME competencies in systems-based practice and practice-based learning. This represents a fresh approach that provides a foundation for a longitudinal quality curriculum.

UNIVERSITY OF ALABAMA SCHOOL OF MEDICINE (UASOM) FACULTY SCHOLARS IN HEALTH DISPARITIES AND CULTURALLY RESPONSIVE CARE Nicole Redmond ${ }^{1}$; Jonathan Waugh ${ }^{2}$; Monika M. Safford ${ }^{1}$; Jeffrey M. Ring ${ }^{3}$. ${ }^{1}$ University of Alabama at Birmingham, Birmingham, AL; ${ }^{2}$ Samford Univeristy, Birmingham, AL; ${ }^{3}$ Health Management Associates, Costa Mesa, CA. (Control ID\#2456713)

NEEDS AND OBJECTIVES: The University of Alabama School of Medicine (UASOM) Faculty Scholars program was developed to train teaching faculty as institutional champions to support the further development, implementation, and evaluation of curricula designed to improve integration of training in health disparities and cultural competence across the entire spectrum of medical education. The aims of the program are to 1) provide education and training on leading, teaching and providing culturally responsive patient care in order to eliminate health disparities; 2) develop knowledge and skills related to curricular design and innovation focused on enhancing the provision of culturally responsive care; and 3) promote leadership and career development in medical education through scholarly productivity such as publications and leading training initiatives for health care providers at all stages of training and clinical practice.

SETTING AND PARTICIPANTS: UASOM faculty of all departments and ranks (tenure or non-tenure track) and staff who are current or prospective clinician-educators 
for trainees, particularly medical students and residents. Participants were selected based on their interest in career development in health disparities and culturally responsive care focused medical education

DESCRIPTION: This 1-year faculty development program began with a half-day orientation in June 2015, followed by twice-monthly meetings. Meetings are with a nationally recognized expert in health disparities curricula via videoconference, alternating with in-person meetings featuring campus experts. All scholars are expected develop an educational innovation project focused on the development, redesign, or evaluation of curricula to enhance learning in health disparities, health equity, social determinants, and/ or culturally responsive care. Participants are expected to present abstracts related to their project during the Annual UAB Research and Innovations in Medical Education (RIME) Week occurring in the fall after the conclusion of the program. It is expected that the project will also culminate in publication in a peer-reviewed journal or repository (e.g. MedEdPORTAL), and enhance their department's curricula.

EVALUATION: Ten faculty members were selected as program participants, each from a different academic unit (department/division). The scholars are $70 \%$ female, $60 \%$ African American, $20 \%$ Hispanic/Latino, $20 \%$ White/Caucasian. On average, the effort allocation is $44 \%$ clinical, $22 \%$ teaching, $21 \%$ administration, and $13 \%$ research. Nine scholars completed the pre-test evaluation. Within the past 2 years, the proportion of faculty that reported teaching about various disparities topics was moderate: racial/ethnic minorities $67 \%$, gender issues $33 \%$, and socioeconomic issues $56 \%$. Only $11 \%$ of faculty endorsed using techniques to create an inclusive environment for diverse students. DISCUSSION / REFLECTION / LESSONS LEARNED: Faculty development is a crucial and often overlooked component of excellence in medical education. Faculty capacity to teach culturally responsive health care, moreover, is comprised of a specific set of teaching skills that are essential for successfully training medical learners to practice medicine toward an eradication of health disparities. This educational program draws on combined local and distance learning models combined with focused curriculum development educational projects to better fulfill AAMC and ACGME competency education. Topics covered range from assessing learners and providing learner feedback to experientially teaching about stereotypes, biases, disparities, and learner self-reflection.

ONLINE RESOURCE URL (OPTIONAL): https://www.uab.edu/medicine/dopm/faculty-scholars-in-hd-overview

USING GROUP REFLECTION TO HELP MEDICAL STUDENTS PROCESS EXPERIENCE IN CARE OF SEXUAL AND GENDER MINORITIES NaOmi Gorfinkle $^{2}$; Jawara Allen ${ }^{2}$; Demere Woolway ${ }^{4}$; James Aluri ${ }^{2}$; Rosalyn W. Stewart ${ }^{1}$; Maura J. McGuire $^{2,3}$. ${ }^{1}$ Johns Hopkins, Baltimore, MD; ${ }^{2}$ Johns Hopkins School of Medicine, Baltimore, MD; ${ }^{3}$ Johns Hopkins Community Physicians, Baltimore, MD; ${ }^{4}$ Johns Hopkins University, Baltimore, MD. (Control ID \#2469661)

NEEDS AND OBJECTIVES: Many medical schools lack a comprehensive curriculum addressing sexual and gender minorities and the unique health care needs of individuals who identify as lesbian, gay, bisexual, transgender or undifferentiated (LGBTQ). In addition, faculty may lack the experience and skills necessary to reinforce what students are learning, resulting in doubt and uneasiness among learners around caring for LGBTQ populations. We modified a required reflective learning activity and provided targeted faculty development to promote understanding of culturally competent care of LGBTQ patients, and to allow students to reflect on related experiences in a safe, supportive space. We expected this activity would help learners: (1) Recognize the benefits of reflective discussion to share and process complex experiences within a group setting. (2) Identify potential knowledge gaps related to care of sexual and gender minority patients. (3) Describe strategies to address barriers to culturally competent care of LGBTQ patients.
SETTING AND PARTICIPANTS: This experience was offered to all 122 second year medical students enrolled in a longitudinal ambulatory clerkship. During this clerkship, students work with community based faculty and provide primary care to patients. The clerkship requires students to write 5 reflections each semester to consolidate their learning about system, social and behavioral aspects of medicine. For this intervention, students were invited to participate in a small group reflection activity to satisfy clerkship requirements. In order to identify facilitators for our LGBTQ small groups we reached out to faculty with special interest in these patients.

DESCRIPTION: We used a group reflection activity to engage faculty and learners in discussing experiences and attitudes related to sexual and gender minorities. Groups consisted of 4-5 students and a facilitator. The activity was completed during a 90-min timeframe: $10 \mathrm{~min}$ for introductions and an explanation of the exercise, $20 \mathrm{~min}$ for writing a 1-2 page reflection, $50 \mathrm{~min}$ for reading and group discussion of each reflection, and $10 \mathrm{~min}$ for wrap up and concluding thoughts. One week prior to the activity, students and faculty received instructions prompting reflection on their experiences in caring for LGBTQ patients; these instructions were given again to group participants during the reflection activity. Facilitators were invited to attend a faculty development session prior to the program.

EVALUATION: Of 122 students invited to participate, 36 (30\%) joined the LGBTQ reflection group activity. Evaluations were completed by 22 students (61\%). Of 20 students who had previously written individual reflections, 19 (95\%) rated the group activity as "significantly more valuable" compared to written reflections, and 1 (5\%) rated it "more valuable". All students suggested that the activity should become a part of the clerkship's formal curriculum. Of 9 facilitators, 8 (89\%) completed the evaluation. All responders felt adequately prepared, and like students thought the activity should become part of the clerkship's formal curriculum. A qualitative analysis of the narrative comments in the evaluation revealed several key themes including (1) benefits of the group reflection process (2) desire for more knowledge, (3) awareness of discomfort and bias, and (4) perceived gaps in preceptor modeling of culturally competent LGBTQ patient care. Despite these gaps, several students indicated that they felt empowered to encourage change through example and by sharing new knowledge.

DISCUSSION / REFLECTION / LESSONS LEARNED: Reflective learning supports professional formation and helps students integrate clinical experience with medical knowledge. To address a need for education related to the sexual orientation of patients, we implemented a group reflective learning activity on caring for patients of sexual and gender minorities. The program appeared to result in affirmation and improved awareness of the ways that health professionals can be more sensitive to the needs of LGBTQ-identifying patients. It also revealed knowledge gaps among both students and faculty in the current curriculum and has spurred a discussion of how to close those gaps moving forward.

\section{USING SIMULATION TO REMEDIATE POOR PROFESSIONAL BEHAVIORS} Jeannette Guerrasio $^{1}$; Kirsten J. Broadfoot ${ }^{2}$; Eva M. Aagaard ${ }^{3} .{ }^{1}$ University of Colorado, Denver, $\mathrm{CO} ;{ }^{2}$ University of Colorado Anschutz Medical Campus, Aurora, $\mathrm{CO} ;{ }^{3}$ University of Colorado School of Medicine, Aurora, CO. (Control ID \#2466042)

NEEDS AND OBJECTIVES: Effective interpersonal interaction is essential for safe independent practice and has been linked to improved patient satisfaction, medical decision making, and patient health outcomes. However, $30 \%$ of residents and faculty referred for remediation have deficits in professionalism, manifested as poor communication and interpersonal behaviors. Methods for providing remedial teaching are often ad hoc and poorly described. The objectives of this innovation are: to implement a consistent approach for the remediation of poor professional behaviors, to assess changes in behavior observed by supervisors and via self assessment for up to 3 years post intervention.

SETTING AND PARTICIPANTS: At the University of Colorado School of Medicine, residents and faculty identified as struggling with unprofessional communicative 
behaviors and interpersonal interactions were referred by program directors, department chairs, and the office of professionalism to a remediation team consisting (two physicians with expertise in remediation and a communication skills educator) and provided the option of participating in simulation based remedial teaching.

DESCRIPTION: Upon referral, collateral information was collected via: multisource evaluations, verbal and email reports from observing faculty and staff, and details on specific events or complaints. At least two members of the remediation team then met with each referred individual to review performance history, the individual's perspective on reported challenges, events related to the referral, the time course, impact on current and prior work performance and personal life, prior attempts at behavior change and any other deficits or mental health issues that may be impacting performance. The individual was asked to describe several interactions and personalities that $\mathrm{s} / \mathrm{he}$ either found challenging or that have gotten him/her in trouble in the past. Examples were also collected from the referring supervisor. The individual was invited to participate in focused, individualized remedial training at the Center for Advancing Professional Excellence (CAPE), which houses a simulation center. The CAPE employs a core group of actors, trained to work with struggling physicians and to provide high quality feedback and coaching. During the training, the individual engaged in 3-4 simulated scenarios. After each scenario, the individual watched the video with the actors and remediation team, and was coached through self reflection, receiving feedback and suggestions for change in an iterative fashion with goal setting for subsequent scenarios. In the last scenario, the physician is presented with a learner with a similar communicative style and professionalism lapse to their own. The individual is asked to give feedback with an action plan to this learner. This allows the remediation team to see how much information the individual has retained, allows for deeper reflection and to help the individual encode the information that they had learned. Shortly after the session, the individual was given a list of specific observations (corrective and reinforcing) made during each scenario, specific behavior recommendations, and then a summary of global recommendations to implement in the clinical environment. The total cost for each remediation event is approximately $\$ 1500$ and takes $4 \mathrm{~h}$, and are generally supported by the individual's department or by the individual themselves.

EVALUATION: Utilization: From July 2013 through Sept 2015, 15 residents and 6 faculty were offered remediation via this method. Eleven residents and 6 faculty completed the described remedial teaching. Four residents did not participate in the teaching, al due to insufficient financial resources to support the remediation. Outcomes: Early feedback is positive from both the individuals participating in the remediation and their departments/programs. To better measure the effectiveness of this program on both shortterm and sustained behavioral change, guided interviews will be conducted separately with both the physician referred and their referring program director, Department Chair, or Office of Professionalism. Review of subsequent evaluations, performance reviews and the presence or absence of recurrent reported events over the duration of 1-3 years will be tracked.

DISCUSSION / REFLECTION / LESSONS LEARNED: Each remediation session is standardized in format, with cases and skill recommendations that are customized to the individual learner. A program that provides struggling residents and faculty with the opportunity to deliberately practice specific skills, receive video and expert feedback, and reflect on their performance has not been previously described in the literature. This method represents a unique approach, grounded in educational and expertise theory, to providing remedial teaching which we hope to validate as we collect more longitudinal data about the individual real world behavior modifications and their sustainability moving forward.

USING SMARTPHONE TECHNOLOGY TO PERFORM FUNDUSCOPIC EXAMINATION: A PROMISING TEACHING TOOL Claudia L. Campos ${ }^{3}$; Carolyn F. Pedley $^{1}$; James L. Wofford ${ }^{3}$; Kirsten Feiereisel ${ }^{2}$; Abigail Koch ${ }^{3}$; Ramon Velez ${ }^{1}{ }^{1}$ Wake Forest, Winston-Salem, NC; ${ }^{2}$ Wake Forest University School of Medicine, WinstonSalem, NC; ${ }^{3}$ Wake Forest University, WInston-Salem, NC. (Control ID \#2468324)
NEEDS AND OBJECTIVES: Funduscopic retinal examination is a useful bedside tool in the diagnosis of many diseases, yet it is underutilized in the primary care setting mainly because of lack of operator proficiency. One important limitation of traditional ophthalmoscopes is the inability to capture images making demonstration of findings to learners difficult. Our objectives were to enhance resident's expertise in interpreting retinal examination findings and to introduce them to new technology that would facilitate the performance of retinal exams at the bedside.

SETTING AND PARTICIPANTS: Our target audience was Internal Medicine Residents in our academic Institution during a scheduled half-day instructional session.

DESCRIPTION: We conducted a 90 min workshop that included 2 Power Point presentations covering normal and pathologic findings of the retina; followed by a hands on portion to practice non dilated retinal examination offering traditional ophthalmoscopes and smartphones with retinal adaptors (iPhone 5 with 2 D-eye adaptor and its application). Minimal $1 \mathrm{~min}$ instruction on how to use the I- phone device was given during the lecture. EVALUATION: Anonymous pre and post workshop surveys with a total of 7 questions were administered to all participants. The 6 initial questions of the survey assessed their perceived abilities to use the portable retinal device and to recognize ophthalmic pathology (hypertensive retinopathy, diabetic retinopathy, glaucoma, macular degeneration, cataracts) using a 5 point Likert scale from (1-extremely unconfident to 5- extremely confident). The last question assessed their enthusiasm on the ability to access new technology to perform retinal exam in the clinic (1 strongly disagree to 5-strongly agree). Survey results:. A total of 28 residents responded the pre course survey and 24 responded the post course survey (out of 30 participants).The majority of residents (96\%) reported a deficiency in the perception of their ability to use a portable retinal camera (extremely and somewhat unconfident) during the pre-course questionnaire. After the workshop, that perception dropped by half (extremely unconfident $4.5 \%$, somewhat unconfident $36.4 \%$ ). As far as the residents' ability to recognize pathology prior to the course, the majority felt unable (extremely and somewhat uncomfortable) to identify hypertensive retinopathy (70\%), diabetic retinopathy (81\%), macular degeneration (92.9\%), glaucoma $(63 \%)$. After the course, they rated that their ability (somewhat and extremely comfortable) to recognize hypertensive retinopathy ( $54 \%$ ), diabetic retinopathy (42\%), macular degeneration (37\%), glaucoma (63\%). Before the course, their enthusiasm regarding the retinal device was moderate ( $48 \%$ agree) and after the course it was good (50\% agree and $45 \%$ strongly agree). Observations: Most residents were able to capture in video the optic disk during their first attempt following the software instructions. None of the residents chose to practice the exam with traditional ophthalmoscopes.

DISCUSSION / REFLECTION / LESSONS LEARNED: The ability to perform and interpret retinal examination findings is a clear deficiency for our Internal Medicine Residents. A short course reviewing retinal pathology and funduscopic technique is a valuable teaching tool to enhance their physical examination skills. The use of smartphones as ophthalmoscopes was received enthusiastically by residents. The I phone with 2 D Eye and its application are user friendly and did not need much instruction from facilitators to capture images. The availability of new Smart phone technology with retinal adaptor is a user friendly and attractive teaching tool that may help revive retinal examination in primary care.

VALUE-ADDED MEDICAL EDUCATION: STUDENTS AS PATIENT NAVIGATORS Jed Gonzalo; Britta M. Thompson; Daniel R. Wolpaw. Penn State College of Medicine, Hershey, PA. (Control ID \#2469456)

NEEDS AND OBJECTIVES: To better align medical education with new demands of healthcare redesign, medical educators have recommended that curricula in UME increase focus on Health Systems Science (HSS) concepts, including population health, social determinants of health, clinical informatics, and teamwork. However, early experiential 
roles still primarily focus on the clinical sciences, limiting student exposure to experiences that will allow them to acquire the knowledge and skills in HSS. Value-added roles, defined as "...experiential roles that can positively impact health outcomes while also enhancing student education," have been proposed as a method to develop students' skills and knowledge in HSS, while also improving patient health. Despite this unmet need, to our knowledge, large-scale programs involving value-added roles in UME that are specifically designed to enhance learner knowledge and skills in HSS have not been described. The objectives of this work were to: (1) build and implement a patient navigator program for 1st-year medical students, and, (2) provide students the opportunity to recognize the importance of HSS through value-added roles.

SETTING AND PARTICIPANTS: From 2013 to 2016, we implemented a program for 1st-year medical students where they are integrated into interprofessional care teams in health systems to serve as patient navigators, which is contextualized in a longitudinal HSS course. A total of 36 clinical sites comprise the network of placement sites, and span a range of patient services, from medicine to surgical specialty clinics, and from inpatient to outpatient-based programs. Specific examples include several primary care patientcentered medical home clinics, inpatient hospital transitions programs, breast cancer survivorship programs, and a Department of Health Tuberculosis Clinic.

DESCRIPTION: In the 2014-15 ( $n=85$ students) and 2015-16 ( $n=138$ students) academic years, 1st-year students were embedded as patient navigators. Students remain at each clinical site for the duration of the academic year, and dedicate a minimum of two half-day sessions per month working with patients and the clinic. Activities include being linked with patients to identify root causes preventing optimal health, and exploring action plans to improve health, including medication reconciliation, home safety assessments, coordinating communication during transitions, linking patients to community-based resources, and motivational interviewing to improve adherence.

EVALUATION: We successfully designed a patient navigator program and clinical site network that has accommodated 223 medical students in several health systems in south-central Pennsylvania. Site mentors for student patient navigators include an array of healthcare team members, including care coordinators, nurse practitioners, navigators, and physicians; over $50 \%$ are in non-physician roles. Students report education in several areas of HSS, including enhanced understanding of patients' barriers and social determinants of health, improvements needed in the healthcare delivery and system to better serve patients, presence and need for interprofessional collaboration and teamwork to solve patient problems, and recognition of the need to have various approaches to communicating with patients. Student evaluations regarding the patient navigator experience are diverse, with numerous success stories and positive experiences, and also concerns related to required time and uncertainty above the importance to their career. Clinical site leadership has demonstrated a positive response to the new program.

DISCUSSION / REFLECTION / LESSONS LEARNED: To our knowledge, the Patient Navigator Program is the first large-scale program dedicated toward early health systems experiences for students that simultaneously contribute to healthcare delivery. Student engagement in patient navigator activities with other responsibilities in the 1styear of medical school has been varied, and merits consideration in future modifications to the program. Many clinical sites are accustomed to traditional "physician-centric" experiences, which requires upfront and regular guidance regarding program goals and objectives. Despite the challenges, these authentic, meaningful workplace roles promise to add depth to classroom learning in patient outcomes, interprofessional collaboration, quality, safety, value, and population health.

WRITE THE RIGHT WAY: WRITING WORKSHOPS AS A WAY TO IMPROVE ACADEMIC WRITING Neda Laiteerapong; Nicole M. Twu; Valerie G. Press. University of Chicago, Chicago, IL. (Control ID \#2465970)
NEEDS AND OBJECTIVES: Effective writing is critical for academic advancement; highly effective writing allows for efficient manuscript publication and grant submissions. However, most faculty do not receive formal training or support in academic writing. Our objective was to provide an internal forum for researchers to receive feedback on the effectiveness of their academic research writing

SETTING AND PARTICIPANTS: Faculty, trainees and staff at an academic medical center

DESCRIPTION: In 2010, a cohort of 14 academic faculty members completed the University of Chicago Writing Program's Little Red Schoolhouse (LRS) course. LRS included formal lectures on academic writing principles and small-group peer review sessions during which students brought writing samples and gave feedback using LRS principles. Based on the small-group sessions, we developed the Research Writing Workshop (RWW) to continue providing the opportunity for health services physician-researchers, clinical educators, and research staff to have an internal peerreview forum. The RWW meets for $1 \mathrm{~h}$ twice per month. Prior to each workshop, one member distributes a manuscript-in-progress to RWW members for review before arriving at the workshop. During the workshop, the RWW participants then provide their review of the manuscript including constructive feedback to the author. The program is run by two faculty co-directors with support from administrative assistants and university leadership. We actively recruit faculty and staff who complete the LRS course and through presentations at faculty events. Although graduates of the LRS are the intended audience, any faculty (or trainee) that wishes to participate is welcomed. In 2014, we widened the scope of the RWW members to include basic science, clinical, and translational researchers. We also sponsor two special guest speaker events annually who discuss academic writing issues. We performed a survey of all members and recruited faculty/staff to examine their attitudes and to elicit feedback about the RWW in 2012, 2013, and 2014 using Likert-scale based responses (strongly disagree-strongly agree).

EVALUATION: The workshop has run continually since 2011 and has reached 41 faculty/staff members. Each year to date, on average, 17 manuscripts were presented, 4.5 faculty/staff attended each workshop, and 24 unique faculty and staff participated. At the special guest speaker events, an average of 19 faculty/staff attends. Survey response rates were $64 \%(N=21 / 33), 73 \%(N=19 / 26)$, and $38 \%(N=12 / 32)$ in 2012,2013 , and 2014 , respectively. Among survey respondents, $52 \%(n=11), 74 \%(n=14)$, and $83 \%$ $(n=10)$ had attended the RWW in 2012, 2013, and 2014. Among attendees, $100 \%$ agreed or strongly agreed that they would recommend the RWW to others. Nearly all attendees (90-100 \%) agreed or strongly agreed that the RWW improved their writing and feedback skills. About half of respondents agreed or strongly agreed that the RWW improved their scholarly productivity.

DISCUSSION / REFLECTION / LESSONS LEARNED: We provide a model for an academic writing program, which provides a supportive environment to enhance faculty development of writing skills. Our Research Writing Workshop has demonstrated success at improving academic writing skills and encouraging productivity. Future work will be focused on maintenance of the small group format as well as piloting a program to encourage the process of writing academic work.

*INNOVATIONS IN CLINICAL PRACTICE (ICP)

A RESIDENT LED QUALITY IMPROVEMENT PROJECT TO REVIEW
YOUTUBE VIDEOS RELATED TO DIABETES EDUCATION WITH THE IN-
TENT TO CREATE A PLAYLIST TO USE FOR PATIENT EDUCATION DUR-
ING THE PATIENT CLINIC VISIT. Scott Joy
${ }^{1,2}$; Brandon Comfort ${ }^{2}$; Benjamin
Capper ${ }^{2}$; Erin Conlin ${ }^{2}$; Dylan R. Rakowski ${ }^{2}$ David Elwell ${ }^{2} .{ }^{1}$ The Colorado Health
Foundation, Denver, CO; ${ }^{2}$ University of Colorado, Denver, CO. (Control ID \#2466658)


STATEMENT OF PROBLEM OR QUESTION (ONE SENTENCE): How can a resident led, academic general internal medicine practice identify high value YouTube videos related to diabetes education to share with population of patients with diabetes during a routine follow up clinic appointment related to diabetes management? OBJECTIVES OF PROGRAM/INTERVENTION (NO MORE THAN THREE OBJECTIVES): Identify the diabetes education videos available on YouTube for specific content areas Develop a method to objectively grade the patient-centeredness and clinical usefulness of these videos Create a YouTube playlist of the most patient-centered videos with the intent to share with patients using a tablet in the exam room

DESCRIPTION OF PROGRAM/INTERVENTION, INCLUDING ORGANIZATIONAL CONTEXT (E.G. INPATIENT VS. OUTPATIENT, PRACTICE OR COMMUNITY CHARACTERISTICS): At the University of Colorado, Internal Medicine residents in the Primary Care track are given $1 / 2$ day to work on quality improvement projects. Residents are on a $4+1$ schedule, so each week, a different team of residents (R1, $\mathrm{R} 2$ and R3) collaborate on a project throughout the year. One team in the practice is focused on diabetes management and decided to focus their efforts to standardize diabetes education during the patient encounter. A previous time study analysis of clinic processes showed that the patients in this clinic spent an average of $36 \mathrm{~min}$ in the clinic when they were not having contact with a provider or member of the clinic team, which is known as Non-Value Added Time (NVAT). The team determined one method to increase the value added time (VAT) for the patient and to standardize diabetes education within the clinic would be to show diabetes education videos to the patient using a secure tablet device in the exam room when the patien was waiting for the resident to sign out to an attending or were waiting for a team member to complete check-in/check-out for their encounter. However, no standardized playlist existed in the clinic for such an intervention. During a group meeting, consensus was obtained that the following topics would be of value to enhance patient education at the point of care: What is Diabetes, Checking Blood Sugar, Giving Insulin, Insulin Pen Use, Hypoglycemia, Diabetic Diet, Preparing for a Diabetic Visit, and Diabetic Foot Exam. Using the YouTube search option and the above search terms, videos were identified, and these videos were further sorted by having residents during their dedicated QI time over a period of 3 months use a set criteria for review, including (1) was the video from a credible source (2) did the videos focus on the topics of interest (3) was the video at a level deemed appropriate for our patients (4) was the video less than $10 \mathrm{~min}$ in duration, and (5) was the video in English or Spanish Videos that met these criteria were evaluated by the residents using The Patient Education Materials Assessment Tool (PEMAT) that is available free online through the AHRQ website and is available for both print and audiovisual materials. A final consensus vote by the residents on the QI team of all the final videos reviewed led to creation of the final YouTube playlist.

MEASURES OF SUCCESS (DISCUSS QUALITATIVE AND/OR QUANTITATIVE METRICS WHICH WILL BE USED TO EVALUATE PROGRAM/ INTERVENTION): Number of diabetes education videos found on YouTube using the defined content search criteria. Number of diabetes education videos chosen for the final playlist after the resident review process. Individual and range of PERMAT scores for diabetes education videos and average PERMAT scores/standard deviation for final videos chosen for the playlist.

FINDINGS TO DATE (IT IS NOT SUFFICIENT TO STATE FINDINGS WILL BE DISCUSSED): Number of videos found on you tube using the following search terms/number of videos on each topic added to the final playlist: What is Diabetes (1,550,000/1), Checking Blood Sugar (69,100/3), Giving Insulin $(8,440 / 2)$, Insulin Pen Use (4,520/1), Hypoglycemia (19,000/1), Diabetic Diet (129,000/2), Preparing for the Diabetic Visit (4,760/1) and Diabetic Foot Exam (12,400/1) PERMAT scores (0-12 range) for videos chosen for final playlist ranged from a low score of 3 for Diabetic Diet to a high score of 9.5 for What is Diabetes. Average PERMAT score for all 12 videos was 5.7, Standard Deviation 1.7 The final playlist was named Uptown Primary Care Diabetes Education.
KEY LESSONS FOR DISSEMINATION (WHAT CAN OTHERS TAKE AWAY FOR IMPLEMENTATION TO THEIR PRACTICE OR COMMUNITY?): Videos related to diabetes education are common on YouTube. However, quality and content vary. High value videos can be identified by a YouTube search, by team consensus, and by a validated scoring tool, and a playlist of these videos can be created on YouTube and used by any outpatient general internal medicine practice. Next steps are to evaluate the logistics of placing electronic devices such as tablets that can be used by patients in the exam room to view these videos to increase the value added time for the patient during the office encounter

A BELLEVUE HOSPITAL OUTREACH PROGRAM: BRINGING LOST TO FOLLOW-UP, POORLY CONTROLLED DIABETIC PATIENTS BACK INTO CARE Natalie K. Levy ${ }^{2}$; Victoria Moynihan ${ }^{2}$; Muhibur Rahman ${ }^{1} .{ }^{1}$ Bellevue Hospital Center, New York, NY; ${ }^{2}$ NYU School of Medicine, Bellevue Hospital, New York, NY. (Control ID \#2465814)

STATEMENT OF PROBLEM OR QUESTION (ONE SENTENCE): Poorly controlled diabetic patients who are lost to follow-up need re-engagement in care. OBJECTIVES OF PROGRAM/INTERVENTION (NO MORE THAN THREE OBJECTIVES): This intervention aims to (1) identify and contact a cohort of high-risk patients with diabetes, (2) re-engage these patients with clinic care through a focused diabetes visit and subsequent clinic follow-up, and (3) show that this registry based approach is an effective tool for a generalist engaging in population health.

DESCRIPTION OF PROGRAM/INTERVENTION, INCLUDING ORGANIZATIONAL CONTEXT (E.G. INPATIENT VS. OUTPATIENT, PRACTICE OR COMMUNITY CHARACTERISTICS): A registry search was used to identify poorly controlled $(\mathrm{HbA} 1 \mathrm{c}>$ or $=9 \%)$ diabetic patients lost to follow-up (no clinic visits in the past 3 months). Letters were mailed inviting patients to come in for a focused diabetes recall visit with our team PA (physician assistant). Patients called our direct team phone number and were generally given this diabetes recall appointment within 3 weeks. During these recall visits our team PA obtained new vital signs, conducted a focused diabetes visit, obtained new labs, updated prescriptions if needed, and attempted to arrange subsequent follow-up visits for these patients.

MEASURES OF SUCCESS (DISCUSS QUALITATIVE AND/OR QUANTITATIVE METRICS WHICH WILL BE USED TO EVALUATE PROGRAM/ INTERVENTION): Outreach Measures: Response rate to the initial mailing Engagement Measures: Show rate of patients booked for a diabetes recall appointment Completion rate of a subsequent clinic follow-up visit $\mathrm{HbAlc}$ Testing: Completion rate of $\mathrm{HbAlc}$ testing at the recall appointment and at subsequent follow-up Median $\mathrm{HbA} 1 \mathrm{c}$ at the recall appointment and at subsequent follow-up Percent of $\mathrm{HbAlc}$ less than $9 \%$ at subsequent follow-up

FINDINGS TO DATE (IT IS NOT SUFFICIENT TO STATE FINDINGS WILL BE DISCUSSED): Outreach We identified 379 patients who met inclusion criteria for the intervention and all were sent an outreach letter. Of the 379 letters sent, approximately $50 \%$ were returned undeliverable. Of the 190 patients whose letters were not returned, 82 responded to the letter ( $43 \%$ response) and 68 of the respondents were booked for a diabetes recall appointment. Diabetes Recall appointments were generally given for the following week, with a maximum lead time of 3 weeks. Reasons for 14 of the 82 respondents not receiving a Diabetes Recall appointment were that patients (1) responded to our letter with a voicemail but could not be reached by our team, (2) had an upcoming appointment with their primary care physician and therefore didn't need a recall appointment, (3) preferred to wait to see their primary care physician. Effectiveness: Engagement levels and $\mathrm{HbAlc}$ Improvement The intervention was effective in bringing patients back to our primary care clinic. Sixty-six percent ( 45 of 68 patients) came for their diabetes 
recall appointment. Eighty percent (36 of 45 patients) returned for a subsequent clinic follow-up visit. Updated $\mathrm{HbAlc}$ values were obtained for $100 \%(45 / 45)$ of patients at the Diabetes Recall appointment and the median value was $10.1 \%$. Thirty-two patients have thus far had follow up labs: 23 of 32 had an improved HbA1c, 16 of the 32 achieved an $\mathrm{HbAlc}<9 \%$, and the median $\mathrm{HbAlc}$ was $9.0 \%$. Conclusion A registry search allowed us to easily identify patients in need and quickly send a large number of template letters inviting overdue poorly controlled diabetic patients back in for care. We were able to engage these lost to follow up patients: $43 \%$ responded to our outreach letter, $66 \%$ of patients that booked a recall appointment came for the session, and $80 \%$ of those returned for subsequent follow up. Patients who returned for a follow-up HbA1c test showed improvement (median HbAlc value declined from 10.1 to $9.0 \%$ ) and $50 \%$ (16/32) achieved a $\mathrm{HbA} 1 \mathrm{c}<9 \%$. Our findings suggest that registry based outreach programs can offer poorly controlled diabetic patients an opportunity for re-engagement in clinic care. KEY LESSONS FOR DISSEMINATION (WHAT CAN OTHERS TAKE AWAY FOR IMPLEMENTATION TO THEIR PRACTICE OR COMMUNITY?): The patient registry was a central component of our intervention and can be an effective tool in population health. A designated staff member to send letters and book appointments as well as access for patients (in our case through our team PA) also made this intervention successful.

A MEDICATION RISK COMMUNICATION AND SURVEILLANCE STRATEGY FOR PRIMARY CARE: INTRODUCING THE EMC2 TRIAL Laurie A. Hedlund $^{1}$; Laura M. Curtis ${ }^{1}$; Amy Moore ${ }^{1}$; Christine Oramasionwu ${ }^{2}$; Danielle M. McCarthy $^{1}$; Bill Adams ${ }^{3}$; Michael Paasche-Orlow ${ }^{3}$; Stacy C. Bailey ${ }^{2}$; Michael S. Wolf ${ }^{1}$. ${ }^{1}$ Northwestern University, Chicago, IL; ${ }^{2}$ University of North Carolina, Chapel Hill, NC; ${ }^{3}$ Boston Medical Center, Boston, MA. (Control ID \#2469116)

STATEMENT OF PROBLEM OR QUESTION (ONE SENTENCE): Research has repeatedly demonstrated that individuals lack essential information pertaining to risks and warnings on how to safely take prescribed $\left(\mathrm{R}_{\mathrm{x}}\right)$ medications, which can lead to unintentional misuse and serious adverse drug events (ADEs).

OBJECTIVES OF PROGRAM/INTERVENTION (NO MORE THAN THREE OBJECTIVES): 1) Improve patient understanding and use of higher-risk medications and increase the detection of medication errors and ADEs in primary care 2) Reduce disparities in understanding and safe Rx use by patient literacy level, English proficiency, and age 3) Create an effective and viable strategy to promote provider counseling, deliver patient Rx information, monitor understanding and use, and inform providers of harms DESCRIPTION OF PROGRAM/INTERVENTION, INCLUDING ORGANIZATIONAL CONTEXT (E.G. INPATIENT VS. OUTPATIENT, PRACTICE OR COMMUNITY CHARACTERISTICS): We have developed a primary care-based, technology-enabled strategy as part of R01DK103684 to improve patient-provider communication about prescribed, higher-risk medications to ensure safe use. Our Electronic health record-based Medication Complete Communication (EMC2) Strategy leverages electronic health record (EHR) and interactive voice response (IVR) technologies to: 1) prompt and guide provider counseling, 2) automate the delivery of Medication Guides at prescribing, 3) follow patients post-visit to confirm Rx understanding and use, and 4) deliver a care alert back to providers to inform them of any potential harms. We target 75 high-risk medications commonly prescribed by clinicians at Federally Qualified Health Centers in Chicago. Our list of study medications includes many that have been identified by the FDA as requiring Medication Guides or REMS and others that our team of clinicians and pharmacists have identified as having safety concerns. The list does not include drugs, such as antibiotics, that are often prescribed for a limited duration, as the patient may have completed their course of treatment before we are able to intervene. The intervention includes the following components: 1) On-screen reminder to counsel
patient-When any of the pre-identified study medications are prescribed, a care alert will pop up to prompt provider to counsel patient about the risks. 2) Med sheet displayed on screen to guide conversation-A one-page summary of the most important information from the FDA-required Medication Guide will be automatically displayed to guide provider counseling to the patient about the medication. 3) Printed med sheet-A copy of the med sheet will print with the After Visit Summary for the patient to take home. 4) IVR call-Two to 3 days after receiving the new prescription, the patient will get an automated phone call that will test knowledge about the drug and probe for any medication concerns. 5) EHR care alert to prompt clinic follow-up-If concern are raised during the IVR call, the system will flag the patient in the EHR with a care alert as a trigger to clinic staff to follow up per standard care. We are testing our intervention in the primary care setting at multiple clinics (diverse by race, ethnicity, language (Spanish, English), age, and socioeconomic status) within two Federally Qualified Health Centers in Chicago, IL. We are randomizing each clinic into one of two study arms: intervention or standard care. All patients prescribed any of the target medicines at an intervention clinic will automatically receive intervention components 1, 2, and 3 during their visit. A research assistant will call each patient within 2 days of receiving the new prescription to invite them to participate in the study; only those who consent will receive components 4 and 5. A total of 1200 patients will be recruited across both arms.

MEASURES OF SUCCESS (DISCUSS QUALITATIVE AND/OR QUANTITATIVE METRICS WHICH WILL BE USED TO EVALUATE PROGRAM/ INTERVENTION): We will measure the effectiveness of our intervention by assessing the following outcomes during patient interviews and chart reviews: 1) Medication knowledge: We have created measures to assess knowledge related to contraindications, risks and benefits, and side effects. Correct answers will align with the content in each drug's med sheet. 2) Medication behavior: We will assess medication behavior in three ways: fill (patient self-report of having picked up medication from pharmacy), proper use (patient ability to correctly state the name, indication, dose, frequency, and total pills per day), and actual use (Patient Medication Adherence Questionnaire (PMAQ) and a phonebased pill count assessment). 3) Adverse drug events (ADEs): Self-reported ADEs will be assessed using a validated questionnaire during the 3-month interview; symptoms will then be reviewed by our pharmacist to ascertain a connection to the prescribed high-risk medication. Detection of confirmed ADEs will be assessed via chart review.

FINDINGS TO DATE (IT IS NOT SUFFICIENT TO STATE FINDINGS WILL BE DISCUSSED): No findings to date. We expect to begin recruitment in the spring of 2016.

KEY LESSONS FOR DISSEMINATION (WHAT CAN OTHERS TAKE AWAY FOR IMPLEMENTATION TO THEIR PRACTICE OR COMMUNITY?): - Increases scope of approaches utilized to increase patient adherence, applying underutilized techniques such as IVR technologies to a health literacy model—Tangible examples of EHR tools, including reminder to counsel and printed patient supplementary materials, that can be integrated into the physician workflow

A NEW PROGRAM OF PRIMARY CARE PHYSICAN INPATIENT CONSULTATION VISITS-MULTIPLE PERSPECTIVES Dana D. Im; Christina Phillips²; Amy Pasternack ${ }^{2}$; Priyank Jain ${ }^{2}$; David Bor ${ }^{2}$; Barbara Ogur ${ }^{1,2}$; Richard Pels ${ }^{2} .{ }^{1}$ Harvard Medical School Center for Primary Care, Boston, MA; ${ }^{2}$ Cambridge Health Alliance, Cambridge, MA. (Control ID \#2466258)

STATEMENT OF PROBLEM OR QUESTION (ONE SENTENCE): The role of primary care physicians (PCPs) in general medical inpatient care has diminished, replaced by hospitalists offering more efficient care with similar quality measures. ${ }^{1,2}$ However, the resulting discontinuity of care raises concerns about fragmented care and patient dissatisfaction. ${ }^{3}$ 
OBJECTIVES OF PROGRAM/INTERVENTION (NO MORE THAN THREE OBJECTIVES): To provide a structured, compensated process for PCPs to visit their patients admitted to the hospital. To ensure that medical residents caring for inpatients of PCPs have ready access to necessary supervision and teaching To determine the acceptability and utility of PCP consult visits to residents, PCPs, and hospitalists

DESCRIPTION OF PROGRAM/INTERVENTION, INCLUDING ORGANIZATIONAL CONTEXT (E.G. INPATIENT VS. OUTPATIENT, PRACTICE OR COMMUNITY CHARACTERISTICS): On January 1, 2015, the Cambridge Health Alliance (CHA) Department of Medicine transitioned from a mixed inpatient model, in which seven PCPs attended on their hospitalized patients and hospitalists cared for all other inpatients, to an all-hospitalist model. A pilot program incorporated these seven PCPs as consultants. PCP consultants visited once or twice during a patient's hospitalization, providing care the PCP considered relevant: a focused assessment, insight into prior care, emotional support based upon a long-term care relationship, discussion of care decisions with the patient and family, and assistance in planning after-hospital care. PCPs were reimbursed for their visits. MEASURES OF SUCCESS (DISCUSS QUALITATIVE AND/OR QUANTITATIVE METRICS WHICH WILL BE USED TO EVALUATE PROGRAM/ INTERVENTION): Surveys of residents pre-intervention and a focus group postintervention A focus group of hospitalists post-intervention A focus group of participating PCPs post-intervention

FINDINGS TO DATE (IT IS NOT SUFFICIENT TO STATE FINDINGS WILL BE DISCUSSED): In the traditional model, CHA residents felt less supported caring for patients of PCPs, compared to patients of hospitalists. Residents reported insufficient supervision of daily care, procedures, and discharge planning. PCPs' clinic schedules often delayed rounding, limiting timely supervision and collaborative decision-making. In comparison, residents have found PCP consultants mainly helpful, especially when the consults are based on long, meaningful relationships with patients. They viewed PCP consultants as having facilitated family meetings, goals of care discussions, and coordination of post-discharge care. Residents did not find the process of communicating with PCP consultants problematic, but did note that sometimes PCPs recommended additional inpatient evaluations that residents felt might have been deferred to the ambulatory setting. In contrast to the residents, hospitalists identified the need for a clear communication mechanism for PCP consultants to close the loop after inpatient visits. Hospitalists also found it difficult to navigate PCP medical recommendations that the hospital team disagreed with. PCP consultants felt that the majority ( $81 \%)$ of the consultations went "okay" to "excellent." They reported contributing to the care of their hospitalized patients by: providing medical or social information, suggesting medical management, supporting transitions of care, facilitating end-of-life discussions, and providing comfort to patients and family members. PCP consultants expressed that the medical and social need for a consultation is often best discerned by PCPs themselves, not the primary team. Some PCPs felt that the term "consultant" did not fit their role, even in inpatient settings. They felt that due to their ongoing relationship with the patient and their responsibility to resume function as physician-of-record after discharge, that the ways that they added value were, therefore, fundamentally different from those of a consultant.

KEY LESSONS FOR DISSEMINATION (WHAT CAN OTHERS TAKE AWAY FOR IMPLEMENTATION TO THEIR PRACTICE OR COMMUNITY?): The "PCP consult" provides a model for PCP involvement that residents view as contributing to care and that may allow effective modeling of the value of long-term patient-doctor relationships. Hospitalists and PCPs also found the PCPs' involvement important and useful, particularly in providing emotional support to patients, but found a number of obstacles, including difficulty in ensuring communication with the team and some concerns about ensuring clear lines of responsibility for the inpatient care. Further research should explore whether PCPs' contributions improve the quality of care, patient satisfaction, and the cost of inpatient care. Identifying specific ways in which PCP consultants add value will help inform how best to structure PCP involvement in their patients' inpatient care.
A NOVEL CARE MODEL INTEGRATING CARDIOLOGISTS IN A PRIMARY CARE PRACTICE IMPROVES COORDINATED CARE AND REFERRING PROVIDER SATISFACTION Muhamad Y. Elrashidi; Paul McKie; Sarah Crane; Jon Ebbert. Mayo Clinic, Rochester, MN. (Control ID \#2467985)

STATEMENT OF PROBLEM OR QUESTION (ONE SENTENCE): The rising costs and fragmentation of healthcare delivery, in which patients with chronic and comorbid cardiovascular disease must engage multiple providers and venues across the spectrum of care while trying to maintain continuity with their primary care home, necessitates development and study of new care delivery models that enhance care coordination and collaboration among primary care and cardiovascular specialty providers

OBJECTIVES OF PROGRAM/INTERVENTION (NO MORE THAN THREE OBJECTIVES): 1 . Describe the model of co-located, integrated cardiology in a primary care, patient-centered medical home. 2 . Report pilot data showing primary care provider referral rate to integrated cardiology vs. non-integrated, cardiology subspecialty practices. 3. Report primary care provider satisfaction data pre- and post- implementation of integrated cardiology model.

DESCRIPTION OF PROGRAM/INTERVENTION, INCLUDING ORGANIZATIONAL CONTEXT (E.G. INPATIENT VS. OUTPATIENT, PRACTICE OR COMMUNITY CHARACTERISTICS): This pilot was conducted with patients empaneled in Mayo Clinic Employee and Community Health (ECH), a multi-site primary care practice located in Rochester, MN. Mayo Clinic is a multispecialty, tertiary-referral group practice with a common electronic health record (EHR). All patients within ECH have an assigned primary care provider and ECH provides care to approximately 140,000 patients. Half of all ECH patients are employees and dependents who receive insurance coverage through Mayo Clinic. Prior to the pilot, ECH patients seeking cardiology care were referred by their primary care provider (PCP) or by self-referral to the non-integrated, cardiology subspecialty practice. On October 1, 2014, a general cardiology practice was co-located within the main location of ECH and provided 1.0 full-time equivalent (FTE) for curbside, electronic (e-consult), and traditional, face-to-face (FTF) consultation. The cardiologists and all ECH providers are compensated on a salary model. Collaboration via curbside (telephone, electronic health record messaging, E-mail) or electronic consultation was encouraged before ordering a FTF consult through physician-led staff meetings, practice-wide electronic communications, and a display of the pager numbers and photographs of the co-located cardiologists in every clinic exam room. Diagnostic testing was not prohibited or constrained. The co-located cardiology schedule allotted two hours each morning for review of emergency department and hospital discharge triage as well as econsults, followed by four, 45-min, new patient FTF visits. Two additional new patient FTF visits were scheduled each afternoon. The remaining unscheduled time was used to provide curbside, electronic, same day FTF consults, and follow up on patient messages and test results. Nonidentifiable, referral volume data of FTF visits to integrated cardiology and the non-integrated, cardiovascular subspecialty practice was accessed through Mayo Clinic Patient Access Management Analytics (PAMA) data warehouse. One month prior to pilot implementation, a 13-question survey was sent electronically to all $\mathrm{ECH}$ primary care providers assessing perceptions of cardiovascular care on a five-point Likert scale, vis-a-vis aspects of referral access, communication, and level of care coordination. The same survey was sent to all ECH providers 14 months after implementation for comparison.

MEASURES OF SUCCESS (DISCUSS QUALITATIVE AND/OR QUANTITATIVE METRICS WHICH WILL BE USED TO EVALUATE PROGRAM/ INTERVENTION): Measures of model success will include: 1. Decreased referral volume of ECH patients to non-integrated, cardiology subspecialty practice. 2. PCP satisfaction on aspects of access, communication, and care coordination with cardiovascular care referrals and specialists. 
FINDINGS TO DATE (IT IS NOT SUFFICIENT TO STATE FINDINGS WILL BE DISCUSSED): Compared to the 2 month average prior to pilot launch, the integrated cardiology model resulted in an average $42.6 \%$ reduction in PCP referrals to the nonintegrated, cardiology subspecialty practice for FTF consultation over the first year of implementation. Integrated cardiology in the primary care practice setting performed half of all PCP referrals for cardiovascular care FTF consultation. Response rates for pre- and post-implementation PCP satisfaction surveys were similar (61.3 and $63.7 \%$, respectively). Overall PCP satisfaction with cardiovascular care improved on all dimensions surveyed. Total satisfaction increased after implementation of the integrated model $(57.1 \%$ vs. $88.1 \%)$. In addition, PCPs reported higher satisfaction with access ( $28.6 \%$ vs. $78.0 \%$ ), avoidance of duplicative or pre-consult testing (68.4\% vs. $90.8 \%$ ), knowledge exchange ( $46.9 \%$ vs. $84.4 \%$ ), and plan of care communication ( $66.3 \%$ vs. $95.4 \%$ ).

KEY LESSONS FOR DISSEMINATION (WHAT CAN OTHERS TAKE AWAY FOR IMPLEMENTATION TO THEIR PRACTICE OR COMMUNITY?): Improving access, communication, and coordination between primary and specialty providers is critical to delivering high value care to patients. The model of a co-located, integrated cardiology practice within a patient centered medical home demonstrates promise for reducing referrals to non-integrated, neurology subspecialty practices, improve communication and care coordination between primary and cardiovascular specialty care. Integrated cardiology has the potential to support achieving the goals of improving population health, care experience, and lowering cost

A NOVEL INTERDISCIPLINARY IMPROVEMENT PROCESS TO ADDRESS DISCHARGE MEDICATION BARRIERS Derek C. Mazique ${ }^{2}$; Parimal A. Patel² Jennifer I. Lee ${ }^{1}{ }^{1}$ NYP/WC, New York, NY; ${ }^{2}$ NewYork-Presbyterian Hospital, New York City, NY. (Control ID \#2466326)

STATEMENT OF PROBLEM OR QUESTION (ONE SENTENCE): Patients discharged from the hospital are frequently unable to fill newly prescribed medications at outpatient pharmacies due to insurance and availability related barriers.

OBJECTIVES OF PROGRAM/INTERVENTION (NO MORE THAN THREE OBJECTIVES): Identify and remove preventable barriers affecting discharge prescriptions that patients would encounter at an outpatient pharmacy.

DESCRIPTION OF PROGRAM/INTERVENTION, INCLUDING ORGANIZATIONAL CONTEXT (E.G. INPATIENT VS. OUTPATIENT, PRACTICE OR COMMUNITY CHARACTERISTICS): Approximately $10 \%$ of hospital discharges are complicated by a medication-related event, with insurance type increasing the risk of a prescription-related issue after discharge. At a large academic medical center in New York City, there is currently no standard system to verify that medications prescribed at discharge are on the patient's insurance formulary or available at outpatient pharmacies. From April to August 2015, we identified patients being discharged during interdisciplinary rounds. Nonclinical staff faxed discharge prescriptions to the patient's preferred pharmacy to identify preventable barriers to acquiring medications: high co-payment, not on formulary, need for prior authorization (PA), and unavailability. Medications with high co-payments or requiring PA were changed to an alternative when appropriate. Alternative pharmacies were called if a medication was unavailable. Patients' prior outpatient prescriptions were also processed if refills were required. All patients received a post-discharge phone call to confirm success in obtaining medications; if unreachable, the team called the pharmacy to confirm.

MEASURES OF SUCCESS (DISCUSS QUALITATIVE AND/OR QUANTITATIVE METRICS WHICH WILL BE USED TO EVALUATE PROGRAM/ INTERVENTION): A resident-driven interdisciplinary improvement process was designed to ensure that at least $95 \%$ of patients discharged home with at least one new prescription were able to obtain their newly prescribed medications. The measure of success was the percent of patients with an identified pre-discharge medication barrier who successfully filled their prescriptions when surveyed after discharge.
FINDINGS TO DATE (IT IS NOT SUFFICIENT TO STATE FINDINGS WILL BE DISCUSSED): Over 20 weeks, 161 patients were included in the improvement process. Nearly a third of patients had Medicare (50/161, $31.1 \%), 46$ (28.6 \%) had Medicaid, 33 (20.5\%) had both Medicare/Medicaid, and 31 (19.3\%) had commercial insurance. Nearly all patients had a preferred outpatient pharmacy (155/161, 96.3\%): 77 patients (47.8\%) used large chain pharmacies, 78 patients (48.4\%) used independent pharmacies. Sixty-one patients $(61 / 161,37.9 \%)$ had at least one identified pre-discharge barrier with their prescriptions. In total, 89 issues were discovered, with several prescriptions having more than one problem requiring intervention. Over a third of the issues (33/ 89, $37.1 \%)$ involved unavailable medications, 26 (29.2\%) needed PA, 16 (18.0 \%) were non-formulary, and six (6.7 \%) had high co-pays. Prescriptions filled under Medicaid required PA (18/26 [69.2\%] Medicaid vs. 8/26 [30.8\%] non-Medicaid, $p=0.06)$ and were non-formulary (11/16 [68.8\%] Medicaid vs. 5/16 [31.3\%] non-Medicaid, $p=0.17$ ) more frequently when compared to under non-Medicaid plans. Medications that required intervention, in order of frequency, were anti-infective medications $(18 / 89,20.2 \%$ of total issues), narcotics/benzodiazepines (13/89, $14.6 \%$ ), and proton pump inhibitors (11/89, $12.4 \%$ ). The incidence of issues for narcotics and benzodiazepines may be higher, as several pharmacies did not release information on controlled substances. Patients included in the improvement process received a post-discharge phone call regardless of whether an issue was identified with their prescriptions during the intervention. Of the 125 patients who responded to follow-up calls, we reached 45 of the 61 patients who had identified predischarge barriers $(73.8 \%)$. Forty $(40 / 45,88.9 \%)$ successfully filled their prescriptions at the pharmacy. Reasons for the inability to obtain prescriptions included unavailability $(2 / 5,40 \%)$, inability to verify controlled substances prior to discharge $(2 / 5,40.0 \%)$, and non-formulary status $(1 / 5,20 \%)$. Barriers around the controlled substances were reported to be availability and high copay.

KEY LESSONS FOR DISSEMINATION (WHAT CAN OTHERS TAKE AWAY FOR IMPLEMENTATION TO THEIR PRACTICE OR COMMUNITY?): Over a third of patients discharged home would have encountered barriers to filling at least one of their medications at their outpatient pharmacy. Anti-infectives, narcotics, anxiolytics, and proton pump inhibitors were more commonly associated with pre-discharge barriers including need for PA and substituting less costly alternatives. Potential issues around controlled substances were difficult to identify prior to discharge for several patients. While need for PA was higher for patients with Medicaid, our improvement process was not powered to reach statistical significance. Through this resident-designed quality improvement process using nonclinical staff, we have shown that early identification and removal of preventable barriers affecting discharge prescriptions allowed nearly $90 \%$ of patients to obtain their discharge medications. Implementation of this process can be adapted throughout the hospital to improve care transitions.

A PATIENT SAFETY PROJECT TO CREATE A CHECKLIST FOR USE IN A GENERAL INTERNAL MEDICINE PRACTICE TO GATHER IMPORTANT INFORMATION DURING A REPORT OF AN UNEXPECTED PATIENT DEATH Scott Joy ${ }^{3,}{ }^{4}$; Shelly Dierking ${ }^{5}$; Brian Dwinnell ${ }^{1,}{ }^{3}$; Michael Masse ${ }^{1}$; Leanne Rupp $^{1,3}$; lynn haley ${ }^{3}$; Judi Hamlin ${ }^{1}$; sheila hancock ${ }^{1}$; Carol A. Stamm ${ }^{4}$; Anjali Dhurandhar ${ }^{2,3}$; Elisabeth Ihler ${ }^{3,4}$. ${ }^{1}$ Presbyterian St. Lukes, Denver, CO; ${ }^{2}$ University of Colorado School of Medicine, Denver, CO; ${ }^{3}$ The Colorado Health Foundation, Denver, $\mathrm{CO} ;{ }^{4}$ University of Colorado, Denver, CO; ${ }^{5}$ Patient Safety Education Partnership, Denver, CO. (Control ID \#2460235)

STATEMENT OF PROBLEM OR QUESTION (ONE SENTENCE): Various staff members within a General Internal Medicine practice often receive unexpected telephone calls reporting a patient death, which can be a stressful time for the caller and for the staff member receiving the call with critically important information not being collected during 
the time that these calls occur, resulting in extra calls between staff, family, and reporting individuals and delays in addressing possible patient safety issues.

OBJECTIVES OF PROGRAM/INTERVENTION (NO MORE THAN THREE OBJECTIVES): To create a standardized guideline for any staff member to use in a General Internal Medicine practice when communicating and documenting details of a telephone conversation related to an unexpected death.

DESCRIPTION OF PROGRAM/INTERVENTION, INCLUDING ORGANIZATIONAL CONTEXT (E.G. INPATIENT VS. OUTPATIENT, PRACTICE OR COMMUNITY CHARACTERISTICS): Our academic outpatient General Internal Medicine practice has a multidisciplinary patient safety team that meets monthly to review patient safety reports which include external telephone calls received by the practice from multiple parties related to unexpected deaths. In reviewing these types of calls, the committee found that additional information was often needed from the caller to better understand the cause of death and the process(es) that had been taken up to that point to notify other relevant parties, such as the county coroner. A procedure and process guiding faculty, resident physicians and staff members on how to handle telephone calls related to unexpected deaths and a checklist of important questions and data to ask and collect during these types of calls did not exist within our practice. Over a period of 3 months in 2015 , our patient safety committee met in person monthly to discuss details of what type of information would be valuable to collect during these types of telephone calls and met with patient safety officers within our medical center to review inpatient hospital policies related to unexpected deaths. Members of the safety committee also shared and edited an online document via e-mail that detailed talking points for all physicians and staff members to use when receiving these types of telephone calls from different categories of individuals (family member/neighbor/landlord, hospice/home health agency, or coroner), listed important data to collect during the call, and described strategies on how to handle difficult conversations that may occur with these types of telephone calls. MEASURES OF SUCCESS (DISCUSS QUALITATIVE AND/OR QUANTITATIVE METRICS WHICH WILL BE USED TO EVALUATE PROGRAM/ INTERVENTION): Creation of an information guideline and checklist for all staff in General Internal Medicine practice to use for calls received related to unexpected deaths FINDINGS TO DATE (IT IS NOT SUFFICIENT TO STATE FINDINGS WILL BE DISCUSSED): Details of the information guideline and checklist are as follows: All calls related to an unexpected death are to occur in a private location, and important to demonstrate compassion at all times. If call is from a family member/neighbor/landlord ask: Date/Time of Event Event Description Was an ambulance called? Was the deceased taken to the hospital? Was the coroner notified? Do you know the cause of death? Do you know what funeral home is handling the arrangements? How are you doing? Is there anything I can do to help you? We have a psychologist and social worker on staff-may we offer you a list of resources? If call from hospice/home health agency ask: Date/Time of Event Event Description Who has been notified? Do you know the cause of death? Do you know what funeral home is handling the arrangements? How are you doing? Is there anything I can do to help you? What information or follow-up can we provide for you? What can we do to support the family? If call from the coroner ask: Date/Time of Event Event Description Who has been notified? What information or follow-up can we provide for you?

KEY LESSONS FOR DISSEMINATION (WHAT CAN OTHERS TAKE AWAY FOR IMPLEMENTATION TO THEIR PRACTICE OR COMMUNITY?): An informational guideline and checklist can be created and used by any outpatient General Internal Medicine practice that can assist all staff in being confident and sensitive when receiving telephone calls from multiple parties who may report an unexpected death to the practice. This process also provides a structured means to collect valuable data during that single call to reduce unnecessary return and follow-up calls between the practice and the reporting individual, and may be used as a method to identify possible trends that may suggest a signal for a systemic patient safety issue.
A POSITIVE DEVIANCE APPROACH SUBSTANTIALLY IMPROVES BLOOD PRESSURE CONTROL IN SAFETY NET POPULATIONS Shari Bolen ${ }^{2,4}$; Thomas Love $^{2,}{ }^{4}$; David Bar-Shain ${ }^{1,}{ }^{1}$; Peter J. Greco ${ }^{2}$; Douglas Einstadter ${ }^{1}$; Mary Behmer ${ }^{2}$; Heather Ways ${ }^{5}$; Aleece Caron ${ }^{3}$; Randall D. Cebul ${ }^{2},{ }^{4}$. ${ }^{1}$ MetroHealth Medical Center, Cleveland, OH; ${ }^{2}$ MetroHealth/Case Western Reserve University, Cleveland, $\mathrm{OH} ;{ }^{3} \mathrm{The}$ MetroHealth System, Cleveland, OH; ${ }^{4}$ Case Western Reserve University, Cleveland, OH; ${ }^{5}$ Neighborhood Family Practice, Cleveland, OH. (Control ID \#2467137)

STATEMENT OF PROBLEM OR QUESTION (ONE SENTENCE): While quality improvement (QI) interventions have been shown to improve blood pressure (BP) control among Health Maintenance Organization (HMO) populations, less is known regarding intervention effectiveness within safety net populations.

OBJECTIVES OF PROGRAM/INTERVENTION (NO MORE THAN THREE OBJECTIVES): Pilot test and evaluate the effectiveness of an adapted hypertension best practice on hypertensive processes and outcomes of care in safety net clinics. DESCRIPTION OF PROGRAM/INTERVENTION, INCLUDING ORGANIZATIONAL CONTEXT (E.G. INPATIENT VS. OUTPATIENT, PRACTICE OR COMMUNITY CHARACTERISTICS): In 2011, 12 clinics of an HMO were identified as having the greatest 2-year improvements in BP control of 51 clinics publicly reporting quality of care through Better Health Partnership (BHP), a regional health improvement collaborative. We adapted the BP protocol of these "positive deviants" and implemented it in diverse clinics of the region's safety net organizations (SNOs). The protocol includes: 1) BP measurement education including performing a second reading when the initial reading is high; 2) a treatment algorithm which prioritizes once daily low-cost medications; 3) outreach to patients with elevated BP using EMR-based patient registries; 4) timely follow-up in nurse or medical assistant (MA)-led BP visits when the BP is high; and 5) a communication curriculum emphasizing building trusting and culturally sensitive relationships with patients We presented the protocol to all practice providers/staff at two safety net clinics (Clinics A and B) in different SNOs. A practice coach met monthly for 6 months with the entire practice to conduct rapid cycle QI, including audit/feedback of process data. At Clinic A, we also introduced an EMR prompt for MAs to conduct a second BP measurement when the first BP was elevated $(>140 / 90 \mathrm{mmHg})$.

MEASURES OF SUCCESS (DISCUSS QUALITATIVE AND/OR QUANTITATIVE METRICS WHICH WILL BE USED TO EVALUATE PROGRAM/ INTERVENTION): The percent of adults with hypertension under good BP control $(<140 / 90 \mathrm{mmHg})$. At clinic A, we also received data on the percent of adults with hypertension and elevated BP who had: 1) a repeat BP measurement at the same office visit, and 2) had a timely follow-up visit scheduled within 35 days.

FINDINGS TO DATE (IT IS NOT SUFFICIENT TO STATE FINDINGS WILL BE DISCUSSED): Among 51 BHP clinics, patients in the two safety-net clinics showed the strongest 2-year improvements in BP control $(<140 / 90)$, rising from $50 \%$ in 2012 to $63 \%$ in 2014 (a $27 \%$ relative increase). Improvements were observed across all patient subgroups in these clinics, including blacks ( 41 to $58 \%$ ), those of Hispanic ethnicity ( 45 to $66 \%$ ), uninsured ( 47 to $64 \%$ ) and those covered by Medicaid (49 to $63 \%$ ). For Clinic A's selected process measures, the proportion of adults with elevated BP who received a second BP measurement increased from 45 to $70 \%$ in the 3 months pre- and 3 months post-implementation of the EMR alert, with results sustained at 12 months postimplementation. At 6 months post implementation, timely follow-up at Clinic A increased from 35 to $58 \%$ and declined to $45 \%$ at 12 months post-implementation.

KEY LESSONS FOR DISSEMINATION (WHAT CAN OTHERS TAKE AWAY FOR IMPLEMENTATION TO THEIR PRACTICE OR COMMUNITY?): Using a "positive deviance" approach to identify and disseminate an adapted BP protocol among SNOs of a regional health improvement collaborative can yield substantial and sustained improvements in BP control for safety net populations. 
A RESIDENT-LED PROGRAM TO INCREASE RATES OF ADMISSION MEDICATION RECONCILIATION AMONG INTERNAL MEDICINE RESIDENTS Hana Lim²; Elaine C. Khoong'; Jessica B. Rubin²; Christine Soran²; Josue Zapata ${ }^{2}$; Andrew D. Auerbach ${ }^{1} .{ }^{1}$ UCSF Division of Hospital Medicine, San Francisco, CA; ${ }^{2}$ University of California - San Francisco, San Francisco, CA. (Control ID \#2469937)

STATEMENT OF PROBLEM OR QUESTION (ONE SENTENCE): Incomplete medication lists at admission can produce medication discrepancies and adverse drug events during hospitalization and afterwards; our internal medicine residency program discovered that only $77 \%$ of patients admitted to the hospital medicine teaching services received a complete medication reconciliation, placing almost a quarter of our patients at risk

OBJECTIVES OF PROGRAM/INTERVENTION (NO MORE THAN THREE OBJECTIVES): The purpose of our intervention was to: 1) Identify strategies to improve rates of admission medication reconciliation among patients admitted to the hospital medicine teaching service 2) Increase the rate of complete medication reconciliation for patients admitted to the hospital medicine teaching service to at least $90 \%$

DESCRIPTION OF PROGRAM/INTERVENTION, INCLUDING ORGANIZATIONAL CONTEXT (E.G. INPATIENT VS. OUTPATIENT, PRACTICE OR COMMUNITY CHARACTERISTICS): The impetus of our program arose from a serious patient safety event that resulted from a medication reconciliation error in the hospital. Using this event as a burning platform to motivate change, we began a housestaff quality improvement project sponsored by the Office of Graduate Medical Education. Our group of Internal Medicine residents organized a project focused on improving housestaff completion of admission medication reconciliation to reduce the risk of future medication errors. At the same time, the Division of Hospital Medicine (DHM) targeted medication reconciliation as a quality improvement priority, allowing for shared resources. DHM supported a weeklong Lean event, during which a multidisciplinary team met to identify barriers and solutions to medication reconciliation. First, we determined best practices and standard work for medication reconciliation, while ensuring that it could still be performed in a timely manner. Next, we observed internal medicine residents perform medication reconciliation in real time to determine barriers to admission medication reconciliation and wasted motion. Using this information, we devised an intervention to increase medication reconciliation rates that contained several components: 1) provider education; 2) electronic medical record (EMR) reminders; 3) team-based approach to medication reconciliation; 4) audit and feedback; and 5) positive incentives. To improve rates of medication reconciliation, housestaff focused on increasing awareness by publicizing the importance of medication reconciliation through monthly resident orientations, emails, and flyers posted in areas frequented by the housestaff. We next built in a systems-level change to make medication reconciliation more visible by adding a "med-rec icon" to teams' patient lists. Our electronic medical record (EMR, Epic) allows individuals to create individualized patient lists including an icon indicating whether medication reconciliation has been completed for each patient. We then educated residents on best practices through presentations at resident didactics, video demonstrations of admission reconciliation using the EMR, and pocket cards as reminders of best practices. To help busy admitting residents with medication reconciliation, the housestaff advocated for a pharmacy student to aid with medication reconciliation in the emergency department. Based on feedback during the initial phases of this intervention, a new note template was developed to formalize the communication between the pharmacy students and the medicine teams regarding the patient's medication regimen. Throughout this process we conducted an audit and feedback program to measure and report performance on medicine reconciliation to our housestaff. Team-based results for admission medication reconciliation were disseminated to inpatient team members on a bi-monthly frequency. The most recent intervention includes providing positive incentives in the form of gift cards to teams achieving $100 \%$ of admissions fully reconciled.
MEASURES OF SUCCESS (DISCUSS QUALITATIVE AND/OR QUANTITATIVE METRICS WHICH WILL BE USED TO EVALUATE PROGRAM/ INTERVENTION): The primary measure of success was the percent of patients discharged from the inpatient medicine teaching service who received all home medications reconciled prior to discharge.

FINDINGS TO DATE (IT IS NOT SUFFICIENT TO STATE FINDINGS WILL BE DISCUSSED): Prior to the implementation of our program, $77 \%$ of all patients admitted to the hospital medicine teaching service had a complete admission medication reconciliation by the time of discharge. By the end of December 2015, the cumulative percentage of all patients from July to December 2015 with a complete admission medication reconciliation increased to $89.1 \%$. For the last two consecutive months (November and December 2015), the percentage was greater than $91 \%$.

KEY LESSONS FOR DISSEMINATION (WHAT CAN OTHERS TAKE AWAY FOR IMPLEMENTATION TO THEIR PRACTICE OR COMMUNITY?): The components of our initiative that we believe most enabled our success were: 1) a multidisciplinary approach to develop best practices; 2) securing buy-in from our entire department; and 3) dynamic data that allowed for quick evaluation of interventions. Despite busy schedules and frequent rotation changes, through education, systems changes, and feedback, housestaff can be encouraged to increase rates of medication reconciliation. Moreover, housestaff value the opportunity to adopt new methods to reduce medication errors.

A SINGLE CENTER STUDY IN REDUCING UNNECESSARY TELEMETRY Marshall Ward; Mark Scaccia. Dartmouth Hitchcock Medical Center, Lebanon, NH. (Control ID \#2469093)

STATEMENT OF PROBLEM OR QUESTION (ONE SENTENCE): Retrospective studies have shown that many patients monitored with remote cardiac telemetry have no evidenced base indication leading to overuse of healthcare resources.

OBJECTIVES OF PROGRAM/INTERVENTION (NO MORE THAN THREE OBJECTIVES): Reduce the unnecessary utilization of telemetry monitoring of patients admitted to inpatient medicine units by at least $50 \%$. Increase the percentage of patients who have appropriate clinical indications for telemetry. Increase staff awareness of current evidenced based guidelines for telemetry.

DESCRIPTION OF PROGRAM/INTERVENTION, INCLUDING ORGANIZATIONAL CONTEXT (E.G. INPATIENT VS. OUTPATIENT, PRACTICE OR COMMUNITY CHARACTERISTICS): Dartmouth-Hitchcock Medical Center (DHMC) is an academic tertiary care hospital with 396 inpatient beds located in Lebanon, New Hampshire which discharges approximately 25,000 patients annually. Baseline measurements were collected of the average number of inpatients being monitored on telemetry. Hospital medicine and house staff were educated on AHA 2004 guidelines for telemetry use, clarifying the AHA guidelines including Class III scenarios that warrant no telemetry monitoring. Nursing staff was also educated about the current evidence-based guidelines for telemetry monitoring in hospitalized patients. Updates were made to the electronic ordering system's telemetry order set to include the AHA guidelines Class I and II indications.

MEASURES OF SUCCESS (DISCUSS QUALITATIVE AND/OR QUANTITATIVE METRICS WHICH WILL BE USED TO EVALUATE PROGRAM/ INTERVENTION): Reduction of telemetry use on inpatient medicine floor by $50 \%$. Increase the percentage of patients with appropriate indication for telemetry based on AHA 2004 guidelines.

FINDINGS TO DATE (IT IS NOT SUFFICIENT TO STATE FINDINGS WILL BE DISCUSSED): At the start of the initiative, baseline measures were obtained by regular review of telemetry records. This revealed that, on average, 12 patients $(35 \%)$ on the unit had 
telemetry monitoring ordered on a daily basis and only $50 \%$ had appropriate clinical indications for monitoring. After the appropriate staff was educated and the electronic order set was updated to reflect current guidelines, the use of telemetry decreased by nearly half, with an average of 7 patients ( $20 \%$ ) on the unit being monitored by telemetry on a daily basis. Additionally random chart review obtained after our interventions showed a $55 \%$ reduction in the number of patients with inappropriate indications for telemetry.

KEY LESSONS FOR DISSEMINATION (WHAT CAN OTHERS TAKE AWAY FOR IMPLEMENTATION TO THEIR PRACTICE OR COMMUNITY?): Educating the staff and altering the electronic ordering system to reflect the appropriate indications for telemetry is a cost effective way to reduce unnecessary telemetry usage while increasing overall appropriate use. Overall, this was an effective way of implementing one of the recommendations of the Choosing Wisely campaign and we believe could be easily replicated at other hospitals.

A SUCCESSFUL STRATEGY FOR IMPLEMENTING ELEMENTS OF A HYPERTENSION BEST PRACTICE IN SAFETY NET CLINICS Shari Bolen ${ }^{3,5}$; James Misak ${ }^{2}$; Aleece Caron ${ }^{4}$; Douglas Einstadter ${ }^{2}$; Steven Lewis ${ }^{1}$; Thomas Love ${ }^{3}, 5$; David Bar-Shain ${ }^{4}$; Peter J. Greco ${ }^{4}$; Randall D. Cebul ${ }^{3,5}$. ${ }^{1} \mathrm{MHMC/CWRU}$, Cleveland, $\mathrm{OH} ;{ }^{2}$ MetroHealth Medical Center, Cleveland, OH; ${ }^{3}$ MetroHealth/Case Western Reserve University, Cleveland, OH; ${ }^{4}$ The MetroHealth System, Cleveland, OH; ${ }^{5}$ Case Western Reserve University, Cleveland, OH. (Control ID \#2467701)

STATEMENT OF PROBLEM OR QUESTION (ONE SENTENCE): Hypertension best practices have been effective in improving blood pressure (BP) control in a Health Maintenance Organization (HMO); less is known regarding effective implementation strategies for elements of the hypertension best practice in safety net clinics.

OBJECTIVES OF PROGRAM/INTERVENTION (NO MORE THAN THREE OBJECTIVES): 1) To improve blood pressure processes and control in safety net clinics 2) To determine strategies for successful implementation of a hypertension best practice within a safety net organization with multiple clinics

DESCRIPTION OF PROGRAM/INTERVENTION, INCLUDING ORGANIZATIONAL CONTEXT (E.G. INPATIENT VS. OUTPATIENT, PRACTICE OR COMMUNITY CHARACTERISTICS): We adapted an existing HMO-based hypertension best practice and implemented it at 10 clinics in a large safety-net health system using limited practice coach support for quality improvement (QI). The best practice includes: 1) accurate BP measurement, including a 2nd BP reading when the 1st is elevated; 2) timely follow-up with a nurse or MA-led visit if the BP is elevated; 3) a treatment algorithm which prioritizes once daily low cost medications; and 4) outreach to patients with elevated BP using EMR-based patient registries. In April 2015, as a part of the implementation, we conducted a cluster randomized trial of an electronic prompt to remind MAs/nurses to repeat the $\mathrm{BP}$ measurement when the $\mathrm{BP}$ was $>140 / 90 \mathrm{mmHg}$ combined with monthly data feedback to the practice on repeat BP measurement. Two sets of similar clinics were randomized to either immediate or 4-month delayed implementation of the prompt plus audit/feedback of practice-level data on repeat BP measurement. Due to pressure to improve accountable care organization metrics combined with early implementation findings, the health system leadership began providing the electronic reminder along with weekly data on repeat BP measurement to all practices in Sept 2015, and required a corrective action plan if a practice did not meet repeat BP measurement goals by Jan 2016 .

MEASURES OF SUCCESS (DISCUSS QUALITATIVE AND/OR QUANTITATIVE METRICS WHICH WILL BE USED TO EVALUATE PROGRAM/ INTERVENTION): • The percent of adults with hypertension under good BP control $(<140 / 90 \mathrm{mmHg})$. The percent of adults with hypertension and elevated BP reading who had: 1) a second BP reading (repeat BP measurement); and 2) a follow-up scheduled in the next 35 days (timely follow-up).
FINDINGS TO DATE (IT IS NOT SUFFICIENT TO STATE FINDINGS WILL BE DISCUSSED): Repeat BP measurement ranged from 10 to $30 \%$ at all clinics in Nov 2014 (baseline) and ranged from 45 to $92 \%$ in Nov 2015. BP control improved at all clinics from Nov 2014 to Nov 2015 (ranging from 5 to 17 absolute percentage points improvement). The five early intervention clinics $(N=10,056$ patients) had a 15 percentage point absolute improvement in repeat BP measurement (95\% CI 13 to 17 percentage points) compared to no improvement in the delayed group ( -2 percentage points; $95 \% \mathrm{CI}$ -4 to $0 ; N=10,044$ patients) across the 3 months where only the early intervention clinics received the intervention. Timely follow-up rates were not statistically different between the two randomized groups in the 3 months post intervention at the early clinics versus 3 month pre implementation. The early intervention clinics had a 7.4 absolute percentage point improvement in the percent of hypertensive adults under BP control compared to an improvement of 6 absolute percentage points for the patients at the delayed clinics (difference of 1.4 percentage points; $p=0.07$ ) during the same time frame.

KEY LESSONS FOR DISSEMINATION (WHAT CAN OTHERS TAKE AWAY FOR IMPLEMENTATION TO THEIR PRACTICE OR COMMUNITY?): Elements of a hypertension best practice can be successfully implemented in other health care systems outside HMOs and yield improvements in BP control. An electronic reminder combined with audit/feedback of practice-level data and strong leadership support can powerfully promote adherence to one of the best practice elements.

ACCELERATING THE PACE OF CHANGE: STANDARDIZING ANTICOAGULATION PRACTICE FOR THE ELDERLY IN THE ERA OF DIRECT ORAL ANTICOAGULANTS Zuzanna Czernik; Dora Cheung; Ethan U. Cumbler. University of Colorado School of Medicine, Aurora, CO. (Control ID \#2469841)

STATEMENT OF PROBLEM OR QUESTION (ONE SENTENCE): Adverse drug events related to anti-thrombotic medicaionts are very common, our hospital reflects this national trend; a 10-month review (Jan-Oct 2014) of 30-day readmissions from our hospital's ACE (Acute Care for the Elderly) service revealed that almost 1 in 10 was related to bleeding or new thromboembolism in patients on antithrombotic medications. OBJECTIVES OF PROGRAM/INTERVENTION (NO MORE THAN THREE OBJECTIVES): Slow adoption of use of DOACs, variations in antithrombotic prescribing patterns, and a lack of consistent follow-up for patients on anticoagulants were felt to contribute to anticoagulation ADEs. We implemented a standardized process for initiation and follow up of newly prescribed anticoagulants for elderly patients admitted on our service. Specifically we aimed to standardize the process for: (1) Which agents to prescribe for pts with newly diagnosed atrial fibrillation and venous thromboembolism (VTE). (2) Time to first outpatient follow-up visit for both new and chronic users of warfarin

DESCRIPTION OF PROGRAM/INTERVENTION, INCLUDING ORGANIZATIONAL CONTEXT (E.G. INPATIENT VS. OUTPATIENT, PRACTICE OR COMMUNITY CHARACTERISTICS): Following stakeholder engagement with hospitalists, geriatricians, cardiologists, hematologists, neurologists, outpatient internal medicine physicians, family medicine physicians, and pharmacists, we created a new algorithm for prescribing and follow-up for anticoagulation in the elderly. We targeted our algorithm and practice guidelines to all geriatric patients admitted to the inpatient ACE unit at the University of Colorado Hospital. It encouraged DOAC prescription for patients with new indications for anticoagulation if patients met inclusion criteria including shared decision making with patients on the cost of medications. For patients on warfarin, we established standards for post discharge INR follow-up (within 3 days post-discharge for most patients). All patients with new anticoagulation were expected to have primary care physician (PCP) appointment within a week of discharge home. After establishing this 
algorithm, education on the protocol was provided to the attending and resident physicians, inpatient pharmacist, and inpatient patient liaisons who assisted with making follow up appointments. Reinforcement of this protocol was included in monthly orientations for physicians newly rotating on the ACE unit. The unit pharmacist was assigned responsibility for encouraging continued adherence to the protocol.

MEASURES OF SUCCESS (DISCUSS QUALITATIVE AND/OR QUANTITATIVE METRICS WHICH WILL BE USED TO EVALUATE PROGRAM/ INTERVENTION): Outcomes for our QI initiative include change in DOAC prescriptions as a percentage of all newly initiated anticoagulation medications, change in percent of post-discharge INR follow-up within target timeframe, and change in percent of PCP follow up within 7 days post discharge and readmission rate. All patients discharged on an anticoagulant from the ACE service were abstracted for a period of 3 months (JulySept 2015) following intervention and compared to baseline.

FINDINGS TO DATE (IT IS NOT SUFFICIENT TO STATE FINDINGS WILL BE DISCUSSED): Initiation of DOACs increased from 0 to $70 \%$ after initiation of our protocol. Percent of patients on warfarin with INR check within target timeframe increased from 23 to $50 \%$. PCP follow-up within a week of discharge increased from 32 to $63 \%$. 30-day readmission rate for patients on anticoagulation decreased from 16 to $13 \%$.

KEY LESSONS FOR DISSEMINATION (WHAT CAN OTHERS TAKE AWAY FOR IMPLEMENTATION TO THEIR PRACTICE OR COMMUNITY?): QI methodology can be successfully used to standardize practice and accelerate the innovation adoption curve.

ADDITIVE UTILITY OF PEAK EXPIRATORY FLOW RATE IN THE ASSESSMENT OF CHRONIC STABLE HEART FAILURE PATIENTS. Fadi Abou Obeid; Anup Kumar; Marc Silver. Advocate Christ Medical Center, Oak Lawn, IL. (Control ID \#2469342)

STATEMENT OF PROBLEM OR QUESTION (ONE SENTENCE): Does PEFR measurement predict the risk of acute heart failure (HF) exacerbation in patients with chronic Congestive HF evaluated in the outpatient setting?

OBJECTIVES OF PROGRAM/INTERVENTION (NO MORE THAN THREE OBJECTIVES): 1) Assess heart failure status in general. 2) Predict risk of acute decompensation of congestive heart failure in stable chronic HF patients. 3) Detect early signs of decompensation, in order to treat early in clinic and minimize the risk for hospitalization and following complications.

DESCRIPTION OF PROGRAM/INTERVENTION, INCLUDING ORGANIZATIONAL CONTEXT (E.G. INPATIENT VS. OUTPATIENT, PRACTICE OR COMMUNITY CHARACTERISTICS): The peak expiratory flow rate (PEFR) is the maximal rate that a person can exhale during a short maximal expiratory effort after a full inspiration. PEFR reflects large airway flow, muscular strength and patient effort and is associated with known indicators of HF status. In patients with asthma, the PEFR percent predicted correlates reasonably well with the percent predicted value for the forced expiratory volume in one second (FEV1). Monitoring the PEFR is useful for detecting changes or trends in a patient's asthma control. PEFR is a tool initially used to differentiate dyspnea caused by COPD exacerbation vs Acute Heart Failure. PEFR has been shown to be an effective tool in assessing heart failure (HF) status and its course of recovery in patients with acute decompensated $\mathrm{HF}$.

MEASURES OF SUCCESS (DISCUSS QUALITATIVE AND/OR QUANTITATIVE METRICS WHICH WILL BE USED TO EVALUATE PROGRAM/ INTERVENTION): - We prospectively enrolled fifty patients with chronic stable HF, AHA Stage C, NYHA functional class I-III, from our outpatient HF clinic. -Patients with HF hospitalization and those with cardiac surgery or myocardial infarction within 3 months were excluded. -PEFR was measured for each subject in triplicate. -Averaged PEFR values were compared to predicted averages (adjusted for age, sex, and height) and expressed as percentages. -Data related to patient demographics, hemodynamics, laboratory values, clinical signs and symptoms, and functional capacity were collected.

FINDINGS TO DATE (IT IS NOT SUFFICIENT TO STATE FINDINGS WILL BE DISCUSSED): Mean patient age was 69 years old with majority being caucasian males. Majority of patients were NYHA class II/ III, chronic stable heart failure with average duration of 6 years. Majority of the patients had ischemic HF and were noted with reduced ejection fraction. Overall mean percentage of predicted PEFR was $81 \%$. Subjects with BNP levels above $100 \mathrm{pg} / \mathrm{ml}$ had significantly lower PEFR compared to those with BNP less than $100 \mathrm{pg} / \mathrm{ml}, p=0.001$. PEFR also tended to be lower in patients with positive clinical findings for HF (JVD, lung crackles, edema, ascites) compared to those without clinical findings, $p=$ 0.06. There was no significant difference in PEFR between patients with NYHA class III compared to NYHA class II, $p=0.3$.

KEY LESSONS FOR DISSEMINATION (WHAT CAN OTHERS TAKE AWAY FOR IMPLEMENTATION TO THEIR PRACTICE OR COMMUNITY?): 1) Our study suggests that there is great variability in normalized PEFR ratios even in stable HF patients. 2) We observed relationships of PEFR with key HF biomarkers like BNP but not between NYHA classes. 3) The ease of performance along with its limited cost and portability suggest that the repeated PEFR testing may be an additive simple clinical tool that distinguishes patients with overlapping clinical scenarios (dyspnea of cardiac vs pulmonary origin) and residual risk for adverse HF since it correlates with BNP that is already a key biomarker and prognostic marker in Congestive HF. 4) So far PEFR did not meet objective number (2) and most likely bigger study with bigger population can be initiated to determine that objective.

ADHERENCE TO USPSTF AND ACIP CLINICAL GUIDELINES IN RESIDENT CLINICS Richard M. Atkins; Victor Poon; Alicia Clark; Joel C. Boggan; Gina-Maria Pomann; Jonathan Bae. Duke University, Durham, NC. (Control ID \#2467851)

STATEMENT OF PROBLEM OR QUESTION (ONE SENTENCE): Adherence to guidelines and recommendations from national organizations and expert committees such as the United States Preventive Services Task Force (USPSTF) and the Advisory Committee on Immunization Practices (ACIP) will become progressively more important as our health system moves further towards a focus on population health and a reimbursement system tied to meeting performance metrics.

OBJECTIVES OF PROGRAM/INTERVENTION (NO MORE THAN THREE OBJECTIVES): 1. Increase resident adherence to USPSTF and ACIP clinical guidelines 2. Engage residents in an audit-and-feedback quality improvement to meet ACGME requirements for 'Practiced Based Learning' and 'Systems Based Practice' DESCRIPTION OF PROGRAM/INTERVENTION, INCLUDING ORGANIZATIONAL CONTEXT (E.G. INPATIENT VS. OUTPATIENT, PRACTICE OR COMMUNITY CHARACTERISTICS): Using an interactive online platform, residents assessed their adherence to grade A and selected grade B USPSTF and ACIP guidelines in their primary care panels in a pre/post quality improvement project. 2nd and 3rd year internal medicine residents were instructed to perform chart reviews of forty patients (twenty patients in 2 phases relative to an action plan). In the first phase, residents completed chart reviews and then received immediate data on their individual performance in adherence to guidelines as well as the performance of their peers aggregated at the clinic and program level. Residents then developed an action plan to set specific goals and methods for improvement. The second phase occurred at least 3 months after the action plan and included a repeat audit to assess improved adherence to guidelines.

MEASURES OF SUCCESS (DISCUSS QUALITATIVE AND/OR QUANTITATIVE METRICS WHICH WILL BE USED TO EVALUATE PROGRAM/ INTERVENTION): For each patient, residents were asked evaluate adherence to each 
of 16 USPSTF and ACIP guidelines if applicable. The primary outcome was the total number of guidelines properly acknowledged (defined as the total number of guidelines recommended to a patient regardless of patient adherence) divided by the total number of patients at risk. Secondary outcomes included: 1) the total number of guidelines completed by the patient divided by the total number at risk and 2) the total number of guidelines completed divided by the total number properly acknowledged by the resident. Each of these outcomes was measured for each resident before and after the resident action plan. A one-sided paired t-test was used to test the outcomes as the same resident was present in both Phase One and Phase Two.

FINDINGS TO DATE (IT IS NOT SUFFICIENT TO STATE FINDINGS WILL BE DISCUSSED): 66 residents completed chart reviews of 2517 patients were included in the analysis (1288 in phase 1 and 1229 in phase 2). Residents properly recommended $77.2 \%$ of guidelines for patients at risk in phase 1 . This increased to $85.1 \%$ in phase 2 (pvalue $<0.001$ ). The percentage of guidelines for at-risk patients that were actually completed in phase 1 and phase 2 were 71.3 and $79.3 \%$, respectively $(p<0.001)$. There was not, however, any evidence that the number of acknowledged guidelines actually completed increased from phase 1 to phase $2: 92.3$ and $93.0 \%$ respectively $(p=.1405)$. This last outcome suggests that the increase in the primary outcome was primarily due to an increase in guideline recommendations actually completed and not just recommended. We are conducting further analyses to determine if these results are primarily due to improvement in a few specific guidelines or due to a general increase in the adherence to all guidelines.

KEY LESSONS FOR DISSEMINATION (WHAT CAN OTHERS TAKE AWAY FOR IMPLEMENTATION TO THEIR PRACTICE OR COMMUNITY?): With our experience showing baseline adherence to USPSTF and ACIP guidelines less than $80 \%$, there certainly is significant opportunity for quality improvement in preventive health. Additionally, our experience further affirms that audit-and-feedback is a valid and beneficial quality improvement tool, and it would behoove experienced clinicians and trainees alike to learn this skill. Finally, we provide the first known benchmark for adherence rate to USPSTF and ACIP recommendations in primary care clinics.

AN INTER-PROFESSIONAL INTERVENTION TO IMPROVE EFFICIENCY IN AN ACADEMIC PRIMARY CARE CLINIC: DIRECT OBSERVATION AND FEEDBACK OF PRIMARY CARE TRAINEE PATIENT VISITS BY HEALTH PSYCHOLOGY COLLEAGUES Yungah Lee ${ }^{2,}{ }^{\text {; }}$ Rebecca Kosowicz ${ }^{2,1}$; Elizabeth A. Hendriks ${ }^{3}$; Kenneth Morford ${ }^{4}$, ${ }^{\text {; }}$ Rachel Laff ${ }^{3}$; Robert Walsh ${ }^{3}$; Danielle Greenman $^{4,}{ }^{1}$; Caroline Falker ${ }^{2},{ }^{1}$; Helena Turner ${ }^{3}$; Rebecca Brienza ${ }^{1}{ }^{1}$ VA Connecticut Healthcare System, West Haven, CT; ${ }^{2}$ Yale New Haven Hospital, New Haven, CT; ${ }^{3}$ VA West Haven, West Haven, CT; ${ }^{4}$ Yale University School of Medicine, New Haven, CT. (Control ID \#2468785)

STATEMENT OF PROBLEM OR QUESTION (ONE SENTENCE): It is challenging to complete patient visits in a timely manner in the primary care clinic setting, especially for resident trainees; trainees often run-over the allotted visit time per patient, exacerbating wait times for subsequent patient visits.

OBJECTIVES OF PROGRAM/INTERVENTION (NO MORE THAN THREE OBJECTIVES): We focused on optimizing trainee factors to improve the efficiency of resident-patient clinical encounters. The study investigated the effect of using health psychology colleagues to provide feedback on resident performance by direct observation in real time during the resident-patient encounter. The hypothesis was that the intervention would improve the clinic flow and reduce patient visit time. The study highlights a potential new role of health psychology as a discipline to enhance primary care resident education by improving efficiency of the resident-patient encounter
DESCRIPTION OF PROGRAM/INTERVENTION, INCLUDING ORGANIZATIONAL CONTEXT (E.G. INPATIENT VS. OUTPATIENT, PRACTICE OR COMMUNITY CHARACTERISTICS): The setting was in the VA Center of Excellence (COE) in Primary Care Education Inter-professional Training Program in West Haven CT. Participants included five MD residents (one PGY-1, two PGY-2, and two PGY-3 level trainees), one Nurse Practitioner (NP) resident trainee, and 1 clinical health psychology postdoctoral fellow. Trainee primary care visits were observed by a clinical health psychology postdoctoral fellow. The fellow used patient centered interviewing models to identify possible targets for intervention for individual trainees. Each trainee was provided with one-on-one feedback following their observed clinical encounter.

MEASURES OF SUCCESS (DISCUSS QUALITATIVE AND/OR QUANTITATIVE METRICS WHICH WILL BE USED TO EVALUATE PROGRAM/ INTERVENTION): Specific time points of the visit were observed and documented by participating trainees for data analysis. The time points included clinic start time, precepting start time, and clinic end time. The primary outcome measure was the difference in the total visit time before and after the intervention. The total visit time is calculated by the difference between the clinic end time and the clinic start time. Secondary measures included (1) trainee visit time, defined by the difference between precepting start time and clinic start time (2) average wait time for trainee patients, and (3) variability of trainee visit time across the different trainee discipline and levels from NP, PGY-1, PGY-2 and PGY-3.

FINDINGS TO DATE (IT IS NOT SUFFICIENT TO STATE FINDINGS WILL BE DISCUSSED): Agenda setting was the most common target identified for intervention. Therefore, much of the feedback given to trainees were focused on improving skills in agenda setting. Pre-intervention data of 69 trainee visits showed total visit time ranging from $14 \mathrm{~min}$ to $90 \mathrm{~min}$ with average of $39 \mathrm{~min}$ and median of $40 \mathrm{~min}$. The allotted time for each trainee visit was $30 \mathrm{~min}$. Preliminary post-intervention data of 25 trainee visits showed reduction of total visit time average and median by $2 \mathrm{~min}$ and $5 \mathrm{~min}$, respectively. The time reduced was seen during the trainee visit time, which was outside of the time spent for precepting

KEY LESSONS FOR DISSEMINATION (WHAT CAN OTHERS TAKE AWAY FOR IMPLEMENTATION TO THEIR PRACTICE OR COMMUNITY?): The data analysis suggested a positive effect of the intervention on improving efficiency of the visit and clinic flow. Limitations of the study included the awareness and self-observation by trainees on their performance, which may have increased bias towards improving efficiency. The study also does not account for the fact that trainees may become more efficient due to gaining more experience over time. There are numerous other potential positive effects of collaborating with health psychology for direct observation and feedback of primary care residents such as improving overall resident satisfaction through inter-professional education, promoting patient-centeredness, and reducing provider burnout by improving efficiency and value added time spent with patients. These are areas for further study.

ASSESSING THE FEASIBILITY AND IMPLEMENTATION OF A BEDSIDE SERVICE HANDOFF ON AN ACADEMIC HOSPITALIST SERVICE: A PHYSICIAN'S PERSPECTIVE Charlie M. Wray ${ }^{1}$; Vineet M. Arora ${ }^{2}$; David Meltzer ${ }^{1}$. ${ }^{1}$ University of Chicago, Chicago, IL; ${ }^{2}$ University of Chicago Medical Center, Chicago, IL. (Control ID \#2457433)

STATEMENT OF PROBLEM OR QUESTION (ONE SENTENCE): With prior studies suggesting the need for more systematic, team-based, and patient-centered handoff models - we hypothesized that performing the service handoff at the patients' bedside may more efficiently transfer patient information between physicians, while further integrating the patient into their hospital care. 
OBJECTIVES OF PROGRAM/INTERVENTION (NO MORE THAN THREE OBJECTIVES): Our objective was to describe and assess the implementation of a bedside handoff (BHO) at hospitalist service transitions from a physician's perspective. DESCRIPTION OF PROGRAM/INTERVENTION, INCLUDING ORGANIZATIONAL CONTEXT (E.G. INPATIENT VS. OUTPATIENT, PRACTICE OR COMMUNITY CHARACTERISTICS): We performed a 4-month prospective study of a $\mathrm{BHO}$ on a non-teaching hospitalist service in which service transitions occurred every 7 days. Each week, 1 out of 3 possible services was scheduled to perform the service handoff at the bedside. Oncoming hospitalists in the BHO group were scheduled to a day admissions service the day prior to taking over a clinical service — which allowed for both oncoming and outgoing hospitalists to be in the hospital at the same time. Oncoming physicians were asked to co-round at the patient's bedside with the outgoing physician during this shift. Both participating physicians were surveyed within 48-h of the BHO. MEASURES OF SUCCESS (DISCUSS QUALITATIVE AND/OR QUANTITATIVE METRICS WHICH WILL BE USED TO EVALUATE PROGRAM/ INTERVENTION): The intervention was evaluated via physician surveys, which assessed for completeness of handoff, communication, missed information, and near misses/adverse events due to incomplete handoffs. Physicians not scheduled to perform the BHO were also surveyed to ascertain differences between the two groups. Responses were dichotomized and chi-square analysis was used to assess differences.

FINDINGS TO DATE (IT IS NOT SUFFICIENT TO STATE FINDINGS WILL BE DISCUSSED): From July to November 2015, 13/17 (76 \%) of scheduled BHO occurred, with 4.2 patients undergoing a $\mathrm{BHO} /$ service transition, with the majority (92\%) of BHO taking 16-60 min to complete. Post-handoff survey response rate was $100 \%$ in both intervention and control groups. Physicians who performed the BHO $(n=13)$ reported statistically higher rates of completeness of information exchange (100 vs. $50 \%, p=0.005)$, less time reviewing the patient's hospital course on the first day of service ( 38 vs. $73 \%, p=0.03$ ), less missed information that should have been discussed at the transition ( 8 vs. $63 \%, p=0.007$ ), and perceived higher patient awareness of the service transition (69 vs. $25 \%, p=$ $0.049)$ compared to physicians who did not perform a BHO $(n=8)$ at the service transition. Narrative feedback from physicians revealed positive attributes that centered on a theme of better knowledge of the patient. The primary negative response was that the $\mathrm{BHO}$ was time-consuming and difficult to coordinate.

KEY LESSONS FOR DISSEMINATION (WHAT CAN OTHERS TAKE AWAY FOR IMPLEMENTATION TO THEIR PRACTICE OR COMMUNITY?): While performing a BHO may be time and resource-intensive, physicians reported improved and more efficient information exchange, along with a perceived improvement in patient awareness of care transitions. Further studies are needed to assess patient-level outcomes and perceptions of a bedside handoff.

ASSESSMENT OF CARE COORDINATION ACTIVITIES FOR HIGH-RISK VETERANS UTILIZING A TEMPLATE INTEGRATED IN THE ELECTRONIC HEALTH RECORD Neha Pathak ${ }^{1,2}$; Anne Tomolo ${ }^{1,2}$; Cam Escoffery ${ }^{3} .{ }^{1}$ Atlanta VA Medical Center, Decatur, GA; ${ }^{2}$ Emory University School of Medicine, Atlanta, GA; ${ }^{3}$ Rollins School of Public Health, Emory University, Atlanta, GA. (Control ID \#2468689)

STATEMENT OF PROBLEM OR QUESTION (ONE SENTENCE): Despite significant interest in promoting care coordination (CC) as part of complex care management programs for high-risk patients, little data exists about the specific activities and time required to provide necessary $\mathrm{CC}$.

OBJECTIVES OF PROGRAM/INTERVENTION (NO MORE THAN THREE

OBJECTIVES): 1) Create a CC Template within the Department of Veterans Affairs (VA) Computerized Patient Record System (CPRS) to facilitate and standardize documentation of CC activities in the electronic health record. 2) Describe types of CC activities performed by care coordinators, modes of communication, collaborators, and time needed to perform $\mathrm{CC}$ by using the $\mathrm{CC}$ Template as an electronic data source. 3) Assess implementation of CC Template at PIM sites with diverse PIM team structures. DESCRIPTION OF PROGRAM/INTERVENTION, INCLUDING ORGANIZATIONAL CONTEXT (E.G. INPATIENT VS. OUTPATIENT, PRACTICE OR COMMUNITY CHARACTERISTICS): Complex care management programs may improve outcomes and experience of care for high-risk patients. In 2013, the Veterans Health Administration (VHA) funded 5 demonstration sites to develop PACT Intensive Management (PIM) programs to target high-risk veterans in diverse VA primary care settings. Similar to interventions outside of VA, a significant component of PIM programs is the provision of CC. While there is significant interest in promoting CC, little data about the specific activities and time required to provide necessary $\mathrm{CC}$ exists. To date, there are very limited tools to obtain descriptive information about $\mathrm{CC}$ services provided in the primary care setting by interdisciplinary teams. In order to identify the CC activities performed by the PIM teams caring for high-risk veterans, the Atlanta VA Medical Center PIM site developed a CC Template with linked codes within CPRS based on CC domains identified in Agency for Healthcare Research and Quality's Care Coordination Measurement Atlas. The aim of this tool is to describe the types of CC activities performed by PIM team members, measure the time it takes to perform these activities, and facilitate documentation of $\mathrm{CC}$ activities in the EHR. The Atlanta PIM team includes 2 Nurse Practitioners, 2 Social Workers, 1 Telehealth RN, and 1 Medical Director and serves 200 high-risk patients identified by risk scores from 10 primary care panels at 1 communitybased outpatient clinic serving both rural and urban veterans. The CC Template has been disseminated to all 5 PIM sites with diverse team structures. .

MEASURES OF SUCCESS (DISCUSS QUALITATIVE AND/OR QUANTITATIVE METRICS WHICH WILL BE USED TO EVALUATE PROGRAM/ INTERVENTION): A mixed methods evaluation of the CC Template will be performed. The quantitative component will include a descriptive analysis of CC activities using the CC Template as a data source. Data collection and analysis for descriptive frequencies will include: 1) Duration of CC encounters 2) PIM Staff CC involvement 3) Collaborators 4) Settings/modes of CC communication 5) Ranges of CC activity types 6) Ranges of related diagnosis The qualitative component will include a survey and semi-interview using REAIM and CFIR frameworks to assess: 1) CC Template implementation process 2) Contextual barriers or facilitators to implementation 3) Acceptability and feasibility of use during day-to-day operations when providing complex care management 4) Improvements for quality of CC Template

FINDINGS TO DATE (IT IS NOT SUFFICIENT TO STATE FINDINGS WILL BE DISCUSSED): Data collection and analysis is in process. Pilot data from 4 months of CC Template use at the Atlanta VA PIM site show $83 \%$ of CC encounters are $<20 \mathrm{~min}$. Of team members utilizing the module to document $\mathrm{CC}$, Nurse Practitioners utilized the module $54.9 \%$ vs. $45 \%$ for social workers. CC by telephone occurred in $56 \%$ of CC episodes, with $24 \%$ of $\mathrm{CC}$ encounters utilizing multiple modes of communication. Among various types of CC activities, CC during transitions of care accounted for $20 \%$ of module use. Other activities documented by the CC Template include determining plan of care (24\%), scheduling appointments (18.2\%), assisting with referrals (10.1\%), and tracking consults ( $2 \%$ ). $83 \%$ of CC encounters were initiated by PIM team vs $16 \%$ initiated by patients or caregivers.

KEY LESSONS FOR DISSEMINATION (WHAT CAN OTHERS TAKE AWAY FOR IMPLEMENTATION TO THEIR PRACTICE OR COMMUNITY?): While there is much interest in promoting $\mathrm{CC}$, many questions remain about the 1) types of CC services provided by care coordinators, 2) time it takes to perform these activities, 3) collaborators within and outside of the healthcare setting, and 4) modes of communication for CC. Standardizing documentation of CC with the EHR allows for precise description of these activities and may have implications for training care coordinators with the 
optimal skill set. A tool such as the CC Template may offer a sustainable way to improve knowledge about and documentation of CC activities, leading to optimization of CC team structure and improvement in the quality of CC.

BUILDING "UC QUITS": A UNIVERSITY OF CALIFORNIA-WIDE SYSTEMS CHANGE TO PROMOTE TOBACCO CESSATION Mai-Khanh Bui-Duy ${ }^{4}$; Robert El-Kareh ${ }^{2}$; Alpesh Amin ${ }^{3}$; Linda Sarna ${ }^{5}$; Elisa Tong ${ }^{1} .{ }^{1}$ University of California Davis, Sacramento, CA; ${ }^{2}$ University of California San Diego, La Jolla, CA; ${ }^{3}$ University of California Irvine, Orange, CA; ${ }^{4}$ University of California San Francisco, San Francisco, CA; ${ }^{5}$ University of California Los Angeles, Los Angeles, CA. (Control ID \#2465530)

STATEMENT OF PROBLEM OR QUESTION (ONE SENTENCE): How can the electronic medical record (EMR) help clinicians address tobacco cessation at every encounter, given that cessation requires multiple quit attempts and assistance with counseling and medication for greater success?

OBJECTIVES OF PROGRAM/INTERVENTION (NO MORE THAN THREE OBJECTIVES): To describe a University of California (UC)-wide network that shares tobacco-related workflow processes using the EMR. To create technological modifications to each UC medical campus' EMR (UC Davis, UCSF, UCLA, UCSD and UC Irvine), including referral to the California Smokers' Helpline. To share campus-specific resources within each UC site for inpatient and outpatient tobacco cessation.

DESCRIPTION OF PROGRAM/INTERVENTION, INCLUDING ORGANIZATIONAL CONTEXT (E.G. INPATIENT VS. OUTPATIENT, PRACTICE OR COMMUNITY CHARACTERISTICS): UC Davis established the first two-way eReferral with the state quitline (California Smokers' Helpline) in 2013. With the twoway eReferral, Helpline counselors proactively call patients within 1-2 business days of receiving the referral, and a Results message is sent back in real time to the ordering provider detailing the Helpline's interventions (e.g. telephone counseling, mailing educational materials, etc). With the patient's verbal consent, the ordering provider enters the patient's preferred contact number, preferred time of day to receive a call, and the preferred language (available in English, Spanish, Korean, Vietnamese, Mandarin and Cantonese) This eReferral, adapted for each of the 5 UC medical campuses, can be used in both inpatient and outpatient settings. Based on discussions with all sites, additional enhancements to the eReferral included a prompt for whether the referral was for a household smoker (e.g. parent of pediatric patient), and functionality to copy Results messages from the Helpline to another provider (e.g. primary care provider). UC Davis, UCSF, UCLA and UCSD are also completing ambulatory and inpatient builds of tobacco cessation order sets, including medication and Helpline eReferral orders, to be used with the Epic EMR UC Irvine, using Allscripts EMR, built a comprehensive workflow across multiple domains: inpatient, ambulatory, ED and psychiatry. The UC Quits network has developed a provider-oriented website (www.ucquits.com) which offers provider and learner tools on tobacco cessation. A series of 5 brief webinars is available for viewing on the website and offers CME credit. Outreach to UC providers has been ongoing for EMR enhancements through emails, educational presentations, and resident handbooks. UC Irvine developed a mandatory training for all providers, including residents, attendings, and nurses. Monthly UC-wide team meetings and internal site meetings have been in place since Spring 2014 to share workflow and ideas.

MEASURES OF SUCCESS (DISCUSS QUALITATIVE AND/OR QUANTITATIVE METRICS WHICH WILL BE USED TO EVALUATE PROGRAM/ INTERVENTION): The California Smokers Helpline maintains a registry of referred patients in order to track the number of eReferrals per UC site every month. The UC Quits website posts workflow maps and local resources for each UC site and clinical setting (inpatient vs ambulatory) to track progress in implementing EMR changes for tobacco cessation.
FINDINGS TO DATE (IT IS NOT SUFFICIENT TO STATE FINDINGS WILL BE DISCUSSED): The UC Quits champion list has grown UC-wide from 8 physician champions and $1 \mathrm{UC}$-wide nurse champion to approximately 50 champions representing internal and family medicine, pediatrics, psychiatry, nursing, pharmacy, respiratory therapy and informatics. All 5 UC sites are live with their eReferral to the Helpline (UCD 3/2013; UCSF \& UCLA 11/2014; UCSD 12/2014; UCI 7/2015). As of December 31, 2015, a total of 2894 referrals have been submitted electronically to the Helpline with 1397 patients reached (48.2\%). Of those patients contacted, 711 patients received a 30min counseling session $(50.8 \%), 171$ patients received materials on tobacco cessation (12.2\%), 503 patients refused services ( $36 \%$ ) and 12 patients did not receive any services. KEY LESSONS FOR DISSEMINATION (WHAT CAN OTHERS TAKE AWAY FOR IMPLEMENTATION TO THEIR PRACTICE OR COMMUNITY?): The eReferral is a powerful tool to connect patients with free tobacco cessation counseling, including household smokers for pediatric patients. A 2002 randomized trial of the Helpline demonstrated that telephone counseling doubled long-term quit rates at 1 year, so connecting patients with counseling support is an important step in achieving tobacco cessation. The feedback that providers receive through the Results message can reinforce the cessation attempt. In 18 months, UC Quits has established a network of multidisciplinary providers on tobacco cessation. Identifying site champions is crucial for local dissemination and provider education. Moreover, interdisciplinary involvement of nursing, pharmacy, and respiratory therapist staff can boost eReferral implementation success by identifying tobacco users at different stages throughout the healthcare process, and can offer multiple opportunities to connect patients to cessation resources when assessing readiness to quit. However, scaling up technology across UC Health depends on individual site workflow and priorities. The UC-wide network has been aligning with quality initiatives to boost and sustain outreach, such as Joint Commission tobacco quality measures and anticipated incentives.

BUNDLED TRAINEE CENTERED INTERVENTIONS DRAMATICALLY IMPROVED ACUTE CARE LENGTH OF STAY IN A LARGE ACADEMIC TERTIARY CARE CENTER Megan Walker ${ }^{1,3}$; Lindsey Jordan²; Glynda Caga-anan ${ }^{2}$; Charlie Lan ${ }^{3,}$; Hans Von Marensdorff ${ }^{3}$, ; Biykem Bozkurt ${ }^{3,1}$. 'Baylor College of Medicine, Houston, TX; ${ }^{2}$ Michael E. Debakey Medical Center and Baylor College of Medicine, Houston, TX; ${ }^{3}$ Michael E. DeBakey VA Medical Center, Houston, TX. (Control ID \#2469745)

STATEMENT OF PROBLEM OR QUESTION (ONE SENTENCE): Problem: The length of stay for acute care medicine patients in our large tertiary care academic Veteran's Affairs (VA) hospital was found to be significantly higher than institutions of comparable volume and complexity.

OBJECTIVES OF PROGRAM/INTERVENTION (NO MORE THAN THREE OBJECTIVES): Objectives: We developed an aim to improve our overall length of stay (LOS) as well as observed minus expected length of stay (OMELOS) for admitted medical patients over the course of 1 year.

DESCRIPTION OF PROGRAM/INTERVENTION, INCLUDING ORGANIZATIONAL CONTEXT (E.G. INPATIENT VS. OUTPATIENT, PRACTICE OR COMMUNITY CHARACTERISTICS): Intervention: Although increased LOS has a significant financial impact for both patients and hospitals, trainees' knowledge of its importance and implications is minimal. Furthermore, additional days in the hospital carry increased risk of adverse events for patients. Our facility is a large tertiary care academic hospital with over 300 inpatient beds, with all inpatients cared for by teaching services. Investigation revealed that in addition to social circumstances, a large contributing factor to our extended LOS was that physician documentation did not accurately reflect the severity and complexity of our patients' illness. Therefore, we created a bundled 
intervention centered on Internal Medicine trainees in an effort to improve length of stay. The most significant interventions were resident education on proper documentation and coding along with implementation of discharge and coding huddles. The coding education consisted of case- based didactics presented by two consistent hospitalists every 4 weeks during resident conferences, particularly aiming to reach the residents in the beginning of their inpatient rotations. Posters and pocket cards with organ system based ICD-10 codes customized to the most commonly seen inpatient diagnoses at our facility were created and distributed to all inpatient medicine teams. In further effort to prevent miscoding, the medicine teams met with their coders for a bi-weekly huddle to clarify documentation in person and consequently also reinforce the education. A standardized form listing the principal diagnosis, secondary diagnoses, complications and comorbidities, the diagnosis related group (DRG), case severity index (CSI), expected LOS and cumulative LOS was used to facilitate this meeting. Lastly, a discharge huddle was created which entailed a brief daily meeting of each inpatient medicine team's residents and attending with their social worker, case manager, pharmacist and utilization management nurse to identify any potential barriers to discharge early in the patient's hospitalization. A unique aspect of the huddle was that it was facilitated by a designated senior attending physician who served as a liaison to hospital leadership to facilitate efficient patient care, flow and disposition.

MEASURES OF SUCCESS (DISCUSS QUALITATIVE AND/OR QUANTITATIVE METRICS WHICH WILL BE USED TO EVALUATE PROGRAM/ INTERVENTION): Measures: Our primary measures were the overall average LOS and the OMELOS for acute care medicine patients. For process measures, we tracked the average case severity index for acute care patients. We also measured resident knowledge of coding through the use of surveys both pre and post intervention. We implemented the bundled intervention in the second quarter of 2014.

FINDINGS TO DATE (IT IS NOT SUFFICIENT TO STATE FINDINGS WILL BE DISCUSSED): Results: Baseline average acute care LOS was 5.9 days prior to our intervention. Following implementation of the bundle, there was significant improvement in average LOS to 5.1 days over 12 months. Furthermore the OMELOS dramatically improved from an average of +0.9 days prior to intervention to -0.9 days during the 1 year time period. The new OMELOS values are now significantly below the average for other VA facilities of similar volume and complexity. Correspondingly the CSI increased from an average of 1.1 prior to the intervention to 1.5 afterwards. We also found that resident knowledge and comfort level in regard to coding improved after the intervention. KEY LESSONS FOR DISSEMINATION (WHAT CAN OTHERS TAKE AWAY FOR IMPLEMENTATION TO THEIR PRACTICE OR COMMUNITY?): Conclusions: We implemented a resident centered bundle that dramatically improved the absolute LOS as well as OMELOS. The key elements of the intervention were providing documentation and coding education to the Internal Medicine residents which was reinforced by a coding huddle, and initiating a discharge huddle facilitated by a senior attending physician that focused on prompt identification of potential discharge delays. To our knowledge the inclusion of trainees in improving these hospital measures is unique in the literature, as is the use of a senior physician as the huddle leader. These strategies could be easily adapted and implemented for use in other facilities to improve length of stay.

CERVICAL CANCER EDUCATION AND SCREENING IN WOMEN'S REFUGEE CLINIC: THE ROLE OF IN-PERSON INTERPRETERS Zehra Hussain; Aba

Y. Barden-Maja; Jennifer Michener. University of Pennsylvania, Philadelphia, PA. (Control ID \#2458770)

STATEMENT OF PROBLEM OR QUESTION (ONE SENTENCE): Do in-person interpreters impact cervical cancer screening outcomes among refugee women in women's health clinic?
OBJECTIVES OF PROGRAM/INTERVENTION (NO MORE THAN THREE OBJECTIVES): The goals of women's refugee clinic and the in-person interpreter program are to: 1) Educate patients about cervical cancer screening and contraception 2) Increase rates of cervical cancer screening among refugee women 3) Promote patients' comfort and acceptance in discussing sensitive women's health topics DESCRIPTION OF PROGRAM/INTERVENTION, INCLUDING ORGANIZATIONAL CONTEXT (E.G. INPATIENT VS. OUTPATIENT, PRACTICE OR COMMUNITY CHARACTERISTICS): Women's health is a particularly challenging area of refugee health care, as refugee women often have had traumatizing experiences which can make invasive screening questions and office exams very sensitive, particularly in the setting of cultural and language barriers. Cervical cancer is still the second most common cause of female cancer mortality worldwide, and most new cases of cervical cancer occur in developing countries, with highest rates in Africa, Asia, and Central and South America. Many refugee women have limited education regarding cervical cancer screening, and the majority have never been screened prior to arrival to the US (1). Even after arrival, the rates of cervical cancer screening among refugee women are lower than for their American counterparts, ranging from 50 to $60 \%(2,3)$. Barriers to cervical cancer screening identified by refugee women in focus groups include lack of accurate knowledge about causes of cervical cancer, language barriers, stigma, fear, lack of time, and embarrassment (4). Penn Center for Primary Care's women's refugee clinic was started in 2013 in response to gaps in counseling related to cancer screening, family planning, and contraception. The women's clinic session consists of both an educational session and a comprehensive women's health screening questionnaire and exam, including pelvic exam and pap smear. The women's health clinic is staffed by only female providers; there is some data that refugee women are more likely to undergo pap smears with female rather than male providers (3). In addition, the clinic initially utilized in-person interpreters to improve patient comfort around sensitive screening questions and exams.

MEASURES OF SUCCESS (DISCUSS QUALITATIVE AND/OR QUANTITATIVE METRICS WHICH WILL BE USED TO EVALUATE PROGRAM/ INTERVENTION): Measures of program success include: 1.) Successfully educating patients about cervical and breast cancer screening 2.) Increasing the percentage of women who undergo a pap smear 3.) Improving patients' comfort in discussing family planning and sexual health issues

FINDINGS TO DATE (IT IS NOT SUFFICIENT TO STATE FINDINGS WILL BE DISCUSSED): In its first 18 months, 34 patients were seen in women's clinic, ranging in age from 19 to 73 years old (mean 39.5, median 36.5), with a range of nationalities, predominantly including Iraqi (35.3\%), Burmese (29.4\%), Bhutanese (20.6\%). Only $17.6 \%$ of patients had had a prior pap smear. After the educational session, $76.5 \%$ of patients reported feeling knowledgeable about the pap smear, and $92.6 \%$ of patients for whom cervical cancer screening was indicated had a pap smear performed during their visit. After the first 18 months, the clinic was no longer able to offer in person interpreters. There were no other major changes to the clinic. During the following 12 months, 29 patients were seen in women's refugee clinic. Of these women, only $48 \%$ felt knowledgeable about pap smears after the educational session. Moreover, only $77.8 \%$ of women for whom cervical cancer screening was indicated underwent a pap smear during their visit.

KEY LESSONS FOR DISSEMINATION (WHAT CAN OTHERS TAKE AWAY FOR IMPLEMENTATION TO THEIR PRACTICE OR COMMUNITY?): Overall the clinic has been successful, with patient comfort and pap smears rates improved from prior to its creation (when women's health assessments were included as part of a follow up visit instead of during dedicated clinic sessions). Other groups may find this clinic model a helpful approach to address comprehensive women's health. Our data also suggests that in personinterpretation was a crucial component of the success of our original women's clinic model. It may be worthwhile to take on the costs of in-person interpretation to improve patient comfort and increase rate of cervical cancer screenings. 
CHALLENGES AND PROGRESS IN FACULTY DEVELOPMENT IN GENERAL INTERNAL MEDICINE Reena H. Hemrajani ${ }^{2}$; Manpreet S. Malik ${ }^{2}$; Laura E. Paletta-Hobbs ${ }^{1} .{ }^{1}$ VCU, Richmond, VA; ${ }^{2}$ Virginia Commonwealth University, Richmond, VA. (Control ID \#2469373)

STATEMENT OF PROBLEM OR QUESTION (ONE SENTENCE): Academic medical centers often struggle with effective faculty development programs due to the broad interests of faculty, the lack of research experience, extensive competing clinical responsibilities, and the institutional challenges that exist in complex systems. OBJECTIVES OF PROGRAM/INTERVENTION (NO MORE THAN THREE OBJECTIVES): 1 . Promote academic productivity and facilitate promotion and tenure 2. Enhance provider engagement and satisfaction 3. Facilitate mentor-mentee relationships DESCRIPTION OF PROGRAM/INTERVENTION, INCLUDING ORGANIZATIONAL CONTEXT (E.G. INPATIENT VS. OUTPATIENT, PRACTICE OR COMMUNITY CHARACTERISTICS): We organized an initial off-campus full day retreat addressing the following topics: icebreaker activity, promotion and tenure tips through varied pathways, advice on how to carry out a research project from start to finish, how to identify and cultivate opportunities into a niche, and creating a shared mental model for personal and divisional goals. Feedback from this retreat and a needs assessment survey led to the creation of a faculty development committee. This committee organized follow-up retreats which included team-building activities, topics focused on developing faculty in areas of technology, mentorship and menteeship, research and education Subsequently, the division organized seminars where senior faculty assisted in faculty writing of abstracts and workshops. The faculty development committee works with the faculty advancement committee, appointed by the division chair, in establishing formal mentorship relationships with faculty who are nearing promotion.

MEASURES OF SUCCESS (DISCUSS QUALITATIVE AND/OR QUANTITATIVE METRICS WHICH WILL BE USED TO EVALUATE PROGRAM/ INTERVENTION): To date, measures of success have included assessment of scholarly output by division faculty and post-retreat surveys of faculty engagement and satisfaction. Future measures will include: ongoing assessment of scholarly output and advancement of faculty, further qualitative evaluation with surveys and focus groups regarding impact of these programs on physician engagement and satisfaction, and collaboration amongst faculty including evaluation of development of mentor-mentee relationships. FINDINGS TO DATE (IT IS NOT SUFFICIENT TO STATE FINDINGS WILL BE DISCUSSED): Surveys were conducted following each retreat and attendees evaluated content and provided feedback for future direction. Greater than $90 \%$ of all survey respondents $(n=18)$ answered that all sessions which delivered content met expectations, held my attention and taught something valuable. While there are no previous data to compare, since 2014, there are currently 11 major projects underway in the division of general internal medicine, which have resulted in multiple manuscripts and abstracts. Forty presentations were made at regional and national meetings in 2015 and multiple manuscripts are being drafted.

KEY LESSONS FOR DISSEMINATION (WHAT CAN OTHERS TAKE AWAY FOR IMPLEMENTATION TO THEIR PRACTICE OR COMMUNITY?): Imme-

diate goals of these development programs include faculty engagement and satisfaction. However, given the complexity and challenges of systems in an academic institution, improvements in faculty clinical care and training will be achieved over a longer timeline. To date, we have initiated episodic retreats which have met faculty expectations for professional development topics. Providing a forum for collegial exchange, including focused sessions and support for scholarly activity, has led to good academic output. Challenges have included balancing the opportunity for professional development with other clinical productivity demands, particularly between varying schedules of inpatient and ambulatory faculty. Division leadership support has been a key component of implementation of these programs. Future direction includes the establishment of a mentorship program to facilitate collaboration amongst faculty members. Meeting the objectives of faculty development is a slow, ongoing process; it requires continuous enthusiasm and engagement of leadership and faculty members with a focus on tailored behavioral interventions that target faculty needs.

CHRONIC OPIATE ANALGESIC THERAPY (COAT) IN A PRIMARY CARE OUTPATIENT CLINIC John Stewart; David Flemig; Judith Daniels; Jimmy Gentry. University of Kentucky, Lexington, KY. (Control ID \#2464956)

STATEMENT OF PROBLEM OR QUESTION (ONE SENTENCE): Can we improve our care for patients receiving COAT in our primary care clinic? OBJECTIVES OF PROGRAM/INTERVENTION (NO MORE THAN THREE OBJECTIVES): 1 . Systematically manage patients on COAT to increase compliance with new legislation. 2. Increase patient satisfaction with pain management. 3. Describe changes in other health care outcomes after implementation of a COAT clinic. DESCRIPTION OF PROGRAM/INTERVENTION, INCLUDING ORGANIZATIONAL CONTEXT (E.G. INPATIENT VS. OUTPATIENT, PRACTICE OR COMMUNITY CHARACTERISTICS): In an effort to decrease opiate abuse in Kentucky the state legislature enacted comprehensive regulations in 2012 intended to ensure patients were monitored to avoid diversion, over-medication, and adverse medication effects. Our practice is a university-run satellite clinic providing primary care in a medically underserved population. Patients and staff were frustrated by the opiate refill process via phone request as prescriptions have to be printed and picked up. We created a novel clinic to improve the management of our patients on COAT. We utilize a nurse to review compliance and prescription fill history and administer screening for substance abuse and depression to offset MD/APRN workload. The MD/APRN reviews pain control, medication adverse effects, and refills medication. Cohorting patients on the same day allows for standardization of chronic pain management care. Eligible patients included established adult patients prescribed controlled substances for chronic pain. A monthly appointment ensures patients can obtain a timely refill without having to call and hopefully improve their satisfaction. The COAT clinic purpose and design was explained to patients and those desiring continued pain management in our practice were enrolled.

MEASURES OF SUCCESS (DISCUSS QUALITATIVE AND/OR QUANTITATIVE METRICS WHICH WILL BE USED TO EVALUATE PROGRAM/ INTERVENTION): One year after implementation, we surveyed our active patients to assess patient satisfaction and pain control. We also performed a chart review of all patients enrolled in the COAT clinic after 1 year of implementation. We compared the number of prescription refill tasks generated by staff. We reviewed documented compliance in the following four areas: family history of substance abuse, narcotic agreement, review of state database of prescription fill history, and annual random urine drug testing. We examined depression scores (PHQ-9) as a pain related health care outcome and vaccination rates for Tdap and influenza as general non-pain health care outcomes.

FINDINGS TO DATE (IT IS NOT SUFFICIENT TO STATE FINDINGS WILL BE DISCUSSED): All current COAT patients capable of providing consent $(n=33)$ were asked to participate in the survey and the response rate was $81.8 \%(n=27)$. Patient satisfaction was rated on a scale of 0 (low) to 10 (high). Patient satisfaction ratings significantly increased after implementation (Mean standard difference $(\mathrm{MSD})=2.0$; $p=0.03$ ). Pain control was rated on a scale of 0 (no pain control) to 10 (complete control). The pain control rating significantly increased after implementation $(\mathrm{MSD}=2.21 ; p=$ 0.0005). A Spearman's correlation coefficient demonstrated a positive correlation between improvement in patient satisfaction and pain control rating $\left(\mathrm{r}_{\mathrm{s}}=0.46, p=0.03\right)$. After 1 year, chart review analysis of all patients enrolled in clinic in the past year was performed 
$(n=43)$. We significantly decreased the number of controlled substance refill tasks handled by staff ( $\mathrm{SMD}=4.91, p<0.0001)$. Compliance in documenting family history of substance abuse increased from 4.7 to $100 \%$, scanned narcotic agreement increased from 44.2 to $100 \%$, urine drug screening from 41.9 to $100 \%$, review of state prescription fill history from 55.8 to $100 \%$. PHQ-9 scores significantly decreased after 1 year (SMD = $2.44(1.0) ; p=0.01)$. Tdap compliance significantly increased from 27.9 to $41.9 \%$ ( $p=$ $0.01)$ and influenza compliance significantly increased from 37.2 to $51.2 \%(p=0.03)$.

KEY LESSONS FOR DISSEMINATION (WHAT CAN OTHERS TAKE AWAY FOR IMPLEMENTATION TO THEIR PRACTICE OR COMMUNITY?): Implementation of COAT clinic within our primary care practice achieved our goal of quality improvement measured by patient satisfaction, compliance with statutory guidelines, and other health care outcomes. Improvement of pain control was positively correlated with the improvement in patient satisfaction. Several factors could influence this correlation including certainty regarding refills, improved depression scores, quick visit workflow, dedicated time to exclusively discuss pain management, recall bias, and other unknown factors. Cohorting these patients to clinic on the same day allowed us to achieve $100 \%$ compliance with new regulations. The clinic change decreased the number of prescription refill tasks, which likely resulted in decreased time spent managing patients between visits. We found improvements in other health care measures of PHQ-9 scores and vaccination compliance. Routine PHQ-9 screening could have allowed for increased recognition of depression and its severity, allowing for improved treatment. Increased vaccination compliance may be secondary to increased opportunities to vaccinate with more frequent COAT visits. The COAT clinic model required little administrative support. It could be replicated in other primary care clinics interested in improving patient satisfaction and health care outcomes of patients managed with chronic controlled substances

COMMUNITYRX: CONNECTING HEALTH CARE TO SELF-CARE IN AN ACADEMIC PRIMARY CARE CLINIC ON CHICAGO'S SOUTH SIDE Elizabeth L. Tung; Jennifer A. Makelarski; Veronica Escamilla; Lisa M. Vinci; Elbert S. Huang; Stacy T. Lindau. University of Chicago, Chicago, IL. (Control ID \#2457665)

STATEMENT OF PROBLEM OR QUESTION (ONE SENTENCE): The Chronic Care Model (CCM) identifies the mobilization of community resources as essential to high-quality chronic disease care; however, a recent systematic review (Stellefson et al. Prev Chronic Dis 2013; 10:26) found few strategies to support practice-based referral to community resources, which may be pivotal to achieving population health management goals in high-risk communities.

OBJECTIVES OF PROGRAM/INTERVENTION (NO MORE THAN THREE OBJECTIVES): To create a dynamic inventory of community-based resources in a high-poverty, urban geography, and to assess the feasibility of integrating this inventory with the EMR workflow to enable automated referrals to community-based resources for common health problems.

DESCRIPTION OF PROGRAM/INTERVENTION, INCLUDING ORGANIZATIONAL CONTEXT (E.G. INPATIENT VS. OUTPATIENT, PRACTICE OR COMMUNITY CHARACTERISTICS): CommunityRx, developed with a U.S. Centers for Medicare \& Medicaid Services Health Care Innovation Award (1C1CMS33099701-00), employed local high school youth to conduct an annual inventory of all businesses and organizations in a 16 ZIP code demonstration area on Chicago's South Side (www. mapscorps.org). These businesses and organizations were surveyed by phone to identify the full range of goods and services offered. The CommunityRx database was integrated with the Epic electronic health record (EHR) workflow, using 7 wellness and 30 condition-specific ontologies to generate personalized, condition-specific referrals (HealtheRx) to community-based resources near the patient's home. A HealtheRx 'prescription' was generated at every visit for patients residing in the demonstration area ( $58 \%$ of households were at or below $200 \%$ federal poverty level). The intervention was implemented in an academic primary care clinic consisting of 140 providers ( 39 attending physicians, 1 nurse practitioner, and 100 resident physicians) seeing over 24,000 unique patients and completing more than 55,000 visits annually on Chicago's South Side. MEASURES OF SUCCESS (DISCUSS QUALITATIVE AND/OR QUANTITATIVE METRICS WHICH WILL BE USED TO EVALUATE PROGRAM/ INTERVENTION): This analysis, using CommunityRx data from 6/1/2014-5/31/ 2015 , describes overall dissemination of the intervention, community resource availability by ZIP code (mean no. resources $\pm \mathrm{SD}$ ), and patterns of community resource referral in an academic primary care clinic. We hypothesize that self-care resources are available in high-poverty communities, and referral to these resources can be feasibly integrated into the primary care workflow.

FINDINGS TO DATE (IT IS NOT SUFFICIENT TO STATE FINDINGS WILL BE DISCUSSED): CommunityRx connected patients to local self-care resources that address chronic disease management goals in a high-risk population. Overall, 23,321 HealtheRx referrals were generated for 10,356 unique patients (68\% female; ages 18 106); $23 \%$ were accessed $\geq 1$ time by a health care provider. Among unique patients, hypertension (63\%), back pain (39\%), and diabetes (31\%) were the most prevalent chronic conditions addressed; $83 \%$ of HealtheRx referrals were generated for patients with $\geq 2$ co-morbidities. A total of 1,664 places and 110 service types were included in the HealtheRx referrals. Although self-care resources could be found in high-poverty communities, availability of resources varied widely by ZIP code. The five most commonly recommended resource types and their mean availability by ZIP code were: group exercise classes ( $92 \%$ of patients; $12 \pm 7$ resources per ZIP code); weight loss classes ( $89 \%$ of patients; $2 \pm 2$ resources per ZIP code), fresh fruits and vegetables ( $89 \%$ of patients; $16 \pm 8$ resources per ZIP code), healthy eating classes ( $88 \%$ of patients; $6 \pm 4$ resources per ZIP code), and individual counseling ( $87 \%$ of patients; $12 \pm 9$ resources per ZIP code).

KEY LESSONS FOR DISSEMINATION (WHAT CAN OTHERS TAKE AWAY FOR IMPLEMENTATION TO THEIR PRACTICE OR COMMUNITY?): Under new payment models and ongoing Health Information Technology for Economic and Clinical Health (HITECH) Act incentives, health systems are being encouraged to engage patients via meaningful use of EHR, reduce overall healthcare costs, and improve population health outcomes. Novel EHR-based programs like Community Rx, that support self-care through automated community-based referrals, may be essential for brokering the challenges inherent to achieving these goals in vulnerable populations. By generating geospatial health resource data and cataloguing these data at the community level, CommunityRx can target populations who are at higher risk for having unmet needs and thereby help high-risk patients to better navigate their neighborhood resource environment. These programs will be critical to achieving population health management goals and reducing health disparities in high-risk communities.

CONFRONTING THE MODERN EXODUS: A SWISS PERSPECTIVE Jeremie Blaser $^{3}$; Patrick Bodenmann ${ }^{3}$; Jacques Cornuz ${ }^{1}$; Elodie Dory ${ }^{3}$; Judith L. Griffin ${ }^{3}$; Yann L'Hostis $^{3}$; Eric Masserey ${ }^{2} .{ }^{1}$ University of Lausanne, Lausanne, Switzerland; ${ }^{2}$ Service of public health, Lausanne, Switzerland; ${ }^{3}$ Vulnerable Population Center, Lausanne, Switzerland. (Control ID \#2462454)

STATEMENT OF PROBLEM OR QUESTION (ONE SENTENCE): Since June 2015, Europe has been faced with the largest movement of refugees since World War II, leading to a pressing need for countries to find new ways to provide health care to this frequently highly vulnerable population.

OBJECTIVES OF PROGRAM/INTERVENTION (NO MORE THAN THREE OBJECTIVES): The objective of this program is to proactively manage the rapidly increasing number of refugees arriving in Switzerland and to provide them with all necessary health care services. 
DESCRIPTION OF PROGRAM/INTERVENTION, INCLUDING ORGANIZATIONAL CONTEXT (E.G. INPATIENT VS. OUTPATIENT, PRACTICE OR COMMUNITY CHARACTERISTICS): Switzerland is a central European country with a resident population of around 8.3 million, and is not a member of the European Union. The Swiss health system is organized at both the national and cantonal (state) levels, and is similar in structure to the Affordable Care Act in the United States, with mandatory individual medical insurance coverage purchased from private companies (including for refugees, purchased by the government). After arriving in Switzerland, refugees are distributed throughout the 26 cantons, in proportion with the population of each canton. In 2014, 23,765 refugees entered the country, while 28,962 had arrived by October 2015. With a population of 755,000, the canton of Vaud should theoretically admit $8.4 \%$ of the total refugee population (2565 in 2014). To date, the health care program for refugees in Vaud has been organized so that all refugees were seen by specialized nurses in one of the 7 dedicated centers, and has been overseen by one of the 5 Swiss university hospitals. The program has the equivalent of 23 full-time employees, with a referral network of 168 participating primary care physicians. These dedicated centers provided the care for $70 \%$ of the refugees, with only $30 \%$ requiring a referral to an outside institution or medical center. Starting in June 2015, with the arrival of up to 400 new refugees every month, this previous system — staffed to care for 80-100 new refugees per month — has not had the capacity to absorb this substantial increase. In order to continue providing the necessary health care to this vulnerable population, a new framework has been developed. First, refugees are seen for a short evaluation by a team of 3 people, comprised of a specialized nurse, an interpreter and an administrative team member. These teams work at the dedicated centers, but also go directly to where the refugees live. The purpose of this intervention is to distinguish individuals who need prompt medical attention, and are referred directly to the appropriate provider for sameday care, from those who may receive care at a later time. During the initial visit, the first series of vaccinations (against diphtheria, tetanus, pertussis, varicella, measles, mumps and rubeola), as well as hepatitis B serology, are all performed. Second, all refugees without urgent conditions are seen by specialized nurses in one of the 7 dedicated centers for the next series of vaccinations, as well as a follow-up evaluation. Based on this second consultation, the patients are divided into 2 groups, those with complex socio-medical issues and those without. The first group is followed at the 7 dedicated centers through a case-management approach, and the latter group is referred to collaborating primary care provider as needed

MEASURES OF SUCCESS (DISCUSS QUALITATIVE AND/OR QUANTITATIVE METRICS WHICH WILL BE USED TO EVALUATE PROGRAM/ INTERVENTION): To evaluate the intervention we will: - Compare the number of refugees evaluated in triage with the total number of new arrivals, as well as to the number of initial medical evaluations during a similar time period under the previous system. Monitor the participation rate of refugees invited to receive medical evaluations, as well as the number of vaccinations and hepatitis B serology. -Monitor the number of people sent to an emergency department and the number with planned follow-up care. FINDINGS TO DATE (IT IS NOT SUFFICIENT TO STATE FINDINGS WILL BE DISCUSSED): In December 2015, 5 months after the initial increase in refugees' arrivals: -241 adult refugees arrived in the canton of Vaud, compared to 53 during the same time period in 2014 (355\% increase). In total, 268 refugees have been seen for an initial triage evaluation, compared to 109 first health evaluations in December 2014. The greater number of evaluations than arrivals includes refugees who arrived in previous months but were seen during this time. -Most of people invited to receive medical evaluation came ( $89 \%$ ). Of those who attended the initial evaluation, we administered vaccinations to $87 \%$, and hepatitis B serology was done for $91 \%$. - Only $2 \%$ needed to be sent to an emergency department and nearly all $(99.6 \%)$ were referred for follow-up care. We expect significant improvements in the coming months, as these findings reflect the early stages of the implementation of the new paradigm.
KEY LESSONS FOR DISSEMINATION (WHAT CAN OTHERS TAKE AWAY FOR IMPLEMENTATION TO THEIR PRACTICE OR COMMUNITY?): This is the first program in Switzerland to modify the structure of its health care system for refugees since the increased influx of refugees in this country. Based on our assessment of this intervention, it could be generalized to other health care systems with a specialized nurse-led program, such as those implemented in other Swiss cantons, or in other countries using a universal health-coverage system.

CONVERT ANSWERS TO QUESTIONS: THE DRIVE TO ALIGN PERFORMANCE Jeffrey Lowenkron. The Villages Health, The Villages, FL. (Control ID \#2470532)

STATEMENT OF PROBLEM OR QUESTION (ONE SENTENCE): Can local physician leaders design a bonus program that aligns with organizational goals to achieve the triple aim (improve quality and service, while reducing cost)?

OBJECTIVES OF PROGRAM/INTERVENTION (NO MORE THAN THREE OBJECTIVES): How to guide engagement and link authority to accountability through questions. Recognize that answers are often easy but good questions require more thought. Use questions to inspire pride of authorship and translate this to performance goals. DESCRIPTION OF PROGRAM/INTERVENTION, INCLUDING ORGANIZATIONAL CONTEXT (E.G. INPATIENT VS. OUTPATIENT, PRACTICE OR COMMUNITY CHARACTERISTICS): The Villages is the largest 55 and older active living community in the country and the fastest growing metropolitan service area in the United States. In 2012 a commitment was made to transform The Villages into America's healthiest hometown. An agreement was reached with United Healthcare to develop a private label Medicare Advantage (MA) program. The Villages Health (TVH) began to build a community based, primary care driven, patient centered medical home model from the ground up. One local family practice group was acquired. The first new primary care center opened in January 2013. Since then, 4 additional primary care centers and a specialty care center have been built. Currently 41 primary care doctors, 11 specialists and 18 nurse practitioners and physician assistants care for 43,000 patients. The patient base is growing by about 1000 new patients per month. With the continuing rapid growth, TVH migrated its leadership and management from centralized to local in the care centers. Recently, local leaders built a bonus plan for physicians and staff to align with organizational goals and be viewed as legitimate measures of performance. The entire program was designed through questions. The time from beginning to implementation was 3 months. TVH expects questions from the care centers will align the workforce to fulfill the commitment to transform The Villages into America's Healthiest hometown. Sample of the questions: What are our organizational goals (think mission and vision)? What are our personal goals? Why do we want our physician work force aligned? What measures legitimately assess performance (by those being measured) and at the same time align with organizational goals? What would we be proud to accomplish? Which goals can we measure? What do you think is the relative value of each of these goals we are trying to accomplish?

MEASURES OF SUCCESS (DISCUSS QUALITATIVE AND/OR QUANTITATIVE METRICS WHICH WILL BE USED TO EVALUATE PROGRAM/ INTERVENTION): Primary care: HEDIS 5 star Categories Patient Satisfaction (PSAT) RAF/HCC Schedule fill-rate/productivity MA program knowledge base FINDINGS TO DATE (IT IS NOT SUFFICIENT TO STATE FINDINGS WILL BE DISCUSSED): 2014 HEDIS 12 of 17 score 5 stars and 2 score 4 stars. In 201512 (or 13) of 15 HEDIS measures are 5 stars PSAT top box scores $98.5 \%$ across the board which are measured and reported monthly. This corresponds with CAHPS score of 9.2 from 2014. CAHPS from 2015 is being measured and will be available at the time of presentation. Reports are being built for Schedule fill rates (currently $80 \%$ but reported 
manually). $\mathrm{RAF} / \mathrm{HCC}$ is requiring an updated build and will be available at the time of the presentation. MA program knowledge base has been internally designed and administered with mastery demonstrated by all providers and staff.

KEY LESSONS FOR DISSEMINATION (WHAT CAN OTHERS TAKE AWAY FOR IMPLEMENTATION TO THEIR PRACTICE OR COMMUNITY?): That which is measured is that which is done. Those who are being measured, if given the opportunity and appropriate guidance through questioning, will rapidly develop a program that is specific, measurable, reportable and usable to align performance through the organization. There is pride of authorship that arises with process and makes explanation of the components to the rest of the group clearer and more accurate. It is a critical first step to think through the right questions.

COORDINATED TEAM APPROACH TO ADDRESS PATIENTS WITH MULTIPLE QUALITY GAPS IN CARE Nisha Basu'; Adam R. Christensen ${ }^{2}$; Scot B. Sternberg ${ }^{3} .{ }^{1}$ BIDMC, Boston, MA; ${ }^{2}$ Beth Israel Deaconess Medical Center, Wakefield, MA; ${ }^{3}$ Beth Israel Deaconess Medical Center, Boston, MA. (Control ID \#2469299)

STATEMENT OF PROBLEM OR QUESTION (ONE SENTENCE): While population health management interventions to achieve quality targets have largely focused on an individual care gap or care gaps related to one condition, a more patient-centered, coordinated, and integrated approach is needed.

\section{OBJECTIVES OF PROGRAM/INTERVENTION (NO MORE THAN THREE} OBJECTIVES): 1 . Create a database of multiple clinical data sources to determine true patient care gaps 2. Coordinate effective outreach programs to economize efforts and engage patients 3 . Engage entire care team members in timely care gap closure DESCRIPTION OF PROGRAM/INTERVENTION, INCLUDING ORGANIZATIONAL CONTEXT (E.G. INPATIENT VS. OUTPATIENT, PRACTICE OR COMMUNITY CHARACTERISTICS): Healthcare Associates is a large academic primary care practice at Beth Israel Deaconess Medical Center, caring for approximately 42,000 patients. This program was designed as a pilot to address care gaps in a subset of the HCA population, 4359 patients, under one payor contract. Using data from our electronic health record, scheduling system, and insurance claims, we identified 883 patients with one or more gaps in care. We combined lists based on conditions or preventive health screenings into a single, patient-focused report, listing all care gaps identified for each patient. The care gaps included preventive and wellness measures for colorectal, breast, and cervical cancer screening; chlamydia screening; adolescent well visits; for patients with diabetes: $\mathrm{A} 1 \mathrm{C}$ testing and control, diabetic retinopathy screening, and urine microalbumin testing; and for patients with hypertension, blood pressure. The population was stratified based on the intervention required to close the specific care gaps - those which required a primary care visit and those only requiring non-primary care appointments. Those patients determined to require appointments in the practice, who did not have an upcoming visit scheduled, received outreach to schedule the appropriate length appointment. Patients who only required follow-up with a specialty (e.g., mammogram) were also called to review gap and facilitate scheduling. Outreach included up to 3 phone calls. During a clinic visit, Medical Assistants (MAs) received a checklist highlighting those care gaps which were due to be addressed. The MAs discussed care gap with the patient, verified it was due, and then communicated this to the provider Providers discussed and, when appropriate, addressed these care gaps. At visit conclusion, clinical administrative assistants (CAAs) reviewed remaining care gaps, facilitating scheduling as needed.

MEASURES OF SUCCESS (DISCUSS QUALITATIVE AND/OR QUANTITATIVE METRICS WHICH WILL BE USED TO EVALUATE PROGRAM/ INTERVENTION): Measures of success include effectiveness of outreach, point of care reminders, and care gap closure. For those patients with care gaps without an upcoming scheduled visit, we measured outreach effectiveness by the percentage of patients outreached who scheduled an appointment, and the percentage of those patients within the cohort who kept their appointments. The impact of point of care reminders effectiveness is measured by the percentage of patients whose care gaps are addressed at the visits. Overall effectiveness was measured by reporting the total rate of care gap closure as well as the total number of patients for whom all care gaps have been closed.

\section{FINDINGS TO DATE (IT IS NOT SUFFICIENT TO STATE FINDINGS WILL} BE DISCUSSED): As of September 2015, we identified 883 patients who had a total of 1489 unique care gaps - ideally to be completed before the end of the year. 577 (65.3\%) patients were missing a single care metric and $306(34.6 \%)$ patients had two or more care gaps. 364 of $883(41.3 \%)$ patients were identified for outreach. For patients needing primary care appointments, 129/235 (54.8\%) patients scheduled appointments. Of those 129 appointments, $89.9 \%$ were kept. There were 129 patients were called for assistance in addressing care gaps by specialty providers. When the claims data is available for these patients, we will assess the care gaps subsequently. As of 12/22/2015, 204 (23.0 \%) patients had closed all care gaps, and total number of care gaps had decreased to 941.413 $(43.9 \%)$ of the remaining care gaps represent procedure need to be done outside of primary care.

KEY LESSONS FOR DISSEMINATION (WHAT CAN OTHERS TAKE AWAY FOR IMPLEMENTATION TO THEIR PRACTICE OR COMMUNITY?): Timely data-driven patient centered care is essential for the success of population health management. Lack of coordinated outreach efforts contributes to patient disengagement. Utilizing only claims is inefficient due to the data's inherent time lags. Conversely, EHR data often fails to capture procedures done external to the respective health network. Efforts directed to address patients' care gaps must integrate both sources of information and then coordinate patient centered strategies to affect them. It is the combined effects of identification of patient care gaps prior to patient visits by using multiple data sources, targeted outreach, introduction of care needs by medical assistants, and ultimately timely and direct provider-patient interactions that leads to success in these measures.

COORDINATING COMPLEX CARE - A HIGH-RISK RESIDENCY CLINIC Rebecca Davis; Riley Lipschitz. Hospital of the University of Pennsylvania, Philadelphia, PA. (Control ID \#2466143)

STATEMENT OF PROBLEM OR QUESTION (ONE SENTENCE): Within many Internal Medicine resident clinics, high risk patients - those with multiple chronic conditions, a high prevalence of behavioral health disorders, and socioeconomic instability - may suffer worse health outcomes due to challenges with resident continuity, inadequate longitudinal care planning and lack of structured inter-disciplinary care teams. OBJECTIVES OF PROGRAM/INTERVENTION (NO MORE THAN THREE OBJECTIVES): 1. Define the high-risk population within an Internal Medicine resident clinic. 2. Utilize an inter-professional care team to develop individualized patient care plans with an emphasis on risk reduction through primary, secondary, and tertiary prevention strategies. 3. Identify targeted health interventions for high-risk patients based on unique social and medical needs and analyze the success of these interventions over time.

DESCRIPTION OF PROGRAM/INTERVENTION, INCLUDING ORGANIZATIONAL CONTEXT (E.G. INPATIENT VS. OUTPATIENT, PRACTICE OR COMMUNITY CHARACTERISTICS): The Penn Center for Primary Care (PCPC) has developed the Coordinated Complex Care (C3) Clinic for high-risk patients as a novel clinical innovation to expose residents to the needs of this patient population, to utilize an inter-disciplinary team to develop integrated care plans, and to offer targeted interventions to improve health outcomes. In order to qualify as "high risk", patients had to have two or more poorly controlled chronic diseases (including diabetes mellitus, hypertension, 
obesity, and COPD/asthma in an active smoker). In addition, patients must have been seen in resident clinic within the past 12 months. Patients with poorly controlled serious mental illness or active substance abuse were excluded from the clinic. Over the course of 6 months, residents invited two of their primary patients to participate in the $\mathrm{C} 3$ clinic. The "C3 Team" includes the resident physician, a nurse practitioner, a social worker, a clinical pharmacist, a community health worker (CHW), and an attending physician. For each patient, there was a "pre-clinic huddle" during which time the patient's overall health challenges were discussed and preliminary goals were established. Following this, the resident physician had a 1 -h clinic visit with his/her patient. This visit was primarily focused on understanding the patient's social determinants of health, evaluating functional health/well-being (using the SF-12), and establishing a 6-month goal to improve chronic disease. The CHW then spent an additional hour with the patient, further evaluating social, cultural and economic barriers to health. A "post-clinic huddle" allowed the entire C3 team to debrief, discuss findings, and identify areas for risk reduction. "Next steps" were established for each patient, which may include follow up clinic visits with the NP, clinical pharmacist or PCP as well as home visits, phone calls, or virtual visits. The CHW plays a crucial role in the clinic structure, engaging with the patient weekly either in person or by phone. CHW visits could incorporate anything that may help the patient achieve his/her health goals, including walking/fitness, identifying community groups for engagement, encouraging clinic follow up, or ensuring medications are refilled. In addition to clinic meetings, the $\mathrm{C} 3$ team has monthly discussions to evaluate patient progress. After development of the longitudinal, multi-disciplinary care plan, high-risk patients were reintegrated into the resident continuity clinic with more clearly defined goals and expectations

MEASURES OF SUCCESS (DISCUSS QUALITATIVE AND/OR QUANTITATIVE METRICS WHICH WILL BE USED TO EVALUATE PROGRAM INTERVENTION): 1. Improving control of previously uncontrolled chronic disease 2 . Reducing hospitalizations, readmissions, and emergency department visits 3. Improving patient satisfaction and engagement within the resident continuity clinic FINDINGS TO DATE (IT IS NOT SUFFICIENT TO STATE FINDINGS WILL BE DISCUSSED): We were able to enroll two patients per resident into our $\mathrm{C} 3$ clinic Each patient has had a least one appointment in the clinic establishing care with the $\mathrm{C} 3$ interdisciplinary team. Additionally, each patient has had a home visit from the community health worker and weekly contact either by phone or in person. The role of the CHW in identifying social barriers to care has greatly impacted our approach to individualizing high-risk patient management. We have found that for many in our high-risk population, social obstacles have far outweighed the organic, diseased-based problems that our patient's face. This knowledge has allowed us to tailor our care planning to focus greater energy and resources on this facet of patient's overall plan. Moreover, it provides a powerful framework for resident physicians to establish appropriate goals for chronic disease management.

KEY LESSONS FOR DISSEMINATION (WHAT CAN OTHERS TAKE AWAY FOR IMPLEMENTATION TO THEIR PRACTICE OR COMMUNITY?): Creating a multi-disciplinary primary care clinic for high-risk patients can allow for improved care and patient buy-in based on more fully assessed needs and appropriate goals setting. CHWs can be valuable assets in the management of this population, especially as high-risk social issues add to the complexity of chronic medical conditions. Providing resident physicians with a structured framework for risk reduction can improve both resident and patient engagement in chronic disease management

CREATING CONVERSATIONS ABOUT LIVING WITH AND TREATING CHRONIC CONDITIONS: THE ICAN DISCUSSION AID Kasey Boehmer; Ian Hargraves; Summer Allen; Victor M. Montori; Christina Maher; Marc Matthews. Mayo Clinic, Rochester, MN. (Control ID \#2468564)
STATEMENT OF PROBLEM OR QUESTION (ONE SENTENCE): Current clinical practice does not regularly create treatment plans for chronic conditions that fit the life of the patient or aligns the work of self-care with the patient's unique capacity to handle it. OBJECTIVES OF PROGRAM/INTERVENTION (NO MORE THAN THREE OBJECTIVES): 1 . To reframe nonadherence as a problem of workload and capacity. 2. To diagnose workload-capacity imbalance using the ICAN Discussion Aid in clinical encounters. 3. To consider next steps in using ICAN in individual practice and team-based care

DESCRIPTION OF PROGRAM/INTERVENTION, INCLUDING ORGANIZATIONAL CONTEXT (E.G. INPATIENT VS. OUTPATIENT, PRACTICE OR COMMUNITY CHARACTERISTICS): Two key issues lie behind the failure to implement effective care in patient daily routines. First, there is significant, underappreciated, patient work necessary to implement interventions. Attending appointments, taking medications, shopping for and preparing healthy food, enacting an active lifestyle, dealing with administrative tasks related to insurance, and selfmonitoring all take time, effort, sense-making, and attention. Second, patients must invest capacity — effort, time, emotion, help, and attention — to implement this work and other important tasks in their lives. Prescribers seldom (a) assess the available capacity patients have to enact new medical and lifestyle interventions, (b) prioritize these interventions, or (c) identify when patients require additional support. ICAN is an encounter discussion aid that is designed to uncover issues of patient work and available capacity. ICAN creates a conversation that: shifts the focus from the medical condition of the person to their situation in life, identifies what the person values doing and being, explores how healthcare and other resources serve or limit this person, and recognizes and cultivates opportunities to advance the person and their situation. By doing so, ICAN seeks to create treatment plans that minimize treatment burden and are feasible for patients and their caregivers to implement, identifies places in which capacity could be bolstered by additional resources, and uncovers areas in which patients' have untapped capacity. The ICAN Discussion Aid was created through a robust user-centered design process, in which prototypes were subjected to real clinical practice settings. ICAN was designed for outpatient settings treating patients with chronic conditions. It has been tried in a variety of geographic locations, covering a diversity of patients and health professionals, with considerable conversational success. ICAN can be implemented in individual patient-clinician encounters by entire teams to orient their care towards the patient's context.

MEASURES OF SUCCESS (DISCUSS QUALITATIVE AND/OR QUANTITATIVE METRICS WHICH WILL BE USED TO EVALUATE PROGRAM/ INTERVENTION): ICAN's initial evaluation should be based upon changes in the clinical encounter, which should contain more contextual information about how healthcare and life are working together or at odds. Furthermore, ICAN should be evaluated based upon its ability to support care plans that positively impact patientimportant outcomes (treatment burden, quality of life), the patient and health professional's satisfaction with its function, and its ability to provide a coordination point for teams around the patient's context (relational coordination).

FINDINGS TO DATE (IT IS NOT SUFFICIENT TO STATE FINDINGS WILL BE DISCUSSED): The final prototype of the ICAN Discussion Aid was informed by 68 clinical encounters with patients and a variety of health professionals. Our key findings that informed ICAN were that the opening questions nature and timing influenced the extent to which life context could be discussed in the encounter, patient-centered language was required to help patients illuminate areas of their capacity and treatment burden, and discussing only concerns was less fruitful than discussion that included both strengths and struggles. Current evaluation on ICAN's impact on patient-clinician encounters and the organization of team-based care is ongoing in primary care encounters, primary care teams, community health worker teams, and wellness coach teams in multiple states. 
KEY LESSONS FOR DISSEMINATION (WHAT CAN OTHERS TAKE AWAY FOR IMPLEMENTATION TO THEIR PRACTICE OR COMMUNITY?): The ICAN Discussion Aid is a clinical conversation tool that requires minimal training for implementation, can be implemented in about $3 \mathrm{~min}$ in busy clinical encounters, and can be used longitudinally with patients to explore life and healthcare's interaction over time. Ultimately, this implementation should result in conversations that allow health professionals to consider patient work and their capacity to handle it to co-create treatment plans that fit the patient's life. ICAN is currently being piloted by a variety of health professionals (physicians, nurses, care coordinators, community health workers, and social workers) as well as by entire healthcare teams to orient care. Exploration of the way in which ICAN-supported care can fit in participant's unique healthcare delivery context are warranted.

CREATION AND EVALUATION OF AN OPIOID REVIEW COMMITTEE IN AN ACADEMIC PRIMARY CARE CLINIC Soraya Azari'; Claire K. Horton ${ }^{3}$; Sahand Ghodrati ${ }^{3}$; RaeAnne Fuller ${ }^{1}$; Melissa Kirkpatrick ${ }^{1}$; Lindsay Evans ${ }^{1}$; Bren Turner ${ }^{1}$; Shin-Yu Lee ${ }^{2} .{ }^{1}$ San Francisco General Hospital, San Francisco, CA; ${ }^{2}$ San Francisco General Hospital \& Trauma Center, San Francisco, CA; ${ }^{3}$ UCSF, San Francisco, CA; ${ }^{4}$ University of California, San Francisco, San Francisco, CA. (Control ID \#2469820)

\section{STATEMENT OF PROBLEM OR QUESTION (ONE SENTENCE): Patients on} chronic opioid therapy for non-cancer pain present various challenges to the primary care provider. We developed a controlled substance review committee in order to offer provider support, develop clinic guidelines, and to improve the safety of opioid prescribing in our community.

\section{OBJECTIVES OF PROGRAM/INTERVENTION (NO MORE THAN THREE} OBJECTIVES): To assemble an opioid review committee that is represented by a diversity of clinic team members. To develop criteria for eligible patients and to create a clinical intake form. To do a needs assessment of clinic providers to understand what functions of a review committee would be of most help.

DESCRIPTION OF PROGRAM/INTERVENTION, INCLUDING ORGANIZATIONAL CONTEXT (E.G. INPATIENT VS. OUTPATIENT, PRACTICE OR COMMUNITY CHARACTERISTICS): In our urban, academic primary care clinic at San Francisco General Hospital, we created an opioid review committee (Controlled Substance Issues committee, or "CSI"). The members of the committee include 1-2 physicians, 1-2 pharmacists, a nurse, and a behavioral health clinician. The group meets monthly and reviews $1-2$ cases that have been submitted by the primary care provider, or by other members of the clinic. Primary care providers complete and submit the intake form and have the option of attending the discussion. Recommendations are sent to providers following the discussion. Given the lack of medical literature about controlled substance review committees, we performed a survey of clinic members to understand the desired functions of the committee and feedback to date on its operations.

MEASURES OF SUCCESS (DISCUSS QUALITATIVE AND/OR QUANTITATIVE METRICS WHICH WILL BE USED TO EVALUATE PROGRAM/ INTERVENTION): Six months following the creation of the review committee, an anonymous,multiple-choice web-based survey was sent to clinic providers to determine perceived importance and desired functions of the committee. With regard to the committee's operations, the major indicators of success include: number of patients reviewed, patient outcomes after recommendations made, and provider satisfaction.

FINDINGS TO DATE (IT IS NOT SUFFICIENT TO STATE FINDINGS WILL

BE DISCUSSED): The survey of clinic providers was conducted 6 months after starting the controlled substance committee. $76 / 85$ clinic providers responded to the voluntary, online survey. The majority of participants were residents ( $55 \%$ ), followed by attendings ( $37 \%$ ), and nurse practitioners $(6.7 \%) .92 \%$ of participants answered that the controlled substance review committee was a good idea either because it decreased the stress of the provider $(50 \%)$ or was good for patient safety (42\%). The desired functions of the review committee were to create clinic-wide policies, provide guidance on opioid tapers, and advice on monitoring of patients. The Controlled Substance Review committee has reviewed 15 patient cases. The outcomes for these patients are currently being monitored. KEY LESSONS FOR DISSEMINATION (WHAT CAN OTHERS TAKE AWAY FOR IMPLEMENTATION TO THEIR PRACTICE OR COMMUNITY?): This is the first description of the creation and desired functions of an opioid review committee in primary care. The basic structure and materials can be adapted to different clinical settings based on need. In our analysis, the committee was positively regarded by providers because of the support it conferred to primary care clinicians and the benefit to patient safety. Given increasing concerns about the safety of long term opioid therapy for chronic non-cancer pain, clinics should consider forming review committees in order to manage complex cases or to devise clinic guidelines.

DELIVERING HIGH VALUE CARE THROUGH CO-LOCATED, INTEGRATED NEUROLOGY IN A PRIMARY CARE PRACTICE Muhamad Y. Elrashidi; Nathan P. Young; Sarah Crane; Jon Ebbert. Mayo Clinic, Rochester, MN. (Control ID \#2467972)

STATEMENT OF PROBLEM OR QUESTION (ONE SENTENCE): The fragmentation of healthcare delivery, rising costs, and access challenges to primary and neurology specialty care necessitates development and study of new care delivery models that enhance care coordination and collaboration among primary care providers and neurologists.

OBJECTIVES OF PROGRAM/INTERVENTION (NO MORE THAN THREE OBJECTIVES): 1 . Describe the model of co-located, integrated neurology in a primary care, patient-centered medical home. 2. Identify consult volume and referral type to an integrated, community-based general neurology practice. 3 . Report pilot data demonstrating avoidance of unnecessary diagnostic testing and decreased primary care provider and patient self-referral to non-integrated, neurology subspecialty practices.

DESCRIPTION OF PROGRAM/INTERVENTION, INCLUDING ORGANIZATIONAL CONTEXT (E.G. INPATIENT VS. OUTPATIENT, PRACTICE OR COMMUNITY CHARACTERISTICS): This pilot was conducted with patients empaneled in Mayo Clinic Employee and Community Health (ECH), a multi-site primary care practice located in Rochester, MN. Mayo Clinic is a multispecialty, tertiary-referral group practice with a common electronic health record (EHR). All patients within ECH have an assigned primary care provider. ECH provides care to approximately 140,000 patients. Prior to the pilot, ECH patients seeking neurology care were referred by their primary care provider (PCP) or by self-referral to the non-integrated, neurology subspecialty practice. On October 1, 2014, a general neurology practice was co-located within the main location of ECH and provided 0.6 full-time equivalent (FTE) for curbside, electronic (e-consult), and traditional, face-to-face (FTF) consultation. The neurologist and all ECH providers are compensated on a salary model. Collaboration via curbside (telephone, EHR messaging, E-mail) or electronic consultation was encouraged before ordering a FTF consult through physician-led staff meetings, practice-wide electronic communications, and a display of the pager number and photograph of the co-located neurologist in every exam room. Diagnostic testing was not prohibited or constrained. Referrals from PCPs directly to tertiary neurology subspecialists were not permitted without integrated neurology review. The co-located neurology schedule allowed for 2-3, 45 min, new patient FTF visits and one, 30-min follow up FTF per day. The remaining unscheduled time was used to provide curbside, electronic, same day FTF consults, and follow up on patient messages and test results. Nonidentifiable, referral volume data of FTF visits to integrated neurology and the non-integrated, neurology subspecialty practice was accessed through Mayo 
Clinic Patient Access Management Analytics (PAMA) data warehouse. Between December 1, 2014 and March 13, 2015, data from consecutive consults including method of contact, provider and consult type, referral reason, and category of neurologic disease were collected by the co-located neurologist. For curbsides and e-consults, PCP requests for FTF consults and diagnostic tests were recorded. The number of FTF consults that could have been completed via curbside or e-consult was also estimated after FTF consultations as well as the potential impact of an earlier FTF consultation on decision to obtain diagnostic testing. A follow up chart review to assess avoided FTF consults and diagnostic testing was performed at 6 months. The study was approved by the Mayo Clinic Institutional Review Board.

MEASURES OF SUCCESS (DISCUSS QUALITATIVE AND/OR QUANTITATIVE METRICS WHICH WILL BE USED TO EVALUATE PROGRAM/ INTERVENTION): Measures of model success will include: 1. Diagnostic tests avoided 2. FTF consultations avoided 3. Decreased PCP referrals to non-integrated, neurology subspecialty practice $<$ span style $=$ "line-height:20.8px" $>$ for FTF consultations FINDINGS TO DATE (IT IS NOT SUFFICIENT TO STATE FINDINGS WILL BE DISCUSSED): During the study period, 429 consultations (359 unique patients) were referred to integrated neurology including 179 curbsides, 68 electronic consultations, and 182 FTF visits. The most common reasons for referral were diagnostic queries, followed by testing and treatment recommendations. The most common disease category for referral was headache (25\%), followed by seizure/spells (11\%), and cerebrovascular disease $(7 \%)$. The integrated model resulted in avoidance of 78 non-integrated, neurology subspecialty practice referrals, 50 electromyograms, 39 brain magnetic resonance imaging studies, and 53 other advanced imaging studies. Compared to the 3 month average prior to pilot, the integrated model resulted in a $61.1 \%$ reduction in PCP referrals to the nonintegrated, neurology subspecialty practice and a $26.5 \%$ decline in overall PCP referrals for FTF visits.

KEY LESSONS FOR DISSEMINATION (WHAT CAN OTHERS TAKE AWAY FOR IMPLEMENTATION TO THEIR PRACTICE OR COMMUNITY?): Improving access, communication and coordination between primary and specialty providers is critical to delivering high value care to patients. The model of a co-located, collaborative neurology practice integrated within a patient centered medical home demonstrates promise for avoiding unnecessary diagnostic testing, reducing referrals for traditional face-to-face visits to non-integrated, neurology subspecialty practices, and has the potential to achieve the goals of improving population health, care experience, and lowering cost.

DEVELOPING A DATA-DRIVEN WORKFLOW FOR POPULATION HEALTH ROUNDING Mark Sendak ${ }^{2,}{ }^{1}$; Blake Cameron ${ }^{1}$; Eugenie Komives ${ }^{1}$; Joseph Futoma ${ }^{1}$; Erich Huang ${ }^{1}$; Katherine Heller ${ }^{1}$; Devdutta Sangvai ${ }^{1}$; L. Ebony Boulware ${ }^{1}$; Uptal D. Patel ${ }^{1}$. ${ }^{1}$ Duke University, Durham, NC; ${ }^{2}$ Duke Institute for Health Innovation, Durham, NC. (Control ID \#2465985)

STATEMENT OF PROBLEM OR QUESTION (ONE SENTENCE): Can Accountable Care Organizations (ACOs) accurately identify patients with progressing Chronic Kidney Disease (CKD) and streamline processes to quickly connect those patients to services? OBJECTIVES OF PROGRAM/INTERVENTION (NO MORE THAN THREE OBJECTIVES): 1) Describe the opportunity for ACOs to improve CKD management by more proactively using data generated as part of routine clinical care 2) Report initial outcomes of deploying analytics on a Medicare Shared Savings Program (MSSP) population to enable interdisciplinary population health rounding to connect patients with progressing CKD to services

DESCRIPTION OF PROGRAM/INTERVENTION, INCLUDING ORGANIZATIONAL CONTEXT (E.G. INPATIENT VS. OUTPATIENT, PRACTICE OR COMMUNITY CHARACTERISTICS): Duke Connected Care is a MSSP that manages healthcare for over 46,000 Medicare patients who are elderly (mean age 71.4 years), mostly female (57.6\%) and who mostly (85\%) live in eleven counties surrounding Duke University Health System. Over 17,000 patients within the MSSP have CKD and over 1,000 have End Stage Renal Disease (ESRD). CKD and ESRD together cause poor health, premature death, and account for a disproportionate share of health care spending. Prompt detection and management of CKD can slow or even halt the progression to ESRD. Medical providers often fail to recognize and treat CKD, contributing to late referral to nephrology and poor clinical outcomes. Prior to our pilot, no dedicated CKD care management services were provided to MSSP patients. A data warehouse combining certain Medicare claims and electronic health record (EHR) data elements existed, but no report identified patients with progressing CKD. Models that accurately predict progression of CKD to ESRD exist and clinical guidelines recommend referral to nephrology for patients with a predicted risk of ESRD progression of 10-20\% or higher. We aggregated lab and diagnosis data across Medicare claims and EHR to deploy two validated models: Tangri's Kidney Failure Risk Equation and rate of estimated glomerular filtration rate (eGFR) decline over 2 years. We identified 1,564 patients who were alive and without evidence of ESRD with predicted risk of progression to ESRD greater than $15 \%$. Of those, structured data enabled the exclusion of 1,068 patients with a past nephrology visit and 83 patients with evidence of acute kidney injury without chronic dysfunction. The remaining 413 patients were eligible for population health rounding. In June 2015, we assembled an interdisciplinary population health rounding team consisting of a primary care physician, nephrologist, pharmacist, and nurse care manager to meet for $1 \mathrm{~h}$ per week to review these patients. During rounds, each patient's name, medical record number, 5-year risk of ESRD progression, and rate of eGFR decline are read aloud. Team members conduct an in-depth EHR review to decide on changes in management (e.g., referral to nephrology, message to primary care physician, medication adjustment, connection to social or care management services).

MEASURES OF SUCCESS (DISCUSS QUALITATIVE AND/OR QUANTITATIVE METRICS WHICH WILL BE USED TO EVALUATE PROGRAM/ INTERVENTION): Operational measures that can drive the optimization of population health rounds (e.g., the number of patients rounded on per month, time-per-case during rounds, and the percent of patients reviewed at rounds that have action taken) and clinical measures that can demonstrate the overall impact on population health (e.g., incidence of ESRD, breakdown of renal replacement modality, and number of dialysis crash-starts) are being collected.

FINDINGS TO DATE (IT IS NOT SUFFICIENT TO STATE FINDINGS WILI BE DISCUSSED): Out of 335 patients rounded upon over 5 months, 73 patients (21.8\%) required changes in management, including 53 patients $(72.6 \%)$ connected to nephrology services, 8 patients $(11.0 \%)$ connected to primary care providers (PCPs), 7 patients $(9.6 \%)$ with lab recommendations, and 4 patients (5.5\%) with medication recommendations. Most of the 262 patients with no action taken met exclusion criteria, but were not effectively screened due to limitations of structured data. Population health rounding revealed 110 patients $(42.0 \%)$ seeing a nephrologist, 35 patients $(13.3 \%)$ recently deceased, and 25 patients $(9.5 \%)$ on dialysis. Six rounding sessions covering a new cohort of 73 patients were timed with an average time-per-case of 2 min and $12 \mathrm{~s}$. In the latest 2 months of the pilot, nearly 100 patients were reviewed per month. Later months were more efficient as the population health rounding team optimized a workflow for surveying data distributed throughout the EHR to support decision-making.

KEY LESSONS FOR DISSEMINATION (WHAT CAN OTHERS TAKE AWAY FOR IMPLEMENTATION TO THEIR PRACTICE OR COMMUNITY?): 1)

Patients with CKD at high risk of progression to ESRD can be identified using validated algorithms applied to structured data that is readily available. 2) The ability to screen out patients with recent nephrology visits, ESRD onset, and death using structured data is limited. To overcome this, we implemented pre-rounding, where a care manager performs chart reviews to quickly screen out patients with evidence of these events. 3) Fast 
decisions can be made during population health rounds to connect patients with progressing CKD to necessary services. Efficiency of population health rounding could be further optimized if this data were aggregated and presented via a rounding dashboard.

DEVELOPING AND SPREADING AN APPROACH FOR POPULATIONBASED PREVENTIVE SERVICES OUTREACH IN A LARGE PRIMARY CARE IMPROVEMENT COLLABORATIVE Shana Ratner ${ }^{1}$; Lindsey Franks ${ }^{1}$; Brooke B. McGuirt ${ }^{2}{ }^{1}$; Christina McMillan ${ }^{1}$; Laura G. Brown ${ }^{2}$; Michael Pignone ${ }^{1,2}$ ${ }^{1}$ UNC Chapel Hill, Chapel Hill, NC; ${ }^{2}$ University of North Carolina, Chapel Hill, NC. (Control ID \#2464599)

STATEMENT OF PROBLEM OR QUESTION (ONE SENTENCE): Our institution lacked a population-based approach to ensure primary care patients were up to date with preventive services.

OBJECTIVES OF PROGRAM/INTERVENTION (NO MORE THAN THREE OBJECTIVES): Develop an effective approach for non-visit-based outreach for patients due for preventive services Develop a collaborative approach between primary care providers and central care managers Spread this approach across the University of North Carolina (UNC) Adult Primary Care Improvement Collaborative

DESCRIPTION OF PROGRAM/INTERVENTION, INCLUDING ORGANIZATIONAL CONTEXT (E.G. INPATIENT VS. OUTPATIENT, PRACTICE OR COMMUNITY CHARACTERISTICS): The program took place in the UNC General Internal Medicine Clinic (GIM) and UNC Adult Primary Care Improvement Collaborative (PCIC), a collaborative of 28 mainly community-based primary care practices owned by UNC that share a common Electronic Health Record (EHR). We first developed and pilottested all processes in GIM, an academic General Internal Medicine practice that cares for 12,000 active patients. The effective approaches were spread throughout the PCIC, which cares for approximately 200,000 patients. Our intervention included developing standard processes to deliver reminder messages (via mail or the EHR portal) to patients due for influenza vaccination, pneumococcal vaccination, breast cancer screening, and colon cancer screening, as well as processes for phone and EHR patient navigation. Patient advisors assisted with writing and modifying the outreach letters. We piloted the EHR bulk outreach capabilities starting with one patient and one letter then made needed modifications to our processes. Three care managers across the collaborative managed the outreach and provided patient navigation for patients who did not respond to the first outreach mailing. For immunizations, outreach was sent to all patients due or overdue based on physician consensus that they did not need to review the lists. For cancer screening, we developed and streamlined a process for primary care providers to review the lists of patients due for outreach. Care managers sent the providers lists of patients due, and providers replied with which patients should be excluded from outreach and why. The care managers then sent bulk outreach through the EHR only to provider-approved patients. We developed standard processes for office staff to reply to messages and calls from patients who had received the letters. The care managers also reviewed the lists of patients excluded by the providers and placed reasons for exclusions in the proper fields in our EHR to remove these patients from future outreach. We created standard work documents documenting each step of these processes to ensure consistent spread of these activities. MEASURES OF SUCCESS (DISCUSS QUALITATIVE AND/OR QUANTITATIVE METRICS WHICH WILL BE USED TO EVALUATE PROGRAM/ INTERVENTION): The primary outcomes are the percent of patients receiving a mailing who subsequently completed the recommended preventive service. Secondary outcomes include: the percent of patients up to date with the recommended preventive service, number of outreach mailings for each preventive service, number of practices participating, number of patients excluded from mailings, and reasons for exclusions.
FINDINGS TO DATE (IT IS NOT SUFFICIENT TO STATE FINDINGS WILL BE DISCUSSED): In total for the PCIC, approximately 60,000 mailings or patent portal reminders have been sent to patients due or overdue for 4 preventive services from 22 practices. For the cancer screenings, providers excluded approximately $25 \%$ of patients from outreach. The most common reasons were: patient received the service outside our system, moved, was deceased, had an exclusion for screening (e.g. bilateral mastectomy), or was too sick to benefit from screening. In 1 year, absolute improvements in vaccination or screening rates were seen across the PCIC in all services. Of the patients who received CRC reminders, $22 \%$ have been screened. Of those who received breast cancer screening reminders, $27 \%$ are now screened. Pneumococcal vaccination for high risk patients below age 65 improved from 42.9 to $47.6 \%$, pneumococcal vaccination for patients 65 and older from 64.4 to $77.5 \%$, breast cancer screening in women (aged 50-74) from 56.3 to $66.6 \%$, colorectal cancer (CRC) screening (aged 50-75) from 55.7 to $64.7 \%$. The social work navigation pilot in GIM showed improved results beyond letters. A higher percent of patients who receive the phone calls ( $38 \%$ ) completed the service than those who received letters alone ( $10 \% 2$ months after mailing). The social worker addressed key barriers to care such as transportation and cost.

KEY LESSONS FOR DISSEMINATION (WHAT CAN OTHERS TAKE AWAY FOR IMPLEMENTATION TO THEIR PRACTICE OR COMMUNITY?): We demonstrated that we can develop and implement a program for preventive care outreach to improve population health. We utilized our academic clinic to pilot, test, and build a program that was then successfully disseminated to multiple other practices. Strengths of this program include: 1) the emphasis on reaching out to appropriate patients; 2) utilizing care managers to standardize the process and provide patient navigation, 3) emphasis on staff training and creation of standard work documents to eliminate the need for more than 20 separate practices to design their own processes

DEVELOPMENT AND IMPLEMENTATION OF AN OPIOID SAFETY CLINIC IN A FEDERALLY QUALIFIED HEALTH CENTER Ingrid A. Binswanger ${ }^{1,3}$; Nicole Joseph ${ }^{2}$; Rebecca Hanratty ${ }^{2}$; Valerie Whitson ${ }^{2}$; Amy J. Witte ${ }^{2} .{ }^{1}$ Kaiser Permanente Colorado, Denver, CO; ${ }^{2}$ Denver Health, Denver, CO ${ }^{3}$ University of Colorado Denver, Denver, CO. (Control ID \#2469117)

STATEMENT OF PROBLEM OR QUESTION (ONE SENTENCE): Given the alarming increase in opioid overdose and death in the United States, we sought to develop, implement, and evaluate an opioid safety clinic for patients on chronic opioid therapy (COT) to improve the safety of COT.

OBJECTIVES OF PROGRAM/INTERVENTION (NO MORE THAN THREE OBJECTIVES): We developed an opioid safety clinic in an academically-affiliated federally qualified health center in Denver, CO: 1) Educate patients on the risks of COT, provide opioid safety training, and offer a prescription/training for intranasal naloxone in the event of an overdose; 2) Ensure that patients have completed structured and guideline concordant questionnaires for function and risk and signed chronic opioid agreements; 3) Standardize documentation of the patient's pain diagnosis, collection of serum or urine toxicology screening, and monitoring of the electronic prescription drug monitoring program.

DESCRIPTION OF PROGRAM/INTERVENTION, INCLUDING ORGANIZATIONAL CONTEXT (E.G. INPATIENT VS. OUTPATIENT, PRACTICE OR COMMUNITY CHARACTERISTICS): A multi-disciplinary team developed the structure, plan, and educational materials for the opioid safety clinic in monthly meetings over approximately 6 months. The clinic was staffed by a medical assistant (MA), nurse and physician during the pilot phase and then by a MA, nurse and advanced practice provider during the ongoing phase, with the support of clerical staff. We invited patients to attend using a standardized letter attached to their monthly opioid prescription. When 
patients attended an opioid safety visit, they completed all paperwork recommended by institutional guidelines for patients receiving COT including: the COT initiation checklist, the COT assessment tool, the Screener and Opioid Assessment for Patients with Pain 14, and an opioid agreement. The provider checked the state prescription drug monitoring program to review all prescriptions for controlled substances that the patient filled in the last calendar year. A serum or urine drug screen for illicit drugs and an opioid confirmatory test was also obtained. We provided all patients information about the risk of illicit drug use with chronic opioids, medications that can put a patient at increased risk for sedation, and the risks of sharing opioids with others. We reviewed safe storage and disposal of controlled substances and encouraged patients to secure their medications in a lock box, specifically highlighting the importance of keeping medications safe from children. Finally, we offered all patients a prescription for intranasal naloxone and educated patients about how to use this medication if overdose is suspected.

MEASURES OF SUCCESS (DISCUSS QUALITATIVE AND/OR QUANTITATIVE METRICS WHICH WILL BE USED TO EVALUATE PROGRAM/ INTERVENTION): We sought to evaluate the feasibility of the program, its reach (number of patients who attended), concordance with institutional guidelines (completion of assessment tools and COT agreements), and receipt of naloxone prescriptions. FINDINGS TO DATE (IT IS NOT SUFFICIENT TO STATE FINDINGS WILL BE DISCUSSED): The opioid safety clinic was successfully implemented and sustained Between July 1, 2014, and December 31, 2015, 433 patients attended an opioid safety visit. All assessment tools, forms, and agreements were completed for each patient who attended. Each patient was given a naloxone prescription. Furthermore, as toxicology screening was systematically performed at each visit, we identified patients with evidence of use of other illicit drugs (mainly cocaine) while on COT. These results were communicated with the primary care providers, who acted on them. Future work will evaluate the number of naloxone prescriptions filled and used and quantify other outcomes among clinic participants.

KEY LESSONS FOR DISSEMINATION (WHAT CAN OTHERS TAKE AWAY FOR IMPLEMENTATION TO THEIR PRACTICE OR COMMUNITY?): An opioid safety clinic can be implemented using existing clinic resources to deliver guideline-concordant care. By separating this visit from a general medical visit with the primary care provider, it allowed the time to provide focused education and safety training. It also provided an opportunity for another provider to evaluate a patient for aberrant behaviors. Although not our primary purpose, we identified patients using substances not previously identified, and primary care providers were able to make appropriate modifications to COT and treatment referrals in response.

DEVELOPMENT OF DENVER HEALTH'S INTENSIVE OUTPATIENT CLINIC TO TARGET SUPER-UTILIZERS Vishnu Kulasekaran; Holly A. Batal; Joshua Blum; Jeremy Long; Deborah Rinehart; Diana Botton; Josh Durfee; Daniel Brewer; Tracy L. Johnson. Denver Health, Denver, CO. (Control ID \#2459943)

STATEMENT OF PROBLEM OR QUESTION (ONE SENTENCE): Interest exists in optimizing programs designed to reduce the total cost of care for patients who use a high level of health care services ("super-utilizers").

OBJECTIVES OF PROGRAM/INTERVENTION (NO MORE THAN THREE

OBJECTIVES): Define super-utilizer population Determine local resources to leverage existing capabilities Recognize importance of continued iteration

DESCRIPTION OF PROGRAM/INTERVENTION, INCLUDING ORGANIZATIONAL CONTEXT (E.G. INPATIENT VS. OUTPATIENT, PRACTICE OR COMMUNITY CHARACTERISTICS): Existing literature suggests that to be successful programs must be responsive to population-specific needs and have clinical buy-in from the delivery system in which the super-utilization is occurring. Denver Health (DH), an integrated safety-net delivery system, received a 2012 Health Care Innovation Award from Centers of Medicare \& Medicaid Services to deliver better care, spend health care dollars more wisely, and make our communities healthier. Identifying super-utilizers and developing an effective clinical intervention was one key strategy to reduce overall costs and improve health. This led to the development of the Intensive Outpatient Clinic, designed to target super-utilizers to provide primary care in an ambulatory-ICU model. MEASURES OF SUCCESS (DISCUSS QUALITATIVE AND/OR QUANTITATIVE METRICS WHICH WILL BE USED TO EVALUATE PROGRAM/ INTERVENTION): This analysis traces the development of an Intensive Outpatient Clinic (IOC) focused solely on adult super-utilizers, and highlights how the capabilities of DH's integrated delivery system are leveraged in program design. Super-utilizer demographic data and utilization patterns, chart reviews and patient interviews, literature review and observation of existing programs, strategic staffing, involvement of stakeholders, and consideration of organizational experience and resources were foundational.

FINDINGS TO DATE (IT IS NOT SUFFICIENT TO STATE FINDINGS WILL BE DISCUSSED): To match allocated resources to projected patient volume, adult superutilizers were defined by $3+$ hospital admissions in the prior 12 months, or $2+$ with a severe mental illness. Through chart reviews and patient interviews, we concluded the best opportunity to engage super-utilizers was during an index hospitalization, made possible through a daily list of patients with qualifying admissions. The IOC was partly modeled after existing DH high intensity clinics. However, IOC patients necessitated more robust social work support, longer visits with same day access, integrated behavioral health, navigation and outreach, smaller patient panels, hospital rounding, and dedicated clinic space located in close proximity to the Emergency Department (ED). The clinic is managed as a primary care clinic, but with significant freedom from organizational staffing ratios and productivity constraints. Staff was hired predominately from DH's primary care and urgent care practices. Strong relationships with the ED and hospitalbased physician were solidified by involving them in the IOC development process. Whereas similarly effective clinics have struggled financially, the IOC has enjoyed broad institutional support, high levels of clinical engagement, and has transitioned from grant to organizational funding.

KEY LESSONS FOR DISSEMINATION (WHAT CAN OTHERS TAKE AWAY FOR IMPLEMENTATION TO THEIR PRACTICE OR COMMUNITY?): Understanding local capabilities along with ascertaining patient population needs are essential in rapidly developing a clinically-viable and effective super-utilizer intervention. If you have seen one super-utilizer program, you have seen one super-utilizer program. Strive for real time patient identification and leverage existing capabilities for rapid implementation. This publication was made possible by Grant Number 1C1CMS331064 from the Department of Health and Human Services, Centers for Medicare \& Medicaid Services. The contents of this publication are solely the responsibility of the authors and do not necessarily represent the official views of the U.S. Department of Health and Human Services or any of its agencies. The research presented here was conducted by the awardee. Findings might or might not be consistent with or confirmed by the findings of the independent evaluation contractor.

EFFECTIVE IMPLEMENTATION OF ANTIBIOTIC STEWARDSHIP PROGRAMS INTO LONG-TERM CARE FACILITIES Theresa A. Rowe ${ }^{1,2}$; Geetha Iyer $^{2}$; Rob Berringer ${ }^{3}$; Jonathan Zenilman ${ }^{2} .{ }^{1}$ Northwestern University, Chicago, IL; ${ }^{2}$ Johns Hopkins University, Baltimore, MD; ${ }^{3}$ AllyAlign, Glen Allen, VA. (Control ID \#2468929)

STATEMENT OF PROBLEM OR QUESTION (ONE SENTENCE): The Centers for Medicare and Medicaid (CMS), Centers for Disease Control and Prevention (CDC) and other agencies have recommended implementing antibiotic stewardship programs 
(ASP) in long-term care facilities (LTCF) to control antimicrobial use, care costs, and reduce the emergence of resistant organisms; however, most LTCFs do not have ready access to resources such as infectious disease physicians or ASP trained pharmacists thus, ASP implementation in these settings requires innovative approaches.

OBJECTIVES OF PROGRAM/INTERVENTION (NO MORE THAN THREE OBJECTIVES): Our objective is to partner an academic hospital based team of clinicians trained in antibiotic stewardship with LTCF management to adapt, implement and evaluate an ASP into a statewide network of LTCFs.

DESCRIPTION OF PROGRAM/INTERVENTION, INCLUDING ORGANIZATIONAL CONTEXT (E.G. INPATIENT VS. OUTPATIENT, PRACTICE OR COMMUNITY CHARACTERISTICS): Our consultative program uses a successful hospital based ASP combining antibiotic stewardship and infection/prevention control measures which are then modified and tailored to meet the needs of each individual LTCF. Introduction and discussion is initially held with key stakeholders, including pharmacy and medical directors of each LTCF. Based on the size, capacity and resources of each facility, the following measures based on the CDC 7 Core elements of ASP are modified and implemented into each facility: Leadership Commitment: Our program engages key stakeholders of each LTCF (e.g., CEO, medical director, director of nursing) and obtains formal commitment from leadership to develop, implement, and evaluate each ASP. Needs Assessment: Conduct an individualized needs assessment of each LTCF using the following parameters: * Determination of antimicrobial burden and resistance * Determination of current antibiotic use and monitoring $*$ Cost assessment Action Plan: Develop and institute ASP into each LTCF using the following measures: * Provide training and education to leadership, staff, patients/families * Development and implementation of Infectious Disease specific clinical pathways: 1. Urinary tract infection/bacteriuria 2. Pneumonia/bronchitis 3. Skin and soft tissue infections 4. Pressure Ulcers * On-site consultation for non-acute infectious disease issues * Infection control support * On-site wound care Monitoring: Provide initial and ongoing support to each LTCF to develop a comprehensive program to monitor antibiotic prescribing and resistance patterns (see below)

MEASURES OF SUCCESS (DISCUSS QUALITATIVE AND/OR QUANTITATIVE METRICS WHICH WILL BE USED TO EVALUATE PROGRAM/ INTERVENTION): * Tracking/Monitoring antibiotic prescribing, use, and resistance: 1. Antibiotic days of therapy/1,000 resident days 2 . New antibiotic starts/1,000 resident days 3. Rates of antibiotic-resistant organisms * Facility Outcomes of Antibiotic Use: 1. Infection rates and length of stay for pneumonia, UTI, Clostridium difficile infection, skin/soft tissue infections, pressure ulcers * Rates of antibiotic adverse events (including hospital and emergency department transfers) * Cost analysis (antibiotic costs, length-of-stay) * Clinical perceptions regarding need and usefulness of the program

FINDINGS TO DATE (IT IS NOT SUFFICIENT TO STATE FINDINGS WILI BE DISCUSSED): We have begun implementation of an ASP to 3 LTCF associated with AllyAlign health. Preliminary data will be available Spring 2016.

KEY LESSONS FOR DISSEMINATION (WHAT CAN OTHERS TAKE AWAY FOR IMPLEMENTATION TO THEIR PRACTICE OR COMMUNITY?): A1though ASP are successful in the hospital setting, many LTCF may not be able to fully implement the CDC's recommended interventions because of limited resources. Providing a consultation service that can modify ASP activities to fit the most urgent needs of each individual LTCF provides an opportunity to successfully reduce unnecessary antibiotic use, prevent emergence of resistant infections, and improve healthcare quality and safety in facilities with limited resources.

ELECTRONIC CIGARETTE USE FOR SMOKING REDUCTION AND CESSATION IN PRIMARY CARE Jean-Paul Humair; Rodrigo Tango. University Hospitals of Geneva, Geneva, Switzerland. (Control ID \#2470049)
STATEMENT OF PROBLEM OR QUESTION (ONE SENTENCE): The electronic cigarette (e-cigarette) might help smokers to reduce or stop tobacco use but the evidence about its acceptability and effectiveness in primary medical care is very limited but plausible.

OBJECTIVES OF PROGRAM/INTERVENTION (NO MORE THAN THREE OBJECTIVES): This exploratory study describes the profile of regular E-cigarette users and assesses whether the e-cigarette is acceptable for patients and helpful for short-term reduction or cessation of tobacco smoking.

DESCRIPTION OF PROGRAM/INTERVENTION, INCLUDING ORGANIZATIONAL CONTEXT (E.G. INPATIENT VS. OUTPATIENT, PRACTICE OR COMMUNITY CHARACTERISTICS): Since mid-2013, e-cigarette was included as a new smoking cessation aid for smokers who attended an outpatient clinic of a Swiss university hospital. All smokers received the usual motivational and behavioral counseling. Those who wished to reduce tobacco use or failed to stop smoking with usual cessation therapy (nicotine replacement, varenicline, bupropion) were offered to use ecigarette containing nicotine.

MEASURES OF SUCCESS (DISCUSS QUALITATIVE AND/OR QUANTITATIVE METRICS WHICH WILL BE USED TO EVALUATE PROGRAM/ INTERVENTION): Acceptability was assessed by proportion of patients using the ecigarette daily for several months. Short-term effectiveness was measured by proportions of e-cigarette users who either reduced by at least $30 \%$ their tobacco consumption or quit smoking completely at the end of follow-up.

FINDINGS TO DATE (IT IS NOT SUFFICIENT TO STATE FINDINGS WILL BE DISCUSSED): This study included 54 e-cigarette users, who were heavy and dependent smokers with a mean daily consumption of 25 cigarettes and smoking on average their first cigarette within 14 min after waking up. Twenty-nine percent were suffering from a somatic tobacco-related illness while $44 \%$ had a mental disorder. All patients but two used the e-cigarette daily with the aim to either reduce their tobacco use (48\%) or to quit smoking (52\%). Most patients (76\%) had a favorable behavior change with either tobacco reduction or cessation during an average follow-up of 5 months. A third of patients (35\%) decreased their mean cigarette consumption by over $30 \%$ while a higher proportion $(41 \%)$ reported tobacco abstinence at the end of follow-up. Pharmacological therapy with nicotine replacement, varenicline or bupropion was prescribed to $67 \%$ of patients in association with ecigarette. Patients reported no significant side effects.

KEY LESSONS FOR DISSEMINATION (WHAT CAN OTHERS TAKE AWAY FOR IMPLEMENTATION TO THEIR PRACTICE OR COMMUNITY?): Ecigarette with nicotine is well accepted and used daily by most patients willing to reduce tobacco consumption or to quit smoking after unsuccessful attempts. Short-term effects on smoking in this small study suggest that e-cigarette might be effective to help smokers attending in primary care to reduce or stop tobacco use. Clinicians may offer the ecigarette to some smokers to widen their smoking cessation options. As stronger evidence is needed, clinical trials with larger samples should test the long-term effectiveness of ecigarette to reduce or quit smoking in primary care settings.

ENHANCED CARE PROGRAM, A MODEL FOR COMPLEX CARE MANAGEMENT: PRELIMINARY OUTCOMES Swati Shroff ${ }^{1,2}$; Jodie Bryk ${ }^{1}$; Gary Fischer ${ }^{1}$; Deborah M. Simak ${ }^{1}$; Dennis Wickline ${ }^{3}$; Pamela Peele ${ }^{3}$; Wishwa N. Kapoor ${ }^{1} .{ }^{1}$ University of Pittsburgh, Pittsburgh, PA; ${ }^{2}$ VA Pittsburgh Healthcare System, Pittsburgh, PA; ${ }^{3}$ UPMC Health Plan, Pittsburgh, PA. (Control ID \#2443879)

STATEMENT OF PROBLEM OR QUESTION (ONE SENTENCE): Five percent of the population accounts for over half of healthcare expenditures in the US. OBJECTIVES OF PROGRAM/INTERVENTION (NO MORE THAN THREE OBJECTIVES): Our innovation is the design and implementation of an Enhanced Care 
Program (ECP) to meet the needs of complex, high-utilizing individuals. The program aims to: reduce unnecessary healthcare utilization, improve continuity and quality of care, and improve the patient experience.

DESCRIPTION OF PROGRAM/INTERVENTION, INCLUDING ORGANIZATIONAL CONTEXT (E.G. INPATIENT VS. OUTPATIENT, PRACTICE OR COMMUNITY CHARACTERISTICS): The ECP is being implemented in the University of Pittsburgh General Internal Medicine practice in Oakland (GIMO) in collaboration with UPMC Health Plan (UPMC-HP), a major insurer in western Pennsylvania. Individuals are invited to participate in the ECP if they meet the following criteria: 1) $>1$ inpatient hospitalization or $>5$ ED visits in the previous year; 2) 18 years of age or older; 3) UPMC-HP member; 4) Receive primary care at GIMO; and 5) Agree to participate. The ECP is embedded within a patient centered medical home primary care practice. The ECP team consists of 2 part-time physicians, 2 full-time nurse care managers, a full-time medical assistant, a part-time psychologist, and a part-time psychiatrist. After a comprehensive assessment, a care plan is developed and agreed upon by the patient and team Patients have regular face-to-face and phone call follow up with the team, including 24/7 direct telephone access. The ECP also offers same day walk-in appointments and home visits. The team communicates with the ED and follows patients in the hospital. Patient care and service needs are discussed during weekly team huddle meetings, and individual care plans are modified as needed.

MEASURES OF SUCCESS (DISCUSS QUALITATIVE AND/OR QUANTITATIVE METRICS WHICH WILL BE USED TO EVALUATE PROGRAM/ INTERVENTION): Data sources include an electronic ECP database, the electronic health record, and UPMC-HP. Our primary outcome of interest is total cost of care per ECP member per month (PMPM). Total cost of care includes medical (e.g. behavioral health, ED, hospital, PCP, specialist visits etc.) and pharmacy (medication) costs. Other utilization measures include number of hospitalizations, ED visits, specialist visits, and ECP primary care provider (ECP-PCP) visits per patient. Continuity measures include the proportion of patient visits with the ECP-PCP out of all providers and number of unique providers who see the patient Quality of care outcomes include adherence to HgbA1C goals, blood pressure goals, and ageappropriate preventive care services. Finally, patient satisfaction will be assessed through patient satisfaction surveys. We will compare outcomes with both pre/post measurements and propensity-matched controls from the UPMC-HP member population. Process measures include number of patients screened, enrolled, and refusing to participate in the ECP, and number of visits and phone calls per ECP member.

FINDINGS TO DATE (IT IS NOT SUFFICIENT TO STATE FINDINGS WILL BE DISCUSSED): From the start of the program in July 2014 to October 2015, 510 patients were screened, and 269 were eligible for the ECP. Of eligible patients, 150 were enrolled, 13 unenrolled and 8 graduated, leaving 129 actively enrolled patients. A preliminary analysis used a propensity matched group (matched by age, sex, chronic medical conditions, behavioral health diagnoses and baseline healthcare costs) to compare to 58 ECP members with at least 6 months of enrollment. Using a difference-indifferences analysis, we found average total cost of care and medical cost PMPM decreased ( $-\$ 126.59$ and $-\$ 250.83$, respectively), but these savings were not significant ( $P=0.90$ and $P=0.48$, respectively). Having a diagnosis of anxiety or depression was more than twice as likely to be associated with cost savings, but this was not significant ( $P=0.26$ and $P=0.31$, respectively). We observed an increase in pharmacy costs, but this was also not significant $(\$ 91.58, P=0.7)$ We plan to evaluate all process and outcome measures discussed above in July 2017.

KEY LESSONS FOR DISSEMINATION (WHAT CAN OTHERS TAKE AWAY FOR IMPLEMENTATION TO THEIR PRACTICE OR COMMUNITY?): After

6 months of ECP enrollment, we see trends towards decreased total and medical costs for ECP members, although it is likely too early to see a full return on program investment. We also saw a trend toward increased pharmacy costs, which may indicate a positive effect of improved medication adherence. We have learned it is essential to keep patients engaged through continuous contact, a large portion of which has been occurring via 24/7 telephone access to the team. However, this level of patient interaction may pose challenges to program scalability. Behavioral health resources are essential given the high rate of mental health illness in these patients, and interim findings indicate targeting these patients may produce greater cost savings. Homelessness is a major issue, but there are few community housing resources, limiting the team's ability to prevent unnecessary healthcare utilization in these patients. Highly coordinated, multidisciplinary care is required for these patients. Staff education on complex patient management is crucial to avoid burnout.

ENSURING ADHERENCE TO COMPLEX COLORECTAL CANCER GUIDELINES IN A VALUE BASED HEALTHCARE SYSTEM - A MOBILE SOLUTION. Rushad Patell; Abhishek Karwa; Rocio Lopez; Carol A. Burke. Cleveland Clinic, Cleveland, OH. (Control ID \#2466522)

STATEMENT OF PROBLEM OR QUESTION (ONE SENTENCE): Although clinicians perceive they know the guidelines for colorectal cancer screening and surveillance, they do not accurately apply them resulting in inappropriate resource utilization. OBJECTIVES OF PROGRAM/INTERVENTION (NO MORE THAN THREE OBJECTIVES): 1 . To determine the utility of smartphones in patient care and the need of a mobile application (app) in assisting clinicians apply colorectal cancer (CRC) screening and surveillance guidelines. 2. To develop and test an evidence based algorithm that is delivered through a simplified mobile app interface to assist clinicians to make quick and accurate $\mathrm{CRC}$ screening and surveillance recommendations. 3. To ultimately compare differences between app generated CRC screening and surveillance recommendations and provider recommendations documented in the electronic medical records using the patient's demographic, endoscopic and pathologic data.

DESCRIPTION OF PROGRAM/INTERVENTION, INCLUDING ORGANIZATIONAL CONTEXT (E.G. INPATIENT VS. OUTPATIENT, PRACTICE OR COMMUNITY CHARACTERISTICS): An IRB approved 9 question anonymous web-based survey was designed that allowed us to determine the general utility of smartphones, and the perceived uptake of an app to generate CRC screening and surveillance according to published guidelines. Program directors of all ACGME approved internal medicine, family medicine, surgery, ob/gyn, urology, gastroenterology and colorectal surgery training programs were sent an email request to forward the survey hyperlink to faculty, fellows and residents in their departments. We are creating a CRC screening and surveillance mobile app designed to work offline as a native stand-alone app (operating system specific) or online through a web-app platform. Ideal users of this technology would be clinicians practicing in the outpatient setting in the fields of primary care, general surgery or gynecology as well as specialty physicians like gastroenterology. To allow for a broad use among clinicians, minimize app complexity, and still be applicable to the majority of the population, patients with hereditary CRC syndromes or incomplete colonoscopy will be excluded. In such scenarios, the User would be recommended to seek specialist guidance. The mobile application poses a series of sequential questions to the health care professional (referred to as "User" from here on) to generate a CRC screening or surveillance recommendation. The algorithm is optimized to allow the User to reach the final recommendation as quickly as possible using current evidence based guidelines avoiding the need for the User to enter all patient history, endoscopic and pathologic variables. Based on 5 initial input variables (age, race, history of previous colonoscopies, IBD, and colorectal cancer) the app will direct the User through one of two pathways known as the Screening or Surveillance pathway.

MEASURES OF SUCCESS (DISCUSS QUALITATIVE AND/OR QUANTITATIVE METRICS WHICH WILL BE USED TO EVALUATE PROGRAM/ INTERVENTION): 1 . To demonstrate a widespread need for a mobile app in assisting clinician recommendations for $\mathrm{CRC}$ screening and surveillance. 2 . The mobile app 
algorithm is being tested using a set of simulated data to cover all screening and surveillance scenarios from factors utilized by evidenced-based off of published guidelines. When $100 \%$ of the algorithm's recommendations match the guideline recommendations, the algorithm will be considered robust for testing on a large patient data set. 3. A large set of patient demographic, endoscopic and pathologic data will be obtained from the Cleveland Clinic Digestive Disease Institute's Datamart. The algorithm's recommended interval will be compared to actual gastroenterologists' recommendations to identify any factors associated with disparity between recommendations. Analysis of variance or the non-parametric Kruskal-Wallis test will be used to compare continuous factors between "correct" and "incorrect" recommendations and Pearson's chi-square tests will be used for categorical factors.

FINDINGS TO DATE (IT IS NOT SUFFICIENT TO STATE FINDINGS WILL BE DISCUSSED): 698 clinicians responded to the survey with $98.7 \%$ indicating they owned a smartphone. Over half of practicing clinicians and nearly $80 \%$ of medical trainees (residents \& fellows) utilized their phone to aid in patient care sometimes $(17.1 \%)$, often $(43.7 \%)$, almost every time $(20 \%)$ and on every encounter $(10.7 \%)$. $83 \%$ of clinicians reported they have interest in a mobile app to aid in ensuring appropriate CRC screening and surveillance recommendations to their patients. Additionally, over a quarter would be willing to pay for an app that helps them in this task

KEY LESSONS FOR DISSEMINATION (WHAT CAN OTHERS TAKE AWAY FOR IMPLEMENTATION TO THEIR PRACTICE OR COMMUNITY?): 1.

Smartphone use is ubiquitous among clinicians. Integration of technology by implementing guideline algorithms and care paths have the potential to assist clinicians in providing accurate recommendations to their patients. 2. Colorectal cancer guidelines have far reaching implications to public health with screening and surveillance recommendations being updated at regular intervals. Recalling these complex CRC recommendations has proven challenging even for gastroenterologists. Smart calculators and algorithms can help alleviate this pitfall and prevent the over and under utilization of resources - a key requirement to sustain our shift to value based healthcare.

FALLS IN THE ELDERLY: HOW TO INCREASE SCREENING IN A RESIDENT PRIMARY CARE CLINIC John L. Elliott; Jennifer Lom. Emory University, Decatur, GA. (Control ID \#2463699)

STATEMENT OF PROBLEM OR QUESTION (ONE SENTENCE): Despite recommendations from numerous societies to screen patients aged 65 and older for falls, residents in clinic frequently do not screen for falls

OBJECTIVES OF PROGRAM/INTERVENTION (NO MORE THAN THREE OBJECTIVES): 1) Analyze the percentage of patients who were eligible and screened for falls by PGY-2 and PGY-3 residents before the educational intervention 2) Reduce the barriers to screening for falls in clinic by providing an education session to the PGY-2 residents 3) Report follow up data on screening rates to ensure improvement was noted after the education session

DESCRIPTION OF PROGRAM/INTERVENTION, INCLUDING ORGANIZATIONAL CONTEXT (E.G. INPATIENT VS. OUTPATIENT, PRACTICE OR COMMUNITY CHARACTERISTICS): Internal Medicine residents working in their outpatient primary care clinics have an increasing number of geriatric patients for which they are providing care. Unfortunately, many do not have a Geriatric Medicine rotation, or what they do have is either so brief or comes so late in training (as in our program) that there is not a positive impact on the patients they care for. Lack of confidence in screening their geriatric patients for falls as well as limited education can lead to many missed opportunities to help prevent falls. Additionally, gaps in the electronic medical record did not allow for ease of documentation of screening during clinic visits. We created an interactive, multi-disciplinary workshop to educate learners during their ambulatory block.
The residents completed an interactive, online case prior to attending the session. The session itself was comprised of a short review of pertinent screening concepts, interactive mini-CEX type cases, instruction on evaluation of gait in the elderly population and demonstration of various assistive devices led by one of our physical therapists. At the same time, a novel documentation tool was created in our electronic medical record and disseminated to the residents. Finally, reminders were posted in clinic rooms.

MEASURES OF SUCCESS (DISCUSS QUALITATIVE AND/OR QUANTITATIVE METRICS WHICH WILL BE USED TO EVALUATE PROGRAM/ INTERVENTION): 1. Increased screening of geriatric age patients (65 years old or greater) for falls by residents in their primary care clinic. 2 . Increased documentation of interventions in those patients who screened positive 3 . Improved self-efficacy of the residents in terms of screening for falls and identifying the appropriate intervention FINDINGS TO DATE (IT IS NOT SUFFICIENT TO STATE FINDINGS WILL BE DISCUSSED): A retrospective chart review of 50 patients aged 65 and older seen by a PGY2 resident from July 2014 to October 2014 found that before the introduction of our education program, only $10 \%$ (5/50) of eligible patients were screened for falls. Of those not screened $24 \%$ were found to have a history of falls on further chart review (11/45). Documentation of interventions when patients were screened and positive for a history of falls was lacking. After residents attended the workshop, the same cohort of residents were reexamined. At that time, $30 \%(15 / 50)$ of eligible patients were screened for falls. Only 7 out of the 35 unscreened patients ( $20 \%$ ) had a history of falls on chart review. Unfortunately, no residents used our documentation tool. Instead, they chose to alter their clinic template note themselves. The residents were asked to take a survey prior to the workshop addressing confidence and attitudes towards screening for falls. 44 residents completed a survey that used a scale of strongly disagree to strongly agree ( 1 being assigned to strongly disagree to 5 being assigned to strongly agree). Confidence in their ability to screen patients, perform a comprehensive falls assessment and prescribe evidence based interventions ranged from 2.58-3.05/5. In terms of importance of the topic, the average score was $4.58 / 5$. Residents will be surveyed again in the spring of 2016 to evaluate long term changes in confidence

KEY LESSONS FOR DISSEMINATION (WHAT CAN OTHERS TAKE AWAY FOR IMPLEMENTATION TO THEIR PRACTICE OR COMMUNITY?): 1. Utilizing an online, web-based case to convey key screening, physical exam and intervention components for a comprehensive falls assessment as well as an interactive educational workshop did increase the rates at which residents screened for falls in their geriatric patients. 2. Placing reminders in the clinic also aided in improving screening rates. 3. Providing a documentation tool was not as effective as hoped, since it appears most residents did not wish to add it to their template. It may be better to form a standardized primary care geriatric progress note which the residents can use in their elderly patients that incorporates falls screening.

FRESH PRESCRIPTION PROGRAM: A PROGRAM TO IMPROVE ACCESS TO FRESH PRODUCTS AMONG UNDERSERVED PATIENTS IN DOWNTOWN DETROIT Jasmine Omar; Zarina Alam. Henry Ford Hospital, Macomb, MI. (Control ID \#2469444)

STATEMENT OF PROBLEM OR QUESTION (ONE SENTENCE): Low income patients living in Detroit have poor access to fresh produce due to poor financial resources and limited knowledge of healthy eating habits.

OBJECTIVES OF PROGRAM/INTERVENTION (NO MORE THAN THREE OBJECTIVES): -Provide patient with baseline nutrition and healthy eating education. -Provide financial assistance and improve access to locations where fresh produce can be purchased. -Provide patients with education and counseling specific to their chronic health conditions. 
DESCRIPTION OF PROGRAM/INTERVENTION, INCLUDING ORGANIZATIONAL CONTEXT (E.G. INPATIENT VS. OUTPATIENT, PRACTICE OR COMMUNITY CHARACTERISTICS): The Fresh Prescription program was launched at Henry Ford Hospital in collaboration with the Community Health and Social Services (CHASS) Center and farmer's markets. The program enrolled patients with a Body Mass Index $>30$ during their Internal Medicine clinic office visits during the months of August-September. Participants met with a dietician who educated them on specific topics based on patient's health conditions, including diabetes education when necessary. The dietician then accompanied them to their first on-site farmer's market visit. To assist in affording the fresh produce, participants were given a rechargeable debit card programmed to be used at the farmer's markets. To recharge the card, patients had to undergo an educational a counseling session of either cooking demonstration, a discussion with a dietician, or other educational activities. After confirming their participation, their cards were recharged to implement new tips and cooking ideas. Additionally, participants were educated about several locations near them providing fresh produce, and were given information on additional financial assistance in order to fully utilize their money. MEASURES OF SUCCESS (DISCUSS QUALITATIVE AND/OR QUANTITATIVE METRICS WHICH WILL BE USED TO EVALUATE PROGRAM/ INTERVENTION): Tracked by pre- and post-surveys, measures of success included a subjective increase in nutrition knowledge and healthy eating habits, as well as change in the amount of fruits/vegetables vs. unhealthy items consumed in the household FINDINGS TO DATE (IT IS NOT SUFFICIENT TO STATE FINDINGS WILL BE DISCUSSED): A total of 8 participants were enrolled in the program so far. Postsurvey information is available for 6 of the 8 participants. We found a mean daily increase of 2.8 cups fruits/vegetables per participant and a daily mean decrease of 2.6 unhealthy food items per partipant. 4/6 participants reported an increase in knowledge of fresh fruits and vegetables and 5/6 reported they were better able to manage their health conditions with their lessons. 5/6 learned new places to buy fresh produce and were comfortable continuing to shop in those location, even after completion of the program.

KEY LESSONS FOR DISSEMINATION (WHAT CAN OTHERS TAKE AWAY FOR IMPLEMENTATION TO THEIR PRACTICE OR COMMUNITY?): Improving access to fresh produce among underserved patients includes enhancing education on healthy eating in addition to providing the necessary financial resources to afford them

GEOGRAPHIC HOSPITALIST UNIT DECREASES LENGTH OF STAY Sameer Qazi; Elizabeth M. Schulwolf; Ryan Mayhew; Elizabeth wantuch. Loyola University Medical Center, Maywood, IL. (Control ID \#2467981)

STATEMENT OF PROBLEM OR QUESTION (ONE SENTENCE): Closed collaborative hospitalist unit decreases length of stay OBJECTIVES OF PROGRAM/INTERVENTION (NO MORE THAN THREE OBJECTIVES): Most academic medical centers, attending rounds serve as a cornerstone for the advancement of patient care. Despite their ubiquity, rounds lack standardized best practices, and they often vary in location, time allotted per patient, inclusion of nursing staff. We wanted to determine whether implementing a multi-disciplinary approach with alternating hospitalists could positively impact our length of stay.

DESCRIPTION OF PROGRAM/INTERVENTION, INCLUDING ORGANIZATIONAL CONTEXT (E.G. INPATIENT VS. OUTPATIENT, PRACTICE OR COMMUNITY CHARACTERISTICS): Beginning from May 2015 to October 2015 we localized two of the four general medicine hospitalist/non-resident covered service team to a localized closed unit. The unit consisted of 21 medical/Telemetry beds. The other two teams continued to work on variable floors as any other typical general medicine service. Over a period of 4 months the physician's would rotate between localized and non localized units. The selection process for physician was random based on schedule. The team that was localized to the unit had to round in the morning daily with patients primary RN. Morning rounds typically started around 7:45-8:AM. In addition, The What is Needed for Discharge (WIND) multidisciplinary team rounds occurred daily at $10 \mathrm{Am}$ and $2 \mathrm{PM}$. The two teams were also assigned a dedicated social worker and case manager. MEASURES OF SUCCESS (DISCUSS QUALITATIVE AND/OR QUANTITATIVE METRICS WHICH WILL BE USED TO EVALUATE PROGRAM/ INTERVENTION): Controlling for case mix index we tracked admissions, discharges, and readmission for greater than 4 months and more than 500 patient's. At week 10 Length of stay for the localized team ( 5 and 7) was noted to be 3.39 and 4.08 for the non-localized team (6 and 8). At the end of week 20 length of stay for the localized teams was 3.64 whereas the non-localized team was at 4.19. After reviewing all patient population that made up the baseline, at 10 Week and 20 week we extrapolated standard deviations for the localized and non-localized teams giving a $P$ value of $<0.0001$. Readmission rate remained constant during this period.

FINDINGS TO DATE (IT IS NOT SUFFICIENT TO STATE FINDINGS WILL BE DISCUSSED): We determined that multidisciplinary collaboration and geographically assigned hospitalists was associated with a statistically significant reduction in length of stay and promotes better communication between physician and RN's. Further review at 1 year will be warranted to track progress and to see if geographically localized model can be applied to all service lines throughout the medical center.

KEY LESSONS FOR DISSEMINATION (WHAT CAN OTHERS TAKE AWAY FOR IMPLEMENTATION TO THEIR PRACTICE OR COMMUNITY?): Reduction in length of stay promoting better communication between physician and RN's. This may ultimately lead to an increase in patient satisfaction scores.

I'VE GOT AN ADMISSION FOR YOU- STANDARDIZING EMERGENCY DEPARTMENT TO INPATIENT MEDICINE ADMISSION HANDOFFS Megan Walker $^{1,3}$; Charlie Lan ${ }^{3}$; Hossam Safar ${ }^{3}$; Jun Ying ${ }^{3}$; Diana Stewart ${ }^{2} .{ }^{1}$ Baylor College of Medicine, Houston, TX; ${ }^{2}$ Baylor College of Medicine/Michael E. DeBakey VA Medical Center, Houston, TX; ${ }^{3}$ Michael E DeBakey VA Medical Center, Houston, TX. (Control ID \#2468826)

STATEMENT OF PROBLEM OR QUESTION (ONE SENTENCE): Problem: Inpatients transferred to the intensive care unit (ICU) from lower acuity units have been shown to have higher mortality and resource utilization compared to patients admitted directly to the ICU from the Emergency Department (ED), even if the transfer occurs early, within the first $48 \mathrm{~h}$ of admission.

OBJECTIVES OF PROGRAM/INTERVENTION (NO MORE THAN THREE OBJECTIVES): Objective: The aim of this study is to reduce the rate of unplanned ICU transfers within the first $48 \mathrm{~h}$ of admission to an Internal Medicine service by $30 \%$ in 4 months by implementing a standardized interspecialty handoff.

DESCRIPTION OF PROGRAM/INTERVENTION, INCLUDING ORGANIZATIONAL CONTEXT (E.G. INPATIENT VS. OUTPATIENT, PRACTICE OR COMMUNITY CHARACTERISTICS): Introduction: We reviewed cases in which unplanned ICU transfers occurred at our facility, which is a large academic tertiary care center. The lack of a standard MD to MD handoff between the ED and inpatient Internal Medicine (IM) physicians was identified as a significant contributing factor to delayed recognition of critical illness. Standardized patient handoffs have been well studied within providers of the same specialty, but there is sparse literature regarding transitions of care from one specialty to another. To achieve our goal we created a standardized admission handoff to be used between the ED and IM physicians which emphasizes patient severity and assignment to the most appropriate level of care. The handoff is based upon a combination of a best practice model suggested in Academic Emergency Medicine and the IPASS system originally developed in pediatrics. 
MEASURES OF SUCCESS (DISCUSS QUALITATIVE AND/OR QUANTITATIVE METRICS WHICH WILL BE USED TO EVALUATE PROGRAM/ INTERVENTION): Measures: Our primary measure was percent of IM admissions requiring an unplanned transfer to the ICU, and specifically transfers within the first 24 and $48 \mathrm{~h}$ after admission. We also chose to track total length of stay for the patients' hospitalizations as well as 30 day mortality. For process measures we tracked use of the handoff. Physician satisfaction was analyzed through the use of surveys administered to the IM hospitalists and residents as well as the ED physicians pre and post intervention. We tracked ED length of stay (LOS) and admission delay time as both a process measure and a balancing measure as increased ER LOS has also been shown to increase morbidity and mortality.

FINDINGS TO DATE (IT IS NOT SUFFICIENT TO STATE FINDINGS WILL BE DISCUSSED): Results: Review of all inpatient Internal Medicine (IM) admissions at our institution over 7 months from January through September $2015(n=3238)$ found that at baseline $17.4 \%$ of patients required transfer to the ICU during their admission; $24 \%$ of these transfers occurred within the first $24 \mathrm{~h}$ and $51 \%$ within the first $48 \mathrm{~h}$. Data analysis tracking the rates of ICU transfers since application of the handoff is ongoing. Preliminary data from physician satisfaction surveys indicated a perceived improvement in the communication between specialties and assignment to the proper level of care since implementation of the handoff. Also the data for ER LOS and admission delay appears stable (mean $315 \mathrm{~min}$ and $175 \mathrm{~min}$ respectively).

KEY LESSONS FOR DISSEMINATION (WHAT CAN OTHERS TAKE AWAY FOR IMPLEMENTATION TO THEIR PRACTICE OR COMMUNITY?): Discussion: The increased mortality observed in transfer of admitted patients to the ICU emphasizes the importance of early recognition of critical illness. We implemented a standardized handoff between the ED and IM physicians in an effort to ensure that patients are efficiently admitted to the appropriate level of care. Literature review demonstrated multiple papers suggesting the need for standardization of this interspecialty communication, but to our knowledge this is the first study implementing and analyzing such a handoff. Preliminary data indicates improved physician satisfaction and does not show any adverse effect of increased ED LOS

IMPACT OF A HOME-BASED PRIMARY CARE INITIATIVE ON EMERGENCY DEPARTMENT AND HOSPITAL UTILIZATION IN MULTIMORBID DISABLED PATIENTS Andrew Schamess ${ }^{2}$; Randi Foraker ${ }^{1}$; Matt Kretovics ${ }^{2}$; Neeraj H. Tayal $^{2}$. ${ }^{1}$ The Ohio State University, Columbus, $\mathrm{OH} ;{ }^{2}$ The Ohio State University Wexner Medical Center, Columbus, OH. (Control ID \#2450142)

STATEMENT OF PROBLEM OR QUESTION (ONE SENTENCE): We hypothesized that, for chronically ill patients with disabilities that limit access to office-based care, the provision of primary care in the home would eliminate an important barrier, allowing for better symptom control, disease self-management, treatment adherence and clinical decision-making, resulting in improved patient quality of life and lower utilization of emergency and inpatient services.

OBJECTIVES OF PROGRAM/INTERVENTION (NO MORE THAN THREE

OBJECTIVES): 1 . Provide home-based primary care (HBPC) to patients meeting program entry criteria (age $>18$, disability creating access barrier, 2 or more chronic conditions, residence within Franklin County). 2. Reduce emergency and inpatient utilization compared with pre-enrollment rates. 3. Improve health-related quality of life (HRQoL), as measured by Rand-36 form, at 6 and 12 months post enrollment, compared with baseline measure obtained upon program entry.

DESCRIPTION OF PROGRAM/INTERVENTION, INCLUDING ORGANIZATIONAL CONTEXT (E.G. INPATIENT VS. OUTPATIENT, PRACTICE OR COMMUNITY CHARACTERISTICS): The OSU Healthy at Home program is based in the Division of General Internal Medicine at the Ohio State University Wexner Medical Center. The Division operates six outpatient clinics serving a total of 40,000 patients in Columbus and surrounding areas. The population is diverse with regard to race, ethnicity and socioeconomic status. Referrals to the Healthy at Home program come from within the Division, from hospitalists and subspecialists at the Medical Center, and from community sources. The Healthy at Home program provides ongoing home-based primary care, including chronic disease management and acute visits, telemedicine, intensive pharmaceutical management, active oversight of home health services such as skilled nursing and rehabilitation, and social work assistance with personal, family, coverage, transportation and housing issues. The program has been operating for 3 years and employs three full-time clinicians (one MD and two CNPs) to make home visits.

MEASURES OF SUCCESS (DISCUSS QUALITATIVE AND/OR QUANTITATIVE METRICS WHICH WILL BE USED TO EVALUATE PROGRAM/ INTERVENTION): 1. Patient profile. Demographics (from EMR), disability (using ACS-6 scale), multimorbidity (using Chronic Conditions Indicator framework from the $\mathrm{CDC}$ ) and polypharmacy (from EMR). These data help to characterize the population that benefits from home-based primary care. 2. Health Service Utilization for 12 months prior to and up to 3 years after initiation of home visits: emergency department visits, hospitalization (frequency of admission and length of stay), 30-day readmission, expressed as rate per 1000 patient-days. 3. HRQoL at patient entry into program, and 6 and 12 months after entry, using Rand-36 form. 4. Program metrics, including patient enrollment, provider productivity, travel times and program costs.

FINDINGS TO DATE (IT IS NOT SUFFICIENT TO STATE FINDINGS WILL BE DISCUSSED): In the first year of operation, 157 patients received HBPC. Mean age for enrolled patients was 60.4. Mean number of medications per patient was 12.2 (SD 4.3). Of the 157 patients, 95 had healthcare utilization data available in our EMR from the period prior to enrollment in the program. For these 95 patients, a decline in admissions at a rate of 10.4 (95\% CI: 8.3-12.6), a reduction in ED visits at a rate of 9.2 (95\% CI: $7.2-$ 11.3), a decrease in length-of-stay days at a rate of 50.1 (95\% CI: 45.4-54.8), and a decline in 30-day readmissions at a rate of 3.4 (95 \% CI: 2.6-4.3) per 1000 patient-days was observed. This represented a $58.6 \%$ decline in ED visits, a $58.4 \%$ decline in hospitalizations, a $59.9 \%$ decline in hospital days and a $91.9 \%$ decline in 30-day readmissions in the year following enrollment in HBPC, compared with the year prior to enrollment. Thirty months post enrollment, utilization remained below pre-intervention baseline: admissions 10.9, ED visits 4.8, length of stay 12.6 (46,398 total follow-up days). We anticipate presenting data on a larger sample at the conference, with more detailed clinical measures. We also hope to have a comparison with controls matched on baseline utilization, demographics and multimorbidity (using the Chronic Conditions Indicator). As of now, 52 patients have completed the Rand-36 baseline survey and 12 have completed 6-month follow up surveys. We will present baseline HRQoL data, and will include 6-month follow up data if a sufficient sample is available to draw meaningful conclusions.

KEY LESSONS FOR DISSEMINATION (WHAT CAN OTHERS TAKE AWAY FOR IMPLEMENTATION TO THEIR PRACTICE OR COMMUNITY?): 1. HBPC is a feasible approach to managing complex, multimorbid patients. 2. HBPC appears to significantly reduce ED and hospital utilization in these patients. Randomized trials are needed to confirm these findings. 3. Disability, in addition to multimorbidity, plays an important role in defining the subset of patients who should be targeted for HBPC. 4. Under the present payment system, costs of providing HBPC exceed revenues. Payment reform is a prerequisite for widespread deployment. 5. We will share insights into the clinical care of these highly complex patients. Our experience suggests that allocating sufficient time for provider-patient communication, problem-based management, a focus on quality of life, recognition of high acute care and between-visit needs, and a multidisciplinary team approach contributed to the success of our program. 
IMPACT OF QUALITY IMPROVEMENT DATA FROM A RESIDENT HOSPITAL DISCHARGE CLINIC ON DISCHARGE PROCESS AND PATIENT OUTCOMES Maria Hamm de Miguel ${ }^{1}$; Jessica Singer ${ }^{1}$; Nancy Chang ${ }^{1}$; Amanda Ramsdell ${ }^{2}$; Christopher Kelly ${ }^{1} .{ }^{1}$ Columbia University, New York, NY; ${ }^{2}$ Cornell University, New York, NY. (Control ID \#2469876)

STATEMENT OF PROBLEM OR QUESTION (ONE SENTENCE): Does a resident-staffed hospital discharge clinic generate quality improvement data that can meaningfully improve the discharge process and patient outcomes post-discharge? OBJECTIVES OF PROGRAM/INTERVENTION (NO MORE THAN THREE OBJECTIVES): To collect measures of quality relating to the hospital discharge process and post-discharge patient outcomes as part of a rapid hospital follow-up appointment during year one of a resident-staffed hospital discharge clinic To use this quality data to inform a hospital discharge process-improvement protocol for the medical residency To measure change in the discharge process and patient outcomes after implementation of the hospital protocol during the second year of the discharge clinic.

DESCRIPTION OF PROGRAM/INTERVENTION, INCLUDING ORGANIZATIONAL CONTEXT (E.G. INPATIENT VS. OUTPATIENT, PRACTICE OR COMMUNITY CHARACTERISTICS): The NYPH-Columbia IM Residency Hospital Discharge Clinic was started in June 2013 to provide rapid hospital follow up in a PCMH residency clinic at the medical center for patients on inpatient medicine teams During the first year of the clinic, quality data on metrics related to the process of discharge (presence and quality of discharge summaries, PMD and specialist follow ups made, communicating labs to be followed up, medication reconciliation) and patient outcomes post-discharge (ability to obtain meds, taking meds correctly, side effects, new symptoms, new functional limitations, falls, ER or urgicare visits, care advancement at the visit) were collected. This information informed a hospital discharge protocol implemented in June 2014, entitled ADIOSSS (Assess understanding, Drugs and Indications, Outpatient followup, Symptoms to expect, Services arranged, Summary in hands). During the subsequent year of discharge clinic, the same process and outcome measures were surveyed and compared to the previous year.

MEASURES OF SUCCESS (DISCUSS QUALITATIVE AND/OR QUANTITATIVE METRICS WHICH WILL BE USED TO EVALUATE PROGRAM/ INTERVENTION): The survey of quality indicators consisted of Yes/No questions, answered by the resident physician during the visit. The quality indicators were collected to assess the value of rapid follow up for clarifying discharge plans, assessing unresolved or new medical or social problems, and reconnecting patients to care. The absolute values of the indicators are useful in qualitatively describing the value of rapid follow up. Furthermore, the responses from the visits in the first year of clinic $(6 / 2013-5 / 2014)$, were compared to answers from visits in the second year $(6 / 2014-5 / 2015)$ in order to assess the impact of the new hospital discharge protocol, informed by the first year of feedback for the discharge process, implemented at the start of the second year of the discharge clinic. The ratio of Yes/No answers for year 1 to year 2 were compared using a Pearson Chi-square test. Questions with missing data and process measures which were marked as "not applicable" were omitted.

FINDINGS TO DATE (IT IS NOT SUFFICIENT TO STATE FINDINGS WILL BE DISCUSSED): Absolute values of quality indicators show that strengths of the discharge process are the presence and quality of the discharge summary (95 and $94 \%$ ), low rates of falls $(1.5 \%)$ and ER/urgicare visits $(6.5 \%)$ in the time since discharge, and low rates of ER transfers $(2.3 \%)$. The discharge clinic shows great value in identifying high rates of errors with medications $(20 \%)$, high rates of new or persistent symptoms ( $37 \%$ ), and by advancing care through medication changes (44\%), new labs/tests (39\%), and re-connection to PMD/specialty (57 \%), and social work care (10 \%). From the first year to the second year of the discharge clinic, significant improvements were found primarily in process rather than outcome measures. Significant improvements ( $p$ value
$<0.05$ ) were found for presence of discharge summary, PMD and specialist follow up appointments made on discharge, and labs to be followed up noted in discharge summary. There were no improvements in process measures of the quality of the discharge summary and patients bringing medications to appointments. The only patient outcome measure that improved significantly was patients taking medications as prescribed. There was no improvement in taking the correct medications, difficulty obtaining medications, medication side effects, new or persistent symptoms, new limitations in functional status, falls, ER visits, urgicare visits, being brought to the ER at the visit, new SW needs, medication changes, new labs/tests reviewed, new labs ordered, or new appointments made at the visit.

KEY LESSONS FOR DISSEMINATION (WHAT CAN OTHERS TAKE AWAY FOR IMPLEMENTATION TO THEIR PRACTICE OR COMMUNITY?): A resident-staffed hospital discharge clinic which incorporates a quality improvement exercise can provide data to meaningfully improve patient care processes through improved protocolized discharge practices and improved continuity with primary care after discharge. Effects on patient outcomes are more difficult to show, possibly given the low prevalence of some (ie, ER visits) and the high prevalence of others (ie, persistent symptoms). Given the lack of improvement in patient outcomes in many areas with a discharge intervention, the on-going need for rapid hospital discharge is vital.

IMPLEMENTATION OF A MEDICAL ASSISTANT- DRIVEN MEDICATION REFILL PROCESS IN AN AMBULATORY TEACHING PRACTICE Elisabeth Ihler $^{2,}{ }^{3}$; Michael Masse ${ }^{1} .{ }^{1}$ High Street Internal Medicine, Denver, CO; ${ }^{2}$ Presbyterian/St. Luke's Medical Center, Denver, CO; ${ }^{3}$ University of Colorado School of Medicine, Aurora, CO. (Control ID \#2467786)

STATEMENT OF PROBLEM OR QUESTION (ONE SENTENCE): Can the process of routinely refilling certain maintenance medications be delegated to a non-provider staff member without compromising patient care?

OBJECTIVES OF PROGRAM/INTERVENTION (NO MORE THAN THREE OBJECTIVES): To measure the impact of a medical-assistant-driven refill protocol on physician workload, appropriate patient monitoring, and physician satisfaction.

DESCRIPTION OF PROGRAM/INTERVENTION, INCLUDING ORGANIZATIONAL CONTEXT (E.G. INPATIENT VS. OUTPATIENT, PRACTICE OR COMMUNITY CHARACTERISTICS): A medication refill protocol was developed at our ambulatory teaching practice ( 6 attending physicians and 18 internal medicine residents at all levels of training). This was developed in response to concern that requiring physicians to perform all medication refills was leading to delays due to competing clinical priorities and potentially to lack of oversight, since the residents' $4+1$ schedule ( 4 weeks inpatient, 1 week outpatient) led to many refills being authorized by physicians other than the patient's PCP. In conjunction with the practice's lead MA, we developed a list of medications that could be refilled by the lead MA under the name of the patient's PCP without physician review under certain conditions related to time of last visit and lab monitoring. Refills made by the MA were reviewed once a month on a random basis by a physician champion.

MEASURES OF SUCCESS (DISCUSS QUALITATIVE AND/OR QUANTITATIVE METRICS WHICH WILL BE USED TO EVALUATE PROGRAM/ INTERVENTION): For this study, we compared prescriptions issued during a 5-week period in May-June 2015 under the protocol system with a similar 5-week period the preceding year (May-June 2014) prior to implementation. We limited the study to refills requested by patients or pharmacies rather than new prescriptions or refills provided during visits. We compared the number of refills, the number of prescriptions eligible for refill under the protocol, and the rank of the authorizing person. We also evaluated turnaround time from request to refill and the number of patients receiving refills who 
were overdue for lab monitoring or visits. We also plan to evaluate physician satisfaction with the process.

FINDINGS TO DATE (IT IS NOT SUFFICIENT TO STATE FINDINGS WILL BE DISCUSSED): During the 2015 study period, 748 refills for individual medications were generated by the practice, compared with 631 in the 2014 comparison period. Of these refills, 237 of the 2015 refills were for protocol-eligible medications (with 113 sent by the MA, 63 by residents, 120 by attendings and 7 by PharmDs with direct physician authorization). During the 2014 comparison period, 244 refills were for protocol-eligible medications, with 13 sent by the MA (at the direct request of an MD), 17 sent by residents and 214 by an attending physician. We measured turnaround time for refill requests made by phone, fax, or in person (due to EMR limitations were unable to measure this metric for e-requests by pharmacies). In 2015 the mean was $5 \mathrm{~h} 10$ min compared with a mean of $6 \mathrm{~h}$ $57 \mathrm{~min}$ and were compared using Student's t test $(P>0.05$, NS) . However, median time for refills in 2015 was $18 \mathrm{~min}$, down from $1 \mathrm{~h} 12 \mathrm{~min}$ in 2014 . We also measured the number of patients who were overdue for lab monitoring or visits. Typical lab montoring might include a basic metabolic panel on patients on diuretics or a hemoglobin Alc on a patient taking diabetes medications. Of patients requiring lab monitoring, 29 of 227 were overdue in the 2015 group compared with 24 of 176 in the 2014 group. Chi-square analysis did not show a significant difference between groups $(p=0.80)$. Visits were required every 6 or 12 months depending on the medication for refills via the protocol. In the 2014 group, 22 of 244 patients receiving refills were overdue for visits compared with 24 of 237 in the 2015 group. Chi-square analysis did not show a significant difference $(p=0.68)$

KEY LESSONS FOR DISSEMINATION (WHAT CAN OTHERS TAKE AWAY FOR IMPLEMENTATION TO THEIR PRACTICE OR COMMUNITY?): Implementation of a protocol-driven refill process that transferred approximately half of the eligible refills to a medical assistant rather than a physician has led to shorter turnaround time for requests, with no evidence of a decrease in clinical care and high physician satisfaction.

IMPLEMENTATION OF A VISIT-BASED REMINDER FOR LUNG CANCER SCREENING IN AN ACADEMIC GENERAL INTERNAL MEDICINE CLINIC Shana Ratner; Caleb Doyle-Burr; Michael Massaro; John Eick; Lindsey Franks; Bailey Minish; Laura P. Cubillos; Alison Brenner; Daniel S. Reuland. UNC Chapel Hill, Chapel Hill, NC. (Control ID \#2467343)

STATEMENT OF PROBLEM OR QUESTION (ONE SENTENCE): Implementing new guidelines for Lung Cancer Screening is complex and requires a systematic, population-based approach in primary care practice to provide high quality decision support, maximize benefit, and minimize harm.

OBJECTIVES OF PROGRAM/INTERVENTION (NO MORE THAN THREE OBJECTIVES): 1) Develop and test a nursing-directed intervention for systematic smoking history collection in the correct EHR discrete queriable fields 2) Develop and test a provider-directed visit based reminder

DESCRIPTION OF PROGRAM/INTERVENTION, INCLUDING ORGANIZATIONAL CONTEXT (E.G. INPATIENT VS. OUTPATIENT, PRACTICE OR COMMUNITY CHARACTERISTICS): The USPSTF now recommends screening with low dose computed tomography $(\mathrm{CT})$ be offered to patients ages $55-80$ with at least 30 pack-years smoking history who currently smoke or quit within 15 years. Medicare coverage requirements include proper patient selection, use of decision aids, and completion of shared decision making (SDM) before ordering a CT. The University of North Carolina is currently invested in high-quality implementation of lung cancer screening, starting in the Academic General Internal Medicine practice (GIM), with plans to spread across the health system. GIM is a Level 3 Patient-Centered Medical Home with Attendings, Residents, Advanced Practice Providers (APPs), and 13 Licensed Practical Nurses. The Electronic Health Record (EHR) employs visit-based reminders called Best Practice Advisories (BPAs) which remind providers of care gaps, and may contain orders, diagnosis codes, and documentation aids. AIM 1: We developed standard work that included questions needed for a complete smoking history, including pack years and quit date (for former smokers), and a process to enter the data in the EHR in discrete fields. Nurses were trained and audited weekly for completion with individual feedback provided, along with recognition of top performers each week on a clinic bulletin board. A care manager flagged nurses to complete smoking histories in the EHR charts. AIM 2: The team worked with EHR programmers to design a BPA to remind providers which patients were eligible for screening based on the smoking history discrete fields. The BPA was designed to include all needed steps for a high quality approach to screening in primary care and to meet Medicare documentation requirements including: a decision aid, an order for a noncontrast low dose CT scan, the correct diagnosis code, and documentation of SDM. As providers were uncomfortable with BPAs, we implemented two types of provider training. First, group training at division meeting included a slide show presentation and review of examples in the EHR training environment. Second, a resident completed a QI project implementing individual BPA training at the point of care using concepts of academic detailing.

MEASURES OF SUCCESS (DISCUSS QUALITATIVE AND/OR QUANTITATIVE METRICS WHICH WILL BE USED TO EVALUATE PROGRAM/ INTERVENTION): AIM 1: outcome measures were the percent of eligible patients with documented complete smoking histories in appropriate, discrete EHR fields. AIM 2: outcome measures were number of BPAs that fired, number and percent of BPAs acted upon, percent of patients with the correct CT order (low-dose, noncontrast chest CT), and percent of randomly assessed charts with provider documentation of SDM and decision aid use. Intervention fidelity measures included the number and type of providers trained on the BPA and the type of training received (group, individual, or both). Providers who received individual training completed an anonymous survey afterward rating their comfort using the BPA before and after each type of training on a 5-point Likert scale ranging from 1 (very uncomfortable) to 5 (very comfortable)

FINDINGS TO DATE (IT IS NOT SUFFICIENT TO STATE FINDINGS WILL BE DISCUSSED): AIM 1: Prior to nurse training, less than $10 \%$ of visits with potentially eligible patients had complete smoking histories documented in the appropriate EHR fields. When care managers began flagging eligible patient charts, results improved to $50 \%$ of visits, and the addition of a nurse "leaderboard" combined with flagging led to further improvements to $75 \%$ of visits with correctly documented smoking histories. AIM 2: In the first 2 weeks after the BPA launch, 75 BPAs fired, and 24/75 (32\%) were acted upon. All ordering providers who used the BPA chose the correct CT. On a baseline chart review, 2/8 (25\%) patients with a screening CT order had documentation of SDM in the chart (and none used a decision aid). After launch of the BPA, chart review showed 9/10 ( $90 \%$ ) had documentation of SDM and decision aid use. Ten providers attended the group training, and Twenty (6 Attendings, 12 Residents, and 2 APPs) were trained individually. Providers trained individually used the BPA in half their visits (14/28), while providers not trained individually used the BPA only $21 \%$ of the time (10/47). Among 20 providers trained individually, baseline mean comfort with the BPA was 2.5 on a 1-5 Likert scale which improved to a mean of 4.4 after individual training.

KEY LESSONS FOR DISSEMINATION (WHAT CAN OTHERS TAKE AWAY FOR IMPLEMENTATION TO THEIR PRACTICE OR COMMUNITY?): A nurse-directed intervention improved fidelity of nurse completion of smoking histories in discrete EHR fields, a key step in driving EHR-programmed visit-based reminders. Early individual provider training improved use of the BPA and all its components including appropriate $\mathrm{CT}$ ordering and SDM. 
IMPLEMENTATION OF THE COMPREHENSIVE UNIT-BASED SAFETY PROGRAM IN AN ACADEMIC PRIMARY CARE PRACTICE, JOHNS HOPKINS GREEN SPRING GENERAL INTERNAL MEDICINE, 2014-2015 Samantha I. Pitts ${ }^{1}$; Melinda Sawyer ${ }^{2}$; Nisa Maruthur ${ }^{1}$; Kimberly Curreri ${ }^{1}$; Renee Grimes ${ }^{1}$; Candace Nigrin ${ }^{1}$; Heather Sateia ${ }^{1}$; Nicklaine Paul ${ }^{1}$; Jeanne M. Clark ${ }^{1}$; Kimberly S. Peairs ${ }^{1} .{ }^{1}$ Johns Hopkins University School of Medicine, Baltimore, MD; ${ }^{2}$ Johns Hopkins University, Baltimore, MD. (Control ID \#2469796)

STATEMENT OF PROBLEM OR QUESTION (ONE SENTENCE): Although the Comprehensive Unit-based Safety Program (CUSP) has been used extensively in inpatient settings, where it has been shown to improve safety climate and clinical patient safety outcomes, no studies to date have examined the use of CUSP to improve patient safety in ambulatory settings.

OBJECTIVES OF PROGRAM/INTERVENTION (NO MORE THAN THREE OBJECTIVES): To improve patient safety through implementation of CUSP in an academic primary care practice.

DESCRIPTION OF PROGRAM/INTERVENTION, INCLUDING ORGANIZATIONAL CONTEXT (E.G. INPATIENT VS. OUTPATIENT, PRACTICE OR COMMUNITY CHARACTERISTICS): CUSP is a structured, team-based approach to identify and address local patient safety concerns. Team members are selected to ensure representation of all the roles in a clinical area and have equal weight in CUSP decisionmaking. All team members receive training in the science of safety. As part of the CUSP process, all staff and providers in a clinical area are asked to identify their greatest safety concern and how it could be addressed. CUSP teams, partnered with an institutional senior executive, meet monthly to identify and address safety issues, using specific tools to identify and correct root causes. Beginning in October 2014, we implemented CUSP in an academic general internal medicine practice.

MEASURES OF SUCCESS (DISCUSS QUALITATIVE AND/OR QUANTITATIVE METRICS WHICH WILL BE USED TO EVALUATE PROGRAM/ INTERVENTION): 1. Change in safety climate as reported on the Johns Hopkins Safety Culture Assessment, which incorporates the safety climate domain of the validated Safety Attitudes Questionnaire. 2. Safety concerns identified and subsequently addressed by the CUSP team.

FINDINGS TO DATE (IT IS NOT SUFFICIENT TO STATE FINDINGS WILL BE DISCUSSED): We initially identified local safety concerns through a 2-question survey of staff and providers. Additional concerns were gathered through our event reporting system and monthly meetings. $100 \%$ of staff completed the 2-question survey. Safety concerns that were prioritized and addressed in the first year of CUSP led to the development of a standardized environmental cleaning protocol, the creation of a nurse triage procedure to address potential delays in care due to limitations in staff communications, and improvement in preparedness for acutely ill patients through tracking of those who required transfer to a higher level of care, identification of essential medical supplies, and additional provider training. Twenty-three of $25(92 \%)$ and 25 of $26(96 \%)$ providers and staff in the practice completed the Safety Culture Assessment prior to and 6 months following implementation of CUSP, respectively. Compared to baseline, following CUSP implementation, survey respondents were more likely to report knowledge of the proper channels for questions about patient safety ( $96 \%$ versus $77 \%$ ), to feel encouraged to report safety concerns ( $76 \%$ versus $52 \%$ ), to believe that the work setting made it easy to learn from the errors of others ( $64 \%$ versus $45 \%$ ), and to report that medical errors were handled appropriately ( $77 \%$ versus $67 \%$ ). The remaining safety climate domains did not change. The overall safety climate domain score increased slightly (64\% versus $57 \%$ ). KEY LESSONS FOR DISSEMINATION (WHAT CAN OTHERS TAKE AWAY FOR IMPLEMENTATION TO THEIR PRACTICE OR COMMUNITY?): Implementation of CUSP likely contributed to observed improvements in safety culture. CUSP provided a structured forum to address safety concerns identified by staff. Engagement of staff and providers and support from key leadership positions were essential to our practice improvements. Including all staff early in the CUSP process and having a strong clinical leader may be key factors to ensure ongoing engagement. Our primary challenges have been difficulty getting data reports, time constraints, and issues with sustaining multiple interventions and improvements in one practice.

IMPLEMENTING A CLINIC-COMMUNITY PARTNERSHIP TO PROMOTE FRUIT AND VEGETABLE CONSUMPTION AMONG FOOD INSECURE PATIENTS WITH HYPERTENSION IN SAFETY NET CLINICS Kakul Joshi ${ }^{1}$; Samantha Smith ${ }^{3}$; Erika Trapl ${ }^{1}$; Shari Bolen ${ }^{2},{ }^{1} .{ }^{1}$ Case Western Reserve University, Cleveland, OH; ${ }^{2}$ MetroHealth/Case Western Reserve University, Cleveland, OH; ${ }^{3} \mathrm{Cuya}-$ hoga County Board of Health, Parma, OH. (Control ID \#2468672)

STATEMENT OF PROBLEM OR QUESTION (ONE SENTENCE): Although prior studies linking patients with farmers' markets (FM) have shown improvements in consumption of fruits and vegetables (FV) in select populations, less is known regarding successful implementation strategies for program delivery within safety net clinics

OBJECTIVES OF PROGRAM/INTERVENTION (NO MORE THAN THREE OBJECTIVES): 1. Leverage hypertension visits, using produce prescriptions, to encourage patient behavior change and linkage to community resources for fruits and vegetables. 2. Evaluate intervention effectiveness on utilization of farmers' markets and change in fruit and vegetable consumption over 2-3 months.

DESCRIPTION OF PROGRAM/INTERVENTION, INCLUDING ORGANIZATIONAL CONTEXT (E.G. INPATIENT VS. OUTPATIENT, PRACTICE OR COMMUNITY CHARACTERISTICS): As part of an innovative communityclinic partnership, members of a county-wide health collaborative (called the Health Improvement Partnership - Cuyahoga) worked with 3 safety net clinics to implement and evaluate a produce prescription program (PRx). Each clinic received assistance to: 1) develop a process flow chart for referral of adults with hypertension and food insecurity to the program, 2) integrate the program into their clinics using the electronic health record (EHR) for easy appointment scheduling and blood pressure (BP) measurement follow-up, and 3) leverage the role of non-physician team members to implement the program. In a $2 \mathrm{~h}$ training, eight non-physician staff (1-3 per clinic), including a pharmacist, pharmacy technician, medical assistants, and care coordinators, were trained on PRx implementation. A subsequent $1 \mathrm{~h}$ meeting collaboratively established a clinic workflow for patient enrollment, scheduling, and follow-up. During the 2015 market season, a convenience sample of 224 patients with diagnosed hypertension who screened positive for food insecurity were enrolled (74-75 per clinic). The intervention included monthly: 1) BP measurement; 2) goal setting of FV consumption; 3) education around purchasing and using FV from farmers' markets; and 4) four \$10 vouchers per visit for purchase of produce at farmers' markets, for 3 months.

MEASURES OF SUCCESS (DISCUSS QUALITATIVE AND/OR QUANTITATIVE METRICS WHICH WILL BE USED TO EVALUATE PROGRAM/ INTERVENTION): - The percent of patients visiting a FM during the program and the average number of FM visits (per redemption data of vouchers redeemed at one of 22 FM) -Total and average produce voucher redemption among program participants Change in participant self-report of FV consumption from baseline to post implementation (2-3 month follow-up) per validated food behavior survey FINDINGS TO DATE (IT IS NOT SUFFICIENT TO STATE FINDINGS WILL BE DISCUSSED): Of the 224 participants, most were female (72\%), African American ( $97 \%$ ), and middle-aged to older adults (mean age 62 years). Over half (62\%) had a high school level education or below. Preliminary results indicate that 186 participants ( $83 \%$ ) 
visited a farmers' market at least once and utilized a total of $\$ 13,000$ worth of PRx vouchers to date. Average number of FM visits among program participants was 2 (range $0-6$ ) and average number of $\$ 10$ vouchers redeemed was 6 (range 0-12). Among the subsample with pre and post survey data $(N=140)$, significant improvement in FV consumption was observed (mean score at baseline $=15.3$, mean score at follow-up $=19.2$; $p<0.001)$

KEY LESSONS FOR DISSEMINATION (WHAT CAN OTHERS TAKE AWAY FOR IMPLEMENTATION TO THEIR PRACTICE OR COMMUNITY?): The PRx program is a powerful and feasible model for linking safety net clinics with local farmers' markets to improve fruit and vegetable consumption among food insecure adult patients with hypertension.

IMPLEMENTING GUIDELINE-BASED INDICATIONS FOR CARDIAC MONITORING AT CEDARS-SINAI MEDICAL CENTER Charles E. Coffey ${ }^{1}$; Robert I. Goodman $^{1}$; Kristy Chang ${ }^{2}$; Francis E. Dailey ${ }^{1}$; Todd Griner ${ }^{1}$; Teryl K. Nuckols ${ }^{1} .{ }^{1}$ Cedars Sinai Medical Center, Los Angeles, CA; ${ }^{2}$ Cedars Sinai Medical Delivery Network, Los Angeles, CA. (Control ID \#2443633)

STATEMENT OF PROBLEM OR QUESTION (ONE SENTENCE): Cardiac monitoring $(\mathrm{CM})$ is often overused, hindering patient mobility, triggering unnecessary cardiac testing, delaying appropriate discharge, and expending resources.

OBJECTIVES OF PROGRAM/INTERVENTION (NO MORE THAN THREE OBJECTIVES): To reduce the days that medical and surgical ward patients spend on $\mathrm{CM}$ without an indication specified in the 2004 American Heart Association (AHA) guidelines for CM.

DESCRIPTION OF PROGRAM/INTERVENTION, INCLUDING ORGANIZATIONAL CONTEXT (E.G. INPATIENT VS. OUTPATIENT, PRACTICE OR COMMUNITY CHARACTERISTICS): At Cedars-Sinai, only $59 \%$ of patients on $\mathrm{CM}$ had an AHA-approved indication for CM. Physicians were able to order CM without specifying an indication or stop date, and could keep patients on $\mathrm{CM}$ indefinitely. Prior efforts to reduce unnecessary $\mathrm{CM}$ use, including academic detailing, had not been effective. Using our electronic medical record, Epic ${ }^{\mathrm{TM}}$, we implemented a modified version of the AHA guidelines throughout the all adult medical-surgical units. Providers now prescribe $\mathrm{CM}$ by selecting a clinical indication, as they would for any diagnostic test. Each clinical indication has an associated duration $-24 \mathrm{~h}, 48 \mathrm{~h}$, or until the provider stops the CM. Four hours prior to the assigned expiration for time-limited CM orders, both the ordering physician and bedside nurse receive an alert. Physicians have the option to renew the $\mathrm{CM}$, discontinue the $\mathrm{CM}$, or defer to another provider. Nurses are offered similar options, as well as instructions to assess clinical stability. If the patient has unstable vital signs or a new or worsening arrhythmia, the nurse can renew the CM. Although a physician's response to the alert overrides the nurse's response, if the patient is stable and the physician has not reordered the $\mathrm{CM}$, the nurse can discontinue it. Nurses may also defer to the patient's primary nurse.

MEASURES OF SUCCESS (DISCUSS QUALITATIVE AND/OR QUANTITATIVE METRICS WHICH WILL BE USED TO EVALUATE PROGRAM/ INTERVENTION): Our measures are: Outcome Measures: 1. Percent of bed days with active "Initiate Cardiac Monitoring (CM)" order 2. Duration of CM Process Measures: 1. Percent of patients on CM that had CM prescribed using CM Order Panel 2. Description of provider type (e.g., Internist, Surgeon, Nephrologist) ordering CM 3. Percent of CM orders by duration category (e.g., $24 \mathrm{~h}, 48 \mathrm{~h}$, or indefinite) and clinical indication provided for CM (e.g., chest pain, syncope) 4. Percent of alerts acted on by Physicians and Nurses and a description of their responses (e.g, renew CM, allow $\mathrm{CM}$ to expire) Balancing Measures: 1. Percent of Rapid Response and Code Blue calls that occur on non-CM patients and $\mathrm{CM}$ patients
FINDINGS TO DATE (IT IS NOT SUFFICIENT TO STATE FINDINGS WILL BE DISCUSSED): To date, physicians have received 403 alerts on 143 patients and nurses have received 1657 alerts on 185 patients that time-limited CM orders were expiring. No alerts resulted in a physician or nurse continuing the CM. Physicians discontinued the CM after 58 alerts (14.4\%), deferred to the primary physician on 72 alerts (17.9\%), and exited 273 alerts (67.7\%) without a response. Nurses discontinued the $\mathrm{CM}$ after 71 alerts (4.3\%), deferred to the primary nurse on 10 alerts $(0.6 \%)$, and exited 1576 alerts $(95.1 \%)$ without a response. In total, this process ensured that CM was timelimited on 129 of 328 patients (39.3\%). Neither physicians nor nurses have voiced concerns about effects on patient safety or a time burden associated with responding to the alerts. Collection of data on reasons for $\mathrm{CM}$, total number of patients on $\mathrm{CM}$, total time patients spend on CM, and safety related to time-limited CM (e.g., codes blue, rapid responses) is ongoing. Further investigation of reasons for exiting the alert without action is needed.

KEY LESSONS FOR DISSEMINATION (WHAT CAN OTHERS TAKE AWAY FOR IMPLEMENTATION TO THEIR PRACTICE OR COMMUNITY?): Based on data obtained to date, using a clinical decision support intervention to implement the AHA guidelines on CM has been acceptable to physicians and nurses, and has triggered clinically appropriate discontinuations of CM, in accordance with the 2004 AHA guidelines.

IMPROVED RATES OF VENOUS THROMBOEMBOLISM PROPHYLAXIS EVALUATION USING FAILURES ANALYSIS, DATA COLLECTION OPTIMIZATION, AND DECISION SUPPORT Sarah Baron ${ }^{2}$; Charles Abam ${ }^{1}$; Tulay Aksoy²; Jeffrey Ceresnak ${ }^{3}$; Regina Cregin ${ }^{3}$; Jessica A. Dekhtyar ${ }^{1}$; Jacqueline T. Hunter ${ }^{1}$; Geetali Mohan $^{3}$; Jacqueline Myrie Weir ${ }^{1}$; Rafael Ruiz ${ }^{1}$; Peter Shamamian ${ }^{1}$; Mark Sinnet ${ }^{3}$; Brandon G. Yongue ${ }^{1}$; William Southern ${ }^{1} .{ }^{1}$ Montefiore, Bronx, NY; ${ }^{2}$ Montefiore, New York, NY; ${ }^{3}$ Montefiore Medical Center, Bronx, NY. (Control ID \#2469084)

STATEMENT OF PROBLEM OR QUESTION (ONE SENTENCE): Can data collection optimization and improved decision support improve our performance on the federal Venous Thromboembolism Prophylaxis evaluation measure?

OBJECTIVES OF PROGRAM/INTERVENTION (NO MORE THAN THREE OBJECTIVES): 1 . To examine our failures of Venous Thromboembolism (VTE) prophylaxis evaluation in order to enhance targeting of interventions 2 . To determine if optimization of data collection with subsequent improved decision support would affect the federally reported rate of VTE prophylaxis evaluation at our institution 3. To determine if these interventions would affect our overall outcome measure, the rate of hospital acquired venous thromboembolism.

DESCRIPTION OF PROGRAM/INTERVENTION, INCLUDING ORGANIZATIONAL CONTEXT (E.G. INPATIENT VS. OUTPATIENT, PRACTICE OR COMMUNITY CHARACTERISTICS): Beginning in 2013, rates of evaluation for VTE prophylaxis became a federally reported Core Measure by the Joint Commission. A patient encounter complied if the patient received pharmacologic or mechanical prophylaxis, had documented contraindications to both of these types of prophylaxis, or was documented to be at low risk for VTE, thereby not requiring any prophylaxis. Reported rates for the Montefiore Medical Center, a large, urban, academic institution in the Bronx, were collected from the three main hospital sites. Our first step was to measure and analyze all failures from 2013 in order to focus our interventions. Informed by this failures analysis, the first intervention was optimization of data collection by our abstraction team, who manually abstract and report data to the federal government. Through meetings and education of this team, we were able to ensure that the abstractors were capturing all current VTE prophylaxis evaluation. The second intervention created high impact changes to existing electronic decision support. We partnered with the Pharmacy Department in 
order to simplify prophylaxis regimens and embed eligibility for each regimen within the decision support itself. Working with our IT Department, we altered the standard language in our decision support so that provider documentation of "Low Risk" assessment was consistently compliant with federal guidelines.

MEASURES OF SUCCESS (DISCUSS QUALITATIVE AND/OR QUANTITATIVE METRICS WHICH WILL BE USED TO EVALUATE PROGRAM/ INTERVENTION): Our main outcome measure was the federally reported VTE prophylaxis evaluation rate.

FINDINGS TO DATE (IT IS NOT SUFFICIENT TO STATE FINDINGS WILL BE DISCUSSED): In the almost 2 years since initiation of our interventions, we were able to increase our rate of Venous Thromboembolism Prophylaxis evaluation from a preintervention baseline of 71-73\% in early 2013 to $86-90 \%$ following data collection optimization in Quarter 4 of 2013 and $85-95 \%$ following the decision support change in Quarter 4 of 2014. While the complete data on rates of Venous Thromboemboli are not yet available, rates of Venous Thromboemboli without evaluation for prophylaxis are down to $0 \%$ for the first time in the first half of 2015 , further supporting the medical validity of this quality improvement initiative.

KEY LESSONS FOR DISSEMINATION (WHAT CAN OTHERS TAKE AWAY FOR IMPLEMENTATION TO THEIR PRACTICE OR COMMUNITY?): Early identification of our largest areas of failure led to a targeted approach, leading to the greatest possible impact from the least resources. Using a multi-step sequential intervention allowed us to visualize our impact while still making improvements throughout the intervention period. The use of a multi-disciplinary team was crucial to our success. This model of failures analysis with subsequent targeted intervention in a multi-disciplinary setting will be applied to future quality improvement interventions in order to maximize impact.

IMPROVING ACCESS TO CARE BY ADMISSION PROCESS REDESIGN IN A VETERANS AFFAIRS NURSING HOME Amy W. Baughman ${ }^{2}$; Marcus D. Ruopp ${ }^{1}$; Juman $\mathrm{Hijab}^{2}$; Thomas A. Parrino ${ }^{2}$; Manuela Ferreira ${ }^{2}$; Steven R. Simon ${ }^{3}{ }^{1}$ VA Boston, Brockton, MA; ${ }^{2}$ VA Boston Healthcare System, Cambridge, MA; ${ }^{3}$ VA Boston Healthcare System, Boston, MA. (Control ID \#2469900)

STATEMENT OF PROBLEM OR QUESTION (ONE SENTENCE): Improve admission process efficiency and enhance access to nursing home care. OBJECTIVES OF PROGRAM/INTERVENTION (NO MORE THAN THREE OBJECTIVES): 1) Decrease response time for admission requests 2) Standardization and automation of the work flow processes 3) Optimize resource utilization: transition screening role from physician to nursing leadership and improve bed tracking system to proactively fill available beds

DESCRIPTION OF PROGRAM/INTERVENTION, INCLUDING ORGANIZATIONAL CONTEXT (E.G. INPATIENT VS. OUTPATIENT, PRACTICE OR COMMUNITY CHARACTERISTICS): This project was initiated in October 2015 in the Veterans Affairs (VA) Boston Community Living Center (CLC) and will end in February 2016. The CLC is a 105 bed facility that includes a 60 bed transitional care unit, a 15 bed hospice unit, and a 30 bed longterm care unit. The CLC admits VA patients from the VA Boston Hospital, other regional VA and non-VA facilities. Admission is by electronic request through the electronic health record (EHR), telephone, fax or pager request. Each approach requires written and/or verbal communication to verify patient readiness and bed availability, a dynamic process requiring continuous update and communication. In addition, the volume of admission requests has risen from an average of 37 per month in 2014 to 65 per month in 2015, further driving the need for efficiency. An admission request has had variable response times, ranging from $10 \mathrm{~min}$ to $3 \mathrm{~h}$, with an additional delay of 1 to 4 days for actual admission completion. The multiple communication modes for requests were difficult to manage, and the process for bed and provider assignment was unstandardized. The process was also managed by a physician, thereby reducing physician availability for direct patient care. Redesign Strategy: We first created a Task Force with provider and nursing leadership that established goals with initial process mapping. We then had biweekly follow up meetings for feedback and process improvement. Initial outcomes: 1. Created a new admission liaison role with clear work flow plan 2. Transitioned prior role from physician to Unit Nurse Managers 3. Created a Provider Capacity Committee to evaluate census and ensure equitable and manageable admission assignment 4 . Simplified documentation: revised admission request tracking list, replaced 16 available admission requests with 1 comprehensive template 5 . Simplified screening process with standardized admission criteria 6 . Daily huddles to review bed flow and admission tracking 7. Creation of 2 pathways: Simple electronic process with immediate response and Complex Interdisciplinary Team Review

MEASURES OF SUCCESS (DISCUSS QUALITATIVE AND/OR QUANTITATIVE METRICS WHICH WILL BE USED TO EVALUATE PROGRAM/ INTERVENTION): Quantitative Evaluation: - Number and type of admissions requests -Time to response for admission requests -Time to admission after request is accepted Days on VA "Delay" List at referring VA hospitals Qualitative feedback: Biweekly task force meetings and discussions to explore challenges and plan additional improvement. FINDINGS TO DATE (IT IS NOT SUFFICIENT TO STATE FINDINGS WILL BE DISCUSSED): 1. Nurses Managers can rapidly learn to be effective screeners for nursing home care (1-2 weeks) 2. Unanticipated outcome of rapid review and patient acceptance resulted in many cancellations (bed requests were being placed before patients were medically ready) - subsequently limited requests to patients who are ready within $48 \mathrm{~h}$. 3. Transitioning screening role from a single physician to a team of rotating nurse managers resulted in a prolonged learning period with high variation in screening practices despite attempted standardization of overall process 4 . Controlling access to a limited resource (transitional care bed) can be highly stressful for screeners who function as gatekeepers; e.g. declining patients who may not be appropriate transitional patients but have no alternative options (e.g. homeless and unable to care for self)

KEY LESSONS FOR DISSEMINATION (WHAT CAN OTHERS TAKE AWAY FOR IMPLEMENTATION TO THEIR PRACTICE OR COMMUNITY?): 1. Early investment to define the problem and set goals as a group enabled clear action plans 2 . Exploring pain points identified new important problems - for example, we learned that provider availability for admissions was a rate limiting step 3. Structured biweekly meetings with task delegation are critical for ongoing process improvement 4 . Building in continuous modification into our process redesign can ease tough transitions

IMPROVING BLOOD PRESSURE CONTROL IN A LARGE URBAN SAFETY NET ACADEMIC CENTER Andrew A. Chang ${ }^{1,2}{ }^{2}$; Teresa Binamira ${ }^{1}$; Anayo Ikeme ${ }^{1}$; Melissa S. Lee ${ }^{1,2}$; Desiree Lyttle ${ }^{1}$; Malcolm Pinkney ${ }^{1}$; David Stevens ${ }^{1,2}$. ${ }^{1}$ Health + Hospitals Kings County, New York, NY; ${ }^{2}$ SUNY Downstate, Brooklyn, NY. (Control ID \#2446682)

STATEMENT OF PROBLEM OR QUESTION (ONE SENTENCE): How can a large urban safety net academic center improve its hypertensive population's blood pressure control rates?

OBJECTIVES OF PROGRAM/INTERVENTION (NO MORE THAN THREE OBJECTIVES): To describe our multi-pronged collaborative team-based approach toward improving blood pressure control in a safety net institution in New York City. DESCRIPTION OF PROGRAM/INTERVENTION, INCLUDING ORGANIZATIONAL CONTEXT (E.G. INPATIENT VS. OUTPATIENT, PRACTICE OR COMMUNITY CHARACTERISTICS): Our adult primary care practice is part of large urban academic safety-net institution in Central Brooklyn, NY serving a primarily Afro-Caribbean population with low socioeconomic status. 15,163 of our patients have hypertension. In March 2015, only $56.4 \%$ of those patients had their blood pressures in 
control, below both national and local safety net benchmarks. At that time, our population based approach to hypertension management included a hypertension registry, RN-driven "Hypertension Treat to Target" program with low rates of patient engagement (revisit show rates of $38 \%$ ), and varied provider prescribing practices. Although patients who worked with RNs in the Treat to Target Program showed improved blood pressure control, low show rates were a barrier to success. To improve revisit show rates, we implemented "warm handoffs" with an RN. Patients met with their team RN at the time of PCP referral. They received education on self-management, including adherence and home blood pressure monitoring. Monitors and blood pressure logs were distributed as needed. To reduce variation in prescribing practices, increase usage of combination pills, and improve communication in PCP cross-coverage, a patient-centered evidence-based hypertension treatment algorithm was developed. Providers and nurses were educated on the usage of the algorithm, and warm handoffs.

MEASURES OF SUCCESS (DISCUSS QUALITATIVE AND/OR QUANTITATIVE METRICS WHICH WILL BE USED TO EVALUATE PROGRAM/ INTERVENTION): Our overall hypertension control rate is calculated using a registry linked to our electronic health record. We also used show rates and patient satisfaction surveys to evaluate success.

FINDINGS TO DATE (IT IS NOT SUFFICIENT TO STATE FINDINGS WILL BE DISCUSSED): Over a period of 6 months, the overall hypertension control improved from 56.4 to $60.5 \%$ (over 750 additional controlled patients), the hypertension revisit show rate improved from 38 to $53 \%$, and patient satisfaction surveys showed unanimously positive feedback with patients emphasizing their feelings of empowerment in controlling their own blood pressures.

KEY LESSONS FOR DISSEMINATION (WHAT CAN OTHERS TAKE AWAY FOR IMPLEMENTATION TO THEIR PRACTICE OR COMMUNITY?): Medication non-adherence is a leading cause of uncontrolled hypertension. Patients who remain non-adherent despite repeated advice from a trusted primary care physician need something more. These patients may have deep personal barriers to engaging in self-management. Our results suggest that establishing a therapeutic relationship with their team RN may be the right approach. Our RNs explored patients' feelings about medication and made a commitment to work with them over time to help them reach their blood pressure target. Just a single brief visit with the RN immediately after the PCP visit led to improved engagement in the program. Conversations about chronic disease allowing for discussions of medication adherence, side effects, goal setting and healthy lifestyle are an integral part of managing illness in a primary care setting. Unfortunately, practices may not support these therapeutic conversations because of limited resources and time pressures, especially in an urban safety net practice. Conversations forged a patient-RN relationship, connecting patients not only to their primary care providers, but also the primary care team. This was an effective way of improving blood pressure control in our population. Our interventions were budget neutral, though did require additional time for nurses to perform the warm handoff encounters. However, decreasing the number of patients with uncontrolled blood pressure has lessened the burden of RN population outreach to this group. On analysis of reasons for missed appointments, we found that the most common issue was work related or forgetting. An even larger proportion of patients were unable to be contacted via phone for follow-up or reminder calls. We are hoping to do a followup analysis of changes in prescribing practices, looking at \#s of combination pills used, and percentage of patients on first line therapies.

IMPROVING CHLAMYDIA SCREENING IN AN ACADEMIC PRIMARY CARE CLINIC Swati Shroff ${ }^{1,}{ }^{2}$; Kwonho Jeong ${ }^{1}$; Doris Rubio ${ }^{1}$; Anna K. Donovan ${ }^{1}$; Elian A. Rosenfeld ${ }^{2}$; Gary Fischer ${ }^{1}$. ${ }^{1}$ University of Pittsburgh, Pittsburgh, PA; ${ }^{2}$ VA Pittsburgh Healthcare System, Pittsburgh, PA. (Control ID \#2431816)
STATEMENT OF PROBLEM OR QUESTION (ONE SENTENCE): The USPSTF recommends chlamydia screening in sexually active women $\leq 24$ years of age; however, only $43 \%$ of sexually active women $\leq 24$ years of age were screened for chlamydia last year in an academic primary care clinic (PCC).

\section{OBJECTIVES OF PROGRAM/INTERVENTION (NO MORE THAN THREE}

OBJECTIVES): Our intervention aims to improve adherence to annual chlamydia screening in sexually active women $\leq 24$ years of age. A secondary aim is to determine if there are any patient or physician characteristics associated with adherence to screening. DESCRIPTION OF PROGRAM/INTERVENTION, INCLUDING ORGANIZATIONAL CONTEXT (E.G. INPATIENT VS. OUTPATIENT, PRACTICE OR COMMUNITY CHARACTERISTICS): In December 2014, we formed a quality improvement (QI) team, including general internists with women's health expertise, a medical assistant, a nurse, and QI experts. From December 2014 through January 2015, our team discussed potential barriers to chlamydia screening and interventions aimed at overcoming these barriers. The team identified and prioritized the top 3 barriers to chlamydia screening: 1) Providers do not think to screen because it is not in the Health Maintenance (HM) tab in the electronic medical record (EMR); 2) Younger patients may be more likely to be seen for acute visits with urgent care providers where prevention is not typically addressed; 3 ) Providers do not know urine screening is available. The team then identified the top 3 potential interventions to address these barriers: 1) Implement a Best Practice Advisory with a built-in order set in the EMR; 2) Add chlamydia screening to the HM tab in the EMR; 3) Collect urine on all women $\leq 24$ years of age at every visit. In February and April 2015, we provided education on chlamydia screening and presented potential interventions to physicians and ancillary staff. Providers preferred the 2nd intervention to add chlamydia screening to the HM tab in the EMR. Because of time and resource limitations, chlamydia screening could not be added automatically to the HM tab. Instead, beginning in May 2015, a team member manually added chlamydia screening to the HM tab through a weekly run report within the EMR for female patients $\leq 24$ years of age with appointments in the upcoming week.

MEASURES OF SUCCESS (DISCUSS QUALITATIVE AND/OR QUANTITATIVE METRICS WHICH WILL BE USED TO EVALUATE PROGRAM/ INTERVENTION): The primary outcome of interest is the rate of sexually active females $\leq 24$ years of age screened for chlamydia 1 year after intervention implementation. We will also determine whether there are any patient (age, race, insurance) or physician (sex, role) characteristics associated with chlamydia screening. As a process measure, we recorded the percentage of female patients $\leq 24$ years of age who were seen in clinic and had chlamydia screening added to the HM tab in the EMR to determine how many patients were missed by the weekly manual intervention.

\section{FINDINGS TO DATE (IT IS NOT SUFFICIENT TO STATE FINDINGS WILL} BE DISCUSSED): An interim analysis was completed after 4 months of intervention implementation. Seventy-five percent of women seen in clinic had the reminder added to the HM tab; the remaining $25 \%$ did not because their appointments were made after the weekly manual intervention. Of 547 women $\leq 24$ years of age seen prior to the intervention, $436(80 \%)$ had a sexual history taken, and of these women, 305 (70 \%) reported they were sexually active. Of the women who reported being sexually active, mean age was 22 $(\mathrm{SD}=1.8)$. Most were white $(65 \%)$ and privately insured $(82 \%)$. Most had a female primary care physician $(80 \%)$ in an attending role $(71 \%)$. About half had only 1 visit to the PCC in the prior year. At baseline 130 (43\%) sexually active women $\leq 24$ years of age were screened for chlamydia in the prior year. Women were significantly more likely to be screened for chlamydia if they were black $(P<0.048)$, had more than 1 visit to the PCC in the prior year $(P=0.006)$, or if they had a gonorrhea test in the prior year $(P<0.001)$. Of 124 sexually active women $\leq 24$ years of age seen after 4 months of intervention implementation, 60 (48 \%) were screened for chlamydia, but the difference from baseline was not significant $(P=0.276)$. Specimen source could be ascertained for $27(33 \%)$ chlamydia tests. Prior to the intervention, 6 tests $(8 \%)$ vs. after the intervention 16 tests (31\%) were urine specimens, but this was not significant $(P=0.056)$. 
KEY LESSONS FOR DISSEMINATION (WHAT CAN OTHERS TAKE AWAY FOR IMPLEMENTATION TO THEIR PRACTICE OR COMMUNITY?): After 4 months of implementation of a QI intervention including physician and ancillary staff education and an EMR HM reminder, there were trends towards increasing chlamydia screening rates and use of urine tests. The weekly manual process of adding chlamydia screening to the HM tab in the EMR missed about $25 \%$ of patients eligible for screening; we plan to automatically add the reminder for all female patients $\leq 24$ years of age in the future. We will also encourage providers to document specimen source when placing chlamydia test orders. After 1 year of intervention implementation, we will reassess all outcomes.

IMPROVING COMMUNICATION ABOUT DELAYS TO PATIENTS IN A BUSY INTERNAL MEDICINE CLINIC Elizabeth Greig $^{3}$; Shana Ratner ${ }^{1}$; Christina McMil$\operatorname{lan}^{2}$; Brandon A. Oliver ${ }^{3}$; Karmen Little ${ }^{3}$; Derek Cain ${ }^{3}$; Tamrah Parker ${ }^{3} .{ }^{1}$ UNC Chapel Hill, Chapel Hill, NC; ${ }^{2} \mathrm{UNC}$ Health Care, Carrboro, NC; ${ }^{3}$ University of North Carolina, Chapel Hill, NC. (Control ID \#2468679)

STATEMENT OF PROBLEM OR QUESTION (ONE SENTENCE): Patient satisfaction surveys routinely assessed our clinic below goal in providing information on provider delays during visits to the clinic.

OBJECTIVES OF PROGRAM/INTERVENTION (NO MORE THAN THREE
OBJECTIVES): 1 . To analyze points of patient contact that could be used as an opportunity to communicate about delays 2 . To create and test a system by which providers, nursing, and staff communicate to each other about delays 3. To identify opportunities for patients to reach out for information about delays

DESCRIPTION OF PROGRAM/INTERVENTION, INCLUDING ORGANIZATIONAL CONTEXT (E.G. INPATIENT VS. OUTPATIENT, PRACTICE OR COMMUNITY CHARACTERISTICS): The UNC Internal Medicine Clinic is a large practice, caring for over 12,000 patients by over 80 mostly part-time providers, including attendings, residents and advanced practice providers. A national standardized survey is used across the hospital system to measure patient satisfaction. The survey is sent to patients following their visit to the clinic and consists questions for patient to rate on Likert Scales and opportunity for free form comments. While scores in the outpatient general medicine clinic are regularly above goal across all topic domains, communication about delays has stably been the clinic's lowest patient satisfaction metric. Numeric results and comments from the surveys were analyzed for specific complaints regarding delays for 1 year prior to implementing systematic solutions. Patients described both waiting in the waiting room, and in clinic rooms after being triaged without further information on wait times. A root cause analysis and brainstorming about solutions to address these root causes was performed at a meeting of all clinic providers, nursing and front desk staff. We purposefully limited the scope of the project to communication strategies. Reducing patient wait times was beyond the scope of the assessment and was not addressed in the intervention. Two types of intervention were determined to be necessary - one that could be initiated by the patient, and one that could be initiated by staff. An initial intervention was signage in both the waiting room and in individual exam rooms, encouraging patients to ask a staff member if they had been waiting longer than 20 min following their scheduled appointment time. Signage in the exam rooms directed patients to place a door hanger on the outside of the exam room door if they wanted information about a delay. Any nurse or provider available was instructed to check on the patient if they saw a door hanger outside a room. A mock interaction was staged by a doctor, nurse and front desk staff. This exercise highlighted the physical barriers between those three roles in our clinic, and an opportunity was recognized to use visual recognition messaging through the electronic health record (EHR) visible to all three job functions. Nursing or the provider could mark the mutual schedule with a yellow dot, communicating a delay of more than
$20 \mathrm{~min}$ to the front desk, which could then be conveyed to patients as they checked in. Finally, a whiteboard was placed facing the waiting room with updated information on expected durations of provider delays.

MEASURES OF SUCCESS (DISCUSS QUALITATIVE AND/OR QUANTITATIVE METRICS WHICH WILL BE USED TO EVALUATE PROGRAM/ INTERVENTION): Provider satisfaction scores are reported as an overall nationwide percentile rank for like institutions, as well as scores by subcategory. The subcategory of focus here is "Moving Through the Visit" which is further broken down into two categories - "Wait Times" and "Information About Delays." Percentile rank for overall score, "Moving through the Visit" and "Information About Delays" was measured at each date of the stepwise rollout of all five components of the intervention and change was monitored. Comments will be mined for specific patient feedback about the new processes as they become available.

FINDINGS TO DATE (IT IS NOT SUFFICIENT TO STATE FINDINGS WILL BE DISCUSSED): Baseline patient satisfaction scores for the category "Information about Delays" have typically remained around 45th percentile for our clinic, under the goal of 66th percentile. A nadir at 40th percentile on 9/21/2015 prompted the root cause analysis. All five components of the intervention were rolled out individually approximately 1 month apart between the date of the analysis and 1/6/2016. Patient satisfaction data is made available weekly, and a corresponding increase in percentile rank from 40th percentile to the 65 th percentile occurred up to $1 / 4 / 2016$, with notable improvements in scores after each component of the intervention was added. Further, the metric encompassing both patient satisfaction on wait times and information about delays increased from the $63 \mathrm{rd}$ percentile to the 77 th percentile, without any intervention on actual wait times occurring over the same period.

KEY LESSONS FOR DISSEMINATION (WHAT CAN OTHERS TAKE AWAY FOR IMPLEMENTATION TO THEIR PRACTICE OR COMMUNITY?): Delays are inevitable, but obtaining patient feedback and performing a root cause analysis specific to the layout, logistics and capabilities of a clinic can help develop a simple yet effective visual management and communication system, without incurring great expense or creating significant disruption to current clinic flow. Patients may value effective communication about delays as highly as a reduction in wait times, as reflected in the improvement in our overall score for both measures.

IMPROVING DIABETIC FOOT EXAM RATES IN A PRIMARY CARE RESIDENT CLINIC Chelsea E. Pearson ${ }^{1}$; Emily D. Fondahn ${ }^{2} .{ }^{1}$ Barnes-Jewish Hospital, St Louis, MO; ${ }^{2}$ Washington University, St Louis, MO. (Control ID \#2469700)

STATEMENT OF PROBLEM OR QUESTION (ONE SENTENCE): The American Diabetes Association recommends a comprehensive foot exam at least yearly to identify high-risk foot conditions and reduce the risk of complications; however, studies have shown that physicians are largely not compliant with these recommendations.

OBJECTIVES OF PROGRAM/INTERVENTION (NO MORE THAN THREE OBJECTIVES): * Improve performance and documentation of the diabetic foot exam to $80 \%$ * Educate residents on the components of a comprehensive foot exam and its importance * Identify impediments to performing the exam and develop strategies to overcome them

DESCRIPTION OF PROGRAM/INTERVENTION, INCLUDING ORGANIZATIONAL CONTEXT (E.G. INPATIENT VS. OUTPATIENT, PRACTICE OR COMMUNITY CHARACTERISTICS): In 2008, our primary care resident clinic began using the NCQA (National Committee for Quality Assurance) Diabetes Recognition Program to benchmark performance for diabetes care. Residents received an email with their patient information compared to clinic averages and NCQA goals. In general, resident performance improved screening for 
nephropathy, meeting LDL targets, and increasing patients receiving a yearly HbA1c. The metric for the foot exam was obtained through random chart audit done by the clinic nurses and only one metric was provided for the entire clinic. In 2012 , the clinic switched to a team structure wherein residents are part of a team of about 16 other residents. Residents were sent quarterly data with the team's information, along with the whole clinic's overall performance. The following year, residents were asked to complete the chart audits for the diabetic foot exam during their QI time on clinic block. This academic year the PGY2 \& PGY3 residents perform the chart audit for patients they have seen; data on completion of the audit and performance on the exam becomes part of their evaluations. We have also focused on identifying barriers to performing and/or documenting the diabetic foot exam. We conducted a random chart audit to identify if the foot exam was done and which, if any, components of the exam were missing. We surveyed the residents to identify barriers to performing the diabetic foot exam. We have been providing education to residents regarding how to complete a foot exam and improve foot care for patients with diabetes.

MEASURES OF SUCCESS (DISCUSS QUALITATIVE AND/OR QUANTITATIVE METRICS WHICH WILL BE USED TO EVALUATE PROGRAM/ INTERVENTION): * Chart audits to determine the percentage of diabetic foot exams performed and documented correctly by the residents on a monthly basis *Ongoing: educational module with pre- and post-tests for residents to test their knowledge on the diabetic foot exam and its importance

FINDINGS TO DATE (IT IS NOT SUFFICIENT TO STATE FINDINGS WILI BE DISCUSSED): *Baseline performance across all teams (determined by whether the exam was both conducted and appropriately documented) was $41 \%$ * Team chart audits (2014): The overall response rate for completing the chart audits was $68.53 \%$ (range $37.93-82.76 \%$ ). The clinic average for foot exams over this year was $60.85 \%$; the teams ranged from 49 to $67 \%$. Individual chart audits (2015): $100 \%$ of PGY2 and PGY3 residents submitted data. The current rate is $56 \%$ (STD $26 \%$, range $0-100 \%$ ). These numbers may be artificially low because in 2015, the definition of a complete foot exam changed to include pulses * Random chart audit (2015): $50 \%$ of charts had a correctly documented foot exam, $25 \%$ documented an exam but in the plan instead of physical exam section of the note, $16.6 \%$ did not document pulses, and $8.4 \%$ had no foot exam in the last year. * Resident survey (2015): $40 \%$ (67/167) of residents completed the survey; the majority identified the following barriers: not having enough time, monofilaments not available in the exam room, and simply forgetting to perform the exam or not knowing when it was due. These future interventions were perceived as most likely to be helpful: $70 \%$ said having nurses ask patients to remove shoes/socks, $44 \%$ said increased access to the monofilaments; and $21 \%$ said provider education. $31 \%$ commented on the requirement to document the exam in the physical exam section of the note as many accidentally placed this information in the plan. $59 \%$ of residents thought that aiding a 'diabetic foot exam' template would be helpful.

KEY LESSONS FOR DISSEMINATION (WHAT CAN OTHERS TAKE AWAY FOR IMPLEMENTATION TO THEIR PRACTICE OR COMMUNITY?): *

Documenting the exam is a barrier. We plan to develop a 'diabetic foot' template for the physical exam to improve documentation. * The majority of residents believe that systems factors drive poor performance more than lack of knowledge or desire to perform the exam, * Most interventions had a measurable and beneficial effect, especially when first implemented, but creating sustained change in practice patterns is difficult

IMPROVING DIAGNOSIS AND MANAGEMENT OF HYPERTENSION: IMPLEMENTATION OF AMBULATORY BLOOD PRESSURE MONITORING IN PRIMARY CARE Jennifer Beach; Scot B. Sternberg; Tarsha Soares; Nabiha Nuruzzaman. Beth Israel Deaconess Medical Center, Boston, MA. (Control ID \#2468897)
STATEMENT OF PROBLEM OR QUESTION (ONE SENTENCE): Although 24-h ambulatory blood pressure monitoring (ABPM) provides a more accurate assessment of true blood pressure and thus cardiovascular risk, it has not historically been available to primary care practices without an outside referral to specialists in either cardiology, nephrology or endocrinology.

OBJECTIVES OF PROGRAM/INTERVENTION (NO MORE THAN THREE OBJECTIVES): 1. Implement a multidisciplinary ABPM clinic at an academic primary care practice. 2. Improve diagnosis and management of hypertension by accurately identifying patients with white coat hypertension $(\mathrm{WCH})$ versus those with sustained hypertension.

DESCRIPTION OF PROGRAM/INTERVENTION, INCLUDING ORGANIZATIONAL CONTEXT (E.G. INPATIENT VS. OUTPATIENT, PRACTICE OR COMMUNITY CHARACTERISTICS): Healthcare Associates (HCA) is a large academic adult internal medicine practice at Beth Israel Deaconess Medical Center (BIDMC) that provides comprehensive primary care for almost 42,000 patients. Approximately $30 \%$ of the patient population has been diagnosed with hypertension. The average rate for patients with hypertension whose blood pressure is in control has been $54 \%$ using JNC 7 guidelines of $<140 / 90$. Using newer JNC 8 guidelines, which stratify patients' goal blood pressures based on their age, our control rates have been $52 \%$ for ages $18-59$ years (target $<140 / 90$ ) and $73 \%$ for patients over age 60 (target $<150 / 90$ ). By offering ABPM to our patients, we hoped to identify those who needed intensification of therapy, either by lifestyle modifications or titrating their antihypertensive regimen, and avoid overtreatment of patients in whose monitoring revealed normotensive blood pressures outside of the office setting. -Available literature on ABPM manufacturers was reviewed and equipment, including monitors with cuffs ranging from small to adult XXL, was purchased along with corresponding software. -Workflow and billing logistics were and discussed with other practices using ABPM at local and national hypertension centers. -The lead physician became certified as a hypertension specialist and received specialized training in ABPM interpretation. -Nursing staff members were trained to appropriately fit patients and provide education on monitor use and function. -Collaborated with Information Support Services to develop a process to integrate results and interpretations within our electronic medical record. -Referral process, patient education materials and activity log were developed and fine-tuned.

MEASURES OF SUCCESS (DISCUSS QUALITATIVE AND/OR QUANTITATIVE METRICS WHICH WILL BE USED TO EVALUATE PROGRAM/ INTERVENTION): Measures include the number of ABPM successfully completed and percent of patients for whom ABPM results in a change in diagnosis and subsequent management. We continuously monitor practice-wide control rates for patients with hypertension to assess for an impact.

FINDINGS TO DATE (IT IS NOT SUFFICIENT TO STATE FINDINGS WILL BE DISCUSSED): We have analyzed the first 100 patients referred for ABPM (50 who were referred for suspected $\mathrm{WCH}$ and 50 who were referred for uncontrolled hypertension). Of the 50 patients referred for $\mathrm{WCH}, 43 \%$ were actually truly hypertensive. Recommendations to initiate antihypertensive medications were made for $32 \%$ of patients referred for suspected $\mathrm{WCH}$; all others received education and recommendations for lifestyle modifications and home BP monitoring. Of the 50 patients referred for uncontrolled hypertension, $47 \%$ were actually shown to have blood pressures within range. Recommendations to change the medication regimen, add medications, or increase dose made for $51 \%$ of patients referred for ABPM for uncontrolled hypertension. A decrease in medication dose was recommended for $10 \%$ of patients. $50 \%$ of patients tested were found to be "non-dippers," i.e., patients whose monitoring does not exhibit the expected $10-15 \%$ nocturnal dipping pattern. This is an independent risk for cardiovascular disease and identifies patients who, when combined with a positive score on a validated sleep apnea screening questionnaire, may benefit from further evaluation for undiagnosed sleep apnea. 
KEY LESSONS FOR DISSEMINATION (WHAT CAN OTHERS TAKE AWAY FOR IMPLEMENTATION TO THEIR PRACTICE OR COMMUNITY?): BP measures during a $24-\mathrm{h}$ cycle provides valuable information to assist providers in accurate diagnosis of hypertension and optimizing management of hypertension as well as identify those needing assessment for secondary causes. ABPM provides a way to reliable distinguish between patients thought to have WCH and patients who are truly hypertensive and need additional therapy. Half of patients thought to have uncontrolled hypertension were actually in control. Further titrating their medication regimen in an effort to lower BPs would likely result in additional side effects and worsening kidney function without providing any clinical benefit. Identifying patients with non-dipping patterns allows more tailored recommendations for medication timing/dosing and highlights patients needing further evaluation for sleep apnea or underlying renal disease. While currently not reimbursed by most major insurers, combining ABPM with a hypertension consult visit allows for interpretation and treatment recommendations to be incorporated into an office visit.

IMPROVING HANDOFF QUALITY: AN ELECTRONIC HANDOFF TOOL INNOVATION FEATURING THE “123, ABC” METHOD Sarah R. Lieber; Eric Goodman. Icahn School of Medicine at Mount Sinai Hospital, New York, NY. (Control ID \#2469716)

STATEMENT OF PROBLEM OR QUESTION (ONE SENTENCE): Many preventable medical errors stem from a breakdown in communication during transitions of care between daytime and nighttime providers; yet, it remains unclear what essential content should be communicated in high quality handoffs, and no standardized handoff tool has yet been associated with a reduction in medical errors among hospitalized adult patients OBJECTIVES OF PROGRAM/INTERVENTION (NO MORE THAN THREE OBJECTIVES): 1) To implement a novel, content-focused handoff tool in the electronic medical record used by Internal Medicine (IM) residents on the teaching services at the Icahn School of Medicine at Mount Sinai. 2) To integrate this novel tool into the electronic medical record with the goal of ensuring standardized and consistent handoffs for adult patients on our teaching services. 3) To evaluate the impact of the handoff tool on perceived handoff quality and perceived preparedness in caring for patients overnight, as well as overall rates of overnight adverse events.

DESCRIPTION OF PROGRAM/INTERVENTION, INCLUDING ORGANIZATIONAL CONTEXT (E.G. INPATIENT VS. OUTPATIENT, PRACTICE OR COMMUNITY CHARACTERISTICS): Prior to development of the new handoff tool, IM residents at our institution were surveyed about the content that should be included in a high quality handoff. $33 \%$ of 87 respondents felt that the existing handoff tool was "very poor" or "bad" and $45 \%$ reported witnessing active medical errors that they felt could have been prevented by a better handoff. Residents felt that a high-quality handoff tool should include information regarding a patient's respiratory status $(92.3 \%)$, cardiac status (84.6\%), mental status $(98.4 \%)$, code status/end-of-life goals $(100 \%)$, emergency contacts (100\%), fluid status (78.5\%), high-risk medications (89.6 \%), infection/fever plans and current antibiotics (96.9\%), as well as vascular access (95.6\%). Beginning in April 2015, we introduced a new electronic handoff tool based on this feedback. The tool uses a novel " $123, \mathrm{ABC}$ " mnemonic, which provides an easy to remember, standardized checklist of key content to be included in every patient handoff. Additionally, the tool is devised to anchor providers engaging in the handoff encounter so that information is conveyed in a more focused and closed-loop fashion. Specifically, the tool reminds providers to include an up-to-date "1-liner," a clear delineation of " 2 to-do items," and "3 must-know facts" for each patient. The "ABC" portion of the tool provides a series of system-specific suggestions regarding anticipatory guidance and important overnight reference material, based on the content suggestions from the resident survey. This mnemonic is now auto-populated in the electronic medical record for each patient in order to promote both verbal and written use of the $123, \mathrm{ABC}$ method during day to night patient handoffs.

MEASURES OF SUCCESS (DISCUSS QUALITATIVE AND/OR QUANTITATIVE METRICS WHICH WILL BE USED TO EVALUATE PROGRAM INTERVENTION): This prospective, quality improvement study of a total $132 \mathrm{IM}$ residents included surveys of overnight providers pre- $(n=71)$ and post- $(n=52)$ implementation of the novel handoff tool. More specifically, providers were asked how prepared they felt to handle specific, content-focused clinical events overnight. Level of preparedness was graded on a Likert scale for different domains (e.g. respiratory status, goals of care, high risk medications). Additionally, providers were asked to enumerate the number of unanticipated events that occurred overnight and the number of unanticipated adverse events including patient deaths and requirements for escalation of care. Ultimately, the target outcome measure is hospital adverse events or errors as measured by transfers to higher levels of care, cardiac arrests, and deaths overnight.

FINDINGS TO DATE (IT IS NOT SUFFICIENT TO STATE FINDINGS WILL BE DISCUSSED): Preliminary data suggests no significant changes in provider comfort level or preparedness before and after the intervention. Moreover, there are no significant differences in rates of perceived adverse events overnight. However, we have yet to quantify actual changes in rates of overnight errors or adverse events. Given the positive feedback we have received from residents, we suspect that these process measures are not fully capturing the intervention effect and that further data needs to be analyzed.

KEY LESSONS FOR DISSEMINATION (WHAT CAN OTHERS TAKE AWAY FOR IMPLEMENTATION TO THEIR PRACTICE OR COMMUNITY?): A survey of IM residents identified large perceived gaps in our institution's previous electronic handoff template and prompted the development of a novel, content-focused handoff tool to fill this need. We hypothesize that this innovation may have substantial impact on overnight patient safety at our institution, despite the preliminary survey data which is likely limited due to recall bias. Future work will investigate whether this intervention reduces actual medical errors and leads to fewer reported adverse events overnight.

IMPROVING HIGH RISK DIABETES CARE MANAGEMENT IN PRIMARY CARE Julia Matthews ${ }^{2}$; Donald Woodbury ${ }^{1}$; Joanna D'Afflitti ${ }^{2}$; Donna Beers ${ }^{1}$; Evonne Yang ${ }^{1}$; Julianne Mcnamara ${ }^{1}$; Rebecca G. Mishuris ${ }^{2}$; Courtney Urick ${ }^{1} .{ }^{1}$ Boston Medical Center, Boston, MA; ${ }^{2}$ Boston University School of Medicine, Boston, MA. (Control ID \#2469658)

STATEMENT OF PROBLEM OR QUESTION (ONE SENTENCE): Uncontrolled diabetes has serious health consequences, and improving care for patients with high risk disease is an imperative for patient health and care delivery efficiency and effectiveness. OBJECTIVES OF PROGRAM/INTERVENTION (NO MORE THAN THREE OBJECTIVES): Improve coordination of care and overall health of our patients with chronic disease, starting with patients with uncontrolled diabetes, through the use of teambased care. Integrate care for high-risk patients with diabetes to create fewer entry points for more comprehensive care for our patients.

DESCRIPTION OF PROGRAM/INTERVENTION, INCLUDING ORGANIZATIONAL CONTEXT (E.G. INPATIENT VS. OUTPATIENT, PRACTICE OR COMMUNITY CHARACTERISTICS): There are more than 7,000 patients with diabetes among the approximate 43,000 patients cared for in the outpatient clinic of General Internal Medicine (divided into 6 teams or suites) at Boston Medical Center (an urban academic safety-net institution). Approximately $20 \%$, or 1,500 , of these patients with diabetes are uncontrolled, with a hemoglobin A1c greater than $9 \%$. A multidisciplinary GIM Team was established in July 2014 to identify areas for improvement, 
brainstorm and implement solutions. This team included clinicians, experts in information technology, administrators and nursing leadership. GIM providers were engaged through clinical advisory groups over lunch and practice meetings for feedback about program development. A team-based care model was implemented encompassing nursing, primary care providers (PCPs), social workers, care coordinators and clinical pharmacists. An initial stratified approach was used with PCPs referring lower risk patients to the suite nurse and higher-risk patients were directed to a highly-skilled RN certified diabetes educator $(\mathrm{CDE})$ for medication management and lifestyle counseling. A patient / provider agreement was signed with each patient enrolling in the program outlining responsibilities of the nurse, care management team and patient. During this initial visit, a refrigerator magnet was given to the patient including care team names, contact information and when to call for help. A nurse education series was completed to provide an update on diabetes care including recommended lifestyle changes, medications, sick day management, and blood sugar monitoring. Early findings resulted in shifting all referrals directly to the RN CDE through a direct referral or warm handoff from the PCP. Visits with patients were organized utilizing a comprehensive assessment tool used to review all aspects of diabetes care, including barriers to care. The RN CDE focused on patient education and medication review including offering group diabetes education at various times. With support from care coordinators, patients were assisted with scheduling specialty care appointments and making connections to other support systems, e.g., behavioral health and clinical pharmacists. Tracking tools and a specific care management note template in the electronic health record were used to guide the work.

MEASURES OF SUCCESS (DISCUSS QUALITATIVE AND/OR QUANTITATIVE METRICS WHICH WILL BE USED TO EVALUATE PROGRAM/ INTERVENTION): The program initially tracked progress based on process metrics including number of patients enrolled in the program, completion of monitoring blood work including $\mathrm{HbA} 1 \mathrm{c}$ and urine microalbumin, and completion of specialty visits (i.e., annual ophthalmology and podiatry exams). As the program progressed, we have also assessed outcome metrics including $\mathrm{HbA} 1 \mathrm{c}$, blood pressure, and cholesterol. Long term quantitative metrics will allow us to see a decrease in ED visits and hospital admissions. We are also focusing on improving qualitative metrics including patient engagement and provider satisfaction

FINDINGS TO DATE (IT IS NOT SUFFICIENT TO STATE FINDINGS WILL BE DISCUSSED): From July to December of 2015, the RN CDE model has been fully in place and has demonstrated positive results. Since July, referrals have come from 37 different providers and the RN CDE has had visits with 111 unique patients. Of these patients, $29 \%$ (32) had a reduction in their HbA1c; $5 \%$ (5) remained stable, and $4 \%$ (4) had an increase in their HbAlc. Of this cohort, $15 \%$ (17) had not followed up for a repeat $\mathrm{HbAlc}$ and $49 \%$ (52) are not yet due for a repeat $\mathrm{HbA} 1 \mathrm{c}$ due to the timing of their enrollment. Of the $29 \%$ who decreased their $\mathrm{HbAlc}$, the mean $\mathrm{HbA1C}$ at time of referral was $11.8 \%$ and the mean $\mathrm{HbA} 1 \mathrm{C}$ after intervention was $8.7 \%$, for a mean decrease of $3.1 \%$ which represents a significant clinical improvement.

KEY LESSONS FOR DISSEMINATION (WHAT CAN OTHERS TAKE AWAY FOR IMPLEMENTATION TO THEIR PRACTICE OR COMMUNITY?): A dedicated RN CDE is required to engage and successfully manage high risk patients. A personal connection is key to patient success. Group visits enhance access to care. Every touch point is an opportunity to engage patients in care and improve health outcomes. Provider communication with the rest of the program team is essential to coordinated care for the patient.

IMPROVING PATIENT SAFETY IN CHRONIC OPIOID THERAPY (COT) MANAGEMENT IN A RESIDENT TEACHING AND FACULTY PRIMARY CARE PRACTICE Jennifer LeComte ${ }^{2}$; Edward Ewen ${ }^{3}$; Sarah Schenck ${ }^{1} .{ }^{1}$ Christiana Care, Wilmington, DE; ${ }^{2}$ Christiana Care Health System, Wilmington, DE; ${ }^{3}$ Christiana Care Health System, Newark, DE. (Control ID \#2470250)
STATEMENT OF PROBLEM OR QUESTION (ONE SENTENCE): Primary care offices have been reported to continue to increase the prescibing of opioid therapy yet there is growing evidence that side effects of long term use especially of high dose opioids are common and significant including accidental overdoses and death.

\section{OBJECTIVES OF PROGRAM/INTERVENTION (NO MORE THAN THREE} OBJECTIVES): Describe processes to identify and evaluated high risk patients using COT. Show how multidisciplinary teams can improve safety in a high risk population. Describe opportunities for educating patients and physicians in the management of COT. DESCRIPTION OF PROGRAM/INTERVENTION, INCLUDING ORGANIZATIONAL CONTEXT (E.G. INPATIENT VS. OUTPATIENT, PRACTICE OR COMMUNITY CHARACTERISTICS): In an outpatient resident teaching and faculty practice, we implemented 5 interventions over 2 years including: (1) the development of a COT registry to identify and track this population, (2) a small group patient education series, (3) mandatory provider education on pain management, (4) standardization of office procedures including risk and functional assessments incorporated into our daily huddle, and (5) the development of a multidisciplinary team for systematic case review to develop written recommendations in order to achieve higher safety.

MEASURES OF SUCCESS (DISCUSS QUALITATIVE AND/OR QUANTITATIVE METRICS WHICH WILL BE USED TO EVALUATE PROGRAM/ INTERVENTION): All demographic information, clinical observations, and office visit activity were obtained from the office electronic medical record. Chronic opioid use was defined as current use and continuous prescribing by the primary care provider of any opioid (excluding tramadol and propoxyphene) for 6 or more months during the study year. COT patients managed outside of the primary care setting were excluded from this analysis. Total number of patients on COT and patients on high dose COT were identified for peer review. A reduction in the total number of patients on COT and a dose reduction for patients on high dose COT were used to measure success. Compliance with mandatory education modules was monitored as well as patient participation in the education series. FINDINGS TO DATE (IT IS NOT SUFFICIENT TO STATE FINDINGS WILL BE DISCUSSED): Approximately 36 cases were reviewed in detail by the multidisciplinary committee over 18 months, 30 patients attended one of the 6 small group education series over 22 months, and $69 \%$ of attending physicians and $56 \%$ of resident physicians completed the COPE-REMS education modules. With this substantial effort, our team was able to reduce the absolute number of COT patients in our practice by $37 \%$, to reduce the number of patients on high dose COT by $21 \%$, and reduce the average daily dose for the high dose patients by $13 \%$. KEY LESSONS FOR DISSEMINATION (WHAT CAN OTHERS TAKE AWAY FOR IMPLEMENTATION TO THEIR PRACTICE OR COMMUNITY?): Peer case review is probably the single most effective intervention in reducing use of high dose COT, but is very time consuming and would benefit from the input of experts in pain management. Informatics support is essential for success. We hope with education of patients and providers, we will see less patients started on opioids for any prolonged period and avoiding high dose opioid therapy whenever possible thereby further improving the safety of our patients who live with chronic pain.

IMPROVING POINT OF CARE LAB WORKFLOW IN AN ACADEMIC INTERNAL MEDICINE CLINIC Sarah E. Smithson ${ }^{1}$; Taylor Harbin ${ }^{1}$; Bobby E. Honeycutt $^{2}$; Amy Legrant ${ }^{1}$; Brooke B. McGuirt ${ }^{1,2}$; Christina McMillan ${ }^{1}$; Tamrah Parker ${ }^{1}$; Shari Sibenge ${ }^{1}$; Eva Wamagata ${ }^{1}$; Vickie Wheeley ${ }^{1}$; Shana Ratner ${ }^{1} .{ }^{1}$ UNC Chapel Hill, Chapel Hill, NC; ${ }^{2}$ University of North Carolina, Chapel Hill, NC. (Control ID \#2466568)

STATEMENT OF PROBLEM OR QUESTION (ONE SENTENCE): A complicated system for point of care Hemoglobin A1C testing, left over from an era of a prior electronic health record, led to long wait times, lost patients, nursing inefficiencies, and missed opportunities for timely management of diabetes. 
OBJECTIVES OF PROGRAM/INTERVENTION (NO MORE THAN THREE OBJECTIVES): 1 . Identify a streamlined process for performing point of care A1C testing to decrease waste. 2. Manage the movement of patients and staff in the clinic. 3. Improve the number of A1C tests delivered to patients due for A1C testing.

DESCRIPTION OF PROGRAM/INTERVENTION, INCLUDING ORGANIZATIONAL CONTEXT (E.G. INPATIENT VS. OUTPATIENT, PRACTICE OR COMMUNITY CHARACTERISTICS): Eight members of the UNC Internal Medicine Clinic (IMC) and one member of McLendon Labs participated in a Lean Six Sigma Purple Belt Training event focused on improving delivery of point of care labs in the IMC, an academic patient-centered medical home and clinical practice site for more than 100 physicians, including residents. The team went to the site of the work to observe the current state, which included provider pre-visit chart review, order entry, and documentation; patient check-in at the front desk, travel to the lab, and back to the waiting room; and nursing retrieval of the patient from the waiting room for nursing assessment. If nurses identified missed A1Cs during the assessment, then the patient would travel to the lab out of sequence, inevitably delaying the provider visit and creating inefficiencies for the patient and nurse. The complicated process often faltered when providers failed to complete pre-visit planning, miscommunication occurred between front desk staff and nurses, and patients were lost in the clinic or struggled physically to navigate the winding halls. Mapping of the entire process revealed significant waste and only a few value-added steps, most importantly collection of the A1C. Consequently, the team tested point of care $\mathrm{A} 1 \mathrm{C}$ collection in the exam rooms by nurses during assessment. The innovation eliminated provider pre-visit planning in favor of an electronic alert in the electronic health record. Point of care A1C testing eliminated all patient and nursing movement to the lab. All exam rooms were stocked with $\mathrm{A} 1 \mathrm{C}$ testing supplies, and the $\mathrm{A} 1 \mathrm{C}$ testing machine was moved to a central location within the clinic. The team created standard work for front desk staff and nurses. A standing order was updated, allowing the nurses to complete the test without an individual provider's order. Individual providers were essentially removed from the process

MEASURES OF SUCCESS (DISCUSS QUALITATIVE AND/OR QUANTITATIVE METRICS WHICH WILL BE USED TO EVALUATE PROGRAM/ INTERVENTION): Patient and provider feedback was measured in a qualitative format Outcome measures included the number of point of care A1Cs performed daily. Process measures were quantitative metrics of patient check-in time from "Arrival" to "Provider Ready" in the electronic health record.

FINDINGS TO DATE (IT IS NOT SUFFICIENT TO STATE FINDINGS WILL BE DISCUSSED): The feedback from patients and providers regarding the A1C being drawn in the exam room was extremely positive. Nurses were surprised at the reduction in time and frustration, and felt rewarded by the positive response from patients. The time from "Arrival" to "Provider Ready" decreased by an average of $10 \mathrm{~min}$ per patient check-in (from $23 \mathrm{~min}$ to $13 \mathrm{~min}$ ). The average number of point of care A1Cs collected each day increased from 5 to 12 , with fewer due or overdue labs being missed. With 12 A1Cs being done per day, this represents $120 \mathrm{~min}$ of saved time per day and $600 \mathrm{~min}$ per week. Overall, changing the location of point of care lab testing positively impacted patient and staff flow, satisfaction, A1C testing, and visit efficiency.

KEY LESSONS FOR DISSEMINATION (WHAT CAN OTHERS TAKE AWAY FOR IMPLEMENTATION TO THEIR PRACTICE OR COMMUNITY?): Engagement of all stakeholders, including patients, staff, providers, clinic leadership, and lab leadership was essential. Going to the site of the work and mapping the process provided invaluable insight into the daily work and waste in the existing process. Simplifying the processes enabled the team to eliminate hundreds of hours of unnecessary work per week while providing improved clinical care at the same time. Nursing autonomy and leadership in the project was a driving force behind successful spread and sustainability.
IMPROVING POPULATION HEALTH IN A SAFETY NET SETTING: A TEAM SPORT Claire K. Horton ${ }^{1}$; Ghezal W. Saffi ${ }^{1}$; Antonella Jimenez ${ }^{1}$; Poneet Dhillon ${ }^{1}$; Daffcar Errol ${ }^{1}$; Rachel Tsolinas ${ }^{1}$; Carol Yam² ${ }^{2}$ Soraya Azari ${ }^{2} .{ }^{1}$ UCSF, San Francisco, CA; ${ }^{2}$ University of California, San Francisco, San Francisco, CA. (Control ID \#2469865)

STATEMENT OF PROBLEM OR QUESTION (ONE SENTENCE): Increasing demands on primary care physicians leave inadequate time to accomplish preventive health care measures during routine clinic visits; this may be particularly true in safety net settings and in residency clinics when resident primary care providers' care may be fragmented and infrequent.

OBJECTIVES OF PROGRAM/INTERVENTION (NO MORE THAN THREE OBJECTIVES): - Describe how a team-based approach to population health can be implemented in a residency clinic with a high-risk, vulnerable patient population Compare and contrast inreach and outreach efforts to improve preventitive health screening -Identify three concrete interventions to implement at your own practice site to improve team-based preventive health screening

DESCRIPTION OF PROGRAM/INTERVENTION, INCLUDING ORGANIZATIONAL CONTEXT (E.G. INPATIENT VS. OUTPATIENT, PRACTICE OR COMMUNITY CHARACTERISTICS): The Richard H. Fine People's Clinic is one of the largest primary care clinic in the San Francisco Health Network. The clinic serves as the continuity clinic site for residents from the University of California / San Francisco, and the patient population is predominantly low-income and diverse, with a high burden of chronic medical disease, psychiatric disease, and substance use disorders. We created a population health program that includes the following elements: - Use of a registry system and continuous refinement of this system -Standardized inreach efforts (created standardized protocols and workflows for medical assistants to administer vaccines, order labs, and schedule cancer screening tests for all patients at all visits) -Robust outreach efforts (trained team-based "quality improvement specialists" to perform outreach to all patients due for cancer screening, pain assessments, hypertension visits, and primary care appointments) -Dedicated "panel review" sessions for trainees -Quarterly provider feedback sessions focused on individual providers' panel data -Stratification of data by race/ethnicity to aide efforts to reduce health care disparities in our patient population Creation of specific women's health sessions for residents to enhance their training as well as our patients' access to cervical cancer screening

MEASURES OF SUCCESS (DISCUSS QUALITATIVE AND/OR QUANTITATIVE METRICS WHICH WILL BE USED TO EVALUATE PROGRAM/ INTERVENTION): We track multiple metrics on a monthly basis, including rates of cervical, colon, and breast cancer screening; hypertension control; smoking cessation referrals; and urine toxicology screens and pain agreements for patients with chronic, non-malignant pain on opiate therapy. Additionally, we seek advice on our outreach efforts from our Patient Advisory Council and modify our initiaves according to the input offered by our patient advisors.

FINDINGS TO DATE (IT IS NOT SUFFICIENT TO STATE FINDINGS WILL BE DISCUSSED): From January 2015 through December 2015, our findings included: -An increase in cervical cancer screening from 65 to $70 \%$-An increase in colorectal cancer screening from 58 to $63 \%$-An increase in patients on chronic opioid therapy with both an updated pain agreement and urine drug screen from 58 to $76 \%$-An increase in the referral of smokers to nicotine cessation services from 30 to $70 \%-$ An increase in our diabetic retinopathy screening rates from 30 to $60 \%$-No change in our rates of hypertension control

KEY LESSONS FOR DISSEMINATION (WHAT CAN OTHERS TAKE AWAY FOR IMPLEMENTATION TO THEIR PRACTICE OR COMMUNITY?): A team-based initiave that combines inreach and outreach efforts can improve population health across multiple domains. Creating standardized protocols for medical assistants and training dedicated QI outreach specialists are critical elements of this approach. Certain 
metrics that involve patient adherence and lifestyle change, such as hypertension, may be harder for health teams to improve with this approach. Involving trainees in these improvement efforts can enhance their education as well as their patient's health. Patient input is an important, often neglected element to improving population health

INCREASING ADHERENCE TO THE UNITED STATES PREVENTIVE SERVICES TASK FORCE (USPSTF) SCREENING AND IMMUNIZATION GUIDELINES AMONG RESIDENTS AT THE INTERNAL MEDICINE CLINIC WITHIN THE GEORGE WASHINGTON UNIVERSITY MEDICAL FACULTY ASSOCIATES GROUP. NEJAT NASER ${ }^{1}$; Talal Alzahrani ${ }^{1}$; Joseph Delio ${ }^{2,1} .{ }^{1}$ GW UNIVERSITY, WASHINGTON, DC; ${ }^{2}$ GWU, Washington, DC. (Control ID \#2466146)

STATEMENT OF PROBLEM OR QUESTION (ONE SENTENCE): How to improve and maintain adherence to USPSTF screening and immunization guidelines among internal medicine residents?

OBJECTIVES OF PROGRAM/INTERVENTION (NO MORE THAN THREE OBJECTIVES): - To improve adhrence to screening guidelines among internal medicine residents -To tease out best method to maintain adherence

DESCRIPTION OF PROGRAM/INTERVENTION, INCLUDING ORGANIZATIONAL CONTEXT (E.G. INPATIENT VS. OUTPATIENT, PRACTICE OR COMMUNITY CHARACTERISTICS): Using the Abdominal Aortic Aneurysm (AAA) screening compliance rate as a surrogate marker for other screening and immunization compliance rates, we observed the screening rates within a group of 99 internal medicine residents. Baseline screening rates were obtained by reviewing medical records of patients who had received a health maintenance exam between June 23rd and August 22nd, 2015. We then introduced intervention methods with the intent to improve screening rates. For our first intervention, residents attended a short educational session, during which a primary care physician outlined the AAA screening recommendation. For the second intervention, an email was sent to all internal medicine residents to reinforce the AAA screening guideline. The third intervention involved sending an email to supervising attendings, informing them of the recommendation and the screening rate among their residents. They were also urged to remind their residents to perform AAA screening when indicated. In our final intervention, we plan to work with the clinic's information technology team to integrate all screening tests and immunization recommendations into the electronic health record.

MEASURES OF SUCCESS (DISCUSS QUALITATIVE AND/OR QUANTITATIVE METRICS WHICH WILL BE USED TO EVALUATE PROGRAM/ INTERVENTION): At the end of each intervention period (each week-long cycle, totaling to 5), retrospective data, including the screening rate, were calculated and compared to both the baseline and prior week's results using the Chi-square test.

FINDINGS TO DATE (IT IS NOT SUFFICIENT TO STATE FINDINGS WILL BE DISCUSSED): The initial AAA screening rate was $2.5 \%$ among internal medicine residents between June 23rd and August 22nd, 2015. Over the next 5 weeks, following the first intervention method, this rate improved significantly from 2.5 to $15 \%$ with a $p$-value $<0.01$. We suspect that this was due to the fact that no previous AAA screening guideline lecture had been given. Conversely, topics such as mammography, colonoscopy and Papanicolaou (Pap) smear screening have been taught consistently. During the next two interventions, the rates were 12.5 and $33.3 \%$, with p-values of 0.48 and $<0.01$, respectively. We suspect these results are due to the complexity of patient concerns, along with high patient volume, which can make it difficult for internal medicine residents to address all necessary screening tests. The final intervention is still underway.

KEY LESSONS FOR DISSEMINATION (WHAT CAN OTHERS TAKE AWAY FOR IMPLEMENTATION TO THEIR PRACTICE OR COMMUNITY?): Effec-

tiveness of various educational and systemic interventions in bringing change.
INFORMED DEVELOPMENT OF DISCHARGE INNOVATIONS TO IMPROVE TRANSITION FROM ACUTE CARE TO SKILLED NURSING FACILITIES. Carly Kuehn $^{2,}{ }^{1}$; Peter Kaboli ${ }^{1}$; Ethan Kuperman ${ }^{3,}{ }^{1}$. ${ }^{1}$ Iowa City VAMC, Iowa City, IA; ${ }^{2}$ University of Iowa, Iowa City, IA; ${ }^{3}$ University of Iowa Carver College of Medicine, Iowa City, IA. (Control ID \#2468378)

STATEMENT OF PROBLEM OR QUESTION (ONE SENTENCE): Patients discharging to skilled nursing facilities (SNFs) are medically complex, often with increased care needs and more post-hospitalization complications than the general population. Identifying and organizing essential information to ensure successful transitions remains challenging, with significant regional variation.

OBJECTIVES OF PROGRAM/INTERVENTION (NO MORE THAN THREE OBJECTIVES): This study sought to improve the transition of patients from the Iowa City VA general medicine service to SNF by identifying essential information for successful discharge and organizing it within the transfer packet.

DESCRIPTION OF PROGRAM/INTERVENTION, INCLUDING ORGANIZATIONAL CONTEXT (E.G. INPATIENT VS. OUTPATIENT, PRACTICE OR COMMUNITY CHARACTERISTICS): We created a novel survey instrument to assess current discharge processes and identify areas of intervention. The survey was guided by review of existing literature on transitions of care and in-person interviews with local SNF staff. SNFs receiving patients from the Iowa City VAMC were contacted via telephone, and the 25 accepting facilities were mailed paper copies of the survey to complete. The survey was distributed to administrators, physicians, nursing supervisors and social work staff and mailed back anonymously. We received responses from 12 facilities and the overall survey completion rate was $27 \%$. SNFs identified medications, wound care, diet, activity and PT/OT recommendations as most important information in the discharge document. They identified cognitive status, peripheral line information, activity orders and wound care orders as not including enough information. The most inaccurate areas involved wound care, cognition and peripheral line information. A multidisciplinary team utilized the survey results focusing on areas SNFs deemed important however inaccurate and/or lacking information: diet orders, therapy orders, wound cares and anticipated appointments to create a discharge checklist as the first innovation. The discharge checklist focuses on succinctly highlighting these areas that are already included within the packet.

MEASURES OF SUCCESS (DISCUSS QUALITATIVE AND/OR QUANTITATIVE METRICS WHICH WILL BE USED TO EVALUATE PROGRAM/ INTERVENTION): A pilot of the novel checklist was sent to 3 facilities. Measures of success for this intervention included ease of compilation of the checklist data by social work and feedback from SNF facilities on the impact the discharge checklist had on initial evaluation or transitioning patients to SNF.

FINDINGS TO DATE (IT IS NOT SUFFICIENT TO STATE FINDINGS WILL BE DISCUSSED): The checklist was found to take less than two minutes for our social worker to complete indicating relative ease. The social worker sent the checklist with initial evaluation for SNF paperwork. Feedback from SNFs indicated this new checklist was not useful to their workflow.

KEY LESSONS FOR DISSEMINATION (WHAT CAN OTHERS TAKE AWAY FOR IMPLEMENTATION TO THEIR PRACTICE OR COMMUNITY?): The checklist was designed for quick completion by the hospital staff and for ease of use by the receiving SNF. The checklist was found to be easy to compile, however, facilities felt the format was not helpful to their intake process. Next steps involve updating the physician discharge summary templates to highlight the areas deemed inaccurate or missing information via the SNF survey data to facilitate communication with SNFs. The update will involve re-designing the discharge templates to populate necessary fields based on the chosen discharge location for each patient; i.e. SNF vs home. 
INITIAL IMPLEMENTATION OF SELF-REPORTED GLOBAL HEALTH MEASUREMENT IN A LARGE ACCOUNTABLE CARE ORGANIZATION Karen J. Blumenthal ${ }^{1}$; Timothy G. Ferris ${ }^{2}{ }^{1}$; Christine Vogeli ${ }^{1}$; Neil W. Wagle ${ }^{2}$; Joshua Metlay $1{ }^{1}$ Massachusetts General Hospital, Boston, MA; ${ }^{2}$ Partners HealthCare, Waban, MA. (Control ID \#2467413)

STATEMENT OF PROBLEM OR QUESTION (ONE SENTENCE): Can patient reported outcome measures (PROMS) be routinely collected in a large Accountable Care Organization (ACO)?

OBJECTIVES OF PROGRAM/INTERVENTION (NO MORE THAN THREE OBJECTIVES): Measures that track self-assessed perceptions of health and healthrelated quality of life have been widely used in research, but adoption in routine clinical settings has been limited. Factors linked to slow adoption include lack of knowledge of how to best use the information and technical challenges in collecting the data. Partners HealthCare (Partners), a large Academic Health System and ACO, instituted collection of patient reported outcome measures (PROMs) in multiple specialty and primary care practices in 2011. The goals were to better understand patient's needs; improve physician-patient communication; assess individual risk; contribute to quality measures; and provide data for comparative effectiveness research. This implementation provides an opportunity to investigate methods for optimizing the use of PROMs, specifically a measure of self-reported global called the PROMIS Global Health (PGH), in routine care.

DESCRIPTION OF PROGRAM/INTERVENTION, INCLUDING ORGANIZATIONAL CONTEXT (E.G. INPATIENT VS. OUTPATIENT, PRACTICE OR COMMUNITY CHARACTERISTICS): Since, 2011, when Partners launched its collection of PROMs, 54 ambulatory practices, both specialty and primary care, have participated in this initiative and currently all participating practices are collecting the $\mathrm{PGH}$ at the point of care. Individuals were asked at the time of check-in to complete the PGH on an iPad at both initial and follow-up visits at participating practice sites, with a minimum of 90 days between completions. The PGH includes 4 questions that generate a Global Physical Health (GPH) score and 4 questions that generate a Global Mental Health (GMH) score. Both the GMH and GPH have T-Score distributions that are standardized such that a score of 50 represents the mean for the US general population with a standard deviation of 10. Higher scores represent higher functioning.

MEASURES OF SUCCESS (DISCUSS QUALITATIVE AND/OR QUANTITATIVE METRICS WHICH WILL BE USED TO EVALUATE PROGRAM/ INTERVENTION): To evaluate this implementation process, we launched an initial study using the PGH score to assess variation in overall health across an ACO, compare the distribution of scores to population standards, and assess how patient and practice level factors influenced the variation in scores. We performed a cross sectional analysis on the 19,664 adults in the Partners ACO/ Risk contract population who completed at least one PGH (from 03/2014 to $09 / 2015$ ) at one of the 54 participating clinics. This population consists of individuals insured by Medicare, commercial plans and a small number of Medicaid patients. Demographic data and insurance data were extracted from the electronic health record and allowed us to look at score distribution across different patient subgroups. Linear regression was used to identify independent predictors of PGH scores.

FINDINGS TO DATE (IT IS NOT SUFFICIENT TO STATE FINDINGS WILL BE DISCUSSED): The overall mean GPH and GMH scores were 49.0 (SD 10) and 52.5 (SD 10.3) respectively. The mean GPH and GMH scores among patients completing the instrument at a primary care visit were 51.6 (SD 8.6) and 52.0 (SD 10.6) compared to 46.4 (SD 10.5) and 48.4 (SD 12.4) for patients completing the instrument at any specialty care visit. Patients with Medicare or Medicaid insurance (vs. private insurance) or seen at specialty clinics (vs. primary care) had lower GPH and GMH scores, even after adjusting for patient demographic characteristics. Female gender and non-white race were also significantly associated with lower GPH and GMH scores on multivariate analyses.
KEY LESSONS FOR DISSEMINATION (WHAT CAN OTHERS TAKE AWAY FOR IMPLEMENTATION TO THEIR PRACTICE OR COMMUNITY?): Despite a history of slow adoption of the collection of PROMs, such as the PGH, in routine clinical care, we have demonstrated that this is feasible in a large integrated health system. Routine collection of PROMs may yield important insights into variations in care across practices and guide future patient and practice level interventions. Our ongoing work will evaluate whether observed differences in scores can be explained by additional clinical variables at the patient level and/or physician and practice level factors. Ultimately, this implementation project will lay the groundwork for future studies looking at the ability of the PGH to predict health outcomes and healthcare utilization in order to maximize value in our delivery system

INTRODUCING PRIMARY CARE TELEPHONE VISITS: AN URBAN SAFETY-NET COMMUNITY CLINIC EXPERIENCE Kelly A. Kyanko'; Antoinette Schoenthaler ${ }^{1}$; Sondra Zabar ${ }^{1}$, $;$ Jennifer Joseph ${ }^{1}$; Peter Davidson ${ }^{1,2}$; William Bateman ${ }^{1,2}$; Kathleen Hanley ${ }^{1,2}$. ${ }^{1}$ New York University School of Medicine, New York, NY; ${ }^{2}$ Gouverneur Health, NYC Health + Hospitals, New York, NY. (Control ID \#2463489)

STATEMENT OF PROBLEM OR QUESTION (ONE SENTENCE): New models of primary care include scheduled, documented telephone visits that are clinical exchanges that occur via telephone between providers and patients. These visits have the potential to increase patient access, and provide a convenient alternative especially for underserved populations with restricted work schedules and limited resources for travel. However, patients and physicians may prefer in-person visits and implementation in a safety-net clinic presents unique challenges. OBJECTIVES OF PROGRAM/INTERVENTION (NO MORE THAN THREE OBJECTIVES): 1) To determine the feasibility of using telephone visits within a diverse safety-net adult ambulatory care practice. 2) To characterize patient and physician acceptability of telephone visits.

DESCRIPTION OF PROGRAM/INTERVENTION, INCLUDING ORGANIZATIONAL CONTEXT (E.G. INPATIENT VS. OUTPATIENT, PRACTICE OR COMMUNITY CHARACTERISTICS): The Gouverneur Health Ambulatory Care (Primary Care) Practice, an urban clinic in New York City serving a largely underserved immigrant population with limited English proficiency, implemented a quality improvement initiative beginning April 2015 to offer telephone visits instead of in-person visits for regular follow-up care. Physicians determined at the initial in-person visit whether or not a patient was eligible for telephone follow-up, and could exclude a patient for reasons such as impaired patient communication or cognition or need for physical exam at follow-up. Physician and patient participation was voluntary.

MEASURES OF SUCCESS (DISCUSS QUALITATIVE AND/OR QUANTITATIVE METRICS WHICH WILL BE USED TO EVALUATE PROGRAM/ INTERVENTION): Within a week of their telephone visit, all patients were contacted via telephone by a research assistant for a brief survey and were also invited to participate in a longer in-depth interview. Physicians were also surveyed after 6 months and focus groups are ongoing.

FINDINGS TO DATE (IT IS NOT SUFFICIENT TO STATE FINDINGS WILL BE DISCUSSED): Since April 2015, 10 (out of 20 eligible) physicians participated in the initiative, 85 telephone visits occurred, and 75 patients completed surveys. Only about a third of scheduled telephone visits were performed. Reasons telephone visits were not completed include: patient showed up in person, the physician was unable to call, or the patient was unreachable. Patient survey results were overall positive: $84 \%$ of patients reported being highly satisfied with receiving telephone visits, $90 \%$ felt they could discuss everything they wanted to on the telephone, and $84 \%$ felt that telephone visits save time travelling to the clinic. Though $50 \%$ reported that they would prefer to discuss their 
medical problems with their doctor in person, $\sim 75 \%$ of patients reported that they would not mind having another telephone visit again. Analysis of in-depth interviews is pending. While only half of physicians chose to participate in the initiative, those who did all $(100 \%)$ felt that patients that participated in telephone visits were satisfied with their care, that they were able to communicate adequately with patients, could safely care for select patients over the phone, and that telephone visits could improve continuity of care for patients. The majority of physicians also felt that patients followed up on recommendations (e.g. blood tests, vaccinations) after telephone visits just as much as they did for inperson visits ( $89 \%$ ) and that telephone visits are more time efficient than in-person followup $(78 \%)$. All physicians would like to continue having appointment slots dedicated to telephone follow-up and the majority ( $89 \%$ ) felt they needed protected, scheduled time for making telephone calls when they are in clinic. Focus group data are pending.

KEY LESSONS FOR DISSEMINATION (WHAT CAN OTHERS TAKE AWAY FOR IMPLEMENTATION TO THEIR PRACTICE OR COMMUNITY?): Several aspects of implementation of the initiative were challenging and should be considered by other facilities planning on translating the practice to their own sites: • Only half of eligible physicians participated. Competing initiatives such as roll out of ICD-10 and eprescribing may have decreased interest in participation. We expect the focus groups to elicit other reasons. • Physicians felt that telephone visits were appropriate for $\sim 27 \%$ of their patients. The following services were considered the most appropriate for telephone visits: 1) lifestyle/behavioral counseling; 2) acute, nonemergency care (e.g., flu symptoms); 3) medication titration; and 4) discussing test results. $\bullet$ Telephone visits are best scheduled at the beginning of a session (e.g. $9 \mathrm{am} / 1 \mathrm{pm}$ ) to ensure that the visits are on time. - Some patients were confused and showed up in person or did not answer at the time of their scheduled call. Patient flyers and FAQ sheets helped mitigate confusion and facilitated rescheduling of missed visits.

LESSONS FROM THE ACADEMIC INNOVATIONS COLLABORATIVE, A PRIMARY CARE PRACTICE TRANSFORMATION LEARNING COLLABORATIVE Jessica Zeidman ${ }^{6,7}$; Jennifer Azzara ${ }^{7}$; Trudy Bearden ${ }^{5}$; Asaf Bitton ${ }^{4,7}$; Lindsay S. Hunt ${ }^{7}$; T'sera M. Mirescu ${ }^{7}$; Gordon D. Schiff ${ }^{1,}{ }^{7}$; Sara Singer ${ }^{3}$, ; Jonathan R. Sugarman ${ }^{5}$; Andrew L. Ellner ${ }^{2} .{ }^{1}$ Brigham and Women's Hospital, Boston, MA; ${ }^{2}$ Harvard Medical School, Boston, MA; ${ }^{3}$ Harvard School of Public Health, Boston, MA; ${ }^{4}$ Northwestern University, Boston, MA; ${ }^{5}$ Qualis Health, Seattle, WA; ${ }^{6}$ The Massachusetts General Hospital, Chelsea, MA; ${ }^{7}$ Harvard Medical School Center for Primary Care, Boston, MA; ${ }^{8}$ Mongan Institute for Health Policy, Massachusetts General Hospital, Boston, MA. (Control ID \#2469936)

STATEMENT OF PROBLEM OR QUESTION (ONE SENTENCE): Primary care practices increasingly incorporate team-based care and quality improvement processes, but do not often have an opportunity to collaborate with other transforming practices or receive ongoing training and feedback on their progress.

OBJECTIVES OF PROGRAM/INTERVENTION (NO MORE THAN THREE

OBJECTIVES): 1. Define elements of a successful primary care practice transformation learning collaborative. 2. Identify predictable barriers to practice transformation and possible strategies to overcome them. 3. Describe measures that can be used to evaluate the impact of a successful collaborative.

DESCRIPTION OF PROGRAM/INTERVENTION, INCLUDING ORGANIZATIONAL CONTEXT (E.G. INPATIENT VS. OUTPATIENT, PRACTICE OR COMMUNITY CHARACTERISTICS): The Academic Innovations Collaborative (AIC) was launched in 2012 by the Harvard Medical School Center for Primary Care in collaboration with Qualis Health and the Institute for Healthcare Improvement to support the transformation of primary care education and practice around Boston by providing longitudinal training, feedback, and a forum for practices to collaborate. Initially, twenty community and hospital-based primary care practices representing six academic medical centers (AMC) and more than 275,000 patients participated in the collaborative. For the second 2 years, one practice dropped out, one additional AMC and nine practices joined, for a total of 28 practices. The first phase of the collaborative lasted 2 years and was organized around the Eight Change Concepts for Practice Transformation, developed by Qualis Health and the MacColl Center for Health Care Innovation. Initial efforts focused on creating systems of team-based care and quality improvement at each clinic. The second phase, which concludes in June 2016, builds on this foundation to increase safety and promote patient engagement, focusing on preventing delayed diagnosis of colon and breast cancer, and improving care of complex patients. To foster practice transformation, the AIC leadership holds three 1-2 day retreats each year, called Learning Sessions, and bi-monthly conference calls for participating clinics to build skills, share best practices, and troubleshoot challenges. Practices also work with a practice coach, engage in monthly data reporting, and participate in annual site visits.

MEASURES OF SUCCESS (DISCUSS QUALITATIVE AND/OR QUANTITATIVE METRICS WHICH WILL BE USED TO EVALUATE PROGRAM/ INTERVENTION): The following measures of success are used to guide the support provided to practices by the central leadership team of the AIC. 1. Patient Centered Medical Home - Assessment (PCMH-A) scores. 2. Practice engagement in the AIC, measured by: a) Participation in conference calls and Learning Sessions. b) Ratings of the conference calls. c) Number of Plan-Do-Study-Act cycles completed by each team. 3 . Monthly Transformation Updates (MTU) submitted by each clinic including: a) Description of the practice's current aims for improvement and progress attaining them. b) Clinic leadership report describing team membership, activities, and challenges which need support. c) Description of current Plan-Do-Study-Act Cycles (PDSA). 4. Identification of key changes to share amongst clinics to improve operations and care. There was also an independent external evaluation of the AIC, but here we focus on measures used by practices and AIC leadership to guide improvement.

FINDINGS TO DATE (IT IS NOT SUFFICIENT TO STATE FINDINGS WILL BE DISCUSSED): 1. PCMH-A scores demonstrated improvement across each change concept, with overall improvement from 6.7 to 9.1 (scale from 1 to 12). 2. Practice Engagement: a) Participation in learning sessions ranged from approximately 175 to 275 people. b) Participation in monthly calls averaged 13.3 of 17 sites, range 10-17 (17 is maximum number possible because some clinics aggregate participation reports). c) Participant ratings of the calls averaged 3.7, range 2.8-4.4 (on a scale of 1-5). d) Number of completed PDSAs per month ranged from 13 to 60.3 . Practices consistently submitted MTUs demonstrating progress in their transformation efforts; these are accessible to all participants to enable shared learning. 4.Through sharing successes across practices, the AIC identified a list of "5 Key Changes to Optimize Colorectal Cancer Screening and Follow-Up" which was disseminated broadly through a Learning Session and conference calls.

KEY LESSONS FOR DISSEMINATION (WHAT CAN OTHERS TAKE AWAY FOR IMPLEMENTATION TO THEIR PRACTICE OR COMMUNITY?): 1.

Central elements of success were: a) Engaging institutional leadership. b) Training practice leadership and staff in transformation concepts and quality improvement processes. c) Providing the structure of ongoing collaboration and feedback for the group over 4 years; this fostered culture change over time. d) Using standardized measures of success allowed practices to compare their progress against that of others, highlighting accomplishments for dissemination. 2. Challenges to supporting change at primary care practices embedded in larger systems include: a) Incentives are still structured around volume which can undermine transformation efforts aligned toward other aims. b) Many processes are dependent on entities outside of primary care and the collaborative, such as the Emergency Department, and these must be engaged for transformation in certain domains to be optimal. c) Data on patient-related outcomes can be difficult to attain from academic institutions, hindering evaluation of the impact of change. 
MAKING DISCHARGE SAFER: A MULTIDISCIPLINARY DISCHARGE TIMEOUT CHECKLIST Brian Eiss ${ }^{3}$; Paul Martin ${ }^{3}$; Michael Gao ${ }^{1}$; Christina Chai ${ }^{1}$; Laura F. Gingras ${ }^{3}$; Julius Motal ${ }^{1}$; Megan E. Maikoff ${ }^{2}$; Nadine Rosenthal ${ }^{1} .{ }^{1}$ New York Presbyterian Hospital - Weill Cornell Medical Center, New York, NY; ${ }^{2}$ NewYork-Presbyterian/Weill Cornell Medical Center, Brooklyn, NY; ${ }^{3}$ Weill Cornell Medical College/ New York Presbyterian Hospital, New York, NY. (Control ID \#2467672)

STATEMENT OF PROBLEM OR QUESTION (ONE SENTENCE): Adverse events after discharge remain common despite national efforts to provide patient education and medication reconciliation; a multidisciplinary "discharge timeout" checklist can increase patient engagement and highlight and counteract potential safety vulnerabilities in the discharge process.

OBJECTIVES OF PROGRAM/INTERVENTION (NO MORE THAN THREE OBJECTIVES): - To use Plan-Do-Study-Act (PDSA) methodology to expand upon the multidisciplinary discharge timeout initiative we launched last year-To increase selfreported provider and nursing awareness of discharge vulnerabilities, including unintended central lines or catheters and medication reconciliation errors, and provide an opportunity to intervene in real time to prevent subsequent related adverse events

DESCRIPTION OF PROGRAM/INTERVENTION, INCLUDING ORGANIZATIONAL CONTEXT (E.G. INPATIENT VS. OUTPATIENT, PRACTICE OR COMMUNITY CHARACTERISTICS): After a series of sentinel adverse events at the time of hospital discharge occurred at our institution in recent years, we formed a multidisciplinary group of physicians, PAs, nurses and care coordinators to create a sixitem discharge timeout checklist with the purpose of identifying potential safety vulnerabilities and reinforcing key medication changes and follow up instructions. Just before discharge, the patient's nurse and MD or PA meet and ask the patient to "teach back" his or her knowledge of the six items: (1) presence of IVs or other catheters, (2) key medication changes, (3) follow up plans, (4) symptoms to watch for, (5) what to do if these symptoms occur, and (6) home care arrangements. They note when a patient is unable to completely teach back these concepts and provide subsequent education at that time. Providers also use the discharge checklist to note "good catches", such as near-miss medication errors or missing follow-up appointments, which are immediately corrected by issuing new prescriptions or making changes to discharge documentation, respectively. Utilizing PDSA methodology, we first refined our initial timeout checklist and standardized a process to coordinate the discharge timeout. Further PDSA cycles expanded the process from just two housestaff medical teams to all housestaff and then to physician assistant teams as well. We also conducted surveys of providers performing the discharge timeout to assess usability and to subjectively weigh the drawbacks (additional time, logistics) against the benefits (improved safety). We will next survey patients and assess unit-specific HCAHPS scores and readmission rates. Pending these results, the six-item discharge timeout checklist may be expanded to other units.

MEASURES OF SUCCESS (DISCUSS QUALITATIVE AND/OR QUANTITATIVE METRICS WHICH WILL BE USED TO EVALUATE PROGRAM/ INTERVENTION): Discharge timeout checklist includes: - \# of good catches (near miss events) $-\%$ of discharge timeouts in which patients were unable to teach back at least 1 of the 6 elements assessed - $\%$ of discharge timeouts in which re-education was provided by either $\mathrm{MD} / \mathrm{PA}, \mathrm{RN}$ or collaboratively $-\%$ of eligible discharge timeouts successfully completed average length of time to complete discharge timeout Staff and patient surveys include: - ease of process -whether the timeout seemed to: delay discharge, make discharge safer, improve communication -whether, taking into account the costs and benefits, it was worth making this a standard part of discharge process Future measures will include: - unit specific HCAHPS/ Press-Ganey scores for questions about discharge, follow-up instructions and medication reconciliation -readmission rates for patients who participated in a discharge timeout FINDINGS TO DATE (IT IS NOT SUFFICIENT TO STATE FINDINGS WILL BE DISCUSSED): Over 6 months: 191 total discharge timeouts (out of 430 eligible discharges, or $44 \%$ ) -First 30 days: 11 out of 54 (20.3\% of eligible) -Final 30 days: 42 out of 80 (52.5\% of eligible) Timeouts took on average $10.9 \pm 9.9 \mathrm{~min}$ There were a total of 20 "good catches" or near-miss events ( $9.4 \%$ of timeouts) -14 involved medication reconciliation errors -5 involved a follow up appointment incorrectly listed on the discharge instructions -1 involved a peripheral intravenous line that the team was unaware of, which was removed before the patient left the hospital-In 55 out of 191 of the timeouts conducted $(28.7 \%)$, the patient was unable to teach back at least 1 of the 6 items in the discharge timeout -In 53 out of these 55 timeouts (96\%), re-education was provided ( $45 \%$ of time by both MD/PA and RN, $34 \%$ by RN alone, $20 \%$ by MD/PA alone) Staff surveys (Likert scale: $1=$ strongly agree; $5=$ strongly disagree) RNs $(n=10)$ : easy to perform $=2$, delays discharge $=3$, improves communication $=2$, should be standard discharge practice $=1.6 \mathrm{MD} / \mathrm{PAs}(n=10)$ : easy to perform $=1.6$, delays discharge $=3.8$, improves communication $=2$, should be standard discharge practice $=1.9$ )

KEY LESSONS FOR DISSEMINATION (WHAT CAN OTHERS TAKE AWAY FOR IMPLEMENTATION TO THEIR PRACTICE OR COMMUNITY?): Nearly $10 \%$ of timeouts over a 6-month period uncovered a "good catch", suggesting that a multidisciplinary intervention can improve patient safety and reduce adverse events on discharge. Providers report that performing discharge timeouts improved communication and offered subjective safety benefits and also felt that these benefits outweighed increased time commitments and logistical complexity.

MAKING PROGRESS ON PROGRESS NOTES Vijay Paryani² ${ }^{2}$ Joseph Chiles²; Drew Burlon ${ }^{2}$; Wycliffe Opii ${ }^{2}$; Clark Sleeth ${ }^{2}$; Laura Fanucchi ${ }^{1}$; Sean M. Lockwood ${ }^{3}{ }^{3}$; John Ragsdale ${ }^{1}$; Joesph R. Sweigart ${ }^{1,3}$. ${ }^{1}$ University of Kentucky, Midway, KY; ${ }^{2}$ University of Kentucky, Lexington, KY; ${ }^{3}$ Lexington VA Medical Center, Lexington, KY. (Control ID \#2470292)

STATEMENT OF PROBLEM OR QUESTION (ONE SENTENCE): Interventions to improve daily progress note quailty have had minimal success, partially because which components of daily progress notes are crucial for effectivel communication are not well established.

OBJECTIVES OF PROGRAM/INTERVENTION (NO MORE THAN THREE OBJECTIVES): 1. Improve the quality of internal medicine resident subsequent daily progress notes 2 . Elucidate which components of daily progress notes correlate with measures of note quality

DESCRIPTION OF PROGRAM/INTERVENTION, INCLUDING ORGANIZATIONAL CONTEXT (E.G. INPATIENT VS. OUTPATIENT, PRACTICE OR COMMUNITY CHARACTERISTICS): We created a note template that best captured the purpose of subsequent day progress notes. This template was informed by results from analysis of the baseline notes, as well as available literature on note quality and consensus of local experts. The template contains all necessary billing information, but minimizes extraneous data. It deliberately encouraged inclusion of a summary statement atop the assessment and plan (A\&P) as well as inclusion of author information. All internal medicine residents received a brief lecture describing the project and encouraging them to use the template. They were also encouraged to focus on the communication function of their notes, rather than other potential functions such as billing, medicolegal, or longitudinal record keeping. Residents received a reminder handout and had the opportunity to ask questions of project leaders during a brief session early in their ward month rotations. The local institutional review board approved this project. Data collection and analysis are ongoing.

MEASURES OF SUCCESS (DISCUSS QUALITATIVE AND/OR QUANTITATIVE METRICS WHICH WILL BE USED TO EVALUATE PROGRAM/ INTERVENTION): We created a note evaluation tool based on one previouslypublished tool and one tool internally derived and validated at the Northwestern University Feinberg School of Medicine. The tool included the presence or absence of specific 
components, as well as gestalt Likert-type assessments of notes. We selected the note from the topmost patient from the electronic team list whose chart contained two consecutive internal medicine resident progress notes. Notes were printed and fully de-identified of both patient and provider information. Notes were extracted once weekly in June 2015 to serve as a baseline. June was selected to minimize bias from house staff maturation. Postintervention notes were selected once weekly from the same resident services and using the same protocol as for baseline notes. All notes were scored independently by a medical student and practicing physician. For numeric items, the average of the two scores was used for all statistical comparisons; for categorical items, the more favorable of the two scores were used. P-values were calculated using t-tests. Best-fit linear trend lines were used to assess correlations between continuous variables.

FINDINGS TO DATE (IT IS NOT SUFFICIENT TO STATE FINDINGS WILL BE DISCUSSED): We compiled a database of 20 baseline and 15 intervention notes as of the time of this writing. Compared to baseline notes, the first three items of the assessment and plan (A\&P) of intervention notes were more likely to contain brief, clear plans (average of 2.73 out of 3 problems v. 2.18 out of 3 problems, $P=0.023$ ). Intervention notes were also rated higher on 5-point Likert scales for being truthful $(4.2$ v. 3.63, $P=$ 0.009 ), updated ( 3.8 v. $3.15, P=0.023$ ), and reasoned (3.7 v. 3.2, $P=0.025$ ). Likert scores for overall quality trended toward improvement, but did not reach statistical significance ( 3.43 v. $3.18, P=0.29)$. Intervention notes also trended toward improvement in reduction of verbatim copying from the prior day's note in the first three problems of the A\&P (average of 2.6 out of 3 problems changed v. 2.23 out of 3 problems changes, $P=0.14$ ). Baseline and intervention notes were not significantly different in terms of note length (109.5 lines v. 108.9 lines, $P=0.96$ ). When baseline and intervention notes were combined, notes with brief summary statements atop the A\&P and those with prioritized problem lists trended toward higher scores for overall quality (3.45 with summary statements v. 3.03 without, $P=0.11 ; 3.39$ with prioritized problem lists v. 2.17 without, $P=0.1$ ). Higher quality scores directly correlated with notes containing more brief, clear plans for the first three problems in the A\&P $\left(\beta=0.43, \mathrm{R}^{2}=0.22\right)$ and with notes that had different text within the first three problems of the $A \& P\left(\beta=0.46, R^{2}=0.25\right)$. Note length was weakly but inversely correlated with Likert-scores for quality $\left(\beta=-0.01, R^{2}=0.28\right)$. KEY LESSONS FOR DISSEMINATION (WHAT CAN OTHERS TAKE AWAY FOR IMPLEMENTATION TO THEIR PRACTICE OR COMMUNITY?): This ongoing project suggests that brief educational interventions combined with evidencebased, consensus-driven note templates can significantly improve the quality of internal medicine resident progress notes. Such efforts are best focused on encouraging brief summary statements atop the A\&P, prioritize problem lists, and aggressive editing of the A\&P text. Encouraging removal of extraneous information may facilitate better attention being paid to content most crucial for effective communication.

MAKING THE DISCHARGE SUMMARY COUNT: A RESIDENT-LED WORKSHOP TO IMPROVE TRANSITION OF CARE Syed W. Haider; Anjeet K. Saini; Angel Mier Hicks; Bernard S. Kadosh; Jamie C. Riches; Erica Bostick; Guido Barmaimon; Deepti Zalavadia; Tamara Goldberg. Icahn School of Medicine at Mount Sinai St. Luke's and West, New York, NY. (Control ID \#2466290)

STATEMENT OF PROBLEM OR QUESTION (ONE SENTENCE): In our modern healthcare system, effective transitions of care often rely on resident physicians' proper EMR documentation at discharge, but without standardization and proper training, such summaries may be inadequate and can result in adverse patient outcomes.

OBJECTIVES OF PROGRAM/INTERVENTION (NO MORE THAN THREE

OBJECTIVES): Through a resident-driven approach, we aim to train our house staff in the composition of high quality discharge summaries in order to improve communication between the inpatient and outpatient setting.
DESCRIPTION OF PROGRAM/INTERVENTION, INCLUDING ORGANIZATIONAL CONTEXT (E.G. INPATIENT VS. OUTPATIENT, PRACTICE OR COMMUNITY CHARACTERISTICS): Our residents rotate in quartiles through our urban underserved primary care clinic every 6 weeks for a 2-week period. During the 2014-2016 academic years, resident quality improvement leaders from each clinic block initiated a longitudinal, resident-driven project to improve provider communication between the inpatient and outpatient setting. We first performed a needs assessment of the existing discharge summary process at our institution that consisted of both a survey of resident attitudes, as well as a retrospective EMR review of discharge summary content. Next, utilizing both the needs assessment and a comprehensive literature search, we developed an ideal discharge summary template. Our educational intervention consisted of a resident-led, $45 \mathrm{~min}$ teaching session that focused on constructing a proper discharge summary using our proposed template. These pilot sessions were mandatory for first year residents rotating on the inpatient ward and were held in place of morning report. Special emphasis was placed on writing a concise hospital course with a problem-based approach (prioritized from most active to least active medical problem), medication reconciliation, and pending labs and referrals. At the end of the workshop, a sample patient from a resident's list was randomly selected and a collaborative group discharge summary was created to provide a real-life example. Pre and post intervention surveys were administered to attendees.

MEASURES OF SUCCESS (DISCUSS QUALITATIVE AND/OR QUANTITATIVE METRICS WHICH WILL BE USED TO EVALUATE PROGRAM/ INTERVENTION): Pre and post intervention survey data was used to assess the educational value of the workshop. In the coming weeks, discharge summaries of attendees will be tracked and scored with an audit tool to objectively assess documentation of various key points taught during the workshop.

FINDINGS TO DATE (IT IS NOT SUFFICIENT TO STATE FINDINGS WILL BE DISCUSSED): Of 18 residents surveyed for our initial needs assessment, $91 \%$ agreed that a change in the discharge summary process was necessary at our institution. Prior to the intervention, an EMR review of 22 discharge summaries showed $75 \%$ lacked information on medication reconciliation and follow-up tests, and $40 \%$ lacked information on post-discharge referrals. Interestingly, $91 \%$ of residents surveyed before the workshop intervention felt their own discharge summaries were useful, and yet, of the 30 post-intervention surveys completed, $93 \%$ felt their discharge summaries would subsequently improve after the resident-led workshop.

KEY LESSONS FOR DISSEMINATION (WHAT CAN OTHERS TAKE AWAY FOR IMPLEMENTATION TO THEIR PRACTICE OR COMMUNITY?): Through a ground-up, resident-led quality improvement effort, we created a promising educational intervention to address transitions of care in our clinic. Our needs assessment results show that the majority of discharge summaries written by residents are lacking important medical information, which can negatively affect outpatient care. However, our residents overwhelmingly felt that our educational workshop will lead to improvement in meaningful documentation. For future directions, an audit tool will help us objectively evaluate the longitudinal impact of this discharge summary workshop.

MARKERS FOR THE WIN: A NOVEL CLINICAL DECISION SUPPORT SYSTEM FOR CHRONIC DISEASE MANAGEMENT Eunice Yu ${ }^{1}$; Leah Marcotte ${ }^{2}$; Andrew Schutzbank ${ }^{1}$; Suzanne Gray ${ }^{1}$. ${ }^{1}$ Iora Health, Detroit, MI; ${ }^{2}$ Iora Primary Care, Seattle, WA. (Control ID \#2469182)

STATEMENT OF PROBLEM OR QUESTION (ONE SENTENCE): Clinical decision support (CDS) for routine chronic disease management suffers from defects in usability including alert fatigue, poor integration into workflow, inability to customize targets, and fragmented data streams. 
OBJECTIVES OF PROGRAM/INTERVENTION (NO MORE THAN THREE OBJECTIVES): - Identify design features that increase usability of CDS tools -Analyze defects in chronic disease management on population levels that could be addressed with marker sets

DESCRIPTION OF PROGRAM/INTERVENTION, INCLUDING ORGANIZATIONAL CONTEXT (E.G. INPATIENT VS. OUTPATIENT, PRACTICE OR COMMUNITY CHARACTERISTICS): Iora Health is comprised of twenty primary care practices across the country. These practices serve a wide range of populations entirely under full-risk, capitated payment systems, partnering with Medicare Advantage programs, insurance programs offered via State Health Insurance Exchanges, unions, and private employers. Many of the populations served by Iora Health are low-income and/or underserved. The electronic health record (EHR), Chirp, is home-grown and was built primarily to support clinicians in documentation and clinical decision making (almost in exclusion of any billing functionality). Iora Health includes a robust information technology team that works closely with clinicians to continue to design and improve the EHR. Clinical marker sets are a core clinical decision support tool embedded into Chirp. Markers are individual measurements that include a value, a goal range, and a default frequency for updating. Values can be discrete ("Yes/No" or PHQ-2 score "0, 1, or 2") or continuous (weight, creatinine, 10-year cardiovascular risk score). Values may be entered by any user on the care team as part of their workflow and will update across marker sets. All marker goals and frequencies are customizable to the individual patient with one click. When marker values are out of goal or out of date, they will turn red to highlight the need for attention. Decision support is embedded in the logic of the parameters as well as user-text at the top of the value entry box. Marker sets are curated sets of clinical indicators organized into preventive care and chronic conditions and displayed in a visually appealing format. They include clean, standardized data feeds from labs, and very soon medications, that update automatically. A clinician selects a relevant marker set for a given condition to embed a list of clinically relevant indicators, including elements of history, vitals, risk scores, and labs, that are visible within one screen. This feature allows for a quick and cognitively easy monitoring of parameters that should be addressed Current marker sets include Prevention, Asthma, Behavioral Health, COPD, Chronic Kidney Disease, Complex Case Management, Congestive Heart Failure, Coronary Artery Disease, Diabetes, Geriatric Assessment, Hyperlipidemia, Hypertension, and Pregnancy. The parameters and logic of marker sets are clinician-guided, written and coded in partnership with the software development team. Marker sets can be developed and integrated into the EHR system in a matter of weeks. The modular design of the marker sets allows them to be quickly written and expandable to an unlimited number of conditions.

MEASURES OF SUCCESS (DISCUSS QUALITATIVE AND/OR QUANTITATIVE METRICS WHICH WILL BE USED TO EVALUATE PROGRAM/ INTERVENTION): Measures of success include user-reported: -understanding about its use -integration into workflow -ability to customize targets to the individual -low alert fatigue

FINDINGS TO DATE (IT IS NOT SUFFICIENT TO STATE FINDINGS WILL BE DISCUSSED): Care teams consistently use clinical marker sets across diverse populations to support guideline-based, high quality care delivery. Further, the demand for the expansion of the clinical marker sets remains high based on the growing number of requests from users (care teams) to develop new marker sets. Although not yet formally studied, the development team works closely with front-line care teams and receives few complaints about usability or clinical workflow integration of marker sets.

KEY LESSONS FOR DISSEMINATION (WHAT CAN OTHERS TAKE AWAY FOR IMPLEMENTATION TO THEIR PRACTICE OR COMMUNITY?): -Clin-

ical marker sets represent an elegant solution to the common problems faced by many ITenabled clinical decision support interventions -Clinical marker sets require clean, integrated data streams for successful implementation -Design principles guiding an iterative building process are critical to developing decision support by allowing for short, manageable timelines and continuous integration into clinical workflow -Integrating clinicians into the development of clinical decision support tools is critical to creating relevant and usable products

NURSE PRACTITIONER IMPACT ON DIABETES OUTCOMES IN AN URBAN ACADEMIC PATIENT-CENTERED MEDICAL HOME. Serge Bebko; Mahmoud Ali; Vallari K. Kothari; Mohamed Altattan; Terri Brown; David E. Willens. Henry Ford Hospital, Detroit, MI. (Control ID \#2469587)

STATEMENT OF PROBLEM OR QUESTION (ONE SENTENCE): Previous studies have shown that nurses specialized in diabetic care using treatment protocols provided equally effective care comparable to standard of care, with less cost and significantly better patient satisfaction. The Henry Ford Health System Diabetes in Active Control (DIAC) program trained and supported one embedded nurse practitioner in our urban academic patient centered medical home (PCMH).

OBJECTIVES OF PROGRAM/INTERVENTION (NO MORE THAN THREE OBJECTIVES): Our objective was to demonstrate that referral of care by a primary care physician (PCP) to a nurse practitioner (NP) specialized in diabetes is an effective method for managing diabetic patients with poor glycemic control in an urban academic PCMH. DESCRIPTION OF PROGRAM/INTERVENTION, INCLUDING ORGANIZATIONAL CONTEXT (E.G. INPATIENT VS. OUTPATIENT, PRACTICE OR COMMUNITY CHARACTERISTICS): We performed a retrospective chart review of all patients diagnosed with Diabetes type II per ICD-9 criteria, in an urban academic patient-centered medical home from $03 / 01 / 2013$ to $01 / 01 / 2015$. Patients were referred to a Diabetes Nurse Practitioner by a clinic PCP if they had at least one of the following criteria: uncontrolled Diabetes, defined as persistent hyperglycemia despite adequate treatment; patients on simultaneous long and short acting insulin treatment; a glycosylated hemoglobin level $(\mathrm{HbA} 1 \mathrm{c})>10 \%$. Patients were included in the present study if they were referred to the NP by their PCP and seen by the NP at least 2 times within a year Each visit with the NP lasted 30 to $60 \mathrm{~min}$. During each visit, the NP helped the patient to identify barriers for Diabetes control. Patients also received Diabetes education using visual aids, teach-back techniques and printed materials. They were educated on eating habits and portion sizes, carbohydrate counting. NP was able to order laboratory exams, titrating diabetes medication and do referral to other specialties

MEASURES OF SUCCESS (DISCUSS QUALITATIVE AND/OR QUANTITATIVE METRICS WHICH WILL BE USED TO EVALUATE PROGRAM/ INTERVENTION): Data collection included demographic information such as gender, age and race, total number and frequency of NP visits and PCP visits. HbAlc levels were collected from 6 months prior to 2 weeks after the time of the first visit (T0), and after 6 months \pm 3 months of T0 (T1). The primary outcome was defined as the change in HBA1c levels between $\mathrm{T} 0$ and $\mathrm{T} 1$

FINDINGS TO DATE (IT IS NOT SUFFICIENT TO STATE FINDINGS WILL BE DISCUSSED): A total of 119 patients were included. The mean age of the sample was $62.1 \pm 11.1$ years, $47.0 \%$ were male and $90.8 \%$ were African American. The mean number of PCP and NP visits was 2.5 and 3.1, respectively. Of 119 patients at T0, $68 \%$ of patients had an $\mathrm{HbA} 1 \mathrm{c}$ level measured at T6. A univariate analysis showed a statistically significant decrease in median $\mathrm{HbAlc}$ levels in patient seen by the NP in a 6 month period. Median HbAlc at T0 was 8.0 (IQR 3.7), compared to a median HbAlc of 7.4 (IQR 2.7) at T6. A multivariate analysis showed that, when controlling for age, number of $\mathrm{PCP}$ and NP visits, patients seen by the NP for a first period of 6 months had a mean decrease of $0.97 \%$ in $\mathrm{HbA1c}$ levels. Age and number of PCP visits were also found to be significant predictors of a decrease in $\mathrm{HbA} 1 \mathrm{c}$ levels. The number of NP visits was not a significant predictor of a decrease in $\mathrm{HbA} 1 \mathrm{c}$ levels after controlling for the aforementioned covariates.

KEY LESSONS FOR DISSEMINATION (WHAT CAN OTHERS TAKE AWAY FOR IMPLEMENTATION TO THEIR PRACTICE OR COMMUNITY?): Our 
results support the findings from previous studies, showing that referral of diabetic care to a nurse practitioner specialized in diabetes may be an effective method for improving diabetes care. However, the adjusted analysis shows that patient age and PCP visits may drive improvement more than NP care.

OPTIMIZING THE VALUE OF HEALTHCARE THROUGH CLINICAL PATHWAY REDESIGN Roselyn Cristelle I. Mateo; Christine Tsai; Amanda Tosto; Grace Lee; Jaini Suttaria; Sean Ryan; Matthew Brugger; Paul Cabanilla; Ishaq Lat; Jordan Dale; Lesley Schmaltz; Phil Zaborowski; Joann Wielosinski; Ruth Kniuksta; Jisu Kim; Amir Jaffer. Rush University Medical Center, Chicago, IL. (Control ID \#2466681)

STATEMENT OF PROBLEM OR QUESTION (ONE SENTENCE): Hospital reimbursement models continue to evolve and payer contracts are becoming increasingly risk based, driving institutions and clinicians to focus their efforts not only on quality but also value. OBJECTIVES OF PROGRAM/INTERVENTION (NO MORE THAN THREE OBJECTIVES): To develop Clinical Care Pathways through a multidisciplinary team in order to detail essential steps in the care of patients with a specific clinical problem. To equip housestaff in an academic center with evidence based management tools aimed to optimize clinical outcomes. To evaluate the impact of multidisciplinary clinical care pathways on outcomes, cost and efficiency for common diagnoses such as chest pain, venous thromboembolism, and pneumonia.

DESCRIPTION OF PROGRAM/INTERVENTION, INCLUDING ORGANIZATIONAL CONTEXT (E.G. INPATIENT VS. OUTPATIENT, PRACTICE OR COMMUNITY CHARACTERISTICS): At Rush University Medical Center we have increasingly focused our efforts on clinical redesign using multidisciplinary clinical pathways to increase the value of care. In this age of electronic medical record (EMR) systems, order sets provide an optimal platform for institutions to standardize evidence based guidelines. Through collaboration with hospitalists, subspecialists, pharmacists, performance improvement experts, information system advisors and financial analysts, we identified the three most commonly encountered diagnoses on the inpatient general medical floors including chest pain, pneumonia and venous thromboembolism. We reviewed the current literature on diagnosis and management of these conditions and the University Health Consortium key performance indicators such as length of stay, cost per case, readmission rates and mortality. We also focused on areas of high cost at Rush, specifically choice of intervention, pharmacotherapy, triage location, and length of stay. We combined the results of our review into order sets that preferentially selected lower cost tests or aimed to decrease length of stay without sacrificing quality, thereby increasing value in patient care. Our new disease-specific evidence-based clinical pathways and order sets incorporated therapies and strategies aimed to increase value for patient care. The essential next step after development was implementation and adoption. This required extensive education of attending hospitalists and internal medicine housestaff to incorporate these order sets into their workflow and practice. We identified many barriers to adoption including institutional culture, ordering patterns, awareness and use of existing information technology and evidence based guidelines and strove to overcome these challenges through various modalitie including but not limited to educational sessions, noontime housestaff conferences, reminder notifications.

MEASURES OF SUCCESS (DISCUSS QUALITATIVE AND/OR QUANTITATIVE METRICS WHICH WILL BE USED TO EVALUATE PROGRAM/ INTERVENTION): Several metrics including the percentage of utilization of the order sets and their effect on length of stay, cost per case, and projected savings for the fiscal year were monitored.

FINDINGS TO DATE (IT IS NOT SUFFICIENT TO STATE FINDINGS WILL BE DISCUSSED): Since the implementation of these pathways, we observed a total cost savings of $\$ 136,137$ in the first quarter or on average $\$ 495$ per case compared to the year prior without an impact on readmission rates. The savings annualized would equal over half a million dollars. Despite the significant savings, the use of order sets remains significantly underutilized likely reflecting a need for additional means of inducing culture change.

KEY LESSONS FOR DISSEMINATION (WHAT CAN OTHERS TAKE AWAY FOR IMPLEMENTATION TO THEIR PRACTICE OR COMMUNITY?): Initial results from our clinical redesign project show promising trends in reducing cost and increasing value. We believe dissemination and adoption of our methods for clinical redesign to deliver value based care has extensive applicability at other medical centers across the country. Every institution can likewise utilizing a multidisciplinary approach in order to identify most commonly encountered diagnoses and areas of potential cost savings, as we transition from volume to value with more risk-based payment models.

OUTCOMES OF AN ATTENDING-ONLY HOSPITALIST BEDSIDE PROCEDURE SERVICE Kierstin C. Kennedy ${ }^{1}$; Nicole Redmond ${ }^{3}$; Benjamin Taylor ${ }^{2}$; Joshua D. Lenchus ${ }^{4} .{ }^{1} \mathrm{UAB}$, Birmingham, AL; ${ }^{2} \mathrm{UAB}$ School of Medicine, Birmingham AL, AL; ${ }^{3}$ University of Alabama at Birmingham, Birmingham, AL; ${ }^{4}$ University of Miami, Miami, FL. (Control ID \#2459884)

STATEMENT OF PROBLEM OR QUESTION (ONE SENTENCE): Academic medical centers with trainee programs have addressed decreasing procedural competency in internists (and increased outsourcing of bedside procedures) by creating procedure services, however this intervention would not benefit hospitalist groups without trainees. OBJECTIVES OF PROGRAM/INTERVENTION (NO MORE THAN THREE OBJECTIVES): To determine the impact of an attending-only hospitalist run procedure consultation service on complication rates, time to procedure, and revenue generation. DESCRIPTION OF PROGRAM/INTERVENTION, INCLUDING ORGANIZATIONAL CONTEXT (E.G. INPATIENT VS. OUTPATIENT, PRACTICE OR COMMUNITY CHARACTERISTICS): Eight hospitalists at a tertiary care academic medical center completed standardized ultrasound guided bedside procedure training for central venous catheter placement, paracentesis, thoracentesis, and lumbar puncture. One nurse clinician assisted with accepting consults, procedure set up, follow up and data collection. One trained hospitalist and the nurse clinician were assigned to the procedure service from August 2014 through October 2015.

MEASURES OF SUCCESS (DISCUSS QUALITATIVE AND/OR QUANTITATIVE METRICS WHICH WILL BE USED TO EVALUATE PROGRAM/ INTERVENTION): For each procedure administrative, clinical, and financial data were collected including: consulting service, patient and clinical characteristics, procedure type, procedure outcomes, complications, and charges.

FINDINGS TO DATE (IT IS NOT SUFFICIENT TO STATE FINDINGS WILL BE DISCUSSED): Between August 2014 and November 2015 there were a total of 586 consults to perform bedside procedures, of which 546 (93\%) were attempted. Of the procedures attempted 499 (91\%) were successful. There was one procedure related complication $(0.18 \%)$ and the average time from consult to procedure completion was 0.01 days. The total number of procedure CPT codes and charges billed for the hospitalist program increased from an average of $5.1 \mathrm{CPT}$ codes per month $(\$ 2707.70$ billed per month) in the year prior to the start of the procedure service to $23.3 \mathrm{CPT}$ codes per month ( $\$ 47,972$ billed per month) after the procedure services' inception.

KEY LESSONS FOR DISSEMINATION (WHAT CAN OTHERS TAKE AWAY FOR IMPLEMENTATION TO THEIR PRACTICE OR COMMUNITY?): A bedside procedure service staffed by hospitalists can yield low wait times for procedures without increasing complication rates and can simultaneously increase revenue. An 'attending-only' staffed procedure service model is feasible and may have broader implications for other Hospitalist programs in settings other than academic medical centers. 
OVERCOMING DIABETES: A RESIDENT-DRIVEN INITIATIVE TO ENGAGE HIGH RISK DIABETICS IN AN UNDERSERVED COMMUNITY. Eric Yudelevich; Gabriela Bambrick-Santoyo; Diego A. Adrianzen Herrera; Regina Belokovskaya; Krishna A. Chokshi; Medha Satyarengga; Stefania Pirrotta; Maryum Hussain; Dipal R. Patel; Tamara Goldberg. Mount Sinai St. Luke's West Hospital Center, New York, NY. (Control ID \#2469584)

STATEMENT OF PROBLEM OR QUESTION (ONE SENTENCE): What is the best way to improve hemoglobin Alc (HbAlc) control among an underserved, uncontrolled diabetic population at an urban community-based primary care practice? OBJECTIVES OF PROGRAM/INTERVENTION (NO MORE THAN THREE OBJECTIVES): 1 . To design a longitudinal, resident-driven quality improvement initiative to address our uncontrolled diabetic patients ( $\mathrm{HbAlc} \geq 9 \%$ ). 2 . To determine the impact on $\mathrm{HbAlc}$ control of a standardized, resident-led "High Risk Diabetic Visit" ("HRDV"). 3. To determine which factors are associated with improved control among this high-risk population.

DESCRIPTION OF PROGRAM/INTERVENTION, INCLUDING ORGANIZATIONAL CONTEXT (E.G. INPATIENT VS. OUTPATIENT, PRACTICE OR COMMUNITY CHARACTERISTICS): From June 2014 to December 2015, our house staff participated in a rigorous, resident-driven quality improvement project at our Federally Qualified Health Center practice site. To address our uncontrolled diabetic population, we first partnered with our clinical care manager and practice IT department to create a registry of our uncontrolled diabetic patients ( $\mathrm{HbAlc} \geq 9 \%$ ). These patients received various interventions from our clinical care team including: proactive care manager outreach to review barriers to care, nursing staff pre-visit phone call reminders, and nutrition visits. After 6 months, we implemented an "adopt-a-patient" intervention in which each of the patients received a personalized provider phone call and an appointment for a standardized, "High Risk Diabetic Visit". The resident-led HRDV included a brief Power Point presentation on the etiology of diabetes, importance of HbAlc control, and discussion of individualized goals. The presentation was geared at an appropriate literacy level for our population and was available in both English and Spanish. Resident providers were educated on the format of the visit during the pre-clinic huddle and encouraged to emphasize the importance of care continuity with the patients.

MEASURES OF SUCCESS (DISCUSS QUALITATIVE AND/OR QUANTITATIVE METRICS WHICH WILL BE USED TO EVALUATE PROGRAM/ INTERVENTION): At the end of the study period, we performed a retrospective chart review to track $\mathrm{HbA} 1 \mathrm{c}$ outcomes. To determine the impact of our HRDV intervention, we compared $\mathrm{HbA} 1 \mathrm{c}$ levels immediately prior to and at least 30 days after the HRDV, and used two-tailed student t-test analysis to compare mean $\mathrm{HbAlc}$ levels between patients who attended the HRDV and those who did not. Finally, using Chi-squared testing $(p<.05)$, we determined the statistical significance of six variables on $\mathrm{HbAlc}$ control among our single arm cohort: number of medical visits, number of nutritionist visits, care manager outreach encounters, MA/RN outreach encounters, provider outreach encounters, and attendance at a High Risk Diabetic Visit.

FINDINGS TO DATE (IT IS NOT SUFFICIENT TO STATE FINDINGS WILL BE DISCUSSED): We identified 106 uncontrolled diabetic patients for our registry, and 77 had a follow-up HbAlc during the study period, of which $48.1 \%(n=37)$ met their goal at some point during the study period and $62 \%$ improved overall. A total of 24 patients had a HRDV with post-visit HbAlc available for comparison. Those who attended their HRDV had a statistically significant mean $\mathrm{HbA1c}$ reduction compared to those who did not $(-0.75$ vs. $+0.85, p=0.001) .79 \%(n=19)$ had some decrease in their HbAlc (range 0.2-5.8) while $21 \%(n=5)$ had an increase in their level (range 0.5-1.6). None of the factors examined (number of medical visits, nutrition visits, care manager outreach encounters, MA/RN outreach encounters, or provider outreach encounters) were found to be significantly associated with $\mathrm{HbA} 1 \mathrm{c}$ control among our cohort.
KEY LESSONS FOR DISSEMINATION (WHAT CAN OTHERS TAKE AWAY FOR IMPLEMENTATION TO THEIR PRACTICE OR COMMUNITY?): The creation of a diabetic registry and the implementation of a standardized 'High Risk Diabetic Visit' are efficient and effective ways to reduce HbAlc among an uncontrolled, poor patient population. We hypothesize that a longer visit duration with a standardized, patient-centered educational component encourages patient engagement in care. Despite our various forms of outreach, we did not find a statistically significant association for $\mathrm{HbAlc}$ control favoring any of the variables examined, highlighting the complexity of patient engagement in care among this population. Future analysis should explore off-site factors such as mental health disorders, level of family support, co-morbid conditions, specialist visits, and financial status to determine other factors that may impact overall control.

PATIENT ALIGNED CARE TEAM INTERDISCIPLINARY CARE UPDATE IMPACT OF A COMPLEX PATIENT CASE CONFERENCE ON QUALITY AND UTILIZATION William G. Weppner ${ }^{2,3}$; C. Scott Smith ${ }^{2,3}$; Rick Tivis ${ }^{1}$; Amber Fisher ${ }^{3}$; India C. King ${ }^{1}$; Timothy Gordon ${ }^{1} .{ }^{1}$ Boise VA Medical Center, Boise, ID; ${ }^{2}$ University of Washington, Boise, ID; ${ }^{3} \mathrm{VA}$, Boise, ID. (Control ID \#2466094)

STATEMENT OF PROBLEM OR QUESTION (ONE SENTENCE): Do interprofessional case conferences for high risk, medically complex patients improve quality of care and utilization of health care resources?

OBJECTIVES OF PROGRAM/INTERVENTION (NO MORE THAN THREE OBJECTIVES): Evaluate the impact of an interprofessional case conference on: 1. Quality of care of chronic diseases 2. Clinical contacts with primary care providers and members of the primary care team 3. Utilization of urgent care, emergency room and hospitalizations

DESCRIPTION OF PROGRAM/INTERVENTION, INCLUDING ORGANIZATIONAL CONTEXT (E.G. INPATIENT VS. OUTPATIENT, PRACTICE OR COMMUNITY CHARACTERISTICS): The Boise VAMC Centers of Excellence in Primary Care Education developed the "PACT ICU" (Patient Aligned Care Team Interdisciplinary Care Update) — an interprofessional case conference to improve care of high risk patients in primary care. Based at our teaching clinic of approximately 32 medica residents and other trainees serving more than 2,000 patients, this conference provides a regularly scheduled opportunity to develop coordinated patient-centered care plans with interprofessional collaboration. On a bimonthly basis, rotating physician and nurse practitioner residents lead discussion of a high risk patient selected from the top five highest risk patients as predicted by the VA-based Care Assessment Needs registry, a score which estimates probability of hospitalization or death in the next 90 days. A clinic-based registered nurse uses a web-based schedule and guide to facilitate selection and review of patients. The nurse and a physician lead coordinates an hour-long conference with interdisciplinary team members, including medicine residents and attendings, nurse practitioner trainees and supervisors, primary care team nurses, pharmacists, psychologists and behavioral health staff, and social workers. Tasks developed in the care plan are shared among team members, who pro-actively coordinate need contacts and consultations with team members. Patients are reviewed 2 months after presentation for progress.

MEASURES OF SUCCESS (DISCUSS QUALITATIVE AND/OR QUANTITATIVE METRICS WHICH WILL BE USED TO EVALUATE PROGRAM/ INTERVENTION): For each patient presented in PACT ICU, a control from the four patients not selected for presentation was assigned using a propensity score based on closest CAN score and age. Patient and control characteristics of gender, age, CAN Score, propensity score and death were recorded. Encounters with primary care providers and associated primary care team members (pharmacy, behavioral health, nursing) were tracked, as were urgent care, emergency room and hospitalizations for PACT ICU patients and controls at baseline and up to 6 months after presentation. Average blood pressure and 
hemoglobin A1c was recorded pre- and post- presentation for patients with a diagnosis of hypertension or diabetes, respectively.

FINDINGS TO DATE (IT IS NOT SUFFICIENT TO STATE FINDINGS WILI BE DISCUSSED): During the observation period, 105 patients were presented in the PACT ICU; 105 controls were selected; there were no significant differences in gender, age, CAN score or propensity score. Of note, $9 \%$ of controls had died in the 6 months after selection, compared to $3 \%$ in the PACT ICU group $(p=0.02)$. At baseline, patients selected for PACT ICU presentation had more PCP visits than controls, which had a non-significant decrease in the following 6 months compared to controls ( $p=0.07$ ). PACT ICU patients had higher team visits (e.g. pharmacy, behavioral health, nursing) than controls, which persisted over time ( $p=0.0002$ ). There was a non-significant trend towards decreased urgent care and emergency visits $(p=0.19)$, and a decreased amount of hospitalizations in the PACT-ICU patients compared to controls $(p=0.05)$. Utilization data from patients was excluded from analysis if they died during observation period, or had been presented previously. Sensitivity analyses done including deceased patient data did not affect conclusions. For hypertension control, PACT ICU patients $(n=67)$ compared with controls $(n=53)$ had a decrease in mean systolic and diastolic blood pressure from 137 to 131 and 81 to 79 , respectively. This was statistically a significant trend when compared to controls for systolic $(p=0.005)$ but not for diastolic $(p=0.05)$. PACT ICU patients with diabetes $(n=12)$ saw a decrease in hemoglobin Alc from 9.0 to 8.6, which was non-significant when compared to trends in controls $(n=12)$.

KEY LESSONS FOR DISSEMINATION (WHAT CAN OTHERS TAKE AWAY FOR IMPLEMENTATION TO THEIR PRACTICE OR COMMUNITY?): These findings suggest that interprofessional case conferences such as PACT ICU may facilitate increased utilization by team members other than the PCP. Such conferences and subsequent team-based care may lead to improvements in chronic disease management, trends towards reduced urgent/emergent care services, and overall reduced hospitalizations. Caveats include the observational nature of the data, as well as lower power to detect differences.

PATIENT-WRITTEN VISIT AGENDAS AND THEIR USE IN PRIMARY CARE: “I FELT LIKE I WAS ABLE TO BE HEARD” Sara L. Jackson ${ }^{3}$; McHale Anderson ${ }^{2}$; Natalia Oster ${ }^{2}$; Sue Peacock ${ }^{2}$; Galen Chen $^{2}$; Janice Walker ${ }^{3}$; Joann G. Elmore ${ }^{1}{ }^{1}$ Univ of WA, Seattle, WA; ${ }^{2}$ University of Washington, Seattle, WA; ${ }^{3}$ Beth Israel Deaconess Medical Center, Harvard Medical School, Boston, MA. (Control ID \#2466169)

STATEMENT OF PROBLEM OR QUESTION (ONE SENTENCE): Are patientwritten visit agendas feasible in a primary care safety-net practice, and will providers and patients perceive that they improve communication?

OBJECTIVES OF PROGRAM/INTERVENTION (NO MORE THAN THREE OBJECTIVES): 1 . To assess if patient-written agendas are feasible in a safety-net primary care practice. 2 . To assess patient and provider perceptions about communication, efficiency, and desire to continue in the future. 3. Utilize free text responses to better understand primary positive and negative experiences with patient-written visit agendas for patients and providers.

DESCRIPTION OF PROGRAM/INTERVENTION, INCLUDING ORGANIZATIONAL CONTEXT (E.G. INPATIENT VS. OUTPATIENT, PRACTICE OR COMMUNITY CHARACTERISTICS): We recruited patients from a large academic teaching clinic at Harborview Medical Center, a safety-net county hospital in Seattle, WA. Participating patients were asked to type their own visit agenda (i.e., a list of questions and/ or concerns they wanted to address during the visit) into their electronic medical record before being seen by their physician. Both patients and providers were surveyed after the clinic visit to assess their perceptions of the patient-typed agenda and patient-provider communication

MEASURES OF SUCCESS (DISCUSS QUALITATIVE AND/OR QUANTITATIVE METRICS WHICH WILL BE USED TO EVALUATE PROGRAM/
INTERVENTION): We assessed the number of patients who are interested in typing visit agendas into the electronic medical record, among those eligible (able to read and write in English and comfortable with typing). We also used 5 point Likert scales to assess patient and provider perceptions of provider preparedness and understanding, efficiency of visit, and desire to use in the future. Qualitative responses were analyzed for primary positive and negative themes for patients and providers experience with patient-written agendas.

FINDINGS TO DATE (IT IS NOT SUFFICIENT TO STATE FINDINGS WILL BE DISCUSSED): One hundred and one patients of 28 primary care clinicians typed an agenda and completed the survey. The response rate was $70.1 \%$ of invited and eligible patients, who were able to read and write English and comfortable typing on a computer. Agendas were an average of 27 words in length (range 1 to 420) and took and average of five minutes for patients to type. Using a 5-point Likert scale, the majority of providers and patients agreed or strongly agreed that the patient-typed agendas improved the provider's understanding of the patient's priorities for the visit ( 75 and $75 \%$, respectively); helped prioritize the visit ( 84 and $79 \%$ ); reported that the doctor seemed more prepared because of the patient agenda ( 82 and $75 \%$ ); viewed the visits as more efficient ( 80 and $67 \%$ ); and reported that patient-typed agendas improved patient-provider communication (75 and $79 \%$ ). Lastly, both providers and patients reported a desire to use them in the future (79 and $72 \%$ ). Qualitative review of survey comments supported these findings with one patient stating, "I felt like I was able to be heard."

KEY LESSONS FOR DISSEMINATION (WHAT CAN OTHERS TAKE AWAY FOR IMPLEMENTATION TO THEIR PRACTICE OR COMMUNITY?): Patients from a safety-net population demonstrated willingness and ability to type their own agendas before clinic visits into the electronic medical records. Both patients and providers agreed that patient-typed agendas improved efficiency and patient-provider communication during visits. Enabling patients to type visit agendas may enhance care by engaging the patient and giving providers a useful and efficient way to prioritize patients' concerns.

PEER AUDITS AND FEEDBACK TO IMPROVE DOCUMENTATION QUALITY Allison C. Heacock ${ }^{2}$; Kristen Lewis ${ }^{2}$; Rashmi Ganith ${ }^{2}$; Jennifer Allen ${ }^{2}$; Kimberly M. Tartaglia ${ }^{1} .{ }^{1}$ The Ohio State University Medical Center, Columbus, OH; ${ }^{2}$ The Ohio State University Wexner Medical Center, Columbus, OH. (Control ID \#2469473)

STATEMENT OF PROBLEM OR QUESTION (ONE SENTENCE): Within our large academic hospitalist division, faculty expressed concern over the variability in quality and consistency of documentation in both progress notes and service hand offs. OBJECTIVES OF PROGRAM/INTERVENTION (NO MORE THAN THREE OBJECTIVES): 1. Create a standard framework for documentation within the electronic medical record regarding progress notes and service hand-offs 2. Provide hospital medicine division members with individual and aggregate feedback regarding the quality of their progress note documentation and handoff communication

DESCRIPTION OF PROGRAM/INTERVENTION, INCLUDING ORGANIZATIONAL CONTEXT (E.G. INPATIENT VS. OUTPATIENT, PRACTICE OR COMMUNITY CHARACTERISTICS): In an attempt to define best practices for documentation within our hospitalist group, we created both a documentation and handoff task force. Through survey data and an iterative process, templates were created for both progress notes and service hand-offs and education to physicians was completed through monthly faculty meetings. However, physicians still reported use of daily progress note and handoff templates to be 88 and $70 \%$, respectively. At the same time $79 \%$ of physicians favored a peer review process to receive feedback on their documentation. In response, we developed a peer review process to audit and provide feedback to individual physicians and the hospitalist group regarding quality of progress note and handoff 
documentation. We selected the PDQI9 as a validated tool by which faculty could review progress note quality. For service hand-offs, we developed a low-inference checklist to assess handoff quality. Our feedback tool was deployed July 2015 and hospitalists are asked to provide anonymous feedback using these tools any time they started on service. To preserve anonymity, feedback is provided to physicians up to quarterly, but only if at least 3 separate reviews have been completed of them. Participation in audit completion is compensated as part of the physician's annual bonus.

MEASURES OF SUCCESS (DISCUSS QUALITATIVE AND/OR QUANTITATIVE METRICS WHICH WILL BE USED TO EVALUATE PROGRAM/ INTERVENTION): Preliminary success of this program is defined by faculty participation through completion of the audit tool and review of aggregated feedback. Ultimately, we intend to track aggregate data and assess potential gaps in documentation and handoff practices to further refine our interventions. We anticipate improved documentation and communication practices by individuals and the division as a whole as measured by improved PDQ19 and handoff survey scores over time.

FINDINGS TO DATE (IT IS NOT SUFFICIENT TO STATE FINDINGS WILI BE DISCUSSED): From July to December 2015, 150 peer reviews of a patient's progress note and handoff have been completed by 56 physicians. For progress notes, physicians have rated their peer's notes as being succinct, organized, and up-to-date with average scores of 4.56, 4.70 and 4.61 out of 5, respectively. Prior to the intervention, when asked physicians about the readability (described as succinct, relevant and organized) of their peer's progress notes, physicians gave an average score of 4.07 out of 5 . The average accuracy score of progress notes was rated a 4.67 out of 5 when baseline survey data show it to be a 4.0 , which is a $13 \%$ increase. With regards to handoffs, use of the handoff template has increased $19 \%$ since instituting peer audits. Handoffs, overall, have been described as easily readable $77 \%$ of the time as opposed to $62 \%$ of the time prior to our peer review process.

KEY LESSONS FOR DISSEMINATION (WHAT CAN OTHERS TAKE AWAY FOR IMPLEMENTATION TO THEIR PRACTICE OR COMMUNITY?): With frequent handoffs among hospitalists, high quality documentation is crucial to maintaining excellence in patient care and preventing errors. We developed templates to improve quality and efficiency of documentation but noted additional opportunities for improvement.By providing hospitalist faculty with the opportunity to review their peers and receive individual feedback regarding the quality of their documentation and handoffs communication, we have given them expectations which are transparent and achievable.We hope to see further improvements with as more physicians receive feedback and plan to expand this intervention to discharge summary documentation.

PICC-ING THE RIGHT IV ACCESS: IMPROVING THE APPROPRIATENESS OF PICC LINE PLACEMENT AT UPMC MONTEFIORE HOSPITAL Andrew Trifan. University of Pittsburgh, Pittsburgh, PA. (Control ID \#2469671)

STATEMENT OF PROBLEM OR QUESTION (ONE SENTENCE): PICC lines are intended for long term ( weeks) access outside the hospital setting, yet are frequently placed in the hospital for various reasons and pulled prior to a patient being discharged home.

OBJECTIVES OF PROGRAM/INTERVENTION (NO MORE THAN THREE OBJECTIVES): 1) Reduce the number of PICC lines not intended for long term outpatient use and pulled prior to a patient being discharged by $15 \%$ 2) Educate housestaff about PICC lines, complications and ultimate indications through handouts 3) Identify the reasons PICC lines are placed on medicine floors at our institution

DESCRIPTION OF PROGRAM/INTERVENTION, INCLUDING ORGANIZATIONAL CONTEXT (E.G. INPATIENT VS. OUTPATIENT, PRACTICE OR COMMUNITY CHARACTERISTICS): This project focused on patients at UPMC
Montefiore Hospital who had PICC lines placed during their hospitalization. Only patients admitted to medicine floors or transferred to medicine floors within 1 day from nonmedicine location (i.e. surgical location, ICU) and had PICC lines placed at the above institution were included. Initial retrospective chart review of PICC lines from OctDec 2013 was undertaken of one general medicine teaching floor. Afterwards, several interventions were conducted throughout Winter/Spring 2014 including: educating house staff teams (medicine, trauma, critical care) about PICC lines through handouts (particularly evidence regarding increased risk infection and blood clots compared to central venous catheters), increasing awareness of when to place PICC lines along with alternative access options (i.e. if transferred out of ICU with internal jugular line that is working, one should continue using it if needed rather than replacing it with PICC line), having a joint IV team leadership under a supervising physician who was directly able to interact with ordering teams about placement of line versus alternatives, improving nurse education and line care (increasing number of peripheral IV requirements for nurses) and finally having increased input from our infectious disease colleagues when considering placing a PICC for antibiotic reasons by way of formal or curbside consult. A similar chart review was then conducted from Oct-Dec 2014 on same medicine floor with same above criteria.

MEASURES OF SUCCESS (DISCUSS QUALITATIVE AND/OR QUANTITATIVE METRICS WHICH WILL BE USED TO EVALUATE PROGRAM/ INTERVENTION): The primary measure was evaluating the number of PICC lines that were pulled prior to a patient being discharged before and after our intervention, with a goal reduction of $15 \%$ less lines pulled. A secondary measure included evaluating effect of ID input and outcome of PICC line removal prior to discharge.

FINDINGS TO DATE (IT IS NOT SUFFICIENT TO STATE FINDINGS WILL BE DISCUSSED): In 2013, there were 22/58 (38 \%) lines pulled prior to discharge. In 2014, there were $16 / 63$ (25\%) lines pulled, an absolute improvement of $13 \%$ less lines removed. Medicine residents were responsible for 70-80\% lines placed, the remainder being ICU locations. Antibiotics were the most common indication for placement at our institution, roughly $66 \%$ cases in both years. In 2013, 18/38 (47\%) of lines placed for antibiotic reasons had ID consult prior to placement, of which 3/18 (17\%) were removed before discharge. 20/38 (52\%) did not have an ID consult of which 8/20 (40\%) were removed prior to discharge. In 2014, 35/42 (83 \%) lines placed for antibiotic reasons had ID consult of which none were removed prior to discharge. 5/7 (71\%) of those without ID consult were removed. Thus, $36 \%$ more ID consults were done in 2014 which resulted in an absolute number of 7 less PICC lines removed in 2014 for antibiotic reasons.

KEY LESSONS FOR DISSEMINATION (WHAT CAN OTHERS TAKE AWAY FOR IMPLEMENTATION TO THEIR PRACTICE OR COMMUNITY?): While there are still many key obstacles, including lack of awareness of complications of PICC lines, ease of placement/difficulty establishing alternative access, and patient/nursing preference regarding blood draws, with basic interventions and raising awareness about PICC lines, $13 \%$ less lines were pulled prior to discharge. Results also show that having an ID consult resulted in dramatic improvement in lines being pulled compared to no ID consult (i.e. may not require long term antibiotics in first place, possible oral alternatives, etc) when considering placement for antibiotic reasons. Our next steps will involve evaluating the effects of an "approval code" on line placement (if patient requires it for antibiotic reasons, then ID attending will evaluate and give approval) as well as a rotating hospitalist to assist housestaff teams with access difficulties such as placement of ultrasound guided IV or external jugular IV.

PILOT OF A LOW-RESOURCE, EHR-BASED PROTOCOL FOR SEPSIS MONITORING, ALERT, AND INTERVENTION Robert L. Fogerty ${ }^{1}$; Christopher Sankey ${ }^{1}$; Kathleen Kenyon ${ }^{2}$; Scott Sussman ${ }^{2}$; Sveinn Sigurdsson ${ }^{3}$; Alan S. Kliger ${ }^{3}$. ${ }^{1}$ Yale School of Medicine, New Haven, CT; ${ }^{2}$ Yale-New Haven Hospital, New Haven, CT; ${ }^{3}$ Yale-New Haven Health System, New Haven, CT. (Control ID \#2467473) 
STATEMENT OF PROBLEM OR QUESTION (ONE SENTENCE): Delays in recognition of clinical deterioration for medical inpatients who develop sepsis during hospitalization have higher mortality than patients who develop sepsis outside of the hospital.

OBJECTIVES OF PROGRAM/INTERVENTION (NO MORE THAN THREE OBJECTIVES): 1) Improved recognition of sepsis-related clinical deterioration in medical inpatients. 2) Reduced delays in the initiation of appropriate sepsis-related therapies, when indicated, in medical inpatients with new onset sepsis. 3) Reduction in mortality, intensive care unit (ICU) utilization, and length of stay (LOS) in medical inpatients with sepsis. DESCRIPTION OF PROGRAM/INTERVENTION, INCLUDING ORGANIZATIONAL CONTEXT (E.G. INPATIENT VS. OUTPATIENT, PRACTICE OR COMMUNITY CHARACTERISTICS): We are using a fully automated, electronic health record (EHR)-based monitoring and alert system for medical inpatients to monitor all medical inpatients for modified SIRS criteria (abnormalities in pulse, respiration rate, temperature, white blood cell count, drop is systolic blood pressure, increase in creatinine). When a patient meets these criteria, the EHR generates a "Sepsis Alert" page to the attending physician on the Rapid Response Team (RRT). The RRT physician performs a chart review, and then intervenes as needed. This protocol is novel in that is uses no new resources, and is designed for sustainability, ease of implementation, and rapid scale-up. MEASURES OF SUCCESS (DISCUSS QUALITATIVE AND/OR QUANTITATIVE METRICS WHICH WILL BE USED TO EVALUATE PROGRAM/ INTERVENTION): In addition to standardized, well-documented outcomes and process measures for sepsis (mortality, time to antibiotics, ICU utilization), the major measure of success will be the sustainability and implementation of this protocol. As this protocol is based on previously published but resource-intensive projects, the goal of this protocol is sustainability, rapid implementation, expansion, and minimal resource outlay while continuing to show clinical and process measure improvements.

FINDINGS TO DATE (IT IS NOT SUFFICIENT TO STATE FINDINGS WILL BE DISCUSSED): To date, our EHR tool has been active for 11,894 adult inpatient hospitalizations. 82 Sepsis Alerts have fired. Preliminary data is largely focused on the validity of the Sepsis Alert, which is novel but adapted from prior studies to improve sustainability. Patients with an Alert fire have a longer non-ICU length of stay (164 h vs $80.8 \mathrm{~h}, p<0.001)$, are more likely to have a sepsis diagnosis at discharge $(0.229 \mathrm{vs} 0.066$, $p<0.0001)$, and have a higher mortality rate $(p<0.0001)$. Furthermore, there is a trend towards longer ICU length of stay in patients with alert fire ( $87.6 \mathrm{~h}$ vs $51.6 \mathrm{~h}, p=0.055)$. Regarding sustainability and ease of implementation, we are currently ahead of schedule in implementation, have successfully trained the majority of RRT physicians and are under budget. In less than 1 year this protocol progressed from idea to fully-implemented in a 950 bed academic medical facility. Our current analysis suggests that our easy-toimplement and low-resource protocol improves sustainability while successfully identifies patients with higher mortality, longer length of stay, and higher risk for sepsis.

KEY LESSONS FOR DISSEMINATION (WHAT CAN OTHERS TAKE AWAY FOR IMPLEMENTATION TO THEIR PRACTICE OR COMMUNITY?): The need for education and communication with floor-based clinical nursing staff was more than anticipated and the communication to the medical housestaff that was needed was less than expected. Keys specific to rapid deployment and sustainability are focusing on low alert fire rates, rotating the staff receiving the Alerts to a small group, and providing specific, easy-to-access 'just in time' education to the RRT physicians.

PILOT OF AN EMBEDDED DISCHARGE SCHEDULER FOR A PATIENTCENTERED DISCHARGE PROCESS Jonathan Weiner ${ }^{2}$; Rebecca E. Berger ${ }^{1}$; Denisa Gace $^{1}$; Nandini Mani ${ }^{1}$; Shaun K. Yang ${ }^{1}$; Kathleen M. Finn ${ }^{1} .{ }^{1}$ Mass General Hospital, Cambridge, MA; ${ }^{2}$ Massachusetts General Hospital, Cambridge, MA. (Control ID \#2433256)
STATEMENT OF PROBLEM OR QUESTION (ONE SENTENCE): In a baseline survey, clinicians identified logistical barriers to successfully making their own postdischarge appointments and revealed that patients are rarely involved in scheduling discharge appointments; only an estimated $66 \%$ of attempted appointments were scheduled by the time of patient discharge.

OBJECTIVES OF PROGRAM/INTERVENTION (NO MORE THAN THREE OBJECTIVES): We designed a pilot to embed a discharge scheduler into general medicine teams. The objectives of the pilot were to reduce administrative burden on clinical staff, incorporate patients' needs and preferences for discharge appointments, and improve patient attendance at post-discharge appointments.

DESCRIPTION OF PROGRAM/INTERVENTION, INCLUDING ORGANIZATIONAL CONTEXT (E.G. INPATIENT VS. OUTPATIENT, PRACTICE OR COMMUNITY CHARACTERISTICS): Baseline data were collected in a timemotion study and clinician survey. Based on these data, a temporary employee was hired for 4 weeks to make discharge appointments. This scheduler worked with a total of four resident-led medical teams, each with a census of 20-24 patients. Each morning, the scheduler met with resident inpatient team leaders, then visited each patient and offered assistance in making appointments. Patients could choose to have the scheduler book the appointment from the patient's room, have the scheduler book the appointment on his own and inform the patient of the appointment date and time, or patients could choose to make the appointment themselves.

MEASURES OF SUCCESS (DISCUSS QUALITATIVE AND/OR QUANTITATIVE METRICS WHICH WILL BE USED TO EVALUATE PROGRAM/ INTERVENTION): The scheduler documented the number of appointments made, patient preferences, and then tracked whether the patient went to the appointment. We also surveyed residents on the impact of this pilot on their workflow. Overall, the scheduler attempted to make 163 appointments for 118 patients, averaging 1.38 appointments per patient.

FINDINGS TO DATE (IT IS NOT SUFFICIENT TO STATE FINDINGS WILL BE DISCUSSED): Of the 163 appointments attempted by the scheduler, $89 \%$ of appointments were successfully made at the time of discharge. For 85/163 (55\%) of appointments, patients preferred that the scheduler make the appointment with them in the room and 54/163 (34\%) wanted to make their own appointments. Only 15/163 (9\%) requested the appointment be made without their input. Based on preliminary data, the percentage of completed appointments rose from $31 \%$ during the time-motion study to $58 \%$ during the intervention period. Clinic no-show rates also decreased in the groups that either made their own appointment $(9 \%)$ or had their appointment made with the scheduler in the room (10\%) compared to the time-motion study (17\%). Resident team leaders stated that the scheduler improved their ability to establish follow-up appointments and care for patients $(9 / 9,100 \%)$; eight residents $(89 \%)$ stated that the scheduler saved them time (up to $45 \mathrm{~min}$ per day).

KEY LESSONS FOR DISSEMINATION (WHAT CAN OTHERS TAKE AWAY FOR IMPLEMENTATION TO THEIR PRACTICE OR COMMUNITY?): The existing discharge process demands valuable clinician time and is not patient-centered despite patients' overwhelming preference to be involved in making their own appointments. Therefore as patients prepare to return to their daily lives, we should engage them in discharge appointment scheduling and recognize the need to partner with patients in order to achieve better appointment attendance. In this pilot, an embedded discharge scheduler was able to involve patients in discharge planning at the bedside, elicit preferences, and improve the percentage of successfully scheduled appointments and actual follow up while reducing clinicians' administrative burden. These findings led our medical center to the hire year-round discharge appointment schedulers for general medical teams. Lastly, in an exit interview, the scheduler noted that the appointmentmaking process seemed unnecessarily complex, decentralized and idiosyncratic suggesting the need for a more streamlined and efficient process for making post-discharge appointments in the future. 
PILOT STUDY TO EXAMINE THE EFFECT OF A SIMPLE INTERVENTION DURING ROUNDS ON LAB-ORDERING PRACTICES AMONG INPATIENT HOUSE-STAFF TEAMS - THE 'LABS TOMORROW' STUDY Aakriti Gupta; Nikhil Sikand; Maxim Polansky; Janine Evans; Scott Sussman. Yale University, New Haven, CT. (Control ID \#2466827)

STATEMENT OF PROBLEM OR QUESTION (ONE SENTENCE): In many cases, test-ordering behavior among physicians lacks clear rationale, resulting in excessive laboratory utilization leading to unnecessary blood draws, patient discomfort and costs. OBJECTIVES OF PROGRAM/INTERVENTION (NO MORE THAN THREE OBJECTIVES): 1 . To examine the longitudinal effect of introducing an intervention on lab ordering practices by housestaff team in a teaching hospital. 2 . To evaluate the impact of this intervention on standardized costs incurred. We performed this pilot study to evaluate whether a simple intervention during daily work rounds can improve conscientious lab-ordering practices thereby promoting cost-effective medicine.

DESCRIPTION OF PROGRAM/INTERVENTION, INCLUDING ORGANIZATIONAL CONTEXT (E.G. INPATIENT VS. OUTPATIENT, PRACTICE OR COMMUNITY CHARACTERISTICS): First, baseline laboratory tests ordered by a housestaff team within 2 weeks for their patients were analyzed. The team was then educated about the intervention called 'Labs tomorrow'. While presenting a patient during daily work rounds, housestaff included another action item called 'Labs tomorrow' in their plan for the patient. This was also incorporated in their daily progress note. This intervention was implemented for the subsequent 2 weeks and laboratory test ordering practices during this period were analyzed and compared with pre-intervention practices.

MEASURES OF SUCCESS (DISCUSS QUALITATIVE AND/OR QUANTITATIVE METRICS WHICH WILL BE USED TO EVALUATE PROGRAM/ INTERVENTION): 1. Total number of laboratory tests—complete blood count (CBC), basic metabolic panel (BMP) and $\mathrm{Ca} / \mathrm{Mg} / \mathrm{Phos}$ ordered pre- and postintervention 2. Standardized costs incurred through laboratory tests ordered by the team pre- and post-intervention 3 . Total amount of blood withdrawn from patients cared by the housestaff team pre- and post-intervention

FINDINGS TO DATE (IT IS NOT SUFFICIENT TO STATE FINDINGS WILL BE DISCUSSED): The mean number of patients that the housestaff team cared for per day during the study period was 8 . The total number of tests ordered (CBC, BMP and $\mathrm{Ca}$ / $\mathrm{Mg} / \mathrm{Phos}$ ) by the housestaff team was significantly reduced post intervention when compared to pre-intervention ( 189 vs. $300, p=0.005$ ). Individually, the numbers of $\mathrm{CBC}, \mathrm{BMP}$ and $\mathrm{Ca} / \mathrm{Mg} / \mathrm{Phos}$ tests ordered were 127,117 and 56 before intervention, and 92,75 and 22 respectively after intervention. Based on standardized costs, total expenditure for laboratory tests ordered significantly reduced post-intervention $(\$ 2339.7$ vs. $\$ 3965.5, p=0.04)$. Moreover, total amount of blood phlebotomized from the patients during the post-intervention was almost half of that during the pre-intervention period (378 cc vs. $600 \mathrm{cc}, p=0.005$ ).

KEY LESSONS FOR DISSEMINATION (WHAT CAN OTHERS TAKE AWAY FOR IMPLEMENTATION TO THEIR PRACTICE OR COMMUNITY?): 1. A

simple practice incorporating planning for laboratory tests to be ordered for patients during daily work rounds can significantly reduce the number of tests ordered. 2 . This simple daily practice promotes cost saving measures for our inpatients. 3. 'Labs tomorrow' promotes patient-centered care by reducing the amount of blood phlebotomized from inpatients on a daily basis. Further studies are indicated to investigate the impact of this intervention on other short-term outcomes.

PREPARED: IMPLEMENTATION OF A PRE-EXPOSURE PROPHYLAXIS (PREP) PROGRAM IN A HOSPITAL-BASED HIV CLINIC Perry J. Tiberio; Kelly Williams; Lydia A. Barakat; E. J. Edelman; Michael Virata; Onyema Ogbuagu. Yale New Haven Hospital, New Haven, CT. (Control ID \#2469012)
STATEMENT OF PROBLEM OR QUESTION (ONE SENTENCE): Despite recommendations and guidelines issued by the United States Centers for Disease Control and Prevention (CDC) and World Health Organization, uptake of pre-exposure prophylaxis for HIV [PrEP] has been low among HIV risk groups. OBJECTIVES OF PROGRAM/ INTERVENTION (NO MORE THAN THREE OBJECTIVES): We established a "PrEP Clinic" at the Yale-New Haven Hospital (YNHH) HIV clinic that would address these needs by 1) providing an evidence-based clinical service consistent with CDC guidelines; 2) community outreach raising awareness regarding PrEP among community members; and 3) educating providers including local primary care physicians and trainees in the medical residency program.

DESCRIPTION OF PROGRAM/INTERVENTION, INCLUDING ORGANIZATIONAL CONTEXT (E.G. INPATIENT VS. OUTPATIENT, PRACTICE OR COMMUNITY CHARACTERISTICS): The PrEP clinic was initiated at two HIV clinics within YNHH at different campuses. The PrEP clinic occurs 1 day a week at the Nathan Smith Clinic, a clinic that provides comprehensive and multidisciplinary care for patients with HIV infection. This clinic also serves as a training site for the HIV training track residents in the Yale Primary Care Residency Program and Infectious Disease fellows. The staff includes trainees, attending physicians, an HIV counselor and nurses. In addition there is a PrEP clinic at the Haelen Center, an Infectious Disease clinic at the St. Raphael's campus of YNHH.

MEASURES OF SUCCESS (DISCUSS QUALITATIVE AND/OR QUANTITATIVE METRICS WHICH WILL BE USED TO EVALUATE PROGRAM/ INTERVENTION): Based on the CDC's 2014 clinical practice guidelines for PrEP, a clinical protocol was developed to guide consistent and best-practices at the two PrEP clinics. Patient referrals to the program are received from the following sources: an AIDS service organization, peer referrals from clinic patients, transitions from HIV postexposure prophylaxis, and by providers from other clinics within the YNHH system. Initially an HIV testing counselor meets patients who receive rapid HIV testing and risk counseling. If they test negative for HIV, they receive an appointment for a clinic evaluation for PrEP. At the initial appointment, the information collected includes: a comprehensive HIV risk assessment with a detailed sexual history including number of sexual partners and their HIV status, type and frequency of sexual acts and condom use; history of sexually transmitted infections (STI); history of drug use, including injection use and needle sharing; assessment of contraindications to PrEP including presence of advanced renal disease and bone fractures; and mental health screening. Vaccinations against HPV and hepatitis A and B are also offered to those who are eligible. Follow-up is scheduled at 3 month intervals for repeat HIV and STI testing, renal function monitoring and ongoing risk reduction counseling and medication adherence monitoring with counseling. All data is entered systematically into the electronic medical record. To raise awareness regarding this novel PrEP clinic, we participated in community events including a PrEP town hall and a local gay men's meeting and have given group lectures to trainees and faculty on how to prescribe and monitor patients while on PrEP.

FINDINGS TO DATE (IT IS NOT SUFFICIENT TO STATE FINDINGS WILL BE DISCUSSED): Established in November 2014, this was the first PrEP Clinic in CT in an academic hospital setting. Between November 2014 and October 2015, we have prescribed PrEP to 33 patients between the two clinic sites. Among these patients, 30 (91\%) are male; 11 (33\%) are between the ages 20 and 29 and $13(39 \%)$ are between ages 30 and 39. Racial/ethnic demographics includes: 19 (58 \%) who are white, $6(18 \%)$ who are black and $5(15 \%)$ who are Hispanic. Sexual identification includes: men who have sex with men-26 (79\%), heterosexual-5 (15\%) with 1 patient (3\%) identifying as bisexual. There was an $18 \%$ incidence of STIs (gonorrhea $[n=3]$ and herpes simplex virus infection $[n=3])$. No HIV infections were diagnosed in anyone prescribed PrEP. KEY LESSONS FOR DISSEMINATION (WHAT CAN OTHERS TAKE AWAY FOR IMPLEMENTATION TO THEIR PRACTICE OR COMMUNITY?): We have established a successful clinic to provide HIV PrEP to high-risk individuals. There 
were no new HIV infections in participants prescribed PrEP. Our clinic serves as linkage to care, providing regular sexual health screening and risk reduction counseling. We have provided education to community members and healthcare providers through town hall events, summit meetings and small group lectures. Future efforts will focus on evaluating PrEP effectiveness, barriers and facilitators to enhancing PrEP implementation within our two sites. We hope that our findings provide other practitioners the confidence to implement similar PrEP programs at their institutions thereby increasing HIV prevention efforts to improve both individual and public health

PREPARING FOR IMPLEMENTATION OF LUNG-CANCER SCREENING WITH LOW DOSE CT: ASSESSING PATIENT VOLUME, SMOKING HISTORY DOCUMENTATION, AND PULMONARY NODULE PREVALENCE IN AGE-ELIGIBLE CURRENT OR FORMER SMOKERS John Eick' ; Alison Brenner ${ }^{1}$; Shana Ratner ${ }^{1}$; Laura P. Cubillos ${ }^{3}$; Daniel S. Reuland ${ }^{1} .{ }^{1}$ UNC Chapel Hill, Chapel Hill, NC; ${ }^{2}$ University of North Carolina, Chapel Hill, NC; ${ }^{3}$ University of North Carolina at Chapel Hill, Chapel Hill, NC. (Control ID \#2468763)

STATEMENT OF PROBLEM OR QUESTION (ONE SENTENCE): Implementation of population-based approaches to lung cancer screening with low dose CT will require practices and health systems to be able to systematically identify eligible patients using tobacco history data drawn from discrete data fields in the electronic health record (EHR), plan workflows around patient volume, and systematically track both existing and new screen-detected lung nodules in high-risk patients.

\section{OBJECTIVES OF PROGRAM/INTERVENTION (NO MORE THAN THREE}

OBJECTIVES): Estimate the percentage and absolute number of current/former smokers in the 55 to 80 -year-old population at our academic internal medicine clinic that may be eligible for screening. Assess the fidelity with which smoking histories are currently documented in discrete smoking history data fields in our institution's EHR. Evaluate the prevalence of extant pulmonary nodules in our clinic's screening eligible population. DESCRIPTION OF PROGRAM/INTERVENTION, INCLUDING ORGANIZATIONAL CONTEXT (E.G. INPATIENT VS. OUTPATIENT, PRACTICE OR COMMUNITY CHARACTERISTICS): The project setting was the academic general internal medicine clinic at the University of North Carolina (UNC) at Chapel Hill. We reviewed medical records of 100 current or former smokers ages 55-80 using the EHR's chart search function. For smoking history, we searched the root word "smok-" for elements needed to identify the eligible population: pack-years smoked and (for former smokers) years since quitting. We reviewed provider notes as well as the discrete EHR smoking history fields. We used USPSTF criteria to categorize patients as eligible, possibly eligible (if there was not enough information in the record to categorize them) or ineligible for screening by smoking history. Using a query to the Carolina Data Warehouse for Health to determine the number of active, age-eligible patients listed as current or former smokers and our calculated prevalence of screening eligible patients, we estimate the volume of these populations in our clinic. For nodule prevalence, we searched "nodule" and reviewed all provider notes and chest imaging reports describing lung nodules.

MEASURES OF SUCCESS (DISCUSS QUALITATIVE AND/OR QUANTITATIVE METRICS WHICH WILL BE USED TO EVALUATE PROGRAM/ INTERVENTION): Our measures included: a) volume of potentially eligible patients as a percentage of age eligible current/ former smokers and in absolute numbers (projecting from warehouse data); b) the percentage of current/former smokers ages 5580 with smoking histories documented in discrete EHR fields; c) percentage of screen eligible patients with existing, incidentally discovered, lung nodules.

FINDINGS TO DATE (IT IS NOT SUFFICIENT TO STATE FINDINGS WILL BE DISCUSSED): Of all subjects, $25 \%(25 / 100)$ were categorized as eligible by smoking history, and an additional $25 \%$ were categorized as possibly eligible. Per the Carolina Data Warehouse for Health, a total of 2,421 patients meeting our study's eligibility criteria were seen in our clinic between April and December 2014. Assuming one third of the possibly eligible patients are also, in fact, eligible, we estimate that approximately $33 \%$ of current/former smokers ages $55-80$ in our practice may be eligible for screening. This translates to approximately 800 screen-eligible patients seen in our clinic over 8 months, or about 100 patients per month. Regarding smoking history documentation, we found that $44 \%$ (44/100) of current/former smokers ages 55-80 had complete smoking histories recorded in the discrete EHR smoking history fields. Of those categorized as eligible for screening, $56 \%(14 / 25)$ were identified by provider notes alone and did not have completed information in the discrete EHR smoking history field. Among all 100 subjects, $30 \%$ (30/100) had lung nodules that had been previously (incidentally) identified. Among the 25 screen eligible participants, 12 (48\%) had previously identified lung nodules.

KEY LESSONS FOR DISSEMINATION (WHAT CAN OTHERS TAKE AWAY FOR IMPLEMENTATION TO THEIR PRACTICE OR COMMUNITY?): In practices such as ours, about a third of 55-80 year olds who are current/former smokers are likely to be eligible for lung cancer screening. However, many of these patients would not be identified in queries or by automated reminders because their smoking histories are not appropriately documented in an easily queried discrete EHR field. For systematic implementation of lung cancer screening, interventions to promote collection and entry of smoking histories in discrete EHR fields are needed. Real world implementation of screening will be made even more challenging because a substantial proportion of patients eligible for lung cancer screening by USPSTF guidelines already have previously identified lung nodules.

PROJECT TO IMPLEMENT RECOMMENDATIONS FOR PCV13 IMMUNIZATION AMONG ADULTS 65 AND OLDER IN A LARGE, UNIVERSITYBASED AMBULATORY CLINIC Christine D. Gladman; Tamrah Parker; Christina McMillan; Thomas M. Miller; Shana Ratner. University of North Carolina - Chapel Hill, Chapel Hill, NC. (Control ID \#2468386)

STATEMENT OF PROBLEM OR QUESTION (ONE SENTENCE): In 2014, the Advisory Committee on Immunization Practices (ACIP) recommended vaccination of all adults 65 and older with the 13-valent pneumococcal conjugate vaccine (PCV13) in addition to the 23-valent pneumococcal vaccine (PPSV23), leaving primary care clinics with the challenge of delivering the new PCV13 and PPSV23 at appropriate intervals based on previous PPSV23 vaccination status.

OBJECTIVES OF PROGRAM/INTERVENTION (NO MORE THAN THREE OBJECTIVES): 1. Design a visual tool that simplified the 2014 ACIP pneumococcal vaccination recommendations for our clinic nurses 2. Describe implementation of 2014 ACIP pneumococcal vaccination recommendations in a large university-based ambulatory care clinic 3 . Evaluate PCV13 vaccination rates after the first 8 months implementation DESCRIPTION OF PROGRAM/INTERVENTION, INCLUDING ORGANIZATIONAL CONTEXT (E.G. INPATIENT VS. OUTPATIENT, PRACTICE OR COMMUNITY CHARACTERISTICS): The University of North Carolina Internal Medicine Clinic is a large academic general internal Medicine practice, serving about 12,000 active patients, of which 3,591 are aged 65 years and older. The clinic is a level 3 patient-centered medical home with a long history of quality improvement. Providers are attendings, residents, and advanced practice providers working side-by-by side. The 12 floor nurses are Licensed Practical Nurses (LPNs) supervised by a Nurse Practitioner. A previously existing pneumococcal vaccination standing order, which allowed nurses to administer vaccines to eligible patients without an individual provider order, was revised to reflect PCV13 recommendations and intervals. We designed a visual tool for nursing 
staff that simplified the new ACIP pneumococcal vaccination recommendations for patients 65 years and older. An educational session was held for nurses to explain the new standing order for pneumococcal vaccination using this visual tool. At the end of the session a case-based post-test was administered to test knowledge and reinforce these recommendations. Within a 2 week period, the standing orders were tested in step-wise approach, first with two nurses, then with four nurses, making necessary edits before they were introduced to all nurses for clinic-wide implementation. After each step, chart reviews on random samples of patients were completed to evaluate nurse performance and the effectiveness of the educational sessions. Repeat nurse trainings were completed when chart reviews showed that vaccination rates were waning. Repeat chart reviews were performed 4 and 8 months later to evaluate effectiveness of the project.

MEASURES OF SUCCESS (DISCUSS QUALITATIVE AND/OR QUANTITATIVE METRICS WHICH WILL BE USED TO EVALUATE PROGRAM/ INTERVENTION): We obtained nurse feedback about the ease of use and difficulties using the visual and new standing orders for pneumococcal vaccinations. We measured nurses' knowledge using a case-based post-test. With several chart reviews, we measured PCV13 vaccination administration rates among sequential samples of patient encounters at various points in time. These were performed at the end of each of the three cycles of implementation and at 4 and 8 months after implementation.

FINDINGS TO DATE (IT IS NOT SUFFICIENT TO STATE FINDINGS WILL BE DISCUSSED): The nurses found the visual tool easy to understand and easy to use They did, however, note several challenges to identifying the population needing vaccination and delivering the vaccine: the need for a chart review to determine PPSV23 vaccine status, variable physician knowledge regarding the updated recommendations, and no automated prompt in the electronic medical record. On the case-based post-test administered after the educational session, scores ranged from $5 / 7$ correct to $7 / 7$ correct. With the first cycle of implementation, two nurses triaged 15 patients eligible for PCV13, and seven patients ( $44 \%$ ) received the vaccine. In the second cycle, four nurses triaged 33 patients eligible to receive PCV13, and 17 (52\%) received the vaccine. After the visual tool was first introduced clinicwide in the third cycle, nurses triaged 74 eligible patients, but delivered vaccines to only six ( $9 \%$ ) of these patients. Another educational session was held to review the new recommendations, obtain feedback, and reinforce standing orders. Four months later, in a chart review of a 432 sequential encounters for patients over the age of 65 and older, 80 (33\%) of 239 patients eligible to receive PCV13 were vaccinated at the visit. Eight months later, in a review of 108 sequential encounters of patients over the age of 65 years, 26 (50\%) of 52 patients eligible to received PCV13 were vaccinated at the visit. When these 26 patients were combined with the 55 who had been vaccinated at a previous visit the total vaccination rate for these patients reached $75 \%$ (81 of 108 patients). Eight months after implementation of the project, 1,539 (43\%) of the 3,591 patients 65 and older in the practice had received the PCV13 vaccine. KEY LESSONS FOR DISSEMINATION (WHAT CAN OTHERS TAKE AWAY FOR IMPLEMENTATION TO THEIR PRACTICE OR COMMUNITY?): This project demonstrates that, using a visual tool to summarize ACIP pneumococcal vaccine recommendations, nurse standing orders, and nurse education, a clinic can achieve substantial immunization rates in less than 1 year.

QUALITY IMPROVEMENT FOR AMELIORATION OF THE RELEVANT USE OF CARDIAC TELEMETRY MONITORING IN A COMMUNITY-BASED TEACHING HOSPITAL somwail rasla ${ }^{1,2}$; Ahmad Abdin ${ }^{1,2}$; farhan ashraf ${ }^{1,2}$; Saira Imran $^{1,2}$; Anais Ovalle ${ }^{1,2}$; Taro Minami ${ }^{1,2}$. ${ }^{1}$ Memorial Hospital, Pawtucket, RI; ${ }^{2}$ AlbertBrown Medical School, Providence, RI. (Control ID \#2454859)

STATEMENT OF PROBLEM OR QUESTION (ONE SENTENCE): Overuse of cardiac telemetry monitoring has led to increased waste in health care system in addition to alarm fatigue and provider workflow interruption .
OBJECTIVES OF PROGRAM/INTERVENTION (NO MORE THAN THREE OBJECTIVES): 1. Exploring effective use of telemetry with accurate monitoring of true positive results 2 . To increase house staff awareness for guidelines of implementing telemetry on a given non-ICU, non-CCU patient. 3. To illustrate the significant cost reduction that follows effective telemetry use.

DESCRIPTION OF PROGRAM/INTERVENTION, INCLUDING ORGANIZATIONAL CONTEXT (E.G. INPATIENT VS. OUTPATIENT, PRACTICE OR COMMUNITY CHARACTERISTICS): We conducted a Quality Improvement (QI) project over a period of 2 weeks at a community-based teaching hospital. Before the intervention, we collected the baseline data for 1 week including: number of patients placed on telemetry per day, number of alarm events on telemetry (excluding false alarms), documentation of these events, and intervention for these events. The timing for checking the monitors was set to be at noon time excluding patients who were admitted on the same day. We also investigated the awareness of appropriate use of telemetry monitoring among residents and nurses. Intervention to improve this included display of the AHA recommendations in posters, multiple teaching sessions held at noon conference, morning reports and daily reminders by electric pagers. During the intervention period, the same data set was collected over 1 week with the same inclusion and exclusion criteria. Patients were categorized according to the AHA guidelines in three categories. We then analyzed the data using unpaired T-test, Fisher's exact test, and descriptive analysis.

MEASURES OF SUCCESS (DISCUSS QUALITATIVE AND/OR QUANTITATIVE METRICS WHICH WILL BE USED TO EVALUATE PROGRAM/ INTERVENTION): (1) Number of patients who meet the criteria to be placed on telemetry (2) Alarm (trend) events on telemetry monitor. (3) Documentation on electronic medical records for telemetry readings, and actions taken based upon telemetry readings. (4) cost reduction associated with appropriate Telemerty use

FINDINGS TO DATE (IT IS NOT SUFFICIENT TO STATE FINDINGS WILL BE DISCUSSED): 98 cases were reviewed prior to the intervention and 100 cases were reviewed during the intervention. The pre-intervention AHA classes I, II, III distribution were $24,35,39$ cases respectively while during the intervention the distribution was 48 , 32,20 cases respectively. At the pre-intervention phase, only 33 cases had documented readings, from a total of 98 cases on telemetry. While during the intervention, 71 cases had a documented reading from a total of 100 cases who were on telemetry $(p<0.0001)$. The number of patients with class III indication during intervention dropped to $20(2.5 \pm 0.93$ per day) from $39(4.87 \pm 2.99$ per day) $(p=0.0011)$ pre-intervention. Average cost reduction per-day was calculated to be $(\$ 3,887 \pm 3,500)$.

KEY LESSONS FOR DISSEMINATION (WHAT CAN OTHERS TAKE AWAY FOR IMPLEMENTATION TO THEIR PRACTICE OR COMMUNITY?): This multi-modal QI project achieved an improvement in the quality of telemetry monitoring in non-ICU units with modest intervention. It reduced the misuse of cardiac telemetry by $31 \%$ with significant cost reduction. Patient safety improved through increased awareness of the house staff of the AHA recommendations and raised the level of documentations associated with cardiac telemetry.

REDUCING CENTRAL LINE ASSOCIATED BLOOD STREAM INFECTION AND CATHETER ASSOCIATED URINARY TRACT INFECTIONS WITH A TEAM OF RESIDENT AND FELLOW DIRECTORS FOR QUALITY IMPROVEMENT Said Ashraf ${ }^{2}$; Amrit Misra ${ }^{2}$; Rick Bloomingdale ${ }^{2}$; Syed A. Hussain ${ }^{1}$; Corinne S. Hamstra ${ }^{1}$; Suzanne White ${ }^{1} .{ }^{1}$ Detroit Medical Center, Detroit, MI; ${ }^{2}$ Detroit Medical Center/ Wayne State University, Detroit, MI. (Control ID \#2470281)

STATEMENT OF PROBLEM OR QUESTION (ONE SENTENCE): Central line associated bloodstream infections (CLABSI) and catheter associated urinary tract infections (CAUTI) are a significant cause of morbidity and mortality in an inpatient setting. 
OBJECTIVES OF PROGRAM/INTERVENTION (NO MORE THAN THREE OBJECTIVES): -To educate physicians and nurses on the appropriate use criteria for indwelling catheters and central lines in the inpatient setting-Recognize that a multi-facet approach to controlling CLABSI and CAUTI is required in order to improve patient outcome, decrease length of stay in hospitals and reduce health care costs. - To reduce the number of central line associated infections and catheter associated urinary tract infections by identifying and reducing the number of inappropriate devices

DESCRIPTION OF PROGRAM/INTERVENTION, INCLUDING ORGANIZATIONAL CONTEXT (E.G. INPATIENT VS. OUTPATIENT, PRACTICE OR COMMUNITY CHARACTERISTICS): Intervention was initiated with widespread education to physicians and nurses regarding indications for central lines and indwelling urinary catheters as well as the care for devices when they are in place. Appropriate use criteria were published and made accessible on all computer workstations, the internet, and a mobile phone widget. Next, residents and fellows at the Detroit Medical Center were assigned to different units and floors of each hospital in the system. Each team member monitored all patient devices on their assigned floors via a computer generated tool on a daily basis. Thorough assessment was undertaken daily to ensure each device meet the Detroit Medical Center appropriate use criteria for central line and urinary catheters. Residents and fellows identified devices that did not meet these accepted, evidencebased criteria and ones that could potentially be removed and replaced with less invasive measures. The resident and fellow directors regularly communicated with the primary physician providers to assess for removal in these cases. Reports regarding these lines were sent daily to physician, hospital, and nursing administrators to help identify common issues to continue the process of systems-based improvement

MEASURES OF SUCCESS (DISCUSS QUALITATIVE AND/OR QUANTITATIVE METRICS WHICH WILL BE USED TO EVALUATE PROGRAM/ INTERVENTION): CAUTI and CLABSI rates at each hospital in the DMC system, as well as the total number of central line days per month, were calculated on a monthly basis to assess for the effectiveness of the intervention.

FINDINGS TO DATE (IT IS NOT SUFFICIENT TO STATE FINDINGS WILL BE DISCUSSED): Prior to start of intervention in May 2015, the rate of CLABSI at the Detroit Medical Center was 1.98 per 1000 central line days and CAUTI rate was 1.97 per 1000 foley catheter days. In October 2015, 6 months following initiation of the intervention, the CLABSI rate decreased $24 \%$ to 1.50 per 1000 central line days and CAUTI rates decreased $35 \%$ to 1.28 per 1000 foley catheter days. Additionally, the number of central line days per month was reduced from 4050 to 3324 .

KEY LESSONS FOR DISSEMINATION (WHAT CAN OTHERS TAKE AWAY FOR IMPLEMENTATION TO THEIR PRACTICE OR COMMUNITY?): The implementation of a team of residents and fellows assessing for inappropriate central venous and urinary catheters in combination with education regarding appropriate use and care of these devices can potentially lead to a decrease in the rates of CAUTI and CLABSI.

SMOKING CESSATION USING INTERACTIVE VOICE RESPONSE AND CARE COORDINATOR CALLS Thomas K. Spain ${ }^{2,}{ }^{1}$; Robert S. Dittus ${ }^{2,}{ }^{1}$; Hilary Tindle ${ }^{2,2}$. ${ }^{1}$ Department of Veterans Affairs, Nashville, TN; ${ }^{2}$ Vanderbilt University School of Medicine, Nashville, TN. (Control ID \#2468314)

STATEMENT OF PROBLEM OR QUESTION (ONE SENTENCE): Care coordinators are increasingly utilized to support health behavior changes such as tobacco cessation, yet proactively engaging patients in effective and cost-effective tobacco cessation during and between clinic visits is challenging in the face of time constraints and competing care management priorities.

OBJECTIVES OF PROGRAM/INTERVENTION (NO MORE THAN THREE OBJECTIVES): We demonstrated the use of automated interactive voice response
(IVR) calls to engage smokers in evidence-based tobacco cessation care including free quitline counseling and use of nicotine replacement therapy (NRT) through an existing care coordination program.

DESCRIPTION OF PROGRAM/INTERVENTION, INCLUDING ORGANIZATIONAL CONTEXT (E.G. INPATIENT VS. OUTPATIENT, PRACTICE OR COMMUNITY CHARACTERISTICS): We implemented a program to engage active tobacco smokers from the Vanderbilt primary care medical home in evidence-based tobacco cessation care through the Vanderbilt MyHealth Team (MHT), a primary care chronic disease management program with over 160 primary care clinicians in 11 clinics across middle Tennessee. A multidisciplinary team was assembled for program development and implementation including clinician, care coordinator, administrator, informatics, tobacco control, and state quitline representation. A panel of "known" smokers who were active Vanderbilt primary care patients (i.e. clinic visit within the past 2 years and smoking status available in structured data fields) was identified from the electronic health record (EHR) and targeted for contact by IVR outreach with up to 3 call attempts. Successful contacts were verified as smokers and given the option of immediate transfer to the Tennessee state quitline or a follow-up call from a care coordinator. Unsuccessful IVR contact prompted a follow-up call from a care coordinator. Smokers who received care coordinator contact were offered a menu of options including: 1) immediate quitline transfer, 2) follow-up call by a care coordinator, and 3) a prescription for NRT, facilitated through the patient's primary care provider. MHT care coordinators are nurses with training and experience in chronic disease management. For this project, the care coordinators received two focused training sessions in basic smoking cessation counseling, motivational interviewing techniques, and the use of nicotine replacement therapy. Care coordinators were prompted to follow-up with each patient after IVR call to discuss smoking cessation. Patients who had unsuccessful IVR call attempts or had declined quitline referral were offered immediate transfer to the quitline by the care coordinators. Patients were also offered NRT consisting of transdermal nicotine patches plus nicotine gum or nicotine lozenges. A template was implemented in the EHR to allow care coordinators to electronically draft NRT prescriptions for primary care provider review and signature utilizing evidence-based dosing regimens.

MEASURES OF SUCCESS (DISCUSS QUALITATIVE AND/OR QUANTITATIVE METRICS WHICH WILL BE USED TO EVALUATE PROGRAM/ INTERVENTION): Patient-level data was collected from the EHR, the IVR operator (WestCorp), and the state quitline operator (IQH). Measures of success included the outcomes of all IVR calls (successful contact, confirmation of smoking status, request for quitline transfer, request for care coordinator call, no interest in tobacco cessation, or call termination) and care coordinator contacts (contact attempted, patient reached, motivation for quit attempt assessed, quitline referrals made, or prescriptions for NRT ordered). In addition, care coordinator documentation was reviewed to identify opportunities for program improvement.

FINDINGS TO DATE (IT IS NOT SUFFICIENT TO STATE FINDINGS WILL BE DISCUSSED): From 5/1/2015 to 5/24/2015, 151/313 eligible patients were successfully contacted by IVR with the following outcomes: 54 (36 \%) confirmed active smoker status, $17(11 \%)$ reported non-smoker status, and 80 (53\%) could not have smoking status confirmed due to unsuccessful IVR authentication or patient termination of the call. Among confirmed smokers, 8 requested automated quitline transfer, 4 requested care coordinator call, 31 expressed no interest in tobacco cessation, and 11 calls were terminated. A total of 25 patients made contact with the quitline through IVR or care coordinator referral. Chart review is ongoing to determine the outcome of care coordinator contacts and all quitline referrals.

KEY LESSONS FOR DISSEMINATION (WHAT CAN OTHERS TAKE AWAY FOR IMPLEMENTATION TO THEIR PRACTICE OR COMMUNITY?): Preliminary results suggest that automated IVR outreach can aid care coordinators to engage confirmed smokers in evidence-based tobacco cessation care. Only about 1 in 3 individuals 
identified as smokers by the EHR confirmed current smoking status in response to IVR outreach. However, IVR was immediately successful in engaging about 1 in 5 confirmed smokers, either via transfer to the state quitline or request for care coordinator follow up. Additional research is needed to determine longer term effects of IVR outreach on care coordinator engagement in smoking cessation efforts, including facilitation of prescriptions for smoking cessation medication and tobacco abstinence.

STANDARDIZING THE ADMISSION PROCESS USING LEAN/SIX SIGMA ONEPIECE-FLOW Escher Howard-Williams ${ }^{2}$; Edmund A. Liles ${ }^{3}$; John Stephens ${ }^{1}$; Kendra Lanza-Kaduce $2 .{ }^{1} \mathrm{UNC}$ Hospitals, Chapel Hill, NC; ${ }^{2} \mathrm{UNC}$ chapel hill, Chapel Hill, NC; ${ }^{3}$ University of North Carolina School of Medicine, Chapel Hill, NC. (Control ID \#2447429)

STATEMENT OF PROBLEM OR QUESTION (ONE SENTENCE): Can a new technique, One-Piece-Flow, as applied to the hospital admission process increase efficiency and flow of admitted patients to the hospital? OBJECTIVES OF PROGRAM/INTERVENTION (NO MORE THAN THREE OBJECTIVES): Evaluate the impact of standardizing the admission process using One Piece Flow by evaluating efficiency and time reduced per admission.

DESCRIPTION OF PROGRAM/INTERVENTION, INCLUDING ORGANIZATIONAL CONTEXT (E.G. INPATIENT VS. OUTPATIENT, PRACTICE OR COMMUNITY CHARACTERISTICS): Safely and efficiently moving patients through the admission process is a key concern for emergency and hospital medicine faculty. Our hospitalist group admits approximately 400 patients per month, each admitting physician evaluates approximately 7.5 patients per shift, utilizing individual approaches. We aimed to improve the quality of our admissions by standardizing our admissions process by utilizing Lean/Six Sigma (LS $\sigma)$ methodology. LS $\sigma$ was developed in the automotive manufacturing industry and is increasingly being applied to quality improvement in the healthcare setting. A basic tenet of LS $\sigma$ is that standardized processes are more efficient and less prone to error when completed from start to finish without interruption, termed "one-piece-flow" (OPF). As contrasted with performing individual parts of a process in groups, termed "batching." Many admitting physicians tend to "batch" admissions, seeing two or three patients sequentially, followed by order entry for those patients, then documentation, all performed in "batch" processing.

MEASURES OF SUCCESS (DISCUSS QUALITATIVE AND/OR QUANTITATIVE METRICS WHICH WILL BE USED TO EVALUATE PROGRAM/ INTERVENTION): Select hospitalist providers committed to performing all patient admissions in OPF, whereby an admitter focused on one patient from initiation of chart review through exam, order entry and documentation, without interruption. Pages and other distractions were addressed only at the completion of this process, unless emergent. To assess the impact of this process on efficiency, we analyzed the time page was received from ER, time to call back to ER, time at initiation of chart review, time of evaluation of patient, time orders were placed, time of H\&P completion, and time of sign-out note completed, before and after implementation of OPF. The times were reported on log sheets completed by attending physicians as admissions were performed. Our pre-intervention data was collected from 11/ 14/15-12/14/15. Our post intervention period data was collected at 30 days, 90 days and 6 months. We reviewed pre and post data encompassing $95 \log$ sheets obtained over 60 days reflecting different eight different providers, averaging 7.5 patients per shift. We then had a second endpoint at 90 days and analyzed $45 \log$ sheets, reflecting 4 different providers. FINDINGS TO DATE (IT IS NOT SUFFICIENT TO STATE FINDINGS WILL BE DISCUSSED): We found a substantial reduction in time of the admission process across all time points. Compared with pre-implementation, at 30 days there was a decrease in time of $30 \mathrm{~min}$ from time page was received from the ER to time of sign-out note completion: from $2 \mathrm{~h} 18 \mathrm{~min}$ to $1 \mathrm{~h} 48 \mathrm{~min}$. In comparing time page received from ER to time orders are placed for the patient, OPF admitting reduced time by $1 \mathrm{~h}$ : from $2 \mathrm{~h} 40 \mathrm{~min}$ to $1 \mathrm{~h} 40 \mathrm{~min}$. And when comparing initiation of chart review to sign-out note completion, OPF achieved a reduction in time of $57 \mathrm{~min}$ : from $1 \mathrm{~h} 27 \mathrm{~min}$ to $30 \mathrm{~min}$. The 90 and 180 day sustainment data showed a consistent decrease of $57 \mathrm{~min}$ over the entire admission process using OPF, from $2 \mathrm{~h} 18 \mathrm{~min}$ to $1 \mathrm{~h} 31 \mathrm{~min}$.

KEY LESSONS FOR DISSEMINATION (WHAT CAN OTHERS TAKE AWAY FOR IMPLEMENTATION TO THEIR PRACTICE OR COMMUNITY?): An OPF method applied to admissions was associated with a sustained and clinically meaningful decrease in time for the admission process. These results may have significant implications for hospitals seeking to improve the efficiency of their admission processes. Further study is needed to examine whether OPF techniques also result in fewer errors during the admissions process.

SUBACUTE REHABILITATION AT HOME: IMPROVING TRANSITIONS FROM HOSPITAL TO HOME WITH A MULTIDISCIPLINARY TEAM AND 30 DAY BUNDLE Christian Escobar; Ramiro Jervis; Gabriel Silversmith; Tacara N. Soones; Linda DeCherrie. Icahn School of Medicine at Mount Sinai, New York, NY. (Control ID \#2469613)

STATEMENT OF PROBLEM OR QUESTION (ONE SENTENCE): Patients with acute illness discharged to subacute rehabilitation (SAR) in skilled nursing homes for functional rehabilitation and medical management of ongoing medical illness experience high rates of adverse events during the transition from institution to community. OBJECTIVES OF PROGRAM/INTERVENTION (NO MORE THAN THREE OBJECTIVES): 1 . To demonstrate the delivery of post-acute rehabilitation and provider led multidisiplanary transitional care in the home setting. 2. To report patient demographics and diagnoses of patients being admitted to Subacute Rehab at Home. 3. To report outcomes data with the goal of equal or reduced rates of adverse events and 30-day readmissions in the intervention relative to control patients discharged to traditional SAR facilities, and decreased costs in the intervention relative to control patients discharged to traditional SAR facilities.

DESCRIPTION OF PROGRAM/INTERVENTION, INCLUDING ORGANIZATIONAL CONTEXT (E.G. INPATIENT VS. OUTPATIENT, PRACTICE OR COMMUNITY CHARACTERISTICS): The Icahn School of Medicine at Mount Sinai is implementing a Hospital at Home program as funded by the Health Care Innovation Award from the Centers for Medicare and Medicaid Innovation (CMMI). The Moun Sinai Hospital at Home program, the Mobile Acute Care Team (MACT) utilizes the expertise of a multidisciplinary team to address acute care needs in the home setting. Other Hospital at Home models provide home based medical services only to acutely ill patients who would otherwise require inpatient hospitalization. An extension of this previously tested model, SAR at Home is a novel model that delivers these home services to patients discharged from the inpatient setting who would otherwise require skilled nursing facility admission for SAR. These patients are eligible if they need intensive physical or occupational rehab. Medicare insured inpatients at the Mount Sinai Hospital requiring post discharge sub-acute services at a traditional skilled nursing facility are identified by the inpatient team (clinicians and social workers) and screened by the MACT providers for eligibility based on (1) geography, (2) social support, (3) home safety and (4) medical appropriateness. Once enrolled, these patients receive multidisciplinary services at home for the duration of their resolving acute medical and rehabilitation needs, for up to 30 days. These services include physical therapy 6 days a week, occupational and speech therapy sessions as needed, skilled nursing by RNs to assist with continuing medical needs after hospitalization (e.g., IV infusions and wound care), social work support, and a physician or nurse practitioner visit on admission and as needed thereafter.. All patients receive daily 24-h on call coverage by the MACT for 30 days regardless of when the patient's rehabilitation is completed. 
MEASURES OF SUCCESS (DISCUSS QUALITATIVE AND/OR QUANTITATIVE METRICS WHICH WILL BE USED TO EVALUATE PROGRAM/ INTERVENTION): The MACT Hospital at Home program, including the SAR at Home arm, is being evaluated though a prospective, non-randomized controlled study. Key measures include: demographics and hospital diagnosis data to better understand the population that fits this model and how it compares with patients discharged to traditional SAR facilities; readmission rates; adverse events; costs; functional status before and after SAR; and patient satisfaction.

FINDINGS TO DATE (IT IS NOT SUFFICIENT TO STATE FINDINGS WILL BE DISCUSSED): During the first 3 months of the program, 36 patients were enrolled in SAR at Home. All patients had fee-for-service Medicare (required for enrollment) and $12 \%$ were dually eligible with Medicaid. Patients were all geriatric, with mean age of 84.6 (range 72 to 97 ) and predominately female (61.1\%). Length of stay for patients was on average 14.7 days (range 5 to 24 days). The most prevalent diagnoses for the index admission preceding SAR were for falls $(12.5 \%)$ and pneumonia ( $8.3 \%)$. Of patients enrolled, 3 required ED visits and 1 patient was readmitted to the hospital. During the SAR at Home episode, 2 patients fell, 1 developed a pressure ulcer, and none died. Data on cost, functional status and patient satisfaction is pending.

KEY LESSONS FOR DISSEMINATION (WHAT CAN OTHERS TAKE AWAY FOR IMPLEMENTATION TO THEIR PRACTICE OR COMMUNITY?): Providing post-hospitalization SAR and medical management in the home at a level of intensity similar to that delivered in skilled nursing facilities appears, in our preliminary experience, to be feasible. This can be accomplished by employing a multidisciplinary team to provide a cohesive transition from acute illness, offering an alternative to an extended institutional stay. Further study is needed to determine safety and effectiveness. Payment models should be explored to allow for these services to be sustained

SYSTEMATIC TRACKING AND FOLLOW UP OF ABNORMAL CANCER SCREENING TESTS UTILIZING A SOCIAL WORK CARE MANAGER TO IMPROVE PATIENT SAFETY IN AN ACADEMIC PRIMARY CARE CLINIC Shana Ratner; Lindsey Franks; Cristin Colford; Daniel S. Reuland; Brooke B. McGuirt; Christina McMillan; Laura G. Brown; Michael Pignone. UNC Chapel Hill, Chapel Hill, NC. (Control ID \#2469016)

STATEMENT OF PROBLEM OR QUESTION (ONE SENTENCE): Many abnormal cancer screening tests completed in primary care do not result in proper follow-up, diagnostic testing, and treatment.

OBJECTIVES OF PROGRAM/INTERVENTION (NO MORE THAN THREE OBJECTIVES): 1) Develop a standardized approach to tracking and follow up of abnormal stool cards and pap smears completed at the UNC General Internal Medicine Practice (GIM) 2) Utilize social work best practices to address underlying barriers to care for patients with abnormal screening tests

DESCRIPTION OF PROGRAM/INTERVENTION, INCLUDING ORGANIZATIONAL CONTEXT (E.G. INPATIENT VS. OUTPATIENT, PRACTICE OR COMMUNITY CHARACTERISTICS): Even when patients complete an initial screening test for cervical cancer and colon cancer, incomplete diagnostic evaluation of abnormal pap smears and positive stool cards eliminate the benefit of screening and are a potential patient safety problem. Many patients have barriers to care that impact their ability to followup with these abnormal tests. We piloted a program in the UNC GIM practice to address this gap in care. GIM is a large attending and resident practice, serving about 12,000 active patients. The practice cares for a diverse group of patients: $20 \%$ are uninsured, and many experience barriers to care due to poverty and rural isolation. A dedicated social work-trained Prevention Care Manager was hired for twofold purpose: 1) to build and deliver outreach for specific preventive services to all patients due (described separately) and 2) to track patients with abnormal screening results performed in our clinic. Providers frequently perform pap smears for cervical cancer screening and order stool cards for colon cancer screening at the clinic. Previously when these results were abnormal, each provider was responsible for follow-up individually, and there was no systematic mechanism to ensure that the patient was notified and follow-through occurred. We developed a process for the care manager to track and follow up all of these abnormal results. The care manager ran monthly reports of abnormal results from the Electronic Health Record (EHR), checked documentation in the EHR to ensure that the patient had been notified of abnormal results, and if this had not occurred, she reminded the PCP to notify the patient and document the interaction. She then ensured that the patient was referred and scheduled for the correct follow up (colposcopy, follow-up pap, or colonoscopy). If no appointment was scheduled, if the patient cancelled or did not attend the diagnostic testing, she reached out to the patient to facilitate scheduling and address barriers to care. She tracked these patients until they had completed diagnostic testing or until the PCP gave permission for a certified letter to be sent notifying the patient of the risks inherent in not following through on these abnormal test results.

MEASURES OF SUCCESS (DISCUSS QUALITATIVE AND/OR QUANTITATIVE METRICS WHICH WILL BE USED TO EVALUATE PROGRAM/ INTERVENTION): Outcome measures are number of patients with successful resolution of abnormal results. Process measures include the number of patients with abnormal pap smears or abnormal stool cards, and the number of patients who would not have been notified of abnormal test results without reminder from the care manager. Qualitatively, we tracked common barriers to care impeding diagnostic follow up.

FINDINGS TO DATE (IT IS NOT SUFFICIENT TO STATE FINDINGS WILL BE DISCUSSED): From April 2014-June 2015, 57 patients were identified with abnormal pap smears or stool cards. Of those, 16/57 (28\%) required care management for completion of follow-up. Four patients were identified who had not been notified of abnormal test results prior to the care manager reviewing the report and contacting the PCP. There were 14 patients with positive stool cards, 8/14 (57\%) of whom required care management to schedule and complete a colonoscopy. There were 43 abnormal pap smears, 8/43 (19\%) of which required care management for completion of referral to gynecology. The most common barriers to care for patients were transportation, finances, and apprehension about follow-up care. To address these barriers, the care manager was able to connect patients to community resources for transportation, facilitate referral to diagnostic centers closer to home, schedule on the same day as other appointments, and provide counseling and education to reduce apprehension. Many patients expressed that calling to schedule the test was a barrier and asked the care manager to schedule on their behalf. We worked closely with UNC Gastroenterology and Gynecology to gain the ability to schedule on patients' behalf. Patients with financial issues were referred to the UNC Charity Care program, resources related to the Affordable Care Act, or to free community resources.

KEY LESSONS FOR DISSEMINATION (WHAT CAN OTHERS TAKE AWAY FOR IMPLEMENTATION TO THEIR PRACTICE OR COMMUNITY?): A practice-based social work care manager was able to successfully ensure that all patients were notified of abnormal screening results that were completed in the clinic. Utilizing social work best practices, we were able to address underlying barriers to care, facilitating diagnostic work-ups in a patient-centered and effective manner. This approach has significant patient safety implications. This pilot will be spread across our primary care improvement collaborative together with a package for outreach to patients about prevention.

SYSTEMATIC TRACKING AND FOLLOW UP OF ABNORMAL CANCER SCREENING TESTS UTILIZING A SOCIAL WORK CARE MANAGER TO IMPROVE PATIENT SAFETY IN AN ACADEMIC PRIMARY CARE CLINIC Shana Ratner; Lindsey Franks; Cristin Colford; Daniel S. Reuland; Brooke B. McGuirt; Christina McMillan; Laura G. Brown; Michael Pignone. UNC Chapel Hill, Chapel Hill, NC. (Control ID \#2469590) 
STATEMENT OF PROBLEM OR QUESTION (ONE SENTENCE): Many abnormal cancer screening tests completed in primary care do not result in proper follow-up, diagnostic testing, and treatment.

OBJECTIVES OF PROGRAM/INTERVENTION (NO MORE THAN THREE OBJECTIVES): 1) Develop a standardized approach to track and follow up abnormal stool cards and pap smears completed at the UNC General Internal Medicine Practice (GIM) 2) Utilize social work best practices to address underlying barriers to care for patients with abnormal screening tests

DESCRIPTION OF PROGRAM/INTERVENTION, INCLUDING ORGANIZATIONAL CONTEXT (E.G. INPATIENT VS. OUTPATIENT, PRACTICE OR COMMUNITY CHARACTERISTICS): Incomplete diagnostic evaluation of abnormal pap smears and positive stool cards eliminates the benefit of screening for cervical and colon cancer and is a patient safety problem. We piloted a program in the UNC GIM practice to address this gap in care. GIM is a large faculty and resident practice, serving about 12,000 active patients. The practice cares for a diverse group of patients. About $20 \%$ are uninsured, and many experience barriers to care due to poverty and rural isolation. A dedicated social work-trained Prevention Care Manager was hired for twofold purpose: 1) to build and deliver outreach to patients due for specific preventive services (described separately) and 2) to track patients with abnormal screening results performed in our clinic. Providers frequently perform pap smears and order stool cards at the clinic. Previously when these results were abnormal, each provider was responsible for follow-up individually, and there was no systematic mechanism to ensure that the patient was notified and appropriate followthrough occurred. We developed a process for the care manager to track and follow up all abnormal results. The care manager ran monthly reports of abnormal results from the Electronic Health Record (EHR), checked documentation in the EHR to ensure that the patient had been notified, and if this had not occurred, she reminded the PCP to notify the patient. She then ensured that the patient was referred and scheduled for the correct follow up (colposcopy, follow-up pap, or colonoscopy). If no appointment was scheduled, if the patient cancelled or did not attend the diagnostic testing, she contacted the patient to facilitate scheduling and address barriers to care. She tracked these patients either until they had completed diagnostic testing, the supervising physician decided that diagnostic evaluation was no longer necessary due to change in patient status, or a decision was made to mail a certified letter notifying the patient of the risks inherent in not following through on these abnormal test results.

MEASURES OF SUCCESS (DISCUSS QUALITATIVE AND/OR QUANTITATIVE METRICS WHICH WILL BE USED TO EVALUATE PROGRAM/ INTERVENTION): We tracked the number of patients with abnormal pap smears or stool cards, number and percentage of patients notified of the abnormal results, and the number and percent of patients who required care management services to resolve the abnormal results by completing diagnostic workup. Qualitatively, we tracked common barriers to care impeding diagnostic workup.

FINDINGS TO DATE (IT IS NOT SUFFICIENT TO STATE FINDINGS WILL BE DISCUSSED): From April 2014-June 2015, 57 patients were identified with abnormal pap smears or stool cards. Of those patients, a quarter (16/57) required care management for completion of follow-up. Four patients were identified who had not been notified of abnormal test results prior to the care manager contacting the PCP. There were 14 patients with positive stool cards, and more than half $(8 / 14)$ required care management to schedule and complete a colonoscopy. There were 43 abnormal pap smears, and $19 \%$ (8/43) required care management for completion of referral to gynecology. Nearly all of the abnormalities were successfully resolved whether through diagnostic workup, PCP decision that workup was no longer necessary, or delivery of a certified letter. The most common barriers to care for patients were transportation, finances, and apprehension about follow-up care. To address these barriers, the care manager was able to connect patients to community resources for transportation, facilitate referral to diagnostic centers closer to home, schedule on the same day as other appointments, and provide counseling and education to reduce apprehension. Many patients expressed that calling to schedule the test was a barrier and asked the care manager to schedule on their behalf, leading us to build stronger ties with UNC Gastroenterology and Gynecology to assist with scheduling. Patients with financial issues were referred to the UNC Charity Care program, the Affordable Care Act, or free community resources.

KEY LESSONS FOR DISSEMINATION (WHAT CAN OTHERS TAKE AWAY FOR IMPLEMENTATION TO THEIR PRACTICE OR COMMUNITY?): A practice-based social work care manager was able to successfully ensure that patients were notified of abnormal screening results and completed appropriate follow up. Utilizing social work best practices, we were able to address underlying barriers to care, facilitating diagnostic evaluation in a patient-centered and effective manner and resolve almost all abnormalities. This approach has significant patient safety implications. This pilot will be spread across our primary care improvement collaborative together with a package for outreach to patients about prevention.

THE COMPLEX CARE PROGRAM: NAVIGATION AND ADVOCACY FOR MEDICALLY COMPLICATED UNDOCUMENTED LATINOS AT PUENTES DE SALUD Robin E. Canada ${ }^{1,2}$; Heather Klusaritz ${ }^{5}$. Joseph M. Garland ${ }^{3}$; Jose Medina ${ }^{6}$; Stephanie Grana Van Decker ${ }^{4}$; Esther Nivasch ${ }^{5}$. ${ }^{1}$ University of Pennsylvania, Philadelphia, PA; ${ }^{2}$ Puentes de Salud, Philadelphia, PA; ${ }^{3}$ Brown University, Providence, RI; ${ }^{4}$ Temple University School of Medicine, Philadelphia, PA; ${ }^{5}$ Perelman School of Medicine, Philadelphia, PA; ${ }^{6}$ Rutgers New Jersey Medical School, Newark, NJ. (Control ID \#2469010)

STATEMENT OF PROBLEM OR QUESTION (ONE SENTENCE): With the recent rapid growth of undocumented in Latinos in Philadelphia, providers at Puentes de Salud are seeing a growing number of patients with complex medical issues that cannot be adequately managed at Puentes.

OBJECTIVES OF PROGRAM/INTERVENTION (NO MORE THAN THREE OBJECTIVES): 1. Facilitate access to specialty services for medically complex, undocumented Latino patients seen at a free clinic, Puentes de Salud (Bridges of Health). 2. Train medical and social work students to become health navigators and advocates for undocumented patients. 3. Provide high-quality, culturally-appropriate, patient education, advocacy, and navigation support.

DESCRIPTION OF PROGRAM/INTERVENTION, INCLUDING ORGANIZATIONAL CONTEXT (E.G. INPATIENT VS. OUTPATIENT, PRACTICE OR COMMUNITY CHARACTERISTICS): Puentes de Salud is an outpatient low cost primary care clinic for undocumented Latinos in Philadelphia. The organization addresses the social determinants of health through providing legal services, psychology, wellness, health counseling with promotoras, extensive tutoring, as well as primary care for adults and children. Due to language barriers, fear, and lack of knowledge, patients with complicated medical issues have difficulty seeking care at more resourced clinics. In order to actively case manage these patients and navigate them to appropriate city resources, the Complex Care Program (CCP) was developed through a collaboration between the School of Social Policy \& Practice at University of Pennsylvania and Puentes de Salud. 1. Students were selected and trained to provide advocacy and patient navigation services. Most patients are referral to the Philadelphia District Health Centers, where Philadelphia residents can be seen, regardless of immigration status. 2. Students were trained to: 1) assist patients with establishing care at a city health center (understanding potential barriers, wait time, documents needed, etc.), 2) triage appropriate referrals to the CCP 3) communicate with the attending physician/NP 4) facilitate specialty referrals and visits. 3. Students were also taught to collaborate with promotoras (community health workers) working at Puentes de Salud to provide additional educational and personal resources for these patients. 4. Providers were educated on the referral process to the CCP, and services available to undocumented patients in Philadelphia 
A protocol was developed for referring patients to the Complex Care Coordinators (CCC) (Up to 2 student chairs, 2 student volunteers/year in medical, social work or nursing school). 1. Provider identifies patient with complex need. (Need to see specialist, obtain specialty radiology or medications); 2. Provider confirms contact information, and explains that the patient will be contacted by a CCC; 3 . Provider contacts CCC and emails the name, contact information, and plan of care; 4 . Coordinator contacts the patient and provides the following: -Calls patients a week before and day before to confirm appointment, inquire about travel plans and address transportation barriers, -Accompanies patients to appointments (if needed), -Follows up on missed appointments to identify any issues, -Serves as point of communication across different care settings, - Obtains medical record release forms to facilitate exchange of health information between care settings, -Interprets during appointments, -Identifies social problems/patient questions and bringing them to the attention of providers. MEASURES OF SUCCESS (DISCUSS QUALITATIVE AND/OR QUANTITATIVE METRICS WHICH WILL BE USED TO EVALUATE PROGRAM/ INTERVENTION): 1 . Successful navigation of needy undocumented patients to the city health centers or specialty care. 2 . Medical student experiential learning about the complexity of health care for the undocumented.

FINDINGS TO DATE (IT IS NOT SUFFICIENT TO STATE FINDINGS WILL BE DISCUSSED): In the past 2 years (February 2014-January 2016), the CCP has had 39 referrals. -13 were successfully navigated to the city health center, obtained specialty care or radiology, and remained as patients at the center. -7 were successfully navigated to the city health center, obtained specialty care, and returned to Puentes for normal care. -1 was referred to the health center for continued management, no referrals. -3 were successfully navigated to the city health center, but were unable to obtain specialty care. -3 obtained Emergency Medical Assistance (EMA) to seek specialty care and treatment independently. - 1 EMA pending. -2 were directly navigated to specialty procedures through links with UPenn. -1 self-paid for outside radiology. -2 lack sufficient data to know outcomes. -2 were unable to be contacted. -4 declined enrollment. In addition, 5 medical and social work students have been immersed in caring for these complicated patients over an entire year KEY LESSONS FOR DISSEMINATION (WHAT CAN OTHERS TAKE AWAY FOR IMPLEMENTATION TO THEIR PRACTICE OR COMMUNITY?): For those of us involved with the care of the most vulnerable- the undocumented or uninsurable-many supports must be offered to ensure appropriate health care for the medically complex. Using student volunteers in care coordinator roles improves access to care and provides vital experiential learning on the nuances of care for undocumented patients.

THE CRITICAL ROLE OF PRIMARY CARE PHYSICIANS IN THE HOSPITAL Syed S. Akhtar ${ }^{1,2}$; Ali M. Khan ${ }^{3}{ }^{1}$ Iora Health, Las Vegas, NV; ${ }^{2}$ National Physicians Alliance, Washington, DC; ${ }^{3}$ Iora Health, San Francisco, CA. (Control ID \#2469978)

\section{STATEMENT OF PROBLEM OR QUESTION (ONE SENTENCE): Does greater} involvement of primary care physicians (PCPs) in the care of patients admitted to the hospital improve provider communication and promote safer transition of care back to the outpatient setting?

OBJECTIVES OF PROGRAM/INTERVENTION (NO MORE THAN THREE OBJECTIVES): 1. Describe a team-based hospital visit program for PCPs designed to facilitate seamless communication. 2. Develop best practices for safe hospital discharge and care transitions for PCPs through an innovative visit program. 3. Identify critical components of a successful hospital visit for PCPs.

DESCRIPTION OF PROGRAM/INTERVENTION, INCLUDING ORGANIZATIONAL CONTEXT (E.G. INPATIENT VS. OUTPATIENT, PRACTICE OR COMMUNITY CHARACTERISTICS): The authors are PCPs at an outpatient clinic dedicated exclusively to the care of a high-risk, blue-collar, primarily Spanish-speaking, urban, super-utilizer population. Reimbursement is based on a capitated, "per member, per month" basis. Teams of physicians and health coaches with expertise in severe chronic disease management care for these patients. Inclusion criteria for patients at the clinic include at least two hospitalizations in the prior 2 years. Given the number of hospitalizations faced by this population, we sought to improve the poor communication that usually occurs between inpatient and outpatient teams. We created a hospital visit program that concentrated on hospitals most commonly visited by the patients. In the group, one physician was primarily responsible for all visits. Comprehensive visits included discussion with inpatient staff regarding the reason for admission, provision of patient history and relevant documentation, reconciliation of medications, and discharge planning. We also met with the patient and family to ensure understanding of the treatment plan, with particular emphasis placed on discharge needs. Finally, we connected with the case manager to ensure provision of necessary resources upon discharge.

MEASURES OF SUCCESS (DISCUSS QUALITATIVE AND/OR QUANTITATIVE METRICS WHICH WILL BE USED TO EVALUATE PROGRAM/ INTERVENTION): We propose several measures for documenting success. The first measure is a qualitative assessment of the patient's understanding of his or her discharge plan and medication list. The measure is based on the "Care Transitions Measure-3," a validated survey tool designed to determine a patient's understanding of their postdischarge health needs and their knowledge of discharge medications. A second measure is the successful completion of a post-discharge visit in a timely manner. We anticipate that clear communication with the patient regarding the need for prompt follow up will result in a higher likelihood of a clinic visit within $72 \mathrm{~h}$ as compared to the period preceding the visit program. A third measure will include patient satisfaction scores as measured by qualitative open-ended questions regarding their hospital visit in addition to net promoter scores, a validated tool already utilized in the clinic to measure patient satisfaction.

FINDINGS TO DATE (IT IS NOT SUFFICIENT TO STATE FINDINGS WILL BE DISCUSSED): Thus far, we have conducted 60 hospital visits involving 29 patients over a period of 2 months. Of the 60 visits, 21 were on or close to discharge days. We found universal patient and family satisfaction with the visits. In approximately half of the visits, patients stated that the outpatient team was the only one providing information regarding the treatment plan. During one notable encounter, a dying patient's family refused to allow the patient's hospitalist to enter the room after noting that the outpatient team was the only one providing clear information about the patient's condition. The outpatient team found medication discrepancies, including duplication of drug classes and incorrect medications, during virtually every examination of discharge medication lists. Patients claimed better understanding of the plan and medication list upon discharge, although it is unclear if long-term medication adherence improved. We have not seen a significant improvement in the time to outpatient followup; however, $83 \%$ of patients completed a discharge follow-up visit.

KEY LESSONS FOR DISSEMINATION (WHAT CAN OTHERS TAKE AWAY FOR IMPLEMENTATION TO THEIR PRACTICE OR COMMUNITY?): Breakdown in provider communication is known to be a leading cause of adverse events, and we therefore sought to improve communication in patients' most vulnerable state—admitted to an inpatient unit. In the short time we have been conducting these visits, we have found that outpatient teams can be useful to inpatient providers by providing much needed context about the patient. They can console ailing patients and families while effectively translating inpatient treatment plans. They can be fierce advocates when patients are subjected to superfluous testing. We also understand that we are able to do hospital visits in a capitated fee environment. In a traditional fee-for-service structure, it is difficult for many PCPs to find the time to perform comprehensive hospital visits; we therefore strongly advocate for reimbursement of these types of visits. In the meantime, we encourage PCPs to reach out to inpatient teams through other means of communications whenever possible. 
THE EFFECT OF A CENTRALIZED TRANSITION CARE DISCHARGE UNIT ON REDUCING HOSPITAL READMISSIONS Danielle M. Candelario ${ }^{1,2}$; Michael P. Carson ${ }^{1,3}$. ${ }^{1}$ Jersey Shore University Medical Center, Neptune, NJ; ${ }^{2}$ Rutgers University, Pistcataway, NJ; ${ }^{3}$ Rutgers-Robert Wood Johnson Medical School, Pistcataway, NJ. (Control ID \#2467198)

STATEMENT OF PROBLEM OR QUESTION (ONE SENTENCE): Ineffective provider handoff and miscommunication can lead to errors in transitions of care resulting in transitional failures, additional costs, and increased hospital readmission rates. OBJECTIVES OF PROGRAM/INTERVENTION (NO MORE THAN THREE OBJECTIVES): Develop and utilize a centralized discharge unit to reduce 30-day readmission rates for patients with high risk medical conditions, to ensure appropriate transitions of care, and mitigate CMS reimbursement penalties.

DESCRIPTION OF PROGRAM/INTERVENTION, INCLUDING ORGANIZATIONAL CONTEXT (E.G. INPATIENT VS. OUTPATIENT, PRACTICE OR COMMUNITY CHARACTERISTICS): In an effort to minimize readmissions and improve care transitions, hospitals have employed roving discharge coordinators who proactively evaluate patients prior to discharge. Jersey Shore University Medical Center (JSUMC) created The Care Transition Center (CTC), the state's first centralized inpatient discharge unit. The CTC operates Monday-Friday during peak hours and is projected to see $66 \%$ of all hospital discharges. Patients admitted with a diagnosis of Heart Failure (HF), Pneumonia (PNA), COPD and Acute Myocardial Infarction (AMI), may be desig nated as "high risk for readmission" via a provider order in the electronic medical record (EMR). Once discharge orders are placed, these patients are transferred to the CTC for further education and final discharge. The multidisciplinary CTC team consists of a pharmacist, two nurses, two patient care assistants, and a unit secretary. The clinica pharmacist reconciles medications, facilitates prescription filling services, clarifies discharge orders, and addresses areas for therapy optimization. The nurses provide disease management education and transitional care services such as: ensuring home care services, follow-up of laboratory tests, scheduling of follow-up appointments, and facilitating communication with the primary care physician. The staff calls patients 3 and 10 days post-discharge to assess continuity of care, medication compliance, and provide continued education.

MEASURES OF SUCCESS (DISCUSS QUALITATIVE AND/OR QUANTITATIVE METRICS WHICH WILL BE USED TO EVALUATE PROGRAM/ INTERVENTION): Percentage of High Risk patients discharged by the CTC Pharmacist Interventions CTC readmission rates versus that of the general medical units Improvement in HCAHPS domains: Medication - Communication and Discharge Information Overall hospital readmission rates

FINDINGS TO DATE (IT IS NOT SUFFICIENT TO STATE FINDINGS WILL BE DISCUSSED): In 2015, 2,111 patients were designated as High Risk; CTC discharged 920 (43.6\%). The clinical pharmacist discharged 748 of the 920 patients $(81.3 \%)$ of whom $23 \%$ required interventions. Addition of therapy and change in therapy (dose, route, frequency or duration) were the two most common interventions. The pharmacist identified and addressed missing new medication prescriptions for $11 \%$ $(n=82)$ of patients. Six Month Data: Respective readmission rates for High Risk patients discharged through the CTC vs. General Units were: COPD 13.5 vs. $30.2 \%$ (Fisher's exact $p=0.06$ ); PNA 6.3 vs. $20.9 \%$ (Fisher's exact $p=0.07$ ); Total 17.2 vs. $20.1 \%$. Rates for AMI and HF were not numerically different. A retrospective chart review of 25 High Risk patients diagnosed with PNA from 11/2014 to 4/2015, found CTC discharged $60 \%$ $(n=15)$. Services to facilitate medication adherence, including filling prescriptions via our hospital ambulatory pharmacy prior to discharge or faxing prescriptions to a patient's retail pharmacy, were provided for $90 \%$ of patients discharged from the CTC vs. $0 \%$ from general units, suggesting that all patients could benefit from this intervention. Follow-up appointments were scheduled for $45.5 \%$ of the CTC group vs $10 \%$ of the General Unit discharges; the rate of ED visits was higher for the general group, and readmission rates were the same. The small sample size of interim data limits the evaluation of program impact on overall PNA hospital readmissions. Pending Outcomes: HCAHPS Hospital Readmission Rates The effect of the CTC on 30-day all-cause readmission rates for all high risk diagnosis is ongoing.

KEY LESSONS FOR DISSEMINATION (WHAT CAN OTHERS TAKE AWAY FOR IMPLEMENTATION TO THEIR PRACTICE OR COMMUNITY?): This centralized discharge unit decreased readmission rates for COPD and PNA, identified areas of transitional failures by correcting medication reconciliation, and improved rates of postdischarge office visit scheduling. Pharmacist oversight during discharge corrected medication errors for $23 \%$ of CTC patients, identified missing prescriptions for $11 \%$, and facilitated prescription filling services for $90 \%$, thus it is possible that pharmacist intervention contributes to a decrease in readmission rates. A centralized unit also assists with patient flow out of the emergency department by freeing up inpatient beds. Limitations: The "High Risk" designation is a subjective assessment with no standardization. Development of an automatic "High Risk" order based on clinical diagnoses in the EMR could ensure a more objective evaluation and improved identification. The current hours of operation limit the percentage of patients who may be evaluated by CTC staff. Off-hour discharges are not eligible for CTC transfer and this inconsistency can lead to process confusion for both staff and patients. CTC staff will be evaluating ways to address this issue.

THE IMPACT OF PHYSICIAN ORDER ENTRY CLERICAL SUPPORT ON PHYSICIAN SATISFACTION AND PRODUCTIVITY. Erin C. Contratto ${ }^{2}$; Carlos Estrada $^{2}$; Katherine G. Romp ${ }^{1}$; April Agne ${ }^{3}$; Lisa L. Willett ${ }^{3}$. ${ }^{1} \mathrm{UAB}$, Birmingham, AL; ${ }^{2}$ University of Alabama Birmingham, Birmingham, AL; ${ }^{3}$ University of Alabama at Birmingham, Birmingham, AL. (Control ID \#2469894)

\section{STATEMENT OF PROBLEM OR QUESTION (ONE SENTENCE): The increasing} burden of time-sensitive clerical duties placed on physicians during an established clinic session forces physicians to either schedule fewer patients per clinic session, thereby decreasing productivity or dedicate more time outside of clinic on required documentation, resulting in decreased physician professional satisfaction.

OBJECTIVES OF PROGRAM/INTERVENTION (NO MORE THAN THREE OBJECTIVES): Practice redesign to address the challenges of the electronic health record (EHR) may alleviate stressors and improve physician satisfaction and burnout. Our aim was to explore the impact of clerical support personnel solely for physician order entry on physician satisfaction, productivity, and efficiency.

DESCRIPTION OF PROGRAM/INTERVENTION, INCLUDING ORGANIZATIONAL CONTEXT (E.G. INPATIENT VS. OUTPATIENT, PRACTICE OR COMMUNITY CHARACTERISTICS): Setting and Participants: We developed a practice redesign with the goal of decreasing the burden of EHR documentation and order entry for one urban academic general internal medicine primary care practice. The practice includes seven board-certified general internists who serve approximately 3,000 patients, of which $96 \%$ are insured. With other academic responsibilities, the physicians provide primary care services ranging from 1 to 4 half-day sessions per week (average of 2.71 sessions per week). Study design: Quasi-experimental (single group, pre- post- design), mixed methods (quantitative, qualitative). Intervention: Full-time clerical support entered tests ordered by the physician, identified incomplete health maintenance measures and preloaded new patient information.

MEASURES OF SUCCESS (DISCUSS QUALITATIVE AND/OR QUANTITATIVE METRICS WHICH WILL BE USED TO EVALUATE PROGRAM/ INTERVENTION): Measures: Physician satisfaction was measured at baseline and at 4 months using a 14 item survey with validated questions assessing quality of life, personal/professional balance, burnout, and depersonalization. Productivity was measured 
using work Relative Value Units (wRVU) from April 2015 through June 2015, compared to April 2014 through June 2014. A post-intervention focus group $(n=9)$ was conducted, the digital recording was transcribed verbatim. Two independent reviewers performed content analysis of the focus group transcript to identify major themes using an iterative, combined deductive -inductive approach

FINDINGS TO DATE (IT IS NOT SUFFICIENT TO STATE FINDINGS WILL BE DISCUSSED): After 4 months of clerical support, physicians reported improvements in quality of life (bad; $14 \%$ pre, $0 \%$ post), personal/professional balance (dissatisfied; $14 \%$ pre, $0 \%$ post), burnout (at least weekly; $43 \%$ pre, $14 \%$ post), and depersonalization (at least weekly; $14 \%$ pre, $0 \%$ post). Five physicians were practicing in 2014 and 2015. Matched for quarter, wRVU per session increased by $20.5 \%$ for these 5 physicians, (before, April-June 2014; after, April-June 2015) (range -9.2 to $27.5 \%$ ). At baseline $100 \%$ of physicians reported rarely or never completing documentation by the end of a clinic session compared to $57 \%$ post-intervention; completion of notes on weekends also decreased ( $86 \%$ pre, $57 \%$ post-). During the focus group, physicians described the EHR as a provider-reliant system. They discussed increased documentation, inability to complete clinic notes during clinic hours, and difficulty ordering specific tests/studies. Before hiring the order entry personnel, physicians described a significant emotional impact of the EHR on workplace satisfaction including mental exhaustion, multi-tasking fatigue, stress over data entry, and resentment to order/re-order tests/studies. Physicians described that the order entry personnel facilitated order entry of tests/studies, referrals, and pre-visit planning. They reported feeling more supported, focused on patient care, and less stressed and fatigued. Physicians stated they ordered more preventive studies, participated in other academic duties, and experienced improved patient interactions.

KEY LESSONS FOR DISSEMINATION (WHAT CAN OTHERS TAKE AWAY FOR IMPLEMENTATION TO THEIR PRACTICE OR COMMUNITY?): Physicians and health care organizations are seeking means to improve care delivery and physician professional satisfaction. ${ }^{1}$ The Healthy Work Place study advocates for tackling practice workflow obstacles and conducting quality improvement projects aligned with physician concerns to improve primary care physician well-being ${ }^{2}$. Our physician-driven study targeted one specific area of physician frustration- delays in practice workflow due to burdensome physician order entry. By improving the order-entry process for physicians, we provide an example of a successful workflow redesign that can easily be implemented in other primary care practices and positively impact physician well-being. In summary, our small study supports the use of physician order entry personnel as a simple, costeffective intervention to improve the work-life health of primary care providers with potential to positively impact each patient's quality of care and experience within the healthcare system. 1. Bodenheimer T and Sinsky C. From triple to quadruple aim: care of the patient requires care of the provider. Ann Fam Med 2014;12:573-576. 2. Linzer M, Poplau S et al. A Cluster Randomized Trial of Interventions to Improve Work Conditions and Clinician Burnout in Primary Care: Results from the Healthy Work Place (HWP) Study. J Gen Intern Med. 2015 Aug. 30(8):1105-11.

THE IMPORTANCE OF REPEAT SCREENING FOR SUBSTANCE MISUSE IN PRIMARY CARE SETTINGS: INCREASED YIELD OF SCREENING, BRIEF INTERVENTION, AND REFERRAL TO TREATMENT FOR PATIENTS WITH MULTIPLE VISITS Sandeep Kapoor ${ }^{1,2}$; Jeanne Morley ${ }^{1,2}{ }^{2}$; Nancy A. LaVine ${ }^{1,2}$; Megan $\mathrm{Yu}^{2}$; Nancy Kwon ${ }^{3,2}$; Jonathan Morgenstern ${ }^{4,2}$; Joseph Conigliaro ${ }^{1,2}{ }^{2}{ }^{1}$ Northwell Health, Manhasset, NY, NY; ${ }^{2}$ Hofstra Northwell Health School of Medicine, Garden City, NY; ${ }^{3}$ Northwell Health, New Hyde Park, NY; ${ }^{4}$ Northwell Health, Great Neck, NY. (Control ID \#2465896)

STATEMENT OF PROBLEM OR QUESTION (ONE SENTENCE): Is the use of repeat screening for substance misuse in primary care settings warranted?
OBJECTIVES OF PROGRAM/INTERVENTION (NO MORE THAN THREE OBJECTIVES): • Appraise variations in screening scores to assess the value of repeat prescreening with primary care practices. - Discuss patient compliance of repeat screening.

DESCRIPTION OF PROGRAM/INTERVENTION, INCLUDING ORGANIZATIONAL CONTEXT (E.G. INPATIENT VS. OUTPATIENT, PRACTICE OR COMMUNITY CHARACTERISTICS): Screening, Brief Intervention, and Referral to Treatment (SBIRT) is a comprehensive, integrated, public health approach to efficiently identify and address substance misuse at the point of service. SBIRT enables the clinical team to deliver early intervention and referral to treatment services for persons with substance use disorders, as well as those who are at risk of developing these disorders. Integration of SBIRT protocols within primary care consists of universally screening for alcohol, drug, and tobacco misuse by asking a 5-item prescreen during vital signs measurement. The use of universal and repeat screening protocols has been a topic of discussion, and sometimes contention, amongst primary care physicians for a variety of reasons including, 1) necessity to repeat screening if a patient was recently seen; 2) patient comfort. Like other medical screenings, the thought process is that annual screening would be sufficient. However, as substance use has no predetermined pattern, and can change either inherently, or in response to social, health, and work factors, repeat screenings allow primary care practices to raise the subject at each visit, and assess and address with patients when indicated. Patients included in this evaluation visited one of two Primary Care practices and had at least one positive SBIRT screen between $1 / 1 / 15$ and $7 / 31 / 15$. We used the AUDIT-C to screen for alcohol and the DAST-1 for drugs. We reviewed the electronic medical records (EMRs) for all SBIRT pre-screens during the same time period to obtain scores and visit time points, and the patient registration system to determine demographics of the overall clinical practice settings. Data collected from the EMR and the patient registration system were combined into one database to conduct this analysis.

MEASURES OF SUCCESS (DISCUSS QUALITATIVE AND/OR QUANTITATIVE METRICS WHICH WILL BE USED TO EVALUATE PROGRAM/ INTERVENTION): • Repeat screened patient population resembles overall general patient population (i.e. not just a certain high risk subsection of patients). $\bullet$ Number of repeat screens highlighting patient compliance, comfort and willingness to be screened repeatedly. $\bullet$ Number of persons that are newly identified as positive.

FINDINGS TO DATE (IT IS NOT SUFFICIENT TO STATE FINDINGS WILL BE DISCUSSED): A total of 477 repeat patients had at least one positive SBIRT score. Although the cohort represented similar demographic makeup as the practice's general patient population, certain distinctions were reveled, such as male repeat patients had higher average AUDIT-C (3.5316 N=212) and greater percentage of positive DAST-1 $(N=53)$. There were 356 patients with at least one positive AUDIT-C. Changes in scores demonstrated that $49.7 \%$ of patients had AUDIT-C scores that decreased $(N=177)$, of which 93 patients had a $>50 \%$ reduction in scores. In addition, $26 \%(n=93)$ had AUDIT-C scores that increased, of which 53 patients had $>50 \%$ increase in scores. The number of visits (ranging from 2 to 7 visits) seemed to be unrelated to the change in scores.

KEY LESSONS FOR DISSEMINATION (WHAT CAN OTHERS TAKE AWAY FOR IMPLEMENTATION TO THEIR PRACTICE OR COMMUNITY?): Repeat patients make up a significant portion of patients receiving SBIRT screenings in these Primary Care settings and the number of patients who agreed to repeat SBIRT services illustrates that patients are willing to participate in repeated SBIRT screening. While the majority of patients decreased their use, almost a quarter increased, indicating that repeat screening may enable the clinical team to newly identify patients at risk, contrasting previous screening results. Fluctuations in AUDIT-C scores over multiple visits demonstrate that there is sound justification for repeat screening in this Primary Care population. Without multiple touch points to identify and gauge substance misuse, risky behavior can go unnoticed and unaddressed. 
THERE'S AN APP FOR THAT! EVALUATION OF A WEB-BASED MOBILE APP TO FACILITATE PATIENT NAVIGATION THROUGH A COMPLEX PRIMARY CARE NETWORK Aparna Sarin ${ }^{2}$; Vernon $\mathrm{Wu}^{1}$; David C. Thomas ${ }^{1}$; Eva Waite $2 .{ }^{1}$ Icahn Scholl of Medicine at Mount Sinai, New York, NY; ${ }^{2}$ Mount Sinai, New York, NY. (Control ID \#2469318)

STATEMENT OF PROBLEM OR QUESTION (ONE SENTENCE): To assess the usability and effectiveness of a mobile app that provides residents and attendings information about all available resources in the outpatient setting, and how to access them e.g. how to refer to a certain specialty, location and hours of various clinics, residents ambulatory schedules, ambulatory curriculum lectures, insurance specific drug formularies, prior authorization protocols, and contact information of key staff like social workers, nurses, medical office assistants.

OBJECTIVES OF PROGRAM/INTERVENTION (NO MORE THAN THREE OBJECTIVES): 1. Educating health care providers (primarily residents, attendings) about available resources for patients in an ambulatory academic practice. 2. Reducing the logistical challenges care providers face, and barriers patients encounter, in accessing health care services 3 . Evaluating the usability of a web-based mobile app in improving awareness and access to resources that exist within a hospital and in the community for patients.

DESCRIPTION OF PROGRAM/INTERVENTION, INCLUDING ORGANIZATIONAL CONTEXT (E.G. INPATIENT VS. OUTPATIENT, PRACTICE OR COMMUNITY CHARACTERISTICS): Rapid access to logistical information necessary for patient care continues to be a challenge for all health care providers. Even common, routine resources for patients tend to have certain requirements e.g. who can be referred to a screening colonoscopy versus a diagnostic colonoscopy in your health center and how to make the referral to the appropriate team. Many resources are gained and lost quickly, based on funding e.g. prediabetes education classes. Adding to that, internal medicine residents are often in the outpatient clinic only every few weeks, which is enough time for things to have changed since their last rotation. Healthcare systems have tackled this problem in the past with paper or electronic survival guides, which can be difficult to use, access and update. Recently, a user-editable knowledge management platform (EHHAPP, East Harlem Software Company, NY) has been described in literature to consolidate logistic information into a web-based app and streamline information flow for trainees. This system's usability and usefulness have never been tested. In June 2015 , Mount Sinai's Internal Medical Associates (IMA) Practice converted paper survival guides (which included specialty referral protocols, contact information, lecture schedules, staff schedules and billing information) onto the EHHAPP platform and deployed it for use by our residents and attendings primarily. The App was designed to aid primary care providers as its primary focus, however the App is accessible to all members of the health care team on their mobiles or on the desktop. After 6 months of use, we conducted a survey based on the technology assessment model (TAM) and Modified Usability Scale (MUS) to assess the effectiveness and usability of the app in helping care providers at IMA to overcome logistical challenges to providing care from the primary care setting. MEASURES OF SUCCESS (DISCUSS QUALITATIVE AND/OR QUANTITATIVE METRICS WHICH WILL BE USED TO EVALUATE PROGRAM/ INTERVENTION): Evaluation of this intervention is ongoing and will include the following: 1. Qualitative feedback from IMA care providers (attendings versus residents) on the utility of the mobile application 2. Quantitative data on the time spent and the time saved in the referrals process using this mobile application 3. Quantitative data on the ease and comfort using this mobile application to make referrals 4. Quantitative data on the effect of the mobile application in enhancing the ability of clinicians to providing effective primary care

FINDINGS TO DATE (IT IS NOT SUFFICIENT TO STATE FINDINGS WILL BE DISCUSSED): To date 35 care providers spanning residents and attendings have completed the QI survey. The application, available as both a mobile app and a webpage, was accessed mainly on a mobile device (70\% respondents did not access the app at all through a desktop). When used on a mobile device, $50 \%$ respondents accessed the app $1-5 \times /$ week with $46 \%$ respondents accessing $>6 \times /$ week. Of the residents and attendings who made referrals in IMA prior to launching of the application, $64 \%$ reported saving $1-2 \mathrm{~h} /$ week and $7 \%$ reported saving $>6-10 \mathrm{~h} /$ week by using this application. Respondents reported positive changes ( $>3$ on a 5 point scale) in productivity $(88 \%$ ), effectiveness as a healthcare provider (89\%), navigating resources for chronic disease (78\%), and getting accurate referral information ( $83 \%$ ). In terms of usability, respondents reported positive experiences ( $86 \%$ ), ease of referrals (72\%), faster referral scheduling (50\%). Additionally respondents reported comfort (79\%) and ease of locating information (50\%) with this application.

KEY LESSONS FOR DISSEMINATION (WHAT CAN OTHERS TAKE AWAY FOR IMPLEMENTATION TO THEIR PRACTICE OR COMMUNITY?): This mobile-phone application allows primary care providers (and outpatient clinic staff) to factor in access-to-care issues when referring patients for a myriad of services in the outpatient setting. Through the creation and updating of this app, care providers can help address barriers to access and learn to navigate complex healthcare systems, particularly for vulnerable patients. Challenges for sustaining this application include keeping the application up-to-date, and updating at the beginning of the academic year due to the yearly turnover of residents.

TRANSFORMING PRIMARY CARE PAYMENT IN HAWAII Amol S. Navathe ${ }^{1,2}$; Andrea B. Troxel ${ }^{1}$; Ezekiel J. Emanuel ${ }^{1}$; Amanda T. Hodlofski ${ }^{1}$; Kristen Caldarella ${ }^{1}$; Kevin G. Volpp ${ }^{1,2}$. ${ }^{1}$ University of Pennsylvania, Philadelphia, PA; ${ }^{2} \mathrm{CMC}$ VA Medical Center, Philadelphia, PA. (Control ID \#2468938)

STATEMENT OF PROBLEM OR QUESTION (ONE SENTENCE): Designing an innovative payment system that improves upon fee-for-service (FFS), incorporates behavioral economic principles, and improves work satisfaction among primary care physicians (PCPs) while improving quality and reducing health spending in Hawaii.

OBJECTIVES OF PROGRAM/INTERVENTION (NO MORE THAN THREE OBJECTIVES): 1. Design and test an innovative risk-adjusted population-based payment model, with stable or greater levels of physician compensation that are linked to quality and total cost of care. 2. Test the incremental effectiveness of two additional interventions: (1) shared financial incentives between PCPs and poorly controlled diabetics and (2) social comparisons ranking PCPs on quality metric performance and total cost of care.

DESCRIPTION OF PROGRAM/INTERVENTION, INCLUDING ORGANIZATIONAL CONTEXT (E.G. INPATIENT VS. OUTPATIENT, PRACTICE OR COMMUNITY CHARACTERISTICS): The goal of this collaborative project with Hawaii Medical Services Association (HMSA), the Blue Cross Blue Shield (BCBS) Plan of Hawaii, is to transform its PCP payment model to better incentivize population health while bending the increasing trend of health spending in the state. This program will be tested within an initial sample of 97 PCPs across 8 Provider Organizations (POs) then rolled out statewide to more than 1,000 PCPs. The model deliberately shifts away from fee-for-service and includes three components: 1) a risk-adjusted per-member, per-month (PMPM) base payment, 2) an enhanced quality incentive program, and 3) a total cost of care incentive at the PO level. We aim to build on the success of the Alternative Quality Contract (AQC) program implemented by BCBS of Massachusetts. While we use AQC as a starting point, we introduce and test a number of innovations using concepts from behavioral economics. First, we move away from the FFS chassis to a PMPM-based capitated payment. Second, $20 \%$ of the PMPM payment is at-risk based on metrics designed to increase engagement between HMSA and physicians. Third, the number of metrics in the quality incentive program is drastically reduced from over 60 metrics to 10 
12 per specialty. Fourth, the scoring of quality incentives incorporates rewards for improvement, rather than exclusively attainment of thresholds, to activate PCPs along the entire performance distribution. In addition to implementing the new payment model, our initial experiment will include a test of two additional behavioral concepts: social comparisons for PCPs and a shared incentive for PCPs and poorly controlled diabetics tied to improved glycemic control. We will perform a three-arm cluster-randomized trial to test these interventions. Arm 1 will be the new payment model. Arm 2 will incrementally add social comparisons, for which providers will be shown comparisons of their own performance against peers within the same PO on specific quality measures and total cost of care. Arm 3 will test the addition of a member incentive for poorly controlled diabetics to the Arm 2 model. The member incentive will be a payment made to diabetes patients with an $\mathrm{A} 1 \mathrm{c}>9 \%$ who experience a reduction of at least $0.5 \%$. Each participating member can receive up to $\$ 75$ per quarter while physicians receive their incentive through the quality program. With 97 PCPs across 76 practice sites serving 4,445 diabetic patients, we will have $80 \%$ power to detect a difference in Alc of $0.65 \%$ between groups.

MEASURES OF SUCCESS (DISCUSS QUALITATIVE AND/OR QUANTITATIVE METRICS WHICH WILL BE USED TO EVALUATE PROGRAM/ INTERVENTION): • Improvement in PCP performance, as measured by improved performance on the reduced set of quality measures (22 total across all primary care specialties) • Improvement in A1c among poorly controlled diabetics

FINDINGS TO DATE (IT IS NOT SUFFICIENT TO STATE FINDINGS WILL BE DISCUSSED): We analyzed trends and relationships in various components of health spending in Hawaii. Findings include significant variation by line of business (commercial vs. Medicare Advantage vs. Managed Medicaid) in the implied PMPM payments. For example, the range of implied PMPM primary care spending was $\$ 7.64-\$ 87.92$ for Commercial, \$13.66-\$212.53 for Medicare Advantage, and \$3.72-\$1008.59 for Managed Medicaid. Risk-adjustment shrinks the range considerably with ranges from $\$ 19.00-\$ 31.88$ for Commercial, \$32.99 -\$64.24 for Medicare Advantage, and $\$ 23.17-\$ 56.17$ for Managed Medicaid. Other results indicate that patient risk is not consistently associated with PMPM and that high PMPM does not consistently imply lower total cost of care. Quality was found to have varying relationships with PMPM by lines of business, with higher PMPM associated with higher quality within the commercial population, but with lower quality within Managed Medicaid and Medicare Advantage.

KEY LESSONS FOR DISSEMINATION (WHAT CAN OTHERS TAKE AWAY FOR IMPLEMENTATION TO THEIR PRACTICE OR COMMUNITY?): Designing a new compensation model that incentivizes alignment between primary care service use, patient risk, quality of care, and total cost of care is challenging. In particular, variation in practice patterns and service offerings (e.g., pediatrics, after hours care, or in-office procedures, introduces complexities in a capitated model. Adequate risk-adjustment and preventing the dumping of high-risk, high-cost patients is of utmost importance. Finally, stakeholder engagement is critical to creating the buy-in to develop a new system and garner provider participation.

UCLA HEALTH ADVANCE CARE PLANNING INITIATIVE Neil Wenger; Anne M. Walling. University of California, Los Angeles, Los Angeles, CA. (Control ID \#2469411)

STATEMENT OF PROBLEM OR QUESTION (ONE SENTENCE): The complexity of illness and broad availability of advanced medical treatments mixed with the drive for clinical advancement create a need for innovative structures and training to promote advance care planning (ACP) that will match clinical care with patient values, prognosis and goals.

OBJECTIVES OF PROGRAM/INTERVENTION (NO MORE THAN THREE OBJECTIVES): The UCLA Health System, in concert with the Coalition for
Compassionate Care of California (CCCC), built on institutional support to develop an ACP model to ensure that patient goals and values guide care with the following objectives: 1 . Develop a model that stratifies patients into groups based on clinical characteristics, readiness and urgency for ACP, and focuses ACP on goals and values concerning future health states, rather than treatments. 2. Develop an infrastructure and training program so that faculty and staff expect ACP, embedded Communication Promoters prompt specialized ACP with advanced illness patients, and special efforts target vulnerable aspects of care continuity such as transitions. 3. Measure processes and outcomes of ACP including targeted denominator definitions and collection of data reflecting the experience of end of life care.

DESCRIPTION OF PROGRAM/INTERVENTION, INCLUDING ORGANIZATIONAL CONTEXT (E.G. INPATIENT VS. OUTPATIENT, PRACTICE OR COMMUNITY CHARACTERISTICS): The initiative is creating a system-wide mechanism to routinely engage patients in considerations about future health care. The ACP process aims to be reproducible across clinicians so that the iterative process of information sharing and decision making can occur over time, across care venues and between providers. The mechanism integrates with CareConnect, the UCLA Epic-based electronic health record (EHR), so patient preferences are elicited, preserved and updated in a fashion that will inform real time care decisions. Guided by a multidisciplinary team of stakeholders, the program disseminates ACP by implementing the following: 1 . A standardized ACP process triggered based on patient condition, readiness to engage and immediacy of need. For patients with advanced disease, this process cultivates prognostic awareness in anticipation of consideration of future health states. 2. Materials to inform patients and promote ACP including an innovative advance directive and materials modified from the CCCC address ACP needs at the readiness level of the patient. 3 . Training mechanism that produces individuals who can reproducibly implement the standardized ACP process in a clinically astute fashion that is built into their job description. 4. CareConnect infrastructure for maintaining ACP preferences including novel Goals of care notes that can be updated and applied at the point of care. 5. Metrics to evaluate the ACP activities and drive quality improvement This infrastructure produced several novel interventions as components of the program: 1. Palliative nurse practitioner embedded in an oncology clinic began taking warm hand-offs from a single oncologist. The NP uses standardized screening instruments for distress and coping and focuses on valued health states and goals. Prognostic awareness and planning is nurtured through a continuity relationship and symptoms addressed. Six oncologists now work with the NP and others want to refer patients. 2. ACP social worker embedded on the heart transplant service sees every pre-heart transplant patient and other high risk cardiac surgery patients to elicit preferences concerning tailored future health states and advance directive completion. 3. Facilitated ACP in primary care: Unlicensed, ACP-trained, care coordinators initiate ACP for moderate to high risk patients to jumpstart the process for PCP completion, including POLST completion, when appropriate. 4. Trained NPs, fellows and physicians on the NeuroOncology service will implement the ACP model in the routine care of their patients. 5. POLST implementation for patients with DNR orders discharged to a nursing facility - Using EHR triggers and an incentive mechanism, promote completion and transition of documents.

MEASURES OF SUCCESS (DISCUSS QUALITATIVE AND/OR QUANTITATIVE METRICS WHICH WILL BE USED TO EVALUATE PROGRAM/ INTERVENTION): Process measures such as advance directives and Goals of care notes and outcome measures such as venue of death, utilization at the end of life, and implementation of a post-mortem family survey to assess end of life care and decision making.

FINDINGS TO DATE (IT IS NOT SUFFICIENT TO STATE FINDINGS WILL BE DISCUSSED): Changes in ACP have been seen for the health system as a whole: Use of Goals of care notes for patients with advanced illness increased from $<1$ to $>10 \%$. Hospice use among decedents increased from 5 to $20 \%$. ICU use and ICU length of stay 
decreased by $20 \%$ among patients with advanced cancer who died. The palliative NP has been embedded in oncology for 18 months and created a new model of practice that has increased hospice use among decedents she touches with advanced cancer from 20 to $85 \%$.

KEY LESSONS FOR DISSEMINATION (WHAT CAN OTHERS TAKE AWAY FOR IMPLEMENTATION TO THEIR PRACTICE OR COMMUNITY?): 1. A guiding stakeholder group is critical to development of a health system wide program. 2 An infrastructure implemented in a graded fashion can tackle specific aspects of complex care and demonstrate success in targeted areas. 3. Continuous measurement is important to evaluate whether interventions are working and modify approach where needed.

UNDERSTANDING AND PROMOTING SHARED DECISION MAKING WITH A NOVEL INTERACTIVE ONLINE TOOL Fredrik Amell ${ }^{2}$; Jacob Solomon ${ }^{4}$; Brian Zikmund-Fisher ${ }^{4}$; Konstantin Boroda ${ }^{1}$; Yaara Zisman Ilani ${ }^{6}$; Caroline Park ${ }^{5}$; Santiago M. Thibaud $^{1}$; Anand D. Jagannath ${ }^{3}$; Frederick Yick ${ }^{1}$; Tina Shah ${ }^{1}$; Taylor Miller ${ }^{7}$; Ramachandra Reddy ${ }^{5}$; Joshua $\mathrm{Cho}^{5}$; Michelle Pong ${ }^{5}$; Shey Mukundan ${ }^{1}$; Esther MIzrachi $^{8}$; Ashley Ceniceros ${ }^{1}$; Manaf Assafin ${ }^{1}$; Hamid Lari ${ }^{1}$; Kenny $\mathrm{Ye}^{5}$; Darlene LeFrancois ${ }^{1}$. ${ }^{1}$ Albert Einstein College of Medicine, Bronx, NY; ${ }^{2}$ Montefiore, Greenwich, CT; ${ }^{3}$ Montefiore Medical Center, New York, NY; ${ }^{4}$ University of Michigan, Ann Arbor, MI; ${ }^{5}$ Einstein School of Medicine, Bronx, NY; ${ }^{6}$ Dartmouth College, Hanover, NH; ${ }^{7} \mathrm{Mt}$ Sinai School of Medicine, New York, NY; ${ }^{8}$ Long Island Jewish Medical Center, Manhasset, NY. (Control ID \#2457361)

STATEMENT OF PROBLEM OR QUESTION (ONE SENTENCE): Central barriers to shared decision making (SDM) at point of care include difficulty estimating or quickly finding absolute harms and benefits of interventions, yielding difficulty identifying preference-sensitive decisions in which risks and benefits are approximately equal and tailoring to an individual patient's values is appropriate.

OBJECTIVES OF PROGRAM/INTERVENTION (NO MORE THAN THREE OBJECTIVES): 1 . Recognize theNNT.com as an effective platform to aid providers in identifying absolute risks and benefits of numerous medical screenings and interventions. 2. Use theNNT.com to help reduce paternalistic decision making, increase intention to share decisions with patients, and improve understanding of absolute risks. 3. Demonstrate that an interactive online tool can perform better than a static online display (the current form of theNNT.com) in achieving the aforementioned goals.

DESCRIPTION OF PROGRAM/INTERVENTION, INCLUDING ORGANIZATIONAL CONTEXT (E.G. INPATIENT VS. OUTPATIENT, PRACTICE OR COMMUNITY CHARACTERISTICS): TheNNT.com is an increasingly comprehensive non-commercial rapid-risk communication website available for physicians across practice settings and serves as the platform for our intervention. It presents statistical evidence from reputable studies in the form of Absolute Risk Reduction (ARR) and the Number Needed to Treat (NNT) for numerous medical screenings and interventions. The site has potential to quickly and efficiently educate providers about explicit absolute risks and harms, making them more likely to share (versus recommend) decisions with patients. Unfortunately, theNNT is limited to ARR/NNT, and does not capture baseline event rates. Furthermore, the ARRs/NNTs expressed are suboptimal for sharing with less numerate patients. A proposed solution to this problem is the use of icon arrays, which are essentially pictograms consisting of 100 cartoon people. A fraction of these people can be colored to demonstrate the effects of a clinical intervention (i.e. ARR) in the context of a baseline event rate. A challenge of icon arrays is that there are often numerous important clinical endpoints to discuss and the display becomes visually overwhelming when more than 3 different arrays are shown at once. As part of a new SDM mode in theNNT, we are developing an interactive Drill Down Array (DDA) technology that can sequentially present a multitude of endpoints in manageable clusters, based on the interests of doctor and patient. To measure the effect, we launched a Qualtrix-based survey, which exposed Internal and Emergency Medicine residents and medical students to theNNT, and subsequently randomized them to multiple static icon arrays (control) vs a DDA containing the same information. We measured interval change of the intention to recommend (paternalistic intention) and/or to share the decision, before and after each exposure, on a $5 \mathrm{pt}$ Likert scale. Results from our evolving trialing efforts will be presented at SGIM before a largescale non-commercial public launch on theNNT.com.

MEASURES OF SUCCESS (DISCUSS QUALITATIVE AND/OR QUANTITATIVE METRICS WHICH WILL BE USED TO EVALUATE PROGRAM/ INTERVENTION): 1: Efficacy of theNNT.com platform in a) reducing paternalistic intention b) increasing intention for SDM. 2: Efficacy of Drill Down Array to a) increase accuracy of baseline event rate perception beyond theNNT.com b) increase intentionality to SDM c) increase physician opinion that patients would benefit from exposure d) reduced paternalistic intentionality

FINDINGS TO DATE (IT IS NOT SUFFICIENT TO STATE FINDINGS WILL BE DISCUSSED): 1. Efficacy of theNNT.com: Multi-center interventional surveys of Internal and Emergency Medicine residents found that simple exposure to numerical ARR a) reduced stated intention to "strongly recommend" test and treatment decisions (1.04 points $p=0.03, n=41) \mathrm{b}$ ) increased intention to share decisions (1.03 points, $p=0.04$ ). This data supports that site exposure helps learners reframe interventions they thought were "effective care" as preference sensitive. 2. Efficacy of Drill Down Array: A digital pilot RCT for novice physicians and students, is showing that complementing theNNT.com with a DDA for Lung Cancer Screening a) improves perception of baseline event rate compared to control $(p=0.004 n=51)$ b) potentially increases stated intention to share decisions compared to control (by 0.32 points $p=0.175 n=24$ ) c) potentially increases belief that their patients' would be able to benefit from exposure (by 0.4 points $p=0.173 n=40)$ d) reduces paternalistic tendency by 0.98 point movement on 5 point likert, over the theNNT $(p=0.04)$, but no difference compared to control.

KEY LESSONS FOR DISSEMINATION (WHAT CAN OTHERS TAKE AWAY FOR IMPLEMENTATION TO THEIR PRACTICE OR COMMUNITY?): 1 TheNNT used alone can increase the medical student and novice physician's stated intention to employ SDM. 2. Both theNNT.com and a novel Drill Down Array (DDA) tool seem equally able to reduce intention towards paternalism by students and novice physicians. 3. The DDA is superior to static icon arrays in communicating baseline risk and may prove superior in increasing tendency towards utilizing SDM with larger sample size. We will continue optimizing our proof-of-concept technology and trialing based on feedback from users.

UNDERSTANDING FREQUENTLY READMITTED PATIENTS IN A SAFETY NET HOSPITAL Jessica Beaman ${ }^{1}$; Elizabeth Imbert ${ }^{2}$; Karishma Oza ${ }^{2}$; Michelle Schneidermann ${ }^{2}$; Larissa Thomas ${ }^{2}$; Elizabeth Davis ${ }^{2}$. ${ }^{1}$ University of California San Francisco, San Francisco, CA; ${ }^{2}$ University of California, San Francisco, San Francisco, CA. (Control ID \#2468081)

STATEMENT OF PROBLEM OR QUESTION (ONE SENTENCE): To analyze the characteristics of frequently readmitted patients in a safety net hospital and identify modifiable health system factors that lead to readmission.

OBJECTIVES OF PROGRAM/INTERVENTION (NO MORE THAN THREE OBJECTIVES): 1. Identify characteristics of frequently readmitted patients 2 . Define modifiable health system factors that lead to readmissions through in-depth chart review 3 . Create recommendations for interventions to decrease readmissions

DESCRIPTION OF PROGRAM/INTERVENTION, INCLUDING ORGANIZATIONAL CONTEXT (E.G. INPATIENT VS. OUTPATIENT, PRACTICE OR 
COMMUNITY CHARACTERISTICS): San Francisco General Hospital (SFGH) is a 598 bed public safety net hospital that serves over 100,000 patients each year and provides $20 \%$ of San Francisco's inpatient care. The hospital provides primary care, specialty, and hospital care for vulnerable and underserved patients. In 2014, the 30 day all-cause readmission rate was $12.7 \%$. Patients who were readmitted disproportionally had Medi$\mathrm{Cal}$, behavioral health diagnoses, and marginal housing. Patient surveys identified difficulty understanding and accessing medications, feeling lost in the system, struggling to get to appointments, and insufficient support from caregivers or family as root causes of preventable readmissions. However, it was not known if there was a subset of patients readmitted frequently who had unique demographic characteristics. We sought to better understand this population using a quantitative and qualitative analysis of patients with 3 or more 30-day readmissions to our safety net hospital in the year of 2014. We analyzed structured data from our electronic medical record as well as conducted chart reviews of 20 patients in this cohort. Using findings from this analysis, we made recommendations for interventions to reduce readmissions.

MEASURES OF SUCCESS (DISCUSS QUALITATIVE AND/OR QUANTITATIVE METRICS WHICH WILL BE USED TO EVALUATE PROGRAM/ INTERVENTION): We will measure our success with the following five outcomes: 1 . Identify key demographic characteristics of patients who had been readmitted to the hospital within 30 days of discharge more than 3 times from January 1, 2014 to December 31, 2014 2. Compare these characteristics to all patients hospitalized in 2014 to identify unique features of frequently readmitted patients 3 . Identify modifiable health system factors that led to readmissions for this high-utilizing population 4. Present our key findings to the SFGH Care Transitions Task Force, our hospital's taskforce to improve transitions of care, in February 2016 5. Recommend a pilot initiative, based on our key findings, to the SFGH Care Transitions Task Force to reduce 30-day readmission rates FINDINGS TO DATE (IT IS NOT SUFFICIENT TO STATE FINDINGS WILL BE DISCUSSED): 138 patients were readmitted to SFGH more than 3 times from January 1, 2014 to December 31, 2014. This represents $1.4 \%$ of hospitalized patients but $37 \%$ of readmissions to SFGH. Their mean age was 52 and the majority of patients were men $(N=89,64.5 \%)$, Black $(N=49,35.5 \%)$ or White $(N=45,32.6 \%)$, and English speaking $(N=113,81.9 \%)$. Twenty percent were homeless. Medi-Cal $(79.4 \%)$ and Medicare (17.9\%) were the primary insurers for these patients. In comparison to the patients who were discharged from SFGH during this same time period, our patients were more likely to be Black or White (35.5 and $32.6 \%$ of high utilizers vs. 20.9 and $28.5 \%$ of all patients discharged, respectively). Patients included in our study were more likely to be men ( $64.5 \%$ of high utilizers vs. $55.3 \%$ of all patients discharged). In addition, they were more likely to be homeless ( $20 \%$ of high utilizers vs. $9.5 \%$ of all patients discharged). High utilizers were more likely to be insured via Medi-cal, Medicare, or both compared to all patients discharged (97.3 vs. $76.5 \%$ ). In our qualitative chart review, we have found that there are themes associated with 30-day hospital readmission. For example, many of the patients that we have defined as high utilizers have multiple comorbidities and thus may have an increased risk of acute health needs due to the severity of their underlying diseases. In addition, substance use, homelessness, and mental illness play a significant role in readmissions for some patients. We also found that some patients struggled with complex medication regimens and were then readmitted when they were unable to follow the medication regimen.

KEY LESSONS FOR DISSEMINATION (WHAT CAN OTHERS TAKE AWAY FOR IMPLEMENTATION TO THEIR PRACTICE OR COMMUNITY?): 1. Our high-utilizer population has unique demographic characteristics as compared to all patients discharged from SFGH. Specifically, our cohort of high-utilizers were more likely to be Black, White, men, and homeless. Future interventions focused on reducing readmission rates could target these unique patient populations. 2. Medi-cal and Medicare are almost universally the primary insurers of our high-utilizer population and thus a partnership between SFGH and these insurers could lead to innovative strategies for helping to reduce readmissions for this patient population. 3. The factors leading to readmission for high utilizers are complex and often patient-specific. From our themes that emerged, transitions in care programs incorporating medication teaching and behavioral health support could have the potential to prevent readmissions.

UNDERSTANDING VALUE FROM THE C-SUITE TO THE BEDSIDE: ONE INSTITUTION'S DEVELOPMENT OF AN INPATIENT PROVIDER VALUE DASHBOARD. Stephanie A. Halvorson ${ }^{1}$; Sudhakar P. Karlapudi ${ }^{2}$; Brian Wheeler ${ }^{2}$; Peter Graven $^{2}$; Ruth Rowland ${ }^{2}$; Thomas Yackel ${ }^{2}$. ${ }^{1}$ OHSU, Portland, OR; ${ }^{2}$ Oregon Health and Science University, Portland, OR. (Control ID \#2465454)

STATEMENT OF PROBLEM OR QUESTION (ONE SENTENCE): Provider engagement is essential to a successful transition from traditional fee-for-service or "volume"-based care, to "value"-based care that emphasizes quality and patient experience, while minimizing unnecessary care and healthcare costs.

OBJECTIVES OF PROGRAM/INTERVENTION (NO MORE THAN THREE OBJECTIVES): Our Department of Clinical Integration (CI) developed the Inpatient Provider Value Dashboard (IPVD) to increase providers' understanding of their own clinical performance as it pertains to value, to provide transparency, and to stimulate provider engagement in the transition to value-based care.

DESCRIPTION OF PROGRAM/INTERVENTION, INCLUDING ORGANIZATIONAL CONTEXT (E.G. INPATIENT VS. OUTPATIENT, PRACTICE OR COMMUNITY CHARACTERISTICS): Our AMC is a 550-bed tertiary and quaternary care center located in Portland, Oregon. Outpatient providers in our institution routinely receive key quality, cost, and utilization metrics via existing dashboards (for example, "Meaningful Use" data). Inpatient providers, however, have not had direct access to their own metrics. The CI team assembled a diverse workgroup including clinical informatics, Epic $^{\circledR}$ (electronic health record), hospital financial services, quality improvement, health systems effectiveness research, and physicians to identify and organize existing data into an electronic dashboard. A needs assessment, consisting of 20 qualitative interviews with physicians across specialties, was performed to assess interest, engagement and priorities for value-related data reporting. The findings of this assessment were used to develop the IPVD, which is now sent out to hospitalist providers quarterly. In its current version, IPVD metrics include patient volume, average length of stay, readmission rate, mortality rate, case mix index, and utilization data (procedures, supplies, laboratories, etc.). Individual provider data is visually displayed with benchmark comparison to their institutional peers and with University Hospital Consortium benchmarks. Data attribution is linked to claims data, usually the discharging attending physician, and as such, are presently not available to residents or physician-extenders.

MEASURES OF SUCCESS (DISCUSS QUALITATIVE AND/OR QUANTITATIVE METRICS WHICH WILL BE USED TO EVALUATE PROGRAM/ INTERVENTION): Measures include pre- and post-surveys of provider perceptions of health care "value" and focus groups with a pilot group of hospitalist physicians to obtain feedback and inform iterative design of the IPVD. The ultimate goal of increased provider engagement, while difficult to directly measure, may be manifested by quality or systemsimprovement projects stimulated by access to these data. Examples will be provided. FINDINGS TO DATE (IT IS NOT SUFFICIENT TO STATE FINDINGS WILL BE DISCUSSED): The dashboard was first piloted with the hospitalist group in August, 2015. Prior to receiving the IPVD, surveyed hospitalists $(n=7 / 9$ meeting attendees, $78 \%$ response rate) were in agreement that access to cost and other value metrics should be considered when making treatment decisions, however, most felt they did not have adequate access to these types of data. Post-survey data will soon be collected.

KEY LESSONS FOR DISSEMINATION (WHAT CAN OTHERS TAKE AWAY FOR IMPLEMENTATION TO THEIR PRACTICE OR COMMUNITY?): Focus 
group feedback suggests the dashboard is stimulating engagement among providers. Acknowledging that most inpatient care is team- based, the main critique of the data is that "discharging MD" does not reflect all care team members.. However, IPVD data do provide an advantage over prior data that, when given, were at the level of MS-DRG or department and lacked relevance for individual providers. Additional critiques of the IPVD include the omission of residents and physician-extenders, lack of patient experience data (currently given at the unit, not provider level), and paradoxical concerns about too much data being released to physicians (i.e., "data fatigue"). Additionally, providers have expressed concern about the potential for punitive use of dashboard data. The next steps for the IPVD is dissemination to all inpatient physicians, planned for February 2016, and future customization as needed for use by different specialty groups. Healthcare leaders have recognized that "for new models of care delivery to succeed, they may need new models of engagement with physicians [SH1] ." The IPVD provides one example of a tool used to engage physicians in the transformation to value-based care

USE OF A PRE-VISIT ASSESSMENT TO AID IN SUCCESSFUL MEDICAL VISITS FOR ADOLESCENTS AND ADULTS WITH AUTISM Christopher Hanks Stephanie Lawrence. The Ohio State University Wexner Medical Center, Hilliard, OH. (Control ID \#2465465)

STATEMENT OF PROBLEM OR QUESTION (ONE SENTENCE): Individuals with autism spectrum disorders experience health care disparities and report difficulty accessing medical care.

OBJECTIVES OF PROGRAM/INTERVENTION (NO MORE THAN THREE OBJECTIVES): Improve access to medical care for individuals with autism spectrum disorders by: 1 . Use a pre-visit phone assessment prior to the first visit to identify barriers to recieving care in a primary care office. 2 . Identify simple modifications to the typical flow of medical visits that can help overcome barriers to care 3 . Evaluate the effectiveness of these modifications.

DESCRIPTION OF PROGRAM/INTERVENTION, INCLUDING ORGANIZATIONAL CONTEXT (E.G. INPATIENT VS. OUTPATIENT, PRACTICE OR COMMUNITY CHARACTERISTICS): The Center for Autism Services and Transition (CAST) currently serves approximately 280 individuals with ASD. CAST is located in an academic multi-specialty office including primary care Internal Medicine and Pediatrics as well as multiple subspecialty practices located in a suburb of Columbus, Ohio. People with autism spectrum disorder (ASD) are less likely to have a primary care physician (PCP) and more likely to report unmet medical needs or dissatisfaction with their care. Additionally, many individuals with ASD experience anxiety or sensory disturbances that can make medical visits overwhelming. Medical providers report lack of comfort caring for adults with ASD. Little is published on how to improve health care for people with ASD, particularly for those transitioning to adult-based settings. CAST was created to address these needs and provides primary care to adolescents and adults with ASD. Each patient undergoes a previsit assessment which identifies barriers to care. The medical visit flow is then changed to address specific needs. Information from the pre-visit assessment including patient and family needs, measures used to overcome barriers, and the outcome of the visit provide useful information for how to improve the healthcare experience of patients with ASD. A retrospective review was done on charts of 93 randomly selected patients who had been seen in the CAST clinic. Data collected included age, vitals, diagnoses, medications, pre-visit assessment findings, specific interventions employed, and outcomes of these interventions. These data were reviewed and are described.

MEASURES OF SUCCESS (DISCUSS QUALITATIVE AND/OR QUANTITATIVE METRICS WHICH WILL BE USED TO EVALUATE PROGRAM/
INTERVENTION): Measures of success will include assessment of concerns identified via phone assessment, frequency of interventions or modifications to visit flow, and outcomes during visits including rates of attendance to appointment to establish care, inability to complete portions of the evaluation or physical exam. We will also evaluate the required interventions to determine if they are generalizable to other medical offices.

FINDINGS TO DATE (IT IS NOT SUFFICIENT TO STATE FINDINGS WILI BE DISCUSSED): Patient characteristics of the 93 charts reviewed included: mean age of 20 years, $52 \%$ with intellectual disability, $48 \%$ with ADHD, 48 with anxiety, and $34 \%$ with a history of aggressive behavior. 87 of 93 patients completed a pre-visit assessment prior to their 1st visit. 27 of 87 (30\%) of patients identified a concern about the office visit or environment with many of them expressing more than one concern. 19 (22\%) had documented changes to standard medical visit format. Specific needs included: difficulty with the waiting room $(n=10)$, difficulty waiting $(n=6)$, history of aggression in a medical facility $(n=5)$, aversion to needles $(n=4)$, not liking loud noises $(n=3)$, concern for anxiety interfering with care $(n=3)$, difficulty being touched $(n=2)$, difficulty hearing children cry $(n=2)$, not liking bright light $(n=2)$, won't allow blood pressure checks ( $n=$ 2 ), and concern for egress from the exam room $(n=1)$. Modifications to the routine visit structure included: rooming the patient immediately upon arrival and completing registration in the exam room instead of utilizing the waiting room $(n=17)$, not performing vital signs at the time of intake ( $n=2)$, notifying the patient prior to touching them $(n=2)$, waiting in the car until the physician was ready to see the patient $(n=2)$, having caregivers plan to bring items to entertain the patient $(n=2)$, turning the lights out in the room prior to rooming $(n=1)$, scheduling them in the 1st appointment of the session $(n=1)$, having a picture of the physician prior to the visit $(n=1)$ and having a security guard present $(n=1)$. All 93 patients were successfully roomed and evaluated by the physician. Of those who underwent a pre-visit assessment, 7 patients had incomplete visits which consisted of being unable to get complete vital signs $(n=5)$ and being unable to complete portions of the physical exam $(n=3)$

KEY LESSONS FOR DISSEMINATION (WHAT CAN OTHERS TAKE AWAY FOR IMPLEMENTATION TO THEIR PRACTICE OR COMMUNITY?): In this sample of patients from the CAST program, $30 \%$ of patients identified concerns or challenges related to medical visits and $22 \%$ required modification of typical visit flow. Patients with identified concerns required relatively simple changes to the routine visit format to allow them to successfully receive care in a General Internal Medicine and Pediatric office setting. These data suggest that patients with ASD can receive patientcentered, individualized care in medical settings designed for the general population with relatively simple interventions

USE OF COMPREHENSIVE SYSTEMS SUPPORT TO IMPROVE CHRONIC DISEASE MANAGEMENT AND CANCER SCREENING Shwetha S. Sequeira ${ }^{1}$; Julianne Mcnamara ${ }^{2}$; Evonne Yang ${ }^{2}$; Joanna D'Afflitti ${ }^{1}$; Rebecca G. Mishuris ${ }^{1} .{ }^{1}$ Boston University School of Medicine, Boston, MA; ${ }^{2}$ Boston University Medical Center, Boston, MA. (Control ID \#2469779)

STATEMENT OF PROBLEM OR QUESTION (ONE SENTENCE): The national focus on high quality, patient centered care, coupled with clinician burnout, makes developing non-provider dependent systems that support point of care chronic disease monitoring and cancer screening a high priority

OBJECTIVES OF PROGRAM/INTERVENTION (NO MORE THAN THREE

OBJECTIVES): Increase the rates of recommended routine monitoring for patients with type 2 diabetes mellitus (hemoglobin A1c testing), influenza vaccination, and routine cancer screening (cervical, breast and colon cancers) in an urban safety net hospital-based adult primary care clinic. 
DESCRIPTION OF PROGRAM/INTERVENTION, INCLUDING ORGANIZATIONAL CONTEXT (E.G. INPATIENT VS. OUTPATIENT, PRACTICE OR COMMUNITY CHARACTERISTICS): We developed a multi-dimensional program that involved medical assistants and clinicians to address these preventive care measures in an urban safety net hospital-based adult primary care clinic. This program is based on evidence from a pilot study which showed that increased operational, navigational and data sharing support at the point of care improved the delivery of preventive services. In addition to the electronic clinical reminders presented to the medical assistants and clinicians in the form of best practice alerts (BPAs) within the electronic medical record (EMR), this program was built around a daily report of scheduled patients due for preventive services. Medical assistants used these opportunity reports as decision support to determine patients who were overdue for cancer screening (based on USPSTF guidelines) and patients with diabetes who were overdue for HbAlc testing (based on ADA guidelines). The opportunity reports were used in pre-visit care team huddles to plan for completion of routine screening tests at the point of care and also triggered non-cliniciandependent workflows such as automatic HbAlc testing by medical assistants for patients who are overdue. Performance reports on rates of patients with up to date HbAlc testing were provided monthly to the entire practice to compare and track performance over time. Cancer screening performance reporting is currently in process.

MEASURES OF SUCCESS (DISCUSS QUALITATIVE AND/OR QUANTITATIVE METRICS WHICH WILL BE USED TO EVALUATE PROGRAM/ INTERVENTION): Number of eligible patients with completed HbA1c testing, number of eligible patients completing influenza vaccination, and number of eligible patients completing cancer screening.

FINDINGS TO DATE (IT IS NOT SUFFICIENT TO STATE FINDINGS WILL BE DISCUSSED): The percentage of eligible diabetic patients who were compliant with guideline recommended $\mathrm{HbA} 1 \mathrm{c}$ testing for the entire practice rose from an average of $84.2 \%$ pre-intervention to $91.7 \%$ with the intervention. Clinicians and Medical Assistants reported improved care team communication, which included agenda setting for patient visits and care team huddles.

KEY LESSONS FOR DISSEMINATION (WHAT CAN OTHERS TAKE AWAY FOR IMPLEMENTATION TO THEIR PRACTICE OR COMMUNITY?): 1 ) Employing non-provider dependent workflows unburdens providers, enhances the nonprovider role in patient care and promotes team based care; 2) Providing up to date, consumable process and outcome metric reporting to the practice allows for identification of best practices and areas to improve, and had the greatest impact on our improvement in rates of $\mathrm{HbAlc}$ monitoring; 3 ) using point of care interventions is more patient -centered and efficient

USING BEHAVIORAL ECONOMICS TO IMPROVE PHYSICIAN PAY-FORPERFORMANCE PROGRAMS Amol S. Navathe ${ }^{1,2}$; Kevin G. Volpp ${ }^{1,2}$; Amanda T. Hodlofski ${ }^{1}$; Kristen Caldarella ${ }^{1}$; Amelia Bond ${ }^{1}$; Ezekiel J. Emanuel ${ }^{1}{ }^{1}$ University of Pennsylvania, Philadelphia, PA; ${ }^{2} \mathrm{CMC}$ VA Medical Center, Philadelphia, PA. (Control ID \#2468841)

STATEMENT OF PROBLEM OR QUESTION (ONE SENTENCE): How can behavioral economic principles be incorporated into the design of physician incentives to improve the impact of pay-for-performance ( $\mathrm{P} 4 \mathrm{P})$ programs?

OBJECTIVES OF PROGRAM/INTERVENTION (NO MORE THAN THREE

OBJECTIVES): 1 . Evaluate the impact of a retrospective intervention using the behavioral economics principles immediacy and relative social ranking on physician performance within a $\mathrm{P} 4 \mathrm{P}$ program 2 . Test the impact of a prospective physician incentive design change using the behavioral economic principles social pressure and loss aversion in a randomized controlled trial 3. Better understand the influence of using these principles in incentive programs on physician attitudes, work satisfaction, and patient experience

DESCRIPTION OF PROGRAM/INTERVENTION, INCLUDING ORGANIZATIONAL CONTEXT (E.G. INPATIENT VS. OUTPATIENT, PRACTICE OR COMMUNITY CHARACTERISTICS): The key goal of this project is to test whether and how using behavioral economic principles in the design of physician incentives can improve quality of care. Behavioral economics principles have been utilized effectively to improve patient well-being and chronic disease management through financial and nonfinancial incentives. However, they have not yet been tested formally or systematically implemented within physician incentive programs such as $\mathrm{P} 4 \mathrm{P}$. Given that $\mathrm{P} 4 \mathrm{P}$ programs have been largely ineffective, utilizing behavioral economics principles may provide an important innovation to make these programs more impactful on the care patients receive. In this program, we are creating a new P4P physician incentive program at a large clinically integrated network, Advocate Physician Partners (APP) in Chicago, Illinois. The first phase of the study includes an evaluation of a recent intervention that uses the behavioral principle immediacy in providing feedback to physicians on their performance. Specifically, APP rolled out a new registry platform in January 2014 that enables real-time feedback and tracks physician/practice use of the registry. We will test the impact of this intervention across a sample of 850 primary care physicians across 400 sites. In the second phase of the program, we have launched a multi-arm randomized controlled trial to test the impact of enhancing the behavioral principle social pressure and the use of the principle loss aversion in the incentive design on physician performance. The third phase of the program will be scaling interventions throughout the entire network. In the trial phase of our program, Arm 1 will serve as the 'usual care' control reflecting the current design. Arm 2 will pre-fund incentive accounts with the entire annual incentive opportunity to create an 'endowment effect' and engender loss aversion among physicians. Physicians will be required to 'pay back' any unearned portion at the end of the program year from this incentive account. To protect physicians against over-withdrawal and avoid excessive friction about the program, physicians are only allowed to access a portion of the prefunded account in advance based on their historic performance. Arm 3 will test enhanced social pressure by increasing the group-based performance component of the individual physician's incentive payment from 30 to $50 \%$ (with other $50 \%$ based on individual performance) and will keep the incentive framing constant. In addition to measuring performance on quality metrics and financial bonus attainment, we are conducting quantitative evaluations of the interventions on changing physician behavior, their attitudes toward the program and the impact on work satisfaction, and the impact on patients through surveys.

MEASURES OF SUCCESS (DISCUSS QUALITATIVE AND/OR QUANTITATIVE METRICS WHICH WILL BE USED TO EVALUATE PROGRAM/ INTERVENTION): The single key measure of success is a composite index of quality metrics currently used by APP. This composite score, called the Clinical Integration (CI) score, reflects HEDIS metrics and other evidence-based clinical guidelines. Secondary measures include incentive bonus earned, patient experience indicators, and scores on the following domains in physician surveys: attitude toward financial incentives, teamwork/ collaboration, financial salience of incentives, practice environment, acceptability, impact on clinical behavior, and unintended consequences.

FINDINGS TO DATE (IT IS NOT SUFFICIENT TO STATE FINDINGS WILL BE DISCUSSED): Retrospective analysis currently indicates that the impact of immediacy resulted in an aggregate $0.5 \%$ change in the CI score. Detailed sub-group analysis is underway. The prospective clinical trial is currently in progress.

KEY LESSONS FOR DISSEMINATION (WHAT CAN OTHERS TAKE AWAY FOR IMPLEMENTATION TO THEIR PRACTICE OR COMMUNITY?): Early evidence suggests that immediacy may be effective in improving the impact of physician incentive programs. Several principles have been anecdotally associated with better physician performance, including better salience of payments through separate bonus 
checks, social pressure through group incentives, and relative social ranking by incorporating transparency. This agenda of program innovation will be the first to rigorously test the incremental impact of using behavioral principles in physician incentive design.

USING POOPULATION MANAGEMENT STRATEGIES TO IMPROVE LIPID MANAGEMENT IN HIGH RISK PATIENTS Eleanor Weinstein; Ingrid Nelson. Jacobi Medical Center, Bronx, NY. (Control ID \#2468908)

STATEMENT OF PROBLEM OR QUESTION (ONE SENTENCE): How can we optimally use population management strategies to improve lipid control in our high risk patients? OBJECTIVES OF PROGRAM/INTERVENTION (NO MORE THAN THREE OBJECTIVES): Major objectives: 1. To provide focused outreach to our highest risk patients using the diabetes and cardiovascular disease patient registries and to develop a tracking process for the population management team. 2 . To improve lipid management and increase the use of the most appropriate statin in these patients. 3 . To enhance provider education in the treatment of hyperlipidemia.

DESCRIPTION OF PROGRAM/INTERVENTION, INCLUDING ORGANIZATIONAL CONTEXT (E.G. INPATIENT VS. OUTPATIENT, PRACTICE OR COMMUNITY CHARACTERISTICS): The ambulatory practice at Jacobi Medical Center is a hospital based outpatient setting in the northeast section of the Bronx in New York City. We serve a vastly diverse population and many patients have complex comorbidities including diabetes (DM), hypertension, obesity and cardiovascular disease Challenges to the control of these chronic diseases includes difficulty with access to care due to staffing shortages in the setting of increasing demand, patients with low health literacy, and nonadherence to medication use. We noted that our lipid control in the patients with DM and ischemic vascular disease was less than optimal and designed this pilot project to address that gap. A list of patients who had not had their lipids checked in > 12 months or who had LDL. > 100 -used as a proxy for suboptimal control likely due to nonadherence or suboptimal statin choice or dose was obtained through the patient registries. Charts were reviewed and if appropriate for intervention, letters were sent to patients asking them to come in for lab work which was ordered by our team nurse practitioner. Results were reviewed and medications were adjusted as appropriate. The patient's provider was also informed of results and medication changes that were made Patients who did not respond to the initial outreach were called by our team RN and medication adherence was assessed and patients were encouraged to come in for testing. Providers were aware of this pilot project and were very supportive. An algorithm for decision support on choice of statin and dose was developed by the clinical leadership and posted in all the exam rooms and teaching areas in the practice. Other barriers to optimal lipid control including need for prior approvals (PA) for high intensity statins or high copays was also addressed during this pilot project. We have recently been informed that one of our managed medicaid plans with the highest enrollment will add atrovastatin to their formulary without need for a PA - in large part due to the advocacy that was made during this lipid management project.

MEASURES OF SUCCESS (DISCUSS QUALITATIVE AND/OR QUANTITATIVE METRICS WHICH WILL BE USED TO EVALUATE PROGRAM/ INTERVENTION): Metrics that were used to evaluate this program included: Number of patients in the intervention group who responded to the outreach - either by letter or by phone. Number of patients in the intervention group who were at goal for their lipid management by the end of the project. Patients in the intervention group who had an improvement in control of hypertension and DM. Htn control, DM control and lipid control in all patients in the diabetes registry through the year.

FINDINGS TO DATE (IT IS NOT SUFFICIENT TO STATE FINDINGS WILL BE DISCUSSED): There were 4409 patients in the Diabetes registry at the beginning of the pilot program. 2345 patients had LDL $>100$ or no lipid panel checked in $>12$ months. 265 of these patients had DM as well as Ischemic Vascular Disease — our target population for intervention. 265 charts were reviewed by the clinical team 121 were not appropriate for intervention (patients had moved, were intolerant to statins or were in control by the beginning of the project) 144 patients were appropriate for the intervention $57 / 144$ patients were at goal with LDL $<100$ - a $39 \%$ improvement rate $16 / 144$ were not at goal outreach continues to assess adherence and proper statin use 9/144 were not at goal but medications had been recently adjusted -lipid panels need to be repeated BP and DM control also improved in the intervention group. BP control, DM control as well as lipid control improved for the entire diabetes registry population over the year with the greatest improvement in lipid control from $39 \%$ in the first quarter to $50 \%$ by end of the fourth quarter

KEY LESSONS FOR DISSEMINATION (WHAT CAN OTHERS TAKE AWAY FOR IMPLEMENTATION TO THEIR PRACTICE OR COMMUNITY?): What worked well: Using population management strategies - patient registries, outreach by letter and phone, tracking process to follow patients through the project was critical to the success of this project Developing an algorithm for decision support and providing performance feedback through registry data was helpful. Advocating for changes in the formulary to include atorvastatin without a prior approval was effective. What did not work as well and what we learned: Mailings often yield in a low result — phone calls were effective but labor intensive Patients often stop their statin and the providers may be unaware until the lipid panel is checked. We need to have other ways to follow adherence and we need to provide education at the proper literacy level about the benefits and safety of statins. We are exploring the use of point of care testing for LDL allowing for real time assessment of lipid control.

UTILIZATION OF CO-LOCATED BEHAVIORAL HEALTH PROVIDERS IN AN ACADEMIC PRIMARY CARE CLINIC Mara Terras; Neda Laiteerapong; Lisa M. Vinci; Daniel Yohanna; Pooja Dave; Nancy Beckman; Erin M. Staab. University of Chicago, Chicago, IL. (Control ID \#2469893)

STATEMENT OF PROBLEM OR QUESTION (ONE SENTENCE): Behavioral healthcare co-located in the primary care setting aims to improve patient access to vital services; however, barriers, such as stigma, may prevent use of available integrated behavioral health providers.

OBJECTIVES OF PROGRAM/INTERVENTION (NO MORE THAN THREE OBJECTIVES): 1) Measure rates of referred, scheduled, and completed appointments for our co-located behavioral health clinic 2) Evaluate the impact of in-person referrals ("warm hand offs") on the rate of completed patient appointments

DESCRIPTION OF PROGRAM/INTERVENTION, INCLUDING ORGANIZATIONAL CONTEXT (E.G. INPATIENT VS. OUTPATIENT, PRACTICE OR COMMUNITY CHARACTERISTICS): Behavioral health specialists began providing co-located care in our academic internal medicine primary care clinic in April 2015. Behavioral health and internal medicine providers shared common workspace to increase visibility and seamless collaboration; the co-located services were also advertised to attendings, residents, and clinical staff through oral presentations by an internal medicine physician "champion" and flyers posted in the clinic. We also carved out dedicated time for "warm hand offs" wherein the primary care provider could introduce the behavioral health provider to their patient at the same visit that the referral for behavioral health services was placed. Our behavioral health specialists were available 4 half-days a week. MEASURES OF SUCCESS (DISCUSS QUALITATIVE AND/OR QUANTITATIVE METRICS WHICH WILL BE USED TO EVALUATE PROGRAM/ INTERVENTION): We measured the monthly rates of referrals to psychiatry/ behavioral health, average wait times for an appointment, rates of scheduled 
appointments, rates of completed appointments overall and for patients who received a "warm hand off."

FINDINGS TO DATE (IT IS NOT SUFFICIENT TO STATE FINDINGS WILL BE DISCUSSED): Of the 811 referrals made to psychiatry/behavioral health from April to December 2015 , only $53 \%$ were scheduled. Of the 428 scheduled appointments, 381 ( $89 \%$ ) were for psychiatry and $47(11 \%)$ were for behavioral health. The wait time for a psychiatry/behavioral health appointment decreased from 45 days (April/May) to 24 days (October/November). For patients who received a warm hand off to a behavioral health provider (49 patients), the wait time for an appointment was 14.3 vs. 32.2 days ( $p=0.01$ ) and the rate of completed appointments was $65 \%$ overall for patients who received a warm hand off compared to only $33 \%(p<.001)$ for patients who did not.

KEY LESSONS FOR DISSEMINATION (WHAT CAN OTHERS TAKE AWAY FOR IMPLEMENTATION TO THEIR PRACTICE OR COMMUNITY?): We found that implementing a co-located behavioral health clinic improved access to care for primary care patients needing psychiatry/behavioral health care. When primary care providers facilitated a "warm hand off" to the behavioral health specialists, there was a significant decrease in wait times and increase in completed appointments. However, nearly half of referrals to psychiatry/behavioral health were not scheduled. Successful implementation of a collaborative co-located model for behavioral health in primary care requires more than just increased proximity between providers to increase access and improve patient outcomes. Next steps include creating workflows to ensure improved communication between primary care and psychiatry/behavioral medicine regarding referrals and also evaluating patient satisfaction with the referral process.

VAMPIRE MEDICINE: DO WE NEED ALL THESE BLOOD TESTS? Megha Garg $^{1}$; Leonard Mermel $^{2}$; Ross W. Hilliard ${ }^{2}$; Lynn McNicoll ${ }^{2}$. ${ }^{1}$ San Francisco VA Medical Center, University of California San Francisco, San Francisco, CA; ${ }^{2}$ Alpert Medical School of Brown University, Providence, RI. (Control ID \#2469654)

STATEMENT OF PROBLEM OR QUESTION (ONE SENTENCE): Unnecessary laboratory testing in the inpatient setting causes patient discomfort and increases cost of care, yet increased diagnostic testing has not been shown to improve mortality or length of stay.

\section{OBJECTIVES OF PROGRAM/INTERVENTION (NO MORE THAN THREE}

OBJECTIVES): 1. Quantify the extent of excess blood testing in a large academic medical center 2. Analyze the impact of an educational and systemic intervention to reduce this excess

DESCRIPTION OF PROGRAM/INTERVENTION, INCLUDING ORGANIZATIONAL CONTEXT (E.G. INPATIENT VS. OUTPATIENT, PRACTICE OR COMMUNITY CHARACTERISTICS): At our 719-bed academic medical center, we performed a prospective observational study and intervention. In collaboration with the Department of Pathology, we obtained lab ordering data for $\mathrm{CBC}$ and $\mathrm{Chem} 7$ tests for medicine and non-medicine patients admitted for $>3$ days for October 2012 (baseline $n=$ 878). Medicine patients included Internal Medicine and subspecialties including the Heme/Onc service, medical and cardiac intensive care units. Non-medical services included neurology and all surgical services. Unnecessary blood tests were defined as 3 consecutive days of normal results, near normal (grossly within $10 \%$ of normal), or abnormal but stable (varying less than $10 \%$ over 3 days) results. We stratified the data by critical care status and teaching versus non-teaching (i.e. hospitalist) service. Our interventions were as follows: 1. Dissemination of the baseline results at Medicine Grand Rounds, hospital committee meetings, and meetings within Divisions of General Internal Medicine and Hospitalist Medicine beginning in October 2013. 2. Change of the evaluation form for faculty and residents for the teaching service to include a competency on proper utilization of blood tests. 3. Implementation of a hospital-wide restriction of the ability to order repeat labs for CBC and CHEM7 for more than 3 consecutive days as of March 1, 2014. 4. Subsequently, our hospital implemented a new electronic medical record system. At the time of this transition in March 2015, we collaborated with the Chief Technology Officer and further restricted the ability to order repeat CBC and Chem7 labs to 1 day only. Lab ordering data for $\mathrm{CBC}$ and Chem7 tests for patients admitted for > 3 days were again collected on April 2014 (post-intervention $n=851$ )

MEASURES OF SUCCESS (DISCUSS QUALITATIVE AND/OR QUANTITATIVE METRICS WHICH WILL BE USED TO EVALUATE PROGRAM/ INTERVENTION): We assessed the difference in unnecessary lab tests at our baseline before intervention in October 2012, and then in April 2014 post intervention. We stratified data according to service (Medicine versus Non-Medicine), acuity of care (Critical vs. NonCritical), and type of medical team (Teaching vs. Non-Teaching). Additionally, we collected physician level data to determine the percentage of doctors who ordered repeat labs on all of their patients. We plan to collect an additional set of data from October 2015 of the same metrics after the new electronic medical record system was implemented.

FINDINGS TO DATE (IT IS NOT SUFFICIENT TO STATE FINDINGS WILL BE DISCUSSED): At baseline overall, $80 \%$ of medical patients had at least 3 consecutive days of repeated $\mathrm{CBC}$, while $79 \%$ had repeat Chem 7 performed. Non-Department of Medicine patients did not routinely have daily blood work. For medicine patients, consecutive ordering decreased to 57 and $59 \%$ respectively (29 and $26 \%$ decline) in April 2014. The decline was driven by non-critical care patients; critical care patients had minimal improvement. The decline was similar for hospitalist and teaching services. Unnecessary blood tests declined from 29 to $13 \%$ for CBCs (52\% decline; $p<0.01$ ) and from 54 to $46 \%$ for Chem7 (14\% decline; $p<0.01)$. This represents 198 patients having fewer repetitive $\mathrm{CBC}$ and 172 patients having fewer repetitive Chem7. The number of physicians who ordered repeat labs on all of their patients reduced from $30 \%$ to less than $10 \%$. By May 2016, we anticipate adding one more set of data from October 2015, which will allow us to further assess the sustainability of our intervention and the effect of the final intervention of limiting lab draw ordering capabilities within our new electronic medical record system.

KEY LESSONS FOR DISSEMINATION (WHAT CAN OTHERS TAKE AWAY FOR IMPLEMENTATION TO THEIR PRACTICE OR COMMUNITY?): We developed a simple method for determining excess repeated blood tests at our hospital. We implemented a multi-step educational and systemic intervention that has led to a significant, sustained decline in repeated and unnecessary blood tests. We added systemic changes - computer order entry restrictions in stepwise fashion, and changes to the medicine evaluation forms - that will be sustainable in the long run. The educational intervention improved the practices of house staff and faculty that may carry through in future practice regardless of future specialty and institution. Significance of computer order entry changes cannot be underestimated - we saw changes across the hospital even in the surgical services that were not a part of our intervention, which were likely due to the systems changes we implemented.

VIRTUAL VISITS IN PRIMARY CARE: A NATIONWIDE STUDY AT THE VETERAN'S HEALTH ADMINISTRATION Leonie Heyworth ${ }^{2}$, 3; Gordon Schectman ${ }^{1}$; Kathryn Corrigan ${ }^{1}{ }^{1}$ Veterans Affairs Central Office, Milwaukee, WI; ${ }^{2}$ VA San Diego Healthcare System, San Diego, CA; ${ }^{3}$ University of California, San Diego, San Diego, CA. (Control ID \#2466037)

STATEMENT OF PROBLEM OR QUESTION (ONE SENTENCE): Does the addition of virtual visits among Veterans receiving traditional primary care improve clinical outcomes, satisfaction and reduce cost of care?

OBJECTIVES OF PROGRAM/INTERVENTION (NO MORE THAN THREE OBJECTIVES): To examine the impact of virtual visits, in addition to traditional primary 
care, on outpatient quality measures, healthcare utilization, cost and satisfaction among Veterans at 4 sites.

DESCRIPTION OF PROGRAM/INTERVENTION, INCLUDING ORGANIZATIONAL CONTEXT (E.G. INPATIENT VS. OUTPATIENT, PRACTICE OR COMMUNITY CHARACTERISTICS): The Veterans Health Administration's (VHA) Patient-Aligned Care Team (PACT), is a team-based approach to delivering patient-centered primary care with the aim of enhanced access and coordinated care management. Core PACT team members include the primary care provider, the registered nurse care manager, the clinical assistant and the clerical assistant. To date, PACT has successfully increased reliance on non-traditional means of accessing care, such as use of secure messaging and teleprimary care into a rural clinic setting. The "Virtual PACT" expands on the concept of convenient virtual access by offering the option of virtual care in the Veteran's home by incorporating the use of Clinical Video Technology (CVT). For this initial proof of concept pilot, four medical centers were invited to participate (two large urban sites, one small urban site and one rural site). Each site identified two physicians with open panel access as Virtual PACT providers. Veterans will be selected for participation in the pilot by their PACT based on several criteria, including willingness to participate, ability to use a computer or tablet, and likelihood to benefit from the pilot (e.g. difficulty accessing care due to physical disability or geographic distance from clinic, frequent ER/hospital admission, multiple psychosocial or medical comorbidities). Veterans will be trained to use the tablet or computer software for the virtual visit by a nurse trained in this technology. Veterans have the option of using a VHA-issued tablet, or the use of their own computer for the pilot. Each tablet is supplied with a stethoscope, wireless thermometer and blood pressure cuff. Each pilot site will enroll between 10 and 15 Veterans to participate in virtual visits, in addition to traditional care, over 6 12 months beginning in October 2015.

MEASURES OF SUCCESS (DISCUSS QUALITATIVE AND/OR QUANTITA TIVE METRICS WHICH WILL BE USED TO EVALUATE PROGRAM/ INTERVENTION): We will assess the impact of virtual visits on clinical quality metrics, such as hemoglobin A1c and blood pressure, and on process measures like cancer screening and receipt of vaccinations. The direct cost of virtual encounters will be compared to face-to-face encounters. Veteran satisfaction will be assessed through administration of a validated survey. We will also report on the process of implementation, as well as provider and staff experience, via in-depth interviews.

FINDINGS TO DATE (IT IS NOT SUFFICIENT TO STATE FINDINGS WILL BE DISCUSSED): Four medical centers have enrolled in the pilot study and all PACT members have completed the necessary training. As of January 2016, 8 Veterans have enrolled and 4 virtual visits have taken place at one site. We anticipate enrolling between 40 and 60 Veterans across 4 sites for the duration of the pilot.

KEY LESSONS FOR DISSEMINATION (WHAT CAN OTHERS TAKE AWAY FOR IMPLEMENTATION TO THEIR PRACTICE OR COMMUNITY?):

Implementing virtual encounters presents a novel opportunity to enhance patientcentered care for organizations interested in leveraging technology to expand access in primary care. The findings from this pilot study will provide valuable information regarding the quality and cost of virtual primary care encounters. It will also identify patients who would benefit most from this technology and inform organizations on processes of implementation and patient experience

WHAT'S OLD IS NEW AGAIN: PCP HOSPITAL ROUNDING ON HIGH-RISK ADULT INPATIENTS Jeremy Long; Vishnu Kulasekaran; Amber Wobbekind; Cynthia Crews; Diana Botton. Denver Health, Denver, CO. (Control ID \#2457061)

STATEMENT OF PROBLEM OR QUESTION (ONE SENTENCE): Ambulatory ICU Clinics have become popular in larger health systems as a way to create a novel medical home for patients categorized as "super-utilizers" - unfortunately, the scope of these clinics is limited by the ability to impact care decisions while patients are admitted to the hospital.

OBJECTIVES OF PROGRAM/INTERVENTION (NO MORE THAN THREE OBJECTIVES): 1) Inpatient rounding will tighten connections between inpatient teams and outpatient providers in an ambulatory ICU. 2) Discharge planning, to include medication reconciliation, disposition, end-of-life planning, and clinic follow-up, will be facilitated through daily hospital rounding from ambulatory ICU medical providers. 3) Quality and utilization metrics, to include readmission, length of stay, patient satisfaction, and staff satisfaction - will be enhanced by this intervention.

DESCRIPTION OF PROGRAM/INTERVENTION, INCLUDING ORGANIZATIONAL CONTEXT (E.G. INPATIENT VS. OUTPATIENT, PRACTICE OR COMMUNITY CHARACTERISTICS): The Intensive Outpatient Clinic (IOC) at Denver Health is an ambulatory ICU clinic devised within the context of greater system-wide efforts to right-size care for patients according to their needs. The IOC has become a medical home for approximately 300 of the sickest and most high-risk patients at Denver Health. The IOC is comprised of an interdisciplinary team, including physicians, a physician assistant, a nurse practitioner, a licensed clinical social worker, registered nurses, a medical assistant, a clerical specialist, and a patient navigator. This team operates M-F from 8-5 to deliver primary health care, including high intensity acute medical and behavioral health care, to patients who suffer from chronic end-stage organ dysfunction, behavioral health problems, chronic pain, substance abuse, and/or homelessness. The IOC operates within a network of primary care clinics that are a part of Denver Health, an integrated health system that includes a tertiary care teaching hospital. In December 2014, a pilot was undertaken to enhance the efforts of inpatient providers by offering bedside rounding from an IOC provider. This has evolved into a daily effort from the MDs who make up approximately half of the IOC provider staff. IOC providers coordinate care across the health system and with outside entities, including home health agencies, insurers, assisted living facilities, and pharmacies. They communicate with inpatient teams and consultant providers to facilitate an expedited discharge, when possible, or a discharge that takes into account pre-hospital characteristics along with post-discharge needs. The project described was supported by Grant Number 1C1CMS331064 from the Department of Health and Human Services, Centers for Medicare $\&$ Medicaid Services. The contents of this publication are solely the responsibility of the authors and do not necessarily represent the official views of the U.S. Department of Health and Human Services or any of its agencies. The research presented here was conducted by the awardee. Findings might or might not be consistent with or confirmed by the findings of the independent evaluation contractor.

MEASURES OF SUCCESS (DISCUSS QUALITATIVE AND/OR QUANTITATIVE METRICS WHICH WILL BE USED TO EVALUATE PROGRAM/ INTERVENTION): 1) Hospital admission data, including length of stay 2) Patient satisfaction 3) Staff satisfaction 4) Patient attendance at hospital follow-up appointments FINDINGS TO DATE (IT IS NOT SUFFICIENT TO STATE FINDINGS WILL BE DISCUSSED): 1) Hospitalist staff report greatly enhanced communication with IOC staff 2) Average daily census for IOC admitted patients has decreased 3) Patient satisfaction has improved - Patients report that seeing outpatient providers at their bedside during hospital admissions makes their stay better

KEY LESSONS FOR DISSEMINATION (WHAT CAN OTHERS TAKE AWAY FOR IMPLEMENTATION TO THEIR PRACTICE OR COMMUNITY?): 1) Medical providers from an ambulatory ICU can enhance care planning for high-risk hospitalized patients. 2) Communication improves when care teams collaborate real-time between the inpatient and outpatient settings. 3) The "Old Model" of generalist physicians providing both inpatient and outpatient care has new relevance in the setting of high-risk patients. 